\section{UEG Week 2017 Poster Presentations}

\author{
MONDAY, OCTOBER 30, 2017
}

LIVER AND BILIARY I - HALL 7 ${ }^{1}$ Faculty Of Medicine, University of Freiburg, Freiburg/Germany

${ }^{2}$ Department Of Pharmaceutical Biology And Biotechnology, University of Freiburg, Freiburg/Germany

Contact E-mail Address: denise.schaffner@uniklinik-freiburg.de

Introduction: Liver cirrhosis is associated with an imbalance between vasodilation and vasoconstriction in the sinusoids. Therefore the investigation of the nitric oxide - cyclic guanosine monophosphate (NO-cGMP) pathway, a key regulator of vascular smooth muscle tone, is essential.

Aims \& Methods: The rat model of thioacetamide (TAA) was used to induce liver fibrosis/cirrhosis and alterations of the NO-cGMP pathway and subsequent liver damage were assessed. 25 male Wistar rats were studied (11 untreated controls and 14 TAA treated animals $[0.03 \mathrm{~g} \mathrm{TAA} / 100 \mathrm{ml}$ drinking water for 16 weeks]). TAA dosage was adjusted weekly based on body weight changes. Hepatic gene expression of endothelial and inducible NO synthase (eNOS and iNOS), phossGCb1) was determined by qRT-PCR. Serum cGMP concentrations were measured by ELISA using blood samples taken from the carotid artery. Likewise liver damage was assessed by liver chemistry (i.e. alanine- and aspartate-aminotransferase (ALAT and ASAT), alkaline phosphatase (AP), albumin and bilirubin). The degree of fibrosis was estimated by histological criteria (i.e. Desmet scores). PDE5-expression was determined by immunohistochemistry. KruskalWallis test was used for statistical analysis of group differences.

Results: $43 \%$ (6/14) of TAA-treated rats developed liver fibrosis (Desmet score of $1-3)$ while $57 \%(8 / 14)$ developed liver cirrhosis (Desmet score of 4). No major differences in ALAT, ASAT, and AP serum concentrations were observed in either group. However, bilirubin was significantly elevated in TAA-treated rats, while albumin concentrations were significantly reduced. Gene expression (7.7fold), and sGCb1 (2.1fold) in fibrotic livers compared to controls. cGMP concentrations in fibrotic animals were slightly decreased (-34\%). Significantly increased expression of eNOS (2.2fold), PDE5 (11fold), sGCal (1.7fold) and sGCb1 (3fold) was observed in cirrhotic livers compared to controls, while cGMP concentrations were significantly decreased $(-40 \%)$. iNOS expression was only detected in fibrotic and cirrhotic livers, but absent in controls. Immunohistochemistry revealed markedly increased PDE5-expression in cirrhotic livers, which was predominantly localized in hepatic stellate cells.

Conclusion: The analysis of the animal model of TAA-induced liver fibrosis cirrhosis revealed alterations of the NO-cGMP pathway, characterized by reduced concentrations of cGMP, a key mediator of vasodilation, due to increased PDE5-expression. These changes reinforce the hypothesis that sinusoids remain in a contractile state in cirrhotic livers, thereby contributing to portal hypertension. Thus, administration of PDE5-inhibitors, possibly combined with sGC-activators, should be further studied in clinical trials as a promising therapeutic approach to target portal hypertension.

Disclosure of Interest: All authors have declared no conflicts of interest.

\section{P0002 NATURAL KILLER CELLS MAY BE THE KEY FACTOR FOR THE AMELIORATION OF THE LIVER FIBROSIS AFTER MESENCHYMAL CELL TRANSPLANTATION}

M. Banzragch ${ }^{1}$, T. Akkoc ${ }^{2}$, N. Zibandeh ${ }^{2}$, M. U. Ugurlu ${ }^{3}$, T. Akkoc ${ }^{4}$, D. Genc ${ }^{2}$, C. A. Celikel $^{5}$, D. G. Duman

${ }_{1}^{1}$ Gastroenterology Division, Internal Medicine, Acibadem University, Izmit/Turkey ${ }^{2}$ Immunology And Allergy, Marmara University, Istanbul/Turkey

${ }^{3}$ General Surgery, Marmara University, Istanbul/Turkey

${ }^{4}$ Tubitak Marmara Research Center, Genetic Engineering and Biotechnology Institute, Kocaeli/Turkey

${ }^{5}$ Gastroenterology, Marmara University, Istanbul/Turkey

\section{Contact E-mail Address: bmutse@ hotmail.com}

Introduction: Mesenchymal stem cell (MSC) therapy is a promising approach that may preclude the need for liver transplantation. Natural killer cells (NK cells) have critical antifibrotic effect in part through killing the activated hepatic stellate cells (HSCs); and the role of NK cells in fibrotic liver treated with MSCs is not thoroughly understood.

Aims \& Methods: To investigate the effects of bone marrow MSC transplantation in rats having hepatic fibrosis produced by common bile duct ligation (CBDL) model with a special focus on the changes of NK population in liver and the supernatant of splenocyte culture. Rats were divided into three groups: 1- CBDL rats that were given MSCs (CBDL + MSC), 2- CBDL rats given phosphatebuffered saline (PBS) (CBDL + PBS), 3- Healthy rats that were sham operated and given MSCs (Healthy + MSC). MSCs or PBS were injected via the tail vein of the rats. MSCs were labeled with GFP to check the localization of stem cells and to get idea for the regenerative capacity in the injured liver. Splenocytes were

\section{P0001 ALTERATIONS OF THE NO-CGMP PATHWAY IN THIOACETAMIDE-INDUCED LIVER FIBROSIS/CIRRHOSIS IN \\ RATS}

D. Schaffner ${ }^{1}$, A. Lazaro ${ }^{1}$, I. Merfort ${ }^{2}$, P. Hasselblatt ${ }^{1}$, P. Deibert ${ }^{1}$, W. Kreisel ${ }^{1}$ phodiesterase 5 (PDE5), soluble guanylate cyclase subunit al and b1 (sGCa1 and analysis revealed significantly increased expression of eNOS (1.5fold), PDE5 2017, Vol. 5(5S) A161-A836

(C) Author(s) 2017

Reprints and permissions:

(c) (i) (8) sagepub.co.uk/journalsPermissions.nav DOI: $10.1177 / 2050640617725676$ journals.sagepub.com/home/ueg

\section{(S)SAGE}

United European Gastroenterology Journal

isolated from spleen and cultured with Anti-CD3 and Anti CD28. Immunologic parameters were analysed with flowcytometry.

Results: Histologically, liver fibrosis developed in CBDL rats while the healthy rats group did not show any alteration in liver architecture. Bilirubin levels were as follows: Grp 1: 6.80 (5.97-8.01), Grp 2: 6.77 (5.95-7.44), Grp 3: 0.15 (0.11-.14) $\mathrm{mg} / \mathrm{dl}$. T-lymphocyte proliferation was suppressed significantly more in $\mathrm{CBDL}+\mathrm{MSC}$ compared to $\mathrm{CBDL}+\mathrm{PBS}$ group. By measuring the cytokine levels in supernatants of cultured lymphocytes, we found that CBDL + PBS group had significantly higher IL- $1 \alpha$, TNF- $\alpha$ and IFN- $\gamma$ levels than health MSCs. Treatment of those rats with MSCs tended to decrease IL- $1 \alpha$, TNF- $\alpha$ and IFN- $\gamma$ levels compared to CBDL + PBS group. The splenocyte supernatants of $\mathrm{CBDL}+\mathrm{MSC}$ rats showed a tendency towards higher $\mathrm{CD} 4{ }^{+} \mathrm{CD} 25^{+}$cell and CD161a cell counts compared to CBDL + PBS. On pathological examination, liver fibrosis was significantly higher in CBDL + PBS compared to CBDL + MSC group. The NK distribution was evaluated both in portal and parenchymal areas of liver. We have found that in the whole liver the NK cells were depressed significantly in CBDL + PBS group compared to healthy + MSC group and furthermore in the CBDL + MSC group, NK cells were significantly increased compared to CBDL + PBS group.

Conclusion: Our findings suggest bone marrow MSCs may be effective in alleviating the hepatic injury by suppressing the $\mathrm{T}$ cell proliferation, increasing the circulating peripheral NK cell population and CD4 + CD25+and suppressing the proinflammatory cytokines in rats. Beneficial effects of MSC treatment is accompanied with significantly alleviated hepatic fibrosis and the significant NK recruitment to liver parenchyme. Thus, MSC injection treatment may appear promising in liver injury and future clinical therapies are warranted. Disclosure of Interest: All authors have declared no conflicts of interest.

\section{References}

Frank Fasbender, Agata Widera, Jan G. Hengstler and Carsten Watzl. Natural Killer Cells and Liver Fibrosis. Front. Immunol., 29 January 2016

Zhigang Tian1, Yongyan Chen and Bin Gao. Natural Killer Cells in Liver Disease. Hepatology. 2013 April; 57(4): 1654-1662. doi:10.1002/hep.26115. Clara Nicolas, Yujia Wang, Jennifer Luebke-Wheeler and Scott L. Nyberg. Stem Cell Therapies for Treatment of Liver Disease. Biomedicines 2016, 4, 2;

\section{P0003 SERPINB3 INVOLVEMENT IN THE STIMULATION OF MACROPHAGE ACTIVATION MARKER SCD163 IN HCV INFECTED PATIENTS}

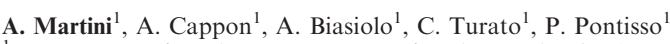

${ }^{1}$ Department Of Medicine, University of Padua, Padova/Italy

Contact E-mail Address: andremartini86@gmail.com

Introduction: In chronic HCV infection disease progression is maintained by sustained necroinflammation and fibrosis in the liver. Upon macrophage activation, the soluble marker CD163 (sCD163) is released in serum and its levels correlate with fibrosis and NASH in the liver. The serin protease inhibitor SerpinB3 (or SCCA1), has been shown to be involved in liver fibrogenesis and the circulating SCCA-IgM complex has been depicted as a marker of liver disease progression and of NASH in patients with chronic hepatitis C. Preliminary data suggest that SerpinB3 activates primary monocytes through the Wnt canonical pathway. The purpose of this study was to evaluate the relationship between SCCA-IgM and sCD163 in serum and the possible effect of SerpinB3 on sCD163 and on pro-inflammatory cytokines expression, in primary monocytes. Aims \& Methods: In 91 patients with biopsy-proven chronic hepatitis C, serum samples were tested for sCD163 $(\mathrm{ng} / \mathrm{ml})$ and SCCA-IgM $(\mathrm{AU} / \mathrm{ml})$ by ELISA. The results were analyzed in relation with clinical and histological parameters. Primary monocytes were isolated from healthy donors, treated with recombinant SerpinB3 $(200 \mathrm{ng} / \mathrm{ml})$ and supernatants analyzed after 2 and 7 days. Expression of sCD163 secreted in the supernatant was evaluated by ELISA. In primary monocytes mRNA expression of IL-12, CXCL-10 and TNF-alpha were also analyzed by PCR at different time points

Results: In patients with chronic hepatitis C sCD163 was found correlated with inflammatory and metabolic alterations (AST, ALT, GGT, HOMA-IR, triglycerides), and was significantly elevated in patients with more advanced histological fibrosis stage (F1-F2 vs. F3-F4 sec. Metavir: $\mathrm{p}<0.04)$. Patients with levels of SCCA-IgM $>200 \mathrm{AU} / \mathrm{ml}$ had more elevated serum levels of sCD163 $(\mathrm{p}<0.05)$. In primary monocytes stimulated with recombinant SerpinB3 "in vitro", sCD163 expression increased of 2.5 times and this finding was parallel to an up-regulation of the inflammatory cytokines IL-12, CXCL-10 and TNFalpha. 
Conclusion: In chronic hepatitis C SerpinB3 is involved in monocyte activation, leading to the release of sCD163. These results support the correlation of these two molecules in serum of patients with more severe liver fibrosis and metabolic alterations.

Disclosure of Interest: All authors have declared no conflicts of interest.

\section{P0004 THE PROTECTIVE EFFECTS OF GROUP 3 INNATE LYMPHOID CELLS ON HEPATITIS B VIRUS RELATED LIVER FIBROSIS COULD BE IMPAIRED BY TH17 CELLS}

S. Wang, W. Ma, L. Cheng, W. Jiang

Gastroenterology, Zhongshan Hospital Fudan University, Shanghai/China

\section{Contact E-mail Address: 15211210012@fudan.edu.cn}

Introduction: Th17 cells have been proved to contribute to hepatitis B virus (HBV) associated liver fibrosis. Group 3 innate lymphoid cells (ILC3s), which have similar profiles of transcription factor and cytokines to that of Th17 cells, were also suggested to be involved in the progression of liver fibrosis.

Aims \& Methods: The study was designed to explore the functions of ILC3s and the relationships between ILC3s and Th17 cells in liver fibrosis. Peripheral blood samples were collected from 60 patients with chronic hepatitis B (CHB), and 50 patients with HBV related liver cirrhosis (LC) as well as 30 healthy controls (HC). The percentages and cytokines secretion of ILC3s $\left(\mathrm{Lin}^{-} \mathrm{CD} 127^{+} \mathrm{CD} 117^{+} \mathrm{CD} 294^{-}\right)$and $\mathrm{Th} 17$ cells $\left(\mathrm{CD} 4^{+} \mathrm{IL}-17^{+}\right)$were detected by flow cytometry. Peripheral blood mononuclear cells (PBMCs) and PBMCs without ILC3s co-cultured with hepatic stellate cells (HSCs)-LX2 in contact and noncontact manners. Then Th17 cells, which were induced from naïve $\mathrm{CD} 4^{+} \mathrm{T}$ cells in vitro, were transferred into $\mathrm{Rag} 1^{-/-}$mice with carbon tetrachloride $\left(\mathrm{CCl}_{4}\right)$ related liver fibrosis. In addition, ILC3s in $\mathrm{Rag}^{-/-}$mice were depleted by injecting with anti-CD90.2 antibody.

Results: Compared with HC, the percentage of ILC3s increased in CHB group. The anti-inflammation cytokines secreted by ILC3s such as IL-22 increased, whereas pro-inflammation cytokines of ILC3s such as IL-17A, TNF- $\alpha$, IFN- $\gamma$ decreased in $\mathrm{CHB}$ patients. However, ILC3s decreased in LC patients with reduced cytokines secretion. Th17 cells frequencies significantly increased both in CHB and LC groups compared with HC. PBMCs without ILC3s, which were collected from CHB and LC patients, promoted the proliferation and activation of HSCs because of less IL-22 secretion. Similarly, compared with wild type mice, ILC3s in spleens and livers of C57BL/6 mice with liver fibrosis increased sequentially at time point of week 2 and week 4 following drug injection. Intriguingly, at week 6 , ILC3s decreased compared with previous. However, Th17 cells increased gradually with $\mathrm{CCl}_{4}$ administration, even at week 6 . Transferring Th17 cells into $\mathrm{Rag}^{-/}$mice with liver fibrosis made the ILC3s in spleens and livers decrease significantly, and the degree of mice liver fibrosis become more severe than control. Furthermore, ILC3s depletion correlated with reduced expression of IL-22 and more severe liver fibrosis. Transferring purified liver ILC3s into recipient mice could alleviate liver inflammation and reverse liver fibrosis. Conclusion: Our study has uncovered the protective role of ILC3s in liver fibrosis, which is through secrete IL-22 to reduce proliferation and activation of HSCs. However, the protective functions of ILC3s could be impaired by Th17 cells. Disclosure of Interest: All authors have declared no conflicts of interest.

\section{P0005 EFFECTS OF INTERNAL AND EXTERNAL BILIARY DRAINAGE ON THE EXPRESSION OF INTESTINAL BILE ACID RECEPTOR AND TLR4/NOD2 IN MICE WITH OBSTRUCTIVE JAUNDICE}

S. $\mathrm{Li}^{1}$, S. Shen ${ }^{2}$, T. Zhang ${ }^{2}$, W. $\mathrm{Li}^{3}$

${ }^{1}$ Gastroenterology-hepatology, People's Liberation Army General Hospital (PLAGH), Beijing/China

${ }^{2}$ The General Hospital of the Chinese People's Liberation Army, Beijing/China ${ }^{3}$ Gastroenterology And Hepatology, The General Hospital of the Chinese People's Liberation Army, Beijing/China

Contact E-mail Address: zhangtt246@sina.com

Introduction: Internal biliary drainage has been confirmed better than external biliary drainage in alleviating the damage of intestinal mucosa barrier caused by obstructive jaundice, but the relevant mechanism is still unclear.

Aims \& Methods: We aimed to investigate the potential relation between the expressions of bile acid receptor and TLR4/NOD2 in intestinal mucosa and its influence on the intestinal mucosal barrier with obstructive jaundice. In this study, we mainly study the expression between FXR and TLR4, TGR5 and NOD2. Sixty male adult Kunming mice were randomly assigned to four groups: SH (shame operation), OJ (obstructive jaundice), ID (internal drainage), ED (external drainage) $(\mathrm{n}=15$ in each group). On the 7 th day from the first operation, the $\mathrm{OJ}$ and $\mathrm{SH}$ mice were executed and specimens of blood and ileal tissue of groups were collected. ED and ID were reoperated on day 8 for biliary drainage procedure. Blood was drawn from heart for liver function test. The terminal ileum specimen was collected for test of histology using haematoxylineosin (HE) staining. Western blot (WB) and real-time polymerase chain reaction (RT-PCR) were used to detect the expression of protein and mRNA of FXRTGR5 TLR4 and NOD2 in intestinal mucosa.

Results: We have successfully established the animal models. The histopathological examination revealed notable inflammatory infiltration and hyperplasia disruption at terminal ileum in OJ mice; significant alleviation of above injures by ID while little improvement by ED. FXR-TLR4: After biliary obstruction, the expression of protein and mRNA of FXR were significantly increased, while the expression of protein and mRNA of TLR4 were significantly decreased compared with SH group's $(\mathrm{P}<0.001)$. After ED, compared with OJ group's, the expression of protein of FXR was decreased while TLR4 were increased. The mRNA of both FXR and TLR4 were increased. After ID, the expression of protein and mRNA of FXR were significantly decreased compared with OJ group's but were still higher than that in SH group and were better than ED group's. And the expression of protein and mRNA of TLR4 were significantly increased compared with OJ group's $(\mathrm{P}<0.001)$, but were still lower than that in $\mathrm{SH}$ group and were better than ED group's. The trend of TLR4 expression was almost the same between vehicle group and no gavage group. After gavage with FXR agonist, the differences of TLR4 expression of four groups disappeared $(\mathrm{P}>0.05)$. TGR5-NOD2: IHC and WB suggested that after OJ surgery, the protein expression of both TGR5 and NOD2 increased obviously compared to that of SH mice; then the level of TGR5 and NOD2 protein fell remarkably after ID surgery close to SH level while in ED group there was only a slightly reduction form OJ level and still with a high expression of TGR5 and NOD2 protein. Detection of RT-PCR found that TGR5 mRNA and NOD2 mRNA level in OJ group increased several times as that of the SH group; after ID surgery, the expression of TGR 5 mRNA significantly reduced, NOD2 mRNA level also fell down consistently, but the effect was not observed in ED mice.

Conclusion: The expression of intestinal FXR and TLR4, TGR5 and NOD2 could be one of the critical mechanism why internal drainage is better than external drainage in restore intestinal barrier function of obstructive jaundice mice.

Disclosure of Interest: All authors have declared no conflicts of interest

\section{P0006 ALTERED SMALL INTESTINAL MICROBIOTA TOWARD} FAMILY LACTOBACILLACEAE IN MIR-21 KNOCKOUT MICE

A. Santos $^{1}$, M. B. Afonso ${ }^{1}$, P. M. Rodrigues ${ }^{2}$, R. S. Ramiro ${ }^{3}$, I. Gordo ${ }^{3}$, R. E. Castro ${ }^{1}$, C.M.P. Rodrigues

${ }^{1}$ Cellular Function And Therapeutic Targeting, iMed.ULisboa - Faculdade de Farmácia, Lisbon/Portugal

${ }^{2}$ Research Institute for Medicines (iMed.ULisboa), Faculty of Pharmacy, Universidade de Lisboa, Lisbon/Portugal

${ }^{3}$ Instituto Gulbenkian de Ciência, Oeiras/Portugal

Contact E-mail Address: afasantos@ff.ulisboa.pt

Introduction: Alterations in the gut microbiota have been correlated to a wide variety of diseases, including liver diseases. Used as probiotics, several strains of Lactobacillus have been associated not only to modulation of intestinal tight junctions but also to amelioration of liver fibrosis. Common bile duct ligation (BDL) results in acute cholestatic injury and secondary biliary fibrosis, associated with early increased intestinal permeability and disturbed bile acid homeostasis. We have demonstrated that the oncogenic microRNA-21 (miR-21) is upregulated in BDL mouse liver, mediating liver fibrosis. We aimed to investigate the role of miR-21 in the response of the small intestinal microbiota to BDL that may explain miR-21 effects in acute liver injury and fibrosis.

Aims \& Methods: Three-month-old C57BL/6 wildtype (WT) and miR-21 whole body knockout $(\mathrm{KO})$ mice were submitted to sham or BDL surgeries. After three days, animals were sacrificed and small intestine and its luminal content were carefully removed and preserved. mRNA expression was analysed by qRT-PCR. Bacterial DNA was purified from the small intestinal lumen samples and analysed by next generation sequencing - metagenome analysis. Liver tissue and serum were also collected for biochemical analysis of hepatic damage and fibrosis.

Results: TNF- $\alpha$ and IL-1- $\beta$ mRNA levels increased in the small intestine of BDLmiR-21 KO mice, compared to WT. TLR- 4 and TGF- $\beta$ expression was increased in both sham- and BDL-miR-21 KO mice which is in accordance with the higher LPS in blood plasma observed. Zona occludens (ZO-1) and occludin mRNA levels were decreased in WT mice after BDL. Strikingly, miR-21 KO reverted mRNA of tight junction proteins to control levels. BDL miR-21 KO mice showed decreased circulating levels of hepatic enzymes, concomitant with decreased fibrogenic gene expression in the liver, in comparison with WT mice, suggesting that miR-21 contributes to BDL-induced liver injury and fibrosis. Further, mir-21 KO not only show a decreased small intestine permeability through a ZO-1 and occludin pathway, as it is associated with development of beneficial strains of Lactobacillaceae that may also contribute to liver protection. Conclusion: These data suggest that miR-21 depletion is associated with increased inflammatory markers in the small intestine and better immune response to bacterial dysbiosis provoked by the BDL surgery, thus halting liver injury and promoting gut microbiota homeostasis. (Supported by PTDC/BIM-MEC/ 089572014, FCT)

Disclosure of Interest: All authors have declared no conflicts of interest. 


\section{P0007 THE EMERGING ROLE OF ZBP-89 IN SENSITIZING}

\section{HEPATIC CANCER STEM CELIS TO SORAFENIB}

N. Wang ${ }^{1}$, Y. $\mathrm{Liu}^{2}$, J. Ren ${ }^{3}$, J. Yu ${ }^{1}$, R. Ho ${ }^{1}$, P.B. Lai ${ }^{1}$, G.G. Chen ${ }^{1}$ Department Of Surgery, The Chinese University of Hong Kong, Hong Kong/Hong Kong Prc

${ }^{2}$ Guangdong Key Laboratory For Research And Development Of Natural Drugs, Guangdong Medical University, Zhanjiang/China

${ }^{3}$ CUHK Shenzhen Research Institute (SZRI), Shenzhen/China

Contact E-mail Address: nwang@surgery.cuhk.edu.hk

Introduction: Sorafenib is the only approved systemic therapy for advanced hepatocellular carcinoma (HCC). However, the application of Sorafenib in therapy has faced increasing challenges due to drug resistance. Drug resistance is known to be associated with cancer stem cells (CSC). The transcription factor ZBP-89 was reported to be involved in cell growth and apoptosis in tumor and acts as a potential tumor suppressor in HCC. It has shown that high levels of ZBP-89 expression were statistically associated with better survival of HCC patients. Unfortunately, the mechanism of ZBP-89 in modulating sensitivity of Sorafenib in CSC remains unknown.

Aims \& Methods: In this study, we investigated the mechanism of Sorafenib resistance in HCC cancer stem cells, and how ZBP-89 reduced drug resistance. The sensitivity of Huh7 and Hep3B parental and sphere-forming cells to Sorafenib was measured by MTT assay. We then examined the expression patterns of Notch1 and liver CSC markers in Huh7 and Hep3B CSC after the treatment with Sorafenib. MTT assay was also used to measure the effects of ZBP-89 overexpression on the sensitivity of Sorafenib in sphere-forming cells. The levels of ZBP-89 and CD44 were measured using q-PCR in human HCC tissue samples. The regulatory effects of ZBP-89 on CSC phenotype were explored by various methods including q-PCR, immunoblotting, tumor sphere formation assay, soft agar formation assay and colony formation assay. Gene expressions and protein interaction in stemness signaling pathways were analyzed.

Results: We found that sphere-forming HCC cells had significant higher resistance to Sorafenib, compared with their parental cells. The expression of Notch1 and EpCAM was increased along with the treatment of low dose of Sorafenib, suggesting that the activation of Notch 1 pathway was associated with the drug resistance in liver CSC. Studies further indicated that ZBP-89 overexpression was able to improve the sensitivity of Sorafenib on sphere-forming HCC cells. Furthermore, we found that ZBP-89 expression was negatively correlated with CSC marker CD44 in human HCC tissues. In vitro study indicated that tumor sphere formation capacity was impaired upon stable overexpression of ZBP-89, suggesting that ZBP-89 was involved in suppression of CSC phenotype. Detailed investigation against control cells showed that overexpression of ZBP-89 resulted in reduced expression of CSC markers EpCAM, CD133, Sox2 and c-myc at both mRNA and protein levels. In addition, the overexpression of ZBP-89 or silencing of Notch 1 reduced the number of colonies formed by sphere-forming HCC cells, demonstrating opposite effects of these two proteins. Mechanistic studies revealed that ZBP-89 was able to repress the expression of Notch 1 and reported Notch1 target genes including HES1, HES6, HEY1 and NRARP. Amino acids $\triangle 6-180$ of ZBP-89 could directly bind to the activated Notch1 in the nucleus, resulting in a negative regulation of CSCs and overcome of Sorafenib resistance. Conclusion: Sphere-forming HCC cells, which contained high levels of Notch1 and EpCAM, were resistant to Sorafenib. The overexpression of ZBP-89 was found to result in the loss of CSC phenotype and improve the sensitivity to Sorafenib in CSC through its interaction with activated Notch1. In conclusion, we believe that targeting ZBP-89 is likely to be a new therapeutic strategy to overcome the resistance to Sorafenib in HCC.

Disclosure of Interest: All authors have declared no conflicts of interest.

\section{References}

1. Tirino, V. et al. Cancer stem cells in solid tumors: an overview and new approaches for their isolation and characterization. The FASEB Journal 27, 13-24, doi:10.1096/fj.12-218222 (2013)

2. Zhang, C. Z. Y., Chen, G. G. \& Lai, P. B. S. Transcription factor ZBP-89 in cancer growth and apoptosis. Biochimica et Biophysica Acta (BBA) Reviews on Cancer 1806, 36-41, doi:10.1016/j.bbcan.2010.03.002 (2010).

\section{P0008 PROTECTIVE EFFECT OF AKKERMANSIA MUCINIPHILA AGAINST IMMUNE-MEDIATED LIVER INJURY IN A MOUSE MODEL}

W. Wu ${ }^{1}$, D. Shi ${ }^{1}$, D. Fang ${ }^{1}$, X. $\mathrm{He}^{2}$, L. Li

${ }^{1}$ State Key Laboratory for Diagnosis and Treatment of Infectious Diseases, The First Affiliated Hospital, School of Medicine, Zhejiang University, Hangzhou, China, Hangzhou/China

${ }^{2}$ Department of Gastroenterology, Sir Run Run Shaw Hospital, Zhejiang

University Medical School, Hangzhou, China, Hangzhou/China

Contact E-mail Address: wwr725@zju.edu.cn

Introduction: Accumulating evidence indicates that gut microbiota participates in the pathogenesis and progression of liver diseases. The severity of immunemediated liver injury is associated with different microbial communities. Akkermansia muciniphila can regulate immunologic and metabolic functions.
However, little is known about its effects on gut microbiota structure and function.

Aims \& Methods: This study investigated the effect of $A$. muciniphila on immunemediated hepatitis and potential underlying mechanisms. Twenty-two C57BL/6 mice were assigned to three groups $(\mathrm{N}=7-8$ per group) and continuously administrated A. muciniphila Muc $^{\mathrm{T}}$ (ATTC BAA-835) or PBS by oral gavage for 10 days. Mouse feces were collected for gut microbiota analysis on the eleventh day, and acute hepatitis was induced by Concanavalin A (Con A, $15 \mathrm{mg} / \mathrm{kg}$ ) injection through the tail vein. Samples (blood, liver, ileum, colon) were assessed for liver injury, systemic inflammation, and intestinal barrier function.

Results: We found that oral administration of A. muciniphila (Akk) decreased serum ALT and AST and alleviated liver histopathological damage induced by Con A. Serum levels of pro-inflammatory cytokines (IL-2, IFN- $\gamma$, IL-12p40, MCP-1, MIP-1a, MIP-1b) were substantially attenuated. Akk significantly decreased hepatic cell apoptosis; Bcl-2 expression increased, but Fas and DR5 decreased. Further investigation showed that Akk enhanced Occludin and Tjp-1, two proteins related to strengthened intestinal barriers. Fecal 16S rRNA sequence analysis indicated that Akk increased microbial richness and diversity. The community structure of the Akk group clustered distinctly from that of the Control and Normal groups. Relative abundance of Firmicutes increased, and Bacteroidetes abundance decreased. Correlation analysis showed that injuryrelated factors (IL-12p40, IFN-g, DR5) were negatively associated with specific genera (Ruminococcaceae UCG-009, Lachnospiraceae UCG-001, Akkermansia), which were enriched in mice pretreated with Akk.

Conclusion: Our results suggested that $A$. muciniphila Muc ${ }^{\mathrm{T}}$ (ATTC BAA-835) had beneficial effects on immune-mediated liver injury by alleviating inflammation and hepatocellular death. These effects may be driven by the protective profile of the intestinal community induced by the bacteria. The results provide a new perspective on the immune function of gut microbiota in host diseases. Disclosure of Interest: All authors have declared no conflicts of interest.

\section{P0009 CLINICAL OBSERVATION ON THE TREATMENT OF NONALCOHOLIC FATTY LIVER WITH PROBIOTICS}

W. Wang

Gastroenterology, Beijing Hospital, Beijing/China

Contact E-mail Address: wangwei-beijingyiyuan@hotmail.com

Introduction: With the improvement of living standards, obesity, the prevalence of insulin resistance (IR), hyperlipidemia and diabetes and other metabolic disorders in nonalcoholic fatty liver (NAFLD) in China had increased. NAFLD refers to the factors caused by exclusion of alcohol and other clear liver damage, clinical pathological syndrome, and genetic liver steatosis as the main feature of the link between insulin resistance and other factors closely for acute liver injury of metabolism. Serious illness can develop into liver cancer. NAFLD has become the leading cause of chronic liver disease in developed countries and China. Chronic liver disease is often accompanied by intestinal micro ecological imbalance; studies have shown that the imbalance of intestinal micro ecology led to the transfer of intestinal endotoxin into the blood, and stimulate the production of inflammatory factors aggravate liver damage, thus chronic liver disease. A series of studies show that changes of intestinal microflora, intestinal bacterial overgrowth (small intestinal bacterial over growth, SIBO) and intestinal endotoxemia in the occurrence and development of NAFLD plays an important role, and the recovery of intestinal micro ecological balance may have assisted treatment of NAFLD. A series of studies show that changes of intestinal microflora, intestinal bacterial overgrowth (SIBO) and intestinal endotoxemia in the occurrence and development of NAFLD plays an important role, and the recovery of intestinal micro ecological balance may have assisted treatment of NAFLD

Aims \& Methods: We aimed to study the clinical effect of probiotics in the treatment of nonalcoholic fatty liver disease. 200 cases of patients with nonalcoholic fatty liver disease were randomly divided into routine treatment group (A group) and combined treatment 3 groups (B, C, D). All 50 cases were given orally Polyene Phosphatidylcholine Capsules, $456 \mathrm{mg}$. TID; The combination therapy group B was given orally the Live Combined Bifidobacterium Lactobacillus and Enterococcus Powder, $420 \mathrm{mg}$. TID; group C, two live combined Bacillus subtilis Enterococcus, $500 \mathrm{mg}$, TID; D group was given orally the both probiotics above. The course was 1 month. All patients were respectively examined before treatment and seven days and thirty days after treatment, for cholesterol (TC), triglyceride (TG), high density lipoprotein cholesterol (HDL-L), low density lipoprotein cholesterol (LDL-L), alanine aminotransferase (ALT), aspartate aminotransferase (AST), fasting blood glucose (FPG), serum high molecular weight adiponectin (HMW APN) and serum TNF-a. The 4 groups were collected faeces samples, that were tested routine detection, bacterial culture. At the same time all patients were checked with liver ultrasound scan.

Results: In terms of blood lipids and blood glucose, each group improved than before, only HDL-Lwas not statistically significant, D group showed significant differences in triglyceride. In liver function, blood ALT, AST were significantly lower in D group than A group. TNF-a levels were decreased after treatment, combined treatment D group was statistically significant; group D more than the group A; serum HMW APN increased after treatment, combined treatment group $\mathrm{D}$ comparing with routine treatment group $\mathrm{A}$ was significant difference. Conclusion: Intestinal probiotics can regulate the intestinal micro ecological imbalance in NAFLD patients, and reduce the level of serum TNF-a, improve the level of adiponectin, which can further improve the blood glucose, lipid metabolism, and then improve the liver injury of non-alcoholic fatty liver disease. Disclosure of Interest: All authors have declared no conflicts of interest. 


\section{Reference}

1. Henao-Mejis, Elinav E, Thaiss CA, et al. Role of the intestinal microbiome in liver disease [J]. J Autoimmunity, 2013, 46(100):66-73.

\section{P0010 FAECALIBACTERIUM ASSOCIATED WITH GUT-} PERMEABILITY IN NONALCOHOLIC FATTY LIVER DISEASE

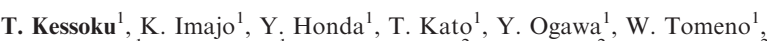
T. Higurashi ${ }^{1}$, M. Yoneda ${ }^{1}$, M. Shimakawa ${ }^{2}$, Y. Tanaka ${ }^{2}$, T. Kawahara ${ }^{2}$, S. Saito ${ }^{1}$, H. Usuda ${ }^{3}$, K. Wada ${ }^{3}$, A. Nakajima

${ }^{1}$ Yokohama City University Graduate School of Medicine, Yokohama/Japan

${ }^{2}$ Biofermin Pharmaceutical Co., Ltd., Kobe/Japan

${ }^{3}$ Shimane University Graduate School of Medicine, Shimane/Japan

\section{Contact E-mail Address: takaomi0027@gmail.com}

Introduction: Despite evidence that the microbiota is involved in the pathogenesis of obesity, the microbiota of patients with nonalcoholic fatty liver disease (NAFLD) has not been well characterized. NAFLD is considered a hepatic manifestation of metabolic syndrome and is particularly associated with insulin resistance, obesity, and gut-driven endotoxin.

Aims \& Methods: The aim of our study is to assess if there are any differences in the microbiota of patients with biopsy-proven NAFLD and healthy controls (HC). In addition, peripheral blood endotoxin (ET) and gut-permeability was analyzed in NAFLD (mild fibrosis vs severe fibrosis) and HC patients. A total of 201 patients were enrolled in this study: $68 \mathrm{HC}$ and 143 biopsy-proven NAFLD (77 mild fibrosis [F0-2] and 56 severe fibrosis [F3-4]). One stool sample was collected from each participant. All NAFLD patients included in this study underwent percutaneous liver biopsy. Healthy controls were volunteers. The composition of gut bacterial communities was determined by 16 S rDNA sequencing. In addition, peripheral blood ET was determined using by endotoxin activity assay (EAA). Gut-permeability was assessed by Lactulose mannitol ratio (LMR). Trial registration: This trial has been registered in the University Hospital Medical Information Network (UMIN) Clinical Trials Registry as UMIN000020917.

Results: Among those taxa with greater than $1 \%$ representation in any of the disease groups, it was significantly decrease in Bacteroidetes at phylum level in NAFLD compared with HC. At genus level, Faecalibacterium prausnitzii (F.P) was significantly decreased in NAFLD compared with HC. F.P is significantly decreased in NAFLD with severe fibrosis compared with those with mild fibrosis patients. In addition, endotoxin levels were increased in NAFLD with severe fibrosis than those with mild fibrosis. Furthermore, occupation ratio of $F . P$ was negative correlation with blood ET levels $\left(\mathrm{R}^{2}=0.327, P<0.0001\right)$. Additionally, it showed a significant correlation among three items of F.B, EAA and LMR (F.B vs EAA: $P<0.0001$, LMR vs EAA: $P<0.0001$, LMR vs F.B: $P<0.0025$ )

Conclusion: Our study indicated that the change of the gut microbiota and pathologic connection were suggested in acknowledgment of the decrease on F.P in the NAFLD patients. The decreased abundance of $F . B$ in NASH with severe fibrosis, elevated blood-endotoxin in NAFLD with severe fibrosis patients suggests a role for ET in the pathogenesis of fibrosis. Moreover, our study showed that the mechanism of fibrotic progression via the endotoxin in NAFLD may relate strongly gut-permeability. We postulate that the distinct composition of the gut microbiota among NAFLD and HC could offer a target for intervention or a marker for disease.

Disclosure of Interest: All authors have declared no conflicts of interest.

P0011 GUT MICROBIOTA COMPOSITION IN EXPERIMENTAL MOUSE MODELS OF NON-ALCOHOLIC FATTY LIVER DISEASE

B. Zhang, L. Fan, J. Ren

Zhongshan Hospital Affiliated To Xiamen University, Department of

Gastroenterology, Xiamen/China

Contact E-mail Address: geebzbz@xmu.edu.cn

Introduction: Non-alcoholic fatty liver disease (NAFLD) has become the most common liver disease worldwide, and is thought to be strongly associated with gut microbiota. Several diet models were therefore built in mice to try to clarify the molecular mechanisms. However, how and to what extent these diet models alter the composition of the gut microbiota have not yet been clearly elucidated. Aims \& Methods: In this study, we developed three mouse models of NAFLD using methionine-choline-deficient (MCD) diet, high-fat (HF) diet, and cholinedeficient- high-fat (CD-HF) diet, evaluated the severity of steatohepatitis, and sequenced the fecal bacteria by targeting 16S V4-V6 regions on Illumina MiSec using PE 300 reagents.

Results: Histological scores showed that MCD induced the severest steatohepatitis, followed by HF and CD-HF diets. Based on OTUs at cutoff of $97 \%$ similarity, there were significant (PERMANOVA, $\mathrm{P}=0.001$ ) differences in overall gut bacterial communities among MCD, HF, CD-HFD, and the Control, forming three major clusters in PCA ordination with HF and CD-HF groups more similar. Furthermore, $\alpha$-diversity of $\mathrm{HF}$ and $\mathrm{CD}-\mathrm{HF}$ groups, including observed OTU numbers, Shannon index, and Pielou evenness were significantly (ANOVA, $\mathrm{P}<0.05$ ) higher than the Control and MCD group. Overall, mouse gut bacteria were mainly composed of Fimicutes (Ruminococcaceae, Lachnospiraceae, Clostridiaceae) and Bacteroidetes (S24-7, Porphyromonadaceae). More specifically, Ruminococcus was significantly reduced in the three NAFLD models than the Control, and was identified as the biomarker of NAFLD in LEfSe analysis. More biomarkers at genus level (Lachnospira, S24-7, etc.) were identified in pairwise comparison of one mouse model with the Control.

Conclusion: In summary, the composition of gut microbiota varied remarkably between mice administrated different experimental diets to induce non-alcoholic fatty liver disease.

Disclosure of Interest: All authors have declared no conflicts of interest.

\section{P0012 PREVALENCE OF METABOLIC SYNDROME AND LIVER STEATOSIS IN A PROSPECTIVE MULTICENTER STUDY O PATIENTS REFERRED FOR HYPERFERRITINEMIA}

A. Castiella Eguzkiza ${ }^{1}$, E. Zapata ${ }^{1}$, I. Urreta ${ }^{2}$, L. Zubiaurre ${ }^{1}$, P. Otazua ${ }^{3}$ J. M. Alustiza ${ }^{4}$, E. Salvador ${ }^{4}$, G. Letamendi ${ }^{5}$, B. Arrizabalaga ${ }^{6}$, L. Mendibil ${ }^{1}$, J.I. Emparanza ${ }^{2}$

${ }^{1}$ Gastroenterology, Mendaro Hospital, Mendaro/Spain

${ }^{2}$ Clinical Epidemiology, Donostia University Hospital, Donostia/Spain

${ }^{3}$ Gastroenterology, Mondragon Hospital, mondragon/Spain

${ }^{4}$ Radiology, Osatek Donostia, Donostia/Spain

${ }^{5}$ Hematology, Galdakao Hospital, Galdakao/Spain

${ }^{6}$ Hematology, Hospital de Cruces, Barakaldo/Spain

Contact E-mail Address: agustincastiella@yahoo.es

Introduction: Aproximately $25 \%$ of adult population in western countries have metabolic syndrome (MS). Hyperferritinemia (HF) is frequently present in patients with MS (dysmetabolic hyperferritinemia). Liver steatosis is often suspected in patients with MS

Aims \& Methods: To study the prevalence of hepatic steatosis determined by MRI in these patients. A prospective study of 312 consecutive with HF ( $>200 \mu \mathrm{g} / \mathrm{L}$ women; $300 \mu \mathrm{g} / \mathrm{L}$ men) and $/$ or TSI $>45 \%$, confirmed in two determinations, was conducted from December 2010 to April 2013. The MS was defined by the presence of three of the following factors: waist circumference $\geq 94 \mathrm{~cm} \mathrm{men} / \geq 80 \mathrm{~cm}$ women; Triglycerides $\geq \geq 150 \mathrm{mg} / \mathrm{dL}$ or treatment for this dislipidemia; HDL $<40 \mathrm{mg} / \mathrm{dL}$ women $/<50 \mathrm{mg} / \mathrm{dL}$ men or treatment for this dislipidemia; glucose $>100 \mathrm{mg} / \mathrm{dL}$ or Type 2 diabetes; hypertension: blood pressure $\geq 130 \mathrm{mmHg} / \geq 85 \mathrm{mmHg}$ or treatment for arterial hypertension (1). LIC was determined by MRI 1.5 Tesla system (SIR method) (2). We systematically performed T1-weighted in-phase and opposed-phase imaging to determine the presence or not of liver steatosis.

Results: 312 patients ( 272 men/40 women) were included. Mean age 55 (SD 13.5); Mean ferritin 729, 6 (SD 449.6), mean TSI 40, 8 (SD 15.8); 276 patients have all the required criteria to determine the MS presence: $115 / 240$ men $(48 \%)$ and $20 / 36$ women $(55.6 \%)$ presented MS: 135 patients with MS (49\%); 141 without MS (NMS) $(51 \%)$. In 286 patients a MR study for the presence of liver steatosis was performed: 196 no steatosis; 90 liver steatosis. 251 patients with MS criteria and MR for steatosis: NMS group (128): no steatosis 103; steatosis 25; MS group (123): no steatosis 72, steatosis 51 (total: no steatosis 175 , steatosis 76 ). When we study if the presence of liver steatosis was more frequent in the MS group, the results obtained were statistically significant, $p=0.000$.

Conclusion: Nearly $50 \%$ of the patients referred for hyperferritinemia to the hospitals of our country had MS; the patients with MS had more frequently liver steatosis than the patients without MS.

Disclosure of Interest: All authors have declared no conflicts of interest.

\section{References}

1. K.G.M.M. Alberti, ; Robert H. Eckel, ; Scott M. Grundy, et al. Harmonizing the Metabolic Syndrome. A Joint Interim Statement of the International Diabetes Federation Task Force on Epidemiology and Prevention; National Heart, Lung, and Blood Institute; American Heart Association; World Heart Federation; International Atherosclerosis Society; and International Association for the Study of Obesity. Circulation 2009; 120: 1640-45.

2. Alústiza JM, Artetxe J, Castiella A, et al. MR quantification of hepatic iron concentration. Radiology 2004; 230: 479-84.

\section{P0013 LIVER IRON CONCENTRATION IN PATIENTS REFERRED FOR HYPERFERRITINEMIA. MULTICENTRE ANALYSIS OF THE DIFFERENT GROUPS ACCORDING TO HFE MUTATIONS AND TRANSFERRIN SATURATION INDEX}

A. Castiella Eguzkiza ${ }^{1}$, E. Zapata ${ }^{1}$, I. Urreta ${ }^{2}$, L. Zubiaurre ${ }^{1}$, P. Otazua ${ }^{3}$, J. M. Alustiza ${ }^{4}$, M. D. De Juan ${ }^{5}$, E. Salvador ${ }^{4}$, G. Letamendi' ${ }^{6}$, B. Arrizabalaga ${ }^{7}$, A. Iribarren ${ }^{1}$, L. Mendibil ${ }^{1}$, J. I. Emparanza ${ }^{2}$

${ }^{1}$ Gastroenterology, Mendaro Hospital, Mendaro/Spain

${ }^{2}$ Clinical Epidemiology, Donostia University Hospital, Donostia/Spain

${ }^{3}$ Gastroenterology, Mondragon Hospital, Mondragon/Spain

${ }^{4}$ Radiology, Osatek Donostia, Donostia/Spain

${ }^{5}$ Immunlogy, Donostia University Hospital, Donostia/Spain

${ }^{6}$ Hematology, Galdakao Hospital, Galdakao/Spain

Hematology, Hospital de Cruces, Barakaldo/Spain

Contact E-mail Address: agustincastiella@yahoo.es

Introduction: In a previous study from our group (1), in a secondary hospital, we did not find differences in the liver iron concentration (LIC) of the different groups, and we can not predict liver iron overload for hyperferritinemia (HF) 
patients with HFE mutations and (transferrin saturation index (TSI) values alone. But we did not have $\mathrm{C} 282 \mathrm{Y} / \mathrm{C} 282 \mathrm{Y}$ patients in the series.

Aims \& Methods: To study the relevance of HFE mutations and TSI in determining LIC for HF patients attending the outpatient clinic at 6 hospitals in the Basque country. Prospective study of 312 consecutive patients with HF. Olynyk et al.(2) described three different groups according to HFE mutations and TSI (Group A: no predisposing mutations (PM) for $\mathrm{HH}$ and TSI $>45 \%$, Group B: PM for HH: C282Y/C282Y; C282Y/H63D, H63D/H63D, and TSI > $45 \%$; Group C: no PM for $\mathrm{HH}$ and normal TSI);Group D: PM and normal TSI. In the Basque country, hereditary hemochromatosis $(\mathrm{HH})$ predisposing mutations differ, with relevance of the H63D/H63D mutation. The LIC was measured by MRI.

Results: In all the patients HFE study was available: C282Y/C282Y 14 (4.49\%); C282Y/H63D 25 (8.01\%); H63D/H63D 47 (15.06\%); H63D/wt 99 (31.73\%); wt/ wt $98(31.41 \%)$; C282Y/S65C $1(0.32 \%) ; \mathrm{H} 63 \mathrm{D} / \mathrm{S} 65 \mathrm{C} 2(0.64 \%) ; \mathrm{C} 282 \mathrm{Y} /$ wt 16 $(5.13 \%)$; S65C/wt $10(3.21 \%)$. LIC was obtained from all the patients by MR. Mean age: $55 \pm 13.5,272$ men and 40 women. Group A: 54, Group B: 32 Group C:160. Group D: 54. The mean LIC in Group A: $37.21 \pm 27.89$, group B: $70.53 \pm 58.67$, group C: $35.23 \pm 22.62$. Group D: $42.67 \pm 22.98$. We compared the LIC mean values of the 4 groups (bonferroni) with significant differences $(\mathrm{p}=0.0000)$.

Conclusion: The LIC in different groups of patients referred for HF are significantly different with different predisposition to $\mathrm{HH}$.

Disclosure of Interest: All authors have declared no conflicts of interest.

\section{References}

1. Castiella A, Zapata E, Zubiaurre L, et al. Liver iron concentration (LIC) in patients referred for hyperferritinemia (HF) to a secondary hospital: analysis of the different groups according to HFE mutations and transferrin saturation index (TSI). UEG journal 2014;2: A293. (P584).

2. Olynyk JP, Gan E, Tan T. Predicting iron overload in hyperferritinemia. Clin Gastroenterol Hepatol 2009; 7: 359-362.

\section{P0014 LIVER IRON CONCENTRATION IN THE METABOLIC SYNDROME WITH HYPERFERRITINEMIA (DYSMETABOLIC HYPERFERRITINEMIA). RESULTS FROM A PROSPECTIVE COHORT OF 312 PATIENTS}

A. Castiella Eguzkiza ${ }^{1}$, E. Zapata ${ }^{1}$, I. Urreta ${ }^{2}$, L. Zubiaurre $^{1}$, P. Otazua ${ }^{3}$, J. M. Alustiza ${ }^{4}$, E. Salvador ${ }^{4}$, G. Letamendi ${ }^{5}$, B. Arrizabalaga ${ }^{6}$, L. Mendibil ${ }^{1}$, J I. Emparanza

${ }^{1}$ Gastroenterology, Mendaro Hospital, Mendaro/Spain

${ }^{2}$ Clinical Epidemiology, Donostia University Hospital, Donostia/Spain

${ }^{3}$ Gastroenterology, Mondragon Hospital, Mondragon/Spain

${ }^{4}$ Radiology, Osatek Donostia, Donostia/Spain

${ }^{5}$ Hematology, Galdakao Hospital, Galdakao/Spain

${ }^{6}$ Hematology, Hospital de Cruces, Barakaldo/Spain

Contact E-mail Address: agustincastiella@yahoo.es

Introduction: Aproximately $25 \%$ of adult population in western countries have metabolic syndrome (MS). Hyperferritinemia (HF) is frequently present in patients with MS (dysmetabolic hyperferritinemia). There are some publications that support that HF is associated with a raised liver iron concentration (LIC) in these patients, but some doubts persist about this subject.

Aims \& Methods: To study the LIC in patients referred for hyperferritinemia to six different hospitals in the Basque Country (multicenter study), Spain, and determine if there are differences between patients with or without metabolic syndrome. A prospective study of 312 consecutive patients with $\mathrm{HF}$ ( $>200 \mathrm{mg}$ / L women, $>300 \mathrm{mg} / \mathrm{L}$ men) was conducted from December 2010 to April 2013. The Metabolic syndrome was defined by the presence of three of the following factors: waist circumference $\geq 94 \mathrm{~cm}$ men/ $\geq 80 \mathrm{~cm}$ women; Triglycerides $\geq 150 \mathrm{mg}$ $\mathrm{dL}$ or treatment for this dislipidemia; HDL $<40 \mathrm{mg} / \mathrm{dL}$ women $/<50 \mathrm{mg} / \mathrm{dL}$ men or treatment for this dislipidemia; glucose $\geq 100 \mathrm{mg} / \mathrm{dL}$ or Type 2 diabetes; hypertension: blood pressure $\geq 130 \mathrm{mmHg} / \geq 85 \mathrm{mmHg}$ or treatment for arterial hypertension (1). LIC was determined by MRI (SIR method) (2).

Results: In 276 of 312 patients we have all the data to determine the MS presence: $115 / 240$ men $(48 \%)$ and $20 / 36$ women $(55.6 \%), 135$ patients, presented MS. In all 276 patients MRI for LIC determination (mean \pm SD) was performed. (We have LIC results $(\mu \mathrm{mol} / \mathrm{g})$ from the 276 patients). The mean LIC was $30.83 \pm 19.38$ (women) and $38.84 \pm 25.50$ (men), with $37.66 \pm 24.79$ (CI 95\%; 33, 44 to 41,88 ) for all the MS group. In 141 patients MS was not diagnosed (NMS): 125/240 were men $(52 \%)$, and $16 / 36$ women $(44.4 \%)$. The mean LIC was $34.88 \pm 16.18$ in women, and $44.48 \pm 38.16$ in men, with $43.39 \pm 36.43$ (CI $95 \%, 37,32$ to 49,46 ) for all the NMS group. We compare the mean values of LIC from both groups (MS vs NMS) by Pearson's Chi square test and Fisher's exact test: no significant differences were seen $(\mathrm{p}=0.12)$.

Conclusion: Patients with HF and MS (dysmetabolic hyperferritinemia) present a mean LIC near normal values and their values do not differ from those of patients with HF and without MS.

Disclosure of Interest: All authors have declared no conflicts of interest.

\section{References}

1. K.G.M.M. Alberti, ; Robert H. Eckel, ; Scott M. Grundy, et al. Harmonizing the Metabolic Syndrome. A Joint Interim Statement of the International Diabetes Federation Task Force on Epidemiology and Prevention; National Heart, Lung, and Blood Institute; American Heart Association; World Heart Federation; International Atherosclerosis
Society; and International Association for the Study of Obesity. Circulation 2009: 120: 1640-45.

2. Alústiza JM, Artetxe J, Castiella A, et al. MR quantification of hepatic iron concentration. Radiology 2004; 230: 479-84.

P0015 INTERLEUKIN-25 PROTECTS AGAINST HIGH-FAT DIETINDUCED HEPATIC STEATOSIS IN MICE BY INDUCING IL-25 AND M2A KUPFFER CELL PRODUCTION

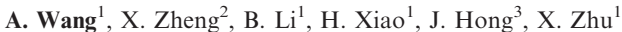

${ }^{1}$ Gastroenterology And Hepatology, The First Affiliated Hospital of Nanchang University, Nanchang/China

${ }^{2}$ Pharmacy, The First Affiliated Hospital of Nanchang University, Nanchang/China ${ }^{3}$ The First Affiliated Hospital of Nanchang University, Nanchang/China

Contact E-mail Address: waj1103b@163.com

Introduction: Alternatively activated anti-inflammatory macrophage (also termed M2 Kupffer cell) is important for prevention of the development of steatosis and liver injury in non-alcoholic fatty liver disease (NAFLD). Our previous studies demonstrated that interleukin (IL)-25 was downregulated in NAFLD mice and exogenous IL-25 protected against NAFLD by inducing M2 Kupffer cells.

Aims \& Methods: We aimed to explore the intracellular signaling pathways of IL25 to regulate macrophage polarization and direct effects of IL-25 on Kupfer cells. Mouse model of NAFLD was induced by feeding a high-fat diet (HFD); In vitro expansion of mouse Kupffer cells, IL-10 and IL-25 were used to induce M2a Kupffer cells; specific siRNAs were used to knockdown IL-25 receptor mRNA for assessing the direct and specific effect of IL-25 on Kupffer cells; IL-25 induced M2a Kupffer cells were back transfusion into the abdomen of NAFLD mouse to assess the efficacy; Dual-luciferase reporter assays and Chromatin immunoprecipitation assays were used to determine the transcription factor of IL-25 promoter.

Results: Exogenous IL-25 induced expression of type 2 cytokine and alternative activation of Kupffer cell in vivo. It could also promote hepatic macrophages to differentiate into M2a Kupffer cells in vitro. Interestingly, IL-25 recovered the expression of IL-25 mRNA in the liver of NAFLD mice. Furthermore, IL-25 could induce the expression of IL-25 in cultured hepatocytes by activation of STAT6, rather than MZF1, AP1 or NF- $\kappa$ B. STAT6 was sufficient and necessary for IL-25 expression. Deletion and site-directed mutagenesis of the IL-25 promoter revealed that IL-25 transcriptional activation depended primarily on a putative STAT-binding sequence between nucleotides $-682 /-674$ upstream of the start site. STAT6 binding to this sequence increased in response to IL-25 treatment in vitro and in vivo. Finally, IL-25 induced M2a Kupffer cells could ameliorate HFD-induced hepatic steatosis by reducing M1 Kupffer cells.

Conclusion: Our results elucidate the molecular mechanisms of IL-25 during amelioration of hepatic steatosis and provide the scientific basis of direct IL-25 treatment or macrophage transfusion therapy for NAFLD.

Disclosure of Interest: All authors have declared no conflicts of interest.

\section{References}

1. Wang AJ, Yang Z, Grinchuk V, Smith A, Qin B, Lu N, Wang D, Wang H, Ramalingam TR, Wynn TA, Urban JF Jr, Shea-Donohue T, Zhao A. IL-25 or IL-17E Protects against High-Fat Diet-Induced Hepatic Steatosis in Mice Dependent upon IL-13 Activation of STAT6. J Immunol. 2015 Nov 15;195(10):4771-80.

2. Natarajan K, Mathialagan GD, Raghavan S, Shanmugam N. The Advanced Lipoxidation end Product Precursor Malondialdehyde Induces IL-17E Expression and Skews Lymphocytes to the th17 Subset. Cell Mol Biol Lett. 2015 Dec;20(4):647-62.

3. Yang Z, Grinchuk V, Urban JF Jr, Bohl J, Sun R, Notari L, Yan S, Ramalingam T, Keegan AD, Wynn TA, Shea-Donohue T, Zhao A. Macrophages as IL-25/IL-33-responsive cells play an important role in the induction of type 2 immunity. PLoS One. 2013;8(3):e59441.

4. Wan J, Benkdane M, Teixeira-Clerc F, Bonnafous S, Louvet A, Lafdil F, Pecker F, Tran A, Gual P, Mallat A, Lotersztajn S, Pavoine C. M2 Kupffer cells promote M1 Kupffer cell apoptosis: a protective mechanism against alcoholic and nonalcoholic fatty liver disease. Hepatology. 2014 Jan;59(1):130-42.

\section{P0016 LONG-TERM BENEFIT OF STATINS USED FOR}

\section{TREATMENT OF NON-ALCOHOLIC STEATOHEPATITIS (NASH)}

A. Suceveanu ${ }^{1}$, A. P. Suceveanu ${ }^{1}$, I. R. Parepa ${ }^{2}$, D. Catrinoiu ${ }^{3}$, F. Voinea ${ }^{3}$, L. Mazilu ${ }^{3}$

${ }^{1}$ Gastroenterology, Ovidius University, Constanta/Romania

${ }^{2}$ Cardiology, Ovidius University, Constanta/Romania

${ }^{3}$ Internal Medicine, Ovidius University, Constanta/Romania

Contact E-mail Address: asuceveanu@yahoo.com

Introduction: NASH is considered an important risk factor for liver fibrosis. Although literature data indicates that statins may be beneficial when given for NASH treatment, recent reports are controversial ${ }^{1}$.

Aims \& Methods: To evaluate if statins independently influence the evolution of fibrosis accompanying NASH using the scales of FibroMax, 120 patients with $\mathrm{NASH}$ and metabolic syndrome were followed-up for a period of 3 years. We excluded patients taking a serie of drugs, with genetic metabolic disorders or impaired intestinal absorption (celiac disease) or alcoholics. Steatosis, fibrosis 
and NASH were quantified by using the FibroMax scales at baseline and after three years of statin treatment. Patients were randomized in two groups: the active group of 60 patients receiving low-dose hydrophilic statin (rosuvastatin $5 \mathrm{mg} /$ day) and the witness group of 60 patients, matched by age, gender and sex, receiving placebo.

Results: $97 \%$ of subjects fulfilled the follow-up period. The FibroMax staging at baseline showed the following results in the active group: S1 $-29 \%, \mathrm{~S} 2-41 \%$ and $\mathrm{S} 3-30 \% ; \mathrm{F} 1-50 \%, \mathrm{~F} 2-30 \%, \mathrm{~F} 3-13 \%$ and $\mathrm{F} 4-7 \%$ of patients, respectively N1$31 \%$ and N2-69\%. The staging according to FibroTest, SteatoTest and NashTest was similar in placebo group. After 2 years of low-dose hydrophilic statin, the mean ALT level from active group decreased from $72.22 \mathrm{IU} / \mathrm{L}$ to $32.80 \mathrm{IU} / \mathrm{L}, \mathrm{p}<0.05$ (ss); in the witness group no significant ALT decrease was noticed $(69.34 \mathrm{IU} / \mathrm{L}$ to $58.17 \mathrm{IU} / \mathrm{L}, \mathrm{p}>0.5)$. The FibroMax showed an important improvement of steatosis and fibrosis in active group, compared with the witness group. After three years of statins, our active group was stratified as follows: $\mathrm{S} 0-27 \%, \mathrm{~S} 1-46 \%, \mathrm{~S} 2-25 \%$, respectively $\mathrm{S} 3-2 \%$ of patients, respectively $\mathrm{F} 0-38 \%, \mathrm{~F} 1-32 \%, \mathrm{~F} 2-28 \%, \mathrm{~F} 3-2 \% ; \mathrm{F} 4-0 \%$ of patients. NashTest also proved a positive evolution under statin treatment, compared with placebo (N0-36\%, N1-40\% respectively N2-26\%, p >0.001, ss) After adjusting for age, BMI, diabetes, LDL-cholesterol and triglyceride levels, statin therapy showed a significant correlation with the steatosis, fibrosis and NASH stages improvement in the active group $(r=0.92, r=0.87$, respectively $r=0.95$, $\mathrm{p}<0.005$, ss).

Conclusion: While statins proved to be safe and efficient for the treatment of NASH in our series, larger cohort studies are needed to further demonstrate this potential positive effect on liver fibrosis.

Disclosure of Interest: All authors have declared no conflicts of interest.

\section{Reference}

1. Geoffrey C. Farrell, Arthur J. McCullough and Christopher P. Non-Alcoholic Fatty Liver Disease: A Practical Guide. Published Online: 8 MAR 2013, DOI: 10.1002/9781118556153.ch26

\section{P0017 THE ROLE OF GENETIC FACTORS IN NON-OBESE NASH PATIENTS}

\section{F. Ates}

Gastroenterology, Mersin University, Mersin/Turkey

\section{Contact E-mail Address: drfehmiates@hotmail.com}

Introduction: Methylenetetrahydrofolate reductase (MTHFR) is the key enzyme in homocysteine metabolism. It is thought that MTHFR A1298C and C677T gene polymorphisms contribute to etiopathogenesis of NASH because of their effects in homocysteine metabolism.

Aims \& Methods: Our aim in this study is to determine the relationship between the NASH and MTHFR C677T and A1298C gene polymorphisms, especially in non-obese NASH patients. Eighty-eight NASH patients whose diagnoses were confirmed by liver biopsies and 90 healthy volunteers as control group were included in the study. We investigated MTHFR A1298C and C677T gene polymorphisms and compared NASH patients and controls. NASH patients were assigned to two groups according to whether they are obese.

Results: Eighty-eight NASH patients (52M, 36F, mean age 45 years), and 90 healthy controls $(53 \mathrm{M}, 37 \mathrm{~F}$, mean age 41 years) were included in the study. According to BMI values of NASH patients, 55 patients were non-obese and 33 patients were obese. There was no statistically significant difference between distribution of MTHFR A1298C polymorphism of NASH patients and controls $(p>0.05)$. The proportion of TT genotype of MTHFR C677T polymorphism of NASH patients was significantly higher than that of controls $(\mathrm{p}<0.01)$. Also the proportion of TT genotype of MTHFR C677T polymorphism of non-obese NASH patients was significantly higher than that of controls $(p<0.01)$. However, the proportion of TT genotype of MTHFR C677T polymorphism of obese NASH patients was not significantly different than the control group $(\mathrm{p}>0.05)$. MTHFR C677T CC (wild) genotype was significantly lower in non-obese NASH patients than controls $(\mathrm{p}<0.05)$

Conclusion: This study revealed that TT genotype of MTHFR C677T polymorphism is more frequent, especially in non-obese NASH patients than in healthy controls. This finding shows that genetic factors are particularly more important in non-obese NASH patients.

Disclosure of Interest: All authors have declared no conflicts of interest.

\section{P0018 EVALUATION OF THE RELATIONSHIP OF LEVELS OF C- REACTIVE PROTEIN AND HOMOCYSTEIN IN PATIENTS WITH ABDOMINAL OBESITY AND PATHOLOGICAL CHANGES IN THE LIVER DEFINED BY BIOPROGNOSTIC TEST STEATOSCREEN}

\section{E. Chernetcova, N. Denisov}

The Second Department Of Therapy Of Improvement Of Doctors, The Federal State Military Educational Institution of Higher Education, Military Medical Academy nam, Saint-Petersburg/Russian Federation

Contact E-mail Address: katemed $@$ list.ru

Introduction: The importance of subclinical inflammation and hyperhomocysteinemia in the development of nonalcoholic fatty liver disease (NAFLD) needs further study. ${ }^{1}$ Recent studies have shown that the development of hyperhomocysteinemia and an increase in the level of C-reactive protein contributes to increased oxidative stress, influences the development of metabolic disorders, which makes it possible to consider these indicators as additional markers for the development of NAFLD in patients with abdominal obesity (AO).

Aims \& Methods: We aimed to study the relationship between CRP and homocysteine (HC) levels with pathological changes in the liver, determined with the non-invasive bioprognostic test Steatoscreen in patients with AO. The study included 60 patients aged 18 to 59 years with joints with a waist circumference $>80 \mathrm{~cm}$ for women, over $94 \mathrm{~cm}$ for men. All patients underwent a bio-prognostic test Steatoscreen. Depending on the severity of the pathological changes in the liver, the Steatoskrin scale divided all the patients into 3 groups: steatosis, fibrosis and steato- fibrosis. Depending on the presence or absence of cytolysis syndrome, 2 subgroups were distinguished in each group: subgroups of nonalcoholic steatohepatitis (ALT values, AST above the norm more than 2 times), and steatosis. In all patients, the levels of CRP and GC were studied. Results: In groups of patients with fibrosis and steato-fibrosis, the levels of CRP and $\mathrm{HC}$ were significantly higher than in patients in the steatosis group, and the highest values of these parameters were recorded in patients in subgroups with non-alcoholic steatohepatitis. In the process of correlation analysis, significantly positive interrelations between the levels of CRP and HC were obtained with the levels of severity of pathological changes in the liver on the Steatoscrin scale: $\mathrm{r}=0.6$ и $\mathrm{p}<0.001 ; \mathrm{r}=0.85$ и $\mathrm{p}<0.0016$ for CRP and HC, respectively.

Conclusion: NAFLD in patients with AO is characterized by the development of a complex of metabolic disorders associated with chronic vascular inflammation. This fact can influence the risk of developing the pathology of not only the liver, but also atherosclerosis and proves the need for a more thorough examination of patients with AO and NAFLD for the purpose of early detection and correction of existing metabolic disorders.

Disclosure of Interest: All authors have declared no conflicts of interest.

\section{Reference}

1. Utility of noninvasive methods for the characterization of nonalcoholic liver steatosis in the family practice. The "VARES" Italian multicenter study. 2016 Jan 29. pii: S0026-0495(16)00027-5.

P0019 INVESTIGATION OF THE RELATIONSHIP BETWEEN THE THICKNESS OF THE INTIMA-MEDIA COMPLEX OF COMMON CAROTID ARTERIES AND PATHOLOGICAL CHANGES IN THE LIVER IN PATIENTS WITH ABDOMINAL OBESITY AND NONALCOHOLIC FATTY LIVER DISEASE

E. Chernetcova, N. Denisov, V. Grinevich, Y. Kravchuk, K. Ivashkin,

L. Kornouchova

The Second Department of Therapy of Improvement of Doctors, The Federal State Military Educational Institution of Higher Education, Military Medical Academy nam, Saint-Petersburg/Russian Federation

\section{Contact E-mail Address: katemed@list.ru}

Introduction: In the last decade, the notion of non-alcoholic fatty liver disease (NAFLD) has undergone noticeable changes. It is shown that in the liver with fatty hepatosis, insulin and glucose utilization is disrupted, conditions are created for the synthesis of atherogenic fractions of cholesterol and triglycerides. This contributes to the development of violations of carbohydrate and lipid metabolism, the early appearance of atherosclerosis and associated cardiovascular complications. Thus, NAFLD can be considered as an independent, additional risk factor for the development of atherosclerosis. Obviously, the studies devoted to clarifying the nature of the relationship between NAFLD and the early manifestations of atherosclerotic vascular wall lesions are relevant.

Aims \& Methods: Study of changes in the vascular wall of the common carotid artery (IMT CCA) and in patients with abdominal obesity (AO) and different forms of nonalcoholic fatty liver disease (NAFLD). The study involved 60 patients with $\mathrm{AO}$ between the ages of 18 to 59 years (waist circumference (WC) $>80 \mathrm{~cm}$ in women and $>94 \mathrm{~cm}$ in men), and NAFLD, in the absence of clinical manifestations, provided written informed consent to participate in the study. All patients underwent an ultrasound examination of the abdominal cavity to determine the size of the liver and signs of steatosis. The level of severity of pathological changes in the liver tissue (fibrosis, steatosis and steatofibrosis) was assessed by non-invasive diagnostic method Steatoskreen. (Biopredictive laboratory, France). Measurement of the CCA IMT was performed according to standard procedures on the machine Voluson 730 Expert, equipped with a linear transducer phased array with a frequency of $7.5 \mathrm{MHz}$. The presence of early signs of atherosclerosis was defined as a local thickening of the IMT CCA more than $0.9 \mathrm{~mm}$ in any point of the carotid artery (CCA IMT max). Depending on the severity of the pathological changes in the liver, the Steatoskreen scale divided all the patients into 3 groups: steatosis, fibrosis and steatofibrosis. Depending on the presence or absence of cytolysis syndrome, 2 subgroups were distinguished in each group: subgroups of non-alcoholic steatohepatitis (ALT values, AST above the norm more than 2 times), and steatosis. In the future, comparative and correlation analysis of the data was carried out.

Results: Signs of early atherosclerosis, in the form of the IMT CCA, were detected in the majority of the patients $(52 \%)$ and differed between the observed groups. The average thickness of the IMT CCA was significantly higher in patients with abdominal obesity and pathological changes in the liver in the form of severe steatofibrosis on the Steatoscreen scale than in groups of patients with less severe changes in hepatic tissue $(0.83 \mathrm{~mm}$ for the steatosis group, 0.89 and $0.97 \mathrm{~mm}$ for fibrosis and steatofibrosis groups respectively, $\mathrm{p}<0.001$ ). At the same time, the maximum thickness of the IMT CCA was recorded in the group of patients with non-alcoholic steatohepatitis in the fibrosis group $(1.14 \mathrm{~mm}, \mathrm{p}=0.002)$. In the process of regression analysis, a direct significantly relationship was found between the thickness of the carotid intima-media 
complex and pathological changes in the liver, determined by the test Steatoscreen $(\mathrm{r}=0.76 ; \mathrm{p}<0.0001)$. The dependence obtained is confirmed by the equation of simple linear regression.

Conclusion: In patients with AO, there is a direct relationship between the presence of pathological changes in the liver and the initial manifestations of atherosclerosis. The results obtained make it possible to evaluate the individual risk of atherosclerosis in this category of patients. Clinical significance of the results is the need for a more thorough examination of patients with $\mathrm{AO}$ and suspicion of liver pathology to assess the development of not only the disease of the liver itself, but also cardiovascular complications.

Disclosure of Interest: All authors have declared no conflicts of interest.

\section{Reference}

1. Targher G, Bertolini L, Padovani R. Relations Between Carotid Artery Wall Thickness and Liver Histology in Subjects With Nonalcoholic Fatty Liver Disease. Diabetes Care. 2006; 29(6):1325-30. expression of TNF- $\alpha$ increased, $\mathrm{P}<0.01$ and IL-10 decreased, $\mathrm{P}<0.05$ ) by 8 th week, which happened to coincide with the presence of hepatic steatosis. Removal of inflamed MAT significantly worsened liver pathology, as well as resulted in hepatic inflammation (mRNA expression of MCP-1 increased, $\mathrm{P}<0.01$ and IL-10 decreased, $\mathrm{P}<0.01$ ) and lipid accumulation (mRNA expression of ACC1 increased, $\mathrm{P}<0.01$ and PPAR- $\alpha$ decreased, $\mathrm{P}<0.01)$. Meanwhile, intestinal permeability was higher in the MAT removal group than that in the Sham group, which was supported by higher lipopolysaccharide $(\mathrm{P}<0.05)$ in serum and lower mRNA expression of ZO-1 $(\mathrm{P}<0.01)$ and occludin $(\mathrm{P}<0.05)$ in small intestine.

Conclusion: These results suggest MAT inflammation arises at the early stage of NAFLD. Removal of inflamed MAT promotes the development of NAFLD and injures the intestinal barrier. Thus, we propose that MAT inflammation seems to be a compensatory response, on the fact that inflamed MAT protects the liver from the gut-derived damage factors via confining them within MAT, rather than aggravates NAFLD.

Disclosure of Interest: All authors have declared no conflicts of interest.
P0020 OVEREXPRESSION OF HEPASSOCIN IN DIABETIC PATIENTS WITH NONALCOHOLIC FATTY LIVER DISEASE MAY FACILITATE INCREASED HEPATIC LIPID ACCUMULATION

S. Abd-Elsalam ${ }^{1}$, S. Khodeir ${ }^{2}$, S. Abou Saif ${ }^{1}$, G. Abdelmoemen ${ }^{3}$

${ }^{1}$ Tropical Medicine Department, Tanta university, Tanta/Egypt

${ }^{2}$ Internal Medicine Department, Tanta university, Tanta/Egypt

${ }^{3}$ Clinical Pathology Department, Tanta university, Tanta/Egypt

Contact E-mail Address: sherif tropical@yahoo.com

Introduction: Insulin resistance is the main pathogenic determinant of both NAFLD and diabetes, and it can facilitate triglyceride accumulation in the liver. Overexpression of hepassocin (HPS) increased hepatic lipid accumulation and NAFLD activity scores (NAS), whereas deletion of HPS improved high fat diet-induced hepatic steatosis and decreased NAS in mice.

Aims \& Methods: The aim of this study was to explore the relationship between hepassocin and diabetic patients with or without NAFLD. The study included 80 patients that were divided into 4 groups: Group I: included 20 patients who were diagnosed as diabetes mellitus type 2, Group II: included 20 patients who were diagnosed as non alcoholic fatty liver disease, Group III: included 20 patients who were diagnosed as diabetes type 2 and non alcoholic fatty liver disease, Group IV (control group): included 20 healthy person who were matched in age and sex with patients group.

Results: There was stastically significant decrease in mean value of serum hepassocin of group 1 and IV on comparing with group II and group III. For group III there was stastically significant increase in mean value of serum hepassocin on comparing with other groups. There was a significant serum hepassocin up regulation in patients with type 2 diabetes and non alcoholic fatty liver diseased patients (Group 3) mostly than diabetic patients (Group 1) and even than non alcoholic fatty liver disease (Group 2).

Conclusion: The present study provides evidence that overexpression of HPS may facilitate increased hepatic lipid accumulation with NAFLD and Type 2 Diabetes mellitus.

Disclosure of Interest: All authors have declared no conflicts of interest.

\section{References}

Marchesini G, Brizi M, Bianchi G, et al. Nonalcoholic fatty liver disease: a feature of the metabolic syndrome. Diabetes. 2001;50:1844-50.

Cao MM, Xu WX, Li CY, Cao CZ, Wang ZD, Yao JW, et al. Hepassocin regulates cell proliferation of the human hepatic cells L02 and hepatocarcinoma cells through different mechanisms. J Cell Biochem. 2011 Oct; 112(10):2882-90. Wu HT, Lu FH, Ou HY, Su YC, Hung HC, Wu JS, et al. The role of hepassocin in the development of non-alcoholic fatty liver disease. J Hepatol. 2013 Nov; 59(5):1065-72.

\section{P0021 MESENTERIC ADIPOSE TISSUE PROTECTS AGAINST NON-ALCOHOLIC FATTY LIVER DISEASE BY IMPROVING INTESTINAL BARRIER}

Z. Wu ${ }^{1}$, J. Tan ${ }^{1}$, F. Zhang ${ }^{1}$, J. Xu ${ }^{2}$, Y. Song ${ }^{1}$, Y. L. Liu ${ }^{2}$

${ }^{1}$ Department Of Gastroenterology, Peking University People's Hospital, Beijing/ China

${ }^{2}$ Department Of Gastroenterology, Peking University People's Hospital, Beijing/ China

Contact E-mail Address: jerry1989@hsc.pku.edu.cn

Introduction: Visceral adipose tissue (VAT) and gut are thought to be the main two sources of damage factors promoting non-alcoholic fatty liver disease (NAFLD). As one part of VAT, mesenteric adipose tissue (MAT) may be unique in VAT for it can affect liver directly via portal vein. However, the relationship of MAT status with different stages of NAFLD is not clear, as well as the role of inflamed MAT in NAFLD.

Aims \& Methods: Mice fed with high fat diet or normal diet were sacrificed in time gradients $(4 \mathrm{w}, 8 \mathrm{w}, 12 \mathrm{w})$. Then, MAT in high fat diet feeding mice was removed or not at 8 th week and mice were sacrificed by 12 th week.

Results: Mice have developed hepatic steatosis at 8th week and progressed to steatohepatitis by 12 th week. Among four parts (mesenteric, epididymal, perirenal and retroperitoneal) of VAT, merely MAT became inflamed (mRNA

\section{P0022 DIAGNOSTIC ACCURACY OF SHEAR WAVE ULTRASOUND ELASTOGRAPHY FOR EARLY DETECTION OF NON ALCOHOLIC STEATOHEPATITIS AMONG PATIENTS WITH TYPE 2 DIABETES MELLITUS}

A. Gameel, E. M. Elhadidy, A. A. Mousa, M. M. Elrakhawy

Internal Medicine, Mansoura University, Mansoura/Egypt

Contact E-mail Address: asmaagameel165@gmail.com

Introduction: Non alcoholic fatty liver disease (NAFLD) is a broad term descriping simple steatosis, non alcoholic steatohepatitis (NASH), NASH cirrhosis and NASH-induced hepatocellular carcinoma (1). Incidence increased in patients with type 2 diabetes mellitus (DM) (2). Different diagnostic modalities have great limitations in differentiating simple staetosis from steatohepatitis (3). Liver biopsy remains the gold standard for diagnosis of NASH, however, it is invasive with potential severe complications (4). Several ultrasound elastography techniques have been descriped incluing transient elastography, acoustic radiation force impulse elastography and shear wave elastography(5). Shear wave elastography shows a stepwise increase of liver stiffness as the severity of liver inflammation increases(6).

Aims \& Methods: We aimed to evaluate the accuracy of shear wave ultrasound elastography in differntiating simple steatosis from steatohepatitis in patients with type $2 \mathrm{DM}$. This was a prospective study including 60 patients 30 males and 30 females who visited our outpatient clinic or inpatient department at Specialized Medical Hospital. These patients were diabetic aged more than 30 years old with ultrasound showing fatty liver. Significant alcohol consumption, drugs causing steatosis and hepatic diseases were excluded by history, laboratory investigations and liver biopsy. All patients underwent full detailed history, examination, laboratory investigations (complete blood count, liver functions, kidney functions, random blood sugar, lipid profile, serology for hepatitis B and $\mathrm{C}$ viruses). Shear wave elastography was performed to all patients and stiffness of the liver was measured from different areas in kilopascal $(\mathrm{kPa})$ then average stiffness by elastography was calculated. Liver biopsy was done and histopathological examination by Hematoxlin, Eosin and Masson Trichrome stains, then NAFLD activity score (NAS) was calculated.

\begin{tabular}{|c|c|c|c|c|c|c|c|c|}
\hline \multicolumn{9}{|c|}{ Roc curve for diagnosis of definitive NASH versus no NASH and probable NASH } \\
\hline Variable & Criterion & AUC & CI & P. & Senstivity & Specificity & PPV & NPV \\
\hline $\begin{array}{l}\text { Average } \\
\text { stiffness by } \\
\text { elastography }\end{array}$ & 8.45 & 0.936 & $0.871-1$ & $<0.001$ & $90 \%$ & $90 \%$ & $81 \%$ & $94 \%$ \\
\hline
\end{tabular}

Results: Correlation between results of stiffness by elastography and NAS by biopsy revealed that: There was a significant positive association between average stiffness by elastography and definitive NASH (NAS 5 and 6) in patients with type $2 \mathrm{DM}$. At a level of $8.45 \mathrm{kPa}$ by shear wave elastography, we can differntiate simple steatosis from steatohepatitis (Area Under Curve 0.936, senstivity $90 \%$, specificity $90 \%$, positive predictive value $81 \%$, negative predictive value $49 \%$ ). Conclusion: Shear wave ultrasound elastography is a promising non invasive technique to differntiate simple steatosis from steatohepatitis in patients with type 2 DM.

Disclosure of Interest: All authors have declared no conflicts of interest. 


\section{References}

1. Mavrogiannaki AN and Migdalis IN. Nonalcoholic fatty liver disease, diabetes mellitus and cardiovascular disease: Newer Data. International Journal of Endocrinology volume 2013 (2013), Article ID 450639, page 8.

2. Loomba, M. Abraham, A. Unalp, et al., Association between diabetes, family history of diabetes, and risk of nonalcoholic steatohepatitis and fibrosis, Hepatology 56 (3) (2012) 943-951.

3. Guha IN, Parkes J, Roderick P et al., Noninvasive markers of fibrosis in nonalcoholic fatty liver disease: validating the European Liver Fibrosis Panel and exploring simple markers. Hepatology 2008;47(2):455-460.

4. Ratizu V, Charlotte F, Heurtier A et al., Sampling variability of liver biopsy in non alcoholic fatty liver disease. Gastroenterology 2005;128:1898-906.

5. Orlaccio A, Bolacchi F, Antonicoli M, Coco I, Costanzo E, Tosti D, Francioso S, Angelico M, Simonetti G. Liver elasticity in NASH patients evaluated with real time elastography (RTE). Ultrasound Med Biol 2012;38:537-544.

6. Chen J, Talwalker JA, Yin M, Glaser KJ, Sanderson SO, Ehman RL. Early detection of nonalcoholic steatohepatitis in patients with nonalcoholic fatty liver disease by using MR elastography. Radiology 2011;259:749756(PMID:21460032 DOI:10.1148/radiol.11101942).

\section{P0024 A NOVEL TOOL FOR THE NON-INVASIVE QUANTITATIVE ASSESSMENT OF HEPATIC STEATOSIS USING B-MODE IMAGE- GUIDED ULTRASOUND ATTENUATION IMAGING: A PROSPECTIVE STUDY}

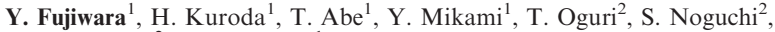
N. Kamiyama ${ }^{2}$, Y. Takikawa ${ }^{1}$

${ }^{1}$ Division Of Hepatology, Department Of Internal Medicine, Iwate Medical University, Morioka/Japan

${ }^{2}$ Ultrasound General Imaging, GE Healthcare, Hino/Japan

\section{Contact E-mail Address: kenyon1266@yahoo.co.jp}

Introduction: Nonalcoholic fatty liver disease is a main cause of chronic liver disease worldwide. A quantitative, non-invasive assessment of hepatic steatosis is desirable. Throughout the path of ultrasound (US), attenuation by liver parenchyma is uneven. This is the basis of the controlled attenuation parameter (CAP). However, further accumulation of data is needed to assess the role of CAP in the diagnosis of steatosis1). We investigated the diagnostic performance of B-mode image-guided ultrasound attenuation imaging and quantification for assessing hepatic steatosis by a liver biopsy (LB, reference standard). It was compared with the liver-to-spleen ratio (L/S ratio) from computed tomography (CT) and CAP.

Aims \& Methods: We prospectively analyzed 112 consecutive patients with chronic liver disease who underwent ultrasound attenuation imaging, CT, and liver biopsy. Ultrasound attenuation imaging was performed using the LOGIQ E9 scanner (GE Healthcare) with a C1-6-D convex array probe (frequency, 4 $\mathrm{MHz}$ ). We acquired a B-mode image of liver parenchyma. RF signals corresponding to the images were compensated by the reference signal previously measured from the uniform phantom (known attenuation, $0.5 \mathrm{~dB} / \mathrm{cm} / \mathrm{MHz}$ ) 2 ). The attenuation coefficient (AC) was calculated from the signals' decay slope. Steatosis, liver fibrosis, and necroinflammatory activity were staged and graded during pathological analysis. The steatosis grade was categorised as follows: S0, $<5 \%$; S1, 5-33\%; S2, 34-66\%; or S3, $>67 \%$. The diagnostic performance of AC for steatosis prediction was assessed using area under the curve (AUC) analysis; it was compared with the L/S ratio or CAP. Univariate and multivariate regression analyses were used to identify variables correlated with AC values.

Results: Patients $(51 \%$ men; $42 \%$ had non-alcoholic fatty liver disease, $58 \%$ had hepatitis $\mathrm{C}$ virus) had a median body mass index of $26 \mathrm{~kg} / \mathrm{m} 2$. Median AC values for grades S0 $(n=38), S 1(n=47), S 2(n=18)$, and S3 $(n=9)$ were $0.49,0.55$, 0.66 , and 0.72 , respectively, demonstrating a stepwise increase with increasing steatosis severity $(\mathrm{P}<0.0001)$. AC was significantly correlated with the steatosis percentage $(\mathrm{r}=0.800, \mathrm{P}<0.0001), \mathrm{L} / \mathrm{S}$ ratio $(\mathrm{r}=-0.670, \mathrm{P}<0.0001)$, and CAP $(\mathrm{r}=0.639, \mathrm{P}<0.001)$. AUCs of AC vs. the $\mathrm{L} / \mathrm{S}$ ratio for identifying grades $\geq \mathrm{S} 1$, $\geq \mathrm{S} 2$, and $\geq \mathrm{S} 3$ were 0.919 vs. $0.856,0.957$ vs. 0.902 , and 0.960 vs. 0.919 , respectively, showing significantly better results than those for the $\mathrm{L} / \mathrm{S}$ ratio and CAP. For the sensitivity and specificity of $\mathrm{AC} \geq 85 \%$, cut-off values were $0.53 \mathrm{~dB} / \mathrm{cm} /$ $\mathrm{MHz}$ for $\geq \mathrm{S} 1,0.60 \mathrm{~dB} / \mathrm{cm} / \mathrm{MHz}$ for $\geq \mathrm{S} 2$, and $0.64 \mathrm{~dB} / \mathrm{cm} / \mathrm{MHz}$ for $\geq \mathrm{S} 3$. Steatosis was the only factor independently affecting $\mathrm{AC}$ values.

Conclusion: Ultrasound attenuation imaging had a high diagnostic accuracy for detecting hepatic steatosis.

Disclosure of Interest: All authors have declared no conflicts of interest.

\section{References}

1. European Association for Study of Liver; Asociacion Latinoamericana para el Estudio del Higado. EASL-ALEH Clinical Practice Guidelines: Non-invasive Tests for Evaluation of Liver Disease Severity and Prognosis. J Hepatol 2015; 63(1):237-264

2. Yao LX, Zaqzebski JA, Madsen EL. Backscatter Coefficient Measurements Using a Reference Phantom to Extract Depth-dependent Instrumentation Factors. Ultrasonic Imaging 1990; 12(1): 58-70.
P0025 SERUM FERRITIN SPECIFICITY IN PREDICTING EARLY MORTALITY OF PATIENTS WITH ALCOHOLIC LIVER CIRRHOSIS

I. Valantiene ${ }^{1}$, A. Kederys ${ }^{2}$, J. Kupcinskas ${ }^{1}$, J. Kondrackiene ${ }^{2}$, J. Šumskienè ${ }^{2}$, V. Petrenkiene ${ }^{2}$, L. Kupcinskas ${ }^{1}$

${ }^{1}$ Gastroenterology, Institute for Digestive Research, Department of

Gastroenterology, Lithuanian University of Health Sciences, Kaunas/Lithuania

${ }^{2}$ Department Of Gastroenterology, Lithuanian University of Health Sciences, Kaunas/Lithuania

Contact E-mail Address: andriuskederys@gmail.com

Introduction: Individuals with chronic liver diseases may have a mild to moderate iron overload, but the mechanism is not fully understood. Increased contents of iron have been attributed to the progression of liver cirrhosis caused by $\mathrm{HCV}$ infection, non-alcoholic fatty liver disease, or alcoholic liver disease. Serum ferritin concentration can be increased in iron overload and shows hepatic necroinflammation. Recently, raised serum ferritin concentration was shown to predict mortality in patients awaiting liver transplantation in decompensated liver cirrhosis, but according to latest research plasma ferritin levels can help to predict the early mortality of patients with alcoholic liver cirrhosis (ALC).

Aims \& Methods: The aim of this study was to determine the association between serum ferritin concentration and the outcomes of patients with ALC. The study included 72 patients with ALC. The diagnosis and etiology of ALC was confirmed by laboratory tests, clinical features, radiological imaging, and percutaneous or tranjugular liver biopsy. Alcoholic liver disease was confirmed when daily consumption of alcohol was $>30 \mathrm{~g} / 20 \mathrm{~g} /$ day for males/females, respectively, as confirmed by at least 1 family member of affected individual. All patients were divided into three groups by serum ferritin concentration: below $200 \mu \mathrm{g} / \mathrm{l}, 200-400 \mu \mathrm{g} / 1$ and above $400 \mu \mathrm{g} / \mathrm{l}$. Statistical analysis was performed using statistical software SPSS 23.0. ROC (Receiver operating characteristic) scale was used to estimate serum ferritin specificity in predicting early mortality. If the area under the curve (AUC) is greater than 0.5, the test is specific.

Results: The first group consisted of 44 patients, the second group 13 patients, the third group 15 patients. The average age was $57.5 \pm 11.7$ years. Serum ferritin concentration in first group was $58.2 \pm 45.9 \mu \mathrm{g} / 1$, second $293.5 \pm 63.5 \mu \mathrm{g} / \mathrm{l}$, third $-599.5 \pm 221.1 \mu \mathrm{g} / \mathrm{l}$. AST concentration in first group was $90.8 \pm 70.2 \mathrm{IU} / 1$, second $-96.7 \pm 58.3 \mathrm{IU} / 1$, third $-133.8 \pm 95.5 \mathrm{IU} / \mathrm{l}$. ALT concentration in first group was $66.7 \pm 66.8 \mathrm{IU} / 1$, second $-64.5+75.5 \mathrm{IU} / 1$, third $-60.1 \pm 49.5 \mathrm{IU} / 1$ Bilirubin concentration in first group was $90.52 \pm 85.7 \mu \mathrm{mol} / 1$, second $111.9 \pm 72.3 \mu \mathrm{mol} / 1$, third $-140.9 \pm 195.0 \mu \mathrm{mol} / 1$. The biggest mortality rate was in third group - 13 of 15 patients $(86.67 \%)(p<0.00001)$. ROC scale shows a $77 \%$ specificity of serum ferritin concentration predicting early mortality in patients with toxic cirrhosis (AUC $0.838, \mathrm{p}<0.0001$ )

Conclusion: Serum ferritin level above $400 \mu \mathrm{g} / 1$, elevated liver enzymes and bilirubin concentration shows a poor outcome of patients with ALC $(p<0.0001)$ Serum ferritin level is a specific indicator for predicting early mortality in ALC. Disclosure of Interest: All authors have declared no conflicts of interest.

\section{P0026 PLASMA RETICULON 4 PROTEIN IS ASSOCIATED WITH} PORTAL HYPERTENSION IN PATIENTS WITH LIVER CIRRHOSIS

S. Gelman ${ }^{1}$, V. Salteniene ${ }^{2}$, A. Pranculis ${ }^{3}$, L. Kupcinskas

${ }^{1}$ Gastroenterology, Lithuanian University of Health Sciences, Medical Academy, Kaunas/Lithuania

${ }^{2}$ Institute For Digestive Research, Lithuanian University of Health Sciencies, Kaunas/Lithuania

${ }^{3}$ Radiology, Lithuanian University of Health Sciences, Medical Academy, Kaunas/ Lithuania

${ }^{4}$ Gastroenterology, Institute for Digestive Research, Department of

Gastroenterology, Lithuanian University of Health Sciences, Kaunas/Lithuania

Contact E-mail Address: sigita.gelman@gmail.com

Introduction: Reticulon 4 (RTN4) protein was first described as a potent neurite growth inhibitor in the central nervous system ${ }^{1}$. However, according to recent research, the protein expression is not limited to the cells of nervous system as it was found in various other tissues, including endothelial cells, fibroblasts, muscle cells, hepatocytes ${ }^{1,2,3}$. The diverse location of the protein accounts for various functions, such as vascular remodeling, inflammation and oxidative stress, cell proliferation, carcinogenesis and atherogenesis $2,3,4,5,6$. The function and expression of the protein in liver disease is still not clear.

Aims \& Methods: In this study we aimed to evaluate plasma levels of RTN4 protein in cirrhotic patients and associate them with clinical parameters and portal hypertension. The pilot study included 72 patients with hepatitis $\mathrm{C}$ or alcoholic liver cirrhosis and 22 healthy controls. Liver cirrhosis was diagnosed by laboratory tests, radiological imaging and/or liver biopsy. Portal pressure was assessed by hepatic venous pressure gradient (HVPG) measurement. Plasma levels of RTN4 were determined by enzyme-linked immunosorbent assay. Association of RTN4 with biochemical parameters, Child-Turcotte-Pugh and Model of End Stage liver disease (MELD) score, transient elastography values, esophagogastric varices, HVPG, clinically significant portal hypertension $(\mathrm{CSPH} ; \mathrm{HVPG} \geq 10 \mathrm{mHg})$ and severe portal hypertension $(\mathrm{SPH} ; \mathrm{HVPG} \geq 12)$ was assessed.

Results: Plasma RTN4 levels were significantly lower in cirrhotic patients than in healthy controls $(\mathrm{p}<0.0001)$. Significant negative correlation was observed between RTN4 and Child-Pugh score $(\mathrm{r}=-0.301 ; \mathrm{p}=0.015)$, MELD score $(\mathrm{r}=-0.311 ; \mathrm{p}=0.026)$, transient elastography values $(\mathrm{r}=-331 ; \mathrm{p}=0.006)$. There was significant correlation between RTN4 and AST $(r=-0.307, p=0.005)$, ALP $(\mathrm{r}=-0.396 ; \mathrm{p}=0.001)$, total bilirubin $(\mathrm{r}=-0.426 ; \mathrm{p}<0.0001)$, thrombocytes $(\mathrm{r}=0.534, \mathrm{p}<0.0001)$, INR $(\mathrm{r}=-0.357, \mathrm{p}=0.002)$ concentrations. RTN4 correlated with HVPG $(r=-0.298 ; p=0.011)$ and predicted CSPH $(p<0.001)$ as well as SPH $(\mathrm{p}<0.0001)$. Using a RTN 4 cut-off value of $\leq 1.7 \mathrm{ng} / \mathrm{ml}$, the AUC for 
detection of CSPH was 0.71 , with positive predictive value of $75 \%$ and negative predictive value of $63 \%$, sensitivity $70 \%$ and specificity $71 \%$. RTN4 value of $\leq 1.1 \mathrm{ng} / \mathrm{ml}$ was associated with esophagogastric varices (odds ratio $[\mathrm{OR}]=3,63$; $\mathrm{p}<0.022)$.

Conclusion: Low levels of RTN4 are associated with liver cirrhosis and portal hypertension. RTN4 correlates with liver function. It may be a surrogate marker of CSPH and presence of esophagogastric varices.

Disclosure of Interest: All authors have declared no conflicts of interest.

\section{References}

Dana A. Dodd et al. Nogo-A, -B, and -C Are Found on the Cell Surface and Interact Together in Many Different Cell Types. J biol chem. 2005; 13:1249412502

Beibei Gao et al. Clinical Implications of Increased Nogo-B Levels in Patients With Acute Coronary Syndromes and Stable Angina Pectoris. Int Heart J 2015; 56:341-344.

Acevedo, L., et al. A new role for Nogo as a regulator of vascular remodeling. (2004) Nat. Med. 10, 382-388.

Rodriguez-Feo JA, Hellings WE, Verhoeven BA, et al. Low levels of Nogo-B in human carotid atherosclerotic plaques are associated with an atheromatous phenotype, restenosis, and stenosis severity. Arterioscler Thromb Vasc Biol 2007; 27: 1354-60.

Oertle T, Schwab ME. Nogo and its paRTNers. Trends Cell Biol 2003; 13: 18794.

Chi C. et al. RTN4/Nogo is an independent prognostic marker for gastric cancer: preliminary results. Eur Rev Med Pharmacol Sci. 2015;19(2):241-6.

\section{P0027 MICRORNAS IN ASCITES AS POTENTIAL BIOMARKERS FOR PERITONEAL CARCINOMATOSIS AND PERITONITIS}

P. Schindler ${ }^{1}$, J. Kupcinskas ${ }^{2}$, S. Juzenas ${ }^{3}$, J. Skieceviciene ${ }^{3}$, V. Salteniene ${ }^{3}$, C. Schulz ${ }^{4}$, J. Weigt ${ }^{4}$, P. Malfertheiner ${ }^{4}$, A. Link ${ }^{4}$

${ }^{1}$ Department Of Gastroenterology, Hepatology And Infectious Diseases, Otto-vonGuericke University, Magdeburg/Germany

${ }^{2}$ Department Of Gastroenterology, Lithuanian University of Health Sciences, Kaunas/Lithuania

${ }^{3}$ Institute For Digestive Research, Lithuanian University of Health Sciences, Kaunas/Lithuania

${ }^{4}$ Department Of Gastroenterology, Hepatology And Infectious Diseases, Otto-vonGuericke University of Magdeburg, Magdeburg/Germany

Contact E-mail Address: alinkmail@gmail.com

Introduction: Peritoneal carcinomatosis (PCA) has a prognostic role in patients with gastrointestinal cancers. Despite the low sensitivity, cytology remains the gold standard in differential diagnosis of PCA to peritonitis (for example spontaneous bacterial peritonitis, SBP) or uncomplicated ascites due to portal hypertension (no SBP/PCA). MicroRNAs (miRNAs) are considered as promising biomarkers and are commonly dysregulated in cancer.

Aims \& Methods: In this proof-of-principle study, we systematically evaluated preanalytical factors and potential of miRNAs as ascites biomarkers. We prospectively examined samples from patients with ascites with benign and malignant conditions including: PCA $(n=15), \operatorname{SBP}(n=15)$ and portal hypertension (no SBP/PCA, $n=15$ ). Various extraction kits were used to compare the total RNA extraction. Furthermore, we systematically evaluated the influence of storage, stability and sample processing (uncentifuged, pelleted etc.) on miRNA expression in ascites. MiRNA expression profiling using TaqMan Low Density Array (TLDA) and quantitative RT-PCR (TaqMan/SYBRgreen) were used to evaluate the expression.

Results: Systematic analysis of miRNAs stability confirms that miRNAs in ascites are well preserved from degradation with good short- $(0 \mathrm{~h}, 12 \mathrm{~h}, 24 \mathrm{~h}$, and $48 \mathrm{~h})$ and long-term stability $\left(-30^{\circ} \mathrm{C},-80^{\circ} \mathrm{C}\right.$ for 2 years). Several miRNAs that were selected for the proof-of-principle analysis (miR-21 and miR-16) were reproducibly detectable in ascites samples. MiRNA expression profiling in patients with PCA compared to those with uncomplicated portal hypertension revealed miR-21, miR-186, miR-222 and miR-483-5p to be up-regulated and miR-26b to be down-regulated. MiRNA expression validation analysis confirmed higher expression of miR-21 (mean delta $\mathrm{CT} \pm \mathrm{SD} ;-11.11 \pm 1.2$ vs. $-8.46 \pm 3.46$ vs. $-9.65 \pm 2.55$ for no SBP/PCA, PCA and SBP, respectively, ANOVA, $\mathrm{p}=0.026$; posttest no SBP $/ \mathrm{PCA}$ vs. PCA $\mathrm{p}<0.05)$ and miR-186 in patients with PCA compared to no SBP/PCA groups, while miR-223 was significantly upregulated in SBP (mean $\pm \mathrm{SD} ;-12.16 \pm 1.56$ vs. $-10.05 \pm 3.19$ vs. $-6.95 \pm 3.56$ for no SBP/PCA, PCA and SBP, respectively, ANOVA, $\mathrm{p}<0.0001$; posttest SBP vs no SBP/PCA and vs. PCA $\mathrm{p}<0.05$ ).

Conclusion: Our data provide novel evidence for the differential expression of miRNAs in ascites in patients with PCA and SBP. Evaluation of ascites-miRNAs may offer an alternative approach for diagnosis of peritoneal carcinomatosis and create an avenue for therapeutic application as well.

Disclosure of Interest: All authors have declared no conflicts of interest.
P0028 ALTERATIONS IN GUT VASCULAR BARRIER IN

EXPERIMENTAL PORTAL HYPERTENSION

M. Sorribas Olivera ${ }^{1}$, I. Spadoni ${ }^{2}$, M. Rescigno ${ }^{2}$, R. Wiest ${ }^{3}$

${ }^{1}$ Department For Clinical Research, University of Bern, Bern/Switzerland

${ }^{2}$ Istituto Europeo di Oncologia, Milan/Italy

${ }^{3}$ Department Of Gastroenterology, University Clinic for Visceral Surgery and Medicine, Bern/Switzerland

Contact E-mail Address: marcel.sorribas@dkf.unibe.ch

Introduction: Pathological bacterial translocation (PBT) in liver cirrhosis (LC) is the pathophysiological hallmark for spontaneous bacterial infections increasing mortality several-fold. Factors known to contribute to PBT in LC are among others an increased intestinal permeability.

Aims \& Methods: A clear route of translocation for luminal intestinal bacteria is yet to be defined but we hypothesize that the recently described gut vascular barrier $(\mathrm{GVB})$ is impaired in experimental portal hypertension leading to protein loss and increased accessibility of the vascular compartment for translocating bacteria. For this purpose two different models of experimental portal hypertension, namely partial portal vein ligation (PPVL) and bile duct ligation (BDL) were used in mice under standardized gnotobiotic conditions (sDMDM2). A novel in vivo confocal endomicroscopy technique was established in order to visualize the intestinal vascular leakage. Briefly, $70 \mathrm{kDa}$ FITC-marked dextran was injected intravenously and confocal probe was placed in the intestinal lumen (terminal ileum) to visualize villus-capillaries. Leakage was measured over time (10 minutes) as a ratio between the mean fluorescent intensity outside the vessel (lamina propria) and inside the vessel. Immunofluorescence (IF) stains of the fenestral diaphragms marker plasmalemma vesicle-associated protein-1 (PV-1) were performed for GVB analysis.

Results: Confocal endomicroscopy data revealed an earlier and significantly increased leakage of $70 \mathrm{kDA}$ through the intestinal vasculature in both BDL and PPVL mice. FITC-70kDA-dextran did only leak in BDL and PPVL but not in control (sham operated) mice. Interestingly GVB stains showed increased expression of PV1 in intestinal vessels $\left(\mathrm{CD} 34^{+}\right)$of BDL but not PPVL.

Conclusion: Portal hypertension per se has an impact on the GVB increasing FITC-70 kDa-dextran leakage from intestinal capillaries to the lamina propria in both BDL and PPVL. However, the IF showed only in BDL an increased PV-1 expression indicative of a wider opening of the fenestral diaphragms than in PPVL. Therefore, different mechanisms appear to be involved in alterations of the gut-vascular barrier in pre-hepatic portal hypertension and biliary cirrhosis. Disclosure of Interest: All authors have declared no conflicts of interest.

\section{Reference}

I. Spadoni et al. Science 350, 830-834 (2015)

\section{P0029 INHIBITION OF CYCLOOXYGENASE-2 AMELIORATES SPLENOMEGALY IN CIRRHOTIC RATS}

C. Tang, L. Zhang, S. Tang, X. Jia, J. Gao

Gastroenterology, West China Hospital, Sichuan University, Chengdu/China

Contact E-mail Address: 812903560@qq.com

Introduction: Splenomegaly is a common finding in liver cirrhosis. However, the precise underlying mechanisms behind this phenomenon have not been elucidated, and its effective therapies are limited.

Aims \& Methods: We aimed to investigate whether cyclooxygenase-2 (COX-2) is involved as a contributing factor in the pathological process of splenomegaly in cirrhotic rats. Thirty-six male Sprague-Dawley rats weighing 200-250 g were randomized into 3 groups with 12 rats in each group. The control group received intraperitoneal injection of normal saline $(1 \mathrm{ml}$, twice a week); the TAA group received intraperitoneal injection of thioacetamide (TAA, $200 \mathrm{mg} / \mathrm{kg}$, twice a week for 16 weeks); the TAA + celecoxib group received TAA intraperitoneally and celecoxib via gastric gavage $(20 \mathrm{mg} / \mathrm{kg} /$ day $)$. The portal pressure was measured by portal venous catheterization. Sections from paraffin-embedded spleens were stained with hematoxylin and eosin and Sirius Red, and immunostained with VEGF and CD31. The protein expressions of COX-2, VEGF, PI3K, p$\mathrm{AKT}$, and AKT in the spleen were assessed by Western blot. The enzymelinked immunosorbent assay was performed to evaluate the expression of TNF-a and IL-1 $\beta$ in the spleen.

Results: The ratio of splenic weight to body weight increased by $73.9 \%$ in TAA group, while in rats treated with celecoxib, the ratio was significantly reduced. While determined by H\&E staining, areas of splenic white pulp in the TAA group enlarged by $27.9 \%$. Yet, compared with that in TAA group, celecoxib obviously decreased the area of splenic white pulp by $37.6 \%$. Besides, the portal pressure elevated by $79.1 \%$ in the TAA group; while significant reduction of the portal pressure was observed in the TAA + celecoxib group (by $28.8 \%$ ). In addition, a considerable amount of collagen was visualized with Sirius Red staining in the splenic red pulp of TAA group. Decreased splenic fibrosis was found in $\mathrm{TAA}+$ celecoxib group. Increased COX-2 protein was detected in the spleen of the TAA group compared with that in the control group. However, celecoxib significantly reduced the expression of $\mathrm{COX}-2$ in the TAA + celecoxib group. Besides, compared with the control group, TNF-a and IL-1 $\beta$, the two proinflammatory cytokines were found to be greatly increased in the TAA group. On the contrary, in TAA + celecoxib group, the protein expression of TNF-a and IL-1 $\beta$ was obviously reduced. We also found that the expression of proangiogenic factor VEGF and the neovascular marker CD31 increased in TAA group by Western blot and immunohistology, which indicated a role of angiogenesis in the pathophysiology of splenomegaly. Furthermore, up-regulation of PI3K and $\mathrm{p}-\mathrm{AKT}$ protein expression was detected in the spleen of TAA group compared 
with that of the control group, demonstrating that PI3K/AKT signal pathway was involved in the development of pathological angiogenesis. However, the treatment with celecoxib strongly decreased the protein expression of VEGF, CD31, PI3K and AKT in the spleen of cirrhotic rats.

Conclusion: The present study indicates that COX-2 contributes to splenomegaly by facilitating angiogenesis, fibrosis and inflammation in the spleen. Moreover, inhibition of COX-2 by celecoxib could ameliorate portal hypertension and splenomegaly

Disclosure of Interest: All authors have declared no conflicts of interest.

P0030 EPITHELIAL BARRIER DESTABILIZATION AND REGULATION OF P53 - A POSSIBLE BACTERIAL DEFENSE MECHANISM IN SPONTANEOUS BACTERIAL PERITONITIS?

M. Haderer ${ }^{1}$, L. Wächter ${ }^{2}$, E. Aschenbrenner ${ }^{3}$, K. Pollinger ${ }^{3}$, J. Middendorf ${ }^{2}$, S. Schlosser ${ }^{2}$, C. Kunst ${ }^{3}$, M. Müller-Schilling ${ }^{3}$

${ }^{1}$ Internal Medicin 1, University hospital Regensburg, Regensburg/Germany ${ }^{2}$ Internal medicine 1, Regensburg/Germany

${ }^{3}$ Department For Internal Medicine I, University Hospital Regensburg,

Regensburg/Germany

Contact E-mail Address: marika.haderer@ukr.de

Introduction: Spontaneous bacterial peritonitis (SBP) is a life-threatening complication in advancing liver cirrhosis. Translocation of intestinal bacteria or bacterial products from the gut to mesenteric lymph nodes is crucial for SBP, with Escherichia coli (E. coli), Klebsiella pneumoniae being the most common germs. Small intestinal bacterial overgrowth and a altered microbiota are so far known as risk factors for SBP. However, the exact mechanisms of bacteria translocation need to be identified as they are supposed to contribute to the development of early recognition systems and initiation of antibiosis.

Aims \& Methods: With regard to the development of early recognition systems, pathomechanisms and signaling pathways of bacterial translocation in SPB were explored. These insights might lead to an initiation of antibiosis on time and reduced mortality in SBP.

Monolayers of human intestinal epithelial cell lines Caco-2 (p53 mutant) and HCT-116 (p53 wildtyp) were cocultured with E. coli with different MOI (MOI $0,1,5$ and 10) for 2 to 4 hours post confluence. Experiments with heat inactivated E. coli were performed as controls. Effects of microbial metabolic products were tested by using the supernatant of an overnight culture. qPCR and Western Blot analysis were performed to analyze changes in mRNA and protein levels of Occludin, E-cadherin and the p53 family including p53 and p73.

Results: E. coli stimulation of HCT-116 cells resulted in a strong decrease of Occludin, E-cadherin, p53 and p73 protein levels. These changes were dependent on incubation time and bacterial concentration. Following bacterial infection, marginal to no effects were detected on mRNA levels of cellular junctions and p53. Caco-2 cells displayed less reduction of Occludin and E-cadherin protein levels compared to p53-wildtyp HCT-116 cells.

Conclusion: By using an in vitro model, we demonstrate destabilizing effects of $E$. coli on intestinal cell junctions, p53 and p73. As far as these effects are dependent on incubation time, microbial concentration and living bacteria, these effects might represent a mechanism to protect bacteria from intestinal immune responses and therefore to promote bacterial translocation in SBP.

Disclosure of Interest: All authors have declared no conflicts of interest.

\section{P0031 INTESTINAL EPITHELIAL BARRIER IN EXPERIMENTAL LIVER CIRRHOSIS - A ROLE FOR BILE SALTS IN THE MUCUS LAYER}

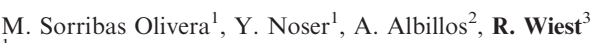

${ }^{1}$ Department For Clinical Research, University of Bern, Bern/Switzerland

${ }^{2}$ Universidad de Alcalá, Alcalá de Henares/Spain

${ }^{3}$ Department Of Gastroenterology, University Clinic for Visceral Surgery and Medicine, Bern/Switzerland

Contact E-mail Address: reiner.wiest@insel.ch

Introduction: Pathological bacterial translocation (PBT) in liver cirrhosis (LC) is the pathophysiological hallmark for spontaneous bacterial infections increasing mortality several-fold. Factors known to contribute to PBT in LC are among others an increased intestinal epithelial permeability.

Aims \& Methods: Since mucus represents one of the major components of this barrier we hypothesize that i) gut mucus is altered in LC and ii) bile could be a modulator of its production. Two different models of experimental LC -namely bile duct ligation (BDL) and the chronic treatment with carbon tetrachloride $(\mathrm{CCl} 4)$ - as well as partial portal vein ligation (PPVL) and sham-operated mice were used. Finally the farnesoid $\mathrm{X}$ receptor (FXR) agonist obeticholic acid (OCA) was used as treatment in CCl4-LC and control-animals. Mucus thickness measurement on gut explants and PAS (Periodic acid-Schiff) staining to visualize and count goblet cells (GC) were utilized.

Results: We have observed a significant reduction in mucus thickness in ileum and colon (Control $101.34 \mu \mathrm{m} \pm 9.54$ vs BDL $77.49 \mu \mathrm{m} \pm 14.31$ in Ileum; control $154.38 \mu \mathrm{m} \pm 12.51$ vs BDL $100.74 \mu \mathrm{m} \pm 0.6$ in proximal colon) and goblet cell numbers in ileum (Control $0.47 \mathrm{GC} / 100 \mu \mathrm{m}$ of villus \pm 0.07 vs BDL $0.29 \mathrm{GC} /$
$100 \mu \mathrm{m}$ of villus \pm 0.04 ) of mice following BDL but not PPVL (Control $0.27 \mathrm{GC}$ / $100 \mu \mathrm{m}$ of villus \pm 0.11 vs PPVL $0.30 \mathrm{GC} / 100 \mu \mathrm{m}$ of villus \pm 0.04$)$. Moreover we have seen that farnesoid $\mathrm{X}$ receptor (FXR) agonist obeticholic acid (OCA) partially restored GC loss in $\mathrm{CCl} 4$ treated animals (Control $0.63 \mathrm{GC} / 100 \mu \mathrm{m}$ of villus \pm 0.08 vs Control OCA $0.65 \mathrm{GC} / 100 \mu \mathrm{m}$ of villus \pm 0.07 vs $\mathrm{CCl} 40.49$ $\mathrm{GC} / 100 \mu \mathrm{m}$ of villus $\pm 0.18 \mathrm{CCl} 4 \mathrm{OCA} 0.57 \mathrm{GC} / 100 \mu \mathrm{m}$ of villus \pm 0.09 ).

Conclusion: All these results suggest that a reduced bile production by the cirrhotic liver and not portal hypertension per se interfere in the goblet cell development and/or maturation. In addition, this effect can be, at least partially, be restored by the FXR agonist OCA. Our study opens the possibility to a, so far, unknown effect of bile salts in the intestinal epithelium development in the context of liver cirrhosis being a clear candidate for mucus layer regulation and hence protective effect against bacterial translocation.

Disclosure of Interest: All authors have declared no conflicts of interest.

\section{P0032 CAPSAICIN AND SULFORAPHANE PREVENT THE ADVANCEMENT OF LIVER FIBROSIS IN AN EXPERIMENTAL MODEL OF LIVER CIRRHOSIS}

E. J. Mendivil ${ }^{1}$, A. Sandoval-Rodriguez ${ }^{1}$, A. Dominguez-Rosales ${ }^{2}$, L. ZuñigaRamos $^{2}$, J. Armendariz-Borunda ${ }^{2}$

${ }^{1}$ Molecular Biology And Genomics, University of Guadalajara, Guadalajara/ Mexico

${ }^{2}$ University of Guadalajara, Guadalajara/Mexico

Contact E-mail Address: edgarmendivil@live.com

Introduction: Liver fibrosis refers to the excessive accumulation of extracellular matrix (ECM) following a chronic liver injury. It is characterised by an increase in gene expression of proinflammatory molecules such as TGF-b1, IL-1 b, IL-6 and TNF-a, as well as excess synthesis in of ECM components such as COL-1. Capsaicin (CAP) is a pungent compound found in chilli peppers which has shown anticarconogenic, antiinflammatory and antifibrotic properties. Moreover, sulforaphane (SFN) is an isothiocyanate which is in cruciferous such as broccoli and it has exhibit an antioxidant effect in several in vitro and in vivo models.

Aims \& Methods: The objective of this project was to evaluate the antifibrogenic and antiinflammatory effects of a daily supplementation with CAP and SFN in a rat model of liver fibrosis due to carbon tetrachloride $\left(\mathrm{CCl}_{4}\right)$ intoxication. 35 male Wistar rats were included ( $\mathrm{n}=7 /$ group); animals were administrated intraperitoneally 3 times per week during 8 weeks with a mix of $\mathrm{CCl}_{4}$ :mineral oil (1:5/ week $1,1: 4$ week 2 and $1: 3$ week $3-8)$. Healthy and $\mathrm{CCl}_{4}$-fibrotic controls received only supplementation vehicle (Tween $2 \%$ in PBS). Treated groups receive SFN $5 \mathrm{ug} / \mathrm{kg}$, or CAP $2 \mathrm{mg} / \mathrm{kg}$, or both supplements daily by oral gavage since the beginning of $\mathrm{CCl}_{4}$-intoxication regimen until sacrifice. Masson staining and PCR was performed in liver samples. Hepatic enzymes were analysed in serum.

Results: Groups treated with CAP and SFN showed a decrease of $\sim 30$ points in percentage of liver fibrosis according to Masson staining $(\mathrm{p}<0.05)$, hepatic function improve since AST and ALT serum levels diminish $(\mathrm{p}<0.01)$ also a lower gene expression of TGF-b1, Col-1, TNF-a, IL-1 b and IL-6 was detected in treated animals when compared with fibrotic controls $(p<0.01)$.

Conclusion: Thus, CAP and SFN seem to exert a hepatoprotective effect in this model of chronic-induced liver damage. These findings suggest that dietary sources of CAP and SFN might be included in dietetic guidelines for the prevention of liver fibrosis

Disclosure of Interest: All authors have declared no conflicts of interest.

\section{P0033 DIAGNOSIS OF GASTRIC VARICES BY ENDOSCOPIC ULTRASONOGRAPHY USING COLOR DOPPLER}

T. Sato

Gastroenterology, Sapporo Kosei Hospital Dept. of Gastroenteroloy, Sapporo/

Japan

Contact E-mail Address: taka.sato@ja-hokkaidoukouseiren.or.jp

Introduction: Gastric variceal bleeding is common complication, and it is associated with higher morbidity and mortality rates than hemorrhage from esophageal varices. Oesophagogastroduodenoscopy is usually the initial investigation in patients with portal hypertension for the purpose of the distinction between gastric varices and gastric folds. The aim of this study was to investigate endoscopic color Doppler ultrasonography (ECDUS) findings of gastric varices.

Aims \& Methods: Two hundred-fifteen patients with gastric varices were evaluated with ECDUS. To begin with, identification of gastric varices was performed with B-mode scanning and then, color flow mapping was done. On B-mode scanning, submucosal gastric varices, and para-gastric collateral veins were obtained as hypoechoic vessels within gastric wall or in the tissue and spaces exterior to the adventitia of gastric wall. ECDUS provides a color display of blood flow and evaluates the flow pattern using fast Fourier transform (FFT) analysis. FFT analysis can indicate the flow pattern and calculate the velocity of blood flow. We monitored the color flow images of gastric varices, and paragastric or peri-gastric collateral veins. Endoscopic findings of gastric varices were evaluated according to the grading system outlined in The General Rules for 
Recording Endoscopic Findings of Esophago-gastric Varices devised by the Japanese Research Committee on Portal Hypertension.

Results: The color flow images of gastric varices and peri-gastric veins were delineated in all 215 patients with ECDUS. Evaluation of blood flow velocity in the 215 gastric varices revealed velocities of $7.7-35.7 \mathrm{~cm} / \mathrm{s}$ (mean, $18.2 \pm 6.0 \mathrm{~cm} / \mathrm{s}$ ). Mean velocity of large, coil-shaped (F3) type gastric varices was $23.7 \pm 6.2 \mathrm{~cm} / \mathrm{s}(\mathrm{n}=52)$, while the mean velocity of enlarged tortuous (F2) type gastric varices was $16.7 \pm 5.0 \mathrm{~cm} / \mathrm{s}(\mathrm{n}=163)$. The velocities of $\mathrm{F} 3$ type gastric varices were significantly higher than those of $\mathrm{F} 2$ type $(\mathrm{P}<0.0001)$. Next, we evaluated the wall thickness to submucosal gastric varices. Two hundred-fifteen of the gastric varices were $1.0-2.2 \mathrm{~mm}(1.6 \pm 0.4 \mathrm{~mm})$ in gastric wall thickness. Mean thickness of red color (RC) or erosion positive varices was $1.2 \pm 0.2 \mathrm{~mm}$ $(\mathrm{n}=42)$, while the mean thickness of $\mathrm{RC}$ or erosion negative varices was $1.7 \pm 0.3 \mathrm{~mm}(\mathrm{n}=173)$. The thickness of $\mathrm{RC}$ or erosion positive varices was significantly thinner than that of the negative cases $(\mathrm{P}<0.0001)$. Seven cases of the 215 patients had the current history of gastric variceal bleeding, and the other three cases had experienced variceal rupture on follow up (bleeding cases, $\mathrm{n}=10$ ), and mean thickness of these bleeding cases were $1.2 \pm 0.2 \mathrm{~mm}$.

Conclusion: ECDUS is a useful modality for the diagnosis of hemodynamics of gastric varices and may allow the stratification of patients into low, high risk for hemorrhage.

Disclosure of Interest: All authors have declared no conflicts of interest.

\section{P0034 PORTAL HYPERTENSIVE COLOPATHY BUT NOT ILEOPATHY IS COMMON IN EGYPTIANS WITH LIVER CIRRHOSIS}

A. Farag ${ }^{1}$, M. Wifi ${ }^{1}$, M. Fawzi ${ }^{1}$, A. Nageeb ${ }^{1}$, M. Alboraie ${ }^{1}$

${ }^{1}$ Internal Medicine Department, Hepatogastroenterology, Cairo University, Kasr Al-Ainy School of Medicine, Cairo/Egypt; Department Of Internal Medicine, AlAzhar University, Cairo/Egypt

Contact E-mail Address: mnwifi@kasralainy.edu.eg

Introduction: Liver cirrhosis and portal hypertension are associated with esophageal varices, gastric varices, small and large intestinal enteropathies.

Aims \& Methods: We aimed to study the prevalence of colopathy and ileopathy in patients with portal hypertension secondary to liver cirrhosis. Chronic hepatitis $\mathrm{C}$ patients with portal hypertension secondary to liver cirrhosis were enrolled. The severity of cirrhosis was classified by the Child-Pugh score. All patients were evaluated by upper endoscopy and colonoscopy for screening of portal hypertensive complications. Esophageal varices were graded as small, moderate and big varices. Portal hypertensive gastropathy was classified as absent or present, and, if present, it was sub-classified as mild or severe. Colonoscopy was done up to terminal ileum in all patients.

Results: Our study included sixty chronic hepatitis $\mathrm{C}$ patients with portal hypertension secondary to liver cirrhosis $(53.33 \%$ females $)$ their mean age $( \pm$ SD) was $54.75( \pm 13.13)$ years. Child-Pugh class was A for $2(3.4 \%)$, B for $33(55.9 \%)$ and $\mathrm{C}$ for $24(40.7 \%) .53(88.33 \%)$ patients had esophageal varices (23 patients had small esophageal varices, 15 had moderate, and 8 had big varices, 2 post-band ligation and 5 obliterated varices). Gastric varices were present in 3 patients $(5 \%)$. Portal hypertensive gastropathy was noted in 43 patients $(71.6 \%)$ and was mild in 38 and severe in 5 patients. Colonoscopy finding up to the terminal ileum revealed that portal hypertensive colopathy was present in 16 patients $(26.7 \%)$. portal hypertensive ileopathy was noted only in one case $(1.7 \%)$. No colonic or ileal varices were noted.

Conclusion: Portal hypertensive colopathy but not ileopathy is common in Egyptians with liver cirrhosis. Ileal varices and ileopathy are not common in patients with cirrhosis and PHT.

Disclosure of Interest: All authors have declared no conflicts of interest.

\section{P0035 PREDICTIVE FACTORS FOR THE DEVELOPMENT OF ACUTE-ON-CHRONIC LIVER FAILURE IN PATIENTS WITH GASTROINTESTINAL BLEEDING}

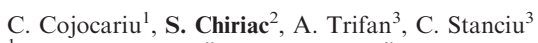

${ }^{1}$ Gastroenterology, "Grigore T. Popa" University of Medicine and Pharmacy Iasi, Iasi/Romania

${ }^{2}$ Gastroenterology, "Grigore T. Popa" University of Medicine and Pharmacy, Iasi/ Romania

${ }^{3}$ Institute of Gastroenterology and Hepatology, Iasi/Romania

Contact E-mail Address: stefannchiriac@yahoo.com

Introduction: Acute-on-chronic liver failure (ACLF) is a specific clinical form of liver failure in patients with liver cirrhosis, referred as acute deterioration of liver function associated with an acute specific complication of liver cirrhosis. ACLF is defined by the presence of renal failure or $\geq 2$ organ failures according to European Association for the Study of the Liver-International Chronic Liver
Failure consortium: 1) circulatory- need for vasopressor support; 2) renalserum creatinine value $\geq 2 \mathrm{mg} / \mathrm{dL} ; 3$ ) cerebral- Grade III or IV hepatic encephalopathy; 4) respiratory- $\mathrm{SpO}_{2} / \mathrm{FiO}_{2} \leq 214$.

Aims \& Methods: We aimed to identify predictive factors for ACLF development in cirrhotic patients admitted for variceal gastrointestinal bleeding. All patients admitted with gastrointestinal bleeding in the Institute of Gastroenterology and Hepatology Iasi (consisting of 8 secondary hepatology centers) between June and December 2016 were evaluated for ACLF (we excluded from the study the patients presenting ACLF diagnosis criteria on admission). We compared cirrhotic patients who developed ACLF after 12 hours of admission with those who did not.

Results: 99 cirrhotic patients with gastrointestinal bleeding were evaluated. $48.5 \%$ of patients admitted with variceal bleeding developed ACLF. Demographic data were similar in patients with ACLF vs. no ACLF in age $(54.2 \pm 7.3$ vs. $56.6 \pm 9.5$ years), male sex (54 vs. 45$)$, and diabetes (56 vs. 43 ) and significant difference was found in alcohol consumption (72 vs. 27). In patients with ACLF, the grade 1 was the most frequent $(56.3 \%)$; grade 2 $(33.3 \%)$ and $3(10.4 \%)$ of ACLF were more rare and no significant differences between the ACLF subgroups was observed. The patients with ACLF were more likely to be admitted with infections and alcohol consumption, when compared to patients without ACLF. Independent predictors for ACLF development included a high admission MELD $(\mathrm{p}<0.05)$, presence of infection and alcohol abuse ( $\mathrm{p}<0.001)$, hospitalization in the last 6 months $(\mathrm{p}<0.05)$. Inhospital and 30 -day mortality were significantly higher in patients with ACLF $(\mathrm{p}<0.0001)$. Conclusion: Patients admitted with variceal bleeding, with alcohol consumption, high MELD on admission, previous admission in $<6$ months are more likely to develop ACLF and need to be monitored closely for the development of ACLF. Disclosure of Interest: All authors have declared no conflicts of interest.

\section{P0036 HAEMOSTASIS IN PORTAL VEIN IN CIRRHOSIS: ROLE OF LOCAL ENDOTHELIAL DAMAGE}

S. Shalaby ${ }^{1}$, P. Simioni ${ }^{2}$, E. Campello ${ }^{2}$, S. Gavasso ${ }^{2}$, A. Zanetto ${ }^{1}$, F. D’Amico ${ }^{3}$, E. Gringeri ${ }^{3}$, U. Cillo ${ }^{3}$, M. Battistel ${ }^{4}$, P. Burra ${ }^{1}$, M. Senzolo ${ }^{1}$

${ }^{1}$ Department Of Surgery, Oncology And Gastroenterology, Multivisceral Transplant Unit, Padua/Italy

${ }^{2}$ Department Of Medicine (dimed), Padua University Hospital, Hemorrhagic and Thrombotic Diseases Unit, Padua/Italy

${ }^{3}$ Department Of Surgery, Oncology And Gastroenterology, Padua University Hospital, Hepatobiliary Surgery and Liver Transplantation Unit, Padua/Italy ${ }^{4}$ Department Of Medicine, Padua University Hospital, University Radiology, Padua/Italy

Contact E-mail Address: sarahshalaby18@gmail.com

Introduction: Cirrhosis is characterized by both bleeding and thrombotic complications due to underlying procoagulative haemostatic imbalance [1]. Among thrombotic events, portal vein thrombosis (PVT) is the most common with annual incidence ranging between $4.6 \%$ and $12.8 \%[2,3]$. Demonstrated associated risk factors are severity of portal hypertension and slowed portal flow [4]. However, data regarding haemostasis in the portal venous system of cirrhotics are lacking.

Aims \& Methods: To evaluate peripheral and portal venous haemocoagulative state in patients with cirrhosis in comparison with controls, through thrombin generation test (TGT), rotational-thrombelastometry (ROTEM) along with evaluation of endothelial dysfunction by quantification of circulating endothelialmicroparticles (MP). Correlate these results with activity levels of local pro and anticoagulant factors. Compare peripheral and portal venous districts in cirrhotics in terms of haemostatic balance. We consecutively enrolled adult patients with liver cirrhosis undergoing liver transplantation (LT) or transjugular intrahepatic portosystemic shunt (TIPS). Patients without liver disease awaiting liver surgery or deceased liver donors were enrolled as controls. The following laboratory tests were performed on citrated peripheral and portal venous blood samples: TGT with and without thrombomodulin (TM), ROTEM, dosage of main pro and anticoagulants factors activity and analysis of circulating endothelial MP.

Results: 25 cirrhotics (15 LT and 10 TIPS) and 6 controls ( 2 undergoing hepatic resection for benign liver lesions and 4 liver donors) were enrolled. Peripheral blood in cirrhotics showed resistance to activation of PC-pathway at TGT (ETP with/without TM $0.89(0.78-0.92)$ vs $0.6(0.3-0.74), \mathrm{p}<0.001)$, lower clot stability at ROTEM (MCF-NATEM mm: $43.5(36-51)$ vs $63(53-69), \mathrm{p}=0.042)$, and significant increase of endothelial-MP (CD62EMP/L: 1391 (651-2301) vs 582 $(380-1161), p=0.046)$, indicative of higher endothelial damage compared to controls. Similar results were obtained comparing portal blood of cirrhotics and controls (ETP with/without TM $0.89(0.78-0.92)$ vs $0.63 \quad(0.33-0.75)$, $\mathrm{p}=0.001$; MCF-NATEM mm: 46 (39-51) vs 62 (49-66), $\mathrm{p}=0.056$; CD62EMP/L: $1606.5(680-1885)$ vs $529.5(266-781), \mathrm{p}=0.069)$. There was a significant correlation between diminished levels of PC, PS, AT, FII and either TGT or ROTEM parameters. Comparing portal and peripheral blood of cirrhotics, we detected endogenous heparinoids in portal blood ( $\alpha$-angle NATEM 51 (46-57) vs 
Abstract No: P0037

Table 1: All-cause ED attendances, with and without admission, pre- and post-RFX initiation

\begin{tabular}{|c|c|c|c|c|c|c|c|c|}
\hline \multirow[b]{2}{*}{ Resource use parameter* } & \multicolumn{4}{|c|}{6 Months $(\mathrm{N}=114)$} & \multicolumn{4}{|c|}{12 Months $(\mathrm{N}=102)$} \\
\hline & $\mathrm{n}^{\#}$ & Pre-RFX initiation & Post-RFX initiation & $\mathrm{P} \dagger$ & $\mathrm{n}^{\#}$ & Pre-RFX initiation & Post-RFX initiation & $\mathrm{P} \dagger$ \\
\hline ED attendances with or without admission & 81 & 264 & 118 & - & 82 & 325 & 217 & - \\
\hline ED attendances with or without admission/patient & 81 & $2.3 \quad(0.3)$ & $1.0 \quad(0.2)$ & $<0.001$ & 82 & $3.2(0.5)$ & $2.1 \quad(0.4)$ & 0.023 \\
\hline ED attendances without admission & 61 & 118 & 60 & - & 62 & 151 & 114 & - \\
\hline ED attendances without admission/patient & 61 & $1.0 \quad(0.2)$ & $0.5 \quad(0.1)$ & $<0.001$ & 62 & $1.5 \quad(0.3)$ & $1.1 \quad(0.2)$ & 0.116 \\
\hline Admissions via ED & 74 & 146 & 58 & - & 76 & 174 & 103 & - \\
\hline Admissions via ED/patient & 74 & $1.3 \quad(0.2)$ & $0.5 \quad(0.1)$ & $<0.001$ & 76 & $1.7 \quad(0.3)$ & $1.0 \quad(0.2)$ & 0.009 \\
\hline Bed days/patient admitted via ED & 74 & $18.2 \quad(2.6)$ & $7.2 \quad(2.0)$ & $<0.001$ & 76 & $23.2 \quad(3.4)$ & $11.4 \quad(2.7)$ & 0.002 \\
\hline
\end{tabular}

*Data are presented for all surviving patients at the end of the 6 months $(\mathrm{N}=114)$ or 12 months $(\mathrm{N}=102)$ as mean (standard error of the mean, SEM) per patient Number of patients with $\geq 1$ ED attendance/admission in the observed periods $\uparrow$ Paired t-test

HEPTEM 57 (50-59), $\mathrm{p}=0.05)$. This finding, together with a decreased concentration of endothelial-MP carrying TM (TM-MP/L: 232 (190-287) vs 377 (218 $493), \mathrm{p}=0.002)$ and endothelial-PC receptor $(\mathrm{EPCR} / \mathrm{CD} 65 \mathrm{E}-\mathrm{MP} / \mathrm{L}: 16$ (14-25) vs $37(24-70), p<0.001)$, demonstrated a local greater endothelial damage in cirrhotics.

Conclusion: In cirrhotics, venous hypercoagulability and portal site specific endothelial damage, associated with hampered antithrombotic properties, may be important local risk factors in the pathogenesis of PVT along with the documented venous stasis.

Disclosure of Interest: All authors have declared no conflicts of interest.

\section{References}

1. Lisman T, Caldwell SH, Burroughs AK, Northup PG, Senzolo M, Stravitz RT, et al. Hemostasis and thrombosis in patients with liver disease: the ups and downs. Journal of hepatology 2010:53:362-371.

2. Nery F, Chevret S, Condat B, de Raucourt E, Boudaoud L, Rautou PE, et al. Causes and consequences of portal vein thrombosis in 1, 243 patients with cirrhosis: results of a longitudinal study. Hepatology 2015;61:660-667.

3. Maruyama H, Okugawa H, Takahashi M, Yokosuka O. De novo portal vein thrombosis in virus-related cirrhosis: predictive factors and long-term outcomes. The American journal of gastroenterology 2013;108:568-574.

4. Zocco MA, Di Stasio E, De Cristofaro R, Novi M, Ainora ME, Ponziani F, et al. Thrombotic risk factors in patients with liver cirrhosis: correlation with MELD scoring system and portal vein thrombosis development. Journal of hepatology 2009;51:682-689.

\section{P0037 RIFAXIMIN-A IS ASSOCIATED WITH REDUCTIONS IN EMERGENCY DEPARTMENT RESOURCE USE IN UK PATIENTS WITH HEPATIC ENCEPHALOPATHY: REAL-WORLD EVIDENCE FROM THE IMPRESS STUDY}

M. Hudson ${ }^{1}$, P. Di Maggio ${ }^{2}$, R. Cipelli ${ }^{3}$, R. Aspinall ${ }^{4}$

${ }^{1}$ Freeman Hospital, The Newcastle Upon Tyne Hospitals NHS Foundation Trust, Newcastle Upon Tyne/United Kingdom

${ }^{2}$ Medical, Norgine UK, Harefield/United Kingdom

${ }^{3} \mathrm{pH}$ Associates, Marlow/United Kingdom

${ }^{4}$ Oueen Alexandra Hospital, Portsmouth Hospitals NHS Trust, Portsmouth United Kingdom

Contact E-mail Address: pdimaggio@norgine.com

Introduction: In clinical trials rifaximin- $\alpha$ (RFX) has been shown to reduce recurrence of episodes of overt hepatic encephalopathy (HE) and HE-related hospitalisations. UK real-world data confirmed reductions in hospital admissions and length of stay with RFX use; however, data on use of emergency department (ED; A\&E in UK) resources are still scarce. This study assessed the impact of RFX on utilisation of ED resources.

Aims \& Methods: Patients from 11 UK hospitals who were prescribed RFX for HE between July-2008 and May-2014 were included in this retrospective observational study. Patient records were reviewed; details of demographic and clinical characteristics, and all-cause ED attendances and admissions were collected in the 6 and 12 months pre- and post-RFX initiation. The analysis included only patients who were alive at the end of the study periods.

Results: Of the 145 patients included, $114(79 \%)$ were alive at 6 months and 102 $(70 \%)$ at 12 months post-RFX initiation. At RFX start, mean age was 61 years, $63 \%$ were male; $67 \%$ had alcohol-related liver disease; for patients with available MELD score $(70 \%)$, the mean was $16 ; 78 \%$ were on lactulose. Use of ED resources in the 6 and 12 months pre- and post-RFX initiation is shown in Table 1. Six patients developed adverse events, none serious.

Conclusion: In UK clinical practice, treatment with RFX for $\mathrm{HE}$ is well-tolerated and associated with significant reductions in ED attendances, with or without admission, both within 6 and 12 months of RFX initiation.

Disclosure of Interest: M. Hudson: Consultant for Norgine; advisory board member; has given sponsored lectures on behalf of Norgine

P. Di Maggio: Employee of Norgine
R. Cipelli: Consultant for Norgine; employee of $\mathrm{pH}$ Associates which was commissioned by Norgine to provide support with study design and management, data analysis and scientific editorial services

R. Aspinall: Consultant for Norgine; advisory board member; has given sponsored lectures on behalf of Norgine

P0038 PREDICTING FACTORS FOR HOSPITAL READMISSON AFTER THE FIRST EPISODE OF HEPATIC ENCEPHALOPATHY

M. Silva ${ }^{1}$, A. Peixoto ${ }^{2}$, H. Cardoso ${ }^{2}$, S. Lopes ${ }^{2}$, G. Macedo $^{2}$

${ }^{1}$ Gastroenterology, Hospital São João, Oporto/Portugal

${ }^{2}$ Centro Hospitalar São João, Porto Medical School, Porto/Portugal

Contact E-mail Address: marcocostasilva87@gmail.com

Introduction: Hepatic encephalopathy (HE) is a frequent complication of liver cirrhosis, with necessity of hospital admission in many cases. The economic burden of HE is substantial. After ascites, HE is the second most common reason for hospitalization of cirrhotic patients. HE is also the most common, possibly preventable, cause for readmission.

Aims \& Methods: We aimed to assess the factors associated with the increased likelihood of hospital readmission for HE after the onset episode.

We completed a retrospective Retrospective analysis of admissions for HE of patients with liver cirrhosis, between October 2010 and October 2015. Only the onset episode was included. Patients were followed for 1 year or until readmission for HE. All payients were discharged under lactulose therapy. Descriptive statistics, uni and multivariate analysis, logistic regression, and ROC curves analysis were performed using IBM SPSS Statistics 22 with $\mathrm{p}<0.05$ deemed to be statistically significant.

Results: In this study 119 patients were included: $78 \%$ men with a mean age of $59 \pm 13$ years; $8 \%$ had hepatocellular carcinoma, and $45 \%$ had Child-Pugh C. The most frequent cirrhosis etiologies were alcoholic disease $(60 \%)$ and $\mathrm{HCV}$ infection $(12 \%)$. The precipitating factors, for the onset episode, more frequently detected were diuretic overdose $(36 \%)$ and infection $(31 \%)$. All patients were treated with standard therapy, with an adequate lactulose dose. The readmission rate after the first episode of $\mathrm{HE}$ was $72 \%(75 \%$ men). The estimated average time to relapse was 18 weeks. The most frequent causes of readmission were also diuretics overdose $(31 \%)$ and infection $(30 \%)$. The patients who were readmitted had a higher MELD score than patients without recurrence (13.9 vs. 11.6 points; $\mathrm{p}=0.015)$. This association was verified in the multivariate analysis $(\mathrm{OR}=1.1$, $\mathrm{p}=0.044)$.

Conclusion: In this cohort, there was a high rate of readmission for HE after the inaugural episode, which carries a great impact on individual health and high socio-economic costs. A higher MELD score was independently associated with a high probability of readmission for HE.

Disclosure of Interest: All authors have declared no conflicts of interest.

\section{Reference}

1. Volk ML, Tocco RS, Bazick J, Rakoski MO, Lok AS. Hospital readmission among patients with decompensated cirrhosis. Am J Gastroenterol. 2012. 107(2):247-52 
P0039 SAFETY, EFFICACY AND RISK OF COMPLICATIONS FOR CIRRHOTIC HCV PATIENTS WITH THROMBOCYTOPENIA AND HYPOALBUMINEMIA TREATED WITH OMBITASVIR/ PARITAPREVIR/R + DASABUVIR+RIBAVIRIN - A REAL-LIFE COHORT

C. Ester ${ }^{1}$, L. Gheorghe ${ }^{1}$, S. Iacob ${ }^{1}$, C. Cijevschi ${ }^{2}$, A. Trifan ${ }^{3}$, C. Stanciu ${ }^{4}$,

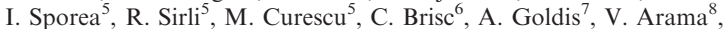
F.A. Caruntu ${ }^{8}$, I. Rogoveanu', A. Seicean ${ }^{10}$, I. Simionov ${ }^{1}$, R. Vadan ${ }^{1}$, I. Pirvulescu ${ }^{1}$, C. Pietrareanu ${ }^{1}$, R.A. Iacob ${ }^{11}$, C. Gheorghe ${ }^{12}$, M. Diculescu ${ }^{1}$ ${ }^{1}$ Department Of Gastroenterology, Fundeni Clinical Institute, Bucharest/Romania ${ }^{2}$ UMF Iasi, Iasi/Romania

${ }^{3}$ Gastroenterology, "Grigore T. Popa" University of Medicine and Pharmacy Iasi, Iasi/Romania

${ }^{4}$ Institute of Gastroenterology and Hepatology, Iasi/Romania

${ }^{5}$ Department Of Gastroenterology And Hepatology, University of Medicine and Pharmacy Victor Babes Timisoara, Timisoara/Romania

${ }^{6}$ Oradea Regional Hospital, Oradea/Romania

${ }^{7}$ Dept. Of Gastroenterology, County Hospital Timisoara Gastroenterology, Timisoara/Romania

${ }^{8}$ Matei Bals Clinical Institute, Bucharest/Romania

${ }^{9}$ UMF Craiova, Craiova/Romania

${ }^{10}$ Third Medical Clinic, Fabrio Turism, Cluj Napoca/Romania

${ }^{11}$ Gastroenterology And Hepatology, Carol Davila University of Medicine and

Pharmacy, Bucharest/Romania

${ }^{12}$ Ist Dept. Of Gastroenterology \& Endoscopy, PetCenter of Gastroenterology and Hepatology, Bucharest/Romania

Contact E-mail Address: carmen.ghidu@gmail.com

Introduction: The regulations for prescribing interferon-free treatment for patients infected with hepatitis $\mathrm{C}$ virus in Romania comprised only patients with F3/F4 fibrosis so the risk of hepatic decompensation and complications was higher compared to other cohorts. In previous interferon-based regimens, thrombocytopenia and hypoalbuminemia as markers for portal hypertension and hepatic synthetic dysfunction, respectively, have been shown to reduce the likelihood of sustained virological response and to increase the rates of serious adverse events.

Aims \& Methods: The aim of this study was to evaluate the impact of thrombocytopenia and hypoalbuminemia on treatment outcome and disease complications. We included in this study $855 \mathrm{HCV}$-infected cirrhotic patients treated with ombitasvir/paritaprevir/r+dasabuvir+ribavirin for 12 weeks in 10 university hospitals in Romania. The following groups were studied: 151 patients $(17.7 \%)$ with albumin $<3.5 \mathrm{~g} / \mathrm{dl}, 239(28 \%)$ with thrombocytopenia (a cutoff of $100000 / \mathrm{mmc}$ was used) and 71 patients $(8.3 \%)$ with both hypoalbuminemia and thrombocytopenia before initiating antiviral treatment. Safety (as AE in $>5 \%$ and SAE), efficacy (defined as HCV RNA undetectable at week 12 post-therapy and complication rate were evaluated using Pearson's correlation, multivariate analysis and Chi-Square test.

Results: Main patient characteristics were: $100 \%$ genotype $1 \mathrm{~b}$, a median age of 60 yo, 466 women $(54.57 \%)$, high rate of previous interferon based-treatment $(36.1 \%)$. End-of-treatment and sustained virological response rate were both $>99 \%$ and there was no correlation with the presence of thrombocytopenia or hypoalbuminemia. The rate of adverse events in the whole cohort was $17.5 \%$ at 2 weeks reaching $18 \%$ at the end of treatment with only $0.8 \%$ severe adverse events with no statistical association with the presence of thrombocytopenia and hypoalbuminemia. The multivariate analysis showed significant asscociation of thrombocytopenia $(<100000 / \mathrm{mmc})$ with higher $(>1)$ degree of oesophageal varices $(p<0.001)$, onset of upper digestive hemorrhage during treatment $(\mathrm{p}=0.011)$, and prior exposure to interferon based regimens $(\mathrm{p}=0.025)$. Low albumin $(<3.5 \mathrm{~g} / \mathrm{dl})$ also correlated with higher $(>1)$ degree of oesophageal varices $(\mathrm{p}<0.001)$ and onset of upper digestive hemorrhage during treatment $(\mathrm{p}=0.002)$

Conclusion: The efficacy and safety of the ombitasvir/paritaprevir/ $\mathrm{r}+$ dasabuvir+ribavirin (as recomended by national regulations) was not different in cirrhotic patients with hypoalbuminemia and thrombocytopenia, but complications rate was higher so close follow-up and profilactic measures should be recommended, especially if previously exposed to interferom containing regimens.

Disclosure of Interest: All authors have declared no conflicts of interest.

\section{P0041 REAL-WORLD IMPACT OF RIFAXIMIN-A USE IN HEPATIC ENCEPHALOPATHY PATIENTS WITH ADVANCED LIVER DISEASE OR CONTINUED ALCOHOL MISUSE: A POST-HOC ANALYSIS OF THE IMPRESS STUDY}

M. Hudson ${ }^{1}$, P. Di Maggio ${ }^{2}$, R. Cipelli ${ }^{3}$, R. Aspinall ${ }^{4}$

${ }^{1}$ Freeman Hospital, The Newcastle Upon Tyne Hospitals NHS Foundation Trust, Newcastle Upon Tyne/United Kingdom

${ }^{2}$ Uk Medical, Norgine, Harefield/United Kingdom

${ }^{3} \mathrm{pH}$ Associates, Marlow/United Kingdom

${ }^{4}$ Queen Alexandra Hospital, Portsmouth Hospitals NHS Trust, Portsmouth

United Kingdom

Contact E-mail Address: pdimaggio@norgine.com

Introduction: In the UK multicentre, retrospective, real-world study, IMPRESS, rifaximin- $\alpha$ (RFX) use in patients with hepatic encephalopathy (HE) significantly reduced hospitalisations and length of stay in the 6 and 12 months post-RFX initiation compared to the respective periods pre-RFX initiation. This post-hoc analysis of the IMPRESS data compared hospital resource use pre- and post-
RFX initiation in 2 sub-groups of difficult-to-treat HE patients: those with advanced liver disease or ongoing alcohol misuse.

Aims \& Methods: Medical records of patients from 11 UK hospitals who were prescribed RFX for HE between July-2008 and May-2014 were retrospectively reviewed; details of demographic and clinical characteristics, and all-cause hospital admissions were collected in the 6 and 12 months pre- and post-RFX initiation. Patients with baseline MELD score $\geq 15$ or not abstinent at the end of the study period were included in this analysis. Statistical significance of the mean change (standard error of the mean, SEM) was calculated using paired ttest or Wilcoxon test.

Results: Only patients alive at the end of the 6 and 12 months RFX-treatment periods were included: 114 and 102, respectively. Amongst these, 33/114 (29\%, for the 6 months) and 26/102 (25\%, for the 12 months) had baseline MELD $>15$; mean age, 63 years; $70 \%$ were male; $66 \%$ had alcohol-related liver disease; mean MELD 24. The mean (SEM) number of bed days/patient reduced from $25(6.0)$ in the 6 months pre- to $15(5.5)$ in the 6 months post-RFX initiation, and from 36 (9.5) in the 12 months pre- to 20 (7.7) in the 12 months post-RFX initiation (p value not significant). At 6 months post-RFX initiation, 15/114 (13\%) patients were still actively drinking. At RFX initiation, mean age was 56 years; $73 \%$ were male, mean MELD was 19. Despite this, the mean (SEM) number of bed days patient decreased from $36(7.9)$ in the 6 months pre- to $15(5.4)$ in the 6 months post-RFX initiation $(\mathrm{p}=0.048)$, and the mean of hospitalisations/patient fell from $2.8(0.8)$ to $1.2(0.4)$ (t-test $\mathrm{p}=0.059$; Wilcoxon test $\mathrm{p}=0.029)$. Too few patients with continued alcohol misuse were alive at 12 months to evaluate. Two patients reported adverse events, none serious.

Conclusion: In UK clinical practice, treatment with RFX for HE for 6 or 12 months suggested trends in reduced hospital length of stay in patients with advanced liver disease and in those with continued alcohol misuse. However, larger studies are needed to strengthen these findings.

Disclosure of Interest: M. Hudson: Consultant for Norgine; advisory board member; has given sponsored lectures on behalf of Norgine

P. Di Maggio: Employee of Norgine

R. Cipelli: Consultant for Norgine; employee of $\mathrm{pH}$ Associates which was commissioned by Norgine to provide support with study design and management, data analysis and scientific editorial services

R. Aspinall: Consultant for Norgine; advisory board member; has given sponsored lectures on behalf of Norgine

\section{P0042 BACTERIAL INFECTION IN PATIENTS WITH DECOMPENSATED CIRRHOSIS - A PREDICTOR OF LONG-TERM MORTALITY INDEPENDENT OF DISEASE SEVERITY}

M. Sousa, S. Fernandes, J. Silva, A. Ponte, J. Rodrigues, J. C. Silva, L. Proença, J. Carvalho

Gastrenterology, Centro Hospitalar de Vila Nova de Gaia e Espinho, Vila Nova de Gaia/Portugal

Contact E-mail Address: mafalda m_p_sousa@hotmail.com

Introduction: Bacterial infections are common in cirrhotic patients and the proinflammatory response superimposed on the hemodynamic dysfunction of portal hypertension predisposes to the development of complications. Some authors suggest that the occurrence of infection should be considered a separate clinical stage, since it alters the natural history of cirrhosis.

Aims \& Methods: Retrospective assessment of patients with cirrhosis hospitalized for first episode of decompensation between 2011-2015. The aim was to evaluate the prognostic significance of bacterial infections regardless of the severity of the underlying liver disease.

Results: Sixty-four patients ( $85 \%$ male, mean age 59 years, mean MELD 15, 72\% alcoholic cirrhosis) were included with a total of 197 hospitalizations. Hospitals admissions were more frequent due to variceal haemorrhage $(42 \%)$ and encephalopathy $(37 \%)$. The incidence of bacterial infection was $25 \%: 41 \%$ respiratory, $31 \%$ spontaneous bacterial peritonitis and $24 \%$ urinary. Of these, $51 \%$ were nosocomial and in $20 \%$ an infectious agent was isolated. The survival rates at 30 days, 3 months, 6 months and 1 year were $65 \%, 55 \%, 34 \%$ and $27 \%$ in patients with infection and $97 \%, 90 \%, 85 \%$ and $78 \%$ in those without infection $(\mathrm{p}<0.001)$. In the multivariate analysis, survival was independently associated with MELD (hazard ratio (HR) 1.073, $\mathrm{p}=0.012)$, age $(H R 1,032, \mathrm{p}=0.012)$ and infection (HR 3, 821, p < 0.001). Bacterial infection remained an independent predictor of mortality, even when excluding patients with in-hospital mortality and at 30 days (HR 3.093, $\mathrm{p}=0.005$ ).

Conclusion: Patients with cirrhosis exposed to a bacterial infection are at increased risk of death. This risk remains in the long term when we exclude patients with in-hospital mortality and at 30 days and regardless of the severity of the underlying disease (MELD).

Disclosure of Interest: All authors have declared no conflicts of interest. 
P0043 A PROPORTIONALLY GREATER ELEVATION IN LIVER

TRANSPLANT CANDIDACY IN PATIENTS WITH NAFLD AND PORTAL VEIN THROMBOSIS

M. Basaranoglu

Gastroenterology, Bezmialem Vakif University, Istanbul/Turkey

Contact E-mail Address: metin_basaranoglu@yahoo.com

Introduction: NASH progresses to cirrhosis and its complications including hepatocellular carcinoma. It is possible that risk factors for NAFLD-associated cirrhosis and HCC in Eastern countries differ from those in the West. Thus, we aimed to document the characteristics of patients with NAFLD-associated cirrhosis from Turkey, a European country sharing 97\% of its borders with Asia. Relative to other Europeans, the Turkish population exhibits a higher rate of obesity that is comparable to that in the United States.

Aims \& Methods: To characterize non-alcoholic fatty liver disease (NAFLD) presentation with esophageal varices. METHODS: We have kept the records of patients at our hepatology unit and affiliated liver center. Data were collected for esophageal varices only at the advanced endoscopy unit. A cohort of patients with esophageal varices from 2003 to 2014 was reviewed. Eligible patients were $\geq 18$ years of age and have had esophageal varices diagnosed by upper gastrointestinal endoscopy examination. They had regular clinical follow-up and endoscopic examinations at our clinic. Efficacy data were based on the last evaluation. Transplanted cases were excluded. The main inclusion criterion was the presence of esophageal varices with or without gastric varices. Only 258 patients with endoscopically defined high-risk varices had reliable data and were included in this study. Each patient was evaluated for fundal varices, PVT, cirrhosis, HCC, and mortality. After the first evaluation, patients were divided into 4 groups: Those with hepatitis B, hepatitis C, NAFLD and others related to autoimmune hepatitis, Wilson Disease, primary biliary cirrhosis, etc.

Results: Primary end-point of the study was to use this cohort of patients with esophageal varices to evaluate the relationship between this disease and several etiologies, including NAFLD, hepatitis B, hepatitis C or other liver-related diseases. Second end-point was to draw this comparison in terms of PVT, HCC survival and mortality. Of the 258 patients with esophageal varices; NAFLD in $39.0 \%$ (101 patients), hepatitis B virus in $29.1 \%$ ( 75 patients) and HCV in $11.2 \%$ ( 29 patients). The mean age of NAFLD was $56.4 \pm 16.0$ years and $62 \%$ of these patients were men. Moreover, 47.5\% had PVT, 5.0\% had HCC and $45.5 \%$ had fundic varices. The mortality rate was $47.5 \%$ during follow-up, but increased to $80 \%$ in the presence of HCC. PVT was observed in $47.5 \%$ of patients with NAFLD, $29.3 \%$ of patients with hepatitis B, $17.2 \%$ of patients with hepatitis $\mathrm{C}$, and $62.3 \%$ of patients with other liver-related diseases $(P<0.0001)$. Of the 111 patients $(43 \%)$ that died during the study period, 72 patients $(64.9 \%)$ had no PVT $(P=0.057)$. HCC was: $5.0 \%$ in patients with NAFLD, $26.7 \%$ in patients with hepatitis B, $34.5 \%$ in patients with hepatitis C, and $5.7 \%$ in other diseases $(P<0.0001)$. Of the 38 patients with HCC, $13 \%$ had PVT. Moreover, HCC increased the mortality rate in almost all the groups. Of the patients, $50.0 \%$ with NAFLD, $33.3 \%$ with hepatitis B, $26.3 \%$ with hepatitis C, and $58.3 \%$ with other diseases were alive at the end of the 5 -year period with a significant difference according to the Kaplan-Meier log Rank test $(\mathrm{P}=0.04)$. Risk for mortality, measured by risk ratio (RR), did not change per gender (RR: male female $=43.3 \% / 42.5 \%, \mathrm{P}>0.05$ ) or with the occurrence of cirrhosis (RR: $44.8 \% / 28.6 \%, \mathrm{P}>0.05)$. However, it changed with the existence of fundic varices (RR: $49.3 / 35.3, P=0.024$ in favor of fundic varices development) and HCC (RR: $78.9 \% / 36.8 \%, \mathrm{P}<0.0001$ in favor of HCC development).

Conclusion: Data revealed a proportionally greater rise in liver transplant candidacy due to NAFLD-associated cirrhosis with portal vein thrombosis. The mortality rate of patients with NAFLD-associated cirrhosis did not differ from that in patients with virally caused cirrhosis. We confirmed that NAFLD was the third leading cause of $\mathrm{HCC}$ on the transplantation waiting list. Older patients were more prone to developing more cirrhosis, HCC and high mortality rates. These findings should constitute a reliable guideline for evaluating patients at the transplant center and for health policy makers to develop better strategic preventive measures against liver diseases.

Disclosure of Interest: All authors have declared no conflicts of interest.

\section{Reference}

Caldwell S, Argo C. The natural history of non-alcoholic fatty liver disease. Dig Dis. 2010;28:162-168.

P0044 RISK FACTORS AND PREDICTIVE MODEL FOR THE DEVELOPMENT OF MULTIDRUG RESISTANT BACTERIAL INFECTIONS AND THE IMPACT ON PROGNOSIS IN HOSPITALIZED DECOMPENSATED LIVER CIRRHOSIS PATIENTS

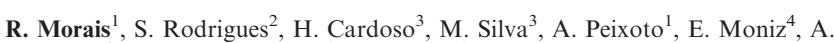
L. Santos ${ }^{2}$, I. Pita ${ }^{5}$, R. Liberal ${ }^{1}$, R. Gaspar ${ }^{1}$, E. Rodrigues-Pinto ${ }^{5}$, G. Macedo ${ }^{5}$

${ }^{1}$ Gastroenterology, Centro Hospitalar São João, Porto/Portugal

${ }^{2}$ Centro Hospitalar De São João, Porto/Portugal

${ }^{3}$ Gastroenterology, Hospital São João, Porto/Portugal

${ }_{5}^{4}$ Clinical Pathology, Centro Hospitalar São João, Porto/Portugal

${ }^{5}$ Centro Hospitalar São João, Porto Medical School, Porto/Portugal

Contact E-mail Address: ruimorais20@gmail.com

Introduction: Bacterial infections are a leading cause of mortality in patients with decompensated cirrhosis.

Aims \& Methods: The objective of this study was to evaluate the prevalence of multiresistant bacterial infections, associated risk factors and their impact on prognosis in hospitalized decompensated liver cirrhosis patients.
This was a retrospective Retrospective study that consecutively evaluated all bacterial infections with confirmed microbiological isolation in patients with decompensated liver cirrhosis admitted to the Gastroenterology ward between January 2009 and May 2016.

Results: There were 308 infections with confirmed microbiological isolates, corresponding to 218 hospitalizations, in a total of 161 patients. The median age of the patients was 63 years (IQR 55-71) and $67 \%$ of them were men. Alcoholic liver disease was the major cause of cirrhosis $(72 \%)$. Among the infections evaluated, $87 \%$ were nosocomial and $13 \%$ community-acquired. Urinary tract infection was the most common infection $(57 \%)$. In $27 \%$ of patients there were at least two concomitant bacterial infections. Multidrug resistant (MDR) bacteria were isolated in over half of patients. In the multivariate analysis, prophylaxis for spontaneous bacterial peritonitis (OR 2.3, $\mathrm{p}=0.009)$, MELD score greater than 19 at admission (OR 1.7, p =0.043), hospitalization in an Intensive/Intermediate Care in the previous month $(\mathrm{OR} 2.8, \mathrm{p}=0.001)$ and antibiotic therapy for infection in the last 6 months (OR 2.4; $\mathrm{p}=0.001$ ) were independently associated with MDR infection. From the variables identified in the multivariate analysis, a predictive model of MDR bacterial infection was created. Assuming a sensitivity of $66 \%$ and a specificity of $72 \%$, we considered the cut-off of -0.0415 as clinically relevant, regarding likelihood of developing a MDR bacterial infection (AUROC $0.723 ; 95 \%$ CI [0.667-0.780]). The occurrence of a MDR infection was associated with a longer duration of hospitalization $(p=0.017)$. In the multivariate analysis there was no independent association between MDR infection and in-hospital mortality and one month after discharge.

Conclusion: The prevalence of MDR bacterial infections in cirrhotic patients is significant and associated with a longer hospital stay. It is possible to identify predictors of its occurrence in order to implement epidemiological strategies to reduce the risk of these infections.

Disclosure of Interest: All authors have declared no conflicts of interest.

\section{Reference}

1. Bajaj JS, O'Leary JG, Wong F, Reddy KR, Kamath PS. Bacterial infections in end-stage liver disease: current challenges and future directions. Gut. 2012;61:1219-1225

\section{P0045 EFFECT OF TREATMENT OF CHRONIC HEPATITIS C WITH SOFOSBUVIR AND DACLATASVIR IN PATIENTS OLDER THAN 60 YEARS}

\section{K. Elnoemany ${ }^{1}$, M. Badr ${ }^{2}$}

${ }_{1}^{1}$ Gastroenterolgy, National Liver Institute (NLI), Elmenofyia gov./Egypt ${ }^{2}$ Internal Medicine, Menofiya University, Shebin Elkom/Egypt

Contact E-mail Address: dr kareemn $@$ yahoo.com

Introduction: Hepatitis $\mathrm{C}$ virus (HCV) diminishes health related quality of life (HRQOL). Currently, there is no published data on assessing of the impact of treatment of chronic hepatitis $\mathrm{C}$ with the new antiviral drugs in old-aged patients.

Aims \& Methods: The aim is to study the effect of treatment of chronic hepatitis $\mathrm{C}$ with the new antiviral drugs in old-aged patients in HRQOL. About 132 patients with chronic hepatitis $\mathrm{C}$ (cirrhotic and non-cirrhotic) were enrolled in the study. Age of patients was sixty years old and older. All patients were treated with sofosbuvir/daclatasvir with or without ribavirin for three months. The HRQOL was assessed with sickness impact profile scoring (SIP) before start of treatment, at end of treatment and after three months of end of treatment.

Results: Old chronic hepatitis $\mathrm{C}$ patients who were treated achieved primary virological response (end of treatment) with percentage $100 \%$ and sustained virological response (SVR) (after 3 months of end of treatment) in about $96 \%$ of treated patients. Before treatment, patients with chronic hepatitis $\mathrm{C}$ had worse scores especially in work, sleep, rest and recreation and pastimes categories. After treatment, patients who received sofosbuvir/daclatasvir with or without ribavirin had significant improve in work, sleep, rest and recreation and pastimes categories with p-value 0.001 . Numerical improvement was observed in total score, physical and psychosocial dimension scores. In patients with SVR, the most improvement was in work and psychosocial dimension scores. There was no significant difference in SIP between scores after end of treatment and after 3 months of end of treatment. Conclusion: Treatment of chronic hepatitis $\mathrm{C}$ in old-aged patients had a significant improvement in HRQOL.

Disclosure of Interest: All authors have declared no conflicts of interest.

\section{Reference}

Umberto Vespasiani-Gentilucci et al., 2015: World J Gastroenterol. 2015 Jun 28; 21(24): $7412-7426$ 
P0046 EGY FIBRO-MARK: A PANEL OF ACCURATE

LABORATORY PARAMETERS FOR THE IDENTIFICATION HEPATIC FIBROSIS PROGRESSION IN PATIENTS WITH CHRONIC HEPATITIS C

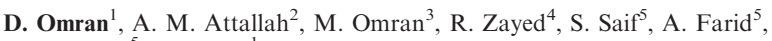
M. Hassany ${ }^{5}$, A. Yosry ${ }^{1}$

${ }^{1}$ Department Of Endemic Medicine And Hepatogastroenterology, Faculty of Medicine, Cairo University, Cairo/Egypt

${ }^{2}$ Research \& Development Department, Biotechnology Research Center, New Damietta/Egypt

${ }^{3}$ Helwan University, Cairo/Egypt

${ }^{4}$ Cairo University, Cairo/Egypt

${ }^{5}$ National Hepatology and Tropical Medicine Research Institute, Cairo/Egypt

Contact E-mail Address: daliaomran@kasralainy.edu.eg

Introduction: Accurate determination of the degree of hepatic-fibrosis is mandatory not only for the diagnosis and prognosis of disease, but also for deciding on the antiviral treatment. Indeed, many studies have been dedicated to the search of non-invasive fibrosis markers capable of providing an accurate information about hepatic fibrosis stage in patients with chronic hepatitis $\mathrm{C}(\mathrm{CHC})$. Direct and indirect markers of hepatic fibrosis are useful for prediction of liver cirrhosis but have limited accuracy for the diagnosis of significant fibrosis. Therefore, the development of more advanced scores combining both direct and indirect markers may improve their diagnostic accuracy.

Aims \& Methods: This work is concerned with determining the levels of some of fibrosis markers, which are directly involved in deposition and removal of extracellular matrix (ECM), together with other indirect fibrosis markers so as to construct a predictive score capable of identifying the presence of significant fibrosis with a high degree of accuracy. Then, we aimed to estimate its performance against that of the other simple noninvasive tests in chronic hepatitis $\mathrm{C}$ patients.

Material and Methods: A total of 148 Egyptian HCV patients were subjected to routine laboratory workup in addition to estimation of serum AFP, hyaluronic acid (HA), platelet-derived growth factor (PDGF), tissue inhibitor of metalloproteinase-1 (TIMP-1) and collagen IV. According to fibroscan, patients were classified into those with non-significant fibrosis $(\mathrm{F}<2)$ and significant fibrosis $(\geq \mathrm{F} 2)$.

Results: Based on univariate analysis, ten variables were significantly higher in patients with significant fibrosis. Patients with F2-F4 had 2.08-fold, 2.14-fold, 1.80-fold and 1.90-fold increase in the concentrations of collagen IV, HA, PDGF and TIMP-1, respectively. Multivariate regression demonstrated that only age, AFP, PDGF, collagen IV and TIMP-1 retained significance. Therefore, a fivemarker score named Egypt (EGY) Fibro-mark (FM) was developed. A significant correlation was found between its candidate markers and liver fibrosis progression. AFP was found to have highest correlation $(\mathrm{r}=0.47, P<0.0001)$ followed by collagen IV $(\mathrm{r}=0.46, P<0.0001)$, Age $(\mathrm{r}=0.43, P<0.0001)$, TIMP-1 $(\mathrm{r}=0.40, P<0.0001)$ and PDGF $(\mathrm{r}=0.40, P<0.0001)$. ROC curve was used to estimate and compare the diagnostic accuracy of these candidate variables. As a consequence, these markers were in a decreasing rank: AFP (AUC 0.79), collagen IV (AUC 0.78), age (AUC 0.76), TIMP-1 and PDGF (AUC 0.75). Additionally, Bivariate Spearman's rank correlation coefficient between EGY-FM and its candidate markers was determined for estimating the impact of each marker on the predictive criteria. The diagnostic value of Egy FM was then assessed by ROC curve showing an AUC of 0.89 for diagnosing significant fibrosis at an optimal cut-off point of 4.05 with $77 \%$ sensitivity, $83 \%$ specificity and $79 \%$ efficiency. Next, the area under the ROC curve (AUC) was used as an index to evaluate and compare the performance characteristics of different non-invasive scores. The AUC was greatest for Fibro-mark (0.89), then BRC (0.83), followed by FRT and King's score (0.82), APRI (0.80), Fibro- $\alpha$ $(0.70)$ and finally FibroQ $(0.63)$

Table 1: The correlation of each score to hepatic fibrosis progression

\begin{tabular}{lllllll}
\hline Index & AUC & Cutoff & Sensetivity & Specificity & Efficiency & $\begin{array}{l}\text { Odds ratio } \\
(95 \% \mathrm{CI})\end{array}$ \\
\hline Fibro-mark & 0.89 & $>4.05$ & 77 & 83 & 79 & $16.18(6.59-39.70)$ \\
BRC score $^{\text {a }}(37)$ & 0.83 & $>7.2$ & 97 & 30 & 76 & $12.86(3.44-48.13)$ \\
FRT $^{\text {a }}(38)$ & 0.82 & $>4.0$ & 99 & 11 & 69 & $10.71(1.21-94.60)$ \\
King's score (41) $^{0}$ & 0.82 & $\geq 12.3$ & 94 & 45 & 79 & $12.25(4.39-34.19)$ \\
APRI a (42) $^{0.80}$ & $>1.5$ & 29 & 94 & 50 & $6.13(1.76-21.30)$ \\
Fibro- $\alpha$ score (43) & 0.70 & $>1.28$ & 95 & 19 & 72 & $4.34(1.33-14.17)$ \\
FibroQ(40) & 0.63 & $>1.6$ & 93 & 13 & 69 & $1.80(0.53-6.04)$ \\
\hline
\end{tabular}

Conclusion: Egy Fibro-mark (FM) score, a more sophisticated score combining 'direct' and 'indirect' markers, is a useful tool to improve the staging of liver fibrosis in CHC patients and seems more efficient than BRC, FRT, King's score, APRI, Fibro- $\alpha$ score and FibroQ in this group of Egyptian patients. Acknowledgment This study was supported by the science and technology development fund (STDF); Project ID: 5380, basic and applied research.
Disclosure of Interest: D. Omran: This study was supported by the science and technology development fund (STDF), Egypt; Project ID: 5380, basic and applied research.

All other authors have declared no conflicts of interest.

\section{Reference}

Attallah AM, Omran MM, Farid K, El-Bendary M, Emran TM, Albannan MS, et al. Development of a novel score for liver fibrosis staging and comparison with eight simple laboratory scores in large numbers of HCV-monoinfected patients. Clinica chimica acta; international journal of clinical chemistry. 2012;413(2122):1725-30.

\section{P0047 EXTRACELLULAR MATRIX PROTEINS CIRCULATING LEVELS SUBSTANTIATE THE EFFECT OF IL-28B RS12979860 T ALLELE ON FIBROSIS STAGE OF CHRONIC HEPATITIS C TYPE 4}

D. Omran ${ }^{1}$, A. M. Attallah ${ }^{2}$, M. Omran ${ }^{3}$, R. Zayed ${ }^{4}$, R. El Essawey ${ }^{4}$, S. Saif ${ }^{5}$, A. Farid ${ }^{5}$, M. Hassany ${ }^{5}$, A. Yosry ${ }^{1}$

${ }^{1}$ Department Of Endemic Medicine And Hepatogastroenterology, Faculty of

Medicine, Cairo University, Cairo/Egypt

${ }^{2}$ Research \& Development Department, Biotechnology Research Center, New Damietta/Egypt

${ }^{3}$ Helwan University, Cairo/Egypt

${ }^{4}$ Cairo University, Cairo/Egypt

${ }^{5}$ National Hepatology and Tropical Medicine Research Institute, Cairo/Egypt

Contact E-mail Address: daliaomran@kasralainy.edu.eg

Introduction: In patients with chronic hepatitis C, host genetics influence liver fibrosis, particularly modifiers in genes controlling the inflammatory and immune response pathways. In this context, interleukin 28B (IL-28B) rs12979860 single-nucleotide polymorphisms (SNP) is considered the most important. Controversial data suggests that IL-28B SNP relate to the severity of hepatic histology. Some studies showed that rs12979860 C allele may be associated with greater hepatic inflammation, higher alanine aminotransferase levels and increased risk of worse clinical outcomes, other studies have not found this association. Furthermore, other studies found that the $\mathrm{T}$ allele affects the severity of liver fibrosis and had a mean staging score higher than other genotypes. Else, none of the previous studies concerned the association between the IL-28B SNP and signs of fibrosis severity.

Aims \& Methods: We aimed to evaluate the cirrhotic development in C/T genotypes using FibroScan, extracellular matrix (ECM) proteins and the model for end-stage liver disease (MELD) in order to resolve conundrum regarding the association between interleukin 28B (IL-28B) rs12979860 and disease severity in chronic hepatitis $\mathrm{C}(\mathrm{CHC})$. So we assessed the allelic and genotypic frequencies of IL-28B rs 12979860 in in $272 \mathrm{HCV}$-infected Egyptian individuals; investigate serum levels of ECM proteins, including hyaluronic acid (HA), laminin, collagen type IV and the N-terminal pro-peptide of collagen type III (PIIINP) as well as its association with liver fibrosis, as assessed by FibroScan, in different IL-28B rs12979860 genotypes; From another view, if C allele has a protective role, we need a precise confirmation, thus we decided to evaluate the difference in cirrhotic behavior using FibroScan, ECM proteins and the MELD score between C/T IL-28B genotypes and evaluate the diagnostic performance of Fibroscan and these ECM proteins in IL-28B rs12979860 genotypes.

Results: IL-28B rs12979860 CT genotype is the commonest genotype among patients constituting $\approx 73 \%$ of the studied sample. The CC and TT genotypes constituted $\approx 18 \%$ and $\approx 9 \%$ respectively. Liver cirrhosis percentage increased with the increasing number of $\mathrm{T}$ alleles as it was $10 \%, 52 \%$ and $96 \%$ in CC, CT and TT genotypes, respectively. FibroScan values $(\mathrm{kPa})$ gave a strong positive correlation $(r=0.6 ; \quad P<0.0001)$ with IL28B polymorphism. Similar to FibroScan, HA $(r=0.5)$, laminin $(r=0.5)$, collagen IV $(r=0.4)$ and PIIINP $(r=0.4)$ serum levels showed significant $(P<0.0001)$ positive associations with IL28B polymorphism. There was stepwise increase in the values of fibroscan and ECM protiens from CC to TT genotypes, so that elevated ECM proteins serum levels were associated with the presence of IL-28B T allele. (Table). In comparison with CC genotype, IL-28B rs12979860 T allele had a significant 2.4-fold increase (in case of CT) and 4.7-fold increase (in case of TT) in Fibroscan score values $(\mathrm{kPa})$. The same was true for ECM proteins serum levels. Interestingly, the characteristics of the cirrhotic patients with TT genotype were completely different from the cirrhotic patients with CT genotype as assessed with FibroScan, ECM proteins and MELD score. Among cirrhotic patients, liver stiffness was $31.13 \pm 2.28 \mathrm{kPa}$ in TT genotype $v s 20.96 \pm 0.74 \mathrm{kPa}$ in CT genotype, MELD was $9.6 \pm 0.73$ in TT genotype vs $3.9 \pm 0.41$ in CT genotype and ECM proteins were significantly $(\mathrm{P}<0.0001)$ higher in patients with TT than CT genotype. AUC values for FibroScan, HA, laminin, collagen IV and PIIINP serum levels to differentiate CC from other IL-28B genotypes were $0.91,0.85$, $0.84,0.82$ and 0.82 , respectively. These values rise to $1.0,0.97,0.93,0.98$ and 0.93 , respectively, when comparing CC to only TT genotype. 
Table 1: Distribution of different fibrosis markers in IL-28B CC, CT and TT genotypes

$$
\text { IL-28B genotypes }
$$

\begin{tabular}{lllll}
\cline { 2 - 4 } Fibrosis marker & $\mathrm{CC}$ & $\mathrm{CT}$ & $\mathrm{TT}$ & $P$ value \\
\hline FibroScan $(\mathrm{kPa})$ & $6.6 \pm 0.4$ & $15.6 \pm 0.6$ & $31.1 \pm 2.3$ & 0.0001 \\
Hyaluronic acid $(\mathrm{ng} / \mathrm{mL})$ & $74.6 \pm 5.2$ & $147.8 \pm 8.2$ & $419.4 \pm 95.2$ & 0.0001 \\
Laminin $(\mathrm{ng} / \mathrm{mL})$ & $60.6 \pm 3.2$ & $106.5 \pm 4.6$ & $282.1 \pm 58.1$ & 0.0001 \\
Collagen $\mathrm{IV}(\mu \mathrm{g} / \mathrm{mL})$ & $6.1 \pm 0.4$ & $11.8 \pm 0.9$ & $30.1 \pm 6.2$ & 0.0001 \\
PIIINP $(\mathrm{ng} / \mathrm{mL})$ & $13.3 \pm 0.7$ & $25.6 \pm 1.7$ & $55.4 \pm 10.4$ & 0.0001 \\
\hline
\end{tabular}

Conclusion: FibroScan and ECM proteins prove that the IL-28B rs12979860 T allele affects the severity of liver disease. Coexistence of $\mathrm{C}$ allele with $\mathrm{T}$ allele reduces cirrhosis severity. This study gives a good deduction that carriage of the IL-28B C allele protects from unfavorable outcomes in CHC. This study shed the light on using FibroScan and ECM proteins as good diagnostic options for liver disease severity in IL-28B genotypes

Disclosure of Interest: D. Omran: This study was supported by the science and technology development fund (STDF) Egypt; Project ID: 5380, basic and applied research

All other authors have declared no conflicts of interest.

\section{P0048 REAL-WORLD EFFECTIVENESS OF FIVE DIFFERENT DIRECT ACTING ANTIVIRAL REGIMENS FOR TREATMENT OF CHRONIC HEPATITIS C WITH NORMAL LIVER ENZYMES: SINGLE-CENTER EGYPTIAN EXPERIENCE}

M. El Kassas ${ }^{1}$, M. Alboraie ${ }^{2}$, M. Salah ${ }^{3}$, A. Eltahan ${ }^{4}$, Y. Abdellatif ${ }^{3}$,

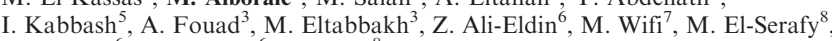

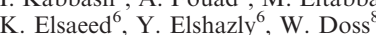

${ }^{1}$ Department Of Endemic Medicine, Faculty of Medicine, Helwan University, Helwan/Egypt

${ }^{2}$ Department Of Internal Medicine, Al-Azhar University, Cairo/Egypt

${ }^{3}$ Tropical Medicine Department, Ain Shams University, Cairo/Egypt

${ }^{4}$ Viral Hepatitis Treatment Unit, New Cairo Hospital, Cairo/Egyp

${ }^{5}$ Department Of Public Health And Community Medicine, Tanta University, Tanta/Egypt

${ }^{6}$ Internal Medicine Department, Ain Shams University, Cairo/Egypt

${ }^{7}$ Internal Medicine Department, Hepatogastroenterology, Cairo University, Kasr Al-Ainy School of Medicine, Cairo/Egypt

${ }^{8}$ Endemic Medicine And Hepatology Department, Cairo University, Cairo/Egypt

Contact E-mail Address: alboraie@azhar.edu.eg

Introduction: Chronic hepatitis $\mathrm{C}$ virus $(\mathrm{CHCV})$ patients with persistently norma transaminases represent a category of patients with mild and slowly progressive disease. Proper management of these patients with direct acting antivirals (DAAs) needs to be investigated in real world treatment settings in Egypt to further validate the accumulating data of the achieved high sustained virologic response (SVR) rates with the use of these drugs in clinical trials.

Aims \& Methods: We aimed to report the efficacy of 5 different DAAs regimens for treatment of $\mathrm{CHCV}$ genotype 4 patients with persistently normal liver enzymes in real-world Egyptian cohort. Data of CHCV genotype 4 patients with normal liver enzymes who started treatment with different DAAs between September 2014 and September 2016 in a single specialized viral hepatitis treatment center in Egypt were retrieved. Treatment regimens included: Pegylated interferon alpha 2b/Sofosbuvir/Ribavirin (PEG/SOF/RBV), Sofosbuvir Daclatasvir (SOF/DCV), Sofosbuvir/Daclatasvir/Ribavirin (SOF/DCV/RBV), Sofosbuvir/Ribavirin (SOF/RBV) and Sofosbuvir/Simeprevir (SOF/SIM). Al regimens were given for 12 weeks except SOF/RBV which was given for 24 weeks. HCV RNA was tested at week 4 of treatment, and 12 weeks after treatment cessation to check for SVR 12.

Results: Our cohort included 1149 patients (562 males and 587 females). 158 patients were treatment experienced $(13.75 \%)$ and 146 patients $(12.7 \%)$ had liver cirrhosis. Regarding the used treatment regimens; $244(21 \%)$ patients were treated with PEG/SOF/RBV, $382(33.5 \%)$ with SOF/DCV, $152(13.5 \%)$ with SOF/DCV/RBV, $117(10 \%)$ with SOF/RBV and $254(22 \%)$ with SOF/SIM The overall SVR rate was $97.5 \%$ while the SVR rates for different regimens were: $94.7 \%, 99.7 \%, 100 \%, 91.5 \%$ and $98 \%$ for (PEG/SOF/RBV), (SOF/DCV), (SOF/DCV/RBV), (SOF/RBV) and (SOF/SIM) respectively.

Conclusion: Different DAAs achieved high SVR rates in treating $\mathrm{CHCV}$ genotype 4 with normal liver enzymes in a real-world cohort from Egypt. SOF/DCV/ RBV combination was the most effective among the studied regimens.

Disclosure of Interest: All authors have declared no conflicts of interest.
P0049 THE SAFETY AND EFFICACY OF THE COMBINATION OF

DACLATASVIR, ASUNAPREVIR, AND BECLABUVIR IN THE

TREATMENT OF CHRONIC HEPATITIS C VIRUS GENOTYPE 1

INFECTION: A SYSTEMATIC REVIEW AND META-ANALYSIS

A.M. Ahmed ${ }^{1}$, M.F. Doheim ${ }^{2}$, O.M. Mattar ${ }^{3}$, N.A. Sherif ${ }^{4}$, D.H. Truong ${ }^{5}$,

P.T.L. Hoa ${ }^{6}$, K. Hirayama ${ }^{7}$, N.T. Huy ${ }^{8}$

${ }^{1}$ Faculty of Medicine, Al-Azhar University, Cairo/Egypt

${ }^{2}$ Faculty of Medicine, Alexandria University, Alexandria/Egyp

${ }^{3}$ Kasr Alainy Faculty of Medicine, Cairo/Egypt

${ }^{4}$ Faculty of Medicine, Mansoura University, Mansoura/Egypt

${ }^{5}$ Ouang Binh Pharmaceutical Joint-Stock Company, Ouang Binh/Viet Nam

${ }^{6}$ Department of Tropical Diseases, University of Medicine and Pharmacy, Ho Chi Minh City/Viet Nam

${ }^{7}$ Institute of Tropical Medicine (NEKKEN), Leading Graduate School Program, and Graduate School of Biomedical Sciences, Nagasaki University, Ngasaki/Japan ${ }^{8}$ Department Of Clinical Product Development, Institute of Tropical Medicine (NEKKEN), Leading Graduate School Program, and Graduate School of Biomedical Sciences, Nagasaki University, Nagasaki/Japan

Contact E-mail Address: med.b.dean $@$ azhar.edu.eg

Introduction: Recently, new direct antiviral agents (DAAs) with different mechanisms of action have been developed to provide much more efficacious and better-tolerated therapeutic strategies for treatment patients with hepatitis $\mathrm{C}$ virus $(\mathrm{HCV})$ infection. Several clinical trials have investigated a 12 -week therapy with fixed dose of all-oral three-drug combination of daclatasvir (DCV), a potent pan-genotypic nonstructural protein $5 \mathrm{~A}$ [NS5A] inhibitor, in $60 \mathrm{mg}$ once daily; asunaprevir (ASV), an NS3 protease inhibitor, in $200 \mathrm{mg}$ twice daily; and beclabuvir (BCV), a non-nucleoside NS5B thumb-1 polymerase inhibitor, in $75 \mathrm{mg}$ twice daily (BCV-TRIO) for treatment of patients with $\mathrm{HCV}$ genotype 1 infection.

Aims \& Methods: This systematic review and meta-analysis aimed to investigate the efficacy and safety outcomes of the three-drug combination of daclatasvir, asunaprevir, and beclabuvir, in treatment of HCV genotype 1 infection. Eleven electronic search engines/libraries, including PubMed, Scopus, Web of Science, Google Scholar, Virtual Health Library (VHL), WHO Global Health Library (GHL), ClinicalTrials, POPLINE, System for Information on Grey Literature in Europe (SIGLE), Cochrane library, and the New York Academy of Medicine (NYAM), were systematically searched for any clinical trial reporting the efficacy and safety of BCV-TRIO for the treatment of HCV genotype 1 infection. Studies were screened for eligibility and data was extracted by two independent reviewers. Sustained virologic response rate after 12 weeks of treatment $\left(\mathrm{SVR}_{12}\right)$ and commonly reported outcomes were pooled as event rate and risk ratio in the comparison meta-analysis (RR). The meta-analysis was conducted using the Comprehensive Meta-analysis (CMA) platform. The protocol was registered in PROSPERO (CRD42017054391).

Results: Among the included five studies, four studies, with low to moderate risk of bias, were included for the meta-analysis $(n=1096)$. The BCV-TRIO showed a high-response rate in naïve patients $\left(\mathrm{SVR}_{12}\right.$ rate $=95.5 \%(95 \% \mathrm{CI}[93.5-96.9])$. This total population was sub-grouped to get $\mathrm{SRV}_{12}$ rates; $89.5 \%(95 \% \mathrm{CI}[85.8-$ 92.4]), 96.2\% (95\% CI [93.0-98.0]), 93.5\% (95\% CI [89.2-96.1]), 91.1\% (95\% CI [87.6-93.7]), 93.9\% (95\% CI [86.6-97.3]), and 91.9\% (95\%CI [89.1-94.0]) for $\mathrm{HCV}$ genotype $1 \mathrm{a}, \mathrm{HCV}$ genotype $1 \mathrm{~b}, I L 28 B$ CC genotype, IL28B non-CC genotype, cirrhotic, and non-cirrhotic respectively. The virologic failure occurred in only 40 patients $(7 \%)(95 \% \mathrm{CI}[5.2-9.4])$. There was no difference when adding ribavirin to this combination $(\mathrm{RR}=0.98,95 \%$ confidence interval $(95 \% \mathrm{CI})$ $[0.90,1.08], P=0.70)$, using this regimen on interferon-experienced patients (RR $=1.03,95 \%$ CI $[0.98,1.08], P=0.30)$, or changing the dose of BCV from $75 \mathrm{mg}$ to $150 \mathrm{mg}$ regardless the genotype 1 subtypes or IL28B genotype. Similarly, the minimal failure of treatment showed no difference regarding the main two comparisons regardless the cause of this failure. Increasing the dose or the duration did not show a significant increase in the efficacy. The rates of serious adverse events (AEs) occurrence were; $(3.4 \%, 95 \% \mathrm{CI}[2.3-5.1]),(6.5 \%$, $95 \%$ CI [3.2-12.7]), $(2.3 \%, 95 \%$ CI [0.6-8.8]), and $(2.9 \%, 95 \%$ CI [0.2-33.6]) for BCV-TRIO, (BCV-TRIO +RBV) for 12 weeks, (DCV + ASV + BCV-150 mg) for 12 weeks, and both (BCV-TRIO) and (DCV + ASV + BCV-150 mg) for 24 weeks respectively. For BCV-TRIO, the most-frequent AEs were headache, diarrhoea, fatigue, and nausea with rates; $(21.2 \%, 95 \%$ CI $[18.4-24.2]),(14.3 \%$, $95 \%$ CI [12.0-16.9]), $(13.8 \%, 95 \%$ CI $[11.2-16.8])$, and $(13.4 \%, 95 \%$ CI $[10.9$ 16.4]) respectively.

Conclusion: This study reported a high SVR, minimal treatment failure rate, and few AEs with fixed-dose three drug combination of daclatasvir, asunaprevir, and beclabuvir for 12-week duration in HCV genotype 1-infected patients, without adding ribavirin, prior interferon-based therapy, restriction on noncirrhotic patients, restriction on certain IL28B genotype, restriction on baseline resistance-associated variants, or expansion the duration of the treatment to 24 weeks.

Disclosure of Interest: All authors have declared no conflicts of interest. 


\section{P0050 GENETIC EPIDEMIOLOGY OF HCV INFECTION IN UPPER}

\section{\&LOWER EGYPT: A MULTICENTRE FAMILY-BASED STUDY}

M. El-Bendary ${ }^{1}$, M. Neamatallah ${ }^{2}$, E. Kamel ${ }^{3}$, H. Elalfy $^{1}$, T. Besheer ${ }^{1}$, H. Elsayed ${ }^{4}$, Y. Ellazeik ${ }^{4}$, A. A. Elmorsy ${ }^{4}$, M. El-Setouhy ${ }^{5}$, A. Eladl ${ }^{6}$, G. Esmat ${ }^{7}$, S.A.T.D.F.(.P.N.1. (Tc/2/health/2009/hep-1.3). ${ }^{8}$

${ }^{1}$ Tropical Medicine And Hepatology, Mansoura Faculty of Medicine-Mansoura University, Mansoura/Egypt

${ }^{2}$ Medical Biochemistry, Mansoura Faculty of Medicine- Mansoura University, Mansoura/Egypt

${ }^{3}$ Mansoura Faculty of Medicine- Mansoura University, Mansoura/Egypt

${ }^{4}$ Mansoura Faculty of Science, Mansoura/Egypt

${ }^{5}$ Ain Shams Faculty of Medicine, Cairo/Egypt

${ }^{6}$ Alazhar Faculty of Medicine -Assuit University, Assuit/Egypt

${ }^{7}$ Department Of Endemic Medicine And Hepatology, Faculty Of Medicine, Cairo University, Bourg El-Dokki El-Edary Dokki/Egypt

${ }^{8}$ Ministry of Research, Cairo/Egypt

Contact E-mail Address: mmelbendary@gmail.com

Introduction: Egypt has the highest prevalence of HCV worldwide. Prevalence of $\mathrm{HCV}$ was reported to be $13.9 \%$ among healthy populations. Adults have higher HCV prevalence $(15.7 \%)$ than children $(4.0 \%)$. Geographically, HCV is highly prevalent in the Nile delta $(15.8 \%)$ than in Upper Egypt $(9.02 \%)$. The household contacts of $\mathrm{HCV}$ seropositive patients had been shown to have a high risk of $\mathrm{HCV}$ infection.

Aims \& Methods: The aim of this study was to determine the prevalence of HCV infection among household contacts of HCV seropositive index patients. We also aimed to compare HCV genotyping distribution in upper and lower Egypt. In this Multicentre hospital case control based study a total of 4894 Egyptian individuals were recruited to the hospitals from different Egyptian population in Upper \& lower Egypt (mainly from Dakahlia, Cairo and Assuit governorates). The index HCV patients were 1106 cases whereas the families or close household contacts of these index cases were 3788 cases. Ideally family was selected on the basis of containing at least one positive $\mathrm{HCV}$ index, one positive $\mathrm{HCV}$ member and other one negative HCV member with no history of any liver complications or disorders(first and second degree consanguinity, living and sharing usual life activity and having at least 15 years of exposure to the index case). The positive cases (whether index or contact cases) in the family were selected with inclusion criteria of 1-HCV positive by PCR RNA $>6$ months, 2-Adults (above 18 years) of both sexes 3-Any stage of HCV related liver diseases. While cases were diagnosed as spontaneously cleared the virus (SVC) based on the following criteria: positive Anti-HCV but negative PCR HCV RNA in 2 successive samples at least 6 months apart with no prior history of antiviral therapy. Each participant was subjected to routine clinical and laboratory investigations in addition to molecular diagnosis and PCR HCV to confirm HCV infection. Sequencing analysis of the 5' UTR of HCV was performed using an ABI Prism 310 Genetic Analyzer (PE Applied Biosystems, Germany). The sequencing reaction was performed using Big Dye Deoxy Terminator method as recommended by the manufacturer (PE Applied Biosystems). Genotypes were determined according to the published reference sequences.

Results: The prevalence of Anti-HCV +ve cases among household contacts was $20.71 \%$ but when PCR HCV was performed only $17.83 \%$ were +ve while $2.9 \%$ were spontaneously cleared the virus (SVC). The HCV prevalence among house hold contacts was $17.29 \% \& 19.17 \%$ while the SVC was $2.49 \% \& 1.55 \%$ in lower \& Upper Egypt respectively. When the genotyping of the positive cases were performed it was found that the following pattern was noticed in the upper \& lower Egypt respectively: (genotype $4 \mathrm{a}(90.3 \%$ \& $70.1 \%), 4 \mathrm{~m}(4.8 \%$ \& $11.8 \%$ ) $4 \mathrm{n}$ $(0.5 \% \& 3.2 \%) 4 \mathrm{o}(0.2 \% \& 2.9 \%) 4 \mathrm{i}(0.5 \% \& 1.9 \%) 4 \mathrm{v}(0.8 \% \& 1.2 \%) \& 1 \mathrm{a}$ $(2.9 \% \& 8.3 \%)$ as shown in the following table.

\begin{tabular}{lll}
\hline HCV genotype & Upper Egypt $(\%)$ & Lower Egypt $(\%)$ \\
\hline $4 \mathrm{a}$ & 90.3 & 70.1 \\
$4 \mathrm{~m}$ & 4.8 & 11.8 \\
$4 \mathrm{n}$ & 0.5 & 3.2 \\
$4 \mathrm{o}$ & 0.2 & 2.9 \\
$4 \mathrm{i}$ & 0.5 & 1.9 \\
$4 \mathrm{v}$ & 0.8 & 1.2 \\
$1 \mathrm{a}$ & 2.9 & 8.3 \\
$1 \mathrm{~g}$ & 0.0 & 0.3 \\
$1 \mathrm{~b}$ & 0.0 & 0.3 \\
\hline
\end{tabular}

Conclusion: The prevalence of HCV was found to be $18.5 \%$ among household contacts of Egyptian families. The genotype 4 was predominant in upper Egypt $(97.1 \%)$ more than lower Egypt $(91.7 \%)$. On the other hand genotype 1a was higher in lower Egypt (8.3\%) more than upper Egypt (2.9\%).

Disclosure of Interest: All authors have declared no conflicts of interest.

\section{References}

Pawlotsky JM. Hepatitis C virus genetic variability: pathogenic and clinical implications. Clin Liver Dis. 2003;7(1):45-66. [PubMed: 12691458]

Youssef A, Yano Y, Utsumi T, abd El-alah EM, abd El-Hameed Ael E, Serwah Ael $\mathrm{H}$, et al. Molecular epidemiological study of hepatitis viruses in Ismailia, Egypt. Intervirology. 2009;52(3):123-31. doi: 10.1159/000219385. [PubMed: 19468235].
Elsadek Fakhr A, Pourkarim MR, Maes P, Atta AH, Marei A, Azab M, et al. Hepatitis C Virus NS5B Sequence-Based Genotyping Analysis of Patients From the Sharkia Governorate, Egypt. Hepat Mon. 2013;13(12):12706. doi: 10.5812/ hepatmon.12706. [PubMed: 24358038].

Elkady A, Tanaka Y, Kurbanov F, Sugauchi F, Sugiyama M, Khan A, et al Genetic variability of hepatitis C virus in South Egypt and its possible clinical implication. J Med Virol. 2009;81(6):1015-23. doi: 10.1002/jmv.21492. [PubMed: 19382263]

Ray SC, Arthur RR, Carella A, Bukh J, Thomas DL. Genetic epidemiology of hepatitis C virus throughout egypt. J Infect Dis. 2000;182(3):698-707. doi: 10.1086/315786. [PubMed: 10950762

\section{P0051 RED BLOOD CELL DISTRIBUTION WIDTH (RDW) AS NON INVASIVE PREDICTOR OF LIVER FIBROSIS IN CHRONIC HEPATITIS C PATIENTS GENOTYPE 4}

M. El-Bendary ${ }^{1}$, K. Farid $^{1}$, D. El-Barber ${ }^{2}$, M. Elnagar ${ }^{3}$

${ }^{1}$ Tropical Medicine And Hepatology, Mansoura Faculty of Medicine-Mansoura University, Mansoura/Egypt

${ }^{2}$ D2fever hospital, Ministry of Health, Domiatte, Domiatte/Egypt

${ }^{3}$ Microbiology Department, Mansoura Faculty of Medicine-Mansoura University, Mansoura/Egypt

Contact E-mail Address: mmelbendary@gmail.com

Introduction: Red blood cell distribution width (RDW) is a numerical measure of the variability in size of red cell It reflects variability in the size of circulating RBCs. RDW can be used as a prognostic marker in heart failure. In hepatic patients it was approved to be an independent predictor of liver fibrosis in patients with chronic HBV infection, and it is higher in patients with alcoholic liver disease and non alcoholic liver cirrhosis. The gold standard for assessing the histological out come of liver disease is liver biopsy. This procedure is costly and carries a small risk of complications due to sampling error, invasiveness and requires hospitalization of at least $6-18 \mathrm{~h}$. These limitations have stimulated the development of non- invasive techniques for assessing the presence and the degree of liver fibrosis. Several laboratory scores composed of routine laboratory markers that are readily available have been proposed for non-invasive prediction of liver fibrosis in chronic hepatitis $\mathrm{C}(\mathrm{CHC})$ patients.

Aims \& Methods: The aim of this work is to use RDW as a marker for noninvasive prediction of the stage of hepatic fibrosis in patients with chronic hepatitis C genotype4. 100 patients with chronic hepatitis $\mathrm{C}$ were subjected to routine laboratory \& radiological investigations in addition to using KX-21 Sysmex automated hematology analyzer to measure RDW\& RPR (RDW\%/Platelet ratio). Comparing with other biomarkers of liver fibrosis like APRI (AST-toPlatelet ratio index) FIB-4 equation (using platelet count, AST, ALT, age) to perform this test. PCR HCV RNA, genotyping \& liver biopsy (using METAVIR scoring system where cases were classified into early fibrosis $(F 1+F 2): 68$ patients \& late fibrosis (F3 + F4): 32 patients]were done.

Results: RDW \& RPR were significantly higher in patients with late fibrosis > early fibrosis $(\mathrm{P}<0.0001)$ while platelets count was significantly lower in late fibrosis $>$ early fibrosis $(\mathrm{p}<0.001)$. By applying ROC curve it was found that the cut off value of RDW was 16.5 , with sensitivity $86.4 \%$ specificity $85.9 \%$ \& accuracy $86 \%$ \& the cut off value of the platelets was 196.5 with sensitivity $81.8 \%$, specificity $62.8 \%$ accuracy $67 \%$, while RPR cut off value was 0.0897 with sensitivity $90.9 \%$, specificity $85.9 \%$ \& accuracy $87 \%$. As regard APRI test it was found that the cut off value was 40.5 with sensitivity $72.7 \%$, specificity $66.7 \%$ \& accuracy $68 \%$ While FIB-4 equation showed cut off value 1.685 with sensitivity $77.3 \%$, specificity $66.7 \%$ and accuracy $69 \%$. In conclusion the area under the ROC curve for, RDW \& RPR were excellent but for platelets, FIB-4 \& APRI were fair. When applying regression analysis it was found that the RDW (OR:3.903, 95\% CI: 1.538-9.904) \& Platelets (OR:0953, 95\% CI: 0.913-0.995), so as the level of RDW increase by one unit the risk late fibrosis will increase by 3.9 on the other hand when the platelets increase by one unit the risk of late fibrosis will decrease by 0.953 .

Conclusion: RDW \& RPR may be used as simple, non-invasive predictors of advanced fibrosis in patients with with chronic HCV genotype-4.

Disclosure of Interest: All authors have declared no conflicts of interest.

\section{Reference}

39(3):862-3; author reply 863.

\section{P0052 CANCER INCIDENCE IN VARIOUS ORGANS OTHER THAN THE LIVER FOLLOWING DIRECT-ACTING ANTIVIRAL (DAA)} THERAPY FOR HEPATITIS C

B. Endoh ${ }^{1}$, N. Esaka ${ }^{2}$, S. Katsushima ${ }^{2}$, K. Chikugo $^{3}$, S. Nakano ${ }^{4}$, T. Shimogama ${ }^{4}$, S. Iwamoto ${ }^{4}$, K. Kasahara ${ }^{4}$, T. Komeda

${ }^{1}$ Department Of Gastroenterology, Kyoto Medical Center, Kyoto/Japan

${ }^{2}$ Dept Of Gastroenterology, Kyoto Medical Center, Japan, Kyoto/Japan

${ }^{3}$ Department Of Gastroenterology, National Hospital Organization Kyoto Medical Center, Kyoto/Japan

${ }^{4}$ Department Of Gastroenterology, Kyoto Medical Center, Japan, Kyoto/Japan

Contact E-mail Address: e-bun@umin.ac.jp

Introduction: The incidence of liver cancer and its recurrence have been reported frequently at an early stage in patients who underwent interferon (IFN)-free direct-acting antiviral (DAA) therapy [1]. The underlying mechanisms of 
cancer incidence following DAA therapy may include the rapid clearance of hepatitis $\mathrm{C}$ virus, reconstitution of the immune system, and reduction of cancer immunosurveillance [2]. These changes may in fact have an impact on the development of cancer in other organs.

Aims \& Methods: We conducted a retrospective analysis to compare the cancer incidence in patients treated with IFN-free DAA therapy with those treated with IFN therapy. All patients who achieved sustained viral response 12 following antiviral therapy between 1992 and 2016 in our hospital were investigated retrospectively. Patient records were examined to identify new cases of cancer, as diagnosed by pathology or medical imaging, in organs other than the liver following antiviral therapy. The date of diagnosis was determined based on the records, and the cancer incidence was compared between patients treated with DAA therapy and those treated with IFN therapy using the Kaplan-Meier method and Cox regression analysis. Patients with recurrent cancer were excluded from the analysis. Propensity score analysis followed by inverse probability of treatment weighting (IPTW) was used to correct for the effects of confounding factors.

Results: There was a significant difference in the age and sex of the patients treated with DAAs $(n=324$, median age: 70 , male: $41 \%)$ and those treated with IFNs $(n=445$, median age: 58 , male: $60 \%)$. Median lengths of the observation period for the DAA and IFN groups were 1.3 and 6.2 years, respectively. There were 12 and 22 cases of cancer occurring in organs other than the liver in the DAA and IFN groups, respectively. These cancer cases occurred the most frequently in the gastrointestinal tract, followed by the urinary organs, hematopoietic organs, biliary tract/pancreas, lungs, and others. The median periods from the start of the antiviral therapy to the time of diagnosis were 0.9 and 6.8 years in the DAA and IFN groups, respectively. Cumulative rates of cancer after 1 and 2 years were 3.0 and $5.0 \%$ for the DAA group, and 0.2 and $0.9 \%$ for the IFN group, respectively. The difference between the groups was significant $(\mathrm{p}=0.02)$ based on Cox regression analysis using IPTW

Table: Cox regression analysis for cancer incidence other than the liver in IPTW samples

$$
\text { Hazard Ratio }
$$$$
95 \% \mathrm{CI}
$$

\section{Treatment}

IFN (Ref.)

DAA

CI: confidence interval, IPTW: inverse probability of treatment weighting, IFN: interferon, DAA: direct acting antiviral

Conclusion: Because cancer detection in organs other than the liver can be challenging in management of hepatitis, some cases with cancer found after the treatment might have been diagnosable before the treatment, possibly leading to an overestimation of the incidence after the treatment. The number of newly diagnosed cancer cases was small in the present study, resulting in a low statistical power. Nevertheless, the cancer incidence in organs other than the liver was significantly higher in patients treated with DAA therapy than those treated with IFN therapy. This difference persisted after correcting for possible confounding factors including the age and sex of the patients. Our findings suggest that patients need to be carefully examined after DAA therapy for the development of cancer in various organs, including but not limited to the liver.

Disclosure of Interest: All authors have declared no conflicts of interest.

\section{References}

1. Reig M, Zoe Mariño Z, Perelló C, et al. Unexpected high rate of early tumor recurrence in patients with HCV-related HCC undergoing interferon-free therapy. $J$ Hepatol 2016;5:719-726.

2. Villani R, Facciorusso A, Bellanti F, et al. DAAs rapidly reduce inflammation but increase serum VEGF level: A rationale for tumor risk during antiHCV treatment. PLOS ONE 2016; 11: e0167934. DOI:10.1371/ journal.pone.0167934

\section{P0053 OPTIMIZATION OF DIRECT ANTI-VIRAL AGENT TREATMENT SCHEDULE: FOCUS ON HCV GENOTYPE 3}

R. Granata ${ }^{1}$, F. Morisco ${ }^{1}$, S. Camera ${ }^{1}$, A. Ippolito ${ }^{2}$, M. Milella ${ }^{3}$, F. Conti ${ }^{4}$, C. Masetti ${ }^{5}$, A. Smedile ${ }^{6}$, P. Tundo ${ }^{7}$, T. Santantonio ${ }^{8}$, M.R. Valvano ${ }^{2}$, A. Termite ${ }^{9}$, P. Gatti ${ }^{10}$, V. Messina ${ }^{11}$, A. Iacobellis ${ }^{2}$, M. Librandi ${ }^{12}$,

N. Caporaso ${ }^{1}$, A. Andriulli ${ }^{2}$

${ }^{1}$ Department Of Clinical Medicine And Surgery, Gastroenterology Unit, University Of Naples "Federico I $i$ ", Naples/Italy

2 Division Of Gastroenterology, Casa Sollievo della Sofferenza" Hospital, IRCCS San Giovanni Rotondo/Italy

${ }^{3}$ Clinics Of Infectious Diseases, University of Bari, Bari/Italy

${ }^{4}$ Centre For The Study Of Hepatitis, department Of Medical And Surgical Sciences (dimec), University of Bologna, Bologna/Italy

${ }^{5}$ Hepatology And Liver Transplantation Unit, University of Tor Vergata, Rome/ Italy

${ }^{6}$ Department Of Medical Sciences, University of Torino, Turin/Italy

${ }^{7}$ Division Of Infectious Diseases, Hospital of Galatina, Galatina/Italy

${ }^{8}$ Clinics Of Infectious Diseases, University of Foggia, Foggia/Italy

${ }^{9}$ Liver Unit, Hospital of Castellaneta, Castellaneta/Italy

${ }^{10}$ Department Of Internal Medicine, Hospital of Ostuni, Ostuni/Italy
${ }^{11}$ Infectious And Tropical Diseases Unit, S. Anna and S. Sebastiano Hospital, Caserta/Italy

${ }^{12}$ Department Of Physiology, faculty Of Pharmacy, La Sapienza University, Rome/Italy

Contact E-mail Address: filomena.morisco@unina.it

Introduction: The shift from therapy with interferon to Direct antiviral agents (DAAs) has been a watershed for the management of HCV-related chronic liver diseases. In fact, treatment with second-generation DAAs cures the great majority of subject with $\mathrm{HCV}$ chronic infectrion, with the exception on genotype 3 cirrhotic patietns

Aims \& Methods: The present report focuses on HCV genotype 3 cirrhotic patients treated with second-generation DAAs in order to identify which of the several treatment schedules recommended for genotype 3 would constitute the best option. Methods: 1. Twenty-four italian centers (ITAL-C consortium) were involved in this real-life study where HCV genotype 3 patients treated with DAAs. Eligible patients were $>18$ years-old with chronic HCV infection, either naïve or treatment-experienced. Patients with any of the following features were excluded: infection with $\mathrm{HCV}$ genotypes other than 3, active $\mathrm{HCC}$ on imaging, $\mathrm{HIV}$ and/or HBV co-infection, liver-transplant recipients, patients with an estimated glomerular filtration rate $<30 \mathrm{ml} / \mathrm{min}$. 2. With the intent to delineate a treatment schedule with the new DAAs that would offer the higher chance of SVR to patients with HCV genotype 3, a systematic search of the literature data was implemented and the retrieved information was pooled and evaluated by a meta-analytical approach. Electronic, systematic review of the available evidence in the published literature was undertaken to identify all studies. The systematic literature review was performed via Medline from 2012 to 2016 by the following search keys: HCV Genotype 3 AND [(DAA)OR (sofosbuvir) OR (daclatasvir) OR (Ledipasvir) OR (Velpatasvir)].

Results: 1. A total of $233 \mathrm{HCV}$ genotype 3 patients were enrolled. In the entire population, the SVR rate was achieved by 205 subjects $(88.0 \%)$. A successful treatment outcome was documented in $79.0 \%$ of patients treated with sofosbuvir in combination with RBV, in $92.0 \%$ of those who received sofosbuvir/daclatasvir with or without RBV, and in all 7 patients treated with sofosbuvir/ledipasvir with or without RBV. At the univariate analysis, baseline predictors of the SVR12 were gender (female patients being more responsive than males), BMI $<30$ and the treatment schedule. Of relevance, age, stage of liver disease (whether advanced fibrosis or cirrhosis), RBV use, and treatment length were irrelevant to SVR12. At the stepwise logistic regression analysis, the only two factors independently associated with SVR 12 were regimens containing sofosbuvir in combination with daclatasvir or ledipasvir $(\mathrm{OR}=4.25 ; 95 \% \mathrm{CI}$ : $1.81-9.97$; $\mathrm{p}=0.001)$, and the $\mathrm{BMI}<30(\mathrm{OR}=2.64 ; 95 \% \mathrm{CI}: 1.04-6.72 ; \mathrm{p}=0.041) .2$. The systematic review of literature provided data of 3311 patients from 17 full text article and two abstracts. The mean weighted SVR12 rate was $84.4 \%$ (CI:80.4-87.8); the rates varied from $79.0 \%$ (CI:70.9-85.3) with sofosbuvir/ribavirin, to $83.7 \%$ (CI:66.2-93.1) with sofosbuvir/ledispavir, and to $88.2 \%$ (CI:83.3-91.7) with sofosbuvir/daclatasvir.

Conclusion: HCV genotype 3-infected patients, and in particular those progressed to cirrhosis, should be no more considered difficult-to-treat individuals, provided that an optimal therapeutic schedule is applied. Patients without cirrhosis should be treated with sofosbuvir and daclatasvir for 12 weeks. Patients with cirrhosis should be treated with sofosbuvir and daclatasvir for 24 weeks with or without ribavirin

Disclosure of Interest: All authors have declared no conflicts of interest.

\section{P0054 IS THERE AN INCREASE IN THE INCIDENCE OF HEPATOCELLULAR CARCINOMA IN CIRRHOTIC PATIENTS WITH HEPATITIS C TREATED WITH THE DIRECT-ACTING} ANTIVIRALS?

J. C. Branco, R. Carvalho, S. Alberto, A. Martins

Gastroenterology, Hospital Professor Doutor Fernando Fonseca, Lisboa/Portugal

Contact E-mail Address: cbranco.joana@gmail.com

Introduction: The impact of the virological cure on the evolution of cirrhotic patients treated with direct-acting antivirals (DAA) is not yet well established. Recently, some papers reported an elevated incidence of recurrence of hepatocellular carcinoma (HCC) ${ }^{1-4}$ and others a possible rise on the de novo incidence of HCC in the first year after treatment with $\operatorname{DAA}^{5-6}$, but not others ${ }^{7-9}$.

Aims \& Methods: This is a prospective study of cirrhotic patients treated with DAA between february/2015 and january/2017, under HCC screening with ultrasonography according to international guidelines. The main endpoint of the study was to determine the incidence of "de novo" and recurrent HCC. The second endpoint was to search for possible predictive factors associated with the occurrence of HCC. Statistical analysis performed on SPSSv.24.

Results: 106 cirrhotics ( $73 \%$ mean; $54.5 \pm 8.8$ years), MELD $7.3 \pm 2.6,60 \%$ with portal hypertension $(\mathrm{n}=64)$ and $22 \%$ with decompensated cirrhosis $(\mathrm{n}=23,22$ Child-Pugh B). Two patients with previous HCC, stage Barcelona Clinic Liver Classification (BCLC) A, inviable after loco-regional treatment. The sustained virological response at week 12 was $89.9 \%(71 / 79)$ : 4 deaths, 1 relapse, 1 therapeutic failure and 2 losses to follow-up (FU). In $11 \pm 7$ months of FU, we registred 5 HCC, 4 "de novo" and 1 recurrence, which corresponded to an incidence of $3.8 \%$ of "de novo" HCC (13\% in decompensated cirrhosis). The BCLC staging was: 2 stage A, 2 stage $B$ and the one with the recurrence was stage D. A Child-Pugh B class $(p=0.004)$, low platelets level $(p=0.001)$ and hospitalization for decompensation $(\mathrm{p}=0.045)$ were associated with the occurrence of $\mathrm{HCC}$; the genotype did not have association. The mean time to HCC development was 7.5 months $(2-14)$ 
Conclusion: In this cohort the "de novo" incidence of $3.8 \%$ of HCC after the treatment with DAA occurred mainly in patients with decompensated cirrhosis, not eligible for treatment with interferon in the past, and in a short interval of time after treatment. These results alert for an eventual need to increase the frequency of screening in the post-treatment period and carefully evaluate the best timing for liver transplantation. We could not conclude about recurrence due to the small number of patients.

Disclosure of Interest: All authors have declared no conflicts of interest.

\section{References}

1 - Reig M, Mariño Z, Perelló C, et al. Unexpected high rate of early tumor recurrence in patients with $\mathrm{HCV}$-related $\mathrm{HCC}$ undergoing interferonfree therapy. J Hepatol 2016;65:719-26.

2 - Conti F, Buonfiglioli F, Scuteri A, et al. Early occurrence and recurrence of hepatocellular carcinoma in HCV-related cirrhosis treated with direct-acting antivirals. J Hepatol 2016;65:727-733

3 - Yang JD, Aqel BA, Pungpapong S et al. Direct acting antiviral therapy and tumor recurrence after liver transplantation for hepatitis-C associated hepatocellular carcinoma. J Hepatol 2016, 65(4):859.

4 - Petta S, Cabibbo G, Barbara M et al. Hepatocellular carcinoma recurrence in patients with curative resection or ablation: impact of HCV eradication does not depend on the use of interferon. Aliment Pharmacol Ther. 2017;45(1):160

5 - Kozbial K, Moser S, Schwarzer R et al. Unexpected high incidence of hepatocellular carcinoma in cirrhotic patients with sustained virologic response following interferon-free direct-acting antiviral treatment. $J$ Hepatol 2016;65:856

6 - Cardoso H, Vale A, Rodrigues S et al. High incidence of hepatocellular carcinoma following successful interferon-free antiviral therapy for hepatitis $\mathrm{C}$ associated cirrhosis. J Hepatol. 2016; 65: 1057.

7 - ANRS collaborative study group on hepatocellular carcinoma (ANRS CO22 HEPATHER, CO12 CirVir and CO23 CUPILT cohorts). Lack of evidence of an effect of direct-acting antivirals on the recurrence of hepatocellular carcinoma: Data from three ANRS cohorts. J Hepatol. 2016;65:734.

8 - Torres HA, Vauthey J-N, Economides MP et al. Hepatocellular carcinoma recurrence after treatment with directacting antivirals: first, do no harm by withdrawing treatment. J Hepatol 2016;65:862

9 - Kobayashi M, Suzuki F, Fujiyama S, Kawamura Y, Sezaki H, Hosaka T, Akuta N, Suzuki Y, Saitoh S, Arase Y, et al. Sustained virologic response by direct antiviral agents reduces the incidence of hepatocellular carcinoma in patients with HCV infection. J Med Virol. 2016;89(3):476.

\section{P0055 MIRNA-506 PROMOTES PRIMARY BILIARY CHOLANGITIS-LIKE FEATURES IN CHOLANGIOCYTES AND IMMUNE ACTIVATION}

O. Erice ${ }^{1}$, P. Munoz-Garrido ${ }^{1}$, J. Vaquero ${ }^{2}$, M.J. Perugorría ${ }^{3}$, M.G. FernandezBarrena $^{4}$, E. Saez ${ }^{4}$, A. Santos-Laso ${ }^{1}$, A. Arbelaiz ${ }^{1}$, R. Jimenez-Agüero ${ }^{1}$, J. Fernandez-Irigoyen ${ }^{5}$, E. Santamaria ${ }^{5}$, V. Torrano ${ }^{6}$, A. Carracedo ${ }^{7}$, M. Ananthanarayanan ${ }^{8}$, M. Marzioni ${ }^{9}$, J. Prieto ${ }^{10}$, U.H.w. Beuers ${ }^{11}$, R. Elferink ${ }^{11}$, N. Larusso ${ }^{12}$, L. Bujanda Fernández De Piérola ${ }^{1}$, J.J. Marin ${ }^{13}$, J.M. Banales ${ }^{1}$

${ }^{1}$ Liver And Gastrointestinal Diseases, Biodonostia Research Institute- Donostia University Hospital- UPV/EHU, San Sebastián/Spain

${ }^{2}$ Sorbonne Universités, UPMC Univ Paris 06, INSERM, Saint-Antoine Research Center, Fondation ARC, Paris/France

${ }^{3}$ Ikerbasque, Basque Foundation for Science, Bilbao/Spain

${ }^{4}$ Division Of Hepatology, CIMA of the University of Navarra, Pamplona/Spain

${ }^{5}$ Proteored-isciii, Proteomics Unit, Navarrabiomed, Navarra Health Department, Public University of Navarra, Navarra Institute for Health Research (IdiSNA), Pamplona/Spain

${ }^{6}$ Cic Biogune, Bizkaia Technology Park, Derio/Spain

${ }^{7}$ Ciberonc, Carlos III National Institute of Health, Madrid/Spain

${ }^{8}$ Liver Center, Yale University School of Medicine, New Haven/United States of America/CT

${ }^{9}$ Department Of Gastroenterology, Università Politecnica delle Marche, Ancona/ Italy

${ }^{10}$ Ciberehd, Carlos III National Institute of Health, Madrid/Spain

${ }^{11}$ Gastroenterology And Hepatology, AMC - Gastroenterology and Hepatology,

AMC; Amsterdam/NL, Amsterdam/Netherlands

${ }^{12}$ Gastroenterology And Hepatology, Mayo Clinic, Rochester/United States of America/MN

${ }^{13}$ Experimental Hepatology And Drug Targeting, Biomedical Research Institute of Salamanca, Salamanca/Spain

Contact E-mail Address: jesus.banales@biodonostia.org

Introduction: Primary biliary cholangitis (PBC) is a chronic cholestatic liver disease associated with autoimmune phenomena targeting intrahepatic bile duct cells (cholangiocytes). Although PBC etiopathogenesis remains still obscure, development of anti-mitochondrial auto-antibodies against pyruvate dehydrogenase complex-E2 (PDC-E2) is a common feature. MicroRNA (miR) dysregulation occurs in liver and immune cells of PBC patients, but their functional relevance is largely unknown. We previously reported that miR-506 is overexpressed in $\mathrm{PBC}$ cholangiocytes and directly targets both $\mathrm{Cl}^{-} / \mathrm{HCO}_{3}{ }^{-}$anion exchanger 2 (AE2) and type III inositol 1, 4, 5-trisphosphate receptor (InsP3R3), leading to cholestasis

Aims \& Methods: The regulation of miR-506 gene expression and its role in cholangiocyte pathophysiology and immune activation was studied. Different sizes of miR-506 promoter were cloned in a luciferase expression vector, which were transfected in human cholangiocytes (H69 cells) and the role of pro- inflammatory cytokines, bile acids, estrogens and glucocorticoids was evaluated on the promoter activities. MiR-506 or a negative control miRNA sequence were also cloned in an expression vector under the regulation of the CMV promoter; these constructs were stably transfected in H69 human cholangiocytes, and cholangiocyte pathophysiology and immune activation were evaluated.

Results: Several pro-inflammatory cytokines overexpressed in PBC livers [such as IL-8, IL-12, IL-17, IL-18 and TNFa] stimulated miR-506 promoter activity in human cholangiocytes, as revealed by luciferase reporter assays. Experimental overexpression of miR-506 in cholangiocytes dysregulated the cell proteomic profile (by mass spectrometry) affecting proteins involved in different biological processes including mitochondrial metabolism. In cholangiocytes, miR-506: $i$ ) induced dedifferentiation with downregulation of biliary and epithelial markers together with upregulation of mesenchymal and pro-inflammatory markers; ii) impaired cell proliferation and adhesion; iii) increased oxidative and endoplasmic reticulum (ER) stress; $i v$ ) caused DNA damage; and v) sensitized to caspase-3dependent apoptosis induced by cytotoxic bile acids. These events were also associated with impaired energy metabolism in mitochondria (proton leak and less ATP production) and PDC-E2 overexpression. Co-culture of miR-506 overexpressing cholangiocytes with $\mathrm{PBC}$ immunocytes induced activation and proliferation of PBC immunocytes.

Conclusion: Different pro-inflammatory cytokines enhance the expression of miR-506 in biliary epithelial cells. MiR-506 induces PBC-like features in cholangiocytes and promotes immune activation, representing a potential therapeutic target for PBC patients.

Disclosure of Interest: All authors have declared no conflicts of interest.

\section{P0056 CLINICAL EXPERIENCE IN THE USE OF TRANSJUGULAR LIVER BIOPSY WITH TRU-CUT NEEDLE: A RETROSPECTIVE EVALUATION OF 265 CASES}

A. Peixoto, M. Silva, A. L. Santos, P. Costa-Moreira, I. Pita, S. Rodrigues, S. Lopes, G. Macedo

Centro Hospitalar De São João, Porto/Portugal

\section{Contact E-mail Address: armandoafp5@gmail.com}

Introduction: Liver biopsy is recognized as the definitive diagnostic tool for the diagnosis and treatment of liver diseases. The transjugular pathway (TJLB) is commonly used in the presence of contraindications to the percutaneous route. Aims \& Methods: The objective was to report the current experience with this technique, as well as its diagnostic acuity and impact in the approach to the patient. Retrospective study of 265 consecutive patients submitted to TJLB between 2010 and 2016 .

Results: We included 265 patients with mean age 56 years $(+/-12.4)$, with the majority $(60.1 \%)$ being male. One hundred and eleven patients $(41.9 \%)$ were hospitalized. In $40.1 \%$ of cases there was previously known liver disease, especially alcoholic disease, hepatitis $\mathrm{C}$ and non-alcoholic steatohepatitis. In $80.5 \%$ of the cases the purpose of TJLB was for diagnosis and in the remaining cases for staging liver disease. Eighty-five patients $(32.1 \%)$ had cirrhosis prior to biopsy. In a fourth of the cases the biopsy was performed in the context of acute hepatitis, being in $12.6 \%$ for diagnosis of alcoholic hepatitis. The reasons for the percutaneous route included: coagulopathy/anticoagulation $(32.2 \%)$, thrombocytopenia $(17.8 \%)$, ascites $(17.8 \%)$, and failed percutaneous liver biopsy $(13.6 \%)$. The technical success was $92.4 \%$. Sampling was considered adequate in $92.2 \%$ of cases, which was associated with diagnostic purpose $(94.9 \%$ vs. $80.4 \%, \mathrm{p}=0.001)$ and evidence of acute hepatitis $(98.4 \%$ vs. $89.8 \%, \mathrm{p}=0.03)$. Most patients $(60.4 \%)$ had histological criteria for cirrhosis. In $76.2 \%$ of patients TJLB allowed a histological diagnosis, mainly alcoholic and non-alcoholic steatohepatitis. Ability to provide a histological diagnosis was associated with adequate sample $(80.8 \%$ vs. $21.1 \%, \mathrm{p}<0.001)$, presence of cirrhosis $(83.9 \%$ vs. $71.6 \%$, $\mathrm{P}=0.023)$ and $>1$ passage $(82.1 \%$ vs. $68.3 \%, \mathrm{p}=0.012)$. Findings were normal in $4.8 \%$. In $70.9 \%$ of the situations the TJLB was considered to have an impact on the subsequent approach. No major complications were recorded.

Conclusion: TJLB is a safe diagnostic tool with high diagnostic acuity, allowing a change in strategy in a high percentage of cases.

Disclosure of Interest: All authors have declared no conflicts of interest.

\section{P0057 OPTIMAL NUMBER OF MEASUREMENTS IN REAL-TIME SHEAR WAVE ELASTOGRAPHY TO ASSESS LIVER FIBROSIS IN PATIENTS WITH CHRONIC HEPATITIS C VIRUS INFECTION}

H. Numao, K. Shimaya, M. Ryoma, S. Igarashi, K. Hasui, K. Kanazawa, M. Munakata, N. Hanabata

Gastroenterology, Aomori Prefectural Central Hospital, Aomori/Japan

Contact E-mail Address: qqqc2c79@celery.ocn.ne.jp

Introduction: Real-time shear wave elastography (SWE) is an established method of evaluating liver stiffness with excellent diagnostic accuracy. However, no consensus exists on the number of measurements or whether mean or median value should be used when reporting results.

Aims \& Methods: In this study, we sought to estimate the most appropriate reporting method for SWE results in practice. Overall, 200 consecutive patients with hepatitis C virus infection who underwent liver biopsy between June 2015 and March 2017 were enrolled. Ten SWE measurements (GE Healthcare, USA) and liver biopsies were performed on the same day. Fibrosis was staged from liver biopsies based on METAVIR scores. SWE results were reported using eight methods: (A) one measurement; (B) the mean of two measurements, (C) three, (D) five, $(E)$ ten; and $(F)$ the median of three, $(G)$ five, and $(H) 10$ measurements. 
Area under the curve and receiver operator characteristic (AUROC) comparisons were used to compare the diagnostic accuracy of the eight methods. The interquartile range (IQR) and median of 10 SWE measurements using body mass index (BMI) and age were analysed using the Mann-Whitney $U$ test.

Results: The study population consisted of 106 men and 94 women with a mean age of $64.7 \pm 10.8$ years and mean BMI of $23.3 \pm 3.64 \mathrm{~kg} / \mathrm{m}^{2}$. Fibrosis severity was $\mathrm{F} 0 / \mathrm{F} 1 / \mathrm{F} 2 / \mathrm{F} 3 / \mathrm{F} 4$ in $7 / 50 / 33 / 24 / 23$ patients, respectively. The median SWE $(\mathrm{m} / \mathrm{s})$ of 10 measurements in patients with F0, F1, F2, F3, and F4 were 1.33, 1.57, $1.73,1.95,1.98$, respectively. The median IQR/median was 0.21 . Furthermore, we found that obesity $(\mathrm{BMI} \geq 25: 0.24,<25: 0.20, p=0.124)$ and older patients (age $\geq 65: 0.22,<65: 0.20, p=0.012)$ indicated significantly greater IQR/median. There was no significant difference in the diagnostic accuracy between using the median or mean of three, five, and 10 measurements. The AUROCs to diagnose patients with significant fibrosis $(\geq \mathrm{F} 2)$ ranged from 0.778 (A) to $0.876(\mathrm{H})$. AUROC increased based on the number of measurements. A significant difference between 1 and $5(p<0.05), 1$ and $10(p<0.01), 2$ and 10 $(p<0.05)$ measurements was observed in pairwise comparison. Likewise, AUROCs to diagnose patients with severe fibrosis $(\geq F 3)$ ranged from 0.782 (A) to $0.923(\mathrm{G})$. A significant difference $(p<0.05)$ was seen between one and 10 measurements. In the cohort of IQR/median $<0.3$, the diagnostic accuracy of $\geq F 2$ and $\geq F 3$ ranged from $0.806(\mathrm{~A})$ to $0.877(\mathrm{H})$, and from 0.832 (A) to 0.928 $(\mathrm{H})$, respectively. In the cohort of obese (BMI $\geq 25$ ) and old patients (age $\geq 65$ ), the diagnostic accuracy of $>\mathrm{F} 2$ and $>\mathrm{F} 3$ ranged from 0.752 (A) to 0.862 (D), and from $0.735(\mathrm{~A})$ to $0.903(\mathrm{H})$, respectively. Comparing the AUROC of one measurement, IQR/median $<0.3$ cohort showed greater AUROC than those of other cohorts, however, the AUROCs of ten measurements were similar in each cohort. Conclusion: No difference was found between reporting mean or median SWE measurements. The diagnostic performance of SWE increased with the number of measurements taken. Our results suggest that 10 measurements are recommended to ensure the accuracy of SWE measurements in a practical setting. Disclosure of Interest: All authors have declared no conflicts of interest.

\section{P0058 APPARENT DIFFUSION COEFFICIENT IN EVALUATING THERAPEUTIC EFFICACY AFTER RADIOFREQUENCY ABLATION FOR HEPATOCELLULAR CARCINOMA: PROMISING RESULTS}

E. Emara ${ }^{1}$, R. Mousa $^{2}$, M. Ghalab ${ }^{3}$, M.H. Emara Elzanan ${ }^{4}$, M. Balbaa ${ }^{1}$, M. Talaat ${ }^{1}$

${ }^{1}$ Radiology, Kafr ElSheikh University Hospital, Kafr Elsheikh/Egypt

${ }^{2}$ Radiology, Zagazig University Hospital, Zagazig/Egypt

${ }^{3}$ Kafr ElSheikh University Hospital, Kafr Elsheikh/Egypt

${ }^{4}$ Tropical Medicine, Zagazig University Dept. of Tropical Medicine Faculty of Medicine, Zagazig/Egypt

\section{Contact E-mail Address: emara 20007@yahoo.com}

Introduction: Percutaneous radiofrequency ablation (RFA) is a commonly used locoregional interventional procedure in treatment of hepatocellular carcinoma (HCC). There is growing evidence that apparent diffusion coefficient (ADC) value can be used in evaluating RFA therapeutic efficacy in treatment of HCC and thus represent a reliable predictor of local HCC recurrence after treatment. Aims \& Methods: We aimed to determine the therapeutic efficacy RFA in patients with hepatocellular carcinomas using ADC value. A total of 52 patients with 58 HCCs were included, and were treated with RFA according to the guidlines. All lesions were evaluated by diffusion weighted imaging (DWI) and ADC value measurement before and after RFA treatment. DWI was obtained using axial a single-shot echoplanar imaging with two $b$-values $\left(500,1000 \mathrm{~mm}^{2} / \mathrm{s}\right)$ using 3 tesla MRI machine. Quantitative ADC maps were calculated using commercially available software and an imaging workstation. Diagnosis of HCC relied on triphasic CT and MRI, showing enhancement at the arterial phase of dynamic contrast enhanced CT or MRI with rapid washout at the portal venous \& delayed phases. Follow-up post ablation by triphasic CT and/or MRI with ADC value measurement was done after one and three months to determine responsive cases (no residual tumoral activity) and non-responsive cases with residual tumor activity.

Results: Forty-eight lesions responded to treatment and 10 lesions had shown no response. ADC values were significantly higher in lesions that responded to RFA than in non-responding lesions. The mean ADC value before treatment was $1.26 \pm 0.16 \times 10^{-3} \mathrm{~mm}^{2} / \mathrm{s} \quad($ mean $\pm \mathrm{SD})$, while after treatment it was $1.46 \pm 0.12 \times 10^{-3} \mathrm{~mm}^{2} / \mathrm{s}$ with a statistically significant difference $(\mathrm{P}=0.003)$ usingb value 500 . The mean $\mathrm{ADC}$ of the lesions using $\mathrm{b}$ value 1000 before treatment was $1.32 \pm 0.28 \times 10^{-3} \mathrm{~mm}^{2} / \mathrm{s}$, and increased after treatment in responding lesions to reach $1.52 \pm 0.1 \times 10^{-3} \mathrm{~mm}^{2} / \mathrm{s}$ with a statistically significant difference $(\mathrm{P}=0.003)$. Using $\mathrm{b}$ value 500 , the mean $\mathrm{ADC}$ value before treatment didn't show significant difference between responding $\left(1.26 \pm 0.16 \times 10^{-3} \mathrm{~mm}^{2} / \mathrm{s}\right)$ and non-responding lesions $\left(1.26 \pm 0.12 \times 10^{-3} \mathrm{~mm}^{2} / \mathrm{s} ; \mathrm{P}=0.97\right)$. While using $\mathrm{b}$ value 1000 , there was a significant difference with higher mean ADC values before RFA in responding lesions than in non-responding $\left(1.32 \pm 0.28 \times 10^{-3} \mathrm{~mm}^{2} / \mathrm{s}\right.$ in responding lesions and $1.09 \pm 0.14 \times 10^{-3} \mathrm{~mm}^{2} / \mathrm{s} \mathrm{in}$ non-responding, respectively; $\mathrm{P}=0.03$ ) The change in ADC in responding lesions is significantly higher than in non-responding lesions, and it was $19.6 \%$ vs. $6.2 \%$, respectively $(\mathrm{P}=0.01)$ using b value 500 and was $23.7 \%$ vs. $21.2 \%$, respectively $(\mathrm{P}=0.001)$ using $\mathrm{b}$ value 1000 .

Conclusion: ADC is a good quantitative measurement allows effective evaluation of the therapeutic efficacy of RFA for treatment of patients with HCC and can be used as good non-contrast alternative to conventional imaging methods in post ablation follow-up.

Disclosure of Interest: All authors have declared no conflicts of interest.
P0059 NON-ALCOHOLIC FATTY LIVER DISEASE (NAFLD) IMPACT ON RESULTS OF SHEAR WAVE ELASTOGRAPHY FOR HEPATIC FIBROSIS STAGING

A. Katrich ${ }^{1}$, A. Okhotina ${ }^{1}$, O. Ponkina ${ }^{2}$, N. Ryabin ${ }^{2}$

${ }^{1}$ Ultrasound Diagnostic, State Public Health Budget Institution 'Scientific

Research Institute - Ochapovsky Regional Clinic H, Krasnodar/Russian Federation

${ }^{2}$ State Public Health Budget Institution 'Scientific Research Institute - Ochapovsky Regional Clinic H, Krasnodar/Russian Federation

Contact E-mail Address: katrich-a1@yandex.ru

Introduction: To study the effect of NAFLD on the results of shear wave elastography (SWE) in patients with chronic diffuse liver disease.

Aims \& Methods: We have performed outcome analysis in 100 patients with diffuse hepatic disease which were treated from 2015 to 2016 . There were 41 male patients $(41 \%)$, and 59 female patients (59\%), age Me (LQ-UQ) 49 (3956), minimal age was 18 years, maximal age was 77 years. All patients were found to have chronic diffuse hepatic diseases and were hospitalized for morphological assessment and diagnosis verification. All patients had shear wave elastography (SWE) with quantitative measure tissue stiffness, Metavir score staging for received results.

Results: Based on the obtained morphological results, we have formed the following subgroups of patients: F0 - F1-31 people, F2-9; F3-15 and F4-45 patients. Given that patients with a degree of fibrosis on the scale METAVIR F0 and F1 do not require active conservative therapy, we combined the data of the group into one F0-F1. The obtained results of shear wave elastography are presented in the form of quantitative variables. Median stiffness with interquartile range (25\%-75\%) in groups: $\mathrm{F} 0$ - F1-5, $4(4,8-7,2) \mathrm{kPa}, \mathrm{F} 2-8,5(8,3-8,9)$ $\mathrm{kPa}, \mathrm{F} 3-13,5(10,1-14,8) \mathrm{kPa}$ and F4-22, $0(18,2-28,5) \mathrm{kPa}$. The parameters of liver elastometry in the various groups on the METAVIR scale differed statistically significantly between $\mathrm{p}<0.05$. When carrying out a correlation analysis between the stiffness indices of the liver parenchyma and the morphological stage of fibrosis, a strong correlation was revealed: the Spearman coefficient was $\mathrm{r}=0.81 \mathrm{p}<0.1 \times 10^{-6}$. Cutoff values in the general group were: for stage F2-7, 75; F3-13, 25; F4-14, $9 \mathrm{kPa}$. In 10 patients, results for SWE differed from morphological conclusion. Analyzing reasons we observed that in $8(80 \%)$ patients there was NAFLD found and in 6 cases more than $66 \%$ was. To study fatty degeneration impact on elastography outcomes we excluded patients with the given disease (16 patients) from the secondary statistical processing. Stiffness median in patients without fatty hepatic degeneration was: F0 - F1-5, $0(4,5-$ $6,5) \mathrm{kPa}, \mathrm{F} 2-8,4(7,55-8,7) \mathrm{kPa}, \mathrm{F} 3-11,7(9,8-14,2) \mathrm{kPa}$ and F4-22 (18, 0 $28,5) \mathrm{kPa}$. According to ROC analyses threshold values were: for stages F2-7, 4; F3-9, 8; F4-14, $85 \mathrm{kPa}$. However, stage correlation for hepatic fatty disease with elastography results was none: $r=0.11 \mathrm{p}=0.246214$.

Conclusion: Quantitative indicators of SWE in patients with diffuse liver disease in combination with steatosis are higher than in patients without it. When interpreting the results of SWE in patients with diffuse liver disease, the possibility of fatty dystrophy should be taken into account, since an underestimation of this fact can lead to false-positive results in the staging of fibrosis by METAVIR. The SWE method can't be recommended for evaluation of the degree of hepatic steatosis, in the absence of correlation with elastometry data.

Disclosure of Interest: All authors have declared no conflicts of interest.

\section{P0060 FEASIBILITY AND REPRODUCIBILITY OF NON-INVASIVE LIVER AND PANCREATIC STIFFNESS ASSESSMENT IN A COHORT OF PATIENTS WITH ALCOHOL-RELATED LIVER DISEASE}

C.B. Conti ${ }^{1}$, N. Weiler ${ }^{2}$, M. Fraquelli ${ }^{1}$, G. Casazza ${ }^{1}$, D. Conte ${ }^{1}$, M. Colombo $^{1}$, S. Zeuzem², M. Friedrich-Rust ${ }^{2}$

${ }^{1}$ Università degli Studi di Milano, Milan/Italy

${ }^{2}$ Department of Internal Medicine 1, J.W. Goethe-University Hospital, Frankfurt am Main/Germany

Contact E-mail Address: benedetta.conti1@gmail.com

Introduction: The estimation of liver stiffness (LS) has recently been evaluating by new elastographic techniques, such as Shear Wave Elastography (SWE), with a wider applicability than transient elastography (1). No studies evaluated LS in patients with alcoholic liver diseases (ALD) by using the elastographic methods. Moreover, exploring the possibility to assess the elasticity of other tissues, few studies evaluated the pancreatic stiffness (PS) by using transabdominal elastography $(2 ; 3)$, observing that chronic pancreatitis and alcoholic etiology had higher PS values (2)

Aims \& Methods: The present study aimed at assessing the feasibility and reproducibility of SWE at measuring LS and PS in a cohort of patients with alcohol abuse and known ALD and investigating the possible correlation between LS or PS with the some clinical and laboratory data. Over a 6-months period 86 patients undergoing LS and PS measurement by SWE were consecutively enrolled. 65 healthy volunteers (HV) were also examined. SWE was blindly performed by two different raters. Liver and pancreatic ultrasound were performed by a single operator. The comparison of stiffness values between patients and $\mathrm{HV}$ was performed. Interobserver agreement for SWE was assessed by intraclass correlation coefficient (ICC). The effect of clinical and imaging data was evaluated on log-transformed PS or LS, with univariate and multivariate linear regression model, with PS or LS as dependent variable. P values $<0.05$ were considered statistically significant.

Results: LS and PS by SWE were obtained in all the patients and HV. No failure was observed. LS and PS were significantly higher in patients than in HV: $22.1 \mathrm{kPa}(95 \% \mathrm{CI}, 16.9-36.2)$ vs $5.7 \mathrm{kPa}(95 \%$ CI $5.2-6.4)$ for LS and $15.4 \mathrm{kPa}$ $(95 \%$ CI $12.2-19.9)$ vs $12.4 \mathrm{kPa}(95 \%$ CI $9.5-13.6)$ for PS, $\mathrm{p}<0.001$. ICC for LS was good: $0.64(95 \% \mathrm{CI}, 0.50-0.45)$. ICC for PS was fair to good: $0.40(95 \% \mathrm{CI}$, 
$0.21-0.57)$. At univariate analysis LS was associated with: liver cirrhosis $(\mathrm{p}<0.0001)$, steatosis $(\mathrm{p}=0.0003)$, liver surface nodularity $(\mathrm{p}=0.0003)$, active alcoholic consume $(\mathrm{p}=0.015)$, alcohol consumption/day $(\mathrm{p}=0.0134)$, diabetes $(p=0.0223)$. At multivariate analysis cirrhosis $(p<0.0001)$ and steatosis $(\mathrm{p}=0.0073)$ were independently associated with LS. At both univariate and multivariate analysis, PS was significantly correlated only with liver cirrhosis $(\mathrm{p}=0.0058)$

Conclusion: The present is the first series assessing LS and PS in ALD patients by using SWE. The feasibility of the technique was excellent. The reproducibility was good for LS and fair for PS. SWE was a good predictor of liver fibrosis in the ALD cohort. Liver cirrhosis was the only independent variable correlating with PS, whose estimation could be useful to detect alcohol-related pancreatic damage in patients with severe ALD.

Disclosure of Interest: All authors have declared no conflicts of interest.

\section{References}

1. Samir AE et al. Radiology, 2015 Mar;274(3):888-96

2. Yashima $\mathrm{Y}$ et al. $J$ Gastroenterol 2012; 47: 427-32

3. Llamoza-Torres CJ et al. Rev Esp Enferm Dig. 2016;108:450-6

\section{P0061 EXOSOMIC MIR-224 REGULATED TUMOR INVASION AND MIGRATION THROUGH IL-6/STAT3 PATHWAY IN HEPATOCELLULAR CARCINOMA}

F. An, D. Chen, Q. Zhan, M. Xia, X. Wu

Gastroenterology, Wuxi People's Hospital Affiliated to Nanjing Medical University, Wuxi/China

\section{Contact E-mail Address: wdf8025@163.com}

Introduction: miR-224 was found regulated progression of liver cancer in our previous studies, IL-6/STAT3 pathway play key role, but the precise underlying mechanism remains to be explored. It was found exosomes are the vesicles released by the tumor cells into tumor microenvironment, they are a powerful diagnostic tool due to relative stability and composition covering the whole range of cancer-related biomarkers including proteins, metabolites, DNA, DNA modifications, coding and non coding RNA. Thus, study the roles of exosomic miRNA could be usefull for therapy and prognosis prediction of hepatocellular carcinoma (HCC).

Aims \& Methods: The expression of miR-224, IL-6, STAT3 and SMAD4 in tumor as well as adjacent tumor tissues of HCC were detected by RT-PCR. Then the role of miR-224 in HCC progression was assessed in vitro as well as in vivo, and the expression of miR-224 and genes related to HCC invasion and migration were detected by RT-PCR and immunochemistry methods in the transplantation tumor of nude mice. Furthermore, the supernatant exosome from HCC cells was isolated and the translocation roles of exosome was studied by confocal. Finally, the expression of miR-224 and genes in STAT3/SMAD4 pathway in the exosome isolated from the supernatant of HCC cells induced by IL-6 were detected.

Conclusion: This study provides the novel mechanism of regulatory roles of miR224 in HCC, and from the study, exosomic miR-224 showed as the novel target and predictor for HCC prevention and treatment in future clinic.

Results: It was found miR-224, IL-6 and STAT3 were up regulated in the tumor tissues of HCC patients but the SMAD4 showed the down regulated when compared with adjacent tumor tissues. Over expression of miR-224 can promote growth, proliferation, migration and invasion capability of $\mathrm{HCC}$ cells in vitro. Furthermore, in the vivo study, miR-224 was found accelerated transplantation tumor growth in nude mice, and the up regulation of miR-224, IL-6/STAT3 and down regulation of SMAD4 were also found in the transplantation tumors which were induced by miR-224. The Exosome from the supernatant of HCC cells can be found translocated into HCC cells conversely, when the HCC cells were induced by IL-6, the overexpression of exosomic miR-224 and STAT3 and decrease of exosomic SMAD4 were detected. It was speculated miR-224 can regulated HCC progression through IL-6/STAT3/SMAD4 pathway by exosomic way.

Disclosure of Interest: All authors have declared no conflicts of interest.

\section{Reference}

1. An F, Olaru AV, Mezey E, Xie Q, Li L, Piontek KB, Selaru FM. PLoS One. 2015 Oct 28;10(10):e0141448. 9. Lou G, Song X, Yang F, Wu S, Wang J, Chen Z, Liu Y. Exosomes derived from miR-122-modified adipose tissuederived MSCs increase chemosensitivity of hepatocellular carcinoma. $J$ Hematol Oncol. 2015 Oct 29;8:122.

\section{P0062 EXPRESSION ANALYSIS OF LIVER-SPECIFIC CIRCULATING MICRORNAS IN HCV-INDUCED HEPATOCELLULAR CARCINOMA IN EGYPTIAN PATIENTS}

L. Mourad $^{1}$, E. El-Ahwany ${ }^{2}$, M. Zoheiry ${ }^{2}$, H. Abu-Taleb ${ }^{2}$, M. Hassan ${ }^{2}$, A. Abdel Rehim $^{2}$, M. Hassan ${ }^{2}$, S. Zada ${ }^{1}$

${ }^{1}$ Biotchnology, The American University in Cairo, Cairo/Egypt

${ }^{2}$ Theodor Bilharz Research Institute, Cairo/Egypt

\section{Contact E-mail Address: lobna.mourad@gmail.com}

Introduction: The prevalence of hepatocellular carcinoma (HCC) in Africa is higher compared to the rest of the world due to the high incidence of chronic infection with hepatitis $\mathrm{C}$ virus (HCV). In Egypt, $\mathrm{HCV}$ infection is the leading cause for the high HCC incidence, which is usually diagnosed at late stages. Due to the absence of reliable and accurate biomarkers for early detection of liver cancer, circulating microRNAs have recently emerged as great candidates for early diagnosis of HCC. These small non-coding RNA molecules are responsible for regulating gene expression and RNA stability. Therefore, the aim of this study is to investigate the potential of liver-specific circulating microRNAs as an accurate non-invasive diagnostic tool for the early detection of HCV-induced HCC.

Aims \& Methods: Seven main miRNAs (miR-125a, miR-139, miR-34a, miR-221, miR-16, miR-145 and miR-199a) were selected due to their expression patterns in $\mathrm{HCC}$ as well as their contribution to the development of hepato-carcinogenesis. A total of 165 patients were enrolled in this study, from which serum samples were collected and categorized into four main patient groups: 42 chronic hepatitis $\mathrm{C}$ (CHC) without cirrhosis, $45 \mathrm{CHC}$ with cirrhosis (LC), $38 \mathrm{HCC}$ with HCV patients, and 40 healthy controls. The expression profile of the seven miRNAs was analyzed using TaqMan real-time reverse transcription-polymerase chain reaction. Additionally, the conventional markers for HCC $\alpha$-fetoprotein (AFP) and des- $\gamma$-carboxyprothrombin (DCP) were measured using commercial kits.

Results: Serum levels of miR-125a, miR-139, miR-145 and miR-199a were significantly decreased $(\mathrm{P}<0.01)$ in HCC than in the CHC and LC groups (Table $1)$. On the other hand, miR-16 and miR-34a were significantly increased $(\mathrm{P}<0.01)$ in $\mathrm{HCC}$ patients compared to the normal group. However, no significant difference was shown in the expression of miR-16, miR-34a, and miR-221 between the CHC, LC, and HCC groups. As a single biomarker, miR-34a showed the highest sensitivity and specificity among all miRNAs investigated, followed by miR-221, miR-125a, miR-139, miR-145, and miR-199a.

Table 1: Expression levels of serum microRNAs of the patient groups

\begin{tabular}{lllll}
\hline MicroRNAs & Normal & CHC & LC & HCC \\
\hline miRNA-16 & $14.26 \pm 0.69$ & $24.09 \pm 0.44^{* *}$ & $23.29 \pm 0.46^{* *}$ & $22.35 \pm 0.54^{* *}$, a \\
miRNA-34a & $27.32 \pm 0.19$ & $32.69 \pm 0.34^{* *}$ & $30.01 \pm 0.54^{* *}, \mathrm{~b}$ & $32.50 \pm 0.94^{* *}$ \\
miRNA-221 & $22.82 \pm 0.38$ & $27.17 \pm 1.44^{* *}$ & $28.22 \pm 0.41^{* *}$ & $28.51 \pm 0.46^{* *}$ \\
miRNA-125 & $20.57 \pm 0.54$ & $96.01 \pm 4.36^{* *}$ & $100.54 \pm 0.81^{* *}$ & $29.96 \pm 0.57^{* *}, \mathrm{~d}$ \\
miRNA-139 & $29.96 \pm 0.57$ & $94.63 \pm 0.38^{* * *}$ & $86.02 \pm 0.40^{* *}, \mathrm{e}$ & $30.03 \pm 0.43$ \\
miRNA-145 & $20.65 \pm 0.52$ & $85.31 \pm 0.53^{* *}$ & $80.74 \pm 0.59^{* *}$ & $20.64 \pm 0.57$ \\
miRNA-199 & $80.23 \pm 0.72$ & $330.38 \pm 0.74^{* *}$ & $311.98 \pm 0.72^{* *}$ & $66.16 \pm 0.44^{\mathrm{c}}, \mathrm{d}$ \\
\hline
\end{tabular}

$* * p<0.01$ significant increase than control; ${ }^{a} p<0.01$ significant decrease than $\mathrm{CHC} ;{ }^{\mathrm{b}} \mathrm{p}<0.01$ significant decrease than $\mathrm{CHC}$ and $\mathrm{HCC} ;{ }^{c} p<0.01$ significant decrease than control; ${ }^{\mathrm{d}} p<0.01$ significant decrease than $C H C$ and $L C ;{ }^{\mathrm{e}} p<0.01$ significant decrease than $\mathrm{CHC}$

Conclusion: These results indicate that measuring the expression levels of liverspecific circulating microRNAs can be used as a reliable diagnostic and prognostic tool for HCC. Our results demonstrated that the up-regulation of miR-16, miR-34a, and miR-221 can differentiate between normal individuals and patients with liver disease ranging from fibrosis, cirrhosis, and HCC. Meanwhile, the noticeable down-regulation of miR-125a, miR-139, miR-145 and miR-199a in the HCC patient group indicates that these microRNAs can differentiate HCC from CHC and LC.

Disclosure of Interest: All authors have declared no conflicts of interest.

\section{References}

Pant, Kishor; Venugopal, Senthil K. (2016). Circulating microRNAs: Possible role as non-invasive diagnostic biomarkers in liver disease. Clin Res Hepatol Gastroenterol, 944: 1-8.

Hayes, C. Nelson; Chayama, Kazuaki. (2016). MicroRNAs as Biomarkers for Liver Disease and Hepatocellular Carcinoma. Int. J. Mol. Sci., 17 (280): 1-17.

\section{P0063 EPIGENETIC INACTIVATION OF METALLOTHIONEIN $1 \mathrm{G}$} IN PATIENTS WITH HEPATOCELLULAR CARCINOMA

X. $\mathbf{L i}^{1}$, N. Zhang ${ }^{2}$, L. Xu' ${ }^{2}$, G. Zhao ${ }^{3}$

${ }^{1}$ State Key Laboratory Of Oncology In South China, Sun Yat-sen University Cancer Center, Guangzhou/China

${ }^{2}$ Sun Yat-sen University, Guangzhou/China

${ }^{3}$ Inner Mongolia People's Hospital, Hohhot/China

Contact E-mail Address: lixiaox@sysucc.org.cn

Introduction: Primary hepatocellular carcinoma (HCC) is one of the most common malignancies all over the world. $\mathrm{HCC}$ is associated with poor prognosis. However, the mechanism of HCC initiation and development remains unclear. In our previous work, high-throughput microarray assay in collected clinical HCC samples followed by bioinformatic analysis suggested that Metallothionein $1 \mathrm{G}$ (MT1G) might be one of the key factors in HCC

Aims \& Methods: We detected the MT1G expression in paired HCC samples and HCC cell lines by RT-qPCR and Western blot. Then MSP (Methylation specific PCR) and BGS (Bisulfite genomic sequencing) were performed to evaluate methylation status of MT1G in HCC. The functional significance of MT1G in HCC was investigated by overexpression or knockdown in HCC cell lines. The effects of MT1G re-expression were also determined by flow cytometry.

Results: MT1G was inactivated in all (6/6) HCC cell lines tested, but was readily expressed in immortalized liver cell line LO2. The expression of MT1G was down 
regulated in cancer tissues compared with the adjacent non-tumor tissues $(\mathrm{P}<0.001)$. The gene expression level of MT1G in the liver cancer cell lines was closely correlated to the promoter hypermethylation status. The MT1G expression in silenced HCC cell lines could be restored by demethylation agent. We generated HCC cell lines overexpressed MT1G. Ectopic re-expression of MT1G by stable transfection in SMMC-7721 and Hep3B cells inhibited colony formation $(\mathrm{P}<0.001)$, suppressed cell motility and invasiveness $(\mathrm{P}<0.05)$, concomitant with up-regulation of E-cadherin; and down-regulation of PCNA, MMP2, MMP13 and Vimentin. The in vivo growth of HCC cells in nude mice was also markedly inhibited after stable expression of MT1G $(\mathrm{P}<0.001)$. MT1G over-expression in HCC cells induced the cell apoptosis $(\mathrm{P}<0.01)$.

Conclusion: Our results demonstrate that MT1G promoter methylation directly mediates the transcription down-regulation and commonly occurs in HCC. MT1G gene can act as a functional tumor suppressor in liver carcinogenesis by playing an important role in depression of cell proliferation, migration, invasion, and induction of cell apoptosis.

Disclosure of Interest: All authors have declared no conflicts of interest.

\section{P0064 THE FXR RECEPTOR PATHWAY IN HEPATOCELLULAR ADENOMA AND FOCAL NODULAR HYPERPLASIA, A PRELIMINARY EXPERIENCE}

B. Van Rosmalen ${ }^{1}$, M. Visentin ${ }^{2}$, J. Verheij ${ }^{3}$, M. Bieze ${ }^{1}$, B. Stieger ${ }^{2}$, T. M. Van Gulik $^{1}$

${ }^{1}$ Surgery, AMC, Amsterdam/Netherlands

${ }^{2}$ Clinical Pharmacology And Toxicology, University Hospital Zurich, Schlieren Switzerland

${ }^{3}$ Pathology, AMC, Amsterdam/Netherlands

Contact E-mail Address: b.v.vanrosmalen@amc.uva.nl

Introduction: Hepatocellular adenoma (HCA) and focal nodular hyperplasia (FNH) may be confused on medical imaging. Both tumours are not connected to the biliary tree, however only FNH accumulates bile salts, suggesting that hepatocellular uptake and secretion of bile constituents differs in FNH and HCA. Therefore, one would anticipate changes in the Farnesoid X receptor (FXR) expression. However, the expression of FXR and its targets in HCA and FNH is relatively unknown. Targets of FXR regulate uptake and excretion of hepatobiliary contrast agents, possibly altering the presentation of FNH and HCA on medical imaging. We studied the expression of FXR and its targets in HCA and FNH and compared this with the appearance of lesions on MRI.

Aims \& Methods: Tumour tissue and normal tissue from 7 patients with HCA and 7 patients with FNH was obtained. Diagnosis was confirmed by histopathological examination in all patients. Reverse transcription of the mRNA to cDNA was performed, using random primers and MultiScribe Reverse Transcriptase (Life Technologies, Carlsbad, Ca). The cDNA was used as template for PCR amplification by Taqman ${ }^{\circledR}$ assay analysis (Applied Biosystems, Foster City, CA). The expression of all the target genes in the FXR pathway (SHP, NTCP OATP1B1, OATP1B3, BSEP, CYP7A1, CYP8B1, BAAT, SLC27A5, CYP3A4, SSULT2A1, UGT2B4, FGFR4, MRP2, MRP3, MDR3) were compared to the expression of UBC which functioned as internal reference in both normal and tumour tissue. Matched case comparisons were made for tumour and normal tissue. DNA expression of FNH and HCA was compared to MRI findings. Results: FXR was downregulated in both HCA and FNH. NTCP was significantly downregulated in $\mathrm{FNH}$, and not significantly in $\mathrm{HCA}$, although showing a trend towards down regulation. Three patients (1 FNH 2 HCA) show aberrant expression of NTCP compared to all the other patients. All these three patients had also an unclear or incorrect diagnosis based on MRI scan with gadoxetic acid as compared to final diagnosis by histopathological examination. OATP1B1 was downregulated in both HCA and $\mathrm{FNH}$, except again for the three patients with the aberrant imaging. Expression of OATP1B8 and SHP in HCA and FNH did not significantly differ from expression in healthy liver tissue. MRP2 was significantly downregulated in HCA, but not in FNH. However, this may again be due to the patients with the aberrant expression pattern. OATP1B3 was significantly downregulated in HCA. CYP3A4 and CYP2A1 were very strong downregulated in HCA, but not in FNH. FGFR 4 was heavily downregulated in HCA, but not in not FNH. BAAT was significantly downregulated in HCA. Conclusion: Limited by sample size, this study suggests that misdiagnosis based on medical imaging might actually correlate with aberrances on hepatocyte transporter level. This seemed to account for NTCP (bile salt importer), OATP1B1 (bile salt importer) MRP2(efflux pump of conjugated compounds). Although FXR itself was downregulated in both FNH and HCA, its downstream targets differed in expression between tumours. FXR receptor activity might be altered even though expression is not different, or downstream targets might be influenced by factors outside the FXR pathway. Future research could provide a more profound insight into this mechanism.

Disclosure of Interest: All authors have declared no conflicts of interest.

\section{P0065 CUX1 CONTROLS ENDOPLASMIC RETICULUM STRESS} AND AUTOPHAGY-RELATED CELL DEATH

G. Metzger ${ }^{1}$, P. Di Fazio ${ }^{1}$, D. Bartsch ${ }^{1}$, T.M. Gress ${ }^{2}$, T. T. Wissniowski ${ }^{2}$ ${ }^{1}$ Department Of Visceral Thoracic And Vascular Surgery, Philipps University Marburg, Marburg/Germany

${ }^{2}$ Klinik Für Gastroenterologie, Endokrinologie, Stoffwechel Und Infektiologie,

Philipps Universität Marburg, Marburg/Germany

Contact E-mail Address: giulia_metzger@web.de

Introduction: CUX1 (CUTL1) is a transcription factor able to promote the expression of several genes implicated in cellular proliferation, differentiation and demise. In normal adult cells, it preferentially favors the expression of proapoptotic genes. Its aberrant expression in tumor turns its role as foe.

Aims \& Methods: Here, we analyze the role exerted by CUX1 during deacetylase inhibitors mediated cell death in liver cancer cells. CUX1, endoplasmic reticulum (ER) stress and autophagy markers were analyzed by RT-qPCR in two liver cancer cell lines HepG2 and Hep3B. Protein level was measured by western blotting. Cells were transfected with siRNA for CUX1 and furthermore treated with deacetylase inhibitors panobinostat, SAHA and trichostatin A Thapsigargin, an endoplasmic reticulum stress inducer, served as positive control.

Results: CUX1 knock down caused a suppression of ER stress and autophagy markers BiP, CHOP, ATF4, ATF6, Beclin1, MAP1LC3B, UVRAG and TFEB at early time point (6 hours) in both cell lines. Prolonged transfection did not alter the expression of the above mentioned markers; $\mathrm{BiP}$ was the only one suppressed in HepG2 after 24 hours. Interestingly, the deacetylase inhibitors are able to promote CUX1 over-expression after 6 hours of treatment, whereas they show to lose this ability after 24 hours. CUX1 knock-down reduced not significantly its protein level after treatment with deacetylase inhibitors. CUX1 knock down counteracts the accumulation of BiP protein after 24 hours of treatment with deacetylase inhibitors. Thapsigargin induced BiP independently from CUX1.

Conclusion: ER stress and autophagy markers are under the control of CUX1. The cell death induced by deacetylase inhibitors is strictly connected with CUX1 expression and activity. Further studies are needed to clarify the exact mechanism exerted by CUX1 in this scenario.

Disclosure of Interest: All authors have declared no conflicts of interest.

\section{P0066 CUX1 CONFERS RESISTANCE TO APOPTOTIC CELL} DEATH IN LIVER CANCER CELLS

E. Hofmann ${ }^{1}$, P. Di Fazio ${ }^{1}$, D. Bartsch ${ }^{1}$, T.M. Gress ${ }^{2}$, T. T. Wissniowski ${ }^{2}$ ${ }^{1}$ Department Of Visceral Thoracic And Vascular Surgery, Philipps University Marburg, Marburg/Germany

${ }^{2}$ Klinik Für Gastroenterologie, Endokrinologie, Stoffwechel Und Infektiologie, Philipps Universität Marburg, Marburg/Germany

Contact E-mail Address: ellihofmann@yahoo.de

Introduction: CUX1 (CUTL1) is a transcription factor able to promote the expression of several genes implicated in cellular proliferation, differentiation and demise. In normal adult cells, it preferentially favors the expression of proapoptotic genes. Its aberrant expression in tumor turns its role as foe.

Aims \& Methods: Here, we analyze CUX1 activity in TRAIL (Tumour necrosis factor related apoptosis inducing ligand) mediated cell death in liver cancer cells. CUX1 was knocked down in HepG2 and Hep3B cells. Cells were further treated for 48 hours with a strong ligand (superkiller) binding DR4 and DR5 (TRAIL death receptors). The cell death events were analyzed by FACS analysis. RTqPCR was performed to detect the expression of apoptotic markers. Caspase activity was measured by luminescence. Apoptosis array was performed. Western blotting was performed for caspase 8 and Flip detection

Results: Treatment with superkiller TRAIL, at 50 and $100 \mathrm{ng} / \mathrm{ml}$, caused cell death in HepG2 and Hep3B cells after $48 \mathrm{~h}$ proven by an accumulation of $40 \%$ of sub-G1 events. CUX1 knock down caused a sensitization of liver cancer cells to TRAIL effect by increasing, significantly, the percentage of subG1 events $(60 \%$ with $100 \mathrm{ng} / \mathrm{ml})$. CUX1 knock down did not change the expression of TP53, KRT18, CDKN1A and CDKN1B. Interestingly, silencing CUX1 increased the activity of caspase $3 / 7$ after treatment with soluble TRAIL. The effect was neutralized by pan-caspase inhibitor zVAD. Apoptosis array evidenced an increased protein level of un-/cleaved caspase 3 after CUX1 knock down. Caspase 8 uncleaved form was down-regulated at protein level after CUX1 knock down and treatment with TRAIL. Its cleaved forms were up-regulated. FlipL decreased in favor of FlipS also.

Conclusion: CUX1 mediates the resistance of liver cancer cells to TRAIL signaling. Knock down of CUX1 restores the potential of TRAIL to trigger cell death. Disclosure of Interest: All authors have declared no conflicts of interest. 
P0067 SERUM SQUAMOUS CELL CARCINOMA ANTIGEN LEVEL

IN CIRRHOTIC CHRONIC HEPATITIS C PATIENTS WITH AND

\section{WITHOUT HEPATOCELLULAR CARCINOMA}

\section{W. I. Ellakany}

Gastroenterology, Hepatology And Infectious Tropical Medicine, Faculty of Medicine, Alexandria University, Egypt, Alexandria/Egypt

\section{Contact E-mail Address: walidellakany@yahoo.com}

Introduction: Hepatocellular carcinoma (HCC) is a primary malignancy of the liver. HCC is the fifth most common malignancy in the world and the third most common cause of cancer-related deaths worldwide. It is a major health problem in Egypt with the incidence expected to rise continuously in the next decade. [1-2] The diagnosis of liver cancer depends on both screening with alpha-fetoprotein (AFP) and radiological imaging studies. Generally, normal levels of AFP are below $10 \mathrm{ng} / \mathrm{ml}$ but AFP greater than $200 \mathrm{ng} / \mathrm{ml}$ is suggestive of HCC . The sensitivity of AFP for liver cancer is about $67 \%$; therefore a normal AFP does not exclude HCC. Searching another tumor marker, that together with AFP could improve the diagnostic utility of HCC.[3]

Squamous cell carcinoma antigen (SCCA), a member of the high molecular weight family of serine protease inhibitors named serpins which are physiologically found in the granular layers of normal squamous epithelium but found to be typically expressed by neoplastic cells of epithelial origin in a number of different cancers for example cancer cervix, lung, and head and neck cancers hence, it can be used as a clinical marker of these malignancies. [4]

The structure of the serpin ovalbumin revealed the archetype native serpin fold that typically have three $\beta$-sheets (termed A, B and C) and eight or nine $\alpha$-helices (hA-hI). Serpins also possess an exposed region termed the reactive centre loop (RCL) that includes the specificity determining region and forms the initial interaction with the target protease.

Recently much attention has been focused on the role of SCCA in HCV cirrhotic patients suggesting that high levels of SCCA can assess HCC development. [5] The aim of this study was to assess the serum level of squamous cell carcinoma antigen (SCCA) in cirrhotic chronic HCV patients with and without hepatocellular carcinoma in relation to alfa feto protein (AFP).

Aims \& Methods: The aim of this study was to assess the serum level of squamous cell carcinoma antigen (SCCA) in cirrhotic chronic HCV patients with hepatocellular carcinoma in relation to alfa feto protein (AFP). These groups were from both sexes who are admitted to the inpatient ward and the outpatient clinic of Tropical Medicine Department, Faculty of Medicine, Alexandria University.

This study was carried out on:

Group A: 100 cases of hepatocellular carcinoma without interventions.

Group B: same 100 cases of group A before and 3 months after successful interventions.

Group C: 100 cases of established cirrhosis.

Group D: 100 cases with chronic hepatitis C virus infection without established cirrhosis.

Group E: 100 healthy individuals as controls.

All patients in this study were subjected to: complete blood picture, liver biochemical profile, serum alanine aminotransferase (ALT), serum aspartate aminotransferase (AST), serum alkaline phosphatase, total and direct serum bilirubin, prothrombin time and activity, serum albumin blood urea nitrogen (BUN), serum creatinine. Fasting blood sugar. Serum alpha fetoprotein (AFP).

Determination of squamous cell carcinoma antigen (SCC-Ag) Sera from selected patients and controls were used for estimation of SCC-Ag using CanAg SCC EIA. The CanAg SCC EIA is a solid phase, non-competitive immunoassay based upon the direct sandwich technique. Calibrators and patient samples are incubated together with biotinylated Anti-SCC monoclonal antibody in Streptavidin coated microstrips. After washing buffered Substrate/Chromogen reagent (hydrogen peroxide and 3, 3', 5, 5' tetra-methylbenzidine) is added to each well and the enzyme reaction is allowed to proceed. During the enzyme reaction a blue colour will develop if antigen is present. The intensity of the colour is proportional to the amount of SCC present in the samples. The colour intensity is determined in a microplate spectrophotometer at $620 \mathrm{~nm}$ (or optionally at $405 \mathrm{~nm}$ after addition of Stop Solution). Calibration curves are constructed for each calibrator. The SCC concentrations of patient samples are the read from the calibration curve.

Results: Table 1 shows a statistical significant difference between different studied groups regarding alpha feto protein $(\mathrm{P}=0.000)$.

Table 1: Comparison Between Different Studied Groups Regarding Alpha Feto Protein

\begin{tabular}{lllll}
\hline & Mean & Std. deviation & Minimum & Maximum \\
\hline Gp. A & 263.0 & 96.02 & 150. & 438 \\
Gp. B & 209.4 & 64.7 & 145. & 380. \\
Gp. C & 154.5 & 48.16 & 75. & 210. \\
Gp. D & 7 & 1.82574 & 5 & 9 \\
Gp. E & 1.22 & 0.27406 & 0.8 & 1.6 \\
F & & & 38.208 & \\
P & & $0.000^{*}$ & \\
\hline
\end{tabular}

Table 2 shows a statistical significant difference between different studied groups regarding SCCA level $(\mathrm{P}=0.000)$.
Table 2: Comparison Between Different Studied Groups Regarding SCCA Score

\begin{tabular}{lllll}
\hline Mean & Std. deviation & Minimum & Maximum & \\
\hline Gp. A & 5.53 & 2.16 & 2.5 & 10. \\
Gp. B & 5.3 & 1.5 & 3.3 & 7.6 \\
Gp. C & 3.3 & 1.6 & 1.2 & 5.6 \\
Gp. D & 0.824 & 0.15897 & 0.6 & 1.05 \\
Gp. E & 0.646 & 0.23172 & 0.3 & 0.95 \\
F & & & 28.897 & \\
P & & & $0.000^{*}$ & \\
\hline
\end{tabular}

Also, Positive significant correlation was found between AFP and SCCA in both groups (Table 3).

Table 3: Correlation Between AFP and SCCA

AFP

HCC without intervention $\mathrm{HCC}$ with intervention

\begin{tabular}{llll}
\hline SCCA & $\mathrm{r}$ & $\begin{array}{l}0.629^{*} \\
<.525^{*} \\
<0.001\end{array}$ \\
\hline
\end{tabular}

Note. r: Pearson coefficient, *: Statistically significant at $\mathrm{p} \leq 0.05$

When combined sensitivity of both markers were calculated in our study at the best-chosen cutoff values (SCCA $3.2 \mathrm{ng} / \mathrm{ml}$ and AFP $200 \mathrm{ng} / \mathrm{ml}$ ) sensitivity improved to $93 \%$ (Table 4).

Table 4: AUC for AFP, SCCA and SCCA + AFP

\begin{tabular}{lll}
\hline & AUC & $\mathrm{p}$ \\
\hline AFP + SCCA & $0.930^{*}$ & 0.001 \\
AFP & $0.890^{*}$ & 0.003 \\
SCCA & $0.820^{*}$ & 0.016 \\
\hline
\end{tabular}

Conclusion: In the present study patients with HCC either with or without therapeutic intervention have significantly higher level of AFP in comparison to chronic HCV, cirrhotic and control groups this is in agreement with Awadallah et al.[6] who reported a statistically highly significant elevation in the serum AFP in HCC group when compared with control group. Moreover, the mean serum level of AFP in group A (HCC before intervention) was $263 \mathrm{ng}$ $\mathrm{ml}$ that decreased to $209.4 \mathrm{ng} / \mathrm{ml}$ in group B after therapeutic intervention and this agreed with Feng et al[7] and Molinari et al.[8] Also, at AFP level of $200 \mathrm{ng} /$ $\mathrm{ml}$, the sensitivity was $90 \%$, while the specificity was $60 \%$.

Our results showed that SCCA level ranged from 2.5-10 with a mean of 5.53 in HCC patients without interventions, 3.3-7.6 with a mean of 5.3 in patients with HCC with therapeutic interventions, 1.2-5.6 with a mean of 3.3 in cirrhotic group, $0.6-1.05$ with a mean of 0.824 in chronic $\mathrm{HCV}$ group while healthy control group had much lower values ranging from $0.3-0.95$ with a mean of 0.646 . Thus, a highly significant increase in serum SCCA level in patients with HCC before and after therapeutic intervention when compared to cirrhotic, chronic $\mathrm{HCV}$ and control groups $(\mathrm{P}<0.001)$. These results were in accordance with Hussein et al. [9] and El Ezawy et al.[10] SCCA was also higher among patients with $\mathrm{HCC}$ before intervention compared to patients with $\mathrm{HCC}$ after intervention as found by Bin et al.[11]

Applying the ROC curves analysis showed the best cut-off value to differentiate HCC patients from cirrhotic patients was $3.2 \mathrm{ng} / \mathrm{ml}$ for SCCA yielded $80 \%$ sensitivity and $90 \%$ specificity. These results were in agreement with Trevisani et al.[12] Patients with $\mathrm{HCC}$, in our study were none randomized selected as BCLC stage B (either one HCC lesion $<5 \mathrm{~cm}$ in size or 3 lesions $<3 \mathrm{cms}$ ) so no statistical correlation was done between serum AFP level and tumor size.

Our results showed a significant positive correlation between serum SCCA and AFP among patients with HCC before and after therapeutic intervention. Our data are in agreement with that of Hussein et al.[9] and El Ezawy et al.[10] who detected that SCCA were positively significantly correlated with AFP level. When combined sensitivity of both markers was calculated in our study at the best-chosen cutoff values (SCCA $3.2 \mathrm{ng} / \mathrm{ml}$ and AFP $200 \mathrm{ng} / \mathrm{ml}$ ) sensitivity improved to $93 \%$. Matching results were found by Gianluigi et al.[4]

Disclosure of Interest: All authors have declared no conflicts of interest.

\section{References}

1 Lok, A., Seeff, L., \& Morgan, T. (2009). Incidence of hepatocellular carcinoma and associated risk factors in hepatitis $\mathrm{C}$ related advanced liver disease. Gastroenterology, 136, 138-48.

2 El-Zayadi, A., \& Badran, H. (2005). Hepatocellular carcinoma in Egypt: A single center study over a decade. World J Gastroenterol, 11, 5193-8.

3 Peng, S.Y., Chen, W., \& Lai, P., et al. (2004). High alpha-fetoprotein level correlates with high stage, early recurrence and poor prognosis of hepatocellular carcinoma: Significance of hepatitis virus infection, age 53 and betacatenin mutations. Int $J$ Cancer, 112, 44-50. 
4 Giannelli, G., \& Antonaci, S. (2011). New frontiers in biomarkers for hepatocellular carcinoma. Dig Liver Disease, 38, 854-9.

5 Biasiolo, A., Chemello, L., \& Quarta, S. (2008). Squamous cell carcinoma antigen (SCCA) detection in patients with HCV infection and rheumatoid factor seropositivity. $J$ Viral Hepat, 15, 246-9.

6 Issa, H., Awadallah, A., \& Soliman, M. (2011). Evaluation of serum chromogranin A as a useful tumor marker for diagnosis of hepatocellular carcinoma. Journal of American Science, 7, 999-1007.

7 Feng, W., Wang Z. B., \& Meng, W. C., et al. (2004). Extracorporeal high intensity focused ultrasound ablation in the treatment of patients with large hepatocellular carcinoma. Surgical Onco, 11, 1061-69.

8 Molinari, M., Kachuray, J., Dixonz, ., Suehiro, Y., Morioka, H., \& Fordtran, B., et al. (2006). Transarterial chemoembolisation for advanced hepatocellular carcinoma: Results from a North American Cancer Centre. Clinical Oncology, 18, 684-92.

9 Hussein, M., Ibrahim, A., \& Abdella, H. (2008). Evaluation of serum squamous cell carcinoma antigen as a novel biomarker for diagnosis of hepatocellular carcinoma in Egyptian patients. Indian J Cancer, 45, 167-72.

10 El Ezawy, H., Shebil, N., \& Mounis, A. (2012). Assessment of serum SCCA and KL-6 as tumor markers and their correlation with tumor size. Journal of American science, 8, 172-9.

11 Bin, X., Fang, G. S., Liu, S, H., Kim, T., Takahashi, S. (2008). SCCA level in peripheral blood in patients with hepatocellular carcinoma before and after TACE. J Huazhong Univ Sci Technol 28, 645-8.

12 Trevisani, F., Daniela, B., \& Gianluca, F. (2012). Serum SCCA as a predictor of hepatocellular carcinoma in patients with liver cirrhosis. Open Journal of Gastroenterology, 2, 56-61.

\section{P0068 HEPATOCELLULAR CARCINOMA MULTIDISCIPLINARY CLINIC - CAIRO UNIVERSITY (HMC-CU) SCORE; A NEW SIMPLE SCORE FOR EARLY DIAGNOSIS OF HCC}

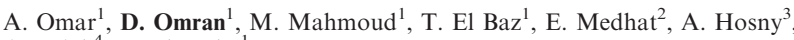
A. Salah ${ }^{4}$, H. Shousha ${ }^{1}$

${ }^{1}$ Department Of Endemic Medicine And Hepatogastroenterology, Faculty of medicine, Cairo University, Cairo/Egypt

${ }^{2}$ Endemic Medicine, Cairo/Egypt

${ }^{3}$ Dep. of Radiodiagnosis, Cairo/Egypt

${ }^{4}$ Department of Surgery, Cairo/Egypt

Contact E-mail Address: daliaomran@kasralainy.edu.eg

Introduction: Hepatocellular carcinoma $(\mathrm{HCC})$ is the first most common primary malignant tumor of the liver, the fifth common cancer and the third most common cause of cancer-related death worldwide. (1) Early detection of HCC provides the best chance for a curative treatment which in turn improves patients survival. However, more than $60 \%$ of HCCs are diagnosed at a late stage (2). This could be explained by poor compliance of cirrhotic patients to the surveillance programs and lack of a sensitive and specific tumor marker. Serum AFP commonly used for $\mathrm{HCC}$ diagnosis has a low sensitivity, and specificity for $\mathrm{HCC}$ detection(3)

Aims \& Methods: The aim of this study was to develop Hepatocellular Multidisciplinary clinic - Cairo University (MHC-CU) score and test its accuracy in HCC detection in comparison to the widely used AFP In the current study, we reviewed the data of 2363 Egyptian patients with HCV genotype-4 related chronic liver disease CLD; 1291 patients were diagnosed to have HCC while 1072 patients were diagnosed to have HCV related liver cirrhosis with no HCC on top. All the patients were recruited from the HCC multidisciplinary clinic, Cairo University Focal hepatic lesions detected by abdominal ultrasound (US) and/or rising AFP assays were further evaluated by tri- phasic multiphase computed tomography (CT) or contrast-enhanced magnetic resonance imaging (MRI). Lesions showing enhancement in the arterial phase were diagnosed as HCC. Diagnosis of HCV related liver cirrhosis was based on clinical, laboratory and imaging evidence of chronic liver disease in addition to detection of HCV antibodies and HCV RNA by PCR technique. Each patient signed an Informed consent and the study was carried out according to the ethical guide-lines of 1975 Helsinki Declaration.

Results: On bivariate analysis, the HCC patients were significantly elder, anemic and showed significant thrombocytopenia, hyper- bilirubinemia, elevated serum AST and AFP serum levels. Serum albumin was significantly lower in HCC patients. Consequently, we entered these significant variables in a multivariate regression model that demonstrated that only age, gender, hemoglobin, albumin, AFP and INR were independently associated with HCC development.

Table: Multivariate analysis for calculation of the HMC-CU score.

\begin{tabular}{rllll}
\hline & \multicolumn{3}{l}{$95 \%$ C.I. } \\
\cline { 3 - 4 } & OR & Lower & Upper & P- value \\
\hline Age Gender Hb INR & 1.164 & 1.141 & 1.188 & $<.001$ \\
Alb AFP Constant & 2.653 & 1.959 & 3.594 & $<.001$ \\
& 0.886 & .819 & .959 & .003 \\
& 0.498 & .267 & .931 & .029 \\
& 0.347 & .268 & .448 & $<.001$ \\
& 1.022 & 1.016 & 1.028 & $<.001$ \\
& 0.080 & & & .010 \\
\hline
\end{tabular}

The best overall formula that could best predict HCC was then constructed Hepatocellular Multidisciplinary clinic - Cairo University (MHC-CU) score is as following: Logit probability of $\mathrm{HCC}=-2.524+0.152 \times$ age $-0.121 \times \mathrm{Hb}-$ $0.696 \times$ INR $-1.059 \times \mathrm{Alb}+0.022 \times \mathrm{AFP}+0.976 \times$ Gender Gender Male $=1$ Female $=0$ The diagnostic value of HMC-CU was then assessed by ROC curve At a cutoff point of $0.56 \mathrm{HMC}-\mathrm{CU}$ enabled the correct identification of patients with HCC with $90 \%$ sensitivity, $80.6 \%$ specificity. AUC was 0.93 and the $95 \%$ confidence interval was $0.917-0.94$. On comparing the diagnostic performance of $\mathrm{HMC}-\mathrm{CU}$ to the performance of serum AFP for early diagnosis of HCC, it was found that serum AFP was able to diagnose HCC at cutoff value of $11.9 \mathrm{ng} / \mathrm{ml}$ with sensitivity of $68 \%$ and specificity $66 \%$. AUC was 0.76 and the $95 \%$ confidence interval was $0.74-0.78$

Conclusion: The HMC-CU score constructed from routine parameters is accurate in early diagnosis of HCC in patients with HCV-related CLD. The elegancy of our score is based on its simplicity, being based on routine laboratory parameters and serum AFP which is being used for screening of patients in many centers all over the world. Our score will not impose extra costs for the patients because it utilizes only routine laboratory parameters. The HMC-CU score may be useful during surveillance programs for HCC. Our study included large number of $\mathrm{HCC}$ and non HCC patients all are Egyptians with a background of HCV type 4 related CLD. A prospective validation study is being planned and further studies are invited to validate this score on patients of other races infected with other HCV genotypes.

Disclosure of Interest: All authors have declared no conflicts of interest.

\section{References}

1. Llovet JM, et al. Hepatocellular carcinoma. Lancet 2003; 362: 1907-17.

2. Singal AG, et al. Utilization of hepatocellular carcinoma surveillance among american patients: a systematic review. J Gen Intern Med 2012;27:861-7

3. Bruix $\mathrm{J}$ and Sherman M. Management of hepatocellular carcinoma. Hepatology 2005; 42:1208-1236

\section{P0069 MULTIPLE BIPOLAR RADIOFREQUENCY ABLATION IN TREATMENT OF MEDIUM TO LARGE HEPATOMAS - EXPERIENCE IN A REGIONAL HOSPITAL}

\section{P. Hsieh, C. Yen}

Division Of Gastroenterology And Hepatology, Chang-Gung Memorial Hospital Keelung branch, Keelung/Taiwan

Contact E-mail Address: b8802050@gmail.com

Introduction: Monopolar radiofrequency ablation (RFA) treatment for hepatomas has been unsatisfactory with local tumor recurrent rates of $8-40 \%$ in tumors $\geq 3 \mathrm{~cm}$. Bipolar RFA devices have been developed to overcome the limitations of monopolar RFA devices.

Aims \& Methods: This study aimed to evaluate the therapeutic effect and long term survival in medium and large hepatomas using multiple bipolar radiofrequency ablation system (Celon). The study subjects had a Child-Pugh classification of A or B. The patients who were ineligible for surgical intervention or preferred RFA over surgery were treated with percutaneous bipolar electrodes. A total of 30 consecutive cirrhotic patients (48 tumors) with hepatomas $>3 \mathrm{~cm}$ were enrolled between January 2011 and November 2012. The follow-up period was 30 months after RFA. The complete necrosis rate, tumor recurrence rate and long-term survival rate were analyzed and compared between medium and large hepatomas using Kaplan-Meier survival and the prognostic factors were using multivariate analysis.

Results: 30 patients were divided equally into two groups with 15 patients in each of medium and large hepatoma groups. 17 patients underwent artificial ascites $(56.3 \%)$. The complete necrosis rate after ablation was $93.3 \%$ (14/15 patients) for either medium or large hepatomas. The local tumor progression rate and distant tumor recurrence rate of $40 \%$ and $60 \%(p=0.098)$ vs $73.3 \%$ and $80 \%(p=0.652)$ for medium and large hepatomas during 30 months follow up were not statistically significant. The overall survival rates of $80 \%, 66.7 \%$ vs $60 \%, 46.7 \%$ at 12 and 30 months, respectively, was not statistically significant $(p=0.390)$. By multivariate analysis, $\mathrm{BCLC}$ stage $(\mathrm{HR}=3.904, p=0.023)$, MELD score $(\mathrm{HR}=1.220$, $p=0.021)$ and pre-treatment AST level $(\mathrm{HR}=1.028, p=0.019)$ were independent prognostic factors for overall survival.

Conclusion: Multiple bipolar RFA system can achieve high complete tumor necrosis rate and low complication rates in treating medium to large hepatomas with shorter duration. Pre-treatment BCLC stage, MELD score and AST level were independent prognostic factors for overall survival. The therapeutic effect and long term survival for large hepatomas $(\geq 5 \mathrm{~cm})$ was not inferior to that of medium hepatomas by multiple bipolar RFA.

Disclosure of Interest: All authors have declared no conflicts of interest.

\section{References}

1. Bruix J, Sherman M, Practice Guidelines Committee AAftSoLD. Management of hepatocellular carcinoma. Hepatology 2005;42:1208-36.

2. Ryder SD, British Society of G. Guidelines for the diagnosis and treatment of hepatocellular carcinoma (HCC) in adults. Gut 2003;52 Suppl 3:iii1-8.

3. Abou-Alfa GK, Johnson P, Knox JJ, et al. Doxorubicin plus sorafenib vs doxorubicin alone in patients with advanced hepatocellular carcinoma: a randomized trial. JAMA 2010;304:2154-60.

4. Buscarini E, Savoia A, Brambilla G, et al. Radiofrequency thermal ablation of liver tumors. Eur Radiol 2005:15:884-94. 
5. Lu DS, Yu NC, Raman SS, et al. Radiofrequency ablation of hepatocellular carcinoma: treatment success as defined by histologic examination of the explanted liver. Radiology 2005;234:954-60.

6. Livraghi T, Goldberg SN, Lazzaroni S, et al. Hepatocellular carcinoma: radio-frequency ablation of medium and large lesions. Radiology 2000;214:761-8

7. Meijerink MR, van den Tol P, van Tilborg AA, et al. Radiofrequency ablation of large size liver tumours using novel plan-parallel expandable bipolar electrodes: initial clinical experience. Eur J Radiol 2011;77:167-71.

8. Osaki Y, Ikeda K, Izumi N, et al. Clinical effectiveness of bipolar radiofrequency ablation for small liver cancers. J Gastroenterol 2013;48:874-83.

9. Cartier V, Boursier J, Lebigot J, et al. Radiofrequency ablation of hepatocellular carcinoma: Mono or multipolar? J Gastroenterol Hepatol 2016;31:654-60

10. Seror O, N'Kontchou G, Ibraheem M, et al. Large ( $>$ or=5.0-cm) HCCs: multipolar RF ablation with three internally cooled bipolar electrodesinitial experience in 26 patients. Radiology 2008;248:288-96.

11. Peng ZW, Liang HH, Chen MS, et al. Conformal radiofrequency ablation of hepatocellular carcinoma with a multi-pin bipolar system. J Surg Oncol 2011;103:69-74.

\section{P0070 RECENT TRENDS IN HEPATOCELLULAR ADENOMAS: CLINICAL FEATURES, DIAGNOSIS AND OUTCOMES}

M. Silva, A. Peixoto, R. Coelho, H. Cardoso, G. Macedo

Gastroenterology, Hospital São João, Oporto/Portugal

\section{Contact E-mail Address: marcocostasilva87@gmail.com}

Introduction: Hepatocellular adenomas (HCA) are rare, benign tumors of presumable epithelial origin, that occur predominantly, but not exclusively, in young women taking oral contraceptives (OC) or other estrogens. The Bordeaux adenoma tumour markers are a promising method of identifying the high-risk HCA of malignant transformation into hepatocellular carcinoma (HCC).

Aims \& Methods: Aims: The authors propose to evaluate the demographics, etiology, clinical manifestations and prognosis of HCA.

We undertook retrospective analysis of patients with HCA, histologically confirmed (by guided biopsy or surgical resection), between 2008 and 2016, in a tertiary referral centre. When feasible, the subtype classification of HCA proposed by the Bordeaux group, was performed. Descriptive statistics, uni and multivariate analysis were performed using IBM SPSS Statistics 22, with $\mathrm{p}<0.05$ deemed to be statistically significant.

Results: In this study 27 patients were included, 2 man and 25 women, with a median age of $38 \pm 11$ years, followed for a mean time of $78 \pm 36$ weeks (lost follow-up in 7 cases). Three cases of hepatic adenomatosis were included. Fortyone percent of the women used OC and $38 \%$ of the patients had dyslipidemia The mean size of the HCA was $70 \pm 42 \mathrm{~mm} ; 63 \%$ of the patients had abnormal liver tests at diagnosis, $46 \%$ were symptomatic and in $21 \%$ the diagnosis was performed due to ruptured HCA. Surgical resection was performed in $88 \%$ of the cases; complete resection was achieved in $75 \%$ of the cases. Of the 19 patients who performed abdominal-CT scan or abdominal-MRI before histological confirmation, only $29 \%$ had an imagiological diagnosis of HCA. In $12(44 \%)$ cases, immunohistochemical analysis was performed. According to the Bordeaux classification of HCA, $8(67 \%)$ cases were classified as inflammatory, $2(17 \%)$ as HNF-1 $\alpha$-mutated, $1(8 \%)$ as $\beta$-catenin mutated and $1(8 \%)$ as unclassified. During follow-up, $1(4 \%)$ patient died due to hemorrhagic shock related with HCA rupture and in $2(10 \%)$ was necessary surgical revision due to incomplete resection. There was no HCC cases diagnosed during the follow-up. The median size of the HCA that weren't completely resected and also of those presenting with HCA rupture was significantly higher: $(110 \mathrm{vs} 55 \mathrm{~mm}[\mathrm{p}=0.035]$ and $105 \mathrm{vs}$ $47 \mathrm{~mm}[\mathrm{p}=0.037]$, respectively). The 2 male patients had inflammatory HCA $(\mathrm{p}=0.011)$.

Conclusion: In this cohort, HCA were prevalent in female taking OC and the inflammatory type HCA was the most common. In many cases, abdominal imaging is insufficient for a correct diagnosis, and biopsy specimen or surgical resection should be performed for a correct diagnosis. Lesion size was associated with the risk of rupture and incomplete surgical resection.

Disclosure of Interest: All authors have declared no conflicts of interest.

\section{Reference}

1. Bioulac-Sage P, Laumonier H, Couchy G, Le Bail B, Sa Cunha A, Rullier A. Hepatocellular Adenoma Management and Phenotypic Classification: the Bordeaux Experience. Hepatology. 2009;50:481-9.

\section{P0071 LASER ABLATION IS SUPERIOR TO TACE IN LARGE SIZED HEPATOCELLULAR CARCINOMA: A CASE-CONTROL STUDY}

S. Camera ${ }^{1}$, G.G. Di Costanzo ${ }^{2}$, R. Tortora ${ }^{3}$, M. Guarino ${ }^{4}$, V. Cossiga ${ }^{4}$, A. Vitiello ${ }^{4}$, G. Cordone ${ }^{2}$, N. Caporaso ${ }^{4}$, F. Morisco ${ }^{4}$, I.1.c. Italian Liver Cancer Group 5

${ }^{1}$ Department Of Clinical Medicine And Surgery, Gastroenterology Unit, AOU

Federico II, NAPLES/Italy

${ }^{2}$ Hepatology Unit, Cardarelli Hospital, Naples/Italy

${ }^{3}$ Hepatology Unit, Cardarelli Hospital, NAPLES/Italy
${ }^{4}$ Department Of Clinical Medicine And Surgery, Gastroenterology Unit AOU FEDERICO II, Naples/Italy

${ }^{5}$ Department Of Medical And Surgical Sciences Semeiotica Medica, Alma Mater Studiorum-University of Bologna, Bologna/Italy

Contact E-mail Address: silvia.camera84@gmail.com

Introduction: Currently, the standard treatment using transarterial chemo-embolisation (TACE) for patients showing solitary large $(\geq 40 \mathrm{~mm})$ hepatocellular carcinoma (HCC) is unsatisfactory with high rate of recurrence. Data from literature suggest the alternative use of thermal ablation.

Aims \& Methods: We aimed to evaluate the efficacy and safety of Laser Ablation (LA) in comparison to TACE in patients with large tumor size HCC. Between January 2009 and December 2012, 41 cirrhotic patients (29/12 M/F; median age 72 yrs, range 54-88, Child-Pugh A/B: $37 / 4$ ) with a single nodule of $\mathrm{HCC} \geq 40$ (median size $46 \mathrm{~mm}$, range 40-75) were enrolled in this study. The patients were treated with multifiber technique of LA. The control group consisting of 41 patients $(29 / 12 \mathrm{M} / \mathrm{F}$; mean age $72 \mathrm{yrs}$, range 49-86; Child-Pugh A/B: 34/7; median size of the nodule $50 \mathrm{~mm}$, range $40-80$, treated with TACE, was obtained from the ITA.LI.CA database and observed in the same period of time. No statistically significant difference between the 2 groups was observed. The diagnosis of HCC was done according to the international guidelines and patients were staged according to BCLC Staging System (BCLC stage A/B: 27/14 and 18 23, for LA and TACE, respectively). Response to therapy was evaluated according to the mRECIST criteria. Survival was calculated from the time of cancer diagnosis to death with values censored at the date of the last follow-up.

Results: Twenty-six $(63.4 \%)$ patients of LA group and $8(19.5 \%)$ patients of TACE group showed a complete response after treatment $(\mathrm{p}<0.001)$. The superior efficacy of LA was confirmed in all categories, also after the stratification of the nodules according to the nodules size $(40-50 \mathrm{~mm}, 51-60 \mathrm{~mm}$ and $>60 \mathrm{~mm}$ ). Disease recurrence, during a mean \pm SD period of follow-up of $37.4 \pm 20.7$ months, was observed in $13(24 \%)$ LA-treated patients $(24 \%)$ and in 24 $(58.5 \%)$ TACE-treated patients $(\mathrm{p}=0.00515)$. Overall survival probability rate at 1 and 3 years was $90.2 \%$ and $55.4 \%$ in LA group and 85.4 and 48.8 . in TACE group.

Conclusion: LA is a more efficacious therapeutic option than TACE in patients with solitary large $\mathrm{HCC}$.

Disclosure of Interest: All authors have declared no conflicts of interest.

\section{References}

1. Di Costanzo GG, Tortora R, G D'Adamo et al. Radiofrequency ablation versus laser ablation for the treatment of small hepatocellular carcinoma in cirrhosis: a randomized trial. J. Gastroenterol. Hepatol. 2015;30:559-565.

2. Di Costanzo GG, D'Adamo G, Tortora R, Zanfardino F, Mattera S, Francica G, Pacella CM. A novel needle guide system to perform percutaneous laser ablation of liver tumors using the multifiber technique. Acta Radiol 2013; 54: 876-881

3. Di Costanzo GG, Francica G, Pacella CM. Laser ablation for small hepatocellular carcinoma: State of the art and future perspectives. World J Hepatol. 2014 Oct 27;6(10):704-15. doi: 10.4254/wjh.v6.i10.704. Review.

\section{P0072 ENDOSCOPIC ULTRASOUND GUIDED BIOPSY FOR LIVER} MASS USING CORE BIOPSY NEEDLE

H.K. Chon ${ }^{1}$, T.H. Kim ${ }^{1}$, K.H. Choi ${ }^{2}$

${ }^{1}$ Internal Medicine, Wonkwang College of Medicine, Iksan/Korea, Republic of ${ }^{2}$ Pathology, Wonkwang College of Medicine, Iksan/Korea, Republic of

Contact E-mail Address: ktw@wku.ac.kr

Introduction: Endoscopic ultrasound (EUS)-guided fine needle aspiration (EUSFNA) is one of the alternative methods for tissue sampling of liver solid mass. However, the diagnostic efficacy using cytology alone was limited.

Aims \& Methods: In this study, we evaluated the feasibility and diagnostic accuracy of EUS-guided fine needle biopsy (EUS-FNB) for hepatic solid masses in patients with suspected malignancy. The EUS-FNB using $20 \mathrm{G}, 22 \mathrm{G}$ or $25 \mathrm{G}$ ProCore needle (PCN) was performed to evaluate the patient with solid liver mass. The primary outcome was the diagnostic accuracy of EUS-FNB for malignancy, and adequacy of the specimen for histology. The secondary outcomes were (1) the proportions of patients in whom immunohistochemical (IHC) stain was possible, and (2) compared diagnostic yield of FNB according to the needle size, and (3) safety of EUS-FNB

Results: Forty-one patients (13 women; mean age, $67.9 \pm 10.3$ years [range, 46 86]) underwent evaluation with EUS and identified hepatic lesions ranging in size from $0.7 \mathrm{~cm}$ to $15 \mathrm{~cm}$. EUS-FNB with $20 \mathrm{G}(\mathrm{n}=10), 22 \mathrm{G}(\mathrm{n}=24)$ or $25 \mathrm{G}$ PCN $(n=7)$ was performed (right lobe: $n=10$, left lobe: $n=31)$. The median number of needle passes was $2.4 \pm 0.8$ (range, 1-5). Technical success rates for tissue acquisition were $97.6 \%$, but both specimen adequacy for histology and available IHC stain was $92.6 \%$. Three $(7.3 \%)$ were non-diagnostic and subsequently proved to be malignant; 2 by smear cytology and 1 after surgical resection. The diagnostic yield, sensitivity and specificity of EUS-FNB for the diagnosis of malignancy were $92.6 \%, 92.6 \%$ and $100 \%$, respectively. The diagnostic yield in $20 \mathrm{G} \mathrm{PCN}$ and $22 \mathrm{G} \mathrm{PCN}$ was significantly superior to the $25 \mathrm{G}$ PCN $(p=0.045)$. There was one bleeding complication, but controlled with endoscopic hemostasis with endoclips.

Conclusion: EUS-FNB with core biopsy needle may be a safe and useful modality in the management of patients with hepatic solid mass. Moreover, 20G and 22G FNB may be adequate for liver biopsy.

Disclosure of Interest: All authors have declared no conflicts of interest. 


\section{References}

1. Lee YN, Moon JH, Kim HK, Choi HJ, Choi MH, Kim DC, et al. Usefulness of endoscopic ultrasound-guided sampling using core biopsy needle as a percutaneous biopsy rescue for diagnosis of solid liver mass: Combined histological-cytological analysis. J Gastroenterol Hepatol. 2015;30(7):1161-6.

2. Wang L, Vuolo M, Suhrland MJ, Schlesinger K. HepPar1, MOC-31, pCEA, mCEA and CD10 for distinguishing hepatocellular carcinoma vs. metastatic adenocarcinoma in liver fine needle aspirates. Acta Cytol. 2006;50(3):257-62.

3. Schulman AR, Thompson CC, Odze R, Chan WW, Ryou M. Optimizing EUS-guided liver biopsy sampling: comprehensive assessment of needle types and tissue acquisition techniques. Gastrointest Endosc. 2017;85(2):419-26.

\section{P0073 COMPARISON OF METHODS TO ESTIMATE LIVER FUNCTION IN NEWLY-DIAGNOSED HEPATOCELLULAR CARCINOMA PATIENTS WITH ASCITES}

Y. Ko

Internal Medicine, Samsung Medical Center, Seoul/Korea, Republic of

\section{Contact E-mail Address: bs2-32@hanmail.net}

Introduction: Liver function is a key element in determining outcome of patients with hepatocellular carcinoma (HCC). For HCC with ascites, estimation of liver function is particularly important, as they already have decreased liver function. Aims \& Methods: We aimed to find out best method to predict outcome of HCC patients with ascites. A total of 437 newly-diagnosed HCC patients with ascites (mean age $=56.0 \mathrm{y}$, male $=74.8 \%$, hepatitis $\mathrm{B}$ virus $=73.2 \%$ ) were analyzed. $\mathrm{We}$ compared Child-Pugh score, Model for End-Stage Liver Disease (MELD) score, MELD-Na score, and the Albumin-bilirubin (ALBI) grade for overall survival. Results: During a median 9.0 months of follow-up (range; $0.1 \sim 154.0$ ), mortality was observed in $373(85.4 \%)$ patients. MELD-Na showed highest time-dependent area under receiver-operating characteristics curves (AUROCs) at 1 year $(0.672)$ that was significantly higher than ALBI grade (0.605), MELD score $(0.580)$, and Child-Pugh score $(0.580)$. The median survival was significantly lower for those with MELD-Na $>12$ than MELD-Na $<12$ (median: 13.6 vs. 3.7 months, $\mathrm{p}<0.001$ ). Overall, 350 patients received treatment, and most commonly used modality was transarterial chemoembolization $(62.3 \%)$, followed by radiofrequency ablation $(15.7 \%)$ and resection $(13.4 \%)$. Overall survival was significantly different for those who received treatment than those who did not (median survival: 13.3 vs. 2.4 months, $\mathrm{p}<0.001$ ). When patients were further stratified by mUICC stage and MELD-Na score, treatment was not associated with better outcome for mUICC stage IV patients with MELD-Na $>12$ (median survival: 2.2 vs. 1.8 months for treatment vs. best supportive care, $\mathrm{p}=0.15$ ), while treatment was associated with better outcome in other subgroups.

Conclusion: In HCC patients with ascites, treatment was associated with better survival, except for subgroup with advanced tumor with decreased liver function, indicating that ascites per se is not absolute contraindication for HCC treatment. For these patients, MELD-Na showed better performance than MELD, ChildPugh score and ALBI score for predicting overall survival.

Disclosure of Interest: All authors have declared no conflicts of interest.

\section{P0074 DIAGNOSTIC AND PROGNOSTIC ROLE OF SQUAMOUS} CELL CARCINOMA ANTIGEN IN HEPATOCELLULAR CARCINOMA: SEROLOGICAL AND TISSUE DETERMINATION

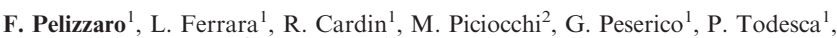
A. Imondi ${ }^{1}$, C. Pozzan ${ }^{3}$, F. Farinati ${ }^{1}$

${ }^{1}$ Department Of Surgery, Oncology And Gastroenterology, University of Padova, Padova/Italy

${ }^{2}$ Uos Sperimentazioni Cliniche, Biostatistica E Nucleo Ricerca Clinica, Istituto Oncologico Veneto IOV-IRCCS, Padova/Italy

${ }^{3}$ Gastroenterology, Ospedale dell'Angelo, Venezia-Mestre/Italy

Contact E-mail Address: filippo.pelizzaro@gmail.com

Introduction: The ideal serological marker in hepatocellular carcinoma (HCC) has not been identified yet since Alphafetoprotein (AFP) has unsatisfactory characteristics. Squamous Cell Carcinoma Antigen (SCCA) is expressed in tissue and serum of HCC patients and when determined immunocomplexed with IgM (SCCA-IgM) has satisfactory diagnostic and prognostic performance. Aims \& Methods: Aim of our study was to evaluate, in HCC patients, the diagnostic and prognostic role of SCCA determination in tissue and in serum samples. SCCA-IgM levels were determined in 409 sera obtained from $196 \mathrm{HCC}$ patients and 213 cirrhotics. SCCA tissue expression was analyzed in a subgroup of 62 patients with biopsy available at diagnosis. Sensitivity, specificity, correlation with clinical and tumor parameters, response to treatment and survival were evaluated.

Results: HCC patients had SCCA-IgM levels significantly higher than cirrhotics $(\mathrm{P}<0.0001)$. Sensitivity, specificity, positive and negative predictive values were $76 \%, 52 \%, 60 \%$ and $70 \%$, respectively. In comparison, sensitivity and specificity of AFP were $54 \%$ and $85 \%$. SCCA-IgM values were correlated to HCC etiology and size. A cut-off of $130 \mathrm{AU} / \mathrm{mL}$ (validated in literature) was used for the prognostic analysis: patients with SCCA-IgM levels $<130 \mathrm{AU} / \mathrm{mL}$ showed a slightly better survival $(\mathrm{p}=\mathrm{ns})$; in long-term survivors $(>24$ months) and in Child A patients the difference in survival was statistically significan ( $\mathrm{p}=0.036$ and $\mathrm{p}=0.01$, respectively). A drop in SCCA-IgM levels after TACE correlated with mRECIST response to treatment. Child-Pugh status was the only independent predictor of survival at Cox multivariate analysis. A better survival trend in HCC with low serpin tissue expression was documented (31 vs 24 months, $\mathrm{p}=\mathrm{ns}$ ). No correlation was found between tissue and serum levels. Stratifying patients by gender, SCCA-IgM levels showed an opposite behavior in the prognostic prediction: in males a better survival was documented with levels $<130 \mathrm{AU} / \mathrm{mL}$ while in females with SCCA-IgM $>130 \mathrm{AU} / \mathrm{mL}$. In males, SCCA-IgM levels and Child-Pugh status were identified as independent predictors of survival. SCCA-IgM levels were directly correlated with tumor size and BCLC stage in females and with etiology in males.

Conclusion: SCCA-IgM is a sensitive marker of HCC but lacks in specificity. As reported in literature, SCCA-IgM levels identify patients with better prognosis (especially in long-term survivors) and the marker confirms its ability in detecting treatment responders. Lower levels of tissue SCCA seem to be related with less aggressive tumors, given that the longer survival of these patients. The genderbased differences observed in our patients, especially with respect to the SCCAIgM prognostic role and correlation with clinical and tumor parameters, are intriguing and focused studies on the point are needed.

Disclosure of Interest: All authors have declared no conflicts of interest.

\section{P0075 ADHERENCE TO BARCELONA CLINIC LIVER CANCER GUIDELINES IN FIELD-PRACTICE: RESULTS OF PROGETTO EPATOCARCINOMA CAMPANIA}

F. Morisco ${ }^{1}$, M. Guarino ${ }^{1}$, A. Vitiello ${ }^{1}$, R. Tortora ${ }^{2}$, G. De Stefano ${ }^{3}$, C. Coppola ${ }^{4}$, A. Salomone Megna ${ }^{5}$, F. Izzo ${ }^{6}$, G. Nardone ${ }^{1}$, L.E. Adinolfi ${ }^{7}$, G. D'Adamo ${ }^{8}$, G.B. Gaeta ${ }^{9}$, V. Messina ${ }^{10}$, P. Guido ${ }^{10}$, G. Francica ${ }^{11}$, V. De Girolamo $^{12}$, N. Coppola ${ }^{9}$, M. Persico ${ }^{13}$, G.G. Di Costanzo

${ }^{1}$ Università Federico II, Napoli/Italy

${ }^{2} A O R N$ Cardarelli, Napoli/Italy

${ }^{3}$ AORN dei Colli- PO Cotugno, Napoli/Italy

${ }^{4}$ OO.RR Area Stabiese, Gragnano/Italy

${ }^{5}$ AO. G. Rummo, Benevento/Ital

${ }^{6}$ IRCCS Fondazione Pascale, Napoli/Italy

${ }^{7}$ Seconda Università di Napoli, Caserta/Italy

${ }^{8}$ P.O. Umberto I Nocera Inferiore/Italy

${ }^{9}$ Seconda Università di Napoli, Napoli/Italy

${ }^{10}$ AORN Sant'Anna e San Sebastiano, Caserta/Italy

${ }^{11}$ PO Pineta Grande, Castelvolturno/Italy

${ }^{12}$ PSI Napoli EST - ASL NA 1, Napoli/Italy

${ }^{13}$ Università di Salerno, Salerno/Italy

Contact E-mail Address: anna-vitiello@hotmail.i

Introduction: The BCLC algorithm is the standard system for clinical management of HCC. Data on adherence to this therapeutic paradigm are scarce. The aim of this field-practice study is to provide a description of HCC patients in Southern Italy, to evaluate the adherence to BCLC guidelines and its impact on patients' survival.

Aims \& Methods: We analyzed the region-wide Italian database of Progetto Epatocarcinoma Campania, which includes data of HCC patients, prospectively collected from January 2013 to December 2015 in 16 regional centers.

Results: Overall 1008 HCC patients were enrolled: $70.6 \%$ patients received therapies recommended by BCLC algorithm, while $29.4 \%$ underwent different treatments. Among patients who were treated in adherence to guidelines, a higher rate of diagnosis on surveillance programs, better liver function, lower rate of AFP $>200 \mathrm{ng} / \mathrm{ml}$, more early stage and monofocal HCC, lower frequency of nodules $>5 \mathrm{~cm}$, portal vein thrombosis and metastases were observed. The multivariate analysis showed that non-adherence to treatment guidelines was independently associated to the BCLC stage B, Child-Pugh classes B-C, and to the presence of neoplastic thrombosis and metastases. The mean overall survival in patients treated according to BCLC indications was 35.5 months, while in patients managed differently was 31.9 months $(\mathrm{p}<0.0001)$.

Conclusion: Adherence to BCLC algorithm in field-practice was high in early and end stage HCC patients, but it was poor in intermediate and advanced patients. This may be due to the wide heterogeneity of intermediate-stage patients, and to the limited use of sorafenib in advanced-stage patients. Strategies to improve treatment and stratification of $\mathrm{HCC}$ patients are required

Disclosure of Interest: All authors have declared no conflicts of interest.

\section{P0076 A QUESTIONNAIRE SURVEY ON QUALITY OF LIFE WITH ANXIETY AND DEPRESSION SELF-RATING IN PATIENTS OF LIVER CIRRHOSIS}

Y. Zhang ${ }^{1}, \mathrm{X} \cdot \mathrm{Li}^{1}, \mathrm{~N} . \mathrm{Cui}^{2}, \mathrm{Y}$. L. Liu

${ }^{1}$ Dept. Of Gastroenterology, Peking University People's Hospital, Beijing/China ${ }^{2}$ Dept. Of Respiratory, Beijing Hospital of Chineses Traditional and Western Medicine, Beijing/China

Contact E-mail Address: medicalyuan@qq.com

Introduction: Liver cirrhosis is a great public health burden for Chinese health system. The most common causese are HBV, HCV, alcohol consumption and non-alcoholic fatty liver disease, et al. The quality of life of liver cirrhosis patients is impacted by the physical symptoms and psychological symptoms such as anxiety as depression.

Aims \& Methods: We aimed to investigate the quality of life of patients with cirrhosis, as well as depression and anxiety. A questionnaire survey was carried out in 95 patients in our gastroenterology department, Peking University People's Hospital from May to August in 2016. The patients were divided into two groups, cirrhosis group and contral group. The patients in cirrhosis group 
were diagnosed liver cirrhosis without complications. The control group included the digestive polyps patients without other diseases. The questionnaire included the World Health Organization Quality of Life (WHOQOL)-BREF, Self-rating Anxiety Scale (SAS) and Self-rating Depression Scale (SPS). The questionnaire scores of the two groups were analysized.

Results: A total of 95 valid questionnaires were collected and divided into cirrhosis group $(n=40)$ and control group $(n=45)$. In the cirrhosis group, there were 22 males and 18 females, average age $57.97 \pm 10.448$ years. In the control group, there were 45 males, 23 males and 22 females, with an average age of $61.47 \pm 13.081$, showing no difference from cirrhosis group. WHOQOL includes four domains: physiological domain, psychological domain, social relationship domain, environment domain. The scores of liver cirrhosis group: physiological field (22.23 \pm 3.312$)$, psychological field $(19.59 \pm 3.925)$, social relationship field $(9.64 \pm 2.497)$, environment domain $(26.23 \pm 7.534)$ and control group $(22.96 \pm 3.275$ in physiological field, $19.87 \pm 3.152$ in psychological field, $10.58 \pm 2.061$ in social relation field and $28.36 \pm 5.091$ in environmental field), they had no significant difference between the two groups $(P>0.05)$. The depression self-rating score of cirrhosis group $(47.86 \pm 10.782)$ was significant higher $(P=0.034)$ than that of control group $(42.61 \pm 11.564)$. Meanwhile, there was no significant difference between the Self-rating Anxiety Scale scores of the cirrhosis group $(38.46 \pm 11.917)$ and control group $(37.00 \pm 12.521)(P>0.05)($ Table 1$)$.

\begin{tabular}{llll}
\hline & cirrhosis group & control group & P value \\
\hline Age & $57.97 \pm 10.448$ & $23.85 \pm 7.406$ & 0.196 \\
Male(n) & 22 & 18 & 0.808 \\
Female(n) & 23 & 22 & 0.736 \\
WHOQOL-BREF & & & \\
physiological domain & $22.23 \pm 3.312$ & $22.96 \pm 3.275$ & 0.317 \\
psychological domain & $19.59 \pm 3.925$ & $19.87 \pm 3.152$ & 0.721 \\
social relationship domain & $9.64 \pm 2.497$ & $10.58 \pm 2.061$ & 0.063 \\
enviroment domain & $26.23 \pm 7.534$ & $28.36 \pm 5.091$ & 0.129 \\
Self-rating Depression Scale(SDS) & $47.86 \pm 10.782$ & $42.61 \pm 11.564$ & $0.034^{*}$ \\
Self-rating Anxiety Scale(SAS) & $38.46 \pm 11.917$ & $37.00 \pm 12.521$ & 0.584 \\
\hline
\end{tabular}

${ }^{*} P<0.05$ : cirrhosis group vs control group

Conclusion: The quality of life and anxiety score in cirrhosis group had no significant difference from the control group, but the depression score was higher than that of the control group.

Disclosure of Interest: All authors have declared no conflicts of interest.

\section{Reference}

1. Dig Dis Sci. 2016 Jun;61(6):1692-9.

\section{P0077 THE IMPORTANCE OF INDIVIDUAL CORRECTION OF EATING BEHAVIOR IN PATIENTS WITH NON-ALCOHOLIC FATTY LIVER DISEASE}

\section{Y. Nikiforova}

Department For The Study Of Diseases Of The Liver And Gastrointestinal Tract (from 05.2016 Year - Department Study Of Digestive Diseases And Their Comorbidity With Non-communicable Diseases), SI «L.T. Mala Therapy Institute of NAMS of Ukraine», Kharkov/Ukraine

\section{Contact E-mail Address: dr.jana@mail.ru}

Introduction: At the present stage, the treatment of patients with non-alcoholic fatty liver disease has insufficient effectiveness due to the simultaneous availability of a number of recommendations and the lack of an individual approach. Not enough attention is paid to the study of nutritional behavior and the role of nutrietics, as additional risk factors for the development of non-alcoholic fatty liver disease. While eating disorders are a modifiable risk factor.

Aims \& Methods: We aimed to study the dynamics of metabolic parameters in patients with non-alcoholic fatty liver disease (NAFLD) as a result of individual correction of eating behavior (EB)

52 patients ( 22 men and 30 women) with NAFLD and visceral obesity were examined. All patients studied EB (questionnaire DEBQ), physical activity (PhA), were determined: waist circumference (WC), lipid and carbohydrate metabolism studies were performed, a visceral obesity index (VOI) was calculated, the body composition was monitored (determination of $\%$ visceral adipose tissue (VAT)) and computed tomography of the abdominal cavity (CT AC) with measurement of the area $(\mathrm{S}, \mathrm{cm} 2)$ of VAT and percutaneous adipose tissue (PAT). Patients were divided into 2 reciprocal comparison groups ( $\mathrm{p}>0.05$ ): 1 st group. $(n=26)$ was assigned individual correction of EB based on nutriogenetic examination (determination of nucleotide polymorphisms of PPARG2 and ADRB3 genes) taking into account the revealed features of EB and $\mathrm{PhA}, 2$ nd group $(n=26)$ - standard non-drug treatment was prescribed according to the general recommendations of the European Association For the Study of the Liver et al. EASL-EASD-EASO Clinical Practice Guidelines for the management of nonalcoholic fatty liver disease (2016).

Results: It was found that after 6 months of compliance with individual recommendations for EB in patients of the 1st group anthropometric and metabolic parameters improved $(\mathrm{p}<0.05)$ : the WC was decreased by $8.9 \%[8.6 ; 9.1]$, the $\%$ of VAT decreased to $8.0 \%[6.0 ; 9.0]$ and $\mathrm{S}$ of VAT -up to $84[82 ; 89] \mathrm{cm} 2$, VOI up to $1,7[1,3 ; 2,4]$, the lipidogram parameters improved (decreased TG, LDL and HDL increased) $(\mathrm{p}<0.05)$, the index of NOMA-IR $(\mathrm{p}<0.05)$ was decreased against the background of a $10.8 \%$ decrease in body weight $[10.1$; 11.2]. $100 \%$ compliance of patients of 1 group was noted to adhere to the appointed recommendations. In the next 6 months in patients 1 st group $(\mathrm{P}<0.05)$ decrease in VAT/PAT (VAT/PAT $<0.6 \%$ in $96.2 \%$ of patients) and VOI (VOI $<1$ in $92.3 \%$ of patients), in contrast to the dynamics of the corresponding parameters in the patients of the 2 nd group.

Conclusion: Thus, the appointment of an individual correction of eating behavior can increase the adherence of patients to treatment and achieve a reliable positive dynamics of metabolic indicators.

With the purpose of increasing the effectiveness of non-drug therapy of patients with non-alcoholic fatty liver disease, methods for studying the characteristics of eating behavior should be applied more widely, which will allow timely appropriate correction. A more extensive study of nutrigenetics will make it possible to designate a personified diet, taking into account the detected polymorphisms, which will make it possible to achieve a significant improvement in metabolic parameters in this category of patients.

Disclosure of Interest: All authors have declared no conflicts of interest.

\section{P0078 NON-GOVERNMENTAL HCV SCREENING IN A VILLAGE IN NILE DELTA IN DAAS ERA IN EGYPT}

A. Sadek ${ }^{1}$, A. Salem ${ }^{2}$, W. Wafy ${ }^{3}$, A. Abdelfattah ${ }^{4}$

${ }_{1}^{1}$ Gastroenterology-Hepatology, Theodor Bilharz Research Institute, Giza/Egypt

${ }^{2}$ Gastroenterology\&hepatology, Naser Institute, Cairo/Egypt

${ }^{3}$ Public Health, Theodor Bilharz Research Institute, Giza/Egypt

${ }^{4}$ Free Physician, Cairo/Egypt

Contact E-mail Address: ahmedsadek48@yahoo.com

Introduction: HCV is highly endemic in Egypt with a prevalence of $12 \%$. The ministry of health through the national committee for hepatic viruses has done an extra-ordinary effort since the beginning of the DAAs era which resulted in treatment of almost one million infected patients till now. Still this looks as the summit of the iceberg and there is still estimated more than 10 million Egyptians infected with the virus, the majority of them are still undetected.

Aims \& Methods: The aim of this work is to give an example of non-governmental efforts which can be done to solve this tough national problem. A group of young medical and non-medical volunteers all from El Salam village in Mansoura, Dakahlia governorate, Egypt have worked together. They contacted mosques and pharmacies in the village and distributed brochures to announce for the free of charge screening for the virus in the village. They contacted businessmen in the village to sponsor the campaign. They contacted the laboratory of the village to do the $\mathrm{HCV} \mathrm{Ab}$ test with nonprofit price.

Results: A total of 2220 citizens have visited the referral lab to do the antibody test. Only 419 persons proved positive. This gives an estimated prevalence in the village of $19.0 \% .119$ patients were insurance patients and they went to treatment and did not complete the study. The rest $300 \mathrm{Ab}+\mathrm{ve}$ patients completed the study. All patients were HBs Ag-ve. 132 PCR -ve (44\%) patients from the 310 $\mathrm{HCV} \mathrm{Ab}+$ ve group. The rest 168 patients were +ve for HCV RNA $(56 \%) .18$ patients of the HCV PCR - ve had previous HCV treatment $(6.5 \%)$. Only 2 patients of the $168 \mathrm{HCV}$ RNA +ve. $(1.2 \%)$ were previously treated. The PCR -ve patients without previous treatment were 114 patients $(38.0 \%)$. So only $62.0 \%$ of the HCV Ab +ve in the community are PCR +ve and require treatment. The total cost for the screening of the 2220 patients was $40.000 \mathrm{~L}$.E. which means 18.0 L.E per person (almost one Dollar).

Conclusion: The screening costs for HCV Ab positivity can be markedly reduced if it is done in each village depending on volunteers from the same village working in related fields like medical, pharmacists, laboratory and social jobs. Only $62.0 \%$ of the $\mathrm{HCV} \mathrm{Ab}+\mathrm{ve}$ in the community are PCR + ve and require treatment.

Disclosure of Interest: All authors have declared no conflicts of interest.

\section{References}

1. Chou R, Cottrell EB, Wasson N, Rahman B, Guise JM. Screening for Hepatitis C Virus Infection in Adults. Comparative Effectiveness Review No. 69. AHRQ Publication No. 12-EHC090-EF. Rockville, MD: Agency for Healthcare Research and Quality; 2012.

2. Chou R, Wasson N. Blood tests to diagnosis fibrosis or cirrhosis in patients with chronic hepatitis C virus infection: a systematic review. Ann Intern Med. 2013;158:807-20.

3. Frank, C, Mohamed, MK, Strickland, GT et al. The role of parenteral antischistosomal therapy in the spread of hepatitis $\mathrm{C}$ virus in Egypt. Lancet. 2000; 355: 887-891.

4. Munier, A, Marzouk, D, Abravanel, F et al. Frequent transient hepatitis C viremia without seroconversion among healthcare workers in Cairo, Egypt. PLoS One. 2013; 8: e57835.

5. WHO. Guidelines for the screening, care and treatment of persons with hepatitis C infection. http://www.who.int/hiv/pub/hepatitis/hepatitis-cguidelines/en/; 2014. 
P0079 EARLY DIAGNOSTICS OF NAFLD: ANALYSIS OF RISK FACTORS AND SCREENING USING ELASTOMETRY. ASSOTIATION BETWEEN THE PREVALENCE STEATOSIS AND COMPONENT COMPOSITION OF THE BODY

I. Bakulin ${ }^{1}$, L. Belousova ${ }^{1}$, L. Evdokimova ${ }^{2}$, M. Serkova ${ }^{1}$, S. Ivanov ${ }^{3}$, D. Korostelev ${ }^{2}$

${ }^{1}$ The Chair Of The Propedeutics Of Internal Diseases, Gastroenterology And Dietology, North-Western State Medical University n.a. I.I. Mechnikov, SaintPetesburg/Russian Federation

${ }^{2}$ North-Western State Medical University n.a. I.I. Mechnikov, Saint-Petesburg/ Russian Federation

${ }^{3}$ Pavlov First Saint Petersburg State Medical University, Saint-Petesburg/Russian Federation

Contact E-mail Address: liya73@mail.ru

Introduction: Non-alcoholic fatty liver disease (NAFLD) is liver disease with histological signs of accumulation of cholesterin excessive amount in hepatocyte in the absence of alcohol consumption by the patient (due to causes other than). The search for accessible, non-invasive and effective methods of screening for this pathology, allowing to detect NAFLD at early, potentially reversible stages of development is relevant. The purpose of the work was frequency estimation of the prevalence steatosis according to elastometry with controlled attenuation parameter $(\mathrm{CAP} \otimes)$ among young people and associated with them specific body composition.

Aims \& Methods: 59 volunteers (students of medical university) at the age of 1928 years (the median age of 20.5) have participated in research. There were 22 $(37.3 \%)$ men and $33(62.7 \%)$ women among them without verified liver diseases. The survey was conducted in order to exclude or detect risk factors. Determining the presence and degree of steatosis and the stage of liver fibrosis was performed with the apparatus FibroScan 502 Touch. The final figures of elasticity of the liver were estimated in $\mathrm{kPa}$ (METAVIR). The controlled attenuation parameter $(\mathrm{CAP} \otimes)$ in $\mathrm{dB} / \mathrm{m}$ was used for the severity of steatosis. Moreover, there was the bioelectrical impedance analysis of body (BIA), evaluated: body mass index (BMI), body fat

Results: The signs of violations of the structure of the liver were diagnosed in 15 people out of $59(25.4 \%)$. The signs of steatosis were founded in $12(20.3 \%)$ students $(\mathrm{CAP}(\mathbb{R})>215 \mathrm{~dB} / \mathrm{m})$. The signs of liver fibrosis were recorded in 7 $(11.9 \%)$ people $(\mathrm{E}>5,8 \mathrm{kPa})$. At the same time the combination of liver fibrosis and steatosis was diagnosed in $4(6.8 \%)$. After analyzing data of BIA it was revealed that body weight above normal in $23(40,3 \%)$; wherein fat body composition above normal values in $19(33,4 \%)$. Results of binary regression analysis showed that the chance of development of hepatic steatosis in case of excess adipose tissue increase 28 times $(\mathrm{p}=0,045)$, influence of BMI, gender, age was statistically insignificant.

Conclusion: Based on the results obtained, it can be concluded that there is high enough level of distribution of liver steatosis among young people. Transient elastography (TE) with controlled attenuation parameter (CAPß) is a fast, reliable, repeatable non-invasive method for the assessment of NAFLD. The development of hepatic steatosis among practically healthy young persons validly associated with the increase the amount of adipose tissue in the body. Confirmed the importance of evaluation of body composition and lack of information of using only BMI when evaluating the chances of development of NAFLD.

Disclosure of Interest: All authors have declared no conflicts of interest.

\section{P0080 SALVAGE TECHNIQUE USING A MICRO GUIDEWIRE FOR DIFFICULT BILIARY CANNULATION IN ENDOSCOPIC RETROGRADE CHOLANGIOPANCREATOGRAPHY}

A. Mori, S. Ito, S. Hayashi, T. Shibuya, M. Sawada, H. Hachiya, T. Yumura, N. Ohashi

Ichinomiya Nishi Hospital Dept. of Gastroenterology, Ichinomiya/Japan

\section{Contact E-mail Address: a-mori@anzu.or.jp}

Introduction: Biliary cannulation is indispensable for therapeutic endoscopic retrograde cholangiopancreatography (ERCP) in patients having biliary disease. Selective biliary cannulation is often difficult due to anatomical constraints. Numerous techniques have been attempted to overcome such problems. Although a wire-guided selective cannulation technique into the bile duct is a useful approach, conventional guidewires $(0.025$ or $0.035 \mathrm{inch})$ are relatively rigid to pass through the long curved narrow distal segment (NDS) or malignant stricture and sometimes get flicked off the NDS in such cases. It may be better to use finer and more flexible guidewire. Hence, we developed a novel guidewire technique (GTWt) using a micro guidewire (GT wire; $0.016 \mathrm{inch}, 300 \mathrm{~cm}$, TERUMO, Japan) designed for super-selective angiography in difficult cases of selective biliary cannulation.

Aims \& Methods: We aimed to assess usefulness of GTWt for salvage technique in biliary cannulation. We studied 240 consecutive ERCP-naïve patients between August 2014 and February 2017. We have tried to perform GTWt to ERCPdifficult-cases that was defined as patients of unsuccessful cannulation despite attempts over $15 \mathrm{~min}$ with conventional techniques including wire-guided cannulation or pancreatic guidewire placement with conventional guidewire $(0.025$ or 0.035 inch). Following the unsuccessful attempts, the guidewire was changed to GT wire and selective biliary cannulation was performed under fluoroscopic control. We assessed the number of patients who benefitted from GTWt and whether overall success rate of biliary cannulation in ERCP was improved.

Results: Among 240 ERCP-naive patients, 40 were ERCP-difficult-cases (success rate with conventional technique: 200/240; 83\%). Among 40 ERCP-difficult cases, GTWt was successful in 32 patients (success rate with GTWt: 32/40;
$80 \%$ ). Overall success rate of ERCP-naive patients improved from $80 \%$ to $97 \%$ (232/240 patients). After ERCP with GTWt, four patients developed mild acute pancreatitis, which resolved in a few days. No serious procedural accidents were reported. The 40 ERCP-difficult cases included 22 patients with a long curved NDS, six patients with juxtapapillary duodenal diverticulum, six patients with malignant stricture, and four patients with difficult front view of papilla. Among eight patients who failed with GTWt, seven were successful performing precut papillotomy or endoscopic ultrasound-guided biliary drainage and one was interrupted because of developed serious condition.

Conclusion: GTWt as a salvage technique for unsuccessful selective biliary cannulation cases improves the success rate of ERCP, and could be attempted before performing a precut papillotomy, endoscopic ultrasound-guided techniques or other cumbersome procedures.

Disclosure of Interest: All authors have declared no conflicts of interest.

\section{P0081 ERCP IN VERY ELDERLY PATIENTS AGE 85 OR OLDER}

M.T. Ayoubi Khajekini ${ }^{1}$, F. Castellino ${ }^{1}$, N. Leone ${ }^{1}$, M. Berrutti ${ }^{1}$, G. Sansoè ${ }^{1}$, L. Framarin ${ }^{1}$, A. Repici ${ }^{2}$

${ }^{1}$ Gi Endoscopy Unit, Humanitas Gradenigo Hospital, Torino/Italy

${ }^{2}$ Dept. Of Gastroenterology, Ist. Clinico Humanitas Rozzano Dept. of Gastroenterology, Milano/Italy

Contact E-mail Address: ayoubi@libero.it

Introduction: To evaluate the safety and effectiveness of this procedure (ERCP) in patients age 85 years and older.

Aims \& Methods: From first January 2010 until end of December 2016 in our digestive endoscopic unit 3153 patients underwent ERCP, including 351 $(11.13 \%)$ patients over 85 . Characteristics of these patients: The mean age was 89 (range 85-99), 117 males and 234 females. 43 (12.2\%) were in treatment with antithrombotic drugs. The initial diagnosis in 218 patients $(62.1 \%)$ was choledocholithiasis, malignant CBD stenosis in 92 patients $(25.9 \%)$, postoperative leak in 11 patients $(3,13 \%)$ and unknown CBD stenosis in 31 patients $(8.83 \%)$. All patients underwent the following clinical evaluation before and after ERCP. 34 patients $(9.4 \%)$ were sedated with Propofol and anesthetists assisted. The rest of the patients $90.6 \%$ were sedated with the intravenous combination of midazolam and Fentanyl with standardized monitoring (pulse oximetry, heart rate, non invasive blood pressure measurements) during the procedure. Drug infusion was performed by the nursing staff and an attending endoscopist.

Results: Deep biliary cannulation was successful in 330 patients (94\%). In 127 patients $(36.18 \%)$ Vater papilla was associated with duodenale diverticula. The post-ERCP complication rates were as follows: Heperamilasemia in 28 patients $(7.9 \%)$, Mild pancreatitis in 12 patients $(3.4 \%)$, Minor bleeding in 10 patients $(2.8 \%)$, Major bleeding in 2 patients $(0,56)$ and cholangitis in 6 patients $(1,7 \%)$. Conclusion: ERCP is an acceptable procedure in patients of 85 or older in terms of safety, success and complication rates.

Disclosure of Interest: All authors have declared no conflicts of interest.

\section{P0082 ALCOHOL CONSUMPTION CAN REDUCE THE RISK OF GALLSTONE DISEASE: A SYSTEMATIC REVIEW WITH DOSE- RESPONSE META-ANALYSIS OF CASE-CONTROL AND COHORT STUDIES}

B.H. Cha ${ }^{1}$, M. Jang ${ }^{2}$

${ }^{1}$ Sheikh Khalifa Specialty Hospital, Ras Al Khaimah/United Arab Emirates ${ }^{2}$ Medical Research Collaborating Center, Seoul National University Hospital, Seoul/Korea, Republic of

Contact E-mail Address: doctorhyo@gmail.com

Introduction: Gallbladder stone (GBS) is a common gastrointestinal disease can progress to severe cholecystitis and is a strong risk factor for gallbladder cancer (GBC). Recently, clinical epidemiologic studies revealed that the alcohol consumption has a preventive effect for development of gallstone diseases.

Aims \& Methods: To evaluate the relative risks of alcohol consumption for the gallbladder stone diseases development. Systematic searching was performed using MEDLINE, EMBASE and Cochrane library from January $1^{\text {st }}, 1996$, to December $31^{\text {st }}, 2016$ for studies assessed the relationship between alcohol consumption and gallbladder stone diseases development risk. The eligibility criteria was included: 1) studies involving the patients with gallbladder stone with or without cholecystitis; 2) cohort or case-control studies investigated the association between alcohol consumption and gallstone disease development. Newcastle-Ottawa Scale was used to assess the methodologic quality of each studies. Data was obtained from each selected studies regarding: 1) baseline characteristics of the study (cohort, case-control); 2) number of participants; 3) participants' clinical features; 4) country; 5) publication year; 6) Risk or odds ratio with confidence intervals for various levels of alcohol consumption and risk of gallstone. The random effect model was used to estimate the pooled relative risks (RR) with $95 \%$ confidence intervals (CIs).

Results: Twenty-five cohort and case-control studies were included, and total 12 , 581 cases with gallstone diseases among those 172, 509 controls. Alcohol consumption indicated a decreased risk of GSD development (Pooled RR $=0.84$ [0.79-0.90], $\mathrm{P}<0.001)$. Subgroup analyses according to the alcohol doses $(\mathrm{g} / \mathrm{d})$ confirmed a gradual risk-reduction effect on GSD compared to non-drinkers (Light: $\mathrm{RR}=0.97[0.94,1.00], \mathrm{p}=0.864 ;$ Moderate: $\mathrm{RR}=0.82[0.79,0.86]$, $\mathrm{p}=0.777 ;$ Heavy: $\mathrm{RR}=0.70[0.62,0.80], \mathrm{p}<0.01)$ 
Conclusion: In this systematic review with meta-analysis, alcohol consumption has dose-dependent negative co-relationship with the risk of gallstone disease development.

Disclosure of Interest: All authors have declared no conflicts of interest.

\section{References}

Stinton LM, Shaffer EA. Epidemiology of gallbladder disease: Cholelithiasis and cancer. Gut and Liver 2012;6:172-187.

Moher D, Liberati A, Tetzlaff J, Altman DG, The PRISMA Group. Preferred Reporting Items for Systematic Reviews and Meta-Analyses: The PRISMA Statement. Int J Surg 2010; doi:10.1016/j.ijsu.2010.02.007

Wells GA, Shea B, O'Connell D, Peterson J, Welch V, Losos M, et al. The Newcastle-Ottawa Scale (NOS) for assessing the quality of nonrandomised studies in meta-analyses. [cited March 2017].

Hamling J, Lee P, Weitkunat R, Ambuhl M (2008) Facilitating meta-analyses by deriving relative effect and precision estimates for alternative comparisons from a set of estimates presented by exposure level or disease category. Stat Med 27: 954-970.

\section{P0083 ERCP WITH SHORT-TYPE SINGLE BALLOON ENTEROSCOPE IN PATIENTS WITH SURGICALLY ALTERED PATIENTS}

M. Kida ${ }^{1}$, H. Yamauchi ${ }^{1}$, Y. Kawaguchi ${ }^{1}$, E. Miyata ${ }^{1}$, R. Hasegawa ${ }^{1}$, T. Kaneko ${ }^{1}$, K. Okuwaki ${ }^{1}$, T. Iwai ${ }^{1}$, H. Kikuchi ${ }^{1}$, H. Imaizumi ${ }^{1}$, W. Koizumi ${ }^{2}$ ${ }^{1}$ Endoscopy \& Gastroenterology, Kitasato University, Sagamihara/Japan ${ }^{2}$ Gastroenterology, Kitasato University School of Medicine, Sagamihara/Japan

Contact E-mail Address: m-kida@kitasato-u.ac.jp

Introduction: Endoscopic retrograde cholangiopancreatography (ERCP) remains challenging in patients who have undergone surgical reconstruction of the intestine. In 2001, double-balloon enteroscope (DBE) was reported by Yamamoto et al to be an effective procedure for the diagnosis and treatment of small intestinal lesions. In 2005, DBE-assisted ERCP was first successfully by Haruta et a used to treat a late anastomotic stricture in a patient who undergone biliary reconstruction by R-Y choledochojejunostomy after liver transplantation. After that, several studies with long enteroscope have reported that balloon enteroscope-assisted ERCP (BEA-ERCP) is a safe and effective procedure with about $69-100 \%$ of reaching the blind end. However long type enteroscope allows us to use limited number of ERCP devices because of its length $200 \mathrm{~cm}$. Then Olympus Co. introduce the prototype of short single balloon enteroscope (SBE) with bigger channel $3.2 \mathrm{~mm}$ in diameter was developed. We will present its usefulness and limitation.

Aims \& Methods: In order to investigate the usefulness and limitation of shorttype SBE, we have performed totally 183 cases 302 procedures of ERCP with short-type SBE in patients with B-II ( 24 cases, 30 procedures), R-Y gastrectomy (RY; 94, 138), Hepatico-Jejunostomy without gastrectomy (HJ; 29, 58), and Child/Whipple (CW; 36, 76) from 2009 to 2016. We have investigated its rate of reaching blind end, time of procedure, success rate of therapeutic procedure, complications and its limitations

Results: Using short type SBE, the rate of reaching blind end is B-II: $97 \%$ ( 29 30), RY: $91 \%$ (126/138), HJ: 72\% (39/58), CW:93\% (71/76), and 88\% (totally). Success rate of therapeutic procedure in reached blind end cases, is B-II: $100 \%$ (27/27), RY: $96 \%(104 / 108), \mathrm{HJ}$ : $94 \%(34 / 36), \mathrm{CW}: 100 \%(67 / 67)$, and $97 \%(232$ 238 ) totally. The average time of procedure is B-II: $38.6 \mathrm{~min}, \mathrm{RY}: 47.2$, HJ: 38.5 , and $\mathrm{CW}: 44.3$, respectively. The reason of unreached cases in HJ without gastrectomy group is not enough scope length: $68 \%(13 / 19)$, stricture: $21 \%(4 / 19)$, adhesion: $11 \%(2 / 19)$. And using long-type SBE, it is practicable to do therapeutic procedure in $85 \%$ in not enough scope length group $(11 / 13)$. Concerning about complication, pancreatitis $3 \%(8 / 302)$, Perforation $1 \%(4 / 302)$, bleeding $0.8 \%(2 / 302)$, and cholangitis $0.8 \%(2 / 302)$.

Conclusion: ERCP with short-type SBE in patients with surgically anatomy is practicable with high success rate, except for cases with $\mathrm{HJ}$ without gastrectomy. However, even in these cases, most of cases can be performed with long-type SBE.

Disclosure of Interest: All authors have declared no conflicts of interest.

P0084 CHOLECYSTECTOMY FOLLOWING ENDOSCOPIC RETROGRADE CHOLANGIOPANCREATOGRAPHY AND DUCT CLEARANCE FOR CHOLEDOCHOLITHIASIS; A SINGLE CENTRE EXPERIENCE FROM UNITED KINGDOM

F. Rana ${ }^{1}$, D. Majumdar ${ }^{1}$, P. Sambaiah ${ }^{1}$, D. Craig ${ }^{1}$, J. Greenaway ${ }^{1}$, V. Mitra ${ }^{2}$ ${ }^{1}$ Dept. Of Gastroenterology, James Cook University Hospital, Middlesbrough/ United Kingdom

${ }^{2}$ Dept. Of Gastroenterology, James Cook University Hospital, Middlesborough United Kingdom

Contact E-mail Address: fahdrana@outlook.com

Introduction: Patients undergoing endoscopic retrograde cholangiopancreatography (ERCP) and duct clearance for common bile duct stones (CBDS) should be followed up with an early cholecystectomy to prevent recurrent biliary complications $(1,2)$ and acute gallstone pancreatitis. Recently the National Confidential Enquiry into Patient Outcome and Death (NCEPOD) recommended that definitive eradication of gallstones by cholecystectomy prevents the risk of a recurrent attack of acute pancreatitis (AP). For patients with an episode of mild acute pancreatitis, early definitive surgery should be undertaken, either during the index admission or within two weeks $(3,4)$.

Aims \& Methods: 1) To determine time frame between ERCP/duct clearance and cholecystectomy (CCX) in non-pancreatitis group. 2) To determine time frame between ERCP/duct clearance and CCX in pancreatitis group. 3) To determine re-admission rate while awaiting CCX. All patients who underwent ERCP for CBDS between $01 / 01 / 2014$ to $31 / 12 / 2014$ were included in the study. Patients who had previously undergone CCX (de novo stones) were excluded. All patients were followed up for a minimum period of 2 years following their ERCP.

Results: A total of 273 patients underwent ERCP for CBDS. Out of these $21.2 \%$ $(\mathrm{n}=58)$ had previously had CCX and were excluded. Out of the remaining 215 with gall bladder (GB) in situ, $87.4 \%(188 / 215)$ underwent successful duct clearance at index or subsequent ERCP. Of these, 47.3\% (89/188) underwent CCX $1 \%(2 / 188)$ patients are currently awaiting CCX and 51.6\% (97/188) did not undergo CCX. The outcomes in remaining $13.4 \%(n=27)$ patients in whom duct clearance was not achieved are discussed later. In the CCX group, the median time between ERCP/duct clearance and CCX was 123 days (range 0820 days). In this group, 15 patients had gall stone pancreatitis (GSP) on presentation and the median time between ERCP and CCX in the GSP group was 136 days (range 35-287 days); 12 of these had mild pancreatitis with a median time to CCX of 140 days (range 60-287 days). 4 patients re-presented with CBDS while awaiting CCX after duct clearance; 1 had pancreatitis on readmission. $51.6 \%(97 / 188)$ patients who did not undergo CCX after duct clearance are referred to as non-cholecystectomy (non-CCX) group. This was mainly secondary to high ASA grade. We compared patient demographics and presentation with the CCX group and the results were as follows:

\begin{tabular}{lll}
\hline Value & $\begin{array}{l}\text { CCX group } \\
(\mathrm{n}=89)\end{array}$ & $\begin{array}{l}\text { Non-CCX group } \\
(\mathrm{n}=97)\end{array}$ \\
\hline Median Age & 61 years & 79 years \\
Median ASA grade & 1 & 2.8 \\
Female patients & $67 \%$ & $56 \%$ \\
Male patients & $33 \%$ & $44 \%$ \\
Pancreatitis on presentation & $17 \%$ & $11.4 \%$ \\
Readmission with CBDS & $4.5 \%$ & $5.4 \%$
\end{tabular}

In 27 patients duct clearance was not achieved; $26 \%(7 / 27)$ underwent surgical management (CBD exploration/on table cholangiogram and CCX). The remaining $74 \%(20 / 27)$ patients were deemed unsuitable for invasive intervention and were either for symptomatic stent change only or conservative management.

Conclusion: The time period between duct clearance and CCX was longer than anticipated, especially in patients with mild acute pancreatitis as none of them underwent CCX during index admission or within 2 weeks of ERCP/duct clearance. Some patients re-presented with CBDS while awaiting CCX. We looked into potential causes of delay in CCX - delayed referral to surgery, long waiting time for elective CCX and patient choice. We propose to develop a local pathway for patients with CBDS and gallstones and induct a robust system for referring patients for CCX following duct clearance. This would help to minimize readmission and potential complications.

Disclosure of Interest: All authors have declared no conflicts of interest.

\section{References}

1. Anandi H. W. Schiphorst, Marc G. H. Besselink, Djamila Boerma, Robin Timmer, Marinus J. Wiezer, Karel J. van Erpecum, Ivo A. M. J. Broeders, Bert van Ramshorst et al. Timing of cholecystectomy after endoscopic sphincterotomy for common bile duct stones; Surg Endosc. 2008 Sep; 22(9): 2046-2050.

2. James Y. W. Lau, Chon-Kar Leow, Terence M. K. Fung, Bing-Yee Suen, Ly-Mee Yu, Paul B. S. Lai, Yuk-Hoi Lam, Enders K. W. Ng, Wan Yee Lau, Sydney S. C. Chung, et al.Cholecystectomy or gallbladder in situ after endoscopic sphincterotomy and bile duct stone removal in Chinese patients. Gastroenterology. 2006 Jan; 130(1): 96-103. doi: 10.1053/j.gastro.2005.10.015

3. http://www.ncepod.org.uk/2016ap.html

4. Tenner S, Baillie J, Dewitt J, Vege SS. American College of Gastroenterology guidelines:management of acute pancreatitis. Am J Gastroenterol 2013; 108: $1400-1415$

P0085 THE DEVELOPMENT OF MULTI-LAYER DRUG ELUTING MEMBRANE USING ULTRASONIC SPRAY COATING TECHNIQUE

J. Park ${ }^{1}$, D.H. Lee

${ }^{1}$ Internal Medicine, Inha University School of medicine, Incheon/Korea, Republic of

${ }^{2}$ Inha University School of medicine, Incheon/Korea, Republic of

Contact E-mail Address: pjsinha @naver.com

Introduction: The placement of self-expandable metallic stent has become the treatment of choice to restore luminal patency in the palliative treatment of unresectable malignant biliary stricture. Currently, drug-eluting stents (DESs) are developed to prevent tumor invasion into the stent and to prolong stent patency. However, the capacity of drug per unit area varies with the position in membrane, and the control of drug release is impossible in current available DESs. 
Aims \& Methods: The aim of current study is to develop the multi-layer drug eluting membrane using ultrasonic spray coating method, which have uniform capacity of drug and be able to control the drug-release capacity.

Methods: The drug eluting membrane was made using ultrasonic spray coating machine (MediCoat-2JX). The membrane consists with two kinds of coating material. One is silicone (MED-6640), that was used to basic structure of membrane and the other coating agent is polyurethane (tecophilic, tecothane, Tecoflex and pellethane). The gemcitabine was used as antitumor drug, and coated to membrane by mixed form with polyurethane (gemcitabine, $250 \mathrm{ug} / \mathrm{ml}$; polyurethane, $500 \mathrm{ug} / \mathrm{ml}$ ). The thickness of membrane and the capacity of drug in membrane were measured at the proximal and distal end, and mid portion. The drug release capacity and duration was measured by using drug releasing test in vitro for 3 days.

Results: The mean thickness of membrane was $50 \mathrm{um}$. The mean capacity of drug per unit area was $100 \mathrm{ug} / \mathrm{cm}^{2}$, and the amount was constant in all tested area (Standard deviation, $5 \mathrm{ug} / \mathrm{cm}^{2}$ ). In drug release test, the capacity of releasing drug was different depended on the kinds of polyurethane. The total amount of released drug in 24 hours was $919 \mathrm{ug}, 836 \mathrm{ug}, 681 \mathrm{ug}$, and $580 \mathrm{ug}$ in tecophilic coating, tecothane coating, tecoflex coating, and pellethane coating. The total of released drug amount depended on polyurethane was described in table 1 .

Table 1: The total of releasing drug amount in 72 hours

\begin{tabular}{llll}
\hline & \multicolumn{2}{l}{ Drug release amount } \\
\cline { 2 - 4 } & \multicolumn{2}{l}{ Gemcitbine (ug) } & \\
\cline { 2 - 4 } Coating Polymer & $24 \mathrm{hrs}$ & $48 \mathrm{hrs}$ & $72 \mathrm{hrs}$ \\
\hline Tecophilic & 919 & 927 & 933 \\
Tecothane & 836 & 859 & 868 \\
Tecoflex & 681 & 690 & 698 \\
Pellethane & 580 & 604 & 622 \\
\hline
\end{tabular}

Conclusion: The ultrasonic spray coating technique could be applied to make multi-layer drug eluting membrane with regular thickness. The membranes contained the uniform capacity of drug in all tested area. The releasing drug capacity is able to control by applying different kind of polyurethane.

Disclosure of Interest: All authors have declared no conflicts of interest.

\section{P0086 THE ANTI-TUMOR EFFECT OF PACLITAXEL, GEMCITABINE AND MITOMYCIN C ELUTING MEMBRANE IN} ANIMAL MODEL

J. Park ${ }^{1}$, D.H. Lee ${ }^{2}$, S. Jeong ${ }^{1}$

${ }^{1}$ Internal Medicine, Inha University School of medicine, Incheon/Korea, Republic of

${ }^{2}$ Inha University School of medicine, Incheon/Korea, Republic of

Contact E-mail Address: pjsinha@naver.com

Introduction: Local treatment of primary bile duct cancer is a challenge and endoscopic stent insertion is widely used to maintain the bile duct patency. Drug eluting stent is currently developed to add the ability of antitumor effect.

Aims \& Methods: We aimed to evaluate the antitumor effect of the paclitaxel, gemcitabine, and mitomycin C-eluting polyurethane membrane in a tumor model. Total of 24 mice were used in current study and divided into four groups, each group had six mice. Membranes containing different antitumor drugs (paclitaxel, gemcitabine, mitomycine C, $100 \mu \mathrm{g} / \mathrm{disc}$ ) were inserted beneath the tumor mass in mouse models. Tumor size and body weight of the tumor model were monitored for 22 days after insertion of the membrane. The results were compared with the tumor model which was inserted only silicone membrane (control).

Results: Tumor volumes on day 22 of membrane treatment were decreased in all drugs, that were significantly different compared to those of control (paclitaxel, $291.77 \mathrm{~mm}^{3}, P$ value $=0.4116$; gemcitabine, $63.38 \mathrm{~mm}^{3}, P$ value $=0.0001 ;$ mitomycin $\mathrm{C}, 119.02 \mathrm{~mm}^{3}$, P value $=0.0029$; control $1362.62 \mathrm{~mm}^{3}$ ). The antitumor effect of gemcitabine was tended to be superior compared to other drugs. However, it was not statistically difference. No significant difference in body weight change was observed among groups.

\begin{tabular}{lllll}
\hline & $\begin{array}{l}\text { Membrane } \\
\text { only }\end{array}$ & Paclitaxel & Gemcitabine & Mitomycin C \\
\hline Mouse weight $(\mathrm{g})$ & 24.28 & 21.05 & 19.56 & 20.67 \\
Tumor volume $\left(\mathrm{mm}^{3}\right)$ & 1362.62 & 291.77 & 63.38 & 119.02 \\
Tumor weight $(\mathrm{mg})$ & 1025 & 524 & 496 & 443 \\
\hline
\end{tabular}

Conclusion: The drug-eluting membrane showed significant antitumor activity. However, the effect was not different according to kinds of the antitumor drugs. Disclosure of Interest: All authors have declared no conflicts of interest.
P0087 ADVANCES IN CYTOLOGY FOR THE EARLY DIAGNOSIS OF PANCREATICO-BILIARY MALIGNANCY

C. Meredith $^{1}$, P. Irandoost ${ }^{2}$, P. Baird ${ }^{2}$

${ }^{1}$ Gastroenterology And Hepatology, Bankstown-Lidcombe Hospital, Bankstown/ Australia $/ N S W$

${ }^{2}$ Cytology, Laverty Pathology, Ryde/Australia/NSW

Contact E-mail Address: drcmeredith@gmail.com

Introduction: Liquid-based sample preparations for cytology have improved the cellular yield in pancreatico-biliary (PB) malignancy ${ }^{(1)}$. The SurePath (SP) methodology produces a pellet of concentrated cellular material which enables additional slides for immunohistochemical (IHC) staining for tumour markers Ki67, p53 and CDX2. The presence of the mitosis-related marker, Ki67, in high concentration and with a specific pattern adds a level of confidence in diagnosing malignancy using cytological preparations. The aim of this study was to assess Ki67 staining in biliary epithelium obtained from patients with bile duct obstruction.

Aims \& Methods: Brushings were obtained from the common bile duct during endoscopic retrograde cholangiopancreatography (ERCP) in patients presenting with biliary obstruction. After collecting the sample, the brush was placed immediately into a SurePath vial and shaken vigorously to fix and suspend the cells. In the cytology laboratory, the vial (with brush included) was agitated on a platform vortex for 10 minutes to shake the cells off the brush into the solution. The high cellular content enabled the preparation of multiple slides for IHC and these slides were reviewed independently by two senior cytopathologists.

Results: Thirty-four (34) consecutive patients with bile duct obstruction were included in the study. The cohort had a mean age of $70.2 ; 41 \%$ were female. Adenocarcinoma was identified in $19(56 \%)$ and atypical/reactive cells in 9 $(26 \%)$. Ki67 positive nuclei were present in $90-100 \%$ of the cells in malignant cell clusters, while sheets of normal cells had positive nuclei in less than $20 \%$ of cells. Atypical cells sheets had an intermediate percentage range.

Conclusion: SP is superior to conventional slide-based cytology preparations in the diagnosis of malignant bile duct strictures. Advantages include ease of collection, no requirement for a cytology technician, a sizable pellet of intact cells for the cytopathologist to examine and the ability to undertake IHC staining. Ki67 is a marker of cell division and cells stained with Ki67 are increased significantly in adenocarcinoma as confirmed by this study. The presence of a large number of cells stained with Ki67 as well as the pattern of intracellular staining adds a level of confidence for the cytopathologist to diagnose malignancy, particularly when there is no clinical or scan evidence of a tumour mass. Early diagnosis is the key for curative surgery and specific cell tumour markers \&/or their pattern may impact significantly on the outcome.

Disclosure of Interest: All authors have declared no conflicts of interest.

\section{Reference}

1. Meredith C, Baird, P. Diagnostic yield of SurePath (SP) and conventional smear preparations (CSP) for brush cytology obtained from the common bile duct (CBD) in patients undergoing endoscopic retrograde cholangiopancreatography (ERCP). Gastroenterology, Vol 150, Issue 4, S516, 2016

\section{P0088 IMPACT OF PALLIATIVE BILIARY DRAINAGE BETWEEN METAL STENT AND PLASTIC STENT ON SURVIVAL RATE IN UNRESECTABLE DISTAL MALIGNANT BILIARY STRICTURE IN SOUTH OF THAILAND}

T. Pattarapuntakul, B. Ovartlanporn, N. Netinatsunton

Faculty Of Medicine, Prince Of Songkla University, NKC Institute of

Gastroenterology and Hepatology, Hadyai/Thailand

Contact E-mail Address: tanawat_kuey@hotmail.com

Introduction: Palliative biliary drainage was used to improving obstructive jaundice, nutritional status, quality of life along with survival rate in unresectable distal malignant biliary stricture patients. The ERCP with biliary drainage with or without systemic chemotherapy are mainstay of treatment in these patients. The benefits of biliary stent type, which are different in cost on survival rate, nutritional status and efficacy of biliary drainage in DMBS patients are still questionable in limit health budget country.

Aims \& Methods: We aimed to assess the impact of endoscopic palliative biliary drainage stents on survival rate, nutritional status and efficacy of biliary drainage of patients in distal biliary malignant stricture patients.

All of the computerized medical records of distal biliary malignant stricture patients, who were undergoing to endoscopic biliary drainage from January 01, 2012 to December 30, 2015 in Songklanagarind hospital were retrospectively review. ERCP with biliary drainage stents was undertaken at the discretion of attending physicians. The overall survival rate, nutritional status (body weight), efficacy of biliary drainage (level of total bilirubin) after biliary drainage between the metal stent group, plastic stent group and plastic stent followed with metal stent group were compared.

Results: Sixty eight patients (45 males, mean age $63.7+/-14.8$ years) were enrolled, 35 patients were classified into the plastic stent group, 18 patients were classified into the metal stent group and 15 patients were classified into the plastic stent followed to metal stent group. Demographic data, primary malignancy, tumor staging and ECOG score, initial total bilirubin and stricture length were similar between 3 groups. The median survival time was 5.4 months $95 \%$ CI (3.2-8.5) and overall survival rate was lowest in the metal stent group (median 3.2 months $95 \%$ CI 1.8-6.9). Mean weight reduction and the declining of total biliary after biliary drainage were not significantly different between biliary 
Abstract No: P0088

Table1.: Demographic data

\begin{tabular}{|c|c|c|c|c|}
\hline Variable & Group 1 Plastic stent & Group 2 Metal stent & Group 3 Plastic - metal stent & $\mathrm{P}$ value \\
\hline Number & 35 & 18 & 15 & \\
\hline Gender (male), $(\%)$ & $26(74 \%)$ & $9(50 \%)$ & $10(66 \%)$ & 0.209 \\
\hline Age (year) & 61.2 & 71.3 & 60.7 & 0.04 \\
\hline $\begin{array}{l}\text { Primary malignancy } \\
\text { Head of pancreas } \\
\text { Cholangiocarcinoma } \\
\text { Ampulla cancer } \\
\text { Neuroendocrine tumor } \\
\text { Hematologic malignancy Metastasis }\end{array}$ & $\begin{array}{l}16(46 \%) \\
4(11.4 \%) \\
10(28.6 \%) \\
1(2.9 \%) \\
2(5.7 \%) \\
2(5.7 \%)\end{array}$ & $\begin{array}{l}11(61 \%) \\
1(5.6 \%) \\
4(22.2 \%) \\
1(5.6 \%) \\
1(5.6 \%)\end{array}$ & $\begin{array}{l}11(73 \%) \\
1(6.7 \%) \\
2(13.3 \%) \\
1(6.7 \%)\end{array}$ & 0.869 \\
\hline Stage of disease 1234 & $\begin{array}{l}6(17.1 \%) \\
12(34.3 \%) \\
6(17.1 \%) \\
11(31.4 \%)\end{array}$ & $\begin{array}{l}5(27.8 \%) \\
3(16.7 \%) \\
2(11.1 \%) \\
8(44.4 \%)\end{array}$ & $\begin{array}{l}3(20 \%) \\
10(66.7 \%) \\
2(13.3 \%)\end{array}$ & 0.076 \\
\hline ECOG pre-treatment 123 & $\begin{array}{l}20(57.1 \%) \\
12(34.3 \%) \\
3(8.6 \%)\end{array}$ & $\begin{array}{l}5(27.8 \%) \\
9(50 \%) \\
4(22.2 \%)\end{array}$ & $\begin{array}{l}11(73.3 \%) \\
4(26.7 \%)\end{array}$ & 0.062 \\
\hline Initial TB $(\mathrm{mg} / \mathrm{dL})$ & 20 & 17.6 & 17.6 & 0.599 \\
\hline Body weight (kg) & $51.8(10.2)$ & $46(7.9)$ & $53(11.6)$ & 0.082 \\
\hline Stricture length $(\mathrm{mm})$ & 23 & 20 & 17.8 & 0.481 \\
\hline CMT (yes, \%) & $6(17.1 \%)$ & $5(27.8 \%)$ & $4(26.7 \%)$ & 0.587 \\
\hline Complications ERCP Biliary stent & 24 & 12 & 13 & 1 \\
\hline Cost of ERCP (bath) & $26,531(9,069)$ & $22,113(7,732)$ & $38,424(10,268)$ & $<0.01$ \\
\hline Cost biliary stents (bath) & $2,480(1,495)$ & $29,539(5,564)$ & $29,965(6,382)$ & $<0.001$ \\
\hline Cost of total procedure (bath) & $28,917(10,018)$ & $55,471(14,398)$ & $69,262(12,135)$ & $<0.001$ \\
\hline
\end{tabular}

stent types. The plastic stent group had lowest cost of total ERCP statistically significance. The complications rate was not different between biliary stent types. Conclusion: Palliative biliary drainage with plastic stent in unresectable dista malignant biliary stricture was slightly better on survival rate and was not different in nutritional status, efficacy to drainage and complication rate compared with metal stent or plastic followed with metal stent. But the plastic stent group had lowest cost of total procedure.

Disclosure of Interest: All authors have declared no conflicts of interest.

\section{References}

Shepherd HA, Royle G, Ross AP, et al. Endoscopic biliary endoprosthesis in the palliation of malignant obstruction of the distal common bile duct. A randomized trial. Br J Surg 1988;75:1166-8.

Davids PH, Groen AK, Rauws EA, et al. Randomised trial of self expandable metal stents versus polyethylene stents for distal malignant biliary obstruction. Lancet 1992;340:1488-92.

Yeoh KG, Zimmerman MJ, Cunningham JT, Cotton PB. Comparative costs of metal versus plastic biliary stent strategies for malignant obstructive jaundice by decision analysis. Gastrointest Endosc 1999;49:466-71

Cotton PB. Metallic mesh stents - is the expanse worth the expense? Endoscopy 1992;24:421-3

Moss AC, Morris E, Leyden J, Macmathuna P. Malignant distal biliary obstruction: A systematic review and meta-analysis of endoscopic and surgical bypass results. Cancer Treat Rev 2007; 33(2): 213-21.

Moss AC, Morris E, Leyden J, Macmathuna P. Malignant distal biliary obstruction: A systematic review and meta-analysis of endoscopic and surgical bypass results. Cancer Treat Rev 2007; 33(2): 213-21.

\section{P0089 PROGNOSTIC VALUE OF EARLY CA19-9 RESPONSE DURING CHEMOTHERAPY IN PATIENTS WITH ADVANCED OR RECURRENT BILIARY TRACT CANCER}

N. Takahara ${ }^{1}$, Y. Nakai ${ }^{1}$, K. Saito ${ }^{1}$, T. Nakamura ${ }^{1}$, T. Sato ${ }^{1}$, T. Takeda ${ }^{1}$, R. Uchino', S. Mizuno ${ }^{1}$, H. Kogure ${ }^{1}$, S. Matsubara ${ }^{1}$, M. Tada' ${ }^{1}$, H. Isayama ${ }^{2}$, K. Koike ${ }^{1}$

${ }^{1}$ Dept. Of Gastroenterology, The University of Tokyo, Tokyo/Japan

${ }^{2}$ Dept. Of Gastroenterology, Juntendo University, Tokyo/Japan

Contact E-mail Address: naminatsu.takahara $@$ gmail.com

Introduction: The most widely used standard for determining the effectiveness of chemotherapy is a set of rules called Response Evaluation Criteria in Solid Tumors, or RECIST. However, it is often difficult to evaluate radiologic response to chemotherapy especially in patients with biliary tract cancer (BTC) mainly because of the pathognomonic tumor progression along bile duct and/or inflammatory reaction induced by indwelling biliary drainage. Therefore, quantitative evaluation using tumor markers is expected to play complementary roles to assess treatment efficacy, however, the prognostic value of carbohydrate antigen 19-9 (CA 19-9) kinetics in patients with advanced or recurrent BTC receiving chemotherapy remains to be elucidated.

Aims \& Methods: Between January 2006 and March 2016, a total of 185 advanced or recurrent BTC patients receiving a first line systemic chemotherapy for at least two cycles were retrospectively studied. Serum CA 19-9 was measured at baseline (CA19-9 Pre) and after two cycles of chemotherapy, and patients were categorized into three groups based on CA19-9 response: CA19-9 decrease group ( $\geq 30 \%$ decrease), stable group $(<30 \%$ decrease and $\leq 20 \%$ increase) and increase group ( $\geq 20 \%$ increase). The Cox proportional hazards model was used to analyze the prognostic factors for OS and PFS, using the landmark method. Results: The primary tumors were located as follows: $68(37 \%)$ in intrahepatic bile duct, $43(23 \%)$ in extrahepatic bile duct, $64(35 \%)$ in gallbladder and $10(5 \%)$ in ampulla. As for chemotherapeutic regimen, single-agent or combination therapy was given in $49(26 \%)$ or $136(74 \%)$, respectively. After 2 cycles of chemotherapy, partial response was achieved in $29(16 \%)$ and stable disease in $112(60 \%)$, giving response rate of $16 \%$ and disease control rate of $76 \%$. The median CA 19-9 levels at baseline and after two cycles were $264 \mathrm{IU} / \mathrm{mL}$ and $194 \mathrm{IU} / \mathrm{mL}$, respectively. After 2 cycles of chemotherapy, CA19-9 decrease was obtained in $77(42 \%)$, stable in $56(30 \%)$, and increase in $52(28 \%)$. The median PFS and OS were 5.62 months $(95 \%$ confidence interval [CI], 5.29-13.28) and 13.71 months $(95 \% \mathrm{CI}, 11.38-22.06)$. There was a statistically significant trend for CA 19-9 and RECIST responses $(\mathrm{p}=0.05)$. Compared with CA19-9 decrease group, hazard ratios for stable and increase groups were $1.22(95 \% \mathrm{CI}, 0.79$ $1.87)$ and $2.42(95 \%$ CI, 1.57-3.72) for PFS, respectively ( $\mathrm{p}$ for trend $<0.001$ ), and $1.04(95 \% \mathrm{CI}, 0.68-1.59)$ and $2.54(95 \% \mathrm{CI}, 1.68-3.85)$ for OS (p for trend 0.060 ). Multivariable analyses showed that CA19-9 response was prognostic both for OS and PFS in addition to CA19-9 Pre and performance status.

Conclusion: CA 19-9 response after two cycles as well as baseline served as a prognostic factor for OS and PFS in patients with advanced and recurrent BTC on systemic chemotherapy.

Disclosure of Interest: All authors have declared no conflicts of interest.

\section{P0090 BILIARY DRAINAGE IN PATIENTS WITH UNRESECTABLE PERIHILAR CHOLANGIOCARCINOMA HAS A VERY HIGH} COMPLICATION AND FAILURE RATE

M. Gaspersz ${ }^{1}$, J. L.a. Van Vugt ${ }^{1}$, E. Roos ${ }^{2}$, R.J. S. Coelen ${ }^{2}$, J. Vugts ${ }^{1}$, E. Belt ${ }^{3}$, J. De Jonge ${ }^{1}$, W. Polak ${ }^{1}$, J.W. Poley ${ }^{4}$, F. E.j.a. Willemssen ${ }^{5}$, L. Hol ${ }^{4}$, T. M. Van Gulik $^{2}$, J. N.m. Ijzermans ${ }^{1}$, B. Groot Koerkamp ${ }^{1}$

${ }^{1}$ Surgery, Erasmus University Medical Center, Rotterdam/Netherlands

${ }^{2}$ Surgery, Academisch Medisch Centrum Dept. of Surgery, Amsterdam

Netherlands

${ }^{3}$ Surgery, Albert Schweitzer ziekenhuis, Dordrecht/Netherlands

${ }^{4}$ Gastroenterology And Hepatology, Erasmus MC University Medical Center

Rotterdam, Rotterdam/Netherlands

${ }^{5}$ Radiology, Erasmus University Medical Cente, Rotterdam/Netherlands

Contact E-mail Address: m.gaspersz@erasmusmc.nl

Introduction: Patients with unresectable perihilar cholangiocarcinoma (PHC) typically present with obstructive jaundice. They require percutaneous or endoscopic biliary drainage for symptom relieve and eligibility for palliative systemic chemotherapy. However, biliary drainage in unresectable PHC is complex with a high failure and complication rate even in tertiary referral centers.

Aims \& Methods: The aim of this study was to investigate the failure and complication rate of the initial drainage procedure in patients with unresectable PHC. Consecutive patients with unresectable $\mathrm{PHC}$ on imaging in two tertiary referral centers between 2002 and 2014 were identified. Patients were included if a biliary drainage procedure was performed. Baseline patient and tumor characteristics 
Abstract No:P0090

Table 1.: Definitions of failure of drainage and other severe drainage related complications

Cholangitis

Acute cholecystitis

Failure of drainage or Reintervention

Acute pancreatitis

Biliary injury

Duodenal perforation

Cardiopulmonary complications
Elevation in temperature more than $38,5^{\circ} \mathrm{C}$ and Leukocytes $\geq 10 * 109 / \mathrm{L}$, thought to have a biliary cause, without concomitant evidence of acute cholecystitis, requiring invasive intervention.

Radiologic evidence of cholecystitis, elevation in temperature more than $38.5^{\circ} \mathrm{C}$ and Leukocytes $\geq 10^{*} 109 / \mathrm{L}$, and requirement of percutaneous drainage or emergency cholecystectomy.

Rising or steady bilirubin level after therapeutic success had initially been obtained, without signs of cholangitis or cholecystitis, requiring new cannulation of the tumor. Bilirubin levels $>50 \mu \mathrm{mol} / \mathrm{L}$ within 14 days after the initial procedure Unsuccessful cannulation during the initial drainage procedure.

Second drainage procedure $\leq 14$ days after the initial procedure

Abdominal pain and a serum concentration of pancreatic enzymes (amylase or lipase) $\geq 3$ times the upper limit of normal, that requires $\geq 1$ one night of hospitalization.

Injury to the bile duct documented by any radiographic technique requiring intervention.

Perforation of the duodenum documented by any radiographic technique requiring intervention.

Any pulmonary or cardiac abnormality occurring during the drainage or in the post-intervention period that produces identifiable disease or dysfunction which is clinically significant and adversely affects the clinical course. and data on the biliary drainage procedure were collected from medical records. Definitions of failure of drainage or other severe drainage related complications are shown in table 1

Results: In total, 187 patients were included. Initial drainage was performed in a non-referral center in 125 patients $(66.8 \%)$. The initial drainage procedure was endoscopic in 158 patients $(84.5 \%)$ and percutaneous in 29 patients $(15.5 \%)$. A stent was placed in 91 patients $(61.5 \%)$ at the initial drainage procedure. The highest bilirubin level in the 2 weeks prior to drainage was 248 (IQR 138-377) umol/L. Only $14(8.1 \%)$ patients had cholangitis prior to the initial drainage procedure. Failure of drainage or other severe complications related to the initial drainage procedure were noted in $117(62.6 \%)$ patients. Failure of drainage or reintervention was most common and was noted in 95 patients $(50.8 \%)$. Bile duct injury occurred in $3(1.6 \%)$ patients, acute pancreatitis in $5(2.7 \%)$ patients and cholangitis in $11(5.9 \%)$ patients. Two $(1.1 \%)$ patients had cardiopulmonary complications and $1(0.5 \%)$ patient had a duodenal perforation. The median period between the initial and second drainage procedure was 13 (5-31) days and the bilirubin level dropped below $50 \mu \mathrm{mol} / \mathrm{L}$ in 27 patients $(14.4 \%)$. After initial drainage, 20 patients $(10.7 \%)$ died within 30-days and 66 patients $(35.3 \%)$ within 90 days. The median OS after initial drainage was 6.6 (95\% CI: $2.0-15.2)$ months.

Conclusion: Patients with unresectable PHC on imaging have a very high failure and complication rate after initial biliary drainage.

Disclosure of Interest: All authors have declared no conflicts of interest.

\section{P0091 BRUSH CYTOLOGY GUIDED BY ENDOSCOPIC RETROGRADE CHOLANGIOPANCREATOGRAPHY OF BILIARY STRICTURES}

A. Peixoto, P. Pereira, M. Silva, F. Vilas-Boas, E. Rodrigues-Pinto,

P. Moutinho-Ribeiro, J. Lopes, G. Macedo

Centro Hospitalar De São João, Porto/Portugal

Contact E-mail Address: armandoafp5@gmail.com

Introduction: Endoscopic retrograde cholangiopancreatography (ERCP)-guided brush cytology has become the most widely used method, although with limitations, in the initial diagnostic evaluation of patients with biliary strictures.

Aims \& Methods: The objective of the study was to evaluate if the systematic use of 10 brush passages improves the diagnostic yield of brush cytology guided by ERCP of bile strictures. ERCPs between 2012 and 2015 involving brush cytology of bile strictures for suspected malignancy were included in the study. Cytologies were obtained using the Brush Master V (Olympus Medical System). Histological evaluation was performed by two experienced cytopathologists.

Results: In total, 62 patients underwent cytology of biliary strictures, with a median age of 69 years (IRQ: 55-81). The cytological analysis was compatible with adenocarcinoma in $30.6 \%$ of the cases, low grade dysplasia in $3.2 \%$, high grade dysplasia in $1.6 \%$ and adenoma in $1.6 \%$. In 26 patients the cytology was negative $(41.9 \%)$ and in 13 cases it was considered inconclusive $(21 \%)$. Fifteen patients were subsequently submitted to surgery $(24.2 \%)$. In 34 cases $(54.8 \%)$ there was a correlation between the cytology and the final diagnosis. In the univariate analysis, previous history of cholecystectomy $(73.7 \%$ vs. $26.3 \%$, $\mathrm{p}=0.047)$, cytology suggestive of malignancy/adenocarcinoma $(89.5 \%$ vs. $10.5 \%, p<0.001)$ and final non-equivocal diagnosis $(14.3 \%$ vs. $85.7 \%$, $\mathrm{p}=0.002)$ were associated with positive correlation. If the cytology and final diagnosis are coded as "malignant, " "benign, " or "inconclusive, " the correlation increases to $67.7 \%$. In this situation, the univariate analysis showed that the presence of malignancy in cytology $(87.5 \%$ vs. $10.5 \%, \mathrm{p}=0.009)$ and final non-equivocal diagnosis $(35.7 \%$ vs. $64.3 \%, \mathrm{p}=0.012)$ were associated with a positive correlation. If the cases identified as undetermined cytology were excluded, there was no increase in correlation with the final diagnosis $(56.5 \%)$. Conclusion: The systematic use of 10 passages in the cytology of the biliary tract modestly increases the accuracy of the detection of malignant versus benign situations.

Disclosure of Interest: All authors have declared no conflicts of interest.

\section{P0093 UNILATERAL VERSUS BILATERAL STENT-IN-STENT PLACEMENT OF METAL STENTS FOR MALIGNANT HILAR BILIARY OBSTRUCTION}

H. Toyonaga, Y. Taniguchi, T. Inokuma

Gastroenterology, Kobe City Medical Center General Hospital, Kobe/Japan

Contact E-mail Address: toyonaga.pc@gmail.com

Introduction: Endoscopic biliary stenting is widely accepted as effective palliation therapy for unresectable malignant hilar biliary obstruction (MHBO). Although draining more than $50 \%$ of liver volume is associated with better outcomes, insertion of multiple stents is technically difficult.

Aims \& Methods: The aim of this study was to evaluate differences in technical feasibility and clinical efficacy between unilateral and bilateral stent-in-stent (SIS) placement of metal stents for MHBO. We retrospectively reviewed 23 consecutive patients with $\mathrm{MHBO}$ who underwent endoscopic biliary drainage with self-expandable metal stents (SEMS) at our institution from March 2012 to March 2017. Unilateral metal stenting was performed in 15 patients (Uni group) and bilateral metal stenting was performed in 18 patients (Bi group). In the Uni group, we placed uncovered SEMS. In the Bi group, we placed crosswired metal stents with the SIS technique. Technical success rates, complication rates and stent patency were compared between groups.

Results: There were no significant differences between the Uni group and the $\mathrm{Bi}$ group in technical success rate $(100 \%$ vs. $94 \%)$, complication rate $(0 \%$ vs. $0 \%)$, stent occlusion rate $(15 \%$ vs. $18 \%)$ or median stent patency period ( 102.5 days vs. 98 days). There was no significant difference in cumulative stent patency between the groups $(\mathrm{p}=0.669)$.

Conclusion: Endoscopic bilateral SIS placement of metal stents for palliative treatment of MHBO had a high technical success rate and low complication rate, similar to those of unilateral placement.

Disclosure of Interest: All authors have declared no conflicts of interest.

\section{P0094 CLINICAL ASSESSMENT OF THE SAFETY AND EFFICACY OF A NOVEL BIODEGRADABLE STENT IN PATIENTS WITH} BILIARY OBSTRUCTION: A PILOT STUDY

H. Othman ${ }^{1}$, N.Y. Yaacob ${ }^{2}$, E.J. Roslan ${ }^{1}$, R. Jarmin ${ }^{1}$, Z. Mohamed ${ }^{2}$

${ }^{1}$ Surgery, UKM Medical Centre, Kuala Lumpur/Malaysia

${ }^{2}$ Radiology, UKM Medical Centre, Kuala Lumpur/Malaysia

Contact E-mail Address: hairol@gmail.com

Introduction: The commonest indication for biliary stent is for the treatment of obstructive jaundice and for the management of bile leak. The currently available stents are made of either plastic or metal alloy. The stents can be inserted endoscopically to provide internal drainage of the bile into the duodenum. Among the disadvantages of plastic stents are recurrences of jaundice due to biofilms formation, which require a repeat ERCP procedure to remove the stent before 3 months. We have embarked to study the safety and feasibility of a biodegradable biliary stents (BBS), which can treat biliary obstruction without the need to undergo a repeat endoscopic procedure to remove the stents.

Aims \& Methods: This is a pilot study enrolling 30 subjects with symptomatic jaundice and pruritus caused by either benign or malignant biliary obstructions that were amenable to treatment by ERCP guided stenting. Primary objective was technical success and safety. Procedural and technical successes were assessed during the stenting procedure. Adverse events or complications were monitored throughout the studies. The secondary endpoints were clinical success, which was measured by a reduction of at least $20 \%$ of the initial serum bilirubin level at Day 7 post stenting. A simple self-assessment scale from 0 to 10 was used to assess quality of life before and after the stenting.

Results: 30 patients had the Biodegradable Biliary Stent (BBS) implanted, 18 patients $(60 \%)$ were males, the mean age was 56.9 years, 26 patients $(86.7 \%)$ had benign biliary duct disease and $4(13.3 \%)$ patients had malignant condition. 
Table 1: Patients' Demography

\begin{tabular}{ll}
\hline & $\mathrm{n}=30(\%)$ \\
\hline $\begin{array}{l}\text { Gender: males, females } \\
\text { Indication: Benign Biliary } \\
\begin{array}{l}\text { Obstruction, Malignant } \\
\text { Biliary Obstruction }\end{array} \\
\text { Age (mean } \pm \mathrm{sd})\end{array}$ & $18(60 \%), 12(40 \%)$ \\
\end{tabular}

9 patients had the fast and medium degradation stents respectively and 12 had the slow degradation stents implanted. All stents were $3.4 \mathrm{~mm}$ in diameter and the length ranges from 60 to $120 \mathrm{~mm}$ depending on the level of obstruction. It took an average of 29.6 minutes to complete each procedures, and the mean stent deployment duration was 6.0 minutes. It ranges from 13.5 minutes in the initial phase and improved to 1.5 minutes in the later phase. Biliary sphincterotomy was not necessary for inserting single biodegradable stent, but however, all patients with biliary stone had sphincterotomy to fascilitate retrival of the stones. Serum bilirubin level (SBL) showed reduction of $52 \%$ from the mean SBL of $54.9 \mu \mathrm{mol} /$ $\mathrm{L}$ prior to stenting to $26.2 \mu \mathrm{mol} / \mathrm{L}$ at Day 7 . Quality of life score improved from 2.0 up to 8.5 after stenting. The BBS ranks high in terms of loadability, trackability over guide-wire, and pushability with push catheter. There was minimal force required to implant it and it has good visibility by fluoroscopy. The BBS is as flexible as the conventional plastic stents and can be accurately deployed under fluoroscopy. Technical success or completion of the ERCP and stent deployment was achieved in all 30 patients.

Conclusion: This pilot study has shown encouraging results. It benefit the patient to avoid the burden of a second ERCP procedure for plastic stent removal. However, these results should be interpreted with caution as this is a pilot study to assess the safety and efficacy of the biodegradable stent on limited number of volunteers with symptomatic jaundice. We plan to conduct a phase 2 study involving a larger number of cohorts with a more specific indication of benign and malignant biliary stricture.

Disclosure of Interest: H. Othman: The Biodegradable Biliary Stents used for this study is sponsored by amg International $\mathrm{GmbH}$, Winsen, Germany. The authors have no financial relationship with the company which could inappropriately influence or bias the content of this presentation.

All other authors have declared no conflicts of interest

\section{References}

1. Dumonceau, J.M., et al., Biliary stenting: indications, choice of stents and results: European Society of Gastrointestinal Endoscopy (ESGE) clinical guideline. Endoscopy, 2012. 44(03): p. 277-298.

2. Antti Siiki, I.R.-K., Juhani Sand, Johanna Laukkarinen, Endoscopic biodegradable biliary stents in the treatment of benign biliary strictures: First report of clinical use in patients. Digestive Endoscopy 2017. 29: p. 118-121.

MONDAY, OCTOBER 30, 2017

09:00-17:00

\section{PANCREAS I - HALL 7}

\section{P0095 TOLL-LIKE RECEPTOR 5 IS ESSENTIAL FOR THE}

\section{ACTIVATION OF LIVER AND PANCREATIC STELLATE CELLS}

I. T. Böhm ${ }^{1}$, D. Bartsch ${ }^{1}$, T.M. Gress ${ }^{2}$, M. Buchholz ${ }^{2}$, T. T. Wissniowski², P. Di

Fazio

${ }^{1}$ Department Of Visceral Thoracic And Vascular Surgery, Philipps University

Marburg, Marburg/Germany

${ }^{2}$ Klinik Für Gastroenterologie, Endokrinologie, Stoffwechel Und Infektiologie,

Philipps Universität Marburg, Marburg/Germany

Contact E-mail Address: Boehmis@students.uni-marburg.de

Introduction: Stellate cells contribute significantly to the development of severa diseases. In particular, liver stellate cells are responsible for liver fibrogenesis and further for cirrhosis that culminates into cancer development eventually. In pancreas, it is known that stellate cells sustain the tumor cells via autophagy mechanism.

Aims \& Methods: This study aimed to clarify the involvement of Toll-like receptor 5 (TLR5) in the activation of human stellate cells. LX-2 liver stellate cells and HPSC (human pancreatic stellate cells) were treated for 48 hours with $2.5 \mathrm{ng} / \mathrm{ml}$ TGF-beta 1. The analysis of activation markers was performed by RT-qPCR, western blotting and immunofluorescence. Real-time cell monitoring with Incucyte was performed. TLRs PCR Array was performed. TLR5 knock down was obtained with commercially validated siRNAs.

Results: Treatment with $2.5 \mathrm{ng} / \mathrm{ml}$ TGF-beta 1 caused the activation of both LX2 and HPSC cells. Over-expression of alpha smooth muscle actin (a-SMA) and collagen 1 (COL1A1) transcripts was observed. The protein level of a-SMA and COL1A1 significantly increased also. Interestingly, SNAIL 1, SLUG, TLR5 and TLR3 transcripts were induced by treatment with TGF-beta 1 in both cell lines. SNAIL 1 was over-expressed at protein level also. Knock down of TLR5 neutralized the activity of TGF-beta 1 by keeping the expression of the above markers at basal level or even not expressed.

Conclusion: TLR5, for the first time, has been identified as key player of the activation of stellate cells. Its contribution represents a new aspect in terms of interaction between immune system and stellate cells and could represent a potential new target for the diseases of the gastrointestinal tract involving the activity of stellate cells. TLR5 and its natural agonist flagellin could be a key link between impairment of microbiota and organo-fibrosis in the gastrointestinal tract.

Disclosure of Interest: All authors have declared no conflicts of interest.

P0096 THE IMPAIRED FUNCTION OF THE PLASMA MEMBRANE $\mathrm{CA}^{2+}$ PUMP RESULTS IN CA ${ }^{2+}$ OVERLOAD AND CELL DAMAGE IN CFTR KNOCK OUT PANCREATIC DUCTAL CELLS

T. Madacsy ${ }^{1}$, J. Fanczal ${ }^{1}$, A. Schmidt ${ }^{1}$, P. Pallagi ${ }^{1}$, Z. Rakonczay ${ }^{2}$, P. Hegyi ${ }^{3}$, Z. Rázga ${ }^{4}$, M.A. Gray ${ }^{5}$, M. Hohwieler ${ }^{6}$, A. Kleger ${ }^{7}$, J. Maléth

${ }^{1}$ First Department Of Medicine, University of Szeged, Szeged/Hungary

${ }^{2}$ Department Of Pathophysiology, University of Szeged, Szeged/Hungary

${ }^{3}$ Centre For Translational Medicine, University of Pecs, Pecs/Hungary

${ }^{4}$ Department Of Pathology, University of Szeged, Szeged/Hungary

${ }^{5}$ University Medical School, Newcastle/United Kingdom

${ }^{6}$ Ulm University Hospital, Ulm/Germany

${ }^{7}$ Internal Medicine 1, Universitätsklinik Ulm, Ulm/Germany

Contact E-mail Address: tamaramadacsy@gmail.com

Introduction: The cystic fibrosis transmembrane conductance regulator (CFTR) has a major role in pancreatic ductal secretion and it's genetic defects damage the pancreas. It is known that intracellular $\mathrm{Ca}^{2+}$ homeostasis is disturbed in bronchial epithelial cells in cystic fibrosis (CF), but the connection of CFTR and the intracellular $\mathrm{Ca}^{2+}$ signaling has never been suggested in pancreatic damage in $\mathrm{CF}$ before.

Aims \& Methods: Our aim was to characterize the $\mathrm{Ca}^{2+}$ homeostasis of CFTRdeficient PDC. Wild type (WT) and CFTR knockout (KO) mouse pancreatic ductal and acinar cells and iPSC (induced pluripotent stem cell) derived human organoids from $2 \mathrm{CF}$ patients and controls, human $\mathrm{CF}$ pancreatic cell line (CFPAC-1; $\triangle$ F508 mutant) were used for intracellular $\mathrm{Ca}^{2+}$ measurements. Mitochondrial membrane potential $\left(\Delta \Psi_{\mathrm{m}}\right)$ and mitochondrial morphology was assessed in isolated pancreatic ducts. Immunofluorescent staining and quantitative PCR measurements were performed to detect changes of mRNA and protein expressions.

Results: The plateau phase of the agonist-induced $\mathrm{Ca}^{2+}$ signal was elevated in CFTR-deficient PDC, which was caused by decreased function of the plasma membrane $\mathrm{Ca}^{2+}$ pump (PMCA). The functional inhibition of CFTR had no effect on the PMCA activity. Human CF organoids have shown decreased PMCA function compared to control while the $24 \mathrm{~h}$ treatment of the CF organoids with VX-809 have restored the PMCA function to the control level. Similarly native CFPAC-1 cells and PDEC treated with siRNA to inhibit the expression of CFTR showed the same PMCA dysfunction. Viral transfection of CFPAC-1 with CFTR gene completely restored PMCA function. Sustained $\left[\mathrm{Ca}^{2+}\right]_{\mathrm{i}}$ levels decreased $\Delta \Psi_{\mathrm{m}}$ and induced cytochrome c release in CFTR KO PDEC without significant alterations in mitochondrial morphology.

Conclusion: Dysfunction of PMCA leads to disturbed $\mathrm{Ca}^{2+}$ homeostasis in CFTR-deficient PDC and the consequent cellular $\mathrm{Ca}^{2+}$ overload impairs mitochondrial function contributing to the pancreatic damage in $\mathrm{CF}$.

Disclosure of Interest: All authors have declared no conflicts of interest.

\section{P0097 EXPDF IMPACTS PANCREATIC DIFFERENTIATION OF HUMAN PLURIPOTENT STEM CELL DERIVED PANCREATIC ORGANOIDS}

M. Breunig ${ }^{1}$, M. Hohwieler ${ }^{1}$, T. Seufferlein ${ }^{2}$, A. Kleger $^{2}$

${ }^{1}$ Ulm University Hospital, Ulm/Germany

${ }^{2}$ Internal Medicine 1, Universitätsklinik Ulm, Ulm/Germany

Contact E-mail Address: alexander.kleger@uni-ulm.de

Introduction: Given their capability to differentiate to every cell type of the human body, human induced pluripotent stem cells (hiPSCs) provide a unique platform for developmental studies and regenerative medicine. The generation of pancreatic progenitor (PP) cells from pluripotent stem cells follows the sequential induction of virtually pure definitive endoderm (DE), foregut endoderm (GTE) and pancreatic endoderm (PE). We have recently reported the generation of a novel three-dimensional pancreatic organoid culture system that generates functional acinar-/ductal-like structures from pluripotent stem cells (Hohwieler et al, GUT, 2016).

Aims \& Methods: In the current study we implemented this culture system to understand the role of exocrine differentiation and proliferation factor (Exdpf), a signalling molecule proposed to be involved pancreatic differentiation in zebrafish. CrisprCas9 technologies were used to ablate Expdf in human embryonic stem cells, while a piggy bac engineering approach allowed us timed expression to study the role of both loss and gain of Exdpf function during pancreatic differentiation.

Results: First, a limited role of Expdf was observed until the PE stage, while PP formation was strongly diminished. Moreover, a dramatically altered organoid morphology was observed upon Expdf knock out leading to mostly cystic structures. Phenotyping for ductal and acinar lineage allowed to investigate these 
changes in more detail and genome wide expression profiling helped us to understand the role of Expdf in more detail.

Conclusion: Thus, we report a novel signalling molecule playing a critical role during human pancreas development based on a pluripotent stem cell differentiation platform

Disclosure of Interest: All authors have declared no conflicts of interest.

\section{Reference}

Human pluripotent stem cell-derived acinar/ductal organoids generate human pancreas upon orthotopic transplantation and allow disease modelling. Hohwieler M, Illing A, Hermann PC, Mayer T, Stockmann M, Perkhofer L, Eiseler T, Antony JS, Müller M, Renz S, Kuo CC, Lin Q, Sendler M, Breunig M, Kleiderman SM, Lechel A, Zenker M, Leichsenring M, Rosendahl J, Zenke M, Sainz B Jr, Mayerle J, Costa IG, Seufferlein T, Kormann M, Wagner M, Liebau S, Kleger A. Gut. 2017 Mar;66(3):473-486.

\section{P0098 MELATONIN METABOLITE; N1-ACETYL-N2-FORMYL-5- METHOXYKYNURAMINE STIMULATES PANCREATIC ENZYME SECRETION VIA CCK RELEASE. STUDY ON THE RATS}

J.M. Jaworek

Dept. Of Medical Physiologyy, Jagiellonian University CM, Krakow/Poland

Contact E-mail Address: jolanta.jaworek@uj.edu.pl

Introduction: $\mathrm{N}^{1}$-acetyl- $\mathrm{N}^{2}$-formyl-5-methoxykynuramine (AFMK), melatonin metabolite, has been demonstrated recently as effective pancreatic protector against acute inflammation. AFMK significantly attenuated acute pancreatitis; however, its effect on pancreatic exocrine function has not been investigated yet. Aims \& Methods: 1 . To investigate the effects of intraduodenal (i.d.) application of AFMK on pancreatic enzyme secretion under basal conditions and following the stimulation of this secretion with diversion of pancreato-biliary juice (DPBJ) and to examine the role of CCK in this process. 2. To assess the effect of AFMK on CCK receptor in pancreatic acinar cell line AR42J. Material and methods: For in vivo study Wistar rats weighing $300 \mathrm{~g}$ were employed. Under pentobarbitane anesthesia the animals were surgically equipped with silicone catheters, inserted into pancreato-biliary duct, and into duodenum. AFMK $(5.10 \mathrm{mg} / \mathrm{kg}$ i.d.) was given to the rats under basal conditions or following stimulation of pancreatic secretion with DPBJ. Lorglumide, the CCK1 receptor antagonist $(1 \mathrm{mg} / \mathrm{kg}$ i.d.) was administered 15 minutes prior to the application of AFMK. Samples of pancreato-biliary juice were collected to measure the amylase outputs. The blood samples were taken for determination of CCK by ELISA kit. For in vitro study pancreatic acinar cells AR42J were incubated in presence of AFMK alone or in combination with CCK. The protein signal of CCK receptor was determined by Western blot.

Results: AFMK given i.d. produced the dose-dependent increases of pancreatic amylase secretions both; unstimulated, as well as that induced by DPBJ. The rises of pancreatic amylase outputs were accompanied by significant increase of CCK plasma levels. Administration of lorglumide, a CCK1 receptor blocker, completely abolished the stimulation of pancreatic exocrine function induced by AFMK. This melatonin metabolite failed to affect protein signal for CCK receptor in AR42J cells.

Conclusion: The stimulatory effect of AFMK on pancreatic enzyme secretion in the rats is indirect and dependent on the release of CCK by this melatonin metabolite.

Disclosure of Interest: All authors have declared no conflicts of interest.

\section{P0099 INVESTIGATION OF THE FUNCTION OF TRPM2 IN MOUSE PANCREATIC ACINAR CELLS}

J. Fanczal ${ }^{1}$, P. Biró ${ }^{1}$, T. Madacsy ${ }^{1}$, P. Hegyi ${ }^{2}$, J. Maléth ${ }^{1}$

${ }^{1}$ First Department Of Medicine, University of Szeged, Szeged/Hungary

${ }^{2}$ Centre For Translational Medicine, University of Pecs, Pecs/Hungary

\section{Contact E-mail Address: julia.fanczal@gmail.com}

Introduction: Aberrant intracellular $\mathrm{Ca}^{2+}$ signaling is the hallmark of acute pancreatitis (AP) inducing mitochondrial damage, intraacinar digestive enzyme activation and cell death. Thus prevention of toxic cellular $\mathrm{Ca}^{2+}$ overload is a promising therapeutic target. The transient receptor potential melastatin 2 (TRPM2) is a non-selective cation channel that plays major role in oxidative stress induced cellular $\mathrm{Ca}^{2+}$ overload in different cell types. Although likely, its role in pancreatic acinar cells and the pathogenesis of AP was not investigated yet.

Aims \& Methods: Our aim was to characterize the functional activity of TRPM2 in pancreatic acinar cells. In our experiments pancreatic acinar cells (PAC) were isolated from wild type (WT) and TRPM2 knockout (KO) mice with enzymatic digestion. The changes of the intracellular $\mathrm{Ca}^{2+}$ level was measured whit fluorescent microscopy using FURA2-AM.

Results: The intracellular $\mathrm{Ca}^{2+}$ signals evoked by $100 \mu \mathrm{M}$ carbachol were not different in WT and TRPM2 KO PAC. On the other hand, $1 \mu \mathrm{M} \mathrm{H}_{2} \mathrm{O}_{2}$ induced significantly higher intracellular $\mathrm{Ca}^{2+}$ elevation in WT PAC compared to the TRPM2 KO. In $\mathrm{Ca}^{2+}$ free extracellular solution the $\mathrm{Ca}^{2+}$ signal in response to $1 \mu \mathrm{M} \mathrm{H} \mathrm{H}_{2} \mathrm{O}_{2}$ was markedly reduced in WT PAC confirming that $\mathrm{H}_{2} \mathrm{O}_{2}$ activates dominantly extracellular $\mathrm{Ca}^{2+}$ influx.
Conclusion: Our result confirmed the functional activity of the TRPM2 channel in pancreatic acinar cells. In our further investigations we aim to clarify the pathogenetic role of TRPM2 in AP.

Disclosure of Interest: All authors have declared no conflicts of interest.

\section{P0100 INVESTIGATION OF THE PANCREATIC DUCTAL ION} SECRETION IN PANCREATIC DUCTAL ORGANOID CULTURES

R. Molnár ${ }^{1}$, L. Alsardi ${ }^{1}$, J. Fanczal ${ }^{1}$, T. Madacsy ${ }^{1}$, P. Hegyi ${ }^{2}$, J. Maléth ${ }^{1}$ ${ }^{1}$ First Department Of Medicine, University of Szeged, Szeged/Hungary ${ }^{2}$ Centre For Translational Medicine, University of Pecs, Pecs/Hungary

\section{Contact E-mail Address: molnar.reka.89@gmail.com}

Introduction: Pancreatic ductal fluid and $\mathrm{HCO}_{3}{ }^{-}$secretion are crucially important in the physiology and pathophysiology of the exocrine pancreas. However, the study of human pancreatic secretory processes is great challenge due to the limited access to human pancreatic ductal cells. The recently developed threedimensional pancreatic organoid cultures (OC) may help to overcome this limitation. However, the ion secretory processes in pancreatic OC is not known. Aims \& Methods: Our aim was to characterize the ion transport processes in mouse pancreatic OCs. Mouse pancreatic ductal fragments were isolated by enzymatic digestion. The isolated ducts were grown in Matrigel on $37^{\circ} \mathrm{C}$ for a week in OC media. Changes of the intracellular $\mathrm{pH}$ was measured to characterize the ion transporter activities of the epithelial cells in OC.

Results: Basolateral administration of $20 \mathrm{mM} \mathrm{NH}_{4} \mathrm{Cl}$ in standard HEPES or $\mathrm{CO}_{2} / \mathrm{HCO}_{3}^{-}$buffered solution resulted in rapid intracellular alkalization, which was followed by a recovery phase. Removal of $\mathrm{NH}_{4} \mathrm{Cl}$ induced rapid acidification followed by regeneration to the resting $\mathrm{pH}$ levels. The regeneration phase was inhibited by the removal of extracellular $\mathrm{Na}^{+}$. The administration of $10 \mu \mathrm{M} \mathrm{CFTR} \mathrm{inh}_{172}$, a selective inhibitor of cystic fibrosis transmembrane conductance regulator decreased the regeneration from alkali load. Basolateral administration of $20 \mathrm{mM}$ amiloride and $20 \mathrm{mM} \mathrm{H}_{2}$ DIDS decreased the intracellular $\mathrm{pH}$ suggesting the activity of $\mathrm{Na}^{+} / \mathrm{H}^{+}$exchanger and $\mathrm{Na}^{+} / \mathrm{HCO}_{3}^{-}$cotransporter on the basolateral membrane.

Conclusion: The ion transport activities in mouse OC are similar to those observed in freshly isolated primary tissue. This suggest that $\mathrm{OC}$ will be suitable to study human ductal epithelial ion transport.

Disclosure of Interest: All authors have declared no conflicts of interest.

P0101 INVESTIGATION OF THE ORAI1 MEDIATED CA2+ ENTRY IN MOUSE PANCREATIC DUCTAL CELLS

M. Görög ${ }^{1}$, A. Grassalkovich ${ }^{1}$, A. Balázs ${ }^{1}$, P. Pallagi ${ }^{1}$, P. Hegyi ${ }^{1}$, J. Maléth ${ }^{1}$

${ }^{1}$ First Department Of Medicine, University of Szeged, Szeged/Hungary

\section{Contact E-mail Address: g.mariet@gmail.com}

Introduction: Acute pancreatitis (AP) is the most common inflammatory disorder in the gastrointestinal tract with an overall mortality of $20-30 \%$ in severe cases. The treatment of AP is not resolved yet, urging the identification of novel drug targets. Toxic cellular $\mathrm{Ca}^{2+}$ overload was highlighted as a key event in pancreatic acinar and ductal cells during the pathogenesis of AP. In addition, the inhibition of Orail in pancreatic acinar cells markedly decreased the $\mathrm{Ca}^{2+}$ toxicity and the severity of AP. However, We have no information regarding the role of Orail in pancreatic ductal physiology or pathophysiology.

Aims \& Methods: Wild type FVB/N mice were used for the isolation of pancreatic ductal fragments. The intracellular $\mathrm{pH}$ and $\mathrm{Ca}^{2+}$ level of the pancreatic ductal cells (PDC) were measured by microfluorimetry. The effect of selective Orail inhibitors provided by CalciMedica was evaluated.

Results: The tested compounds dose-dependently inhibited $\mathrm{Ca}^{2+}$ influx during the carbachol induced $\mathrm{Ca}^{2+}$ signal in PDC. Inhibition was complete at a concentration of $10 \mu \mathrm{M}$ (CM-B: 99.87\%, CM-C: 95.29\%). Next, endoplasmic reticulum $\mathrm{Ca}^{2+}$ stores were depleted with cyclopiazonic acid and the inhibition of store-operated $\mathrm{Ca}^{2+}$ entry (SOCE) was investigated after the re-addition of extracellular $\mathrm{Ca}^{2+}$. Under these conditions $\mathrm{CM}-\mathrm{B}$ and $\mathrm{CM}-\mathrm{C}$ significantly, but not completely, decreased SOCE in PDC $(55.96 \%$ and $55.03 \%$ respectively). The removal of extracellular $\mathrm{Na}^{+}$to abolish activity of the $\mathrm{Na}^{+} / \mathrm{Ca}^{2+}$ exchanger had no effect on the inhibition of SOCE by CM-B or CM-C. We also showed that the inhibition of Orail has no effect on the basal secretion of $\mathrm{HCO}_{3}{ }^{-}$by PDC, which is the main physiological function of these cells.

Conclusion: We showed that Orail has a significant role in the $\mathrm{Ca}^{2+}$ signaling of PDC. In the next step we will evaluate the pathophysiological relevance of the channel.

Disclosure of Interest: All authors have declared no conflicts of interest.

\section{P0102 ACUTE PANCREATITIS OF UNKNOWN ORIGIN AND} IDIOPATHIC JUVENILE PANCREATITIS IN SWEDEN

M. Vujasinovic ${ }^{1}$, F. Lindgren ${ }^{2}$, S. Haas ${ }^{1}$, R. Valente $^{1}$, S. Ghazi ${ }^{3}$, N. Kartalis ${ }^{4}$, R. Pozzi ${ }^{4}$, M. Del Chiaro ${ }^{5}$, P. Bauer ${ }^{6}$, C. Verbeke ${ }^{3}$, H. Witt ${ }^{7}$, U. Arnelo ${ }^{1}$, J.Löhr ${ }^{8}$

${ }^{\mathrm{i}}$ Center For Digestive Diseases, Karolinska University Hospital, Stockholm/ Sweden 
${ }^{2}$ Astrid Lindgrens Childrens Hospital, Karolinska University Hospital, Stockholm/ Sweden

${ }^{3}$ Department Of Pathology, Karolinska University Hospital, Stockholm/Sweden ${ }^{4}$ Department Of Radiology, Karolinska University Hospital, Stockholm/Sweden ${ }^{5}$ Karolinska Institutet, Karolinska Institutet Division of Surgery CLINITEC, Stockholm/Sweden

${ }^{6}$ Department Of Genetics, Centogene AG, Berlin/Germany

${ }^{7}$ Department Of Pediatrics, Technical University Munich, Munich/Germany

${ }^{8}$ Center For Digestive Diseases And Department Of Clinical Sciences, Innovation

And Technology (clintec), Karolinska University Hospital, Stockholm/Sweden

Contact E-mail Address: miroslav.vujasinovic@karolinska.se

Introduction: Acute pancreatitis (AP) is among the most difficult diseases faced by gastroenterologists and surgeons. In some cases it is difficult to understand etiology of AP. Hereditary pancreatitis (HP) is an autosomal dominant genetic disorder characterized by recurrent attacks of acute pancreatitis.

Aims \& Methods: We analyzed medical records of patients who were diagnosed with juvenile pancreatitis and pancreatitis of unknown etiology (PUE) at the Center for Digestive Diseases at Karolinska University Hospital from January 2008 to December 2016.

Results: During the observation period, 44 patients ( 17 male and 27 female) were registered with the ICD code chronic or relapsing pancreatitis, and onset of symptoms before the age of twenty. At time of first visit, the mean age was $36.7 \pm 26.9$ years, range $24-57$. The average period between the occurrence of first symptoms and diagnosis was 14.6 years (range 1-39 years). All patients $(100 \%)$ clinically presented with recurrent acute pancreatitis. There were 28 $(63.7 \%)$ patients with genetic mutations. Five out of 28 genetic positive patients $(17.9 \%)$ had a definitive diagnosis of genetic etiology of pancreatitis. Seven out of 28 genetic positive patients $(25 \%)$ had complications: in five patients endoscopic treatment due to pancreatic duct stenosis was performed; one patient had pancreatic and bile duct stenosis and one patient (female, age 28, CFTR heterozygous mutation) a pancreatic tumor (mucinous cystadenoma with high dysplasia that was successfully surgically treated with $\mathrm{R} 0$ resection). One patient died due to non-pancreatic related disease (kidney cancer). None of the patients reported alcohol overconsumption. Four out of 28 genetic positive patients $(14.3 \%)$ were active smokers. Fecal elastase-1 (FE-1) was tested in $28(63.6 \%)$ patients: $16(57.1 \%)$ in genetic positive and $12(75 \%)$ in genetic negative group of patients. Pancreatic exocrine insufficiency (PEI) was found in 5 out of $12(41.7 \%)$ of genetic negative patients and in 5 out of $16(31.2 \%)$ genetic positive patients Average age at onset of PEI was 38 years (range 27-53). Diabetes mellitus (DM) was diagnosed in one patient in group with genetic alterations and 2 patients in group without genetic alterations.

Conclusion: We found high proportion of genetic alterations in patients with juvenile pancreatitis and PUE. In patients in whom pancreatitis remains unexplained after excluding of the most often etiologies and presence of genetic alteration, hereditary pancreatitis seems as reasonable explanation even in patients with mutations in other genes than PRSS1. Routine follow-up of patients with regular testing on pancreatic exocrine insufficiency and diabetes mellitus and pancreatic cancer surveillance is necessary.

Disclosure of Interest: All authors have declared no conflicts of interest.

P0103 THE FACTORS FOR PREDICTING HOSPITAL MORTALITY IN EARLY STAGE OF SEVERE ACUTE PANCREATITIS

M. Horibe ${ }^{1}$, M. Sasaki ${ }^{2}$, M. Sanui ${ }^{3}$, H. Sawano ${ }^{4}$, T. Goto ${ }^{5}$, T. Ikeura ${ }^{6}$,

T. Hamada ${ }^{7}$, E. Iwasaki ${ }^{1}$, T. Kanai ${ }^{1}$, T. Mayumi ${ }^{8}$

${ }^{1}$ Keio University School of Medicine, Tokyo/Japan

${ }^{2}$ National Cancer Center Hospital, Tokyo/Japan

${ }^{3}$ Jichi Medical University Saitama Medical Center, Saitama/Japan

${ }^{4}$ Osaka Saiseikai Senri Hospital, Osaka/Japan

${ }^{5}$ Hiroshima City Hiroshima Citizens Hospital, Hiroshima/Japan

${ }^{6}$ Kansai Medical University, Osaka/Japan

${ }^{7}$ Gastroenterology, The University of Tokyo, Tokyo/Japan

${ }^{8}$ School of Medicine University of Occupational and Environmental Health

Fukuoka/Japan

Contact E-mail Address: aries24sirius@yahoo.co.jp

Introduction: Severe acute pancreatitis has high mortality and needs intensive care. However it is difficult to stratify the severity of acute pancreatitis in early stage because revised Atlanta classification requires persistent organ failure lasting at least 48 hours.

Aims \& Methods: We searched factors to predict hospital mortality in early stage of severe acute pancreatitis. This was a retrospective cohort study of all consecutive patients with severe acute pancreatitis who admitted at 44 institutions between June 1, 2009 and December 31, 2013. We evaluated ten factors which associated with mortality in previous study.

\begin{tabular}{llc}
\hline & $\begin{array}{l}\text { Odds ratios and 95\% } \\
\text { confidence intervals }\end{array}$ & P value \\
\hline Respiratory failure & $3.13(2.01-4.89)$ & $<0.001$ \\
Advanced age & $2.64(1.78-3.93)$ & $<0.001$ \\
Renal failure & $2.02(1.29-3.15)$ & 0.002 \\
High lactate dehydrogenase & $1.63(1.03-2.58)$ & 0.04 \\
\hline
\end{tabular}

Results: The mortality was $12.7 \%$ (142/1114 patients). All ten factors were associated with mortality in univariable analysis. In multivariable analysis, four factors, namely " partial pressure of oxygen in blood $\leq 60 \mathrm{mmHg}$ (room air) or mechanical ventilation", "age $\geq 70$ years", " blood urea nitrogen $\geq 40 \mathrm{mg} / \mathrm{dL}$ (or creatinine $\geq 2.0 \mathrm{mg} / \mathrm{dL}$ ) or oliguria (daily urine output $<400 \mathrm{~mL}$ even after adequate intravenous fluid resuscitation)", and " lactate dehydrogenase $\geq 2$ times upper limit of the normal range" were associated with mortality. The other factors, namely "base excess $\leq-3 \mathrm{mEq} / \mathrm{L}$ ", "platelet count $\leq 100,000 / \mathrm{mm} 3$ ", "serum calcium $\leq 7.5 \mathrm{mg} / \mathrm{dL}$ ", " c-reactive protein $>15 \mathrm{mg} / \mathrm{dL}$ ", "number of positive measures in systemic inflammatory response syndrome criteria $\geq 3$ " and " computed tomography grade" were not associated with mortality.

Conclusion: Advanced age, respiratory failure, renal failure and high lactate dehydrogenase could predict mortality in early stage of severe acute pancreatitis. The patients with these factors require transport to a hospital with intensive care unit.

Disclosure of Interest: All authors have declared no conflicts of interest.

\section{References}

Yokoe M, Takada T, Mayumi T, et al. Japanese guidelines for the management of acute pancreatitis: Japanese guidelines 2015. J Hepatobiliary Pancreat Sci 2015; 22: 405-32

Horibe M, Sasaki M, Sanui M, et al. Continuous Regional Arterial Infusion of Protease Inhibitors Has No Efficacy in the Treatment of Severe Acute Pancreatitis: A Retrospective Multicenter Cohort Study. Pancreas. 2017 Apr;46(4):510-517.

\section{P0104 EARLY OR LATE CHOLECYSTECTOMY IN MILD} GALLSTONE PANCREATITIS? RESULTS FROM RANDOMIZED TRIAL

R. Noel ${ }^{1}$, U. Arnelo ${ }^{1}$, L. Enochsson ${ }^{2}$, G. Sandblom ${ }^{1}$

${ }^{1}$ Department Of Clinical Science, Intervention And Technology, Karolinska Institute, Stockholm/Sweden

${ }^{2}$ Department Of Surgical And Perioperative Sciences, Umeå University, Division of Surgery, Luleå/Sweden

Contact E-mail Address: rozh.noel@sll.se

Introduction: Cholecystectomy during the index admission may reduce risk of recurrent biliary events but concerns have been raised about complications if surgery is performed to early. The objectives of this study were to compare gallstone- and cholecystectomy-related complications and patient reported quality-of-life and pain if cholecystectomy was performed before discharge or scheduled 6 weeks from the initial episode.

Aims \& Methods: Patients admitted with biliary pancreatitis at the Karolinska University Hospital were assessed for eligibility. Patients with mild pancreatitis were enrolled into either index- or scheduled cholecystectomy (IC vs. SC). IC was performed when patients showed recovery and within 48 hours from randomization. SC was planned after 6 week from randomization. The primary outcome was recurrent biliary events. Secondary endpoints were cholecystectomy complications within 1 months, the proportion of common bile duct stones at cholecystectomy requiring ERC and patientś reported quality-of-life and pain.

Results: Sixty-four patients between May 2009 and March 2017 were randomized into IC $(n=31)$ or SC $(n=33)$. Recurrent pancreatitis occurred in one and six patients in the IC and SC groups respectively $(3.2 \%$ vs. $18.7 \%, \mathrm{P}=0.049)$ Higher proportion of common bile duct stones were found in the IC group during cholecystectomy requiring ERC $(23.3 \%$ vs. $10.7 \%, \mathrm{P}=0.30)$. There was no difference in pain measure between the groups at randomization and followup.

Conclusion: Delaying cholecystectomy in mild gallstone pancreatitis is associated with increased risk for recurrent gallstone-related events. Cholecystectomy performed at the index admission is safe and feasible.

Disclosure of Interest: All authors have declared no conflicts of interest.

\section{P0105 COMPARISON OF PREDICTIVE SYSTEMS TO PREDICT DEATH, SEVERE AND MILD ACUTE PANCREATITIS ACCORDING TO THE REVISED ATLANTA CLASSIFICATION}

R. Pique Becerra, F.J. García Alonso, F. García Durán, M. Hernández Tejero, A. Granja Navacerrada, P. Bernal Checa, P. Bernal Checa, F. Bermejo Aparato Digestivo, Hospital Universitario de Fuenlabrada, Fuenlabrada/Spain

Contact E-mail Address: ruben.pique@salud.madrid.org

Introduction: The course of acute pancreatitis (AP) ranges from life threatening to mild disease, so accurately predicting its outcome is important. The revised Atlanta classification breaks the previous mild/severe dichotomy, so the absence of predictors of severity does not preclude a mild course. Studies designed according to the new classification evaluating existing predictors are still scarce. Aims \& Methods: Our study aims at evaluating the diagnostic accuracy of easily available prognostic scores to predict mortality, persistent organ failure (severe AP) and mild AP. We analyzed a single-center retrospective cohort including all adult patients admitted between 2010 and 2015. Patients with a previous episode of AP in the six months before admission, with other primary diagnosis at discharge and those partially attended at other institutions were excluded. Severity and local complications were defined according to the 2012 Atlanta classification. Four different scores (BISAP, SIRS, APACHE II and HAPS) and the following predictors: $\mathrm{C}$ reactive protein $(\mathrm{CPR})$ at $24 \mathrm{~h}$, hematocrit and $\mathrm{BUN}$ at admission 
and their evolution after $24 \mathrm{~h}$ were evaluated. Accuracy was measured using diagnostic tests and receiver operating characteristic analyses.

Results: Of the 817 eligible patients, 118 were excluded, most for a previous episode before admission. We analyzed 699 patients with a median age of 57.5 years (IQR: 45.1-72.7), 57.4\% males. Most frequent comorbidities were: diabetes $(16 \%)$, ischemic heart disease $(8.6 \%)$ and COPD $(7.7 \%)$. Median length of stay was 7 (5-10) days. Most common causes were: biliary $(53.9 \%)$, idiopathic $(21.8 \%)$ and alcoholic pancreatitis $(14.3 \%)$. A CT scan was performed in $56.1 \%$ identifying local complications in $36.2 \%$ of them, acute fluid collections in $30.1 \%$ and acute necrotic collections in $16.8 \%$. There were $42(6 \%)$ severe and $196(28 \%)$ moderately severe cases. Overall mortality was $2.4 \%(1,5-3,9 \%)$, $35.7 \%(23-50.8 \%)$ among severe cases. BUN at admission AUC: 0.88 [0.850.90]), BISAP score (AUC: 0.88 [0.85-0.90]) and APACHE II (AUC: 0.87 [0.83-0.90]) outperformed all other evaluators in predicting death. APACHE II presented the highest sensitivity, $100 \%(81.6-100 \%)$, while the BISAP score presented the highest specificity, 93.1\% (90.6-94.8\%). BUN at admission (AUC: 0.89 [0.86-0.91]) and the BISAP score (AUC: 0.87 [0.84-0.89]) also presented the best performance for severe AP. The BISAP score presented the highest specificity, $94.2 \%(92.2-95.8 \%)$, although with a low PPV, 32.1\% (21.4-45.2\%). On the other hand, diagnostic accuracy for mild AP was poor, as all predictors presented an AUC $<0.7$. The HAPS score reached the highest specificity, $87.8 \%(83-91.4 \%)$, but presented a very poor sensitivity $(28.9 \%[24.3-33.9 \%])$.

Conclusion: The revised Atlanta classification accurately identifies those patients at higher risk of death. Among the available predictors of severity, BISAP and BUN at admission presented an excellent performance, with an AUC of nearly 0.9 . New scores are needed to predict a mild course, as none of the available indexes presented an AUC $>0.7$.

Disclosure of Interest: All authors have declared no conflicts of interest.

\section{P0106 PANCREATIC DUCT ASCARIASIS}

\section{P. Somani, M. Sharma}

Department Of Gastroenterology, Jaswant Rai Speciality Hospital, Meerut/India

\section{Contact E-mail Address: dr piyushsomani $@$ yahoo.co.in}

Introduction: Although uncommon in the West, Ascaris lumbricoides is a common cause of acute pancreatitis in developing countries. The mechanism of acute pancreatitis in ascariasis may be due to obstruction of papilla of Vater, invasion of common bile duct (CBD), or pancreatic duct (PD). The invasion of pancreatic duct occurs rarely owing to its smaller calibre. Ultrasonography (USG) is an effective tool for the diagnosis of biliary and pancreatic ascariasis; however, the diagnosis may be false negative in up to $30 \%$ of cases. Pancreatic ascariasis is a rare entity. Only case reports has been described in literature. We present our retrospective data of last 10 years of 15 cases of pancreatic ascariasis. Aims \& Methods: During a study period of 10 years, 15 cases of pancreatic ascariasis were diagnosed by USG or endoscopic ultrasonogrpahy(EUS). EUS was performed with a linear or radial echoendoscope. 13 patients presented with symptoms of acute pancreatitis. Out of 13 patients, 9 presented with first episode of idiopathic pancreatitis while 4 presented with idiopathic recurrent acute pancreatitis (IRAP). One patient had biliary colic and one patient presented with acute cholangitis. 12 patients had mild pancreatitis while only one had moderate pancreatitis. Patients were treated with parenteral fluids, analgesics, antibiotics, and albendazole. Only 2 cases were diagnosed with USG while 13 patients were diagnosed with EUS. The patients underwent side viewing endoscopy/ERCP under the same sedation after EUS if EUS revealed biliary/pancreatic ascariasis. Out of 15 patients, 14 underwent side viewing endoscopy with removal of live single/multiple worms with rat tooth forceps/biopsy forceps/dormia basket in 13 patients. 2 patients were managed conservatively with repeat USG showing absence of ascariasis. There were no complications.

Results: The patient characteristics are described in table. 2 patients had associated bile duct ascariasis. Sonographic characteristics of $A$. lumbricoides were single or multiple echogenic nonshadowing linear, tubular structures and curved strips with anechoic tubular central lines. EUS features were single or multiple linear hyperechoic structure without acoustic shadowing in the PD or CBD ("single-tube sign" or "strip sign") or with central hypoechoic tube representing alimentary canal of the worm ("double tube sign" or "inner tube sign") and movements of worms inside the duct. Live roundworms were removed from PD without undertaking sphincterotomy. In endemic areas, sphincterotomy facilitates the risk of migration of worms into the CBD or PD

Conclusion: Ascariasis-induced acute pancreatitis is usually mild and EUS is the investigation of choice. The recurrence is rare and treatment is side viewing endoscopy with removal of worms. EUS significantly improves the diagnostic yield for idiopathic acute pancreatitis (IAP). Our retrosceptive study shows that EUS is a highly accurate method to diagnose the etiology of IAP with reference to biliopancreatic ascariasis. Although USG is quite sensitive for diagnosing BPA, its sensitivity significantly falls when the worm is thin, in the PD, or the CBD is non-dilated. EUS is more sensitive for diagnosis of ascariasis in the pancreatic duct than other radiologic investigations. The probable reasons are excellent imaging of pancreas by EUS and in and out movement of ascaris which might me missed by other investigations. Endoscopic retrograde cholangiopancreatography, considered the gold standard for diagnosis of biliary ascariasis, should be reserved for therapeutic rather than diagnostic use as papillotomy can lead to reentry of the worm into the common bile duct. EUS should be used early in the work-up of IAP after the first episode. We propose EUS to be investigation of choice for PD ascariais. Most of the episodes are of mild pancreatitis with no mortality.

Disclosure of Interest: All authors have declared no conflicts of interest.

\section{References}

1. Somani P, Sharma M, Pathak A et al. Endoscopic ultrasound imaging of pancreatic duct ascariasis. Endoscopy 2016; 48: E33-E34.

2. Sharma M, Somani P, Patil A. Double Trouble in Acute Pancreatitis. Gastroenterology 2016; 150:833-834.

\section{P0107 LARGE-VOLUME FLUID RESUSCITATION IN PATIENTS WITH SEVERE ACUTE PANCREATITIS IS ASSOCIATED WITH REDUCED MORTALITY: A MULTI-CENTRE RETROSPECTIVE STUDY}

T. Yamashita ${ }^{1}$, M. Horibe ${ }^{2}$, M. Sanui ${ }^{3}$, M. Sasaki ${ }^{4}$, H. Sawano ${ }^{5}$, T. Goto ${ }^{6}$, T. Ikeura ${ }^{7}$, T. Hamada ${ }^{8}$, T. Oda ${ }^{9}$, T. Mayumi ${ }^{10}$

${ }^{1}$ Emergency Medical Center, Fukuyama City Hospital, Hiroshima/Japan ${ }^{2}$ Division Of Gastroenterology And Hepatology Department Of Internal Medicine, Keio University School of Medicine, Tokyo/Japan

${ }^{3}$ Department Of Anesthesiology And Critical Care Medicine, Jichi Medical University Saitama Medical Center, Saitama/Japan

${ }^{4}$ Department Of Hepatobiliary And Pancreatic Oncology, National Cancer Center Hospital, Tokyo/Japan

${ }^{5}$ Senri Critical Care Medical Center, Osaka Saiseikai Senri Hospital, Osaka/Japan ${ }^{6}$ Department Of Anesthesiology And Intensive Care, Hiroshima City Hiroshima Citizens Hospital, Hiroshima/Japan

${ }^{7}$ The Third Department Of Internal Medicine, Kansai Medical University, Osaka/ Japan

${ }^{8}$ Gastroenterology, The University of Tokyo, Tokyo/Japan

${ }^{9}$ Department Of General Internal Medicine, Iizuka Hospital, Fukuoka/Japan

${ }^{10}$ Department Of Emergency Medicine, School of Medicine University of

Occupational and Environmental Health, Fukuoka/Japan

Contact E-mail Address: bluenoble21@gmail.com

Introduction: The severity of acute pancreatitis varies widely, from a mild selflimited disease to one with a severe clinical course complicated by multiple organsystem failure $^{1}$. No pharmacologic therapy has been shown to improve the prognosis of patients with severe acute pancreatitis, while the quality of supportive care including early fluid resuscitation is critically important ${ }^{1,2}$. Fluid resuscitation maintains adequate intravascular volume by compensating for fluid shifts to the third space ${ }^{3}$. However, there is a lack of consensus regarding the details of optimal fluid administration such as the type of fluid, infusion rate and volume of administration, and the physiologic goals of fluid resuscitation ${ }^{4}$

Aims \& Methods: The aim of this study is to evaluate the association between the volume of fluid administered and clinical outcomes in patients with severe acute pancreatitis. We conducted a secondary analysis of data from a multi-centre retrospective study of patients with severe acute pancreatitis in Japan, which was registered with the University Hospital Medical Information Network Clinical Trials Registry (Registry number 000012220) and approved by the Institutional Review Board or the Medical Ethics Committee at each institution ${ }^{5}$. The diagnosis of severe acute pancreatitis was based on criteria of the Japanese Ministry of Health, Labour and Welfare (Japanese Severity Score) ${ }^{6}$. Patients were stratified into two groups: administered fluid volume $<6000 \mathrm{ml}$ and $\geq 6000 \mathrm{ml}$ in the first 24 hours. We evaluated the association between the two groups and clinical outcomes using multivariable logistic regression analysis. The primary outcome was in-hospital mortality. As a sensitivity analysis, we conducted an identical analysis for subgroup patients with severe acute pancreatitis diagnosed according to the revised Atlanta classification ${ }^{7}$.

Results: We analysed 1097 patients, and the mean fluid volume administered was $5618 \pm 3018 \mathrm{ml}$ (mean \pm standard deviation), with 708 and 389 patients stratified into the fluid $<6000 \mathrm{ml}$ and fluid $\geq 6000 \mathrm{ml}$ groups, respectively. Overall in-hospital mortality was $12.3 \%$. The fluid $>6000 \mathrm{ml}$ group had significantly higher mortality than the fluid $<6000 \mathrm{ml}$ group $(15.9 \%$ vs. $10.3 \%$, p $<0.05)$. However, in multivariable logistic regression analysis, conversely fluid $\geq 6000 \mathrm{ml}$ within the first 24 hours was significantly associated with reduced mortality (OR 0.58 , $95 \%$ CI $0.34-0.98$ ). We performed subgroup analyses for patients diagnosed with severe acute pancreatitis based on the revised Atlanta classification ${ }^{7}$. One hundred sixty-seven patients were classified in the fluid $<6000 \mathrm{ml}$ group, and 201 patients classified in the fluid $\geq 6000 \mathrm{ml}$ group. There were no significant differences between the two groups with regard to in-hospital mortality (fluid $<6000 \mathrm{ml}: 35.3 \%$ vs. fluid $\geq 6000 \mathrm{ml}: 28.4 \%, \mathrm{p}=0.18$ ). In multivariable logistic regression analysis, administered fluid $>6000 \mathrm{ml}$ within the first 24 hours was associated with significantly less mortality (OR $0.56,95 \%$ CI $0.32-0.98$ ).

Conclusion: In patients with severe acute pancreatitis, administration of fluid $>6000 \mathrm{ml}$ within the first 24 hours is associated with decreased mortality.

Disclosure of Interest: All authors have declared no conflicts of interest.

\section{References}

1. Pandol SJ, Saluja AK, Imrie CW, et al. Acute pancreatitis: bench to the bedside. Gastroenterology 2007; 132: 1127-51.

2. Gardner TB, Vege SS, Pearson RK, et al. Fluid resuscitation in acute pancreatitis. Clin Gastroenterol Hepatol 2008; 6: 1070-6.

3. Frossard JL, Steer ML, Pastor CM. Acute pancreatitis. Lancet 2008; 371: 143-52.

4. Haydock MD, Mittal A, Wilms HR, et al. Fluid therapy in acute pancreatitis: anybody's guess. Ann Surg 2013; 257: 182-8.

5. Horibe M, Sasaki M, Sanui M, et al. Continuous Regional Arterial Infusion of Protease Inhibitors has no Efficacy in the Treatment of Severe Acute Pancreatitis: A Retrospective Multi-center Cohort Study. Pancreas 2017; 46: $510-7$ 


\section{Abstract No:P0109}

Table 1a: AUC, sensitivity, specificity, positive predictive values and negative predictive values of scoring systems and biomarkers in predicting Severe Acute Pancreatitis. (SAP: Severe acute pancreatitis. AUC: Area under the curve. PPV: positive predictive value. NPV: Negative predictive value.

\begin{tabular}{lllllll}
\hline SAP & Cut-off values & AUC (95\% CI) & Sensitivity $(95 \%$ CI $)$ & Specificity $(95 \%$ CI $)$ & PPV (95\% CI) \\
BISAP & $\geq 3$ & $0.9(0.83-0.97)$ & $70.6 \%(46.9 \%-86.7 \%)$ & $93.3 \%(89.5 \%-95.7 \%)$ & $41.4 \%(25 \%-59.3 \%$ & $97.9 \%(95.3 \%-99.1 \%)$ \\
RANSON & $\geq 4$ & $0.85(0.76-0.95)$ & $88.2 \%(65.7-96.7 \%)$ & $79 \%(73.5 \%-83.5 \%)$ & $22.1 \%(13.8 \%-33.3 \%)$ & $99 \%(96.4 \%-99.7 \%)$
\end{tabular}

Table 1b AUC, sensitivity, specificity, positive predictive values and negative predictive values of scoring systems and biomarkers in predicting Mortality. (AUC: Area under the curve. PPV: positive predictive value. NPV: Negative predictive value.

\begin{tabular}{|c|c|c|c|c|c|c|}
\hline Mortality & Cut-off values & $A U C(95 \% C I)$ & Sensitivity (95\% CI) & Specificity $(95 \% \mathrm{CI})$ & $P P V(95 \% C I)$ & $N P V(95 \% C I)$ \\
\hline BISAP & $\geq 3$ & $0.97(0.95-0.99)$ & $100 \%(67.6 \%-100 \%)$ & $92 \%(88 \%-94.7 \%)$ & $27.6 \%(14.7 \%-45.7 \%)$ & $100 \%(98.4 \%-100 \%)$ \\
\hline RANSON & $\geq 4$ & $0.94(0.89-0.99)$ & $100 \%(67.6-100 \%)$ & $77 \%(71.5 \%-81.7 \%)$ & $11.8 \%(6.1 \%-21.5 \%)$ & $100 \%(98.1 \%-100 \%)$ \\
\hline
\end{tabular}

6. Yokoe M, Takada T, Mayumi T, et al. Japanese guidelines for the management of acute pancreatitis: Japanese guidelines 2015. J Hepatobiliary Pancreat Sci 2015; 22: 405-32.

7. Banks PA, Bollen TL, Dervenis C, et al. Classification of acute pancreatitis2012: revision of the Atlanta classification and definitions by international consensus. Gut 2013; 62: 102-11.

\section{P0108 SERUM PHOSPHATE AS A PROGNOSTIC FACTOR IN POST-ENDOSCOPIC RETROGRADE CHOLANGIOPANCREATOGRAPHY PANCREATITIS}

Y.H. Choi ${ }^{1}$, D.K. Jang ${ }^{2}$, Y. Kim ${ }^{1}$, J.K. Ryu ${ }^{1}$, W.H. Paik ${ }^{1}$, J.W. Kang ${ }^{1}$, J.H. Choi ${ }^{1}$, S.H. Lee ${ }^{1}$

${ }^{1}$ Department Of Internal Medicine And Liver Research Institute, Seoul National University College of Medicine, Seoul/Korea, Republic of

${ }^{2}$ Department Of Internal Medicine, Dongguk University Ilsan Medical Center,

Goyang-si, Gyeonggi-do/Korea, Republic of

Contact E-mail Address: crzyzs@naver.com

Introduction: There is no definite prognostic marker of post-endoscopic retrograde cholangiopancreatography (ERCP) pancreatitis (PEP). Previous animal study reported that serum phosphate correlated with severity of acute pancreatitis.

Aims \& Methods: In this study, we aimed to evaluate the efficacy of serum phosphate as a prognostic factor for PEP in human. Between January 2005 and December 2016, 176 patients who underwent serum phosphate test among patients with PEP were included in the study. Serum phosphate was measured between 12 and 24hours after ERCP. PEP was defined as new abdominal pain with at least 3 -fold increase in serum amylase or lipase levels. The severity of the pancreatitis was determined according to consensus guidelines.

Results: A total of 176 patients with mild $(n=69 ; 39.2 \%)$, moderate $(n=80$ $45.5 \%)$, or severe $(n=27 ; 15.3 \%)$ PEP were included. Serum phosphate was associated with severity of PEP. Serum phosphate levels in mild, moderate and severe PEP were $2.9,3.0$, and $3.3 \mathrm{mg} / \mathrm{dL}$, respectively $(\mathrm{P}=0.035)$. In the linear regression analysis, only the serum phosphate was associated with the duration of hospitalization $(\mathrm{P}=0.016)$. Hematocrit, blood urea nitrogen, and high sensitivity C-reactive protein were not significantly associated with admission duration. Conclusion: According to our retrospective data, serum phosphate is associated with the severity of PEP, and its role as a prognostic factor for PEP can be considered.

Disclosure of Interest: All authors have declared no conflicts of interest.

\section{References}

1. Mazzini GS, Jost DT, Ramos DB, et al. High phosphate serum levels correlate with the severity of experimental severe acute pancreatitis: insight into the purinergic system. Pancreas. 2015;44:619-25.

2. Cotton PB, Lehman G, Vennes J, et al. Endoscopic sphincterotomy complications and their management: an attempt at consensus. Gastrointest Endosc. 1991;37:383-93.

3. Freeman ML, Guda NM. Prevention of post-ERCP pancreatitis: a comprehensive review. Gastrointest Endosc. 2004;59:845-64.

4. Freeman ML, Nelson DB, Sherman S, et al. Complications of endoscopic biliary sphincterotomy. N Engl J Med. 1996;335:909-18.

5. Ranson JH. Etiological and prognostic factors in human acute pancreatitis: a review. Am J Gastroenterol 1982;77:633-8.

6. Blamey SL, Imrie CW, O'Neill J, et al. Prognostic factors in acute pancreatitis. Gut 1984;25:1340-6.

7. Knaus WA, Zimmerman JE, Wagner DP, et al.APACHE-acute physiology and chronic health evaluation: a physiologically based classification system. Crit Care Med 1981;9:591-7.

8. Balthazar EJ, Robinson DL, Megibow AJ, et al. Acute pancreatitis: value of CT in establishing prognosis. Radiology 1990;174:331-6.

9. Luo H, Zhao L, Leung J, et al. Routine pre-procedural rectal indometacin versus selective post-procedural rectal indometacin to prevent pancreatitis in patients undergoing endoscopic retrograde cholangiopancreatography: a multicentre, single-blinded, randomised controlled trial. Lancet. 2016;387:2293-301.

10. Elmunzer BJ, Scheiman JM, Lehman GA, et al. A randomized trial of rectal indomethacin to prevent post-ERCP pancreatitis. $N$ Engl $\mathrm{J}$ Med. 2012;366:1414-22.
11. Kannan S. E-NTPase/NTPDase: potential role as a regulatory element in inflammation. Med Hypotheses. 2002;58:527-8.

12. Longhi MS, Robson SC, Bernstein SH, et al. Biological functions of ectoenzymes in regulating extracellular adenosine levels in neoplastic and inflammatory disease states. $J$ Mol Med (Berl). 2013;91:165-72.

13. Csoka B, Nemeth ZH, Rosenberger P, et al. A2B adenosine receptors protect against sepsis-induced mortality by dampening excessive inflammation. J Immunol. 2010;185:542-50.

14. Birkenfeld AL, Gollasch M, Göbel U, et al. The phosphorus connection-a puzzling business. Nephrol Dial Transplant. 2004;19:1643-5.

15. Sitkovsky MV, Lukashev D, Apasov S, et al. Physiological control of immune response and inflammatory tissue damage by hypoxia-inducible factors and adenosine A2A receptors. Anпu Rev Immunol. 2004;22:657-82.

\section{P0109 BISAP AND RANSON AS PREDICTORS OF SEVERE ACUTE PANCREATITIS AND MORTALITY IN A EUROPEAN COHORT}

F. Valverde López ${ }^{1}$, E. Fernández-Fernández ${ }^{1}$, R. Jiménez-Rosales ${ }^{1}$,

A.M. Matas-Cobos ${ }^{1}$, E. Ortega-Suazo ${ }^{2}$, E. Redondo-Cerezo ${ }^{1}$

${ }^{1}$ Gastroenterology And Hepatology, University Hospital Virgen de las Nieves,

Granada/Spain

${ }^{2}$ Gastroenterology, "Virgen de las Nieves" University Hospital, Granada/Spain

Contact E-mail Address: fcovalverde89@gmail.com

Introduction: The 2012 revised Atlanta Classification in 2012 has established an important change in Acute Pancreatitis (AP) definitions, comprising 3 grades of severity depending on the presence and persistence of organic failure (1). Thereby, patients with severe acute pancreatitis (SAP) include the vast majority of mortality in this disease (2-4).

Aims \& Methods: Our goal was to know the predicting ability of BISAP and Ranson in a European cohort with the latest Atlanta Classification. Patients diagnosed of AP in University Hospital Virgen de las Nieves from June 2010June 2012 were included. BISAP was calculated on admission and Ranson was calculated with parameters of the first 48 hours. We assessed the presence of SAP and mortality, receiving operating curves (ROC) and area under the curve (AUC) were calculated, selecting the best cut-off value in terms of sensibility, specificity, positive predictive value (VPP) and negative predictive value (NPV)

Results: 269 patients were included, $52.8 \%$ female and $47.2 \%$ male, 17 presented SAP $(6.3 \%), 8$ deaths $(3 \%)$. Etiology: $65 \%$ biliary, $10.4 \%$ alcoholic, post ERCP $3 \%$, drugs related in $0.7 \%$, metabolic in $1.1 \%$, idiopathic $15.2 \%$, mixed (alcoholic + biliary) $1.1 \%$ and others in $2.6 \%$ BISAP showed an AUC 0.9 , with sensitivity of $70 \%$, specificity of $94 \%$, PPV $41.4 \%$, NPV $97.9 \%$ and overall accuracy of $91.8 \%$ for the prediction of SAP. Regarding mortality prediction, it exhibited an AUC of 0.97 , sensitivity of $100 \%$, specificity $92 \%$, PPV $27.6 \%$, NPV $100 \%$ and accuracy $92.2 \%$ (Tables $1 \mathrm{a}$ and $1 \mathrm{~b}$ ) RANSON showed AUC 0.85 , sensitivity of $88 \%$ and specificity of $79 \%$, PPV $22.1 \%$, NPV $99 \%$ and accuracy of $79.6 \%$ for SAP prediction. With the respect of mortality prediction, it presented an AUC of 0.94 , sensitivity of $100 \%$ and specificity of $77 \%$, PPV $11.8 \%$, NPV $100 \%$ and accuracy of $77.7 \%$ for mortality prediction (Tables 1a and $1 \mathrm{~b}$ )

Conclusion: Our study analyzed the prediction ability of BISAP and Ranson in a European cohort using de updated classification of Atlanta 2012 and showed good results in terms of sensitivity and specificity in both scores when predicting SAP and death. As they are low prevalent outcomes in our sample, PPVs are low but it gives interesting information, since $41 \%$ of patients with BISAP $\geq 3$ on admission will develop SAP. On the other hand, NPVs are very high so in patients with BISAP $<3$ or Ranson $<4$, SAP and mortality can be considered very unlikely. Although both scores have similar results, Ranson needs more than 48 hours to be completed, so it does not provide enough information on admission to warrant the patient aggressive therapies or ICU admission in the earliest stage of the disease. Multicenter studies in European cohorts are needed to confirm our findings.

Disclosure of Interest: All authors have declared no conflicts of interest.

\section{References}

1. Banks PA, Bollen TL, Dervenis C, et al Classification of acute pancreatitis 2012: revision of the Atlanta classification and definitions by international consensus. Gut 2013; 62(1):102-11

2. Buter A, Imrie CW, Carter CR, et al. Dynamic nature of early organ dysfunction determines outcome in acute pancreatitis. Br J Surg 2002;89:298302. 
Table 1a. AUC, sensitivity, specificity, positive predictive values and negative predictive values of scoring systems and biomarkers in predicting Severe Acute Pancreatitis. (SAP: Severe acute pancreatitis. AUC: Area under the curve. PPV: positive predictive value. NPV: Negative predictive value. BUN: Blood urea nitrogen measured on admission. CRP: C-reactive protein measured on admission.

\begin{tabular}{lllllll}
\hline SAP & Cut-off values & AUC $(95 \%$ CI) & Sensitivity $(95 \%$ CI) & Specificity $(95 \%$ CI) & PPV (95\% CI) \\
Lactate & $>2.8 \mathrm{mEq} / \mathrm{L}$ & $0.79(0.71-0.88)$ & $58.8 \%(36 \%-78.4 \%)$ & $83.3 \%(78.2 \%-87.4 \%)$ & $19.2 \%(10.8 \%-31.9 \%)$ & $96.8 \%(93.5 \%-98.4 \%)$ \\
Creatinine & $>1.4 \mathrm{mg} / \mathrm{Dl}$ & $0.82(0.71-0.93)$ & $64.7 \%(41.3 \%-82.7 \%)$ & $86.1 \%(81.3 \%-89.8 \%)$ & $23.9 \%(13.9 \%-37.9 \%)$ & $97.3 \%(94.3 \%-98.8 \%)$ \\
BUN & $>28 \mathrm{mg} / \mathrm{dL}$ & $0.83(0.73-0.93$ & $64.7 \%(41.3 \%-82.7 \%$ & $86.9 \%(82.2 \%-90.5 \%)$ & $25 \%(14.6 \%-39.4 \%)$ & $97.3 \%(94.3 \%-98.8 \%)$ \\
CRP & CRP $>47 \mathrm{mg} / \mathrm{L}$ & $0.72(0.60-0.83)$ & $70.6 \%(46.9 \%-86.7 \%)$ & $69.7 \%(63.8 \%-75.1 \%)$ & $13.6 \%(8 \%-22.3 \%)$ & $97.2 \%(93.7 \%-98.8 \%$
\end{tabular}

Table 1b. AUC, sensitivity, specificity, positive predictive values and negative predictive values of scoring systems and biomarkers in predicting Mortality. (AUC: Area under the curve. PPV: positive predictive value. NPV: Negative predictive value. BUN: Blood urea nitrogen measured on admission. CRP: C-reactive protein measured on admission.

\begin{tabular}{|c|c|c|c|c|c|c|}
\hline Mortality & Cut-off values & $A U C(95 \% C I)$ & Sensitivity (95\% CI) & Specificity $(95 \%$ CI $)$ & $P P V(95 \% C I)$ & $N P V(95 \% C I)$ \\
\hline Lactate & $>2.8 \mathrm{mEq} / \mathrm{L}$ & $0.87(0.78-0.96)$ & $87.5 \%(52.9 \%-97.2 \%)$ & $82.7 \%(77.6 \%-86.8 \%)$ & $13.5 \%(6.7 \%-25.3 \%)$ & $99.5 \%(97.4 \%-99.9 \%)$ \\
\hline Creatinine & $>1.4 \mathrm{mg} / \mathrm{dL}$ & $0.85(0.70-0.99)$ & $75 \%(40.9 \%-92.9 \%)$ & $84.7 \%(79.8 \%-88.5 \%)$ & $13 \%(6.1 \%-25.7 \%)$ & $99.1 \%(96.8 \%-99.8 \%)$ \\
\hline BUN & $>28 \mathrm{mg} / \mathrm{dL}$ & $0.83(0.68-0.98)$ & $75 \%(40.9 \%-92.9 \%)$ & $85.4 \%(80.6 \%-89.2 \%)$ & $13.6 \%(6.4 \%-26.7 \%)$ & $99.1 \%(96.8 \%-99.8 \%)$ \\
\hline CRP & $\mathrm{CRP}>47 \mathrm{mg} / \mathrm{L}$ & $0.62(0.41-0.82)$ & $62.5 \%(30.6 \%-86.3 \%)$ & $68.1 \%(62.2 \%-73.4 \%)$ & $5.7 \%(2.5 \%-12.6 \%)$ & $98.3 \%(95.2 \%-99.4 \%)$ \\
\hline
\end{tabular}

3. Johnson CD, Abu-Hilal M. Persistent organ failure during the first week as a marker of fatal outcome in acute pancreatitis. Gut 2004;53:1340

4. Mofidi R, Duff MD, Wigmore SJ, et al. Association between early systemic inflammatory response, severity of multiorgan dysfunction and death in acute pancreatitis. Br J Surg 2006;93:738-44

\section{P0110 POST-ENDOSCOPIC RETROGRADE CHOLANGIOPANCREATOGRAPHY PANCREATITIS: MORBIDITY AND PREDICTORS OF SEVERITY}

E. El-Hanafy ${ }^{1}$, A. El Nakeeb ${ }^{2}$

${ }^{1}$ Facultu Of Medicin, Mansoura University, gastroenterology surgical center. Cairo/Egypt

${ }^{2}$ Facultu Of Medicin, Mansoura University, gastroenterology surgical center, Cairo/Egypt

Contact E-mail Address: dr_ehab_elhanafy@yahoo.com

Introduction: Endoscopic retrograde cholangiopancreatography (ERCP) is increasingly used for therapeutic management of various biliary and pancreatic diseases[1]. However, ERCP is not a procedure without morbidities[2]. PostERCP pancreatitis (PEP) remains the most common and serious complication after ERCP[3]

Aims \& Methods: To detect risk factors for post-endoscopic retrograde cholangiopancreatography (ERCP) pancreatitis (PEP) and investigate the predictors of its severity. This is a prospective cohort study of all patients who underwent ERCP. Pre-ERCP data, intraoperative data, and post-ERCP data were collected Results: The study population consisted of 996 patients. Their mean age at presentation was $58.42( \pm 14.72)$ years, and there were 454 male and 442 female patients. Overall, PEP occurred in $102(10.2 \%)$ patients of the study population; eighty $(78.4 \%)$ cases were of mild to moderate degree, while severe pancreatitis occurred in $22(21.6 \%)$ patients. No hospital mortality was reported for any of $\mathrm{PEP}$ patients during the study duration. Age less than 35 years $(\mathrm{P}=0.001$, $\mathrm{OR}=0.035)$, narrower common bile duct $(\mathrm{CBD})$ diameter $(\mathrm{P}=0.0001)$ and increased number of pancreatic cannulations $(\mathrm{P}=0.0001)$ were independent risk factors for the occurrence of PEP.

Conclusion: In conclusion, PEP is the most frequent and devastating complication after ERCP. PEP is associated with higher morbidity and mortality beside its effect in increasing the consumption of hospital resources. Age less than 35 years, narrower median CBD diameter and increased number of pancreatic cannulations are independent risk factors for the occurrence of PEP. Patients with these risk factors are candidates for prophylactic and preventive measures against PEP.

Disclosure of Interest: All authors have declared no conflicts of interest.

\section{References}

1 Jeurnink SM, Siersema PD, Steyerberg EW, Dees J, Poley JW, Haringsma J, Kuipers EJ. Predictors of complications after endoscopic retrograde cholangiopancreatography: a prognostic model for early discharge. Surg Endosc 2011; 25: 2892-2900. [PMID: 21455806 DOI: 10.1007/s00464-011-1638-9]

2 Cotton PB, Garrow DA, Gallagher J, Romagnuolo J. Risk factors for complications after ERCP: a multivariate analysis of 11, 497 procedures over 12 years. Gastrointest Endosc 2009; 70: 80-88. [PMID: 19286178 DOI: 10.1016/ j.gie.2008.10.039]

3 Yang D, Draganov PV. Indomethacin for post-endoscopic retrograde cholangiopancreatography pancreatitis prophylaxis: is it the magic bullet? World $J$ Gastroenterol 2012; 18: 4082-4085 [PMID: 22919238 DOI: 10.3748/ wjg.v18.i31.4082]

\section{P0111 LACTATE AND OTHER BIOMARKERS AS PREDICTORS OF SEVERE ACUTE PANCREATITIS IN A EUROPEAN COHORT}

F. Valverde López ${ }^{1}$, E. Fernández-Fernández ${ }^{1}$, F. Vadillo-Calles ${ }^{1}$, A.M. MatasCobos $^{1}$, P. Abellán-Alfocea ${ }^{2}$, E. Redondo-Cerezo ${ }^{1}$

${ }^{1}$ Gastroenterology And Hepatology, University Hospital Virgen de las Nieves, Granada/Spain

${ }^{2}$ Gastroenterology, "Virgen de las Nieves" University Hospital, Granada/Spain

Contact E-mail Address: fcovalverde89@gmail.com

Introduction: The revision of Atlanta Classification in 2012 has established an important change in Acute Pancreatitis (AP) definitions, comprising 3 grades of severity depending on the presence and persistence of organic failure (1). Thereby, patients with severe acute pancreatitis (SAP) include the vast majority of deaths in this disease (2-4).

Aims \& Methods: Our aim was to know the predicting ability of lactate and other single biomarkers in a European cohort with the revised Atlanta Classification in terms of severe acute pancreatitis (SAP) and mortality. Patients diagnosed of AP in the University Hospital "Virgen de las Nieves" from June 2010 to June 2012 were included. Blood sample including hematological parameters, biochemical test, BUN, CRP and venous gasometry including lactate were performed on admission. We assessed the presence of SAP and mortality during admission, receiving operating curves (ROC) and area under the curve (AUC) were calculated, selecting the best cut-off value in terms of sensibility, specificity, positive predictive value (VPP) and negative predictive value (NPV).

Results: 269 patients were included, $52.8 \%$ female and $47.2 \%$ male, 17 presented SAP $(6.3 \%), 8(3 \%)$ patients died. Etiology: $65 \%$ biliary, $10.4 \%$ alcoholic, post ERCP $3 \%$, drugs related in $0.7 \%$, metabolic in $1.1 \%$, idiopathic $15.2 \%$, mixed (alcoholic + biliary) $1.1 \%$ and other in $2.6 \%$ The results in terms of sensitivity, specificity, PPV, NPV and AUC are summarized in Table 1a and 1b. BUN had the highest AUC in terms of SAP predictions whereas Lactate had was the best in terms of mortality prediction.

Conclusion: Biomarkers are quick but incomplete tools for SAP prediction, which can be easily obtained at any moment throughout the disease. Our study shows good values of specificity and AUC for BUN, $\mathrm{Cr}$ and lactate but not for CRP concerning SAP and mortality prediction on admission, but since they are low prevalent outcomes in our sample, PPVs are low and not very reliable. Although many of these parameters has been analyzed in previous studies, we present lactate as a new biomarker with similar performance than that of $\mathrm{Cr}$ and BUN, suggesting a possible role for scores building or outcome monitoring. Disclosure of Interest: All authors have declared no conflicts of interest.

\section{References}

1. Banks PA, Bollen TL, Dervenis C, et al. Classification of acute pancreatitis 2012: revision of the Atlanta classification and definitions by international consensus. Gut 2013; 62(1):102-11

2. Buter A, Imrie CW, Carter CR, et al. Dynamic nature of early organ dysfunction determines outcome in acute pancreatitis. Br J Surg 2002;89:298302.

3. Johnson CD, Abu-Hilal M. Persistent organ failure during the first week as a marker of fatal outcome in acute pancreatitis. Gut 2004;53:1340

4. Mofidi R, Duff MD, Wigmore SJ, et al. Association between early systemic inflammatory response, severity of multiorgan dysfunction and death in acute pancreatitis. Br J Surg 2006;93:738-44. 


\section{P0112 COTTON VS. REVISED ATLANTA CRITERIA TO DEFINE}

\section{SEVERITY OF POST-ERCP PANCREATITIS}

X. Smeets ${ }^{1}$, A.C. Tan $^{2}$, J.P.h. Drenth ${ }^{1}$, E. Van Geenen

${ }^{1}$ Gastroenterology \& Hepatology, Radboudumc, Nijmegen/Netherlands

${ }^{2}$ Canisius Wilhelmina Hospital Dept. of Hepatogastroenterology, Nijmegen Netherlands

Contact E-mail Address: xaviersmeets@gmail.com

Introduction: The Cotton criteria (1) and the revised Atlanta classification (2) are advocated to define post-endoscopic retrograde cholangiopancreatography (ERCP) pancreatitis (PEP) severity (3). Whereas Cotton puts the emphasis on length of hospitalisation. Atlanta focuses on the presence of local (necrosis) and systemic (organ failure) complications. The number of hospitalization days may not be a proper representation of PEP severity, because it is influenced by other diagnoses such as ERCP complications and comorbidity. The goal of this retrospective cohort study is to compare the Cotton and Atlanta criteria for the severity of PEP

Aims \& Methods: All ERCP procedures from a Dutch university medical centre and a teaching hospital between 2012 and November 2016 were checked retrospectively for patients with PEP. Patients were eligible if they met the Cotton criteria or Atlanta criteria for acute pancreatitis. All records were checked up to 48 hours after ERCP to capture delayed PEP. Patients were excluded if they had acute pancreatitis prior to ERCP or had chronic pancreatitis. In the primary analysis, mild/moderate and severe PEP were compared between Cotton and Atlanta with Fischer's exact test. Atlanta was regarded as the golden standard. Secondarily, we compared the sensitivity and specificity of both definitions for mortality.

Results: Out of a total 2156 ERCPs, 66 patients (3\%) had PEP. Two patients were excluded due to missing data for hospital stay. Of the 64 patients analysed, $39(60.9 \%)$ were female, mean age was 60.6 years and the most common indication for ERCP was choledocholithiasis $(n=33,51.6 \%)$. Four patients $(6.3 \%)$ developed organ failure, 3 patients $(4.7 \%)$ died. The table below depicts the PEP severity distribution according to Cotton and Atlanta. No significant differences were found $(p=0.64)$. Of the 25 severe patients according to Cotton, 23 were categorized due to hospital stay exceeding 10 days, but were mild according to Atlanta. In 11 patients $(44 \%)$, concomitant disease (syndrome of inappropriate antidiuretic hormone secretion, cholangitis, pneumonia, perforation or biliary leakage) was the cause for prolonged stay.

Severity of PEP according to Cotton and Atlanta

\begin{tabular}{lllll}
\hline & & $\begin{array}{l}\text { Atlanta } \\
\text { Mild-moderate }\end{array}$ & Severe & Total \\
\hline \multirow{2}{*}{ Cotton } & Mild-moderate & $37(62 \%)$ & $2(50 \%)$ & $39(61 \%)$ \\
& Severe & $23(38 \%)$ & $2(50 \%$ & $25(39 \%)$ \\
\multirow{2}{*}{ Total } & & $60(94 \%)$ & $4(6 \%)$ & $64(100 \%)$ \\
\hline
\end{tabular}

All 3 patients that died had severe PEP according to Atlanta due to persistent organ failure. Cotton classified them as mild, moderate and severe based on hospitalization. Two patients died within 10 days of early multiple organ failure. No other Cotton criterion for severity was met. Thus, the Cotton criteria due not capture early deaths due to multiple organ failure. The sensitivity and specificity of Atlanta and Cotton for mortality were $100 \%, 98.4 \%, 33.3 \%$ and $60.7 \%$, respectively.

Conclusion: The Cotton criteria for PEP overestimate disease severity, but underestimate mortality. Therefore, the Atlanta criteria should be used for defining PEP severity.

Disclosure of Interest: All authors have declared no conflicts of interest.

\section{References}

1. Cotton PB, et al. Gastrointest Endosc. 1991; 37(3):383-93.

2. Banks PA, et al. Gut. 2013; 62(1):102-11

3. Dumonceau JM, et al. Endoscopy. 2014; 46(9):799-815.

P0113 RISKS FACTORS AND OUTCOMES OF INFECTED

PANCREATIC NECROSIS: RESULTS FROM A COHORT OF 148 PATIENTS ADMITTED IN ICU FOR SEVERE ACUTE PANCREATITIS

C. Garret ${ }^{1}$, M. Péron ${ }^{2}$, A. Le Thuaut ${ }^{1}$, M. Le Rhun ${ }^{2}$, C. Guitton ${ }^{3}$, J. Gournay ${ }^{2}$, J. Reignier ${ }^{1}$, E. Coron ${ }^{2}$

${ }^{1}$ Loire Atlantique, $\mathrm{CHU}$ de Nantes, nantes/France

${ }^{2}$ Digestive Diseases Institute, University of Nantes Institute of Digestive Disease -

Digestive Diseases Institute, University of $\mathrm{Na}$, Nantes/France

${ }^{3} \mathrm{CH}$ Le Mans, Le Mans/France

Contact E-mail Address: charlotte.garret@chu-nantes.fr

Introduction: Acute pancreatitis (AP) is a common but potentially lethal pathology due to the multiplicity and severity of its complications ${ }^{1}$. Infected pancreatic necrosis (IPN) occurs in $30 \%$ of patients with necrotizing AP and is associated with an increase in mortality ranging from $15 \%$ to $39 \%{ }^{1}$. While interventional drainage and/or removal of the infected tissue remain the mainstay of therapy of IPN, important progresses have been achieved over the last decade and miniinvasive interventional treatments have been developed. The aim of this study was to identify factors associated with IPN and to describe outcomes and management.

Aims \& Methods: This was a retrospective study of collected data from all patients admitted in Intensive Care Unit (ICU) in a single centre from 2012 to 2015 for a severe AP. Baseline characteristics of the overall population were expressed as frequencies (percentages) for categorical variables, as mean \pm standard deviation (SD) for continuous data. For the analysis of mortality, multivariate analysis with Cox proportional hazards regression modeling was used to identify independent predictors. Association between IPN and patients' characteristics at baseline was evaluated using logistic regression.

Results: In total, 148 patients were included in this study. Overall mortality was $17 \%$. Body mass Index, computed Tomography Severity Index (CTSI) ans persistent $(>48 \mathrm{H})$ organ failure $(\mathrm{OF})$ were independently associated with overall mortality. $4 / 16$ patients died of documented mesenteric ischemia during the early phase ( $\leq 8$ days) and $3 / 10$ patients during the late phase ( $>8$ days). IPN was present in 62 patients $(43 \%)$, all requiring an intervention (i.e. radiological, endoscopical, and/or surgical). $35 \%$ of patients $(22 / 62)$ had only one modality of drainage (radiologic or transgastric) and did not required any necrosectomy. For 30 patients $(48 \%)$, additional necrosectomy was needed because of lack of improvement after drainage alone. 10 patients $(17 \%)$ had only necrosectomy without prior drainage procedure. Complications such as hemorrhage and perforation of visceral organ occurred more frequently in the IPN group $(1.4 \%$ vs $19.4 \%, \mathrm{p}<0.001$ and $0 \%$ vs $8.5 \%, \mathrm{p}=0.02$ respectively). The late phase mortality ( $>8$ days) was significantly higher in the IPN group $(14.5 \%$ vs $1.4 \%$, $\mathrm{p}<0.01)$. In multivariate analysis, factors associated with IPN were number of $\mathrm{OF}$ and portosplenomesenteric venous thrombosis (table 1). 39 patients $(68 \%)$ received anticoagulants with a median time of 6 [3-6] months and among them. 25 patients developed cavernoma, irrespective of whether or not they receive systemic anticoagulation $(\mathrm{p}=0.31)$

Table 1: Multivariate analysis of factors associated with infected pancreatic necrosis

\begin{tabular}{lcc}
\hline & OR $_{\text {adjust }[\text { IC95\%] }}$ & $\mathrm{p}$ \\
\hline Cause of pancreatitis & & \\
Alcohol abused Biliary Others & $12.43[0.79-7.45]$ & 0.02 \\
& $5.36[1.59-18.12]$ & \\
Number of organ failure (OF) & & \\
No OF 1 or 2 OF multiple OF $(\geq 3)$ & $14.44[1.07-18.40]$ & $<0.001$ \\
& $28.67[6.23-131.96]$ & \\
Postosplenomesenteric vein thrombosis & $8.16[3.06-21.76]$ & $<0.001$ \\
\hline
\end{tabular}

Conclusion: In conclusion, this study performed in routine practice conditions showed that IPN occurs in almost half of patients hospitalized in ICU for severe $\mathrm{AP}$, and is associated with increased mortality and complications rates. Overall mortality was $17.6 \%$, and factors associated with mortality were a high BMI, CTSI and persistent OF. Those results are consistent with previous studies ${ }^{1,2}$, but we reported a high rate of mesenteric ischemia ( $7 / 26$ patients deceased) while this complication is occasionally described. IPN patients required an intervention for drainage of infected tissue removal, which was performed using minimally invasive techniques in the vast majority of cases, with no complication or severe side effect. $35 \%$ of patients were treated with drainage alone without any additional necrosectomy. Finally, PSMVT and early OF appeared to be associated with the risk of developing an IPN but anticoagulation for PSMVT did not protect for cavernoma occurrence and can expose to intestinal bleeding. Our results also suggest that the optimal and early management of OF and detection of PVSMT might prevent IPN and/or its complications. Such hypothesis will need to be tested in large multicentre prospective studies.

Disclosure of Interest: All authors have declared no conflicts of interest.

\section{References}

1. Forsmark, C. E., et al. Acute Pancreatitis. N. Engl. J. Med. 375, 1972-1981 (2016)

2. Johnson, C. D. \& Abu-Hilal, M. Persistent organ failure during the first week as a marker of fatal outcome in acute pancreatitis. Gut 53, 1340 1344 (2004)

\section{P0114 EARLY PREDICTORS AND OUTCOMES OF FLUID SEQUESTRATION IN ACUTE PANCREATITIS}

S. Goyal

Gastroenterology, Banaras Hindu University, VARANASI/India

Contact E-mail Address: drsundeepgoyal@gmail.com

Introduction: Although it is well known that some patients with AP have an increased need for fluid therapy, it is not clear who should get fluids aggressively. Changes in hematocrit, BUN and serum creatinine, has been documented to limit necrosis and improve outcome. The early prediction of fluid sequestration may help to select patients for more or less aggressive fluid resuscitation ${ }^{1}$.

Aims \& Methods: 1) To determine early variables as predictors of fluid sequestration in the first 48 hours. 2) To determine outcome associated with fluid sequestration. In this prospective cohort study 300 consecutive patients of acute pancreatitis were included. Fluid sequestration was calculated by adding the total amount of fluid administered and subtracting the total amount of fluid lost in the first 48 hours of hospitalization. Local complications were defined 
according to the revised Atlanta classification. Univariate and multivariate analysis were performed.

Results: The median fluid sequestration in the first $48 \mathrm{~h}$ after hospitalization was 4.7 liter (2.8-6.8 L). It was $3.21(1.4-51), 6.41(3.6-9.51)$ in those without necrosis and those with necrosis, and 7.5 1 (4.4-121) in those with persistent organ failure. The univariate and multivariate analysis showed that alcohol etiology, an increasing number of SIRS criteria and Hematocrit were significantly associated with increased fluid sequestration (Table). Body mass index, APACHE II score, sodium, creatinine and blood urea nitrogen levels did not help predict fluid sequestration. Patients with and without acute fluid collections had a median sequestration of 7.2 and $4.2 \mathrm{~L}(\mathrm{p}<0.001)$, respectively. 22 patients died $(7.33 \%)$; median fluid sequestration in the patients who died was $6.5 \mathrm{~L}$ compared to $4.2 \mathrm{~L}$ among the patients who survived (p.0.05). Increased fluid sequestration was associated with prolonged hospital stay $(\mathrm{p}<0.01)$. (table) Association between variables determined at admission and fluid sequestration.

\begin{tabular}{|c|c|c|c|c|}
\hline Variable & & $\begin{array}{l}\text { Fluid } \\
\text { sequestration } \\
\text { (48 hours) (L) }\end{array}$ & $\begin{array}{l}\text { Univariate } \\
\text { analysis, } \\
\text { p value }\end{array}$ & $\begin{array}{l}\text { Multivariate } \\
\text { analysis, } \\
\text { p value }\end{array}$ \\
\hline \multirow[t]{5}{*}{ Age (years) } & $<35$ & $5.3(2.7-8.9)$ & \multirow[t]{5}{*}{0.06} & \multirow[t]{5}{*}{$<0.05$} \\
\hline & $35-45$ & $5.6(2.9-7.6)$ & & \\
\hline & $45-55$ & $4.9(3.1-7.9)$ & & \\
\hline & $55-65$ & $5.3(2.7-6.9)$ & & \\
\hline & $>65$ & $4.5(2.7-6.2$ & & \\
\hline \multirow[t]{2}{*}{ Sex } & Male & $5.1(2.7-8.9)$ & \multirow[t]{2}{*}{$<0.01$} & \multirow[t]{2}{*}{$>0.05$} \\
\hline & Female & $4.8(2.8-7.4)$ & & \\
\hline \multirow[t]{4}{*}{ Etiology } & Alcohol & $5.5(2.7-8.8)$ & \multirow[t]{4}{*}{$<0.001$} & \multirow[t]{4}{*}{$<0.001$} \\
\hline & Biliary & $5.2(2.4-8.1)$ & & \\
\hline & Idiopathic & $4.8(2.8-7.2)$ & & \\
\hline & Others & $4.6(2.7-6.4)$ & & \\
\hline \multirow[t]{4}{*}{ Hematocrit $(\%)$} & $<35$ & $3.6(2.7-4.9)$ & \multirow[t]{4}{*}{$<0.001$} & \multirow[t]{4}{*}{$<0.01$} \\
\hline & $35-40$ & $3.3(2.7-5.1)$ & & \\
\hline & $40-45$ & $3.8(3.1-7.5)$ & & \\
\hline & $>45$ & $4.7(3.8-8.9)$ & & \\
\hline \multirow[t]{5}{*}{ SIRS score } & 0 & $5.3(2.7-4.1)$ & \multirow[t]{5}{*}{$<0.001$} & \multirow[t]{5}{*}{$<0.01$} \\
\hline & 1 & $5.3(2.2-4.4)$ & & \\
\hline & 2 & $5.3(2.9-6.5)$ & & \\
\hline & 3 & $5.3(3.1-7.2)$ & & \\
\hline & 4 & $5.3(3.8-8.9)$ & & \\
\hline
\end{tabular}

Conclusion: Alcohol etiology, increased number of SIRS criteria, hemoconcentration and younger age were independent predictors of increased fluid loss. Patients with increased sequestration of fluid are at higher risk of local complications and prolonged stay.

Disclosure of Interest: All authors have declared no conflicts of interest.

\section{Reference}

1. de-Madaria E, Soler-Sala G, Sánchez-Payá J, Lopez-Font I, Martínez J, Gómez-Escolar L, Sempere L, Sánchez-Fortún C, Pérez-Mateo M. Infuence of fluid therapy on the prognosis of acute pancreatitis: a prospective cohort study. Am J Gastroenterol 2011; 106: 1843-1850

\section{P0115 LONGER CANNULATION TIME AND PANCREATIC GUIDEWIRE DURING ERCP CAN BE A GOOD CANDIDATE FOR PROPHYLACTIC PANCREATIC STENT TO PREVENT POST-ERCP PANCREATITIS: A MATCHED PROPENSITY ANALYSIS}

S. Hayashi, T. Nishida, R. Tomita, Y. Higaki, H. Shimakoshi, A. Shimoda N. Osugi, A. Sugimoto, K. Takahashi, D. Nakamatsu, K. Mukai, T. Matsubara, M. Yamamoto, K. Fukui, M. Inada

Gastroenterology And Hepatology, Toyonaka municipal hospital, Toyonaka/Japan

Contact E-mail Address: hayashishiro1976@yahoo.co.jp

Introduction: Post ERCP pancreatitis (PEP), that can potentially result in procedure-related death is still great concern in pancreato-biliary endoscopists because it may be a potentially preventable complication but it is difficult to prevent. Among reported prophylactic procedures for PEP, placement of prophylactic pancreatic stent (PPS) is known as a promising one to decrease the risk of PEP in high-risk patients. It is, however, still unclear what high-risk patient is a good candidate for PPS. We have previously reported the two predictive factors for PEP; the cannulation time longer than $13 \mathrm{~min}$ and 2-hour amylase level over 264 IU/L (World $J$ Gastrointest Endosc 2016; 8: 777-784). In the present study, we evaluated if PPS for PEP was useful in high-risk patients with using the propensity score matching method.

Aims \& Methods: This is a retrospective single center cohort study of consecutive patients that underwent ERCP from January 2010 to December 2015. A total of 2298 patients (average age $73 \pm 11$, female $41.2 \%$ ) were enrolled. Of them, 174 cases with the following conditions were excluded: 1) gallstone pancreatitis, 2) unreachable to papilla, 3) missing data of procedure time or serum amylase levels. Finally, 2124 cases were analyzed. Overall, 86 of 2124 cases developed PEP $(4.0 \%)$. Pancreatic guidewire in addition to the cannulation time longer than 13 min were revealed as significant high-risk factor of PEP using a univariate analysis. There were a total of 216 patients with above two risk factors (HR group). Of them, 30 patients developed PEP (13.9\%). Using logistic regression, propensity scores were used to prevent selection bias between with and without PPS. The covariates entered in the propensity model were age, gender, native papilla, endoscopic biliary stent, endoscopic metallic stent, pancreatic guidewire, pancreatic injection, endoscopic sphincterotomy, precut, endoscopic papillary balloon dilation, pancreatic duct brush. Subsequently, 1-to-1 matched PPS and non-PPS group (each group $\mathrm{N}=64$ ) were extracted from the cohort using the closet matching score.

Results: After matching, mean propensity matching score in PPS and non-PPS group were $0.46 \pm 0.2$ and $0.46 \pm 0.2$ respectively. There were 62 female $(48.4 \%)$ with a mean age of 71 in each group. A total of 17 patients with HR developed PEP $(13.2 \%)$. The PEP rate in PPS group was significantly lower than in nonPEP group $(6.3 \%$ vs $20.0 \%$ : $\mathrm{p}=0.019)$. Mean post-ERCP $2 \mathrm{~h}$ amylase levels were also lower in PPS group than non-PPS group (178 vs 352: $p=0.0214$ ).

Conclusion: Using a propensity analysis revealed that PPS significantly reduce the risk of developing PEP in high-risk patients with longer cannulation time and pancreatic guidewire. Consequently, we considered patients with above two risk factors be a good candidate for PPS.

Disclosure of Interest: All authors have declared no conflicts of interest.

\section{Reference}

Combination of two-hour post-endoscopic retrograde cholangiopancreatography amylase levels and cannulation times is useful for predicting post-endoscopic retrograde cholangiopancreatography pancreatitis Shiro Hayashi et al. World $J$ Gastrointest Endosc 2016; 8: 777-784

\section{P0116 AUTOIMMUNE PANCREATITIS CLASSIFIED AS NOT- OTHERWISE SPECIFIED (NOS) ACCORDING TO THE INTERNATIONAL CONSENSUS DIAGNOSTIC CRITERIA: CLINICAL FEATURES AND OUTCOMES IN 47 PATIENTS}

N. De Pretis, A. Brandolese, L. Brozzi, F. Vieceli, A. Amodio, M. Ruffini, S. Crinò, A. Gabbrielli, L. Frulloni

Gastroenterology, University of Verona, Verona/Italy

Contact E-mail Address: martina.ruffini@libero.it

Introduction: Autoimmune pancreatitis (AIP) is a well-recognized fibro-inflammatory disease of the pancreas characterized by a drammatic response to steroid therapy.(1) Three different types have been identified according to the International Consensus Diagnostic Criteria: type 1, type 2 and type not-otherwise specified (NOS).(2) Despite the significant number of studies published on AIP type 1 and $2,(3,4)$ no studies have been focused on AIP type NOS and therefore very little is known about clinical features and long-term outcomes of these patients.

Aims \& Methods: Aim was to investigate clinical features, risk of other organ involvement, risk of relapse and long-term outcomes of AIP type NOS-patients. Patients classified by International Consensus Diagnostic Criteria (ICDC) as AIP type NOS at clinical onset included in our database prospectively maintained since 1995 have been evaluated. AIP type 1 (168 patients) and AIP type 2 (63 patients) were excluded. Epidemiological and clinical data have been collected and analyzed.

Results: 47 patients fulfilled inclusion criteria. Symptoms at clinical onset were mainly weight-loss $(40,5 \%)$, jaundice $(34 \%)$ and pancreatitis $(29.8 \%)$. Six patients $(12,8 \%$ ) had other organs involvement (5 proximal biliary involvement and 1 salivary involvement) and only two $(4,3 \%)$ patients had serum IgG4 levels $>140 \mathrm{mg} / \mathrm{dL}$ at clinical onset. Six patients $(12,8 \%)$ developed ulcerative colitis (UC) during follow-up and were therefore reclassified as AIP type 2. The mean time between the clinical onset of AIP-NOS and development of UC was 14 months (range 4-48). Eight patients $(17 \%)$ experienced a relapse after steroid treatment and two $(4,3 \%)$ needed immunosuppressive drugs (Azathioprine) because of recurrent relapses. None underwent resective surgery despite 30 patients $(63,8 \%)$ had a focal pancreatic involvement of the pancreas at imaging. Conclusion: Patients suffering from AIP type NOS have own clinical features. The risk of relapse is low $(17 \%)$ but not irrelevant, as well as the risk of developing UC (12, 8\%) during follow-up switching the diagnosis to AIP type 2.

Disclosure of Interest: All authors have declared no conflicts of interest.

\section{References}

1. Hart PA, Zen Y, Chari ST. Recent advances in autoimmune pancreatitis. Gastroenterology 2015;149:39-51.

2. Shimosegawa T, Chari S, Frulloni L et al. International consensus diagnostic criteria for autoimmune pancreatitis: guidelines of the International Association of Pancreatology. Pancreatology 2011;40:352-358.

3. Kamisawa T, Chari ST, Lerch MM et al. Recent advances in autoimmune pancreatitis: type 1 and type 2. Gut 2013;62:1373-1380.

4. Ikeura T, Manfredi R, Zamboni G et al. Application of International consensus diagnostic criteria to an italian series of autoimmune pancreatitis. United European Gastroenterol J 2013;1276-1284. 
P0117 698 CASES OF PANCREATIC DISEASES TREATED BY EPDBD (ENDOSCOPIC PANCREATIC DUCT BALLOON DILATION) - ITS USEFULNESS AND SAFETY

T. Tsuji

Gastroenterology, Saitama Kyoudou Hospital, Kawaguchishi/Japan

Contact E-mail Address: t-tsuji@mcp-saitama.or.jp

Introduction: We started our original EPDBD (endoscopic pancreatic duct balloon dilation) therapy for pancreatic diseases in 1996. In these 22 years, 698 cases were treated by this method. We would like to show its usefulness and safety. Aims \& Methods: The balloon (6 mm diameter, $15 \mathrm{~mm}$ long -Boston Scientific) was inflated in the stenotic portion of the pancreas duct at $6 \mathrm{~atm}$. pressure for 1 minute several times. Then stone removal or EPS (endoscopic pancreatic stenting) was done. Following were evaluated; 1 . The stone free rate and stone relapse rate in 568 pancreatic stone cases treated by EPDBD 2. The prognoses of 114 EPS-successful pseudocyst cases treated by EPDBD 3. The prognoses of 16 EPSsuccessful divisum cases (complete type 6, incomplete type 10) trerated by EPBDB respectively. Complications after these therapies are also evaluated.

Results: The purpose of EPDBD therapy for pancreatic stone was to ease endoscopic procedures in the pancreatic duct and stone removal, and to reduce stone relapse rates. 568 cases of pancreatic stone were treated by EPDBD. They consisted of 90 cases treated by endoscopic method alone (via major papilla 62, minor papilla 28), and 478 cases treated by ESWL+ endoscopic method (via major papilla 381, minor papilla 97). After EPDBD therapy, the stone free rate was $75.3 \%$, the pain free rate $97.1 \%$. The stone relapse rate was $5.7 \%$ this is a much lower result compared to other reports. We think that EPDBD contributes to this good result. Complications of EPDBD therapy were only minor bleeding from orifice at the therapy and mild pancreatitis after therapy for several days. Case A; 22 y/o male. idiopathic chronic pancreatitis, pancreas stone: After $4^{\text {th }}$ ESWL, small stones remained in the head duct which can't be removed by basket catheter and severe pain continued, so EPDBD was done under good informed consent. After several dilation of the orifice and the head duct, stones were removed easily. This is our first case of EPDBD. In our hospital, EPS and ENPD (endoscopic nasal pancreatic drainage) are the preferred choice for pancreatic pseudocyst therpy after dilation of the stenotic duct. 114 cases were successfully treated without major complications, and their prognoses were good. Case B; 29 y/o female. alchoholic chronic pancreatitis, pancreatic stone: ERP via minor papilla revealed the duct rupture in the body portion and contrast medium flowed out into thoracic and abdominal cavity. After dilation of the stenotic duct in the body, EPS placement was done and she recovered quickly. The 16 cases of divisum consisted of 6 complete and 10 incomplete type. After EPDBD, EPS was successful in 2 cases via major papilla, and 14 cases via minor papilla with good prognoses. Case C; $72 \mathrm{y} / \mathrm{o}$ male. alcoholic chronic pancreatitis, pancreatic stone: ERP revealed type 2 incomplete divisum. After ESWL and EPDBD, stones were removed via minor papilla, then EPS was placed into minor papilla successfully.

Conclusion: By EPDBD therapy, the relapse rate of pancreatic stone decreased, and the success rate of endoscopic drainage and stenting in pseudocyst and divisum increased markedly with minor complications, and their prognoses were good. EPDBD is a safe and favorable procedure for pancreatic diseases.

Disclosure of Interest: All authors have declared no conflicts of interest.

\section{P0118 ENDOSCOPIC ULTRASOUND AS A PREDICTOR AND GUIDE TO SUCCESSFUL ENDOTHERAPY IN CHRONIC PANCREATITIS}

\section{Sharma, P. Somani}

Department Of Gastroenterology, Jaswant Rai Specialty Hospital, Meerut/India

Contact E-mail Address: sharmamalay@hotmail.com

Introduction: Pancreatic calculi (PC) are a sequelae of chronic pancreatitis (CP) and may obstruct and produce ductal hypertension leading to pain, the cardinal feature of CP. The rationale for endoscopic treatment of obstructing PC is based on the observation that pain subsides when the stone(s) is removed and drainage of pancreatic secretion is restored. Indications for endotherapy include stones $<5 \mathrm{~mm}$ size, stones in head of pancreas which are not impacted and absence of downstream strictures. The assessment prior to the procedure is done by MRCP or CT. However, problems are encountered during ERCP clearance which are not anticipated despite $\mathrm{MRCP} / \mathrm{CT}$. The problems are, possible impacted stones, hard stones, indeterminate stricture, and change of finding during ERCP. Hence, controversy exists. EUS can help by providing concordance or discordance with MRCP images and may help in further clarification.

Aims \& Methods: We aimed to evaluate the role, feasibility and management changes of EUS prior to ERCP in patients planned for endotherapy in CP. Another objective was to evaluate whether EUS features of pancreatic duct (PD) stones can serve as a predictor of successful removal during ERCP. The data of 412 patients during the study period (2009-2016) with CP was retrospectively analysed. 143 were associated with stones in head/papillary region of pancreas. Out of these, 75 were excluded and remaining 68 were evaluated by EUS using a linear/radial echo endoscope prior to ERCP.

Results: Out of 68 cases, 48 were associated with hard stones with acoustic shadowing while 20 were associated with soft stones without acoustic shadowing. In 20 soft stones cases, ERCP was successful in 18 patients. In 48 patients with hard stones, there was failure of endotherapy in 40 patients which required ESWL/surgery. The remaining 8 patients required multiple sessions of ERCP for successful removal. Three patients had ampullary/papillary stones which were removed with precut sphincterotomy with immediate relief of pain. Four patients had ventral duct obstruction by calculi and hence underwent ERCP through minor papilla with successful removal of stones in 3 patients. In three patients, there were calculi in pancreatic parenchyma and pancreatic duct simultaneously (ducto-parenchymal stones) and hence endotherapy was avoided. Three patients had pancreas divisum diagnosed on EUS and hence underwent minor papillotomy with stone removal. Three patients also had biliary obstruction with CBD stone/sludge and underwent biliary endotherapy. Four patients had pancreatic mass in head and underwent EUS-FNA with two patients diagnosed with pancreatic cancer who were referred for surgery. The remaining two were also referred for surgery. Four patients were found to have strictures on EUS and hence were referred for surgery. The presence of large $(\geq 5 \mathrm{~mm})$, hard, immobile stones were negative predictors of successful endotherapy. Small $(<5 \mathrm{~mm})$, ampullary/papillary stones were positive predictors.

Conclusion: Present study suggests that EUS can differentiate "soft PD stones" (without an acoustic shadow) from "hard PD stones"(with an acoustic shadow). This differentiation can help in predicting successful removal during ERCP. "Hard PD stones" diagnosed on EUS are better managed by ESWL. An EUS can influence important therapeutic decisions before endotherapy and can prevent unsuccessful attempts at ERCP and thus improve overall success/prognosis. An EUS has an additional advantage of making a diagnosis of ampullary/papillary stones and biliary obstruction which can be treated endoscopically. It can guide whether endotherapy needs to be performed through major or minor papilla. EUS by diagnosing pancreatic tumour/strictures missed on other imaging modalities allows early surgical reference and hence improves long-term prognosis. It can prevent unsuccesful attempts at endotherapy and its possible risks/costs. We conclude that EUS before endotherapy plays an important role regarding further management decisions in patients with $\mathrm{CP}$.

Disclosure of Interest: All authors have declared no conflicts of interest.

\section{P0119 UTILITY OF SERUM APOPTOSIS INHIBITOR OF MACROPHAGE FOR DIFFERENTIATING IGG4-RELATED DISEASE FROM MALIGNANT DISEASE}

S. Tanoue, S. Hashimoto, S. Arima, Y. Nasu, F. Sasaki, S. Kanmura, A. Ido School Of Medical And Dental Sciences, Kagoshima University, Kagoshima/Japan

Contact E-mail Address: tanoue@m.kufm.kagoshima-u.ac.jp

Introduction: IgG4-related disease (IgG4-RD) is characterized by the infiltration of inflammatory cells, such as plasma cells and macrophages, and fibrosis in tissues. We previously reported that apoptosis inhibitor of macrophage (AIM), which is secreted by macrophages, is related to the progression of hepatic fibrosis in chronic hepatitis C. Some studies have observed a relationship between IgG4$\mathrm{RD}$ and malignancy. IgG4-RD is considered to represent a premalignant state or paraneoplastic condition

Aims \& Methods: To clarify the significance of the serum AIM levels in patients with IgG4-RD, we measured these levels in 22 healthy controls, 32 patients with IgG4-RD, and 36 patients with other pancreatic diseases (chronic pancreatitis $[\mathrm{CP}]$, intraductal papillary mucinous neoplasm [IPMN], pancreatic cancer [PC]). We also analyzed the prevalence of malignancy, the relationship between the appearance of malignancy and the diagnosis of 42 IgG4-RD, the type of cancer, and related factors, and we compared the age, gender, laboratory data, and AIM level.

Results: Fifteen malignancies were seen in 12 of 42 patients $(28.6 \%)$. These diagnoses were made before the diagnosis of IgG4-RD for 10 malignancies in 8 patients (mean 4.8 years earlier, range $1-16$ years), and after the diagnosis of IgG4-RD for 3 malignancies in 2 patients (mean 2 years later, range $1-3$ years). Two malignancies in 2 patients were diagnosed at the same time as IgG4-RD. The AIM level was significantly higher in the IgG4-RD patients with malignancy than in those without malignancy. No significant differences were seen in the mean age or gender between patients with and without malignancies. The IgG4RD group had significantly higher serum AIM levels than the healthy control or IPMN groups, and the levels tended to be higher than in the PC group.

Conclusion: Macrophages are reportedly related to IgG4 class switching in B cells and fibrosis in IgG4-RD. The serum level of AIM, which is secreted by macrophages, is considered a useful biomarker for evaluating the pathology and differentiating IgG4-RD and malignant diseases.

Disclosure of Interest: All authors have declared no conflicts of interest.

\section{Reference}

Mera K. et al Serum levels of apoptosis inhibitor of macrophage are associated with hepatic fibrosis in patients with chronic hepatitis C. BMC gastroenterology 2014

P0120 ENDOSONOGRAPHY FOR SUSPECTED PANCREATIC NEOPLAMS: OUTCOME AND FOLLOW-UP OF PANCREATIC LESIONS IN THE SETTING OF A NORMAL OR INFLAMED PANCREAS

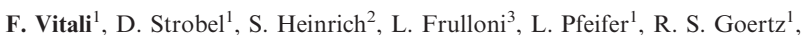

M.F. Neurath ${ }^{1}$, D. Wildner

${ }^{1}$ Internal Medicine 1, UK-Erlangen, Erlangen/Germany

${ }^{2}$ Radiology, UK Erlangen, Erlangen/Germany

${ }^{3}$ Gastroenterology, University of Verona, Verona/Italy

Contact E-mail Address: francesco.vitali@uk-erlangen.de

Introduction: Endosonography (EUS) is one of the main diagnostic tools for the differential diagnosis of pancreatic masses. The aim of our study was to determine pitfalls of this technique in patients suspected of having pancreatic cancer. 
This was done in consideration of the current knowledge about lesions mimicking cancer in the setting of a normal pancreatic parenchyma or existence of signs for pancreatitis

Aims \& Methods: Retrospective analysis of prospectively collected data in our tertiary University center. From March 2007 to October 2015, 218 (124 men, 94 women; mean age $60 \pm 14$ years) patients underwent EUS for suspected solid pancreatic neoplasm because of cross sectional imaging results, idiopathic acute pancreatitis, weight loss, pancreatic hyperenzymemia, painless jaundice and elevated $\mathrm{Ca}$ 19-9 values. Cystic pancreatic lesions, pseudocysts and cystic pancreatic neoplasms were excluded from the analysis.

Results: Malignant lesions were diagnosed in 98 (45\%) patients. 54 patients $(24.8 \%)$ underwent surgery and 61 patients $(28 \%$ of all patients) underwent clinical follow-up ( $16.5 \pm 27.3$ months, 18 needed surgery). 43 lesions not undergoing surgery needed EUS follow-up before achieving final diagnosis: pancreatic cancer $(n=6,9.8 \%)$, neuroendocrine tumor (NET) $(n=10,16.4 \%)$, paraduodenal pancreatitis $(\mathrm{n}=5,8.9 \%)$, chronic pancreatitis $(\mathrm{n}=13,21.3 \%)$, necrosis $(\mathrm{n}=3,4.9 \%)$, autoimmune pancreatitis (AIP) $(\mathrm{n}=3,4.9 \%)$, microcystic serous neoplasia $(n=1)$, ventral/dorsal split $(n=1)$, lipomatosis $(n=1)$. EUS showed sensitivity and specificity for malignancy of $91.4 \%$ and $97.7 \%$, respectively, in the non-pancreatitis group $(n=121)$ and $44 \%$ and $87.1 \%$ in the pancreatitis group $(n=97)$. Ca 19-9 elevation, rapid onset jaundice, double duct sign are useful indicators of malignancy both in the setting of normal and inflamed pancreas. Patients without pancreatitis the presence of enlarged lymphnodes or a mass in EUS, weight loss and worsening diabetes are predictor of malignancy. In patients without pancreatitis and without jaundice Ca 19-9 sensitivity for malignancy was $95 \%$ and specificity was $39 \%$. In the pancreatitis group, Ca 19-9 sensitivity for malignancy (in patients without jaundice) was $45 \%$ and specificity was $86 \%$. IgG4 elevation presented a sensitivity of $83.3 \%$ and a specificity of $77.8 \%$ for AIP, where one false elevation was seen in a distal cholangiocellular carcinoma.

Conclusion: Diagnostic accuracy of EUS is lower in the presence of pancreatitis. Focal autoimmune pancreatitis and paraduodenal pancreatitis are still confused with pancreatic cancer, also in the setting of a not inflamed pancreatic parenchyma. EUS in the setting of a normal parenchyma is an excellent tool to exclude pancreatic cancer. Tumor markers like Ca 19-9 and IgG4 values should be measured in the evaluation of pancreatic masses, also in the setting of chronic pancreatitis. In unclear cases, clinical and imaging follow-up with more than one modality (usually EUS with CT) were necessary for achieving a definitive diagnosis. If there is a high clinical suspicion of pancreatic neoplasm in a patient that does not undergo surgery and if EUS or other available imaging modalities are negative, repeated EUS after 2-3 months may be useful for detecting an occult pancreatic neoplasm.

Disclosure of Interest: All authors have declared no conflicts of interest.

\section{References}

Dietrich CF, Sahai AV, D'Onofrio M et al. Differential diagnosis of small solid pancreatic lesions. Gastrointest Endosc 2016; 84: 933-940

Cieslak KP, van Santvoort HC, Vleggaar FP et al. The role of routine preoperative EUS when performed after contrast enhanced CT in the diagnostic work-up in patients suspected of pancreatic or periampullary cancer. Pancreatology 2014; 14: $125-130$

Bhutani MS, Gress FG, Giovannini M et al. The No Endosonographic Detection of Tumor (NEST) Study: a case series of pancreatic cancers missed on endoscopic ultrasonography. Endoscopy 2004; 36: 385-389

\section{P0121 GALECTIN-1 EXPRESSED IN PANCREATIC STELLATE CELLS PROMOTES TUMOR PROGRESSION IN PANCREATIC CANCER VIA UPREGULATION OF SDF-1 AND ACTIVATION OF NF- KB}

T. $\mathrm{Yu}^{1}$, D. Qian ${ }^{2}$

${ }^{1}$ Department, the First Affiliated Hospital of Nanjing Medical University, Nanjing/ China

${ }^{2}$ Jiangsu Province Hospital of Traditional Chinese Medicine, Nanjing/China

Contact E-mail Address: njmuqiandong@163.com

Introduction: Pancreatic cancer is characterized by a high density of stroma. Interactions between tumor and stromal cells play a critical role in tumor progression and there is increasing evidence that pancreatic stellate cells (PSC), a main component of the stroma, may participate in the development of pancreatic cancer[1]. However, details of the mechanism underlying the interaction between PSCs and pancreatic cancer cells (PCC) are poorly understood.

Stromal cell-derived factor-1 (SDF-1 or CXCL12) belongs to the CXC chemokine family and is the ligand of CXCR4 [2]. It has been implicated in promoting the metastatic potential of breast, gastric, ovarian, prostate, lung and pancreatic cancer cells. Although SDF-1 reports of SDF-1 in pancreatic cancer cell lines are rare, it has been identified in pancreatic cancer tissue samples [3], suggesting that SDF-1 produced in activated PSCs may be an integral factor in tumor-stromal interactions.

Galectin-1 mediates communication between cells by binding to glycol-conjugated proteins on the cell surface. Studies have shown that it is involved in multiple cancer-related processes, including immunosuppression, angiogenesis and metastasis [4]. We previously reported that Galectin-1 was highly expressed in pancreatic cancer tissues; furthermore, the primary source of Galectin-1 was in activated PSCs within the stroma of cancer cells [5]. It has previously been hypothesized that Galectin-1 may also induce activation of PSCs and stimulate secretion of chemokines [6]; however, the biological mechanism and its activities in PCCs are unclear.
Aims \& Methods: The purpose of this study was to identify the effect and elucidate the biological mechanism of Galectin-1 in PSCs and its role in tumor progression in pancreatic cancer.

Results: By conducting a chemokine antibody array assay and transwell invasion and migration assays we were able to show that Galectin-1 induced secretion of SDF-1 in PSCs leading to increased migration and invasion of PCCs. An NF- $\kappa$ B activity assay indicated that the mechanism involved activation of NF- $\kappa \mathrm{B}$. These observations were confirmed by qRT-PCR, ELISA and immunofluorescent assays, and were further verified by knockdown of Galectin-1 through lentiviral transfection and by employing inhibitors to block SDF-1, its ligand CXCR4 and $\mathrm{NF}-\kappa \mathrm{B}$. The in vitro findings were supported in an in vivo mouse model. In combination, our results demonstrated that Galectin-1 stimulated production of SDF-1 in PSCs through activation of $\mathrm{NF}-\kappa \mathrm{B}$, and that SDF-1 promoted invasion and migration of PCCs.

Conclusion: This study suggested that enhanced expression of Galectin-1 in stromal PSCs promotes metastasis in PDAC, thereby offering a potential target in the treatment of pancreatic cancer.

Disclosure of Interest: All authors have declared no conflicts of interest.

\section{References}

1. Xu Z, Vonlaufen A, Phillips PA, et al. Role of pancreatic stellate cells in pancreatic cancer metastasis. Am J Pathol. 2010; 177(5):2585-2596.

2. Wu PF, Lu ZP, Cai BB, et al. Role of CXCL12/CXCR4 signaling axis in pancreatic cancer. Chinese medical journal. 2013; 126(17):3371-3374.

3. Marchesi F, Monti P, Leone BE, et al. Increased survival, proliferation, and migration in metastatic human pancreatic tumor cells expressing functional CXCR4. Cancer Res. 2004; 64(22):8420-8427.

4. Tang D, Yuan Z, Xue X, et al. High expression of Galectin-1 in pancreatic stellate cells plays a role in the development and maintenance of an immunosuppressive microenvironment in pancreatic cancer. International Journal of Cancer. 2012; 130(10):2337-2348.

5. Xue X, Lu Z, Tang D, et al. Galectin-1 secreted by activated stellate cells in pancreatic ductal adenocarcinoma stroma promotes proliferation and invasion of pancreatic cancer cells: an in vitro study on the microenvironment of pancreatic ductal adenocarcinoma. Pancreas. 2011; 40(6):832-839.

6. Masamune A, Satoh M, Hirabayashi J, et al. Galectin-1 induces chemokine production and proliferation in pancreatic stellate cells. American journal of physiology Gastrointestinal and liver physiology. 2006; 290(4):G729-736.

\section{P0122 WHOLE-GENOME SEQUENCING OF PANCREATIC TUMOR BY NEXT-GENERATION SEQUENCING USING EUS-FNA SPECIMENS}

M. Matsukawa ${ }^{1}$, M. Nishimura ${ }^{2}$, Y. Fujii ${ }^{3}$

${ }_{1}^{1}$ Gastroenterology, Tokyo Metropolitan Geriatric Hospital, Itabashi-ku/Japan ${ }^{2}$ Gastrointestinal Endoscopy, Tokyo Metropolitan Geriatric Hospital, Tokyo/ Japan

${ }^{3}$ Tokyo Metropolitan Geriatric Hospital and Institute of Gerontology, Tokyo/ Japan

Contact E-mail Address: 1110mmatsukawa@gmail.com

Introduction: Intraductal papillary mucinous neoplasms (IPMN) are common pancreatic cystic neoplasms often detected by chance, and are sometimes precursors to invasive pancreatic cancer, resulting in a poor prognosis. These neoplasms are distinct from pancreatic ductal adenocarcinoma (PDA), conventional pancreatic cancer. In some IPMN cases, they cannot be detected before invasive and metastatic carcinoma develops. Knowledge of genetic backgrounds may lead to the discovery of IPMN with poor prognosis. Although whole-genome sequencing by next-generation sequencing (NGS) has become popular, the use of minute samples obtained by EUS-FNA is scarce. The value of NGS of pancreatic cystic fluids or walls has not been clear relative to imaging impressions. On the other hand, in some kinds of cancer, genetic analysis by NGS was used to select the treatment method. In this study, we analysed whether minute clinical samples from EUS-FNA are useful for genetic analysis and whether that aids in deciding the adequate treatment.

Aims \& Methods: We analysed IPMN or PDA tissues using NGS and assessed the impact of NGS on clinical diagnosis. From January to December 2015, 14 tissue samples of undiagnosed pancreatic tumours (from 8 male patients, 6 female patients; mean age, 79.3 years; age range, $85-100$ years; mean tumour size, $29.6 \times 17.9 \mathrm{~mm}$; tumour size range, $10-66 \mathrm{~mm}$ ) were collected by EUS-FNA. The locations of the tumours were the head (3), body (4), and tail (5); we obtained their samples through the stomach or duodenum. EUS-FNA was performed using a curvilinear echoendoscope (GF-UCT 260; Olympus Medical Systems, Tokyo, Japan) and 22- or 25-gauge needles (ProCore; Cook Japan, Tokyo, Japan/Expect; Boston Scientific, Tokyo, Japan). Both slow-pull and negative pressure techniques were used to obtain specimens. We performed whole-genome sequencing of DNA obtained from IPMN or PDA using the Comprehensive Cancer panel, and compared their genetic mutations. Additionaly, in the case of IPMN, we compared the differences in results between mural nodules and cystic fluids or walls.

Results: Pathological diagnoses showed 2 adenocarcinomas, 3 intraductal papillary mucinous carcinomas, 7 intraductal papillary mucinous adenomas; the remaining 2 cases could not be diagnosed. We used minute specimens obtained from EUS-FNA, and analysed genetic mutations of 10 cases using NGS. Homogenous genetic mutations were approximately $18 \%$ and heterogenous genetic mutations were approximately $25 \%$. Five of the cases had mural nodules inside the cysts. We could analyse genetic mutations of cystic fluids or walls by the same way as of mural nodules. For the gradually growing IPMN without 
mural nodules, it is useful to analyse genetic mutations of cystic fluids or walls. Currently, adequate samples from EUS-FNA were unavailable, and in some cases, we could not make a pathological diagnosis. Even in such cases, genetic analysis and the subsequent diagnoseis of malignant or benign tumours may be possible. We could identify several cancer-related genes, such as GNAS, KRAS, $T P 53$, and $B R A F$.

Conclusion: In this study, we performed whole-genome sequencing of samples obtained from IPMN or PDA using EUS-FNA. Consequently, genetic analysis by NGS may be effective in addition to pathological diagnosis when deciding the management of pancreatic tumours.

Disclosure of Interest: All authors have declared no conflicts of interest.

\section{References}

1. Furukawa, T. et al. Whole-exome sequencing uncovers frequent GNAS mutations in intraductal papillary mucinous neoplasm of the pancreas. Scientific Reports. 1:161 (2011)

2. Jones, $\mathbf{M}$ et al. Impact of next-generation sequencing on the clinical diagnosis of pancreatic cysts. Gastrointest Endosc. 83(1):140-8 (2016)

3. Amato, E et al. Targeted next-generation sequencing of cancer genes dissects the molecular profiles of intraductal papillary neoplasm of the pancreas. $J$ pathol. 233(3):217-27 (2014)

4. Kubota, Y. et al. CTNNB1 mutational analysis of solid-pseudopapillary neoplasm of he pancreas using endoscopic ultrasound-guided fine-needle aspiration and next-generation deep sequencing. J Gastroenterol. 50:203$210(2015)$

\section{P0123 PTPN11 DRIVES TUMOR DEVELOPMENT AND DEFINES A \\ NOVEL THERAPEUTIC TARGET IN KRAS-MUTANT CANCERS}

D. A. Ruess ${ }^{1}$, G. J. Heynen ${ }^{2}$, K. Ciecielski ${ }^{1}$, H. Algül ${ }^{1}$

${ }^{1}$ II. Med. Klinik, TU Munich, Klinikum rechts der Isar, Munich/Germany

${ }^{2}$ Max Delbrück Center for Molecular Medicine, Berlin/Germany

\section{Contact E-mail Address: dietrich.ruess@uniklinik-freiburg.de}

Introduction: The ubiquitously expressed non-receptor protein tyrosine phosphatase SHP2, encoded by PTPN11, is involved in the regulation of multiple signaling cascades. SHP2 was the first reported oncogenic tyrosine phosphatase, although more recently demonstrated tumor suppressive properties as well. SHP2 has been proven to be required for proper wild-type RAS activation, yet studies addressing the relevance of SHP2 for mutated KRAS dependent cancers, such as pancreatic and lung cancer are lacking.

Aims \& Methods: Employing mutant Kras ${ }^{\mathrm{G} 2 \mathrm{D}}$ driven genetically engineered murine models (GEMM) of pancreatic ductal adenocarcinoma (PDAC) and non-small cell lung cancer, CRISPR/Cas9 mediated gene knockout in vitro, and patient derived tissue xenografts.

Results: In mutant Kras ${ }^{\mathrm{G} 12 \mathrm{D}}$ driven genetically engineered murine models (GEMM) of pancreatic ductal adenocarcinoma (PDAC) we detected a pivotal requirement of Shp2 for pancreatic carcinogenesis. Spontaneous as well as cerulein-triggered accelerated formation of premalignant lesions, and consequently PDAC, was almost completely abrogated in GEMM lacking Ptpn11 in the pancreas. Even in Ink4a/Arf and Trp53 deficient mutant Kras-driven tumors PDAC formation was dependent on Shp2 expression. Survival in these mice was substantially prolonged. Functional and transcriptional analyses revealed Shp2dependent regulation of Ras-activity and its main downstream effectors, and of the mutant Kras related oncogenic transcriptional program. Importantly, oncogenic transformation of the pancreas by constitutive active mutants of Map2k1 or Pik3ca was not impaired by deletion of Ptpn11, confirming a striking dependency of oncogenic Kras on Shp2 expression. These findings were corroborated in mutant Kras-driven lung adenocarcinoma GEMMs, in which the lack of Ptpn11 significantly delayed carcinogenesis suggesting a central role for Shp2 across organs. In contrast, spatiotemporal genetic deletion or pharmacologic inhibition of SHP2 in established Kras-mutant pancreatic and lung tumors or tumor cells had limited effect on growth capacity indicating that SHP2 is dispensable for tumor maintenance. However, focused pharmacologic screening using various substances revealed synergistic effects of SHP2 and MEK-inhibitors. In fact, co-inhibition of SHP2 and MEK prevented tumor relapse upon MEK monotherapy in endogenous GEMM and demonstrated synergistic potency in the treatment of patient-derived PDAC organoids and tissue xenografts.

Conclusion: Thus, as an integrator of RTK-Ras-MEK-ERK signaling downstream of almost all RTKs, SHP2 may hold promise as a therapeutic target not only in RTK-driven, but also in Kras-mutant tumors.

Disclosure of Interest: All authors have declared no conflicts of interest.

\section{P0124 CIRCHIPK3 PROMOTES PANCREATIC CARCINOMA CELLS BXPC3 PROLIFERATION BY TARGETING MIR-124/IL6R/ STAT3 PATHWAY}

G. Chen ${ }^{1}$, Y. Zhang ${ }^{1}$, J. Sun ${ }^{2}$

${ }^{1}$ Department Of Gastroenterology, Zhongshan Hospital, Fudan University, Shanghai/China

${ }^{2}$ Department Of Gastroenterology, Zhongshan Hospital, Fudan University Hospital, Fudan University, Shanghai/China

Contact E-mail Address: gwchen15@fudan.edu.cn
Introduction: Circular RNAs (circRNAs) are a novel class of noncoding RNAs that formed by a junction of the 5' end and 3'end. Increasing reports have shown that circRNA is dysfunction in neuro system diseases, cardiological heart diseases, human cancers and many other diseases. CircRNA have been demonstrated involving in tumorigenicity, proliferation, apoptosis, angiogenesis, migration, invasion and metastasis in human carcinoma. CircRNA can act as microRNA (miRNA) sponge and regulate the targets of miRNA. Circular RNA HIPK 3 (circHIPK3) is originated from second exon of HIPK 3 gene, which is upregulated in gastric, liver, esophageal. However, the mechanism remains unclear. Previous studies revealed that signal transducer and activator of transcription 3 (STAT3) as an oncogene that was activated in pancreatic carcinoma. Phosphorylation of STAT3 (p-STAT3) is a downstream target of interleukin 6 receptor (IL6R). Activation of STAT3 leads to malignancy of tumorgenesis, cell proliferation and migration. Knockdown STAT3 induces cell apoptosis by Bcl$\mathrm{xL}, \mathrm{c}-\mathrm{Myc}$, cyclinD1, etc. CircHIPK3 regulates BxPC3 cell proliferation through IL6R/STAT3 pathway. It may be a new target for the therapy of pancreatic carcinoma.

Aims \& Methods: Our research is to study whether circHIPK3 can promote proliferation of pancreatic carcinoma cell line, BxPC3, and to explore the mechanism of circHIPK 3 in cell proliferation. Cell viability was determined by cell counting kit-8 (CCK-8). Transient knockdown of circHIPK3 using specific siRNA targeting the conjunction of circHIPK3. Overexpression of miR-124 was transfected with synthetic miRNA mimic. Real-time quantitative reverse transcription-polymerase chain reaction (qRT-PCR) was performed to detect circHIPK3, miR-124 and mRNAs. The expressions of STAT3, p-STAT3, IL6R were measured by Western blot. Overexpression of STAT3 was transfected with STAT3 plasmid. Dual-Luciferase Reporter Assay was performed to detect the interaction of circHIPK 3 and miR-124.

Results: CircHIPK3 was upregulated in $\mathrm{BxPC} 3$ compared to human pancreatic duct epithelial cells (HPDE6-C7). Knockdown of circHIPK3, which didn't affect the linear transcript, significantly decreased cell viability of BxPC3. Bioinformatical analysis and luciferase assay demonstrated that circHIPK3 interacted with miR-124. QRT-PCR and Western blot confirmed that knockdown of circHIPK3 caused decreasing of mRNA levels of STAT3 and IL-6R and protein levels of STAT3, p-STAT3 and IL-6R. Previous studies confirmed that miR-124 negatively regulates STAT3, IL-6R via interacting with 3'-UTR (untranslated region). Ectopic expression of miR-124 decreased the expression of STAT3, pSTAT3, IL-6R. Indeed, miR-124 repressed BxPC3 cell proliferation which was consistent with si-circHIPK3. In addition, overexpression STAT3 abolished the si-circHIPK3 and miR-124 mimic induced cell suppression. QRT-PCR and Western blot confirmed that circHIPK3 and both STAT3, p-STAT3 and IL6R were upregulated in BxPC3 cells than HPDE6-C7 cells while miR-124 was downregulated. MiR-124 was negatively correlated with circHIPK3 and STAT3, p-STAT3 and IL-6R

Conclusion: In this study, we identified circHIPK 3 promotes BxPC3 pancreatic carcinoma cell proliferation by targeting miR-124 and its target genes STAT3 and IL-6R. We found that miR-124 was a negative regulator of proliferation in BxPC3. And overexpression of STAT3 could attenuate the anti-proliferation of si-circHIPK 3 and miR-124. These results demonstrated that circHIPK 3 regulates BxPC3 cell proliferation by acting as miR-124 sponge.

Disclosure of Interest: All authors have declared no conflicts of interest.

\section{References}

1. Scholz A, Heinze S, Detjen KM, Peters M, Welzel M, Hauff P, et al. Activated signal transducer and activator of transcription 3 (STAT3) supports the malignant phenotype of human pancreatic cancer. Gastroenterology. 2003;125(3):891-905.

2. Hatziapostolou M, Polytarchou C, Aggelidou E, Drakaki A, Poultsides GA, Jaeger SA, et al. An HNF4 alpha-miRNA Inflammatory Feedback Circuit Regulates Hepatocellular Oncogenesis. Cell. 2011;147(6):1233-47.

3. Zheng Q, Bao C, Guo W, Li S, Chen J, Chen B, et al. Circular RNA profiling reveals an abundant circHIPK 3 that regulates cell growth by sponging multiple miRNAs. Nature Communications. 2016;7.

\section{P0125 CONCOMITANT PANCREATIC CANCERS ARISING ADJACENT TO INDEX INTRADUCTAL PAPILLARY MUCINOUS NEOPLASMS SHARE IDENTICAL KRAS MUTATIONS AND ARE ASSOCIATED WITH A FAVORABLE PROGNOSIS}

Y. Omori ${ }^{1}$, Y. Ono ${ }^{2}$, M. Tanino ${ }^{3}$, H. Karasaki ${ }^{2}$, T. Shinohara ${ }^{1}$, S. Tanaka ${ }^{3}$, H. Maguchi ${ }^{4}$, Y. Mizukami ${ }^{5}$

${ }^{1}$ Department Of Pathology, Teine-Keijinkai Hospital, Sapporo/Japan

${ }^{2}$ Center For Clinical And Biomedical Research, Sapporo Higashi Tokushukai Hospital, Sapporo/Japan

${ }^{3}$ Department Of Cancer Pathology, Hokkaido University Graduate School of

Medicine, Sapporo/Japan

${ }_{5}^{4}$ Center For Gastroenterology, Teine-Keijinkai Hospital, Sapporo/Japan

${ }^{5}$ Department Of Medicine, Asahikawa Medical University, Asahikawa/Japan

Contact E-mail Address: yukoom@gmail.com

Introduction: Intraductal papillary mucinous neoplasms (IPMNs) are precursors of pancreatic ductal adenocarcinoma (PDA) and are also associated with multicentric lesions (field defect), where concurrent de novo PDA, independent of index IPMN lesion, can also develop. However, there are cases where PDAs arise adjacent to the index IPMNs, and occasionaly they are pathologically indistinguishable whether the carcinoma developed from IPMN or was coincidental to the IPMN. A genetic approach can be useful to clarify the origin of each tumor compartment to determine if they shared molecular signatures. 
Aims \& Methods: Twenty concomitant PDAs and IPMNs (39 samples, including cuncurrent lesions) from surgically resected patients were enrolled in this study. Resected pancreata were sliced at 5-mm intervals for whole-section histological analysis, and the distance between PDA and IPMNs was measured after precise pathological mapping. Target amplicon sequencing that covers 18 PDA-associated genes including KRAS, GNAS, TP53, SMAD4, CDKN2A/p16, CTNNB1 and RNF43, was performed using Ion PGM ${ }^{\mathrm{TM}}$ system (Thermo Fisher Scientific). Protein expression of TP53, SMAD4, p16, b-catenin, and RNF43 was also analyzed immunohistochemically.

Results: KRAS mutations were detected in 19/20 (95\%) of PDAs and in 38/39 $(97 \%)$ of IPMNs. "Adjacent" concomitant PDAs, defined as those that are $5 \mathrm{~mm}$ or less away from the IPMN $(\mathrm{n}=11)$, tended to harbor identical KRAS mutations as the index IPMNs (KRAS identical; $\mathrm{n}=8,72 \%, K R A S$ different; $\mathrm{n}=3$, $27 \%$ ). All three cases with contiguous neoplastic lesions via the main pancreatic duct between PDAs and IPMNs had identical KRAS mutations. In contrast, 7 of 9 "distant" concomitant PDAs, defined as those greater than $5 \mathrm{~mm}$ away from the IPMN $(\mathrm{n}=9)$, possessed distinct KRAS mutations from the index IPMNs $(78 \%)$. Mutations in GNAS were demonstrated in $14 / 20(70 \%)$ of index IPMNs and in 29/39 (74\%) of all IPMNs, but not in PDAs, supporting de novo carcinogenesis rather than progression from the IPMNs. PDAs harboring identical mutations in KRAS as IPMNs were significantly closer to the IPMNs (KRAS identical $\mathrm{n}=16,0-35 \mathrm{~mm}$, average $9 \mathrm{~mm}$ ) relative to cases with distinct $K R A S$ mutations in the PDAs and IPMNs $(\mathrm{n}=23,0-75 \mathrm{~mm}$, average $20 \mathrm{~mm}$, $\mathrm{p}=0.0397)$. The KRAS identical group had a better prognosis than the KRAS different group (disease-free survival $\mathrm{p}=0.0245$, overall survival $\mathrm{p}=0.205$ ) Curiously, the molecular signature of 18 PDA-associated genes was not significantly different between two groups.

Conclusion: Multiple clones with distinct KRAS mutations were identified in pancreata during initiation and progression of IPMNs, and a subset of PDAs arising within the field defect share the same $K R A S$ mutation with index IPMN lesions. Interestingly, PDAs adjacent to IPMN tend to have identical KRAS mutations, suggesting PDAs and index IPMNs may arise from a common founder. The KRAS identical group appears to have better prognosis relative to the $K R A S$-different group, implying distinct molecular programs may govern their biological behavior.

Disclosure of Interest: All authors have declared no conflicts of interest.

\section{Reference}

Patra KC, Bardeesy N, Mizukami Y. Diversity of Precursor Lesions For Pancreatic Cancer: The Genetics and Biology of Intraductal Papillary Mucinous Neoplasm. Clin Transl Gastroenterol 2017;8:e86.

\section{P0126 POLYMORPHISM OF TP53 GENE, LEVELS OF INSULIN AND PROINFLAMMATORY CYTOKINES IN PATIENTS WITH PANCREATIC CANCER}

T. I. Romanova ${ }^{1}$, I. N. Grigoreva ${ }^{1}$, V. N. Maximov ${ }^{1}$, O. V. Efimova ${ }^{1}$, Y I. Ragino, T. S. Suvorova ${ }^{2}$, N. L. Tov ${ }^{2}$

${ }^{1}$ Gastroenterology, Federal State Budgetary of Scientific Institution «Institution of Internal and Preventive Medicine», Novosibirsk/Russian Federation

${ }^{2}$ NSMU, Novosibirsk/Russian Federation

\section{Contact E-mail Address: igrigorieva@ngs.ru}

Introduction: The pancreatic cancer is a leading cause of death in cancer carriers worldwide.

Aims \& Methods: To study the polymorphism of the TP53 gene on the suppressor in the blood serum and to evaluate proinflammatory cytokines (IL-1 $\beta, \mathrm{TNF}-\alpha$ ), insulin blood serum levels at patients with various pathologies of the pancreas (cancer $(\mathrm{PCa})$, acute and chronic pancreatitis (OP and $\mathrm{CP})$ ) with various genotypes of TP53. 150 patients were followed in a one-stage clinical trial (42 patients with $\mathrm{OP}, 81$ with $\mathrm{CP}$, and 27 with $\mathrm{PCa}$ ). The diagnosis has been verified by clinic methods, ultrasonography, CT. The mean age of patients with PCa was $63.6 \pm 4$, 9 years. In patients with OP, blood sampling was carried out in the first 5 days after admission to hospital, patients with $\mathrm{CP}$ were examined at the stage of exacerbation. The concentration of IL- $1 \beta$, TNF- $\alpha$, serum insulin was determined by ELISA using the ELISA kit and Monobind Inc., USA. The frequencies of genotypes and alleles of the TP53 gene by exon (exon 4, Arg72Pro) polymorphism were studied by PCR.

Results: The incidence of Arg/Arg genotypes of the TP53 gene was 65\% in patients with $\mathrm{PCa}, 49 \%$ in the control group. In patients with $\mathrm{PCa}$ there was no homozygotic genotype Pro/Pro, in the comparison group - $13 \%, \mathrm{p}<0.05$. The frequency of Arg/Pro genotypes was $35 \%$ in patients with $\mathrm{PCa}$ and $38 \%$ in the comparison group. The frequency of alleles of the TP53 gene in patients with PCa and in the comparison group was: Arg $(82.5 \%$ and $68 \%)$, Pro $(17.5 \%$ and $32 \%$ ). The concentration of insulin in different genotypes in patients with $\mathrm{PCa}$ did not differ significantly and was $7.5 \pm 2.2 \mu \mathrm{U} / \mathrm{ml}$ in $\mathrm{Arg} / \mathrm{Arg}$, Arg/Pro $11.0 \pm 5.9 \mu \mathrm{U} / \mathrm{ml}, \mathrm{p}>0.05$. In the comparison group, the serum level of insulin was $5.7 \pm 1.8 \mu \mathrm{U} / \mathrm{ml}$. In patients with $\mathrm{PCa}$, the glucose level was significantly higher, compared with patients with $\mathrm{OP}$ and $\mathrm{CP}(8.5 \pm 1.4 \mathrm{mlMol} / 1,5.4 \pm 0.3$ and $5.1 \pm 0.1 \mathrm{mlMol} / 1$, respectively, $\mathrm{P}<0.05$ ). The level of IL-1 $\beta$ was significantly higher in patients with $\mathrm{OP}$ than in patients with $\mathrm{CP}$ and $\mathrm{PCa}(5.1 \pm 1.7$, $2.0 \pm 0.3$ and $1.3 \pm 0.2 \mathrm{pg} / \mathrm{ml}$, respectively), $\mathrm{p}<0.05$. The level of TNF- $\alpha$ in the serum of patients with $O P$ was $3.5 \pm 0.5 \mathrm{pg} / \mathrm{ml}$, and did not significantly differ from the serum level of patients with $\mathrm{CP}$ and $\mathrm{PCa}-4.3 \pm 0.7$ and $1.1 \pm 0.2 \mathrm{pg} / \mathrm{ml}$, respectively. In patients with $\mathrm{PCa}$, the level of TNF- $\alpha$ was significantly lower than in patients with CP, $p<0.05$. The levels of IL- $1 \beta$ in the serum of patients with PCa with different genotypes of the TP53 gene did not differ significantly and amounted to $1.1 \pm 0.2 \mathrm{pg} / \mathrm{ml}$ in patients with the Arg/Arg genotype, with
Arg/Pro genotypes of $1.2 \pm 0.3 \mathrm{pg} / \mathrm{ml}, \mathrm{p}>0.05$. The level of TNF- $\alpha$ in the serum of patients with PC with the Arg/Arg genotype was $-1.2 \pm 0.2 \mathrm{pg} / \mathrm{ml}$, and did not significantly differ from the level in the serum of patients with the Arg/Pro genotype $-1.3 \pm 0.1 \mathrm{pg} / \mathrm{ml}$.

Conclusion: The Pro/Pro genotype of the TP53 gene was significantly more common in the comparison group than in the patients with $\mathrm{PCa}$. We detected significant differences in serum insulin levels in the comparison group and in patients with heterozygous genotypes, $\mathrm{p}<0.05$. The level of TNF- $\alpha$ in patients with $\mathrm{PCa}, \mathrm{CP}$ was significantly lower than in patients with $\mathrm{OP}$, and the level of IL-1 $\beta$ was significantly lower in patients with PCa than in patients with $\mathrm{CP}$. Disclosure of Interest: All authors have declared no conflicts of interest.

\section{P0127 VALIDATION OF SERUM/PLASMA METABOLOMIC BIOMARKERS AGAINST PANCREATIC CANCER BY QUANTITATIVE TARGETED GC/MS/MS}

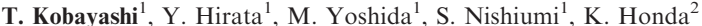
${ }^{1}$ Gastroenterology, Kobe university, Kobe/Japan

${ }^{2}$ Division Of Biomarker For Cancer Early Detection, National Cancer Center Research Institute, Tokyo/Japan

Contact E-mail Address: kobatak@med.kobe-u.ac.jp

Introduction: Pancreatic cancer (PC) is one of the most lethal diseases due to the difficulty of early detection. There is no effective blood biomarker for screening. Recently metabolomics is considered to be a promising approach to discover disease biomarkers. We previously reported that the serum/plasma levels of some metabolites in PC patients were significantly changed compared with those of healthy individuals.

Aims \& Methods: The aim of this study is to confirm and develop our candidate metabolomic biomarkers in blood of PC patients. Blood samples from PC patients and healthy volunteers (HV) were collected by two independent groups consisting of multiple institutions. The $1^{\text {st }}$ set was included $55 \mathrm{PC}$ in stage I and II and $58 \mathrm{HV}$. The $2^{\text {nd }}$ set was included $16 \mathrm{PC}$ and $16 \mathrm{HV}$. Sixteen candidate metabolites were selected from previous report. Quantitative analyses were performed by gas chromatography/tandem mass spectrometry (GC MS/MS) together with their corresponding stable isotopes. In the $1^{\text {st }}$ set, diagnostic models were constructed via multivariate logistic regression analysis. These results were validated using the $2^{\text {nd }}$ set.

Results: In the $1^{\text {st }}$ set, the levels of 11 metabolites differed significantly between PC and HV. Model Y consisting of 2 metabolites; i.e., histidine and xylitol showed high sensitivity $(70.4 \%)$ than CA19-9. Furthermore, combination of model $\mathrm{Y}$ with CA19-9 increased its sensitivity $(90.7 \%)$ and specificity $(89.5 \%)$. In the $2^{\text {nd }}$ set, combination of model Y with CA19-9 demonstrated high sensitivity $(81.3 \%)$ and specificity $(93.8 \%)$. In particular, it displayed very high sensitivity $(100 \%)$ for $\mathrm{PC}$ in a resectable state.

Conclusion: Quantitative analysis using GC/MS/MS confirmed the possibility of metabolomics-based screening methods for PC.

Disclosure of Interest: All authors have declared no conflicts of interest.

P0128 COMBINED HISTO-CYTOLOGICAL ANALYSIS OF EUS-
FNA SAMPLES FROM SOLID LESIONS USING STANDARD FNA
NEEDLES GIVES BETTER DIAGNOSTIC YIELD AND ACCURACY

A. K. Banerjee ${ }^{1}$, A. Cairns ${ }^{2}$, L. Sanni ${ }^{2}$, B. Paranandi ${ }^{1}$, M. Huggett ${ }^{1}$

${ }_{1}^{1}$ Gastroenterology, St James University Hospital, Leeds, Leeds/United Kingdom

${ }^{2}$ Pathology, St James University Hospital, Leeds, Leeds/United Kingdom

Contact E-mail Address: ashwinibanerjee@gmail.com

Introduction: Diagnostic yield from EUS-FNAC (fine needle aspiration cytology) has improved in the past few years with better tissue acquisition techniques. Core biopsy needles are now available but are more expensive than FNAC needles. We assessed the diagnostic yield and accuracy of FNAC samples processed for both cytology and histology

Aims \& Methods: EUS-FNA samples of solid lesions (from September 2014 to September 2016) were included in a prospective audit of practice. Between September 2014 and January 2016 the standard practice was to send FNAC samples for cytology only. After this date, FNAC samples were filtered through a cassette. The solid material was sent in formalin to histology and the effluent to cytology for cytospin and cell block as previously. Cellularity was graded as adequate or inadequate. Final diagnosis was obtained from MDT outcomes

Results: A total of 211 patients (118 male) were included. Samples were sent to cytology ( $\mathrm{n}=135 ; 107$ pancreas, 10 biliary, 7 lymph nodes, 11 other), or cytology \& histology $(\mathrm{n}=76 ; 56$ pancreas, 12 biliary, 5 lymph nodes, 3 other $)$. Sample adequacy was $80.7 \%$ and $98.7 \%(\mathrm{p}=0.0004)$. Diagnostic yield $(64.4 \%, 94.7 \%)$ and accuracy $(81.3 \%, 96.1 \%)$ was significantly better in the combined (histology $\&$ cytology) group $(\mathrm{p}<0.0001, \mathrm{p}=0.003)$. Within the combined group, diagnostic yield and accuracy improved by $20.5 \%(\mathrm{p}=0.007)$ and $26 \%(\mathrm{p}=0.0002)$ respectively when the sample was processed for both cytology and histology.

Conclusion: Our study confirms significant improvement in diagnostic yield and accuracy when samples were sent for both cytology and histology using standard FNAC needles.

Disclosure of Interest: All authors have declared no conflicts of interest. 
P0129 VERIFICATION OF INTERNATIONAL CONSENSUS GUIDELINES 2012 - SURGICAL INDICATION FOR BRANCH DUCT INTRADUCTAL PAPILLARY MUCINOUS NEOPLASM OF THE PANCREAS (BD-IPMN) WITH WORRISOME FEATURES

Y. Shimizu ${ }^{1}$, H. Yamaue ${ }^{2}$, H. Maguchi ${ }^{3}$, S. Hirono ${ }^{2}$, S. Hijioka ${ }^{4}$, K. Hara ${ }^{4}$, T. Sano ${ }^{5}$, Y. Senda ${ }^{6}$, S. Natsume ${ }^{6}$, A. Yanagisawa ${ }^{7}$

${ }^{1}$ Gastroenterological Surgry, Aichi Cancer Center Hospital, nagoya/Japan

${ }^{2}$ The Second Department Of Surgery, Wakayama Medical University, Wakayama/ Japan

${ }^{3}$ Center For Gastroenterology, Teine-Keijinkai Hospital, Sapporo/Japan

${ }^{4}$ Department Of Gastroenterology, Aichi Cancer Center Hospital, Nagoya/Japan ${ }^{5}$ Department Of Gastroenterological Surgery, Aichi Medical University Hospital, Nagoya/Japan

${ }^{6}$ Department Of Gastroenterological Surgery, Aichi Cancer Center Hospital, Nagoya/Japan

${ }^{7}$ Department Of Pathology, Japanese Red Cross Kyoto Daiichi Hospital, Kyoto/ Japan

\section{Contact E-mail Address: yshimizu@aichi-cc.jp}

Introduction: In the revised international consensus guidelines of 2012 for the management of IPMN of the pancreas, resection is recommended for all main pancreatic duct IPMN. While in branch pancreatic duct IPMN (BD-IPMN), the indications for resection are more conservative. Cyst size $>30 \mathrm{~mm}$ withou "high-risk stigmata" can be observed without immediate resection. And EUS observation is recommended to decide a treatment strategy.

Aims \& Methods: The present study was a retrospective investigation of surgica indication for BD-IPMN with worrisome features (WF). 466 patients with IPMN underwent pancreatic resection at 3 high volume centers in Japan between 1996 and 2014. Among them, 156 patients with BD-IPMN were enrolled this study. The investigation of predictors of malignancy was done for 10 factors: age at time of surgery, sex, presence or absence of symptoms, serum amylase, CA19-9, CEA, tumor location, size of mural nodules (MN), diameter of main pancreatic duct (MPD), and cyst size of branch pancreatic duct (BPD). In preoperative examination, endoscopic ultrasonography (EUS) and computed tomography (CT) were considered to be essential. As for size of MN, EUS measurements were used in all 156 cases. For diameter of MPD and cyst size of BPD, the CT measurement values were used in all cases. In this study, BD-IPMN was defined as cases with cystic dilatation of BPD and the MPD diameter was considered $<5 \mathrm{~mm}$ (International Consensus Guidelines 2012). According to the WHO histological classification of IPMN (2000), pathological diagnosis is classified as adenoma (IPMA), borderline (IPMB), and noninvasive and invasive carcinoma (IPMC). A central review of pathological diagnosis was done in the cases of IPMB and IPMC. Patients were categorized as benign (IPMA and IPMB) or malignant (IPMC).

Results: Pathological diagnosis was benign IPMN in 91cases (58\%) and malignant in $65(42 \%)$. In univariate analysis CA19-9, MPD diameter, the sizes of MN and BPD cysts were significant factors. In multivariate analyses, size of MN $(p<0.0001)$ and cyst size of BPDs $(p=0.0004)$ were independent predictors of malignancy, and in ROC analysis AUC for these factors was 0.74 and 0.72 , respectively. Among 78 cases with WF (BPD cyst size $\geq 30 \mathrm{~mm}$ ), 45 cases $(58 \%)$ had malignant IPMN. The rate of malignancy was significantly higher than that of patients without $\mathrm{WF}(26 \%)(\mathrm{p}<0.0001)$. Among $78 \mathrm{WF}$ patients, 54 cases had MN with EUS observation. The rate of malignancy in patients with $\mathrm{MN}$ was significantly higher than that of WF patients without MN (69\% vs 33\%). With $7 \mathrm{~mm}$ taken as the cutoff value for the size of $\mathrm{MN}$, the diagnosis of malignant IPMN had sensitivity of $76 \%$, specificity of $73 \%$ and accuracy of $74 \%$. Carcinoma without MN was present in 8 patients $(8 / 45=17 \%)$ among $78 \mathrm{WF}$ patients. Pathological findings of these patients were noninvasive carcinoma in 6 , invasive carcinoma 2.

Conclusion: Algorithm for the management of BD-IPMN of International Consensus Guidelines 2012 was acceptable. Mural nodules observed with EUS showed high predictive ability in BD-IPMN patients with WF. However, about $15 \%$ of carcinoma patients did not have nodules, and the handling of the diagnosis in such cases is a problem for the future.

Disclosure of Interest: All authors have declared no conflicts of interest.

\section{Reference}

Tanaka M, Fernandez-del Castillo, C, Adsay, V et al. International consensus guidelines 2012 for the management of IPMN and MCN of the pancreas. Pancreatology 2012;12:183-197

\section{P0130 USE OF A NOVEL THROUGH-THE-NEEDLE MICRO- BIOPSY FORCEPS IN DIAGNOSING PANCREATIC CYSTS - A MULTICENTER FEASIBILITY STUDY}

B. Kovacevic ${ }^{1}$, J. G. Karstensen ${ }^{2}$, R. F. Havre ${ }^{3}$, K. D. Pham ${ }^{3}$, M. Giovannini ${ }^{4}$, E. Dabizzi ${ }^{5}$, P.G. Arcidiacono ${ }^{5}$, E. Vazquez Sequeiros ${ }^{6}$, P. Klausen ${ }^{1}$, C. V. Rift ${ }^{7}$, J. P. Hasselby ${ }^{7}$, A. Toxværd ${ }^{8}$, E. Kalaitzakis ${ }^{1}$, C. P. Hansen ${ }^{9}$, P. Vilmann ${ }^{1}$ ${ }^{1}$ Gastro Unit, Division Of Endoscopy, Copenhagen University Hospital Herlev, Herlev/Denmark

${ }^{2}$ Department Of Gastrointestinal Surgery, Slagelse Hospital, Slagelse/Denmark ${ }^{3}$ Department Of Medicine, Haukeland University Hospital, Bergen/Norway ${ }^{4}$ Gastroenterology And Endoscopy, AP-HM, Marseille/France

${ }^{5}$ Pancreato-biliary Endoscopy And Endosonography Division, San Raffaele

Scientific Institute Vita Salute San Raffaele University, Milan/Italy

${ }^{6} \mathrm{MD}$ Anderson, Madrid/Spain

${ }^{7}$ Department Of Pathology, Rigshospitalet, Copenhagen/Denmark
${ }^{8}$ Department Of Pathology, Copenhagen University Hospital Herlev, Herlev/ Denmark

${ }^{9}$ Department Of Gastrointestinal Surgery, Copenhagen University Hospital Rigshospitalet, Copenhagen/Denmark

Contact E-mail Address: bojan.k@dadlnet.dk

Introduction: Cystic lesions of the pancreas represent a diagnostic dilemma as some of the lesions are non-neoplastic or benign, whereas others are malignant or have potential for malignant transformation. As indicated in a recent meta-analysis on diagnostic accuracy of endoscopic ultrasound (EUS) guided fine-needle aspiration (FNA) cytology, it is often impossible to obtain sufficient cellular material when diagnosing these lesions with current modalities [1]. Recently, a novel biopsy forceps (Moray ${ }^{\mathrm{TM}}$, US Endoscopy, Mentor, USA) has become available. It can be introduced through a 19 G FNA-needle, enabling the endoscopists to obtain histological specimens from the pancreatic cyst wall for the first time [2].

Aims \& Methods: The aim of this study was to evaluate the use of the novel micro-forceps in a multicenter clinical setting. The patients referred for EUS evaluation of pancreatic cysts were included retrospectively from five European tertiary centers. Inclusion criteria were age of 18 or above and a pancreatic cyst of a size that allowed for FNA puncture. Exclusion criteria were pregnant or lactating females. A standardized data collection sheet including the information about patient demographics, cyst size, EUS/FNA findings, technical and clinical success, and the results of the biopsies taken was sent to the collaborating centers. Technical success was defined by successful puncture of the pancreatic cyst, subsequent successful mounting of the micro-biopsy forceps, and extraction of at least one micro-biopsy. Clinical success was defined by obtaining useful histological results.

Results: Twenty patients were included. There was a slight overrepresentation of female patients $(\mathrm{n}=12,60 \%)$ and the median age was 65 (range: $41-80)$. The patients had a median cyst size of $30 \mathrm{~mm}$ (range: $15-130 \mathrm{~mm}$ ) and a median procedural time was $30,5 \mathrm{~min}$ (range: $17-58 \mathrm{~min}$ ). We report a technical success rate of $85 \%(\mathrm{n}=17)$ - technical failure was only seen in transduodenal puncture $(\mathrm{n}=3,15 \%)$. Biopsies were generally of good quality and contributed to the diagnosis in 14 patients (clinical success of $82 \%$ ). Among these, there were ten cases of intraductal papillary mucinous neoplasia, two serous cystic adenomas, one mucinous cystic adenoma, one mucinous cystadenocarcinoma, and one pseudocyst. Two mild adverse events were recorded $(10 \%)$, a case of re-admission due to non-specific abdominal pain and a mild acute pancreatitis.

Conclusion: The use of micro-biopsy forceps was until now only reported in case reports. This is a first larger-scale feasibility study. We conclude that the use of the micro-forceps seems feasible and safe with acceptable rates of technical and clinical success. However, prospective studies are needed in order to determine diagnostic potential of this instrument compared to the other modalities currently used.

Disclosure of Interest: All authors have declared no conflicts of interest.

\section{References}

1. Suzuki R, Thosani N, Annangi S et al. Diagnostic yield of EUS-FNA-based cytology distinguishing malignant and benign IPMNs: a systematic review and meta-analysis. Pancreatology 2014;14(5):380-384.

2. Barresi L, Tarantino I, Ligresti D et al. A new tissue acquisition technique in pancreatic cystic neoplasm: endoscopic ultrasound-guided through-theneedle forceps biopsy. Endoscopy 2015; 47 Suppl 1 UCTN:E297-298.

\section{P0131 PANCREATIC DUCTAL CYTOLOGY: AN UNDERUSED DIAGNOSTIC TOOL}

A. Peixoto, P. Pereira, E. Rodrigues-Pinto, F. Vilas-Boas, J. Lopes, G. Macedo Centro Hospitalar De São João, Porto/Portugal

Contact E-mail Address: armandoafp5@gmail.com

Introduction: The diagnosis of pancreatic malignancy can be performed by brush cytology of the common bile duct or main pancreatic duct (MPD) during endoscopic retrograde cholangiopancreatography (ERCP). Preliminary data suggest that cytologic brushing of the MPD is safe and has a sensitivity equal to or slightly higher to that of bile duct cytology, although its clinical application is not defined.

Aims \& Methods: In this work we report our experience in the execution of MPD brush cytology. ERCPs between 2014 and 2015 that involved brush cytology of pancreatic strictures were included. Cytologies were obtained using the Brush Master V (Olympus Medical System). Histological evaluation was performed by two experienced cytopathologists.

Results: Of the 18 patients evaluated, 16 were men and 2 women, with a median age of 62 years (range: 43-89). All patients underwent abdominal computed tomography and 3 patients had magnetic resonance imaging. In addition to pancreatic strictures, abdominal CT revealed cephalopancreatic lesions in $61 \%$, findings suggestive of chronic pancreatitis in $28 \%$, pancreas divisum in $6 \%$, and pancreatic inflammatory features in $6 \%$. The distribution of the strictures was: head - 16, head and body - 1, tail - 1 . The pancreatic duct was dilated in 16 patients with a median of $7.5 \mathrm{~mm}$ (IQR: 6-15). The diagnostic values of MPD brush cytology for pancreatic cancer were: sensitivity $-81.8 \%$, specificity $-100 \%$, positive predictive value $-100 \%$, negative predictive value $-77.8 \%$, acuity $88.9 \%$. Sixty-one percent $(n=11)$ of the patients had a final diagnosis of pancreatic adenocarcinoma, $5.6 \%(\mathrm{n}=1)$ of neuroendocrine tumor and $33.3 \%$ $(\mathrm{n}=6)$ inflammatory stricture. All the adenocarcinomas lead to strictures in the head of the pancreas. The diagnosis of neuroendocrine tumor was made by 
endoscopic ultrasonography fine needle aspiration. One patient developed mild pancreatitis $(5.6 \%)$.

Conclusion: In patients with suspected cephalopancreatic adenocarcinoma referred for ERCP, MPD brush cytology may be performed beyond biliary cytology, as it may improve cytologic diagnosis of malignancy without increasing complications rate.

Disclosure of Interest: All authors have declared no conflicts of interest.

\section{P0132 ANALYSIS OF PROGNOSTIC FACTORS IN PANCREATIC} METASTASES, A MULTICENTER RETROSPECTIVE ANALYSIS

T. Ito ${ }^{1}$, R. Takada ${ }^{2}$, S. Omoto ${ }^{3}$, M. Tsuda ${ }^{4}$, D. Masuda ${ }^{5}$, H. Kato ${ }^{6}$, H. Ishii ${ }^{7}$, I. Moriyama ${ }^{8}$, K. Tanaka ${ }^{9}$, H. Kawamoto ${ }^{10}$, S. Yazumi

${ }^{1}$ Division Of Gastroenterology And Hepatology, Digestive Disease Center, Kitano Hospital, Osaka/Japan

${ }^{2}$ Department Of Hepatobiliary And Pancreatic Oncology, Osaka International Cancer Institute, Osaka/Japan

${ }^{3}$ Department Of Gastroenterology And Hepatology, Kindai University, Sayama/ Japan

${ }^{4}$ Department Of Gastroenterology And Hepatology, Graduate School of Medicine Kyoto University, Kyoto/Japan

${ }^{5}$ 2nd Department Of Internal Medicine, Osaka Medical College, Takatsuki/Japan ${ }^{6}$ Department Of Gastroenterology And Hepatology, Okayama University Graduate School of Medicine, Okayama/Japan

${ }^{7}$ Department Of Gastroenterology, National Hospital Organization Shikoku Cancer Center, Matsuyama/Japan

${ }^{8}$ Department Of Internal Medicine 2, Shimane University School of Medicine, Izumo/Japan

${ }^{9}$ Department Of Gastroenterology, Kyoto Second Red Cross Hospital, Kyoto/ Japan

${ }^{10}$ Department Of Internal Medicine, Kawasaki Medical School General Medical Center, Kawasaki Hospital, Kawasaki Medical School, Okayama/Japan

\section{Contact E-mail Address: kmus0416@yahoo.co.jp}

Introduction: Pancreatic metastases (PM) account for $1-2 \%$ of pancreatic tumors. Several cancer types metastasize to the pancreas, but even recently developed cross-sectional imaging modalities have difficulties distinguishing PM from primary pancreatic tumors. Moreover, their prognostic significance is poorly defined.

Aims \& Methods: The aims of this study were to clarify the incidence of primary tumors leading to PM, the clinical characteristics, and prognoses, and to define the prognostic factors for survival. A retrospective analysis was performed at 39 Japanese tertiary referral hospitals between January 2005 and August 2015, after receiving approval from the institutional review board of each hospital. We identified the patients based on data obtained from each institutional database, and analyzed patient and tumor characteristics, and survival time. All the patients enrolled in the analysis were histopathologically or cytopathologically diagnosed with PM. Kaplan-Meier analysis and Cox's proportional hazard models were applied to evaluate overall survival and survival analysis, respectively.

Results: We enrolled 159 patients (median age 74.5 years) with a pathologic diagnosis of PM. The most common primary tumor was renal cell carcinoma $(38.4 \%, \mathrm{n}=61)$, followed by lung cancer $(24.5 \%, \mathrm{n}=39)$, colorectal cancer $(11.3 \%, \mathrm{n}=18)$, sarcoma $(6.3 \%, \mathrm{n}=10)$, breast cancer $(6.3 \%, \mathrm{n}=10)$, and other cancer $(n=21)$. At the time of the diagnosis of PM, 38 patients $(24 \%)$ had at least one tumor-related symptom. Additional extra-pancreatic metastases were diagnosed in 94 patients $(59 \%)$. Sixty-four patients $(40 \%)$ underwent surgical resection, and no surgical resection was performed in 95 patients $(60 \%)$. Additional therapies were chemotherapy $(n=69)$, chemoradiation $(n=4)$, radiation $(n=3)$, palliative care, and unclear $(n=2)$. Eight patients were lost during follow-up and 151 patients were included in the statistical analysis. All patients with PM had a median overall survival of 43.0 months, with 3- and 5-year survival rates of $52.5 \%$ and $42.6 \%$, respectively. Among the five frequent primary sites of PM, prognoses of RCC, breast cancer, and colorectal were better than those of lung cancer and sarcoma. Univariate Cox proportional regression analysis identified four prognostic factors: pancreatic resection (hazard ratio [HR] $0.31,95 \%$ confidence interval [CI] $0.18-0.57, p<0.001$ ), extra-pancreatic metastases (HR 3.07, 95\% CI 1.71-5.51, $p<0.001$ ), tumor-related symptoms at PM diagnosis (HR 3.88, 95\% CI 2.29-6.56; $p<0.001$ ), and pathologic diagnosis of primary tumors $(p<0.001)$. Multivariate Cox proportional regression analysis identified three independent prognostic factors: extra-pancreatic metastases (HR 2.13,95\% CI 1.11-4.07, $p=0.02$ ), tumor-related symptoms at diagnosis (HR $5.39,95 \%$ CI $2.92-9.91, p<0.001)$, and pathologic diagnosis of primary tumors $(p<0.001)$

Conclusion: Treatment strategies and prognoses for PM completely differ according to the primary tumor type. A definitive pathologic diagnosis of PM is essential for selecting the appropriate treatment.

Disclosure of Interest: All authors have declared no conflicts of interest.

\section{References}

The role of surgery in the management of isolated metastases to the pancreas. The Lancet Oncology 2009;10:287-93.
Analysis of prognostic factors in metastatic tumors of the pancreas: a singlecenter experience and review of the literature. Pancreas 2010;39:135-43.

Secondary tumors of the pancreas diagnosed by endoscopic ultrasound-guided fine-needle aspiration: a 10-year experience. Diagn Cytopathol 2014;42:738-43. Clinical Impact of Pancreatic Metastases from Renal Cell Carcinoma: A Multicenter Retrospective Analysis. PLoS One 2016;11:e151662.

\section{P0133 LUNG METASTASIS IN PANCREATIC CANCER: SHOULD STAGING CHEST CT BE ROUTINELY PERFORMED?}

S. Singh ${ }^{1}$, B. Rao ${ }^{1}$, S. Umar ${ }^{1}$, M. Chowdhry ${ }^{1}$, A. Kulkarni ${ }^{2}$, K. Farah ${ }^{1}$, S. Morrissey ${ }^{1}$, M. Dhawan ${ }^{1}$, S. Thakkar ${ }^{1}$

${ }^{1}$ Gastroenterology, Allegheny Health Network, Pittsburgh/United States of AmericalPA

${ }^{2}$ Gastroenterology, Allegheny Health Network, pittsburgh/United States of AmericalPA

Contact E-mail Address: shail121@gmail.com

Introduction: National Comprehensive Cancer Network (NCCN) guidelines recommend chest x-ray or chest computed tomography (CT) for the staging of potential resectable pancreatic adenocarcinoma (PDA). However, there is limited data supporting these guidelines, and the prevalence of lung metastasis is not well defined on staging CT scans. We report our findings of patients with lung metastasis during initial staging and follow-up of patients with PDA.

Aims \& Methods: Data was prospectively collected from May 2013 to September 2016 for PDA patients who were presented at a multidisciplinary pancreas conference (MDPC) at a large tertiary care center. All patients were staged with CT pancreatic protocol, CT chest and Endoscopic Ultrasound. Patients with findings of lung lesions on initial staging chest CT were followed prospectively. Metastatic lung lesions were determined based on definite imaging characteristics with clinical consensus or lung biopsy results.

Results: A total 278 PDA patients referred to MDPC were staged with CT chest (Table 1). Out of these, $36(12.6 \%)$ patients were found to have either malignant $(\mathrm{N}=6)$ or indeterminate $(\mathrm{N}=30)$ lung lesions on initial staging CT chest. Out of the six malignant lung lesions, $5(1.8 \%)$ patients had metastatic PDA lesions, and $1(0.35 \%)$ patient had incidental primary lung cancer. On a follow-up of 30 patients with indeterminate lung lesions, 8 patients $(26.7 \%)$ were later determined to be lung metastasis. The overall prevalence of definite lung metastasis was at least $4.8 \%(13 / 278)$. The prevalence of lung metastasis in pancreatic head cancer was $3.0 \%$, while body and tail masses was $10.5 \%$. Lung metastasis was almost four times more likely in the body, and tail masses $(\mathrm{OR}=3.83$, CI $1.2-11.8$, $\mathrm{p}=0.02$ ) compared to head. Overall CT chest resulted in change in management plan in $9(2.9 \%)$ patients due to change in the stage to metastatic (8) and diagnosis primary lung cancer (1). Staging with CT chest changed otherwise resectable disease to unresectable/metastatic in 5 patients $(1.8 \%)$ and borderline resectable to metastatic disease in $2(0.7 \%)$ patients. Prevalence of isolated PDA lung metastasis without any other metastasis was $2.8 \%(8 / 278)$.

Table 1: Comparison of patient and tumor characteristics.

\begin{tabular}{llll}
\hline & $\begin{array}{l}\text { Patients without } \\
\text { Lung metastasis } \\
\mathrm{N}=265\end{array}$ & $\begin{array}{l}\text { Patients with } \\
\text { LungMetastasis } \\
\mathrm{N}=13\end{array}$ & P value \\
\hline Age (yrs), mean (S.D) & 68.6 & 64.8 & 0.22 \\
Male (\%) & 48.4 & 69.2 & 0.14 \\
Race, Caucasian (\%) & 90.2 & 100 & 0.36 \\
Mass size (mm), mean (S.D) & 26.9 & 31.1 & 0.16 \\
Mass Location & & & \\
Head (\%) & 76.7 & 46.2 & 0.01 \\
Body/Tail (\%) & 23.3 & 53.8 & 0.90 \\
CA 19-9, mean (S.D) & $899(1528)$ & $961(482)$ & \\
\hline
\end{tabular}

Conclusion: Our study showed that the prevalence of pulmonary metastasis in PDA was clinically relevant to mandate routine staging with CT chest. Prevalence was significantly higher for pancreatic body and tail cancers compared to the head. Staging CT chest resulted in a change in the stage of PDA and management decisions.

Disclosure of Interest: All authors have declared no conflicts of interest.

\section{P0134 VALUE OF EUS IN EARLY DETECTION OF TUMOR LESION} IN THE REMNANT PANCREAS

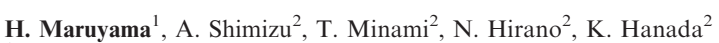
${ }^{1}$ Dept. Of Gastroenterology, Onomichi General Hospital, Onomichi/Japan ${ }^{2}$ Gastroenterology, Hiroshima onomichi city, onomichi/Japan

Contact E-mail Address: hiromaruyama99@gmail.com 
Introduction: New lesions (metachronous pancreatic cancer) and recurrence may develop in the remnant pancreas after initial resection for pancreatic cancer and Intraductal Papillary Mucinous Neoplasm (IPMN). Endoscopic ultrasonography (EUS) is proved as a more specific and sensitive method for pancreatic lesion. However, there is no report about EUS after pancreatectomy. If it is possible to observe from the anastomotic part to remnant pancreas under the EUS, remnant pancreatic cancer may be pointed out an early stage.

Aims \& Methods: The aim of this study was retrospectively to investigate the observation ability of EUS for remnant pancreas. In this retrospective study, 44 patients who underwent EUS for remnant pancreas were enrollment. The definition of observation under the EUS for remnant pancreas was as follows, total observation for remnant pancreas observed from liner white line (anastomotic part) to opposite side pancreas, otherwise it was insufficient observation. We compared the detection rate of EUS findings and that of CT or MRI findings. Results: Among the 395 patients who underwent pancreatectomy at the JA Onomichi General Hospital between December 2002 and March 2016, the enrolled patients were 44 who underwent EUS for remnant pancreas. In the surgical procedure, pancreaticoduodenectomy (PD) including pylorus-preserving PD (PPPD) and subtotal stomach-preserving PD (SSPPD) was 20 cases and distal pancreatectomy (DP) was 24 cases. Total observation of remnant pancreas was possible in 41 cases $(93 \%)$. Seven of 44 cases showed the lesion of recurrence in the remnant pancreas. Although CT or MRI was able to point out it in only 2 cases, EUS was able to point out it in the remnant pancreas of all cases. Stage of six cases were as follows, 1 case of stage 0,2 cases of stage Ia, 3 cases of stage IIb. The other case was IPMN. We were able to perform EUS-FNA for lesion in the remnant pancreas in all cases. Pathological results were positive in 5 cases. One of the other 2 cases was negative (class III), but it was a recurrence by surgery. The other case was strongly suspected to recurrence by Positron emission tomography (PET). Second pancreatectomy was performed in 4 out of 7 cases. The sensitivity of EUS-FNA was $71.4 \%(5 / 7)$, the specificity was $85.7 \%(6 / 7)$ and the accuracy was $71.4 \%(5 / 7)$. In addition, a comparison of detection ability of EUS and CT or MRI findings showed that EUS was significantly superior to CT or MRI $(P<0.01)$

Conclusion: EUS was able to observe remnant pancreas in almost cases. We were able to perform EUS-FNA for lesion in the remnant pancreas. In addition, the detection ability of EUS was significantly superior to that of CT or MRI. We believe that EUS and EUS-FNA should be underwent for lesion in remnant pancreas, and that remnant pancreatic cancer may be pointed out an early stage. Disclosure of Interest: All authors have declared no conflicts of interest.

\section{P0135 PATIENTS WITH INTRADUCTAL PAPILLARY MUCIONOUS NEOPLASMS OF PANCREAS (IPMNS) ARE AT INCREASED RISK OF RENAL CELL CARCINOMA, PROSTATE, COLORECTAL AND BREAST CANCER: A SINGLE CENTER ITALIAN EXPERIENCE}

N. Panic ${ }^{1}$, M. Bulajic ${ }^{1}$, F. Macchini ${ }^{1}$, S. Solito ${ }^{1}$, S. Boccia ${ }^{2}$, E. Leoncini ${ }^{2}$, D. Berretti ${ }^{1}$, S. Pevere ${ }^{1}$, S. Vadala $^{1}$, M. Marino ${ }^{1}$, E. Pinese $^{1}$, M. Zilli ${ }^{1}$ ${ }^{1}$ Department For Gastroenterology And Digestive Endoscopy, Academic Center of Udine, Udine/Italy

${ }^{2}$ Public Health Department, Catholic University, Rome/Italy

\section{Contact E-mail Address: nikola.panicmail@gmail.com}

Introduction: Number of studies reported that patients with intraductal papillary mucionous neoplasms of pancreas (IPMNs) are at the increased risk for occurrence of extrapancreatic malignancies (EPMs). We have conducted a study in order to assess the prevalence and incidence of EPMs in a cohort of Italian patients diagnosed with IMPN and to identify risk factor for their occurrence. Aims \& Methods: A hospital based single-centre study was conducted in hospital Santa Maria della Misericordia, Udine, Italy. Hospital records were screened in order to identify all new diagnosed IPMN cases in period January $1^{\text {st }} 2009$ December $31^{\text {st }} 2015$, as well as those seen during the follow-up. Data were extracted for all the cases identified on demographics, clinical characteristics, treatment and outcomes. Furthermore we searched for any EPMs diagnosed previous, synchronous or metachronous in relation to IPMN diagnosis. We compared the distribution of the demographics clinical characteristics, treatment and outcomes among patients with and without EPMs by univariate analysis. The ratio of the observed $(\mathrm{O})$ number of patients with EPMs to the expected $(\mathrm{E})$ number was calculated along with $95 \%$ confidence interval (CI).

Results: Number of 198 patients with IPMN was included in the study. Patients were predominantly female $(59.1 \%)$ and age $70-79(47.2 \%)$. IPMNs were predominantly brunch duct $(79.2 \%)$, multifocal $(66.2 \%)$ and were not subjected to surgical treatment $(89.9 \%)$. We identified 72 EPMs in 63 patients with IPMN $(31.8 \%)$. Eight patients have had 2 EPMs and 2 patients have had 3 EPMs. Among identified EPMs, $51(70.8 \%)$ were diagnosed previous to IPMN, 17 $(23.6 \%)$ synchronous to IPMN and $4(5.6 \%)$ metachronous to IPMN. Among most frequently diagnosed were colorectal cancer (12 patients, $6.1 \%$ ), breast cancer ( 8 patients, $4.0 \%$ ), renal cell cancer ( 8 patients, $4.0 \%$ ) and prostate cancer ( 7 patients, $3.5 \%)$. O/E ratios for EPMs were significantly increased for cancer in general (3.66, CI 95\%: 2.39-5.37) as well as for renal cell carcinoma (9.62, CI 95\%: 1.98-28.10) and prostate cancer (4.91, CI 95\%: 1.59-11.45), while borderline significance was observed for colorectal carcinoma (3.59, CI 95\%: $0.98-9.19)$ and breast cancer (3.16, CI 95\%: 1.03-7.37) (Table 1). We did not identify any of the demographics and clinical characteristics to significantly influence EPM occurrence among patients with IPMN

Table 1: Observed and expected number of patients with extrapancreatic malignancies in 60 patients with intraductal papillary mucinous neoplasms

\begin{tabular}{|c|c|c|c|c|}
\hline & Observed & Expected & $\mathrm{O} / \mathrm{E}$ & CI $95 \%$ \\
\hline ALL & 26 & 7, 096 & 3,66 & $2.39-5.37$ \\
\hline prostate cancer & 5 & 1,019 & 4,91 & $1.59-11.45$ \\
\hline breast cancer $^{\mathrm{a}}$ & 5 & 1,583 & 3,16 & $1.03-7.37$ \\
\hline colorectal cancer & 4 & 1,115 & 3,59 & $0.98-9.19$ \\
\hline renal cell cancer & 3 & 0,312 & 9,62 & $1.98-28.10$ \\
\hline
\end{tabular}

${ }^{a}$ Calculated for females.

Conclusion: We report an increased prevalence of EPMs in Italian patients with IPMN, especially for renal cell carcinoma, prostate, colorectal and breast cancer. A systematic surveillance of IPMN cases for such cancer types would be advised. Disclosure of Interest: All authors have declared no conflicts of interest.

\section{P0136 CLINICAL CHARACTERISTICS OF SECOND PRIMARY PANCREATIC CANCER}

J.H. Jo, I.R. Cho, H.S. Lee, M.J. Chung, S. Bang, S.W. Park, S.Y. Song, J. Chung, J.Y. Park

Department Of Internal Medicine And Institute Of Gastroenterology, Yonsei University Health System Dept. of Internal Medicine Dept. of Gastroenterology, Seoul/Korea, Republic of

Contact E-mail Address: woforce@ naver.com

Introduction: Pancreatic ductal adenocarcinoma (PDAC) is known to have an extremely poor prognosis. Several studies reported the increased risk of second primary pancreatic ductal adenocarcinoma (2nd PDAC) in cancer survivors. However, data on the characteristics of 2 nd PDAC are insufficient. Studies of PDAC in the setting of second primary cancer can provide etiologic clues to understand PDAC

Aims \& Methods: The aim of this study was to investigate the clinical characteristics of the patients with second primary PDAC compared to patients with first primary PDAC. This retrospective cohort study included 1759 patients with PDAC. They were classified as having 2nd PDAC or first primary PDAC (1st PDAC) according to a prior diagnosed cancer that originated from different organ and diagnosed at least 6 months before the diagnosis of PDAC. Comparative analysis and multivariated survival analysis were used to evaluate the characteristics of the 2nd PDAC.

Results: A total of 1759 patients with PDAC were included in the cohort. Fortythree patients were classified as having synchronous 2nd PDAC and excluded from the analysis. There were 110 patients $(6.4 \%)$ with 2 nd PDAC and 1606 $(93.6 \%)$ patients with 1st PDAC. The median interval between the diagnosis of the 2nd PDAC and the diagnosis of the prior cancer was 8.4 years (range 0.7-31.4 years) in the 2nd PDAC group. In the comparison of baseline characteristics between the 1st PDAC and 2nd PDAC groups, patients with 2nd PDAC presented significantly older age at diagnosis ( 66.5 vs. 62.2 years, $\mathrm{p}<0.001)$, lower rate of alcohol consumption $(25.5$ vs. $36.8 \%, \mathrm{p}=0.017)$, higher rate of resectability of PDAC (26.4 vs. $15.9 \%, \mathrm{p}=0.004)$, and higher rate of receiving surgery as initial treatment $(26.4$ vs. $15.9 \%, p=0.018)$ than patients with 1 st PDAC. The most common origin of prior cancers was the stomach (22 of 110, 20.0\%), followed by the thyroid $(21$ of $110,19.1 \%)$, breast ( 19 of $110,17.3 \%)$, colon $(12$ of $110,10.9 \%)$, and others. The overall survival (OS) was slightly longer in patients with 2nd PDAC; however, the difference was not significant (11.8 vs. 12.3 months, $\mathrm{p}=0.068)$. Multivariate analysis without resectable status showed that 2nd PDAC (HR $0.73,95 \%$ CI $0.56-0.94, \mathrm{p}=0.016$ ), age at diagnosis (HR $1.02,95 \%$ CI $1.01-1.02, \mathrm{p}<0.001$ ), and alcohol consumption (HR $1.28,95 \%$ CI $1.13-1.47, \mathrm{p}<0.001$ ) were significantly related to OS (Table 1$)$. When resectable status was included in multivariate analysis, age at diagnosis (HR $1.02,95 \% \mathrm{CI}$ $1.01-1.02, \mathrm{p}<0.001$ ), alcohol consumption (HR $1.25,95 \%$ CI 1.11-1.42, $\mathrm{p}=0.001$ ), and resectable status at diagnosis (HR $0.30,95 \%$ CI $0.25-0.36$, $\mathrm{p}<0.001$ ) were significantly associated with OS. However, 2nd PDAC (HR $0.85,95 \%$ CI $0.66-1.09, \mathrm{p}=0.198$ ) was no longer significantly associated with OS after adjusting for resectable status. This analysis suggested that the association between 2nd PDAC and survival was owing to the higher resectability rate. When subgroups were separately analyzed according to initial treatment modality, the effectiveness of surgery and chemotherapy were similar between 2 nd and 1 st PDAC. In the subgroup of patients who received curative surgery, the median OS was 28.5 months (95\% CI, 23.0-34.1) in the 1st PDAC group com- 
pared with 33.1 months (95\% CI, 9.0-27.2) in the 2nd PDAC group (N: 259 vs. $29, \mathrm{p}=0.860$ ). In the subgroup of patients who received chemotherapy, the median OS was 10.7 months (95\% CI, 10.0-11.4) in 1st PDAC compared with 10.8 months $(95 \%$ CI, 9.2-12.3) in 2nd PDAC (N: 1094 vs. $66, \mathrm{p}=0.952)$.

Table 1: Cox proportional analysis for the contribution of clinical factors to overall survival

\begin{tabular}{|c|c|c|c|c|c|c|}
\hline & \multicolumn{2}{|l|}{ Univariate } & \multicolumn{2}{|l|}{ Multivariate } & \multicolumn{2}{|l|}{ Multivariate } \\
\hline & $\operatorname{HR}(95 \% \mathrm{CI})$ & $\mathrm{p}$-Value & $\mathrm{HR}(95 \% \mathrm{CI})$ & $\mathrm{p}$-Value & $\operatorname{HR}(95 \% \mathrm{CI})$ & p-Value \\
\hline $\begin{array}{l}\text { Second } \\
\text { PDAC }\end{array}$ & $0.81(0.63-1.04)$ & 0.093 & $0.73(0.56-0.94)$ & 0.016 & $0.85(0.66-1.09)$ & 0.198 \\
\hline $\begin{array}{l}\text { Age, mean } \\
\text { (SD) }\end{array}$ & $1.02(1.01 \sim 1.02)$ & $<0.001$ & $1.02(1.01-1.02)$ & $<0.001$ & $1.02(1.01-1.02)$ & $<0.001$ \\
\hline Male sex & $1.12(0.99 \sim 1.27)$ & 0.056 & $1.04(0.90-1.19)$ & 0.627 & $1.03(0.90-1.19)$ & 0.645 \\
\hline Alcohol & $1.23(1.08 \sim 1.39)$ & 0.001 & $1.28(1.13-1.47)$ & $<0.001$ & $1.25(1.11-1.42)$ & $<0.001$ \\
\hline $\begin{array}{l}\text { Resectable } \\
\text { status }\end{array}$ & $0.30(0.25-0.35)$ & $<0.001$ & Not included & & $0.30(0.25-0.36)$ & $<0.001$ \\
\hline
\end{tabular}

Abbreviations: PDAC, pancreatic ductal adenocarcinoma; SD, standard deviation; HR, hazard ratio; $\mathrm{CI}$, confidence interval

Conclusion: Second primary pancreatic cancer had a higher rate of resectability, and there was no difference in the effectiveness of curative surgery and chemotherapy between 2nd and 1st PDAC. Therefore, when curative surgery for 2nd PDAC is possible, it should be conducted similarly to curative surgery for $1 \mathrm{st}$ PDAC. Considering the increased risk of 2 nd PDAC in cancer survivors and the fact that surgery is the only curative treatment for this fatal cancer, more efforts are needed to develop screening programs for second primary pancreatic cancer in cancer survivors.

Disclosure of Interest: All authors have declared no conflicts of interest.

\section{P0137 ADEQUACY ASSESSMENT OF EUS-FNAB SAMPLES OF PANCREATIC CANCER FOR "PRECISION MEDICINE": A COMPARISON OF 22-GAUGE AND 25-GAUGE NEEDLES}

N. Yoshizawa ${ }^{1}$, R. Yamada ${ }^{1}$, H. Miura ${ }^{1}$, T. Takeuchi ${ }^{1}$, T. Harada ${ }^{1}$, H. Inoue ${ }^{1}$, H. Okuse ${ }^{1}$, T. Sakuno ${ }^{1}$, M. Nakamura ${ }^{1}$, Y. Hamada ${ }^{1}$, M. Katsurahara ${ }^{1}$, K. Tanaka ${ }^{2}$, N. Horiki ${ }^{2}$, Y. Takei ${ }^{1}$

${ }^{1}$ Gastroenterology And Hepatology, Mie University Hospital, Tsu, Mie/Japan

${ }^{2}$ Endoscopy, Mie University Hospital, Tsu, Mie/Japan

Contact E-mail Address: lasxia@gmail.com

Introduction: The development of new technology including next-generation sequencing has accelerated seeking new biomarkers and implementation "precision medicine". Generally, formalin-fixed paraffin-embedded tumor tissues obtained by surgery are used for molecular testing. The problem is that surgery is invasive, therefore, acquisition of adequate specimen by less-invasive procedure is getting significant factor. Endoscopic ultrasound-guided fine-needle aspiration and biopsy (EUS-FNAB) is the standard technique for diagnosing pancreatic solid tumor. There are several studies that have assessed the diagnostic yield of EUS-FNAB, comparing different needle sizes. Whereas, few reports had evaluated the sample volume of EUS-FNAB depends on the needle size. Therefore, we investigated the accuracy and assessed the adequacy of sample volume for "precision medicine", evaluating EUS-FNAB specimens obtained by 22 -gauge needles $(22 \mathrm{G})$ and 25 -gauge needles $(25 \mathrm{G})$.

Aims \& Methods: The aim of our study was to verify the accuracy and sample volume obtained by EUS-FNAB using $22 \mathrm{G}$ and $25 \mathrm{G}$, evaluating the feasibility for immunohistochemistry (IHC) staining. This was a retrospective study in a single tertiary referral center. Between October 2006 and November 2015, we investigated 153 patients of pancreatic ductal adenocarcinoma (PDAC) undergone diagnostic EUS-FNAB before neoadjuvant gemcitabine-based chemoradiotherapy. EUS-FNAB was performed with rapid on-site evaluation. We reviewed the sampling rate, the accuracy rate, and the success rate of IHC staining between $22 \mathrm{G}$ and $25 \mathrm{G}$.

Results: 153 patients underwent EUS-FNAB; 70 patients in $22 \mathrm{G}$ group and 83 patients in $25 \mathrm{G}$ group. The overall sampling rates on cytology and histology were $100 \%(153 / 153)$ and $98.0 \%(150 / 153)$, respectively. Both $22 \mathrm{G}$ and $25 \mathrm{G}$ groups had high sampling rates on cytology and histology, and the sampling rate did not differ between $22 \mathrm{G}$ and $25 \mathrm{G}$ groups. The overall diagnostic accuracy rates on cytology and histology were $94.8 \%(145 / 153)$ and $79.7 \%(122 / 153)$, respectively. The accuracy rates of $22 \mathrm{G}$ and $25 \mathrm{G}$ on cytology were $94.3 \%(66 / 70)$ and $95.2 \%$ $(79 / 83)$, whereas those on histology were $80.0 \%(56 / 70)$ and $79.5 \%(66 / 83)$, respectively. The overall accuracy rate on combined analysis both cytology and histology was $96.7 \%(148 / 153)$. The accuracy rates on combined analysis of $22 \mathrm{G}$ and $25 \mathrm{G}$ were better than the accuracy rates on cytology or histology alone; $97.1 \%(68 / 70)$ for $22 \mathrm{G}$ and $96.4 \%(80 / 83)$ for $25 \mathrm{G}$. The diagnostic accuracy on cytology, histology and combined analysis did not differ significantly between $22 \mathrm{G}$ and $25 \mathrm{G}$ groups. We performed hENT1 IHC analysis using the remaining cell blocks followed by cytological/histological diagnosis for PDAC. $69 \%$ (106 153) of PDAC provided sufficient specimens for IHC staining. The success rate of IHC staining did not differ significantly between $22 \mathrm{G}(67 \%, 47 / 70)$ and $25 \mathrm{G}$ $(71 \%, 59 / 83)(\mathrm{p}=0.60)$.

Conclusion: In the present study, both $22 \mathrm{G}$ and $25 \mathrm{G}$ diagnostic yields were high; the accuracy rate on histology of $25 \mathrm{G}$ was equivalent to that of $22 \mathrm{G}$. Moreover, EUS-FNAB specimens obtained by both $22 \mathrm{G}$ and $25 \mathrm{G}$ can be adequate for IHC analysis. Further investigations such as EUS-FNAB needle design and novel cell block preparation are needed for acquiring of adequate material not only for diagnosis, but for "precision medicine".

Disclosure of Interest: All authors have declared no conflicts of interest.

\section{MONDAY, OCTOBER 30, 2017} ENDOSCOPY AND IMAGING I - HALL 7

09:00-17:00

\section{P0138 FEASIBILITY OF GASTRIC ENDOSCOPIC SUBMUCOSAL DISSECTION WITH CONTINUOUS LOW-DOSE ASPIRIN FOR PATIENTS ON DUAL ANTIPLATELET THERAPY}

H. Harada, S. Suehiro, D. Murakami, R. Nakahara, T. Ujihara, T. Shimizu, Y. Katsuyama, K. Hayasaka

Gatroenterology, New Tokyo Hospital, Matsudo, Chiba/Japan

Contact E-mail Address: nerimaendo@hotmail.co.jp

Introduction: Endoscopic submucosal dissection (ESD) for gastric neoplasms with continuous low-dose aspirin (LDA) is comparatively acceptable according to recent guidelines [1-3]. This study aimed to evaluate risk factors for postoperative bleeding after gastric ESD with continuous LDA for patients on dual antiplatelet therapy (DAPT).

Aims \& Methods: This retrospective study was conducted at New Tokyo Hospital. A total of 597 gastric neoplasms (496 with early gastric cancers and 101 with gastric adenomas) in 571 consecutive patients were treated with gastric ESD between January 2010 and October 2016. A total of 102 lesions were excluded from this study: 51 lesions due to anticoagulation therapy; 25 lesions in patients receiving antiplatelet therapy excluding single-LDA and DAPT; and 26 lesions in patients who underwent ESD for more than two lesions at the same time. Thus, a total of 495 patients were enrolled in this study. The patients were categorized according to antiplatelet therapy (APT). APT was defined as follows: oral administration of single-LDA (aspirin [100 mg/day]) or DAPT (aspirin [100 $\mathrm{mg}$ /day] plus clopidogrel [75 mg/day]). Logistic regression analysis was performed for risk factors of bleeding after gastric ESD.

Results: The patients were categorized into two groups: no APT $(n=370)$ and APT $(n=125)$. APT included single-LDA $(n=74)$ and DAPT (LDA plus clopidogrel; $\mathrm{n}=51$ ). Among them, 46 received continuous LDA on single-LDA and 40 received continuous LDA on DAPT. The postoperative bleeding rate in the APT group was significantly higher than that in the no APT group $(16.0 \%$ vs. $5.9 \% ; P=0.001)$. Postoperative bleeding occurred in seven and nine patients in the continuous single-LDA group $(15.2 \%)$ and the continuous LDA on DAPT group $(22.5 \%)$, respectively. In multivariate analysis, specimen size of $\geq 40 \mathrm{~mm}$ (odds ratio [OR] $3.19 ; 95 \%$ confidence interval [CI], 1.65-6.16; $P<0.001$ ) was a sole independent risk factor for postoperative bleeding (Table.1). In subgroup univariate analysis among continuous LDA users, continuous single-LDA and continuous LDA on DAPT were not related to postoperative bleeding.

Table.1 Multivariate analysis for postoperative bleeding after ESD.

\begin{tabular}{llll}
\hline & Odds ratio & $95 \%$ CI & P-value \\
\hline Coronary artery disease & 1.52 & $0.61-3.78$ & 0.370 \\
CKD with hemodialysis & 3.21 & $0.97-10.60$ & 0.056 \\
Continuous LDA & 2.13 & $0.83-5.45$ & 0.116 \\
Specimen size $\geq 40 \mathrm{~mm}$ & 3.19 & $1.65-6.16$ & $<0.001$ \\
\hline
\end{tabular}

Conclusion: This study suggests that continuous LDA may be acceptable for gastric ESD in patients on DAPT. However, patients with continuous LDA on DAPT should be monitored carefully for postoperative bleeding after gastric ESD because the rate of postoperative bleeding in the continuous LDA on DAPT group was higher than that in the other groups.

Disclosure of Interest: All authors have declared no conflicts of interest.

\section{References}

1. Sanomura Y, Oka S, Tanaka S, et al. Continued use of low-dose aspirin does not increase the risk of bleeding during or after endoscopic submucosal dissection for early gastric cancer. Gastric Cancer 2014; 17: 489-96.

2. Lim JH, Kim SG, Kim JW, et al. Do antiplatelets increase the risk of bleeding after endoscopic submucosal dissection of gastric neoplasms? Gastrointest. Endosc. 2012; 75: 719-27.

3. Tounou S, Morita Y, Hosono T. Continuous aspirin use does not increase post-endoscopic dissection bleeding risk for gastric neoplasms in patients on antiplatelet therapy. Endosc. Int. Open 2015; 03: E31-8.

P0139 OUTCOMES OF PERORAL ENDOSCOPIC MYOTOMY FOR TREATMENT OF ESOPHAGEAL ACHALASIA WITH A MEDIAN FOLLOW-UP OF 4 YEARS

Q. Li ${ }^{1}$, Q. Wu ${ }^{2}$, X. Zhang ${ }^{1}$, P. Zhou ${ }^{2}$

${ }^{1}$ Endoscopy Center, Zhongshan Hospital, Fudan University, Zhongshan Hospital, Fudan University, Shanghai/China

${ }^{2}$ Endoscopy Center, Zhongshan Hospital, Fudan University, Shanghai/China

Contact E-mail Address: liquanlin321@126.com 
Introduction: Peroral endoscopic myotomy (POEM) has received wide acceptance as a highly effective and safe treatment for esophageal achalasia. Short-term and small-scale studies are ample but long-term large-scale studies are few.

Aims \& Methods: The aim of this study was to systematically analyze our longterm results of POEM, with particular emphasis on POEM failures and associated risk factors. This is a single center study. Consecutive POEM patients between Aug, 2010 and Dec, 2012 were included. Kaplan-Meier survival function was used to estimate clinical success rate at each year. The Cox proportional hazards model was used to analyze risk factors related to recurrence.

Results: A total of 564 patients were included. Mucosa injuries happened in 93 patients $(16.5 \%)$ and 36 patients $(6.4 \%)$ experienced major perioperative adverse events. The Eckardt score and lower esophageal sphincter (LES) pressure were significantly decreased after POEM (median Eckardt score 8 to 2, p $<0.05$; median LES pressure $29.7 \mathrm{~mm} \mathrm{Hg}$ to $11.9 \mathrm{~mm} \mathrm{Hg}, \mathrm{p}<0.05$ ). During a median follow-up period of 49 months (range 3-67 months), fifteen failures occurred within 3 months, 23 between 3 months and 3 years, and 10 after 3 years. The estimated clinical success rates at $1,2,3,4$, and 5 years were $94.2 \%, 92.2 \%$, $91.1 \%, 88.6 \%$ and $87.1 \%$, respectively. Multivariate Cox regression revealed long disease duration ( $>10$ years) and history of prior interventions to be risk factors for recurrence. Clinical reflux occurred in 37.3\% (155/416) patients.

Conclusion: POEM is a highly safe and effective treatment for esophageal achalasia with favorable long-term outcomes.

Disclosure of Interest: All authors have declared no conflicts of interest.

\section{P0140 PATIENTS WITH CHRONIC GASTROINTESTINAL ISCHEMIA HAVE AN ALTERED SUBLINGUAL MICROCIRCULATION}

J. Harki ${ }^{1}$, M. Suker ${ }^{2}$, M.S. Tovar Doncel ${ }^{3}$, L. J.d. Van Dijk ${ }^{1}$, D. Van Noord ${ }^{1}$, C.H.j. Van Eijck ${ }^{2}$, M.J. Bruno ${ }^{1}$, E.J. Kuipers ${ }^{4}$, C. Ince

${ }^{1}$ Gastroenterology \& Hepatology, Erasmus Medisch Centrum, Rotterdam/

Netherlands

${ }^{2}$ Surgery, Erasmus Medisch Centrum, Rotterdam/Netherlands

${ }^{3}$ Anesthesiology, University Hospital Rio Hortega, Valladolid/Spain

${ }_{4}^{4}$ Internal Medicine, Erasmus MC University Medical Centre, Rotterdam

Netherlands

${ }^{5}$ Intensive Care, Erasmus Medisch Centrum, Rotterdam/Netherlands

Contact E-mail Address: j.harki@erasmusmc.nl

Introduction: Chronic gastrointestinal ischemia (CGI) results of insufficient blood supply to the gastrointestinal tract ${ }^{1}$. The majority of CGI patients has systemic disorders of the circulatory system including hypertension, diabetes and other cardiovascular risk factors ${ }^{2}$. Studies in patients with acute gastrointestinal ischemia found a correlation between intestinal ischemia and sublingual microcirculatory alterations ${ }^{3}$. However, little is known about microcirculatory alterations in patients with CGI. We hypothesized that patients with CGI may reveal sublingual microcirculation alterations. We further hypothesized that such alterations will be amplified when challenging the patient to enteral caloric challenges. This would provide a patient-friendly means to identify CGI.

Aims \& Methods: Consecutive patients with CGI and healthy controls were prospectively included between September 2014 and August 2015. All patients received the standard work-up for CGI, consisting of assessment of medical history and symptoms, radiological imaging of the gastrointestinal arteries, and endoscopic visible light spectroscopy (VLS) for assessment of gastroduodenal mucosal oxygen saturation. The sublingual microcirculation was evaluated before (T0) and 20 minutes after enteral feeding (T1). Total vessel density (TVD $\left(\mathrm{mm} / \mathrm{mm}^{2}\right)$ ), perfused vessel density (PVD $\left(\mathrm{mm} / \mathrm{mm}^{2}\right)$, proportion of perfused vessels (PorPV (\%)), and microvascular flow index (MFI (AU)) were assessed. Results: We included 12 consecutive patients (63.2 (IQR 48.8-70.4) years, 67\% male) with CGI and 12 controls (32.7 (IQR 27.7-38.1) years, 42\% male). At baseline, patients with CGI had a decreased PORPV of the sublingual small vessels (median $84.77 \%$ vs $95.70 \%, p=0.006$ ), PORPV of all vessels (median $85.38 \%$ vs $95.27 \%, p=0.007$ ) and MFI of all vessels (median 3.00 vs 2.80 , $p=0.039$ ) compared to healthy controls. After caloric challenge, PVD increased significantly in both in small $\left(\mathrm{PVD}_{\mathrm{s}}\right)$ vessels and all vessels $\left(\mathrm{PVD}_{\mathrm{a}}\right)$ in patients with CGI (PVDs (T0) median 16.3 (IQR 13.3-22.1) $\mathrm{mm} / \mathrm{mm}^{2}$ vs (T1) 19.9 (IQR 14.2-26.2) $\mathrm{mm} / \mathrm{mm}^{2}, p=0.008$; PVDa (T0) median 19.1 (IQR 16.2-23.6) $\mathrm{mm}$ $\mathrm{mm}^{2}$ vs (T1) $22.2(16.5-28.9) \mathrm{mm} / \mathrm{mm}^{2}, p=0.02$; PorPVs (T0) median $84.8 \%$ (IQR 75.3-90.4) vs (T1) $91.0 \%(80.1-93.8), p=0.01)$. In contrast, no significant changes in microcirculatory parameters were observed after caloric challenge in the healthy controls.

Conclusion: Patients with CGI have impaired sublingual microcirculation compared to healthy controls. They also show significant alterations in the sublingual microcirculation after oral caloric challenge compared to healthy controls. Sublingual microcirculation visualization may offer a fast non-invasive diagnostic opportunity to diagnose patients with CGI.

Disclosure of Interest: All authors have declared no conflicts of interest.

\section{References}

1. Mensink PB, Moons LM, Kuipers EJ. Chronic gastrointestinal ischaemia: shifting paradigms. Gut. 2011 May;60(5):722-37.
2. Sana A, van Noord D, Mensink PB, Kooij S, van Dijk K, Bravenboer B, et al. Patients with chronic gastrointestinal ischemia have a higher cardiovascular disease risk and mortality. Atherosclerosis. 2012 Sep;224(1):235-41.

3. Chierego M, Verdant C, De Backer D. Microcirculatory alterations in critically ill patients. Minerva Anestesiol. 2006 Apr;72(4):199-205.

\section{P0141 CONVENTIONAL NARROW BAND IMAGING HAS GOOD CORRELATION WITH OLGA STAGING OF GASTRITIS}

A. Gawish ${ }^{1}$, A. Elbahrawy ${ }^{1}$, S. Mabrouk ${ }^{1}$, M. Abdellah ${ }^{1}$, M. El Fayoumie ${ }^{1}$,

A. Aboelfotoh ${ }^{1}$, M. Eldahshan ${ }^{1}$, F. Al Ghamry ${ }^{1}$

${ }^{1}$ Internal Medicine, Al Azhar University, Cairo/Egypt

Contact E-mail Address: dr.ahmedgawish1988@gmail.com

Introduction: The operative link of gastritis assessment (OLGA) staging system is widely used to assess the risk for gastric cancer on the basis of several biopsy samples taken from the antrum and corpus.

Aims \& Methods: In this study we attempted to evaluate whether gastritis staging using conventional narrow band imaging (NBI) endoscopy is equivalent to that determined by histopathology. Fifty (50) consecutive patients with Helicobacter Pylori (H. Pylori) related gastric atrophy selected according to NBI endoscopic findings 1 . The diagnosis of $\mathrm{H}$. Pylori based on direct detection of the organism by histopathology assessment. The NBI grade of lower gastric atrophy scored from 0 to 3 . The histopathological assessment of lower gastric atrophy was based on OLGA scoring system. Furthermore, we assessed the presence or absence of antral intestinal metaplasia. The NBI and histology stages of gastric atrophy were assessed using a combination of scores for the antrum and corpus. These stages further classified into low risk (stage 0, I and II) and high risk (stage III and IV). Finally the degree of correspondence between NBI and histopathology, in prediction of gastric cancer risk, was assessed.

Results: The mean age of included patients was $38.7 \pm 15.6$ years, they were 21 $(42 \%)$ males and $29(58 \%)$ females. $38(76 \%)$ and $13(26 \%)$ patients have pseudopyloric and intestinal metaplasia respectively. Overall $41(82 \%)$ and $9(18 \%)$ patients have low and high gastric cancer risk respectively. The sensitivity of NBI in diagnosis of Helicobacter Pylori infection, gastric atrophy and intestinal metaplasia were $96 \%(n=48 / 50) .100 \%$ and $61.5 \%(n=8 / 13)$ respectively. The degree of correspondence between the scores obtained by NBI and by histology was $58 \%(29 / 50)$ for the lower gastric body atrophy and $86 \%(n=43 / 50)$ for the antral intestinal metaplasia. The degree of correspondence between the high risk and low risk groups determined on the basis of NBI endoscopy on one hand and histopathology on the other hand was $80 \%(n=40 / 50)$.

Conclusion: NBI is able to approximate histopathological staging of gastritis to good extent. More studies and training will further improve the performance of our suggested new staging method.

Disclosure of Interest: All authors have declared no conflicts of interest.

\section{Reference}

Alaboudy et al. Conventional Narrow-Band Imaging Has Good Correlation with Histopathological Severity of Helicobacter pylori Gastritis. Digestive Diseases and Sciences 2011; 56(4):1127-30.

\section{P0142 SAFETY ADVANTAGE OF THE NEW DEVICE (SPLASH-M KNIFE®) FOR ENDOSCOPIC SUBMUCOSAL DISSECTION OF EARLY GASTRIC CANCER}

M. Esaki ${ }^{1}$, K. Hayashi ${ }^{2}$, S. Suzuki ${ }^{2}$, C. Kusano ${ }^{2}$, S. Itonaga ${ }^{1}$, S. Abe ${ }^{1}$, Y. Hayashi ${ }^{1}$, A. Yokoyama ${ }^{1}$, T. Hosokawa ${ }^{1}$, H. Ogino ${ }^{3}$, H. Akiho ${ }^{1}$, T. Gotoda ${ }^{2}$ ${ }^{1}$ Department Of Gastroenterology, Kitakyushu Municipal Medical Center, Fukuoka/Japan

${ }^{2}$ Division Of Gastroenterology And Hepatology, Department Of Medicine, Nihon University School of Medicine, Tokyo/Japan

${ }^{3}$ Medicine And Bioregulatory Science, Kyushu University, Fukuoka/Japan

Contact E-mail Address: esaki_saiseikai@yahoo.co.jp

Introduction: Endoscopic submucosal dissection (ESD) is a standard treatment for early gastric cancer. Development of the ESD device has been conducted recently. Splash M-Knife $\mathbb{R}$, the new multi-functional ESD device was invented to achieve complete ESD with a single device. It achieves clear marking, better hemostasis and smoother operation during a procedure without replacing the knife.

Aims \& Methods: The aim of this study was to investigate clinical outcome of ESD for early gastric cancer with a new device (Splash M-knife $\left.{ }^{\circledR}\right)$. In total, early gastric cancer treated by ESD with a needle-type knife between January 2012 and August 2016 at Kitakyushu Municipal Medical Center were retrospectively reviewed. Lesions treated by ESD with a conventional needle-knife (ESD-C, $\mathrm{n}=76)$ and by ESD with a new device (ESD-N, $\mathrm{n}=73$ ) were compared. Multivariate analyses and propensity score matching were used to compensate for the differences in age $(>75$ years vs $<75$ years), sex (male vs female), underlying disease (none vs with cardiopathy or cirrhosis), antithrombotic drugs (not receiving or discontinuation vs continuation), tumor size ( $\geq 21 \mathrm{~mm}$ vs $<21 \mathrm{~mm}$ ), lesion location (in the upper or middle third of the stomach vs in the lower stomach), lesion position (in the lesser curvature of the stomach vs others), macroscopic type (flat or depressed vs elevated), presence of ulceration (presence vs absence) and operator level (experience of $>50$ vs experience $<50$ ). As primary endpoint, the rate of the lesions that need hemostatic forceps was compared 
among two groups. As sub-analyses, the cutting time, rate of en-block/complete resection and rates of adverse events were evaluated among two groups.

Results: Propensity score matching analysis created 46 matched pairs. Adjusted comparisons between two groups showed a significantly smaller usage rate of hemostatic forceps in ESD-N than that in ESD-C (4.35\% vs $84.8 \%, \mathrm{p}<0.001)$, and similar treatment outcomes (en-block resection rate: $100 \%$ in both groups; complete resection rate: $97.8 \%$ vs $100 \%, p=1$; cutting time: $84.6 \mathrm{~min}$ vs $83.0 \mathrm{~min}$, $\mathrm{p}=0.89$; perforation during ESD: $0 \%$ in both groups).

Conclusion: Splash M-Knife ${ }^{\circledR}$ achieved better hemostasis and safer ESD for early gastric cancer. It may contribute to reduce cost for ESD by reducing usage of hemostatic forceps during ESD procedure.

Disclosure of Interest: All authors have declared no conflicts of interest.

\section{P0143 INTUBATION FAILURE DURING GASTROSCOPY -} INCIDENCE, PREDICTORS AND FOLLOW-UP FINDINGS

K. Siau ${ }^{1}$, J. $\mathrm{Li}^{2}$, N. C. Fisher ${ }^{2}$, C.J.j. Mulder ${ }^{3}$, S. Ishaq ${ }^{2}$

${ }^{1}$ Royal College Of Physicians, JAG Clinical Fellow, London/United Kingdom ${ }^{2}$ Department Of Gastroenterology, Dudley Group Hospitals NHS Foundation Trust, Dudley/United Kingdom

${ }^{3}$ Dept. Of Gastroenterology, VU University Medical Center, Amsterdam/ Netherlands

\section{Contact E-mail Address: keith@ siau.org}

Introduction: Intubation failure (IF) occurs when a trained endoscopist is unable to progress into the upper oesophagus via the oropharynx. The incidence is unknown, but estimated at $1.8 \%{ }^{[1]}$ There have been no studies exploring IF and follow-up findings. We aimed to assess the incidence, causes of IF, predictors of pathology in patients with IF, and follow-up findings.

Aims \& Methods: We retrospectively identified all gastroscopies performed at a district general hospital between August 2010-August 2016 from an endoscopy database, and reviewed cases with IF. We excluded patients who had achieved oesophageal intubation. Data on sedation use, endoscopist status, indications, radiological and endoscopic findings were recorded. Procedural limitations were classified into 2 groups: 'failure to tolerate' (e.g. pulling out scope, anxiety) and 'failure to progress'. Statistical analyses were made using Pearson's chi ${ }^{2}$ and Wilcoxon signed rank test.

Results: The incidence of IF was $0.95 \%$ (248/26130). 238 patients were identified, with a mean age of 63.2 (SD 16.1), with 'failure to progress' in 41 and 'failure to tolerate' in 197. Subsequent investigations included barium radiology $(59.7 \%$, $\mathrm{n}=142)$, CT $(21 \%, \mathrm{n}=50)$, repeat gastroscopy $(29.4 \%, \mathrm{n}=70)$ and no further investigations $(19.7 \%, \mathrm{n}=47)$. Structural pharyngeal abnormalities were detected in $41(28.9 \%)$, comprising of cricopharyngeal hypertrophy $(\mathrm{CPH})$ [49\%], Zenker's diverticulum (ZD) [14.6\%], pharyngeal web $(12.2 \%)$, ZD with $\mathrm{CPH}(9.8 \%)$, cervical spondylosis $(7.3 \%)$ and other $(7.3 \%)$. Endoscopist status was a predictor of IF (OR for medical vs. non-medical endoscopist $0.7,95 \%$ CI: $0.5-0.9, \mathrm{p}=0.007)$. Within the IF cohort, predictors of structural causes on barium radiology included: dysphagia (OR 5.5, 95\% CI: $2.5-11.8, \mathrm{p}<0.001$ ), failure to progress (OR 5.2, 95\% CI: $2.3-12.0, \mathrm{p}<0.001$ ) and age $>65$ (OR 4.0, $95 \%$ CI: $1.8-8.9, \mathrm{p}<0.001$ ). Repeat gastroscopy was successful in $63 / 70$ (2 using nasendoscope) after increasing midazolam dosage (mean increase $=1.5 \mathrm{mg}, 95 \%$ CI: $1.0-2.0 \mathrm{mg}, \mathrm{p}<0.001)$. Diagnostic yield for barium radiology, CT and repeat gastroscopy were $69.0 \%, 54.0 \%$ and $64.3 \%$ respectively. The concordance of endoscopic indication and pathology on further investigation for IF was 110 / $192(57.3 \%)$. In patients undergoing barium radiology and repeat gastroscopy, the false negative rate for endoscopy was $17 / 30(56.7 \%)$, consisting of pharyngeal pathology $(n=9)$, dysmotility $(n=4)$ and significant reflux $(n=4)$.

Conclusion: We present novel data regarding IF, and report an incidence of $\approx 1 \%$. Patients should be investigated further owing to the high risk of underlying pathology, particularly if associated with age $\geq 65$, dysphagia, and failure of endoscopic progression. Barium radiology is comparable to repeat gastroscopy in terms of diagnostic yield, and may be more helpful in evaluating pharyngeal and functional pathology.

Disclosure of Interest: All authors have declared no conflicts of interest.

\section{Reference}

1. Ponchon T, GIE, April 2000; 51(4): AB275

\section{P0144 DIAGNOSTIC CAPABILITY OF ENDOSCOPY FOR HELICOBACTER PYLORI INFECTION}

Y. Shinji ${ }^{1}$, K. Mabe ${ }^{2}$, M. Kato ${ }^{2}$, N. Sakamoto ${ }^{3}$

${ }^{1}$ Department Of Gastroenterology, Sapporo Medical Center Ntt Ec, Sapporo

Japan

${ }^{2}$ Department Of Gastroenterology, Hakodate National Hospital, Hakodate/Japan ${ }^{3}$ Department Of Gastroenterology And Hepatology, Hokkaido University Hospital, Sapporo/Japan

Contact E-mail Address: shinjiyoshii@yahoo.co.jp

Aims \& Methods: The aim of this study was therefore to determine the usefulness of the Kyoto classification for diagnosis of HP infection status. A total of 498 subjects were recruited during the period from January to October in 2015 for this study after providing informed consent in writing. HP infection status was determined by the presence of HP-IgG antibody (E-plate II H. pylori antibody, Eiken Chemical Co., Ltd., Tokyo, Japan) and history of eradication therapy. HP infection status was judged to be "eradicated" if there was a definite history of eradication therapy. Without a history of eradication therapy, HP infection status was judged to be "uninfected" for an HP antibody titer of less than 3 $\mathrm{U} / \mathrm{ml}$, "eradicated" for an HP antibody titer of 3-10 U/ml and "infected" for an $\mathrm{HP}$ antibody titer of more than $10 \mathrm{U} / \mathrm{ml}$. Seven endoscopists (5 well-experienced endoscopists and 2 trainees) who were blinded to history of eradication therapy performed the examinations. The following endoscopic findings were assessed according to the Kyoto gastritis classification: diffuse redness, regular arrangement of collecting venules (RAC), fundic gland polyp (FGP), atrophy, xanthoma, hyperplastic polyp, map-like redness, intestinal metaplasia, nodularity, mucosal swelling, white and flat elevated lesion, sticky mucus, depressive erosion, raised erosion, red streak, and enlarged fold. HP infection status was diagnosed on the basis of the findings. An Olympus H260 and Xp260NS were used for endoscopy. The diagnostic accuracy rate of the Kyoto classification and the sensitivity, specificity, positive predictive value (PPV), negative predictive value (NPV) and diagnostic odds ratio (DOR) for endoscopic findings were determined. This study was registered as a clinical trial in UMIN (UMIN000016674) and was conducted with the approval of the ethics committee in our institution.

Introduction: Since Helicobacter pylori (HP) eradication therapy is necessary to prevent the development of gastric cancer, evaluation of HP infection status (uninfected, infected, eradicated) by endoscopy has become important. For that purpose, the Japan Gastroenterological Endoscopy Society proposed the Kyoto classification for gastritis. However, the usefulness of the classification in daily clinical practice has not been sufficiently evaluated.

Results: The 498 subjects included 376 males and 122 females with a mean age of 53.1 years. HP status was unaffected in 315 subjects, eradicated in 104 subjects and infected in 79 subjects. The diagnostic accuracy rate was $82.9 \%$. The sensitivity, specificity, PPV, NPV and DOR were $88.3 \%, 92.9 \%, 95.5 \%, 82.1 \%$ and 98.6, respectively, for uninfected status, $78.8 \%, 90.0 \%, 69.5 \%, 94.2 \%$ and 37.0 , respectively, for eradicated status, and $67.1 \%, 91.4 \%, 59.6 \%, 93.6 \%$ and 21.7 , respectively, for infected status. High DORs were obtained for the following endoscopic findings: 32.2 for RAC, 7.7 for FGP and 4.7 for red streak in subjects with uninfected status, 12.9 for map-like redness in subjects with eradicated status, and 26.8 for diffuse redness, 13.3 for mucosal swelling, 10.2 for sticky mucus and 8.6 for enlarged fold in subjects with infected status.

Conclusion: The Kyoto classification is useful for diagnosis of HP infection status. Particularly important findings are RAC, FGP and red streak for uninfected status, map-like redness for eradicated status, and diffuse redness, mucosal swelling, sticky mucus and enlarged fold for infected status.

Disclosure of Interest: All authors have declared no conflicts of interest.

\section{References}

1. Kato M, Terao S, Adachi K, et al. Changes in endoscopic findings of gastritis after cure of $H$. pylori infection: Multicenter prospective trial. Digestive Endoscopy 2013; 25: 264-273

2. Kato T, Yagi Y, Kamada T, et al. Diagnosis of Helicobacter pylori infection in gastric mucosa by endoscopic features: A multicenter prospective study. Digestive Endoscopy 2013; 25: 508-518

\section{P0145 A RETROSPECTIVE AUDIT OF OUTCOMES AND CURRENT CLINICAL PRACTICE POST-BALLOON TAMPONADE FOR ACUTE SEVERE VARICEAL BLEEDING: HAVE THINGS IMPROVED OVER} TIME?

C. Keung ${ }^{1}$, A. Jackson ${ }^{2}$, M. Swan

${ }^{1}$ Department Of Gastroenterology And Hepatology, Monash Health, Melbourne/ Australia

${ }^{2}$ Department Of General Medicine, Monash Health, Melbourne/Australia/VIC

Contact E-mail Address: c.keung1@gmail.com

Introduction: Balloon tamponade, such as with Sengstaken-Blakemore tubes (SBT), remains the main immediate salvage therapy for acute variceal bleeding uncontrolled by variceal ligation or injection therapy. Previous cohort studies from the 1970-1980s report success rates of $40-94 \%$ for initial haemostasis but high re-bleeding rates of $40-70 \%$ on removal [1-3]. Despite guidelines recommending balloon tamponade as initial therapy in treating endoscopically uncontrollable variceal bleeding, specialists and trainees feel uncomfortable with SBT insertion [4] given the perceived difficulties and complications [5].

Aims \& Methods: We aimed to describe the current practices surrounding insertion of SBT for acute variceal bleeding, the outcomes and to identify areas requiring improvement. A retrospective audit of all patients from 2008-2016 who required SBT insertion for control of acute variceal bleeding was undertaken at Monash Health, a large tertiary Australian centre. These patients were identified from coding classifications. Details regarding their admission were obtained via electronic records.

Results: Approximately $14 \%$ of all patients with variceal bleeding required insertion of a SBT. Of these 42 patients, the majority were male $(71 \%)$ with a median age of 55 years (range 34-78). Alcohol was the most common aetiology for cirrhosis $(62 \%)$, with $65 \%$ actively drinking. Most patients had cirrhosis severity scores of Child-Pugh B $(67 \%)$ or Child-Pugh C $(29 \%)$ and a median MELD score of 16 (range 8-39). At the index variceal bleed, $67 \%$ were haemodynamically unstable and $29 \%$ were encephalopathic. All received standard medical therapy with octreotide or terlipressin, antibiotics and blood products as required. The time to initial endoscopy from $1^{\text {st }}$ onset bleeding was prompt (median 6.62 hours). Most bleeding varices were oesophageal $(90 \%)$. Initial ligation/injection was performed in $64 \%$ with the remaining patients having such large volumes of blood in the UGI tract that satisfactory views were unable to be obtained. The current practice surrounding SBT insertion is shown in the table 
below. Re-look endoscopy post-SBT insertion was performed in $86 \%$ patients at a median of 39 hours after insertion with further endoscopic therapy in $47 \%$. Complications of SBT insertion occurred in $31 \%$ and included minor oesophageal ulceration (9), significant oesophageal ulceration (3), aspiration pneumonia (4) and oesophageal perforation (1).

Current practice surrounding Sengstaken-Blakemore Tube insertion

\begin{tabular}{lc}
\hline Variable & Results $(\mathrm{n}=42)$ \\
Indication for SBT insertion & Incomplete haemostasis \\
& $74 \%$, poor view $50 \%$ \\
SBT insertion site & Oral 32, nasal 8 \\
Confirmation of position & Direct endoscopic visualisation 13, \\
& imaging 25 , none 6 \\
Volume of balloon inflation & Gastric balloon -306 ml mean \\
& $(60-450$ ml) Oesophageal \\
buration of balloon & Gastric balloon $-35.1(1-140.3)$ \\
inflation (median hours) & Oesophageal balloon $-16(1-62.8)$ \\
Time to re-look endoscopy & $39.3(11.5-348.2)$ \\
after SBT (median hours) &
\end{tabular}

Re-bleeding occurred in $45 \%$ patients during the admission despite SBT insertion, of which $79 \%$ did not survive. Seven other patients subsequently underwent a TIPS procedure but 4 of these still died. Survival at discharge and at 12 months was $50 \%$ and $41 \%$ respectively. The median duration of hospitalisation, intensive care and mechanical ventilation was 13 days (1-56), 6.2 days (0.3-36.2) and 120 hours (1-708) respectively.

Conclusion: Primary haemostasis was achieved in $93 \%$ of patients; however, rebleeding occurred in $45 \%$ and was associated with a poor survival rate of $20 \%$. Short and longer-term survival overall has not significantly improved since studies in the 1970s-1980s despite advances in pharmacological therapy. Current practices surrounding SBT insertion are variable and would benefit from further education. Rates of direct visualisation of balloon position prior to inflation with endoscopy should be improved as with referrals for early TIPS

Disclosure of Interest: All authors have declared no conflicts of interest.

\section{References}

1. Chojkier, M. and H.O. Conn, Esophageal tamponade in the treatment of bleeding varices. A decadel progress report. Dig Dis Sci, 1980. 25(4): p. 26772 .

2. Haddock, G., et al., Esophageal tamponade in the management of acute variceal hemorrhage. Dig Dis Sci, 1989. 34(6): p. 913-8.

3. Panes, J., et al., Efficacy of balloon tamponade in treatment of bleeding gastric and esophageal varices. Results in 151 consecutive episodes. Dig Dis Sci, 1988. 33(4): p. 454-9.

4. Bajaj, J.S., A. Ananthakrishnan, and K. Saeian, Survey of attitudes of AASLD members toward balloon tamponade. Hepatology, 2005. 41(6): p. 1435-6.

5. Nielsen, T.S. and A.V. Charles, Lethal esophageal rupture following treatment with Sengstaken-Blakemore tube in management of variceal bleeding: a 10-year autopsy study. Forensic Sci Int, 2012. 222(1-3): p. e19-22.

\section{P0146 CONSCIOUS SEDATION FOR ENDOSCOPIC SUBMUCOSAI RESSECTION BY USING DEXMEDETOMIDINE}

M. Esaki, M. Hattori, I. Matsuzaki, K. Hirose, C. Izumi, M. Yoshikawa, H. Yamauchi, S. Nakazawa

Department of Gastroenterology, Yamashita Hospital, Ichinomiya/Japan

Contact E-mail Address: e.m.6089@gmail.com

Introduction: To evaluate the feasibility and safety of the dexmedetomidine (DEX) for conscious sedation during endoscopic submucosal dissection (ESD). Aims \& Methods: This study was a prospective trial, and was conducted at the Yamashita Hospital in Japan. Between January 2016 and December 2016, all 50 patients were enrolled in this study. The inclusion criteria for the study was the presence of esophageal, gastric or duodenal tumors. The criteria for exclusion from this study is as follows: patients who were allergic to the drugs used, a baseline heart rate less than 50 beats/minutes, patients who could not use anticholinergic drugs, and lack of patient's consent. A total 50 patients who underwent ESD by using DEX for esophageal, gastric or duodenal tumor. The patients were sedated with DEX (an initial bolus infusion of $3.0 \mu \mathrm{g} / \mathrm{kg} / \mathrm{hour}$ intravenously over 10 minutes, followed by a continuous infusion at $0.4 \mu \mathrm{g} / \mathrm{kg} / \mathrm{hour}$ titrated). During sedation, midazolam $(0.03 \mathrm{mg} / \mathrm{kg})$ and pethidine $(17.5 \mathrm{mg})$ were added intravenously as needed. If the level of sedation dropped to less than RASS -3, the intravenous DEX injection rate was reduced by $0.1 \mu \mathrm{g} / \mathrm{kg} /$ hour. If heart rate was less than 50 beats/minutes or systolic blood pressure was less than $80 \mathrm{mmHg}$, the intravenous DEX injection rate was reduced by $0.1 \mu \mathrm{g} / \mathrm{kg} / \mathrm{hour}$ If the patient's heart rate was less than 50 beats/minutes, the patient was intravenously infused with atropine sulfate hydrate $(0.125 \mathrm{mg}-0.25 \mathrm{mg})$. During procedure, the following parameters were measured continuously, and recorded every 5 minutes: heart rate, blood pressure, hemoglobin oxygen saturation $\left(\mathrm{SpO}_{2}\right)$, respiratory rate and RASS. As the achievement rate of conscious sedation during procedure, the percentage of the time that the depth of sedation from
RASS -1 to -3 during procedure were evaluated. Body movement leading to the interruption of ESD were recorded appropriately. After the procedure, all patients were intravenously infused with flumazenil $(0.3 \mathrm{mg})$ and observed until the Aldrete score reached 9 points

Results: During this study period, 50 patients with esophageal, gastric and duodenal tumors were identified as potentially eligible for participation. There were 37 males and 13 females, and the mean age was $67.5 \pm 8.6$ y. 27 patients regularly consumed alcohol and 5 patients use sleeping drugs regularly. Tumors were located in the following locations: 9 cases in the esophagus, 38 cases in the stomach, 3 cases in the duodenum. The mean tumor size was $23.8 \pm 16.9 \mathrm{~mm}$ and the procedure time was $88.0 \pm 59.5$ minutes. The histologic results of ESD were squamous cell carcinoma $(n=9)$, adenoma $(n=17)$ and adenocarcinoma $(\mathrm{n}=24)$. ESD by using DEX were successfully performed in all 50 tumors. No adverse events that were the results of procedures occurred. The mean achievement rate of conscious sedation during procedure was $84.7 \pm 16.5 \%$. The median frequency of disturbance by patient's movement was 0 times (range $0-3$ times). 33 cases reduced and 14 cases discontinued a continuous infusion of DEX. In 23 cases, there was a decreased heart rate less than 50 beats/minutes. In 16 cases, the patient required an infusion of atropine sulfate hydrate. Thirteen cases of 16 cases required an infusion of atropine sulfate hydrate after the procedure and 3 cases required it during procedure. The median time that the final administration of an atropine sulfate hydrate was $35 \mathrm{~min}$ (range $5-140 \mathrm{~min}$ ) after procedure. There was no case in lowering of systolic blood pressure less than $80 \mathrm{mmHg}$. Although in 7 cases, the patient's $\mathrm{SpO}_{2}$ level dropped to less than $90 \%$, however, they recovered after the administration of oxygen into the nasal cannula. The mean time that the Aldrete score reached 9 or over 9 was $7 \pm 4$ minutes. The median endoscopist and patient's satisfaction score was 9 points (range 2-10 points) and 8 points (range $2-10$ points). There were 30 patients $(60 \%)$ who have a memory during procedure.

Conclusion: Conscious sedation with DEX is effective, safe and a high level of satisfaction for endoscopists and patients for upper gastrointestinal ESD. Disclosure of Interest: All authors have declared no conflicts of interest.

\section{P0147 DIAGNOSTIC ACCURACY OF BLUE LASER IMAGING WITH MAGNIFYING ENDOSCOPY FOR INVASION DEPTH OF SUPERFICIAL ESOPHAGEAL SQUAMOUS CELL CARCINOMA}

T. Ueda ${ }^{1}$, O. Dohi ${ }^{1}$, K. Ogita ${ }^{1}$, S. Takayama ${ }^{1}$, K. Terasaki ${ }^{1}$, T. Nakano ${ }^{1}$

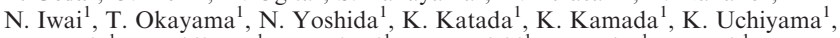
O. Handa ${ }^{1}$, T. Ishikawa ${ }^{1}$, T. Takagi ${ }^{1}$, H. Konishi ${ }^{1}$, Y. Naito ${ }^{1}$, Y. Itoh ${ }^{1}$ ${ }^{1}$ Gastroenterology And Hepatology, Kyoto Prefectural University of Medicine, Kyoto/Japan

\section{Contact E-mail Address: tm-ueda@koto.kpu-m.ac.jp}

Introduction: Preoperative diagnosis of invasion depth of superficial esophageal squamous cell carcinoma (SESCC) is very important to select appropriate therapeutic procedure. The Japan Esophageal Society (JES) classification using narrow-band imaging with magnification (M-NBI) was efficient for predicting invasion depth of SESCC ${ }^{1)}$. Blue laser imaging (BLI) is an image enhanced endoscopy consisted of two different lasers with wavelength 410 and $450 \mathrm{~nm}$ as light source, which can enhance mucosal vascular and surface structure. In previous study, BLI with magnification (M-BLI) was useful for evaluating gastrointestinal neoplasms such as predicting invasion depth or tumor detection ${ }^{2}$.

Aims \& Methods: We aim to investigate the diagnostic value of M-BLI by comparing that of M-NBI. Our study was single center retrospective study and approved by the Ethical Review Committee of Kyoto Prefectural University of Medicine, and performed in accordance with the World Medical Association's Declaration of Helsinki. All patients provided informed consent for undergoing both M-NBI and M-BLI. Consecutive 166 patients underwent endoscopic submucosal dissection (ESD) for esophageal tumor at Kyoto Prefectural University of Medicine between April 2014 and March 2016. Endoscopic images of SESCCs were recorded by both M-BLI and M-NBI prior to ESD. SESCCs were pathologically diagnosed by ESD specimens. Three endoscopists with no information of the lesions evaluated invasion depth of SESCCs using M-BLI and M-NBI images according to JES classification. The diagnostic value of each procedure was evaluated.

Results: 124 SESCCs were analyzed in this study. The numbers of male/female were $104 / 20$, respectively. Median age was 68.5 years old. Median size of tumor was $17.6 \mathrm{~mm}$. The proportion of tumor location at $\mathrm{Ut} / \mathrm{Mt} / \mathrm{Lt}$ was $13 / 70 / 17(\%)$ respectively. The proportion of macroscopic type for $0-\mathrm{I} / 0-\mathrm{IIa} / 0-\mathrm{IIb} / 0-\mathrm{IIc}$ was $1 /$ $10 / 66 / 23(\%)$, respectively. The proportion of invasion depth of the lesions subclassified as EP or LPM, MM or SM1, and SM2 were 80, 13 and 7(\%) respectively. The overall diagnostic accuracy of BLI and NBI were $88.7 \%$ and $83.9 \%$ $(\mathrm{P}=0.35)$, respectively. The interobsever variability of three endscopists with BLI and NBI was $0.679 / 0.560 / 0.559$ and $0.568 / 0.822 / 0.560$, respectively. The intraobserver variability with BLI and NBI was $0.839 / 0.718 / 0.531$ and $0.517 / 0.514$ 0.441 , respectively.

Conclusion: M-BLI was efficient for diagnosing invasion depth of SESCC according to JES classification, similar to M-NBI

Disclosure of Interest: O. Handa: I received lecture fee from Astrazeneka Co. Dai-ichi sankyo Co.

Y. Naito: I received collaboration research funding from Fujifilm Co. Y. Itoh: I am affiliated with a department that was partially funded by Fujifilm Medical Co., Ltd.

All other authors have declared no conflicts of interest. 


\section{References}

1. Oyama, T., et al., Prediction of the invasion depth of superficial squamous cell carcinoma based on microvessel morphology: magnifying endoscopic classification of the Japan Esophageal Society. Esophagus, 2016.

2. Yoshida, N., et al., The ability of a novel blue laser imaging system for the diagnosis of invasion depth of colorectal neoplasms. J Gastroenterol, 2014. 49(1): p. 73-80.

\section{P0148 IMPACT OF NEEDLE-BASED CONFOCAL LASER ENDOMICROSCOPY (NCLE) IN IMPROVING DIAGNOSIS OF PANCREATIC CYSTIC NEOPLASMS: SINGLE CENTER EXPERIENCE}

F. Shariati ${ }^{1}$, E. Verter ${ }^{1}$, M. Lucas ${ }^{1}$, C. Molina ${ }^{1}$, J. Adams ${ }^{2}$, V. Joshi ${ }^{1}$ Ochsner Medical Center, New Orleans/United States of America

${ }^{2}$ Poindexter Labs, Dallas/United States of America

Contact E-mail Address: vjoshi@ochsner.org

Introduction: Endoscopic Ultrasound (EUS) has been found to be an effective tool in diagnosing pancreatic cystic neoplasms (PCN). Carcinoembryonic antigen (CEA) tumor marker has also been used to differentiate PCN and is the most accurate marker of mucinous cystic neoplasms. Recently, needle-based conofocal laser endomicroscopy (nCLE) has been increasingly used for the diagnosis of PCN. nCLE allows for evaluation of pancreatic cysts with results similar to that of a pathological diagnosis. In this study, we will compare our standard of care, EUS, with combined CEA and nCLE to determine which combination of diagnostic modalities is a better predictor of PCN.

Aims \& Methods: In this retrospective chart review, 22 patients with pancreatic cysts were evaluated. Specificity and Negative Predictive Value (NPV) of EUS alone or EUS with CEA and nCLE combined were evaluated and diagnostic accuracy was compared with pathology using McNemar's test. Worrisome features (increased cyst size, wall thickness, main pancreatic duct size, and presence of non enhanced mural nodules, abrupt changes, distal atrophy and lymphadenopathy) were tested by determining dissimilar calculations using Euclidean distance and later were used in hierarchical clustering to create two clusters based on Euclidean distance.

Results: Diagnosis of PCN using EUS alone had a specificity of 0.75 and a NPY of 0.88. EUS and CEA combined had a specificity of 0.95 and a NPV of 0.90 Finally, EUS with CEA and nCLE combined had a specificity of 0.80 and a NPV of 0.94 . Worrisome features clustering was able to predict pathology, $\mathrm{p}=.000289$.

Conclusion: We concluded that specificity and NPV of EUS predicting PCN are positively impacted by the addition of CEA and nCLE. We also found that clustering of worrisome factors predicts pathology, however, a larger cohort is required for future studies.

Disclosure of Interest: All authors have declared no conflicts of interest.

\section{References}

1. Spinelli K, Fromwiller T, Daniel R, Kiely J, Nakeeb A, Komorowski R, Wilson S, Pitt H. Cystic pancreatic neoplasms: observe or operate. Ann Surg. 2004;239(5):651-7.

2. Müller $\mathrm{M}$, Meyenberger $\mathrm{C}$, Bertschinger $\mathrm{P}$, Schaer $\mathrm{R}$, Marincek $\mathrm{B}$. Pancreatic tumors: evaluation with endoscopic US, CT, and MR imaging. Radiology. 1994;190(3):745-51.

3. Banafea O, Mghanga F, Zhao J, Zhao R, Zhu L. Endoscopic ultrasonography with fine-needle aspiration for histological diagnosis of solid pancreatic masses: a meta-analysis of diagnostic accuracy studies. BMC Gastroenterol. 2016 Aug 31;16:108. doi: 10.1186/s12876-016-0519-z.

4. Rockacy M, Khalid A. Update on pancreatic cyst fluid analysis. Ann Gastroenterol. 2013;26(2):122-127.

5. Kawaguchi Y, Mine T. Endoscopic approach to the diagnosis of pancreatic cystic tumor. World J Gastrointest Oncol. 2016;15;8(2):159-64. doi: 10.4251/ wjgo.v8.i2.159

\section{P0149 FULL-SPECTRUM ENDOSCOPY FOR UPPER GASTROINTESTINAL SCREENING INCLUDING PRECISE OBSERVATION OF THE AMPULLA OF VATER AND THE ANAL SIDE OF THE PYLORIC RING}

H. Yamada ${ }^{1}$, T. Shibata ${ }^{1}$, T. Kawamura ${ }^{1}$, N. Horiguchi ${ }^{1}$, M. Okubo ${ }^{1}$,

T. Tahara ${ }^{1}$, M. Nagasaka ${ }^{1}$, Y. Nakagawa ${ }^{1}$, N. Ohmiya ${ }^{1}$

${ }^{1}$ Gastroenterology, Fujita Health University, Toyoake/Japan

Contact E-mail Address: hyugayama1988@yahoo.co.jp

Introduction: Full-spectrum esophagogastroduodenoscopy (FUSE-EGD) provides a 245-degree field of view with double CCE imagers on the front and left side of the endoscope.

Aims \& Methods: The aim of this study is to determine the usefulness of FUSEEGD for upper gastrointestinal screening. The primary endpoint was comparison of the success rates of entire visualization of the ampulla of Vater and the secondary endpoint was comparison of success rates of visualization of the anal side of the pyloric ring between FUSE-EGD and conventional forward-viewing EGD. The additional endopoint was possibility of close-up visualization of the squamo-columnar junction (SCJ). Between April 2016 and September 2016, 88 healthy individuals ( 46 male and 42 women; median age of examination, 68 years; range, 26-91) underwent FUSE-EGD, and 818 healthy individuals (423 male and 395 women; median age of examination, 67 years; range, 19-97) underwent conventional EGD for upper gastrointestinal screening. Among them, 40 individuals who underwent FUSE-EGD with a previous history of conventional EGD were interviewed about the degree of pain at insertion, during and after the FUSE-EGD examinations using the visual analog scale (VAS) (0, comfort; 50 , equal to the last conventional EGD; and 100, painful). Furthermore, we evaluated 3 patients with familial adenomatous polyposis (FAP) and 2 with the ampullary tumors using FUSE-EGD.

Results: Entire visualization of the ampulla of Vater was successful at $90.4 \%$ (47/ 52) with FUSE-EGD, compared to $41.2 \%$ (174/422) with conventional EGD $(P<0.001)$. Visualization of the anal side of the pyloric ring was successful at $88.5 \%(46 / 52)$ with FUSE-EGD, compared to $8.5 \%(6 / 70)$ with conventional EGD $(P<0.001)$. Close-up visualization of SCJ was successful at $92.0 \%(81 / 88)$ with FUSE-EGD. VASs at insertion, during and after examinations were 51.2 (10-75), 46.9 (0-75), and 45.2 (10-50) points, respectively, which were equivalent to conventional EGD. The ampullary adenoma was observed in all 3 patients with FAP, and the openings of the biliary and pancreatic ducts of the ampulla of Vater were observed in all 2 cases with the ampullary tumors with FUSE-EGD. There were no adverse events associated with FUSE-EGD.

Conclusion: FUSE-EGD seems safe and effective for upper gastrointestinal screening, especially when the precise observation of the ampulla of Vater and/ or the anal side of the pyloric ring is necessary.

Disclosure of Interest: All authors have declared no conflicts of interest.

\section{P0150 PREDICTIVE FACTORS OF PROCEDURAL DIFFICULTIES IN ENDOSCOPIC SUBMUCOSAL DISSECTION OF EARLY-STAGE GASTRIC CANCER}

H. Yamada ${ }^{1}$, T. Shibata ${ }^{1}$, T. Kawamura ${ }^{1}$, N. Horiguchi ${ }^{1}$, M. Okubo ${ }^{1}$,

T. Tahara ${ }^{1}$, M. Nagasaka ${ }^{1}$, Y. Nakagawa ${ }^{1}$, N. Ohmiya

${ }^{1}$ Gastroenterology, Fujita Health University, Toyoake/Japan

Contact E-mail Address: hyugayama1988@yahoo.co.jp

Introduction: Endoscopic submucosal dissection (ESD) is accepted as the treatment of intestinal-type early-stage gastric cancer. However, ESD occasionally results in unfavourable outcome due to technical difficulties. Therefore, predictions of difficulties in ESD would preclude complications associated with ESD. Aims \& Methods: The aim of this study is to determine the predictive factors of procedural difficulties in ESD. Between January 2009 and July 2016, 577 consecutive patients who underwent ESD for gastric neoplasms were enrolled. These patients were classified into 3 groups: group S, group L, and others. Group S comprised 30 patients who underwent ESD for the shortest duration (10-16 min). Group L comprised 30 patients who underwent ESD for the longest duration (149-215 min). Multivariate analysis was performed between Groups L and S using the following factors: location (cardia, posterior wall of angle, lesser curvature of lower gastric body and others), macroscopic type (protruded, depressed or mixed), size of the resected specimen, preoperative scar, number of preoperative biopsies, /others), and as predictor for submucosal fat tissue, body mass index, waist circumference, visceral fat tissue measurements on CT, blood test findings (glycated hemoglobin, triglyceride and total cholesterol), blood pressure, and heart rate before ESD.

Results: Significant differences were found regarding the number of biopsies (group L, 8.5; group S, 6.8, $P=0.0211$ ), (group L, $616.7 \mathrm{~mm}^{2}$; group $\mathrm{S}$, $\left.99.2 \mathrm{~mm}^{2}, P=0.0286\right)$, biopsy diagnosis $(P=0.0292)$, and location $(P=0.0086)$. Based on these factors and odds ratio, we prioritized sensitivity to avoid missing cases with removal difficulties during ESD and suggested predictive factors for removal difficulties during ESD. Preoperative scar: 3 points, the size of the resected specimen $>800 \mathrm{~mm}^{2}: 3$ points, difficult location: 2 points, the number of biopsies $>7$ pieces: 1 point, Group 5 on biopsy diagnosis: 1 point. Cases of 6 points or more was regarded as difficult to remove that takes over 70 minutes. When examining 43 patients who underwent ESD for gastric neoplasms between August to November 2016 the sensitivity was $87.5 \%$ and the specificity was $80 \%$. Conclusion: Our results suggest that the number of biopsies, size of the resected specimen, biopsy diagnosis, preoperative scar and biopsy location are predictive factors for difficulties in ESD.

Disclosure of Interest: All authors have declared no conflicts of interest.

\section{P0151 LOCATION FEATURES OF EARLY GASTRIC CANCER TREATED WITH ENDOSCOPIC SUBMUCOSAL DISSECTION}

C.W. Choi, D.H. Kang, H.W. Kim, S.J. Kim, J.S. Lee, C.W. Yeo, S.H. Choi, H.J. Kim, J.H. Ko

Pusan National University Yangsan Hospital, Yangsan/Korea, Republic of

Contact E-mail Address: luckyace@hanmail.net

Introduction: Timely detection of early gastric cancer (EGC) is important in performing endoscopic submucosal dissection (ESD). We attempted to know the location characteristics where EGC is frequently detected and analyzed EGC characteristics associated with the location.

Aims \& Methods: We retrospectively reviewed the medical records of patients with EGC treated by ESD between November 2008 and August 2016. We retrospectively investigated and analyzed 647 EGC lesions.

Results: The patients' mean age was $66.7 \pm 10.8$ years. The patient population was predominantly male $(77.1 \%, 499 / 647)$. A well to moderately differentiated carcinoma was observed in $97.2 \%$ of patients. The common site was the lower part of the stomach $(89.6 \%, 580 / 647)$. The highest percentage of EGC was found 
in the lesser curvature $(43.9 \%, 284 / 647)$. Posterior EGC was more frequent in the mid-to-upper part of the stomach than the anterior part (20.4\%, 31/67 vs. $16.4 \%$, $11 / 67$, respectively). For EGC characteristics compared between the lower and mid-to-upper parts, submucosal invasive EGC was found to be significantly different (odds ratio, 1.919; confidence interval, 1.014-3.623; $\mathrm{p}=0.045$ ).

Conclusion: Most of the EGCs resectable with ESD were found in the lower part of the stomach and lesser curvature of the stomach. The incidence of the posterior part in the mid-to-upper part of the stomach was higher than that of anterior part. The EGC located in the mid-to-upper part of the stomach was found to have a higher incidence of invasive cancer.

Disclosure of Interest: All authors have declared no conflicts of interest

\section{P0152 A STUDY OF THE RECOGNITION OF ENDOSCOPIC IMAGES BY MACHINE LEARNING WITH CONVOLUTIONAL NEURAL NETWORK AND DEEP LEARNING}

R. Yamakawa, M. Harada, K. Kawauchi, S. Nuzuki, M. Iwata

Gastroenterology, Kaetsu Hospital, Niigata/Japan

\section{Contact E-mail Address: yamakawa_r@niigata-min.or.jp}

Introduction: The recognition of general images by machine learning (ML) with the convolutional neural network (CNN) and deep learning (DL) is good. However, the possibility of the recognition of endoscopic images by ML with CNN and DL is undetermined.

Aims \& Methods: The aim of this study was to clarify the possibility of the recognition of endoscopic images by $\mathrm{ML}$ with $\mathrm{CNN}$ and DL.

We selected 816 endoscopic images of 8 categories which include laryngopharynx (LP), thoracic esophagus (TE), abdominal esophagus (AE), gastric fundus (GF), gastric body (GB), gastric antrum (GA), duodenal bulb (DB) and descending part of the duodenum (DD). Each category had approximately 100 images.

These images were randomly separated into two groups, $60 \%$ (489 images) for learning and 40\% (327 images) for testing. We increased the learning group images to 8313 by adding additionally rotated images of each five degrees. We made an ML model with three CNN layers, three Activation Function layers, two Max-Pooling layers and two Dens layers by TensorFlow and Keras.

We trained the ML model with the learning group images $(n=8313)$ and then tested it with the testing group images $(n=327)$ to determine whether it can recognize the endoscopic site. Two members of our hospital staff performed the same test utilizing the same images.

Results: It took 73 minutes for the ML model to learn and 6 seconds to answer the test. The percentage of correct answers of the ML model was $70.6 \%$ in all categories $(\mathrm{n}=327), 77.1 \%$ in LP $(\mathrm{n}=48), 91.5 \%$ in TE $(\mathrm{n}=47), 64.4 \%$ in AE $(\mathrm{n}=45), 73.7 \%$ in GF $(\mathrm{n}=38), 61.5 \%$ in GB $(\mathrm{n}=39), 52.8 \%$ in GA $(\mathrm{n}=36)$, $65.6 \%$ in DB $(n=32)$ and $71.4 \%$ in DD $(n=42)$. The average percentage of correct answers of humans was $95.4 \%$ in gastroenterologists $(n=5), 85.2 \%$ in junior residents $(n=2), 81.2 \%$ in endoscopy nurses $(n=5), 54.4 \%$ in medical clerks $(n=5)$ and $51.8 \%$ in floor nurses $(n=4)$.

The percentage of correct answers of the ML model was lower than those of humans who have knowledge about endoscopic images. However, it was higher than those of other humans who do not.

Conclusion: The results suggest the possibility of the recognition of endoscopic images by ML with CNN and DL. Further study is necessary to confirm the ability of it because this study was conducted in a simple ML model with three CNN layers and a small number of images.

Disclosure of Interest: All authors have declared no conflicts of interest

\section{P0153 CONVENTIONAL VERSUS TRACTION-ASSISTED ENDOSCOPIC SUBMUCOSAL DISSECTION FOR GASTRIC NEOPLASMS (CONNECT-G): A MULTICENTER, RANDOMIZED CONTROLLED TRIAL}

S. Suzuki ${ }^{1}$, M. Yoshida ${ }^{2}$, K. Takizawa ${ }^{2}$, Y. Koike ${ }^{3}$, S. Nonaka ${ }^{4}$, Y. Yamasaki ${ }^{5}$, T. Minagawa ${ }^{6}$, C. Sato ${ }^{7}$, C. Takeuchi ${ }^{8}$, K. Watanabe ${ }^{9}$, K. Miura ${ }^{10}$ H. Morimoto ${ }^{11}$, T. Yano ${ }^{12}, \mathrm{~K}$. Sudo ${ }^{13}, \mathrm{~K}$. Mori ${ }^{2}, \mathrm{~T}$. Gotoda ${ }^{14}, \mathrm{H}$. Ono ${ }^{2}$

${ }^{1}$ Division Of Gastroenterology And Hepatology, Department Of Medicine, Nihon University School of Medicine, Tokyo/Japan

${ }^{2}$ Division Of Endoscopy, Shizuoka Cancer Center, Shizuoka/Japan

${ }^{3}$ Department Of Gastroenterology, Sendai City Medical Center, Sendai/Japan

${ }^{4}$ Division Of Endoscopy, National Cancer Center Hospital, Tokyo/Japan

${ }^{5}$ Department Of Gastrointestinal Oncology, Osaka Medical Center For Cancer And Cardiovascular Diseases, Osaka/Japan

${ }^{6}$ Gastroenterology, Tonan Hospital, Sapporo/Japan

${ }^{7}$ Division Of Endoscopy, Yokohama City University Medical Center, Yokohama/ Japan

${ }^{8}$ Dept. Of Gastroenterology, University of Tokyo, Tokyo/Japan

${ }^{9}$ Department Of Endoscopy, Fukushima Medical University Hospital, Fukushimal Japan

${ }^{10}$ Department Of Gastroenterology And Hepatology, Okayama University Graduate School of Medicine, Dentistry, and Pharmaceutical Sciences, Okayama/ Japan

${ }^{11}$ Department Of Gastrointestinal Oncology And Endoscopy, National Cancer Center Hospital East, Chiba/Japan

${ }^{12}$ Gastroenterology, Kitasato University School of Medicine, Sagamihara/Japan

${ }^{13}$ Department Of Gastroenterology, Saitama Medical University International

Medical Center, Saitama/Japan
${ }^{14}$ Division Of Gastroenterology And Hepatology, Department Of Medicine, Nihon University School of Medicine, Tokyo/Japan

Contact E-mail Address: s.sho.salubriter.mail@gmail.com

Introduction: To clarify whether traction assist improves technical outcomes of endoscopic submucosal dissection (ESD).

Aims \& Methods: A superiority, randomized phase 3 trial was conducted at 14 institutions across Japan. Patients with single gastric neoplasm meeting the indication of the Japanese gastric treatment guidelines were enrolled and assigned to conventional ESD or dental floss clip traction ESD (DFC-ESD) by a computergenerated random sequence with stratification by institution, tumor location, tumor size and operator's proficiency. The primary endpoint was ESD procedure time, which was defined as the time from the start of submucosal injection to the time, which was defined

Results: Between July 2015 and September 2016, 640 patients underwent randomization. 316 patients assigned to conventional ESD and 319 patients assigned to DFC-ESD were included in the analysis set. Mean ESD procedure time was 60.7 minutes in the conventional ESD group and 58.1 minutes in the DFC-ESD group $(\mathrm{p}=0.45)$. Perforation was less frequent in the DFC-ESD group (conventional ESD vs. DFC-ESD: $2.2 \%$ vs. $0.3 \%, p=0.04$ ). Among the lesions in the greater curvature of the upper or middle stomach, mean procedure time in the DFCESD group was shorter than the conventional ESD group (conventional ESD vs. DFC-ESD: 104.1 vs. 57.2 minutes, $\mathrm{p}=0.01$ ).

Conclusion: This study reveals that traction-assisted ESD does not result in quicker procedures in the entire population, but it can reduce the risk of perforation. Selectively applied to the lesions in the greater curvature of the upper or middle third stomach, traction assist contributes to a remarkable reduction of procedure time.

Disclosure of Interest: All authors have declared no conflicts of interest.

\section{P0154 MACHINE LEARNING-BASED AUTOMATIC DETECTION} SYSTEM FOR DEMARCATION LINE OF GASTRIC CANCER WITH NBI IMAGES

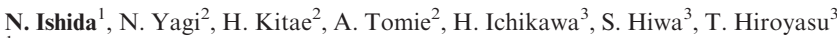
${ }^{1}$ Life And Medical Science, Graduate School of Doshisha University, Kyoto, Kyotanabe/Japan

${ }^{2}$ Gastroenterology, Murakami Memorial Hospital, Asahi University, Gifu/Japan ${ }^{3}$ Life And Medical Science, Doshisha University, Kyoto, Kyotonabe, Japan, Kyotanabe/Japan

Contact E-mail Address: nishida@mis.doshisha.ac.jp

Introduction: The vessel plus surface (VS) classification system proposed by Yao is widely used for endoscopic diagnosis of early gastric cancer ${ }^{1}$. However, this diagnosis is performed by visual observation and no quantitative index exists.

Aims \& Methods: In this study, a method for automatically detecting early gastric cancer lesions by narrow-band imaging (NBI) using a magnifying endoscopic image in the stomach is proposed to support diagnosis. The proposed system quantitatively shows the demarcation line (DL) of lesions in narrow-band images. Machine learning is introduced into the VS classification and image processing is performed. In the literature, texture analysis, which is a type of image processing, is used to assess the mucosal surface ${ }^{2}$. In addition, the proposed system uses a color feature that quantitatively expresses mucosal vessels. Furthermore, the narrow-band image contained 200 superpixels, and each superpixel contained texture and color features; a superpixel is a collection of pixels with similar features. Finally, lesions were identified by a support vector machine, which is a model for machine learning, and DL was detected. In this computational experiment, the utility of the system was verified by identifying 25 early-stage gastric cancer lesions (50 endoscopic images) using NBI-magnified observation at the Department of Gastroenterology, Murakami Memorial Hospital, Asahi University, Gifu.

Results: The average detection rate of the lesion area greatly improved to $63.0 \%$ with the proposed method compared with $28.8 \%$ with the conventional method. In addition, the obtained DL was similar to that indicated by an experienced medical physician. Based on these results, the proposed system enabled the automatic detection of early gastric cancer DL in narrow-band images, suggesting that the proposed system is useful for the determination of DL.

Conclusion: In this study, a method to assess features of gastric lesions combined with the use of superpixels was proposed. The average detection rate of the lesion range using the proposed method greatly improved compared with that using the existing method, enabling the detection of DL without depending on a physician's experience.

Disclosure of Interest: All authors have declared no conflicts of interest.

\section{References}

1 Yao, K., G. K. Anagnostopoulos, and K. Ragunath. "Magnifying endoscopy for diagnosing and delineating early gastric cancer." Endoscopy 2009; 41.05: $462-467$.

2 Hiroyasu, T., et al. Preprocessing with image denoising and histogram equalization for endoscopy image analysis using texture analysis." Engineering in Medicine and Biology Society (EMBC), 2015 37th Annual International Conference of the IEEE. IEEE, 2015; 789-792. 
P0155 ENDOSCOPIC TREATMENT OF FISTULAS AFTER SLEEVE GASTRECTOMY: ASSESSMENT FOR SWITCHING TOWARDS INTERNAL DRAINAGE IN A REFERENCE CENTER

D. Lorenzo ${ }^{1}$, T. Guilbaud ${ }^{1}$, J. Gonzalez ${ }^{2}$, A. Benezech ${ }^{2}$, S. Berdah ${ }^{2}$, T. Bège ${ }^{2}$, M. Barthet ${ }^{3}$

${ }^{1}$ Hopital Nord, Marseille/France

${ }^{2}$ Dept. De Gastroenterologie, APHM - North Hospital, Marseille/France

${ }^{3}$ Gastroenterology, Hopital Nord, Marseille/France

Contact E-mail Address: diane.lorenzo@gmail.com

Introduction: Post-sleeve gastrectomy fistulas (PSGF) are major complication of bariatric surgery. Endoscopic management evolved from a fistula closure to an internal drainage (ID) strategy within the 2013 year. The main objective of this study was to evaluate these different endoscopic approaches.

Aims \& Methods: This retrospective study included all patients treated for PSGF in a referral center. "Closure" management was defined as: initial treatment using covered-metal-stent and endoclips. ID management was defined as: initial treatment by nasocystic drain and/or double-pigtail-stent. The failure was defined as: need for surgery, or death.

Results: Between 2007 and 2015, 100 patients (women: $\mathrm{N}=78$; mean age: $42 \pm 12$ years) were included. The mean delay between SG and the first endoscopy was $82 \pm 125$ days. Overall success of endoscopic treatment was $86 \%$ within $6 \pm 27$ months. Two patients died. Primary success of ID and closure management occurred in $19 / 22(86 \%)$ and $49 / 77(63 \%)$ patients, respectively. Among patients in failure of closure management, 22 had secondary ID (18 being successful). Success of initial management was significantly higher for ID $(p<0.05)$. Factors associated with failure of closure management were in multivariate analysis: collection $>5 \mathrm{~cm}(\mathrm{p}=0.013, \mathrm{OR}=3.895 \% \mathrm{CI}[1.3-10.9])$. Factors associated with duration of management over 6 months were in multivariate analysis: reoperation before endoscopy $(\mathrm{p}=0.044, \mathrm{OR}=3.995 \% \mathrm{CI}[1.0-14.9])$ and purulent flowing at endoscopy $(\mathrm{p}=0.043, \mathrm{OR}=4.695 \% \mathrm{CI}[1.0-20.4])$. Factors associated with post-2013 management were in multivariate analysis: first endoscopy within 30 days $(\mathrm{p}=0.016)$, Clavien-Dindo-type 4 and $5(\mathrm{p}=0.016)$, and absence of glue sealing $(\mathrm{p}=0.027)$

Conclusion: Endoscopic management of PSGF healed in $86 \%$ of cases. In case of collection greater than $5 \mathrm{~cm}$, an internal drainage should be proposed first. A second surgical procedure before endoscopy is associated with longer care. Management in our center has changed over time with earlier first endoscopy and management of more severe patients.

Disclosure of Interest: M. Barthet: Boston scientific consultant

All other authors have declared no conflicts of interest.

\section{P0156 CLOSURE BY USING OVER-THE-SCOPE CLIPS AFTER ENDOSCOPIC FULL-THICKNESS RESECTION \\ J. Guo \\ Endoscopy Center, Shengjing Hospital of China Medical University, Shenyang China}

Contact E-mail Address: guojt@sj-hospital.org

Introduction: Endoscopic full-thickness resection (EFTR) is a mini-invasive technique for gastrointestinal subepithelial tumors, which enables a full-thickness resection of tumors and can provide a complete basis for pathological diagnosis. Gastrointestinal fistula closure after EFTR is a challenge for endoscopists. In this study we introduced EFTR with fistula closure using the over-the-scope clip (OTSC) system for gastrointestinal subepithelial tumors originating from the muscularis propria.

Aims \& Methods: We aimed to evaluate the feasibility and safety of fistula closure with OTSC by a retrospective analysis on the cases of EFTR with defect closure using OTSC for gastrointestinal subepithelial tumors in our hospital. The patients were selected who underwent EFTR for gastrointestinal subepithelial tumors originating from the muscularis propria (tumor diameter $\leq 3 \mathrm{~cm}$ ) in our hospital from May 2014 to December 2016. After a full-thickness resection of tumors, one or two OTSCs were released to close the defect. The success rate of defect closure with OTSC was observed and the endoscopic follow-up was performed at 1 week, 1 month, 6 months, 24 months after operation to check OTSC closure.

Results: In total 49 patients were included into the study. The full-thickness resection rate of gastrointestinal tumors in the muscularis propria was $100 \%(49 / 49)$, the success rate of defect closure was $100 \%$, and the average time of defect closure was $7.3 \mathrm{~min}$ (range: $3-27 \mathrm{~min}$ ). All patients experienced no postoperative complications such as bleeding and perforation. The postoperative follow-up time was 1-24 months (mean: 11 months), and no late complications was found.

Conclusion: OTSC can be used to perform EFTR with defect closure for gastrointestinal tumors in the muscularis propria (tumor diameter $\leq 3 \mathrm{~cm}$ ). It is simple, convenient, safe and effective.

Disclosure of Interest: All authors have declared no conflicts of interest.

\section{References}

1 Hwang JH, Rulyak SD, Kimmey MB et al. American Gastroenterological Association Institute technical review on the management of gastric subepithelial masses. Gastroenterology 2006; 130: 2217-228

2 Weiland T, Fehlker M, Gottwald T, Schurr MO. 2014 May;28(5): 1447-53.

6 von Renteln D, Rudolph HU, Schmidt A, Vassiliou MC, Caca K. Endoscopic closure of duodenal perforations by using an over-the-scope clip: a randomized, controlled porcine study. Gastrointest Endosc 2010; 71: $131-138$
7 Baron TH, Song LM, Ross A, Tokar JL, Irani S, Kozarek RA. Use of an over-the-scope clipping device: multicenter retrospective results of the first U.S. experience (with videos). Gastrointest Endosc 2012; 76: 202-208

8 Albert JG, Friedrich-Rust M, Woeste G, Strey C, Bechstein WO, Zeuzem S, Sarrazin C. Benefit of a clipping device in use in intestinal bleeding and intestinal leakage. Gastrointest Endosc 2011; 74: 389-397

9 von Renteln D, Denzer UW, Schachschal G, Anders M, Groth S, Rösch T. Endoscopic closure of GI fistulae by using an over-the-scope clip (with videos). Gastrointest Endosc 2010; 72: 1289-1296

10 Parodi A, Repici A, Pedroni A, Blanchi S, Conio M. Endoscopic management of GI perforations with a new over-the scope clip device (with videos). Gastrointest Endosc 2010; 72: 881-886

P0157 EFFICACY OF ORAL MIXTURE OF HYDROCORTISONE SODIUM SUCCINATE AND ALUMINUM PHOSPHATE GEL FOR THE PREVENTION OF STRICTURE AFTER $\geq 2 / 3$ CIRCUMFERENTIAL ENDOSCOPIC SUBMUCOSAL DISSECTION (ESD) FOR ESOPHAGEAL CANCER-A SINGLE CENTER PILOT STUDY FROM CHINA

Y. Huang, X. Yan, H. Chang, Y. Zhang, W. Yao, K. Li Gastroenterology And Hepatology, Peking University Third Hospital, Beijing/ China

Contact E-mail Address: 13911765322@163.com

Introduction: ESD has been performed on many patients with early stage esophageal cancer. However, postoperative stricture after $\geq 2 / 3$ circumferential ESD is the most important issues for quality of life in patients which is drastically decreased and repeat, periodic endoscopic balloon dilatation (EBD) is usually required over long periods. It is well known that hormone for external use is more easily absorbed in broken skin. Accordingly, We explore an innovative strategy with oral mixture of hydrocortisone sodium succinate and Aluminum phosphate gel for prevention of the stricture

Aims \& Methods: To evaluate the efficacy of this mixture in single center of Beijing, China.

Patients and Methods: In total, 13 patients who underwent more than $2 / 3$ circular or complete circular ESD for esophageal superficial squamous cell carcinoma were included in this study. They all received preventative strategy for stricture and were divided into three groups chronologically. Four patients received systemic steroid treatment (ST group), three patients received endoscopic intralesional steroid (triamcinolone acetonide $80 \mathrm{mg}$ ) injection accompanied with systemic steroid treatment (IT + ST group), six patients received oral mixture of hydrocortisone sodium succinate and aluminum phosphate gel (OHA group). We compared the two groups in terms of stricture rate and total number of endoscopic balloon dilatation (EBD) sessions. ST groups started with $30 \mathrm{mg} /$ day prednisolone on the second day post-ESD, and continued with a gradually tapering prednisolone dose, finally discontinuing systemic steroid administration 8 weeks later. IT + ST group started with $80 \mathrm{mg}$ introlesional steroid at the end of ESD procedure, and $30 \mathrm{mg} /$ day prednisolone on the second day post-ESD which exactly was the same as ST group of tapering process. OHA group started with mixture of hydrocortisone sodium succinate $50 \mathrm{mg}$ and aluminum phosphate gel $20 \mathrm{~g}$, qid for 2 weeks and continued with a gradually tapering OHA dose on the second day post-ESD. Esophagogastroduodenoscopy (EGD) was performed on demand whenever patients complained of dysphagia. Among those cases, EBD was performed when patients experienced persistent dysphagia. If the patient had no complaint of dysphagia, EGD was performed 8 weeks after ESD to evaluate any possible stricture. The primary end point in this study was the stricture rate after ESD followed by oral prednisolone. The secondary end point was the number of EBD sessions required to resolve the stricture. A stricture was defined as a difficulty in swallowing solids or an inability to pass an EGD (9.2 mm diameter endoscope).

Results: There were two complete and two $75 \%$ circular ESD in ST group, one complete and two $75 \%$ circular ESD cases in IT + ST group, and one complete and five $75 \%$ circular ESD cases in OHA group. 12 cases were resected en bloc with tumor free lateral and basal margins. No complications were seen after this procedure. The stricture rates of ST, IT + ST, OHA group after ESD were $100 \%$ (4 of 4 patients), 33.\% (one of three patients), $0 \%$ (none of six patients), respectively. One patient with stricture after ESD had lateral recurrence at the margin of ulcer. One EBD was performed in three patients in ST group and one patient in IT + ST group with esophageal stricture. One patient in ST group underwent operation in response to patient demand

Conclusion: Short period, oral 1 mixture of hydrocortisone sodium succinate and aluminum phosphate gel showed promising results for the prevention of stricture after ESD for early stage esophageal cancers.

Disclosure of Interest: All authors have declared no conflicts of interest.

\section{References}

1 Risk factors for postoperative stricture after endoscopic submucosal dissection for superficial esophageal carcinoma. Endoscopy, 2014, 46(8): 640-644.

2 Usefulness of oral prednisolone in the treatment of esophageal stricture after endoscopic submucosal dissection for superficial esophageal squamous cell carcinoma. Gastrointestinal Endoscopy, 2011, 73(6): 1115-1121.

3 Intralesional steroid injection to prevent stricture after endoscopic submucosal dissection for esophageal cancer: a controlled prospective study. Endoscopy, 2012, 44(11): 1007-1011. 
P0158 LONG-TERM OUTCOMES OF COLD POLYPECTOMY FOR NONAMPULLARY DUODENAL ADENOMAS

D. Maruoka, M. Arai, N. Akizue, K. Okimoto, T. Matsumura, T. Nakagawa, T. Katsuno, N. Kato

Department Of Gastroenterology, Graduate School of Medicine, Chiba University, Chiba City/Japan

Contact E-mail Address: d-maruoka@ biscuit.ocn.ne.jp

Introduction: Endoscopic resection for superficial nonampullary duodenal epithelial tumors (SNADETs) has a high incidence of complications such as perforation in endoscopic mucosal resection (EMR) as well as in endoscopic submucosa dissection (ESD), compared with resection of other parts of the digestive tract. We had written the first report on the safety and efficacy of cold polypectomy (cold forceps polypectomy [CFP] and cold snare polypectomy [CSP]) for sporadic SNADETs. However, there is no report on the long-term outcomes of cold polypectomy for sporadic SNADETs. In this study, we aimed to assess the long-term outcomes of cold polypectomy for sporadic SNADETs.

Aims \& Methods: Patients without polyposis syndrome who underwent cold polypectomy for one or more SNADETs $\leq 6 \mathrm{~mm}$ in size and were diagnosed with adenomas between March 2015 and November 2016, and were followed up by endoscopy for more than 1 year were analyzed. All patients subsequently underwent upper gastrointestinal endoscopy 3 months after the intervention. The presence of residual tumors was evaluated by conducting endoscopic examinations and histopathological tests with tissue samples obtained from the cold polypectomy scars. Subsequently, patients underwent upper gastrointestinal endoscopy annually, and when residual tumors could not be denied, biopsies were taken from the scars.

Results: A total of 43 lesions in 33 patients were removed using cold polypectomy. Twenty-four lesions in 20 patients were followed up for more than 1 year. The mean follow-up period by upper gastrointestinal endoscopy was 13.1 (12-18) months. Of these 20 patients, $12(60 \%)$ were men and the mean age of the subjects was $63 \pm 11$ years. The number of lesions were 5,16 , and $3(21 \%$, $67 \%, 13 \%$ ) per lesion location (1st, 2 nd, and 3rd portion), respectively, and 2 , $3,13,4$, and $2(8 \%, 13 \%, 54 \%, 17 \%, 8 \%)$ per macroscopic appearance (Isp, Is, IIa, IIa + IIc, and IIc), respectively. Nine lesions in 8 patients were resected using CFP, while 15 lesions in 12 patients were resected using CSP. Seven of $9(78 \%)$ and 14 of $15(93 \%)$ lesions were removed en bloc using CFP and CSP, respectively; the other 3 lesions were removed by piecemeal resection in 2 pieces. All specimens resected using both CFP and CSP were successfully retrieved. Histopathologic analysis showed that 21 of 24 lesions $(88 \%)$ were low-grade adenomas, and $3(13 \%)$ were high-grade adenomas. The mean size of the adenomatous lesions was $4.0 \pm 1.3 \mathrm{~mm}(2-6 \mathrm{~mm})$. Eleven of 24 adenomas $(46 \%)$ were R0 resections; 3 of $9(33 \%)$ and 8 of $15(53 \%)$ were R0 resections using CFP and CSP, respectively. Delayed bleeding and intraprocedural/delayed perforation were not observed in any case. The scars were identified and biopsied at follow-up endoscopy performed 3 months after cold polypectomy, and no residual or recurrent tumor was detected morphologically or histopathologically. Although there was no recurrent case during the follow-up period, one patient died 6 months after resection because of heart failure.

Conclusion: Cold polypectomy is a safe and effective treatment for diminutive and small sporadic SNADETs that have been subjected to long-term follow-up. Disclosure of Interest: All authors have declared no conflicts of interest.

P0159 ENDOSCOPIC TREATMENT OF BOERHAAVE SYNDROME: A SURPRISINGLY QUICK HEALING

G. Sakizlis, D. Karagiannis

Gastroenterology, Iatrikon Medical Center, Athens/Greece

Contact E-mail Address: sakizlis@gmail.com

Introduction: Endoscopic Treatment of Boerhaave Syndrome: A Surprisingly Quick Healing

Aims \& Methods: A 48-year-old male was presented at the emergency room complaining of severe chest pain, which began after several episodes of vomiting, following a meal. Upon his arrival a severe episode of haematemesis was reported. The electrocardiogram showed tachycardia with a ventricular rate of $128 \mathrm{bpm}$. The computed tomography of the chest demonstrated a hydrothorax, as well as mediastinal free air. Due to the hemorrhage we performed an emergency upper endoscopy after we obtained the patient's consent. We observed an esophageal opening, $11 \mathrm{~cm}$ long, with a Mallory-Weiss tear at the distal end. We clipped the visible vessel and decided to close the opening with clips (Figure 1). We used twelve clips and successfully stopped the bleeding while also closing the esophageal opening. Thoracic surgeons placed a thoracic drainage tube. The patient received a conservative therapy, complemented by a withdrawal of oral intake and administration of broad spectrum antibiotics.

Results: The next day we performed a follow-up upper endoscopy to inspect the closure and hemostasis. We observed an amazing healing (Figure 2). The chest tube was removed at the fourth day and the patient started oral feeding on the sixth day. The patient was discharged from the hospital with no complications on the eight day. We performed an upper endoscopy after one month with no strictures

Conclusion: Boerhaave syndrome has an estimated mortality rate of $20 \%$ to $40 \%$. The standard of care is multidisciplinary; surgical, endoscopic or conservative approaches are acceptable. No consensus exists regarding the best strategy. Endoscopic treatment consists of closure with endoscopic clips - the throughthe-scope (TTS) clip and the over the-scope clip (OTSC) - or stenting with removable stents. This paper explores the case of a spontaneous esophageal rupture after vomiting, which was treated with TTS clips. We note the extremely fast rate of healing of the wound.
Disclosure of Interest: All authors have declared no conflicts of interest.

\section{Reference}

Esophageal perforation: Continuing challenge to treatment, Gastrointestinal intervention, June 2013, Pages 1-6

\section{P0160 ARTIFICIAL INTELLIGENCE DIAGNOSIS OF HELICOBACTER PYLORI INFECTION USING LINKED COLOR} IMAGING

H. Nakashima ${ }^{1}$, H. Kawahira ${ }^{2}$, H. Kawachi ${ }^{3}$, N. Sakaki ${ }^{1}$

${ }^{1}$ Foundation For Detection Of Early Gastric Carcinoma, Tokyo/Japan ${ }^{2}$ Center For Frontier Medical Engineering, Chiba University, Chiba/Japan ${ }^{3}$ Department Of Pathology, The Cancer Institute Hospital, Japanese Foundation for Cancer Research, Tokyo/Japan

Contact E-mail Address: naka2515@gmail.com

Introduction: Esophagogastroduodenoscopy (EGD) is of growing importance in the diagnosis of Helicobacter pylori (HP) gastritis, because HP infection is strongly associated with gastric carcinogenesis ${ }^{1)}$. However, the accuracy of endoscopic diagnosis of HP infection may vary according to the experience and technique of the attending endoscopist. Here, we challenged to establish a computer aided endoscopic diagnosis system for HP infection using two novel technologies. First is a Linked color imaging (LCI). It is a new Image-enhanced endoscopy (IEE) using a LASER light source to enhance slight differences in mucosal color. Second is an Artificial Intelligence (AI) technology. Deep Learning has attracted attention in diagnostic imaging. It is a type of AI which imitates neural network in the brain.

Aims \& Methods: The aim of this study was to establish an AI diagnosis of HP infection using LCI. We designed a prospective study of all patients who underwent EGD and were tested for serum anti-HP IgG antibodies at our medical clinic. Subjects who had a history of HP eradication therapy were excluded in this study. A total of 220 examinees were candidates who underwent EGD and serum test of anti-HP IgG antibodies (HP-positive examinees were 112). The HP IgG antibody titer of each subject was taken as the gold standard for HP infection status for this study. During EGD an enodoscopist took 3 LCI pictures of the lesser curvature, greater curvature and anturum of the stomach by EG-L580NW (FUJIFILM Co., Japan). Finally, we used a total of 639 LCI pictures in the study. The specifications of the AI used in this study were as follows: Operating system: Linux (Ubuntu 14.04 LTS), Neural network: GoogLeNet ${ }^{2}$, Framework: Caffe $^{3)}$, and Graphic processor unit: Geforce GTX TITAN X (NVIDIA Co. USA), and we used R (version 3. 3. 2.) for all statistical analyses.

Results: The area under the curve (AUC) of receiver operating characteristics (ROC) was 0.95 for the lesser curvature. Compared to this, the AUC of the greater curvature and anturum was 0.81 and 0.67 , respectively. The AUCs obtained in the lesser curvature was significantly larger than that in the greater curvature and anturum $(P<0.01)$.

Conclusion: The results demonstrate that the AI has excellent ability to diagnose HP infection using LCI in the lesser curvature. The authors believe that AI technology with IEEs is likely to become a useful image diagnostic tool. Disclosure of Interest: All authors have declared no conflicts of interest.

\section{References}

1. IARC Helicobacter pylori Working Group (2014). Helicobacter pylori Eradication as a Strategy for Preventing Gastric Cancer. Lyon, France: International Agency for Research on Cancer (IARC Working Group Reports, No. 8).

2. Szegedy C, Liu W, Jia Y et al. Going deeper with convolutions. The IEEE Conference on Computer Vision and Pattern Recognition (CVPR) 2015: 1-9. 3. Jia Y, Shelhamer E, Donahue J et al. Caffe: Convolutional Architecture for Fast Feature Embedding. ACM Int Conf Multimed [Internet]. 2014; 675-8.

P0161 ENDOSCOPIC GRADING FOR GASTRIC INTESTINAL METAPLASIA (EGGIM): A MULTICENTRE VALIDATION STUDY

G. Esposito ${ }^{1}$, P. Pimentel-Nunes ${ }^{2}$, S. Angeletti ${ }^{1}$, R. Castro $^{2}$, D. Libanio ${ }^{2}$, G. Galli ${ }^{1}$, E. Lahner ${ }^{1}$, E. Di Giulio ${ }^{1}$, B. Annibale ${ }^{1}$, M. Dinis-Ribeiro ${ }^{2}$ ${ }^{1}$ Department Of Medical-surgical Sciences And Translational Medicine,

Sant'Andrea Hospital, University Sapienza, Rome/Italy

${ }^{2}$ Dept. Of Gastroenterology, IPO Porto, Porto/Portugal

Contact E-mail Address: gle.esposito@gmail.com

Introduction: Current guidelines (MAPS) suggest that intestinal metaplasia (IM) should be staged using OLGIM ${ }^{1}$ (Operative Link on Gastric Intestinal Metaplasia) and that patients with stages OLGIM 3 and 4 should be followed$u^{2}$. High-resolution narrow band imaging (HR-NBI) was previously shown to be accurate to diagnose $\mathrm{IM}^{3}$. Recently a new endoscopic classification (Endoscopic Grading of Gastric Intestinal Metaplasia - EGGIM) has been proposed to assess the risk phenotype of patients by the evaluation of IM in the antrum and in the corpus with the use of HR-NBI scopes ${ }^{4,5}$

Aims \& Methods: We aimed at determining the accuracy of EGGIM classification, compared with the pathological evaluation of gastric biopsies expressed according to OLGIM classification. Two centers (Italy, Portugal) consecutively included 78 adult patients (female $56 \%$; median age $61(20-84$ ) years; 8 with 1 st degree family history of gastric cancer). All patients were evaluated by High- 
Resolution White Light Endoscopy (HR-WLE) followed by HR-NBI. A careful evaluation of the antrum and corpus mucosa was performed and EGGIM score was calculated. Five different areas were considered (lesser and greater curvature in the antrum, lesser and greater curvature in the corpus and incisura) and in each area 0 (no IM), 1 (focal IM, less or equal than $30 \%$ of the area) or 2 points (extensive IM in that area, more than $30 \%$ of the area) were attributed for a total of 10 points. Biopsies were taken where the endoscopists observed IM and, if IM was not present, random biopsies were taken using the updated Sydney System protocol. Biopsies from the different sites were sent for histopathologic evaluation in separate jars. The diagnostic performance of EGGIM was then compared to OLGIM (gold standard) and sensibility, specificity, positive predictive value (PPV) and negative predictive value (NPV) were calculated.

Results: IM was staged as OLGIM 0, 2, 3 and 4, respectively: $32(41.0 \%), 23$ $(29.5 \%), 17(21.8 \%)$, and $6(7.7 \%)$ pts (no patients with OLGIM 1 were found). Table 1 shows detailed the EGGIM scores compared to OLGA. Compared to OLGIM as gold standard for the evaluation of IM, sensitivity, specificity, PPV and NPV of EGGIM classification were $97.8 \%, 81.2 \%, 88.2 \%$ and $96.3 \%$, respectively. Three of the 6 patients with false positive results using the EGGIM classification were $\mathrm{H}$. pylori positive. Analyzing the subgroup of patients with OLGIM 3 and 4 , the diagnostic performance of EGGIM was: sensibility $95.6 \%$, specificity $90.9 \%$, PPV $81.5 \%$ and NPV $98.0 \%$. Two of the 5 patients who resulted false positive using the EGGIM classification were $\mathrm{H}$. pylori positive. A high agreement between EGGIM and OLGIM scores was observed $(83.3 \%)$

\begin{tabular}{lllllll}
\hline $\begin{array}{l}\text { \% of total (within } \\
\text { each OLGIM grade) }\end{array}$ & $\begin{array}{l}\text { EGGIM } \\
\text { score } \\
0\end{array}$ & $1-2$ & $3-4$ & $5-7$ & $8-10$ \\
\hline OLGIM & 0 & $33(81)$ & $5(12)$ & $3(7)$ & 0 & 0 \\
& I & 0 & 0 & 0 & 0 & 0 \\
& II & $1(4)$ & $8(26)$ & $14(48)$ & $4(13)$ & $3(9)$ \\
& III & 0 & 0 & $1(6)$ & $17(76)$ & $4(18)$ \\
Extent of intestinal & IV & 0 & 0 & 0 & $4(50)$ & $4(50)$ \\
metaplasia & & Absent & Focal & Moderate & Extensive & Extensive \\
& & & & & & \\
\hline
\end{tabular}

Conclusion: The EGGIM classification showed a high diagnostic performance compared to OLGIM, in particular in patients with OLGIM 3 and 4. A possible confounding factor leading to overestimation of presence of intestinal metaplasia might be the presence of $H$. pylori infection. This approach could be used to simplify the surveillance of these patients.

Disclosure of Interest: All authors have declared no conflicts of interest.

\section{References}

1. Dinis-Ribeiro $\mathrm{M}$ et al. Management of precancerous conditions and lesions in the stomach (MAPS): guideline from the European Society of Gastrointestinal Endoscopy (ESGE), European Helicobacter Study Group (EHSG), European Society of Pathology (ESP), and the Sociedade Portuguesa de Endoscopia Digestiva (SPED). Endoscopy 2012.

2. Capelle LG et al. The staging of gastritis with the OLGA system by using intestinal metaplasia as an accurate alternative for atrophic gastritis. Gastrointest Endosc 2010.

3. Bansal A et al. Correlation between narrow band imaging and nonneoplastic gastric pathology: a pilot feasibility trial. Gastrointest Endosc 2008.

4. Pimentel-Nunes $\mathbf{P}$ et al. A multicenter prospective study of the real-time use of narrow-band imaging in the diagnosis of premalignant gastric conditions and lesions. Endoscopy 2016.

5. Lage $\mathbf{J}$ et al. Light-NBI to identify high-risk phenotypes for gastric adenocarcinoma: do we still need biopsies? Scand J Gastroenterol 2016.

\section{P0162 PERCUTANEOUS TRANSESOPHAGEAL GASTROTUBING (PTEG) IN THE DECOMPRESSION TREATMENT FROM THE GASTROINTESTINAL TRACT AS THE IDEAL PALLIATIVE CARE FOR THE PATIENTS WITH MALIGNANT DISEASE}

M. Murakami ${ }^{1}$, K. Nishino ${ }^{1}$, T. Okamoto $^{1}$, S. Murakami ${ }^{1}$, K. Mori ${ }^{1}$, B. Murakami ${ }^{1}$, M. Azuma ${ }^{2}$, S. Tanabe ${ }^{3}$, M. Kida ${ }^{4}$, W. Koizumi ${ }^{2}$ ${ }^{1}$ Dept. Of Internal Medicine, Murakami Memorial Hospital, Saijo/Japan ${ }^{2}$ Gastroenterology, Kitasato University School of Medicine, Sagamihara/Japan ${ }^{3}$ Research And Development Center For New Medical Frontiers, Kitasato University, Sagamihara/Japan

${ }^{4}$ Endoscopy \& Gastroenterology, Kitasato University, Sagamihara/Japan

\section{Contact E-mail Address: masato@murakami-kinen.or.jp}

Introduction: Percutaneous transesophageal gastrotubing (PTEG) was developed as an alternative route to access the gastrointestinal tract for the patients that Percutaneous Endoscopic Gastrostomy was contraindicated with conditions such as prior gastrectomy, gastric anterior wall malignancies, or massive ascites. PTEG will be an ideal method as the palliative care for the patients with malignant disease.

Aims \& Methods: The aim of this study is to evaluate the clinical usefulness of PTEG supported by endoscopy for the patients who need decompression from gastrointestinal tract due to malignancies. A rupture-free balloon (RFB) catheter is inserted into the upper esophagus. Percutaneous balloon puncture with a specialized needle is then performed from the left side of patient's neck under ultrasonographic control. A guide wire is inserted through the needle into the RFB, followed by a dilator and sheath. A placement tube is then inserted through the sheath, and the sheath is removed. Double Balloons equipped Over tube type RFB were used instead of primary RFB in five cases that the puncture needle is punctured into the over tube trough the balloon. We perform PTEG in a total of 62 patients (37 men and 25 women, mean age 61.8 years) in whom PEG was not feasible for decompression. Every patient suffered discomfort to the prior insertion of nasal tube. Fifty-three patients had peritoneal dissemination and 7 patients had gastrointestinal tract obstruction, and 2 patients need biliary drainage,

Results: Satisfactory results were achieved in all 62 patients. Median follow-up was 60.5 days in patients who received decompression because of the obstruction due to malignancies. All patients could free from nasal tube prior insertion. Oral ingestion could be achieved in $48.6 \%$ and home care could achieved in $63.9 \%$ of those patients. Complications were minor oozing bleeding in three patients that did not require blood transfusion, thyroid puncture in three patients, which were managed conservatively. No patient required surgical treatment or died after PTEG

Conclusion: PTEG is feasible, safe, and useful. PTEG could be an only procedure to be free from a nasal tube especially for the patients with carcinomatous peritonitis. PTEG is the ideal solution for the patients with malignant gastrointestinal obstruction. PTEG is one of the best tool in palliative care for the patients with malignant disease.

Disclosure of Interest: All authors have declared no conflicts of interest.

\section{P0163 COMPARISON OF PERCUTANEOUS ENDOSCOPIC VERSUS RADIOLOGIC GASTROSTOMY IN TERMS OF INDICATIONS, EFFICACY, COMPLICATIONS; A RETROSPECTIVE ANALYSIS \\ D. Strijbos ${ }^{1}$, D. Keszthelyi ${ }^{1}$, L. P.1. Gilissen ${ }^{2}$, M. W. De Haan ${ }^{3}$, C. Van Der Leij ${ }^{3}$, R. De Ridder ${ }^{1}$, J. Kruimel $^{1}$, J. Conchillo ${ }^{1}$, A. A.m. Masclee ${ }^{1}$ ${ }^{1}$ Gastroenterology And Hepatology, Maastricht University Medical Center, Maastricht/Netherlands \\ ${ }^{2}$ Gastroenterology And Hepatology, Catharina Ziekenhuis, Eindhoven/Netherlands ${ }^{3}$ Radiology, Maastricht University Medical Center, Maastricht/Netherlands}

Contact E-mail Address: denise.strijbos@catharinaziekenhuis.nl Introduction: Gastrostomy is the current method of choice for medium and longterm enteral feeding. Available techniques include Percutaneous Endoscopic Gastrostomy (PEG) and Percutaneous Radiologic Gastrostomy (PRG). Both techniques are preferred over surgical gastrostomy. Previous studies that have compared outcomes between PEG and PRG are limited due to small sample sizes, high risk of confounding and selection bias. Our primary aim was to retrospectively analyse data from our centre with respect to complications and mortality between PEG and PRG procedures in relation to indications. These data may help to predict which technique is best for an individual patient.

Aims \& Methods: A retrospective analysis including all adult patients receiving initial PRG (January 2010 until April 2016) and PEG (January 2008 until April 2016) placement in our university hospital, was performed.

Outcomes were complications (early ( $\leq 30$ days) and late), success rates and mortality (procedure related, 30-day, and overall). Chi ${ }^{2}$, Fisher's exact and ttests were used. Multivariate logistic regression and Cox proportional hazards regression analysis were performed.

Results: A total of 760 initial procedures (469 PRG and 291 PEG) were included in the analysis $(62.9 \%$ male, mean age $62.8 \mathrm{yrs}$ [SD 12.64]). Most common indications were head and neck malignancy (HN, PEG 38.8\%, PRG 69.9\%, $\mathrm{p}=<0.001$ ), Cerebrovascular Accident (CVA, PEG 13.7\%, PRG 2.1\%, $\mathrm{p}<0.001$ ) and Motor Neuron Disease (MND, PEG 2.7\%, PRG 9.8\%, $\mathrm{p}=<0.001$ ). Success rates for placement were $91.2 \%$ for PEG (failure mostly due to absence of transillumination, $n=14)$ and $97.1 \%$ for PRG $(p=0.001)$. Major complications (e.g. abscess, buried bumper, peritonitis) and infections did not differ amongst groups, neither did procedure-related mortality, which was $1.7 \%$ in PEG $(n=5)$ vs. $0.4 \%$ in PRG $(n=2, p=0.113)$. One case of tumour (HN) seeding occurred after PRG placement. Tube related complications (including dislocation, obstruction, leak and tube defects) were lower in PEG than PRG, both within 30 days $(2.7 \%$ vs. $26.4 \%$ of patients, $\mathrm{p}=<0.001$ and after 30 days $(8.6 \%$ vs. $31.5 \%, \mathrm{p}=<0.001)$.

The 30-day mortality was higher in patients who received PEG $(11.3 \%: 27$ deaths due to underlying conditions, 5 related to the procedure ( 1 massive bleeding, 4 aspiration pneumonia)) compared to those with PRG $(4.48 \%, 19$ deaths due to underlying conditions, 2 related to the procedure (both aspiration pneumonia), $\mathrm{p}=<0.001$ ). The 30 -day mortality was related more to the patients' general condition, than to the procedure itself

Overall survival was $46.7 \%$ vs. $44.1 \%$ (PEG vs. PRG; $p=0.049$, OR 1.224[1.000 1.498]). Positive predictive factors for overall survival were PEG, ALS and a higher BMI before placement (adjusted OR for all three factors together $1.292[1.027-1.626])$.

Conclusion: PRG appears favourable with respect to 30 day mortality while PEG appears favourable over PRG in terms of complications (such as dislocation, pain, and irritation). A higher initial success rate and the possibility of placement in case of a narrow lumen favour PRG placement. More adequate patient selection and more thorough procedure selection prior to gastrostomy is therefore required.

Disclosure of Interest: All authors have declared no conflicts of interest. 
P0165 COMPREHENSIVE EVALUATION OF THE LEARNING CURVE FOR PERORAL ENDOSCOPIC MYOTOMY: LESSONS FROM 1346 PATIENTS

Z. Liu ${ }^{1}$, X. Zhang ${ }^{1}$, Q. Li ${ }^{1}$, P. Zhou

${ }^{1}$ Zhongshan Hospital, Endoscopy Center and Endoscopy Research Institute, Zhongshan Hospital, Fudan University - Zhongshan, Shanghai/China

Contact E-mail Address: liuzq16@fudan.edu.cn

Introduction: Peroral endoscopic myotomy (POEM) is being increasingly performed worldwide. However, studies on its learning curve are limited. A comprehensive evaluation based on risk factors is needed.

Aims \& Methods: This study was aimed to evaluate the impact of various factors on the learning curve of POEM. From August 2010 to July 2015, 1346 POEM performed in Zhongshan Hospital were analyzed. The primary outcome of the study was a composite outcome of aborted procedures and complication. The secondary outcomes included procedure time and hospital stay. The impact of risk factors was assessed by backward conditional logistic regression on primary and secondary outcomes. The risk-adjusted CUSUM and moving average methods were used to evaluate the outcomes.

Results: Fifty-four (4\%) patients had the composite outcome with 10 aborted procedures and 44 adverse events. The composite outcome was related to case number, full-thickness myotomy and procedure time in the multivariate logistic regression. Adjusted for these risk factors, the CUSUM analysis showed that the composite outcome gradually decreased after 150 cases. The procedure time was higher in the early stage and decreased after 71 cases. Case number, in representative of the operative experience, is also an independent risk factor for a longer procedure time and hospital stay.

Conclusion: For POEM operators, seventy cases might be considered a threshold for faster operation, i.e., technical proficiency. A hundred-and-fifty cases might be considered a threshold for the decrease of aborted procedures and adverse events, i.e., technical reliability.

Disclosure of Interest: All authors have declared no conflicts of interest

\section{P0166 CLINICAL CURATIVE EFFECT ANALYSIS OF 162 GASTRIC STROMAL TUMORS RESECTED BY ENDOSCOPIC TREATMENTS}

\author{
L. Liu, W. Liu, Z. Fan \\ Digestive Endoscopy Center, the First Affiliated Hospital of Nanjing Medical \\ University, Nanjing/China
}

\section{Contact E-mail Address: kit9178@sina.com}

Introduction: Gastrointestinal stromal tumor (GIST) is one of the most common tumors originating from mesenchymal tissue of gastrointestinal tract, which accounts for about $0.2 \%$ of gastrointestinal tumors. Gastric stromal tumors are more common, accounting for about $40 \% \sim 70 \%$ of GIST. At present, the endoscopic treatments of gastric stromal tumors includes endoscopic submucosal dissection (ESD), endoscopic full-thickness resection (EFR) and combined endoscopic and laparoscopic surgery.

Aims \& Methods: Our study is aimed to assess the safeness and effectiveness of endoscopic treatments for gastric stromal tumor. Clinical data of 162 patients with gastric stromal tumor who underwent endoscopic treatments from June 1st 2011 to July 31 st 2015 were analyzed retrospectively. The mean diameter of the tumors was $1.5 \mathrm{~cm}(0.3 \sim 5.0 \mathrm{~cm}) .104$ patients received endoscopic submucosal dissection, 58 patients received endoscopic full-thickness resection. Among them, 4 operations were completed under the monitor of laparoscopy.

Results: Complications were observed in 8 patients $(4.9 \%)$ : bleeding during operations: 3 patients, post-operation perforation: 3 patients, respiratory tract infection: 2 patients. The mean post-operation feeding time was 2.67 days (range $1-9$ days) and post-operation hospital stays were 5.39 days (range 2-10 days). The mean time of follow-up was 26.4 months (range 5-51months). The follow-up showed that 6 patients kept on treating with oral administration of imatinib. No patient was found recurrence or death.

Conclusion: Endoscopic treatments were demonstrated as safe and effective ways to resect gastric stromal tumors in this study.

Disclosure of Interest: All authors have declared no conflicts of interest.

\section{References}

1. Catalano F, Rodella L, Lombardo F, et al. Endoscopic submucosal dissection in the treatment of gastric submucosal tumors: results from a retrospective cohort study. Gastric Cancer, 2013, 16 (4): 563-570.

2. Zhao XH, Yue CJ. Gastrointestinal stromal tumor. Gastrointest Oncol, 2012, 3(3): 189-208.

3. Cho JW, the Korean ESD Study Group. Current guidelines in the management of upper gastrointestinal subepithelial tumors. Clin Endosc, 2016, 49:235-240.

4. Wilhelm D., Delius S. v., Burian M. et al. Simultaneous use of laparoscopy and endoscopy for minimally invasive resection of gastric subepithelial masses:analysis of 93 interventions. World J Surg, 2008, 32:1021-1028.

5. Zhang Y, Wang X, Xiong GY, et al. Complete defect closure of gastric submucosal tumors with purse-string sutures. Surg Endosc, 2014, 28:1844-1851.

6. Casali PG, Jost L, Reichardt P, et al. Gastrointestinal stromal tumours:ESMO clinical recommendations for diagnosis, treatment and follow-up. Ann Oncol, 2009, 20(Supp14):4 64-67.
P0167 GASTROENTEROLOGY REGISTRAR OF THE WEEK: A

SOLUTION FOR AUGIB ENDOSCOPY TRAINING?

S. Budihal ${ }^{1}$, P. Wurm

${ }^{1}$ Digestive Diseases Centre, University Hospitals Leicester, GB/United Kingdom

Contact E-mail Address: shivbudihal@yahoo.co.uk

Introduction: Much concern surrounds Gastroenterology Specialist Registrar (StR) endoscopy training, especially in regards to endoscopic management of Acute Upper Gastrointestinal Bleeding (AUGIB). Recent evidence suggests there has been a decline in exposure and experience in AUGIB endoscopy ${ }^{1}$. In July 2013 our University Hospital introduced a Consultant-led and Registrarsupported Monday to Friday, 9 to $5 \mathrm{pm}$ in-reach service. It comprises of a morning visit to the acute medical units and a daily inpatient emergency list. This study looked at registrar AUGIB endoscopy training after its implementation.

Aims \& Methods: Endoscopy reports of patients presenting with haematemesis, melena or both who had undergone gastroscopy during the period of 1st of March 2015 to 31st August 2015 were retrieved using the endoscopy reporting tool Unisoft and analysed. Reports where StRs were the primary operator were considered. Number of procedures, haemostatic intervention and nature of haemostasis was analysed. This was then compared to data from the year before implementation $(01 / 03 / 2012$ to $31 / 08 / 2012)$

Results: A total of 7 StRs (5 Full Time and 2 Less than Full Time) performed gastroscopies on AUGIB patients as first operators under Consultant supervision. Over the 6-month period a total of 166 gastroscopies were undertaken (Mean 24). On 26 occasions, endoscopic intervention (EI) was performed (Mean 4). On average, $16 \%$ of the AUGIB patients required EI. In cases of Non Variceal Bleeding, Dual therapy was applied in $87.5 \%$ of the cases. In the remaining cases Haemospray was used. On average each StR was able to perform one case of oesophageal variceal banding and one case where Haemospray was utilised. Data from the 2012 cohort in comparison showed a total of 66 gastroscopies over 6 months with 13 EI. On average 13 procedures and 2.6 EIs were performed by each StR. Dual therapy was applied in only $28.5 \%$ of the cases

Conclusion: The introduction of the Registrar of the Week Service provides a valuable opportunity for StRs to be trained in endoscopic haemostasis and acquire exposure to AUGIB patients. As per this study each StR on an average performed endoscopy on 24 AUGIB patients. If this is extrapolated, each StR will be able to perform 48 procedures in 1 year and 240 procedures over 5 years. In the case of EI, on average a StR can perform around 4 interventions over 6 months, which comes to 8 per year and 40 in a 5 year programme which is significantly better than in the previous cohort and other centres ${ }^{1}$. Hospitals should consider developing similar services not only to meet demands for $24 / 7$ Consultant led AUGIB endoscopy service but provide adequate endoscopic training provision for current specialist registrars in order to ensure future competent and confident consultants.

Disclosure of Interest: All authors have declared no conflicts of interest.

\section{Reference}

1. Penny HA, Kurien M, Wong E, et al. Frontline Gastroenterology 2016;7:6772.

P0168 HIGH PERCENTAGE OF VISIBLE LESIONS IN PATIENTS WITH BARRETT'S ESOPHAGUS REFERRED WITH DYSPLASIA IN RANDOM BIOPSIES

I. C. Noordzij ${ }^{1}$, W. L. Curvers ${ }^{1}$, I. Van Lijnschoten ${ }^{2}$, C. J. Huysentruyt ${ }^{2}$, E.J. Schoon

${ }^{1}$ Gastroenterology And Hepathology, Catharina Hospital, Eindhoven/Netherlands ${ }^{2}$ Laboratory Of Pathology And Medical Microbiology, PAMM, Eindhoven/ Netherlands

Contact E-mail Address: irma.noordzij@catharinaziekenhuis.nl

Introduction: Endoscopic recognition of dysplasia or early cancer in Barrett's esophagus (BE) is difficult. Experience in recognition of early neoplastic lesions is thought to increase the detection of visible dysplastic lesions. A previous study reported that endoscopists in community hospitals detect neoplastic lesions at a significant lower rate than referral centres. The aim of the study we want to assess the significance of dysplasia in random biopsies in BE, in the absence of reported visible lesions as well as the final outcome of pathology.

Aims \& Methods: We retrospectively analysed all patients referred from 19 community hospitals to our tertiary referral centre with the diagnosis of BE with dysplasia or early adenocarcinoma (EAC) between February 2008 and April 2016. All patients underwent a dedicated imaging endoscopy with high-definition endoscopy supplemented with virtual chromoendoscopy and/or acetic acid staining at the discretion of the endoscopist. All procedures were performed by an endoscopist with extensive experience in the detection of early neoplastic lesions in BE. During endoscopy all visible lesions were noted and biopsied and/or removed by endoscopic resection (ER). Patients were included for analysis in case of absence of reporting visible lesions at referral.

Results: In total 184 patients were referred with dysplasia or EAC of which 82 patient $(80.5 \%$ male, age $42-81$ years (median (68)) did not show a visible lesion upon referral endoscopy. Referral diagnosis of these 82 patients was 32 low-grade dysplasia (LGD), 43 high-grade dysplasia (HGD) and 7 EAC, In three of 32 patients $(9.4 \%)$ referred with LGD, a visible lesion during imaging endoscopy was detected. Two cases of histology proved EAC and one confirmed LGD. In twenty-six of 43 patients $(60.5 \%)$ referred with HGD, a visible lesion with histology specimens corresponding to HGD (10) and EAC (16) were found, 
respectively. All cases of EAC were detected (7/7). In 18/75 (24\%) patients referred with dysplasia (LGD/HGD) without a visible lesion, the referral diagnosis was thus upstaged to EAC. Overall, 41/82 (50\%) lesions were found additionally.

Conclusion: The presence of any grade of dysplasia in random biopsies in BE screening in community hospitals is a potential marker for more severe final pathology after endoscopic work-up in an expert centre. Training in Barrett imaging is mandatory for non-expert endoscopists.

Disclosure of Interest: All authors have declared no conflicts of interest.

P0169 ENDOSCOPIC MANAGEMENT OF BENIGN ESORESPIRATORY FISTULAS

A. Debourdeau, J. Gonzalez, A. Benezech, M. Barthet

Gastroenterology, Aix Marseille University, AP-HM, Hôpital Nord, Marseille/ France

Contact E-mail Address: antoinedebourdeau@ $@$ hotmail.com

Introduction: Nonmalignant esophago-respiratory fistulas (ERF) are rare but frightening clinical situations. They usually involve surgery, but the morbidity and the mortality is high. The knowledges about the modalities and outcomes after endoscopic management of ERF remain limited.

Aims \& Methods: The aim of this study was to describe and assess the endoscopic management of benign ERF in our center. This was a retrospective study involving patients manage for benign ERF in our tertiary center between July 2012 and December 2016. The inclusion criterion was the presence of communication between esophagus and bronchial tree diagnosed confirmed and treated by endoscopy, and malignant ERFs were excluded. The ERFs were classified into three groups of sizes: punctiform (if the orifice was no larger than a straight catheter), medium and large (with visibility of bronchial tree). The primary endpoint was to document the clinical success defined as the closure of the fistula confirmed by endoscopy and persisting $>6$ months. The secondary endpoints were to document the characteristics of endoscopic treatment, the functional success and death, and to identify factors associated with success and death.

TABLE 1: - Demographics \& Results

\begin{tabular}{|c|c|c|c|}
\hline & $\begin{array}{l}\text { Clinical } \\
\text { failure }\end{array}$ & $\begin{array}{l}\text { Clinical } \\
\text { success }\end{array}$ & $\mathrm{p}$ value \\
\hline Number of patients $n=22$ & $\mathrm{n}=12$ & $\mathrm{n}=10$ & \\
\hline \multicolumn{4}{|l|}{ Gender } \\
\hline Male/Female & $6 / 6$ & $7 / 3$ & 0.342 \\
\hline \multicolumn{4}{|l|}{ Etiology } \\
\hline Post-surgery & 7 & 6 & 0.665 \\
\hline Post-dilatation & 0 & 3 & 0.062 \\
\hline Post-radiation & 2 & 0 & 0.138 \\
\hline Post-invasive ventilation & 3 & 2 & 0.534 \\
\hline \multicolumn{4}{|l|}{ Pulmonary location } \\
\hline Trachea & 10 & 7 & 0.127 \\
\hline Right bronchus & 2 & 1 & 0.534 \\
\hline Left bronchus & 0 & 2 & 0.138 \\
\hline \multicolumn{4}{|l|}{ Orifice size } \\
\hline Punctiform & 3 & 6 & 0.193 \\
\hline Medium & 3 & 3 & 1 \\
\hline Large & 6 & 1 & 0.17 \\
\hline \multicolumn{4}{|l|}{ Timing of closure } \\
\hline Resolution at 3 monts & 0 & 7 & $<10^{\wedge}-3$ \\
\hline Resolution at 6 months & 0 & 8 & $<10^{\wedge}-3$ \\
\hline No resolution at 6 months & 12 & 2 & $<10^{\wedge}-3$ \\
\hline \multicolumn{4}{|l|}{ Endoscopic treatment } \\
\hline Mean number of esophageal stents & $3.6( \pm 3.9)$ & $2.3( \pm 2.7)$ & 0.069 \\
\hline Mean number of OTSc & $1.2( \pm 1.8)$ & $0.4( \pm 0.7)$ & 0.082 \\
\hline At least one esophageal stent & 11 & 9 & 0.892 \\
\hline At least one OTSc & 6 & 2 & 0.146 \\
\hline
\end{tabular}

Results: A total of 22 patients were included and analyzed. The etiologies of ERF were esophageal surgery in 12 patients $(54.5 \%)$, esophageal dilatation in 3 $(13.6 \%)$, invasive ventilation in $3(13.6 \%)$, radiation therapy in $2(9.1 \%)$ and tracheostomy in $2(9.1 \%)$. A total of 93 procedures were performed with a mean of number of endoscopies of $4.2 \pm 4.5$ per patient. At some point of the management, twenty-one patients $(95 \%)$ had esophageal stents placement, eight patients $(36 \%)$ had over the scope clips (OTSC) placement and seven had OTSC associated with esophageal stent. The clinical success rate was $45.5 \%(n=10)$, and $55 \%$ of the patients had a functional success $(n=12)$. Serious adverse events occurred in 9 patients $(40.9 \%)$ such as gastrointestinal bleeding (4 patients, $18.2 \%$ ), stent migration ( 4 patients, $18.2 \%$ ), thoracic spondylodiscitis ( 2 patients, $9.1 \%$ ) alimentary esophageal impaction (1 patient, $4.5 \%)$, stent mucosal impaction (1 patient, $4.5 \%)$, major chest pain (1 patient, $4.5 \%)$. Six patients died $(27 \%)$. Clinical success was reached for $67 \%$ of punctiform ERF $(p=0.193)$, $50 \%$ of medium ERF $(\mathrm{p}=1)$ and $14 \%$ of large ERF $(\mathrm{p}=0.17)$. The factor associated with the failure of endoscopic treatment was the persistence of the fistula after 6 months $(\mathrm{OR}=44$; IC95: 3.38-573, 4; $\mathrm{p}=0.004$ multivariate analysis). The orifice's size was associated with the mortality with $71 \%$ of death among large fistulas ( $\mathrm{p}=0.001$ univariate analysis).

Conclusion: Conclusions: Endoscopic treatment of ERF can lead to $45.5 \%$ of clinical success and $55.5 \%$ of functional success. However, this outcome appears to depend on the size of the fistula. Moreover, the absence of resolution after 6 months of endoscopic treatment dramatically decreases the chance for ERF healing. In conclusion, the endoscopic approach seems reasonable for small or medium orifices, and has to be attempted during six months. After this time or for larger orifices, surgery or palliative therapy should be considered.

Disclosure of Interest: All authors have declared no conflicts of interest.

\section{P0170 CLINICAL UTILITY OF NARROW BAND IMAGING MAGNIFYING ENDOSCOPY FOR MM/SM1 ESOPHAGEAL SQUAMOUS CELL CARCINOMA}

M. Inoue ${ }^{1}$, T. Omori ${ }^{1}$, K. Nastu ${ }^{1}$, K. Aiura ${ }^{1}$

${ }^{1}$ Department Of Surgery, Kawasaki Municipal Kawasaki Hospital, Kawasaki/ Japan

Contact E-mail Address: masaz.inoue@gmail.com

Introduction: Esophageal squamous cell carcinoma (ESCC) is common in Asia. Predicting invasion depth of superficial ESCC is crucial in determining the precise treatment because the rate of lymph node metastasis increases in proportion of the invasion depth of the carcinoma. According to Japanese guidelines for diagnosis and treatment of esophageal cancer, superficial invasions are divided into 5 categories: carcinoma in situ (EP), tumors invades lamina propria mucosa (LPM), lamina muscularis mucosa (MM), the submucosa to a depth of $200 \mu \mathrm{m}$ or less from the muscularis mucosa (SM1), and the submucosa to a depth more than $200 \mu \mathrm{m}$ (SM2). The rate of lymph node metastasis is extremely low in EP/LPM tumors, and endoscopic resection (ER) is certified as precise treatment. On the other hand, the rate of lymph node metastasis in MM/SM1 tumors are reported to $10-20 \%$, and both operation and ER are considered as their treatment. Accurate pretherapeutic diagnosis of MM/SM1 tumor is very important for selection of appropriate treatment and interests of patients. In this point, endoscopic diagnosis is very important diagnostic approach.

Aims \& Methods: The purpose of this study is to investigate the utility of Narrow Band Imaging (NBI) magnifying endoscopy for the diagnosis of MM/SM1 ESCC. From January 2011 to April 2017, 23 patients were diagnosed as pathologically MM/SM1 ESCC in our hospital. We retrospectively analyzed their endoscopic findings and pathological findings. The depth of invasion was diagnosed by NBI magnifying endoscopy according to the Japan Esophageal Society (JES) magnifying endoscopic classification ${ }^{1}$. Diagnostic criteria are based on the degree of microvascular irregularity in the target lesion observed by NBI magnifying endoscopy. Microvessels are grouped into 2 types. Type A microvessels are normal intrapapillary capillary loops or abnormal microvessels without severe irregularity. Type B microvessels are abnormal vessels with severe irregularity or highly dilated abnormal vessels, and subclassified into B1, B2, and B3 based on the running pattern or degree of dilation of severely irregular microvessels. When target lesions have B1 vessels, the invasion depth is predicted as EP or LPM. When B2 or B3 vessels are seen, the invasion depth is predicted as MM or SM1 and SM2 or deeper, respectively.

Results: In 23 pathologically MM/SM1 cases, clinical type diagnosed by endoscopy was 0 -IIc in 16 cases $(70 \%), 0$-IIa in 3 cases $(13 \%), 0$-IIb in 3 cases $(13 \%)$, and 0 -Ip in 1 case $(4 \%)$. Predicted depth of invasion by NBI magnifying endoscopy based on the JES classification was EP in 2 cases (9\%), LPM in 7 cases $(30 \%), \mathrm{MM}$ in 10 cases $(43 \%), \mathrm{SM} 1$ in 3 cases $(13 \%)$ and SM2 in 1 case $(4 \%)$. Total diagnostic accuracy of MM/SM1 was $57 \%(13 / 23)$. When the B2 vessels were observed, diagnostic accuracy of MM/SM1 was $90 \%(9 / 10)$. In the cases that MM or SM1 invasion remained pathologically quite localized, B2 vessels could not be observed by NBI magnifying endoscopy. And also, in the cases with inflammation or keratinizing epithelium, precise diagnosis of microvessels were difficult.

Conclusion: Our data indicate that diagnosis of MM/SM1 ESCC by NBI magnifying endoscopy based on the JES classification is useful when the abnormal microvessels are observable, and NBI magnifying endoscopy is essential method for pretherapeutic examination.

Disclosure of Interest: All authors have declared no conflicts of interest.

\section{Reference}

1. Oyama T et al. Esophagus. 2017;14:105-112

P0171 PER-ORAL ENDOSCOPIC PYLOROMYOTOMY (POEP) IN THE TREATMENT OF REFRACTORY GASTROPARESIS - A SINGLE CENTRE EXPERIENCE

R. Hustak ${ }^{1}$, Z. Vackova ${ }^{1}$, J. Spicak ${ }^{2}$, L. Jurgoš ${ }^{3}$, J. Martinek $^{2}$

${ }^{1}$ Department Of Hepatogastroenterology, Institute for Clinical and Experimental Medicine, Prague/Czech Republic

${ }^{2}$ Dept. Of Hepatogastroenterology, Institute for Clinical and Experimental

Medicine, Prague/Czech Republic

${ }^{3}$ Poliklinika Mytna, Bratislava/Slovak Republic

Contact E-mail Address: rhustak@gmail.com

Introduction: Gastroparesis is a chronic, debilitating motility disorder. Effective treatment is challenging especially in patients with severe symptoms. POEP is an 
emerging modality for refractory gastroparesis with promising preliminary results.

Aims \& Methods: The aim of this prospective case series was to assess our first (single center) experience with POEP. Main outcomes were: 1) the efficacy defined by improvement of GCSI score; 2) gastric emptying evolution and 3) safety. Since Nov 2015, a total of 7 patients underwent POEP. The etiology of gastroparesis was post-operative in 4 , diabetic in 2 and idiopathic in 1 patient. One patient underwent POEP for gastroparesis following a multivisceral transplantation; one patient underwent both POEP and POEM (as a single procedure) for coexisting refractory idiopathic gastroparesis and achalasia. All patients had severe gastroparesis as defined by elevated GCSI score and delayed gastric emptying scintigraphy. Follow visit at 3, 6, 12-months were completed in 7/7 (100\%), $5 / 7(71 \%)$ and $1 / 7(14 \%)$ patients, respectively. Upper GI endoscopy and scintigraphy were performed 3 months after the procedure.

Results: POEP was successfully performed in all patients. Mean procedure time was 70 minutes (range 63-106). After POEP, mean GCSI decreased from $3.0 \pm 1.2$ to $0.8 \pm 0.7$ (at 3 -months) and $0.9 \pm 0.8$ (at 6 -months). One women finished the 12-months follow maintaining excellent outcome. Treatment success was reached in $6 / 7(85 \%)$ of patient, one female patient with diabetic gastroparesis did not have a major symptomatic improvement despite normalisation of gastric emptying study. Gastric scintigraphy normalized in all patients, mean half emptying time decreased from $108 \pm 30 \mathrm{~min}$ to $62 \pm 23 \mathrm{~min}$; and mean bolus retention at 4 hours decreased from $17 \pm 9.2 \%$ to $2.0 \pm 2.0 \%$. One patient developed bleeding ulcer 10 days after POEP, this adverse event was successfully managed endoscopically (clips) and by parenteral proton pump inhibitor.

Conclusion: We report our first experiences with POEP for refractory gastroparesis, demonstrating its feasibility and safety with promising clinical efficacy. Disclosure of Interest: All authors have declared no conflicts of interest.

\section{P0172 BLUE LIGHT IMAGING FOR BARRETT'S NEOPLASIA CLASSIFICATION (BLINC): THE DEVELOPMENT AND VALIDATION OF A NEW ENDOSCOPIC CLASSIFICATION SYSTEM TO IDENTIFY BARRETT'S NEOPLASIA}

S. Subramaniam ${ }^{1}$, K. Kandiah ${ }^{1}$, F. Chedgy ${ }^{2}$, R. Bhattacharyya ${ }^{3}$, P. Basford ${ }^{4}$, G. Longcroft-Wheaton ${ }^{5}$, P. Bhandari ${ }^{1}$

${ }^{1}$ Gastroenterology, Queen Alexandra Hospital, Portsmouth/United Kingdom ${ }^{2}$ Gastroenterology, Brighton \& Sussex University Hospitals NHS Trust, Brighton/ United Kingdom

${ }^{3}$ Gastroenterology, Royal Hampshire County Hospital, Winchester/United Kingdom

${ }^{4}$ Gastroenterology, St Richard's Hospital, Chichester/United Kingdom

${ }^{5}$ Gastroenterology, Portsmouth Hospitals NHS trust, Hampshire/United Kingdom

\section{Contact E-mail Address: shar811@googlemail.com}

Introduction: Neoplasia in Barrett's can be subtle and difficult to identify. Blue light imaging (BLI) by Fujifilm is a novel advanced endoscopic technology that provides high-intensity contrast imaging for superior visualisation of mucosal surface and vessel patterns. This can improve the identification of Barrett's neoplasia. To date there is no formal classification system that enables the characterisation of neoplastic and non-neoplastic Barrett's for BLI.

Aims \& Methods: The aim of our study was to develop and validate a classification to identify Barrett's neoplasia using BLI. 3 expert endoscopists formed a working group to identify criteria characterising neoplastic and non-neoplastic Barrett's on BLI using a modified Delphi method. A simple classification system utilising pit, vessel pattern and colour was developed using a database of 40 images. 6 experienced endoscopists then assessed a library containing 45 images of neoplastic and non-neoplastic Barrett's using the proposed criteria. Sensitivity, specificity, positive (PPV) and negative predictive values (NPV) were calculated to assess its performance. The same parameters were then evaluated for each component criteria.

Results: The BLINC classification descriptors are as follows: Non Neoplastic Barrett's: Pit pattern: circular, tubular or branching with normal density; Vessel pattern: regular, pericryptal non dilated vessels with normal density; Colour: pale Neoplastic Barrett's: Pit pattern: irregular, crowded with increased density; Vessel pattern: irregular, non cryptal, dilated vessels with increased density; Colour: focal darkness The overall sensitivity and specificity, negative and positive predictive values with corresponding $95 \%$ confidence intervals are as follows: $96.7(92.4-98.9) \%, 96.7(91.2-99.1) \%, 97.3(93.3-99.0) \%$ and 95.9 (90.7-98.2)\% respectively in the classification of Barrett's neoplasia. When each category in the classification was analysed separately the predictive values of pit and vessel pattern in neoplasia characterisation were high compared to colour (as shown in the table below)

\begin{tabular}{lllll}
\hline & $\begin{array}{l}\text { Sensitivity } \\
(95 \% \mathrm{CI})\end{array}$ & $\begin{array}{l}\text { Specificity } \\
(95 \% \mathrm{CI})\end{array}$ & $\begin{array}{l}\text { PPV } \\
(95 \% \mathrm{CI})\end{array}$ & $\begin{array}{l}\text { NPV } \\
(95 \% \mathrm{CI})\end{array}$ \\
\hline Pit pattern & $96.0(91.5-98.5) \%$ & $98.3(94.1-99.8) \%$ & $98.6(94.8-99.7) \%$ & $95.2(89.9-97.7) \%$ \\
Vessel Pattern & $94.7(89.8-97.7) \%$ & $93.3(87.3-97.1) \%$ & $94.7(90.1-97.2) \%$ & $95.2(89.9-97.7) \%$ \\
Colour & $86.7(80.2-91.7) \%$ & $78.3(69.9-85.3) \%$ & $83.3(78.0-87.6) \%$ & $82.5(75.6-87.7) \%$ \\
\hline
\end{tabular}

Conclusion: We have developed the first internally validated simple classification system for the diagnosis of Barrett's neoplasia using BLI. The classification criteria demonstrated high sensitivity and specificity particularly with regards to mucosal pit and vessel patterns. We aim to use the proposed classification in future studies for real time optical diagnosis of Barrett's neoplasia.
Disclosure of Interest: P. Bhandari: Educational grants for research received from Olympus, Pentax and Fujifilm

All other authors have declared no conflicts of interest.

\section{P0173 TREATMENT OF MULTIPLE GASTROINTESTINAL SUBMUCOSAL TUMORS BY SUBMUCOSAL TUNNELING ENDOSCOPIC RESECTION}

J. Liu, W. Qin, Y. Huang, Z. Ren, P. Zhou

Endoscopy Center And Endoscopy Research Institute, Fudan University Zhongshan Hospital, Shanghai/China

\section{Contact E-mail Address: liu.jingzheng@zs-hospital.sh.cn}

Introduction: Submucosal tunneling endoscopic resection (STER) is a novel technique to remove the gastrointestinal submucosal tumors. Previous studies mainly focused on technical feasibility for patients with one single gastrointestinal submucosal tumor. No systematic studies about multiple upper gastrointestinal submucosal tumors synchronously removed by STER are addressed. The aim of this study was to evaluate the safety and outcome of STER in treatment of multiple gastrointestinal submucosal tumors.

Aims \& Methods: From January 2011 to January 2017, 42 patients with multiple gastrointestinal submucosal tumors undergoing STER were included. Variables of each tumor and patient were analyzed. Detailed tumor characteristics included max size, sum of max size and number of tumors, and longest distance of tumor. While detailed technique information included number of tunnels, tunnel length, hospital stay, procedure time, complication, follow-up, recurrence, and metastasis.

Results: Among all the cases, 96 lesions of upper gastrointestinal submucosal tumors were removed by STER. The median procedure time was $50 \mathrm{~min}$ (range $13.6-84.9 \mathrm{~min})$. The median number of tumors was $2(2-4)$. The median max size of each tumor was $1.8 \mathrm{~cm}$ (range $0.7-3.5 \mathrm{~cm}$ ) and the median sum of max size of each tumor of each patient was $3 \mathrm{~cm}$ (range $1.3-8 \mathrm{~cm}$ ). Six patients had perioperative complications $(14.2 \%)$, with 3 pneumothorax/hydrothorax $(7.2 \%), 1$ mucosal injury $(2.4 \%), 1$ pneumonia $(2.4 \%)$, and 1 major bleeding $(2.4 \%)$. Patients with different number of tunnels had similar tumor characteristics and techniques. There were significant differences in longest distance of tumors comparing two groups $(\mathrm{p}<0.001)$. No local recurrence or distant metastasis was detected with a median follow-up of 33 months.

Conclusion: STER is a safety and feasible technique for multiple upper gastrointestinal submucosal tumors no matter in one tunnel or two tunnels resection. Based on the longest distance of tumors, different number of tunnels can be performed with similar procedure technique and prognosis.

Disclosure of Interest: All authors have declared no conflicts of interest.

\section{References}

1. American Gastroenterological Association Institute. American Gastroenterological Association Institute medical position statement on the management of gastric subepithelial masses. Gastroenterology 2006; 130: 2215-2216.

2. Ponsaing LG, Kiss K, Hansen MB. Classification of submucosal tumors in the gastrointestinal tract. World J Gastroenterol 2007;13:3311-3315.

3. Lee IL, Lin PY, Tung SY, et al. Endoscopic submucosal dissection for the treatment of intraluminal gastric subepithelial tumors originating from the muscularis propria layer. Endoscopy 2006;38:1024-1028

4. Chatzipantelis P, Salla C, Karoumpalis I, et al. Endoscopic ultrasoundguided fine needle aspiration biopsy in the diagnosis of gastrointestinal stromal tumors of the stomach. A study of 17 cases. J Gastrointestin Liver Dis. 2008;17:15-20.

5. Meng FS, Zhang ZH, Hong YY, et al. Comparison of endoscopic submucosal dissection and surgery for the treatment of gastric submucosal tumors originating from the muscularis propria layer: a single-center study (with video). Surg Endosc. 2016 Nov;30(11):5099-5107

6. Zhang Y, Ye LP, Zhu LH, et al. Endoscopic muscularis excavation for subepithelial tumors of the esophagogastric junction originating from the muscularis propria layer. Dig Dis Sci. 2013;58:1335-1340.

7. Reinehr R. Endoscopic submucosal excavation (ESE) is a safe and useful technique for endoscopic removal of submucosal tumors of the stomach and the esophagus in selected cases. Z Gastroenterol. 2015;53(6):573-578

8. Zhou PH, Yao LQ, Qin XY, et al. Endoscopic full-thickness resection without laparoscopic assistance for gastric submucosal tumors originated from the muscularis propria. Surg Endosc. 2011 Sep;25(9):2926-2931

9. Xu MD, Cai MY, Zhou PH et al. Submucosal tunneling endoscopic resection: a new technique for treating upper GI SMTs originating from the muscularis propria layer (with videos). Gastrointest. Endosc. 2012; 75: 195 199.

10. Chen $\mathrm{T}, \mathrm{Zhou} \mathrm{PH}, \mathrm{Chu} \mathrm{Y}$, et al Long-term Outcomes of Submucosal Tunneling Endoscopic Resection for Upper Gastrointestinal Submucosal Tumors. Ann Surg. 2017 Feb;265(2):363-369 
P0174 COMPARISON OF THE LINKED COLOR IMAGING (LCI) TECHNOLOGY AND CHROMOENDOSCOPY WITH ACETIC ACID FOR DIAGNOSIS OF BARRETT'S ESOPHAGUS

H. Neumann ${ }^{1}$, H. Neumann $\mathrm{Sen}^{2}$, P. Grimminger ${ }^{3}$, F. Corvinus ${ }^{3}$, F. Rahman ${ }^{4}$, F. Thieringer ${ }^{4}$, G.E. Tontini ${ }^{5}$, P. R. Galle ${ }^{4}$

${ }^{1}$ Universitätsmedizin Johannes Gutenberg University Mainz, Mainz/Germany

${ }^{2}$ Gastroenterologische Schwerpunktpraxis, Bad Salzuflen/Germany

${ }^{3}$ Department Of General, Visceral And Transplant Surgery, University Medical Center Mainz, Mainz/Germany

${ }^{4}$ Interdisciplinary Endoscopy, University Medical Center Mainz, Mainz/Germany ${ }^{5}$ Gastroenterology And Digestive Endoscopy Unit, IRCCS Policlinico San Domato, San Donato Milanese/Italy

Contact E-mail Address: helmut.neumann@unimedizin-mainz.de Introduction: LCI is a new imaging technique based on 4 independently acting LEDs that is enhancing the mucosal vascular pattern and surface pattern morphology. To date, chromoendoscopy with acetic acid is considered the gold standard for diagnosis of Barrett's esophagus.

Aims \& Methods: The aim of this prospective study was to evaluate the recently introduced LCI technique compared to conventional dye spraying with acetic acid for diagnosis of Barrett's esophagus. Therefore, consecutive patients with Barrett's esophagus were categorized according to the Prague classification and prospectively included. All Barrett segments were carefully evaluated by using high-definition white-light imaging, followed by LCI and acetic acid spraying. At each examination targeted biopsies were taken from all visible lesions, followed by random four-quadrant biopsies were applicable.

Results: The diagnostic yield of conventional dye spraying was significantly higher for diagnosis of Barrett's esophagus compared to high-definition whitelight endoscopy $(\mathrm{P}<0.05)$. Of note, no significant difference for diagnosis of Barrett's esophagus was noted between acetic acid chromoendoscopy and the LCI technique. LCI diagnosis was always consistent to traditional dye spraying $(100 \%$ concordance). The random four-quadrant biopsy protocol did not add any new information to the one already obtained by using LCI.

Conclusion: The newly introduced LCI technique is superior to high-definition white light endoscopy for diagnosis of Barrett's esophagus and equally effective to acetic acid dye spraying. Therefore, the LCI technique has the potential to facilitate the diagnosis of Barrett's esophagus and to overcome the limitations of a random 4-quadrant biopsy protocol.

Disclosure of Interest: All authors have declared no conflicts of interest.

\section{P0175 ALBERTA FAMILY PRACTICE ELECTRONIC ENDOSCOPY STUDY (AFPEE)}

M. Kolber ${ }^{1}$, L. Green ${ }^{1}$, N. Olivier ${ }^{1}$, R. Torrie ${ }^{2}$, O. Babenko ${ }^{1}$

${ }^{1}$ Family Medicine, University of Alberta, Edmonton/Canada/AB

${ }^{2}$ Taber Medical Clinic, Taber/Canada/AB

\section{Contact E-mail Address: mkolber@ualberta.ca}

Introduction: In Canada, gastroenterologists and general surgeons perform 97\% of the colonoscopies. There are a small number of rural Canadian Family Physicians who perform colonoscopies. These endoscopists may improve access for rural patients who require endoscopy and help improve provincial endoscopy wait times. Although some studies demonstrate that adequately trained Family Physicians are able to perform quality endoscopy, other studies question the quality of colonoscopies performed by non-gastroenterologists.

Aims \& Methods: The Alberta Family Physician Electronic Endoscopic (AFPEE) study aimed to examine the quality of colonoscopies performed by Family Physicians in Alberta, Canada. Primary outcomes include the proportion of successful cecal intubations; proportion of patients 50 years and older having a first time colonoscopy with an adenoma; and immediate complication rates. Prospective, multi-centred observational study with real-time electronic data collection and pathological reconciliation. Using IPADs, ${ }^{\mathrm{TM}}$ data was inputted in real time into an REDCap ${ }^{\mathrm{TM}}$ based electronic record developed by the study team. Individual physician and group results of key quality indicators were compared to standard colonoscopy quality benchmarks.

Results: In this six-month study, 9 Family Physicians performed 1769 colonoscopies in 11 rural Alberta sites. The proportion of successful cecal intubations was $97.9 \%$ (95\% CI: 97.2, 98.5). All physicians had over $90 \%$ successfully completed colonoscopies (ranging from 95.2 to $100 \%$ ) The proportion of males and females $>50$ years old with an adenoma on a first-time colonoscopy was $67.4 \%$ $(95 \% \mathrm{CI} ; 62.4,72.7)$ and $51.1 \%(95 \% \mathrm{CI} ; 45.5,56.7)$ respectively. All physicians achieved benchmarks of $30 \%$ of males and $20 \%$ for females having at least one adenoma. From all colonoscopies in the study there were 2099 pathologically confirmed adenomas or SSAs, 628 advanced adenomas and 17 cancers. corresponding to 120 adenomas, 36 advanced adenomas and 1 colon cancer per 100 colonoscopies. There were 2 post-polypectomy bleeds, no perforations and no deaths.

Conclusion: Alberta Family Physician colonoscopists are meeting benchmarks in colonoscopy quality. Ongoing electronic collection of endoscopy quality markers should be encouraged. Supporting and training rural Family Physicians who perform endoscopy may help alleviate current wait times and improve access for rural Canadian patients.

Disclosure of Interest: All authors have declared no conflicts of interest.

\section{References}

Hilsden RJ, Tepper J, Moayyedi P, Rabeneck L. Who provides gastrointestinal endoscopy in Canada? Can J Gastroenterol 2007;21[12]:843-846.
Kolber MR, Wong CKW, Fedorak RN, Rowe BH, on behalf of the APC-Endo Study Physicians. Prospective Study of the Quality of Colonoscopies Performed by Primary Care Physicians: The Alberta Primary Care Endoscopy [APC-Endo] Study. PLoS ONE 2013; 8[6]: e67017. doi:10.1371/journal.pone.0067017

Wilkins T, LeClair B, Smolkin M, Davies K, Thomas A, Taylor ML, et al. Screening colonoscopies by primary care physicians: a meta-analysis. Ann Fam Med 2009; 7: 56-62.

Rabeneck L, Paszat LF, Saskin R [2010] Endoscopist specialty is associated with incident colorectal cancer after a negative colonoscopy. Clin Gastroenterol Hepatol 8: 275-279.

Bressler B, Paszat LF, Chen Z, Rothwell DM, Vinden C, et al. Rates of new or missed colorectal cancers after colonoscopy and their risk factors: a populationbased analysis. Gastroenterology 2007; 132: 96-102.

Rex DK, Schoenfeld PS, Cohen J, Pike IM, Adler DG, Fennerty MB et al, on behalf of the American Society for Gastrointestinal Endoscopy. Quality Indicators for Colonoscopy. Gastrointestinal Endoscopy 2015; 81 (1); 31-53 Valori R on behalf of the Joint Advisory Group. A guide to auditing quality and safety items of Endoscopy Global Rating Scale. Available at: www.thejag.org.uk

\section{P0176 PREDICTORS OF ADENOMA DETECTION AT COLONOSCOPY AFTER BOWEL SCOPE SURVEILLANCE: RESULTS FROM A UK PILOT SCREENING CENTRE}

A. Yew ${ }^{1}$, K. Siau ${ }^{2}$, S. Jewes ${ }^{1}$, A. Veitch ${ }^{1}$, S. Ishaq ${ }^{3}$, M. Brookes ${ }^{1}$, B. Mckaig ${ }^{1}$, A. Veitch ${ }^{1}$, S. Shetty ${ }^{3}$, A. Murugananthan ${ }^{1}$

${ }^{1}$ Department Of Gastroenterology, Royal Wolverhampton Hospitals NHS Trust, Wolverhampton/United Kingdom

${ }^{2}$ Royal College Of Physicians, JAG Clinical Fellow, London/United Kingdom ${ }^{3}$ Gastroenterology, Dudley Group Hospitals NHS Foundation Trust, Dudley/ United Kingdom

Contact E-mail Address: keith@siau.org

Introduction: In a randomised controlled trial, flexible sigmoidoscopy (bowel scope) reduced colorectal cancer incidence and mortality in a population aged 55-64.[1] Patients progressed to colonoscopy based on 'high risk' features (Table 1).[1] Based on these pivotal findings, the UK bowel scope (BS) surveillance programme was introduced in 2013 to individuals aged 55 . The Wolverhampton Bowel Cancer Screening Centre was the first UK site to fully roll out the programme. The correlation between BS findings and subsequent colonoscopy has not previously been evaluated in this specific cohort.

Aims \& Methods: We prospectively collated data from all BS patients at our centre and identified those undergoing colonoscopy between August 20132016. We assessed conversion rates, compliance with BS protocol and correlated endoscopic and histological findings to identify predictors for detection of pathology at colonoscopy. Univariate analysis was performed using Pearson's $\mathrm{chi}^{2}$

Results: 11,711 bowel scopes were performed, with an adenoma detection rate (ADR) of $8.5 \%$, and conversion to colonoscopy in 421 patients $(3.6 \%) .386$ were included for analysis after excluding incomplete colonoscopy/histology. All patients were aged $55(64.8 \%$ male $)$. Additional ADR at colonoscopy was $35.2 \%$, with malignant diagnoses in $1.5 \%$ (all detected at BS). The adenoma miss rate at BS was $5.2 \%$. On univariate analysis (Table 1 ), polyp $\geq 10 \mathrm{~mm}$ was the only indication associated with increased ADR at colonoscopy (OR 2.13, $\mathrm{p}<0.001$ ). Additional predictors identified included villous (not tubulovillous) histology (OR 4.41, p=0.02), and male gender (OR 2.35, p < 0.001). These factors also significantly predicted new $>10 \mathrm{~mm}$ adenoma. $57(14.8 \%)$ underwent colonoscopy outside protocol, which reduced ADR (OR 0.29, p=0.03). After excluding high risk indications, changing the conversion criteria from any villous to villous only histology altered sensitivity from $27.2 \%$ to $83.3 \%$, and specificity from $84.5 \%$ to $80.5 \%$.

Table 1: Indications for progression from BS to colonoscopy (in bold), ${ }^{[1]}$ and likelihood of new adenoma detection. ${ }^{*}$ Patients in multiple categories are included multiple times. ${ }^{* *} p<0.05$

\begin{tabular}{lllll}
\hline Indication & $N^{*}$ & New adenoma & OR $(95 \% \mathrm{CI})$ & $p$-value \\
\hline At least 3 polyps & 78 & $45(57.7 \%)$ & $1.46(0.88-2.43)$ & 0.14 \\
Size at least 10 mm & 196 & $86(43.9 \%)$ & $2.13(1.39-3.27)$ & $<0.001^{* *}$ \\
High grade dysplasia & 16 & $5(31.3 \%)$ & $0.82(0.28-2.41)$ & 0.72 \\
Any villous component & 190 & $69(36.3 \%)$ & $1.09(0.72-1.67)$ & 0.66 \\
$>$ 20 hyperplastic polyps & 3 & 0 & NA & NA \\
None of the above & 57 & $9(15.8 \%)$ & $0.29(0.14-0.62)$ & $0.001^{* *}$ \\
Villous only histology & 10 & $7(70.0 \%)$ & $4.41(1.12-17.36)$ & $0.02^{* *}$ \\
\hline
\end{tabular}

Conclusion: At BS, male gender, $\geq 10 \mathrm{~mm}$ polyps, and villous histology are predictors of proximal colonic pathology. Further analyses are required to clarify the benefits of converting low-risk tubulovillous adenomas at BS to colonoscopy. Disclosure of Interest: All authors have declared no conflicts of interest.

\section{Reference}

1. WS Atkin, Lancet 2010, 375:1624-33 


\section{P0177 COLD SNARE POLYPECTOMY WITH SUBMUCOSAL PRE-} LIFT VERSUS ENDOSCOPIC MUCOSAL RESECTION FOR 6-10MM COLORECTAL POLYPS: A RANDOMIZED NON-INFERIORITY TRIAL

V. Papastergiou', M. Fragkaki², I. Dimas ${ }^{2}$, A. Mpitouli², E. Vardas ${ }^{2}$, A. Theodoropoulou ${ }^{2}$, N. Mathou ${ }^{1}$, A. Giannakopoulos ${ }^{1}$, K. Karmiris ${ }^{2}$, D. Apessou ${ }^{3}$, L. Giannikaki ${ }^{4}$, G. Chlouverakis ${ }^{5}$, K. Paraskeva ${ }^{1}$, G. Paspatis ${ }^{2}$ ${ }^{1}$ Gastroenterology, Konstantopoulio Hospital, Athens/Greece

${ }^{2}$ Gastroenterology, Venizeleion General Hospital, Heraklion/Greece ${ }^{3}$ Histopathology, Konstantopoulio Hospital, Athens/Greece

4. Histopathology, Venizeleion General Hospital, Heraklion/Greece

${ }^{5}$ University of Crete Medical School, Heraklion/Greece

\section{Contact E-mail Address: gpaspatis@gmail.com}

Introduction: Cold snare polypectomy is an established method for the resection of small colorectal polyps (SCPs); however, significant incomplete resection rates still leave room for improvement. We aimed to assess the efficacy of cold snare polypectomy with submucosal lift (SL-CSP), compared to endoscopic mucosal resection (EMR), for nonpedunculated polyps 6-10 $\mathrm{mm}$ (ClinicalTrials.gov NCT02678663).

Aims \& Methods: Dual-center, randomized, noninferiority trial. Consecutive adult patients with at least one nonpedunculated polyp $6-10 \mathrm{~mm}$ were enrolled. Eligible polyps were randomized $(1: 1)$ to be treated with either SL-CSP or EMR. The primary noninferiority endpoint was histologic eradication, with a noninferiority margin of $-10 \%$. Evaluation of histologic complete resection relied on a postpolypectomy biopsy protocol (4 biopsies obtained in a 4-quadrant fashion from the polypectomy site margins; 1 biopsy from the base). Secondary outcomes included occurrence of intraprocedural bleeding (IPB; defined as any immediate episode requiring endoscopic haemostasis), clinically-significant postprocedural bleeding (CSPPB; any episode requiring emergency department presentation, hospitalization, or reintervention within 30 days of the procedure) and perforation.

Results: Among 689 patients screened, 155 patients with 164 eligible polyps (SLCSP $; n=83$, EMR $;=81$ ) were included. The overall rate of histologic complete resection was $92.8 \%(77 / 83)$ in the SL-CSP group and $96.3 \%(78 / 81)$ in the EMR group (difference $3.5 \% ; 95 \% \mathrm{CI},-4.15$ to 11.56 ), showing noninferiority of SLCSP compared with EMR. The SL-CSP technique was noninferior to EMR both for polyps measuring 6-7 mm (SL-CSP, 93.3\%; EMR, 100\%; 95\% CI, -7.95 to 21.3 ) and those $8-10 \mathrm{~mm}$ (SL-CSP, 92.5\%; EMR, 94.7\%; 95\%CI, -7.91 to 13.16). By multivariate analysis, female gender (OR, $0.15 ; 95 \% \mathrm{CI}, 0.02-1.06$; $\mathrm{P}=0.06)$ and Paris 0 -IIa morphology $(\mathrm{OR}, 0.12 ; 95 \% \mathrm{CI}, 0.01-1.19 ; \mathrm{P}=0.07)$ were marginally significant predictors correlating negatively with complete resection. Rates of IPB were similar between the two groups (SL-CSP; 3.6\%, EMR; $1.2 \%, \mathrm{p}=0.3$ ). No CSPPB or perforation occurred in either group.

Conclusion: SL-CSP appears to be an effective modification of standard cold snare technique, obviating the need to use diathermy for $6-10 \mathrm{~mm}$ colorectal polyps.

Disclosure of Interest: All authors have declared no conflicts of interest.

\section{P0178 COLONOSCOPIC POLYPECTOMY PRACTICE AMONGST SPANISH CLINICAL GASTROENTEROLOGISTS. RESULTS OF A NATIONAL SURVEY FROM THE SPANISH ENDOSCOPY SOCIETY ENDOSCOPIC RESECTION GROUP}

C.J. Gargallo Puyuelo ${ }^{1}$, E. Albéniz Arbizu ${ }^{2}$, M. Fraile González ${ }^{2}$, C. Guarner Argente $^{3}$, M. Pellisé Urquiza ${ }^{4}$, M.A. Alvarez ${ }^{5}$, F. Ramos ${ }^{6}$, M. Rodriguez Tellez ${ }^{7}$, D. Martínez-Ares ${ }^{8}$, J. Santiago ${ }^{9}$, A. Sanchez Yague ${ }^{10}$, F. Gonzalez Huix ${ }^{11}$, J. Merlo ${ }^{12}$

${ }^{1}$ Gastroenterology, Hospital Clínico Lozano Blesa, Zaragoza/Spain

${ }^{2}$ Gastroenterology, Complejo Hospitalario de Navarra, Pamplona/Spain

${ }^{3}$ Servei De Patologia Digestiva, Hospital de la Santa Creu i Sant Pau, Barcelona Spain

${ }^{4}$ Hospital Clinic, Barcelona/Spain

${ }_{6}^{5}$ Hospital del Mar, zaragoza/Spain

${ }^{6}$ Hospital Universitario Madrid Monteprincipe, Madrid/Spain

${ }^{7}$ Hospital Universitario Virgen de la Macarena, Malaga/Spain

${ }^{8}$ Complexo Hospitalario Universitario de Vigo, Vigo/Spain

${ }^{9}$ Hospital Puerta de Hierro, Madrid/Spain

${ }^{10}$ Hospital Costa del Sol, marbella/Spain

${ }^{11}$ Clinica Gerona, Gerona/Spain

${ }^{12}$ Centro Médico Quirúrgico Servidigest SL, Barcelona/Spain

\section{Contact E-mail Address: carlajerusalen@hotmail.com}

Introduction: Colonoscopic polipectomy effectively reduce the incidence of colorectal cancer. Variations in the technique have been implicated in the effectiveness of the resection and in the complication rates. However, there is no consensus regarding the optimal polypectomy technique for diminutive ( $\leq 5 \mathrm{~mm}$ ) and small (6 to $9 \mathrm{~mm})$ polyps. There are scarce of data about polypectomy practices among European endoscopists

Aims \& Methods: To determine the different techniques used by Spanish endoscopists for resection of sessile or flat colorectal polyps smaller than $20 \mathrm{~mm}$.

A 70-item survey was sent by email to all Gastroenterology Departments in all Spanish hospitals (1678 gastroenterologists). The survey was conducted from December 2015 to February 2016.

Results: The rate of participation was $20.3 \%$ (341/1678). All physicians indicated they were practicing gastroenterologist (none were trainees). Most respondents $(60 \%)$ were males and $50 \%$ had more than 14 years in practice. The mean number of colonoscopies per week was $21 \pm 12.3$. Half of participants performed endoscopies for $\geq 3$ days per week and $49.6 \%$ did not performed advanced therapeutic endoscopy. The techniques used for the excision of polyps smaller than $20 \mathrm{~mm}$ by Spanish clinicians are summarized in Table 1. Significant differences were noted in the polypectomy techniques used for the resection of polyps 1-3 $\mathrm{mm}, 6-9 \mathrm{~mm}$ and $10-19 \mathrm{~mm}$ in diameter; being cold forceps, hot snare and endoscopic mucosal resection (EMR), the preferred techniques respectively for the different sizes. However, for polyps $4-5 \mathrm{~mm}$ in size, both the cold snare and cold forceps were the most commonly used techniques, though no method was use more often than the other. Years in practice, colonoscopy volume per week and performing advanced therapeutic endoscopy were associated with different choices of polypectomy technique. For polyps measuring 4-5 mm, cold snare was the favourite method among endoscopists who performed $\geq 20$ colonoscopies per week $(\geq 20$ colonoscopies/week vs. $<20$ colonoscopies/week, $42.4 \%$ vs. $26.6 \%$, $\mathrm{p}<0.05$ ), among those who performed endoscopies $\geq 3$ days/week $(\geq 3$ days week vs. $<3$ days $/$ week, $40 \%$ vs. $25.7 \%, \mathrm{p}<0.05$ ) and among those who performed advanced endoscopy (advanced endoscopy vs no advanced endoscopy, $40.4 \%$ vs. $29.2 \%, p<0.05$ ). However, cold forceps was the preferred technique among endoscopists who performed endoscopies $<3$ days per week $(38.9 \%$ vs. $29.2 \%, \mathrm{p}<0.05)$ and among those who did not perform advanced endoscopy $(37.3 \%$ vs. $28.1 \%, \mathrm{p}<0.05)$. For small polyps $(6-9 \mathrm{~mm})$, cold snare polypectomy was most frequent among endoscopists who performed more colonoscopies $(\geq 20$ colonoscopies/week vs. $<20$ colonoscopies/week, $28 \%$ vs. $13 \%, \mathrm{p}<0.05$ ) and among those with less than 10 years in practice $(<10$ years vs. $\geq 10$ years, $27.2 \%$ vs. $17.7 \%, p<0.05$ ). However, hot snare polypectomy was most frequent among endoscopists who performed less than 20 colonoscopies/week $(<20$ colonoscopies/week vs. $\geq 20$ colonoscopies/week, $56.7 \%$ vs. $44.7 \%, \mathrm{p}<0.05)$ and among those with more than 10 years in practice $(\geq 10$ years vs. $<10$ years, $53.8 \%$ vs. $44.7 \%, \mathrm{p}<0.05)$.

Table 1: The techniques used for the excision of colorectal polyps smaller than $20 \mathrm{~mm}$

\begin{tabular}{lllll}
\hline Resection Method & $1-3 \mathrm{~mm}$ & $4-5 \mathrm{~mm}$ & $6-9 \mathrm{~mm}$ & $10-19 \mathrm{~mm}$ \\
\hline Standard cold biopsy forceps & $70.5 \%$ & $32.7 \%$ & $3.3 \%$ & $0.3 \%$ \\
Jumbo biopsy forceps & $7.3 \%$ & $6.8 \%$ & $1.9 \%$ & $0 \%$ \\
Hot biopsy forceps & $1 \%$ & $1.9 \%$ & $2.5 \%$ & $2 \%$ \\
Cold snare & $16.2 \%$ & $34.8 \%$ & $21.2 \%$ & $1.1 \%$ \\
Hot snare & $4.1 \%$ & $19.6 \%$ & $50.4 \%$ & $38.9 \%$ \\
Endoscopic mucosal resection & $0.6 \%$ & $3.9 \%$ & $19.7 \%$ & $57 \%$ \\
\hline
\end{tabular}

Conclusion: There is a remarkable heterogeneity in the techniques used for removal of polyps $<20 \mathrm{~mm}$ among Spanish endoscopists. Cold forceps, hot snare and EMR are the preferred methods for removing diminutive polyps, small polyps and polyps measuring 10 to $19 \mathrm{~mm}$, respectively. The use of cold snare for removing small and diminutive polyps is most frequent among gastroenterologists with a greater dedication to endoscopy (colonoscopy volume per week and performing advanced endoscopy) and among endoscopists with less than 10 years in practice.

Disclosure of Interest: All authors have declared no conflicts of interest.

\section{P0179 ASSESSMENT OF TRAINING NEEDS AND DEVELOPMENT OF A SIMULATION BASED TRAINING PROGRAMME FOR SEMI- AUTOMATED ROBOTIC COLONOSCOPY}

M. Kopczynska ${ }^{1}$, R. Hopps ${ }^{1}$, S. Smits ${ }^{1}$, N. Warren ${ }^{2}$, S. Goddard ${ }^{3}$, X. Ye ${ }^{4}$, S. Dolwani ${ }^{5}$

${ }^{1}$ Cardiff University School of Medicine, Cardiff/United Kingdom

${ }^{2}$ Welsh Institute For Minimal Access Therapy, Cardiff University School of Medicine, Cardiff/United Kingdom

${ }^{3}$ School Of Postgraduate Medical, Cardiff University, Cardiff/United Kingdom

${ }^{4}$ University of Lincoln, Lincoln/United Kingdom

${ }^{5}$ Population Medicine, Cardiff University, Cardiff/United Kingdom

Contact E-mail Address: dolwanis@cardiff.ac.uk

Introduction: Early diagnosis of colorectal cancer whether through a symptomatic or screening pathway results in better outcomes for patients. Various studies have reported barriers to screening amongst non-responders as well as delays in diagnostic pathways. Current constraints in the NHS in colonoscopy capacity are a compounding factor and all these factors contribute to delays in diagnosis. We hypothesise that changing the diagnostic paradigm through community or primary care based semi-automated robotic colonoscopy is a method of improving access to and engagement with non-responders $(1,2)$.

Aims \& Methods: This project forms part of the development of a training programme with the use of a simulation based training model to understand the specific training needs and methods of fulfilling these for the potential ultimate users of community-based robotic colonoscopy. This study involved participants with varying degree of skills and background knowledge in the field of colonoscopy. We enrolled three expert endoscopists, three trainee endoscopists, two novices and two experienced video gamers. All participants performed colonoscopies on a validated 'colonoscopy suitcase' model developed at Welsh Institute for Minimal Access Therapy (WIMAT) centre. Before testing each participant received both verbal and written instructions on the goals of the study and information about the semi-automated robotic endoscope along with a familiarisation period with the device. Quantitative parameters were recorded related to procedure times and lesion detection during the procedure. Afterwards participants filled out a questionnaire evaluating the robotic 
colonoscope. Some operators participated in a follow up session in order to analyse the learning curve of the robotic endoscopy.

Results: On average, experts required the shortest time to reach the caecum, followed by video gamers, trainees then novices. Polyp detection rate (as a proportion of total number in the model simulator colon) was the highest in the novice group $(91.67 \%)$ followed by the experts $(86.11 \%)$, then equally, trainees and video gamers $(79.17 \%)$. Four out of nine participants attended the second session where they were asked to repeat the procedure from the first session. Each participant had a lower caecal intubation time during session 2 in comparison with session 1, with range of improvement between $30 \%$ and $70 \%$. Each of the participants also had the same or higher polyp detection rate with range of improvement between $0 \%$ and $25 \%$. Qualitative assessment of feedback from all participants indicated that most operators felt that the role of the novel test was likely to be greater as a diagnostic procedure in an out of hospital setting. Expert operators felt that training in the device was easier but also provided less ability to torque steer due to automated sequences.

Conclusion: This project is the first step in identifying specific training needs and potential roles of robotics in early diagnosis of cancer. This study also shows a potential to reduce the length of time for skills acquisition associated with standard colonoscopy training through the use of semi-automated robotic devices. Disclosure of Interest: All authors have declared no conflicts of interest.

\section{References}

1. Alazmani A, Hood A, Jayne D, Neville A, Culmer P. Quantitative assessment of colorectal morphology: Implications for robotic colonoscopy. Medical Engineering and Physics.38(2):148-54.

2. Tapia-Siles SC, Coleman S, Cuschieri A. Current state of micro-robots/ devices as substitutes for screening colonoscopy: assessment based on technology readiness levels. Surgical Endoscopy. 2016;30(2):404-13.

\section{P0180 WHITE OPAQUE SUBSTANCE, A NEW OPTICAL MARKER ON MAGNIFYING ENDOSCOPY: USEFULNESS IN DIAGNOSING COLORECTAL EPITHELIAL NEOPLASMS}

K. Yamasaki ${ }^{1}$, T. Hisabe ${ }^{1}$, K. Yao ${ }^{1}$, H. Ishihara ${ }^{1}$, T. Ueki ${ }^{1}$, T. Matsui ${ }^{1}$, H. Tanabe ${ }^{2}$, A. Iwashita ${ }^{2}$

${ }^{1}$ Gastroenterology, Fukuoka University Chikushi Hospital, chikushino/Japan ${ }^{2}$ Pathology, Fukuoka University Chikushi Hospital, chikushino/Japan

\section{Contact E-mail Address: ky875416@yahoo.co.jp}

Introduction: Yao et al. observed gastric epithelial neoplasms and chronic gastritis mucosa using magnifying endoscopy with narrow-band imaging (M-NBI), and reported a phenomenon in which a white opaque substance (WOS) present in the epithelium did not allow passage of the projected light and obscured the subepithelial microvasculature (1). Furthermore, the morphology of the WOS is a useful marker for differentiating between adenoma and carcinoma in gastric epithelial neoplasms (1). Recently, we reported for the first time that WOS is also detected in colorectal epithelial neoplasms (2). However, it is unclear whether the morphology of the WOS in colorectal epithelial neoplasms is useful in the differential diagnosis of adenoma and carcinoma. We, therefore, conducted a prospective observational study to determine whether it is possible to differentiate between carcinoma and adenoma based on the morphology of the WOS in colorectal epithelial neoplasms (UMIN000021167).

Aims \& Methods: The subjects were consecutive patients with colorectal epithelial neoplasms (adenoma, early colorectal cancer) who underwent endoscopic or surgical resection at Fukuoka University Chikushi Hospital from December 2015 to November 2016. Prior to treatment, the entire lesion was observed using M-NBI, and endoscopic images were taken and recorded in a filing system. After the endoscopy was completed, a determination was made regarding the presence or absence of WOS on the endoscopic images. The morphology of the WOS was determined for cases in which WOS was present and in whom WOS was seen in more than half of the region before the results of histopathological examination of the lesions were known. The morphological characteristics of the WOS were classified as regular WOS or irregular WOS according to our previous report (1). The primary endpoint was the diagnostic performance of morphological analysis of the WOS (accuracy, sensitivity, specificity) for early colorectal cancer taking irregular WOS as an indicator. The secondary endpoint was the difference in the prevalence of irregular WOS between mucosal (M) or SM-s cancer (the depth of submucosal invasion is limited 1000 micrometers) and SM-m (the depth of submucosal invasion is over 1000 micrometers).

Results: Five hundred twenty-nine lesions in 296 patients were included in this study. Of these lesions, 404 were excluded, according to the following conditions: 268 negative for WOS, 72 in which WOS was seen in less than half of the region 34 that were diagnosed histologically as hyperplastic polyp, and 30 that could not be investigated because of rich mucus. The analysis was conducted using 125 lesions from 96 patients. Of these 125 lesions, 33 were determined to have irregular WOS and 92 to have regular WOS. Of the 33 lesions showed irregular WOS, $30(90.9 \%)$ were early cancer and $3(9.1 \%)$ were adenoma. Of the 92 lesions demonstated regular WOS, $13(14.1 \%)$ were early cancer and 79 $(85.9 \%)$ were adenoma. The prevalence of irregular WOS in early cancer was significantly higher than that in adenoma $(\mathrm{p}<0.001$, chi-square test). The diagnostic accuracy for the differentiation between early cancer and adenoma using irregular WOS as an indicator of cancer was $87 \%$. The sensitivity was $91 \%$ and specificity was $86 \%$. The frequency of irregular WOS in M or SM-s cancer and SM-m cancer was $67.8 \%(21 / 31)$ and $75 \%(9 / 12)$, respectively. There was no significant difference in the prevalence between irregular WOS in M or SM-s cancer and SM-m cancer ( $\mathrm{p}=0.727$, chi-square test).
Conclusion: These findings suggest that in colorectal epithelial neoplasms in which the microvasculature cannot be visualized because of WOS, the morphology of the WOS may be a useful marker in the differential diagnosis of adenoma and carcinoma using magnifying endoscopy.

Disclosure of Interest: All authors have declared no conflicts of interest.

\section{References}

Yao K, Iwashita A, Tanabe H, et al. White opaque substance within superficial elevated gastric neoplasia as visualized by magnification endoscopy with narrowband imaging: a new optical sign for differentiating between adenoma and carcinoma. Gastrointest Endosc 2008; 68: 574-580.

Hisabe T, Yao K, Imamura K, et al. White opaque substance visualized using magnifying endoscopy with narrow-band imaging in colorectal epithelial neoplasms. Dig Dis Sci 2014; 59: 2544-2549.

\section{P0182 NARROW BAND IMAGING OPTICAL DIAGNOSIS OF SMALL COLORECTAL POLYPS. LEARNING CURVE AND SUBSEQUENT DIAGNOSTIC ACCURACY IN UNEXPERIENCED EVALUATORS}

F.J. Garcia-Alonso ${ }^{1}$, I. Manzano Santamaría ${ }^{2}$, A. Guardiola Arévalo ${ }^{2}$, A. Bermejo ${ }^{2}$, M. Molinero García ${ }^{2}$, S. Sevo Spahiu ${ }^{2}$, L. García Martín ${ }^{2}$ I. Gómez-Pastrana ${ }^{2}$, I. Carrascosa Del Álamo ${ }^{2}$, G. Martínez Izquierdo ${ }^{2}$, M.J. Motta Molina ${ }^{2}$, A. Gutiérrez Martín ${ }^{2}$, M. Alguacil Cuéllar ${ }^{2}$, A. Zubillaga De Diego ${ }^{2}$, F.J. Parra Villanueva ${ }^{2}$, P. Plaza Roig ${ }^{2}$, M.J. Reyes Larriba ${ }^{2}$, A. Posada Restrepo ${ }^{2}$, F. Bermejo ${ }^{3}$

${ }^{1}$ Hospital Universitario Río Hortega, Valladolid/Spain

${ }^{2}$ Hospital Universitario de Fuenlabrada, Fuenlabrada/Spain

${ }^{3}$ Aparato Digestivo, Hospital Universitario de Fuenlabrada, Fuenlabrada/Spain

Contact E-mail Address: fj.garcia.alonso@gmail.com

Introduction: To reduce costs of colorectal cancer screening, a resect and discard strategy has been proposed for small polyps. The American Society of Gastrointestinal Endoscopy (ASGE) recommends achieving a diagnostic accuracy $\geq 90 \%$, before implementing it (1). Narrow band imaging (NBI) is increasingly available and the NICE classification is frequently used to reach real time histologic classification. Endoscopists usually rely on short sessions, online courses or self-education for training in NBI, so we evaluated the learning curve in subjects with no previous endoscopic experience and their subsequent performance in patients with polyps $\leq 7 \mathrm{~mm}$.

Aims \& Methods: Participants ( $4^{\text {th }}$ or $5^{\text {th }}$ year medical students) attended one training session regarding NBI and NICE classification. Learning curves were posteriorly evaluated employing LC-CUSUM curves, with each participant individually assessing 100 lesions. Polyps were ordered by a computer-generated random sequence and divided into 6 sets of images (presenting 2 sets every week) consisting of 16-17 polyps. After evaluating each polyp, they received the correct diagnosis and a short explanation. The main outcome was a correct NICE diagnosis. A $90 \%$ accuracy defined an adequate performance and $80 \%$ was considered inadequate. A virtual cohort of 15000 evaluators was used to estimate a cut-off of 3, 45 for the LC-CUSUM curve. To assess the diagnostic performance, consecutive patients with lesions $\leq 7 \mathrm{~mm}$ were included. Patients with polyps lacking histological assessment, polyps $>7 \mathrm{~mm}$ or incomplete polypectomies were excluded. All participants received the same white light and NBI still images from 10 patients every week, including all polyps excised. Colonoscopies were presented in the same order as they were included in the study. Histological results were available with a 2-week delay. Evaluators predicted each polyp's histology, giving their level of confidence, and recommended a surveillance interval for each patient. Diagnostic accuracy was evaluated at polyp level and for follow-up intervals.

Results: Thirty-eight evaluators (median age: 22.1 years, $71.1 \%$ women) completed the study. Of the 100 lesions $(8.5 \mathrm{~mm}$, IQR: 5-17.5) included in the learning curve evaluation 19 were NICE 1.71 were NICE 2 and 10 NICE 3. Median diagnostic accuracy was $86 \%$ (IQR: $84-91 \%$, range: $76-96 \%$ ), increasing from $81.8 \%$ (IQR: $78.8-87.9 \%$ ) in the first 33 lesions to $87.9 \%$ (IQR: $84.8-93.9 \%$ ) in the last 33 . According to the LC-CUSUM curve, 20 evaluators $(52.6 \%)$ reached diagnostic competence. During the diagnostic performance assessment, a total of 180 patients were included, presenting 307 polyps (range: $1-8$ polyps/patient), with a median size of $3 \mathrm{~mm}$ (IQR: $2-5$ ); $71.3 \%$ were adenomas, $21.8 \%$ hyperplastic $2.6 \%$ serrated adenomas and $4.2 \%$ presented other diagnoses. Overall diagnostic accuracy was $76.9 \%(76.2-77.7 \%)$, reaching $81.3 \%(80.5-82.1 \%)$ in high-confidence diagnoses. Sensitivity for adenomas was $83 \%(82.2-83.8 \%)$, while for hyperplastic polyps it was $62.6 \%(60.8-64.5 \%)$. Surveillance intervals were correct in $78 \%(77-79 \%)$ of patients, $81.3 \%(80.2-82.4 \%)$ in those presenting highly confident diagnoses. Only $2(5.3 \%)$ evaluators reached the $90 \%$ recommended threshold. Larger polyp size was associated with higher accuracy $(\mathrm{p}<0.001)$, but neither competence according to the LC-CUSUM test $(\mathrm{p}=0.99)$ nor frequent review of the histological results $(\mathrm{p}=0.74)$ improved the evaluators' accuracy

Conclusion: Self-formation after a single training session did not allow most evaluators to reach the ASGE suggested thresholds, mainly due to the low sensitivity for hyperplastic polyps. Further studies are required to address if specific training for small polyps or different formation methods might make NBI assisted optical diagnosis a plausible option for small polyps outside expert centers.

Disclosure of Interest: All authors have declared no conflicts of interest. 


\section{Reference}

1. ASGE Technology Committee., Abu Dayyeh BK, Thosani N, Konda V et al. ASGE Technology Committee systematic review and meta-analysis assessing the ASGE PIVI thresholds for adopting real-time endoscopic assessment of the histology of diminutive colorectal polyps. Gastrointest Endosc. 2015 Mar;81(3):502.e1-502.e16.

\section{P0183 HIGH CLEANSING EFFICACY OF NER1006 ALSO IN THE ELDERLY: POST HOC SUBGROUP ANALYSIS OF RANDOMISED PHASE 3 TRIALS}

R. Jover ${ }^{1}$, R. Ng Kwet Shing ${ }^{2}$, P. Uebel ${ }^{3}$

${ }^{1}$ Hospital General Universitario de Alicante, Alicante/Spain

${ }^{2}$ Clinical Development, Norgine Ltd, Harefield/United Kingdom

${ }^{3}$ Internistische Gemeinschaftspraxis, Ludwigshafen/Germany

Contact E-mail Address: rodrigojover@gmail.com

Introduction: Effective colonoscopy requires effective bowel cleansing. Inadequate cleansing may decrease diagnostic sensitivity, necessitate repeat procedures and delay appropriate treatment. Successful colon cleansing is harder to achieve in patients aged over 65 than in younger patients. NER1006 is the first $1 \mathrm{~L}$ polyethylene glycol (PEG) -based bowel preparation, a patented combination optimised for effective bowel cleansing. Here, the efficacy of NER1006 at cleansing the colon in preparation for colonoscopy is compared to three active comparators, with attention to their efficacies in patients aged $\leq 65$ years and $>65$ years who had a readable colonoscopy.

Aims \& Methods: Colon cleansing efficacy of NER1006 was compared to three currently used bowel preparations in patients aged $\leq 65$ years and in patients aged $>65$ years. NER 1006 was compared to sodium picosulfate + magnesium citrate $(\mathrm{NaPic}+\mathrm{MgCit})$, trisulfate and $2 \mathrm{~L}$ PEG with ascorbate $(2 \mathrm{~L} \mathrm{PEG}+\mathrm{Asc})$, in three multicentre randomised Phase 3 clinical trials: DAYB ${ }^{1}, \mathrm{NOCT}^{2}$ and $\mathrm{MORA}^{3}$, respectively. 2L PEG + Asc was administered over 2 days and in the MORA trial, the doses of NER 1006 were administered either both in 1 day morningonly (N1D) or, as with $2 \mathrm{~L} \mathrm{PEG}+\mathrm{Asc}$, split over 2 days (N2D). In the DAYB study, NER1006 was administered evening-only the day before colonoscopy (NDB). Treatment-blinded central readers rated colon cleansing according to the Harefield Cleansing Scale. Following segmental scoring, overall colon cleansing was graded from A to D. Grades A and B were judged as successful cleansing; grades $\mathrm{C}$ and $\mathrm{D}$ were judged as failed cleansing.

Results: Pooling the data from the three trials to assess colon cleansing in the two age groups showed successful cleansing in $80.5 \%(1158 / 1438)$ of patients aged $\leq 65$ and in $79.6 \%(277 / 348)$ of patients aged $>65$ (difference of $0.9 \% ; \mathrm{P}=0.698$; $95 \%$ CI: $-3.7-5.6 \%)$. Within each trial the difference in colon cleansing in the age groups indicated that the effect of increased age on cleansing efficacy was lesser in the NER1006-treated patients than in patients treated with the active comparators (Table 1). For example, in patients treated with NER1006 the rate of successful colon cleansing in patients aged $>65$ was $5.2 \%$ higher than in patients aged $\leq 65$, whereas in patients treated with $\mathrm{NaPic}+\mathrm{MgCit}$, there was $3.5 \%$ lower successful cleansing rate in patients aged $>65$ than in patients aged $\leq 65$.

Conclusion: NER1006 was efficacious in successful colon cleansing in patients aged $>65$ (in whom successful colon cleansing is harder to achieve) as well as in patients aged $\leq 65$. Statistical significance was not reached in these comparisons. Disclosure of Interest: R. Jover: Recieved grants support from MSD; Advisory board participation for Norgine

R. Ng Kwet Shing: Employee of Norgine

All other authors have declared no conflicts of interest.

\section{References}

1. Schreiber S et al. United European Gastroenterology J 2016; 4(5S): A157A720. P1266

2. DeMicco M et al. United European Gastroenterol J 2016; 4(5S): A1-156. OP375

3. Bisschops R et al. Gastroenterology 2016; 150(4): S1269-70. Abstract Tu2084
P0184 ACHIEVING ADEQUATE LEVEL BOWEL PREPARATION

WITH EVENING/MORNING OR MORNING-ONLY SPLIT-DOSING REGIMENS OF NER1006 VERSUS STANDARD 2L PEG WITH ASCORBATE: POST HOC ANALYSIS OF A PHASE 3 TRIAL

R. Bisschops ${ }^{1}$, L. Clayton ${ }^{2}$

${ }^{1}$ Gastroenterology, Katholieke Universiteit Leuven, Leuven/Belgium

${ }^{2}$ Clinical Development, Norgine Ltd., Harefield, Uxbridge/United Kingdom

Contact E-mail Address: raf.bisschops@uzleuven.be

Introduction: Effective colonoscopy requires effective bowel preparation. For detection of polyps larger than $5 \mathrm{~mm}$, an 'adequate' segmental cleansing level has recently been defined as 2 or more on the Boston Bowel Preparation Scale (BBPS). ${ }^{1}$ The Phase 3 trial MORA compared NER1006 as an evening/morning split-dosing or a morning-only dosing regimen, against 2 L PEG with ascorbate as an evening/morning split-dosing regimen ( $2 \mathrm{~L}$ PEG + Asc). Treatment-blinded central readers assessed the bowel cleansing efficacy using both the Harefield Cleansing Scale (HCS) and the BBPS. This post hoc analysis shows the BBPS scores for the two primary endpoints, in those patients who had a readable colonoscopy.

Aims \& Methods: In the MORA trial, ${ }^{2} 849$ patients aged $18-85$ were randomised to bowel preparation with morning-only or evening/morning split-dosing using either NER 1006 or 2L PEG + Asc. Adequate level cleansing success was assessed according to the BBPS for both overall colon (all segments $\geq 2$ ) and right colon cleansing (segmental score $\geq 2$ ). The analysis includes all subjects for whom colonoscopy videos were available for assessment by central readers.

Results: A total of 792 patients were analysed. When using an evening/morning split-dosing, 249/262 (95\%) patients on NER1006 achieved adequate level overall colon cleansing compared to $232 / 260(89 \%)$ on $2 \mathrm{~L}$ PEG + Asc (Table 1). Using morning-only dosing, 243/270 $(90 \%)$ patients on NER1006 achieved the same. Using evening/morning split-dosing, 254/262 (97\%) patients on NER1006 achieved adequate level right colon cleansing compared to $242 / 260(93 \%)$ on 2L PEG + Asc. Using morning-only dosing, 253/270 (94\%) patients on NER1006 achieved adequate level right colon cleansing. Adequate level cleansing success was achieved significantly more often with NER1006 evening/morning split-dosing than $2 \mathrm{~L}$ PEG + Asc, both in the overall colon $(\mathrm{P}=0.013)$ and in the right colon $(\mathrm{P}=0.042)$. The slight improvement seen with NER1006 morningonly dosing in the cleansing rate of the overall colon and right sided colon was not statistically significant. Table 1: Adequate level cleansing of the overall colon and right colon (BBPS segmental scores 2-3) as determined by treatment-blinded central readers

Conclusion: NER1006 demonstrated an exceptionally high adequate level bowel cleansing efficacy. A significant improvement was shown for both the overall colon and the clinically relevant right sided colon versus $2 \mathrm{~L}$ PEG + Asc when administered using the same dosing regimen. The morning-only dosing regimen of NER 1006 delivered a very high success rate, similar to that of 2L PEG + Asc. Disclosure of Interest: R. Bisschops: Norgine, self: salary, speaking and teaching; funded attendance by Norgine for Investigator's Meeting trip for the MORA trial

L. Clayton: Employee of Norgine

\section{References}

1. Clark BT et al. Gastroenterology 2016; 150(2): 396-405

2. Bisschops R et al. Gastroenterology 2016; 150(4): S1269-70. Abstract Tu2084

\section{P0185 ASSESSMENT OF COLONOSCOPY QUALITY IN CLINICAL \\ PRACTICE COMPARED WITH EUROPEAN SOCIETY OF} GASTROINTESTINAL ENDOSCOPY PERFORMANCE INDICATORS

E. Toth ${ }^{1}$, R. Jover ${ }^{2}$, C. Spada ${ }^{3}$, A. Agrawal ${ }^{4}$, P. Amaro 5 , L. Brink ${ }^{6}$, W. Fischbach ${ }^{7}$, M. Hünger ${ }^{8}$, A. Ono ${ }^{9}$, L. Petruzziello ${ }^{10}$, A. Naidoo ${ }^{11}$, J.F. Riemann ${ }^{12}$

${ }^{1}$ Skåne University Hospital, Malmo/Sweden

${ }^{2}$ Hospital General Universitario de Alicante, Alicante/Spain

${ }^{3}$ Digestive Endoscopy Unit, Catholic University Rome, Rome/Italy

${ }^{4}$ Doncaster Royal Infirmary, Doncaster/United Kingdom

${ }^{5}$ Gastroenterology, Coimbra University Hospital, Coimbra/Portugal

${ }^{6} \mathrm{R} 1$ Gastrounit, Herlev Hospital Gastro/Surgical, Herlev/Denmark

${ }^{7}$ Medizinische Klinik Ii, Klinikum Aschaffenburg II. Med, Aschaffenburg/Germany

${ }^{8}$ Aschaffenburg Hospital, Aschaffenburg/Germany

\begin{tabular}{|c|c|c|c|c|c|c|c|}
\hline & \multicolumn{7}{|c|}{ Patients with successful cleansing, n (\%) } \\
\hline & \multicolumn{2}{|l|}{ DAYB $(1: 1)$} & \multicolumn{2}{|l|}{ NOCT $(1: 1)$} & \multicolumn{3}{|l|}{ MORA (1:1:1) } \\
\hline & NER1006 (NDB) & $\mathrm{NaPic}+\mathrm{MgCit}$ & NER1006 & Trisulfate & NER1006 (N1D) & NER1006 (N2D) & $2 \mathrm{~L} \mathrm{PEG}+\mathrm{Asc}$ \\
\hline Aged $\leq 65 \quad \mathrm{~N}(\%)$ & $127 / 196(64.8)$ & $115 / 205(56.1)$ & $192 / 208(92.3)$ & $197 / 213(92.5)$ & $191 / 210(90.9)$ & $184 / 192(95.8)$ & $198 / 214(92.5)$ \\
\hline Aged $>65 \quad \mathrm{~N}(\%)$ & $28 / 40(70.0)$ & $20 / 38(52.6)$ & $43 / 47(91.4)$ & $41 / 47(87.2)$ & $54 / 60(90.0)$ & $69 / 70(98.6)$ & $40 / 46(86.9)$ \\
\hline Difference $(\%)$ & -5.2 & 3.5 & 0.9 & 5.3 & 0.9 & -2.8 & 5.6 \\
\hline $\mathrm{P}$-value & 0.522 & 0.699 & 0.856 & 0.320 & 0.828 & 0.179 & 0.301 \\
\hline $95 \%$ CI $(\%)$ & $-21.4-11$ & $-14.4-21.3$ & $-8.1-9.8$ & $-5.2-15.7$ & $-7.7-9.6$ & $-6.7-1.2$ & $-5.1-16.2$ \\
\hline
\end{tabular}




\begin{tabular}{|c|c|c|c|}
\hline & $\begin{array}{l}\text { NER1006 } \\
\text { evening/morning split dosing }\end{array}$ & $\begin{array}{l}\text { NER } 1006 \\
\text { morning only dosing }\end{array}$ & $\begin{array}{l}\text { 2L PEG }+ \\
\text { Ascevening/morning split dosing }\end{array}$ \\
\hline Patients $(\mathrm{N})$ & 262 & 270 & 260 \\
\hline $\begin{array}{l}\text { Patients with an adequate level } \\
\text { cleansing success of the overall colon, } \mathrm{n}(\%)\end{array}$ & $249(95)$ & $243(90)$ & $232(89)$ \\
\hline $\begin{array}{l}\text { Patients with an adequate level cleansing } \\
\text { success of the right colon, } \mathrm{n}(\%)\end{array}$ & $254(97)$ & $253(94)$ & $242(93)$ \\
\hline P vs. $2 \mathrm{~L}$ PEG + Asc (overall colon) & 0.013 & 0.772 & - \\
\hline P vs. $2 \mathrm{~L}$ PEG + Asc (right colon) & 0.042 & 0.772 & - \\
\hline
\end{tabular}

${ }^{9}$ Gastroenterology, Virgen de la Arrixaca, Murcia/Spain

${ }^{10}$ Endoscopia Digestiva Chirurgica, Policlinico A. Gemelli, Rome/Italy

${ }^{11}$ Medical Affairs, Norgine Ltd, Harefield/United Kingdom

${ }^{12}$ Stiftung LebensBlicke, Ludwigshafen/Germany

Contact E-mail Address: ervin.toth@med.lu.se

Introduction: The European Colonoscopy Quality Investigation (ECQI) Group comprises expert colonoscopists and investigators from Europe and aims to raise awareness of the need for improvement in colonoscopy standards across Europe. Recently, the European Society of Gastrointestinal Endoscopy (ESGE) has developed key performance measures for lower gastrointestinal colonoscopy. ${ }^{1}$

Aims \& Methods: To assess the quality of colonoscopy in current clinical practice, through the use of online questionnaires, compared with recently published ESGE performance indicators. The development of the online practitioner and procedure questionnaires has been previously described. ${ }^{2,3}$ Data collection is an ongoing process. We analysed data collected between $2 / 6 / 16$ and $17 / 4 / 17$ and compared with the ESGE performance measures.

Results: 40 of 50 practitioners completed the practitioner questionnaire. 2094 colonoscopies were documented across 8 European countries by 47 practitioners. The ESGE sets a minimum standard of $\geq 90 \%$ of patients with adequate bowel preparation, defined as Boston Bowel Preparation Scale score $\geq 6$. From our data, $81 \%(n=1692)$ of procedures had adequate bowel cleansing (data unavailable for $96.4 .6 \%$ ). The ESGE recommends a minimum standard of $\geq 90 \%$ of all diagnostic and screening colonoscopies visualize the whole caecum (excluding those that have no indication to reach the caecum). Only $55 \%$ of practitioners routinely record caecal intubation rate. The caecum was the intended endpoint in $66 \%$ of procedures. For those diagnostic and screening colonoscopies where the caecum was the intended endpoint $(n=1390), 93 \%$ reached the endpoint but only $70 \%$ had this endpoint photo-documented. The ESGE recommends that adenoma detection rate (ADR) should be used as a measure of adequate inspection at screening or diagnostic colonoscopy in patients aged 50 years or more. ADR was routinely recorded by only $18 \%$ of practitioners. Polyp removal rate is routinely recorded by $30 \%$ of practitioners, and polyp retrieval rate by $23 \%$. Conclusion: Our findings indicate that some important performance measures recommended by ESGE are not currently being achieved in practice. By providing a self-assessment tool and as a next step, by individual consultations with national Group members, ECQI hopes to improve clinical practice standards. Further information on the ECQI Group initiative can be found on www.ecqigroup.eu

Disclosure of Interest:

E. Toth: Consultancy and Advisory Board participant for Norgine

R. Jover: Consultancy and Advisory Board participant for Norgine

C. Spada: Consultant fee by Norgine

A. Agrawal: Consultancy and Advisory Board participant for Norgine

P. Amaro: Consultancy and Advisory Board participant for Norgine

L. Brink: Consultancy and Advisory Board participant for Norgine

W. Fischbach: Consultancy and Advisory Board participant for Norgine

M. Hünger: Consultancy and Advisory Board participant for Norgine

A. Ono: Consultancy and Advisory Board participant for Norgine

L. Petruzziello: Consultancy and Advisory Board participant for Norgine

A. Naidoo: Employee of Norgine

J.F. Riemann: In terms of ECQI, consultant to Norgine, otherwise no conflict of interest

\section{References}

1. Kaminski MF et al. Endoscopy 2017;49(4):378-397

2. Jover R et al. Poster P0165: UEGW 2016 Oct 15-19: Vienna 3. Riemann JF et al. Poster P0160: UEGW 2015 Oct 24-28: Barcelona
P0186 SEDATION IN GASTROINTESTINAL ENDOSCOPY: CURRENT PRACTICES OF GREEK GASTROENTEROLOGISTS

A. Protopapas, E. Stournaras, G. Neokosmidis, A. Protopapas 1st Department Of Propaedeutic Internal Medicine, Ahepa Hospital, Thessaloniki/ Greece

Contact E-mail Address: adoprot@hotmail.com

Introduction: When it comes to gastrointestinal endoscopy, considerable heterogeneity is observed between gastroenterologists regarding the use of sedation and the preferred sedative agents. The sedation protocol used by a gastroenterologist may have a significant effect on endoscopic quality, patient cooperation and both the doctor's and the patient's satisfaction with the procedure.

Aims \& Methods: The aim of this study was to document current endoscopic practices of Greek gastroenterologists and investigate whether they use sedation to perform gastrointestinal endoscopy and which pharmaceutical agents are usually involved. A 39-item online questionnaire was devised, addressing demographic data, use of sedation in endoscopy and monitoring practices. It was subsequently made available to 509 Greek gastroenterologists by e-mail.

Results: A total of 195 questionnaires were successfully completed (38.3\%). 49 gastroenterologists did not use sedation to perform esophagogastroduodenoscopy (EGD) or colonoscopy $(25.1 \%)$. The younger gastroenterologists were more likely to use sedation $(\mathrm{p}=0.005)$. Among those using sedation, midazolam was the most frequently used agent in EGD (50\%) and the combination of midazolam/fentanyl was the most frequently used in colonoscopy $(24.6 \%)$, followed by midazolam $(21.9 \%)$. Out of 137 physicians using benzodiazepines (midazolam, diazepam) as part of their endoscopic sedation regimen, $91(66.4 \%)$ routinely used flumazenil to facilitate pharmacological antagonism after the completion of the endoscopy. In total, 45 physicians, $23.1 \%$ of the participants and $30.8 \%$ of those using sedation, used propofol or a combination of propofol and other agents. 30 gastroenterologists routinely administered propofol without the aid of an anesthesiologist $(66.6 \%)$. Medicolegal issues $(33 \%)$, inadequate training in the use of propofol $(26.4 \%)$ and risk of cardiopulmonary complications $(23.6 \%)$ were cited as the main reasons for not using propofol. As far as monitoring practices go, the majority of gastroenterologists observed heart rate and oxygen saturation ( $96 \%$ and $97 \%$ respectively). Regarding the safety equipment available to the gastroenterologists, $160(82 \%)$ reported having access to regimens of pharmaceutical resuscitation, $145(74 \%)$ to oropharyngeal airway devices or laryngeal airway masks, $92(47 \%)$ to endotracheal intubation equipment and $86(44 \%)$ to a defibrillator. When asked to rate their level of satisfaction with their preferred sedation regimen (or with not using sedation) in a scale of 1 to 10,72 physicians rated their satisfaction level as 9 or $10(36.9 \%)$ and 92 as 7 or $8(47.1 \%)$. While there was no significant difference in terms of satisfaction between the doctors that used sedation and those who did not, there was a statistically significant difference between the gastroenterologists that used propofol (alone or in combination with other agents) and those who used other sedative agents $(\mathrm{p}=0.003)$. When asked on their preferred method of sedation, if they were themselves subjected to gastrointestinal endoscopy, 104 physicians opted for propofol-based sedation regimens $(53.3 \%)$.

Conclusion: Gastrointestinal endoscopy is performed with the use of sedation by the majority of Greek gastroenterologists. Propofol-based regimens are seldom used in everyday clinical practice, despite a vast number of Greek gastroenterologists identifying them as their preferred regimen, in case they themselves should undergo endoscopy. Compared to a past survey, Greek gastroenterologists are still hesitant about using propofol. However, an increasing tendency towards administering propofol without the aid of an anesthesiologist is observed. Also, physicians using propofol seem to be more satisfied with their sedation practices than the doctors using other sedation regimens. Absence of a distinct legal framework, inadequate training and fear of cardiopulmonary complications are identified as the main reasons preventing Greek gastroenterologists from using propofol.

Disclosure of Interest: All authors have declared no conflicts of interest.

\section{Reference}

Paspatis, G.A. et al. Endoscopic sedation in Greece: results from a nationwide survey for the Hellenic Foundation of gastroenterology and nutrition. Digestive and Liver Disease, 2009, 41.11: 807-811. 
P0187 QUALITY MEASURE IN COLONOSCOPY: IMPLEMENTATION OF COLONOSCOPY QUALITY MONITORING IN A BELGIAN UNIVERSITY HOSPITAL

S. Ouazzani ${ }^{1}$, A. Lemmers ${ }^{1}$, F. Martinez ${ }^{1}$, M. Delhaye ${ }^{1}$, M. Arvanitakis ${ }^{1}$, O. Le Moine ${ }^{1}$, P. Demetter ${ }^{2}$, J. Deviere ${ }^{1}$, P. Eisendrath

${ }^{1}$ Department Of Gastroenterology, Hepatopancreatology And Digestive Oncology, Erasme Hospital, Université Libre de Bruxelles, ULB, Brussels/Belgium

${ }^{2}$ Department Of Pathology, Erasme Hospital, Université Libre de Bruxelles, ULB, Brussels/Belgium

Contact E-mail Address: souazzan@ulb.ac.be

Introduction: Indicators for colonoscopy quality assessment were developed and promoted during this last decade. However technical and human resources constraints limit local implementation of continuous recording of endoscopic quality indicators (QI). Automatic system of data extraction and presentation could help endoscopy units in their seek for quality improvement. We hereby report our local experience in implementing colonoscopy QI record trough an automatic data extraction from two separate databases (DB), and assess the colonoscopy quality at unit and individual levels.

Aims \& Methods: We locally adapted a company reporting system for colonoscopy by adding in a dedicated tab, selected procedure indicators. Endoscopic QI data from reporting system DB and pathological results from another DB were extracted and merged together in a separated DB. On a regular period basis or on request, key QI are calculated and extracted. It includes adenoma detection rate (ADR), polyp detection rate, caecal intubation rate, quality of bowel preparation (using the Boston bowel preparation scale) and type of sedation. During a first period of 6 months starting in January 2016, endoscopists were encouraged to fulfill the dedicated tab on a voluntary basis. In a second period, filling of QI was turned to be mandatory. The completeness of QI recording was evaluated across both periods, and results from second period are presented. Performance measures of all endoscopists were compared to global results of our department and to published targets.

Results: During the 6 months "mandatory-filling" period (July-December 2016), 1802 colonoscopies were performed with a QI tab fully filled in $100 \%$ of cases compared to $63.1 \%$ after the "free-filling period" $(\mathrm{p}<0.0001)$. The global caecal intubation rate for screening colonoscopy was $92.9 \%$. Mean Boston bowel preparation score was $7.2 \pm 0.76$ with $86.9 \%$ of cases with adequate preparation (Boston score $>5 ; 89.9 \%$ among outpatients and $81.9 \%$ among inpatients). Colonoscopies were performed under propofol sedation in $94.1 \%$. During this second period, the global ADR was $32.4 \%$ (range: $0 \%-55.7 \%$ ). The polyp detection rate was $44.4 \%$ with a mean of 1.19 polyp removed by colonoscopy. Conclusion: This study illustrates that quality indicators for colonoscopy assessment in a Belgian tertiary hospital endoscopy unit could be easily implemented with limited human resources by adapting a company reporting system and link it to the pathology department database. Mandatory filling of QI items is the key for system implementation success. Our results were consistent with goals required by international guidelines. This system allows giving feedback to individual endoscopists for self-performance assessment and might be easily adapted in the future following guidelines updates.

Disclosure of Interest: All authors have declared no conflicts of interest.

P0188 LARGE RECTAL ADENOMAS - EMR VERSUS ESD: WHAT IS THE BEST APPROACH?

\section{H.H. Nietsch}

Department Of Gastroenterology, St. Elisabeth Krankenhaus Med. Klinik 1, Halle Germany

Contact E-mail Address: nietsch2@yahoo.com

Introduction: Since the advent of screening colonoscopy we diagnose a lot more asymptomatic large rectal polyps $>5 \mathrm{~cm}$ in diameter. With the introduction of EMR (endoscopic mucosal resection) and ESD (endoscopic submucosal dissection) we have now endoscopic means to resect those lesions effectively. It is however still unclear whether EMR or ESD is superior in terms of complete resection and safety

Aims \& Methods: We will compare the outcome (perforation, bleeding, complete resection, recurrence rate) in two cohorts of patients with lateral spreading rectal adenomas $>5 \mathrm{~cm}$ who were either treated by EMR or ESD in our centre.

Results: 62 large laterally spreading rectal adenomas in consecutive patients (age 45-84 years) were completely resected endoscopically. ESD en-bloc resection was performed in 23 patients. All other lesions were excised by piecemeal EMR. Prior to resection a careful examination under white light and NBI was performed using the Paris classification. In comparison ESD took about three times as long as EMR (average procedure time $98 \mathrm{~min}$. vs $37 \mathrm{~min}$ ) with comparable intra- and postoperative bleeding rates (5\% in each group). No perforations were encountered. All en-bloc ESD specimens were R0 on histology. In one patient deep submucosal invasion of the submucosa $(>1000 \mu \mathrm{m})$ was present. The patient underwent surgical resection 6 weeks after the endoscopy without evidence of remaining tumor or lymph node invasion. The most striking difference in the two groups was seen regarding recurrence of adenomas during 6 months follow-up: $0 \%$ in the ESD cohort and $12 \%$ in the piecemeal EMR group. All recurrent adenomas after EMR could however be easily resected by snare polypectomy without any further recurrence over the next 12 months. The calculated average equipment cost for ESD (hook knife, dual knife, injectate, transparent cap) was significantly higher compared to EMR (687\$ vs. $39 \$$ ). Conclusion: EMR and ESD are equally effective in resecting large lateral spreading adenomas of the rectum. The higher rate of post-EMR adenoma recurrence did not impact the patients overall cure rate after one year. ESD is significantly more time consuming and costly.

Disclosure of Interest: All authors have declared no conflicts of interest.

P0189 COMPUTED TOMOGRAPHY COLONOGRAPHY VERSUS COLONOSCOPY FOR THE DIAGNOSIS OF COLORECTAL CANCER: A SYSTEMATIC REVIEW AND META-ANALYSIS

R. B. Duarte, W. M. Bernardo, C. M. Sakai, G. L.R. Silva, H. G. Guedes, R. Kuga, E. Ide, R. K. Ishida, P. Sakai, E. G.H. De Moura Gastrointestinal Endoscopy Unit, Clinical Hospital of São Paulo University Medical School, Sao Paulo/Brazil

\section{Contact E-mail Address: ralphbduarte $@$ hotmail.com}

Introduction: Colorectal cancer (CRC) is a significant cause of morbidity and mortality; it is third in terms of incidence and cause of death among both men and women in the United States. In Brazil, CRC incidence has reached 19-21 cases per 100,000 individuals. CRC has several precursory lesions, and patient survival depends largely on disease stage at diagnosis. Thus, CRC screening is vital for decreasing CRC incidence and disease-specific mortality. Optical colonoscopy $(\mathrm{OC})$ is the first-choice investigation for colonic conditions and it is excellent for CRC screening and surveillance. Newer technologies such as computed tomography colonography (CTC) may also be useful in CRC screening. Aims \& Methods: This systematic review compares the benefits of CTC and OC for $\mathrm{CRC}$ screening. This systematic review includes all available randomized clinical trials available comparing CTC and OC for CRC screening in asymptomatic patients. We assessed study quality using the revised version of the Quality Assessment of Diagnostic Accuracy Studies. In this meta-analysis, we compared, in the form of Forest Plots, patient participation rate and the detection rates for advanced colorectal neoplasia (ACN) between the two methods. The positive predictive value (PPV) of CTC in terms of the outcome "detection of ACN" was also calculated, comparisons were made after considering patients in all included studies.

Results: A total of 386 articles were identified in the initial search. Of these, three were included in the systematic review and submitted for meta-analysis. A total of 16,592 patients were invited to undergo screening programs, but only 3881 underwent the procedures. In the analysis of participation rates, only 2333 of 8104 patients invited underwent CTC and only 1486 of the 7310 patients invited underwent OC. The absolute risk difference in participation rate in the two procedures was $0.1(95 \% \mathrm{CI}, 0.05-0.14)$ in favor of CTC. In the analysis of ACN detection rates, 2357 patients undergoing CTC and 1524 patients undergoing OC were included. Of these, 135 patients who underwent a CTC and 130 patients who underwent an $\mathrm{OC}$ were diagnosed with ACN. The absolute risk difference in ACN detection rate in the two procedure types was -0.02 (with a $95 \%$ CI between -0.04 and -0.00 ) in favor of OC.

Conclusion: CTC is an option for the exercise of CRC screening in asymptomatic patients. However, as a CTC has proved inferior in detecting advanced colorectal neoplasia, the method should not replace a OC, wich remains as gold standard. Disclosure of Interest: All authors have declared no conflicts of interest.

\section{References}

1. Lin JS, Piper MA, Perdue LA, et al. Am J Gastroenterol. 2004;99(6):11451151

2. Sali L, Mascalchi M, Falchini M, et al; SAVE study investigators. Am J Gastroenterol. 2004;99(6):1145-1151.

3. Stoop EM, de Haan MC, de Wijkerslooth TR, et al. Am J Gastroenterol. 2004;99(6):1145-1151.

4. Scott RG, Edwards JT, Fritschi L, Foster NM, Mendelson RM, Forbes GM. Am J Gastroenterol. 2004;99(6):1145-1151.

5. Pickhardt PJ, Hassan C, Halligan, Marmo R. Colorectal Câncer: CT Colonography and Colonoscopy for detection-systematic review and metaanalysis. Radiol 2011;259(2):393-405.

6. Haan MC, van Gelder RE, Graser A, Bipat S, Stoker J. Diagnostic value of CT-Colonography as compared to coloscopy in a asymptomatic screening population: a meta-analysis. Eur Radiol 2011;21(8):1747-1763.

\section{P0190 AUTOMATED POLYP DETECTION FOR COLONOSCOPY} USING DEEP LEARNING TECHNOLOGY: PRELIMINARY RESULTS

M. Misawa ${ }^{1}$, S. Kudo ${ }^{1}$, Y. Mori ${ }^{1}$, K. Takeda ${ }^{1}$, K. Ichimasa ${ }^{1}$, Y. Maeda ${ }^{1}$, Y. Ogawa ${ }^{1}$, S. Kataoka' ${ }^{1}$, H. Nakamura', T. Ishigaki ${ }^{1}$, Y. Yagawa ${ }^{1}$, S. Matsudaira ${ }^{1}$, N. Toyoshima ${ }^{1}$, N. Ogata ${ }^{1}$, T. Kudo $^{1}$, T. Hayashi $^{1}$

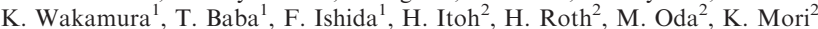
${ }^{1}$ Digestive Disease Center, Showa University Northern Yokohama Hospital, Yokohama/Japan

${ }^{2}$ Graduate School Of Informatics, Nagoya University, Nagoya/Japan

Contact E-mail Address: misawaanny@gmail.com

Introduction: High adenoma detection rates (ADRs) for colonoscopy are associated with a lower risk of colorectal cancer. ADR is therefore treated as a quality indicator of colonoscopy. However, ADR varied among endoscopists and depend on their skill. To facilitate high-quality clinical practice that is not dependent on endoscopists' skill, we have been researching a computer-aided diagnosis system $^{1,2}$. This time, we developed an automated polyp detection system based on deep learning (one of the latest artificial intelligence technologies). 
Aims \& Methods: The aim of this study was to develop a computer-aided detection (CAD) algorithm for colonoscopy using deep learning. To evaluate the developed CAD algorithm, we retrospectively viewed colonoscopy videos from a previous randomized controlled study (UMIN000017083) conducted from April 2015 to October 2015. All examinations were performed using CFHQ290ZI (Olympus Corp., Tokyo, Japan). Each case was recorded with full high-definition quality using HVO-1000MD (Sony Corp., Tokyo, Japan). In this study, two endoscopists (M.M, Y.M.) manually annotated 43 colonoscopy videos with 238 min of $17,903,967$ frames. These videos included 75 polyps (48 neoplasms, 27 non-neoplasms), and annotations were made on the presence or absence of polyps in every frame. Forty-three videos were divided into 300 short video for machine learning and validation process. Among 300 short videos, 246 were used for the machine-learning process. The remaining 54 (33 included a lesion) were used to validate the CAD algorithm. A modified version of Caffe with 3-Dimensional Convolutional Networks (a kind of deep learning) was used for the CAD algorithm. The validation samples were analyzed using the CAD algorithm and its output as the probability of the presence of a lesion in each validation video. A receiver operating characteristic (ROC) analysis was performed to evaluate the efficacy of the CAD algorithm.

Results: The mean probability of a polyp-positive video was $62.1 \pm 27.9 \%$, whereas that of a polyp-negative video was $18.1 \pm 24.6 \%(\mathrm{P}<0.001)$. The area under the ROC curve was 0.887 . If a cutoff probability was set at $20.4 \%$, the present CAD algorithm could detect a polyp with $90.9 \%$ sensitivity and $76.2 \%$ specificity.

Conclusion: Our preliminary results showed that state-of-the-art artificial intelligence has the potential for achieving automatic detection of colorectal polyps. A prospective study is now planned after more machine-learning sessions.

Acknowledgment: This study was supported by JSPS KAKENHI Grant Number JP $17 \mathrm{~K} 15971$

Disclosure of Interest: K. Mori: Kensaku Mori received research founding from Cybernet System Company and Olympus Company.

All other authors have declared no conflicts of interest.

\section{References}

1. Misawa, M., et al., Characterization of Colorectal Lesions Using a Computer-Aided Diagnostic System for Narrow-Band Imaging Endocytoscopy. Gastroenterology, 2016. 150(7): p. 1531-1532 e3.

2. Mori, Y., et al., Impact of an automated system for endocytoscopic diagnosis of small colorectal lesions: an international web-based study. Endoscopy, 2016. 48(12): p. $1110-1118$

\section{P0191 MOTORIZED SPIRAL COLONOSCOPY (MSC) - A FIRST FEASIBILITY TRIAL}

T. Beyna, M. Schneider, D. Pullmann, H. Neuhaus

Department Of Internal Medicine, Evangelisches Krankenhaus Düsseldorf, Düsseldorf/Germany

Contact E-mail Address: torsten.beyna@evk-duesseldorf.de

Introduction: Colonoscopy is widely accepted for the diagnosis and treatment of colonic diseases. Accepted quality parameters for colonoscopy include a cecal intubation rate of $>=90 \% .10 \%$ of all colonoscopies are difficult and intubation of the cecum can sometimes be impossible. The novel motorized endoscope was recently developed (Olympus Medical Systems Corporation, Tokyo, Japan) and represents a disruptive new technology in endoscopy. It is currently being evaluated for anterograde enteroscopy. Spiral technique offers the potential to overcome some of the limitations of standard colonoscopy with push technique by actively pleating the bowel onto the endoscope with motorized rotation of the spiral overtube. This may have advantages in cases of difficult standard colonoscopy to facilitate cecal intubation on the one hand and in all colonoscopies in terms of patients comfort, sedation and ease of use also in therapeutic situations. Aims \& Methods: To evaluate feasibility and safety of MSC for diagnostic colonoscopy. Secondary endpoints were ileum intubation rate, procedure time, need for sedation and external compression, patients' pain and satisfaction, adenoma detection rate (ADR) and feasibility of therapeutic interventions. 30 consecutive patients with indication for colonoscopy meeting the inclusion criteria at a single tertiary referral center were enrolled in the trial between December 2016 and January 2017. The study was conceived as proof of concept trial with the primary aim to achieve a cecal intubation rate of at least $90 \%$ according to quality guideline recommendations

Results: 13 male and 17 female patients were enrolled. Mean age was 68.9 years $(30-90)$, health status was ASA-1: $16.7 \%$, ASA-2: $36.7 \%$ and ASA-3: $46.6 \%$ $43.3 \%$ of the patients had diverticula. Indications for colonoscopy were clarification of indeterminate iron deficiency anemia (IDA, $n=5$ ), lower gastrointestinal bleeding $(\mathrm{GIB}, \mathrm{n}=6)$, surveillance after previous polypectomy $(\mathrm{n}=6)$, screening for polyps and colorectal cancer $(n=11)$ and others $(n=2)$. Sedation level (ESGE-Guideline, Level 1-3) in all patients was 3 (deep sedation). Mean amount of propofol was 305 [130-880] mg. Mean procedure time was 20.8 [11.4-55.3] min. Cecal intubation rate (technical success) was 96.7\% (29/30). One incomplete colonoscopy occurred due to an unexpected postinflammatory stricture of the sigmoid. All colonoscopies reaching the cecum also successfully intubated the ileum $(96.7 \%)$. Only in one case external compression was needed. Adenoma detection rate was $46.7 \%$. EMR was performed in 9 cases, 5 patients had forceps polypectomy. One case of incidental finding of submucosal invasive adenocarcinoma in EMR specimen was histologically proven to have R0 en-bloc resection. All other therapeutic interventions could also successfully be conducted (clip $\mathrm{n}=3$, argon plasma coagulation $\mathrm{n}=1$, tissue sampling $\mathrm{n}=2$ ). Two mild adverse events were recorded (mild superficial mucosal lesions without clinical symptoms). No severe adverse occured.

Conclusion: This study represents the first clinical evaluation of the novel motorized spiral endoscope for examination of the colon. Our data show that it is effective and safe for diagnostic and therapeutic colonoscopy. It may also have potential advantages over standard colonoscopy technique in terms of effectiveness and convenience of colonoscopy.

Disclosure of Interest: H. Neuhaus: Honoraria and consultancy fees from

Olympus Medical Systems Corporation

All other authors have declared no conflicts of interest.

\section{P0192 TREATMENT OUTCOMES OF COLD FORCEPS POLYPECTOMY FOR PATIENTS WITH DIMINUTIVE POLYPS: A PROSPECTIVE FOLLOW-UP STUDY}

H. Hasegawa ${ }^{1}$, S. Bamba ${ }^{1}$, H. Ban ${ }^{1}$, H. Imaeda ${ }^{1}$, A. Nishida ${ }^{1}$, O. Inatomi ${ }^{1}$, M. Sasaki ${ }^{1}$, M. Sugimoto ${ }^{1}$, A. Andoh ${ }^{1}$

${ }^{1}$ Dept. Of Gastroenterology, JCHO Shiga Hospital, Otsu/Japan

\section{Contact E-mail Address: hirohase@belle.shiga-med.ac.jp}

Introduction: The results of the National Polyp Study are premised on the removal of all adenomatous lesions. Cold forceps polypectomy (CFP) using jumbo biopsy forceps is a simple and safe technique used for diminutive polyps $(\leq 5 \mathrm{~mm})$. The recurrence rate after CFP for patients with diminutive polyps has not been elucidated

Aims \& Methods: We have prospectively enrolled patients with diminutive polyps treated by CFP from June 2015 to March 2017. Magnifying colonoscopy was used for all procedures. The location, size, endoscopic findings and procedures were recorded. The patients who have undergone CFP had their follow-up colonoscopy in one year after CFP

Results: CFP was performed for total 515 polyps from 277 patients. The size of the polyps was $<3 \mathrm{~mm} / 4 \mathrm{~mm} / 5 \mathrm{~mm}=379 / 101 / 35$. The rate of one-bite polypectomy for adenoma was $<3 \mathrm{~mm} / 4 \mathrm{~mm} / 5 \mathrm{~mm}=79 \% / 54 \% / 33 \%$. There was no significant difference in the one-bite rate between endoscopists' experience. No cancer was observed in histology. Rates of delayed bleeding after CFP was $0.19 \%(1 / 515)$. Concomitant use of anticoagulation use of antiplatelet drugs was found in $14 \%(72 / 458)$, and none of them experienced delayed bleeding. No perforation occurred. Seventy-five patients had their follow-up colonoscopy so far. There are no polyps suspecting residual or recurrent lesions. Among 75 patients, 62 patients had less than two polyps removed at their first colonoscopy (Group A). On the other hand, 13 patients had more than three polyps removed at their first colonoscopy (Group B). Follow-up colonoscopy revealed that the rates of newly discovered polyps in the same segment were $8 \%$ and $23 \%$ in groups $\mathrm{A}$ and $\mathrm{B}$, respectively. The rates of newly discovered polyps in the different segment were $27 \%$ and $61 \%$ in groups A and B, respectively. When the initial CFP was performed by the endoscopist with the experience of $<5$ years $/ 5-9$ years/more than ten years, the rates of newly discovered polyps found at follow-up colonoscopy was $54 \%(14 / 26) / 42 \%(8 / 18) / 37 \%(11 / 30)$, respectively. Conclusion: The rate of one-bite polypectomy was significantly higher for diminutive polyps especially less than $3 \mathrm{~mm}$. Importantly there are no polyps suspecting residual or recurrent lesions in the follow-up colonoscopy. CFP is a safe and effective option for diminutive polyps $(5 \mathrm{~mm})$. Although the rate of onebite polypectomy was not related to the endoscopists' experience, adenoma detection rate is seemed to be low in young endoscopists. Since achievement of "clean colon" is difficult with a single colonoscopy, multiple colonoscopic examinations are necessary to achieve "clean colon" especially if the patients have more than two polyps at the first examination.

Disclosure of Interest: All authors have declared no conflicts of interest.

\section{References}

1. Michael J. O'brien, Sidney J. Winawer, ANN Graham Zauber, Leonard S. Gottlieb, Stephen S. Sternberg, Barbara Diaz, G. Richard Dickersin, Stephen Ewing, Stephen Geller, Dennis Kasimian, Richard Komorowski, Arnold Szporn, and the National Polyp Study Workgroup. The National Polyp Study: Patient and Polyp Characteristics Associated With High-Grade Dysplasia in Colorectal Adenomas. Gastroenterology 1990:98:371-379.

2. Sidney J. Winawer, Ann G. Zauber, Michael J. O'Brien, May Nah Ho, Leonard Gottlieb, Stephen S. Sternberg, Jerome D. Waye, John Bond, Melvin Schapiro, Edward T. Stewart, Joel Panish, Fred Ackroyd, Robert C. Kurtz, Moshe Shike, and the National Polyp Study Workgroup. Randomized Comparison of Surveillance Intervals after Colonoscopic Removal of Newly Diagnosed Adenomatous Polyps. N Engl J Med 1993; 328:901-906.

\section{P0193 EFFICACY OF CIMETROPIUM BROMIDE ON POLYP DETECTION DURING COLONOSCOPIC WITHDRAWAL: A DOUBLE-BLIND, RANDOMIZED, PLACEBO-CONTROLLED, CLINICAL TRIAL}

D.H. Kang, H.W. Kim, C.W. Choi, S.J. Kim, J.S. Lee, H.S. Nam, S.H. Choi, B.J. Kwon, C.W. Yeo

Pusan National University Yangsan Hospital, Yangsan/Korea, Republic of

Contact E-mail Address: sulsulpul@naver.com

Introduction: Colonoscopy is the most effective method for preventing colorectal cancer, as it offers easy detection and resection of polyps. Cimetropium bromide 
results in colonic spasmolysis and may improve polyp detection. We studied the effect of cimetropium bromide on polyp detection during colonoscopic withdrawal.

Aims \& Methods: Patients undergoing colonoscopy for screening examinations were included and randomized at cecal intubation to receive either $5 \mathrm{mg}$ cimetropium bromide or placebo. We evaluated the polyp detection rate (PDR), adenoma detection rate (ADR), and advanced ADR (AADR) in the right side colon as well as in the colorectum.

Results: A total of 181 patients were analyzed in this study. Cimetropium group consisted of 91 patients, and control group consisted of 90 patients. PDR, ADR, and AADR were not significantly different in cimetropium and control groups $(62.6 \%$ vs. $66.6 \%, \mathrm{P}=0.571 ; 51.6 \%$ vs. $47.7 \%, \mathrm{P}=0.603 ; 3.2 \%$ vs. $7.7 \%$, $\mathrm{P}=0.187$; respectively). Similarly, $\mathrm{PDR}$ and $\mathrm{ADR}$ in the right side colon were not significantly different between the groups $(46.1 \%$ vs. $47.7 \%, \mathrm{P}=0.827$; $32.9 \%$ vs. $35.5 \%, \mathrm{P}=0.714$; respectively).

Conclusion: Cimetropium bromide does not improve the PDR or ADR in the right side colon or the colorectum. Thus, administration of cimetropium bromide can be used selectively in screening colonoscopy.

Disclosure of Interest: All authors have declared no conflicts of interest.

\section{P0194 ADHERENCE TO EUROPEAN SOCIETY OF GASTROENTEROLOGY ENDOSCOPY (ESGE) POLYPECTOMY GUIDELINES: AN IRISH EXPERIENCE}

N. O'Morain ${ }^{1}$, V. Parihar ${ }^{1}$, O. O'Dwyer ${ }^{1}$, P. Maheshwari ${ }^{1}$, L. Kumar ${ }^{1}$, S. Fennessy ${ }^{1}$, N. Breslin ${ }^{2}$, B. Ryan ${ }^{2}$, D. Mcnamara ${ }^{2}$

${ }^{1}$ Gastroenterology \& Hepatology, Tallaght Hospital, Dublin/Ireland

${ }^{2}$ Gastroenterology Trinity Academic Gastroenterology Group (TAGG), AMNCH Tallaght, Dublin/Ireland

\section{Contact E-mail Address: neil.moran $@$ amnch.ie}

Introduction: Colorectal cancer (CRC) accounts for up to $11 \%$ of all cancers in women and $14 \%$ of men in Ireland, and is the second most common cancer across sexes. The adenoma-carcinoma sequence of colorectal carcinogenesis lends itself to screening with the aim of complete excision of polyps. It has been estimated that incomplete resections of polyps are involved in $19-31 \%$ of interval cancers. ESGE guidelines state that polyps $5 \mathrm{~mm}$ or greater should be removed by snare resection. Previous studies report inappropriated resection techniques employed in up to $46 \%$ of cases. The aim of this study was to investigate polypectomy techniques and to assess adherence to guidelines in a tertiary referral, University teaching hospital in Dublin, Ireland. We also investigated the differences between subspecialty and consultants versus trainees.

Aims \& Methods: The study included all patients who underwent colonoscopy in Tallaght Hospital (Dublin, Ireland) between January 2012 and December 2015 for any indication. From this, a list of patients with colonic polyps was compiled. Demographics and other information including number and site of polyps, resection and retrieval rates, method of resection and specialty of endoscopist were included. Results: 11,400 colonoscopies were performed during the study period. To date, the records of approximately $7000(61 \%)$ procedures have been reviewed. 2337 $(22.5 \%)$ patients were identified with polyps, with 1027 females $(43.4 \%), 1310$ males $(56.6 \%)$ and a mean age of 60.6 years. The indication for colonoscopy included symptoms $(47.2 \%)$, polyp surveillance $(22.3 \%)$, CRC cancer screening $(9.2 \%)$, family history of CRC $(4.4 \%)$ IBD surveillance $(3.8 \%)$, with no indication noted in $12.95 \%$. The mean number of polyps per patient was $2.2 .47 .7 \%$ of patients had one polyp identified. In $52.5 \%(n=1227)$, polyps were left-sided, and were right-sided in $23.1 \%(\mathrm{n}=540)$. In all $15.72 \%$ of polyps $>5 \mathrm{~mm}$ were resected by forceps and not by snare resection. Non-adherence to guidelines was observed in $17.56 \%$ and $17.3 \%$ of procedures completed by medical and surgical trainees respectively. Medical $(12.3 \%)$ and surgical $(12.1 \%)$ consultants had a higher adherence rate.

Conclusion: European guidelines have yet to be universally implemented. Previous cohort studies report non-adherence rates of up to $46 \%$. Our study shows a non-adherence rate of $12-18 \%$ amongst trainees and Consultants. The low polyp detection rate likely represents the varied case mix. A greater emphasis on ESGE guidelines could improve awareness and enhance compliance which is suboptimal. A follow up study to determine the effect on interval cancers in these groups is planned.

Disclosure of Interest: All authors have declared no conflicts of interest.

\section{References}

1. Monika Ferlitsch, Alan Moss, Cesare Hassan, Pradeep Bhandari, Jean-Marc Dumonceau, Gregorios Paspatis et al. Colorectal polypectomy and endoscopic mucosal resection (EMR): European Society of Gastrointestinal Endoscopy (ESGE)

2. Britto-Arias, M., Waldmann, E., Jeschek, P., Gessl, I., Sallinger, D., Bannert, C., Weber, M., Trauner, M., Weiss, W., Ferlitsch, A. and Ferlitsch, M. (2015) "Forcepsversus snare polypectomies in colorectal cancer screening: are we adhering to the guidelines?", Endoscopy, 47(10), pp. 898-902. doi: 10.1055/s-0034-1392328.

3. Lee, S. P., Sung, I.-K., Kim, J. H., Lee, S.-Y., Park, H. S., \& Shim, C. S. (2015). Risk Factors for Incomplete Polyp Resection during Colonoscopic Polypectomy. Gut and Liver, 9(1), 66-72. http://doi.org/10.5009/gnl13330.

4. Bonnington, S. N., \& Rutter, M. D. (2016). Surveillance of colonic polyps: Are we getting it right? World Journal of Gastroenterology, 22(6), 1925-1934. http://doi.org/10.3748/wjg.v22.16.1925
P0195 WOMEN AWAKE FASTER THAN MEN AFTER EEG

MONITORED PROPOFOL SEDATION - FIRST PROSPECTIVE OBSERVATIONAL STUDY OF GENDER DIFFERENCES IN PROPOFOL DOSES AND RECOVERY TIMES FOR COLONOSCOPY

A. Riphaus ${ }^{1}$, M. Slottje ${ }^{1}$, J. Bulla ${ }^{2}$, C. Keil ${ }^{1}$, C. Mentzel $^{1}$, V. Limbach ${ }^{1}$,

B. Schultz ${ }^{3}$, C. Unzicker ${ }^{1}$

${ }^{1}$ Department of Medicine, Laatzen/Germany

${ }^{2}$ Department of Mathematics, Bergen/Norway

${ }^{3}$ Department of Anaesthesia MHH, Hannover/Germany

\section{Contact E-mail Address: ariphaus@web.de}

Introduction: Sedation for colonoscopy by using intravenous propofol has become standard in many Western countries.

Aims \& Methods: While gender-specific differences have been shown for general anaesthesia used in dentistry, no such data exist as yet for gastrointestinal endoscopy. In a prospective observational study at an Academic teaching hospital of Hannover Medical School 219 patients (108 women and 111 men) scheduled for colonoscopy were included. Propofol sedation using EEG monitoring during a constant level of sedation depth (D0 to D2) performed by trained nurses or physicians after bodyweight adjusted loading-dose.

Main outcome measures: Primary endpoint was the presence of gender-specific differences in wake-up time (time from end of sedation to eye - opening and the complete orientation of the patient); secondary outcome parameters analysed were total dose of propofol, sedation associated complications (bradycardia, hypotension, hypoxia, apnoea), patient cooperation and patient satisfaction. Multivariate analysis was performed to correct confounding factors such as age and BMI.

Results: Women awake significantly faster compared to men with a time to eye opening of $7.26 \pm 3.69$ versus $8.39 \pm 3.43 \mathrm{~min} .(\mathrm{p}=0.005)$ and time until complete orientation $9.14 \pm 3.88$ versus $10.4 \pm 13.71 \mathrm{~min}(\mathrm{p}=0.008)$; propofol dosage was not significantly different, with some trend towards more propofol per kg body weight in women $(3.98 \pm 1.81 \mathrm{mg}$ versus $3.72 \pm 1.75 \mathrm{mg}, \mathrm{p}=0.232$, n.s.).

Conclusion: The effect of gender aspects should be taken into account upon propofol induced sedation for gastrointestinal endoscopy. That includes adequate dosing for female as well as cautiousness regarding potential overdosing of male patients.

Trial registration: ClinicalTrials.gov (Identifier: NCT02687568). Data were presentet at a national meeting (DGEBV) in Germany.

Disclosure of Interest: All authors have declared no conflicts of interest.

\section{References}

Dumonceau JM, Riphaus A, Schreiber et al. Non-anesthesiologist administration of propofol for gastrointestinal endoscopy: European Society of Gastrointestinal Endoscopy, European Society of Gastroenterology and Endoscopy Nurses and Associates Guideline - Updated June 2015. Endoscopy 2015; 47: 1175-1189

Maeda S, Tomoyasu Y, Higuchi H, et al. Female Patients Require a Higher Propofol Infusion Rate for Sedation. Anesth Prog 2016 63: 67-70

Haensch K, Schultz A, Krauß T et al. Women need more propofol than men during EEG-monitored total intravenous anaestesia. Biomed Tech 2009; 54: 76 82

Schultz B, Schultz A, Grouven U. Sleeping stage based systems (Narcotrend). In: Bruch HP, Köckerling F, Bouchard R, Schug-Pass C, editors. New aspects of high technology in medicine. Bologna: Monduzzi Editore; 2000. p. 285-91

Kugler J. Elektroenzephalography in Klinik und Praxis. Stuttgart: Thieme; 1981. P. $120-46$

Schultz B, Grouven U, Schultz A. Automatic classification algorithms of EEGmonitor Narcotrend to routinely recorded EEG-data from general anaesthesia: a validation study. Biomed Eng 2002; 47: 9-13

\section{P0196 EFFECT OF PREOPERATIVE COLONOSCOPIC TATTOOING USING BOTH SIDE INJECTION OF INDOCYANINE GREEN FOR IMPROVEMENT OF LYMPH NODE HARVEST IN COLORECTAL CANCER}

H.W. Kim, J.S. Lee, D.H. Kang, C.W. Choi, S.J. Kim, H.S. Nam, D.G. Ryu, C.W. Yeo, S.H. Choi, B.J. Kwon

Pusan National University Yangsan Hospital, Yangsan/Korea, Republic of

Contact E-mail Address: mdkhwook@gmail.com

Introduction: Consensus guidelines suggest to assess at least 12 lymph nodes for adequate staging of colorectal cancer and the correlation between number of lymph nodes retrieved and the patient survival has been formerly reported. To facilitate the retrieval of lymph nodes, preoperative endoscopic tattooing to mark the site of the tumor has been proposed. In this study, we aimed to evaluate the effect of preoperative colonoscopic tattooing (PCT) using indocyanine green (ICG) for lymph node harvest in colorectal cancer. Additionally, we evaluated the effect of both side injection of ICG for improving the rate of adequate lymph node harvest.

Aims \& Methods: 1023 patients who underwent curative resection for colorectal cancer between Jan 2013 and Aug 2016 at the Pusan National University Yangsan Hospital in Korea were retrospectively divided into the tattooing group and the non-tattooing group depending on whether PCT using ICG was done. Pathological findings and lymph node harvest were compared between the two groups.

Results: The rate of adequate lymph node harvest (retrieval of more than 12 lymph nodes) was similar in tattooing group and non-tattooing group $(91.9 \%$ vs. $91.4 \%$ ). However, when comparing the both side injection group and 
nontattooing group, both side injection group was better result $(96.2 \%$ vs. $91.4 \%$, OR 2.348, p-value 0.229 ). Most results did not have statistical association with higher lymph node yield in colorectal cancer. But in T1 cancer, the rate of adequate lymph node harvest was higher in the both side injection group, statistically $(94.7 \%$ vs. $81.0 \%$, OR 4.235 , pvalue 0.047$)$

Conclusion: PCT using ICG was associated with higher lymph node harvest in colorectal cancer, especially in T1 cancer. And both side injection of ICG increased the rate of adequate lymph node harvest. Further studies and methods are needed to harvest adequate lymph nodes in colorectal cancer.

Disclosure of Interest: All authors have declared no conflicts of interest.

\section{P0197 THE EFFICACY OF COLD SNARE POLYPECTOMY IN ACHIEVING COMPLETE RESECTION OF SUBCENTIMETRE COLORECTAL POLYPS: A MULTICENTRE RANDOMISED CONTROLLED TRIAL (CRESCENT STUDY)}

T. Kawamura ${ }^{1}$, Y. Takeuchi ${ }^{2}$, S. Asai ${ }^{3}$, I. Yokota ${ }^{4}$, E. Akamine ${ }^{3}$, M. Kato ${ }^{5}$ T. Akamatsu ${ }^{6}$, K. Tada ${ }^{7}$, Y. Komeda ${ }^{8}$, M. Iwatate ${ }^{9}$, K. Kawakami ${ }^{10}$ M. Nishikawa ${ }^{11}$, D. Watanabe ${ }^{12}$, A. Yamauchi ${ }^{13}$, N. Fukata ${ }^{14}$, M. Shimatani ${ }^{14}$,

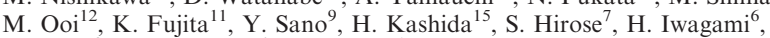
N. Uedo ${ }^{2}$, S. Teramukai ${ }^{4}$, K. Tanaka

${ }^{1}$ Department Of Gastroenterology, Kyoto Second Red Cross Hospital, Kyoto Japan

${ }^{2}$ Gastrointestinal Oncology, Osaka International Cancer Center, Osaka/Japan ${ }^{3}$ Department Of Gastroenterology, Tane General Hospital, Osaka/Japan ${ }^{4}$ Department Of Biostatistics, Kyoto Prefectural University of Medicine, Kyoto/ Japan

${ }^{5}$ Osaka International Cancer Center, Osaka/Japan

${ }^{6}$ Department Of Gastroenterology, Japanese Red Cross Society Wakayama Medical Center, Wakayama/Japan

${ }^{7}$ Department Of Gastroenterology, Bellland General Hospital, Osaka/Japan ${ }^{8}$ Gastorenterology And Hepatology, Kindai University, Faculty of Medicine, Osaka/Japan

${ }^{9}$ Sano Hospital Gastrointestinal Center, Kobe/Japan

${ }^{10}$ Second Department Of Internal Medicine, Osaka Medical Collage, Osaka/Japan ${ }^{11}$ Department Of Gastroenterology, Yodogawa Christian Hospital, Osaka/Japan ${ }^{12}$ Department Of Gastroenterology, Kobe University, Kobe/Japan

${ }^{13}$ Division Of Gastroenterology And Hepatology, Kitano Hospital, Osaka/Japan

${ }^{14}$ The Third Department Of Internal Medicine, Kansai Medical University 3rd Dept. of Internal Medicine, Hirakata/Japan

${ }^{15}$ Dept. Of Gastorenterology And Hepatology, Kindai University Faculty of Medicine, Osaka/Japan

Contact E-mail Address: kawamurat@kyoto2.jrc.or.jp

Introduction: Cold snare polypectomy (CSP) has grown in popularity worldwide due to its ease and safety with a low incidence of adverse events, such as hemorrhage and post-polypectomy syndrome ${ }^{1-3}$. However, there are concerns regarding tumor residue with CSP because it does not use electrocautery, thereby eliminating its burning effect. European Society of Gastrointestinal Endoscopy (ESGE) clinical guideline suggests CSP for subcentimetre sessile polyps because of its safety profile, despite lack of evidence for efficacy compared to $\mathrm{HSP}^{4}$. The aim of this study was to investigate the success rate of CSP for complete resection of subcentimetre colorectal adenomatous polyps compared to that of HSP.

Aims \& Methods: This was a prospective, multicentre, randomised controlled, non-inferiority trial conducted in 12 Japanese endoscopy units. Patients aged $\geq 20$ years, undergoing elective colonoscopy/polypectomy, and who provided written informed consent were included. Patients who were taking anti-thrombotic agents and undergoing hemodialysis were excluded, as well as those with inflammatory bowel diseases, polyposis, and pregnancy. Endoscopically diagnosed sessile adenomatous polyps, $4-9 \mathrm{~mm}$ in size, were randomly assigned to the CSP or HSP group. After complete removal of the polyp using the allocated technique, biopsy specimens from the resection margin after polypectomy were obtained. The primary endpoint was the complete resection rate, defined as no evidence of adenomatous tissue in the biopsied specimens, among all pathologically confirmed adenomatous polyps (full analysis set: FAS). Pre-planned subgroup analyses for the size of polyp were also conducted. The sample size was calculated according to the incomplete resection rate seen in previous articles and the required sample size was estimated at 780 lesions, including drop out cases. The protocol was approved by the institutional review board in each institution.

Results: A total of 796 eligible polyps were detected in 538 of 912 patients screened for eligibility between September 2015 and August 2016. Three hundred and ninety-four lesions were assigned to the CSP group and 402 lesions were assigned to the HSP group. One hundred and nine lesions (56 in the CSP group and 53 in the HSP group) were excluded for FAS analysis. Background characteristics of the lesions (size, location, morphology, and institution) were similar in both groups. The complete resection rate for CSP was $98.2 \%$, compared to $97.4 \%$ for HSP. The between group difference in complete resection rate was $+0.8 \%$, favouring CSP $(90 \%$ C.I. of -0.8 .2 .7 ; p- $<0.0001)$. Resection time, overall, was significantly shorter with CSP than with HSP ( $60 \mathrm{~s}$ versus $83 \mathrm{~s}$, respectively; $\mathrm{p}<0.001)$. Postoperative bleeding requiring endoscopic haemostasis occurred only in the HSP group $(0.5 \%, 2$ of 402 cases). Subgroup analysis according to the size of the polyp (4-5 mm and 6-9 $\mathrm{mm}$ ) showed a comparable complete resection rate for CSP and HSP for both subgroups of polyps.

Conclusion: The complete resection rate of CSP is not inferior to that of HSP CSP can be one of the standard techniques for subcentimetre colorectal polyps. (Study registration: UMIN000018328)

Disclosure of Interest: All authors have declared no conflicts of interest

\section{References}

1. Repici A, Hassan C, Vitetta E, et al: Safety of cold polypectomy for $<10 \mathrm{~mm}$ polyps at colonoscopy: a prospective multicenter study. Endoscopy 44:27-31, 2012

2. Horiuchi A, Nakayama Y, Kajiyama M, et al: Removal of small colorectal polyps in anticoagulated patients: a prospective randomized comparison of cold snare and conventional polypectomy. Gastrointest Endosc 79:417-423, 2014

3. Ichise Y, Horiuchi A, Nakayama Y, et al: Prospective randomized comparison of cold snare polypectomy and conventional polypectomy for small colorectal polyps. Digestion 84:78-81, 2011

4. Ferlitsch M, Moss A, Hassan C, et al: Colorectal polypectomy and endoscopic mucosal resection (EMR): European Society of Gastrointestinal Endoscopy (ESGE) Clinical Guideline. Endoscopy 49:270-297, 2017

\section{P0198 EFFICACY OF PLATELET-RICH PLASMA (PRP) ON ENDOSCOPIC RESECTION TECHNIQUES: CLINICAL STUDY IN 15 PATIENTS}

V. Lorenzo-Zúñiga García ${ }^{1}$, V. Moreno De Vega ${ }^{1}$, I. Marín ${ }^{2}$, N. Caballero ${ }^{2}$, R. Bartolí ${ }^{1}$, I. Bon ${ }^{1}$, J. Boix ${ }^{1}$

${ }^{1}$ Endoscopy/ter Group, Germans Trias/IGTP, Badalona/Spain

${ }^{2}$ Endoscopy Unit, University Hospital Germans Trias, Badalona/Spain

Contact E-mail Address: vlorenzo.germanstrias@gencat.cat

Introduction: Prevention of complications secondary to endoscopic resection techniques (EMR or ESD) requires avoiding deep thermal damage and increase mucosal healing. Platelet-rich plasma (PRP) has demonstrated efficacy in preclinical endoscopic resection models [1]. The EndoPRP study was a prospective single-center study to assess the efficacy of PRP on endoscopic resection of large sessile lesions (larger than $35 \mathrm{~mm}$ ). (Study registered at ClinicalTrials.gov: NCT02931149)

Aims \& Methods: In the EndoPRP study 15 patients (males and females, aged 52 $80)$ were assigned to receive PRP $(6-18 \mathrm{~mL})$ : i) Endoscopic Shielding Technique $(\mathrm{EST}, \mathrm{n}=4)$, applying PRP as a shield after standard resection technique, or ii) Submucosal injection (SMI, $\mathrm{n}=11$ ), performing a submucosal injection of PRP prior to EMR or ESD. Patients were informed and accepted to participate with a written consent. PRP was obtained from a sample of patient's blood (18-36 mL) drawn at the time of endoscopy. Patients underwent endoscopic follow-up at 4 weeks. The efficacy of PRP was assessed by the incidence of adverse events (delayed bleeding or perforation). Mucosal healing rate (MHR) was defined as a percentage of mucosal restoration after 4 weeks.

Results: Shielding technique with PRP was performed in 4 lesions at rectum ( $Æ$ $53.7 \pm 20.6 \mathrm{~mm}$, range $35-80 \mathrm{~mm}$ ). Submucosal injection of PRP was used in 11 lesions ( 2 at antrum, 3 at rectum, and 8 at colon) (Æ $41.6 \pm 9.6 \mathrm{~mm}$, range 35 $70 \mathrm{~mm}$ ). Delayed bleeding occurred after EMR of 1 lesion (no required blood transfusion or endoscopic treatment; $6.6 \%$ of all lesions: 1 patient at EST group, 0 patients at SMI group). MHR was significantly higher in patients treated with SMI than EST $(87.5 \%$ vs. $78.6 \% ; \mathrm{p}=0.03)$

Conclusion: PRP applied as a shield over the scar or as submucosal fluid cushion has proved clinical efficacy in endoscopic resection of large lesions. Submucosal injection of PRP has showed better mucosal healing rate as comparison with shielding technique.

Disclosure of Interest: All authors have declared no conflicts of interest.

\section{Reference}

1 Lorenzo-Zúñiga, et al. Endoscopy International Open 2016; 04:E1-E6.

\section{P0199 INTERVENTIONS AND COSTS ASSOCIATED WITH SIVA- DEFINED ADVERSE EVENTS DURING PROCEDURAL SEDATION} IN FIVE COUNTRIES

R. Saunders ${ }^{1}$, J. Davis ${ }^{1}$, R. Weissbrod ${ }^{2}$, D. Whitaker ${ }^{3}$, P. Kranke ${ }^{4}$, J.

R. Lightdale ${ }^{5}$

${ }^{1}$ Health Economics, Coreva Scientific, Freiburg/Germany

${ }^{2}$ Medtronic, Jerusalem/Israel

${ }^{3}$ Manchester Royal Infirmary, Manchester/United Kingdom

${ }^{4}$ University of Würzburg, Würzburg/Germany

${ }^{5}$ University of Massachusetts, Worcester/United States of America

Contact E-mail Address: rhodri@coreva-scientific.com

Introduction: Procedural sedation is commonly used during gastrointestinal endoscopy procedures to improve patient comfort. It comes, however, with the risk of sedation-related adverse events (AEs) as defined by World SIVA. ${ }^{1}$ The cost of AEs and their impact on healthcare resource use and efficiency is currently unknown

Aims \& Methods: The aim of our study is to quantify and compare the costs of AEs during procedural sedation in France, Germany, Italy, UK, and USA. Online interviews were conducted with providers (nurses, physicians, and anaesthesiologists) and payers. Respondents were screened to ensure that they had the expertise and experience to complete the survey. The provider survey covered topics such as guidelines, sedation agents, monitoring, and patient outcomes following AEs. Each provider reported on current clinical practice, the incidence of AEs, and the standard treatment and outcomes for 100 hypothetical patients 
with a defined AE. Treatment options, including none required, were taken from SIVA-defined lists. Outcomes examined included delayed procedures, unplanned hospital admission, and death. Payers were surveyed about costs for interventions, provider time, hospital administration, and admissions. Outliers were identified using Dixon's Q test. The mean treatments and outcomes per AE per country were calculated, with responses weighted by the AE frequency reported by physicians and the outliers replaced by global means. Mean costs were calculated per intervention and outcome, with outliers removed.

Results: 101 providers and 26 payers completed the surveys, with a minimum of 20 providers and 5 payer responses per country. Over $62 \%$ of providers were gastroenterologists and anaesthesiology nurses. Local guidelines determined practice in most cases, and propofol and midazolam were the main sedation agents employed. The most common AEs reported were hypotension and bradycardia, with $9 \%$ and $4 \%$ of respondents, respectively, estimating each to occur during $>10 \%$ of procedures. Mean provider time required to treat AEs ranged from 1.7 minutes for mild desaturation in Germany to 31.0 minutes for cardiac arrest in the USA. Accounting for interventions and provider time, the mean direct cost per AE ranged from EUR 12 for bradycardia in Germany to USD 3, 877 for cardiac arrest in the USA (Table). When costs were "fully loaded" these became EUR 39 and USD 19, 722, respectively. Although of low direct cost, bradycardia in Germany was reported to cause procedure termination or substantial delay in $3.8 \%$ of cases. In Euro countries, the median of mean direct costs for an AE was EUR 40 (IQR: 29-67). When costs of outcomes of AEs were included the median "fully loaded" cost reached EUR 301 (IQR: 115-738).

Table: Costs of select adverse events by country. FL: Fully-loaded (costs including hospital administration, time, inpatient stays, delays, and cancellations, but excluding legal costs)

\begin{tabular}{|c|c|c|c|c|c|c|}
\hline $\begin{array}{l}\text { Country, } \\
\text { currency }\end{array}$ & $\begin{array}{l}\text { Hypotension } \\
\text { Direct; FL }\end{array}$ & $\begin{array}{l}\text { Mild } \\
\text { desaturation } \\
\text { Direct; FL }\end{array}$ & $\begin{array}{l}\text { Severe } \\
\text { desaturation } \\
\text { Direct; FL }\end{array}$ & $\begin{array}{l}\text { Bradycardia } \\
\text { Direct; FL }\end{array}$ & $\begin{array}{l}\text { Prolonged } \\
\text { apnoea } \\
\text { Direct; FL }\end{array}$ & $\begin{array}{l}\text { Cardiac } \\
\text { arrest } \\
\text { Direct; FL }\end{array}$ \\
\hline France, EUR & $32 ; 173$ & $23 ; 471$ & $79 ; 1994$ & $17 ; 131$ & $53 ; 490$ & $137 ; 11,936$ \\
\hline $\begin{array}{c}\text { Germany, } \\
\text { EUR }\end{array}$ & $23 ; 193$ & $18 ; 212$ & $92 ; 1268$ & $12 ; 39$ & $118 ; 807$ & $274 ; 4765$ \\
\hline Italy, EUR & $41 ; 111$ & $32 ; 98$ & $59 ; 201$ & $33 ; 93$ & $43 ; 99$ & $101 ; 1195$ \\
\hline UK, GBP & $69 ; 537$ & $34 ; 606$ & $93 ; 1258$ & $35 ; 362$ & $80 ; 631$ & $658 ; 8984$ \\
\hline US, USD & $247 ; 841$ & $463 ; 1456$ & $529 ; 1715$ & $83 ; 358$ & $394 ; 1262$ & $3877 ; 19,722$ \\
\hline
\end{tabular}

Conclusion: Costs of sedation-related AEs can be substantial regardless of country of origin. Disruption of patient flow and provider efficiency may add to the cost burden. Even relatively minor events may prompt additional intervention, increasing the overall cost of care.

Disclosure of Interest:

R. Saunders: Rhodri Saunders is the owner of Coreva Scientific GmbH \& Co $\mathrm{KG}$, which received consultancy fees for designing and performing this research J. Davis: Jason Davis is an employee of Coreva Scientific GmbH \& Co KG, which received consultancy fees for designing and performing this research

R. Weissbrod: Rachel Weissbrod is an employee of Medtronic

D. Whitaker: David Whitaker did not receive any remuneration for work on this research project. He has previously consulted for Medtronic and Covidien

P. Kranke: Peter Kranke did not receive any remuneration for work on this research project. He has previously consulted for Medtronic and Covidien

J.R. Lightdale: Jenifer Lightdale did not receive any remuneration for work on this research project. She has previously consulted for Medtronic Inc

\section{Reference}

1. Mason et al. BJA. 2012;108(1):13-20

\section{P0200 COLORECTAL ENDOSCOPIC SUBMUCOSAL DISSECTION (CR-ESD), KNIFE-ASSISTED SNARE RESECTION (KAR) AND RECURRENCE RATE: A WESTERN EUROPEAN EXPERIENCE IN SPAIN}

J. C. Marín-Gabriel ${ }^{1}$, J.B. Díaz Tasende ${ }^{1}$, P. Cancelas-Navia ${ }^{2}, \mathrm{~S}$. Rodríguez-

Muñoz ${ }^{3}$, A.J. Del Pozo-García ${ }^{1}$, M. Alonso-Riaño ${ }^{4}$, Y. Rodríguez-Gil ${ }^{4}$,

C. Ibarrola-De Andrés ${ }^{4}$, G. Castellano-Tortajada ${ }^{5}$

${ }^{1}$ Gastroenterology. Endoscopy Unit, University Hospital "12 de Octubre", Madrid/ Spain

${ }^{2}$ Research Institute " $i+12 "$, Hospital Universitario "12 de Octubre", Madrid/Spain ${ }^{3}$ Gastroenterology, Endoscopy Unit, Hospital Ruber, Madrid/Spain

${ }^{4}$ Pathology, University Hospital "12 de Octubre", Madrid/Spain

${ }^{5}$ Gastroenterology, University Hospital "12 de Octubre", Madrid/Spain

Contact E-mail Address: josecarlos.marin@salud.madrid.org

Introduction: Performing CR-ESD remains challenging in Western countries and surveillance studies in this setting are not fully described. KAR has been advised as a reasonable strategy for non-expert endoscopists and difficult lesions. However, some KAR eventually requires a piecemeal resection (p-KAR). A direct comparison between these two techniques is lacking. Additionally, when the specimen is resected en bloc regardless of what procedure is used, and the only pathological risk factor for recurrence is lateral margin (LM) involvement, its implications concerning the recurrence rate should be assessed.

Aims \& Methods: 1.) To compare the recurrence rate after R0 and R1/Rx endoscopic resection (ER), on an ESD "intention-to-treat" basis, in a Western European setting where CR-ESD is performed by non-experts. 2.) To evaluate the impact of LM involvement on local recurrence when neoplasms without risk factors for lymph node metastasis are resected en bloc. We prospectively included 89 consecutive CR neoplasms planned for ESD from September 2008 to December 2015. When technical difficulties arose or for patient's safety reasons, we performed a KAR. Kaplan-Meier survival curves were used to assess the recurrence rate over time. The end of follow-up was considered when a local recurrence occurred or at the end of the surveillance period in those patients who did not develop the event. Comparisons were made using the log-rank test. The recurrence rate during follow-up was stratified considering advanced histology, en bloc resection and $\mathrm{R} 0$ resection.

Results: The ER was aborted in 5 cases (perforation $n=3$; technical difficulties $\mathrm{n}=2$ ). Surgical intervention was needed after ER because of submucosal or linfovascular invasion in 4 patients. Five out of the remaining 80 cases, were lost to follow-up. Finally, $75 \mathrm{CR}$ neoplasms were included in 74 patients (43 male; $58.1 \%$ ). Median age was 71 years (range: $37-93$ ). Median size of the lesions was $32 \mathrm{~mm}$ (range 10-100). Histology was $26(34.7 \%$ ) Vienna category 3; 46 $(61.3 \%)$ Vienna 4 and $3(4 \%)$ sm1-Vienna 5. En bloc resections were obtained in 44 cases (58.7\%): 33 ESD (44\%) and 11 KAR (14.7\%). The ER finished as pKAR in the 31 remaining lesions $(41.3 \%)$. R0 resections $(n=23 ; 30.7 \%)$ were achieved in $18 / 33$ ESD and 5/42 KAR [OR $=8.9$ (CI 95\%: 2.8-28.3); $\mathrm{p}<0.0001]$. The median follow-up period was 16 months $(1-91)$. Local recurrence occurred in 11 cases: 9 of the latter throughout the first year $(81.8 \%)$. No surgery was needed because of recurrence. The overall recurrence rate at 36 months was $15 \%$. The recurrence rate at 3 years showed a statistical significant difference when R0 resections were compared with $\mathrm{R} 1 / \mathrm{Rx}: 0 \%$ vs. $21.5 \%$ $(p=0.03)$. When results were stratified according to histology and en bloc resections, no significant differences were found in the recurrence rate. When en bloc resections in $\mathrm{pT} 1 \mathrm{a} / \mathrm{T} 1 \mathrm{~b}(\mathrm{sm} 1)$; ly $(-) ; \mathrm{v}(-) ; \mathrm{pVM} 0$ lesions $(\mathrm{n}=44)$ were analysed separately, LM distribution was: 23 LM0 (52.3\%); 18 LM1 (40.9) and 3 LMx (6.8). There was a non-significant trend concerning the recurrence rate when LM0 $(n=23)$ lesions were compared with LM1/LMx $(n=21): 0 \%$ vs. $14.8 \%$ at 3 years; $\mathrm{p}=0.06$.

Main characteristics of the resected lesions by procedure

\begin{tabular}{lllll}
\hline & ESD & en bloc KAR & p-KAR & p \\
\hline $\begin{array}{l}\text { Mean tumor size, mm; } \\
\text { median (range) }\end{array}$ & $28(11-50)$ & $20(11-65)$ & $42.1(17-100)$ & $\mathbf{0 . 0 0 2}$ \\
$\begin{array}{l}\text { Length of procedure, min; } \\
\quad \text { median (range) }\end{array}$ & $25(62-340)$ & $175(60-300)$ & $270(75-400)$ & 0.11 \\
$\begin{array}{l}\text { Rectal location; n (\%) } \\
\begin{array}{l}\text { Vienna category } \geq 4 ; \\
\text { n (\%) }\end{array}\end{array}$ & $22(68.7)$ & $5(41.6)$ & $21(67.7)$ & 0.38 \\
$\begin{array}{l}\text { Follow up - months; } \\
\text { median (range) }\end{array}$ & $16.3(2.6-62.7)$ & $16.9(8.0-91.3)$ & $13.8(1.1-44.6)$ & 0.59 \\
\hline
\end{tabular}

Conclusion: ESD R0 resections were 9 times greater than that of KAR on an ESD "intention-to-treat" basis. R0 resections were associated with lower recurrence rates in comparison with $\mathrm{R} 1 / \mathrm{Rx}$ resections. $\mathrm{LM}$ involvement increased the recurrence rate but without a statistical significance when it was the only pathological risk factor for recurrence and the specimen was resected en bloc.

\section{References}

1. Bhattacharyya R, Chedgy FJ, Kandiah K, Longcroft-Wheaton G, Bhandari $P$. Knife-assisted snare resection (KAR) of large and refractory colonic polyps at a Western centre: Feasibility, safety and efficacy study to guide future practice. United European Gastroenterol J. 2016;4(3):466-73.

2. Makazu M, Sakamoto T, So E, Otake Y, Nakajima T, Matsuda T, Kushima $\mathrm{R}$, Saito Y. Relationship between indeterminate or positive lateral margin and local recurrence after endoscopic resection of colorectal polyps. Endosc Int Open. 2015;3(3):E252-7.

\section{P0201 ASSOCIATION BETWEEN SIZE, LOCATION AND HISTOLOGICAL CHARACTERISTICS OF COLORECTAL LATERALLY SPREADING TUMORS}

F. L. Mota ${ }^{1}$, J. F. Loureiro ${ }^{1}$, L. S.N. Da Costa ${ }^{2}$

${ }^{1}$ Digestive Endoscopy, Hospital Sírio-Libanês, SAO PAULO/Brazil

${ }^{2}$ Digestive Endoscopy Department, Hospital Sirio Libanes, Sao Paulo/Brazil

Contact E-mail Address: lucasnde@gmail.com

Introduction: Laterally spreading tumors (LST) are important precursors of colorectal cancer $(\mathrm{CRC})^{1}$. The endoscopic characteristics of the LSTs, such as size and location, appear to correlate with the histological findings ${ }^{2,3}$, which is an essential data for the decision of the best therapeutic procedure to be carried out $^{4,5}$

Aims \& Methods: To determine the association between size, location and the histological characteristics of colorectal LSTs by reviewing of the colonoscopy and histopathological reports of the LSTs endoscopically removed between October 2013 and June 2015 at the digestive endoscopy department of a tertiary hospital. The Vienna revised classification was used for the adenomatous lesions $^{6}$, and the World Health Organization (WHO) classification for the "sessile serrated adenomas" (SSA) 7,8 . The regions of the colon were referred to 
as either "proximal" or "distal" colon. Thereafter the division into six anatomical segments was considered (cecum, ascending, transversal, descending, sigmoid and rectum).

Results: A total of 218 LSTs were included in this study. Most patients (59.4\%) were female. The mean age was 66.1 years, and the average size of the LSTs included was $1.69 \mathrm{~cm}$. The proximal colon was the most common site $(73.4 \%)$ of occurrence of the LSTs, with $34 \%$ being at the ascending colon. The most common histological type was the low grade dysplasia adenoma (Vienna 3), followed by the SSA without dysplasia with $21.6 \%$. There was significant correlation between size and histology $(\mathrm{p}<0.005)$, where the adenomatous lesions were found to be larger than the other categories. The SSAs, however, did not show this association. We identified association between location and histological type $(\mathrm{p}<0.005)$ : the adenomas with low grade dysplasia were most prevalent in the proximal colon. However, when the subdivision of the colon into anatomical segments was considered, the SSA without dysplasia was the most common type at the ascending colon.

Conclusion: There is association between the size and the histological characteristics of colorectal LSTs. Adenomas with high grade dysplasia were found to be larger than the other classifications. This association, however, is not observed between SSAs lesions. There is association between location and histology; with the SSAs without dysplasia being the predominant type at the ascending colon. Disclosure of Interest: All authors have declared no conflicts of interest.

\section{References}

1. Lambert R, Tanaka S. Laterally spreading tumors in the colon and rectum. Eur J Gastroenterol Hepatol. 2012; 24(10):1123-32.

2. Saito Y, Fuijii T, Kondo H, Mukai H, Yokota T, Kozu T, Saito D. Endoscopic treatment for laterally spreading tumors in the colon. Endoscopy. 2001; 33(8):682-86.

3. Rotondano G, Bianco MA, Buffoli F, Cipolletta L. The cooperative italian FLIN study group: prevalence and clinic-pathological features of colorectal laterally spreading tumors. Endoscopy. 2011; 43(10):856-61.

4. Facciorusso A, Antonio M, Maso MD, Barone M, Muscatiello N. Nonpolypoid colorectal neoplasms: classification, therapy and follow-up. World J Gastroenterol. 2015; 21(17):5149-57.

5. Ross AS, Waxman I. Flat and depressed neoplasm of the colon in the western populations. Am J Gastroenterol. 2006; 10(1)1:172-80.

6. Dixon MF. Gastrointestinal epithelial neoplasia: Vienna revisited. Gut. 2002; 51(1):130-1

7. Snover D, et al. WHO classification of tumours. Pathology and genetics. Tumours of the digestive system. $4^{\mathrm{a}}$ ed. Berlin: Springer-Verlag, 2010

8. Snover DC. Update on the serratedpathway to colorectal carcinoma. Hum Pathol. 2011; 42(1):1-10.

\section{P0202 SAFE AND SUCCESSFUL RESECTION OF DIFFICULT GI LESIONS USING A NOVEL SINGLE-STEP FULL-THICKNESS RESECTION DEVICE (FTRD)}

P.V. Valli, J. Mertens, P. Bauerfeind

Division Of Gastroenterology And Hepatology, University Hospital Zurich, Zurich/Switzerland

Contact E-mail Address: piero.valli@usz.ch

Introduction: Endoscopic mucosal resection (EMR) and endoscopic submucosal dissection (ESD) are well-established and effective techniques for the endoscopic resection of mucosal neoplasms along the gastrointestinal (GI) tract. However, these procedures are limited to superficial lesions. In the case of deeper ingrowth into the gut wall as well as anatomic sites prone to perforation, the novel fullthickness resection device (FTRD $\left.{ }^{\circledR}\right)$ opens a new dimension of possibilities for thickness resection devic
endoscopic resection.

Aims \& Methods: Sixty patients underwent therapeutic endoscopic full-thickness resection (eFTR) at our institution. The procedures were carried out as follows: First, the target lesion is marked with electrocautery and the endoscope is then retracted. The full-thickness resection device (FTRD, Ovesco ${ }^{\circledR}$ Endoscopy AG, Tübingen), is fitted onto a therapeutic endoscope. The endoscope with the FTRD ${ }^{\circledR}$ is advanced to the previously marked lesion. Grasping forceps are used to take hold of the target lesion and carefully pull it into the plastic cap

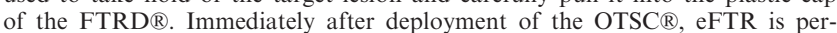
formed using the hyperthermic snare within the plastic cap. The full-thickness specimen is retrieved and processed for histopathological examination. Safety, learning curve, R0 resection rate and clinical outcome of all 60 interventions were studied.

Results: EFTR was performed for the following indications: 1. Recurrent adenomas $(n=22.37 \%)$ with a non-lifting sign after previous incomplete polypectomy and adenomas with a primary non-lifting sign on saline injection $(\mathrm{n}=2.3 \%) .2$ Non-lifting base after extensive piecemeal resection of a spreading adenoma $(\mathrm{n}=10.17 \%) .3$. Polyps in a diverticulum $(\mathrm{n}=2.3 \%) .4$. Polyps the cecal appendix $(4.6 .7 \%)$. 5. Submucosal lesions $(n=5.8 .3 \%)$. 6. Early carcinoma $(n=7.12 \%) .7$. Follow-up resection of a malignant polyp $(\mathrm{n}=6.10 \%)$ had. 8 . EFTR over endoloop resection $(\mathrm{n}=2.3 \%)$. In $97 \%(58 / 60)$ of the interventions, the FTRD $\AA$-mounted endoscope reached the previously marked lesion and eFTR was performed (technical success). Full-thickness resection was achieved in $88 \%$ of the cases, with an $\mathrm{R} 0$ resection on histological examination in $79 \%$. The clinical success rate based on follow-up histology was even higher $(88 \%)$. The following adverse events occurred: Appendicitis of the residual cecal appendix after eFTR of an appendicular adenoma $(1 / 58.2 \%)$. Minor bleeding at the eFTR site $(2 / 58.3 \%)$. EFTR performed accidently without proper prior deployment of the OTSC ${ }^{R}(1 / 58.2 \%)$. There was no secondary perforation or eFTR-associated mortality.
Conclusion: In conclusion, after specific training, endoscopic full-thickness resection is a feasible, safe and promising resection technique. It allows complete resection of lesions affecting layers of the gut wall beneath the mucosa, without the risk of perforation. In the future, eFTR may become a valuable alternative to a surgical approach in cases where endoscopic resection was previously thought impossible.

Disclosure of Interest: All authors have declared no conflicts of interest.

\section{P0203 VASCULAR AND PIT-PATTERN ANALYSIS ACCORDING TO KUDO, SANO AND NICE CLASSIFICATIONS SIGNIFICANTLY IMPROVES AFTER AN IMAGE-BASED TRAINING PROGRAM}

F. Desideri ${ }^{1}$, G. Esposito ${ }^{2}$, S. Angeletti ${ }^{2}$, F. Iacopini ${ }^{3}$, M. Haefner ${ }^{1}$, E. Di Giulio ${ }^{2}$ ${ }^{1}$ Gastroenterology, S. Maurizio Hospital, Bolzano/Italy

${ }^{2}$ Endoscopy Unit, S. Andrea Hospital, Rome/Italy

${ }^{3}$ Endoscopy Unit, S. Giuseppe Hospital, Albano/Italy

Contact E-mail Address: federico.desideri@gmail.com

Introduction: Narrow Band Imaging (NBI) and chromoendoscopy with methylene blue are enhancing techniques which are helpful in differentiating vascular and pit patterns of colorectal neoplasms. Therefore, they have a key-role for the adequate management of the lesions which might be candidates for endoscopic resection.

Aims \& Methods: The aim of our study was to measure the interobserver agreement and the diagnostic accuracy in an endoscopy unit using methylene blue and NBI for the evaluation of the pit and vascular pattern according to the Kudo, Sano and NICE classifications of colo-rectal neoplasms, before and after an image-based training program. We retrospectively collected consecutive endoscopic images (NBI and with methylene blue) of colo-rectal neoplasms from the internal database. The image set was then evaluated by our gold standard composed by two expert endoscopists. Their evaluation resulted confident with histology reports in $88 \%$ of cases. The images set was then evaluated by the 9 endoscopists of the unit, before and after a 30-minutes image-based training program on enhancing techniques and surface colorectal patterns. NBI and colonic neoplasms' surface and vascular patterns. Interobserver agreement was calculated using the kappa statistic by Cohen. By using the gold standard evaluation as criterion standard, the accuracy of colo-rectal neoplasms' evaluation before and after the training was also calculated using the McNemar test. A value of $\mathrm{p}<0.05$ was considered statistically significative.

Results: A total of 30 images were obtained (see Table). Before the training process, the interobserver agreement was minimal for Kudo $(0.10 \pm 0.03)$ and Sano $(0.12 \pm 0.04)$, and poor for the NICE classification $(0.24 \pm 0.05)$ Diagnostic accuracy was $0.33 \pm 0.07,0.54 \pm 0.12$ and $0.60 \pm 0.10$ for Kudo, Sano and NICE classifications, respectively. After the image-based training program, interobserver agreement moved to moderate for the Kudo classification $(\mathrm{p}<0.0001)$ and to good for Sano and NICE classifications $(\mathrm{p}<0.0001)$. Diagnostic accuracy increased significantly, too, with values of $0.60 \pm 0.05$, $0.76 \pm 0.05,0.80 \pm 0.05$ for Kudo, Sano and NICE classifications, respectively $(\mathrm{p}<0.0001)$

\begin{tabular}{lll}
\hline Classification & Type & $\mathrm{N}^{\circ}$ \\
\hline Kudo & I & 0 \\
& II & 2 \\
& III 1 II s & 1 \\
& III & 6 \\
& IV & 12 \\
& V i & 5 \\
SANO & V n & 4 \\
& I & 2 \\
& II & 19 \\
& III a & 5 \\
NICE & III b & 4 \\
& I & 2 \\
& II & 19 \\
& III & 9 \\
\hline
\end{tabular}

Conclusion: To the best of our knowledge, we present the first study on the ability of an image-based training program in increasing the interobserver agreement and diagnostic accuracy in differentiating pit and vascular patterns of colo-rectal neoplasms using all the available endoscopic classifications (Kudo, Sano and NICE classifications). Such training seems mandatory for endoscopists using enhancing techniques especially when advanced lesions are planned to be treated endoscopically.

Disclosure of Interest: All authors have declared no conflicts of interest. 


\section{References}

1. Higashi R et al. Diagnostic accuracy of narrow-band imaging and pit pattern analysis significantly improved for less-experienced endoscopists after an expanded training program. Gastrointestinal endoscopy 2010; 72, 127-135

2. Ignjatovic A et al. Development and validation of a training module on the use of narrow-band imaging in differentiation of small adenomas from hyperplastic colorectal polyps. Gastrointestinal endoscopy 2011; 73, 128-133

3. McKill S et al. Endoscopists can sustain high performance for the optical diagnosis of colorectal polyps following standardized and continued training. Endoscopy 2015; 47: 200-206

4. Sikong $\mathrm{Y}$ et al. Effectiveness of systematic training in the application of narrow-band imaging international colorectal endoscopic (NICE) classification for optical diagnosis of colorectal polyps: experience from a single center in China. Digestive Endoscopy 2016; 28: 583-591

\section{P0204 YIELD OF 2ND SURVEILLANCE COLONOSCOPY IN "INTERMEDIATE RISK" PATIENTS. COULD SURVEILLANCE INTERVALS BE REFINED?}

M. Balakrishnan, B. Adeoti, S. Cerys, S. Catnach, A. King, B. Macfarlane, J. Landy

Gastroenterology, West Hertfordshire Hospitals NHS Trust, Watford/United Kingdom

Contact E-mail Address: jonathan.landy@whht.nhs.uk

Introduction: Data regarding the yield of 2nd surveillance colonoscopy after index procedure findings of advanced colonic neoplasia (ACN) are limited. The yield of ACN at 2nd surveillance is associated with high risk index or 1st surveillance findings (1). However, previous studies are heterogenous and definitions of ACN include characteristics of both "intermediate" (IR, >3 adenomas or any adenoma $>10 \mathrm{~mm}$ ) and "high risk" groups (HR, $>5$ adenomas or $>3$ adenomas with at least $1>10 \mathrm{~mm}$ ) as defined by BSG guidelines.

Aims \& Methods: We aimed to evaluate the differences in yield of advanced colonic neoplasia at $2^{\text {nd }}$ surveillance colonoscopy (S2) between "intermediate" and "high" risk patients at index colonoscopy in our unit. ACN was defined as $\geq 5$ adenomas, any adenoma $\geq 1 \mathrm{~cm}$, tubulovillous histology or high grade dysplasia, or cancer. Patients with HR or IR index procedures undertaken by 3 experienced, accredited bowel cancer screening colonoscopists and at least 2 surveillance colonoscopies, were identified from our local database between 2008 and 2016. Findings at $1^{\text {st }}$ and $2^{\text {nd }}$ surveillance procedures were assessed for the presence of ACN. Statistical analysis was undertaken using Graphpad Prism 5 using Fisher's exact test. All tests were two tailed and a p value of $\leq 0.05$ was considered significant. ORs with a $95 \%$ CI were calculated for significant findings.

Results: 218 patients meeting inclusion criteria were identified. $53 \%$ of patients had IR index findings. The median time to S2 was 49 months (IQR $48-49 \mathrm{~m}$ ) for HR index patients and $72 \mathrm{~m}$ (IQR $70-73 \mathrm{~m}$ ) for IR index patients. $11 \%$ of all patients had ACN at S2. $4 \%$ of IR patients v $18 \%$ of HR patients had ACN at S2, OR 0.4 (95\% CI $0.2-0.6) .3 \%$ of IR patients without ACN at S1 had ACN at S2 v $15 \%$ of IR patients with ACN at S1 (ns). $11 \%$ of HR patients without ACN at $\mathrm{S} 1$ v $37 \%$ with $\mathrm{ACN}$ at $\mathrm{S} 1$ had $\mathrm{ACN}$ at S2; OR 0.2 (95\% CI 0.07-0.6). Conclusion: Stratification of high-risk index findings into HR and IR groups as per BSG guidelines differentiates a low-risk group at second surveillance colonoscopy. The second surveillance interval for IR patients without ACN at first surveillance might be increased as ACN is infrequently detected in this group. Disclosure of Interest: J. Landy: Educational support from Norgine.

\section{References}

1. Morelli MS et al. Yield of the second surveillance colonoscopy based on the results of the index and first surveillance colonoscopies. Endoscopy. 2013 Oct;45(10):821-6.

2. Cairns $\mathrm{S}$ et al. Guidelines for colorectal cancer screening and surveillance in moderate and high risk groups (update from 2002). Gut. 2010 May;59(5):666-89.

\section{P0205 SAFETY OF COLD SNARE COLON POLYPECTOMY IN PATIENTS ON ANTITHROMBOTIC MEDICATION}

M. Matsumoto

Dept. Of Gastroenterology, Hokkaido Medical Center, Sapporo/Japan

Contact E-mail Address: miosakra@outlook.jp

Introduction: Cold snare polypectomy (CSP) has been increasingly used in recent years because post- polypectomy bleeding is less common with this technique than with conventional polypectomy. According to the 2012 update of the Japanese guideline for periprocedural management of antithrombotic medications issued by the Japan Gastroenterological Endoscopy Society, procedures with a low risk of hemorrhage may be performed with a short interruption or continuation of antithrombotic medication. However, the guideline does not refer to periprocedural antithrombotic management for CSP.

Aims \& Methods: The objective of this study was to determine the safety of CSP in patients on antithrombotic medication. The subjects were patients who underwent CSP at this hospital between April 2014 and March 2016. Post-CSP bleeding rates were examined in relation to the use of antithrombotic medication. CSP was indicated for non-pedunculated polyps smaller than $10 \mathrm{~mm}$, excluding lesions with submucosal invasion and suspected of being cancerous at the preprocedural diagnostic evaluation.

Results: CSP was performed to remove 2466 polyps in 1003 patients; cancerous lesions accounted for $0.2 \%$ of them, but all had negative margins. There were 549 polyps $(22.3 \%)$ in 186 patients who had been taking antithrombotic medication before CSP (antithrombotic group), and 1917 (77.7\%) in 817 patients not taking antithrombotic medication (non-antithrombotic group). In the antithrombotic group, 106 patients with 283 polyps continued taking the antithrombotic medication; specifically, aspirin in 41 patients with 113 polyps, clopidogrel in 13 patients with 17 polyps, dual antiplatelet therapy (DAPT) in 13 patients with 18 polyps, antiplatelet agents other than clopidogrel in 17 patients with 68 polyps, anticoagulant agents in 20 patients with 56 polyps, and antiplatelet plus anticoagulant combination therapy in 2 patients with 11 polyps. Heparin bridging was used in 13 patients with 38 polyps. Post-CSP bleeding occurred in $0.54 \%(3 / 549)$ of the interventions in the antithrombotic group and in $0.10 \%(2$ 1917) of those in the non-antithrombotic group, showing no significant difference $(\mathrm{p}=0.08)$. Endoscopic hemostasis was successful in all cases of bleeding, without requiring blood transfusion. As for the 3 cases of post-CSP bleeding in the antithrombotic group, the specific antithrombotic medication being used was aspirin in 1 patient with 1 polyp $(0.88 \%, 1 / 113)$, and aspirin and clopidogrel a patient with 2 polyps $(11.1 \%, 2 / 18)$. No post-CSP bleeding occurred in patients on other antiplatelet or anticoagulant agents, or on heparin bridging. Clipping after CSP was more likely used in the antithrombotic group (i.e., $13.5 \%$ vs. $4.6 \%$; $\mathrm{p}<0.01)$. No significant difference in post-CSP bleeding rate was observed between lesions with and without clipping ( $0 \%$ with clipping vs. $0.34 \%$ without clipping; $\mathrm{p}=0.55$ )

Conclusion: CSP is a safe procedure even in patients on antithrombotic medication. The risk of bleeding after CSP was not high compared with that after biopsy in patients on antithrombotic medication (post-procedural bleeding rate, $0.09-0.61 \%$ ), suggesting that CSP can be virtually categorized as a procedure with a low risk for hemorrhage in the guideline for periprocedural antithrombotic management.

Disclosure of Interest: All authors have declared no conflicts of interest.

\section{P0206 OPTICAL ENHANCEMENT FOR THE IN VIVO PREDICTION OF COLORECTAL POLYP HISTOLOGY}

E. Klenske, C. Neufert, A. Nägel, S. Zopf, M. F. Neurath, T. Rath Department Of Medicine ${ }^{1}$ University Hospital of Erlangen, Erlangen/Germany

Contact E-mail Address: entcho.klenske@uk-erlangen.de

Introduction: Diminutive polyps are a common finding among surveillance colonoscopies without having high prevalence of advanced histology, making their standardized removal cost-, time- and risk-intensive. Based on these considerations, the American Society of Gastrointestinal Endoscopy (ASGE) proposed the so called PIVI statement, in which diagnostic thresholds are defined that new technologies used for the real-time assessment of colorectal polyp histology should meet. Optical enhancement (OE) is a novel endoscopic pre-processing optical filter technology, in which the spectrum of the emitted wavelengths is reduced, thereby leading to enhanced visualization of the mucosal and vascular pattern.

Aims \& Methods: In this study we aimed to assess whether OE can accurately predict the histology of diminutive colorectal polyps according to the ASGE PIVI criteria. A total of 106 colorectal polyps from 49 patients undergoing diagnostic or surveillance colonoscopy were included. The in vivo histology prediction using OE was compared to results of histopathology as a reference standard.

Results: The overall accuracy of OE for real-time prediction of polyp histology was $94.3 \%$ with a sensitivity, specificity, positive (PPV) and negative prediction value (NPV) of $100 \%, 95.3 \%, 85.4 \%$ and $100 \%$, respectively. When including only high confidence (HC) predictions, the accuracy of OE increased to $96.5 \%$. Sensitivity, specificity, PPV and NPV were $100 \%, 94.5 \%, 91.2 \%$ and $100 \%$, respectively. In distal colorectal polyps the accuracy was $93.3 \%$ with sensitivity, specificity, PPV and NPV being $100 \%, 91.3 \%, 80 \%$ and $100 \%$, respectively. The post-polypectomy colonoscopy surveillance intervals were predicted correctly in $>90 \%$ of patients with $\mathrm{OE}$

Conclusion: Optical enhancement allows to accurately predict the histology of diminutive colorectal polyps in vivo in real-time and meets the PIVI thresholds for resecting and discarding diminutive polyps without histological assessment and for leaving distal diminutive colorectal polyps in place. Hence, optical enhancement can potentially reduce time, risk and costs associated with removal and histopathological assessment of diminutive polyps.

Disclosure of Interest: All authors have declared no conflicts of interest. 
P0207 BLUE LASER IMAGING OPTICAL DIAGNOSIS OF COLORECTAL POLYPS: ACCURACY OF THE NICE, SANO AND WASP CLASSIFICATIONS

S. Ribiere, J. Dreanic, M. Barret, M. Camus, M. Dior, B. Brieau, S. Leblanc, F. Prat, R. Coriat, S. Chaussade

Department Of Gastroenterology, Cochin Hospital, Assistance Publique-Hôpitaux de Paris, Paris/France

Contact E-mail Address: sophieclem.ribiere@gmail.com

Introduction: Blue Laser Imaging (BLI) is a new image-enhanced endoscopic technique, meant, in association with magnification endoscopy, to help differentiating between neoplastic and non-neoplastic colorectal polyps. A variety of endoscopic classifications have been developed to guide optical diagnosis of colorectal polyps. The aim of our study was to evaluate NICE, WASP and Sano classifications for the optical diagnosis of colorectal polyps using Blue Laser Imaging and magnification.

Aims \& Methods: Between May 2014 and December 2015, 181 colorectal polyps in 65 patients were imaged and resected in our single center study. Each polyp was evaluated using white light endoscopy, BLI with and without magnification. An independant expert reviewed the pictures and the videos of the polyps and staged them using NICE, Sano and WASP classifications: his conclusions were compared with the actual histology of the polyps. Diagnostic performances of BLI and magnification were calculated with each endoscopic classification.

Results: 181 polyps were studied, among which 125 adenomas, 24 sessile serrated adenomas/polyps, 25 hyperplastic polyps, 2 adenocarcinomas and 11 norma colorectal mucosal samples. The median polyp size was $7 \mathrm{~mm}$. Overall, the NICE, Sano and WASP classifications were comparable in terms of diagnostic performances for the optical diagnosis of colorectal adenomas $(p=0.7)$. However, the WASP classification provided the best results with a sensitivity, specificity, positive and negative predictive value, and diagnostic accuracy for the diagnosis of adenoma of 0.93 ( $95 \%$ CI 0.86 to 0.97 ), 0.80 (95\% CI 0.66 to 0.91 ), $0.93,0.80$ and 0.9 . In the rectosigmoid, negative predictive values for the diagnosis of adenoma were 0.77 ; 0.91 ; and 1.0 using NICE, Sano and WASP classifications.

Conclusion: Our work suggests that BLI with magnification is a promising technique for the optical diagnosis of colorectal polyps with a diagnostic accuracy of $86-90 \%$. Our study did not establish significant difference between the three classifications. However, the ASGE criteria for the implementation of the "resect and discard" strategy were met for the classifications of Sano and WASP with a negative predictive value for the diagnosis of adenoma beyond $90 \%$ in the rectosigmoid.

Disclosure of Interest: J. DREANIC: HOSPIRA Congress invitation M. Barret: 3D Matrix scientific work, Life partners europe training sessions,

M. Camus: Life partners europe, Medwork scientific work. Cook medical, Fujifilm France, Ipsen Pharma, Life partners europe, MSD, Olympus: training sessions.

M. Dior: Roche: congress invitation

B. Brieau: Amgen Ipsen pharma: congress invitation

S. Leblanc: Boston scientific, Cook medical: scientific work. Ibsen pharma, Olympus, Life partners europe: training sessions. Cook medical, Olympus: congress invitation.

F. Prat: Boston scientific, Medtronic, Olympus: expert. Olympus: training sessions. Cook medical: congress invitation. Medwork: patent application.

R. coriat: Amgen, Ipsen pharma, Novartis oncology Celgene, Lilly, Mayoly spindler, Pfizer, Roche, sanofi

S. Chaussade: Mayoli Spindler, Medtronic: scientific work. Bouchara recordati, Fujifilm france, Medtronic, Norgine: training sessions. Bouchara recordati congress invitation.

All other authors have declared no conflicts of interest.

\section{References}

Sano Y, Horimatsu T, Fu KI, Katagiri A, Muto M, Ishikawa H. Magnifying Observation of Microvascular Architecture of Colorectal Lesions using a Narrow-band Imaging System. Dig Endosc. 2006 Jul 1;18:S44-51.

IJspeert JEG, Bastiaansen BAJ, van Leerdam ME, Meijer GA, van Eeden S, Sanduleanu S, et al. Development and validation of the WASP classification system for optical diagnosis of adenomas, hyperplastic polyps and sessile serrated adenomas/polyps. Gut. 2016 Jun;65(6):963-70.

Sano Y, Tanaka S, Kudo S-E, Saito S, Matsuda T, Wada Y, et al. Narrow-band imaging (NBI) magnifying endoscopic classification of colorectal tumors proposed by the Japan NBI Expert Team. Dig Endosc Off J Jpn Gastroenterol Endosc Soc. 2016 Jul;28(5):526-33.

Ignjatovic A, East JE, Suzuki N, Vance M, Guenther T, Saunders BP. Optical diagnosis of small colorectal polyps at routine colonoscopy (Detect InSpect ChAracterise Resect and Discard; DISCARD trial): a prospective cohort study. Lancet Oncol. 2009 Dec;10(12):1171-8

ASGE Technology Committee, Abu Dayyeh BK, Thosani N, Konda V, Wallace MB, Rex DK, et al. ASGE Technology Committee systematic review and metaanalysis assessing the ASGE PIVI thresholds for adopting real-time endoscopic assessment of the histology of diminutive colorectal polyps. Gastrointest Endosc. 2015 Mar;81(3):502.e1-502.e16.

Yoshida N, Yagi N, Inada Y, Kugai M, Okayama T, Kamada K, et al. Ability of a novel blue laser imaging system for the diagnosis of colorectal polyps. Dig Endosc Off J Jpn Gastroenterol Endosc Soc. 2014 Mar;26(2):250-8.

Rees CJ, Rajasekhar PT, Wilson A, Close H, Rutter MD, Saunders BP, et al. Narrow band imaging optical diagnosis of small colorectal polyps in routine clinical practice: the Detect Inspect Characterise Resect and Discard 2 (DISCARD 2) study. Gut. 2017 May;66(5):887-95.
P0208 AN INNOVATIVE 3D COLONOSCOPE SHAPE IMAGING SYSTEM BASED ON FIBER BRAG GRATING ARRAY

I.K. Yoo ${ }^{1}$, B. Keum ${ }^{1}$, W. Kim ${ }^{1}$, S.J. Choi ${ }^{1}$, G. $\mathrm{Min}^{1}$, S.H. Kim ${ }^{1}$, J.M. Lee ${ }^{1}$, H.S. Choi ${ }^{1}$, E.S. Kim ${ }^{1}$, Y.T. Jeen ${ }^{1}$, H.J. Chun ${ }^{1}$, H.S. Lee ${ }^{1}$, C.D. Kim ${ }^{1}$, J.M. Lee ${ }^{1}$, J. Kim², M.S. Jang 2 , S. Yang

${ }_{1}^{1}$ Division of Gastroenterology and Hepatology, Department of Internal Medicine, Korea University College of Medicine, Seoul/Korea, Republic of

${ }^{2}$ Center for Bionics, Korea Institute of Science and Technology, Seoul/Korea, Republic of

Contact E-mail Address: borakeum@hanmail.net

Introduction: Colonoscopy is difficult procedure, largely due to unpredictable looping during insertion. If the endoscopist is able to see the colonoscope on the image display, fewer attempts are needed to straighten the shaft of the scope. A prototype Fiber Brag Grating(FBG) scope guided endoscopy provides a facility for continuous viewing on a monitor of the position of the colonoscope during examination.

Aims \& Methods: The aim of this study was to evaluate the accuracy and feasibility of the innovative 3D Colonoscope using FBG. In the first part of the study, the FBG sensor was inserted into the working channel of a routine colonoscope in the first $70 \mathrm{~cm}$ from the tip of the scope. Then, the scope was placed in front of the monitor to confirm movement of the scope in all three dimensions. We evaluated loop formation such as $\mathrm{N}$ loop, alpha loop, reverse alpha loop, with the $3 \mathrm{D}$ imaging monitor. In the second part of the study, 5 patients underwent colonoscopy with a FBG sensor, the colonoscope can be displayed in anteroposterior or lateral view, or in both positions together. Fluoroscopy was used in all investigations for comparison.

Results: In the first part of the study, the results showed that the shape sensor was reliable at a maximum bending curvature of $80 \mathrm{~mm}^{-1}$. The average tip position error was $1.722 \pm 1.678 \mathrm{~mm}$, which corresponds to $1.50 \pm 1.46 \%$ of the total length of the sensor. Scope movement and loops were detected correctly in all cases through the monitor. The prototype used in the second part of the study showed a high correlation and little discrepancy with the comparative findings at fluoroscopy.

Conclusion: Scope-guided endoscopy using FBG sensor can be successfully used to display colonoscope configuration. This flexible, thin and almost weightless shape sensor would be a novel technique for identification of colonoscope shape. Disclosure of Interest: All authors have declared no conflicts of interest.

\section{P0209 REAL-TIME MORPHOLOGIC CHANGE ON NUCLEUS \& MITOCHONDRIA OF COLON CANCER CELL BY IRREVERSIBLE ELECTROPORATION: DETECTED BY PROBE-BASED MULTIPHOTON MICROSCOPY}

I.K. Yoo ${ }^{1}$, B. Keum ${ }^{1}$, W. Kim ${ }^{1}$, S.J. Choi ${ }^{1}$, G. Min $^{1}$, J.M. Lee ${ }^{1}$, S.H. Kim ${ }^{1}$, J.M. Lee ${ }^{1}$, H.S. Choi ${ }^{1}$, E.S. Kim ${ }^{1}$, Y.T. Jeen ${ }^{2}$, H.J. Chun ${ }^{2}$, H.S. Lee ${ }^{2}$, C.D. Kim ${ }^{2}$ ${ }_{1}^{1}$ Division of Gastroenterology and Hepatology, Department of Internal Medicine, Korea University College of Medicine, Seoul/Korea, Republic of

${ }^{2}$ Department Of Internal Medicine, Institute of Digestive Disease and Nutrition, Korea University College of Medicine, Seoul/Korea, Republic of

Contact E-mail Address: borakeum@hanmail.net

Introduction: Irreversible electroporation (IRE) is a promising novel technique for tumor ablation using energy current pulses. IRE can effectively remove unwanted cells without thermally damaging surrounding tissues. We used multiphoton microscopy for evaluated the response of cancer cell to IRE ablation and found apoptotic process after applying IRE.

Aims \& Methods: By using multi-photon (MP) probes, that is ABI-Nu for nucleus and PMT for mitochondria, we were focusing on these two vital intracellular organelles for examining the real-time phenomenon of IRE-induced apoptosis. The study was conducted in three stages. Colon cancer cell lines and normal colon mucosa and colon neoplasm tissues obtained during colonoscopic biopsy from 10 patients were stained with multi-photon (MP) probes that is ABI-Nu for nucleus and PMT for mitochondria. We evaluated the feasibility of using multiphoton microscopy (MPM) to observe IRE response. First, the IRE responses of colon cancer cell lines were compared before and after IRE. Electrical pulses were administered with a Harvard apparatus, and the changes in the intensity of the nucleus and mitochondria were observed with time. Second, the IRE response of normal colon and colon cancer tissue obtained from same patient were evaluated before and after IRE same with previous method. Also, the 3-D images of the tissues co-labelled with ABI-Nu and PMT were reconstructed. Third, to assess apoptosis, colon cancer cells were stained with the fluorescent dye Annexin V or propidium iodide (PI) after applying electroporation at the same energy used earlier. Also, in order to determine whether IRE induce apoptosis, membrane blebbing of colon cancer cell lines were examined after apply IRE.

Results: MPM images of cancer cells stained with MP probes revealed that ABI$\mathrm{Nu}$ stained quicker after IRE ablation. At the tissue level, nuclear staining was present earlier and was more prominent after IRE was applied. In addition, IRE had a relatively stronger effect on cancer than normal. We obtained MPM images for each tissue slice, including four MP images for every 150 sections at a depth of $90-150 \mu \mathrm{m}$ along the z-direction. Using the same electroporation energy, staining was positive for Annexin V and PI, providing the evidence of apoptosis. Also, blebs, which are distinctive of apoptotic cells were developed after IRE in the colon cancer cell. However, bleb formation was not appeared in the control group.

Conclusion: Here, we observed using MPM that nuclear staining occurred quickly due to increased cell membrane permeability and bleb was formed after electric pulse exposure. These results are expected to challenge the understanding of the 
permeability process after IRE by providing the real-time images. Additionally, MPM can replace other apoptosis assessment methods, including Annexin VFITC and PI staining. This MP probe protocol would dramatically increase the accuracy of diagnostic techniques by providing in vivo cell images.

Disclosure of Interest: All authors have declared no conflicts of interest.

P0211 LARGE ( $>$ 30MM) POLYP ENDOSCOPIC MUCOSAL RESECTION: OUTCOMES AND PREDICTORS OF SUCCESS

K. Siau ${ }^{1}$, E. Karunadasa ${ }^{2}$, A. Kawesha ${ }^{2}$, S. Ishaq ${ }^{1}$

${ }_{1}^{1}$ Department Of Gastroenterology, Dudley Group Hospitals NHS Foundation Trust, Dudley/United Kingdom

${ }^{2}$ Department Of Surgery, Dudley Group Hospitals NHS Foundation Trust, Dudley/ United Kingdom

Contact E-mail Address: keith@siau.org

Introduction: Endoscopic mucosal resection (EMR) is an established therapeutic option for large $(>30 \mathrm{~mm})$ colonic polyps. We aimed to assess characteristics and outcomes of this cohort. Primary outcomes consisted of rates, predictors and durability of EMR success, whilst secondary outcomes included complications, malignant risk, and conversion to surgery.

Aims \& Methods: We prospectively identified patients referred for large polyp EMR from a polyp multidisciplinary team meeting between August 2008-2016) in a district general hospital with tertiary EMR expertise. Data on demographics, polyp site, morphology, size, accessibility (SMSA), histology and follow-up endoscopy were retrospectively collected. Binary logistic regression modelling was performed using SPSS, with components comprising of year, individual SMSA components, and histology. The Kaplan-Meier approach was used to measure durability of EMR success.

Results: Large polyp EMR was performed in 91 patients out of 125 MDT referrals $(73 \%)$. Patients had a median age of 72 (interquartile range [IQR] 14.4), and were predominantly male $(60 \%)$. Polyps were sessile $(46 \%)$, flat $(49 \%)$ or pedunculated $4(4 \%)$, with a median size of $40 \mathrm{~mm}$ (IQR $20.5 \mathrm{~mm}$ ), and were leftcolonic in $81 \%$. Bleeding occurred in $16.5 \%$, all of whom achieved haemostasis. The 30-day complication rate was $1.1 \%$ (delayed bleeding in 1 patient). $54(59 \%)$ were fully resected in one session, with overall EMR successful in $75(81.5 \%)$ after an average of 1.5 sessions. On multivariable analysis, significant predictors of complete resection at first attempt (Table 1) included: increasing year, sessile vs. flat morphology, and non-malignant histology. Malignant histology $(\mathrm{p}<0.001)$ predicted overall EMR failure, but not age, gender, year of EMR, SMSA score, or concomitant argon plasma coagulation. Of the EMR failure group, $11 / 16(69 \%)$ underwent surgical resection, of which $7 / 11(64 \%)$ harboured adenocarcinoma. Of the EMR success group, 4/80 were malignant polyps with $\mathrm{R} 0$ endoscopic resection. The overall malignant histology rate in this cohort was $11 / 91(12 \%)$. In this cohort, the R0 EMR success rates was $4 / 11(36 \%)$, with no recurrence after 60 months of follow-up. The overall 12-month recurrence rates following complete EMR was $1.5 \%$, with no significant factors affecting EMR durability identified.

Table 1: Predictors of complete resection on first EMR attempt. p-values derived from bivariate regression, with bold values significant if $<0.05$. *Increase in OR for each increase in year. $* *$ p-value $<0.05$ considered statistically significant.

\begin{tabular}{llll}
\hline Factor & Odds Ratio & $\begin{array}{l}95 \% \text { Confidence } \\
\text { Interval }\end{array}$ & p-value \\
\hline Year & $1.41^{*}$ & $1.04-1.90$ & $0.048^{* *}$ \\
Size $(3-3.9$ cm vs. $>4.0 \mathrm{~cm})$ & 2.96 & $0.85-10.3$ & 0.088 \\
Site (left vs. right colon) & 0.46 & $0.09-2.48$ & 0.367 \\
Access (easy vs. difficult) & 1.39 & $0.38-5.14$ & 0.619 \\
Morphology (sessile vs. flat) & 3.38 & $1.04-11.0$ & $0.043^{* *}$ \\
Non-malignant histology & 41.5 & $3.74-461$ & $0.002^{* *}$ \\
\hline
\end{tabular}

Conclusion: Large polyp EMR is a safe and effective alternative to surgical resection of large polyps. Endoscopist experience, polyp morphology, and benign histology predict complete resection at index EMR. Further data are required to evaluate the longer-term outcomes of malignant polyps.

Disclosure of Interest: All authors have declared no conflicts of interest.

\section{P0212 PROSPECTIVE RANDOMIZED CONTROLLED TRIAL COMPARING EFFICACY OF 1-L PEG-ASC WITH PRUCALOPRIDE AND 2-L PEG-ASC FOR BOWEL PREPARATION}

S.J. Choi ${ }^{1}$, Y.T. Jeen ${ }^{2}$, G. Min ${ }^{2}$, W. Kim ${ }^{2}$, J.M. Lee ${ }^{2}$, I.K. Yoo ${ }^{2}$, S.H. Kim ${ }^{2}$, J.M. Lee ${ }^{2}$, H.S. Choi ${ }^{2}$, E.S. Kim ${ }^{2}$, B. Keum ${ }^{2}$, H.S. Lee ${ }^{2}$, H.J. Chun ${ }^{2}$, C.D. Kim ${ }^{2}$ ${ }^{1}$ Gastroenterology And Hepatology, Korea University Anam Hospital, Seoul/ Korea, Republic of

${ }^{2}$ Division of Gastroenterology and Hepatology, Department of Internal Medicine, Korea University College of Medicine, Seoul/Korea, Republic of

Contact E-mail Address: drcoolandy@gmail.com

Introduction: Though numerous research has enabled decrease of the bowel preparation solution volume, it is still a major complaint of patients preparing colonoscopy. There have been studied that additional administration of laxatives could lessen the amount of aqueous formula with prokinetic effect. Prucalopride is a serotonin (5-HT4) receptor agonist which stimulate colonic mass movements and provide main propulsive force for defecation.

Aims \& Methods: The aim of this study is to compare 2-L PEG-Asc and 1-L PEG-Asc plus prucalopride for quality of bowel cleansing while preparing for colonoscopy and patient compliance. Two hundred patients were prospectively enrolled. Patients referred for colonoscopy were divided into group A (the splitdose 2-L PEG-Asc) and group B (1-L PEG-Asc+trucalopride) randomly. During colonoscopy, each patient's bowel preparation quality was evaluated with The Boston Bowel Preparation Scale (BBPS) and Aronchick Preparation Scale (APS). The tolerability and satisfaction of patients was determined based on a questionnaire-based survey.

Results: One hundred patients received either 2-L PEG-Asc or 1-L PEG-Asc with prucalopride. Regarding colon cleansing outcome (BPPS and APS), the 1-L PEG-Asc with prucalopride group showed similar, but non-inferior results compared to the 2-L PEG-Asc group on both BBPS (7.65 \pm 1.27 vs $7.52 \pm 1.40$, $\mathrm{p}=0.586)$ and APS scales $(93.3 \%$ vs $95 \%, \mathrm{p}=0.717)$. Tolerability was similar for both 1-L PEG-Asc with prucalopride and 2-L PEG-Asc.

Conclusion: 1-L PEG-Asc plus prucalopride preparation showed comparable result to traditional 2-L PEG-Asc preparation. 1-L PEG-Asc plus prucalopride preparation method could be an alternative method for bowel preparation which can relieve patient discomfort.

Disclosure of Interest: All authors have declared no conflicts of interest.

\section{P0213 IMPROVING SURVEILLANCE FOLLOW UP RATES AFTER COLONOSCOPY ENDOSCOPIC MUCOSAL RESECTION: A} QUALITY IMPROVEMENT PROJECT

M. E. Werlang ${ }^{1}$, P. Kandel $^{1}$, I. Ahn ${ }^{1}$, B. Brahmbhatt ${ }^{2}$, M. Raimondo ${ }^{1}$, E. Bouras ${ }^{1}$, T. Woodward ${ }^{1}$, M.B. Wallace ${ }^{1}$, V. Gomez

${ }^{1}$ Gastroenterology And Hepatology, Mayo Clinic, Jacksonville/United States of America

${ }^{2}$ Gastroenterology, Mayo Clinic, Jacksonville/United States of America

Contact E-mail Address: werlang.monia@mayo.edu

Introduction: Endoscopic mucosal resection (EMR) is an effective and safe treatment for large $(\geq 20 \mathrm{~mm})$ laterally spreading colorectal lesions. Although colon EMR has been established as a minimally invasive technique for treatment of large colorectal lesions, risk of adenoma recurrence is the main limitation. Current guidelines recommend first follow-up at 3-6 months; however, there are no well-designed prospective-studies published establishing an optimal system to ensure SC1 (first surveillance colonoscopy after initial exam) is scheduled and executed in the appropriate timeline. Inadequate or absent scheduling of $\mathrm{SCl}$ can have an impact on the detection of recurrent adenomas, increasing potential patient morbidity, adding unnecessary costs and potentially leading to repeated and avoidable procedures. The aim of this study was to conduct a quality improvement initiative aimed at increasing compliance in SC1 by understanding the current scheduling process and developing strategies to standardize our endoscopy center practices.

Aims \& Methods: Single tertiary referral center quality improvement project started in January 2017 and currently still in progress. We present here the interim data. Consecutive patients who had undergone or would have EMR of lesions $\geq 20 \mathrm{~mm}$ were eligible for inclusion. The process of following-up patients after EMR was divided at two levels: A dedicated team member generated a monthly report identifying patients who underwent colon EMR using our endoscopy procedure documentation program. The appropriate timeline for SC1 for each patient that underwent colonoscopy EMR was identified and orders and scheduling for the colonoscopy follow-up were carried out. Evaluation of followup: A dedicated team member reviewed the status of patients who underwent colon EMR six months prior to the start of the QI study. If patient did not show up on their scheduled follow-up, phone calls were placed to contact the patients. Patients who had followed-up with their local gastroenterologists were recorded. All the data in intervention group was compared retrospectively with nonintervention group who were not tracked through quality improvement process. Mean follow-up time and follow-up rate (\%) at 6-9 months at $\mathrm{SC1}$ after index EMR was compared between the two groups.

Results: Per-protocol 25 patients included in intervention group were compared to 60 patients in the nonintervention group. Mean age was 62 years in intervention group and 66 years in non-intervention group $(\mathrm{p}=0.04)$. There were no differences in distribution in size of lesion, gender, EMR site, and polyp histology between two groups (Table 1). The mean follow-up time in intervention group was 8.2 months $( \pm 2.6)$ and nonintervention group was 10.4 months $( \pm 9.1)$. There was increase in rate of 6-9 months follow-up in intervention group when compared to the nonintervention group $(88 \%, 95 \%$ CI $[0.80 \%-0.94 \%]$ vs $64 \%, 95 \%$ CI $[0.54 \%-0.73 \%])($ Table 1$)$

Table 1: Demographic, clinical characteristics, follow up rates

\begin{tabular}{lll}
\hline Variables & $\begin{array}{l}\text { Intervention } \\
\text { group }(\mathrm{n}=25)\end{array}$ & $\begin{array}{l}\text { Non-intervention } \\
\text { group }(\mathrm{n}=60)\end{array}$ \\
\hline Age, Mean (SD) & $62(8.7)$ & $66(10.5)$ \\
Sex, Male (\%) & $38 \%(10)$ & $58 \%(35)$ \\
$\begin{array}{l}\text { Size of polyp }(\mathrm{mm}), \\
\quad \text { Mean (SD) }\end{array}$ & $35(18)$ & $30(12)$ \\
\hline
\end{tabular}


Table 1 Continued

\begin{tabular}{|c|c|c|}
\hline Variables & $\begin{array}{l}\text { Intervention } \\
\text { group }(\mathrm{n}=25)\end{array}$ & $\begin{array}{l}\text { Non-intervention } \\
\text { group }(\mathrm{n}=60)\end{array}$ \\
\hline \multicolumn{3}{|l|}{ Site of polyp resection } \\
\hline Rectum & $8 \%(2)$ & $5 \%(3)$ \\
\hline Sigmoid & $4 \%(1)$ & $7 \%(4)$ \\
\hline Recto-sigmoid & $0 \%$ & $2 \%(1)$ \\
\hline Descending colon & $0 \%$ & $3 \%(2)$ \\
\hline Transverse colon & $15 \%(4)$ & $12 \%(7)$ \\
\hline Hepatic flexure & $15 \%(4)$ & $8 \%(5)$ \\
\hline Ascending colon & $23 \%(6)$ & $37 \%(22)$ \\
\hline Mid ascending colon & $0 \%$ & $5 \%(3)$ \\
\hline Cecum & $23 \%(6)$ & $13 \%(8)$ \\
\hline Cecum with appendicle orifice & $8 \%(2)$ & $0 \%$ \\
\hline Ileocecal valve & $4 \%(1)$ & $8 \%(5)$ \\
\hline \multicolumn{3}{|l|}{ Polyp histology } \\
\hline Sessile serrated adenoma & $23 \%(6)$ & $30 \%(18)$ \\
\hline Tubular adenoma & $35 \%(9)$ & $35 \%(21)$ \\
\hline Tubular adenoma with HGD & $8 \%(2)$ & $2 \%(1)$ \\
\hline Tubulovillous adenoma & $31 \%(8)$ & $32 \%(19)$ \\
\hline $\begin{array}{l}\text { Tubulovillous adenoma } \\
\text { with HGD }\end{array}$ & $4 \%(1)$ & $0 \%$ \\
\hline Adenocarcinoma & $0 \%$ & $2 \%(1)$ \\
\hline \multicolumn{3}{|l|}{ Follow-up Rates } \\
\hline Median(range) & $\begin{array}{l}7.3 \text { months } \\
\quad(6-15 \text { months })\end{array}$ & $\begin{array}{l}7.3 \text { months } \\
\quad(4-66 \text { months })\end{array}$ \\
\hline Mean $( \pm$ SD) & 8.2 months $(2.6)$ & $10.4(9.1)$ \\
\hline $\begin{array}{l}\text { Colon EMR follow-up rate } \\
\text { of } 6-9 \text { months, } \% \text { (n) }\end{array}$ & $\begin{array}{l}88 \%, 95 \% \\
\quad \text { CI }[0.8 \%-0.94 \%] \\
\quad(22)\end{array}$ & $\begin{array}{l}64 \%, 95 \% \\
\quad \text { CI }[0.54 \%-0.73 \%] \\
\quad(35)\end{array}$ \\
\hline
\end{tabular}

Conclusion: These preliminary results suggest significant improvement in SC1 compliance with our intervention. We believe that continuing these efforts and further refining the intervention process, requiring less personnel resources, may be helpful to improve the follow-up time until 3-6 months interval while also enduring as a sustainable change for our practice.

Disclosure of Interest: M.B. Wallace: Michael Wallace reports grant support from Boston Scientific, Medtronic, Cosmo pharmaceuticals, and equity interest in iLumen. Dr Wallace is a consultant to Aries Pharmaceuticals and Lumendi Inc.

\section{References}

1. Saunders BP, Tsiamoulos ZP. Endoscopic mucosal resection and endoscopic submucosal dissection of large colonic polyps. Nat Rev Gastroenterol Hepatol. 2016

2. Repici A, Pellicano R, Strangio G, Danese S, Fagoonee S, Malesci A. Endoscopic mucosal resection for early colorectal neoplasia: pathologic basis, procedures, and outcomes. Dis Colon Rectum. 2009;52(8):1502-1515.

3. Tanaka S, Kashida H, Saito Y, et al. JGES guidelines for colorectal endoscopic submucosal dissection/endoscopic mucosal resection. Digestive endoscopy: official journal of the Japan Gastroenterological Endoscopy Society. 2015;27(4):417-434

4. Rex DK, Schoenfeld PS, Cohen J, et al. Quality indicators for colonoscopy. Gastrointest Endosc. 2015;81(1):31-53.

\section{P0214 META-ANALYSIS SUGGESTS: INSPECT TWICE TO INCREASE RIGHT COLON ADENOMA DETECTION RATE}

K. Triantafyllou, P. Gkolfakis, G. Tziatzios, G. D. Dimitriadis

2nd Dept Of Internal Medicine And Research Institute, National and Kapodistrian University of Athens, Medical School, Athens/Greece

Contact E-mail Address: ktriant@med.uoa.gr

Introduction: Missed adenomas in the right colon are of major concern for interval colon cancer (CRC) development. There is evidence from cohort and randomized controlled studies (RCTs) that a second examination of the right coloneither in direct view or in retroflexion- increases the diagnostic yield of the procedure. However, data are not accepted unanimously.

Aims \& Methods: The aim of this meta-analysis was to examine the effect of a second, back-to-back mucosa inspection on the diagnostic yield of colonoscopy in the cecum and the ascending colon. We performed literature searches in MEDLINE to identify studies evaluating the effect of a second pass endoscopic examination on adenoma detection rate (ADR) and advanced ADR (AADR) in the right colon. Study outcomes effect sizes were calculated using RevMan 5.3 software fixed or random effect model, as appropriate, and they are presented as $\mathrm{OR}[95 \% \mathrm{CI}]$. Heterogeneity was measured using the $\mathrm{I}^{2}$ statistics. Publication bias was assessed by Funnel plots inspection and the quality of the meta-analyzed studies was assessed using the Jadad criteria.

Results: We identified 8 studies ( 5 cohort and 3 RCT, with 9 sets of data and 5639 subjects - mixed CRC screening/surveillance and symptomatic population) that reported on the aforementioned outcomes. Two sets of data examined the yield of the second direct view as compared to that of a single inspection, one set examined the cumulative yield of two passes compared to that of an extended (timely) inspection of the right colon and six sets of data evaluated the yield of the second examination of the right colon with scope retroflexion compared to that of the single direct view. They were moderate risk of bias studies; suspicion for publication bias was detected in the direct view arm of the analysis. As compared to a single pass, the second right colon inspection significantly increased ADR (1.31 [1.15-1.49], I2 $=49 \%)$. The effect size of ADR was higher in the direct view second pass arm $(1.73[1.41-2.12], \mathrm{I} 2=0 \%)$ as compared to the retroflexion arm $(1.17[1.06-1.29, \mathrm{I} 2=0 \%)$. Sensitivity analysis with removal of one study each time did not identify a single study responsible for the detected heterogeneity. Our analysis did not show significant increase in right colon AADR $(1.5[0.76-1.56], \mathrm{I} 2=0 \%)$ after the second exam.

Conclusion: In comparison to a single pass, the second inspection of the right colon either in direct view or with scope retroflection increases ADR in this colon segment. However, results should be interpreted cautiously due to the small number of meta-analyzed studies with mixed indications populations, and the detected moderate levels of heterogeneity and risk for bias.

Disclosure of Interest: All authors have declared no conflicts of interest.

\section{P0215 ENDOSCOPIC FULL-THICKNESS RESECTION FOR T1 EARLY RECTAL CANCER: A CASE SERIES (WITH VIDEO)}

S. Vavassori ${ }^{1}$, P. Soriani ${ }^{1}$, G.E. Tontini ${ }^{1}$, H. Neumann ${ }^{2}$, G. De Nucci ${ }^{3}$, D. De Toma $^{4}$, B. Bruni ${ }^{5}$, L. Pastorelli ${ }^{6}$, M. Vecchi ${ }^{6}$, P. Lagoussis ${ }^{7}$

${ }^{1}$ Gastroenterology And Digestive Endoscopy Unit, IRCCS Policlinico San Donato, San Donato Milanese/Italy

${ }_{2}^{2}$ Interventional Endoscopy Center, I. Medizinische Klinik Und Poliklinik,

Universität Erlangen-Nürnberg, Mainz/Germany

${ }_{3}^{3}$ Asst Rhodense, Garbagnate Milanese/Italy

${ }^{4}$ Oncology, IRCCS Policlinico San Donato, San Donato Milanese/Italy

${ }^{5}$ Pathology And Citodiagnostic Unit, IRCCS Policlinico San Donato, San Donato Milanese/Italy

${ }^{6}$ Department Of Biomedical Sciences For Health, University of Milan, Milan/Italy ${ }^{7}$ Division Of General Surgery I, IRCCS Policlinico San Donato, San Donato Milanese/Italy

\section{Contact E-mail Address: paola.soriani@gmail.com}

Introduction: Endoscopic treatment of malignant colorectal polyps is often challenging, especially for early rectal cancer (ERC) localized close to the dentate line. Conversely, the surgical approach may result in temporary or definitive stoma and in frequent post-surgical complications [1-2]. Endoscopic Full Thickness resection (EFTR) is a novel technique that, besides having other indications, appears to be promising for wall-thickness excision of intestinal T1 carcinoma following incomplete endoscopic resection [3-4].

Aims \& Methods: Follow-up data on patients treated with this device are scarce, particularly for ERC. We enrolled six consecutive patients with T1-ERC. They were treated with the EFTR, after appropriate staging, and their long-term outcomes were evaluated based on a detailed clinical and instrumental assessment. Results: The endoscopic en bloc full-thickness resection was technically feasible in all patients. The histopathologic analysis showed a complete endoscopic resection in all cases, and a full-thickness excision in four. Neither complication, nor disease recurrence was observed during the one-year follow-up performed. Conclusion: EFTR is a promising tool for treating ERC featuring a residual risk of disease recurrence after incomplete endoscopic mucosal resection in patients unfit for surgery or refusing surgical approach.

Disclosure of Interest: All authors have declared no conflicts of interest.

\section{References}

1 Wasif N, Etzioni D, Maggard MA et al. Trends, patterns, and outcomes in the management of malignant colonic polyps in the general population of the United States. Cancer 2011;117:931-7.

2 Ueno H, Mochizuki H, Hashiguchi Y et al. Risk factors for an adverse outcome in early invasive colorectal carcinoma. Gastroenterology 2004;127:38594.

3 Schimdt A, Damm M, Caca K et al. Endoscopic full-thickness resection using a novel over-the-scope device. Gastroenterology 2014;147:740-42.

4 Richter-Schrag HJ, Walker C, Thimme R et al. Full thickness resection device (FTRD): experience and outcome for benign neoplasms of the rectum and colon. Chirurg 2016;87(4):316-25. 
Abstract No:P0215.

Table 1: T1 early rectal cancer features, indications to endoscopic full-thickness resection, and follow-up.

\begin{tabular}{|c|c|c|c|c|c|c|c|}
\hline \# & Rectal site & Endoscopic features & $\begin{array}{l}\text { Positive Ueno's criteria } \\
\text { after en bloc EMR }\end{array}$ & Indication to EFTR & Pre-EFTR staging & Histology following EFTR & Follow-up after EFTR \\
\hline 1 & Distal & $\begin{array}{l}30 \mathrm{~mm} \text {, Is, Kudo } \mathrm{V} \text {, } \\
\text { negative lifting sign }\end{array}$ & $\begin{array}{l}\text { Tumor budding, exci- } \\
\text { sion margin, } \\
\text { Kikuchi's level, } \\
\text { width of submuco- } \\
\text { sal invasion }\end{array}$ & $\begin{array}{l}\text { unfit for surgery (ASA } \\
\text { IV) }\end{array}$ & T0, N0 & $\begin{array}{l}\text { R0, full-thickness resection; histology } \\
\text { negative for residual disease }\end{array}$ & $\begin{array}{l}\text { Endoscopy, EUS, and } \\
\text { CT negative at } 3 \\
\text { and } 12 \text { months; } \\
\text { Endoscopy and } \\
\text { EUS negative at } 18 \\
\text { months. }\end{array}$ \\
\hline 2 & Distal & $\begin{array}{l}20 \text { mm, I sp, Kudo } \\
\text { IIIL, negative lift- } \\
\text { ing sign }\end{array}$ & $\begin{array}{l}\text { Tumor budding, } \\
\text { Haggitt's level, } \\
\text { excision margin, } \\
\text { depth and width of } \\
\text { submucosal } \\
\text { invasion }\end{array}$ & $\begin{array}{l}\text { refusing surgery (ASA } \\
\text { II) }\end{array}$ & T0, N0 & $\begin{array}{l}\text { R0, full-thickness resection; histology } \\
\text { negative for residual disease }\end{array}$ & $\begin{array}{l}\text { Endoscopy, EUS and } \\
\text { CT negative at } 6 \\
\text { and } 12 \text { months }\end{array}$ \\
\hline 3 & Distal & $\begin{array}{l}18 \text { mm, I sp, Kudo } \\
\text { IIIL, negative lift- } \\
\text { ing sign }\end{array}$ & $\begin{array}{l}\text { Haggitt's level, excision } \\
\text { margin, depth and } \\
\text { widht of submuco- } \\
\text { sal invasion }\end{array}$ & $\begin{array}{l}\text { refusing surgery (ASA } \\
\text { III) }\end{array}$ & T0, N0 & $\begin{array}{l}\text { R0, complete submucosal resection } \\
\text { but no muscolaris propria layer } \\
\text { in the specimen; histology nega- } \\
\text { tive for residual disease }\end{array}$ & $\begin{array}{l}\text { Endoscopy, EUS and } \\
\text { CT negative at } 6 \\
\text { and } 12 \text { months }\end{array}$ \\
\hline 4 & Proximal & $\begin{array}{l}0.6 \mathrm{~mm} \text {, I s, Kudo } \mathrm{V} \text {, } \\
\text { negative lifting sign }\end{array}$ & $\begin{array}{l}\text { Haggitt's level, excision } \\
\text { margin }\end{array}$ & $\begin{array}{l}\text { unfit for surgery (ASA } \\
\text { IV) }\end{array}$ & T1, N0 & $\begin{array}{l}\text { R0, full-thickness resection; histology } \\
\text { positive for adenocarcinoma }\end{array}$ & $\begin{array}{l}\text { Endoscopy, EUS and } \\
\text { CT negative at } 6 \\
\text { month. Patient } \\
\text { died for severe car- } \\
\text { diac disease at } 8^{\circ} \\
\text { follow-up month. }\end{array}$ \\
\hline 5 & Distal & $\begin{array}{l}0.7 \mathrm{~mm}, \mathrm{I} \mathrm{s}, \text { Kudo IV, } \\
\text { negative lifting sign }\end{array}$ & $\begin{array}{l}\text { Low tumor differentia- } \\
\text { tion grade, excision } \\
\text { margin }\end{array}$ & $\begin{array}{l}\text { unfit for surgery (ASA } \\
\text { IV) }\end{array}$ & T0, N0 & $\begin{array}{l}\text { R0, full-thickness resection; histology } \\
\text { negative for residual disease }\end{array}$ & $\begin{array}{l}\text { Endoscopy, EUS and } \\
\text { CT negative at } 6 \\
\text { and } 12 \text { months }\end{array}$ \\
\hline 6 & Distal & $\begin{array}{r}18 \mathrm{~mm}, \mathrm{I} \text { s, Kudo IIIL, } \\
\text { negative lifting sign }\end{array}$ & $\begin{array}{l}\text { Tumor budding, exci- } \\
\text { sion margin, width } \\
\text { of submucosal } \\
\text { invasion }\end{array}$ & $\begin{array}{l}\text { refusing surgery (ASA } \\
\text { III) }\end{array}$ & T0, N0 & $\begin{array}{l}\text { R0, complete submucosal resection } \\
\text { but no muscolaris propria layer } \\
\text { in the specimen; histology nega- } \\
\text { tive for residual disease }\end{array}$ & $\begin{array}{l}\text { Endoscopy, EUS and } \\
\text { CT negative at } 6 \\
\text { and } 12 \text { months }\end{array}$ \\
\hline
\end{tabular}

P0216 UNTUTORED LEARNING CURVE ANALYSIS FOR COLORECTAL ENDOSCOPIC SUBMUCOSAL DISSECTION: PREDICTIVE FACTORS FOR COMPLEX TECHNIQUE

F. Ramos-Zabala ${ }^{1}$, J. Vásquez-Guerrero ${ }^{2}$, A. Alzina-Pérez ${ }^{1}$, M. García-Mayor ${ }^{2}$,

A. Domínguez-Pino ${ }^{3}$, J.M. Cárdenas-Rebollo ${ }^{4}$, F.J. Pérez-Rodríguez ${ }^{1}$,

J. Rodríguez-Pascual ${ }^{1}$, L. Moreno-Almazán ${ }^{1}$

${ }^{1}$ Servicio De Aparato Digestivo, Hospital Universitario HM Montepríncipe,

Boadilla del Monte (Madrid)/Spain

${ }^{2}$ Servicio De Aparato Digestivo, Hospital Universitario HM Puerta del Sur,

Móstoles (Madrid)/Spain

${ }^{3}$ Servicio De Anestesiología Y Reanimación, Hospital Universitario HM

Montepríncipe, Boadilla del Monte (Madrid)/Spain

${ }^{4}$ Facultad de Medicina. Universidad CEU San Pablo, Madrid/Spain
Contact E-mail Address: jorgevasquez $81 \mathrm{md} @$ gmail.com

Introduction: Colorectal Endoscopic Submucosal Dissection (CR-ESD) is technically difficult, time-consuming, and has a long learning curve for Western endoscopists. Several factors related with greater difficulty while performing this technique have been described. Generally, during the learning curve phase, we select simple lessions while initiating the technique.

Aims \& Methods: Our goal was to assess those factors associated with greater difficulty during untutored DSE-CR without prior selection of less difficult lessions. All patients who attended the complex colorectal polyps consultation were included consecutively. No polyps regardless of their size, morphology, location or any characteristic of greater technical difficulty were ruled out. All CR-ESDs were performed by an endoscopist with previous animal model experience. The demographic and clinical characteristics of the patient, the morphology of the lesion and factors related to the technique were collected. A complex technique

Abstract No:P0216

\begin{tabular}{|c|c|c|c|c|c|c|}
\hline & Complex $(n=27)$ & No complex $(n=27)$ & $P$ Univ. & Odds ratioUniv. & PMultiv. & Odds ratioMultiv \\
\hline \multicolumn{7}{|l|}{ SEX, n $(\%)$} \\
\hline Male Female) & $16(59.2) 11(40.8)$ & $49(60.4) 32(39.6)$ & 0.910 & $0.95(0.39-2.31)$ & & \\
\hline \multicolumn{7}{|l|}{ AGE, $n(\%)$} \\
\hline$<70$ years old $\geq 70$ years old & $17(63) 10(37)$ & $51(63) 30(37)$ & 1 & $1.00(0.41-2.46)$ & & \\
\hline \multicolumn{7}{|l|}{ SMOKER, n(\%) } \\
\hline No Yes Former smoker & $\begin{array}{l}12(44.4) \\
4(14.8) \\
11(40.8)\end{array}$ & $\begin{array}{l}30(37) \\
18(22.2) \\
33(40.8)\end{array}$ & $\begin{array}{l}0, \\
3620 \\
708\end{array}$ & $\begin{array}{l}11, \\
8(0.50-6.43) 1, \\
2(0.46-3.12)\end{array}$ & & \\
\hline \multicolumn{7}{|c|}{$\begin{array}{l}\text { ANTICOAGULANT/ANTIAGGREGANT/ } \\
\text { COAGULATION DEFICIT, } \mathrm{n}(\%)\end{array}$} \\
\hline $\begin{array}{l}\text { No } \\
\text { Yes }\end{array}$ & $\begin{array}{l}22(81.4) \\
5(18.6)\end{array}$ & $\begin{array}{l}61(75.3) \\
20(24.7)\end{array}$ & $\begin{array}{l}0, \\
510\end{array}$ & $\begin{array}{l}0 \\
70(0.23-2.07)\end{array}$ & & \\
\hline \multicolumn{7}{|l|}{ Body Mass Index (obese), $\mathrm{n}(\%)$} \\
\hline$<30 \geq 30$ & $22(81.4) 5(18.6)$ & $72(88.9) 9(11.1)$ & 0.510 & $1.82(0.55-6.00)$ & & \\
\hline \multicolumn{7}{|l|}{ Body Mass Index (overweight), $\mathrm{n}(\%)$} \\
\hline$<25 \geq 25$ & $10(37) 17(63)$ & $36(44.4) 45(55.6)$ & 0.500 & $1.36(0.56-3.33)$ & & \\
\hline \multicolumn{7}{|l|}{ ANESTHETIC RISK, n(\%) } \\
\hline Low (ASA I-II) High (ASA III) & $18(66.6) 9(33.3)$ & $59(72.9) 22(27.1)$ & 0.539 & $1.34(0.53-3.43)$ & & \\
\hline \multicolumn{7}{|c|}{ PREVIOUS COLORECTAL SURGERY, n(\%) } \\
\hline No Yes & 25 (92.6) 2 (7.4) & $68(84.0) 13(16.0)$ & 0.261 & $0.418(0.09-1.99)$ & & \\
\hline \multicolumn{7}{|l|}{$\mathrm{CO} 2$ insufflation, $\mathrm{n}(\%)$} \\
\hline No Yes & $15(55.6) 12(44.4)$ & $16(19.8) 65(80.2)$ & $<0.001$ & $5.08(1.99-12.94)$ & 0.030 & $6.34(1.20-33.57)$ \\
\hline \multicolumn{7}{|l|}{ Size, $n(\%)$} \\
\hline $\begin{array}{l}<35 \mathrm{~mm} \geq 35 \mathrm{~mm} \\
\text { LOCATION, } \mathrm{n}(\%)\end{array}$ & \multicolumn{6}{|c|}{ LOCATION, n(\%) } \\
\hline Right Colon Left Colon Rectum & $17(63) 5$ (18.5) 5 (18.5) & $55(67.9) 17(21) 9(11.1)$ & 0.9320 .342 & $11.05(0.3-3.2) 0.56(0.1-1.8)$ & & \\
\hline \multicolumn{7}{|l|}{ MORPHOLOGY, n(\%) } \\
\hline LST-G LST-NG No LST & $16(59.3) 9(33.3) 2(7.4)$ & $36(44.4) 43(53.1) 2(2.5)$ & 0.1080 .587 & $12.12(0.8-5.3) 0.44(0.06-3.4)$ & & \\
\hline \multicolumn{7}{|l|}{ SEVERE FIBROSIS, n(\%) } \\
\hline No $\mathrm{Si}$ & $14(51.9) 13(48.1)$ & 75 (92.6) 6 (7.4) & $<0.001$ & $11.61(3.78-35.69)$ & 0.039 & $7.42(1.11-49.65)$ \\
\hline \multicolumn{7}{|l|}{ FATTY TISSUE, n(\%) } \\
\hline No $\mathrm{Si}$ & $11(40.7) 16(59.3)$ & $63(77.8) 18(22.2)$ & $<0.001$ & $5.09(2.01-12.90)$ & 0.035 & $5.78(1.13-29.53)$ \\
\hline Time dissection Mean, min (range) & $180(80-280)$ & $131.9(45-290)$ & not applicable & & & \\
\hline
\end{tabular}


was defined as that dissection that is not done en block and/or had complications.

Results: 112 lesions were selected, discarding 4 due to deep invasion. We evaluated in this study 108 DSE-CR, $27(25 \%)$ of which were compatible with our definition of "complex" ESD. In Table 1 you can see the characteristics of each group. Univariate analysis showed that variables such as size over $35 \mathrm{~mm}[63 \%$ vs. $27.2 \%$; OR 4.56 (95\% CI: $1.81-11.46) ; \mathrm{P}=0.001]$, absence of $\mathrm{CO} 2$ [55.6\% vs. $19.8 \%$; OR 5.08 (95\% CI: $1.99-12.94) ; \mathrm{P}<0.001]$, presence of serious fibrosis in the submucosa [48.1\% vs. $7.4 \%$; OR $11.61(95 \%$ CI: 3.78-35.69); P $<0.001]$ and presence of fatty tissue in the submucosa $[59.3 \%$ vs. $22.2 \%$; OR 5.09 (95\% CI: 2.01-12.90); $\mathrm{P}<0.001]$ were related to a "complex" ESD. Finally, in the multivariate analysis, those variables were associated with a complex technique with an Odds Ratio of 7.42 for severe fibrosis $(\mathrm{p}=0.039), 6.34$ for non-CO2 insufflation $(p=0.030), 5.78$ in the presence of fatty tissue in the submucosa $(p=0.035)$ and 5.74 in size greater than $35 \mathrm{~mm}(\mathrm{p}=0.025)$. There was no relation with the complexity of the technique the demographic-clinical characteristics of the patient, nor the location-morphology of the lesions. The duration of the technique was an average of 48 minutes longer in cases of a complex technique.

Conclusion: In our series the difficulty of CR-ESD was associated with factors described in other studies such as the size, the non-insufflation of $\mathrm{CO} 2$ and the presence of severe fibrosis in the submucosa. Our results describe the presence of fatty tissue in the submucosa as a new predictor of technical difficulty. In our study, we did not select the location to begin the technique, and in our learning curve we did not find significant differences in the performance of ESD in the proximal colon, distal or rectum.

Disclosure of Interest: All authors have declared no conflicts of interest.

\section{P0217 PERSISTENT PAIN AFTER COLONIC ENDOSCOPIC MUCOSAL RESECTION: PREDICTORS, A MANAGEMENT ALGORITHM AND OUTCOMES}

L. Desomer, D. J. Tate, H. Awadie, L. Pillay, G. Ahlenstiel, M.J. Bourke Department Of Gastroenterology And Hepatology, Westmead Hospital, Sydney/ Australia/NSW

Contact E-mail Address: lobkedesomer@gmail.com

Introduction: Endoscopic mucosal resection (EMR) of large ( $\geq 20 \mathrm{~mm}$ ) laterally spreading colonic lesions (LSL) is safe, effective and superior to surgery. This advantage is based on a day stay model of care; however, the most common adverse event is abdominal pain and this is a major impediment to its efficiency. No prospective data exist on the optimal selection of analgesics, the necessary recovery period or the triggers that should alert the practitioner to a more serious trajectory and the need for escalation of care.

Aims \& Methods: We aimed to characterise potential predictors for PP and develop a simple and effective management algorithm for patients with PP based on the need for analgesics in recovery. Data on consecutive patients with a LSL referred for EMR at a single, tertiary referral centre were included. Patient and lesion characteristics and peri-procedural data were prospectively collected. Standard post EMR care included 2 hours in first stage recovery followed by 1 hour in $2^{\text {nd }}$ stage recovery where clear fluids were given and discharge after if the patients were well. Persistent post-procedural pain (PP) was graded from 0 to 10 using a Visual Analogue Scale (VAS). If PP occurred $>5$ minutes, 1 gram of acetaminophen was administered parenterally and outcomes were monitored. If pain settled the patient was transferred to second stage recovery after medical review. PP $>30$ minutes lead to clinical review and upgrade of analgesics to fentanyl, with a starting dose of 25 micrograms (mcg) up to a maximum of $100 \mathrm{mcg}$. Investigations, admission and interventions for PP were recorded.

Results: 166 patients with 166 lesions were included between February and April 2017. 34/166 (20.5\%) of patients had PP requiring intervention (median VAS 5, IQR 3-6). 27/34 (79.4\%) had resolution of pain with acetaminophen only and were ultimately discharged without sequelae. $7 / 34$ patients $(20.6 \%)$ required fentanyl ( $25 \mathrm{mcg}$ of fentanyl in 3 patients, $50 \mathrm{mcg}$ in $1.75 \mathrm{mcg}$ in 1 and $100 \mathrm{mcg}$ in 2).
A CT scan was performed in the 2 patients requiring $100 \mathrm{mcg}$ of fentanyl, showing serositis in 1 patient and no abnormalities in the other. Both patients were admitted and managed conservatively (discharge day 6 and 2 respectively). The other 5 patients were discharged home on the same day after extended recovery. Predictors of PP were lesion size $\geq 45 \mathrm{~mm}(P=.003)$, Paris classification $(P=.022)$ and intra-procedural bleeding requiring endoscopic control (IPB, $P=.042$ ). Lesion size $\geq 45 \mathrm{~mm}$ and IPB were also independent variables on multivariate analysis with an odds ratio of 2.8 (95\% confidence interval $1.3-6.3$, $\mathrm{p}=.012)$ and $2.3(95 \%$ confidence interval $1.0-5.2, \mathrm{p}=.042$ respectively (Table 1).

Conclusion: Pain after EMR occurs in $20 \%$ of patients and is associated with larger lesion size and intraprocedural bleeding requiring endoscopic control in a multivariate analysis. If pain subsides after parenteral acetaminophen and does not recur the patient can be safely and confidently discharged to the step down recovery area and after medical review allowed to leave hospital. PP despite parenteral acetaminophen heralds a more serious scenario and imaging should be considered when stronger analgesics do not relieve the pain.

Disclosure of Interest: All authors have declared no conflicts of interest.

\section{P0218 QUALITY OF SINGLE-SESSION COLONOSCOPIC} EXAMINATIONS INTENDING TO REMOVE ALL NEOPLASTIC POLYPS USING COLD POLYPECTOMY IN OUTPATIENT SETTING: RESULTS FROM CLINICAL PRACTICE DATA OF SINGLE CANCER CENTER HOSPITAL IN JAPAN

K. Hotta, K. Imai, S. Ito, Y. Kishida, M. Yoshida, N. Kawata, N. Kakushima, K. Takizawa, M. Tanaka, H. Ishiwatari, H. Matsubayashi, H. Ono Division Of Endoscopy, Shizuoka Cancer Center, Nagaizumi/Japan

\section{Contact E-mail Address: k.hotta@scchr.jp}

Introduction: Some high-quality, large-scale cohort studies proved removals of colorectal neoplasms achieved prevention of colorectal cancer incidence and deaths. We introduced a strategy of removing all neoplastic polyps in single session colonoscopic examinations using cold polypectomy was started.

Aims \& Methods: The aim of this retrospective study was to investigate about achievement of colorectal polyp remove in our clinical practice setting. Scheduled colonoscopic examinations for 40-79 years outpatients who had at least one colorectal neoplasm between January 2015 and December 2016 were collected from our endoscopic data base. Exclusion criteria were as follows; patients who had colorectal neoplasm larger than $20 \mathrm{~mm}$, pre-examination of colorectal surgery or endoscopic submucosal dissection, inflammatory bowel disease, familial adenomatous polyposis, uncontrolled malignancies, by trainee endoscopists ( $<500$ colonoscopies), no agreements of polyp removal, and/or patients with continuation of anti-thrombotic agents. Outcome measurements were polyp removal rate (per-lesion analysis), complete polyp removal rate (per-patient analysis) and complications. Proportions of each endoscopic removal method according to size were also analyzed.

Results: A total of 2527 patients (mean age 66.8 y, 799 females) with 8203 colorectal neoplasms (CRNs) (7675 adenomas, 423 serrated polyps and 105 Tis and T1 cancers) who met inclusion and exclusion criteria were analyzed. Mean number of CRNs per patients was 3.2. Mean size was $4.7( \pm 2.9) \mathrm{mm}$. Polyp removal rate (per-lesion) and complete polyp removal rate (per-patient) were 97.0\% (7955 8203 ) and $94.7 \%$ (2394/2527), respectively. Post-polypectomy bleeding requiring endoscopic hemostasis occurred in 7 patients $(0.27 \%)$ and all origins of bleeding were endoscopic mucosal resection (EMR) and hot snare polypectomy (HSP). Post electrocoagulation syndrome requiring admission was occurred in one patient $(0.04 \%)$ after pre-cutting EMR. Mean procedure time was $27.4( \pm 13.3)$ min. Proportions of each endoscopic removal method according to size were presented in an attached table. In 1-4 mm CRNs, both cold snare polypectomy (CSP) $(51.8 \%)$ and cold forceps polypectomy (CFP) $(45.8 \%)$ for $1-4 \mathrm{~mm}$ CRNs were main methods. In 5-9 m CRNs, CSP was a leading method $(73.8 \%)$ and EMR was the second one $(24.1 \%)$. CRNs larger than $10 \mathrm{~mm}$ were almost removed by EMR $(94.4 \%)$

\section{Abstract No:P0217}

Table 1.: Baseline and lesion characteristics. Comparison between the patients with and without pain post endoscopic mucosal resection of a large ( $\geq 20$ mm) laterally spreading lesion. SD: standard deviation, IQR: interquartile range, *left colon: distal to hepatic flexure, "\# using thermal therapy.

\begin{tabular}{|c|c|c|c|c|}
\hline & No pain $(\mathrm{n}=132)$ & Pain $(n=34)$ & P-value & Multivariate \\
\hline \multicolumn{5}{|l|}{ Baseline characteristics } \\
\hline Age (years, mean, SD) & $69.2(10.6)$ & $69.6(10.7)$ & .944 & \\
\hline Sex $(\%)$ Male Female & $73(55.3) 59(44.7)$ & $14(41.2) 20(58.8)$ & .141 & \\
\hline \multicolumn{5}{|l|}{ Lesion characteristics } \\
\hline Size $(\mathrm{mm}, \%) 20-44 \mathrm{~mm} \geq 45 \mathrm{~mm}$ & $103(78.0) 29(22.0)$ & $18(52.9) 16(47.1)$ & .003 & .012 \\
\hline Location $(\%)$ Left colon* Right colon & $53(40.2) 79(59.8)$ & $17(50.0) 17(50.0)$ & .300 & \\
\hline Paris classification $(\%)$ 0-Is 0 -IIa 0 -IIa + Is Others & $6(4.5) 84(63.6) 40(30.3) 2(1.5)$ & $1(2.9) 14(41.2) 16(47.1) 3(8.8)$ & .022 & \\
\hline $\begin{array}{l}\text { Procedural data } \\
\text { Submucosal fibrosis (\%) }\end{array}$ & $38(28.8)$ & $9(26.5)$ & .789 & \\
\hline Intra-procedural bleeding requiring endoscopic control ${ }^{\#}(\%)$ & $59(44.7)$ & $23(67.6)$ & .017 & 0.042 \\
\hline Intra-procedural perforation $(\%)$ & $6(4.5 \%)$ & $0(0 \%)$ & .348 & \\
\hline
\end{tabular}


Proportions of each endoscopic removal method according to size

\begin{tabular}{lllll}
\hline Size $(\mathrm{mm})$ & CFP & CSP & HSP & EMR \\
\hline $1-4(\mathrm{~N}=5046)$ & $45.8 \%$ & $51.8 \%$ & $0.5 \%$ & $1.9 \%$ \\
$5-9(\mathrm{~N}=2294)$ & $0.6 \%$ & $73.8 \%$ & $1.5 \%$ & $24.1 \%$ \\
$10-20(\mathrm{~N}=612)$ & $0 \%$ & $4.9 \%$ & $0.7 \%$ & $94.4 \%$ \\
\hline
\end{tabular}

Conclusion: In our clinical practice setting, the polyp removal rates were satisfactory level in single session colonoscopic examinations using cold polypectomy. Disclosure of Interest: All authors have declared no conflicts of interest.

\section{P0219 META-ANALYSIS AND OWN EXPERIENCE IN THE TREATMENT OF RECTO-UROGENITAL FISTULA USING THE OVER-THE-SCOPE CLIP (OTSC)}

M. Raithel ${ }^{1}$, M. Vetter ${ }^{2}$, A. Braun ${ }^{1}$, T. Vasilakis ${ }^{2}$, A. F. Hagel ${ }^{2}$

${ }^{1}$ St. Marien Waldkrankenhaus, Erlangen/Germany

${ }^{2}$ University Clinical Center Erlangen, Erlangen/Germany

Contact E-mail Address: martin.raithel@waldkrankenhaus.de

Introduction: The transmural OTSC is used to achieve a full-thickness, serosa-toserosa apposition (emergency \& elective cases) for closure of GI wall defects (perforation, leak, fistula) with reported mean closure rates of $62-100 \%$ (range $0-100 \%$ ), depending on the size of perforation, type and nature of lesion and the endoscopists' experience ${ }^{1-3}$. However, recto-urogenital fistula may arise from a variety of etiologies and are mostly leaks or fistula of chronic nature, rarely acute perforations with vital wound tissue. They may occur in Crohn's disease, but can also be a consequence of abdominal surgery, traumatic lesions or post-radiation damage.

Aims \& Methods: To further explore the role of the OTSC in this particular type of fistula we analyzed own cases and 21 reports from the literature dealing with any type of recto-urogenital fistula. In total, 25 patients. were identified with closure of a recto-urogenital fistula using the OTSC, but there was considerable heterogeneity, because of the fistula location (rectocutaneous $n=2$, rectovaginal $\mathrm{n}=10$, rectovesical $\mathrm{n}=7$, rectourethral $\mathrm{n}=2$, other rectal fistula $\mathrm{n}=3$ ).

Results: In most situations a previous interdisciplinary discussion was reported before an OTSC attempt, or patients refused to undergo re-operation. However, special characteristics of these leaks were reported to make more difficult the OTSC procedure compared with other GI locations, e.g. the site of the fistula is nearby located to L. dentata and anal sphincter, it includes a localization with little space for endoscopic manipulation, fibrous and scarry tissue is around the fistula in rectum or anastomosis and there may be sometimes suture material in situ. Thus, the tissue is often fixed and there is not so much tissue for grasping tissue into the OTSC.

The diagnosis of recto-urogenital fistula was usually made by endoscopic visualization and radiologically documented extravastion of contrast media into the vagina, urethra, bladder or into other adjacent tissue. For fistula closure traumatic OTSC was mostly used, but sometimes other adjuvant therapeutic modalities were also combined locally (e.g. histoacryl injection, fibrin glue, argon plasma coagulation, brushing etc) or systemically (e.g. ascorbic acid $7.5 \mathrm{~g}$ i.v.) to stimulate wound healing. The procedural success of occluding various types of recto-urogenital fistulae by the OTSC system was found to be successful in $71 \%$ $(0-100 \%)$ in the short-term, while a durable clinical success was found in only $52 \%(0-100 \%)$ of all 25 patients. The success rate was lower in cases of highly fibrotic chronic fistulae, after dislocation of clips and when using the atraumatic clip. Thus, even in this distal problematic site usage of a traumatic OTSC should be tried in experienced hands before surgical repair.

Conclusion: In conclusion, recto-urogenital fistula may be a potential indication for OTSC application, after interdisciplinary consensus, when re-operation is avoided, deemed to be too risky or cumbersome. Although this type of fistula carries some difficulties because of little space, tissue tension and fibrous or postop changes, long-term success may be achieved in around half of all patients. Further improvements should focus on increasing healing potency of the fistula or better after performing anastomosis creation (ascorbic acid?), to avoid postoperative recto-urogenital leaks.

Disclosure of Interest: All authors have declared no conflicts of interest.

\section{References}

1. Weiland T, Fehlker M, Gottwald T, Schurr MO. Performance of the OTSC System in the endoscopic closure of gastrointestinal fistulae - a meta-analysis. Minim Invasive Ther Allied Technol. 2012 Jul;21(4):249-58.

2. Prosst RL, Joos AK, Ehni W, Bussen D, Herold A. Prospective pilot study of anorectal fistula closure with the OTSC Proctology. Colorectal Dis. 2015 Jan;17(1):81-6.

3. Raithel, M., Albrecht, H., Scheppach, W. et al. Outcome, comorbidity, hospitalization and 30-days mortality after closure of acute perforations and postoperative anastomotic leaks by the over-the-scope-clip (OTSC) in an unselected cohort of patients. Surg Endosc (2016). doi:10.1007/s00464-0165242-x.
P0220 QUALITY INDICATORS FULFILLMENT AT QUALISCOPIA

STUDY

C. Mangas ${ }^{1}$, E. Santana Rocamora ${ }^{1}$, A. Suárez González ${ }^{2}$, I. Portillo Villares ${ }^{3}$, A. Seoane ${ }^{4}$, M. Ponce ${ }^{5}$, P. Diez ${ }^{6}$, E. Quintero Carrion ${ }^{7}$, M. Herraiz ${ }^{8}$, M. Pellisé Urquiza $^{9}$, Á. Ferrández Arenas ${ }^{10}$, V. Hernández ${ }^{11}$, Á.E. Pizarro Moreno ${ }^{12}$, R. Jover ${ }^{1}$

${ }^{1}$ Hospital General Universitario de Alicante, Alicante/Spain

${ }^{2}$ Servicio De Aparato Digestivo, Hospital Universitario Central de Asturias, Oviedo/Spain

${ }^{3}$ Hospital Universitario de Donostia, San Sebastián/Spain

${ }^{4}$ Hospital del Mar-Parc de Salut Mar Barcelona, Barcelona/Spain

${ }^{5}$ Hospital Universitari i Politecnic de la Fe, Valencia/Spain

${ }^{6}$ Gastroenterology, Hospital Universitario Rio Hortega, Valladolid/Spain

${ }^{7}$ Gastroenterology, Hospital Univ. de Canarias, Santa Cruz de Tenerife/Spain

${ }^{8}$ Clinica Universitaria de Navarra, Pamplona/Spain

${ }^{9}$ Gastroenterology, Hospital Clinic Barcelona, Barcelona/Spain

${ }^{10}$ Hospital Clínico Universitario Lozano Bleza, Zaragoza/Spain

${ }^{11}$ Instituto de Investigación Biomédica, Xerencia de Gestión Integrada de Vigo,

Vigo/Spain

${ }^{12}$ Gastroenterology, Hospital Virgen del Rocio, Sevilla/Spain

Contact E-mail Address: cmangassanjuan@gmail.com

Introduction: Colonoscopy plays a key role in the prevention and diagnosis of colorectal cancer (CRC), and the quality of it influences interval cancer. However, we have little information on compliance with quality standards in Spain.

Aims \& Methods: Knowing quality indicators fulfillment may lead to apply measures to improve the efficiency in the colonoscopy. Hence the aim of this study was to know the quality indicators of colonoscopies in a sample of centers in Spain. A total of 6912 colonoscopies performed between January and November 2016 were prospectively included in the QUALISCOPIA project, an observational, multicenter and prospective study, developed in 12 centers in Spain. Patients between the ages of 40 and 80 years with indication for colonoscopy due to digestive symptoms, post-polypectomy surveillance, positive fecal immunochemical test (FIT + ) and direct colonoscopy of colorectal cancer were included. The exclusion criteria were patients with diagnosis of colorectal cancer or adenomas in the last 6 months, incomplete excision or post-excised adenomas, treatment of colon stenosis, abdominal or rectal mass, inflammatory bowel disease and hereditary cancer syndrome.

Results: $51.9 \%$ (3586) patients were men and the median age was 61 years Regarding the colonoscopies indications, thirty one percent (2971) of the patients presented gastrointestinal symptoms, $20.3 \%$ (1398) were admited due to postpolypectomy surveillance, $28.3 \%$ (1949) presented positive fecal inmmunochemical test (FIT + ), and $8.4 \%$ (578) due to direct screening. $70.4 \%$ (4869) of the explorations were performed in the morning shift. Respecting the bowel preparation, 48.4\% used polyetilenglycol (PEG) 2L, 27.5\% (1902) used sodium picosulphate/magnesium citrate, and 19.5\% (1347) PEG4L. Digital chromoendoscopy was used for lesion seen in $4.7 \%$ (322) and panchrome in $0.1 \%$ (7). CO2 was used in $42 \%$ (2906) of the procedures. Colon cleansing was good-excellent in $86.1 \%$ (5512) and cecal intubation was performed in 95.8\% (6590). $92.8 \%(6260)$ of the colonoscopies were performed under sedation, and the gastroenterologist was responsible for it in $80.1 \%$ (4995). The most used drugs for sedation were propofol in $63.9 \%(4417)$, midazolam in $44.1 \%$ (3045) and fentanyl in $31.1 \%(2149)$. Olympus was used in $69.2 \%$ (4732), Pentax in $21.8 \%$ (1492) and Fuji in $8.3 \%$ (569). Polyps were found in $50.9 \%$ (3515) of the procedures, and CRC was found in $4.1 \%(281)$. The total number of adenomas was 6249 , and the total number of traditional and sessile serrated adenomas was 224 . The adenomas detection rate (ADR) was $39.6 \%$. The sessile and traditional serrated polyps detection rate (SDR) was $2.2 \%$. And for advanced adenomas, detection rate (AADR) was $21.5 \%$ and for colorectal cancer $4.1 \%$.

Conclusion: In Spain, there is good compliance with the quality indicators of colonoscopies.

Disclosure of Interest: All authors have declared no conflicts of interest.

\section{P0221 ENDOSCOPIC SUBMUCOSAL DISSECTION (ESD) IN THE COLORECTUM: FEASIBILITY IN AN EUROPEAN SINGLE CENTER CASE SERIES}

R.M. Lackermeier ${ }^{1}$, M. Meiborg ${ }^{1}$, S. Schürle ${ }^{1}$, C. Aubele ${ }^{1}$, E. Tsegai-Eh ${ }^{1}$ M. Edelmann ${ }^{1}$, H. Kitterer ${ }^{1}$, S. Leykauf ${ }^{1}$, M. Hack ${ }^{2}$, G. Kleber ${ }^{3}$

${ }^{1} 1$ st Department Of Medicine, Ostalb-Klinikum, Aalen/Germany

${ }^{2}$ Ostalb-klinikum Aalen, Institute of Pathology, Aalen/Germany

${ }^{3} 1$ st Department Of Medicine, Ostalb-Klinikum Aalen, Acad. Teaching Hospital, University of Ulm, Aalen/Germany

Contact E-mail Address: regina.lackermeier@uni-ulm.de

Introduction: While ESD in the upper GI tract is well established, it is as yet not standard of care in the colorectum. Contrary to Japan, western experience is limited and only relatively few case series have been published in Europe (e.g. Dessain et al. 2017).

Aims \& Methods: For the period 5/2012-1/2017 the first fifty-one consecutive patients with colorectal $(\mathrm{n}=18 / 17 / 16 \mathrm{rectum} / \mathrm{left} / \mathrm{right}$ hemicolon $)$ neoplasias (diameter $27 \pm 13 \mathrm{~mm}, \mathrm{x} \pm \mathrm{SD}$; low grade dysplasia: $\mathrm{n}=8 / 9 / 14$ serrated/tubular/villous; high grade dysplasia: $\mathrm{n}=12$; malignant: $\mathrm{n}=4$ ) or hyperplastic polyps $(n=4)$ receiving ESD according to a predefined protocol (hook or dual knife, Olympus Medical Systems, Hamburg, Germany; procedure time $162 \pm 63 \mathrm{~min} ; \mathrm{x} \pm \mathrm{SD}$ ) are reported. ESD was performed by a single investigator (G.K.) trained previously by Japanese experts and in experimental models. ESD was complete $(\mathrm{n}=22)$ or complemented by snare resection of a remaining stalk $(n=25)$ or cancelled $(n=4)$ with subsequent alternative treatment. 
Results: According to endoscopic or pathologic judgment resection was complete in 40 or 30 patients, respectively. During hospital follow-up (2-14; median 4 days) abdominal pain, fever or local peritonitis were noted in 6 and bleeding in 3 patients (hypotension in 1) with antibiotics/transfusions/surgery needed in $4 /$ $0 / 0$ patients. There was no hospital mortality. Among those with histologic incomplete resection $(\mathrm{n}=21)$, surgery or FTR was performed in 5 patients, endoscopic follow-up is pending in 7 and revealed no residual neoplasia in 9. Among those with cancelled ESD or endoscopic incomplete resection $(n=11)$, surgery or FTR was performed in 5, endoscopic follow-up is pending in 2 and revealed no residual neoplasia in 4

Conclusion: After appropriate training, even in low volume European case series ESD in the colorectum appears to be safe and partially effective.

Disclosure of Interest: G. Kleber: Activity as tutor in ESD learning courses sponsored by Olympus Medical Systems, Hamburg, Germany

All other authors have declared no conflicts of interest.

\section{Reference}

Dessain A. et al. 2017; Virchows Arch 470:165

\section{P0222 CLINICAL USABILITY QUANTIFICATION OF A REAL-TIME POLYP DETECTION METHOD IN VIDEOCOLONOSCOPY}

Q. Angermann ${ }^{1}$, J. Bernal ${ }^{2}$, C. Sánchez-Montes ${ }^{3}$, M. Hammami ${ }^{1}$, G. FernándezEsparrach $^{3}$, O. Romain ${ }^{1}$, J. Sánchez ${ }^{2}$, X. Dray ${ }^{4}$, A. Histace

${ }^{1}$ University Paris-seine, University Of Cergy-pontoise, Ensea, Cnrs, ETIS UMR 8051, Cergy/France

${ }^{2}$ Center Computer Vision, Universitat Autònoma de Barcelona, Barcelona/Spain ${ }^{3}$ Endoscopy Unit, Hospital Clínic, University of Barcelona, Barcelona/Spain ${ }^{4}$ Department Of Digestive Diseases, APHP Saint Antoine Hospital, Paris/France

Contact E-mail Address: aymeric.histace@ensea.fr

Introduction: Colorectal cancer is the second leading cause of cancer death in US [1]. Its incidence can be mitigated by detecting its precursor lesion, the polyp, before it develops into cancer. Coloscopy is still the gold standard for colon screening though some polyps are still missed. This can be explained by technical limitations of colonoscopes (camera orientation, field of view, etc.), but also by human factors (such as experience). Several computational systems, being the majority still-frame-based, have been proposed to assist clinicians in this task [2] but, to the best of our knowledge, none of them is being used in the exploration room due to not meeting real-time constraints ( $40 \mathrm{~ms} \max$ per image). In this abstract, we present a methodology to adapt and evaluate a real-time still framebased method [3] to video analysis.

Aims \& Methods: The still frame detection system used as reference [3] was based on an active learning method. We base the adaptation to video analysis on two aspects: (i) influence of the type of information used for polyp candidate characterisation, and (ii) introduction of spatio-temporal coherence. The former studies whether the combination of different types of information may lead to improve system performance whereas the latter fosters stability in the position of the detector output between consecutive frames. The learning stage of the method used a public still-frame database (CVC-Clinic, 612 images) whereas the testing was done on a new set of 18 sequences with a polyp $(10,294$ images) collected with an Olympus colonoscope CIF-H190 at Hospital Clinic, Barcelona. Performance was evaluated using two groups of metrics: (i) standard image/video metrics: Precision, Recall and F1-Score (ii) ad-hoc clinical metrics (assessing the clinical usability). Among the latter group we define: a) Polyp Detection Rate (PDR), checking if a method is able to detect polyp at least in $10 \%$ of the sequence, b) Mean Processing Time per frame (MPT), c) Mean Number of False Alarms per frame (MNFP), number of false alarms that can disturb the physician and d) Reaction Time (RT), representing the delay between first appearance of a polyp in the sequence and the first correct detection provided by the method.

Results: Table 1 shows the influence of local features on the overall performance and how the combination of both types of features can lead to an overall improvement in Recall and RT, which we interpret as local descriptors complementing each other.

\begin{tabular}{llllllll}
\hline Method & PDR & MPT & MNFP & Prec & Rec & F1 & RT \\
\hline $\begin{array}{c}\text { Texture } \\
\quad \text { Local Binary }\end{array}$ & $100 \%$ & $162 \mathrm{~ms}$ & 0.7 & $29.88 \%$ & $34.96 \%$ & $32.22 \%$ & $45.9[1.8 \mathrm{sec}]$ \\
$\begin{array}{c}\text { Patterns [3]) } \\
\begin{array}{c}\text { Shape (Haar } \\
\text { features [5]) }\end{array}\end{array}$ & $100 \%$ & $21 \mathrm{~ms}$ & 0.6 & $39.14 \%$ & $42.56 \%$ & $40.78 \%$ & $27.3[1.1 \mathrm{sec}]$ \\
\begin{tabular}{c} 
Combination \\
\hline
\end{tabular} & $100 \%$ & $185 \mathrm{~ms}$ & 1.0 & $30.72 \%$ & $51.00 \%$ & $38.34 \%$ & $17.4[0.7 \mathrm{sec}]$ \\
\hline
\end{tabular}

Conclusion: Work presented in this abstract shows how a real-time still-framebased polyp detection method can be successfully adapted to video analysis. Clinical usability metrics along with a new fully annotated video database were introduced to completely assess method performance. Results show methodology potential regarding clinical deployment as it detects all different polyps with a small RT. Results show that the sole use of shape features allows to meet realtime constraints but that a combination with a computationally efficient texture descriptor might improve frame-based performance.

Disclosure of Interest: X. Dray: Xavier Dray has received consultance fees from Covidien GI solutions
All other authors have declared no conflicts of interest.

\section{References}

1 ACS2016 (2016) Key statistics for colorectal cancer. Online

2 Bernal J, Tajbakhsh N, Sanchez FJ, Matuszewski B, Chen H, Yu L, Angermann Q, Romain O, Rustad B, Balasingham I, Pogorelov K, Choi S, Debard Q, Maier-Hein L, Speidel S, Stoyanov D, Brandao P, Cordova H, Sanchez-Montes C, Gurudu S, Fernandez-Esparrach G, Dray X, Liang J, Histace A (2017) Comparative Validation of Polyp Detection Methods in Video Colonoscopy: Results from the MICCAI 2015 Endoscopic Vision Challenge, IEEE Trans. On Medical Images (TMI), published on line: DOI: $10.1109 /$ TMI.2017.2664042

3 Angermann Q, Histace A, Romain O (2016) Active learning for real time detection of polyps in videocoloscopy. Procedia Computer Science 90, 182187

4 Bernal J, Sanchez FJ, Fernandez-Esparrach G, Gil D, Rodriguez C, Vilarino F (2015) WM-DOVA maps for accurate polyp highlighting in colonoscopy: validation vs. saliency maps from physician. Computerized Medical Imaging and Graphics. 43, 99-111

5 Pham, M. T., \& Cham, T. J. (2007). Fast training and selection of Haar features using statistics in boosting-based face detection. In ICCV 2007 (pp. 1-7)

\section{P0223 RESECT AND DISCARD/DIAGNOSE AND DISREGARD STRATEGY FOR COLONIC POLYPS: ARE WE READY TO START IT?}

R. Azevedo, J. Pinto, H. Ribeiro, F. Pereira, C. Leitão, A. Caldeira, E. Pereira, R. Sousa, J. Barros, A. Banhudo

Gastroenterology, Amato Lusitano Hospital, Castelo Branco/Portugal

Contact E-mail Address: richardazevedo13@gmail.com

Introduction: The use of Narrow Band Imaging (NBI) technology for in vivo histological prediction of colorectal polyps presents high accuracy in Referral Centers, particularly for diminutive polyps, which could be managed by the "resect and discard" strategy and, for sigmoid and rectum polyps, the "diagnose and disregard" strategy. However, the applicability of this practice in Community Hospitals still needs to be determined.

Aims \& Methods: We aimed to determine the accuracy of NBI in predicting histology, according to NICE and WASP classifications, in a Center without previous NBI experience. This was a prospective study including patients submitted to colonoscopy between June 2016 and July 2017. Polyps characteristics: location, size, morphology (Paris Classification), NICE/WASP classification (hyperplastic, sessile serreated, adenoma, invasive carcinoma) and degree of confidence (low: $<90 \%$ vs. high $>90 \%$ ). Comparison between NBI classification and histology SPSS 23 .

Results: 163 polyps included (71 patients); mean polyp dimension of $6.1 \mathrm{~mm}$ $(61.3 \%<5 \mathrm{~mm}) ; 91.4 \%$ sessile polyps; $62.6 \%$ on the left colon. Polyps classification according to NICE/WASP vs. histology: hyperplastic 49.7 vs $42.9 \%$; sessile serrated polyps $4.9 \mathrm{vs} 9.8 \%$; adenoma 44.2 vs. $43.6 \%$; carcinoma 1.2 vs. $0 \%$; inflammatory reaction on histology $-3.7 \%$. Adenoma diagnosis using NICE WASP classification presents an accuracy, sensitivity, specificity, positive predictive value and negative predictive value of $80.9 \%, 78.1 \%, 84.2 \%, 85 \%$ and $77.1 \%$, respectively. For left polyps $\leq 5 \mathrm{~mm}(\mathrm{n}=61)$ the accuracy and negative predictive value were of $81.2 \%$ and $82.3 \%$, respectively, with $79.4 \%$ high confidence classifications. Multivariate analysis showed that high confidence prediction and $\geq 3$ polyps/exam had a significant association with correct NBI classification $(\mathrm{p}<0.05)$.

Conclusion: NBI utilization by inexperienced endoscopists presented moderate acuity in histological prediction. Despite promising results, acuity and confidence levels were lower than the thresholds recommended in guidelines $(\geq 90 \%)$. These results justify implementing additional training and monitoring.

Disclosure of Interest: All authors have declared no conflicts of interest.

\section{References}

1. Wilson AI, Saunders BP. New paradigms in polypectomy: resect and discard, diagnose and disregard. Gastrointest Endosc Clin N Am. 2015;25(2):287-302. doi:10.1016/j.giec.2014.12.001.

2. Vu HT, Sayuk GS, Hollander TG, et al. Resect and discard approach to colon polyps: real-world applicability among academic and community gastroenterologists. Dig Dis Sci. 2015;60(2):502-508. doi:10.1007/s10620-0143376-z.

3. IJspeert JEG, Bastiaansen BAJ, van Leerdam ME, et al. Development and validation of the WASP classification system for optical diagnosis of adenomas, hyperplastic polyps and sessile serrated adenomas/polyps. Gut. 2016;65(6):963-970. doi:10.1136/gutjnl-2014-308411. 
P0224 THE EFFICACY AND SAFETY OF JUMBO FORCEPS BIOPSY USING NARROW-BAND IMAGING ENDOSCOPY IN PATIENTS WITH DIMINUTIVE POLYPS

T. Yamada ${ }^{1}$, T. Kuwai ${ }^{2}$, T. Toyokawa ${ }^{3}$, H. Iwase ${ }^{4}$, T. Kudo ${ }^{5}$, N. Esaka ${ }^{6}$, H. Ota ${ }^{7}$, H. Yamashita ${ }^{8}$, Y. Hosoda ${ }^{9}$, N. Watanabe ${ }^{10}$, N. Harada ${ }^{11}$

${ }^{1}$ Gastroenterology \& Hepatology, Osaka National Hospital, Osaka/Japan ${ }^{2}$ Gastroenterology, Kure MC \& Chugoku CC, Kure/Japan

${ }^{3}$ Department Of Gastroenterology, National Hospital Organization Fukuyama Medical Center, Fukuyama/Japan

${ }^{4}$ Gastroenterology, Nagoya Medical Center, Nagoya/Japan

${ }^{5}$ Gastroenterology, Takasaki General Medical Center, Takasaki/Japan

${ }^{6}$ Kyoto Medical Center, Kyoto/Japan

${ }^{7}$ Kanazawa Medical Center, Kanazawa/Japan

${ }^{8}$ Okayama Medical Center, Okayama/Japan

${ }^{9}$ Saitama National Hospital, Saitama/Japan

${ }^{10}$ Mie Chuo Medical Center, Tsu/Japan

${ }^{11}$ Gastroenterology, Clinical Research Institute, Kyushu Medical Center, Fukuoka/ Japan

Contact E-mail Address: yamtak1973@gmail.com

Introduction: Cold forceps polypectomy (CFP) is commonly used to remove diminutive colorectal polyps $(\leq 5 \mathrm{~mm})$. In addition, jumbo biopsy forceps are superior to standard forceps for removing colorectal polyps. However, problems remain for CFP with regard to residual adenomatous tissue on histological evaluation after a complete endoscopic cold forceps polyp resection.

Aims \& Methods: The aim of this study was to evaluate the efficacy and safety of jumbo forceps biopsy using narrow-band imaging endoscopy in patients with diminutive polyps. In addition, we evaluated the factors related to one-bite resection. This is a multicenter, prospective, single-arm observational study conducted at 11 institutes of the National Hospital Organization between January 2015 and September 2016. Patients aged 20 to 75 years with diminutive polyps were enrolled in this study. When lesions were found, we used magnification endoscopy with narrow-band imaging (NBI) in all the cases. CFP was performed until no polyps were visible under magnified endoscopy with NBI. We evaluated the patients' characteristics, clinicopathological features of the polyps, adverse events, and complete resection rates of the lesions. Additionally, we studied the factors of one-bite resection using Cox's regression model.

Results: A total of 503 patients were prospectively assessed, and 1015 polyps were resected. The median age of the patients was 65 years. The patients comprised 329 men $(65 \%)$ and 174 women $(35 \%)$. The polyp morphologies were 0 -Is lesions in 886 cases $(87.2 \%)$, 0-IIa lesions in $65(6.4 \%), 0$-Isp lesions in $63(6.2 \%)$, and 0 Ip lesion in $1(0.1 \%)$. Polyps were most often resected in the ascending colon (289 lesions) or transverse colon (262 lesions). Of all the polyps, 88\% (896 lesions) were adenomas, $10 \%$ (100 lesions) were hyperplastic, and $0.3 \%$ were adenocarcinomas. The mean procedure and treatment times were 26.5 and $20.4 \mathrm{~min}$, respectively. The complete resection rate was $99.3 \%$. The rate of one-bite polypectomy was $71.8 \%$, which included rates of $100 \%, 91.5 \%, 81.8 \%, 56.9 \%$, and $40.5 \%$ for lesions $1,2,3,4$, and $5 \mathrm{~mm}$ in diameter, respectively. Delayed bleeding that required endoscopic hemostasis occurred in only one case, but no other adverse events occurred. The most important factor rerated to one-bite polypectomy was polyp size ( $\leqq 3 \mathrm{~mm}$; OR: 5.58$)$, followed by macroscopic type of polyps (non-IIa; OR: 1.95).

Conclusion: In this large-scale multicenter prospective study, $99.3 \%$ of all diminutive polyps were completely resected by using jumbo forceps biopsy and magnified endoscopy with NBI. In addition, we was able to do one-bite polypectomy for more smaller polyps ( $\leqq 3 \mathrm{~mm}$ ). Jumbo forceps biopsy appears to be adequate for resecting diminutive polyps if no residual tissue is visible by using magnified endoscopy with NBI.

Disclosure of Interest: All authors have declared no conflicts of interest.

P0225 PERIOPERATIVE MANAGEMENT OF ORAL ANTICOAGULANTS WITHOUT HEPARIN BRIDGING THERAPY FOR PATIENTS UNDERGOING ENDOSCOPIC SURGERY: A PILOT STUDY

S. Ono ${ }^{1}$, M. Kato ${ }^{2}$, M. Kato ${ }^{3}$, K. Matsuda ${ }^{3}$, M. Tsuda ${ }^{3}$, S. Abiko ${ }^{4}$ S. Miyamoto ${ }^{3}$, K. Yamamoto ${ }^{3}$, T. Kudo ${ }^{3}$, Y. Shimizu ${ }^{1}$, N. Sakamoto ${ }^{3}$ ${ }^{1}$ Division Of Endoscopy, Hokkaido University Hospital, Sapporo/Japan

${ }^{2}$ Department Of Gastroenterology, Hakodate National Hospital, Hakodate/Japan ${ }^{3}$ Department Of Gastroenterology And Hepatology, Hokkaido University Graduate School of Medicine, Sapporo/Japan

${ }^{4}$ Department Of Gastroenterology And Hepatology, Hokkaido University Graduate School of Medicine, Sapporo/Japan

Contact E-mail Address: onosho@med.hokudai.ac.jp

Introduction: Heparin bridging therapy (HBT) is recommended for patients administered anticoagulants who have a high thrombotic risk and who undergo a high bleeding-risk procedure such as endoscopic submucosal dissection (ESD) or endoscopic mucosal resection (EMR) $)^{1,2)}$. However, HBT is actually related to a high frequency of delayed bleeding ${ }^{3,4)}$.

Aims \& Methods: Our aim is to analyze bleeding and coagulation markers in the management of anticoagulants without HBT during the perioperative periods of ESD and EMR. Patients who underwent ESD or EMR and received warfarin or a direct oral anticoagulant (DOAC) during the period from January 2013 to March 2017 were analyzed. Generally, administration of warfarin was continued within the therapeutic range of the international normalized ratio (INR) during the perioperative periods and DOACs were not administered on the day of the procedure. HBT was conducted only for patients who had a hypercoagulable condition. The rates of delayed bleeding in patients who received warfarin and patients who received DOACs were compared, and coagulation molecular markers including soluble fibrin (SF), thrombin-antithrombin complex (TAT), prothrombin fragment $1+2(\mathrm{~F} 1+2)$ and D-dimer (DD) were compared before and after the procedures in 13 patients who received DOACs.

Results: Among the patients who underwent ESD or EMR during the study period, 51patients received warfarin and 49 received DOACs. Delayed bleeding occurred in 6 patients $(11.8 \%)$ in the warfarin group and in 8 patients $(16.3 \%)$ in the DOAC group, and there was no significant difference. Only one patient with continued administration of antiplatelet agents had delayed bleeding among the patients in whom administration of warfarin was continued within the therapeutic range $(5.3 \%, 1 / 19)$. Six $(15 \%)$ of the 40 patients in the DOAC group for whom the DOAC was not administered only on the day of the procedure had delayed bleeding, and $23.8 \%(5 / 21)$ of the patients who received HBT had delayed bleeding. No thrombotic events occurred from one month after the procedures. One patient in whom the DOAC was not administered on the day of the procedure became positive for TAT, F1 +2 and DD after EMR and had a hypercoagulable condition.

Conclusion: For perioperative management of anticoagulants in patients undergoing ESD or EMR, continuous use of warfarin within the therapeutic range is better than HBT. However, DOACs should be carefully managed with attention to hemorrhagic risk and coagulable condition.

Disclosure of Interest: All authors have declared no conflicts of interest.

\section{References}

1. Acosta RD, Abraham NS, Chandrasekhara V, et al. The management of antithrombotic agents for patients undergoing GI endoscopy. Gastrointest Endosc. 2016; 83(1): 3-16.

2. Veitch AM, Vanbiervliet G, Gershlick AH, et al. Endoscopy in patients on antiplatelet or anticoagulant therapy, including direct oral anticoagulants: British Society of Gastroenterology (BSG) and European Society of Gastrointestinal Endoscopy (ESGE) guidelines. Endoscopy. 2016;48(4): 385-402.

3. Matsumoto M, Mabe k, Tsuda M, et al. Multicenter study on hemorrhagic risk of heparin bridging therapy for periendoscopic thromboprophylaxis. BMC Gastroenterol. 2015;15: 89

4. Jaruvongvanich V, Assavapongpaiboon B, Wijarnpreecha K, Ungprasert P. Heparin-bridging therapy and risk of post-polypectomy bleeding: A metaanalysis of data reported by Japanese colonoscopists. Dig Endosc. 2017 Mar 31. doi: $10.1111 /$ den. 12882

\section{P0226 ENDOSCOPIC FEATURE OF DEPRESSED TYPE COLORECTAL NEOPLASMA IN MAGNIFYING ENDOSCOPY AND ENDOCYTOSCOPY}

S. Kudo ${ }^{1}$, K. Mochizuki ${ }^{1}$, T. Okumura ${ }^{1}$ S. Matsudaira ${ }^{1}$, Y. Kouyama ${ }^{1}$

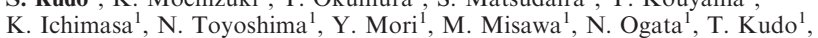

T. Hisayuki ${ }^{1}$, T. Hayashi ${ }^{1}$, K. Wakamura ${ }^{1}$, E. Hidaka ${ }^{1}$, T. Baba ${ }^{1}$, S. Hamatani ${ }^{2}$, F. Ishida ${ }^{1}$

${ }^{1}$ Digestive Disease Center, Showa University Northern Yokohama Hospital, Yokohama/Japan

${ }^{2}$ Department Of Pathology, Jikei University School of Medicine, Tokyo/Japan

Contact E-mail Address: kudos@med.showa-u.ac.jp

Introduction: Colorectal cancers are generally recognized to develop from"polyps". This "adenoma-carcinoma sequence" theory has been in the mainstream of development of colorectal cancers. But recently the existence of many depressed-type cancers has been revealed, which are considered to emerge directly from normal epithelium, not through the adenomatous stage. This theory is called "de novo" pathway. Now, it is possible to presume the histology of colorectal lesions using magnifying endoscopy(pit pattern classification) and endocytoscopy(EC classification). We can observe not only the structural atypia but also the cellular atypia in living colorectal lesions.

Aims \& Methods: The aim is to clarify the endoscopic characteristics of depressed-type colorectal neoplasms, demonstrating the validity of pit pattern diagnosis and EC classification. A total of 29,030 colorectal neoplasms excluding advanced cancers were resected endoscopically or surgically in our unit from April 2001 to December 2016. Of these, 17,761 lesions were low-grade dysplasia, 2922 were high-grade dysplasia and 1077 were submucosally invasive (T1) carcinomas. According to the developmental morphology classification, they were divided into 3 types: depressed, flat and protruded-type. We investigated the rate of T1 carcinomas and the characteristics of depressed- type neoplasms concerning pit pattern and EC classification.

Results: The rate of T1 carcinomas in depressed-type lesions reached to $62.0 \%$, meanwhile that in flat-type and protruded-type lesions was $3.3 \%$ and $2.8 \%$, respectively. Within less than $5 \mathrm{~mm}$ in diameter, that was $10.6 \%, 0 \%$ and $0.04 \%$, respectivel. Most $(93.0 \%$ and $93.1 \%)$ of the flat-type and protrudedtype lesions showed typeIIIL or IV pit pattern corresponding to adenomas, whereas $91.3 \%$ of the depressed-type lesions were characterized by type IIIS, VI or VN pit patterns correspondings to carcinomas. As for endocytoscopy, most of the flat-and protruded-type lesions showed EC2 corresponding to adenomas. In contrast, the depressed-type lesions were observed as EC3a (38.9\%) and $\mathrm{EC} 3 \mathrm{~b}(58.0 \%)$ corresponding to invasive carcinomas

Conclusion: This study revealed the diagnostic characteristics of depressed-type lesions. They show typically type IIIS, VI or VN pit patterns in magnifying 
endoscopy and type EC3a or EC3b in endocytoscopy. These lesions tend to invade the submucosal layer even when they are small. Therefore, it is important to consider deeply and examine the developmental morphology of colorectal neoplasms.

Disclosure of Interest: All authors have declared no conflicts of interest.

\section{P0227 COLONOSCOPY INDICATION INFLUENCE ON THE COMPLIANCE OF QUALITY INDICATORS}

C. Mangas ${ }^{1}$, E. Santana Rocamora ${ }^{1}$, A. Suárez González ${ }^{2}$, I. Portillo Villares ${ }^{3}$, A. Seoane ${ }^{4}$, M. Ponce 5 , P. Diez 6 , E. Quintero Carrion ${ }^{7}$, M. Herraiz ${ }^{8}$, M. Pellisé Urquiza $^{9}$, A. Ferrández Arenas ${ }^{10}$, V. Hernández ${ }^{11}$, Á.E. Pizarro Moreno ${ }^{12}$, R. Jover ${ }^{13}$

${ }^{1}$ Hospital General Universitario de Alicante, Alicante/Spain

${ }^{2}$ Servicio De Aparato Digestivo, Hospital Universitario Central de Asturias, Oviedo/Spain

${ }^{3}$ Hospital Universitario de Donostia, San Sebastián/Spain

${ }^{4}$ Hospital del Mar-Parc de Salut Mar Barcelona, Barcelona/Spain

${ }^{5}$ Hospital Universitari i Politecnic de la Fe, Valencia/Spain

${ }^{6}$ Gastroenterology, Hospital Universitario Rio Hortega, Valladolid/Spain

${ }^{7}$ Gastroenterology, Hospital Univ. de Canarias, Santa Cruz de Tenerife/Spain

${ }^{8}$ Clinica Universitaria de Navarra, Pamplona/Spain

${ }^{9}$ Gastroenterology, Hospital Clinic Barcelona, Barcelona/Spain

${ }^{10}$ Hospital Clínico Universitario Lozano Bleza, Zaragoza/Spain

${ }^{11}$ Instituto de Investigación Biomédica, Xerencia de Gestión Integrada de Vigo,

Vigo/Spain

${ }^{12}$ Gastroenterology, Hospital Virgen del Rocio, Sevilla/Spain

${ }^{13}$ Unidad De Gastroenterología, Hospital General Universitario, Alicante/Spain

Contact E-mail Address: cmangassanjuan@gmail.com

Introduction: It remains unknown if colonic lesions detection rates as quality indicators of the colonoscopy, behave in the same way in relation to colonoscopy indication.

Aims \& Methods: The aim of this study was to evaluate the adenoma detection rate (ADR), serrated polyp detection rate (SDR), advanced adenoma detection rate $(A A D R)$ and colorectal cancer detection rate (CRCDR) depending on the colonoscopy indication. A total of 6912 colonoscopies have been prospectively included in the QUALISCOPIA project, an observational, multicenter and prospective study, developed in 12 centers in Spain. The ADR, SDR, AADR and CRCDR have been calculated. These data were analysed according to the colonoscopy indication adjusted by sex, age, cecal intubation, adequate cleansing (Boston scale score of 2-3 in all segments) and sedation use.

Results: The results can be seen in table 1 . In colonoscopies performed due to positive fecal immunochemical test (FIT +$)$, the ADR was $54.0 \%(\mathrm{p}<0.001$, aOR 3.0, 95\% CI 2.6-3.4). In those performed because of post-polypectomy surveillance, was $49.3 \%$ ( $<<0.001$, aOR $2.2,95 \%$ CI $1.9-2.5)$, and those due to direct screening, the ADR was $31.6 \%(\mathrm{p}<0.005$, aOR $1.4,95 \% \mathrm{CI} 1.1-1.7)$ compared to $28 \%$ in patients with gastrointestinal symptoms. Regarding the serrated polyps, in procedures performed due to FIT+, the SDR was $1.9 \%$ $(\mathrm{p}=0.094$, aOR $1.5,95 \% \mathrm{CI} \quad 0.9-2.5), 4.2 \%$ in surveillance colonoscopies $(\mathrm{p}<0.001, \mathrm{aOR} 3.4,95 \% \mathrm{CI} 2.2-5.3)$, and $3.3 \%$ in direct screening $(\mathrm{p}<0.001$, aOR $2.8,95 \%$ CI $1.6-5.0$ ), compared to $1.2 \%$ of patients with digestive symptoms. Moreover, the AADR in colonoscopies performed due to FIT+ was $36.8 \%$ ( $p<0.001$, aOR $3.9,95 \%$ CI $3.3-4.6$ ), compared to $23.1 \%$ in surveillance colonoscopies ( $\mathrm{p}<0.001$, aOR $1.8,95 \%$ CI $1.5-2.2)$, and $14.9 \%$ in direct screening $(\mathrm{p}=0.023$, aOR $1.3,95 \%$ CI $1.1-1.8)$. However, the SDR was $12.8 \%$ in patients with digestive symptoms. Finally, the CRCDR was $5.8 \%$ in patients with gastrointestinal symptoms ( $\mathrm{p}<0.001$, aOR $11.6,95 \% \mathrm{CI} 4.7-28.7), 4.8 \%$ in those with FIT $+(\mathrm{p}<0.001, \mathrm{aOR} 13.4,95 \% \mathrm{CI} 5.4-33.2)$, and $1.8 \%$ in direct screening $(\mathrm{p}=0.005, \mathrm{aOR} 5.1,95 \% \mathrm{CI} 1.6-15.6)$, compared to $0.5 \%$ in post-polypectomy surveillance.

\section{TABLE 1: DETECTION RATES BY INDICATION}

ADR

\begin{tabular}{|c|c|c|c|c|c|}
\hline & $\%(n)$ & OR $(95 \% \mathrm{CI})$ & p-value ${ }^{1}$ & $\mathrm{aOR}^{2}(95 \% \mathrm{CI})$ & p-value ${ }^{1}$ \\
\hline $\begin{array}{l}\text { Post-polypectomy } \\
\text { surveillance }\end{array}$ & $49.3(629 / 1275)$ & $2.5(2.2-2.9)$ & $<0.001$ & $2.2(1.9-2.5)$ & $<0.001$ \\
\hline FIT+ & $54.0(928 / 1718)$ & $3.0(2.7-3.4)$ & $<0.001$ & $3.0(2.6-3.4)$ & 0.001 \\
\hline Direct screening & $31.6(174 / 550)$ & $1.2(1.0-1.5)$ & 0.085 & $1.4(1.1-1.7)$ & 0.005 \\
\hline Digestive symptoms & $28.0(793 / 2832)$ & 1.0 & & 1.0 & \\
\hline $\begin{array}{l}\text { SDR } \\
\text { Post-polypectomy } \\
\text { surveillance }\end{array}$ & 4.2( & $3.5(2.6-5.3)$ & 0.001 & $.2-5.3)$ & $<0.001$ \\
\hline FIT + & $1.9(32 / 1718)$ & $1.5(0.9-2.5)$ & 0.091 & $1.5(0.9-2.5)$ & 0.094 \\
\hline Direct screening & $3.3(18 / 550)$ & $2.7(1.5-4.8)$ & $<0.001$ & $2.8(1.6-5.0)$ & 0.001 \\
\hline Digestive symptoms & $1.2(35 / 2832)$ & 1.0 & & 1.0 & \\
\hline $\begin{array}{l}\text { AADR } \\
\text { Post-polypectomy } \\
\text { surveillance }\end{array}$ & $23.1(294 / 1275)$ & $2.0(1.7-2.4)$ & $<0.001$ & $1.8(1.5-2.2)$ & $<0.001$ \\
\hline FIT + & $36.8(632 / 1718)$ & $4.0(3.4-4.6)$ & $<0.001$ & $3.9(3.3-4.6)$ & $<0.001$ \\
\hline Direct screening & $14.9(82 / 550)$ & $1.2(0.9-1.5)$ & 0.177 & $1.3(1.1-1.8)$ & 0.023 \\
\hline Digestive symptoms & $12.8(362 / 2832)$ & 1.0 & & 1.0 & \\
\hline $\begin{array}{l}\text { CRCDR } \\
\text { Digestive symptoms }\end{array}$ & $5.8(165 / 2832)$ & $13.1(5.8-29.6)$ & $<0.001$ & $11.6(4.7-28.7)$ & $<0.001$ \\
\hline
\end{tabular}

TABLE 1 Continued

ADR

\begin{tabular}{|c|c|c|c|c|c|}
\hline & $\%(\mathrm{n})$ & OR $(95 \% \mathrm{CI})$ & p-value ${ }^{1}$ & $\mathrm{aOR}^{2}(95 \% \mathrm{CI})$ & p-value ${ }^{1}$ \\
\hline FIT + & $4.8(83 / 1718)$ & $10.7(4.7-24.7)$ & 0.001 & $13.4(5.4-33.2)$ & $<0.001$ \\
\hline Direct screening & $1.8(10 / 550)$ & $3.9(1.4-10.8)$ & 0.009 & $5.1(1.6-15.6)$ & 0.005 \\
\hline $\begin{array}{l}\text { Post-polypectomy } \\
\text { surveillance }\end{array}$ & $0.5(6 / 1275)$ & 1.0 & & 1.0 & \\
\hline
\end{tabular}

surveillance

${ }^{1}$ p-value: significance level; ${ }^{2} \mathrm{aOR}$ : adjusted Odds Ratio

Conclusion: The indication of colonoscopy has a very important influence on the different quality indicators such as detection rates of lesions.

Disclosure of Interest: All authors have declared no conflicts of interest.

\section{P0228 THERAPEUTIC ERCP USING A SHORT SINGLE-BALLLOON ENTEROSCOPE IN PATIENTS WITH SURGICALLY ALTERED ANATOMY}

K. Masu, K. Ito, T. Ohira, S. Koshita, Y. Kanno, T. Ogawa

Gastroenterology, Sendai City Medical Center, Sendai/Japan

Contact E-mail Address: k-masu@openhp.or.jp

Introduction: Recently, we have performed therapeutic ERCP using a newly developed short single-balloon enteroscope (sSBE) (working length of $152 \mathrm{~cm}$, working channel of $3.2 \mathrm{~mm}$ ) in patients with surgically altered anatomy.

Aims \& Methods: We aimed to evaluate the usefulness and safety of sSBE for therapeutic ERCP in patients with surgically altered anatomy. One hundred four patients with surgically altered anatomy who underwent therapeutic ERCP using a sSBE between August 2011 and February 2017 were included in this study. Patient anatomy consisted of Roux-en-Y anastomosis $(R-Y)(n=82)$, hepaticojejunostomy $(\mathrm{HJ})(\mathrm{n}=11)$, subtotal stomach-preserving pancreaticoduodenectomy (SSPPD) $(\mathrm{n}=11)$. The indications for ERCP were choledocholithiasis (68: R-Y cases), malignant biliary strictures (20: R-Y 14, HJ 7, SSPPD 4), intrahepatic stones (9: HJ 7, SSPPD 2), and anastomotic stenosis (7: SSPPD 5, HJ 2). The success rate of reaching the target site, the technical success rate, and the adverse event rate were retrospectively evaluated.

Results: The success rate of reaching the target site was $91 \%(95 / 104)$, and the overall technical success rate was $79 \%(80 / 104)$. Biliary interventions included 64 stone extraction (R-Y 58, HJ 5, SSPPD 1), and 12 metallic biliary stent placement (R-Y 7, HJ 1, SSPPD 4). Of 17 unsuccessful cases, nine with choledocholithiasis underwent surgical operation (R-Y 6, HJ 2, SSPPD 1) and EUS-guided drainage was successfully performed in six with anastomotic stenosis (SSPPD 3, R-Y 2, HJ 1). The remaing two with malignant bile duct stricture (R-Y) underwent PTCD. The adverse event rate was $10 \%$ (10: cholangitis 4 , mild pancreatitis 3 , perforation 2, aspiration pneumonitis 1). The two perforation cases required urgent operation but remaing eight cases were managed conservatively.

Conclusion: Therapeutic ERCP using a sSBE in patients with surgically altered anatomy was considered to be safe and effective.

Disclosure of Interest: All authors have declared no conflicts of interest.

\section{P0229 USEFULNESS OF SPYGLASS PERORAL}

\section{CHOLANGIOSCOPY FOR THE DIAGNOSIS AND TREATMENT OF} BILE-DUCT DISORDERS: EXPERIENCE FROM A LARGE-VOLUME

\section{CENTER}

X. Tang, D. Zhang, C. Xu, X. Zhang

Department Of Gastroenterology, Hangzhou First People's Hospital, Nanjing Medical University, Hangzhou/China

\section{Contact E-mail Address: solitude5834@hotmail.com}

Introduction: SpyGlass single-operator peroral cholangioscopy (SOC) has been designed to overcome some limitations of conventional cholangioscopy, and demonstrated improved diagnostic and therapeutic abilities of complex pancreaticobiliary disease.

Aims \& Methods: To assess the clinical efficacy and safety of the SpyGlass system for diagnosis and treatment of bile-duct disorders in a large-volume center. All patients undergoing SOC in our department between January 2013 and May 2016 were retrospectively identified from a prospectively collected database. The baseline characteristics, including age, gender, presenting symptoms, indication and others were recorded. Procedure-related parameters of SOC for detecting malignant lesions and the stone clearance rate were calculated.

Results: During the study period, a total of 68 patients underwent 78 SOC procedures: $26(38.2 \%)$ with indeterminate strictures, $7(10.3 \%)$ with indeterminate filling defects, $31(45.6 \%)$ with difficult bile stones, and $4(5.9 \%)$ with cystic lesions. SpyGlass was technically successful in 63 of 68 patients $(92.6 \%)$. The mean total SpyGlass procedure time was $12 \mathrm{~min}$. In patients with indeterminate biliary strictures, 6 cases of definite diagnosis (stones, varices) was made by SOC evaluation. Twenty patients underwent SOC-directed biopsy, and samples were adequate for histological diagnosis in 17 patients $(85 \%)$. The preliminary accuracy of SpyGlass-directed biopsy to diagnose malignancy were $76 \%$. For the patients with biliary stone, SpyGlass-guided holmium laser lithotripsy or electrohydraulic lithotripsy succeeded in 15 of 15 patients $(100 \%)$. There were 6 procedure-related adverse events occurred $(8.8 \%)$, and resolved uneventfully. 
Conclusion: SpyGlass cholangioscopy system can be safe and useful for definite diagnosis with high accuracy in patients with indeterminate biliary lesions, and successfully guided stone therapy. Further prospective multicenter trials of the system are warranted in the future.

\section{P0230 DIAGNOSTIC AND THERAPEUTIC ENDOSCOPIC} RETROGRADE CHOLANGIOPANCREATOGRAPHY (ERCP) IN INFANT AND CHILDREN: A LARGE RETROSPECTIVE STUDY

X. Tang, D. Zhang, C. Xu, X. Zhang

Department Of Gastroenterology, Hangzhou First People's Hospital, Nanjing

Medical University, Hangzhou/China

Contact E-mail Address: solitude5834@hotmail.com

Introduction: Endoscopic retrograde cholangiopancreatography (ERCP) is increasingly being used in the diagnosis and management of biliary and pancreatic disorders in pediatric patients.

Aims \& Methods: To evaluate the indications, success rate, diagnostic and therapeutic yields, and complications of ERCP performed in Chinese children. A retrospective study was conducted in an academic, tertiary care, medical center, in which all children undergoing ERCP between 2005 to 2016 were identified from endoscopy databases. Data on demographics, indication, ERCP findings, ERCP interventions performed and complications were collected.

Results: A total of 288 children (mean age 9.3 years, range 1 month to 18 years) underwent 312 ERCP procedures. General anesthesia and sedation were performed in $48 \%$ and $52 \%$ of procedures, respectively. Indications for ERCP were common bile duct obstructions $(n=153,54.2 \%)$, recurrent or chronic pancreatitis $(\mathrm{n}=64,22.2 \%)$ and others. ERCP was successfully performed in 267 of 288 cases $(92.7 \%)$. The most common ERCP findings was choledocholithiasis $(\mathrm{n}=146,50.7 \%)$. A therapeutic intervention was performed in $70.8 \%$ patients $(\mathrm{n}=204)$, including sphincterotomy $(\mathrm{n}=97)$, stone extraction $(\mathrm{n}=55)$, and stent insertion $(\mathrm{n}=52)$. Complications occurred for only 13 patients $(4.5 \%)$, including 12 cases of post-ERCP pancreatitis and 1 case of bleeding. No severe pancreatitis, or perforation was noted.

Conclusion: Diagnostic and therapeutic ERCP is effective and safe in the children population, with the high rates of technical success and low rates of complication.

Disclosure of Interest: All authors have declared no conflicts of interest.

\section{P0231 ENDOSCOPIC RETROGRADE}

CHOLANGIOPANCREATOGRAPHY IN PATIENTS WITH

SURGICALLY ALTERED GASTROINTESTINAL ANATOMY: 11 YEARS' EXPERIENCE AT A LARGE CENTER IN CHINA

X. Tang, D. Zhang, C. Xu, X. Zhang

Department Of Gastroenterology, Hangzhou First People's Hospital, Nanjing Medical University, Hangzhou/China

\section{Contact E-mail Address: solitude5834@hotmail.com}

Introduction: It is technically challenging to perform endoscopic retrograde cholangiopancreatography (ERCP) in patients with surgically altered gastrointestinal anatomy

Aims \& Methods: The aims of this study were to investigate the yield, efficacy and safety of ERCP in surgically altered anatomy patients at a single tertiary-care center with a high volume of endoscopy. All patients with altered surgical anatomy who underwent ERCP at our center from September 2005 to July 2016 were retrospectively reviewed. Data regarding to patients baseline characteristics, procedure-related details and adverse events was recorded and analyzed.

Results: A total of 304 procedures were performed in 236 patients, including 108 cases $(45.8 \%)$ with Billroth II gastrectomy, 45 cases $(19.1 \%)$ with Billroth gastrectomy, 52 cases $(22.0 \%)$ with hepaticoduodenostomy, 18 cases $(7.6 \%)$ with esophagogastrostomy and 13 cases $(5.5 \%)$ with Roux-en-Y reconstruction. The most common indication was cholelithiasis $(58.1 \%, 137 / 236)$. The overall technique success rate of reaching the papilla was $90.8 \%(276 / 304)$, including 91.3\% (126/138) for Billroth II gastrectomy, 94.5\% (52/55) for Billroth I gastrectomy, $89.9 \%(71 / 79)$ for hepaticoduodenostomy, $100 \%(19 / 19)$ for esophagogastrostomy and $61.5 \%(8 / 13)$ for Roux-en-Y reconstruction. The clinical success rate was $88.2 \%(268 / 304)$. Therapeutic interventions were performed in 194 patients successfully, including stone extraction $(\mathrm{n}=146)$, sphincterotomy $(n=44)$, stent placement $(n=57)$, papillary balloon dilatation $(n=27)$ and mechanical lithotripsy $(n=25)$. The adverse event rate was $7.2 \%(17 / 236)$. Mild pancreatitis occurred in 3\% (7/236) of cases, perforation occurred in $2.5 \%(6 / 236)$ of cases, and asymptomatic hyperamylasemia occurred in $1.7 \%$ $(4 / 236)$ of cases.

Conclusion: ERCP can be performed in surgically altered anatomy patients with a high success rate.

Disclosure of Interest: All authors have declared no conflicts of interest.
P0232 IMPACT OF HIGH DEFINITION, NEAR FOCUS-IMAGING AND SYDNEY RECURRENCE TOOL (SERT) AFTER COLORECTAL ENDOSCOPIC MUCOSAL RESECTION: A PROPENSITY SCORE ANALYSIS

D. Guerrero Vinsard ${ }^{1}$, R. Lennon ${ }^{2}$, P. Kandel ${ }^{1}$, L.K. Mejia ${ }^{1}$, R. Bingham ${ }^{1}$, T. Woodward ${ }^{1}$, V. Gomez ${ }^{1}$, M. Raimondo ${ }^{1}$, E. Bouras ${ }^{1}$, M.B. Wallace ${ }^{1}$ ${ }^{1}$ Gastroenterology And Hepatology, Mayo Clinic, Jacksonville, Florida/United States of America/FL

${ }^{2}$ Biostatistics, Mayo Clinic, Rochester/United States of America/MN

Contact E-mail Address: danielaguerrerotorres@gmail.com

Introduction: Risk factors for colorectal adenoma recurrence after Endoscopic Mucosal Resection (EMR) such as size $\geq 20 \mathrm{~mm}$, high grade dysplasia, use of argon plasma coagulation (APC) and intraprocedural bleeding (IPB), have been well documented in literature. However, it is unknown if the latest generation dual-focus (DF) colonoscopes ability to visualize subtle residual neoplasia, has improved the rate of complete EMR.

Aims \& Methods: We aimed to assess the efficacy of the newer 190 colonoscopes versus standard 180 colonoscopes for complete resection of lateral spreading lesions (LSL) $\geq 20 \mathrm{~mm}$. A secondary aim was to identify risk factors for recurrence and the applicability of the Sydney EMR recurrence tool (SERT score) in our cohort.

This was a single-center retrospective study of patients who underwent EMR with 180 or 190 colonoscope series from 2010 to 2016 . Lesions $\geq 20 \mathrm{~mm}$ resected in a piecemeal fashion and patients with a surveillance colonoscopy after index EMR were included. A propensity score approach with inverse probability weighting (IPW) was used to control potential confounders affecting adenoma recurrence. Each lesion was graded according to SERT score and associations with recurrence were analyzed.

Results: 291 patients met inclusion criteria for the study. The rate of adenoma recurrence at the EMR site was $23.3 \%$ for the 180 colonoscope cases and $25.2 \%$ for the 190 colonoscope cases. Odds ratio (OR) for recurrence with 190 series was $1.06(\mathrm{p}=0.85)$. Adenoma size $(\mathrm{p}=0.002)$ and concomitant need for supplemental APC $(\mathrm{p}<0.001)$ were risk factors for recurrence. SERT $>0$ lesions had a higher risk of recurrence during follow-up (OR $1.71 ; 95 \%$ CI 1.00-2.92; $p=0.048)$ and a higher cumulative incidence for recurrence. Conversely, SERT $=0$ lesions reached a plateau for recurrence after 12 and 18 months in Kaplan Meier curves. Odds ratio estimates for 190 colonoscope effect on adenoma recurrence at different stages of adjustment.

\begin{tabular}{llllc}
\hline Model & Odds Ratio & $\begin{array}{l}\text { Lower } \\
95 \% \text { CI }\end{array}$ & $\begin{array}{l}\text { Upper } \\
95 \% \text { CI }\end{array}$ & P-value \\
\hline $\begin{array}{c}\text { Unadjusted all-subject } \\
\text { (en-bloc and piecmeal) }\end{array}$ & 1.58 & 0.94 & 2.65 & 0.08 \\
$\begin{array}{c}\text { Unadjusted-piecemeal } \\
\text { resection only }\end{array}$ & 1.11 & 0.64 & 1.92 & 0.71 \\
$\begin{array}{l}\text { IPW adjustment-piecemeal } \\
\text { resection }\end{array}$ & 1.06 & 0.60 & 1.86 & 0.85 \\
\hline
\end{tabular}

Cumulative incidence of recurrence at SC1 by SERT score in 291 lesions.

\begin{tabular}{lllll}
\hline Months & $\begin{array}{l}\text { N-events } \\
{[\text { SERT }=0]}\end{array}$ & $\begin{array}{l}\text { Event rate, } \% \\
{[\text { SERT }=0]}\end{array}$ & $\begin{array}{l}\text { N-events } \\
{[\text { SERT }>0]}\end{array}$ & Event rate, \% [SERT > 0] \\
\hline 6 & 26 & $16.4 \%$ & 21 & $21.9 \%$ \\
12 & 34 & $23.3 \%$ & 32 & $37.6 \%$ \\
18 & 34 & $23.3 \%$ & 35 & $43.6 \%$ \\
24 & 35 & $25.4 \%$ & 36 & $47.1 \%$ \\
36 & 36 & $27.5 \%$ & 37 & $51.5 \%$ \\
\hline
\end{tabular}

CI: Confidence intervals; IPW: Inverse probability weighting; SERT: Sydney EMR recurrence tool; SC1: First surveillance colonoscopy.

Conclusion: In this study, recurrence was significantly associated with adenoma size and complementary use of APC for EMR. The use of the latest generation DF colonoscopes (CF-HQ190L/I) did not measurably affect adenoma recurrence at the EMR site during first surveillance colonoscopy ( $\mathrm{SC} 1)$. Lesions with SERT $>0$ were associated with higher recurrence rates. In our cohort, $\mathrm{SERT}=0$ lesions that remain negative for recurrence at 18 months, may return to routine surveillance.

Disclosure of Interest: All authors have declared no conflicts of interest.

P0233 INCIDENCE AND RISK FACTORS FOR PANCREATITIS IN EMERGENCY ENDOSCOPIC RETROGRADE CHOLANGIOPANCREATOGRAPHY: A PROSPECTIVE MULTICENTER STUDY

N. Yoshitaka ${ }^{1}$, K. Kusumoto ${ }^{1}$, Y. Itokawa ${ }^{1}$, B. Endoh $^{2}$, K. Chikugo ${ }^{2}$, A. Suzuki ${ }^{3}$, T. Kawakami ${ }^{3}$, T. Suzuki ${ }^{3}$, O. Inatomi ${ }^{4}$, S. Bamba ${ }^{4}$, T. Kusaka ${ }^{1}$ H. Kokuryu ${ }^{1}$, Y. Mizumoto ${ }^{2}$, K. Tanaka ${ }^{3}$ 
${ }^{1}$ Digestive Disease Center, Department Of Gastroenterology And Hepatology, Kyoto Katsura Hospital, Kyoto/Japan

${ }^{2}$ Department Of Gastroenterology, Kyoto Medical Center, Kyoto Medical Center, Kyoto/Japan

${ }^{3}$ Department Of Gastroenterology, Kyoto Second Red Cross Hospital, Kyoto

Japan

${ }^{4}$ Division Of Gastroenterology, Shiga University of medical science, Shiga/Japan

Contact E-mail Address: digestivenews@yahoo.co.jp

Introduction: Post-endoscopic retrograde cholangiopancreatography (ERCP) pancreatitis (PEP) is a potentially serious complication, and some risk factors for PEP have been reported in general ERCP in previous studies. Emergency ERCP is different from normal state ERCP, and the risk factors for PEP in emergency ERCP are not clear.

Aims \& Methods: This study aimed to identify the incidence and risk factors for PEP in emergency ERCP. We performed a prospective study of 2078 cases undergoing diagnostic and therapeutic ERCP at five Japanese institutions between April 2015 and May 2016. Exclusion criteria were active pancreatitis, choledochojejunostomy, inability to approach a papilla, and inspection aimed at only the pancreatic duct (PD). Emergency ERCP indicated unscheduled inspections performed within and outside duty hours in this study. PEP was considered when two of the following three conditions were met: (1) serum amylase level more than three times the upper limit of the normal range in each institution, (2) continuous abdominal pain for over 24 hours, and (3) presence of pancreatitis findings on computed tomography. The first study involved comparison of the incidence of PEP and its characteristics between emergency and elective ERCP. The second study involved determining the predictive risk factors for PEP in emergency ERCP using univariate and multivariate analyses.

Results: A total of 1677 cases were enrolled in this study. $<$ Study $1>$ PEP developed in 20 of 429 cases $(4.7 \%)$ from the emergency group and in 101 of 1248 cases $(8.1 \%)$ from the elective group. The incidence of PEP was significantly lower in the emergency group than in the elective group (odds ratio [OR]: 0.56, $95 \%$ confidence interval $[\mathrm{CI}]: 0.32-0.92, \mathrm{P}=0.017$ ). Endoscopic sphincterotomy, stone removal, papillary balloon dilatation, and intraductal ultrasound sonography were performed significantly more often in the elective group than in the emergency group $(\mathrm{P}<0.001)$. Placement of a biliary stent was significantly more common in the emergency group than in the elective group. In addition, the procedure time was significantly longer $(\mathrm{P}<0.001)$ and the number of endoscopists who had more than five years of experience was significantly higher $(\mathrm{P}=0.04)$ in the elective group than in the emergency group. $<$ Study $2>$ Cases with no naïve papilla $(n=181)$ were excluded from the analysis of risk factors for PEP because no PEP was observed in these cases. Only cases with naïve papilla $(\mathrm{n}=248)$ were analyzed. Univariate analysis showed that contrast injection into the PD (OR: $4.20,95 \% \mathrm{CI}: 1.64-10.80, \mathrm{P}=0.0028)$, more than four cannulation attempts (OR: 2.85, 95\% CI: $1.00-8.09, \mathrm{P}<0.05)$ increased and placement of a biliary stent (OR: $0.028,95 \% \mathrm{CI}: 0.11-0.88, \mathrm{P}=0.028)$ decreased the risk of PEP in emergency ERCP. Multivariate analysis showed that contrast injection into the PD (OR: 4.51, 95\% CI: $1.64-12.40, \mathrm{P}=0.0035)$ increased the risk of PEP in emergency ERCP

Conclusion: The incidence of PEP was lower in emergency ERCP than in elective ERCP, and it was largely unaffected by the endoscopist's experience and the procedure time. This may be associated with a tendency to avoid invasive procedures in emergency cases, and it is considered that only placement of a biliary stent contributes to a decrease in the development of PEP. Close attention should be paid for contrast injection into the PD, particularly when attempt of cannulation for naïve papilla are required.

Disclosure of Interest: All authors have declared no conflicts of interest.

\section{P0234 WALLED-OFF NECROSIS (WON): OUTCOMES OF AN}

\section{ALGORITHMIC APPROACH TO NECROSECTOMY}

J.Y. Bang, U. Navaneethan, M. Hasan, R. Hawes, S. Varadarajulu Center For Interventional Endoscopy, Florida Hospital, Orlando/United States of America/FL

Contact E-mail Address: jybang213@gmail.com

Introduction: Endoscopic necrosectomy (EN) in walled-off necrosis (WON) is a labor intensive, high-risk, non-standardized technique that is associated with significant morbidity and mortality.

Aims \& Methods: We aimed to compare the clinical outcomes of patients with WON treated by conventional EN versus an algorithmic approach that is tailored to the extent and location of WON.

This observational study included 45 consecutive patients with WON who had suboptimal treatment response to EUS-guided transluminal drainage and subsequently underwent necrosectomy. The conventional technique using a diagnostic or therapeutic gastroscope involved removal of necrotic debris using any $15-20 \mathrm{~mm}$ polypectomy snare, $7.5 \mathrm{~mm}$ rat tooth or tripod grasping forceps and retrieval nets. Normal saline was used for intra-procedural irrigation and lavage of the necrotic cavity. The algorithmic technique, using a cap-fitted therapeutic gastroscope, was tailored to the extent and location of the WON: While WON extending to the flanks and more proximate $(<20 \mathrm{~cm})$ to the abdominal wall were treated by percutaneous necrosectomy, those in close proximity to the stomach and without extension to the flanks were treated by transluminal EN. Adherent debris were removed using $15-25 \mathrm{~mm}$ round, braided-wire snares and non-adherent debris were removed using 15 $30 \mathrm{~mm}$ oval snares. A large $14.9 \mathrm{~mm}$ diameter, rat tooth forceps were used for debridement when the necrotic debris were not amenable for snaring. The necrotic cavity was evacuated by sucking the debris within the gastroscope cap using snares and/or forceps. In order to avoid frothing that obscures visibility, intra-procedural lavage of the necrotic cavity was performed using normal saline mixed with $120 \mathrm{mg}$ gentamicin and irrigation with hydrogen peroxide was reserved for sterilizing the cavity at the end of the procedure. The primary outcome measure was to compare the treatment success and number of reinterventions performed between the two groups. Treatment failure was defined as death from underlying disease or need for open surgical necrosectomy.

Results: Of the 45 WON patients, 23 were treated using conventional techniques and 22 using the algorithmic approach. Treatment success was significantly higher for patients treated using the algorithmic approach, 100 vs. $69.6 \%$, $\mathrm{p}=0.009$. Of the 7 patients who had treatment failure in the conventional technique cohort, 6 required open necrosectomy and 1 died of multi-organ failure. The median number of reinterventions required to achieve treatment success was significantly lower for the algorithmic approach, $1(\mathrm{IQR}=1-1)$ vs. $2(\mathrm{IQR}=1-$ $2), p=0.003$. Multivariable logistic regression analysis revealed that the algorithmic approach was the only variable associated with treatment success $(\mathrm{OR}=60.4, \mathrm{p}=0.02)$ when adjusted for patient demographics, lab parameters and disease/WON characteristics.

Conclusion: A structured, algorithmic approach to endoscopic necrosectomy results in successful treatment outcomes.

Disclosure of Interest: R. Hawes: Consultant for Boston Scientific Corporation and Olympus America Inc.

S. Varadarajulu: Consultant for Boston Scientific Corporation and Olympus America Inc.

All other authors have declared no conflicts of interest.

\section{P0235 ENDOSCOPIC RETROGRADE}

CHOLANGIOPANCREATOGRAPHY IN THE MANAGEMENT OF PANCREAS DIVISUM ASSOCIATED WITH RECURRENT ACUTE PANCREATITIS IN CHILDREN: EXPERIENCE FROM A SINGLE CENTER IN CHINA

G. Cui, J. Yang, X. Tang, J. Yang, X. Zhang

Gastroenterology And Hepatology, Hangzhou First People's Hospital, Hangzhou China

\section{Contact E-mail Address: zxf837@tom.com}

Introduction: Pancreas divisum( PD) is the most common congenital anomaly of the pancreas. Most PD individuals are asymptomatic, but a few may present symptoms in the form of recurrent acute pancreatitis (RAP), chronic pancreatitis (CP) or pancreatic-type pain. It is imperative to treat PD associated with RAP as early as possible to prevent it from developing CP. Unfortunately, to date, most PD-related studies have been concentrated on adults. Researches of PD in children is rare.

Aims \& Methods: To evaluate the safety and efficacy of endoscopic retrograde cholangiopancreatography (ERCP) for the treatment of pancreas divisum (PD) associated with recurrent acute pancreatitis (RAP) in children. We retrospectively analyzed patients of PD associated with RAP who were younger than 18 years old from January 2011 to December 2015 in our center. All the patients were diagnosed and treated with ERCP. Patients of complete PD associated with RAP underwent endoscopic minor sphincterotomy combined with dorsal duct stenting (ESCS). Patients of incomplete PD underwent bipapilla endoscopic sphincterotomy combined with dorsal duct stenting (BiESCS). ERCP-related data, complications and other relevant data were collected. Long time follow up was conducted after removal of the last dorsal duct stent, and then to observe children's recovery, as well as their weight, growth and intelligence.

Results: A total of 227 pediatric ERCPs were performed for 117 pediatric patients during this period. Of which 24 were PD cases. The endoscopic detection rate of PD was $20.5 \%$. Of the 24 patients, 12 were PD associated with RAP, among which 10 were complete PD and 2 were incomplete PD. A total of 21 therapeutic ERCPs were performed for these cases. All procedures were successful with $100 \%(21 / 21)$ of cannulation rate of the minor papilla. The mean interval of changing pancreatic dorsal duct stent is 3 months (from 2 to 6 months). ERCP-related complications were mild with a rate of $9.5 \%(2 / 21)$. One was acute mild pancreatitis and the other was hyperamylasemia, both of which were managed conservatively. During follow up from 15 to 74 months (mean 33.9 months), all patients had pain relief with a relief rate of $100 \%$, of which 10 were asymptomatic with no longer onset of acute pancreatitis. During follow-up, MRCP or CT scan showed no more dilation of dorsal ducts in all children and all presented normal in weight, growth and intelligence.

Conclusion: The techniques of ESCS and Bi-ESCS under ERCP are safe and effective methods to manage PD associated with RAP in pediatric patients. It seems very vital for such children to undergo endoscopic interventions as early as possible in order to avoid developing $\mathrm{CP}$.

Disclosure of Interest: All authors have declared no conflicts of interest.

P0236 ENDOSCOPIC BILIARY SPHINCTEROTOMY IN MALIGNANT BILIARY OBSTRUCTION: IS IT INDICATED IN CASE OF STENT PLACEMENT? A META-ANALYSIS

B. Mangiavillano ${ }^{1}$, A. Montale ${ }^{2}$, L. Frazzoni ${ }^{2}$, M. Bianchetti ${ }^{1}$, A. Repici $^{3}$, L. Fuccio ${ }^{4}$

${ }^{1}$ Gastrointestinal Endoscopy Unit, Humanitas - Mater Domini, Castellanza (VA) Italy 
${ }^{2}$ Department Of Medical And Surgical Sciences, S. Orsola-Malpighi Hospital, Bologna/Italy

${ }^{3}$ Dept. Of Gastroenterology, Ist. Clinico Humanitas Rozzano Dept. of Gastroenterology, Milano/Italy

${ }^{4}$ Deprtment Of Medical And Surgical Sciences, S. Orsola-Malpighi University Hospital, Bologna/Italy

\section{Contact E-mail Address: b_mangiavillano@hotmail.com}

Introduction: Endoscopic biliary stenting is the treatment of choice in presence of malignant biliary obstruction (MBO), especially if palliative treatment. The role of endoscopic biliary sphincterotomy (EBS) before stent insertion is not clearly defined. The primary outcome of our meta-analysis was to assess the technical success of biliary (plastic or metal) stent insertion. Secondary outcomes included early complications within 30 days from ERCP and late complications which (from 30 days since ERCP).

Aims \& Methods: We performed a literature search by using PubMed, SCOPUS, Google Scholar and the Cochrane Central Register of Clinical Trials (up to February 2017) to identify full-text studies evaluating the efficacy and safety of stent positioning, with and without EBS, in patients with MBO not suitable to surgery.

Results: 14 papers were assessed via full text for eligibility. 8 articles were excluded leaving 6 prospective studies (total of 711 patients). Technical success: The overall rate of biliary stent insertion was not significantly different: $384 / 392$ patients $(98 \%)$ in the no-EBS group versus $331 / 339(97.6 \%)$ in the EBS arm (OR: $1.05 ; 95 \%$ CI, 0.42-2.63). Early complications: The overall early AEs developed in $43 / 392(11 \%)$ of patients whithout EBS versus $68 / 339(20.1 \%)$ of patients who received EBS, with a significantly difference (OR: 0.55; 95\%CI: 0.33-0.92). PostERCP pancreatitis (PEP) was no significantly different in the two groups: $24 / 392$ $(6.1 \%)$ in no-EBS group versus $17 / 339(5 \%)$ in EBS group (OR: 1.33 ; $95 \% \mathrm{CI}$ : $0.68-2.59)$. The bleeding was significantly different in patients without EBS: $0 /$ 351 of patients in no-EBS group versus 15/298 (5\%) in the EBS group (OR: 0.12; $95 \% \mathrm{CI}, 0.03-0.45)$. The rate of duodenal perforation was not significantly different: $1 / 320(0.3 \%)$ in no-EBS versus $4 / 260(1.5 \%)$ in EBS (OR: $0.52 ; 95 \% \mathrm{CI}$ : $0.09-2.88)$. Early cholangitis was significantly lower in patients who didn't receive EBS: $13 / 392(3.3 \%)$ patients in no-EBS group vs $25 / 339(7.4 \%)$ subjects in the EBS group (OR: $0.38 ; 95 \% \mathrm{CI}: 0.17-0.83$ ). Early mortality rate was $0 \%$ in both groups. Late complications: No significantly difference occurred in the overall late adverse events in the two groups: $50 / 251$ patients $(19.9 \%)$ in no-EBS group vs $38 / 201$ subjects $(18.9 \%$ ) in the EBS group (OR: 0.93 ; $95 \%$ CI: 0.56 $1.53)$. No significantly differences in stent occlusion (11.6\% patients in no-EBS vs 11.4\%in EBS - OR: $0.90 ; 95 \% \mathrm{CI}$ : $0.4-2.0)$. No significantly differences in stent migration ( $4 \%$ in no-EBS group vs $5.5 \%$ - OR: 0.81 ; $95 \%$ CI: $0.29-2.25)$. No significantly differences in late cholangitis $(2.6 \%$ in no EBS vs $0 \%$ in EBS group OR: $1.83 ; 95 \%$ CI: $0.17-19.85)$. Long-term mortality was not significantly different $(2.5 \%$ in no-EBS group and $2.9 \%$ in the EBS arm - OR: 1.18 ; $95 \%$ CI: 0.22 6.29).

Conclusion: Our meta-analysis showed no significantly differences in technical success and in PEP. In consideration of the significantly increase of the overall AEs in the EBS group, and in particularly of the bleeding and cholangitis, the EBS seems not be recommended in patients not suitable to surgery undergone biliary stenting.

Disclosure of Interest: All authors have declared no conflicts of interest.

\section{P0237 ENDOSCOPIC PANCREATIC SPHINCTEROTOMY COMBINED WITH PANCREATIC DUCT STENT CAN EFFECTIVELY PREVENT RECURRENCE OF ACUTE RECURRENT PANCREATITIS CAUSED BY BILIARY MICROLITHIASIS - A SINGLE-CENTER STUDY FROM BEIJING, CHINA}

Y. Huang, X. Yan, W. Liu, Y. Zhang, W. Yao, K. Li

Gastroenterology And Hepatology, Peking University Third Hospital, Beijing China

\section{Contact E-mail Address: 13911765322@163.com}

Introduction: Acute recurrent pancreatitis (ARP) refers to a clinical entity characterized by episodes of acute pancreatitis which occurs on more than one occasion. Biliary microlithiasis plays an important role in the etiology of ARP. Bile sludge may induce acute pancreatitis as a consequence of transient papillary edema that can obstruct the pancreatic juice flow. The established treatments of microlithiasis with ARP include endoscopic sphincteropapillotomy (EST) and empirical cholecystectomy. However, EST may increase the morbidity of biliary reflux or cholelithiasis recurrence. We hypothesized that endoscopic pancreatic sphincterotomy (EPS) can save the function of biliary sphincter and prevent the recurrence of ARP.

Aims \& Methods: The aim of the study is to evaluate the effectiveness of EPS combined pancreatic duct stent for preventing ARP caused by biliary microlithiasis. 67 patients with ARP from 2005 to 2016 were diagnosed as biliary microlithiasis by endoscopic retrograde cholangiopancreatography (ERCP), bile microscopy or intraductal ultrasonography (IDUS). The whole was divided into two groups according to endoscopic therapy by EST or EPS with pancreatic stent. Rate of pancreatitis recurrence, early complication of post ERCP pancreatitis (PEP), and late complications (3 months after treatment) which included cholangitis, cholecystitis or cholelithiasis were compared between the two groups. Results: (1) 38 and 29 patients were included in EST and EPS group, respectively. The mean age and follow-up duration of EST and EPS were $48.4 \pm 15.1 \mathrm{yrs}$, $45.7 \pm 36.5$ months and $45.6 \pm 15.2 \mathrm{yrs}, 24.1 \pm 26.3$ months, respectively. (vary from 2 months to 115 months). (2) The mean episodes of ARP in EST and EPS group before endoscopic therapy were $3.9 \pm 3.3$ times and $7.9 \pm 11.8$ times. (3) Four patients in EST group and 6 patients in EPS group suffered PEP after the endoscopic therapy $(P=0.418)$. (4) 15 patients in EST group and 3 in EPS group suffered recurrent pancreatitis. The efficiency in EST group and EPS group is $68.4 \%$ and $89.6 \%$ respectively $(P=0.039)$. (5) The incidence of late complications is $18.4 \%$ in EST group and $10.3 \%$ in EPS group $(\mathrm{P}=0.567)$.

Conclusion: EPS combined with pancreatic stent is a promising strategy to prevent recurrence of ARP due to biliary microlithiasis.

Disclosure of Interest: All authors have declared no conflicts of interest.

\section{References}

1 Testoni P A. Acute recurrent pancreatitis: Etiopathogenesis, diagnosis and treatment. World J Gastroenterol, 2014, 20(45):16891-16901.

2 Parsi M A, Stevens T, Dumot J A, et al. Endoscopic therapy of recurrent acute pancreatitis. Cleveland Clinic Journal of Medicine, 2009, 76(4):225-233.

3 Da Costa D W, Schepers N J, Römkens T E H, et al. Endoscopic sphincterotomy and cholecystectomy in acute biliary pancreatitis. The Surgeon, 2016, 14(2):99-108

$4 \mathrm{Kim} \mathrm{H} \mathrm{S,} \mathrm{Moon} \mathrm{J} \mathrm{H,} \mathrm{Choi} \mathrm{H} \mathrm{J,} \mathrm{et} \mathrm{al.} \mathrm{The} \mathrm{role} \mathrm{of} \mathrm{intraductal} \mathrm{US} \mathrm{in} \mathrm{the}$ management of idiopathic recurrent pancreatitis without a definite cause on ERCP. Gastrointestinal Endoscopy, 2011, 73(6):1148-1154.

5 Doi S, Yasuda I, Mukai T, et al. Comparison of long-term outcomes after endoscopic sphincterotomy versus endoscopic papillary balloon dilation: a propensity score-based cohort analysis. Journal of gastroenterology, 2013, 48(9):1090-1096.

\section{P0238 OUTCOME OF ENDOSCOPIC RETROGRADE CHOLANGIOPANCREATOGRAPHY IN PATIENTS WITH PERIAMPULLARY DIVERTICULUM}

M.A. Baig ${ }^{1}$, M. Salih ${ }^{1}$, N.H. Shah ${ }^{1}$, M. Fatima ${ }^{2}$

${ }_{1}^{1}$ Gastroenterology, Shifa international hospital, islamabad/Pakistan

${ }^{2}$ shifa college of medicine, islamabad/Pakistan

Contact E-mail Address: muhammadasifbaig81@gmail.com

Introduction: Periampullary diverticulum (PAD) is frequently asymptomatic, usually encountered in patients undergoing endoscopic retrograde cholangiopancreatography(ERCP).

Aims \& Methods: The aim of this study was to investigate the association of PAD with bile duct stones, biliary cannulation success and

different types of PAD.

A total of 1164 ERCP procedures were performed in 833 patients in a single center by single operator from January 2012 to October 2016 after excluding post liver transplant, emergency procedures. Out of 1164 ERCP procedure 49 patients were encountered with PAD, they were compared with 635 controls without PAD in terms of age, sex, CBD cannulation success and complications of ERCP.

\begin{tabular}{|c|c|c|c|}
\hline \multicolumn{4}{|c|}{ BASELINE CHARACTERISTICS AND COMPARISON OF FINDINGS } \\
\hline & $\begin{array}{l}\text { PAD GROUP } \\
(\mathrm{n}=49)\end{array}$ & $\begin{array}{l}\text { NON-PAD } \\
\text { GROUP } \\
\text { P-VALUE } \\
(\mathrm{n}=635)\end{array}$ & P-VALUE \\
\hline $\operatorname{Age}($ mean $\pm \mathrm{SD})$ & $59.10 \pm 15.16$ & $52.74 \pm 17.94$ & $<0.05$ \\
\hline Sex (male/female) & $21 / 28$ & $315 / 320$ & $\begin{array}{l}\text { NOT } \\
\text { SIGNIFICANT }\end{array}$ \\
\hline $\begin{array}{l}\text { Patients with bile duct stones } \\
\quad(\mathrm{n}=\text { number of patients) } \%\end{array}$ & $35(71.4 \%)$ & $210(33.1 \%)$ & $<0.05$ \\
\hline $\begin{array}{l}\text { CBD cannulation (easy/difficult) } \\
\mathrm{n}=\text { numbar of patients } \%\end{array}$ & $35 / 14(75.5 \%)$ & $263 / 72(82.3 \%)$ & $<0.05$ \\
\hline $\begin{array}{l}\text { Complications(bleedi } \\
\text { ng/pancreatitis/perfor ation) } \%\end{array}$ & $2 \% / 2 \% / 2 \%$ & $0.1 \% / 0.1 \% / 0.1 \%$ & $<0.05$ \\
\hline
\end{tabular}

Results: PAD identified in $49(4.2 \%)$ cases, PAD type 1 (inside the diverticulum) was found in 7 pts $(14.3 \%)$, Type 11 (at edge/brim) in 34 pts $(69.4 \%)$, Type 111 (adjacent/near diverticulum) in 8 pts $(16.3 \%)$. Patients with PAD had mean age 59.10 years (range 18 to 84 years) 17 were $<50$ yrs while $32>50$ yrs, compared to controls mean age 52.74 yrs (range 12 to 95 yreas) 230 were $<50$ yrs while remaining more than 50 yrs. P-value $(<0.5)$. PAD predominantly occurred in females 28/49 cases.

Patients with PAD had increased prevalence of gallstone/biliary stone disease compared with controls, $71.4 \%$ vs $33.1(\mathrm{p}<0.01)$ compared with controls. Easy cannulation of CBD without difficulty (PRECUT/Pacreatic cannulation/stenting) was more frequent in patients controls $(82.3 \%)$ compared to PAD group $(75.5 \%) \mathrm{p}<0.05$. However, CBD clearance was same in both groups $>90 \%$ (p value not significant) Incidence of complications in PAD group bleeding $(2 \%)$, Pancreatitis $(2 \%)$ and one small retroduodenal perforation $(2 \%)$ all managed conservatively. In without PAD group bleeding $0.6 \%$, pancreatitis $0.7 \%$ and no perforation.

Conclusion: PAD is seen with advanced age, predominantly in female and frequently associated with bile duct stones. In this case control study PAD did not appear to be a barrier for successful ERCP with acceptable complication rates. Disclosure of Interest: All authors have declared no conflicts of interest. 


\section{References}

1. Perdikakis E, Chryssou EG, Karantanas A. Diagnosis of periampullary duodenal diverticula: the value of new imaging techniques. Annals of gastroenterology: quarterly publication of the Hellenic Society of Gastroenterology 2011;24(3):192-99

2. Lobo DN, Balfour TW, Iftikhar SY. Periampullary diverticula: consequences of failed ERCP. Annals of the Royal College of Surgeons of England 1998;80(5):326-31

3. Zoepf T, Zoepf DS, Arnold JC, et al. The relationship between juxtapapillary duodenal diverticula and disorders of the biliopancreatic system: analysis of 350 patients. Gastrointestinal endoscopy 2001;54(1):56-61 doi: 10.1067/mge.2001.115334 [published Online First: Epub Date]|

4. Tyagi P, Sharma P, Sharma BC, et al. Periampullary diverticula and technical success of endoscopic retrograde cholangiopancreatography. Surgical endoscopy 2009;23(6):1342-5 doi: 10.1007/s00464-008-0167-7 [published Online First: Epub Date]|.

5. Zippi M, Traversa G, Pica R, et al. Efficacy and safety of endoscopic retrograde cholangiopancreatography (ERCP) performed in patients with Periampullary duodenal diverticula (PAD). La Clinica terapeutica 2014;165(4):e291-4 doi: 10.7417/CT.2014.1745 [published Online First: Epub Date].

6. Kim KH, Kim TN. Endoscopic papillary large balloon dilation in patients with periampullary diverticula. World journal of gastroenterology: WJG 2013;19(41):7168-76 doi: 10.3748/wjg.v19.i41.7168 [published Online First: Epub Date]|.

7. Yildirgan MI, Basoglu M, Yilmaz I, et al. Periampullary diverticula causing pancreaticobiliary disease. Digestive diseases and sciences 2004;49(1112):1943-5

8. Mohammad Alizadeh AH, Afzali ES, Shahnazi A, et al. ERCP features and outcome in patients with periampullary duodenal diverticulum. ISRN gastroenterology 2013:217261 doi: 10.1155/2013/217261 [published Online First: Epub Date]|.

9. C.W. Kim, J. H. Chang, J.H. Kim, T.H. Kim, I. S. Lee, and S.W. Han. Size and type of priampullary duodenal diverticula are associated with bile duct diameter and recurrence of bile duct stones. Journal of gastroentrology and Hepatology 2013; 28: 893-898.

\section{P0239 COMPARISON OF DIGITAL VS FIBEROPTIC CHOLANGIOSCOPY IN PATIENTS REQUIRING EVALUATION OF BILE DUCT DISEASE OR TREATMENT OF BILIARY STONES}

I. D. Dimas, E. Vardas, M. Fragkaki, A. Mpitouli, E. Voudoukis, A. Theodoropoulou, G. Paspatis

Department Of Gastroenterology, Venizeleion General Hospital, Heraklion/Greece

Contact E-mail Address: gpaspatis@gmail.com

Introduction: Since the emergence of the fiberoptic single-operator cholangioscopy, the sensitivity for detecting bile duct lesions has been increased and the management of difficult stones is facilitated, establishing its superiority over standard endoscopic retrograde cholangio-pancreatography and often altering the clinical management. Digital cholangioscopes provide higher-resolution imaging of the pancreatobiliary tract compared with the fiberoptic instruments

Aims \& Methods: The aim of the present study was to assess the frequency of digital cholangioscopy (DC) to alter the diagnosis and clinical management of bile duct disease compared with fiberoptic cholangioscopy (FC). A retrospective review of 68 cases needing cholangioscopy, and which were performed in our department, was conducted. Patients enrolled exhibited stenosis of the biliary tract $(67.6 \%)$, stones $(20.6 \%)$, primary sclerosing cholangitis (PSC $4.4 \%$ ) or other rare cause for cholangioscopy (e.g. stent migration, guidewire passage) All patients underwent endoscopic retrograde cholangiography (ERC) before cholangioscopy. Aim of cholangioscopy was to confirm ERC diagnosis, obtain adequate biopsy specimens for histological evaluation and remove biliary stones. From $5 / 2009$ to $8 / 2015$ all cholangioscopies were performed with the fiberoptic scope. From $9 / 2015$ to $3 / 2017$ all cholangioscopies were performed with the digital scope.

Results: 30 women and 38 men with a mean age of 61years underwent cholangioscopy. Fiberoptic cholangioscope was used in 39 cases and digital cholangioscope was performed in 29 cases respectively. Cholangioscopy-guided biopsies for malignancy were obtained in 11 and 15 cases respectively. In only one case of FC $(9.1 \%)$ biopsy confirmed the endoscopic diagnosis, in contrast with 10 cases of DC-guided biopsies $(66.7 \%)$ confirming the diagnosis. In 13 patients who underwent DC (44.8\%) the initial diagnosis and clinical management was altered after cholangioscopy (e.g. cancer diagnosis, successful EHL lithotripsy), in contrast with 11 cases of FC $(28.2 \%)$. Moreover it was unanimously felt by our staff that DC was an easier procedure compared to FC.

Conclusion: Our data suggest that DC has overcome the impediment of fiberoptic technology, providing increased sensitivity and specificity for visual impression diagnosis of malignancy and successful therapy of biliary stones. Moreover DC has the ability to alter more often the initial ERC diagnosis or management compared to FC and provides a new sophisticated and easy to use equipment. Disclosure of Interest: All authors have declared no conflicts of interest
P0240 EMERGENCY ENDOSCOPIC RETROGRADE

CHOLANGIOPANCREATOGRAPHY IN SUPER-ELDERLY PATIENTS WITH SEVERE ACUTE CHOLANGITIS: CAN WE PERFORM THE PROCEDURE SAFELY?

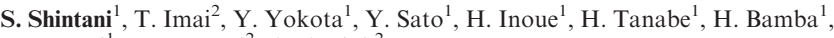
Y. Komai ${ }^{1}$, O. Inatomi ${ }^{2}$, A. Andoh ${ }^{2}$

Gastroenterology, Nagahama Red Cross Hospital, Nagahama/Japan

${ }^{2}$ Gastroenterology, Shiga University of Medical Science Hospital, Otsu/Japan

Contact E-mail Address: mtr_bs@yahoo.co.jp

Introduction: Tokyo Guidelines 2013 (TG13) have been used worldwide to assess the diagnostic criteria and severity grading of acute cholangitis. Acute cholangitis is a life-threatening disease, and the emergency biliary drainage procedure is necessary for moderate or severe cases, according to TG13. In recent years, with an aging society, necessity to perform endoscopic retrograde cholangiopancreatography (ERCP) in elderly patients are increasing. However, few studies have examined the efficacy and safety of emergency ERCP in super-elderly patients with severe cases.

Aims \& Methods: In this study, we examined the efficacy and safety of emergency ERCP in super-elderly patients with moderate to severe acute cholangitis, according to TG13. We performed 178 emergency ERCP procedures in 132 patients during 3 years (June 2014-December 2016). We determined patients $>90$ years as "super-elderly" and those $<90$ years as "non-super-elderly". Evaluation criteria included comorbidities, oral administration of anticoagulants, cause of cholangitis, ERCP procedure (examination time, endoscopic biliary sphincterectomy (EST)/pre-cut papillotomy, treatment success rate, presence or absence of peripapillary diverticula and papilla after EST, sedation dosage), ERCP-related complications (bleeding, perforation, post-ERCP pancreatitis, post-ERCP pneumonia, death within 30 days after ERCP procedure), and anesthesia-related complications (blood pressure decrease, pulse reduction, respiratory depression)

Results: We examined 69 males $(52.3 \%)$ and 63 females $(47.7 \%)$. Women accounted for a larger proportion in the super-elderly group ( $71 \%$ vs $40 \%$ ). The average age was 92.5 years (range, 90-97) in the super-elderly group and 77.9 years (range, $50-89$ ) in the non-super-elderly group. The super-elderly group comprised 54 ERCP procedures (moderate, 32; severe, 22) against 124 ERCP procedures (moderate, 104; severe, 20) in non-super-elderly group, and severe cases observed in the super-elderly group were statistically significant $(\mathrm{p}<0.001)$. Regarding comorbidities, chronic heart and renal failure were statistically dominant in the super-elderly group. However, no difference was seen in the presence of other diseases and receiving anticoagulant medication between the two groups. The causes of acute cholangitis were common in both groups with common bile duct stone ( $46 \%$ vs $46 \%$ ), followed by malignant obstruction $(9 \%$ vs $12 \%)$ and benign stenosis $(0 \%$ vs $5 \%)$, but no difference was found Regarding the ERCP procedure, the examination time was longer in the superelderly group $(37.5 \pm 28.1 \mathrm{~min}$ vs $29.2 \pm 24.0 \mathrm{~min}, \mathrm{p}=0.044)$, but there was no difference in the procedure success rate $(93 \%$ vs $97 \%, p=0.249)$ and the presence of peripapillary diverticula. The patients were sedated using midazolam (MDZ) plus pentazocine (PTZ). The amount of anesthetic used was less in the superelderly group (MDZ: 2.2 vs $3.3 \mathrm{mg}, \mathrm{p}<0.001$, PTZ: 3.1 vs $5.4 \mathrm{mg}, \mathrm{p}=0.005$ ). Regarding (i) ERCP-related and (ii) anesthesia-related complications, these were higher in the super-elderly group [(i) $15 \%$ vs $9 \%, \mathrm{p}=0.293$, (ii) $17 \%$ vs $7 \%$, $\mathrm{p}=0.0504)$ ]. Every patient with complication improved with conservative treatment, and no intravascular or surgical treatment was required.

Conclusion: Acute cholangitis in super-elderly patients was more likely to become severe and the complications were higher than that in non-super-elderly patients. When performing an emergency ERCP procedure in super-elderly patients, we should particularly pay attention to developing complications in patients with moderate or higher acute cholangitis, according to TG13.

Disclosure of Interest: All authors have declared no conflicts of interest.

\section{P0241 EFFICACY AND SAFETY OF ENDOBILIARY}

RADIOFREQUENCY ABLATION FOR THE ERADICATION OF RESIDUAL NEOPLASIA AFTER ENDOSCOPIC AMPULLECTOMY. RESULTS OF A MULTICENTER PROSPECTIVE STUDY

M. Camus ${ }^{1}$, B. Napoléon ${ }^{2}$, A. Vienne ${ }^{3}$, M. Le Rhun ${ }^{4}$, S. Leblanc ${ }^{1}$, M. Barret ${ }^{5}$, N. Kaddour ${ }^{6}$, F. Robin ${ }^{7}$, S. Chaussade ${ }^{8}$, F. Prat ${ }^{9}$

${ }^{1}$ Gastroenterology, University Paris 5 APHP Hopital Cochin, Paris/France

${ }^{2}$ 69. Hôpital Privé Jean Mermoz, Lyon/France

${ }^{3}$ Gastroenterology And Digestive Endoscopy, Georges-Pompidou European Hospital, Paris/France

${ }^{4}$ Institut des Maladies de l'Appareil Digestif, CHU Nantes, Nantes/France

${ }^{5}$ Gastroenterology, Cochin Hospital, Paris/France

${ }^{6}$ URC/CIC Paris Descartes Necker-Cochin, Paris/France

${ }^{7}$ ARC SFED, Hépato-Gastroentérologie, HCL, Hôpital Edouard Herriot, Lyon, Lyon/France

${ }^{8}$ Service De Gastro-entérologie, CHU Cochin Dept. de Gastroenterologie, Paris/ France

${ }^{9}$ Gastroenterology, Hôpital Cochin Dept. of Gastroenterology, Paris/France

Contact E-mail Address: marine.camus@gmail.com

Introduction: Dysplasia may persist at the termination of the common bile duct (CBD) after endoscopic ampullectomy. Radiofrequency ablation (RFA) could be an interesting alternative to surgery to reduce the risk of invasive cancer with less morbi-mortality.

Aims \& Methods: The aim of the study was to evaluate the efficacy and morbidity of endo-biliary RF for the treatment of residual endo-biliary dysplastic lesions after endoscopic ampullectomy. A prospective open-label multicenter study 
included 20 patients with low-grade dysplasia (DBG) or high grade (DHG) lesions in the CBD, confirmed by a double anatomopathological lecture, in relation to a residual adenomatous bud after endoscopic ampullectomy for ampullary adenoma. The lesions should extend to a maximum length of $20 \mathrm{~mm}$ in the CBD. Endoscopic retrograde cholangio-pancreatectomy (ERCP) was performed under general anesthesia with the Habib ${ }^{\mathrm{TM}}$ EndoHPB probe (EMcision, UK) (effect 8 , power 10 Watts, $30 \mathrm{~s}$ ). Biliary \pm pancreatic stent were placed at the end of the procedure. The primary endpoint was the rate of residual neoplasia (eg, DBG, DHG or invasive carcinoma) at 1 year after treatment. Secondary endpoints were: 1) residual neoplasia at 6 months after treatment; 2) rate of surgery at 12 months; 3) adverse events.

Results: The mean age $( \pm$ SD) was 67 years $( \pm 11)$, with 12 men and 8 women. RFA was performed on average $( \pm$ SD) 1.9 years $( \pm 3.5)$ after ampullectomy. The mean resected ampullary adenoma size $( \pm \mathrm{SD})$ was $24.9 \mathrm{~mm}( \pm 10.2)$, and 7 patients had adjacent duodenal mucosectomy at the time of ampullectomy. The histology of the resected ampullary adenoma was DBG for 7 patients, DHG for 12 patients, and in situ carcinoma for 1 patient. Lateral margins were healthy for 13 patients. CBD recurrence was diagnosed predominantly on ERCP and/or endoscopic ultrasonography surveillance procedures with an estimated mean infiltration height $( \pm \mathrm{SD})$ of $11.2 \mathrm{~mm}( \pm 4.5)$. The passage of the RFA probe was judged to be easy in $100 \%$ of cases with visibility of the radiopaque markers judged satisfactory to very satisfactory in $80 \%$ of the cases. All patients included had RFA without any technical problems. All patients had biliary stent (4 SEMS $10 \mathrm{~mm}, 16$ plastic stents 10 French) implanted following RFA and $5(25 \%)$ had a pancreatic stent. The residual rate of DBG, DHG, invasive carcinoma at 6 months and at 12 months after treatment were $25 \%$ $(5 / 20, \mathrm{DBG}$, carcinoma) and $45 \%(9 / 20, \mathrm{DBG}$, carcinoma) respectively. The adverse events were as follows: 4 benign pancreatitis all medically treated, 2 patients had angiocholitis requiring biliary stent replacement, 1 patient had an episode of unexplained spontaneously resolved abdominal pain (normal CT scan, colonoscopy and biological tests). At M12, one patient presented with a biliary scar stricture resolved by dilation and a calibration biliary stent.

Conclusion: Endobiliary RFA performed on residual endo-biliary dysplastic buds after ampullectomy is an alternative to surgery, with a rate $55 \%$ dysplasia eradication at 12 months after a single RFA session. Regular monitoring of these patients is still necessary considering recurrence rate. Multiple RFA sessions may be proposed in case of incomplete results.

Disclosure of Interest: All authors have declared no conflicts of interest.

\section{P0242 EXPERT VALIDATION OF A NOVEL MECHANICAL CUTTING PAPILLA}

S. E. Van Der Wiel ${ }^{1}$, A.D. Koch ${ }^{2}$, M.J. Bruno ${ }^{1}$

${ }^{1}$ Gastroenterology And Hepatology, Erasmus University Medical Center,

Rotterdam/Netherlands

${ }^{2}$ Erasmus MC Rotterdam Gastroenterology and Hepatology, Rotterdam/ Netherlands

Contact E-mail Address: s.e.vanderwiel@erasmusmc.nl

Introduction: Simulation-based training has become an important pillar in competence-based learning in medicine, especially in training novice endoscopists. Several simulators have been validated and implemented in training curricula pertaining gastrointestinal endoscopy. Surprisingly, limited data are available on simulators in ERCP training, despite the fact that ERCP seems to be an ideal platform for simulator-based training due to its technical complexity. The available simulators are difficult to implement in training settings due to the lack of realism or use of live animals or ex-vivo components. Recently, the BoškoskiCostamagna ERCP Trainer was validated as a very realistic training model by our study group. A novel mechanical papilla has been designed allowing to train (precut) sphincterotomy. The mechanical papilla is inserted into the simulator. A specific alloy allows for electrical conduction and cutting of the material using standard sphincterotomes and needle knives.

Aims \& Methods: The aim of our study was to determine the expert validity of this cutting papilla and its didactic value for training sphincterotomy, as judged by experts. Expert participants with more than 2500 ERCPs lifetime were invited to perform a sphincterotomy and fill out a questionnaire on the realism of the sphincterotomy procedure and it's didactic value.

Results: A total of 40 ERCP experts were included. All experts were men, originating from 16 different countries with a mean age of 49.6 years (range 37-65). Thirty-seven participants were gastroenterologists $(92.5 \%), 3$ participants were surgeons $(7.5 \%)$. The mean number of years of endoscopic experience was 20.9 (range 10-40). Experts' opinion on realism of performing a sphincterotomy was rated 6.98 on a ten-point Likert scale, resemblance of the performed maneuvers 7.60 and tactile feedback 6.78 . When asked if the cutting was perceived as expected, experts rated 6.35 and the cutting result was rated 7.30 on a tenpoint scale. The potential as a training tool of the cutting papilla in training novices was rated 3.93 on a four-point scale, and there was a high agreement among the experts to include the papilla in the training of novices (3.93 on a fourpoint scale).

Conclusion: This is the first mechanical papilla available for training sphincterotomy on the Boškoski-Costamagna ERCP Trainer and demonstrates good expert validity. ERCP experts highly agree on the didactic value and added value of this papilla in the training curriculum of novice endoscopists.

Disclosure of Interest: M.J. Bruno: We have received a unrestricted research grant by Cook Medical, Limerick, Ireland

All other authors have declared no conflicts of interest.
P0243 MEDICO-LEGAL CLAIMS IN GASTROINTESTINAL ENDOSCOPY: DOES PROCEDURE RISK RELATE TO SUCCESSFUL OUTCOMES?

S. Budihal, J. F Mayberry

Digestive Diseases Centre, University Hospitals Leicester, GB/United Kingdom

Contact E-mail Address: shivbudihal@yahoo.co.uk

Introduction: Complications in endoscopy can lead to adverse clinical events. The likelihood of developing a complication depends on the degree of risk associated with a certain procedure. It is generally noted that riskier the procedure larger is the chance for a complication and higher the likelihood for medico legal issues. This is relevant as in the event of the risk materialising, patients make seek legal redress. The aim of this study was to investigate the degree of success of medico legal claims based on the nature of the endoscopic procedure and the outcome of the claims.

Aims \& Methods: The National Health Service Litigation Authority (NHSLA) database in U.K. was searched using a Freedom of Information request (F/2405) to investigate endoscopic claims notified to the NHSLA between 2010/11 and 2014/15. The terms "Gastroscopy", "Sigmoidoscopy", "Colonoscopy", "PEG" and "ERCP" were used to search the database. They were then analysed for procedure type, characteristics and outcomes. StatsDirect statistical software was used for statistical analysis.

\begin{tabular}{llll}
\hline OUTCOME OF ENDOSCOPY CLAIMS & \\
\hline PROCEDURE & OPEN & SUCCESSFUL & UNSUCCESSFUL \\
\hline Gastroscopy & 6 & 10 & 7 \\
PEG & 12 & 19 & 12 \\
Sigmoidoscopy & 17 & 13 & 5 \\
ERCP & 22 & 26 & 24 \\
Colonoscopy & 50 & 40 & 28 \\
\hline
\end{tabular}

Results: A total of 291 claims were notified to the NHSLA during this period. $107(36.7 \%)$ of claims still remain 'open'. Analysing outcomes by procedures reveals a success rate of $44 \%, 44 \%, 37 \%, 36 \%$ and $34 \%$ (rounded up to the nearest whole figure) for Gastroscopy, PEG, Sigmoidoscopy, ERCP and Colonoscopy claims respectively. There is no statistical difference between the proportions comparing Gastroscopy and Colonoscopy (StatsDirect software used)

Conclusion: A significant number of claims remain open leading to concern and worry among endoscopists. The impact on practitioners after a successful claim is unknown and merits further investigation. Procedures considered as dangerous like ERCP and Colonoscopy have the least successful claims. It is imperative that clinicians remain vigilant. Performing Gastroscopy is dangerous and so is undertaking a Percutaneous Endoscopic Gastrostomy. Endoscopists should tighten their approach to all procedures.

Disclosure of Interest: All authors have declared no conflicts of interest.

\section{P0244 VISUALIZATION OF INTRA-AMPULLARY CHOLEDOCHOCELE WITH CONTRAST MEDIUM FOR EVALUATING TECHNICAL DIFFICULTY IN ERCP}

N. Nishino

Gastroenterology Center, Southern Tohoku Hospital, Koriyama/Japan

Contact E-mail Address: nishinon@tim.hi-ho.ne.jp

Introduction: Choledochocele has been rarely recognized. We focus on intraampullary choledochocele (IAC). We had experienced some cases with IAC as refractory access to bile duct (BD). IAC has small cyst within ampulla regulated by Oddi's sphincter, so the BD axis has changed via IAC. The cases with IAC would require a high technical skill for axis alignment or alternative strategy such as infundibulotomy or precut. We propose advantage of conventional ERCP (cERCP) with contrast medium, which provides images of IAC and leads to evaluate the difficulties in cannulation.

Aims \& Methods: This study aims to recognize the morphology of intra-ampullary bifurcation of bile duct (BD) and pancreatic duct (PD). Its variation allows the elucidation of the reason for difficulties in cannulation. The current study is a retrospective consecutive case study that is conducted in a single facility, with a study period of 8 years. Our strategy for ERCP was carried out with the contrast medium injected via a catheter, but without guide wire $(\mathrm{GW})$ seeking. Intraampullary bifurcation was particularly visualized with the contrast medium, and X-ray images were magnified sequentially 5-10 times each. The eligibility criteria were: it must be naive papilla and both of BD and PD must be visualized. The following factors were evaluated: ampulla shape, number of orifices, angle of intra-ampullary bifurcation and presence of IAC. The Location, size and shape of IAC were observed. The requirement of GW placement on PD to access BD were evaluated. The shapes of the ampulla were divided into three groups: long nose $(\mathrm{L} / \mathrm{N})$, meaning a long protrusion, Dome $(\mathrm{D})$, meaning a hemisphere and flat $(\mathrm{F})$. The IAC location were classified into BD (Ab), PD (Ap) and common channel (Ac). The choledochocele shapes were divided into three groups: spindle $(\mathrm{Sp})$, sphere (Sh) and oval (Ov). IAC was also recognized on magnetic resonance cholangiopancreatography (MRCP). We analyzed our results of image database with descriptive epidemiology. 
Results: There were cases of 1223 naive papilla out of 2226 cases in total. The success rate to access BD with naive papillae was $97.7 \%(1195 / 1223)$ and overall post-ERCP pancreatitis (PEP) was 1.3\% (29/2226). The eligible patients were 908 (505 male and 403 female), among whom IAC was identified in $6.0 \%(54 / 908)$ The prevalence of IAC in the $\mathrm{L} / \mathrm{N}, \mathrm{D}$ and $\mathrm{F}$ types were $8.9 \%(48 / 542), 1.2 \%(4 /$ $339)$ and $7.4 \%(2 / 27)$, respectively. IAC was significantly higher in the $\mathrm{L} / \mathrm{N}$ $(\mathrm{p}<0.01)$ and $\mathrm{F}(\mathrm{p}<0.05)$ types than in the $\mathrm{D}$ type. The choledochocele shapes of $\mathrm{Sp}, \mathrm{Sh}$ and $\mathrm{Ov}$ were $59.3 \%, 13.0 \%, 27.8 \%$, respectively. The average size was $8.1 \mathrm{~mm}(3.7-18.3)$ in diameter. The location of IAC in Ac and Ab were $63.0 \%$ and $37.0 \%$. The IAC in $\mathrm{Ab}$ was found with $\mathrm{L} / \mathrm{N}$ shape only. Patients of $53.7 \%(29 / 54)$ required GW placement on PD to access BD. IAC was alternatively seen on MRCP in $10 \%(3 / 30)$.

Conclusion: Choledochocele is rarely seen on even cERCP, in addition the visualization of IAC has been rarely reported. IAC could be actually visualized with prudent contrast medium injection. Our results showed miscellaneous variations in the intra-ampullary images. IAC would require refractory pursuit of the axis alignment due to its unexpected pathway within ampulla to access BD. Moreover $6.0 \%$ prevalence of IAC should not be ignored. IAC can be one of the factor of refractory cannulation. cERCP with focus on ampulla could the difficulty in cannulation. On the other hand, WGC would not do. The previous randomized control trials showed no difference to access to BD between WGC and cERCP. However, both procedures still remained cases with refractory cannulation. It has been reported that refractory cannulation might cause PEP1). Therefore careful attention should be paid while passing through IAC to avoid PEP. According to ampulla shapes, especially of $\mathrm{L} / \mathrm{N}$ and $\mathrm{F}$, cERCP would be recommended to identify the presence of IAC. It will be a warrant strategy to choose.

Disclosure of Interest: All authors have declared no conflicts of interest.

\section{Reference}

Risk factors for post-ERCP pancreatitis: a prospective, multicenter study. Freeman ML et al, Gastrointest Endosc.2001 54(4):425-34.

\section{P0245 ENDOTHERAPIES FOR DUCT-TO-DUCT BILIARY ANASTOMOTIC STRICTURE AFTER LIVER TRANSPLANTATION (BASALT STUDY GROUP): INTERIM ANALYSIS AND MEDIUM- TERM OUTCOMES OF A RETROSPECTIVE NATIONWIDE ITALIAN SURVEY}

P. Cantù ${ }^{1}$, I. Parzanese ${ }^{1}$, R. Rosa ${ }^{1}$, G. Santi ${ }^{1}$, F. Barbaro ${ }^{2}$, E. Forti ${ }^{3}$, G. Lombardi ${ }^{4}$, E. Nadal ${ }^{5}$, V. Boarino ${ }^{6}$, A. Pisani ${ }^{7}$, A. Di Sario ${ }^{8}$, H. Bertani ${ }^{9}$, M. Bulajic ${ }^{10}$, D. Ghinolfi ${ }^{11}$, T. Staiano ${ }^{12}$, D. Ligresti ${ }^{13}$, L. Barresi $^{13}$, G. Catalano ${ }^{14}$, A. Baldan ${ }^{15}$, A. Cerofolini ${ }^{16}$, L. De Carlis ${ }^{17}$, M. Monteleone ${ }^{18}$, A. Tringali ${ }^{2}$, G. Costamagna ${ }^{2}$, M. Mutignani ${ }^{3}$, A. Fantin $^{5}$, A. Merighi ${ }^{6}$, S. Traini ${ }^{8}$, R. Conigliaro ${ }^{9}$, M. Zilli ${ }^{10}$, F. Filipponi ${ }^{11}$, E. Masci ${ }^{12}$, I. Tarantino ${ }^{13}$, M. Traina ${ }^{13}$, M. Salizzoni ${ }^{14}$, P. Ravelli ${ }^{15}$, L. Rodella ${ }^{16}$, V. Mazzaferro ${ }^{18}$, M. F. Donato ${ }^{19}$, U. Maggi ${ }^{20}$, L. Caccamo ${ }^{20}$, G. Paone ${ }^{20}$, P. Reggiani ${ }^{20}$, G. E. Rossi ${ }^{20}$, D. Conte ${ }^{1}$, R. Penagini

${ }^{1}$ Department Of Pathophysiology And Transplantation, Università Degli Studi Di Milano, Milan- Italy, Gastroenterology and Endoscopy Unit, Fondazione IRCCS Ca' Granda Ospedale Maggiore Policlinico, Milan/Italy

${ }^{2}$ Digestive Endoscopy Unit, Catholic University, Gemelli University Hospital, Rome/Italy

${ }^{3}$ Diagnostic and Interventional Digestive Endoscopy, Niguarda Ca' Granda Hospital, Milan/Italy

${ }^{4}$ Digestive Endoscopy Unit, A. Cardarelli Hospital, Naples/Italy ${ }^{5}$ Division of Gastroenterology, Department of Surgical, Oncological and Gastroenterological Sciences, Azienda Ospedaliera - University of Padua, Padua/ Italy

${ }^{6}$ Gastroenterology and Endoscopy Unit, Azienda Ospedaliero-Universitaria Policlinico di Modena, Modena/Italy

${ }^{7}$ Gastroenterology Section, Department of Emergency and Organ Transplantation, University of Bari, Bari/Italy

${ }^{8}$ Gastroenterology Clinic, Department of Gastroenterology and Transplantation,

Polytechnic Marche University - United Hospitals of Ancona, Ancona/Italy

${ }^{9}$ U.O.C. Gastroenterology and Digestive Endoscopy Unit, Nuovo Ospedale Civile

Sant'Agostino Estense, Modena/Italy

${ }^{10}$ University Clinical Hospital "Santa Maria della Misericordia, Udine/Italy

${ }^{11}$ Hepatobiliary Surgery and Liver Transplantation Unit, Department of Oncology,

Transplants and Advances in Medicine, University of Pisa Medical School

Hospital, Pisa/Italy

${ }^{12}$ Diagnostic and Therapeutic Endoscopy Unit, Fondazione IRCCS Istituto

Nazionale Tumori, Milan/Italy

${ }^{13}$ Endoscopy Service, Department of Diagnostic and Therapeutic Services, IRCCSISMETT (Istituto Mediterraneo per i Trapianti e Terapie ad Alta

Specializzazione), Palermo/Italy

${ }^{14}$ Liver Transplant Center and General Surgery, A.O.U. Città della Salute e della

Scienza di Torino, Molinette Hospital, University of Turin, Turin/Italy

${ }^{15}$ Digestive Endoscopy Unit, Department of Gastroenterology, Papa Giovanni

XXIII Hospital, Bergamo/Italy

${ }^{16}$ Emergency Endoscopy Unit, Borgo Trento Hospital, Verona/Italy

${ }^{17}$ Department of General Surgery and Transplantation, Niguarda Ca' Granda Hospital, Milan/Italy

${ }^{18}$ Liver Surgery, Transplantation and Gastroenterology, University of Milan and Istituto Nazionale Tumori Fondazione IRCCS, Milan/Italy

${ }^{19}$ Gastroenterology and Hepatology Unit, Fondazione IRCCS Ca' Granda

Ospedale Maggiore Policlinico, Università degli Studi di Milano, Milan/Italy
${ }^{20}$ General Surgery and Liver Transplantation Unit, Department of Pathophysiology and Transplantation, University of Milan and Fondazione IRCCS Ca' Granda,

Ospedale Maggiore Policlinico, Milan/Italy

Contact E-mail Address: roberto.penagini@unimi.it

Introduction: Most appropriate endotherapy of biliary anastomotic strictures (AS) remains to be defined.

Aims \& Methods: Aim is to retrospectively report the endotherapy for duct-toduct AS in 2013, procedure related complications and medium-term outcome results in Italy. A questionnaire was sent to the Endoscopy Units working with Italian Liver Transplantation Centers (BASALT study group).

Results: At present sixteen of the 19 Units $(84 \%)$ returned the questionnaire. Complete endotherapy data and follow-up are available for 182 pts. One-hundred and two patients have been treated with plastic multistenting (PM), 27 with fully covered SEMS and 53 with single stenting (SS). Radiological success was achieved in 144 pts $(79 \%)$, i.e $86 \%$ of PM, $89 \%$ of fully covered SEMS and $60 \%$ of SS ( $p<0.01$ vs PM). Recurrence occurred in 31 pts, i.e. $21 \%$ of pts in whom radiological success was achieved: $11 \%$ of PM ( $p<0.0001$ vs SEMS and $\mathrm{p}<0.05$ vs SS), $41 \%$ of fully covered SEMS and $17 \%$ of SS. After failure of first-line endotherapy (36) or recurrence (31), patients were re-treated with endotherapy $(75 \%)$, surgery $(21 \%)$ or percutaneous balloon dilation $(3 \%)$; one patient dropped out because of death unrelated to endotherapy. Second-line endotherapy was PM for $26 \%$, fully covered SEMS for $52 \%$ and SS for $22 \%$ of pts and radiological success was achieved in $82 \%$ of them (in $86 \%, 89 \%$, and $60 \%$ with PM, SEMS and SS respectively). Procedure-related complications occurred in $7.8 \%(51 / 656)$, i.e. $2.6 \%$ pancreatitis (1 severe leading to death), $4.1 \%$ cholangitis and $0.9 \%$ bleeding. Overall clinical success was achieved in $83 \%$ after a median f-up of 25 mos and no need of surgery in $92 \%$ of patients. Conclusion: Endotherapy is confirmed as the preferred first-line and rescue option for AS. Progressive plastic multi-stenting is most frequently used. Single stenting has suboptimal results and should be abandoned. Use of SEMS is effective, but recurrences seem to be frequent, although a larger patients' sample needs to be evaluated.

Disclosure of Interest: All authors have declared no conflicts of interest.

\section{P0246 COMPARATIVE EVALUATION OF TWO PORCINE EX-VIVO MODELS FOR TRAINING IN ENDOSCOPIC ULTRASOUND-} GUIDED DRAINAGE OF PANCREATIC FLUID COLLECTIONS

F. Moryoussef ${ }^{1}$, S. Leblanc ${ }^{2}$, A. Bertucat ${ }^{3}$, A. Laquière $^{4}$, E. Coron ${ }^{5}$, L. Mangialavoria ${ }^{2}$, J.C. Duchmann ${ }^{2}$, Y. Le Baleur ${ }^{6}$, F. Prat ${ }^{7}$ ${ }^{1}$ Gastroenterology, Hôpital Pitié Salpêtrière, Paris/France

${ }^{2}$ Gastroenterology, Hôpital Cochin, Paris/France

${ }^{3}$ Life Partners Europe, Bagnolet/France

${ }^{4}$ Hôpital Saint Joseph, Marseille/France

${ }^{5}$ Digestive Diseases Institute, University of Nantes Institute of Digestive Disease Digestive Diseases Institute, University of $\mathrm{Na}$, Nantes/France

${ }^{6}$ Gastroenterology, Hôpital Henri Mondor, Créteil/France

${ }^{7}$ Gastroenterology, Hôpital Cochin Dept. of Gastroenterology, Paris/France

Contact E-mail Address: fr.moryoussef@gmail.com

Introduction: EUS-guided Cysto-Enterostomy (EUCE), technique indicated for drainage of symptomatic pancreatic pseudocysts and other peri-enteric fluid collections, requires specific skills for which dedicated models are needed. Based on a compact EASIE model (Erlangen Active Simulator for Interventional Endoscopy) we developed two ex-vivo porcine models of retrogastric cysts and evaluated learning performance within the frame of a structured training program.

Aims \& Methods: The first model was made of porcine colon (i.e. "natural cyst"), and second one was made with an ostomy bag (i.e. "artificial cyst"). All procedures were achieved with EUS scope under fluoroscopy. Both models were evaluated prospectively over a 2-days session involving 14 students and 5 experts.

Results: "Natural cyst" and "artificial cyst" were prepared respectively within 10 and 16.5 minutes $(\mathrm{p}=0.78)$. More than 10 EUCE procedures were done in each model. Model grading (analogic scale) showed no significant difference for primary endpoint of global satisfaction $(\mathrm{p}=0.06)$. Regarding secondary endpoints, difference was not significant for overall impression of realism $(p=0.75)$ whereas it was significant favoring "artificial cyst" in terms of ability to teach procedural steps $(\mathrm{p}=0.01)$ and ease of puncture $(\mathrm{p}=0.03)$ because of less elasticity. Moreover, experts considered ability to improve students' proficiency superior with "artificial cyst" $(\mathrm{p}=0.008)$

Conclusion: Both "artificial and natural cysts" are efficient for EUCE training in terms of global satisfaction. However, the "artificial cyst" model appears to make procedure easier and better to teach procedural steps improving students' proficiency. Larger applications of this model are needed to validate as a standard of training.

Disclosure of Interest: All authors have declared no conflicts of interest.

P0247 A COMPARATIVE STUDY OF SUCTION METHODS DURING ENDOSCOPIC ULTRASOUND-GUIDED FINE-NEEDLE ASPIRATION (CONVENTIONAL SUCTION VERSUS CAPILLARY SUCTION)

K. Yamakita ${ }^{1}$, Y. Kitano ${ }^{2}$, H. Iwamoto ${ }^{2}$, K. Takahashi ${ }^{2}$, K. Wada ${ }^{2}$, S. Otake ${ }^{2}$,

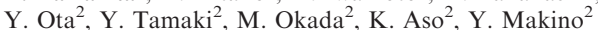


${ }^{1}$ Division Of Metabolism And Biosystemic Science, Department Of Internal Medicine, Asahikawa Medical University, Asahikawa/Japan

${ }^{2}$ Asahikawa Medical University, Asahikawa/Japan

Contact E-mail Address: keisuke_yamakita@yahoo.co.jp Introduction: Endoscopic ultrasound-guided fine-needle aspiration (EUS-FNA) is an established procedure for obtaining a pathological specimen. However, detailed techniques of EUS-FNA vary substantially across medical centers. There is a need to establish more efficient method for better diagnostic accuracy and adequate sample. In the present study, we investigated the suction method for collecting specimens by EUS-FNA.

Aims \& Methods: The aim of our study was to compare conventional suction using a $20 \mathrm{~mL}$ syringe and capillary suction during EUS-FNA. Patients who referred to EUS-FNA for solid mass lesion were prospectively enrolled. We performed EUS-FNA with two needle passes and applied each pass of different techniques which were randomly allocated. The diagnostic accuracy, the quantity of samples (0-5.5 represents sufficient material for adequate for histological interpretation and a high-quality sample), the degree of contamination, and the amount of blood (0-2.2 represents significant amount of blood) were compared between conventional suction and capillary suction. Further analysis was carried out in the patients with pancreatic cancer. For patients with negative EUS-FNA, surgical specimen evaluation, results of other diagnostic investigations and/or long term clinical follow-up (6 months) were used to establish the definitive diagnosis.

Results: During the study period, 96 patients underwent EUS-FNA were enrolled and 7 patients were excluded due to loss of follow-up. Finally 89 patients (averaged $68.5 \mathrm{y} / \mathrm{o}, \mathrm{M} ; \mathrm{F}=47 ; 42)$ were analyzed. There were 60 pancreatic lesions (42 pancreatic cancer, 6 neuroendocrine tumor, etc.), 17 lymph adenopathy, 6 submucosal lesions, 6 other lesions biopsied, with a mean diameter of $28 \pm 3 \mathrm{~mm}$. Although there was no significant difference in the diagnostic accuracy $(86.5 \%$ vs. $79.8 \%, \mathrm{p}=0.32$ ), the quantity of samples (3.64 vs.3.43, p=0.21) and the degree of contamination, the samples obtained by conventional suction contained more blood compared to those obtained by capillary suction $(0.17$ vs. $0.02, \mathrm{p}<0.05)$ ). Moreover, conventional suction showed favorable diagnostic accuracy $(83.3 \%$ vs. $69.0 \%, \mathrm{p}=0.20)$ and significantly higher quality of samples $(3.38$ vs. $2.74, \mathrm{p}<0.05)$ than capillary suction, in the patients with pancreatic cancer.

Conclusion: Capillary suction was effective in the EUS-FNA sampling and associated with less contamination with blood, but conventional suction is recommended in case of fibrotic pancreatic cancer for obtaining high-quality sample. Disclosure of Interest: All authors have declared no conflicts of interest.

\section{P0248 A PROSPECTIVE COMPARISON OF EUS-GUIDED TISSUE ACQUISITION USING A 25G-GAUGE CORE BIOPSY NEEDLE WITHOUT AND WITH A STYLET FOR INTRAABDOMINAL SOLID MASS LESIONS}

J. Kim, M.J. Yang, J.C. Hwang, B.M. Yoo

Dept. Of Gastroenterology, Ajou University Hospital, Suwon/Korea, Republic of

Contact E-mail Address: jinhkim@ajou.ac.kr

Introduction: The effectiveness of endoscopic ultrasound (EUS) guided tissue acquisition using a $25 \mathrm{G}$-gauge core biopsy needle without and with a stylet has never been compared.

Aims \& Methods: The aims of this study were to evaluate the feasibility, safety, and diagnostic yield for malignancy of the 25-gauge core biopsy needle without (S-) a stylet and to compare its performance with that of the 25-gauge core biopsy needle with $(\mathrm{S}+)$ a stylet. This study was a single-center randomized clinical trial. From March 2013 to January 2016, a total of 114 patients (57 in a S- group and 57 in a S+ group) with solid lesions were included. S- or S+ EUS guided tissue acquisition was performed using a 25 -gauge core biopsy needle without an on-site cytopathologist.

Results: There were no significant differences in technical success $(100 \%$ vs. $100 \%, \mathrm{P}=1.000)$, the mean number of needle passes $(6.8$ vs. $7.0, \mathrm{P}=0.556)$, or complication $(0 \%$ vs. $3.5 \%, \mathrm{P}=0.496)$ between the $\mathrm{S}$ - group and the $\mathrm{S}+$ group. The $\mathrm{S}$ - and $\mathrm{S}+$ groups exhibited comparable outcomes with respect to cytologic diagnostic yield $(91.2 \%$ vs. $93.0 \%, P=1.000)$, histologic diagnostic yield $(87.7 \%$ vs. $86.0 \%, \mathrm{P}=1.000)$, and needle malfunction $(7 \%$ vs. $0 \%, \mathrm{P}=0.118)$. The procedure time was significantly shorter in the $\mathrm{S}$ - group than in the $\mathrm{S}+$ group $(32.4$ vs. $39.7 \mathrm{~min}, \mathrm{P}<0.001$ )

Conclusion: EUS guided tissue acquisition using a 25G-gauge core biopsy needle without a stylet did not decrease the diagnostic yield for malignancy and is associated with shorter procedure time.

Disclosure of Interest: All authors have declared no conflicts of interest.

\section{P0249 DIAGNOSTIC EFFICACY OF ENDOSCOPIC ULTRASOUND- GUIDED FINE-NEEDLE ASPIRATION FOR A PANCREATIC MASS USING THE CELL BLOCK METHOD WITHOUT RAPID ON-SITE CYTOLOGY}

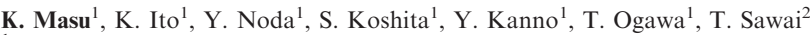

${ }^{1}$ Gastroenterology, Sendai City Medical Center, Sendai/Japan

${ }^{2}$ Pathology, Sendai City Medical Center, Sendai/Japan

Contact E-mail Address: k-masu@openhp.or.jp

Introduction: Endoscopic ultrasound-guided fine-needle aspiration (EUS-FNA)

has been shown to be efficient for diagnosis of pancreatic masses. Only with the smear method, however, its diagnostic efficacy may vary greatly depending on the level of proficiency of the cytopathologists. On the other hand, the cell block (CB) method allows cytological and/or histological evaluation with hematoxylin and eosin (HE) staining and with immunostaining for serial sections if necessary.

Aims \& Methods: The aim of this study was to evaluate the diagnostic efficacy of EUS-FNA for a pancreatic masse using the CB method without rapid on-site cytology retrospectively. A total of 206 patients with pancreatic masses (head: 87 , body: 86, tail: 33) who underwent EUS-FNA using a GF-UL240P or GFUCT260 (Olympus Medical Systems, LTd., Tokyo, Japan) between June 2005 and November 2016 were included in this study. The needles used were $22 / 25 \mathrm{G}$ needles. At least two passes were made during the procedure (mean $3.0 \pm 0.9$ passes). Adequate specimens were regarded to be those in which whitish flagments were macroscopically achieved. The samples were immediately fixed in $10 \%$ formalin and processed by the cell block method using sodium alginate. Rapid on-site cytology was not performed. All samples were stained by hematoxyline and eosin, periodic acid Schiff and Alcian-blue, and immunohistochemical stainings if necessary. Class IIIb, IV, and V were defined as malignant. The final diagnosis was based on histological findings of surgicaly resectioned specimen, image diagnosis and clinical course for more than six months.

Results: The final diagnosis was malignancy in 184 patients (pancreatic ductal cancer (PDC), 171; neuroendocrine tumor (NET), 9; malignant lymphoma, 2; metastasis of malignant melanoma, 1; solid pseudopapillary neoplasm, 1), and benignancy in 23 patients (autoimmune pancreatitis (AIP), 11; chronic pancreatitis, 7; organizing pancreatic pseudocyst, 4; IPMN, 1). Adequate tissue sampling was successfully achieved in $97 \%(199 / 206)$. Of 199 patients with successful tissue sampling, sensitivity, specificity and accuracy for malignancy were $98 \%(175 / 179), 95 \%(19 / 20)$ and $97 \%(194 / 199)$. The diagnostic accuracy of PDC only by HE and PAS-AB staining was $76 \%$ (129/170) (Class V 91; IV 36; IIIb 2), but by adding immunochemical staining, the accuracy of PDC was $98 \%(167 / 170)$ (Class V 112; IV 50; IIIb 5). In the cases of highly suspicious NET, all samples were stained by chromogranin A or synaptophysin and the diagnostic accuracy of NET was $100 \%(6 / 6)$. No procedure-related complications occurred.

Conclusion: EUS-FNA of a pancreatic mass with the CB method without rapid on-site cytology showed high accuracy for definitive diagnosis.

Disclosure of Interest: All authors have declared no conflicts of interest.

\section{P0250 A COMPARATIVE STUDY BETWEEN EUS-GUIDED BILIARY DRAINAGE AND PERCUTANEOUS BILIARY DRAINAGE IN PATIENTS WITH MALIGNANT BILIARY OBSTRUCTION AND FAILED ERCP}

L. Lu, J. Yang, H. Jin, X. Tang, J. Yang, X. Zhang

Department Of Gastroenterology, Hangzhou First People's Hospital, Hangzhou China

Contact E-mail Address: zacharylu@126.com

Introduction: Patients with malignant biliary obstruction conventionally undergo percutaneous transhepatic biliary drainage (PTBD) after failed endoscopic retrograde cholangiopancreatography (ERCP) (1). While PTBD is effective, it is associated with significant morbidity, such as bile leakage, bleeding, and pneumothorax, and involves uncomfortable external drainage (2). Endoscopic ultrasound-guided biliary drainage (EUS-BD) is a minimally invasive endoscopic option increasingly offered as an alternative to PTBD for malignant biliary obstruction after failed ERCP (3). Although a recent meta-analysis reported better clinical efficacy and superior safety of EUS-BD when compared to PTBD, data from mainland China is sporadic (4)

Aims \& Methods: We aimed to compare efficacy and safety of EUS-BD to PTBD in patients with malignant biliary obstruction after failed ERCP at a single tertiary referral center from mainland China. From November 2011 through December 2015, consecutive patients undergoing EUS-BD or PTBD for malignant biliary obstruction after failed ERCP were included. Demographical, biochemical, and outcome data were registered for each group. The primary outcomes included technical success rate and incidence of complications, the second outcomes were clinical success rates and re-intervention rate.

Results: A total of 93 patients (mean age $68 \pm 13.5$ years, 49 males) were included, 33 in the EUS-BD group and 60 in the PTBD group. Both groups were similar in terms of age, gender, baseline bilirubin and functional status. Technical success was achieved in $32(97 \%)$ of 33 patients in the EUS-BD group and in $57(95 \%)$ of 60 patients $(\mathrm{p}>0.05)$ in the PTBD group. The clinical success (jaundice relief: reduction in serum bilirubin by $50 \%$ within the first month) was achieved in all patients with technical success (32/32, 100\% EUS-BD vs. 57/57, 100\% PTBD). Procedure-related complication rates were higher in the PTBD group (18/57, $31.6 \%$ : 5 bleeding, 5 catheter site infection, 4 bile leaks, 2 cholangitis, and 2 tube malposition) than in the EUS-BD group $(3 / 32,9.4 \%: 2$ bleeding and 1 cholangitis $)(p=0.018)$. Rate of re-intervention appeared to be lower in the EUS-BD group $(2 / 32,6.3 \%$ : 2 stent occlusion) than in the PTBD group (17/ $57,29.8 \%$ : 6 stent occlusion, 5 catheter site infection, 4 bile leaks, and 2 tube malposition $)(\mathrm{p}=0.009)$.

Conclusion: Despite similar high technical and clinical success rates compared with PTBD, EUS-BD was associated with reduced rate of complications and required fewer re-interventions. EUS-BD seems a better alternative than PTBD for malignant biliary obstruction after failed ERCP.

Disclosure of Interest: All authors have declared no conflicts of interest. 


\section{References}

1. Voegeli DR, Crummy AB, Weese JL. Percutaneous transhepatic cholangiography, drainage, and biopsy in patients with malignant biliary obstruction. An alternative to surgery. Am J Surg 1985; 150 (2): 243-7.

2. Nennstiel S, Weber A, Frick G, et al. Drainage-related Complications in Percutaneous Transhepatic Biliary Drainage: An Analysis Over 10 Years. $J$ Clin Gastroenterol 2015; 49(9): 764-70.

3. Lee TH, Choi JH, Park do H, et al. Similar Efficacies of Endoscopic Ultrasound-guided Transmural and Percutaneous Drainage for Malignant Distal Biliary Obstruction. Clin Gastroenterol Hepatol 2016; 14 (7): 1011 1019.

4. Sharaiha RZ, Khan MA, Kamal F, et al. Efficacy and safety of EUS-guided biliary drainage in comparison with percutaneous biliary drainage when ERCP fails: a systematic review and meta-analysis. Gastrointest Endosc 2017.

P0251 A PROSPECTIVE COMPARATIVE STUDY OF EFFICACY OF EUS GUIDED FNA VERSUS ERCP GUIDED BRUSH CYTOLOGY IN ATTAINMENT OF HISTOPATHOLOGY OF DISTAL CBD MASSES

P.N. Desai, M. Kabrawala

Endoscopy, Surat Institute Of Digestive Sciences, Surat/India

Contact E-mail Address: drp_desai@hotmail.com

Introduction: Distal CBD masses have always been a diagnostic dilemma. They are difficult to diagnose with any modality used. Brush cytology under ERCP guidance was used uptill now and also intraductal biopsies were used. The yield was hardly around $60 \%$ using all together. We started doind EUS localization of these difficult to identify distal CBD masses and took FNA from them. We devised a protocol to see the results of EUS FNA and brush cytology in the diagnosis of these masses.

Aims \& Methods: We aimed to study the efficacy of EUS guided FNA for attaining tissue from distal CBD masses and comparing it to ERCP guided brush cytology from the same masses. 56 cases with distal bile duct mass with obstructive jaundice in the last 3 years were taken for the study. The protocol we followed First - EUS was done using a linear echoendoscope, mass identified and FNA performed with a $25 \mathrm{G}$ needle making 2 to 5 passes and material sent for cytology. Same patients subjected to ERCP. A wide papillotomy was performed and over the wire cytology brush was used and brush cytology was obtained. Two passes were made and material taken on a slide and sent for cytology after wet fixation.

Results: Total number of cases 56 Age (range) 57.2 13.6 Male to Female 40: 16 Total Serum Bilirubin $90 \mathrm{mg} / \mathrm{dl}) 5.9 \pm 6.4$ Mean size of the mass $12 \mathrm{~mm}(7 \mathrm{~mm}$ to $30 \mathrm{~mm}$ ). Mean Number of passes with FNA needle 2.5 (2 to 5 passes). Mean number of passes with cytology brush 2 (2to 5). Positive diagnosis obtained with FNA $47(83.9 \%)$. Positive Diagnosis obtained by brush $34(60.7 \%)$

Diagnosis in Positive Cases

\begin{tabular}{lll}
\hline & FNA (47) & Brush Cytology (34) \\
\hline Malignancy & $38(80.8 \%)$ & $23(67.6 \%)$ \\
Suspicious Of Malignancy & $5(10.6 \%)$ & $7(20.5 \%)$ \\
Benign & $4(8.5 \%)$ & $4(8.5 \%)$ \\
\hline
\end{tabular}

With EUS the tumors were sometimes difficult to locate and identify. But giving some time and instilling water in duodenum were useful techniques to identify the masses. Only a $25 \mathrm{G}$ needle was used as the FNA had to be taken almost always from the duodenum and with difficult angles. But we succeeded in taking FNA from all cases.

Conclusion: EUS FNA is a very effective method for diagnosis of distal bile duct masses with a certain diagnosis in almost $81 \%$ and a suspicious diagnosis in around $11 \%$ cases. Its efficacy is better than ERCP guided brush cytology. Even small masses are amenable to FNA using EUS guidance. Male over 57 years with jaundice and distal bile duct obstruction has a very likelihood of have a distal CBD cholangio carcinoma.

Disclosure of Interest: All authors have declared no conflicts of interest.

\section{Reference}

Krasinskas A, Pawlik TM, Mino-Kenudson M, Vauthey J-N. Distal bile duct. In: AJCC Cancer Staging Manual, 8th, Amin MB. (Ed), AJCC, Chicago 2017. p.317.

P0252 RANDOMIZED TRIAL COMPARING THE LUMENAPPOSING METAL STENTS (LAMS) AND PLASTIC STENTS FOR EUS-GUIDED DRAINAGE OF WALLED-OFF NECROSIS (WON)

J.Y. Bang, M. Hasan, U. Navaneethan, R. Hawes, S. Varadarajulu Center For Interventional Endoscopy, Florida Hospital, Orlando/United States of America/FL

Contact E-mail Address: jybang213@gmail.com
Introduction: Although lumen-apposing metal stents (LAMS) are being increasingly used for drainage of walled-off necrosis (WON), their advantage over plastic stents is unclear.

Aims \& Methods: We aimed to compare the efficacy of LAMS and plastic stents for drainage of WON.

Symptomatic patients with WON ( $>20 \%$ necrosis) were randomized to undergo transmural drainage using LAMS (Hot AXIOS, $15 \mathrm{~mm} \times 10 \mathrm{~mm}$ ) or two double pigtail plastic stents $(7 \mathrm{Fr} \times 4 \mathrm{~cm})$. Reintervention in persistently symptomatic patients included additional stent placement, percutaneous drainage and/or endoscopic necrosectomy. Treatment success was defined as symptom relief in conjunction with resolution of WON on CT at 6-week follow-up. Main outcome measure was to compare the no. of reinterventions. Secondary outcome measures were to compare treatment success, procedural duration, resolution of systemic inflammatory response syndrome (SIRS), clinical and stent-related adverse events, readmissions and length of hospital stay. Sample size to detect a difference of 1 in the no. of reinterventions performed at $90 \%$ power was calculated at 58 patients.

Results: 60 patients were randomized to LAMS $(n=31)$ or plastic stent $(n=29)$ placement. While there was no significant difference in the no. of reinterventions (median 1 [IQR 1-2] for both stent types, $\mathrm{p}=0.78$ ), the procedural duration was significantly shorter ( 15 vs. 42.5 mins, $\mathrm{p}<0.001)$ and stent-related adverse event rate was significantly higher with LAMS placement $(32.3$ vs. $6.9 \%, p=0.02)$. At an interim audit, significant adverse events (delayed bleeding [n $=3$ ], buried stent $[n=2]$, biliary stricture $[n=3]$ ) were observed in the LAMS cohort after 3 weeks post-intervention. This necessitated an amendment to the study protocol whereby a CT scan was obtained at 3 weeks followed by LAMS removal if the WON had resolved. After protocol amendment, no difference in stent-related adverse events was observed between the cohorts (LAMS 6.5 vs. plastic $6.9 \%$, $\mathrm{p}=0.99$ ). Also, there was no significant difference in treatment success, SIRS resolution, clinical adverse events, readmissions or length of hospital stay between the cohorts (Table).

\begin{tabular}{lllll}
\hline & & $\begin{array}{l}\text { Metal } \\
(\mathrm{n}=31)\end{array}$ & $\begin{array}{l}\text { Plastic } \\
(\mathrm{n}=29)\end{array}$ & p-value \\
\hline $\begin{array}{l}\text { Procedure duration (mins): } \\
\begin{array}{l}\text { Resolution of } \\
\text { pre-intervention SIRS (\%): }\end{array}\end{array}$ & Median [IQR] & $15(8-25)$ & $42.5(21-55)$ & $<0.001$ \\
Length of hospital stay (days): & Median [IQR] & $3(0-5)$ & $3.5(2-11)$ & 0.16 \\
Treatment success (\%): & & 96.3 & 88.0 & 0.34 \\
Adverse events (\%): & Overall & 41.9 & 20.7 & 0.10 \\
& Stent-related & 32.3 & 6.9 & 0.02 \\
& Other & 9.7 & 13.8 & 0.70 \\
No. of reinterventions (n): & Median [IQR] & $1(1-2)$ & $1(1-2)$ & 0.78 \\
Readmissions (\%): & & 29.0 & 34.5 & 0.78 \\
\hline
\end{tabular}

Conclusion: Except for shorter procedural duration, there was no significant difference in treatment outcomes between patients treated with LAMS or plastic stents. Given the faster resolution of WON, to minimize adverse events, patients undergoing LAMS placement should undergo post-intervention imaging at 3 weeks followed by stent removal if the WON has resolved.

Disclosure of Interest: R. Hawes: Consultant for Boston Scientific Corporation and Olympus America Inc.

S. Varadarajulu: Consultant for Boston Scientific Corporation and Olympus America Inc.

All other authors have declared no conflicts of interest.

P0253 TRANS AORTIC ENDOSCOPIC ULTRASOUND GUIDED FNA IN THE DIAGNOSIS OF LUNG CANCERS AND MEDIASTINAL LYMPH NODES

P. Somani, M. Sharma

Department Of Gastroenterology, Jaswant Rai Speciality Hospital, Meerut/India

Contact E-mail Address: dr piyushsomani@yahoo.co.in

Introduction: Obtaining a tissue diagnosis from a lung tumour or a mediastinal lymph node located lateral to the aorta (para-aortal) is a diagnostic challenge because of the interposition of the aorta. Invasive surgical procedures like mediastinotomy, thoracotomy, or video-assisted thoracic surgery is required for the diagnosis of these lesions. Lymph node stations immediately anterior to the aortic arch and lateral to the descending aorta are difficult to access. Lymph nodes on the "far-side" of major blood vessels can be visualized by endoscopic ultrasound(EUS), however Fine needle aspiration(FNA) is avoided due to concern for bleeding complications. Tumours and mediastinal lymph nodes located in the para-aortic region can easily be visualized by esophageal EUS, because the aorta provides an excellent medium to transfer ultrasound waves. 
Aims \& Methods: We aimed to evaluate the feasibility, yield, and safety of EUSguided transaortic FNA of lung tumours and para-aortic lymph nodes We undertook a retrospective case series of 12 consecutive patients with suspected lung cancer or tuberculosis who underwent transaortic FNA during a study period of 7 years. In all cases, the para-aortal lesion was the only site suspicious for malignancy/tuberculosis (other lesion/lymph node if present were negative). Based on CT/PET imaging, a transesophageal FNA performed through the aorta was considered as the only option to diagnose or stage these patients by means of a minimally invasive procedure. Seven patients had leftsided lung mass (mean size $30 \mathrm{~mm}$ ). Four were located in left lower lobe and three in left upper lobe. Four patients has enlarged para-aortic lymph node (mean size $18 \mathrm{~mm}$, range $8-22 \mathrm{~mm})$, suspicious for IASLC stations $5(\mathrm{n}=1)$ and $6(\mathrm{n}=3)$. One patient had anterior mediastinum mass. EUS was performed with a linear echoendoscope. All aspirates were obtained under real-time US guided FNA by using a 22/25-gauge needle. A single real-time FNA of the lung mass or lymph node was performed. The para-aortal area was observed on EUS for 5 minutes to assess for immediate procedure-related complications.

Results: The final diagnosis was known in 11 patients (5 non-small-cell lung carcinoma [NSCLC], 2 small-cell lung carcinoma [SCLC], 3 tuberculosis and one thymolipoma). EUS-FNA established diagnosis in 9 of 12 patients $(75 \%)$ (4 NSCLC, 1 SCLC, 3 tuberculosis and 1 thymolipoma). One aspirate revealed reactive nodal tissue, and one demonstrated nonrepresentative material. One procedure was abandoned due to complication. Three patients in whom diagnosis was not established by transaortic FNA underwent subsequent surgical staging (1 thoracotomy, 1 mediastinotomy, and 1 VATS), and malignancy was found in 2 of the 3 patients. Trans aortic FNA was found to be safe. In one patient, EUS images after FNA were suspicious for a small para-aortic hematoma. This patient recovered without any adverse event.

Conclusion: This case series demonstrates the feasibility and probable safety of single EUS guided transaortic aspiration in para-aortic lesions. The diagnostic yield is 75 percent. Clearly, further study and very careful selection by expert EUS operators is needed before this procedure can be routinely recommended. Advantages of this procedure includes day care procedure, less invasive than surgical procedures, low cost, good diagnostic yield and can be performed in poor surgical candidate. Limitations includes single centre study, require EUS expertise, more data is required. At present, Transaortic FNA should only be performed in the absence of alternative minimally invasive diagnostic procedures. Disclosure of Interest: All authors have declared no conflicts of interest.

\section{P0254 ULTRASOUND-GUIDED ENDOSCOPIC TRANSGASTRIC DRAINAGE OF PANCREATIC FLUID COLLECTIONS}

M. Silva ${ }^{1}$, S. Lopes $^{1}$, A. Peixoto ${ }^{1}$, F. Vilas-Boas ${ }^{1}$, P. Moutinho-Ribeiro ${ }^{1}$, G. Macedo ${ }^{2}$

${ }^{1}$ Gastroenterology, Hospital São João, Oporto/Portugal

${ }^{2}$ Centro Hospitalar São João, Porto Medical School, Porto/Portugal

Contact E-mail Address: marcocostasilva87@gmail.com

Introduction: Ultrasound-guided endoscopic transgastric drainage (EUSTD) of pancreatic fluid collections (PFCs) by using double-pigtail plastic stents (DPPS) requires placement of multiple stents and can be restricted by inadequate drainage and leakage risk. Recently, the use of fully covered self-expanding metal stents (FCSEMSs) has been reported as an effective alternative.

Aims \& Methods: We aimed to evaluate the successful placement of stents, the complete resolution of PFCs at 6-months, adverse events, and recurrence of EUSTD of PFCs using DPPS and FCSEMSs.

This was a single-centre retrospective study (2012-2016) on 67 patients with symptomatic/infected PFCs, who underwent EUSTD. PFCs were drained using DPPS and FCSEMSs (Hanarostent BCF and Hot AXIOS system).

Results: We included 42 cases of pancreatic pseudocysts (PP), and 25 walled-off necrosis (WON), drained using FCSEMSs in $51 \%$ of cases. The remaining cases $(49 \%)$ were drained using DPPS exclusevely. Eighteen $(27 \%)$ patients had a prior failed drainage attempt. Median follow-up time was 88 weeks (IQR: 42-140); 19 patients were lost to follow-up (6 deaths during follow-up). The median size of lesions was $92 \mathrm{~mm}$ (IQR: 74-120). The overall technical success rate was $78 \%$ $(74 \%$ for PP and $84 \%$ for WON; $p>0.05)$. Six patients required additional drainage with a different modality (surgery/percutaneous drainage) and 8 cases required another EUSTD attempt. Complete resolution of PFCs with DPPS was lower compared with those that underwent drainage with FCSEMSs $(58 \%$ vs. $84 \% ; \mathrm{p}<0.05)$. Complete resolution of PP was lower than in the cases of WON $(65 \%$ vs. $79 \% ; \mathrm{p}>0.05)$. Procedure-related adverse events were noted in $18 \%$ of cases of the DPPS group and $21 \%$ in the FCSEMS group $(p>0.05)$; overall 1 procedure-related death was registred (massive bleeding after a FCSMES placement). Recurrence occurred in $27 \%$ cases of PP and in none of WON ( $p>0.05$ ); the recurrence was higher in the DPPS group than in the FCSMES group ( $43 \%$ vs. $13 \% ; p>0.05)$.

Conclusion: EUSTD of PFCs using FCSEMSs improves clinical outcomes with similar adverse event rates compared with the cases drained with DPPS Disclosure of Interest: All authors have declared no conflicts of interest.
P0255 ENDOSCOPIC ULTRASOUND-GUIDED TISSUE SAMPLING WITH A NEW 20G BIOPSY NEEDLE FOR THE CHARACTERIZATION OF GASTROINTESTINAL SUBEPITHELIAL LESIONS

F. Antonini ${ }^{1}$, G. Delconte ${ }^{2}$, L. Fuccio ${ }^{3}$, G. De Nucci ${ }^{4}$, C. Fabbri ${ }^{5}$, E. Armellini ${ }^{6}$, L. Frazzoni ${ }^{7}$, A. Fornelli ${ }^{5}$, A. Magarotto ${ }^{2}$, E. Mandelli ${ }^{4}$, P. Occhipinti ${ }^{6}$, E. Masci ${ }^{2}$, G. Manes ${ }^{4}$, G. Macarri ${ }^{1}$

${ }_{1}^{1}$ Gastroenterology, Augusto Murri Hospital, Fermo/Italy

${ }^{2}$ Gastroenterology, Fondazione IRCCS Istituto Nazionale Tumori, Milano/Italy ${ }^{3}$ Department Of Medical And Surgical Sciences, S. Orsola-Malpighi University Hospital, Bologna/Italy

${ }^{4}$ Asst Rhodense, Garbagnate Milanese/Italy

${ }^{5}$ Bellaria-Maggiore Hospital, Bologna/Italy

${ }^{6}$ Azienda Ospedaliero Universitaria Maggiore della Carità, NovaralItaly

${ }^{7}$ Department Of Medical And Surgical Sciences, S. Orsola-Malpighi Hospital, Bologna/Italy

Contact E-mail Address: filippore@yahoo.it

Introduction: Histological examinations and immunohistochemical stains (IHC) are necessary for the differential diagnosis of gastrointestinal subepithelial lesions (SELs). Endoscopic ultrasound-guided fine needle biopsy (EUS-FNB) is the primary modality in the diagnosis of SELs, but still has limited accuracy. A new 20 gauge $(G)$ biopsy needle with a core-trap technology (EchoTip ProCore $\AA$, Cook Medical) has been developed with a large core size and enhanced flexibility.

Aims \& Methods: The aim of this multicenter study was to determine the feasibility, efficacy and safety of EUS-FNB with the new $20 \mathrm{G}$ needle in diagnosing SELs. Data retrieved from a prospectively collected database at five medical centers were analyzed and all consecutive patients with SELs undergoing EUSFNB with the $20 \mathrm{G}$ needle were included in the present study. The reference standards for the final diagnosis were histology on surgical specimen or clinical follow-up.

Results: A total of 50 SELs in 50 patients ( 22 males, mean age $61.5 \pm 14.8$ years) were included. The mean lesion size was $43.1 \pm 17.5 \mathrm{~mm}$. The lesion locations were esophagus $(n=1)$, stomach $(n=37)$, distal duodenum $(n=5)$, rectum $(n=6)$, and colon $(n=1)$. The procedure was technically feasible in all patients. Mean number of passes required to reach a diagnosis was 2.2 (range 1-4). Definitive diagnosis with full histological assessment including IHC was obtained in $88 \%(44 / 50)$ of the patients. Diagnosis of EUS-FNB showed $36(72 \%)$ malignant SELs (32 GISTs, 1 metastasis from breast cancer, 1 leiomyosarcoma, 1 carcinoid, 1 SEL-like adenocarcinoma), 8 (16\%) benign SELs (3 leiomyomas, 4 schwannomas, and 1 lipoma), and $6(12 \%)$ indeterminate SELs. Considering malignant vs. benign lesions, the sensitivity, specificity, PPV, and NPV were $85 \%$ (95\% CI 70.2-94.3), 100\% (95\% CI 58.7-100\%), 100\% (95\% CI 85.1-100\%), and $62.5(95 \%$ CI $27.7-84.8)$, respectively. No major complications requiring additional care have been observed.

Conclusion: In this multicenter study, we found that EUS-FNB with the new 20G core needle is an effective and safe method for the diagnosis of SELs with a high rate of producing adequate histological material and high diagnostic accuracy even from difficult-to-approach anatomical locations. Comparative studies with different needle sizes are awaited.

Disclosure of Interest: All authors have declared no conflicts of interest.

\section{References}

Hwang JH, Rulyak SD, Kimmey MB. American Gastroenterological Association Institute technical review on the management of gastric subepithelial masses. Gastroenterology. 2006; 130: 2217-2228.

Akahoshi K, Sumida Y, Matsui N, et al. Preoperative diagnosis of gastrointestinal stromal tumor by endoscopic ultrasound-guided fine needle aspiration. World J Gastroenterol. 2007;13:2077-2082.

Hoda KM, Rodriguez SA, Faigel DO. EUS-guided sampling of suspected GI stromal tumors. Gastrointest Endosc. 2009;69:1218-1223.

Philipper M, Hollerbach S, Gabbert HE, et al. Prospective comparison of endoscopic ultrasound-guided fine-needle aspiration and surgical histology in upper gastrointestinal submucosal tumors. Endoscopy. 2010;42:300-305.

Dumonceau JM, Polkowski M, Larghi A, et al. Indications, results, and clinical impact of endoscopic ultrasound (EUS)-guided sampling in gastroenterology: European Society of Gastrointestinal Endoscopy (ESGE) Clinical Guideline. Endoscopy. 2011;43:897-912.

\section{P0256 COMPARISON OF NATURAL COURSE VERSUS EUS-} GUIDED ETHANOL ABLATION FOR PANCREATIC CYSTIC LESIONS

J.H. Choi ${ }^{1}$, S.H. Lee ${ }^{1}$, Y.H. Choi ${ }^{1}$, J.W. Kang ${ }^{1}$, W.H. Paik ${ }^{1}$, D. Ahn ${ }^{1}$, J.K. Ryu ${ }^{1}$, Y. Kim ${ }^{1}$

${ }^{1}$ Department Of Internal Medicine And Liver Research Institute, Seoul National University College of Medicine, Seoul/Korea, Republic of

Contact E-mail Address: pseudo.jh $@$ gmail.com

Introduction: Endoscopic ultrasonography(EUS)-guided ethanol ablation for pancreatic cystic lesions (PCLs) is a recently introduced treatment option for PCLs. The aim of this study was to compare the clinical outcomes of EUSguided ethanol ablation with those of the natural course of PCLs.

Aims \& Methods: We performed retrospective study of patients with PCLs divided in two groups: EUS-guided ethanol ablation group $(\mathrm{n}=118$, performed between June 2006 to August 2015) and natural course group $(n=458$, diagnosed between January 1993 to August 2015). The propensity score-matching analysis 
between the two groups was applied in order to minimize the effect of selection bias. The primary outcome was the rate of significant reduction in size $(<20 \%$ of initial size). The secondary outcomes were the rate of significant growth in size $(>10 \mathrm{~mm})$, complete remission rate, and surgical resection rate.

Results: In a propensity matched analysis of 88 pairs, the mean initial cystic size of EUS-guided ethanol ablation group and natural course group was $23.72 \pm 10.99, \quad 23.16 \pm 13.15 \mathrm{~mm}$ and the mean follow-up duration was $75.45 \pm 38.12,82.12 \pm 59.06$ months respectively. Significant reduction in size was detected in $53(60.2 \%)$ of the EUS-guided ethanol ablation group and 17 $(19.3 \%)$ of the natural course group $(\mathrm{p}<0.001)$. Significant growth in size was detected in $6(8.9 \%)$ of ablation group and $11(12.5 \%)$ of natural course group. $(\mathrm{p}=0.202)$. Seven patients $(7.95 \%)$ underwent surgical resection in the EUSguided ethanol ablation group and 17 patients $(19.3 \%)$ in the natural course group ( $\mathrm{p}=0.028)$ during follow-up. Overall $28.8 \%$ patients (34 of 118 ) who underwent EUS-guided ethanol ablation had achieved the complete remission.

Conclusion: PCLs that underwent EUS-guided ethanol ablation can be seen the likelihood of getting clinical benefits such as reduction of the cystic size, the chance for surgical resection in comparison to the natural course of them. It is also expected to achieve a certain level of complete remission for PCLs. Disclosure of Interest: All authors have declared no conflicts of interest.

\section{P0257 RANDOMIZED TRIAL COMPARING THE FRANSEEN AND} FORK-TIP NEEDLES FOR EUS-GUIDED FINE NEEDLE BIOPSY

J.Y. Bang, S. Hebert-Magee, M. Hasan, U. Navaneethan, R. Hawes, S. Varadarajulu

Center For Interventional Endoscopy, Florida Hospital, Orlando/United States of America/FL

\section{Contact E-mail Address: jybang213@gmail.com}

Introduction: Histological tissue comprising tumor and desmoplastic stroma is required for molecular profile-based personalized chemotherapy in pancreatic cancer. Recently, a three-plane symmetric needle with Franseen geometry and a Fork-tip biopsy needle have been developed for histological tissue procurement.

Aims \& Methods: We aimed to compare tissue acquisition between the $22 \mathrm{G}$ Franseen and 22G Fork-tip needles in patients undergoing EUS-guided sampling of pancreatic masses.

Patients with pancreatic masses were randomized to undergo EUS-guided sampling using the $22 \mathrm{G}$ Franseen and $22 \mathrm{G}$ Fork-tip needles. Two dedicated passes were first performed using both needles in individual patients for cell block. Subsequent passes were then performed for rapid onsite evaluation (ROSE) using both needles alternately until a diagnosis was established. The main outcome measures were to compare total tissue volume and presence of desmoplastic stroma in pancreatic cancer. Secondary outcome measures were to compare rates of diagnostic cell block and diagnostic adequacy at ROSE.

Results: In 50 patients randomized to undergo EUS-guided sampling, the diagnosis was pancreatic cancer in 43, neuroendocrine tumor in 2 , lymphoma in 1 and chronic pancreatitis in 4 patients. There was no significant difference in total tissue area (median $6.1 \mathrm{~mm}^{2}$ [IQR 3.5-10.5] vs. $8.2 \mathrm{~mm}^{2}$ [IQR 4.0-13.0], $\mathrm{p}=0.53$ ), presence of desmoplastic stroma in tumor $(100 \mathrm{vs} .83 .3 \%, \mathrm{p}=0.23)$, rates of diagnostic cell block $(96.0$ vs. $92.0 \%, \mathrm{p}=0.68)$ and diagnostic adequacy at ROSE ( 94.0 vs. $98.0 \%, \mathrm{p}=0.62)$ between the Franseen and Fork-tip needles, respectively.

Conclusion: Both the Franseen and Fork-tip needles appear equally effective in yielding histological tissue. By virtue of their ability to yield a diagnostic cell block in greater than $90 \%$ of patients, the new generation FNB needles may obviate the need for ROSE during EUS-guided tissue sampling.

Disclosure of Interest:

S. Hebert-Magee: Consultant for Boston Scientific Corporation

R. Hawes: Consultant for Boston Scientific Corporation and Olympus America Inc.

S. Varadarajulu: Consultant for Boston Scientific Corporation and Olympus America Inc.

All other authors have declared no conflicts of interest.

\section{P0258 THE SUCCESS RATE OF DOUBLE BALLOON ENDOSCOPIC CHOLANGIOGRAPHY IN PATIENTS WHO UNDERWENT THEIR INITIAL SURGERY AS INFANT IS SIGNIFICANTLY LOWER THAN OTHER PATIENTS}

K. Yokoyama ${ }^{1}$, T. Yano ${ }^{1}$, J. Ushio ${ }^{1}$, K. Tamada $^{1}$, A. K. Lefor ${ }^{2}$, H. Yamamoto ${ }^{1}$ Department Of Gastroenterology, Jichi medical university, Tochigi/Japan ${ }^{2}$ Department Of Surgery, Jichi Medical University, Tochigi/Japan

Contact E-mail Address: r0760ky@jichi.ac.jp

Introduction: To evaluate the success rate of double-balloon endoscopic retrograde cholangiography (DBERC) to reach the anastomosis in patients with surgically altered gastrointestinal anatomy.

Aims \& Methods: We review 346 patients with surgically altered anatomy who underwent DBERC from April, 2002 to December, 2016 (47 patients with biliary atresia(BA) after living donor liver transplantation (LDLT), 33 with LDLT without BA, 45 with biliary resection and choledochojejunostomy, 111 with gastric resection and Roux-en-Y bypass, 48 with gastric resection and BillrothIIremnant, 18 with pylorus-preserving pancreatoduodenectomy, and 42 others). We evaluate the success rate according to the type of gastrointestinal anastomosis, age, and age at surgery.

Results: The success rate for reaching the biliary anastomosis (or papilla of Vater) in all 346 patients $(66$ y.o (3-91)) was $83 \%$. The rate in 47 patients with BA after
LDLT (12y.o (3-39)) was 57\%. In the remaining 299 patients the rate was $87 \%$. The success rate of reaching the biliary anastomosis in patients with BA after LDLT was significantly lower than other patients $(\mathrm{p}<0.01)$. There was no significant difference between the success rate in the patients over or under 13 years at the time of ERCP $(50 \%$ vs $56 \%, \mathrm{p}=0.70)$. The success rate was lower in patients who underwent initial surgery as an infant (Kasai hepatoportoenterostomy) than in those past infancy $(54 \%$ vs $88 \%, \mathrm{p}<0.01)$. When reaching the biliary anastomosis is successful, the success rate of cannulation in the patients after LDLT is high $(92 \%)$

Conclusion: The success rate for reaching the biliary anastomosis in patients with BA after LDLT is significantly lower than other patients. The age at the time of ERCP did not affect the success rate of reaching the biliary anastomosis, but the success rate was lower in patients who underwent their initial surgery as infants. Disclosure of Interest: All authors have declared no conflicts of interest.

\section{P0259 EUS-GUIDED RADIOFREQUENCY ABLATION OF} DIFFICULT SITES IN THE LIVER: A PRECLINICAL STUDY

N. Junya, T. Tamura, M. Itonaga, R. Shimizu, Y. Ida, M. Kitano Second Department Of Internal Medicine, Wakayama Medical University, Wakayama/Japan

Contact E-mail Address: jswnq633@yahoo.co.jp

Introduction: Liver tumors such as hepatocellular carcinoma and liver metastases sometimes occur in positions in which treatment using percutaneous radiofrequency ablation (RFA) is difficult, such as the caudate lobe and surface of the left lobe. EUS-guided RFA (EUS-RFA) can offer an alternative treatment by accessing these tumors through the stomach or duodenum. To the best of our knowledge, only one report has described EUS-RFA of the liver in an animal model, using a 19-gauge EUS-FNA needle with an umbrella-shaped array at the needle.

Aims \& Methods: We examined whether a novel 19-gauge RFA needle can be introduced to ablate the liver in a porcine model under EUS guidance. Two pigs were used in this study. All procedures were carried out under general anesthesia. EUSRA $^{\mathrm{TM}}$ 19-gauge needles and a VIVA combo ${ }^{\mathrm{TM}}$ generator (TaeWoong Medical, Gimpo city, Korea) were used for the procedures. Three kinds of RFA needles (10-, 15-mm, and 20-mm exposed tips) were used. After the echoendoscope was advanced to the stomach, the RFA needle was inserted into the surface of the left lobe. EUS-RFA was performed at 5-40 W for 2-6 min in general mode. In each pig, three RFA needles with 10-, 15-, or 20-mm exposed tips were serially used for insertion and ablation. Subsequently, the RFA needle with the $10-\mathrm{mm}$ exposed tip was used in the quadrate lobe of the gallbladder through the bulb of the duodenum.

Results: All procedures were technically successful. After the procedure, the liver of the pig was removed, and visible RFA effect were evaluated macroscopically. Histology with hematoxylin and eosin (HE) staining showed coagulative necrosis in the ablated area, corresponding with the macroscopic ablated area.

Conclusion: In this experimental study, EUS-RFA could be performed technically not only in the surface of the left lobe, but also in the adjacent to the gallbladder of the porcine liver. Further studies are required to confirm the efficacy and safety.

Disclosure of Interest: All authors have declared no conflicts of interest.

\section{Reference}

Varadarajulu S, Jhara NC, et al. EUS-guided radiofrequency ablation with a prototype electrode array system in an animal model (with video). Gastrointestinal Endoscopy 2009; 70: 372-376

\section{P0260 CYANOACRYLATE INJECTION THERAPY OF SMALL BOWEL VARICES BY DOUBLE-BALLOON ENTEROSCOPY (DBE): A TERTIARY CENTRE EXPERIENCE}

A. Murino ${ }^{1}$, N. Koukias ${ }^{1}$, E. Vlachou ${ }^{1}$, K. Planche ${ }^{2}$, D. Patch ${ }^{1}$, E. J. Despott ${ }^{1}$ The Royal Free Unit For Endoscopy, The Royal Free Hospital and University College London (UCL) Institute for Liver and Digestive Health, London/United Kingdom

${ }^{2}$ Department Of Radiology, The Royal Free Hospital and University College London (UCL) Institute for Liver and Digestive Health, London/United Kingdom

Contact E-mail Address: albertomurino@yahoo.it

Introduction: Small bowel varices (SBV) occur as a consequence of portal hypertension and may result in life-threatening mid-gut bleeding. First line management usually involves radiological intervention (RI) (e.g. TIPSS, stenting of occluded mesenteric veins $+/-$ embolisation of culprit varices). In cases where RI is impossible, management options become very limited.

Aims \& Methods: This case series evaluated the usefulness of DBE facilitated cyanoacrylate injection of SBV. Retrospective review of DBE facilitated cyanoacrylate injection of SBV at our institution (December 2015 to August 2016). Demographic, clinical, endoscopic and radiological findings, interventions and follow-up data were analysed.

Results: Seven DBEs were performed in 5 patients (3 women, median age: 73 years). Four patients had previous surgery (hemi-hepatectomy $(n=2)$; SB resection $(\mathrm{n}=2)$ ); one patient had a history of intra-abdominal sepsis in childhood causing portal vein thrombosis. No radiological or surgical options were deemed feasible in any case. SBV were diagnosed at capsule endoscopy and triple phase CT mesenteric angiography. At DBE, a total of 10 nests of SBV were identified 
and injected with cyanoacrylate glue. There were no haemorrhagic or embolic complications but 1 patient developed an infection of a congenital urachal cyst, which was treated successfully with antibiotics. All patients underwent DBEs via the anterograde route and 1 patient required bi-directional DBE for treatment of both proximal and distal SBV and another patient required a $2^{\text {nd }}$ anterograde DBE for treatment of further patent proximal SBV. At 30-day follow-up posttherapy, only 1 patient had experienced a mild recurrence of mid-gut bleeding. Conclusion: Cyanoacrylate injection therapy of SBV at DBE appears to be a safe and effective management strategy for this condition when other first-line options are not feasible.

Disclosure of Interest: E. Vlachou: I have received a research \& education grants from Fujifilm \& Aquilant Medical.

E.J. Despott: I have received a research \& education grants from Fujifilm \& Aquilant Medical.

All other authors have declared no conflicts of interest.

\section{P0261 MAGNIFYING NARROW-BAND IMAGING FINDINGS EFFICACY FOR INFLAMMATORY ACTIVITY EVALUATION IN SMALL INTESTINAL CROHN'S DISEASE WHEN USING NEWLY DEVELOPED MAGNIFYING ENTEROSCOPY: A PILOT STUDY}

N. Ogata ${ }^{1}$, K. Ohtsuka ${ }^{2}$, S. Sasanuma ${ }^{1}$, M. Misawa ${ }^{1}$, Y. Mori ${ }^{1}$, T. Kudo ${ }^{1}$, T. Hisayuki ${ }^{1}$, T. Hayashi ${ }^{1}$, K. Wakamura ${ }^{1}$, S. Kudo ${ }^{1}$

${ }^{1}$ Digestive Disease Center, Showa University Northern Yokohama Hospital, Yokohama/Japan

${ }^{2}$ Gastroenterology And Hepatology, Tokyo Medical and Dental University, Tokyo/ Japan

Contact E-mail Address: n.ogata@ hotmail.co.jp

Introduction: The development of balloon endoscopy and capsule endoscopy has made observation of the small intestine possible in clinical practice. The usefulness of magnifying endoscopy has already been reported in observing the pharynx, esophagus, stomach and colon. A single-balloon enteroscopy (SBE) with 80x magnification has been recently developed.

Aims \& Methods: The aim of this pilot study was to assess the efficacy of narrowband imaging (NBI) magnifying findings for evaluating the severity of inflammation in small intestinal crohn's disease (CD). The study was conducted in Showa University Northern Yokohama Hospital. We included CD patients who underwent enteroscopy with magnification from September 2013 to February 2015. NBI images and a biopsy specimen were obtained from small intestinal mucosa for CD patients with use of SBE (Y-0007, Olympus, Tokyo). Magnifying NBI was performed, and the images were evaluated by assessing visibility, increased vascularization, and the increased caliber of capillaries into three grades as follows: Normal, Visible and Irregular. Normal was indicative of inactive disease, while Visible and Irregular were indicative of acute inflammation in our study. The outcome measures included the diagnostic ability of magnifying NBI findings to distinguish active CD from inactive $C D$ on the basis of histological activity.

Results: Twenty-four patients were enrolled. There was a correlation between magnifying NBI findings and the histological assessment (Spearman's $|\mathrm{r}|=0.549)$. The sensitivity, specificity, positive predictive value, negative predictive value, and accuracy of magnifying NBI findings for diagnosing acute inflammation were $88.2 \%, 71.4 \%, 88.2 \%, 71.4 \%$, and $83.3 \%$, respectively.

Conclusion: The NBI magnifying findings in the small intestinal mucosa had a correlation with histological inflammation and could help in distinguishing between active and inactive CD.

Disclosure of Interest: All authors have declared no conflicts of interest.

P0262 SINGLE-INCISION LAPAROSCOPIC-ASSISTED DOUBLE BALLOON ENTEROSCOPY: A NOVEL TECHNIQUE TO MANAGE SMALL BOWEL PATHOLOGY

I. Stasinos ${ }^{1}$, N. Kamperidis ${ }^{1}$, R. Rameshshanker ${ }^{1}$, A. Murino ${ }^{1}$, C. Fraser ${ }^{1}$, J. Warusavitarne ${ }^{2}$, A. Humphries ${ }^{1}$

${ }^{1}$ The Wolfson Unit For Endoscopy, St Mark's Hospital, London/United Kingdom ${ }^{2}$ Surgery, St Mark's Hospital and Academic Institute, London/United Kingdom

Contact E-mail Address: Ioannisstasinos@nhs.net

Introduction: Double balloon enteroscopy (DBE) has revolutionised the diagnosis and treatment of small intestinal conditions. However, even in expert hands, deep small bowel (SB) insertion can be challenging, especially in patients with a history of abdominopelvic surgery. Moreover, if the findings at DBE are not amenable to endoscopic therapy, a further surgical procedure is usually required to provide definite treatment. Laparoscopic-assisted DBE (LA-DBE) using a standard multi-port technique has previously only been reported in a small series of 3 patients with Peutz -Jeghers Syndrome (PJS) ${ }^{1}$

Aims \& Methods: This case series reports the development of LA-DBE using single-incision laparoscopic surgery (SILS) applied to a wide range of clinical indications. Retrospective review of LA-DBE procedures performed in a single tertiary centre over a 6 year period. Demographics, indication, findings, diagnosis and therapeutic interventions were recorded. Completion, complication rates and hospital length of stay were also captured.

Results: 17 procedures were performed over 6 years in 17 patients who had failed standard DBE. Mean (range) age was $40(17-73)$ and $41 \%$ of patients were male. The enteroscopic approach was oral in $13 / 17$ patients and rectal in $4 / 17$. Laparoscopic approach was standard (multiport) in the first 4 cases, SILS was then used in all subsequent patients $(13 / 17)$. The mean (range) procedure time was $147(84-210)$ mins. Indications were PJS $(n=10)$, suspected submucosal/ polypoid lesion at small bowel imaging $(n=5)$ and obscure gastrointestinal bleed (OGIB) with vascular abnormalities seen at capsule endoscopy $(n=2)$. In $15 / 17$ procedures the target pathology was reached using laparoscopic assistance only and $1 / 17$ was converted to intraoperative enteroscopy (IOE). In 1/17 the suggested pathology at mangnetic resonance enterography (MRE) was not identified. Therapy was applied in $15 / 17(88 \%)$ cases. 7 underwent endoscopic therapy of which 6 polypectomy and 1 ablation with argon plasma coagulation (APC). 4 required limited SB resection and 4 underwent both endoscopic polypectomy and small bowel resection for a second polyp that could not be removed endoscopically. A total number of 57 polyps were removed with the largest measuring $40 \mathrm{~mm}$. The range of length of surgically resected SB was $4-17 \mathrm{~cm}$. Diagnoses were PJS polyps $(\mathrm{n}=9)$, neuroendocrine tumour (NET) $(\mathrm{n}=2)$, PJS polyps and $\operatorname{NET}(\mathrm{n}=1)$, transmural arteriovenous malformation $(\mathrm{n}=1)$, angioectesia $(\mathrm{n}=1)$, inflammatory polyp $(\mathrm{n}=1)$, leiomyoma $(\mathrm{n}=1)$, Meckel's diverticulum $(\mathrm{n}=1)$. Median length of stay post procedure was $2(1-19)$ days $8 / 17$ patients were discharged at 24 hours. $3 / 17$ patients developed complications: 1 post procedure ileus, 1 pelvic collection that was managed with antibiotics and 1 patient that was readmitted 8 days post procedure with subacute SB obstruction which resolved with conservative management.

Conclusion: LA-DBE appears to be a safe, effective and minimally invasive procedure that can be applied for the management of a wide range of small bowel pathology. A SILS approach allows all therapeutic modalities to be available as needed during the procedure, including conversion to IOE, laparoscopic small bowel resection and laparotomy.

Disclosure of Interest: All authors have declared no conflicts of interest.

\section{Reference}

1. AS. Ross et al. Laparascopic-assisted double-balloon enteroscopy for smallbowel polyps surveillance and tretament in patients with Peutz-Jeghers syndrome. Gastrointestinal Endoscopy 2006; 64: 6: 984-988

\section{P0263 GASTRIC EMPTYING IN CROHN'S DISEASE -}

\section{EVALUATION BY SMALL BOWEL CAPSULE ENDOSCOPY}

A.M. Singeap ${ }^{1}$, A. $\operatorname{Trifan}^{1}$, S. Chiriac ${ }^{1}$, I. Girleanu ${ }^{1}$, T. Cuciureanu ${ }^{1}$, C. Stanciu ${ }^{2}$ ${ }^{1}$ Gastroenterology, "Grigore T. Popa" University of Medicine and Pharmacy, Iasi/ Romania

${ }^{2}$ Institute of Gastroenterology and Hepatology, Iasi/Romania

Contact E-mail Address: anamaria.singeap@yahoo.com

Introduction: The complex relationship between inflammatory bowel disease (IBD) and motility disorders of the digestive tract is a complex area of study, so far incompletely elucidated. The association between Crohn's disease and gastric emptying time modification has been relatively less studied. However, there is no single standardized method to study gastric emptying, one particular investigation that could bring direct information in this field being the small bowel capsule endoscopy (SBCE).

Aims \& Methods: We aimed to study gastric emptying by small bowel capsule endoscopy in patients with suspected and confirmed Crohn's disease. We evaluated gastric passage time showed by SBCE in patients with small bowel Crohn's disease, compared to patients without IBD, investigated by SBCE (PillCam), following recognized indications, in the Institute of Gastroenterology and Hepatology of Iasi, tertiary center in North-East of Romania.

Results: 144 SBCE studies were included, 24 were cases of suspected and confirmed Crohn's disease. The mean time of gastric passage in patients with Crohn's disease was $51 \pm 21.9$ minutes, longer than in patients without inflammatory bowel disease, in which the mean gastric passage time was $24 \pm 16.6$ minutes.

Conclusion: Gastric passage time, evaluated by SBCE, is prolonged in patients with Crohn's disease compared to patients without IBD, suggesting a relationship between chronic inflammation and gastric motor disorders. Globally, the values correlated with those considered as physiological by other exploration methods. SBCE studies may provide additional data on gastric motility (and in general gut motor disorders), with special usefulness in some individual cases, as: particular symptoms or variations in the bioavailability of small bowel- released drugs.

Disclosure of Interest: All authors have declared no conflicts of interest.

P0264 META-ANALYSIS SHOWS THAT PURGATIVE

PREPARATION INCREASES SMALL BOWEL VIDEO CAPSULE ENDOSCOPY DIAGNOSTIC YIELD AND IMPROVES THE QUALITY OF SMALL BOWEL MUCOSA VISUALIZATION

P. Gkolfakis, G. Tziatzios, G. D. Dimitriadis, K. Triantafyllou

2nd Dept Of Internal Medicine And Research Institute, National and Kapodistrian University of Athens, Medical School, Athens/Greece

Contact E-mail Address: ktriant@med.uoa.gr

Introduction: The value of purgative preparation (PBP) before small bowel video capsule endoscopy (VCE) remains controversial and it has been recently challenged.

Aims \& Methods: The aim of this meta-analysis was to examine the effect of PBP on small bowel VCE outcomes. We performed literature searches in MEDLINE and Cochrane Library to identify randomized-controlled trials (RCTs) evaluating the effect of small bowel preparation -purgative (PEG, sodium phosphate, 
others) vs. clear liquids diet- on VCE outcomes. Examination's diagnostic yield (DY) was meta-analysis primary end point; small bowel visualization quality (VQ), VCE completion rate (CR), gastric (GTT) and small bowel (SBTT) transit times comprised the secondary endpoints. Study outcomes effect sizes were calculated using RevMan 5.3 software fixed or random effect model, as appropriate, and they are presented as $\mathrm{OR}[95 \% \mathrm{CI}]$ or mean difference $[95 \% \mathrm{CI}]$, respectively. Heterogeneity was measured using the $\mathrm{I}^{2}$ statistics. Publication bias was assessed by Funnel plots inspection and the quality of the meta-analyzed studies was assessed using the Jadad criteria.

Results: We identified 9 eligible RCTs with 12 sets of data, including 1029 subjects. They were low risk of bias trials and no publication bias was detected. As compared to clear liquids diet, PBP significantly increased small bowel VCE DY $\left(1.44\right.$ [1.14-1.83], $\left.\mathrm{I}^{2}=39 \%\right)$. However, a sensitivity analysis that excluded studies with sodium phosphate preparation (abandoned nowadays) and PBPs different (not widely used) than PEG showed no benefit of PEG preparation over clear liquids $\left(1.31[0.79-2.17], \mathrm{I}^{2}=54 \%\right)$. VQ significantly improved after PBP $(2.05$ $\left.[1.49-2.82), \mathrm{I}^{2}=45 \%\right)$, without any significant effect on VCE CR $(0.98$ [0.73$\left.1.33], \mathrm{I}^{2}=0 \%\right)$ and GTT $\left(-1.21[-7.21-4.79] \mathrm{min}, \mathrm{I}^{2}=94 \%\right)$. On the contrary, VCE SBTT was statistically lower by $-15.06[-25.52--6.61]$ min $\left(I^{2}=90 \%\right)$ after PBP.

Conclusion: In comparison to clear liquids diet, PBP increases small bowel VCE DY and improves VQ without affecting exam's CR. However, the positive effect of PBP on VCE DY is mainly derived from two relative small, old (2004 and 2009) RCTs and disappears if only PEG preparation studies are meta-analyzed. Disclosure of Interest: All authors have declared no conflicts of interest.

\section{P0265 INTER-OBSERVER AGREEMENT IN BROTZ CLEANING SCALES FOR CAPSULE ENDOSCOPY}

M. Sousa, R. Pinho, A. Ponte, J. Silva, J. Rodrigues, J. C. Silva, A. Rodrigues, L. Proença, J. Carvalho

Gastrenterology, Centro Hospitalar de Vila Nova de Gaia e Espinho, Vila Nova de Gaia/Portugal

Contact E-mail Address: mafalda_m_p_sousa@hotmail.com

Introduction: The diagnostic yield of capsule endoscopy (CE) depends on the adequate visualization of the mucosa. As with colonoscopy, cleaning scales should be described in the report in order to better interpret results. In 2009, Brotz et al proposed and validated 3 different cleansing scales in 40 patients.

Aims \& Methods: A hundred CE videos (Mirocam $\left.{ }^{\circledR}\right)$ were reviewed by 2 authors at a fixed frame rate of 100 frames per second in quadruple view (Miroview Client). The CE were evaluated according to Brotz scales: (1) Overall adequacy assessment (adequate/inadequate) (2) Qualitative scale (excellent, good, fair, poor) and (3) Quantitative scale (0-10 score, graded from 0-2 visualization of the mucosa, fluids, bubbles, bile and luminosity). The aim of this study was to evaluate the inter-observer variability of this cleaning scales. The kappa coefficient was used to calculate the inter-observer agreement in overall adequacy assessment and the intra-class correlation coefficient was used to evaluate the concordance of the qualitative and quantitative scales.

Results: In overall adequacy assessment, the quality of bowel preparation was classified as adequate by observer 1 in $67 \%$ and by observer 2 in $73 \%$, with an inter-observer kappa index of $0.76(\mathrm{p}<0.001)$ suggesting strong agreement. In the qualitative scale, most of the bowel preparations were considered reasonable $(40 \%$ observer 1 vs $36 \%$ observer 2$)$, with an intra-class coefficient of 0.89 $(\mathrm{p}<0.001)$. In the quantitative scale, the mean score of the two observers was 6.5 and 6.7, resulting in an intra-class agreement of $0.78(\mathrm{p}<0.001)$.

Conclusion: The optimization of quality of bowel preparation and the diagnostic yield of the CE requires, first, a well-validated cleaning scale. Brotz's rating scales have strong inter-observer agreement. The qualitative scale is easier to apply and has better inter-observer agreement, so the authors propose that it should be used routinely in the CE report.

Disclosure of Interest: All authors have declared no conflicts of interest.

\section{Reference}

Brotz C, Nandi N, Conn $\mathrm{M}$ et al. A validation study of 3 grading systems to evaluate small-bowel cleansing for wireless capsule endoscopy: a quantitative index, a qualitative evaluation, and an overall adequacy assessment. Gastrointest Endosc 2009; 69:262-270

\section{P0266 PILOT STUDY OF THE EFFECTS OF IMAGE QUALITY ON LESION VISUALISATION IN SMALL BOWEL CAPSULE ENDOSCOPY}

D. E. Yung ${ }^{1}$, X. Dray ${ }^{2}$, E. Toth ${ }^{3}$, E. Rondonotti ${ }^{4}$, R. Sidhu ${ }^{5}$, U. Kopylov ${ }^{6}$, M. Mcalindon ${ }^{5}$, M. Pennazio ${ }^{7}$, J. N. Plevris ${ }^{1}$, A. Koulaouzidis ${ }^{1}$

${ }^{1}$ The Royal Infirmary of Edinburgh, Edinburgh/United Kingdom

${ }^{2}$ Hôpital Saint-Antoine, Paris/France

${ }^{3}$ Skåne University Hospital, Malmo/Sweden

${ }^{4}$ Ospedale Valduce, Como/Italy

${ }^{5}$ Royal Hallamshire Hospital, Sheffield/United Kingdom

${ }^{6}$ Gastroenterology, Chaim sheba Medical Center, Ramat Gan/Israel

${ }^{7}$ San Giovanni Battista University Teaching Hospital, Turin/Italy

Contact E-mail Address: diana.e.yung@gmail.com

Introduction: Capsule endoscopy (CE) is the prime mode of investigation for small bowel (SB) pathology. However, as an entirely visual medium it depends heavily on image quality. The definition of optimal image quality remains unstandardised between studies and poses significant limitations to the quality of study reporting. As yet, there is no widely-accepted or integrated method for scoring SB cleanliness during CE reporting. This pilot study aims to quantify the image properties contributing to adequate visualisation quality in $\mathrm{CE}$ images.

Aims \& Methods: Five clear images of SB pathology were obtained using MiroCam ${ }^{\circledR}$ (Intromedic, South Korea), image resolution $320 \times 320$ pixels(px): $\mathrm{P} 1$ and P2 angioectasias, ulcer, aphtha and polyp. Each image was processed using GIMP2 image editing software (www.gimp.org) for 3 parameters: (1) opacity (opacity filter matched in colour to commonly-seen SB contents, $10-90 \%$ in $10 \%$ increments), (2) blur (Gaussian blur, radius $1-10 \mathrm{px})$, (3) contrast $(-50 \%$ to $50 \%$ in $10 \%$ increments). Gaussian blur was used to simulate the effects of rapid capsule movement as well as to affect image definition. A set of 5 original and 190 edited images was obtained. A web-based survey was created using Google Forms and 9 expert CE readers were asked to indicate whether each image was adequate or not for diagnosis. The order of images was randomised for each reader. For each type of pathology, we determined the threshold of image quality which was deemed adequate for diagnosis.

Results: For image opacity, both angioectasias and the polypoid lesion were adequately visualised below $40 \%$ opacity whereas the threshold was lower for both the ulcer and aphtha (10\% opacity). Increasing blur radius significantly impacted the acceptability of images for reaching a diagnosis with confidence; for most images, blur radius $3 \mathrm{px}$ was the threshold for adequate visualisation but even $1 \mathrm{px}$ of blur radius decreased the visualisation quality of the aphtha image. The aphtha image was also affected the most by decreased contrast; conversely the ulcer was deemed more inadequately visualised with higher contrast. The other images were generally adequately visualised at $\pm 10 \%$ contrast. Results are detailed in the table below.

Percentages of respondents finding each image adequate

\begin{tabular}{|c|c|c|c|c|c|}
\hline Image parameter & $\begin{array}{l}\text { P2 } \\
\text { Angioectasia }\end{array}$ & $\begin{array}{l}\text { P1 } \\
\text { Angioectasia }\end{array}$ & Ulcer & Aphtha & $\begin{array}{l}\text { Polypoid } \\
\text { lesion }\end{array}$ \\
\hline Original image & $100 \%$ & $100 \%$ & $100 \%$ & $88.9 \%$ & $100 \%$ \\
\hline \multicolumn{6}{|l|}{ Opacity } \\
\hline $10 \%$ opacity & $100 \%$ & $100 \%$ & $100 \%$ & $77.8 \%$ & $100 \%$ \\
\hline $20 \%$ opacity & $100 \%$ & $100 \%$ & $88.9 \%$ & $77.8 \%$ & $100 \%$ \\
\hline $30 \%$ opacity & $100 \%$ & $100 \%$ & $88.9 \%$ & $33.3 \%$ & $100 \%$ \\
\hline $40 \%$ opacity & $100 \%$ & $100 \%$ & $77.8 \%$ & $33.3 \%$ & $100 \%$ \\
\hline $50 \%$ opacity & $100 \%$ & $88.9 \%$ & $77.8 \%$ & $22.2 \%$ & $100 \%$ \\
\hline $60 \%$ opacity & $77.8 \%$ & $75 \%$ & $66.7 \%$ & $11.1 \%$ & $77.8 \%$ \\
\hline $70 \%$ opacity & $66.7 \%$ & $44.4 \%$ & $22.2 \%$ & $0 \%$ & $44.4 \%$ \\
\hline $80 \%$ opacity & $33.3 \%$ & $22.2 \%$ & $22.2 \%$ & $0 \%$ & $22.2 \%$ \\
\hline $90 \%$ opacity & $0 \%$ & $0 \%$ & $0 \%$ & $0 \%$ & $0 \%$ \\
\hline \multicolumn{6}{|l|}{ Blur radius } \\
\hline $1 \mathrm{px}$ & $100 \%$ & $100 \%$ & $100 \%$ & $88.9 \%$ & $100 \%$ \\
\hline $2 \mathrm{px}$ & $100 \%$ & $100 \%$ & $100 \%$ & $77.8 \%$ & $100 \%$ \\
\hline $3 \mathrm{px}$ & $100 \%$ & $100 \%$ & $100 \%$ & $77.8 \%$ & $100 \%$ \\
\hline $4 \mathrm{px}$ & $77.8 \%$ & $100 \%$ & $100 \%$ & $77.8 \%$ & $77.8 \%$ \\
\hline $5 \mathrm{px}$ & $33.3 \%$ & $66.7 \%$ & $100 \%$ & $22.2 \%$ & $44.4 \%$ \\
\hline $6 \mathrm{px}$ & $33.3 \%$ & $55.6 \%$ & $88.9 \%$ & $33.3 \%$ & $11.1 \%$ \\
\hline $7 \mathrm{px}$ & $22.2 \%$ & $22.2 \%$ & $55.6 \%$ & $25 \%$ & $0 \%$ \\
\hline $8 \mathrm{px}$ & $11.1 \%$ & $22.2 \%$ & $33.3 \%$ & $0 \%$ & $0 \%$ \\
\hline $9 \mathrm{px}$ & $22.2 \%$ & $0 \%$ & $33.3 \%$ & $11.1 \%$ & $0 \%$ \\
\hline $10 \mathrm{px}$ & $0 \%$ & $0 \%$ & $33.3 \%$ & $0 \%$ & $0 \%$ \\
\hline \multicolumn{6}{|l|}{ Contrast } \\
\hline$-50 \%$ & $66.7 \%$ & $77.8 \%$ & $66.7 \%$ & $0 \%$ & $88.9 \%$ \\
\hline$-40 \%$ & $100 \%$ & $85.7 \%$ & $55.6 \%$ & $11.1 \%$ & $100 \%$ \\
\hline$-30 \%$ & $100 \%$ & $100 \%$ & $88.9 \%$ & $11.1 \%$ & $100 \%$ \\
\hline$-20 \%$ & $100 \%$ & $100 \%$ & $88.9 \%$ & $22.2 \%$ & $100 \%$ \\
\hline$-10 \%$ & $100 \%$ & $100 \%$ & $100 \%$ & $44.4 \%$ & $100 \%$ \\
\hline$+10 \%$ & $88.9 \%$ & $100 \%$ & $100 \%$ & $88.9 \%$ & $100 \%$ \\
\hline$+20 \%$ & $100 \%$ & $100 \%$ & $75 \%$ & $100 \%$ & $77.8 \%$ \\
\hline$+30 \%$ & $100 \%$ & $100 \%$ & $62.5 \%$ & $88.9 \%$ & $66.7 \%$ \\
\hline$+40 \%$ & $100 \%$ & $100 \%$ & $66.7 \%$ & $88.9 \%$ & $66.7 \%$ \\
\hline$+50 \%$ & $66.7 \%$ & $55.6 \%$ & $11.1 \%$ & $88.9 \%$ & $66.7 \%$ \\
\hline
\end{tabular}

Conclusion: This pilot study shows that image quality is a defining factor in accurate diagnosis in CE. Image quality is commonly affected by the opacity of luminal fluid/residue, and the quality of imaging delivered by the CE system. More subtle mucosal lesions such as aphthae are affected more by decreased contrast. Interestingly, a relatively high level of image opacity can be tolerated by $\mathrm{CE}$ readers whereas blurriness seems to have a greater effect on visualisation quality and reviewer confidence in the diagnosis. The effects of these aspects in combination merit further investigation.

Disclosure of Interest: All authors have declared no conflicts of interest. 
P0267 EVALUATION OF A NEW PAN ENTERIC CAPSULE SYSTEM

IN PATIENTS WITH SUSPECTED OR ESTABLISHED

INFLAMMATORY BOWEL DISEASE - ASSESSING THE SYSTEM

FUNCTIONALITY TO VISUALIZE AND ASSESS THE SMALL AND LARGE BOWELS

R. Eliakim ${ }^{1}$, C. Spada ${ }^{2}$, I. Fernández-Urién Sainz ${ }^{3}$, H. Yanai ${ }^{4}$, A. Lahat ${ }^{1}$, Y. Ron ${ }^{4}$, S. Pecere ${ }^{2}$, G. Costamagna ${ }^{2}$, A. Schwartz ${ }^{5}$, I. Eyal ${ }^{6}$, A. Lapidus ${ }^{6}$, S. N. Adler ${ }^{5}$

${ }^{1}$ Dept. Of Gastroenterology, Sheba Medical Center, Tel-Aviv/Israel

${ }^{2}$ Policlinico Universitario "A Gemelli", UO di Endoscopia Digestiva Chirurgica,

Rome/Italy

${ }^{3}$ Servicio de Digestivo - Complejo Hospitalario de Navarra, Pamplona/Spain

${ }^{4}$ Souraski Medical Center, Tel-Aviv/Israel

${ }^{5}$ Share Zedek Medical Center, Jerusalem/Israel

${ }^{6}$ Medtronic, Yoqneam/Israel

\section{Contact E-mail Address: abraham.eliakim@sheba.health.gov.il}

Introduction: Inflammatory bowel diseases (IBDs) are chronic inflammatory diseases that may affect the whole gastrointestinal (GI) tract, mainly the small bowel and colon. Endoscopic evaluation of these parts is essential to assess disease extent and severity. The small bowel colon capsule endoscopy (SBC-CE) system is a new system composed of a two-headed capsule with a panoramic field of view and adaptive frame rate, customized for complete coverage of IBD lesions in the entire bowel, data recorder and new disease specific software, allowing assessment and follow-up over time of disease severity and extent.

Aims \& Methods: The aim was to evaluate SBC-CE system functionality in suspected or established IBD (Crohn's disease [CD] and Ulcerative Colitis [UC]) patients. This was a prospective 5 center feasibility study assessing the performance of the new capsule and software. Subjects enrolled into the study ingested the new capsule after standard bowel preparation plus boosts. Contraindications for its use included obstruction, dysphagia or swallowing disorders, pacemakers etc. GI patency was assured using the patency capsule. The primary endpoint was successful procedure in terms of video creation and report generation in accordance to the video reading methodology. Secondary endpoints were subjective coverage of SBC, subjective duration of total and segmental reading time, over all video quality and occurrence/severity of adverse events. Results: 68 patients were screened, of which 54 were enrolled and 49 ingested the capsule (14 patency failure, 5 withdrew consent). Mean age was 40.1 years, $51 \%$ were males. $69 \%$ of patients had established CD, $10 \%$ UC and $21 \%$ suspected $\mathrm{CD}$. The disease was active in $62 \%$ of known IBD patients. One patient who underwent extensive colon resection was excluded from all colon analyses. Overall cleansing was regarded good or excellent in $96 \%$ of patients. All 49 videos met the primary endpoint, i.e. video was created and report generated with all included information and in accordance to the video reading methodology. All capsules reached the rectum/toilet with no retention, most while photographing. Gastroenterologists were satisfied with small bowel coverage $6.6 \pm 0.6$ (mean \pm standard deviation) and colon coverage of $6.0 \pm 1.2$ in a scale of $1-7$ (unconfident to confident), image quality $6.1 \pm 0.8$ ( $1-7$, poor to excellent), and subjective video reading time $3.9 \pm 1.4$ (1-7, very short to very long). There were 4 patients with small interferences related to video continuity (gaps).

Conclusion: The new SBC capsule is a friendly, minimally invasive capsule allowing complete evaluation of the entire gut of IBD patients. The system may be used to assess disease severity and extent and for follow up of IBD patients.

\section{Disclosure of Interest:}

R. Eliakim: I have received consultant fee from Medtronic. I am in the advisory committee for PhotoPill, Tarus medical

C. Spada: consultant and speaker fees for Medtronic.

I. Fernández-Urién Sainz: Receive Consulting fee from Medtronic

H. Yanai: I received consulting, advisory, lectures and speaker's fees from: Abbvie, Janssen, and Takeda

I. Eyal: Employee at Medtronic

A. Lapidus: Employee at Medtronic

S.N. Adler: Received consulting fee from Medtronic

All other authors have declared no conflicts of interest.

\section{P0268 THE UTILITY OF A NOVEL TRANSPAPILLARY DILATION TECHNIQUE WITH A DIATHERMIC CATHETER FOR SEVERE MAIN PANCREATIC DUCT STRICTURE DUE TO CHRONIC PANCREATITIS}

S. Kato, M. Kuwatani, R. Sugiura, I. Sano, K. Kawakubo, N. Sakamoto Gastroenterology And Hepatology, Hokkaido University Hospital, Sapporo/Japan

\section{Contact E-mail Address: shinchan1231@gmail.com}

Introduction: Transpapillary dilation for severe main pancreatic duct (MPD) stricture is sometime difficult and diathermic dilation is now getting attention as a salvage technique for severe stricture; however its efficacy and safety remains unclear.

Aims \& Methods: To evaluate the efficacy and safety of a novel transpapillary dilation technique with a diathermic catheter for severe MPD stricture due to chronic pancreatitis. Between April 2011 and March 2017, 143 patients with chronic pancreatitis underwent endoscopic transpapillary stent placement for MPD. MPD dilatation was indicated in 18 patients, and diathermic dilation was required in nine patients. We evaluated (1) the patients' characteristics, (2) procedure characteristics, (3) clinical outcomes, (4) adverse events.

Results: (1) Six patients were men and three were women (mean age, 50.1 years). Alcohol 8, unknown 1. The strictures were in the head of pancreas: 8 , body: 1 .
The mean length of stricture was $20.2 \mathrm{~mm}$ (range, 10-30.8). The mean MPD diameter on the distal side of stricture was $6.2 \mathrm{~mm}$ (range, 3-12.7). Five patients $(55.6 \%)$ among them had no former procedure for MPD including stenting. (2) A wire-guided 6 Fr diathermic catheter with $30 \mathrm{~W}$ power was used for all cases. All cases underwent diathermic dilation as salvage procedure subsequent to conventional dilation. One to 7 diathermy procedures (mean 2.9) were required to pass through each stricture. (3) Passage of the diathermic catheter and stent placement was successful in all patients $(100 \%)$. After diathermy and stent placement, 8 $(88.9 \%)$ showed improvement of clinical symptoms (abdominal pain). Recurrence of stricture was observed in 2 patients $(22.2 \%)$. One of them needed diathermic dilatation again. (4) Two adverse events $(22.2 \%)$ were observed and both of them were mild pancreatitis. Multiple diathermy procedures (6 times and 4 times, respectively) and relatively long duration of total diathermy ( $39 \mathrm{sec}$. and $25 \mathrm{sec}$. respectively) were observed in cases with pancreatitis.

Conclusion: Transpapillary diathermic dilation is a relatively safe and effective salvage procedure for severe MPD stricture due to chronic pancreatitis. However, care should be taken in cases that require multiple times and long duration diathermy procedures because of a risk of pancreatitis.

\section{P0269 CLINICAL OUTCOMES OF BILIARY DRAINAGE DURING NEOADJUVANT CHEMOTHERAPY/CHEMORADIOTHERAPY IN MULTICENTER PANCREATIC CANCER STUDIES: METAL STENT VERSUS PLASTIC STENT}

M. Kuwatani, T. Nakamura, T. Hayashi, Y. Kimura, M. Ono, K. Yamakita, T. Goto, K. Takahashi, M. Motoya, H. Maguchi, S. Hirano, N. Sakamoto

\section{Hokkaido Pancreatic Cancer Study Group (HOPS), Sapporo/Japan}

Contact E-mail Address: mkuwatan $@$ med.hokudai.ac.jp Introduction: It is controversial whether preoperative biliary drainage is necessary or not in patients with pancreatic head cancer. Meanwhile, some reports indicated the necessity of biliary drainage in the case of severe jaundice and neoadjuvant chemotherapy/chemoradiotherapy (NAC/NACRT) ${ }^{(1,2)}$

Aims \& Methods: We aimed to clarify clinical outcomes of biliary drainage with metal stent (MS) or plastic stent (PS) during neoadjuvant chemotherapy/chemoradiotherapy in patients with pancreatic cancer who underwent subsequent pancreatoduodenectomy Patients and methods: From Oct. 2013 to Apr. 2016, 96 patients with pancreatic cancer were registered to NAC (S-1 $\left[80 \mathrm{mg} / \mathrm{m}^{2}\right.$, twice daily], 2 cycles) or NACRT (a total dose of 50.4 Gy in 28 fractions and S-1 [80 $\mathrm{mg} / \mathrm{m}^{2}$, twice daily on radiation day alone] for 1 month and subsequent chemotherapy with gemcitabine for 3 cycles) studies conducted by by Hokkaido Pancreatic Cancer Study Group (HOPS) (UMIN000013031/UMIN000012293). Of them 29 patients who underwent biliary drainage with a MS or PS before NAC/NACRT and subsequent pancreatoduodenectomy were analyzed. Examination items were 1) patient characteristics, 2) preoperative recurrent biliary obstruction (RBO: stent obstruction, migration, tumor ingrowth/overgrowth) occurrence rate, 3) NAC/NACRT delay or discontinuation rate, 4) surgical time, volume of hemorrhage, and postsurgical adverse event rate.

Results: 1$)$ The median of age was 67 years $(50-83) ; \mathrm{M} / \mathrm{F}$ ratio was $13 / 16$. The distribution of pathological stages (based on the General Rules for the Study of Pancreatic Cancer by the Japan Pancreas Society, 6th edition) included 2 patients with stage I, 1 with II, 13 with III and 13 with IV disease. NAC and NACRT were performed for 14 and 15 patients, respectively. MS and PS were used in 17 and 12 patients, respectively. The distribution of used MS and PS diameters included 17 patients with $10 \mathrm{~mm}, 9$ with $7 \mathrm{~F}, 2$ with $8.5 \mathrm{~F}$ and 1 with $10 \mathrm{~F}$. 2) RBO occurred in $5.9 \%$ of patients in the MS group and $83.3 \%$ in the PS group with a significant difference $(\mathrm{p}<0.0001)$. The RBO included the following conditions: stent obstruction, 1 patient in the MS group and 8 in the PS group; stent migration, 0 in the MS group and 2 in the PS group. 3) NAC/NACRT delay was observed in $35 \%$ of patients in the MS group and $50 \%$ in the PS group. NAC/NACRT discontinuation was observed in $12 \%$ of patients in the MS group and $17 \%$ in the PS group. There were no significant differences between both groups in rates of delay and discontinuation of the neoadjuvant therapies. 4) The operative time of the MS group was significantly longer than that of the PS group (610 $\mathrm{min}$ vs $495 \mathrm{~min}, \mathrm{p}=0.036$ ), while blood losses $(870 \mathrm{ml}$, the MS group vs. $658 \mathrm{ml}$, the PS group) and postoperative morbidity rates (35\%, the MS group vs. $33 \%$, the PS group) of both groups were not significantly different.

Conclusion: MS is better than PS from the viewpoint of the prevention of RBO. Meanwhile, MS was similar to PS in the postoperative outcome. For standardization of biliary stenting during NAC/NACRT, further large-scale and prospective studies are needed.

Disclosure of Interest: All authors have declared no conflicts of interest.

\section{References}

1. Tol JAMG, van Hooft JE, Timmer R, et al. Metal or plastic stents for preoperative biliary drainage in resectable pancreatic cancer. Gut 2016;65:1981-1987.

2. Crippa S, Cirocchi R, Partelli S, et al. Systematic review and meta-analysis of metal versus plastic stents for preoperative biliary drainage in resectable periampullary or pancreatic head tumors. Eur J Surg Oncol. 2016; 42:1278-85. 
P0270 OUTCOME OF ENDOSCOPIC REINTERVENTION FOR MALIGNANT HILAR OBSTRUCTION TREATED BY STENT-INSTENT DEPLOYMENT

D.H. Kang, H.W. Kim, C.W. Choi, S.J. Kim, J.S. Lee, C.W. Yeo, H.S. Nam, S.H. Choi, J.H. Ko, B.J. Kwon, D.G. Ryu

Pusan National University Yangsan Hospital, Yangsan/Korea, Republic of

\section{Contact E-mail Address: sulsulpul@naver.com}

Introduction: Endoscopic biliary decompression is widely used for advanced hilar cholangiocarcinoma. Bilateral stenting has become more feasible with more experienced endoscopists and the development of new devices. However, stent dysfunction develops in 3\% to $45 \%$ because of tumor ingrowth, overgrowth, or debris as disease progresses. Endoscopic reintervention is difficult and complex with worsening bile duct strictures. The present study aimed to evaluate a suitable reintervention procedure for stent malfunction after stent-in-stent (SIS) deployment for malignant hilar obstruction.

Aims \& Methods: From September 2009 to June 2016, a total of 52 patients who underwent endoscopic bilateral stenting at Pusan National University Yangsan Hospital were enrolled in this study. Among them, 20 patients who underwent reintervention due to stent malfunction were analyzed. Reintervention was performed endoscopically or percutaneously. Technical and functional success rates were evaluated retrospectively.

Results: Technical and functional success rates of endoscopic reintervention were $83 \%(10 / 12)$ and $80 \%(8 / 10)$, respectively. Endoscopic bilateral and unilateral reintervention success rates were $75 \%(6 / 8)$ and $100 \%(4 / 4)$, respectively. Functional success was observed in 8 out of 10 patients $(80 \%)$ who achieved technical success. For bilateral reintervention, either plastic or plastic and metal stents were used. PTBD was performed in 8 patients because of duodenal stenosis (2 patients) and poor conditions.

Conclusion: Endoscopic reintervention could be considered in the case of stent malfunction and fair patient conditions after SIS placement for malignant hilar obstruction. Decisions regarding bilateral or unilateral drainage and types of stents should depend on the conditions of the disease and the patient.

Disclosure of Interest: All authors have declared no conflicts of interest.

\section{P0271 LONG-TERM OUTCOMES OF ENDOSCOPIC ULTRASOUND-GUIDED RIGHT INTRAHEPATIC DUCT DRAINAGE WITH TRANSMURAL COVERED METAL STENT}

D.H. Cho, D.H. Park, D. Oh, T.J. Song, S.S. Lee, D.W. Seo, S.K. Lee, M. Kim Gastroenterology, Asan Medical Center, Seoul/Korea, Republic of

\section{Contact E-mail Address: dhcho81@daum.net}

Introduction: Endoscopic ultrasound-guided biliary drainage (EUS-BD) has been regarded as an effective alternative in cases of endoscopic retrograde cholangiopancreatography (ERCP) failure or inaccessible papilla. However, EUS-BD for right intrahepatic duct obstruction (EUS-BDR) remains challenging, although recent studies showed promising result. The aim of current study was to evaluate the feasibility and long-term outcomes of EUS-BD with transmural coved metal stents for right intrahepatic duct obstruction.

Aims Methods: In this single-center, retrospective study, a total of 24 consecutive patients who underwent EUS-BDR after failed ERCP were enrolled. The patients were consisted of 12 cases of benign strictures and 12 cases of malignant strictures. The biliary stents used in this study was covered metal stent with antimigration properties (fully covered metal with anchoring flaps or partially covered metal stent with anchoring flaps). The technical success rate, clinical success rate and adverse events were evaluated

Results: The target bile duct was right anterior in 6 patients and right posterior segmental duct in 18 patients. Among them, percutaneous transhepatic biliary drainage assisted EUS-BDR was performed in three patients. The technical and clinical success rate was $95.8 \%(23 / 24)$ and $95.6 \%(22 / 23)$. Mean diameter of right intrahepatic duct was $6.5(4-30) \mathrm{mm}$. A fully covered metal stent was used in 22 patients and partial covered stent in 2 patients. Early adverse events developed after EUS-BDR in 2 patients ( 1 case of cholangitis and 1 case of liver abscess in patients with malignant biliary stricture). Late adverse event that stent occlusion was observed in 5 patients. Neither proximal peritoneal stent migration nor spontaneous distal stent migration was observed during followup periods. The stent patency duration was 275.2 (147.8-402.7) days. During follow-up period, stent revision via fistula tract was successful and additional percutaneous biliary drainage for right intrahepatic duct obstruction was not required in all patients who achieved clinical success.

Conclusion: EUS-BD using transmural covered metal stent with antimigration properties for right intrahepatic duct obstruction may be technically feasible, effective and relatively safe for both benign and malignant biliary strictures by expert hands. Furthermore, the route of hepaticoduodenostomy created by covered metal stent was durable and endoscopically easily managed.

Disclosure of Interest: All authors have declared no conflicts of interest.

\section{P0272 UTILITY OF EUS-GUIDED HEPATICOGASTROSTOMY WITH ANTEGRADE STENTING FOR MALIGNANT BILIARY OBSTRUCTION OF ERCP INABILITY}

H. Imai, M. Takenaka, M. Kudo

Gastroenterology And Hepatology, Kindai University, Osakasayama/Japan

Contact E-mail Address: codenamegenchan1023@gmail.com
Introduction: Recently, EUS-guided biliary drainage (EUS-BD) techniques such as EUS-guided choledochoduodenostomy (CDS), hepaticogastrostomy (HGS) antegrade stenting (AGS) and rendezvous stenting (RVS) are useful for biliary drainage methods after unsuccessful ERCP. Among these procedures, CDS and RVS require the echoendoscope reaching duodenum. Therefore, HGS or AGS are indicated in cases with inaccessible duodenum. However, HGS is associated with a higher risk of adverse events, compared with the other methods. When the stent dysfunction occurs, re-intervention is more difficult after AGS alone than after HGS or CDS. Thus, we started to add AGS during HGS in a single session from Sep. 2011.

Aims \& Methods: The aim of this study to assess the efficacy and safety of HGS combined with AGS for malignant biliary stricture-induced obstructive jaundice. Between Jan. 2006 and Dec. 2014, ERCP was attempted in patients with obstructive jaundice, which was successful in 641 patients and impossible in 154 patients (101 cases due to post-surgical altered anatomy or duodenal stenosis, 53 cases due to difficult cannulation). A total of 145 patients received EUS-BD; HGS and HGS with AGS were attempted in 42 (Group A; from Jan 2006 to Aug 2011) and 37 patients (Group B; from Sep 2011 to Dec. 2014), respectively. The assessed outcomes were the technical and functional success rates, adverse events rate, reintervention rate, procedure time, overall patient survival time, and time to stent dysfunction or patient death. In Group A, technical success of HGS was defined as successful stent deployment between the left hepatic bile duct and the stomach. In Group B, technical success of HGS with AGS was defined as successful stent deployment at bile duct stricture (AGS) in addition to success of HGS. Functional success for obstructive jaundice was defined as a decrease in bilirubin levels to $<40 \%$ of the pretreatment value within 2 weeks. The incidence rate of adverse events such as peritonitis, bile leakage, bleeding, stent migration, and stent occlusion was assessed. The re-intervention was defined as any endoscopic, surgical, or percutaneous procedure that was required to improve symptoms after placement of the stent. Time to stent dysfunction or patient death was defined as the time from stent deployment to biliary re-intervention due to stent dysfunction or the time from stent deployment to patient death. Groups $\mathrm{A}$ and $\mathrm{B}$ were compared in terms of the technical success, clinical success, and adverse events outcomes, the procedure time, the overall patient survival and time to stent dysfunction or patient death. For subgroup analysis in patients who underwent chemotherapy the Groups A and B were compared in the overall patient survival, time to stent dysfunction or patient death and adverse events rate.

Results: Technical success rate of Group A was significantly higher than Group B (97.6 vs $83.8 \%, p=0.03$ ). The two groups were comparable for the functional success rate $(90.2 \%$ vs $90.3 \%$ ), although the rate of adverse events tended to be higher in Group A than in Group B $(26.1 \%$ vs $13.5 \%, p=0.10)$. The re-intervention rate tended to be higher in Group A than in Group B $(16.7 \%$ vs $8.1 \%$, $P=0.25$ ). Groups $A$ and $B$ did not differ significantly in terms of median overall patient survival ( 75 days vs. 61 days, $P=0.70$ ), and median time to stent dysfunction or patient death ( 68 vs 63 days, $\mathrm{P}=0.08$ ) In patients who underwent chemotherapy, there were no difference in the overall patient survival time (121 vs 157 days, $\mathrm{p}=0.08$ ) between the two groups although the time to stent dysfunction or patient death was significantly shorter in Group A than in Group B (71 vs 95 days, $\mathrm{p}=0.02$ ).

Conclusion: Technical success rate of HGS with AGS was lower than HGS, although HGS with AGS is superior to HGS in terms of stent patency in patients receiving chemotherapy.

Disclosure of Interest: All authors have declared no conflicts of interest.

\section{P0273 EUS-GUIDED GALLBLADDER DRAINAGE REDUCES LATE ADVERSE EVENT AND NEED FOR RE-INTERVENTION COMPARED WITH PERCUTANEOUS CHOLECYSTOSTOMY IN PATENTS WHO ARE NOT ELIGIBLE FOR SURGERY}

D.H. Cho, S.S. Lee, D. Oh, T.J. Song, D.H. Park, D.W. Seo, S.K. Lee, M. Kim Gastroenterology, Asan Medical Center, Seoul/Korea, Republic of

Contact E-mail Address: dhcho81@daum.net

Introduction: Endoscopic ultrasound guided transmural gall-bladder drainage (EUS-GBD) with covered metal stent has become increasingly used to treat patients with acute cholecystitis who are not a candidate for surgical treatment. However, there are limited data comparing long-term outcomes of EUS-GBD with covered metal stent and conventional percutaneous cholecystostomy.

Aims \& Methods: This is a single-center, retrospective study comparing long-term outcomes of EUS-GBD and percutaneous cholecystostomy in patients who are not suitable for cholecystectomy. Data about the patient who underwent EUSGBD for acute cholecystitis is obtained from prospective collected EUS database of our institute. In percutaneous cholecystostomy group, electrical medical record of patients who underwent percutaneous cholecystostomy was reviewed and analyzed. Demographics and procedure related outcomes including early, late adverse events and need for re-intervention in each group was compared. Results: A total of 181 patients (74 in EUS-GBD group and 107 in percutaneous cholecystostomy group) were enrolled in this study. The cause of cholecystitis and ASA class were similar in both groups. The technical/clinical success rate was $100 \% / 98.6 \%$ in EUS-GBD group and $99.1 \% / 97.2 \%$ in percutaneous cholecystostomy group $(\mathrm{P}=0.591$ and 0.646$)$ respectively. Early adverse event rate was also similar between two groups $(6.8 \%$ in EUS-GBD group vs. $15.0 \%$ in percutaneous cholecystostomy group, $\mathrm{P}=0.103$ ). However, late adverse events including migration of stent or dislodgement of drainage tube, stent or tube occlusion, tract inflammation around percutaneous tube, bile leakage and recurrence of cholecystitis was more frequently observed in percutaneous cholecystostomy group (5/74 in EUS-GBD group and 21/107 in percutaneous cholecyststomy group, $\mathrm{P}=0.017)$. Percutaneous cholecystostomy tube was indwelled for 
median 20 days (14.0-45.2) after the procedure. A total of 7 patients in EUSGBD group received re-intervention for adverse events and all of them were conducted successfully. The patients who underwent percutaneous cholecytostomy more frequently received re-intervention for adverse event or recurrence of cholecystitis after removal of cholecystostomy. (7/74 vs. $23 / 106, \mathrm{P}=0.041)$.

Conclusion: EUS-GBD and percutaneous cholecystostomy were both effective interventions to urgent drainage for acute cholecystitis. However, EUS-GBD might be beneficial than percutaneous cholecystostomy in long term management for the patients with acute cholecystitis who are not suitable for cholecystectomy. Disclosure of Interest: All authors have declared no conflicts of interest.

\section{P0274 ENDOSCOPIC TREATMENT OF ANASTOMOTIC BILIARY STRICTURES IN PATIENTS WITH LIVING DONOR LIVER TRANSPLANTATION: MULTIPLE PLASTIC STENTS VS FULLY COVERED SELF-EXPANDABLE METALLIC STENTS}

A.T. Eminler, E. Parlak, A. S. Koksal, B. Toka, M. I. Uslan Gastroenterology, Sakarya University, Sakarya/Turkey

\section{Contact E-mail Address: drerkanparlak@gmail.com}

Introduction: The "fully covered self-expandable metallic stents"(fcSEMSs) were found to be non-inferior to multiple plastic stents (MPSs) for the treatment of anastomotic biliary strictures after orthotopic liver transplantation (OLT). However, there is scarce data about their efficacy in the treatment of anatomotic biliary strictures after living donor liver transplantation (LDLT). We aimed to compare the efficacy of fcSEMS and MPSs for the treatment of anastomotic biliary strictures after LDLT.

Aims \& Methods: We retrospectively analyzed the data of LDLT patients with duct-to-duct anastomotic biliary strictures who underwent endoscopic treatment at our center within the last 3 years. FcSEMSs were inserted in 23 patients (13 men, mean age: $53 \pm 10$ years) (Group-1). Thirty-two patients (23 men, mean age:51 \pm 9 years) were managed with MPSs insertion (Group-2). In Group-1, secondary branch ducts were prophylactically drained with insertion of plastic stent(s) in order to prevent the development of cholangitis due to their occlusion. FcSEMS and plastic stent(s) were left in place for 2 months. In Group-2, maximum number of plastic stents were inserted and replaced every 3 months. Patients with a follow-up duration of at least 3 months after stenting were included to the study. Primary end-points were the number of endoscopic procedures and the time required for stricture resolution. The secondary end-point was the recurrence rate of the stricture.

Results: FcSEMSs were successfully deployed in all cases. The diameter of the Fc-SEMSs were $10 \mathrm{~mm}$ in 22 patients and $8 \mathrm{~mm}$ in 1 patient. The length of the Fc-SEMSs were $8 \mathrm{~cm}$ in 13 patients, $10 \mathrm{~cm}$ in 8 , and $6 \mathrm{~cm}$ in 2 patients. Secondary branch ducts were prophylactically drained with a single plastic stent in 12 patients, 2 plastic stents in 8 patients, and 3 plastic stents in 3 patients. The median number of endoscopic procedures was 2 (2-4) in Group-1 and 4 (2-9) in Group-2 $(\mathrm{p}<0.001)$. The time required for stricture resolution was shorter in Group-1 $(65.7 \pm 18.2$ days $)$ than in Group-2 $(240.1 \pm 183.4$ days $)(\mathrm{p}<0.001)$. The recurrence rates were similar in Group-1 (17.4\%) and Group-2 (15.6\%) $(\mathrm{p}=0.57)$ after a follow-up period of $515 \pm 290$ and $378 \pm 86$ days, respectively. Conclusion: FcSEMS insertion is an effective method for the treatment of anastomotic biliary strictures after LDLT, with a less number of endoscopic sessions and a shorter stenting duration required for the resolution compared to MPS. Disclosure of Interest: All authors have declared no conflicts of interest.

\section{P0275 PROSPECTIVE RANDOMIZED STUDY FOR EFFICACY OF AN DOUBLE BARE STENT COMPARED A DOUBLE COVERED STENT IN MALIGNANT COLORECTAL OBSTRUCTION}

\author{
A. Vodoleev ${ }^{1}$, V. Duvanskiy ${ }^{1}$, V. Malyuga ${ }^{2}$ \\ ${ }^{1}$ Endoscopy, endoscopical And Lazer Medicine, RUDN-University, Moscow/ \\ Russian Federation \\ ${ }^{2}$ Eramishanzev Clinic, Moscow/Russian Federation
}

\section{Contact E-mail Address: asvodoleev@list.ru}

Introduction: Colorectal stenting is a minimally invasive, reliable and effective intervention in patients with malignant colonic obstruction, associated with a low complication rate and mortality compared with traditional surgical treatment. One of the actual problems associated with colorectal stenting is the recurrence of symptoms of obstruction. The most common cause is migration of covered stents and ingrown of uncovered stents ${ }^{1,2,3}$. The aim of our study was to compare the results of the use of stents of a new design, the development of which was aimed at preventing these complications.

Aims \& Methods: We aimed to evaluate the results of the use of stents of the new design (double uncoated and dual coated colorectal stents). Between December 2012 and April 2017, 77 patients with colonic malignant obstruction were implanted 78 stents (39 bare, 39 covered EGIS Colorectal stent, S\&G Biotech Inc., South Korea). A double uncovered stent has a two-layer structure created by crossing two stents, resulting in a smaller cell size. Such a design theoretically can prevent migration and reduce tumor ingrowth. The covered stent is coated with a silicone membrane between two layers of a metal mesh with distal, uncoated ends of $5 \mathrm{~mm}$ each. The role of the membrane is to prevent the ingrowth of the tumor, localization and uncoated edges prevent the migration of the stent. All interventions are performed by one operator using endoscopic and radiological control. Groups of patients using coated and uncovered stents were comparable in terms of sex, age, duration of symptoms of obstruction, and stenosis localization. The reasons for the obstruction were primary tumors of the colon $97.4 \%$. The localization of the tumor is most common in the sigmoid colon - $54 \%$ patients. Palliative care was provided to $43(55.8 \%)$ patients, «bridge to surgery» $-34(44.7 \%)$ patients.

Results: Clinical success was achieved in $74(96.1 \%)$ patients. In two cases, when using covered stents, the symptoms of obstruction could not be regressed, the patients were operated. In one case, 18 hours after stenting with an uncovered stent, was diagnosed perforation due to obstructive colitis. The average stay in hospital after the intervention was 3 days; the difference between the groups was statistically insignificant. 30 day mortality was $5.2 \%$, the difference was statistically insignificant. Complications were detected in 3 patients in the group of bare stents and in 1 patient in the group of covered stents, the difference was statistically insignificant. One patient with the carcinoma of a sigmoid colon with invasion in anterior abdominal wall noted the appearance of subcutaneous emphysema without pneumoperitoneum, in $3(3.8 \%)$ patients the occlusion of the stents. The reasons for obstruction of the stents were occlusion by the obstruction from stool (fibers) on the 83rd day (the endoscopic recanalization was performed) and tumor overgrowth by 165 days (endoscopic «stent-in-stent» placement). In one patient, the cause of occlusion is unknown, operated in another hospital 34 days after stenting - a transversostomy was performed, died on the 4th day after the operation.

Conclusion: Double bare and double covered colorectal stents were feasibility and efficacy for relieving malignant colorectal obstruction. Reobstruction was rare complication and not different in both groups stent groups. Necessary to continue to research for the accumulation of material from other centers.

Disclosure of Interest: All authors have declared no conflicts of interest.

\section{References}

1. Matsuzawa T, Ishida H, Yoshida S, A Japanese prospective multicenter study of self-expandable metal stent placement for malignant colorectal obstruction: short-term safety and efficacy within 7 days of stent procedure in 513 cases. Gastrointest Endosc. 2015 Oct;82(4):697-707

2. Lee KM, Shin SJ, Hwang JC, Cheong JY, . Comparison of uncovered stent with covered stent for treatment of malignant colorectal obstruction. Gastrointest Endosc 2007; 66: 931-936

3. Park S, Cheon JH, Park JJ, Comparison of efficacies between stents formalignant colorectal obstruction: a randomized, prospective study. Gastrointest Endosc 2010; 72: 304-310

\section{P0276 THE FEASIBILITY OF NEW ENDOSCOPIC GASTROINTESTINAL BYPASS STENT FOR WEIGHT REDUCTION: EXPERIMENTAL STUDY}

S.J. Choi ${ }^{1}$, C.D. Kim ${ }^{2}$, W. Kim², G. Min $^{2}$, J.M. Lee ${ }^{2}$, I.K. Yoo ${ }^{3}$, S.H. Kim ${ }^{2}$, J.M. Lee ${ }^{2}$, H.S. Choi ${ }^{2}$, E.S. Kim ${ }^{2}$, B. Keum ${ }^{2}$, Y.T. Jeen ${ }^{2}$, H.J. Chun ${ }^{2}$, H.S. Lee ${ }^{2}$ ${ }^{1}$ Gastroenterology And Hepatology, Korea University Anam Hospital, Seoul/ Korea, Republic of

${ }^{2}$ Division of Gastroenterology and Hepatology, Department of Internal Medicine, Korea University College of Medicine, Seoul/Korea, Republic of

${ }^{3}$ Department Of Internal Medicine, Institute of Digestive Disease and Nutrition, Korea University College of Medicine, Seoul/Korea, Republic of

Contact E-mail Address: kumcge@korea.ac.kr

Introduction: Endoscopic therapy has been emerged as alternative treatment to bariatric surgery for reducing weight. We developed a new endoscopic gastrointestinal (GI) bypass stent and designed a preclinical study to assess the safety in a porcine model.

Aims \& Methods: The aim of this study is to investigate the feasibility of our GI bypass device in animal. Before animal study, we performed an experimental study for durability test under simulated intestinal fluid flow. And next, we performed an animal study with 10 Yorkshire pigs. The stents were placed on pylorus with fixation by clippings or on duodenal bulb without fixation. Follow up endoscopy was done per one week after implantation. After they were sacrificed, gastric, duodenal, and jejunal tissues were harvested and examined for histologic assessment of any device or procedure-related effects.

Results: Our new GI bypass stent showed a good durability in simulated solution flow. No breakage or migration of stent occurred under continuous water flow in simulation system setting. In animal study, the mean starting weight was $30.1 \pm 1.5 \mathrm{~kg}$. Delivery of the implant took an average of $19.8 \mathrm{~min}$ (range, 11 $32 \mathrm{~min}$ ) in pylorus stent group and $11.2 \mathrm{~min}$ (range, 6-18 min) in duodenal bulb stent group. It required an average clipping time of $10.8 \mathrm{~min}$ (range, $8-14 \mathrm{~min}$ ). Followed for stent migration after implantation, the mean patency duration was $1.5 \pm 0.7$ weeks. One pig was died due to small bowel perforation and peritonitis after stenting. In histologic finding, there were moderate degree of mucosal erosions, but no definite ulceration on pylorus and duodenum.

Conclusion: New GI bypass stent has a good physicochemical properties in simulated intestinal system. In animal, all stents were successfully deployed but migration at short term follow-up were shown in this study. Proper fixation method such as endoscopic suture machine and modification of stent would be required. Disclosure of Interest: All authors have declared no conflicts of interest. 
P0277 LONG TERM EFFICACY OF AN ENDOSCOPIC DILATION PROGRAM ON POST-RADIATION AND ANASTOMOTIC FARINGOESOPHAGIC STRICTURES

D. Martins ${ }^{1}$, S. Pires ${ }^{2}$, C. Camila Dias ${ }^{3}$, P. Pimentel-Nunes ${ }^{4}$, R. Silva ${ }^{4}$, M. DinisRibeiro ${ }^{4}$

${ }^{1}$ Gastroenterology, Tondela - Viseu Hospital Center, Viseu/Portugal

${ }^{2}$ Gastroenterology, Espirito Santo Hospital, ÉvoralPortugal

${ }^{3}$ Department of Community Medicine, Information and Health Decision Sciences, Faculty of Medicine of the University of Porto, Porto/Portugal

${ }^{4}$ Dept. Of Gastroenterology, IPO Porto, Porto/Portugal

Contact E-mail Address: dianacpmartins@gmail.com

Introduction: Dysphagia may occur due to benign pharingo-esophageal strictures, often requiring repeated dilations. The aim of the study was to access long-term efficacy of pharingo-esophageal dilations due to anastomotic or post-radiation strictures.

Aims \& Methods: Retrospective study of patients suffering of dysphagia due to radiation (Group I) or anastomotic (Group II) induced pharingo-esophagea benign strictures submitted to endoscopic dilation between January 2013 and December 2015. The long-term efficacy (after a minimum follow-up of 12 months) was prospectively assessed by telephone interview by: a) dysphagia improvement or b) resolution (grade 0 or 1 of Mellow-Pinkas scale), c) absence of further dilations and d) absence of PEG. Additional therapy (PEG or prothesis placement, electroincision or surgery) was considered inefficiency criteria and these patients were excluded from the interview. Post-procedure complications were registered. Efficacy predictive factors were assessed.

Results: Forty-eight patients (296 dilations) were evaluated (median of 4 dilations/patient): $85 \%$ were male, mean age of 62 years-old, $60 \%$ belonging in Group I. The median interval between dilations was of 5 weeks. Pre-dilation dysphagia Mellow-Pinkas score and luminal calibre were $3 \pm 1$ and $7 \pm 2$, $8 \mathrm{~mm}$, respectively. Twenty-eight patients (out of 30 live patients non-submitted to additional therapies) answered to the interview: a) $96 \%$ had improved, b) $60 \%$ had resolved dysphagia, c) $75 \%$ didńt need further dilations, d) $89 \%$ without PEG, with a combined efficacy of $58,3 \%$. Nine patients required additional therapy (6 PEGs, 2 prothesis, 1 eletroincision). Overall, 17 (out 21 with previous PEG) were able to resume feeding per os. Fifteen and $29 \%$ presented Kochman criteria for refractory and recurrent strictures, respectively. There were two postprocedure complications $(<1 \%)$ : one deep laceration and one pharingo-esophageal fistula. Overall mortality was of $20 \%$ (10 patients died of non related procedure causes). Mean follow-up was 29, $2 \pm 11,2$ months. Number of dilations and initial lumen calibre were significant predictors of combined efficacy in the univariated analysis; radic strictures predicted a greater final dysphagia in the uni- and multivariate analysis; recurrent/refractory stenosis didn't significantly predict global efficacy.

Conclusion: Our dilation programme presents relevant benefit to these patients and a low rate of complications. Patients with post-radiation strictures presented a worse prognosis. Even though retrospective we present the longest follow-up and focusing not only in objective measures but also in patient perception of relief.

Disclosure of Interest: All authors have declared no conflicts of interest.

\section{References}

Chapuy CI, Annino DJ, Tishler RB, et al. Success of endoscopic pharyngoesophageal dilation after head and neck cancer treatment. Laryngoscope. 2013;123(12):3066-3073

Rodrigues-Pinto E, Pereira P, Ribeiro A, et al. Risk factors associated with refractoriness to esophageal dilatation for benign dysphagia. Eur $J$ Gastroenterol Hepatol. 2016;28(6):684-688.

Tuna Y, Koçak E, Dinçer D, et al. Factors affecting the success of endoscopic bougia dilatation of radiation-induced esophageal stricture. Dig Dis Sci. 2012;57(2):424-428.

\section{P0278 OUTCOME AFTER THE USE OF SX-ELLA DANIS BLEEDING STENTS FOR REFRACTORY VARICEAL BLEEDING - A VIENNA MULTICENTER EXPERIENCE}

N. Pfisterer ${ }^{1}$, T. Pachofszky ${ }^{1}$, W. Dolak ${ }^{2}$, M. Schöniger-Hekele ${ }^{2}$, B. Schuster ${ }^{1}$,

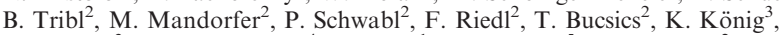
L. Kramer ${ }^{3}$, M. Gschwantler ${ }^{4}$, C. Madl $^{1}$, M. Trauner ${ }^{5}$, T. Reiberger ${ }^{2}$ ${ }^{1}$ Gastroenterology And Hepatology, Krankenanstalt Rudolfstiftung, Vienna/ Austria

${ }^{2}$ Division Of Gastroenterology \& Hepatology, Department Of Internal Medicine III, Medical University of Vienna, Vienna/Austria

${ }^{3}$ Krankenhaus Hietzing mit Neurologischem Zentrum Rosenhügel, Wien/Austria ${ }^{4} 4$ th Department Of Internal Medicine, Wilhelminenspital, Vienna/Austria ${ }^{5}$ Department Of Medicine Iii, Head of Division of Gastroenterology and Hepatology - Department of Medicine III, Head of Division o, Wien/Austria

Contact E-mail Address: nikolauspfisterer@hotmail.com Introduction: Current guidelines favour the use of bleeding stents over balloon tamponade for refractory esophageal variceal bleeding (EVB). However, data on the efficacy of and outcomes after the placement of an SX-ELLA "Danis-Stent" are limited.

Aims \& Methods: Retrospective multicenter study including cirrhotic patients receiving Danis-Stents for massive/refractory EVB at 4 tertiary care centers in Vienna (Medical University of Vienna, Krankenanstalt Rudolfstiftung, Wilhelminenspital and Krankenhaus Hietzing). Rates of bleeding control (5 days), bleeding-related mortality (6 weeks) and overall mortality were assessed.
Results: Among 35 patients, 13 patients had an unsuccessful endoscopic band ligation (EBL) prior to Danis-Stent placement. Danis-Stent controlled EVB in $80 \%(28 / 35)$ of patients. In the remaining uncontrolled bleeders $(n=7), 3$ patients had subsequent EBL, while in 3 patients the stent had to be replaced and 1 patient received a Linton-tube. Among these patients with initial DanisStent failure, 4 died of uncontrollable EVB, 2 experienced early bleeding-related mortality, and only 1 patient achieved a successful long-term bleeding control. In total, early-rebleeding within 6 weeks occurred in $14.3 \%$ (including $\mathrm{n}=1$ while Danis-Stent was still in place and $n=5$ after Danis-Stent was removed): 3 underwent EBL, 1 received a subsequent Danis-Stent, and 1 patient was treated with a Sengstaken tube. Moreover, among $n=14$ patients without early rebleeding within 6 weeks, only $\mathrm{n}=3(21.4 \%)$ showed rebleeding later during follow-up: $\mathrm{n}=2$ patients were treated with a Sengstaken-Tube (both experienced bleedingrelated death) and $n=1$ had another Danis-Stent placed (successful bleeding control). Only $\mathrm{n}=11(31.4 \%)$ patients did not experience any rebleeding after Danis-Stent removal, while $\mathrm{n}=8$ patients died with the Danis-Stent in situ. Notably, no "early-TIPS" was performed in this study, but $4(11.4 \%)$ received an elective TIPS during follow-up. $\mathrm{N}=6$ patients $(17.1 \%)$ died due to uncontrolled bleeding ( $\leq 5$ days) and $n=10$ died within 6 weeks (bleeding-related mortality: $28.6 \%)$. Overall, $\mathrm{n}=22 / 35(62.9 \%)$ patients died. The median survival was 10.5 (IQR82) days after Danis-Stent placement. Median Danis-Stents dwell time was 5 (range: $0-13$ ) days. The most common adverse events were stent dislocations $(\mathrm{n}=13 ; 37.1 \%)$, while ulcers/necrosis of the esophageal mucosa were seen in only $4(11.4 \%)$ patients.

Conclusion: Danis-Stent controlled refractory/massive EVB in $80 \%$ of patients but bleeding-related mortality was as high as $45 \%$. While stent dislocations are frequent, ulcers/necrosis of the esophagus were rare with a dwell time of 5 days. The implementation of an early-TIPS strategy might improve the overall outcome after Danis-Stent placement.

Disclosure of Interest: All authors have declared no conflicts of interest.

\section{References}

Escorsell A, Pavel O, Cardenas A, Morillas R, Llop E, Villanueva C, et al. Esophageal balloon tamponade versus esophageal stent in controlling acute refractory variceal bleeding: A multicenter randomized, controlled trial. Hepatology 2016, 63:1957-1967.

de Franchis R, Baveno VIF. Expanding consensus in portal hypertension: Report of the Baveno VI Consensus Workshop: Stratifying risk and individualizing care for portal hypertension. J Hepatol 2015, 63:743-752.

Escorsell A, Bosch J. Self-expandable metal stents in the treatment of acute esophageal variceal bleeding. Gastroenterol Res Pract 2011, 2011:910986. Wright G, Lewis H, Hogan B, Burroughs A, Patch D, O’Beirne J. A self-expanding metal stent for complicated variceal hemorrhage: experience at a single center. Gastrointest Endosc 2010, 71:71-78.

D'Amico M, Berzigotti A, Garcia-Pagan JC. Refractory acute variceal bleeding: what to do next? Clin Liver Dis 2010, 14:297-305.

Zehetner J, Shamiyeh A, Wayand W, Hubmann R. Results of a new method to stop acute bleeding from esophageal varices: implantation of a self-expanding stent. Surg Endosc 2008, 22:2149-2152.

Wright G, Lewis H, Hogan B, Burroughs A, Patch D, O'Beirne J. A self-expanding metal stent for complicated variceal hemorrhage: experience at a single center. Gastrointest Endosc 2010, 71:71-78.

\section{P0279 A NOVEL METHOD WITH SELF-EXPANDABLE METALLIC STENT FASTENED WITH CLIP AND LOOP FOR THE TREATMENT OF ANASTOMOTIC STRICTURE AFTER SUBTOTAL GASTRECTOMY}

J.K. Kang, S.G. Lim, K.M. Lee, S.J. Shin, B.M. Yoo, J. Kim, C. Noh Gastroenterology, Ajou university school of medicine, Suwon/Korea, Republic of

Contact E-mail Address: pterion1@naver.com

Introduction: Benign anastomotic strictures are common adverse events of gastrointestinal tract surgery. And, they are difficult to be managed conservatively. The first choices of treatment of anastomotic stricures are balloon dilatation and bougination. But, they are requiring repeated sessions. Self-expandable metalic stent (SEMS) placement has continous expanding effect for a long period. But, It has problem of frequent stent migration, because of slow stent expanding, 2-3 days. Therefore the new mehod to inhibit stent migration is needed for more successful management of anastomotic stenosis.

Aims \& Methods: The aim of this study was to evaluate the clinical feasibility of new method to inhibit stent migration in postoperative anastomotic stricture. From May 2013 until February 2015, patients with benign anastomotic stricture after subtotal gastrectomy were enrolled at a single tertiary referral hospital, prospectively. The Niti-S ComVi pyloric stents (Taewoong Medical, Korea), double-layered covered, were inserted. We made two nylon thread loops at the proximal bared section of the stents. After stent placement, stent fastening with loop and clip method was performed. Patients' symptoms and oral intake were assessed once or twice a week with a clinical check-up or telephone interview. After two weeks, the loop and stent removals were done.

Results: In all patients, stents were successfully inserted and patency were confirmed (technical success rate, $100 \%$ ). The obstructive symptoms were subsided in 12 of 13 patients (clinical success rate, $92.3 \%$ ). Stent migration was found in a follow-up endoscopy on 14 days ( 1 of 13, 7.7\%). Anastomotic restenosis occurred in two patients 14 and 20 days after stent removal. However, obstructive symptoms were relieved by stent reinsertion.

Conclusion: The new method with fastening the stent with loop and clip can reduce the risk of stent migration. 
Our fastening method can be feasible and useful technique for postoperative anastomotic stricture after subtotal gastrectomy.

A large-scale prospective research is needed to validate the clinical effectiveness of our method and to determine the appropriateness.

Disclosure of Interest: All authors have declared no conflicts of interest.

\section{P0280 EFFICACY AND TOLERABILITY OF BIODEGRADABLE STENTS FOR RECURRENT BENIGN OESOPHAGEAL STRICTURES: THE LEEDS EXPERIENCE}

N. Rabb, H. Procter, N. Burr, V. Appleby, S. Everett

Leeds Centre For Digestive Diseases, Leeds Teaching Hospitals Trust, Leeds/

United Kingdom

\section{Contact E-mail Address: n.rabb@nhs.net}

Introduction: The optimum management of refractory or recurrent oesophageal strictures (RBES) despite repeated attempts at dilatation is controversial ${ }^{1}$. An accepted method includes temporary stent placement. Most commonly temporary fully covered self-expanding metal stents (FC-SEMS) are used but these require removal some $8-12$ weeks later. Oesophageal biodegradable stents (EBS) have the advantage that they do not require removal. However, there is a lack of good-quality evidence in support of their use $\mathrm{e}^{2,3}$. We aimed to review the safety and efficacy of a large series of single and multiple EBS insertions for benign RBES in a single tertiary referral centre.

Aims \& Methods: A retrospective review of insertion of EBS (SX-ELLA) between April 2008 and October 2016 was conducted. Patients with one or more EBS insertion for benign RBES were included and clinical data extracted from the hospital database. 30 day safety and efficacy for all stent insertions and 12 month efficacy and median time to further intervention (MTFI) for first stents were analysed using the Stata package.

Results: 20 patients ( $13 \mathrm{~m}, 7 \mathrm{f}$; age 44-93; Charlson index range 2-8, median 4) with 37 stents were included. Stricture aetiologies included peptic $(55 \%)$, radiotherapy $(20 \%)$, post-surgical $(20 \%)$ and post EMR $(5 \%)$. Dysphagia score ranged from 2-4(median 3 ). The total median number of dilatations prior to first EBS was 6 (range $0-17) .15(75 \%$ ) patients had previously had a FC-SEMS placed with subsequent recurrence of symptoms. 30-day technical success and symptom improvement was $100 \%$. 30 day adverse events included $4(11 \%)$ stent migrations and $12(32 \%)$ with significant pain, 3 patients requiring in-patient pain control ( $<3$ days). There were no significant bleeds or perforations. 12 months following first EBS insertion $18(90 \%)$ required further endoscopic intervention due to recurrent symptoms; $5(25 \%)$ had further dilatation, $9(45 \%)$ had either a FC-SEMS (2 patients) or a further EBS (7 patients), $3(15 \%)$ had $\mathrm{NG}$ tube insertion and $1(5 \%)$ patient died of unrelated illness. $2(10 \%)$ were symptom free after one stent at $12 \mathrm{~m}$. MTFI was 139 days (range 75-517) and was not dependent on aetiology (peptic vs. nonpeptic; 135 vs. 127 days, $\mathrm{p}=0.54$ ). There was a significant reduction in median number of interventions in the $12 \mathrm{~m}$ following EBS insertion compared to the preceding $12 \mathrm{~m}$ ( 2 vs. 7 respectively, $\mathrm{p}=0.0003$ ). $7(35 \%)$ patient received multiple EBS (range of stent insertions $2-9$ ). 3 of these $(43 \%$ ) have subsequently required no further intervention, $3(43 \%)$ continue to receive intermittent EBS, inserted on a symptomatic basis, 1(14\%) patient continues to receive treatment for RBES, but has declined further EBS after 2 EBS resulted in discomfort.

Conclusion: Use of EBS for RBES in our centre was safe and well tolerated but there was short-lived pain in a significant number. In this highly selected cohort of difficult strictures, EBS led to short-term benefit in all patients but sustained benefit in only $10 \%$. However, there was a significant reduction in intervention frequency $12 \mathrm{~m}$ after stent insertion and repeated EBS insertion appears a reasonable strategy for the most resistant strictures. The role of EBS earlier in the treatment pathway for RBES needs exploring in future studies.

Disclosure of Interest: All authors have declared no conflicts of interest.

\section{References}

1. Repici A, Kochman ML. Natural history and management of refractory benign esophageal strictures. Gastrointest Endosc. 2016;84(2):222-228

2. Fuccio L, Repici A et al. Clinical oucomes following stent placement in refractory benign esophageal stricture: a systematic review and meta-analysis. Endoscopy 2016;48(2):141-148

3. Spaander M, Bruno $\mathbf{M}$ et al. Esophageal stenting for benign and malignant disease: European Society of Gastrointestinal Endosocpy (ESGE) Clinical Guideline. Published online 2016

\section{P0281 OUTCOMES OF PATIENTS UNDERGOING PLACEMENT OF FULLY COVERED SELF EXPANDING METAL STENTS FOR MALIGNANT OESOPHAGEAL STRICTURES}

M. A. Everson, C. Magee, P. Galloway, A. Nelson, R. Sweis, M. Banks, S. Bown, D. Graham, L. Lovat, R. Haidry

Department Of Gastroenterology, University College London Hospital, London/ United Kingdom

Contact E-mail Address: martin.everson@nhs.net

Introduction: Fully covered self-expanding metallic stents (SEMS) have an established role in the palliation of oesophageal strictures, particularly in those with malignancy. SEMS are advantageous as they reduce the rate of tumour ingrowth, in doing so reduce the need for repeated endoscopic interventions in a cohort of patients with severe comorbidity. A drawback to SEMS include their ability to migrate distally, with some studies estimating the rate at around $20 \%{ }^{(1-3)}$. We report a single-centre, retrospective cohort study on the use if SEMS, and aimed to establish risk factors for stent migration.

Aims \& Methods: Case note review was undertaken retrospectively on all patients who had fully covered SEMS inserted at a high-volume tertiary oesophageal cancer centre between Jul 13 to Feb 17. All SEMS were placed under fluoroscopic guidance by experienced endoscopists. Stent migration was confirmed endoscopically or radiologically and was defined as displacement of the stent into the stomach or distal to the baseline stenosis with subsequent loss of the recannalised lumen. Shapiro-Wilks testing showed non-normal distribution of data. Non-parametric testing by logistic regression was therefore performed

Results: 188 stents were inserted to palliate malignant strictures, $75 \%$ in males. We observed a migration rate of $20 \%$ (39) in our cohort. We observed a significant association between predilation (within 7 days of the stent procedure) and subsequent stent migration (31\% in dilated vs $13 \%$ in undilated [OR 2.89 CI: $1.33-6.30,(\mathrm{p}=0.005)$ ]. This also met Bonferroni-corrected significance. We demonstrate a trend towards shorter strictures being associated with an increased risk of migration [OR 1.14 CI:1.122-1.164 $(\mathrm{p}=0.052)]$. There were no significant associations between migration and whether patients received previous chemo-radiotherapy, or whether the stent crossed the GOJ.

Conclusion: Endoscopic placement of SEMS is a safe and effective procedure in the management of malignant dysphagia with a low risk of complications. Predilation of lesions within 7 days of SEMS insertion carries a risk of subsequent stent slippage - suggesting that a cautious approach to dilation may be prudent. Further, larger studies may demonstrate an association with shorter strictures and migration. The migration rate at our centre is consistent with those found in other studies.

Disclosure of Interest: All authors have declared no conflicts of interest.

\section{References}

1. Vleggaar FP, Siersema PD. Expandable stents for malignant esophageal disease. Gastrointest Endosc Clin N Am. 2011;21:377-88, vii.

2. Schoppmann SF, Langer FB, Prager G, Zacherl J. Outcome and complications of long-term self-expanding esophageal stenting. Dis Esophagus. 2013;26:154-158.

3. Song HY, Lee DH, Seo TS, Kim SB, Jung HY, Kim JH, Park SI Retrievable covered nitinol stents: experiences in 108 patients with malignant esophageal strictures. J Vasc Interv Radiol. 2002;13:285-293.

MONDAY, OCTOBER 30, 2017

09:00-17:00

SURGERY I - HALL 7

\section{P0282 COMBINED RESECTION IN ESOPHAGEAL CANCER: MYTH OR REALITY}

D. Rusanov ${ }^{1}$, K. Pavelets ${ }^{1}$, M. Antipova ${ }^{1}$, M. Protchenkov ${ }^{1}$, M. Pavelets ${ }^{1}$, U. Drozd ${ }^{1}$, A. Sokolova ${ }^{1}$, G. Florovsky

${ }^{1}$ Faculty Surgery Named After Prof. A.a. Rusanov, Saint-Petersburg State

Pediatric Medical University, Saint-Petersburg/Russian Federation

\section{Contact E-mail Address: Rusanov.vergeltung@yandex.ru}

Introduction: Esophagus cancer is one of the 10 most common malignant diseases of the digestive tract and ranks 7 th in the structure of mortality. The indicators of 5 -year survival, at the present stage, rarely overcome the threshold of $15-20 \%$ in the I-III stage of the disease. Most patients are considered inoperable by the time of admission to hospital due to the spread of the tumor process. Local-regional spread of the tumor is the reason for refusing to perform radical surgical intervention. Taking into account the data for the last 10 years, only $1.6 \%$ of patients had different types of combined and surgical treatment. According to various authors, chemoradiotherapy is offered as an alternative to surgical intervention in locally advanced forms of esophageal cancer. In addition, there is no consensus between "western" and "eastern" authors about the issues of lymphodissection in esophageal cancer. Thus, for the optimal primary staging of esophageal carcinoma and planning of surgical treatment, the surgeon needs a preoperative, spatial picture of the prevalence of the tumor process in patients suffering from esophageal cancer.

Aims \& Methods: To analyze the probation of tumor using computer 3D-model of mediastinum and long-term results of treatment. Describes the experience of treatment of 190 patients with esophageal cancer from 2010 to 2015. Of them to $52(27.3 \%)$ performed CT, to $37(19.5 \%)$ EUS. Besides, CT to $101(53.1 \%)$ and MRI $7(0.1 \%)$ with subsequent 3D-modeling. I group- $123(64.7 \%)$ patients, performed surgical treatment based on resection of thoracic part of esophagus, fundamental part of stomach and two-field lymphodissection. To $15(7.9 \%)$ performed combined operations. The data obtained were compared with intraoperative findings and pathological examination. II group- 67 patients $(35.3 \%)$, used palliative treatments: $31(16.3 \%)$ - esophageal stenting, $26(13.7 \%)$-argontumor recanalization, $10(5.3 \%)$ - argon-tumor recanalization with esophageal stenting.

Results: On data of all 59 (100\%) 3D-models, were estimated, localization and length of esophageal tumor, it's relationship with the structures of the mediastinum, severity of intraabdominal and intrathoracic lymphadenopathy. In most cases, according to the data 3D- model, as well as intraoperative, met defeat of middle and lower esophagus. Severity of involvement of mediastinal structures rated at 48 patients $(81.4 \%)$. At $35(59.3 \%)$ and $37(62.7 \%)$ cases amared fiber mediastinum, at $5(8.5 \%)$ patients - invasion to the main bronchi, At $13(22.0 \%)$ and $11(18.6 \%)$ combination of anatomical structures of mediastinum defeat. 
Severity of intrathoracic and intraabdominal lymphadenopathy: mediastinal lymphadenopathy marked at $19(32.2 \%)$ and $20(33.9 \%)$ patients, combined of $38(64.4 \%)$ and $35(59.3 \%)$. At pre-operated staging mostly met advanced form of cancer: T4N1 at $16(27.1 \%)$, T4N2 at $23(38.9 \%)$. Sensitivity in staging of tumor $89.8 \%$. Long-term results: 1-year survival at I group $96.1 \%$, 3-year is $42.3 \%$, 5-year 19.6\%; at II group 1-year survival $6.45 \%$.

Conclusion: The use of 3D-modeling performed using MRI, spiral CT and EUS, allows to planning the optimal surgery and lymph node for locally common form of esophageal cancer, and improve the results of survival.

The scope of surgical intervention is advisable to plan taking into account the constructed 3D-models, which helps to solve the problem of the possibility of surgical intervention in esophageal cancer.

Disclosure of Interest: All authors have declared no conflicts of interest.

\section{P0283 INFLUENCE OF CONTINUOUS ADMINISTRATION OF LOW-DOSE ASPIRIN FOR INTRAOPERATIVE BLEEDING ON GASTRIC ENDOSCOPIC GASTRIC DISSECTION: A PROPENSITY SCORE MATCHING ANALYSIS}

\section{Y. Horikawa, N. Mimori, H. Mizutamari, Y. Kato, S. Fushimi, S. Okubo,}

S. Sato

Gastroenterology, Hiraka General Hospital Dept. of Gastroenterology, Yokote/ Japan

\section{Contact E-mail Address: horikawa 01@me.com}

Introduction: Endoscopic submucosal dissection (ESD) was a promising method for the resection of superficial gastric neoplasms. The patient with antithrombotic agents has increased for first or secondary prevention of cardiovascular or cerebral disease. Continuous administration of low-dose aspirin (LDA) during ESD was recommended in American, British and Japanese guidelines. However, the influence of this drug for the hemostasis condition during ESD procedure is still unclear. Therefore, we performed this study for addressing intraoperative bleeding risk without cessation of LDA.

Aims \& Methods: In this retrospective study, we assessed the hemostasis condition during ESD that were treated for superficial gastric lesions between January 2014 and March 2017. Patients with antithrombotic therapy by LDA $(n=42)$ and those with no antithrombotic therapy $(n=187$; Control) were compared using propensity score matching. Primary outcome was frequency of intraoperative major bleeding. Secondary outcomes included procedure time, $\mathrm{Hb}$ reduction rate, En bloc resection rate, and adverse events rate.

Results: The propensity score analysis yielded 39 matched pairs. Adjusted comparison between the two groups showed similar with regards to major bleeding, median [range] (times): 1.0 [0.0-4.0] vs. $1.0[0.0-4.0], p=0.621$. Procedure time was prolonged in Aspirin group by $16.7 \%$ without significant differences. Other aspects were the same in both groups with low incidence of adverse events; perforation $(0 \%)$, thromboembolism $(0 \%)$

Conclusion: This study indicated the feasibility of gastric ESD with continuous administration of LDA including little intraoperative bleeding and adverse events.

Disclosure of Interest: All authors have declared no conflicts of interest.

\section{P0284 WEEKDAY OF CANCER SURGERY IN RELATION TO PROGNOSIS}

P. Lagergren, F. Mattsson, J. Lagergren

Department Of Molecular Medicine And Surgery, Karolinska Institutet,

Stockholm/Sweden

\section{Contact E-mail Address: pernilla.lagergren@ki.se}

Introduction: Later weekday of surgery seems to reduce the prognosis in oesophageal cancer, while any such influence on other cancer sites is unknown. This study aimed to test whether weekday of surgery influences prognosis following commonly performed cancer operations.

Aims \& Methods: This nationwide Swedish population-based cohort study from 1997-2014 analysed weekday of elective surgery for 10 major cancer groups in relation to disease-specific and all-cause mortality. Cox regression provided hazard ratios with $95 \%$ confidence intervals (CI) adjusted for the covariates age, sex, comorbidity, hospital volume, calendar year, and tumour stage.

Results: Included were 228,927 patients. Later weekday of surgery (Thursdays and even more so Fridays) was associated with increased mortality rates for gastrointestinal cancers. The adjusted hazard ratios for disease-specific mortality comparing surgery on Friday with Monday were 1.57 (95\% CI 1.31-1.88) for oesophago-gastric cancer, 1.49 (95\% CI 1.17-1.88) for liver-pancreatic-biliary cancer, and $1.53(95 \%$ CI $1.44-1.63)$ for colorectal cancer. Excluding mortality during the initial 90 days of surgery made little change to these findings, and the all-cause mortality was similar to the disease-specific mortality. The associations were similar in analyses stratified for covariates. No consistent associations were found between weekday of surgery and prognosis for cancer of the head-andneck, lung, thyroid, breast, kidney-bladder, prostate, or ovary-uterus.

Conclusion: Later weekday of surgery (Thursday-Friday) seems to negatively influence the prognosis for cancer of the gastrointestinal tract, indicating a need for re-scheduling of these operations.

Disclosure of Interest: All authors have declared no conflicts of interest.
P0285 IMPACT OF POSTOPERATIVE COMPLICATIONS AND PERIOPERATIVE ONCOLOGICAL TREATMENTS FOR GASTRIC CANCER PATIENTS AFTER GASTRECTOMY

S. Kamiya ${ }^{1}$, F. Klevebro ${ }^{2}$, I. Rouvelas ${ }^{2}$, M. Lindblad ${ }^{2}$, L. Lundell ${ }^{2}$, M. Nilsson ${ }^{2}$ ${ }^{1}$ Surgical Gastroenterology, Karolinska University Hospital, Stockholm/Sweden ${ }^{2}$ Karolinska University Hospital, Stockholm/Sweden

\section{Contact E-mail Address: satoxi_k@hotmail.com}

Introduction: Recently, multidisciplinary treatments such as perioperative chemo radio treatments have been introduced to improve their prognosis in gastric cancer surgery. Besides that, the postoperative severe complications are thought to be the poor prognostic factor. Present study assessed the prognostic impacts of severe postoperative complications and perioperative oncological treatments in gastric cancer patients.

Aims \& Methods: Consequent gastric cancer patients who underwent curative gastrectomy in Karolinska University Hospital between 2006 and 2016 were enrolled. Patients' characteristics, surgical data, postoperative courses and prognosis were examined retrospectively. Complications were evaluated according to Clavien-Dindo classification. The significance of postoperative severe complications and perioperative oncological treatment for overall survival (OS) was evaluated by the Cox proportional hazard model.

Results: Hundred-sixty-nine patients were examined in this study. $89(52.7 \%)$ and $66(39.1 \%)$ patients had neoadjuvant and adjuvant treatment, $85(50.3 \%)$ and 84 $(49.7 \%)$ underwent distal and total gastrectomy, respectively. $24(14.2 \%), 16$ $(9.5 \%)$ and $5(3.0 \%)$ were diagnosed as grade III, IV, V complications. The prognosis of the patients with grade III or higher complication was significantly worse (3-year OS: $66.6 \%$ vs $47.3 \%, \mathrm{P}=0.001$ ). Subgroup analysis by pathological stage showed that the prognosis of pStage III/IV patients with postoperative grade III or higher complication was significantly poorer than the patients without grade III or higher complications (3-year OS: $45.3 \%$ vs $7.5 \%, \mathrm{P}<0.001$ ). For the patients who had either neoadjuvant or adjuvant treatment, however, no obvious prognostic worsening were seen by the existence of complications (3-year OS: $66.5 \%$ vs $52.9 \%, P=0.13$ ). Multivariate analysis identified that severe complication was independent risk factor for OS (hazard ratio $1.82 ; 95 \%$ confidence interval 1.08-3.05), especially in pStage III/IV gastric cancer (hazard ratio 3.00; $95 \%$ confidence interval $1.53-5.86$ )

Conclusion: Postoperative severe complications had considerable impact on the OS, especially for pStage III/IV gastric cancer patients. Perioperative oncological treatment may be able to prevent the prognosis from deteriorating due to postoperative complications.

Disclosure of Interest: All authors have declared no conflicts of interest.

\section{P0286 ENDOSCOPIC PAPILLECTOMY OF DUODENAL PAPILLARY TUMOR: A REPORT OF 75 CASES}

Z. Wang ${ }^{1}$, E. Linghu ${ }^{2}$, F. Cai ${ }^{2}$, Y. Yang ${ }^{2}$, G. Sun ${ }^{1}$, M. Li ${ }^{1}$, S. $\mathrm{Li}^{1}$, X. Wang ${ }^{2}$, J. Meng ${ }^{1}$, H. Du ${ }^{2}$, J. Zhu ${ }^{1}$, W. Li ${ }^{1}$

${ }^{1}$ Gastroenterology And Hepatology, The General Hospital of the Chinese People's Liberation Army, Beijing/China

${ }^{2}$ The General Hospital of the Chinese People's Liberation Army, Beijing/China

Contact E-mail Address: wangzikai301@126.com

Introduction: Duodenal papillary tumor as rare gastrointestinal neoplasm is essential for curative therapy due to its malignant potential. Endoscopic papillectomy to the treatment of duodenal papillary tumor has developed and is accepted as an alternative approach to surgery in select cases. Endoscopic papillectomy as a relatively difficult endoscopic technique mainly performed by experienced endoscopists. Moreover, the indications and standard endoscopic procedures for endoscopic papillectomy have not been established.

Aims \& Methods: We aimed to investigate the clinical value of endoscopic papillectomy for duodenal papillary tumor based on the endoscopic and clinical characteristics. Between 2006 and 2017, seventy-five patients with duodenal papillary tumor under endoscopic papillectomy in the gastrointestinal endoscopic center of Chinese PLA General Hospital were included. These patients were diagnosed of duodenal papillary tumor by the clinical manifestation, laboratory tests, CT, MRCP, endoscope, EUS, ERCP along with biopsies and histopathologic tests. During the detailed clinical assessment combined with patients' wishes, endoscopic papillectomy and followed ERCP procedures were performed successfully, and the clinical data of these patients were retrospectively summarized.

Results: 75 patients (50 males and 25 females) with a median age of 58.6 yrs (range 27 to $82 \mathrm{yrs}$ ) were evaluated. The main clinical symptoms were predominated by abdominal pain followed by cholestasis and cholangitis, but nine cases with no abdominal symptoms. Endoscopic papillectomy was technically feasible in all these patients, and was mainly performed by four experienced endoscopists. The majority of excised tumors were exogenous $(90.7 \%, 68 / 75)$, and the tumor size ranged between 8 and $55 \mathrm{~mm}$. The final histopathological diagnosis included adenoma $(37.3 \%, 28 / 75)$, adenoma with high-grade intraepithelial neoplasia $(18.7 \%, 14 / 75)$, adenoma with low-grade intraepithelial neoplasia $(26.7 \%, 20$ / $75)$, adenoma combined with local carcinoma $(16 \%, 12 / 75)$, and neuroendocrine tumor $(1.3 \%, 1 / 75)$. En bloc resection was achieved in 53 cases $(70.7 \%)$ and the piecemeal resection was performed in 22 cases $(29.3 \%)$. After endoscopic papillectomy, the ERCP procedures were performed in 70 cases $(93.3 \%)$. The prophylactic pancreatic duct stent was placed in 30 cases $(40 \%)$ for preventing pancreatitis, the biliary plastic stent or nasobiliary drainage tube in $16 \%(12 /$ $75)$, the combined of both in $17.3 \%(13 / 75)$, and no stent placement in $26.7 \%$ $(20 / 75)$. Moreover, intraoperative hemostasis was performed in 47 cases $(62.7 \%)$, including pure endoscopic clip placement, followed by injection therapy, thermal therapy or in combination. Regarding to the postoperative adverse events, 
hemorrhage was identified in 11 patients $(14.6 \%)$ but mainly cured by endoscopic hemostasis, followed by pancreatitis $(9.3 \%, 7 / 75)$ but cured with medical treatment.

Conclusion: Endoscopic papillectomy can be considered as a feasible and reasonable treatment option for suitable patients with tumors of duodenal papilla. Disclosure of Interest: All authors have declared no conflicts of interest.

\section{P0287 PROPHYLACTIC COLECTOMY WITH EXTENDED INDICATION OF RECTAL PRESERVATION IN RELATED APC FAMILIAL ADENOMATOUS POLYPOSIS: SYSTEMATIC ADENOMA TREATMENT DRAMATICALLY CHANGES THE NATURAL HISTORY OF POLYPOSIS}

A. Pasquer ${ }^{1}$, G. Poncet ${ }^{1}$, M. Pioche ${ }^{2}$, J. Rivory ${ }^{2}$, O. Vinet ${ }^{2}$, N. Benech ${ }^{2}$, J.C. Saurin ${ }^{2}$

${ }^{1}$ Digestive Surgery, Hopital Edouard Herriot, Lyon/France

${ }^{2}$ Hepatogastroenterology, Hopital Edouard Herriot, Lyon/France

Contact E-mail Address: arnaud.pasquer@chu-lyon.fr

Introduction: Prophylactic surgery of familial adenomatous polyposis (FAP) ranges from total colectomy with ileorectal anastomosis (IRA) to proctocolectomy with ileoanal anastomosis and J pouch (IAA). Rectal preservation is based on studies that did not include systematic endoscopic treatment that we perform. The objective was to compare IRA to IAA in terms of oncological safety and quality-of-life.

Aims \& Methods: Between January 1965 and November 2015, all consecutive patients who underwent prophylactic surgery for FAP with aggressive endoscopic follow up in our unit: systematic endoscopic treatment of adenomas (argon, mucosectomie), were prospectively included. MYH-related polyposes and patients who underwent abominoperineal resection were excluded from analysis.

Results: 296 patients were included: 92 proctocolectomy with IAA (31.1\%), 197 total colectomies with IRA $(66.5 \%)$, and 7 abdominoperineal resections $(2.4 \%)$. Mean (SD) number of preoperative rectal adenomas was 24.7 (33.9) in the IRA group vs. $52.8(27.8)$ in the IAA group $(\mathrm{p}=0.0001)$. Rectal cancer prevalence was $3.1 \%(\mathrm{n}=9)$. Mean (SD) follow-up was $16.6(11.9)$ years, during which the mean (SD) number of lower endoscopies was 3.4 (2.5) in the IRA group vs. 3.4 (2.5) in the IAA group $(\mathrm{p}=0.91)$; mean $(\mathrm{SD})$ number of treated adenomas was 17.8 $(20.8)$ and $12.9(18.8)$, respectively $(\mathrm{p}=0.06)$; secondary cancer incidence was $6.1 \%$ vs. $1.1 \%(\mathrm{p}=0.06)$. The 15 -year recurrence-free and overall survival (IR vs. IAA) were respectively $99.5 \%$ vs. $100 \%(\mathrm{p}=0.09)$ and $98.9 \%$ vs. $98.8 \%$ $(\mathrm{p}=0.82)$.

Conclusion: Combination of aggressive endoscopic treatment and extended rectal preservation appears to be a safe alternative to ileoanal anastomosis and $\mathbf{J}$ pouch. Disclosure of Interest: All authors have declared no conflicts of interest.

P0288 ANAL PROBLEMS DURING PREGNANCY AND POSTPARTUM: A PROSPECTIVE COHORT STUDY

K. Ferdinande, Y. Dorreman, K. Roelens, W. Ceelen, D. De Looze University of Ghent, Ghent/Belgium

Contact E-mail Address: kymentie.ferdinande@ugent.be

Introduction: Many pregnant women have anal symptoms during pregnancy and postpartum. The most common proctological problems reported are haemorrhoids, anal fissures and anal incontinence. Literature about this problem is scarce.

Aims \& Methods: The aim of this study is to determine the prevalence of anal problems and constipation during the second and third trimester of pregnancy, in the immediate postpartum and up to three months after childbirth. We also want to identify the risk factors for the development of anal symptoms. This is a prospective cohort study. Women between their 19th and 25th week of pregnancy are included. High-risk pregnancy and non-Dutch speaking are exclusion criteria. Ninety-four women were followed with a symptom questionnaire in the second and third trimester, in the immediate postpartum (within 3 days) and three months postpartum. Descriptive data were obtained from the patient files. A specific proctological diagnosis was presumed on the basis of combined symptoms (rectal bleeding, anal pain and swelling). Constipation was defined by the Rome III criteria. Statistical analysis was performed with SPSS and risk factors were identified using multivariate analysis with binary logistic regression.

Results: Sixty-eight percent of the women developed anal symptoms during the whole study period. Anal symptoms occurred in $50 \%$ of the women during pregnancy, in $56.2 \%$ in the immediate postpartum and in $62.9 \%$ during the three months postpartum. The most prevalent symptom was anal pain. Constipation was reported by $60.7 \%$ during the whole study period. Most prevalent diagnoses were: hemorrhoidal thrombosis (immediate postpartum), hemorrhoidal prolapse (3rd trimester and immediate postpartum) and anal fissure (not episode-related). Anal incontinence was only reported in $2 \%$ during the postpartum. Multivariate analysis identified constipation and a history of anal problems as significant risk factors for the development of anal complaints prepartum and postpartum.

Conclusion: Two-thirds of pregnant women deal with anal symptoms during pregnancy or postpartum, especially hemorrhoidal complications and anal fissure. This high prevalence emphasises the clinical importance of this problem. The most important risk factor is constipation. Therefore, prevention of constipation in pregnant women is recommended.

Disclosure of Interest: All authors have declared no conflicts of interest.
P0289 SURGICAL TREATMENT OF DIVERTICULITIS AND ITS COMPLICATIONS: A SYSTEMATIC REVIEW AND METAANALYSIS OF RANDOMIZED CONTROL TRIALS

A.M. Ahmed ${ }^{1}$, A.T. Moahammed ${ }^{1}$, O.M. Mattar ${ }^{2}$, E.M. Mohamed ${ }^{2}$,

E.A. Faraag ${ }^{2}$, A.M. Alsafadi ${ }^{2}$, N.T. Huy ${ }^{3}$, K. Hirayama ${ }^{4}$

${ }^{1}$ Faculty of Medicine, Al-Azhar University, Cairo/Egypt

${ }^{2}$ Kasr Alainy Faculty of Medicine, Cairo/Egypt

${ }^{3}$ Department Of Clinical Product Development, Institute of Tropical Medicine

(NEKKEN), Leading Graduate School Program, and Graduate School of

Biomedical Sciences, Nagasaki University, Nagasaki/Japan

${ }^{4}$ Institute of Tropical Medicine (NEKKEN), Leading Graduate School Program, and Graduate School of Biomedical Sciences, Nagasaki University, Ngasaki/Japan

Contact E-mail Address: med.b.dean@azhar.edu.eg

Introduction: Diverticulitis is a common gastrointestinal disease in developed countries, especially among elders. It is classified into five stages according to the severity of the inflammation with stage 5 involving peritonitis as a consequence of perforation and organ dysfunction with 30-day-mortality reaching $32 \%$. This indicates that acute diverticulitis is an emergency case requiring rapid management. However, the surgical interventions of diverticulitis vary according to its grade and severity, there is a controversy about the preferable and suitable of these different surgical procedures for each grade or complication.

Aims \& Methods: We aimed to systematically review and meta-analyze randomized controlled trials (RCTs) comparing outcomes and complications between different surgical approaches for acute diverticulitis and its complications. Nine electronic databases, including PubMed, Scopus, Google Scholar, ISI Web of science, WHO Global health library (GHL), POPLINE, Virtual health library (VHL), NYAM (New York Academy of Medicine), and SIGLE (System for information on grey literature in Europe), were searched for RCTs comparing different surgical procedures for different grades of diverticulitis. Out of 1738 articles, we included 14 studies with 1076 patients. the primarily assessed outcomes were post-surgical mortality rate besides short- and long-term post-surgical major and minor complications. The risk of bias was assessed using the Cochrane Collaboration tool. The pooled risk ratio (RR) and $95 \%$ confidence interval (CI) were calculated in the meta-analysis using the RevMan platform. The protocol was registered in PROSPERO (CRD42015032290).

Results: Outcome data were analyzed from five RCTs comparing laparoscopic sigmoid resection $(\mathrm{LSR})(\mathrm{n}=247)$ versus open sigmoid resection $(\mathrm{OSR})(\mathrm{n}=237)$ for treatment of acute complicated diverticulitis with minimal heterogeneity. For short-term outcomes, there was no significant difference in postoperative overall morbidity $(\mathrm{RR}=0.89,95 \%$ CI $[0.61-1.31] ; \mathrm{P}=0.56)$, all major postoperative morbidity $(\mathrm{RR}=0.79,95 \% \mathrm{CI}[0.12-5.07] ; \mathrm{P}=0.80)$, and all minor postoperative complications $(\mathrm{RR}=0.98 ; 95 \% \mathrm{CI}[0.62-1.57] ; \mathrm{P}=0.94)$. Similarly, there was no difference between the two procedures regarding the long-term postoperative overall morbidity $(\mathrm{RR}=0.83,95 \% \mathrm{CI}[0.57-1.21] ; \mathrm{P}=0.34)$, all major morbidity $(\mathrm{RR}=0.78,95 \% \mathrm{CI}[0.46-1.31], \mathrm{P}=0.34)$, and mortality $(\mathrm{RR}=0.95,95 \% \mathrm{CI}$ [0.04-24.59]; $\mathrm{P}=0.98)$. In other four RCTs compared laparoscopic lavage with resection (sigmoidectomy) for treatment of perforated diverticulitis with peritonitis, the postoperative mortality rate was non-significant in both short-term $(\mathrm{RR}=1.55,95 \% \mathrm{CI}[0.79-3.04] ; \mathrm{P}=0.21)$ and long-term $(\mathrm{RR}=0.67,95 \% \mathrm{CI}$ $[0.29-1.58] ; \mathrm{P}=0.36)$ follow up. Interestingly, the short-term reoperation rate and long-term precense of intra-abdominal abscesses were significantly higher in $\mathrm{LL}$ group $(\mathrm{RR}=1.74,95 \% \mathrm{CI}[1.01-3.02], \mathrm{P}=0.05)$ and $(\mathrm{RR}=2.47,95 \% \mathrm{CI}$ $[1.03-5.92] ; \mathrm{P}=0.04)$ respectively. The remaining five RCTs compared between different procedures, like primary anastomosis versus non-restorative resection, RP-LASR versus NRP-LASR, and primary versus secondary resection, for different situations and reviewed qualitatively.

Conclusion: The superiority of LSR over OSR was non-significant in the treatment of acute symptomatic diverticulitis regarding postoperative complications, operational time, and cost. However, the cosmetic aspect still gives LSR more advantage. Regarding perforated diverticulitis with purulent peritonitis, our results showed that LL is as safe as resection (either Hartmann's operation or sigmoidectomy with primary anastomosis) regarding short- and long-term postoperative outcomes especially in long-term, hospital stay, and operational duration. Hence, LL is feasible and can act as definitive treatment. Further RCTs are still needed to make a decision regarding these and other procedures. Disclosure of Interest: All authors have declared no conflicts of interest.

\section{P0290 NLR AND PLR IN DIAGNOSING SYNCHRONOUS LIVER} AND LYMPH NODE METASTASES IN PATIENTS WITH CRC

M. Stojkovic Lalosevic ${ }^{1}$, A. Pavlovic Markovic ${ }^{1}$, V. Markovic ${ }^{2}$, I. Dimitrijevic ${ }^{2}$, M. Stojkovic ${ }^{1}$, S. Kiurski ${ }^{3}$, Z. Krivokapic ${ }^{2}$

${ }^{1}$ Clinic Of Gastroenterology And Hepatology, Clinic of gastroenterology, Clinical center Serbia, Belgrade/Serbia

${ }^{2}$ First Surgery Clinic, Clinical center Serbia, Belgrade/Serbia

${ }^{3}$ School of medicine, Belgrade/Serbia

Contact E-mail Address: drmilicastojkovic@gmail.com

Introduction: There has been enormous progress in diagnosing and treatment of colorectal cancer (CRC), however a great number of patients is nevertheless diagnosed in advanced disease stages. It is of great importance to develop noninvasive, inexpensive, prognostic, diagnostic, and treatment predicting biomarkers in early diagnostics of CRC considering its incidence worldwide. There are studies suggesting that the systemic inflammation play an important role in CRC tumor stage development, which can be reflected by the levels of neutrophil to lymphocyte ratio (NLR) and platelet to lymphocyte ratio (PLR).

Aims \& Methods: This study was designed to investigate the efficiency of preoperative NLR, PLR as a tool for the assessment of synchronous lymph nodes 
and liver metastases in newly diagnosed patients with CRC. Three hundred patients with I-IV stage CRC undergoing surgical resection were included in this cross sectional study. Complete blood counts with automated differential counts were performed preoperatively. The NLR was calculated by dividing the absolute neutrophil count by the absolute lymphocyte count; also PLR was calculated by dividing the absolute platelet count by the absolute lymphocyte count. The diagnostic performance of NLR and was estimated by ROC curve. Results: Our results suggest that there was high statistically significant difference between NLR ( $p=0.003)$, PLR $(\mathrm{p}=0.002)$ and tumor stages (I to IV). ROC curve analysis showed high diagnostic efficacy of NLR (AUC 0.774, $95 \% \mathrm{CI}=0.683-0.790$ ) and PLR (AUC $0.698,95 \% \mathrm{CI}=0.663-0.742$ ) for synchronous lymph node and liver metastases. Also combination of NLR and PLR improved diagnostic efficacy (AUC $0.841,95 \% \mathrm{CI}=0.811-0.863$ ) for synchronous liver and lymph node metastases.

Conclusion: Our results suggest that NRL and PLR could be useful diagnostic CRC biomarkers, and could have potential use in early recognition of different stages of CRC.

Disclosure of Interest: All authors have declared no conflicts of interest.

\section{References}

1. Song L-L, Li Y-M. Current noninvasive tests for colorectal cancer screening: An overview of colorectal cancer screening tests. World Journal of Gastrointestinal Oncology. 2016;8(11):793-800. doi:10.4251/wjgo.v8.i11.793.

2. Elinav E, Nowarski R, Thaiss CA, Hu B, Jin C, Flavell RA. Inflammationinduced cancer: crosstalk between tumours, immune cells and microorganisms. Nat Rev Cancer. 2013;13:759-771.

3. Liu H, Wu Y, Wang Z, Yao Y, Chen F, Zhang H, Wang Y, Song Y. Pretreatment platelet-to-lymphocyte ratio (PLR) as a predictor of response to first-line platinum-based chemotherapy and prognosis for patients with non-small cell lung cancer. $J$ Thorac Dis.2013;5:783-789.

4. Zhang Z, Li C, Gao W, et al. A Nomogram to Predict Adequate Lymph Node Recovery before Resection of Colorectal Cancer. St-Pierre Y, ed. PLOS ONE. 2016;11(12):e0168156. doi:10.1371/journal.pone.0168156.

5. Wu Y, Li C, Zhao J, et al. Neutrophil-to-lymphocyte and platelet-to-lymphocyte ratios predict chemotherapy outcomes and prognosis in patients with colorectal cancer and synchronous liver metastasis. World Journal of Surgical Oncology. 2016;14:289. doi:10.1186/s12957-016-1044-9.

\section{P0291 DEVELOPING AND VALIDATING OF RAMATHIBODI APPENDICITIS SCORE (RAMA-AS) FOR DIAGNOSIS OF APPENDICITIS IN SUSPECTED APPENDICITIS PATIENTS}

C. Wilasrusmee $^{1}$, B. Siribumrungwong ${ }^{1}$, S. Phuwapraisirisan ${ }^{1}$, N. Poprom ${ }^{1}$, A. Thakkinstian ${ }^{2}$

${ }^{1}$ Surgery, Faculty of Medicine Ramathibodi Hospital, Mahidol University, Bangkok/Thailand

${ }^{2}$ Section For Clinical Epidemiology And Biostatistics, Faculty of Medicine

Ramathibodi Hospital, Mahidol University, Bangkok/Thailand

Contact E-mail Address: chumpon.wil@mahidol.ac.th

Introduction: Diagnosis of appendicitis is still clinically challenge where resource is limited. The purpose of this study is to develop and externally validate Ramathibodi Appendicitis Score (RAMA-AS) in aiding diagnosis appendicitis. Aims \& Methods: Two-phase cross-sectional study (i.e., derive and validation) was conducted at Ramathibodi Hospital (for derive), Thammasat University Hospital and Chaiyaphum Hospital (for validation). Patients with abdominal pain and suspected of having appendicitis were enrolled. Multiple logistic regression was applied to develop parsimonious model. Calibration and discrimination performances were assessed. In addition, our RAMA-AS was compared with Alvarado's score performances using ROC curve analysis. The study was conducted and reported according to Transparent Reporting of a Multivariable Prediction Model for Individual Prognosis Or Diagnosis (TRIPOD) statement. Results: The RAMA-AS consisted of 3 domains 7 predictors including symptoms (i.e. progression of pain, aggravation of pain, and migration of pain), signs (i.e. fever and rebound tenderness), and laboratory (i.e. white blood cell count (WBC) and neutrophil). The model fitted well with data and it performed better discriminate than the Alvarado score with C-statistic of 0.842 (95\% CI: $0.804,0.881$ ) versus $0.760(0.710,0.810)$. Internal validation by bootstrap yielded Sommer's D of $0.686(0.608,0.763)$ and C-statistics of $0.848(0.846,0.849)$. The C-statistics of two external validations were $0.853(0.791,0.915)$ and $0.813(0.736,0.892)$ with fair calibrations.

\begin{tabular}{llllll}
\hline Risk group & Sensitivity & Specificity & LR+ & LR- & $\begin{array}{l}\text { Post-positive } \\
\text { test odds }\end{array}$ \\
\hline Very low & 100 & 0 & 1 & 0 & 61.8 \\
Low & 89.75 & 54.97 & 1.98 & 0.19 & 76.00 \\
& $(85.25-93.26)$ & $(46.67-63.06)$ & $(1.65-2.37)$ & $(0.13-0.28)$ & $(73.00-79.00)$ \\
Moderate & 64.08 & 88.08 & 5.25 & 0.41 & 89.00 \\
& $(57.73-70.09)$ & $(81.82-92.78)$ & $(3.39-8.13)$ & $(0.34-0.49)$ & $(85.00-93.00)$ \\
High & 37.96 & 95.36 & 8.36 & 0.65 & 93.00 \\
& $(31.86-44.36)$ & $(90.68-98.12)$ & $(3.96-18.00)$ & $(0.59-0.72)$ & $(86.00-97.00)$ \\
\hline
\end{tabular}

Conclusion: RAMA-AS is a useful tool for diagnosis of appendicitis. It has good discrimination performance and good calibration performance after revision.
Practitioners should be encouraged to use the score in clinical practice in order to conforming diagnosis and choosing the patient who should undergo imaging or surgical management.

Disclosure of Interest: All authors have declared no conflicts of interest.

\section{References}

Steyerberg EW, Moons KG, van der Windt DA, Hayden JA, Perel P, Schroter S, et al. Prognosis Research Strategy (PROGRESS) 3: prognostic model research. PLoS Med. 2013;10(2):e1001381.

Moons KG, Altman DG, Reitsma JB, Collins GS. New Guideline for the Reporting of Studies Developing, Validating, or Updating a Multivariable Clinical Prediction Model: The TRIPOD Statement. Adv Anat Pathol. 2015 Sep;22(5):303-5.

\section{P0292 IS HAEMORRHOIDECTOMY SAFE IN PATIENTS WITH ULCERATIVE COLITIS?}

S. Lee ${ }^{1}$, Y.S. Choi ${ }^{1}$, E. Lee ${ }^{1}$, J.B. Lee ${ }^{1}$, D.S. Lee ${ }^{1}$, E.G. Youk ${ }^{1}$, D.H. Lee ${ }^{1}$, D.S. Kim ${ }^{1}$

${ }^{1}$ Dept. Of Surgery, Daehang Hospital Dept. of Surgery, Seoul/Korea, Republic of

\section{Contact E-mail Address: vocalcord@hanmail.net}

Introduction: Haemorrhoidectomy in ulcerative colitis (UC) have been considered to be potentially dangerous, but the evidence is poor.

Aims \& Methods: A study was conducted to ascertain the safety of haemorrhoidectomy in patients with UC. Retrospective review of 44 UC patients from 2004 to 2014. Patient demographics and clinical characteristics (anorectal symptoms, prior non operative haemorrhoidal therapy, whether proven UC preoperatively (BD) and unproven preoperatively (AD), whether to use azathioprine, presence of other perianal disease, and activity, anatomic location of UC) were recorded. Postoperative complications, and differences between BD and AD were analysed. Results: The patients were 29 males $(65.9 \%)$, median age 44 (range, 19-72) years. Predominant symptoms were bleeding and prolapse $(n=24 ; 54.5 \%)$, prolapse only $(\mathrm{n}=6 ; 13.6 \%)$, bleeding only $(\mathrm{n}=14 ; 31.8 \%) .17$ patients $(\mathrm{BD}, 38.6 \%)$ were diagnosed with UC prior to surgery. 4 patients $(9.1 \%)$ had azathioprine therapy before surgery. There was no other perianal disease. Disease was limited to the rectum $(\mathrm{n}=33 ; 75 \%)$, left-sided $(\mathrm{n}=9 ; 20.5 \%)$, and extended to rightsided $(\mathrm{n}=2 ; 4.5 \%)$. During follow-up, there were no complications such as sepsis, anal stenosis, abscess and fistula formation, and recurrence. There was no difference in complications and other clinical characteristics between BD and AD. There was no difference in complications according to disease extent $(\mathrm{p}=0.158)$.

Conclusion: Our data suggest that haemorrhoidectomy may be performed safely in UC patients.

Disclosure of Interest: All authors have declared no conflicts of interest.

\section{P0293 EARLY VERSUS DELAYED CLOSURE OF TEMPORARY LOOP ILEOSTOMY AFTER COLORECTAL SURGERIES: A PROSPECTIVE RANDOMIZED STUDY}

S. Gavini, V. Vutukuru, D. Annareddy, C. Chandramaliteeswaran, V. Dasari, N. Pagadala

Gi Surgery, SVIMS, Tirupathi/India

Contact E-mail Address: srkgavini@gmail.com

Introduction: Temporary loop ileostomies are commonly performed to protect a distal anastomosis in colorectal surgeries. Although they have been shown to reduce the number of leaks requiring surgery, they remain a source of complications and have an adverse effect on the quality of life. A few non-randomized studies have shown the feasibility of early stoma closure.

Aims \& Methods: To compare the outcomes of early and delayed closure of temporary loop ileostomy in terms of operative parameters, morbidity, mortality, and quality of life. The study was conducted from May 2014 to September 2015. Following creation of loop ileostomy after colorectal surgeries, distal loop contrast study was done on POD 7. Patients who had no leak were randomized to either early closure (8-13 days) or delayed closure (after 6 weeks) group. Patient demographics, operative parameters, morbidity, mortality and quality of life data were recorded in both groups.

Results: There were 24 patients in each group. Both groups were comparable in terms of demographic data except for age, which was significantly higher $(\mathrm{p}=0.012)$ in the early closure group. Incidence of stoma related complications $(\mathrm{p}=0.01)$ and Pittman ostomy complication severity index $(\mathrm{p}<0.01)$ were significantly higher in the delayed group. Operative time $(\mathrm{p}=0.033)$ and Surgeons assessment score $(\mathrm{p}=0.0012)$ for the stoma closure surgery were significantly lower for the early closure group. There was no significant difference in the duration of hospital stay and the incidence of postoperative complications in the two groups. Quality of life as calculated by the Ostomy Adjustment Index score (OAI 23) was better in the early closure group $(\mathrm{p}=0.014)$.

Conclusion: Early closure of a temporary loop ileostomy is feasible with the advantages of decreased stoma related morbidity, operative difficulties without increased morbidity and mortality when compared with conventional delayed ileostomy closure.

Disclosure of Interest: All authors have declared no conflicts of interest. 


\section{P0294 FULL-SCALE INTRODUCTION OF RADICAL}

LAPAROSCOPIC SURGERY FOR INGUINAL HERNIA EMPLOYING THE TRANSABDMINAL PREPERITONEAL (TAPP) REPAIR AND EARLY OUTCOMES

T. Saito, T. Sano, S. Komatsu, T. Arikawa, S. Ishiguro, K. Komaya Gastroenterological Surgery, Aichi Medical University, nagakute/Japan

\section{Contact E-mail Address: tsaito0726@gmail.com}

Introduction: Ger reported the first laparoscopic hernia repair in 1982 by approximating the internal ring with stainless steel clips. The laparoscopic trans-abdominal preperitoneal (TAPP) repair was a revolutionary concept in hernia surgery and was introduced by Arregui and Dion in the early 1990s. Institutions performing radical laparoscopic surgery for inguinal hernia have been rapidly increasing since the NHI point was amended in Japan. However, in the 12th JAPAN SOCIETY FOR ENDOSCOPIC SURGERY questionnaire survey, the recurrence rate after surgery employing the TAPP method was reported to be $4 \%$, posing a problem regarding the thoughtless introduction of the TAPP method. Our hospital performed surgery employing the TAPP method only occasionally until April 2015, but treatment of inguinal hernia was integrated, the indication was established in May 2015, and laparoscopic surgery employing the TAPP method has been performed for the indicated cases. In this study, we investigated the current state of inguinal hernia treatment at our hospital. Surgical indication of inguinal hernia in our department is as follows. Symptomatic inguinal hernia is treated using the TAPP method when there is only one POSSUM score-based risk factor. When 2 or more risk factors are present or the patient has undergone surgery of the prostate, the anterior approach is employed (the UHS and Mesh Plug methods for internal and external inguinal hernia, respectively). Treatment under local anesthesia is prioritized for patients aged 90 years or older and patients with PS2 or higher. Arrangement in operating room is that the operator and assistant stand on the left and right sides of the patient, respectively, an anesthesiologist stands at the patient's head, and a nurse stands caudal to the assistant.

Aims \& Methods: In this study, we investigated the current state of inguinal hernia treatment at our hospital. The subjects were 120 patients who underwent radical surgery for inguinal hernia before and after the full-scale introduction of the TAPP method (early period: October 2014-April 2015 (7 months), late period: May 2015-November 2015 (7 months), 47 and 73 patients were treated in the early and late periods, respectively). Changes in the surgical procedure, complications, and duration of hospital stay were investigated in 120 patients.

Results: The median age was 70 years old (19-91years old). There were 114 male and 6 female patients, with a total of 132 lesions (unilateral in 108 (right: 60, left: 48 ) and bilateral in 12). The hernia classification (Japanese Hernia Society) was 1 , $2,3,4$, rec in $91,37,0,3$, and 1 lesions. Surgery was performed under local anesthesia in 43, lumbar anesthesia in 1, and general anesthesia in 76. A laparoscope was used in 70 and not used in 50. TAPP, mesh plug, and UHS and others were used in 58, 54, 17, 3 lesions. There was no change for both the Early period and the Late Period about the median operative time (Early period: 93 minutes, Late period: 102 miniutes). Early Period was 4 days (4-24), Late Period was 3 days (3-9) for median duration of hospital stay. In Early period, complications which required treatment (Clavien Dindo Grade3 or higher) were intestinal obstruction and recurrence in one each. In Late period, the recurrence and complications of Clavien Dindo Grade3 or higher did not occur.

Conclusion: No severe complication or recurrence has occurred since integrating treatment for inguinal hernia and introduction of the TAPP method. Application after carefully deciding the indication may be important for laparoscopic surgery for inguinal hernia. A randomized prospective exploratory study on analgesic methods for laparoscopic radical surgery for inguinal hernia aiming at introducing day surgery (approved by the Ethics Committee) is being performed.

Disclosure of Interest: All authors have declared no conflicts of interest.

\section{References}

1. Ger R: The management of certain abdominal herniae by intra-abdominal closure of the neck of the sac. Preliminary communication. Ann R Coll Surg; 1982; 64: 342-4.

2. Arregui ME, Davis CJ, Yucel O, et al.: Laparoscopic mesh repair of inguinal hernia using a pre-peritoneal approach: A preliminary report. Surg Laparopsc Endosc; 1992; 2: 53-8.

3. Dion YM, Morin J: Laparoscopic inguinal herniorraphy. Can J Surg; 1992; 35:209-12.

\section{P0295 EFFECTIVENESS OF PURE LAPAROSCOPIC LEFT COLECTOMY WITH PRIMARY ANASTOMOSIS AND LOOP ILEOSTOMY FOR THE TREATMENT OF COMPLICATED HINCHEY 3 DIVERTICULITIS}

F. Roscio, A. De Luca, I. Scandroglio

Galmarini Hospital, ASST Sette Laghi, Tradate/Italy

Contact E-mail Address: francesco_roscio@yahoo.it

Introduction: To evaluate the effectiveness of laparoscopic left colectomy with primary anastomosis and loop ileostomy in the treatment of complicated acute diverticulitis with diffuse purulent peritonitis (Hinchey 3), also considering the lack of evidence about this topic due to the difficulty of carrying out comparative trials with the laparoscopic washing/drainage technique.

Aims \& Methods: A consecutive unselected series of 44 patients undergone emergency surgery for acute complicated Hinchey 3 diverticulitis from January 2012 to December 2016 was retrospectively evaluate. All patients were treated by pure laparoscopic left colectomy with primary colorectal anastomosis and temporary loop ileostomy. All the procedures were performed by the same surgeons (IS, ADL, FR). Perioperative care plan, operative steps and surgical instrumentations were standardized. We collected patients-, surgery- and hospital stay-related data, as well as short-term outcomes. Complications were classified using the Clavien-Dindo classification system (CDCS).

Results: There were 31 men $(70.4 \%)$ and 13 women $(29.6 \%)$ with a mean age of $57.8 \pm 11.9$ years. The mean body mass index was $28.3 \pm 3.1 \mathrm{~kg} / \mathrm{m} 2$. No conversion to open surgery was registered. The mean operative time and estimated blood loss were $184.3 \pm 32.7$ minutes and $81.2 \pm 72.7 \mathrm{ml}$ respectively. All the specimens showed diverticulitis with peridiverticulitis. Length of hospital stay was $7.8 \pm 2.8$ days and we have not recorded any readmissions in patients discharged within 60 days after surgery. The rates of postoperative complications were $6.8 \%$ and $2.3 \%$ for grades 3 and 5 according to the CDCS respectively. Conclusion: Laparoscopic left colectomy with primary anastomosis and loop ileostomy seems to be a good technique that resulted in encouraging shortterm outcomes. In expert hands it represents an effective technique for the treatment of acute diverticulitis complicated by diffuse purulent peritonitis.

Disclosure of Interest: All authors have declared no conflicts of interest.

\section{P0296 LAPAROSCOPIC COMPLETE LATERAL LYMPH NODE DISSECTION FOR LOW RECTAL CANCER}

T. Kinoshita, K. Komori, T. Oshiro, A. Ouchi, Y. Shimizu Gastroenterological Surgery, Aichi Cancer Center Hospital, NAGOYA/Japan

Contact E-mail Address: t-kinoshita@aichi-cc.jp

Introduction: Total mesorectal excision (TME) with lateral pelvic lymph node dissection (LLND) is a standard procedure for low rectal cancer in Japan. However, ME alone is the international standard surgical procedure for rectal cancer. Complete LLND is difficult because of the narrow visual field in the pelvic cavity and the complicated anatomical structure and invasive procedure which needs longer operative time and greater blood loss. Herein we introduce laparoscopic LLND as our new procedure securing equivalent range of lymph node dissection and report the clinical outcomes.

Aims \& Methods: After laparoscopic ME, first, the external iliac artery was exposed and the external iliac nodes were completely removed from inguinal ligament. Obturator nodes were completely dissected while preserving the obturator nerve, resecting the obturator artery and vein, and confirming lateral pelvic wall, bladder wall and sciatic nerve. Subsequently, proximal internal iliac nodes were removed and superior vesical artery was separated. Distal internal iliac nodes from the coccygeal muscle (Alcock's canal) were completely dissected while preserving the superior vesical artery and the pelvic plexus, and transecting several inferior vesical arteries. Finally, bilateral hypogastric nerves were separated to be preserved. Common iliac nodes were dissected; aortic bifurcation nodes and presacral nodes were also dissected by exposing the aortic bifurcation and the pelvic surface of the sacrum.

Results: Between 2015 and 2016, we performed laparoscopic ME with LLND for 10 patients with cT2 or deeper low rectal cancer. The median operative time was $502 \mathrm{~min}$ (420-679 min), and the median blood loss was $90 \mathrm{ml}(5-500 \mathrm{ml})$. Postoperative complications developed in $4(40 \%)$ patients, and the most frequent one was temporary urination disorder in $2(20 \%)$ patients. The median number of harvested lateral lymph node was 20 (14-23). So far there are no recurrent cases.

Conclusion: Our laparoscopic LLND provides good visual field and reduces an amount of operative bleeding and results in favorable clinical outcomes. Disclosure of Interest: All authors have declared no conflicts of interest.

\section{P0297 TRANSGASTRIC-NOTES SIGMOID RESECTION IN AN ANIMAL SURVIVAL MODEL USING THE ANUBIS-SYSTEM}

J. Bernhardt ${ }^{1}$, H. Steffen ${ }^{2}$, P. Koehler ${ }^{3}$, S. Schneider-Koriath ${ }^{2}$, K. Ludwig ${ }^{2}$

${ }^{1}$ Dept. Of Endoscopy, Klinikum Suedstadt Rostock, Rostock/Germany

${ }^{2}$ Dept. Of Surgery, Klinikum Suedstadt Rostock, Rostock/Germany

${ }^{3}$ Friedrich Loeffler Institute, Neustadt/Germany

\section{Contact E-mail Address: joern.bernhardt@kliniksued-rostock.de}

Introduction: Natural orifice translumenal endoscopic surgery (NOTES) proposed advantages should be established by comparison with standard procedures. Using a single side transluminal access, the feasibility of performing advanced surgical procedures is still limited, especially for a single endoscope. We used the ANUBIS-system for sigmoid resection with a transgastric access to the abdominal cavity assisted by colonoscopy and one trocar.

Aims \& Methods: The experimental study was conducted in a porcine model in general anesthesia. After operation in an acute model, we started the study with 5 pigs in a survival model. Using Anubisscope, a transgastric access was reached by needle-knife incision and balloon dilatation. $\mathrm{CO}_{2}$ peritoneum was achieved by insufflation via a working channel. By steering the colonoscope, the colon was maneuvered endoluminally and the colic mesentery was exposed. Bowel-close mesocolic preparation was performed with a coagulating hook-knife with simultaneously assistance by a grasper via the Anubiscope. Both instruments have the possibility of two-directional movements. The access angle for preparation and visualisation could be altered during the procedure by manoeuvring the colonoscope and the movements of the flexible endoscopic instruments. To prepare anastomosis, circular stapler anvil was introduced transluminally and penetration of the colon wall was carried out. Subsequently, proximal resection of the sigmoid colon was performed using a linear stapler inserted through a trocar at the left 
lower abdomen. The bowel extraction was performed by invagination transrectally. After extracorporeal distal linear stapling of the sigmoid, the colorectal anastomosis was completed by applying a circular stapling device transrectally, assisted by a transcutaneous inserted grasper. Function testing was performed by the colonoscope. Gastric access closure was performed by OTSC clip.

Results: The procedure was successful in all animals with operation time ranging from 4.5 to 6 hours. After weight gain in all cases, the animals were sacrificed after postoperative day 42 and the workup showed competent anastomotic healing with a stenosis and consecutive prestenotic dilatation in one case. These animal had a small peritoneal abscess beside the anastomosis. Gastric closure was healed and the OTSC clip still in situ in all animals. In one case we used two OTSC clips for gastric closure, there were severe adhesions with two perigastric abscesses.

Conclusion: The use of an operating platform like the Anubisscope has the advantage of flexible preparation in opposite position of the instruments. The disadvantages are the only two degrees of freedom of the flexible instruments and the rotation-like movements. Flexible colonoscopy provided a fixed reference frame that enabled preparation and dissection. For resection and anastomosis an additional transcutaneous access was necessary.

Disclosure of Interest: All authors have declared no conflicts of interest.

\section{P0298 ASCITES, COMPLEX ADNEXAL MASSES AND RAISED CA- 125 IN POST-MENOPAUSAL WOMEN: OVARIAN CANCER OR TUBERCULOSIS?}

S. Jardak ${ }^{1}$, H. Kchir ${ }^{2}$, N. Maamouri ${ }^{1}$, H. Chaabouni ${ }^{1}$, N. Ben Mami ${ }^{1}$ ${ }^{1}$ Tunis, rabta B, Tunis/Tunisia

${ }^{2}$ rabta B, Tunis/Tunisia

\section{Contact E-mail Address: sondajardak1@gmail.com}

Introduction: Peritoneal tuberculosis (TB) and advanced ovarian cancer, two conditions with different management and prognosis, have many similarities: ascites, complex adnexal mass, peritoneal deposits, and raised CA-125 level. Symptoms such as weight loss, reduced appetite, and dull abdominal pain are also common to these two entities.

Aims \& Methods: The aim of this study was to analyze patients' characteristics, laboratory investigations, radiological and surgical findings in post-menopausal women with pelvic TB who were diagnosed after laparotomy or laparoscopy for suspected ovarian cancer. We report twenty-one cases of pelvic-peritoneal TB in post-menopausal women who presented with features mimicking ovarian malignancy from 2004 to 2014 in a Tunisian center.

Results: The mean age was 59.8 (46-87 years). Three patients have personal or family history of TB. All women presented with abdominal pain and distension of varying duration of 1 month to 6 months. Eleven patients had reduced appetite and weight loss, and four women gave a history of low-grade fever. A CT scan showed the presence of solid-cystic adnexal masses ranging rom $3 \mathrm{~cm}$ to $12 \mathrm{~cm}$ in $100 \%$ and ascites in $90.4 \%$. Ascitic fluid analysis was done in 19 patients which showed a lymphocytic predominant pattern, and absence of malignant cells. Ascitic fluid cultures was negative in all. CA-125 was elevated in all and ranged between 185 and $1300 \mathrm{IU} / \mathrm{ml}$. CA-125 >600 IU/L was found in three women. Quantiferon-TB Gold (QFT-G) performed in 3 patients was positive in two. A laparoscopic evaluation with biopsies was performed in 16 patients and an exploratory laparotomy in 4 women for suspected ovarian cancer. Intraoperative findings of tubercles on the pelvic organs and peritoneal surfaces suggested TB in 18 patients. In two, TB was not suspected intraoperatively. The diagnosis of TB was confirmed by histopathology in $95.2 \%$. Response to therapeutic trial of anti-tubercular drugs was the basis of diagnosis in one case because of a high anesthetic risk.

Conclusion: It is a diagnostic challenge to differentiate pelvic-peritoneal TB from ovarian cancer which has entirely different management and prognosis. Ascitic fluid showing lymphocytic predominance and no malignant cells and positive(QFT-G) are pointers to obtain a histopathological diagnosis by laparoscopic biopsy or frozen section at laparotomy.

Disclosure of Interest: All authors have declared no conflicts of interest.

MONDAY, OCTOBER 30, 2017

09:00-17:00

IBD I - HALL 7

\section{P0299 INSULINLIKE GROWTH FACTOR IGF-I AND INFLAMMATORY RESPONSE IN THE COLONIC MUCOSA IN ULCERATIVE COLITIS}

V. Pavlenko, F. Urusova, G. Eseneeva, A. Pavlenko, N. Korablina

Stavropol State Medical University, Stavropol/Russian Federation

\section{Contact E-mail Address: pavlenkovv@yandex.ru}

Introduction: Peptide growth factors including the IGF family are expressed in the intestine and modulate proliferative activity of the intestinal epithelium in ulcerative colitis (UC) and Crohn's disease (CD). At the same time, IL- 8 is one of the main triggers behind the immunoinflammatory process in the colonic mucosa $(\mathrm{CM})$ in $\mathrm{UC}$, and its level may be of prognostic value in determining the illness course.

Aims \& Methods: The aim of the study was to identify the role that IGF-I plays in colonic inflammation in 35 patients with different clinical (Rahmilevich index) and endoscopic (Mayo index) activity of UC. The treatment was administered in view of the severity of UC. 20 healthy volunteers were the control group. IGF-I levels in peripheral blood were determined by ELISA (Mediagnost, Germany). The results were expressed as nmol/l. The spontaneous and E. Coli LPS-induced synthesis of IL-8 in rectal bioptic samples were studied via ELISA. The results were expressed as picograms per $1 \mathrm{mg}$ of wet tissue $(\mathrm{pg} / \mathrm{mg})$. The severity of the inflammation in the CM was studied by the method of Avtandilov G.G. counting with inflammatory infiltrate (in \%) in the lamina propria. The study implied investigating the cellular composition of the inflammatory infiltrate in the lamina propria. Depending on the density of the inflammatory infiltrate, the patients were divided into two groups: Group 1 revealed a weak inflammatory response $(20.2 \pm 0.8 \%)$, while Group 2 demonstrated prominent infiltration intensity $(39.0 \pm 2.09 \%, \mathrm{P}<0.05$ to Group 1$)$. Statistical processing was performed using the SPSS 13.0 software.

Results: It has been shown that in acute UC stages the amount of IGF-I in the blood plasma decreased $(15.16 \pm 1.35 \mathrm{nmol} / 1$ ( $\mathrm{p}<0.05$ to control)), while spontaneous production of IL- 8 chemokine in rectal bioptic samples went up $(300.0 \pm 6.0 \mathrm{pg} / \mathrm{ml}, \mathrm{P}<0.05)$. In case of the LPS stimulation the production of IL-8 in the CM went up more than 2 times $(750.0 \pm 7.0 \mathrm{pg} / \mathrm{mg}, \mathrm{P}<0.05)$. There was significant inverse correlation (rs) detected between the IGF-I levels in the blood plasma, on one hand, and indicators of the UC clinical, endoscopic activity and the intensity of the inflammatory infiltrate in the CM, on the other. Direct relationship was found between the levels of spontaneous and stimulated production of the IL- 8 chemokine and the density of the inflammatory infiltrate in the CM of patients with active UC. Through the period of the clinical remission development (an average of 8 weeks) the IGF-I levels increased up to $94.125 \pm 28.18 \mathrm{nmol} / 1 \quad(\mathrm{P}<0.05)$, yet have not reached the control value $(\mathrm{P}<0.05$ to control). Induction of the clinical remission was associated with a decrease to the level of the control values for spontaneous and LPS-induced IL-8 production, regardless of UC activity.

Conclusion: The intensity of the inflammatory process in the $\mathrm{CM}$ depends on the level of IL-8 produced by respective cells. Evidently, IL- 8 has a capacity of inhibiting the production of IGF-I at the peak of inflammation (acute UC). Increased synthesis of IGF-I and reduced IL-8 in remission facilitates regeneration of the damaged mucosa.

Disclosure of Interest: All authors have declared no conflicts of interest.

\section{P0300 REGULATORY B CELLS CONTRIBUTE TO THE ALLEVIATION OF COLITIS INDUCED BY DEXTRAN SULPHATE SODIUM AFTER H.PYLORI INFECTION}

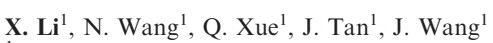

${ }^{1}$ Department Of Gastroenterology, Peking University, People's Hospital, Beijing/ China

Contact E-mail Address: lixialjay@163.com

Introduction: Epidemiological studies have showed that there was an inverse association between Helicobacter pylori (H.pylori) infection and the incidence of inflammatory bowel disease (IBD). Our previous research indicated that the regulatory immune responses induced by $H$.pylori infection were not limited to gastric mucosa, IL-10-producing Breg cells and Foxp $3^{+}$Treg cells expanded in spleen and mesenteric lymph nodes (MLN), the balance of intestinal mucosal immunity was influenced to a skewed regulatory immune response.

Aims \& Methods: A murine model with H.pylori infection and acute and chronic colitis induced by dextran sulphate sodium (DSS) was established to explore the function of the Breg cells in the effect of H.pylori infection on acute and chronic colitis induced by DSS.A C57BL/6 mice model of acute and chronic colitis was induced by $3 \%$ DSS with or without H.pylori infection in advance, the colitis performances were assessed by disease acitive index (DAI), colon length and colonic histological inflammatory scores. The CD19+ IL- $10^{+}$Breg cells and $\mathrm{CD} 4{ }^{+} \mathrm{CD} 25^{+} \mathrm{Foxp}^{+}$Treg cells in blood spleen MLN PP and gastrointestinal mucosa were measured by flow cytometry, immunohistochemistry and immunofluorescence. The anti- and pro-inflammatory cytokines were also detected at mRNA level by real-time PCR.

Results: Compared with the DSS treated acute colitis group, H.pylori/DSS cotreated acute colitis group: (1) DAI and colonic histological scores reduced $(9.25 \pm 4.42$ vs $16.00 \pm 2.00, \quad \mathrm{P}=0.025)$ and colon length shortened less $(6.56 \pm 0.63$ vs $5.40 \pm 0.54, \mathrm{P}<0.001)$. (2) The percentages of $\mathrm{CD} 19^{+} \mathrm{IL}-$ $10^{+}$Breg cells detected by flow cytometry expanded in different tissues: blood: $5.05 \pm 0.68$ vs $2.89 \pm 0.55, \mathrm{P}=0.001$; spleen: $4.32 \pm 0.56$ vs $3.17 \pm 0.20, \mathrm{P}=0.05$; MLN:5.89 \pm 0.54 vs $4.94 \pm 0.65, \quad \mathrm{P}=0.047 ; \mathrm{PP}: 6.95 \pm 1.67$ vs $5.39 \pm 0.88$, $\mathrm{P}=0.005$; respectively. (3) The percentages of $\mathrm{CD} 4^{+} \mathrm{CD} 25^{+} \mathrm{Foxp} 3^{+}$Treg cells expanded in different tissues: spleen: $13.50 \pm 1.37$ vs $10.73 \pm 1.13, \mathrm{P}=0.008$; MLN: $17.50 \pm 0.82$ vs $14.87 \pm 1.53, \mathrm{P}=0.001 ; \mathrm{PP}: 12.70 \pm 3.24$ vs $8.58 \pm 1.71$, $\mathrm{P}=0.028$. The numbers of Foxp $3+$ cells per $\mathrm{HPF}$ in colonic mucosa: $3.44 \pm 0.40$ vs $2.58 \pm 0.34, \mathrm{P}=0.004$. (4) $\mathrm{mRNA}$ expression in colonic mucosa: IL-10 $(\mathrm{P}=0.001)$ mRNA relative expression upregulated and IFN- $\gamma(\mathrm{P}=0.048)$ mRNA relative expression downregulated significantly. Compared with DSS treated chronic colitis group, H.pylori/DSS co-treated chronic colitis group: (1) DAI and colonic histological scores reduced $(9.00 \pm 1.73$ vs $14.67 \pm 1.53$, $\mathrm{P}=0.001)$, and colon length shortened less $(6.82 \pm 0.41$ vs $5.98 \pm 0.15$, $\mathrm{P}=0.001)$. (2) The percentages of $\mathrm{CD} 19^{+} \mathrm{IL}-10^{+}$Breg cells expanded which were consisted with the acute colitis groups: spleen: $7.05 \pm 0.28$ vs $5.39 \pm 0.72$, $\mathrm{P}=0.024 ; \mathrm{MLN}: 4.78 \pm 0.73$ vs $3.02 \pm 0.83, \mathrm{P}=0.047 ; \mathrm{PP}: 7.14 \pm 1.04$ vs $4.34 \pm 1.03, \quad \mathrm{P}=0.005$; respectively. (3) The percentages of $\mathrm{CD} 4{ }^{+} \mathrm{CD} 25^{+} \mathrm{Foxp}^{+}$Treg cells decreased dramatically in different tissues:blood: $4.66 \pm 0.22$ vs $7.37 \pm 0.87, \quad \mathrm{P}<0.001$; spleen: $9.07 \pm 2.88$ vs $13.10 \pm 1.19$, $\mathrm{P}=0.018$; PP: $7.33 \pm 1.07$ vs $12.80 \pm 0.96, \mathrm{P}<0.001$; The numbers of Foxp3+ cells per HPF in colonic mucosa: $3.66 \pm 0.99$ vs $4.08 \pm 0.56, P=0.392$. (4) mRNA expression in colonic mucosa: IL-10 $(\mathrm{P}=0.023)$. Foxp3 $(\mathrm{P}=0.000) \mathrm{mRNA}$ relative expression upregulated significantly, and IFN- $\gamma(\mathrm{P}=0.001)$. TNF$\alpha(\mathrm{P}=0.005)$. IL-17A $(\mathrm{P}=0.019)$ and $\mathrm{IL}-23(\mathrm{P}=0.000)$ mRNA relative expression downregulated significantly. 
Conclusion: (1) H.pylori infection can alleviate the acute and chronic colitis induced by DSS. (2) $\mathrm{CD} 19^{+} \mathrm{IL}-10^{+}$Breg cells and $\mathrm{CD} 4^{+} \mathrm{CD} 25^{+}$Foxp $3^{+}$Treg cells expanded significantly in H.pylori/DSS co-treated acute colitis mice. (3) $\mathrm{CD} 19^{+} \mathrm{IL}-10^{+}$Breg cells expanded while $\mathrm{CD} 4^{+} \mathrm{CD} 25^{+} \mathrm{Foxp}^{+}$Treg cells reduced significantly in H.pylori/DSS co-treated chronic colitis mice. The potential protective effect of H.pylori infection on acute and chronic colitis induced by DSS may through the expansion and function of CD $19^{+}$IL- $10^{+}$Breg cells.

Disclosure of Interest: All authors have declared no conflicts of interest.

P0301 FUNGAL COMPOSTION AND FUNGI-BACTERIA CORRELATION IN IBD PATIENTS WITH DIFFERENT TREATMENT STRATEGIES

J. Xu ${ }^{1}$, Z. Wu ${ }^{1}$, Y. Song ${ }^{1}$, N. Wu ${ }^{2}$, F. Zhang ${ }^{1}$, H. X. Ren ${ }^{1}$, N. Chen ${ }^{1}$, Y. L. Liu ${ }^{1}$ ${ }^{1}$ Department Of Gastroenterology, Peking University People's Hospital, Beijing/ China

${ }^{2}$ Institute Of Clinical Molecular Biology \& Central Laboratory, Peking University People's Hospital, Beijing/China

Contact E-mail Address: jerry1989@hsc.pku.edu.cn

Introduction: The microbial dysbiosis plays a pivotal role in the pathogenesis of inflammatory bowel disease (IBD), however, the role of fungal microbiota in IBD was unclear. The aim of our study was to clarify the gut fungal composition in IBD patients with different treatment strategies.

Aims \& Methods: 73 IBD patients were divided into three groups, Untreatment $(\mathrm{n}=21)$, Antiinflammation $(\mathrm{n}=43)$ and Immunosuppression $(\mathrm{n}=9)$. Antiinflammation was defined as treatment with 5-aminosalicylic acid (5ASA)/salazosulfapyridine (SASP) and Immunosuppression as treatment with Glucocorticoid (GC), azathioprine (AZA), biologics and thalidomine. Noninflamed and inflamed mucosae were acquired for 16S and ITS sequencing to investigate the bacterial and fungal composition. Inflamed mucosa was used for RNA extraction and real-time PCR to detect the expression of IBD-associated biomarkers, such as TNF-alpha, IL-17A, MCP-1, etc. Analysis of Spearman's correlation was performed to estimate the fungi-bacteria and microbiota-biomarkers correlation.

Results: Compared with noninflamed mucosa, lower diversity and evenness were observed in inflamed mucosa in all IBD patients, but no significance in noninflamed (or inflamed) mucosa of different treatment strategies. Beta diversity showed a treatment-dependent clustering in inflamed mucosa. Fungal microbiota was constituted by fungi from Ascomycota, Basidomycota and Zygomycota phyla. There was a higher proportion of Zygomycota in inflamed mucosa than noninflamed mucosa in untreated IBD patients, and Antiinflammation and Immunosuppression didn't significantly alter its abundance. Mortierella from Zygomycota was the richest fungi in both inflamed and noninflamed mucosae of all patients. To analyze the effects of treatment strategies on fungal microbiota in IBD patients, we found Immunosuppression decreased abundance of Ascomycota in both noninflamed and inflamed mucosae, while antiinflammation increased Ascomycota in noninflamed mucosa but decreased it in inflamed mucosa. Both Antiinflammation and Immunosuppression increased Zygomycota in inflamed mucosa, but not in noninflamed mucosa. Fungi-bacteria correalation analysis showed a weak correlation in noninflamed mucosa of untreated IBD patients, and Antiinflammation and Immunosuppression didn't significantly alter fungi-bacteria correlation patterns in noninflamed mucosa. However, after Immunosuppression, fungi including Candida, Chaetomium, Cladosporium and Cryptococcus were positively correlated to bacteria such as Coprococcus and Lachnospira. Futhermore, Mortierella was negatively correlated with Blautia and Lachnospira in inflamed mucosa of immunosuppression. In analysis of bacteria-biomarkers correlation, we found bacteria such as Clostridiales, Lachnospiraceae, Parabacteroides, Blautia, Coprococcus, Roseburia, Faecalibacterium, Ruminococcus and Megamonas were significantly correlated to several biomarkers (such as IFN-gamma, IL-10, IL-17A, IL-22, TNF-alpha, etc). Notably, there were different correlation patterns in different treatment strategies, especially, IL-17A was extensively correlated to bacteria such as Enterococcus, Corprococcus, Faecalibacterium and Klebsiella in inflammed mucosa of Immunosuppression, but only correlated to Faecalibacterium in inflammed mucosa of Antiinflammation. Additionally, we found a weak fungi-biomarkers correlation in IBD patients, but fungi such as Asterotremella and Verticillium were correlated to biomarkers such as IL-17A, IL-22, IL-8 and MCP-1, and treatment altered microbiota-biomarkers correlation patterns.

Conclusion: Treatment strategies affect fungal composition. To some extent, immunosuppression may aggravate gut fungal dysbiosis in IBD patients, but antiinflammation partially ameliorate it. The bacteria-fungi and microbiota-biomarkers correlation displayed a treatment-specific feature, and treatments change these correlation patterns. Additionally, IL-17A tended to be the main mediator for bacteria to induce inflammation in IBD.

Disclosure of Interest: All authors have declared no conflicts of interest.

\section{P0302 INVESTIGATING THE MICROBIOME IN A PHASE 1B \\ STUDY OF ANDECALIXIMAB IN ULCERATIVE COLITIS}

\section{B. Lamere ${ }^{1}$, E. R. Wendt ${ }^{2}$, B. Kanwar ${ }^{3}$, S. V. Lynch}

${ }^{1}$ Division Of Gastroenterology, UCSF, San Francisco/United States of America/ $C A$

${ }^{2}$ Biomarker Sciences, Gilead Sciences, Inc, Foster CIty/United States of America/ $C A$

${ }^{3}$ Gilead Sciences, Foster City/United States of America

Contact E-mail Address: Brandon. Lamere@ucsf.edu

Introduction: MMP9 is involved in the degradation of the extracellular matrix and its expression is elevated in the inflamed tissue of patients with ulcerative colitis (UC) [1-3]. Pre-clinical models of colitis demonstrate a therapeutic benefit of anti-MMP9 in preventing and ameliorating colitis [4]. Andecaliximab (previously GS-5745) is a high-affinity IgG4 monoclonal antibody against human Matrix Metallopeptidase 9 (MMP9). In a 36-day Phase 1b study in UC, andecaliximab demonstrated clinical efficacy relative to placebo treatment [5]. Here we describe bacterial microbiota analysis of stool samples collected during the Phase $1 \mathrm{~b}$ study of andecaliximab in UC.

Aims \& Methods: The objective of this study is to examine changes to the bacterial microbiota pre- and post-andecaliximab treatment and relative to therapeutic response. Stool was collected prior to treatment (Baseline) and at the end of the study (Day 36). Clinical response was defined as a Mayo score reduction $\geq 3$ point and $\geq 30 \%$ reduction from baseline score; accompanying decrease in rectal bleeding sub-score of $\geq 1$ or an absolute rectal bleeding sub-score of 0 or 1. DNA was extracted from fecal samples using a modified CTAB method and 16S rRNA amplicon sequencing was performed on 59 samples (27 paired and 5 unpaired samples). Alpha diversity, beta diversity (calculated in QIIME), and taxonomic differences were examined between placebo and andecaliximab-treated patients and between responders and non-responders.

Results: Compared to placebo-treated patients, those who received andecaliximab trended towards decreased alpha diversity $(\mathrm{p}=0.06)$ at 36 days post-treatment. These changes in alpha diversity were not dose related. At Day 36, a trend towards a significant difference in community beta-diversity was observed between the andecaliximab-treated group relative to placebo $(p=0.07)$ Andecaliximab treatment was also associated with differences in bacterial taxonomy relative to placebo $(\mathrm{p}=0.07)$. Specifically, the genera Clostridia and Akkermansia represented some of the top organisms enriched post andecaliximab treatment relative to placebo. Andecaliximab treatment exhibited a non-significant expansion of Akkermansia from Baseline to Day $36(\mathrm{p}=0.15)$. Amongst andecaliximab-treated patients, responders exhibited a trend of increased relative abundance of Akkermansia muciniphilia $(\mathrm{p}=0.08)$.

Conclusion: Akkermansia muciniphilia is the most consistently enriched organism for subjects treated with andecaliximab who respond to treatment. The presence of Akkermansia muciniphilia at Baseline and/or an increase in Akkermansia relative abundance may be associated with response to andecaliximab. Analysis of a larger study population would be required to verify these observations. Recent Phase 2 studies of andecaliximab in UC and Crohn's disease failed to demonstrate clinical efficacy. Therefore these microbiome results are unlikely to be pursued in relation to andecaliximab treatment effects, but may be beneficial as a reference for future trials in inflammatory bowel disease.

Disclosure of Interest: B. LaMere: Microbiome data was analyzed and interpreted by UCSF and funded by Gilead Sciences.

E.R. Wendt: Employee of Gilead Sciences, Inc.

B. Kanwar: Employee of Gilead Sciences, Inc.

S.V. Lynch: Consultant for Theravance Sponsored research projects from Sloan Foundation, CF Foundation, Broad Foundation, J\&J, Jannsen Pharmaceuticals and Gilead Royalties for IP licensed by KaloBios Inc. Founder and Board of Directors, Siolta Therapeutics

\section{References}

1. Baugh, M.D., et al., Matrix metalloproteinase levels are elevated in inflammatory bowel disease. Gastroenterology, 1999. 117(4): p. 814-22.

2. Gao, Q., et al., Expression of matrix metalloproteinases-2 and -9 in intestinal tissue of patients with inflammatory bowel diseases. Dig Liver Dis, 2005. 37(8): p. $584-92$

3. Hu, J., et al., Matrix metalloproteinase inhibitors as therapy for inflammatory and vascular diseases. Nat Rev Drug Discov, 2007. 6(6): p. 480-98.

4. Marshall, D.C., et al., Selective Allosteric Inhibition of MMP9 Is Efficacious in Preclinical Models of Ulcerative Colitis and Colorectal Cancer. PLoS One, 2015. 10(5): p. e0127063.

5. Sandborn, W.J., et al., Randomised clinical trial: a phase 1, dose-ranging study of the anti-matrix metalloproteinase-9 monoclonal antibody GS-5745 versus placebo for ulcerative colitis. Aliment Pharmacol Ther, 2016. 44(2): p. 157-69.

\section{P0303 MUCOSAL CYTOKINE PROFILE IN INFLAMMATORY BOWEL DISEASE PATIENTS: A LASER CAPTURE MICRODISSECTION APPROACH}

G. Mazzarella ${ }^{1}$, V. Rotondi Aufiero ${ }^{1}$, P. Marena ${ }^{1}$, A. Venezia ${ }^{1}$, R. Melina ${ }^{2}$, N. Giardullo ${ }^{2}$, B. Perugini ${ }^{3}$, F.S. Taccone ${ }^{4}$, S. Iaquinto ${ }^{5}$, G. Iacomino ${ }^{1}$, G. Iaquinto ${ }^{3}$

${ }^{1}$ Immunomorphology, Institute of Food Sciences-CNR, Avellino/Italy

${ }^{2}$ Gastroenterology, S.G. Moscati Hospital, Avellino/Italy

${ }^{3}$ Gastroenterology, Clinic Santa Rita, N. Taccone Foundation, Atripalda/Italy

${ }^{4}$ Department Of Intensive Care Unit, Hopital Erasme, Université Libre de

Bruxelles, Brussels/Belgium

${ }^{5}$ Gastroenterology, San Filippo Neri Hospital, Roma/Italy

Contact E-mail Address: gmazzarella@isa.cnr.it

Introduction: Crohn's Disease (CD) and Ulcerative Colitis (UC) are Inflammatory Bowel Diseases (IBD) with a complex etiology, including an immune response against microbial and autologous antigens and an imbalance between pro-inflammatory and anti-inflammatory mediators. Different approaches have been used to study the pattern of cytokines in IBD and few data are available on cytokines production in different intestinal compartments. Laser Capture Microdissection (LCM) is a powerful tool for the isolation of specific tissue compartments (1). 
Aims \& Methods: This work was designed to investigate the pattern of cytokines that regulate the mucosal immune response occuring in different intestinal compartments of IBD patients, using LCM technology (1). Frozen sections of colonic biopsies were obtained from 5 patients with active $\mathrm{CD}, 5$ patients with active UC and 5 controls. None of the patients with $\mathrm{CD}$ or UC had been ever undergone medical therapy. Epithelium (EP) and lamina propria (LP) were isolated by LCM, RNAs from EP and LP samples were extracted and, after a reverse transcription, RNA levels of TNF- $\alpha$, IFN- $\gamma$, IL-17, IL-10 and TGF- $\beta$ were determined by quantitative PCR, using glyceraldehyde 3-phosphate dehydrogenase (GAPDH) as reference gene.

Results: We observed a significant increase in gene expression level of IL-17 in the lamina propria of UC patients respect to $\mathrm{CD}$ and controls $(\mathrm{p}<0.05)$. TNF- $\alpha$, IFN- $\gamma$, IL-10 and TGF- $\beta$ levels were significantly higher in the LP of CD as compared to UC and controls $(\mathrm{p}<0.05)$. All the cytokines investigated were not significantly up-regulated in the surface EP of both CD and UC patients, when compared to controls

Conclusion: Our data show that the LP compartment play a key role in the mucosal immune response in IBD patients. In particular, CD seems to be prominently an innate immune response-mediated disease, which is characterized by an increased production of IFN- $\gamma$ and TNF-a. On the opposite, UC seems to be prominently a CD4 lymphocyte-mediated disease, which is characterized by an increased production of IL-17. Concomitantly with the pro-inflammatory response, high amounts of the anti-inflammatory cytokines IL-10 and TGF- $\beta$ are also produced in $\mathrm{CD}$ compared to UC patients, suggesting that in UC patients the immune-regulatory mechanisms could be impaired. This work underlines the importance of LCM as a valuable tool to determine potential inflammatory components involved in IBD pathogenesis.

Disclosure of Interest: All authors have declared no conflicts of interest.

\section{Reference}

1. Iacomino G, Marano A, Stillitano I, Aufiero VR, Iaquinto G, Schettino M, Masucci A, Troncone R, Auricchio S, Mazzarella G. Mol Cell Biochem. 2016 Jan;411(1-2):341-9.

P0304 ALTERATIONS IN THE MUCOSA-ASSOCIATED FUNGAL MICROBIOTA IN PATIENTS WITH ULCERATIVE COLITIS

\section{Qiu, H. Zhang}

Department Of Gastroenterology, Jiangsu Province Hospital and Nanjing Medical University, Nanjing/China

Contact E-mail Address: qiuxinyun2819@126.com

Introduction: The gut microbiota play important roles in the development of the ulcerative colitis (UC). Enough evidence has proven the role of intestinal bacterial microbiota in UC pathogenesis. However, the role of intestinal fungal microbiota in UC pathogenesis has not been fully demonstrated.

Aims \& Methods: Fungal microbiota from the descending colon mucosal samples of 14 active UC patients and 15 healthy subjects (HS) were analyzed by highthroughput sequencing method. The expressions of pro-inflammatory cytokines(IL-I $\beta$, TNF- $\alpha$, INF- $\gamma$, IL-6, IL-17A, and IF-23) in intestinal mucosa tissues were examined. The Baron and Mayo scores of UC patients were evaluated, and the correlations between intestinal fungal composition and intestinal inflammatory status were analyzed.

Results: The number of fungi decreased significantly in inflamed mucosal tissue compared to HS counterpart while the shannon diversity did not show significant differences. Fifteen major genera were examined, among which Wickerhamomyces, unidentified genus of Saccharomycetales, Aspergillus, Sterigmatomyces, and Candida showed increasing trends, whereas Exophiala, Alternaria, Emericella, Epicoccum, Acremonium, Trametes, and Penicillium showed decreasing trends in UC patients compared to HS. The pro-inflammatory cytokines (IL-I $\beta$, TNF- $\alpha$, INF- $\gamma$, IL-6, IL-17A, and IF-23) were up-regulated in the UC patients. The genera Wickerhamomyces. Sterigmatomyces, and Penicilium were positively correlated with the expression of several pro-inflammatory cytokines in the colonic mucosa, whereas Nigrospora was negatively correlated. Nigrospora and Sterigmatomyces were positively correlated with the Baron and/or Mayo score.

Conclusion: The fungal microbiota in the colonic mucosa of UC patients was different from that of the HS. Alterations in gut fungal composition might be associated with mucosal inflammation and pathogenesis of UC. Further studies are needed to define the fungal composition in detail and identify the role of different fungi in the gut, and determine the mechanism of the host-fungal interaction underlying the development of UC.

Disclosure of Interest: All authors have declared no conflicts of interest.

\section{P0305 CD16 POSITIVE CELLS, EXPRESSING WNT LIGANDS, ACCUMULATE IN THE MUCOSA AND MEDIATE INTESTINAL FIBROSIS}

D. Ortiz Masia ${ }^{1}$, P. Salvador ${ }^{1}$, D. Macias-Ceja ${ }^{2}$, S. Coll-Puig ${ }^{1}$, L. GisbertFerrándiz $^{1}$, C. Hernández ${ }^{2}$, S. Calatayud ${ }^{1}$, M.D. Barrachina ${ }^{1}$

${ }^{1}$ Dept. De Farmacologia, CIBERehd-Univ. de Valencia, Valencia/Spain

${ }^{2}$ Fisabio, Valencia/Spain

Contact E-mail Address: mdorma@uves

Introduction: STAT6 plays a crucial role in M2a macrophage polarization in vitro and these cells mediate mucosal healing in an acute model of TNBS-colitis through the expression of Wnt ligands (Mucosal Immunology, 2016). We have recently reported that STAT6 deficiency favours fibrosis in a murine model of TNBS colitis (P031, ECCO 2016).

Aims \& Methods: We aim to characterize here the functional relevance of the macrophage phenotype in fibrosis development. WT or STAT6 (-/-) mice were given TNBs $(0.5,0.5,0.75,0.75,1$, and $1 \mathrm{mg}$, intrarectally) or saline weekly and they were sacrificed 3,5 or 7 weeks after the first TNBs administration. The percentage of CD206, CD16, and CD86 positive cells was analyzed by flow cytometry in $\mathrm{F} 4 / 80+$ macrophages isolated from the intestinal mucosa. The mRNA expression of Wnt ligands was evaluated in F4/80+ CD16+ macrophages isolated from the mucosa, 7 weeks after the first TNBs administration and results are expressed as fold induction $v s$ vehicle-treated mice. The mRNA expression of CD16 and fibrosis markers were evaluated in the colonic mucosa. Data are expressed as Mean \pm SEM with $n \geq 8$ in all groups. In all cases results were expressed as fold induction vs vehicle $\left({ }^{*} \mathrm{p}<0.05\right.$ vs TNBS WT) and correlations between data were analyzed using Pearson's correlation coefficient $(* \mathrm{p}<0.05)$.

Results: TNBs increased the percentage of CD206 positive macrophages in the mucosa of TNBS-WT animals while it failed to do that in TNBS-STAT6 (- $/-$ mice. The percentage of CD16 positive macrophages increased in a time-dependent manner only in the mucosa of STAT6 (-/-)-TNBS-treated mice. The percentage of CD86+ cells was similar between TNBS-WT and TNBS-STAT6 (-/-) mice. In $\mathrm{CD} 16+$ macrophages isolated from TNBS-STAT6 (-/-) mice the mRNA expression of canonical and non-canonical Wnt ligands was significantly increased compared with cells isolated from TNBS-WT mice (Table). A positive and significant correlation between CD16 and Vimentin $\left(p=0.0088^{*}, r=0.51\right)$, $\alpha$-SMA $\left(\mathrm{p}=0.0044^{*}, \mathrm{r}=0.55\right)$ and MMP2 $\left(\mathrm{p}=0.0002^{*}, \mathrm{r}=0.67\right)$ was detected in TNBS-STAT6 (-/-) mice but not in WT animals. Table

\begin{tabular}{llllll}
\hline & 2B & $5 \mathrm{~A}$ & 6 & $7 \mathrm{~B}$ & $10 \mathrm{~A}$ \\
\hline WT & $1,6 \pm 0,2$ & $2,1 \pm 0,5$ & $1,2 \pm 0,1$ & $2,7 \pm 0,6$ & $3,3 \pm 0,8$ \\
STAT6 $(-/-)$ & $1,9 \pm 0,4$ & $6,4 \pm 0,6^{*}$ & $2,7 \pm 0,4^{*}$ & $15,3 \pm 2,2^{*}$ & $2,9 \pm 1,0$
\end{tabular}

Conclusion: The expression of Wnt ligands from CD16 positive cells, which are accumulated in the mucosa, may be involved in murine intestinal fibrosis Disclosure of Interest: All authors have declared no conflicts of interest.

\section{Reference}

Cosín-Roger J, Ortiz-Masiá D, Calatayud S, Hernández C, Esplugues JV, Barrachina MD. Mucosal Immunol. 2016 Jul;9(4):986-98. doi: 10.1038/ mi.2015.123. P031. STAT6 deficiency alters macrophage polarization and promotes fibrosis in a murine model of chronic inflammation. ECCO. 2016.

\section{P0306 CHARACTERIZATION OF GUT MICROBIOME} ASSOCIATED WITH IMPROVEMENT OF ULCERATIVE COLITIS AFTER ANTIBIOTIC COMBINATION THERAPY USING FECAL METAGENOMIC ANALYSIS

K. Kato ${ }^{1}$, T. Sekizuka ${ }^{2}$, T. Sugiyama ${ }^{3}$, Y. Ishii ${ }^{1}$, M. Kuroda ${ }^{2}$, T. Ohkusa ${ }^{4}$ ${ }^{1}$ Division Of Research Planning And Development, Nihon University School of Medicine, Tokyo/Japan

${ }^{2}$ Pathogen Genomics Center, National Institute of Infectious Diseases, Tokyo Japan

${ }^{3}$ Department Of Gastroenterology And Hematology, Graduate School of Medicine and Pharmaceutical Sciences, University of Toyama, Toyama/Japan

${ }^{4}$ Department Of Microbiota Research, Juntendo University School of Medicine, Tokyo/Japan

Contact E-mail Address: katou.kimitoshi@nihon-u.ac.jp

Introduction: Although the etiology of ulcerative colitis (UC) has yet to be characterized, it is increasingly accepted that the cause of UC might well be related to commensal enteric bacteria in a genetically susceptible patient. Anti-inflammatory drugs and immune system suppressors are usually prescribed for UC treatment, and we previously demonstrated that triple antibiotic combination therapy with oral amoxicillin $(1500 \mathrm{mg} /$ day $)$, tetracycline $(1500 \mathrm{mg} /$ day $)$ or fosfomycin $(3000 \mathrm{mg} /$ day), and metronidazole $(750 \mathrm{mg} /$ day) (ATM/AFM), for two weeks, induces remission in more than $27 \%$ of patients with active UC including those with steroid-refractory or dependent disease, suggesting ATM/AFM to possibly be effective for achieving UC remission.

Aims \& Methods: Thirty-two patients with UC given ATM/AFM therapy for two weeks on average were enrolled in this study. The clinical conditions of these UC patients were evaluated by Mayo score. Fecal samples were obtained prior to, after therapy and at three months after treatment completion. Gut microbiota were compared employing metagenomic analysis of fecal samples.

Results: Of the 32 patients, 17 and eight, respectively, experienced complete and partial remission over three months in response to ATM/AFM therapy, whereas ATM/AFM showed no efficacy in seven patients. The metagenomic analysis revealed abundant human DNA to correlate positively with the disease activity indicated by the Mayo score. Furthermore, dramatic gut microbiota changes were observed at an early stage, i.e. just two weeks after starting ATM/AFM therapy. Comparison of the metagenomic data suggested that the dysbiosis 
before treatment in the active stage to possibly be associated with increased populations of Bacteroides, Parabacteroides, Rickenella, Clostridium, Flavonifractor, Pelagibacter, Bordetella, Massilia and Piscrickettsia species. In responders after treatment, populations of Bifidobacterium and Lactobacillus species were significantly increased. In this study, there was an especially strong negative correlation between Bacteroides and Bifidobacterium both before and after treatment.

Conclusion: These results suggested metagenomic analysis results to be associated with a remarkable change in gut microbiota after antibiotic combination treatment. In responders, remission is associated with increases in Bifidobacterium and Lactobacillus species and a decrease in Bacteroides.

Disclosure of Interest: All authors have declared no conflicts of interest.

\section{References}

Ohkusa T, et al. American J Gast 2010;105:1820-9.

Kato K, et al. $A P T$ 2014; 39: 949-56

\section{P0307 GLP-1 EXPRESSING ENTEROENDOCRINE CELL NUMBERS ARE REDUCED AT THE SITE OF ACTIVE DISEASE IN VARIOUS MOUSE MODELS OF INTESTINAL INFLAMMATION}

E. Rath $^{1}$, N. Waldschmitt ${ }^{1}$, M. Ahmed ${ }^{1}$, S. Khaloian Sarnaghi ${ }^{1}$, M. Schaubeck ${ }^{1}$, G. Hörmannsperger ${ }^{1}$, J. Planchais ${ }^{2}$, H. Sokol ${ }^{3}$, D. Haller

${ }^{1}$ Chair Of Nutrition And Immunology, Technische Universität München, FreisingWeihenstephan/Germany

${ }^{2}$ UMR 1319 Micalis, Jouy-en-Josas/France

${ }^{3}$ Avenir Team Gut Microbiota and Immunity, INSERM U1157/UMR CNRS 7203 UPMC; UMR 1319 Micalis; APHP, St Antoine, Department of Gastroenterology, Paris/France

\section{Contact E-mail Address: eva.rath@tum.de}

Introduction: Classically, enteroendocrine cells (EEC) are renowned for regulating gastrointestinal motility, secretion, and insulin levels by release of peptide hormones. Yet, via receptors and transporters, EEC are capable of sensing the lamina propria and luminal environment, including the microbiota, and also mediate immune-related signals. In particular, the L-cell-derived incretin hormone glucagon-like peptide 1 (GLP-1) is increasingly recognized to exert direct effects on immune cells and to orchestrate a metabolic-inflammatory response. In inflammatory bowel disease (IBD), a role for EEC in disease pathogenesis is indicated by a risk-associated SNP and autoantibodies affecting EEC function as well as general disease symptoms like insulin resistance and altered intestinal motility. However, the total number of studies investigating EEC number and function in IBD and mouse models of intestinal inflammation is limited and results are conflicting.

Aims \& Methods: To characterize alterations in GLP-1-expressing EEC numbers under intestinal inflammatory conditions, immunostainings for GLP-1 and ChgA as well as mRNA expression analysis was performed in intestinal tissue samples. Mouse models of intestinal inflammation used include genetic models, Il-10 $0^{-1-}$ mice (colitis), an adoptive transfer model, $\mathrm{Rag}^{-/-}$mice reconstituted with $\mathrm{CD}^{+} \mathrm{T}$ cells (colitis), chemically (DSS)-induced colitis and an infection model (Citrobacter rodentium).

Results: Numbers of $\mathrm{GLP}_{-}{ }^{+}$and $\mathrm{ChgA}^{+}$cells were consistently reduced in all mouse models of intestinal inflammation. These changes were confined to the site of active inflammation. Neither absence of mature B and T cells in Rag2 ${ }^{-/-}$mice alone was associated with reduced numbers of EEC, nor adoptive transfer of $\mathrm{CD}^{+} \mathrm{T}$ cells per se, since transfer of non-colitogenic $\mathrm{CD}^{+}{ }^{+} \mathrm{CD} 25^{+} \mathrm{T}$ cells did not lead to changes in $\mathrm{ChgA}^{+}$cell numbers in the colon. The reduction of GLP$1^{+}$and $\mathrm{ChgA}^{+}$cells observed by immunohistochemistry was reflected by diminished levels of Gcg and Chga mRNA expression, whereby mRNA levels of the Lcell derived-hormone Pyy remained unaltered.

Conclusion: To our knowledge, this work provides the first comprehensive study of $\mathrm{GLP}_{-}{ }^{+}$and $\mathrm{ChgA}^{+}$cell numbers in different mouse models of intestinal inflammation. A reduction of GLP-1-expressing EEC seems to be a general feature of small as well as large intestinal inflammation. Further research will clarify if these alterations represent a consequence or causatively contribute to intestinal inflammation and elucidate the functional consequences on immune responses.

Disclosure of Interest: All authors have declared no conflicts of interest.

\section{P0308 ACTIVE ULCERATIVE COLITIS IS CHARACTERIZED BY THE FORMATION OF THROMBOGENIC TISSUE FACTOR- EXPRESSING NEUTROPHIL EXTRACELLULAR TRAPS}

I. Angelidou ${ }^{1}$, A. Mitsios ${ }^{1}$, A. Chrysanthopoulou ${ }^{1}$, K. Kambas ${ }^{1}$,

A. Arampatzioglou ${ }^{1}$, E. Dellaporta ${ }^{2}$, S. Arelaki ${ }^{3}$, V. Dalla ${ }^{4}$, K. Ritis ${ }^{4}$,

G. Kouklakis ${ }^{4}$, P. Skendros ${ }^{4}$

${ }^{1}$ Laboratory Of Molecular Hematology, University Hospital of Alexandroupolis, Democritus University of Thrace, Alexandroupolis/Greece

${ }^{2}$ Laboratory For The Study Of Gastrointestinal And Liver Diseases, University Hospital of Alexandroupolis, Democritus University of Thrace, Alexandroupolis/ Greece

${ }^{3}$ Department Of Pathology, University Hospital of Alexandroupolis, Democritus University of Thrace, Alexandroupolis/Greece

${ }^{4}$ First Department Of Internal Medicine, University Hospital of Alexandroupolis, Democritus University of Thrace, Alexandroupolis/Greece
Contact E-mail Address: pskendro@med.duth.gr

Introduction: Neutrophil extracellular traps (NETs) are fibrous structures released by activated neutrophils upon stimulation. NETs consist of chromatin and cytoplasmic proteins, which are released extracellularly during a process called NETosis. Tissue factor (TF) is the main in-vivo initiator of coagulation and is expressed on NETs in a plethora of neutrophil-mediated thromboinflammatory conditions [1-3]. Ulcerative colitis (UC) is characterized by infiltration of neutrophils into the affected mucosa and increased risk of thromboembolic events; however the mechanism behind the thrombophilic state of UC has not been clearly elucidated yet $[4,5]$.

Aims \& Methods: We aimed to investigate for the first time the role of neutrophils/NETs through TF expression in the pathophysiology of UC. Neutrophils, sera and colon biopsies were obtained from 10 naïve patients with active UC (6 male and 4 female, mean age $34.6 \pm 19.1$ years, mean Mayo score $8.5 \pm 1.4$ ) and from 10 naïve patients suffered from active Crohn disease (CD) (7 male, 3 female, mean age $34.2 \pm 18.5$, CDAI > 220). Additionally, 10 sex- and age-matched healthy subjects, were served as control subjects ( 5 male, 5 female, mean age $38.5 \pm 12.5$ ). Ex-vivo findings regarding peripheral blood neutrophils and colonic tissue specimens were verified using appropriate in-vitro stimulations of control neutrophils with corresponding sera and intestinal tissue-conditioned media, respectively. Identification and quantification of NETs were performed with immunofluorescence confocal microscopy (ICM), flow cytometry and MPO DNA complex ELISA. The expression of TF on neutrophils/NETs was determined using ICM, qRT-PCR and western blot analysis. The bioactivity of TF on NETs was assessed by measuring thrombin-antithrombin complex levels with ELISA.

Results: Neutrophils from patients with active UC are characterized by increased NET formation in both the peripheral blood and affected colonic mucosa, compared to CD. Furthermore, NETs in UC are decorated with functionally active TF and the amount of TF-bearing NETs was reduced from the inflamed to normal colon. In-vitro stimulations of controls neutrophils with sera or intestinal tissue-conditioned media corroborated the findings obtained ex-vivo.

Conclusion: NETs expressing bioactive TF may be involved in the induction of intestinal inflammation and systemic thrombosis in UC probably constituting a novel candidate diagnostic and/or therapeutic target.

Disclosure of Interest: All authors have declared no conflicts of interest.

\section{References}

1. Martinod K, Wagner DD. Blood. 2014;123:2768-76.

2. Kambas K, Chrysanthopoulou A, Vassilopoulos D, et al. Ann Rheum Dis. 2014;73:1854-63.

3. Stakos DA, Kambas K, Konstantinidis T, et al. Eur Heart J 2015;36:140514.

4. Brazil JC, Louis NA, Parkos CA. Inflamm Bowel Dis. 2013;19:1556-65

5. Zezos P, Kouklakis G, Saibil F. World J Gastroenterol. 2014;20:13863-78.

\section{P0309 IMPAIRED MITOCHONDRIAL PROTEOSTASIS IS ASSOCIATED WITH MITOCHONDRIAL DYSFUNCTION AND INDUCES PHENOTYPIC TRANSITION OF LGR5 $^{+}$STEM CELLS INTO PANETH CELLS}

S. Khaloian Sarnaghi, E. Rath, N. Waldschmitt, I. Gosch, E. Berger, D. Haller Chair Of Nutrition And Immunology, Technische Universität München, FreisingWeihenstephan/Germany

Contact E-mail Address: sevana.khaloian@tum.de

Introduction: The intestinal epithelium is a multicellular interface that is completely renewed every 3-5 days. Pluripotent stem cells reside at the crypt bottom giving rise to transient amplifying cells and subsequently differentiated intestinal epithelial cells (IEC) of all subtypes. Phases of cellular and functional transitions are characterized by distinct metabolic identities, reflected by changes in mitochondrial activity. Alterations in mitochondrial function and mitochondrial unfolded protein response (MT-UPR) activation are associated with various chronic pathologies including inflammatory bowel diseases (IBD) and cancer. We have previously shown that MT-UPR and mitochondrial function itself is involved in the regulation of cell cycle progression and intestinal stemness. Here, we present evidence that impaired mitochondrial proteostasis is sufficient to drive differentiation of $\mathrm{Lgr}^{+}$stem cells (ISC) into Paneth cells.

Aims \& Methods: To depict the impact of imbalances in mitochondrial proteostasis on ISC, we used mice with a tamoxifen-inducible ISC or IEC-specific conditional knockout allele for the mitochondrial chaperone Hsp60 and the mitochondrial protease ClpP. Molecular consequences of the gene deletions in the different models were further characterized ex vivo using intestinal organoid culture. In situ hybridization, IHC and combinations of both as well as IF were performed to illustrate alterations of IEC subtypes. Readouts were complemented by mRNA expression analysis and biochemical approaches.

Results: ClpP-knockout as well as chemical inhibition of the respiratory chain in intestinal organoids led to diminished Lgr5 expression confirming our results from IEC and ISC specific Hsp60-deletion. In vivo, the ISC-specific loss of HSP60 resulted in a transient drop in $\mathrm{Lgr}^{+}$cells with $\mathrm{Lgr} 5$ expression being reduced from day 2 after end of tamoxifen treatment and signals reappearing from day 4. Cells positive for the stem cell markers Olfm 4 and Hopx expanded at day 2 , indicating reserve stem cell populations compensating for the $\mathrm{Lgr} 5^{+}$ISC loss. Minimal numbers of $\mathrm{Lgr} 5^{+}$stem cells at day 2 were further paralleled by increased numbers of $\mathrm{Lgr}^{+}, \mathrm{Lyz}^{+}$Paneth cells and no signs of enhanced cell death, indicating differentiation of ISC into Paneth cells. $\mathrm{Lgr}^{+}, \mathrm{Lyz}^{+}$Paneth cells displayed a premature phenotype with diffuse Lyz staining in the cytoplasm. 


\begin{tabular}{|c|c|c|c|c|c|c|}
\hline \multicolumn{7}{|c|}{ Abstract No:P0310 } \\
\hline \multicolumn{7}{|l|}{ HILIC } \\
\hline $\begin{array}{l}\text { Mass/RT } \\
\text { (mins) }\end{array}$ & Biofluid & $\begin{array}{l}\text { Univariate (UV) } \\
\text { or Multivariate (MV) }\end{array}$ & Change* & p-value & Compound & Pathway \\
\hline $136.061 / 2.13$ & Faecal & UV & Increased & 0.0049 & Adenine & Protein digestion and absorption small bowel \\
\hline $166.084 / 3.65$ & Faecal & UV & Increased & 0.0218 & L-phenylalanine & Release of gut hormones from endocrine system \\
\hline $205.1 / 3.61$ & Faecal & UV & Increased & 0.0058 & L-tryptophan & Protein digestion and absorption small bowel \\
\hline $229.153 / 5.78$ & Faecal & MV & Increased & 0.0075 & 1-Leucylproline & Related to leucine pathway \\
\hline $114.065 / 2.53$ & Faecal & MV & Increased & 0.0049 & Creatinine & Arginine and proline metabolism \\
\hline $132.099 / 4.89$ & Urine & UV & Decreased & 0.0215 & Creatine & Facilitate recycling of ATP \\
\hline $166.119 / 3.62$ & Urine & UV & Decreased & 0.0066 & L-phenylalanine & Release of gut hormones from endocrine system \\
\hline $180.063 / 0.99$ & Urine & UV & Decreased & 0.0021 & Hippuric acid & Product of microbial metabolism of dietary aromatic compounds \\
\hline $268.103 / 1.72$ & Urine & UV & Increased & 0.0011 & Adenosine & Purine biosynthesis \\
\hline $148.032 / 4.57$ & Urine & MV & Increased & 0.0014 & Glutamic acid & Tyrosine metabolism \\
\hline $247.142 / 3.88$ & Urine & MV & & & Unassigned & \\
\hline $249.149 / 3.87$ & Urine & MV & & & Unassigned & \\
\hline \multicolumn{7}{|c|}{ BILE ACIDS } \\
\hline $375.28 / 10.79$ & Faecal & UV & Decreased & 0.0046 & Lithocholic acid & Secondary bile acid from bacterial metabolism of chenodeoxycholic acid \\
\hline $375.28 / 10.81$ & Serum & UV & Decreased & 0.0041 & Lithocholic acid & \\
\hline $391.28 / 10.35$ & Serum & UV & Decreased & 0.0072 & Deoxycholic acid & Secondary bile acid from metabolism of cholic acid \\
\hline $407.28 / 8.33$ & Serum & UV & Decreased & 0.0045 & Cholic acid & Primary bile acid \\
\hline $432.314 / 9.91$ & Serum & UV & Decreased & 0.0230 & Glycolithocholic acid & Sulphur ester of lithocholic acid \\
\hline $373.272 / 10.17$ & Serum & MV & Decreased & 0.0007 & 5-Cholenic Acid-3B-ol & \\
\hline $391.283 / 10.34$ & Serum & MV & Decreased & 0.0018 & Deoxycholic acid & \\
\hline
\end{tabular}

Concomitantly, these cells were positive for the WNT ligand WNT10A and autophagy/mitophagy- associated LC3, suggesting autoregulatory mechanisms for the maintenance of the stem cell niche and mitochondria-associated functional alterations, respectively.

Conclusion: Our results indicate that mitochondrial function not only reflects IEC phenotypic changes but seems to be the driving force in differentiation processes. Mitochondrial function might therefore represent a key player at the edge of intestinal tissue homeostasis and repair/healing processes in the context of diseases.

Disclosure of Interest: All authors have declared no conflicts of interest.

\section{P0310 METABONOMIC PROFILING OF ULCERATIVE COLITIS PATIENTS; RESULTS FROM AN INCEPTION COHORT TIME SERIES ANALYSIS}

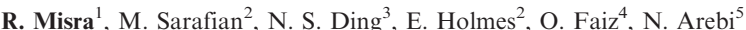

${ }^{1}$ Gastroenterology, St. Marks Hospital, London/United Kingdom

${ }^{2}$ Centre For Computational And Systems Medicine, Imperial College, London/

United Kingdom

${ }^{3}$ Gastroenterology, St. Vincents Hospital, Melbourne/Australia

${ }^{4}$ Surgical Epidemiology Trials And Outcomes Unit (SETOC), St. Marks

Academic Institute, London/United Kingdom

${ }^{5}$ Gastroenterology, St. Mark's Academic Institute, London/United Kingdom

Contact E-mail Address: rm399@ic.ac.uk

Introduction: Previous studies have shown differences in disease phenotype of ulcerative colitis (UC) in South Asian (SA) migrants compared to Caucasians with pan-colonic phenotype predominant. ${ }^{1}$ The gut microbiota differs in Caucasian and SA patients with $\mathrm{UC}^{2}$ however, there is limited evidence on how this translates to the metabolome.

Aims \& Methods: We aimed to examine the metabolic profile in a newly diagnosed cohort of UC patients recruited from St. Marks Hospital, London, UK. Patients were stratified by ethnicity (SA, Caucasian, Other), treatment (None, 5ASA, Azathioprine and Steroids) and disease duration. Healthy controls (HC) were recruited locally among the staff at St. Marks Hospital. Biofluids (urine, faeces and serum) were collected at diagnosis (time point 1 ; months $0-3$ ) and 2 further time points over one year (time point 2 : months 4-8, time point 3 : month 9-12) Metabonomics approach was applied using two different UPLC-MS profiling methods; for small metabolites (hydrophilic liquid chromatography, HILIC) and for bile acids (BA) platforms. Univariate (UV) and multivariate (MV) data analysis was implemented to build models using principle component analysis (PCA) and orthogonal partial least squares -discriminant analysis (OPLS-DA) to find metabolites that were expressed in significantly different amounts between the two populations, time points ( 1 vs 3 ) and treatment groups. Results: Fifty patients with UC of SA and Caucasian backgrounds were recruited. A total of 309 samples were collected. Sample collection was completed for all time points for $18 \mathrm{SA}(11 \mathrm{UC}$ and $7 \mathrm{HC}$ ) and 21 Caucasians ( $9 \mathrm{UC}$ and 12 $\mathrm{HC}$ ). There was no significant difference between SA and Caucasian at time points 1,2 and 3 and treatment groups Significant differences were observed between HC vs. disease, SA HC vs. Caucasian HC and SA UC vs Caucasian UC. For the UC cohort, robust models were obtained by OPLS-DA between SAs and Caucasians; Faecal HILIC $\left(\mathrm{R}^{2} \mathrm{Y} 0.869, \mathrm{Q}^{2} \mathrm{Y} 0.451, \mathrm{p}<0.0003\right)$ and urine HILIC $\left(R^{2} Y 0.783, Q^{2} Y 0.526, p<0.0001\right)$ and serum BA $\left(R^{2} Y 0.702, Q^{2} Y\right.$
0.517, $\mathrm{p}<0.0001)$ and faecal $\mathrm{BA}\left(\mathrm{R}^{2} \mathrm{Y} \quad 0.832, \mathrm{Q}^{2} \mathrm{Y} \quad 0.395, \mathrm{p}<0.0001\right)$. Combined analysis revealed 1611 significant features (faecal HILIC 60, urine HILIC 189, serum BA 489 and faecal BA 873). The assigned features are shown in Table 1. Faecal HILIC showed significantly higher essential amino acids (Adenine, L-phenylalanine, L-tryptophan) levels on UV and higher 1-leucylproline and creatinine levels on MV analysis for SAs. Urine HILIC showed lower creatine, L-phenylalanine and hippuric acid levels. Serum primary (Cholic and chenodeoxycholic acid) and secondary bile acids (BA modified by the gut) were significantly reduced in SAs. Table 1: Significant metabolites in OPLS-DA model between South Asian (SA) and Caucasians with UC. *compound is increased or decreased in SA compared with Caucasians respectively Conclusion: This study highlights the promise of UPLC-MS profiling to differentiate between SA and Caucasian groups. There are several possible reasons but two important factors are differing microbial metabolism and diet between the two groups. We are conducting further studies incorporating dietary data and $16 \mathrm{~S}$ microbial analysis in this cohort. In combination with matching disease extent (left-sided vs colonic disease) may help to identify possible explanations for the different disease phenotype in this group.

Disclosure of Interest: All authors have declared no conflicts of interest.

\section{References}

1. Carr I., Mayberry JF. The effects of migration on ulcerative colitis: A threeyear prospective study among Europeans and first- and second-generation South Asians in Leicester (1991-1994). Am $J$ Gastroenterol 1999;94(10):2918-22. Doi: 10.1016/S0002-9270(99)00494-3.

2. Mar JS., LaMere BJ., Lin DL., Levan S., Nazareth M., Mahadevan U., et al. Disease Severity and Immune Activity Relate to Distinct Interkingdom Gut Microbiome States in Ethnically Distinct Ulcerative Colitis Patients. MBio 2016;7(4). Doi: 10.1128/mBio.01072-16.

\section{P0311 BASELINE CLINICAL AND ENDOSCOPIC FEATURES OF ULCERATIVE COLITIS PATIENTS ARE RELEVANT GUIDE FOR SELECTING RESPONDERS TO SELECTIVE DEPLETION OF MYELOID LINEAGE LEUCOCYTES AS REMISSION INDUCTION THERAPY}

T. Tanaka ${ }^{1}$, M. Akagi ${ }^{2}$, A. R. Saniabadi ${ }^{3}$, H. Goishi ${ }^{1}$, T. Iiboshi ${ }^{1}$, T. Kajihara ${ }^{1}$, T. Miura ${ }^{1}$

${ }^{1}$ Akitsu Prefectural Hospital, Hiroshima/Japan

${ }^{2}$ Akitsu Hospital, Hiroshima/Japan

${ }^{3}$ JIMRO, Takasaki/Japan

\section{Contact E-mail Address: tomotaka@c.do-up.com}

Introduction: Patients with active inflammatory bowel disease have elevated myeloid lineage leucocytes ${ }^{1}$ including the $\mathrm{CD} 14+\mathrm{CD} 16+\mathrm{DR}++$ phenotype known as proinflammatory monocytes, and a major source of tumour necrosis factor- $\alpha{ }^{2}$ Accordingly selective depletion of myeloid leucocytes by granulocyte/monocyte apheresis (GMA) is expected to promote remission or enhance drug efficacy. However, studies in ulcerative colitis (UC) patients have reported contrasting efficacy outcomes, ranging from an $85 \%^{3}$ to statistically insignificant level. Patients' baseline demographic features may guide to selecting responder patients. 
Aims \& Methods: In a retrospective and single-centre setting we aimed to understand if patients' baseline clinical and endoscopic features were relevant guide for identifying likely responders and non-responders to adsorptive GMA. The subjects were 145 consecutive UC patients who had undergone GMA with the Adacolumn as remission induction therapy between 2012 and 2016. Seventythree patients were steroid naive, 70 were steroid dependent, and 2 patients were steroid refractory. Patients had received up to an 11 GMA sessions over 10 weeks. At entry and week 12 , patients were clinically and endoscopically evaluated, allowing each patient to serve as her or his own control. Clinical activity index $(\mathrm{CAI}) \leq 4$ was defined as remission. Biopsies from endoscopically detectable inflamed large intestinal mucosa were processed to see the impact of GMA on leucocytes within the mucosal tissue.

Results: At entry, the average CAI was 12.8 , range $10-17$. Ninety-three patients $(64.1 \%)$ had responded to GMA, 52 of 73 steroid naïve $(71.2 \%), 40$ of 70 steroid dependent $(57.1 \%)$, and 1 of the 2 steroid refractory cases. On average remission was sustained for 8.6 months in steroid naïve patients and for 10.4 months in steroid dependent subgroup. Observations on mucosal tissue biopsies showed that infiltrating leucocytes were mostly neutrophils and monocytes. There was a marked reduction of infiltrating leucocytes in responder patients. Patients with extensive deep UC lesions together with loss of the mucosal tissue at the lesions were non-responders. Patients with the first UC episode were identified as the best responders $(100 \%)$, followed by steroid naive patients. Additionally, a short duration of active UC prior to GMA marked a patient as a likely responder. Further, all patients who achieved remission were steroid free at week 12

Conclusion: First-episode and steroid-naïve cases who responded well to GMA attained a favourable long-term clinical course. Additionally, GMA was more effective if applied immediately after a relapse than after a lag time. In general, GMA is favoured by patients for its safety profile and for being a non-drug remission induction therapy. Patients with extensive deep ulcers, with long duration of UC refractory to multiple pharmacologicals are unlikely to benefit from GMA. In therapeutic settings, knowing baseline clinical and endoscopic features, which may identify GMA responder patients should guide to stop futile use of medical resources.

Disclosure of Interest: A.R. Saniabadi: Dr. Saniabadi has a non-regular employment position at JIMRO

All other authors have declared no conflicts of interest.

\section{References}

1. Saniabadi AR, et al. World J Gastroenterol 2014; 20: 9699-715.

2. Belge KU, et al. J Immunol 2002; 168: 3536-3542.

3. Cohen RD. Gastroenterology 2005; 128: 235-236.

4. Sands BE, et al. Gastroenterology 2008; 135: 400-409.

\section{P0312 THE EFFECTS OF GLIAL -DERIVED NEUROTROPHIC FACTOR PRODUCED BY ENTERIC GLIAL CELLS ON DENDRITIC CELL AND ITS ROLES IN DEXTRAN SULPHATE SODIUM INDUCED COLITIS}

\section{Y. Huan}

West China School of Medicine of Sichuan University, Chengdu/China

\section{Contact E-mail Address: yuhuan86@126.com}

Introduction: Much research has demonstrated that tolerogenic dendritic cell (DC) with the capacity to induce regulatory $\mathrm{T}$ cells (Treg) play an important role in maintaining immune tolerance and has been proposed for treatment of inflammatory bowel disease (IBD). In this study, we report on the use of glialderived neurotrophic factor (GDNF) produced by Enteric glial cells (EGCs) as a new approach to induce tolerogenic DCs with capacity to generate Treg, to restore immune tolerance in vivo, and to ameliorated experimental colitis.

Aims \& Methods: DCs were generated from rat bone marrow (BMDC) in the absence $\left(\mathrm{DC}_{\mathrm{Control}}\right)$ or presence of GDNF (DC $\left.\mathrm{DCNF}_{\mathrm{G}}\right)$ and additionally stimulated with LPS to induce maturation (mDC). The expression of major histocompatibility complex II (MHC-II), CD40, CD80, and CD86 was determined by flow cytometry. Levels of IFN- $\gamma$, interleukin-4 (IL-4), and IL-10 in the culture supernatants were determined by ELISA. The location of GDNF receptor- $\alpha 1$ (GFR$\alpha 1$ ) in DCs was detected by immuno-fluorescence staining. The expression of GFR- $\alpha 1$ and ERK1/ERK2 in DCs was tested by Western Blot. SD rats were fed with $5 \%$ Dextran Sulphate Sodium (DSS) to induce experimental colitis, the therapeutic action of $\mathrm{DC}_{\mathrm{GDNF}}$ were examined. Diverse clinical signs of the disease including weight loss, diarrhea, colitis, histopathology were evaluated, and the mechanisms involved in the potential therapeutic effect of $\mathrm{DC}_{\mathrm{GDNF}}$, such as inflammatory cytokines and chemokines (IL- 4 , IFN- $\gamma$, TNF- $\alpha$, IL-1 $\beta$, IL-17), the generation of IL-10 -secreting Treg (Treg1), and the level of Treg1/Th2. Treg1/ Th17 were investigated.

Results: GFR $-\alpha 1$ was expressed in BMDC, and the expression of ERK1/ERK2 were significantly up-regulated after treatment with GDNF. DC ${ }_{\text {GDNF }}$ did not upregulate MHC-II, CD40, CD80, CD86, and produced very low levels of proinflammatory cytokines (IFN- $\gamma$, IL-4) but secreted significant levels of the antiinflammatory cytokine IL-10 after LPS stimulation, as compared with DC $\mathrm{Control}_{\text {. }}$ CD4 $\mathrm{T}$ cells primed with $\mathrm{DC}_{\mathrm{GDNF}}$ resulted in weak proliferation and exhibited a Tr1-like phenotype, which characterized by IL-10, whereas $D_{\text {Control }}$ induced a strong proliferation, exhibited a phenotype characterized by IFN- $\gamma$, IL-4. $\mathrm{DC}_{\mathrm{GDNF}}$ injection significantly ameliorated body weight loss, diarrhea, and colonic histopathologic injury in rat, and $\mathrm{DC}_{\mathrm{GDNF}}$ injection strikingly reduced the production of inflammatory factors such as IL-4, IFN- $\gamma$, TNF- $\alpha$, IL-1 $\beta$, and IL17, generated Treg1 with suppressive capacity on autoreactive $\mathrm{T}$ cells, and demonstrated a higher level of Treg1/Th2 Treg1/Th17.
Conclusion: GDNF could induce tolerogenic DCs through ERK1/ERK2 signal pathway, and $\mathrm{DC}_{\mathrm{GDNF}}$ could alleviate the severity of DSS induced colitis in rats. The mechanism may be related to down-regulation of inflammatory and immune response, generating Treg1 and restoring immune tolerance.

Disclosure of Interest: All authors have declared no conflicts of interest.

\section{P0313 DETERMINANTS OF REDUCED GENETIC CAPACITY FOR BUTYRATE SYNTHESIS BY THE GUT MICROBIOME IN CROHN'S DISEASE AND ULCERATIVE COLITIS}

E. J. Laserna-Mendieta ${ }^{1}$, A. G. Clooney ${ }^{1}$, J. F. Carretero-Gomez ${ }^{2}$, J. Nolan ${ }^{1}$, C. Hill ${ }^{1}$, C. G. Gahan ${ }^{3}$, S. A. Joyce ${ }^{4}$, C. Moran ${ }^{5}$, D. Sheehan ${ }^{5}$, F. Shanahan ${ }^{5}$, M. J. Claesson

${ }^{1}$ School Of Microbiology, APC Microbiome Institute-University College Cork, Cork/Ireland

${ }^{2}$ Department Of Laboratory Medicine, Virgen de la Salud Hospital, Toledo/Spain ${ }^{3}$ School Of Microbiology \& School Of Pharmacy, APC Microbiome InstituteUniversity College Cork, Cork/Ireland

${ }^{4}$ School Of Biochemistry \& Cell Biology, APC Microbiome Institute-University College Cork, Cork/Ireland

${ }^{5}$ School Of Medicine, APC Microbiome Institute-University College Cork, Cork/ Ireland

Contact E-mail Address: emilio.lasernamendieta@ucc.ie

Introduction: Alterations in short chain fatty acid (SCFA) metabolism have been reported in inflammatory bowel disease (IBD). Among SCFA, butyrate has been described as a potent communicator to the immune system eliciting an antiinflammatory response and other positive effects to human health ${ }^{1}$. A reduction of faecal butyrate levels has been reported in IBD but results have been conflicting or discrepant because of small study numbers and failure to distinguish disease type, activity or other variables such as diet. Microbiota is receiving increasing attention as a key environmental factor influencing $\mathrm{IBD}^{2}$, and butyryl-CoA:acetate CoA-transferase (BCoAT) is considered the main enzyme involved in butyrate synthesis by gut microbiota ${ }^{3}$

Aims \& Methods: We performed a comparative assessment of the capacity of the microbiota for butyrate synthesis by quantifying BCoAT gene content in stool from patients with Crohn's disease (CD; $n=71)$, ulcerative colitis (UC; $n=58$ ) and controls $(n=75)$, and determined whether it was related to disease activity, inflammation, microbial diversity and composition and/or dietary habits. BCoAT gene content was quantified by $\mathrm{qPCR}^{4}$. Disease activity was assessed clinically and faecal calprotectin concentration measured as biomarker of inflammation in the gut. Microbial composition was determined by sequencing $16 \mathrm{~S}$ rRNA gene. Dietary data were collected using an established food frequency questionnaire.

Results: Reduced butyrate-synthetic capacity was found in patients with active and inactive $C D(p<0.001$ and $p<0.01$, respectively), but only in active UC $(\mathrm{p}<0.05)$. In patients with $\mathrm{CD}$, low BCoAT gene content (below $9.5 \log 10$ copies $\mathrm{BCoAT} / \mathrm{g}$ ) was associated with active disease, increased inflammation, lower microbial diversity, greater microbiota compositional change and decreased butyrogenic taxa, while no major changes were observed between patients with UC grouped according to BCoAT gene levels. Reduced BCoAT gene content in patients with CD was, in part, linked with lower intake of certain foods containing fibre (vegetables, fruits, high-fibre cereals, brown/wholemeal bread and nuts).

Conclusion: Reduced butyrate-synthetic capacity by the microbiota is more evident in CD than UC and may relate to reduced fibre intake. The results suggest that simple replacement of butyrate per se may be therapeutically inadequate, whereas manipulation of microbial synthesis perhaps by dietary means may be more appropriate and profitable for patients with CD.

Disclosure of Interest: C. Hill: Prof Colin Hill has received research funding from Jansenn and Artugen Therapeutics.

F. Shanahan: Prof Fergus Shanahan has been a collaborator and has received research funding from Janssen, Abbvie, Alimentary Health Ltd, Sigmoid, 4dPharma and Second Genome.

M.J. Claesson: Dr Marcus Claesson has received research funding from Second Genome.

All other authors have declared no conflicts of interest.

\section{References}

1. Ríos-Covián D, Ruas-Madiedo P, Margolles A, Gueimonde M, de Los Reyes-Gavilán CG, Salazar N. Intestinal short chain fatty acids and their link with diet and human health. Front Microbiol. 2016;7:185.

2. Sheehan D, Moran C, Shanahan F. The microbiota in inflammatory bowel disease. J Gastroenterol. 2015;50(5):495-507.

3. Louis P, Flint HJ. Development of a semiquantitative degenerate real-time PCR-based assay for estimation of numbers of butyryl-coenzyme A (CoA) CoA transferase genes in complex bacterial samples. Appl Environ Microbiol. 2007;73(6):2009-12.

4. Vital M, Howe AC, Tiedje JM. Revealing the bacterial butyrate synthesis pathways by analyzing (meta)genomic data. mBio. 2014;5(2):e00889. 
P0314 A COMBINED ADMINISTRATION OF AMPICILLIN AND VANCOMYCIN INDUCES MILD COLITIS WITH DECREASED DIVERSITY OF GUT MICROBIOTA AND PERTURBATION OF GLUTAMINE AND SHORT CHAIN FATTY ACID METABOLISMS

A. Sonoda ${ }^{1}$, N. Sachi ${ }^{2}$, Y. Gendo ${ }^{2}$, T. Ozaki ${ }^{2}$, N. Kamiyama ${ }^{2}$, S. Hidano ${ }^{2}$, K. Mizukami ${ }^{3}$, T. Okimoto ${ }^{1}$, K. Murakami ${ }^{3}$, T. Kobayashi ${ }^{2}$

${ }^{1}$ Oita University Dept. of Gastroenterology, Yufu/Japan

${ }^{2}$ Infectious Disease Control, Oita University, Yufu/Japan

${ }^{3}$ Gastroenterology, Oita University, Yufu/Japan

Contact E-mail Address: load2akr@oita-u.ac.jp

Introduction: Antibiotics sometimes have an influence on colitis negatively. Although it is well known that dysbiosis is one of the major disturbances to the gut environment, the molecular mechanisms underling the pathogenesis remains unclear.

Aims \& Methods: We aimed to clarify how antibiotics affect the gut microbiota and the pathology of colitis. Mice were gavaged with ampicillin (ABPC), vancomycin (VCM), metronidazole, neomycin, or a combination of ABPC and VCM (A-V) for three consecutive days. Colitis was assessed by fecal occult blood test (FOBT) and mRNA level of cytokines. Metabolites and short chain fatty acid (SCFA) in the feces were measured by a chromatography-tandem mass spectrometry. Fecal microbiota was characterized by 16 rRNA sequencing. In the glutamine (Gln) treatment exam, the mice were given $30 \mathrm{mg} / \mathrm{mL}$ of Gln in drinking water ad lib for six days, and additionally gavaged $\mathrm{A}-\mathrm{V}$ for the last three days. In vitro experiments, mouse colon carcinoma cell line CMT93 and macrophage cell line RAW267.4 were stimulated by butyric acid.

Results: ABPC and VCM, but not other antibiotics, resulted in FOBT-positive in some cases. All A-V treated mice were FOBT-positve and IL-6, IL-12p40 and MIP-1a were significantly increased. The caecums were enlarged and dun-colored in the A-V mice. Moreover, Gln metabolites and SCFA including butyric acid were decreased and family S24-7 and order Clostridiales were less abundant in the feces of $\mathrm{A}-\mathrm{V}$ mice. Interestingly, Gln treatment improved the antibioticsinduced colitis. Butyric acid reduced mRNA expression of pro-inflammatory cytokines in RAW267.4 and induced expression of antimicrobial peptides (secretory leukocyte protease inhibitor and lactoferrin) in CMT93.

Conclusion: A-V treatment induced mild colitis with reduced abundance of family S24-7 and order Clostridiales, which might disturb Gln and SCFA metabolisms. Disclosure of Interest: All authors have declared no conflicts of interest.

\section{P0315 ANTI-INFLAMMATORY EFFECTS OF G PROTEIN- COUPLED RECEPTOR 18 - A NOVEL POTENTIAL THERAPEUTIC TARGET IN INFLAMMATORY BOWEL DISEASE}

A. Fabisiak ${ }^{1}$, N. Fabisiak ${ }^{1}$, M. Zielińska ${ }^{1}$, A. Mokrowiecka ${ }^{2}$, E. Malecka-Panas ${ }^{2}$, R. Kordek ${ }^{3}$, K. Kieć-Kononowicz ${ }^{4}$, J. Fichna

${ }^{1}$ Department Of Biochemistry, Medical University of Lodz, Lodz/Poland ${ }^{2}$ Department Of Digestive Tract Diseases, Medical University of Lodz, Lodz/ Poland

${ }^{3}$ Department Of Pathology, Medical University of Lodz, Lodz/Poland

${ }^{4}$ Department Of Technology And Biotechnology Of Drugs, Jagiellonian University, Krakow/Poland

Contact E-mail Address: adam.fabisiak@stud.umed.lodz.pl

Introduction: Inflammatory bowel disease (IBD) is a group of gastrointestinal tract diseases consisting mainly of Crohn's Disease (CD) and Ulcerative Colitis (UC). Various etiological factors contribute to the pathogenesis of IBD, including modulation of microbiota, epithelial barrier disruption, genetics and environmental factors. Currently, the treatment of IBD comprises several groups of drugs the choice of which is made based on disease activity and extent. The treatment options include: analogs of 5-aminosalicylic acids, glucocorticoids and other immunosuppressive drugs such as azathioprine. Biological therapy with anti-tumor necrosis factor alpha (TNF $\alpha$ ) are considered when conventional therapy fails. In portion of patients surgical approach is necessary. Therefore novel pharmacological targets are sought in order to improve the remission rate and avoid the disabling procedures. G protein-coupled receptor 18 (GPR18) belongs to the endogenous cannabinoid system which already earned its place in the pathogenesis of IBD. GPR18 was found to be implicated in protection against bacterial infection and organ injury. Reports indicate that it may also be connected to the obesity/diabetes-related inflammation. As we lack data regarding its association with intestinal inflammation we attempted to shed some light on this phenomenon.

Aims \& Methods: The aim of the study was to assess the anti-inflammatory and anti-nociceptive actions of GPR18 in the intestinal inflammation. The antiinflammatory activity of GPR18 agonist PSB-KK-1415 and antagonist PSBCB5 (1-5 mg/kg, i.c., once daily) was characterized in two mouse models of colitis, induced by 2, 4, 6-trinitrobenzenesulfonic acid (TNBS) and dextran sulfate sodium (DSS). The extent of inflammation was evaluated based on the macroscopic score, microscopic score, quantification of myeloperoxidase (MPO) activity and alpha (TNF $\alpha$ ) in colonic tissue. The expression of GPR18 gene in colonic samples from patients with IBD was quantified using real-time RT PCR and Western blot analysis. GPR 18 was localized in human colonic samples using immunohistochemistry methods.

Results: The results of our study with lower doses of GPR18 ligands in the semichronic TNBS model of colitis showed a non-significant decrease in macroscopic score, ulcer score and a significant decrease in MPO activity $(\mathrm{p}<0.05)$ in mice injected with PSB-KK-1415 compared with TNBS-treated mice. PSB-CB5 increased inflammation indices. Study in the mouse model of TNBS-induced colitis with higher dose of PSB-KK-1415 presented a pronounced reduction in the indices after treatment with the agonist. We also showed that GPR18 is expressed in the colon of patients with IBD.

Conclusion: We demonstrated potential ability of the GPR18 agonist PSB-KK1415 to alleviate inflammation in the mouse models of colitis and showed that GPR18 is expressed in the human colon. We conclude that GPR18 is another receptor of the endogenous cannabinoid system family which may be implicated in the pathogenesis of IBD and intestinal inflammation overall. Disclosure of Interest: All authors have declared no conflicts of interest.

\section{P0316 THE MECHANISM OF PROTECTIVE ROLE OF D3 DOPAMINE RECEPTORS IN PATHOGENESIS OF ULCERATIVE COLITIS}

A. Prysiazhniuk, T. Dovbynchuk, Y. Holota, I. Vareniuk, L. Garmanchuk, G. Tolstanova

Taras Shevchenko National University of Kyiv, ESC 'Institute of Biology and Medicine', Kyiv/Ukraine

Contact E-mail Address: alona.prysiazhniuk@gmail.com

Introduction: Our previous study showed that activation of D3 dopamine receptors (D3R) had the beneficial effect in experimental colitis treatment while the mechanism of this effect is unclear [1]. The disruption of surface colon mucosa layer with subsequent activation of local immune response by the bacterial infiltration into the inner layer of the mucosa are the key pathogenic mechanisms of ulcerative colitis progression and perpetuation. We found the localization of $\mathrm{D} 3 \mathrm{R}$ on the Goblet cells in colonic mucosa [2]

Aims \& Methods: In present study we tested the hypothesis that activation of D3R improves colonic mucus secretion during experimental colitis. Study was done on male Wistar rats (180-230). Experimental colitis was induced by $6 \%$ iodoacetamide (IA) $(0,1 \mathrm{ml}$, enema). Selective D3R agonist 7-OH-DPAT $(0.02 \mathrm{mg} / 100 \mathrm{~g}$, s.c. $)$ was injected $0,5 \mathrm{~h}$ prior to IA enema. Rats were euthanized 0,5 and $2 \mathrm{~h}$ after IA enema. During the autopsy $7 \mathrm{~cm}$ colon from the anus has been removed. Surface mucus layer was separated from epithelial cells with Nacetyl-L-cysteine and glycoproteins were measured by periodic acid/Schiff (PAS) staining or by the reaction with Folin reagent. The content of hexose, fucose and hexosamine were determined by standard biochemical assays. Morphometric analysis was performed to evaluate the histological changes of colonic epithelial and Goblet cells. Oxidative metabolism and arginase activity (analyzed by colorimetric method) in peritoneal macrophages were investigated.

Results: Pre-treatment with 7-OH-DPAT did not affect the glycoprotein levels in normal mucosa, but significantly increased total levels of glycoprotein $(1,6$-fold, $\mathrm{p}<0.05)$ and hexose $(1,1$-fold, $\mathrm{p}<0.05)$ during IA-colitis. Furthermore, 7-OHDPAT significantly increased functional reserve of peritoneal macrophages in 0 , $5 \mathrm{~h}(1,6$-fold, $\mathrm{p}<0.05)$ and in $2 \mathrm{~h}(1,3$-fold, $\mathrm{p}<0.05)$ after IA enema. Pretreatment with 7-OH-DPAT decreased the mucosal layer thickness 1, 1-fold $(\mathrm{p}<0.05)$, crypt depth 1,1 -fold $(\mathrm{p}<0.05)$ and Goblet cell intersection area 1 , 2 -fold $(\mathrm{p}<0.05)$ after IA enema

Conclusion: Pre-treatment with D3R-agonist increased levels of mucus secretion and activated natural immune response by macrophage activation during experimental colitis, which could indicate about the protective role of D3R

Disclosure of Interest: All authors have declared no conflicts of interest.

\section{References}

1. Prysiazhniuk A., Dziubenko N., Kernychnyi V., Szabo S., Tolstanova G. The role of D3-dopamine receptors in the ulcerative colitis pathogenesis// Book of abstracts of '9th International Symposium on Cell/Tissue Injury and Cytoprotection/Organoprotection', 15-17 September. Cracow, 2016. P. 45.

2. Prysiazhniuk A., Nesteruk K., Chervinska T., Tolstanova G. Expression and localization of D3 receptors during experimental ulcerative colitis [in Ukrainian]//Bulletin of Taras Shevchenko National University of Kyiv, series 'Problems of Physiological Functions Regulation'. 2015. №19. P. 10-13.

\section{P0317 INFLAMMATION AT DISTAL ILEOCAECAL RESECTION MARGINS INCREASE THE RISK OF POSTOPERATIVE CROHN'S RECURRENCE}

K. Wasmann ${ }^{1}$, J. Van Amesfoort ${ }^{1}$, L. Koens ${ }^{2}$, W.A. Bemelman ${ }^{1}$, C.J. Buskens ${ }^{1}$ Surgery, Academic Medical Centre, Amsterdam/Netherlands

${ }^{2}$ Pathology, Academic Medical Center, Amsterdam/Netherland.

Contact E-mail Address: k.a.wasmann@amc.nl

Introduction: Guidelines advise limited resection for Crohn's terminal ileitis, as previous literature did not demonstrate clinical benefit from more extensive resections. Recently, some cohort studies identified positive resection margin as an independent risk factor for postoperative Crohn's recurrence. But it is difficult to draw clinical conclusions, as non-uniform pathological definitions have been used. The aim of this study was to assess the incidence of non-radical resections by a validated pathological score, and correlate pathological findings to clinical outcome.

Aims \& Methods: H\&E stained slides from proximal (ileum) and distal (colon) resection sites were scored according to the modified Geboes score from all consecutive patients undergoing primary ileocaecal resection between January 2002 and September 2009. Endpoints were clinical recurrence (defined as recurrent disease activity demonstrated by endoscopy or MRI) requiring upscaling medical treatment or surgical recurrence. 
Results: Of 104 ileocaecal resections, 30 (29\%) and $15(14 \%)$ had inflammation at the proximal and distal resection margins, respectively. After a median follow-up of 8.6 years, clinical recurrence was seen in $57 \%$, and surgical recurrence in $26 \%$. A significantly increased recurrence rate was seen in patients with active inflammation at the distal resection margin whereas recurrence rates were comparable for inflammation at the proximal site and radical resections $(87 \%, 61 \%$, and $50 \%$ resp., $\mathrm{p}<0.001)$. Active inflammation at the distal resection margin (HR: $3.189(1.635-6.220) ; \mathrm{p}=0.001)$ and smoking (HR: $2.502(1.331-4.703) ; \mathrm{p}=0.004)$ were the only independent predictors for clinical recurrence. The incidence of surgical recurrence was too small to perform statistics.

Conclusion: Inflammation at the distal (colon) resection margin, and not the proximal ileum, after ileocecal resection was associated with significantly increased risk of clinical recurrence. This unexpected finding suggests that radicality does not play a role in correctly diagnosed terminal ileitis (L1 disease), while it is of crucial importance to exclude colonic activity (L3 disease). As this different phenotype is unlikely to benefit from more extensive surgery, pathological finding of positive distal resection margin should be regarded as a risk factor, warranting prophylactic drugs or close monitoring.

Disclosure of Interest: All authors have declared no conflicts of interest.

\section{References}

1. Botti F, Carrara A, Antonelli B, et al. [The minimal bowel resection in Crohn's disease: analysis of prognostic factors on the surgical recurrence]. Ann Ital Chir 74:627-33. Available at: http://www.ncbi.nlm.nih.gov/pubmed/ 15206803 [Accessed April 26, 2017].

2. Fazio VW, Marchetti F, Church M, et al. Effect of resection margins on the recurrence of Crohn's disease in the small bowel. A randomized controlled trial. Ann Surg 1996;224:563-71-3. Available at: http://www.ncbi.nlm.nih.gov/pubmed/8857860 [Accessed April 26, 2017].

3. Gionchetti P, Dignass A, Danese S, et al. 3rd European Evidence-based Consensus on the Diagnosis and Management of Crohn's Disease 2016: Part 2: Surgical Management and Special Situations. J Crohn's Colitis 2017;11:135-149. Available at: https://academic.oup.com/ecco-jcc/articlelookup/doi/10.1093/ecco-jcc/jjw169 [Accessed April 26, 2017].

4. Geboes K, Riddell R, Ost A, et al. A reproducible grading scale for histological assessment of inflammation in ulcerative colitis. Gut 2000;47:404-9. Available at: http://www.ncbi.nlm.nih.gov/pubmed/10940279 [Accessed April 26, 2017].

5. Mowat C, Arnott I, Cahill A, et al. Mercaptopurine versus placebo to prevent recurrence of Crohn's disease after surgical resection (TOPPIC): a multicentre, double-blind, randomised controlled trial. Lancet Gastroenterol Hepatol 2016;1:273-282. Available at: http://www.ncbi.nlm.nih.gov/ pubmed/28404197 [Accessed April 26, 2017].

6. Rutgeerts P, Geboes K, Vantrappen G, et al. Predictability of the postoperative course of Crohn's disease. Gastroenterology 1990;99:956-63. Available at: http://www.ncbi.nlm.nih.gov/pubmed/2394349 [Accessed April 26, 2017].

\section{P0318 APPLICATION OF NEW SINGLE NUCLEOTIDE POLYMORPHISMS TO THE IDENTIFICATION OF ADHERENT- INVASIVE E. COLI (AIEC)}

C. Camprubí-Font, M. Lopez-Siles, M. Ferrer-Guixeras, L. Niubo-Carulla, C. Abellà-Ametller, L.J. Garcia-Gil, M. Martinez-Medina

Biology Department, Universitat de Girona, Girona/Spain

Contact E-mail Address: c.camprubi4@gmail.com

Introduction: The genetic basis that constitutes an adherent-invasive Escherichia coli (AIEC), a pathotype associated with Crohn's disease, still needs to be deciphered. Obtaining new molecular tools for AIEC identification would be of great significance, as current techniques based on phenotypic screening of cultured bacteria are time consuming (1).

Aims \& Methods: Our aims were: (i) to search for genetic elements putatively involved in the AIEC phenotype in order to find specific signature sequences and (ii) to determine the distribution of these elements to assess their usefulness in AIEC identification. AIEC-specific sequences were identified through comparative genomics of three E. coli strain pairs (from different phylogroups) displaying different AIEC phenotype but identical pulsotype. Paired-end libraries of Illumina HiSeq and PacBio of the six strains' genomes were combined de novo and assembled by SPAdes. Differences in gene content between pairs were accomplished with OrthoVenn. Harvest was performed to compare strain pairs against an AIEC reference genome (UM146). Only non-synonymous single nucleotide polymorphisms (SNPs) in coding regions were selected. We performed Sanger sequencing to confirm the presence of SNPs and to evaluate the distribution of the SNPs in a collection of 22 AIEC and 29 non-AIEC isolates. Nucleotides for each SNP were analysed taking into account AIEC phenotype, adhesion and invasion indices by $\chi 2$ test or Kruskal-Wallis test, as required. Binary logistic regression was applied to assess the usefulness of particular variants for AIEC screening. Isogenic mutants were generated with a PCR product using the method previously described $(2,3)$ to assess the effect of the novel candidate genes in AIEC phenotype. In this collection, point mutations or prevalence of previously AIEC-associated genes has also been examined.

Results: A total yield of 5209 gene clusters was obtained, 3327 of which were common for the six strains and none was found to be common in the three AIEC strains, not even exclusively in two of them. Comparative genomics identified 18 SNPs between the strains of the D-phylogroup pair, 17 in the B2-pair and 30 in the B1-pair that met the selection criteria. Of those, 24 SNPs (found in 13 genes) were confirmed and further analysed in the strain collection. Three of the SNPsencompassing genes were related with adhesion/invasion and two with stress tolerance. Three SNPs resulted in differential nucleotide distribution between AIEC and non-AIEC strains $(\mathrm{p}<0.016)$. These SNPs also presented association with adhesion and invasion strain capacities $(\mathrm{p}<0.006)$. Interestingly, one of these polymorphisms can predict the AIEC phenotype with a $71 \%$ of specificity $(\mathrm{p}=0.008)$. No differences according to pathotype were reported in the prevalence of previously AIEC-related genes, nor in point mutations frequency.

Conclusion: Our study corroborates the absence of AIEC-specific genetic markers widely distributed across all AIEC strains. Nonetheless, three SNPs putatively involved with the AIEC phenotype have been described and one of them could be applied in AIEC screening.

Disclosure of Interest: All authors have declared no conflicts of interest.

\section{References}

1. Darfeuille-Michaud A, et al. 2004. Gastroenterology, no.127(2), p.412-421.

2. Datsenko K \& Wanner B. 2000. PNAS, no.97(12), p.6640-6645. 3. Chaveroche M, et al. 2000. NAR, no.28(22), p.E97.

\section{P0319 LIPOSOMAL FORMULATION AS A NEW DRUG DELIVERY SYSTEM FOR CROHN'S DISEASE - VALIDATION IN THE MOUSE MODEL OF TNBS-INDUCED COLITIS}

J. B. Krajewska, P. Pietruszka, D. Tomczyk, J. Fichna

Department Of Biochemistry, Medical University of Lodz, Lodz/Poland

Contact E-mail Address: julia.krajewska@stud.umed.lodz.pl

Introduction: Crohn's Disease (CD), one of the two main types of inflammatory bowel disease (IBD), constitutes a significant healthcare burden, especially in the developed societies. Current methods of treatment are only partially effective and/or associated with major adverse effects. New therapeutic solutions are required to ameliorate the effects of medical therapy, reducing complications and improving patients' quality of life.

Aims \& Methods: The objective of the study was to assess the effectiveness of delivery of anti-inflammatory drugs encapsulated in the liposomal formulation. Liposomes were prepared using thin-lipid hydration method. $0.9 \%$ sodium chloride was used as a solvent. The hydration solutions contained an aminosalicylate mesalazine (5-ASA), two recently validated plant-derived anti-inflammatories with low bioavailability, chlorogenic acid (CGA) and berberine, and pure solvent as negative control. Colitis was induced in male $\mathrm{BALB} / \mathrm{c}$ mice by a single intracolonic (i.c.) administration of 2, 4, 6-trinitrobenzene sulphonic acid (TNBS) on Day 0. Liposomal suspensions containing 5-ASA (5 mg/kg), CGA $(20 \mathrm{mg} / \mathrm{kg})$, berberine $(5 \mathrm{mg} / \mathrm{kg})$ and the solvent were administered i.c. twice daily from Day 3 to Day 6. Mice were sacrificed on Day 7 and colonic damage was evaluated. Results: The macroscopic scoring system included the evaluation of the colon length and bowel thickness as well as the presence of ulcers, haemorrhage, faecal blood and diarrhea. Additionally, tissue myeloperoxidase (MPO) activity was determined and body weight was measured. The obtained results indicate that the best anti-inflammatory effect was obtained when liposomal suspension with berberine was used, while the treatment with 5-ASA was less effective. Surprisingly, CGA administration caused a detrimental effect as demonstrated by higher macroscopic score and increased MPO activity.

Conclusion: Drug-loaded liposomes may enhance the penetration of active compounds through the gut wall and therefore allow for increased bioavailability and effectiveness of the treatment at lower doses. We also showed that plant-derived compounds with low bioavailability, such as berberine and CGA, provide a new direction in the search of anti-inflammatory substances. However, increased bioavailability of anti-inflammatories due to liposomal formulation may result in adverse effects.

Disclosure of Interest: All authors have declared no conflicts of interest.

\section{P0320 EPIDEMIOLOGY OF MICROSCOPIC COLITIS IN}

\section{NOTTINGHAM: A CONTEMPORARY COHORT STUDY}

N. R. Lewis ${ }^{1}, \mathrm{~T}$. Archer ${ }^{1}, \mathrm{P} . \mathrm{Kaye}^{2}$

${ }^{1}$ Gastroenterology, Nottingham University Hospitals NHS Trust, Nottingham/ United Kingdom

${ }^{2}$ Histopathology, Nottingham University Hospitals NHS Trust, Nottingham United Kingdom

Contact E-mail Address: nina.lewis@nuh.nhs.uk

Introduction: Diarrhoea is a common indication for colonoscopy. International guidelines recommend colonic biopsies should be routinely taken at lower GI endoscopy in patients with diarrhoea $[1,2]$ to look for microscopic colitis. The epidemiology of contemporary microscopic colitis is largely unknown with published data based on an earlier time period pre-2001 and in small retrospective case series. We have systematically collected a large and contemporary cohort of patients with incident microscopic colitis to help further our understanding of this disease.

Aims \& Methods: Colonic biopsies demonstrating microscopic colitis between 2005-2016 were identified from electronic pathology records. GI endoscopy procedures taking place during this time period were identified from an electronic database. Incidence rates were calculated using the total population for Nottinghamshire derived from UK mid-year population estimates [3]. Poisson regression models were used to determine rate ratios.

Results: 843 people were diagnosed with microscopic colitis of which $60.7 \%$ were female $(\mathrm{n}=512)$. The mean age at diagnosis was $65.0(95 \% \mathrm{CI} 64.0,65.9)$ years. $57.5 \%(n=485)$ had collagenous colitis and $42.5 \%$ had lymphocytic colitis. The 
incidence rate of microscopic colitis appeared to increase with time (Table). The incidence rate of microscopic colitis in 2016 was twice of that observed in 2009 (incidence rate ratio $1.86 ; 95 \% \mathrm{CI} 1.41,2.46$ ). There was a strong, independent graded association between the incidence of microscopic colitis and the number of lower GI endoscopy procedures undertaken $(\mathrm{p}=0.03)$.

\begin{tabular}{lllll}
\hline & $\begin{array}{l}\text { N } \\
\text { microscopic } \\
\text { colitis }\end{array}$ & $\begin{array}{l}\text { Incidence per } \\
100,000\end{array}$ & $\begin{array}{l}\text { Incidence rate } \\
\text { ratio }[95 \% \mathrm{CI}]\end{array}$ & $\begin{array}{l}\text { N lower } \\
\text { GI endoscopy }\end{array}$ \\
\hline 2005 & 3 & 0.39 & & \\
2006 & 4 & 0.52 & & \\
2007 & 5 & 0.65 & & \\
2008 & 23 & 2.96 & 1.0 & \\
2009 & 76 & 9.74 & $0.97[0.71,1.34]$ & \\
2010 & 74 & 9.44 & $1.22[0.91,1.66]$ & \\
2011 & 94 & 11.95 & $1.35[1.01,1.82]$ & 10124 \\
2012 & 104 & 13.16 & $1.15[0.84,1.56]$ & 10100 \\
2013 & 89 & 11.18 & $1.51[1.13,2.01]$ & 11026 \\
2014 & 118 & 14.72 & $1.32[1.01,1.78]$ & 13225 \\
2015 & 104 & 12.91 & $1.86[1.41,2.46]$ & 13031 \\
2016 & 147 & 18.15 & & \\
\hline
\end{tabular}

Conclusion: Microscopic colitis diagnosis is becoming more common. It is unclear whether microscopic colitis itself is increasing or greater numbers of lower GI endoscopy are being undertaken causing an ascertainment bias. Further work is required to explore environmental exposures such as drugs associated with microscopic colitis and to observe its natural history.

Disclosure of Interest: All authors have declared no conflicts of interest.

\section{Reference}

1. Gut 2003;52:v1-v15 2. Gut 2016;0:1-7 3.www.ons.gov.uk

\section{P0321 EXTRA-INTESTINAL MANIFESTATIONS AT DIAGNOSIS IN PAEDIATRIC- AND ELDERLY-ONSET ULCERATIVE COLITIS ARE ASSOCIATED WITH A MORE SEVERE DISEASE OUTCOME: A POPULATION-BASED STUDY}

D. Duricova ${ }^{1}$, A. Leroyer ${ }^{1}$, G. Savoye ${ }^{2}$, H. Sarter ${ }^{1}$, B. Pariente ${ }^{3}$, D. Aoucheta ${ }^{4}$, L. Armengol-Debeir ${ }^{2}$, D. Ley ${ }^{5}$, D. Turck ${ }^{5}$, L. Peyrin-Biroulet ${ }^{6}$, C. GowerRousseau ${ }^{1}$, M. Fumery ${ }^{7}$

${ }^{1}$ Public Health, Epidemiology And Economic Health, Registre Epimad, Maison Régionale De La Recherche Clinique, Lille University and Hospital, Lille/France ${ }^{2}$ Gastroenterology Unit, Epimad Registry, Hôpital Charles Nicolle, Rouen University Hospital, Rouen/France

${ }^{3}$ Gastroenterology Unit, Hôpital Huriez, Lille University Hospital, Lille/France

${ }^{4}$ Associated Medical Director Gastro-Immunology at MSD, Paris/France

${ }^{5}$ Division of Gastroenterology, Hepatology and Nutrition, Department of

Paediatrics, Lille University Jeanne de Flandre Children's Hospital, University of Lille, Lille/France

${ }^{6}$ Gastroenterology Unit, Inserm U954, Nancy University and Hospital, Nancy/

France

${ }^{7}$ Gastroenterology Unit, Epimad Registry, CHU Amiens Sud, Avenue Laennec-

Salouel, Amiens University Hospital, Amiens/France

\section{Contact E-mail Address: mathurinfumery@gmail.com}

Introduction: Data on extra-intestinal manifestations (EIM) and their impact on disease course of ulcerative colitis (UC) in population-based cohorts are scarce, particularly in paediatric- and elderly-onset UC patients.

Aims \& Methods: The aims of this population-based study were to assess 1) the occurrence of EIM in paediatric- and elderly-onset UC; and 2) their impact on long-term disease outcome. Paediatric-onset $(<17$ years at diagnosis) and elderly-onset UC patients ( $>60$ years) from a French prospective populationbased Registry (EPIMAD) were included. Data on EIM and other clinical factors at diagnosis and at maximal follow-up were collected.

Results: 158 paediatric- and 470 elderly-onset patients were included (median age at diagnosis 14.5 and 68.8 years; median follow-up 11.2 and 6.2 years, respectively). EIM occurred in $8.9 \%$ of childhood- and $3 \%$ of elderly-onset patients at diagnosis and in $16.7 \%$ and $2.2 \%$ of individuals during follow-up $(\mathrm{p}<0.01)$. The most frequent EIM was joint involvement $(15.8 \%$ of paediatric-onset and $2.6 \%$ of elderly-onset). Presence of EIM at diagnosis was associated with more severe disease course (need for immunosuppressive or biologic therapy or colectomy) in both paediatric- and elderly-onset UC $(\mathrm{HR}=2.0,95 \% \mathrm{CI}: 1.0-4.2$ and $\mathrm{HR}=2.8$, 0.9-7.9). Extensive colitis was another independent risk factor in both age groups.
Conclusion: Elderly-onset UC patients had lower risk of EIM either at diagnosis or during follow-up than paediatric-onset individuals. EIM at diagnosis predicted more severe disease outcome including need for immunosuppressive or biologic therapy or surgery in both paediatric- and elderly-onset UC.

Disclosure of Interest: All authors have declared no conflicts of interest.

P0322 LONG-TERM NATURAL HISTORY OF MICROSCOPIC COLITIS: A POPULATION-BASED STUDY

J. Loreau ${ }^{1}$, D. Duricova ${ }^{2}$, C. Gower-Rousseau ${ }^{3}$, G. Savoye ${ }^{4}$, H. Sarter ${ }^{3}$, C. Yzet ${ }^{1}$, M. Kohut ${ }^{5}$, F. Brazier ${ }^{1}$, D. Chatelain ${ }^{6}$, E. Nguyen-Khac ${ }^{1}$, J. Dupas ${ }^{7}$, M. Fumery ${ }^{7}$

${ }^{1}$ Amiens University Hospital, Amiens/France

${ }^{2}$ Ibd Clinical And Research Center, ISCARE I.V.F. a.s., Prague/Czech Republic ${ }^{3}$ Public Health, Epidemiology And Economic Health, Registre Epimad, Maison Régionale De La Recherche Clinique, Lille University and Hospital, Lille/France ${ }^{4}$ Gastroenterology Unit, Epimad Registry, Hopital Charles Nicolle, Rouen University Hospital, Rouen/France

${ }^{5}$ Clinique St Isabelle, Abbeville/France

${ }^{6} \mathrm{CHU}$, Amiens, France, Amiens/France

Gastroenterology Unit, Epimad Registry, CHU Amiens Sud, Avenue LaennecSalouel, Amiens University Hospital, Amiens/France

Contact E-mail Address: jloreau@yahoo.com

Introduction: Data on long-term natural history of microscopic colitis (MC), including collagenous (CC) and lymphocytic colitis (LC) are lacking.

Aims \& Methods: All new cases of MC diagnosed in the Somme area, France between January 1st, 2005 and December 31th, 2007 were prospectively included. Colonic biopsies from all patients were reviewed by a group of 4 expert gastrointestinal pathologists to assess the diagnosis of CC or LC. Demographic and clinical data were retrospectively collected from diagnosis to February 31th, 2017.

Results: One hundred and thirty cases of MC, 87 CC and 43 LC, were included (median age at diagnosis 70 and 48 years, respectively). The median follow-up was 9.6 years $(\mathrm{Q} 1=7.6 ; \mathrm{Q} 3=10.6)$. By the end of follow-up, 37 patients $(28 \%)$ relapsed after a median time of 3.9 years $(1.2 ; 5)$ since diagnosis, without significant difference between CC and CL ( $30 \%$ vs $26 \%, \mathrm{p}=0.47)$. Twenty patients $(15 \%)$ were hospitalized for a disease flare and $32(25 \%)$ presented with another autoimmune disease. Budesonide was the most widely used treatment $(n=74$ $59 \%)$, followed by 5 -aminoslaycilic acid $(n=31,25 \%)$. Median duration of budenoside treatment was 92 days $(70 ; 168)$ and no adverse event to budesonide were reported. Sixteen patients $(22 \%)$ developed steroid-dependency and $4(5 \%)$ were corticoresistant. Only one patient was treated by immunosuppressants (azathioprine). No colorectal cancer was reported during follow-up. Any of the death $(\mathrm{n}=25)$ observed during follow-up were linked to MC. In multivariate analysis, age at diagnosis (HR 1.03; 95\% CI, 1.00-1.06; $\mathrm{p}=0.02$ ) and budesonide exposure (HR $0.40 ; 95 \% \mathrm{CI}, 0.18-0.90 ; \mathrm{p}=0.03$ ) were significantly associated with relapse.

Conclusion: This population-based study showed that after diagnosis, two third of patients with MC observed long term clinical remission. Age at diagnosis and budesonide exposure were associated with a risk of relapse.

Disclosure of Interest: M. Fumery: Lecture feers or consultant fees: Abbvie, Ferring, MSD, Takeda

All other authors have declared no conflicts of interest.

\section{P0323 IBD-INFO QUESTIONNAIRE: A MULTICENTER FRENCH UP-TO-DATE SURVEY OF PATIENT KNOWLEDGE IN INFLAMMATORY BOWEL DISEASE}

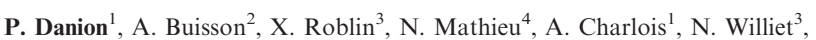
E. Del Tedesco ${ }^{3}$, B. Flourie ${ }^{1}$, S. Nancey ${ }^{1}$, G. Boschetti ${ }^{1}$

${ }_{1}^{1}$ Gastroenterology, Lyon-Sud University Hospital, Pierre Benite/France

${ }^{2}$ Dept. Of Gastroenterology, CHU Estaing Clermont-Ferrand, Clermont-ferrand/ France

${ }^{3}$ CHU Saint-Etienne, Saint-Etienne/France

${ }^{4} \mathrm{CHU}$ Grenoble, Grenoble/France

Contact E-mail Address: pauline.danion@chu-lyon.fr

Introduction: It has been demonstrated in many chronic conditions, including inflammatory bowel disease (IBD), that better patients' knowledge about pathology and treatment improves the course and management of disease. The aim of this study was to develop an updated self-questionnaire to assess patients' level of knowledge of IBD

Aims \& Methods: The IBD-INFO included 3 parts: an original part (Q1), and 2 parts from the translation of the pre-existing questionnaires Crohn's and Colitis Knowledge score (CCKNOW) (Q2) and Crohn's and Colitis Pregnancy Knowledge score (CCPKNOW) (Q3). The reliability and discriminatory ability of the questionnaire were validated with 3 groups of non-IBD volunteers with various theoretical knowledge levels. The final questionnaire (64 validated questions) was then tested on 364 in- and out- IBD patients from 4 French university hospitals. The score for each part of the questionnaire was calculated and factors associated with low scores were identified by uni- and multivariate logistic regression analyses.

Results: The scores obtained by the 3 non-IBD volunteer groups differed significantly $(\mathrm{p}<0.0001)$ and the IBD-INFO questionnaire showed excellent internal reliability and consistency $(\alpha=0.98)$. The median total score obtained by the IBD patients was 27/64 [0-59], and scores for Q1, Q2 and Q3 were, respectively, 10/23 
[0-21], 11/24 [0-23] and 4/17 [0-16]. In multivariate analysis, lack of a university degree, not being a member of a patient association, not receiving anti-TNF $\alpha$ treatment, duration of $\mathrm{IBD} \leq 3$ years, male sex and age $>38$ years and were independent risk factors of poor IBD-INFO knowledge score. The areas of knowledge least mastered were vaccination, IBD-related cancers, treatments and pregnancy.

Conclusion: Using the IBD-INFO, an updated self-administered questionnaire built to assess IBD patients' knowledge, several risk factors have been highlighted that allow better targeting of patients and areas requiring an improvement in the level of information.

Disclosure of Interest: All authors have declared no conflicts of interest.

\section{P0324 IMPACT OF PRIMARY SCLEROSING CHOLANGITIS ON THE DISEASE COURSE IN PATIENTS WITH INFLAMMATORY BOWEL DISEASE - EVIDENCE FROM A LARGE RETROSPECTIVE STUDY WITH MATCHED COHORTS}

F. Cordes ${ }^{1}$, T. Laumeyer ${ }^{1}$, M. Brückner ${ }^{1}$, T.M. Nowacki ${ }^{1}$, J. Gerß ${ }^{2}$, T. Kucharzik ${ }^{3}$, D. Bettenworth ${ }^{1}$

${ }^{1}$ Department Of Medicine B, University Hospital Münster, Münster/Germany ${ }^{2}$ Institute Of Biostatistics And Clinical Research, University of Münster, MünsterGermany

${ }^{3}$ Department Of Internal Medicine And Gastroenterology, University Teaching

Hospital Lüneburg, Lüneburg/Germany

Contact E-mail Address: annafriederike.cordes@ukmuenster.de

Introduction: Inflammatory bowel diseases (IBDs), comprising Crohn's disease (CD) and Ulcerative colitis (UC), are characterized by chronic remittent intestinal inflammation and carry the risk for extraintestinal manifestations including primary sclerosing cholangitis (PSC). Available data on the impact of PSC on the disease course in IBD patients is conflicting. Therefore, we assessed the impact of coincidental PSC on the disease course in a large IBD patient cohort.

Aims \& Methods: In total 1814 patients with histologically confirmed IBD were evaluated. Medical records from $705 \mathrm{UC}$ and $1022 \mathrm{CD}$ patients as well as from 77 UC-PSC and $10 \mathrm{CD}$-PSC patients were assessed. Data were evaluated using standard statistical methods. In matched-pair analyses, IBD patients with and without PSC were matched at the ratio of $3: 1$ by sex, disease entity, age at diagnosis, time from diagnosis to first presentation, and duration of follow-up. Time to event analysis was performed using survival analytic methods including Kaplan-Meier method and Log-rank test.

Results: PSC was diagnosed in 77 and 10 patients out of $781 \mathrm{UC}(9.8 \%)$ and $1033 \mathrm{CD}$ patients $(1.0 \%)$, respectively. Age at UC onset was significantly lower in UC-PSC patients than in patients without PSC (23.3 vs. 29.3 years; $p<0.001$ ). Extensive disease manifestation was observed in $46 \%$ of UC patients, whereas pancolitis was more frequently diagnosed in UC-PSC patients $(75 \%, p<0.001)$. Concerning $\mathrm{CD}$, all patients with coincident PSC showed colonic involvement, while only $69 \%$ of the CD patients without PSC had colonic manifestation $(p=0.044)$. Interestingly, IBD patients without PSC presented more frequently with active disease, as compared to IBD-PSC patients $(1.1 /$ year vs. $0.7 /$ year; $p=0.055)$. Conveniently, average disease activity assessed by complete Mayo score, was significantly higher in UC patients with acute flare as compared to UC-PSC patients (7.3 vs. $6.2 ; p<0.001)$. Investigation of the average disease activity in 5-year-intervals in UC patients revealed a stronger disease activity in UC patients without PSC especially within the first 10 years after UC onset. Furthermore, biological therapy including vedolizumab and anti-TNF antibodies was initiated more frequently $(35 \%$ vs. $22 \%, p=0.043)$ and earlier ( 20.4 vs. 28.6 years after onset, $p=0.087)$ in UC without PSC than in those with coincident PSC. Colorectal high grade intraepithelial neoplasia (HGIEN) and CRC were detected in 25 IBD patients without PSC and in 7 IBD-PSC (4 UC and $3 \mathrm{CD})$ patients $(1.45 \%$ vs. $8.05 \%$ ). Of note, in IBD-PSC patients, HGIEN/CRC occurred significantly earlier than in IBD patients without PSC (20-year-risk: 9.6\% vs. 5.6\%; $p=0.003)$

Conclusion: In our large cohort study, IBD patients with coincident PSC showed a distinct disease course with less relapse, lower disease activity, but earlier disease onset and higher risk for extensive disease manifestation as well as increased risk for colorectal dysplasia development.

Disclosure of Interest: All authors have declared no conflicts of interest.

\section{P0325 UNCHANGED SURGERY AND HOSPITALIZATION RATES IN AN EAST-WEST EUROPEAN INCEPTION COHORT DESPITE DIFFERENCES IN USE OF BIOLOGICALS - 5-YEAR FOLLOW-UP OF THE ECCO-EPICOM COHORT}

J. Burisch ${ }^{1}$, E. Langholz ${ }^{2}$, A. Goldis ${ }^{3}$, R. D'Inca ${ }^{4}$, L. Kupcinskas ${ }^{5}$,

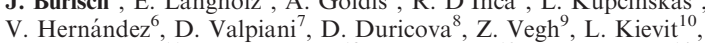
I. Kaimakliotis ${ }^{11}$, J.F. Dahlerup ${ }^{12}$, R. Salupere ${ }^{13}$, J. Halfvarson ${ }^{14}$, K. R. Nielsen ${ }^{15}$, M. Giannotta ${ }^{16}$, K. H. Katsanos ${ }^{17}$, N. Pedersen ${ }^{18}$, P. Ellul ${ }^{19}$, M. Fumery ${ }^{20}$, D. Schwartz ${ }^{21}$, S. Turcan ${ }^{22}$, S. Čuković-Cavka ${ }^{23}$, P. Oksanen ${ }^{24}$, F. Magro ${ }^{25}$, N. Arebi ${ }^{26}$, P.L. Lakatos ${ }^{9}$, P. Munkholm ${ }^{1}$

${ }^{1}$ Department Of Gastroenterology, North Zealand University Hospital,

Frederikssund/Denmark

${ }^{2}$ Herlev University Hospital, Herlev/Denmark

${ }^{3}$ Dept. Of Gastroenterology, County Hospital Timisoara Gastroenterology,

Timisoara/Romania
${ }^{4}$ Department Of Surgery, Oncology And Gastroenterology, Azienda Ospedaliera di Padova, Padova/Italy

${ }^{5}$ Lithuanian University of Health Sciences Inst. for Digestive Research, Kaunas/ Lithuania

${ }^{6}$ Instituto de Investigación Biomédica, Xerencia de Gestión Integrada de Vigo, Vigo/Spain

${ }^{7}$ Department Of Gastroenterology And Digestive Endoscopy, Morgagni Hospital, Forli/Italy

${ }^{8}$ IBD Clinical And Research Center, ISCARE I.V.F. a.s., Prague/Czech Republic ${ }^{9} 1$ st Department Of Medicine, Semmelweis University Faculty of Medicine 1st Dept. of Medicine - 1st Department of Medicine, Semmel, Budapest/Hungary ${ }^{10}$ Department Of Medicine, Herning Central Hospital, Herning/Denmark

${ }^{11}$ American Gastroenterology Center, Nicosia/Cyprus

${ }^{12}$ Department Of Hepatology And Gastroenterology, Aarhus University Hospital, Aarhus/Denmark

${ }^{13}$ Department Of Gastroenterology, Tartu University Hospital, Tartu/Estonia

${ }^{14}$ Dept. Of Gastroenterology, Faculty Of Medicine And Health, Örebro University, Örebro/Sweden

${ }^{15}$ Medical Department, The National Hospital of the Faroe Islands, Torshavn Faroer Islands

${ }^{16}$ Gastroenterology Department, OU Careggi Regional Referral Center for Inflammatory Bowel Disease, Florence/Italy

${ }^{17} 1$ st Division Of Internal Medicine And Hepato-gastroenterology Unit, University Hospital, Ioannina/Greece

${ }_{18}^{18}$ Gastroenterology Department, Slagelse Hospital, Slagelse/Denmark

${ }^{19}$ Medicine, Mater Dei Hospital, Msida/Malta

${ }^{20}$ Gastroenterology Unit, Epimad Registry, CHU Amiens Sud, Avenue Laennec-

Salouel, Amiens University Hospital, Amiens/France

${ }^{21}$ Soroka Hospital, Beer Sheva/Israel

${ }^{22}$ Gastroenterology, The State University of Medicine and Pharmacy «N.

Testemitanu», Chisinau/Moldova

${ }^{23}$ Division Of Gastroenterology And Hepatology, University Hospital Center

Zagreb, University of Zagreb School of Medicine, Zagreb/Croatia

${ }^{24}$ Department Of Gastroenterology And Alimentary Tract Surgery, Tampere University Hospital, Tampere/Finland

${ }^{25}$ Centro Hospitalar São João, Amadora/Portugal

${ }^{26}$ St. Mark's Hospital, Imperial College London, London/United Kingdom

Contact E-mail Address: burisch@gmail.com

Introduction: The EpiCom-cohort is a European prospective population-based cohort of unselected, uniformly diagnosed patients with inflammatory bowel disease (IBD) diagnosed in 2010 in centres from Western and Eastern European countries. The cohort aims at describing differences in occurrence, treatment strategies, disease course and prognosis within Europe.

Aims \& Methods: Patients were followed each $3^{\text {rd }}$ month for the first year after diagnosis and then according to the treating physician for the $2-5^{\text {th }}$ year of follow-up. Clinical data on surgery, hospitalizations and medical treatment incl. biological therapy were captured prospectively throughout the follow-up period and entered in a validated web-based database. The aim of the study was to investigate differences in disease outcome and the use of biologicals between Eastern and Western Europe, from diagnosis and during the first 5 years of follow-up. Associations between outcomes and multiple covariates were analysed by Cox regression analyses.

Results: A total of 1289 patients aged 15 years or older from 29 centres in 13 Western and 8 Eastern European countries were followed prospectively of whom $717(56 \%)$ had ulcerative colitis (UC), $488(38 \%)$ had Crohn's disease (CD), and $84(6 \%)$ had IBD unclassified (IBDU). Crude annual rates for CD and UC patients regarding surgery, biological treatment and hospitalization are shown in Table 1. Significantly more CD patients in Western Europe received biological therapy $(\mathrm{p}<0.05)$, while surgery and hospitalization rates did not differ between the regions $(\mathrm{p}>0.05)$. In $\mathrm{UC}$, surgery rates did not differ between the regions, while hospitalization rates were higher in Western Europe $(\mathrm{p}<0.05)$. Cox regression analysis showed that in CD stricturing (B2) or penetrating disease (B3) and progressing from luminal disease to $\mathrm{B} 2 / \mathrm{B} 3$ increased while early $(<6$ months from diagnosis) treatment with immunosuppressives reduced the risk of surgery and hospitalization. In UC, progressing to extensive colitis increased the risk of colectomy while females, extensive disease, need for prednisolone at diagnosis, and progressing to extensive colitis carried the highest risk for hospitalization. The cumulative probability of $\mathrm{CD}$ patients receiving treatment with 5-ASA was $90 \%$ in Eastern Europe and $56 \%$ in Western Europe, $69 \%$ and $75 \%$ for prednisolone, $54 \%$ and $66 \%$ for immunomodulators, respectively. For UC patients the cumulative probability of receiving treatment with 5-ASA within the first year of disease was $100 \%$ in Eastern Europe and $91 \%$ in Western Europe, $44 \%$ and $52 \%$ for prednisolone, $27 \%$ and $30 \%$ for immunomodulators, respectively.

Conclusion: In an era of early and aggressive immunological therapy, surgery and hospitalization rates for CD and surgery rates for UC patients were similar in Eastern and Western Europe. Overall, surgery and hospitalization rates were comparable to population-based cohorts from the past decade and pre-biological era. This similar disease course was in spite of more early and aggressive treatment with biologics and immunomodulators, with significantly more CD and UC patients in Western Europe receiving biologics.

Disclosure of Interest: All authors have declared no conflicts of interest. 
Abstract No: P0325

Table 1: Crude rates for surgery, biological therapy and hospitalization after 1 and 5 years follow-up in the EpiCom-cohort

\begin{tabular}{|c|c|c|c|c|c|c|c|c|c|c|}
\hline & & \multicolumn{3}{|c|}{ Biological therapy } & \multicolumn{3}{|l|}{ Surgery } & \multicolumn{3}{|c|}{ Hospitalization } \\
\hline & & 1 year & 3 years & 5 years & 1 year & 3 years & 5 years & 1 year & 3 years & 5 years \\
\hline \multirow[t]{2}{*}{ Crohn's disease } & Eastern Europe & $4(5 \%)$ & $6(7 \%)$ & $12(14 \%)$ & $12(14 \%)$ & $16(19 \%)$ & $18(21 \%)$ & $22(26 \%)$ & $26(31 \%)$ & $26(33 \%)$ \\
\hline & Western Europe & $80(20 \%)$ & $110(27 \%)$ & $132(33 \%)$ & $50(12 \%)$ & $70(17 \%)$ & $89(22 \%)$ & $91(23 \%)$ & $122(30 \%)$ & $147(36 \%)$ \\
\hline \multirow[t]{2}{*}{ Ulcerative colitis } & Eastern Europe & $1(1 \%)$ & $6(5 \%)$ & $10(8 \%)$ & $2(2 \%)$ & $2(2 \%)$ & $3(2 \%)$ & $8(6 \%)$ & $16(13 \%)$ & $21(17 \%)$ \\
\hline & Western Europe & $28(5 \%)$ & $53(9 \%)$ & $70(12 \%)$ & $21(4 \%)$ & $33(6 \%)$ & $40(7 \%)$ & $85(14 \%)$ & $124(21 \%)$ & $142(24 \%)$ \\
\hline
\end{tabular}

P0326 OPIATE USE IN INFLAMMATORY BOWEL DISEASE. PRESCRIPTION TRENDS AND MORTALITY IN AN ENGLISH PRIMARY CARE COHORT FROM 1990-2014

N. Burr ${ }^{1}$, C. Smith ${ }^{1}$, R. West ${ }^{1}$, M. A. Hull ${ }^{1}$, V. Subramanian ${ }^{1}$

${ }^{1}$ Gastroenterology, Leeds Teaching Hospitals NHS Trust, Leeds/United Kingdom

Contact E-mail Address: nick.burr@nhs.net

Introduction: Chronic abdominal pain is a common symptom in inflammatory bowel disease (IBD). Pain management is complicated by clinically important gastrointestinal side effects of many of the available analgesics, particularly opiates. Opiate prescribing for cancer and non-cancer pain has increased dramatically in recent years but there is a paucity of data on prescription trends for individuals with IBD. The only population-based study is from Canada where $5 \%$ of subjects with IBD became heavy opiate users after 10 years of diagnosis and there was a strong association between heavy opiate use and mortality (OR $2.91,95 \%$ CI 1.58-5.02). In this study we explore trends in the prescription of opiate medications and assess the association between opiate prescription and mortality in English primary care cohort of patients with IBD.

Aims \& Methods: We used the English primary care database ResearchOne for this study which holds records from approximately 6 million individuals $(>10 \%$ of the total population). We extracted relevant clinical codes and prescription data on all patients with IBD, and separated out those with ulcerative colitis (UC) and Crohn's disease (CD). We created 4 categories of opiate medication use, namely; any opiate medication, codeine only, tramadol, and strong opiates. We defined 3 groups of prescription density as none/infrequent users, moderate and heavy users as $<1,1-3$ and $>3$ prescriptions per calendar year respectively. We examined the trend in opiate prescriptions for all IBD patients in 4 year blocks from 1990-2014 using chi $^{2}$ for trend as a significance. Separate trends were produced for each of our opiate classes. We calculated a propensity score estimating the conditional probability of being prescribed an opiate medication based on pre-defined characteristics which may influence the prescription of opiates. All-cause mortality in opiate users and non-users was compared in a propensity score matched, Cox proportional hazards regression analysis to produced hazard ratios (HR) and 95\% confidence intervals (CI). All analyses were performed for each opiate medication class in $\mathrm{CD}$ and UC patients.

Results: We included 3517 patients with CD and 5349 with UC. Opiate prescriptions increased from $10 \%$ in 1990 to $30 \%$ in 2014 (chi2 for trend $p<0.005$ ). There was a similar, significant increase for codeine $\left(\mathrm{chi}^{2}\right.$ for trend, $\left.\mathrm{p}=0.008\right)$, tramadol $(\mathrm{p}<0.005)$ and strong opiates $(\mathrm{p}<0.005)$ when analyzed separately. Table 1 shows the association between opiate use and all-cause mortality in patients with IBD. Any opiate use in patients with UC was associated was associated with increased mortality ((HR 1.67, 95\% CI 1.25-2.23). The strongest associations were for heavy users of strong opiates in patients with CD (HR 2.18, $95 \%$ CI $1.20-3.95$ ) and UC (HR 3.30, 95\% CI 1.77-6.18). There was no associations for prescriptions of tramadol at any prescription density in CD or UC.

Table 1: - The association between opiate prescriptions and all-cause mortality in patients with inflammatory bowel disease in the English primary care database Research One. Propensity score matched, Cox proportional hazards regression analysis.

\begin{tabular}{lll}
\hline & $\begin{array}{l}\text { Crohn's disease } \\
\text { Hazard ratio } \\
(95 \% \mathrm{CI})\end{array}$ & $\begin{array}{l}\text { Ulcerative colitis } \\
\text { Hazard ratio } \\
(95 \% \mathrm{CI})\end{array}$ \\
\hline Any opiate medication & & \\
$\begin{array}{l}\text { None/infrequent use }(<1 \text { prescription per year }) \\
\text { Moderate use (1-3 prescriptions per year) }\end{array}$ & 1 & 1 \\
Heavy use ( $>3$ prescriptions per calendar year) & $0.94(0.64-1.39)$ & $0.83(0.56-1.21)$ \\
Codeine only & $1.15(0.85-1.55)$ & $1.67(1.25-2.23)$
\end{tabular}

Codeine only

(continued)
Table 1 Continued

\begin{tabular}{|c|c|c|}
\hline & $\begin{array}{l}\text { Crohn's disease } \\
\text { Hazard ratio } \\
(95 \% \text { CI })\end{array}$ & $\begin{array}{l}\text { Ulcerative colitis } \\
\text { Hazard ratio } \\
(95 \% \text { CI })\end{array}$ \\
\hline None/infrequent use ( $<1$ prescription per year) & 1 & 1 \\
\hline Moderate use (1-3 prescriptions per year) & $1.66(0.89-3.09)$ & $0.72(0.35-1.47)$ \\
\hline Heavy use ( $>3$ prescriptions per calendar year) & $0.70(0.35-1.39)$ & $1.83(1.10-3.05)$ \\
\hline \multicolumn{3}{|l|}{ Tramadol alone or in combination with codeine } \\
\hline None/infrequent use ( $<1$ prescription per year) & 1 & 1 \\
\hline Moderate use (1-3 prescriptions per year) & $0.69(0.27-1.72)$ & $0.50(0.19-1.36)$ \\
\hline Heavy use ( $>3$ prescriptions per calendar year) & $0.79(0.35-1.81)$ & $1.39(0.66-2.94)$ \\
\hline \multicolumn{3}{|l|}{ Strong opiates } \\
\hline None/infrequent use ( $<1$ prescription per year) & 1 & 1 \\
\hline Moderate use (1-3 prescriptions per year) & $1.34(0.67-2.70)$ & $2.44(1.16-5.15)$ \\
\hline Heavy use ( $>3$ prescriptions per calendar year) & $2.18(1.20-3.95)$ & $3.30(1.77-6.18)$ \\
\hline \multicolumn{3}{|l|}{ Strong opiates versus weak opiates } \\
\hline None/infrequent use ( $<1$ prescription per year) & 1 & 1 \\
\hline Moderate use (1-3 prescriptions per year) & $1.36(0.69-2.69)$ & $1.81(0.91-3.62)$ \\
\hline Heavy use ( $>3$ prescriptions per calendar year) & $2.04(1.14-3.65)$ & $2.47(1.41-4.33)$ \\
\hline
\end{tabular}

Conclusion: Our study is the largest population based study of opiate use in patients with IBD. We have shown a significant increase in the prescription of opiates since 1990 , with $30 \%$ being prescribed an opiate medication between 2010 and 2014. Prescriptions of codeine in UC and strong opiates in both CD and UC were associated with increased all-cause mortality. There appears to be a dose association as heavy users of strong opiates had the largest association with mortality. Observational studies are not proof of causality and there may be residual confounding. A dose reponse is a strong indicator that opiates could be responsible for the associations seen, which is consistent with other studies investigating opiates used for non-cancer pain in chronic disease. Randomised controlled trials would be unethical and not faesible to investigate this potential effect so population-based observational studies may provide the best estimate. Opiate prescriptions are increasing worldwide for chronic non-cancer pain, and individuals with IBD can now be included. Clinicians managing pain in individuals with IBD should consider the potential implications of prescribing, or continuing with opiate prescriptions as they are a marker for increased mortality. Disclosure of Interest: All authors have declared no conflicts of interest.

\section{P0327 PATIENTS WITH INFLAMMATORY BOWEL DISEASE HAVE AN INCREASE RISK OF PERIODONTITIS CORRELATED WITH DISEASE ACTIVITY}

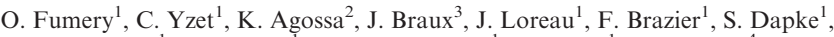
B. Devauchelle ${ }^{1}$, S. Testelin ${ }^{1}$, E. Nguyen-Khac ${ }^{1}$, J. Dupas ${ }^{1}$, M. Fumery ${ }^{4}$

${ }^{1}$ Amiens University Hospital, Amiens/France

${ }^{2}$ Faculté Dentaire de Lille, France/France

${ }^{3}$ Faculté dentaire Reims, Amiens/France

${ }^{4}$ Gastroenterology Unit, Epimad Registry, CHU Amiens Sud, Avenue LaennecSalouel, Amiens University Hospital, Amiens/France

Contact E-mail Address: mathurinfumery@gmail.com

Introduction: We aimed to: (1) evaluate the prevalence of periodontitis in patient with inflammatory bowel disease (IBD), (2) assess the impact of IBD activity and IBD therapy on parodontal outcomes. 
Aims \& Methods: In a prospective 6-months study, dental examination was performed in 54 patients with IBD and in 19 healthy controls. IBD related variables were prospectively collected, as well as markers for periodontitis including gingival bleeding (BOP index, marker of periodontal inflammation), gingival recession (REC index, marker of cumulative periodontal destructions) and probing depth (PPD as a marker of the severity of the disease). Additional dental examination was proposed 3 months after to all patients diagnosed with periodontitis. Results: Among the 54 included patients, 44 had Crohn disease $(81 \%)$ and 31 were women $(55 \%)$. At the time of dental examination, median age was 33 years $(\mathrm{Q} 1=26 ; \mathrm{Q} 3=41), 20(36 \%)$ were smokers and the median IBD duration was 8.4 years $(3.4-16.3)$. Eleven $(20 \%)$ were treated by corticosteroids, $27(49 \%)$ by antiTNF, $6(10 \%)$ by other biologics and 8 had no IBD treatment. IBD was significantly associated with periodontitis $(81 \%$ vs $27 \%$; Odds Ratio 2.9, 95\%CI1.3-6.2). Mild, moderate and severe periodontitis were respectively observed in $34(63 \%), 8(15 \%)$ and $3(5 \%)$ IBD patients. As compared to healthy controls, IBD patients had significant increase of BOP index $(p=0.008)$, probing death $(\mathrm{p}=0.03)$, and REC index $(\mathrm{p}=0.01)$. Patients with active IBD (Harvey Bradshaw $>4$ or Mayo Score $>1$ ) had a significant increase of BOP index $(\mathrm{p}=0.007)$ as compared to patients with inactive disease. A significant correlation between BOP and Harvey-Bradshaw index was observed $(r=0.44$, $\mathrm{p}=0.0018)$. Anti-TNF therapy was significantly associated with lower BOP index $(p=0.02)$. All patients with diagnosis of periodontitis were treated by periodontal debridement and subgingival irrigation with povidone-iodine which led to a significant decrease of BOP index three months after diagnosis

Conclusion: Inflammatory bowel diseases were associated with an increase risk of periondotitis. Gingival inflammation was correlated to disease activity and antiTNF therapy was associated with a lower risk of active parodontal disease. Disclosure of Interest: All authors have declared no conflicts of interest.

\section{P0328 DETECTION OF MUTATIONS IN NOD2/CARD15 GENE IN ARAB PATIENTS WITH CROHN'S DISEASE}

I. Siddique $^{1}$, I. Khan ${ }^{2}$, R. Sulaiman ${ }^{3}$, N. Kadungothayil ${ }^{3}$, M. Al-Tarrah ${ }^{4}$, A.S. Mustafa

${ }^{1}$ Medicine, Faculty of Medicine, Kuwait University, Safat/Kuwait

${ }^{2}$ Biochemistry, Faculty of Medicine, Kuwait University, Safat/Kuwait

${ }^{3}$ Research Core Facility, Omics Research Unit, Faculty of Medicine, Kuwait

University, Safat/Kuwait

${ }^{4}$ Thunayan Al-ghanim Gastroenterology Center, Al-Amiri Hospital, Safat/Kuwait

${ }^{5}$ Microbiology, Faculty of Medicine, Kuwait University, Safat/Kuwait

Contact E-mail Address: isiddique@hsc.edu.kw

Introduction: Crohn's disease is a chronic, immune mediated inflammatory condition which affects the gastrointestinal tract. NOD2/Card15 mutations have been linked to an increased risk of Crohn's disease and to some of its phenotypes. This study aimed to determine the presence of the above mutations in Arab patients suffering from Crohn's disease in Kuwait.

Aims \& Methods: Blood samples were obtained from 103 Arab patients with Crohn's disease and 100 Arab control subjects. The genomic DNA was isolated from the samples using Qiagen DNA Blood mini kit. The isolated DNA were used in Polymerase Chain Reaction (PCR) using four sets of primers specific for each mutation in the NOD2/Card15 (rs2066842, SNP5, Exon 802C $>\mathrm{T}$; rs2066844, SNP8, Exon4 2104C > T; rs2066845, SNP12, Exon 8 2722G > C; and, rs2066847, SNP13, Exon11 3020insC). The PCR-amplified DNA were sequenced using ABI 3130xl Genetic analyzer, and specific mutations were detected by using the sequence analysis software.

Results: Table 1 shows the results of all homozygous and heterozygous mutations found in the patients and control subjects. The mutation in rs2066842 (SNP5, Exon4 $802 \mathrm{C}>\mathrm{T})$ was detected in the NOD2/Card15 sequence of $17(16.5 \%)$ Arab patients with Crohn's disease compared to $32(32.0 \%)$ normal controls $(\mathrm{p}<0.05)$. This difference was statistically significant if the mutation was heterozygous $(\mathrm{p}<0.05)$ but not in homozygous. The mutation in rs2066845 (SNP12, Exon $82722 \mathrm{G}>\mathrm{C})$ was found in $24(23.3 \%)$ patients and $10(10.0 \%)$ controls $(\mathrm{p}<0.05)$. This difference was statistically significant if the mutation was homozygous $(\mathrm{p}<0.05)$ but not in heterozygous. The mutation in rs2066844 (SNP8, Exon4 $2104 \mathrm{C}>\mathrm{T}$ ) was found only in one patient and no controls and rs 2066847 (SNP13, Exon11 3020insC) was not detected in any of the patients or controls. Table 1. Mutations in SNP5, SNP8, SNP12 and SNP13 of the NOD2/Card15 gene in Arab patients with Crohn's disease and control subjects.

Conclusion: This study suggests that mutation in rs2066845 (SNP12, Exon 8 $2722 \mathrm{G}>\mathrm{C}$ ) occurs more frequently in Arab patients with Crohn's disease compared to controls, but the disease is associated only with the homozygous mutation. Mutation in rs2066842 (SNP5, Exon4 802C > T) occurs more frequently in controls compared to patients and the heterozygous mutation appears to have a protective effect against Crohn's disease in the Arab population. Mutations in rs2066844 (SNP8, Exon4 2104C > T) and rs2066847 (SNP13, Exon11 3020insC) are not seen in this population.

Disclosure of Interest: All authors have declared no conflicts of interest.

\section{References}

1.B Khor, A Gardet, RJ Xavier. Genetics and pathogenesis of inflammatory bowel disease. Nature 2011; 474 (2011): 307-317.

2.C.S. Probert, V. Jayanthi, D.S. Rampton, J.F. Mayberry. Epidemiology of inflammatory bowel disease in different ethnic and religious groups: limitations and aetiological clues. Int J Colorectal Dis. 1996; 11: 25-28.

3.J.C. Barrett et al. Genome-wide association defines more than 30 distinct susceptibility loci for Crohn's disease. Nat Genet. 2008; 40: 955-962.

4.R.B. Sartor. Mechanisms of disease: pathogenesis of Crohn's disease and ulcerative colitis. Nat Clin Pract Gastroenterol Hepatol. 2006; 3: 390-407.

5.T.R. Orchard, J. Satsangi, D. Van Heel, D.P. Jewell. Genetics of inflammatory bowel disease: a reappraisal. Scand J Immunol. 2000; 51: 10-17.

6.V. Binder, M. Orholm. Familial occurrence and inheritance studies in inflammatory bowel disease. Neth J Med. 1996; 48: 53-56.

7.J.P. Hugot, P. Laurent-Puig, C. Gower-Rousseau, J.M. Olson, J.C. Lee, et al. Mapping of a susceptibility locus for Crohn's disease on chromosome 16. Nature 1996; 379 (6568): 821-823.

8.T. Ahmad, A. Armuzzi, M. Bunce, K. Mulcahy-Hawes, S.E. Marshall, et al. The molecular classification of the clinical manifestations of Crohn's disease. Gastroenterology 2002; 122: 854-866.

\section{P0329 GENETIC ASSOCIATIONS OF INFLAMMATORY BOWEL DISEASE IN SRI LANKA: A CASE-CONTROL STUDY OF PHENOTYPES AND SELECTED GENETIC POLYMORPHISMS}

M. A. Niriella ${ }^{1}$, K. S. Kodisinghe ${ }^{1}$, A. P. De Silva ${ }^{1}$, N. Rajapakshe ${ }^{1}$, S D. Nanayakkara', D. P. Luke ${ }^{1}$, T. M. De Silva ${ }^{1}$, N. M. Navarathne ${ }^{2}$, U. Kalubovila ${ }^{3}$, R. S. Kumarasena ${ }^{4}$, V. H. Dissanayake ${ }^{5}$, R. W. Jayasekara ${ }^{5}$, H. J. De Silva ${ }^{1}$

${ }^{1}$ Faculty of Medicine, University of Kelaniya, Ragama/Sri Lanka

${ }^{2}$ National Hospital of Sri Lanka, Colombo/Sri Lanka

${ }^{3}$ Teaching Hospital, Kandy/Sri Lanka

${ }^{4}$ Teaching Hospital Karapitiya, Galle/Sri Lanka

${ }^{5}$ Human Genetics Unit, Faculty of Medicine, University of Colombo, Colombo/Sri Lanka

Contact E-mail Address: maduniln@yahoo.co.uk Introduction: There is limited data on genetics of inflammatory bowel disease (IBD) in populations where the condition is emerging, especially from South Asia.

Aims \& Methods: A case [histologically confirmed ulcerative colitis (UC) and Crohn disease (CD) of $\geq 1$ year duration] control [unrelated, healthy, gender matched] study was conducted at four major gastroenterology centres in Sri Lanka. Phenotypic data (type, location, severity, treatment types, response to treatment and complications) of cases were obtained. Cases and controls were genotyped for 16 selected variants with known IBD disease associations in Western and East Asian populations [IL12B:rs1045431, IL23R:rs11805303, ARPC2:rs12612347, IRGM:rs13361189, IL26/IL22:rs1558744, CDH1:rs1728785, IL10:rs3024505, FCGR2A:rs3737240, PTGER4:rs4613763, IL17REL/PIM3:rs5771069, HNF4a:rs6017342, STAT3:rs744166, SMURF1·rs7809799, LAMB1·rs886774, HLA DRB5, DQA1, DRB1, DRA:rs9268853, MST1, UBA7, APEH:rs9822268]. Genotypes of all variants were in Hardy-Weinberg Equilibrium. $\left(\mathrm{P}>10^{-3}\right)$. To account for multiple hypothesis testing associations that had a p-value of $<0.003$ were considered significant.

Results: There were 411 (males $=49.6 \%)$ cases [UC-258 $(62.8 \%)$, males-47.7\%), CD-153 (37.2\%), males-50.3\%] and 465 (males-50.5\%) controls. The following variants were associated with corresponding phenotypes: IL23R:rs11805303 with IBD (all cases) $(\mathrm{p}=0.001)$; IL12B:rs1045431 with upper gastrointestinal (UGI) CD $(p=0.001) ;$ IL17REL/PIM3:rs5771069 with relapsing IBD $(p=0.003)$ MST1, UBA7, APEH:rs9822268 with maintained remission $(\mathrm{p}<0.001)$ IL17REL/PIM3:rs5771069 with disease complications in all cases $(p=0.001)$; HLA-DRB5, DQA1, DRB1:rs9268853 with immunomodulator use in all cases $(\mathrm{p}=0.001)$. Two variants showed a non-significant trend toward s associationPTGER4:rs4613763 with ileal CD ( $\mathrm{p}=0.005)$; IL17REL/PIM3:rs5771069 with treatment-refractory IBD (all cases) $(\mathrm{p}=0.005)$

Conclusion: This first study, confirms the association of genetic variants of IBD previously reported in other populations, with clinical, prognostic and treatment phenotypes of IBD in a Sri Lankan (South Asian) population.

Disclosure of Interest: All authors have declared no conflicts of interest.

Abstract No: P0328

\begin{tabular}{|c|c|c|c|c|c|c|c|c|c|}
\hline Mutation & Patients (n 103) & $\begin{array}{l}\text { Any mutation } \\
\text { Controls (n 100) }\end{array}$ & $P$-value & Patients (n 103) & $\begin{array}{l}\text { Homogyzous mutation } \\
\text { Controls (n 100) }\end{array}$ & $P$-value & Patients (n 103) & $\begin{array}{l}\text { Heterogyzous mutation } \\
\text { Controls (n 100) }\end{array}$ & $P$-value \\
\hline SNP5 & 17 & 32 & $<0.05$ & 6 & 10 & NS & 11 & 22 & $<0.05$ \\
\hline SNP8 & 1 & 0 & NS & 0 & 0 & NS & 1 & 0 & NS \\
\hline SNP12 & 24 & 10 & $<0.05$ & 10 & 0 & $<0.05$ & 14 & 10 & NS \\
\hline SNP13 & 0 & 0 & NS & 0 & 0 & NS & 0 & 0 & NS \\
\hline
\end{tabular}


P0330 VDR GENE BSM I POLYMORPHISM AND ULCERATIVE

\section{COLITIS}

D. Kuznetsova ${ }^{1}$, A. Razumov ${ }^{1}$, M. Merzlyakov ${ }^{2}$, G. Vavin ${ }^{3}$

${ }^{1}$ Department Of Pathophysiology, Medical And Clinical Biochemistry, Kemerovo State Medical University, Kemerovo/Russian Federation

${ }^{2}$ Department Of Endoscopy, Kemerovo Regional Clinical Hospital, Kemerovo Russian Federation

${ }^{3}$ Kemerovo Regional Clinical Hospital, Kemerovo/Russian Federation

Contact E-mail Address: lariwar@mail.ru

Introduction: Among the numerous genetic factors associated with ulcerative colitis (UC), an increasing attention has been paid to the polymorphisms of the vitamin D receptor gene $(V D R)$ associated with disorders of innate and adaptive immunity, as well as the barrier function of the intestinal epithelium. However, the results of studies on the prevalence, clinical, diagnostic and prognostic significance of polymorphisms of the $V D R$ gene in different populations are ambiguous and contradictory. In particular, associations of Bsm I polymorphism of the VDR gene with UC in the Chinese population and in the Jewish Ashkenazi has been found, while in the Irish population, with a sufficient prevalence of Bsm I polymorphism, this association is absent [1-3]. In the Russian Federation, there is no data on the prevalence, clinical, diagnostic and prognostic significance of Bsm I polymorphism of the $V D R$ gene with UC. These circumstances determined the purpose and objectives of this study.

Aims \& Methods: The purpose is to assess the clinical, diagnostic and prognostic significance of the Bsm I polymorphism of the VDR gene (rs1544410) at UC among the residents of the Kemerovo region of the Russian Federation. The study included 76 patients with UC and 85 controls. Genotyping was performed by PCR method ("SNP-express" reagents, Lytech Co. Ltd., Russia) with electrophoretic detection of amplification products. Statistical analysis was performed using the $\mathrm{X}^{2}$ and Mann-Whitney tests. In the presence of statistically significant differences $(\mathrm{p}<0.05)$, odds ratios $(\mathrm{OR})$ with $95 \%$ confidence interval (CI) were calculated.

Results: It was found that the frequency of the allele B polymorphism of the $V D R$ Bsm I gene was higher among patients with UC than in the control group (44\% vs. $26 \%, \mathrm{p}=0.02)$, which increases the risk of this pathology by $2.2 \%(95 \% \mathrm{CI}$ : $1.2-4.1$ ). In the case of carriers of the $\mathrm{B} / \mathrm{B}$ genotype, the risk of developing $\mathrm{UC}$ increased up to 3.5 times in comparison with the control group ( $21 \% \mathrm{vs} .7 \%$, $\mathrm{p}=0.02,95 \% \mathrm{CI}: 1.4-8.6$ ), whereas in $\mathrm{b} / \mathrm{b}$ genotype the risk of UC decreased $(33 \%$ and $54 \%$, respectively, $\mathrm{p}=0.02, \mathrm{OR}=0.4,95 \% \mathrm{CI}: 0.2-0.7)$. Significant differences between carriage of the B allele Bsm I polymorphism and the features of the clinical course of the UC have not been established. However, it has been shown that in carriers of allele B, the clinical implementation of UC develops significantly later than in patients with the b/b genotype (43 and 28.5 years, respectively, $\mathrm{p}=0.04$ )

Conclusion: For the carriers of the B allele Bsm I polymorphism of the $V D R$ gene is a predictor of a high risk of ulcerative colitis with an increase in the age of diagnosis. Genotype $\mathrm{b} / \mathrm{b}$ Bsm I polymorphism of the $V D R$ gene has a protective effect in the development of ulcerative colitis among the residents of the Kemerovo region of the Russian Federation.

Disclosure of Interest: All authors have declared no conflicts of interest.

\section{References}

1. Pei F.H., Wang Y.J., Gao S.L. et al. Vitamin D receptor gene polymorphism and ulcerative colitis susceptibility in Han Chinese. J Dig Dis. 2011; 12(2):9098.

2. Dresner-Pollak R., Ackerman Z., Eliakim R. et al. The Bsm I vitamin D receptor gene polymorphism is associated with ulcerative colitis in Jewish Ashkenazi patients. Genet Test. 2004; 8(4):417-420.

3. Hughes D.J., McManus R., Neary P. et al. Common variation in the vitamin $\mathrm{D}$ receptor gene and risk of inflammatory bowel disease in an Irish casecontrol study. Eur J Gastroenterol Hepatol. 2011; 23(9):807-812.

\section{P0331 GENETIC AND SEROLOGICAL PROFILE AS MARKERS OF DISEASE SUSCEPTIBILITY IN SIBLINGS OF CHILDREN WITH INFLAMMATORY BOWEL DISEASE}

G. D’Arcangelo ${ }^{1}$, M. Bramuzzo ${ }^{2}$, G. Catassi ${ }^{1}$, C. Romano ${ }^{3}$, E. Miele ${ }^{4}$, G. Morabito ${ }^{5}$, S. Pellegrino ${ }^{6}$, C. Cuzzupè ${ }^{6}$, C. Strisciuglio ${ }^{7}$, S. Martelossi ${ }^{2}$, S. Cucchiara ${ }^{1}$, M. Aloi ${ }^{1}$

${ }_{1}^{1}$ Pediatric Gastroenterology And Liver Unit, Sapienza University of Rome, Rome/ Italy

2 Institute For Maternal And Child Health, Irccs "burlo Garofalo,", Pediatric Department, Gastroenterology and Nutrition Unit, Trieste/Italy

${ }^{3}$ Pediatric Gastroenterology And Endoscopy, Pediatric Department, Pediatric

Department, Messina/Italy

${ }^{4}$ Department Of Translational Medical Science, University of Naples "Federico II", Naples/Italy

${ }^{5}$ Pediatric Gastroenterology And Endoscopy, Pediatric Department, G. Martino Hospital, Messina/Italy

${ }^{6}$ Pediatric Department, Gastroenterology Unit, University of Messina, Messina Italy

${ }^{7}$ Department $O f$ Women, Child And General And Specialist Surgery, SUN, Napoli Italy

Contact E-mail Address: giuliadarcangelo87@gmail.com

Introduction: Having a family history for inflammatory bowel disease (IBD) is the only known risk factor for disease development. Indeed, up to $30 \%$ of IBD patients report at least 1 first-degree relative with IBD, and siblings carry the highest risk. Recent data have shown that genetic and serological markers may predict IBD development. However, there are only few studies evaluating a genetically well-characterized population and at high risk for disease, such as siblings and twins. Therefore, aim of this study was to evaluate genetic and serological findings as markers of disease susceptibility in healthy siblings and twins of children with IBD

Aims \& Methods: This is the first phase of a prospective, longitudinal, multicenter, case-control study. Serum was collected from 80 siblings and twins of children with IBD and 77 healthy controls with no family history for IBD. Genotyping (TaqmanMGB) for variants of ATG16L1 (SNP rs2241880), STAT3 (SNP rs744166), ECM1 (SNP rs3737240), NKX2-3 (SNP rs10883365), was performed. Serological titers of anti-Saccharomyces cerevisiae (ASCA IgG and ASCA IgA), perinuclear anti-neutrophil cytoplasmic antibodies (pANCA), anti-outer membrane porin $\mathrm{C}$ antibody (anti-OmpC), and antibacterial flagellin antibody (anti-CBir1), were determined by specific enzyme-linked immunosorbent assay (ELISA)

Results: Fifty-nine out of 80 cases $(74 \%)$ and $50 / 77$ controls $(65 \%)$ were positive for at least 1 of the serum autoantibodies $(p=0.29)$; a combination of any 4 of them was found in 3 cases $(4 \%)$ and no controls $(p=0.28)$. No significant difference was shown for any of the studied autoantibodies between cases and controls. Homozigosity for any susceptibility gene variant was found in 60 out of 80 cases $(75 \%)$ and in $52 / 77$ controls $(67.5 \%)(p=0.37)$, with no significant association between family history and genotype status. No combination of gene variants significantly differed between cases and controls.

Conclusion: Our preliminary results argue against a role of commonly recognized genetic polymorphisms and microbial antibodies as markers of disease susceptibility in siblings of children with IBD. However, data from larger and prospective studies, possibly including microbial characterization, are warranted before drawing definite conclusions.

Disclosure of Interest: All authors have declared no conflicts of interest.

\section{P0332 ROLE OF DNMT3A IN INTESTINAL EPITHELIAL CELLS}

A. Fazio, J. Kuiper, A. Sinha, R. Sheibani-Tezerji, P. Rosenstiel Institute of Clinical Molecular Biology, Kiel/Germany

Contact E-mail Address: a.fazio@ikmb.uni-kiel.de

Introduction: DNA methylation is an important epigenetic modification mechanism of gene expression. Several studies have shown an association of impaired methylation with inflammatory bowel diseases (IBD) pathogenesis. DNMT3A and DNMT3B are two of the three members of the family of de novo DNA methyltransferases. Variants in these proteins are responsible for the establishment of de novo genomic DNA methylation patterns and are involved in the normal development as well as in many diseases. However, it is unknown if DNMT3A may play a role during the mechanism involved in this abnormal methylation pattern and consequently the development of diseases.

Aims \& Methods: To assess the function of DNMT3A in intestinal epithelial cells (IECs), human Caco-2 colonic carcinoma cells were transfected with siRNA targeting DNMT3A, DNMT3B and DNMT3L. Gene expression analysis and DNA methylation analysis using qRT-PCR, RNA sequencing and $850 \mathrm{k}$ methylation chip assay were performed. For long-term experiments, we used a CRISPR/Cas9 genome editing to delete DNMT3A gene in Caco-2 cells. DNMT3A knockout Caco-2 cells were grown in a 3D-Matrigel culture system and after 2 weeks, spheroids cells were stained for actin/nuclei and subjected to confocal microscopy analysis.

Results: From the RNA sequencing data, approximately 1000 genes were found to be differentially expressed between cells lacking DNMT3A and controls. The KEGG pathway analysis identifies differentially regulated genes associated with several functional categories comprising extracellular matrix receptor interaction, focal adhesion and MAPK signalling pathway. In contrast, we observed no difference in DNA methylation between the groups. Furthermore, loss of DNMT3A induces abnormal spheroids formation by reducing spheroids diameter and defect in actin organization and lumen formation. Thus, the observed morphological phenotype may be linked to the differentially regulated genes involved in the previous analysed pathways.

Conclusion: Rapidly inactivation of DNMT3A in IECs promotes changes in gene expression, but does not change global DNA methylation. The absence of DNMT3A induces abnormal/defective spheroids formation in $3 \mathrm{D}$ culture system. Hence, our data provide a preliminary overview of DNMT3A in intestinal epithelial cells and its potential role in maintaining intestinal homeostasis. Disclosure of Interest: All authors have declared no conflicts of interest.

\section{P0333 ORMDL PROTEINS: CRITICAL REGULATORS OF FINE- TUNING OF THE UNFOLDED PROTEIN RESPONSE IN} INTESTINAL INFLAMMATION

M. Jentzsch, M. Falk-Paulsen, A. Luzius, S. T. Stengel, J. Kuiper, B. Messner, F. Tran, K. Aden, P. Rosenstiel

Institute Of Clinical Molecular Biology, Christian-Albrechts-University and University Hospital Schleswig-Holstein, Kiel/Germany

Contact E-mail Address: s.stengel@ikmb.uni-kiel.de

Introduction: The endoplasmic reticulum (ER) plays a crucial role in maintaining cellular homeostasis by coordinating the processing and folding of secretory and membrane proteins. The accumulation of unfolded or misfolded proteins induces ER stress. The unfolded protein response (UPR) aims at restoring ER function and is comprised of three signaling branches via inositol-requiring enzyme 1 
(IRE1), double-stranded RNA-dependent protein kinase (PKR)-like ER kinase (PERK) and activating transcription factor $6 \alpha$ (ATF6 $\alpha$ ). Defects in the ER stress response have been shown to predispose to chronic inflammatory bowel disease (IBD). Genome-wide association studies identified disease susceptibility loci in or adjacent to several UPR related genes including $X B P 1$ and $O R M D L 3$.

Aims \& Methods: The objective of this study was to determine the function of ORMDL3 in UPR signaling to gain insights into the molecular mechanisms promoting chronic intestinal inflammation. Using molecular cell biology approaches, we studied the effect of ORMDL3 on ATF $6 \alpha$, PERK and IRE signaling. The role of ORMDL3 in intestinal inflammation was determined in vivo in both an acute and chronic DSS-colitis model using Ormdl3-deficient mice.

Results: Our in vitro studies demonstrate that ORMDL3 facilitates ER stressinduced ATF6 $\alpha$ activation. Overexpression of ORMDL3 resulted in increased cleavage of ATF6 $\alpha$ and augmented ERSE promoter activity. Mechanistically, we show that ORMDL3 colocalizes and directly interacts with ATF6 $\alpha$. Furthermore, ORMDL3 overexpression induced the PERK pathway by elevating p-eIF $2 \alpha$ and CHOP protein levels. In contrast, we observed an inhibition of IRE1 signaling exerted by ORMDL proteins as shown by reduced XBP1 splicing and decreased UPRE promoter activity. Ormdl3-deficient mice showed an increased susceptibility to acute DSS-induced colitis compared to their wildtype littermates, as indicated by a more severe body weight loss and higher disease activity indices (DAI). In contrast, Ormdl3-deficiency was observed to be beneficial in the course of chronic colitis: compared to their wild-type littermates, Ormdl3 $3^{-1-}$ mice showed less body weight loss and an improved survival rate.

Conclusion: This study demonstrates for the first time the modulatory functions of ORMDL proteins as regulators of all three UPR signaling pathways. Additionally, our in vivo studies suggest that ORMDL proteins constitute a precise fine-tuning mechanism of the UPR determining cell fate decisions in response to ER stress.

Disclosure of Interest: All authors have declared no conflicts of interest.

\section{P0334 THE USE OF LÉMANN SCORE TO EVALUATE THE DAMAGE TO THE DIGESTIVE TRACT CAUSED BY CROHN'S DISEASE IN AN EGYPTIAN COHORT}

H. M. Saad ${ }^{1}$, O. E. Salem ${ }^{1}$, M. A. Salem ${ }^{1}$, M. E. Ibrahim ${ }^{2}$, S. M. El Kady ${ }^{1}$

${ }^{1}$ Internal Medicine, Alexandria University, Alexandria/Egypt

${ }^{2}$ Radiology, Alexandria University, Alexandria/Egypt

Contact E-mail Address: hussienmahmoud7@hotmail.com

Introduction: Several studies have assessed Crohn's disease (CD) according to disease activity using clinical, laboratory \& radiological activity indexes; but few have analyzed the damage the disease bring about on the GI tract. Lémann score was designed to develop a comprehensive assessment of the structural bowel damage, including stricturing lesions, penetrating lesions (fistulas and abscesses), and surgical resection. ${ }^{(1)}$

Aims \& Methods: To calculate Lémann score in a cohort of Egyptian patients to determine its ability to assess the structural damage caused by CD in Egyptian population. Lémann score was calculated using specific computer software based on the original paper by Pariente. B et al ${ }^{(1)}$ in a cohort of Egyptian patients with CDf ollowed from April 2013 - August 2015. The temporal relation between Lémann score and disease duration was also assessed.

Results: A total of 100 Egyptian patients with CD were enrolled, $69 \%$ males and $31 \%$ females. Median age was 32 years, $36 \%$ were smokers. The clinical presentation varied between abdominal pain occurring in $90 \%$ followed by $69 \%$ with chronic diarrhea, $52 \%$ with weight loss. Few patients $(26 \%)$ presented with extraintestinal manifestations. According to ECCO classification of disease severity $73 \%$ of our patients had mild disease, $17 \%$ had moderate disease, $10 \%$ had severe disease. According to Montreal classification, $25 \%$ of patients were A $1,55 \%$ A $2,20 \%$ A $3,79 \%$ L1, $9 \%$ L2 \& $12 \%$ L3, $63 \%$ B $1,7 \%$ B2, $26 \%$ B3, \& only $4 \%$ had perianal disease. According to CDAI, $83 \%$ were in clinical remission, $11 \%$ mild "CDAI $150-220 ", 4 \%$ moderate " $220-450$ ", $2 \%$ severe "CDAI > 450" disease activity. When assessing structural damage of upper GIT, $4 \%$ had stricturing lesions, $2 \%$ grade $1 \& 2 \%$ grade 2 lesions. The small bowel showed stricturing lesions in $65 \%: 39 \%$ grade $1,23 \%$ grade $2,3 \%$ grade 3 lesions. $25 \%$ of patients had grade 3 penetrating lesions, $5 \%$ had surgery which was resection in all the cases. As regards the assessment of stricturing lesion in the colon/rectum, the cecum showed stricturing lesions in 3\%, 2\% grade 2 and $1 \%$ grade 3 lesions; ascending colon showed stricturing lesions in $20 \%$, with $7 \%$ grade 1 lesions, $11 \%$ grade $2 \& 2 \%$ grade 3 lesions; transverse colon showed stricturing lesions in $1 \%$; sigmoid colon showed stricturing lesions in $2 \%$; rectum showed stricturing lesions in 1 ; while in only $1 \%$ whole colon showed grade 1 stricturing lesions affecting the 6 segments. In addition, $12 \%$ had grade 3 penetrating lesions while $9 \%$ had history of surgery mainly intestinal resection. In the anal canal, only $3 \%$ of patients had grade 3 penetrating lesions. The mean Lémann score was $4.02 \pm 4.19 \&$ the median was 2 . Increased median Lémann score was statistically positively correlated with disease duration of $\leq 2$ years \& $>2-<10$ years and $\geq 10$ years respectively. $\left(\mathrm{r}_{\mathrm{s}}=0.343 \& \mathrm{p}<0.001\right)$ $(\mathrm{KW}=9.235(0.010))$. The current study showed that affection of the GI tract was $(5 \%, 92 \%, 41 \%$, and $3 \%$ with upper tract, small bowel, colon/rectum, \& anus CD location, respectively). There is an increase in median Lémann score with increase in disease duration (global test $p<0.001$ ): $0.60,2.50,6.40$ for disease duration of $\leq 2$ years $\&>2-<10$ years and $\geq 10$ years respectively. These results are in concordance with what was published by Pariente B et al. ${ }^{(1)}$ Conclusion: Crohn's disease affection pattern in our Egyptian Cohort is mainly in the small bowel $(92 \%)$ followed by the colon $(41 \%)$ with the upper GI and the anal canal representing only $5 \%$ and $3 \%$ respectively. Lémann score designed by and derived from the linear mixed model showed a good fit with the investigator organ damage evaluations in Egyptian population with CD. Lémann score could strongly assess the damage to the digestive tract caused by CD in a cohort of Egyptian patients. Despite calculating Lémann score in Egyptian cohort reflects the same positive trend with western cohort but with relatively smaller figures could reflect that Egypt is a young population for CD.

Disclosure of Interest: All authors have declared no conflicts of interest.

\section{Reference}

1. Pariente B, Mary JY, Danese S, Chowers Y, De Cruz P, D'Haens G, et al. Development of the Lémann index to assess digestive tract damage in patients with Crohn's disease. Gastroenterology. 2015 Jan;148(1):52-63.

\section{P0335 FINAL RESULTS ON IMMUNOGENICITY PROFILE AND PREDICTORS OF ADA DEVELOPMENT OF BIOSIMILAR INFLIXIMAB DURING THE FIRST 12 MONTHS OF THE THERAPY: RESULTS FROM A PROSPECTIVE NATIONWIDE COHORT}

P.A. Golovics ${ }^{1}$, B.D. Lovasz ${ }^{1}$, Z. Kurti ${ }^{1}$, M. Rutka ${ }^{2}$, Z. Vegh ${ }^{1}$, K.B. Gecse ${ }^{1}$, K. Farkas ${ }^{2}$, J. Banai ${ }^{3}$, L. Bene ${ }^{4}$, L. Gonczi ${ }^{1}$, B. Gasztonyi ${ }^{5}$, T. Kristof ${ }^{6}$, L. Lakatos ${ }^{7}$, P. Miheller ${ }^{8}$, K. Palatka ${ }^{9}$, A. Patai ${ }^{10}$, M. Papp ${ }^{11}$, A. Salamon ${ }^{11}$, T. Szamosi ${ }^{3}$, Z. Szepes ${ }^{2}$, G.T. Toth ${ }^{12}$, A. Vincze ${ }^{13}$, E. Biro ${ }^{14}$, T. Molnar ${ }^{2}$, P.L. Lakatos ${ }^{1}$

${ }^{1} 1$ st Department Of Medicine, Semmelweis University Faculty of Medicine 1st Dept. of Medicine - 1st Department of Medicine, Semmel, Budapest/Hungary ${ }^{2}$ First Department Of Internal Medicine, University of Szeged, Szeged/Hungary ${ }^{3}$ Military Hospital - State Health Centre, Budapest/Hungary

${ }^{4} 1$ st Department Of Medicine, Peterfy Hospital, Budapest/Hungary

${ }^{5}$ 2nd Department Of Medicine, Zala County Hospital, Zalaegerszeg/Hungary

${ }^{6}$ 2nd Department Of Medicine, $B-A-Z$ County and University Teaching Hospital, Miskolc/Hungary

${ }^{7}$ Department Of Internal Medicine, Csolnoky Ferenc Regional Hospital, Veszprem/ Hungary

${ }^{8}$ Second Department Of Internal Medicine, Semmelweis University, Budapest/

Hungary

${ }^{9}$ Department Of Gastroenterology, University of Debrecen, Debrecen/Hungary

${ }^{10}$ Department Of Medicine And Gastroenterology, Markusovszky Hospital,

Szombathely/Hungary

${ }^{11}$ Department Of Gastroenterology, Tolna County Teaching Hospital, Szekszard/ Hungary

${ }^{12}$ Department Of Gastroenterology, Janos Hospital, Budapest/Hungary

${ }^{13} 1$ st Department Of Medicine, University of Pecs, Pecs/Hungary

${ }^{14}$ Department Of Laboratory Medicine, Semmelweis University, Budapest/

Hungary

Contact E-mail Address: golovics.petra@gmail.com

Introduction: Biosimilar infliximab CT-P13 received EMA approval in June 2013 for all indications of the originator product and its use is mandatory in all antiTNF naïve IBD patients in Hungary since May 2014.

Aims \& Methods: In the present study we aimed to prospectively evaluate the immunogenicity profile of the biosimilar infliximab and predictors of TDM in IBD during the first year of therapy in a nationwide, multicentre cohort. Demographic data were collected and a harmonized monitoring strategy was applied. Clinical and biochemical activity were evaluated at weeks 14, 30 and 54. Routine therapeutic drug monitoring (TDM) was applied. Trough level (TL) and anti-drug antibody (ADA) concentration were measured by ELISA (LT-005, Theradiag, France) at baseline and at 2, 6, 14, 30 and 54 weeks right before antiTNF administration during the induction treatment.

Results: 353 consecutive IBD patients (209 CD patients and 144 UC patients) were included in the present cohort. $23.4 \%$ of CD patients and $19.4 \%$ of UC patients had received previous anti-TNF therapy. None of the patients had received infliximab within 12 months prior to initiation of the biosimilar infliximab. $60 / 51 \%$ of $\mathrm{CD} / \mathrm{UC}$ patients received concomitant immunosuppressives at baseline. Mean TLs were 18.9, 17.3, 7.4, 4.3 and $5.3 \mu \mathrm{g} / \mathrm{ml}$ at weeks $2,6,14,30$

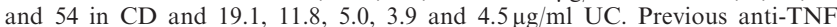
therapy was associated with lower early TL-s in both CD (week 2, 14, and 30, $\mathrm{p}<0.05$ ) and UC (week 2 and $6, \mathrm{p}=0.03$ ). ADA positivity rates were $4.3 \%$, $12.0 \%, 20.9 \%$ and $28.6 \%$ in naïve patients at weeks $0,14,30$ and 54 (ntotal $=266,312,290$ and 210). ADA positivity at week 14 was associated with lower TLs in all CD (week 2, 14 and 30, p $<0.007$ for all) and UC (week 6,14 and $30, \mathrm{p}<0.001$ for all) patients. Concomitant IS use prevented ADA formation in anti-TNF-naïve patients (week 14, 30 and 54, p=0.01, 0.02 and 0.004 ) in $\mathrm{CD}$ but not in UC and did not affect clinical remission or response rates. $32(8.9 \%)$ patients had infusion reactions during induction or maintenance treatment, of which 16 patients had received previous infliximab treatment.

Conclusion: Drug TLs and ADAs in IBD patients until week 54 were in line with results reported for the originator in previous studies. Patients with previous exposure to anti-TNFs had lower early TL coupled with ADA positivity and were more likely to develop infusion reactions. Concomitant IS use prevented ADA development in anti-TNF naïve patients.

Disclosure of Interest: All authors have declared no conflicts of interest. 


\section{P0336 RECONSIDERING THE PROGNOSTIC VALUE OF} TRADITIONAL SEROLOGIC ANTIBODIES IN CROHN'S DISEASE IMMUNOGLOBULIN CLASSES TO TAKE THE CENTRE STAGE

N. Sipeki ${ }^{1}$, B. Suga ${ }^{1}$, G.L. Norman ${ }^{2}$, Z. Shums ${ }^{2}$, G.L. Veres ${ }^{3}$, P.L. Lakatos ${ }^{4}$, P. Antal-Szalmas, M. Papp

${ }^{1}$ Department Of Internal Medicine, Division Of Gastroenterology, University of Debrecen, Faculty of Medicine, Debrecen/Hungary

${ }^{2}$ Inova Diagnostics, San Diego/United States of America/CA

${ }^{3}$ I. sz. Gyermekklinika, Semmelweis Egyetem, Budapest/Hungary

${ }^{4}$ Department Of Gastroenterology, McGill University Health Center, Montreal

General Hospital, Montreal/Canada/OC

${ }^{5}$ Department Of Laboratory Medicine, University of Debrecen, Faculty of Medicine, Debrecen/Hungary

Contact E-mail Address: norasipeki@gmail.com

Introduction: The most relevant scope of serologic antibodies in Crohn's disease $[C D]$ is to stratify the risk of complicated disease course. Significance of distinct antibody classes and their characterisation was rarely considered. We aimed to address these concerns.

Aims \& Methods: Sera of 266 well-characterized CD patients (m/f:112/154, median age: 25 yrs, B1:80.1\%, P1:18.0\%) and 155 controls were assayed for traditional anti-microbial antibodies (ASCA $\mathrm{IgA} / \mathrm{IgG}$, anti-OMP $\operatorname{IgA}$ ). Endotoxin core IgA (EndoCAb) and a panel of non-specific immunoglobulin A (IgA) antibodies (IgA1, IgA2 and secretory [s] IgA) were also assessed by ELISA. An observational follow-up study [median, 143 months] was conducted to assess possible associations between serologic antibodies and the development of various complications and subsequent surgical interventions. A novel flow cytometry test system was established for characterisation of IgA type ASCA to reveal possible origin of the antibody.

Results: A total of $65.7 \%$ and $46.2 \%$ of the CD patients were positive for ASCA IgA/IgG and anti-OMP antibodies. Both ASCA types occurred equally. EndoCAb $\mathrm{IgA}$ positivity was more frequent $(15.4 \%$ vs. $5.4 \%$, $\mathrm{p}<0.01$ ) and sIgA levels were increased [median, 51 vs. $29 \mu \mathrm{g} / \mathrm{ml}$, $\mathrm{p}<0.001]$ in $\mathrm{CD}$ compared to controls. They were also associated with presence of IgA type anti-microbial antibodies. Contrary, ratio of IgA2/A1 in CD corresponded with the value of the controls. In Kaplan-Meier analysis, development of internal penetrating and/or stenosing (IP/S) complications and resective surgery (SR) was significantly associated with $\operatorname{IgA}$ type $\left(\mathrm{pLogRank}, 0.001\right.$ and $\mathrm{p}_{\text {LogRank }}=0.025$ respectively), while development of perianal penetration (PP) with $\mathrm{IgG}$ type ASCA $\left(\mathrm{p}_{\text {LogRank }}=0.008\right)$. Performance OMP IgA was equal to ASCA IgA, however sIgA not. Antimicrobial antibodies remained independent predictors in multivariate Coxregression analysis comprising relevant clinical factors.

Summary of multivariate Cox regression analysis for the association of serologic antibodies with complicated disease course

\begin{tabular}{llll}
\hline HR [95\% CI]; & IP/S in B1 pts & SR in B1 pts & PP in P0 pts \\
\hline ASCA IgA & $2.92[1.85-4.62] ;<0.001$ & $1.77[1.09-2.87] ; 0.021$ & \\
ASCA IgG & & & $2.77[0.36-5.63] ; 0.005$ \\
ASCA IgA/IgG & $1.76[1.09-2.87] ; 0.022$ & $1.45[0.86-2.45] ; 0.163$ & $2.07[0.98-4.39] ; 0.057$ \\
Omp IgA & $1.66[1.09-2.54] ; 0.019$ & $2.08[1.28-3.38] ; 0.003$ & $1.13[0.63-2.01] ; 0.692$ \\
sIgA & $1.54[0.97-2.44] ; 0.066$ & $1.37[0.82-2.28] ; 0.23$ & $1.25[0.67-2.34] ; 0.475$ \\
Endocab IgA & $2.60[1.62-4.17] ;<0.001$ & $1.66[0.96-2.87] ; 0.071$ & $0.74[0.33-1.68] ; 0.475$
\end{tabular}

Without uncoupling of Ig antibody classes yielded clearly inferior performance. ASCA IgA subtyping assays revealed marked increase in the proportion of IgA2 subtype $(29 \%)$ and presence of the secretory component $(89 \%$ of total ASCA IgA) concurrently.

Conclusion: Consideration of antibody classes is an important novel parameter in serology-based prediction in CD. Involvement of gut mucosal immune system is in center of $\operatorname{IgA}$ type antibody formation reflecting sustained exposure and dysregulated immunresponse to bacterial constituents.

Disclosure of Interest: G.L. Norman: Gary L. Norman is employed by Inova Diagnostics, Inc., San Diego, California and are getting personal fees from the company

Z. Shums: Zakera Shums is employed by Inova Diagnostics, Inc., San Diego, California and are getting personal fees from the company

All other authors have declared no conflicts of interest.

\section{P0337 UTILITY OF MAGNETIC RESONANCE EVALUATION FOR SMALL BOWEL ENDOSCOPIC HEALING IN PATIENTS WITH CROHN'S DISEASE}

K. Takenaka, K. Ohtsuka, Y. Kitazume, T. Fujii, K. Matsuoka, M. Kimura, M. Nagahori, M. Watanabe

Tokyo Medical And Dental University, Tokyo/Japan

Contact E-mail Address: ktakenaka.gast@tmd.ac.jp

Introduction: Achievement of endoscopic healing is a key treatment goal in patients with Crohn's disease (CD). We previously reported that magnetic resonance enterography (MRE) could exactly assess small bowel (SB) active lesions such as ulcers using balloon assisted enteroscopy (BAE) reference $[1,2]$

Aims \& Methods: We aimed to evaluate whether MRE could predict patient prognosis in prospective observational study. From July 2012 to December 2015, $139 \mathrm{CD}$ patients in clinical and serological remission were followed up after BAE and MRE procedure. Two endoscopists performed BAE and assessed the endoscopic findings, while two radiologists assessed the MRE findings. Both the endoscopists and radiologists were blinded to the patient's clinical presentation and results of other studies. We used Simple Endoscopic Score for CD (SESCD) for endoscopic evaluation and Magnetic Resonance Index of Activity (MaRIA) for MR evaluation. Endoscopic healing was defined as the absence of ulcerative disease (SES-CDa $<5$ ). Primary endpoints were clinical relapse and serological relapse. The relationship between endoscopic SB lesions and endpoints was evaluated. Moreover, whether MRE findings could predict patient outcomes was assessed.

Results: The median duration of follow-up was 27 months (range, 12-48). Clinical and serological relapse occurred in $30(21.6 \%)$ and $62(44.6 \%)$ patients, respectively. SB endoscopic healing (SES-CDa $<5$ ) was achieved in $76(54.7 \%)$ patients. Multiple regression analysis of BAE findings showed that the absence of SB endoscopic healing (ulcerative disease; SES-CDa $\geq 5$ ) was an independent risk factor for clinical relapse (Hazard ratio $[\mathrm{HR}]=4.78 ; 95 \%$ CI: $1.94-11.80 ; \mathrm{P}=0.001)$ and serological relapse $(\mathrm{HR}=2.84 ; 95 \% \mathrm{CI}: 1.63-$ $4.95 ; \mathrm{P}<0.001)$, respectively. On Kaplan-Meier analysis, patients who did not achieve endoscopic healing were at a significant risk of worse outcomes (clinical relapse, $\mathrm{P}<0.001$; serological relapse, $\mathrm{P}<0.001$ ). $\mathrm{MR}$ ulcer healing (MaRIA score $<11)$ showed a high sensitivity $(82.5 \%$; $95 \%$ confidence intervals [CIs]: $74.9 \%-88.2 \%)$ and specificity $(85.5 \% ; 95 \%$ CIs: $79.2 \%-90.2 \%)$ for endoscopic healing. On Cox's proportional hazards analysis, MRE findings of ulcer healing were associated with a low risk of clinical relapse (Hazard Ration [HR]: $0.24 ; 95 \%$ CIs: $0.111 \%-0.54 \% ; \quad \mathrm{P}=0.001)$ and serological relapse (HR: $0.47 ; 95 \%$ CIs: $0.29 \%-0.78 \%$; $\mathrm{P}=0.003$ ).

Conclusion: The absence of endoscopic healing (ulcerative disease) were seen in a considerable number of $\mathrm{CD}$ patients who were in clinical-serological remission, and these lesions were at risk factor for worse prognosis. MRE could evaluate SB endoscopic healing with a high diagnostic accuracy and could predict patient outcomes.

Disclosure of Interest: All authors have declared no conflicts of interest.

\section{References}

1. Takenaka K, Ohtsuka K, Kitazume Y, et al. Comparison of Magnetic Resonance and Balloon Enteroscopic Examination of the Small Intestine in Patients With Crohn's Disease. Gastroenterology 2014;147:334-342

2. Takenaka K, Ohtsuka K, Kitazume Y, et al. Correlation of the Endoscopic and Magnetic Resonance Scoring Systems in the Deep Small Intestine in Crohn's Disease. Inflamm Bowel Dis. 2015; 8: 1832-1838.

\section{P0338 USEFULNESS OF DOUBLE BALLOON ENDOSCOPY IN DIAGNOSIS AND TREATMENT OF SMALL BOWEL CROHN'S DISEASE}

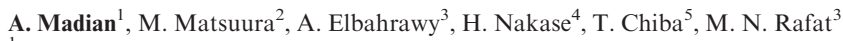
${ }_{1}^{1}$ Internal Medicine, Al Azhar University, Assiut/Egypt

${ }^{2}$ Gastroenterology And Hepatology, Kyoto University, Kyoto/Japan

${ }^{3}$ Internal Medicine, Al-Azhar University, Cairo/Egypt

${ }^{4}$ Gastroenterology And Hepatology, Sapporo Medical University, Sapporo/Japan

${ }^{5}$ Gastroenterology And Hepatology, Kyoto University, Kyoto/Japan

Contact E-mail Address: a.madian@azhar.edu.eg

Introduction: In Crohn's disease (CD), accurate evaluation of location and small bowel involvement are necessary at the time of diagnosis for prognostic concern and planning of treatment strategy. Since Double Balloon Endoscopy (DBE) enables us to examine deep small bowel either oral or anal it could be of great utility in the management of Crohn's disease patients.

Aims \& Methods: We aimed to evaluate the diagnostic yield and therapeutic impact of DBE on small bowel CD. The medical records of $180 \mathrm{CD}$ patients, from October 2009 to April 2015, were retrospectively analysed. Patients were included if they had known CD based on clinical, colonoscopic and histological findings and had been subjected to DBE. If one patient underwent more than one DBE examination only the first evaluation was considered. The primary end point of our study was to evaluate small bowel involvement that is beyond the reach of conventional colonoscopy. The secondary endpoints were to determine the impact of DBE findings on management strategy of CD. The diagnostic yield of DBE in small bowel CD was determined. In addition, the changes in medical treatment, endoscopic intervention and surgical procedures, within three months after DBE, were analysed.

Results: Among 180 patients with CD, 90 patients underwent 168 DBE examinations and were included. The mean age of included patients was $40 \pm 13.6$ years. They were 63 males and 27 females. Eighty-two $(91 \%)$ patients with established CD underwent DBE for evaluation of small bowel involvement and $8(9 \%)$ patients underwent $\mathrm{DBE}$ because of suspicion of $\mathrm{CD}$ and had been newly diagnosed. The overall diagnostic yield of DBE was $69 \%$.DBE revealed small bowel involvement proximal to the terminal ileum in 40 $(64.5 \%)$ patients; of them $17(42.5 \%)$ patients had isolated small bowel CD. Within 3 months after DBE examination the management strategy of CD changed in $47(52.2 \%)$ patients, based on DBE findings. The medical treatment escalated in $20(32 \%)$ patients, and decreased in $7(11 \%)$. Fourteen $(24 \%)$ patients underwent DBE-assisted balloon dilatation, and $6(9.6 \%)$ patients underwent CD-related surgery.

Conclusion: DBE is able to detect small bowel involvement in a significant proportion of CD patients. The DBE findings modified the management strategy in at least one half of $\mathrm{CD}$ patients.

Disclosure of Interest: All authors have declared no conflicts of interest. 


\section{References}

D'Haens, G., Mucosal healing in pediatric Crohn's disease. The goal of medical treatment. Inflammatory bowel diseases, 2004. 10(4): p. 479-480.

Jang, H.J., et al., Clinical usefulness of double balloon enteroscopy in suspected Crohn's disease: the KASID multi-center trial. Hepato-gastroenterology, 2013. 61(133): p. 1292-1296

Kondo, J., et al., Roles of double-balloon endoscopy in the diagnosis and treatment of Crohn's disease: a multicenter experience. J Gastroenterol, 2010. 45(7): p. 713-20.

Manes, G., et al., Use of double-balloon enteroscopy in the management of patients with Crohn's disease: feasibility and diagnostic yield in a high-volume centre for inflammatory bowel disease. Surg Endosc, 2009. 23(12): p. 2790-5.

May, A., et al., Push-and-pull enteroscopy using the double-balloon technique: method of assessing depth of insertion and training of the enteroscopy technique using the Erlangen Endo-Trainer. Endoscopy, 2005. 37(1): p. 66-70.

Mensink, P.B., et al., Double-balloon enteroscopy in Crohn's disease patients suspected of small bowel activity: findings and clinical impact. $J$ Gastroenterol, 2009. 44(4): p. 271-6

Mensink, P.B., et al., Double-balloon enteroscopy in Crohn's disease patients suspected of small bowel activity: findings and clinical impact. J Gastroenterol, 2009. 44(4): p. 271-6.

Mensink, P.B., et al., Impact of double-balloon enteroscopy findings on the management of Crohn's disease. Scand J Gastroenterol, 2010. 45(4): p. 483-9. Sugano, K. and N. Marcon, The First International Workshop on Double Balloon Endoscopy: a consensus meeting report. Gastrointest Endosc, 2007. 66(3 Suppl): p. S7-11.

Xin, L., et al., Indications, detectability, positive findings, total enteroscopy, and complications of diagnostic double-balloon endoscopy: a systematic review of data over the first decade of use. Gastrointest Endosc, 2011. 74(3): p. 563-70.

P0339 AGE AND SMOKING KEY TO ADHERENCE IN INFLAMMATORY BOWEL DISEASE: LOW ADHERENCE CAN SERIOUSLY LIMIT DRUG EFFECTIVENESS IN YOUNG PATIENTS

C.J. Gargallo Puyuelo ${ }^{1}$, P. Alarcon ${ }^{2}$, E. Alfambra ${ }^{3}$, T. Arroyo Villarino ${ }^{1}$, J.M. Nerin De La Puerta ${ }^{1}$, A. Lué ${ }^{1}$, F. Gomollon ${ }^{1}$

${ }^{1}$ Gastroenterology, Hospital Clínico Lozano Blesa, Zaragoza/Spain

${ }^{2}$ Universidad de Zaragoza, Zaragoza/Spain

${ }^{3}$ ISS Aragon, Zaragoza/Spain

Contact E-mail Address: carlajerusalen@hotmail.com

Introduction: Therapeutic adherence is crucial in the management of patients with inflammatory bowel disease (IBD). Poor adherence may lead to suboptimal control of the disease, decreased quality of life and increased health care costs. Aims \& Methods: 1) To evaluate the prevalence of non-adherence to treatment in Spanish patients with IBD and 2) To identify factors associated with low, medium and high adherence. We performed a cross-sectional study that included consecutive patients with IBD attending our adult IBD clinic in a three-month period. Consenting patients completed a survey performed by two researches not involved in the care in our patients. Activity was assessed with Harvey-Bradhsaw index in Crohn's disease (CD) and Partial Mayo Score in Ulcerative colitis (UC). Anxiety and depression were estimated by the Goldberg Anxiety and Depression Scale and modified Morisky Medication Adherence Scale (MMAS-8) was used to assess adherence $(<6,6-7$ and 8 points indicate low, medium and high adherence, respectively). In statistical analyses, Chi-square and Student's t-test were used for side by side comparisons and logistic regression for multivariant analysis.

Results: A total of 181 patients were evaluated. The median age was $47 \pm 16$ years; $98(54.1 \%)$ were males and $32(17.7 \%)$ were active smokers. $82(45.3 \%)$ patients had CD and $99(54.7 \%)$ had UC. The mean disease duration was $10.21 \pm 8.59$ years. Most patients were in remission $(87.8 \%)$. In relation to the treatment, $35.9 \%$ were taking mesalazine, $2.8 \%$ steroids, $29.3 \%$ immunomodulators and $30.4 \%$ biologics. The oral route was the most frequent $(52.5 \%)$, following by rectal route $(17.1 \%)$ and subcutaneous or intravenous $(30.4 \%)$. Based on MMAS- 8 , almost half of our patients $84(46.4 \%)$ had high adherence to IBD treatment, $56(30.9 \%)$ had medium and $41(22.7 \%)$ had low adherence. In relation to factors associated with adherence, univariate analyses showed that patients with high adherence were older $(52.0 \pm 16.1$ years vs. $42.5 \pm 14.4$ $p<0.001)$ and their disease had longer duration $(12.2 \pm 9.7$ years vs $8.5 \pm 7.1$; $\mathrm{p}=0.004)$ than patients with medium/low adherence. However, smokers had a low adherence $(\mathrm{p}=0.007)$. Multivariate analysis confirmed that age was associated with high adherence (OR:1.04, CI95\% 1.01-1.06, $\mathrm{p}=0.002)$ and being smoker with low adherence (OR:3.47, CI95\% 1.36-8.90, p < 0.01). Also, multivariate analysis showed that $\mathrm{CD}$ was associated with low adherence (OR:2.54, CI95\% 1.11-5.79, $\mathrm{p}<0.05$ ). Other factors as sex, anxiety, depression, quality of life, disease activity, type of drugs or administration route were no significantly associated with adherence.

Conclusion: Only active smoking and age were predictors of insufficient adherence to drugs in IBD. Efforts for reinforce adherence should be especially directed to young patients. Quitting tobacco could improve adherence.
P0340 IS THE CDMRIS USEFUL TO MONITOR PATIENTS WITH CROHN'S DISEASE BY MAGNETIC RESONANCE IMAGING?

C. Le Berre ${ }^{1}$, M. Tahora ${ }^{2}$, C. Trang ${ }^{1}$, M. Flamant ${ }^{3}$, P. Hallouin ${ }^{3}$, S. Bruley Des Varannes $^{4}$, A. Bourreille ${ }^{1}$, E. Frampas ${ }^{2}$

${ }^{1}$ Hépato-gastro-entérologie, CHU de Nantes Hôtel Dieu, NANTES CEDEX/ France

${ }^{2}$ Radiologie Centrale, CHU de Nantes Hôtel Dieu, NANTES CEDEX/France ${ }^{3}$ Clinique Jules Verne, Nantes/France

${ }^{4}$ Institut Des Maladies De L'appareil Digestif, Hopital Hotel Dieu, Nantes/France

Contact E-mail Address: catherine@1eberre.org

Introduction: Magnetic resonance enterography is now recognized by the European Crohn's and Colitis Organization (ECCO) as a reference procedure to assess the intestinal involvement of Crohn's disease (CD), including extramural complications, as well as to monitor patients under treatment. A new MRI index of severity was developed in 2015 by the GETAID consortium, specifically to evaluate lesions located in the small intestine. This score, labeled CDMRIS (Crohn's disease magnetic resonance index of inflammation severity), considers, for each $20-\mathrm{cm}$ small bowel segment, the intensity of relative contrast enhancement (mild-moderate or severe), deep ulceration without fistula, "comb sign", any fistula, and abscess. Although well standardized, this index has not yet been validated, either for the initial assessment of CD at diagnosis, or for monitoring patients under treatment. Its feasibility in routine practice has never been tested.

Aims \& Methods: The aims of this study were to evaluate the feasibility of applying the CDMRIS score in clinical practice, to evaluate its variability after the initiation or optimization of an anti-TNF treatment, and to measure its correlation with an evaluation of clinical activity. Patients with known small bowel CD who underwent two MRI examinations at a maximal interval of 30 months were included between 2010 and 2015. Each exam was interpreted twice and the CDMRIS score was calculated on both exams in addition to classical criteria. All patients had a clinical evaluation over time, separating them in two groups: "active" and "inactive" disease.

Results: Seventy-two patients were included, with a mean CDMRIS of 3.4 at baseline, decreasing to $2.6(\mathrm{p}=0.052)$ independently of clinical disease activity. The mean interval between the two MRIs was 15.4 months, and there was a significant, larger decrease in the CDMRIS score when the interval was above 12 months. Two other radiological parameters decreased significantly: the rate of patients with a mural T2-hyperintensity $(36.1 \%$ to $20.8 \% \mathrm{p}=0.042)$, with a good clinicoradiological correlation, and mean wall thickness $(5.5$ to $4.4 \mathrm{~mm}$, $\mathrm{p}=0.047)$.

Conclusion: This study demonstrated the feasibility of applying the CDMRIS in clinical practice, but sensitivity was too low to detect early changes. Accuracy for a long-term monitoring needs to be evaluated. Wall thickness and mural T2hyperintensity emerged as two sensitive radiological factors, significantly associated with the disease activity, allowing monitoring of the short-term efficacy of biotherapies.

Disclosure of Interest: All authors have declared no conflicts of interest.

\section{References}

Van Assche G, Dignass A, Panes J, Beaugerie L, Karagiannis J, Allez M, Ochsenkühn T, Orchard T, Rogler G, Louis E, Kupcinskas L, Mantzaris G, Travis S, Stange E; European Crohn's and Colitis Organisation (ECCO). The second European evidence-based Consensus on the diagnosis and management of Crohn's disease: Definitions and diagnosis. J Crohns Colitis Feb;4(1):7-27: published online. Jan 15. DOI: 10.1016/j.crohns.2009.12.003.

Zappa M, Stefanescu C, Cazals-Hatem D, Bretagnol F, Deschamps L, Attar A, Larroque B, Tréton X, Panis Y, Vilgrain V, Bouhnik Y. Which magnetic resonance imaging findings accurately evaluate inflammation in small bowel Crohn's disease? A retrospective comparison with surgical pathologic analysis. Inflamm Bowel Dis. 2011 Apr;17(4):984-93: published online 2010 Aug 18. DOI: 10.1002 ibd.21414.

Bouhnik Y. Etude GETAID 2009-5. Développement d'un score radiologique de sévérité des lésions inflammatoires du grêle au cours de la maladie de Crohn: Crohn's disease magnetic resonance index of inflammatory severity (CDMRIS). (Accessed at: https://www.getaid.org/etudes/etudes-terminees-en-cours-d-analyse/99-etude-cdmris-getaid-2009-5.html)

Y. Bouhnik, M. Zappa, V. Abitbol, M. Lewin, M. Boudiaf, J. Cosnes, J.Y. Mary. P247 Development of a new tool to assess Crohn's disease magnetic resonance inflammation severity. (Accessed at: https://www.ecco-ibd.eu/index.php/publications/congress-abstract-s/abstracts-2014/item/p247-development-of-a-new-toolto-assess-crohn-s-disease-magnetic-resonance-inflammation-severity.html and from http://www.scumc.ro/wp-content/uploads/2013/12/UEG-Week-AbstractBook-2013.pdf)

\section{P0341 CONCORDANCE OF STOOL FREQUENCY AND ABDOMINAL PAIN MEASURES WITH SIMPLE ENDOSCOPIC SCORE FOR CROHN'S DISEASE}

J. D. Lewis ${ }^{1}$, P. Rutgeerts ${ }^{2}$, G. D'Haens ${ }^{3}$, J. Colombel ${ }^{4}$, W. Sandborn ${ }^{5}$, W. Reinisch ${ }^{6}$, J. Butler ${ }^{7}$, J.F. Maa ${ }^{8}$, A.M. Robinson ${ }^{7}$

${ }^{1}$ Center For Clinical Epidemiology And Biostatistics, Perelman School of Medicine at the University of Pennsylvania, Philadelphia/United States of America/PA

${ }^{2}$ University of Leuven, Leuven/Belgium

${ }^{3}$ Academic Medical Center, Amsterdam/Netherlands

${ }^{4}$ Icahn School of Medicine at Mount Sinai, New York/United States of America

${ }^{5}$ University of California San Diego, La Jolla/United States of America/CA 
${ }^{6}$ Medical University of Vienna, Vienna/Austria

${ }^{7}$ AbbVie Inc., North Chicago/United States of America

${ }^{8}$ Dept. R436, Bldg. Ap9a-2, AbbVie Inc, North Chicago/United States of America

Contact E-mail Address: lewisjd@mail.med.upenn.edu

Introduction: Crohn's Disease Activity Index (CDAI) has been shown to correlate poorly with endoscopic measures of mucosal inflammation/ulceration. ${ }^{1,2}$ There is a movement towards using components of the CDAI, namely stool frequency (SF) and abdominal pain (AP), rather than the total score, to evaluate a patient's disease activity in registration trials. This post hoc analysis assessed the association between individual components of the CDAI with the Simple Endoscopic Score for Crohn's Disease (SES-CD) from EXTEND.

Aims \& Methods: All patients randomized in the EXTEND study ${ }^{3}$ with both CDAI and SES-CD values at the time point evaluated (week 12 and week 52) were analyzed. Correlations between SES-CD and CDAI, SES-CD and individual components of CDAI, and mean changes from baseline in both SES-CD and CDAI were assessed at weeks 12 and 52 using Pearson correlation coefficient. Endoscopies were scored by a central reader. Data are reported as observed.

Results: A total of 121 patients at week 12 and 80 patients at week 52 had both CDAI and SES-CD values. Mean (SD) CDAI and SES-CD values at week 12 were at 193.8 (116.4) and 8.0 (7.4), respectively, and 132.5 (97.6) and 5.9 (6.6), respectively, at week 52. A significant, but weak correlation was observed between CDAI and SES-CD at week $12(\mathrm{r}=0.31[P<0.001])$ and at week 52 $(\mathrm{r}=0.31[P=0.005])$. Similar results were observed for correlations between mean changes from baseline in CDAI and SES-CD at weeks $12(\mathrm{r}=0.35$ $[P<0.001])$ and $52(\mathrm{r}=0.31[P=0.005])$. Correlations between individual components of CDAI and SES-CD at weeks 12 and 52 are shown in the table. SF, $\mathrm{AP}$, extra-intestinal manifestations, and $\mathrm{SF}+\mathrm{AP}$ were significantly correlated with SES-CD at week 12; the strongest correlation was for SF $(r=0.46)$ and the addition of AP to SF did not increase the correlation (Table). At week 52, SF, hematocrit, and SF + AP were significantly correlated with SES-CD. At week 12, the correlation of SES-CD with SF was similar regardless of whether the patient had disease of the ileum $(\mathrm{r}=0.44[P<0.001]$ with ileal disease; $\mathrm{r}=0.48$ $[P<0.001]$ without ileal disease), while SES-CD correlated more strongly with AP in those with ileal disease $(\mathrm{r}=0.27[P=0.036])$ than those without ileal disease $(\mathrm{r}=0.16[P=0.233])$

Table: Correlation of components of the CDAI with SES-CD

\begin{tabular}{|c|c|c|c|c|c|c|}
\hline \multirow[b]{2}{*}{ Variable } & \multicolumn{3}{|c|}{ Week $12 \mathrm{n}=121$} & \multicolumn{3}{|c|}{ Week $52 \mathrm{n}=80$} \\
\hline & Mean (SD) & $\mathrm{r}$ & $P$-value & Mean (SD) & $\mathrm{r}$ & $P$-value \\
\hline Stool frequency* & $47.1(35.2)$ & 0.46 & $<0.001$ & $33.7(32.1)$ & 0.35 & 0.002 \\
\hline Abdominal pain & $41.0(29.6)$ & 0.21 & 0.020 & $21.8(25.6)$ & 0.06 & 0.597 \\
\hline General well-being & $63.3(50.2)$ & 0.16 & 0.073 & $42.2(46.7)$ & 0.17 & 0.123 \\
\hline Extra-intestinal manifestations & $14.0(16.3)$ & 0.22 & 0.017 & $11.5(13.8)$ & 0.11 & 0.317 \\
\hline Diarrhea/pain medications & $3.2(9.3)$ & 0.01 & 0.927 & $1.9(7.3)$ & 0.08 & 0.465 \\
\hline Abdominal mass & $0.8(4.0)$ & -0.50 & 0.588 & $0.0(0.0)$ & NA & NA \\
\hline HCT & $21.1(21.3)$ & 0.09 & 0.355 & $17.7(20.6)$ & 0.40 & $<0.001$ \\
\hline Weight $(\mathrm{kg})$ & $3.2(6.1)$ & -0.03 & 0.770 & $3.8(7.0)$ & -0.07 & 0.568 \\
\hline Stool frequency + Abdominal pain & $88.1(56.6)$ & 0.40 & $<0.001$ & $55.5(48.1)$ & 0.26 & 0.018 \\
\hline
\end{tabular}

*the number of liquid or very soft stools per day. SES-CD, Simple Endoscopic Score for Crohn's Disease. HCT, hematocrit. NA, not applicable.

Conclusion: Data from patients with moderate to severe $\mathrm{CD}$ and evidence of mucosal ulceration in EXTEND supported previous findings that the CDAI was only weakly correlated with SES-CD, as assessed at 12 and 52 weeks. Only SF was significantly correlated with SES-CD at both time points. At 12 weeks, the correlation of SES-CD with SF was not affected by the presence of ileal disease, while correlation with AP was stronger with ileal disease than without it.

Disclosure of Interest: J.D. Lewis: consultant for AbbVie, Johnson \& Johnson, Janssen Pharmaceuticals, Samsung Bioepis, Takeda, and Merck. He has served on Data and Safety Monitoring Boards for Pfizer, Gilead, and UCB. He has received research support from Takeda and Nestle Health Science.

P. Rutgeerts: Consultancy fees from AbbVie, Bristol-Myers Squibb, Centocor,

Merck, Takeda, and UCB Pharma; speaker fees and research support from AbbVie, Centocor, MSD, and UCB Pharma.

G. D'Haens: Consulting/speaker/research: AbbVie, ActoGeniX, AIM, Boehringer Ingelheim, Centocor, Chemo Centryx, Cosmo Tech, Dr Falk Pharma, Elan, enGene, Ferring, Galapagos, Giuliani SpA, Given Imaging, GSK, Janssen, MSD, Neovacs, Novo Nordisk, Norgine, etc

J. Colombel: consultant/advisory board/speaker for AbbVie, Bristol-Myers Squibb, Ferring, Genentech, Giuliani SPA, Given Imaging, Merck, Millennium, Pfizer, Prometheus Labs, Sanofi, Schering-Plough Corp, Takeda, Teva Pharma, and UCB.

W. Sandborn: Consult/speaker/research: AbbVie, ActoGeniX, AGI, Alba, Albireo, Alfa Wasserman, Amgen, AM-Pharma, Anaphore, Astellas, Athersys, Atlantic Healthcare, Aptalis, BioBalance, Boehringer Ingelheim, Bristol-Myers Squibb, Celgene, Celek, Cellerix, Cerimon, etc

W. Reinisch: speaker/consult/research/ad board: Abbott, Abbvie, Aesca, Amgen, AM Pharma, Aptalis, Astellas, Astra Zeneca, Avaxia, Bioclinica, Biogen, Boehringer-Ingelheim, Bristol-Myers Squibb, Centocor, Celltrion, Cellerix, Chemocentryx, Celgene, Centocor, etc

J. Butler: Abbvie employee; may own AbbVie stock and/or options

J.F. Maa: Abbvie employee; may own AbbVie stock and/or options
A.M. Robinson: Abbvie employee; may own AbbVie stock and/or options

\section{References}

1. Schoepfer AM et al., Am J Gastroenterol. 2010 Jan;105(1):162-9.

2. Sipponen T et al., Inflamm Bowel Dis. 2010 Dec;16(12):2131-6. 3. Rutgeerts $\mathrm{P}$ et al., Gastroenterology 2012;142:1102-1111.

\section{P0342 DECREASED CD8 ${ }^{+} \mathrm{CD}^{2} 8^{+} / \mathrm{CD8}^{+} \mathrm{CD}^{-} 8^{-} \mathrm{T}$ CELLS' RATIO CAN PREDICT THE POOR OUTCOME SENSITIVELY FOR PATIENTS WITH COMPLICATED CROHN'S DISEASE}

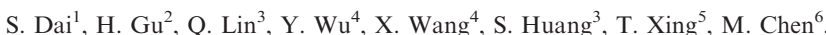
Q. Zhang ${ }^{1}$, Z. Zheng ${ }^{1}$, W. Sha

${ }^{1}$ Department Of Gastroenterology, Guangdong General Hospital and Guangdong Academy of Medical Sciences, Guangzhou/China

${ }^{2}$ Department Of Gastroenterology, Nanfang Hospital, Southern Medical University, Guangzhou/China

${ }^{3}$ The First Clinical College, Southern Medical University, Guangzhou/China ${ }^{4}$ Zhongshan School of Medicine, Sun Yat-sen University, Guangzhou/China ${ }^{5}$ Brody School of Medicine, East Carolina University, NC/United States of America/NC

${ }^{6}$ School of Public Health, Southern Medical University, Guangzhou/China

Contact E-mail Address: xrayxjc@126.com

Introduction: Crohn's disease (CD) with complications such as penetrating, structuring, and perianal disease are called complicated CD. However, no validated, inexpensive, or sensitive models for prediction of risk are available in complicated $\mathrm{CD}$. We have found that a novel immunological balance, the $\mathrm{CD} 8^{+} \mathrm{CD} 28^{+} /$ $\mathrm{CD} 8^{+} \mathrm{CD} 28^{-}$, consisting of $\mathrm{CD} 8^{+} \mathrm{CD} 28^{+}$and $\mathrm{CD} 8^{+} \mathrm{CD} 28^{-} \mathrm{T}$ cells, can predict the prognosis for patients with inflammatory bowel disease (IBD). Thus, we hypothesize that the $\mathrm{CD} 8^{+} \mathrm{CD} 28^{+} / \mathrm{CD} 8^{+} \mathrm{CD} 28^{-}$balance (ratio) can predict the poor outcome for patients with complicated $\mathrm{CD}$.

Aims \& Methods: To test the efficiency of $\mathrm{CD} 8^{+} \mathrm{CD} 28^{+} / \mathrm{CD} 8^{+} \mathrm{CD} 28^{-}$balance to predict a subsequent active stage, and to explore the correlation between the balance and the risk factors, for the newly diagnosed complicated CD. Seventeen patients with complicated CD were enrolled as the observation group, while the other $48 \mathrm{CD}$ patients with no complications were enrolled as the control group. Peripheral blood samples were drawn from all the 65 newly diagnosed $\mathrm{CD}$ patients for $\mathrm{CD} 8^{+} \mathrm{T}$ cells testing through flow cytometry (FCM) when enrolling. The potential risk factors, including demographic, pathophysiological, and therapeutic factors were compared between the two groups. A 30week follow-up was performed and the $\mathrm{CD} 8^{+} \mathrm{T}$ cells testing were repeated. The sensitivity and specificity of the $\mathrm{CD} 8^{+} \mathrm{T}$ cells' level and balance in predicting were analyzed through receiver operator characteristic (ROC) curves. The cumulative remission lasting rates (CRLRs) under the different risk factors were analyzed using the Kaplan-Meier method.

Results: I. Risk factors: compared with the control CD group, patients with complicated $\mathrm{CD}$ had a larger proportion in male $(P=0.001)$, younger in age $(P=0.019)$, lower body mass index (BMI) $(P<0.0001)$, higher Crohn's disease activity index $(\mathrm{CDAI})(P<0.0001)$, higher prescription rates in immunosuppressants $(P=0.029)$ and steroids $(P=0.015)$, as well as a significant higher surgical rate $(P<0.0001)$. Pearson and Spearman correlation analysis showed that $\mathrm{CD} 8^{+} \mathrm{CD} 28^{+} / \mathrm{CD} 8^{+} \mathrm{CD} 28^{-}$balance was associated with BMI, CDAI, steroids, and surgery (all $P<0.005$ ). II. Follow-up and dynamic changes of the balance: the ratios of $\mathrm{CD} 8^{+} \mathrm{CD} 28^{+} / \mathrm{CD} 8^{+} \mathrm{CD} 28^{-}$reached the bottom at the $30^{\text {th }}$ week and were significantly lower at the $0,6^{\text {th }}, 22^{\text {nd }}$, and $30^{\text {th }}$ week during follow-up, in the complicated CD patients when compared to the control ones (all $P<0.05$ ). A shorter lasting time of remission (LTR) was found in complicated CD patients $(P=0.044)$. ROC curve showed that $\mathrm{CD} 8^{+} \mathrm{CD} 28^{+} / \mathrm{CD} 8^{+} \mathrm{CD} 28^{-}$ratio could accurately predict the active stage for the complicated $\mathrm{CD}$ patients [with area under curve (AUC) of 0.890 , and $95 \%$ CI of 0.822 to 0.958$]$, and the best sensitivity of $89.2 \%$ and specificity of $85.3 \%$ were found when the ratio was 1.03. III. Kaplan-Meier analysis: Undergoing of steroids and surgery was closely related to worse outcome for the complicated CD patients, and patients who underwent steroids and surgery had the significantly lower $\mathrm{CD} 8^{+} \mathrm{CD} 28^{+}$ $\mathrm{CD} 8^{+} \mathrm{CD} 28^{-}$ratio and lower CRLRs (all $P<0.05$ ).

Conclusion: Depending on steroids and surgery stands for a more severe disease activity and thus disequilibrate the immunological balance, which could be the main reason for lower $\mathrm{CD} 8^{+} \mathrm{CD} 28^{+} / \mathrm{CD} 8^{+} \mathrm{CD} 28^{-}$ratio, and this ratio can predict the active stage sensitively for patients with complicated $\mathrm{CD}$. More strategies should be taken when the ratio is to be lower than 1.03 .

Disclosure of Interest: All authors have declared no conflicts of interest.

\section{References}

1 Maguire L H, Alavi K, Sudan R, et al. Surgical Considerations in the Treatment of Small Bowel Crohn's Disease. Journal of Gastrointestinal Surgery 2017; 21: 398-411.

2 Fumery M, Seksik P, Auzolle C, et al. Postoperative Complications after Ileocecal Resection in Crohn's Disease: A Prospective Study From the REMIND Group. American Journal of Gastroenterology 2017; 112: 337-45.

3 Fumery M, Dulai P S, Meirick P, et al. Systematic review with meta-analysis: recurrence of Crohn's disease after total colectomy with permanent ileostomy. Aliment Pharmacol Ther 2017; 45: 381-90.

$4 \mathrm{Li} \mathrm{Y}$, Stocchi L, Mu X, et al. Long-term Outcomes of Sphincter-Saving Procedures for Diffuse Crohn's Disease of the Large Bowel. Diseases of the Colon \& Rectum 2016; 59: 1183-90. 
5 Van Der Sloot K W, Joshi A D, Bellavance D R, et al. Visceral Adiposity, Genetic Susceptibility, and Risk of Complications Among Individuals with Crohn's Disease. Inflammatory Bowel Diseases 2017; 23: 82-8.

6 Rinawi F, Assa A, Hartman C, et al. Incidence of Bowel Surgery and Associated Risk Factors in Pediatric-Onset Crohn's Disease. Inflammatory Bowel Diseases 2016; 22: 2917-23.

7 Ong M S, Grand R J, Mandl K D. Trends in Pharmacologic Interventions for Preventing Recurrence of Crohn's Disease After Ileocolonic Surgery. Inflammatory Bowel Diseases 2016; 22: 2432-41.

8 Lemmens B, de Buck V O A, Arijs I, et al. Submucosal Plexitis as a Predictive Factor for Postoperative Endoscopic Recurrence in Patients with Crohn's Disease Undergoing a Resection with Ileocolonic Anastomosis: Results from a Prospective Single-centre Study. Journal of Crohns \& Colitis 2017; 11: 212-20.

9 Ng S C, Leung W K, Shi H Y, et al. Epidemiology of Inflammatory Bowel Disease from 1981 to 2014: Results from a Territory-Wide Population-Based Registry in Hong Kong. Inflammatory Bowel Diseases 2016; 22: 1954-60.

10 Torres J, Caprioli F, Katsanos K H, et al. Predicting Outcomes to Optimize Disease Management in Inflammatory Bowel Diseases. Journal of Crohns \& Colitis 2016; 10: 1385-94.

11 Chapman C G, Yamaguchi R, Tamura K, et al. Characterization of T-cell Receptor Repertoire in Inflamed Tissues of Patients with Crohn's Disease Through Deep Sequencing. Inflammatory Bowel Diseases 2016; 22: 1275-85.

12 Kugathasan S, Denson L A, Walters T D, et al. Prediction of complicated disease course for children newly diagnosed with Crohn's disease: a multicentre inception cohort study. Lancet 2017.

13 Irwin J, Ferguson E, Simms L A, et al. A rolling phenotype in Crohn's disease. PLoS One 2017; 12: e174954.

14 Drastich P, Oliverius M. Crohn's Disease and Intestinal Transplantation. Dig Dis 2017; 35: 127-33.

15 Kugathasan S, Denson L, Walters T, et al. O-002 Predicting a Complicated Course for Children Newly Diagnosed with Crohn's Disease: A Multicenter Inception Cohort Study. Inflammatory Bowel Diseases 2017; 23 Suppl 1: S1.

16 Heerasing N, Thompson B, Hendy P, et al. Exclusive enteral nutrition provides an effective bridge to safer interval elective surgery for adults with Crohn's disease. Aliment Pharmacol Ther 2017; 45: 660-9.

17 Ng Siew C., Tang Whitney, Ching Jessica Y., et al. Incidence and Phenotype of Inflammatory Bowel Disease Based on Results From the Asia-Pacific Crohn's and Colitis Epidemiology Study[J]. Gastroenterology, 2013, 145(1):158-165.

18 Ng S. C., Bernstein C. N., Vatn M. H., et al. Geographical variability and environmental risk factors in inflammatory bowel disease[J]. Gut, 2013, 62(4):630-649

19 Allez M., Mayer L. Regulatory T cells: peace keepers in the gut[J]. Inflamm Bowel Dis, 2004, 10(5):666-676.

20 Dai Shixue, Gu Hongxiang, Lin Qianyi, et al. Disequilibrium in the CD $8+C D 28+/ C D 8+C D 28-T$ Lymphocyte Balance Is Related to Prognosis in Rats with Trinitrobenzenesulfonic Acid-Induced Colitis[J]. Digestive Diseases and Sciences, 2017, 62(3):639-651.

21 Dai Shi-Xue, Wu Gang, Zou Ying, et al. Balance of CD8+CD28+ CD8+CD28- T Lymphocytes Is Vital for Patients with Ulcerative Colitis[J]. Digestive Diseases and Sciences, 2013, 58(1):88-96.

22 Chachu K. A., Osterman M. T. How to Diagnose and Treat IBD Mimics in the Refractory IBD Patient Who Does Not Have IBD[J]. Inflamm Bowel Dis, 2016, 22(5):1262-1274.

23 Molodecky Natalie A., Soon Ing Shian, Rabi Doreen M., et al. Increasing Incidence and Prevalence of the Inflammatory Bowel Diseases With Time, Based on Systematic Review[J]. Gastroenterology, 2012, 142(1):46-54.

24 Zhao J., Ng S. C., Lei Y., et al. First prospective, population-based inflammatory bowel disease incidence study in mainland of China: the emergence of "western" disease[J]. Inflamm Bowel Dis, 2013, 19(9):1839-1845.

25 Song Xin-ming, Gao Xiang, Li Ming-zhe, et al. Clinical Features and Risk Factors for Primary Surgery in 205 Patients With Crohn's Disease: Analysis of a South China Cohort[J]. Diseases of the Colon \& Rectum, 2011, 54(9):1147-1154.

26 Ahmed Ishfaq, Roy Badal, Khan Salman, et al. Microbiome, Metabolome and Inflammatory Bowel Disease[J]. Microorganisms, 2016, 4(2):20

27 Ananthakrishnan Ashwin N. Epidemiology and risk factors for IBD[J]. Nature reviews. Gastroenterology \& hepatology, 2015, 12(4):205.

28 Burisch J., Pedersen N., Čuković-Čavka S., et al. East-West gradient in the incidence of inflammatory bowel disease in Europe: the ECCO-EpiCom inception cohort[J]. Gut, 2014, 63(4):588-597.

29 John Elizabeth S., Katz Kristina, Saxena Mark, et al. Management of Inflammatory Bowel Disease in the Elderly[J]. Current Treatment Options in Gastroenterology, 2016, 14(3):285-304.

30 Ananthakrishnan Ashwin N., Shi Hai Yun, Tang Whitney, et al. Systematic Review and Meta-analysis: Phenotype and Clinical Outcomes of Older-onset Inflammatory Bowel Disease[J]. Journal of Crohn's and Colitis, 2016, 10(10):1224-1236

31 Lichtenstein G. R., Feagan B. G., Cohen R. D., et al. Serious infection and mortality in patients with Crohn's disease: more than 5 years of follow-up in the TREAT registry[J]. Am J Gastroenterol, 2012, 107(9):1409-1422.

32 Campbell N. A., Park M. S., Toy L. S., et al. A Non-class I MHC Intestinal Epithelial Surface Glycoprotein, gp180, Binds to CD8[J]. Clinical Immunology, 2002, 102(3):267-274

33 Mayer L., Shlien R. Evidence for function of Ia molecules on gut epithelial cells in man[J]. J Exp Med, 1987, 166(5):1471-1483.

34 Brandeis J. M., Sayegh M. H., Gallon L., et al. Rat intestinal epithelial cells present major histocompatibility complex allopeptides to primed $\mathrm{T}$ cells[J]. Gastroenterology, 1994, 107(5):1537-1542.
35 Cortesini R., LeMaoult J., Ciubotariu R., et al. CD8+CD28- T suppressor cells and the induction of antigen-specific, antigen-presenting cell-mediated suppression of Th reactivity[J]. Immunol Rev, 2001, 182:201-206.

36 Mayer L., Eisenhardt D. Lack of induction of suppressor T cells by intestinal epithelial cells from patients with inflammatory bowel disease[J]. J Clin Invest, 1990, 86(4):1255-1260.

37 Das G., Augustine M. M., Das J., et al. An important regulatory role for CD4+CD8 alpha alpha $T$ cells in the intestinal epithelial layer in the prevention of inflammatory bowel disease[J]. Proc Natl Acad Sci U S A, 2003, 100(9):5324-5329.

38 Tom M. R., Li J., Ueno A., et al. Novel CD8+ T-Cell Subsets Demonstrating Plasticity in Patients with Inflammatory Bowel Disease[J]. Inflamm Bowel Dis, 2016, 22(7):1596-1608.

39 Funderburg N. T., Stubblefield Park SR, Sung H. C., et al. Circulating $\mathrm{CD} 4(+)$ and $\mathrm{CD} 8(+) \mathrm{T}$ cells are activated in inflammatory bowel disease and are associated with plasma markers of inflammation[J]. Immunology, 2013, 140(1):87-97.

40 Sahami Saloomeh, Buskens Christianne J., Fadok Tonia Young, et al. Defunctioning Ileostomy is not Associated with Reduced Leakage in Proctocolectomy and Ileal Pouch Anastomosis Surgeries for IBD $[\mathrm{J}]$. Journal of Crohn's and Colitis, 2016, 10(7):779-785.

41 Ruemmele F. M., Veres G., Kolho K. L., et al. Consensus guidelines of ECCO/ESPGHAN on the medical management of pediatric Crohn's disease[J]. Journal of Crohn's and Colitis, 2014, 8(10):1179-1207.

42 Iskandar Heba N., Dhere Tanvi, Farraye Francis A. Ulcerative Colitis: Update on Medical Management[J]. Current Gastroenterology Reports, 2015, 17(11):44.

\section{P0343 BOWEL PREPARATION QUALITY OF NER1006 VERSUS STANDARD 2L PEG WITH ASCORBATE AS ASSESSED BY COLONOSCOPISTS AT SITE: A POST HOC ANALYSIS FROM A RANDOMISED CONTROLLED TRIAL}

\section{J. Manning ${ }^{1}$, L. Clayton ${ }^{2}$, V. Lorenzo-Zúñiga García ${ }^{3}$}

${ }_{1}^{1}$ Borders General Hospital, Melrose/United Kingdom

${ }^{2}$ Clinical Development, Norgine Ltd., Harefield, Uxbridge/United Kingdom

${ }^{3}$ Department Of Medicine, Universitat Autònoma de Barcelona, Barcelona/Spain

Contact E-mail Address: Jmanning1@nhs.net

Introduction: Successful colonoscopy requires effective bowel cleansing. NER1006 is the first 1L polyethylene glycol (PEG)-based bowel preparation, a patented combination optimised for effective bowel cleansing. The MORA study was a multicentre randomised Phase 3 clinical trial using blinded central readers to assess the cleansing efficacy of the overall colon and high-quality cleansing of the ascending colon by NER1006 vs standard 2L PEG with ascorbate (2L PEG + Asc) [1]. This post hoc analysis shows the cleansing assessment by site colonoscopists, who typically guide clinical decision-making; hence this analysis may be more relevant for clinical practice than previous analyses.

Aims \& Methods: In the MORA study 849 patients (males and females, aged 18 85) were randomly assigned in a 1:1:1 ratio to receive i) NER1006 in an evening/ morning split-dose (N2D), or ii) NER1006 in a morning-only dose (N1D) or iii) 2L PEG + Asc in an evening/morning split-dose. The 796 subjects who underwent a colonoscopy and were assessed by a treatment-blinded site colonoscopist were included in this analysis. Cleansing was assessed according to the Harefield Cleansing Scale [2]; following segmental scoring, cleansing of the overall colon was graded from $\mathrm{A}$ to $\mathrm{D}$; grades $\mathrm{A}$ and $\mathrm{B}$ were judged as successful cleansing. Cleansing of the ascending colon and caecum was scored from 0 to 4 ; scores of 3 and 4 were judged as high-quality cleansing.

Results: The bowel preparation quality of NER 1006 showed a statistically significant improvement over $2 \mathrm{~L}$ PEG + Asc for the overall colon when both treatments were administered as an evening/morning split-dose $(\mathrm{P}=0.003 ; 95 \% \mathrm{CI}$ : $2.0-10.1 \%$ ) (Table 1). NER1006 administered either as an overnight split-dose or morning-only dose produced high-quality cleansing of the ascending colon in a statistically significantly higher proportion of patients compared to $2 \mathrm{~L}$ PEG + Asc $(\mathrm{P}<0.001 ; 95 \%$ CI: $7.2-23.1 \%$ and $\mathrm{P}<0.001 ; 95 \%$ CI: 7.2 $23.0 \%$ respectively). The morning-only dose showed no difference compared to 2L PEG + Asc as an evening/morning split-dose for overall colon cleansing quality $(\mathrm{P}=0.924 ; 95 \% \mathrm{CI}:-5.1-4.6 \%)$. Table 1 . A comparison of bowel cleansing efficacy as assessed by site colonoscopists between NER1006 (administered in a N1D or N2D regimen) or $2 \mathrm{~L}$ PEG + Asc.

Conclusion: Colonoscopists assessed both dosing regimens of NER1006 as having a significantly increased rate of high-quality cleansing of the ascending colon when compared with $2 \mathrm{~L}$ PEG + Asc; this cleansing is important in ensuring the detection of lesions in the ascending colon. When comparing similar overnight split-dose treatment regimens, NER1006 showed a significantly increased rate of overall bowel cleansing compared to $2 \mathrm{~L}$ PEG + Asc.

Disclosure of Interest: J. Manning: Received funding to attend MORA study Investigator Meeting

L. Clayton: Employee of Norgine

All other authors have declared no conflicts of interest.

\section{References}

1. Bisschops R et al. Gastroenterology 2016; 150(4): S1269-70. Abstract Tu2084

2. Halphen $\mathrm{M}$ et al. Gastrointest Endosc 2013; 78(1): 121-31 


\begin{tabular}{|c|c|c|c|c|}
\hline & NER1006 N2D & $2 \mathrm{~L} \mathrm{PEG}+\mathrm{Asc}$ & NER1006 N1D & 2L PEG + Asc \\
\hline \multicolumn{5}{|l|}{ Overall colon } \\
\hline $\mathrm{N}$ & 263 & 263 & 270 & 263 \\
\hline Patients with successful cleansing N (\%) & $255(97)$ & $239(91)$ & $246(91)$ & $239(91)$ \\
\hline Difference $(\%)$ & 6 & & 0 & \\
\hline P-value & 0.003 & & 0.924 & \\
\hline $95 \%$ CI $(\%)$ & $2.0-10.1$ & & $-5.1-4.6$ & \\
\hline \multicolumn{5}{|l|}{ Ascending colon and caecum } \\
\hline $\mathrm{N}$ & 263 & 263 & 270 & 263 \\
\hline Patients with successful (high quality) cleansing N (\%) & $196(74)$ & $156(59)$ & $201(75)$ & $156(59)$ \\
\hline Difference $(\%)$ & 15 & & 16 & \\
\hline P-value & $<0.001$ & & $<0.001$ & \\
\hline $95 \%$ CI $(\%)$ & $7.2-23.1$ & & $7.2-23.0$ & \\
\hline
\end{tabular}

N.B. successful cleansing defined here as a Harefield Cleansing Scale grade of A or B (overall colon) or score of 3 or 4 (ascending colon, high quality)

\section{P0344 BOWEL PREPARATION QUALITY OF NER1006 VERSUS ORAL TRISULFATE SOLUTION AS ASSESSED BY COLONOSCOPISTS AT SITE: A POST HOC ANALYSIS FROM A RANDOMISED CONTROLLED TRIAL}

R. Ng Kwet Shing', P. Bekal ${ }^{2}$

${ }^{1}$ Clinical Development, Norgine Limited, Harefield/United Kingdom

${ }^{2}$ Ohio GI and Liver Institute, Cincinnati/United States of America/OH

Contact E-mail Address: rng@norgine.com

Introduction: The success of colonoscopy is dependent on efficient bowe cleansing. Inadequate bowel cleansing may decrease diagnostic sensitivity, necessitate repeat procedures and potentially delay appropriate treatment The increasing frequency of the incidence of colorectal cancer arising in the ascending colon necessitates effective cleansing of this area; additionally these cancers are often associated with poorer prognoses. Data suggest that detection in the ascending colon is more dependent on higher grades of cleansing, perhaps due to the nature of polyps present, which may be more likely to be sessile or serrated. NER1006 is the first 1L polyethylene glycol (PEG)-based bowel preparation, a patented combination optimised for effective bowe cleansing. The NOCT study (a multicentre randomised Phase 3 clinical tria investigating bowel cleansing efficacy of NER1006 vs trisulfate solution) reported bowel preparation quality assessed by central readers. ${ }^{1}$ This post hoc analysis shows the cleansing assessment by site colonoscopists, who typically guide clinical decision making; hence this study may be more relevant for clinical practice than previous studies.

Aims \& Methods: In the NOCT study 621 patients (males and females, aged 1885 ) were randomly assigned in a 1:1 ratio to receive either NER1006 or trisulfate solution, each administered as an overnight split-dose. Data from the 523 patients who underwent a colonoscopy and had a site colonoscopist assessment were used in this analysis. Colonoscopists were blinded to the preparation administered. Cleansing was assessed according to the Harefield Cleansing Scale; ${ }^{2}$ following segmental scoring, cleansing of the overall colon was graded from A to $\mathrm{D}$; grades $\mathrm{A}$ and $\mathrm{B}$ were judged as successful cleansing. Cleansing of the ascending colon was graded from 0 to 4 ; grades 3 and 4 were judged as highquality cleansing.

Results: As Table 1 shows, the bowel preparation quality of NER1006 when assessed by site colonoscopists did not show a statistically significant difference to trisulfate for the overall colon $(93 \%$ vs $94 \%, \mathrm{P}=0.681 ; 95 \% \mathrm{CI}:-5.1-3.3 \%)$ or ascending colon ( 80 vs $74 \%, \mathrm{P}=0.079 ; 95 \% \mathrm{CI}:-0.7-13.6 \%$ ). There was, however, a numerical advantage in favour of NER1006 on the proportion of patients achieving high-quality cleansing success in the right colon.

Table 1: Successful colon cleansing rates when treated with NER1006 or trisulfate solution.

\begin{tabular}{|c|c|c|c|c|c|}
\hline $\begin{array}{l}\text { Bowel } \\
\text { preparation }\end{array}$ & $\mathrm{N}$ & $\begin{array}{l}\text { Patients with } \\
\text { successful } \\
\text { cleansing n }(\%)\end{array}$ & Difference $(\%)$ & P-value & $95 \% \mathrm{CI}(\%)$ \\
\hline \multicolumn{6}{|l|}{ Overall colon } \\
\hline NER 1006 & 259 & $241(93)$ & -1 & 0.681 & $-5.1-3.3$ \\
\hline Trisulfate & 264 & $248(94)$ & & & \\
\hline \multicolumn{6}{|c|}{ Ascending colon } \\
\hline NER1006 & 259 & $208(80)$ & 6 & 0.079 & $-0.7-13.6$ \\
\hline Trisulfate & 264 & $195(74)$ & & & \\
\hline
\end{tabular}

N.B. successful cleansing defined here as a Harefield Cleansing Scale grade of A or B (overall colon) or 3 or 4 (ascending colon, high quality)
Conclusion: For both preparations, site colonoscopist findings demonstrated similar very high rates of cleansing success for the overall colon $(>93 \%)$ and high rates of high-quality cleansing of the ascending colon $(>73 \%)$, however, statistical significance was not met in either comparison. The rates of cleansing success in the ascending colon reported by the site colonoscopists are notably higher than those previously reported by central readers.

Disclosure of Interest: R. Ng Kwet Shing: Employee of Norgine

All other authors have declared no conflicts of interest.

\section{References}

1. DeMicco M et al. United European Gastroenterol J 2016; 4(5S): A1-156. OP375

2. Halphen M, et al. Gastrointest Endosc 2013; 78: 121-131

P0345 BOWEL PREPARATION QUALITY OF NER1006 VERSUS SODIUM PICOSULFATE + MAGNESIUM CITRATE AS ASSESSED BY COLONOSCOPISTS AT SITE: A POST HOC ANALYSIS FROM A RANDOMISED CONTROLLED TRIAL

S. Lewis ${ }^{1}$, J.P.h. Drenth ${ }^{2}$, C. Santander ${ }^{3}$, C. Pediconi ${ }^{4}$, B. Amlani ${ }^{5}$, A. Repici ${ }^{6}$ ${ }^{1}$ Gastroenterology, General (internal) Medicine, Derriford Hospital, Plymouth/ United Kingdom

${ }^{2}$ Gastroenterology And Hepatology, Radboud University Nijmegen Medical Centre - Gastroenterology and Hepatology, Radboud University Nij, Nijmegen/

Netherlands

${ }^{3}$ Aparato Digestivo, Hospital Universitario de la Princesa, Madrid/Spain

${ }^{4}$ Clinical Development, Norgine Ltd, Harefield/United Kingdom

${ }^{5}$ Medical Affairs, Norgine Ltd, Harefield/United Kingdom

${ }^{6}$ Dept. Of Gastroenterology, Ist. Clinico Humanitas Rozzano Dept. of Gastroenterology, Milano/Italy

Contact E-mail Address: sjl@doctors.org.uk

Introduction: The efficacy of colonoscopy is dependent on the quality of bowel cleansing. NER1006 is the first 1L polyethylene glycol (PEG)-based bowel cleansing solution and is a patented combination optimised for effective bowel cleansing. The DAYB study was a European multicentre, randomised trial that tested the hypothesis that NER1006 would be non-inferior to sodium picosulfate and magnesium citrate $(\mathrm{NaPic}+\mathrm{MgCit})$ in terms of overall bowel cleansing and high-quality cleansing of the ascending colon plus caecum [1]. Bowel cleansing was assessed using the Harefield Cleansing Scale (HCS) [2]. The primary endpoints of the study were assessed by video review by a central reader. Bowel cleansing on the HCS was also assessed by the site colonoscopist and this post hoc analysis assessed the cleansing grades as determined by the site colonoscopists.

Aims \& Methods: In the DAYB study, 515 patients (aged 18-85, median age: 55.0 years) underwent screening, surveillance, or diagnostic colonoscopy and were randomly assigned in a 1:1 ratio to receive either NER1006 or $\mathrm{NaPic}+\mathrm{MgCit}$, each on the day before colonoscopy. In this analysis, data from 479 patients who underwent a colonoscopy and had a completed assessment by the site colonoscopist were included. Colonoscopists were blinded to the preparation administered. Cleansing was assessed according to the HCS; cleansing of each segment of the colon was scored from 0 to 4 . Scores 3 and 4 of the ascending colon were judged as high-quality cleansing. Cleansing of the overall colon was graded from A to D; grades A (all segments scored 3 or 4 ) and B ( $\geq 1$ segments scored 2, no sections scored 1 or 0 ) were judged as successful cleansing.

Results: As indicated in Table 1, in the overall colon, successful cleansing was achieved in $12 \%$ more patients who received NER1006 than who received $\mathrm{NaPic}+\mathrm{MgCit}(73 \%$ vs $61 \%, \mathrm{P}=0.003,95 \% \mathrm{CI}: 4.0-20.7)$. In the ascending colon, high-quality cleansing was achieved in $20 \%$ more patients who received 
NER 1006 than who received $\mathrm{NaPic}+\mathrm{MgCit}(34 \%$ vs $14 \%, \mathrm{P}<0.001,95 \% \mathrm{CI}$ : 12.7-27.8).

Table 1: A comparison of bowel cleansing efficacy as assessed by site colonoscopists between NER1006 and $\mathrm{NaPic}+\mathrm{MgCit}$

\begin{tabular}{|c|c|c|c|c|c|}
\hline $\begin{array}{l}\text { Bowel } \\
\text { preparation }\end{array}$ & $\mathrm{N}$ & $\begin{array}{l}\text { Patients with } \\
\text { successful } \\
\text { cleansing n }(\%)\end{array}$ & Difference $(\%)$ & P-value & $95 \% \mathrm{CI}$ \\
\hline \multicolumn{6}{|l|}{ Overall colon } \\
\hline NER1006 & 236 & $173(73)$ & 12 & 0.003 & $4.0-20.7$ \\
\hline $\mathrm{NaPic}+\mathrm{MgCit}$ & 243 & $148(61)$ & & & \\
\hline $\begin{array}{l}\text { Ascending colon } \\
\text { NER1006 }\end{array}$ & 236 & $82(34)$ & 20 & $<0.001$ & $12.7-27.8$ \\
\hline $\mathrm{NaPic}+\mathrm{MgCit}$ & 243 & 35 (14) & & & \\
\hline
\end{tabular}

Conclusion: NER1006 was shown to provide significantly better cleansing of the overall colon and high-quality cleansing of the ascending colon compared to $\mathrm{NaPic}+\mathrm{MgCit}$, when both treatments were administered the day before colonoscopy. The cleansing efficacy rate of the comparator was within its previously reported cleansing rates for day before administration, suggesting the improvement seen with NER1006 is of clinical relevance.

Disclosure of Interest: J.P.H. Drenth: DAYB investigator; no other conflicts of interest

C. Pediconi: Employee of Norgine

B. Amlani: Employee of Norgine

All other authors have declared no conflicts of interest.

\section{References}

1. Schreiber S et al. United European Gastroenterol J 2016; 4(5): A157-A720. P1266

2. Halphen M et al. Gastrointest Endosc 2013; 78(1): 121-31

\section{P0346 LOW VITAMIN D LEVELS ARE RELATED TO CLINICAL ACTIVITY, MUCOSAL INFLAMMATION, AND INTESTINAL FIBROSTENOSIS IN CROHN'S DISEASE}

T. Sawada ${ }^{1}$, O. Watanabe ${ }^{1}$, M. Nakamura ${ }^{1}$, T. Yamamura ${ }^{2}$, M. Matsushita ${ }^{1}$, M. Saito ${ }^{1}$, R. Matsuura ${ }^{1}$, Y. Mizutani ${ }^{1}$, Y. Niwa ${ }^{1}$, E. Ishikawa ${ }^{1}$, G. Uchida ${ }^{1}$, H. Otsuka ${ }^{1}$, H. Suzuki ${ }^{1}$, T. Nishikawa ${ }^{1}$, T. Ishida ${ }^{1}$, T. Kuno ${ }^{1}$, S. Hattori ${ }^{1}$, K. Yamada ${ }^{1}$, T. Ishikawa ${ }^{1}$, K. Furukawa ${ }^{2}$, K. Funasaka ${ }^{1}$, E. Ohno ${ }^{1}$, R. Miyahara ${ }^{1}$, H. Kawashima ${ }^{1}$, H. Goto ${ }^{1}$, Y. Hirooka ${ }^{2}$

${ }^{1}$ Gastroenterology And Hepatology, Nagoya University Graduate School of Medicine, Nagoya City, Aich-Pref./Japan

${ }^{2}$ Endoscopy, Nagoya University Hospital, Nagoya/Japan

Contact E-mail Address: t.sawada@med.nagoya-u.ac.jp

Introduction: Several studies in recent decades have revealed new roles for vitamin D. For example, vitamin D plays a role in regulating skeletal muscle, as well as in cardiovascular and renal physiology, producing anticancer effects, suppressing fibrosis, and as a regulator of the immune system. In light of these new roles - especially as a regulator of the immune system and suppressor of fibrosis - vitamin D deficiency is considered to be related to disease activity and intestinal fibrosis, including that seen in Crohn's disease (CD). Several reports have demonstrated a relationship between vitamin D deficiency and CD activity according to clinical parameters such as Crohn's disease activity index (CDAI) and quality of life (QoL). However, no reports have demonstrated this relationship by using endoscopic parameters such as endoscopic activity, mucosal inflammation, and intestinal fibrostenosis.

Aims \& Methods: The aim of this study was to clarify the relationship between vitamin $\mathrm{D}$ deficiency and $\mathrm{CD}$ by using endoscopic parameters, as well as clinical parameters. Of the CD patients visiting Nagoya University Hospital from May 2011 to February 2016, 82 patients were enrolled in this study. Serum 25-hydroxyvitamin D (25[OH]D) levels, disease activity, and clinical factors of the subjects were investigated prospectively. Endoscopic findings of 52 of the 82 total patients enrolled were investigated retrospectively from endoscopic records. This study design was approved by the ethics committee of Nagoya University Hospital. Clinical remission was defined as $\mathrm{CDAI} \leq 150$. Mucosal healing was defined as a simple endoscopic score for Crohn's disease (SES-CD) $\leq 1$. Moreover, to evaluate endoscopic activity from two aspects (mucosal inflammation and fibrotic stenosis), we divided SES-CD score into endoscopic "mucosal inflammation score" and "narrowing score". No mucosal inflammation was defined as mucosal inflammation score $\leq 1$, fibrostenosis was defined as narrowing score 0 . The primary endpoint of this study was the relationship between $25(\mathrm{OH}) \mathrm{D}$ and clinical activity. Secondary endpoint was the relationship between $25(\mathrm{OH}) \mathrm{D}$ and endoscopic findings, including mucosal healing, mucosal inflammation, and intestinal fibrostenosis.

Results: Mean age of the subjects was 41.11, and the male/female proportion was $64 / 18$. The mean serum 25(OH)D level of subjects was $17.1 \mathrm{ng} / \mathrm{mL}$, and 61 cases $(74.4 \%)$ were classified as severe deficiency or deficiency. Mean serum 25(OH)D levels of the clinical remission and clinically active groups were $18.7 \pm 8.1 \mathrm{ng} / \mathrm{mL}$ and $12.4 \pm 3.6 \mathrm{ng} / \mathrm{mL}$, respectively $(\mathrm{P}<0.001)$. In a multivariate analysis, low levels of serum $25(\mathrm{OH}) \mathrm{D}$ and serum albumin and positive C-reactive protein
(CRP) results were correlated with clinical activity. Mean serum 25(OH)D levels of the mucosal healing and no mucosal healing groups were $24.0 \pm 9.8 \mathrm{ng} / \mathrm{mL}$ and $15.1 \pm 6.6 \mathrm{ng} / \mathrm{mL}$, respectively, $(\mathrm{P}<0.001)$. Mean serum $25(\mathrm{OH}) \mathrm{D}$ levels for the no mucosal inflammation and mucosal inflammation groups were $21.6 \pm 9.6 \mathrm{ng} / \mathrm{mL}$ and $14.3 \pm 5.5 \mathrm{ng} / \mathrm{mL}$, respectively, $(\mathrm{P}<0.001)$ and those of the no fibrostenosis and fibrostenosis groups were $20.2 \pm 8.9 \mathrm{ng} /$ $\mathrm{mL}$ and $14.2 \pm 6.7 \mathrm{ng} / \mathrm{mL}$, respectively, $(\mathrm{P}<0.001$; Mann-Whitney $\mathrm{U}$ test $)$. In a multivariate analysis, low serum $25(\mathrm{OH}) \mathrm{D}$ levels were related with mucosal inflammation and intestinal fibrotic stenosis of $\mathrm{CD}(\mathrm{P}<0.05$; logistic regression analysis).

Conclusion: This study demonstrated the relationship between vitamin D level and disease activity in $\mathrm{CD}$ patients. The disease pathology of CD consists of repetitive intestinal inflammation and intestinal fibrostenosis formed during healing of the inflammation. We consider it important to demonstrate this relationship not only using clinical parameters, but also using endoscopic parameters such as mucosal inflammation and intestinal fibrostenosis.

Disclosure of Interest: All authors have declared no conflicts of interest.

\section{References}

Kabbani TA et al. Association of Vitamin D Level With Clinical Status in Infl ammatory Bowel Disease: A 5-Year Longitudinal Study. Am J Gastroenterol 2016; 111:712-719.

Jørgensen SP et al. Active Crohn's disease is vitamin D levels. J Crohns Colitis 2013; 7: 407-13.

\section{P0347 PATIENT SATISFACTION WITH HOME MONITORING OF DISEASE ACTIVITY AND FECAL CALPROTECTIN IN ADULT PATIENTS WITH INFLAMMATORY BOWEL DISEASE- INTERIM ANALYSIS OF 68 PATIENTS}

P. Weimers, D. Marker, D. V. Ankersen, J. Burisch, P. Munkholm Department Of Gastroenterology, North Zealand University Hospital, Frederikssund/Denmark

Contact E-mail Address: zld651@alumni.ku.dk

Introduction: Inflammatory Bowel Disease (IBD), mainly represented by Crohn's Disease (CD) and Ulcerative Colitis (UC), is a chronic, relapsing and remitting disease impairing patients' quality of life (QOL). To maintain a high QOL and to decrease the inflammation burden, it is important to tightly monitor the disease and promptly treat relapses when they occur. The quality of care perceived by IBD patients play an important role in the management of IBD. An eHealth web application consisting of a validated Fecal Calprotectin (FC) home testing kit (Calpro Smart ${ }^{\mathrm{TM}}$ ), questionnaires regarding disease activity and QOL has been developed to improve disease monitoring, patient empowerment and patientcaregiver communication.

Aims \& Methods: The aim of this study was to evaluate patient satisfaction with an eHealth home monitoring solution during the participation in a one year trial. The trial includes 120 adult IBD patients which have been randomized into two groups; one performing a disease activity screening procedure every 3 months $(3 \mathrm{M})$ and one screening only at the patient's discretion, on demand (OD). Both groups used the web-program where they were requested to fill out a disease activity questionnaire, Harvey-Bradshaw Index (HBI) for CD or Simple Clinical Colitis Activity Index (SCCAI) for UC, and perform a home testing of FC. The results from disease activity questionnaire and FC score are combined in the webprogram rendering a Total Inflammation Burden Scoring (TIBS) which is visualized to the patient in a traffic light manner for instant recommendation of individualized treatment strategies. At baseline and upon completion of the trial the patients were requested to fill out a QOL questionnaire (ShortInflammatory Bowel Disease Questionnaire (s-IBDQ)) as well as a questionnaire regarding their overall satisfaction with the trial and the home monitoring solution.

Results: To date, 83 patients have been included, 15 patients have dropped out ( 7 in OD-group and 8 in 3M-group) and 68 (3M-group: $\mathrm{n}=32,47 \%$; OD-group: $\mathrm{n}=36,53 \%)$ patients have fulfilled the first year of follow-up and were included in the analysis. The trial lived up to the expectations in $\mathrm{n}=63,93 \%$ (3M-group: $\mathrm{n}=29,91 \%$; OD-group: $\mathrm{n}=34,94 \%$ ) of the patients and the support given to the patients was estimated to be sufficient by $n=67,99 \%$ (3M-group: $n=31$, $97 \%$; OD-group: $\mathrm{n}=36,100 \%$ ). Only $\mathrm{n}=14,21 \%$ (3M-group: $\mathrm{n}=6,19 \%$; ODgroup: $\mathrm{n}=8,22 \%$ ) of the patients experienced difficulties with the application or the home testing kit and $\mathrm{n}=64,94 \%$ (3M-group: $\mathrm{n}=29,91 \%$; OD-group: $\mathrm{n}=35,97 \%$ ) wanted to continue to be monitored in an eHealth setting in the future. The mean s-IBDQ scores at baseline were 58 (95\% CL: 55-61) in the 3Mgroup and $54(95 \%$ CL: 50-58) in the OD-group as well as 58 (95\% CL: 54-62) in the $3 \mathrm{M}$ group and 61 (95\% CL: 58-64) in the OD group at one year follow up. No difference in s-IBDQ measured QOL was found between the two groups. However, patients in the OD group had a significant increase in mean S-IBDQ score at follow up $(\mathrm{p}=0.04)$.

Conclusion: Patients in both groups were generally satisfied by the home monitoring set up. Patients in the on-demand group also presented a significant increase in quality of life over time.

\section{Disclosure of Interest:}

P. Weimers: Calpro Inc. Norway has provided all fecal Calprotectin home testing kits used in this study.

D. Marker: Calpro Inc. Norway has provided all fecal Calprotectin home testing kits used in this study.

D.V. Ankersen: Calpro Inc. Norway has provided all fecal Calprotectin home testing kits used in this study. 
J. Burisch: Calpro Inc. Norway has provided all fecal Calprotectin home testing kits used in this study.

P. Munkholm: Calpro Inc. Norway has provided all fecal Calprotectin home testing kits used in this study.

\section{Reference}

Burisch and Munkholm 2015, Telemonitoring and self-care in patients with IBD (pp. 85-99) in Telemanagement of inflammatory Bowel Disease. $1^{\text {st }}$ edition. Edited by Cross, R. K and Watson A. R, Springer.

\section{P0348 SKELETAL MUSCLE ATROPHY IS A PREDICTIVE FACTOR FOR INTESTINAL RESECTION IN PATIENTS WITH CROHN'S DISEASE}

S. Bamba ${ }^{1}$, M. Sasaki ${ }^{2}$, A. Takaoka ${ }^{2}$, A. Nishida ${ }^{1}$, O. Inatomi ${ }^{1}$, M. Sugimoto ${ }^{3}$, A. Andoh ${ }^{1}$

${ }^{1}$ Division Of Gastroenterology, Shiga Univ. of Medical Sciences, Otsu/Japan

${ }^{2}$ Division Of Clinical Nutrition, Shiga Univ. of Medical Sciences, Otsu/Japan

${ }^{3}$ Division Of Endoscopy, Shiga Univ. of Medical Sciences, Otsu/Japan

Contact E-mail Address: sb@belle.shiga-med.ac.jp

Introduction: Inflammatory bowel diseases (IBD), such as ulcerative colitis (UC) and Crohn's disease (CD), are chronic gastrointestinal diseases that are associated with protein-energy malnutrition (PEM). Although the frequency of altered body composition, such as reduced fat-free mass or skeletal muscle volume, has been shown to be high in patients with IBD, the relationships between skeletal muscle volume and the prognosis are yet to be elucidated.

Aims \& Methods: We have conducted a retrospective study on 61 IBD patients who have admitted due to exacerbation of the disease. We have enrolled IBD patients who had abdominal computed tomography and assessed the nutritional indices, such as the Onodera's prognostic nutritional index (O-PNI) and controlling nutritional status (CONUT). O-PNI was calculated based on the serum albumin and total lymphocyte count, using the following equation: O$\mathrm{PNI}=10 \times[$ serum albumin $(\mathrm{g} / \mathrm{dl})+0.005 \times$ total lymphocyte count $(/ \mathrm{ml})]$. The L3 skeletal muscle index (SMI) which is the cross-sectional area of the skeletal muscle at the level of the third lumbar (L3) vertebra normalized by the height squared is used to identify sarcopenia.

Results: Sarcopenia defined as low SMI were observed in 44\% of all IBD patient $(29 \%$ in CD, $54 \%$ in UC). In UC patients, the O-PNI, CONUT, height and albumin were significantly lower than the $C D$ patients. Spearman's rank correlation revealed that the SMI has a strong correlation to body weight and O-PNI in IBD patients. Multivariate analysis using Cox regression model demonstrated the presence of sarcopenia $(\mathrm{P}=0.022)$, disease type (CD or $\mathrm{UC})(\mathrm{P}=0.034)$, and $\mathrm{C}$ reactive protein $(P=0.047)$ were the significant factors predicting intestinal resection. The cumulative operation-free survival rate was significantly lower among sarcopenic patients in all IBD patients $(\mathrm{P}=0.039)$ and stratified analysis in $C D$ patients $(\mathrm{P}=0.032)$ using Kaplan-Meier method and log-rank test.

Conclusion: The L3 skeletal muscle area can be a prognostic factor of intestinal resection in $\mathrm{IBD}$, especially in $\mathrm{CD}$. The results may originate from the fact that $\mathrm{CD}$ presents the gastrointestinal diseases which accumulate intestinal deformity. Disclosure of Interest: All authors have declared no conflicts of interest.

\section{P0349 A PROSPECTIVE STUDY TO PREDICT A MILD COURSE OF CROHN'S DISEASE AN INTERIM ANALYSIS OF THE PROGNOS STUDY}

W. Kruis ${ }^{1}$, L. Leifeld ${ }^{2}$, N. Hoepffner ${ }^{3}$, M. Hoesl ${ }^{4}$, P. Jessen ${ }^{5}$, M. Mroß ${ }^{6}$, T. Klugmann ${ }^{7}$, S. Ceplis-Kastner ${ }^{8}$, B. Reimers ${ }^{8}$, B. Bokemeyer ${ }^{9}$ ${ }^{1}$ Innere Medizin, Evangelisches Krankenhaus Köln-Kalk, Köln/Germany

${ }^{2}$ St. Bernward Krankenhaus, Hildesheim/Germany

${ }^{3}$ Centrum Gastroenterologie Bethanien, Frankfurt/Germany

${ }^{4}$ Internistische Gemeinschaftspraxis- Praxisklinik, Nürnberg/Germany

${ }^{5}$ Gemeinschaftspraxis im Medicum, Altenholz/Germany

${ }^{6}$ Gastroenterologie in Berlin-Karlshorst, Berlin-Karlshorst/Germany

${ }^{7}$ Internistische Gemeinschaftspraxis, Leipzig/Germany

${ }^{8}$ Ferring Arzneimittel GmbH, Kiel/German

${ }^{9}$ Gastroenterologische Gemeinschaftspraxis Minden, Minden/Germany

Contact E-mail Address: wolfgang.kruis@googlemail.com

Introduction: Crohn's Disease (CD) spans a wide range of severity, from mild to severe. To avoid under- as well as overtreatment is challenging. While factors determining bad prognosis are studied in detail, factors predicting a mild course with the chance of simple treatments are less known. Here we show first results of a prospective evaluation of a retrospectively created score (JCC 2013;7:e263) for prediction of mild $\mathrm{CD}$ which consists of age at diagnosis, CRP, an endoscopy score, presence of perianal lesions and complications.

Aims \& Methods: This is a prospective, ongoing study performed in 12 IBDspecialized private gastroenterology practices (outpatients only) in Germany. All consecutive newly diagnosed CD patients (diagnosis $\leq 6$ weeks) are included. At screening ileocolonoscopy with histology, investigation of the perianal area, laboratory tests including CRP are performed and CD complications (stenosis, fistula, extraintestinal manifestations or fever $>38^{\circ} \mathrm{C}$ ) evaluated to complete of the above quoted score. Patients are treated at the discretion of the physician. In case of a score indicating a good prognosis $(\leq 2)$ or of mild clinical appearance mesalamine is started. In all other cases patients are treated according to guidelines. 5 year follow up is planned for all patients. If initial therapy fails, treatment is escalated. Source data verification is performed by external monitors. Primary aim of the study is to confirm the previously identified score and to test its power to predict a mild disease course as indicated by the need of not more than mesalamine therapy. Additional analyses include the percentage of patients with a score indicating a severe disease and their characteristics at diagnosis. This interim analysis presents preliminary data.

Results: Currently, 78 patients ( 33 male, 45 female; age 16-72, mean 35 years) with newly diagnosed CD are enrolled. $56 \mathrm{CD}$-patients with follow up $>8$ weeks (mean 8.5 months), mean age 35 years, 35 female, 21 male, mean CRP $12.2 \mathrm{mg} / 1$ were included into the interim analysis. In 28 patients with a score from $0-2$ stepup treatment occurred in $7 \%$, whereas in 28 patients with a score $>2$, step-up rate was $43 \%(\mathrm{p}=0.0043)$. Differences between patients with a score $0-2$ and $>2$ were age ( 41 vs. 28 years, $\mathrm{p}=0.0011), \mathrm{CRP}<2 \mathrm{mg} / 1(17 / 28$ patients vs. $0 / 28$, $\mathrm{p}<0.0001$ ), endoscopic score 1.4 vs. $2.7, \mathrm{p}<0.0001$ ), perianal lesions $0 / 28$ vs. $4 /$ 28 , stenosis $1 / 28$ vs. $6 / 28$. There were no differences in terms of sex, fistula, extraintestinal manifestations and fever.

Conclusion: In this early analysis of a prospective study planned with a 5-year follow-up a significant proportion of patients with mild CD and simple mesalamine therapy can be identified. These initial results encourage to continue and expand this prospective long-term study on the predictability of a mild CD course.

Disclosure of Interest:

W. Kruis: Financial Support for Research/Consultancy: Falk, Ferring, Genetic Analysis, Institut Allergosan, Nikkiso, Otsuka, Shire, Tigenix; Lecture fee(s): AbbVie, Ardeyoharm, Falk, Ferring, Genetic Analysis, Institut Allergosan, Nikkiso, Otsuka, Recordati

L. Leifeld: Financial Support for Research: Boehringer, Olympus, DCCV; Lecture fee(s): Falk, AbbVie, MSD; Merckle, Falk, Takeda

N. Hoepffner: Lecture fee(s): AbbVie, Biogen, Consultancy: Invendo medicals

P. Jessen: Consultancy: Takeda

S. Ceplis-Kastner: employee of Ferring Arzneimittel GmbH

B. Reimers: employee of Ferring Arzneimittel GmbH

B. Bokemeyer: Consultancy/Speaker Fee(s): AbbVie, MSD, Shire, Ferring, UCB, Hospira, Takeda, Movetis, Shield, Janssen, Pfizer, Hexal, Boehringer, Biogen, Merckle, Falk, HLR, Mundipharma, Celltrion

All other authors have declared no conflicts of interest.

\section{P0350 USEFULNESS OF REPEATING TESTING FOR LATENT TUBERCULOSIS INFECTION IN PATIENTS WITH INFLAMMATORY BOWEL DISEASE (IBD). CORRELATION BETWEEN TUBERCULIN SKIN TEST (TST)/BOOSTER AND QUANTIFERON-TB (QFT)}

A.M. Fuentes Coronel ${ }^{1}$, P. Fradejas Salazar ${ }^{1}$, C. López Ramos ${ }^{1}$, C. Ochoa Sangrador $^{2}$, G. Rascarachi ${ }^{1}$, L. Julian Gómez ${ }^{1}$, C. Bailador ${ }^{1}$, E. Martín ${ }^{1}$,

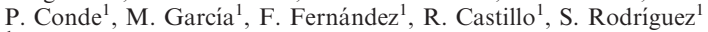
${ }^{1}$ Gastroenterology, Hospital Virgen Concha, Zamora/Spain

${ }^{2}$ Pediatrics, Hospital Virgen Concha, Zamora/Spain

Contact E-mail Address: amfcoronel@gmail.com

Introduction: The Spanish Working Group on Crohn's Disease and Ulcerative Colitis (GETECCU) and other international guidelines recommend testing of latent tuberculosis infection (LTI) before anti TNF therapy by screening with tuberculin skin test (TST) and, in a potential state of anergy, double screening by TST and interferon-gamma release assays (IGRAs) or two-time tuberculin test (TST/booster). Routine repetition is not recommended.

Aims \& Methods: We aimed to assess the correlation between (TST/booster) and IGRAs using QuantiFERON-TB (QFT) and the usefulness of repeating periodic (annual or biannual) screening in a population of IBD patients of Zamora (Spain). In a single cohort of IBD patients attended in the department of gastroenterology of Zamora Hospital, we implemented a questionnaire and collected TST/booster performed previously to February 2015 . Afterwards, prospectively, between February 2015 to February 2017, TST and QFT were performed at the same day, and the TST/booster 7 days after. Finally we compared the results of the LTI screening performed prospectively with the screening of the retrospective cohort.

Results: A total of 404 patients were included with a mean age of 51.5 (SD 16.6), $225(55.7 \%)$ male and $179(44.3 \%)$ female. 227 patients $(56.2 \%)$ were ulcerative colitis, $167(41.3 \%)$ were Crohn disease and $10(2.5 \%)$ were diagnosed of indeterminate colitis. 160 patients live in rural areas $(40.6 \%) .60 / 355(16.9 \%)$ were smokers. The prevalence of LTI and the correlation between TST/booster and QFT is shown in table 1

Table 1: Prevalence of LTI and correlation between TST/booster and QFT.

\begin{tabular}{llll}
\hline & & $\begin{array}{l}\text { Prevalence of LTI } \\
\text { in patients on } \\
\text { immunomodulator } \\
\text { treatment }\end{array}$ & $\begin{array}{l}\text { Prevalence of } \\
\text { LTI in patients } \\
\text { on anti-TNF } \\
\text { therapy }\end{array}$ \\
\hline TST/booster or QFT (+) & $\begin{array}{l}130 / 399(32.6 \%) \\
(95 \% \text { CI }[27.8-37.3 \%])\end{array}$ & $47 / 239(28.5 \%)$ & $9 / 49(18.4 \%)$ \\
TST/booster positives & $116 / 371(31.3 \%)$ & $40 / 163(24.5 \%)$ & \\
TST $(-) /$ booster $(+)$ & $28 / 272(10.3 \%)$ & $12 / 135(8.9 \%)$ & $3 / 42(7.1 \%)$ \\
QFT positives & $40 / 264(15.5 \%)$ & $6 / 105(5.7 \%)$ & $0 / 12$ \\
TST/booster(-)/QFT (+) & $12 / 264(4.5 \%)$ & $3 / 89(0.8 \%)$ & $0 / 11$ \\
TST/booster(+)/QFT(-) & $24 / 370(6.4 \%)$ & $4 / 162(2.4 \%)$ & $3 / 49(6.1 \%)$ \\
\hline
\end{tabular}


Prevalence of LTI in retrospective testing was of 54/246 (22.0\%). Prospective testings were positive in 72/261 (27.6\%). During the follow up, 30/191 (15.7\%) patients who were negative for screening before 2015 were converted in positive for LTI (95\% CI [10.2-21.1]).

Conclusion: The prevalence of LTI in our area is high $(32.6 \%)$. The simultaneous performance of the TST/booster and QFT increases the detection of LTI. The TST/booster increases the detection of LTI even when is performed in patients without immunosuppressive treatments, in whom is not routinely recommended. The QFT is more useful in patients without immunosuppressive therapy. The repeated screening (annual or every two years) is useful in this population with high prevalence of LTI, since it may detect LTI in patients with previous negatives test $(15.7 \%)$. The TST/booster is essential due to the possible false negatives of QFT when screening patients on anti-TNF therapy.

Disclosure of Interest:

All authors have declared no conflicts of interest

\section{References}

Cabriada JL, Vera I, Domènech E, Barreiro-de Acosta M, Esteve M, Gisbert JP, et al. [Recommendations of the Spanish Working Group on Crohn's Disease and Ulcerative Colitis on the use of anti-tumor necrosis factor drugs in inflammatory bowel disease]. Gastroenterol Hepatol. 2013 Mar;36(3):127-46.

Rahier JF, Magro F, Abreu C, Armuzzi A, Ben-Horin S, Chowers Y, et al. Second European evidence-based consensus on the prevention, diagnosis and management of opportunistic infections in inflammatory bowel disease. $J$ Crohns Colitis. 2014 Jun;8(6):443-68.

\section{P0351 MAGNETIC RESONANCE ENTEROGRAPHY GLOBAL SCORE ALLOWS FOR ACCURATE QUANTIFICATION OF SMALL BOWEL INFLAMMATION IN CROHN'S DISEASE- A COMPARISON WITH CAPSULE ENDOSCOPY}

E. Klang ${ }^{1}$, M.M. Amitai ${ }^{1}$, A. Lahat ${ }^{2}$, D. Yablecovitch ${ }^{3}$, S. Neuman ${ }^{3}$, N. Levhar ${ }^{2}$, N. Rozendorn ${ }^{1}$, B. Avidan ${ }^{2}$, S. Ben-Horin ${ }^{2}$, R. Eliakim ${ }^{2}$, U. Kopylov ${ }^{2}$

${ }^{1}$ Diagnostic Imaging, Sheba Medical center, Ramat Gan/Israel

${ }^{2}$ Gastroenterology Department, Sheba Medical Center, Ramat Gan/Israel

${ }^{3}$ Chaim Sheba Medical Center, Tel Aviv University, Tel Aviv/Israel

Contact E-mail Address: ukopylov@gmail.com

Introduction: Magnetic resonance enterography (MRE) and capsule endoscopy (CE) are prime modalities for evaluation of small bowel in patients with Crohn's disease (CD). However, detection of proximal (jejunum and proximal ileum) small bowel inflammation by MRE is challenging. Current quantitative scores such as Magnetic Resonance Index of Activity (MaRIA) do not incorporate proximal small bowel data and were validated against ileocolonoscopy. Magnetic resonance enterography global score (MEGS) was designed for quantitative evaluation of the entire digestive tract; however, it was only validated against ileocolonoscopy and its accuracy in the proximal small bowel was not assessed. CE allows for accurate assessment of the entire small bowel and is the modality of choice for evaluation of the proximal small bowel.

Aims \& Methods: We aimed to compare the quantitative evaluation of the small bowel inflammation by MEGS score and the Lewis capsule endoscopy score. Patients with known quiescent small bowel (CD) for at least 3 months $($ CDAI $<150)$ were prospectively recruited and underwent magnetic resonance enterography (MRE) and capsule endoscopies (CE). MEGS score was calculated for each bowel segment and the entire small bowel. MEGS is based on the involved segment length, wall thickness, mural enhancement, mural and perimural edema and extra-intestinal findings. In addition, MARIA score was calculated for the terminal ileum. Small bowel inflammation on CE was quantified using the Lewis score (LS) ( $\mathrm{LS}<135$ - mucosal healing; $\mathrm{LS} \geq 790$ - moderate to severe inflammation). Proximal small bowel was defined as jejunum and duodenum on MRE and as $1^{\text {st }}$ and $2^{\text {nd }}$ tertiles LS on CE. Distal small bowel was defined as terminal ileum on MRE and $3^{\text {rd }}$ tertile LS on CE. Fecal calprotectin (FCP) levels were measured and correlated with all scores.

Results: Fifty-two patients were included in the study. There was a strong correlation between MEGS and LS (Pearson correlation $-r=0.61 . p=0.001$ ) for the entire small bowel. In the proximal small bowel, the correlation was moderate (for duodenum vs LS $1^{\text {st }}$ tertile and proximal ileum vs $2^{\text {nd }}$ tertile LS - both $\mathrm{r}=0.54, \mathrm{p}=0.001)$. In the terminal ileum, there was a strong correlation between MEGS and MaRIA score $(\mathrm{r}=0.74, \mathrm{p}=0.001)$ while both MRE based scores were moderately correlated with $3^{\text {rd }}$ tertile $\operatorname{LS}(\mathrm{r}=0.5, \mathrm{p}=0.001$ for both). The correlation with FC was stronger for MEGS $(r=0.68 \mathrm{p}=0.001)$ than for MaRIA $(\mathrm{p}=0.40 \mathrm{p}=0.01)$ or for $\operatorname{LS}(\mathrm{r}=0.38, \mathrm{p}=0.02)$.

Conclusion: MEGS score provides accurate evaluation of the small bowel in CD and is strongly correlated with FC levels; the main advantage of MEGS is the accurate quantification of proximal small bowel inflammation that is unavailable for other quantitative MRE scores.

Disclosure of Interest: U. kopylov: The study was supported by a generous grant from the Helmsley Charitable Trust

All other authors have declared no conflicts of interest.
P0352 WHICH ONE IS BETTER FOR ASSESSMENT OF ESTABLISHED CROHN'S DISEASE BY CAPSULE ENDOSCOPY: THE LEWIS SCORE OR THE CAPSULE ENDOSCOPY CROHN'S DISEASE ACTIVITY INDEX?

D. Yablecovitch ${ }^{1}$, A. Lahat ${ }^{2}$, S. Neuman ${ }^{1}$, N. Levhar ${ }^{2}$, B. Avidan ${ }^{2}$, S. BenHorin $^{2}$, R. Eliakim ${ }^{2}$, U. Kopylov ${ }^{2}$

${ }^{1}$ Chaim Sheba Medical Center, Tel Aviv University, Tel Aviv/Israel

${ }^{2}$ Gastroenterology, Chaim Sheba Medical Center, Ramat Gan/Israel

Contact E-mail Address: ukopylov@gmail.com

Introduction: Small-bowel capsule endoscopy (CE) is a prime modality for evaluation of the small bowel. The Lewis score (LS) and the Capsule Endoscopy Crohn's Disease Activity Index (CECDAI) are validated endoscopic indices for quantification of small bowel inflammation on CE. It is unclear whether these indexes are interchangeable for evaluation of mucosal inflammation in established Crohn's disease (CD).

Aims \& Methods: We aimed to prospectively compare the quantitative evaluation of the small bowel inflammation by both scores. Patients with known quiescent small bowel $(\mathrm{CD})$ for at least 3 months $(\mathrm{CDAI}<150)$ were prospectively recruited and underwent CE. LS was calculated using the capsule reading software (RAPID 8) and CECDAI was calculated manually, by 2 independent experienced gastroenterologists (one for each score) unaware of each other's results. Mucosal healing was defined as $\mathrm{LS}<135 ; \mathrm{LS} \geq 790$ signified moderate to severe inflammation. Fecal calprotectin (FCP) and $\bar{C}$-reactive protein (CRP) levels were measured and correlated with the scores.

Results: Fifty patients were included in the study. There was a strong correlation between LS and CECDAI (Pearson's $r=0.67, p=0.001$ ). CECDAI $<7.2$ corresponded to mucosal healing $(\mathrm{LS}<135)$, while CECDAI $>11.1$ corresponded to moderate to severe inflammation $(\mathrm{LS} \geq 790)$ by linear regression. There was a moderate correlation between both scores and FCP levels that was somewhat stronger for CECDAI $(r=0.39, p=0.002$ vs $r=0.53, p=0.001$ for both). There was a weak correlation between LS and CRP levels $(r=0.27, p=0.04)$ and none for CECDAI and CRP $(r=0.21, p=0.1)$.

Conclusion: In our prospective study, CECDAI and LS strongly correlated and performed similarly for quantitative assessment of mucosal inflammation in established CD.

Disclosure of Interest: U. kopylov: The study was suopported by a generous grant by the Helmsley Charitable fund

All other authors have declared no conflicts of interest.

\section{P0353 CONCORDANCE BETWEEN TUBERCULIN SKIN TEST AND INTERFERON GAMMA RELEASE ASSAY FOR LATENT TUBERCULOSIS SCREENING IN INFLAMMATORY BOWEL DISEASE (META-ANALYSIS)}

S. Alrajhi ${ }^{1}$, P. Germain ${ }^{2}$, W. Afif ${ }^{2}$, M. Martel $^{2}$

${ }_{1}^{1}$ Internal Medicine, McGill University, Montreal/Canada/QC

${ }^{2} M c$ Gill University, Montreal/Canada/QC

Contact E-mail Address: saad.alrajhi@mail.mcgill.ca

Introduction: Screening for latent tuberculosis infection (LTBI) is mandatory prior to initiating anti-tumor necrosis factor (anti-TNF) medications. New guidelines recommend interferon-gamma release assays as first line screening method for the general population. Studies have provided conflicting evidence on the performance of interferon-gamma release assays (IGRAs), compared to tuberculin skin test (TST) in inflammatory bowel disease (IBD) patients. We assessed the concordance of these two tests in IBD patients and the effect of immunosuppression on their performance.

Aims \& Methods: We performed a systematic search of MEDLINE, EMBASE and Cochrane Library databases, from 2011 to 2016, for relevant studies testing both TST and IGRA in IBD patients. The primary outcome was concordance between TST and IGRA. Secondary outcomes were effects of immunosuppressive therapy on both TST and IGRA. Immunosuppression was defined as either steroids more than $5 \mathrm{mg}$ for at least two weeks, thiopurine, methotrexate or cyclosporine. We used the Mantel-Haenszel method for a pooled random effects model, given heterogeneity of studies included. We also compared the fixed effects model to exclude any effect of smaller studies. Heterogeneity between studies was analysed using the statistical $\mathrm{I}^{2}$, Q and Tau 2 tests. The quality of included studies was evaluated using a modified QUADAS-2 method.

Results: Sixteen studies, including 2488 patients with IBD, were included for the analysis. The pooled concordance between the TST and IGRA was $85 \%(95 \%$ confidence interval $[\mathrm{CI}] 81 \%-88 \%, \mathrm{p}=0.01)$. Effects of immunosuppression on both tests were reported in eight studies including 814 patients with IBD. The odds ratio of testing positive by IGRA decreased to 0.57 if immunosuppressed $(95 \%$ confidence interval $[\mathrm{CI}] 0.31-1.03, \mathrm{p}=0.06)$. The odds ratio of testing positive by TST if immunosuppressed was $1.14(95 \%$ confidence interval [CI] $0.61-2.12, p=0.69)$. Using the fixed effect model yielded similar results, however the negative effect of immunosuppression on IGRA reached statistical significance $(\mathrm{p}=0.06$ to 0.01$)$.

Conclusion: While concordance was $85 \%$ between TST and IGRA, the performance of IGRA seems to be negatively affected by immunosuppression. Given the importance of detecting latent TB prior to anti-TNF initiation, using only IGRA should be avoided in immunosuppressed IBD patients.

Disclosure of Interest: W. Afif: Abbvie, Janssen, Takeda, Merck, Pfizer, Shire, Ferring, Theradiag All other authors have declared no conflicts of interest. 
P0354 THIOPURINE MAINTENANCE THERAPY FOR IBD: WHICH IS THE BEST METHOD TO MEASURE MEDICATION ADHERENCE?

\author{
A. Ochieng, V. George, C. Selinger \\ Gastroenterology, St James Hospital, Leeds/United Kingdom
}

\section{Contact E-mail Address: odouri.ochieng@nhs.net}

Introduction: For the majority of patients with IBD long-term therapy is required to maintain remission, yet $30-45 \%$ of patients do not adhere to their IBD medication. Medication adherence can be assessed with prescription refill rates, biological measures (metabolites, trough levels, etc) and patient self-report tools. There is currently no accepted gold standard and the feasibility and utility of different adherence assessment tools in the routine outpatient clinic setting have not been fully examined. The aim of this service improvement project was to test the acceptability of self-report tools assessing thiopurine adherence in the IBD clinic and to correlate the results with thioguanine-nucleotide (TGN) levels.

Aims \& Methods: Consecutive outpatients on thiopurine maintenance therapy for IBD for $>3$ months were recruited from clinic. Patients selfreported adherence using a visual analogue scale (VAS), the validated Morisky adherence tool (MOR) and the validated Medication Adherence Report Scale (MARS). TGN levels were classed as complete non-adherence $(<100$ and MMP low), partial adherence (TGN 100-235 and MMP low) or full adherence ( $>235$ or MMP high). Correlation analysis was performed using Pearson tests.

Results: Of 100 approached patients none refused participation and TGN levels were available for 69 . These included 38 women. Diagnoses were Crohn's disease in 27, ulcerative colitis in 41 and IBD-U in 1 cases. Concomitant therapy included 5-ASA ( 25 cases), anti-TNF (13 cases) and Vedolizumab (2 cases). The proportion of adherent patients was according to the relevant report tool $71 \%$ (TGN), 87\% (VAS), 87\% (Morisky) and 77\% (MARS). VAS (Pearson $0.315 ; \mathrm{p}=0.005$ ) and Morisky (Pearson $-0.363 ; \mathrm{p}=0.001$ ) correlated moderately with TGN, but MARS (Pearson $0.09 ; \mathrm{p}=0.39$ ) did not. The 7 patients, who were non-adherent by TGN were detected by VAS in 3, Morisky in 6 and MARS in 3 cases. However, patients showing non-adherence according to self-report tools had normal TGN levels in 6 of 10 cases for VAS, 10 of 26 for Morisky and 4 of 15 for MARS.

Conclusion: Self-report tools provided a patient-friendly and inexpensive way of assessing adherence, but the correlation with TGN levels was only moderate. While providing a more objective assessment TGN levels are problematic for routine use in all patients. TGN require a more invasive and expensive approach. Furthermore, TGN cannot detect "white coat adherence" (patients take medication only around appointments), which is the most likely explanation for normal TGN levels in patients reporting to be poorly adherent. Neither TGN levels nor self-report tools can be seen as the gold standard at present.

Disclosure of Interest: All authors have declared no conflicts of interest.

\section{References}

A. Orlando, M. Principi, M.L. Scribano et al., Medication adherence in patients with active Crohn's disease: results from a large Italian multicenter survey. IBDclinical presentation-2014(ECCO)

R. Goldberg, G. Cunningham, G. Moore, et al., Thiopurine metabolite testing in inflammatory bowel disease, Dept. of Gastroenterology, Melbourne, Australia, 2St Vincent's Hospital \& University of Melbourne, Gastroenterology, Melbourne, Australia, IBD - clinical presentation -2014(ECCO)

Selinger C, Eaden J, Jones B, et al. Modifiable Factors Associated with Nonadherence to Maintenance Medication for Inflammatory Bowel Disease. Inflamm Bowel Dis. 2013; 19(10)2199-2206.

Peel at al., Factors associated with non-adherence to oral IBD medication: a systematic review of the literature 1980-2013, Gastrointestinal Nursing 2015 $13: 9,17-24$

M. Severs at el., Non-adherence to medical therapy is associated with hospitalisations and the development of active disease in inflammatory bowel disease, Dutchy study-clinical presentation -2016(ECCO)

\section{P0355 ROLE FOR THERAPEUTIC DRUG MONITORING IN ASSESSING SECONDARY LOSS OF RESPONSE TO MAINTENANCE ANTI-TNFA THERAPY IN INFLAMMATORY BOWEL DISEASE}

D. Tighe ${ }^{1}$, R. Hession ${ }^{2}$, A. Naqeem ${ }^{2}$, S. Smith ${ }^{3}$, A. O'Connor ${ }^{1}$, N. Breslin ${ }^{1}$, B. Ryan ${ }^{1}$, D. Mcnamara ${ }^{1}$

${ }^{1}$ Gastroenterology Trinity Academic Gastroenterology Group (TAGG), AMNCH Tallaght, Dublin/Ireland

${ }^{2}$ School Of Medicine, Trinity College Dublin, Dublin/Ireland

${ }^{3}$ Trinity Academic Gastroenterology Group, Trinity College Dublin, Dublin/Ireland

Contact E-mail Address: donaltighe83@gmail.com

Introduction: Anti-TNFa therapies have improved outcomes in patients with inflammatory bowel disease. Their use has been associated with improved clinical endpoints, reduced hospitalisation and rates of surgery. However secondary loss of response (LOR) to both infliximab (IFX) and adalimumab (ADA) is a significant problem, leading to further flares of disease, disease progression and poorer outcomes. Therapeutic drug monitoring (TDM), which involves measurement of an individual's anti-TNFa trough and antibody levels, offers the opportunity of exploring an immune basis behind LOR, and potentially adjusting doses or switching therapies to help regain clinical response.

Aims \& Methods: The aim of this study was to evaluate whether TDM can help predict secondary LOR to infliximab and adalimumab and whether dose adjustments based on this information can help patients regain clinical response. This was a prospective, single-centre study, performed from September 2015 to April 2016, at our institution. Patients with Ulcerative colitis (UC) and Crohn's disease (CD) were enrolled, if they were clinically (based on Harvey-Bradshaw (HBI) or partial Mayo scores) felt to be experiencing a secondary LOR to either infliximab or adalimumab maintenance therapy. Patients had serum biochemical markers measured, including CRP and albumin. In addition assessment of inflammation took place, with measurement of SES-CD and Mayo scores, for $\mathrm{CD}$ and UC respectively. Anti-TNFa trough and antibody levels were performed using standard ELISA techniques. Patients were followed for a one-year period, from their initial assessment for secondary LOR to assess outcomes.

Results: 46 patients were recruited, $40 \mathrm{CD}$ with Harvey-Bradshaw Index (HBI) $>4$ points and 6 patients with UC with Partial Mayo Score (PMS) $>2$ points. Mean age for the cohort was 40.9 years, $26(56.5 \%)$ were female, mean duration of disease was 9.8 years, $3(8.3 \%)$ were smokers, $7(15.2 \%)$ on immunomodulators, and $11(23.9 \%)$ had prior anti-TNFa exposure. $17(48.6 \%)$ were on an adalimumab maintenance dose of $40 \mathrm{mg}$ every other week. Overall HBI for the group was 11.9, partial Mayo 6.5. In terms of markers of inflammation, mean SES-CD was 8.5, Mayo endoscopy score 2.7. Mean adalimumab trough level was $4.5 \mathrm{ug} / \mathrm{ml}$, and $15 / 36(42.9 \%)$ had a sub-therapeutic trough level less than $1 \mathrm{ug}$ ml. 9 patients $(25.7 \%)$ had antibodies to adalimumab. Mean infliximab trough level was $8.1 \mathrm{ug} / \mathrm{ml}$ and $2 / 10(20 \%)$ had sub-therapeutic trough levels, none with antibodies. Higher baseline adalimumab trough levels, were noted in patients who were well or who had functional symptoms at one-year follow-up compared to patients, who were unwell, and required change in therapy, mean trough 6.4 $\mathrm{ug} / \mathrm{ml}$ (IQR 2.1-10.3) compared to $2.9 \mathrm{ug} / \mathrm{ml}$ (IQR 0.3-6.3) ( $\mathrm{p}$ value $=0.0265$ $95 \%$ C.I. 0.4 to 6.5 ). The area under the curve [AUC] for association of ADA level at secondary LOR with a good outcome was $0.766, p=0.037$. In addition a trough level of $3.5 \mathrm{ug} / \mathrm{ml}$ predicted clinical response at one year, following secondary LOR to adalimumab, with a sensitivity of $85.7 \%$ and a specificity of $81.8 \%$. Similarly for infliximab higher baseline trough levels, were noted in patients who were well at one-year follow-up compared to those who required change in treatment, $16.0 \mathrm{ug} / \mathrm{ml}$ (IQR 15-16.8) versus $4.6 \mathrm{ug} / \mathrm{ml}$ (IQR 1.1-6.1), p value $=0.00595 \%$ CI 4.5 to 18.3 . The therapeutic strategy chosen for each group was: $23.9 \%$ no change in treatment, $26.1 \%$ increase anti-TNFa dose or decrease infusion interval, $19.6 \%$ switch to another anti-TNFa drug, $15.2 \%$ switch to non anti-TNFa (ustekinumab). For patients who had doses adjusted, clinical response (decrease of $\mathrm{HBI} \geq 3$ points for $\mathrm{CD}$ ) was reached in $77.8 \%$ of patients and remission $(\mathrm{HBI} \leq 4$ for $\mathrm{CD}$ ) in $55.6 \%$ at the end of follow-up.

Conclusion: Secondary LOR to anti-TNFa therapy has a significant impact on patient outcomes. Therapeutic drug monitoring is helpful for predicting secondary LOR and for facilitating dose adjustment or switch in therapy in a clinically guided fashion.

Disclosure of Interest: All authors have declared no conflicts of interest.

\section{P0356 COMPARATIVE ACCURACY OF BOWEL ULTRASOUND VERSUS MAGNETIC RESONANCE ENTEROGRAPHY AND COLONOSCOPY IN ASSESSING DISEASE ACTIVITY AND COMPLICATIONS AND INFLUENCING THE DECISION-MAKING PROCESS IN CROHN'S DISEASE}

M. Allocca ${ }^{1}$, G. Fiorino ${ }^{1}$, C. Bonifacio ${ }^{2}$, F. Furfaro ${ }^{1}$, D. Gilardi ${ }^{1}$, S. Radice ${ }^{1}$, L. Peyrin-Biroulet ${ }^{3}$, S. Danese ${ }^{1}$

${ }^{1}$ Istituto Clinico Humanitas-irccs In Gastroenterology, Istituto Clinico Humanitas IBD Center - Istituto Clinico Humanitas-IRCCS in Gastroenterology, Istitu,

Rozzano/Italy

${ }^{2}$ Radiology, IRCCS Humanitas, Rozzano/Italy

${ }^{3}$ Gastroenterology Unit, Inserm U954, Nancy University and Hospital, Nancy/

France

Contact E-mail Address: mariangela.allocca@humanitas.it

Introduction: Bowel Ultrasound (US) and Magnetic Resonance Enterography (MRE) are accurate in assessing disease activity and complications in Crohn's disease (CD) patients. The comparative accuracy of US versus $\mathrm{MRE}+$ Colonoscopy (CS) in assessing disease activity and complications and influencing the decision-making process in $\mathrm{CD}$ is unknown.

Aims \& Methods: Ileo-colonic CD consecutive patients seen in a tertiary referral Center (Humanitas Research Hospital, Milan, Italy) were prospectively assessed by MRE, CS and US, within 1 week. Sensitivity, specificity, accuracy, positive and negative predictive values (PPV and NPV) of US in assessing localization and extension (bowel wall thickening $>3 \mathrm{~mm}$ ), bowel wall enhancement (increase of vascularization at power Doppler), strictures (narrowing of the lumen), fistulas and abscesses, and active disease (presence of ulcers at colonoscopy) were calculated using CS in combination with MRE findings as a reference standard. Two independent blinded IBD specialists reviewed separately MRE and US findings, and were asked to decide the therapeutic strategy (continue therapy vs. optimize/change therapy). Kappa agreement between MRI and US was also investigated.

Results: Forty-one consecutive CD patients, irrespectively of disease activity and current therapy, were enrolled. Twenty-three patients had active disease as assessed by MRE and CS (56\%), 20/41 (49\%) had CD-related complications. Sensitivity, specificity, accuracy, PPV and NPV of US are showed in Table 1. Based on US findings alone, compared to MRE $+\mathrm{CS}$, management of IBD patients (continuing or changing/optimizing therapy) was judged accurate in $85 \%$ of patients, compared to $75 \%$ managed by MRI only $(p<0.001)$. Agreement between MRE and US was $80 \%$. 


\begin{tabular}{llll}
\hline Parameter & Sensitivity $\%$ Specificity $\%$ Accuracy $\%$ & PPV $\%$ & NPV $\%$
\end{tabular}

Localization + extension $80(0.50-0.93) 94(0.70-0.99) 85(0.70-0.94) 95(0.76-0.99) 75(0.50-0.91)$ Bowel wall enhancement $80(0.58-0.94) 88(0.65-0.98) 84(0.69-0.94) 89(0.66-0.98) 80(0.56-0.94)$ Strictures $\quad 76(0.50-0.93) 79(0.57-0.93) 78(0.62-0.89) 72(0.46-0.90) 82(0.61-0.95)$

Fistulas

Abscesses

Active disease $100(0.16-1) \quad 97(0.86-0.99) 97(0.87-0.99) 66(0.09-0-99) 100(0.90-1)$ $100(0.02-1) \quad 95(0.83-0.99) 95(0.83-0.99) 33(0.01-0.90) 100(0.90-1)$ $83(0.58-0.96) 100(0.85-1) \quad 93(0.80-0.98) 100(0.78-1) \quad 88(0.69-0.97)$

Conclusion: US was as accurate as the combination CS + MRI in assessing disease activity and complications in CD patients. Therapeutic decisions based on US findings alone were appropriate in the vast majority of CD patients. US is a non-invasive, easy-to-use tool to manage CD patients in clinical practice. Disclosure of Interest: All authors have declared no conflicts of interest.

\section{P0357 DIAGNOSTIC DELAY IN PATIENTS WITH INFLAMMATORY BOWEL DISEASE - A STUDY OF THE AUSTRIAN IBD STUDY GROUP (ATISG)}

G. Novacek ${ }^{1}$, H.P. Gröchenig ${ }^{2}$, T. Haas ${ }^{3}$, H.H. Wenzl ${ }^{4}$, P. Steiner ${ }^{5}$, R. Koch ${ }^{6}$, T. Feichtenschlager ${ }^{7}$, G. Eckhardt ${ }^{8}$, A. Mayer ${ }^{9}$, A. Kirchgatterer ${ }^{10}$, O. Ludwiczek ${ }^{11}$, R. Platzer ${ }^{12}$, P. Papay ${ }^{13}$, J. Gartner ${ }^{14}$, H. Fuchssteiner ${ }^{15}$, W. Miehsler ${ }^{16}$, P. Peters ${ }^{17}$, G. Reicht ${ }^{18}$, H. Vogelsang ${ }^{1}$, C. Dejaco ${ }^{1}$, T. Waldhör ${ }^{19}$ ${ }^{1}$ Department Of Internal Medicine III, Medical University of Vienna, Viennal Austria

${ }^{2}$ Innere Medizin, Krankenhaus Barmherzige Brüder, St. Veit an der Glan/Austria ${ }^{3}$ Darmpraxis, Salzburg/Austria

${ }^{4}$ Department Of Gastroenterology And Hepatology, Medical University Graz, Graz/Austria

${ }^{5}$ Abteilung Für Innere Medizin I, Klinikum Wels-Grieskirchen, Wels/Austria ${ }^{6}$ Department Of Internal Medicine I, Medical University Innsbruck, Innsbruck/ Austria

${ }^{7}$ Innere Medizin IV, Krankenanstalt Rudolfstiftung, Vienna/Austria

${ }^{8}$ Innere Medizin, Landeskrankenhaus Oberpullendorf, Oberpullendorf/Austria

${ }^{9}$ 2. Medizinische Abteilung, Universitätsklinikum St. Pölten, St. Pölten/Austria

${ }^{10}$ Abteilung Für Innere Medizin V, Klinikum Wels-Grieskirchen, Grieskirchen/ Austria

${ }^{11}$ Innere Medizin, Landeskrankenhaus Hall in Tirol, Hall in Tirol/Austria

${ }^{12}$ Abteilung Für Innere Medizin I, Landeskrankenhaus Wiener Neustadt, Wiener Neustadt/Austria

${ }^{13}$ Innere Medizin, Hartmannspital, Vienna/Austria

${ }^{14}$ Innere Medizin, Hanusch Krankenhaus, Vienna/Austria

${ }^{15}$ Innere Medizin IV, Krankenhaus Elisabethinen Linz, Linz/Austria

${ }^{16}$ Innere Medizin, Krankenhaus Barmherzige Brüder, Salzburg/Austria

${ }^{17}$ Innere Medizin, Landeskrankenhaus Feldkirch, Feldkirch/Austria

${ }^{18}$ Innere Medizin, Krankenhaus Barmherzige Brüder, Graz/Austria

${ }^{19}$ Department Of Epidemiology, Medical University of Vienna, Vienna/Austria

Contact E-mail Address: gottfried.novacek@meduniwien.ac.at

Introduction: Diagnostic delay seems to be common in inflammatory bowel disease (IBD), especially in Crohn's disease (CD). We sought to investigate the diagnostic delay in Austrian IBD patients and to identify associated risk factors as well as the impact of delayed diagnosis on the risk of intestinal surgery in CD. Aims \& Methods: In a multicentre cohort study adult patients with IBD (CD, ulcerative colitis UC, inflammatory bowel disease unclassified IBDU) attending 18 Austrian outpatient clinics were recruited between May 2014 and July 2015 to complete a multi-item questionnaire, which recorded medical and socioeconomic characteristics. Study outcome was the diagnostic delay defined as the time period from first symptom onset to diagnosis of IBD. A multivariable proportional hazard regressions model based on interval censored latency times was calculated

Results: 1217 patients (CD 779, UC 400, IBD 21, missing 17; females 615) with a median age of 40 years (interquartile range (IQR) 31-52 years) and a median disease duration of 10 years (IQR 4-18 years) were analysed. The median diagnostic delay was 0.53 years (IQR $0.20-1.92$ years) in CD and 0.28 years (IQR $0.11-0.86$ years) in $\mathrm{UC}$, respectively $(\mathrm{p}<0.001)$. In the multivariable regression analysis patients with $\mathrm{CD}$ had a significantly longer diagnostic delay than patients with UC (HR 1.56; 95\% CI 1.34-1.82; p < 0.0001) and a quadratic effect of age leading to higher risk of delayed diagnosis in older patients $(\mathrm{p}<0.0001)$ was found. Diagnostic delay did not differ significantly between patients with intestinal CD-related surgery (53\% of all CD patients) and those without surgery. However, in the Kaplan-Meier curve for the probability of being diagnosed after symptom onset a trend of a difference between both groups was seen after 10 months $(\mathrm{p}=0.13)$.

Conclusion: The median diagnostic delay was longer in CD (6 months) than in UC patients ( 3 months) and was associated with older age at diagnosis. Disclosure of Interest: All authors have declared no conflicts of interest.

\section{References}

Vavricka SR, et al. Systematic evaluation of risk factors for diagnostic delay in inflammatory bowel disease. Inflamm Bowel Dis 2012;18:496-505

Schöpfer A, et al. Diagnostic delay in Crohn's disease is associated with a complicated disease course and increased operation rate. Am $J$ Gastroenterol 2013;108:1744-53
Burisch J, et al. East-west gradient in the incidence of inflammatory bowel disease in Europe: the ECCO-EpiCom inception cohort. Gut 2014;63:588-97

Chouraki V, et al. The changing pattern of Crohn's disease incidence in northern France: a continuing increase in the 10- to 19-year-old age bracket. Aliment Pharmacol Ther 2011;33:1133-42

Nahon S, et al. Diagnostic delay in a French cohort of Crohn's disease patients. $J$ Crohns Colitis 2014;8:964-9

Zaharie R, et al. Diagnostic delay in Romanian patients with inflammatory bowel disease: risk factors and impact on the disease course and need for surgery. J Crohns Colitis 2016;10:306-14

P0358 IDENTIFICATION OF NON-INVASIVE BIOMARKERS TO DETECT ILEAL CEACAM-6 OVEREXPRESSION AND ADHERENT AND INVASIVE $E$. COLI (AIEC) INFECTION IN CROHN'S DISEASE PATIENTS: RESULTS FROM THE CEALIVE MULTICENTER STUDY

E. Vazeille ${ }^{1}$, X. Hebuterne ${ }^{2}$, M. Fumery ${ }^{3}$, B. Pariente ${ }^{4}$, S. Nancey ${ }^{5}$, P. Seksik ${ }^{6}$, L. Peyrin-Biroulet ${ }^{7}$, M. Allez ${ }^{8}$, A. Dubois ${ }^{1}$, N. Ballet ${ }^{9}$, J. Filippi ${ }^{2}$, J. Dupas ${ }^{10}$, M. Nachury ${ }^{11}$, G. Boschetti ${ }^{5}$, M. Goutte ${ }^{1}$, G. Bommelaer ${ }^{1}$, B. Pereira ${ }^{1}$,

N. Barnich ${ }^{1}$, A. Buisson

${ }^{1}$ Dept. Of Gastroenterology, CHU Estaing Clermont-Ferrand, Clermont-ferrand/ France

${ }^{2}$ Service de Gastroentérologie et Nutrition Clinique, Nice/France

${ }^{3}$ Amiens University Hospital, Amiens/France

${ }_{5}^{4}$ Gastroenterology Unit, Hopital Huriez, Lille University Hospital, Lille/France ${ }^{5}$ university of Lyon, lyon/France

${ }^{6}$ Gastroenterology And Nutrition Unit, Gastroenterology \& Nutrition Department, Paris/France

${ }^{7}$ Department Of Gastroenterology, Nancy University Hospital Inserm U954 Dept. of Hepato-Gastroenterology, Vandoeuvre les Nancy/France

${ }^{8}$ Gastroenterology, Hopital Saint-Louis APHP, Université Denis Diderot Paris 7 Paris/France

${ }^{9}$ Lesaffre, Marcq-en-baroeul/France

${ }^{10} \mathrm{CHU}$ Nord Amiens, Amiens/France

${ }^{11} \mathrm{CHU}$ Lille, Lille/France

Contact E-mail Address: emilie.vazeille@udamail.fr

Introduction: Enterobacteria, especially adherent and invasive E. coli (AIEC), are suspected to play a key role in Crohn's disease (CD). These bacteria are able to highly adhere to the ileal mucosa of CD patients through the CEACAM6 receptor (Carcinoembryonic antigen-related cell adhesion molecule 6). It has been shown that therapies targeting enterobacteria and/or AIEC could be more effective in mice overexpressing CEACAM6. In this line, the overexpression of CEACAM6 in the ileum as well as the presence of AIEC in the ileum could be potential biomarkers to select the patients who could benefit from drugs targeting the host-pathogen interaction. Unfortunately, the identification of these biomarkers is time-consuming and invasive highlighting the need for more convenient alternative.

Aims \& Methods: We aimed to assess the correlation between the level of CEACAM6 in the saliva and the level of CEACAM6 in the ileum in CD patients and to define the best threshold of CEACAM6 in the saliva to detect overexpression of ileal CEACAM6. In addition, we attempted to identify non-invasive biomarkers of AIEC infection. In this prospective multicentre study ( 8 centers), all the patients requiring ileocoloscopy, regardless the indication, were consecutively included between September 2015 and September 2016. Clinical and endoscopic data were collected on the day of colonoscopy. Blood samples, stool samples (before bowel cleansing), saliva and ileal biopsies from healthy and ulcerated areas were also collected. CEACAM6 from ileal biopsies and saliva were measured (duplicates) using ELISA assays. AIEC were identified using phenotypical assays.

Results: Overall, 102 patients were enrolled in the study (Table 1).

Table 1: Baseline characteristics of the $102 \mathrm{CD}$ patients included in the study.

\begin{tabular}{ll}
\hline Female gender n, $\%$ & $56(56.6 \%)$
\end{tabular}

Active smokers n, \% $34(34.3 \%)$

Montreal classification

Disease location

L1

L2

L3

L4

Disease behaviour

$\mathrm{B} 1$

B2

Table 1: Baseline characteristics of the $102 \mathrm{CD}$ patients included in the study.

Perianal lesions

Prior intestinal

Current therapies
$26(27.7 \%)$

$18(19.1 \%)$

$20(21.3 \%)$

$27(28.4 \%)$

$58(61.1 \%)$ $7(7.4 \%)$

(continued) 


\section{5-ASA}

Corticsteroids

Immunosuppressive therapies

Anti-TNF agents

Other biologics

CDAI, mean \pm standard deviation

CRP, g/L mean \pm standard deviation

Fecal calprotectin, $\mu \mathrm{g} / \mathrm{g}$ mean \pm standard deviation

Ileal CEACAM6 level did not depend on disease severity or the site of biopsies as the median level of ileal CEACAM6 was $854.9 \mathrm{pg} / \mathrm{mg}[570.2$; $1646]$ and there was no difference in healthy or ulcerated zone $(756 \mathrm{pg} / \mathrm{mg}$ [487; 1617] vs $947 \mathrm{pg} / \mathrm{mg}[604 ; 1820], \mathrm{p}=0.86)$. The median level of CEACAM6 from saliva was $3837 \mathrm{pg} / \mathrm{mg}[1889 ; 7338]$. There was a positive correlation between the levels of CEACAM6 in saliva and CEACAM6 in the ileum $(\rho=0.47 ; \mathrm{p}<0.0001)$ in both macroscopically healthy areas $(\rho=0.53$, $\mathrm{p}<0.0001)$ and ulcerated zone $(\rho=0.39, \mathrm{p}=0.0082)$. Using a ROC curve, we determined the best threshold of CEACAM6 in saliva for detecting ileal CEACAM6 overexpression. Using a ROC curve (area under the curve $(A U R O C)=0.73$ ), the cut-off value of $3800 \mathrm{pg} / \mathrm{mg}$ demonstrated the best performances to detect ileal CEACAM6 overexpression with substantial specificity $(76.0 \%$ [54.9-90.6]) and positive predictive value $(87.5 \%$ [74.8-95.3]) The number of enterobacteria was increased in $\mathrm{CD}$ patients with prior intestinal resection $(562[20 ; 1674]$ vs. $116[0 ; 752] \mathrm{pg} / \mathrm{mg}, \mathrm{p}=0.03)$. Interestingly, the number of enterobacteria was also increased in AIEC positive-patients (640 $[184 ; 2392]$ vs. $60[0 ; 1028] \mathrm{pg} / \mathrm{mg}, \mathrm{p}=0.004)$. Using a ROC curve, we determined the best threshold of enterobacteria in the ileum to detect the presence of ileal AIEC bacteria. We found an area under the curve (AUROC) of 0.70 $[0.61 ; 0.77]$. The cut-off value of $60 \mathrm{cfu} /$ biopsy demonstrated the best performances to detect the presence of ileal AIEC bacteria. The number of enterobacteria associated to ileal mucosa (cut-off value $>60 \mathrm{cfu}$ /biopsy) strongly predicted the presence of AIEC and then is a reliable test for AIEC screening with very high negative predictive value $(94.1 \%$ [80.3-99.3]) and high sensitivity $(91.7 \%$ [73.0-99.0])

Conclusion: CEACAM6 measurement in the saliva is feasible, non time-consuming and non-invasive. It could be a reliable test to detect the overexpression of CEACAM6 in the ileum from CD patients and could then be proposed as a non-invasive biomarker to select patients who might benefit from anti-adhesive therapies. In addition, we identify that the number of enterobacteria associated to the ileum is a convenient and reliable test to screen CD patients for AIEC bacteria.

Disclosure of Interest: A. Buisson: The study was funded by LESAFFRE company. I declare lecture fees for Abbvie, Takeda, Hospira, MSD, Vifor Pharma, SAnofi-Aventis and Ferring. I declare consulting fees for Abbvie, Takeda, Hospira.

All other authors have declared no conflicts of interest.

\section{P0359 INCREASED LEVEL OF FIBROBLAST GROWTH FACTOR 19 IN PATIENTS WITH ULCERATIVE COLITIS IN REMISSION}

M. Panek-Jeziorna, M. Jasinska, B. Marczak-Karpina, A. Mulak

Department Of Gastroenterology And Hepatology, Wroclaw Medical University, Wroclaw/Poland

\section{Contact E-mail Address: panekm@wp.pl}

Introduction: Fibroblast growth factor 19 (FGF19) is the ileal hormone providing feedback inhibition of bile acid (BA) synthesis in the liver. BAs are actively reabsorbed in the terminal ileum by the apical sodium-dependent BA transporter (ASBT). FGF19 is produced by the ileal enterocytes in response to absorbed BAs binding and activating the nuclear $\mathrm{BA}$ receptor, also known as farnesoid $\mathrm{X}$ receptor (FXR) [1]. The FXR activation may enhance expression of anti-inflammatory genes and down-regulate pro-inflammatory genes [2]. The glucocorticoid budesonide has been shown to increase the expression of ASBT and BA absorption inducing FGF19 release [3]. Disturbances in BA enterohepatic circulation may be associated with colonic inflammation, but little is known about the mechanisms of BA dysregulation in ulcerative colitis (UC).

Aims \& Methods: The aim of the study was to evaluate serum FGF19 level in patients with active and inactive phase of UC. Fasting serum FGF19 level was measured using ELISA test in 14 patients with active UC ( 7 F, 7 M; mean age 44), $16 \mathrm{UC}$ patients in remission ( $10 \mathrm{~F}, 6 \mathrm{M}$; mean age 39), and 7 healthy controls (4 F, $3 \mathrm{M}$; mean age 35). Disease activity was assessed based on the clinical evaluation and fecal calprotectin level. Non-parametric statistics were used and results are expressed as median with interquartile ranges. The Mann-Whitney U test was used for the comparison of differences between the groups. Spearman's rank correlation coefficient was also calculated

Results: Median fecal calprotectin level in UC patients with active and inactive phase of the disease amounted to 1675.1 vs $142.8 \mu \mathrm{g} / \mathrm{g}$, respectively $(\mathrm{p}=0.004)$. Median serum FGF19 level was significantly higher in UC patients in remission (median $192.6 \mathrm{pg} / \mathrm{ml}$, range 155.1-351.2) than in UC patients with active disease (median $82.6 \mathrm{pg} / \mathrm{ml}$, range 64.0-151.8), $\mathrm{p}=0.004$. Median FGF19 level in healthy controls (median $151.6 \mathrm{pg} / \mathrm{ml}$, range 121.6-216.1) was lower than in UC patients in remission and higher than that in patients with active $\mathrm{UC}$, but the differences did not reach statistical significance. A negative correlation between serum FGF19 and fecal calprotectin levels was observed, but it was not statistically significant $(\mathrm{R}=-0.328, \mathrm{p}=0.077)$.
Conclusion: The increased FGF19 level in UC patients in remission, compared to the patients with active UC, may be influenced by the treatment, in particular corticosteroids used to induce remission. Steroid-induced increase in FGF19 synthesis may be associated with anti-inflammatory effect. The stimulation of FGF19 release during UC remission may also have beneficial effect by the inhibition of BA synthesis and reduction of excess colonic BAs.

Disclosure of Interest: All authors have declared no conflicts of interest.

\section{References}

1. Walters JR. Bile acid diarrhoea and FGF19: new views on diagnosis, pathogenesis and therapy. Nat Rev Gastroenterol Hepatol 2014;11:426-34.

2. Ding L, Yang L, Wang Z, Huang W. Bile acid nuclear receptor FXR and digestive system diseases. Acta Pharm Sin B 2015;5:135-44.

3. Jung D, Fantin AC, Scheurer U, Fried M, Kullak-Ublick GA. Human ileal bile acid transporter gene ASBT (SLC10A2) is transactivated by the glucocorticoid receptor. Gut 2004;53:78-84.

\section{P0360 DOUBLE-BALLOON ENDOSCOPIC EVALUATION OF} FECAL CALPROTECTIN AS A BIOMARKER FOR SMALL INTESTINAL INFLAMMATION IN CROHN'S DISEASE

R. Matsuura ${ }^{1}$, O. Watanabe ${ }^{1}$, M. Nakamura ${ }^{1}$, T. Yamamura ${ }^{2}$, M. Matsushita ${ }^{1}$,

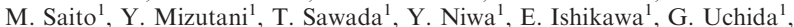
H. Otsuka ${ }^{1}$, H. Suzuki ${ }^{1}$, T. Nishikawa ${ }^{1}$, T. Ishida ${ }^{1}$, T. Kuno ${ }^{1}$, S. Hattori ${ }^{1}$, K. Yamada ${ }^{1}$, T. Ishikawa ${ }^{1}$, K. Furukawa ${ }^{2}$, K. Funasaka ${ }^{1}$, E. Ohno ${ }^{1}$, H. Kawashima ${ }^{1}$, R. Miyahara ${ }^{1}$, Y. Hirooka ${ }^{2}$, H. Goto $^{1}$

${ }^{1}$ Gastroenterology And Hepatology, Nagoya University Graduate School of Medicine, Nagoya City, Aich-Pref./Japan

${ }^{2}$ Endoscopy, Nagoya University Hospital, Nagoya/Japan

\section{Contact E-mail Address: mrinsato@med.nagoya-u.ac.jp}

Introduction: Crohn's disease (CD) has a progressive course and often causes mucosal injury throughout the gastrointestinal tract. Mucosal healing (MH) has been proposed as a treatment goal for patients with CD. Endoscopy, computed tomography, magnetic resonance imaging, and other examinations are used to evaluate $\mathrm{MH}$. However, repeated examinations require considerable effort and are highly invasive. Recently, fecal calprotectin (FC) has attracted attention as a new biomarker. The correlation between FC levels and CD activity is well established in ileocolonic or colonic $\mathrm{CD}$, but few reports have described this correlation in ileal CD alone.

Aims \& Methods: This study evaluated the correlation between FC levels and endoscopic activity in ileal CD. Fifteen patients with ileal CD who underwent double-balloon endoscopy (DBE) between May 2016 and February 2017 at our hospital were included in this study. The entire small intestine was examined with DBE and radiological enteroclysis. We evaluated the correlations of FC levels, Creactive protein (CRP) levels, erythrocyte sedimentation rates (ESRs), and CD activity index (CDAI) scores with the endoscopic activity. To evaluate the endoscopic activity of the small intestine, we used Double-Balloon Endoscopic Score for CD (DES-CD), which is a modified version of the Simple Endoscopic Score for CD (SES-CD). To determine the DES-CD, the small intestine was divided into four segments (upper jejunum, lower jejunum, upper ileum, and lower ileum), and four variables were evaluated in each segment (ulcer size, extent of ulcerated surface, extent of affected surface, and stenosis) in accordance with the SES-CD. For the evaluation of mucosal injury, the partial DES-CD (pDES-CD) was calculated by excluding "stenosis" from the DES-CD. The DES-CD and DES-CD ranges were $0-46$ and $0-24$, respectively.

Results: Fifteen patients (11 males and four females) with a median age of 42 (range, 27-71) were assessed. No colitic lesions were observed in any of the patients. The DES-CD correlated with FC $(\gamma=0.688, P=0.005)$ and CRP $(\gamma=0.765, P=0.001)$ levels. In addition, the pDES-CD correlated with FC levels $(\gamma=0.803, P=0.001)$, CRP levels $(\gamma=0.673, P=0.006)$, and ESRs $(\gamma=0.704, \mathbf{P}=0.003)$. The CDAI scores did not correlate with either of the endoscopic scores

Conclusion: In this study, the endoscopic activity in ileal CD correlated with FC levels. In addition, pDES-CD scores exhibited a stronger correlation with FC levels than did DES-CD scores. This might be because FC levels reflect mucosal injury and not stenosis. Our findings suggest that FC can be used for monitoring mucosal injuries in the small intestine and as a biomarker for evaluating $\mathrm{MH}$. Disclosure of Interest: All authors have declared no conflicts of interest.

\section{P0361 CLINICIANS' ATTITUDES TO PHOTO PROTECTION IN INFLAMMATORY BOWEL DISEASE PATIENTS ON IMMUNOSUPPRESSION}

C. Gallagher ${ }^{1}$, M. Hussey ${ }^{1}$, D. Tighe ${ }^{2}$, A. Ridge ${ }^{3}$, M. Kennedy ${ }^{4}$, Y. Bailey ${ }^{5}$, D. Kevans ${ }^{3}$, A.M. Tobin', D. Mcnamara ${ }^{2}$

${ }^{1}$ Trinity Academic Gastroenterology Group, Trinity College Dublin, Dublin/Ireland ${ }^{2}$ Gastroenterology Trinity Academic Gastroenterology Group (TAGG), AMNCH Tallaght, Dublin/Ireland

${ }^{3}$ Gastroenterology, St James' Hospital, Dublin/Ireland

${ }^{4}$ Gastroenterology, AMNCH, Dublin/Ireland

${ }^{5}$ Gastroenterology, Tallaght Hospital, Dublin/Ireland

${ }^{6}$ Dermatology, Tallaght Hospital, Dublin/Ireland

Contact E-mail Address: gallagc6@tcd.ie 
Introduction: Increasingly, immunosuppressive medications such as azathioprine and biologics are being used in order to promote clinical remission in inflammatory bowel disease (IBD) patients. It has been reported that such treatments increase the risk of developing all types of skin cancer. Education of these patients is key in order to promote their awareness of their increased risk and it is vital for gastroenterologists to counsel patients on sun protection strategies on initiating therapy. We recently performed a pilot study in this group which highlighted gaps in their knowledge of the increased risk and prevention strategies. We speculate clinician's lack of knowledge was partly to blame.

Aims \& Methods: Our aim was to determine Irish IBD clinicians' knowledge of the skin cancer risk and advised photoprotective behaviours in this cohort. Cross-sectional descriptive study. We invited IBD clinicians via email to fill in an anonymous online survey designed to assess knowledge of skin cancer risk and prevention methods as recommended by the "Sunsmart" guidelines, as currently gastroenterology based guidelines are lacking. In addition their grade of training and clinical experience was noted.

Results: To date, 45 Irish Gastroenterology clinicians completed the online questionnaire. Of these, fifteen $(33 \%)$ were consultants, fourteen $(31 \%)$ gastroenterology trainees, four $(9 \%)$ general medical trainees and twelve $(27 \%)$ IBD nurse specialists. Overall, clinician's knowledge of general factors associated with increased risk of skin cancer was reassuring; with all $45(100 \%)$ knowing sun beds increased skin cancer risk and almost $100 \%(44,98 \%)$ knew working outdoors incurred increased risk. $42(93 \%)$ knew a personal history of skin cancer and previous blistering sunburn were risks; however, only $34(79.1 \%)$ recognised family history as a risk. Regarding gender associated risk; only $67.4 \%(n=29)$ knew men were greater risk than women of non-melanoma skin cancer (NMSC). Their knowledge of specific immunosuppressant risk was suboptimal; while many $(37,82 \%)$ recognized azathioprine was a risk factor for developing NMSC, only $53 \%(n=24)$ knew anti-TNF medications were strongly associated with an increased risk of malignant melanoma. Regards prevention strategies; the majority knew what changes to look for in a suspicious mole; $100 \%(\mathrm{n}=45)$ knew to be suspicious of changing color and $84 \%(n=38)$ of an irregular border, but shockingly only five $(11 \%)$ perform yearly skin checks on their patients on immunosuppressants. Their knowledge of preventative measures was also lacking; $37(86 \%)$ knew patients should wear SpF 50 but almost half $(47 \% \mathrm{n}=20)$ thought it should be applied twice daily rather than two hourly $(51 \% \mathrm{n}=23)$ and only $47 \%(\mathrm{n}=20)$ knew patients should stay in the shade from $11 \mathrm{am}-3 \mathrm{pm}$. Regards their own practice; 39 (87\%) report they do emphasise the importance of sun protection in their patients; however, worryingly only $24(55.8 \%)$ had heard of our national skin cancer prevention guidelines "Sunsmart". Of interest; while physicians had a greater understanding of patient risk factors $(\mathrm{p}<0.03)$, nurse specialists were more likely to emphasise the need for sunprotection in clinic $(\mathrm{p}<0.0003)$, and of physicians, trainees had a more complete knowledge of all advised preventative measures $(\mathrm{p}<0.03)$

Conclusion: Our study highlights IBD clinicians' suboptimal knowledge of immunosuppression risk and their lack of emphasis on preventative measures and skin examination in clinics. A targeted educational and awareness programme may address this.

Disclosure of Interest: All authors have declared no conflicts of interest.

\section{P0362 HOME MONITORING OF DISEASE ACTIVITY AND FECAL CALPROTECTIN IN ADULT PATIENTS WITH INFLAMMATORY BOWEL DISEASE - INTERIM ANALYSIS OF 68 PATIENTS}

\section{V. Ankersen, D. Marker, P. Weimers, J. Burisch, P. Munkholm} Department Of Gastroenterology, North Zealand University Hospital, Frederikssund/Denmark

\section{Contact E-mail Address: dorit.vedel.ankersen@regionh.dk}

Introduction: Due to the chronic and progressive nature of inflammatory bowel disease (IBD) it is of significant importance to detect and treat a relapse as soon as possible in order to decrease the total inflammation burden and avoid progression of intestinal damage, and possibly improve the disease course. A validated Fecal Calprotectin (FC) home testing kit and smart phone application CalproSmart ${ }^{\mathrm{TM}}$ have been added to an existing eHealth web-application, enabling patients to monitor their disease activity using clinical scores and FC from home with results shown on their smart phone. eHealth allows for tight monitoring of disease activity, however, the frequency of an optimal screening procedure for adult IBD patients has not yet been determined.

Aims \& Methods: The aim of this one-year randomized controlled trial of 120 adult IBD patients, was to determine if an eHealth screening procedure for disease activity should be implemented in clinical practice 'every 3 months, $3 \mathrm{M}$ ' or according to patients own gut feeling, 'on demand, OD'. Both groups used the ehealth-program to tightly monitor their disease activity either OD or every $3 \mathrm{M}$. Patients randomized to screen every $3 \mathrm{M}$ were also allowed to monitor themselves OD if they were feeling a flare was coming. The web-algorithm consists of a short disease questionnaire either Harvey-Bradshaw Index (HBI) for Crohn's disease (CD) or Simple Clinical Colitis Activity Index (SCCAI) for Ulcerative Colitis plus home monitoring of FC, which together gives a total inflammation burden scoring (TIBS). Based on longitudinal FC and disease activity scores area under the curve (AUC) were calculated by an algorithm taking the inclusion date and the first observation 357 days after inclusion into the calculation.

Results: To date, 83 patients have been included, 15 patients have dropped out (7 in OD-group and 8 in 3M-group) and 68 (3M-group: $\mathrm{n}=32,47 \%$; OD-group: $\mathrm{n}=36,53 \%$ ) patients have fulfilled the first year of follow-up and were included in the analysis. There were no statistical difference between the two groups OD vs. $3 \mathrm{M}$ on the following indices: Active disease $(\mathrm{FC}>600 \mathrm{mg} / \mathrm{kg})$ at inclusion $\mathrm{n}=17,25 \%$ (3M-group: $\mathrm{n}=6,19 \%$; OD-group: $\mathrm{n}=10,29 \%, \mathrm{p}=0.26$ ), active disease at least once throughout the whole year: 41 patients $(61 \%)$ (3M-group: $\mathrm{n}=21,66 \%$; OD-group: $\mathrm{n}=20,57 \%$, OD, $\mathrm{p}=0.32$ ). Patients screened themselves with regards to FC 233 times in the OD-group and 232 times in the 3Mgroup. The mean no of FC home measurements pr. patient was similar in the two groups: OD 6.7 (95\% CL: 5.2-8.2) vs. 3M 7.3 (95\% CL:5.9-8.5), p=0.54. Median (range) AUC for FC and disease active scores were not significantly different in the groups (OD, 3M): 62016 (3782-541338) vs 89730 (10286$353881) \mathrm{p}=0.71,549(0-3726)$ vs $610(0-2.884) \mathrm{p}=0.61$.

Conclusion: In this preliminary analysis, no difference between the two screening procedures was found in regards to disease course and resource utilization. A slightly but non-significant reduction in no of FC measurements pr. patient used in OD-group relative to $3 \mathrm{M}$-group could argue for an economical benefit of choosing the OD screening procedure. Long-term results are awaited.

\section{Disclosure of Interest:}

D.V. Ankersen: Calpro Inc. Norway has provided all fecal Calprotectin home testing kits used in the study

D. Marker: Calpro Inc. Norway has provided all fecal Calprotectin home testing kits used in the study

P. Weimers: Calpro Inc. Norway has provided all fecal Calprotectin home testing kits used in the study

J. Burisch: Calpro Inc. Norway has provided all fecal Calprotectin home testing kits used in the study

P. Munkholm: Calpro Inc. Norway has provided all fecal Calprotectin home testing kits used in the study

All authors have declared no conflicts of interest.

\section{Reference}

Burisch and Munkholm 2015, Telemonitoring and self-care in patients with IBD (pp. 85-99) in Telemanagement of inflammatory Bowel Disease. $1^{\text {st }}$ edition. Edited by Cross, R. K and Watson A. R, Springer.

\section{P0363 S100A4 PROTEIN IN INFLAMMATORY BOWEL DISEASE: RESULTS OF A SINGLE CENTRE PROSPECTIVE STUDY}

\section{P. Moravkova ${ }^{1}$, D. Kohoutova ${ }^{1}$, J. Vavrova ${ }^{2}$, J. Bures}

${ }^{1}$ 2nd Department Of Internal Medicine - Gastroenterology, Charles University, Faculty of Medicine and University Hospital in Hradec Kralove, Hradec Kralove Czech Republic

${ }^{2}$ Institute Of Clinical Biochemistry And Diagnostics, Charles University, Faculty of Medicine and University Hospital in Hradec Kralove, Hradec Kralove/Czech Republic

\section{Contact E-mail Address: darina.kohoutova@seznam.cz}

Introduction: Ulcerative colitis (UC) and Crohn's disease (CD) represent a serious medical and socio-economic problem worldwide. The family of S100 proteins represents a total of at least 25 relatively small calcium binding proteins. S100 proteins have a broad range of functions: they play a role in the regulation of cell proliferation, differentiation, apoptosis, energy metabolism, cellular signalling, and calcium homeostasis. S100A4 (metastatin-1, calvasculin) strongly contributes to a process of metastasizing. Still, the role of S100A4 seems to be more complex as its profibrotic effect has been confirmed in the myocardium, liver parenchyme and in the intestine. Fibroblasts represent the key cell type in the pathogenesis of fibrostenosing/stricturing CD.

Aims \& Methods: The aim of this prospective study was to assess serum concentration of S100A4 protein in UC and CD. Study included 118 subjects: 93 patients with CD (44 men, 49 women, aged $22-79$, mean $44 \pm 14), 16$ patients with UC ( 8 men, 8 women, aged 20-74, mean 39 \pm 15 ) and 9 controls (average risk population with normal findings on colonoscopy and with negative history of colorectal neoplasia and/or inflammatory bowel disease; 2 men, 7 women, aged 23-74, mean 52 \pm 17 ). CD group was divided according to the Montreal classification: 20/93 (22\%) patients had B1 phenotype, 19/93 (20\%) B2, 20/93 $(21 \%) \mathrm{B} 3$ and $34 / 93(37 \%) \mathrm{B} 2+\mathrm{B} 3$. Perianal involvement was present in $27 / 93$ $(29 \%)$. L1 involvement was present in 15/93 (16\%), L2 in 14/93 $(15 \%)$ and L3 in $64 / 93(69 \%)$. Serum concentration of S100A4 protein was investigated by means of Human Protein S100-A4 ELISA kit, the quantitative sandwich enzyme immunoassay technique (purchased from My Biosource, San Diego, California, USA). Results: Serum concentration of S100A4 protein was significantly higher in UC (mean $158.6 \pm 56.2 \mu \mathrm{g} / \mathrm{L}$ ) compared to controls (mean $104.8 \pm 40.5 \mu \mathrm{g} / \mathrm{L}$ ), $\mathrm{p}=0.019$ and in $\mathrm{CD}($ mean $154.4 \pm 52.1 \mu \mathrm{g} / \mathrm{L}$ ) compared to controls, $\mathrm{p}=0.007$. No difference in S100A4 was revealed between UC and CD, p>0.05. In CD group, serum concentration of S100A4 in each subgroup (divided according to behaviour of CD) was significantly higher compared to controls, $p<0.05$. No differences in S100A4 were documented between each CD phenotypes. Serum concentration of S100A4 was significantly higher in L2 (mean $144.6 \pm 44.2 \mu \mathrm{g} / \mathrm{L}$ ) compared to controls, $\mathrm{p}=0.041$ and in $\mathrm{L} 3$ (mean $163.0 \pm 52.8 \mu \mathrm{g} / \mathrm{L}$ ) compared to controls, $\mathrm{p}=0.002$. Serum concentration of $\mathrm{S} 100 \mathrm{~A} 4$ was significantly higher in $\mathrm{L} 3$ (mean $163.0 \pm 52.8 \mu \mathrm{g} / \mathrm{L}$ ) compared to $\mathrm{Ll}$ (mean $126.9 \pm 47.6 \mu \mathrm{g} / \mathrm{L}$ ), $\mathrm{p}=0.017$. No difference in S100A4 was observed between patients with and without perianal involvement, $\mathrm{p}>0.05$. 
Conclusion: Association of serum S100A4 protein with UC and CD was confirmed. In CD, disease behaviour did not have impact on serum concentration of S100A4 protein. In CD, higher levels of serum S100A4 were observed in patients with ileo-colonic and colonic involvement compared to those with isolated small bowel involvement.

Acknowledgement: The study was supported by the Research Project PROGRES Q40-15 from Charles University.

\section{P0364 SEVERE VITAMIN D DEFICIT IN ACTIVE INFLAMMATORY BOWEL DISEASE}

G. Burrelli Scotti ${ }^{1}$, M.T. Afferri ${ }^{1}$, A. De Carolis ${ }^{1}$, V. Vaiarello ${ }^{1}$, V. Fassino ${ }^{2}$, S. Minisola ${ }^{2}$, P. Vernia

${ }^{1}$ Gastroenterology, Department Of Internal Medicine And Medical Specialties, Sapienza University of Rome, Rome/Italy

${ }^{2}$ Department Of Internal Medicine And Medical Specialties, Sapienza University of Rome, Rome/Italy

Contact E-mail Address: piero.vernia@uniroma1.it

Introduction: Hypovitaminosis D is common in Inflammatory Bowel Disease (IBD) patients. Some studies suggest that the finding may be related to severity of the disease. ${ }^{1,2}$

Aims \& Methods: The aim of the study was to determine the Vitamin D (VitD) status in an Italian IBD cohort in relation to disease activity. Serum 25-hydroxyvitamin D was measured in 260 IBD outpatients, not supplemented with VitD (110 Crohn's Disease (CD) and 150 Ulcerative Colitis (UC); 145 males and 115 females; mean age $50.7 \pm 15$ years), and compared to those of 205 healthy blood donors, matched by sex, age $(+/-2$ years $)$ and month in which the blood sample was collected. VitD levels were correlated to C-reactive protein (CRP), erythrocyte sedimentation rate (ESR), Harvey Bradshaw Index (HBI) and Crohn's Disease Activity Index (CDAI) for CD and Mayo partial score for UC. Chi square, $\mathrm{T}$ test and linear correlation were used when appropriate.

Results: IBD patients were at higher risk of VitD deficiency (defined as $<20 \mathrm{ng} /$ $\mathrm{ml}$ ) than controls (OR 4.5, 95\% CI 2.9-6.9, p < 0.0001). Of 260 IBD patients, 156 $(60 \%)$ had VitD deficiency, more often in CD than in UC $(72.7 \%$ vs $48 \%$ respectively, $\mathrm{p}<0.0001)$. Age $<40$ and $\geq 60$ years, winter/spring season, CRP $\geq 0.5 \mathrm{mg} / \mathrm{dl}, \mathrm{ESR} \geq 20 \mathrm{~mm} / \mathrm{h}$, previous intestinal surgery and HBI $\geq 5$ were significant risk factors for VitD deficiency. No differences were observed in relation to sex, smoking status, BMI, age at diagnosis, localization and behavior of disease, and need of steroids. There was a weak negative correlation between CRP values, HBI scores and VitD levels $(R=-0.13, p=0.037$ and $R=-0.26, p=0.006$ respectively).

Conclusion: VitD deficiency is significantly more common in IBD patients than in controls, more so in CD. Patients with active disease are more likely to have VitD severe deficiency than those in remission. The correlation with activity indexes should be confirmed in larger series.

Disclosure of Interest: All authors have declared no conflicts of interest.

\section{References}

1. Mouli VP, Ananthakrishnan AN. Review article: Vitamin D and inflammatory bowel diseases. Aliment Pharmacol Ther 2014, 39(2), 125-136.

2. Kabbani TA et al. Association of Vitamin D Level With Clinical Status in Inflammatory Bowel Disease: A 5-Year Longitudinal Study. Am J Gastroenterol 2016, 111(Jan), 1-8.

\section{P0365 MAGNETIC RESONANCE OF THE SMALL BOWEL WITH EARLY (70S) AND LATE (7MINS) PHASE POST GADOLINIUM IMAGING TO IDENTIFY FIBROSIS IN STRICTURING SMALL BOWEL CROHN'S DISEASE}

R. Stack ${ }^{1}$, C. O’Brien ${ }^{2}$, T. Nuzum ${ }^{1}$, S. Cummins ${ }^{1}$, N. O'Morain ${ }^{1}$, A. O'Connor ${ }^{1}$, N. Breslin ${ }^{1}$, B. Ryan ${ }^{1}$, I. Murphy ${ }^{1}$, D. Mcnamara ${ }^{1}$

${ }^{1}$ Gastroenterology, Tallaght Hospital, Dublin/Ireland

${ }^{2}$ Radiology, Tallaght Hospital, Dublin/Ireland

Contact E-mail Address: stack.roisin@gmail.com

Introduction: Small bowel (SB) Crohn's disease (CD) strictures can comprise of both inflammatory and fibrotic elements. An accurate tool to discriminate fibrosis and inflammation would be clinically useful to guide therapy and predict response. While the magnetic resonance index of activity (MaRIA) is a validated means to assess activity, to date, no specific tool has been developed to identify fibrotic from inflammatory disease. Lesions with a dense fibrotic matrix exhibit delayed gadolinium enhancement on MRI. The role of delayed enhancement in assessment of SB CD strictures is unclear. Recent evidence suggests relative contrast enhancement (REC) of $>24 \%$ on delayed MRI sequences may accurately detect fibrosis.

Aims \& Methods: To determine the feasibility of MRE SB stricture assessment with early $(70 \mathrm{~s})$ and late $(7 \mathrm{mins})$ phase post gadolinium imaging comparing MaRIA, RCE and biochemical activity in patients with ileal CD. We performed a retrospective review of 208 consecutive MREs with known and suspected SBCD. MRE was performed as standard with additional coronal T1 sequences 7 minutes post gadolinium administration. Demographics, MRE findings and biochemical markers were recorded. Patients with stricturing disease were further assessed. Two independent blinded Radiologists calculated RCE and MaRIA's at $70 \mathrm{sec}$ and $7 \mathrm{~min}$.
Results: Median age 40.5 years; male $n=83(39.9 \%) .117,72$ and 19 patients had known $\mathrm{CD}$, suspected $\mathrm{CD}$ and indeterminate IBD, respectively. In total, $119(57.2 \%)$ MREs were normal. Ileitis, strictures and fistulas were found in $40(19.2 \%), 49(23.6 \%)$ and $1(0.5 \%)$ patients, respectively. While there was no difference in $\mathrm{Hb}$ between patient groups (Normal, Inflammatory and Stricturing CD); Albumin and CRP were statistically different between normal subjects and those with disease; albumin $42 \mathrm{~g} / \mathrm{L}$ v $38.9 \mathrm{~g} / \mathrm{L}$ in normal v stricturing disease $(\mathrm{p}<0.018195 \% \mathrm{CI}-0.23-0.02)$. CRP $8.8 \mathrm{mg} / \mathrm{L}$ v $18.3 \mathrm{mg} / \mathrm{L}(\mathrm{p}<0.003$ $95 \% \mathrm{CI}-0.46-0.10)$ and $\vee 29 \mathrm{mg} / \mathrm{L}(\mathrm{p}<0.00295 \% \mathrm{CI}-0.43-0.11)$ amongst normal $v$ inflammation and strictures respectively. Neither parameter could differentiate between inflammatory and stricturing disease. 26 MREs performed with ileal $\mathrm{CD}$ have been further assessed; median age $=41 \mathrm{yrs}$, male $=10(38 \%)$. RCE $>24 \%$ and high T2 signal intensity (SI); $6 / 26(23 \%)$ and $11 / 26(42.3 \%)$. REC $>24 \%$ occurred in only $1 / 10$ with a visible stenosis. Average MaRIAs: $2 /$ $26(7.7 \%)<7$ mild; $3 / 26(11.5 \%) 7-11$ moderate; $21 / 26(80.7 \%)>11$ severe. MaRIAs did not change significantly between $70 \mathrm{sec}$ and $7 \mathrm{~min}$. As expected T2 SI increased with MaRIAs $>11,26$ v 13 (p $<0.001,95 \%$ CI 7.73-17.27). RCE did not correlate with MaRIA group, suggesting MaRIA is not a predictive factor for fibrosis. Consistent with MRE findings, CRP was higher in patients with MaRAI $>11(13.3$ v 5.2) and lower in patients with RCE $>24 \%(3.9$ v 14$)$, $\mathrm{p}<0.0495 \%$ CI $0.37-15.71$ and $\mathrm{p}<0.0195 \%$ CI $2.5-19.05$ respectively. Conclusion: Unlike biochemical markers, MRE may be a useful means to differentiate between inflammatory and structuring disease. Further study is required to assess the long-term predictive value. RCE may be a useful adjunct to current MRE and help detect fibrosis in small bowel lesions and warrants further investigation.

Disclosure of Interest: All authors have declared no conflicts of interest.

\section{P0366 GASTRODUODENAL INVOLVEMENT IN PATIENTS WITH CROHN'S DISEASE - UPPER ENDOSCOPY ONLY IN SYMPTOMATIC PATIENTS?}

M. Sousa, L. Proença, J. Silva, A. Ponte, J. Rodrigues, J. C. Silva, J. Carvalho Gastrenterology, Centro Hospitalar de Vila Nova de Gaia e Espinho, Vila Nova de Gaia/Portugal

Contact E-mail Address: mafalda_m_p_sousa@hotmail.com

Introduction: The need for upper endoscopy in patients with Crohn's disease (CD) without symptoms is controversial. The aim of this study was to establish the prevalence of gastroduodenal involvement, regardless of symptoms, and its prognostic implications.

Aims \& Methods: Patients from a single centre with established CD $(n=347)$ were retrospectively evaluated - inclusion criteria: upper endoscopy without treatment. Gastroduodenal involvement was defined by considering macroscopic (erosions, ulcers or stenosis) and microscopic criteria (focal gastritis, cryptic irregularity, erosion/ulceration and granuloma in the absence of Helicobacter pylori (HP) infection).

Results: We included 140 patients - phenotype: 50\% inflammatory, 31\% stricturing and 19\% penetrating; Location: $42 \%$ ileal, $45 \%$ ileocolic and $13 \%$ colic. Upper endoscopy was performed in $19 \%$ for symptoms and in $81 \%$ for staging. Gastric macroscopic findings were detected in $49 \%(69 / 140)$; the most common were erosions $(21 \%)$ and erythematous mucosa $(18 \%)$. Biopsies were performed in $56 \%$ of patients: chronic gastritis $66 \%$, normal $23 \%$, granuloma $5 \%$, focal gastritis $2 \%$ and cryptic microabcess in $2 \%$. HP was positive in $25 \%$ of patients. In the duodenum, endoscopic lesions were observed in $33 \%$ of the patients (46/ $140)$; the most common were erosions $(16 \%)$ and ulcers $(9 \%)$. Biopsies were performed in $32 \%$ and the most prevalent findings were chronic non-specific inflammation $62 \%$, ulcers $17 \%$, granuloma $3 \%$ and erosion $3 \%$. Applying macro/microscopic criteria, gastroduodenal involvement by CD was considered in $18 \%$ of the patients and was not correlated with the presence of symptoms, phenotype or localization of the disease. The presence of gastroduodenal involvement was a significant predictor of hospitalization $(p=0.009)$.

Conclusion: The prevalence of gastroduodenal involvement by $\mathrm{CD}$ in this sample was $18 \%$, and a larger percentage have macro/microscopic findings that are not disease specific. The presence of symptoms does not predict gastroduodenal involvement due to $\mathrm{CD}$ that is associated with a worse prognosis.

Disclosure of Interest: All authors have declared no conflicts of interest.

\section{P0367 INCIDENTAL FINDINGS AT CT ENTEROGRAPHY IN PATIENTS WITH CROHN'S DISEASE: CLINICAL SIGNIFICANCE AND IMPACT ON TARGETED THERAPY}

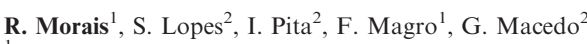

${ }^{1}$ Gastroenterology, Centro Hospitalar São João, Porto/Portugal

${ }^{2}$ Centro Hospitalar São João, Porto Medical School, Porto/Portugal

Contact E-mail Address: ruimorais20@gmail.com

Introduction: CT enterography is one of the most accurate imaging methods for evaluating Crohn's disease (CD) extent and intestinal involvement.

Aims \& Methods: The aim of this study was to determine the frequency and clinical impact of the incidental findings in CD patients who underwent CT enterography.

This was a retrospective study that evaluated patients with $\mathrm{CD}$ who underwent CT enterography between January 2012 and December 2016. Incidental findings were defined as previously unknown extraintestinal lesions. The orientation of the patients after their detection was evaluated. 
Results: A total of 520 patients who underwent CT enterography were identified, with incidental findings being reported in $276(53 \%)$. The median age was 43 (3253 ) years and $53 \%$ were women. The main indication for CT enterography was CD staging $(81 \%)$. A total of 531 incidental findings were detected (median of 2 [1-3] per patient). The main findings identified were hepatic nodules $(\mathrm{n}=59)$, hepatic cysts $(n=55)$ and sacroiliitis $(n=46)$. The findings implicated orientation to another medical specialty in 80 patients $(29 \%)$, the main ones being Urology $(\mathrm{n}=14)$ and Gynecology $(\mathrm{n}=11)$. The findings implied additional exams in 59 patients $(21 \%)$. Five $(2 \%)$ underwent subsequent surgical intervention. Clinically relevant findings were found in 38 patients $(14 \%)$, including 2 renal tumors, 2 ovarian teratomas and 3 cases of primary sclerosing cholangitis. The detection of incidental findings implied a change in $\mathrm{CD}$ therapy in 9 patients $(3 \%)$ : one suspended biologic therapy, 2 suspended immunomodulator therapy and 6 initiated biologic therapy.

Conclusion: Incidental findings are relatively common in patients with $\mathrm{CD}$ who undergo CT enterography. A significant proportion is clinically relevant and may involve change $C D$ therapy. A risk stratification may be important to avoid morbidity associated with unnecessary examinations to assess benign situations. Disclosure of Interest: All authors have declared no conflicts of interest.

\section{Reference}

1. Dignass A, Van Assche G, Lindsay JO, et al. The second European evidencebased Consensus on the diagnosis and management of Crohn's disease: current management. J Crohns Colitis. 2010;4:28-62.

\section{P0368 CLINICAL SIGNIFICANCE OF ASYMPTOMATIC CLOSTRIDIUM DIFFICILE CARRIAGE IN PATIENTS ON IMMUNOMODULATOR FOR INFLAMMATORY BOWEL DISEASE}

\section{S.T. Law, W.M. Yip, K.K. Li}

Department Of Medicine And Geriatrics, Tuen Mun Hospital, Hong Kong/Hong Kong Prc

Contact E-mail Address: st1168@hotmail.com

Introduction: Clinical significance of asymptomatic Clostridium difficile (C. difficile) carriage in patients on immunomodulator for inflammatory bowel disease (IBD) is largely unknown. $[1,2]$

Aims \& Methods: The aim of this study was to investigate the clinical implication of asymptomatic carriage of $\mathrm{C}$ diff in IBD patients.

Consecutive IBD patients on immunomodulators in clinical remission for the past six months were prospectively recruited from the IBD clinic since 2013 .

Those cases were excluded if they had past history of total colectomy, the dosage of their immunodulators were titrated according to their disease activity in the past six months or the types of their immunomodulators were other than azathioprine, mercaptopurine or methotrexate.

Stool specimen for C. difficile cytotoxin real-time polymerase chain reaction (RT-PCR) assay was obtained to all eligible patients at the time of enrollment and every follow-up during the study period. Patients were monitored for any IBD flare-up in which if happened, an additional stool specimen for C. difficile cytotoxin RT-PCR assay was obtained.

The primary outcomes were the disease activity which was graded by Crohn Disease Activity Index (CDAI) in Crohn disease (CD) (graded as follows: $<150$ : remission; 150-<220: mild-moderate; 220- $<450$ : moderate-severe; $>450$ : severe/fulminant) and Ulcerative colitis Disease Activity Index (UCDAI) in ulcerative colitis (UC) (graded as follows: the total index score ranges from 0-12; 0-2: remission; 3-6: mild; 7-10: moderate; >10: severe UC). The secondary outcome was proportion with $C$. difficile diarrhea which was defined in the patients with active lower gastrointestinal symptoms accompanying with positive RT-PCR assay of $C$. difficile at that instant.

Statistical inference of the variables was examined by Mann-Whitney $U$ and $\chi 2$ test for numerical and categorical parameters respectively

Results: Of 197 IBD patients (CD: 98 (49.75\%); male: 132(67.01\%); age (yrs): median 43, minimum 17, maximum 79), 9(4.57\%; CD: 6 patients) patients were found to be asymptomatic carriage of $C$. difficile during the study period. The demographic features, including age, gender ratio, smoking history and the duration of IBD, of the patient group with and without asymptomatic carriage of $C$. difficile were comparable each other. Four UC patients in the non-carriage group had prior history of anti-TNF exposure in which three were treated as maintenance therapy for the active disease activity, associated axial spondyloarthroapthy and rectovaginal fistula while the other two patients (one from each group) had received three doses of anti-TNF as rescue therapy for severe disease flare-up.

Incidence rates of the disease flare-up were comparable (11.17 vs. $22.22 \%$, p 0.313 ) between the non-carriage and carriage groups in which all these flares were under-controlled by course of high-dose prednisolone.
The asymptomatic carriage group had a significant higher rate (33.33 vs. $7.45 \%$, p 0.007) and earlier onset (18.78 vs. 34.42 months, log rank p 0.009; MannWhitney $\mathrm{U}, \mathrm{p}$ 0.037) in evolving into clinical $C$. difficile infection as compared with the non-carriage group.

No other serious complications, such as toxic megacolon, colonic perforation, sepsis, and death, were reported in the both groups during the study period.

Clinical characteristics of the IBD patients with and without asymptomatic carriage of $C$. difficile

\begin{tabular}{llll}
\hline & $\begin{array}{l}\text { Non C. } \\
\text { difficile } \\
\text { carrier } \\
(\mathrm{n}=188)\end{array}$ & $\begin{array}{l}\text { C. difficile } \\
\text { carrier } \\
(\mathrm{n}=9)\end{array}$ & P value \\
\hline Age (Yr) & $43(26)$ & $44(33)$ & 0.788 \\
Sex (m:f) & $128: 60$ & $4: 5$ & 0.159 \\
Smoker (n, \%) & $24(12.77)$ & $1(11.11)$ & 0.881 \\
Year of Diagnosis (Yr) & $7(9)$ & $7(13)$ & 0.940 \\
Crohn disease (n, \%) & $92(48.94)$ & $6(66.67)$ & 0.298 \\
Prior exposure of Anti-TNF (n, \%) & $4 *(2.13)$ & $1 * *(11.11)$ & 0.095 \\
Flare up (n, \%) & $21(11.17)$ & $2(22.22)$ & 0.313 \\
mild/moderate/severe & $16 / 5 / 0$ & $2 / 0 / 0$ & $0.165 / 0.617 / \mathrm{ns}$ \\
C. difficile infection (n, \%) & $14(7.45)$ & $3(33.33)$ & 0.007 \\
\hline
\end{tabular}

Data were expressed as median(interquartile range)

*: all are UC cases and 3 for maintenance therapy with indications as follows: refractory control, spondyloarthropathy, rectovaginal fistula

**: case of UC received 3 doses of anti-TNF for severe flare

Abbreviation: IBD, inflammatory bowel disease; C. difficile, Clostridium difficile; ulcerative colitis, UC; ns, non-significant

Conclusion: The incidence of asymptomatic carriage of $C$. difficile in the IBD patients on imunomodulators was not common. It did not associate with the disease flare-up but a significant portion of them could evolve subsequently into clinical infection.

Disclosure of Interest: All authors have declared no conflicts of interest.

\section{References}

1. Issa M, et al. Impact of Clostridium difficile on inflammatory bowel disease. Clin Gastroenterol Hepatol. 2007;5:345-351.

2. Rodemann JF, et al. Incidence of Clostridium difficile infection in inflammatory bowel disease. Clin Gastroenterol Hepatol. 2007;5:339-344.

\section{P0369 BOWEL ULTRASOUND IS USEFUL IN DISEASE} MONITORING OF ULCERATIVE COLITIS PATIENTS: FIRST ANALYSIS FROM THE TRUST\&UC STUDY IN GERMANY

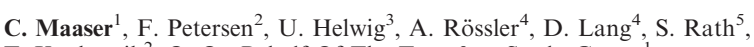
T. Kucharzik ${ }^{2}$, O. On Behalf Of The Trust\&uc Study Group ${ }^{1}$

${ }_{1}^{1}$ Ambulanzzentrum Gastroenterologie, University Teaching Hospital Lueneburg, Lueneburg/Germany

${ }^{2}$ Department Of Internal Medicine And Gastroenterology, University Teaching Hospital Lüneburg, Lüneburg/Germany

${ }^{3}$ Gastroenterology Practice, Oldenburg/Germany

${ }^{4}$ Medical Department, AbbVie Deutschland GmbH \& Co. KG, Wiesbaden/ Germany

${ }^{5}$ Medical Area Immunology, AbbVie Deutschland GmbH \& Co. KG, Wiesbaden Germany

Contact E-mail Address: christian.maaser@klinikum-lueneburg.de

Introduction: Due to the relapsing and highly variable nature of ulcerative colitis (UC), it would be desirable to have reliable tools for measuring parameters of disease activity in order to monitor response to therapy and to detect relapse. In Crohn's disease it has already been shown that ultrasound (US) is a useful to monitor the disease activity. ${ }^{1}$ The hypothesis of the TRUST\&UC (TRansabdominal UltraSonography of the bowel To monitor disease activity in subjects with Ulcerative Colitis) study is that transabdominal US is an easy to use, easily repeatable, and accurate diagnostic tool in the assessment of UC activity, in monitoring the disease course, and response to therapy.

Aims \& Methods: TRUST\&UC is a German ongoing prospective, observational multi-center study in patients with active UC. The primary objective of this study is the prospective evaluation of bowel wall US in response to therapy in order to assess its value in monitoring UC patients in routine medical practice. Clinical parameters (e.g. CRP, fecal calprotectin) and the Simple Colitis Clinical Activity Index (SCCAI) were used for routine assessment of disease activity.

Results: 176 patients with active UC have been enrolled in 37 German IBD study group (GISG) centres until February 2017. 47.2\% of the patients were female, median age was 38.9 years (range 19-77) with median disease duration of 152.2 days (range -8-1017). Of all the patients with a clinical flare defined by SCCAI $90.3 \%$ showed a bowel wall thickening (BWT), and only $9.7 \%$ showed no US signals. At US examination, a BWT in the colon sigmoideum was present in $87.5 \%$ of the patients, in the colon descendens in $83.7 \%$, in the colon transversum in $42.3 \%$ and in the colon ascendens in $18.3 \%$. Loss of bowel wall 
stratification was the case in $20.6 \%$ of the patients, mesenteric fibro-fatty proliferation in $40.0 \%$ and more than $50 \%$ had an increased signal in the color Doppler US. At baseline systemic steroids were used in $62.1 \%$, azathioprine in $36.2 \%$, and TNF antagonists in $40.0 \%$ of patients $(\mathrm{N}=174)$.

All follow-up patients $(\mathrm{N}=104)$ displayed acute inflammatory symptoms at baseline, with at least moderate disease activity which required an introduction or escalation of treatment. After 12 weeks, the US examination showed significant improvements of the following parameters: BWT in colon sigmoideum $(87.5 \%$ vs $33.7 \%, \quad \mathrm{p}=0.034)$ and colon transversum $(42.3 \%$ vs $15.4 \%$, $\mathrm{p}=0.012)$, loss of haustration $(54.8 \%$ vs $33.7 \%, \mathrm{p}<0.001)$, ascites $(9.7 \%$ vs $2.9 \%, \mathrm{p}<0.001)$, mesenteric lymphadenopathy $(31.6 \%$ vs $14.3 \%, \mathrm{p}=0.005)$, mesenteric fibro-fatty proliferation $(40.0 \%$ vs $10.0 \%, \mathrm{p}=0.041)$ and increased signal in color Doppler US $(56.7 \%$ vs $23.1 \%, \mathrm{p}=0.039)$. A decrease of BWT was significantly accompanied by a decrease in SCCAI $(8.0$ to 1.5 points, $\mathrm{p}<0.001)$. Conclusion: In this real-life cohort almost $90 \%$ of the patients showed a BWT, a pivotal IBD symptom and within 12 weeks selected bowel US parameters improved significantly from treatment intensification. Therefore, US examination is a useful tool to monitor disease activity and response to therapy in UC patients. Disclosure of Interest:

C. Maaser: C. Maaser has received lecture and consulting fees from AbbVie. F. Petersen: F. Petersen has received lecture and consulting fees from AbbVie. U. Helwig: U. Helwig has received lecture and consulting fees from AbbVie. A. Rössler: A. Rössler is AbbVie employee and may own AbbVie stock or options.

D. Lang: D. Lang is AbbVie employee and may own AbbVie stock or options. S. Rath: S. Rath is AbbVie employee and may own AbbVie stock or options. T. Kucharzik: T. Kucharzik has received lecture and consulting fees from AbbVie. All other authors have declared no conflicts of interest.

\section{Reference}

1. Kucharzik T. et al., Clin Gastroenterol Hepatol. 2017 Apr;15(4):535-542.

\section{P0370 THE GUT MICROBIOME IN IBD IS CHARACTERIZED BY IMPAIRED METABOLIC COOPERATIVITY AND CAN BE RESTORED UPON ANTI-TNFA THERAPY}

K. Aden ${ }^{1}$, A. Rehman ${ }^{2}$, W.H. Pan ${ }^{2}$, S. Waschina ${ }^{3}$, J. Zimmermann ${ }^{3}$, J. Bethge ${ }^{1}$, A. Franke ${ }^{2}$, S. Nikolaus ${ }^{1}$, J. O. Schröder ${ }^{1}$, R. Zeuner ${ }^{1}$, C. Kaleta ${ }^{3}$, S. Schreiber ${ }^{1}$, P. Rosenstiel ${ }^{2}$

${ }^{1}$ I Medical Department, University of Kiel, Kiel/Germany

${ }^{2}$ Institute Of Clinical Molecular Biology, University of Kiel, Kiel/Germany

${ }^{3}$ Institute For Experimental Medicine, University of Kiel, Kiel/Germany

\section{Contact E-mail Address: k.aden $@$ ikmb.uni-kiel.de}

Introduction: Blocking TNF $\alpha$ is an important treatment option for inflammatory bowel disease (IBD). The etiology of the disorder comprises a permanent activation of immune cascades and imbalanced cytokine networks. Evidence has been put forward that alteration of the human gut microbiome may play a critical role in the pathogenesis of IBD. However, the impact of targeted cytokine blockade on dysbiosis of intestinal microbial communities is poorly understood. Here, we investigate the effect of anti-TNF $\alpha$ treatment on gut microbial community structures in a prospective, longitudinal study for 30 weeks. The study compares IBD as a disorder, which primarily affects the gut, with seropositive and -negative rheumatoid arthritis and ankylosing spondylitis as a rheumatic disorder (RD) as an inflammatory disease complex, which usually does not affect the intestine.

Aims \& Methods: anti-TNF $\alpha$ naive patients suffering from IBD $(n=12)$ or RD $(n=17)$, subject to first-time anti-TNF $\alpha$ therapy were recruited for longitudinal stool sampling at baseline and 2, 6 and 30 weeks after therapy induction. Intestinal microbiota communities were studied by $16 \mathrm{~S}$ rRNA gene (V4) sequencing. Changes in microbiota before and after therapeutic interventions were assessed in terms of alpha and beta diversity, indicator species and prediction of metabolic cooperative interactions. Samples from healthy controls $(n=19)$ were included as a benchmark of healthy microbial profiles.

Results: Intestinal microbial diversity and cooperativity are decreased in both disease entities, IBD and RA. In IBD, anti-TNF $\alpha$ therapy is able to restore microbial diversity and cooperativity. More over cooperative metabolic interaction is significantly increased only in anti-TNF $\alpha$ responders. In RA, anti-TNF $\alpha$ therapy did not significantly restore microbial community structures.

Conclusion: We show that anti-TNF $\alpha$ treatment increases the gut microbial diversity and coupling of cross feeding metabolic interactions towards the state of healthy individuals. Assessment of metabolic interactions of intestinal microbiota may serve as a marker for clinical response in IBD patients.

Disclosure of Interest:

All authors have declared no conflicts of interest.

\section{References}

Schreiber, S., Rosenstiel, P., Albrecht, M., Hampe, J. \& Krawczak, M. Genetics of Crohn disease, an archetypal inflammatory barrier disease. Nat Rev Genet 6 , 376-388 (2005).

McInnes, I. B. \& Schett, G. Cytokines in the pathogenesis of rheumatoid arthritis. Nat Rev Immunol 7, 429-442 (2007).

de Lange, K. M. et al. Genome-wide association study implicates immune activation of multiple integrin genes in inflammatory bowel disease. Nat Genet 49, 256-261, doi:10.1038/ng.3760 (2017).

Franke, A. et al. Genome-wide meta-analysis increases to 71 the number of confirmed Crohn's disease susceptibility loci. Nat Genet42, 1118-1125, (2010).
Anderson, C. A. et al. Meta-analysis identifies 29 additional ulcerative colitis risk loci, increasing the number of confirmed associations to 47 . Nat Genet $\mathbf{4 3}, 246$ 252, (2011).

Ellinghaus, D. et al. Analysis of five chronic inflammatory diseases identifies 27 new associations and highlights disease-specific patterns at shared loci. Nat Genet advance online publication, (2016)

\section{P0371 SELF-MONITORING OF THE COLONIC INFLAMMATORY BOWEL DISEASE BY A RAPID HOME BASED FEACAL} CALPROTECTIN TEST AND A SYMPTOM QUESTIONNAIRE

A. Puolanne ${ }^{1}$, M.A. A. Färkkila ${ }^{1}$, K. Kolho ${ }^{2}$

${ }^{1}$ Clinic Of Gastroenterology, Helsinki University Hospital, Helsinki/Finland

${ }^{2}$ Pediatric Gastroenterology, University of Helsinki Childreńs Hospital, Helsinki/ Finland

Contact E-mail Address: anna-maija.puolanne@hus.fi

Introduction: Faecal calprotectin (FC) is a most reliable noninvasive means to distinguish remission from active inflammation in inflammatory bowel disease (IBD). Commercially available FC tests are time-consuming, and consequently new rapid tests have been validated. As the incidence of IBD is increasing, selfmonitoring and eHealth technologies have been evaluated in managing patients with this life-long disease.

Aims \& Methods: The aim of this prospective study was to evaluate the feasibility and cost-effectiveness of a semi-quantitative rapid FC home test and a validated symptom questionnaire, in patients with colonic IBD. The influence of the selfmonitoring to the course of the disease will also be evaluated. Between April 2015 and December 2016, 180 patients with colonic IBD (126 with UC, 47 with CD, and 7 with IBD unclassified) were included in the study and randomized in a study group and control group. Patients in the study group were instructed to perform the FC home test and fill in a symptom questionnaire every other month and with increasing of the symptoms, and sent the results to the study/IBD nurse by e-mail. The control group patients filled in the symptom questionnaire at baseline and at 12 months and with the appointment to the outpatient clinic according to normal practice. The patients were not reminded of performing the stool tests or filling in the questionnaires. The study period was 12 months, and it is still ongoing.

Results: By the end of February 2017, 134 of the 180 included patients had completed the 12 months' follow-up. In the study group, 20/91 (22\%) patients had performed the stool tests and filled in the symptom scores according to the study protocol for 6 months, and $14 / 91(15 \%)$ patients for 12 months. In the control group, $14 / 89(16 \%)$ patients had filled in the symptom score at baseline and at $12 \mathrm{kk}$. There was no significant difference of the adherence between patients stratified for IBD-diagnosis, age, or sex. The satisfaction of the patients with the program as well as the reasons for the discontinuation of the study and influence of self-monitoring in the number of relapses, phone calls, e-mails, and appointments to the outpatient clinic in both groups will be evaluated.

Conclusion: The self-monitoring of IBD activity with a rapid FC home test provides an option for individualized treatment for increasing amount of IBD patients. However, in this study the adherence to the self-monitoring program was low. The patients need to be reminded of performing the stool tests and filling in the questionnaires in time. Also, the selection and education of the patients, as well as the easy accessibility of the monitoring program are crucial and need further consideration.

Disclosure of Interest: All authors have declared no conflicts of interest.

\section{References}

Damms A, Bischoff SC. Validation and clinical significance of a new calprotectin rapid test for the diagnosis of gastrointestinal diseases. Int $J$ Colorectal Dis 2008;23:985-992.

Rogler G, Aldeguer X, Kruis W, et al. Concept for a rapid point-of-care calprotectin diagnostic test for diagnosis and disease activity monitoring in patients with inflammatory bowel disease: Expert clinical opinion. J Crohn's Colitis 2013;7:670-677.

Simrén M, Axelsson J, Gillberg R, et al. Quality of life in inflammatory bowel disease in remission: the impact of IBS-like symptoms and associated psychological factors. Am J Gastroenterol 2002;97:389-396.

\section{P0372 CLINICAL CHARACTERISTICS IN ULCERATIVE COLITIS PATIENTS WITH COLITIS ASSOCIATED DYSPLASIA/CANCER AND SPORADIC TUMOR}

M. Mutaguchi ${ }^{1}$, M. Naganuma ${ }^{1}$, Y. Iwao ${ }^{2}$, T. Fukuda ${ }^{1}$, S. Sugimoto ${ }^{1}$, K. Nanki ${ }^{1}$, S. Mizuno ${ }^{1}$, H. Ogata ${ }^{3}$, T. Kanai ${ }^{1}$

${ }^{1}$ Division Of Gastroenterology And Hepatology, Department Of Internal Medicine, Keio University, Tokyo/Japan

${ }^{2}$ Center For Preventive Medicine, Keio University School of Medicine, Tokyo Japan

${ }^{3}$ Center For Diagnostic And Therapeutic Endoscopy, Keio University School of Medicine, Tokyo/Japan

Contact E-mail Address: ma.mutaguchi@gmail.com

Introduction: Although the incidence of ulcerative colitis (UC)-related colorectal cancer (CAC) is increased in cases with long duration of disease, it should also be recognized that sporadic tumors (ST) develop as older. Various studies have been conducted on CAC, but there are few clinical studies on ST merged with UC. In 
this study, the clinical and endoscopic features of CAC and ST, treatment method, and prognosis are compared.

Aims \& Methods: Among 261 UC patients who underwent colonoscopy (CS) and had neoplastic lesions, the clinical features, treatment and prognosis were compared between 71 patients (88 lesions) with CAC (including HGD; CAC group) and 47 patients (63 lesions) who underwent local excision (surgical or endoscopic resection) within the presence of the past/present inflammation of UC (ST group). Definition of CAC and ST was performed by conventional pathological and immunohistochemical findings.

Results: The age of UC onset (29.8 vs. 39.0$)$ and tumor detection (45.5 vs. 57.3$)$ in the CAC group were significantly higher than those in ST group $(\mathrm{p}<0.01)$. The CAC group $(47.1 \%)$ has a higher percentage of chronic persistent type than the ST group $(2.3 \%)$, and the Mayo endoscopic score is also significantly higher $(\mathrm{p}<0.01)$ in the CAC group (1.43) than ST group (0.38). The percentage of advanced cancer $(35.2 \%$ vs. $7.9 \%)$ was higher in CAC group than ST group. In patients with intraepitherial neoplasia (IEN) or submucosal lesions, flat lesion was found in 15 lesions of CAC group and whereas no flat lesion was observed in ST group. Only one lesion in ST group could not distinguish the lesions from the surrounding mucosa without magnifying colonoscopy. In ST group who received resections, 4 patients after resections observed ectopic CAC or low-grade dysplasia during follow-up. In CAC group, 50, 5, 4 patients received total colectomy, local colectomy, ESD, respectively, whereas in ST group, 1, 7, 40, 15 patients received total colectomy, local colectomy, EMR and polypectomy, ESD, respectively. Although mortality from cancer was $11.4 \%$ (8/70 cases) in CAC group, group, no death due to cancer observed in patients whose lesions were found as IEN. On the other hand mortality from cancer was $2.1 \%$ (1/47 cases) in ST group

Conclusion: Most sporadic lesions were endoscopically distinct and local resection was safe if inflammation was controlled. After the sporadic lesions were resected in remitting UC patients, regular surveillance colonoscopy is necessary because $8.5 \%(4 / 47)$ of patients was found CAC/dysplasia. Even in CAC group, prognosis is well in patients with IEN.

Disclosure of Interest: All authors have declared no conflicts of interest.

\section{P0373 CAN WE PREDICT THE LACK OF RESPONSE TO CYCLOSPORINE AS SECOND LINE THERAPY IN PATIENTS WITH ACUTE SEVERE COLITIS REFRACTORY TO CORTICOSTEROIDS?}

H. Aya, B.S. Aida, H. Bassem, J. Hanen, E. Nour, B.M. Imed, A. Salem, B. Ahlem, K. Mehdi, J. Ali

Gastroenterology, University Hospital Sahloul, Sousse/Tunisia

Contact E-mail Address: aya_med@hotmail.fr

Introduction: Acute severe colitis (ASC) is a dangerous clinical condition that requires intensive intravenous (iv) corticoisteroids treatment. Nevertheless, about $30-40 \%$ of patients fail to response. Intravenous cyclosporine is an effective rescue therapy in steroid-refractory patients.

Aims \& Methods: The aim of our study was to identify the clinical and biological predictive factors of lack of response to cyclosporine as second-line therapy in patients with ASC recfractory to IV corticosteroids.

Results: Our study included 52 females and 38 males, with a mean age of 35 years [14-70 years]. There were 34 patients with Crohn's disease and 56 diagnosed with ulcerative colitis. Among the 90 patients enrolled, 68 patients $(75.5 \%)$ had a good reponse to cyclosporine. Eleven petients were non responders and underwent colectomy. In univariate analysis, more than 6 bloody stools per day before initiation of cyclosporine therapy, a C-Reactive Protein (CRP) greater than $45 \mathrm{mg} / \mathrm{l}$ prior to treatment, and at day 3 and 7 of treatment by ciclosporine $(\mathrm{p}=0.007 ; 0.002$ and 0.001 respectively), ESR greater than $30 \mathrm{~mm}$ at day 3 of treatment $(\mathrm{p}=0.05)$, thrombocytosis at day 3 of treatment $(\mathrm{p}=0.05)$, a Lichtiger colitis activity index scoring greater than 10 at day 3 of treatment $(\mathrm{p}=0.001)$ and the need for blood transfusion $(\mathrm{p}<0.0001)$ were significantly correlated with the lack of response to cyclosporine therapy. In a multiple linear regression analysis, only a CRP greater than $45 \mathrm{mg} / 1$ on day 7 of treatment, and the necessity of transfusion were predictive factors of no-response to cyclosporine $(\mathrm{p}=0.008)$. Conclusion: Cyclosporine therapy is rapidly effective in preventing surgery in patients with ASC with a response rate of $75.5 \%$. A high CRP on day 7 of treatment with cyclosporine and the need for transfusion, predispose to poor reponse to intravenous cyclosporine.

Disclosure of Interest: All authors have declared no conflicts of interest.

\section{P0374 CHANGES IN THERAPEUTIC STRATEGY AND OUTCOMES IN NEWLY DIAGNOSED PATIENT WITH CROHN'S DISEASE IN THE BIOLOGICAL ERA IN HUNGARY: A NATIONWIDE STUDY BASED ON THE NATIONAL HEALTH INSURANCE FUND DATABASE}

Z. Kurti ${ }^{1}$, L. Gonczi ${ }^{1}$, Z. Vegh ${ }^{1}$, P.A. Golovics ${ }^{1}$, P. Fadgyas-Freyler ${ }^{2}$, J. GimesiOrszagh $^{2}$, G. Korponai ${ }^{2}$, B.D. Lovasz ${ }^{1}$, K.B. Gecse ${ }^{3}$, P.L. Lakatos ${ }^{1}$

${ }^{1} 1$ st Department Of Medicine, Semmelweis University Faculty of Medicine 1st Dept. of Medicine - 1st Department of Medicine, Semmel, Budapest/Hungary ${ }^{2}$ Strategic Analysis Department, National Health Insurance Fund (OEP), Budapest/Hungary

${ }^{3} 1$ st Department Of Medicine, Semmelweis University, Budapest/Hungary

Contact E-mail Address: zsuzsa.kurti@gmail.com
Introduction: Accelerated treatment strategy, including tight disease control and early aggressive therapy with immunomodulators (IM) and biological agents have become increasingly common in IBD.

Aims \& Methods: The aim of the present study was to estimate the early treatment strategy and outcomes in newly diagnosed patients with Crohn's disease (CD) diagnosed between 2004-2015 in Hungary based on the administrative database of the National Health Insurance Fund (OEP). We used the administrative database of the National Health Insurance Fund (OEP), the only nationwide state-owned health insurance provider in Hungary. Newly diagnosed CD patients were identified through previously reported algorithms using the ICD-10 codes for Crohn's disease in the out-, inpatient (medical, surgical) non-primary care records and drug prescription databases between 2004-2015. Patients were stratified according to the year of diagnosis and maximum treatment step during the first 3-years after the diagnosis.

Results: A total of 6173 (male/female: $46.12 \% / 53.87 \%$ ) newly diagnosed CD patients were identified during the observational period. Maximum treatment steps did not differ in patients diagnosed before and after 2009 (5-ASA: 11.7\% vs, $13.5 \%$, steroid $31 \%$ vs. $30.5 \%$, IM $40.4 \%$ vs. $40.2 \%$, biologicals $16.5 \%$ vs. $15.6 \%)$. Probability of hospitalizations during the first 3 -years from diagnosis was lower according to the maximal treatment step in patients diagnosed after 2009 (at $36 \times 30$-day period: overall $55.7 \%$ vs. $47.4 \%(\mathrm{p}=0.000)$, anti-TNF: $73 \%$ vs. $66.7 \%(\mathrm{p}=0.103)$, IS: $64.6 \%$ vs. $56.1 \%(\mathrm{p}=0.000)$, steroid: $44.2 \%$ vs. $36.8 \%$ $(\mathrm{p}<0.007), 5$-ASA: $32.6 \%$ vs. $26.7 \% \mathrm{p}=0.157)$ ), respectively. In contrast, surgery rates were not different according to the maximum treatment step (at $36 \times 30$-day period: overall $16.0 \%$ vs. $15.3 \%(\mathrm{p}=0.672)$ anti-TNF $26.7 \%$ vs. $27.2 \% \quad(p=0.993)$, IS: $24.1 \%$ vs $22.2 \% \quad(p=0.565)$, steroid $8.1 \%$ vs. $7.9 \%$ $(\mathrm{p}=0.896), 5$-ASA $10 \%$ vs. $11 \%(\mathrm{p}=0.816))$

Conclusion: Distribution of maximal treatment steps and surgery rates was not different in patients diagnosed before and after 2009, although immunosuppressive and was commenced earlier. Of note, steroid and 5-ASA remained high after 2009. Maximal treatment steps were associated to hospitalization and surgery rates, suggesting that maximal treatment steps can be used as proxy marker of severity in CD. Hospitalization rates during the first 3-years after the diagnosis decreased in all treatment groups, suggesting a change in the patient management.

Disclosure of Interest: All authors have declared no conflicts of interest.

\section{P0375 RELATIVE FREQUENCY OF RELAPSES IN PATIENTS WITH ULCERATIVE COLITIS AND CROHN'S DISEASE TREATED WITH MESENCHYMAL STROMAL CELLS - 5 YEARS OF FOLLOW-UP}

O. Knyazev ${ }^{1}$, A. Kagramanova ${ }^{1}$, N. Fadeeva $^{1}$, A. Lishchinskaya ${ }^{1}$, I. Ruchkina ${ }^{2}$, A. Konoplyannikov ${ }^{3}$, A. Parfenov ${ }^{1}$

${ }_{1}^{1}$ Ibd, Moscow Clinical Research Center, Moscow/Russian Federation

${ }^{2}$ State Scientific Center of Gastroenterology, Moscow/Russian Federation

${ }^{3}$ Medical Radiological Research Cente, Obninsk/Russian Federation

\section{Contact E-mail Address: chuevana@mail.ru}

Introduction: Numerous studies have shown that mesenchymal stromal cells (MSCs) have a high potential for differentiation and immunosuppressive properties. Currently under phase I-III clinical trials evaluating the efficacy and safety of MSCs in the treatment of patients with inflammatory bowel disease - ulcerative colitis and Crohn's disease.

Aims \& Methods: We aimed to compare the frequency of relapses and duration of remission for 5 years of follow up in patients with luminal Crohn's disease (CD) and the total defeat of ulcerative colitis (UC) receiving therapy with mesenchymal stromal cells (MSCs), bone marrow. We compared the frequency of relapses in patients with luminal form CD (colitis and ileokolit), with a group of patients with UC (total lesion) receiving MSCs. A group of patients (CD) aged 22 to 56 years $(\mathrm{Me}-28)(\mathrm{n}=24)$ received MSC culture scheme $(0-1-2$ weeks, then every 26 weeks). The second group of patients with UC $(n=26)$ aged 20 to 62 years $(\mathrm{Me}-$ 28) received the culture of MSCs in a similar way. Evaluation of the effectiveness of therapy for relapse frequency was carried out at 12, 24, 36, 48 and 60 months after initiation of therapy.

Results: Among the patients in 1st group relapse in the 12 months of observation occurred in $2 / 24$ patients $(8.3 \%)$ in 2 nd group, relapse occurred in $3 / 26(11.5 \%)$ (OR-0.72; 95\% CI $0,13-3,96, \mathrm{p}=0.92)$. After 24 months in the group of patients (group 1) receiving MSC, relapse occurred in 5/24 $(20.8 \%)$ in group 2 patients with recurrent disease in $7 / 26(26.9 \%)(\mathrm{OR}-0.77 ; 95 \% \mathrm{CI} 0.13-3.96, \mathrm{p}=0.92)$. After 36 months in group 1 patients with a relapse of the disease in $8 / 24(33.3 \%)$ in group 2 relapsed in 14/26 (53.8\%) (OR-0.62; 95\% CI $0.32-1.21 ; \mathrm{p}=0.24)$. After 48 months in group 1 receiving MSC, relapsed in 11/24 $(45.8 \%)$ in group 2 relapsed in $18 / 26(69.2 \%$ ) (OR-0.6; 95\% CI $0.37-0.97, \mathrm{p}=0.048$ ). After 60 months in 1st relapse in $16 / 24(66.6 \%)$ in group 2 relapsed in $22 / 26$ $(84.6 \%)(\mathrm{OR}-0.63 ; 95 \%$ CI $0.44-090, \mathrm{p}=0.013)$.

Conclusion: MSCs transplantation longer contributes to clinical remission in patients with Crohn's disease luminal shape compared to a group of patients suffering from ulcerative colitis.

Disclosure of Interest: All authors have declared no conflicts of interest.

\section{P0376 CELL THERAPY FOR PERIANAL CROHN'S DISEASE}

O. Knyazev ${ }^{1}$, A. Kagramanova ${ }^{1}$, N. Fadeeva ${ }^{1}$, A. Lishchinskaya ${ }^{1}$, N. Belyakov ${ }^{1}$, A. Konoplyannikov ${ }^{2}$, A. Parfenov

${ }^{1}$ Ibd, Moscow Clinical Research Center, Moscow/Russian Federation

${ }^{2}$ Medical Radiological Research Cente, Obninsk/Russian Federation 
Contact E-mail Address: belyakov.n.i@gmail.com

Introduction: Perianal fistulas are the most widespread and common types of fistulas in Crohn's disease (CD). They are difficult to treat, worsen the quality of life of the patient and increase the risk of bowel resection. Despite the significant effect of anti-cytokine therapy fistulous forms of CD, treatment of these patients remains a difficult task with high risk of relapse of $\mathrm{CD}$. Mesenchyma stromal cells have immunomodulatory properties and a large regenerative potential, at present also used for treatment of fistulous CD and perianal fistulas of different etiologies.

Aims \& Methods: We aimed compare the efficacy of combined therapy (local and systemic) mesenchymal stromal cells (MSCs) of bone marrow, infliximab (IFX) and antibiotics/immunosupression (IS) on the rate of healing of simple perianal fistulas in Crohn's disease. 36 patients with Crohn's disease with perianal lesions were divided into three groups depending on the method of therapy. The first group of patients aged from 19 to 58 years $(M e-29)(n=12)$ received culture of MSCS systemically via the scheme and locally: on the perimeter of the fistulous introduced 40 million MSCs - 4 point of inject and $1 \mathrm{ml}$ of saline containing 10 million MSCs. Then after 4 and 8 weeks re-injected 40 million MSCs in the area of the fistula. The second group of patients with $C D(n=10)$ aged 20 to 68 years (Me-36) were receiving anti-cytokine therapy of IFX. The 3-rd group of patients with $C D(n=14)$ aged 20 to 62 years $(M e-28)$ received antibiotics and is. In the dynamics evaluated the closure of the external opening of the fistula. Ano- and rectosigmoscopy carried out after 3, 6, 12 and 36 months from start of therapy. Results: After 12 weeks among patients of the 1st group simple healing of fistulas was observed in $10 / 12$ patients $(83.3 \%)$, in the 2 nd group healing simple fistulas have a $8 / 10(80.0 \%)(\mathrm{OR}-0.83 ; 95 \%$ CI $0.14-4.9 ; \mathrm{p}=0.72)$. In the 3 rd group - in $5 / 14$ patients $(35.7 \%)(\mathrm{OR} 0.26 ; 95 \%$ CI $0.07-0.97 ; \mathrm{p}=0.04$ in comparison with the 1st group). After 6 months in the 1st group of patients receiving MSCs, healing of simple fistulas persisted in $8 / 12(66.6 \%)$ with the 2 nd group $-7 / 10$ $(70.0 \%)(\mathrm{OR}-1.11 ; 95 \%$ CI $0.32-3.84 ; \mathrm{p}=0.76)$. In the $3 \mathrm{rd}$ group - patients $4 / 14$ $(28.6 \%)(\mathrm{OR}-0.47 ; 95 \%$ CI $0.2-1.11 ; \mathrm{p}=0.12$ in comparison with the 1 -st group). After 12 months in the 1st group receiving MSCs, healing of simple fistulas persisted in $7 / 12(58.3 \%)$, in the 2 nd group - in $6 / 10(60.0 \%)(\mathrm{OR}$ $1.25,95 \%$ CI $0.48-3.22 ; \mathrm{p}=0.69)$. In the 3 rd group - in $2 / 14$ patients $(14.3 \%)$ (OR- $0.49 ; 95 \%$ CI 0.24 to about $0.98 ; \mathrm{p}=0.03$ in comparison with the 1 st group). After 36 months among the patients of the 1st group, the closure of the fistula was preserved in $5 / 12$ patients $(41.6 \%)$, in the 2 nd group $-5 / 10(50.0 \%)(\mathrm{OR}$ $-1.17 ; 95 \%$ CI $0.53-2.55 ; \mathrm{p}=0.96)$. In the $3 \mathrm{rd}$ group - in $0 / 14$ patients $(0.0 \%)$ of (OR - 0.58; 95\% CI $0.36-0.94 ; \mathrm{p}=0.01$ in comparison with the 1 st group).

Conclusion: Combined stem cell and anti-cytokine therapy of CD with perianal lesions significantly contributes to more frequent and prolonged closure of simple fistula, compared with antibiotics/immunosuppressant.

Disclosure of Interest: All authors have declared no conflicts of interest.

P0377 DYNAMICS OF PROINFLAMMATORY CYTOKINES IN PATIENTS WITH CROHN'S DISEASE TREATED WITH MESENCHYMAL STROMAL CELLS OF BONE MARROW AND AZATHIOPRINE

O. Knyazev ${ }^{1}$, N. Fadeeva ${ }^{1}$, K. Noskova ${ }^{2}$, A. Kagramanova ${ }^{1}$, A. Konoplyannikov ${ }^{3}$, R. Gudkova ${ }^{4}$, E. Dobrolyubova ${ }^{1}$, A. Parfenov ${ }^{1}$ IBD, Moscow Clinical Research Center, Moscow/Russian Federation

${ }^{2}$ Department Of Labaratory, Moscow Clinical Research Center, Moscow/Russian Federation

${ }^{3}$ Medical Radiological Research Cente, Obninsk/Russian Federation

${ }^{4}$ Immunology, Moscow Clinical Research Center, Moscow/Russian Federation

Contact E-mail Address: chuevana@mail.ru

Introduction: Mesenchymal stromal cells (MSC) are used for the treatment of chronic inflammatory and autoimmune diseases in recent years, including rheumatoid arthritis (RA) and inflammatory bowel disease (IBD). In most cases, together with the MSC, patients receiving concomitant immunosuppressive therapy. It is found that immunomodulatory drugs (azathioprine (AZA), methotrexate, 6-mercaptopurine, infliximab (IPF)), regardless of the concentration, do not affect the viability, differentiation, phenotype, and ability to inhibit proliferation of MSCs peripheral blood mononuclear cells [1]. However, studies conducted by Huang HR et al. demonstrate that IPF rendered minimal impact on the MSC proliferation, apoptosis and cell cycle, while, azathioprine inhibited cell proliferation and induced apoptosis of MSCs in vitro [2]

Aims \& Methods: We aimed to evaluate the effectiveness of therapy mesenchymal stromal cells (MSCs) from the bone marrow of patients with Crohn's disease (CD) receiving azathioprine. 34 patients with inflammatory (luminal) form CD were divided into two groups. The first group of patients aged 19 to 58 years old $(\mathrm{Me}-29)(\mathrm{n}=15)$ was treated with anti-inflammatory therapy with MSCs culture in combination with AZA. The second group of patients with CD $(n=19)$ aged 23 to 60 years old (Me-31) received MSCs according to the recommended scheme without AZA. To assess the effectiveness of anti-inflammatory therapy was determined by the dynamics of the level of pro-inflammatory interleukins (IL) - TNF- $\alpha$, IFN- $\gamma$ and IL-1 $\beta$ at 2, 6 and 12 months from the start of MSC therapy. Initial level of IFN- $\gamma$ in the 1st group was $110.4 \pm 12.5 \mathrm{pg} / \mathrm{ml}$, in the 2 nd $450.8 \pm 22.4 \mathrm{pg} / \mathrm{ml}(\mathrm{p}<0.05), \mathrm{TNF}-\alpha$ in 1 st group $-13.9 \pm 1.9 \mathrm{pg} / \mathrm{ml}$, in the 2nd $-66.7 \pm 14.2 \mathrm{pg} / \mathrm{ml}(\mathrm{p}<0.05)$, IL- $1 \beta$ in 1 st group $-5.7 \pm 0.28 \mathrm{pg} / \mathrm{ml}$, in the 2nd $-9.2 \pm 0.2 \mathrm{pg} / \mathrm{ml}(\mathrm{p}<0.05)$.

Results: After 2 months of therapy MSCs level of IFN- $\gamma$ in 1st group was significantly decreased from baseline and was $86.5 \pm 9.1 \mathrm{pg} / \mathrm{ml}$, the $2 \mathrm{nd}$ was $96.9 \pm 12.1 \mathrm{pg} / \mathrm{ml}$ (between groups $\mathrm{p}=0.5$ ), TNF- $\alpha$ in 1 st group decreased to $39.6 \pm 8.4 \mathrm{pg} / \mathrm{ml}$, in the $2 \mathrm{nd}-56.5 \pm 10.7 \mathrm{pg} / \mathrm{ml}$ (between groups $\mathrm{p}=0.2$ ), IL-1 in 1 st group $-50.7 \pm 9.3 \mathrm{pg} / \mathrm{ml}$, in the $2 \mathrm{nd}-56.2 \pm 10.2 \mathrm{pg} / \mathrm{ml}$ (between groups $\mathrm{p}=0.7)$. After 6 months of therapy MSCs level of IFN- $\gamma$ in 1st group decreased and amounted to $79.4 \pm 8.5 \mathrm{pg} / \mathrm{ml}$, in the $2 \mathrm{nd}-80.8 \pm 7.3 \mathrm{pg} / \mathrm{ml}$ (between groups $\mathrm{p}=0.9)$, TNF $-\alpha$ in 1 st group decreased to $44.9 \pm 6.3 \mathrm{pg} / \mathrm{ml}$, in the $2 \mathrm{nd}$ $49.7 \pm 10.4 \mathrm{pg} / \mathrm{ml}$ (between groups $\mathrm{p}=0.7$ ). IL- $1 \beta$ in 1 st group $-45.6 \pm 7.3 \mathrm{pg} /$ $\mathrm{ml}$, in the $2 \mathrm{nd}-54.2 \pm 9.2 \mathrm{pg} / \mathrm{ml}$ (between groups $\mathrm{p}=0.45$ ). After 12 months of initiation of therapy MSCs level of IFN- $\gamma$ in 1st group decreased and amounted to $90.8 \pm 6.5 \mathrm{pg} / \mathrm{ml}$, in the $2 \mathrm{nd}-128.8 \pm 12.3 \mathrm{pg} / \mathrm{ml}$ (between groups $\mathrm{p}=0.02$ ), TNF- $\alpha$ in the 1 st group $-78.9 \pm 10.5 \mathrm{pg} / \mathrm{ml}$, in the $2 \mathrm{nd}-116.9 \pm 13.2 \mathrm{pg} / \mathrm{ml}$ (between groups $\mathrm{p}=0.04$ ), IL- $1 \beta$ in 1 st group $-68.7 \pm 8.9 \mathrm{pg} / \mathrm{ml}$, in the $2 \mathrm{nd}-$ $96.9 \pm 9.6 \mathrm{pg} / \mathrm{ml}$ (between groups $\mathrm{p}=0.03$ )

Conclusion: After one year of observation concomitant treatment with AZA when administered MSCs significantly reduces the level of pro-inflammatory cytokines, which could have a more pronounced anti-inflammatory therapeutic effect.

Disclosure of Interest: All authors have declared no conflicts of interest.

\section{References}

1. Duijvestein M, Molendijk I, Roelofs H, et al. Mesenchymal stromal cell function is not affected by drugs used in the treatment of inflammatory bowel disease. Cytotherapy. 2011;13:1066-1073.

2. Huang HR, Zan H, Lin Y, Zhong YQ Effects of azathioprine and infliximab on mesenchymal stem cells derived from the bone marrow of rats in vitro. Mol Med Rep. 2014 Mar;9(3):1005-12. doi: 10.3892/mmr.2014.1905. Epub 2014 Jan 17.

\section{P0378 EFFICACY AND SAFETY OF RECTAL 5-AMINOSALICYLIC ACID VERSUS CORTICOSTEROIDS IN ACTIVE DISTAL ULCERATIVE COLITIS: A SYSTEMATIC REVIEW AND NETWORK META-ANALYSIS}

\section{Zhao, H. Zhang}

Department Of Gastroenterology, Jiangsu Province Hospital and Nanjing Medical University, Nanjing/China

Contact E-mail Address: zhaoxj91718@163.com

Introduction: Ulcerative colitis (UC) is characterized by diffuse and continuous inflammation of the colon. Currently, the etiology and pathogenesis remain unclear. According to a previous epidemiological study, approximately $75 \%$ of newly diagnosed UC patients have active distal UC. Topical 5-aminosalicylic acid (5-ASA) and corticosteroids are used frequently in the treatment of active distal UC.

Aims \& Methods: Our study aimed to determine the efficacy and safety of different topical drugs used to treat active distal UC. A random-effects model within a Bayesian framework was utilized to compare treatment effects and safety as odds ratios (ORs) with corresponding 95\% credible intervals $(\mathrm{CrI})$. The surface under the cumulative ranking area (SUCRA) and median rank (MR) with corresponding 95\% CrI were calculated to rank the treatment outcomes.

Results: In the induction of clinical and endoscopic remission, most regimens showed significant advantages over placebo except topical budesonide $0.5 \mathrm{mg} / \mathrm{d}$ and hydrocortisone $100 \mathrm{mg} / \mathrm{d}$. According to SUCRA and MR values, rectal 5 ASA 1.5 to $2.0 \mathrm{~g} / \mathrm{d}$ + Beclomethasone dipropionate (BDP) $3 \mathrm{mg} / \mathrm{d}$ rendered the highest probability of being the best regimen to achieve clinical and endoscopic remission, followed by the separate use of 5-ASA $4 \mathrm{~g} / \mathrm{d}$ and BDP $3 \mathrm{mg} / \mathrm{d}$. The occurrence of adverse events was not significantly different between each treatments and placebo.

Conclusion: In conclusion, the combined use of topical 5-ASA and BDP proved to be the best choice for active distal UC and further well-designed researchers are warranted to assess its efficacy and safety.

Disclosure of Interest: All authors have declared no conflicts of interest.

\section{References}

1. Regueiro, M.D. Diagnosis and treatment of ulcerative proctitis. $J$ ClinGastroenterol 38, 733-40 (2004).

2. Ordás, I., Eckmann, L., Talamini, M., Baumgart, D.C., Sandborn, W.J. Ulcerative colitis. Lancet 380, 1606-19 (2012).

3. Ayres, R.C., Gillen, C.D., Walmsley, R.S., Allan, R.N. Progression of ulcerative proctosigmoiditis: incidence and factors influencing progression. Eur J Gastroenterol Hepatol 8, 555-8 (1996).

4. Campieri, M. et al. Optimum dosage of 5-aminosalicylic acid as rectal enemas in patients with active ulcerative colitis. Gut 32, 929-31 (1991).

5. Kornbluth, A. Sachar, D.B. Practice Parameters Committee of the American College of Gastroenterology. Ulcerative colitis practice guidelines in adults: American College Of Gastroenterology, Practice Parameters Committee. Am J Gastroenterol 105:501-23 (2010).

6. Cohen, R.D. Woseth, D.M., Thisted, R.A., Hanauer, S.B.A. Meta-analysis and overview of the literature on treatment options for left-sided ulcerative colitis and ulcerativeproctitis. Am J Gastroenterol 95, 1263-76 (2000). 
P0379 A PROTEASE-STABILISED ORAL DOMAIN ANTIBODY TO

TNF DELIVERS HIGH CONCENTRATIONS OF ACTIVE COMPOUND IN ILEAL FLUID OF SUBJECTS WITH AN ILEOSTOMY

J. Robinson ${ }^{1}$, S. Crowe ${ }^{1}$, G. Whale ${ }^{1}$, K. Roberts ${ }^{1}$, M. West ${ }^{1}$, J. Ritter ${ }^{2}$, P. Irving ${ }^{3}$, S. Nurbhai ${ }^{1}$

${ }^{1}$ VHsquared Ltd, Cambridge/United Kingdom

${ }^{2}$ Kings College London, London/United Kingdom

${ }^{3}$ Guýs and St Thomaś Hospital Dept. of Gastroenterology - Guýs and St Thomaś

Hospital Dept. of Gas, London/United Kingdom

Contact E-mail Address: suhail.nurbhai@vhsquared.com

Introduction: The oral delivery of therapeutic concentrations of anti-TNF to affected mucosa of patients with inflammatory bowel disease (IBD) has remained challenging despite advances in protein engineering, the attractions of oral dosing for chronic therapies, and the acknowledged benefit of anti-TNF monoclonal antibodies in the management of IBD. As the ileum is commonly involved in Crohn's disease (CD), it is important to deliver drug there if treatment is to be effective. This is the first report of a domain antibody to TNF, V565, engineered to be resistant to intestinal proteases, delivering high concentrations of active compound in the ileal fluid following oral administration to human volunteers. The oral domain antibodies (Vorabodies) are delivered via enteric coated minitablets (MTs) designed to release active drug at $\mathrm{pH} 6.5$.

Aims \& Methods: Following prior placebo-controlled demonstration of the safety and tolerability of high single and multiple doses of V565, this open label assessment was performed to confirm the delivery of active domain antibody to the terminal ileum of human subjects. Four subjects with a terminal ileostomy were given a single oral dose of V565 and ileostomy bags were collected hourly for the first 12 hours post dose with further collections 16, 20 and $24 \mathrm{~h}$ post dose. Contents were analysed for V565 concentrations by competitive ELISA. In addition serial blood samples were taken for determination of V565 serum concentrations over $24 \mathrm{~h}$.

Results: Four subjects with an ileostomy ( 3 with UC; 1 with a prior history of colonic obstruction) were given a single $1665 \mathrm{mg}$ dose of V565. The dose was selected based on the prior demonstration of safety and tolerability of this dose and the likely maximum daily dose for initial clinical efficacy assessment. High concentrations of active V565 were demonstrated in the ileal fluid of all four subjects as shown in Table 1 below.

\begin{tabular}{lllllllll}
\hline & \multicolumn{7}{l}{ Micromolar concentration of V565 in ileal fluid } \\
\cline { 2 - 9 } Subject & 1 & 2 & 3 & 4 & 5 & 6 & $7-16$ & $17-24$ \\
\cline { 2 - 9 } 31001 & - & 406 & 306 & 0.8 & 0 & 0 & 0 & 0 \\
31002 & - & 33 & 1130 & 792 & 82 & 13 & 5 (ave) & 0 \\
31003 & - & - & 1060 & 496 & 7 & 0 & 38 (ave) & 0 \\
31004 & - & 126 & 0.2 & 11 & 4 & 7 & 0 & 0 \\
\hline
\end{tabular}

In addition to the V565 concentrations in ileal fluid, partially dissolved MTs were recovered from the ileostomy bags of all subjects. Each $1665 \mathrm{mg}$ dose contained a total of 135 MTs. 50 MTs were recovered $2 \mathrm{~h}$ post dose from Subject 31001; these were not analysed for V565 as this was a post hoc analysis and the MTs were not stored in a way to enable reliable analysis. 64MTs (containing $135 \mathrm{mg}$ V565) were recovered $3 \mathrm{~h}$ post dose from subject $31002 ; 78 \mathrm{MTs}(458 \mathrm{mg}) 3 \mathrm{~h}$ post dose from subject 31003 ; and $125 \mathrm{MTs}(1260 \mathrm{mg}) 2 \mathrm{~h}$ post dose from subject 31004 . Overall, $66-82 \%$ of an administered dose was recovered from ileostomy bags when MT quantities were added to ileal fluid concentrations. V565 was not detected in any serum sample (LLoQ $62.5 \mathrm{ng} / \mathrm{ml}$ ).

Conclusion: V565, an oral domain antibody (Vorabody) to TNF engineered to be resistant to intestinal proteases, resulted in high concentrations of active drug in ileal fluid and was not detected in serum following oral administration. In patients with no ileostomy the partially dissolved MTs seen in this study are expected to provide active V565 to lesions distal to the ileum. This profile may be beneficial for IBD and merits further investigation as a potential oral treatment.

Disclosure of Interest: J. Robinson: J Robinson is an employee of the Sponsor company

S. Crowe: S Crowe is an employee of the Sponsor company

G. Whale: G Whale is an employee of the Sponsor company

$\mathrm{K}$. Roberts: K Roberts is an employee of the Sponsor company

M. West: M West is an employee of the Sponsor company

J. Ritter: J Ritter was a salaried employee of Quintiles at the time of the study; he has no other significant relationships.

P. Irving: Lecture: AbbVie, Warner Chilcott, Ferring, Falk, Takeda, MSD, Janssen, Shire Research Support: MSD, Takeda Advisor: AbbVie, Warner Chilcott, Takeda, MSD, Vifor, Pharmacosmos, Topivert, Genentech, Hospira, Samsung Bioepis, VH2, Janssen, Pfizer

S. Nurbhai: S Nurbhai is an employee of the Sponsor company
P0380 ULCERATIVE COLITIS (UC) WITH IBS - LIKE DISORDERS: PARTICULAR QUALITIES OF CLINICAL MANIFESTATIONS AND MEDICAL THERAPY

E. Dobrolyubova ${ }^{1}$, I. Ruchkina ${ }^{2}$, A. Parfenov ${ }^{3}$, O. Knyazev

${ }^{1}$ Department Of Health Of Moscow, Moscow Clinical Scientific - Practical Center, Central Scientific - Research Institute of Gastroente, Moscow/Russian Federation ${ }^{2}$ State Scientific Center of Gastroenterology, Moscow/Russian Federation

${ }^{3}$ Ibd, Moscow Clinical Research Center, Moscow/Russian Federation

Contact E-mail Address: dobroljubova.ekaterina@rambler.ru

Introduction: In recent years publications of different countries paid great attention to the symptoms, that specific for the functional bowel disease in patients with UC in remission. Our aim was to determine the incidence of IBS - like disorders in UC, especially their clinical manifestations and medical therapy. Aims \& Methods: The study included 162 patients with UC a year after the last attack of the disease, that were on maintenance therapy with 5-ASA dosage of 2 gram per day for the last 12 months. Study group was dominated by women - 94 patients $(58 \%)$, average the age of the subjects was $38 \pm 3$ years. Localization of the UC in $83 \%$ of cases revealed left-sided and $17 \%$ - subtotal lesion of the colon. All patients underwent a standard examination of the definition of the level of $\mathrm{C}$ reactive protein (CRP), and faecal calprotectin (FCP), and exclude the acute intestinal clostridial infection, faecal microbiota was determined. The clinical activity of the general condition of the patient and endoscopic subscale were evaluated using the Mayo score. Quality of life of patients was calculated on a Gastrointestinal Simptom Rating Scale (GSRS), where the minimum score was 15 and the maximum 105. Evaluation of the severity of IBS symptoms was calculated using a visual - analogue scale (VAS), where 0 was the absence of clinical manifestations IBS, and 10 - the maximum clinical manifestations. Results: Of the 162 explored patients in 122 cases (77.8\%) clinical and endoscopic remission in UC was determined: a total Mayo score $<2$, physician' $s$ global assessment $=0$, rectal bleeding $=0$, endoscopic subscale $=0$, the normal structure of the colon mucosa by histological study of biopsies, CRP $<5, \mathrm{FCP} \leq 50 \mathrm{mg} / \mathrm{g}$, the amount of points on the scale GSRS $=15$, VAS $=0$. In $36(22.2 \%)$ patients intestinal symptoms were determined: mushy stools $2-4$ times per day at daytime, bloating and rumbling in the abdomen. In the clinical picture of 5 patients of these group $(3.1 \%)$ there dominated loose stools 3-4 times a day, sometimes streaked with blood. The examination revealed the exacerbation of UC, leftsided lesion, moderate activity (a total Mayo score $=5$, physiciań s global assessment $=1$, rectal bleeding $=1$, endoscopic subscale $=2$, intense lymphoplasmacytic infiltration on histological studies of colon mucosa biopsies, $\mathrm{CRP}=11 \pm 0.9 \mathrm{mg} / 1, \mathrm{FCP}=250 \pm 40 \mathrm{mg} / \mathrm{g}$, quality of life GSRS lowered to $35 \pm 5$ points. For these patients the dose of mesalazine was increased to $3 \mathrm{~g}$ per day and rectal forms of mesalazine were added. In $31(19.1 \%)$ patients in the clinical picture pain syndrome was dominated (daily abdominal pain, worse before stool, severity of pain according to VAS $=7 \pm 1$ points), abdominal distension (VAS $=6 \pm 1$ points), rumbling, frequency stool reached 2-4 times per day, sometimes with mucus, but without blood. The examination confirmed the remission of UC (total Mayo score $\leq 2$, physiciań s global assessment $=0$, rectal bleeding $=0$, endoscopic subscale $=0$, the minimum colitis histological study biopsies of colon mucosa, CRP $=2.5 \pm 2.5 \mathrm{mg} / 1, \mathrm{FCP}=125.0 \pm 25.0 \mathrm{mg} / \mathrm{g}$, the level of quality of life was reduced by GSRS to $75 \pm 5$ points. In this group of patients showed changes in faecal microbiota: reducing the number of bifidobacilles $<107$ CFU (colony-forming unit) and lactobacilles $<105$ CFU per 1 g. of feces, the growth performance of opportunistic microbiota: Klebsiella $>104$ CFU per $1 \mathrm{~g}$. of feces, Proteus $>104 \mathrm{CFU}$ per $1 \mathrm{~g}$. of feces, Citrobacter $>104$ $\mathrm{CFU}$ per $1 \mathrm{~g}$. of feces, Coccious microflora, Escherichia coli hemolitica $>0 \mathrm{CFU}$ per $1 \mathrm{~g}$. of feces. Attempt to increase the dosage of 5-ASA and accession to treatment the rectal forms of mesalazine in this group of patients have not led to the disappearance of the existing symptoms. This group of patients was appointed by pathogenetic therapy of functional bowel disease: myotropic antispasmodics (mebeverin $405 \mathrm{mg} /$ day), a course of rifaximin $(1650 \mathrm{mg} /$ day) and combination probiotics on the background maintenance therapy mesalazine $2 \mathrm{~g} /$ day. As a result of therapy regression of IBS - like symptoms was reached: severity of pain according to $\mathrm{VAS}=0$, bloating $\mathrm{VAS}=0$, stool frequency was 1 time per day without mucus, improved quality of life by GSRS to 15 points.

Conclusion: In $19.1 \%$ of the studied patients with UC in remission IBS-like disorders was found. In the clinical picture pain sindrome, bloating, rumbling, moderate diarrhea syndrome with mucus, a sharp decline in quality of life level GSRS were determined. The development of clinical manifestations of IBS in the target group of patients with UC is associated with persistent changes in faecal microbiota. Remission of UC with IBS-like disorders reached by the appointment of pathogenetic therapy of functional bowel disease on the background of mesalazine maintenance therapy.

Disclosure of Interest: All authors have declared no conflicts of interest.

\section{P0381 A PROSPECTIVE RANDOMISED STUDY:} ELECTROACUPUNCTURE VS. SHAM PROCEDURE FOR THE TREATMENT OF FATIGUE IN PATIENTS WITH QUIESCENT INFLAMMATORY BOWEL DISEASE

D. Horta ${ }^{1}$, M. Sanchez-Lloansi ${ }^{1}$, A. Lira ${ }^{1}$, A. Villoria ${ }^{1}$, M. Teggiachi ${ }^{1}$,

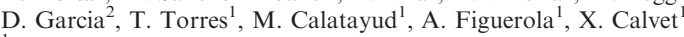

${ }^{1}$ Gastroenterology, Hopsital Universitari Parc Tauli, Sabadell/Spain

${ }^{2}$ Urology, Hopsital Universitari Parc Tauli, Sabadell/Spain

Contact E-mail Address: diana.horta.s@gmail.com

Introduction: Fatigue is a common symptom in inflammatory bowel disease (IBD) and persists despite clinical remission. Acupuncture has been shown to be effective for treating fatigue in many chronic diseases. Fatigue can be 
objectively evaluated by the IBD validated Functional Assessment of Chronic Illness Therapy-Fatigue Scale (FACIT-F).

Aims \& Methods: The main objective was to assess the efficacy of electroacupuncture (EAc) vs. sham EAc and no treatment for treating fatigue in patients with quiescent IBD in a single-blind randomized trial. Secondary objectives were to assess changes in quality of life, depression, anxiety and sleepiness after treatment with EAc.

Methods: Fifty-two patients with quiescent IBD and severe fatigue (FACIT$\mathrm{F}<40)(65.3 \%$ female, mean age 42 years $)$ were randomized to EAc vs sham acupuncture vs control group. Patients in EAC and sham EAC groups performed a total of 9 acupuncture sessions during eight weeks ( 2 sessions/first week and one session per week during 7 weeks). Fatigue was evaluated with IBD validated Functional Assessment of Chronic Illness Therapy-Fatigue Scale (FACIT-F). Patients also completed validated questionnaires to assess quality of life in IBD, depression, anxiety and sleepiness during and after the treatment periods. Results: Both EAc and Sham group improved the FACIT-F score post-treatment (EAP -9.53 points, 95\% CI $\left(-12.3\right.$ to -6.75 , Basal Vs $9^{\text {th }}$ session $\left.\mathrm{p}<0.001\right)$; Sham -5.46 points, $95 \%$ CI $\left(-9.7\right.$ to -2.7 , Basal Vs $9^{\text {th }}$ session $\left.p=0.003\right)$. No significant changes were observed in control group $(\mathrm{p}=0.339)$. We found a trend for better response in the EAP than in the sham group although the difference in FACIT-F score was not significant $(\mathrm{p}=0.09)$. EAc also improved quality of life $\left(-5.17\right.$ points, $95 \%$ CI $\left(-8.2\right.$ to -2.06 , Basal Vs $9^{\text {th }}$ session $\left.\mathrm{p}=0.003\right)$; depression ( 8.9 points, $95 \%$ CI ( 4 to 13.8 , Basal Vs $9^{\text {th }}$ session $\left.\mathrm{p}=0.002\right)$, anxiety $(10.6$ points, 95\% CI (3.6 to 17.6, Basal Vs $9^{\text {th }}$ session $\mathrm{p}=0.006$ ) and sleepiness scores (1.46 points, $95 \%$ CI $\left(0.096\right.$ to 2.83 , Basal Vs $9^{\text {th }}$ session $\left.\mathrm{p}=0.038\right)$. However, the differences in between EAc and sham and control groups were not significant $(\mathrm{p}>0.05)$.

Conclusion: Both targeted and sham electroacupuncture are effective in managing fatigue in patients with quiescent IBD. NCT02733276.

Disclosure of Interest: All authors have declared no conflicts of interest.

\section{References}

Bager P, Befrits R, Wikman O, et al. Fatigue in out-patients with inflammatory bowel disease is common and multifactorial. AlimentPharmacolTher 2012; 35:133-141.

Czuber-Dochan W, Ream E, Norton C. Review article: description and management of fatigue in inflammatory bowel disease. AlimentPharmacolTher 2013; 37:505-516.

Jelsness-Jorgensen LP, Bernklev TB, Henriksen M, et al. Chronic fatigue is more prevalent in patients with inflammatory bowel disease than in healthy controls. InflammBowelDis 2010;17:1564-72.

Tessa EH, Römkens, Maria WJ, et al. High prevalence of fatigue in inflammatory bowel disease: A case control study. Journal of Crohn's and Colitis 2011;5: 332-337. 2004;69(3):131-9. Epub 2004 Apr 26.

\section{P0382 EFFICACY AND SAFETY OF GOLIMUMAB IN CROHN'S DISEASE: A FRENCH NATIONAL RETROSPECTIVE STUDY}

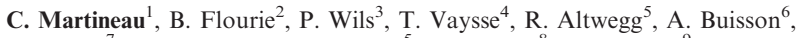
A. Amiot ${ }^{7}$, G. Pineton De Chambrun ${ }^{5}$, V. Abitbol ${ }^{8}$, M. Fumery ${ }^{9}$,

X. Hebuterne ${ }^{10}$, S. Viennot ${ }^{11}$, D. Laharie ${ }^{12}$, L. Beaugerie ${ }^{1}$, S. Nancey ${ }^{2}$, H. Sokol $^{1}$ ${ }^{1}$ St Antoine Hospital, Paris/France

${ }^{2}$ Gastroenterologie, CH Lyon Sud Gastro secteur Jules Courmont, Pierre Benite Cedex/France

${ }^{3}$ Hurriez Hospital, Lille/France

${ }_{5}^{4}$ Bicetre Hospital, Kremlin-Bicetre/France

${ }^{5}$ Hopital Saint Eloi Hepatologie Gastro enterologie, Montpellier/France

${ }^{6}$ Dept. Of Gastroenterology, CHU Estaing Clermont-Ferrand, Clermont-ferrand/

France

${ }^{7}$ Dept. Of Gastroenterology, Henri Mondor Hospital, APHP Dept. of Gastroenterology, Creteil/France

${ }^{8}$ Hopital Cochin Gastroentérologie, Paris/France

${ }^{9}$ Amiens University Hospital, Amiens/France

${ }^{10}$ Hospital Arche 2, Nice/France

${ }^{11}$ Caen Hospital, Caen/France

${ }^{12} \mathrm{CHU}$ de Bordeaux Hopital Haut-Leveque Dept. de Gastroenterologie, Pessac/ France

\section{Contact E-mail Address: klomartineau@gmail.com}

Introduction: Anti-TNF, such as adalimumab (ADA) and infliximab (IFX), have improved the therapeutic care of Crohn's disease (CD). However their use may be associated with loss of efficacy, adverse events and sometimes primary failure. After the first anti-TNF discontinuation, it is possible to switch to another antiTNF. In France, three anti TNF are available in ulcerative colitis (IFX, ADA and golimumab), but only the first two are approved in $\mathrm{CD}$, because golimumab has not been studied in this indication. The aim of this study was to report golimumab efficacy and safety in CD.

Aims \& Methods: This national multicenter retrospective study included patients with $\mathrm{CD}$ from 12 French tertiary centers who received golimumab and analyzed: clinical response, duration of treatment, tolerance, reasons for discontinuation of treatment, disease phenotype, and treatments preceding and associated with golimumab. The main endpoint was the efficacy of golimumab defined by the duration of treatment before failure (need for therapeutic optimization or cessation). Predictive factors of therapeutic response were determined (log rank and Cox model), and the tolerance was evaluated.

Results: One hundred and fifteen patients with a median duration of the disease of 13.5 years received on average golimumab in 3, 6 th line of biotherapy. The overall clinical response assessed by the physician was $55.8 \%$ at the time of the re-evaluation (on average, at 3.8 months [0.6-24] after initiation of therapy). The mean duration of treatment was 12.3 months (0.55-44). Sixty-seven percent of patients received treatment for more than 6 months and $48.7 \%$ of patients were still treated with golimumab at the end of the follow-up. At 12 months, $34.9 \%$ of patients still received golimumab without optimization. At 24 months, this figure was $19.3 \%$. In univariate analysis, the factors associated with a longer golimumab treatment duration without stopping or optimizing were the active smoking status $(\mathrm{p}=0.043)$, the absence of anoperineal lesions $(\mathrm{p}=0.012)$, the presence of extra-intestinal symptoms $(\mathrm{p}=0.035)$, the presence of a co-immunosuppression of more than 6 months $(\mathrm{p}<0.001)$ and discontinuation of the first anti-TNF $\alpha$ for intolerance $(\mathrm{p}=0.022)$.) In multivariate analysis, discontinuation of the first anti-TNF $\alpha$ for intolerance and the presence of co-immunosuppression with thiopurine derivatives or methotrexate over 6 months were independently associated with golimumab efficacy (OR 2.16, 95\% CI [1.25-3.86], p=0.005 and OR $3.98 .95 \%$ CI [2.3-7.1], $\mathrm{p}<0.001$, respectively). Side effects led to discontinuation of treatment in $6 \%$ of patients. These were paradoxical psoriasis in three patients, paresthesia $(n=1)$, lower extremity edema $(n=1)$, injection site reaction $(n=1)$ and not reported reason for one patient.

Conclusion: After failure of the other anti-TNF agents, golimumab is well tolerated and results in sustained clinical response in one in two patients with Crohn's disease, particularly when associated with a co-immunosuppression, and if the reason for discontinuation of the first anti $-\mathrm{TNF} \alpha$ was an intolerance.

Disclosure of Interest: H. Sokol: consulting fee: Tillotts, Abbvie, MSD, Enterome, Maat

All other authors have declared no conflicts of interest.

\section{P0383 BIOLOGICS AND BIOSIMILARS: WHAT MATTERS TO PHYSICIANS?}

A. Molinari ${ }^{1}$, A. Loaiza-Bonilla ${ }^{2}$, D. Charles ${ }^{1}$

${ }^{1}$ Global Alliance for Patient Access, Washington, DC/United States of America

${ }^{2}$ Cancer Treatment Centers of America, Philadelphia, PA/United States of America/AL

Contact E-mail Address: pdavidcharles $@$ gmail.com

Aims \& Methods: The purpose of this survey was to determine physicians' familiarity and comfort level with prescribing biosimilars to patients. The survey was sent to physicians residing in the European Union and specializing in the following clinical fields: dermatology, endocrinology, gastroenterology, neurology, oncology, and rheumatology.

Introduction: Biologic medicines and their biosimilar counterparts are effective therapies for many conditions, including inflammatory bowel disease, Crohn's disease, and ulcerative colitis. The European Medicines Agency (EMA) has approved twenty-two biosimilar medicines, which are derivatives of eight original biologics, and four more biosimilar are scheduled to be reviewed this year. As the number of approved biosimilars rises, regulatory agencies must closely monitor their safety and efficacy.

Results: The majority of survey respondents specialized in endocrinology (19\%) and gastroenterology $(19 \%)$. Respondents were recruited almost equally from the five countries, with France being the most represented country $(22 \%)$ and the UK being the least represented $(18 \%)$. The majority of respondents $(55 \%)$ indicated that safety and efficacy is the most important factor in determining whether a patient should be switched from a prescribed biologic therapy to its approved biosimilar. Thirty percent of respondents indicated that clinical trials related to the disease or condition being treated is the most important factor to switching. Only $12 \%$ of respondents indicated that cost to the government or insurance companies is a primary concern, and only $3 \%$ were primarily concerned with immunogenicity.

Conclusion: This survey suggests that the safety and efficacy of biosimilar medicines is of paramount importance to physicians and that physicians highly value clinical trial data for biosimilars. Given that biosimilars are structurally distinct from their original innovator biologic counterparts, the EMA should consider requiring more stringent clinical trials data for biosimilars seeking approval. Specifically, the EMA should require clinical trials for each proposed indication and should provide physicians with this data so that physicians can make informed prescribing choices for the safety of their patients.

Disclosure of Interest: D. Charles: David Charles receives income from Medtronic, Allergan, Ipsen, and the Alliance for Patient Access for education or consulting services.

This data was generated from a SERMO Poll. SERMO is the largest global social network exclusively for doctors.

All other authors have declared no conflicts of interest.

\section{P0384 ARE STEROIDS STILL USEFUL IN THOSE INFLAMMATORY BOWEL DISEASE PATIENTS UNDER IMMUNOSUPPRESSION? A RETROSPECTIVE POPULATION- BASED STUDY}

L. Arias García ${ }^{1}$, G. Hontoria Bautista ${ }^{1}$, E. Badia Aranda ${ }^{1}$, F. Saez-Royuela Gonzalo $^{1}$, F. Gomollon ${ }^{1}$, B. Sicilia Aladrén ${ }^{1}$

${ }^{1}$ Servicio De Aparato Digestivo, H. Universitario Burgos, Burgos/Spain

Contact E-mail Address: bsicilia4@gmail.com

Introduction: Oral steroids are effective in inducing remission of moderate flares of patients with either ulcerative colitis (UC) or Crohn's disease (CD). However, we know little about their efficacy in immunosuppressed patients or their possible role in reducing biologics and/or surgical needs in these patients 


\section{Abstract No: P0384}

\begin{tabular}{|c|c|c|c|}
\hline IMM $>6$ MONTHS & CD $260(65 \%)$ & UC $138(35 \%)$ & TOTAL $392(100 \%)$ \\
\hline Gender (male) & $136(52 \%)$ & $71(54 \%)$ & $207(53 \%)$ \\
\hline Location & $46 \% \mathrm{~L} 1 / 18 \% \mathrm{~L} 2 / 36 \% \mathrm{~L} 317 \% \mathrm{~L} 4$ & $52 \% \mathrm{E} 3 / 45 \% \mathrm{E} 2 / 3 \% \mathrm{E} 1$ & \\
\hline Behaviour & $57 \% \mathrm{~B} 1 / 43 \% \mathrm{~B} 2-325 \%$ Perianal disease & & \\
\hline Appendectomy & $91(77 \%)$ & $6(5 \%)$ & $97(25 \%)$ \\
\hline Extraintestinal manifestations & $49(19 \%)$ & $23(18 \%)$ & $72(19 \%)$ \\
\hline Smoke habit & $\begin{array}{l}\text { Smoker } 84(32 \%) \\
\text { Ex-smoker } 50(19 \%) \\
\text { Non smoker } 126(48 \%)\end{array}$ & $\begin{array}{l}\text { Smoker } 17(13 \%) \\
\text { Ex-smoker } 12(9 \%) \\
\text { Non smoker } 103(78 \%)\end{array}$ & $\begin{array}{l}\text { Smoker } 101(26 \%) \\
\text { Ex-smoker } 62(16 \%) \\
\text { Non smoker } 229(58 \%)\end{array}$ \\
\hline Steroids at diagnosis & $200(77 \%)$ & $104(79 \%)$ & $304(78 \%)$ \\
\hline Biological before IMM & $4(2 \%)$ & $0(0 \%)$ & $4(2 \%)$ \\
\hline Surgery before IMM & $62(24 \%)$ & $2(16 \%)$ & $64(16 \%)$ \\
\hline Steroids post-IMM & $\begin{array}{l}\text { Classical } 38(63 \%) \\
\text { Low biodisp } 22(37 \%)\end{array}$ & $\begin{array}{l}\text { Classical } 18(62 \%) \\
\text { Low biodisp } 11(38 \%)\end{array}$ & Classical $56(63 \%)$ Low biodisp $33(37 \%)$ \\
\hline Surgery at follow-up & $13(5)$ & $1(0.8 \%)$ & $14(3.5 \%)$ \\
\hline Biologic at follow-up & $27(10 \%)$ & $9(7 \%)$ & $35(9 \%)$ \\
\hline
\end{tabular}

Aims \& Methods: We aimed to determine the efficacy of systemic or low bioavailability oral steroid treatment for moderate flares of patients with at least 6 months of immunosuppressive treatment, and describe long-term follow-up Inflammatory bowel disease (IBD) immunosuppressed patients (thiopurines or methotrexate) from our population-data registry were analyzed. For statistical analysis, Chi-square test, U Mann-Whitney test and Kaplan Meier survival analysis were used.

Results: 392 IBD patients with a median of 82 (6-271) months of immunosuppressive (IMM) treatment were identified (table 1$) .89$ patients $(23 \%)(33 \%$ UC and $67 \% \mathrm{CD}$ ) needed at least one steroid treatment during follow-up (63\% systemic steroid and $37 \%$ low bioavailability oral steroid) with a median time of steroid treatment of $4(1-168)$ months. Average time from IMM to steroid treatment was $26(6-207)$ months. In IBD patients there were no differences regarding sex, age, disease, location, perianal disease, extra intestinal manifestations, appendectomy, smoke habit, need for steroids at diagnosis and previous abdominal surgery between patients with no need of steroids and patients with steroid treatment during follow-up. In CD patients, biological treatment for perianal disease before IMM $(\mathrm{p}=0.0039)$ and fibrostenotic (B2) or fistulizating (B3) behavior ( $\mathrm{p}=0.005$; OR 2.284) were risk factors for using steroids after IMM treatment. In UC patients, no statistically significant variables were identified. 49 of these 89 steroid treatment patients $(55 \%)$ needed biological treatment or surgery after a median of 13 months $(0-178) ; 19(21 \%)$ needed more than one steroid treatment $(2-5)$ and just 31 patients $(35 \%)$ did not need any other treatment. CD patients had higher risk $(\mathrm{p}=0.007$; OR: 3.529) to receive biological treatment or surgery versus UC patients. Otherwise, the more months using steroids in UC patients, the greater risk for biological or surgery treatment $(\mathrm{p}=0.009)$. During follow-up, though it's not statistically significant $(\mathrm{p}=0.078)$, we observe that $75 \%$ probability of rescue treatment for UC patients is 62 months versus 36 months for CD patients.

Conclusion: $23 \%$ of IBD immunosuppressive patients needed at least one steroid treatment after 6 months of IMM. Previous biological treatment and B2-B3 behavior predicted steroid treatment in CD patients, who had 3.5 times more risk to receive biological treatment or surgery after steroid treatment using it earlier than UC patients. Just $1 / 3$ of patients who needed steroid treatment after IMM did not need any other rescue treatment

Disclosure of Interest: All authors have declared no conflicts of interest.

\section{P0385 ADALIMUMAB LONG-TERM EFFECTIVENESS IN ADALIMUMAB-NAIVE PATIENTS WITH CROHN'S DISEASE: FINAL DATA FROM PYRAMID REGISTRY}

E. V. Loftus $\mathbf{J r}^{1}$, G. D'Haens ${ }^{2}$, W. Reinisch ${ }^{3}$, J. Satsangi ${ }^{4}$, R. Panaccione ${ }^{5}$, S. Berg ${ }^{6}$, G. Alperovich ${ }^{7}$, M. Bereswill ${ }^{8}$, J. Kalabic ${ }^{9}$, M. Skup ${ }^{10}$, J. Petersson ${ }^{11}$, A.M. Robinson ${ }^{10}$

${ }^{1}$ Mayo Clinic College of Medicine, Rochester/United States of America/MN

${ }^{2}$ Academic Medical Center, Amsterdam/Netherlands

${ }^{3}$ Medical University of Vienna, Vienna/Austria

${ }^{4}$ University Of Edinburgh, Gastrointestinal Unit, Edinburgh/United Kingdom

${ }^{5}$ University of Calgary, Calgary/Canada

${ }^{6}$ AbbVie, Solna/Sweden

${ }^{7}$ Medical, Abbvie, Madrid/Spain

${ }^{8}$ AbbVie Deutschland GmbH \& Co. KG, Ludwigshafen/Germany

${ }^{9}$ AbbVie Deutschland GmbH \& Co, Ludwigshafen/Germany

${ }^{10}$ AbbVie Inc., North Chicago/United States of America

${ }^{11}$ Global Medical Affairs, AbbVie Inc, North Chicago/United States of America

Contact E-mail Address: loftus.edward $@$ mayo.edu

Introduction: PYRAMID was an international multi-center non-interventional postmarketing registry assessing long-term safety and effectiveness of adalimumab (Humira $\left.{ }^{\circledR}[\mathrm{ADA}]\right)$ as used in routine clinical practice. Patients with and without prior ADA experience were allowed to enroll. The final long-term effectiveness of ADA is reported in adult ADA-naïve patients (those who had not received ADA before registry enrollment) with moderate to severe Crohn's disease $(C D)$ who were treated according to the local product label.

Aims \& Methods: All patients entering the registry were followed for up to 6 years. Effectiveness of ADA was measured using Physician's Global Assessment (PGA; [a composite of Harvey Bradshaw Index and rectal bleeding score]), Short Inflammatory Bowel Disease Questionnaire (SIBDQ), and 4 components of the Work Productivity and Activity Impairment (WPAI) questionnaire, including absenteeism, presenteeism, overall work impairment, and activity impairment. Effectiveness measures, captured in all patients who received at least 1 dose of ADA in the registry and had at least 1 post-enrollment measurement, were summarized descriptively by the number of observations that were not missing at each registry visit; data were reported as observed. Values at enrollment are considered as baseline values.

Results: Among 5025 patients evaluated in the registry, 2057 (40.9\%) were ADAnaïve. Of these, 1199 patients $(58.3 \%)$ were female; mean age 37.1 years at enrollment. Mean \pm SD ADA exposure for the ADA-naïve subgroup during the registry was $1118.5 \pm 842.3$ days. A total of 1082 patients $(52.6 \%)$ had prior exposure to at least 1 anti-TNF/biologic; $853(41.5 \%)$ and 831 patients $(40.4 \%)$ used immunomodulators and corticosteroids, respectively, at enrollment. Mean change from baseline in effectiveness measures for patients with $\mathrm{CD}$ is shown in the table. Mean PGA score and SIBDQ as well as WPAI domains improved in ADA-naïve patients from enrollment to 1 year and were sustained for up to 6 years (table). No new safety signals were identified in the registry.

Conclusion: At 1 year after entering the international postmarketing registry of ADA use in routine clinical practice, clinically meaningful improvements in disease activity, work productivity, and activity impairment were achieved in ADAnaïve patients with moderately to severely active $C D$. These improvements were maintained for up to 6 years of the registry among the patients who remained in the study.

Disclosure of Interest: E.V. Loftus Jr: consultant and/or research support: AbbVie, UCB, Janssen, Takeda, Eli Lilly, Mesoblast, Amgen, Pfizer, CVS Caremark, Salix, Genentech, Robarts Clinical Trials, Gilead, Receptos, Seres Pharmaceuticals, Celgene, and Medimmune.

G. D'Haens: consulting and/or lecture fees and/or research grants and/or speaking honoraria from AbbVie, Dr Falk Pharma, Ferring, Given Imaging, Janssen Biologics, MSD, Shire Pharmaceuticals, Tillotts Pharma, UCB Pharma, and others.

W. Reinisch: speaker/consultant/advisory board member and has received research funding from Abbott Laboratories, AbbVie, AESCA, Centocor, Falk Pharma GmbH, Immundiagnostik, and MSD and others.

J. Satsangi: speaker, consultancy, or travel support from AbbVie, MSD, Takeda, Shire, Ferring

R. Panaccione: consultant and/or lecture fees from AbbVie, Amgen, AstraZeneca, Axcan Pharma (now Aptalis), Biogen Idec, Bristol-Myers Squibb, Centocor, ChemoCentryx, Eisai Medical Research Inc, Elan Pharmaceuticals, Ferring, Genentech, GlaxoSmithKline, and others.

S. Berg: AbbVie employee; may own AbbVie stock and/or options G. Alperovich: AbbVie employee; may own AbbVie stock and/or options M. Bereswill: AbbVie employee; may own AbbVie stock and/or options J. Kalabic: AbbVie employee; may own AbbVie stock and/or options M. Skup: AbbVie employee; may own AbbVie stock and/or options J. Petersson: AbbVie employee; may own AbbVie stock and/or options A.M. Robinson: AbbVie employee; may own AbbVie stock and/or options 
Abstract No: P0385

Table: Change from enrollment (baseline) in effectiveness measure scores in ADA-naïve patients with CD (N=2057)

\begin{tabular}{|c|c|c|c|c|c|c|c|}
\hline \multirow[b]{2}{*}{ Effectiveness measure } & \multirow[b]{2}{*}{ Enrollment, mean (SD) } & \multicolumn{6}{|c|}{ Change from enrollment, mean (SD) } \\
\hline & & Month 12 & Month 24 & Month 36 & Month 48 & Month 60 & Month 72 \\
\hline PGA & $8.0(5.6) \mathrm{n}=1969$ & $-3.6(5.4) \mathrm{n}=1305$ & $-3.7(5.5) \mathrm{n}=1109$ & $-4.2(5.3) \mathrm{n}=1036$ & $-4.2(5.2) \mathrm{n}=962$ & $-4.2(5.5) \mathrm{n}=858$ & $-4.1(6.1) \mathrm{n}=809$ \\
\hline SIBDQ* & $40.7(12.7) \mathrm{n}=1422$ & $11.0(12.4) \mathrm{n}=689$ & $10.2(14.1) \mathrm{n}=505$ & $11.3(13.4) \mathrm{n}=430$ & $11.5(13.1) \mathrm{n}=388$ & $11.4(13.1) \mathrm{n}=337$ & $11.1(12.9) \mathrm{n}=309$ \\
\hline WPAI Absenteeism** & $21.9(33.5) \mathrm{n}=802$ & $-12.2(33.8) \mathrm{n}=314$ & $-10.3(32.8) n=217$ & $-15.5(35.3) n=186$ & $-11.6(39.3) \mathrm{N}=165$ & $-10.2(34.3) \mathrm{n}=146$ & $6-10.7(31.6) n=119$ \\
\hline WPAI Presenteeism** & $41.1(30.0) n=833$ & $-18.9(32.4) \mathrm{n}=338$ & $-19.3(35.7) n=248$ & $-22.0(33.2) \mathrm{n}=207$ & $-18.7(37.8) n=181$ & $-18.4(31.3) \mathrm{n}=160$ & $-19.6(30.4) n=134$ \\
\hline WPAI Overall Work Impairment** & $49.8(33.4) \mathrm{n}=800$ & $-23.4(34.8) \mathrm{n}=310$ & $-22.7(39.2) n=214$ & $-27.5(36.2) n=181$ & $-22.7(39.1) \mathrm{n}=164$ & $-20.2(35.2) \mathrm{n}=145$ & $5-23.0(35.7) \mathrm{n}=117$ \\
\hline WPAI Activity Impairment** & $50.3(30.1) \mathrm{n}=1386$ & $-21.9(31.7) \mathrm{n}=664$ & $-22.5(34.4) n=491$ & $-23.4(32.9) \mathrm{n}=411$ & $-22.2(34.1) \mathrm{n}=371$ & $-22.2(30.9) \mathrm{n}=320$ & $-21.3(29.6) n=292$ \\
\hline
\end{tabular}

*Total SIBDQ ranges from 1 (poor health-related quality of life) to 70 (optimum health-related quality of life). A 9-point change in total SIBDQ correlates with a 100point change in Crohn's Disease Activity Index (Irvine EJ, Zhou Q, Thompson AK Am J Gastroenterol 91:1571-8, 1996). ** A 7-percentage point change in WPAI represents the minimum clinically important difference. (Reilly MC, et al. Gut 2007. 56Suppl3 A159).

P0386 EFFECT OF ADALIMUMAB ON CLINICAL AND HEALTHRELATED QUALITY OF LIFE OUTCOMES BY DISEASE SEVERITY AND PRIOR TUMOUR NECROSIS FACTOR INHIBITOR USE IN PATIENTS WITH ULCERATIVE COLITIS IN A CLINICAL PRACTICE SETTING: SUBGROUP ANALYSES FROM INSPIRADA

S. Travis ${ }^{1}$, B. G. Feagan ${ }^{2}$, L. Peyrin-Biroulet ${ }^{3}$, R. Panaccione ${ }^{4}$, S. Danese ${ }^{5}$, J. Petersson ${ }^{6}$, A. Lazar ${ }^{7}$, A.M. Robinson ${ }^{6}$, N. Chen ${ }^{6}$, M. Skup ${ }^{6}$, W. Lee ${ }^{6}$ ${ }^{1}$ Oxford University Hospitals, Oxford/United Kingdom

${ }^{2}$ Robarts Research Institute, London/Canada

${ }^{3}$ University Hospital of Nancy, Les Nancy/France

${ }^{4}$ University of Calgary, Calgary/Canada

${ }^{5}$ Istituto Clinico Humanitas, Milan/Italy

${ }^{6}$ AbbVie Inc., North Chicago/United States of America

${ }^{7}$ AbbVie Deutschland GmbH \& Co. KG, Ludwigshafen/Germany

Contact E-mail Address: Simon. Travis@ndm.ox.ac.uk

Introduction: Adalimumab (ADA) has been shown to improve clinical outcomes and health-related quality of life (HRQoL) significantly in patients (pts) with ulcerative colitis (UC) in a clinical practice setting. ${ }^{1}$ Evidence is limited about benefits of ADA among UC pts with different characteristics.

Aims \& Methods: The aim was to examine clinical and HRQoL effects of ADA in pts with UC based on disease severity and prior use of tumour necrosis factor inhibitor (TNFI). InspirADA details have been presented. ${ }^{1}$ Pts received ADA $160 / 80 \mathrm{mg}$ at week (wk) $0 / 2$ followed by ADA $40 \mathrm{mg}$ eow at wks 4 through 26 . Pts who did not respond to ADA by wk 8 were to discontinue. Pts who lost response at or after wk 8 could escalate to ADA $40 \mathrm{mg}$ weekly. UC pts were categorized into subgroups based on physician global assessment (PGA) of disease severity (moderate [baseline PGA = 2] vs severe [baseline PGA =3]) and previous TNFI use (naïve vs experienced). Proportions of pts with Simple Clinical Colitis Activity Index (SCCAI) response (defined as a decrease of $\geq 2$ points vs baseline) and remission (defined as an SCCAI $\leq 2$ ) were calculated for each cohort at wks 2, 8 and 26. Change from baseline in HRQoL outcomes was calculated, including Short Inflammatory Bowel Disease Questionnaire (SIBDQ), European Quality of Life-5 Dimensions-5 Level (EQ-5D-5L), Treatment Satisfaction Questionnaire for Medication (TSQM) and Work Productivity and Activity Impairment (WPAI). Missing data were imputed using nonresponder imputation for response/remission and last observation carried forward for all other outcomes. Comparisons of remission rate (using logistic regression) and HRQoL outcomes (using linear regression) were conducted in moderate vs severe UC and in TNFI naïve vs experienced cohorts.

Results: Among pts with moderate UC $(n=386)$ and severe UC $(n=74)$, SCCA response rates were $74.6 \%$ vs $74.3 \%, 80.1 \%$ vs $71.6 \%$, and $67.1 \%$ vs $64.9 \%$ at wk $2,8,26$, respectively. Although remission rates were similar between moderate and severe pts at wk $26(49.5 \%$ vs $40.5 \%, \mathrm{p}=0.16)$, ADA provided greater disease control for moderate pts at wk $2(29.8 \%$ vs $9.5 \%$, odds ratio [OR] $4.1 .95 \%$ confidence interval $[\mathrm{CI}] 1.8-9.1 ; \mathrm{p}<0.001)$ and wk $8(52.3 \%$ vs $31.1 \%$, OR $2.4,95 \%$ CI $1.4-4.4 ; \mathrm{p}=0.01$ ) compared to severe pts (table). The rate of dose escalation (ADA $40 \mathrm{mg}$ weekly) was $28.0 \%$ in moderate and $28.4 \%$ in severe UC pts. HRQoL outcomes were similar between the moderate and severe cohorts. Among pts who were naïve $(\mathrm{n}=389)$ and those experienced to TNFIs $(\mathrm{n}=72)$, response rates were $74.0 \%$ vs $76.4 \%, 79.2 \%$ vs $75.0 \%$, and $66.3 \%$ vs $68.1 \%$ at wk $2,8,26$, respectively. No significant difference was observed in remission rates for naïve vs experienced pts at wk $2(28.0 \%$ vs $19.4 \%, \mathrm{p}=0.43)$ and wk $26(49.4 \%$ vs $41.7 \%, \mathrm{p}=0.39)$, but naïve pts showed a significantly higher remission rate than those experienced to TNFIs at wk 8 $(52.2 \%$ vs $31.9 \%$, OR $2.1,95 \%$ CI: $1.2-3.7$; p < 0.001$)$. The rate of dose escalation was $26.5 \%$ in naïve pts vs $36.1 \%$ in experienced pts $(\mathrm{p}=0.09)$. In general, HRQoL outcomes were similar between naïve and experienced TNFI pts.

Table: Remission rate by disease severity and previous use of TNFIs

\begin{tabular}{lllll}
\hline $\begin{array}{l}\text { Remission rate, } \\
\mathrm{n}(\%)\end{array}$ & $\begin{array}{l}\text { Moderate UC } \\
(\mathrm{n}=386)\end{array}$ & $\begin{array}{l}\text { Severe UC } \\
(\mathrm{n}=74)\end{array}$ & $\begin{array}{l}\text { Odds ratio } \\
(95 \% \mathrm{CI})\end{array}$ & P value \\
\hline Wk 2 & $115(29.8 \%)$ & $7(9.5 \%)$ & $4.06(1.81-9.12)$ & $<0.001$ \\
Wk 8 & $202(52.3 \%)$ & $23(31.1 \%)$ & $2.43(1.43-4.40)$ & 0.001 \\
Wk 26 & $191(49.5 \%)$ & $30(40.5 \%)$ & $1.44(0.87-2.38)$ & 0.16 \\
\hline
\end{tabular}

Table Continued

\begin{tabular}{lllll}
\hline $\begin{array}{l}\text { Remission rate, } \\
\mathrm{n}(\%)\end{array}$ & $\begin{array}{l}\text { Moderate UC } \\
(\mathrm{n}=386)\end{array}$ & $\begin{array}{l}\text { Severe UC } \\
(\mathrm{n}=74)\end{array}$ & $\begin{array}{l}\text { Odds ratio } \\
(95 \% \mathrm{CI})\end{array}$ & P value \\
\hline $\begin{array}{l}\text { Remission rate, } \\
\mathrm{n}(\%)\end{array}$ & $\begin{array}{l}\text { Nä̈ve to TNFIs } \\
(\mathrm{n}=389)\end{array}$ & $\begin{array}{l}\text { Experienced to } \\
\text { TNFIs }(\mathrm{n}=72)\end{array}$ & $\begin{array}{l}\text { Adjusted } \\
\text { odds ratio } \\
(95 \% \mathrm{CI})^{*}\end{array}$ & P value \\
Wk 2 & $109(28.0 \%)$ & $14(19.4 \%)$ & $\begin{array}{l}1.30(0.68-2.51) \\
2.09(1.19-3.65)\end{array}$ & 0.43 \\
Wk 8 & $203(52.2 \%)$ & $23(31.9 \%)$ & 2.01 \\
Wk 26 & $192(49.4 \%)$ & $30(41.7 \%)$ & $1.26(0.75-2.11)$ & 0.38 \\
\hline
\end{tabular}

*Comparison between naïve and experienced groups was adjusted for baseline SCCAI. Because baseline SCCAI is highly correlated with PGA, adjustment for baseline SCCAI in moderate and severe UC pts defined by PGA was not performed

Conclusion: ADA treatment achieved clinically relevant rates of SCCAI response and remission even in pts who had severe UC and those who were more treatment-refractory (experienced to TNFIs), in clinical practice. In addition, ADA was associated with greater disease control in the induction period for pts with moderate than severe UC and for naïve pts than those experienced to TNFIs.

Disclosure of Interest: S. Travis: Adviser, grants, lecturer: AbbVie; Asahi; Boehringer; BMS; Cosmo; Elan; Ferring; FPRT Bio; Genentech/Roche; Genzyme; Glenmark; GW; Lilly; Merck; Novartis; Novo Nordisk; Ocera; Pfizer; Shire; Santarus; SigmoidPharma; Synthon; Takeda; Tillotts; Topivert ... Funding Statement Financial support for the study was provided by AbbVie. AbbVie participated in the interpretation of data, review, and approval of the abstract. All authors contributed to the development of the publication and maintained control over the final content.

Acknowledgement: Medical writing support was provided by Joann Hettasch, Fishawack Group of Companies, Conshohocken, PA; this support was funded by AbbVie.

B.G. Feagan: Research support: Centocor, Merck, UCB, Abbott; Lecturer: Centocor, Merck, Abbott; Consultancy: Centocor, Merck, UCB, Abbott, Millennium/Takeda, Genentech/Hoffman LaRoche, Neovacs, Merck/Serono, Bristol Myers Squibb, Robarts, Tillotts, Pfizer, Falk Pharma

L. Peyrin-Biroulet: Consultant: Merck, AbbVie, Janssen, Genentech, Mitsubishi, Ferring, Norgine, Tillotts, Vifor, Therakos, Pharmacosmos, Pilège, BMS, UCB, Hospira, Celltrion, Takeda, Biogaran, Boehringer, Lilly, Pfizer, HAC, Index, Amgen, Sandoz. Lecturer: Merck, AbbVie.

R. Panaccione: Consultant, lecture fees: AbbVie, Amgen, AstraZeneca, Axcan, Biogen, BMS, Centocor, ChemoCentryx, Eisai, Elan, Ferring, Genentech, GSK, Janssen, MSD, Millennium, Ocera, Otsuka, Pfizer, Shire, Prometheus, ScheringPlough, Synta, Teva, UCB, Warner Chilcott

S. Danese: Board membership fees: Merck Sharp \& Dohme; Consulting fees: Schering Plough, AstraZeneca, AbbVie, Takeda Millennium; Lecture fees, including fees for service on speakers' bureaus: UCB Pharma, Ferring, Merck Sharp \& Dohme

J. Petersson: Employee, stockholder: AbbVie

A. Lazar: Employee, stockholder: AbbVie

A.M. Robinson: Employee, stockholder: AbbVie

N. Chen: Employee, stockholder: AbbVie

M. Skup: Employee, stockholder: AbbVie

W. Lee: Employee, stockholder: AbbVie

\section{Reference}

1. Travis S et al. Poster P574. ECCO, Amsterdam, Netherlands, 2016. 
P0387 SUBCUTANEOUS ADMINISTRATION OF A NOVEL FORMULATION OF CT-P13 (INFLIXIMAB BIOSIMILAR) IS SAFE AND ACHIEVES PROJECTED THERAPEUTIC DRUG LEVELS: A PHASE I STUDY IN HEALTHY SUBJECTS

R. Westhovens ${ }^{1}$, D.H. Yoo ${ }^{2}$, S. Schreiber ${ }^{3}$, W. Reinish ${ }^{4}$, S. Ben-Horin ${ }^{5}$,

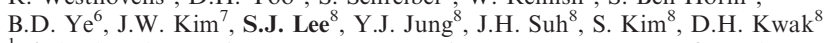
${ }^{1}$ Skeletal Biology and Engineering Research Center, Department of Development and Regeneration KU Leuven, Leuven/Belgium

${ }^{2}$ Hanyang University Hospital for Rheumatic Disease, Seoul/Korea, Republic of ${ }^{3}$ University Hospital Schleswig-Holstein, Department for Internal Medicine, Schittenhelmstr. 1224105 , Kiel/Germany

${ }^{4}$ Medical University of Vienna, Waehringer Guertel 18-201 090, VIENNA/Austria ${ }^{5}$ Sheba Medical Center and Sackler School of Medicine, Shederot Habroshim, Ramat Efal/Israel

${ }^{6}$ Department of Gastroenterology and Inflammatory Bowel Disease Center, University of Ulsan College of Medicine, Asan Medical Center, Seoul/Korea, Republic of

${ }^{7}$ Clinical Trial Center, Chungnam national university hospital, Daejeon/Korea, Republic of

${ }^{8}$ CELLTRION, Incheon/Korea, Republic of

\section{Contact E-mail Address: SangJoon. Lee@celltrion.com}

Introduction: Treatment with intravenous (IV) CT-P13, a biosimilar infliximab (INX) licensed for use in 80 countries, is highly effective and well tolerated. To increase treatment modalities with CT-P13 for patients, a new subcutaneous (SC) formulation was developed.

Aims \& Methods: This phase I and open label study, conducted at a single site in Korea, was designed to evaluate safety and pharmacokinetics (PK) of SC administration of CT-P13 in healthy subjects. In a single dose escalation study, 38 subjects received either SC injection or IV infusion of CT-P13 on Day 0 and were followed for 12 weeks ( 20 subjects in 3 different dosages of SC; 18 subjects in 2 different dosages of IV). After reviewing safety data observed for 48 hours, the next cohort was conducted subsequently from low dose to high dose. The PK profile of $\mathrm{SC}$ and IV formulation was evaluated by measuring the $\mathrm{AUC}_{0 \text {-last }}$, $\mathrm{C}_{\max }, \mathrm{T}_{\max }$ and $\mathrm{T}_{1 / 2}$

Results: A total of 38 male subjects with median age of 23 years (range 19, 30 years) had no treatment-emergent serious adverse events or systemic hypersensitivity reaction. In SC cohort, two subjects experienced mild injection site reactions, and both have resolved without any treatment. Mean $\mathrm{AUC}_{0 \text {-last }}$ and $\mathrm{C}_{\text {max }}$ ranged from 5016.4 to $14253.6 \mathrm{~h} * \mathrm{ug} / \mathrm{mL}$ and 10.0 to $23.1 \mathrm{ug} / \mathrm{mL}$, respectively, after a single SC injection of CT-P13. SC CT-P13 formulation was absorbed slower into the systemic circulation (median $\mathrm{T}_{\max }$ ranging from 7.0 to 7.1 days) in comparison with IV formulation (median $T_{\max }$ ranging from 2.2 to 3.2 hours) but the drug elimination measured by half-life $\left(\mathrm{T}_{1 / 2}\right)$ was similar (mean range 11.3 to 13.7 days vs. 11.7 to 12.2 days) between SC and IV formulations, respectively. Bioavailability of CT-P13 SC was approximately $60.6 \%$, when comparing across all CT-P13 SC cohorts to CT-P13 IV cohorts.

Conclusion: PK profiles after a single SC injection were linear by dose levels. SC administration of CT-P13 is feasible in terms of bioavailability and safety profiles.

\section{Disclosure of Interest:}

R. Westhovens: Grant: BMS, Roche Other: Advisory Board Galapagos/Gilead as well as CELLTRION, Inc and Janssen

D.H. Yoo: Consulting fee: CELLTRION, Inc (for consulting of study design) Support for travel to meetings for the study or other purposes: CELLTRION, Inc (Payment for travel and hotel to attend investigator's meetings) W. Reinish: fees for consultation and lecturing from CELLTRION, Inc S. Ben-Horin: Grant: CELLTRION, Inc, Takeda, Abbvie, Janssen Consulting fee or honorarium: MSD, Ferring, CELLTRION, Inc, Takeda, Abbvie, Novartis, Pfizer, Janssen

B.D. Ye: Lecture fee(s): Abbvie Korea, Janssen Korea, CELTRION, Inc. Consultancy: Shire Korea, Abbvie Korea, Kuhnil Pharm., CELLTRION, Inc., Takeda Korea, Kangstem Biotech, Robarts Clinical Trials Inc., Quintiles J.W. Kim: Grant: CELLTRION, Inc Consulting fee: CELLTRION, Inc Support for travel to meetings for the study or other purpose: CELLTRION, Inc S.J. Lee: Employee of CELLTRION, Inc

Y.J. Jung: Employee of CELLTRION, Inc

J.H. Suh: Employee of CELLTRION, Inc

S. Kim: Employee of CELLTRION, Inc

D.H. Kwak: Employee of CELLTRION, Inc

All other authors have declared no conflicts of interest.

\section{Reference}

1. Westhovens et al. The Journal of Rheumatology. 2006; 33:5

\section{P0388 REAL-WORLD HEALTHCARE UTILISATION IN PATIENTS WITH INFLAMMATORY BOWEL DISEASE NEWLY TREATED WITH VEDOLIZUMAB AND ANTI-TUMOUR NECROSIS FACTOR AGENTS}

M. T. Osterman ${ }^{1}$, A. Afzali ${ }^{2}$, X. Song ${ }^{3}$, N. Shi ${ }^{3}$, M. Skup ${ }^{4}$, W. Lee ${ }^{4}$

${ }^{1}$ University of Pennsylvania, Philadelphia/United States of America/PA

${ }^{2}$ University of Washington-Harborview Medical Center, Seattle/United States of

America/WA

${ }^{3}$ Truven Health Analytics, Cambridge/United States of America/MA

${ }^{4}$ AbbVie Inc., North Chicago/United States of America/IL
Introduction: Biological therapy has been highly effective for inflammatory bowel disease (IBD). In addition to anti-tumour necrosis factor (anti-TNF) drugs, a gut-selective anti-integrin biologic, vedolizumab (VDZ), has been approved since 2014. However, the real-world comparative effectiveness of VDZ and anti-TNF has not been fully investigated.

Aims \& Methods: This study aimed to evaluate all-cause and IBD-related healthcare utilisation in IBD patients (pts) newly treated with VDZ and anti-TNFs. Crohn's disease (CD) and ulcerative colitis (UC) pts $\geq 18$ years old with $\geq 2$ claims for VDZ or $\geq 2$ claims for anti-TNF from 20/5/2014 to $31 / 12 / 2016$ were identified from a large, de-identified administrative claims database in the US (Truven MarketScan ${ }^{\circledR}$ Commercial and Medicare Supplemental Databases). The date of the first VDZ/anti-TNF claim was defined as index date. New VDZ/antiTNF treatment was defined as no claims for these agents in the 1 year before index date. All pts had $\geq 12$-month continuous enrolment prior to and $\geq 6$-month following index date. All-cause and IBD-related healthcare utilisation including hospitalisation, emergency department (ED) visits and outpatient visits during 6 months post-index were examined for the IBD cohort overall, as well as in CD and UC cohorts. Multivariable logistic regression was employed to estimate the odds ratio (OR) for hospitalisation and ED visits, while Poisson regression was used to examine the rate ratio (RR) for outpatient visits with VDZ compared to anti-TNF use, controlling for demographic characteristics, index year, baseline Charlson comorbidity index (CCI) and baseline hospitalisation and ED visits 1 year prior to index date. In sensitivity analyses, outpatient visits related to infusion administration for VDZ or anti-TNF were excluded to examine the RR associated with outpatient visits that were not related to the visits for infusion procedure.

Results: A total of 652 and 6974 IBD pts newly treated with VDZ and anti-TNF were identified, respectively (mean age [year]: $45 \mathrm{VDZ}$ vs 42 anti-TNF; male: $47 \%$ vs $48 \%$ ). VDZ pts had a higher CCI than anti-TNF pts (0.7 vs 0.5$)$. During the 6 month follow-up, compared to anti-TNF, use of VDZ had significantly higher rates of all-cause hospitalisation $(16.3 \%$ vs $13.1 \%$, OR $1.30,95 \%$ confidence interval [CI] 1.03-1.64), all-cause outpatient visits (mean visits 45.1 vs 31.0, RR 1.39, 95\% CI 1.32-1.37) and IBD-related outpatient visits (mean visits 28.1 vs 17.5 , RR $1.60,95 \%$ CI 1.51-1.69). A sensitivity analysis excluding outpatient visits related to infusion administration showed similar results for IBDrelated outpatient visits (RR $1.53,95 \%$ CI $1.44-1.62$ ). In pts with $C D$, the magnitude of risk of IBD-related hospitalisation was even higher $(\mathrm{OR} 1.67,95 \% \mathrm{CI}$ $1.17-2.38$ ), but no significant difference in IBD-related hospitalisation (OR 0.57, $95 \%$ CI $0.30-1.06$ ) was observed in pts with UC. The difference in IBD-related ED visits between VDZ and anti-TNF pts was not significant in CD (OR 1.32, $95 \%$ CI $0.96-1.82$ ) or UC (OR $0.72,95 \%$ CI $0.40-1.29$ ). However, the rates of outpatient visits were consistently higher in VDZ vs anti-TNF across CD (RR $1.82,95 \% \mathrm{CI} 1.69-1.96)$ and UC cohorts (RR $1.29,95 \% \mathrm{CI} 1.18-1.42$ ). The results were similar in the sensitivity analyses when infusion-related visits were excluded.

Conclusion: In this real-world setting, VDZ treatment was shown to be associated with higher all-cause hospitalisations and outpatient services compared to antiTNF for pts with IBD. A higher risk of IBD-related hospitalisation associated with VDZ use was observed in CD but not UC pts. Outpatient visit rates were consistently higher for VDZ users, regardless of taking into account the infusionrelated visits for biologics. These results should be interpreted with caution as disease activity was not fully accounted for in this claims data analysis.

Funding Statement Financial support for the study was provided by AbbVie. AbbVie participated in the interpretation of data, review, and approval of the abstract. All authors contributed to the development of the publication and maintained control over the final content. Acknowledgement: Editorial support was provided by Fishawack Communications, Conshohocken, PA; this support was funded by AbbVie.

Disclosure of Interest: M.T. Osterman: Consultant fees: AbbVie, Janssen, Lycera, Merck, Pfizer, Takeda, and UCB. Research grant support: UCB

A. Afzali: Consultant: AbbVie, Takeda, and UCB. Research grant: UCB

X. Song: Employee: Truven Health Analytics, an IBM Company, Cambridge, MA, USA and received payment from AbbVie to assist with the analyses of this study

N. Shi: Employee: Truven Health Analytics, an IBM Company, Cambridge, MA, USA and received payment from AbbVie to assist with the analyses of this study

M. Skup: Employee, stockholder: AbbVie

W. Lee: Employee, stockholder: AbbVie

\section{P0389 SAFETY OF ANTI-TNF TREATMENT IN ELDERLY PATIENTS WITH INFLAMMATORY BOWEL DISEASE}

L. Maia, P. Lago, Â. Rodrigues, M.S. Rodrigues, C. Caetano, D. Ferreira, M. Rocha, I. Pedroto

Gastroenterology, Centro Hospitalar do Porto, Porto/Portugal

Contact E-mail Address: luismaia.gastro@gmail.com

Introduction: Due to population ageing and improved survival, the percentage of elderly patients with inflammatory bowel disease (IBD) is increasing. The safety 
of tumour necrosis factor antagonists (anti-TNF) treatment in this populations is ill-studied.

Aims \& Methods: To assess the main adverse events (AE) of this therapy in the elderly population in comparison with the younger patients, we performed a retrospective cohort study of patients with IBD that initiated treatment with anti-TNF between 2003 and 2014, with follow-up until December 2016. Demographic, clinical and medication data were collected. AE (infectious including opportunistic, malignancy, dermatologic, neurologic, cardiac and vascular, hepatic, infusion reactions and others) occurring during anti-TNF treatment in elderly and younger patients were analysed and both groups were compared. The severe AE definitions from Food and Drug Administration (FDA) and European Medicines Agency (EMA) were used.

Results: Of the 219 patients $(55.3 \%$ women; average disease duration $13.60+/-$ 7.74 years), 25 were more than 65 years-old (elderly group, mean age 70.0 years vs. younger group, mean age 41.77 years). Infliximab was used in 174 patients (on average 1585 days) and adalimumab in 93 (on average 1379 days), with a total 1106 years of anti-TNF exposure. In the elderly, azathioprine was used less frequently ( 68.0 vs. $95.4 \%, \mathrm{p}=0.000$ ). There were 46 severe AE overall, including 18 cancers and 16 opportunistic infections ( 5 tuberculosis). Malignancy $(20.0 \%$ vs. $6.7 \%, p=0.039)$ and cardiovascular events $(16.0$ vs. $4.1 \%, p=0.036)$ occurred more frequently in the elderly, whereas dermatologic AE were more common in the younger group $(4.0$ vs. $19.1 \%, p=0.044)$. The total number of severe $\mathrm{AE}(24.0$ vs. $20.1 \%, \mathrm{p}=0.794)$ including death $(4.0$ vs. $2.6 \%, \mathrm{p}=0.521)$ was not significantly different between groups.

Conclusion: Despite being at higher risk of malignancy and cardiovascular events, the total number of severe adverse events was not significantly increased in elderly patients. Particular attention to malignancy surveillance and treatment of cardiovascular comorbidities is advised in this population.

Disclosure of Interest: All authors have declared no conflicts of interest.

\section{Reference}

Lobatón T et al. Efficacy and safety of anti-TNF therapy in elderly patients with inflammatory bowel disease. Aliment Pharmacol Ther 2015; 42: 441-451

\section{P0390 SWITCHING FROM REFERENCE INFLIXIMAB TO CT-P13 IN PATIENTS WITH INFLAMMATORY BOWEL DISEASE: 12 MONTHS RESULTS}

M.F. Guerra Veloz ${ }^{1}$, F. Argüelles Arias ${ }^{1}$, R. Perea Amarillo ${ }^{1}$, L. Castro Laria ${ }^{1}$, B. Maldonado Pérez ${ }^{1}$, D. Chaaro ${ }^{1}$, A. Benítez Roldan ${ }^{1}$, V. Merino ${ }^{1}$, G. Ramirez ${ }^{1}$, A. Caunedo Álvarez ${ }^{1}$, M. Romero Gómez ${ }^{2}$

${ }^{1}$ Hospital Universitario Virgen Macarena (Sevilla), Seville/Spain

${ }^{2}$ Hospital Universitario Virgen del Rocio (Sevilla), Seville/Spain

\section{Contact E-mail Address: maferguerrita@hotmail.com}

Introduction: Over the past twenty years, the introduction of biological agents into clinical practice has radically improved outcomes in patients with inflammatory bowel disease (IBD), Crohn's disease (CD) and ulcerative colitis (UC). Tumor necrosis factor (TNF) antagonists, such as infliximab, act by preventing TNF from binding to its receptor, neutralizing its activity and alleviating mucosal inflammation. However, biological agents are much more expensive than traditional treatments, and the high cost of these drugs in the treatment of IBD imposes a considerable burden on the national healthcare system. As a result, interest in biosimilars has grown as biosimilar agents are highly similar in terms of quality, efficacy, and safety to already licensed biologics but are associated with lower development costs. CT-P13 (Remsima ${ }^{\circledR}$ and Inflectra ${ }^{\circledR}$ ) is a biosimilar of infliximab (Remicade $(\mathbb{R})$, which is its reference product (RP). Both CT-P13 and infliximab RP are chimeric IgG1 monoclonal antibodies produced in cell lines derived from the same cell type of murine hybridoma. CT-P13 was authorized by the EMA in 2013 for several indications, including IBD, based on two pivotal clinical trials in patients with rheumatoid arthritis (RA) and ankylosing spondylitis (AS). However, a number of observational studies of CT-P13 in clinical practice in both anti-TNF-naïve patients and those who have been switched from infliximab RP have been published with good results. Aims \& Methods: We aimed to assess the effectiveness and safety of switching to CT-P13 from infliximab reference product (RP) in patients with inflammatory bowel disease. This was a prospective single-center observational study in patients with moderate to severe Crohn's disease (CD) and ulcerative colitis (UC). All patients were switched from infliximab RP (Remicade $\left.{ }^{\circledR}\right)$ to CT-P13 treatment and followed for up to 12 months. The efficacy endpoint was the change in clinical response assessed at 3-monthly intervals, according to the Harvey-Bradshaw (HB) score and partial Mayo score for patients with $\mathrm{CD}$ and UC, respectively. C-reactive protein (CRP) was also measured. The Cochrane's Q and Friedman tests were used to analyze the change in clinical scores and CRP. Adverse events were monitored and recorded throughout the study.

Results: A total of 98 patients with inflammatory bowel disease (67 CD/31 UC) were included. $83.6 \%(56 / 67)$ of patients with $\mathrm{CD}$ were in remission at the time of the switch and $62.7 \%$ were in remission at 12 months. The HB score showed a significant change at 12 months $(P=0.007)$ but no significant change was observed in median $\mathrm{CRP}$ at this timepoint $(\mathrm{P}=0.364) .80 .6 \%(25 / 31)$ of patients with UC were in remission at the time of the switch and $65.3 \%(18 / 28)$ were in remission at 12 months. No significant changes in the median partial Mayo score $(\mathrm{P}=0.058)$ or $\mathrm{CRP}(\mathrm{P}=0.329)$ were observed at 12 months. Serious adverse events related to medication were reported in $11(11.2 \%)$ patients. In this group, six patients discontinued treatment because of these AEs.

Conclusion: Switching from infliximab RP to CT-P13 is efficacious and well tolerated in patients with CD or UC for up to 12 months.
Disclosure of Interest: F. Argüelles Arias, : Advisory boards and has received financial support to attend scientific meetings from Kern Pharma All other authors have declared no conflicts of interest.

\section{References}

1 Cote-Daigneault J, Bouin M, Lahaie R, Colombel JF, Poitras P. Biologics in inflammatory bowel disease: what are the data? United European Gastroenterol $J$ 2015; 3(5): 419-428 [PMID: 26535119 DOI: 10.1177/ 2050640615590302]

2 European Medicines Agency. Committee for Medicinal Products for Human Use (CHMP). Assessment report: Remsima (infliximab). 2013. Available at: http://www.ema.europa.eu/docs/en_GB/document_library/EPAR_-

_Public_assessment_report/human/002576/WC500151486.pdf. Accessed on January 23, 2017

3 European Medicines Agency. EPAR summary for the public. Remsima. 2013. Available at: http://www.ema.europa.eu/docs/en_GB/document_library/ EPAR_-_Summary_for_the_public/human/002576/WC500150872.pdf. Accessed on January 23, 2017.

4 Jung YS, Park DI, Kim YH, Lee JH, Seo PJ, Cheon JH, Kang HW, Kim JW. Efficacy and safety of CT-P13, a biosimilar of infliximab, in patients with inflammatory bowel disease: A retrospective multicenter study. $J$ Gastroenterol Hepatol 2015; 30(12): 1705-1712 [PMID: 25974251 DOI: $10.1111 /$ jgh.12997]

\section{P0391 CLINICAL RESPONSE TO VEDOLIZUMAB IN IBD PATIENTS IS ASSOCIATED WITH THE CONCOMITANT USE OF IMMUNOMODULATORS}

I. Parisi, R. Vega, L. Whitley, S. Bloom, S. Mccartney

Gi Medicine, University College London Hospitals, London/United Kingdom

Contact E-mail Address: ioannaparis@hotmail.com

Introduction: The role of biologics in medical management of inflammatory bowel disease (IBD) has been established since anti-TNF agents invaded the market several years ago. Vedolizumab, an anti-integrin gut-selective molecule, is a more recent biologic treatment which has been approved for the management of both Crohn's disease and ulcerative colitis. Its efficacy in inducing and maintaining remission was shown in GEMINI studies, although a good percentage of the trial participants had previously failed anti-TNFs. We conducted this study in order to describe outcomes in a real-life cohort of IBD patients who were treated with Vedolizumab, consisting both of previously anti-TNF exposed but also antiTNF naive patients. Multivariate analysis searched for factors associated with response to treatment.

Aims \& Methods: All patients with IBD who received at least three doses of Vedolizumab in UCLH since the drug was offically licensed in the UK were included in the study. Demographics, clinical and endoscopic response rates were recorded and analysis was conducted in the whole cohort and in the subgroups of Crohn's and UC patients separately. Univariate analysis and logistic regression were conducted in order to identify important associations with clinical response.

Results: 59 patients with IBD were treated with vedolizumab from May 2015 to October 2016. $28(47 \%)$ had Crohn's disease and the majority $(\mathrm{n}=43,73 \%)$ had mainly colonic inflammation (12 colonic Crohn's, 29 UC, 2 IBDU). Median time from diagnosis to Vedolizumab initiation was 8 years. $17(29 \%)$ were anti-TNF naïve (all UC) and $28(67 \%)$ had previously failed both Infliximab and Adalimumab. $36(61 \%)$ were on a concomitant immunomodulator (IM), either a thiopurine or methotrexate. $41(70 \%)$ patients had a clinical response to Vedolizumab based on a reduction of Harvey-Bradshaw index (HBI) from baseline $\geq 3$ points for Crohn's patients or a reduction of partial Mayo score $\geq 2$ points for UC patients. The rates of response were similar in Crohn's and UC patients while there was no difference in response according to gender, previous anti-TNF exposure, disease duration or location of inflammation. Patients on no concomitant IM were less likely to respond to Vedolizumab (Odds ratio 0.26, $95 \%$ CI $0.07-0.91, \mathrm{p}=0.036) .11(18.6 \%)$ patients experienced adverse events while treated with Vedolizumab, five of which were related to active IBD. There were two minor allergic reactions and two mild infections.

Conclusion: Clinical response to Vedolizumab was observed in two-thirds of our IBD patients, similarly in Crohn's disease and ulcerative colitis. Concomitant IM were the only factor which was importantly associated with a higher response rate. Overall there were no serious adverse events.

Disclosure of Interest: All authors have declared no conflicts of interest.

\section{References}

Feagan B, Rutgeerts P, Sands B, et al. Vedolizumab as induction and maintenance therapy for ulcerative colitis. New Engl J Med. 2013;369(8):699-710.

Sandborn W, Feagan B, Rutgeerts P, et al. Vedolizumab as induction and maintenance therapy for Crohn's disease. New Engl J Med. 2013;369(8):711-721. Sands B, Feagan B, Rutgeerts P et al. Effects of Vedolizumab Induction Therapy for Patients With Crohn's Disease in Whom Tumor Necrosis Factor Antagonist Treatment Failed. Gastroenterology 2014; 147:618-627 


\section{P0392 CORRELATION OF RELATIONSHIP BETWEEN}

INFLIXIMAB AND ADALIMUMAB TROUGH AND ANTIBODY LEVELS WITH CLINICAL RESPONSE RATES AT COMPLETION OF INDUCTION THERAPY

D. Tighe ${ }^{1}$, S. Smith ${ }^{2}$, A. O'Connor ${ }^{1}$, B. Ryan ${ }^{1}$, N. Breslin ${ }^{1}$, D. Mcnamara ${ }^{1}$ ${ }^{1}$ Gastroenterology Trinity Academic Gastroenterology Group (TAGG), AMNCH Tallaght, Dublin/Ireland

${ }^{2}$ Trinity Academic Gastroenterology Group, Trinity College Dublin, Dublin/Ireland

Contact E-mail Address: donaltighe83@gmail.com

Introduction: Anti-TNFa therapies have helped improved response rates, reduced complication rates, and quality of life for patients with inflammatory bowel disease (IBD). However primary loss of response (LOR) is still a big concern. Therapeutic drug monitoring (TDM) potentially offers the opportunity of adjusting doses of anti-TNFa in a treat to target fashion. The end of anti-TNFa induction therapy is a key time point in the management of IBD. TDM is a useful method to help explore an immune basis behind LOR. In addition antiTNFa trough and antibody levels, are a significiant predictor of future liklihood of clinical response and mucosal healing.

Aims \& Methods: The aim of this study was to explore the relationship between infliximab (IFX) and adalimumab (ADA) trough and antibody levels with clinical response rates, at the end of anti-TNFa induction therapy. This was a prospective, single-centre study. Patients were recruited from the gastroenterology department at our centre, from July 2015 to August 2016. Inclusion criteria were all patients older than 17 years old with IBD who started treatment with antiTNFa drugs, either infliximab or adalimumab, during the study period. Patient demographics, medication and clinical history were collected from the electronic hospital information system. Baseline clinical disease activity indexes were performed (Harvey-Bradshaw Index for Crohn's disease (CD), and partial Mayo scores for Ulcerative colitis (UC)). Clinical response was defined as reduction in HBI $\leq 3$ or reduction in partial Mayo score $\leq 4$ and $<30 \%$ from baseline. AntiTNFa trough and antibody levels were measured using standard ELISA techniques. Blood was taken just prior to their first maintenance infusion. Only patients who completed induction therapy were included. Standard induction were regimes used, $5 \mathrm{mg} / \mathrm{kg}$ week 0,2 and 6 and $160 \mathrm{mg}, 80, \mathrm{mg}$ and $40 \mathrm{mg}$ every other week.

Results: 35 patients were recruited; $23 \mathrm{CD}, 12 \mathrm{UC} .18$ patients were treated with ADA, 17 with IFX. Mean age was 40.3 years, $22(62.9 \%)$ were female, 12 $(34.3 \%)$ were on thiopurines and $9(25.7 \%)$ had prior anti-TNFa exposure. Overall response rate was $51.4 \%(n=18), 33.3 \%(n=6)$ for ADA, and $70.6 \%(\mathrm{n}=12)$ for IFX. Overall trough levels were $12.5 \mathrm{ug} / \mathrm{ml}$ for IFX, and $4.4 \mathrm{ug} / \mathrm{ml}$ for ADA, $71.4 \%$ had therapeutic trough levels $>1 \mathrm{ug} / \mathrm{ml}$. There was a clear link between higher anti-TNFa trough levels at the end of induction with clinical response rates. For infliximab, mean trough levels in responders was 16.4 $\mathrm{ug} / \mathrm{ml}$ (IQR 8.4-22.7) versus $5.3 \mathrm{ug} / \mathrm{ml}(0.5-8.8)$ for non-responders ( $\mathrm{p}$ value $0.02695 \%$ CI: $1.50-20.7)$. The area under the curve [AUC] for association of IFX level at end of induction with clinical response was $0.864, p=0.0001$. In addition a trough level of $4.8 \mathrm{ug} / \mathrm{ml}$ predicted clinical response at end of induction, with a sensitivity of $90.91 \%$ and a specificity of $67 \%$. Similarly there was a link between higher ADA levels with clinical response, though not statistically significant (Responders mean trough $6.6 \mathrm{ug} / \mathrm{ml}$ (IQR 4.9-8.7) versus non-responders $3.0 \mathrm{ug} / \mathrm{ml}$ (IQR $0.1-2.7$ ) p value $0.13595 \%$ CI $1.24-8.43$ ). Antibody formation occurred in 6 patients $(17.1 \%)$. Of the patients who had primary nonresponse, $18 / 35(51.4 \%)$ had doses of anti-TNFa escalated, $7 / 17(41.1 \%)$ for infliximab, and $11 / 18(61.1 \%)$ for adalimumab. 4 patients required surgical intervention, including 1 colectomy

Conclusion: Higher trough levels at the end of anti-TNFa induction are associated with improved response rates. Ongoing work will define optimal targets at this key timeframe.

Disclosure of Interest: All authors have declared no conflicts of interest

\section{P0393 BUDESONIDE IS SUPERIOR TO MESALAZINE AND PLACEBO FOR INDUCTION OF REMISSION IN LYMPHOCYTIC COLITIS}

S. Miehlke ${ }^{1}$, D. Aust ${ }^{2}$, E. Mihaly ${ }^{3}$, P. Armerding ${ }^{4}$, G. Boehm ${ }^{5}$, O.K. Bonderup ${ }^{6}$, F. Fernández-Bañares ${ }^{7}$, J. Kupcinskas ${ }^{8}$, L.K. Munck ${ }^{9}$, K. Rehbehn ${ }^{10}$, T. Nacak ${ }^{11}$, R. Greinwald ${ }^{12}$, A. Münch ${ }^{13}$

${ }^{1}$ Cooperation Of Internal Medicine, Center for Digestive Diseases, Hamburg/ Germany

${ }^{2}$ Institute of Pathology, University Hospital, Dresden/Germany

${ }^{3}$ Semmelweis Egyetem, Budapest/Hungary

${ }^{4}$ Practice for Gastroenterology, Berlin/Germany

${ }^{5}$ Internist practice, Ludwigshafen/Germany

${ }^{6}$ Department Of Gastroenterology, Regional Hospital Silkeborg, Silkeborg/ Denmark

${ }^{7}$ Hospital Mutua de Tarrassa, Barcelona/Spain

${ }^{8}$ Hospital of Lithuanian University of Health Sciences Kaunas Clinics, Kaunas/ Lithuania

${ }^{9}$ Internal Medicine, Koge Hospital Dept. of Medicine, Koge/Denmark

${ }^{10}$ Community Practice Gastroenterology, Solingen/Germany

${ }^{11}$ Dr. Falk Pharma GmbH, Freiburg/Germany

${ }^{12}$ Forschung Und Entwicklung, R\&D Dr. Falk Pharma GmbH, Freiburg/Germany

${ }^{13}$ Linköping University, Linköping/Sweden

Contact E-mail Address: prof.miehlke@mdz-hamburg.de
Introduction: Lymphocytic colitis (LC) is a common cause of chronic, nonbloody diarrhea. Budesonide appears to be effective based on small studies. Mesalazine has been proposed as a treatment option but no placebo-controlled trials have been reported. Thus, we performed a placebo-controlled, multicenter study to evaluate budesonide and mesalazine as induction treatments for lymphocytic colitis.

Aims \& Methods: Patients with active lymphocytic colitis were randomly assigned to either budesonide $9 \mathrm{mg}$ once daily or mesalazine $3 \mathrm{~g}$ once daily, or placebo for 8 weeks in a double-blind, double-dummy design. The primary endpoint was clinical remission defined by the Hjortswang-Criteria (1). Secondary endpoints included histopathology and safety.

Results: Based on an interim analysis the trial was stopped in accordance with the pre-specified adaptive design. The final analysis included 57 patients (19 per group). Most patients were of female gender $(72 \%)$ and mean age was 59 years. The proportion of patients in clinical remission at week 8 was significantly higher in the budesonide group than in the placebo group (intention-to-treat (ITT) $79 \%$ vs $42 \% ; \mathrm{p}=0.01)$. The difference in clinical remission at week 8 between mesalazine $(63 \%)$ and placebo failed statistical significance $(p=0.09)$. The proportion of patients with histological remission at week 8 was higher with budesonide $(68 \%)$ than with mesalazine $(26 \% ; \mathrm{p}=0.02)$ and placebo $(21 \%$; $\mathrm{p}=0.008)$. The rate of adverse events did not differ among groups.

Conclusion: Oral budesonide $9 \mathrm{mg}$ once daily is highly effective and safe for induction of clinical and histological remission in lymphocytic colitis, while oral mesalazine $3 \mathrm{~g}$ once daily was only numerically, but not statistically significant better than placebo.

Disclosure of Interest: S. Miehlke: Prof. Miehlke receives lecture fees and travel costs

T. Nacak: I am employee at Dr. Falk Pharma GmbH.

R. Greinwald: Dr. Greinwald is employee at Dr. Falk Pharma GmbH

All other authors have declared no conflicts of interest.

\section{Reference}

1. Hjortswang H, Tysk C, Bohr J, Benoni C, Kilander A, Larsson L, et al Defining clinical criteria for clinical remission and disease activity in collagenous colitis. Inflamm Bowel Dis. 2009;15(12):1875-81.

\section{P0394 PREGNANCY OUTCOMES IN THE TOFACITINIB ULCERATIVE COLITIS OCTAVE STUDIES}

U. Mahadevan ${ }^{1}$, D.C. Baumgart ${ }^{2}$, M. C. Dubinsky ${ }^{3}$, N. Lawendy ${ }^{4}$, G. Friedman ${ }^{4}$, G. G. Konijeti ${ }^{5}$, H.P. Gröchenig ${ }^{6}$, T. V. Jones ${ }^{4}$, A. Marren ${ }^{4}$, A. J. Thorpe ${ }^{4}, \mathrm{C}$. Nduaka ${ }^{4}, \mathrm{C} . \mathrm{Su}^{4}$

${ }^{1}$ University of California, San Francisco/United States of America/CA

${ }^{2}$ Department Of Gastroenterology And Hepatology, Charité Medical Center

Virchow Hospital and Medical School of the Humboldt, University of Berlin, Berlin/Germany

${ }^{3}$ Icahn School of Medicine at Mount Sinai, New York/United States of America/ NY

${ }^{4}$ Pfizer Inc, Collegeville/United States of America/PA

${ }^{5}$ Division Of Gastroenterology, Scripps Clinic, La Jolla, San Diego/United States of America/CA

${ }^{6}$ Krankenhaus der Barmherzigen Brüder, Salzburg/Austria

\section{Contact E-mail Address: daniel.baumgart@charite.de}

Introduction: A pregnant woman with ulcerative colitis (UC), compared with agematched controls, is at higher risk of adverse outcomes including spontaneous abortion, preterm birth and low birth weight. ${ }^{1,2}$ Tofacitinib is an oral, small molecule Janus kinase inhibitor that is being investigated for UC. Tofacitinib has been shown to be foeticidal and teratogenic in both rats and rabbits at exposures 146 times and 13 times, respectively, the human dose of $5 \mathrm{mg}$ twice daily (BID). There are no adequate and well-controlled studies of tofacitinib in pregnant women.

Aims \& Methods: We report the pregnancy outcomes from three randomised, placebo-controlled studies (OCTAVE Induction 1, NCT01465763; OCTAVE Induction 2, NCT01458951; OCTAVE Sustain, NCT01458574) and one ongoing open-label extension study (OCTAVE long-term study, NCT01470612) of tofacitinib monotherapy in patients (pts) with moderate to severe UC. ${ }^{3,4}$ Pregnancy outcomes following maternal or paternal exposure to tofacitinib 5 or $10 \mathrm{mg}$ BID were identified from Pfizer's internal safety database up to 23 March, 2017, and categorised as: healthy newborn, medical termination, foetal death, congenital malformation, spontaneous abortion or pending/lost to follow-up. Trial protocols required use of highly effective contraception for females of childbearing potential, and study drug to be discontinued in any female pts who became pregnant.

Results: A total of 1139 unique pts (incl. placebo) enrolled in the UC OCTAVE trials, of whom 296 were females of childbearing age. There were a total of 25 pregnancies reported with exposure to tofacitinib. Of these, 11 were cases of maternal exposure, all during the $1^{\text {st }}$ trimester, including: $2(18.2 \%)$ spontaneous abortions ( $5 \mathrm{mg}$ BID, $\mathrm{n}=1 ; 10 \mathrm{mg}$ BID, $\mathrm{n}=1), 2(18.2 \%)$ medical terminations (both $10 \mathrm{mg}$ BID), $4(36.4 \%)$ healthy newborns (all on $10 \mathrm{mg}$ BID) and $3(27.3 \%)$ cases lost to follow up (all on $10 \mathrm{mg}$ BID). Out of the 14 cases of paternal exposure, $11(78.6 \%)$ were healthy newborns $(5 \mathrm{mg}$ BID, $\mathrm{n}=2 ; 10 \mathrm{mg}$ BID, $\mathrm{n}=9)$ and $3(21.4 \%)$ were pending/lost to follow-up (5 mg BID, $\mathrm{n}=1 ; 10 \mathrm{mg}$ BID, $n=2)$. Overall, there were no cases of foetal death or congenital malformation. 
Table: Summary of pregnancy outcomes in the OCTAVE programme

Foetal Congenital Spontaneous Healthy Medical Pending/los death malformation abortion newborn termination to follow-up

Maternal exposure $(\mathrm{n}=11)$

Tofacitinib $5 \mathrm{mg}$ BID 0

Tofacitinib $10 \mathrm{mg}$ BID 0

Paternal exposure $(\mathrm{n}=14)$

Tofacitinib $5 \mathrm{mg}$ BID 0

Tofacitinib $10 \mathrm{mg}$ BID 0

0
0
0

$\begin{array}{llll}1 & 0 & 0 & 0 \\ 1 & 4 & 2^{\dagger} & 3 \\ 0 & 2 & 0 & 1 \\ 0 & 9 & 0 & 2\end{array}$

$\doteqdot$ Case 1: the subject decided to terminate pregnancy based on potential risks of tofacitinib; Case 2: reason unknown;

BID, twice daily

Conclusion: Based on the limited data and follow up available, pregnancy and newborn outcomes among pts with UC with prenatal (maternal/paternal) exposure to tofacitinib appear to be similar to those reported for the UC population, as well as those previously reported in pts with RA and PsO. ${ }^{5}$ Larger, long-term follow-up studies are needed to examine safety of tofacitinib during pregnancy. Disclosure of Interest: U. Mahadevan: Consultant: Pfizer, Janssen, AbbVie, Takeda, Celgene

D.C. Baumgart: Grants/personal fees/non-fin supp: Shire, AbbVie, MSD,

Takeda, Biogen, Foreward, Dr. Falk, Ferring, Recordati, Genentech, Janssen,

TiGenix, Shield, Pfizer, BMS. Activities conform to FSA-Kodex Fachkreise, checked by legal Dpt Charité Universitätsmedizin

M.C. Dubinsky: Consultant for, Pfizer, AbbVie, Takeda, Janssen, UCB, Celgene, BMS, Gilead

N. Lawendy: Employee and shareholder of Pfizer Inc

G. Friedman: Employee and shareholder of Pfizer Inc

G.G. Konijeti: Previously received honoraria from Pfizer (advisory board),

Abbvie (advisory board), Janssen (advisory board), and Takeda (speaker), but

is not funded by them for any research.

T.V. Jones: Employee and shareholder of Pfizer Inc

A. Marren: Employee and shareholder of Pfizer Inc

A.J. Thorpe: Employee and shareholder of Pfizer Inc

C. Nduaka: Employee and shareholder of Pfizer Inc

C. Su: Employee and shareholder of Pfizer Inc

All other authors have declared no conflicts of interest.

\section{References}

1. Cornish J et al. Gut 2007;56:830-7

2. de Lima-Karagiannis A et al. Am J Gastroenterol 2016;111:1305-12

3. Sandborn WJ et al. J Crohns Colitis 2016;10(S1):S15, Abstract OP019

4. Sandborn WJ et al. J Crohns Colitis 2017:11(S1):S19-20, Abstract OP032

5. Clowse ME et al. Drug Saf 2016;39:755-62

P0395 SAFETY AND EFFICACY OF GRANULOCYTE AND MONOCYTE ADSORPTIVE APHERESIS IN 363 PATIENTS WITH INFLAMMATORY BOWEL DISEASE WHO HAVE SPECIAL SITUATIONS: AN INTERIM ANALYSIS OF A POST-MARKETING SURVEILLANCE STUDY

S. Motoya ${ }^{1}$, H. Tanaka ${ }^{2}$, T. Shibuya ${ }^{3}$, T. Osada ${ }^{3}$, H. Hongo ${ }^{4}$, T. Kimura ${ }^{4}$, D. Saito ${ }^{5}$, A. Sakuraba ${ }^{5}$, C. Mizuno ${ }^{6}$, A. Ito ${ }^{7}$, T. Kobayashi ${ }^{8}$, D. Abukawa ${ }^{9}$, S. Kokuma ${ }^{10}$, E. Hosoi ${ }^{10}$

${ }^{1}$ Sapporo Kosei Hospital, Sapporo/Japan

${ }^{2}$ IBD Center, Sapporo Kosei General Hospital, Sapporo/Japan

${ }^{3}$ Department Of Gastroenterology, Juntendo University Faculty of Medicine,

Tokyo/Japan

${ }^{4}$ Fujita Gastroenterological Hospital, Takatsuki/Japan

${ }^{5}$ The Third Department Of Internal Medicine, Kyorin University School of

Medicine, Mitaka/Japan

${ }^{6}$ Department Of Gastroenterology, Saiseikai Suita Hospital, Suita/Japan

${ }^{7}$ Department Of Gastroenterology, Tokyo Women's Medical University, Tokyo Japan

${ }^{8}$ Department Of Gastroenterology, Hakodate Goryoukaku Hospital, Hakodate/ Japan

${ }^{9}$ Department Of General Pediatrics, Miyagi Children's Hospital, Sendai/Japan

${ }^{10}$ JIMRO Co., Ltd., Tokyo/Japan

\section{Contact E-mail Address: sa-motoya@nifty.com}

Introduction: Granulocyte and monocyte adsorptive apheresis (GMA) has been shown to be effective and safe in patients with inflammatory bowel disease (IBD). We report an interim analysis of a post-marketing surveillance study of granulocyte and monocyte adsorptive apheresis using Adacolumn ${ }^{\mathbb{B}}$ for patients with inflammatory bowel disease who have special situations (PARTICULAR).

Aims \& Methods: The aim of the PARTICULAR study was to assess the safety and efficacy of GMA treatment in patients with IBD who have special situations This study was an interim analysis of the multi-centre observational study conducted at 80 institutions in Japan. Data were collected from patients with ulcerative colitis (UC) or Crohn's disease (CD) who received GMA between November 2013 and September 2016. Patients who had at least one special situation were included in the study. GMA was performed using Adacolumn ${ }^{\circledR}$ (JIMRO, Takasaki, Japan). Each patient received up to a maximum of 11 GMA sessions. Safety assessments were performed on all patients in this study. All adverse events (AEs) during the study period were recorded. AEs for which the causality of GMA could not be ruled out were defined as side effects (SEs). Feasibility problems (FPs) included blood withdrawal difficulty, venous pressure elevation, coagulation in the apheresis system and venous access difficulty. The safety of GMA was investigated in the following six special situation subgroups: the elderly ( $\geq 65$ years), concomitant treatment with multiple immunosuppressants, retreatment with GMA, anaemia (haemoglobin $<10 \mathrm{~g} / \mathrm{dl}$ ), paediatric ( $\leq 18$ years) and other groups. We also compared AEs, SEs and FPs between the subgroups with/without each special situation by univariate analysis. The efficacy of GMA was also assessed in patients with UC. Patients with a partial UC disease activity index score (pUC-DAI) of $<3$, those with missing pUC-DAI scores and those receiving concomitant treatment with infliximab, adalimumab, tacrolimus or cyclosporine were excluded from efficacy assessment. pUC-DAI scores were calculated at baseline and then after the final GMA session or when GMA therapy had to be discontinued because of AEs or FPs. Remission was defined as a pUC-DAI score of $\leq 2$ with no individual sub-score exceeding 1 point. Patients who received additional treatment by the final GMA session, including steroids, infliximab, adalimumab, tacrolimus and cyclosporine, were considered non-responders to GMA.

Results: This study included 363 patients (304 UC, 59 CD). Among these patients, SEs, AEs and FPs were observed in $3.0 \%, 10.7 \%$ and $16.3 \%$ of the patients, respectively. There were 105, 112, 103, 89, 43 and 39 patients in the elderly, concomitant treatment with multiple immunosuppressants, retreatment with GMA, anaemia, paediatric and other groups, respectively. The incidence of AEs was significantly higher in patients on multiple concomitant immunosuppressants compared with those not receiving them. Likewise, the incidence of AEs was significantly higher in patients of the anaemia group compared with patients with haemoglobin of $\geq 10 \mathrm{~g} / \mathrm{dl}$. The incidence of FPs was significantly lower in patients of the retreatment with GMA group than in those who received GMA for initial treatment (Table 1). The efficacy of GMA was assessed in 209 UC patients. The numbers of patients administered prednisolone, infliximab, adalimumab, tacrolimus and cyclosporine were 24, 6, 3, 6 and 1, respectively, and these patients were considered to be non-responders to GMA. The mean pUC-DAI score significantly decreased from 6.2 at baseline to 3.4 after the final GMA session $(P<0.001)$ and the remission rate at the final GMA session was $43.5 \%$.

Conclusion: This multi-centre observational study showed that GMA has an acceptable safety profile in IBD patients and sufficient effectiveness in UC patients who have special situations. However, care should be taken when GMA is used in patients with anaemia or those who have received concomitant treatment with multiple immunosuppressants.

Disclosure of Interest: H. Tanaka: Lecture Fee(s) from JIMRO Co., Ltd.

T. Shibuya: Unrestricted grant from JIMRO Co., Ltd. Financial support for research from JIMRO Co., Ltd.

T. Osada: Unrestricted grant from JIMRO Co., Ltd. Financial support for research from JIMRO Co., Ltd.

D. Saito: Unrestricted grant from JIMRO Co., Ltd.

A. Sakuraba: Unrestricted grant from JIMRO Co., Ltd.

S. Kokuma: Employee of JIMRO Co., Ltd

E. Hosoi: Employee of JIMRO Co., Ltd.

All other authors have declared no conflicts of interest.

\section{P0396 INTEGTRATING PSYCHOLOGICAL SUPPORT INTO ROUTINE CARE FOR PEOPLE WITH INFLAMMATORY BOWEI DISEASE}

T. Lores ${ }^{1}$, C. Goess ${ }^{1}$, C. Hrycek ${ }^{2}$, R. Grafton ${ }^{1}$, J. Hughes ${ }^{1}$, L. Cronin ${ }^{1}$, A. Mikocka-Walus ${ }^{3}$, A. Chur-Hansen ${ }^{4}$, A. L. Burke ${ }^{5}$, K. L. Collins ${ }^{5}$, J M. Andrews

${ }^{1}$ Department Of Gastroenterology \& Hepatology, Royal Adelaide Hospital - SA Health, Adelaide/Australia

${ }^{2}$ Department Of General Medicine, Royal Adelaide Hospital - SA Health,

Adelaide/Australia

${ }^{3}$ School Of Psychology, Deakin University, Melbourne/Australia

${ }^{4}$ School Of Psycholoy, University of Adelaide, Adelaide/Australia

${ }^{5}$ Clinical Psychology, Royal Adelaide Hospital - SA Health, Adelaide/Australia

Contact E-mail Address: charlotte.goess@sa.gov.au

Introduction: People with Inflammatory Bowel Disease (IBD) commonly experience mental health issues (MHIs) such as anxiety and depression. MHIs reduce quality of life and are associated with poor medication adherence and worse disease course. However, psychological support is not routinely provided to people with IBD. There are scant prospective, systematically gathered data on MHIs in IBD, despite solid evidence of the value of psychological input for people with other chronic diseases.

Aims \& Methods: The current study is investigating: the prevalence of MHIs in an IBD cohort; the acceptability and uptake of psychological screening; the acceptability and uptake of psychological support and treatment; whether MHIs correlate with higher healthcare utilisation; and potential benefits of integrated psychological care to patients' mental health, physical health, and/or healthcare utilisation. Potential participants were prospectively recruited from the IBD service of a large tertiary hospital in South Australia via post and in-person at scheduled/routine outpatient appointments. Data were collected at two timepoints - at baseline screening and at 12 month follow-up. Mental health, medication adherence, and quality of life were measured by questionnaires: the Hospital Anxiety and Depression Scale (HADS), the Kessler 6 Scale of General Psychological Distress (K6), the Morisky Medication Adherence Scale (MMAS-8), and the Assessment of Quality of Life measure (AQoL-8D). Demographic and healthcare utilisation data were collected by electronic, 
Table1: Safety profile of GMA in each group

\begin{tabular}{|c|c|c|c|}
\hline & AEs (No. of patients) $\mathrm{P}$ value & SEs (No. of patients) $\mathrm{P}$ value & FPs (No. of patients) P value \\
\hline All subjects $(\mathrm{n}=363)$ & $10.7 \%(n=39)$ & $3.0 \%(\mathrm{n}=11)$ & $16.3 \%(\mathrm{n}=59)$ \\
\hline Elderly $(\mathrm{n}=105)$ & $9.5 \%(\mathrm{n}=10) \mathrm{p}=0.6284 *$ & $0 \%(\mathrm{n}=0) \mathrm{p}=0.0690 * *$ & $17.1 \%(\mathrm{n}=18) \mathrm{p}=0.7650^{*}$ \\
\hline Multiple immunosuppressants $(\mathrm{n}=112)$ & $17.0 \%(\mathrm{n}=19) \mathrm{p}=0.0134 *$ & $5.4 \%(\mathrm{n}=6) \mathrm{p}=0.1011 * *$ & $19.6 \%(\mathrm{n}=22) \mathrm{p}=0.2685^{*}$ \\
\hline Retreatment $(\mathrm{n}=103)$ & $9.7 \%(\mathrm{n}=10) \mathrm{p}=0.6859 *$ & $2.9 \%(\mathrm{n}=3) \mathrm{p}=1.0000^{* *}$ & $8.7 \%(\mathrm{n}=9) \mathrm{p}=0.0172 * *$ \\
\hline Anaemia $(\mathrm{n}=89)$ & $18.0 \%(\mathrm{n}=16) \mathrm{p}=0.0158^{*}$ & $4.5 \%(\mathrm{n}=4) \mathrm{p}=0.4743^{*}$ & $21.4 \%(\mathrm{n}=19) \mathrm{p}=0.1548^{*}$ \\
\hline Paediatric $(n=43)$ & $18.6 \%(\mathrm{n}=8) \mathrm{p}=0.1096^{* *}$ & $7.0 \%(\mathrm{n}=3) \mathrm{p}=0.1027 * *$ & $23.3 \%(\mathrm{n}=10) \mathrm{p}=0.2136^{*}$ \\
\hline Others $(\mathrm{n}=39)$ & $2.6 \%(\mathrm{n}=1) \mathrm{p}=0.1001 * *$ & $0 \%(\mathrm{n}=0) \mathrm{p}=0.6159 * *$ & $15.4 \%(n=6) p=1.0000 * *$ \\
\hline
\end{tabular}

GMA; Granulocyte monocyte adsorptive apheresis *Chi-squared test **Fisher's exact tests AEs; Adverse events SEs; Side effects FPs; Feasibility problems

state-wide hospital records. Psychological support was offered where scores on HADS and/or K6 indicated likely need.

Results: 500 patients were approached during the 12-month screening phase; $50.6 \%$ were male, $70.8 \%$ had Crohn's disease, mean age of 40 years, mean disease duration of 11 years, $43 \%$ in clinical remission, and $9.8 \%$ current smokers (Australia's average $13.3 \%$ ). Of these $500,67 \%$ participated in psychological screening, $38 \%$ scored within the clinical range, and $17 \%$ accepted psychological support. Gender was a significant predictor of participation in psychological screening: women were $62 \%$ more likely to participate than men. Analgesics and/or mental health medication increased the likelihood of scoring within the clinical range nearly fivefold (analgesic use $O R=5.32, p=030$; psych $O R=6.04$, $p=.001)$. Significant predictors of accepting psychological treatment included older age $(O R=1.03, p=.041)$, anxiety $(O R=1.09, p=.045)$, general distress $(O R=1.11, p=.003)$ and lower quality of life $(O R=.93, p=.042)$. At baseline, anxiety and depression were both negatively correlated with medication adherence (anxiety $r=-.323, p=.000$, depression $r=-.200, p=.000$ ) and overall quality of life (anxiety $r=-.708, p=.000$; depression $r=-.787, p=.000$ ). Depression and general distress were related to overall healthcare utilisation (depression $r=.131, p=.018$, general distress $r=.124, p=.026)$. Anxiety was not related to overall healthcare utilisation, but was positively correlated with numbers of emergency department presentations $(r=.124, p=.024)$, outpatient appointments $(r=.119, p=.030)$, and appointment cancellations $(r=.155, p=.005)$. Currently, approximately half of the twelve month follow-up data has been collected. Preliminary analysis shows improvements for patients' mental health, quality of life and medication adherence (see table below).

Table1: Outcomes of psychological support

\begin{tabular}{llllllll}
\hline \multirow{2}{*}{ Variable } & $\begin{array}{l}\text { Screening } \\
\text { (Mean) }\end{array}$ & SD & $\begin{array}{l}\text { Follow-Up } \\
\text { (Mean) }\end{array}$ & SD & t value & p value & Eta $^{2}$ \\
\hline Anxiety & 12 & 3.6 & 9 & 4.1 & 4.87 & $.000 * * *$ & 0.36 \\
Depression & 8.8 & 3.9 & 6.4 & 5.0 & 4.34 & $.000 * * *$ & 0.30 \\
Distress & 18.2 & 4.8 & 13.9 & 5.1 & 7.47 & $.000 * * *$ & 0.56 \\
Mental QoL & 51.1 & 15.9 & 60.6 & 18.5 & -4.91 & $.000 * * *$ & 0.39 \\
Physical QoL & 72.5 & 14.9 & 75.0 & 17.7 & -1.50 & .142 & 0.06 \\
Total QoL & 57.6 & 14.6 & 65.1 & 17.4 & -4.39 & $.000 * * *$ & 0.34 \\
Medication adherence & 5.1 & 2.0 & 5.7 & 2.2 & -2.03 & $.049 *$ & 0.09 \\
\hline
\end{tabular}

$* p<.05, * * p<.01, * * * p<.001$

Conclusion: Psychological issues are prevalent in patients with IBD and associated with lower quality of life and reduced medication adherence. Women are more likely to participate in psychological screening, and in general the screening approach was widely accepted. In addition, high proportions of patients reported clinical levels of distress (irrespective of their IBD activity) and went on to accept psychological intervention. All of which demonstrates a widespread need for support in this cohort. Furthermore, preliminary data of treatment outcomes are promising. At study completion we will be better able to clarify the extent to which patients with IBD benefit from this new integrated approach.

Disclosure of Interest: All authors have declared no conflicts of interest.

P0397 LONG-TERM EFFICACY, SAFETY, AND IMMUNOGENICITY

DATA FROM A PHASE III CONFIRMATORY STUDY COMPARING GP2017, A PROPOSED BIOSIMILAR, WITH REFERENCE ADALIMUMAB
A. Blauvelt ${ }^{1}$, J. Lacour ${ }^{2}$, J. F. Fowler ${ }^{3}$, E. Schuck ${ }^{4}$, J. Jauch-Lembach ${ }^{4}$ A. Balfour ${ }^{4}$, C. L. Leonardi ${ }^{5}$
${ }^{1}$ Oregon Medical Research Center, Portland/United States of America
${ }^{2}$ University of Nice Sophia Antipolis, Nice/France
${ }^{3}$ Dermatology Specialists, Louisville/United States of America
${ }^{4}$ Hexal $A G$, Holzkirchen/Germany
${ }^{5}$ Central Dermatology, St Louis/United States of America

Contact E-mail Address: julia.jauch-lembach@sandoz.com

Introduction: Demonstration of biosimilarity is based on the evaluation of physicochemical, biological, preclinical, and clinical data. Based on this totality of evidence, a biosimilar may be approved for use in the same indications for which the reference medicine is approved without conducting a clinical trial in each indication. A prerequisite for this extrapolation is clinical confirmation of biosimilarity in a patient population sensitive enough to detect potential differences in efficacy, safety, or immunogenicity between the proposed biosimilar and the reference medicine. GP2017, a proposed biosimilar to adalimumab, was investigated in patients with chronic plaque psoriasis.

Aims \& Methods: To evaluate long-term efficacy, safety, and immunogenicity in patients continuously treated with either GP2017 or reference adalimumab from initial randomization to Week 51. Eligible patients with moderate-to-severe chronic plaque psoriasis were randomized to receive an initial dose of $80 \mathrm{mg}$ subcutaneous GP 2017 or reference adalimumab, followed by $40 \mathrm{mg}$ every other week, starting one week after the initial dose, up to Week 17. At Week 17 , patients with $\geq 50 \%$ improvement in Psoriasis Area and Severity Index (PASI 50 ) at Week 16 were re-randomized in a 2:1 ratio to either remain on their initial study treatment or undergo a sequence of three treatment switches between GP 2017 and reference adalimumab until Week 35. Thereafter, patients were returned to their originally randomized treatment up to Week 51 .

Results: From randomization to Week 51, 168 and 171 patients received continuous treatment with GP 2017 or reference adalimumab, respectively. In the perprotocol analysis set, PASI 75 response rates for continual GP2017/reference adalimumab at Weeks 17 and 51 were $75.2 \% / 67.8 \%$ and $84.5 \% / 79.6 \%$ respectively. Investigator's global assessment (IGA) response rates (IGA score of 0 [clear] or 1 [almost clear] and $\geq 2$ point improvement from baseline) were also similar between the continual GP2017/reference adalimumab groups, increasing over time and remaining stable from Week $17(60.0 \% / 53.9 \%)$ to Week 51 $(59.8 \% / 55.1 \%)$. There were no clinically relevant differences between the continual GP2017/reference adalimumab groups in the frequency of adverse events (AEs) $(61.3 \% / 64.9 \%)$, treatment-related AEs $(17.9 \% / 18.7 \%)$, serious AEs $(3.0 \% / 8.8 \%)$, or AEs leading to discontinuation of study drug $(4.8 \% / 7.0 \%)$. Infections/infestations were the most commonly reported AEs, with nasopharyngitis most frequently reported by $8.9 \% / 10.5 \%$ of patients treated with continual GP2017/reference adalimumab. Between Weeks 1 and 51, binding antidrug antibodies were detected in $38.8 \% / 45.3 \%$ of patients treated with continual GP2017/reference adalimumab, $88.7 \% / 84.7 \%$ of which were neutralizing.

Conclusion: Efficacy was similar and sustained in patients with psoriasis continuously treated with GP 2017 or reference adalimumab for up to 51 weeks. Safety profiles and immunogenicity were generally similar in both groups. Clinical data add to the totality of evidence suggesting GP 2017 could be used as a biosimilar for the treatment of the same indications for which reference adalimumab is approved, including inflammatory bowel disease.

Disclosure of Interest: A. Blauvelt: Investigator for Sandoz

J. Lacour: Investigator for AbbVie, Amgen, BMS, BI, Celgene, Galderma, Janssen, LEO Pharma, Lilly, MSD, Novartis, Pfizer, Regeneron, Roche, Sandoz; consultant/speaker for AbbVie, BMS, Celgene, Galderma, LEO Pharma, Lilly, Novartis, Regeneron, Roche and Sanofi

J.F. Fowler: Investigator for Sandoz

E. Schuck: Paid employee of Hexal AG, a Sandoz company

J. Jauch-Lembach: Paid employee of Hexal AG, a Sandoz company

A. Balfour: Paid employee of Hexal AG, a Sandoz company

C.L. Leonardi: Consultant for Abbvie, Amgen, BI, Dermira, Janssen, Eli-Lilly, Leo, Sandoz, UCB, Pfizer and Vitae and member of the Speaker bureau for Abbvie, Celgene, Novartis and Eli Lilly.

\section{P0398 PREDICTIVE FACTORS OF RESPONSE TO GRANULOCYTE - MONOCYTE APHERESIS IN INFLAMMATORY BOWEL DISEASE}

I. Rodríguez - Lago', J.M. Benítez-Cantero ${ }^{2}$, V. García-Sánchez ${ }^{2}$, L. Sempere ${ }^{3}$, A. Gutiérrez ${ }^{3}$, I. Galdona ${ }^{1}$, A. Rodríguez-Pescador ${ }^{1}$, E. Fernández ${ }^{4}$, I. Lafuente ${ }^{5}$, A. Loroño ${ }^{5}$, J.L. Cabriada ${ }^{1}$

${ }^{1}$ Gastroenterology, Hospital de Galdakao, Galdakao/Spain

${ }^{2}$ Gastroenterology, Hospital Universitario Reina Sofía, Córdoba/Spain

${ }^{3}$ Gastroenterology, Hospital General Universitario de Alicante, Alicante/Spain ${ }^{4}$ Dialysis Unit, Hospital de Galdakao, Galdakao/Spain

${ }^{5}$ Research Unit. Red De Investigación En Servicios De Salud En Enfermedades Crónicas (redissec)., Hospital de Galdakao, Galdakao/Spain

Contact E-mail Address: iago.r.lago@gmail.com

Introduction: Granulocyte-monocyte apheresis (GMA) can be employed for the treatment of inflammatory bowel disease (IBD), especially for ulcerative colitis (UC). The usual treatment schedule is a weekly session for 5 weeks processing $1800 \mathrm{ml}$ in 60 minutes. It has been described that different factors of the disease and the technique can improve the response to this treatment. 
Aims \& Methods: We performed a retrospective study of all patients treated with GMA (Adacolumn) in 3 IBD Units in Spain. The clinical and analytical data were assessed before and 1 month after the end of the GMA. The Ethics Committee of Euskadi approved the study protocol. The aim of our study was to evaluate the presence of clinical, analytical of technique-related factors associated to a better response to GMA.

Results: A total of 105 patients were included [51 female (49\%), age 35.7 (SD 16.5)]. Ninety-three had UC (50\% extensive, $45 \%$ left-sided), 10 Crohn's disease (90\% ileocolonic) and 2 IBD-U. Mayo score at baseline was 3.5 (SD 4.6) and Harvey - Bradshaw was 10.1 (SD 3.8). The Mayo endoscopic subscore was 1 $(16 \%), 2(56 \%)$ or $3(27 \%)$. Almost all patients $(97 \%)$ have been previously treated with steroids and $42 \%$ were exposed to biologics. At baseline, $85 \%$ were on steroids, $38 \%$ thiopurines and $18 \%$ biologics. None of the previous or concomitant treatments were associated with a better response to GMA. Fifty-six subjects received weekly sessions for 5 weeks processing $1800 \mathrm{ml} / \mathrm{session}$ in 60 minutes. Forty patients received an intensive GMA regimen: biweekly sessions with a mean of 8 sessions (SD 2.6), processing $3886 \mathrm{ml} /$ session (SD 1729) and lasting 91 minutes (SD 24). The intensive group showed a slightly higher response rate to GMA as compared with those in the standard regimen (response rate $67 \%$ vs $55 \%, \mathrm{p}=0.28)$. Those subjects treated with $>5$ sessions showed higher remission $(24 \%$ vs $13 \%)$ and response rates $(47 \%$ vs $24 \%)$ as compared to $<5$ sessions $(\mathrm{p}=0.004)$. A mean duration of $>60 \mathrm{~min} / \mathrm{session}$ also showed better results in terms of remission ( $22 \%$ vs $16 \%$ ) and response ( $45 \%$ vs $27 \%$ ) when compared to $<60 \mathrm{~min} / \mathrm{session}(\mathrm{p}=0.04)$. There was also a trend towards higher remission rates in those with higher processed blood volume. Thirty-nine percent were able to wean off steroids completely one month after GMA. We observed a decrease in the mean platelet volume and the platelet to lymphocyte ratio after GMA in those cases who did not respond

Conclusion: GMA showed its clinical efficacy in this clinical practice study. Increasing the number of sessions or its length were associated with a better response to GMA. The mean platelet volume and the platelet to lymphocyte ratio could help to predict the response.

Disclosure of Interest: All authors have declared no conflicts of interest.

\section{References}

Sakuraba A, Motoya S, Watanabe K, Nishishita M, Kanke K, Matsui T, et al. An open-label prospective randomized multicenter study shows very rapid remission of ulcerative colitis by intensive granulocyte and monocyte adsorptive apheresis as compared with routine weekly treatment. Am J Gastroenterol. 2009;104:2990-5

Yoshimura N, Yokoyama Y, Matsuoka K, Takahashi H, Iwakiri R, Yamamoto $\mathrm{T}$, et al. An open-label prospective randomized multicenter study of intensive versus weekly granulocyte and monocyte apheresis in active Crohn's disease. BMC Gastroenterol. 2015;15:163.

Yoshimura N, Tadami T, Kawaguchi T, Sako M, Yoshimoto H, Yamaka T, et al. Processed blood volume impacts clinical efficacy in patients with ulcerative colitis undergoing adsorptive depletion of myeloid lineage leucocytes. $J$ Gastroenterol. 2012;47:49-55.

\section{P0399 ANDECALIXIMAB (ANTI-MMP9) INDUCTION THERAPY FOR ULCERATIVE COLITIS: A DOUBLE-BLIND, RANDOMIZED, PLACEBO-CONTROLLED, PHASE 2 STUDY}

W.J. Sandborn ${ }^{1}$, B. R. Bhandari ${ }^{2}$, C. Randall ${ }^{3}$, Z. Younes ${ }^{4}$, T. Romańczyk ${ }^{5}$, H. Chai ${ }^{6}$, M. Mckevitt ${ }^{7}$, S. Zhao ${ }^{6}$, B. Kanwar ${ }^{6}$, J. Sundy ${ }^{6}$, S. Keshav ${ }^{8}$, S. Danese ${ }^{9}$

${ }^{1}$ University of California, San Diego/United States of America

${ }^{2}$ Delta Research Partners, Monroe/United States of America/LA

${ }^{3}$ Gastroenterology Research of America/U. of Texas, San Antonio/United States of America/AL

${ }^{4}$ GastroOne, Germantown/United States of America/TN

${ }^{5}$ Takeda Polska Sp. z o.o., Tychy/Poland

${ }^{6}$ Gilead Sciences, Foster City/United States of America

${ }^{7}$ Clinical Research, Inflam/resp, Gilead Sciences, Foster City/United States of America

${ }^{8}$ Translational Gastroenterology Unit, University of Oxford, DU/United Kingdom ${ }^{9}$ Istituto Clinico Humanitas IBD Center, Milan/Italy

Contact E-mail Address: wsandborn@ucsd.edu

Introduction: Circulating and mucosal concentrations of matrix-metalloproteinase-9 (MMP-9) are upregulated in patients with ulcerative colitis (UC) and correlate with disease activity. Selective inhibition or deletion of MMP-9 reduced disease severity in murine models of colitis. Accordingly, MMP-9 has been proposed as a biomarker for disease activity and/or as a treatment target in UC. Andecaliximab (GS-5745) is a monoclonal antibody that selectively binds and inhibits MMP-9. A Phase 1 dose-ranging study of andecaliximab was conducted in UC patients, demonstrating clinical response and remission. We conducted a phase 2 study to further evaluate the safety and efficacy of andecaliximab in subjects with UC.

Aims \& Methods: This was a double-blind, randomized, placebo-controlled 8week induction study in adult subjects with UC with moderate to severe disease activity; defined as an endoscopy score $\geq 2$, rectal bleeding score $\geq 1$, stool frequency score $\geq 1$ and physician global assessment of 2 or 3 (using the Mayo score). Subjects were required to have an inadequate response to, or loss of response or intolerance to at least 1 of the following treatments in the last 5 years: corticosteroids, immunomodulators, TNF-alpha antagonist or vedolizumab. Subjects were randomized 1:1:1 to receive subcutaneous (SC) injections of: placebo, $150 \mathrm{mg}$ andecaliximab every 2 weeks (Q2W) or $150 \mathrm{mg}$ andecaliximab weekly (QW). Centrally-read sigmoidoscopies/colonoscopies were performed at baseline and week 8. The primary outcome was EBS clinical remission, defined as an Endoscopic subscore of $\leq 1$, rectal Bleeding subscore of 0 , and $>1$ point decrease in Stool frequency from baseline to achieve a subscore of 0 or 1 .

Results: A total of 165 subjects from 23 countries were enrolled. The percentage (confidence intervals) of subjects achieving EBS clinical remission was similar between subjects treated with andecaliximab Q2W, andecaliximab QW and placebo: $7.4 \%(2.1-17.9 \%), 1.8 \%(0-9.6 \%)$ and $7.3 \%(2.0-17.6 \%)$, respectively. Confidence intervals overlap for all groups and no single EBS component subscore appears to have driven the results. No concerning imbalances occurred between the treatments groups (Table 1).

\begin{tabular}{llll}
\hline & $\begin{array}{lll}150 \mathrm{mg} \text { Q2W } \\
\mathrm{N}=54\end{array}$ & $\begin{array}{l}150 \mathrm{mg} \text { QW } \\
\mathrm{N}=56\end{array}$ & $\begin{array}{l}\text { Placebo } \\
\mathrm{N}=55\end{array}$ \\
\hline Mayo Clinical Score, mean (SD) & $9(1.3)$ & $9(1.3)$ & $9(1.3)$ \\
TNF-alpha experienced & $53.7 \%$ & $55.4 \%$ & $54.5 \%$ \\
Corticosteroid use at baseline & $37 \%$ & $39.3 \%$ & $30.9 \%$ \\
\hline
\end{tabular}

The frequency of adverse events (AEs) was similar in the andecaliximab Q2W, andecaliximab QW and placebo groups: $53.7 \%, 58.9 \%$ and $60 \%$, respectively. Common AEs included anemia, abdominal pain and nausea. Three AEs led to discontinuation in the andecaliximab QW group compared to one each in the andecaliximab Q2W and placebo groups. Two serious AEs occurred in the andecaliximab QW group (anemia and angina pectoris) compared to one in placebo. Frequency of arthralgia and musculoskeletal pain was similar between andecaliximab and placebo groups.

Conclusion: SC $150 \mathrm{mg}$ andecaliximab was well tolerated; however, neither dosing regimen demonstrated a treatment effect in subjects with UC.

Disclosure of Interest: W.J. Sandborn: Consulted for Gilead Sciences.

Z. Younes: Consulted for Gilead Sciences

H. Chai: Employee of Gilead Sciences.

M. McKevitt: Employee of Gilead Sciences

S. Zhao: Employee of Gilead Sciences.

B. Kanwar: Employee of Gilead Sciences.

J. Sundy: Employee of Gilead Sciences.

S. Keshav: Consulted with Gilead Sciences

S. Danese: Consulted for Gilead Sciences.

All other authors have declared no conflicts of interest.

\section{P0400 ANDECALIXIMAB (ANTI-MMP9) INDUCTION THERAPY FOR CROHN'S DISEASE: A DOUBLE-BLIND, RANDOMIZED, PLACEBO-CONTROLLED, PHASE 2 STUDY}

S. Schreiber ${ }^{1}$, C. Siegel ${ }^{2}$, K. Friedenberg ${ }^{3}$, U. Seidler ${ }^{4}$, B. R. Bhandari ${ }^{5}$

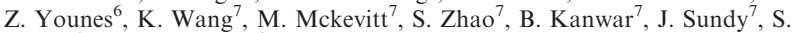

D. Lee ${ }^{8}$, E. V. Loftus $\mathrm{Jr}^{9}$

${ }^{1}$ Christian-Albrechts-University Kiel, Kiel/Germany

${ }^{2}$ Dartmouth-Hitchcock Medical Center, Lebanon/United States of America/NH

${ }^{3}$ Great Lakes Gastroenterology, Mentor/United States of America/OH

${ }^{4}$ Hannover Medical School, Hannover/Germany

${ }^{5}$ Delta Research Partners, Monroe/United States of America/LA

${ }^{6}$ GastroOne, Germantown/United States of America/TN

${ }^{7}$ Gilead Sciences, Foster City/United States of America

${ }^{8}$ University of Washington, Seattle/United States of America/WA

${ }^{9}$ Mayo Clinic College of Medicine, Rochester/United States of America

Contact E-mail Address: s.schreiber@mucosa.de

Introduction: Elevated levels of matrix-metalloproteinase-9 (MMP-9) and its degradation products are detected in patients with active Crohn's disease (CD). Selective inhibition of MMP-9 reduced fibrosis in a murine model of intestinal fibrosis, suggesting that MMP-9 may contribute to intestinal complications in CD. Accordingly, MMP-9 has been proposed as a therapeutic target for CD. Andecaliximab (GS-5745) is a monoclonal antibody that selectively binds and inhibits MMP-9. It was found to be safe in a phase 1 dose-ranging study in UC subjects, where it showed clinical response and remission compared to placebo. The aim of this phase 2 study was to evaluate the safety and efficacy of andecaliximab in subjects with moderately to severely active CD.

Aims \& Methods: This was a double-blind, randomized, placebo-controlled 8 week induction study in adult CD subjects with moderate to severe disease activity (defined as: CDAI total score 220-450, weighted PRO2 score $>11$ [standard CDAI weightings: abdominal pain $0-3 \times 7$ plus mean number of daily stools $\times 2]$ and SES-CD total score $\geq 6$ [or $\geq 4$ score if disease limited to ileum and/or right colon or ulcer presence and size score $>2]$ ). Subjects were required to have an inadequate response, or loss of response or intolerance to at least 1 of the following treatments in the last 5 years: corticosteroids, immunomodulators, TNFalpha antagonist or vedolizumab. Subjects were randomized 1:2:2:2 to receive subcutaneous (SC) injections of: placebo, $150 \mathrm{mg}$ andecaliximab every 2 weeks (Q2W), $150 \mathrm{mg}$ andecaliximab weekly (QW) or $300 \mathrm{mg}$ andecaliximab QW Centrally-read colonoscopies were performed at baseline and week 8. Co-primary outcomes were clinical response (PRO2 score of $\leq 8$ at week 8 ) and endoscopic response (SES-CD decrease of $\geq 50 \%$ from baseline at week 8 ).

Results: A total of 187 subjects were enrolled from 13 countries. No concerning baseline imbalances occurred between the treatment groups, overall: $17.6 \%$ of subjects had evidence of fistula at screening, mean (SD) disease duration was 12 (9.5) years, $84.5 \%$ of subjects had previous exposure to TNF-alpha antagonist and $44.4 \%$ of subjects were taking oral corticosteroids at baseline. The 


\begin{tabular}{|c|c|c|c|c|}
\hline $\begin{array}{l}\text { Number }(\%) \text { of Subjects } \\
\text { Achieving [confident intervals] }\end{array}$ & $150 \mathrm{mg}$ Q2W $\mathrm{N}=53$ & $150 \mathrm{mg} \mathrm{QW} \mathrm{N}=53$ & $300 \mathrm{mg} \mathrm{QW} \mathrm{N}=53$ & placebo $\mathrm{N}=28$ \\
\hline PRO2 score of $\leq 8$ & $9(17.0 \%)[8.1-29.8]$ & $7(13.2 \%)[5.5-25.3]$ & $6(11.3 \%)[4.3-23.0]$ & $4(14.3 \%)[4.0-32.7]$ \\
\hline$\geq 50 \%$ decrease in SES-CD & $6(11.3 \%)[4.3-23.0]$ & $7(13.2 \%)[5.5-25.3]$ & $4(7.5 \%)[2.1-18.2]$ & $3(10.7 \%)[2.3-28.2$ \\
\hline $\mathrm{CDAI} \leq 150$ & $11(20.8 \%)[10.8-34.1]$ & $9(17.0 \%)$ [8.1-29.8] & $6(11.3 \%)[4.3-23.0]$ & $6(21.4 \%)[8.3-41.0$ \\
\hline
\end{tabular}

The frequency of adverse events (AEs) was similar between the treatment groups: placebo (67.9\%), 150 mg Q2W (60.4\%), 150 mg QW (62.3\%), 300 mg QW (69.8\%). Common AEs included abdominal pain, nausea, fatigue, anemia and pyrexia. One AE led to study discontinuation in the placebo group (3.6\%) compared to 2 in the $150 \mathrm{mg}$ QW group (3.8\%) and 4 in the $300 \mathrm{mg}$ QW group (7.5\%). Three serious AEs occurred in the placebo group (10.7\%) compared to 1 in the $150 \mathrm{mg}$ Q $2 \mathrm{~W}$ group $(1.9 \%), 6$ in the $150 \mathrm{mg}$ QW group $(11.3 \%)$ and 8 in the $300 \mathrm{mg}$ QW group $(15.1 \%)$. Frequency of arthralgia and musculoskeletal pain was similar or lower in andecaliximab groups compared to placebo.

percentage of subjects achieving clinical response/remission and endoscopic response was similar between treatment groups (Table 1).

Conclusion: SC andecaliximab was well tolerated; however, none of the treatment regimens demonstrated a treatment effect in subjects with $\mathrm{CD}$.

\section{Disclosure of Interest:}

S. Schreiber: Consulted with Gilead Sciences.

Z. Younes: Consulted with Gilead Sciences.

K. Wang: Employed by Gilead Sciences.

M. McKevitt: Employee of Gilead Sciences.

S. Zhao: Employee of Gilead Sciences.

B. Kanwar: Employee of Gilead Sciences

J. Sundy: Employee of Gilead Sciences.

E.V. Loftus Jr: Consulted with Gilead Sciences.

All other authors have declared no conflicts of interest.

\section{P0401 TUBERCULIN SKIN TEST CONVERSION RATE IN INFLAMMATORY BOWEL DISEASE PATIENTS RECEIVING ANTI- TNF ALPHA AGENTS}

M. Fragkaki, A. Mpitouli, I. Dimas, G. Paspatis, K. Karmiris Gastroenterology, Venizeleion General Hospital, Heraklion/Greece

\section{Contact E-mail Address: gpaspatis@gmail.com}

Aims \& Methods: Few data exist regarding the kinetics of this test during therapy. Therefore, our study investigated the conversion rate of PPD-TST in IBD patients under anti-TNFalpha treatment. Anti-TNFalpha-treated IBD patients followed up in our centre with a baseline PPD-TST underwent a second one during therapy. Those with a positive PPD-TST either at baseline or during therapy $(\mathrm{d}>10 \mathrm{~mm}$ in naïve and $\mathrm{d}>5 \mathrm{~mm}$ in those exposed to immunomodulators [IMS]) received $300 \mathrm{mg}$ isoniazid orally for 9 months.

Introduction: Anti-TNFalpha therapy increases the risk of tuberculosis (TB) (re)activation in inflammatory bowel disease (IBD) patients. Purified protein derivative tuberculin skin test (PPD-TST) is considered a pre-requisite at baseline. Results: Sixty-eight IBD patients have currently been enrolled (males: $51.47 \%$, Crohn's disease: $82.35 \%$ ). Median age at IBD diagnosis was 33.1 years [IQR: 20.3, range: 16.7-66.7]. Median duration of IBD was 7.7 months [IQR: 9.8, range: $1.4-32.7]$. Nine patients $(13.23 \%)$ had a positive PPD-TST at baseline. 48 patients have undergone a $2^{\text {nd }}$ PPD-TST (median time between the $1^{\text {st }}$ and $2^{\text {nd }}$ PPD-TST: 44.26 months [IQR: 42.8, range: 6.3-190.1]). Twenty patients were under combination therapy with an IMS at the $2^{\text {nd }}$ PPD-TST. Six patients with a positive baseline PPD-TST remained positive (in 5 patients the diameter was decreased $\&$ in one increased $7 \mathrm{~mm}$ ). Out of the remaining 42 patients with a negative baseline PPD-TST, eight (19\%) exhibited a positive $2^{\text {nd }}$ PPD TST; three of them were receiving infliximab for less than 3 years and five of them adalimumab ( 2 for less and 3 for more than 3 years). Only $2 / 8$ were under combination therapy. There was no case of active tuberculosis during the study. All patients with a PPD-TST conversion received anti-tuberculous treatment.

Conclusion: A positive PPD-TST followed by anti-TB treatment before the initiation of anti-TNFalpha in IBD patients was not associated with an increased rate of TB infection during therapy. One-fifth of the patients with a negative baseline PPD-TST demonstrated a conversion but without any undesirable consequence if so treated. Therefore, the kinetic of PPD-TST in IBD patients under antiTNFalpha treatment should be monitored.

\section{P0402 THE TEMPORAL EVOLUTION OF IMMUNOGENICITY IN INFLAMMATORY BOWEL DISEASE PATIENTS TREATED WITH ADALIMUMAB}

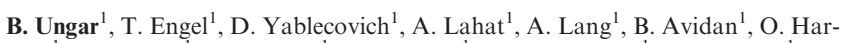
Noy $^{1}$, N. Levhar ${ }^{1}$, L. Selinger ${ }^{1}$, S. Neuman ${ }^{1}$, O. Haj Natour ${ }^{1}$, M. Yavzori ${ }^{1}$, E. Fudim ${ }^{1}$, O. Picard ${ }^{1}$, U. Kopylov ${ }^{1}$, Y. Chowers ${ }^{2}$, T. Naftali ${ }^{3}$, E. Broide $^{4}$, E. Shachar ${ }^{5}$, R. Eliakim ${ }^{1}$, S. Ben-Horin ${ }^{1}$

${ }^{1}$ Gastroenterology Institute, - Gastroenterology institute, ;/IL, Ramat Gan/Israel ${ }^{2}$ Gastroenterology, Rambam Health Care Campus, Haifa/Israel

${ }^{3}$ Gastroenterology, Meir Medical Center, Kfar Saba/Israel

${ }^{4}$ The Kamila Gonczarowski Institute Of Gastroenterology, Assaf Harofeh Medical Center, Zerifin/Israel
${ }^{5}$ Assaf Haroffe medical center Clalit Health Services Gastroenterology, Zerifin/ Israel

Contact E-mail Address: bellageyshis@gmail.com

Introduction: Adalimumab and anti-adalimumab-antibodies (AAA) levels have been associated with clinical outcome of Crohn's disease (CD). Nevertheless, because adalimumab is usually self-injected at home, prospective serial-sampling studies are scarce. Thus, data on the temporal evolution of adalimumab immunogenicity is still limited, and the validity of comparisons of adalimumab versus infliximab immunogenicity remains questionable.

Aims \& Methods: Our aim was to assess trends in adalimumab and AAA levels over time and their clinical implications. CD patients starting adalimumab therapy were followed prospectively in three participating medical centers in Israel, by establishing a program for home-visits by physicians at induction and every 3 months, or in case of relapse. At each home visit, patients' clinical activity scores were determined and blood tests obtained for CRP, drug and AAA trough levels. AAA levels were determined by a drug-tolerant assay. A comparison with temporal evolution of infliximab immunogenicity in a previously reported cohort using the same assay and methodology was additionally performed.

Results: $102 \mathrm{CD}$ patients starting adalimumab were prospectively followed. Fourteen $(14 \%)$ experienced primary non-response and $20(20 \%)$ lost response to adalimumab therapy during maintenance. Thirty-three $(32 \%)$ developed AAA, which were more common among those previously exposed to adalimu$\mathrm{mab}(\mathrm{p}=0.002)$ but were not affected by co-treatment with immunomodulators or not $(\mathrm{p}=0.28)$. AAA developed as early as week 2 in $18 / 33(55 \%)$ of AAA positive patients ( $7 / 18$ with history of interrupted therapy), and in 26/33 (79\%) within week 14. Patients with week 2 AAA had $34 \%$ of primary non-response compared to $9 \%$ in patients without early AAA $(\mathrm{OR}=4.8, \mathrm{p}=0.009)$. In $92.6 \%$ of cases, AAA preceded loss-of-response or occurred simultaneously (median interval - 4 weeks). As compared to antibodies-to-infliximab (ATI), AAA formation rate over time was significantly lower $(\mathrm{p}=0.01, \log$ rank test), and some patients developed AAA even after one year of therapy. Transient AAA were much less common than transient ATI $(7 \%$ vs $32 \%, \mathrm{p}<0.0001)$, and $85 \%$ of AAA events were associated with loss of response compared with $58 \%$ rate for $\operatorname{ATI}(\mathrm{p}=0.01)$

Conclusion: AAA formation often occurs earlier than appreciated, and associates with primary non-response to adalimumab induction. Overall rate of immunogenicity is lower for adalimumab compared to infliximab. However, once they occur, AAA are more specific than ATI.

Disclosure of Interest: B. Ungar: This study was supported in part by a grant from "Abbvie". In addition, BU received consultation fees from "Abbvie" and from "Jannsen".

U. Kopylov: Speaker fees - abbvie Research support, speaker and advisory fees jannsen

Y. Chowers: Abbvie - grant support, lecture and advisory fees Janssen - lecture and advisory fees Takeda - grant support lecture and advisory fees Medtronics advisory fees

S. Ben-Horin: SBH has received consultancy and/or advisory board fees from Schering-Plough, AbbVie, Celltrion, Pfizer, Janssen and Takeda; and has received research support from Celltrion, AbbVie \& Takeda

All other authors have declared no conflicts of interest.

\section{P0403 GOLIMUMAB IN ULCERATIVE COLITIS. REAL-LIFE PROSPECTIVE COHORT STUDY FROM A SINGLE REFERRAL CENTRE OF MIDDLE ITALY}

L. Grossi ${ }^{1}$, V. Vecchione ${ }^{1}$, S. Cocciolillo ${ }^{2}$, R. Tavani ${ }^{3}$, M. Di Berardino ${ }^{1}$, A. Sepe ${ }^{4}$, C. Di Giacomo ${ }^{1}$, N. Russo ${ }^{1}$, V. D'Addetta ${ }^{1}$, L. Marzio

${ }^{1}$ Digestive Sciences, G. D'Annunzio University, Pescara/Italy

${ }^{2} G$ dAnnunzio University, Digestive Physiopathology Unit, Ospedale Spirito Santo, Pescare/Italy

${ }^{3} G$ dAnnunzio University, School of Gastroenterology, Digestive Physiopathology Unit, Ospedale Spirit, Pescara/Italy

${ }_{5}^{4} G$ d'Annunzio University, Pescara/Italy

${ }^{5}$ Dipartimento Medicina - Ospedale Civile Pescara, Università G. DAnnunzio, Pescara/Italy

Contact E-mail Address: 1.grossi@unich.it

Introduction: Golimumab (GLB) has been the last anti-TNF agent authorized for the treatment of Ulcerative Colitis (UC). Results from registratory trial (PURSUIT) documented a clinical response in $51 \%$ of patients after 6 weeks 
of GLB with $47-49 \%$ of patients maintaining the effect after one year. Due to its relatively recent use there are still few real-life data on clinical outcomes of patients receiving golimumab in the routinely activities. In our region GLB became available starting July 2015.

Aims \& Methods: Aim of this study has been to prospectively evaluate the efficacy and safety of golimumab for the treatment of UC in the real-life setting of our referral centre. 13 patients ( 7 male, 4 female) with moderate-to-severe UC were enrolled in the study from June 2015 to December 2016. Patients received an induction dose of GLB $200 \mathrm{mg}$ s.c. at baseline, $100 \mathrm{mg}$ at week 2 and then a monthly dose of $50 \mathrm{mg}$ or $100 \mathrm{mg}$ for a body weight $<$ or $>80 \mathrm{~kg}$, respectively, with no optimization allowed. Partial Mayo score was computed at baseline and every 2 weeks for the first 6 weeks of therapy, then every 4 weeks throughout the maintenance period. Follow-up is still ongoing. Primary end point has been the clinical response at the end of the induction phase (intended as the reduction of Partial Mayo score $>30 \%$ and $>3$ points vs baseline) and in the maintenance period, the secondary end point being the steroid-free clinical remission (Partial Mayo score $<2$ with all subscores $<1$ ) at the end of the induction phase and throughout the maintenance phase. Complete follow-up is available for all patients at week 30 , with 4 patients reaching the week 54 of monitoring.

Results: At the time of GLB starting, localization of the disease according to Montreal classification was left-sides colitis (E2) in $70 \%$, pancolitis (E3) in $23 \%$ and proctitis (E1) in 7\% of patients. Ten patients $(77 \%)$ were anti-TNF naïve, 3 patients $(23 \%)$ had already received one anti-TNF in the past. Clinical response was obtained in $6 / 13(46 \%)$ at week 6 and in 2 further patients at week 10, for a total of $8 / 13(62 \%)$. Three patients resulted in complete clinical steroid-free remission after 6 weeks. At week 30, 5 patients still showed a clinical response $(38 \%)$, one of them $(7 \%)$ resulted in complete steroid-free remission. Among the 4 patients reaching week 54, 2 experienced a flare of disease whereas 2 were still in remission $(16 \%)$; the last patient is in remission at week 42 , potentially accounting for a total of $3 / 13$ patients in remission after one year $(23 \%)$. No differences were found between naïve and non-naïve patients. No significant adverse events were reported in the study period.

Conclusion: Our real-life data seem to suggest that Golimumab, as compared to registratory trials, is able to induce a better initial clinical response but shows a higher secondary loss of response in the long term. Whether this really reflects a lower efficacy of GLB or could depend on the unavailability of dose optimization, not allowed in the Italian prescription rules for GLB, needs to be evaluated. Disclosure of Interest: All authors have declared no conflicts of interest.

\section{P0404 FIVE-YEAR SAFETY DATA FROM THE OBSERVATIONAL POSTMARKETING ULCERATIVE COLITIS STUDY, A EUROPEAN REGISTRY FOR ADULTS WITH ULCERATIVE COLITIS TREATED WITH ORIGINATOR INFLIXIMAB OR STANDARD THERAPY}

J. Panés ${ }^{1}$, J. Lindsay ${ }^{2}$, N. Teich ${ }^{3}$, S. Lindgren ${ }^{4}$, J. Colombel $^{5}$, F. Cornillie ${ }^{6}$, H.A. Flynn ${ }^{7}$, S. Huyck ${ }^{7}$, P. Stryszak ${ }^{7}$, R. Yao ${ }^{7}$, G. Philip ${ }^{7}$, W. Reinisch ${ }^{8}$

${ }^{1}$ Dept. Of Gastroenterology, Hospital Clínic Barcelona Dept. of Gastroenterology Dept. of Gastroenterology, Hospital Clínic Bar, Barcelona/Spain

${ }^{2}$ Dept. Of Digestive Diseases, Digestive Disorders Clinical Academic Unit, Barts \& The London School of Medicine, London/United Kingdom

${ }^{3}$ Internistische Gemeinschaftspraxis, Leipzig/Germany

${ }^{4}$ Lund University, Malmo/Sweden

${ }^{5}$ Icahn School of Medicine at Mount Sinai, New York/United States of America ${ }^{6}$ MSD Switzerland, Lucerne/Switzerland

${ }^{7}$ Merck \& Co., Inc., Kenilworth/United States of America

${ }^{8}$ Dep.of Internal Medicine IV, Division Of Gastroenterology And Hepatology, Medizin. Universität Wien Abt. für Innere Medizin III - Dep.of Internal Medicine IV, Division of Gas, Wien/Austria

Contact E-mail Address: jpanes@ clinic.ub.es

Introduction: The Observational Postmarketing Ulcerative colitis Study (OPUS) registry was conducted to collect long-term (5 years) real-world clinical practice safety data in patients with moderate to severe ulcerative colitis (UC) treated with originator infliximab and to compare this safety profile to that of UC patients treated with standard therapies.

Aims \& Methods: The OPUS registry was a prospective, non-randomized, observational study that followed patients with UC (in routine practice in 14 European countries) who were enrolled to receive treatment with either originator infliximab or standard therapy (defined as initiation or dose-increase of corticosteroids and/or immunosuppressants), as determined by their treating physician. Adverse events (AEs) were recorded during the 5-year follow-up period; at any time during the 5 years of observation, the patient's therapy could be changed to any other UC therapy, based on the physician's clinical judgment. Frequency of events was evaluated in nine pre-specified categories (serious infection, infusionrelated reaction, fatality, worsening or new case of congestive heart failure $(\mathrm{CHF})$, central and peripheral demyelinating neurological disorder, hematologic condition, malignancy/lymphoproliferative disorder, autoimmune disorder, or hepatobiliary event). Multivariable Cox proportional hazards $(\mathrm{PH})$ models assessed time-to-first AE for the originator infliximab and standard therapy groups, using an intent-to-treat approach, in eight of the pre-specified categories (infusion-related reactions were not compared between originator infliximab and standard [i.e., generally oral] therapies); p-values were not adjusted for multiple comparisons. Safety data were collected every 6 months.

Results: Data for 2239 patients were available: 1180 patients enrolled to standard therapy (including 296 patients who switched to originator infliximab during follow-up) and 1059 patients enrolled to originator infliximab. Patients who received originator infliximab, standard therapy, or who were switched to originator infliximab during follow-up on standard therapy, were followed for medians of $59.4,50.6$, and 49.8 months, respectively. However, median exposure to infliximab was 19.8 and 15.9 months in the originator infliximab and switchedto-originator infliximab groups, respectively. Patients enrolled in the originator infliximab group, compared with the standard therapy group, had more severe disease at baseline, based on partial Mayo score (PMS): $46.0 \%$ of patients in the originator infliximab group had severe disease (PMS of 7-9 [out of 9]), compared with $30.5 \%$ in the standard therapy group. In time-to-event analyses adjusting for risk factors/confounders, enrollment into the originator infliximab group was associated with a higher risk of serious infection (hazard ratio $=2.08,95 \%$ confidence interval $[\mathrm{CI}] 1.42,3.06 ; \mathrm{p}<0.001]$ ) compared with enrollment into the standard therapy group (Table). No notable risk differences between groups were identified for hematologic condition, autoimmune disorder, malignancy/lymphoproliferative disorder, hepatobiliary event, and fatality (Table). Because of very low incidence of AEs in the categories of $\mathrm{CHF}$ and demyelinating disorder $(£ 0.3 \%$ in each group), meaningful multivariable time-to-event analyses could not be performed for these categories.

Conclusion: Data from 5-year safety follow-up of patients with moderate to severe UC in this non-randomized registry population demonstrate that, compared with the standard therapy group, the patients enrolled to receive originator infliximab had an increased risk of serious infection. This finding is consistent with the previously established safety profile for originator infliximab in the treatment of UC. In this large registry, the originator infliximab group, compared with the standard therapy group, did not have a significantly increased risk of a hematologic condition, autoimmune disorder, malignancy/lymphoproliferative disorder, hepatobiliary event, $\mathrm{CHF}$, demyelinating disorder, or fatality. No new safety concerns were observed with originator infliximab in the OPUS registry.

Disclosure of Interest: J. Panés: J.P. has received consultant or speaker fees from Merck \& Co., Inc.

J. Lindsay: J.L. has received consultant and speaker fees from Merck \& Co., Inc. N. Teich: N.T. is a scientific advisor for and has received speaker fees from Merck \& Co., Inc.

S. Lindgren: S.L. has received consultant and lecture fees from Merck \& Co., Inc. J. Colombel: J-FC. has served as a consultant, advisory board member, or speaker for Merck \& Co., Inc.

F. Cornillie: F.C. is an employee of MSD Switzerland, the sponsor of the study. H.A. Flynn: HAF. is an employee of Merck \& Co., Inc., the sponsor of the study. S. Huyck: S.H. is an employee of Merck \& Co., Inc., the sponsor of the study. P. Stryszak: P.S. is an employee of Merck \& Co., Inc., the sponsor of the study. R. Yao: R.Y. is an employee of Merck \& Co., Inc., the sponsor of the study. G. Philip: G.P. is an employee of Merck \& Co., Inc., the sponsor of the study. W. Reinisch: W.R. has served as a speaker and advisory board member for Merck \& Co., Inc.

\section{P0405 DIRECT INTESTINAL PH-TRANSIT ASSESSMENT IN PATIENTS WITH ULCERATIVE COLITIS AS A STRATEGY FOR PREDICTING SUCCESS OF REGIONAL DELIVERY OF TOPICALLY- ACTING DRUGS}

C. K. Yao ${ }^{1}$, R. E. Burgell ${ }^{1}$, K. M. Taylor ${ }^{2}$, M. G. Ward ${ }^{3}$, J. S. Barrett ${ }^{1}$, J. G. Muir ${ }^{1}$, P.R. Gibson ${ }^{3}$

${ }^{1}$ Department Of Gastroenterology, Monash University, Melbourne/Australia/VIC

${ }^{2}$ Dept Gastroenterology, Monash University and Alfred Hospital, Melbourne/ Australia/VIC

${ }^{3}$ Department Of Gastroenterology, Alfred Hospital, Melbourne/Australia/VIC

Contact E-mail Address: chu.yao@monash.edu

Introduction: Several oral mesalazine utilise $\mathrm{pH}$-dependent release coating to optimise mucosal delivery of active drug. Little data currently exist on concurrent intestinal $\mathrm{pH}$ and transit times in patients with $\mathrm{UC}$ to predict likely efficacybased delivery profiles. No studies have accounted for acute variations in dietary fibre intake, which might affect regional transit and $\mathrm{pH}$ profiles.

Aims \& Methods: This study aimed to (1) to examine regional luminal $\mathrm{pH}$ and transit after acute changes in fibre intake in quiescent UC patients; and (2) deduce delivery of topically-acting drugs using published pharmacokinetic (PK) data. In this randomised, double-blind study, patients with UC in clinical remission (partial Mayo Score $\leq 1+$ faecal calprotectin $<150 \mu \mathrm{g} / \mathrm{g}$ ) without recent antibiotic (including sulfasalazine), probiotic or fibre use were recruited After a 7-day run-in, subjects were supplied with study meals containing high $(13 \mathrm{~g}$ oligosaccharides and resistant starch) $(\mathrm{HF})$ or low $(<1 \mathrm{~g})$ fermentable fibre (LF) over a 12-h period prior to the ingestion of a $\mathrm{pH}$-motility capsule (Smart Pill). Telemetric recordings were made for 5 days or until the capsule had been excreted. After a $\geq 3$-day washout period, they crossed-over to the other dietary arm and $\mathrm{pH}$-motility test. Small and large intestinal $\mathrm{pH}$ profiles were defined as the length in time $(\mathrm{h})$ of different intraluminal $\mathrm{pH}$ ranges $-\mathrm{pH}<6, \geq 6$ to $<7$ and $\geq 7$ and compared between diets. Using published $\mathrm{PK}$ of $\mathrm{pH}$-dependent coated mesalazine preparations, the patterns of dissolution in UC patients were estimated.

Results: In 15 patients (aged 24-72 y; 9 males - 5 extensive, 5 distal and 5 proctitis), acute HF intake significantly increased median (IQR) time for colonic $\mathrm{pH}<6$ [HF: $4.5(2.4-10.2)$ vs LF: $0.9(0.2-3.1) \mathrm{h} ; p=0.004$, Wilcoxon], tended to increase colonic $\mathrm{pH} \geq 6$ to $<7$ [HF $7.5(6.3-13.5)$ vs LF: 7.1(3.4-15.2) h] and had no impact on colonic $\mathrm{pH} \geq 7$ [HF: $4.8(2.1-8.1)$ vs LF: $1.2(1.0-11.4)$ ]. Table 1 summarises hypothetical dissolution profiles for $\mathrm{pH}$-coated mesalazine in patients with UC. Considerable variations across release mechanisms were evident, but, despite alterations in $\mathrm{pH}$ and transit, the patterns were not affected by diet for Eudragit L alone or with slow release mechanisms. Only minor differences were found for Eudragit S and MMX.

Conclusion: Current delivery mechanisms for mesalazine lead to a proportion of quiescent UC patients having incomplete release and suboptimal regional 
Pre-specified Category of Adverse Event

\begin{tabular}{|c|c|c|c|c|c|}
\hline Serious Infection & $\begin{array}{l}\text { Hematologic } \\
\text { Condition }\end{array}$ & $\begin{array}{l}\text { Autoimmune } \\
\text { Disorder }\end{array}$ & \multicolumn{3}{|c|}{ Malignancy/Lympho-proliferative Disorder Hepatobiliary Event Fatality } \\
\hline \multicolumn{6}{|c|}{ Number of patients and incidence (\%) } \\
\hline $97(9.2 \%)$ & $44(4.2 \%)$ & $23(2.2 \%)$ & $39(3.7 \%)$ & $47(4.4 \%)$ & $8(0.8 \%)$ \\
\hline $40(3.4 \%)$ & $28(2.4 \%)$ & $13(1.1 \%)$ & $34(2.9 \%)$ & $43(3.6 \%)$ & $15(1.3 \%)$ \\
\hline \multicolumn{6}{|c|}{$\begin{array}{l}\text { Hazard Ratio }(95 \% \mathrm{CI}) \text { and } \\
\text { p-value for time to event analysis } \\
\text { (multivariable Cox PH modeling) }\end{array}$} \\
\hline $2.08(1.42,3.06) \mathrm{p}<0.001$ & $\begin{array}{l}1.51(0.94,2.42) \\
\mathrm{p}=0.089\end{array}$ & $\begin{array}{l}1.42(0.70,2.88) \\
p=0.326\end{array}$ & $\begin{array}{l}1.25(0.78,1.99) \\
p=0.359\end{array}$ & $\begin{array}{l}1.04(0.69,1.58) \\
\mathrm{p}=0.850\end{array}$ & $\begin{array}{l}0.63(0.26,1.56 \\
p=0.321\end{array}$ \\
\hline \multicolumn{2}{|c|}{$\begin{array}{l}\text { age, disease severity at baseline (none) } \\
\text { y (partial Mayo score), } \\
\text { history of previous dysplasia }\end{array}$} & \multicolumn{2}{|c|}{$\begin{array}{l}\text { disease duration at age, disease duration at baseline, } \\
\text { baseline history of previous dysplasia }\end{array}$} & (none) & age \\
\hline
\end{tabular}

delivery. An acute high fermentable fibre intake delays drug dissolution in the colon, but had little influence over total release. These findings have implications for optimising drug selection in maintenance of remission in UC.

Disclosure of Interest: C.K. Yao: CK Yao received research support to conduct the study from Ferring Pharmaceuticals. The Department of Gastroenterology at Monash University benefits financially from the sales of a digital app and booklets on the low FODMAP diet.

R.E. Burgell: Rebecca has received consultancy fees from Allergan. The Department of Gastroenterology at Monash University benefits financially from the sales of a digital app and booklets on the low FODMAP diet. J.S. Barrett: The Department of Gastroenterology at Monash University benefits financially from the sales of a digital app and booklets on the low FODMAP diet.

J.G. Muir: The Department of Gastroenterology at Monash University benefits financially from the sales of a digital app and booklets on the low FODMAP

P.R. Gibson: PG has served as consultant or advisory member for AbbVie, Ferring, Janssen, Merck, Allergan, Pfizer, Celgene \& Takeda; research support from AbbVie \& Janssen; speaking honoraria for his institution from AbbVie, Janssen, Ferring, Takeda, Mylan \& Pfizer.

All other authors have declared no conflicts of interest.

\section{P0406 HIGHER EXPOSURE TO GOLIMUMAB IS ASSOCIATED WITH ENDOSCOPIC RESPONSE IN PATIENTS WITH ULCERATIVE COLITIS: RESULTS FROM THE GO-KINETIC TRIAL}

S. Berends ${ }^{1}$, A. Strik ${ }^{2}$, R. Mathot ${ }^{1}$, G. R. D'Haens ${ }^{2}$, M. Lowenberg ${ }^{2}$

${ }^{1}$ Hospital Pharmacy, Academic Medical Center, Amsterdam/Netherlands

${ }^{2}$ Gastroenterology, Academic Medical Centre, Amsterdam/Netherlands

Contact E-mail Address: s.e.berends@amc.uva.nl

Introduction: Golimumab (GLM) is a subcutaneously administered anti-tumor necrosis factor (anti-TNF) antibody that is approved for the treatment of moderate to severe ulcerative colitis (UC). We investigated the association between systemic exposure (area under the curve (AUC)) of GLM during induction therapy and endoscopic response in moderate-severe UC.

Aims \& Methods: In this prospective observational trial, patients with moderate to severe UC (Mayo endoscopy score $\geq 2$ ) received induction treatment with GLM $200 \mathrm{mg} \mathrm{SQ}$ (at week 0) and $100 \mathrm{mg}$ (at week 2) followed by 50 or $100 \mathrm{mg}$ at week 6 , in patients with a bodyweight of less or more than $80 \mathrm{~kg}$, respectively. Serum GLM concentrations were measured at day $0,4,7,14,18,28,42$ and 56 , as well as antiGLM antibody levels, C-reactive protein (CRP) and albumin serum concentrations. Serum GLM concentrations were measured with an enzyme-linked immunoassay and anti-GLM antibody levels were measured with a drug-sensitive antigen binding test, both developed by Sanquin laboratories. Endoscopic response was defined as $\geq 1$ point reduction in endoscopic Mayo score at week 8-10 compared to baseline. AUCs were calculated using nonlinear mixed effect modelling (NONMEM $®$ ) and were compared using the non-parametric Mann-Whitney U test. Correlation analysis was performed using Pearson's correlation coefficient. A receiver-operating characteristic (ROC) curve reported the predictive performance of GLM serum trough levels at week 2 and 6 for endoscopic response.

Results: A total of 20 patients were enrolled of which 19 patients underwent an endoscopy at baseline and 8-10 weeks after start of treatment. Median age ([interquartile range]) was 46 years [36-57], median baseline CRP serum concentration was $4.5 \mathrm{mg} / \mathrm{L}[1.1-13.7]$ and median baseline albumin serum concentration was $44 \mathrm{~g} / \mathrm{L}$ [40-45]. None of the patients developed antibodies against GLM during induction treatment. After the induction phase, 12 out of 19 patients $(63 \%)$ achieved an endoscopic response. Median AUC at week 2 and 6 was higher in endoscopic responders compared to non-responders. Median GLM trough concentrations at week 2 and 6 were higher in endoscopic responders compared to non-responders (Table 1). Correlations between GLM trough concentrations and AUCs at week 2 (Pearson correlation coefficient: 0.86 , $\mathrm{P}<.0001$ ) and week 6 (Pearson correlation coefficient: 0.81, P $<.0001$ ) were statistically significant. Despite a low area under the ROC-curve (AUROC), a GLM serum trough concentration $\geq 6.6 \mathrm{mg} / \mathrm{L}$ at week 2 (AUROC: $0.68,95 \%$ CI: 0.43-0.927, sensitivity: $75 \%$, specificity: $57 \%$ ) was associated with endoscopic response after the induction phase. At week 6, a GLM serum trough concentration $\geq 3.3 \mathrm{mg} / \mathrm{L}$ (AUROC: $0.75,95 \%$ CI: $0.526-0.974$, sensitivity: $67 \%$, specificity: $71 \%$ ) was associated with endoscopic response after the induction phase.

Table 1: Median golimumab trough concentrations and AUCs at week 2 and 6

\begin{tabular}{llll}
\hline & $\begin{array}{l}\text { Endoscopic } \\
\text { responders } \\
\text { median [IQR] }\end{array}$ & $\begin{array}{l}\text { Endoscopic } \\
\text { non-responders } \\
\text { median [IQR] }\end{array}$ & P-value \\
\hline AUC $(\mathrm{mg} / \mathrm{L} /$ day), week 2 & $134[102-170]$ & $94[80-169]$ & .48 \\
AUC $(\mathrm{mg} / \mathrm{L} /$ day), week 6 & $333[250-476]$ & $212[206-417]$ & .21 \\
Serum trough concentration $(\mathrm{mg} / \mathrm{L})$, week 2 & $9.1[6.1-10]$ & $6.1[5.2-9.0]$ & .20 \\
Serum trough concentration $(\mathrm{mg} / \mathrm{L})$, week 6 & $3.8[2.6-6.0]$ & $2.4[1.1-3.8]$ & .08
\end{tabular}

Conclusion: Serum trough concentrations of GLM and AUCs at week 2 and 6 were higher in endoscopic responders compared to patients without an endoscopic response. A significant correlation was found between GLM trough concentrations and AUC. A GLM trough level $\geq 3.3 \mathrm{mg} / \mathrm{L}$ at week 6 is associated with improved endoscopic outcomes.

Disclosure of Interest: S. Berends: Has received lecture fees from Johnson and Johnson, and Merck Sharp \& Dohme.

A. Strik: Has received lecture fees from Biogen, Johnson and Johnson, Merck Sharp \& Dohme, Mundipharma, Takeda, and Tillots.

R. Mathot: Has received consulting fees from MSD and research grants from Bayer, UCB Pharma, Shire and Roche

G.R. D'Haens: Has received speaker fees from Abbvie, Ferring, Johnson and Johnson, Merck Sharp \& Dohme, Mundipharma, Norgine, Pfizer, Shire, Millenium/Takeda, Tillotts and Vifor.

M. Lowenberg: Has received speaking fees from Abbvie, Covidien, Dr. Falk, Ferring Pharmaceuticals, Merck Sharp \& Dohme, Receptos, Takesa, Tillotts and Tramedico. He has received research grants from AbbVie, Merck Sharp \& Dohme, Achmea healthcare and ZonMW.

\section{P0407 COMPARATIVE EFFICACY AND SAFETY OF TOFACITINIB AND BIOLOGICS AS INDUCTION THERAPY FOR MODERATELY TO SEVERELY ACTIVE ULCERATIVE COLITIS: A SYSTEMATIC REVIEW AND NETWORK META-ANALYSIS}

D. T. Rubin ${ }^{1}$, A. O. Ashaye ${ }^{2}$, Y. Zhang ${ }^{3}$, Y. $\mathrm{Xu}^{2}$, K. Fahrbach ${ }^{2}$, L.A. Chen ${ }^{4}$, A. Manuchehri ${ }^{5}$, C. Kayhan ${ }^{6}$, J. Woolcott ${ }^{7}$, J. C. Cappelleri ${ }^{6}$

${ }^{1}$ Inflammatory Bowel Disease Center, University of Chicago Medicine, Chicago, United States of America/IL

${ }^{2}$ Evidera Inc, Waltham/United States of America/MA

${ }^{3}$ Evidera Inc, London/United Kingdom

${ }^{4}$ Division Of Gastroenterology, New York University School of Medicine, New York/United States of America/NY

${ }^{5}$ Pfizer Ltd, Walton Oaks/United Kingdom

${ }^{6}$ Pfizer Inc, Groton/United States of America/CT

${ }^{7}$ Pfizer Inc, Kirkland/Canada/QC

Contact E-mail Address: drubin@medicine.bsd.uchicago.edu

Introduction: Tofacitinib is an oral, small molecule Janus kinase inhibitor being investigated for moderately to severely active ulcerative colitis (UC). We performed a systematic literature review (SLR) and network meta-analysis (NMA) to compare the efficacy and safety of tofacitinib to available tumour necrosis factor inhibitors (TNFi) and integrin receptor antagonists for induction therapy of adults with moderately to severely active UC.

Aims \& Methods: Using indexing and free-text terms, searches were conducted in the EMBASE, MEDLINE, CENTRAL, DARE and CINAHL databases to identify RCTs published as of January 2015. Proceedings of relevant conferences from 2012-2014 were also reviewed. Comparators of interest were infliximab, golimumab, adalimumab and vedolizumab. Two reviewers independently assessed studies for inclusion, and extracted and validated the study/patient data. Fixed- and random-effects Bayesian NMAs were conducted to compare efficacy outcomes and rates of adverse events (AEs) at 6-12 weeks in the overall population (TNFi-naïve or exposed) and by prior TNFi exposure. 
Abstract No: P0405

Table 1: Hypothetical dissolution profiles UC patients $(\mathrm{n}=15)$

\begin{tabular}{|c|c|c|c|c|}
\hline Dissolution characteristics & Eudragit L & Eudragit L with slow release & Eudragit $\mathrm{S}$ & Multi-matrix (MMX) \\
\hline Hypothetical complete dissolution & $p H \geq 6$ for $1 h$ & $p H \geq 6$ for $4-5 h$ & $p H \geq 7$ for $2-4 h$ & $p H \geq 7$ for $6 h$ \\
\hline Complete in small intestine & $67 \%$ & $100 \%$ & HF $25 \%$; LF $19 \%$ & HF $0 \% ;$ LF $13 \%$ \\
\hline Complete dissolution & $100 \%$ & HF $97 ;$ LF $100 \%$ & HF $80 ;$ LF $87 \%$ & HF 53; LF 67\% \\
\hline
\end{tabular}

Results: Twelve induction trials were identified from the SLR (ACT 1 \& 2 , EUCALYPTUS, GEMINI-I, PURSUIT SC, TOFACITINIB PHASE 2, Feagan $2005,{ }^{1}$ Probert 2003, ${ }^{2}$ UC-SUCCESS, ULTRA 1, ULTRA 2, Suzuki 2014 ${ }^{3}$ ) and included in the NMA. Unpublished data from tofacitinib Phase 3 induction trials (OCTAVE $1 \&$ 2) were also used in the analysis. Fixed-effects NMA showed that tofacitinib $10 \mathrm{mg}$ twice daily (BID) is associated with a higher rate of mucosal healing vs adalimumab $160 / 80 / 40 \mathrm{mg}$ in the overall population (odds ratio [OR] $1.82[95 \%$ credible interval (CrI) $1.06,3.14]$ ), and vs vedolizumab $300 \mathrm{mg}$ (OR $3.71[95 \%$ CrI 1.37, 10.64]) and etrolizumab $300 \mathrm{mg}$ (OR 12.09 [95\% CrI 1.68 , 122.73]) in TNFi-exposed patients. A higher rate of clinical remission was seen with tofacitinib $10 \mathrm{mg}$ BID vs adalimumab in TNFi-exposed patients (OR 11.93 [95\% CrI 1.84, 154.78]). AE rates were similar between tofacitinib $10 \mathrm{mg}$ BID and comparators in the overall and TNFi-naïve populations when analysed individually, but tofacitinib $10 \mathrm{mg}$ BID was found to be associated with a higher rate of disaggregated AEs ("any AE") than etrolizumab $300 \mathrm{mg}$ in the overall population (OR $2.78[95 \%$ CrI $1.08,7.41])$. There were no statistically conclusive differences in the rates of specific AEs between tofacitinib $10 \mathrm{mg}$ BID and comparators

Conclusion: This NMA suggests that tofacitinib may be more effective as induction therapy in moderately to severely active UC than adalimumab and vedolizumab in TNFi-exposed patients, and is associated with a higher rate of mucosal healing than adalimumab in the overall population. Rates of specific safety events were similar between tofacitinib and other treatments.

Disclosure of Interest: C. Kelly: travel support and fees for serving on advisory boards from Seres Therapeutics, Summit Pharmaceuticals, and Synthetic Biologics, lecture fees from Seres Therapeutics, and grant support from Institut Mérieux, ntera Health, and Merck

D.T. Rubin: Consulting fees: AbbVie, Amgen, Janssen, Pfizer Inc, Takeda, UCB. Research grants: AbbVie, Genentech, Janssen, Takeda, UCB

A.O. Ashaye: Employee of Evidera, who provide consulting and research services to pharmaceutical and related organisations. In salaried positions, work with a variety of companies, and are precluded from receiving payment or honoraria directly for services rendered.

Y. Zhang: Employee of Evidera, who provide consulting and research services to pharmaceutical and related organisations. In salaried positions, work with a variety of companies, and are precluded from receiving payment or honoraria directly for services rendered.

Y. Xu: Employee of Evidera, who provide consulting and research services to pharmaceutical and related organisations. In salaried positions, work with a variety of companies, and are precluded from receiving payment or honoraria directly for services rendered.

K. Fahrbach: Employee of Evidera, who provide consulting and research services to pharmaceutical and related organisations. In salaried positions, work with a variety of companies, and are precluded from receiving payment or honoraria directly for services rendered.

L.A. Chen: Employee of New York University School of Medicine, which is contracted by Pfizer Inc to perform consultative services. LA Chen's husband is a shareholder of Pfizer Ltd.

A. Manuchehri: Employee and shareholder of Pfizer Ltd.

A. Manuchehri: Employee and shareholder of Pfizer Ltd

C. Kayhan: Employee and shareholder of Pfizer Inc.

J. Woolcott: Employee and shareholder of Pfizer Inc.

J.C. Cappelleri: Employee and shareholder of Pfizer Inc.

\section{References}

1. Feagan BG et al. N Engl J Med 2005;352:2499-507

2. Probert CS et al. Gut 2003;52:998-1002

3. Suzuki Y et al. J Gastroenterol 2014;49:283-94

\section{P0408 CHARACTERISTICS AND OUTCOMES IN PATIENTS WITH C. DIFFICILE INFECTION AND INFLAMMATORY BOWEL} DISEASE: BEZLOTOXUMAB VERSUS PLACEBO

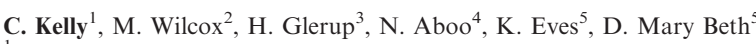
${ }^{1}$ BIDMC \& Harvard Medical School, Boston/United States of America/MA ${ }^{2}$ Leeds Teaching Hospitals \& University of Leeds, Leeds/United Kingdom ${ }^{3}$ Regional Hospital Silkeborg, Silkeborg/Denmark

${ }^{4}$ Parklands Medical Centre, Overport/New Zealand

${ }^{5}$ Merck \& Co., Inc., Kenilworth/United States of America/NJ

Contact E-mail Address: ckelly2@bidmc.harvard.edu

Introduction: Patients with inflammatory bowel disease (IBD) experience higher rates of $C$. difficile infection (CDI) than the overall population, often lack typical risk factors, and frequently experience severe and recurrent episodes. MODIFY I/II were global trials of the efficacy and safety of bezlotoxumab (bezlo: a human monoclonal antibody against $C$. difficile toxin $\mathrm{B}$ ), in which bezlo was superior to placebo at preventing $\mathrm{CDI}$ recurrence ( $\mathrm{rCDI}$ ) in participants with primary or recurrent CDI given antibacterial drug treatment for CDI. Participants with IBD could be enrolled if, in the opinion of the investigator, symptoms were more likely due to CDI than IBD.

Aims \& Methods: The objective of this post-hoc subgroup analysis was to summarize CDI-related outcomes, including initial clinical cure and rCDI, through 12 weeks in participants with IBD enrolled in the MODIFY trials. CDI-related outcomes through 12 weeks in the subset of IBD participants enrolled in the MODIFY trials included: initial clinical cure (no diarrhea during the 2 consecutive days following completion of $<14$ days of an antibacterial drug treatment for CDI); and rCDI (new episode of diarrhea associated with a positive stool test for toxigenic $C$. difficile in participants who had achieved initial clinical cure). For this post-hoc analysis, participants randomized to bezlo or actoxumab + bezlo were pooled and are referred to as the "bezlo" group and participants randomized to placebo or actoxumab were pooled and are referred to as the "no bezlo" group.

Results: Overall, 2559 participants were included in the mITT population; 1554 participants were randomized to a bezlo group and 1005 were randomized to a no bezlo group. There were 44 participants with IBD: $23(52.3 \%)$ had ulcerative colitis; $18(40.9 \%)$ had Crohn's disease; and $3(6.8 \%)$ had non-characterized IBD. Compared with participants without IBD, participants with IBD tended to be younger, were more often treated as outpatients, were more often immunocompromised, and a smaller percentage had severe CDI. Among IBD participants, a higher proportion had initial clinical cure in the no bezlo group compared with the bezlo group and there was a higher proportion of participants with rCDI in the no bezlo group compared with the bezlo group. In IBD participants who did not receive bezlo, most of the recurrences (5 of 7) occurred within 4 weeks after study infusion, while most of the recurrences among participants who received bezlo occurred after week 4 (3 of 4).

Conclusion: Participants with IBD and CDI enrolled in the MODIFY trials were younger, more likely to be diagnosed with CDI as an outpatient, to be immunocompromised, and to develop rCDI compared with non-IBD participants. Bezlo yielded a $27.2 \%$ absolute reduction $(50 \%$ relative reduction) in the incidence of rCDI in participants with IBD. The efficacy of bezlo in preventing rCDI may extend to patients with IBD, but additional data are needed due to the limited cohort size.

\begin{tabular}{lll}
\hline & IBD n-44 & No IBD n=2515 \\
\hline Female, n (\%) & $25(56.8)$ & $1419(56.4)$ \\
Mean age, yrs (SD) & $50.3(18.9)$ & $63.5(17.5)$ \\
Severe CDI & $4(9.1)$ & $416(16.5)$ \\
Inpatient & $20(45.5)$ & $1711(68.0)$ \\
Immunocompromised & $18(40.9)$ & $531(21.1)$ \\
Initial Clinical Cure, n/m (\%) & Bezlo & No Bezlo \\
rCDI, n/m (5) & $15 / 28(53.6)$ & $13 / 16(81.3$ \\
\hline
\end{tabular}

\section{Disclosure of Interest}

C. Kelly: travel support and fees for serving on advisory boards from Seres Therapeutics, Summit Pharmaceuticals, and Synthetic Biologics, lecture fees from Seres Therapeutics, and grant support from Institut MÕrieux, ntera Health, and Merck

M. Wilcox: consult/grant/lect fees: Alere, Abbott., Actelion, Astellas, Cerexa, Cubist, Optimer, Sanofi Pasteur, Summit, bio-Mérieux, Da Volterra Qiagen, Astra Zeneca, Pfizer, Durata Therap, Merck, Seres Therap, Valneva, Nabriva Thera, Roche, Medicines Company

K. Eves: K. Eves - an employee of Merck Sharp \& Dohme Corp., a subsidiary of Merck \& Co., Inc., Kenilworth, NJ, USA, who may own stock and/or hold stock options in the Company.

D. Mary Beth: MB Dorr - an employee of Merck Sharp \& Dohme Corp., a subsidiary of Merck \& Co., Inc., Kenilworth, NJ, USA, who may own stock and/or hold stock options in the Company.

All other authors have declared no conflicts of interest. 
P0409 SIMILAR TRIAL - EFFICACY OF INFLIXIMAB-BIOSIMILAR COMPARED TO INFLIXIMAB-BIOLOGICAL IN PATIENTS WITH INFLAMMATORY BOWEL DISEASE IN REMISSION - A RANDOMIZED, CONTROLLED, DOUBLE BLIND, PHASE 4 NONINFERIORITY TRIAL

A. G. Volkers ${ }^{1}$, J.M. Jansen ${ }^{2}$

${ }^{1}$ Gastroenterology And Hepatology, OLVG, Amsterdam/Netherlands

${ }^{2}$ Departement Of Gastroenterology And Hepatology, Onze Lieve Vrouwe Gasthuis, Amsterdam/Netherlands

\section{Contact E-mail Address: a.g.volkers@amc.uva.nl}

Introduction: Crohn's disease (CD) and ulcerative colitis (UC) are the main entities of inflammatory bowel disease (IBD). For most patients, medical treatment is sufficient to keep the disease in remission. In both diseases tumor necrosis factoralpha (TNF- $\alpha$ ) inhibitors, such as Infliximab (IFX), are a major component in inducing and maintaining remission in more refractory patients. Recently IFXbiosimilars have been introduced for the treatment of IBD, these are less expensive than IFX-biologicals. Efficacy of IFX-biosimilar and IFX-biological have been compared for ankylosing spondylitis and rheumatoid arthritis showing no difference between efficacy and safety. So far, no double blind randomized clinical trial has been published that compared IBD patients who used IFX-biological or IFX-biosimilar. Cohort studies in IBD patients showed switching from IFX-biologicals to IFX-biosimilar had no impact on short-term clinical outcomes. The aim of this study was to examine non-inferiority in safety and efficacy of IFX-biosimilar compared with IFX-biologicals and to evaluate adverse effects and pharmacokinetics.

Aims \& Methods: We randomized patients with CD or UC in clinical and biochemical remission to either switch to IFX-biosimilar or to continue using IFXbiological. Randomization was performed in a $2: 1$ ratio (65\% to IFX-biosimilar). Patients in both arms received 4 to 6 doses of $5 \mathrm{mg} / \mathrm{kg}$ to $10 \mathrm{mg} / \mathrm{kg}$ during the 30 week study period. Patients eligible for inclusion had to be in clinical remission $(\mathrm{HBI}<5$ and MAYO $<2)$ and have a fecal calprotectin $<250 \mathrm{mg} / \mathrm{g}$. The primary endpoint was number of patients in remission at week 30 . We measured Creactive protein [CRP], fecal calprotectin [FCP], infliximab trough level [TL] and anti-drug antibodies [ADAs]. Patients were asked to fill in SIBDQ and Mayo or $\mathrm{HBI}$ questionnaires three times during the study period. Adverse events (AE) and serious adverse events (SAE) that patients experienced were documented.

Results: So far, we included 47 patients from 6 secondary Dutch Teaching hospitals. 35 patients had CD and 12 had UC. 27 patients were female, 20 were male. Mean age at inclusion was 42 years. 21 patients have finished the 30 -week followup. 15 received IFX-biosimilar, 6 IFX-biological. One patient experienced relapse of IBD, this patient received IFX-biosimilar. 2 patients experienced a SAE, none were related to the study drug.

Conclusion: This is the first double blind randomized clinical trial that compares treatment of IBD with IFX-biological or IFX-biosimilar. The preliminary results show that switching from IFX-biological to IFX-biosimilar is feasible and safe. Disclosure of Interest: All authors have declared no conflicts of interest.

\section{P0411 ENCAPSULATED FECAL MICROBIOTA TRANSFER IN PATIENTS WITH CHRONIC, ANTIBIOTIC-REFRACTORY POUCHITIS}

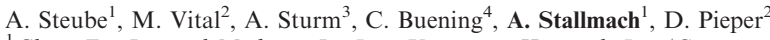
${ }^{1}$ Clinic For Internal Medicine Iv, Jena University Hospital, Jena/Germany ${ }^{2}$ Microbial Interactions And Processes, Helmholtz Centre for Infection Research, Braunschweig/Germany

${ }^{3}$ Department Of Internal Medicine (gastroenteriology), DRK Kliniken Berlin I Westend, Berlin/Germany

${ }^{4}$ Department Of Internal Medicine, Krankenhaus Waldfriede, Berlin-Zehlendorf/ Germany

Contact E-mail Address: andreas.stallmach@med.uni-jena.de

Introduction: We analyzed the success of fecal microbiota transfer (FMT) via encapsulated or endoscopic jejunal application to 14 patients with chronic, antibiotic refractory pouchitis.

Aims \& Methods: FMT was performed either via encapsulated cryopreserved microbiota or via endoscopic jejunal application to 14 patients. Stool samples for FMT preparation derived from three unrelated healthy donors. Patients were treated by FMT every 4 weeks according to the individual therapeutic outcome. Stool samples before FMT and during follow-up were subjected to microbial community structure analysis through high throughput sequencing of the V1-2 regions of the $16 \mathrm{~S}$ rRNA (1), clinical response and mucosal inflammation was assessed by fecal calprotectin (FCP) levels.

Results: Clinical response occurred in 7 of 14 patients after two to four FMTs. 4 patients showed clinical worsening and 3 patients showed no improvement. FCP dropped in responders from $536 \mathrm{mg} / \mathrm{kg}$ stool (med.; min-max: 116-3000) to $150 \mathrm{mg} / \mathrm{kg}$ (191-1409), whereas in patients with flare FCP values increased from $1005 \mathrm{mg} / \mathrm{kg}(529-1579)$ to $1450 \mathrm{mg} / \mathrm{kg}$ (1221-1778). Microbiota analysis of 10 patients and two donors revealed a significantly lower diversity in pouchitis patients compared to healthy donors as assessed by the total phylotype number, the Shannon diversity and Pieloús evenness. In patients showing response, typically a significant increase in diversity was observed. This increase in diversity was due to the successful establishment of the donor microbiota as assessed by the analysis of sample-similarity matrices constructed using the Bray-Curtis algorithm and a detailed analysis of the taxonomic composition. The encapsulated fecal microbiota was as effective as the endoscopic jejunal application in its competence to restructure the patients microbiota. However, an increase in the diversity and an overall restructuring of the microbiota into a composition resembling the donor not necessarily correlated with clinical outcome and clinical worsening was observed during three FMTs where the established microbiome structure resembled that of the healthy donor in diversity and composition. Interestingly, a high abundance of Ruminococcaceae was associated with remission in a recent study on ulcerative coltis (2). However, two patients showed worsening here despite a high increase in the relative abundance of Ruminococcaceae after FMT.

Conclusion: Fecal microbiome transfer in patients with chronic pouchitis is a promising therapeutic option and donor microbiomes could successfully be transferred via capsules or via jejunoscopy delivering fresh stool filtrate. However, a simple increase in microbial diversity and successful establishment of members of the butyrate producing Lachnospiraceae and Ruminococcaceae families is not sufficient for a successful outcome of FMT.

Disclosure of Interest: All authors have declared no conflicts of interest.

\section{References}

1. Camarinha-Silva A, Jáuregui R, Chaves-Moreno D, Oxley AP, Schaumburg F, Becker K, Wos-Oxley ML, Pieper DH. 2014. Comparing the anterior nare bacterial community of two discrete human populations using Illumina amplicon sequencing. Environ Microbiol. 16:2939-2952.

2. Rossen NG, Fuentes S, van der Spek MJ, Tijssen JG, Hartman JH, Duflou A, Lowenberg M, van den Brink GR, Mathus-Vliegen EM, de Vos WM, Zoetendal EG, D'Haens GR, and Ponsioen CY. 2015. Findings from a randomized controlled trial of fecal transplantation for patients with Ulcerative Colitis. Gastroenterology 149:110-118.

P0412 HISTOLOGIC MEASURES OF MUCOSAL HEALING CORRELATE WITH ENDOSCOPIC MEASURES OF DISEASE ACTIVITY AT BASELINE AND FOLLOWING INDUCTION THERAPY WITH THE JAK1 INHIBITOR FILGOTINIB IN ACTIVE CROHN'S DISEASE: RESULTS FROM FITZROY STUDY

W. Reinisch ${ }^{1}$, G. R. D’Haens ${ }^{2}$, G. De Hertogh ${ }^{3}$, A. Van Der Aa ${ }^{4}$, J. Zhang ${ }^{5}$, C. Tasset $^{6}$, C. Yun ${ }^{7}$, A. Serone ${ }^{7}$, B. G. Feagan ${ }^{8}$, W.J. Sandborn ${ }^{9}$, S. Vermeire ${ }^{10}$ ${ }^{1}$ Medical University of Vienna, Vienna/Austria

${ }^{2}$ Gastroenterology, Academic Medical Centre, Amsterdam/Netherlands

${ }^{3}$ Department Of Pathology, University Hospitals Leuven, Leuven/Belgium

${ }^{4}$ Galapagos NV, Mechelen/Belgium

${ }^{5}$ Biostatistics, Gilead Sciences, Inc., Foster City/United States of America ${ }^{6}$ Development, Galapagos NV, Mechelen/Belgium

${ }^{7}$ Clinical Research, Gilead Sciences, Inc, Foster City/United States of America

${ }^{8}$ Robarts Research Institute, London/Canada

${ }^{9}$ University of California, San Diego/United States of America

${ }^{10}$ Dept. Of Gastroenterology, University Hospital Leuven - Dept. of

Gastroenterology, University Hospital Leuven; Leuven/BE, Leuven/Belgium

Contact E-mail Address: walter.reinisch@meduniwien.ac.at

Introduction: Mucosal healing ( $\mathrm{MH})$ has been established as co-primary treatment target in Crohn's disease, predominantly defined by the absence of ulceration. However, even in patients with $\mathrm{MH}$, inflammation may persist on histologic examination ${ }^{1}$. Filgotinib (FIL), a selective inhibitor of JAK1 that blocks cytokine signaling through inhibition of STAT phosphorylation, has recently shown efficacy in a double-blind, placebo (PBO)-controlled Phase 2 study in CD $\left(\right.$ FITZROY ${ }^{2}$ ). Effects of filgotinib versus placebo have been demonstrated on centrally read endoscopy and histopathology assessments after a 10-week induction treatment.

Aims \& Methods: In this post hoc analysis, we explored the correlation between histologic and endoscopic disease activity at baseline (BL) and following FIL induction therapy by comparison of total ileal Global Histology Activity Score (IGHAS)/colonic GHAS (CGHAS) score or IGHAS/CGHAS activity subscores (a; activity items: presence of epithelial damage, polymorphonuclear leukocytes in lamina propria, neutrophils in epithelium, erosion or ulceration, granuloma ${ }^{3}$ ${ }^{4}$ ) versus total ileal Simplified Endoscopic Score for CD (ISES-CD)/colonic SESCD (CSES-CD) score or ISES-CD/CSES-CD ulcer subscores (u; sum of size and $\%$ affected surface). CD patients were randomized 3:1 to receive $200 \mathrm{mg}$ FIL or PBO QD for 10 weeks $^{2}$. Intestinal biopsies were collected at BL and Week 10 (W10) from the most affected areas of each predefined bowel segment (ileum, ascending, transverse, descending, sigmoid colon and rectum). Biopsies were formalin fixed and paraffin embedded. The mean changes from baseline for each treatment group were compared to zero using a one-sample t-test.

Results: Baseline values were comparable across treatment groups, although CGHAS and CSES-CD were numerically higher in the PBO group (Table 1). Following 10 weeks of treatment with FIL $200 \mathrm{mg}$, histologic measures of colonic mucosal inflammation (CGHAS and aCGHAS) were significantly improved and were coupled with macroscopic changes in both CSES-CD and uCSES-CD. Changes in histology score for ileal segments were numerically greater after FIL treatment versus placebo. Histology total and subscores were significantly associated with total and endoscopic ulcer subscores at both time points, and more pronounced when looking into the colonic segments versus the ileal segments (IGHAS v ISES-CD: Corr $=.0 .62, p<0.001$; Corr $=.0 .65, p<0.001 ; \mathrm{BL}$ and W10 respectively)(aIGHAS $\mathrm{v}$ uISES-CD: Corr $=0.53, \quad \mathrm{p}<0.001$; 
Abstract No: P0412

\begin{tabular}{|c|c|c|c|c|c|c|c|c|c|}
\hline & \multirow[b]{2}{*}{ Mean (SD) } & \multicolumn{2}{|l|}{ IGHAS } & \multicolumn{2}{|l|}{ CGHAS } & \multicolumn{2}{|l|}{ ISES-CD } & \multicolumn{2}{|l|}{ CSES-CD } \\
\hline & & PBO & FIL & PBO & FIL & PBO & FIL & PBO & FIL \\
\hline \multirow[t]{3}{*}{ Total Score } & Baseline & $4.10(3.94)$ & $3.94(3.74)$ & $21.10(19.26)$ & $14.69(12.25)$ & $4.13(3.32)$ & $4.41(3.53)$ & $11.91(8.31)$ & $9.63(8.04)$ \\
\hline & $\Delta \mathrm{W} 10$ & $0.24(3.66)$ & $-0.37(3.69)$ & $-0.81(14.72)$ & $-4.83(11.07)$ & $-0.30(1.72)$ & $-0.18(2.27)$ & $-2.09(6.62)$ & $-2.68(4.90)$ \\
\hline & $\mathrm{N}$ & 21 & 52 & 21 & 52 & 23 & 76 & 23 & 76 \\
\hline \multirow[t]{3}{*}{ Subscore* } & Baseline & $2.57(2.56)$ & $2.27(2.67)$ & $13.76(13.05)$ & $9.33(8.47)$ & $2.39(1.90)$ & $2.55(2.07)$ & $7.22(5.23)$ & $6.09(5.34)$ \\
\hline & $\Delta \mathrm{W} 10$ & $0.10(2.84)$ & $-0.23(2.95)$ & $-0.86(10.79)$ & $-3.48(8.28)$ & $0.00(0.95)$ & $-0.21(1.51)$ & $-1.70(4.37)$ & $-1.76(3.64)$ \\
\hline & $\mathrm{N}$ & 21 & 52 & 21 & 52 & 23 & 76 & 23 & 76 \\
\hline
\end{tabular}

Only subjects with non-missing data for all segments at BL and W10 were included in the calculation;*Subscore for GHAS data is activity subscore and for SES-CD data is ulcer subscore; bolded texts indicate p-value $<0.05$ from t-test

Corr $=0.43, \mathrm{p}<0.001 ; \mathrm{BL}$ and $\mathrm{W} 10$ respectively) (CGHAS $\mathrm{v}$ CSES-CD: Corr $=.0 .80, \mathrm{p}<0.001 ;$ Corr $=.0 .77, \mathrm{p}<0.001 ; \mathrm{BL}$ and $\mathrm{W} 10$ respectively) (aCGHAS v uCSES-CD: Corr $=0.77, \mathrm{p}<0.001$; Corr $=0.70, \mathrm{p}<0.001 ; \mathrm{BL}$ and $\mathrm{W} 10$ respectively).

Conclusion: Improvements in endoscopic severity induced by filgotinib are paralleled by reductions of histologic scores. In line with previous findings from antiTNF therapies ${ }^{4,5}$ colonic mucosa is more prone to improve than ileal disease. Spontaneous reductions of histologic activity under placebo were not observed. Disclosure of Interest: G.R. D'Haens: Abbvie, Ablynx, Biogen, BMS, BoehringerIng., Celgene, Celltrion, Ferring, Galapagos, Gilead, GSK, Hospira, Immunic, J\&J, Lycera, Millen./Takeda, MitsubishiPh., MSD, Mundiph., NovoNordisk, Pfizer, Protagonist, Robarts, Salix, Sandoz, Shire, Teva, Tigenix, Tillotts, a.o.

G. De Hertogh: has received fees for central pathology review from Genentech, Centocor, and Galapagos.

W. Reinisch: Abbvie, Amgen, Astellas, Astra Zeneca, Bioclinica, Biogen IDEC, Boehringer-Ing.BMS, Celgene, Celltrion, Covance, Galapagos, Genentech, Gilead, Grünenthal, JNJ, LipidTher., MedImmune, MSD, Novartis, Otsuka, Pfizer, P\&G, Robarts, Sandoz, SP, Takeda, Tigenix, UCB, a.o.

A. Van der Aa: employee of Galapagos NV

J. Zhang: employee of Gilead Sciences Inc

C. Tasset: employee of Galapagos NV

C. Yun: employee of Gilead Sciences Inc

A. Serone: employee of Gilead Sciences Inc

B.G. Feagan: AbbVie, Amgen, AstraZeneca/MedImmune, Baxter, BoehringerIng., BMS, Celgene, Elan/Biogen, EnGene, Ferring, Roche/Genentech, Galapagos, Gilead, GSK, JnJ, Lilly, MSD, Novartis, Novo Nordisk, Pfizer, Sanofi, Shire, Takeda, Teva, TiGenix, UCB, VertexWyeth, Robarts a.o.

W.J. Sandborn: AbbVie, Vertex, Gilead, BoehringerIng., UCB, Baxter, Ferring, Amgen, Takeda, Novo Nordisk, Shire, MedImmune/AstraZeneca, Teva, Eli Lilly, TiGenix, Adherion, Immune Pharmaceuticals, Celgene, BMS, Genentech, GSK, Pfizer, Tillots, American College of Gastroenterology a.o.

S. Vermeire: research funding from Abbvie, Galapagos, MSD, and Takeda; speaker fees from Abbie; and consultancy fees from Abbvie, MSD, Takeda, Ferring, Genentech/Roche, Shire, Pfizer, Galapagos, Mundipharma, Hospira, Celgene, Second Genome, and Janssen.

\section{References}

1. Neurath MF. Mucosal immunology 2014; 7: 6-19

2. Vermeire, S, Schreiber, S, et al. Lancet. 2016; 389(10066):266-275

3. D'Haens, G R, Geboes, K, et al. Gastroenterology. 1998;114(2):262-7.

4. Geboes, K, Rutgeerts, P, et al. Curr Med Res Opin. 2005;21(11):1741-54.

5. Reinisch, W, Colombel, J, et al. J Crohns Colitis. 2016 doi: 10.1093/ecco-jcc/ jjw178

\section{P0413 RESPONSE AND REMISSION AFTER 16 WEEKS OF USTEKINUMAB- AN ALL PATIENTS ANALYSIS FROM THE UNITI CROHN'S STUDIES}

J. Colombel ${ }^{1}$, S. Sloan ${ }^{2}$, C. Gasink ${ }^{2}$, L. Gao ${ }^{2}$, D. Jacobstein ${ }^{3}$, S. D. Lee ${ }^{4}$, S. R. Targan $^{5}$

${ }^{1}$ Icahn School of Medicine at Mount Sinai, New York/United States of America

${ }^{2}$ Janssen Scientific Affairs, LLC, Horsham/United States of America/PA

${ }^{3}$ Janssen Research \& Development, LLC, Spring House/United States of America/ $P A$

${ }^{4}$ University of Washington Medical Center, Seattle/United States of America/WA

${ }^{5}$ Cedars-Sinai Medical Center, Los Angeles/United States of America/CA

Contact E-mail Address: jean-frederic.colombel@mssm.edu

Introduction: Ustekinumab (UST) has been shown to induce and maintain clinical response and remission in moderate to severe Crohn's disease (CD) in 2 induction [(UNITI-1 (anti-TNF failures) and UNITI-2 (anti-TNF non-failures) and 1 maintenance (IM-UNITI)] randomized, placebo controlled Phase 3 trials. We evaluated the efficacy (response and remission) for all patients who received an intravenous (IV) induction dose of approximately $6 \mathrm{mg} / \mathrm{kg}$, including responders (CDAI decrease $>100$ ) and non-responders, 8 weeks after the first UST maintenance dose of $90 \mathrm{mg}$ subcutaneous (SC), i.e. 16 weeks from the IV induction dose.

Aims \& Methods: Patients achieving clinical response 8 wks after a single IV induction dose were randomized to SC placebo (PBO), UST $90 \mathrm{mg}$ every 12 weeks $(\mathrm{q} 12 \mathrm{w})$ or every 8 weeks $(\mathrm{q} 8 \mathrm{w})$. UST patients not in clinical response 8 weeks after the IV induction dose were given UST $90 \mathrm{mg}$ SC and if in clinical response 8 weeks later were continued on $90 \mathrm{mg} \mathrm{SC} \mathrm{q} 8 \mathrm{w}$ dosing. A total of 458 patients were exposed to an IV induction dose of $6 \mathrm{mg} / \mathrm{kg}$ (UNITI-1, N $=249$ and in UNITI-2, $\mathrm{N}=209$ ) with a response rate at week 8 of $37.8 \%$ and $57.9 \%$ vs. PBO response rate of $20.2 \%$ and $32.1 \%$ respectively. The remission rate at week 8 in UNITI- 1 and UNITI-2 was $20.9 \%$ and 40.7 vs. PBO of $7.3 \%$ and $19.6 \%$ respectively. For this evaluation, the response and remission status of the entire population exposed to an IV induction dose of $6 \mathrm{mg} / \mathrm{kg}$ of UST was evaluated 8 weeks after the first subcutaneous maintenance dose of UST. All patients who received $6 \mathrm{mg} / \mathrm{kg}$ IV UST induction were included, including responders randomized to SC PBO (who did not receive SC UST at week 8).

Results: Of the 219 patients not in clinical response in UNITI $1 \& 2,37.6 \%$ and $60.5 \%$ respectively were in clinical response 8 weeks after the first maintenance UST dose $(90 \mathrm{mg} \mathrm{SC})$. Evaluating all patients exposed to $6 \mathrm{mg} / \mathrm{kg}$ IV UST induction, response rates 8 weeks after the first subcutaneous injection (16 weeks after the IV induction dose) for UNITI1\&2 are $47.4 \%$ and $73.7 \%$ respectively (see table for response and remission rate). Similar assessments were calculated in the sub-population who were anti-TNF naïve upon enrolment into UNITI-2.

Response rates and Remission rates for all patients 16 weeks after induction of $6 \mathrm{mg} / \mathrm{kg}$ IV UST

\begin{tabular}{llll}
\hline Study & IV UST (n) & $\begin{array}{l}\text { \% Clinical } \\
\text { Response }\end{array}$ & $\begin{array}{l}\text { \% Clinical } \\
\text { Remission }\end{array}$ \\
\hline UNITI-1 & 249 & 47.4 & 24.1 \\
UNITI-2 & 209 & 73.7 & 55.5 \\
UNITI-2 TNF Naive & 144 & 72.9 & 60.4 \\
\hline
\end{tabular}

Conclusion: These numbers at week 16 are expected to reflect real-world experience in patients who receive the induction dose and one additional maintenance dose 8 weeks later. The resulting rates of response and remission are higher than previously reported in the induction studies across all populations (anti-TNF non-failures and anti-TNF failures). About $73 \%$ of anti-TNF non-failures attain clinical response and over half are in remission. The data support the clinical rationale for providing at least one SC maintenance dose of ustekinumab irrespective of clinical response 8 weeks after IV induction.

Disclosure of Interest: J. Colombel: Investigator for Janssen Scientific Affairs, LLC

S. Sloan: Janssen Scientific Affairs, LLC employee

C. Gasink: Janssen Scientific Affairs, LLC employee

L. Gao: Janssen Scientific Affairs, LLC employee

D. Jacobstein: Janssen Research \& Development, LLC employee S.D. Lee: Investigator for Janssen Research \& Development, LLC S.R. Targan: Investigator for Janssen Research \& Development, LLC 
P0414 REAL-WORLD PATTERNS OF TREATMENT

DISCONTINUATION, FLARES, AND HOSPITALISATIONS AMONG

INFLAMMATORY BOWEL DISEASE PATIENTS WITHIN 12

MONTHS OF INITIATION OF VEDOLIZUMAB OR INFLIXIMAB

H. Patel ${ }^{1}$, M. Raluy Callado ${ }^{2}$, A. Berger ${ }^{3}$, R. Curtis ${ }^{1}$, M. J. Khalid ${ }^{1}$

${ }^{1}$ Takeda Development Centre Europe Ltd, London/United Kingdom

${ }^{2}$ Evidera, London/United Kingdom

${ }^{3}$ Evidera, Waltham/United States of America

Contact E-mail Address: hari.patel@takeda.com

Introduction: Biologics such as infliximab (IFX) (an anti-TNF) and vedolizumab (VDZ) (anti-integrin) are treatment options for patients with moderate-toseverely active inflammatory bowel disease (IBD), who have failed conventional therapy.

Aims \& Methods: Our aim was to compare time to treatment discontinuation, flares, and hospitalisations among patients with IBD initiating VDZ versus IFX who were biologic-naïve. All patients with IBD (ulcerative colitis or Crohn's disease [CD]) who initiated biologic treatment with VDZ or IFX between 01 / $05 / 2014$ and $22 / 02 / 2016$ were identified in the US Explorys Universe database; the first infusion was deemed the index date. Analyses focused on patients who: (1) successfully completed induction therapy ( $\geq 3$ infusions within 98 days of index date); (2) were $\geq 18$ years of age at index; (3) had $>365$ days of medical history prior to index ("baseline"); and (4) had 365 days of follow-up after the index date. VDZ initiators were matched to IFX initiators (1:3) using propensity scores. Kaplan-Meier Method was used to compare median time to discontinuation of VDZ and IFX during follow-up, defined as the first of either: no receipt of biologic $\leq 90$ days of previous infusion, or switch to another biologic. Similar method was also used to compare median time to IBD-related hospitalisations, surgeries, and flares (defined as use of intravenous steroids), respectively. Interquartile range (IQR) was also calculated.

Results: 105 VDZ initiators were matched to 315 IFX initiators. Baseline characteristics of both cohorts are described in Table 1 . CD accounted for $\sim 60 \%$ of patients in each cohort. In the baseline period, $\sim 70 \%$ of patients in both cohorts had received corticosteroids; $20 \%$ of VDZ vs. $38 \%$ of IFX initiators received an immunosuppressive therapy. Median time since diagnosis was 2.4 years for VDZ initiators and 1.9 years for IFX initiators. Median time to treatment discontinuation was 244 (IQR for VDZ: 194-307 and IFX: 190-300) days in both cohorts. Median time to first IBD-related hospitalisation was 153 (IQR: 78-209) days for VDZ initiators vs. 98 (IQR: 45-168) days for IFX initiators. For IBD-related flares, median time was 111 (IQR: 40-226) days for VDZ initiators vs. 93 (IQR: 35-182) days for IFX initiators.

Table 1. Baseline characteristics of propensity-score matched IBD patients initiating therapy with vedolizumab or infliximab

\begin{tabular}{llll}
\hline & $\begin{array}{l}\text { Vedolizumab } \\
(\mathrm{N}=105)\end{array}$ & $\begin{array}{l}\text { Infliximab } \\
(\mathrm{N}=315)\end{array}$ & p-value \\
\hline Mean (SD) age, years & $46(16.0)$ & $44(16.8)$ & 0.297 \\
Female, \% & 52.4 & 52.7 & $>0.999$ \\
Caucasian, \% & 89.5 & 84.1 & 0.180 \\
Insurance type, \% & & & 0.202 \\
Medicaid & 6.7 & 11.1 & \\
Medicare & 23.8 & 14.3 & \\
Private & 63.8 & 65.7 & \\
Other & 5.7 & 8.9 & \\
Patients with Crohn's Disease, \% & 60.0 & 60.9 & \\
Mean (SD) time from diagnosis, years & $3.6(3.5)$ & $3.1(3.6)$ & 0.667 \\
Comorbidities, \% & & & \\
Congestive heart failure & 3.8 & 2.9 & 0.745 \\
Rheumatic disease & 5.7 & 2.9 & 0.221 \\
Mild liver disease & 11.4 & 10.2 & 0.715 \\
Malignancies & 6.7 & 4.1 & 0.295 \\
IBD-related measures (during the baseline period), \% & & & \\
Surgery & 5.7 & 7.3 & 0.663 \\
Hospitalisations & 37.1 & 32.7 & 0.407 \\
Corticosteroids & 70.5 & 71.1 & 0.902 \\
Immunosuppressives & 20.02121 & 37.8 & 0.001 \\
\hline
\end{tabular}

Conclusion: Among biologic-naïve IBD patients, there was a trend toward prolonged median times to first IBD-related hospitalization or first flare with VDZ compared to IFX. The median time to discontinuation was comparable between the therapies. Future studies should examine comparative effectiveness outcomes in a larger cohort over a longer follow-up period.

Disclosure of Interest: H. Patel: I am currently an employee of Immensity Consulting, Inc., which received funding from Takeda Development Centre Ltd. M. Raluy Callado: Mireia Raluy Callado is a full-time employee of Evidera.

A. Berger: Ariel Berger is a full-time employee of Evidera.

R. Curtis: Employee of Takeda Development Centre Ltd.

M.J. Khalid: Employee of Takeda Development Centre Ltd. 
MONDAY, OCTOBER 30, 2017

OTHER LOWER GI DISORDERS I - HALL 7

09:00-17:00

\section{P0415 OXIDATIVE STRESS ENHANCES THE ANTIGEN}

PRESENTING FUNCTION OF COLONIC EPITHELIAL CELLS BY

INDUCING CD80 IN THE EARLY STAGES OF COLONIC

CARCINOGENESIS

C. Marchiori ${ }^{1}$, A. Kotsafti ${ }^{2}$, I. Angriman ${ }^{3}$, C. Castoro ${ }^{2}$, A. Porzionato ${ }^{4}$,

P. Brun ${ }^{5}$, M. Scarpa ${ }^{2}$, I. Castagliuolo ${ }^{1}$, M. Scarpa ${ }^{2}$

${ }^{1}$ Molecular Medicine, University of Padua, Padova/Italy

${ }^{2}$ Esophageal And Digestive Tract Surgical Unit, Regional Centre For Esophageal

Disease, Veneto Institute of Oncology (IOV-IRCCS), Padova/Italy

${ }^{3}$ Dept. Of General Surgery, University of Padova, Padova/Italy

${ }^{4}$ Neurosciences, University of Padua, Padova/Italy

${ }^{5}$ Dept. Of Molecular Medicine, University of Padova Dept. of Molecular Medicine, Padova/Italy

Contact E-mail Address: chiara.marchiori.1@studenti.unipd.it

Introduction: Cancer development has been linked to oxidative stress by increasing DNA mutations or inducing DNA damage, genome instability and cell proliferation. Interestingly, reactive oxygen species (ROS) seem to modulate antigen presentation, a crucial event in the immune surveillance mechanisms ${ }^{1}$. We recently showed that expression of the co-stimulatory molecule CD80 on epithelial cells has a critical role during the immune surveillance process occurring in colon carcinogenesis ${ }^{2}$. Remarkably, ROS have been involved in the transcriptional regulation of CD 80 gene expression ${ }^{3}$; in addition, oxidative DNA damage was directly correlated to CD80 expression in colon mucosa dysplasia ${ }^{4}$.

Aims \& Methods: Therefore, the aim of this work was to examine the role of ROS on CD80 expression in colonic epithelial cells using an in vitro and an in vivo model of colonic carcinogenesis. A mouse colorectal cancer cell line, CT26, was used to quantify the expression of CD80 in response to pro-oxidant (such as Antimycin $\mathrm{A}$ and $\mathrm{H}_{2} \mathrm{O}_{2}$ ) and antioxidant (N-acetyl cysteine) stimuli in presence or absence of pharmacological inhibitors of p38MAPK (SB203580 and BIRB 796) and NF-kB (JSH-23). C57BL/6 mice (wild type, WT) received azoxymethane (AOM) i.p. at a dose of $10 \mathrm{mg} / \mathrm{kg}$ body weight weekly for 6 weeks to induce colorectal cancer (CRC) and then sacrificed four months after the first $\mathrm{AOM}$ injection to perform flow cytometry analysis on colonocytes and mucosal lymphocytes. Mice were also randomized to received $5 \mathrm{mM} \mathrm{N}$-acetyl cysteine (NAC) in drinking water ( $\mathrm{pH} 7)$ ad libitum after AOM treatment.

Results: In CT26 cells ROS-generating agents (Antimycin A and $\mathrm{H}_{2} \mathrm{O}_{2}$ ) caused a significant CD80 up-regulation at mRNA $(p=0.01)$ and protein level $(\mathrm{p}<0.001) . \mathrm{H}_{2} \mathrm{O}_{2}$-induced $\mathrm{CD} 80$ up-regulation in colonic epithelial cells was significantly inhibited by $\mathrm{N}$-acetyl cysteine $(\mathrm{p}<0.001)$ and by $\mathrm{p} 38 \mathrm{MAPK}$ $(\mathrm{p}=0.001)$ but not by NF-kB pharmacological inhibition. In vivo administration of N-acetylcysteine significantly reduced AOM-induced CD80, MHC-I and MHC- II up-regulation in colonic epithelial cells $(\mathrm{p}<0.001, \mathrm{p}<0.001$ and $\mathrm{p}<0.001$ respectively). Moreover, CD8+CD28+, CD8+CD69+, $\mathrm{CD} 4+\mathrm{CD} 25+\mathrm{T}$ cells rate in colonic mucosa was significantly lower in AOMNAC mice than AOM mice ( $\mathrm{p}=0.08, \mathrm{p}=0.03$ and $\mathrm{p}=0.01$, respectively). Conclusion: All in all, our data support the hypothesis that oxidative stress promote the antigen presenting function of colonic epithelial cells by inducing CD80 in the early stages of colonic carcinogenesis. ROS-mediated CD80 up-regulation in colonic epithelial cells relies on the p38MAPK pathway in vitro.

Disclosure of Interest: All authors have declared no conflicts of interest.

\section{References}

1. Maemura K, Zheng Qizhi, Wada T, Ozaki M, Takao S, Aikou T, Bulkley G, Klein A, Sun Z. Reactive oxygen species are essential mediators in antigen presentation by Kupffer cells. 2005 Aug;83(4):336-43.

2. Scarpa M, Brun P, Scarpa M, Morgan S, Porzionato A, Kotsafti A, Bortolami M, Buda A, D'Incà R, Macchi V, Sturniolo GC, Rugge M, Bardini R, Castagliuolo I, Angriman I, Castoro C. CD80-CD28 signaling controls the progression of inflammatory colorectal carcinogenesis. Oncotarget 2015;6(24):20058-69.

3. Donepudi, M., Raychaudhuri, P., Bluestone, J. A. \& Mokyr, M. B. Mechanism of Melphalan-Induced B7-1 Gene Expression in P815 Tumor Cells. J. Immunol. 166, 6491-6499 (2001).

4. Scarpa M, Cardin R, Bortolami M, Kotsafti A, Scapa M C, Pozza A, Maran G, Picciocchi M, Ruffolo C, D'Inca R, Sturniolo G C, Castagiuolo I, Castoro C, Angriman I. Mucosal immune enviroment in colonic carcinogenesis: CD80 expression is associated to oxidative DNA damage and TLR4NF-kB signalling. Elsevier 2013 Jan;49(1):254-63.
P0416 ALTERED INTESTINAL EXPRESSION PROFILES OF ANTI-

MICROBIAL GENES IN IRRITABLE BOWEL SYNDROME ARE

LINKED TO BACTERIAL COMPOSITION AND IMMUNE ACTIVATION

J. Sundin ${ }^{1}$, S. M. P. Bennet ${ }^{2}$, M. K. Magnusson ${ }^{3}$, J. Tap ${ }^{4}$, M. Derrien ${ }^{5}$, B. Le Nevé ${ }^{5}$, J. Doré, H. Törnblom ${ }^{7}$, M. Simrén ${ }^{8}$, L. Öhman ${ }^{3}$

${ }^{1}$ Sahlgrenska Academy, Inst. of Medicine, Gothenburg/Sweden

${ }^{2}$ Inst. of Medicine, Gothenburg/Sweden

${ }^{3}$ Dept. Of Microbiology Immunology, University of Gothenburg, Göteborg/Sweden

${ }^{4}$ Microbiology, INRA, Jouy enJosas, France, Palaiseau/France

${ }^{5}$ Life Science, Danone Nutricia Research, Plaiseau/France

${ }^{6}$ Metagenopolis, INRA - MICALIS, Jouy-en-Josas/France

${ }^{7}$ Department Of Internal Medicine And Clinical Nutrition, Sahlgrenska Academy, University of Gothenburg, Gothenburg/Sweden

${ }^{8}$ Dept Of Internal Medicine, Sahlgrenska University Hospital - Dept of Internal Medicine, Sahlgrenska University Hospital; Gothe, Gothenburg/Sweden

Contact E-mail Address: johanna.sundin@gu.se

Introduction: Altered immune activity and gut microbiota alterations are proposed to be important factors in the pathophysiology of irritable bowel syndrome (IBS), but the relevance for symptoms is unclear.

Aims \& Methods: We aimed to determine if colonic antimicrobial gene expression profiles differ between IBS and healthy subjects and if potential alterations are linked to immune activity or gut microbiota composition. The expression of 84 key antimicrobial genes in sigmoid colon biopsies from patients with IBS, defined as being either immuno-active or immuno-norm based on systemic and mucosal cytokine profiles (Bennet et. al Am J Gastro, 2016), and healthy subjects was assessed by Human Antibacterial Response RT ${ }^{2}$ Profiler PCR Array. Targeted 16S rRNA pyrosequencing was performed on faecal microbiota. To identify discrimination profiles based on multiple variables between IBS patients and healthy subjects, orthogonal partial least squares discriminant analysis (OPLSDA) with a cut off for Variable Importance for the Projection $>0.7$ was performed.

Results:

Table 1: Differences in mucosal antimicrobial mRNA expression between IBS (Immuno-active and Immuno-norm) and healthy subjects.

\begin{tabular}{|c|c|c|c|c|}
\hline $\begin{array}{l}\text { Gene } \\
(2 \Delta \mathrm{Ct})\end{array}$ & $\begin{array}{l}\text { IBS }(n=31) \\
\text { v Healthy } \\
(n=16)\end{array}$ & $\begin{array}{l}\text { Immuno-active } \\
(\mathrm{n}=16) \mathrm{v} \\
\text { Healthy }\end{array}$ & $\begin{array}{l}\text { Immuno-norm } \\
(\mathrm{n}=15) \mathrm{v} \\
\text { Healthy }\end{array}$ & $\begin{array}{l}\text { Immuno-active } \\
\text { v Immuno-norm }\end{array}$ \\
\hline AKT1 & 0.01 & 0.01 & - & - \\
\hline IRF7 & 0.0002 & 0.0008 & 0.004 & - \\
\hline MAP2K4 & 0.002 & 0.006 & 0.01 & - \\
\hline TICAM1 & 0.002 & 0.01 & 0.007 & - \\
\hline TNFRSF1A & 0.003 & 0.005 & 0.03 & - \\
\hline SUGT1 & 0.004 & 0.05 & 0.002 & 0.01 \\
\hline LYZ & 0.004 & 0.01 & 0.02 & - \\
\hline LTF & 0.008 & 0.009 & - & - \\
\hline CHUK & 0.01 & 0.002 & - & - \\
\hline IRAK1 & 0.02 & 0.02 & - & - \\
\hline MAP2K1 & 0.02 & - & 0.02 & - \\
\hline ZBP1 & 0.04 & 0.05 & - & - \\
\hline TLR4 & 0.04 & - & - & - \\
\hline IL1B & 0.05 & - & 0.04 & - \\
\hline RIPK1 & 0.05 & - & 0.03 & - \\
\hline XIAP & - & - & - & 0.03 \\
\hline TIRAP & - & - & - & 0.04 \\
\hline NFKB1 & - & - & 0.01 & 0.05 \\
\hline CARD9 & - & - & - & - \\
\hline IL -18 & - & 0.05 & - & - \\
\hline IRF5 & - & 0.01 & - & - \\
\hline CXCL1 & - & - & 0.03 & - \\
\hline TOLLIP & - & - & 0.04 & - \\
\hline
\end{tabular}

Data pressented as p-values (Mann-Whitney t-test) '-' = non significant. We included 31 IBS patients ( 16 females, median age 32 (25-44) years) and 16 healthy subjects ( 8 females, median age $27(24-30)$ years). An OPLS-DA model demonstrated that the antimicrobial profiles differed between IBS and healthy subjects $\left(\mathrm{R}^{2}=0.54, \mathrm{Q}^{2}=0.16\right)$. The mucosal mRNA expression of 14 antimicrobial genes was downregulated, while one gene was upregulated in IBS patients compared to healthy subjects (Table 1). Antimicrobial profiles did not differ between IBS patients subtyped according to their predominant bowel habit $\left(\mathrm{R}^{2}=0.16\right.$, $\left.\mathrm{Q}^{2}=-0.02\right)$. An OPLS-DA model showed discrimination between immunoactive $(n=16)$ and immuno-norm $(n=15)$ IBS patients based on their mucosal antimicrobial profiles $\left(\mathrm{R}^{2}=0.91, \mathrm{Q}^{2}=0.61\right)$. This finding was corroborated by four antimicrobial genes being altered between the two IBS groups (Table 1). Adding healthy subjects to the model revealed three differing antimicrobial profiles for each respective group $\left(\mathrm{R}^{2}=0.44, \mathrm{Q}^{2}=0.30\right)$. Compared to healthy subjects, 19 genes in the immuno-active and immuno-norm IBS groups were differently expressed $(p<0.05$, Table 1$)$. Only one of the antimicrobial genes differently expressed between IBS patients and healthy subjects was associated with faecal microbiota in immuno-norm IBS patients (Conserved Helix-LoopHelix Ubiquitous Kinase (CHUK) with Anaerovorax $r=-0.76, p<0.01)$. In the immuno-active IBS group 11 associations were identified, including TNF 
Receptor Superfamily Member 1A (TNFRSF1A) with Bifidobacterium $(\mathrm{r}=-0.80, \mathrm{p}<0.01)$.

Conclusion: The intestinal antimicrobial gene profiles differ between subsets of IBS patients and healthy subjects. An altered ability to recognise microbiota associated with immune activity and the relative abundance of gut bacteria may play a role in the complex pathophysiology of IBS.

Disclosure of Interest: All authors have declared no conflicts of interest.

\section{P0417 THE EXPOSURE OF ANTIBIOTICS IS ASSOCIATED WITH INCREASED RISK OF COLORECTAL CANCER- A SYSTEMATIC REVIEW AND META-ANALYSIS}

X. $\mathrm{He}^{1}, \mathrm{~W} \cdot \mathrm{Wu}^{2}, \mathrm{Y} . \mathrm{Ding}^{3}, \mathrm{~L} \cdot \mathrm{Sun}^{1}$

${ }^{1}$ Department Of Gastroenterology, Sir Run Run Shaw Hospital, Zhejiang University, Hangzhou/China

${ }^{2}$ The First Affiliated Hospital, School Of Medicine, State Key Laboratory for Diagnosis and Treatment of Infectious Diseases, hangzhou/China

${ }^{3}$ Department Of Gastroenterology, Sir Run Run Shaw Hospital, hangzhou/China

\section{Contact E-mail Address: hexingkang1990@163.com}

Introduction: Recently, accumulating evidence suggested that the dysbiosis of the intestinal microbiota was associated with increased risk of colorectal cancer and might play a significant role in the colorectal carcinogenesis [1-2]. Many environmental factors (e.g. diet and lifestyle) that altered the gut microbiota had been reported in the development of colorectal cancer[3]. Antibiotics are able to shift the gut microbiota by altering bacterial composition and functions. The overuse of antibiotics has been associated with several adverse effects, such as severe infections, obesity, inflammatory bowel disease. Similarly, it is plausible to hypotheses that overuse of antibiotic might be linked to CRC by altering the colonic microbiota. However, the relationship between antibiotic and CRC was unclear and studies regarding this topic were limited [4-7].

Aims \& Methods: To evaluate the association between use of antibiotic and the risk of developing colorectal cancer, a systematic literature search was conducted using PubMed, EMBASE, Web of science and Cochrane library to identify related studies published before October 2016. Two independent investigators screened and extracted data from included articles. A random-effects model was adopted to calculate overall odds ratio (OR) and $95 \%$ confidence interval (CI).

Results: From initial search, we identified four case-control studies and finally included in the meta-analysis. Compared with no/low use of antibiotics, high prescriptions of antibiotics were significantly associated with an excess cancer risk $(\mathrm{OR}=1.11,95 \% \mathrm{CI} 1.01-1.21)$. There was a significant heterogeneity across studies $\left(\mathrm{I}^{2}=62.1 \%, \mathrm{P}=0.048\right)$. Longer duration of antibiotics was also significantly linked to increased risk of CRC $(\mathrm{OR}=1.14,95 \% \mathrm{CI} 1.03-1.25)$ with no significant heterogeneity $\left(\mathrm{I}^{2}=39.6 \%, \mathrm{P}=0.191\right)$. There was no evidence of significant publication bias among this meta-analysis.

Conclusion: Higher prescriptions and longer duration exposure of antibiotics were associated with increased risk of developing colorectal cancer. Further studies are needed to verify our results and explore underlying mechanisms.

Disclosure of Interest: All authors have declared no conflicts of interest.

\section{References}

1. Nakatsu G, Li X, Zhou H, Sheng J, Wong SH, Wu WK, Ng SC, Tsoi H, Dong Y, Zhang N, He Y, Kang Q, Cao L, Wang K, Zhang J, Liang Q, et al. Gut mucosal microbiome across stages of colorectal carcinogenesis. Nat Commun. 2015; 6:8727.

2. Louis P, Hold GL and Flint HJ. The gut microbiota, bacterial metabolites and colorectal cancer. Nat Rev Microbiol. 2014; 12(10):661-672.

3. Guinane $\mathrm{CM}$ and Cotter PD. Role of the gut microbiota in health and chronic gastrointestinal disease: understanding a hidden metabolic organ. Therap Adv Gastroenterol. 2013; 6(4):295-308.

4. Kilkkinen A, Rissanen H, Klaukka T, Pukkala E, Heliovaara M, Huovinen $\mathrm{P}$, Mannisto S, Aromaa A and Knekt P. Antibiotic use predicts an increased risk of cancer. Int J Cancer. 2008; 123(9):2152-2155.

5. Boursi B, Haynes K, Mamtani R and Yang YX. Impact of antibiotic exposure on the risk of colorectal cancer. Pharmacoepidemiol Drug Saf. 2015; 24(5):534-542.

6. Dik VK, van Oijen MG, Smeets HM and Siersema PD. Frequent Use of Antibiotics Is Associated with Colorectal Cancer Risk: Results of a Nested Case-Control Study. Dig Dis Sci. 2016; 61(1):255-264.

7. Wang JL, Chang CH, Lin JW, Wu LC, Chuang LM and Lai MS. Infection, antibiotic therapy and risk of colorectal cancer: a nationwide nested casecontrol study in patients with Type 2 diabetes mellitus. Int J Cancer. 2014; 135(4):956-967.
P0418 EFFECT OF INTERNAL AND EXTERNAL BILIARY DRAINAGE ON INTESTINAL MUCOSAL BARRIER FUNCTION IN BILIARY OBSTRUCTION RATS

S. $\mathrm{Li}^{1}$, X. $\mathrm{Su}^{2}, \mathrm{~K} . \mathrm{Luo}^{3}, \mathrm{C} \cdot \mathrm{Ge}^{4}, \mathrm{~W} \cdot \mathrm{Li}^{5}$

${ }^{1}$ Gastroenterology-hepatology, People's Liberation Army General Hospital (PLAGH), Beijing/China

${ }^{2}$ Beijing Youan Hospital Capital Medical University, Beijing/China

${ }^{3}$ The General Hospital of the Chinese People's Liberation Army, Beijing/China

${ }^{4}$ Yichang Central's Hospital, Yichang/China

${ }^{5}$ Gastroenterology And Hepatology, The General Hospital of the Chinese People's Liberation Army, Beijing/China

Contact E-mail Address: 1183573491@qq.com

Introduction: Internal biliary drainage has been confirmed better than external biliary drainage in alleviating the damage of intestinal mucosa barrier caused by obstructive jaundice, but the relevant mechanism is still unclear.

Aims \& Methods: We aimed to investigate the effect of internal and external drainage of obstructive jaundice rats on intestinal mucosal barrier function with special reference of intestinal immune-related index expression. Sixty male Sprague-Dawley rats were randomly assigned to four groups: OJ, sham operation (SH), internal biliary drainage (ID) and external biliary drainage (ED). All animals underwent surgical ligation of the bile duct, except SH was produced by separating common bile duct locally but not dividing on day 1. Then ED and ID were reoperated on day 8 for biliary drainage procedure. Blood from inferior vena cava were collected for the test of DAO and slgA activities by the method of ELASA. The terminal ileum specimens of each groups were collected for observation of the morphological changes with haematoxylin-eosin (HE) staining. The expression of IgA mRNA, pIgR mRNA, GP-BAR1mRNA, RD-5mRNA were measured by reverse transcription polymerase chain reaction (RT-PCR). GPBAR1 of the ileum mucosa was analyzed by Western blot.

Results: After bile duct ligation, the injures of the intestinal mucosa were obvious in OJ group with thinner mucosa, sparser villi, destruction of the epithelial integrity and accompanied by inflammatory cell infiltration. The mucosal lesions evaluated by Chiu's method was $2.158 \pm 0.579$, while the impaired intestinal mucosa have different degrees of recovery and ID group was more similar to $\mathrm{SH}$ group in intestinal mucosal morphology. The levels of the DAO in OJ group were increased more dramatically than that in SH, ID and ED groups while slgA were decreased $(p<0.01)$, and the activities of the DAO, slgA in ID group were similar to the level of SH group ( $p>0.05$ ), different to the level of ED group $(\mathrm{p}<0.01)$. The changes of the plasma DAO and slgA activities were significantly correlated with the conditions of intestinal mucosa $(\mathrm{P}<0.01)$. The expression of RD-5 mRNA in OJ group were decreased significantly than that in SH, ID and ED groups while GP-BAR1 mRNA, IgA mRNA, pIgR mRNA were increased $(\mathrm{p}<0.01)$. Interestingly enough, after external bile drainage, there is no improvement in IgA mRNA and plgR mRNA $(P>0.05)$. But in ID, the relative expression of IgA mRNA and pIgR mRNA reduced markedly $(\mathrm{P}<0.01)$. while the mRNA expression of GP-BAR 1 and RD-5 mRNA in ED group was changed less than that in ID which were more similar to SH group. The protein expression of GP-BAR1 was increased significantly in the intestinal mucosal of OJ group, which was higher than that of in SH group $(\mathrm{P}<0.01)$. After internal and external biliary drainage to alleviate OJ respectively, the GP-BAR1 expression was decreased significantly in ID group, similar with $\mathrm{SH}$ group (ID vs OJ, $\mathrm{P}<0.01$; ID vs SH, $\mathrm{P}>0.05)$, and lower than that of in ED group $(\mathrm{P}>0.05)$. Conclusion: The differential expression of IgA mRNA, pIgR mRNA, GP-BAR1 mRNA, RD-5 mRNA and activities of DAO and slgA in OJ, ID, ED and SH reflect internal biliary drainage better than external biliary drainage. There may be a regulatory mechanism between GP-BAR1 and intestinal immune-related index, which thus appears to be a key factor in maintaining function of intestinal mucosa barrier.

Disclosure of Interest: All authors have declared no conflicts of interest

\section{P0419 COMPARATIVE EFFECT OF XYLOGLYCAN ASSOCIATIONS WITH COMPOUNDS FROM ANIMAL OR ALGAE ORIGIN ON LPS-INDUCED ENTERITIS IN RATS}

H. Eutamene, C. Harkat, V. Theodorou

Unité De Neuro-gastroenterologie \& Nutrition, Toxalim UMR 1331 INRA/UPS/ INPT-EI-Purpan, Toulouse/France

Contact E-mail Address: helene.eutamene@inra.fr

Introduction: Xyloglucan (XG) is a film-forming agent exhibiting protective effects against diarrhea linked to infectious gastroenteritis in humans; further in animal models, xyloglucan efficacy against cholera-toxin-induced diarrhea was timely prolonged when this mucoprotectant agent is associated with gelatin from animal origin. The use of compounds from animal source in galenic formulations is nowadays questionable.

Aims \& Methods: Thus, in this study, we aimed at comparing the efficacy of XG associated with gelatin vs XG associated with gelose a moiety from algae origin on LPS-induced enteritis in rats. Since LPS-induced enteritis is characterized by increased intestinal epithelial permeability and mucosal inflammation, the efficacy of xyloglucan associations was evaluated by measurement of these two parameters. Male Wistar rats $(200-225 \mathrm{~g})$ were orally treated with either XG $(10 \mathrm{mg} / \mathrm{kg})+$ gelatin $(25 \mathrm{mg} / \mathrm{kg})$ or XG $(10 \mathrm{mg} / \mathrm{kg})+$ gelose $(25 \mathrm{mg} / \mathrm{kg})$ or XG $(10 \mathrm{mg} / \mathrm{kg})+$ gelose $(50 \mathrm{mg} / \mathrm{kg})$ or vehicle $(\mathrm{NaCl} 0.9 \%) 3 \mathrm{~h}$ before intraperitoneal (IP) administration of LPS from E. coli $(1 \mathrm{mg} / \mathrm{kg})$. Six hours later after LPS administration, the animals were sacrificed and strips of jejunum were collected in order to evaluate (i) intestinal epithelial paracellular permeability to FITCdextran $4 \mathrm{Kd}$ in Ussing chambers and (ii) mucosal inflammatory response by myeloperoxidase (MPO) activity measurement. 
Results: Compared with control, LPS administration induced a significant increase $(p<0.05)$ of intestinal paracellular permeability $(53.0 \pm 4.9 v$ s $181.6 \pm 21.1 \mathrm{pmol} / \mathrm{cm}^{2}$, respectively) associated with jejunal mucosal inflammation $(302.1 \pm 9.5$ vs $655.6 \pm 108.9 \mathrm{U} \mathrm{MPO} / \mathrm{g}$ protein, respectively). XG $(10 \mathrm{mg} /$ $\mathrm{kg})+$ gelose at the lowest dose $(25 \mathrm{mg} / \mathrm{kg})$ failed to reverse the intestinal hyperpermeability and mucosal inflammation induced by LPS. In contrast, XG $(10 \mathrm{mg} / \mathrm{kg})+$ gelatin $(25 \mathrm{mg} / \mathrm{kg})$ and $X G(10 \mathrm{mg} / \mathrm{kg})+$ gelose at $50 \mathrm{mg} / \mathrm{kg}$ significantly $(\mathrm{p}<0.01)$ and equally prevented LPS-induced hyperpermeability $\left(34.8 \pm 2.8,38.7 \pm 3.9\right.$ vs $181.6 \mathrm{~V} 21.1 \mathrm{pmol} / \mathrm{cm}^{2}$ respectively) and jejunal inflammation $(277.0 \pm 32.2 ; \quad 286.2 \pm 28.8$ vs $655.6 \pm 108.9$ U $\mathrm{MPO} / \mathrm{g}$ protein respectively).

Conclusion: This study shows that oral treatment with xyloglucan associated with gelose at $50 \mathrm{mg} / \mathrm{kg}$ has similar protective effects on LPS-induced enteritis in rats than xyloglucan associated with gelatin. These data demonstrate that algae is an effective and safe substitute for replacing compounds from animal origin in xyloglucan mucoprotectant formulations.

Disclosure of Interest: All authors have declared no conflicts of interest.

\section{P0420 RISK FACTORS ASSOCIATED WITH RECURRENCE OF CLOSTRIDIUM DIFFICILE INFECTION IN THE ELDERLY}

M.S. You ${ }^{1}$, D.H. Lee ${ }^{2}$

${ }^{1}$ Department Of Internal Medicine, Seoul National University Hospital, Seoul| Korea, Republic of

${ }^{2}$ Department Of Internal Medicine, Seoul National University Bundang Hospital, Seongnam-si/Korea, Republic of

Contact E-mail Address: bass105@hanmail.net

Introduction: The old age is one of the most important risk factors for recurrent C. difficile Infection (CDI). However, risk factors among the elderly patients are largely unknown.

Aims \& Methods: The purpose of this study was to investigate risk factors associated with recurrent CDI in the elderly. Patients 65 years of age or older with positive CDI toxin test between January 2005 and December 2016, who received either oral metronidazole or oral vancomycin therapy were included Recurrent CDI was defined as another positive laboratory result for $C$. difficile toxin between 15 days and 90 days after initial positive diagnostic test. Clinical charts of relevant factors in 633 patients with positive CDI toxin tests were reviewed. Continuous variables were tested via Student's t-test, and categorical data was analyzed via Chi-Square test. All variables with $P<0.1$ in the univariate analysis were included in the multivariable logistic regression analysis.

Results: The overall mean age was $77.0 \pm 7.0$ years. In $96(15.2 \%)$ of 633 patients, C. difficile toxin was detected again after the initial test. The length of hospital stay was longer in recurrent CDI group than in non-recurrent group $(80.54 \pm 89.44$ vs. $43.81 \pm 65.42, P<.001)$. Patients with eGFR $<60 \mathrm{ml} / \mathrm{min}$ $1.73 \mathrm{~m}^{2}$ were at higher risk for the development of recurrent CDI than those with normal renal function (OR 1.844; 95\% CI, 1.139-2.985, $P=.013$ ). There were no significant differences on mean age $(77.21 \pm 6.65$ in recurrent CDI group vs. $77.01 \pm 7.04$ in non-recurrent $\mathrm{CDI}$ group, $P=.799$ ) and proton pump inhibitor therapy (OR $1.277 ; 95 \% \mathrm{CI}, 0.825$ to $1.977, P=.272$ ) between both groups. Renal function and length of hospital stay were significantly associated with recurrence of CDI.

Conclusion: In this study, impaired renal function and prolonged hospitalization were related to the increased risk of recurrent CDI.

Disclosure of Interest: All authors have declared no conflicts of interest.

\section{References}

1. Bartlett JG, Gerding DN. Clinical recognition and diagnosis of Clostridium difficile infection. Clin Infect Dis. 2008:46 Suppl 1:S12-8.

2. Simor AE, Bradley SF, Strausbaugh LJ, Crossley K, Nicolle LE, Committee SL-T-C. Clostridium difficile in long-term-care facilities for the elderly. Infect Control Hosp Epidemiol. 2002;23(11):696-703.

3. Freedberg DE, Salmasian H, Friedman C, Abrams JA. Proton pump inhibitors and risk for recurrent Clostridium difficile infection among inpatients. Am J Gastroenterol. 2013;108:1794-801.

4. Thongprayoon C, Cheungpasitporn W, Phatharacharukul P, Mahaparn P, Bruminhent J. High Mortality Risk in Chronic Kidney Disease and End Stage Kidney Disease Patients with Clostridium Difficile Infection: A Systematic Review and Meta-analysis. J Nat Sci. 2015;1(4).

\section{P0421 CLINICAL CHARACTERISTICS OF CYTOMEGALOVIRUS COLITIS: 15 YEAR-EXPERIENCE IN A TERTIARY MEDICAL CENTER}

P. Le ${ }^{1}$, R. Wu ${ }^{2}$, C. Chiu ${ }^{1}$, C. Kuo ${ }^{1}$, M. Su ${ }^{1}$, C. $\operatorname{Lin}^{1}$, J. Hsu ${ }^{3}$

${ }^{1}$ Department Of Gastroenterology And Hepatology, Linkou Chang Gung Memorial Hospital, Taoyuan/Taiwan

${ }^{2}$ Department Of Pathology, Linkou Chang Gung Memorial Hospital, Taoyuan/ Taiwan

${ }^{3}$ Department Of General Surgery, Linkou Chang Gung Memorial Hospital, Taoyuan/Taiwan

Contact E-mail Address: puohsien@gmail.com

Introduction: Cytomegalovirus (CMV) colitis in adults is mostly described in immunocompromised patients (solid organ or hematopoietic stem cell transplant recipients, patients with human immunodeficiency virus (HIV) infection, use of immunosuppressive drugs, including steroid or chemotherapeutic agents), and often has poorer outcome than in children. Besides, it was also frequently presented in patients with known or subsequent new diagnosis inflammatory bowel disease $[1,2]$. However, there are only case reports and few case series with limited patients (below 15 cases) among immunocompetent individuals without steroid use or inflammatory bowel disease [3-5]. The largest meta-analysis study of cytomegalovirus colitis in immunocompetent hosts included 44 patients and noted advanced age, male gender, presence of immune-modulating comorbidities and need of surgical intervention negatively influencing survival in 2005 [6]. The case number of CMV colitis in immunocompetent patients seemed increasing in our hospital these years. There was no single study showing comprehensive clinical characteristics, identifying the independent factors of in-hospital mortality and comparing the differences between immunocompetent and compromised patients with CMV colitis. Therefore, we tried to clarify the issue in this study. Aims \& Methods: We enrolled 42 immunocompetent patients and 27 immunocompromised patients with CMV colitis diagnosed by immunohistochemistry stain between April 2002 and December 2016 in Linkou Chang Gung Memorial Hospital, a 3383-bed tertiary medical center and referral center in Taiwan. We analyzed the risk factors of in-hospital mortality and overall survival. Furthermore, we compared the clinical differences between immunocompetent and immunocompromised patients with CMV colitis.

Results: Early diagnosis (before 9 days) was independent predictor of in-hospital mortality in CMV colitis patients. ICU admission $(\mathrm{P}=0.010)$, requisite days of diagnosis $\geqq 9$ days after admission $(P=0.018)$, shock $(P=0.001)$, respiratory failure $(\mathrm{P}=0.033)$, hemoglobin $<10 \mathrm{~g} / \mathrm{dL} \quad(\mathrm{P}=0.002), \quad$ Creatinine $\geqq 1.37 \mathrm{mg} / \mathrm{dL}$ $(\mathrm{P}=0.004)$ and $\mathrm{CRP} \geq 59 \mathrm{mg} / \mathrm{dL}(\mathrm{P}=0.011)$ negatively impacted on overall survival. There were older and more comorbidities in immunocompetent group. However, the in-hospital mortality rate and overall survival rate was similar to immunocompromised group. Besides, Clostridium difficile infection or steroid use didn't affect in-hospital mortality rate and overall survival rate nether. Melena was first and most common symptom in immunocompetent group, but diarrhea in the other.

Analysis of the clinical factors associated with in-hospital mortality in all patients

\begin{tabular}{llll}
\hline Characteristic & Odd ratio & $95 \% \mathrm{CI}$ & P-value
\end{tabular}

Univariate analysis

Age $\geqq 65 y$

Gender (male/female)

Immunocompromised status

Intensive care unit admission

0.545

0.545
0.986

6.871

Requisite time of diagnosis

1.034

$0.691 \sim 6.209$

0.194

(day after admission)

General condition

Sepsis

Shock

Respiratory failure

Operation before diagnosis

Underlying diseases

Inflammatory bowel disease

Systemic lupus erythematosus

Solid organ transplantation

Solid organ malignancy

Hematological malignancy

Liver cirrhosis

Chronic kidney disease

End stage renal disease

Diabetes mellitus

$0.184 \sim 1.619$

0.275

$0.328 \sim 2.969 \quad 0.981$

$2.068 \sim 22.833 \quad 0.002 *$

HIV infection

$1.002 \sim 1.066 \quad 0.034 *$

Immunosuppressive medication

Immunosuppressant

Chemotherapy

Steroid

Steroid over 1 month

$1.039 * 10^{9} \quad 0.000 \sim>10^{12} \quad 0.998$

$5.714 \quad 1.793 \sim 18.210 \quad 0.003 *$

$4.062 \quad 1.309 \sim 12.610 \quad 0.015^{*}$

$3.200 \quad 0.583 \sim 17.553 \quad 0.180$

Laboratory data

Total WBC count (/mcL)

ANC $(/ \mathrm{mcL})$

ALC (/mcL)

Hemoglobin level $(\mathrm{g} / \mathrm{dL})$

0.000

4.900

0.000

0.999

2.941

$0.747 \sim 32.123 \quad 0.098$

0.941

$0.174 \sim 49.636 \quad 0.454$

$0.092 \sim 9.671 \quad 0.959$

2.941

$0.174 \sim 49.636 \quad 0.454$

$0.941 \quad 0.092 \sim 9.671 \quad 0.959$

$2.067 \quad 0.576 \sim 7.421 \quad 0.265$

$3.357 \quad 0.742 \sim 15.181 \quad 0.116$

$1.682 \quad 0.543 \sim 5.205 \quad 0.367$

$0.000 \quad 0.000 \sim>10^{12} \quad 0.999$

Platelet count $(\times 1000 / \mathrm{mm} 3)$

Creatinine (mg/dL)

ALT (IU/L)

Bilirubin (mg/dL)

$3.200 \quad 0.583 \sim 17.553 \quad 0.180$

$4.846 * 10^{9} \quad 0.000 \sim>10^{12} \quad 1.000$

$1.124 \quad 0.336 \sim 3.764 \quad 0.849$

$2.350 \quad 0.472 \sim 11.708 \quad 0.297$

Albumin $(\mathrm{g} / \mathrm{dL})$

C-reactive protein $(\mathrm{mg} / \mathrm{dL})$

1.000

$1.000 \sim 1.000 \quad 0.419$

1.000

0.999

$1.000 \sim 1.000 \quad 0.254$

$0.998 \sim 1.000 \quad 0.018^{*}$

$0.668 \quad 0.485 \sim 0.918 \quad 0.013 *$

$0.995 \quad 0.990 \sim 1.001 \quad 0.100$

$1.448 \quad 1.059 \sim 1.978 \quad 0.020$ *

$0.995 \quad 0.958 \sim 1.033 \quad 0.787$

$1.370 \quad 0.965 \sim 1.944 \quad 0.078$

$0.625 \quad 0.231 \sim 1.687 \quad 0.354$

$1.009 \quad 1.000 \sim 1.018 \quad 0.047^{*}$

Viral markers 
Continued

Analysis of the clinical factors associated with in-hospital mortality in all patients

\begin{tabular}{llll}
\hline Characteristic & Odd ratio & $95 \% \mathrm{CI}$ & P-value \\
\hline CMV pp65 antigenemia & 0.656 & $0.140 \sim 3.079$ & 0.593 \\
CMV IgG positive & 0.286 & $0.016 \sim 5.095$ & 0.394 \\
CMV IgM positive & 3.125 & $0.547 \sim 17.841$ & 0.200 \\
Clostridium difficile infection & 0.889 & $0.077 \sim 10.300$ & 0.925 \\
Ganciclovir or valganciclovir treatment & 2.286 & $0.579 \sim 9.026$ & 0.238 \\
Treatment duration & 0.989 & $0.953 \sim 1.026$ & 0.563 \\
Surgical treatment & 1.840 & $0.392 \sim 8.630$ & 0.439 \\
Perforation & 1.441 & $0.123 \sim 16.920$ & 0.771 \\
Multivariate analysis & & & \\
Intensive care unit admission & 4.726 & $0.374 \sim 59.747$ & 0.230 \\
Requisite time of diagnosis & 1.075 & $1.005 \sim 1.149$ & $0.035^{*}$ \\
$\quad$ (day after admission) & & & \\
Shock & 4.905 & $0.596 \sim 40.362$ & 0.139 \\
Respiratory failure & 0.661 & $0.053 \sim 8.269$ & 0.748 \\
ALC (/mcL) & 0.998 & $0.996 \sim 1.001$ & 0.157 \\
Hemoglobin level (g/dL) & 0.787 & $0.459 \sim 1.349$ & 0.383 \\
Creatinine (mg/dL) & 0.618 & $0.306 \sim 1.246$ & 0.179 \\
C-reactive protein (mg/dL) & 1.009 & $0.998 \sim 1.021$ & 0.106 \\
\hline
\end{tabular}

Conclusion: Immunocompromised patients or steroid users did not have higher in-hospital mortality rate. Early diagnosis was only independent factor for lower in-hospital mortality in patients with CMV colitis.

Disclosure of Interest: All authors have declared no conflicts of interest.

\section{References}

1. Weng MT, Tung CC, Lee YS, et al. Cytomegalovirus colitis in hospitalized inflammatory bowel disease patients in Taiwan: a referral center study. $B M C$ Gastroenterol 2017; 17(1): 28.

2. Khan TV, Toms C. Cytomegalovirus Colitis and Subsequent New Diagnosis of Inflammatory Bowel Disease in an Immunocompetent Host: A Case Study and Literature Review. Am J Case Rep 2016; 17: 538-43.

3. Paparoupa M, Schmidt V, Weckauf H, Ho H, Schuppert F. CMV Colitis in Immunocompetent Patients: 2 Cases of a Diagnostic Challenge. Case Rep Gastrointest Med 2016; 2016: 4035637.

4. Inayat $\mathrm{F}$, Hussain $\mathrm{Q}$, Shafique $\mathrm{K}$, Tasleem SH, Hurairah A. Cytomegalovirus Colitis in Immunocompetent Patients. Cureus 2016; 8(11): e869.

5. Harano Y, Kotajima L, Arioka H. Case of cytomegalovirus colitis in an immunocompetent patient: a rare cause of abdominal pain and diarrhea in the elderly. Int J Gen Med 2015; 8: 97-100.

6. Galiatsatos P, Shrier I, Lamoureux E, Szilagyi A. Meta-analysis of outcome of cytomegalovirus colitis in immunocompetent hosts. Dig Dis Sci 2005; 50(4): 609-16.

\section{P0422 FIRST CASE SERIES OF FECAL MICROBIOTA TRANSPLANTATION FOR RECURRENT CLOSTRIDIUM DIFFICILE INFECTION IN BALTIC COUNTRIES}

R. Gedgaudas ${ }^{1}$, M. Urba ${ }^{1}$, V. Petkevicius ${ }^{2}$, L. Jonaitis ${ }^{1}$, G. Kiudelis ${ }^{1}$, L. Kupcinskas ${ }^{1}$, J. Kupcinskas ${ }^{2}$

${ }^{1}$ Gastroenterology, Lithuanian University of Health Sciences, Kaunas/Lithuania

${ }^{2}$ Lithuanian University Of Helath Sciences, Kaunas, Kaunas/Lithuania

Contact E-mail Address: R.gedgaudas@gmail.com

Introduction: Clostridium difficile infection (CDI) is one of the most common hospital-acquired infections. Faecal microbiota transplantation (FMT) is used for complicated recurrent CDI treatment; however, to date no data on the efficacy of this method in Eastern Europe have been published.

Aims \& Methods: The aim of this study was to assess effectiveness of FMT for recurrent CDI therapy in the hospital of Lithuanian University of Health Sciences (LUHS KK, Kaunas, Lithuania). Clinical data of patients who were treated for recurrent ( $>2$ times) CDI using FMT in the Department of Gastroenterology of LUHS KK during 2015-2016, were analyzed. All patients were monitored for disease relapse for six months. Clinical data, the use of antibiotics and immunosuppressive drugs were included in analysis. Statistical analysis was performed using statistical software package SPSS version 17.

Results: FMT was used for 18 patients with recurrent CDI. The mean age of patient was $60.4 \pm 8.4$ years. The patients were treated with antibiotics 14.8 days on average before manifestation of CDI. FMT procedure was performed using naso-enteral tube. After the first procedure, the positive clinical effect was observed in 15 patients with a cure rate of $83.3 \%$. FMT procedure was repeated for two out of the three patients without positive effect (one patient refused repetitive FMT). Normal stool habits were restored in both patients leading to the increase of cure rate to $94.4 \%$ ( 17 out of 18 patients). Seventeen patients that were successfully treated with one or two FMT procedures in the short term also remained asymptomatic $(100 \%)$ at 6 months of follow up. All patients without positive effect of first FMT procedure were on immunosuppressive drugs (3/3;
$100 \%)$, as compared to only one patient in the group with positive effect $(1 / 15$; $7.1 \%), p=0.028$

Conclusion: FMT is an effective therapy for recurrent CDI infection both in short and long term follow-up. Effectiveness of primary FMT treatment could be associated with the use of immunosuppressive drugs.

Disclosure of Interest: All authors have declared no conflicts of interest.

P0423 A RANDOMISED CONTROLLED TRIAL OF RIFAXIMIN TO PREVENT RELAPSE OF CLOSTRIDIUM DIFFICILE ASSOCIATED DIARRHOEA AFTER RESOLUTION WITH STANDARD THERAPY

G. Major ${ }^{1}$, L. Bradshaw ${ }^{2}$, N. Boota ${ }^{3}$, K. Sprange ${ }^{2}$, A. Jawhari ${ }^{4}$, M. Diggle ${ }^{4}$, A. Montgomery ${ }^{2}$, R. Spiller

${ }^{1}$ NIHR Nottingham Digestive Diseases Biomedical Research Centre, University of Nottinghan, Nottingham/United Kingdom

${ }^{2}$ Nottingham Clinical Trials Unit, University of Nottingham, Nottingham/United Kingdom

${ }^{3}$ Warwick Clinical Trials Unit, University of Warwick, Coventry/United Kingdom

${ }^{4}$ Nottingham University Hospitals NHS Trust, Nottingham/United Kingdom

Contact E-mail Address: Giles.Major@nottingham.ac.uk

Introduction: Clostridium difficile associated diarrhoea (CDAD) is a common nosocomial infection. The most commonly prescribed treatments, metronidazole and vancomycine, have a primary cure rat of $90 \%$ but 1 in 4 cases suffer a relapse in the following months. A disrupted microbiota is thought to increase the risk of relapse. Rifaximin is a non-absorbable antibiotic that suppresses C.difficile proliferation. In a trial of 68 patients Garey et al. found that a course of rifaximin after standard therapy reduced relapse rate though not significantly ${ }^{1}$

Aims \& Methods: We aimed to further investigate the efficacy of rifaximin to prevent CDAD relapse in a parallel group, randomised, placebo controlled trial in 23 hospitals in England. Population: age $\geq 18$ with resolution of CDAD after treatment with metronidazole or vancomycin, defined as cessation of diarrhoea for $\geq 2$ days. CDAD diagnosis required evidence of toxin production or pseudomembranes at endoscopy. Exclusion criteria were pregnancy or breast feeding; life expectancy $<4$ weeks; unable to take intervention (hypersensitivity or swallowing disorder); $>5$ days elapsed since treatment. Randomisation was stratified by hospital using a remote, internet-based system. Participants, clinicians and researchers were blind to allocation. Intervention: Rifaximin $1200 \mathrm{mg}$ daily for two weeks then $600 \mathrm{mg}$ daily for two weeks, in three divided doses. Comparator: identical placebo. Primary Outcome: relapse $\leq 12$ weeks after treatment initiation, defined as diarrhoea ( $\geq 3$ type 6 or 7 stools per day) for 2 days with evidence of toxin production. Sample size: The planned sample size was 180 to detect a difference in relapse of $20 \%$ (30\% placebo, $10 \%$ rifaximin) with $80 \%$

power, allowing for loss to follow-up of 20\%. EudraCT 2012-003205-10; www.clinicaltrials.gov NCT01670149; ISRCTN 65163992

Results: Recruitment occurred December 2012-March 2016. Of 2157 patients screened, 151 were eligible, willing and randomised before funding limits were reached ( 74 placebo, 77 rifaximin). Primary outcome data were available on 130 . Mean age was 71.9 (SD 15.3).

$36 \%$ were in-patients at start of intervention. $13 \%$ had a prior recorded episode of CDAD. $26 \%$ were using proton pump inhibitors pror to CDAD diagnosis, with a higher rate of use in the rifaximin group (32\% vs. $20 \%) .18 / 61(29.5 \%)$ on placebo relapsed within 12 weeks compared to $11 / 69(15.9 \%)$ on rifaximin, a difference between groups of $-13.7 \%(95 \% \mathrm{CI}-28.1 \%$ to $0.7 \%, \mathrm{p}=0.06)$. The risk ratio was $0.54(95 \%$ CI 0.28 to $1.05, \mathrm{p}=0.07)$. During 6-month safety follow up 9 participants died in each group $(12 \%)$. Adverse event rates were similar between groups.

Conclusion: CDAD relapse rate was $13.7 \%$ lower than on placebo. The confidence interval means that lack of effect remains possible but the estimated effect size is similar to Garey's trial ${ }^{1}$ with meta-analysis of the trials showing a statistically significant effect. The effect size is similar to that reported for fidaxomicin at 40 days $^{2}$, or for bezlotoxumab at 3 months ${ }^{3}$. Age and mortality rate were higher in our trial which may reflect greater similarity to the population at risk. Comparative trials of the effectiveness and cost effectiveness of alternative treatment strategies should follow.

Disclosure of Interest: R. Spiller: Trial funded through National Institute for Health Research RfPB grant PB-PG-0110-21041. Norgine Pharmaceuticals Ltd supplied product and comparator free of cost.

All other authors have declared no conflicts of interest.

\section{References}

1. Garey $\mathrm{KW}$ et al. A randomized, double-blind, placebo-controlled pilot study to assess the ability of rifaximin to prevent recurrent diarrhoea in patients with Clostridium difficile infection. J Antimicrob Chemother 2011 Dec;66(12):2850-5

2. Crook DW et al. Fidaxomicin versus vancomycin for Clostridium difficile infection: meta-analysis of pivotal randomized controlled trials. Clin Infect Dis 2012 Aug;55 Suppl 2:S93-103.

3. Wilcox $\mathrm{MH}$ et al. Bezlotoxumab for Prevention of Recurrent Clostridium difficile Infection. NEJM 2017 Jan 26;376(4):305-317. 
P0424 A SYSTEMATIC REVIEW AND META-ANALYSIS OF INHOSPITAL DELAY BEFORE SURGERY AS A RISK FACTOR FOR COMPLICATIONS IN PATIENTS WITH ACUTE APPENDICITIS

S. Van Dijk ${ }^{1}$, A. Van Dijk ${ }^{1}$, M.G.w. Dijkgraaf ${ }^{2}$, M.A. Boermeester ${ }^{3}$ ${ }^{1}$ Dept. Of Surgery, Academic Medical Center, Amsterdam/Netherlands ${ }^{2}$ Clinical Research Unit, Academic Medical Center, Amsterdam/Netherlands ${ }^{3}$ Dept. Of Surgery, Academic Medical Center Amsterdam, Amsterdam/ Netherlands

Contact E-mail Address: stefanvandijk@amc.nl

Introduction: The traditional fear that every acute appendicitis will eventually perforate leads to prompt surgery, but this fear may be outdated. In-hospital delay of surgery for acute appendicitis has been subject of a large number of studies. However, consensus about the consequences of delaying appendectomy is lacking, which is reflected in variety or absence of recommendations in guidelines.

Aims \& Methods: The aim of this study was to assess in-hospital delay of surgery as potential risk factor for complicated appendicitis and complications in patient with acute appendicitis. PubMed and EMBASE were searched from 1990 to July 2016. Outcome measures of interest were complicated appendicitis, surgical site infections and postoperative morbidity. All studies reporting surgically treated patients with one of these outcome measures in two or more predefined time intervals were included. Adjusted odds ratios were pooled using forest plots if possible. All unadjusted data was pooled using generalized linear mixed models. Results: Forty-five studies with 152, 314 patients were included. Pooled adjusted odds ratios revealed no significantly higher risk for complicated appendicitis when delaying appendectomy for 6 to 12 hours or 13 to 24 hours; odds ratio 1.07 (95\% CI 0.98-1.17) and 1.09 (95\% CI 0.95-1.24), respectively. For a delay of more than 24 hours, insufficient adjusted data was available for meta-analysis. Pooled unadjusted data showed a decreased risk for complicated appendicitis when appendectomy was delayed for 24 to 48 hours, however statistical uncertainty in this interval increased considerably compared to the first 24 hours. Conclusion: Delaying appendectomy for up to 24 hours after admission did not result in higher rates of complicated appendicitis, surgical site infections or morbidity. When prompt surgery is hampered by logistic or personal reasons, delaying appendectomy up to 24 hours is an acceptable alternative for patients with no preoperative signs of complicated appendicitis.

Disclosure of Interest: All authors have declared no conflicts of interest.

\section{P0425 VANCOMYCIN FOLLOWED BY FECAL MICROBIOTA TRANSPLANTATION VERSUS VANCOMYCIN FOR INITIAL CLOSTRIDIUM DIFFICILE INFECTION: AN OPEN-LABEL RANDOMISED CONTROLLED TRIAL}

S.C. C. Ng$^{1}$, S.H. Wong ${ }^{1}$, R. N. Lui ${ }^{2}$, K. Cheung ${ }^{1}$, J.Y.L. Ching ${ }^{1}$, W. Tang ${ }^{1}$, M. Kyaw ${ }^{1}$, Z. Tao ${ }^{1}$, K.T. Ho ${ }^{1}$, M. Ip ${ }^{2}$, P. Chan ${ }^{2}$, F.K.L. Chan ${ }^{1}$, J.J.Y. Sung ${ }^{1}$, J. C. Wu ${ }^{1}$, L.Y.K. Lam ${ }^{1}$

${ }^{1}$ Medicine And Therapeutics, Chinese University Of Hong Kong, Hong Kong/Hong Kong PRC

${ }^{2}$ Microbiology, Chinese University of Hong Kong, Hong Kong/Hong Kong PRC

\section{Contact E-mail Address: siewchienng@cuhk.edu.hk}

Introduction: Fecal microbiota transplantation (FMT) is effective for the treatment of recurrent Clostridium difficile infection (CDI) but its role as an initial therapy for patients with CDI has not been studied.

Aims \& Methods: We assessed the efficacy of FMT in patients with an initial episode of CDI compared with standard vancomycin regimen. In a single center, open-label, randomised study, we assigned 30 patients with an initial episode of CDI to receive either: oral vancomycin $(500 \mathrm{mg}$ four times daily) followed by FMT consisting of a single infusion of donor feces through a nasoduodenal tube; or a standard oral vancomycin regimen ( $500 \mathrm{mg}$ four times daily for 10 days). The primary end point was resolution of diarrhea associated with CDI without relapse within 10 weeks after initiation of therapy. Secondary outcomes included 30-day and 6-month mortality, 30-day colectomy rates, length of hospital stay, adverse effects and alteration of fecal microbiota after FMT using metagenomic sequencing.

Results: Baseline characteristics including age, gender and co-morbidities were comparable between the vancomycin and FMT arm. $60 \%$ and $47 \%$ of subjects in the vancomycin and FMT arm, respectively, had severe CDI. Resolution of $C$ difficile infection occurred in 10 of 15 patients $(66.7 \%)$ receiving vancomycin and 11 of 15 patients $(73.3 \%)$ receiving FMT $(\mathrm{p}=1.00)$. Two deaths occurred in the vancomycin group and none in the FMT group within 30 days. Nine $(60 \%)$ and three deaths $(20 \%)$ occurred in the vancomycin and FMT arms, respectively, within 6 months. None of the patients had a colectomy. Median length of hospital did not differ between both arms (13 vs 15 days; $p=0.95)$. No serious adverse events attributed to FMT were observed. A restoration of healthy control enriched bacteria in recipients was observed after FMT, with a decrease in abundance of CDI-enriched bacteria. FMT, but not vancomycin treatment, resulted in marked virome alterations.

Conclusion: In this pilot randomised controlled trial, FMT was not superior to vancomycin in patients with an initial episode of CDI. 30-day and 6-month mortality was higher in the vancomycin arm. A restoration of healthy control enriched bacteria in recipients was observed after FMT, with a decrease in abundance of CDI-enriched bacteria (Clinical Trial registry, NCT02570477; Funded by the Hong Kong Society of Gastroenterology Society).

Disclosure of Interest: All authors have declared no conflicts of interest.
P0426 A 10-YEAR REVIEW OF ABDOMINAL TUBERCULOSIS EXPERIENCE IN A MULTI-ETHNIC SECONDARY CARE POPULATION

U. Shivaji ${ }^{1}$, T. Critchlow ${ }^{1}$, S. Pathmakanthan ${ }^{1}$, S. Ghosh ${ }^{2}$, M. Iacucci ${ }^{3}$, N. Sharma ${ }^{4}$, R. Cooney ${ }^{3}$, T. Iqbal ${ }^{3}$, P. Glynn ${ }^{1}$, N. Bhala ${ }^{3}$

${ }^{1}$ Gastroenterology, University Hospitals Birmingham NHS Foundation Trust Queen Elizabeth Hospital, Birmingham/United Kingdom

${ }^{2}$ University Of Birmingham, Institute of Translational Medicine, Birmingham United Kingdom

${ }^{3}$ Gastroenterology, University Hospitals Birmingham NHS Foundation Trust, Birmingham/United Kingdom

${ }^{4}$ Gastroenterology, Heart of England NHS Foundation Trust, Birmingham/United Kingdom

Contact E-mail Address: Neeraj.Bhala@uhb.nhs.uk

Introduction: Tuberculosis (TB) carries significant morbidity globally and in Europe: it is most commonly pulmonary but it can also affect the gastrointestinal (GI) tract $(6 \%$ of total cases in UK). Abdominal tuberculosis (A-TB) is a rare disease which can also present an unique diagnostic challenge, mimicking various GI diseases leading to late diagnosis and treatment. There is limited clinical data on its presentation and clinical features in Europe - in the UK it is registered via regional Public Health registries.

Aims \& Methods: We reviewed a 10-year cumulative regional TB database in an university teaching hospital covering an urban multi-ethnic population to report clinical details of A-TB and results on this unusual condition. A clinical review of patients diagnosed for A-TB between 2006 to 2016 in a single tertiary centre in South Birmingham covering a multi-ethnic urban population of $\sim 750,000$ was conducted. A central suveillance database managed by Respiratory Physicians and Public Health England were used to identify patients with A-TB. We reviewed clinical data from electronic records including radiology, chemical pathology, histopathology, endoscopy databases, surgical notes and letters.

Results: Of 41 patients $[\mathrm{M}=22(54 \%)$; mean age $42 \mathrm{y}(\mathrm{SD} \pm 17 \mathrm{y})]$ identified with A-TB, $41 \%$ (17) were Pakistani, 15\% (6) were other Asian, $19 \%$ (8) were AfroCaribbean. Thirty-three $(80 \%)$ were residents of economically deprived areas which were among the $10 \%$ of most deprived constituencies in the UK. The most frequently reported symptoms were abdominal pain $(\mathrm{n}=23 ; 56 \%)$, weight loss $(n=17 ; 41 \%)$, fever $(n=10 ; 24 \%)$ \& vomiting $(n=9 ; 22 \%)$. Twelve $(29 \%)$ patients were first seen in a surgical clinic and $9(22 \%)$ in a medical gastroenterology clinic. A-TB was confirmed on tissue biopsy in $22(54 \%)$, of which two were diagnosed post-bowel resection. Seventeen $(41 \%)$ patients had positive cultures with full drug sensitivity and $5(12 \%)$ patients had polymerase chain reaction (PCR) tests. Eight patients $(20 \%)$ had concurrent pulmonary TB. Thirty seven patients $(90 \%)$ received full, successful treatment for A-TB

Conclusion: Asian ethnicity and low socioeconomic status appear to be risk factors for A-TB in a single UK tertiary centre. Histological diagnosis at endoscopic or surgical biopsy is a reliable diagnostic tool for confirming TB. Both gastroenterologists and surgeons need to consider A-TB in their differentials, as once diagnosed, most are successfully treated.

Disclosure of Interest: All authors have declared no conflicts of interest.

\section{References}

1. Debi U, Ravisankar V, Prasad KK, Sinha SK, Sharma AK. Abdominal tuberculosis of the gastrointestinal tract: Revisited. World J Gastroenterol. 2014 Oct 28; 20(40): 14831-14840.

2. Mamo JP, Brij SO, Enoch DA. Abdominal tuberculosis: a retrospective review of cases presenting to a UK district hospital. QJM. 2013 Apr:106(4):347-54

3. Addison NV. Abdominal tuberculosis-a disease revived. Ann $R$ Coll Surg Engl. 1983 Mar;65(2):105-11.

\section{P0427 INCREASING INCIDENCE OF CLOSTRIDIUM DIFFICILE INFECTION AND THE USE OF PROTON-PUMP INHIBITORS: RESULTS FROM A TERRITORY-WIDE POPULATION STUDY IN HONG KONG}

S. Wong ${ }^{1}$, J. $\mathrm{Ho}^{2}$, R. Dai ${ }^{1}$, L.Y.K. Lam ${ }^{1}$, S.C. C. $\mathrm{Ng}^{1}$, J. C. Wu ${ }^{1}$, J.J.Y. Sung ${ }^{1}$, W. $\mathrm{Wu}^{2}$

${ }^{1}$ Institute Of Digestive Disease, The Chinese University Of Hong Kong, Hong Kong/Hong Kong PRC

${ }^{2}$ Department Of Anaesthesia And Intensive Care, The Chinese University of Hong Kong, Hong Kong/Hong Kong PRC

Contact E-mail Address: wonghei@ $@$ cuhk.edu.hk

Introduction: Clostridium difficile infection represents a major burden in Europe and North America. However, data on its disease epidemiology remain sparse in Asia. This study aims to investigate the burden, risk factors and clinical outcomes of $C$. difficile infection in Hong Kong, using a large territory-wide population database.

Aims \& Methods: This is a population-based study conducted from 1 January 2006 to 31 December 2014, and included all in-patient $C$. difficile infections in public hospitals in Hong Kong. Cases were identified from a territory-wide electronic database, and were defined as the isolation of $C$. difficile, or positive test for either toxin or molecular assay from the fecal specimens. The disease incidence, mortality, risk factors and clinical outcomes were analyzed.

Results: A total of 15,753 cases were identified, including $14,402(91.4 \%)$ healthcare-associated and $817(5.1 \%)$ community-associated infections. The crude incidence increased from 16.21 in 2006 to 49.36 in 2014 per 100,000 population $\left(P_{\text {trend }}<0.001\right)$, representing an over $30 \%$ increase annually. This rise was 
notably contributed by elderly patients, of which the incidence has increased by three-fold over the period. Recurrence at 60 days increased from $5.7 \%$ in 2006 to $9.1 \%$ in $2014\left(P_{\text {trend }}<0.001\right)$. The increased use of proton-pump inhibitors accounted for $58.8 \%$ of the surge.

Conclusion: The incidence of $C$. difficile infection has increased more than threefold, and was associated with an increased disease recurrence and use of protonpump inhibitors. Our results suggest need for further surveillance in Asia which habors over half of the world's population.

Disclosure of Interest: All authors have declared no conflicts of interest.

\section{References}

1. Lessa FC, Mu Y, Bamberg WM, et al. Burden of Clostridium difficile infection in the United States. N Eng J Med. 2015; 372 (24): 825-834.

2. Kotila SM, Mentulla S, Ollgren J, Virolainen-Julkunen A, Lyytikainen O. Community- and healthcare-associated Clostridium difficile infections, Finland, 2008-2013. Emerg Infect Dis. 2015; 22 (10): 1747-1753.

3. Dingle KE, Didelot X, Quan TP, et al. Effects of control interventions on Clostridium difficile infection in England: an observational study. Lancet Infect Dis. 2017; 17(4): 411-421.

4. Cohen SH, Gerding DN, Johnson S, et al. Clinical Practice Guidelines for Clostridium difficileinfection in adults: 2010 update by the Society for Healthcare Epidemiology of America (SHEA) and the Infectious Diseases Society of America (IDSA). Infect Control Hosp Epidemiol. 2010; 31(5): 431-455.

5. Wong SH, Ip M, Hawkey PM, et al. High morbidity and mortality of Clostridium difficile infection and its associations with ribotype 002 in Hong Kong. J Infect. 2016; 73(2): 115-122.

\section{P0428 THE PROPHYLACTIC CLIP APPLICATION BEFORE SNARE POLYPECTOMY DECREASES IMMEDIATE POST-POLYPECTOMY BLEEDING IN LARGE PEDUNCULATED POLYPS}

J.S. Soh ${ }^{1}$, S.H. Park ${ }^{2}$, Y. Song ${ }^{2}$, K. Kim ${ }^{3}$

${ }^{1}$ Department Of Internal Medicine, University of Hallym College of Medicine, Hallym University Sacred Heart Hospital, Anyang/Korea, Republic of ${ }^{2}$ University of Ulsan College of Medicine, Asan Medical Center, Seoul/Korea, Republic of

${ }^{3}$ Konkuk University School of Medicine, Konkuk University Chungju Hospital, Chungju/Korea, Republic of

Contact E-mail Address: jssoh@hanmail.net Introduction: Post-polypectomy bleeding (PPB) is the most common complication following polypectomy, especially in cases with large pedunculated polyps. Although the clip application before snare polypectomy may decrease PPB, there were few prospective randomized studies to confirm the efficacy of prophylactic clip. This present study was conducted to investigate whether prophylactic clip application for large pedunculated colorectal polyps could decrease PPB and to evaluate associated risk factors of PPB.

Aims \& Methods: We enrolled 137 pedunculated polyps ( $\geq 1 \mathrm{~cm}$ in size) in 116 patients. The polyps were randomized into the two groups with or without prophylactic clips application. Immediate PPB was defined as bleeding that continued for over 30 seconds from the polypectomy site and graded from grade 1 to 4 , and delayed bleeding was defined as a history of hematochezia from the day of procedure to the day of first visit of outpatient clinic.

Results: Sixty-seven polyps were included in the clip group and 70 polyps in the control group respectively. Immediate PPB occurred in 6 cases $(9.0 \%)$ of the clip group and 22 cases $(31.4 \%)$ of the control group $(P=0.001)$. The occurrence of immediate PPB of grade 3-4 which needed endoscopic treatment was lower in the clip group than in the control group $(4.5 \%$ vs. $20.0 \%, P=0.008)$. However, that of grade $1-2$ was not different in both groups $(4.5 \%$ vs. $11.4 \%, P=0.208)$. Delayed bleeding occurred in five polyps in both groups $(P=0.943)$. The prophylactic clip application was a significant factor for lowering immediate PPB in the univariate (OR $0.215,95 \% \mathrm{CI} 0.081-0.571, P=0.002)$ and multivariate analysis (OR $0.210,95 \%$ CI $0.074-0.591, P=0.003$ ). In addition, polyp size $\geq 20 \mathrm{~mm}$ and stalk diameter $\geq 4 \mathrm{~mm}$ were significant risk factors of immediate PPB compared with polyp size $10-19 \mathrm{~mm}$ and stalk diameter $1-3 \mathrm{~mm}$.

Conclusion: The prophylactic clip application in large pedunculated polyps $\geq 1 \mathrm{~cm}$ is effective in reducing immediate PPB. Polyp size and stalk diameter are associated with PPB.

Disclosure of Interest: All authors have declared no conflicts of interest.

\section{References}

1. Dominitz JA, Eisen GM, Baron TH, Goldstein JL, Hirota WK, Jacobson $\mathrm{BC}$, et al. Complications of colonoscopy. Gastrointest Endosc 2003; 57:441-5.

2. Kim HS, Kim TI, Kim WH, Kim YH, Kim HJ, Yang SK, et al. Risk factors for immediate postpolypectomy bleeding of the colon: a multicenter study. Am J Gastroenterol 2006;101:1333-41.

3. Kapetanos D, Beltsis A, Chatzimavroudis G, Katsinelos P. Postpolypectomy bleeding: incidence, risk factors, prevention, and management. Surg Laparosc Endosc Percutan Tech 2012;22:102-7.

4. Gimeno-Garcia AZ, de Ganzo ZA, Sosa AJ, Perez DN, Quintero E. Incidence and predictors of postpolypectomy bleeding in colorectal polyps larger than $10 \mathrm{~mm}$. Eur J Gastroenterol Hepatol 2012;24:520-6.

5. Iida Y, Miura S, Munemoto Y, Kasahara Y, Asada Y, Toya D, et al. Endoscopic resection of large colorectal polyps using a clipping method. Dis Colon Rectum 1994;37:179-80.
6. Boo SJ, Byeon JS, Park SY, Rew JS, Lee DM, Shin SJ, et al. Clipping for the prevention of immediate bleeding after polypectomy of pedunculated polyps: a pilot study. Clin Endosc 2012;45:84-8

7. Quintanilla E, Castro JL, Rabago LR, Chico I, Olivares A, Ortega A, et al. Is the use of prophylactic hemoclips in the endoscopic resection of large pedunculated polyps useful? A prospective and randomized study. $J$ Interv Gastroenterol 2012;2:99-104.

\section{P0429 DOES CONTINUATION OF WARFARIN BECOME A PERIPROCEDURAL ALTERNATIVE METHOD TO HEPARIN REPLACEMENT IN COLONIC POLYPECTOMY/ENDOSCOPIC MUCOSAL RESECTION?}

I. Saito ${ }^{1}$, S. Ono ${ }^{2}$, Y. Takeda ${ }^{3}$, K. Takemura ${ }^{3}$, H. Doyama ${ }^{3}$, Y. Tsuji ${ }^{2}$, K. Niimi ${ }^{4}$, S. Kodashima ${ }^{2}$, N. Yamamichi ${ }^{2}$, M. Fujishiro ${ }^{1}$, K. Koike ${ }^{2}$

${ }^{1}$ Department Of Endoscopy And Endoscopic Surgery, Graduate School of

Medicine, the University of Tokyo, Tokyo/Japan

${ }^{2}$ Department Of Gastroenterology, The University of Tokyo Hospital, Tokyo/

Japan

${ }^{3}$ Department Of Gastroenterology, Ishikawa Prefectural Central Hospital,

Ishikawa/Japan

${ }^{4}$ Center For Epidemiology And Preventive Medicine, Graduate School of Medicine, the University of Tokyo, Tokyo/Japan

Contact E-mail Address: isaitou-nms@umin.ac.jp

Introduction: Heparin replacement (HR) during periprocedural periods is described in various guidelines as the recommended method while discontinuing warfarin. However, the rate of post-colonic polypectomy bleeding in patients undergoing HR has been reported to be as high as $20 \%$. As an alternative method to HR, colonic polypectomy without discontinuation of warfarin may be feasible, however there is still insufficient evidence. The aim of this study was to assess the safety of colonic polypectomy/endoscopic mucosal resection (EMR) without discontinuation of warfarin during periprocedural periods.

Aims \& Methods: This is a prospective multicenter single-arm exploratory study in Japan. Patients who received warfarin for the purpose of prevention of thrombosis were prospectively enrolled and underwent colonic polypectomy or EMR without discontinuation of warfarin. Conventional clip closure of the resection site was performed in all cases and oral diet was resumed 2 days after the procedure. The primary outcome was post-polypectomy/EMR bleeding that was confirmed by emergency endoscopy or a decrease in the hemoglobin level of $>2 \mathrm{~g} / \mathrm{dl}$ with hematochezia even if the bleeding site was not identified.

Results: Between January 2015 and November 2016, a total of 30 consecutive patients (M/F: 26/4, 69.9 \pm 8.0 years) were enrolled in this study after written informed consent was obtained. A total of 81 lesions (tumor diameter: $5.4 \pm 2.9 \mathrm{~mm}$, adenoma 70 , others 10 , lost lesion 1 , number of prophylactic clippings $4.9 \pm 2.2$ ) were treated by polypectomy/EMR. Four patients experienced post-polypectomy/EMR bleeding $(4 / 30: 13.3 \%)$ in 3-11 days after the procedure although no cases required blood transfusion. In 3 of these bleeding cases, a single responsible site was identified by emergency endoscopy. Therefore, the confirmed rate of post-polypectomy/EMR bleeding based on the number of resected lesions was $3.9 \%(3 / 76)$, but may range to $9.9 \%(8 / 81)$. There were no other adverse events.

Conclusion: The rate of post-colonic polypectomy/EMR bleeding in patients without discontinuation of warfarin single therapy was comparable to that in patients undergoing HR. Colonic polypectomy/EMR without discontinuation of warfarin is feasible and may reduce the hospitalization associated with HR. Disclosure of Interest: All authors have declared no conflicts of interest.

P0430 EFFECTS OF HEPARIN BRIDGING THERAPY ON POSTPOLYPECTOMY BLEEDING AND THROMBOEMBOLIC RISKS IN PATIENT UNDERGOING COLONOSCOPIC POLYPECTOMY

N. Hayashi ${ }^{1}$, H. Kataoka ${ }^{1}$, T. Katano ${ }^{2}$, K. Ozeki ${ }^{1}$, Y. Okamoto ${ }^{2}$, T. Mizoshita ${ }^{2}$, T. Shimura ${ }^{3}$, E. Kubota ${ }^{2}$, S. Tanida ${ }^{2}$, T. Joh

${ }^{1}$ Nagoya City University Gastroenterology and Metabolism, Nagoya/Japan

${ }^{2}$ Nagoya City University Graduate School of Medical Sciences Gastroenterology and Metabolism, Nagoya/Japan

${ }^{3}$ Nagoya City University Graduate School of Medical Sciences, Nagoya/Japan

Contact E-mail Address: nori53412@yahoo.co.jp

Introduction: Warfarin and direct oral anticoagulants (DOACs) rivaroxaban, apixaban, dabigatran and edoxaban are approved for stroke prevention in patients with atrial fibrillation (AF). The Japan Gastrointestinal Endoscopy Society guidelines recommend that anticoagulants should be discontinued at a low thromboembolic risk, or replaced by heparin bridging therapy at a high thromboembolic risk, for colonoscopic polypectomy. However, safety and efficacy of heparin bridging therapy for colonoscopic polypectomy remains scare. Aims \& Methods: The aim of the present study was to evaluate the risk of postpolypectomy bleeding (PPC) in patients who take anticoagulants. We retrospectively collected data of patients who underwent colonoscopic polypectomy between January 2014 and February 2017 at Nagoya City University Hospital. Polyp characteristics (number of polyps removed per patient, size, morphology) and patient characteristics (age, sex, comorbidities, medication) was analyzed. Results: A total of 1007 patients underwent colonoscopic polypectomy. 67 patients were in the anticoagulants group and 808 patients were in the normal group (taking no antiplatelet agents and anticoagulants). The incidence of PPC was significantly higher in the anticoagulants group than in the normal group 
(2.98\% vs $1.49 \%$, respectively). No patients experienced recurrent bleeding and a blood transfusion. The mean age $(71.3 \pm 8.2$ years $)$ and male $(82.1 \%)$ in the anticoagulants group were higher than those in the normal group $(67.7 \pm 11.2$ years and $64.1 \%$ ). There was no difference between the groups in size of polyp and morphology. In the anticoagulants group, 34 patients received heparin bridging therapwarfarin 30 , warfarin + antiplatelet 3 , rivaroxaban 1)and 33 patients discontinued anticoagulants (warfarin 8 , warfarin + antiplatelet 1 , rivaroxaban 10 , apixaban 8 , dabigatran 4 , edoxaban 2). The incidence of PPC was no difference between two groups (1,1 patient, respectively). There was no difference between the groups in age, sex, size of polyp and morphology). Recurrent bleeding didn't occur. In the discontinued group, 1 patient developed acute myocardial infarction in next day after colonoscopic polypectomy.

Conclusion: Patients taking anticoagulants have an increased risk of PPB compared with the control, even if the anticoagulants are discontinued. Heparinbridge therapy might be responsible for increased PPB in patients taking anticoagulants.

Disclosure of Interest: All authors have declared no conflicts of interest.

\section{P0431 ARTERIOSCLEROSIS IS A SIGNIFICANT CONTRIBUTOR TO COLON DIVERTICULUM RECURRENT BLEEDING}

T. Aoyama ${ }^{1}$, S. Nagata ${ }^{1}$, T. Sakamoto ${ }^{2}$, Y. Shimohara ${ }^{1}$, H. Tamari ${ }^{1}$, Y. Ogawa ${ }^{1}$, K. Shigita ${ }^{1}$, N. Asayama ${ }^{1}$, A. Fukumoto ${ }^{1}$, S. Mukai ${ }^{1}$

${ }^{1}$ Gastroenteorolgy, Hiroshima City Asa Citizens Hospital, Hiroshima City/Japan ${ }^{2}$ Radiology, Hiroshima City Asa Citizens Hospital, Hiroshima City/Japan

\section{Contact E-mail Address: t-aoyama@asa-hosp.city.hiroshima.jp}

Introduction: Intermittent bleeding from colon diverticulum has a significant clinical impact with some cases experiencing recurrent bleeding episodes for several years. No report has directly evaluated the association between arteriosclerosis and diverticulum bleeding recurrence.

Aims \& Methods: We sought to assess the degree of arteriosclerosis in cases with diverticulum bleeding as well as the patients' clinical characteristics. We conducted a retrospective cohort study in a group of 79 consecutive patients with colon diverticulum bleeding ( 51 men) who underwent both colonoscopy and computed tomography (CT) between August 2007 and March 2014. The mean age of the patient population was 69.5 years (range 29-91 years) and mean ( \pm standard deviation) follow-up time was $6.2( \pm 2.0)$ years (range 3.1-9.7 years). Patients were divided into two groups: the recurrent bleeding episode group and the single bleeding episode group. Recurrent bleeding episodes were defined as bleeding intervals of $>1$ month. Cases that underwent successful therapy during the initial bleeding episode were excluded. We compared Agatston scores (total calcium score) between the two groups of patients to assess the degree of arteriosclerosis. A calcified lesion was defined as an area of at least 2 connected pixels with $>120$ Hounsfield units (HU) on the unenhanced CT scan. Aortic mural calcified area $\times$ cofactor $(1: 120-199 H U, 2: 200$ $299 \mathrm{HU}, 3: 300-399 \mathrm{HU}, 4:>400 \mathrm{HU})$ was determined. The sums of the scores for every calcified speck were calculated across all lesions in a slice from the level of the diaphragm to the aortoiliac bifurcation to obtain the total calcium score. Moreover, the relationship between recurrent bleeding episodes and the patients' clinical characteristics, including age, sex, smoking habit, comorbidities (hypertension, cerebro-cardiovascular disease, diabetes mellitus, hyperlipidaemia, chronic liver disease, chronic kidney disease, and chronic obstructive pulmonary disease), internal medicine (antithrombotic drug, non-steroidal anti-inflammatory drug, and proton pump inhibitor), shock vital on hospitalization, and transfusion need, was determined.

Results: Overall, 39 (49\%) cases had recurrent bleeding episode and 40 (51\%) had single bleeding episode. The cumulative recurrent bleeding rate in the recurrent bleeding episode group was $33 \%$ and $59 \%$ at 1 and 3 years, respectively. Agatston scores in the recurrent bleeding episode group were significantly higher than those in the single bleeding episode group (5902 \pm 7187 vs $2912 \pm 4687$, $\mathrm{P}=0.031)$. Clinical characteristics associated with recurrent bleeding episodes were cerebro-cardiovascular disease $(\mathrm{P}=0.0044)$, chronic kidney disease $(\mathrm{P}=0.031)$, and antithrombotic drug $(\mathrm{P}=0.048)$ in univariate analysis. Subsequent multivariate analysis determined that cerebro-cardiovascular disease was an independent contributor to recurrent bleeding episode (OR: $5.48 ; 95 \% \mathrm{CI}$ : 1.11-40.7).

Conclusion: Arteriosclerosis along with cerebro-cardiovascular disease may be a significant contributing factor for colon diverticulum recurrent bleeding.

Disclosure of Interest: All authors have declared no conflicts of interest.

\section{Reference}

Fujino Y, et al. Risk factors for early re-bleeding and associated hospitalization in patients with colonic diverticular bleeding. Colorectal Dis 2013;15:982-6.

\section{P0432 EARLY VERSUS STANDARD COLONOSCOPY - A RANDOMIZED CONTROLLED TRIAL IN PATIENTS WITH ACUTE LOWER GASTRO-INTESTINAL BLEEDING: RESULTS OF THE BLEED STUDY}

I. Van Rongen ${ }^{1}$, B. Thomassen ${ }^{2}$, L. Perk

${ }_{1}^{1}$ Gastroenterology, Haaglanden MC, The Hague/Netherlands

${ }^{2}$ Landsteiner Institute, Haaglanden Medical Centre, The Hague/Netherlands

Contact E-mail Address: inge.van.rongen@gmail.com
Introduction: The incidence of acute lower gastro-intestinal bleeding (LGIB) is estimated at 21 adults per 100,000 person years and is increasing with the ageing of the population [1]. Diagnostic management of LGIB has been extensively debated in recent literature, especially whether colonoscopy within 24 hours of presentation is feasible and safe [2-4].

Aims \& Methods: The aim of our study was to examine differences in length of hospital stay in patients with LGIB receiving either early colonoscopy (within 24 hours of presentation) or standard colonoscopy (within 1-3 days).

We performed a single-centre, non-blinded randomized controlled trial, including patients presenting at the emergency department with acute hematochezia and excluding patients with an upper bleeding source. Primary outcome was the length of hospital stay. Secondary outcomes included yield of colonoscopy, blood transfusion requirements, recurrent bleedings, complications, diagnostic and therapeutic interventions related to complications and 30-day mortality. The follow-up period was one month.

Results: In total, 132 patients were randomized: 63 for early colonoscopy and 69 for standard colonoscopy. Baseline characteristics of both groups were comparable. In the intention-to-treat (ITT) analysis, the length of hospital stay was significantly lower in patients that underwent an early colonoscopy, compared to the standard colonoscopy group: median 2.0 days [IQR 2.0-4.0] vs. median 3.0 days [IQR 2.0-4.0] $(\mathrm{p}=0.009)$. Recurrent bleedings and hospital re-admissions were significantly more frequent in the early colonoscopy group: $13 \%$ vs. $3 \%$ $(\mathrm{p}=0.04)$ and $11 \%$ vs. $2 \%(\mathrm{p}=0.02)$ respectively. The reason for more recurrent bleedings could not be established, although use of anti-thrombotic therapy might be a factor. No difference was observed regarding the number of patients diagnosed with either a confirmed active bleeding or presumptive bleeding source. In both groups, blood transfusion rate was similar and thirty-day mortality was zero.

Conclusion: In patients with LGIB, early colonoscopy reduces the length of hospital stay compared to standard colonoscopy. However, more recurrent bleedings are observed and no improvement of diagnostic yield could be established.

Disclosure of Interest: All authors have declared no conflicts of interest.

\section{References}

1. Lanas, A., Garcia-Rodriguez, L.A., Polo-Tomas, M. et al. Time trends and impact of upper and lower gastrointestinal bleeding and perforation in clinical practice. Am J Gastroenterol 2009;104:1633-41

2. Laine, L. and Shah, A. Randomized trial of urgent vs. elective colonoscopy in patients hospitalized with lower GI bleeding. Am $J$ Gastroenterol 2012;105:2636-41

3. Green, B.T., Rockey D.C., Portwood, G. et al. Urgent colonoscopy for evaluation and management of acute lower gastrointestinal haemorrhage: a randomized controlled trial Am J Gastroenterol 2005;100:2394-402

4. Sengupta, N., Tapper, E.B. and Feuerstein, J.D. Early versus delayed colonoscopy in hospitalized patiens with lower gastrointestinal bleeding - a metaanalysis. J Clin Gastroenterol 2017;51(4):352-59.

\section{P0433 THE COMPARISON OF DIRECT ORAL ANTICOAGULANTS (DOAC) AND WARFARIN FOR ANTICOAGULATION IN THE PATIENTS WITH GASTROINTESTINAL BLEEDING}

K. Kojima, N. Toda, J. Arai, K. Kurokawa, C. Shibata, S. Kurosaki, K. Funato, M. Kondo, T. Ohki, M. Seki, K. Tagawa

Gastroenterology, Mitsui Memorial Hospital, Tokyo/Japan

Contact E-mail Address: kenken.kojiken.529@gmail.com

Introduction: Direct oral anticoagulants (DOAC) are now popularly used as anticoagulation for atrial fibrillation and deep vein thrombosis, as well as Warfarin. But, direct comparison of DOAC and warfarin in the patients with gastrointestinal bleeding was little reported.

Aims \& Methods: We retrospectively analyzed 18 on DOAC and 60 cases on Warfarin of the patients with gastrointestinal bleeding from January 2011 to March 2017 on the basis of single-center experience in Japan. We analyzed concentrated red cell (CRC) and fresh frozen plasma (FFP) transfusion rate, rebleeding rate during hospitalization, the duration from bleeding to endoscopy, from endoscopy to discharge and from bleeding to discharge in both group. In DOAC group, each 6 patients took Dabigatran, Rivaroxaban and Apixaban.

\begin{tabular}{llll}
\hline & $\begin{array}{l}\text { DOAC } \\
(\mathrm{N}=18)\end{array}$ & $\begin{array}{l}\text { Warfarin } \\
(\mathrm{N}=60)\end{array}$ & p value \\
\hline Male sex, n (\%) & $15(83.3 \%)$ & $41(68.3 \%)$ & 0.20 \\
Age, years & $74.0 \pm 2.2$ & $74.4 \pm 1.2$ & 0.87 \\
Anti-platelet therapy, n (\%) & $8(47.1 \%)$ & $22(36.7 \%)$ & 0.44 \\
Lower gastrointestinal bleeding, n (\%) & $12(66.7 \%)$ & $29(48.3 \%)$ & 0.17 \\
Bleeding after endoscopic procedure, n (\%) & $11(61.1 \%)$ & $22(36.7 \%)$ & 0.07 \\
Hemoglobin, g/dl & $11.1 \pm 0.7$ & $9.6 \pm 0.4$ & 0.06 \\
PT-INR & $1.51 \pm 0.36$ & $2.50 \pm 0.19$ & 0.02 \\
Fresh frozen plasma transfusion, n (\%) & $3(16.7 \%)$ & $20(33.3 \%)$ & 0.16 \\
Concentrated red cell transfusion, n (\%) & $9(50.0 \%)$ & $32(53.3 \%)$ & 0.80 \\
Re-bleeding during hospitalization, n (\%) & $1(5.6 \%)$ & $12(20.0 \%)$ & 0.11 \\
\hline
\end{tabular}

(continued) 
Continued

\begin{tabular}{llll}
\hline & $\begin{array}{l}\text { DOAC } \\
(\mathrm{N}=18)\end{array}$ & $\begin{array}{l}\text { Warfarin } \\
(\mathrm{N}=60)\end{array}$ & p value \\
\hline The duration from bleeding to endoscopy, days & $0.8 \pm 0.5$ & $1.2 \pm 0.2$ & 0.52 \\
The duration from endoscopy to discharge, days & $9.0 \pm 5.5$ & $23.0 \pm 3.0$ & 0.03 \\
The duration from bleeding to discharge, days & $9.8 \pm 5.4$ & $24.2 \pm 3.0$ & 0.02 \\
Thrombotic embolism during hospitalization, $\mathrm{n}(\%)$ & $0(0.0 \%)$ & $1(1.7 \%)$ & 0.47 \\
\hline
\end{tabular}

Results: Patient characteristics such as sex, age, anti-platelet therapy, location of bleeding and bleeding after endoscopic procedure had no significant difference in both groups. Upper gastrointestinal bleeding occurred $6(33.3 \%)$ of DOAC group and $31(51.7 \%)$ of Warfarin group. Hemoglobin tended to be lower $(11.1 \pm 0.7 \mathrm{~g} / \mathrm{dl}$ vs $9.6 \pm 0.4 \mathrm{~g} / \mathrm{dl}, \mathrm{p}=0.06)$ and international normalized ratio of prothrombin time (PT-INR) was significantly prolonged in Warfarin group $(1.51 \pm 0.36$ vs $2.50 \pm 0.20, \mathrm{p}=0.02)$. CRC transfusion rate had no significant difference in both group, but FFP tended to be transfused at high rate in Warfarin group $(16.7 \%$ vs $33.3 \%, p=0.16)$. Re-bleeding rate during hospitalization had no significant difference in both group, but tended to be higher in Warfarin group $(5.6 \%$ vs $20.0 \%, \mathrm{p}=0.11)$. The duration from bleeding to endoscopy had no significant difference in both group $(0.8 \pm 0.5$ days vs $1.2 \pm 0.2$ days, $\mathrm{p}=0.52$ ), but the duration from endoscopy to discharge was significantly longer in Warfarin group $(9.0 \pm 5.5$ days vs $23.0 \pm 3.0$ days, $\mathrm{p}=0.03)$. Also, the duration from bleeding to discharge was significantly longer in Warfarin group $(9.8 \pm 5.4$ days vs $24.2 \pm 3.0$ days, $\mathrm{p}=0.02)$. Thrombotic embolism during hospitalization occurred only $1(1.7 \%)$ of Warfarin group.

Conclusion: The duration of hospitalization was significantly shorter in DOAC group of the patients with gastrointestinal bleeding, and the rate of FFP transfusion and re-bleeding tended to be lower in DOAC group. This study showed that DOAC may be more superior to Warfarin as anticoagulation for atrial fibrillation and deep vein thrombosis at the quality of life (QOL) in the patients with gastrointestinal bleeding.

Disclosure of Interest: All authors have declared no conflicts of interest.

\section{P0435 INCREASED INCIDENCE OF OVARIAN CANCER FOLLOWING COLORECTAL CANCER: A KOREAN NATIONWIDE COHORT STUDY}

D.W. Shin ${ }^{1}$, D.H. Lee ${ }^{1}$, H.S. Kim ${ }^{1}$

${ }^{1}$ Internal Medicine, SNUBH, Seongnam/Korea, Republic of

Contact E-mail Address: delight0618@naver.com

Introduction: In Korea, colorectal cancer is the most common cancer among old aged women over 65 years old. The incidence of colorectal cancer, in particular, is dramatically increasing due to environmental factors such as the westernized eating habits. Furthermore, due to advances in medicine, the survival rate of those with advanced colon cancer is increasing. An increased risk of malignant tumors associated with colorectal cancer has been suggested recently, but adequate studies have not been conducted. The purpose of the study is to determine whether ovarian cancer is more common in the patients diagnosed with colorectal cancer than in the general population. If a woman diagnosed with colorecta cancer indeed has a high incidence of ovarian cancer, a screening test can be performed on high-risk patients.

Aims \& Methods: This is a retrospective cohort study using data registered in the National Health Insurance Corporation as a cancer diagnostic code since 2007. In Korea, once cancer is diagnosed, this information is recorded by the National Health Insurance Corporation with a relevant code, and this system provides every patient's data for medical research purposes. The colorectal cancer group includes patients newly enrolled with the corresponding diagnostic code (ICD-10 code $\mathrm{C} 18, \mathrm{C} 19$, and $\mathrm{C} 20$ ). The 56,682 colorectal cancer patients and 288,119 sexand age- matched general population was collected. Propensity score methods were used to facilitate the creation of comparison group that is similar. Each cancer patient was matched to five individuals in the unexposed cohort. All data was followed up by a new diagnostic code of ovarian cancer (ICD-10 code C56) was given. After adjusting for sex, age, smoking, drinking, exercise and comorbidities (diabetes mellitus, hypertension, and hyperlipidemia), further analysis was performed. The hazard ratios and $95 \%$ confidence intervals were calculated via Cox proportional hazards regression models. Statistical analysis will be performed with SPSS version 24.0. When $\mathrm{P}<0.05$, the result was defined as statistically significant.

Results: Patients with colorectal cancer were followed up for an average of 4.4 years until the occurrence of ovarian cancer. During the follow-up period, 338 out of $56,682(0.60 \%)$ colorectal cancer patients and 258 out of $288,119(0.09 \%)$ people in the general population were diagnosed with ovarian cancer. Ovarian cancer was more common in the colorectal cancer group than the general population [Hazard ratio (HR) 7.13, 95\% Confidence interval $(\mathrm{CI})=5.06-10.05]$. The additional analysis was conducted only for those who had medical checkup data within one year $(14,190$ patients in colorectal cancer group, 71,933 people in the control group). Even though the subjects in this group were adjusted for several factors (age, sex, smoking, drinking, exercise, diabetes, hypertension, and hyperlipidemia), the incidence of ovarian cancer was also higher in colorectal cancer group [HR 7.12, 95\% CI $=5.05-10.04]$. Colorectal cancer patients had a higher risk of ovarian cancer across all age groups including patients under the age of 55 years [HR 10.69, 95\% CI $=6.26-18.26$ ] and patients older than 55 years [HR $5.17,95 \% \mathrm{CI}=3.26-8.19]$.

Conclusion: In conclusion, data from the National Health Insurance Corporation revealed that the incidence of ovarian cancer in colorectal cancer patients was higher than that of the general population. In woman diagnosed with colorectal cancer, the screening test should be done to monitoring the occurrence of ovarian cancer. Further research is necessary to determine the interactive association between the development of ovarian cancer and colorectal cancer, and large prospective studies are needed.

Disclosure of Interest: All authors have declared no conflicts of interest.

\section{P0436 WORLD ENDOSCOPY ORGANISATION CONSENSUS STATEMENTS ON POST-COLONOSCOPY/POST-IMAGING COLORECTAL CANCER}

I. Beintaris ${ }^{1}$, S. Sanduleanu ${ }^{2}$, H.M. Chiu ${ }^{3}$, D. Corley $^{4}$, M. Cuatrecasas ${ }^{5}$, E. Dekker ${ }^{6}$, A. Forsberg ${ }^{7}$, U. Haug ${ }^{8}$, M.F. Kaminski ${ }^{9}$, T. Matsuda ${ }^{10}$, G. A. Meijer ${ }^{11}$, E. Morris ${ }^{12}$, A. Plumb ${ }^{13}$, L. Rabeneck ${ }^{14}$, D. Robertson ${ }^{15}$ R. Schoen ${ }^{16}$, H. Singh ${ }^{17}$, J. Tinmouth ${ }^{18}$, R. Valori ${ }^{19}$, G. Young ${ }^{20}$, M.D. Rutter ${ }^{1}$ ${ }^{1}$ Gastroenterology, University Hospital North Tees NHS Dept. of Gastroenterology, Stockton-on-Tees/United Kingdom

${ }^{2}$ Gastroenterology And Hepatology, Maastricht Hospital, Maastricht/Netherlands ${ }^{3}$ Division Of Gastroenterology, Department Of Internal Medicine, National Taiwan University, Taipei City/Taiwan

${ }^{4}$ California And San Francisco Medical Center, Kaiser Permanente Division of Research, Oakland/United States of America

${ }^{5}$ Pathology Department - Centre De Diagnòstic Biomèdic (cdb), Hospital Clínic Barcelona, University of Barcelona, Barcelona/Spain

${ }^{6}$ Gastroenterology \& Hepatology, AMC - Gastroenterology \& Hepatology, AMC; Amsterdam/NL, Amsterdam/Netherlands

${ }^{7}$ Kep. Karolinska Institutet, Institution of Medicine Solna, Stockholm/Sweden

${ }^{8}$ Clinical Epidemiology, Leibniz Institute for Prevention Research and

Epidemiology, Bremen/Germany

${ }^{9}$ Department Of Gastroenterology, Maria Sklodowska-Curie Memorial Center and Institute of Oncology - Department of Gastroenterology, M, Warsaw/Poland

${ }^{10}$ Cancer Screening Center, National Cancer Center Hospital, Tokyo/Japan

${ }^{11}$ Pathology, Netherlands Cancer Institute, Amsterdam/Netherlands

${ }^{12}$ Cancer Epidemiology Group, University of Leeds, Leeds/United Kingdom

${ }^{13}$ Radiology And Imaging, University College London Hospital, London/United

Kingdom

${ }^{14}$ Prevention And Cancer Control, Cancer Care Ontario, University of Toronto, Toronto/Canada

${ }^{15}$ Gastroenterology, VAMC, White River Junction, United States, Vermont/United States of America

${ }^{16}$ University of Pittsburgh and the University of Pittsburgh Cancer Institute, Pittsburgh/United States of America

${ }^{17}$ Section Of Gastroenterology, University of Manitoba and the University of Manitoba IBD Clinical and Research Centre, Winnipeg/Canada

${ }^{18}$ Division of Gastroenterology, Sunnybrook Health Sciences Centre, Toronto/ Canada

${ }^{19}$ Medicine, Gloucestershire NHS Foundation Trust - Medicine, Gloucestershire NHS Foundation Trust; Cheltenham/GB, Cheltenham/United Kingdom

${ }^{20}$ FCIC, Flinders University, Templestowe Lower/Australia

Contact E-mail Address: iosif.beintaris@nhs.net

Introduction: Colonoscopy is an imperfect tool. Several publications confirm colorectal cancer may manifest after a negative colonoscopy(1-3). The term "interval cancer" has often been used for cancers appearing after a negative colonoscopy. However, this is primarily a screening term(1). Post-colonoscopy colorectal cancer (PCCRC) is a broader term for cancers detected after a negative colonoscopy in any setting, including screening(2). Although there is overlap between these two terms, they are not synonymous. PCCRC can be thought of as the overarching term. PCCRCs can be subcategorised into interval cancers (identified prior to the next recommended screening or surveillance procedure) and non-interval cancers (identified at or after a recommended screening or surveillance interval, or where no subsequent screening or surveillance interval was recommended, up to 10 years following the colonoscopy).

Aims \& Methods: The goal of this consensus process was to provide a framework for the terminology, identification, analysis and reporting of cancers appearing after a negative colonoscopy or computed tomographic colonography (postcolonoscopy/post-imaging colorectal cancers- PCCRC/PICRC respectively). We based our methodology on The Appraisal of Guidelines for Research and Evaluation (AGREE II) tool(4). An international multidisciplinary team (gastroenterologists, pathologists, epidemiologists, a radiologist and a patient representative) were summoned by the World Endoscopy Organisation (WEO); the final panel consisted of 20 voting members. The following topics were addressed by 2 working groups (WGs):

1. Aetiology WG

a. Terminology of aetiology categories

b. Risk factors/potential explanations of PCCRC

c. How to ascribe potential explanations

d. Minimal colonoscopy, histology and radiology datasets to examine PCCRC

e. Molecular tests to be performed to examine PCCRC

f. Prevention of PCCRC in high-risk groups

2. Performance WG

a. PCCRC calculation \& reporting

b. PCCRC monitoring

c. PCCRC papers peer-review

d. Post-imaging CRC A literature search was performed in MEDLINE and Cochrane using terms "colorectal cancer AND interval cancer", "healthcare quality assurance AND colorectal cancer" and "healthcare quality assurance AND colorectal cancer AND interval cancer". The final output consisted of 391 articles. Proposed statements were subjected to anonymous voting via ecorrespondence. Each statement was scored on an scale of 1 (strongly agree) to 
5 (strongly disagree). A modified Delphi process was followed; consensus required at least $80 \%$ agreement. In areas of continuing disagreement, a recommendation for or against a particular statement required both $>50 \%$ of participants in favour and $<20 \%$ preferring the comparator. Failure to meet this resulted in no recommendation. The GRADE system for rating evidence and strength of recommendations was applied to final statements.

Results: The final output consists of 21 statements providing guidance on key aspects of PCCRC/PICRC, namely definitions, terminology, qualitative review/ aetiology attribution and quantitative assessment of cases. A Root-Cause Analysis checklist as well as a PCCRC/PICRC manuscript peer-review checklist were also developed.

Conclusion: This is the first consensus aiming to standardise terminology around PCCRC. Each previous study defined PCCRC differently, making its use for benchmarking purposes impossible. This consensus presents a methodology for analysis of causation of PCCRC/PICRC and defines its potential role as a key quality indicator, providing recommendations for future investigators, policy makers, services and patients.

Disclosure of Interest: E. Dekker: Research grant from Olympus and endoscopic equipment on loan from Olympus and Fujifilm.

A. Plumb: I have no conflicts related to the present project. Other disclosures (not related to the present project): I have received payment for educational lectures organized by Warner Chilcott, a pharmaceutical company, and the medical device company Acelity.

H. Singh: No direct conflicts of interest. In terms of industry funding, disclosure includes Advisory Board for Pendopharm and research funding from Merck Canada

J. Tinmouth: Lead Scientist for the ColonCancerCheck program, the CRC screening program in Ontario. I am paid a salary for this work

R. Valori: I am joint director of a Limited Liability Partnership (Quality Solutions for Healthcare) which provides advice and support for quality improvement and QA within and outside of endoscopy, mostly in the UK and Ireland, as well as training internationally.

M.D. Rutter: Research grant from Olympus, speaker fees/travel reimbursement from Falk, Abbvie

All other authors have declared no conflicts of interest.

\section{References}

1. Sanduleanu $\mathrm{S}$ et al. Definition and taxonomy of interval colorectal cancers: a proposal for standardising nomenclature. Gut. 2015;64(8):1257-67.

2. Rabeneck L, Paszat LF. Circumstances in which colonoscopy misses cancer. Frontline Gastroenterol. 2010;1(1):52-8.

3. Robertson DJ et al. Colorectal cancers soon after colonoscopy: a pooled multicohort analysis. Gut. 2014;63(6):949-56.

4. Brouwers MC et al. AGREE II: advancing guideline development, reporting and evaluation in health care. CMAJ: Canadian Medical Association journal. 2010;182(18):E839-42.

\section{P0437 EXCESS RISK OF SECOND PRIMARY CANCERS IN YOUNG- ONSET COLORECTAL CANCER SURVIVORS}

\section{X. $\mathrm{He}^{1}, \mathrm{~W} \cdot \mathrm{Wu}^{2}, \mathrm{~L} \cdot \mathrm{Sun}^{1}, \mathrm{Y} \cdot \mathrm{Ding}^{1}, \mathrm{~J} \cdot \mathrm{Si}^{1}$}

${ }^{1}$ Department Of Gastroenterology, Sir Run Run Shaw Hospital, hangzhou/China ${ }^{2}$ The First Affiliated Hospital, School Of Medicine, State Key Laboratory for Diagnosis and Treatment of Infectious Diseases, hangzhou/China

Contact E-mail Address: hexingkang@zju.edu.cn

Introduction: Colorectal cancer (CRC) is still the third most common malignancies in the US according to Colorectal Cancer Statistics, 2017[1]. During past decades, the incidence and mortality of CRC among individuals aged over 50 years are declining significantly, while the rate of CRC in the young is sharply on the rise curiously [2-3]. Escalating rate of young-onset CRC, coupled with increased survival relate, would definitely lead to accumulation of young survivors considerably. There is a growing study reporting the risk of second primary cancers (SPCs) in certain cancer survivors, including CRC. Several populationbased studies revealed that patients with a history of CRC were at high risk of SPCs than the general population [4-6]. However, to the best of our knowledge, very little is known regarding the risk and sites of SPCs following prior diagnosis of $\mathrm{CRC}$ in the young (aged $\leq 50$ ).

Aims \& Methods: To address this important gap, we aimed to quantify the relative risk of SPCs after a diagnosis of CRC in the young CRC survivors. We conducted this retrospective study by utilizing the Surveillance, Epidemiology, and End Results (SEER) database and identified primary CRC patients with subsequent cancers between 1973 and 2013. We excluded cases with less than 6-months latency restriction. Standardized incidence ratios (SIR) and absolute excess risk (AER) were calculated to assess the relative risk for SPCs. SIRs for subgroup analysis were further stratified by gender, race, calendar year, latency period, SEER stage, cancer subsite, radiotherapy. All statistical tests were performed by SEER*Stat version 8.3 and a $P$ value $<0.05$ was considered statistically significant. Our study was approved by the review board of Zhejiang Institute of Gastroenterology, Sir Run Run Shaw Hospital, China.

Results: Among 340,992 CRC patients, there were 44,472 survivors who developed 51,084 SPCs during the follow-up, including 3283 young (young aged $\leq 50$ ) and 41,189 (old $>50$ ) old survivors. The SIR of all sites significantly decreased with increased age. Compared with the general population, SIRs of all solid tumors and hematological disease were significantly increased in the young. There was significant $43 \%$ risk of SPCs in young survivors (SIR $=1.43$, $95 \mathrm{CI} \%=1.39-1.48, \quad \mathrm{AER}=33.85$ ) and slight increases in old survivors $(\mathrm{SIR}=1.02,95 \mathrm{CI} \%=1.01-1.03, \mathrm{AER}=4.20)$. For young survivors, small intestine $(\mathrm{SIR}=8.36)$, colon $(\mathrm{SIR}=3.77)$, rectum $(\mathrm{SIR}=3.56)$, bile ducts $(\mathrm{SIR}=3.70)$ were the most common sites. This trend was persisted regardless of other factors, such as gender, race, calendar year, stage, subsites, radiation, latency. For young patients with second cancers, $36.4 \%$ died of their initial cancer, but $44.6 \%$ died of their second primary malignancy.

Conclusion: Excess risk of developing a second malignancy existed in young-onset CRC survivors and this trend was consistent among different subgroups. About $44.6 \%$ young patients died of their SPCs. We hope our results may provide some implication for future surveillance and prevention strategies for young CRC survivors.

Disclosure of Interest: All authors have declared no conflicts of interest.

\section{References}

1. Siegel RL, Miller KD and Jemal A. Cancer Statistics, 2017. CA Cancer J Clin. 2017; 67(1):7-30.

2. Siegel RL, Fedewa SA, Anderson WF, Miller KD, Ma J, Rosenberg PS and Jemal A. Colorectal Cancer Incidence Patterns in the United States, 1974 2013. J Natl Cancer Inst. 2017; 109(8).

3. Siegel RL, Miller KD, Fedewa SA, Ahnen DJ, Meester RG, Barzi A and Jemal A. Colorectal cancer statistics, 2017. CA Cancer J Clin. 2017.

4. Guan X, Jin Y, Chen Y, Jiang Z, Liu Z, Zhao Z, Yan P, Wang G and Wang $X$. The Incidence Characteristics of Second Primary Malignancy after Diagnosis of Primary Colon and Rectal Cancer: A Population-Based Study. PLoS One. 2015; 10(11):e0143067.

5. Yang J, Li S, Lv M, Wu Y, Chen Z, Shen Y, Wang B, Chen L, Yi M and Yang J. Risk of subsequent primary malignancies among patients with prior colorectal cancer: a population-based cohort study. Onco Targets Ther. 2017; 10:1535-1548.

6. Liang YH, Shao YY, Chen HM, Lai CL, Lin ZZ, Kuo RN, Cheng AL, Yeh $\mathrm{KH}$ and Lai MS. Young patients with colorectal cancer have increased risk of second primary cancers. Jpn J Clin Oncol. 2015; 45(11):1029-1035.

\section{P0438 INCIDENCE OF FECAL OCCULT BLOOD TEST INTERVAL CANCERS IN COLORECTAL CANCER SCREENING; A SYSTEMATIC REVIEW AND META-ANALYSIS}

E. Wieten ${ }^{1}$, E. Schreuders ${ }^{1}$, E.J. Grobbee ${ }^{1}$, D. Nieboer ${ }^{2}$, I. Lansdorp-Vogelaar ${ }^{2}$, M.J. Bruno ${ }^{1}$, E.J. Kuipers ${ }^{1}$, M.C.w. Spaander ${ }^{1}$

${ }^{1}$ Gastroenterology \& Hepatology, Erasmus MC University Medical Center,

Rotterdam/Netherlands

${ }^{2}$ Public Health, Erasmus MC University Medical Center, Rotterdam/Netherlands

Contact E-mail Address: e.wieten@erasmusmc.nl

Introduction: Worldwide, many organized colorectal cancer (CRC) screening programs use non-invasive fecal occult blood tests (FOBTs). Although the interval colorectal cancer (iCRC) rate is an important performance indicator of a screening program, data on iCRC after negative FOBTs are limited.

Aims \& Methods: In this systematic review and meta-analysis we compared the incidence of iCRCs following a negative fecal immunochemical test (FIT) or guaiac fecal occult blood test (gFOBT). Second, we assessed if screening-related or patient-related factors are associated with FOBT iCRCs. Ovid Medline, Embase, The Cochrane Library, the Science Citation Index, PubMed publisher and Google scholar were searched up to May, 2016. All studies reporting on the incidence of FIT or gFOBT iCRCs in average CRC screening populations were included, without language restrictions. Main outcome was pooled incidence rate of iCRCs per 100, 000 person-years (p-y). FOBT iCRC was according to international standards defined as cancer that developed after a negative FOBT and before the next FOBT was due. Pooled incidence rates were obtained by fitting random effect poisson regression models. The between-study heterogeneity of effect-size was quantified using the $\mathrm{I}^{2}$.

Results: We identified 5,873 records, of which 413 full-text articles were assessed for eligibility and 30 studies were included in both qualitative and quantitative syntheses. Meta-analyses comprised data of 5,252,563 screening participants, in which 14,030 screen-detected CRCs and 5398 FOBT iCRCs were documented. Pooled incidence rates of iCRC following FIT and gFOBT were $20(95 \%$ CI 14 $\left.28 ; \mathrm{I}^{2}=94 \%\right)$ and $40\left(95 \% \mathrm{CI} 26-61 ; \mathrm{I}^{2}=93 \%\right)$ per $100,000 \mathrm{p}-\mathrm{y}$, respectively. The pooled incidence rate ratio of FIT iCRC compared to gFOBT iCRC was 0.50 $(95 \%$ CI $0.30-0.84, \mathrm{n}=30$ studies $)$. For every FIT iCRC, three CRCs were found with FIT, while for gFOBT the ratio between iCRC and screen-detected CRC was $1: 1.3$, Table 1 . No significant differences were found between the relative risk of FOBT iCRC in the second and third screening round compared to the first, with $1.03(95 \%$ CI $0.94-1.13)$ and $1.08(95 \%$ CI $0.93-1.22)$, respectively. Incidence rate ratio of FOBT iCRC was $1.2(95 \% \mathrm{CI} 0.8-1.7)$ for males relative to females and $5.0(95 \% \mathrm{CI} 1.2-21)$ for screenees aged $\geq 60$ relative to $<60$ years.

Table 1: Baseline data of 30 studies included in quantitative meta-analyses displayed per test type

\begin{tabular}{lllll}
\hline & $\begin{array}{l}\text { Screening } \\
\text { participants } \\
\mathrm{n}=5,252,563\end{array}$ & $\begin{array}{l}\text { Screen-detected } \\
\text { CRCs n=14,030 }\end{array}$ & $\begin{array}{l}\text { FOBT iCRCs } \\
\mathrm{n}=5,398\end{array}$ & $\begin{array}{l}\text { Ratio } \\
\text { screen-detected } \\
\text { CRC to FOBT iCRCs }\end{array}$ \\
\hline $\begin{array}{l}\text { FIT n, (\%) } \\
\text { gFOBT n, (\%) }\end{array}$ & $\begin{array}{l}4,774,516(91) \\
478,047(9)\end{array}$ & $12,172(87)$ & $4003(80)$ & 3.0 \\
\hline
\end{tabular}

Conclusion: This is the first study to report on the pooled incidence of FIT and gFOBT iCRC in screening setting. The incidence rate of iCRC after a negative 
FOBT is two-fold higher in gFOBT than in FIT, which supports the use of FIT over gFOBT as screening stool test. However, for every three FIT-detected CRCs, still one CRC is missed, which highlights the importance to adequately inform screenees about the risk of developing a colorectal carcinoma after a negative FIT.

Disclosure of Interest: E. Wieten: I declare no competing interests.

All other authors have declared no conflicts of interest.

\section{P0439 MEASURES OF BODY COMPISITION AND GENDER DIFFERENCES IN RISK FOR COLORECTAL CANCER - A POPULATION-BASED COHORT STUDY}

A. Forsberg ${ }^{1}$, F. Sköldberg ${ }^{2}$, P. Thelin Schmidt ${ }^{1}$, A. Carlsson ${ }^{3}$, K. Önnerhag ${ }^{4}$, H. Hagström ${ }^{1}$, A. Andreasson ${ }^{1}$

${ }^{1}$ Karolinska Institutet, Institution of Medicine Solna, Stockholm/Sweden

${ }^{2}$ Uppsala University, Department of Surgical Sciences, Uppsala/Sweden

${ }^{3}$ Karolonska Institutet Huddinge, Division of Family Medicine, Department of

Neurobiology, Care Science and Society, Huddinge/Sweden

${ }^{4}$ Skåne University Hospital Malmö, Department of Gastroenterology and

Hepatology, Malmö/Sweden

Contact E-mail Address: anna.forsberg@ki.se

Introduction: Age and family history of colorectal cancer (CRC) are the strongest risk factors for CRC. Obesity, commonly assessed based on body mass index (BMI), is associated with an increased risk for CRC in men but the association is weaker in women and differs between studies. We investigated which of the following body composition measures: BMI, waist circumference (WC), waisthip ratio (WHR), weight-height-ratio (WHtR), weight-hip-height ratio (WHHR), A Body Shape Index (ABSI) and percent body fat that best predict the development of CRC in men and women.

Aims \& Methods: We used data from Malmö Diet and Cancer cohort in Sweden, including 16,840 women and 10,903 men (mean age, 58.1 years at baseline), followed for a median of 19.8 years. We identified cases with CRC until the end of 2014 using national Swedish registers. Hazard ratios (HR) for CRC, colonic cancer $(\mathrm{CC})$ and rectal cancer $(\mathrm{RC})$ per one standard deviation increase in each body composition measure respectively were calculated using Cox regression models, stratified by sex and adjusted for age, alcohol consumption, smoking, education and physical activity. Likelihood ratio tests and C-statistics were calculated to identify the anthropometric measure that improves the null model the most.

Results: Incident CRC occurred in 880 individuals (477 women) during followup. All body composition measures apart from WHHR significantly predicted CRC in men and waist circumference (WC) was the best predictor based on C-statistics and LR-test (HR per standard deviation [SD] increment, 1.19; 95\% CI, 1.08-1.31, LR-test $\mathrm{p}<.001, \mathrm{C}$-statistics 0.6278$)$. The association between WC and CRC was only found in men with a BMI above 25 . All body composition measures apart from WHHR and percent body fat significantly predicted $\mathrm{CC}$ in men, again WC was the best predictor (HR 1.25; 95\% CI, 1.11-1.42, LR-test $\mathrm{p}<.001, \mathrm{C}$-statistics 0.6444$)$. ABSI was the only measure significantly associated with risk for $\mathrm{RC}$ in men (HR, 1.24; 95\% CI, 1.05-1.47). In women neither of the measures was significantly associated with an increased risk for CRC, CC nor RC.

Conclusion: In this Swedish population-based cohort study on well-characterized participants, body composition measures predicted CRC in men but not in women after adjusting for age, education and health behaviors. WC was the best predictor of CRC and CC in men and the association was only significant in overweight/obese men in stratified analyses. Gender difference in the interplay between sex and metabolic hormones in the adipose tissue may explain the lack of associations in women.

Disclosure of Interest: All authors have declared no conflicts of interest.

\section{Reference}

Kim H, Giovannucci E L. Sex differences in the association of obesity and colorectal cancer. Cancer Causes Control 2017; 28:1-4. PubMed PMID: 27878394

\section{P0440 TH17 CELLS INDUCE EPITHELIAL-MESENCHYMAL TRANSITION VIA IL-17/PI3K/AKT/SNAIL PATHWAY IN COLORECTAL CANCER}

\section{Q. $\mathbf{L i}^{1}, \mathrm{X} \cdot \mathrm{Xu}^{2}, \mathrm{P} \cdot \mathrm{Zhou}^{2}$}

${ }^{1}$ Endoscopy Center, Zhongshan Hospital, Fudan University, Zhongshan Hospital, Fudan University, Shanghai/China

${ }^{2}$ Endoscopy Center, Zhongshan Hospital, Fudan University, Shanghai/China

Contact E-mail Address: liquanlin321@126.com

Introduction: T helper 17 (Th17) cells participate in the progression of various cancers. Both tumor-promoting and tumor-suppressing effect have been reported. The role of Th17 cells in colorectal cancer (CRC) remains controversial and the specific mechanism of how Th17 cells affect the development of CRC remains to be explored.

Aims \& Methods: The study aimed to clarify the role of Th17 cells in CRC and identify the underlying molecular mechanisms. The percentage of Th17 cells and
IL-17 expression were evaluated via flow cytometry, enzyme-linked immunosorbent assay, and immunohistochemistry in tissue samples and peripheral blood. Effects and underlying molecular mechanisms of IL-17 cells on epithelialmesenchymal transition (EMT) process were explored in vitro using IL-17 transfection and in nude mice by implanting IL-17 overexpressed CRC cells. To detect the direct role of Th17 cells on EMT process, SW480 cells were co-cultured with Th17 cells via transwell system. Cancer signaling phospho antibody microarray was used to explore the potential signaling pathway. The clinical significance of Th17 cells was investigated in tissue microarrays containing CRC tissues from 90 patients following surgery using immunohistochemistry.

Results: A higher percentage of Th17 cells and serum IL-17 level were found in CRC patients than healthy controls, and Th17 cells presented a gradual upward trend in normal epithelium-adenoma-carcinoma sequence. The overexpression of IL-17 significantly promoted tumor proliferation and invasion, and inhibited apoptosis in vitro and in vivo. IL-17 overexpression reduced the expression of E-cadherin and induced the expression of Snail, $\beta$-catenin, and Vimentin in both SW480 cells and tumor xenografts, suggesting that IL-17 could induce the EMT process in CRC. When co-cultured SW480 cells with Th17 cells, we found Th17 cells could directly promote the EMT process of tumor cells. Furthermore, using cancer signaling phospho antibody microarray, we found that PI3K/AKT/Snail signaling pathway played a key role in the regulation of EMT. EMT process could be reversed by LY294002 and IL-17 mAb intervention, suggesting that IL17/PI3K/AKT/Snail pathway played a vital role in Th17 cells-induced EMT in CRC. Supporting these findings, in human CRC tissues, immunostaining indicated that the percentage of Th17 cells was significantly associated with E-cadherin expression and AKT phosphorylation. The clinical significance of Th17 cells was authenticated by revealing that the combination of intratumoral Th17 cells and E-cadherin served as a better prognosticator for postoperative tumor recurrence than either marker alone.

Conclusion: Th17 cells promote EMT process and facilitate tumor progression via activating IL-17/PI3K/AKT/Snail signaling pathway in CRC.

Disclosure of Interest: All authors have declared no conflicts of interest.

\section{P0441 MICRORNA EXPRESSION PROFILE IN RECTAL CANCER}

J. Král $^{1}$, V. Rusnakova ${ }^{2}$, L. Langerova ${ }^{2}$, V. Korenkova ${ }^{2}$, P. Vodicka $^{3}$, J. Spicak ${ }^{1}$, J. Slyskova ${ }^{3}$

${ }^{1}$ Dept. Of Hepatogastroenterology, Institute for Clinical and Experimental Medicine, Prague/Czech Republic

${ }^{2}$ Institute of Biotechnology AS CR, v.v.i., Prague/Czech Republic

${ }^{3}$ Department Of The Molecular Biology Of Cancer, Institute of Experimental

Medicine AS CR, v.v.i., Prague/Czech Republic

Contact E-mail Address: jan.kral@centrum.cz

Introduction: Colorectal cancer (CRC) remains the second most common cancer in women and third in men worldwide, with more than 1.3 million patients diagnosed every year (1-3). Still more than $50 \%$ of patients are diagnosed with advanced disease (stage III and IV), which has worse prognosis and survival. There is a need for new biomarkers for early diagnostics, predicting patient's treatment response and follow-up. Recent data suggest that microRNAs (miRNAs) might be utilized as these biomarkers $(4,5)$.

Aims \& Methods: We focused on specifying differences in expression profile between rectal tumor and adjacent healthy mucosa. Individual miRNA levels were analyzed in relation to patient's treatment response and post-treatment survival, in order to identify miRNAs with possible role of predictive and prognostic markers in rectal cancer. At first, we screened 20 pairs of rectal tumors and healthy surrounding mucosae for the expression levels of 2555 microRNAs using 3D-Gene TORAY microarray system. We have identified 71 candidate miRNAs with different expression profile in tumor and healthy tissue. These 71 miRNAs were further explored and verified in larger cohort of 101 rectal tumors and 105 colon tumors compared to matched healthy tissues by qPCR (Fluidigm BioMark). Results were analyzed in relation to different clinico-pathological characteristics of tumors, to individual patient's treatment response and overall or disease-free survival.

Results: We confirmed 18 miRNAs to be differently expressed in rectal tumors as compared to healthy mucosa. This expression profile was observed in tumors resected prior to neoadjuvant therapy, but not in those that were neoadjuvantly treated. Only 2 miRNAs were dysregulated irrespectively on neoadjuvant therapy. We have further investigated whether this signature is specific for rectal cancer, or is observed also in colon cancer. We have found that 14 out of 18 miRNAs were indeed commonly dysregulated in whole CRC, and 4 miRNAs were dysregulated in rectal tumor only. In order to identify miRNAs with any association to treatment, we have analyzed miRNA expression profile of rectal tumors in relation to patient's treatment response and their long-term survival. Thirteen miRNAs were differently expressed between tumors that responded to therapy and those that relapsed.

Conclusion: We have identified miRNA expression patters specific for rectal cancer and identified miRNA candidates that might be used as a predictive and prognostic marker in patient's follow-up.

Disclosure of Interest: All authors have declared no conflicts of interest.

\section{References}

1. Ferlay J, S.I., Ervik M, Dikshit R, Eser S, Mathers C, Rebelo M, Parkin DM, Forman D, Bray, F. GLOBOCAN 2012 v1.0, Cancer Incidence and 
Mortality Worldwide: IARC CancerBase No. 11 [Internet]. The following article reviews the sources and methods used in compiling the national cancer incidence and mortality estimates in GLOBOCAN 2012, and briefly describes the key results by cancer site and in 2020 large areas of the world. (Lyon, France: International Agency for Research on Cancer; 2013. Available from:, accessed on day/month/year., 2013).

2. El Zoghbi, M. \& Cummings, L.C. New era of colorectal cancer screening. World journal of gastrointestinal endoscopy 8, 252-258 (2016).

3. Lansdorp-Vogelaar, I., Knudsen, A.B. \& Brenner, H. Cost-effectiveness of colorectal cancer screening. Epidemiologic reviews 33, 88-100 (2011).

4. S. Hrasovec et al., Front Genet 3, 180 (Sep 24, 2012).

5. B. Kusenda et al., Biomed Pap Med Fac Univ Palacky Olomouc 150, 205-15 (Apr 12, 2007).

\section{P0442 MUSCARINIC-3 RECEPTOR TARGETED MIRNAS ARE INVOLVED IN BILE ACID-INDUCED PROLIFERATION ON H508 COLON CANCER CELL LINE}

F. Tekin, C. Aktan, N. Oruc, O. Ozutemiz

Gastroenterology, Ege University Medical School, Izmir/Turkey

Contact E-mail Address: drtekinfatih@gmail.com

Introduction: Studies with the colon cancer cell lines which express muscarinic-3 (M3) receptors showed that taurine conjugates of lithocholic acid, but not other bile acids, bind to M3 receptors, and stimulate an increase in cell proliferation. On the other hand, many microRNAs (miRNAs) are involved in colon carcinogenesis. However, the interaction of bile acid-M3 receptors and miRNAs and their potential effects in colon carcinogenesis remains to be elucidated.

Aims \& Methods: For the first time in the literature, we examined the possible role of $\mathrm{M} 3$ receptor-targeted miRNAs on two human colon cancer cell lines: H508, which expresses M3 receptors, and SNU-C4, which does not. Cell proliferation for 6 days after sodium taurolithocholat (ST) and atropin (A) treatment was analysed by WST-1 method. Expression of M3 receptor gene at mRNA level was analysed by qPCR, and at protein level by Western Blot method. Apoptotic experiments were analysed by Annexin V assay. MiRNAs which possibly targeted M3 receptors were identified by in silico analyses. The methods were repeated three times, and the average values were calculated.

Results: When compared to SNU-C4 cells, M3 receptor gene expression was found to be increased 70-fold on $\mathrm{H} 508$ cells. After a 6-day incubation, maximum H508 cell proliferation $(300 \%)$ was achieved on 5th day with a dose of $300 \mu \mathrm{M}$ ST, inhibited by a dose of $1 \mu \mathrm{M}$ A. In contrast, the SNU-C4 cells showed no significant change in cellular proliferation. Treatment of H508 cells with ST caused a decrease (2.53-fold) of M3 receptor gene expression, however, no change of M3 receptor at protein level was seen. No changes in apoptosis on both colon cancer cell lines were observed. Of $25 \mathrm{M} 3$ receptor-targeted miRNAs, expression levels altered in 9; 6 of them were up-regulated (hsa-miR-129-5p, hsamiR-30c-5p, hsa-miR-224-5p, hsa-miR-30b-5p, hsa-miR-522-3p, hsa-miR-1246) and 3 of them (hsa-miR-30e-5p, hsa-miR-147b, hsa-miR-885-3p) were downregulated on $\mathrm{H} 508$ cells $(\mathrm{p}<0.05)$

Conclusion: ST interact with M3 receptors which modulate colon cancer cell proliferation on $\mathrm{H} 508$ cells. M3 receptor-targeted miRNAs are involved in ST induced proliferation. Whether the use of ursodeoxycholic acid, selective antimiRNAs, anti-cholinergic agents or other approaches to blocking potential interactions of bile acids/salts with neoplastic colonic epithelium may be a useful adjunct to colon cancer prevention or treatment remains to be determined.

Disclosure of Interest: All authors have declared no conflicts of interest.

\section{P0443 COLORECTAL CANCER AND DYSLIPIDAEMIA: CAUSE OR CONFOUNDING? A MENDELIAN RANDOMIZATION STUDY}

G. Ibáñez ${ }^{1}$, A. Díez-Villanueva ${ }^{1}$, M. Riera-Ponsati ${ }^{1}$, E. Guinó ${ }^{1}$, B. PérezGómez $^{2}$, M. Bustamante ${ }^{3}$, V. Martin ${ }^{4}$, J. Llorca ${ }^{5}$, P. Amiano 6 , E. Ardanaz, A. Tardón ${ }^{8}$, J.J. Jimenez-Moleon ${ }^{9}$, R. Peiró ${ }^{10}$, J. Alguacil ${ }^{11}$, C. Navarro ${ }^{12}$, P. Pablo Fernández Navarro ${ }^{2}$, A. Espinosa ${ }^{3}$, V. Verónica Dávila-Batista ${ }^{4}$, A.J. Molina ${ }^{4}$, C. Palazuelos 5 , G. Castaño-Vinyals ${ }^{3}$, N. Aragonés ${ }^{2}$, M. Kogevinas ${ }^{3}$, M. Pollán ${ }^{2}$, V. Moreno ${ }^{1}$

${ }^{1}$ Unit Of Biomarkers And Susceptibility, Catalan Institute of Oncology-IDIBELL, L'Hospitalet de Llobregat/Spain

${ }^{2}$ Environmental And Cancer Epidemiology, Instituto de Salud Carlos III, Madrid/ Spain

${ }^{3}$ ISGlobal Centre for Research in Environmental Epidemiology, Barcelona/Spain ${ }^{4}$ Grupo De Investigación En Interacciones Gen Ambiente Y Salud, Universidad de León, León/Spain

${ }^{5}$ Universidad de Cantabria - IDIVAL, Santander/Spain

${ }^{6}$ Public Health Division Of Gipuzkoa, Biodonostia Research Institute, San

Sebastián/Spain

${ }^{7}$ Navarra Public Health Institute, Navarra/Spain

${ }^{8}$ University Institute of Oncology of Asturias, Oviedo/Spain
${ }^{9}$ Instituto de Investigación Biosanitaria de Granada (ibs.GRANADA), Granada/ Spain

${ }^{10}$ Fundación para el Fomento de la Investigación Sanitaria y Biomédica de la Comunitat Valenciana FISABIO, Valencia/Spain

${ }^{11}$ Centre for Research in Health and Environment (CYSMA), Huelva/Spain

${ }^{12}$ Imib-arrixaca And Department Of Health And Social Sciences, Universidad de Murcia, Murcia/Spain

Contact E-mail Address: gibanesa@gmail.com

Introduction: Dyslipidaemia and statin use have been associated to colorectal cancer (CRC), but prospective studies have shown controversial results. Dyslipidaemia has been thought to have an important role in inflammatory pathways, oxidative stress and insulin resistance, which could contribute to the pathogenesis of cancer. However, findings from prospective studies that have examined the association between serum dyslipidaemia (low density lipoprotein cholesterol (LDL), HDL or TG) and colorectal neoplasia have been inconsistent. [1-4] It is unknown whether lipids and lipoproteins cause cancer or are intermediate or correlated factors within carcinogenic pathways. Epidemiological studies could be confounded by 3-Hydroxy-3-methylglutaryl-coenzyme A reductase inhibitors (statins) use, which might also have a protective effect to CRC. It is unclear whether it is statin use or dyslipidaemia that prompted statin use, which may be associated with CRC. Indeed, a large number of epidemiological studies have examined the effect of statins on colorectal cancer risk, with often inconsistent results.[5-6] A Mendelian randomization approach could help to establish a causal relationship between dyslipidaemia and CRC.

Aims \& Methods: We aimed at determining whether dyslipidaemia is causally linked to CRC risk and to explore association of statins with CRC. A casecontrol study was performed including 1336 CRC cases and 2744 controls (MCC-Spain) between 2008 and 2013. Subjects were administered an epidemiological questionnaire that included lifetime regular use of prescription drugs. Also, subjects were genotyped with an exome array supplemented with 5000 custom SNPs. We applied the Mendelian randomization approach. The array included 136 SNPs previously shown to be associated with blood lipids levels in GWAS, that were used to build three genetic lipid scores, as the count of risk alleles. The scores were specific for low density lipoprotein cholesterol (LDL), high density lipoprotein cholesterol (HDL) or triglycerides (TG). We tested the association on regular statin use and the genetic lipid scores with logistic regression models, adjusted for potential confounders.

Results: The LDL genetic risk score was significantly associated with statin consumption $(\mathrm{OR}=1.07,95 \% \mathrm{CI} 1.05-1.10, \mathrm{p}=4.4 \mathrm{e}-11)$. The dyslipidaemia genetic risk score was not significantly associated with CRC for either of the target lipids studied. Cases had the same average alleles as controls in all the lipids traits. Statin use was a borderline significant protective factor for CRC (multivariate adjusted $\mathrm{OR}=0.83 ; 95 \% \mathrm{CI} 0.69-1.00, \mathrm{p}=0.049$ ).

Conclusion: Using the Mendelian randomization approach, our study does not support the hypothesis that lipid levels are associated with the risk of CRC. This study does not rule out, however, a possible protective effect of statins in CRC by a mechanism unrelated to lipid levels.

Disclosure of Interest: All authors have declared no conflicts of interest.

\section{References}

1. Esposito K, Chiodini P, Capuano A, Bellastella G, Maiorino MI, Rafaniello $\mathrm{C}$, et al. Colorectal cancer association with metabolic syndrome and its components: a systematic review with meta-analysis. Endocrine. 2013;44:634-47.

2. Yao X, Tian Z. Dyslipidemia and colorectal cancer risk: a meta-analysis of prospective studies. Cancer Causes Control. 2015;26:257-68.

3. Tian Y, Wang K, Li J, Wang J, Wang Z, Fan Y, et al. The association between serum lipids and colorectal neoplasm: a systemic review and metaanalysis. Public Health Nutr. 2015;18:3355-70.

4. Coppola JA, Shrubsole MJ, Cai Q, Smalley WE, Dai Q, Ness RM, et al. Plasma lipid levels and colorectal adenoma risk. Cancer Causes Control. 2015;26:635-43.

5. Liu Y, Tang W, Wang J, Xie L, Li T, He Y, et al. Association between statin use and colorectal cancer risk: a meta-analysis of 42 studies. Cancer Causes Control. 2014;25:237-49.

6. Lytras T, Nikolopoulos G, Bonovas S. Statins and the risk of colorectal cancer: an updated systematic review and meta-analysis of 40 studies. World J Gastroenterol. 2014;20:1858-70. 
P0444 LINC00152 LONG NON-CODING RNA FACILITATES CELL PROLIFERATION IN SW480 COLON CARCINOMA CELLS THROUGH REGULATION OF CELL CYCLE AND WNT SIGNALING PATHWAY

O. Galamb ${ }^{1}$, B.K. Bartak ${ }^{1}$, A. Kalmár ${ }^{1}$, A. Sebestyén ${ }^{2}$, T. Dankó2 ${ }^{2}$ C. TolnaiKriston ${ }^{2}$, G. Valcz ${ }^{1}$, K. Szigeti ${ }^{3}$, Z.B. Nagy ${ }^{3}$, G. Barna ${ }^{2}$, Z. Tulassay ${ }^{4}$, P. Igaz ${ }^{3}$, B. Molnar ${ }^{4}$

${ }^{1}$ Semmelweis University, 2nd Departemnt of Internal Medicine, Cell Analysis Laboratory, Budapest/Hungary

${ }^{2} 1$ st Department Of Pathology And Experimental Cancer Research, Semmelweis University, Budapest/Hungary

${ }^{3} 2 n d$ Department Of Internal Medicine, Semmelweis University, Budapest/ Hungary

${ }^{4}$ 2nd Department Of Medicine, SE II. Belgy. Klinika Belgyogyaszat, Budapest/ Hungary

Contact E-mail Address: orsg1@yahoo.com

Introduction: Long non-coding RNAs (lncRNAs) contribute to different cancers including colorectal cancer (CRC) through influencing cancer-related processes such as cell proliferation, apoptosis, and invasion. Previous studies have shown altered LINC00152 expression in CRC, but the detailed mechanism of its effects during colorectal carcinogenesis and cancer progression is not well studied.

Aims \& Methods: We aimed to study the effects of LINC00152 to the cell cycle regulation and promoter DNA methylation of several CRC-associated tumor suppressor genes in colon cancer cells. We also analyzed the expression and promoter DNA methylation of LINC00152 and of its regulated molecules in human colonic tissue samples. LINC00152 were silenced in SW480 colon carcinoma cells using Stealth siRNAs. Cells were harvested 48 or 72 hours after transfection. Flow cytometric cell cycle analysis was performed using propidium-iodide DNA staining. Cyclin D1 protein expression was detected using flow cytometry after labeling with anti-cyclin D1 antibody. The effect of LINC00152 silencing to DNA methylation levels of SFRP1, SFRP2, SDC2 and PRIMA1 tumor suppressor gene promoters was studied using MethyLight technology. Promoter methylation and expression of the above molecules were also studied on human colonic tissue samples.

Results: LINC00152 expression was successfully silenced in SW480 cells with 93$98 \%$ efficiency. Silencing of LINC00152 suppressed cell growth, induced apoptosis and decreased cyclin D1 protein expression. LINC00152 knockdown did not affect the promoter methylation status of SFRP1, SDC2 and PRIMA1 genes, while reduced the DNA methylation level of SFRP2 promoter. Remarkable hypomethylation of LINC00152 promoter was detected in CRC compared to normal samples $(\mathrm{p}<0.01)$, which correlated with increased expression $(\mathrm{R}=0.90)$. SFRP2 promoter hypermethylation and decreased expression were measured in CRC and adenoma tissues compared to normal samples $(\mathrm{p}<0.05)$. Conclusion: Our results support that LINC00152 lncRNA can contribute to CRC development by facilitating cell proliferation through upregulation of cyclin D1 cell cycle progression gene and by affecting promoter methylation of SFRP2 tumor suppressor gene. On human tissue level, similar signaling pathway alterations were detected in CRC.

Disclosure of Interest: All authors have declared no conflicts of interest.

\section{P0445 GENETIC PROFILE OF POLYPS AND RISK OF ADVANCED METACHRONOUS LESIONS}

O. Murcia ${ }^{1}$, M. Juárez ${ }^{2}$, E. Hernández-Illán ${ }^{2}$, M. Rodríguez-Soler ${ }^{1}$, C. Egoavil ${ }^{2}$, C. Alenda ${ }^{1}$, A. Martínez-García ${ }^{1}$, C. Mangas ${ }^{1}$, M. Giner-Calabuig ${ }^{2}$, M. Alustiza ${ }^{2}$, J.R. Aparicio ${ }^{1}$, F.A. Ruiz ${ }^{1}$, J.F. Martínez ${ }^{1}$, J.A. Casellas ${ }^{1}$, J.L. Soto ${ }^{3}$, P. Zapater ${ }^{1}$, R. Jover ${ }^{1}$

${ }^{1}$ Unidad De Gastroenterología, Hospital General Universitario, Alicante/Spain ${ }^{2}$ Instituto De Investigación Sanitaria Isabial, Hospital General Universitario de Alicante, Alicante/Spain

${ }^{3}$ Hospital General Universitario de Elche, Alicante/Spain

\section{Contact E-mail Address: omp_89@hotmail.com}

Introduction: Colonoscopy surveillance of polyps is based on their size, number and pathological features. The role of their genetic profile to predict advanced metachronous lesions (AML) remains unknown.

Aims \& Methods: To study the relation between genetic profile of polyps and both risk of AMLs and time to develop them in surveillance. 308 patients with colonic polyps were consecutively enrolled between 2007 and 2009 for this cohort study, and followed up to 2014. Variables as age, sex, smoking, weight, number of colonoscopies and number and characteristics of polyps were collected. 995 polyps were analyzed for somatic mutations on BRAF and KRAS genes using allelic discrimination by real-time PCR and direct DNA sequenciation, respectively. High level of methylation on $\mathrm{CpG}$ islands (CIMP-H) was also tested using MS-MLPA. AML was defined by a size higher than $9 \mathrm{~mm}$, high-grade dysplasia or villous component in adenomatous lesions. Regarding serrated lesions, AML was defined by a size higher than $9 \mathrm{~mm}$, any grade of dysplasia and proximal location. Risk of developing AML for individual genetic markers was studied using Chi-square tests and logistic regression for univariate and multivariate analysis, respectively. Log-rank test with Kaplan Meier survival curves for univariate analysis and Cox-regression model for multivariate analysis were also performed. As logistic as Cox regression were adjusted by sex, age, familial colorectal cancer, smoking and features of AML in first colonoscopy. P value of 0.05 was considered significant.

Results: Patients were followed up for a median of 26 months (range 63). 21\% of polyps in first colonoscopy were CIMP-H. KRAS and BRAF mutations accounted for $25 \%$ and $17 \%$ of polyps, respectively. In univariate analysis, KRAS-mutated polyps were related to higher risk of AML in surveillance (52\% KRAS-mutated polyps vs $31 \%$ non-mutated; $\mathrm{RR}=1.8,95 \%$ CI $1.2-2.9$; $\mathrm{p}=0.01)$. Similar results were obtained regarding CIMP-H (77\% CIMP-H polyps vs $38 \%$ non-CIMP; $\mathrm{RR}=3.7,95 \%$ CI $1.3-10.3 ; \mathrm{p}=0.005)$ but no association was found with BRAF-mutated ones. Logistic regression showed CIMP$\mathrm{H}$ as the unique genetic marker of risk for AML (OR 11.41, 95\% CI 2.04-63.70; $\mathrm{p}=0.006$ ). Regarding time to develop AML, shorter intervals were found related to CIMP-H (median of 31 vs 48 months in non-CIMP-H; $\mathrm{p}=0.002$ ) and KRASmutations (median of 36 vs 49 months in non-mutated; $p=0.029$ ) in univariate analysis. There were no significant results concerning BRAF status. Multivariate analysis highlighted CIMP-H as the unique independent marker associated to shorter time to develop AML (HR 4.01, 95\% CI 1.36-10.46; $\mathrm{p}=0.01$ ).

Conclusion: Presence of CIMP-H in polyps associates higher risk of subsequent AML and shorter interval to their development. Genetic profile of polyps emerges as useful tool for colonoscopy surveillance.

Disclosure of Interest: All authors have declared no conflicts of interest.

\section{P0446 MITOCHONDRIAL GLUTAMATE TRANSPORTER (SLC25A22) MEDIATES DNA AND HISTONE HYPERMETHYLATION AND PROMOTES THE TUMORIGENICITY OF KRAS-MUTANT COLORECTAL CANCER}

C.C. Wong ${ }^{1}$, J. Wu ${ }^{2}$, J. Xu ${ }^{1}$, W. Kang ${ }^{3}$, Y. Qian ${ }^{4}$, H. Chen ${ }^{1}, \mathrm{X} \mathrm{Li}^{5}$, Z. Cai ${ }^{5}$, J. Jao-Yiu Sung 6 , J. Yu ${ }^{7}$

${ }^{1}$ Chinese University of Hong Kong, Hong Kong/Hong Kong PRC

${ }^{2}$ Macau University of Science and Technology, Macau/Macau PRC

${ }^{3}$ Anatomical And Cellular Pathology, Chinese University of Hong Kong, Hong Kong/Hong Kong PRC

${ }^{4}$ Department Of Gastroenterology, Sir Run Run Shaw Hospital, School of Medicine, Zhejiang University, Hangzhou/China

${ }^{5}$ Hong Kong Baptist University, Hong Kong/Hong Kong PRC

${ }^{6}$ Department of Medicine and Therapeutics, The Chinese University of Hong Kong, Hong Kong, Hon, Hong Kong/Hong Kong PRC

${ }^{7}$ Institute Of Digestive Disease, Partner State Key Laboratory Of Digestive

Disease, Chinese University of Hong Kong, Hong Kong/Hong Kong PRC

Contact E-mail Address: chichun.wong@cuhk.edu.hk

Introduction: Colorectal cancer (CRC) frequently harbours concomitant mutations in KRAS and APC that promote carcinogenesis. Epigenetic dysregulation plays essential roles in the tumorigenicity of KRAS mutant CRC. Our preliminary data demonstrated that simultaneous KRAS gain-of-function mutations in APC-null CRC cells induced the hypermethylation of DNA and histones, an effect driven by metabolic rewiring of glutamine metabolism. We recently unveiled that glutamine metabolism in KRAS-mutant CRC could be rewired by the mitochondrial glutamate transporter, SLC25A22, a synthetic lethal gene in KRAS-mutant CRC in vitro and in vivo. In this study, we investigate the potential role of SLC25A22-mediated glutaminolysis in regulating DNA and histone methylation in $\mathrm{CRC}$, its underlying mechanisms, and the association of SLC25A22 with epigenetic dysregulation in human CRC cohorts.

Aims \& Methods: We aim to 1) evaluate the impact of mutant KRAS on DNA and histone methylation in CRC; 2) examine the role of SLC25A22 in DNA and histone methylation in KRAS-mutant CRC; 3) elucidate the underlying mechanisms that underlies SLC25A22-mediated epigenetic dysregulation; and 4) investigate the clinical association between SLC25A22 and epigenetic dysregulation in CRC patients. DNA methylation was determined by the Illumina $860 \mathrm{~K}$ methylation array and Methyl Light qMSP assays. Histone methylation was determined by Histone H3 Modification Multiplex Assay Kit and Western blot. U-13C5-Glutamine metabolic labelling and analysis of glutamine metabolism via the TCA cycle was determined by liquid chromatography-mass spectrometry analysis. In clinical samples, SLC25A22 mRNA and protein expression was determined and their correlation with $\mathrm{CpG}$ Island Methylator Phenotype (CIMP) status and histone methylation mark (H3K36me2) was evaluated.

Results: Using three pairs of isogenic cell lines harbouring wild-type and mutant KRAS (DKS8(WT) vs DLD1(mutant); HKE3(WT) vs HCT116(mutant); ICT(WT) vs ICT-KRAS(mutant)), we demonstrated that significant DNA and histone $\mathrm{H} 3$ hypermethylation in cell lines expressing mutant KRAS. DNA hypermethylation was associated with the up-regulation of 5 -hmC, indicating suppressed DNA demethylation in KRAS mutant CRC cell lines. Metabolomic analysis revealed that KRAS mutation modified glutaminolysis via TCA cycle leading to high succinate and fumarate to $\alpha$-ketoglutarate $(\alpha \mathrm{KG})$ ratio, which was to pivotal in suppressing the enzymatic activity of dioxygenases such as DNA and histone demethylases, thereby leading to aberrant methylation. Interestingly, simultaneous APC-loss and KRAS activating mutations synergistically up-regulated the expression of SLC25A22, a key regulator of glutamine metabolism via the TCA cycle. CRISPR-Cas9 mediated knockout of SLC25A22 suppressed glutaminolysis in KRAS-mutant CRC cell lines, which in turn, reduced the ratio of succinate and fumarate to $\alpha$-ketoglutarate. The impact of SLC25A22 knockout on glutamine metabolism had a profound effect on epigenetic regulation, as DNA methylation profiling revealed that SLC25A22 knockout increased 5-hmC levels and decreased DNA hypermethylation in KRASmutant CRC cell lines. Moreover, histone H3 methylation was reduced at multiple histone marks after the knockout of SLC25A22. These data implied that SLC25A22 inhibited DNA and histone demethylases by promoting the production of succinate and fumarate. Indeed, succinate supplementation reversed the effect of SLC25A22 knockout on DNA and histone methylation in KRASmutant CRC cell lines. In addition, succinate restored cell growth in SLC25A22 knockout cell lines, suggesting that epigenetic dysregulation was closely associated with tumorigenicity. In human CRC, SLC25A22 expression was positively associated with CIMP $(\mathrm{P}<0.0001)$ and histone H3K36me2 methylation status $(\mathrm{P}<0.0001)$ 
Conclusion: SLC25A22 promotes the tumorigenicity of KRAS mutant CRC by driving aberrant DNA and histone hypermethylation, an effect mediated by increased production of TCA cycle intermediates succinate and fumarate, which inhibits DNA and histone demethylases. SLC25A22 is correlated with CIMP and histone hypermethylation in CRC patients.

Disclosure of Interest: All authors have declared no conflicts of interest.

\section{P0447 FOLLISTATIN-LIKE PROTEIN 1 SUSTAINS COLON CANCER CELL GROWTH AND SURVIVAL}

G. Bevivino $^{1}$, V. De Simone ${ }^{1}$, R. Izzo ${ }^{1}$, M. Di Giovangiulio ${ }^{1}$, S. Sedda ${ }^{1}$, E. Franzè ${ }^{1}$, A. Rizzo $^{1}$, P. Rossi ${ }^{2}$, C. Stolfi ${ }^{1}$, G. Monteleone ${ }^{1}$

${ }^{1}$ Systems Medicine, University of Rome "Tor Vergata", Rome/Italy

${ }^{2}$ Surgery, University of Rome "Tor Vergata", Rome/Italy

\section{Contact E-mail Address: bevivino@med.uniroma2.it}

Introduction: Follistatin-like protein 1 (FSTL1) is a secreted glycoprotein, widely expressed in human tissues, which plays key functions in the regulation of cell survival, proliferation, differentiation and migration. Moreover, deregulated expression of FSTL1 has been described in malignancies but its contribution to carcinogenesis remains controversial.

Aims \& Methods: We here investigated the expression and role of FSTL1 in sporadic colorectal cancer (CRC). FSTL1 was evaluated in human CRC samples and cell lines by immunohistochemistry, Western blotting and real-time PCR. Cell proliferation and survival and cell cycle were evaluated in human CRC cell lines (i.e., HCT-116, DLD-1) treated with a specific FSTL1 antisense (AS) or control oligonucleotide by flow-cytometry. By Western blotting, we assessed expression of proteins involved in cell cycle progression, poly ADP-ribose polymerase (PARP), caspase-9 and active caspase-3. Moreover, the effect of FSTL1 knockdown on cell death was evaluated in cells cultured in the presence or absence of the pan-caspase inhibitor Q-VD-OPh by flow-cytometry.

Results: FSTL1 was significantly increased in both epithelial and lamina propria compartments of human CRC specimens as compared to controls. In CRC cell lines, FSTL1 knockdown caused accumulation of cells in G1 phase of the cell cycle and reduced CRC cell proliferation. FSTL1-deficient CRC cells had reduced levels of proteins involved in late $\mathrm{G} 1$ cell cycle phase, such as phosphorylated retinoblastoma protein $(\mathrm{pRb}), \mathrm{E} 2 \mathrm{~F}-1$, cyclin $\mathrm{E}$ and cyclin-dependent kinase-2 (Cdk2), with no modification of early G1 phase proteins (i.e. cyclin D). Treatment of CRC cells with FSTL1 AS increased the percentages of apoptotic cells and this effect was associated with activation of PARP, caspase-9 and caspase-3. Pre-incubation of HCT-116 and DLD-1 cells with Q-VD-OPh abolished the FSTL1 AS-induced cell death and reduced PARP and caspase activation, thus indicating that FSTL1 silencing induces CRC cell death through a caspase-dependent mechanism

Conclusion: Our data indicate that FSTL1 is over-expressed in CRC cells and suggest a role for this protein in promoting intestinal tumorigenesis.

Disclosure of Interest: All authors have declared no conflicts of interest.

\section{P0448 TP53 MUTATION ACQUIRES HIGHER MALIGNANT POTENTIAL IN HUMAN COLON CANCER CELLS}

S. Watanabe ${ }^{1}$, K. Tsuchiya ${ }^{2}$, T. Shirasaki ${ }^{1}$, S. Hibiya ${ }^{1}$, S. Ooshima ${ }^{1}$,

T. Nakamura ${ }^{1}$, M. Watanabe ${ }^{2}$, R. Nishimura ${ }^{1}$, R. Okamoto ${ }^{3}$

${ }^{1}$ Gastroenterology, Tokyo Medical And Dental University, Tokyo/Japan

${ }^{2}$ Tokyo Medical And Dental University, Tokyo/Japan

${ }^{3}$ Tokyo Medical and Dental University Gastroenterology and Hepatology, Tokyo Japan

Contact E-mail Address: swatanabe.gast@tmd.ac.jp

Introduction: In sporadic colon cancer, TP53 mutation is well known to occur in the late phase of colon carcinogenesis as adenoma-carcinoma sequence. Although numerous reports about clinical information of the patients with colon cancer have suggested that TP53 mutation might be related to various malignant potentials, direct effect of TP53 mutation on malignant potential in colon cancer is still unknown. Notably, there is no report about a relationship between TP53 mutation and cancer stemness. We therefore aimed to assess the function of TP53 mutation in colon cancer cells, by using recently established lentiviral CRISPR Cas9 system.

Aims \& Methods: Two types of TP53 mutation were generated in LS174T cells, which are derived from human colon adenocarcinoma with wild-type TP53 (WTTP53), by using lentiviral CRISPR Cas9 system. The guide RNAs were designed to bind exon3 or exon 10 of TP53, respectively. TP53 mutation in LS174T was confirmed by direct sequencing. The expression of TP53 protein was assessed by immunohistochemistry. Loss of function of TP53 was assessed by Nutlin-resistance and the expression of TP53 target genes. Malignant potentials of TP53mutated cells were assessed by MTS Assay, 3D sphere formation assay and cell migration assay for cell proliferation, cancer stemness and cell migration, respectively. Chemo-resistance was also assessed by the treatment with 5-FU and LOHP.

Results: We first selected LS174T cells with WT-TP53 because TP53 gene has already been mutated in almost colon cancer cell lines. We then successfully established 2 types of TP53 mutation in LS174T cells due to high effectiveness of gene-mutating by lentiviral system. Mutation in exon3 (TP53Ex3) and exon 10 (TP53Ex10) of TP53 created the shorter form of TP53 (TP53Ex3; 55a.a. and TP53Ex10; 377a.a., respectively) compared to WT-TP53 (393a.a.). Mutant TP53 (TP53Ex10) is strongly expressed in nuclei as often shown in colon cancer region, whereas both WT-TP53 and mutant TP53 (TP53Ex3) are not expressed in LS174T cells. In contrast, both TP53 mutants (TP53Ex3 and TP53Ex10) showed Nutlin-resistance and the down-regulation of TP53 target genes, suggesting that both mutants induced loss of function of TP53. We then assessed the effect of both TP53 mutants on various malignant potentials, resulting equally in accelerated cell growth, enhanced invasiveness and the resistance against 5-FU treatment compared to WT-TP53. Moreover, both mutants showed more frequent formation of 3D sphere and more expression of Lgr5 than WTTP53, suggesting the promotion of cancer stemness by TP53 mutation even after being adenocarcinoma.

Conclusion: We for the first time showed the direct effect of TP53 mutation on malignant potential in colon cancer cells. Loss of function of TP53 induced by not only TP53Ex10 but also TP53Ex3 mutation, might promote malignant potentials including cancer stemness at the late phase of carcinogenesis. In general, nuclear accumulation of TP53 protein in cancer region is represented as TP53 mutation. However, negative staining of TP53 might also be careful for TP53 mutation to estimate malignant potential in colon cancer, since $\mathrm{N}$-terminal mutation of TP53 in colon cancer has already been reported.

Disclosure of Interest: All authors have declared no conflicts of interest.

\section{P0449 PROTECTIVE EFFECT OF OPIOID RECEPTOR ACTIVATION IN THE DEVELOPMENT OF COLITIS-ASSOCIATED COLORECTAL CANCER IN MICE}

A. Jarmuz ${ }^{1}$, D. Jacenik ${ }^{2}$, H. Zatorski ${ }^{1}$, R. Kordek ${ }^{3}$, W. M. Krajewska ${ }^{2}$, J. Fichna ${ }^{1}$, M. Zielińska ${ }^{1}$

${ }^{1}$ Department Of Biochemistry, Medical University of Lodz, Lodz/Poland

${ }^{2}$ Department Of Cytobiochemistry, Faculty Of Biology And Environmental

Protection, University of Lodz, Lodz/Poland

${ }^{3}$ Department Of Pathology, Medical University of Lodz, Lodz/Poland

Contact E-mail Address: agat.jarmuz@gmail.com

Introduction: Endogenous opioid system is involved in the maintenance of the intestinal homeostasis. Recently, we proved that stimulation of opioid receptors using P-317 - a novel cyclic morphiceptin analog with mu- and kappa- opioid receptor affinity, resulted in alleviation of acute phase of experimental colitis (induced by dextran sodium sulfate [DSS]) in mice. Chronic inflammation is associated with increased risk of colitis-associated colorectal cancer. Stimulation of opioid receptors produces different effects on cancer progression depending on the cancer type and stage of disease.

Aims \& Methods: The aim of our studies was to characterize the role of the endogenous opioid system in pathogenesis and treatment of colitis-associated colorectal cancer using P-317. Colitis-associated colorectal cancer was induced by a single intraperitoneal injection of azoxymethane [AOM] $(10 \mathrm{mg} / \mathrm{kg})$ and subsequent addition of DSS $(1.5 \% \mathrm{w} / \mathrm{v})$ into drinking water (week $2,6,9)$. From week 3, P-317 was injected intraperitoneally at the dose of $0.1 \mathrm{mg} / \mathrm{kg}$ twice per week and the body weight and clinical score (rectal bleeding, stool consistency) were assessed. After 14 weeks, the macroscopic colon damage score was assessed and the samples were collected and used for biochemical, molecular and histological studies.

Results: A significant difference in colorectal tumor development was observed between vehicle- and P-317-treated mice. P-317 significantly decreased total number of colonic tumors as well as colon thickness and width after 14 weeks of disease induction. Myeloperoxidase activity, a marker of neutrophil infiltration, was inhibited by P-317 injections. Hematoxylin and eosin staining confirmed anti-tumor activity of P-317 as indicated by histological score connecting the following features: muscle thickness, damage of the intestinal wall, immune cell infiltration, invasion depth, crypt hyperplasia and disruption. The expression of IL- $1 \beta$ and TNF- $\alpha$ at mRNA level was decreased in P-317treated mice as compared to vehicle-treated group.

Conclusion: P-317 may become an important pharmacological tool to study the factors that determine the development of inflammatory bowel disease and to define the role of the endogenous opioid system in chronic colitis and colorectal cancer.

Disclosure of Interest: All authors have declared no conflicts of interest.

\section{P0450 INCREASED HMGB1 EXPRESSION CORRELATES WITH HIGHER EXPRESSION OF C-IAP2 AND PERK IN COLORECTAL CANCER}

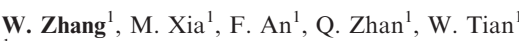

'Gastroenterology, Wuxi People's Hospital Affiliated to Nanjing Medical University, Wuxi/China

Contact E-mail Address: wenjiazhang1221@163.com

Introduction: Colorectal cancer (CRC) is the third most common type of cancer in the world, and it's incidence continues to rise ${ }^{[1,2]}$. The probability of recurrence and subsequent death due to colorectal cancer is associated with its stage ${ }^{[1,}$ 2]. Because of its insidious onset, the diagnosis of CRC is usually delayed. However, serological markers can be a relatively easier and cheaper alternative to colonoscopy for screening an average risk population ${ }^{[1]}$. Several recent studies have shown that high-motility group box 1 (HMGB1) plays a critical role in tumorigenesis, disease progression and metastasis by activation of cancer cells, promotion of tumor angiogenesis, suggesting that HMGB1 may be useful as a new biomarker of cancer ${ }^{[1,2,3,4]}$. Studies have shown that HMGB1 is overexpressed in various types of cancers, include CRC, and those cases with higher expression of HMGB1 are associated with lymphatic metastasis, distant metastasis and poor prognosis ${ }^{[5]}$. Several reports have demonstrated that HMGB1 is 
secreted by cancer cells may be involved in occurrence of tumor metastasis ${ }^{[6,7]}$ In a study by Luo et al, the authors found that HMGB1 secreted by the primary tumors had an apoptotic effect on the Kupffer cells which promoted development of liver ${ }^{[6,7]}$. Furthermore, some researchers showed that increased levels of c-IAP2 and pERK, the downstream effector molecules of HMGB1 are found in tumors ${ }^{[8]}$. The current studies suggest that HMGB1 may be useful for diagnosis and treatment of CRC. However, whether HMGB1 has any role in the development of CRC metastasis is not clear. In this study, we investigated the effects of HMGB1 on CRC, and the possible underlying mechanisms were examined.

Aims \& Methods: To investigate the relationship between high-mobility group B1 (HMGB1) and colorectal cancer (CRC) and the probable underlying pathogenic mechanism. In this prospective study, patients with CRC undergoing primary surgery and healthy subjects (control group) were included from July 2013 to December 2014. Serum HMGB1 concentration was determined using ELISA and HMGB1 mRNA expression was detected by RT-PCR method. Immunohistochemical analysis was performed to determine HMGB1, pERK and c-IAP2 protein expressions in the cancer tissues.

Results: 144 patients with CRC and 50 healthy subjects underwent serum HMGB1 testing. Resected specimens of 50 patients were used for HMGB1 mRNA and protein expression analysis. Serum HMGB1 levels in CRC patients were higher than that of the control group $(8.42$ vs. $1.79 \mu \mathrm{g} / \mathrm{L}, \mathrm{p}<0.05)$. Preoperative serum HMGB1 concentrations were significantly higher than the postoperative values $(8.42 \pm 5.67$ vs $1.64 \pm 1.89 \mu \mathrm{g} / \mathrm{L}, \mathrm{p}<0.05)$. Serum HMGB1 levels in CRC patients with distant metastasis were significantly higher $(13.32 \pm 6.12$ vs $7.37 \pm 5.17 \mu \mathrm{g} / \mathrm{L}, \mathrm{p}<0.05)$. HMGB1 mRNA and protein expression in $\mathrm{CRC}$ tissues was significantly higher than in the adjacent normal mucosa. HMGB1 protein expression positively correlated with the lymph node metastasis. There was positive correlation between HMGB1 and c-IAP2 $(r=0.457$, $\mathrm{P}<0.05)$, HMGB1 and pERK $(\mathrm{r}=0.461, \mathrm{P}<0.05)$ as well as pERK and $\mathrm{c}-$ IAP2 $(r=0.399, \mathrm{P}<0.05)$

Conclusion: HMGB1 expression in CRC correlates with distant and lymph nodal metastasis. It may inhibit apoptosis by inducing activation of pERK and cIAP2. Disclosure of Interest: All authors have declared no conflicts of interest.

\section{References}

1. Lee H, Song M, Shin N, et al. Diagnostic significance of serum HMGB1 in colorectal carcinomas[J]. PloS One, 2012, 7(4): e34318.

2. Mou K, Liu W, Han D, et al. HMGB1/RAGE axis promotes autophagy and protects keratinocytes from ultraviolet radiation-induced cell death[J]. Journal of Dermatological Science, 2016. [Epub ahead of print]

3. Pang X, Zhang Y, Zhang S. High-mobility group box 1 is overexpressed in cervical carcinoma and promotes cell invasion and migration in vitro[J]. Oncology Reports, 2016.

4. Milena Schiraldi, Angela Raucci, Laura Martínez Muñoz, et al. HMGB1 promotes recruitment of inflammatory cells to damaged tissues by forming a complex with SDF-1 $\alpha$ and signaling via CXCR4[J]. J Exp Med, 2012, 209(3):551-63.

5. Varfolomeev E, Goncharov T, Vucic D. Roles of c-IAP proteins in TNF receptor family activation of $\mathrm{NF}-\kappa \mathrm{B}$ signaling[J]. Methods Mol Biol. 2015;1280:269-82.

\section{P0451 THE POSSIBILITIES OF APPLICATION OF METABOLIC PROFILES OF BLOOD SERUM AND RED BLOOD CELLS IN DIAGNOSING OF COLORECTAL CANCER}

M. V. Kruchinina ${ }^{1}$, A. A. Gromov ${ }^{1}$, Y. I. Prudnikova ${ }^{1}$, S.A. Kurilovich ${ }^{1}$, V N. Kruchinin ${ }^{2}$, M. V. Shashkov ${ }^{3}$, A. S. Sokolova ${ }^{4}$, S. E. Peltec

${ }^{1}$ Federal State Budgetary of Scientific Institution "Institution of Internal and

Preventive Medicine", Novosibirsk/Russian Federation

${ }^{2}$ Rzhanov's Institute of Semiconductor Physics Siberian Branch of Russian

Academy of Sciences, Novosibirsk/Russian Federation

${ }^{3}$ Boreskov Institute of Catalysis Siberian Branch of Russian Academy of Sciences, Novosibirsk/Russian Federation

${ }^{4}$ N.N. Vorozhtsov Novosibirsk Institute of Organic Chemistry Of Siberian Branch of Russian Academy of Sciences, Novosibirsk/Russian Federation

${ }^{5}$ Institute of Cytology and Genetics of Siberian Branch of Russian Academy of Sciences, Novosibirsk/Russian Federation

\section{Contact E-mail Address: kruchmargo@yandex.ru}

Introduction: Colorectal cancer (CRC) is often diagnosed at an intermediate or late stage with poor prognosis. Hence, there is an urgent need for new biomarkers for the early detection of colorectal cancer. The aim of the work was to investigate the lipidomic profile of serum, red blood cells (RBCs), the serum metabolomic profile from patients with CRC to identify the stage of the disease and the presence of metastasis of various localizations.

Aims \& Methods: Sera, RBCs from 64 patients ( $52 \pm 7$ years old) with colorectal adenocarcinoma were analyzed by 1 H NMR spectroscopy, gas chromatography. The metabolomics, lipidomic profiles generated from each platform were compared between the following groups: locoregional CRC $(\mathrm{N}=28$-with $\mathrm{T} 2 \mathrm{n}=12$, with T3 $\mathrm{n}=16)$; liver-only metastases $(\mathrm{N}=19)$; and extrahepatic metastases $(\mathrm{N}=17)$. The control group consisted of 26 healthy people.

Results: Complex of serum metabolites as myristic acid, palmitic amide, oleamide, hexadecanedioic, octadecanoic, eicosatrienoic acids, LPC(18:2), $\operatorname{LPC}(20: 4), \operatorname{LPC}(22: 6), \operatorname{LPC}(16: 0)$ contributed to the differentiation of the early CRC patients from healthy controls (AUC of 0.97), while lysophosphatidylcholine $(18: 0,16: 0)$, lysophosphatidic acid $(18: 0,16: 0)$, phosphatidylcholine (34:1) were significantly increased with the CRC progression, which allowed us to distinguish early stages from late ones (AUC of 0.82). The erythrocyte increased levels of stearic $(p=0,03)$, oleic acid $(p=0,02)$ and decreased total phospholipid fraction $(\mathrm{p}<0,05)$, arachidonic, eicosapentaenoic, docosahexaenoic acids $(\mathrm{p}<0,01)$, polyunsaturated:saturated fatty acid ratio $(\mathrm{p}<0,001)$ occurred in cancer patients. The linoleic acid proportion in the RBCs phospholipid fraction was lower in patients with $\mathrm{CRC}(\mathrm{p}=0,02)$, but no changes were found in the serum phospholipid fraction. Changes in fatty acid composition may affect different aspects of cell structure and function, including proliferation. By ${ }^{1} \mathrm{H}$ NMR spectroscopy 55 metabolites were detected, with 25 found to be differentially abundant in the initial data filtering process $(\mathrm{p}<0.30)$. The serum metabolomic profile associated with locoregional CRC was distinct from that associated with liver-only metastases, based on $1 \mathrm{H}$ NMR spectroscopy $\left(\mathrm{p}=4.43 \times 10^{-7}\right)$. Similarly, the serum metabolomic profile differed significantly between patients with liver-only metastases and with extrahepatic metastases $(\mathrm{p}=0.04)$. So, we revealed that isoleucine and 2-oxoglutarate were more abundant in sera from patients with extrahepatic metastases, while methionine and fumarate were more abundant in liver-only metastases.

Conclusion: Investigation of blood serum and erythrocyte metabolites should be considered as promising for verification of colorectal cancer, the detection of metastases of various localizations.

Disclosure of Interest: All authors have declared no conflicts of interest.

\section{P0452 THE MICRORNAS EXPRESSION PROFILES OF MULTIPLE} COLORECTAL TUMORS

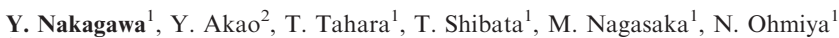
${ }^{1}$ Gastroenterology, Fujita Health University, Toyoake/Japan

${ }^{2}$ United Graduated School Of Drug Discovery And Medical Information Sciences, Gifu University, Gifu/Japan

Contact E-mail Address: yo-hi@fujita-hu.ac.jp

Introduction: Accumulating data indicate that some microRNAs (miRNAs or miRs) function as tumor suppressors or oncogenes in cancer development. We previously reported that certain miRNAs (miR-143, -145, -7, and -34a) were differently expressed in samples of tumors and paired non-tumorous samples taken from the same patients with colorectal tumors, and there was a relation close to adenoma-carcinoma sequence for these miRNAs expression.

Aims \& Methods: In this study, we focus on the miRNA expression profiles of multiple colorectal adenomas comparing between sporadic colorectal adenoma and familial adenomatous polyposis (FAP). We examined the miRNA expression profiles (miRs-143, -145, -7, and -34a) and morphological appearance of 102 sporadic colorectal adenomas (SA), 27 tumors of multiple colorectal adenoma (over 10 adenomas/one patient, MA), 21 tumors of FAP and 114 sporadic cancer (SC).

Results: The expression levels of miRs-143 and -145 were reduced in all tumors compared with the paired non-tumorous samples in the same patient. Especially, these miRNAs were significantly reduced in MA $(\mathrm{P}=0.042$ and $\mathrm{P}=0.004)$ and FAP $(P=0.027$ and $\mathrm{P}=0.022)$ compared with $\mathrm{SA}$. The expression levels of miR7 were significantly up-regulated in cancers compared with adenomas $(\mathrm{P}<0.001)$. The expression levels of miR-34a were significantly down-regulated in CA $(\mathrm{P}<0.001)$, MA $(\mathrm{P}<0.001)$, and FAP $(\mathrm{P}=0.006)$ compared with SA. Conclusion: These findings suggest that the malignant potential of MA and FAP was higher than SA, therefore MA needs strict follow-up like FAP. Disclosure of Interest: All authors have declared no conflicts of interest.

\section{Reference}

Nakagawa Y, Akao Y, Ohmiya N, et. al. Relationship between Expression of Onco-Related miRNAs and the Endoscopic Appearance of Colorectal Tumors. Int J Mol Sci., 2015 Jan; 16(1):1526-1543.

\section{P0453 UTILITY OF MEAN PLATELET VOLUME, PLATELETCRIT, PLATELET-LYMPHOCYTE RATIO AND NEUTROPHIL LYMPHOCYTE RATIO IN THE DIFFERENTIATION OF COLON CANCER AND COLONIC POLYPS IN OLDER PATIENTS}

F. Karakaya, Ç. Kalkan, I. Soykan

Gastroenterology, Ankara University, Ankara/Turkey

Contact E-mail Address: mfkarakaya@yahoo.com

Introduction: Colorectal carcinoma (CRC) is an important cause of mortality and morbidity and mostly encountered in anemic and non-anemic elderly symptomatic patients, irrespective of the iron status. There is a close association between increased mean platelet volume (MPV) and the presence of many solid tumors such as hepatocellular carcinoma, pancreatic carcinoma, lung cancer, endometrial cancer and gastric cancer. Neutrophil/lymphocyte ratio (NLR) and platelet/ lymphocyte ratio (PLR) are markers of systemic inflammatory response and their elevation is closely some studies to be associated with progression of colorectal carcinoma. Although colonoscopic examination is the standart way of colorectal cancer screening, screening for patients between 75 and 85 years of age should be tailored on the basis of the presence of coexisting conditions because the risk of serious complications from colonoscopy also increase with age.

Aims \& Methods: The aim of this study was to investigate whether MPV, plateletcrit, PLR and NLR may have a role in the discrimination of CRC and colonic polyps in older patients. 418 patients aged $>65$ years with colorectal carcinoma ( $\mathrm{n}=93$ ) (Group I) and colonic polyps $(\mathrm{n}=325)$ (Group II) were included into the study. Also 601 (Group III) patients aged $>65$ years with normal colonoscopic findings served as a control group. All study subjects were investigated by using MPV, plateletcrit, PLR and NLR in order to establish sensitivity and specificity for predicting colorectal carcinoma and colonic polyps for each parameter studied. 
Results: MPV, PCT, NLR and PLR were significantly higher in Group III compared to Groups II\&III. However, only MPV was significantly higher in Group II compared to group I $(8.6 \pm 1.1$ vs $8.2 \pm 1, \mathrm{p}<0.001)$. The cut-off value of MPV in predicting CRC from patients with normal colonoscopic findings was $9.15 \mathrm{fL}$ with a sensitivity and specifity of $80 \%$ and $91 \%$ respectively $(\mathrm{r}=0.892)$. MPV and PCT were also significantly higher in patients with neoplastic polyps compared to patients with non-neoplastic polyps (MPV: $8.7 \pm 1.1$ vs $8 \pm 1$, $\mathrm{p}<0.001$ ande PCT: $0.23 \pm 0.07$ vs $0.19 \pm 0.05, \mathrm{p}=0.003)$.

Conclusion: MPV and PCT may have a role as useful and simple markers in the distinguisment of patients with colorectal cancer from patients with normal colonoscopic findings. In the clinical settings, these simple markers may be useful in selecting older patients for colonoscopic examination.

Disclosure of Interest: All authors have declared no conflicts of interest.

\section{References}

Kilincalp S et al. 2015;24(4):328-33.

Karaman $\mathrm{H}$ et al. Relationship between Colonic Polyp Type and the Neutrophil/ Lymphocyte Ratio as a Biomarker. Asian Pacific J Cancer Prev 2013;14;31593161 .

Inadomi JM. Screening for colorectal neoplasia. N Engl J Med 2017;376:149-56.

\section{P0454 IMPROVING THE SELECTION OF COMPLETE RESPONDERS FOR WATCHFUL WAITING AFTER CHEMORADIOTHERAPY FOR RECTAL CANCER: WHAT CAN WE LEARN FROM THE 'MISSED' PATHOLOGIC COMPLETE RESPONDERS AFTER SURGERY?}

D.M. Lambregts ${ }^{1}$, M. Maas ${ }^{1}$, M. Van Der Sande ${ }^{2}$, B. Hupkens ${ }^{3}$, M. Martens ${ }^{3}$, F. Bakers ${ }^{4}$, R. G.h. Beets-Tan ${ }^{1}$, S.O. Breukink ${ }^{3}$, G.L. Beets ${ }^{2}$

${ }^{1}$ Department Of Radiology, The Netherlands Cancer Institute, Amsterdam

Netherlands

${ }^{2}$ Surgery, The Netherlands Cancer Institute, Amsterdam/Netherlands

${ }^{3}$ Surgery, Maastricht University Medical Centre, Maastricht/Netherlands

${ }^{4}$ Radiology, Maastricht University Medical Centre, Maastricht/Netherlands

Contact E-mail Address: doenja.lambregts@gmail.com

Introduction: Rectal cancer patients with clinical evidence of a complete response after chemoradiotherapy may be selected for watchful waiting instead of surgical resection (although currently mainly within the scope of clinical trials). The clinical selection of potential candidates for watchful waiting to date is mainly done using a combination of imaging (MRI) and endoscopy. However, it is known that with this strategy up to $29 \%$ of complete responders may still be missed $^{1}$. Failure to identify complete responders will deny these patients the option of watchful waiting as a result of which they will receive potentially unnecessary surgery. Investigating the reasons why some complete responders are missed with current selection tools can help to further optimize our selection strategy and help improve the identification of potential candidates for watchful waiting in the future.

Aims \& Methods: Aim of this study was to assess what can be learned from operated ('missed') pathologic complete responders by re-evaluating their postCRT appearance on imaging and endoscopy.

Patients with a pathologic complete response (pCR) after CRT and surgery were retrospectively selected from our hospital's database (2005-2015). The MRIs (including diffusion weighted images) + endoscopic images performed postCRT were re-evaluated by a team consisting of an expert radiologist and expert surgeon. The MRI morphology (signal abnormalities, diffusion restriction, volume, shape, aspect of lymph nodes) was assessed on T2-weighted MRI and diffusion-weighted MRI (DWI). On endoscopy the aspect of the scar (normal/teleangiectatic, ulcer, polypoid) was assessed. Additionally, the likelihood for a complete response was re-scored by the expert team according to updated selection criteria and classified as (1) residual tumour or (2) (near) CR Results: 41 patients with ypT0 after surgery were identified: $9 / 41(22 \%)$ had ypT0N+ disease, $32(78 \%)$ were true complete responders (ypT0N0). Upon reassessment by the expert team, $20 / 32$ ypT0N0 patients $(63 \%)$ were still deemed to have residual tumour (non-cCR group), the remaining $12(37 \%)$ were deemed to be possible clinical complete responders (cCR-group). In the non-cCR group (vs. the non-CR group) patients more often showed heterogeneous signal on T2WMRI $(75 \%$ vs. $25 \%, \mathrm{p}=0.01)$, massive/spicular fibrosis $(90 \% \mathrm{vs} .58 \%, \mathrm{p}=0.07)$ and residual high signal on DWI $(58 \%$ vs. $27 \%, \mathrm{p}=0.11)$. On endoscopy the majority of patients still had mucosal abnormalities (see Table 1). Eight ypN0 patients were still overstaged as being $\mathrm{yN}+$ at re-assessment, mainly based on irregular nodal morphology.

Table 1: Endoscopic features of patients with 'missed' complete response (pCR after surgery)

Feature Present in $(\%)$ of patients

Gross residual tumour

$33 \%$

Polypoid tissue

$38 \%$

Ulcer with irregular border

$29 \%$

Flat ulcer

$25 \%$

White scar (with teleangiectasia)

$33 \%$
$\mathrm{ycN}+$ disease and residual mucosal abnormalities at endoscopy. Knowledge about these features may serve as a teaching reference to help improve the selection of patients for watchful waiting in the future.

Disclosure of Interest: All authors have declared no conflicts of interest.

\section{Reference}

1. Maas M, Lambregts DM, Nelemans PJ, Heijnen LA, Martens MH, Leijtens JW, Sosef M, Hulsewé KW, Hoff C, Breukink SO, Stassen L, Beets-Tan RG, Beets GL. Ann Surg Oncol. 2015 Nov;22(12):3873-80.

P0455 RADIOMICS AS A NOVEL TOOL FOR PRE-TREATMENT RESPONSE PREDICTION IN RECTAL CANCER

J. J.m. Van Griethuysen ${ }^{1}$, S. Trebeschi ${ }^{1}$, D.M. Lambregts ${ }^{2}$, M. Lahaye ${ }^{2}$, F. Bakers ${ }^{3}$, R.f.a. Vliegen ${ }^{4}$, E. Voest ${ }^{2}$, G.L. Beets ${ }^{5}$, R. G.h. Beets-Tan ${ }^{1}$, H. J.w.l. Aerts ${ }^{6}$

${ }^{1}$ Radiology, The Netherlands Cancer Institute, Amsterdam/Netherlands

${ }^{2}$ The Netherlands Cancer Institute, Amsterdam/Netherlands

${ }^{3}$ Radiology, Maastricht University Medical Centre, Maastricht/Netherlands

${ }^{4}$ Zuyderland Medical Centre, Heerlen/Netherlands

${ }^{5}$ GROW School for Oncology and Medical Biology - Maastricht University, Maastricht/Netherlands

${ }^{6}$ Dana Farber Institute, Boston/Netherlands

Contact E-mail Address: doenja.lambregts@gmail.com

Introduction: In patients with locally advanced rectal cancer (LARC) that show a very good response to neoadjuvant treatment, organ-preserving treatments such as watchfull waiting may be a good alternative to surgical resection and can improve functional outcome and quality of life. If we can predict upfront (i.e. before onset of CRT) how patients will respond to treatment this may create opportuinities to further personalize and optimize the neoadjuvant treatment to enhance the chance of a good response, thereby ultimately offering more patients the chance of organ preservation. A promising new tool in this regard is 'Radiomics'. Radiomics refers to a collection of analytical methods to convert images into high dimensional data via a set of quantitative descriptors called "features". These features have the potential to uncover disease characteristics that cannot be detected by means of conventional (visual) imaging evaluation. Aims \& Methods: We aimed to assess the Radiomics signature ${ }^{1}$ of patients with LARC and evaluate its potential value for pre-treatment prediction of the response to neoadjuvant chemoradiotherapy.

We retrospectively assessed the primary staging MRIs (1.5T) of 124 LARC patients treated with CRT. The standard MRI protocol included T2-weighted (T2W) and diffusion-weighted imaging (DWI) sequences, as well as quantitative apparent diffusion coefficient (ADC) maps derived from the DWI scans. For each patient, the whole volume of the rectal tumour was delineated on pretreatment MRI: [1] by 2 expert pelvic radiologists, [2] using a semi-automatic (region growing) software algorithm, and [3] by manual adjustment of the semiautomatic delineations by two non-expert readers, adding up to 5 delineations per tumor/patient in total. Radiomics signatures were extracted from the images for each of the five delineations (3802 features in total). Features showing both sufficient stability (ICC $\geq 0.75$ ) as well as reproducible performance (Wilcoxon test, False Detection Rate (FDR) 10\%) across different readers/delineations were selected to test their performance in predicting response to CRT by means of receiver operator characteristics (ROC) curve analysis. The main outcome was complete tumour regression (yT0) versus residual tumour (yT1-4) using histology and/or long-term FU as the standard of reference.

Results: Out of 3802 initially identified Radiomics features, 1853 proved stable across different readers/delineations. For the four manual delineations \pm 300 / 3802 features per reader remained significantly performant after FDR correction. However, these features did not sustain after FDR correction for the fully automated segmentation. A final subset of 266 features remained stable and performant across all five readers/delineations. These features resulted in a mean AUC of 0.67 (range $0.64-0.73$ ) to predict a complete response and a mean ICC of 0.81 (range $0.75-0.95$ ). Best results were obtained for textural features measuring the heterogeneity of the tumor on DWI (all top 20 features). In contrast, more 'common' radiological features such as volume showed inferior performance (highest rating 56) Results derived from delineations performed by the two expert radiologists and non-expert readers resulted in comparable diagnostic performance.

Conclusion: 1) Various Radiomics features extracted from pre-treatment MRI correlate to neoadjuvant treatment response and may be used as imaging biomarkers to predict the response to chemoradiotherapy in rectal cancer.

2) Best results are obtained for textural features (representing tumour heterogeneity) derived from diffusion-weighted MR sequences

3) Features extracted from semi-automated (software generated) delineations show inferior performance compared to features extracted from manual delineations, emphasizing the need for adequate tumour delineation. Interestingly, however, delineations from expert and non-expert readers rendered similar good results, suggesting that the selected features are robust and do not necessarily require highly expert input.

Disclosure of Interest: All authors have declared no conflicts of interest. 


\section{Reference}

1. Aerts HJWL, et al. Decoding tumour phenotype by noninvasive imaging using a quantitative radiomics approach. Nature Communications $2014 ; 5: 4006$

\section{P0456 CORRELATION OF ELECTRICAL AND VISCOELASTIC PARAMETERS OF ERYTHROCYTES WITH FATTY ACID COMPOSITION OF THEIR MEMBRANES AND SERUM IN PATIENTS WITH COLORECTAL CANCER}

\section{Prudnikova}

Institution of Internal and Preventive Medicine, Federal State Budgetary and Scientific Institution, Novosibirsk, Novosibirsk/Russian Federation

\section{Contact E-mail Address: dr.prudnikova@mail.ru}

Introduction: An analysis of the efficient implementation of the guidelines for screening colorectal cancer (CRC) in patients with first diagnosed CRC (according to the archival case histories of the two medical institutions in Novosibirsk) was performed in 2013-2016, and leading reasons of late CRC diagnostics were identified.

Aims \& Methods: We aimed to investigate the correlation of the electrical and viscoelastic parameters of erythrocytes with the fatty acid composition of their membranes and blood serum in patients with colorectal cancer (CRC) of different stages. 46 patients (median age of $53+9$ years old) with CRC of various localizations and stages and 16 conditionally healthy patients were examined. Electrical and viscoelastic parameters of erythrocytes have been studied by dielectrophoresis. Fatty acid composition of erythrocyte membranes and serum has been studied using GC/MS system triple quad Agilent 7000B (USA).

Results: Erythrocytes of patients with CRC were characterized by increasing the proportion of deformed cells with reduced strain amplitude and surface charge (low levels of cell velocity to electrodes and dipole moment) $(\mathrm{p}<0.001-0.05)$ Metastasis was associated with an increase in the cell electrical conductivity, a sharp decrease of polarizability, and an increase in the tendency to hemolysis $(\mathrm{p}<0.0001-0.03)$. Saturated fatty acids prevailed in composition of erythrocyte membranes in patients with CRC; omega 6/omega 3 fatty acid index was decreased, while the level of linoleic acid was significantly increased as related to oleinic acid in serum of the patients with CRC compared to the healthy people $(\mathrm{p}<0.01-0.04)$. The observed shifts correlated with a disease stage $(\mathrm{r}=0.64$ $\mathrm{p}=0.04)$. The erythrocyte strain amplitude was associated with the level of unsaturated fatty acids in erythrocyte membranes $(r=0.58 ; \mathrm{p}<0.05)$, as well as with summarized viscosity $(\mathrm{r}=-0.47 ; \mathrm{p}=0.03)$ and rigidity $(\mathrm{r}=-0.41 ; \mathrm{p}<0.05)$. While the level of hemolysis of erythrocytes and their tendency to aggregate correlated with the level of lysofractions of fatty acids $(r=0.54, p=0.04$ $r=0.42, p<0.05)$. The surface charge of erythrocytes more closely correlated to the level $(\mathrm{C} 16: 2$ and $\mathrm{C} 18: 1)$ in the blood serum $(\mathrm{r}=-0.42 ; \mathrm{p}=0.04)$. There was a shift of the crossover frequency to the high-frequency range $(p=0.03)$, a decrease of the cell capacity $(\mathrm{p}<0.01)$, and a decrease of polarizability at high frequencies $(106,0.5 \times 106 \mathrm{~Hz})(\mathrm{p}=0.02-0.05)$, as well as a decrease of $\mathrm{C} 18: 3$, C20:2, C20:3, C20:5 and C22:6 levels in erythrocyte membranes (but not in blood serum) $(\mathrm{p}<0.01)$ at the initial stages of CRC.

Conclusion: Revealing changes in the parameters of erythrocytes and fatty acid composition in blood serum associated with a stage of the disease can be promising for diagnostics and the case follow-up of patients with CRC.

Disclosure of Interest: All authors have declared no conflicts of interest.

\section{References}

Вергасова Е.В., Пиманов С.И. Методика скрининговой ультразвуковой лиагностики рака ободочной кишки//Новости .лучевой лиагностики. - 2002 - № 1. - С. 42-46.

Израильский онкологический журнал [Электронный ресурс]. - Режим доступа: Colorectal Cancer: Genetics is Changing Everything// GastroenterolClin North Am. 2016. - Vol. 45. - № 3. - P. 459-476

Stoffel E.M., Yurgelun M.B. Genetic predisposition to colorectal cancer: Implications for treatment and prevention//Seminars in Oncology. 2016. - Vol. 43.- № 5. - P. 536-542.

Winawer.S. Скрининг колоректального рака. Практическое руководство Всемирного гастроэнтерологического общества (ВГО) и Международного союза по профилактике рака пищеварительной системы. WGO. - 2008. - С. $1-16$.

Jemal A., Bray F., Ferlay J. et al. Global Cancer Statistics//CA CANCER J CLIN. - 2011. - V. 61. - P. 69-90.

Nugent F.W., Marks J.W. Colon cancer (colorectal cancer) [Электронный peсурс]. - Режим доступа:. Colorectal Cancer: Genetics is Changing Everything//GastroenterolClin North Am. 2016. - Vol. 45. - № 3. - P. 459-476 Parkin D. M., Boyd L., Walker L. C. The fraction of cancer attributable to lifestyle and environmental factors in the UK in 2010. Summary and conclusions.//Br. J. Cancer. - 2011. - Vol. 105. - P. 77-81.

\section{P0457 CLINICOPATHOLOGICAL STUDY OF LATERALLY} SPREADING TUMORS OF THE COLORECTUM

M. Iwabuchi, M. Sugimura, S. Toda, K. Ukai

Gastroenterology, Sendai Medical Center, Sendai/Japan
Introduction: Laterally spreading tumors (LSTs) of the colorectum are classified into the following four subtypes according to their morphology; granular homogeneous type (LST-GH), granular nodular mixed type (LST-GM), non-granular flat-elevated type (LST-NGF), and non-granular pseudo-depressed type (LSTNGPD). Clinical features of each subtype of LSTs have not been fully evaluated. Aims \& Methods: The aim of this study was to clarify the clinicopathological features of colorectal LSTs focusing on their subtypes. We reviewed clinical charts and surgical pathology files of 5352 endoscopically resected specimens during January 2007 and December 2016 at our institution. A total of 422 LSTs were detected. We examined the clinical features (mean age, male to female ratio, size, location, Incidence of concomitant carcinoma) according to their subtypes.

Results: Of these 422 lesions, a total of $151(35.8 \%)$ were LST-GH, $34(8.1 \%)$ LST-GM, $209(49.5 \%)$ LST-NGF, and $28(6.6 \%)$ LST-NGPD. Mean age of patients with each subtype was 68.3 years old for LST-GH, 67.1 for LST-GM, 67.9 for LST-NGF, and 67.2 for LST-NGPD. Male to female ratio (M/F) was 1.21 for LST-GH, 2.05 for LST-GM, 1.95 for LST-NGF, and 1.65 for LSTNGPD. Mean size of LST-GH $(21.2 \mathrm{~mm})$ and LST-GM $(25.8 \mathrm{~mm})$ were significantly larger than that of LST-NGF $(17.0 \mathrm{~mm})$ and LST-NGPD $(15.1 \mathrm{~mm})$. All subtypes were located predominantly in the proximal colon. Incidences of concomitant carcinomas in LST-GH, LST-GM, LST-NGF, and LST-NGPD were $19.2 \%$ ( 29 out of 151 ), $55.9 \%$ (19 out of 34 ), $16.3 \%$ (34 out of 209 ), and $57.1 \%$ (16 out of 28), respectively. Incidences of concomitant submucosal carcinomas in LST-GH, LST-GM, LST-NGF, and LST-NGPD were $0 \%$ ( 0 out of 151 ), $14.7 \%$ ( 5 out of 34 ), $1.0 \%$ ( 2 out of 209 ), and $25.0 \%$ (7 out of 28 ), respectively. Conclusion: Each subtype of LSTs has distinct clinical features. LST-GM and LST-NGPD have higher malignant potentials than other subtypes. Especially LST-NGPD has the highest risk of invasive carcinoma regardless of its size. Therefore we should carefully detect these lesions and choose appropriate treatment according to the subtypes.

Disclosure of Interest: All authors have declared no conflicts of interest.

P0458 POST-INVESTIGATION COLORECTAL CANCER RATES INCLUDING POST COLONOSCOPY COLORECTAL CANCER RATES IN A DISTRICT GENERAL HOSPITAL: THE POOLE EXPERIENCE MARCH 2015 TO FEBRUARY 2017

S.D. Parry, T. Deacy, E. J. Williams

Dept. Of Gastroenterology, Poole Hospital NHS, Poole Dorset/United Kingdom

Contact E-mail Address: sally.parry@poole.nhs.uk

Introduction: Post-colonoscopy colorectal cancer (PC-CRC) rates are proposed as a quality indicator of a colonoscopy service. Extrapolating the data is challenging but important to assess local practise and to compare with recent published National Data. We aimed to calculate the PC-CRC and the post CT (Colonoscopy + abdomen) CRC rate at Poole Hospital using the number of colonoscopies or CT scans done within 3 years of a CRC diagnosis as the denominator for post-investigation PI -CRC calculations as outlined in a previous study ${ }^{1}$

Aims \& Methods: Retrospective audit of all patients diagnosed with CRC during a two year period 1st March 2015-28th February 2017 inclusive identified via the Somerset Cancer registry database for Poole Hospital using Crystal software. Previous colonoscopy and CT Colonoscopy (CTC) or CT abdomen results in the 3 years preceding the diagnostic investigations were reviewed across two neighbouring hospitals sharing the same electronic patient records. If patients had multiple surveillance colonoscopies the latest was counted as false negative as in previous studies

Results: 416 patients were identified, 67 were excluded (39 non adenocarcinoma, 3 out of area, 12 patients where earlier decision was best supportive care, 6 patients diagnosed at laparotomy, 2 patients with abnormal PET scans and 6 with incomplete datasets). 348 patients were included for analysis. Colorectal cancer was diagnosed by colonoscopy in 200 patients and by CTC or CT abdomen in 148 cases. In the colonoscopy diagnosed group, 55 were via Bowel Cancer Screening Programme (BCSP), 138 via the symptomatic service and 7 from the surveillance programme. In the BCSP group 1 patient had a preceding colonoscopy within the previous 3 years $(6-36 \text { months })^{1}$. In the symptomatic service groups there were 7 preceding colonoscopies and in the surveillance group there were 5 preceding ("false negative') colonoscopies within the previous 3 years. The overall PC-CRC rate was $6.5(+3.6,-2.2) \%(90 \%$ confidence interval). In the CT diagnosed group, 1 patient from the BCSP group, 10 patients from the symptomatic service and 4 from the surveillance group underwent CT scans within the preceding 6-36 months. All bar one of these 15 patients had undergone preceding CT abdomen scans with one elderly patient from the symptomatic group having undergone a prior CT colonoscopy. The post CT-CRC rate was $10.2(+5.0,-3.2) \%$ and the overall combined post investigation colorectal cancer (PI-CRC) rates during this two year period was $8.0(+2.8,-2.0) \%$.

Conclusion: Our findings offer the opportunity for further review of individual cases at local governance level and within the limitations of data collection (NHS hospital records reviewed) our estimated PC-CRC rate of $6.5 \%$ compares favourably to the published National PC-CRC rate of $8.6 \%$ between $2001-2007^{1}$. However, we question the validity of a post CT-CRC rate as a true quality indicator as a CT abdomen scan is a non-colon dedicated study. In addition, the method of calculating PI- CRC rates needs ratification as well as more robust IT systems to capture and analyse the data if it is indeed a measure that is to become part of endoscopic quality assurance. Finally our numbers are small and methods suitable for retrospective observational population based studies may not transfer to assessing quality indicators at a local level

Disclosure of Interest: S.D. Parry: None

E.J. Williams: None 
All other authors have declared no conflicts of interest.

\section{Reference}

1. Morris EJA, Rutter MD, Finan PJ, et al. Post- colonoscopy colorectal cancer (PCCRC) rates vary considerably depending on the method used to calculate them: a retrospective observational population-based study of PCCRC in the English National Health Service. Gut 2015;64:1248-1256

\section{P0459 RISK OF DETECTION OF GASTROINTESTINAL NEOPLASMS AND DEATH IN SYMPTOMATIC PATIENTS WITH A POSITIVE FECAL IMMUNOCHEMICAL TEST WITHOUT COLORECTAL CANCER}

N. Pin, M.J. Iglesias, D. Remedios, P. Vega, J. Cubiella

Servicio De Aparato Digestivo, Complejo Hospitalario Universitario de Ourense, Ourense/Spain

\section{Contact E-mail Address: noel.pin.vieito@sergas.es}

Introduction: The fecal immunochemical test (FIT) has a high diagnostic accuracy for the detection of colorectal cancer (CRC) in symptomatic patients. However, we do not know the risk of other gastrointestinal neoplasms associated with a false positive test.

Aims \& Methods: To calculate the risk of detection of gastrointestinal tract tumors (GITT) and death in symptomatic patients with a positive FIT determination and without a CRC in a complete colonoscopy with an adequate bowel preparation. We designed a prospective cohort study with follow-up. Patients from the COLONPREDICT study with complete colonoscopy without CRC were included. Two cohorts were defined: FIT positive and negative according to the $\geq 20 \mu \mathrm{g}$ hemoglobin/g of feces threshold. We performed a descriptive analysis of the GITT detected during follow-up and mortality. We estimated the differences in the risk of GITT detection and mortality between the two cohorts by logistic regression and proportional hazards after adjusting for age, sex, and significant colonic lesions (CSL) detection at baseline colonoscopy.

Results: We included 1061 patients without CRC and a complete baseline colonoscopy; $320(30.2 \%)$ with a positive FIT and 741 with a negative FIT. The median follow-up was of $36.0 \pm 8.9$ months with no difference between both groups $(\mathrm{p}=0.2)$. There were significant differences regarding age $(67.5 \pm 12.7$ years vs $64.8 \pm 13.5$ years, $\mathrm{p}=0.04)$ and sex $(45.9 \%$ vs $52.0 \%$ females, $\mathrm{p}=0.04)$ between both cohorts. We detected a GITT in $14(4.4 \%)$ patients with a positive FIT: 5 CRC, 6 gastric, one esophageal, 1 small intestinal lymphoma and one patient with a CRC and a small intestine adenocarcinoma; and in $12(1.6 \%)$ with a negative FIT: 4 CCR, 4 gastric, 2 small intestine adenocarcinoma, one esophageal, and one patient with a gastric and a CRC. Patients with a positive FIT had a non-significant increase in the risk of GITT detection (OR 2.1, 95\% CI 0.9-4.8) after adjusting for age, sex and SCL. The overall risk of death in both groups was $8.8 \%$ and $6.7 \%$, respectively, with no significant differences between both groups in the survival analysis (HR 1.3, $95 \%$ CI $0.8-2.1)$. However, the risk of death due to a GITT was $3.1 \%(10$ deaths) in the positive FIT group and $0.8 \%$ (6 deaths) in the negative FIT group., with a significant difference after adjusting for age, sex and SCL (HR $3.295 \%$ CI $1.2-8.9$ ).

Conclusion: Symptomatic patients with a positive FIT and complete colonoscopy without CRC are at increased risk of death due to GITT regardless of age, sex or the presence of SCL.

Disclosure of Interest: All authors have declared no conflicts of interest.

P0460 LONG-TERM OUTCOMES OF TRANSANAL COLORECTAL TUBE PLACEMENT FOR DISTAL STAGE II/III COLORECTAL CANCER WITH ACUTE COLORECTAL OBSTRUCTION

T. Yamada ${ }^{1}$, Y. Okuda ${ }^{2}$, R. Yamaguchi ${ }^{2}$, E. Sakamoto ${ }^{1}$, Y. Hirata ${ }^{2}$, T. Shimura ${ }^{3}$ ${ }^{1}$ Gastroenterology, Japanese Red Cross Nagoya Daini Hospital, Nagoya/Japan ${ }^{2}$ Kasugai Municipal Hospital, Kasugai/Japan

${ }^{3}$ Nagoya City University Graduate School of Medical Sciences, Nagoya/Japan

Contact E-mail Address: yamadatomonori@mac.com

Introduction: Emergency surgery is a standard treatment for colorectal cance (CRC) with acute colorectal obstruction (ACO). Transanal colorectal tube (TCT) placement is an alternative endoscopic treatment for ACO; however, the oncological outcomes of TCT placement for the curative treatment of CRC remain unknown.

Aims \& Methods: Data were retrospectively reviewed from patients with distal stage II/III CRC who underwent surgery between January 2007 and December 2011 at two Japanese affiliate hospitals with an interexchange of endoscopists and surgeons. One hospital conducted emergency surgery and the other performed TCT placement as the standard treatment for CRC with ACO. To analyze the efficacy of TCT placement, we compared long-term outcomes for stage II/III CRC with ACO among patients in the two institutions.

Results: In total, 764 patients with distal stage II/III CRC were identified for this study. Among the 764 patients, 690 did not have ACO (non-ACO group), and 74 had ACO (ACO group). In the non-ACO group, we confirmed that the surgical quality was equivalent between the two institutions, with no significant differences in overall survival (OS) $(\mathrm{P}=0.271)$ or disease-free survival (DFS) $(\mathrm{P}=0.184)$. Among the 74 patients with ACO, 27 underwent emergency surgery (surgery group) and 47 underwent TCT placement (TCT group). The rate of primary resection/anastomosis was higher in the TCT group than in the surgery group $(91.5 \%$ vs. $22.2 \% ; \mathrm{P}<0.001)$. No significant differences were noted between the two groups in OS (surgery vs. TCT: 5 -year OS, $65.9 \%$ vs. $58.1 \%$; $\mathrm{P}=0.452$ ) or DFS (surgery vs. TCT: 5 -year DFS, $47.6 \%$ vs. $43.1 \% ; \mathrm{P}=0.755$ ). Subset analysis also showed no significant differences in OS and DFS between patients with stage II and stage III CRC

Conclusion: TCT placement can achieve similar long-term outcomes to those of emergency surgery, with a high rate of primary resection and anastomosis for distal stage II/III CRC with ACO.

Disclosure of Interest: All authors have declared no conflicts of interest.

\section{Reference}

Yamada T, Shimura $\mathrm{T}$, et al. Preoperative drainage using a transanal tube enables elective laparoscopic colectomy for obstructive distal colorectal cancer. Endoscopy. 2013;45(4):265-71.

\section{P0461 FACTORS ASSOCIATED WITH THE TECHNICAL DIFFICULTY OF DOUBLE-WIRE WOVEN UNCOVERED SELF- EXPANDABLE METALLIC STENT PLACEMENT FOR MALIGNANT COLORECTAL OBSTRUCTION}

K. Tominaga ${ }^{1}$, I. Maetani ${ }^{1}$, S. Yoshida ${ }^{2}$, H. Isayama ${ }^{3}$, T. Yamada ${ }^{4}$, Y. Sumida ${ }^{5}$ R. Kyo ${ }^{6}$, T. Kuwai ${ }^{7}$, M. Tomita ${ }^{8}$, Y. Kushiyama ${ }^{9}$, M. Kikkawa ${ }^{10}$, S. Saito ${ }^{11}$ Y. Saida ${ }^{12}$

'Division Of Gastroenterology, Department Of Internal Medicine, Toho University Ohashi Medical Center, Tokyo/Japan

${ }^{2}$ Department Of Endoscopy And Endoscopic Surgery, Graduate School of

Medicine, The University of Tokyo, Tokyo/Japan

${ }^{3}$ Department Of Gastroenterology, Graduate School of Medicine, The University of Tokyo, Tokyo/Japan

${ }^{4}$ Department Of Gastroenterology, Japanese Red Cross Nagoya Daini Hospital, Nagoya/Japan

${ }^{5}$ Department Of Gastroenterology, Kyushu Medical Center, Fukuoka/Japan ${ }^{6}$ Department Of Gastroenterology, Saiseikai Yokohamashi-Nanbu Hospital, Kanagawa/Japan

${ }^{7}$ Department Of Gastroenterology, National Hospital Organization Kure Medical Center and Chugoku Cancer Center, Hiroshima/Japan

${ }^{8}$ Department Of Surgery, Kishiwada Tokushukai Hospital, Osaka/Japan

${ }^{9}$ Department Of Gastroenterology, Matsue Red Cross Hospital, Shimane/Japan

${ }^{10}$ Digestive Disease Center, Akita Red Cross Hospital, Akita/Japan

${ }^{11}$ Division Of Surgery, Gastrointestinal Center, Yokohama Shin-Midori General Hospital, Kanagawa/Japan

${ }^{12}$ Department Of Surgery, Toho University Ohashi Medical Center, Tokyo/Japan

\section{Contact E-mail Address: kenjitominaga@hotmail.com}

Introduction: Self-expandable metallic stent placement for malignant colorectal obstruction has been widely used; however, factors affecting the technical difficulty of stenting remain unclear.

Aims \& Methods: The aim of this study was to clarify the factors associated with the technical difficulty of stenting for malignant colorectal obstruction. We established the Colonic Stent Procedure Research Group to provide instructions on how to safety perform stent placement, and then, we conducted this prospective, single-arm, observational, multicenter clinical trial between October 2013 and May 2014 in Japan. Thirty-two facilities participated in this study. A double-wire woven uncovered stent was placed by using a standard through-thescope colonoscopic placement technique in each patient. Stent deployment time was defined as the time from reaching a lesion with a colonoscope to finish stenting. Technically difficult cases of stenting were defined as those that had a longer than $75 \%$ o deployment time. We examined the clinical data and extracted factors associated with the technical difficulty of stenting by using univariate and multivariate analyses.

Results: A total of 205 consecutive patients were enrolled in this study. Nine patients including 3 patients with technical failure of stenting, 5 patients with non-stenting and 1 patient with stenting for benign lesion were excluded. The remaining all 196 patients were succeeded in stenting. Of these, 100 were men $(51 \%)$, and the median age was 72 years old (interquartile range (IQR), 62-82 years old). One hundred eleven patients $(57 \%)$ underwent stenting as a bridge to surgery, and $85(43 \%)$ underwent stenting for palliation. The technical and clinical success rates were $98.5 \%$ and $97.0 \%$, respectively. None of the patients experienced colorectal perforation. The median total procedure time in the cohort with technical success was 30 minutes (IQR, 18-42 minutes). The median deployment time was 21 minutes (IQR, 11-31 minutes). Forty-nine patients with a deployment time longer than 31 minutes were regarded as technically difficult cases of stenting. The following were identified as independent factors of the technical difficulty in stent placement: presence of ascites (odds ratio, 2.483; 95\% confidence interval $[95 \% \mathrm{CI}], 1.17-5.29 ; p=0.02$ ), placement of $>1$ stent (odds ratio, 4.80; 95\% CI, $1.10-21.1 ; p=0.04$ )

Conclusion: This study demonstrated the high technical and clinical success rate of double-wire woven uncovered stent placement for malignant colorectal obstruction. Clinicians should perform this procedure carefully in patients with presence of ascites.

Disclosure of Interest: I. Maetani: Lecture fee: Century medical inc., Boston Scientific Japan., Piolax Medical Device, MC Medical

S. Yoshida: personal fees: Century Medical Inc., Boston Scientific Japan, ZEON H. Isayama: Donation \& Lecture fee:Century Medical Inc., Boston Scientific Corp., Taewoong Medical Co, Ltd

T. Yamada: personal fees: Century Medical Inc

T. Kuwai: personal fees: Boston Scientific Japan, Century Medical Inc

S. Saito: personal fees: Century Medical Inc., Boston Scientific Japan 
Y. Saida: grants and personal fees: Century Medical Inc., Boston Scientific Japan, Olympus Medical Systems

All other authors have declared no conflicts of interest.

\section{P0462 PROGNOSIS AND CLINICOPATHOLOGICAL FACTORS OF PATIENTS WHO SELECTED THE FOLLOW-UP OPTION AMONG HIGH-RISK T1 COLORECTAL CANCER PATIENTS AFTER ENDOSCOPIC RESECTION BASED ON JAPANESE CLINICAL PRACTICE GUIDELINE: A RETROSPECTIVE OBSERVATIONAL STUDY}

Y. Nishikawa ${ }^{1}$, T. Horimatsu ${ }^{2}$, S. Minamiguchi ${ }^{3}$, H. Seno ${ }^{4}$, Y. Sakai $^{5}$, T. Nakayama

${ }^{1}$ Health Informatics, School of Public Health, Kyoto University, Kyoto/Japan ${ }^{2}$ Therapeutic Oncology, Kyoto University, Kyoto/Japan

${ }^{3}$ Diagnostic Pathology, Kyoto University, Kyoto/Japan

${ }^{4}$ Gastroenterology And Hepatology, Kyoto University, Kyoto/Japan

${ }^{5}$ Surgery, Kyoto University, Kyoto/Japan

Contact E-mail Address: yoshitakanishikawa@gmail.com

Introduction: Colorectal cancer is the third most common cancer in the world and the fourth leading cause of cancer death ${ }^{1}$. Treatment strategy for colorectal cancer is selected considering clinical stages. T1 colorectal cancer (T1CRC) can be treated with endoscopic resection. If patients have pathological risk factors such as deep submucosal invasion, budding, por/muc pathological features and lymphovascular invasion, they considered to be at high risk of lymph node metastasis based on the indication of Japanese Society for Cancer of the Colon and Rectum guideline ${ }^{2}$. In such cases, the selection of subsequent option is important and has been frequently decided by clinicians' customs and preferences. However, it is not clear whether these risk factors adequately predict patients' prognosis in the clinical practice.

Aims \& Methods: This research aims at revealing the prognosis and clinicopathological features of pathologically high-risk T1CRC patients (the high-risk group) with and without additional surgery; followed up by computed tomography, ultrasound, endoscopy, and tumor marker (CEA: carcinoembryonic antigen). 1. To evaluate the difference of overall survival (OS), cancer specific survival (CSS) and recurrent-free survival (RFS) between the patient performed additional colectomy with lymph node dissection (AS) and the patient followed up without additional surgery (FU). 2. To reveal what clinicopathological factors are considered in the selection of subsequent option, whether AS or FU, in the high-risk group. We retrieved the clinical data of 162 patients who had diagnosed and treated as T1 colorectal cancer at Kyoto University Hospital (Kyoto, Japan) between February 2005 and February 2015. Treatment strategy after diagnosed as "high-risk" and clinicopathological features, presence or absence of recurrence and the final state as of Fubruary 2017. We used the Kaplan-Meier product limit method and the Log-rank test to compare OS, CSS, and RFS between AS and FU groups. In clinical setting, based on the guideline indication, the clinician offered subsequent options and described their risks and benefits, and the patient expresses his or her preferences and values. Factors considered through selecting treatment strategy were extracted from informed consent and provider's note of electronic medical records

Results: Among 162 T1CRC patients, 78 cases were treated with endoscopic resection for the first time. Of them, 46 patients had at least one pathological risk factor (high-risk patients). Among 46 high-risk patients, 22 patients were carefully followed up (FU), 20 patients were performed additional surgery with lymph node dissection (AS). Four patients treated with additional radiation therapy were excluded. Median survival time was 39 (FU) and 62 (AS), respectively. No difference was detected in OS $(p$ value $=0.99)$, CSS $(p$ value $=0.44)$ and RFS ( $\mathrm{p}$ value $=0.81$ ) between FU and AS groups. From provider's notes in electric medical records, factors considered in selection of FU were extracted as follows: Patient's values and preferences 8 , sole presence of "depth" risk factor 7 , possible intensive surgery due to T1CRC located in the lower rectum 3, other advanced malignancy 1 , and perioperative risks; advanced age 5, past history of abdominal surgery or radiation 2 and severe comorbidities 12 (chronic heart failure 4, chronic kidney disease 2, connective tissue disease 2, lung disease 1 etc). Together with absence of significant difference of prognosis in this study and the burden of surgery, the subsequent option should be selected through shared decision making between the clinician and the patient ${ }^{3}$.

Conclusion: Among high-risk group after endoscopic resection of T1CRC, no significant difference in OS, CSS nor RFS are detected between FU and AS groups. Factors considered in selecting FU were patient values and preferences, sole presence of "depth" risk factor and severe comorbidity including perioperative risk. Shared decision making should be achieved subsequent option in the high-risk group.

Disclosure of Interest: All authors have declared no conflicts of interest.

\section{References}

1 Int $J$ Cancer 2014; 136: E359-86.

2 Int J Clin Oncol 2015; 20: 207-39.

$3 \quad$ N Engl J Med 2012; 366: 780-1.
P0463 LONG-TERM OUTCOMES AFTER TREATMENT FOR T1

CARCINOMA OF LATERALLY SPREADING TUMORS: A MULTICENTER RETROSPECTIVE STUDY OF HIROSHIMA GI ENDOSCOPY RESEARCH GROUP

K. Yamashita, S. Tanaka, S. Oka, S. Nagata, T. Kuwai, A. Furudoi, T. Tamura, M. Kunihiro, H. Okanobu, K. Nakadoi, H. Kanao, M. Higashiyama,

Y. Tamaru, F. Shimamoto, K. Chayama

Hiroshima GI Endoscopy Research Group, Hiroshima/Japan

Contact E-mail Address: kenyama5@hiroshima-u.ac.jp

Introduction: Laterally spreading tumors (LSTs) are defined as colorectal tumors defined by their characteristic laterally spreading growth pattern larger than $10 \mathrm{~mm}$ in size. LSTs are divided into 4 four subtypes based on different surface morphologies morphology [LST-granular $(\mathrm{G})$ /homogeneous: LST-G-H and nodular mixed: LST-G-M, LST-nongranular (NG)/flat elevated: LST-NG-F and pseudodepressed: LST-NG-PD]. The aim of this study was to analyze the long-term outcomes, including recurrence, 5-year disease-free survival (DFS), 5-year disease-specific survival (DSS), and 5-year overall survival rates (OS) after treatment of T1 LSTs carcinoma.

Aims \& Methods: We examined 169 patients with T1 LSTs carcinoma of LSTs treated between January 1992 and December 2008 at the Hiroshima GI Endoscopy Research Group (Hiroshima University Hospital and 9 nine affiliated hospitals) with a mean follow-up period of $>5$ years. 62 Sixty-two patients who were diagnosed with curable T1 LST carcinoma of LSTs after en bloc endoscopic resection (ER), according to the Japanese Society for Cancer of the Colon and Rectum (JSCCR) criteria, were defined as e-curable patients. One hundred seven patients who did not meet the criteria were defined as non-e-curable patients. According to the clinical and pathological diagnosis of LSTs, patients' backgrounds, and lesion location, the treatments were selected from among the following: ER alone (excluded histological incomplete resection), ER and an additional surgical resection with lymph node (LN) dissection, and surgical resection with LN dissection alone.

Results: Tumor size in Group A was significantly larger (A: $36.6 \pm 22.6 \mathrm{~mm}$ vs B $21.6 \pm 7.2 \mathrm{~mm}$ vs $\mathrm{C}: 24.6 \pm 7.6 \mathrm{~mm}, \mathrm{p}<0.01)$ than in the other two groups. The adenomatous component was more frequent in Group A than in the other two groups. The rates of $\mathrm{LN}$ metastasis in Groups A, B, and C were $10 \%(5 / 49), 20 \%$ $(3 / 15)$, and $5 \%(1 / 21)$, respectively. There were no recurrences among the $62 \mathrm{e}-$ curable patients. On the other hand, five recurrences $(5 \%)$ were found in non-ecurable patients, and they were all in Group A. They consisted of local recurrence (one patient who also had lung metastasis), LN metastasis (two patients), lung metastasis (three patients), and liver metastasis (one patient who also had LN metastasis). There were no significant differences in DSS between Group A and Group B + C (LST-NG). However, OS was $93 \%$ in Group A, which was significantly lower than that $(96 \%)$ in Group B $+\mathrm{C}(\mathrm{p}<0.05)$. DFS in Group A was $90 \%$, which was significantly lower than that $(100 \%)$ in Group B $+\mathrm{C}(\mathrm{p}<0.05)$. The prognosis of patients with non-e-curable disease after ER alone showed no significant differences in OS, DFS, and DSS between Group A and Group B + C The prognosis of patients with non-e-curable disease after surgical resection showed no significant differences in DFS or DSS. However, OS in Group A was $94 \%$, which was significantly lower than that $(97 \%)$ in Group B $+C(p<0.05)$. Conclusion: Long-term outcomes supported the JSCCR criteria for e-curable patients after ER for T1 LSTs. All recurrences occurred in patients with T1 LST-G-M carcinoma. OS and DFS in the LST-G-M group were significantly shorter than in the LST-NG group.

The non-e-curable patients were divided into 3 groups: Group A (61 patients were non-e-curable patients diagnosed with LST-G-M), Group B (23 patients were non-e-curable patients diagnosed with LST-NG-F), and Group C (23 patients were non-e-curable patients diagnosed with LST-NG-PD). There were no patients diagnosed with T1 LST-G-Hcarcinoma of LST-G-H.

Disclosure of Interest: All authors have declared no conflicts of interest.

\section{P0464 ASSESSMENT OF THE EFFECTS OF A PACLITAXEL-} ELUTING SELF-EXPANDING METAL STENT ON NAÏVE PORCINE BILIARY TISSUE

J. T. Favreau ${ }^{1}$, G. Haber ${ }^{2}$, S. Alkaade ${ }^{3}$, M. Arain ${ }^{4}$, T.H. Baron ${ }^{5}$, S. Branch ${ }^{6}$, P. V. Draganov ${ }^{7}$, J. H. Lee ${ }^{8}$, D. K. Mullady ${ }^{9}$, B. T. Petersen ${ }^{10}$, R. J. Shah ${ }^{11}$ S. Sherman ${ }^{12}$, D. Amos ${ }^{1}$, R. Bennett ${ }^{13}$, J. Hemerick ${ }^{13}$, S. Dassner ${ }^{13}$, A. Foss ${ }^{13}$, D. Ross ${ }^{13}$, A. Pearlman ${ }^{1}$, J. Saunders ${ }^{1}$, M. Rivera-Bermudez ${ }^{1}$, C. O. Clerc ${ }^{1}$, L. Swanson ${ }^{14}$, J. Scutti ${ }^{1}$

${ }^{1}$ Boston Scientific, Marlborough/United States of America/MA

${ }^{2}$ New York University Medical Center, New York/United States of America

${ }^{3}$ Saint Louis University School of Medicine, St. Louis/United States of America/ $\mathrm{MO}$

${ }^{4}$ University of California San Francisco, San Francisco/United States of America/ $C A$

${ }^{5}$ Gastroenterology \& Hepatology, University of North Carolina, North Carolina United States of America

${ }^{6}$ Duke University Medical Center, Durham/United States of America/NC

${ }^{7}$ Gastroenterology, University of Florida, Gainesville/United States of America/FL

${ }^{8}$ MD Anderson Cancer Center, Houston/United States of America/TX

${ }^{9}$ Washington University School of Medicine, St. Louis/United States of America/ MO

${ }^{10}$ Mayo Clinic, Rochester/United States of America/MN

${ }^{11}$ University of Colorado Denver, Denver/United States of America

${ }^{12}$ Indiana University Medical Center, Indiana/United States of America

${ }^{13}$ Boston Scientific, Maple Grove/United States of America/MN

${ }^{14}$ Boston Scientific, Saint Paul/United States of America/MN 
Contact E-mail Address: john.favreau@bsci.com

Introduction: Cholangiocarcinoma and pancreatic adenocarcinoma account for over 190,000 new clinical cases of pancreatobiliary malignancy worldwide annually. For palliation of obstructive jaundice in these patients, plastic or self-expanding metal stent (SEMS) are placed. However, re-occlusion rates for currently available stents range as high as $36 \%$ for uncovered metal stents, $25 \%$ for covered metal stents and $52 \%$ for plastic stents. Tissue ingrowth accounts for up to $76 \%$ of occlusions of bare metal stents. ${ }^{1,2,3,4}$ Stent occlusion can result in recurrent obstruction and typically requires endoscopic re-intervention. Therefore there is a real clinical need to reduce tissue ingrowth and improve biliary stent patency rates.

Aims \& Methods: In this study we developed and tested a controlled-release paclitaxel-eluting SEMS designed to prevent tissue hyperplasia and stent occlusion. A $4 \mathrm{~cm}$ length uncovered, laser-cut nitinol stent was coated with a polymer matrix allowing slow release of paclitaxel. Naïve Yucatan swine were assigned to one of three stent groups: bare control $(\mathrm{n}=3$, no polymer), standard dose paclitaxel $(n=6,149.4 \mu \mathrm{g}$ paclitaxel $)$ and challenge dose $(n=3,538.0 \mu \mathrm{g}$ paclitaxel. Two stents were endoscopically implanted in each swine from its assigned group, one in the intrahepatic/hilar region and a second in the common bile duct placed proximal to the papilla. Stents were assessed for migration via digital radiographs for the first 2 weeks and then monthly via endoscopy using SpyGlass ${ }^{\mathrm{TM}}$ DS cholangioscopy and cholangiography with a targeted 6 month study endpoint. Results: At 30 days post-implant, no significant tissue reaction to any stent was observed. However, all animals displayed mild biofilm formation and increased intraductal mucus production. Substantial dilation of the common bile duct was observed in 5/11 animals with no apparent relationship between drug coating and duct dilation. At 60 days post-implant, moderate mucus and biofilm formation was observed within the stent, however in only 3 animals biliary ductal dilation persisted and the majority of stents were fully apposed to the duct wall. Although some animals displayed minimal tissue hyperplasia at the proximal end of the stents, no tissue overgrowth or stent embedding was observed in any animal. Up to 60 days post-implant, no persistent clinical symptoms were observed in any animal. Stents in one standard dose animal migrated out of the bile duct between days 15 and 30, this animal is not included in patency results reported. At both 30 and 60 day timepoints, no apparent differences in outcome were observed among the three study groups.

Conclusion: At this mid-study follow-up, paclitaxel-eluting stents appear to be safe for use in naïve tissue and do not negatively impact function of the biliary system, even at challenge condition doses. Although the cause of bile duct dilation observed in all stent groups has not been conclusively identified, we hypothesize the cause may be distal stent impaction and intermittent (clinically insignificant) obstruction of the papilla and/or stent, resulting in retained mucus and bile. Bile duct dilation, in turn, has reduced the opportunity for tissue overgrowth in all stent groups, which was expected to occur in the bare stent control group by 60 days post-implant ${ }^{5}$. Given the observed $60 \%$ reduction in number of dilated ducts between days 30 and 60 , we expect increased rate of apposition. Ongoing efforts include continued follow-up for an additional 120 days, and in a second cohort, determination of in vivo drug release rates in the bile duct over a 30 day period. Future cholangioscopic and histopathological assessment of these swine will further clarify the safety and effectiveness of paclitaxel stent coatings to mediate bile duct tissue ingrowth.

Disclosure of Interest: J.T. Favreau: John Favreau is an employee of Boston Scientific.

G. Haber: Gregory Haber is a consultant of Boston Scientific

S. Alkaade: Samer Alkaade is a consultant for Boston Scientific

M. Arain: Mustafa Arain is a consultant for Boston Scientific

T.H. Baron: Todd Baron is a consultant for Boston Scientific

S. Branch: Stan Branch is a consultant for Boston Scientific

P.V. Draganov: Peter Draganov is a consultant for Boston Scientific

J.H. Lee: Jeffrey Lee is a consultant for Boston Scientific

D.K. Mullady: Daniel Mullady is a consultant for Boston Scientific

B.T. Petersen: Bret Petersen is a consultant for Boston Scientific

R.J. Shah: Raj Shah is a consultant of Boston Scientific

S. Sherman: Stuart Sherman is a consultant of Boston Scientific

D. Amos: Devon Amos is an employee of Boston Scientific.

R. Bennett: Ryan Bennett is an employee of Boston Scientific.

J. Hemerick: Jim Hemerick is an employee of Boston Scientific.

S. Dassner: Sarah Dassner is an employee of Boston Scientific.

A. Foss: Aaron Foss is an employee of Boston Scientific.

D. Ross: Dan Ross is an employee of Boston Scientific.

A. Pearlman: Allison Pearlman is an employee of Boston Scientific.

J. Saunders: Jennifer Saunders is an employee of Boston Scientific.

M. Rivera-Bermudez: Moises Rivera-Bermudez is an employee of Boston Scientific.

C.O. Clerc: Claude Clerc is an employee and consultant for Boston Scientific.

L. Swanson: Lynne Swanson is an employee of Boston Scientific.

J. Scutti: James Scutti is an employee of Boston Scientific.

\section{References}

1. Sawas et al Gastrointest Endosc 2015 382(2): 256-267

2. Lee et al Gastrointest Endosc 2013 78(2): 312-324

3. Kitano et al Am J Gastroenterol 2013 108(11): 1713-1722

4. Gardner et al Gastrointest Endosc 2016 84(3): 460-466

5. Haber et al, Gastrointest Endosc, 83(5): Supp AB238-AB239

\section{P0465 COMPLETE ENDOSCOPIC MUCOSAL RESECTION OF}

MALIGNANT COLONIC SESSILE POLYPS AND CLINICAL

\section{OUTCOME OF 51 CASES}

M. Fragkaki ${ }^{1}$, E. Chliara ${ }^{2}$, I. Dimas ${ }^{1}$, A. Mpitouli ${ }^{1}$, E. Vardas ${ }^{1}$

A. Theodoropoulou ${ }^{1}$, K. Karmiris ${ }^{1}$, E. Voudoukis ${ }^{1}$, L. Giannikaki ${ }^{2}$, G. Paspatis ${ }^{1}$

${ }^{1}$ Gastroenterology, Venizeleion General Hospital, Heraklion/Greece

${ }^{2}$ Histopathology, Venizeleion General Hospital, Heraklion/Greece

\section{Contact E-mail Address: gpaspatis@gmail.com}

Introduction: Meta-analyses and guidelines recommend that deep submucosal invasion $(>1 \mathrm{~mm})$ in malignant colonic polyps is an important risk factor for residual malignant disease. However, the existing data are based on small retrospective studies with marked heterogeneity.

Aims \& Methods: The aim of this study is to test the correlation between the submucosal invasion depth and the rate of residual malignant disease in complete endoscopic mucosal resection (EMR) of malignant colonic sessile polyps. The secondary outcomes include risk factors such as: lymphovascular invasion, tumor differentiation, resection margin status and the presence of tumor budding. A retrospective review of the endoscopy charts for the period 2000-2016 was conducted. All patients enrolled exhibited a malignant colonic sessile polyp which was endoscopically completely resected. Histological findings of the polyps were also recorded. Thorough computed or magnetic scanning was performed in all patients before deciding on further management. All patients were advised for the option of surgical treatment or endoscopic follow-up.

Results: 51 patients with confirmed adenocarcinoma in sessile colonic polyps undergoing endoscopic mucosal resection (EMR) were retrospectively included in this study. A total of $33(64.7 \%)$ patients underwent subsequent surgery after EMR, and $18(35.3 \%)$ chose endoscopic follow up. The histological characteristics are shown in Table 1 . In $44(86.3 \%)$ patients the submucosal invasion was $>1 \mathrm{~mm}$. Residual malignant disease was identified in the surgical pathological specimen of only one patient. With a median follow-up of 25.12 months (IQR: 31.5; range 1.84-144.92), no local recurrences or lymph node metastasis were identified. 49 were alive without evidence of disease and 2 died of other causes (without evidence of disease at last follow-up). The data regarding the other risk factors are presented in Table 1 .

Table 1: Histological characteristics of the patients

\begin{tabular}{|c|c|c|c|}
\hline Factors & $\begin{array}{l}\text { Total }(\mathrm{N}=51), \\
\mathrm{n}(\%)\end{array}$ & $\begin{array}{l}\text { EMR only, } \\
(\mathrm{n}=18) \\
\mathrm{n}(\%)\end{array}$ & $\begin{array}{l}\text { EMR }+ \text { Surgery, } \\
(\mathrm{n}=33), \\
\mathrm{n}(\%)\end{array}$ \\
\hline $\begin{array}{l}\text { Submucosal invasion } \\
\leq 1 \mathrm{~mm}>1 \mathrm{~mm}\end{array}$ & $\begin{array}{l}7(13.7) \\
44(86.3)\end{array}$ & $\begin{array}{l}2(11.1) \\
16(88.9)\end{array}$ & $\begin{array}{l}5(15.2) \\
28(84.8)\end{array}$ \\
\hline $\begin{array}{l}\text { Resection margin status } \\
\text { (mm) median (IQR; } \\
\text { range) }\end{array}$ & $1(2 ; 0-7)$ & $1(1 ; 0-4)$ & $0,8(1.55 ; 0-7)$ \\
\hline Lymphovascular invasion & $7(13.7)$ & 0 & $7(21.2)$ \\
\hline $\begin{array}{l}\text { Tumor differentiation: } \\
\text { well-differentiated; } \\
\text { moderate-differentiated; } \\
\text { poor-differentiated }\end{array}$ & $\begin{array}{l}14(27.5) \\
28(54.9) \\
9(17.6)\end{array}$ & $\begin{array}{l}6(33.3) \\
9(50) \\
3(16.7)\end{array}$ & $\begin{array}{l}8(24.2) \\
19(57.6) \\
6(18.2)\end{array}$ \\
\hline Tumor budding & $9(17.6)$ & $4(26.7)$ & $5(15.2)$ \\
\hline
\end{tabular}

Conclusion: Our data suggest that even in cases with submucosal invasion $>1 \mathrm{~m}$ and the presence of other high-risk features (lymphovascular invasion, tumour budding), complete EMR in malignant colonic sessile polyps supported by the histological findings predicts for a good clinical outcome.

Disclosure of Interest: All authors have declared no conflicts of interest.

\section{P0466 IMPACT OF EVALUATION OF PRECISION CHEMOTHERAPY FOR COLORECTAL CANCER (CRC) BASED ON COLLAGEN GEL DROPLET-EMBEDDED DRUG SENSITIVITY TEST (CD-DST)}

T. Ochiai ${ }^{1}$, K. Nishimura ${ }^{1}$, T. Watanabe ${ }^{1}$, M. Kitajima ${ }^{1}$, A. Nakatani ${ }^{1}$, K. Nagayasu ${ }^{1}$, N. Sakuyama ${ }^{1}$, T. Sato ${ }^{1}$, K. Kishine ${ }^{1}$, Y. Abe ${ }^{1}$, C. Hara ${ }^{1}$, K. Fujiwara ${ }^{1}$, I. Nagaoka ${ }^{2}$

${ }^{1}$ Dept. Of Surgery, Tobu Chiiki Hospital Dept. of Surgery, Tokyo/Japan ${ }^{2}$ Host Defense And Biochemical Research, Juntendo University School of Medicine, Tokyo/Japan

Contact E-mail Address: takumi_ochiai@tokyo-hmt.jp

Introduction: The leucovorin (FOL) and fluorouracil (5-FU) plus oxaliplatin (1OHP; FOLFOX) or FOL and 5-FU plus irinotecan (SN-38; FOLFIRI) are widely used as first-line chemotherapy in the treatment of advanced CRC. However, second-line chemotherapy must be abandoned in certain cases due to disease progression, adverse effects or high medical cost in clinical setting. Firstline chemotherapy is usually administered over a long period of time. In addition, the response rate of the first-line chemotherapy is typically higher than the second-lines. Therefore, the most effective regimen should be selected as firstline chemotherapy. We have reported that individualization of first-line treatment (FOLFOX/FOLFIRI/Dual/Poor responder) was possible using CD-DST and individualized first-line therapy with the CD-DST may improve the prognosis of patients with unresectable CRC (UEGW: 2014; P1538, 2015; P1681, 2016; P0929). 
Aims \& Methods: In this prospective study, we evaluated the overall survival (OS) of appropriate first-line chemotherapy group and inappropriate first-line chemotherapy group based on CD-DST. Moreover, we evaluated additional effects of EGFR (Cetuximab; Cmab, Panitumumab; Pmab) to FOLFOX/FOLFIRI using CD-DST. Between Mar. 2008 and Aug. 2016, we obtained tumor specimens from 131 CRC patients without preoperative chemotherapy. Informed consent for measurement of individual chemosensitivity was obtained from all patients in writing. Approval for the present study was obtained from the Tobu Chiiki Hospital Institutional Review Board (No: 02.03.29. \#1). The growth inhibition rate (IR\%) was determined by CD-DST. The regimens were as follow: FOLFOX, FOLFIRI, Cmab, Pmab, and FOLFOX/FOLFIRI + Cmab. The incubation conditions were as follow: FOLFOX; 5-FU and 1-OHP (6.0 and $3.0 \mu \mathrm{g} / \mathrm{ml}$, respectively) for $24 \mathrm{~h}$. FOLFIRI; 5-FU and SN-38 (6.0 and $0.2 \mu \mathrm{g} /$ $\mathrm{ml}$, respectively) for $24 \mathrm{~h}$. Cmab; Cmab $250 \mu \mathrm{g} / \mathrm{ml}$ for $144 \mathrm{~h}$. Pmab; Pmab $200 \mu \mathrm{g} / \mathrm{ml}$ for $144 \mathrm{~h}$. FOLFOX $+\mathrm{Cmab} ; \mathrm{Cmab} 250 \mu \mathrm{g} / \mathrm{ml}$ for $120 \mathrm{~h}$ after FOLFOX/FOLFIRI incubation process. The cumulative distribution of IR values under each condition was evaluated on the basis that the clinical response to FOLFOX and FOLFIRI is equivalent (approximately 50\%). The OS between the group treated with appropriate first-line chemotherapy and the group treated with inappropriate first-line chemotherapy were evaluated Kaplan-Meier method. Additional effects of Cmab to FOLFOX/FOLFIRI were also evaluated. Results: There was strongly relationship between the IR\% of the FOLFOX and FOLFIRI regimen $\left(\mathrm{R}^{2}=0.7415\right)$. The median of the $\mathrm{IR} \%$ with the FOLFOX and FOLFIRI regimen were 58.6 and 69.1, respectively. FOLFOX responder, FOLFIRI responder, dual responder, and poor responder were $8,10,53$, and 60 , respectively. There were 42 unresectable CRC patients with chemotherapy. The median survival time of appropriate first-line chemotherapy group (n: 28) and inappropriate first-line chemotherapy group (n: 14) were 1128 and 506 days, respectively $(\mathrm{P}=0.01)$. There was positive relationship between the $\mathrm{IR} \%$ of $\mathrm{Cmab}$ and that of Pmab $\left(\mathrm{R}^{2}=0.468\right)$. Additional rates $(\%)$ of $\mathrm{Cmab}$ to FOLFOX between poor responder and other responder were 19.81 and 5.46, respectively $(\mathrm{P}=0.020)$. Additional rates of $\mathrm{Cmab}$ to FOLFIRI between poor responder and other responder were 16.50 and -1.29 , respectively $(P=0.005)$. There was significantly more additional effect of Cmab to FOLFOX/FOLFIRI in poor responder than in other responders.

Conclusion: Administration of the recommended first-line regimen using CDDST for unresectable CRC patient is important for improvement in the further prognosis. Moreover, especially in poor responder, Cmab should be administrated to FOLFOX/FOLFIRI regimen.

Disclosure of Interest: All authors have declared no conflicts of interest.

\section{Reference}

Ochiai T, Nishimura K, Watanabe T, et al: Individualized chemotherapy for colorectal cancer based on the collagen gel droplet-embedded drug sensitivity test. Oncol Lett 4: 621-624, 2012.

\section{P0467 WAIT-AND-SEE STRATEGY IN LOW RECTAL CANCER}

J. Cortez-Pinto ${ }^{1}$, R. Oom ${ }^{2}$, I. Rosa ${ }^{1}$, C. Travancinha ${ }^{3}$, G. Fernandez ${ }^{3}$, I. Marques ${ }^{1}$, I. Miguel ${ }^{4}$, J. Freire ${ }^{4}$, J. Pereira Da Silva ${ }^{1}$, J. Venâncio ${ }^{5}$, L. D' orey ${ }^{2}$, L. Mirones ${ }^{3}$, M. Limbert ${ }^{2}$, P. Chaves ${ }^{6}$, P. Pereira ${ }^{3}$, R. Fonseca ${ }^{6}$, R. Barroca ${ }^{2}$,

T. Ferreira ${ }^{7}$, T. Marques $^{8}$, A. Dias Pereira ${ }^{1}$

${ }^{1}$ Gastroenterology, IPOLFG, EPE, Lisboa/Portugal

${ }^{2}$ Surgery, IPOLFG, EPE, Lisboa/Portugal

${ }^{3}$ Radiotherapy, IPOLFG EPE, Lisboa/Portugal

${ }^{4}$ Oncology, IPOLFG EPE, Lisboa/Portugal

${ }^{5}$ Radiology, IPOLFG EPE, Lisboa/Portugal

${ }^{6}$ Pathology, IPOLFG EPE, Lisboa/Portugal

${ }^{7}$ Nuclear Medicine, IPOLFG EPE, Lisboa/Portugal

${ }^{8}$ IPOLFG EPE, Lisboa/Portugal

Contact E-mail Address: joao_cpinto@hotmail.com

Introduction: The standard treatment for locally advanced low rectal adenocarcinoma (ADC) is to conduct surgical resection after neoadjuvant chemoradiotherapy (CRT). In the wait-and-see $(W \& S)$ strategy, those who achieve clinical complete response (cCR) after CRT undergo regular clinical, radiologic and endoscopic surveillance, with surgery being reserved for tumor "regrowth", Aims \& Methods: To evaluate the impact of a $W \& S$ strategy for low rectal ADC, regarding overall and disease-free survival. Single-center prospective observational study. All patients with low rectal (up to $6 \mathrm{~cm}$ from the anal verge) ADC, stage I to III, discussed in a multidisciplinary colorectal cancer clinic since the implementation of the $W \& S$ strategy $(11 / 2014-11 / 2016)$ were evaluated. Demographic data, post-CRT evaluation and decision, recurrence rates, "regrowth" and mortality were analyzed.

Results: 56 patients were evaluated [ $54 \%$ males; mean age at diagnosis 64,4 years (34-90)], of which 53 had already completed CRT. Stage III prevailed $(79 \%)$ in the initial evaluation. From the 53 patients who completed CRT, $77 \%$ were clinically reevaluated and submitted to pelvic MRI and sigmoidoscopy, after a mean of 6.7 weeks. 17\% (7/41) displayed cCR and entered $W \& S$ surveillance program. In this subgroup, with a maximum follow-up of 25 months, there was only one $(14 \%)$ "regrowth", which was clinically detected and then confirmed by the other surveillance methods. This patient underwent a R0 low anterior resection and there were no complications. There were no distant recurrences or deaths. In the subgroup of patients with no cCR: pathologic complete response was observed in $20 \%$; there was one lymphatic recurrence; the overall survival was $95.2 \%$.
Conclusion: Preliminary results of our series confirm that the $W \& S$ strategy is associated with overall and disease-free survival not inferior to those of the traditional approach, favoring its implementation.

Disclosure of Interest: All authors have declared no conflicts of interest.

\section{P0468 LONG-TERM COLONOSCOPIC SURVEILLANCE BETWEEN PATIENTS AFTER SURGICAL RESECTIONS OF COLORECTAL INVASIVE CANCER AND THOSE AFTER ENDOSCOPIC RESECTIONS OF COLORECTAL ADENOMA AND INTRAMUCOSAL} CANCER

S. Kimura ${ }^{1}$, M. Tanaka ${ }^{2}$

${ }^{1}$ Gastroenterology And Endoscopy, Aomori Rousai Hospital, Hachinohe/Japan ${ }^{2}$ Pathology And Laboratory Medicine, Hirosaki Municipal Hospital, Hirosaki Japan

Contact E-mail Address: saint4road@gmail.com

Introduction: Patients after surgical resection of colorectal invasive cancer and those after endoscopic resections of colorectal adenoma and intramucosal cancer, both of them have the risk of metachronous advanced neoplasia during followup. The present study aimed to compare the risk of metachronous neoplasia during a long-term follow-up between patients with colorectal invasive cancer resected by surgery and those having colorectal adenoma and intramucosal cancer resected at initial colonoscopy.

Aims \& Methods: A total of 1078 patients were colonoscopically followed-up during a long-term period in our hospital. They were divided into group A, B, and $\mathrm{C}$ as follows; 445 in group $\mathrm{A}$ (mean age $64.7 \mathrm{yr}, \mathrm{M}: \mathrm{F}=2.37: 1$ ) with lowgrade adenoma colonoscopically resected at baseline, 245 in group B (66.1 yr, 2.31:1) with high-grade adenoma or intramucosal cancer colonoscopically resected at baseline, 388 in group C $(65.1 \mathrm{yr}, 1.54: 1)$ with invasive cancer resected by surgery at baseline. During follow-up colonoscopies detected metachronous neoplasms were resected and pathologically evaluated into non-index lesion (lowgrade adenoma) or index lesion (high-grade adenoma or cancer). The cumulative incidences of metachronous colorectal neoplasms were compared with each other group by Logrank test.

Results: Median follow-up periods and frequencies of colonoscopy were 64.3 months and 3.7 times in group A, 52.0 months and 3.5 times in group B, and 74.6 months and 3.9 times in group $\mathrm{C}$, respectively. The cumulative incidences of metachronous non-index lesion were 24.5\% (109 patients with 289 low-grade adenomas) in group A, 26.1\% (64 with 184) in group B, and $19.3 \%$ ( 75 with 229 ) in group $C$, respectively. The prevalence of metachronous non-index lesion was lower in group $\mathrm{C}$ compared to that in group $\mathrm{A}(\mathrm{p}=0.07)$, and group $\mathrm{B}$ $(\mathrm{p}<0.05)$. The cumulative incidences of metachronous index lesion were $7.0 \%$ (31 patients with 34 high-grade adenmas or cancers) in group A, 6.9\% (17 with 17 ) in group B, and $12.3 \%$ (48 with 55 ) in group $\mathrm{C}$, respectively. The prevalence of metachronous index lesion was higher in group $\mathrm{C}$ compared to that in group $\mathrm{A}$ $(\mathrm{p}<0.05)$ and group $\mathrm{B}(\mathrm{p}<0.05)$. The cumulative incidences of metachronous invasive cancer were $0.9 \%$ (4 patients with 4 invasive cancers) in group $\mathrm{A}, 1.2 \%$ (3 with 3 ) in group $\mathrm{B}$, and $3.6 \%$ (14 with 14) in group $\mathrm{C}$, disclosing highest prevalence in group $\mathrm{C}(\mathrm{p}<0.05)$. Logrank test revealed that the cumulative incidence of non-index lesion was lowest in group $\mathrm{C}$, and statistical significances were observed between group A and C $(\mathrm{p}<0.01)$, and between group B and C $(\mathrm{p}<0.001)$. Logrank test also revealed that the cumulative incidence of index lesion was highest in group $\mathrm{C}$, but no significant differences were observed compared to those in group A and B.

Conclusion: Significant higher prevalence of metachronous index lesion including invasive cancer and, in contrast, significant lower prevalence of metachronous non-index lesion were observed in patients after resections of colorectal invasive cancer compared to those after endoscopic resections of colorectal adenoma and intramucosal cancer.

Disclosure of Interest: All authors have declared no conflicts of interest.

\section{P0469 LONG-TERM COLONOSCOPIC SURVEILLANCE BETWEEN PATIENTS WITH UNRESECTED DIMINUTIVE POLYPS AND THOSE WITH COLORECTAL ADENOMAS > 5MM IN SIZE RESECTED AT INITIAL COLONOSCOPY}

S. Kimura ${ }^{1}$, M. Tanaka ${ }^{2}$

${ }^{1}$ Gastroenterology And Endoscopy, Aomori Rousai Hospital, Hachinohe/Japan ${ }^{2}$ Pathology And Laboratory Medicine, Hirosaki Municipal Hospital, Hirosaki Japan

Contact E-mail Address: saint4road@gmail.com

Introduction: A long-term risk of colorectal advanced neoplasia among patients having diminutive polyps at initial colonoscopy has been unknown. The present study aimed to compare the risk of metachronous advanced neoplasia during follow-up between patients with untreated diminutive colorectal polyps and those with small or large adenoma resected at baseline colonoscopy.

Aims \& Methods: A total of 1595 patients were colonoscopically followed-up during a long-term period in our hospital. They were divided into group A, B, and $\mathrm{C}$ as follows; 581 in group A (mean age $65.0+-8.9 \mathrm{yr}, \mathrm{M}: \mathrm{F}=411: 170$ ) with colorectal adenoma more than $5 \mathrm{~mm}$ in size resected at baseline, 495 in group B $(65.2+-9.6 \mathrm{yr}, 328: 167)$ with diminutive polyps left untreated at baseline, and 519 in group C $(62.5+-10.7 \mathrm{yr}, 255: 264)$ with no polyps at baseline. During follow-up colonoscopies detected metachronous neoplasms more than $5 \mathrm{~mm}$ in diameter were resected and pathologically evaluated into non-index lesion (lowgrade adenoma) or index lesion (high-grade adenoma or cancer). The cumulative 
incidences of metachronous colorectal neoplasms were compared with each other group by Logrank test.

Results: Median follow-up periods and frequencies of colonoscopy were 61.9 months and 3.6 times in group A, 61.6 months and 3.4 times in group B, and 72.3 months and 2.7 times in group $\mathrm{C}$, respectively. The cumulative incidences of metachronous non-index lesion were $24.1 \%$ (140 patients with 375 low-grade adenomas) in group A, $14.7 \%$ (73 with 168 ) in group B, and $6.6 \%$ (34 with 56 ) in group $\mathrm{C}$, respectively. The prevalence of metachronous non-index lesion was highest in group $\mathrm{A}$ followed by those in group $\mathrm{B}$ and $\mathrm{C}$, with significan differences between group A and B $(\mathrm{p}<0.0005)$, and B and C $(\mathrm{p}<0.0001)$. The cumulative incidences of metachronous index lesion were $7.2 \%$ (42 patients with 45 high-grade adenmas or cancers) in group A, 5.1\% (25 with 27) in group B, and $2.3 \%$ (12 with 13) in group $\mathrm{C}$, respectively. The prevalence of metachronous index lesion was highest in group $\mathrm{A}$ followed by those in group $\mathrm{B}$ and $\mathrm{C}$, and significant difference was observed between group $\mathrm{A}$ and $\mathrm{C}(\mathrm{p}<0.001)$, and $\mathrm{B}$ and $\mathrm{C}(\mathrm{p}<0.05)$. The cumulative incidences of metachronous invasive cancer were $1.0 \%$ (6 patients with 6 invasive cancers) in group A, 1.4\% (7 with 7) in group $\mathrm{B}$, and $0.2 \%$ (1 with 1 ) in group $\mathrm{C}$ with no significant difference. Logrank test revealed that the cumulative incidence of non-index lesion was highest in group A, and statistical significances were observed between group A and B $(\mathrm{p}<0.0001)$, and between group B and C $(\mathrm{p}<0.0001)$. Logrank test also revealed that the cumulative incidence of index lesion was highest in group $\mathrm{A}$, and statistical significances were observed between group A and B $(p<0.05)$, and between group B and C $(\mathrm{p}<0.005)$.

Conclusion: The results of a long-term colonoscopic follow-up disclosed a significantly higher prevalence of metachronous advanced neoplasms in patients with adenoma $>5 \mathrm{~mm}$ in size resected at baseline compared to those with diminutive polyps left untreated at baseline. Persons with no polyps at baseline colonoscopy were at very low risk of advanced neoplasia within five years during follow-up.

Disclosure of Interest: All authors have declared no conflicts of interest.

\section{P0470 A NEW SCORING MODEL FOR PREDICTING ADVANCED COLORECTAL NEOPLASIA IN ASYMPTOMATIC SCREENING POPULATION AND COMPARISON WITH THE MODIFIED ASIA- PACIFIC COLORECTAL SCREENING SCORE}

M. Sekiguchi ${ }^{1}$, T. Matsuda ${ }^{1}$, Y. Kakugawa ${ }^{2}$, M. Matsumoto ${ }^{2}$, Y. Saito ${ }^{2}$ ${ }^{1}$ Cancer Screening Center, National Cancer Center Hospital, Tokyo/Japan ${ }^{2}$ Endoscopy Division, National Cancer Center Hospital, Tokyo/Japan

\section{Contact E-mail Address: masekigu $@$ ncc.go.jp}

Introduction: Colorectal cancer (CRC) is still a major cause of death even in countries with a CRC screening program, indicating the need for improved screening methods. Risk-stratification of populations is one strategy that might satisfy this requirement. Currently, in the Asia-Pacific region, the use of the modified Asia-Pacific Colorectal Screening (APCS) score [age 50-59: 1 point, age $\geq 60: 2$, male sex: 1, presence of a first-degree relative (FDR) with CRC: 1 , current or past smoker: 1, body mass index $\left.(\mathrm{BMI}) \geq 23 \mathrm{~kg} / \mathrm{m}^{2}: 1\right]$ has been proposed for risk-stratification. ${ }^{1-4}$ However, further validation studies are required to appraise its usefulness, and considering the reported limited discriminatory capability of the score for advanced colorectal neoplasia (ACN), ${ }^{4}$ the development of a more useful scoring model is expected.

Aims \& Methods: The aim of this study was primarily to develop and validate a new scoring model for predicting ACN in asymptomatic screening populations that is more useful than the APCS score. We externally validated the APCS score in a Japanese screening population and compared its discriminatory capability with that of our new scoring model. Data were reviewed from 5218 consecutive asymptomatic screened individuals who underwent colonoscopy for their first time at the Cancer Screening Center, National Cancer Center Hospital, Tokyo between February 2004 and March 2013. Multivariate logistic regression was used to investigate the associations between clinical variables and the presence of ACN in the subjects, and then a new scoring model was developed based on these associations. Scores were weighted according to the beta coefficient obtained from the logistic regression model. Thereafter, the discriminatory capability of the new model was assessed using the c-statistics in the development set. Performance of the new model was internally validated using bootstrapping with 1000 replicates. The discriminatory capability of the modified APCS score in the 5218 subjects was also assessed using the c-statistics. The value obtained was compared with that of the new scoring model using the DeLong test, and this comparison was also performed in the 1000 bootstrapped replicates.

Results: A total of 225 individuals $(4.3 \%$ of 5218 subjects) had ACN ( $n=257)$, including 116 proximal lesions $(45.1 \%)$ and 76 lesions with flat-type morphology $(29.6 \%)$. An 8-point scoring model to predict ACN was developed by using five identified independent risk factors for ACN as scoring items. These included sex (male: 1 point, female: 0 ), age (40-49 years: 0, 50-59: 2, 60-69: 3, >70:3.5), CRC family history (presence of $\geq 2$ FDRs with CRC: 2 , others: 0$)$, BMI $(\leq 22.5$ : 0 , $>22.5: 0.5)$ and smoking ( $\leq 18.5$ pack-years: $0,>18.5: 1)$. Presence of one FDR with CRC was not detected as an independent risk factor for ACN and was not assigned any score. Using the scoring model, the proportion of subjects with $\mathrm{ACN}$ increased with the order of scores. The proportions were $1.6 \%(34 / 2172)$, $5.3 \%(127 / 2419)$ and $10.2 \%(64 / 627)$ in the groups with scores of $<3, \geq 3$ and $<5$, and $\geq 5$, respectively. The c-statistic of the score in the development set was $0.70(95 \%$ CI, $0.67-0.73)$ and this was the same in the internal validation set. The value of the modified APCS score was 0.68 (95\% CI, 0.65-0.71). The c-statistics of the new score were significantly higher than those of the modified APCS score, both in the 5,218 subjects $(P=0.03)$ and in 1,000 bootstrapped replicates $(P=0.03)$.
Conclusion: An 8-point scoring model to predict ACN in asymptomatic screening population that might have a higher discriminatory capability than the modified APCS score was developed and internally validated in this study. Our simple scoring model could stratify the screened population into low-, moderate-, and high-risk groups. Of the detected $\mathrm{ACN}$, a substantial number were proximal or flat; therefore, primary screening with total colonoscopy may be advisable for high-risk individuals.

Disclosure of Interest: All authors have declared no conflicts of interest.

\section{References}

1. Yeoh KG, et al; Asia-Pacific Working Group on Colorectal Cancer. Gut 2011; 60: 1236-41.

2. Sung JJ, et al; Asia Pacific Working Group. Gut. 2015; 64: 121-32.

3. Sung JJ, et al. Gastroenterology 2014; 146: S-730.

4. Wong MC, et al. Sci Rep 2016; 6: 20080.

\section{P0471 ASSOCIATION BETWEEN PARAMETERS OF THE RECTOANAL INHIBITORY REFLEX AND THRESHOLD FOR FIRST RECTAL SENSATION ESTABLISHED BY HIGH-RESOLUTION ANORECTAL MANOMETRY (HRAM) AND ITS SIGNIFICANCE FOR FECAL INCONTINECE DIAGNOSTICS}

O. Storonova ${ }^{1}$, A. Trukhmanov ${ }^{2}$, V. T. Ivashkin ${ }^{3}$

${ }^{1}$ Neurogastroenterology \&motility, Sechenov University, Moscow, Russian Federation, Moscow/Russian Federation

${ }^{2}$ Dept. Of Gastroenterology, Secretary General, Sechenov University, Moscow/ Russian Federation

${ }^{3}$ Dept. Of Gastroenterology, director Of The Clinic, Sechenov University, Moscow/ Russian Federation

Contact E-mail Address: storonova@yandex.ru

Introduction: Previous studies have shown that increase of threshold for first rectal sensation can be a predictor of fecal incontinence. However, significance of percentage range of rectoanal inhibitory reflex (\%RAIR) in development of this disease remains unknown.

Aims \& Methods: To determine association between \% RAIR and threshold for first rectal sensation in healthy adults and its significance in development of fecal incontinence. 26 asymptomatic healthy volunteers (18 women, 8 men) median age was 35.03 years (19-59) were studied. We performed them a high-resolution anorectal manometry (HRAM) using a 20 channels silicone water-perfused catheter (Solar GI, MMS, Netherlands). The following HRAM parameters were analyzed: threshold for RAIR and \%RAIR (automatically calculated as the ratio of the amplitude of the relaxation of the anal sphincter (AS) to the basal pressure AS * $100 \%$ ), threshold for first rectal sensation (RS) and for desire to defecate. The statistical analyses were performed using Statistica for Windows 6.0 (StatSoft Inc.)

Results: Threshold for RAIR and \%RAIR were $22.3 \mathrm{ml}(10.0 ; 30.0), 74.4 \%$ (38; 99.5) respectively. Threshold for first RS was $30,07 \mathrm{ml}(11.1 ; 58.3)$ and desire to defecate $-65.12 \mathrm{ml}(33.5 ; 182.0)$. Threshold for RAIR was not associated with threshold for first RS $(r=-0.07)$ and for desire to defecate $(r=0.02) . \%$ RAIR was weak positively correlated with threshold for first RS $(r=0.26)$ and was not associated with threshold for desire to defecate $(r=-0.03)$.

Conclusion: Threshold for RAIR and \%RAIR are not associated with first rectal sensation. So, these parameters of RAIR cannot be predictors of fecal incontinence.

Disclosure of Interest: All authors have declared no conflicts of interest.

P0472 AVOIDANT COPING AND SOMATIZATION PARTLY EXPLAIN THE RELATIONSHIP BETWEEN NEUROTICISM AND GASTROINTESTINAL SYMPTOM BURDEN

M.P. P. Jones ${ }^{1}$, A. P. Beath ${ }^{2}$, A. Ejova ${ }^{2}$, P. Mahoney ${ }^{2}$, J. M. Koch ${ }^{2}$

${ }^{1}$ Psychology, Macquarie University, North Ryde/Australia

${ }^{2}$ Psychology, Macquarie University, North Ryde/Australia/NSW

Contact E-mail Address: mike.jones@mq.edu.au

Introduction: Trait neuroticism has been consistently found to be associated with more severe functional gastrointestinal (GI) symptoms (1). One explanation for this is that high neuroticism predisposes individuals towards avoidant coping by leading them to view negatively-valenced situations as catastrophic (2) and, therefore, unsolvable (3). Avoidant coping, in turn, increases the risk of developing GI discomfort because over-reliance on "flight" strategies in stressful situations overstimulates the sympathetic nervous system at the expense of parasympathetic activity necessary for digestion (4). Evidence for this proposed chain of associations comes largely from studies that have observed relationships between each pair of the chain's components: neuroticism and avoidant coping (e.g., 3), and avoidant coping and higher GI symptom burden (e.g., 1). Across two studies, this paper integrates these findings, while also exploring the role of somatisation. Somatisation is consistently observed in people suffering from functional GI Conditions (e.g., 1) and refers to a subtype of avoidant coping the expression of psychological stress through complaints about bodily symptoms (6). Should evidence emerge that somatisation is an intermediary between avoidant coping and GI symptom burden in the relationship between neuroticism, coping styles and GI symptom burden, the dominant explanation for how avoidant coping and GI symptoms are connected would need to be expanded to 
predict that somatisation has deleterious consequences for GI conditions - possibly because it encourages reduced physical activity (7).

Aims \& Methods: In Study 1, 147 undergraduate students completed measures of neuroticism, 14 coping styles (including avoidant styles such as denial and disengagement), somatisation and GI symptom burden. In Study 2, where participants were undergraduates and hospital outpatients (pooled $\mathrm{N}=250$ ), the variables investigated in Study 1 were measured alongside hypochondriasis, which was included to measure the aspect of somatisation that involves worry independently of any actual physical symptoms. Statistical analysis was based on path modeling. It involved fitting a model to test a priori hypothesised indirect relationships between neuroticism and GI symptom severity via the selected coping styles and somatisation. Direct effects were also estimated, meaning that the path analysis provided information regarding the significance of any indirect effects once a range of direct effects were accounted for. Only six coping styles found to correlate with both neuroticism and GI symptom severity were included (see Results table). Coping styles were assumed to covary, and the model in Study 2 assumed a covariance relationship between somatisation and hypochondriasis.

\begin{tabular}{|c|c|c|c|c|}
\hline & $\begin{array}{l}\text { Study } 1 \text { direct } \\
\text { effects on row } \\
\text { variables }\end{array}$ & $\begin{array}{l}\text { Study } 1 \\
\text { indirect effects } \\
\text { on symptom } \\
\text { burden via } \\
\text { row variables }\end{array}$ & $\begin{array}{l}\text { Study } 2 \text { direct } \\
\text { effects on } \\
\text { row variables }\end{array}$ & $\begin{array}{l}\text { Study } 2 \\
\text { indirect effects } \\
\text { on symptom } \\
\text { burden via } \\
\text { row variables }\end{array}$ \\
\hline Self-distraction & $.33(.07)^{* * * *}$ & n.s. & $.22(.07)^{* * *}$ & n.s. \\
\hline Denial & $.24(.07)^{* * *}$ & n.s. & $.28(.05)^{* * *}$ & n.s. \\
\hline Venting & $.36(.07)^{* * *}$ & n.s. & $.32(.06)^{* * *}$ & n.s. \\
\hline Substance-use & $.40(.07)^{* * * *}$ & n.s. & $.19(.06)^{* *}$ & n.s. \\
\hline Disengagement & $.52(.06)^{* * *}$ & n.s. & $.55(.06)^{* * *}$ & n.s. \\
\hline Self-blame & $.53(.06)^{* * *}$ & n.s. & $.62(.04)^{* * *}$ & n.s. \\
\hline Somatisation & $.42(.09)^{* * *}$ & $.16(.05)^{* *}$ & $.40(.07)^{* * *}$ & $.17(.04)^{* * *}$ \\
\hline Hypochondriasis & Not applicable & Not applicable & $.30(.08)^{* * *}$ & $.08(.03)^{* *}$ \\
\hline Symptom burden & n.s. & Not applicable & n.s. & Not applicable \\
\hline $\begin{array}{l}\text { Substance-use and } \\
\text { somatisation }\end{array}$ & Not applicable & $.03(.02)^{*}$ & Not applicable & n.s. \\
\hline $\begin{array}{l}\text { Disengagement and } \\
\text { somatisation }\end{array}$ & Not applicable & $.04(.02)^{*}$ & Not applicable & n.s. \\
\hline $\begin{array}{l}\text { Self-blame and } \\
\text { somatisation }\end{array}$ & Not applicable & $-.05(.02)^{*}$ & Not applicable & n.s. \\
\hline Denial and somatisation & Not applicable & n.s. & Not applicable & $.02(.01)^{*}$ \\
\hline $\begin{array}{l}\text { Denial and } \\
\text { hypochondriasis }\end{array}$ & Not applicable & Not applicable & Not applicable & $.02(.01)^{*}$ \\
\hline
\end{tabular}

Results: Significant standardised path model coefficients involving neuroticism across the two studies. In Study 1, neuroticism exerted indirect effects on symptom burden through substance-use-based coping and somatisation, as well as through disengagement-based coping and somatisation. In Study 2, neuroticism affected GI symptom burden through denial-based coping and somatisation, as well as through denial-based coping and hypochondriasis. An indirect effect of neuroticism through self-blame and somatisation, with the two intermediary variables relating negatively to each other, was observed in Study 1. (Note: $* * * \mathrm{p}<.001, * * \mathrm{p}<.01, * \mathrm{p}<.05$. n.s. denotes non-significant coefficients). Conclusion: Somatisation and hypochondriasis were found to be intermediaries in the relationship between neuroticism, avoidant coping (through substance-use, disengagement and denial) and GI symptom burden. Two interpretations of the findings are: (1) avoidant coping can stimulate somatisation, leading to reduced physical activity, which can interfere with digestion; and (2) GI symptoms are among the wide range of functional somatic symptoms that can arise from avoidant coping. These findings open new avenues for multidisciplinary treatment of FGIDs.

Disclosure of Interest: All authors have declared no conflicts of interest.

\section{References}

1 Koloski et al. 2006. Eur J Gastroenterol Hepatol, p1101

2 Affleck et al. 1992. J Consult Clin Psychol, p119

3 Coen et al. 2011. Gastroenterology, p909

4 Van Oudenhove \& Aziz 2009. Dig Liver Dis, p781

5 Jones et al. 2006. Clin Gastroenterol Hepatol, p474

6 Kroenke et al. 2002. Psychosom Med, p258

7 Daley et al. 2008. Int J Sports Med, p778

\section{P0473 POTENTIAL REGULATORY EFFECTS OF CORTICOTROPIN-RELEASING FACTOR ON TIGHT JUNCTION- RELATED INTESTINAL EPITHELIAL PERMEABILITY ARE PARTIALLY MEDIATED THROUGH CK8 UPREGULATION}

Y. $\mathrm{Hu}^{1}, \mathrm{~B} \cdot \mathrm{Lv}^{1}$

${ }^{1}$ Gastroenterology, First Affiliated Hospital of Zhejiang Chinese Medical University, Hangzhou/China

Contact E-mail Address: jiuyueqingxuan@163.com

Introduction: This study aimed to investigate the regulatory effects of corticotropin-releasing factor (CRF) on the permeability of human intestinal epithelial cells through CK8-mediated tight junction.
Aims \& Methods: The expression of CRFR1 and CRFR2 on HT29 cell surfaces were detected by immunofluorescence, RT-PCR, and Western blotting. After treatment with $100 \mathrm{nM}$ CRF for $72 \mathrm{~h}$, the transmission of FITC-labeled Dextran was measured by using a transwell chamber; the structural changes of tight junctions were observed under transmission electron microscopy; the expression of $\mathrm{CK} 8$, F-actin and tight junction proteins $\mathrm{ZO}-1$, claudin-1, and occludin were detected by immunoblotting and immunofluorescence. The activity of RhoA was detected by immunoprecipitation. Furthermore, effects of CRF on intestinal epithelial permeability were examined in CK8-silenced HT29 cells, which were constructed by shRNA interference.

Results: CRF treatment increased FITC-labeled Dextran permeability, caused opening of tight junctions, induced increased fluorescence intensity of CK8 and decreased intensity of ZO-1, claudin-1, and occluding, together with structural disruption. The expression of F-actin, occludin, claudin-1, and ZO-1 were downregulated. RhoA activity peaked at $30 \mathrm{~min}$ after CRF treatment. The increased permeability and the downregulation of claudin-1 and occludin induced by CRF treatment were not blocked by CK 8 silencing. Nevertheless, CK8 silencing blocked the effects of CRF with regard to decrease in the expression of F-action and ZO-1 and increase in RhoA activity.

Conclusion: CRF may increase intestinal epithelial permeability by upregulating CK8 expression, activating the RhoA signaling pathway, promoting intestinal epithelial actin remodeling, and decreasing the expression of the tight junction protein ZO-1. Other CK8-independent pathways may lead to decreased expression of claudin-1 and occludin, which also contributes to increased intestinal epithelial permeability.

Disclosure of Interest: All authors have declared no conflicts of interest.

P0474 REGULATING EFFECTS OF TONGXIE-YAOFANG FORMULA ON COLONIC EPITHELIAL SECRETION IN RATS WITH DIARREHEA-PREDOMINANT IRRITABLE BOWEL SYNDROME

Y. Cheng ${ }^{1}$, Z. Qiang ${ }^{1}$, Z. Q. Lin ${ }^{1}$, S. Jing ${ }^{1}$, L. X. Ling ${ }^{2}$

${ }^{1}$ Digestive Medicine, Wuxi People's Hospital, wu xi/China

${ }^{2}$ Digestive Medicine, Beijing Hospital of TCM, Beijing/China

Contact E-mail Address: zhanq33@163.com

Introduction: Diarrhea-predominant irritable bowel syndrome (D-IBS) is a chronic functional gastrointestinal disease. Its clinical manifestations are characterized by diarrhea and abdominal pain or discomfort in the absence of a demonstrable pathology. The diagnosis of D-IBS is based on symptom assessment and the Rome III Diagnostic Criteria. According to an epidemiological study, D-IBS mainly affects young adults of 20-40 years old, and the quality of their lives is seriously affected. The pathogenesis of D-IBS has not been fully clarified. Consequently, the usual treatment of the disease in Western medicine involves symptomatic therapy, which is unsatisfactory for patients while simultaneously increasing the use of health-care resources. Because traditional Chinese medicine (TCM) can significantly improve patients' symptoms and quality of life, increasing numbers of patients have begun to seek treatment with TCM.A series of randomized, double-blind, placebocontrolled trials had shown that TongXieYaoFang(TXYF) formula can significantly improve the clinical symptoms, such as diarrhea and abdominal pain or discomfort, of patients with D-IBS and improve the quality of their lives. However, the specific mechanism of it has not been completely elaborated. The purpose of this paper is to observe the regulating effects of TXYF-formula on colonic epithelial secretion via relevant ion channels.

Aims \& Methods: We aimed to investigate the pharmacological effect of TongXie-YaoFang (TXYF) formula, a Chinese herbal formula, on Diarrheapredominant irritable bowel syndrome (D-IBS) rats. In a neonatal maternal separation plus restraint stress(NMS + RS) model of D-IBS, male Sprague Dawley rats were randomly divided into two groups (NMS + RS group and TXYF-formula group) with no handlings were used as controls (NH group). Starting from postnatal day 60, rats in TXYF-formula group were administered TXYF-formula ( $4.92 \mathrm{~g} / 100 \mathrm{~g}$ bodyweight) orally twice a day for 14 consecutive days while NH group and NMS + RS group were given distilled water. Using short-circuit current technology, we observed 5-HT-induced changes of current across ion channels, such as cystic fibrosis transmembrane conductance regulator (CFTR) $\mathrm{Cl}^{-}$channel, epithelial $\mathrm{Na}^{+}$channel $(\mathrm{ENaC}), \mathrm{Ca}^{2+}$-dependent $\mathrm{Cl}^{-}$channel (CACC), $\mathrm{Na}^{+}-\mathrm{K}^{+}-2 \mathrm{Cl}^{-}$co-transporter (NKCC), and $\mathrm{Na}^{+}-\mathrm{HCO}_{3}{ }^{-}$ co-transporter (NBC), in the colonic epithelium of three groups after exposure to drugs and specific blockers with a Power Lab System (AD Instruments International).

Results: Under basal conditions, the changes of short-circuit current (DIsc, mA/ $\mathrm{cm}^{2}$ ) induced by 5-HT were similar in NH group and TXYF-formula group, and both higher than NMS + RS group $\left(70.86 \pm 12.32 \mathrm{~mA} / \mathrm{cm}^{2}, 67.67 \pm 11.68 \mathrm{~mA} / \mathrm{cm}^{2}\right.$ VS $38.8 \pm 7.25 \mathrm{~mA} / \mathrm{cm}^{2}, \mathrm{P}<0.01$, respectively). When CACC was blocked by DIDS, 5-HT-induced DIsc was smaller in NMS + RS group than in NH group and TXYF-formula group, respectively $\left(48.41 \pm 13.15 \mathrm{~mA} / \mathrm{cm}^{2}\right.$ VS $74.62 \pm 10.73 \mathrm{~mA} / \mathrm{cm}^{2}, 69.22 \pm 11.7 \mathrm{~mA} / \mathrm{cm}^{2}, \mathrm{P}<0.05$, respectively). The similar result could be obtained when $\mathrm{ENaC}$ was blocked by Amiloride $\left(44.69 \pm 12.58 \mathrm{~mA} / \mathrm{cm}^{2} \quad\right.$ VS $\quad 62.05 \pm 11.26 \mathrm{~mA} / \mathrm{cm}^{2}, \quad 62.11 \pm 12.01 \mathrm{~mA} / \mathrm{cm}^{2}$, $\mathrm{P}<0.05$, respectively). However, when CFTR $\mathrm{Cl}^{-}$channel was blocked by DPC, 5-HT-induced DIsc did not significantly differ in three groups $\left(42.28 \pm 10.61 \mathrm{~mA} / \mathrm{cm}^{2}\right.$ VS $51.48 \pm 6.56 \mathrm{~mA} / \mathrm{cm}^{2}$ VS $47.75 \pm 7.99 \mathrm{~mA} / \mathrm{cm}^{2}$, $\mathrm{P}>0.05$, respectively). The similar results could also be obtained in three groups when NBC and NKCC were respectively blocked by their blockers. Conclusion: TXYF-formula can regulate the $\mathrm{Cl}^{-}$and $\mathrm{HCO}_{3}{ }^{-}$secretion of colonic mucosa via CFTR $\mathrm{Cl}^{-}$channel, $\mathrm{Cl}^{-} / \mathrm{HCO}_{3}^{-}$exchanger, $\mathrm{NBC}$ and $\mathrm{NKCC}$ cotransporters.

Disclosure of Interest: All authors have declared no conflicts of interest. 


\section{References}

1 Ford AC, Bercik P, Morgan DG, et al. Validation of the Rome III criteria for the diagnosis of irritable bowel syndrome in secondary care. Gastroenterology 2013;145: 1262-1270

2 Shalaby SA, Sayed MM, Ibrahim WA, et al. The prevalence of coeliac disease in patients fulfilling Rome III criteria for irritable bowel syndrome. Arab $J$ Gastroenterol 2016; S1687-1979: 30028-4

3 Marquis P, Lasch KE, Delgado-Herrera L, et al. Qualitative development of a patient-reported outcome symptom measure in diarrhea-predominant irritable bowel syndrome. Clin Transl Gastroenterol 2014; 5:e59

\section{P0475 DIOSMECTITE CHRONIC TREATMENT SUPPRESSES GUT VISCERAL HYPERSENSITIVITY AND INTESTINAL TRANSIT ACCELERATION INDUCED BY CHRONIC STRESS IN RAT}

H. Eutamene ${ }^{1}$, C. Beaufrand ${ }^{1}$, Mathiex-FortunetH. ${ }^{2}$, V. Theodorou ${ }^{1}$

${ }^{1}$ Unité De Neuro-gastroenterologie \& Nutrition, Toxalim UMR 1331 INRA/UPS

INPT-EI-Purpan, Toulouse/France

${ }^{2}$ IPSEN Pharma, Boulogne Billancourt/France

\section{Contact E-mail Address: helene.eutamene@inra.fr}

Introduction: Stressful life events may trigger the symptoms of irritable bowel syndrome (IBS). Preclinical chronic stress models have been developed in animals to mimic changes in visceral sensitivity in response to gut wall distension seen in IBS patients. In the rat, chronic passive water avoidance stress (WAS) is associated with hypersensitivity to colorectal distension. Diosmectite, a purified silicate clay, is an adsorbent widely used for the treatment of several gastrointestinal diseases, mainly diarrhoea but also the functional abdominal pain experienced in chronic IBS. However, the effect of diosmectite treatment on IBS visceral hypersensitivity has never been investigated.

Aims \& Methods: The aims of the present study were to evaluate the effect of diosmectite on gut transit time and visceral hypersensitivity induced by WAS in rats. Wistar rats $(175-200 \mathrm{~g})(\mathrm{n}=9-10)$ were randomized to one of four groups: diosmectite $(500 \mathrm{mg} / \mathrm{kg})$, diosmectite $(500 \mathrm{mg} / \mathrm{kg}$, WAS procedure), water $(0.5 \mathrm{ml} /$ rat) or water $(0.5 \mathrm{ml} / \mathrm{rat}$, WAS procedure). Treatment was for 5 days, with the WAS procedure conducted once daily. The test apparatus consisted of a Plexiglas tank with a block affixed to the center of the floor. The tank was filled with fresh room temperature water to within $1 \mathrm{~cm}$ of the top of the block. Rats were placed on the block for a period of $1 \mathrm{~h}$ every day. For both conditions (basal and after WAS), intestinal transit was evaluated by fecal output measurement Visceral sensitivity in response to colorectal distension (CRD) was assessed both in basal conditions and $30 \mathrm{~min}$ after the last WAS session.

Results: Under basal conditions, chronic oral treatment with diosmectite did not modify visceral sensitivity in response to CRD $(20 \pm 2$ vs. $23 \pm 1 \mathrm{cramps} / 5 \mathrm{~min}$ for vehicle at $0.8 \mathrm{~mL} ; 20 \pm 4$ vs. $24 \pm 2$ for vehicle at $1.2 \mathrm{~mL} ; \mathrm{p}=0.97$ and $\mathrm{p}=0.75$ respectively) or intestinal transit in comparison with control group $(\mathrm{p}=0.33)$. WAS treatment significantly increased the number of abdominal contractions at both 0.8 and $1.2 \mathrm{ml}$ of CRD $v s$ vehicle values $(30.1 \pm 2.5$ vs. $19.78 \pm 2.8$ at $0.8 \mathrm{~mL}$ [p $<0.05$ ]; $34.4 \pm 2.4$ vs. $23.2 \pm 1.1$ at $1.2 \mathrm{~mL}[\mathrm{p}<0.05]$ ). One hour after the beginning of the last WAS session a significant increase of the fecal output in comparison with vehicle non-stressed rat $(6.3 \pm 1.1$ vs. $0.3 \pm 0.3 ; p<0.05)$ was observed. Chronic administration of diosmectite significantly $(\mathrm{p}<0.05)$ reduced the number of abdominal contractions (a visceral sensitivity index) induced by WAS: the number of abdominal contractions at 0.8 and $1.2 \mathrm{ml}$ of CRD in comparison with vehicle was $(30 \pm 3$ vs. $24 \pm 2$ contractions at $0.8 \mathrm{~mL} ; 34 \pm 2$ vs. $27 \pm 2$ contractions at $1.2 \mathrm{~mL}$ ). Diosmectite also tended to improve stress-induced intestinal transit acceleration $(6.3 \pm 1.1$ vs $4.9 \pm 1.2$ number of feces for $1 \mathrm{~h}$; $\mathrm{p}=0.38$ ).

Conclusion: For the first time, these data illustrate in wistar rat, that diosmectite treatment is able to suppress WAS-induced visceral hypersensitivity to colorecta distension. This study adds relevant evidence to the use of diosmectite treatment in the management of IBS

Disclosure of Interest: H. Mathiex-Fortunet: Ipsen employee

All other authors have declared no conflicts of interest.

P0476 METABOLOMIC SIGNATURE OF THE POSTPRANDIAL

\section{EXPERIENCE}

C. Malagelada ${ }^{1}$, T. Pribic ${ }^{1}$, N. Cañellas ${ }^{2}$, J. Gomez $^{2}$, N. Amigo ${ }^{2}$, B. Ciccantelli ${ }^{1}$, A. Accarino ${ }^{1}$, J.R. Malagelada ${ }^{1}$, F. Azpiroz

${ }^{1}$ Digestive And Liver Diseases, Hospital Vall d'Hebron, Barcelona/Spain

${ }^{2}$ Metabolomics Platform, Iispv, Universitat Rovira i Virgili, Tarragona/Spain

Contact E-mail Address: cmalagelada@gmail.com

Introduction: We have recently shown that postprandial sensations correlate with changes in circulating metabolites after a meal ingestion; however this phenomenon was demonstrated with a meal load up to the level of tolerance which involved an unpleasant fullness sensation.

Aims \& Methods: In the present study we aimed to evaluate the relation between the hedonic and the metabolomic responses to a palatable meal. Healthy men $(\mathrm{n}=32 ; 18-44$ yrs range) were evaluated after a $5 \mathrm{~h}$ fast. Perception measurements and blood samples were taken before and 20 minutes after ingestion of a palatable probe meal (juice and warm ham and cheese sandwich, total $300 \mathrm{ml}$, $440 \mathrm{kcal}$ ). Homeostatic (satiety, desire of eating a food of choice and abdominal fullness) and hedonic sensations (digestive well-being and mood) were measured by $10 \mathrm{~cm}$ analogical scales. NMR spectroscopy was performed to determine plasmatic Low Molecular Weight Metabolites (LMWM) and to characterize lipoprotein profiles (number of particles, lipid content and size of the main three lipoprotein classes (VLDL, LDL and HDL)).

Results: Meal ingestion induced a pleasant sensation involving mild fullness $(2.7 \pm 0.4$ score increment; $p<0.001$ vs baseline $)$ and an increase in digestive well-being and mood $(3.1 \pm 0.3$ and $2.0 \pm 0.3$ score increment respectively; $\mathrm{p}<0.001$ vs baseline). Distinctive changes in the spectrum of circulating metabolites induced by meal ingestion were detected. A non-supervised PCA model of the metabolomic response correctly discriminated between the pre and postprandial state. The main variables determining the differences were glucose, triglycerides, number of VLDL, HDL and LDL particles, alanine, isoleucine, acetate, formate, creatine and lactate. The increase in the sensation of fullness correlated with the levels of glucose, alanine and lactate $(R=0.44 ; p=0.011$ and $R=0.37$; $\mathrm{p}=0.036$ and $\mathrm{R}=0.44 ; \mathrm{p}=0.008$ respectively). Desire of eating a food of choice decreased after meal ingestion and correlated with the increase in HDL-triglycerides $(\mathrm{R}=0.35 ; \mathrm{p}=0.047)$. The increase in the concentration of VLDL- triglycerides correlated with the increase in sensation of digestive wellbeing $(R=0.36$; $\mathrm{p}=0.044$ ).

Conclusion: Homeostatic and hedonic sensations in response to a pleasant meal correlate with changes in circulating metabolites. Metabolomic changes in the response to a meal could serve as objective biomarkers of the postprandial experience.

Disclosure of Interest: All authors have declared no conflicts of interest.

\section{P0477 NEGATIVE EFFECTS OF BIFIDOBACTERIUM BIFIDUS ON THE RAT WITH COLONIC VISCERAL HYPERSENSITIVITY INDUCED BY ACETIC ACID PERFUSION}

X. Wang, W. Zhang, K. Wang, L. Zhang, Q. Sun, L. Duan

Department Of Gastroenterology, Peking University Third Hospital, Beijing/China

Contact E-mail Address: 15035126210@163.com

Introduction: Bifidobacterium with appropriate doses has been suggested to reduce the visceral hypersensitivity in IBS. But different treatment effects have been reported. The negative effect of Bifidobacterium has been rarely studied and reported.

Aims \& Methods: We aimed to study the effects of gavage administration with Bifidobacterium bifidus for two weeks on the visceral hypersensitivity of rats. Colonic visceral hypersensitivity $(\mathrm{CVH})$ was induced by colonic injection of $0.5 \%$ acetic acid (AA) in 10-day old rats while control (NS) induced with $0.9 \%$ normal saline. The abdominal withdrawal reflexes (AWR), induced by colorectal distention (CRD), was used to quantify the level of colonic sensitivity in adult rats. The $\mathrm{CVH}$ rats in 42-day old were treated by gavage administration with Bifidobacterium bifidus $\left(1 * 10^{9} \mathrm{CFU} /\right.$ day) for two weeks (CVH-Bifi). Other $\mathrm{CVH}$ rats were treated with $0.9 \% \mathrm{NaCL}$ (CVH-NS). A group of control with normal sensitivity was treated with sham gavage (Con-sham). In day $56^{\text {th }}$, another AWR was assessed, and the hippocampus and prefrontal cortex (PFC) were separated and used to analyze the c-fos, NMDAR 2A, NMDAR2B with western-blot.

Results: After two-week gavage, the CVH-Bifi presented lower volume than that of $\mathrm{CVH}-\mathrm{NS}$ in $\mathrm{CRD}$, though without statistical difference $(2.35 \pm 0.28$ vs. $2.40 \pm 0.64, p=0.11$ ). No significant difference was found between $\mathrm{CVH}$ Bifi and Con-sham as well. In hippocampus, c-fos of CVH-Bifi was higher than that of Con-sham $(0.77 \pm 0.23$ vs. $0.31 \pm 0.08, \mathrm{p}=0.032)$ and $\mathrm{CVH}-\mathrm{NS}$ $(0.77 \pm 0.23$ vs. $0.48 \pm 010, p=0.171)$. The NMDAR2A of CVH-Bifi was higher than that of Con-sham $(1.04 \pm 0.22$ vs. $0.51 \pm 0.16, \mathrm{p}=0.055)$. In PFC, the NMDAR2A in CVH-Bifi was significantly higher than that of CVH-NS $(0.63 \pm 0.14$ vs. $0.21 \pm 0.05, \mathrm{p}=0.004)$ and Con-sham $(0.63 \pm 0.14$ vs. $0.20 \pm$ $0.07, \mathrm{p}=0.011)$

Conclusion: We reported the negative effects of Bifidobacterium bifidus gavage, which induced higher activation of c-fos and higher expression of NMDAR 2A in hippocampus and PFC. The roles of Bifidobacterium bifidus and its metabolites on visceral sensitivity needs further study to clarify.

Disclosure of Interest: All authors have declared no conflicts of interest.

\section{P0482 DA-9701 IMPROVES COLONIC TRANSIT TIME AND SYMPTOMS IN PATIENTS WITH FUNCTIONAL CONSTIPATION: A PROSPECTIVE STUDY}

S. Kim, H.S. Woo, S.H. Choi, D.K. Park, K.A. Kwon, J. Chung, Y.J. Kim, J.H. Kim, S.J. Kim, K.O. Kim

Dept. Of Gastroenterology, Gachon University Gil Hospital, Incheon/Korea,

Republic of

Contact E-mail Address: gesung11@naver.com

Introduction: DA-9701, a newly developed prokinetic agent formulated with Pharbitis Semen and Corydalis Tuber, has been shown to effectively treat functional dyspepsia. Recently, it has also been suspected to improve gastrointestinal motor function.

Aims \& Methods: The aims of this study were to assess the effect of DA-9701 on colonic transit time (CTT) and symptoms of functional constipation. We prospectively enrolled 33 patients with functional constipation based on the Rome III criteria. The patients received $30 \mathrm{mg}$ DA-9701 three times a day for 24 days. CTT was estimated initially and at the end of treatment. We also analyzed symptoms such as spontaneous bowel movements (SBMs), straining, stool form, feeling of incomplete emptying and anorectal blockage, abdominal discomfort and pain, overall defecation satisfaction, and incidence of adverse events. 
Results: Twenty-seven patients completed the study. DA-9701 was associated with a significantly reduced CTT from $34.9 \pm 17.6$ to $23.7 \pm 19.1$ hours $(P=0.001)$. Segmental CTT also significantly decreased after treatment (right CTT: from $14.0 \pm 8.2$ to $7.5 \pm 7.4$ hours, $P<0.001$; rectosigmoid transit time: from $14.2 \pm 11.9$ to $9.5 \pm 10.9$ hours, $P=0.021$ ). In addition, all constipationrelated subjective symptoms, including SBM frequency, significantly improved compared to those before treatment. Serious adverse events did not occur. Conclusion: DA-9701 accelerates colonic transit and safely improves symptoms in patients with functional constipation. Therefore, we suggest that this novel agent could help to treat patients with this condition.

Disclosure of Interest: All authors have declared no conflicts of interest.

\section{References}

1. Talley NJ, Weaver AL, Zinsmeister AR, Melton LJ, 3rd. Functional constipation and outlet delay: a population-based study. Gastroenterology. 1993; 105: 781-90.

2. Emmanuel A, Cools M, Vandeplassche L, Kerstens R. Prucalopride improves bowel function and colonic transit time in patients with chronic constipation: an integrated analysis. Am J Gastroenterol 2014; 109: 887-94.

3. Kwon YS, Son M. DA-9701: A New Multi-Acting Drug for the Treatment of Functional Dyspepsia. Biomol Ther (Seoul) 2013; 21: 181-9.

4. Hussain Z, Rhee KW, Lee YJ, Park H. The Effect of DA-9701 in Opioidinduced Bowel Dysfunction of Guinea Pig. J Neurogastroenterol Motil 2016; 22: 529-38.

5. Lee SP, Lee OY, Lee KN, Lee HL, Choi HS, Yoon BC, et al. Effect of DA9701, a Novel Prokinetic Agent, on Post-operative Ileus in Rats. $J$ Neurogastroenterol Motil 2017; 23: 109-116.

\section{P0483 INDEPENDENT VALIDATION OF THE ROME IV CRITERIA FOR IRRITABLE BOWEL SYNDROME REVEALS THEIR MODEST PERFORMANCE AND RESTRICTIVE NATURE}

\section{O. Craig ${ }^{1}$, C. Black ${ }^{1}$, L. Houghton ${ }^{2}$, A.C. Ford ${ }^{1}$}

${ }^{1}$ Leeds Gastroenterology Institute, St. James's University Hospital, Leeds/United Kingdom

${ }^{2}$ Leeds Institute Of Biomedical \& Clinical Sciences, St. James's University

Hospital, Leeds/United Kingdom

\section{Contact E-mail Address: ofcraig@gmail.com}

Introduction: Previous symptom-based criteria to diagnose irritable bowel syndrome (IBS) performed only modestly. The Rome IV criteria are the current gold-standard, and dispensed with the symptom of abdominal discomfort, as well as making slight modifications to the symptom frequency threshold and associated symptoms required for a diagnosis of IBS. Epidemiological surveys suggest that these modifications reduce the overall prevalence of IBS in the community, but the effect on their performance in referral populations is uncertain, as independent validation studies are lacking.

Aims \& Methods: We collected complete symptom data from consecutive, unselected patients referred to secondary care with lower GI symptoms, and seen in a specialist IBS clinic. All participants completed the validated Rome IV questionnaire for IBS, and underwent investigation to the level deemed appropriate by two clinicians with considerable experience in diagnosing and managing IBS. The final clinical diagnosis, after a limited panel of investigations, was recorded. Coeliac disease was excluded in all individuals, via serological testing. The reference standard used to define the presence of true IBS was a physician's clinical diagnosis of IBS, after appropriate limited investigation to exclude relevant organic disease. Sensitivity, specificity, and positive and negative likelihood ratios (LRs) were calculated for the Rome IV criteria.

Results: We recruited 104 adults referred with lower GI symptoms (81 (77.9\%) female, mean age 35.8 years (range 16 to 77 years)). Among 91 individuals with IBS according to the reference standard, 72 met the Rome IV criteria (sensitivity $=79.1 \%$ ), and 19 did not. There were 13 individuals who did not have IBS according to the reference standard, of whom 11 did not meet Rome IV criteria (specificity $=84.6 \%$ ). The other two individuals who met Rome IV criteria, but who did not have IBS after appropriate investigation to exclude relevant organic disease, both had bile acid diarrhoea. Positive and negative LRs for the Rome IV criteria were 5.14 and 0.25 respectively. Among the 19 patients who had IBS according to the reference standard, but who did not meet the Rome IV criteria for IBS, seven $(36.8 \%)$ did not meet the minimum symptom duration of 6 months, six $(31.6 \%)$ did not meet the minimum pain frequency threshold of once per week, and six $(31.6 \%)$ did not report two or more of pain in relation to defaecation, pain in association with a change in stool frequency, or pain in association with a change in stool form.

Conclusion: The Rome IV criteria performed modestly in a specialist IBS clinic. One-in-five patients felt to have IBS according to an experienced clinician, and after limited investigation to exclude relevant organic disease, did not meet the Rome IV criteria, suggesting they may be too restrictive for use in clinical practice.

Disclosure of Interest: All authors have declared no conflicts of interest.
P0484 WHAT DETERMINES WHETHER INDIVIDUALS WITH IRRITABLE BOWEL SYNDROME IN THE GENERAL POPULATION SEEK MEDICAL CARE FOR THEIR DISORDER?

N. Jossan ${ }^{1}$, H. Törnblom ${ }^{2}$, M. Simrén ${ }^{3}$, I. Aziz ${ }^{1}$, O.S. Palsson ${ }^{4}$, A. Sperber ${ }^{5}$ W.E. Whitehead ${ }^{6}$

${ }^{1}$ Department Of Internal Medicine And Clinical Nutrition, Institute of Medicine, Sahlgrenska University hospital, Gothenburg/Sweden

${ }^{2}$ Department Of Internal Medicine And Clinical Nutrition, Sahlgrenska Academy, University of Gothenburg, Gothenburg/Sweden

${ }^{3}$ Dept Of Internal Medicine, Sahlgrenska University Hospital - Dept of Internal Medicine, Sahlgrenska University Hospital; Gothe, Gothenburg/Sweden

${ }^{4}$ Dept. Of Medicine, University of North Carolina, Chapel Hill, Chapel Hill, NC/ United States of America/NC

${ }^{5}$ Ben-gurion University Of The Negev, Faculty of Health Sciences, Beer-Sheval Israel

${ }^{6}$ Dept. Of Medicine, University of North Carolina at Chapel Hill, Chapel Hill, NC/ United States of America

Contact E-mail Address: navkiran.jossan@gu.se

Introduction: No studies have investigated how commonly individuals meeting the new Rome IV IBS criteria seek medical care for their bowel symptoms, or what factors determine medical consultation.

Aims \& Methods: We aimed to characterize health care-seeking behaviour in Rome IV IBS subjects in a large multi-national population sample. Data was retrieved from a large Internet survey. The survey was completed by 6300 individuals distributed equally between United States, United Kingdom and Canada. Equal sex, age and education distribution across the countries was ensured by use of quota-based sampling. The survey included questions on demographics, the Rome IV diagnostic questionnaire, the Patient Health Questionnaire (PHQ-12), the 8-item Short Form (SF-8) quality of life (QOL) questionnaire, health care utilization and past gastrointestinal (GI) disease diagnoses by doctors. Respondents with an organic GI disease were excluded from the IBS population. IBS consulters were defined as individuals meeting Rome IV IBS criteria who had visited a doctor for GI symptoms.

Results: 6300 individuals completed the survey, 369 were excluded due to inconsistent responses, leaving 5931 (49.2\% female; mean age $47.4 \pm 17.1$ years) to be included for analysis (1949 US, 1994 UK, 1988 Canada). After excluding 36 individuals due to lower GI organic disease, 305 subjects $(5.1 \%$; $66 \%$ female; mean-age $44.7 \pm 14.5$ years) fulfilled diagnostic criteria for IBS. From these, 195 $(64 \%)$ had consulted a doctor for GI problems. IBS consulters had equal sex distribution $(63.6 \%$ vs. $69 \%$ female $(\mathrm{p}=0.4)$ and somatization scores $(\mathrm{p}=0.8)$ compared to non-consulters, but were older (mean age $47.1 \pm 14.8$ vs. $40.5 \pm 13.1$ years), more concerned about their bowel function $(\mathrm{p}<0.001)$, more frequently bloated $(\mathrm{p}=0.01)$, and experienced greater impact on social activities $(\mathrm{p}=0.008)$. The distribution of the most bothersome symptom was similar $(\mathrm{p}=0.38)$, and abdominal pain was the predominant symptom in both groups. See table for details. The frequency of doctor visits for non-GI health issues did not differ $(p=0.15)$, but IBS consulters had undergone more abdominal surgery $(p=0.04)$. IBS consulters also reported higher consumption of GI related $(\mathrm{p}<0.001)$, prescribed pain $(p<0.001)$, and anti-depressive medications $(p=0.03)$, but had similar consumption of anxiety $(\mathrm{p}=0.11)$ and over the counter pain medications $(p=0.34)$ as non-consulters. See table for details.

IBS consulters IBS non-consulters p-value
GI symptoms

Most bothersome symptom Abdominal pain Loose stools/high frequency

Hard stools/low fre-

quency Bloating None of the above Frequency Abdominal pain $>3$ times/week Bloating $>3$ bowel function Not at all Somewhat Very

Somitization

PHQ-12 score 7 or above

Quality of life

Overall estimation of health past 4 weeks ( $S F-8$ ). Very poor/poor Fair good Very good/ excellent

Bodily pain past 4 weeks (SF-8). None/very mild Mild/moderate Severe very severe

Limitation in social activities due to physical health or emotional problems past 4 weeks $(S F-8)$. Not at

all Very little/somewhat times/month Concern of
$75(38.5)$

$48(24.6)$

$40(20.5)$

$24(12.3)$

8 (4.1)

$72(36.9)$

$158(81.0)$

$12(6.2)$

$106(54.4)$

77 (39.5)

147 (65.4)

$59(30.3)$

$118(60.5)$

$18(9.2)$

22 (11.3)

$110(56.4)$

63 (32.3)

$24(12.3)$

$98(50.3)$

73 (37.4)
48 (43.6)

$22(20.0)$

$16(14.5)$

$20(18.2)$

4 (3.6)

$31(28.2)$

$74(67.3)$

$25(22.7)$

$70(63.3)$

$15(13.6)$

85 (77.3)

0.82

31 (28.2)

$64(58.2)$

15 (13.6)

19 (17.3)

$58(52.7)$

$33(30.0)$

$29(26.4)$

$46(41.8)$

35 (31.8)
0,5

0.35

0.008 
Continued

IBS consulters IBS non-consulters p-value

Quite a lot/could do no

social activity

Frequency of doctor visits

At least one visit/year

Medication use

For GI related issues For pain, over the counter For pain, prescribed by doctor For depression

For anxiety

Surgery

Cholecystectomy

Appendectomy

Hysterectomy Other

abdominal surgery

Conclusion: Among individuals who meet Rome IV criteria for IBS in the general population, those who are older, have more frequent bloating, have greater concern about their bowel function, and who are more socially affected by their bowel symptoms, are more likely to consult doctors about their bowel symptoms. In contrast, IBS consulters and non-consulters do not differ in their abdominal pain severity or extra intestinal symptom burden. [Support: The Rome Foundation].

Disclosure of Interest: All authors have declared no conflicts of interest.

\section{P0485 PERFORMANCE OF A DIAGNOSTIC ALGORITHM FOR FUNCTIONAL GASTROINTESTINAL DISORDERS}

E. C. Linedale ${ }^{1}$, A. Mikocka-Walus ${ }^{2}$, P.R. Gibson ${ }^{3}$, J. M. Andrews ${ }^{4}$ ${ }^{1}$ Department Of Medicine, The University of Adelaide, Adelaide/Australia/SA ${ }^{2}$ School Of Psychology, Deakin University, Melbourne/Australia ${ }^{3}$ Gastroenterology, Monash University, Melbourne/Australia/VIC ${ }^{4}$ Department Of Gastroenterology \& Hepatology, Royal Adelaide Hospital - SA Health, Adelaide/Australia

Contact E-mail Address: ecushla.linedale@adelaide.edu.au

Introduction: Non-urgent referrals to specialist gastroenterology exceed capacity, resulting in long waiting lists and poor patient outcomes. New models of care are needed to translate specialist knowledge of functional gastrointestinal disorders (FGID) into primary care practice.

Aims \& Methods: This study aimed to evaluate the safety and performance of an algorithm-based approach to the diagnosis and management of FGID. Consecutive patients triaged to the 'routine waitlist' of an Australian public hospital Gastroenterology Department over 2 years, with non-specific GI symptoms (no alarms) were randomised to waitlist control or intervention $(2: 1)$. Intervention patients were screened for alarms and abnormal blood/stool tests without an in-person consultation, to exclude organic disease (full blood count, C-reactive protein, biochemistry, thyroid function tests, iron studies, coeliac serology, $+/-$ H. pylori serology, $+/-$ faecal calprotectin and elastase) and classified according to the Rome III criteria. Information from patients with clinical alarms was reviewed by a gastroenterologist (GE) and, where judged appropriate, a prompt GE appointment offered. Elsewise patients received a letter stating FGID diagnosis and management options. Referrals were analysed for quality according to current triage practices.

Results: 89 intervention patients (61\% female, mean 42, [SD 14] y) and 21 control $(75 \%$ female, mean 42 , [SD 16] y) patients completed intake. 35 intervention patients warranted prompt GE review after active screening. Organic disease was diagnosed in 10 (diagnosed: 19 FGID, 2 IBD, 1 neoplasm, 1 pancreatic insufficiency, 1 reflux oesophagitis, iron deficiency 1, 7 did not attend), and 4 had additional clinically significant findings (polyps, iron deficiency). 45 were diagnosed with a FGID (9 had another non-urgent diagnosis). At follow up (mean $2.7 \mathrm{yrs}$ [SD $0.5 \mathrm{yrs}$ ] post-referral), none of the 45 patients diagnosed with FGID had received a gastroenterology consult based on the original referral (six received a specialist appointment via duplicate referrals within the system). Confirmation of diagnoses at follow-up was received by patients and/or referring doctors in $42 / 45$ cases (three no longer contactable). The majority of patients (37/ $42,88 \%$ ) had received no alternate diagnosis to account for their gastrointestinal symptoms since the study. Whilst two had additional diagnoses (FGID plus diverticulitis, FGID plus prostatitis), and two - incidental yet clinically significant findings (FGID plus polyps).

Conclusion: The quality of referrals of patients with clinically suspected FGIDs was insufficient to allow the safe triage of patients according to urgency, with a third warranting more urgent review. Organic disease was diagnosed in a significant proportion of patients who would otherwise were unlikely to have been seen in tertiary care. Use of this specialist-independent diagnostic algorithm in primary or tertiary care may facilitate more timely and accurate diagnosis of organic gastrointestinal disease, and improve patient care.

Disclosure of Interest: E.C. Linedale: Abbott's Pathology provided a small untied grant covering the cost of faecal calprotectin tests.

A. Mikocka-Walus: JMA has served as a speaker, a consultant and/or an advisory board member for Abbott, Abbvie, Allergan, Celgene, Ferring, Takeda,
MSD, Shire, Janssen, Hospira and Pfizer, and has received research funding from Abbott, Abbvie, Ferring, MSD, Shire, Janssen.

P.R. Gibson: Consultant/advisory board member/speaker/research grants from AbbVie, Ferring, Janssen, Merck, Nestle Health Science, Danone, Allergan, Pfizer, Fresenius Kabi, Mylan and Takeda, Falk Pharma, Danone and A2 Milk Company.

J.M. Andrews: JMA has served as a speaker, a consultant and/or an advisory board member for Abbott, Abbvie, Allergan, Celgene, Ferring, Takeda, MSD, Shire, Janssen, Hospira and Pfizer, and has received research funding from Abbott, Abbvie, Ferring, MSD, Shire, Janssen.

P0486 SCOTTISH GUT MOTILITY DISORDER CLINIC: REVIEW OF ACTIVITY OVER 1-YEAR PERIOD

F. Moroni ${ }^{1}$, M. P. Eugenicos ${ }^{2}$

${ }^{1}$ University of Edinburgh, Edinburgh/United Kingdom

${ }^{2}$ Gastroenterology, Western General Hospital, Edinburgh/United Kingdom

Contact E-mail Address: francesca.moroni@nhs.net

Introduction: IBS is common worldwide. In UK it exceeds the $20 \%$ of the population ${ }^{1}$. Burden of Irritable Bowel Syndrome (IBS) on UK healthcare has been estimated around 1800 £per patient per year ${ }^{2}$. NICE guideline ${ }^{3}$ IBS provides a systematic approach to symptoms and therapies available to GPs and general gastroenterologists. This creates a good asset to minimize referrals to tertiary centres and address costs.

Aims \& Methods: We retrospectively reviewed, via electronic records, all the patients seen at the Scottish Gut Motility Disorder Clinic between January and December 2016 included, focusing on original referral, diagnosis and treatment to evaluate the need for specialist input.

Results: In 2016, 378 patients attended the Motility Clinic; total of 459 visits; 333 females. Mean age was 51.4 (age range: 16 to 95 years). $60 \%$ of referrals originated via secondary care $(40 \%$ GI, $50 \%$ Surgery, $10 \%$ other disciplines). The commonest reason for referral was IBS $(40 \%)$; IBS-Constipation $(58 \%)$, IBSDiarrhoea $(21 \%)$ or IBS-MixType $(21 \%) .16 \%$ were referred with faecal incontinence and $37 \%$ with chronic constipation. $35 \%$ of patient didn't receive any therapy at time of referral. $44 \%$ were prescribed treatment but not followed up for assessment of successful response to therapy prior to referral to the specialist clinic. In $28 \%$ of patients the diagnosis changed following Motility clinic assessment. Diagnosis at clinic is based on ROMEIII questionnaire, depression and anxiety score, thorough history taking and physical examination (including per rectum exam), ad hoc psychiatry input and referral to specialist investigation. In $30 \%$ of patients referred with chronic constipation the diagnosis was changed to IBS-Constipation, $8 \%$ changed from IBS-D to IBS-M, $8 \%$ referred with faecal incontinence had Obstructive Defecation Syndrome, $6 \%$ referred as IBS-M were diagnosed with IBS-C, $5 \%$ referred as IBS-D were diagnosed as IBS-M, 3\% referred with IBS-D had bile acid malabsorption. $56 \%$ of patients underwent specialist investigations including anorectal physiology (70\%).33\% of patients attending the Specialist clinic received 1st -line therapy and life style advice, albeit $57 \%$ of them, who received 2 nd -line treatment, having failed 1st-line management.

Conclusion: The above data indicate the need for education and expansion of resources available in primary care to optimise patients' management. Furthermore, it highlights the necessity for the introduction of a formal Neurogastroenterology curriculum in the general Gastroenterology training. Disclosure of Interest: All authors have declared no conflicts of interest.

\section{References}

1. Canava C, et al. The epidemiology of Irritable Bowel Syndrome. Clin Epidemiol. 2014b;6:71-80

2. Quinley EMM, et al. Irritable Bowel Syndrome: the burden and unmet needs in Europe. Digest Liver Dis. 2006;38(10):717-23

3. National Institute for Health and Clinical Excellence. Irritable bowel syndrome in adults: diagnosis and management. Clinical guidelineCG061. February 2008; Updated February 20151

\section{P0487 ALTERED EXPRESSION OF MEMBRANE TRANSPORTERS} IN COLONIC MUCOSA OF PATIENTS WITH IRRITABLE BOWEL SYNDROME (IBS) AND POST-INFECTIOUS (PI)-IBS COMPARED TO HEALTHY SUBJECTS

R. Wall ${ }^{1}$, T. M. Marques ${ }^{1}$, H. Edebol-Carlman ${ }^{1}$, J. Sundin $^{2}$, R. Vumma ${ }^{3}$, I. Rangel ${ }^{1}$, R. J. Brummer ${ }^{4}$

${ }^{1}$ Nutrition-gut-brain Interactions Research Centre, Medical Sciences, Örebro University, Örebro/Sweden

${ }^{2}$ Sahlgrenska Academy, Inst. of Medicine, Gothenburg/Sweden

${ }^{3}$ Department Of Chemistry And Biomedical Sciences, Linnaeus University, Växjö/ Sweden

${ }^{4}$ Nutrition-gut-brain Interactions Research Centre, School Of Medical Sciences, Örebro University, Örebro/Sweden

Contact E-mail Address: rebecca.wall@oru.se

Introduction: Irritable bowel syndrome (IBS) affects 5-15\% of adults in the general population, and is characterized by chronic recurrent abdominal pain and discomfort and associated with altered bowel habits. The pathophysiology of IBS is complex and not fully understood. Hence, treatment is often based on symptomatology rather than underlying physiological aberrancies. 
Aims \& Methods: The aim of this study was to compare the expression of membrane transporters in mucosal biopsies of healthy subjects, IBS patients and postinfectious (PI)-IBS patients. Mucosal biopsies were obtained from the unprepared sigmoid colon in 18 IBS patients, 9 PI-IBS patients and 10 healthy subjects. Total RNA was isolated and prepared for gene expression analyses using quantitative reverse-transcription polymerase chain reaction (qRT-PCR). We compared the expression of genes encoding membrane-spanning transporters, using GAPDH as a reference gene, and by using the comparative $2^{-\Delta \Delta \mathrm{Ct}}$ method.

Results: Colonic expression of SLC7A5 and SLC3A2 (together comprising the amino acid transporter LAT1 +4F2hc) was significantly lower in IBS patients, but not in PI-IBS patients, compared to healthy controls $(\mathrm{P}<0.001)$. The expression of SLC7A8 (LAT2) tended to be lower in IBS patients compared to controls $(\mathrm{P}=0.06)$. Mucosal gene expression of the short chain fatty acid transporter SMCT1 (SLC5A8) was lower in both IBS-patients and PI-IBS patients compared to healthy subjects $(\mathrm{P}<0.01)$.

Conclusion: The amino acid transporters LAT1 and LAT2 appeared to be affected in IBS patients, but not in PI-IBS patients, compared to healthy subjects, suggesting a possible alteration in amino acids transport in this patient group. Furthermore, our results suggest a lower uptake of short chain fatty acids in both IBS- and PI-IBS patients. Altered expression of these transporters may be involved in the pathophysiology of IBS as well as being a potential biomarker of this aberration, and therefore deserves further study in IBS.

Disclosure of Interest: All authors have declared no conflicts of interest.

\section{P0488 DIVERTICULITIS IN THE SIGMOID COLON HAS THE} HIGHEST RISK FOR INTESTINAL COMPLICATION OF COLONIC DIVERTICULITIS IN JAPANESE PATIENTS

H. Takayama, Y. Shimodate, A. Doi, N. Nishimura, H. Mouri, K. Matsueda, H. Yamamoto, M. Mizuno

Gastroenterology And Hepatology, Kurashiki Central Hospital, Kurashiki/Japan

Contact E-mail Address: hi7.takayama@gmail.com

Introduction: Most colonic diverticulitis can be conservatively treated, but some need surgical intervention due to intestinal complications. Risk factors associated with complications of diverticulitis have been reported mainly from Western countries, but few from Asian countries including Japan.

Aims \& Methods: In this study, we aimed to determine risk factors for complications of colonic diverticulitis in Japan. Two hundreds and eighty-two patients with acute diverticulitis who were hospitalized from November 2011 to November 2016 in our hospital were studied. Diagnosis of diverticulitis was based on symptoms, physical examination, blood tests, and results of computed tomography. We retrospectively collected data of medical history, examinations, and therapy. Risk factors associated with complications were analyzed by using logistic regression.

Results: Of the 282 patients, $183(64.9 \%)$ patients had right-sided diverticulitis, and $70(24 \%)$ had complications; perforation $(n=53)$, fistula $(n=8)$, abscess $(n=5)$ and stenosis $(n=4)$. The rate of complication was highest in sigmoid colon $(88.6 \%)$ when compared with other locations; ascending colon $(10 \%)$, transverse colon $(1.4 \%)$, and descending colon $(0 \%)$. Multivariate analysis identified the location of sigmoid colon (odds ratio $62.2,95 \%$ confidence interval $21.8-178.0)$ as a significant independent factor for complications of diverticulitis. Among 70 patients with complicated diverticulitis, $55(78.6 \%)$ patients underwent emergent surgery; most of them (54 patients, $98.2 \%$ ) were with diverticulitis in the sigmoid colon.

Table. Risk factors associated with complications of colonic diverticulitis (univariate and multivariate analysis)

\begin{tabular}{|c|c|c|c|c|c|}
\hline \multirow[b]{2}{*}{ Factor } & & \multicolumn{2}{|l|}{ Univariate } & \multicolumn{2}{|l|}{ Multivariate } \\
\hline & & $\begin{array}{l}\text { Odds ratio } \\
(95 \% \mathrm{CI})\end{array}$ & p-value & $\begin{array}{l}\text { Odds ratio } \\
(95 \% \mathrm{CI})\end{array}$ & p-value \\
\hline Age & $\begin{array}{l}\text { Per 10-year } \\
\text { increment }\end{array}$ & NA & & $1.37(0.99-1.89)$ & 0.055 \\
\hline \multirow[t]{2}{*}{ Sex } & Male & $\begin{array}{l}1.72 \\
(0.95-3.19)\end{array}$ & 0.07 & & \\
\hline & Female & 1 & & & \\
\hline \multirow[t]{2}{*}{ Body mass index } & $\geqq 25$ & $\begin{array}{l}2.13 \\
(1.1-4.09)\end{array}$ & 0.019 & $\begin{array}{l}1.79 \\
(0.57-5.61)\end{array}$ & 0.32 \\
\hline & $<25$ & 1 & & & \\
\hline \multirow[t]{2}{*}{$\begin{array}{l}\text { Time from symptom } \\
\text { onset to diagnosis }\end{array}$} & $\geqq 3$ & $\begin{array}{l}2.13 \\
(1.13-4.02)\end{array}$ & 0.017 & $\begin{array}{l}2.60 \\
(0.97-6.91)\end{array}$ & 0.056 \\
\hline & $<3$ & 1 & & & \\
\hline \multirow[t]{2}{*}{ Fever } & $\geqq 38$ & $\begin{array}{l}1.36 \\
(0.71-2.55)\end{array}$ & 0.349 & & \\
\hline & $<38$ & 1 & & & \\
\hline \multirow[t]{2}{*}{ Current smoking } & Yes & $\begin{array}{l}0.82 \\
(0.38-1.67)\end{array}$ & 0.616 & & \\
\hline & No & 1 & & & \\
\hline \multirow[t]{2}{*}{ Current drinking } & Yes & $\begin{array}{l}0.99 \\
(0.51-1.91)\end{array}$ & 1 & & \\
\hline & No & 1 & & & \\
\hline \multirow[t]{2}{*}{ High blood pressure } & Yes & $\begin{array}{l}4.97 \\
(2.66-9.39)\end{array}$ & $<0.001$ & $\begin{array}{l}0.48 \\
(0.14-1.62)\end{array}$ & 0.24 \\
\hline & No & 1 & & & \\
\hline
\end{tabular}

\begin{tabular}{|c|c|c|c|c|c|}
\hline \multirow[b]{2}{*}{ Factor } & & \multicolumn{2}{|l|}{ Univariate } & \multicolumn{2}{|l|}{ Multivariate } \\
\hline & & $\begin{array}{l}\text { Odds ratio } \\
(95 \% \mathrm{CI})\end{array}$ & p-value & $\begin{array}{l}\text { Odds ratio } \\
(95 \% \mathrm{CI})\end{array}$ & p-value \\
\hline \multirow[t]{2}{*}{ Hyperlipidemia } & Yes & $\begin{array}{l}2.39 \\
(1.07-5.21)\end{array}$ & 0.023 & $\begin{array}{l}1.22 \\
(0.31-4.73)\end{array}$ & 0.77 \\
\hline & No & 1 & & & \\
\hline \multirow[t]{2}{*}{ Diabetes } & Yes & $\begin{array}{l}1.27 \\
(0.34-4.07)\end{array}$ & 0.77 & & \\
\hline & No & 1 & & & \\
\hline \multirow[t]{2}{*}{$\begin{array}{l}\text { Non-steroidal anti- } \\
\text { inflammatory drugs }\end{array}$} & Yes & $\begin{array}{l}0.6 \\
(0.06-2.89)\end{array}$ & 0.736 & & \\
\hline & No & 1 & & & \\
\hline \multirow{2}{*}{$\begin{array}{l}\text { Steroid/ } \\
\text { Immunosuppressive } \\
\text { drugs }\end{array}$} & Yes & $\begin{array}{l}4.84 \\
(1.11-24.1)\end{array}$ & 0.017 & $\begin{array}{l}1.81 \\
(0.26-12.4)\end{array}$ & 0.55 \\
\hline & No & 1 & & & \\
\hline \multirow[t]{2}{*}{$\begin{array}{l}\text { Antiplatelet/ } \\
\text { Anticoagulant drugs }\end{array}$} & Yes & $\begin{array}{l}4.95 \\
(1.0-12.7)\end{array}$ & $<0.001$ & $\begin{array}{l}2.68 \\
(0.59-12.2)\end{array}$ & 0.2 \\
\hline & No & 1 & & & \\
\hline \multirow[t]{2}{*}{$\begin{array}{l}\text { Location } \\
\text { diverticulitis }\end{array}$} & Sigmoid colon & $\begin{array}{l}85.8 \\
(34.3-243)\end{array}$ & $<0.001$ & $\begin{array}{l}62.2 \\
(21.8-178)\end{array}$ & $<0.001$ \\
\hline & The other locations & 1 & & & \\
\hline
\end{tabular}

Conclusion: The sigmoid colon was a significant risk factor for complication of colonic diverticulitis in Japanese patients. Acute colonic diverticulitis in the sigmoid colon should carefully be treated with surgical interventions in mind. Disclosure of Interest: All authors have declared no conflicts of interest.

\section{References}

James P.L. Tan, Ahmed W.H. Barazanchi, et al. Predictors of acute diverticulitis severity: A systematic review. International Journal of Surgery 2016;26:43-52.

Noriaki Manabe, Ken Haruma, et al. Characteristics of Colonic Diverticulitis and Factors Associated With Complications: A Japanese Multicenter, Retrospective, Cross-Sectional Study. Diseases of the Colon \& Rectum 2015;58:1174-1181.

Sun Young Kim, Tae Hoon Oh, et al. The Clinical Factors for Predicting Severe Diverticulitis in Korea: A Comparison with Western Countries. Gut and Liver 2012;6:78-85.

Massimo Sartelli, et al. WSES Guidelines for the management of acute left sided colonic diverticulitis in the emergency setting. World Journal of Emergency Surgery 2016;37:1-15.

\section{P0489 A VARIANT OF COL3A1 (RS3134646) IS ASSOCIATED WITH} RISK OF DEVELOPING DIVERTICULOSIS IN CAUCASIAN MALES

M. C. Reichert ${ }^{1}$, J. Kupcinskas ${ }^{2}$, M. Krawczyk ${ }^{1}$, C. Jüngst ${ }^{1}$, B. Appenrodt ${ }^{1}$, S. N. Weber ${ }^{1}$, V. Zimmer ${ }^{1}$, A. Tamelis ${ }^{3}$, J. I. Lukosiene ${ }^{2}$, N. Pauziene ${ }^{4}$,

G. Kiudelis ${ }^{2}$, L. Jonaitis ${ }^{2}$, C. Schramm ${ }^{5}$, T. Goeser ${ }^{5}$, M. Glanemann', L. Kupcinskas ${ }^{2}$, F. Lammert ${ }^{1}$

${ }^{1}$ Department Of Medicine II, Saarland University Medical Center, Homburg/ Germany

${ }^{2}$ Department Of Gastroenterology, Lithuanian University of Health Sciences, Kaunas/Lithuania

${ }^{3}$ Department Of Surgery, Lithuanian University of Health Sciences, Kaunas/ Lithuania

${ }^{4}$ Institute Of Anatomy, Lithuanian University of Health Sciences, Kaunas/ Lithuania

${ }^{5}$ Clinic For Gastroenterology And Hepatology, University Hospital of Cologne, Cologne/Germany

${ }^{6}$ Department Of General, Visceral, Vascular And Pediatric Surgery, Saarland

University, Homburg/Saar/Germany

Contact E-mail Address: j_kupcinskas@yahoo.com

Introduction: Colonic diverticulosis is one of the most common gastroenterological disorders. Though diverticulosis is typically benign, many individuals develop diverticular disease (DD). DD is thought to stem from a complex interaction of environmental, dietary and genetic factors; however, the exact pathogenesis remains unknown.

Aims \& Methods: The aim of our present study was to determine the role of genetic variation within genes encoding for collagens of the connective tissue in the development of diverticulosis. Genetic polymorphisms COL3A1 (rs3134646, rs 1800255) and COL1A1 (rs1800012) were genotyped in 422 patients with diverticulosis and 285 controls of Caucasian descent using TaqMan assays.

Results: All genotype distributions did not deviate from the Hardy-Weinberg equilibrium. Overall, rs3134646, rs1800255 and rs1800012 were associated with diverticulosis. After multivariate logistic regression analysis, they were not linked with the risk of developing colonic diverticulosis in general; when selectively analyzing genders, the minor allele (AA) in rs3134646 remained significantly associated with diverticulosis in men $(\mathrm{p}=0.037)$

Conclusion: Our study shows that a variant of COL3A1 rs3134646 is associated with risk of developing colonic diverticulosis in Caucasian men, while COL3A1 rs1800255 and COL1A1 rs1800012 were not associated with this condition in our cohort of patients after adjusting for confounding factors.

Disclosure of Interest: All authors have declared no conflicts of interest. 
P0490 THE USE OF ENDOSCOPIC CLASSIFICATION "DICA" MAY HAVE A SIGNIFICANT COST-SAVING ON THE BURDEN OF DIVERTICULAR DISEASE OF THE COLON

A. Tursi ${ }^{1}$, W. Elisei ${ }^{2}$, M. Picchio ${ }^{3}$, G. Nasi ${ }^{4}$, A.M. Mastromatteo ${ }^{4}$, F. Di Mario ${ }^{5}$, E. Di Rosa ${ }^{6}$, M.A. Brandimarte ${ }^{6}$, C. Cassieri ${ }^{7}$, P.G. Lecca ${ }^{7}$, G. Brandimarte ${ }^{8}$ ${ }_{1}^{1}$ Gastroenterology Service, ASL BAT Gastroenterology Service, Andria/Italy ${ }^{2}$ Division of Gastroenterology, ASL RM6, Albano Laziale/Italy

${ }^{3}$ Division of Surgery, "P. Colombo" Hospital, ASL RM6, Velletri/Italy

${ }^{4}$ Clinical Management Staff, "Cristo Re" Hospital, Rome/Italy

${ }^{5}$ University Of Parma, Department of Clinical and Experimental Medicine, section of Gastroenterology, Parma/Italy

${ }^{6}$ Department of Hygiene and Public Health, ASL RM1, Rome/Italy

${ }^{7}$ Division of Internal Medicine and Gastroenterology, "Cristo Re" Hospital, Rome/ Italy

${ }^{8}$ Division of Internal Medicine and Gastroenterology, "Cristo Re” Hospital, Rome, Italy, Roma/Italy

\section{Contact E-mail Address: giovannibrandimarte56@gmail.com}

Introduction: Although symptoms occurs in only $20 \%$ of patients harbouring diverticula, Diverticular Disease (DD) of the colon DD represents the $8^{\text {th }}$ disease as burden in USA. Several treatment are currently advised in managing those patients, but their impact on the burden of the disease is unknown. The recent DICA endoscopic classification has been developed and validated for the classification of DD, founding that treatment of DICA 1 and DICA 3 patients did not impact significantly in terms of acute diverticulitis occurrence/recurrence and surgery occurrence. Our aim was to assess the impact of using DICA classification on the burden of DD in Italy.

Aims \& Methods: We assessed retrospectively the overall and the cost/year of treatments (mesalazine and/or rifaximin, or any other treatment, including probiotics, fibers, systemic antibiotics and spasmolithics) in DICA 1, DICA 2 and DICA 3 population. Analysis of diverticulosis prevalence was estimated according to data population provided by Italian Institute of Statistics (ISTAT). Cost of treatments were calculated according to data on drugs' consumption collected during the DICA study.

Results: According to 2015 ISTAT population data, we estimated that $>8$ million of Italian people $>60$ years may have diverticulosis. According to our enrolled population, we estimated that about $75 \%$ of diverticular population are on DICA 1 , about $30 \%$ on DICA 2, and about $13 \%$ on DICA 3 . According to the drugs' consumption recorded during our study, we estimated that overall about 679 million of euros could be spent in Italy in treating those patients. In particular, $>387$ million of euros are spent in DICA 1 population, $>203$ million of euros in DICA 2 population, and $>88$ million of euros in DICA 3 population. Considering that medical treatments did not show any significant advantage when treating DICA 1 and DICA 3 people in terms of prevention of acute diverticulitis occurrence/recurrence and surgery occurrence, we can estimated that $>475$ million of euros could be spent in Italy without any significant benefit for DD population.

Conclusion: DD has a significant burden for National Health System in Italy. DICA endoscopic classification may have a significant impact of this burden, helping to select DD people who effectively need treatments in terms of prevention of acute diverticulitis occurrence/recurrence and surgery occurrence.

Disclosure of Interest: All authors have declared no conflicts of interest.

\section{P0491 NATURAL HISTORY OF SYMPTOMATIC UNCOMPLICATED DIVERTICULAR DISEASE: A 13-YEAR PROSPECTIVE STUDY}

A. Tursi ${ }^{1}$, S. Scida ${ }^{2}$, C. Miraglia ${ }^{2}$, C. Scarpignato ${ }^{2}$, M. Franceschi ${ }^{3}$, W. Elisei ${ }^{4}$, M. Picchio $^{5}$, G. Brandimarte ${ }^{6}$, F. Di Mario

${ }^{1}$ Gastroenterology Service, ASL BAT Gastroenterology Service, Andria/Italy

${ }^{2}$ Department Of Medicine And Surgery, University Of Parma, Italy, University of Parma, Parma/Italy

${ }^{3}$ Digestive Endoscopy Unit, ULSS4 Alto Vicentino, Santorso/Italy

${ }^{4}$ Division of Gastroenterology, ASL RM6, Albano Laziale/Italy

${ }^{5}$ Division of Surgery, "P. Colombo" Hospital, ASL RM6, Velletri/Italy

${ }^{6}$ Division of Internal Medicine and Gastroenterology, "Cristo Re" Hospital, Rome, Italy, Roma/Italy

${ }^{7}$ University Of Parma, Department of Clinical and Experimental Medicine, section of Gastroenterology, Parma/Italy

Contact E-mail Address: francesco.dimario@unipr.it

Introduction: Symptomatic Uncomplicated Diverticular Disease (SUDD) affects about $20 \%$ of patients having diverticulosis. However, SUDD natural history is not completely understood yet. Our aim was to assess the outcome of a cohort of SUDD patients during a 13-year follow-up.

Aims \& Methods: 185 patients suffering from SUDD were enrolled during 2000 2002, and followed-up until 2015. Symptoms assessed were abdominal pain, meteorism, bowel movement/day, and each of them was scored from 0 (min) to $3(\max )$, with a sum ranging from $0(\min )$ to $12(\max )$. Also Visual Analogic Scale (VAS) was provided in order to assess patients' quality of life. Patients were treated according to physician convenience (rifaximin, mesalazine, probiotics, spasmolithics) only when symptoms occurred during the follow-up. Follow-up visit was performed every 6 months or whenever patients consider it necessary. Results: During the observational period, 47 patients were lost to follow-up. Among them, 9 deceased for causes not related to diverticular disease. Acute diverticulitis occurred in 14 patients ( $7.56 \%$ of the overall population): 6 patients (3.24\% of the overall population) underwent to surgery, and 2 patients $(1.08 \%$ of the overall population) deceased for peritonitis. The mean symptoms' score was 7.5 at baseline, ranged between 6.6 and 9 during the follow-up, and was 8.4 at the end of the observation. VAS score was 4 at baseline, ranged between 4 and 9 during the follow-up, and was 4.5 at the end of observation.

Conclusion: SUDD is a clinical entity that, although benign, significantly affects quality of life of patients. Acute diverticulitis may occur in those patients, sometimes needs of surgical treatment, and may cause mortal complications, although not frequent

Disclosure of Interest: All authors have declared no conflicts of interest.

P0492 IMPACT OF TREATMENTS ON FECAL MICROBIOTA AND FECAL METABOLIC PROFILING IN SYMPTOMATIC UNCOMPLICATED DIVERTICULAR DISEASE OF THE COLON

A. Tursi ${ }^{1}$, P. Mastromarino ${ }^{2}$, D. Capobianco ${ }^{2}$, W. Elisei ${ }^{3}$, L. Laghi $^{4}$, C. $_{\text {Zhu }}{ }^{5}$, M. Picchio ${ }^{6}$, G. Giorgetti ${ }^{7}$, F. Fabiocchi ${ }^{7}$, F. Di Mario ${ }^{8}$, G. Brandimarte ${ }^{9}$ ${ }^{1}$ Gastroenterology Service, ASL BAT Gastroenterology Service, Andria/Italy ${ }^{2}$ Department of Public Health, Section of Microbiology, "Sapienza" University, Rome/Italy

${ }^{3}$ Division of Gastroenterology, ASL RM6, Albano Laziale/Italy

${ }^{4}$ Inter-Departmental Centre for Industrial Agri-Food Research, Alma Mater Studiorum University of Bologna, Cesena/Italy

${ }^{5}$ Inter-Departmental Centre for Industrial Agri-Food Research, Alma Mater

Studiorum University of Bologna, Cesena/Italy

${ }^{6}$ Division of Surgery, "P. Colombo" Hospital, ASL RM6, Velletri/Italy

${ }^{7}$ Digestive Endoscopy and Nutrition Unit, "S. Eugenio" Hospital, Rome/Italy

${ }^{8}$ University Of Parma, Department of Clinical and Experimental Medicine, section of Gastroenterology, Parma/Italy

"Division of Internal Medicine and Gastroenterology, "Cristo Re" Hospital, Rome, Italy, Roma/Italy

Contact E-mail Address: antotursi@tiscali.it

Introduction: Fecal microbiota and metabolome may be altered in patients with Symptomatic Uncomplicated Diverticular Disease (SUDD). In particular, we found that Akkermansia muciniphila species were significantly increased in SUDD patients when compared with asymptomatic diverticulosis and healthy people, as well as PLS-DA analysis of NMR-based fecal metabolomics showed significant discrimination between $\mathrm{HC}$ and AD patient. Our aim was to assess the effect of current treatments for SUDD on fecal microbiota and fecal metabolic profiling in those patients.

Aims \& Methods: Thirteen consecutive female patients, living in the same district and suffering from SUDD, were studied. Patients were treated with a 2 -week course of $30 \mathrm{~g}$ /day fiber supplementation (3 patients), 1.6 grams/day of mesalazine ( 3 patients), 900 billion/day of probiotic mixture VSL\#3 (currently available in Europe as VivoMixx ${ }^{(\mathbb{R}}, 3$ patients), and $800 \mathrm{mg} /$ day of rifaximin (4 patients). Stool samples were collected at entry (T0), at the end of the 2-week course of treatment (T1), and after 30 (T2) and therefore after 60 days at the end of the therapeutic course (T3). Real-time PCR was used to quantify targeted microorganisms. High-resolution proton nuclear magnetic resonance (NMR) spectroscopy associated to Multivariate Analysis with partial least square discriminant analysis (PLS-DA) were applied on the metabolite data set.

Results: The overall bacterial quantity did not differ before and after treatment $(\mathrm{p}=0.449)$. The overall amount of Akkermansia muciniphila species was significantly reduced at $\mathrm{T} 1(\mathrm{p}=0.017)$ and $\mathrm{T} 2(\mathrm{p}=0.026)$, while at $\mathrm{T} 3$ it became similar to that of T0 $(\mathrm{p}=0.09)$. The amount of Lactobacilli group was increased in all groups but not significantly at T1 and T2, while at T3 it became similar to that of T0. All treatments showed the same results except for probiotic group, who had higher and persistent amount of Lactobacilli up to T3. PLS-DA analysis of NMR-based fecal metabolomics showed significant changes at T1 and T2, while at T3 it became similar to that of T0. All treatment were showed the same behaviour in influencing fecal metabolome except for rifaximin group, in which we did not find any metabolic change neither at the end of treatment nor during the washout period.

Conclusion: This preliminary study confirms that Akkermansia muciniphila may play a pathogenetic role in the occurrence of SUDD. We found also that current treatments for SUDD patients are able to influence metabolic activity in those patients except for rifaximin.

Disclosure of Interest: All authors have declared no conflicts of interest.

P0493 5-YEARS ITALIAN REGISTER OF DIVERTICULOSIS AND DIVERTICULAR DISEASE (REMAD): A LOW PROGRESSION RATE OF DISEASE DURING THE FIRST YEAR OF FOLLOW-UP

M. Carabotti ${ }^{1}$, R. Cuomo ${ }^{2}$, G. Barbara ${ }^{3}$, F. Pace ${ }^{4}$, P. Andreozzi ${ }^{5}$, R. Benini ${ }^{6}$, B. Annibale

${ }^{1}$ University Sapienza, Rome/Italy

${ }^{2}$ University of Naples'Federico II, Naples/Italy

${ }^{3}$ Department Of Medical And Surgical Sciences, University of Bologna, Bologna/ Italy

${ }^{4}$ Gastroenterology, Bolognini Hospital, Seriate (BG)/Italy

${ }^{5}$ Clinical Medicine And Surgery, University Of Naples " Federico Ii", Naples/Italy ${ }^{6}$ CD Pharma, Milano/Italy

${ }^{7}$ Department Of Medical-surgical Sciences And Translational Medicine,

Sant'Andrea Hospital, University Sapienza, Rome/Italy

Contact E-mail Address: mcarabotti@yahoo.it

Introduction: Natural history of colonic diverticulosis and diverticular disease (DD) is poorly known, and available data derived mostly from retrospective cohort studies. 
Aims \& Methods: Aim of this study was to assess, in a cohort of patients with colonic diverticula, the incidence of new cases of symptomatic uncomplicated diverticular disease (SUDD) and diverticulitis, and recurrence of diverticulitis after 1-year of follow-up. GRIMAD (Italian Diverticular Disease Group) promoted the creation of REMAD (Register of Diverticular Disease) a prospective, 5-years, no-profit, cohort study involving 47 Italian centers. Each center enrolled at least 20 consecutive patients during a period of two months. Inclusion criteria were: informed consent; age $>18$ years and endoscopic/radiological-confirmed colonic diverticula. Outpatient/telephone visits were scheduled every 6 months. The clinical data (patients' characteristics and habits, characteristics of DD, comorbidities and therapies) collected by participating centers were reported on an electronic Case Report Form managed by CD Pharma, Milan. At entry, patients were categorized according to the following criteria: i) diverticulosis (presence of diverticula in the absence of abdominal symptoms); ii) SUDD (recurrent abdominal symptoms as abdominal pain and/or changes in bowel habit, in the absence of overt inflammation); iii) PD (patients who experienced at least one episode of acute diverticulitis in the past). Patients were allowed to continue their therapy for DD, if any. Logistic regression was performed to identify patients' features associated with new occurrence of SUDD and diverticulitis.

Results: Overall, at baseline 1217 [556 (45.7\%) female, median years 67 (28-95), BMI $25.6 \mathrm{Kg} / \mathrm{m}^{2}$ (16.2-43.4)] patients were enrolled: $707(58.1 \%), 300(24.7 \%)$, and $210(17.3 \%)$ with diverticulosis, SUDD, and PD, respectively. At 12 months, 922 patients $(53.1 \%, 29.8 \%$, and $17.1 \%$ with diverticulosis, SUDD, and PD) were followed, and $27.4 \%$ of patients were lost at follow-up. In the 12 months follow-up, $33(6 \%)$ and $4(0.7 \%)$ of diverticulosis patients developed SUDD and acute diverticulitis, respectively; $4(1.6 \%)$ of SUDD patients developed acute diverticulitis, and in $14(9.4 \%)$ of PD patients a new episode of acute diverticulitis occurred. Overall, only 3 patients developed a complication, without need of surgery. One year of follow-up logistic regression, showed that only female gender was associated with subjects who changed subgroup from diverticulosis to SUDD (OR 2.26, 95\% CI 0.97-5.22). No specific features associated with recurrence of diverticulitis could be identified.

Conclusion: These preliminary data suggested that, during an observation period of one year, progression from diverticulosis to SUDD occurred in less than a tenth of patients, and was associated with female gender. Overall incidence of diverticulitis was very low $(2.3 \%)$, whereas recurrent diverticulitis was not uncommon. This observational study suggested, that although the vast majority of patients did not show progression of disease, in those who progressed one in ten tended to relapse during follow-up.

Disclosure of Interest: R. Cuomo: Speaker and consultant for Alfa Wassermann, G. Barbara: Speaker and consultant for Alfa Wassermann

F. pace: Speaker and consultantfor Alfa Wassermann,

B. Annibale: Speaker and consultantfor Alfa Wassermann

All other authors have declared no conflicts of interest.

\section{P0494 CLINICAL FEATURES ASSOCIATED WITH SYMPTOMATIC UNCOMPLICATED DIVERTICULAR DISEASE AND DIVERTICULITIS PATIENTS: RESULTS FROM THE ITALIAN REGISTER OF DIVERTICULAR DISEASE (REMAD)}

M. Carabotti ${ }^{1}$, R. Cuomo ${ }^{2}$, G. Barbara ${ }^{3}$, F. Pace ${ }^{4}$, P. Andreozzi ${ }^{5}$, R. Benini ${ }^{6}$, B. Annibale

${ }^{1}$ University Sapienza, Rome/Italy

${ }^{2}$ University of Naples'Federico II, Naples/Italy

${ }^{3}$ Department Of Medical And Surgical Sciences, University of Bologna, Bologna/ Italy

${ }^{4}$ Gastroenterology, Bolognini Hospital, Seriate (BG)/Italy

${ }^{5}$ Clinical Medicine And Surgery, University Of Naples " Federico I ${ }^{\prime \prime}$, Naples/Italy ${ }^{6} \mathrm{CD}$ Pharma, Milano/Italy

${ }^{7}$ Department Of Medical-surgical Sciences And Translational Medicine,

Sant'Andrea Hospital, University Sapienza, Rome/Italy

Contact E-mail Address: mcarabotti@yahoo.it

Introduction: In the vast majority of patients colonic diverticula remain asymptomatic (diverticulosis), while about $20 \%$ develop recurrent abdominal symptoms (symptomatic uncomplicated diverticular disease, SUDD) or complications (diverticulitis), with subsequent impact on quality of life (QoL). To date, clinical features associated with each subgroup are not fully understood.

Aims \& Methods: Aim of this study was to assess, in a cohort of patients with colonic diverticula, the clinical features and QoL scores associated with each subgroup of patients. GRIMAD (Italian Diverticular Disease Group) promoted the creation of REMAD [(Register of Diverticular Disease (DD)] a 5-years prospective, no-profit, cohort study involving 47 Italian centres. Each centre enrolled at least 20 consecutive patients during a period of 2 months. Inclusion criteria were: informed consent; age $>18$ years; endoscopic/radiological-confirmed colonic diverticula. The clinical data [patients' characteristics and habits, characteristics of DD, treatment, and QoL (SF-12 questionnaire with evaluation of physical and mental component summary scores (RPCS12 and RMCS12)] collected by participating centres were reported on an electronic Case Report Form managed by CD Pharma, Milan. At entry, patients were categorized into subgroups according to the following criteria: i) diverticulosis (presence of diverticula in the absence of abdominal symptoms); ii) SUDD (recurrent abdominal symptoms as abdominal pain and/or changes in bowel habit, in the absence of overt inflammation); iii) PD (patients who experienced at least one episode of acute diverticulitis in the past). Patients were allowed to continue their therapy for DD, if any. T test was used to compare QoL scores. Logistic regression was performed to identify patients' features associated with the presence of subtypes of DD. A p value $<0.05$ was considered statistically significant.
Results: 1217 patients were enrolled. Characteristics of each subgroup of patients are reported in the table.

\begin{tabular}{|c|c|c|c|c|}
\hline $\mathrm{N}(\%)$ & $\begin{array}{l}\text { Diverticulosis } \\
\mathrm{n}=707 \\
(58.1)\end{array}$ & $\begin{array}{l}\text { SUDD } \\
\mathrm{n}=300 \\
(24.7)\end{array}$ & $\begin{array}{l}\mathrm{PD} \\
\mathrm{n}=210 \\
(17.3)\end{array}$ & $\begin{array}{l}\text { OR } \\
(95 \% \mathrm{CI})\end{array}$ \\
\hline Female & $\begin{array}{l}276 \\
(39)\end{array}$ & $\begin{array}{l}174 \\
(58)\end{array}$ & $\begin{array}{l}106 \\
(50.5)\end{array}$ & $\begin{array}{l}* 2.18 \\
(1.60-2.95){ }^{\#} 2.06 \\
(1.43-2.98)\end{array}$ \\
\hline $\begin{array}{l}\text { Age years median } \\
\text { (range) }\end{array}$ & $\begin{array}{l}67 \\
(34-93)\end{array}$ & $\begin{array}{l}67 \\
(37-90)\end{array}$ & $\begin{array}{l}65 \\
(28-95)\end{array}$ & $\begin{array}{l}{ }_{2} 2.33 \\
(1.53-3.57)\end{array}$ \\
\hline $\begin{array}{l}\mathrm{BMI} \mathrm{Kg} / \mathrm{m}^{2} \text { median } \\
\text { (range) }\end{array}$ & $\begin{array}{l}25.6 \\
(16.2-40.8)\end{array}$ & $\begin{array}{l}26 \\
(18-41)\end{array}$ & $\begin{array}{l}25.3 \\
(16.6-43.4)\end{array}$ & ns \\
\hline Family history for DD & $\begin{array}{l}129 \\
(18.2)\end{array}$ & $\begin{array}{l}65 \\
(21.7)\end{array}$ & $\begin{array}{l}60 \\
(28.6)\end{array}$ & $\begin{array}{l}{ }^{\#} 1.73 \\
(1.15-2.60)\end{array}$ \\
\hline $\begin{array}{l}\text { Gastrointestinal } \\
\text { (GI) comorbidities }\end{array}$ & $\begin{array}{l}224 \\
(31.7)\end{array}$ & $\begin{array}{l}134 \\
(44.6)\end{array}$ & $\begin{array}{l}69 \\
(32.8)\end{array}$ & $\begin{array}{l}* 1.65 \\
(1.21-2.25)\end{array}$ \\
\hline Charlson Index $>3$ & $108(15.3)$ & $41(13.6)$ & $26(12.3)$ & $\begin{array}{l}\mathrm{ns} \\
\mathrm{p} \text { value }\end{array}$ \\
\hline RPCS12 (mean) & 48.5 & 46.1 & 47.0 & $\begin{array}{l}* 0.0002 \\
\# 0.0302\end{array}$ \\
\hline RMCS12 (mean) & 48.2 & 45.0 & 45.9 & $\begin{array}{l}*<0.0001 \\
\# 0.0048\end{array}$ \\
\hline
\end{tabular}

"diverticulosis vs SUDD; \#diverticulosis vs PD; ns: not significant

Conclusion: These data showed that, with respect to diverticulosis, female gender and presence of GI comorbidities are associated with SUDD, whereas younger age, family history for DD and female gender are associated with PD. Furthermore, patients with diverticulosis have higher physical and mental scores compared both to patients with SUDD and PD, suggesting that SUDD and PD reduced QoL of the affected patients.

Disclosure of Interest: R. Cuomo: Speaker and consultant for Alfa Wassermann G. Barbara: Speaker and consultant for Alfa Wassermann F. pace: Speaker and consultant for Alfa Wassermann B. Annibale: Speaker and consultant for Alfa Wassermann All other authors have declared no conflicts of interest.

\section{P0495 FEATURES OF ABDOMINAL PAIN MAY DISTINGUISH PATIENTS WITH PREVIOUS DIVERTICULITIS FROM PATIENTS WITH SYMPTOMATIC UNCOMPLICATED DIVERTICOLAR DISEASE: RESULTS FROM THE ITALIAN REGISTER OF DIVERTICULAR DISEASE (REMAD)}

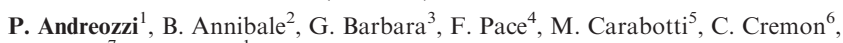
R. Benini ${ }^{7}$, R. Cuomo

${ }^{1}$ Clinical Medicine And Surgery, University Of Naples " Federico I $i "$, Naples/Italy ${ }^{2}$ Department Of Medical-surgical Sciences And Translational Medicine,

Sant'Andrea Hospital, University Sapienza, Rome/Italy

${ }^{3}$ Department Of Medical And Surgical Sciences, University of Bologna, Bologna/ Italy

${ }^{4}$ Gastroenterology, Bolognini Hospital, Seriate (BG)/Italy

${ }^{5}$ University Sapienza, Rome/Italy

${ }^{6}$ University Of Bologna, St. Orsola-Malpighi Hospital, Bologna/Italy

${ }^{7} \mathrm{CD}$ Pharma, Milano/Italy

Contact E-mail Address: paoloandre.85@gmail.com

Introduction: Patients with symptomatic uncomplicated diverticular disease (SUDD) and those with diverticulits share similar clinical patterns characterized by abdominal pain or changes of bowel habits. In clinical practice, differential diagnosis between the two conditions may be useful in the diagnostic approach and therapeutic management.

Aims \& Methods: Our aim was to assess the features of abdominal pain in patients with SUDD and previous diverticulitis (PD). GRIMAD (Italian Diverticular Disease Group) promoted the creation of REMAD (Registry of Diverticular Disease), a prospective, 5-years, no-profit, cohort study involving 47 Italian centers. All patients were enrolled during a 2 months-period. At entry, patients were categorized according to the following criteria: i) diverticulosis (presence of diverticula in the absence of abdominal symptoms); ii) SUDD (recurrent abdominal symptoms in the absence of overt inflammation); iii) PD (patients who experienced at least one episode of acute diverticulitis). Patients who fulfilled criteria for diagnosis of SUDD and PD were asked to fill in two questionnaires assessing features of short pain $(<24 \mathrm{~h})$ and long-acting pain $(>24 \mathrm{~h})$ in the last 6 months. Both questionnaires assessed patterns of pain, including severity (evaluated by means of a 10-cm VAS), localization, association with clinical parameters (fever, confinement to bed, medical consultation, antibiotic therapy and hospital admission). Data were expressed as percentages and means \pm standard deviations. $\mathrm{P}<0.05$ was considered significant.

Results: 1217 [556 (45.7\%) female, age 66.1 $\pm 9.9 \mathrm{y}$ ] consecutive patients were enrolled in the study. $300(24.7 \%)$ and $210(17.3 \%)$ fulfilled criteria for SUDD and PD, respectively. Among them, 223 patients with SUDD (74.3\%) and 154 patients with PD $(73.3 \%)$ accepted to fill in short-lasting pain questionnaire, whereas 156 patients with SUDD $(52.0 \%)$ and 140 patients with PD $(66.6 \%)$ 
filled in long-lasting pain questionnaire. Abdominal pain lasting $<24 \mathrm{~h}$ was reported by $192 / 223$ patients with SUDD (86.1\%) and $119 / 154$ with PD $(77.3 \%)(\mathrm{p}=0.026)$. Symptom severity score was higher in PD group than in SUDD group, but this difference was not statistically significant $(5.5 \pm 2.4$ vs $5.1 \pm 2.2 \mathrm{~cm} ; \mathrm{p}=0.130$ ). Patients with PD had short-lived pain located more frequently in left lower abdomen $(50.6 \%$ vs $39.9 \%$; $=0.002)$, whereas abdominal diffuse pain was more prevalent in patients with SUDD $(29.6 \%$ vs $20.8 \% ; \mathrm{p}=0.058$ ). Pain lasting $>24 \mathrm{~h}$ was more prevalent in PD group compared to SUDD group $(62.1 \%$ vs $52.6 \% ; p=0.029)$. Pain severity was higher in patients with PD, but this difference was not significant $(6.4 \pm 2.6$ vs $5.9 \pm 2.4 ; \mathrm{p}=0.069)$. Long-lasting pain was more frequently located in left lower abdomen in patients with PD $(53.6 \%$ vs $23.7 \%$; $<0.01)$, whereas more frequently was diffuse in SUDD patients $(17.3 \%$ vs $7.1 \% ; \mathrm{p}<0.001)$. Moreover, in patients with PD, pain lasting $>24 \mathrm{~h}$ was more often associated to fever $(28.6 \%$ vs $6.4 \%$; $\mathrm{p}<0.001)$, confinement to bed $(35.7 \%$ vs. $18.6 \% ; \mathrm{p}=0.002)$, medical consultation $(38.6 \%$ vs $23.7 \%$; $=0.013)$, need for therapy $(42.9 \%$ vs $19.2 \%$; $<0.001)$, and hospitalization $(28.6 \%$ vs $8.3 \%$; $<<0.001)$

Conclusion: SUDD and PD patients show some peculiar clinical features of abdominal pain. SUDD patients frequently complained abdominal diffuse and short lasting pain. In contrast patients with PD frequently complained pain located in the left lower abdomen lasting for more than $24 \mathrm{~h}$. Our results suggest that these features are useful indicators to distinguish patients with SUDD and PD and should be carefully assessed in clinical work-up of diverticular disease. Disclosure of Interest: B. Annibale: Speaker and consultant for Alfa Wasserman G. Barbara: Speaker and consultant for Alfa Wasserman

F. pace: Speaker and consultant for Alfa Wasserman

R. Cuomo: Speaker and consultant for Alfa Wasserman

All other authors have declared no conflicts of interest.

\section{P0496 MUSCULAR INFLAMMATORY STATE AND PHENOTYPIC SWITCH IN DIVERTICULOSIS AND COMPLICATED DIVERTICULAR DISEASE}

L. Pallotta ${ }^{1}$, A. Scirocco ${ }^{2}$, A. Ignazzi $^{2}$, M.A. Maselli ${ }^{2}$, M. Carabotti ${ }^{1}$,

A. Cicenia ${ }^{1}$, G. De Toma ${ }^{1}$, F. Pezzolla ${ }^{2}$, E.S. Corazziari ${ }^{1}$, C. Severi $^{3}$

${ }^{1}$ University Sapienza of Rome, Roma/Italy

${ }^{2}$ Scientific Institute of Gastroenterology "S. De Bellis", Castellana Grotte/Italy

${ }^{3}$ Internal Medicine And Medical Specialties, University Sapienza of Rome,

Roma/Italy

Contact E-mail Address: carola.severi@uniroma1.it

Introduction: Colonic diverticulosis, as well as diverticular disease, is a multifactorial disorder characterized by neuro-muscular alterations consisting in impaired contraction, inflammation and fibrosis. Mesenchymal smooth muscle cells (SMC) are able to switch from a contractile phenotype to a less mature synthetic phenotype, characterized by a loss of differentiation with decreased expression of contractile markers as well as synthesis and release of several pro-inflammatory cytokines. Different organ specific pathways have been demonstrated to induce this mesenchymal transition. Renal fibrosis is driven by transforming growth factor- $\beta$ (TGF- $\beta$ ) through inverse regulation of Smad2/3 while vascular fibrosis by PDGF- $\beta$, ending in downregulation of marker gene Trb3 expression.

Aims \& Methods: Aim of this study was to determine, both in human uninvolved and involved tracts of asymptomatic diverticulosis $\left(\mathrm{AD}^{-}, \mathrm{AD}^{+}\right)$and in stenotic segments of complicated diverticular disease (CDD), the alterations intrinsic to smooth muscle. Circular and longitudinal smooth muscle strips and cells (SMC) were isolated separately from surgical colon specimen of 18 patients $(58<$ age $<80$ years) affected either by sigmoid AD (6) or CDD (6) and patients $(61<$ age $<80$ years) submitted to surgery for cancer (6) (CTR). Contraction was tested in response to carbachol and relaxation in response to VIP. qPCR analysis, expressed as Relative Quantification, was performed for transcription of mRNA encoding for TNF- $\alpha$, inflammasome components (NLRP3, ASC, CASP1, IL-1 $\beta$ ) and for SMC phenotypic switch molecules (Collagen I, $\alpha$-SMA, TGF- $\beta$, PDGF$\beta, \operatorname{Trb} 3, \operatorname{Smad} 2 / 3)$. Data were normalized to $\beta$-actin mRNA and expressed as mean \pm SE. In addition, the activation of inflammasome complex was indirectly tested trough quantification of IL-1 $\beta$ secretion by commercial ELISA kit.

Results: In both muscle layers, $\mathrm{AD}^{-}$and $\mathrm{AD}^{+} \mathrm{SMC}$, compared to $\mathrm{CTR}$, showed an overall increase in inflammatory gene expression, with a trend of decrease from $\mathrm{AD}^{-}$to $\mathrm{AD}^{+}$, the lowest expression been observed in $\mathrm{CCD}$. This inflammatory state was associated with an increase in IL- $1 \beta$ secretion in SMC culture medium compared to CTR and a progressive inhibition of contraction to carbachol, already in $\mathrm{AD}^{-}$in circular strips and SMC. In contrast relaxation in response to VIP resulted significantly decreased only in $\mathrm{AD}^{+}$both on strips and SMC with no alteration in $\mathrm{AD}^{-}$and $\mathrm{CDD}$. Peculiarity of circular SMC was a progressive increase in Coll1 expression from AD to CDD compared to CTR ( 3 hundred fold increase) paralleled to about $50 \%$ decrease in the contractile protein $\alpha$-SMA. Differently, longitudinal SMC, both in AD and CDD, presented a homogenous increased Colll expression, decrease in $\alpha$-SMA and reduction of contraction. VIP-induced relaxation was significantly decreased in CCD. Phenotypic switch was only observed in CDD, driven in circular layer, by a TGF- $\beta$-dependent pathway (increased expression for TGF- $\beta$ : $2.88 \pm 0.6$ and Smad3: $1.67 \pm 0.12$ and reduced Smad2: $0.39 \pm 0.11$ ) while in longitudinal driven by PDGF- $\beta$-dependent pathway (increase of PDGF- $\beta$ : $2.27 \pm 0.44$ and parallel decrease of Trb3: $0.58 \pm 0.13$ )

Conclusion: Intrinsic myogenic alterations are present in colonic asymptomatic diverticulosis and complicated diverticular disease, both in the circular and longitudinal layers characterized by a myogenic pro-inflammatory state and an impaired contractile activity that, in complicated diverticular disease, ended in a muscular synthetic pro-fibrotic switch.

Disclosure of Interest: All authors have declared no conflicts of interest.

MONDAY, OCTOBER 30, 2017

09:00-17:00 OESOPHAGEAL, GASTRIC AND DUODENAL DISORDERS I - HALL

\section{P0497 THE ONCOGENIC MIR-491-5P/MIR-875-5P-NOTCH3-}

\section{PHLDB2 AXIS IN GASTRIC TUMORIGENESIS}

T. Huang ${ }^{1}$, Y. Zhou ${ }^{1}$, J. Zhang ${ }^{1}$, C.C. Wong ${ }^{2}$, Y. Dong ${ }^{2}$, A.S.l. Cheng ${ }^{3}$, J. Yu ${ }^{2}$, K.F. To ${ }^{1}$, W. Kang ${ }^{1}$

${ }^{1}$ Anatomical And Cellular Pathology, Chinese University of Hong Kong, Hong Kong/Hong Kong PRC

${ }^{2}$ Institute Of Digestive Disease, Partner State Key Laboratory Of Digestive Disease, Chinese University of Hong Kong, Hong Kong/Hong Kong PRC ${ }^{3}$ School Of Biomedical Sciences, Chinese University of Hong Kong, Hong kong/ Hong Kong PRC

Contact E-mail Address: huangtingting0531@gmail.com

Introduction: Aberrant Notch activation has been implicated in multiple malignancies, including gastric cancer (GC). However, the clinical significance of Notch receptors and their functional role in gastric carcinogenesis remain unclear.

Aims \& Methods: We aim to delineate the dysregulated Notch signaling in GC and comprehensively reveal its activation by silenced microRNAs (miRNAs) in gastric carcinogenesis. The expression clinical relevance of NOTCH1-4 in GC were achieved from online available dataset. The mRNA and protein expression of NOTCH3 were examined by qRT-PCR and Western blot. The biological function of NOTCH3 in GC was demonstrated by MTT proliferation, monolayer colony formation, cell invasion and migration assays through siRNAmediated knockdown. The prediction of miRNAs which potentially target NOTCH3 was performed by www.microRNA.org and TargetScan. The regulation of NOTCH3 by putative miRNAs was confirmed by qRT-PCR, Western blot and dual luciferase activity assays through ectopic overexpression of miR491-5p and miR-875-5p. The functional downstream targets of NOTCH3 were identified by gene expression microarray.

Results: NOTCH3, but not NOTCH1, 2, 4, is uniformly up-regulated and significantly correlated with poor survival in multiple GC datasets. Knockdown of NOTCH3 in AGS and MKN28 cells exhibited significant anti-oncogenic effect in vitro. NOTCH3 downregulation suppressed cell proliferation, reduced monolayer colony formation, and inhibited cell invasion ability. Moreover, NOTCH3 knockdown significantly promoted cleaved caspase- 3 and cleaved-PARP expression to induce apoptosis, which was further revealed by the gene set enrichment analysis (NCBI/GEO/GSE57303 and NCBI/GEO/GSE57303 database). NOTCH3 was further confirmed to be a direct target of tumor-suppressive miRNAs, miR-491-5p and miR-875-5p. Enforced overexpression of miR-491$5 \mathrm{p}$ and $\mathrm{miR}-875-5 \mathrm{p}$ in GC cells also exerted tumor-suppressive function by inhibiting cell proliferation and inducing apoptosis. More importantly, the expression of miR-491-5p showed negative correlation with NOTCH3 mRNA expression in primary GC samples and re-overexpression of NOTCH3 rescued the inhibitory effect of miR-491-5p and miR-875-5p. Pleckstrin Homology Like Domain Family B Member 2 (PHLDB2) is identified as the functional downstream of NOTCH3 in GC. Concordantly, PHLDB2 knockdown significantly inhibited cell proliferation and promoted apoptosis, which phenocopied NOTCH3 knockdown or miR-491-5p/miR-875-5p overexpression. In primary GC samples, the expression of PHLDB2 and NOTCH3 showed positive correlation in The Cancer Genome Atlas (TCGA) cohort. Thus, the miR-491-5p/miR875-5p-NOTCH3-PHLDB2 oncogenic cascade was constructed.

Conclusion: NOTCH3 is over-expressed and plays an oncogenic role in gastric carcinogenesis through its direct downstream PHLDB2. The activation of NOTCH3 in GC is partly due to the silence of tumor-suppressive miRNAs, miR-491-5p and miR-875-5p. These findings comprehensively revealed the activation of Notch signaling pathway and provided clinical translational potential for GC.

Disclosure of Interest: All authors have declared no conflicts of interest.

P0498 FOXF2 SUPPRESSES WNT SIGNALING PATHWAY IN GASTRIC CARCINOGENESIS THROUGH TRANSCRIPTIONALLY UPREGULATING E3 LIGASE IRF2BPL AND PROMOTING BCATENIN DEGRADATION

Y. Dong ${ }^{1}$, A. Higashimori ${ }^{2}$, Y. Zhang ${ }^{1}$, S. S.m. Ng ${ }^{3}$, T. Arakawa ${ }^{2}$, F.K.L. Chan ${ }^{1}$, J.J.Y. Sung ${ }^{1}$, J. Yu ${ }^{1}$

${ }^{1}$ Institute Of Digestive Disease And Department Of Medicine And Therapeutics, State Key Laboratory Of Digestive Disease, Li Ka Shing Institute Of Health Sciences, The Chinese University of Hong Kong, Hong Kong/Hong Kong PRC ${ }^{2}$ Department Of Gastroenterology, Osaka city University Graduate School of Medicine, Osaka/Japan

${ }^{3}$ Department Of Surgery, The Chinese University of Hong Kong, HONG KONG/ Hong Kong PRC

Contact E-mail Address: yujuan_dong@yahoo.com

Introduction: We found that tumor suppressor gene FOXF2 was silenced in gastric cancer (GC) through promoter hypermethylation. Restoration of FOXF2 suppressed GC tumorigenicity through inhibition of canonical Wnt 
signaling pathway. However, the molecular mechanism of FOXF2 in GC is still unknown.

Aims \& Methods: We hypothesize that FOXF2 transcriptional upregulates a novel E3 ligase that targets $\beta$-catenin for degradation. We aim to investigate the molecular mechanism of FOXF2 in GC and identify such E3 ligase by PCR array, Chromatin Immunoprecipitation (ChIP) assay and luciferase assay. Results: FOXF2 significantly decreased both nuclear and cytosolic levels of $\beta$ catenin in a GSK-3 $\beta$ independent manner and promoted $\beta$-catenin degradation via ubiquitin-proteasome pathway in gastric cells. Using Human Ubiquitin Ligases $\mathrm{RT}^{2}$ Profiler PCR Array and western blot, we identified that IRF2BPL was upregulated upon FOXF2 overexpression and was a promising E3 ligase for $\beta$-catenin. Overexpression of IRF2BPL suppressed the TOP-flash luciferase reporter and reduced Wnt target gene c-myc expression in GC cells. Overexpression of IRF2BPL significantly increased $\beta$-catenin ubiquitination and reduced $\beta$-catenin protein without alteration of its mRNA level. Conversely, knockdown of IRF2BPL significantly decreased endogenous $\beta$-catenin ubiquitination. Immunoprecipitation assay suggested that IRF2BPL interacted with $\beta$ catenin. These results reveal that IRF2BPL is a potential E3 ligase that targets $\beta$ catenin for degradation. To investigate whether FOXF2 directly regulates IRF2BPL transcription, we performed ChIP assay and found that FOXF2 bound on to the IRF2BPL promoter region. We cloned the IRF2BPL promoter region $(-2700 \mathrm{bp}$ to TSS) into pGL3-basic plasmid and performed a luciferase activity assay. Wild-type FOXF2 but not the mutant $\triangle F O X F 2$ significantly activated the luciferase reporter in AGS and $293 \mathrm{ft}$ cells, suggesting that FOXF2 directly activated IRF2BPL transcription. Moreover, FOXF2 significantly increased the level of $\mathrm{H} 3 \mathrm{~K} 27 \mathrm{Ac}$ (a marker to distinguish active from inactive enhancer element) on the 5'-flanking region of IRF2BPL gene, suggesting that FOXF2 positively regulated IRF2BPL gene transcription. In addition, IRF2BPL was significantly downregulated in human GC tissues compared to the adjacent normal tissues $(\mathrm{N}=30, P<0.01)$ by real-time PCR analysis. IRF2BPL mRNA showed a positive correlation with FOXF2 in gastric cancer in a Chinese cohort $(\mathrm{N}=30$, Spearman's rho $=0.42, P<0.05)$ and in the TCGA cohort $(\mathrm{N}=421$, Spearman's rho $=0.38, P<0.001)$.

Conclusion: We reported a novel FOXF2-IRF2BPL- $\beta$-catenin signaling axis in gastric cells. FOXF2 is a critical tumor suppressor in gastric carcinogenesis through promoting $\beta$-catenin degradation by transcriptionally upregulating E3 ligase IRF2BPL.

Disclosure of Interest: All authors have declared no conflicts of interest.

P0499 CLINICOPATHOLOGICAL AND COMPREHENSIVE ANALYSIS BY NEXT GENERATION SEQUENCING IN GASTRIC ADENOCARCINOMA WITH ENTEROBLASTIC DIFFERENTIATION

Y. Akazawa ${ }^{1}$, T. Saito ${ }^{2}$, T. Hayashi², Y. Yanai², S. Tsuyama², K. Akaike², T. Murakami ${ }^{1}$, T. Yao ${ }^{2}$

${ }^{1}$ Department Of Gastroenterology, Juntendo University School of Medicine, Tokyo/Japan

${ }^{2}$ Department Of Human Pathology, Juntendo University School of Medicine, Tokyo/Japan

Contact E-mail Address: yakazawa@juntendo.ac.jp

Introduction: Gastric adenocarcinoma with enteroblastic differentiation (GAED) is a rare variant of gastric carcinoma. It is well known that GAED is associated with poor prognosis $[1,2]$. However, the clinicopathological and molecular biological features have not been elucidated enough. We performed comprehensive analysis by next generation sequencing (NGS) to clarify the clinicopathological features of GAED and to find new therapeutic targets.

Aims \& Methods: Among 1003 patients with primary gastric cancer who underwent surgery or endoscopic resection in our hospital between April 2008 and February 2017, we enrolled 52 cases (early:17, advanced:35) of GAED defined as having tubular to papillary or solid structure with clear cytoplasm and immunohistochemical positivity for at least one of the following antibodies; AFP, Glypican-3 and SALL4. NGS was performed for 24 cases of formalin-fixed paraffin-embedded samples (early: 2, advanced: 22 ) using Ion PGM $^{\mathrm{TM}}$ system with cancer hotspot panel v2 targeting 50 genes (Thermo Fisher Scientific). Immunohistochemical staining (IHC) was carried out to validate genetic alterations. We also analyzed the association among these genetic alterations, clinicopathological factors and prognosis.

Results: As a previous study has shown, lymphatic and venous invasion, lymph nodes metastasis and liver metastasis were frequently observed in this tumor $(67.3,67.3 \%, 69.2 \%$ and $32.7 \%$, respectively). Immunohistochemically, even though AFP positive rate was low $(32.7 \%)$, most cases were diagnosed by positivity of either of other two markers; glypican-3 (80.8\%), SALL4 (80.8\%). There were significant association between lymphatic invasion, liver metastasis and prognosis. NGS revealed that most cases harbored TP53 mutation (19/24, $79.2 \%)$, and other mutations such as CTNNB1 $(2 / 24,8.3 \%)$, APC $(1 / 24$, $4.2 \%)$, ATM $(1 / 24,4.2 \%)$, FBXW7 $(1 / 24,4.2 \%)$ were also detected. Compared with the public data of conventional gastric carcinoma (CGA) [3], TP53 mutation rate was significantly higher in GAED $(79.2 \%$ vs. $47.8 \%$, $\mathrm{p}<0.01)$. However, there was no association between TP53 mutation and clinicopathological factors including patients' prognosis. With regard to the correlation between TP53 mutation and p53-IHC, all cases with TP53 missence mutation $(\mathrm{n}=12)$ showed $\mathrm{p} 53$ overexpression. We found $\mathrm{p} 53$ overexpression by IHC in 29 out of 52 GAED cases $(55.8 \%)$, however, this did not affect patients' prognosis. We also detected ERBB2 amplification in 9 out of 24 cases $(37.5 \%)$ by analyzing copy number variation, and this rate was significantly higher than that reported in CGA $(13.0 \%)(\mathrm{p}<0.01)$ [3]. Most of GAED with ERBB2 amplification showed HER2 overexpression by IHC.
Conclusion: GAED shows generally aggressive behavior characterized by frequent lymphatic invasion and liver metastasis. TP53 mutation rate and ERBB2 amplification rate in GAED is significantly higher than those of CGA. HER2 overexpression associated with ERBB2 amplification could be a new therapeutic target in GAED.

Disclosure of Interest: All authors have declared no conflicts of interest.

\section{References}

1. Murakami T, et al., Gastric Cancer, 2016.

2. Matsumoto K, et al., World J Gastroenterol, 2016.

3. Cancer Genome Atlas Research Network. Nature 2014

\section{P0500 CO-OCCURRENCE OF MODERATE RISK ALLELES IN THE GERMLINE OF FAMILIAL INTESTINAL GASTRIC CANCER SYNDROME}

J. Carvalho ${ }^{1}$, C. São José2 ${ }^{2}$ H. Pinheiro ${ }^{1}$, P. Oliveira ${ }^{2}$, J. Senz ${ }^{3}$, S. Hansford ${ }^{3}$, A.S. Valente ${ }^{2}$, D. Lemos ${ }^{1}$, V. Pascale ${ }^{4}$, F. Roviello ${ }^{4}$, D. Huntsman ${ }^{3}$, C. Oliveira ${ }^{1}$ ${ }^{1}$ i3S - Instituto de Investigação e Inovação em Saúde, Universidade do Porto, Portugal, Porto/Portugal

${ }^{2}$ Ipatimup - Institute of Molecular Pathology and Immunology, University of Porto, Portugal, Porto/Portugal

${ }^{3}$ Department Of Pathology And Laboratory Medicine, University of British Columbia, Vancouver, British Columbia, Canada, Vancouver/Canada/BC ${ }^{4}$ Department Of General Surgery And Surgical Oncology, University of Siena, Italy, Siena/Italy

Contact E-mail Address: jcarvalho@ipatimup.pt

Introduction: Ten percent of all gastric cancers show familial aggregation and may account for at least three syndromes ${ }^{1,2}$ : hereditary diffuse gastric cancer (HDGC), gastric adenocarcinoma and proximal polyposis of the stomach (GAPPS $)^{3}$, and familial intestinal gastric cancer (FIGC) ${ }^{4}$. Whilst germline defects at the $C D H 1^{5},{ }^{6}$ and $A P C^{7}$ genes have been found for HDGC and GAPPS families, respectively, FIGC remains genetically unexplained.

Aims \& Methods: We hypothesised that the rare FIGC syndrome is caused by germline co-occurrence of moderate-risk alleles and represents a polygenic, rather than a classical monogenic disease. Therefore, this study aimed at dissecting the germline and somatic landscapes of the largest FIGC cohort ever studied. Constitutional and tumour DNA from probands of 53 families, fulfilling clinical criteria of FIGC, were screened for 55 candidate gastrointestinal cancer-associated genes with Illumina's MiSeq-platform, and classified according to the American College of Medical Genetics and Genomics (ACMG) guidelines. Somatic second hits, such as second mutation, loss of heterozygosity and promoter methylation, were searched for in FIGC tumours at potentially causative genes by PCR-Sequencing.

Results: Twenty-five out of 53 (47\%) FIGC families harboured germline variants, and co-occurrence of germline moderate-risk alleles was found in ten families. From these ten families, seven harboured one pathogenic or likely pathogenic variant combined with one or more unclassified novel variants. The remaining three families carried solely clusters of novel unclassified variants. Moderate-risk alleles of BRCA2, MAP3K6, MSH6, MSR1, SDHB and SDHD were the most frequently found in this cohort. In addition, tumours arising in these 10 families were enriched in somatic variants within DNA repair genes and often display microsatellite instability phenotype.

Conclusion: The clinical homogeneity and relatively high number of FIGC families herein studied allowed supporting the hypothesis that FIGC may be a polygenic syndrome caused by moderate-risk alleles in gastrointestinal cancerassociated genes.

This work is funded by: 1) FEDER/COMPETE, FCT/MEC/FEDER/PT2020 and FCT funds (projects "PEst-C/SAU/LA0003/2013"; project 007274 (UID/ BIM/04293); 2) ON.2-O Novo Norte/FEDER/QREN (projects NORTE-070162-FEDER-000118 and NORTE-07-0162-FEDER-000067); 3) No Stomach for Cancer Foundation; 4) FCT Fellowships (SFRH/BPD/89764/2012 to PO; SFRH/BPD/86543/2012 to JC; SFRH/BPD/79499/2011 to HP). Disclosure of Interest: All authors have declared no conflicts of interest.

\section{References}

1. Fitzgerald $\mathrm{R}$ and the IGCLC, JMG 2010

2. Oliveira $\mathrm{C}$ et al, Lancet Oncol 2015

3. Worthley et al, Gut 2012

4. Corso G et al, Biomed Res Int 2013

5. Guilford P et al, Nat Genetics 1999

6. Oliveira C et al, Hum Mutat 2002

7. Jun Li et al, Am J Hum Gen 2016

P0501 PREVENTION OF STENOSIS WITH ENDOSCOPIC TRANSPLANTATION OF CULTURED AUTOLOGOUS ORAL MUCOSAL EPITHELIAL CELL SHEETS AFTER ESOPHAGEAL ENDOSCOPIC SUBMUCOSAL DISSECTION

D. Murakami ${ }^{1}$, H. Harada ${ }^{2}$, S. Suehiro ${ }^{2}$, T. Ohki ${ }^{3}$, M. Yamato ${ }^{1}$ Gastroenterology, New Tokyo Hospital, Matsudo, Chiba/Japan ${ }^{2}$ Gatroenterology, New Tokyo Hospital, Matsudo, Chiba/Japan

${ }^{3}$ Institute Of Advanced Biomedical Engineering And Science, Tokyo Women's Medical University, Shinjyuku-ku, Tokyo/Japan 
Contact E-mail Address: daisuke.murakami@gmail.com Introduction: Endoscopic submucosal dissection (ESD) is widely accepted to treat large superficial esophageal neoplasms. However, esophageal stenosis after ESD has become a key complication. To prevent such stenosis, we developed new regenerative therapies that suppresses contracture and stenosis. About two weeks prior to the esophageal ESD, autologous oral mucosal epithelial cell sheets were fabricated from the patient's own oral mucosal tissue. The cell sheets were then endoscopically transplanted onto the ulcer surface immediately after ESD. To date, this cell sheet treatment, which resembles cultured epidermal cell sheet transplantation in severe burn cases, has been clinically applied, and successful outcomes have been obtained at several institutions in Japan and Sweden. In addition to preventing stenosis by the simple dressing of the ulcer's surface area, anti-inflammatory and wound-heal promoting functions of the cell sheets were expected.

Aims \& Methods: For understanding the therapeutic effects of the cell sheet transplantation, we investigated the morphological and histological features of the human oral mucosal epithelial cell sheets, in addition to the cellular gene and protein expressions. From a small biopsy of human volunteer donors' oral mucosal tissue, we isolated and cultured epithelial cells, which were then fabricated into transplantable cell sheets using a previously described procedure. The cell sheets were examined by immunohistochemistry (IHC) and electron microscopy as well as the gene and protein expression analysis by RT-PCR, with a DNA array. Furthermore, the culture supernatant obtained immediately before the transplantation, were analyzed with a cytokine array.

Results: Histological and morphological analyses revealed that tissue-engineered stratified epithelial cell sheets have an apical-basal polarity, and that the junctions between the basal cells of the sheets were significantly loose, due to dissociating desmosomes, which were also fewer in numbers. IHC showed that the expression levels of E-cadherin and desmosomal cadherins were downregulated in the basal cells of the epithelial cell sheets, but mesenchymal markers (N-cadherin, vimentin, and fibronectin) were upregulated. Taken together, these findings implied that epithelial mesenchymal transition (EMT) was induced in the basal cells. The results of the cytokine array showed that the cell sheets secreted EMT-related proteins, growth factors (progranulin and epiregulin), and antimicrobial proteins (b-defensin)

Conclusion: The EMT of the basal cell layer of epithelial cell sheets may contribute to the improved engraftment after cell sheet transplantation. The secretion of antibacterial peptides and various growth factors from the cell sheets would exhibit anti-inflammatory effects, and promote wound-healing compared to reinforcement of esophagus with absorbable synthetic materials such as polymer membrane of polyglycolic acid and polylactic acid.

Disclosure of Interest: All authors have declared no conflicts of interest.

\section{References}

Ohki T, Yamato M, Murakami D, et al. Treatment of oesophageal ulcerations using endoscopic transplantation of tissue-engineered autologous oral mucosa epithelial cell sheets in a canine model. Gut 2006;55(12):1704-10.

Ohki T, Yamato M, Ota M, et al. Prevention of esophageal stricture after endoscopic submucosal dissection using tissue-engineered cell sheets. Gastroenterology 2012;143(3):582-8

\section{P0502 THE OBESTATIN/G PROTEIN-COUPLED RECEPTOR 39 SYSTEM REGULATES PEPSINOGEN I SECRETION IN HUMAN STOMACH}

B. Otero-Alen ${ }^{1}$, S. Leal-López ${ }^{1}$, L. S. Estevez-Perez ${ }^{1}$, M. Otero-Alen ${ }^{2}$, J. BaltarBoileve $^{3}$, J.E. Dominguez-Muñoz ${ }^{4}$, R. Gallego-Gomez ${ }^{5}$, T. Garcia-Caballero ${ }^{5}$, J. P. Perez-Camiña ${ }^{2}$, Y. Pazos-Randulfe ${ }^{1}$

${ }^{1}$ Gastroenterology, Health Research Institute of Santiago (IDIS), Santiago de Compostela/Spain

${ }^{2}$ Health Research Institute of Santiago (IDIS), Santiago de Compostela/Spain ${ }^{3}$ Surgery, University Hospital of Santiago, Santiago de Compostela/Spain

${ }^{4}$ Gastroenterology, University Hospital of Santiago, Santiago de Compostela/Spain

${ }^{5}$ Morphological Sciences, University Hospital of Santiago, Santiago de

Compostela/Spain

Contact E-mail Address: info@fienad.org

Introduction: Obestatin, a 23-amino acid peptide derived from the ghrelin peptide precursor, was originally isolated from stomach and characterized to bind selectively to the G protein-coupled receptor GPR39 (1). We have recently described the expression of obestatin/GPR39 system in healthy human stomach as well as in human gastric adenocarcinoma. In healthy human stomachs, obestatin expression was observed in the neuroendocrine cells and GPR39 expression was localized mainly in the chief cells of the oxyntic glands. In human gastric adenocarcinomas, the reported data strongly suggest the involvement of the obestatin/GPR39 system in the pathogenesis and/or clinical outcome, and highlight the potential usefulness of GPR39 as a prognostic marker in gastric cancer (2). However, the physiological role of the obestatin/GPR39 system in healthy stomach remains unknown.

Aims \& Methods: To investigate the implication of the obestatin/GPR39 system in the regulation of pepsinogen secretion. GPR39, obestatin and pepsinogen I (PGI) expression was determined in the AGS cell line by immunocytochemistry; and in endoscopic biopsies of human healthy stomachs by immunohistochemistry and immunofluorescence. PGI secretion was measured after exogenous administration of obestatin (hemoglobin method, immunoblot) in AGS cells and in an in vitro explant culture of human stomach tissues obtained from surgical specimens after bariatric surgery. The influence of the acute GPR39 deficiency on PGI secretion was determined using siRNA GPR39.

Results: AGS cells expressed obestatin, GPR39 and PGI. In these cells, exogenous administration of obestatin $(200 \mathrm{nM}, 40 \mathrm{~min})$ stimulated PGI secretion $(60 \%$ over control). This effect was exerted via the GPR39 receptor. In the human healthy stomach, GPR39 expression was detected mainly in the chief cells of the oxyntic glands but also in a few cells of the neck section (pre-chief cells). This expression co-localized with PGI expression in both cell types. The mucous neck cells were positive for PGI and negative for GPR39. Obestatin also exerted a dose-dependent stimulatory effect on PGI secretion in the in vitro explant culture of human stomach, being significant for 100 and $200 \mathrm{nM}$ compared to the control sample at $20 \mathrm{~min}$ (39\% and $66 \%$ over control, respectively), for $200 \mathrm{nM}$ at $40 \mathrm{~min}$ $(51 \%$ over control) and $100 \mathrm{nM}$ at $60 \mathrm{~min}(64 \%$ over control).

Conclusion: The obestatin/GPR39 system is physiologically involved in the stimulation of pepsinogen secretion in the healthy human stomach.

Disclosure of Interest: All authors have declared no conflicts of interest.

\section{References}

1. Zhang JV, et al. Science. 2005;310:996-9.

2. Alen BO, et al. Oncotarget. 2016;7:5957-71.

\section{P0503 SERUM EXOSOMAL MIRNAS EXPRESSION AS NOVEL BIOMARKERS FOR DETECTION OF ESOPHAGEAI ADENOCARCINOMA}

H. Chen

Jiangsu Procince Hospital, Nanjing/China

Contact E-mail Address: chenhan068@hotmail.com

Introduction: Novel biomarkers for the diagnosis of esophageal adenocarcinoma (EAC) are urgently required. Currently, there is increasing evidence suggesting that serum exosomal miRNAs may be potential noninvasive biomarkers for certain diseases. The objective of the present study was to find and investigate whether exosomal miRNAs could be effective biomarkers for EAC.

Aims \& Methods: In the present study, exosomes were isolated from the serum of both EAC patients and normal controls. Total RNA was extracted from exosomes and miRNA levels were compared between EAC and control patients in serum exosomes. We also sought to investigate the relevance of exosomal miRNA expression to clinicopathological factors in EAC.

Results: We measaured levels of several exosomal miRNAs, including miR-21, miR-16, miR-25, miR-155, miR-192, miR-92a, in 9 EAC patents and 9 controls. Theses miRNAs were chosen because they have been shown to function as oncomiRs in previous studies. Levels of miR-21, miR-16, miR-25 and miR-155 were significantly higher in EAC patients than in controls (fold- change 35.36, 30.87, 9.24 and 2.26, respectively). The level of miR-192 was significantly lower in EAC patients than in controls (Fold-change: 0.35 ). We did not observe a significant fold-change in miR-92a expression levels between EAC and controls. P-values did not achieve statistical significance, possible due to large standard deviations and relatively small sample sizes. We also visualized exosomes isolated from both cell culture medium and sera of EAC patients and control subjects, with diameter ranging from 30 to $100 \mathrm{~nm}$ using transmission electron microscopy.

Conclusion: Serum exosomal miRNAs can be isolated, measured, and may serve as potential biomarkers in EAC patients. miRNA microarray or next-gen sequencing analyses and larger sample sizes are needed to validate these early results.

Disclosure of Interest: All authors have declared no conflicts of interest.

\section{P0504 COMPARATIVE STUDY BETWEEN THE EFFICACY OF REBAMIPIDE, SUCRALFATE AND PANTOPRAZOLE IN TREATMENT OF POST-BANDING VARICEAL ULCERS}

M. Yousry ${ }^{1}$, A. A. Wahib ${ }^{2}$, G. M.M. Soliman

${ }^{1}$ Tropical Medicine \& Gastroenterology, Faculty of Medicine Al-Azhar university, Gharbia, Almehalla Saftturab/Egypt

${ }^{2}$ Tropical Medicine \& Gastroenterology, Faculty of Medicine Al-Azhar university, Cairo/Egypt

${ }^{3}$ Tropical Medicine \& Gastroenterology, Faculty of Medicine Al-Azhar university, Cairo/Egypt

Contact E-mail Address: doctor gemy@yahoo.com

Introduction: Endoscopic variceal band ligation (EVL) is an effective procedure to control and prevent variceal bleeding in patients with liver cirrhosis. Although EVL has some complications, yet these complications are related to post-EVL ulcers. Few data exist regarding therapy of post-ligation ulcer and treatment been mostly empirical with drugs used for peptic ulcer diseases.

Aims \& Methods: We aimed to compare between the efficacy of rebamipide, sucralfate and pantoprazole in treatment of post banding variceal ulcers. Seventy-five patients with oesophageal varices eligible for elective band ligation represented the population of the study. The patients were allocated into three groups; rebamipide group, they received rebamipide $100 \mathrm{mg} 3$ times daily; pantoprazole group, they received pantoprazole $40 \mathrm{mg}$ /day orally at morning; sucralfate group, they received sucralfate 1 gm every 6 hours, for 14 days beginning at the next day of band ligation. Subjects underwent EGD 14 days after banding. 
Primary outcomes included the size and number of ulcers and the subjects' reports of bleeding, dysphagia, chest pain and vomiting.

Results: At follow-up endoscopy, the number of patients with post-band ulcers and size of ulcers were similar in the three groups. However, the number of ulcers for each patient is statistically significant less in rebamipide group when compared to pantoprazole and sucralfate $(\mathrm{P}<.001)$. Chest pain, dysphagia and vomiting scores were not significantly different. Dysphagia was by far the most common symptom with no case of bleeding was reported in all patients of the studied groups.

Conclusion: Rebamipide is effective in decreasing the post banding complication and reducing size of ulcer as well as the number of ulcers with no significant effect on post banding ulcer formation. Rebamipide can be used routinely in settings of post-EVL as a good alternative to pantoprazole and sucralfate.

Disclosure of Interest: All authors have declared no conflicts of interest.

\section{P0505 MULTICENTRE EVALUATION OF FIRST-LINE ENDOSCOPIC TREATMENT WITH THE OTSC IN ACUTE NON- VARICEAL UPPER GASTROINTESTINAL BLEEDING AND COMPARISON WITH THE ROCKALL COHORT - THE FLETROCK- STUDY}

E. Wedi ${ }^{1}$, A. Fischer ${ }^{2}$, J. Hochberger ${ }^{3}$, C. Jung ${ }^{1}$, H. Richter-Schrag ${ }^{2}$ ${ }^{1}$ Gastroenterology And GI Oncology, University Hospital Göttingen, Göttingen Germany

${ }^{2}$ Department Of Medicine II, University of Freiburg, Freiburg/Germany ${ }^{3}$ Departement Of Gastroenterology, Vivantes Klinikum in Friedrichshain, Berlin/ Germany

\section{Contact E-mail Address: edris1@web.de}

Introduction: The over-the-scope-clip (OTSC) overcomes limitations of standard clips and achieves a more efficient and reliable haemostasis in non-variceal upper gastrointestinal bleeding (NVUGIB). The study aims to evaluate mortality, rebleeding and mortality after rebleeding of patients in whom the OTSC was used as first-line endoscopic treatment (FLET) of NVUGIB. Data on OTSC use for NVUGIB in high-risk patients is currently limited.

Aims \& Methods: In total, 118 patients (FLET cohort) with a median age of 73.5 years (range $29-93$ years; mean $( \pm \mathrm{SD}) 71.39 \pm 12.39$ years) were included. The distribution of patients with respect to risk category revealed a median Rockall score (RS) of 7 (range 3-10; mean $( \pm \mathrm{SD}) 7.25 \pm 1.51$ ). For hypothesis testing, the FLET cohort was categorized into 3 risk groups taking into account the Rockall score: low risk (Rockall risk category $(\mathrm{RRC}) \leq 3$ ), moderate risk (RRC 4-7), and high-risk $(\mathrm{RRC}>8)$. Event rates (mortality, re-bleeding and mortality after rebleeding) observed per risk group were compared to predicted event rates (Rockall cohort) using Fisher's Exact Test.

Results: Primary successful haemostasis (PSH) was achieved in $92.4 \%$ either by FLET alone or in combination with an additional haemostasis technique in $1.7 \%$ ( $\mathrm{SCS}=$ secondary clinical success). In $7.5 \%$ of the FLET cohort PSH couldn't be achieved. Mortality in the FLET cohort was in the high-risk group (RRC $\geq 8$ ) lower compared to RRC prediction, but no significant difference. However mortality after re-bleeding was significantly reduced from $27.9 \%$ to $10.9 \%$ in the high-risk group $(\mathrm{RRC} \geq 8)$ treated with FLET $(\mathrm{p}<0.011)$. Furthermore, occurrence of re-bleeding or continued bleeding was significantly lower in the moderate risk group (RRC 4-7) with $4.9 \%$ as well as in the high-risk group ( $\mathrm{RCC} \geq 8$ ) with $21.4 \%$ compared to RCC $24.0 \%$ and $53.2 \%$, respectively, as predicted by the Rockall scoring system $(\mathrm{p}<0.001)$.

Conclusion: Our study shows that OTSC is superior to standard clipping techniques and FLET of OTSC reduces significantly re-bleeding and re-bleeding associated mortality in NVUGIB and it is for this reason we recommend FLET for NVUGIB in high-risk patients.

Disclosure of Interest: All authors have declared no conflicts of interest.

\section{P0506 A NEW PREDICTION RULE FOR UPPER GASTROINTESTINAL BLEEDING USING THE GLASGOW BLATCHFORD SCORE AND CT NUMBER}

M. Tsudome, K. Fujita, A. Sugahara

Department Of Gastroenterology, Yodogawa Christian Hospital, Osaka/Japan

Contact E-mail Address: marie_t@zpost.plala.or.jp

Introduction: Upper gastrointestinal bleeding can have various severities, with some cases needing for intensive care while, others allow for patient to go home. Thus, it is important to detect the patients who need endoscopic treatment. The Glasgow Blatchford score (GBS) is currently regarded as the best score for predicting endoscopic treatment (area under the receiver operating characteristic curve (AUROC) 0.75)[1]. Contrast-enhanced CT is also useful for patients with gastrointestinal bleeding [2]. However, it is a complicated procedure and it cannot be used for patients with renal failure.

Aims \& Methods: The aim is to analyze the accuracy of the GBS and nonenhanced CT in predicting the necessity of endoscopic treatment for patients with upper gastrointestinal bleeding. This study was designed as a hospitalbased observation study. Patients who were hospitalized for upper gastrointestinal bleeding and had an endoscopy performed within 24 hours from April 2013 to March 2014 at Yodogawa Christian Hospital were studied. The clinical utility of the GBS and CT were assessed separately and in combination
Results: A total of 113 patients were included in the study. The median GBS was 10 (interquartile range (IQR) 7-12). The score did not discriminate well with AUROC of 0.63 . The median CT number was $48 \mathrm{HU}$ (IQR 26.7-60.7). The CT number also did not discriminate well with AUROC of 0.65 . We set the cut off for GBS at 4 , and a CT number at $50 \mathrm{HU}$. The endoscopic treatment percentage of the group of GBS $\geqq 4$ and CT number $\geqq 50 \mathrm{HU}$ was $63.5 \%$, GBS $\geqq 4$ and $\mathrm{CT}$ number $<50 \mathrm{HU}$ was $30.6 \%, \mathrm{GBS}<4$ and $\mathrm{CT}$ number $\geqq 50 \mathrm{HU}$ was $33.3 \%, \mathrm{GBS}<4$ and $\mathrm{CT}$ number $<50 \mathrm{HU}$ was $11.1 \%$. We counted that GBS $\geq 4$ was 1 point, CT number $\geq 50 \mathrm{HU}$ was 1 point. The points were added up to a total score that predicts the necessity for endoscopic treatment. Those scoring 2 points was about 60 percentage for the necessity for endoscopic treatment, 1 points was about 30 percentage, 0 point was about 10 percentage. AUROC of this model was 0.69

Conclusion: Using both the GBS and CT in combination performed better for predicting the necessity of endoscopic treatment for patients presenting with upper gastrointestinal bleeding.

Disclosure of Interest: All authors have declared no conflicts of interest.

\section{References}

1. Comparison of risk scoring systems for patients presenting with upper gastrointestinal bleeding. Adrian J Stanley et al, BMJ 2017; 356: i6432

2. Usefulness of CT angiography in diagnosing acute gastrointestilnal bleeding. Lian-Ming Wu et al, W J Gastroenterology 16(31):3957-3963, 2010

\section{P0507 FIBRIN GLUE CAN PREVENT BLEEDING AFTER ENDOSCOPIC SUBMUCOSAL DISSECTION}

L. Yu, Y. Wang

Jiangsu Province Hospital, Nanjing/China

Contact E-mail Address: ylianzhen@126.com

Introduction: Fibrin glue can prevent bleeding after endoscopic submucosal dissection.

Aims \& Methods: We aimed to explore the utility of fibrin glue in prevention of bleeding after endoscopic submucosal dissection and to investigate the risk factors for bleeding after endoscopic submucosal dissection. 330 patients who underwent ESD between July 2015 and June 2016 in Jiangsu Province Hospital were enrolled in this study, the patients were randomly divided into two groups, fibrin glue was sprayed during the operation in 161 patients of Group A, while 169 patients in group B were treated with routine hemostasis method postoperative bleeding was defined as hematemesis or melena or $\mathrm{Hb}$ $>2 \mathrm{~g} / \mathrm{dL}$ decrease after the end of ESD within 30 days. Delayed postoperative bleeding was defined as bleeding 7 to 30 days after operation. Then bleeding rate after ESD, average length of stay and average hospitalization expenses were compared between the two groups to explore the clinical effect of fibrin glue. 37 patients experienced bleeding after ESD were grouped into bleeding group, the remaining 293 patients were divided into non-bleeding group, several factors were compared between the two groups to analyse the risk factors of bleeding after ESD.

Results: There are no differences in age, gender, lesion location, underlying disease, taking anticoagulant drugs or not, invasive depth, pathological type and procedure time between Group A and Group B. Bleeding rate after ESD was lower in group A. $(\mathrm{P}<0.05)$. And Intraoperative hemostasis rate was higher in group A. ( $\mathrm{P}<0.05) .2$ patients in group $\mathrm{B}$ experienced delayed postoperative bleeding, while none in group A experienced delayed postoperative bleeding. Patients in group B spent more time staying in hospital than group A, while the average hospitalization expense was higher in Group B.37 patients experienced bleeding after ESD, among whom 22 occurred within 24 hours after ESD, 13 within 1 week and 2 after 1 week. Univariate analysis revealed that resection size and procedure time over 120 minutes are risk factors for bleeding after ESD. The use of fibrin glue can reduce the bleeding rate after ESD. Multivariate analysis revealed that resection size greater than or equal to $3 \mathrm{~cm}$ and the absence of fibrin glue are independent risk factor for bleeding after ESD.

Conclusion: The use of fibrin glue is safe, effective and economical in the procedure of ESD. Resection size and procedure time over 120 minutes are risk factors for bleeding after ESD. The use of fibrin glue can reduce the bleeding rate after ESD. Resection size greater than or equal to $3 \mathrm{~cm}$ and the absence of fibrin glue are independent risk factor for bleeding after ESD. So adequate preparation and close monitoring are needed during the procedure of ESD.

Disclosure of Interest: All authors have declared no conflicts of interest.

\section{References}

1. Oka, S., et al., Advantage of endoscopic submucosal dissection compared with EMR for early gastric cancer. Gastrointestinal Endoscopy, 2006. 64(6): p. 877883

2. Takao, T., et al., Tissue shielding with polyglycolic acid sheets and fibrin glue on ulcers induced by endoscopic submucosal dissection in a porcine model. Endoscopy International Open, 2015. 03(02): p. E146-E151.

3. Nakanishi, H., et al., Pretreatment Gastric Lavage Reduces Postoperative Bleeding after Endoscopic Submucosal Dissection for Gastric Neoplasms. PLOS ONE, 2016. 11(2): p. e0149235.

4. Zhang, Y., Effects of medical adhesives in prevention of complications after endoscopic submucosal dissection. World Journal of Gastroenterology, 2013. 19(17): p. 2704. 
5. Hiroyuki, T., et al., A basic study of the effect of the shielding method with polyglycolic acid fabric and fibrin glue after endoscopic submucosal dissection. Endosc Int Open, 2016. 4(12): p. E1298-E1304.

6. Tan, E.S., et al., Fibrin Glue Spray as a Simple and Promising Method to Prevent Bleeding after Gastric Endoscopic Submucosal Dissection. Digestive Surgery, 2016. 33(6): p. 455-461.

7. Tsuji, Y., et al., Polyglycolic acid sheets and fibrin glue decrease the risk of bleeding after endoscopic submucosal dissection of gastric neoplasms (with video). Gastrointest Endosc, 2015. 81(4): p. 906-12.

\section{P0509 A COMPARATIVE STUDY OF THERAPEUTIC EFFECT BY VONOPRAZAN AND ESOMEPRAZOLE ON BLEEDING AFTER GASTRIC ENDOSCOPIC SUBMUCOSAL DISSECTION}

Y. Sakata ${ }^{1}$, T. Yamazaki ${ }^{1}$, Y. Yasui ${ }^{1}$, K. Yamamoto ${ }^{1}$, M. Suo ${ }^{2}$, A. Kimura ${ }^{1}$, A. Nakata ${ }^{1}$, M. Yamamura ${ }^{1}$, T. Suekane ${ }^{1}$, T. Nakai ${ }^{2}$, E. Sasaki ${ }^{1}$, K. Sano ${ }^{1}$, K. Watanabe ${ }^{1}$, Y. Kawasaki ${ }^{2}$, K. Kioka ${ }^{2}, \mathrm{H}$. Nebiki ${ }^{3}$

${ }^{1}$ Gastroenterology, Osaka City General Hospital, Osaka/Japan

${ }^{2}$ Hepatology, Osaka City General Hospital, Osaka/Japan

${ }^{3}$ Dept. Of Gastroenterology, Osaka City General Hospital, Osaka/Japan

Contact E-mail Address: saka_t.med@icloud.com

Introduction: Proton pomp inhibitors (PPIs) have been widely used for the treatment of endoscopic submucosal dissection-induced gastric ulcers. However, postoperative bleeding is still one of the most important adverse side effects., Vonoprazan (VPZ), a potaissium-competitive acid blocker, is a new class of acid-suppressing agents, and it is expected to reduce bleeding after gastric endoscopic submucosal dissection (ESD) by strongly inhibiting gastric acid secretion compared with PPIs. ${ }^{3}$

Aims \& Methods: We compared the incidence of bleeding after gastric ESD between subjects treated with VPZ and those treated with esomeprazole (EPZ). Data for 101 patients who underwent gastric ESD from December 1, 2014 to December 31, 2016 in Osaka City General Hospital and started to take VPZ $(\mathrm{n}=22)$ or EPZ $(\mathrm{n}=79)$ by the day before ESD was reviewed. Twelve of them (3 in the VPZ group, 9 in the EPZ group) were excluded for simultaneous resection of two or more sites. A case in which active bleeding or exposed vessels were observed on the bottom of ulcers with hematemesis, melena or a drop of not less than $2 \mathrm{~g} / \mathrm{dl}$ of Hemoglobin within 4 weeks after ESD was defined as "post-ESD bleeding". In addition, we perform second-look endoscopy on the day after ESD. A case in which hemostasis was needed with hemorrhage of Forrest IIa or more when we underwent second-look endoscopy was defined as "next-day hemostasis case". We investigated retrospectively post-ESD bleeding rate and next-day hemostasis rate in the VPZ group and the EPZ group.

Results: Gender, age, resected specimen diameter, oral antithrombotic drug administration, and dialysis were not significantly different in both groups. Two of the 19 patients in the VPZ group $(10.5 \%)$ and 6 of the 70 patients in the EPZ group (8.6\%) had Post-ESD bleeding (Table). In addition, 6 patients in the VPZ group (31.6\%) and 37 patients in the EPZ group $(52.9 \%)$ had next-day hemostasis. There was no significant difference in both groups regarding postESD bleeding $(\mathrm{p}=0.678)$ and next-day hemostasis $(\mathrm{p}=0.197)$. However, nextday hemostasis rate was somewhat higher in the EPZ group than that in the VPZ group. That is possibly because EPZ or VPZ was first administered mostly from the day before ESD in both groups in our hospital, moreover, VPZ rapidly inhibited gastric acid secretion after administration compared with PPIs. ${ }^{3}$

Table: Incidence of post-ESD bleeding and next-day hemostasis

\begin{tabular}{llll}
\hline & $\begin{array}{l}\text { Vonoprazan } \\
\text { group, n }(\%)\end{array}$ & $\begin{array}{l}\text { Esomeprazole } \\
\text { group, n }(\%)\end{array}$ & P-Value \\
\hline Total & 19 & 70 & \\
Post-ESD bleeding & $2(10.5)$ & $6(8.6)$ & 0.678 \\
Next-day hemostasis & $6(31.6)$ & $37(52.9)$ & 0.197 \\
\hline
\end{tabular}

Conclusion: VPZ didn't significantly reduce post-endoscopic submucosal dissection bleeding compared with EPZ.

Disclosure of Interest: All authors have declared no conflicts of interest.

\section{References}

1. Toyokawa $\mathrm{T}$, Inaba $\mathrm{T}$, Omote $\mathrm{S}$, et al. Risk factor for perforation and delayed bleeding associated with endoscopic submucosal dissection for early gastric neoplasms: analysis of 1123 lesions. J Gastroenterol Hepatol 2012; 27: 907-12.

2. Mannen K, Tsunada S, Hara M, et al. Risk factors for complications of endoscopic submucosal dissection in gastric tumors: analysis of 478 lesions. $J$ Gastroenterol 2010; 45: 30-6

3. Sakurai Y, Mori Y, Okamoto H, et al. Acid-inhibitory effects of vonoprazan $20 \mathrm{mg}$ compared with esomeprazole $20 \mathrm{mg}$ or rabeprazole $10 \mathrm{mg}$ in healthy adult male subjects - a randomized open-label cross-over study. Aliment Pharmacol Ther 2015; 42: 719-30.

4. Kagawa T, Iwamoto M, Ishikawa S, et al. Vonoprazan prevents bleeding from endoscopic submucosal dissection-induced gastric ulcers. Aliment Pharmacol Ther 2016; 44: 583-91.
P0510 OUTCOMES FROM AN INTERNATIONAL MULTICENTRE REGISTRY OF PATIENTS WITH GASTROINTESTINAL BLEEDING UNDERGOING ENDOSCOPIC TREATMENT WITH HEMOSPRAY

D. Alzoubaidi ${ }^{1}$, S. Gulati ${ }^{2}$, A. Murino ${ }^{3}$, L.H. Eusebi ${ }^{4}$, D. Graham ${ }^{5}$, A. Haji ${ }^{6}$, J.J. G.h.m. Bergman ${ }^{7}$, M. Goetz ${ }^{8}$, E. J. Despott ${ }^{9}$, M. Banks ${ }^{5}$, B. Hayee ${ }^{2}$, R. Haidry ${ }^{1}$ ${ }^{1}$ Division Of Surgery And Interventional Science, University College London,

London/United Kingdom

${ }^{2}$ Department Of Gastroenterology, Kings College Hospital, London/United Kingdom

${ }^{3}$ The Royal Free Unit For Endoscopy, The Royal Free Hospital and University College London (UCL) Institute for Liver and Digestive Health, London, United Kingdom, London/United Kingdom

${ }^{4}$ Department of Medical and Surgical Sciences, University of Bologna, Bologna Italy

${ }^{5}$ Gastroenterology, University College London Hospital, London/United Kingdom ${ }^{6}$ Gastroenterology, Kings College Hospital, London/United Kingdom

${ }^{7}$ Gastroenterology \& Hepatology, Academic Medical Centre - Gastroenterology \& Hepatology, Academic Medical Centre; Amsterdam/NL. Amsterdam/Netherlands ${ }^{8}$ Interdisziplinäre Endoskopie, Universitätsklinikum Tübingen, Tübingen/Germany ${ }^{9}$ Royal Free Unit For Endoscopy \& Centre For Gastroenterology, Ucl Institute For Liver \& Digestive Health, Royal Free Hospital \& UCL School of Medicine,

London/United Kingdom

Contact E-mail Address: d.alzoubaidi@ucl.ac.uk

Introduction: Acute gastrointestinal bleeding (AGIB) can carry poor outcomes unless prompt endoscopic haemostasis is achieved. Hemospray is a novel proprietary mineral blend that forms a mechanical barrier over the bleeding site when applied endoscopically.

Aims \& Methods: The primary aim of this international multicentre registry is to collect data on the successful cessation of GI bleeding following application with Hemospray. Secondary outcomes of recurrent bleeding (within 72 hours), 30 day mortality, disease and procedure specific outcomes were also collected.

Method: Data was collected prospectively (January 2016 - April 2017) on the use of Hemospray in acute upper and lower GI bleeding, from 3 initial centres in the international registry. The use of Hemospray in GI bleeding was at the endoscopist's discretion at the time of endoscopy. Hemospray use was either as monotherapy, as dual-therapy with standard endoscopic techniques or as rescue therapy once standard methods had failed.

Results: To date 56 cases have been recruited ( 39 male and 17 female). The Forrest Classification of the bleeding lesions were in $5(9 \%)$ cases Forrest Ia bleed, $41(73 \%)$ Ib, $3(5 \%)$ IIa, $4(7 \%)$ IIb and $3(5 \%)$ Forrest III bleed Sources of GI bleed included Peptic ulcer disease $24(43 \%)$, post endoscopic therapy $9(16 \%)$, malignancy $11(20 \%)$, inflammation $3(5 \%)$, Mallory Weiss tear $2(4 \%)$, angiodysplasia $1(2 \%)$, bleeding polyp $2(4 \%)$, duodenal diverticular bleed $1(2 \%)$, oesophageal variceal bleed $1(2 \%)$, post radiation $1(2 \%)$, and bleed post NGT insertion $1(2 \%)$. A total of 48 patients $(86 \%)$ achieved immediate haemostasis after Hemospray endoscopic therapy. 8 patients did not achieve haemostasis. 2 managed conservatively, 1 treated by radiological intervention and 5 died. Hemospray was used in 25 patients $(45 \%)$ as monotherapy [haemostasis achieved in $22 / 25(88 \%)]$, in 22 patients $(39 \%)$ in combination with other modalities [haemostasis achieved in $17 / 22(77 \%)$ ] and in 9 patients $(16 \%)$ used as rescue therapy where other modalities failed [haemostasis achieved in $9 / 9(100 \%)$ ]. 9 patients $(16 \%)$ were anticoagulated at the time of emergency endoscopy. Haemostasis was achieved in all anticoagulated patients. There were 6 cases of delayed re-bleeding of which 1 occurred in less than 24 hours post initial endoscopy, 2 at 24-72 hours, 1 at 4-7 days, 1 at 7-14 days and 1 more than 14 days after the initial endoscopy. There was no prior use of anticoagulation in any of these patients. There were no reported immediate or delayed complications from the treatment.

Conclusion: Early data from our registry show a high rate of immediate haemostasis $(86 \%)$ with Hemospray and an excellent safety profile. The imminet expansion of this registry to other centres in Europe will provide invaluable data on the efficacy of Hemospray in various disease and patient types over the coming years. Disclosure of Interest: All authors have declared no conflicts of interest.

\section{P0511 RISK FACTORS OF GASTROINTESTINAL BLEEDING IN} PATIENTS RECEIVED DUAL ANTIPLATELET THERAPY

W. Yingyongthawat ${ }^{1}$, A. Pulsombat ${ }^{2}$

${ }^{1}$ Department Of Medicine, Faculty of Medicine Ramathibodi Hospital, Bangkok/ Thailand

${ }^{2}$ Department Of Medicine, Faculty of Medicine Ramathibodi Hospital, Bangkok/ Thailand

Contact E-mail Address: Dr.worapoth@gmail.com

Introduction: Current guidelines suggest dual antiplatelet therapy (DAPT), clopidogrel or ticagrelor with aspirin, for patients with acute coronary syndrome. Other indications of DAPT include recurrent ischemic stroke and peripheral vascular disease. Gastrointestinal bleeding (GIB) is one of the most common adverse effects of DAPT, potentially causing hospital admission and death. Scanty information regarding safety of DAPT in Thailand is available.

Aims \& Methods: The objectives were to determine cumulative incidence and risk factors of GIB in patients received DAPT, clopidogrel with aspirin and ticagrelor with aspirin among Thai patients.

A retrospective cohort study was conducted. We reviewed the medical records of patients received clopigogrel with aspirin or ticagrelor with aspirin between January 2013 and June 2015 at Ramathibodi hospital. In patients with GIB, the endoscopic finding with stigmata of recent hemorrhage was also recorded. 
Results: A total of 201 patients received clopidogrel with aspirin and 199 patients received ticagrelor with aspirin were recruited. Mean \pm standard deviation age was $66.2 \pm 11.3$ years and $63.3 \%$ of patients were male. The most common indication of DAPT was acute coronary syndrome $(85.4 \%$ in clopidogrel group vs. $100 \%$ in ticagrelor group). Duration of treatment with clopidogrel and ticagrelor were 121.5 days vs. 231 days, respectively $(\mathrm{p}=0.216)$. There were $20(10.1 \%)$ GIB events in clopidogrel group and $11(5.5 \%)$ in ticagrelor group. The most endoscopic findings of GIB was gastric erosion $(44 \%$ in clopidogrel group vs. $66.7 \%$ in ticagrelor group). Risk ratio (RR) of GIB event of clopidogrel compared to ticagrelor was 1.84 [95\% confidence interval (CI) 0.9 $3.7, \mathrm{p}=0.093]$. By multivariate logistic regression analysis, duration of DAPT $<180$ days (RR 3.28; 95\% CI 1.89-5.69, $\mathrm{p}<0.001$ ) and history of previous GIB were associated with GIB events (RR 10.35; 95\% CI 6.04-17.71, p < 0.001).

Conclusion: Risk of GIB is almost two times higher among patients received clopidogrel with aspirin compared to those received ticagrelor with aspirin. Closed monitoring patients who had duration of DAPT $<180$ days and previous GIB might be minimized the risk of GIB event after receiving DAPT.

Disclosure of Interest: All authors have declared no conflicts of interest.

\section{References}

1. Baigent C, Blackwell L, Collins R, et al. Aspirin in the primary and secondary prevention of vascular disease: collaborative meta-analysis of individual participant data from randomized trials. Lancet 2009; 373: 1849-1860.

2. Wright RS, Anderson JL, Adams CD, et al. 2011 ACCF/AHA Focused Update of the Guidelines for the Management of Patients With Unstable Angina/Non-ST-Elevation Myocardial Infarction (Updating the 2007 Guideline). J Am Coll Cardiol 2011; 57(19): 1920-59.

3. Serebruan VL, DiNicolantonio JJ, Can MM, et al. Gastrointestinal Adverse Events after Dual Antiplatelet Therapy: Clopidogrel Is Safer than Ticagrelor, but Prasugrel Data Are Lacking or Inconclusive. Cardiology 2013; 126: 35-40.

4. James S, Akerblom A, Cannon CP, et al. Comparison of ticagrelor, the first reversible oral $\mathrm{P} 2 \mathrm{Y}(12)$ receptor antagonist, with clopidogrel in patients with acute coronary syndromes: rationale, design, and baseline characteristics of the PLATelet inhibition and patient Outcomes (PLATO) trial. Am Heart $J$ 2009; 157: 599-605.

P0512 REAL-LIFE ANALYSIS OF FREQUENCY, LOCATIONS AND BLEEDING SOURCES IN UNSELECTED EMERGENCY PATIENTS DURING NON-VITAMIN K ANTICOAGULANT (NOAC) THERAPY AND COMPARISON TO CONTROLLED APPROVAL STUDIES

M. Raithel ${ }^{1}$, H. Albrecht ${ }^{2}$, L. S. Maass ${ }^{2}$, S. Peter ${ }^{1}$, A.K. Kluger ${ }^{1}$, M.F. Neurath ${ }^{3}$, A. F. Hagel ${ }^{2}$

${ }^{1}$ St. Marien Waldkrankenhaus, Erlangen/Germany

${ }^{2}$ University Clinical Center Erlangen, Erlangen/Germany

${ }^{3}$ Department Of Internal Medicine 1, University Clinical Center Erlangen,

Erlangen/Germany

Contact E-mail Address: martin.raithel@waldkrankenhaus.de

Introduction: Non-vitamin K direct oral anticoagulants (NOAC) are increasingly used in thromboembolic disorders due to an efficacy at least equally as vitamin K antagonists (VKA) and/or significantly higher saftey for intracerebal bleeding or major bleedings of any source. In the approval studies, there was no generally increased bleeding rate for all types of bleeding, but different gastrointestinal bleeding (GIB) rates for apixaban, dabigatran, edoxaban and rivaroxaban.

Aims \& Methods: The patient cohort included consecutive unselected patients manifesting with a GI bleeding under anticoagulation in 2014. All patients who were diagnosed with a GI bleeding under NOAC or VKA therapy in the emergency department of the University Hospital Erlangen were analyzed. Their data were entered in a registry and evaluated in terms of bleeding type, localization, use of proton pump inhibitor and frequencies. These real-life results were then compared with the published data from important approval studies, reporting each on the above mentioned NOACs.

Results: In 213 patients with GI bleeding, 31 patients received VKA $(14.5 \%)$ and 23 patients $(10.8 \%$, n.s. ) had NOAC with major bleeding rates of $68 \%$ and $61 \%$, resp.. In patients with VKA $87 \%$ had an upper GIB, $12 \%$ a lower GIB, and none had a rectal bleeding $(0 \%)$. During NOAC therapy, a similar distribution was found with $71 \%$ and $17 \%$, but the proportion of rectal bleeding was higher with $10 \%$.

This frequency of GIB rates in unselected emergency patients is significantly higher than reported in the controlled NOAC approval studies that included selected patients $(1.8-3.6 \%$ GIB!). In these NOAC studies a lower rate of upper GIB $(55 \%, 0-71 \%)$, a higher rate for lower GIB $(32 \%, 17-84 \%)$ and rectal bleeding $(15 \%, 10-47 \%)$ was found. Although NOACs are associated with a lower rate for GIB than VKA in the setting of emergency patients, NOACs show a shift of the type of bleeding to lower GIB or rectal bleeding sources in our analysis from emergency patients and in the NOAC approval studies. Only $50 \%$ of patients with NOAC were on proton pump inhibitor therapy.

Conclusion: The frequency of GIB in everyday life is approximately $10 \%$ higher than reported from the controlled NOAC studies, irrespective of the type of anticoagulation used. NOACs were associated with a non-significantly lower bleeding rate compared with VKA, but major GIB rates were similar. VKA with a bioavailability of $100 \%$ after oral ingestion showed a tendency of higher rates of upper GIB, while NOACs with a reduced GI absorption rate of $7-68 \%$ were found to occur more frequently at lower GIB sites. Thus, prior to any anticoagulation, a pre-therapeutic risk analysis for the occurrence of GIB is still required. Certain patient groups (anemia, aortic valve stenosis, renal insufficiency, NSAIDs, etc.) can benefit from proton pump inhibitor therapy, early endoscopy with intervention, or NOAC differential therapy.

Disclosure of Interest: All authors have declared no conflicts of interest.

\section{P0513 REDUCED INTERSTITIAL CELLS OF CAJAL DENSITY AND INCREASED INTRAEPITHELIAL LYMPHOCYTES ARE ASSOCIATED WITH DEVELOPMENT OF SMALL INTESTINAL BACTERIAL OVERGROWTH IN POST-INFECTIOUS IBS MOUSE MODEL}

B. Chen ${ }^{1}$, L. Du ${ }^{1}$, H. He ${ }^{1}$, S. Zhu ${ }^{1}$, J.J. Kim ${ }^{1}$, N. Dai ${ }^{2}$

${ }^{1}$ Department Of Gastroenterology, Sir Run Run Shaw Hospital, School of

Medicine, Zhejiang University, Hangzhou/China

${ }^{2}$ Gastroenterology, Department of Gastroenterology, Sir Run Run Shaw Hospital, School of Medicine, Zhejiang University, Hangzhou, Zhejiang Province, China,

Hangzhou/China

Contact E-mail Address: binrui@zju.edu.cn

Introduction: Small intestinal bacteria overgrowth (SIBO) is common in patients with irritable bowel syndrome (IBS), and symptoms of IBS improve after treatment of SIBO with antibiotics. Although the pathogenesis of SIBO specifically in IBS patients is not well defined, intestinal dysmotility and immune activation are likely involved.

Aims \& Methods: We aimed to investigate the role of interstitial cells of Cajal (ICC) and intestinal inflammation in the development of SIBO using a postinfectious IBS (PI-IBS) mouse model. Sixty NIH mice were randomly assigned in a 2:1 fashion into T.spiralis infected PI-IBS vs. healthy control group. Visceral sensitivity was evaluated by measuring abdominal withdrawal reflex (AWR) in response to colorectal distension (CRD) before infection and at 8-weeks postinfectious (PI). Fecal consistency and the number of stool pellets were evaluated by visual inspection. Intestinal bacteria counts from jejunum and ileum were measured by quantitative real-time PCR to evaluate the presence of SIBO. ICC density in ileum was evaluated by immunohistochemistry and expressed as number of DMP-ICC per villus. Intraepithelial lymphocytes (IELs) in sections of bowel was counted in hematoxylin and eosin (H\&E) staining slice and expressed as number of lymphocytes per 100 epithelial cells. Intestinal cytokine levels (IL1- $\beta$, IL-6, Toll-like receptor-4 (TLR-4), IL-10) in the ileum were also examined.

Results: PI-IBS mice demonstrated increased mean AWR scores, decreased pain and volume thresholds compared with the control group suggested that infected group as a good model of PI-IBS with visceral hyperalgesia. One-third of the PIIBS mice developed SIBO (SIBO+/PI-IBS) and was more likely to have altered stool form compared with SIBO negative PI-IBS (SIBO-/PI-IBS) mice but without difference in mean number of fecal pellets and visceral sensitivity. There was reduced number of ICC in SIBO+/PI-IBS compared with SIBO-/PI-IBS mice $(0.47 \pm 0.35$ vs. $1.58 \pm 0.89, \mathrm{P}=0.01)$ and control mice $(1.44 \pm 0.77, \mathrm{P}=0.02)$. IELs counts were higher in SIBO+/PI-IBS mice compared with SIBO-/PI-IBS mice in ileum $(24.2 \pm 6.5$ vs. $15.0 \pm 4.4, \mathrm{P}=0.01)$ and rectum $(12.7 \pm 3.2$ vs. $8.2 \pm 1.8, \mathrm{P}=0.01$ ). In duodenum, jejunum, ileum, left colon and rectum, IELs counts were significantly increased in PI-IBS group compared with control group. No difference in inflammatory cytokine expression levels were detected between the groups except for increased TLR-4 among PI-IBS mice compared with the control group.

Conclusion: The development of SIBO in PI-IBS mice was associated with reduced ICC density and increased IELs counts in the ileum. Our findings support the role of intestinal dysmotility and inflammation in the pathogenesis of SIBO in IBS and may provide potential therapeutic targets.

Disclosure of Interest: All authors have declared no conflicts of interest.

\section{Reference}

1. Pimentel M. Review of rifaximin as treatment for SIBO and IBS. Expert opinion on investigational drugs 2009: 18; 349-358. 
P0514 CLINICAL CHARACTERISTICS OF FUNCTIONAL DYSPEPSIA PATIENTS DEPENDING ON WHETHER THEY ARE CHEMOSENSITIVE OR NOT

V. Hammer, K. Hammer, M. Führer, J. Hammer

Department Of Gastroenterology And Hepatology, Medical University of Vienna, Wien/Austria

Contact E-mail Address: jcapsaicin@yahoo.com.au

Introduction: Augmented chemosensitivity to capsaicin has been demonstrated in approximately half of functional dyspepsia patients, as can be determined by the oral capsaicin capsule test (Hammer et al, NGM 2008). Sensations induced by gastric capsaicin are distinct from sensations induced by stimulation of mechanoreceptors (Hammer \& Vogelsang, NGM 2007).

Aims \& Methods: The aim of the study was to determine clinical characteristics of FD patients with and without chemical hypersensitivity at baseline and after capsaicin ingestion for 4 weeks. $\mathrm{N}=49$ outpatients with confirmed FD received an oral sensitivity test with $0.75 \mathrm{mg}$ capsaicin at two occasions, before and after ingesting $0.25 \mathrm{mg}$ capsaicin tid for four weeks. Symptomatic response to capsaicin at the initial test allowed stratification to a capsaicin positive (chemosensitive) and a capsaicin negative (not chemosensitive) patient group. Symptom diaries for upper and lower gastrointestinal symptoms (visual analogue scales) were completed in the week before and during capsaicin ingestion and weekly aggregate symptom scores were calculated. Results are given as median; $25 \% / 75 \%$, $\mathrm{p}<0.05$ was considered significant.

Results: $53 \%$ FD had a positive capsaicin test. Basic clinical characteristics (age, gender, FD subtype, medication, psychological profile) were comparable in capsaicin positive and negative FD, but median daily aggregate upper gastrointestinal symptoms scores were significantly higher in capsaicin positive (median: 9.4; $5.4 / 11.7)$ than in capsaicin negative patients $(6.6 ; 4.1 / 8.1)(\mathrm{p}<0.05)$. Median scores for epigastric pain, nausea and epigastric distension were similar in capsaicin positive and negative patients $(\mathrm{p}>0.05)$. On the contrary, capsaicin negative patients had significantly lower scores for satiety $(\mathrm{p}<0.001)$ and epigastric bloating $(\mathrm{p}<0.01)$ than capsaicin positive patients. Lower abdominal symptoms were comparable in capsaicin positive and negative patients at baseline (NS). After capsaicin ingestion, aggregate upper gastrointestinal symptoms scores were reduced by $-3.3(-4.9 /-1.9 ; \mathrm{p}<0.001)$ in capsaicin positive and -2.6 $(-3.8 /-0.3 ; \mathrm{p}<0.05)$ in capsaicin negative patients. Lower abdominal symptoms scores after capsaicin ingestion were reduced by $-1.0 \quad(-1.8 /-0.1$; $\mathrm{p}<0.05)$ in capsaicin positive but not significantly altered $(-0.6 ;-1.7 /+0.9$; NS) in capsaicin negative patients. After long-term capsaicin ingestion, the capsaicin test turned negative in $53 \%$ of chemosensitive patients $(\mathrm{p}<0.01)$

Conclusion: Differences in upper GI symptoms distinguished capsaicin positive and negative patients at baseline. Symptom improvement after long-term capsaicin ingestion was indirect proportional to the result during the initial capsaicin test. Sensitivity to orally ingested capsaicin decreases after long-term capsaicin ingestion.

Disclosure of Interest: All authors have declared no conflicts of interest.

\section{References}

Hammer J, Führer M, et al. Neurogastroenterol Motil 2008; 20:125

Führer M, Vogelsang H, Hammer J. Neurogastroenterol Motil 2011;23:918

Hammer J, Vogelsang H. Neurogastroenterol Motil 2007;19:279

\section{P0515 NUCLEAR LORICRIN AND A DYSREGULATION OF BARRIER PROTEINS OBSERVED IN GASTRO-OESOPHAGEAL REFLUX DISEASE AFFECTED OESOPHAGEAL EPITHELIUM}

L. A. Mcginty, J. Ooi, P. Woodland, D. Kelsell, D. Blaydon Blizard Institute, London/United Kingdom

\section{Contact E-mail Address: 1.a.mcginty@qmul.ac.uk}

Introduction: Gastro-oesophageal reflux disease (GORD) is one of the most common disorders encountered in clinical gastroenterology. GORD patients can be categorised as having erosive oesophagitis or non-erosive reflux disease (NERD), with the latter being more frequently encountered in clinical practice. In NERD, even though the endoscopic presentation of the oesophageal mucosa appears visibly normal, histological changes within the oesophageal epithelium such as dilated intercellular spaces and basal cell hyperplasia are present, which are indicative of an altered epithelial barrier (Tobey et al 1996).

Aims \& Methods: Here, via immunohistochemical staining and subsequent immunoflourescent (IF) microscopic analysis, we investigate differences in the expression intensity, localisation and activity of key barrier function proteins present within the stratified squamous epithelium of proximal and distal biopsies derived from 13 patients with typical GORD symptoms. We compared this to biopsies taken from 5 healthy controls. Endoscopic oesophageal mucosal biopsies were taken from $5 \mathrm{~cm}$ above the LOS (distal) and at $20 \mathrm{~cm}$ from the incisors (proximal). Where no erosions were seen, NERD was confirmed by wireless $\mathrm{pH}$ capsule.

Results: IF analysis of loricrin (LOR), exhibits upregulated expression levels in 11 out of 13 GORD biopsies when compared to controls. This is also accompanied by an increased level of specific nuclear localisation, away from its more frequently observed membranous or cytoplasmic site. In addition, IF analysis of involucrin (INV), revealed sporadic regions of cytoplasmic localisation in multiple GORD biopsies, different from the specific membranous localisation observed in all control samples analysed. The transglutaminase-1 (TGM-1) enzyme functions to reinforce barrier function through its ability to crosslink LOR and INV during terminal differentiation in epithelial tissues at the cellular membrane. Analysis of TGM-1 enzymatic activity was performed in all GORD and control biopsies using a targeted assay combined with subsequent IF analysis. When compared to controls, we observed a dysregulation in TGM-1 activity levels throughout our GORD biopsies. This result also correlates with an observed reduction in the expression of ADAM17, a regulator of TGM-1 activity.

Conclusion: The observed increase in nuclear LOR combined with the sporadic cytoplasmic INV localisation, dysregulation of TGM-1 activity and reduced A17 expression levels in our GORD epithelial samples are all indicative of an altered epithelial barrier present within these individuals. For the first time, we highlight the importance of these barrier proteins in GORD pathogenesis. Further investigation into these barrier proteins and their associated signalling pathways could provide possibilities for the development of novel therapies designed to specifically target patients suffering from GORD.

Disclosure of Interest: All authors have declared no conflicts of interest.

\section{Reference}

Tobey, N. A., et al. (1996). "Dilated intercellular spaces: a morphological feature of acid reflux-damaged human esophageal epithelium." Gastroenterology 111(5): $1200-1205$.

\section{P0516 INFLUENCE OF PRUCALOPRIDE ON SECONDARY PERISTALSIS IN REFLUX PATIENTS WITH INEFFECTIVE ESOPHAGEAL MOTILITY}

C. Chen, W. Lei, C. Yi, T. Liu, J. Hung

Department Of Medicine, Hualien Tzu Chi Hospital, Buddhist Tzu Chi Medical Foundation and Tzu Chi University, Hualien/Taiwan

Contact E-mail Address: harry.clchen@msa.hinet.net

Introduction: Ineffective esophageal motility (IEM) is frequently found in patients with gastroesophageal reflux disease (GERD). Secondary peristalsis functions to maintain an empty esophagus by clearing refluxed gastric contents. Prucalopride, a high-affinity 5-hydroxytrypatamine 4receptors agonist, is useful in the treatment of chronic constipation by improving colon motility. Prucalopride also promotes secondary peristalsis in healthy adults (Clin Transl Gastroenterol. 2016).

Aims \& Methods: We aimed to determine whether prucalopride would augment secondary peristalsis in reflux patients with IEM. After a baseline recording of primary peristalsis, secondary peristalsis was stimulated by slow and rapid midesophageal injections of air in 15 patients. Two separate sessions with $4 \mathrm{mg}$ oral prucalopride or placebo were randomly performed.

Results: Prucalopride significantly increased primary peristaltic wave amplitude $(68.1 \pm 10.0$ vs. $55.5 \pm 8.8 \mathrm{mmHg}, \mathrm{p}=0.02)$. The threshold volume for triggering secondary peristalsis was significantly decreased by prucalopride during slow $(9.3 \pm 0.8$ vs. $12.0 \pm 0.8 \mathrm{~mL} ; \mathrm{p}=0.04)$ and rapid air injection $(4.9 \pm 0.3$ vs. $7.1 \pm 0.1 \mathrm{~mL} ; \mathrm{p}=0.01)$. Secondary peristalsis was triggered more frequently after application of prucalopride $(55 \%[43-70 \%])$ than placebo $(45 \%$ $[33-50 \%])(\mathrm{p}=0.008)$. Prucalopride didn't change pressure wave amplitudes during slow air injection $(84.6 \pm 8.1$ vs. $57.4 \pm 13.8 \mathrm{mmHg} ; \mathrm{p}=0.19)$ or pressure wave amplitudes during rapid air injection $(84.2 \pm 8.6$ vs. $69.5 \pm 12.9 \mathrm{mmHg}$; $\mathrm{p}=0.09$ ).

Conclusion: Prucalopride enhances mechanosensitivity of secondary peristalsis and promotes motor properties of primary peristalsis in IEM patients. Our study suggests that prucalopride could be a therapeutic option in the management of GERD patients with significant esophageal hypomotility.

Disclosure of Interest: All authors have declared no conflicts of interest.

\section{Reference}

CH Yi, WY Lei, JS Hung, TT Liu and CL Chen. Effects of prucalopride on esophageal secondary peristalsis in humans. Clinical and Translational Gastroenterology (2016) 7, e202

P0517 EFFECTS OF PRIOR JEJUNAL FEEDING ON GASTRIC EMPTYING AND SYMPTOMS IN PATIENTS WITH DIABETIC GASTROPARESIS (J4G STUDY): A RANDOMIZED, DOUBLE BLIND CONTROLLED CLINICAL TRIAL

H. L. Parker ${ }^{1}$, L. Carneiro ${ }^{2}$, C. L. Hoad $^{2}$, E. Tucker ${ }^{2}$, J. R. White ${ }^{2}$, L. Marciani ${ }^{3}$, P. Mansell ${ }^{4}$, T. Gazis ${ }^{4}$, M. M. Walker ${ }^{5}$, B. Scurry ${ }^{5}$, M. Fox ${ }^{6}$

${ }^{1}$ School Of Medicine, Pharmacy And Health, Durham University, Stockton-onTees/United Kingdom

${ }^{2} \mathrm{Nihr}$ Biomedical Research Unit In Gastrointestinal And Liver Diseases, Nottingham University Hospitals NHS Trust and the University of Nottingham Nottingham/United Kingdom

${ }^{3}$ Nottingham Digestive Diseases Centre, School Of Medicine, University of

Nottingham, Nottingham/United Kingdom

${ }^{4}$ Department Of Diabetes And Endocrinology, Nottingham University Hospitals NHS Trust, Nottingham/United Kingdom

${ }^{5}$ School Of Medicine And Public Health, The University of Newcastle Australia, Callaghan/Australia/NSW

${ }^{6}$ Abdominal Center: Gastroenterology, St. Claraspital, Basel/Switzerland

Contact E-mail Address: helen.1.parker@durham.ac.uk

Introduction: Symptoms compatible with diabetic gastroparesis (DG) affect up to 1 in 5 patients with type I diabetes mellitus. Those affected suffer postprandial 
nausea, vomiting, abdominal pain and impaired glycaemic control. Repeated hospital admissions are common. Endoscopy is normal in most patients. Impaired gastric function is thought to cause the condition. DG does not respond reliably to intensive insulin regimes or prokinetic medications. Jejunal nutrition $(\mathrm{JN})$ is an option in patients that cannot maintain their weight. The benefits are thought to follow improved nutrition and glycaemia; however, we have observed that some DG patients eat normally during and after JN (i.e. a quasi-pharmacological effect). One explanation could be that DG represents a failure of oral nutrition to "switch" the stomach from the fasted to the fed state. According to this hypothesis, nutrition delivered direct to the small bowel triggers the release of peptide hormones that induce normal gastric function.

Aims \& Methods: The study tests the hypothesis that JN prior to a test meal improves postprandial symptoms (primary outcome) and gastric function. Diabetic patients with severe symptoms (gastroparesis cardinal symptom index (GCSI) >27), diabetic controls (GCSI <14) and healthy controls entered a randomized, double blind, controlled trial. An insulin/glucose infusion controlled glycaemia. A JN feeding tube was placed at endoscopy with biopsies taken from the stomach and duodenum. Either liquid nutrient $(2 \mathrm{kcal} / \mathrm{min})$ or water was infused for $60 \mathrm{~min}$. Afterwards the Nottingham Test Meal was ingested (NTM liquid: $400 \mathrm{~mL}, 300 \mathrm{kcal}$; solid: 12 non-nutrient agar beads) ${ }^{1}$. Symptoms were documented by VAS, gastric function by MRI and the GI-peptide response was monitored over $120 \mathrm{~min}$ using published methodology. Bayesian methods provided $95 \%$ posterior ("credible") intervals and mixed model analysis compared response to intervention and between groups.

Results: 9 DG patients, 9 diabetic and 12 healthy controls were recruited. There was no difference in sex distribution, age, weight, medical history (e.g. duration of disease) or endoscopic findings (including histology) between groups. DG patients had more psychiatric co-morbidity and reported higher satiety, bloating and pain after ingestion of the NTM than diabetic and healthy controls $(\mathrm{p}<0.05)$. Sensations were not affected by JN in the controls; however, fullness, bloating and pain were reduced by JN in DG patients $(\mathrm{p}<0.05)$. Compared to water, JN induced a greater GI-peptide response (e.g. PP, GLP-1) and initial liquid GE was slower (gastric content volume after meal: $\mathrm{GCV}_{0} 31 \pm 13 \mathrm{~mL}$ higher, $\mathrm{p}=0.019$ ). Subsequent liquid GE was similar in both study conditions $\left(\mathrm{T}_{50}+3 \pm 8 \mathrm{~min}, \mathrm{p}=0.727\right)$. Antral contraction wave $(\mathrm{ACW})$ frequency was 2.7 $(2.6-2.9) / \mathrm{min}$ in health and was highest in diabetic controls $(3.1(2.7$ to 3.3$) / \mathrm{min})$. Solid GE was more rapid after JN than water (2 (1 to 3) beads emptied @60 min) and, again, was highest in diabetic controls (3 (1 to 7) beads emptied @60min). Numerically the GI-peptide response was less pronounced in both diabetic groups than healthy controls; however, the difference was not significant and a correlation with postprandial symptoms or gastric function was not identified.

\begin{tabular}{|c|c|c|c|c|c|c|}
\hline \multicolumn{7}{|c|}{$\mathrm{J} 4 \mathrm{G}$ study results from mixed model analysis } \\
\hline Measurement & $\begin{array}{l}\text { DG } \\
\text { Patient } \\
\text { JN }\end{array}$ & $\begin{array}{l}\text { DG } \\
\text { Patient } \\
\text { Water }\end{array}$ & $\begin{array}{l}\mathrm{DM} \\
\text { control } \\
\text { JN }\end{array}$ & $\begin{array}{l}\text { DM } \\
\text { control } \\
\text { Water }\end{array}$ & $\begin{array}{l}\mathrm{HV} \\
\text { control JN }\end{array}$ & $\begin{array}{l}\text { HV } \\
\text { control } \\
\text { Water }\end{array}$ \\
\hline $\begin{array}{l}\text { median GCSI } \\
\text { (range) }\end{array}$ & $\begin{array}{l}30 \\
(27-39)\end{array}$ & & $\begin{array}{l}4 \\
(1-12)\end{array}$ & & $\begin{array}{l}7 \\
(1-9)\end{array}$ & \\
\hline $\begin{array}{l}\text { Liquid GE } \\
\qquad\left(T_{50}\right) \pm \mathrm{SE}\end{array}$ & $68 \pm 7$ & $72 \pm 11$ & $55 \pm 7^{*}$ & $59 \pm 11^{*}$ & $75 \pm 7$ & $79 \pm 11$ \\
\hline $\begin{array}{l}\text { Solid GE } \\
\quad(\text { beads } / \mathrm{hr}) \pm \mathrm{CI}\end{array}$ & $\begin{array}{l}3 \\
(1-6)\end{array}$ & $\begin{array}{l}2 \\
(1-3)\end{array}$ & $\begin{array}{l}3 \\
(1-5)^{*}\end{array}$ & $\begin{array}{l}4 \\
(2-8)^{*}\end{array}$ & $\begin{array}{l}3 \\
(1-5)\end{array}$ & $\begin{array}{l}2 \\
(1-3)\end{array}$ \\
\hline $\mathrm{ACW}$ frequency $\pm \mathrm{CI}$ & $\begin{array}{l}2.9 \\
(2.7-3.1)\end{array}$ & $\begin{array}{l}2.9 \\
(2.7-3.1)\end{array}$ & $\begin{array}{l}3.1 \\
(2.8-3.3)^{*}\end{array}$ & $\begin{array}{l}3.1 \\
(2.8-3.3)^{*}\end{array}$ & $\begin{array}{l}2.7 \\
(2.6-2.9)\end{array}$ & $\begin{array}{l}2.7 \\
(2.6-2.9)\end{array}$ \\
\hline $\begin{array}{l}\text { Fullness } \\
\quad(\text { VAS }) \pm S E\end{array}$ & $16 \pm 7^{\S}$ & $27 \pm 11^{*}$ & $15 \pm 11$ & $23 \pm 11$ & $20 \pm 11$ & $20 \pm 7$ \\
\hline $\begin{array}{l}\text { Nausea } \\
\quad(\text { VAS }) \pm S E\end{array}$ & $4 \pm 9^{\S \S}$ & $8 \pm 9 *$ & $2 \pm 9$ & $4 \pm 9$ & $2 \pm 6$ & $2 \pm 6$ \\
\hline $\begin{array}{l}\text { Bloating } \\
\quad(\text { VAS }) \pm \text { SE }\end{array}$ & $16 \pm 12^{\S}$ & $27 \pm 11^{*}$ & $4 \pm 6$ & $5 \pm 7$ & $5 \pm 7$ & $5 \pm 7$ \\
\hline $\begin{array}{l}\text { Pain } \\
\quad(V A S) \pm S E\end{array}$ & $2 \pm 6^{\S \S}$ & $12 \pm 6^{*}$ & $0 \pm 6$ & $0 \pm 6$ & $0 \pm 6$ & $0 \pm 6$ \\
\hline
\end{tabular}

$\overline{\text { VAS - visual analogue score over course of study } * \mathrm{p}<0.05 * * \mathrm{p}<0.01 \mathrm{vs} \text {. HV; }{ }^{\S}}$ $\mathrm{p}<0.05^{\S \S} \mathrm{p}<0.01 \mathrm{JN}$ vs. water infusion

Conclusion: This clinical study demonstrates beneficial effects of prior $\mathrm{JN}$ on fullness, bloating and pain after a $400 \mathrm{~mL}$ test meal in diabetic patients with moderate-severe symptoms compatible with gastroparesis (GCSI > 27). Additionally, solid GE was accelerated after JN; however, this effect was not limited to DG patients and, thus, the treatment effect that improved symptoms could not be identified. Few patients in the DG group had objective evidence of abnormal gastric motor function and the benefit of "prior JN" on symptoms was not limited to patients with slow GE. However, it was observed that diabetic controls had relatively rapid ACW and solid GE. Future studies will identify patients likely to benefit from this novel approach to treatment. (ClinicalTrials.gov Identifier: NCT01919021, NCT00944593)

Disclosure of Interest: All authors have declared no conflicts of interest.

\section{Reference}

1. Parker, H. L., et al. (2016). Neurogastroenterol Motil 28(4): 554-568.
P0518 PER-ORAL ENDOSCOPIC MYOTOMY IN TREATMENT NAIIVE VERSUS PRIOR TREATMENT FAILURE CASES -OUTCOME IN OVER 500 PATIENTS

Z. Nabi, M. Ramchandani, R. Chavan, S. Darisetty, R. Kalapala, D.N. Reddy Gastroenterology, Asian Institute of Gastroenterology, Hyderabad/India

Contact E-mail Address: zaheernabi1978@gmail.com

Introduction: Per-oral endoscopic myotomy (POEM) has emerged as an efficacious treatment modality for achalasia cardia (AC). Prior treatment (PT) may affect the outcomes of subsequent. The impact of prior treatment on technical and clinical success of POEM is not well known. Small studies with short followup indicate that POEM is safe and feasible in PT failure cases. However, there is paucity of large studies with long-term follow-up.

Aims \& Methods: In this study we aim to compare the safety and efficacy of POEM in treatment naïve (TN) cases versus prior treatment (PT) failure cases. The data of consecutive patients with AC who underwent POEM at a single tertiary care center from (January 2013 to November 2016) was analysed retrospectively. A comparative analysis was performed between TN and PT failure cases. Technical and clinical success, adverse events (AE), operative time (OT) for POEM were compared between TN versus PT failure cases.

Results: Overall, 502 patients with AC underwent POEM during the study period. 260 patients $(51.8 \%)$ were TN and $242(48.2 \%)$ patients had PT. Type II AC was the most common subtype in both the groups (TN $-63.5 \%$ vs PT $57.8 \%$ ) followed by type I and type III. There was no significant difference with regards to AC subtypes between the two groups. The distribution of patients according to prior treatment history is as follows - PBD (205), LHM (23), LHM and PBD both (7), botulinum toxin injection (4) and POEM (3). Significantly more patients in the PT group had sigmoid oesophagus (47 vs 18). Mean OT was significantly more in the PT group when compared to the TN group (PT vs TN $74.9 \pm 30.6$ vs $67.0 \pm 27.1 \mathrm{~min} ; \mathrm{P}=0.002$ ). On multivariate analysis- type of $\mathrm{AC}$, dilated esophagus $(>6 \mathrm{~cm})$ and type of knife used were significant predictors of OT. Technical $(98.1 \%$ vs $97.1 \%, \mathrm{P}>0.05)$ and clinical success $(94.9 \%$ vs $91.9 \%)$ of POEM procedure was similar in TN and PT cases. Gas related events and mucosotomies were equal in both groups (TN-35.7\% vs PT-33.1\%; p- 0.57 ). Objective evidence of gastroesophageal reflux was found in $17 / 53$ patients $(32.1 \%)$ in PT group as compared to $11 / 44(25 \%)$ in TN group (p-0.50).

\begin{tabular}{llllll}
\hline \multicolumn{2}{l}{ Predictors of operative time on multivariate analysis } & & & \\
\hline & Factor & OR & p value & $95 \%$ & CI \\
\hline 1. & Type of AC $(\mathrm{III} / \mathrm{I}-\mathrm{II})$ & 16.24 & 0.002 & 2.66 & 99.1 \\
2. & Esophageal diameter $(>6 /<6 \mathrm{~cm})$ & 2.76 & 0.012 & 1.24 & 6.13 \\
3. & Knife(TT/TTJ) & 14.41 & 0.001 & 5.68 & 36.5 \\
4. & Adverse events & 0.98 & 0.941 & 0.59 & 1.62 \\
5. & Prior treatment & 1.16 & 0.480 & 0.75 & 1.81 \\
6. & Pediatric Achalasia & 0.71 & 0.544 & 0.23 & 2.13 \\
\hline
\end{tabular}

Conclusion: POEM is equally efficacious and safe in treatment naïve and prior treated cases. POEM should be considered in treatment failure cases. Disclosure of Interest: All authors have declared no conflicts of interest.

\section{References}

Smith CD, Stival A, Howell DL, et al. Endoscopic therapy for achalasia before Heller myotomy results in worse outcomes than heller myotomy alone. Ann Surg 2006:243:579-84; discussion 584-6.

Jones EL, Meara MP, Pittman MR, et al. Prior treatment does not influence the performance or early outcome of per-oral endoscopic myotomy for achalasia. Surg Endosc 2016;30:1282-6.

Ngamruengphong S, Inoue H, Ujiki MB, et al. Efficacy and Safety of Peroral Endoscopic Myotomy for Treatment of Achalasia After Failed Heller Myotomy. Clin Gastroenterol Hepatol 2017.

\section{P0519 ESOPHAGEAL REFLUX BURDEN IN RELATIONSHIP TO ESOPHAGOGASTRIC JUNCTION (EGJ) AND ESOPHAGEAL BODY FUNCTION ON HIGH RESOLUTION MANOMETRY (HRM)}

A. Rengarajan ${ }^{1}$, S. Roman ${ }^{2}$, E. Savarino ${ }^{3}$, R. Yadlapati ${ }^{4}$, M. Tye ${ }^{4}$, F. Mion ${ }^{5}$, J. Pandolfino ${ }^{4}$, C.P. Gyawali ${ }^{1}$

${ }^{1}$ Washington University in St. Louis, St Louis/United States of America/MO

${ }^{2}$ Digestive Physiology, Hôpital Edouard Herriot, Lyon/France

${ }^{3}$ Department Of Surgery, Oncology And Gastroenterology, University of Padua, Padua/Italy

${ }^{4}$ Northwestern University, Chicago/United States of America/IL

${ }^{5}$ Digestive Physiology, Hospices Civils de Lyon, Lyon/France

Contact E-mail Address: rengara@wustl.edu

Introduction: Both EGJ and esophageal body motor abnormalities are found on esophageal HRM in reflux disease, and contribute to reflux burden assessed using acid exposure time (AET) on ambulatory reflux monitoring. Mean nocturnal baseline impedance (MNBI) represents a new paradigm that may assess longitudinal esophageal reflux burden, but its precise role in clinical esophagology, particularly in relationship to AET, remains unclear. 
Aims \& Methods: Our aim was to evaluate the complex interrelationships between EGJ and esophageal motor abnormalities, and esophageal reflux burden in this ongoing multicenter collaboration. Esophageal function studies from patients with persisting reflux symptoms were reviewed from four centers (2 each in Europe and US) for this preliminary report. EGJ morphology was categorized using HRM into hypotensive (EGJ-CI $<46 \mathrm{mmHg} . \mathrm{cm}$ ), hiatus hernia (HH, manometric separation between lower esophageal sphincter and crura diaphragm) and intact EGJ (normotensive EGJ-CI, no HH). Esophageal body metrics were characterized using Chicago Classification v 3.0 into intact, ineffective esophageal motility (IEM) and absent contractility. Total and supine AET were extracted from ambulatory $\mathrm{pH}$-impedance studies. Baseline impedance was calculated at the $5 \mathrm{~cm}$ impedance channel (to correspond to AET) at three stable 10 -min time periods $(1,2$, and $3 \mathrm{AM})$ during the ambulatory $\mathrm{pH}$-impedance study, and averaged to yield MNBI (normal > $2292 \mathrm{ohms}$ ). Univariate and multivariate analyses were performed to assess EGJ and esophageal body predictors of esophageal reflux burden, and to discern the value of MNBI in comparison to AET.

Results: 1244 patients $(53.4 \pm 0.4 \mathrm{yr}, 59.6 \% \mathrm{~F})$ undergoing esophageal motor testing using HRM (Medtronic, Duluth, GA) and ambulatory $\mathrm{pH}$ or $\mathrm{pH}$-impedance monitoring studies performed off antisecretory therapy were included. A hypotensive EGJ was noted in $70.9 \%$, HH in $34.0 \%$, IEM in $26.3 \%$ and absent contractility in $3.5 \%$. A disrupted EGJ and absent contractility had the highest proportions with AET $>6 \%$; combinations thereof raised the proportions even higher (Table, $\mathrm{p}<0.001$ for each comparison to intact EGJ and/or esophageal body). Compared to an intact EGJ, the odds ratio (OR) of total AET $>6 \%$ with $\mathrm{HH}$ was $2.0(95 \%$ CI $1.1-3.9, \mathrm{p}=0.04)$. Supine AET $>2 \%$ was even more impacted (HH: OR 2.4, 95\% CI 1.3-4.5, p =0.007; $\mathrm{HH}+$ hypotensive EGJ OR 3.3, 95\% CI 2.1-5.2, p < 0.001). A hypotensive EGJ was not discriminative of AET or MNBI values. Concordance between AET and MNBI thresholds was noted in 401 of 596 studies $(67.2 \%$; both abnormal in $24.8 \%$, both normal in $42.4 \%$ ). When concordant and abnormal, proportions with EGJ and esophageal body abnormalities were highest $(\mathrm{p} \leq 0.005)$ compared to concordant and norma AET/MNBI, and discordant studies. On multivariate regression with categorical and linear EGJ and esophageal body motor findings as dependent variables, presence of HH $(\mathrm{p}<0.0001)$, IEM $(\mathrm{p}=0.032)$, and absent contractility $(\mathrm{p}=0.0012)$ were independent categorical predictors of AET $>6 \% ; \mathrm{HH}$ size $(\mathrm{p}<0.0001)$, lower EGJ-CI $(\mathrm{p}=0.002)$, and increasing numbers of ineffective swallows $(\mathrm{p}=0.043)$ were independent linear predictors. Only the presence of $\mathrm{HH}$ was an independent categorical predictor of abnormal MNBI $(\mathrm{p}<0.0001)$, increasing $\mathrm{HH}$ size $(\mathrm{p}<0.0001)$ and proportions of ineffective swallows $(\mathrm{p}<0.0001)$ were independent linear predictors.

Proportions with abnormal reflux burden in relationship to EGJ and esophageal body motor findings on high resolution manometry

\begin{tabular}{|c|c|c|c|}
\hline & $\begin{array}{l}\text { AET }>6 \\
n=431\end{array}$ & $\begin{array}{l}\quad \mathrm{AET}<4^{\circ} \\
n=642\end{array}$ & $\begin{array}{l}\text { MNBI }<2292 \\
\text { ohms } n=596\end{array}$ \\
\hline \multicolumn{4}{|l|}{ EGJ findings } \\
\hline Intact EGJ $(n=280)$ & $25.7 \%$ & $60.7 \% * *$ & $58.3 \%(63 / 108)$ \\
\hline Hypotensive EGJ ( $n=862$ ) & $36.5 \% *$ & $49.2 \% * *$ & $56.3 \%(259 / 460)$ \\
\hline Hiatus hernia $(n=422)$ & $49.0 \% *$ & $36.5 \% * *$ & $69.8 \% *(138 / 199)$ \\
\hline Both $(n=342)$ & $49.4 \% *$ & $34.8 \% * *$ & $70.9 \% *(124 / 175)$ \\
\hline $\begin{array}{l}\text { Esophageal body motor findings } \\
\text { Intact esophageal body }(n=686)\end{array}$ & $31.0 \%$ & $56.9 \% * *$ & $46.5 \%(158 / 340)$ \\
\hline $\operatorname{IEM}(n=326)$ & $41.4 \% *$ & $44.8 \%$ & $69.6 \% *(94 / 135)$ \\
\hline Absent contractility $(n=43)$ & $53.5 \% *$ & $39.5 \%$ & $88.2 \% *(15 / 17)$ \\
\hline \multicolumn{4}{|l|}{$\begin{array}{l}\text { Combined EGJ \& esophageal } \\
\text { body motor findings }\end{array}$} \\
\hline Intact EGJ and body $(n=170)$ & $25.3 \%$ & $61.2 \% * *$ & $49.3 \%(36 / 73)$ \\
\hline Hypotensive EGJ, HH, IEM $(n=105)$ & $56.2 \% *$ & $24.8 \% * *$ & $83.0 \% *(44 / 53)$ \\
\hline $\begin{array}{l}\text { Hypotensive EGJ, HH, } \\
\text { absent contractility }(n=7)\end{array}$ & $71.4 \% *$ & $14.3 \%$ & $100 \% *(5 / 5)$ \\
\hline
\end{tabular}

${ }^{*} \mathrm{p}<0.05$ compared to intact EGJ and/or esophageal body function $* * \mathrm{p}<0.05$ compared to AET $>6 \%$ EGJ: esophagogastric junction; AET: acid exposure time; MNBI: mean nocturnal baseline impedance; IEM: ineffective esophageal motility, $\mathrm{HH}$ : hiatus hernia

Conclusion: A disrupted EGJ and IEM on esophageal HRM are independent predictors of elevated esophageal reflux burden. Hierarchical HRM evaluation of EGJ and esophageal body metrics adds confidence to categorization of esophageal reflux burden.

Disclosure of Interest: S. Roman: consulting fees: Medtronic research support: Sandhill, Crospon

E. Savarino: Consulting fee from: Medtronic, Sofar, Malesci, Takeda, Abbvie, MSD

J. Pandolfino: Medtronic, Sandhill, Torax- Consultants/Speaker Astra Zeneca, Takeda- speaker Ironwood, Impleo- consultant

C.P. Gyawali: Research support, speaker bureau for Medronic, Inc; Consultant for Torax, Ironwood, Quintiles; Speaker bureau for Allergan

All other authors have declared no conflicts of interest.

\section{References}

Roman S, Gyawali CP, Savarino E, et al. Neurogastroenterol Motil 2017. Gyawali CP, Roman S, Bredenoord AJ, et al. Classification of esophageal motor findings in gastroesophageal reflux disease. Neurogastroenterol Motil 2017.

\section{P0520 MEASURING THE ACTIVE AND PASSIVE CHARACTERISTICS OF CONTRACTILE SMOOTH MUSCLE IN PORCINE INTESTINE MODEL}

G. Min ${ }^{1}$, W. Kim ${ }^{2}$, S.J. Choi ${ }^{1}$, I.K. Yoo ${ }^{3}$, S.H. Kim ${ }^{2}$, J.M. Lee ${ }^{1}$, H.S. Choi ${ }^{2}$, E.S. Kim ${ }^{2}$, B. Keum ${ }^{2}$, H.S. Lee', H.J. Chun ${ }^{2}$, C.D. Kim ${ }^{2}$, Y.T. Jeen ${ }^{2}$

${ }^{1}$ Division Of Gastroenterology And Hepatology, Department Of Internal Medicine, Korea University Anam Hospital, Seoul/Korea, Republic of

${ }^{2}$ Division of Gastroenterology and Hepatology, Department of Internal Medicine, Korea University College of Medicine, Seoul/Korea, Republic of

Department Of Internal Medicine, Institute of Digestive Disease and Nutrition, Korea University College of Medicine, Seoul/Korea, Republic of

Contact E-mail Address: doctordrum@naver.com

Introduction: Electrical stimulation therapy is a new way to treat digestive disorders such as constipation, colonic inertia. It is necessary to understand the physiology of smooth muscle contraction in developing novel medical devices related with electrical stimulation therapy. The aims of this study were to measure the active characteristics of smooth muscle with acetylcholine in porcine intestine segment.

Aims \& Methods: We used five female pigs and obtained ten centimeters of each porcine small intestine. To measure passive characteristics of small intestine, a universal testing machine with a tensile rate of $30 \mathrm{~mm} / \mathrm{min}$. To estimate the active characteristic parameters of smooth muscle and isometric and isotonic intestinal motility of smooth muscle, muscle contraction was induced by applying the stimulation solution (HTK solution containing $1 \mathrm{mM}$ of acetylcholine chloride). Then, we obtained the maximum muscle contractile force of the specimens to measure the isometric and isotonic intestinal motility.

Results: In tensile test, the maximum repulsive force, that indicate passive muscle force of smooth muscle $0.702 \mathrm{~N}$, was measured. In the isometric and isotonic contractions in the porcine small intestine, the maximum myotility, $12.35 \mathrm{mN}$, was obtained in isometric experiments, and the maximum velocity of muscular contraction, $0.4476 \mathrm{~mm} / \mathrm{min}$, was obtained in isotonic experiments. We demonstrated that in equal lengths, the muscle contraction velocity of the smooth muscle is $10-100$ times slower than that of the skeletal muscle indicating forcevelocity relationship of smooth muscle. And we obtained that the maximum contraction force from each individual percentage of active force $(25 \%, 50 \%$, and $100 \%$ ) was achieved at $L / L_{\mathrm{opt}}=1$.

Conclusion: We straighten out the active and passive property of porcine intestinal smooth muscle. Our study may be helpful for developing novel medical devices and understanding the physiology of smooth muscle in the porcine small intestine.

Disclosure of Interest: All authors have declared no conflicts of interest.

\section{P0521 MOTILITY PATTERNS AFTER PER-ORAL ENDOSCOPIC MYOTOMY (POEM) IN PATIENTS WITH ACHALASIA}

Z. Vackova ${ }^{1}$, J. Krajciova ${ }^{1}$, L. Zdrhova ${ }^{2}$, P. Loudova $^{3}$, P. Stirand ${ }^{1}$, T. Hucl ${ }^{1}$, J. Spicak ${ }^{1}$, J. Martinek ${ }^{1}$

${ }^{1}$ Department Of Hepatogastroenterology, Institute for Clinical and Experimental Medicine, Prague/Czech Republic

${ }^{2}$ Department Of Internal Medicine, University Hospital Plzen, Plzen/Czech Republic

${ }^{3}$ Department Of Gastroenterology, Hospital Kolin, Kolin/Czech Republic

Contact E-mail Address: vackova.zuz@gmail.com

Introduction: Partial recovery of esophageal peristalsis has been reported in up to $47 \%$ of achalasia patients treated by myotomy (either per-oral endoscopic myotomy (POEM) or laparoscopic Helleŕs myotomy) in several rather small studies. The aim of our study was to assess motility patterns focusing on possible "recovery" of esophageal peristalsis in a large cohort of patients after POEM.

Aims \& Methods: We performed a retrospective analysis of prospectively collected data of patients undergoing POEM at our tertiary referral center. All patients in whom high-resolution manometry (HRM) studies were performed prior to and 3 months after POEM and who completed at least 6-month follow-up were included. All HRM studies were reviewed and the Chicago Classification (CC) v3.0 of motility disorders was applied to characterize both pre- and post-POEM motility patterns.

Results: From 192 patients who underwent POEM since 2012, 127 patients met the inclusion criteria. The initial CC diagnoses before POEM were as follows: type I achalasia - 20 pts $(16 \%)$, type II achalasia - 100 pts $(79 \%)$, type III achalasia -5 pts $(4 \%)$, other (esophago-gastric junction outflow obstruction (EGJOO) and Jackhammer) - 2 pts $(1 \%)$. Only 6 patients $(5 \%$; type III achalasia, Jackhammer, EGJOO) had had some signs of esophageal contractility before POEM. After POEM, peristaltic fragments were present in $28 / 127$ patients $(22 \%)$ - 9x ineffective esophageal motility, $5 \mathrm{x}$ fragmented peristalsis, $2 \mathrm{x}$ distal esophageal spasm, 5x EGJOO, 7x type III achalasia. Thus, the partial "recovery" of esophageal peristalsis was observed in $22 / 121$ patients $(18 \%)$ and it only occurred in patients with type II achalasia; contractile activity was not detected in any patient with type I achalasia after POEM $(22 / 100$ vs. $0 / 20, p=0.023)$. Panesophageal pressurization completely resolved in 88 patients $(88 \%)$ with 
achalasia type II. The mean integrated-relaxation pressure (IRP) decreased from $27( \pm 13) \mathrm{mmHg}$ to $13( \pm 5) \mathrm{mmHg}(\mathrm{p}<0.0001)$. The presence of partial peristaltic recovery was neither associated with normalization of IRP (IRP normalized in $17 / 28(61 \%)$ patients with peristaltic recovery and in $72 / 99(73 \%)$ patients without, $\mathrm{p}=0.25$ ), nor with overall treatment success of POEM (Eckardt score $<3)$.

Conclusion: In this so far largest case-series investigating the rate of peristaltic recovery after POEM this was present in $18 \%$ of patients, therefore, the rate may be lower than previously reported. Peristaltic recovery seems to have no clinical impact on post-POEM symptomatology. Esophageal contractility after POEM was not observed in any patient with achalasia type I.

Disclosure of Interest: All authors have declared no conflicts of interest.

\section{References}

Roman $\mathrm{S}$ et al. Partial recovery of peristalsis after myotomy for achalasia; more the rule than the exception. JAMA Surg: 2013;148(2):157-64

Teitelbaum EN et al. Symptomatic and physiologic outcomes one year after peroral esophageal myotomy (POEM) for treatment of achalasia. Surg Endosc: 2014;28(12):3359-65.

\section{P0522 WHAT IS THE EFFECT OF MYOTOMY SITE ON PER-ORAL ENDOSCOPIC MYOTOMY? COMPARISON OF ANTERIOR AND POSTERIOR MYOTOMY}

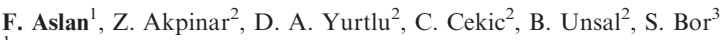
${ }^{1}$ Gastroenterology, Koc University School of Medicine, Istanbul/Turkey

${ }^{2}$ Izmir Ataturk Training and Research Hospital, Izmir/Turkey

${ }^{3}$ Ege University Medical School, Izmir/Turkey

Contact E-mail Address: drfatihaslan@hotmail.com

Introduction: Medical treatments, endoscopic balloon dilatation, Botox and Heller myotomy are treatment modalities for managing achalasia. Recently per-oral endoscopic myotomy (POEM) has became a new option for achalasia patients and since 2010 it has became widespread. Earlier, anterior myotomy was used in this technique but in the last few years there are studies reporting that posterior myotomy is more effective. However, there are limited numbers of publications comparing anterior and posterior myotomy. This study aimed to investigate the effect of myotomy site on POEM, to our knowledge it is the first time in Europe and our country.

Aims \& Methods: Between May 2014 and January 2017, POEM was performed to 225 achalasia patients at the gastroenterology clinics under general anesthesia by an endoscopist experienced at endoscopic submucosal dissection and trained for POEM. Demographic data, previous history for balloon dilatation and results of the procedure were recorded prospectively. Patients with anterior myotomy were grouped as "group A" and those with posterior myotomy as "group P", and the results were compared.

Demographic features and results of POEM procedure

\begin{tabular}{llll}
\hline & $\begin{array}{l}\text { Group Anterior } \\
\mathrm{N}=114\end{array}$ & $\begin{array}{l}\text { Group Posterior } \\
\mathrm{N}=111\end{array}$ & $\mathrm{p}$ \\
\hline Sex (Male/Female), $\mathrm{n}$ & $56 / 58$ & $58 / 53$ & 0.639 \\
Age mean (SD) & $41.05 \pm 14.89$ & $42.24 \pm 13.52$ & 0.905 \\
$\quad$ (median; range) year & $(41.5 ; 12-73)$ & $(41 ; 18-75)$ & \\
Prior achalasia treat- & $48 / 66$ & $36 / 75$ & 0.087
\end{tabular}

ment, $\mathrm{n}$ (yes or $\mathrm{No}$ )

Achalasia sub-type; Unknown I/II/III, n

Tunnel length, mean (SD) (median; range) $\mathrm{cm}$

Myotomy length, mean (SD) (median;

range) $\mathrm{cm}$

Procedure Time, mean (SD) (median; range) $\mathrm{min}$

Tunnel time, mean (SD) (median; range) min

Myotomy time, mean (SD) (median; range) $\mathrm{min}$

Dysphagia Score preoperative/postoperative (median; range)

Eckardt Score, preoperative/postoperative (median; range)

$\begin{array}{lll}3 / 6 / 94 / 11 & 0 / 17 / 86 / 8 & 0.029 \\ 17.07 \pm 2.63 & 17.32 \pm 2.49 & 0.278 \\ (17 ; 12-27) & (17 ; 12-25) & \\ & & \\ 13.79 \pm 2.46 & 14.04 \pm 2.44 & 0.235 \\ (14 ; 10-25) & (14 ; 8-21) & \end{array}$

$34.60 \pm 14.67$

\section{$27.02 \pm 9.74$}

(25; 10-68)

$10.08 \pm 4.89$

$12.11 \pm 6.67$

(9; 3-30)

$(3 ; 3-4) /(0 ; 0-2)$

$(3 ; 3-4) /(0 ; 0-1)$

$(8 ; 6-12) /(0 ; 0-2) \quad(8 ; 5-12) /(0 ; 0-2)$

383

330

0.176
Continued

Demographic features and results of POEM procedures

\begin{tabular}{lll}
\hline Group Anterior & Group Posterior & \\
$\mathrm{N}=114$ & $\mathrm{~N}=111$ & $\mathrm{p}$
\end{tabular}

Adverse events, $\mathrm{n}$ -

Capnoperitoneum -

Mucosal injury

Results: There were 114 patients in group A, 111 patients in group P. There was no statistical difference between the groups in regards of tunnel length, myotomy length, tunnel entrance time and frequency of homeostatic forceps use $(\mathrm{p}>0.05)$. However duration of opening the tunnel, myotomy, closure of the tunnel and total procedure time were significantly shorter in group $\mathrm{P}(\mathrm{p}<0,05)$. Eckardt and dysphagia scores before the procedure were similar in both groups. After the procedure Eckardt scores were significantly low in all patients $(\mathrm{p}<0.005)$. Demographic features and data of the procedures are shown in the table below. Mucosal damage during the procedure occurred in 3 patients and capnoperitoneum developed in 71 patients. All complications were treated endoscopically. Control endoscopy was performed to 151 patients at $3 \mathrm{rd}$ month; that revealed esophagitis grade A in 24, grade B in 6 and grade C in 3 patients.

Conclusion: According to our results posterior approach can shorten the procedure time in POEM compared to anterior myotomy. This may be due to a better angle of approach with endoscopic equipments for posterior myotomy. We believe that long-term results will also show the effects of myotomy site on clinical outcome of patients.

Disclosure of Interest: All authors have declared no conflicts of interest.

\section{P0523 LONG-TERM RESULTS OF PERORAL ENDOSCOPIC} MYOTOMY (POEM) FOR ACHALASIA

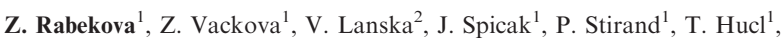
E. Kieslichova ${ }^{3}$, J. Martinek

${ }^{1}$ Department Of Hepatogastroenterology, Institute for Clinical and Experimental Medicine, Prague, Czech Republic, Prague/Czech Republic

${ }^{2}$ Institute for Clinical and Experimental Medicine, Prague/Czech Republic

${ }^{3}$ Anaesthesiology And Resuscitation, Institute for Clinical and Experimental Medicine, Prague/Czech Republic

Contact E-mail Address: zuzana.rabekova@ikem.cz

Introduction: Peroral endoscopic myotomy (POEM) has gained trust by proven safety and short-term efficacy and at present, it is considered to be a standard method for treatment of esophageal achalasia. However, long-term data concerning the efficacy and safety especially with regard to post-POEM reflux are still awaited.

Aims \& Methods: The aim of this prospective single-center case series was to assess the long-term clinical outcome of POEM with emphasis on post-POEM reflux evaluated by $\mathrm{pH}$ monitoring, endoscopy findings, reflux symptoms and use of proton pump inhibitors (PPIs). Since 2012, a total of 192 patients with achalasia underwent 202 POEM procedures. Follow-up visits at 3, 12, 24 and 36 months were completed in 160, 116, 70 and 27 patients. Upper GI endoscopy, high-resolution manometry (HRM) and 24-hour $\mathrm{pH}$ monitoring were performed 3 months after POEM; endoscopy was then repeated between 24-36 months. Main outcomes were treatment success defined as Eckardt score $<3$, recurrence rate and post-POEM reflux.

Results: At 3, 12, 24 and 36 months, treatment success was achieved in $97 \%(95 \%$ CI: 94-100), 95\% (CI 91-99), 88\% (CI 82-95) and 81\% (CI 69-93) of patients. A total of 14 patients experienced treatment failure $(n=5)$ or recurrence $(n=9)$. The recurrences occurred most often in patients with HRM type I achalasia (4 out of $26,15.4 \%$ ) followed by type II ( 3 out of $113,2.6 \%$ ) vs. none in type III achalasia $(0$ out of $10,0 \%) ; \mathrm{p}=0.022$. At 3 months, reflux esophagitis was diagnosed in $63 / 160$ patients $(39.4 \%$; severe esophagitis LA C or D in 8 patients). Abnormal acid exposure on $\mathrm{pH}$-metry studies was detected in 58/146 (39.7\%). At 24-36 months, endoscopy was performed in 41 patients and reflux esophagitis was present in 9 patients $(21.9 \%$; none of the patients has been treated with PPIs). At 3 and $24 \mathrm{M}$, a proton pump inhibitor was administered to $33.5 \%$ and $31.4 \%$ of patients.

Conclusion: POEM is effective treatment modality for achalasia with treatment success around $90 \%$ at 2 years, slightly dropping down to $81 \%$ at 3 years. Generally mild reflux esophagitis and abnormal esophageal acid exposure are diagnosed in about $40 \%$ of patients 3 months after POEM but are successfully manageable with proton pump inhibitors. Occurrence of reflux esophagitis tends to decrease with time.

Disclosure of Interest: All authors have declared no conflicts of interest.

\section{P0524 BEER EFFECTS ON POSTPRANDIAL DIGESTIVE} SYMPTOMS AND GASTROESOPHAGIC PHYSIOLOGY

B. Serrano Falcón, M. Megía Sánchez, A. Ruiz De León, E. Rey Gastroenterology Department, Hospital Clínico San Carlos, Madrid/Spain

Contact E-mail Address: blan.serrano.falcon@gmail.com Introduction: Beer has been related to gastroesophageal reflux (GER) and dyspepsia $(1,2)$, based on its alcohol and gas content. Main objective was to evaluate if moderate regular and non-alcohol beer consumption is related to postprandial 
Abstract No: P0524

Table 1

BEER CONSUMPTION

AND DYSPEPTIC

SYMPTOMS

Mean increasement of dys-

peptic symptoms compared

to Minute (Min) 0

Min 5- Min 0

Min 10- Min 0

Min 15- Min 0

Min 20- Min 0

Min 25- Min 0

Min 30- Min 0

Min 33- Min 0
SUBSTUDY 1 (Regular beer)

SUBSTUDY 2 (Non alcohol beer)

$\begin{array}{llllll}\text { Control visit (Mean) } & \text { Intervention visit (Mean) } & \mathrm{p} \text { value } & \text { Control visit (Mean) } & \text { Intervention visit (Mean) } & \mathrm{p} \text { value } \\ -0,1(0,73(-2-1)) & 0 & 0,655 & 0 & 0,1(0,3(0-1)) & 0,157 \\ -0,1(0,73(-2-1)) & 0,2(0,42(0-1)) & 0,18 & 0,3(0,47(0-1)) & 0,55(0,6(0-2)) & 0,132 \\ 0,2(0,63(0-2)) & 0,4(0,51(0-1)) & 0,317 & 0,65(0,67(0-2)) & 0,8(0,76(0-2)) & 0,454 \\ 0,5(0,97(0-3)) & 0,8(0,63(0-2)) & 0,18 & 1,15(0,93(0-3)) & 1,35(0,98(0-4)) & 0,521 \\ 1,1(1,19(0-3)) & 1,2(0,78(0-2)) & 0,655 & 1,75(1,12(0-4)) & 1,85(1,26(0-4)) & 0,685 \\ 1,3(1,41(0-4)) & 1,3(0,82(0-2)) & 1 & 2,15(1,06(0-4)) & 2,3(1,45(0-5)) & 0,38 \\ 1,2(1,31(0-4)) & 1,5(1,08(0-3)) & 0,18 & 2,57(1,21(0-5)) & 2,8(1,24(0-5)) & 0,131\end{array}$

dyspeptic symptoms after a controlled meal. Secondary objectives were to evaluate its relation with postprandial GER and gastric acommodation and to evaluate its relation with daily digestive symptoms under real conditions.

Aims \& Methods: Healthy people over 18 years old, free of frequent digestive symptoms (<once a week) and GER disease (GERD), were included. Basal symptoms were assessed through PAGI-SYM(3) and QOLRAD (4) questionnaires, both validated to Spanish. Study was divided in two substudies based on the study intervention: $33 \mathrm{cl}$ of regular beer (substudy 1) and the same ammount of non-alcohol beer (substudy 2). Mineral water ( $33 \mathrm{cl}$ ) was the control intervention in both substudies. Each participant was its own control. The study lasted two weeks (control study week and intervention study week). Each week started with a visit to the laboratory at 7:30 h am, when a pHimpedance catheter was placed and taken off 24 hours later. Gastric acommodation was assessed through the maximum tolerated volume during a nutrient drink test (ENSURE ${ }$ $\mathrm{HN}, 500 \mathrm{ml}$ ) in a rithm of $15 \mathrm{ml} /$ minuts, after the ingestion of beer (intervention) or water (control). It was defined as the volume after which the test finished or the participant reported the maximum puntuation for any dyspeptic symptoms (early satiety, bloating, epigastric pain and nausea), which were asked every 5 minutes ( 1 meant no symptom and 5 meant the highest perception). GER was evaluated in the postprandial period and during 24 hours through pHimpedance register. Weekly symptoms evaluation was made though a diary adapted from PAGYSYM questionnaire and sum of symptoms was used for analysis. Data were collected daily through email. Variables were compared between both visits and weeks in both substudies using a non parametric test for matching data Participants should drink $33 \mathrm{cl}$ of beer before lunch and dinner during the intervention week. Other alcohol drinks were prohibited during the study.

Results: Ten participants were enrolled in substudy 1, mean aged 24 years old (SD 4, 1 (18-32)); 80\% were men. Twenty participants were enrolled in substudy 2 , mean aged 23.4 years (SD $5.5(20-38)$ ); $65 \%$ were men. No significant differences were detected in the increase of symptoms during the nutrient drink test between control and intervention visits in both substudies (table 1). Maximum tolerated volume did not show any difference between visits in both substudies. Reflux episodes after nutrient drink test and reflux episodes registered in 24 hours did not show significant differences between control and study visits. The sum of weekly symptoms did not show any difference between control and intervention weeks in both substudies.

Conclusion: Moderate beer consumption (regular and non-alcohol beer) does not cause an increasement of dyspeptic symptoms and reflux in healthy people. It has been shown in a controlled situation (nutrient drink test and $\mathrm{pH}$ impedance register) as well as real life (diary weekly symptoms). Gastric acommodation and reflux episodes have either shown to be affected by moderate beer consumption.

Disclosure of Interest: All authors have declared no conflicts of interest.

\section{References}

1. Pehl C, Wendl B, Pfeiffer A. White wine and beer induce gastro-oesophageal reflux in patients with reflux disease. Alimentary pharmacology \& therapeutics. 2006;23(11):1581-6.

2. Seidl H, Gundling F, Schepp W, Schmidt T, Pehl C. Effect of low-proof alcoholic beverages on duodenogastro-esophageal reflux in health and GERD. Neurogastroenterology and motility: the official journal of the European Gastrointestinal Motility Society. 2011;23(2):145-50, e29.

3. Tobon S VS, Sandin B. Estudio preliminar de validez y confiabilidad del cuestionario PAGI-SYM para determinar síntomas de dispepsia acorde con la calidad de vida. Rev Col Gastroenterol. 2006;21(4).

4. Nuevo J, Tafalla M, Zapardiel J. [Validation of the Reflux Disease Questionnaire (RDQ) and Gastrointestinal Impact Scale (GIS) in patients with gastroesophageal reflux disease in the Spanish population]. Gastroenterologia y hepatologia. 2009;32(4):264-73.

\section{P0526 DUODENAL PATHOLOGY IN PATIENTS WITH RUMINATION SYNDROME - EOSINOPHILIA AND} INTRAEPITHELIAL LYMPHOCYTOSIS

M. Halland ${ }^{1}$, N.J. Talley ${ }^{2}$, J.A. Murray ${ }^{1}$, R. Cameron ${ }^{3}$, M. M. Walker ${ }^{3}$ ${ }^{1}$ Gastroenterology And Hepatology, Mayo Clinic, Rochester/United States of America

${ }^{2}$ University of Newcastle Faculty of Health PVC Office, Callaghan/Australia ${ }^{3}$ School Of Medicine And Public Health, The University of Newcastle Australia, Callaghan/Australia/NSW

Contact E-mail Address: halland.magnus@mayo.edu Introduction: Rumination syndrome is a functional gastrointestinal disorder characterized by effortless, post-prandial regurgitation of food. In addition to regurgitation, a large proportion of patients report functional dyspepsia (FD) symptoms including post-prandial discomfort, early satiety and nausea ${ }^{1,2}$ Recently, duodenal eosinophilia has been described both in adult and pediatric patients with $\mathrm{FD}^{3-5}$. Because of the significant symptomatic overlap between FD and rumination syndrome we hypothesized that histological changes similar to those described in FD might exist among patients with rumination syndrome. Aims \& Methods: We therefore aimed to assess histology of duodenal biopsies from patients with rumination syndrome and compared these to healthy controls. Rumination syndrome was diagnosed with post-prandial esophageal high resolution impedance manometry (HRIM) and/or fulfilled ROME II/III criteria. This study was approved by the Institutional Review Board. We included persons aged 18 and above with a diagnosis of rumination syndrome in whom we had also obtained 4-6 duodenal biopsies from diagnostic upper endoscopy. Normal controls were aged 18 and above without any gastrointestinal symptoms in whom 4-6 duodenal biopsies were obtained for research purposes. Cases and controls with a personal history of an eosinophilic disorder, gastric or esophageal surgery, recent (within 30 days) intake of NSAIDS and pregnant and/or lactating females were excluded. Duodenal biopsies obtained were routinely processed to formalinfixed paraffin-embedded tissue blocks which were cut at $3 \mu \mathrm{m}$, stained with $\mathrm{H} \& \mathrm{E}$ and scanned to digital images (Aperio). The pathologist, blinded to the casecontrol status, analyzed de-identified digital images of the biopsy specimens and assessed for eosinophil counts/mm2 in sections. Individual sections were also assessed for the presence of Brunner's glands (BG) and intraepithelial lymphocyte counts (IEL)/100 enterocytes. This was done in order to distinguish the first part of the duodenum with BG, D1 and the second part, generally without BG, (D2) and intraepithelial lymphocyte counts (IEL)/100 enterocytes.

Results: Patients with rumination syndrome (22) had a mean age of 39.2 years (range 19-71) and 77\% were female. Controls (10) had a mean age of 34.3 (range 19-69) and $80 \%$ were female. The mean eosinophil counts/biopsy fragment $+/-$ Brunners glands (BG), showed no difference in counts in the sections $+/-$ BG (D1 vs D2), $\mathrm{p}=0.8$. No overt pathology was noted, but IEL counts were significantly higher in rumination patients (mean 15, range 8-29, and 2 cases had lymphocytic duodenosis) vs controls (mean 11 , range $11-18$ ), $\mathrm{p}=0.02$. Compared to controls, there was a significant increase in the mean eosinophil count among the patients with rumination syndrome rumination, 26 per $\mathrm{mm}^{2}$ (range 16-42), vs $18 \mathrm{~mm}^{2}$ (range $\left.10-28\right), \mathrm{p}=0.006$

Conclusion: These findings demonstrate that patients with rumination syndrome have duodenal eosinophilia and increased IEL counts compared to healthy controls. To our knowledge, histopathological changes among patients with rumination syndrome compared to controls have not previously been described. Therefore, a potential role of duodenal immune mechanisms in the pathophysiology of rumination syndrome warrants further enquiry.

Disclosure of Interest: All authors have declared no conflicts of interest.

\section{References}

1. O'Brien, M.D., B.K. Bruce, and M. Camilleri, The rumination syndrome: clinical features rather than manometric diagnosis. Gastroenterology, 1995 108(4): p. 1024-29

2. Soykan I, Chen J, Kendall BJ, McCallum RW. The rumination syndrome: clinical and manometric profile, therapy and long term outcome. Dig Dis Sci 1997;42:1866-1872 
3. Vanheel, H., et al., Impaired duodenal mucosal integrity and low-grade inflammation in functional dyspepsia. Gut, 2014. 63(2): p. 262-271.

4. Walker, M.M., et al., Duodenal eosinophilia and early satiety in functional dyspepsia: confirmation of a positive association in an Australian cohort. Journal of Gastroenterology and Hepatology, 2014. 29(3): p. 474-479.

5. Wauters, L., et al., Functional dyspepsia is associated with duodenal eosinophilia in an Australian paediatric cohort. Alimentary pharmacology \& therapeutics, 2017. 45(10): p. 1358-1364.

\section{P0527 CHRONIC POSTSTROKE OROPHARYNGEAL DYSPHAGIA IS ASSOCIATED WITH IMPAIRED CORTICAL ACTIVATION TO PHARYNGEAL SENSORY INPUTS}

O. Ortega ${ }^{1}$, C. Cabib ${ }^{1}$, N. Vilardell ${ }^{1}$, L. Mundet ${ }^{1}$, P. Clavé $^{2}$, L. Rofes ${ }^{1}$

${ }^{1}$ GI Physiology Laboratory, Hospital de Mataró, Consorci Sanitari del Maresme, Mataró/Spain

${ }^{2}$ Centro De Investigación Biomédica En Red De Enfermedades Hepáticas $Y$ Digestivas., Instituto de Salud Carlos III, Barcelona/Spain

\section{Contact E-mail Address: oortega@csdm.com}

Introduction: The role of afferent sensory pathways in the pathophysiology of post-stroke oropharyngeal dysphagia (OD) is not known [1]. We hypothesized that chronic post-stroke patients with OD (PSD) would show impaired sensory cortical activation in the afected hemisphere.

Aims \& Methods: We studied 28 chronic unilateral post-stroke patients (17 PSD and 11 nondysphagic [PSnD]) and 11 age-matched healthy volunteers (HV). Electroencephalography was used to assess event-related sensory evoked potentials to pharyngeal stimulation (pSEP) and sensory thresholds with a naso-pharyngeal catheter with two electrodes passed through the nostrils $14-15 \mathrm{~cm}$ until the pharynx (Gaeltec Ltd, Dunvegan, Scotland) [2]. We analyzed pSEP peak-latency and amplitude (N1, P1, N2, P2) and neurotopographic stroke characteristics from brain MRI.

Results: HV presented a highly symmetric bi-hemispheric cortical pattern of brain activation at centro-parietal areas (N1-P1, N2-P2) to pharyngeal stimuli. In contrast, an asymmetric pattern of reduced ipsilesional activation was found in PSD (N2-P2; $\mathrm{p}=0.026)$ but not in PSnD. PSD presented impaired safety of swallow (Penetration- Aspiration score: $4.3 \pm 1.6$ ) and delayed laryngeal vestibule closure $(360.0 \pm 70.0 \mathrm{~ms})$, and higher NIHSS $(7.0 \pm 6.2$ vs. $1.9 \pm 1.4, \mathrm{p}=0.001)$ and Fazekas scores $(3.0 \pm 1.4$ vs. $2.0 \pm 1.1 ; \mathrm{p}<0.05)$ than PSnD. pSEP showed a unilateral delay at stroke site exclusively for PSD (peak-latency inter-hemispheric difference vs. PSnD: N1, $6.5 \pm 6.7$ vs. $1.1 \pm 1.0 \mathrm{~ms} ; \mathrm{N} 2,32.0 \pm 15.8$ vs. $4.5 \pm 4.9 \mathrm{~ms} ; \mathrm{p}<0.05)$

Conclusion: Chronic post-stroke OD is associated with stroke severity and degree of leukoaraoisis. Impaired conduction and cortical integration of pharyngeal sensory inputs at stroke site is a key feature of chronic PSD. These findings highlight the role of sensory pathways in the pathophysiology of post-stroke $\mathrm{OD}$ and offer a potential target for future treatments.

Disclosure of Interest: All authors have declared no conflicts of interest.

\section{References}

1. Cabib C, et al. Neurorehabilitation strategies for poststroke oropharyngeal dysphagia: from compensation to the recovery of swallowing function. Ann N Y Acad Sci. 2016; 1380(1):121-138.

2. Rofes L, et al. Spatiotemporal characteristics of the pharyngeal event-related potential in healthy subjects and older patients with oropharyngeal xddysfunction. Neurogastroenterol Motil. 2017; 29(2).doi:10.1111/nmo.12916.

\section{P0528 PERCUTANEOUS ENDOSCOPIC GASTROSTOMY WITH JEJUNAL EXTENSION FOR GASTROPARESIS: THE ULTIMATE SOLUTION?}

D. Strijbos ${ }^{1}$, D. Keszthelyi ${ }^{1}$, J. Kruimel ${ }^{1}$, L. P.l. Gilissen ${ }^{2}$, R. De Ridder ${ }^{1}$, J. Conchillo ${ }^{1}$, A. A.m. Masclee ${ }^{1}$

${ }^{1}$ Gastroenterology And Hepatology, Maastricht University Medical Center, Maastricht/Netherlands

${ }^{2}$ Gastroenterology And Hepatology, Catharina Hospital Eindhoven, Eindhoven/ Netherlands

Contact E-mail Address: denise.strijbos@catharinaziekenhuis.nl

Introduction: Gastroparesis is characterized by abnormal gastric motor function with delayed gastric emptying in the absence of mechanical obstruction. In our tertiary referral centre, patients are treated with a stepwise approach, starting with dietary and lifestyle advice and prokinetics followed by pyloric botulinum toxin. When these initial measures fail, in the presence of malnutrition, one of the following interventions are considered: three months nasoduodenal tube feeding with 'gastric rest' and placement of a percutaneous endoscopic gastrostomy with jejunal extension (PEG-J). Our primary aim was to evaluate the effect of nutritional treatment entities in patients with gastroparesis who fail previous treatments, on weight and symptoms.

Aims \& Methods: Prospectively collected data of all referred gastroparesis patients between 2008 and 2016 were reviewed.
Results: A total of 101 gastroparesis patients (71\% female, 20-86yrs, mean 55yrs) were analyzed. Etiologies were idiopathic $(37 \%)$, diabetes mellitus $(30 \%)$, postsurgical $(27 \%)$ and other $(7 \%)$. Of the 101 patients, 51 patients had adequate responses to dietary advice and prokinetics, not requiring further therapeutic interventions. For the remaining 60 patients, various treatments were used. With respect to nutritional interventions, 36 patients were treated with three months of gastric rest via complete nasoduodenal tube feeding. Enteral tube feeding was well accepted, occlusion occurred in $8 \%$ of patients. Mean weight gain in symptom responders was $3.5 \%(2.4 \mathrm{~kg}, \mathrm{p}=0.02)$, in non-responders $3.7 \%$ $(2.4 \mathrm{~kg}, \mathrm{p}=0.01)$. These 19 patients with insufficient symptomatic response after 3 months gastric rest continued treatment with enteral feeding through PEG-J. A significant weight gain of $8.2 \%$ was seen (mean $5.0 \mathrm{~kg}$, range $-6 \%$ to $+29 \%$ ), $\mathrm{p}=0.003$ ) within 3-6 months after PEG-J placement. Thereafter only 3 patients $(16 \%)$ have been able to resume complete oral intake, the PEG-J was removed after a mean treatment time of 11 months. In $84 \%$ of patients the PEG-J is still in use, with a mean treatment time of 894 days. Over $75 \%$ of patients report adequate effect on symptoms. Most frequent complication was luxation of the jejunal extension to the stomach ( $32 \%$ of patients). Other complications were peristomal infection ( $11 \%$ within 30 days, $16 \%$ after 30 days) and buried bumper $(16 \%)$.

Conclusion: This study describes the sequelae of a large group of tertiary referral gastroparesis patients treated with PEG-J treatment. In gastroparesis patients who failed all previous treatment, PEG-J is an excellent option to regain and maintain adequate nutritional status with marked symptom control.

Disclosure of Interest: All authors have declared no conflicts of interest.

\section{P0529 RELEVANCE OF SLEEP DISTURBANCE TO FUNCTIONAL GASTROINTESTINAL SYMPTOMS, CLINICAL CHARACTERISTICS, AND PSYCHOLOGICAL DISTRESS}

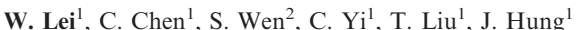

${ }^{1}$ Department Of Medicine, Hualien Tzu Chi Hospital, Buddhist Tzu Chi Medical Foundation and Tzu Chi University, Hualien/Taiwan

${ }^{2}$ Department Of Public Health, College of Medicine, Tzu Chi University, Hualien/ Taiwan

Contact E-mail Address: aquarious@seed.net.tw

Introduction: Reduced sleep quality has been linked to gastroesophageal reflux disease (GERD) and functional gastrointestinal disorders. It is unknown whether GERD, functional dyspepsia (FD) and irritable bowel syndrome (IBS) are more prevalent in subjects with significant sleep disturbance (SD) than those without SD.

Aims \& Methods: The aim of the study was to investigate gastrointestinal symptoms, clinical characteristics, and psychological factors in subjects with and without SD in a general population undergoing health checkups. We enrolled 2752 consecutive subjects who received upper gastrointestinal endoscopy and colonoscopy during their health checkups. All participants underwent an evaluation with questionnaires including Reflux Disease Questionnaire score, Pittsburgh Sleep Quality Index (PSQI), Taiwanese Depression Questionnaire, and StateTrait Anxiety Inventory before receiving endoscopic exam. Demographic characteristics and biochemical data were also recorded. FD and IBS were based on Rome III diagnostic criteria, and metabolic syndrome was defined by the National Cholesterol Education Program Adult Treatment Panel III definition. Sleep disturbance was confirmed when PSQI score was greater than 5. We compared the clinical and psychological factors between subjects with and without sleep disturbance.

Results: Among the study population $(\mathrm{n}=2674), 956(36 \%)$ individuals had SD. SD subjects had more female gender, older age, lower level of education, higher systolic blood pressure, higher serum high-density lipoprotein levels, and higher prevalence of FD and IBS than those without SD. In addition, SD patients also had more depression, more anxiety, more severe GERD symptoms, and higher prevalence of non-erosive reflux disease (NERD) $(\mathrm{p}<0.001)$. Multivariate analysis revealed that female sex $(O R=1.75, p<0.001)$, older age $(O R=1.03$, $\mathrm{p}<0.001)$, more severe GERD symptoms $(\mathrm{OR}=1.03, \mathrm{p}<0.033)$, NERD $(\mathrm{OR}=1.63, \mathrm{p}=0.023)$, IBS $(\mathrm{OR}=1.48, \mathrm{p}=0.05)$, and depression $(\mathrm{OR}=1.16$, $\mathrm{p}<0.001$ ) were positive predictive factors for SD, whereas higher level of education $(\mathrm{OR}=0.57, \mathrm{p}<0.001)$ was negative predictive factor for SD.

Conclusion: Our study demonstrates that SD is associated with female sex, older age, lower education level, greater GERD symptom burden, greater depression, and higher prevalence of NERD and IBS. Future studies will be needed to clarify the relationship between functional gastrointestinal diseases and sleep disorders. Disclosure of Interest: All authors have declared no conflicts of interest.

\section{References}

1. Orr WC, Chen CL. Sleep and the gastrointestinal tract. Neurol Clin. 2005; 23(4):1007-24.

2. Futagami S, Yamawaki H, Hashimoto S, et al. Sleep Disturbances in Functional Gastrointestinal Disorders. Intern Med. 2016;55(12):1509-10.

3. Dent J, Holloway RH, Eastwood PR. Systematic review: relationships between sleep and gastro-oesophageal reflux. Aliment Pharmacol Ther. 2013; 38(7):657-73. 
P0530 ESOPHAGEAL SYMPTOMS ARE COMMON AND RELATED TO OTHER FUNCTIONAL GASTROINTESTINAL DISORDERS (FGIDS) IN A WESTERN POPULATION

A. Josefsson ${ }^{1}$, O.S. Palsson ${ }^{2}$, M. Simrén ${ }^{3}$, A. Sperber ${ }^{4}$, H. Törnblom ${ }^{5}$, W.E. Whitehead ${ }^{6}$

${ }^{1}$ Department Of Gastroenterology, Sahlgrenska University Hospital, Göteborg/ Sweden

${ }^{2}$ Dept. Of Medicine, University of North Carolina, Chapel Hill, Chapel Hill, $\mathrm{NC} /$ United States of America $/ \mathrm{NC}$

${ }^{3}$ Dept Of Internal Medicine, Sahlgrenska University Hospital - Dept of Internal Medicine, Sahlgrenska University Hospital; Gothe, Gothenburg/Sweden

${ }^{4}$ Ben-gurion University Of The Negev, Faculty of Health Sciences, Beersheba Israel

${ }^{5}$ Dept Of Gastroenterology And Hepatology, Sahlgrenska Academy Faculty of Medicine, Gothenburg/Sweden

${ }^{6}$ Dept. Of Medicine, University of North Carolina at Chapel Hill, Chapel Hill, NC United States of America

Contact E-mail Address: axel.josefsson@vgregion.se

Introduction: The prevalence and frequency of esophageal symptoms suggestive of a functional esophageal disorder according to the Rome IV criteria are unknown. This study aimed to describe the general population prevalence and risk factors for esophageal symptoms compatible with functional esophageal disorders

Aims \& Methods: Data from an online survey of 6300 individuals age $\geq 18$ years in the United States, United Kingdom and Canada (2100 in each country) including the Rome IV diagnostic questionnaire for adults and demographic questions was used. Quota-based sampling ensured equal proportions of sex, age groups, and education distributions across countries. Prevalence and frequency of esophageal symptoms in the past 3 months and putative functional esophageal disorders were retrieved from the Rome IV questionnaire. Symptoms were considered present if they occurred at least weekly for dysphagia, chest pain, and globus, and at least twice weekly for heart burn. Variables with a $p \leq 0.1$ in univariate analyses were entered into a multivariate analysis (logistic regression) to identify factors independently related to esophageal symptoms. As endoscopy and $\mathrm{pH}$ measurement are parts of the clinical diagnosis of esophageal disorders in the Rome IV criteria, we only describe esophageal symptoms compatible with functional esophageal disorders. Somatization was assessed with the Patient Health Questionnaire (PHQ)-12.

Results: Data from 5177 participants $(47.8 \%$ female; mean age 46.7 (range 18-92) years; 1645 US, 1734 UK, 1798 Canada) were retained for analysis after 369 inconsistent responders and 754 previously diagnosed with gastroesophageal reflux disease (GERD) were excluded. Esophageal symptom prevalence was: feeling of a lump or something stuck in the throat (globus) $8.1 \% \quad(n=420)$, heartburn $6.5 \%(\mathrm{n}=334)$, dysphagia $4.5 \% \quad(\mathrm{n}=233)$ and chest pain $5.2 \%$ $(n=269)$. Independent predictors for increased risk of esophageal symptoms included younger age, symptoms consistent with other FGIDs, using medications for gastrointestinal symptoms, somatization, cannabis use, and certain foods (see table 1). When individuals with GERD were included in re-analyses, GERD was a significant predictor for chest pain and heartburn (OR 1.939 and 1.525), but not globus and dysphagia. The prevalence of symptom constellations consistent with Rome IV esophageal diagnoses was $1 \%$ for globus, $3.2 \%$ for functional dysphagia, $0.7 \%$ for reflux hypersensitivity, $0.8 \%$ for functional chest pain, and $1.1 \%$ for functional heartburn. There was a significant difference in symptom consistent with any functional esophageal disorder between USA $7.0 \%$, UK $4.3 \%$ and Canada $5.9 \%(\mathrm{p}=0.003)$.

Table 1: Factors independently associated with presence of esophageal symptoms compatible with a functional esophageal disorder

\begin{tabular}{lllll}
\hline \multirow{2}{*}{ Symptom } & Factor & $\begin{array}{l}\text { Odds } \\
\text { ratio }\end{array}$ & $\begin{array}{l}\text { 95\% Confidence } \\
\text { interval }\end{array}$ & $\mathrm{R}^{2}$ value \\
\hline Globus & PHQ12 & 1.138 & $1.105-1.172$ & 0.189 \\
& Medication for constipation & 1.718 & $1.146-2.577$ & \\
& Diet rich in pasta & 1.068 & $1.000-1.139$ & \\
& Gastroduodenal disorder & 2.616 & $2.006-3.412$ & \\
& Bowel disorder & 1.318 & $1.032-1.684$ & \\
Chest pain & Anorectal disorder & 1.699 & $1.243-2.321$ & 0.301 \\
& Cannabis consumption & 1.949 & $1.215-3.128$ & \\
& Previously diagnosed with & 6.492 & $1.342-31.417$ & \\
& $\quad$ functional dyspepsia & & & \\
& Medication for acid/ & 1.488 & $1.039-2.130$ & \\
& $\quad$ heartburn & & & \\
& PHQ12 & 1.214 & $1.171-1.259$ & \\
& Gastroduodenal disorder & 2.820 & $2.051-3.877$ & \\
& Bowel disorder & 1.791 & $1.311-2.446$ & \\
& Anorectal disorder & 1.536 & $1.058-2.230$ & \\
& Diet rich in tofu & 1.192 & $1.058-1.343$ & \\
& Home with electricity as a & 2.509 & $1.256-5.011$ & \\
child & & & &
\end{tabular}

(continued)
Table 1 Continued

\begin{tabular}{lllll}
\hline \multirow{2}{*}{ Symptom } & Factor & $\begin{array}{l}\text { Odds } \\
\text { ratio }\end{array}$ & $\begin{array}{l}95 \% \text { Confidence } \\
\text { interval }\end{array}$ & $\mathrm{R}^{2}$ value \\
\hline $\begin{array}{l}\text { Medication for acid/ } \\
\text { heartburn }\end{array}$ & 11.427 & $8.602-15.179$ & \\
& $\begin{array}{l}\text { Gastroduodenal disorder } \\
\text { Dowel disorder }\end{array}$ & 2.789 & $2.049-3.798$ & \\
& Diet rich in pasta & 2.165 & $1.632-2.872$ & \\
& Age & 0.990 & $0.980-1.000$ & 0.242 \\
& Medication for diarrhea & 1.882 & $1.100-3.221$ & \\
& Medication for acid/ & 1.456 & $1.007-2.106$ & \\
$\quad$ heartburn & & & \\
& Medication for gas/bloating & 1.913 & $1.178-3.106$ & \\
& Gastroduodenal disorder & 4.368 & $3.146-6.065$ & \\
& Anorectal disorder & 1.585 & $1.072-2.343$ & \\
& Diet rich in rice & 1.097 & $1.006-1.196$ & \\
& PHQ12 & 1.110 & $1.069-1.154$ &
\end{tabular}

Variables with a p-value of 0.1 or less in univariate analysis were entered into a multivariate analysis (logistic regression) in order to identify factors independently associated with esophageal symptoms (up to 33 variables).

Conclusion: Esophageal symptoms compatible with a functional esophageal disorder are common in the Western population. Age and presence of other GI and non-GI symptoms are associated with reporting esophageal symptoms.

Disclosure of Interest: All authors have declared no conflicts of interest.

\section{P0531 DETERMINANT FACTORS OF QUALITY OF LIFE IN ADULT PATIENTS WITH EOSINOPHILIC ESOPHAGITIS}

A. J. Lucendo Villarin ${ }^{1}$, L. Arias-González ${ }^{1}$, J. Molina-Infante ${ }^{2}$, Á. Arias ${ }^{1}$ ${ }^{1}$ Dept. Of Gastroenterology, Hospital General de Tomelloso, Tomelloso/Spain ${ }^{2}$ Gastroenterology, Hospital San Pedro de Alcántara, Cáceres/Spain

Contact E-mail Address: ajlucendo@hotmail.com

Introduction: Eosinophilic esophagitis (EoE) affects children and young adults and has rapidly grown over the past decade, especially in developed countries. Presently, it represents the second leading cause of chronic esophagitis after gastroesophageal reflux disease (GERD) and the main cause of dysphagia in children and young adults. EoE affects health-related quality of life (HRQoL). Data on determinant factors and the influence of dietary interventions are scarce. Aims \& Methods: In this study we aimed (1) to determine for the first time the health-related QoL in a representative sample of Spanish adults with EoE, and (2) to identity determinants of impaired HRQoL, including the effect of dietary restrictions. Multicentre observational, cross-sectional study in eight Spanish centers attending adult EoE patients throughout several Spanish Regions. A validated Spanish version of the self-administered Adult Eosinophilic Esophagitis Quality of Life (EoE-QoL-A) questionnaire was used, as well as a survey of demographic and clinical data. Multiple linear regression was used to identify and quantify determinant factors of HRQoL.

Results: Responses provided by 170 patients were assessed $(73.5 \%$ male; mean age $33.5 \pm 11.4 \mathrm{y}$ ). Overall mean score for the EoE-QoL-A index was $1.4 \pm 0.8$, with no differences between patients on dietary or pharmacological therapy $(1.82 \pm 0.8$ vs. $1.62 \pm 0.8 ; p=0.132)$. Disease anxiety showed the highest. The mean score $(2.13 \pm 0.9$ points $)$, followed by choking anxiety $1.97 \pm 1.1$; social impact, $1.77 \pm 1.1$, and diet/eating impact $1.68 \pm 0.9$ points. Emotional impact had the lowest rating $(1.15 \pm 0.9)$, and the only with a significantly worse score in patients under dietary restrictions. Recurrent food impaction, a higher educational level, dietary interventions and symptom duration were all independent determinant factors significantly impairing HRQoL. Female gender and empiric elimination diets negatively influenced on diet/eating impact.

Conclusion: HRQoL is impaired in adult EoE patients, especially disease and chocking anxiety. Recurrent food impaction, dietary interventions and symptom duration are the most important factors influencing the perception of HRQOL in EoE.

Disclosure of Interest: All authors have declared no conflicts of interest.

\section{P0532 THE ASSOCIATION BETWEEN ATOPIC MANIFESTATIONS AND EOSINOPHILIC ESOPHAGITIS: A SYSTEMATIC REVIEW AND META-ANALYSIS}

A. J. Lucendo Villarin ${ }^{1}$, J. González-Cervera ${ }^{2}$, Á. Arias ${ }^{3}$, O. Redondo-González ${ }^{3}$, I.T. Terreehorst ${ }^{4}$, M.M. Cano-Mollinedo ${ }^{2}$

${ }^{1}$ Dept. Of Gastroenterology, Hospital General de Tomelloso, Tomelloso/Spain

${ }^{2}$ Allergy, Hospital General de Tomelloso, Tomelloso/Spain

${ }^{3}$ Research Support Unit, Hospital General La Mancha Centro, Alcázar de San Juan/Spain

${ }^{4}$ Department $O f$ ENT And Pediatrics, AMC, Amsterdam, Amsterdam/Netherlands

Contact E-mail Address: ajlucendo@hotmail.com

Introduction: Eosinophilic oesophagitis (EoE) is a chronic, immune-mediated, inflammatory disorder, defined symptomatically by esophageal dysfunction and histologically by eosinophil-predominant inflammation of the esophagus. 
Several studies have provided information on the prevalence of different atopic conditions in paediatric and adult EoE patients and compared them with several groups of control subjects. The findings indicate that, overall, EoE patients show a higher frequency of asthma, rhinoconjunctivitis, eczema, and food allergies than control groups; however, definitions for the associated atopic conditions have not always been provided and the selection process for the controls has not been such that they can be considered universally representative of the general population without EoE. These two limitations have hampered researchers in their efforts to clearly assess the magnitude of the association between atopy and EoE. Weaim to conduct a systematic review of the literature and to perform a meta-analysis in order to evaluate the presence of atopic diatheses in patients with EoE as well as to summarize the prevalence of atopic conditions in both paediatric and adult EoE patients in comparison with the non-EoE control population.

Aims \& Methods: A highly sensitive search strategy was designed to identify and retrieve all documents dealing with the relationship between atopy and EoE in children and adults. This systematic literature search was performed independently by two researchers (AA and AJL) on April $6^{\text {th }}, 2016$ in three major bibliographic databases (PubMed, EMBASE, and Scopus) for the period up to March 2016. The search was not restricted with regard to the language of publication. A predetermined protocol was used in accordance with the quality standards for reporting meta-analyses of observational studies in epidemiology. Four reviewers (JG-C, AA, MM-CM, and AJL) independently extracted relevant information from each eligible study using a standardized data extraction sheet and then proceeded to cross-check the results. Estimates for the prevalence of each atopic manifestation in EoE patients and controls were summarized with the aid of a fixed- or random-effects meta-analysis, depending on intra-study heterogeneity, weighted for inverse variance following the method elaborated by DerSimonian and Laird. Summary estimates, including $95 \%$ confidence intervals $(\mathrm{CI})$, were calculated for each season and month, whenever possible.

Results: Of the 2954 references identified, data was collected from 21 studies including a total of 53,542 EoE patients and 54,759 controls. The criteria for defining a diagnosis of atopy in either EoE patients or controls was not structurally considered in most of the studies. The frequency or prevalence of the different atopic manifestations among EoE patients was compared with that observed in several types of control populations, including series of patients with gastroesophageal reflux disease (GORD), other patients, and healthy volunteers, all of whom were endoscopically assessed with a diagnosis of EoE specifically ruled out. In all cases, EoE was considered as independent from GORD and other upper GI tract diseases. Some studies included database-registered subjects as control groups. The criteria for defining a diagnosis of atopy among EoE patients and control subjects varied widely across the different studies, from self-reported/parent-reported atopic background to strict allergist/immunologist-provided diagnoses. Overall, allergic rhinitis was significantly more common among EoE patients compared to control subjects (OR 5.58; 95\% CI, $\left.3.27,9.53 ; \mathrm{I}^{2}=86 \%\right)$ as were bronchial asthma (OR $3.06(95 \% \mathrm{CI}, 2.01,4.66$; $\mathrm{I}^{2}=83.4 \%$ ) and eczema (OR 2.86; $95 \%$ CI, 1.88, 4.36; $\left.\mathrm{I}^{2}=57.2 \%\right)$. Food allergies and other atopic conditions were also assessed. No significant publication bias was found for studies dealing with allergic rhinitis and eczema in EoE. Finally, our search uncovered two papers that reported on the frequency of drug allergy in EoE patients compared to controls, showing no significant differences between these two populations $(\mathrm{OR}=0.981 ; 95 \% \mathrm{CI}, 0.07,14.72)$.

Conclusion: The present study shows that an accurate diagnosis of atopy is lacking in most of the research evaluating the prevalence of asthma, rhinitis, and eczema among EoE patients. Still, the prevalence of these three conditions seems to be significantly higher in children and adults with EoE as compared to control subjects representative of the general population. Further research should clearly document and use standard definitions of allergic rhinitis, asthma (including its severity and level of control), skin allergy, and food allergy (rather than mere sensitization) when assessing and documenting concurrent allergic diseases in patients with EoE.

Disclosure of Interest: All authors have declared no conflicts of interest.

\section{P0534 A PHASE 1 STUDY TO ASSESS THE PHARMACOKINETICS, SAFETY, AND TOLERABILITY OF SINGLE DOSE APT-1011 ADMINSTERED UNDER FED OR FASTED CONDITIONS OR AT BEDTIME IN A RANDOMISED THREE-WAY, CROSSOVER DESIGN}

\section{G. M. Comer, B. A. Meltzer}

Adare Pharmaceuticals, Inc., Lawrenceville/United States of America/NJ

\section{Contact E-mail Address: brian.meltzer@adarepharma.com}

Introduction: APT-1011 is an orally disintegrating tablet (ODT) formulation of fluticasone propionate (FP) that provides a novel approach for the treatment of eosinophilic esophagitis (EoE).

Aims \& Methods: A randomised, single-dose, three-way, crossover study was conducted in healthy volunteers to compare the pharmacokinetics (PK), safety, and tolerability of APT-1011 when administered under morning (AM) fed or fasted conditions or before bedtime (HS). Participants were administered $6 \mathrm{mg}$ APT-1011 following these three different administration schedules, with a 7-day washout separating the three periods. Serial plasma samples for FP PK were collected predose and up to 72 hours following each dose. Noncompartmental and statistical analyses were performed for PK parameters.

Results: A total of 24 participants enrolled and $22(92 \%)$ completed the study. A summary of the PK parameters by test group is presented in Table 1. AM dosing was associated with a higher rate of absorption under fed compared to fasted conditions. Following a high fat meal, there was a higher peak concentration $\left\{\mathrm{C}_{\max }\right.$ : ratio $[90 \% \mathrm{CI}$ (confidence interval) $\left.]=120.65 \%[99.84 \%-145.79 \%]\right\}$ and a faster time to peak concentration compared to the fasted state ( $\mathrm{T}_{\max }$ : $\mathrm{fed}=5.00 \mathrm{~h}$, fast $=10.00 \mathrm{~h}$ ). However, lower total exposure in the fed compared to the fasted state A AUC [area under the curve] $]_{\text {last }}$ : ratio $(90 \% \mathrm{CI})=76.97 \%$ $[67.64 \%-87.59 \%]\}$ was observed. HS dosing was found to slow the rate of absorption compared to AM dosing conditions. Specifically, the time to peak concentration with HS dosing $\left(\mathrm{T}_{\max }=14 \mathrm{~h}\right)$ was longer than with AM dosing $\left(\mathrm{T}_{\max }\right.$ : fast $=10 \mathrm{~h}$, fed $\left.=5 \mathrm{~h}\right)$. HS dosing was associated with higher overall exposure $\left(\mathrm{AUC}_{\text {inf: }}\right.$ ratio $\left.[90 \% \mathrm{CI}]=122.36 \%[107.02 \%-139.88 \%]\right)$ and lower $\mathrm{C}_{\max }$ $\left(\mathrm{C}_{\max }\right.$ : ratio $\left.[90 \% \mathrm{CI}]=67.79 \%[56.29 \%-81.64 \%]\right)$ versus the fed dosing regimen. Compared to the fasted regimen, HS dosing yielded lower overall exposure $\left(A C_{\text {inf }}\right.$ : ratio $\left.[90 \% \mathrm{CI}]=87.00 \%[75.24 \%-100.59 \%]\right)$ and lower $\mathrm{C}_{\max }\left(\mathrm{C}_{\max }\right.$ : ratio $[90 \% \mathrm{CI}]=81.78 \%[67.93 \%-98.47 \%])$. Across all dosing regimens, the $\mathrm{C}_{\max }$ of FP with APT-1011 ranged from 5.97-200 pg/mL. Seven subjects (29\%) reported 12 treatment-emergent adverse events over the course of the study; all were mild in severity. No serious adverse events or deaths were reported.

Conclusion: Oral APT-1011 was safe and well-tolerated when administered under AM fasted, AM fed, or HS conditions to healthy subjects. While there was faster absorption of APT-1011 under fed conditions and slower absorption at bedtime, overall absorption of FP with this ODT formulation is low $(<200 \mathrm{pg} / \mathrm{mL})$. Slower absorption with HS dosing suggests a potential for longer dwell times in the esophagus; the relationship of HS dosing with histological efficacy in both the proximal and distal portions of the esophagus will be explored in future studies of APT-1011 in EoE.

Table 1: Plasma FP PK Parameters

\begin{tabular}{llll}
\hline \multirow{2}{*}{ Parameter } & \multicolumn{3}{l}{ AM Fast Geometric AM Fed Geometric HS Geometric } \\
& Mean $(\mathrm{CV} \%)$ & Mean $(\mathrm{CV} \%)$ & Mean $(\mathrm{CV} \%)$ \\
\hline $\mathrm{C}_{\max }(\mathrm{pg} / \mathrm{mL})$ & $31.1(103.6)$ & $34.2(102.3)$ & $23.8(111.9)$ \\
$\mathrm{T}_{\max }(\mathrm{h})^{*}$ & $10.00(2.00-30.00)$ & $5.00(1.00-10.00)$ & $14.00(2.00-20.00)$ \\
$\mathrm{AUC}_{0-24}(\mathrm{pgh} / \mathrm{mL})$ & $366.607(115.8)$ & $361.277(105.5)$ & $359.541(100.5)$ \\
$\mathrm{AUC}_{\text {inf }}(\mathrm{pgh} / \mathrm{mL})$ & $1044.308(90.1)$ & $587.890(107.2)$ & $726.451(100.2)$ \\
\hline
\end{tabular}

$\mathrm{CV} \%=$ percentage coefficient of variation. $*$ Median and range are presented.

Disclosure of Interest: G.M. Comer: Dr. Gail M. Comer is a paid consultant for Adare Pharmaceuticals, Inc.

B.A. Meltzer: Dr. Brian A. Meltzer is an employee of Adare Pharmaceuticals, Inc.

\section{P0535 EOSINOPHILLIC ESOPHAGITIS: RATIONALISING THERAPY}

M. A. Everson, I. Parisi, S. Mankodi, A. Shah, D. Grant, M. Rodriguez-Justo, M.R. Novelli, R. Sweis

Department Of Gastroenterology, University College London Hospital, London United Kingdom

\section{Contact E-mail Address: martin.everson@nhs.net}

Introduction: Eosinophilic esophagitis (EE) is a chronic condition of the esophagus with pathognomonic clinical, endoscopic and histologic features. We aimed to prognosticate which cohort of patients respond best to proton pump inhibitor monotherapy or topical steroids based on index endoscopy and histology in conjunction with symptoms at presentation and on follow-up.

Aims \& Methods: All patients referred with dysphagia or with an incidental histology finding of $>15$ eosinophils/high power field (eos/hpf) between Feb 2013 and Dec 2015 were analyzed by retrospective case note review and patient communication. Univariate analysis and binary logistic regression was used to identify associations between patient characteristics, presentation, endoscopic findings, degree of eosinophilia and subsequent response to treatment. Associations were assessed by Fishers exact test, t-test and Mann-Whitney for nominal, continuous parametric and non-parametric variables respectively.

Results: 1653 patients fulfilled the entry criteria. 544 with previous cancer, achalasia, post-operative strictures or Barrett's endotherapy were excluded. 95 patients had histological eosinophilia in keeping with EE; 85 with dysphagia and 10 with reflux as their presenting complaint $(67$ male, mean age $42 \pm 17$ years $) .31(32 \%)$ of these had at least one presentation with food bolus obstruction (FBO). There was a trend towards a higher eos/hpf in patients who presented with FBO $(47 \pm 21)$ compared to dysphagia $(38 \pm 17)$ and reflux $(38 \pm 17)(\mathrm{p}=0.073)$. Endoscopic evidence of chronic stricturing disease was associated with a higher eos/hpf than those with no strictures (mean 50.3 vs 38.6; $\mathrm{p}=0.04)$. 31 patients had endoscopic biopsies taken at least 3 months post therapy. Those with index features of chronic disease were more likely to be associated with failure of the eosinophilia to normalize regardless of medical treatment compared to those with acute changes (furrows, exudates) $(33 \%$ vs. $75 \% ; \mathrm{p}=0.02)$. Furthermore, patients with dysphagia or FBO demonstrated a reduced normalisation of eos/hpf following either steroid or PPI therapy compared to those not presenting with these symptoms at a minimum of 3 months ( $46 \%$ vs. $100 \% ; p=0.03$ ). Overall, there were no significant associations between concentration of eos/hpf and response to steroids or PPI. On the other hand, regardless of endoscopic findings, patients presenting with dysphagia and/or FBO demonstrated a higher response to steroids than those with reflux symptoms $(50 \%$ vs $9 \% \mathrm{p}=0.018)$ who responded best to PPI $(91 \%) .78$ patients were contactable a minimum of 3 months following initiation of treatment. Patients with chronic EE findings at initial endoscopy were less likely to respond symptomatically to PPI monotherapy compared to those with normal or acute endoscopic findings $(32 \%$ vs. $68 \% ; \mathrm{p}=0.003)$ while they were more likely to respond 
to steroids $(64 \%$ vs $36 \%$; $=0.002$. Specifically, the presence of strictures indicated a more likely clinical response to steroids compared to PPI alone. $(\mathrm{p}=0.007)$.

Conclusion: A higher eos/hpf was found in patients with chronic EE features at index endoscopy than those with normal or acute endoscopic signs. In those with normal or acute EE changes and without dysphagia as a presenting complaint, a clinical response was noted with PPI therapy alone. In those with chronic EE changes or with dysphagia/FBO, steroids appear to be the preferred therapeutic option, although at 3 months follow up a clinical response might precede a histological one.

Disclosure of Interest: All authors have declared no conflicts of interest.

\section{P0536 ESOMEPRAZOLE, RABEPRAZOLE AND PANTOPRAZOLE ARE EQUALLY EFFECTIVE IN INDUCING ENDOSCOPIC AND HISTOLOGIC REMISSION IN PATIENTS WITH PROTON PUMP INHIBITOR-RESPONSE ESOPHAGEAL EOSINOPHILIA}

M. Della Coletta ${ }^{1}$, S. Tolone ${ }^{2}$, N. De Bortoli ${ }^{3}$, O. Bartolo $^{4}$, G. Bodini ${ }^{5}$, E. Marabotto ${ }^{6}$, P. Zentilin ${ }^{7}$, A. Mauro ${ }^{8}$, R. Penagini ${ }^{9}$, V. Savarino ${ }^{10}$,

\section{E. Savarino}

${ }^{1}$ Division Of Gastroenterology, Department Of Surgery, Oncology And

Gastroenterology, University of Padua, Padua/Italy

${ }^{2}$ Surgery, Second University of Naples, Naples/Italy

${ }^{3}$ Division Of Gastroenterology, Department Of Internal Medicine, University of Pisa, Pisa/Italy

${ }^{4}$ Dept. Of Gastroenterology, University of Padova, Padova/Italy

${ }^{5}$ Department Of Internal Medicine, IRCCS San Martino DIMI, Genova/Italy

${ }^{6}$ University of Genoa, Genoa, Italy, Genoa/Italy

${ }^{7}$ Dept. Of Internal Medicine, University of Genoa, Genova/Italy

${ }^{8}$ Gastroenterology And Endoscopy Unit, University of Milan, Milano/Italy

${ }^{9}$ Dipartimento Di Scienze Mediche, Università degli Studi di Milano Dipto. di

Gastroenterologia, Milan/Italy

${ }^{10}$ Dept Internal Medecine, Universita di Genova, Genova/Italy

\section{Contact E-mail Address: edoardo.savarino@unipd.it}

Introduction: Proton Pump Inhibitor-response esophageal eosinophilia (PPI$\mathrm{REE}$ ) is an emerging condition characterized by a constellation of clinical, endoscopic, and histopathologic features in the setting of eosinophilic inflammation on esophageal biopsies responding to a course of proton pump inhibitor (PPI) therapy. A recent meta-analysis explored the role of different PPIs in inducing clinical and histological remission in patients with esophageal eosinophilia without observing significant differences among them due to several limitations (i.e. limited number of studies using each PPI drug, small sample size, and heterogeneity among the various studies).

Aims \& Methods: We aimed to prospectively compare the effect of different PPIs in inducing endoscopic and histologic remission in patients with PPI-REE. Consecutive patients with symptoms suggestive of EoE underwent upper endoscopy to assess the presence of at least $15 \mathrm{eos} / \mathrm{hpf}$ on oesophageal biopsies at mid proximal esophagus and, then, were treated with twice-daily PPI for at least 8 weeks. Patients were assigned to receive esomeprazole $20 \mathrm{mg}$ bid, rabeprazole $20 \mathrm{mg}$ bid or pantoprazole $40 \mathrm{mg}$ bid in a $1: 1: 1$ ratio. Thereafter, patients repeated upper endoscopy and PPI-REE was identified in case of less than 15 eos/hpf and a 50\% decrease from baseline. Endoscopic, according to Endoscopic Reference Score (EREFS), and histologic features were blindly reviewed for each patient and treatment

Results: Twenty-eight patients [23M/5F; mean age 35] reporting dysphagia $(93 \%)$, bolus impaction $(68 \%)$ and chestpain $(25 \%)$ were diagnosed with PPIREE. According to treatment allocation, $8(29 \%)$ patients received esomeprazole, $9(32 \%)$ rabeprazole and $11(39 \%)$ pantoprazole. At baseline, demographic and clinical data, including age, sex, BMI, $H$. pylori infection, concomitant allergy conditions and latency from diagnosis were similar $(\mathrm{p}=\mathrm{ns})$. Moreover, no differences were found in terms of frequency of symptoms reported $(\mathrm{p}=\mathrm{ns})$. Endoscopic and histologic features at baseline and after PPI therapy are shown in the Table. Esomeprazole, rabeprazole and pantoprazole reached the same degree of efficacy in inducing endoscopic and histologic changes in PPI-REE patients $(\mathrm{p}=\mathrm{ns})$.

Table: Endoscopic and histologic features at baseline and after PPI therapy in PPI-REE

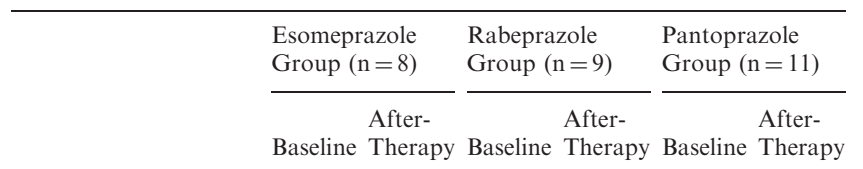

Endoscopic Features, \%

Rings

Linear furrows

$75 \%$

$26 \%$

$67 \%$

$22 \%$

$63 \%$

$18 \%$

Whitish exudates

$62 \%$
$50 \%$

$13 \%$

$55 \%$

$11 \%$

$54 \%$

$9 \%$

Edema

$26 \%$

$13 \%$

$44 \%$

$11 \%$

$54 \%$

$18 \%$

Crepe paper

$13 \%$

$0 \%$

$0 \%$

$11 \%$

$27 \%$

$9 \%$

Strictures

$13 \%$

$13 \%$

$22 \%$

$11 \%$

$18 \%$

$0 \%$

$9 \%$

Mean EREFS Score
Table Continued

\begin{tabular}{|c|c|c|}
\hline Esomeprazole & Rabeprazole & Pantoprazole \\
\hline Group $(\mathrm{n}=8)$ & Group $(n=9)$ & Group $(n=11)$ \\
\hline $\begin{array}{l}\text { After- } \\
\text { Therapy }\end{array}$ & $\begin{array}{c}\text { After- } \\
\text { seline Therapy }\end{array}$ & $\begin{array}{cl}\text { After- } \\
\text { Baseline Therapy }\end{array}$ \\
\hline
\end{tabular}

Histologic Features, $n$ or

Median maximum eos 75 count*

Median eos count (of 555 HPFs)*

Degranulation present $\quad 88 \% \quad 13 \% \quad 100 \% \quad 11 \% \quad 90 \% \quad 18 \%$

$\begin{array}{llllll}\text { Microabscess present } \quad 63 \% & 13 \% & 55 \% & 11 \% & 48 \% & 9 \%\end{array}$

Basal layer present $\quad 100 \% \quad 26 \% \quad 100 \% \quad 22 \% \quad 100 \% \quad 18 \%$

$\begin{array}{lllllll}\text { Spongiosis present } & 88 \% & 26 \% & 88 \% & 11 \% & 90 \% & 18 \%\end{array}$

Subepithelial tissue $\quad 75 \% \quad 13 \% \quad 55 \% \quad 11 \% \quad 81 \% \quad 9 \%$ present

Lamina propria fibrosis $0 \% \quad 0 \% \quad 0 \% \quad 0 \% \quad 9 \% \quad 0 \%$ presen

Eos $=$ esosinophils $*$ Calculated for an HPF area $=0.24 \mathrm{~mm}^{2}$

Conclusion: Esomeprazole, rabeprazole and pantoprazole administered at double daily dose were equally effective in determining endoscopic and histologic remission in patients with PPI-REE. These data, although obtained in a small sample of patients, suggest that the pharmacokinetic and pharmacodynamic differences among these drugs do not affect their effect on PPI-REE patients.

Disclosure of Interest: V. Savarino: Consulting fee from Malesci, Reckitt, AlfaWasserman, Abbvie

E. Savarino: Consulting fee from Medtronic, Sofar, Takeda, Abbvie, MSD

All other authors have declared no conflicts of interest.

P0537 THE « GARD (GASTRO-ESOPHAGEAL ANTI-REFLUX DEVICE»: A NEW ENDOSCOPIC MEDICAL DEVICE TO DIAGNOSE, MANAGE AND TREAT GERD

N. Godin

Private Practice, Geneva/Switzerland

Contact E-mail Address: dr.godin68@gmail.com

Introduction: The "GARD» (Gastroesophageal Anti-Reflux Device) is an antireflux tubular valve placed in the lower esophagus under endoscopic control allowing normal ingestion of food and beverages but blocking all gastro-esophageal refluxate mechanically (fluid, solids and gas)

Aims \& Methods: The "GARD» has an upper ring sized to the diameter of the patient's esophagus with an accessory called the " calibration device " placed through the $2.8 \mathrm{~mm}$ working channel of a standard gastroscope. The GARD is held in place by pressure. The upper ring holds an anti-reflux thin-walled tubular valve moulded in one piece under the ring. After placement of a standard guidewire, the "GARD» is placed through the patient's mouth and is released in the lower esophagus. The procedure is performed under sedation on an ambulatory basis in about 15 minutes when experienced. The "GARD» was placed in the esophagus of 8 pigs during 7 days to evaluate placement, feeding, weight gain, absence of migration as well as removal. Deployment of the "GARD » was easy, there was no migration in the stomach and removal of the GARD with the developed accessories was easily achieved. However, pigs have a strong lower esophageal sphincter and have no reflux so gastro-esophageal reflux could not be evaluated. In a human volunteer with very severe gastro-esophageal reflux who had previously failed anti-reflux surgery and had an unsatisfactory response to clinical and $\mathrm{pH}$ metric measurement under $80 \mathrm{mg}$ of esomeprazole, the «GARD» was placed preoperatively. Hereafter are this patient's $\mathrm{pH}$ metric results without PPIs (table 1); with the patient taking $80 \mathrm{mg}$ /day PPIs (table 2) and after GARD placement and PPIs stopped for 10 days, table 3 .

Results: Fig 1. First $\mathrm{pH}$ metric study (no PPIs for 10 days): The patient has very severe reflux with $63 \%$ of the time with a pH under 4 (normal less than $4 \%$ of the time at a $\mathrm{pH}$ under 4) after PPIs were stopped for 10 days. Fig 2. Patient is taking $80 \mathrm{mg}$ of esomeprazole a day. $\mathrm{pH}$ metric measurement remains highly pathological at $23 \%$ of the time under $\mathrm{pH} 4$ once PPIs have been resumed at $80 \mathrm{mg}$ of PPIs daily. Fig 3. After placement of the GARD and PPIs have been stopped for 10 days, there is no longer any reflux measured. The $\mathrm{pH}$ tracing does not drop under the red line at $\mathrm{pH} 4$

Conclusion: a new medical device blocking GERD is presented. A first clinical trial is scheduled to start in 2018 to help diagnose and manage Refractory GERD. Further clinical indications are at the planning stage including treatment of Refractory GERD and LPR.

Disclosure of Interest: All authors have declared no conflicts of interest. 
P0538 SYMPTOM PATTERNS AND TYPES OF

GASTROESOPHAGEAL REFLUXES SIGNIFICANTLY DIFFER IN GROUPS OF EROSIVE ESOPHAGITIS AND NON-EROSIVE FORM OF GASTROESOPHAGEAL REFLUX DISEASE (GERD) PATIENTS

M. Konovalova, S. Morozov, V. Isakov

Gastroenterology And Hepatology, Federal Research Center of Nutrition and Biotechnology, Moscow/Russian Federation

Contact E-mail Address: sunnysister@mail.ru

Introduction: Patients with gastroesophageal reflux disease (GERD) demonstrate a range of different symptoms (esophageal and extraesophageal) however the relationship between symptoms and types of reflux was not evaluated.

Aims \& Methods: The aim of the study was to assess the relationship between GERD patients' symptoms with characteristics of refluxes obtained by 24-h esophageal $\mathrm{pH}$-impedance. One hundred fifty eight GERD patients (68 men, 89 women, age $(\mathrm{M} \pm \mathrm{m}) 42 \pm 4.8$ yrs) and 49 controls (22 men, 27 women, age $(\mathrm{M} \pm \mathrm{m}) 46 \pm 6.7 \mathrm{yrs})$ were examined using 24-hours esophageal $\mathrm{pH}$-impedance recordings (Ohmega, MMS, the Netherlands; 2pH-6 impedance channels catheters, UnisensorAG, USA) and validated GERD-Q questionnaire. According to baseline endoscopy 91 patients were classified as non-erosive reflux disease (NERD) and 67 as erosive reflux disease (ERD) patients. Patients' symptoms were classified according to Montreal classification.

Results: Extraesophageal symptoms as well as weak acid gastroesophageal refluxes were found significantly more often in patients with NERD compared to ERD group (table 1). However higher number of acid refluxes, higher GERDQ score and DeMeester score were present in ERD. The total number of gastroesophageal refluxes didn't differ between ERD and NERD groups of patients.

Table 1: Results of the study

\begin{tabular}{|c|c|c|c|c|}
\hline & $\begin{array}{l}\text { Controls } \\
(\mathrm{n}=49)\end{array}$ & $\begin{array}{l}\text { NERD } \\
(\mathrm{n}=91)\end{array}$ & $\begin{array}{l}\text { ERD } \\
(n=67)\end{array}$ & $\mathrm{p}$ \\
\hline $\begin{array}{l}\text { Number of refluxes/ } \\
\text { day, } \mathrm{n}\end{array}$ & $17 \pm 1.3$ & $55 \pm 3.07^{*}$ & $55 \pm 4.7^{*}$ & ${ }^{*} \mathrm{p}=0.729$ \\
\hline $\begin{array}{l}\text { Number of acid } \\
\quad \text { refluxes/day, n }\end{array}$ & $6 \pm 1.0$ & $27 \pm 2.2^{*}$ & $33 \pm 3.7^{*}$ & ${ }^{*} \mathrm{p}=\mathbf{0 . 0 4 0}$ \\
\hline $\begin{array}{l}\text { Number of weak acid } \\
\text { refluxes/day, n }\end{array}$ & $7 \pm 0.93$ & $22 \pm 2.1^{*}$ & $15 \pm 2.3^{*}$ & ${ }^{*} \mathrm{p}=\mathbf{0 . 0 3 8}$ \\
\hline $\begin{array}{l}\text { Number of high gastro- } \\
\text { esophageal refluxes/ } \\
\text { day, } \mathrm{n}\end{array}$ & $2 \pm 0.47$ & $15 \pm 1.4^{*}$ & $12 \pm 2.2^{*}$ & ${ }^{*} \mathrm{p}=0.347$ \\
\hline DeMeester score & $3.16 \pm 1.75$ & $13.3 \pm 2.0^{*}$ & $26.92 \pm 6.2^{*}$ & $*_{p}=0.0001$ \\
\hline GERD-Q score & $5 \pm 0.31 \times \dagger$ & $10 \pm 0.24 * \times$ & $13.1 \pm 0.22 * \dagger$ & $*_{x} \dagger p=0.0001$ \\
\hline $\begin{array}{l}\text { Extraesophageal symp- } \\
\text { toms (cough, laryn- } \\
\text { gitis, etc.) (presence, } \\
\% \text { in group) }\end{array}$ & 0 & $61.5^{*}$ & $31.3^{*}$ & ${ }^{*} \mathrm{p}=\mathbf{0 . 0 0 0 1}$ \\
\hline
\end{tabular}

Conclusion: ERD and NERD groups of patients are charachterized by different symptom patterns and types of gastroesophageal refluxes registered with 24hours esophageal $\mathrm{pH}$-impedance monitoring. These findings could reflect differences in pathogenesis and clinical manifestations of mentioned forms of GERD. Disclosure of Interest: All authors have declared no conflicts of interest.

\section{P0539 LARYNGEAL DISORDERS AND CHRONIC COUGH IN ADULTS WITH AND WITHOUT EROSIVE ESOPHAGITIS: A CASE- CONTROL STUDY IN ALBANIA}

B. Kraja ${ }^{1}$, I. Këlliçi ${ }^{2}$, I. Mone ${ }^{3}$, F. Kraja ${ }^{4}$, S. Prifti ${ }^{5}$, G. Burazeri ${ }^{6}$

${ }^{1}$ University Clinic of Gastrohepatology, University Hospital Center Mother Teresa, Tirana/Albania

${ }^{2}$ Endoscopy Unit, Service of Surgery, Regional Hospital Durres, Durres/Albania ${ }^{3}$ Department of Laboratory, University Hospital Center Mother Teresa, Tirana/ Albania

${ }^{4}$ University Clinic of Oncology, University Hospital Center Mother Teresa, Tirana/ Albania

${ }^{5}$ University Clinic of Gastrohepatology, University Hospital Center Mother Theresa, Tirana/Albania

${ }^{6}$ Department of International Health, School for Public Health and Primary Care (CAPHRI), Faculty of Health, Medicine and Life Sciences, Maastricht University, Maastricht/Netherlands

Contact E-mail Address: bledarkraja@yahoo.com

Introduction: Several clinical-based studies from Western countries have investigated the prevalence of extra-esophageal symptoms in various degrees of reflux erosive esophagitis. However, the independent factors related to the development extra-esophageal manifestations remain unclear.

Aims \& Methods: Our aim was to assess the prevalence of extra-esophageal symptoms (laryngeal disorders and chronic cough) in adults with (cases) and those without (controls) erosive esophagitis in Albania, a developing Southeast European country. A case-control study was conducted at the Regional Hospital of Durres, the second main district in Albania, a transitional country in South Eastern Europe, including 248 patients with erosive esophagitis (aged $46.5 \pm 16.3$ years) and 273 controls (aged $46.4 \pm 16.0$ years; response rate: $70 \%$ ) enrolled during the period January 2013 - June 2014. Both cases and controls underwent upper endoscopy. Information on socio-demographic characteristics and lifestyle factors was also collected. Binary logistic regression was used to assess the association of erosive esophagitis and extra-esophageal symptoms.

Results: Patients with erosive esophagitis had a higher prevalence of excessive alcohol consumption, smoking, sedentarity and obesity compared to their control counterparts $(9 \%$ vs. $5 \%, 70 \%$ vs. $49 \%, 31 \%$ vs. $17 \%$ and $22 \%$ vs. $9 \%$, respectively). The prevalence of hiatal hernia was higher in cases than in controls $(21 \%$ vs. $8 \%$, respectively), whereas the prevalence of gastric-duodenal ulcer was similar in both groups (13\% vs. 14\%, respectively). Upon adjustment for all socio-demographic characteristics and lifestyle/behavioral factors, there was evidence of a strong association of erosive esophagitis with chronic cough $(\mathrm{OR}=3.1,95 \% \mathrm{CI}=1.7-5.7)$, and even more so with laryngeal disorders $(\mathrm{OR}=4.4,95 \% \mathrm{CI}=2.6-7.4)$. In all models, the association of erosive esophagitis with any extra-esophageal symptoms was strong and mainly consistent with each of the symptoms separately (fully-adjusted model: $\mathrm{OR}=4.6,95 \% \mathrm{CI}=2.9-7.3$ ). Conclusion: Our findings indicate that the prevalence of extra-esophageal symptoms is higher among patients with erosive esophagitis in a transitional country characterized conventionally by the employment of a Mediterranean diet. Therefore, the upper endoscopy should be part of the evaluation in patients with suspected reflux-related chronic cough and laryngeal disorders. Disclosure of Interest: All authors have declared no conflicts of interest.

\section{P0540 ASSESSMENT OF EXHALED BREATH CONDENSATE FOR NON-INVASIVE DIAGNOSIS OF GASTROESOPHAGEAL REFLUX DISEASE IN CORRELATION WITH MII-PH AND PEPTEST}

S. Konecny ${ }^{1}$, J. Dolina ${ }^{2}$, M. Doubkova ${ }^{3}$, J. Skrickova ${ }^{3}$, D. Kindlova ${ }^{3}$ E. Pokojova ${ }^{3}$, P. Durc ${ }^{4}$, J. Lacna ${ }^{4}$, M. Gregus ${ }^{4}$, F. Foret ${ }^{4}$, P. Kuban ${ }^{4}$ ${ }^{1}$ Internal Gastroenterology Clinic, FN Brno, Brno/Czech Republic

${ }^{2}$ Internal Gastroenterology Department, FN Brno, Brno/Czech Republic

${ }^{3}$ Department Of Pulmonary Diseases And Tuberculosis, FN Brno, Brno/Czech Republic

${ }^{4}$ Department Of Bioanalytical Instrumentation, CEITEC Masaryk University, Brno/Czech Republic

Contact E-mail Address: stefankonecny@gmail.com

Introduction: Gastroesophageal reflux disease (GERD) is a disease caused by backflow of gastric contents into the esophagus due to the failure of physiological antireflux mechanisms and can lead to esophageal and extraesophageal symptomatology. Extraesophageal reflux (EER) is a condition where refluxate penetrate above the upper esophageal sphincter (UES) in to the oral cavity, pharynx, upper and lower respiratory tract and leads to pathological changes like e.g. chronic cough, globus pharyngis, laryngitis, pharyngitis, rhinosinusitis, otitis media, bronchial asthma, COPD, sleep apnea and noncardiac chest pain. Currently there is no suitable, non-invasive diagnostic method applicable for GERD in clinical practice. Exhaled breath condensate (EBC) and saliva are two easily obtained samples that could be used in monitoring of patients suffering from extraesophageal symptoms of GERD. The aim of this study was to compare the $\mathrm{pH}$ and total ionic profile of EBC with 24-hour multichannel intraluminal impedance and $\mathrm{pH}$ monitoring $(\mathrm{MII}-\mathrm{pH})$ and salivary Peptest in a group of patients with acid reflux $(\mathrm{pH}<4)$, weakly acid reflux $(\mathrm{pH} \mathrm{4-7)}$ and healthy controls.

Aims \& Methods: A portable EBC sampler was used for collection of EBC. $10 \mu \mathrm{L}$ sample aliquots of EBC were analyzed. For $\mathrm{pH}$ measurement, the $\mathrm{CO}_{2}$ from $\mathrm{EBC}$ was removed with $\mathrm{N}_{2}$ gas for $5 \mathrm{~min}$. The $\mathrm{pH}$ of $\mathrm{EBC}$ was measured with a $\mathrm{pH}$ microelectrode and total ionic profile (anions, cations, organic acids $-\mathrm{NH}_{4}{ }^{+}, \mathrm{K}^{+}$, $\mathrm{Ca}^{2+}, \mathrm{Na}^{+}, \mathrm{Mg}^{2+}, \mathrm{Cl}^{-}, \mathrm{NO}_{2}^{-}, \mathrm{NO}_{3}{ }^{-}, \mathrm{SO}_{4}{ }^{2-}$, acetate, lactate, propionate, butyrate) was analyzed by capillary electrophoresis in each sample. Saliva was collected using the commercial Peptest sampling containers, applied to the Peptest lateral flow devices and analyzed using the device reader. The data from EBC were compared with MII-pH and Peptest. In total the study comprised of 39 participants. The patients were divided by dominant findings from MII-pH in to groups with acid reflux $(\mathrm{n}=17)$, weakly acid reflux $(\mathrm{n}=8)$ and without reflux $(\mathrm{n}=14)$.

Results: The values of $\mathrm{pH}$ (after $\mathrm{CO}_{2}$ removal with $\mathrm{N}_{2}$ ) were significantly higher in the group with acid reflux $(\mathrm{p}<0.01)$, (mean $\mathrm{pH} 7.13$, interquartile ranges 6.83-7.47) and in the group with weakly acid reflux $(\mathrm{p}<0.01)(7.37$, (7.18$7.57)$ ) vs. healthy controls $(6.8,(6.65-6.99))$. Butyric acid (BA) was the second most significant parameter that was significantly elevated $(\mathrm{p}<0.01)$ in both patient groups (acid reflux- mean BA $2.29 \mu \mathrm{M}$, weakly acid reflux- mean BA $3.33 \mu \mathrm{M}$ ) compared to healthy subjects (mean BA $0.69 \mu \mathrm{M}$ ).

Further statistically significant differences were found in chloride $\left(\mathrm{Cl}^{-}\right)$, nitrate $\left(\mathrm{NO}_{3}{ }^{-}\right)$and sodium $\left(\mathrm{Na}^{+}\right)$ions concentration. $\mathrm{Cl}^{-}$was elevated $(\mathrm{p}<0.01)$ in group with acidic reflux vs. healthy controls and $\mathrm{NO}_{3}{ }^{-}$and $\mathrm{Na}^{+}$were elevated $(\mathrm{p}<0.01)$ in group with weakly acidic reflux vs. healthy controls. Peptest for saliva sampling and pepsin analysis showed no statistically significant differences within the groups. In the groups of patients with acid reflux, the incidence of high pepsin concentration (above $75 \mathrm{ng} / \mathrm{ml}$ ) was found only in $50 \%$ of the patients. Conclusion: We found statistically significant difference in $\mathrm{pH}$ and selected ions from EBC between different groups of patients and healthy controls. The analysis of selected parameters in EBC could provide a fast and non-invasive diagnostic method for GERD patients with EER symptoms in the future. This can 
potentially reduce the diagnostic cost and avoid unnecessary invasive MII-pH testing in future. Unlike the EBC, pepsin analysis using Peptest did not provide any diagnostic value.

Disclosure of Interest: This work was supported by Ministry of Health of the Czech Republic, grant nr. 17-31945A. All rights reserved.

\section{P0541 GASTRIN 17 MEASUREMENTS IN SINGLING OUT PATIENTS WITH DIFFERENT PATTERNS OF REFLUXATE: A PILOT STUDY USING IMPEDANCE-PH AS REFERENCE STANDARD}

M. Della Coletta ${ }^{1}$, E. Savarino ${ }^{2}$, N. De Bortoli ${ }^{3}$, S. Speroni ${ }^{4}$, S. Landi $^{5}$, S. Grillo ${ }^{6}$, A. Bertelè ${ }^{4}$, N. Dal Bò ${ }^{7}$, F. Di Mario ${ }^{8}$

${ }^{1}$ Division Of Gastroenterology, Department Of Surgery, Oncology And

Gastroenterology, University of Padua, Padua/Italy

${ }^{2}$ Department Of Surgery, Oncology And Gastroenterology, University of Padua, Padua/Italy

${ }^{3}$ Division Of Gastroenterology, Department Of Internal Medicine, University of Pisa, Pisa/Italy

${ }^{4}$ Department Of Clinical \& Experimental Medicine, Clinical Pharmacology \&

Digestive Pathophysiology Unit, University of Parma, Parma/Italy

${ }^{5}$ University of Parma, Parma/Italy

${ }^{6}$ Gastroenterology, University of Parma, Parma/Italy

${ }^{7}$ Gastroenterological Unit, Treviso Hospita, Treviso/Italy

${ }^{8}$ University Of Parma, Department of Clinical and Experimental Medicine, section of Gastroenterology, Parma/Italy

Contact E-mail Address: marcodellacoletta@gmail.com

Introduction: Impedance-pH testing is actually considered the gold standard diagnostic tool for reflux assessment. In fact, it allows to characterize any type of gastro-esophageal reflux (GER), namely acid and non-acid, and therefore permits - in presence of typical reflux symptoms - to diagnose functional heartburn (FH) based on the lack of abnormal acidic or non-acidic refluxate (i.e. normal number of reflux episodes and negative reflux-symptom association). Gastrin-17 (G17) has been proposed as a non-invasive marker of GERD, due to the negative feedback between acidic output and this hormone. Indeed, preliminary data showed that intermediate values of G17, between very low to normal levels, may identify GERD subjects with abnormal non-acid reflux.

Aims \& Methods: We aimed to correlate various patterns of refluxate (i.e. predominant acidic refluxate, predominant non-acidic refluxate and no reflux at all), as assessed by impedance-pH, with different levels of G17 in endoscopy-negative subjects with heartburn. Thirty-five consecutive patients $(19 \mathrm{~F} / 16 \mathrm{M}$, mean age 47 years, range 31-56 years), all reporting heartburn since 6 months with at least 3 episodes/week, entered the study. All patients underwent upper endoscopy offmedication and in case of no lesions were classified as endoscopy-negative. In all subjects, impedance-pH was performed off-therapy and blood determination of G17® (Biohit Oji, Finland; normal values: 1-9 pmol/l) was evaluated on the same day of reflux monitoring. Impedance-pH tracings were blindly and manually analyzed to detect the distal acid exposure time (AET abnormal if $\geq 3.2 \%$ over 24 hours) and the number as well as the characteristics of reflux episodes (acidic/ weakly acidic refluxate). Patients were then divided in three groups according to the results of impedance-pH: a) Group A: subjects with increased number of acid refluxes or abnormal AET; b) Group B: subjects with increased episodes of nonacid reflux and normal AET; c) Group C: subjects without increased of any kind of reflux and normal AET.

Results: According to impedance-pH results, 14 out of 35 patients had increased acid reflux episodes or abnormal AET (Group A), 14 patients showed increased non-acid reflux episodes (Group B) and 7 patients had no increased number of any kind of GER (Group C). In the Group A, the mean value of G17 was $0.9 \mathrm{pmol} / 1$, in the Group B was $2.6 \mathrm{pmol} / 1$, and, in the Group C was $5.1 \mathrm{pmol} / 1$ $(\mathrm{p}<0.01)$. Very low G17 levels were significantly associated with increased acid reflux episodes or abnormal AET, confirming the background of the negative feedback between acidic output and G17 levels. Interestingly, when impedance$\mathrm{pH}$ showed a feature of non-acid reflux pattern (Group B), levels of G17 were always low, but higher than in case of acid reflux pattern (Group A) $(\mathrm{p}<0.05)$. Finally, when impedance-pH did not show any kind of abnormal reflux, suggesting a diagnosis of $\mathrm{FH}, \mathrm{G} 17$ levels were always normal.

Conclusion: In this preliminary study, G17 levels well correlated with the three different categories of patients suffering of heartburn and included in the NERD umbrella (i.e. NERD patients with increased acid reflux episodes or abnormal AET, endoscopy-negative patients with increased non-acidic reflux and subjects with $\mathrm{FH}$ ), suggesting its use as surrogate marker of NERD or non-acid reflux disease, without the need of performing invasive tests.

Disclosure of Interest: E. Savarino: Consulting fee from Medtronic, Sofar, Takeda, Abbvie, MSD

All other authors have declared no conflicts of interest.

\section{P0542 ANTI REFLUX MUCOSECTOMY (ARMS) FOR REFRACTORY GASTRO ESOPHAGEAL REFLUX DISEASE (GERD) - ARE WE THERE YET?}

R. Shah, A. P. Maydeo, V. Dhir

Gastroenterology, Baldota Institute of Digestive Sciences, Global Hospital, Mumbai/India

Contact E-mail Address: rahulhshah@hotmail.com

Introduction: As a treatment for gastro esophageal reflux disease (GERD), proton pump inhibitors (PPIs) are the mainstay of medical therapy. Laparoscopic fundoplication is generally advised when symptoms are poorly controlled with PPIs and is regarded as a gold standard of treatment, with excellent control in the short- and midterm. Long-term results, however, remain equivocal. Following on from the principles of surgical fundoplication, a variety of endoscopic procedures for GERD have been proposed to achieve non-surgical control. Linx procedure, Stretta have been proposed as less invasive options.

Aims \& Methods: We recruited all patients who had GERD refractory to standard medical therapy to see whether anti reflux mucosectomy prevents acid reflux into the esophagus. We screened all GERD patients who were refractory to proton pump inhibitors, hydrogen 2 receptor blockers and alginates and had an endoscopy suggestive of a lax cardia with mucosal flap valve grading of 1 to 3 . We performed a baseline screening endoscopy to rule out a hiatus hernia and to exclude helicobacter infection. A GERDQ questionnaire was filled by all the patients indicative of severity of reflux All patients had a high resolution manometry (Sandhill scientific) to exclude significant dysmotility and 24 hour $\mathrm{pH}$ measurements using Zephyr $\mathrm{pH}$ probe (Sandhill scientific) on therapy to demonstrate significant acid reflux. Only patients with mucosal flap valve grading 1,2 or 3 were selected for anti reflux mucosectomy.

Results: Technique: Crescentic ARMS of the esophagogastric junctional (EGJ) mucosa was conducted with the standardized technique of endoscopic mucosal resection (EMR) of at least $3 \mathrm{~cm}$ length in the stomach, with the length of mucosal resection at the cardia measured in retroflexion from the gastric side. ARMS was conducted along the lesser curve of the stomach, thus preserving a sharp mucosal valve at gastric cardia. All the patients who underwent ARMS had a significant reduction in the DeMeester score, with predominant decrease in the recumbent acid exposure. 7/12 patients were able to discontinue all the medical therapy. PPI dose reduction was possible in the other patients with a mean reduction of $50 \%$, alginates were stopped in all patients and H2RA were also discontinued

Conclusion: Results suggest a potential anti-reflux effect of ARMS. The mechanism is presumed to be due to scar formation after healing of the mucosal defect. On the gastric side, this induces narrowing of the gastric cardia opening, while preserving and/or re-creating a robust his angle. After ARMS, the lesser curve of the gastric cardia takes on an almost "mechanically-stitched" appearance. The mucosal flap is rebuilt and looks well-defined. Furthermore, the lesser curve side

\begin{tabular}{|c|c|c|c|c|c|c|c|c|c|c|c|c|c|}
\hline & $\begin{array}{l}\text { PRE } \\
\text { DEMEESTER }\end{array}$ & $\begin{array}{l}\text { POST } \\
\text { DEMEESTER }\end{array}$ & $\begin{array}{l}\text { UPRIGHT-ACID } \\
\text { EXP-PRE } \\
\text { ARMS (\%) }\end{array}$ & $\begin{array}{l}\text { POST } \\
\text { ARMS (\%) }\end{array}$ & $\begin{array}{l}\text { RECUMBENT } \\
\text { ACID EXP - } \\
\text { PRE } \\
\text { ARMS }(\%)\end{array}$ & $\begin{array}{l}\text { POST } \\
\text { ARMS } \\
(\%)\end{array}$ & $\begin{array}{l}\text { LONGEST } \\
\text { REFLUX } \\
\text { EPISODE } \\
\text { PRE ARMS } \\
\text { (minutes) }\end{array}$ & $\begin{array}{l}\text { POST } \\
\text { ARMS } \\
\text { (minutes) }\end{array}$ & $\begin{array}{l}\text { TOTAL } \\
\text { REFLUX } \\
\text { TIME - PRE } \\
\text { ARMS } \\
\text { (minutes) }\end{array}$ & $\begin{array}{l}\text { POST } \\
\text { ARMS } \\
\text { (minutes) }\end{array}$ & $\begin{array}{l}\text { TOTAL } \\
\text { REFLUX } \\
\text { EPISODES - } \\
\text { PRE ARMS } \\
\text { (number) }\end{array}$ & $\begin{array}{l}\text { POST } \\
\text { ARMS } \\
\text { (number) }\end{array}$ & $\begin{array}{l}\text { MUCOSAL } \\
\text { FLAP VALVE } \\
\text { GRADING }\end{array}$ \\
\hline A & 255 & 72 & 89 & 66 & 94 & 5 & 252 & 5 & 90 & 52 & 138 & 29 & 1 \\
\hline B & 234 & 29 & 69 & 5 & 71 & 6 & 473 & 57 & 70 & 9 & 126 & 27 & 1 \\
\hline $\mathrm{C}$ & 40 & 22 & 13 & 10 & 8 & 4 & 36 & 7 & 10 & 7 & 134 & 101 & 3 \\
\hline $\mathrm{D}$ & 112 & 32 & 48 & 4 & 12 & 11 & 382 & 32 & 32 & 8 & 93 & 73 & 2 \\
\hline $\mathrm{E}$ & 28 & 23 & 13 & 12 & 2 & 0 & 8 & 7 & 9 & 7 & 145 & 103 & 3 \\
\hline $\mathrm{F}$ & 44 & 5 & 15 & 1 & 7 & 1 & 5 & 1 & 10 & 6 & 268 & 43 & 1 \\
\hline G & 68 & 2 & 21 & 1 & 7 & 0 & 13 & 1 & 13 & 1 & 494 & 16 & 1 \\
\hline $\mathrm{H}$ & 196 & 21 & 33 & 3 & 12 & 5 & 45 & 7 & 50 & 4 & 256 & 118 & 2 \\
\hline I & 36 & 5 & 6 & 2 & 8 & 0 & 8 & 3 & 6 & 7 & 237 & 38 & 2 \\
\hline $\mathbf{J}$ & 21 & 6 & 10 & 2 & 0 & 0 & 79 & 3 & 7 & 5 & 79 & 58 & 2 \\
\hline $\mathrm{K}$ & 40 & 5 & 27 & 1 & 4 & 0 & 61 & 2 & 12 & 4 & 59 & 43 & 2 \\
\hline $\mathrm{L}$ & 26 & 4 & 8 & 6 & 1 & 0 & 3 & 1 & 5 & 1 & 212 & 31 & 2 \\
\hline
\end{tabular}


of the EGJ is shortened with scar formation, and greater curve of EGJ (his angle side) is kept non-scarred and therefore retains its flexibility as a mucosal flap valve. The quantity of mucosa to be resected to induce appropriate ("not too tight and not too loose") scar formation is a key issue in this procedure. Total circumferential resection causes stricturing as demonstrated in previous studies, while subtotal dissection, which we have termed crescentic, produces better results in this regard, while still resulting in symptom control. Mucosal flap valve grading is not only a good predictor of reflux in these patients but also is a prognostic marker of effectiveness of ARMS, i.e. higher the grade worse the outcome. Perhaps tailoring the extent and type of mucosal resection (EMR vs. ESD) according to the mucosal flap valve grading may be a better predictor of outcome than a box standard procedure. This technique has a potential role in people with oesophageal dysmotility wherein Nissen's fundoplication is relatively contraindicated.

Disclosure of Interest: All authors have declared no conflicts of interest

\section{Reference}

Inoue $\mathrm{H}$, Ito $\mathrm{H}$, Ikeda $\mathrm{H}$, Sato $\mathrm{C}$, Sato $\mathrm{H}$, Phalanusitthepha C, Hayee B, Eleftheriadis N, Kudo SE.

\section{P0543 A RANDOMISED, DOUBLE-BLIND, PLACEBO- CONTROLLED, MULTICENTRE 26-WEEK STUDY ON THE EFFECTS OF DEXLANSOPRAZOLE AND ESOMEPRAZOLE ON BONE HOMEOSTASIS IN HEALTHY POSTMENOPAUSAL WOMEN \\ K. E. Hansen ${ }^{1}$, J. W. Nieves ${ }^{2}$, S. Nudurupati ${ }^{3}$, D. C. Metz ${ }^{4}$, M. C. Perez ${ }^{3}$} ${ }^{1}$ School Of Medicine And Public Health, University of Wisconsin, Madison, Madison/United States of America

${ }^{2}$ Mailman School Of Public Health, Columbia University, new york/United States of America

${ }^{3}$ Medical Affiars, Takeda Development Center Americas, Inc, Deerfield/United States of America/IL

${ }^{4}$ Perelman School Of Medicine, University of Pennsylvania, Philadelphia/United States of America/PA

\section{Contact E-mail Address: maria.perez@takeda.com}

Introduction: Observational and epidemiologic data have suggested an association between proton pump inhibitor (PPI) use and osteoporotic fractures. To evaluate potential mechanisms for this association, we measured bone turnover, bone mineral density (BMD), true fractional calcium absorption (TFCA), and serum and urine mineral levels in healthy postmenopausal women taking PPIs or placebo for 26 weeks.

Aims \& Methods: Postmenopausal women aged 45-75 were randomised to daily oral 60-mg dexlansoprazole (DEX), 40-mg esomeprazole (ESO), or PBO for 26 wks with follow-up at wk 52. Primary endpoints were 26 -wk \% change vs placebo in procollagen type $1 \mathrm{~N}$-terminal propeptide (P1NP) and C-terminal telopeptide of type 1 collagen (CTX). Additional endpoints included changes in BMD (26 and $52 \mathrm{wks}$ ) and serum and urine mineral levels (26 wks). Fractures between baseline and wk 26 were recorded as adverse events. TFCA $(0$ and 26 wks) was measured in a subset $(\mathrm{n}=34)$ of patients.

Results: Excluding 1 disqualified site, 115 women were randomised and 93 completed the study. There were no substantial differences in age, BMI, baseline serum calcium, or vitamin D levels between groups. The bone turnover markers P1NP and CTX remained within normal range during 26 wks of PPI therapy. Within each group, there was no statistically significant 26-wk change in bone turnover, except a small increase in CTX levels with DEX $(0.12 \mathrm{ng} / \mathrm{mL}, 95 \%$ CI $0.03-0.23$ ). The $26-w k$ median \% increases in P1NP from baseline vs PBO (difference in median \% change $[95 \% \mathrm{CI}$ ] were $19 \%(7 \%-30 \%)$ for DEX and $18 \%$ $(7 \%-30 \%)$ for ESO. CTX levels increased vs PBO by $27 \%(13 \%-43 \%)$ for DEX and $22 \%(8 \%-36 \%)$ for ESO. PPI effects on BMD, serum and urine mineral levels, and parathyroid hormone were not statistically different vs $\mathrm{PBO}$. Median \% change from baseline in TFCA vs PBO was not statistically significant for DEX, but was significant for ESO $(6 \%, 95 \%$ CI $2 \%-11 \%)$. No spontaneous fractures occurred during treatment; 1 traumatic foot fracture (DEX) and 1 humerus fracture (circumstance unknown; ESO) occurred during follow-up.

Conclusion: 26 wks of DEX or ESO therapy increased bone turnover markers, but did not reduce BMD, TFCA, or serum or urine mineral levels. ESO increased TFCA by $<1 \%$. Although bone turnover markers increased with PPI therapy, levels remained within the normal ranges. No clear explanation for an association between PPI therapy and fracture risk was found in this study.

Disclosure of Interest: K.E. Hansen: Takeda paid me for my work as a consultant in the design of the study, and for my work in conducting the study at my medical center.

D.C. Metz: Takeda - access to writing and data analysis for the purposes of this protocol

M.C. Perez: Employee of Takeda Pharmaceuticals

All other authors have declared no conflicts of interest.

Trial Registration: This study has the ClinicalTrials.gov identifier NCT01216293.
P0544 ALGINATE EFFECT ON POSTPRANDIAL REFLUXES AND PH OF STOMACH CONTENT: RESULTS OF PH-IMPEDANCE MONITORING COMBINED WITH STOMACH PH MONITORING IN REFLUX PATIENTS

I. Paliy ${ }^{1}$, S. Zaika ${ }^{1}$, O. Ksenchyn ${ }^{1}$

${ }^{1}$ Internal And Family Medicine, Vinnytsya National Pirogov Memorial Medical University, Vinnytsya/Ukraine

Contact E-mail Address: dpaliy@mail.ru

Introduction: Raft-forming alginates may inhibit gastroesophageal reflux; however, remains unexplored effect of alginates on postprandial processes in the stomach.

Aims \& Methods: 25 patients (14 F, age 23-69) with typical GERD symptoms, participated in this study. All patients underwent a 3-hour combined gastroesophageal $\mathrm{pH}$-impedance monitoring with standardized breakfast (muffin and coffee). To determine the effect of alginates on postprandial reflux and $\mathrm{pH}$ in the esophagus and stomach, all patients underwent a repeat of similar monitoring the next day but in this case, they took alginate after breakfast. The difference in $\mathrm{pH}$ in the esophagus, in two parts of the stomach and the different types of gastroesophageal reflux (acid, low acid, alkaline, liquid, gas \& mixed) was estimated. To evaluate the effects of raft-forming alginate on the severity of postprandial reflux in patients with GERD and for the postprandial stomach content.

Results: Monitoring with alginate showed significantly $(\mathrm{P}<0.05)$ less number of acid [average values $5.42 \pm 0.69(\mathrm{M} \pm \mathrm{m})$ vs. $3.33 \pm 0.43$ during 1 st postprandial hour and $3.96 \pm 0.8$ vs. $1.82 \pm 0.57$ during 2 nd postprandial hour] and gas $[0.26 \pm 0.06$ vs. $0.08 \pm 0.05$ during 1 st postprandial hour and $0.47 \pm 0.35$ vs. 0 during 2nd postprandial hour] gastroesophageal reflux, but increased of number low acid reflux $[2.52 \pm 0.46$ vs. $3.91 \pm 0.82$ during 1 st postprandial hour and $1.00 \pm 0.42$ vs. $2.16 \pm 0.45$ during 2 nd postprandial hour; $\mathrm{P}>0.05$ ] Also noted is a significant $(\mathrm{P}<0.05)$ increase in the $\mathrm{pH}$ in the esophagus for 120 minutes after ingestion [average $\mathrm{pH}$ values $6.04 \pm 0.27$ vs. $4.86 \pm 0.23$ during $0-60 \mathrm{~min}$., and $5.93 \pm 0.25$ vs. $4.15 \pm 0.26$ during $60-120 \mathrm{~min}$.]. $\mathrm{pH}$ in the gastric cardia (a typical place of formation of postprandial acid pocket) showed significant $(\mathrm{P}<0.05)$ higher values for the first 60 minutes after intake of alginate $[\mathrm{pH}$ $4.3 \pm 0.37$ vs. $3.04 \pm 0.25$ ], during $60-90 \mathrm{~min}$. $\mathrm{pH}$ values wasn't significantly $(\mathrm{P}>0.05)$ different $[2.75 \pm 0.45$ vs. $2.43 \pm 0.20]$. In the stomach body no significant effect of the drug on $\mathrm{pH}$ was recorded [average $\mathrm{pH}$ values for stomach $2.56 \pm 0.46$ vs. $2.1 \pm 0.18$ during 1 st postprandial hour and $2.29 \pm 0.49$ vs. $2.09 \pm 0.18$ during 2 nd postprandial hour; $\mathrm{P}>0.05$ ].

Conclusion: Our findings demonstrate that raft-forming alginate is an effective means for the prevention of postprandial acid and gas reflux and to reduce the injurious effect of acid in the esophagus. At the same time alginate showed no effect on stomach content in the postprandial period, it means that the main mechanism of action is through the movement of postprandial acid contents of the stomach from the lower esophageal sphincter, but not the neutralization of stomach acid, unlike nonraft-forming antacids and PPIs.

Disclosure of Interest: All authors have declared no conflicts of interest.

P0545 EFFICACY OF S-PANTOPRAZOLE 10 MG IN THE SYMPTOM CONTROL OF NON-EROSIVE REFLUX DISEASE: A PHASE III PLACEBO-CONTROLLED TRIAL

M. Choi ${ }^{1}$, Y.K. Cho ${ }^{1}$, Y. Bak ${ }^{2}$, P. Rhee ${ }^{3}$, S.G. Kim ${ }^{4}$, H. Jung ${ }^{5}$, S.Y. Seol ${ }^{6}$ ${ }_{1}^{1}$ Dept. Of Internal Medicine, The Catholic University of Korea, Seoul/Korea, Republic of

${ }^{2}$ Korea University College of Medicine, Seoul/Korea, Republic of

${ }^{3}$ Dept. Of Internal Medicine, Sungkyunkwan University School of Medicine, Seoul Korea, Republic of

${ }^{4}$ Internal Medicine, Seoul National University, Seoul/Korea, Republic of ${ }_{6}^{5}$ Asan Medical Center, Ulsan University, Seoul/Korea, Republic of

${ }^{6}$ Inje University College of Medicine, Pusan/Korea, Republic of

Contact E-mail Address: choim@catholic.ac.kr

Introduction: S-isomer (S) pantoprazole is more bioavailable and less dependent on cytochrome $2 \mathrm{C} 19$ than is racemic pantoprazole.

Aims \& Methods: We aimed to evaluate the efficacy and safety of $10 \mathrm{mg}$ S-pantoprazole for treatment of non-erosive reflux esophagitis (NERD). This study was designed as a multicenter, randomized, double-blind, placebo controlled trial.NERD was defined as reflux symptoms and normal endoscopy findings. Patients were allocated to take either $10 \mathrm{mg}$ S-pantoprazole or placebo once daily for 4 weeks, after which reflux symptoms were reassessed. Recurrence of symptoms was assessed at 4 weeks after cessation of medication. The efficacy endpoints were complete relief of symptoms, improvement of reflux symptoms, and safety.

Results: Eighty-eight patients were randomly assigned to the S-pantoprazole group (25 males, 43.7 years old) and 86 to the placebo group (32 males, 43 years old), and 163 patients were subjected to a per protocol analysis. A higher proportion of patients in the S-pantoprazole group had complete symptom relief $(34 \%$ vs. $14 \%, \mathrm{P}<0.001)$. In both groups, symptoms of heartburn, acid regurgitation and epigastric discomfort significantly improved after treatment compared with baseline; however, improvement of all symptoms was greater in the spantoprazole group compared to placebo group. Therapeutic gains in controls of heartburn, acid regurgitation and epigastric discomfort were $0.66,1.82$, and 0.57 . The factors associated with poor symptom responsiveness to PPI were older age, female sex, greater body mass index and symptom severity in both groups. Conclusion: S-pantoprazole $(10 \mathrm{mg})$ was more efficacious than placebo in providing reflux symptom relief in patients with NERD, especially acid regurgitation Disclosure of Interest: All authors have declared no conflicts of interest. 
P0546 TAILORED THERAPY GUIDED BY MULTICHANNEL

INTRALUMINAL IMPEDANCE PH MONITORING FOR REFRACTORY NON EROSIVE REFLUX DISEASE: PRELIMINARY EXPERIENCE

N. Ranaldo, G. Losurdo, A. Morelli, A. Iannone, M. Barone, M. Principi, E. Ierardi, A. Di Leo

Emergency And Organ Transplantation, University of Bari, Bari/Italy

Contact E-mail Address: giuseppelos@alice.it

Introduction: Refractory non erosive reflux disease (NERD) is defined by absence of clinical response to a 12 -week course of proton pump inhibitors (PPI) at full dose in absence of esophagitis. It accounts for about $20 \%$ of all NERD cases. 24hour multichannel intraluminal impedance $\mathrm{pH}(\mathrm{MII}-\mathrm{pH})$ monitoring should give useful patho-physiological information about refractory NERD. Therefore, our aim was to assess whether this technique could be useful to guide a "tailored" therapy to refractory NERD patients.

Aims \& Methods: We retrospectively recruited patients undergoing MII-pH monitoring for refractory NERD. All patients had undergone upper endoscopy, and cases of esophagitis were excluded. No patient received PPI during MII-pH monitoring. Subjects were subgrouped into 3 categories according to Zerbib's classification: i) Acid reflux (exposure to $\mathrm{pH}<4$ for at least $1.1 \%$ of record time), ii) Non acid reflux (symptom association probability to $\mathrm{pH}>4$ reflux episodes $>95 \%$ ) and iii) Functional heartburn (no pathologic reflux, with symptom association probability $<50 \%$ ). MII-pH guided therapy was performed as follows: patients with acid reflux received PPI at double dose, patients with non acid reflux PPI at full dose plus alginates and patients with functional heartburn levosulpiride $75 \mathrm{mg} /$ day for 4 weeks. A visual analogue scale (VAS) ranging 0100 was administered before and after such tailored therapy to evaluate overall symptoms. Responders were defined by VAS improvement of at least $10 \%$. Comparisons between continuous variables were performed by ANOVA or paired/unpaired t-test where required, and Fisher's exact test was applied to categorical variables. Variables with statistical significance $p<0.10$ at univariate analysis were analyzed by binomial multivariate regression analysis, aimed to investigate factors predictive of response to tailored therapy.

Results: Thirty-four patients with refractory NERD were selected (female:male ratio 20:14, mean age $47.4 \pm 12.8$ ). Twelve had acid reflux, 7 non acid reflux and 15 functional heartburn. Overall effectiveness of tailored therapy was $82.3 \%$ ( 28 out of 34$)$, and it did not differ between subgroups $(91.7 \%$ acid reflux, $71.4 \%$ non acid reflux, $80.0 \%$ functional heartburn, $\mathrm{p}=0.51$ ). At univariate analysis, therapy failure directly correlated with dysphagia $(\mathrm{OR}=0.15, \mathrm{p}=0.10)$ and inversely with sensation of slow digestion $(\mathrm{OR}=7.70, \mathrm{p}=0.05)$. However, at multivariate analysis, these parameters were not statistically significant. We found a mean VAS reduction of $30.2 \pm 24.9$, which was similar between acid reflux $(36.7 \pm 22.7)$, non acid reflux $(30.0 \pm 27.7)$ and functional heartburn $(25.0 \pm 25.7), \mathrm{p}=0.49$.

Conclusion: A tailored approach to refractory NERD, guided by MII-pH monitoring, demonstrated to be effective, independently from disease subtype. Therefore it should be advised to patients who complain of symptom persistence despite PPI therapy.

Disclosure of Interest: All authors have declared no conflicts of interest

\section{P0547 STW5 MODULATES TIGHT-JUNCTION GENE AND PROTEIN EXPRESSIONS IN REFLUX-ESOPHAGITIS - POSSIBLE RELEVANCE FOR TUMORIGENESIS}

H. Abdel-Aziz ${ }^{1}$, G. Ulrich-Merzenich ${ }^{2}$, A. Shcherbakova ${ }^{3}$, O. Kelber ${ }^{1}$

${ }^{1}$ Innovation \& Development, Steigerwald Arzneimittelwerk GmbH, Bayer Consumer Health, Darmstadt/Germany

${ }^{2}$ Medical Clinic III, University Clinic Centre, Bonn/Germany

${ }^{3}$ Volga State Technical University, Yoshkar-Ola/Russian Federation

Contact E-mail Address: gudrun.ulrich-merzenich@ukb.uni-bonn.de

Introduction: STW5, a herbal combination preparation of nine different plant extracts (Iberis amara (L.), Menthae piperitae (L.) Chamomilla recutita (L.), Glycyrhiza glabra (L.), Angelica archangelica (L.), Carum Carvi (L.), Silybium marianum (L.) Gaertn. Melissa officinalis (L.) und Chelidonium majus (L.)) has been extensively studied for the treatment of gastrointestinal disorders resulting in an recommendation in the German therapy guidelines for both upper and lower functional gastrointestinal disorders (1). We investigated the mode of action of STW5 in a rat model of reflux-esophagitis (RE) (2). RE is the most common condition treated by gastroenterologists with possible long-term implications such as Barrett's esophagus and esophageal adenocarcinoma.

Aims \& Methods: We were especially interested in the tight junction proteins (TJ), which are multi-protein complexes in epithelial and endothelial cells known to contribute to the barrier-function, but recently discovered to play an important role in tumorigenesis (3). TJ proteins like ZO-1 and 2 participate in cell-cycle regulation, occludin (OCCL) regulates the mitotic entry via centrosome separation and correlates with decreased cell proliferation, claudins (CLDNs) are made responsible for increased proliferation of the crypt cells (3). CLDN 1, 3, 4, 5, 7, 10 and 16 have been found altered in various types of cancer and OCCL is regarded an anti -transformation protein (3).

Results: In our model, RE was induced by surgical methods and rats $(n=6$ per group) were treated with STW5 $(0.5$ or $2 \mathrm{ml} / \mathrm{kg})$ or the proton pump inhibitor (PPI) omeprazole (O) $(30 \mathrm{mg} / \mathrm{kg})$ as described earlier (2). RNA was isolated from defined tissue areas of the esophagi. In the inflamed tissue we found a simultaneous transcript down regulation of claudin $3(\mathrm{p}<0.0001)$, confirmed by RTPCR. Also claudin 4, claudin 23, OCCL, MARVELD2 and ZO1 were significantly downregulated $(\mathrm{p}<0.001)$. The down regulation of $\mathrm{ZO} 1$ was seen by Western Blot, too. Transcripts of MarvelD1 and JAM 2 were increased. The treatment of rats with STW5 $(2 \mathrm{ml} / \mathrm{kg})$ or O (partially) normalized the esophageal tissue transcripts of ZO1, CLDN3, CLDN23, OCCL, MARVELD1, 2 and JAM. Conclusion: Data demonstrate a reversal of inflammatory changes in the TJproteins by STW5 and O. They also suggest that inflammatory changes in TJ are consistent with TJ-protein modulations in tumorigenesis. Subsequent studies are necessary to better define the complex role of $\mathrm{TJ}$ in inflammatory processes and in tumorigenesis and the potential benefit arising from a treatment with STW5 or O.

Disclosure of Interest: $\mathrm{H}$. Abdel-Aziz: Fully employed by Steigerwald Arzneimittelwerk $\mathrm{GmbH}$

G. Ulrich-Merzenich: Received Research funds from Steigerwald Arzneimittelwerk $\mathrm{GmbH}$

O. Kelber: Fully employed by Steigerwald Arzneimittelwerk GmbH

All other authors have declared no conflicts of interest.

\section{References}

1. Layer P, Andresen V, Pehl C, et al. S3 Leitlinie Reizdarmsyndrom. AWMFRegistriernummer:021/016, 2011. Z Gastroenterol. 2011:49:237-93.

2. Abdel-Aziz H, Schneider M, Neuhuber W, Kassem AM, Khailah S, Müller J, Gamaleldeen H, Khairy A, Khayyal MT, Thomas Efferth T, UlrichMerzenich G. GPR84 and TREM-1 signaling contribute to the pathogenesis of reflux esophagitis. Mol Med. 2015 Nov 24. doi: 10.2119/ molmed.2015.00098.

3. Runkle EA, Mu D. Tight junction proteins: from barrier to tumorigenesis. Cancer Lett. 2013;337(1):41-48.

\section{P0548 THE EFFECT OF SPECIFIC REFLUXATE COMPONENTS ON BILE RECEPTOR SIGNALING AND DEVELOPMENT OF METAPLASTIC BARRETT-LIKE GLANDS IN MICE}

D. Straub ${ }^{1}$, L. Mari ${ }^{1}$, S. Van De Graaf ${ }^{2}$, K.K. Krishnadath ${ }^{3}$

${ }^{1}$ Center For Experimental And Molecular Medicine, Academic medical center, Amsterdam/Netherlands

${ }^{2}$ Gastroenterology And Hepatology, Tytgat institute for liver and intestinal research, Amsterdam/Netherlands

Gastroenterology And Hepatology, AMC, Amsterdam/Netherlands

Contact E-mail Address: k.k.krishnadath $@$ amc.uva.nl

Introduction: Chronic reflux, a risk factor for the development of Barrett's esophagus (BE), causes damage to the normal squamous epithelium of the esophagus, which eventually is replaced by a columnar type of epithelium. It has been shown that bile acids (BAs) play a role in the development of BE. The effect of different BAs in the refluxate has been poorly studied with respect to the pathogenesis of BE. Insights in the effects of the bile components in reflux can help provide more efficacious targets for preventing the development of metaplasia and the progression from BE to EAC. Before being released into the duodenum, BAs produced by the liver are conjugated with either taurine or glycine. BAs not only have detergent properties, but can also act as signaling molecules by activating the farnesoid X receptor (FXR) and G-protein coupled bile acid receptor (TGR5). Both receptors are upregulated in BE and EAC compared to normal squamous mucosa, suggesting a possible role in the progression of BE (De Gottardi 2006; Hong 2010).

Aims \& Methods: The aim of the study was to analyze refluxates of BE patients and evaluate the effect of the different bile components on metaplastic gland development and bile receptor activation.

Results: Determination of the bile composition in refluxate of BE patients by HLPC showed the presence of both glyco-conjugated $(67.6 \pm 8.8 \%)$ and tauroconjugated BAs $(31.5 \pm 9.1 \%)$. Only a low percentage of unconjugated BAs was found in the patient refluxates $(0.5 \pm 0.4 \%)$. Although unconjugated BAs were absent in our refluxates, others have shown their presence in GERD patients (Nehra 1999). Deoxycholic acid (DCA), a secondary bile, is formed through deconjugation by microbial enzymes present in the colon, but the increase in $\mathrm{pH}$ due to PPI use can cause gastric bacterial overgrowth, resulting in the presence of DCA in reflux (Theisen 2000). Each of the six conjugated bile components [glycocholic-, glycodeoxycholic-, glycochenodeoxycholic-, taurocholic-, taurodeoxycholic- and taurochenodeoxychoic acid] and DCA were tested individually in vivo for 16 weeks at $10 \mathrm{mM}$. All mice developed multi-layered glands at the SCJ (36/36). Overall, mice treated with glyco-conjugated BAs developed more glands compared to DCA treated or tauro-conjugated BAs. Also alcian blue expression, representing mucin production, was higher compared to DCA treated or tauro-conjugated BAs treated group. To investigate which bile components are able to activate bile receptors we transfected primary mouse esophageal keratinocytes with either TGR5 or FXR and treated the cells with different BAs. Activation of the receptors result in conformational changes, which can be detected by measuring fluorescence resonance energy transfer (FRET). Both DCA and all conjugated BAs were able to activate the membrane bound TGR5 receptor, as shown by a change in signal. DCA was able to activate FXR, while the addition of all conjugated BAs did not result in a signal change. Conclusion: The possibility of DCA to activate the FXR may result in the onset of different genes, which might result in the progression from BE to EAC. Disclosure of Interest: All authors have declared no conflicts of interest. 


\section{P0549 REMODELING BARRETT'S METAPLASIA IN A NOVEL IN} VIVO HUMANORGANOID MODEL

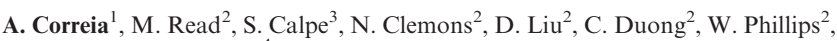
K. Kausilia Krishnadath ${ }^{4}$

${ }^{1}$ CEMM, Academic Medical Center Amsterdam, Amsterdam/Netherlands ${ }^{2}$ Surgical Oncology Research Laboratory, Peter MacCallum, Melbourne/Australia ${ }^{3}$ CEMM, Academic Medical Center, Amsterdam/Netherlands

${ }^{4}$ Medical Center, University Of Amsterdam, Amsterdam/Netherlands

Contact E-mail Address: a.c.pachecocorreia@amc.uva.nl

Introduction: Barrett's esophagus (BE) is a metaplastic abnormality in patients with Gastro-esophageal reflux disease in which the normal stratified squamous epithelium in the esophagus is replaced by columnar epithelium. BE predisposes for esophageal adenocarcinoma. One underlying mechanism of BE is that columnar stem cells residing in the esophageal mucosa (e.g., in submucosal glands) or multipotent stem cells give rise to the columnar lining. Bone Morphogenetic Proteins (BMPs) are a family of growth factors that control tissue architecture, homeostasis and stem cell differentiation. In BE, BMP4 is upregulated in columnar cells.

Aims \& Methods: Organoid cultures are widely used because they mimic in vivo differentiation of self-organizing stem cells and represent the perfect model to study stem cell interaction in basic and translational research. We developed an in vivo organoid model of human $\mathrm{BE}$ to investigate the potential to modulate the metaplastic process using an innovative anti-BMP2/4 llama-derived Dwarfbody ${ }^{\mathbb{B}}$ (DB). Endoscopic BE biopsies were implanted into immunocompromised mice intramuscularly and grown for a period of three months with DB or control. These structures were assessed histologically and immunohistochemically (IHC) using panels of squamous, intestinal and stem cell markers.

Results: BE organoids were lined by a columnar epithelial layer containing goblet cells and recapitulated the crypt and villous regions seen within BE glands. IHC validation confirmed that the xenograft structures were of human origin and expressed markers of intestinal differentiation (CK8, CDX2 and villin). In contrast, treatment with the BMP inhibitor lead to the formation of multi-layered squamous epithelium expressing both the stem cell marker p63 and the squamous marker CK5.

Conclusion: Preliminary results demonstrate that inhibition of BMP $2 / 4$ in this model enhanced the amount of squamous epithelium. These pre-clinical results may be translated to the clinical setting in order improve treatment of BE and as such prevent the development of esophageal adenocarcinoma.

Disclosure of Interest: All authors have declared no conflicts of interest.

\section{References}

Calpe, S., Correia, A. C., Sancho-Serra Mdel, C. \& Krishnadath, K. K. 2016. Comparison of newly developed anti-bone morphogenetic protein 4 llamaderived antibodies with commercially available BMP4 inhibitors. MAbs, 8, 678-88.

Read, M., Liu, D., Duong, C. P., Cullinane, C., Murray, W. K., Fennell, C. M., Shortt, J., Westerman, D., Burton, P., Clemons, N. J. \& Phillips, W. A. 2016. Intramuscular Transplantation Improves Engraftment Rates for Esophageal Patient-Derived Tumor Xenografts. Ann Surg Oncol, 23, 305-11.

\section{P0550 GASTRIC CARDIA GLANDS MANIFEST APPARENT BROAD DIFFERENTIATION POTENTIAL, AS EVIDENCED BY HOXA13 EXPRESSION, IMPLICATIONS FOR THE ORIGIN OF BARRETT'S ESOPHAGUS}

V. T. Janmaat, M.j.m. Smits, M.J. Bruno, M.C.w. Spaander, M. P. Peppelenbosch

Gastroenterology \& Hepatology, Erasmus Medisch Centrum, Rotterdam Netherlands

Contact E-mail Address: v.janmaat@erasmusmc.nl

Introduction: Metaplastic phenomena in the upper gastrointestinal tract are still poorly understood. A prominent theory is that Barrett's esophagus originates from gastric glandular epithelium. However, in absence of evidence that the gastric stem cell has broad differentiation potential, this theory remains controversial. The gastroesophageal junction is a high prevalence area for metaplasia and subsequent cancer. Characteristic of this area are the gastric cardia glands which cover only the most proximal part of the anatomic gastric cardia. Recent evidence from human and mouse studies has shown Barrett's esophagus can originate from these gastic cardia glands. ${ }^{1,2}$ If it can be shown that gastric cardia glands contain elements associated with positional misspecification, this theory could be substantially bolstered. $H O X$ genes are a family of transcription factors that convey positional information. The 3' to 5' sequence of $H O X$ genes corresponds to the sequence in which they act along the anterior to posterior axes of the gut. This property is termed collinearity and links clustering to function. In gastrointestinal physiology, $H O X A 13$, a 5' member of the $H O X A$ cluster, has an expression pattern restricted to the colonic epithelium. However, pathological metaplastic lesions of the esophagus and stomach are also characterized by HOXA13 expression. This in parallel with the similarities of these lesions with physiological colonic morphology. Hence, investigating HOXA13 expression in gastric cardia glands appears a rational strategy in assessing the potential of this gastric cardia epithelium to serve as the origin of Barrett's esophagus.

Aims \& Methods: We aimed to determine HOXA13 expression in physiological gastric cardia glands. Firstly, strips of tissue from surgical specimens containing squamous esophageal epithelium, gastric cardia glands, and oxyntic stomach glands, were collected. These were continuous strips, from proximal to distal, to preserve morphological information. Material from three patients was selected, they suffered from either a neuroendocrine tumor, or decompensated achalasia, or an adenocarcinoma. Antibodies against HOXA13 were found not to be specific. Therefore, RNA in situ hybridization by RNA-scope was performed to visualize $H O X A 13$ RNA. Secondly, a Hoxa13GFP x C57BL/6J heterozygous mutant mouse model was used. In these animals, the cardiac glands were analyzed directly for GFP expression using a fluorescence confocal microscope.

Results: All three patients showed HOXA13 expression of a portion of gastric cardia epithelial cells. The squamous epithelium, the oxyntic epithelium, and the stroma did not show any signal. The signal is located relatively close to the base of the crypts of the cardiac glands. The Hoxa13GFP x C57BL/6 J heterozygous mice showed GFP expression localized to the nucleus of some of the epithelial cells of the cardiac gland. No nuclear signal was detected in the squamous or oxyntic epithelium, nor in the stroma. The colonic epithelium of the mouse showed nuclear GFP signal. Rectal squamous epithelium was negative as well as ileal epithelium, in accordance with HOXA13 collinearity in mouse and human. A littermate negative for Hoxa13GFP was analyzed and showed no nuclear GFP signal in either gastric cardia gland cells or in colon epithelial cells. Conclusion: Gastric cardia gland epithelial cells in both human and mouse exhibit HOXA13 expression. All other physiological upper gastrointestinal tract tissues are HOXA13 negative in line with HOX gene collinearity in the gut. This dichotomy proves positional information in these glands is discordant with their actual location. These findings suggest that gastric cardia glands have a broad differential potential. This is consistent with an origin of Barrett's metaplasia in the gastric cardia and might be indicative of Barrett's not being a true transdifferentiation.

Disclosure of Interest: All authors have declared no conflicts of interest.

\section{References}

1. Quante M, Bhagat G, Abrams JA, et al. Bile acid and inflammation activate gastric cardia stem cells in a mouse model of Barrett-like metaplasia. Cancer Cell 2012;21:36-51.

2. McDonald SA, Lavery D, Wright NA, et al. Barrett oesophagus: lessons on its origins from the lesion itself. Nat Rev Gastroenterol Hepatol 2015;12:50 60

\section{P0551 ACTIVE HUMAN PAPILLOMAVIRUS INVOLVEMENT IN BARRETT'S DYSPLASIA AND OESOPHAGEAL ADENOCARCINOMA IS CHARACTERIZED BY WILD-TYPE P53 AND ABERRATIONS OF THE RETINOBLASTOMA PROTEIN PATHWAY}

S. Rajendra ${ }^{1}$, T. Yang ${ }^{2}$, W. Xuan ${ }^{3}$, P. Sharma ${ }^{4}$, D. Pavey ${ }^{5}$, C. S. Lee ${ }^{2}$, S. Le $^{5}$, J. Collins 5 , B. Wang

${ }_{1}^{1}$ Gastroenterology \& Hepatology, Bankstown-Lidcombe Hospital, Bankstown, Sydney, NSW/Australia

${ }^{2}$ Department Of Anatomical Pathology, South Western Sydney Area Pathology Service, Sydney/Australia/NSW

${ }^{3}$ Ingham Institute for Applied Medical Research, Sydney/Australia ${ }^{4}$ Division Of Gastroenterology And Hepatology, Veterans Affairs Medical Center and University of Kansas City, Kansas City/United States of America/MO ${ }^{5}$ Gastroenterology And Hepatology, Bankstown-Lidcombe Hospital, Sydney Australia/NSW

${ }^{6}$ Ingham Institute for Applied Medical Research, Sydney/Australia/NSW

Contact E-mail Address: shan.rajendra@sswahs.nsw.gov.au

Introduction: We have previously demonstrated that transcriptionally active highrisk HPV (hr-HPV) is strongly incriminated in Barrett's dysplasia (BD) and oesophageal adenocarcinoma (OAC) using mainly fresh frozen tissue ${ }^{1-4}$ This study aimed to identify biomarkers of active HPV infection in Barrett's metaplasia, (BM)/BD/OAC by immunohistochemical staining (IHC) of formalin-fixed paraffin embedded (FFPE) tissue for aberrations of $\mathrm{p} 53$ and the retinoblastoma $(\mathrm{pRb})$ pathway which are targets for the viral oncoproteins, E6/E7 respectively. Aims \& Methods: Prospectively, $\operatorname{BM}(\mathrm{n}=81) / \mathrm{BD}(\mathrm{n}=72) / \mathrm{OAC}(\mathrm{n}=65)$ FFPE specimens were subjected to IHC staining for pRb, p16INK4A, cyclin D1, p53 and RNA in-situ hybridization (ISH) for E6/E7 transcripts. HPV DNA was determined via PCR in fresh frozen specimens. Viral load measurement (realtime PCR) and Next Generation Sequencing of TP53 was also performed.

Results: Of 218 patients, 56 were HPV DNA positive [HPV16 $(n=42), 18$ $(\mathrm{n}=13), 6(\mathrm{n}=1)]$. Viral load was low. Transcriptionally active HPV (DNA+/ RNA+) was only found in the dysplastic and adenocarcinoma group $(n=21)$. The majority of HPV DNA+/RNA $+\mathrm{BD} / \mathrm{OAC}$ were characterized by pl6INK4Ahigh $(14 / 21,66.7 \%)$, pRblow $(15 / 21,71.4 \%)$ and p53low $(20 / 21$, $95 \%$ ) and was significantly different to controls [combination of HPV DNA-/ RNA- $(\mathrm{n}=94)$ and HPV DNA+/RNA- cohorts $(\mathrm{n}=22)]$ p53low had the strongest association with $\mathrm{DNA}+\mathrm{RNA}+$ oesophageal lesions $(\mathrm{OR}=23.5,95 \%$ $\mathrm{CI}=2.94-187.8, \mathrm{p}=0.0029)$. Seventeen HPV DNA + RNA + BD/OAC identified as p5low, were sequenced and all but one exhibited wild-type status. $\mathrm{pRblow} / \mathrm{p} 53$ low provided the best balance of strength of association $(\mathrm{OR}=8.0$, $95 \% \mathrm{CI}=2.6-25.0, \mathrm{p}=0.0003)$ and sensitivity $(71.4 \%)$ /specificity $(71.6 \%)$ for $\mathrm{DNA}+/ \mathrm{RNA}+\mathrm{BD} / \mathrm{OAC}$. Transforming infections i.e viral positive BD/OAC lesions exhibiting $\mathrm{p} 16_{\text {high }} / \mathrm{pR}_{\text {blow }}$ was found in $47.6 \%$ of the $\mathrm{RNA}^{+}$patients and $31.8 \%$ of $\mathrm{RNA}^{-}$subjects.

Conclusion: Active HPV involvement in BD/OAC is characterized by wild-type p53 and aberrations of the retinoblastoma protein pathway. Transforming infections which are a feature of HPV driven lesions was identified in a significant proportion of virally positive $\mathrm{BD} / \mathrm{OAC}$.

Disclosure of Interest: All authors have declared no conflicts of interest. 


\section{References}

1. Rajendra S, Wang B, Snow ET, Sharma P, Pavey D, Merrett N, Ball MJ, Brain T, Fernando R, Robertson IK. Transcriptionally active human papillomavirus is strongly associated with Barrett's dysplasia and esophageal adenocarcinoma. Am J Gastroenterol 2013;108: 1082-93.

2. Wang B, Rajendra S, Pavey D, Sharma P, Merrett N, Wu X, Snow ET, Kumbhari V, Ball MJ, Robertson IK. Viral load and integration status of high-risk human papillomaviruses in the Barrett's metaplasia-dysplasia-adenocarcinoma sequence. Am J Gastroenterol 2013;108: 1814-6.

3. Rajendra S, Wang B, Pavey D, Sharma P, Yang T, Lee CS, Gupta N, Ball MJ, Gill RS, Wu X. Persistence of Human Papillomavirus, Overexpression of p53, and Outcomes of Patients After Endoscopic Ablation of Barrett's Esophagus. Clin Gastroenterol Hepatol 2015;13: 1364-8.

4. Rajendra S, Wang B, Merrett N, Sharma P, Humphris J, Lee HC, Wu J. Genomic analysis of HPV-positive versus HPV-negative oesophageal adenocarcinoma identifies a differential mutational landscape. J Med Genet 2016;53: 227-31

\section{P0552 CHARACTERIZING DIPEPTIDYL PEPTIDASE SPECIFIC ACTIVITY IN HUMAN BARRETT'S OESOPHAGUS AND IN A PANEL OF OESOPHAGEAL METAPLASIA, DYSPLASIA AND CANCER CELL LINES}

S. Jaenisch ${ }^{1}$, D. J. Hussey ${ }^{2}$, D. Watson ${ }^{1}$, C. A. Abbott ${ }^{3}$, M. Squire ${ }^{1}$, R. Yazbeck $^{2}$ ${ }^{1}$ Surgery, Flinders University, Adealaide/Australia

${ }^{2}$ Flinders Centre for Innovation in Cancer, Adelaide/Australia

${ }^{3}$ School Of Biological Science, Flinders University, Adelaide/Australia

Contact E-mail Address: roger.yazbek@flinders.edu.au

Introduction: Barrett's oesophagus is defined as the replacement of the normal stratified oesophageal squamous epithelium with a metaplastic columnar intestinal-like epithelium. Dipeptidyl peptidase-4 (DPP4) is an integral membrane glycoprotein that is highly expressed on the small intestinal brush border. DPP4 is part of a larger enzyme family that also includes DPP8, DPP9 and fibroblast activation protein (FAP).

Aims \& Methods: We aimed to characterise DPP4 enzyme activity in a panel of oesophageal metaplasia, dysplasia and cancer cell lines, and in Barrett's oesophagus patient biopsies. FLO-1, OE33 and JH-EsoAd1 (oesophageal adenocarcinoma), OE21 and TE7 (oesophageal squamous cell carcinoma), GihTERT, GohTERT, ChTERT (dysplastic Barrett's) and QhTERT (non-dysplastic Barretts) were grown to confluence in T75 flasks using standard cell culture techniques. Oesophageal squamous tissue biopsies were collected from nonBarrett's patients participating in the Barrett's screening endoscopy program at Flinders Medical Centre $(n=6)$. Duodenal and gastric tissue biopsies were also collected as positive and negative control tissue respectively. Membrane and soluble enzyme activity was determined in cell and tissue extracts by colorimetric enzyme assay using the DPP selective substrate H-Gly-Pro-pNA. Sitagliptin (a DPP4 selective inhibitor) and 1G244 (DPP8/9 selective inhibitor) were used to define specific enzyme activity. Final enzyme activity was expressed as nm pNA released $/ \mathrm{min} / \mathrm{mg}$ protein. Data is expressed as mean \pm standard error of the mean.

Results: Relatively high DPP activity was detected on the membrane of OE33 (13.92 $\mathrm{nm} \mathrm{pNA} / \mathrm{min} / \mathrm{mg}$ protein), GihTERT (6.93 nm $\mathrm{pNA} / \mathrm{min} / \mathrm{mg}$ protein), GohTERT $(2.65 \mathrm{~nm} \mathrm{pNA} / \mathrm{min} / \mathrm{mg}$ protein) and QhTERT $(1.80 \mathrm{~nm} \mathrm{pNA} / \mathrm{min}$ $\mathrm{mg}$ protein) compared to all other cell lines, where activity was $<1 \mathrm{~nm}$ pNA/ $\mathrm{min} / \mathrm{mg}$ protein). Addition of $1 \mathrm{mM}$ sitagliptin completely inhibited enzyme activity in OE33 cells, indicating that membrane enzyme activity was specific to DPP4. Cytoplasmic DPP activity was highest in FLO-1 $(3.77 \mathrm{nM}$ pNA/min $\mathrm{mg}$ protein) and ChTERT $(2.39 \mathrm{nM} \mathrm{pNA} / \mathrm{min} / \mathrm{mg}$ protein). Addition of $1 \mathrm{mM}$ sitagliptin did not reduce cytoplasmic DPP activity in FLO-1 cells, suggesting the presence of other peptidases. High DPP4 activity was detected in the membrane fraction of duodenal biopsies ( $45.90 \pm 8.55 \mathrm{~nm} \mathrm{pNA} / \mathrm{min} / \mathrm{mg}$ protein) compared to gastric $(3.35 \pm 1.59 \mathrm{~nm} \mathrm{pNA} / \mathrm{min} / \mathrm{mg}$ protein) and oesophageal biopsies $(0.65 \pm 0.71 \mathrm{~nm} \mathrm{pNA} / \mathrm{min} / \mathrm{mg}$ protein). In contrast, differential DPP activity was detected in the soluble fraction of all duodenal, gastric and oesophageal tissue biopsies. Using 1uM sitagliptin, this was found to be specific to DPP4 in oesophageal samples, but likely derived from other peptidases in gastric and duodenal samples.

Conclusion: Our preliminary in vitro findings suggest a differential pattern of DPP activity in Barrett's metaplasia and dysplasia, which could have potentia significance as a biomarker target for the continuum of Barrett's oesophagus. Human tissue sample collection is ongoing to define specific DPP enzyme activity and expression in tissue specimens from Barrett's metaplasia and dysplasia subgroups compared to normal, non-Barrett's tissue.

Disclosure of Interest: All authors have declared no conflicts of interest.

P0553 AN ANTI-INFLAMMATORY ENVIRONMENT CHARACTERISES BARRETT'S OESOPHAGUS ASSOCIATED ADENOCARCINOMAS AND INFLUENCES PATIENT SURVIVAL

K. Karstens ${ }^{1}$, J. Kempski ${ }^{2}$, A. Giannou ${ }^{2}$, S. Steurer ${ }^{3}$, G. Wolters-Eisfeld ${ }^{1}$, T. Rösch ${ }^{4}$, J.R. Izbicki ${ }^{1}$, A. W. Lohse ${ }^{2}$, N. Gagliani ${ }^{1}$, S. Huber ${ }^{2}$

${ }^{1}$ General, Visceral And Thoracic Surgery, University Medical Center Hamburg-

Eppendorf (UKE), Hamburg/Germany
${ }^{2}$ I. Department Of Medicine, University Medical Center Hamburg-Eppendorf, Hamburg/Germany

${ }^{3}$ Institute Of Pathology, University Medical Center Hamburg-Eppendorf (UKE), Hamburg, Germany, Hamburg/Germany

${ }^{4}$ Department Of Interdisciplinary Endoscopy, University Medical Center Hamburg-Eppendorf, Hamburg/Germany

Contact E-mail Address: k.karstens@uke.de

Introduction: Barrett's Oesophagus (BE) is an acquired condition resulting from oesophageal reflux that causes intestinal metaplasia. These mucosal alterations can progress to dysplastic lesions, which ultimately lead to oesophageal adenocarcinoma (EAC). The incidence of EACs is rising in the Western world and 5 -year survival rates are below $20 \%$. Hence, new diagnostic and prognostic markers are needed. Since elevated levels of interleukin (IL-) 22 and IL-17A producing cells are associated with a poor prognosis in colorectal cancer we wanted to investigate their influence in EACs.

Aims \& Methods: None of the patients enrolled in this study received chemoradiation therapy prior to surgery and all patients had pathologically confirmed BE. mRNA expression levels of interleukins in tumour tissue and non-malignant peritumoral samples of 39 patients were measured. Immunohistochemical analysis was conducted to investigate interleukins during the progression from BE to EAC. Interleukins were investigated by flow cytometry in freshly resected esophageal cancer specimens and in its non-malignant peritumoral tissue $(n=11)$ During routine endoscopy samples from healthy volunteers were obtained and analysed by flow cytometry as controls $(n=5)$.

Results: During progression from BE to EAC an increase of CD4+ cells was observed. The number of IL-22+ cells and IL-17A + cells as well as the number of FOXP3 + cells/mg tissue increased in oesophageal cancer and in its peritumoral tissue as compared to healthy controls. The relative amounts of IL-22+ and IL-17A + cells decreased while an increase of FOXP3 + cells was observed. Also more IL-10+ cells/mg tissue were observed in the tumours. In accordance to the latter finding high IL-10 mRNA expression levels were associated with poor survival in EAC patients. Interestingly, high mRNA expression levels of IL-10 in the non-malignant peritumoral tissue also correlated with poor survival.

Conclusion: During progression from BE to EAC the infiltration of CD4+ immune cells increases. While the relative amount of pro-inflammatory cells decreases an accumulation of immunosuppressive cells was observed. Our data suggest that especially regulatory $\mathrm{T}$ cells influence overall patient survival in EAC. Notably, high levels of IL-10 in the non-malignant peritumoral tissue also demonstrated to be unfavourable for patient survival. Thus, a pro-tumorigenic field effect in yet unaltered oesophageal mucosa might contribute to local recurrences despite radical endoscopic or surgical resection.

Disclosure of Interest: All authors have declared no conflicts of interest.

\section{P0554 DIFFERENT GLAND PHENOTYPES ARE CLONALLY RELATED IN BARRETT'S EOSOPHAGUS AND GLAND PHENOTYPIC DIVERSITY IS INCREASED IN PATIENTS WHO HAVE PROGRESSED TO DYSPLASIA}

J. Evans ${ }^{1}$, E. Carlotti ${ }^{1}$, M. Rodriguez-Justo ${ }^{2}$, S. L. Preston ${ }^{3}$, N. Wright ${ }^{1}$, T. Graham ${ }^{1}$, M. Jansen ${ }^{4}$, S. A.C. Medonald ${ }^{1}$

${ }^{1}$ Centre For Tumour Biology, Barts Cancer Institute, London/United Kingdom ${ }^{2}$ Histopathology, University College London, London/United Kingdom ${ }^{3}$ Gastroenterology, Barts Health NHS Trust, London/United Kingdom ${ }^{4}$ UCL Cancer Institute, University College London, London/United Kingdom

Contact E-mail Address: s.a.mcdonald@qmul.ac.uk

Introduction: A range of complex glandular phenotypes exist in Barrett's esophagus (BE), but how these phenotypes are related and evolve is unknown. Genetic diversity is an established risk factor for cancer development in BE, yet as phenotype not genotype is selected for and as the gland is the unit of selection, we suggest that phenotypic diversity may confer a increased risk of cancer progression. Exploiting mutations in the itochondrial genome (MtDNA) as a marker of clonality we have demonstrated that the glandular phenotypes seen in Barrett's represent an evolutionary pathway and that phenotypic diversity also leads to an increased cancer risk.

Aims \& Methods: Biopsies from patients with a new diagnosis or surveillance for $\mathrm{BE}$ were obtained at endoscopy and frozen. Immunohistochemistry was performed on frozen sections for proteins including MUC5AC, MUC2, MUC6 and $\mathrm{H}^{+} \mathrm{K}^{+}$ATPase to distinguish intestinal from gastric cell lineages. Individual glands were laser capture microdissected and DNA was extracted using the ARCTURUS ${ }^{\circledR}$ PicoPure ${ }^{\circledR}$ DNA Extraction Kit. Sanger sequencing of the entire mitochondrial genome was performed to determine the clonal relationships between different Barrett's gland phenotypes.

Gland phenotypes and phenotypic diversity was counted in archived FFPE cases of $\mathrm{BE}$ who had progressed to dysplasia versus those who had not $(\mathrm{n}=10$ in each group - counts performed in a blinded fashion). Using the Shannon index as a measure of diversity, 5 distinct $\mathrm{BO}$ gland phenotypes were counted to determine if there was a significant increase in gland phenotype diversity in those biopsies taken adjacent to dysplastic glands compared to biopsies from patients who did not have dysplasia.

Results: In 2 separate cases specialised mature intestinal type glands were shown to be present expressing both MUC2 and MUC5AC. Furthermore, in the same section MUC5AC+ MUC2- cardia-like Barrett's glands and MUC5ACMUC2+ mature intestinal-like Barrett's glands were also present. LCM sequencing of DNA extracted from each of these glands yielded an $\mathrm{m} .7148 \mathrm{C}>\mathrm{T}$ mutation and $\mathrm{m} .10492 \mathrm{~T}>\mathrm{C}$ mutation respectively in each gland that was not present in the stroma. This is the first time that different gland phenotypes have been demonstrated to be clonal and suggests that Barrett's displays phenotypic as well 
as genotypic evolution. Patients with dysplasia also show a significant increase in gland phenotype diversity (Shannon) per biopsy in adjacent to dysplasia glands compared with patients who do not have dysplasia.

Conclusion: BE is phenotypically diverse with a range of glandular phenotypes that are clonally related. An increase in phenotypic diversity may be a potential biomarker for predicting which patients are at risk of progressing from $\mathrm{BE}$ to cancer with implications for diagnostic and surveillance policy.

Disclosure of Interest: All authors have declared no conflicts of interest.

\section{P0555 A DEDICATED BARRETT'S OESOPHAGUS ENDOSCOPY LIST IMPROVES THE ACCURACY OF ENDOSCOPIC REPORTING AND QUALITY OF BIOPSIES}

H. Al-Hasani, T. Sharp, S.M. Ha, L. Matsuka, N. Siddique

Gastroenterology, Queen Elizabeth the Queen Mother Hospital, Margate/United Kingdom

\section{Contact E-mail Address: hannahalhas@gmail.com}

Introduction: The importance of skilled endoscopic assessment of Barrett's oesophagus (BO) has been clearly established and forms part of the British Society of Gastroenterology guidelines ${ }^{1}$. Use of Prague classification when reporting on areas of BO improves standardisation, and adherence to the Seattle biopsy protocol (quadrantic biopsies every $2 \mathrm{~cm}$ ) when sampling Barrett's mucosa is thought to improve dysplasia detection ${ }^{2}$. In East Kent Hospitals NHS Foundation Trust we have created a dedicated nurse-led BO surveillance endoscopy list with the aim of improving compliance with guidelines and the quality of biopsies taken. Here we present a retrospective observational study of patients who underwent upper GI endoscopy on a general endoscopy (GE) list compared with the dedicated BO endoscopy (DBO) list.

Aims \& Methods: We searched our endoscopy software for patients who had had an indication for gastroscopy documented as BO and who had an endoscopy on a GE list from 2012-2013. The same search was performed for patients who were scoped on the DBO list from 2014-2016. Endoscopy reports were reviewed to assess the use of Prague classification and determine numbers of biopsies taken. Biopsy results were reviewed on our electronic pathology database.

Results: One hundred procedures for BO surveillance on GE lists were audited, comprising $65 \%$ male patients with median age 68 years; $60 \%$ were performed by a consultant gastroenterologist and the remainder were performed by other operators including surgical consultants and gastroenterology registrars. Of the 105 procedures on the DBO lists, $63 \%$ of patients were male, median age 70 years. Prague classification was used in $94 \%$ of endoscopy reports on the DBO lists compared with $5 \%$ on the GE lists. The Seattle biopsy protocol was observed in $70 / 94(74 \%)$ cases on the DBO lists as opposed to $30 / 100(30 \%)$ on the GE lists. Dysplasia detection rate (low grade, high grade or indefinite) was similar in both groups: $8 / 105(7.6 \%)$ on the DBO list and $6 / 100(6 \%)$ in the GE group. All $(100 \%)$ of the dysplasia detected on the GE lists occurred in procedures performed by consultant gastroenterologists.

Conclusion: Our comparison shows that observance of Prague classification is significantly higher on the DBO lists when compared with GE lists (94\% vs 5\%), and compliance with the Seattle biopsy protocol is similarly higher $(74 \%$ vs $30 \%$ ). These are indicators of higher quality endoscopic surveillance on DBO lists. However, this did not translate to a different dysplasia detection rate which appeared to be more influenced by the endoscopy operator since all of the dysplasia detected on GE lists was identified by consultant gastroenterologists. We believe that this reflects the additional familiarity of guidelines and possible greater experience of endoscopists regularly taking BO biopsies. We therefore recommend that all Barrett's oesophagus patients have their surveillance endoscopies performed on dedicated BO endoscopy lists.

Disclosure of Interest: All authors have declared no conflicts of interest.

\section{References}

1 Fitzgerald RC et al. British Society of Gastroenterology guidelines on the diagnosis and management of Barrett's oesophagus. Gut 2014;63:7-42.

2 Levine DS et al. Safety of a systematic endoscopic biopsy protocol in patients with Barrett's esophagus. Am J Gastroenterol 2000;95:1152-7

\section{P0556 ADHERENCE TO QUALITY INDICATORS AND SURVEILLANCE GUIDELINES IN THE MANAGEMENT OF BARRETT'S ESOPHAGUS: A RETROSPECTIVE ANALYSIS}

D. Westerveld ${ }^{1}$, V. Khullar ${ }^{2}$, M. B. Riverso ${ }^{2}$, Y. Perbtani ${ }^{2}$, M. Agarwal ${ }^{1}$, J. J. Forde ${ }^{1}$, J. Chakraborty ${ }^{1}$, T. S. Brar ${ }^{1}$, F. Ayoub ${ }^{1}$, A. Gupte ${ }^{2}$, C. E. Forsmark ${ }^{2}$, P. V. Draganov ${ }^{2}$, D. Yang

${ }^{1}$ Medicine, University of Florida, Gainesville/United States of America/FL

${ }^{2}$ Gastroenterology, University of Florida, Gainesville/United States of America

Contact E-mail Address: dennis.yang@medicine.ufl.edu

Introduction: Adherence to quality indicators and surveillance guidelines in the management of Barrett's esophagus (BE) promotes high-quality cost-effective care.

Aims \& Methods: The aims of this study were to evaluate (1) adherence to standardized classification (Prague Criteria) and a systematic four-quadrant biopsy protocol, (2) identify predictors of practice patterns, and (3) to assess adherence to surveillance guidelines for non-dysplastic BE (NDBE).

This was a Single-center retrospective study of endoscopies (EGDs) performed for BE between June 2008 to December 2015. Data on patient demographics, procedure characteristics and histology results were obtained from a prospectively collected endoscopy database and chart review. Adherence to the use of Prague Criteria and systematic biopsy protocol were assessed based on operative report documentation. Guideline adherent surveillance EGD was defined as those performed within 6 months of the recommended 3-5 year interval. Uni- and multivariate analysis were performed to identify predictors of practice patterns.

Results: A total of 397 patients (66.5\% male; mean age $60.1 \pm 12.5$ years) had an index EGD during the study period. Adherence to the use of Prague Criteria and systematic biopsies were $27.4 \%$ and $24.1 \%$, respectively. Endoscopists who perform therapeutic interventions for BE were more likely to use the Prague Criteria (OR: 3.16 ; 95\% CI: 1.47-6.82) than those who do not. Longer time in practice (in years) was positively associated with adherence to Prague Criteria (OR 1.07; 95\% CI: $1.02-1.12 ; \mathrm{p}<0.01)$ but with a lower likelihood of performing systematic biopsies (OR 0.91 ; 95\% CI: $0.85-0.97$; p $<0.01$ ). Nearly $41 \%$ of patients with NDBE (11/27) underwent surveillance EGD sooner (range 1-24 months) than the recommended interval.

Conclusion: Adherence to quality indicators and surveillance guidelines in BE is low. Operator characteristics, including experience with endoscopic therapy for $\mathrm{BE}$ and time in practice predicted adherence to the use of Prague Criteria and systematic biopsies. Future efforts are needed to reduce variability in practice and promote high-value care.

Disclosure of Interest: All authors have declared no conflicts of interest.

\section{References}

De Jonge PJ, van Blankenstein M, Looman CW et al. Risk of malignant progression in patients with Barrett's oesophagus: a Dutch nationwide cohort study. Gut 2010; 59: 1030-1036

Hvid-Jensen F, Pedersen L, Drewes AM, et al. Incidence of adenocarcinoma among patients with Barrett's esophagus. N Engl J Med 2011; 365: 1375-1383. El-Serag HB, Naik AD, Duan Z, et al. Surveillance endoscopy is associated with improved outcomes of oesophageal adenocarcinoma detected in patients with Barrett's oesophagus. Gut 2016; 65: 1252-1260.

Sharma P, Katzka DA, Gupta N, et al. Quality indicators for the management of Barrett's esophagus, dysplasia, and esophageal adenocarcinoma: international consensus recommendations from the American Gastroenterological Association Symposium. Gastroenterology 2015; 149: 1599-1606.

\section{P0557 EXPRESSION OF TGF-B AND CD-44 IN AGE SPECIFIC SUBGROUP OF PATIENTS WITH ADENOCARCINOMA OF GASTRIC CARDIA}

N. Lukavetskyy, T. Fetsych

Oncology Department, Lviv Medical University, Lviv/Ukraine

\section{Contact E-mail Address: lukavetskyy@ukr.net}

Introduction: Adenocarcinoma near the esophagogastric junction is one of the most lethal GI malignancies known. Surgical treatment of these cancers stay determinative factors of patient survival. Older persons often differ from the younger adult population in terms of biological and functional perspectives; as such, they may have particular needs which require an interdisciplinary approach and intervention, especially when faced with a cancer diagnosis.

Aims \& Methods: The aim of this study was to detected expression of TGF- $B$ and CD-44 in age specific subgroup. The expressions of TGF- $B$ and CD-44 were evaluated immunohistochemically in 23 patients with adenocarcinoma of gastric cardia who underwent curative surgery (R0) without any neo/adjuvant therapy. Additionally we analyzed control group of patients with non-cancer lesion or normal tissue of upper digestive tract (13 patients). We divided the patients into two groups. Group A consisted of 13 cancer patients and 7 control patients 65 years of age or older, while Group B consisted of 10 cancer and 6 control patients younger than 65 years of age. The two groups were comparable - there were no differences between the two groups regarding tumor stage.

Results: Elderly patients have statistically significant better survival (median 20.2 month) compared with younger patients (median 15.4 month $)(p=0.045)$. The median survival rate of patients without TGF- $B$ and/or CD-44 expression was significantly lower $(7 \mathrm{~m})$ than that of patients with positive expression $(>15 \mathrm{~m})$ $(p=0.003)$. Regardless of patients age, CD-44 was significantly higher in the cancer tissue of elderly patients than in younger $(p<0,035)$. But no significant difference was observed in the TGF- $B$ expression between group A and group B cancers tissue $(\mathrm{p}=0.005)$.

Conclusion: The biology of tumors may be different in elderly patients, leading to a lower rate of tumor-related mortality.

Disclosure of Interest: All authors have declared no conflicts of interest.

\section{P0558 BARRETT'S OESOPHAGUS PROFILE AND OUTCOMES IN} A LARGE COHORT

N. Bar ${ }^{1}$, N. Fliss Isacov², M. Nisim², R. Kariv ${ }^{2}$

${ }^{1}$ Gastroenterology, Tel Aviv Sourasky Medical Centre, Tel Aviv/Israel

${ }^{2}$ Gasteroenterology and Hepatology, Tel Aviv Medical center, Tel-aviv/Israel

Contact E-mail Address: nirbar7@gmail.com

Introduction: Barrett's oesophagus (BO) is considered a premalignant condition for oesophageal adenocarcinoma (OAC). Once diagnosed, interval endoscopic surveillance is recommended to promote early detection of dysplasia and cancer. Occurrence and incidence of dysplasia and cancer among BO vary across populations. Recent studies show BO patients mortality is mainly related to nonoesophageal cancer and cardiovascular morbidity. 
Abstract No: P0556

Table: Factors associated with the adherence to the use of Prague Criteria and systematic four-quadrant biopsies for BE evaluation

\begin{tabular}{|c|c|c|c|c|c|c|c|c|}
\hline \multirow{3}{*}{ Clinical Variable } & \multicolumn{4}{|l|}{ Prague Criteria } & \multicolumn{4}{|c|}{ Systematic Biopsies } \\
\hline & Univariate & & Multivariable & & Univariate & & Multivariate & \\
\hline & OR $(95 \% \mathrm{CI})$ & $\mathrm{P}$ value & OR $(95 \% \mathrm{CI})$ & $\mathrm{P}$ value & OR $(95 \% \mathrm{CI})$ & $P$ value & OR $(95 \% \mathrm{CI})$ & $\mathrm{P}$ value \\
\hline Age & $1.02(0.99-1.04)$ & 0.06 & $1.00(0.98-1.04)$ & 0.53 & $1.02(0.99-1.05)$ & 0.06 & $1.01(0.97-1.05)$ & 0.42 \\
\hline BMI & $1.00(0.95-1.04)$ & 0.84 & $0.98(0.93-1.04)$ & 0.60 & $0.96(0.90-1.03)$ & 0.23 & $0.96(0.89-1.05)$ & 0.40 \\
\hline Sex (male) & $2.34(1.38-3.94)$ & 0.001 & $2.07(0.99-4.31)$ & 0.05 & $1.32(0.73-2.40)$ & 0.35 & $1.18(0.46-3.01)$ & 0.73 \\
\hline GERD & $1.31(0.71-2.43)$ & 0.40 & $0.64(0.17-2.40)$ & 0.51 & $1.74(0.78-3.89)$ & 0.18 & $1.75(0.34-8.96)$ & 0.50 \\
\hline PPI use & $1.48(0.90-2.47)$ & 0.13 & $1.29(0.49-3.39)$ & 0.61 & $1.25(0.68-2.28)$ & 0.72 & $0.45(0.15-1.33)$ & 0.15 \\
\hline $\begin{array}{l}\text { Smoking history } \\
\text { Current smoker } \\
\text { Previous smoker }\end{array}$ & $\begin{array}{l}1.53(0.77-3.00) \\
0.97(0.57-1.70)\end{array}$ & $\begin{array}{l}0.22 \\
0.91\end{array}$ & $\begin{array}{l}2.16(0.81-5.80) \\
0.82(0.37-1.81)\end{array}$ & $\begin{array}{l}0.17 \\
0.62\end{array}$ & $\begin{array}{l}1.98(0.86-4.60) \\
1.91(0.97-3.8)\end{array}$ & $\begin{array}{l}0.11 \\
0.06\end{array}$ & $\begin{array}{l}1.59(0.45-5.65) \\
1.29(0.46-3.63)\end{array}$ & $\begin{array}{l}0.47 \\
0.63\end{array}$ \\
\hline Short segment BE & $1.28(1.09-2.84)$ & 0.02 & & & $0.22(0.18-0.82)$ & 0.01 & & \\
\hline Long segment $\mathrm{BE}$ & $0.78(0.44-1.37)$ & 0.40 & $0.61(0.27-1.34)$ & 0.22 & $4.54(2.37-8.7)$ & 0.001 & $2.36(0.96-5.88)$ & 0.06 \\
\hline 1 experience year $\%$ & $1.06(1.02-1.11)$ & 0.001 & $1.07(1.02-1.12)$ & $<0.01$ & $0.91(0.87-0.94)$ & 0.001 & $0.91(0.85-0.97)$ & 0.001 \\
\hline BE therapeutics* & $3.82(2.31-6.33)$ & $<0.001$ & $3.16(1.47-6.82)$ & $<0.01$ & $1.40(0.78-2.50)$ & 0.26 & $1.11(0.43-2.88)$ & 0.83 \\
\hline Attending only & $0.70(0.43-1.14)$ & 0.157 & $0.77(0.36-1.64)$ & 0.50 & $1.40(0.77-2.56)$ & 0.27 & $1.41(0.54-3.63)$ & 0.48 \\
\hline 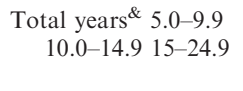 & $\begin{array}{l}0.94(0.47-1.88) \\
2.16(1.09-4.26) \\
2.54(1.22-5.31)\end{array}$ & $\begin{array}{l}0.866 \\
0.03 \\
0.01\end{array}$ & $\begin{array}{l}0.93(0.44-1.95) \\
3.25(1.52-6.96) \\
2.84(1.27-6.36)\end{array}$ & $\begin{array}{l}0.85 \\
0.002 \\
0.01\end{array}$ & $\begin{array}{l}0.93(0.47-1.84) \\
0.32(0.13-0.77) \\
0.28(0.10-0.80)\end{array}$ & $\begin{array}{l}0.84 \\
0.01 \\
0.02\end{array}$ & $\begin{array}{l}0.74(0.33-1.66) \\
0.35(0.13-0.95) \\
0.29(0.10-0.91)\end{array}$ & $\begin{array}{l}0.47 \\
0.04 \\
0.03\end{array}$ \\
\hline
\end{tabular}

Aims \& Methods: In this cross-sectional study, our aims were to describe the local BO clinical, endoscopic and histologic profile in our tertiary referral centre, and discover whether the Prague classification reporting requirements are filled. We identified and included all consecutive patients with oesophageal intestinal metaplasia (identified by the presence of goblet cells) from March 2009 to May 2015. All endoscopies and biopsy reports were reviewed: BO segment length, use of the Prague classification, endoscopic abnormalities, treatment modalities, and histologic findings of dysplasia. Participants were sent a clinical questionnaire, via which pertinent clinical data including personal and familial cancer history, were collected.

Results: Clinical profile- Our cohort consists of 406 patients, with a mean age of $60.9 \pm 13.3$ years, $69 \%$ were male. Endoscopic profile- Mean maximal BO length (Prague classification $\mathrm{M}$ ) was $2.8 \pm 1.9 \mathrm{~cm}$ (reported in $49.6 \%$ of endoscopies) Mean circumferential BO (Prague classification C) was $4.9 \pm 3.1 \mathrm{~cm}$ (reported in $18.1 \%$ of endoscopies). Histologic profile- Low-grade dysplasia(LGD) was seen in $4.4 \%$ of patients, high-grade dysplasia (HGD) in $3 \%$, intramucosal carcinoma (IMC) in $0.7 \%$, and $\mathrm{OAC}$ in $2 \%$. A subgroup of 250 patients underwent more than one endoscopy, allowing for prospective incidence analysis. They had 914 years of follow-up, with a mean number of endoscopies of $4.7 \pm 3$. The incidence rates of LGD, HGD, IMC, OAC per 1000 patient years were 20.8, 15.3, 2.2, and 7.6 respectively.

One hundred and fifty-five patients returned the questionnaire. In this subgroup analysis, we learned BO was diagnosed at $57.8 \pm 12.5$ years of age, with a mean duration of $4.9 \pm 5.7$ years. A personal history of non-oesophageal malignancy was reported in $15.4 \%$. A family history of $\mathrm{BO}, \mathrm{OAC}$ and non-oesophageal cancer were reported in $5.2 \%, 4.5 \%$, and $35.4 \%$, respectively.

Conclusion: Compared to the information gathered by Katz et.al (1), we demonstrated a lower rate of LGD, but comparable rates of HGD and OAC. The personal and familial history of non-oesophageal malignancy was higher than the oesophageal malignancy rates. Our findings may support the importance of age appropriate non-oesophageal malignancy screening in BO patients. Physician compliance in reporting $\mathrm{BO}$ according to the Prague classification is lacking. Factors associated with our local BO profile as well as the implication of family history requires further prospective studies

Disclosure of Interest: All authors have declared no conflicts of interest.

\section{Reference}

1. Katz, D. et al. The development of dysplasia and adenocarcinoma during endoscopic surveillance of Barrett's esophagus. Am. J. Gastroenterol. 93, $536-541$

P0559 THE EFFICACY OF ENDOSCOPIC MUCOSAL RESECTION IN MANAGING EARLY NEOPLASIA IN BARRETT'S OESOPHAGUS, EXPERIENCES OF A TERTIARY REFERRAL CENTER IN THE UK

B. Eross ${ }^{1}$, C. Clisby $^{2}$, B. Foria ${ }^{3}$, C. Gordon ${ }^{1}$

${ }^{1}$ Gastroenterology, Royal Bournemouth Hospital, Bournemouth/United Kingdom

${ }^{2}$ Endoscopy, Royal Bournemouth Hospital, Bournemouth/United Kingdom

${ }^{3}$ Pathology, Royal Bournemouth Hospital, Bournemouth/United Kingdom

Contact E-mail Address: xerobal@yahoo.it

Introduction: Endoscopic mucosal resection (EMR) is an established diagnostic and treatment tool in the management of Barrett's oesophagus (BO) with early neoplasia. Our objectives were to demonstrate EMR's efficacy in removing early neoplasia within BO and its usefulness in obtaining comprehensive histological specimens to accurately stage the early neoplasia and effectively deliver therapy.

Aims \& Methods: We have conducted a retrospective analysis using our electronic database for endoscopic procedures for patients with BO, who underwent EMR from October 2010 to December 2016. We analysed the resection margins for both en bloc and piecemeal EMRs. We compared the original histology from the referral endoscopy to the histology obtained from the EMR, to analyse any deviation and to demonstrate the importance of histological staging of the early neoplasia in BO. We also investigated the three-year survival in patients, who have had their EMR longer than 3 years ago, along with causes of death. Results: A total of 99 patients underwent 134 EMR procedures and 259 EMRs, $84 \%$ were male, the mean age at first EMR was 71 years $(S D=8.2) .24$ patients underwent 2 EMR procedures, 2 patients underwent 3 EMR procedures and 2 patients underwent 4 EMR procedures. The median length of the circumferential and maximum extent of the $\mathrm{BO}$ segments were 3 and $4 \mathrm{~cm}$ respectively (interquartile range (IQR) 2-4). 44 patients underwent 60 en bloc resections. After histologic assessment of these EMR specimens, 34 (56.7\%) had clear deep and radial resection margins, $14(23.3 \%)$ showed at least low-grade dysplasia at the radial margin, $2(3.3 \%)$ at the deep margin and $8(13.4 \%)$ at both margins. 2 $(3.3 \%)$ were clear at the deep margin, but due to thermal damage the radial margins were indeterminate. Following the 60 EMRs there was no visible residual early neoplasia on the follow up endoscopy in 37 cases $(61.7 \%) .55$ patients underwent 74 piecemeal EMRs, of which $52(70.3 \%)$ had clear deep margins on histologic assessment and $38(51.4 \%)$ had no visible residual neoplasia on the follow up endoscopy. Pre EMR histology was available in 82 patients and it showed high-grade dysplasia (HGD) in 49 (59.8\%), mucosal adenocarcinoma in $24(29.2 \%)$ and low-grade dysplasia in 9 patients $(11 \%)$. However the EMR histology resulted in altered grading in $59(72 \%)$ patients, with $47(57 \%)$ upgraded and $12(14 \%)$ downgraded from the pre EMR histology and unchanged only in 23 patients $(28 \%)$. The EMR histologies in the 82 patients showed HGD in $16(19.5 \%)$ patients, intramucosal adenocarcinoma in 33 $(40.2 \%)$, adenocarcinoma with submucosal invasion in $20(24.4 \%)$ and LGD in $13(15.9 \%)$. The remaining 52 EMRs were performed for visible lesions within $\mathrm{BO}$ without pre EMR histology result, predominately for patients in the radiofrequency ablation program. The 3 year survival rate for 42 patients was $81 \%, 8$ patients died, 5 due to cardiac failure, 1 due to a PE and 2 due to advanced oesophageal adenocarcinoma.

Conclusion: In this moderately sized retrospective study EMR has been proven to be an effective tool in managing early neoplasia within BO. It not only allows the potential complete resection of the visible early neoplasia $(61.7 \%$ for en bloc and $51.4 \%$ for piecemeal EMR), but also gives a histological specimen with a more accurate grading of the neoplasia (upgraded in $57 \%$, downgraded in $14 \%$ and unchanged in only in $23 \%$ of all EMRs), both aspects culminating in more effective and precise patient treatment. In all patients managed and assessed by EMR we have seen $19 \%$ mortality of which only $4.8 \%$ was cancer related.

Disclosure of Interest: All authors have declared no conflicts of interest.

\section{P0560 ADHERENCE TO BARRETT'S ESOPHAGUS SURVEILLANCE GUIDELINES: A SYSTEMATIC REVIEW AND META-ANALYSIS}

C. A.m. Roumans ${ }^{1}$, E. W. Steyerberg ${ }^{2}$, D. Rizopoulos ${ }^{3}$, I. Lansdorp-Vogelaar ${ }^{4}$, M.C.w. Spaander ${ }^{5}$, M.J. Bruno ${ }^{5}$

${ }^{1}$ Department Of Gastroenterology \& Hepatology, Erasmus University Medical Center, Rotterdam/Netherlands

${ }^{2}$ Department Of Medical Statistics And Bioinformatics, Leiden University Medical Center, Leiden/Netherlands

${ }^{3}$ Department Of Biostatistics, Erasmus University Medical Center, Rotterdam/ Netherlands

${ }^{4}$ Department Of Public Health, Erasmus University Medical Center, Rotterdam Netherlands 
${ }^{5}$ Erasmus University Medical Center, Both authors contributed equally/ Netherlands

Contact E-mail Address: c.roumans@erasmusmc.nl

Introduction: Barrett esophagus (BE) is a premalignant condition for esophageal adenocarcinoma (EAC) where recommended. Guidelines aim to reduce treatment variation and improve quality of care. Data on the extent to which level BE surveillance guidelines are followed are scarce.

Aims \& Methods: The objectives of this systematic review and meta-analysis were to (1) quantify adherence to guidelines in BE surveillance and (2) identify factors that are associated with adherence. A systematic literature research was performed using EMBASE, MEDLINE, PubMed and Web of Science up to September 2016. Studies reporting adherence in at least one of the following four domains were selected: surveillance interval, biopsy protocol, landmark identification and histopathological information. Relevant publications were assessed using the STROBE statement for observational studies (http://strobestatement.org/). Adherence was considered a prevalence ratio and reported as a percentage. Pooled data for the associated factors were reported as odds ratios with analysis of heterogeneity $\left(\mathrm{I}^{2}\right.$ statistic).

Results: From a total of 373 studies, 49 were eligible for this meta-analysis. For $\mathrm{BE}$ surveillance in non-dysplastic BE patients a prevalence ratio for adherence of $54 \%(95 \%$ confidence interval (CI) $45 \% ; 63 \%)$ was found and $41 \%(95 \% \mathrm{CI}$ $37 \% ; 45 \%$ ) in patients with low-grade dysplasia, but with large heterogeneity $\left(\mathrm{I}^{2}=98 \%\right.$ and $\left.\mathrm{I}^{2}=100 \%\right)$. Adherence to the Seattle protocol was $47 \%(95 \%$ CI $33 \% ; 61 \%, \mathrm{I}^{2}=100 \%$ ), length of $\mathrm{BE}$ was reported according to the Prague classification in $34 \%\left(95 \%\right.$ CI $\left.23 \% ; 44 \%, \mathrm{I}^{2}=98 \%\right)$ and in $51 \%(95 \% \mathrm{Cl}$ $27 \% ; 75 \%, \mathrm{I}^{2}=99 \%$ ) of patients with a dysplastic BE the histology samples were reviewed by a second pathologist. Shorter BE length, an academic practice setting, younger age and the involvement of the clinical set-up incorporating a multifaceted intervention program were associated with better adherence. Endoscopists following the Seattle protocol detected more dysplasia.

Conclusion: Adherence to guidelines for the surveillance of BE is far from optima and highly heterogeneous. A clinical set-up of a dedicated BE surveillance program is needed, for example follow-up within a research program, involvement of a dedicated nurse managing the program or guideline promotion by using posters stating recommendations. Besides initiating more and better studies to facilitate the creation of evidence based guidelines, more effort should be directed to stimulate and monitor the implementation of guidelines in clinical practice. Disclosure of Interest: All authors have declared no conflicts of interest.

\section{P0561 LONG-TERM OUTCOMES OF ENDOSCOPIC RESECTION VERSUS SURGICAL RESECTION FOR MM-SM1 ESOPHAGUS SQUAMOUS CELL CARCINOMA}

K. Yamauchi ${ }^{1}$, M. Iwamuro ${ }^{2}$, Y. Obayashi ${ }^{2}$, Y. Baba ${ }^{2}$, H. Sakae ${ }^{2}$, T. Gotoda ${ }^{2}$, Y. Kouno ${ }^{2}$, H. Kanzaki ${ }^{2}$, S. Kawano ${ }^{2}$, Y. Kawahara ${ }^{2}$, H. Okada

${ }^{1}$ Departments Of Gastroenterology, Mitoyo general hospital, Kanonji/Japan

${ }^{2}$ Departments Of Gastroenterology And Hepatology, Okayama university hospital, Okayama/Japan

Contact E-mail Address: yamauchi.kenji@hotmail.com

Introduction: Since squamous cell carcinoma in the esophagus confined to the muscularis mucosae (MM) or submucosa up to $200 \mu \mathrm{m}$ (SM1) has a risk of lymph node metastasis, it is defined as relative indication for endoscopic submucosal dissection (ESD) by the Japan esophageal society guideline. Although additional surgical treatment after ESD is recommended, long-term outcomes of ESD compared with surgery has not been clarified.

Aims \& Methods: This study aimed to evaluate the long-term outcomes of ESD and surgery for $\mathrm{cN} 0 \mathrm{M} 0$ relative indication lesions of ESCC. Between 2006 and 2016, patients with relative indication lesions of ESCC treated endoscopically or surgically in Okayama University Hospital were retrospectively analyzed. We evaluated risk factors for mortality using cox regression analysis, adjusted hazard ratios for ESD compared with surgery, the survival curves stratified with risk factor, and perioperative comlication rate.

Results: 54 lesions in the ESD group and 51 lesions in the surgery group met the pathological criteria of relative indication for endoscopic resection. 10 patients underwent additional chemoradiation in the ESD group. 8 patients underwent additional chemotherapy and 1 patient underwent additional chemoradiation in the surgery group. Lymphovascular invasion, submucosal invation, and ASA-PS was significantly associated with mortality using Cox analysis. Adjusted for lymphovascular invation, submucosal invation, and ASA-PS, multivariate Cox proportional hazard ratio of mortality for ESD compared with surgery was not significantly dfferent (hazard ratio [HR], 0.76; 95\% confidence interval [CI], $0.26-2.2 ; \mathrm{P}=0.61)$. The survival curves for ESD and surgery stratified with each risk factor were not significantly different. Perioperative comlication rate were significantly low in ESD compared to surgey $(29.6 \%$ vs $49.1 \% ; \mathrm{P}=0.047)$. Conclusion: ESD dose not compromise the long-term outocome compared to surgery. Further large number randomized controlled trials are necessary to confirm these resluts.

Disclosure of Interest: All authors have declared no conflicts of interest.

\section{References}

Kuwano H, Nishimura Y, Oyama T, et al. Guidelines for Diagnosis and Treatment of Carcinoma of the Esophagus April 2012 edited by the Japan Esophageal Society. Esophagus 2015;12:1-30.
P0562 ESOPHAGEAL REFLUX DISEASE AND ESOPHAGEAL

SQUAMOUS CELL CANCER IN PATIENTS WITH FANCONI ANEMIA UNDERGOING ENDOSCOPIC SURVEILLANCE

D. Itskoviz ${ }^{1}$, H. Tamary ${ }^{2}$, T. Krasnov ${ }^{3}$, N. Sahar ${ }^{1}$, N. Zevit ${ }^{4}$, O. Ben-Bassat ${ }^{1}$, Y. Leibovici Wiseman ${ }^{1}$, D. Boltin ${ }^{1}$, Y. Goldberg ${ }^{5}$, Z. Levi ${ }^{1}$

${ }^{1}$ Gastroenterology, Rabin Medical Center, Petach Tiqva/Israel

${ }^{2}$ Pediatrics Hematology Unit, Schneider's Children Medical Center, Petach Tiqva/ Israel

${ }^{3}$ Felsenstein Medical Research Center Beilinson Campus, Petach Tiqva/Israel ${ }^{4}$ Institue Of Gastroenterology, Nutrition And Liver Disease, Schneider Children's Medical Center of Israel, Petach Tiqva/Israel

${ }^{5}$ Genetic Department, Rabin Medical Center, Petach-Tiqva, Israel, Petach Tiqval Israel

Contact E-mail Address: mditskov@gmail.com

Introduction: Patients with Fanconi anemia (FA) have an increased risk of developing esophageal squamous cell carcinoma. Data regarding endoscopic findings in patients with FA are lacking. Furthermore, there are no clear guidelines for endoscopic surveillance of patients with FA.

Aims \& Methods: We aimed to describe the endoscopic findings among subjects with FA undergoing endoscopic surveillance and to determine the interval to development of esophageal cancer.

Results: Eight FA subjects with a median age of 22.2 years at first endoscopy (range 16-41) were identified. The median upper endoscopies number per patient was 5.5 (range 2-14) with a median time of follow-up of 4.5 years (range 1-9 years). All subjects $(100 \%)$ had an endoscopic evidence of reflux esophagitis: 3 $(37.5 \%)$ had mild and $5(62.5 \%)$ had moderate-severe reflux esophagitis. Three subjects $(37.5 \%)$ had complicated esophageal reflux disease (two subjects developed Barrett's esophagus and one subject had an esophageal stricture). Two subjects $(25 \%)$ developed esophageal squamous cell carcinoma during followup, with interval time of 8 and 18 months from previous upper endoscopy. Both had tumor expression of p16 protein suggesting human papilloma virus (HPV) infection. The calculated standardized incidence ratio (SIR) for the development of esophageal squamous cell carcinoma is 5107 .

Conclusion: FA patients are at an increased risk for developing esophageal cancer and reflux esophageal disease with associated complications. Larger, prospective studies are needed to determine the optimal interval for endoscopic screening in these patients.

Disclosure of Interest: All authors have declared no conflicts of interest.

\section{References}

Mehta PA, Tolar J. GeneReviews: Fanconi Anemia. 2016 09.2016 [cited 2016 11.2016]; Available from: Smith AR, Wagner JE. Current clinical management of Fanconi anemia. Expert Review of Hematology 2012;5:513-522

Schneider M, Chandler K, Tischkowitz M, et al. Fanconi anaemia: genetics, molecular biology, and cancer - implications for clinical management in children and adults. Clin Genet 2015;88:13-24

\section{P0563 RISK FACTORS FOR THE DEVELOPMENT OF DYSPLASTIC} SQUAMOUS EPITHELIUM IN THE ESOPHAGUS

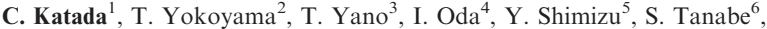
H. Doyama ${ }^{7}$, T. Koike ${ }^{8}$, K. Takizawa ${ }^{9}$, M. Hirao ${ }^{10}$, H. Okada $^{11}$, T. Yoshii ${ }^{12}$, K. Konishi ${ }^{13}$, T. Yamanouchi ${ }^{14}$, T. Tsuda ${ }^{15}$, T. Omori ${ }^{16}$, N. Kobayashi ${ }^{17}$, H. Ishikawa ${ }^{18}$, A. Yokoyama ${ }^{19}$, M. Muto ${ }^{20}$

${ }^{1}$ Kitasato University, Sagamihara/Japan

${ }^{2}$ National Institute of Public Health, Wako/Japan

${ }^{3}$ National Cancer Center Hospital East, Kashiwa/Japan

${ }^{4}$ National Cancer Center Hospital, Tokyo/Japan

${ }^{5}$ Hokkaido University, Sapporo/Japan

${ }^{6}$ Kitasato University School of Medicine, Sagamihara/Japan

${ }^{7}$ Ishikawa prefectural central hospital, Kanazawa/Japan

${ }^{8}$ Tohoku University, Sendai/Japan

${ }^{9}$ Shizuoka Cancer Center, Shizuoka/Japan

${ }^{10}$ Osaka National Hospital, Osaka/Japan

${ }^{11}$ Okayama University, Okayama/Japan

${ }^{12}$ Kanagawa Cancer Center, Kanagawa/Japan

${ }^{13}$ Showa University, Tokyo/Japan

${ }^{14}$ Kumamoto Regional Medical Center, Kumamoto/Japan

${ }^{15}$ St. Marianna University, Kawasaki/Japan

${ }^{16}$ Kawasaki Municipal Kawasaki Hospital, Kawasaki/Japan

${ }^{17}$ Tochigi Cancer Center, Utsunomiya/Japan

${ }^{18}$ Kyoto Prefectural University of Medicine, Kyoto/Japan

${ }^{19}$ Kurihama Medical and Addiction Center, Yokosuka/Japan

${ }^{20}$ Therapeutic Oncology, Kyoto University, Kyoto/Japan

Contact E-mail Address: ckatada@med.kitasato-u.ac.jp

Introduction: Multiple development of squamous cell carcinoma (SCC) in the upper aero-digestive tract is known as the "field cancerization phenomenon," and alcohol is a definite carcinogen. Multiple dysplastic lesions in the esophagus are a useful predictor of the risk for the field cancerization. However, what causes the development of dysplastic squamous epithelium in the esophagus is still unclear.

Aims \& Methods: The aim of this prospective cohort study was to identify associations between patients' lifestyle, including alcohol consumption, the genetic trait of aldehyde dehydrogenase-2 (ALDH2), and the development of dysplastic squamous epithelium in the esophagus. This is a post hoc analysis of the Japan Esophgeal Cohort (JEC) study (UMIN1676). Patients with superficial SCC 
treated by endoscopic resection were prospectively recruited from 16 hospitals throughout Japan. This cohort study was approved by the institutional review board at each hospital, and we obtained written informed consent from all patients. Using Lugol chromoendoscopy, we evaluated the dysplastic squamous epithelium in the esophagus. Lugol voiding lesion (LVL) was graded into 3 categories $(\mathrm{A}=$ no lesion; $\mathrm{B}=1$ to 9 lesions; $\mathrm{C}=>10$ lesions per endoscopic view). Endoscopic images obtained from eligible patients at study entry were centrally reviewed in a blinded fashion by three endoscopists to determine the grade of LVL. ALDH2 status was determined by questionnaire facial flushing after alcohol drinking (present and past flushing = inactive ALDH2, never flushing = active ALDH2). Lifestyle surveys were conducted using a self-assessment questionnaire. Data collected between July 2000 and Dec 2001 from a different cross-sectional cohort $(n=1042 ; M / F=610 / 432)$ were used as an historical control.

Results: Between Sep 2005 and May 2010, 330 patients $(\mathrm{M} / \mathrm{F}=278 / 52)$ were registered. The proportions of the different grades of LVL were $\mathrm{A}=50$ $(15.2 \%), \mathrm{B}=174(52.7 \%)$, and $\mathrm{C}=106(32.1 \%)$. After adjusting for sex and age, controls and the LVL grade was associated with progressively higher proportions of heavy drinkers $(8.4 \%, 24.8 \%, 26.2 \%$, and $52.5 \%$, respectively, $\mathrm{p}<0.0001)$, frequently strong alcoholic beverages $(2.3 \%, 7.2 \%, 11.8 \%$, and $11.6 \%$, respectively, $\mathrm{p}<0.0001)$, heavy smokers $(34.6 \%, 38.7 \%, 65.7 \%$, and $70.8 \%$, respectively, $\mathrm{p}<0.0001)$, liking high-temperature food $(4.6 \%, 19.6 \%$, $20.8 \%$, and $20.7 \%$, respectively, $\mathrm{p}<0.0001$ ), not eating green-yellow vegetables almost every day $(55.0 \%, 48.9 \%, 54.9 \%$, and $71.1 \%$, respectively, $\mathrm{p}<0.0001)$, and not eating fruit almost every day $(51.6 \%, 74.3 \%, 68.0 \%$, and $75.3 \%$, respectively, $\mathrm{p}<0.0001)$. The risk of LVL grade $\mathrm{B}$ and $\mathrm{C}$ was strongly associated with the amount of alcohol consumption especially in inactive ALDH2. Odds ratio (OR) of LVL grade B associated with heavy drinking was significantly stronger in inactive ALDH2 $(\mathrm{OR}=47.5)$ than active ALDH2 $(\mathrm{OR}=12.8)(\mathrm{p}<0.05)$. OR of LVL grade $\mathrm{C}$ associated with heavy drinking was significantly stronger in inactive ALDH2 $(\mathrm{OR}=358)$ than active ALDH2 $(\mathrm{OR}=138)(\mathrm{p}<0.05)$.

\begin{tabular}{llllll}
\hline Alcohol drinking & $\begin{array}{l}\text { Controls } \\
(\mathrm{n}=1042)\end{array}$ & $\begin{array}{l}\text { Grade A } \\
(\mathrm{n}=50)\end{array}$ & $\begin{array}{l}\text { Grade B } \\
(\mathrm{n}=174)\end{array}$ & $\begin{array}{l}\text { Grade C } \\
(\mathrm{n}=106)\end{array}$ & $\mathrm{P}$ \\
\hline Never/rare & $38.2 \%$ & $25.1 \%$ & $7.9 \%$ & $4.7 \%$ & \\
Light & $30.5 \%$ & $15.0 \%$ & $21.4 \%$ & $8.9 \%$ & \\
Moderate & $21.4 \%$ & $30.1 \%$ & $30.9 \%$ & $25.7 \%$ & \\
Heavy & $8.4 \%$ & $24.8 \%$ & $26.2 \%$ & $52.5 \%$ & \\
Ex-drinker & $1.4 \%$ & $5.1 \%$ & $13.6 \%$ & $8.3 \%$ & $<.0001$ \\
P, vs. controls & - & 0.002 & $<.0001$ & $<.0001$ & \\
Units/week (Mean $\pm \mathrm{SE})$ & $5.9 \pm 0.4$ & $9.2 \pm 1.5$ & $13.9 \pm 0.8$ & $20.1 \pm 1.0$ & $<.0001$ \\
P, vs. controls & - & 0.037 & $<.0001$ & $<.0001$ & \\
\hline
\end{tabular}

Conclusion: The development of dysplastic squamous epithelium in the esophagus was associated with the amount of alcohol consumption and genetic trait of inactive ALDH2.

Disclosure of Interest: All authors have declared no conflicts of interest

\section{Reference}

Katada C, et al. Alcohol consumption and multiple dysplastic lesions increase risk of squamous cell carcinoma in the esophagus, head, and neck. Gastroenterology 2016;151:860-9.

\section{P0564 EVALUATION OF THE RISK OF METACHRONOUS SQUAMOUS CELL CARCINOMA OF THE OESOPHAGUS AND THE HEAD AND NECK AFTER ENDOSCOPIC RESECTION FOR SQUAMOUS CELL CARCINOMA OF THE ESOPHAGUS BASED ON THE GENETIC POLYMORPHISMS OF ADH1B AND ALDH2}

S. Abiko, Y. Shimizu, T. Mizushima, M. Kato, K. Matsuda, S. Miyamoto, M. Tsuda, K. Yamamoto, S. Ono, T. Kudo, N. Sakamoto

Department Of Gastroenterology And Hepatology, Hokkaido University Graduate School of Medicine, sapporo/Japan

\section{Contact E-mail Address: abiko1982@gmail.com}

Introduction: Metachronous multiple squamous cell carcinoma (SCC) of the oesophagus and the head and neck often occurs in patients who previously underwent endoscopic resection (ER) for SCC of the oesophagus. This has become a problem regarding the curability of ER. Katada et al reported that alcohol abstinence significantly decreased the risk of developing a secondary SCC of the oesophagus, based on a prospective study of 330 patients from 16 hospitals ${ }^{1}$. However, there are few studies that have investigated the risk of developing a secondary SCC of the oesophagus and the head and neck based on the genetic polymorphisms of alcohol dehydrogense-1B (ADH1B) and aldehyde dehydrogenase-2 (ALDH2) which are closely associated with developing oesophageal SCC. No studies have evaluated the risk of developing a third (or more) SCC after ER for SCC of the oesophagus.

Aims \& Methods: The study group included patients who underwent ER for SCC of the oesophagus at Hokkaido University Hospital. All patients were followed up by using endoscopic examination for $\geq 2$ years. Overall, 126 patients were included in the study. The drinking and smoking histories before and after ER were carefully documented. To examine two single nucleotide polymorphisms
(SNPs) on ADH1B and ALDH2 genotyping, we obtained approximately $1 \mathrm{ml}$ of saliva with a pipette or cotton swab before the endoscopic examination. The subjects were classified as rare drinkers who consumed $<1$ units/week, current drinkers who consumed 1 to 8.9 units/week (light drinkers), 9 to 17.9 units/week (moderate drinkers), or $\geq 18$ units/week (heavy drinkers); alcohol consumption ( 1 unit $=22 \mathrm{~g}$, the ethanol content of one serving of sake). The physicians recommended all subjects to temperate in drinking and smoking. We retrospectively evaluated the risk of metachronous SCC of the oesophagus and the head and neck after ER for SCC of the oesophagus, based on the genetic polymorphisms for ADH1B and ALDH2 and the drinking and smoking histories.

Results: During a median follow-up period of 80 months (range, 24-228 months), a secondary SCC of the oesophagus and the head and neck was detected in 46 patients $(36.5 \%)$. The high incidence groups had inactive heterozygous ALDH2 (rs671 GA, 36/83; p < 0.05), moderate and heavy drinkers (42/93; $\mathrm{p}<0.01)$, continuous moderate and heavy drinkers after ER $(21 / 35 ; \mathrm{p}<0.01)$. Multivariate analysis revealed that the inactive heterozygous ALDH2 $(\mathrm{OR}=2.24$ $\mathrm{p}<0.05)$, moderate and heavy drinkers $(\mathrm{OR}=3.54 ; \mathrm{p}<0.05)$ and continuous moderate and heavy drinkers after $\mathrm{ER}(\mathrm{OR}=2.73 ; \mathrm{p}<0.05)$ were independently associated with the risk of developing a secondary SCC after ER. A third SCC was detected in 19 patients $(15.1 \%)$, and the high incidence groups had inactive heterozygous ALDH2 (17/83; p < 0.05), moderate and heavy drinkers $(19 / 93$; $\mathrm{p}<0.01)$ and continuous heavy drinkers after ER $(10 / 16 ; \mathrm{p}<0.01)$. A fourth SCC was detected in seven patients $(5.6 \%)$, who were all cases of continuous moderate and heavy drinkers after ER. Six of the seven patients had an inactive heterozygous ALDH2. We analysed the 63 patients with inactive heterozygous ALDH 2 and moderate and heavy drinkers before ER based on their temperance history and found 38 patients in the temperance group (ฏlight drinkers after ER) and 25 patients in the non-temperance group. The 3-year cumulative incidence rate of secondary SCC in the temperance and non- temperance groups revealed an incidence of $16.2 \%$ vs $49.8 \%$, respectively $(\mathrm{p}<0.05)$. The 5 -year cumulative incidence rate of a third SCC revealed an incidence of $0 \%$ vs $28.3 \%$, respectively $(\mathrm{p}<0.01)$. The 7 -year cumulative incidence rate of a fourth SCC revealed an incidence of $0 \%$ vs $16.9 \%$, respectively $(\mathrm{p}<0.05)$.

Conclusion: Among the patients who underwent ER for oesophageal SCC, an inactive heterozygous ALDH2 with a continue drinking habit were the significant risk factors of developing metachronous multiple SCC. These are the greater risk factors for developing a third (or more) SCC. Patients with an inactive heterozygous ALDH2 and a drinking habit should receive strict instruction for temperance.

Disclosure of Interest: All authors have declared no conflicts of interest.

\section{Reference}

1. Katada C, Yokoyama T, Yano T, et al. Alcohol Consumption and Multiple Dysplastic Lesions Increase Risk of Squamous Cell Carcinoma in the Esophagus, Head, and Neck. Gastroenterology 2016.

\section{P0565 IS IT FEASIBLE TO TREAT ESOPHAGEAL SQUAMOUS CELL CARCINOMA WITH CLINICAL DEPTH OF MM/SM1 BY ENDOSCOPIC RESECTION?}

K. Namikawa, T. Yoshio, A. Ishiyama, T. Tsuchida, J. Fujisaki Gastroenterology, Cancer Institute Hospital Ariake, Tokyo/Japan

Contact E-mail Address: ken.namikawa@jfcr.or.jp

Introduction: According to the Japanese guidelines for the treatment of esophageal cancer, T1a-EP or T1a-LPM squamous cell carcinoma (SCC) is definitive indication for endoscopic resection (ER) which is the first line treatment for them. T1a-MM or T1b-SM1 is a relative indication of ER, because $10-20 \%$ of the cases has metastasis in the previous analysis of operation cases. Recent advances in ER such as endoscopic submucosal dissection (ESD) and steroid injection for the prevention of stricture, provide us increasing opportunity to treat clinical $\mathrm{MM} / \mathrm{SM} 1$ which means preoperative predicted invasion depth of $\mathrm{MM} / \mathrm{SM} 1$. Recently we start to treat them because MM without lymphovascular invasion were reported to have very low risk of metastasis and occupy majority part of MM/SM1. There is no report about long term outcome of ER for clinical $\mathrm{MM} / \mathrm{SM} 1$.

Aims \& Methods: This study aimed to evaluate the clinical outcomes in patients having esophageal SCC with a predicted invasion depth of MM/SM1. We retrospectively reviewed 45 patients having esophageal SCC with a predicted invasion depth of MM/SM1. We predicted the invasion depth using conventional endoscopy, magnifying endoscopic classification of Japan esophageal society, and endoscopic ultrasound (EUS). The patients were diagnosed and treated at our hospital from 2010 to 2013. If pathological diagnosis after ER reveals T1b-SM2 or lymphovascular invasion, we recommended CRT or esophagectomy as additional therapy.

Results: The median age was 67 years (range, 39-81 years), including 37 males and 8 females. The median diameter of the tumor was $27 \mathrm{~mm}$ (range, $4-70 \mathrm{~mm}$ ). The tumors were located in the cervical esophagus $(n=2)$, upper esophagus $(n=9)$, mid-esophagus $(n=30)$, and lower esophagus $(n=4)$. The number of tumors subclassified as types 0 -I, 0 -IIa, 0 -IIb, and 0 -IIc was $0,7,1$, and 37, respectively. Forty cases were diagnosed as MM/SM1 using the Japanese Esophageal Society classification for magnifying endoscopy, and its accuracy was $62.5 \%(25 / 40)$. EUS was performed for 21 patients, and its accuracy was $68.8 \%(16 / 21)$. The patients underwent endoscopic mucosal resection $(\mathrm{n}=5)$ or $\operatorname{ESD}(n=40)$. In pathological diagnosis, LPM invasion was found in 14 patients, $\mathrm{MM} / \mathrm{SM} 1$ invasion in 28 patients, and SM2 invasion in 3 patients. The overall accuracy rate of diagnosing MM/SM1 invasion was $62 \%(28 / 45)$. Among patients with $\mathrm{MM} / \mathrm{SM} 1$ invasion, 20 had no lymphovascular invasion, and 
they were not recommended additional therapy. The remaining eight patients had lymphovascular invasion, and they were recommended additional therapy. In total 33 cases were treated only by ER without additional therapy. Besides $97 \%(32 / 33)$ of those cases had no recurrence except for one case with a lymph node recurrence, which was successfully treated by additional CRT. The other 12 cases including cases with lymphovascular invasion or SM2 were recommended additional therapy. They underwent CRT $(n=4)$, radiotherapy $(n=3)$, or esophagectomy $(n=3)$. No recurrence was observed after the abovementioned treatments. At the end of the follow-up, the 3-year overall and disease-specific survival rates were $91.1 \%$ and $100 \%$, respectively. Considering ER for clinical $\mathrm{MM} / \mathrm{SM} 1,76 \%$ of all cases were completed their treatment only with ER, and $24 \%$ of high risk case for metastasis were treated appropriately with additional therapy.

Conclusion: Conclusions: Our study suggests that ER is a valid treatment for esophageal SCC with a preoperative predicted invasion depth of MM/SM1.

Disclosure of Interest: All authors have declared no conflicts of interest.

\section{References}

1. Japan Esophageal Society. Japanese classification of esophageal cancer. 10th English edn. Tokyo: Kanehara \& Co Ltd; 2008.

2. Araki K, Ohno S, Egashira A, et al. Pathologic features of superficial esophageal squamous cell carcinoma with lymph node and distal metastasis. Cancer. 2002;94:570-5.

3. Oyama T, Inoue H, Arima M, et al. Prediction of the invasion depth of superficial squamous cell carcinoma based on microvessel morphology: magnifying endoscopic classification of the Japan Esophageal Society. Esophagus. 2017;14:105-112.

\section{P0566 TRIAMCINOLONE INJECTION AND SHIELDING WITH POLYGLYCOLIC ACID SHEETS AND FIBRIN GLUE FOR THE PREVENTION OF POSTOPERATIVE STRICTURE AFTER ESOPHAGEAL ENDOSCOPIC SUBMUCOSAL DISSECTION: A RETROSPECTIVE COMPARISON OF TWO PILOT STUDIES AND A HISTORICAL COHORT}

Y. Sakaguchi, Y. Tsuji, Y. Kataoka, I. Saito, K. Niimi, S. Ono, S. Kodashima, M. Fujishiro, K. Koike

Dept. Of Gastroenterology, University of Tokyo, Tokyo/Japan

\section{Contact E-mail Address: sakaguchi-tky@umin.ac.jp}

Introduction: Triamcinolone injection is an effective and widely used method for preventing postoperative stricture after wide-spreading esophageal endoscopic submucosal dissection (ESD), but even with this method there is a stricture risk of $10-62 \%$, and there is a need for an even more effective method. The objective of our study was to evaluate the efficacy of combining this method with the shielding method with polyglycolic acid (PGA) sheets and fibrin glue, another novel and effective method for the prevention of postoperative stricture. Aims \& Methods: 2 consecutive single-arm pilot studies were performed, and retrospectively compared with the results of a historical control group. After approval by the Institutional Review Board and trial registry, we enrolled patients with a diagnosis of superficial esophageal squamous cell carcinoma covering over half the circumference of the esophagus. In study group A (UMIN000014642), performed October 2014 to July 2015, immediately after the enrolled patients underwent esophageal ESD, a total of $40 \mathrm{mg}$ of triamcinolone acetonide was injected into the submucosal layer of the ESD defect, followed by adhesion of a PGA sheet to the post-ESD defect with fibrin glue. In study group B (UMIN000011058), performed July 2013 to September 2014, immediately after the enrolled patients underwent esophageal ESD, only adhesion of a PGA sheet to the post-ESD defect with fibrin glue was performed. As historical control group C, all patients at our institute who had undergone ESD for superficial esophageal squamous cell carcinoma covering over half the circumference of the esophagus during 2002 to June 2013 were extracted. After exclusion of patients who did not undergo followed-up for at least 3 months, and patients who underwent salvage surgery after non-curative ESD, statistical analysis of the incidence of postoperative stricture and required endoscopic balloon dilation (EBD) sessions in each of these 3 groups was performed.

Results: During the study period, 15 patients were enrolled in study A, 13 patients in study $\mathrm{B}$, and 37 patients were extracted as group C. After exclusion, 11 cases in group A ( 2 circumferential, 9 semi-circumferential), 11 cases in group B (11 semicircumferential), and 30 cases in group C (3 circumferential, 27 semi-circumferential) were analyzed. Although postoperative stricture occurred in all circumferential cases in group $\mathrm{C}$, it was successfully prevented in 1 case of circumferential ESD in group A. A sub-analysis comparing the semi-circumferential ESD cases was performed. There were no significant differences in tumor size (A: $34.9 \pm 8.1$, $\mathrm{B}: 31.5 \pm 7.0, \mathrm{C}: 34.8 \pm 13.8 \mathrm{~mm}$ ) and operation times (A: $95.4 \pm 23.8, \mathrm{~B}$ : $108.2 \pm 33.1, \mathrm{C}: 112.1 \pm 50.3 \mathrm{~min})$ in the 3 groups. Group A demonstrated the most effective results in both the incidence of postoperative stricture (A: $11.1 \%$, $\mathrm{B}: 36.4 \%$, C: $55.6 \%$ ), and the number of EBD sessions required (median A: 0, B: 0, C: 3 )

Conclusion: The combination of triamcinolone injection and the shielding method with PGA sheets and fibrin glue is a promising method for preventing stricture after esophageal ESD. Prospective studies for confirmation of efficacy and safety are required.

Disclosure of Interest: All authors have declared no conflicts of interest.
P0567 MULTICENTRIC ASSESSMENT OF THE ENDOSCOPIC MANAGEMENT OF SUPERFICIAL ESOPHAGEAL SQUAMOUSCELL CARCINOMA IN WESTERN POPULATION

A. Berger ${ }^{1}$, G. Rahmi ${ }^{2}$, G. Perrod ${ }^{3}$, M. Pioche ${ }^{4}$, J. Canard $^{5}$, E. Cesbron

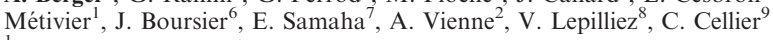
${ }^{1} \mathrm{CHU}$ Angers, Angers/France

${ }^{2}$ Gastroenterology And Digestive Endoscopy, Georges-Pompidou European Hospital, Paris/France

${ }^{3}$ Gastroenterology, Hopital Européen Georges Pompidou, Paris/France ${ }^{4}$ Gastroenterology And Endoscopy, Hospices civils de Lyon, Lyon/France ${ }^{5}$ Trocadero Clinic, Hopital Euopeen Georges Pompidou, Paris/France ${ }^{6}$ Service D'hépato-gastroentérologie, Centre Hospitalier Universitaire, Angers/ France

${ }^{7}$ HEGP, Paris/France

${ }^{8}$ Hopital prive Jean Mermoz, Lyon/France

${ }^{9}$ Gastroenterology, European Georges Pompidou Hospital Dept. of Gastroenterology, Paris/France

Contact E-mail Address: arthur.berger.bx@gmail.com

Introduction: Endoscopic mucosal resection (EMR) and endosopic submucosal dissection (ESD) are the first line treatment for superficial esophageal squamouscell carcinoma (SCC). Comparatively to surgery, endoscopic resection is mini invasive and associated with a lower morbidity and mortality.

Aims \& Methods: Evaluation of the endoscopic resection efficiency for superficial esophageal SCC and long-term outcome. Primary outcome was recurrence rate after endoscopic resection defined as local recurrence or metastatic evolution. We conducted a retrospective multicenter study in 5 french tertiary care hospitals. All patients treated by EMR or ESD for histologically proven SCC were consecutively included. Esophageal SCC were defined as superficial after macroscopic evaluation including Lugol staining and endoscopic ultrasonography (EUS). Curative resection was defined as pTla with free resection margins, without lympho-vascular embols.

Results: Between 1998 to 2016, 132 patients were enrolled and 148 tumors were resected $(E M R=80, E S D=68)$. The mean age was $63.9[35.7-86.0]$ years-old and $108(73 \%)$ patients were male. Mean tumor size was $15.0 \mathrm{~mm}$ in the EMR group and $35.0 \mathrm{~mm}$ in the ESD group $(\mathrm{p}<0.001)$. The complete resection rate in the EMR and ESD groups were respectively $30 \%(24 / 80)$ and $100 \%(68 / 68)$ $(\mathrm{p}<0.0001)$. The mean follow-up period was 22 months. The recurrence rate was $14.2 \%(19 / 80$ in the EMR group and $2 / 68$ in the ESD group, $p=0.001)$. At 12 months, recurrence-free survival rate was $84.4 \%$ and $74.6 \%$ at 24 months. Factors associated with recurrence in univariate analysis were: tumors size $(\mathrm{p}=0.013)$, resection by EMR $(\mathrm{p}=0.001)$, piecemeal resection $(\mathrm{p}=0.016)$, and microscopic positive margins $(\mathrm{p}=0.044)$. In multivariate analysis, risks factors for recurrence were: resection by EMR $(\mathrm{OR}=7.315$; IC $[1.685-31.762] ; p=0.008)$ and tumor deep infiltration $\geq \mathrm{m} 3(\mathrm{OR}=2.635$; IC [1.065-6.519]; $\mathrm{p}=0,036)$. At 24 months, recurrence-free survival rate were $95.2 \%$ in ESD group, versus $59.8 \%$ in EMR group $(p=0.001)$. For infiltrating tumors $>m 3$, metastasis free survival rate at 24 motnhs were $100.0 \%$ after complementary treatment by radiochemotherapy, and $62.2 \%$ without complementary treatment $(\mathrm{p}=0.042)$

Conclusion: Endoscopic resection of superficial esophageal SCC is safe and efficient. According to our results, ESD should be preferred to EMR because it is associated with a higher curative rate and an increased recurrence free survival rate.

Disclosure of Interest: All authors have declared no conflicts of interest.

P0568 CURATIVE CONDITIONS AFTER ENDOSCOPIC RESECTION FOR MM/SM1 OESOPHAGEAL SQUAMOUS CELL CARCINOMA BASED ON LONG-TERM OUTCOMES

T. Mizumoto ${ }^{1}$, S. Oka ${ }^{2}$, S. Tanaka ${ }^{3}$, T. Hiyama ${ }^{4}$, K. Kuroki ${ }^{2}$, M. Kurihara ${ }^{2}$, Y. Yoshihuku ${ }^{2}$, Y. Sanomura ${ }^{3}$, Y. Urabe ${ }^{2}$, K. Chayama ${ }^{2}$

${ }^{1}$ Dept. Of Endoscopy, Hiroshima University Hospital, Hiroshima/Japan ${ }^{2}$ Department Of Gastroenterology And Metabolism, Hiroshima University Hospital, Hiroshima/Japan

${ }^{3}$ Dept. Of Endoscopy, Hiroshima University Dept. of Endoscopy, Hiroshima/Japan ${ }^{4}$ Health Service Center, Hiroshima University, Higashihiroshima/Japan

Contact E-mail Address: mizumoto@hiroshima-u.ac.jp

Introduction: Oesophageal squamous cell carcinoma (ESCC) with invasion into the muscularis mucosae (MM) or submucosae up to 200- $\mu$ m (SM1) has approximately $10 \%$ lymph node metastasis and is a relative indication for endoscopic resection (ER) as per the Japanese Esophageal Society (JES) guidelines. The consideration criteria for additional treatment of MM/SM1 ESCC are as follows: (1) lymphovascular invasion, (2) SM1, (3) positive vertical margin, and (4) diffuse pattern of infiltration (INFc). However, the clinical validity of the JES guidelines has not been established. We evaluated the curative conditions after ER for MM SM1 ESCC based on long-term outcomes.

Aims \& Methods: We recruited 98 patients with MM/SM1 ESCC who underwent ER between August 1992 and October 2013 and were followed up for more than 3 years at Hiroshima University Hospital. As per the JES guidelines, the e-curable group was characterised by en bloc resection lesions with pathological MM, tumor infiltration pattern (INF) a/b, VM0, ly0 and v0. We evaluated the clinicopathological characteristics of patients and lesions and the rates of overall survival, disease-specific survival, recurrence-free survival, lymph node recurrence and local recurrence in the e-curable and non-e-curable groups.

Results: We enrolled 98 consecutive MM/SM1 ESCC patients ( 88 males; mean age, $67 \pm 9$ years; e-curable group, 39 patients; non-e-curable group, 59 patients; mean follow-up period, $75 \pm 44$ months). There were no significant differences in the clinicopathological characteristics of the patients and lesions between the 2 
groups. The proportion of patients with additional treatment after ER was significantly higher in the non-e-curable group $(66 \%, 39 / 59)$ than in the e-curable group $(23 \%, 9 / 39)(p<0.05)$. Operation, radiotherapy, and chemoradiotherapy were administered to $3(8 \%), 4(10 \%)$, and $12(5 \%)$ patients, respectively in the ecurable group and to $7(12 \%), 22(37 \%)$, and $10(17 \%)$ patients, respectively in the non-e-curable group. The 5-year overall survival rates in the e-curable and non-e-curable groups were $97 \%$ and $75 \%(p<0.05)$, respectively. The overall survival rate was significantly higher in the e-curable group. Three deaths $(10 \%)$ occurred due to primary cancer. The other reasons were as follows: other organ cancer, 10 cases; heart failure, 4 cases; pneumonia, 3 cases; and others, 11 cases. The 5-year disease-specific survival rates in the e-curable and non-e-curable groups were $100 \%$ and $98 \%$, respectively. The lymph node recurrence rates in the e-curable and non-e-curable groups were 3\% (1/39) and 7\% (4/59), respectively. The local recurrence rates in the e-curable and non-e-curable groups were $0 \%(0 / 39)$ and $7 \%(4 / 59)$, respectively. The 5 -year recurrence-free survival rates in the e-curable and non-e-curable groups were $97 \%$ and $98 \%$, respectively. The 5 -year recurrence-free survival rates in the group with INFa and no lymphovascular invasion and in the group with INFb, INFc, or lymphovascular invasion were $100 \%$ and $87 \%$, respectively. The recurrence-free survival rate was significantly higher in the group with INFa and no lymphovascular invasion than in the group with INFb, INFc or lymphovascular invasion.

Conclusion: Our outcome data support the clinical validity of the e-curative conditions after ER for MM/SM1 ESCC of the JES guidelines. However, $\mathrm{MM} / \mathrm{SM} 1$ ESCC with INFa and no lymphovascular invasion may have more possible curative conditions after ER without additional treatment.

Disclosure of Interest: All authors have declared no conflicts of interest.

\section{P0569 ENDOSCOPIC SUBMUCOSAL DISSECTION COMPARED TO LAPAROSCOPIC GASTRECTOMY FOR TREATMENT OF EARLY GASTRIC CANCER - A PROSPECTIVE RANDOMIZED TRIAL}

P.W.Y. Chiu ${ }^{1}$, A. Y. Teoh ${ }^{1}$, E. $\mathrm{Ng}^{1}$, V. Wong ${ }^{1}$, H.C. Yip ${ }^{1}$, J. C. Wu ${ }^{1}$, S. Liu ${ }^{1}$, S. K. Wong ${ }^{1}$, F.K.L. Chan ${ }^{2}$, J. Jy Sung ${ }^{3}$, J.Y. Lau ${ }^{1}$

${ }^{1}$ Department Of Surgery, Chinese University of Hong Kong, Hong Kong/Hong Kong PRC

${ }^{2}$ Medicine And Therapeutics, Institute of Digestive Disease, The Chinese University of Hong Kong, Hong Kong/Hong Kong PRC

${ }^{3}$ Institute Of Digestive Disease, The Chinese University of Hong Kong, Hong Kong/Hong Kong PRC

Contact E-mail Address: philipchiu@surgery.cuhk.edu.hk

Introduction: The development of endoscopic submucosal dissection (ESD) allows en-bloc resection of early gastric cancer (EGC) with wide resection margins $[1,2]$. En-bloc resection resulted in adequate resection margins and reduced local recurrence. When compared to gastrectomy, endoscopic resection should theoretically result in better perioperative outcomes and quality of life [3]. There is currently no study in the literature which directly compares laparoscopic assisted gastrectomy against ESD for treatment of intramucosal EGC. The objective of the study is to compare clinical, oncological and immunological outcomes of endoscopic submucosal dissection (ESD) against laparoscopic assisted gastrectomy (LAG) for treatment of early gastric cancer.

Aims \& Methods: All patients with endoscopic diagnosis of early gastric cancer (EGC) and biopsy confirmed to be high grade dysplasia or adenocarcinoma were recruited. They received staging investigations including image enhanced endoscopy, EUS and CT and those predicted to be Tla (intramucosal) neoplasia were randomly assigned to receive ESD or LAG. ESD were performed according to the previously reported procedure, while LAG were performed with D1 + B lymphadenectomy. The baseline demographics, clinical perioperative outcomes, immunological and oncological outcomes were compared between the two groups. Primary outcome was rate of complication after operation.

Results: From 2011 to 2016,36 patients with early gastric cancers were randomly assigned to receive $\operatorname{ESD}(n=18)$ or $\operatorname{LAG}(n=18)$. There was no difference between the two groups in terms of age, gender, ASA grade and baseline demographics (Table 1). ESD was associated with significantly shorter operative time $(109.4 \pm 55.4$ vs $266.2 \pm 47.8$ mins, $p<0.001)$, hospital stay (4 (3-6) vs $8(4-14)$ days; $\mathrm{p}<0.001)$ and lower complication rate $(1(5.6 \%)$ vs $7(38.9 \%) ; \mathrm{p}=0.041)$ There was no mortality at 30 days for the two groups, while those in ESD group tolerated full diet earlier $(2(1-5)$ vs $5(3-12)$ days; $p<0.001)$. Patients who received ESD had significantly lower level of CRP as well as VAS pain scores on postoperative days 1, 2, 3 and 7 when compared to LAG. The median followup was 41.5 months for ESD group and 36 months for LAG group, and there was no difference in the cancer recurrence and overall survival. $27.8 \%$ of patients required re-intervention after ESD

Table 1: - Outcomes of ESD vs Lap Gastrectomy

\begin{tabular}{llll}
\hline Parameters & Lap Gastrectomy & ESD & p value \\
\hline Male (\%) & $7(38.9)$ & $11(61.1)$. & 0.317 \\
Age (mean +/- SD) & $62.5+/-10.4$ & $61.7+/-11.2$ & 0.899 \\
ASA (I/II/III) & $5 / 8 / 5$ & $5 / 8 / 5$ & 1.0 \\
Comorbidities (Median) & $2(0-5)$ & $1(0-4)$ & 0.505 \\
Smoker (No/Ex/Current) & $10 / 3 / 3$ & $11 / 3 / 4$ & 0.534 \\
\hline
\end{tabular}

Table 1 Continued

\begin{tabular}{llll}
\hline Parameters & Lap Gastrectomy & ESD & p value \\
\hline $\begin{array}{l}\text { Drinker (No/Social/Heavy) } \\
\text { Operation time (mins) }\end{array}$ & $12 / 1 / 3$ & $14 / 2 / 2$ & 0.480 \\
$\quad$ (Median(range) & $263.5(165-365)$ & $97.5(30-195)$ & $<0.001 \dagger$ \\
$\begin{array}{l}\text { Hospital stay (days) } \\
\quad \text { Median(range) }\end{array}$ & $8(4-14)$ & $4(3-6)$ & $<0.001 \dagger$ \\
$\begin{array}{l}\text { Days to resume full diet } \\
\text { Complications (\%) }\end{array}$ & $5(3-12)$ & $2(1-5)$ & $<0.001 \dagger$ \\
30 days mortality & $7(38.9)$ & $1(5.6)$ & $0.041 \dagger$ \\
Reintervention & 0 & 0 & - \\
$\quad$ (Gastrectomy/ESD/ & $0(0)$ & $5(27.8)$ & $<0.001 \dagger$ \\
$\quad$ Reoperation)(\%) & & & \\
Postop Day 1 VAS pain & $49(8-80)$ & $23(0-57)$ & $0.013 \dagger$ \\
Postop Day 3 VAS pain & $39.5(6-65)$ & $5(0-37)$ & $<0.001 \dagger$ \\
Postop Day 7 VAS pain & $21(0-53)$ & $0(0-10)$ & $0.003 \dagger$ \\
Postop CRP Day 1 & $81.1(54-249)$ & $11.7(2-46)$ & $<0.001 \dagger$ \\
Postop CRP Day 3 & $110.3(39.1-229.4)$ & $22.8(1.4-60.0)$ & $<0.001 \dagger$ \\
Pathology T1a & 12 & 14 & \\
Pathology T1b & 3 & 3 & \\
Pathology T2 & 0 & 1 & \\
Pathology T3 & 3 & 0 & \\
\hline
\end{tabular}

Conclusion: Our prospective randomized study showed that patients treated by ESD had significantly lower complication rate and better perioperative outcomes when compared laparoscopic gastrectomy. ESD should be the first line treatment for intramucosal early gastric cancers.

Disclosure of Interest: P.W.Y. Chiu: I serve as chairman of Asia Novel BioImaging \& Intervention Group which received sponsorship from Olympus Co Ltd

All other authors have declared no conflicts of interest.

\section{References}

1. Chiu PW, Chan KF, Lee YT, Sung JJ, Lau JY, Ng EK. Endoscopic submucosal dissection used for treating early neoplasia of the foregut using a combination of knives. Surg Endosc. 2008 Mar;22(3):777-83.

2. Chiu PW. Novel endoscopic therapeutics for early gastric cancer. Clin Gastroenterol Hepatol. 2014 Jan;12(1):120-5.

3. Chiu PW, Teoh AY, To KF, Wong SK, Liu SY, Lam CC, Yung MY, Chan FK, Lau JY, Ng EK. Endoscopic submucosal dissection (ESD) compared with gastrectomy for treatment of early gastric neoplasia: a retrospective cohort study. Surg Endosc. 2012 Dec;26(12):3584-91.

\section{P0570 PREVALENCE OF PRE-MALIGNANT LESIONS IN BIOPSIES TAKEN FROM GASTRIC MUCOSA ENDOSCOPICALLY NORMAL OR WITH GASTRITIS}

V. Yep-Gamarra ${ }^{1}$, P. Rojas-Perez ${ }^{1}$, A. Aldave ${ }^{1}$, C. Rodriguez-Ulloa ${ }^{2}$, C. DíazVeléz $^{3}$, H. Mariños ${ }^{1}$, H. Calvo ${ }^{1}$, J. Pelaez ${ }^{1}$, G. Flores-Trujillo ${ }^{4}$

${ }^{1}$ Gastroenterologia, Hospital Regional de Trujillo, Trujillo/Peru

${ }^{2}$ Gastroenterologia, Hospital Central de la Fuerza Aérea del Perú, Lima/Peru

${ }^{3}$ Gastroenterologia, Hospital Nacional Almanzor Aguinaga-Essalud, Chiclayo/

Peru

${ }^{4}$ Instituto Regional de Enfermedades Neoplasicas, Trujillo/Peru

Contact E-mail Address: vyg77@yahoo.com

Introduction: Pre-malignant conditions and lesions of the stomach (PCLS): atrophy, intestinal metaplasia and dysplasia are risk factors for the development of stomach cancer; therefore, its diagnosis is very important to identify patients with greater probability of this malignant neoplasm. The Clinical Guidelines recommend that during endoscopy procedure, to avoid an under diagnosis, biopsies of different areas of the stomach should be taken even when no lesion is evident in order to identify PCLS that are generally multi-focal. The initial identification of patients with this type of lesions and their subsequent stratification with the OLGA and OLGUIM systems allows defining the subgroup of patients that merit follow-up because they have a higher risk of developing gastric cancer. However, there is a discrepancy between endoscopists, because it is now preferred to take biopsies directed at the lesions and not to do them systematically at fixed sites, so that no lesion is observed.

Aims \& Methods: We aimed to evaluate and compare the prevalence of PCLS in biopsies taken from gastric mucosa with or without lesion during the endoscopic examination. A retrospective, cross-sectional study was performed on 356 dyspeptic patients. We reviewed the reports of esophagogastroduodenoscopies at the Trujillo Regional Teaching Hospital-Perú from October 2016 to March 2017. This study included reports which were consigned diagnosis with biopsies of different areas from the stomach. These biopsies were sent in different vials. Permission was obtained from the Hospital's research committee. Those reports that had a diagnosis of stomach cancer, gastrectomy, and those with not biopsy 
or those in which the biopsies were still taken from the different anatomical areas were sent in a single vial were excluded.

Results: Only 148 patients were admitted to the study. The mean age was $49.7+/$ -3 years, $60 \%$ were female (CI: $52-67,95 \%$ ). 264 vials were sent with biopsies of the different areas of the stomach distributed as follows: 148 of antrum, 54 of angle and 62 of body. Only $48(32.4 \%)$ patients had biopsies of antrum, angle and body. From 148 patients, $116(78.4 \%)$ had an endoscopic diagnosis of normal gastritis or mucosa and $32(21.6 \%)$ had endoscopic diagnosis of PCLS From 116 patients with endoscopic diagnosis of gastritis or normal mucosa, LCPM was identified in 46 patients $(39.6 \%)(\mathrm{p}<0.001)$ and 1 of them were low-grade dysplasia. From 32 of patients with suspected endoscopic PCLS, the diagnosis was confirmed with histology in 26 patients $(81.2 \%)$. A total of 72 patients had PCLS vs. 32 who were initially suspected $(\mathrm{p}<0.01)$, with a total prevalence of $48 \%$ (CI $40.7-56.6,95 \%$ ).

Conclusion: Pre-malignant conditions and lesions of the stomach (PCLS) can show as normal mucosa or gastritis during endoscopic procedure. $39.9 \%$ of patients who underwent endoscopic procedure with presumptive gastritis had PCLS. PCLS may be under-diagnosed if random biopsies are not taken. Therefore, taking biopsies from areas without suspected PCLS causes a change in the clinical management of patients, both for the initial diagnosis and for the staging according to OLGA and OLGUIM systems.

Disclosure of Interest: All authors have declared no conflicts of interest.

\section{References}

Dinis-Ribeiro M, Areia M, de Vries AC, et al. Management of precancerous conditions and lesions in the stomach (MAPS): guideline from the European society of gastrointestinal endoscopy (ESGE), European helicobacter study group (EHSG), European society of pathology (ESP), and the sociedade Portuguesa de endoscopia digestiva (SPED). Endoscopy. 2012;44:74-94.

Lahner E, Esposito G, Zullo A, et al. Gastric precancerous conditions and Helicobacter pylori infection in dyspeptic patients with or without endoscopic lesions. Scand J Gastroenterol. 2016 Nov;51(11):1294-8

\section{P0571 CORRELATION BETWEEN THE COMBINATION OF HELICOBACTER PYLORI ANTIBODY AND PEPSINOGEN AND OLGA/OLGIM STAGING FOR RISK ASSESSMENT OF GASTRIC PRECANCEROUS LESIONS}

X. Wang ${ }^{1}$, B. $\mathrm{Lv}^{2}, \mathrm{Z}_{\mathrm{Ji}} \mathrm{Ji}^{1}$

${ }^{1}$ The First Hospital of Jiaxing, Jiaxing/China

${ }^{2}$ First Affiliated Hospital of Zhejiang Chinese Medical University, Hangzhou China

\section{Contact E-mail Address: rainbowlove44@126.com}

Introduction: Prognosis of GC has a noticeable relation with its clinical stage. Atrophic gastritis (AG), intestinal metaplasia (IM) and dysplasia are well-recognized risk factors for intestinal type GC (GC). A large cohort study demonstrated that the annual incidence of GC were approximately $0.1 \%$ for patients with AG, $0.25 \%$ for IM and $6 \%$ for high-grade dysplasia, which were much higher than those with normal mucosal. In addition, long-term follow-up studies have confirmed that the extent/topography of mucosal atrophy parallels the risk of GC and it is on this ground that a system for staging gastritis, known as the Operative Link for Gastritis Assessment (OLGA) was created. It is widely accepted that serum PG level reflects the functional and morphologic status of gastric mucosal. While the gold standard for atrophy assessment is histology, the combination of serum pepsinogen and Helicobacter pylori $(\mathrm{Hp})$ antibody, known as the ABC method, has been suggested as a predictive marker for patients with GC.

Aims \& Methods: We aimed to discuss the correlation between the combination of Helicobacter pylori antibody and pepsinogen and OLGA/OLGIM staging system in gastric precancerous lesions risk assessment. A total of 331 patients were enrolled after the examination of endoscopy at Endoscopy Center, the First Affiliated Hospital of Zhejiang Chinese Medical University from October 2014 to December 2015. According to the result of gastroscope, gastric secretion and serum Helicobacter pylori antibody test, patients were divided into four groups: Group A: Hp (-)PG (-), Group B: Hp (+)PG (-), Group C: Hp (+)PG $(+)$ and Group D: Hp (-)PG (+). PG positive was defined as PGI $\leq 70 \mu \mathrm{g} / \mathrm{L}$ and $\mathrm{PGR} \leq 7.0$. According to the range and degree of atrophy/intestinal metaplasia, patients were divided into five groups on the basis of OLGA/OLGIM staging system. The levels of $\mathrm{Hp}$ infection rate, PG I, PG II and PGR were compared between different groups, and the correlation between ABC method and OLGA/ OLGIM staging system were evaluated. Statistical analysis was accomplished by chi-square test and logistic regression modeling analysis.

Results: A total of 331 patients were enrolled. 214 patients were classified into group A, 106 patients into group B, 4 patients into group C and 7 patients into group D, respectively. According to the pathological results, 177 cases were nonatrophic gastritis and 154 cases atrophic gastritis. For OLGA staging system, 177 patients were divided into stage- 0 group, 82 patients into stage-I group, 49 patients into stage-II group, 16 patients into stage-III group and 7 patients into stage-IV groups. The Hp infection rate was significant higher in patients with higher OLGA stages $\left(\chi^{2}=13.42, P=0.006\right)$. PGI and PGR level correlated inversely with the rising OLGA stages $(F=2.127, P=0.041, F=4.350, P=0.002)$. For OLGIM staging system, 172 patients were divided into stage-0 group, 81 patients into stage-I group, 47 patients into stage-II group, 19 patients into stageIII group and 12 patients into stage-IV group. The $\mathrm{Hp}$ infection rate was significant higher in patients with higher OLGIM stage $\left(\chi^{2}=16.09, P=0.003\right)$ and the PGR level correlated inversely with the rising OLGIM stages $(F=3.207$,
$P=0.013)$. Logistic regression modeling showed significant correlations between ABC methods and OLGA/OLGIM staging system $(P<0.001)$.

Conclusion: Serological ABC method and histological OLGA/OLGIM staging system are closely linked in gastric precancerous lesions risk assessment. Serum pepsinogen test could be applied for high risk population identifying and provide a recommendation for further endoscopy examination.

Disclosure of Interest: All authors have declared no conflicts of interest.

\section{References}

1. Ajani JA, Bentrem DJ, Besh S, et al. Gastric cancer, version 2.2013: featured updates to the NCCN Guidelines. J Natl Compr Canc Netw, 2013, 11(5):53146.

2. Rugge M, Meggio A, Pennelli G, et al. Gastritis staging in clinical practice: the OLGA staging system [J]. Gut, 2007, 56(5): 631-636.

3. Miki K. Gastric cancer screening by combined assay for serum antiHelicobacter pylori IgG antibody and serum pepsinogen levels - "ABC method". ProcJpnAcadSer B Phys Biol Sci. 2011;87(7):405-14.

4. Leung WK, Wu MS, Kakugawa Y, et al. Screening for gastric cancer in Asia: current evidence and practice. Lancet Oncol 2008;9:279-287.

\section{P0572 ENDOSCOPIC MUCOSAL RESECTION FOR SPORADIC NON AMPULLARY DUODENAL ADENOMA (SDA): CAN WE REDUCE THE RISK OF RECURRENCE AND COMPLICATIONS IN TERTIARY CENTERS?}

S. Hoibian ${ }^{1}$, E. Bories ${ }^{2}$, F. Caillol ${ }^{3}$, C. Pesenti ${ }^{4}$, J.P. Ratone ${ }^{5}$, J. Gonzalez ${ }^{6}$, M. Giovannini ${ }^{7}$, M. Barthet ${ }^{8}$

${ }^{1}$ Service D'hépato-gastro-enterologie, Centre hospitalier universitaire Nord de Marseille, Marseille/France

${ }^{2}$ Paoli Calmettes Institute, marseille/France

${ }^{3}$ Endoscopy, Institut Paoli Calmette, marseille/France

${ }^{4}$ Dept. De Gastroenterologie, APHM - North Hospital, Marseille/France

${ }^{5}$ Institut paoli Calmettes, Marseille/France

${ }^{6}$ Centre hospitalier universitaire Nord, Marseille/France

Contact E-mail Address: solene.hoibian@hotmail.fr

Introduction: Endoscopic treatment of sporadic duodenal adenoma is mainly performed in tertiary centers because it is technically challenging and associated with major complications (perforation $1-5 \%$ and delayed bleeding $10-15 \%$ ). The aim of this study was to evaluate the safety and efficacy of the endoscopic treatment for non ampullary sporadic duodenal adenomas (SDA) in two tertiary centers in a large series and to try to determine the predictive factors of outcomes with a long follow-up.

Aims \& Methods: This retrospective study was conducted in two tertiary centers between $12 / 2003$ to $03 / 2016$. All the patients who underwent at least one endoscopic treatment by EMR for SDA histologically proven were included. Patients with PAF and ampullary adenoma were excluded. All the following outcomes were systematically recorded in both centers: complete endoscopic resection, resection with negative lateral and vertical margins, recurrence, success of the endoscopic treatment and adverse events (Perforation, intra-procedural bleeding, delayed bleeding, others). There were analysed with multivariate analysis.

Results: 134 procedures were performed. The mean patient age was 65 years ( 33 $85), 50.7 \%$ were women. The mean SDA size was of $20.7 \mathrm{~mm}(5-50 \mathrm{~mm})$, mostly located in the second duodenum $(61.2 \%) .64 .9 \%$ of the adenomas had a villous component, $34.3 \%$ with high grade dysplasia and $7.5 \%$ with in situ or intramucosal. Discrepancy between biopsies and the final histology was demonstrated, $32 \%$ of the lesions being upgraded and $10.6 \%$ being downgraded. An EMR was performed in $98.5 \%$ of the cases with a complete endoscopic resection rate of $96.2 \%$ which was associated in multivariate analysis with the lesion size and depressed relief. The en-bloc resection's rate was of $44 \%$. Vertical margins were negative in $91.8 \%$ of the cases. Negative lateral and vertical margins was associated in multivariate analysis with the lesion size and its en-bloc resection. Intraprocedural bleeding occurred in $5.9 \%$ of the case and was associated in multivariate analysis. Delayed bleeding occurred in $13.4 \%$ of the cases and was associated with a larger lesion size and the presence of high-grade dysplasia or adenocarcinoma. usual use of antiplatelet or anticoagulant did not increase the risk of bleeding after pre operative management. A prophylactic hemostasis was realized for $61.9 \%$ of the procedure, by clips alone or associated in $72.3 \%$ of the cases. Prophylactic clipping reduced significantly the risk of delayed bleeding Perforation occurred in $3.7 \%$ of case. Median follow-up was of 31.2 months with at least one follow-up endoscopy $(78.3 \%)$. Final success of endoscopic treatment occurred in $83.8 \%$ of the case. 30 patients had a recurrence $(28.6 \%) .13$ among them being successfully retreated with endoscopy, 12 still receiving endoscopic treatment with multiple sessions, and 5 were referred to surgery. In multivariate analysis, the main risk factor for recurrence was the lesion size.

Conclusion: Endoscopic treatment of SDA appears to be effective and relatively safe in tertiary centers. All the bleeding complications were endoscopically controlled and their occurrence could be reduced with clipping prophylactic hemostasis. Perforation is rare. Recurrence rate is frequent and associated with piecemeal resection but can be endoscopically managed.

Disclosure of Interest: All authors have declared no conflicts of interest. 
P0573 CAN BE THE PATIENT WITH NON-CURATIVE ESD FOR

EARLY GASTRIC CANCER RESCUED BY SURGERY AFTER RECURRENCE?

K. Takizawa ${ }^{1}$, W. Hatta ${ }^{2}$, T. Gotoda ${ }^{3}$, N. Kawata ${ }^{4}$, M. Nakagawa ${ }^{5}$,

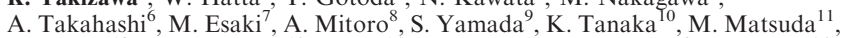
J. Takada ${ }^{12}$, Y. Yoshifuku ${ }^{13}$, H. Ito ${ }^{14}$, K. Ohnita ${ }^{15}$, R. Shimoda ${ }^{2}$, S. Hoteya ${ }^{16}$, T. Oyama ${ }^{2}$, T. Shimosegawa ${ }^{2}$

${ }^{1}$ Division Of Endoscopy, Shizuoka Cancer Center, Shizuoka/Japan

${ }^{2}$ EAST study group, Nagaizumi/Japan

${ }^{3}$ Division Of Gastroenterology And Hepatology, Department Of Medicine, Nihon University School of Medicine, Tokyo/Japan

${ }^{4}$ Division Of Endoscopy, Shizuoka Cancer Center, Nagaizumi/Japan

${ }^{5}$ Hiroshima City Hospital, Hiroshima/Japan

${ }^{6}$ Saku Central Hospital Advanced Care Center, Saku/Japan

${ }^{7}$ Kitakyushu Municipal Medical Center, Kitakyushu/Japan

${ }^{8}$ Nara Medical University, Nara/Japan

${ }^{9}$ Japanese Red Cross Society Kyoto Daiichi Hospital, Kyoto/Japan

${ }^{10}$ Shinshu University School of Medicine, Nagano/Japan

${ }^{11}$ Toyama Prefectural Central Hospital, Toyama/Japan

${ }^{12}$ Gifu University Graduate School of Medicine, Gifu/Japan

${ }^{13}$ Hiroshima University, Hiroshima/Japan

${ }^{14}$ Osaki Citizen Hospital, Osaki/Japan

${ }^{15}$ Department Of Gastroenterology And Hepatology, Nagasaki University

Hospital, Nagasaki/Japan

${ }^{16}$ Dept. Of Gastroenterology, Toranomon Hospital, Tokyo/Japan

Contact E-mail Address: k.takizawa@scchr.jp

Introduction: Additional surgery should be recommended in patients with noncurative endoscopic resection for early gastric cancer (EGC). However, this decision has been often hesitated according to patient' condition such as advancedage or comorbidities. After the recognition of recurrence, the salvage surgery has been considered difficult. However, little has been reported on it.

Aims \& Methods: The aim of this study was to clarify the results of salvage surgery for recurrence after non-curative ESD for EGC using data from multicenter retrospective study (EAST study*). Of 15,785 patients who underwent ESD for EGC at 19 participating institutions from January 2000 to August 2011, 1969 patients who failed to meet the current curative criteria for ESD were retrospectively reviewed. Among 1969 patients enrolled into EAST study, 1064 patients underwent additional surgery, and 905 patients were observed without any additional treatment. We evaluated first site of recurrence, clinical course after salvage surgery, and long-term survival on non-treatment group. Recurrence was classified regional LNM, and distant metastasis.

Results: Over a median follow-up period of 64 months, recurrence was detected in 27 patients. Among them, 2 patients were excluded from this study due to missing data. First sites of recurrence were only local site (intra-gastric relapse 3, regional LNM 7), and distant metastasis $15(60 \%)$. The first treatments for recurrence were endoscopic treatment 1 , salvage surgery 7 (28\%), chemotherapy 6 , and best supportive care 11 . Only one patient was alive without any recurrence for 31 months after salvage surgery. And one patient died of acute myocardial infarction just one month after salvage surgery. In the remaining 5 patients, recurrence was detected at $0,2,3,5,30$ months after salvage surgery, and al of them died of gastric cancer. Median survival time of all 25 patients with recurrence was 5 months from recurrence. And median survival time of 7 patients who underwent salvage surgery was only 7 months from salvage surgery.

Conclusion: More than half of recurrence after non-curative ESD without additional surgery were distant metastasis, and the survival rate after salvage surgery was quite low.

Disclosure of Interest: All authors have declared no conflicts of interest.

\section{Reference}

* Hatta W, et al. Is radical surgery necessary in all patients who do not meet the curative criteria for endoscopic submucosal dissection in early gastric cancer? A multi-center retrospective study in Japan. Journal of Gastroenterology 2016

\section{P0574 BLEEDING AFTER ENDOSCOPIC RESECTION FOR EARLY GASTRIC LESIONS IN PATIENTS ON ANTITHROMBOTIC THERAPY}

T. Nagai, S. Matsui, H. Kashida, Y. Komeda, T. Sakurai, M. Kudo Dept. Of Gastorenterology And Hepatology, Kindai University Faculty of Medicine, Osaka-sayama/Japan

Contact E-mail Address: tomoyukinagai@mac.com

Introduction: Due to the increase of elderly patients who are often receiving antithrombotic therapy for cardio- and cerebrovascular diseases, postprocedure bleeding after endoscopic treatments for early gastric lesions has become one of the major concerns of therapeutic endoscopists. The Japan Gastroenterological Endoscopy Society (JGES) and other related associations published the Guidelines for Gastroenterological Endoscopy in Patients Undergoing Antithrombotic Treatment in $2012^{1}$. According to the guideline it is not necessary to suspend an antiplatelet agent before endoscopic treatments including endoscopic mucosal resection (EMR) or endoscopic submucosal dissection (ESD) if the agent is not combined with other antithrombotic drugs (monotherapy). On the other hand it is recommended that anticoagulants should be substituted with heparin before EMR/ESD. The aim of this study is to clarify the efficacy of the recommendations of the guideline.

Aims \& Methods: In this study 888 early gastric lesions in 783 patients who underwent EMR/ESD at our hospital between January 2012 and March 2017 were retrospectively analysed. Postprocedure bleeding was defined as: (1) hematemesis or melena for which an emergency endoscopy was required and (2) bleeding which were confirmed with a repeat endoscopy after a drop $\geq 2 \mathrm{~g} / \mathrm{dL}$ of haemoglobin level.

Results: The total number of patients undergoing antithrombotic therapy was 78 , out of which 38 patients were taking antiplatelet agents only, 29 were taking anticoagulants only, and 11 were taking the both. The antithrombotics were suspended in 22 cases (Group A), substituted with heparin in 18 (Group B), and kept continued in 38 (Group C). Postprocedure bleeding was encountered in 31 out of 783 cases $(4.0 \%), 21$ of which occurred in patients on antithrombotic therapy (21/78: $27 \%$ ) whereas 10 of which occurred in those without (10/752: $1.3 \%$ ). A univariate analysis between the patients with postprocedure bleeding and those without concerning such variables age, gender, the diameter and number of the resected lesions, use of antithrombotics, and the expertise of the operating endoscopist revealed that only the use of antithrombotics was significant risk factor for the postprocedure bleeding (odds ratio $=15.926,95 \%$ confidence interval: 7.415-34.208, $\mathrm{p}<0.01$ ). However, the rate of postprocedure bleeding was not significantly different among Group A, B and C. Among the 21 bleeding patients with antithrombotics, the agent had been suspended or substituted with heparin before EMR/ESD in 10 and had been continued without suspension in 11. There was no significant difference of bleeding rate between the two groups.

Conclusion: The use of antithrombotics was a significant risk factor for the postprocedure bleeding after EMR/ESD for early gastric lesions. The rate of bleeding was not significantly different regardless if the antithrombotics were suspended, substituted with heparin, or continued without suspension.

Disclosure of Interest: All authors have declared no conflicts of interest.

\section{Reference}

1. Fujimoto K, Fujishiro M, Kato M, et al. Guidelines for gastroenterological endoscopy in patients undergoing antithrombotic treatment. Dig Endosc.2014;26(1):1-14.

\section{P0575 FACTORS AFFECTING CURATIVE ENDOSCOPIC RESECTION OF EARLY GASTRIC CANCER IN THE ENVIRONMENT OF POPULATION-BASED SCREENING}

Y.G. Mun ${ }^{1}$, H.H. Lee ${ }^{2}$, D.H. Kang ${ }^{1}$, J.M. Park ${ }^{1}$, K.Y. Song ${ }^{3}$, M. Choi ${ }^{1}$

${ }^{1}$ Internal Medicine, Catholic Medical Center, the Catholic university of Korea,

College of Medicine, Seoul/Korea, Republic of

${ }^{2}$ Internal Medicine, Seoul ST. Mary's Hospital, Seoul/Korea, Republic of

${ }^{3}$ Surgery, Catholic Medical Center, the Catholic university of Korea, College of Medicine, Seoul/Korea, Republic of

Contact E-mail Address: myg0988@naver.com

Introduction: Since population-based screening for gastric cancer in Korea was implemented in 2002, endoscopic treatment of early gastric cancer (EGC) has been popularized. Most patients with early neoplasm have no alarming symptoms/signs. In addition, the strategy for detecting factors predicting curative endoscopic resection of EGC is becoming important, because the general population is aging and considering the quality of life after treatment.

Aims \& Methods: This study investigates factors affecting curative endoscopic resection of EGC in the era of population-based screening for gastric cancer. The subjects consisted of patients newly diagnosed with stomach cancer at Seoul St. Mary's Hospital between May 2011 and May 2016. All patients completed questionnaires about symptoms, social history, family history, knowledge of national cancer screening program, the reason for screening and the interval between endoscopy screening examinations for gastric cancer.

Results: Of a total of 469 patients, $147(31.3 \%)$ had a curative endoscopic resection, $260(55.4 \%)$ had a curative surgical resection and $62(13.3 \%)$ were in noncurative surgical resection or an inoperable state. The patients with curative endoscopic resection had minimal abdominal symptoms and fewer alarm symptoms/signs (a family history of gastric cancer, anemia, and clinically important weight loss), whereas alarm symptoms were more common in patients with advanced cancer. In multivariate analysis, regular surveillance endoscopy was only the factor predicting the curative endoscopic resection [Odd ratio $95 \%$ CI) $6.099(2.532 \sim 14.933), \mathrm{p}=0.000]$. In addition, the proportion of curative endoscopic resection was significantly higher in the 1 -year [(Odds ratio $(95 \%$ CI $10.381(4.081 \sim 26.405), p=0.000)]$, 2-year endoscopy interval groups [(Odds ratio $(95 \%$ CI $) 3.161(1.106 \sim 9.035), \mathrm{p}=0.032)]$ than patients who had no endoscopy within 2 years.

Conclusion: Most patients with the curative endoscopic resection have minimal abdominal symptoms and no alarming symptoms/signs. Regular surveillance endoscopy was the only factor predicting the curative endoscopic resection of gastric cancer. In addition, more frequent endoscopic surveillance could help to early detect gastric cancers with curative endoscopic resection.

Disclosure of Interest: All authors have declared no conflicts of interest. 
P0576 USEFULNESS OF OPERATIVE LINK ON GASTRITIS

ASSESSMENT (OLGA) AND OPERATIVE LINK ON GASTRIC

INTESTINAL METAPLASIA (OLGIM) FOR DIAGNOSIS OF

HELICOBACTER PYLORI-ASSOCIATED GASTRIC CANCER

REGARDLESS OF TISSUE TYPE IN KOREA

C. Yun ${ }^{1}$, N. Kim ${ }^{2}$, Y. Hwang ${ }^{2}$, H. Lee ${ }^{2}$, M. Kwon ${ }^{3}$, J. Lee ${ }^{3}$, Y.J. Choi ${ }^{5}$,

H. Yoon ${ }^{1}$, C.M. Shin ${ }^{1}$, Y.S. Park ${ }^{1}$, D.H. Lee ${ }^{2}$

${ }^{1}$ Internal Medicine, Seoul National University Bundang Hospital, Seongnam

Korea, Republic of

${ }^{2}$ Department Of Internal Medicine And Institute Of Liver Research, Seoul

National University College of Medicine, Seoul/Korea, Republic of

${ }^{3}$ Division of Statistics in Medical Research Collaborating Center, Seoul National

University Bundang Hospital, Seongnam, Republic of Korea, Seongnam/Korea,

Republic of

Contact E-mail Address: ycyhiphop@hanmail.net

Introduction: Atrophic gastritis and intestinal metaplasia are the cancerization field in which gastric cancer (GC) develops in case of intestinal type. The OLGA and OLGIM staging systems have been suggested to provide risk estimation for GC.

Aims \& Methods: The aim of this study is to evaluate the usefulness of OLGA and OLGIM staging according to Laurens's histological classification of GC in considering with other risk factors of gastric cancer. From January 2006 to December 2015, 607 GC patients and 677 control subjects were enrolled who underwent esophagogastroduodenoscopy. Biopsies were taken from the greater and lesser curvatures of the antrum and mid-body, respectively. The OLGA and OLGIM stage $(0-$ IV) was recorded by combining antral with body atrophy scores using the Updated Sydney System. Stage III and IV OLGA or OLGIM was classified as high-risk stage group and Stage 0-II as low-risk group. H. pylori infection was assessed by modified Giemsa stain, rapid urease test and culture and was defined by a positive result on any of these tests. Multivariate logistic analysis was performed for the age, sex, smoking, alcohol and family history of GC.

Results: GC patients had more high-risk OLGA stages $(25.9 \%)$ than controls $(6.8 \%, \mathrm{P}<0.001)$ and high-risk OLGIM stages $(18.3 \%)$ than controls $(4.9 \%$, $\mathrm{P}<0.001$ ). In the multivariate logistic analysis, Old age [odds ratios (ORs), 1.932; $\mathrm{P}=0.004$ and 2.584; $\mathrm{P}<0.001$ for ages in the $40 \sim 59$ and $>60$, respectively], family history of GC (OR, 2.119; $\mathrm{P}<0.001)$, and H. pylori infection (OR, $1.963 ; \mathrm{P}<0.001)$ were independent risk factors for $\mathrm{GC}$ in the diffuse type as well as intestinal type (Table). High-risk OLGA stages were significantly associated with increased risk of $\mathrm{GC}$ in comparison to low-risk $(\mathrm{OR}, 3.778 ; \mathrm{P}<0.001)$ : intestinal-type $(\mathrm{OR}, 4.318 ; \mathrm{P}<0.001)$ and diffuse-type $(\mathrm{OR}, 2.920 ; \mathrm{P}<0.001)$ (Table). High-risk OLGIM stages were also significantly associated with increased risk of $\mathrm{GC}$ in comparison to low-risk ( $\mathrm{OR}, 3.306 ; \mathrm{P}<0.001)$ : intestinal-type $\mathrm{GC}(\mathrm{OR}, 3.051 ; \mathrm{P}<0.001)$ and diffuse-type $\mathrm{GC}(\mathrm{OR}, 3.981$; $\mathrm{P}<0.001)$.

Conclusion: High-risk OLGA and OLGIM stages were useful for intestinal type as well as diffuse type. This usefulness will be increased when combined with $H$. pylori status and family history of GC in regions with high prevalence of GC. Analysis regarding specific interaction among these three factors is undergoing.

Disclosure of Interest: All authors have declared no conflicts of interest.
P0577 BODY MASS INDEX AND DIGESTIVE CANCER

MORTALITY IN THE KOREAN GENERAL POPULATION: A NATIONWIDE COHORT STUDY

P. Kim ${ }^{1}$, Y.J. Kim ${ }^{1}$, M.K. Baeg ${ }^{1}$, S.H. Jeong ${ }^{1}$, S. Yi ${ }^{2}$

${ }^{1}$ Divsion Of Gastroenterology, Department Of Internal Medicine, Catholic Kwandong University International St. Mary's Hospital, Incheon/Korea, Republic of

${ }^{2}$ Preventive Medicine And Public Health, Catholic Kwandong University College of Medicine, Gangwon-do/Korea, Republic of

Contact E-mail Address: pumsoo.kim@gmail.com

Introduction: The association between body mass index (BMI) and digestive cancer mortality is not conclusive in East Asians.

Aims \& Methods: We evaluated the relationship between BMI and digestive cancer mortality, using prospective cohort data by the National Health Insurance Service in Korea, which consisted of more than one million subjects. A total of 510, 148 Korean adults were followed-up until 2010. The adjusted hazard ratios (HRs) of cancer mortality were calculated using a Cox model.

Results: During follow-up, 7774 total deaths occurred from digestive cancer. HR for digestive cancer mortality across seven BMI categories. Below $25 \mathrm{~kg} / \mathrm{m}^{2}$, the HRs of death for each $5 \mathrm{~kg} / \mathrm{m}^{2}$ increase in BMI were $0.43(95 \%$ confidence interval $[\mathrm{CI}]=0.32-0.58)$ for esophagus cancer, $0.70(0.62-0.79)$ for stomach cancer, and $0.77(0.65-0.90)$ for colorectal cancer. Over $25 \mathrm{~kg} / \mathrm{m}^{2}$, the HRs of death for each $5 \mathrm{~kg} / \mathrm{m}^{2}$ increase in BMI were $1.30(95 \% \mathrm{CI}=1.04-1.64)$ for colorectal cancer, $1.28(1.07-1.53)$ for liver cancer, and $1.28(0.96-1.71)$ for gallbladder cancer and biliary tract cancer. BMI were not associated mortality from small intestine cancer and pancreatic cancer.

Conclusion: Low BMI were predictors of mortality from esophageal cancer and stomach cancer. High BMI were predictors of mortality from liver cancer and gallbladder cancer and biliary tract cancer. Both low and high BMI were predictors of mortality from colorectal cancer. Further research is needed to evaluate whether interventions involving weight change (loss or gain) reduce the risk of cancer or improve the survival.

Disclosure of Interest: All authors have declared no conflicts of interest.

\section{References}

1. Smith M, Zhou M, Whitlock G, et al. Esophageal cancer and body mass index: Results from a prospective study of 220,000 men in China and a metaanalysis of published studies. Int. J. Cancer: 122, 1604-1610.2008.[18059032]

2. Hong JS, Yi SW, Yi JJ et al. Body Mass Index and Cancer Mortality Among Korean Older Middle-Aged Men: A Prospective Cohort Study. Medicine (Baltimore). 2016 May;95(21):e3684.[27227928]

3. Minami Y, Kawai M, Fujiya T, et al. Family history, body mass index and survival in Japanese patients with stomach cancer: a prospective study. Int $J$ Cancer. 2015;136:411-424. [24890283]

4. Parr CL1, Batty GD, Lam TH et al. Body-mass index and cancer mortality in the Asia-Pacific Cohort Studies Collaboration: pooled analyses of 424, 519 participants. Lancet Oncol. 2010 Aug;11(8):741-52. [20594911]

5. Renehan A G, Tyson M, Egger M, et al. Body-mass index andincidence of cancer: a systematic review and meta-analysis of prospective observational studies. Lancet. 2008;371:569-578.

\begin{tabular}{|c|c|c|c|c|c|c|c|c|c|}
\hline & \multicolumn{2}{|c|}{ Gastric cancer patients $(n=607)$} & \multirow[b]{2}{*}{ p-value } & \multicolumn{2}{|c|}{ Intestinal-type $(\mathrm{n}=354)$} & \multirow[b]{2}{*}{ p-value } & \multicolumn{2}{|c|}{ Diffuse-type $(\mathrm{n}=233)$} & \multirow[b]{2}{*}{ p-value } \\
\hline & OR & $95 \% \mathrm{CI}$ & & OR & $95 \% \mathrm{CI}$ & & OR & $95 \% \mathrm{CI}$ & \\
\hline \multicolumn{10}{|l|}{ Sex } \\
\hline Female & 1 & & & 1 & & & 1 & & \\
\hline Male & 1.193 & $0.847-1.679$ & 0.312 & 2.833 & $1.833-4.381$ & $<0.001$ & 0.474 & $0.295-0.761$ & 0.002 \\
\hline \multicolumn{10}{|c|}{ Age(year, mean \pm sd $)$} \\
\hline$<40$ & 1 & & & 1 & & & 1 & & \\
\hline $40 \sim 59$ & 1.932 & $1.229-3.036$ & 0.004 & 7.266 & $2.570-20.544$ & $<0.001$ & 1.238 & $0.748-2.047$ & 0.406 \\
\hline$\geq 60$ & 2.584 & $1.647-4.056$ & $<0.001$ & 14.706 & $5.244-41.243$ & $<0.001$ & 0.908 & $0.538-1.535$ & 0.720 \\
\hline \multicolumn{10}{|l|}{ Smoking status } \\
\hline Never-smoker & 1 & & & 1 & & & 1 & & \\
\hline Ever-smoker & 1.467 & $0.974-1.918$ & 0.050 & 1.012 & $0.589-1.351$ & 0.289 & 2.194 & $1.355-3.553$ & 0.001 \\
\hline \multicolumn{10}{|c|}{ Alcohol consumption } \\
\hline Never-drinker & 1 & & & 1 & & & 1 & & \\
\hline Ever-drinker & 1.322 & $1.010-1.731$ & 0.042 & 1.183 & $0.852-1.643$ & 0.317 & 1.402 & $0.970-2.026$ & 0.072 \\
\hline \multicolumn{10}{|c|}{ Family history of gastric cancer } \\
\hline Negative & 1 & & & 1 & & & 1 & & \\
\hline Positive & 2.119 & $1.521-2.953$ & $<0.001$ & 1.995 & $1.344-2.960$ & 0.001 & 2.436 & $1.600-3.709$ & $<0.001$ \\
\hline \multicolumn{10}{|l|}{ H. pylori status } \\
\hline Positive & 1.963 & $1.540-2.503$ & $<0.001$ & 1.604 & $1.193-2.157$ & 0.002 & 2.800 & $1.991-3.937$ & $<0.001$ \\
\hline $\begin{array}{l}\text { OLGA } \\
\text { low risk }\end{array}$ & 1 & & & 1 & & & 1 & & \\
\hline high risk & 3.778 & $2.612-5.465$ & $<0.001$ & 4.318 & $2.899-6.431$ & $<0.001$ & 2.920 & $1.779-4.793$ & $<0.001$ \\
\hline
\end{tabular}


6. Chen Y, Liu L, Wang X, et al. Body mass index and risk of gastric cancer: a meta-analysis of a population with more than ten million from 24 prospective studies. Cancer Epidemiol Biomarkers Prev. 2013;22:1395-1408.

7. Bhaskaran K, Douglas I, Forbes H, et al. Body-mass index and risk of 22 specific cancers: a population-based cohort study of 5.24 million UK adults. Lancet. 2014;384:755-765.

\section{P0578 RISK FACTORS FOR LYMPH NODE METASTASIS OF ULCERATIVE TYPE INTRAMUCOSAL EGC}

T. Kim, T.J. Kim, H. Lee, J.H. Lee, J.J. Kim

Department Of Medicine, Samsung Medical Center, Sungkyunkwan University School of Medicine, Seoul/Korea, Republic of

Contact E-mail Address: attack836@naver.com

Introduction: Endoscopic submucosal dissection (ESD) is not currently accepted as an alternative treatment to surgery in ulcerative type EGC due to relatively higher probability of lymph node metastasis (LNM). This present retrospective analysis examined the correlation of various histologic factors with the presence of lymph node metastasis in ulcerative type EGC.

Aims \& Methods: A retrospective analysis on 200 patients with ulcerative type EGC who underwent radical gastrectomy with D2 lymph node dissection Several clinicopathologic factors were investigated to identify predictive factors for LNM: tumor size, histopathologic type of tumor, lymphovascular invasion and depth of invasion. Multivariate logistic regression analysis was performed to evaluate the risk factors for LNM.

Results: The total rate of LNM was $15.5 \%$ (31/200). The rate of LNM was $2.1 \%$ $(2 / 95)$ in the lesions confined to the mucosa and $27.6 \%(27 / 105)$ in those that had infiltrated the submucosa On univariate analysis, depth of invasion $(\mathrm{p}=0.047)$ and lymphovascular invasion $(\mathrm{p}<0.001)$ were significant associated with LNM However, there was no significant association between tumor size, histopathologic type of tumor and LNM. On multivariate analysis, only lymphovascular invasion $(\mathrm{p}<0.001)$ was significantly associated with LNM. There was no significant association between tumor size and lymph node metastasis in ulcerative type EGC.

Conclusion: Ulcerative EGC confined to the mucosa could be considered for candidate for curative ESD due to the low risk of LNM. This finding should be confirmed by more data from other centers, which focus on LNM after ESD in ulcerative type intramucosal EGC.

Disclosure of Interest: All authors have declared no conflicts of interest.

\section{P0579 THE FEASIBILITY STUDY USING KUMC ROBOTIC MANIPULATOR IN ENDOSCOPIC SUBMUCOSAL DISSECTION}

G. Min ${ }^{1}$, S.J. Choi ${ }^{1}$, W. Kim², J.M. Lee ${ }^{1}$, I.K. Yoo ${ }^{3}$, S.H. Kim ${ }^{2}$, J.M. Lee ${ }^{2}$, H.S. Choi ${ }^{2}$, E.S. Kim ${ }^{2}$, B. Keum ${ }^{2}$, H.S. Lee ${ }^{2}$, H.J. Chun ${ }^{2}$, C.D. Kim ${ }^{2}$, Y.T. Jeen ${ }^{2}$ ${ }^{1}$ Division Of Gastroenterology And Hepatology, Department Of Internal Medicine Korea University Anam Hospital, Seoul/Korea, Republic of

${ }^{2}$ Division of Gastroenterology and Hepatology, Department of Internal Medicine, Korea University College of Medicine, Seoul/Korea, Republic of

${ }^{3}$ Department Of Internal Medicine, Institute of Digestive Disease and Nutrition, Korea University College of Medicine, Seoul/Korea, Republic of

Contact E-mail Address: mdkorea@gmail.com

Introduction: Gastrointestinal cancers are one of the most common malignancy worldwide. Especially endoscopic submucosal dissection (ESD) for early gastrointestinal cancers have been considered as the current standard cancer treatment. However, lack of counter traction during ESD procedure is one of major difficulty. To overcome this problem, we developed new endoscopic technique using robotic manipulator and conducted study about efficacy and safety in vitro animal study.

Aims \& Methods: A novel robotic suture manipulator is composed of control panel and a working arm, which grasp and move objects at the end of scope. A total of 10 porcine stomachs were used for the test. Porcine stomachs were assigned randomly to 2 groups and ESD was performed on mucosa of stomach using conventional technique and new endoscopic technique with robotic manipulator. Endoscopic experts and novice endoscopists performed ESD in 2 parts (antrum \& body) of stomach. During procedure, robotic manipulator lifts up dissected tissue of stomach to make better visibility. Procedure time, complete resection rate, and complication such as perforation was recorded

Results: The average procedure time for the robotic manipulator and conventional ESD was 42 minutes and 45.9 minutes. In novice endoscopists, the average procedure time using robotic manipulator is faster than conventional ESD group ( $47.5 \mathrm{~min}$ vs $55.3 \mathrm{~min}, \mathrm{p}<0.05$ ). Both endoscopic expert and novice endoscopist completed the ESD procedure for en bloc resection of target lesions using KUMC robotic manipulator. There was no difference in complete resection rates between two groups. No complication such as perforation was occurred in both groups during the procedures. There was no difference depending on resected location in stomach.

Conclusion: The robotic manipulator, which can perform ESD more easily showed feasible result comparing with conventional ESD. ESD using robotic manipulator could be helpful, especially in novice endoscopists. This research proposes a novel approach for safe and feasible method during ESD.

Disclosure of Interest: All authors have declared no conflicts of interest.

\section{P0580 DEVELOPMENT OF NOVEL ENDOSCOPIC IRREVERSIBLE} ELECTROPORATION ABLATION DEVICE

G. Min ${ }^{1}$, S.J. Choi ${ }^{1}$, W. Kim ${ }^{1}$, I.K. Yoo ${ }^{1}$, S.H. Kim ${ }^{1}$, J.M. Lee ${ }^{1}$, H.S. Choi ${ }^{1}$, E.S. Kim ${ }^{1}$, B. Keum ${ }^{1}$, H.S. Lee ${ }^{1}$, H.J. Chun ${ }^{1}$, C.D. Kim ${ }^{1}$, Y.T. Jeen ${ }^{1}$

${ }^{1}$ Division Of Gastroenterology And Hepatology, Department Of Internal Medicine, Korea University Anam Hospital, Seoul/Korea, Republic of

Contact E-mail Address: mdkorea $@$ gmail.com

Introduction: Irreversible electroporation (IRE) is a promising novel technique for the ablation of tumors. An advantage of IRE is its mechanism to remove undesired cells by affecting the cell membrane without thermally destructing blood vessels, nerves and the surrounding tissues. Several clinical trials for applying IRE to human organs such as liver, pancreas, and kidney are conducted and studies about IRE ablation for gastrointestinal tumors also have been conducted recently. Here, we developed new endoscopic IRE device, and studied about its effectiveness and feasibility in animal model.

Aims \& Methods: Newly developed endoscopic IRE ablative catheter works with single channel of endoscope. A pair of dipolar electrodes consist of pre-shaped $f$ $0.63 \mathrm{~mm}$ nitinol wire and the distance between each electrode is $10 \mathrm{~mm}$. The electrodes are loaded within braided tube for stent delivery system then deployed when IRE catheter put in stomach through the endoscope. We performed endoscopy and IRE ablation was done on pig's stomach mucosa by using endoscopy with newly developed IRE catheter. We divided pig's stomach into 2 parts (antrum \& body), and IRE ablation was applied on each part of the stomach. Pigs were sacrificed after 24 hours, and we collected their stomachs with surgical technique. Following fixation, tissues were stained with $\mathrm{H} \& \mathrm{E}$

Results: Ten male Yorkshire pigs and in vitro stomachs were used in this study. The tissue with H\&E stain showed diffuse cell death $24 \mathrm{hr}$ after IRE ablation.

Abstract No: P0577

Digestive cancer mortality associated with baseline BMI according to BMI ranges

\begin{tabular}{|c|c|c|c|c|c|c|c|c|c|c|c|c|}
\hline \multirow[b]{3}{*}{ Digestive cancer } & \multicolumn{4}{|c|}{ All participants $\left(12-47 \mathrm{~kg} / \mathrm{m}^{2}\right)$} & \multicolumn{4}{|c|}{$12-24.9 \mathrm{~kg} / \mathrm{m}^{2}$} & \multicolumn{4}{|c|}{$25-47 \mathrm{~kg} / \mathrm{m}^{2}$} \\
\hline & \multirow[b]{2}{*}{ Deaths } & \multicolumn{3}{|c|}{ per $5 \mathrm{~kg} / \mathrm{m}^{2}$ increase inBMI } & \multirow[b]{2}{*}{ Deaths } & \multicolumn{3}{|c|}{ per $5 \mathrm{~kg} / \mathrm{m}^{2}$ decrease inBMI } & \multirow[b]{2}{*}{ Deaths } & \multicolumn{3}{|c|}{ per $5 \mathrm{~kg} / \mathrm{m}^{2}$ increase in BMI } \\
\hline & & P-value & $\mathrm{HR}^{\mathrm{a}}$ & $(95 \% \mathrm{CI})$ & & $\mathrm{P}$-value & $\mathrm{HR}^{\mathrm{a}}$ & $(95 \% \mathrm{CI})$ & & $\mathrm{P}$-value & $\mathrm{HR}^{\mathrm{a}}$ & $(95 \% \mathrm{CI})$ \\
\hline Esophagus & 310 & $<0.001$ & 0.53 & $(0.43-0.65)$ & 252 & $<0.001$ & 0.43 & $(0.32-0.58)$ & 58 & 0.491 & 1.28 & $(0.64-2.57)$ \\
\hline Stomach & 2,032 & $<0.001$ & 0.77 & $(0.72-0.83)$ & 1488 & $<0.001$ & 0.70 & $(0.62-0.79)$ & 544 & 0.244 & 1.14 & $(0.91-1.43)$ \\
\hline Colon and rectum & 1328 & 0.845 & 1.01 & $(0.92-1.11)$ & 866 & 0.002 & 0.77 & $(0.65-0.90)$ & 462 & 0.024 & 1.30 & $(1.04-1.64)$ \\
\hline Colon & 835 & 0.347 & 1.06 & $(0.94-1.19)$ & 536 & 0.070 & 0.82 & $(0.66-1.02)$ & 299 & 0.089 & 1.28 & $(0.96-1.71)$ \\
\hline Rectum & 493 & 0.366 & 0.93 & $(0.80-1.08)$ & 330 & 0.006 & 0.69 & $(0.53-0.90)$ & 163 & 0.136 & 1.34 & $(0.91-1.97)$ \\
\hline Small intestine & 61 & 0.049 & 0.64 & $(0.41-1.00)$ & 49 & 0.863 & 0.94 & $(0.46-1.92)$ & 12 & 0.231 & 0.26 & $(0.03-2.36)$ \\
\hline Liver & 2365 & 0.601 & 1.02 & $(0.95-1.09)$ & 1577 & 0.323 & 0.94 & $(0.82-1.07)$ & 788 & 0.007 & 1.28 & $(1.07-1.53)$ \\
\hline Pancreas & 929 & 0.937 & 1.00 & $(0.90-1.12)$ & 603 & 0.626 & 0.95 & $(0.77-1.17)$ & 326 & 0.504 & 0.90 & $(0.67-1.22)$ \\
\hline GB and Biliary tract & 749 & 0.012 & 1.16 & $(1.03-1.31)$ & 471 & 0.644 & 1.06 & $(0.84-1.34)$ & 278 & 0.221 & 1.28 & $(0.96-1.71)$ \\
\hline
\end{tabular}

BMI, body mass index; CI, confidence interval; GB, gallbladder; HR, hazard ratio. ${ }^{\text {a }}$ Hazard ratios were calculated using Cox proportional hazards models after adjustment for age at baseline (continuous variable), smoking status (current smoker, former smoker, never-smoker, and missing smoking status), alcohol consumption (frequency; five or more times/week, one to four times/week, less than one times/week, past drinker [no alcohol for a year], never-drinker, or missing information),

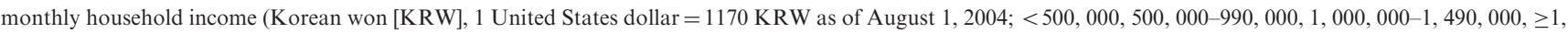
500, 000, missing information), and physical activity (yes, no). HRs were not presented for causes with less than 10 deaths. 
Consistent with the mechanism of action of IRE on the cell membrane only, there was complete cell death within the IRE lesions without intervening live cells. But there was no difference in histology depending on gastric part in which ablation was applied. During the study, no complication was observed in pigs in 24 hours after ablation.

Conclusion: The new endoscopic IRE device, which can perform IRE ablation on gastrointestinal tract using endoscopy showed safe and feasible result.

Disclosure of Interest: All authors have declared no conflicts of interest.

P0581 DIAGNOSIS OF MICROVASCULAR PATTERN IS MORE IMPORTANT THAN MICROSURFACE PATTERN TO DELINEATE GASTRIC CANCERS DETECTED AFTER H. PYLORI ERADICATION BY MAGNIFYING ENDOSCOPY

T. Iwasaki, K. Uchita, K. Kojima, S. Iwamura

Kochi Red Cross Hospital, kochi/Japan

Contact E-mail Address: space-rendez-vous@sings.jp

Introduction: It is difficult to delineate gastric cancer that is detected after successful eradication of Helicobacter pylori. One reason is reportedly the difficulty in identifying the demarcation line between the cancerous lesion and non-cancerous gastric mucosa due to a mixture of non-neoplastic epithelial-lined structure inside the neoplasm. However, almost all previous studies have only used magnification endoscopy (ME) at low power magnification which could evaluate microsurface pattern (MSP) but could not evaluate microvascular pattern (MVP)1). The highest power magnification was necessary to evaluate MVP accurately2), and the diagnostic efficacy of ME in delineating the gastric cancers might not have been accurately assessed in these studies.

Aims \& Methods: The aim of this study was investigating diagnostic efficacy of $\mathrm{ME}$ with narrow band imaging (NBI) in delineating the gastric cancers after eradication $\mathrm{HP}$, using ME at highest power magnification, and classifying ME features of the marginal area according to the vessel plus surface classification system (VSCS) to realize which was more important ME findings MSP or MVP to detect demarcation line. Endoscopic examination was performed using a magnification endoscope (GIF-H260Z; Olympus Medical Systems Co, Tokyo, Japan) and NBI system (EVIS LUCERA Spectrum system; Olympus Medical Systems Co, Tokyo, Japan). Endoscopic imaging procedures were performed at lowpower magnification followed by highest power magnification. On the day of ESD, the resection line was marked 3-5 mm outside of the margin of the lesion. A lesion meeting all of the following criteria was defined as a lesion with successful delineation: (1) the demarcation line of the lesion is endoscopically identified with a high level of confidence; (2) According to histopathological findings, the lesion is confined within the resection line. The diagnostic accuracy of ME-NBI in delineating the lesions was evaluated. On the other hand the ME findings of the marginal area in each lesion were classified in terms of microsurface pattern (MSP) and microvascular pattern (MVP) according to the VSCS to identify the findings that were useful in delineating the lesions in patients with differentiatedtype early gastric cancers. The classification according to the VSCS was made in the marginal area with the least irregular findings.

Results: Of 178 consecutive lesions of differentiated-type early gastric cancer treated with endoscopic submucosal dissection (ESD) between August 2013 and March 2017, the study included 59 lesions that were detected after successful $H$. pylori eradication. The result of ME-MBI findings are summarized in the table. Gastric cancer was successfully delineated in $98.3 \%(58 / 59)$ of the lesions with irregular MSP and/or irregular MVP with a demarcation line. Among the ME findings of the demarcation line, irregular MSP and irregular MVP were present in $67.7 \%(40 / 59)$ and $93.2 \%(55 / 59)$, respectively, according to the VSCS, with a higher percentage of lesions with irregular MVP than those with irregular MSP. In addition, there was no finding which was irregular MSP and regular MVP, but $27.1 \%(16 / 59)$ was regular MSP and irregular MVP, which indicated that the ME findings of MVP was more important than MSP. One lesion showed regular MSP or regular MVP without a demarcation line in a portion of the marginal area, resulting in unsuccessful delineation.

\begin{tabular}{llll}
\hline & Regular MVP & Irregular MVP & Absent MVP \\
\hline Regular MSP & $1(1.6 \%)$ & $16(27.1 \%)$ & $0(0 \%)$ \\
Irregular MSP & $0(0 \%)$ & $37(62.7 \%)$ & $3(5 \%)$ \\
Absent MSP & $0(0 \%)$ & $2(3.3 \%)$ & $0(0 \%)$ \\
\hline
\end{tabular}

Conclusion: The accuracy of ME with NBI in delineating gastric cancer detected after H. Pylori eradication was $98.3 \%$, which was higher than the values reported previously. In addition, the MVP as visualized by ME appeared to be a more reliable marker than MSP. These results indicate a meticulous observation of the MVP under maximal magnification to be crucial for the delineation of gastric cancer detected after eradication of $H$. pylori.

Disclosure of Interest: All authors have declared no conflicts of interest.

\section{References}

1. Kobayashi M, Hashimoto S, Nishikura K, et al. Magnifying narrow-band imaging of surface maturation in early differentiated-type gastric cancers after Helicobacter pylori eradication. J Gastroenterol 48:1332-1342, 2013
2. Uchita $\mathrm{K}$, Yao K, Uedo N, et al. Highest power magnification with narrowband imaging is useful for improving diagnostic performance for endoscopic delineation of early gastric cancers. BMC Gastrorntrology 15:155, 2015

\section{P0582 ENDOSCOPIC SMALL CAPACITY FORCEPS INCREASE THE PATHOLOGICAL DIAGNOSIS OF GASTRIC INDEFINITE NEOPLASIA}

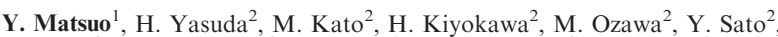
Y. Ikeda ${ }^{2}$, M. Yamashita ${ }^{2}$, T. Fujino ${ }^{3}$, H. Yamamoto ${ }^{2}$, M. Takagi ${ }^{3}$, F. Itoh ${ }^{2}$ ${ }^{1}$ St. Marianna University School of Medicine, Kawasaki/Japan

${ }^{2}$ Gastroenterology And Hepatology, St. Marianna Medical University, Kawasaki/ Japan

${ }^{3}$ Pathology, St. Marianna Medical University, Kawasaki/Japan

Contact E-mail Address: yasumasa_matsuo@marianna-u.ac.jp

Introduction: Endoscopic forceps biopsy (EFB) is the gold standard for gastric epithelial tumor diagnosis. However, definitive diagnosis is often difficult, and some cases are diagnosed as gastric indefinite neoplasia (GIN), which corresponds to category 2 in the revised Vienna classification. GIN lesions require short periods of follow-up. ${ }^{1}$ The most appropriate forceps size for gastric biopsy has yet to be determined. In the Japanese Classification of Gastric Cancer, diagnoses of GIN are attributed, at least partly, to the small size of biopsy specimens. ${ }^{2}$ Since specimens yielded by small biopsy forceps are small, the use of small biopsy forceps is expected to increase the rate of GIN diagnoses.

Aims \& Methods: The relationship between forceps size and the frequency of GIN was investigated. The patients in this cohort were divided into two historical groups. The first group comprised patients evaluated during the period when standard biopsy forceps (StF) were used (April 2010-March 2011), and the second comprised patients evaluated during the period when small biopsy forceps (SmF) were used (April 2011-March 2013). Standard caliber endoscopy was used for all esophagogastroduodenoscopy(EGD). We count the number of GIN and gastric carcinoma lesions. Patient characteristics, lesion characteristics (e.g., site, macroscopic appearance, and color tone), endoscopist experience level, biopsy sample size were investigated in both groups diagnosed as GIN. The clinical courses of GIN cases were followed for 3 years, and the timing of EGD after the GIN diagnosis and the final pathological result were investigated.

Results: Among the 8420 patients who underwent EGD in the first period, 2, 584 $(30.7 \%)$ underwent gastric biopsy with StF. Among the 15,968 patients who underwent EGD in the second period, $4204(26.3 \%)$ underwent gastric biopsy with SmF. Gastric carcinoma was diagnosed in $7.93 \%(205 / 2584)$ and $7.54 \%(317$ $4204)$ of the $\mathrm{StF}$ and $\mathrm{SmF}$ groups, respectively $(P=0.556)$. GIN was diagnosed in $0.73 \%(19 / 2583)$ and $1.25 \%(52 / 4204)$ of the StF and $\mathrm{SmF}$ groups, and the difference was significant $(P=0.048)$. The two groups diagnosed as GIN did not differ significantly in terms of the patient characteristics, the lesion characteristics, endoscopist experience level and biopsy related hemorrhage. The mean minoraxis lengths of the biopsy samples were $1.50 \pm 0.50 \mathrm{~mm}$ and $1.38 \pm 0.40 \mathrm{~mm}$ in the $\mathrm{StF}$ and $\mathrm{SmF}$ groups, respectively. The $\mathrm{SmF}$ group samples tended to be shorter $(P=0.088)$. In both groups, $40 \%$ of the final diagnoses were epithelial neoplasias; no significant differences were observed.

Conclusion: SmF use may increase the rate of GIN. Thus, SmF use should be avoided with a standard caliber endoscopy.

Disclosure of Interest: All authors have declared no conflicts of interest.

\section{References}

1. Dixon MF. Gastrointestinal epithelial neoplasia: Vienna revisited. Gut 2002;51:130-131.

2. Japanese Gastric Cancer Association. Japanese classification of gastric carcinoma: 3rd English edition. Gastric Cancer 2011 Jun; 14:101-112.

\section{P0583 THE ROLE OF STRESS AND NITROSAMINES IN THE DEVELOPMENT OF GASTRIC CANCER: A NEW MODEL OF ADENOCARCINOMA FORMATION WITH METASTASES IN RATS}

A. Khorovodov ${ }^{1}$, I. Agranovich ${ }^{2}$, N. Shushunova ${ }^{1}$, N. Navolokin ${ }^{3}$, A. Telegin ${ }^{1}$, A. Shnitenkova ${ }^{1}$, M. Sagatova ${ }^{1}$, I. Trishkina ${ }^{1}$, M. Ulanova $^{1}$, E. Borisova ${ }^{4}$, O. Semyachkina-Glushkovskaya ${ }^{1}$

${ }^{1}$ Biological, Saratov State University, Satatov/Russian Federation

${ }^{2}$ Biology, Saratov State University, Saratov/Russian Federation

3 Anatomy, Saratov Medical University, Saratov/Russian Federation

${ }^{4}$ Institute of Electronics, Bulgarian Academy of Sciences, Sofia/Bulgaria

Contact E-mail Address: khorovodov2012@yandex.ru

Introduction: Stomach cancer is a leading cause of cancer-related deaths in the world. It well knows that stress play an important role in the cancer. However, for the role of stress in cancer initiation is contradicted and debatable. Other natural factors such as nitrites, which are widely presented in daily food, are actively discussed as carcinogenic to humans. But, there is no clinical and epidemiological evidences that nitrosamines itself can induce the stomach cancer. Aims \& Methods: For the better understanding of carcinogenic effects of daily stress and nitrites in development of stomach cancer, here we studied the role of these factors in adenocarcinoma in stomach of rats. The experiments were carried out with male adult rats $(n=200)$. To examine the role of stress and nitrosamines in gastric mucosal injuries we used: 1) the model of chronic social stress (overpopulation during 9 months); 2) the daily using of toluidine $(2 \mathrm{~g} / \mathrm{kg})$ in food and water with nitrites $(2 \mathrm{~g} / 1) ; 3)$ the combined effects of stress + nitrosamines. The 
upper endoscopy was performed using our in-house custom-made multichannel endoscopy system. Histological assay performed to analyze the changes in the gastric tissues.

Results: Using upper gastroscopy, we studies the stomach tissues during 9 months of lining of rats in chronic stress. There were no changes in the gastric mucosa during the first two months. In the third month 35\% (7 of 20) of animals demonstrated multiple small peptic ulcer $(n=11)$. These changes progressed during other time of observation. 9 months of experiment. So, this time all rats showed peptic ulcers both types with significant increase in the number of ulcers (small, $n=21$ and large, $n=9$ ). Thus, this series of experiments clearly showed that chronic stress plays provoking role in the peptic ulcer formation in the stomach of rats. The deleterious effects of nitrosamines on the gastric mucosa observed 4 months after the beginning of daily using of toluidine and nitrites in $75 \%$ of rats ( 15 of 20$)$. These rats showed symptoms of atrophic gastritis. Other $25 \%$ (5 of 20) of animals did not demonstrate any changes in gastric mucosa. Thus, this series of experiments markedly showed that effect of long-term eating low-dose nitrosamines induced of atrophic gastritis in the stomach of majority of rats. Using similar protocol of the first and second parts of experiments, we observed the changes in the stomach tissues during 9 months. The same scenarios of typical gastric injuries induced by stress and nitrosamines were observed in rats, i.e. they showed development of peptic ulcers and atrophic gastritis. But, 7 months after the start of experiment, these pathological changes of gastric tissues were associated with intestinal metaplasia of goblet cells, which is the pre-cancer symptom. In 9 months of study, symptoms of gastric adenocarcinoma were observed in $82 \%$ of rats (131 of 160$)$. Tumor lesions was accompanied by the migration of metastatic tumor cells through the bloodstream in the liver. The number of metastatic nodes varied from 1 to 5 .

Conclusion: Thus, in our research we clearly show that only combination of two pro-cancer factors such as stress and nitrosamines cause development of gastric cancer with metastasis in the liver while the presence of these factors alone contribute mucosal injuries without oncological changes in the stomach. Disclosure of Interest: All authors have declared no conflicts of interest.

\section{References}

1. Torre, Lindsey A., et al. "Global cancer statistics, 2012." CA: a cancer journal for clinicians 65.2 (2015): 87-108.

2. Sintara, K., and D. Thong-Ngam. "Gastric cancer: the experimental models." Thai J Physiol Sci 21.1 (2008): 31-38.

3. Moreno-Smith, M., Lutgendorf, S. K., \& Sood, A. K. (2010). Impact of stress on cancer metastasis. Future oncology, 6(12), 1863-1881.

4. Jakszyn, Paula, and Carlos Alberto González. "Nitrosamine and related food intake and gastric and oesophageal cancer risk: a systematic review of the epidemiological evidence." World journal of gastroenterology 12.27 (2006): 4296

5. Semyachkina-Glushkovskaya, O. V., et al. "Adrenergic mechanism responsible for pathological alteration in gastric mucosal blood flow in rats with ulcer bleeding." Twelfth International Conference on Photonics and Imaging in Biology and Medicine (PIBM 2014). International Society for Optics and Photonics, 2014

\section{P0585 RELEVANCE OF PTGS1 AND PTGS2 GENE POLYMORPHISMS TO GASTRIC CANCER RISK AND PHENOTYPE IN CAUCASIANS}

M.A. García-González ${ }^{1}$, L. Bujanda Fernández De Piérola ${ }^{2}$, E. Quintero Carrion $^{3}$, M. Strunk ${ }^{4}$, F. Sopeña-Biarge ${ }^{5}$, S. Santolaria ${ }^{6}$, R. Benito ${ }^{7}$, E. Piazuelo ${ }^{8}$, C. Thomson ${ }^{9}$, Á. Pérez-Aísa ${ }^{10}$, M. Badía $^{11}$, D. Nicolás-Pérez ${ }^{3}$ E. Hijona ${ }^{12}$, J. Espinel ${ }^{13}$, R. Campo ${ }^{14}$, M. Pellisé ${ }^{15}$, M. Manzano ${ }^{16}$, F. Geijo ${ }^{17}$ F. González-Huix ${ }^{18}$, M. Zaballa ${ }^{19}$, J. Espinós ${ }^{20}$, L. Titó $^{21}$, L. Barranco ${ }^{22}$, Á. Lanas

${ }^{1}$ Instituto de Investigación Sanitaria Aragón (IIS Aragón), Instituto Aragonés de Ciencias de la Salud (IACS), CIBER de enfermedades hepáticas y digestivas

(CIBERehd), Zaragoza/Spain

${ }^{2}$ Gastroenterology, Hospital Donostia/Instituto Biodonostia, CIBERehd,

Universidad del País Vasco (UPV/EHU), San Sebastian/Spain

${ }^{3}$ Gastroenterology, Hospital Universitario de Canarias, Instituto Universitario de Tecnologías Biomédicas (ITB), Centro de Investigación Biomédica de Canarias (CIBICAN), Tenerife/Spain

${ }^{4}$ Instituto Aragonés de Ciencias de la Salud (IACS)., Zaragoza/Spain

${ }^{5}$ Gastroenterology, Hospital Clínico Universitario Lozano Blesa, IIS Aragón, CIBERehd., Zaragoza/Spain

${ }^{6}$ Gastroenterology, Hospital San Jorge, Huesca/Spain

${ }^{7}$ Microbiology, Faculty of Medicine, Hospital Clínico Universitario Lozano Blesa, IIS Aragón, CIBERehd, Zaragoza/Spain

${ }^{8}$ IIS Aragón-IACS, CIBERehd, Zaragoza/Spain

${ }^{9}$ Gastroenterology, Hospital Obispo Polanco, Teruel/Spain

${ }^{10}$ Gastroenterology, Hospital Costa del Sol, Marbella/Spain

${ }^{11}$ Gastroenterology, Hospital General de la Defensa, Zaragoza/Spain

${ }^{12}$ Gastroenterology, Hospital Donostia/Instituto Biodonostia, CIBERehd., San

Sebastián/Spain

${ }^{13}$ Gastroenterology, Complejo Hospitalario de León, León/Spain

${ }^{14}$ Gastroenterology, Hospital Parc Tauli, Sabadell/Spain

${ }^{15}$ Gastroenterology, Hospital Clinic, Institut d Investigacions Biomèdiques August Pi i Sunyer (IDIBAPS). Universidad de Barcelona, CIBERehd., Barcelona/Spain ${ }^{16}$ Gastroenterology, Hospital 12 de Octubre, Madrid/Spain

${ }^{17}$ Gastroenterology, Hospital Clínico Universitario, Salamanca/Spain

${ }^{18}$ Gastroenterology, Hospital Josep Trueta, Girona/Spain
${ }^{19}$ Gastroenterology, Hospital de Cruces, Barakaldo/Spain

${ }^{20}$ Gastroenterology, Hospital Mutua Terrassa, Barcelona/Spain

${ }^{21}$ Gastroenterology, Hospital de Mataró, Mataró/Spain

${ }^{22}$ Gastroenterology, Hospital del Mar, Barcelona/Spain

Contact E-mail Address: asgarcia@unizar.es

Introduction: Cyclooxigenases 1 (COX-1) and 2 (COX-2) are key enzymes in mediating the conversion of arachidonic acid into prostaglandins which are important regulators of the immune inflammatory response in the gastrointestinal mucosa $a^{1,2}$. COX-1 is constitutively expressed in most cell types and involved in maintaining gastric mucosal homeostasis, whereas COX-2 is induced by proinflammatory and mitogenic stimuli and involved in several relevant mechanisms of gastric cancer (GC) development.

Aims \& Methods: Since variants in the PTGS1 and PTGS2 genes may modify the expression or function of their encoded enzymes COX-1 and COX-2, the aim of our study was to assess the relevance of environmental factors and several functional PTGS1 and PTGS2 gene polymorphisms on GC risk and phenotype in a Caucasian population in Spain. Methods: DNA from 603 unrelated Spanish patients with primary GC and 603 sex- and aged- ( \pm 5 years) matched cancerfree healthy controls was typed for 10 single nucleotide polymorphisms (SNPs) in the PTGS1 $(+50 \mathrm{C} / \mathrm{T}$, and $+644 \mathrm{C} / \mathrm{A})$ and PTGS2 $(-1195 \mathrm{~A} / \mathrm{G},-765 \mathrm{G} / \mathrm{C},-297 \mathrm{C} /$ $\mathrm{G},+3050 \mathrm{G} / \mathrm{C},+8473 \mathrm{~T} / \mathrm{C},+9850 \mathrm{~A} / \mathrm{G},+10335 \mathrm{G} / \mathrm{A}$, and Val511Ala) genes by PCR, RFLP and TaqMan assays. H. pylori infection and CagA/VacA antibody status were also determined by western blot in patients and controls.

Results: H. pylori infection with CagA strains (OR: 1.99; 95\% CI: 1.55-2.54), smoking habit (OR: $1.77 ; 95 \%$ CI: $1.22-2.57$ ) and positive family history of GC (OR: 3.02; 95\% CI: 1.99-4.60) were identified as independent risk factors for GC. Concerning gene polymorphisms, no differences in carriage, genotype, and allele frequencies of the PTGS1 and PTGS2 gene polymorphisms were found between GC patients and controls. However, when GC patients were stratified according to the localization of the tumor, we found that carriers of the PTGS2 -1195 (rs689466) $\mathrm{G}$ allele were significantly more frequent in the group of cardia-GC patients than in controls $(46.8 \%$ vs. $32.8 \%$; OR: $1.77 ; 95 \%$ CI: $1.06-2.95)$. Moreover, carriers of the PTGS2 GGGTAG haplotype that contains the PTGS2-1195G allele, were also more frequent in the group of cardia-CG patients than in controls $(41.3 \%$ vs. $32.1 \%$; OR: 1.73 ; $95 \%$ CI: $0.86-3.49)$. Finally, no differences in carriage, genotype, and allele frequencies of the PTGS1 and PTGS2 gene polymorphisms were found when GC patients were categorized according to gender, age, smoking habit, H. pylori infection, CagA/VacA antibody status, family history of GC, and histological subtype of the tumor.

Conclusion: Our data show that the PTGS2-1195A/G (rs689466) gene polymorphism is involved in defining the genetic basis of the susceptibility to cardia-gastric cancer in a South-European population.

Disclosure of Interest: All authors have declared no conflicts of interest.

\section{References}

1. Dubois RN, Abramson SB, Crofford L, Gupta RA, Simon LS, et al. (1998) Cyclooxygenase in biology and disease. FASEB J. 12: 1063-1073.

2. Fujimura T, Ohta T, Oyama K, Miyashita T, Miwa K (2006) Role of cyclooxygenase- 2 in the carcinogenesis of gastrointestinal tract cancers: a review and report of personal experience. World J Gastroenterol. 12: 13361345 .

\section{P0586 GASTRIC JUICE FREE AMINO ACID PROFILING AS A METHOD FOR DISCOVERING POTENTIAL BIOMARKERS OF GASTRIC CANCER}

J. Liu ${ }^{1}$, Y. L. Zhou ${ }^{1}$, R. S. Lin ${ }^{1}$, Y. Xue ${ }^{1}$, E. X. Yan ${ }^{1}$, M. L. Meng ${ }^{1}$, J. B. Suo ${ }^{1}$, J. J. $\mathrm{Lu}^{1}$

${ }^{1}$ Department Of Gastroenterology, Peking University Third Hospital, Beijing/

China

Contact E-mail Address: liujian-825@163.com

Introduction: Gastric cancer (GC) contributes a heavy burden to the global health, especially in Asian countries. Early diagnosis is crucial to improve patients' outcome, but reliable biomarkers are desperately needed. In our previous studies, we established several endogenous fluorescence spectra of gastric juice for GC diagnosis and screening [1,2], isolated and identified three fluorescence candidates (aromatic amino acids, AAAs), which can be used to distinguish GC from non-neoplastic gastric diseases (NGD) regardless of the stage [3, 4]. However, the characteristic biosignature for the whole metabolic spectra of gastric juice free amino acids (GJFAAs) in GC patients remain unclear. Although many investigators had reported on the changes of amino acids' concentrations in the peripheral blood, urine and tissues of GC patients $[5,6]$.

Aims \& Methods: In order to determine the metabolic patterns of GJFAA in GC and NGD patients, gastric juice samples were collected from GC patients $(n=47)$ and age-matched NGD patients $(\mathrm{n}=83)$ from December 2015 to May 2016, and then measured by an automatic amino acid analyzer. Orthogonal partial least squares discriminant analysis (OPLS-DA) and Mann-Whitney U test are used for data analysis. The diagnostic value of GJFAAs was evaluated by ROC curve. Furthermore, significantly altered metabolic pathways were identified by pathway analysis using public databases such as KEGG and MetaboAnalyst 3.0.

Results: GJFAA profiles significantly differed between the GC and NGD patients. A total of 14 kinds of GJFAAs, whose first principal component of variable importance in the projection (VIP) value exceeding 1 and $P$-value less than 0.05 , were screened as differential GJFAAs. Compared with the NGD patients, GC patients had higher levels of threonine, serine, alanine, valine, methionine, isoleucine, leucine, tyrosine, phenylalanine, lysine and arginine, 
together with lower levels of phosphoserine, ethanolamine phosphate and urea (Table 1). The 14 GJFAAs revealed diagnostic values with AUC from 0.666 to 0.868 , and the combined AUC of them reached to 0.902 (95\% CI, 0.846-0.959) for the diagnosis of GC. Importantly, their AUCs were from 0.649 to 0.857 , and the combined AUC reached to 0.880 (95\% CI, 0.792-0.969) for the diagnosis of early GC. Particularly, leucine, threonine and serine are the most altered three GJFAAs between the two groups, whose fold change more than 2 and AUC value greater than 0.8. Moreover, the combined AUC of the 3 non-AAAs was 0.869 (95\% CI, 0.805-0.934) for the diagnosis of GC. It was slightly higher than that combined with 3 AAAs 0.841 (95\% CI, 0.773-0.908). Additionally, the pathway of aminoacyl-tRNA biosynthesis metabolism was excessively activated, which significantly responsible for the above metabolic alternations in GC.

Table 1: Differential GJFAAs between GC and NGD patients and their discriminating performance

\begin{tabular}{lllllllll}
\hline \multirow{2}{*}{ Number } & Abbreviation & $\begin{array}{l}\text { Median } \\
\text { GC }\end{array}$ & $\begin{array}{l}\text { Median } \\
\text { NGD }\end{array}$ & P-value & VIP & FC & AUC & $95 \%$ CI \\
\hline AA01 & PSer & 0.028 & 0.037 & 0.002 & 1.054 & 0.768 & 0.666 & $0.561-0.771$ \\
AA03 & PEtN & 0.007 & 0.018 & $<0.001$ & 1.028 & 0.606 & 0.718 & $0.615-0.820$ \\
AA04 & Urea & 0.178 & 0.604 & $<0.001$ & 1.058 & 0.486 & 0.804 & $0.729-0.880$ \\
AA06 & Thr & 0.022 & 0.007 & $<0.001$ & 1.489 & 2.431 & 0.835 & $0.764-0.907$ \\
AA07 & Ser & 0.016 & 0.005 & $<0.001$ & 1.420 & 2.671 & 0.831 & $0.759-0.903$ \\
AA12 & Ala & 0.033 & 0.016 & $<0.001$ & 1.238 & 1.973 & 0.783 & $0.702-0.865$ \\
AA15 & Val & 0.025 & 0.013 & $<0.001$ & 1.025 & 1.763 & 0.717 & $0.621-0.814$ \\
AA17 & Met & 0.017 & 0.007 & $<0.001$ & 1.178 & 2.148 & 0.797 & $0.718-0.877$ \\
AA18 & Ile & 0.026 & 0.007 & $<0.001$ & 1.343 & 2.674 & 0.812 & $0.736-0.887$ \\
AA19 & Leu & 0.075 & 0.020 & $<0.001$ & 1.626 & 2.697 & 0.868 & $0.803-0.933$ \\
AA20 & Tyr & 0.066 & 0.026 & $<0.001$ & 1.580 & 1.926 & 0.833 & $0.765-0.902$ \\
AA21 & Phe & 0.069 & 0.032 & $<0.001$ & 1.415 & 1.754 & 0.802 & $0.720-0.883$ \\
AA31 & Lys & 0.044 & 0.015 & $<0.001$ & 1.091 & 2.321 & 0.804 & $0.725-0.883$ \\
AA32 & Arg & 0.036 & 0.008 & $<0.001$ & 1.332 & 2.722 & 0.772 & $0.686-0.859$ \\
\hline
\end{tabular}

$P$-value, Statistically significant difference using Mann-Whitney $U$ test; VIP, variable importance in the projection; FC, Fold Change; AUC, area under the ROC curve; $95 \% \mathrm{CI}, 95 \%$ confidence interval.

Conclusion: GJFAA profiles may be helpful for improving GC diagnosing even in the early stage and for providing more information about its metabolism. Leucine, threonine and serine, three non-AAAs, warrant further validation as alternative metabolic biomarkers for GC.

Disclosure of Interest: All authors have declared no conflicts of interest.

\section{References}

1. Zhou LY, Lin SR, Li Y, et al. The intrinsic fluorescence spectrum of dilute gastric juice as a novel diagnostic tool for gastric cancer. J Dig Dis 2011, 12(4):279-285.

2. Deng K, Zhou LY, Lin SR, et al. A novel approach for the detection of early gastric cancer: fluorescence spectroscopy of gastric juice. J Dig Dis 2013, 14(6):299-304

3. Deng K, Lin S, Zhou L, et al. Three aromatic amino acids in gastric juice as potential biomarkers for gastric malignancies. Anal Chim Acta 2011, 694(12):100-107.

4. Deng K, Lin S, Zhou L, et al. High levels of aromatic amino acids in gastric juice during the early stages of gastric cancer progression. PLoS One 2012, 7(11):e49434.

5. Gu Y, Chen T, Fu S, et al. Perioperative dynamics and significance of amino acid profiles in patients with cancer. $J$ Transl Med 2015, 13:35.

6. Fan J, Hong J, Hu JD, et al. Ion chromatography based urine amino Acid profiling applied for diagnosis of gastric cancer. Gastroenterol Res Pract 2012, 2012:474907.

\section{P0587 THE ASSOCIATION BETWEEN MMP-2/9 AND TYPE IV COLLAGEN AND THE LEVELS OF AROMATIC AMINO ACIDS IN GASTRIC JUICE OF GASTRIC CANCER PATIENTS}

J. Liu, Y. L. Zhou, R. S. Lin, Z. Jin, J. Y. Han, Y. Xue, E. X. Yan, M. L. Meng Department Of Gastroenterology, Peking University Third Hospital, Beijing/China

Contact E-mail Address: liujian-825@163.com

Introduction: It is reported that aromatic amino acids (AAAs) in gastric juice could be used as potential diagnostic biomarkers to screen gastric cancer (GC) [1-3]. However, the underlying mechanism remain elusive [4]. Our group had conducted a series of explorations on the reasons to cause such phenomenon before. The candidate molecules: 1) L- type amino-acid transporer $1\left(\mathrm{LAT}_{1}\right)$, which is involved in the enhancement transport of amino acids and the accumulation of AAAs near cancer foci; 2) intracellular amino-acid-metabolizing enzymes, such as indoleamine 2, 3-dioxygenase (IDO) and monoamine oxidase (MAO); 3) proteins involved in intracellular protein degradation or autophagy, (e.g., SQSTM1/p62) had been examined [5]. However, because of the difference in the expression of the above proteins in different pathological classifications of GC tissues, it is impossible to explain the phenomenon that the elevation of AAAs' levels in gastric juice in almost all types of GC patients.

Aims \& Methods: To investigate the role of proteolytic enzymes matrix metalloproteinase $-2 / 9$ (MMP-2/9) in the abnormal elevation of AAAs' concentrations in gastric juice of GC patients, gastric mucosal specimens and gastric juice samples were simultaneously collected from 29 GC patients and 91 age- and gendermatched non-neoplastic gastric disease (NGD) patients. The expression levels of MMP-2/9 and type IV collagen (Col IV) in gastric mucosal tissues were examined by immunohistochemical staining while the levels of AAAs in gastric juice were measured by liquid chromatography-tandem mass spectrometry (LCMS/MS). Furthermore, the association between them was evaluated by Spearman correlation analysis.

Results: On the one hand, the expression intensity of MMP-2/9 in GC group were significantly higher than those in NGD group, while Col IV was markedly lower than that in NGD group $(P<0.001$ for all). Moreover, there was a positive correlation between the expression level of MMP-2 and MMP-9 (rho $=0.439$, $P<0.01$ ), but they were both negatively correlated with Col IV (rho $=-0.454$, $P<0.01 ;$ rho $=-0.392, P<0.01)$. On the other hand, significantly higher levels of AAAs in gastric juice were observed in GC patients than those in NGD individuals $(P<0.001$ for all $)$. Ultimately, the expression levels of MMP-2/9 in gastric mucosal tissues were both positively correlated with the concentrations of AAAs in gastric juice (MMP-2 rho $=0.262,0.295$, and 0.293 , respectively, $P<0.01$ for all; MMP-9 rho $=0.457,0.455$, and 0.417 , respectively, $P<0.001$ for all), but Col IV was negatively correlated with them $($ rho $=-0.283,-0.280$, and -0.273 , respectively, $P<0.01$ for all) (Table 1$)$.

Table 1: Relationship between the expression levels of MMP-2/9 and Col IV in gastric tissues and the levels of AAAs in gastric juice

\begin{tabular}{llll}
\hline Variable & MMP-2 & MMP-9 & Col IV \\
\hline Tyrosine & $0.262^{* *}$ & $0.457^{* * *}$ & $-0.283^{* *}$ \\
Phenylalanine & $0.295^{* *}$ & $0.455^{* * *}$ & $-0.280^{* *}$ \\
Tryptophan & $0.293^{* *}$ & $0.417^{* * *}$ & $-0.273^{* *}$
\end{tabular}

${ }^{* *}$ represents significant correlation using Spearman correlation analysis when the confidence level was $0.01 ; * *$ represents significant correlation using Spearman correlation analysis when the confidence level was 0.001 .

Conclusion: The overexpression of MMP-2/9 resulting in the degradation of $\mathrm{Col}$ IV in basement membrane and extracellular matrix may lead to the variation of AAAs' levels in gastric juice of GC patients.

Disclosure of Interest: All authors have declared no conflicts of interest.

\section{P0588 MISSING RATE OF GASTRIC CANCER DURING UPPER GASTROINTESTINAL ENDOSCOPY AND INFLUENCE ON THE NATURAL HISTORY OF THE DISEASE}

R. Salmoral ${ }^{1}$, J. Rodriguez-Sanchez ${ }^{2}$, F. Martín Dávila ${ }^{2}$, B. López Viedma ${ }^{3}$, P. Olivencia Palomar ${ }^{3}$, E. De La Santa Belda ${ }^{3}$, J. Olmedo Camacho ${ }^{3}$ ${ }^{1}$ Gastroenterology, Hospital General Universitario Ciudad Real, Ciudad Real/ Spain

${ }^{2}$ Hospital General Universitario de Ciudad Real, Ciudad Real/Spain ${ }^{3}$ Endoscopy Unit, Hospital General Universitario de Ciudad Real, Ciudad Real Spain

Contact E-mail Address: salmora172@gmail.com

Introduction: Gastric cancer (GC) is the fourth most common type of cancer and the second leading cause of cancer related death. The gold standard for diagnosis is the esophagogastroduodenoscopyy (EGD) with targeted biopsies.

Aims \& Methods: Retrospective, observational and descriptive study in patients diagnosed of gastric cancer from January 2013 to December 2016 in the area of Ciudad Real (Spain). Missing rate of gastric cancer was defined as the percentage patients who had a negative EGD three years before the diagnosis of CG. A survival analysis was performed with Kapplan Meier curves, mainly focussing on the influence of missing rate for gastric cancer. We studied the features related to EGD that could lead this issue.

Results: 162 patients were included, $65 \%$ male with a mean age at diagnosis of 72 years. Intestinal type was the most common histology $(76 \%)$. A rate of $6.8 \%$ missing of gastric cancer was detected with an average of 20 months in delay of diagnosis. However, the survival rate was similar between patients with and without a previous EGD (7.08 vs 5.05 months $\mathrm{p}=0.60)$. Among the patients who passed away, a longer delay period was observed comparing to patients who were still alive ( 6 months vs. 25 months; $p=0.006$ ). In the aforementioned subgroup, biopsias were taken in $72 \%$ with gastric atrophy in all these cases. Helicobacter pylori infection was detected in $50 \%$ of them. $55.6 \%$ of the EGDs were carried out without sedation. At no point chromoendoscopy was performed, pictures were taken and withdrawal times were not reflected.

Conclusion: Despite the fact that EGD is by far the most effective method to diagnose gastric cancer, 1 out of 10 cancers or premalignant lesions are not figured out. Therefore, it is of utmost importance to put in place quality protocols in EGD that may help to increase the diagnosis of early gastric cancer, and by this way, improve the survival rate of these patients.

Disclosure of Interest: All authors have declared no conflicts of interest.

\section{References}

1. Voutilainen ME and Juhola MT. Evaluation of the diagnostic accuracy of gastroscopy to detect gastric tumors: clinicopathological features and prognosis of patients with gastric cancer missed on endoscopy. European Journal of Gastroenterology and Hepatology 2005, 17:1345-1349. 
2. Pimenta-Melo AR, Monteiro-Seores M, Libânio D and Dinis-Ribeiro M. Missing rate for gastric cancer during upper gastrointestinal endoscopy: a systematic review and meta-analysis. European Journal of Gastroenterology and Hepatology. 2016; 28: 1041-1049.

\section{P0589 INTERFERENCE OF PG2 TATA BOX REGION WITH THE SERUM PG2 LEVEL IN GASTRIC CANCER}

V. De Re ${ }^{1}$, R. Magris ${ }^{2}$, M. De Zorzi ${ }^{1}$, S. Maiero ${ }^{2}$, L. Caggiari ${ }^{1}$, M. Fornasarig ${ }^{2}$, O. Repetto ${ }^{1}$, E. Buscarini ${ }^{3}$, F. Di Mario ${ }^{4}$, R. Cannizzaro ${ }^{2}$

${ }^{1}$ Sos Bioimmunoterapia Dei Tumori Umani/bioproteomics Facility, Centro di Riferimento Oncologico di Aviano, Aviano/Italy

${ }^{2}$ Oncological Gastroenterology, Centro di Riferimento Oncologico di Aviano S.O.C. di Gastroenterologia, Aviano/Italy

${ }^{3}$ Dept. Of Gastroenterology, Gastroenterology Dept Maggiorehospital, Crema/Italy

${ }^{4}$ University Of Parma, Department of Clinical and Experimental Medicine, section of Gastroenterology, Parma/Italy

\section{Contact E-mail Address: rcannizzaro@cro.it}

Introduction: Several studies have demonstrated serum PGII level as a marker of the functional gastric mucosa, and a marker of some tumor including the gastric cancer. However, the modulation of the protein and its role in cancer is not fully understood. The aim of this study was to analyse the polymorphisms in the TATA BOX region, which provides a binding site for the transcription factor for the PG2 gene, in association with the PG2 circulating level and clinical parameters in population at risk for GC and in GC patients.

Aims \& Methods: Gastric function of 180 patients (67 GC, 71 first-degree relatives of GC patients (FDR-GC) and 42 autoimmune chronic AG (ACAG)) was assessed by gastropanel test. We investigated the PG2 TATA BOX polymorphism frequencies in relation to serum PG2 (sPG2) expression level, HP-positivity and risk for GC. TATA BOX DNA fragments were amplified by PCR and analyzed by the capillary-electrophoresis (GeneMapper software). Association among clinical data and PG2 polymorphisms were estimated by Receiver operating characteristic (ROC) curve and linear regression analyses.

Results: After ROC curve analysis, the sensitivity to discriminate GC at $15 \mathrm{ng} / \mathrm{mL}$ PG2 cut-off was $70.15 \%$ and $79,65 \%$ sensibility and sensitivity, respectively (AUC: $758, \mathrm{p}<0.0001$ ). We obtained 26 different PG2 TATA box fragments (from $308 \mathrm{bp}$ to $479 \mathrm{bp}$ ). These fragments were grouped into 4 sized categories $(1=308-400 \mathrm{bp} ; 2=401-436 \mathrm{bp} ; 3=437-438 \mathrm{bp} ; 4=439-479 \mathrm{bp})$. A positive correlation among the increase of PG2 sized fragments and the SPG2 level was found in the GC group (linear regression $\mathrm{y}=16,4381+2,6846 \mathrm{x}, \mathrm{p}=0.02$ )

Conclusion: Accordantly to the literature, we confirm sPG2 level as a marker discriminating between GC and individuals at risk for GC (i.e ACAG and FDR) in our series. In addition we reported a correlation between the shortest PG2 TATA BOX fragments and the lower PG2 level. Since highest PG2 level was related to the GC condition, our data suggest that carriers having longer TATA BOX region may produce higher sPG2 level than patients with shorter region. The clinical significance of the differences in PG2 level associated with the TATA BOX fragments, by interfering with the transcriptional factor and then with the expression of the corresponding gene, are under investigation. This study will be important to deeper understand the physiopathological PG2 role in GC.

Disclosure of Interest: All authors have declared no conflicts of interest.

MONDAY, OCTOBER 30, 2017

09:00-17:00

\section{H. PYLORI I - HALL 7}

\section{P0590 HELICOBACTER PYLORI INFECTION ASSOCIATED WITH NONALCOHOLIC FATTY LIVER DISEASE: A LARGE-SCALE COHORT STUDY}

T. Kim, T.J. Kim, H. Lee, J.J. Kim

Department Of Medicine, Samsung Medical Center, Sungkyunkwan University

School of Medicine, Seoul/Korea, Republic of

Contact E-mail Address: attack836@naver.com

Introduction: Previous studies suggested a link between Helicobacter pylori $(H$. pylori) infection and nonalcoholic fatty liver disease (NAFLD), yet large-scale longitudinal studies are lacking to elucidate this association.

Aims \& Methods: A cohort study of 17,028 adults without NAFLD at baseline, who participated in a repeated health screening examination including an $H$. pylori-specific immunoglobulin $\mathrm{G}$ antibody test, was conducted to evaluate the association between $H$. pylori and NAFLD development. Fatty liver was diagnosed by ultrasonography.

Results: During the 83,130 person-years follow-up, participants with $H$. pylori infection had a higher rate of incident NAFLD than those who were uninfected. In a multivariable model adjusted for age, sex, body mass index, smoking status, alcohol intake, regular exercise, year of screening exam, and education level, the hazard ratio (HR) for NAFLD development in participants with $H$. pylori-infection compared to those without infection was 1.21 (95\% confidence interval [CI], $1.10-1.34)$. The association persisted after further adjustment for metabolic variables, inflammatory marker, and liver enzymes. The association between $H$. pyloriand NAFLD was still evident in an analysis using fatty liver index as a surrogate marker of NAFLD. In addition, the association between $H$. pylori infection and incident NAFLD did not differ across clinically relevant subgroups evaluated.
Table 1: Development of nonalcoholic fatty liver disease (NAFLD) by $H$. pylori status

\begin{tabular}{llllll}
\hline & $\begin{array}{l}\text { Person- } \\
\text { years }\end{array}$ & $\begin{array}{l}\text { Number of } \\
\text { Incident } \\
\text { cases }\end{array}$ & $\begin{array}{l}\text { Incidence } \\
\text { density } \\
\text { (per 1, 000 } \\
\text { person-years) }\end{array}$ & $\begin{array}{l}\text { Age- and } \\
\text { sex- } \\
\text { adjusted HR } \\
(95 \% \mathrm{CI})\end{array}$ & $\begin{array}{l}\text { Multivariable- } \\
\text { adjusted } \\
\mathrm{HR}^{\mathrm{a}}(95 \% \mathrm{CI})\end{array}$ \\
\hline H. pylori (-) & $34,960.7$ & 1301 & 37.2 & 1.00 (reference) & 1.00 (reference) \\
H. pylori (+) & $48,169.3$ & 2080 & 43.2 & $1.14(1.06-1.22)$ & $1.21(1.10-1.34)$
\end{tabular}

${ }^{a}$ Estimated from Cox proportional hazard models adjusted for age, sex, body mass index, year of screening exam, smoking status, alcohol intake, regular exercise, and education level. H. pylori, helicobacter pylori; HR, hazards ratio; $\mathrm{CI}$, confidence intervals.

Conclusion: $H$. pylori infection was significantly associated with the development of NAFLD, independent of metabolic and inflammatory risk factors. H. pylori infection may play a pathophysiologic role in NAFLD development, indicating that $H$. pylori eradication might play a role in reducing risk of NAFLD Disclosure of Interest: All authors have declared no conflicts of interest.

\section{P0591 HELICOBACTER PYLORI INFECTION STATUS IN HUMAN} IMMUNODEFICIENCY VIRUS-POSITIVE PATIENTS

S. Kato, T. YamadaY. Fujii, A. Shoji, R. Kiyota, K. Shinkai, T. Tashiro, A. Ishihara, T. Iwasaki, S. Tanaka, H. Hasegawa, Y. Sakakibara, S. Nakazuru, H. Ishida, E. Mita

Gastroenterology\&hepatology, Osaka National Hospital, Osaka/Japan

Contact E-mail Address: seiya weckle_0728@yahoo.co.jp

Introduction: Helicobacter pylori infects the gastric mucosa and causes chronic gastritis via the immunoreaction of the host. By contrast, the human immunodeficiency virus (HIV) infects CD4-positive T lymphocytes and destroys the immune system of the host. Some studies pointed out that the H.pylori infection rate is lower in HIV-positive patients. This is because in these patients, H. pylor is incidentally eradicated by the course of antibiotic therapy for HIV infection and because the supply of nutrients to $H$. pylori is prevented by the decrease in the number of CD4 lymphocytes.

Aims \& Methods: We enrolled 290 HIV-positive patients who underwent esophagogastroduodenoscopy in our Hospital between January 2013 and September 2016. As end points of $H$. pylori infection examination, we retrospectively examined for the presence of gastric mucosa atrophy, H. pylori infection, H. pylori eradication and comorbidity. As end points of HIV infection examination, we quantified the number of CD4 lymphocytes and the titer of HIV and investigated the presence of acquired immunodeficiency syndrome (AIDS). Based on these data, we examined the relationship between $H$. pylori and HIV infections.

Results: Of the 290 patients, 281 were men and 9 were women, whose median age was 46 years (range, 22-82 years). Ninety patients had atrophic gastritis or stomach or duodenal ulcer, of whom 40 underwent examination for $H$. pylori infection. The median number of CD4 lymphocytes in the $21 \mathrm{H}$. pylori-positive cases was $505 / \mu \mathrm{L}$ (range, $108-952 / \mu \mathrm{L}$ ). The titer of HIV ranged from non-detection to 90,900 copies/mL, and one patient had AIDS. Meanwhile, the median number of CD4 lymphocytes in the $19 \mathrm{H}$. pylori-negative cases was $331 / \mu \mathrm{L}$ (range, 15-998/ $\mathrm{LL}$ ), the titer of HIV ranged from non-detection to $1,590,000$ copies/mL, and three patients had AIDS. H. pylori eradication therapy was applied in 18 of $21 \mathrm{H}$. pylori-positive cases. The success rate of primary H. pylori eradication was $37.5 \%$ (6/16 patients) and that of secondary eradication was $70 \%$ (7/10 patients). In addition, $2(7.7 \%)$ of the 26 patients with stomach or duodenal ulcer needed urgent hemostasis. Five $(6.7 \%)$ of the 74 cases of atrophic gastritis had gastric cancer, of which two were undifferentiated stomach cancers. Conclusion: In our study, the number of CD4 lymphocytes was higher in the HIV-positive patients with $H$. pylori infection, implying that the high CD4 count was suggested to be associated with persistent $H$. pylori infection. In addition, the success rate of $H$. pylori eradication was shown to be insufficient in HIV-positive patients.

Disclosure of Interest: All authors have declared no conflicts of interest.

\section{References}

1. Nevin DT, Morgan CJ, Graham DY, Genta RM: Helicobacter pylori gastritis in HIV-infected patients: a review. Helicobacter. 2014 Oct;19(5):323-9.

2. Romanelli F, Smith KM, Murphy BS: Does HIV infectioin alter the incidence or pathology of Helicobacter pylori infection?. AIDS Patient Care STDS. 2007 Dec;21(12):908-19.

3. Battan R, Raviglione MC, Palagiano A, Boyle JF, Sabatini MT, Sayad K, Ottaviano LJ: Helicobacter pylori infection in patients with acquired immune deficiency syndrome. Am J Gastroenterol. 1990 Dec;85(12):1576-9. 
P0592 HELICOBACTER PYLORI INFECTION REDUCES THE RISK OF BARRETT'S OESOPHAGUS AND IT IS INDEPENDENT FROM THE GEOGRAPHICAL LOCATION, A META-ANALYSIS

B. Eross ${ }^{1}$, N. Farkas ${ }^{2}$, K. Márta ${ }^{3}$, P. Hegyi ${ }^{1}$

${ }^{1}$ Centre For Translational Medicine, University of Pecs, Pecs/Hungary

${ }^{2}$ Institute Of Bioanalysis, Universitiy of Pécs, Pécs/Hungary

${ }^{3}$ Institute For Translational Medicine, University of Pécs, Pécs/Hungary

Contact E-mail Address: xerobal@yahoo.it

Introduction: In Western European and Northern American populations a decreasing prevalence of $H$. pylori infection has been observed, along with an increasing prevalence of Barrett's oesophagus and an increasing incidence and prevalence of the adenocarcinoma of the oesophagus and gastro-oesophageal junction. In previous meta-analyses, $\mathrm{H}$ pylori infection has been proven a protective factor against Barrett's oesophagus, but some individual studies suggested the opposite.

Aims \& Methods: Our aim was to scrutinise all data available on the relationship between $\mathrm{H}$ pylori infection and Barrett's oesophagus prevalence, to see, if $\mathrm{H}$ pylori has a protective role for Barrett's oesophagus and if it is dependent from the geographical location. A meta-analysis was performed using the preferred reporting items for systematic review and meta-analysis protocols (PRISMA-P). We conducted a systematic search in PUBMED, EMBASE and COCHRANE databases from inception to December 2016, for the keywords of Barrett's, Barrett's metaplasia, Barrett metaplasia, Barrett's oesophagus, Barrett's oesophagus, Barrett oesophagus, Barrett oesophagus, Helicobacter pylori, H pylori, H. pylori and Helicobacter. We also used information from the references of relevant publications to find further eligible studies. We have conducted a meta-analysis of the data from all studies included. We used the random effect modell by DerSimonian and Laird.

Results: We have found 568 articles in PUBMED, 741 in EMBASE and 15 in COCHRANE databases. After exclusion of the articles without sufficient data on the prevalence of $\mathrm{H}$ pylori and Barrett's oeosphagus, we have identified 83 articles with detailed data, suitable for statistical analysis. This meta-analysis involved 98665 patients with Barrett's oesophagus and 720800 patients without Barrett's oesophagus. The statistical analysis from all 83 studies from five continents and 27 countries showed a protective effect of $\mathrm{H}$ pylori for Barrett's oesophagus on four continents, but not in Africa, overall Odds Ratio $=0.63$ (95\% CI:0.55, 0.71). The OR and $95 \%$ CI values were $0.34(0.17,0.67)$ for Asia, $0.71(0.55,0.91)$ for Europe, $3.05(0.59,15.73)$ for Africa, $0.60(0.51$, $0.71)$ for North-America, $0.95(0.56,1.64)$ for South- America and $0.56(0.39$, $0.80)$ for Australia. The OR and $95 \%$ CI values were $0.84(0.43,1.64)$ for Eastern Europe; $0.68(0.52,0.90)$ for Western Europe and $0.71(0.55,0.91)$ for all of Europe, suggesting that the protective role of $\mathrm{H}$ pylori infection is not different across Europe.

Conclusion: This large meta-analysis has given further evidence, that Helicobacter pylori infection has a protective role for Barrett's oesophagus and this protective role is independent from the geographical location, apart from Africa. In view of the decreasing prevalence of $\mathrm{H}$ pylori in developed countries and the epidemiological rise of Barrett's oesophagus and adenocarcinoma, it would be important to conduct further large, prospective, multinational studies on the effect of H. pylori infection on Barrett's oesophagus.

Disclosure of Interest: All authors have declared no conflicts of interest.

\section{References}

The reference list for all studies involved in this meta-analysis will be presented at the conference, if this abstract was to be accepted, as the reference list is too long for the constraints on the number of characters in the abstract.

\section{P0593 THE IMPACT OF HELICOBACTER PYLORI ON MORTALITY AND OTHER OUTCOMES IN PATIENTS WITH HEPATIC ENCEPHALOPATHY: A NATIONWIDE ANALYSIS}

P.T. Kroener ${ }^{1}$, M. S. Abougergi ${ }^{2}$, V. Popov ${ }^{3}$, C. C. Thompson ${ }^{4}$ ${ }^{1}$ Mt. Sinai St. Luke's/West, New York/United States of America ${ }^{2}$ Catalyst Medical Consulting, Simpsonville/United States of America/SC

${ }^{3}$ New York University School of Medicine, New York/United States of America

${ }^{4}$ Division Of Gastroenterology, Hepatology, And Endoscopy, Brigham and

Women's Hospital, Boston/United States of America

Contact E-mail Address: thomaskroner@gmail.com

Introduction: Helicobacter Pylori (H. Pylori) has been implicated in worsening outcomes in patients with hepatic encephalopathy. This is believed to be the result of its urease enzyme that increases the production of ammonia. Small studies so far have yielded contradictory results on whether the presence of $H$. pylori worsens treatment outcomes in hepatic encephalopathy. Therefore, the aim of this study was to assess the impact of $H$. pylori on mortality, morbidity and resource utilization among patients with hepatic encephalopathy using a national database.

Aims \& Methods: This was a case-control study using the National Inpatient Sample 2013, the largest publically available inpatient database in the United States. All patients with an ICD-9 CM code for a principal diagnosis of hepatic encephalopathy were included. There were no exclusion criteria. Patients positive for $H$. pylori were identified using the appropriate ICD-9CM codes. The primary outcome was all cause mortality. The secondary outcome was resource utilization as measure by use of abdominal imaging (CT scan and ultrasound of the abdomen), length of hospital stay (LOS), total hospitalization charges and costs.
Multivariate regression analyses were used to adjust for the following confounders: Age, sex, race, income in patients' zip code, Charlson Comorbidity Index, hospital region, location, size and teaching status.

Results: A total of 55,360 patients with hepatic encephalopathy were included in the study, of which 20 had $H$. pylori infection. The mean patient age was 60 years and $42 \%$ were female. After adjusting for confounders using multivariate analysis, patients with and without $H$. pylori had similar adjusted odds of mortality (adjusted Odds Ratio (aOR): 1.71, 95\% CI: 0.62-4.74, p=0.30). As far as resource utilization, patients with and without $H$. pylori had similar adjusted odds of abdominal imaging (aOR: $3.02,95 \% \mathrm{CI}$ : $0.88-10.40, \mathrm{p}=0.08$ ), LOS (adjusted mean difference: 1.7 days, $95 \%$ CI: $-0.02-3.42, \mathrm{p}=0.52$ ), and total hospitalization charges (adjusted mean difference: \$16588, 95\% CI: -\$4499 $\$ 37675, \mathrm{p}=0.12$ ). However, patients with $H$. pylori had higher adjusted total hospitalization costs compared with patients without $H$. pylori (adjusted mean difference: $\$ 6128,95 \%$ CI: $\$ 1141$ - $\$ 11115, \mathrm{p}=0.01$

Conclusion: Presence of Helicobacter Pylori has no impact on inpatient mortality among patients with liver cirrhosis and hepatic encephalopathy. In addition, the presence of Helicobacter Pylori is not associated with any increase in resource utilization among this patient population, with the exception of total hospitalization costs. It is surprising to note that, although total hospitalization costs differed between the two groups, they received the same total hospitalization charges from admitting hospitals.

Disclosure of Interest: All authors have declared no conflicts of interest.

\section{P0594 ERADICATION THERAPY FOR HELICOBACTER PYLORI- AN IMPORTANT TOOL TO IMPROVE THE HEART FUNCTION IN PATIENTS WITH CORONARY HEART DISEASE}

A. Suceveanu ${ }^{1}$, A. P. Suceveanu ${ }^{2}$, I. R. Parepa $^{3}$, F. F. Voinea ${ }^{4}$, D. Catrinoiu ${ }^{5}$, L. Mazilu ${ }^{6}$

${ }^{1}$ Gastroenterology, Ovidius University, Constanta/Romania

${ }^{2}$ Gastroenterology, Ovidius University, c/Romania

${ }^{3}$ Cardiology, Ovidius University, Constanta/Romania

${ }^{4}$ Gastroenterology, Ovidius University, constanta/Romania

${ }^{5}$ Internal Medicine, Ovidius University, constanta/Romania

${ }^{6}$ Internal Medicine, Ovidius University, Constanta/Romania

Contact E-mail Address: andrasuceveanu $@$ yahoo.com

Introduction: Literature data involves Helicobacter Pylori (HP) as a pro-inflammatory and pro-coagulant risk factor for ischemic coronary heart disease ${ }^{1,2}$, but the benefit of the curative treatment against HP infection and its repercussions on heart function were not explored sufficiently.

Aims \& Methods: We aimed to investigate the role of HP eradication therapy in patients with different stages of heart insufficiency (HI) and coronary heart disease (CHD). 290 patients with CHD admitted in Cardiology and Gastroenterology Departments of St. Andrew Apostle Constanta were explored for HP infection status and found positive. The infection was determined by HP$\mathrm{Ag}$ in faces, urea breath test or endoscopic biopsy. The CHD was assessed by electrocardiography (ECG) and HI was staged according to NYHA classification. According to the implementation of eradication therapy for HP, we divided the patients in two equal groups, matched by age, gender and severity of $\mathrm{HI}$ : one group of 145 patients with treatment and the other group of 145 patients without eradication therapy. We compared the heart function improvement after treatment for HP in both groups. We also compared the evolution of PCR, fibrinogen, IL-6 and other inflammatory cytokine.

Results: The eradication therapy for HP with one PPI and two antibiotics associated with cardiac treatment, improved the clinical status based on NYHA criteria in 118 patients with at least 1 stage decrease in NYHA staging compared with only 52 patients from the group with cardiac treatment but without eradication therapy $(\mathrm{p}=0.03)$. The laboratory finding also revealed a favorable course of pro-inflammatory tests in treated patients compared with untreated patients, regarding protein C-reactive, leucocytes, Il-6 and fibrinogen $(\mathrm{p}=0.019$, $\mathrm{p}=0.037, \mathrm{p}=0.08$, respectively $\mathrm{p}=0.005, \mathrm{ss}$ )

Conclusion: HP should be one of the treatment targets when managing infected patients with CHD, the eradication therapy being along with cardiac treatment important steps to improve the cardiac function.

Disclosure of Interest: All authors have declared no conflicts of interest.

\section{References}

1. Yingsong Lin, Yuki Obata, Shogo Kikuchi, Akiko Tamakoshi, Hiroyuki Iso, JACC Study Group. Helicobacter Pylori Infection and Risk of Death From Cardiovascular Disease Among the Japanese Population: a Nested Case-Control Study within the JACC Study. Journal of Atherosclerosis and Thrombosis Vol. 22 (2015) No. 11 p. 1207-12133.

2. Jamshid Vafaeimanesh. Seyyed Fakhroldin Hejazi, Vahid Damanpak, Mostafa Vahedian, Mohammadamin Sattari, Mohammadreza SeyyedmajidiAssociation of Helicobacter pylori Infection with Coronary Artery Disease: Is Helicobacter pylori a Risk Factor? The Scientific World Journal Volume 2014 (2014), Article ID 516354, 6 pages. 
P0595 RANDOMIZED CONTROLLED STUDY OF A NOVEL

TRIPLE NITAZOXANIDE (NTZ) CONTAINING THERAPEUTIC

REGIMEN VERSUS THE TRADITIONAL REGIMEN FOR

ERADICATION OF HELICOBACTER PYLORI INFECTION

S. Abd-Elsalam ${ }^{1}$, M. A.H. Shehata ${ }^{1}$, H. Elmesseri ${ }^{1}$, R. Talaat ${ }^{2}$

${ }^{1}$ Tropical Medicine Department, Tanta university, Tanta/Egypt

${ }^{2}$ Microbiology Department, Tanta university, Tanta/Egypt

Contact E-mail Address: sherif tropical@yahoo.com

Introduction: Helicobacter pylori infection has become more and more resistant to conventional first-line treatment regimens. So, there is a considerable interest in evaluating new antibiotic combinations and regimens. Nitazoxanide is an antiinfective drug with demonstrated activity against protozoa and anaerobic bacteria including Helicobacter pylori.

Aims \& Methods: This work is designed to evaluate the efficacy and safety of a unique triple Nitazoxanide containing regimen as a treatment regimen in Egyptian patients with Helicobacter pylori infection.

Methods: Two hundred and twenty four patients with upper Gastro-intestinal tract (GIT) dyspeptic symptoms in whom Helicobacter pylori induced GIT disease was confirmed were included in the study. They have been randomized to receive either Nitazoxanide $500 \mathrm{mg}$ bid, Clarithromycin $500 \mathrm{mg}$ bid and Omeprazole $40 \mathrm{mg}$ twice daily for 14 days or Metronidazole $500 \mathrm{mg}$ bid, Clarithromycin $500 \mathrm{mg}$ bid and Omeprazole 40mg twice daily for 14 days. Laboratory evaluation for Helicobacter pylori antigen within the stool was done 6 weeks after cessation of Helicobacter pylori treatment regimens to assess the response.

Results: The response to treatment was significantly higher in group 1 of Nitazoxanide treatment regimen than group 2 of traditional treatment regimen. One hundred and six cases $(94.6 \%)$ of 112 patients who completed the study in group 1 showed complete cure while only 63 cases $(60.6 \%)$ of 104 patients who completed the study in group 2 showed the same response according to perprotocol $(\mathrm{PP})$ analysis $(\mathrm{p}<0.001)$. The regimen was well tolerated by all the patients enrolled in the study.

Conclusion: Nitazoxanide-containing triple therapy is a promising therapy for the first-line eradication of Helicobacter pylori. (ClinicalTrials.gov Identifier: NCT02422706)

Disclosure of Interest: All authors have declared no conflicts of interest.

\section{References}

Guttner Y, Helen MW, Charlie V, et al. (2003): Nitazoxanide in Treatment of Helicobacter pylori: a Clinical and In Vitro Study. Antimicrob Agents Chemother; 47(12): 3780-3783

Basu PP, Rayapudi K, Pacana T, et al. (2011): A randomized study comparing levofloxacin, omeprazole, nitazoxanide, and doxycycline versus triple therapy for the eradication of Helicobacter pylori. Am J Gastroenterol; 106: 1970-1975.

\section{P0596 PREVIOUS INTAKE OF MACROLIDES PREDICTS FAILURE TO ERADICATE HELICOBACTER PYLORI WITH CLARITHROMYCIN-CONTAINING REGIMENS}

P. Muñoz-Gómez ${ }^{1}$, A. Jordán-Castro ${ }^{2}$, M. Abanades-Tercero ${ }^{1}$, J. BlancoGonzález ${ }^{1}$, J. Valle-Muñoz ${ }^{1}$

${ }^{1}$ Gastroenterology, Complejo Hospitalario de Toledo, Toledo/Spain

${ }^{2}$ Gastroenterology, Hospital del Bierzo, Ponferrada/Spain

Contact E-mail Address: juliov@ sescam.jccm.es

Introduction: There is some evidence that prior use of macrolides is a usefu predictor of the likelihood of standard triple therapy failure in $H$. pylori eradication (Lim SG, et al. Dig Liver Dis 2016). The goal of this study is to evaluate whether previous intake of various macrolide antibiotics can predict failure to eradicate $H$. pylori using first-line clarithromycin-containing regimens.

Aims \& Methods: Between February 2014 and June 2016 a total of 250 patients with $H$. pylori infection were prospectively included in a study whose goal was to assess the eradication rates obtained with first-line clarithromycin-containing regimens in our health area. Patients were randomly assigned to one of two regimens: A/Triple therapy (PPI, amoxicillin, clarithromycin, optimized with a double dose of PPI) for 10 days; and B/Concomitant therapy (PPI, amoxicillin, clarithromycin, and metronidazole administered concurrently) for 10 days. The eradication was evaluated by the Stool antigen test or with the Urease test in those patients with a gastroscopy performed after the treatment. A total of 113 patients in the group A (58 women; Median age: 54 years; Range: 21-79) and 106 patients in the group B (56 women; Median age: 49 years; Range: 18-81) completed the study protocol. The electronic medical records of the patients, which contain information regarding all the medication prescribed to the patient during the previous 12 years, were reviewed and the intake of macrolides (clarithromycine, azithromycine and erythromycine) was registered.

Results: $89 / 219$ patients $(40,6 \%)$ had received at least one treatment with macrolides during the previous 12 years. H. pylori eradication rates with the two treatment regimens are shown in Table 1.
Table 1: H. pylori eradication rates in patients with and without previous intake of macrolides.

\begin{tabular}{llll}
\hline & $\begin{array}{l}\text { Previous use } \\
\text { of Macrolides }\end{array}$ & $\begin{array}{l}\text { No previous use } \\
\text { of Macrolides }\end{array}$ & $\mathrm{p}$ \\
\hline A: Triple therapy $(\mathrm{n}=113)$ & $24 / 45(53,3 \%)$ & $65 / 68(95,5 \%)$ & $<0,0001$ \\
B: Concomitant $(\mathrm{n}=106)$ & $37 / 44(84,1 \%)$ & $61 / 62(98,4 \%)$ & 0,0085 \\
Total $(\mathrm{n}=219)$ & $61 / 89(68,5 \%)$ & $126 / 130(96,9 \%)$ & $<0,0001$ \\
\hline
\end{tabular}

Conclusion: Previous use of macrolide antibiotics predicts a low response to triple and to concomitant clarithromycin-containing regimens. In addition, our study shows that in patients without previous use of macrolides triple therapy achieves per-protocol eradication rates over $90 \%$.

Disclosure of Interest: All authors have declared no conflicts of interest.

\section{Reference}

1. Lim SG, et al. The relationship between the failure to eradicate Helicobacter pylori and previous antibiotics use. Dig Liver Dis 2016; 48:385-390.

\section{P0597 EFFICACY OF THREE-IN-ONE CAPSULE BISMUTH QUADRUPLE THERAPY FOR HELICOBACTER PYLORI ERADICATION IN CLINICAL PRACTICE IN A MULTINATIONAL} PATIENT POPULATION

S. Miehlke ${ }^{1}$, D. Frederking ${ }^{1}$, T. Guenther ${ }^{2}$, E. Glocker $^{3}$, B. Eisele ${ }^{4}$, V. Andresen ${ }^{5}$, S. Schroeder ${ }^{2}$, A. Morgner ${ }^{6}$

${ }^{1}$ Center for Digestive Diseases, Internal Medicine Center Eppendorf, Hamburg Germany

${ }^{2}$ Institute of Pathology, Lademannbogen, Hamburg/Germany

${ }^{3}$ Institute of Laboratory Medicine, Brandenburg Hospital, Brandenburg Medical School, Brandenburg/Germany

${ }^{4}$ Department of Medical Microbiology and Hygiene, University Hospital, Freiburg/ Germany

${ }^{5}$ Dept. of Medicine, Israelitic Hospital, Hamburg/Germany

${ }^{6}$ University Cancer Center, University Hospital Eppendorf, Hamburg/Germany

Contact E-mail Address: prof.miehlke@mdz-hamburg.de

Introduction: Due to increasing prevalences of clarithromycin resistance in $\mathrm{H}$ pylori infection, current guidelines recommend quadruple therapies as first-line therapy $^{1,2}$. Bismuth quadruple therapy (BQT) has been proven superior to standard triple therapy in clinical trials ${ }^{3}$, however little is known about the efficacy of BQT in clinical routine practice.

Aims \& Methods: In a prospective single center cohort study we analyzed consecutive patients in whom three-in-one capsule BQT (Pylera ${ }^{\circledR}+$ omeprazole) has been prescribed between $1 / 2013$ and $12 / 2016$. All patients were instructed in a standardized fashion and a prospective follow-up was planned. In a subgroup of patients, genotypic susceptibility testing for clarithromycin and levofloxacin by PCR was carried out on gastric biospies before treatment. Treatment outcome was assessed by $13 \mathrm{C}$ urea breath test or by histology not earlier than 4 weeks after end of treatment.

Results: Three-in-one capsule BQT has been prescribed in 322 patients (mean age 41 years (18-80), $65 \%$ female, $26 \%$ active smoker). $71 \%$ of patients had a migrational background including Southern-/Eastern Europe, Eurasia/Central Asia, Southeast-Asia, Africa, and Central/South America. PCR results were available in 163 patients $(50.6 \%)$ and identified resistance to clarithromycin and levofloxacin in $29(17,8 \%)$ and $20(12,3 \%)$ of cases, respectively. BQT was administered as firstline, secondline and rescue treatment in $74 \%, 17 \%$ and $9 \%$ of cases, respectively. 5 patients discontinued treatment prematurely due to side effects $(1,8 \%)$ and 43 patients were lost to follow-up $(13,4 \%)$. By modified intentionto-treat and per-protocol analysis the $H$. pylori eradication rates were $94,9 \%$ (95\% CI: $92.1-97.5 \%)$ and $96.7 \%$ (95\% CI: $94.4-98.8 \%)$, respectively. The low number of treatment failures $(n=9)$ did not allow to identify risk factors for failure.

Conclusion: Three-in-one capsule bismuth quadruple therapy is highly effective and safe for treatment of $H$. pylori infection in clinical routine practice, irrespective of the patient's migrational background or the number of previous treatment failures.

Disclosure of Interest: S. Miehlke: Speakers honoraria: Allergan, Kibion, Olympus

All other authors have declared no conflicts of interest.

\section{References}

1. Fischbach W, et al. S2k-guideline Helicobacter pylori and gastroduodenal ulcer disease. Z Gastroenterol 2016;54:327-63.

2. Malfertheiner $\mathbf{P}$ et al. Management of Helicobacter pylori infection-the Maastricht V/Florence Consensus Report. Gut 2017;66:6-30

3. Malfertheiner P, et al. Helicobacter pylori eradication with a capsule containing bismuth subcitrate potassium, metronidazole, and tetracycline given with omeprazole versus clarithromycin-based triple therapy: a randomised, openlabel, non-inferiority, phase 3 trial. Lancet 2011;377:905-13. 
P0598 ERADICATION OF HELICOBACTER PYLORI INFECTION

WITH A COMBINATION FORMULATION OF BISMUTH,

METRONIDAZOLE AND TETRACYCLINE WITH ESOMEPRAZOLE: A REAL-LIFE STUDY

E. Pérez Arellano' ${ }^{1}$, M.I. Rodriguez Garcia ${ }^{2}$, A.B. Galera ${ }^{3}$, E. De La Morena ${ }^{4}$ ${ }^{1}$ Digestive, Hospital, Madrid/Spain

${ }^{2}$ Digesetivo, Hospital Zarzuela, Madridmail.com/Spain

${ }^{3}$ Digestivo, Hospital Zarzuela, Madrid/Spain

${ }^{4}$ Hospital Zarzuela, Madrid/Spain

\section{Contact E-mail Address: eperezarellano@telefonica.net}

Introduction: Background: Eradication of Helicobacter pylori (H. pylori) infection represents a clinical challenge. The current requirements demand eradication rates above the $90 \%$, which has made that the use of triple treatment including clarithromycin or metronidazole had been gave up on those countries, such as Spain, with high resistance rates. Quadruple therapy with a proton pump inhibitor (PPI) plus a single three-in-one capsule containing bismuth subcitrate potassium, metronidazole, and tetracycline (BMT) have shown high eradication rates in clinical trials.

Aims \& Methods: We aimed to evaluate the efficacy and safety of a PPI-bismuth based quadruple therapy in patients diagnosed of $\mathrm{H}$ pylori infection in a clinical setting of a Private Hospital, located at the North of Madrid (Spain). A prospective and real-life study was conducted, between March 2016 to February 2017, on consecutive patients with confirmed $\mathrm{H}$ pylori infection eradication indication. Patients were treated for ten days with a galenic preparation containing bismuth subcitrate potassium $140 \mathrm{mg}$, metronidazole $125 \mathrm{mg}$, and tetracycline $125 \mathrm{mg}$, three capsules four times daily, and esomeprazole $40 \mathrm{mg}$ twice daily and probiotic during 30 days. The primary endpoint was $H$. pylori eradication rate, defined as one negative urea breath test performed, at least 28 days, after the end of treatment. Intent-to-treat (ITT) efficacy analyses included all patients who received study medication and took at least one dose of study medication; patients without an observed outcome were considered as treatment failures. Per protocol (PP) analyses, which excluded patients who did not complete the study or who had major protocol violations, were also conducted to confirm the ITT results.

Results: A total of 100 patients, $60(60.0 \%)$ women and $40(40.0 \%)$ men, who fulfilled the respective demands of the inclusion and exclusion criteria, were enrolled consecutively. Five of these were lost to follow-up. Mean (standard deviation) [95\% confidence interval] age was $47.1(15.4)$ [ 44.0 to 50.2$]$ years. Twenty-five $(25.0 \%)$ patients had a prior history of using medications to treat H. pylori most often clarithromycin, amoxicillin, and PPI. In the ITT population, the eradication rates were the $90.7 \%(68 / 75)$ and the $80.0 \%(20 / 25)$ depending on whether the PPI-BMT treatment was administered as first-line or as rescue therapy, respectively. In the PP population, the eradication rates were the $98.6 \%$ $(68 / 69)$ and the $95.2 \%(20 / 21)$ in those patients treated with PPI-BMT as firstline or as rescue therapy, respectively. Eighteen $(18.0 \%)$ patients reported at least one adverse event.

Conclusion: In patients with confirmed $\mathrm{H}$ pylori infection, 10 days of treatment with a quadruple regimen of bismuth, metronidazole and tetracycline plus esomeprazole provides high eradication rates not only as first-line but also as rescue therapy, with an acceptable safety profile.

Disclosure of Interest: All authors have declared no conflicts of interest.

\section{P0599 COST EFFECTIVENESS OF HELICOBACTER PYLORI POPULATION SCREENING: ECONOMIC EVALUATION ALONGSIDE A RANDOMIZED CONTROLLED TRAIL WITH 13- YEARS FOLLOW-UP (THE HEP-FYN STUDY)}

M. Bomme Høgh ${ }^{1}$, C. Kronborg ${ }^{2}$, J.M. Hansen ${ }^{1}$, M. Wildner-Christensen ${ }^{1}$, J. Hallas ${ }^{3}$, O.B. Schaffalitzky De Muckadell ${ }^{1}$

${ }^{1}$ Dept. Of Gastroenterology, Odense University Hospital, Odense/Denmark ${ }^{2}$ Centre Of Health Economic Research, University of Southern Denmark, Odense M/Denmark

${ }^{3}$ Institute Of Clinical Pharmacology, University of Southern Denmark, Odense M/ Denmark

\section{Contact E-mail Address: maria_bomme@ $@$ hotmail.com}

Introduction: Most Helicobacter pylori $(\mathrm{Hp})$ infections are asymptomatic but $15 \%$ of those infected with $\mathrm{Hp}$ will eventually experience dyspepsia symptoms or peptic ulcer.

Aims \& Methods: We aimed to evaluate the cost effectiveness of population screening and eradication for Helicobacter pylori $(\mathrm{Hp})$. This was a cost effectiveness analysis and cost utility analysis alongside randomized controlled trial with 13-years follow-up, with a random sample of the general population from the county of Funen, Denmark. 20,011 individuals aged 40-65 were randomized and invited in 1998-99; 12,530 were enrolled and of these 8658 individuals have been successfully followed up at 1,5 , and 13 years after intervention. Questionnaires included Gastrointestinal Symptom Rating Scale and the quality of life instrument SF-36. From SF-36 responses an SF-6D score was derived and used for calculation of quality adjusted life years (QALY). EQ-5D-5L was incorporated in the last follow-up. The intervention was an invitation to Hp screening by in-office blood test; positive tests were validated by $13 \mathrm{C}$-Urea Breath test. Those with a positive 13C-Urea Breath test received eradication therapy. The $\mathrm{Hp}$ prevalence was $17.5 \%$. Main outcome measure was incremental cost per quality adjusted life year (QALY) and Life-years gained. The evaluation has a National Health Sector perspective.

Results: There was no significant difference in index scores and in mean QALY between groups. Hp population screening and eradication with 13-years followup was not effective in regards to quality of life and the cost of screening was higher than not screening $(14,327 \mathrm{DKK}(95 \% \mathrm{CI}$ : 4155-24,499)). The probability of being cost-effective was $80 \%$ with a willingness to pay of 400,000 DKK per life-year gained. When including only peptic ulcer disease related costs the probability was $85 \%$ a willingness to pay of $100,000 \mathrm{DKK}$ per life-year gained. Conclusion: Hp population screening and eradication with 13-years follow-up was not effective in regards to quality of life and the cost of screening was higher than not screening.

Disclosure of Interest: All authors have declared no conflicts of interest.

\section{P0600 A SEVEN-DAY TRIPLE THERAPY CONTAINING A} POTASSIUM-COMPETITIVE ACID BLOCKER COMPARED WITH PROTON PUMP INHIBITORS, AMOXICILLIN AND CLARITHROMYCIN FOR FIRST-LINE HELICOBACTER PYLORI ERADICATION IN JAPAN: A SINGLE-CLINIC RETROSPECTIVE STUDY

S. Yojiro

Sadamoto GI Clinic, Kitakyushu/Japan

\section{Contact E-mail Address: sadamotoy@yahoo.co.jp}

Introduction: This study was evaluated the effectiveness and safety of Vonoprazan, a potassium-competitive acid blocker (P-CAB) compared with proton pump inhibitors (PPIs) for a first-line Helicobacter Pylori (H. pylori) eradication.

Aims \& Methods: We retrospectively analyzed data from first-line $H$. pylori eradication treatment (vonoprazan or PPIs with $400 \mathrm{mg}$ clarithromycin and $750 \mathrm{mg}$ amoxicillin twice daily for 7-day) $(n=1393)$ between April 2008 and February 2017 at Sadamoto GI clinic, Japan. Patients who received 7-day PCAB therapy (vonoprazan $20 \mathrm{mg}$ twice daily; $n=498$ ) were compared with those who received 7-day PPI therapy (lansoprazole $30 \mathrm{mg} ; n=216$, rabeprazole $10 \mathrm{mg} ; n=331$, esomeprazole $20 \mathrm{mg} ; n=348$ twice daily). Eradication rates were calculated by intention-to-treat (ITT) and by per-protocol (PP). Compliance and adverse events were also assessed for each study group.

Results: ITT and PP analysis of the first-line $H$. pylori eradication for vonoprazan, lansoprazole, rabeprazole, and esomeprazole were $75.5 \% / 86.8 \%, 63.9 \% / 76.2 \%$, $68.0 \% / 79.5 \%$, and $63.2 / 70.8 \%$, respectively. The vonoprazan eradication rates were significantly higher than those of these PPIs $(P<0.05)$, respectively. There was no significant difference in the adverse events between the two therapies.

Conclusion: 7-day P-CAB based triple therapy is more effective than 7-day PPI based triple therapy as a first-line $H$. pylori eradication without differences in tolerability.

Disclosure of Interest: All authors have declared no conflicts of interest.

\section{P0601 AN OPEN-LABEL, RANDOMIZED CONTROLLED TRIAL OF VONOPRAZAN VERSUS ESOMEPRAZOLE AS PART OF FIRST- LINE TRIPLE THERAPY FOR HELICOBACTER PYLORI INFECTION}

H. Tamaki, M. Morita, A. Omura, T. Noda, A. Kubo, C. Ogawa, T. Matsunaka, M. Shibatoge

Gastroenterology And Hepatology, Takamatsu Red Cross Hospital, Takamatsu/ Japan

Contact E-mail Address: h-tama@gc4.so-net.ne.jp

Introduction: Vonoprazan (VPZ) is a novel, orally bioavailable, potassium-competitive acid blocker for the treatment and prevention of acid-related gastrointestinal diseases. A phase III study revealed that VPZ is superior to lansoprazole as part of first-line therapy for Helicobacter pylori (HP) infection when combined with 400 or $800 \mathrm{mg} /$ day clarithromycin (CAM).

Aims \& Methods: The aim of the current study was to evaluate the efficacy and safety of VPZ, CAM $(400 \mathrm{mg} /$ day), and amoxicillin (ABPC; $1500 \mathrm{mg} /$ day) triple therapy in post-marketing use in Japan. A randomized, open-label, single-center study was conducted to verify the superiority of VPZ to esomeprazole (EPZ) as part of first-line triple therapy in patients with HP infection. Three hundred and forty-nine Japanese patients with HP infection diagnosed using a rapid urease test were enrolled between June 2015 and October 2016. The patients were randomly allocated to VPZ group (VPZ $40 \mathrm{mg} /$ day, ABPC $1500 \mathrm{mg} /$ day, CAM $400 \mathrm{mg} /$ day) or EPZ group (EPZ $40 \mathrm{mg} /$ day, ABPC $1500 \mathrm{mg} /$ day, CAM $400 \mathrm{mg} /$ day) with stratification according to endoscopic findings of gastric/duodenal ulcer/scar and CAM resistance determined via a microbial sensitivity test. The eradication rates were evaluated using the urea breath test 8 to 12 weeks after cessation of therapy.

Results: Three hundred and forty-five patients (177 men, 168 women; mean age 64.7 years [range 27-89 years]; VPZ group, 169; EPZ group, 176) completed the study. One patient in the VPZ group and three patients in the EPZ group discontinued the treatment because of adverse events. One patient in the VPZ group and three patients in the EPZ group were lost to follow-up. There was no significant difference in the overall first-line eradication rate between the two groups $(81.7 \%[138 / 169]$ in the VPZ group vs. $74.4 \%[131 / 176]$ in the EPZ group $[P=0.10])$. Furthermore, there was no significant difference in the first-line eradication rate in patients with CAM-sensitive HP $(87.2 \%$ [82/94] versus $84.6 \%$ $[77 / 91]$ in the VPZ and EPZ groups, respectively, $[\mathrm{P}=0.60])$, although the eradication rate was significantly higher among patients with CAM-resistant HP in the VPZ group than that in the EPZ group $(73.6 \%$ [39/53] vs. 55.6\% [35/63], $[P=0.044])$. The first-line eradication rate in the patient with high estimated glomerular filtration rate $\left(\mathrm{eGFR} ; \geq 100 \mathrm{ml} / \mathrm{min} / 1.73 \mathrm{~m}^{2}\right)$ was significantly lower than that in the patients with low eGFR $\left(<60 \mathrm{ml} / \mathrm{min} / 1.73 \mathrm{~m}^{2} ; 86.4 \%[32 / 37]\right.$ in 
the patients with low eGFR, 65.3\% [34/53] in the patients with high eGFR [P= 0.034]), and it was significantly higher in the VPZ group than that in the EPZ group $(79.3 \%$ [23/29] versus $50 \%$ [11/13], respectively, $[\mathrm{P}=0.025])$. The first-line eradication rate in continuous smokers was significantly lower than that in nonsmokers $(81.0 \%$ [187/231] in non-smokers vs. $64.3 \%$ [27/42] in continuous smokers $[\mathrm{P}=0.016])$. However, there were no significant differences between the VPZ and EPZ groups in non-smokers $(84.2 \%$ [96/114] versus $77.8 \%$ [91/117], respectively, $[\mathrm{P}=0.21])$ and in continuous smokers $(84.2 \%[12 / 16]$ versus $57.7 \%[15 /$ $26]$, respectively, $[P=0.33])$. Furthermore, the first-line eradication rates in both groups were not influenced by age, sex, body mass index, drinking habit, and the endoscopic findings of gastric/duodenal ulcers/scars. There were no significant differences with regard to adverse effects between the two groups.

Conclusion: In contrast to the previous reports, the first-line eradication rate of VPZ-based triple therapy with $400 \mathrm{mg} /$ day CAM and $1500 \mathrm{mg} /$ day ABPC was similar to that of EPZ-based triple therapy in all groups except in patients with CAM-resistant HP and high eGFR. It is necessary to determine the most appropriate conditions that will maximize the therapeutic effect of VPZ-based triple therapy.

Disclosure of Interest: All authors have declared no conflicts of interest.

\section{Reference}

Vonoprazan, a novel potassium-competitive acid blocker, as a component of first-line and second-line triple therapy for Helicobacter pylori eradication: a phase III, randomised, double-blind study. Murakami K, et al. Gut 2016;65:1439-1446.

\section{P0602 THE IMPACT OF AMOXICILLIN RESISTANCE ON THE EFFICACY OF AMOXICILLIN-CONTAINING REGIMENS FOR HELICOBACTER PYLORI ERADICATION- A POST-HOC ANALYSIS OF FIVE RANDOMIZED TRIALS}

J. Liou ${ }^{1}$, M. Chen ${ }^{1}$, J. $\operatorname{Lin}^{2}$, M. Wu ${ }^{1}$

${ }^{1}$ Dept. Of Internal Medicine, National Taiwan University Hospital Dept. of Internal Medicine Taiwan Helicobacter Consortium, Taipei/Taiwan

${ }^{2}$ College Of Medicine, Fu-Jen Catholic University, New Taipei City/Taiwan

Contact E-mail Address: dtmed046@pchome.com.tw

Introduction: The impact of amoxicillin resistance on the efficacy of regimens containing amoxicillin for Helicobacter pylori $(H$. pylori) eradication remains inconclusive. Therefore, we aimed to investigate whether the efficacy of amoxicillin containing regimen is affected by amoxicillin resistance and to identify the optimal breakpoint of amoxicillin resistance.

Aims \& Methods: This was a post-hoc analysis of five randomized trials conducted in Taiwan from 2007 to 2016. Patients who received amoxicillin-containing regimens were recruited. The minimum inhibitory concentrations (MICs) were determined by agar dilution test. Meta-analysis was performed to assess the risk ratio of eradication failure in amoxicillin resistant strains compared to susceptible strains of seven different regimens. We further performed pooled analysis and logistic regression in patients treated with clarithromycin triple therapy to identify the optimal breakpoint of amoxicillin resistance.

Results: A total of 2339 patients with available data of amoxicillin MICs were enrolled. Meta-analysis showed that the presence of amoxicillin resistance was consistently associated with increased risk of treatment failure of amoxicillincontaining regimens at different breakpoints (RR [Risk ratio]: $1.41,95 \% \mathrm{CI}$ [confidence interval]: $1.12-1.78, \mathrm{P}=0.004$ when cut at $0.5 \mathrm{mg} / \mathrm{ml}$ ). The heterogeneity was low $(\mathrm{I} 2=0 \%, \mathrm{p}=0.615)$. Pooled analysis also showed that amoxicillin resistance was an independent risk factor of treatment failure of clarithromycin triple therapy at different breakpoints. The best correlation was observed when the breakpoint of amoxicillin resistance was ${ }^{3} 0.125 \mathrm{mg} / \mathrm{ml}$ (Kappa coefficient 0.298$)$, at which the resistance rate was $11.1 \%(110 / 990)$.

Conclusion: The efficacies of amoxicillin containing regimens are affected by amoxicillin resistance and the optimal breakpoint of MIC is ${ }^{3} 0.125 \mathrm{mg} / \mathrm{ml}$. Disclosure of Interest: All authors have declared no conflicts of interest.

\section{References}

Liou JM, Fang YJ, Chen CC, et al. Concomitant, bismuth quadruple, and 14day triple therapy in the first-line treatment of Helicobacter pylori: a multicentre, open-label, randomised trial. Lancet 2016;388:2355-2365.

Liou JM, Chen CC, Chen MJ, et al. Sequential versus triple therapy for the firstline treatment of Helicobacter pylori: a multicentre, open-label, randomised trial. Lancet 2013;381:205-13.

Liou JM, Chen CC, Chang CY, et al. Sequential therapy for 10 days versus triple therapy for 14 days in the eradication of Helicobacter pylori in the community and hospital populations: A randomised trial. Gut 2016;65:1784-1792.
P0603 ROLE OF POLYMORPHISMS IN TLR1 AND PRKAA1 GENES IN THE DEVELOPMENT OF ATROPHIC GASTRITIS AND GASTRIC CANCER

V. Petkevicius ${ }^{1}$, G. Dargiene ${ }^{1}$, M. Leja ${ }^{2}$, A. Link ${ }^{3}$, L. Jonaitis ${ }^{1}$, G. Kiudelis ${ }^{1}$, G. Streleckiene ${ }^{4}$, J. Skieceviciene ${ }^{4}$, L. Kupcinskas ${ }^{1}$, P. Malfertheiner ${ }^{3}$, J. Kupcinskas 1

${ }^{1}$ Department Of Gastroenterology, Lithuanian University of Health Sciences, Kaunas/Lithuania

${ }^{2}$ Institute For Clinical And Preventive Medicine, University of Latvia, Riga/Latvia ${ }^{3}$ Department Of Gastroenterology, Hepatology And Infectious Diseases, Otto-vonGuericke University of Magdeburg, Magdeburg/Germany

${ }^{4}$ Institute For Digestive Research, Lithuanian University of Health Sciences, Kaunas/Lithuania

Contact E-mail Address: vytenis.petkevicius@gmail.com

Introduction: Previous genome-wide association studies showed that genetic polymorphisms in toll-like receptor (TLR) 1 and protein kinase AMP-activated alpha 1 catalytic subunit (PRKAA1) were associated with gastric cancer (GC) or increased Helicobacter pylori (H. pylori) infection susceptibility.

Aims \& Methods: The aim of this study was to evaluate associations between TLRI and PRKAAI genes polymorphisms and $H$. pylori infection, atrophic gastritis (AG) or GC in European population. Single-nucleotide polymorphisms (SNPs) were analyzed in 1178 subjects ( 511 controls, 340 AG patients and 327 GC patients) from 3 gastroenterology centers in Germany, Lithuania and Latvia. Patients with AG and controls were from the out-patient departments, who underwent upper endoscopy because of dyspeptic symptoms and had no history of malignancy. GC patients had histopathological verification of gastric adenocarcinoma and were recruited from out-patient and stationary departments. Genomic DNA was extracted from peripheral blood mononuclear cells. TLR1 $\mathrm{C}>\mathrm{T}(\mathrm{rs} 4833095)$ and PRKAAl C > T (rs13361707) were genotyped by the real-time polymerase chain reaction. $H$. pylori status was determined by testing for anti-H. pylori IgG antibodies in sera. Associations between SNPs and presence of $H$. pylori infection were evaluated using logistic regression analysis. The same analysis with adjustment for sex, age and $H$. pylori infection status was used for evaluation of associations between gene polymorphisms and AG or GC. The Bonferroni-corrected alpha level was set at $0.025(0.05 / 2 \mathrm{SNPs})$.

Results: We observed similar distribution of $T L R I$ and $P R K A A l$ genotypes in $H$. pylori positive and negative cases. Moreover, no significant differences in the frequencies of all polymorphisms between $\mathrm{AG}$ patients and controls were found. TC genotype of TLRI gene was more prevalent in GC patients compared to control group $(22.3 \%$ and $29.7 \%$ respectively, $\mathrm{P}=0.018)$. Logistic regression analysis revealed that polymorphism in $T L R 1$ gene was associated with increased risk of GC. Carriers of TC genotype had higher odds of GC when compared to TT genotype $(\mathrm{OR}-1.89,95 \%$ PI $1.26-2.83, \mathrm{P}=0.002)$. Similar association was observed in a dominant model for TLRl gene, where comparison of CC + TC vs TT genotypes showed an increased risk of GC (OR - 1.86, 95\% PI 1.26-2.75, $\mathrm{P}=0.002$ ). No association between genetic polymorphism in PRKAAl gene and GC was observed.

Conclusion: TLR1 rs4833095 SNP is associated with increased risk of GC in population of European descent, while polymorphism in PRKAAl gene is not linked with the presence of GC. Both genetic polymorphisms showed no associations with $H$. pylori infection susceptibility and risk of AG.

Disclosure of Interest: All authors have declared no conflicts of interest.

\section{P0604 ASSOCIATION BETWEEN GASTRIC ADENOCARCINOMA OF THE FUNDIC GLAND TYPE AND $H$. PYLORI INFECTION}

M. Matsueda, H. Kinugasa, K. Miyahara, M. Nakagawa

Internal Medicine, Hiroshima City Hiroshima Citizens Hospital, Hiroshima/Japan

Contact E-mail Address: maaaaayu0917@gmail.com

Introduction: Gastric adenocarcinoma of the fundic gland type (GAFG) is gastric adenocarcinoma with low-grade atypia occurring in the mucosa of the fundic gland without atrophy, and is recognized that it is not related to H.pylori $(\mathrm{Hp})$ infection. However, GAFG is also found in $H p$-infected and past $H p$-infected patients, as well as in Hp-uninfected patients. It can not deny the relationship between the progress of GAFG and $H p$ infection.

Aims \& Methods: Ten lesions of GAFG resected endoscopically or surgically in our hospital from December 2010 to October 2016 were classified as $H p$-uninfected $(\mathrm{n}=3) /$ past $H p$-infected $(\mathrm{n}=5) / H p$-infected $(\mathrm{n}=2)$, and each endoscopic and clinicopathological features were examined.

Results: Median age of $H p$-infected/past $H p$-infected/Hp-uninfected were $65 / 71 /$ 54.5 years old, respectively, male ratio were $100 / 80 / 0 \%$, occupied site U area were $33 / 80 / 50 \%$. There was no difference between the 3 groups in these parameters. However, a significant difference in the rate of submucosal invasion is recognized between the $H p$-uninfected and past $H p$-infected group and the $H p$ uninfected group (submucosal invasion was found at $0 / 80 / 100 \%, p=0.035$ ). According to endoscopic features, background mucosa of gastric fundus gland mucosa without atrophic change was found in $100 / 100 / 100 \%$, whitish color in $67 / 60 / 50 \%$, submucosal tumor shape in $67 / 60 / 0 \%$, dilated vessels with branching architecture in $100 / 100 / 50 \%$. The association with $\mathrm{Hp}$ infection was not clear. In immunohistochemical staining, MUC6 positive, Pepsinogen-I positive, MUC2 negative, CD 10 negative in all cases, whereas the rate of MUC5AC positive was significantly higher in the $H p$-infected group as $0 / 20 / 100 \%(P=0.045)$. We reported that black pigmentation is recognized in GAFG (stomach and intestine 50: 1521-1531, 2015), but no association between $H p$ infection and black pigmentation was observed. On the other hand, the rate of PPI administration divided pigmentation $(n=6) /$ no pigmentation $(n=4)$ are $50 / 0 \%$, and it is suggested that the pigmentation in GAFG may relate with PPI $(\mathrm{p}=0.091)$. 
Conclusion: $\mathrm{Hp}$ infection is suggested to be related to the invasion depth of GAFG and the MUC5AC prevalence, which is also an important profile in GAFG cases. Pigmentation in GAFG is found frequently, but it may be related to taking PPI.

Disclosure of Interest: All authors have declared no conflicts of interest.

\section{References}

Ueyama H, et al. Am J Surg Pathol 34:609-619, 2010

Ueyama H, et al. Endoscopy 46:153-157, 2014

MONDAY, OCTOBER 30, 2017

09:00-17:00

SMALL INTESTINAL I - HALL 7

P0605 MUCIN EXPRESSION IN THE SMALL BOWEL OF CELIAC

DISEASE - A SYSTEMATIC REVIEW AND META-ANALYSIS

Y. Niv ${ }^{1}$, R. Shamir ${ }^{2}$

${ }^{1}$ Dept. Of Gastroenterology, Rabin Medical Center, Tel Aviv University

Gastroenterology, Petach Tikva/Israel

${ }^{2}$ Gatroenterology, Schneider Children Hospital, Petah-Tikva/Israel

Contact E-mail Address: yniv@clalit.org.il

Introduction: Mucins, heavily glycosylated glycoproteins, synthesized by mucosal surfaces and have an important role in healthy and malignant states. Changes in mucin synthesis, expression and secretion may be a primary event or may be secondary to inflammation and carcinogenesis.

Aims \& Methods: Since untreated celiac disease (CD) is associated with intestinal malignancy, we aimed to assess the current knowledge about mucin expression in the small bowel of CD patients, and to look for a possible association between mucin profile and gluten-free diet. English Medical literature searches were conducted for "mucin" and "celiac". Observational studies were included. Metaanalysis was performed by using Comprehensive meta-anaslysis software. Pooled odds ratios and $95 \%$ confidence intervals were calculated.

Results: Out of 18 titles initially generated by the literature searches, 3 observational studies that fulfilled the inclusion criteria remained eligible for meta-analysis. They included 58 patients and 68 controls from 3 countries (Finland, Japan, USA). Mucin expression was significantly increased in small bowel mucosa of $\mathrm{CD}$ patients than in normal small bowel mucosa [OR $10.789,95 \% \mathrm{CI}$ $(1.062-109.634), \mathrm{p}=0.044]$ (random-effect model). Heterogeneity was significant: $\mathrm{Q}=9.470$, df $(\mathrm{Q})=3, \mathrm{P}=0.024, \mathrm{I} 2=68.323 \%$. ORs for MUC2 and MUC5AC expression in the small bowel mucosa of untreated CD patients were 1.143, $95 \%$ CI $0.060-21.870, \mathrm{P}=0.929$ and $21.429,95 \%$ CI $3.883-118.255, \mathrm{P}<0.0001$, respectively.

Conclusion: We found that expression of certain mucin genes in the small bowel mucosa of CD patients may serve as a diagnostic tool, and assist in surveillance programs.

Disclosure of Interest: All authors have declared no conflicts of interest.

\section{P0606 HEPATIC ABNORMALITIES ASSOCIATED WITH CELIAC} DISEASE

D. Tagzout $^{1}$, A. Tebaibia $^{2}$, N. Oumnia ${ }^{3}$, N. Benfenatki ${ }^{3}$

${ }^{1}$ Medicine, University Hospital Center, Tizi Ouzou/Algeria

${ }^{2}$ Medicine, University Hospital Center, Algiers/Algeria

${ }^{3}$ Medicine, Hospital University center, Algiers/Algeria

Contact E-mail Address: tagzout.d@gmail.com

Introduction: Celiac disease (CD) is a gluten-sensitive enteropathy that resolves with gluten-free diet (GFD). It's now considered as multisystem disorder. A number of studies have shown the occurrence of liver diseases at a higher frequency in patients with $\mathrm{CD}$ compared with that in the general population. Cryptogenetic hypertransaminasemia is observed in about half of celiac patients not following GFD with reversibility in the majority of cases after 6 to 12 months of GFD (1). Other liver abnormalities such as autoimmune hepatitis, cholestatic liver diseases, cirrhosis can be observed.

Aims \& Methods: The aims of our study are to report the prevalence of liver diseases during $\mathrm{CD}$ and to describe the characteristics, etiologies and response to GFD of these liver disorders. Prospective multicentric study including 154 celiac patients $(42 \mathrm{H} ; 112 \mathrm{~F})$; middle age: 36,1 ans $\pm 13,6$, recruited over a period of 18 months from 01-01-2013 to 30-06-2014 and followed at least over a period of 12 months. Hepatic abnormalities were investigated with an etiologic assessment. The evolution of liver disorers after GFD was assessed.

Results: The prevalence of hepatic disorders in celiac patients is estimated at $18.1 \%(\mathrm{n}=28)$. A cryptogenetic hypertransaminase is found in $12.3 \%$ of cases $(\mathrm{n}=19)$. This diagnosis was retained after a negative etiological assessment of hepatic cytolysis in patients at a diagnosis of CD. Severe hepatic pathologies are associated with CD in $5.8 \%(\mathrm{n}=9)$ of cases, and in almost all cases in the hepatic cirrhosis stage. Cirrhosis is cryptogenetic in 55.5\% (5/9) of cases and secondary to an autoimmune origin in $44.5 \%(4 / 9)$. Chronic hepatopathy revealed CD in $1.9 \%$ of the celiac population $(3 / 154)$, two cases of autoimmune cirrhosis, one associated with pituitary insufficiency, and one case of cryptogenetic cirrhosis. The diagnosis of cirrhosis preceded the diagnosis of $\mathrm{CD}$ in $55.6 \%(5 / 9)$ of cases, and was contemporary and revealing the CD in $33.3 \%(3 / 9)$. In a single case $(11.1 \%, 1 / 9)$, the diagnosis of cryptogenetic cirrhosis was made in a celiac patient diagnosed in childhood without the concept of GFD followed, 7 years after the diagnosis of CD. It's noted that hepatic cirrhosis occurred in the celiac population at a young age, on average 31.1 years. Cirrhosis is most often complicated $(77.8 \%)$, with bleeding esophageal varices in $22.2 \%(n=2)$ of the cases and ascitic decompensation in slightly more than half of cases $55.6 \% \quad(n=5)$. Hepatocellular insufficiency was noted in $66.7 \%$ of cases. Specific management of hepatic cirrhosis has been established with ligation of esophageal varices in 2 patients after bleeding esophageal varices until eradication of varices. A treatment with $\beta$ blockers was prescribed in primary prophylaxis of digestive bleeding by rupture of oesophageal varices in 4 patients. Diuretic therapy and aspiration of the ascites fluid are performed in cirrhotic patients with ascites. A GFD is established in addition to the specific management of liver cirrhosis. Liver tests were standardized in $100 \%$ of patients with cryptogenetic transaminasemia followed GFD. The chronic liver disease was often decompensated and the hepatic signs for cirrhosis were in the foreground masking the response to GFD. Conclusion: It is recommended to look for hepatic abnormalities during CD and even to think of the diagnosis of CD in front of liver cytolysis syndrome without obvious etiology and in the presence of a chronic cryptogenetic or dysmimunes liver diseases.

Disclosure of Interest: All authors have declared no conflicts of interest.

\section{Reference}

1. Rostami-Nejad $\mathrm{M}$ et al. The role of celiac disease in severity of liver disorders and effect of a gluten free diet on diseases improvement. Hepat Mon 2013; 13(10): el1893, Oct 2013.

\section{P0607 CELIAC DISEASE ASSOCIATED WITH VASCULAR} THROMBOSIS

D. Tagzout $^{1}$, A. Tebaibia ${ }^{2}$, N. Oumnia ${ }^{2}$, N. Benfenatki ${ }^{2}$

${ }^{1}$ Medicine, Hospital University Center, Tizi Ouzou/Algeria

${ }^{2}$ Medicine, Hospital University Center, Algiers/Algeria

Contact E-mail Address: tagzout.d@gmail.com

Introduction: Celiac disease (CD) is a life-long autoimmune disease affecting multiple organs of genetically susceptible individuals. One of the extra intestinal manifestations of the disease is thromboembolic events like strokes and veins' thrombosis.

Aims \& Methods: The aim of this work is to determine the prevalence and clinical characteristics of the thrombosis observed during CD. Prospective multicenter work involving 154 adult celiac patients $(42 \mathrm{H}, 112 \mathrm{~F})$, with an average age of 36.1 years \pm 13.6 , recruited between 01-01-2013 and 30-06-2014 with a minimum follow-up of 12 months. The diagnosis of CD was in all cases based on clinical, serological and histological arguments. Thrombotic complications were noted as well as their modalities of occurrence.

Results: Vascular thrombosis was noted in 13 patients $(8.4 \%)$, and occurred almost exclusively in women $(84.6 \%)(11 \mathrm{~F}-2 \mathrm{H})$. There are 6 cases of portal cavernoma, one associated with lower limb thrombosis, 4 cases of stroke and 3 cases of thrombosis of the lower limbs. The diagnosis of thrombosis revealed the diagnosis of MC in 8 patients $(61.5 \%)$ with an average delay of 11.6 months and extreme delays of 1 to 43 months. These include 4 cases of a portal cavernoma, one associated with deep limb thrombosis, 3 cases of stroke, and one case of deep thrombosis of the lower limbs. The diagnosis of $\mathrm{CD}$ was made on average 72 months after that of thrombosis in 4 patients $(30.8 \%)$. In one case, thrombosis was complicated 39 years after diagnosis of CD diagnosed in childhood at the age of 5 years without gluten-free diet. The thrombophilia assessment was carried out in all patients and. The thrombophilia assessment was was negative in 11 cases $(84.6 \%)$. A S protein deficiency associated with the $\mathrm{CD}$ in one case and an antithrombin III deficiency in another case were detected. The use of oral contraceptives and in all cases a micro-dosed oestro-progestin was found in 7 women ( $63.6 \%$ of the women involved in thrombosis).

Conclusion: The diagnosis of CD must be evoked when there is a thrombotic disease without obvious cause, factors of thrombophilia may be present during the CD. Early CD diagnosis with respect to the gluten-free diet may prevent the development of this complication.

Disclosure of Interest: All authors have declared no conflicts of interest.

P0608 DIFFERENT PROFILES OF TLR 2, 4, 7 AND 9 MRNA IN PBMC AND BIOPSY SPECIMENS OF PATIENTS WITH CELIAC DISEASE

H. Ghasiyari ${ }^{1}$, M. Rostami-Nejad ${ }^{2}$, D. Amani ${ }^{3}$, M.A. Pourhosseingholi ${ }^{4}$ E. Aghamohammadi ${ }^{2}$, K. Rostami ${ }^{5}$, H. Asadzadeh ${ }^{1}$, M.R. Zali

${ }^{1}$ Basic and Molecular Epidemiology of Gastrointestinal Disorders Research Center, Research institute for Gastroenterology and Liver Diseases, Shahid Beheshti University of Medical Sciences, Tehran/Iran

${ }^{2}$ Celiac Disease, Gastroenterology and Liver Disease Research Institute, Tehran Iran

${ }^{3}$ Immunology Department, School of Medicine, Shahid Beheshti University of Medical Sciences, Tehran/Iran

${ }^{4}$ Gastroenterology and Liver Diseases Research Center, Research Institute for Gastroenterology and Liver Diseases, Shahid Beheshti University of Medical Sciences, Tehran/Iran

${ }^{5}$ Milton University Hospital, birmingham/United Kingdom

Contact E-mail Address: m.rostamii@gmail.com

Introduction: Celiac disease (CD) is an organ-specific autoimmune disease, and both adaptive and innate immunity are involved in its development. Recent studies suggest the dysregulation Toll-like receptors (TLRs) in innate immunity can confer risk to autoimmune diseases such as CD. 
Aims \& Methods: In this study we investigated the TLRs 2, 4, 7, 9 genes expression in the Iranian patients with celiac disease comapred with healthy control. Blood samples from $120 \mathrm{CD}$ patients diagnosed according to the Iranian Society for Gastroenterology were collected and 120 healthy individuals were served as a control group during 2016. Also, among them, 20 duodenal biopsy specimens were collected randomly. Total RNA for both boold samples and biopsy specimens was isolated using a standard commercial kit. The mRNA expression of TLRs were quantified by relative qPCR with B2M as a reference gene.

Results: Significantly higher expression of TLR4 and TLR9 mRNA was observed in blood samples of $C D$ patients compared to the healthy controls $(P<0.05)$; but there were no significant differences between expression of TLR2 and TLR7 mRNA compared to the controls. Furthermore, TLR4 and TLR2 expression level was increased in CD biopsy specimens compared to controls, whereas expression of TLR9 mRNA was decreased in CD patients. No significant differences in expression of TLR7 was observed in biopsy specimens.

Conclusion: The result of this study show that the alteration of TLR4 and TLR9 genes expression in intestinal mucosa of CD can be detected in PBMs in peripheral blood. Further, this data supports the potential implication of innate immune system in the pathomechanism of celiac disease.

Disclosure of Interest: All authors have declared no conflicts of interest.

\section{P0609 STUDY OF BONE DENSITY IN CELIAC DISEASE}

\author{
A. $\operatorname{Ravaux}^{1}$, G. Malamut ${ }^{1}$, C. Cellier ${ }^{2}$ \\ ${ }^{1}$ HEGP, paris/France \\ ${ }^{2}$ Gastroenterology, European Georges Pompidou Hospital Dept. of \\ Gastroenterology, Paris/France
}

Contact E-mail Address: agathe.ravaux@live.fr

Introduction: Low bone mineral density (BMD) is common in celiac disease (CD). Aims \& Methods: The goals of the study were to identify the predictive factors of low BMD and of bone density variations. The medical files of 607 patients with CD at Europeen Georges Pompidou Hospital were reviewed (2000-2015). Four hundred and four had an osteodensitometry and 133 had one before or until six months after beginning a gluten free diet (GFD). An univariate and multivariate analysis were performed for the different factors. The speed of variation of $T$ and $\mathrm{Z}$-score was calculated by the ratio between $\mathrm{T}$ and $\mathrm{Z}$-score variation and the duration between these two measures.

Results: $46 \%$ of patients had osteopenia and $17.5 \%$ had osteoporosis. The predictive factors of low BMD in celiac patients were, in lumbar spine and forearm tests, male gender $(\mathrm{p}<0.001)$, in lumbar spine and femoral neck tests, low body mass index $(\mathrm{BMI})(\mathrm{p}<0.001)$ and in the three sites, old age at the diagnosis $(\mathrm{p}<0.001)$. In the femoral neck, secondary hyperparathyroidism was a predictive factor of low BMD at celiac disease diagnosis $(p=0.016)$. The factors with an impact on gain of bone density were, in the forearm, female gender $(p=0.034)$ and in the femoral neck, the HLA-DQ2/DQ2 status $(\mathrm{p}=0.008)$

Conclusion: $64 \%$ of celiac patients had low BMD. The predictive factors were male gender, old age at diagnosis, low BMI and secondary hyperparathyroidism. The gain of bone density was better in woman and in HLA-DQ2/DQ2 patients whereas GFD had no impact.

Disclosure of Interest: All authors have declared no conflicts of interest.

\section{References}

Sollid LM, and al. Evidence for a primary association of celiac disease to a particular HLA-DQ alpha/beta heterodimer. J Exp Med.1989 Jan

Marsh MN, and al. Gluten, major histocompatibility complex, and the small intestine. A molecular and immunobiologic approach to the spectrum of gluten sensitivity ('celiac sprue'). Gastroenterology.1992 jan

Newnham ED, and al. Adherence to the gluten-free-diet can achieve the therapeutic goals in almost all patients with coeliac disease: A 5-year longitudinal study from diagnosis. J Gastroenterol Hepatol. 2016 Feb

Molteni N, and al. Bone mineral density in adult celiac patients and the effect of gluten-free diet from childhood. Am J Gastroenterol. 1997 Aug

Tau C, and al. Bone mineral density in children with celiac disease. Effect of Gluten-free diet. Eur J Clin Nutr. 2006 Mar

Pantaleoni S, and al. Bone mineral density at diagnosis of celiac disease and after 1 year of gluten-free diet. ScientificWorldJournal. 2014

Valdimarsson T, and al. Reversal of osteopenia with diet in adult coeliac disease. Gut. 1996 Mar

Mcfarlane XA, and al. Effect of a gluten free diet on osteopenia in adults with newly diagnosed coeliac disease. Gut. 1996 Aug

Kemppainen T, and al. Osteoporosis in adult patients with celiac disease. Bone. 1999 Mar

\section{P0610 GLUTEN-FRIENDLY BREAD (GFB) INCREASES GUT MUCUS PRODUCTION AND MONOLAYER PERMEABILITY IN VITRO}

G. Corona ${ }^{1}$, M. Henderson ${ }^{1}$, T. Bergillos-Meca ${ }^{1}$, M. Roul $^{1}$, D. Musaico ${ }^{2}$, C. Lamacchia ${ }^{2}$ A. Costabile

${ }^{1}$ Life Sciences, University of Roehampton, London/United Kingdom

${ }^{2}$ Science Of Agriculture, Food And Environment, University of Foggia, Foggia/ Italy

Contact E-mail Address: adele.costabile@roehampton.ac.uk
Introduction: The mucus layer, covering the gastrointestinal mucosa, is considered the first line of defence against mechanical, chemical, or microbiological aggressions arising from the luminal contents ${ }^{1}$. Among all different cell types of the intestinal epithelium, the goblet cells are specialised in the secretion of mucus constituent ${ }^{2}$. As an example of failing barrier function, patients with coeliac disease $(C D)$ have been reported to have altered intestinal barrier ${ }^{3}$. Human colonic mucus-secreting cells HT-29-16E are valuable tools to explore the effect of a specific treatment on permeability and mucus production by the human intestinal epithelium.

Aims \& Methods: We investigated the new and innovative gluten detoxified bread (GFB; patent PCT/IB2013/000797 ${ }^{4}$ ) effects on mucus production by means of Alcian blue staining in comparison to the control bread (CB). In addition, MUC2 and MUC3 were quantified by ELISA and the permeability of the intestinal epithelium monolayer was evaluated by trans-epithelial electrical resistance (TEER) measurement. The statistical analysis was conducted by one-way ANOVA followed by a Bonferroni post-hoc t-test.

Results: Mucin production by Alcian blue staining was expressed as \% black pixels obtained from Image Tool software. GFB increased the secretion of mucin after 24 hours by Alcian blue staining $(10.28 \pm 1.82$; $\mathrm{P} \leq 0.01)$, whereas $\mathrm{CB}$ did not $(9.94 \pm 0.67 ; \mathrm{P}=0.05)$. Higher $\mathrm{MUC2}$ concentrations expressed as $\mathrm{ng} / \mathrm{ml}$ were found on cells treated with GFB $(10.82 \pm 1.35 ; \mathrm{P}=0.01)$ compared to control, which was not found for $\mathrm{CB}(9.54 \pm 0.18 ; \mathrm{P}=0.05)$. Additionally, greater TEER values, expressed as a percentage of initial TEER, were observed after 24 hours of incubation with GFB in comparison to the control (163.2 \pm 33.8 , $\mathrm{P}=0.01)$ which was not observed or $\mathrm{CB}(139.4 \pm 28.8, \mathrm{P}=0.05)$.

Conclusion: It could be concluded that GFB has a potential of inducing MUC2 secretion by intestinal epithelial cells and improving intestinal epithelium permeability in vitro. Such observed potential may effectively contribute to consequent benefits such as higher gut barrier defence, decreased susceptibility to infections and better absorption regulation, thus ameliorating such alterations in coeliac patients.

Disclosure of Interest: All authors have declared no conflicts of interest.

\section{References}

1. Atuma C, Strugala V, Allen A, et al. The adherent gastrointestinal mucus gel layer: thickness and physical state in vivo. Am J Physiol Gastrointest Liver Physiol 2001; 280, 922-929.

2. Alaedini A and Green PH. Narrative review: celiac disease: understanding a complex autoimmune disorder. Ann Intern Med 2005; 142, 289-298.

3. Augeron $\mathrm{C}$ and Laboisse CL. Emergence of permanently differentiated cell clones in a human colonic cancer cell line in culture after treatment with sodium butyrate. Cancer Res 1984; 44, 3961-3969.

4. Lamacchia C, Di Luccia A and Gianfrani C, inventors. University of Foggia, assignee. Method for the detoxification of gluten proteins from grains of cereals. Patent Cooperation Treaty CT/IB2013/000797. 2013.

\section{P0611 IMMUNOHISTOCHEMICAL LYMPHOCYTE PATTERN OF NON CELIAC GLUTEN SENSITIVITY MAY SUPPORT THE INVOLVEMENT OF INNATE IMMUNITY}

G. Losurdo, F. Pezzuto, C. Covelli, F. Fortarezza, A. Marra, A. Iannone, A. Amoruso, M. Barone, M. Principi, E. Ierardi, A. Di Leo Emergency And Organ Transplantation, University of Bari, Bari/Italy

Contact E-mail Address: giuseppelos@alice.it

Introduction: Non celiac gluten sensitivity (NCGS) is a gluten-related enteropathy with distinct features compared to celiac disease. Patients with NCGS experience symptoms, such as diarrhea or abdominal pain, shortly after gluten exposure. Differently from celiac disease, serology is negative, and histological picture is characterized by no lesion or a mild enteropathy (Marsh 1 stage). Few studies have investigated possible histopathological characteristics of NCGS. An immune response against gluten elicited exclusively by innate immunity has been hypothesized. On these bases, we attempted to depict an immunohistochemical trait of NCGS by exploring markers of lymphocyte and innate immunity activation.

Aims \& Methods: Duodenal biopsy samples of patients diagnosed of NCGS according to Salerno criteria were retrieved. Duodenal biopsy samples of positive controls (overt seropositive celiac disease at Marsh 1 stage) and negative controls (functional dyspepsia and normal microscopic picture) were selected. Immunohistochemistry for CD3 (intraepithelial lymphocytes), CD4 (T-helper lymphocytes), CD8 (T-cytotoxic lymphocytes) and CDla (Langerhans cells) was performed. Cell count was carried out both in the epithelial layer (expressed as positive cells $/ 100$ enterocytes) and in the lamina propria (positive cells $/ \mathrm{mm}^{2}$ ). Comparison of means was performed by ANOVA test with Bonferroni's posthoc analysis.

Results: Twenty NCGS, 12 celiac patients (positive controls) and 16 negative controls were selected. CD3 positive intraepithelial lymphocytes in NCGS were expressed at intermediate levels $(18.5 \pm 6.4)$ between negative controls $(11.9 \pm 2.8)$ and celiac disease $(40.8 \pm 8.1, \mathrm{p}<0.0001)$. CD4 T-helper lymphocytes were present only in lamina propria and NCGS had a lower level $(31.0 \pm 22.1)$ than controls $(72.5 \pm 29.5)$ and celiac disease $(103.7 \pm 15.7$, $\mathrm{p}<0.0001)$. Intraepithelial CD $8+$ cells were similar between NCGS and negative controls $(14.0 \pm 7.4$ versus $17.8 \pm 4.2)$, but lower than celiac disease $(34.0 \pm 7.1, \mathrm{p}<0.0001)$. CDla + Langerhans cells were over-expressed in the lamina propria of NCGS $(1.9 \pm 1.1)$ in comparison to celiac disease and negative controls (respectively $0.3 \pm 0.8$ and $0.4 \pm 0.5, \mathrm{p}<0.0001$ ).

Conclusion: NCGS is characterized by a mild immunologic reaction, as shown by the slight increase in CD3 intraepithelial lymphocytes. The over-expression in the 
lamina propria of Langerhans cells, which are antigen presenting cells mainly involved in innate immunity, seems to confirm previous hypotheses on the pathogenesis of NCGS. Additionally, this finding could represent an useful pathological feature of NCGS.

Disclosure of Interest: All authors have declared no conflicts of interest.

\section{P0612 FUNCTIONAL DYSPEPSIA SYMPTOMS ARE STRONGLY ASSOCIATED WITH COELIAC DISEASE: RESULTS FROM A POPULATION-BASED STUDY}

M. Potter ${ }^{1}$, M. M. Walker ${ }^{2}$, M.P. P. Jones ${ }^{3}$, N. Koloski ${ }^{1}$, G. Brogan ${ }^{1}$, S. Keely ${ }^{1}$, N.J. Talley 1

${ }^{1}$ Faculty Of Health And Medicine And Priority Research Center For Digestive

Health And Neurogastroenterology, The University of Newcastle Australia,

Callaghan/Australia/NSW

${ }^{2}$ Anatomical Pathology, University of Newcastle, Newcastle/Australia/NSW

${ }^{3}$ Psychology, Macquarie University Psychology, North Ryde/Australia

Contact E-mail Address: Michael. Potter@hnehealth.nsw.gov.au

Introduction: Coeliac disease (CD) is estimated to affect up to 1 in 100 Australians (1). Although $\mathrm{CD}$ has a wide range of clinical manifestations, patients frequently present with gastrointestinal (GI) symptoms which overlap with functional GI disorders, particularly irritable bowel syndrome (IBS) and functional dyspepsia (FD); the prevalence of biopsy proven CD is higher in IBS (2) and in dyspepsia (3). Patients with CD have been shown to experience persistent GI symptoms despite long term treatment with a gluten-free diet (4).

Aims \& Methods: The aim of this study was to define GI symptoms reported in an Australian cohort with a doctor diagnosis of CD and compare with those not reporting CD. A total of 3825 people (mean age 58.4 years, age range 18-100 years and $47.5 \%$ males) randomly selected from the Australian population returned a mail survey (Digestive Health \& Wellbeing Survey, response rate$=45 \%$ ) which contained questions on whether the participant had ever been told by a physician that they had CD, and questions regarding GI symptoms to establish whether they had co-existent functional GI disorders. Adherence to a gluten-free diet was not assessed. Prevalence of CD, FD and IBS are reported with $95 \%$ exact confidence intervals. The difference between symptoms in those with $C D$ compared with the unaffected population was tested for significance by the Pearson chi-square test.

Results: The prevalence of doctor-diagnosed CD was $1.2 \%$ (95\% CI $0.84-1.59)$ in this cohort. Subjects with CD reported significantly higher levels of GI symptoms than unaffected individuals, including abdominal pain associated with abnormal bowel habit, diarrhoea, bloating, distention, epigastric burning and early satiety (see Table). There was no significant difference observed in symptoms of postprandial fullness, nausea, constipation, abnormal stool consistency, or straining with defecation. The prevalence of FD as defined by Rome III criteria in the CD cohort was $37.5 \%(95 \%$ CI $22.7-54.2)$ compared to $13.9 \%$ (95\% CI $12.8-15.1)$ in the non-affected population (OR 3.7, 95\% CI 1.9-7.1, p $<0.001$ ). There was no significant difference in the prevalence of IBS in the affected compared with the non- affected cohort $(30.8 \%$ versus $22.2 \%, \mathrm{p}=0.2)$.

Table: Gastrointestinal symptoms reported by patients with and without self reported coeliac disease (CD). Items reported as greater than one day per week $(*)$ or greater than or equal to "often" $(* *)$

\begin{tabular}{llll}
\hline Symptoms & $\begin{array}{l}\text { self report } \\
\text { CD - Yes }\end{array}$ & $\begin{array}{l}\text { self report } \\
\text { CD - No }\end{array}$ & p value \\
\hline $\begin{array}{c}\text { Abdominal pain associated with } \\
\text { loose bowel motions ** }\end{array}$ & $16 / 3743.2 \%$ & $600 / 323418.6 \%$ & $\mathrm{P}<0.001$ \\
$\begin{array}{c}\text { More bowel motions } \\
\quad \text { associated with pain ** }\end{array}$ & $13 / 3834.2 \%$ & $504 / 324815.5 \%$ & $\mathrm{P}=0.002$ \\
$\begin{array}{l}\text { Bloating * } \\
\text { Distention * }\end{array}$ & $13 / 4032.5 \%$ & $436 / 338112.9 \%$ & $\mathrm{P}<0.001$ \\
Abdominal pain * & $12 / 4030 \%$ & $395 / 337111.7 \%$ & $\mathrm{P}<0.001$ \\
$>3$ bowel motions per day ** & $9 / 3923.1 \%$ & $362 / 337810.7 \%$ & $\mathrm{P}=0.014$ \\
$\begin{array}{l}\text { Epigastric burning * } \\
\text { Early satiety * }\end{array}$ & $8 / 4017.5 \%$ & $7 / 4017.5 \%$ & $\mathrm{P}=0.046$ \\
\hline
\end{tabular}

Conclusion: The prevalence of gastrointestinal symptoms and in particular functional dyspepsia are significantly higher in patients with a doctor diagnosis of CD than in the unaffected population. Studies show that biopsy proven coeliac disease in IBS is higher in IBS cohorts than healthy controls (2) and the value of screening with duodenal biopsy testing for CD in FD is concluded to be useful (3), this study supports these views

Disclosure of Interest: All authors have declared no conflicts of interest.

\section{References}

1. Anderson RP, Henry MJ, Taylor R, et al. A novel serogenetic approach determines the community prevalence of celiac disease and informs improved diagnostic pathways. BMC Med. 2013;11:188.

2. Ford AC, Chey WD, Talley NJ, et al. Yield of diagnostic tests for celiac disease in individuals with symptoms suggestive of irritable bowel syndrome: systematic review and meta-analysis. Arch Intern Med. 2009;169:651-8.

3. Ford AC, Ching E, Moayyedi P et al. Meta-analysis: yield of diagnostic tests for coeliac disease in dyspepsia. Aliment Pharmacol Ther. 2009;30:28-36.
4. Laurikka P, Salmi T, Collin P, et al. Gastrointestinal Symptoms in Celiac Disease Patients on a Long-Term Gluten-Free Diet. Nutrients. 2016:Jul $14 ; 8(7)$.

P0613 MALE GENDER AND UNDERWEIGHT ARE ASSOCIATED WITH OSTEOPOROSIS IN PATIENTS WITH NEW DIAGNOSIS OF COELIAC DISEASE

G. Galli, E. Lahner, L. Conti, G. Esposito, M.C. Sacchi, E. Di Giulio, B. Annibale

Department Of Medical-surgical Sciences And Translational Medicine, Sant'Andrea Hospital, University Sapienza, Rome/Italy

Contact E-mail Address: gloria.galli5@gmail.com

Introduction: Osteoporosis is a systemic skeletal disorder characterized by low bone mass and micro-architectural deterioration with increase of bone fragility and consequent fracture risk. About $50-75 \%$ of patients (pts) with untreated coeliac disease (CD) suffer from bone mass loss (osteopenia or osteoporosis). Despite this strong correlation, guidelines do not express with certainty on the need to undergo a dual-energy X-ray absorptiometry (DEXA) scan in every patient with new diagnosis of CD. Recently, the DEXA screening was suggested for CD peri-post menopausal females, males over 55 years, pts with overt malabsorption or with a history of fragility fractures. Studies on bone mineral density (BMD) in $\mathrm{CD}$ pts led to discrepant results, probably due to heterogeneous designs.

Aims \& Methods: The aim of this study was to evaluate, in a cohort of consecutive newly diagnosed CD adults, the prevalence of BMD alterations at diagnosis time and to evaluate associated clinical features. From January 2004 to December 2016, 258 consecutive pts $(F=72.4 \%)$ were diagnosed with $C D$. All pts were adults (median age 38 , range 18-72 years), had atrophic disease and were invited to undergo a DEXA within 3 months from diagnosis to screen for osteoporosis (T-score $<-2.5$ ) or osteopenia (T-score $<-1$ and $>-2.5$ ). A total of $214(82.9 \%)$ pts underwent the DEXA scan and were included in the study ( $F=71.5 \%$, median age 38 , range $18-72$ years). On the basis of DEXA results (codified according to WHO classification) pts were divided into 3 groups: pts with normal BMD, with osteopenia, and with osteoporosis. For each patient, reported risk factors for low BMD (underweight, alcohol intake, drugs, menopause, smoke) and serological PTH values were assessed. The signs/symptoms leading to $\mathrm{CD}$ and their duration before diagnosis, autoimmune/not-autoimmune comorbidities, familiarity for $\mathrm{CD}$, previous fractures and serological assays (specific antibodies for CD, ferritin, cholesterol, triglycerides, and albumin) were also assessed. All the variables described were analyzed and compared between the 3 groups. Logistic regression was performed including into the model those independent variables which showed a significant difference at univariate analysis.

Results: At the DEXA scan, $85(39.7 \%)$ and $129(60.3 \%)$ pts had normal or low $\mathrm{BMD}$, respectively. Among pts with low BMD, 91 (42.5\%) had osteopenia and $38(17.8 \%)$ osteoporosis. At logistic regression, clinical features significantly associated with osteoporosis were male gender (OR 4.7; 95\% CI 1.3 to 17.4), underweight (OR 8.1; 95\% CI 1.8 to 35.3 ) and increased PTH values (OR 5.1; $95 \%$ CI 1.4 to 18.8 ), while age $\geq 40$ years and gastrointestinal symptoms at diagnosis time, menopause, alcohol intake and previous fractures were not associated. Clinical features significantly associated with osteopenia were underweight (OR 4.0, 95\% CI 1.4 to11.2) and increased PTH values (OR 2.6, $95 \%$ CI 1.1 to 6.4 ).

Conclusion: In newly diagnosed coeliac pts, the overall prevalence of BMD alterations was more than $60 \%$, with osteoporosis in nearly $1 / 5$. Osteoporosis was significantly associated with male gender, underweight and increased PTH. This study suggests that at CD diagnosis, DEXA scan might be of benefit, in particular in male underweight pts.

Disclosure of Interest: All authors have declared no conflicts of interest.

\section{References}

M.A. Fouda, A.A. Khan, M.S. Sultan, L.P. Rios, K. McAssey, D. Armstrong. Evaluation and management of skeletal health in celiac disease: Position statement. Can J Gastroenterol. 2012

M. Di stefano, C. Mengoli, M. Bergonzi, G.R. Corazza. Bone mass and mineral metabolism alterations in adult celiac disease: Pathophysiology and clinical approach. Nutrients. 2013

\section{P0614 SELF-REPORTED WHEAT SENSITIVITY IN AN AUSTRALIAN POPULATION STUDY}

M. Potter ${ }^{1}$, M. M. Walker ${ }^{2}$, M.P. P. Jones ${ }^{3}$, N. Koloski ${ }^{1}$, G. Brogan ${ }^{1}$, S. Keely ${ }^{1}$, N.J. Talley ${ }^{1}$

${ }^{1}$ Faculty Of Health And Medicine And Priority Research Center For Digestive

Health And Neurogastroenterology, The University of Newcastle Australia,

Callaghan/Australia/NSW

${ }^{2}$ Dept. Of Anatomical Pathology, University of Newcastle Dept. of Anatomical

Pathology, Newcastle/Australia

${ }^{3}$ Psychology, Macquarie University Psychology, North Ryde/Australia

Contact E-mail Address: Michael.Potter@hnehealth.nsw.gov.au Introduction: Coeliac disease $(\mathrm{CD})$ affects $0.6-1 \%$ of the population worldwide (1). Wheat avoidance in the absence of $\mathrm{CD}$ is common, and studies report a population prevalence of self-reported wheat or gluten sensitivity (SRWS) of 
up to $13 \%(2,3)$. SRWS is defined as gastrointestinal (GI) or extra intestinal symptoms on ingestion of wheat or gluten-containing food $(2,3)$.

Aims \& Methods: The aim of this study was to determine the prevalence of SRWS in an Australian population, define associated GI symptoms, and relate the diagnosis to demographic, lifestyle and medical factors. A total of 3825 people (mean age 58.4 years, age range $18-100$ years and $47.5 \%$ males) randomly selected from the Australian population returned a mail survey (Digestive Health \& Wellbeing Survey, response rate $=45 \%$ ) which contained questions on wheat avoidance, GI symptoms, demographic, medical and lifestyle factors. We defined SRWS as people who reported gastrointestinal symptoms on ingestion of wheat based foods, but did not suffer from doctor diagnosed coeliac disease, inflammatory bowel disease or bowel cancer. Prevalence of SRWS is reported with $95 \%$ exact confidence intervals. The association between SRWS prevalence and potential risk factors was reported using unconditional logistic regression. The degree of differentiation of SRWS from health was evaluated through the area under the receiver-operator-characteristic curve.

Results: The prevalence of SRWS in this cohort was $13.5 \%(455 / 3331,95 \%$ CI $12.5-14.9 \%)$. Only $11 \%(50 / 455)$ of these subjects had received a doctor diagnosis of wheat or gluten intolerance. The most commonly reported GI symptoms (reported as more than weekly or often) associated with SRWS included abdominal pain relieved by bowel movements $(54.5 \%)$, bloating $(37.6 \%)$ and abdominal distention $(30.8 \%)$. In a multivariate analysis, a diagnosis or SRWS was significantly associated with irritable bowel syndrome (IBS) and functional dyspepsia (FD) (Rome III criteria), female gender, and food allergy (see Table). Older age was negatively associated with SRWS. In this multivariate model, factors with no observed association included body mass index, depression, anxiety, sleep problems, proton pump inhibitor use, gastrointestinal infection, rheumatoid arthritis, scleroderma, migraine, Parkinson's disease, asthma, pollen allergy, animal allergy or psoriasis, diabetes and recent antibiotic use. The model provided useful although imperfect differentiation of SRWS from health $(\mathrm{AUC}=0.76)$.

Table: Medical and demographic factors associated with a diagnosis of selfreported wheat sensitivity (SRWS).

Odds

SRWS -YES SRWS -NO ratio $95 \%$ CI, p value

\begin{tabular}{|c|c|c|c|c|}
\hline Age: mean (SD) & $\begin{array}{l}51.9 \\
(14.9)\end{array}$ & $\begin{array}{l}59.3 \\
(16.5)\end{array}$ & 0.98 & $0.98-0.99, \mathrm{p}<0.001$ \\
\hline $\begin{array}{l}\text { Female gender } \\
\quad(\text { total }=52.5 \%)\end{array}$ & $\begin{array}{l}336 / 450 \\
(74.7 \%)\end{array}$ & $\begin{array}{l}1392 / 2854 \\
(48.8 \%)\end{array}$ & 1.86 & $1.51-2.30, \mathrm{p}<0.001$ \\
\hline Functional dyspepsia & $\begin{array}{l}128 / 466 \\
(27.5 \%)\end{array}$ & $\begin{array}{l}327 / 2865 \\
(11.4 \%)\end{array}$ & 1.74 & $1.38-2.20, \mathrm{p}<0.001$ \\
\hline $\begin{array}{l}\text { Irritable bowel } \\
\text { syndrome }\end{array}$ & $\begin{array}{l}248 / 453 \\
(54.8 \%)\end{array}$ & $\begin{array}{l}571 / 2866 \\
(19.9 \%)\end{array}$ & 3.96 & $3.23-4.86, \mathrm{p}<0.001$ \\
\hline Food allergy & $\begin{array}{l}56 / 455 \\
(12.3 \%)\end{array}$ & $\begin{array}{l}151 / 2878 \\
(5.3 \%)\end{array}$ & 2.05 & $1.50-2.80, \mathrm{p}<0.001$ \\
\hline
\end{tabular}

Conclusion: SRWS has a prevalence of $13.5 \%$ in this Australian cohort. Those with SRWS are likely to report abdominal symptoms, including abdominal pain associated with abnormal bowel habits, bloating, or abdominal distention. SRWS is significantly associated with IBS and FD, younger age, female gender, and food allergy.

Disclosure of Interest: All authors have declared no conflicts of interest.

\section{References}

1. Fasano A, Catassi C. Clinical practice. Celiac disease. $N$ Engl J Med. 2012;367:2419-26.

2. Molina-Infante J, Santolaria S, Sanders DS, Fernandez-Banares F. Systematic review: noncoeliac gluten sensitivity. Aliment Pharmacol Ther. 2015;41:807-20.

3. Volta U, Caio G, Karunaratne TB, Alaedini A, De Giorgio R. Non-coeliac gluten/wheat sensitivity: advances in knowledge and relevant questions. Expert Rev Gastroenterol Hepatol. 2017;11:9-18.

\section{P0615 INSUFFICIENCY OF THE SMALL INTESTINAL ENZYMES MAY BE ONE OF THE CAUSES OF FUNCTIONAL BOWEL DISEASE}

O. Akhmadullina ${ }^{1}$, A. Parfenov ${ }^{1}$, N. Belostotsky ${ }^{2}$, E. Sabelnikova ${ }^{1}$, S. Bykova ${ }^{1}$, S. Khomeriki

${ }^{1}$ Small Intestine, Moscow Clinical Scientific Center, Moscow/Russian Federation

${ }^{2}$ Moscow Clinical Scientific Center, Moscow/Russian Federation

Contact E-mail Address: olgaakh@inbox.ru

Introduction: Pathogenesis of functional bowel disease is usually associated with disorders of visceral sensitivity and intestinal motility, which result from a dysfunction of the central nervous system, intestinal microflora and immune system Aims \& Methods: We aimed to highlight importance of intestinal enzymes (glucoamylase, maltase, sucrase and lactase) in the etiology and pathogenesis of functional bowel disease. 74 patients with functional bowel diseases in age from 18 to 50 years ( 36 men and 38 women) were examined. According to Rome IV criteria (2016), 21 had irritable bowel syndrome (IBS) with predominance of diarrhea, 33 - functional diarrhea, 6 - IBS with predominant constipation, 4 - functional constipation and 10 - mixed type of IBS. Activity of the mucosa enzymes of the small intestine was determined by Dahlquist-Trinder method in duodenal biopsies obtained during esophagogastroduodenoscopy.
Results: Lactase deficiency was identified in $87.8 \%$ of patients, maltase deficiency - in $48.6 \%$, sucrase deficiency - in $51.3 \%$, the glucoamylase deficiency - in $85.1 \%$. The activity of all investigated enzymes was reduced in $23(31.1 \%)$ patients with functional bowel diseases, failure of 1 to 3 enzymes detected in $47(63.5 \%)$. Normal activity of enzymes was observed in $4(5.4 \%)$ patients.

Conclusion: In 70 of $74(94.5 \%)$ patients with functional bowel disease and a disorder of the stool, abdominal pain and flatulence, there was a decrease in the activity of intestinal enzymes, which may be a cause of intestinal symptoms.

Disclosure of Interest: All authors have declared no conflicts of interest.

\section{P0616 WHEAT PROTEIN ALLERGY OR SENSITIZATION TO WHEAT PROTEIN IN A CELIAC POPULATION}

C. Caruso ${ }^{1}$, V. Gerardi ${ }^{2}$, A. Armuzzi ${ }^{2}$, L. Guidi ${ }^{3}$, A. Papa ${ }^{2}$, S. Ennas ${ }^{3}$,

A. Romano ${ }^{1}$, G. Rapaccini ${ }^{2}$, I. De Vitis ${ }^{3}$

${ }_{1}^{1}$ Allergology, A.Gemelli Foundation, Rome/Italy

${ }^{2}$ Complesso Integrato Columbus, Internal Medicine and Gastroenterology -

Complesso Integrato Columbus Catholic University, Rome/Italy

${ }^{3}$ Gastroenterology \& Internal Medicine-celiac Cisease Unit, A.gemelli Foundation, Catholic University of Sacred Heart, Rome/Italy

Contact E-mail Address: italodev@tin.it

Introduction: Epidemiological studies estimate a worldwide prevalence of CD of approximately 1:100 individuals, with a considerable proportion of patients remaining undiagnosed and untreated.

According to a study performed by the National Health and Nutrition Examination Survey in the United States, the prevalence of self-prescribed GFD in an unselected population of subjects aged 6 years or older was $0.5 \%$. Epidemiological studies report a prevalence of WA in American population of around $0.4 \%$ untill $0.6 \%$

The diagnosis of WA is classically based on skin prick tests (SPT), in vitro specific Immunoglobulin E (sIgE) assays and functional assays. SPTs and sIgE in vitro assays are the first-level diagnostics for WA. However, they are affected by a low predictive value. In particular, their low sensitivity can be explained by the fact that the commercial test reagents are mixtures of water/salt-soluble wheat proteins that lack allergens from the insoluble gluten fraction. The association between food allergy and celiac disease (CD) is still to be clarified. Gluten-related disorders are an epidemiologically relevant phenomenon whit a global prevalence that is estimated around $5 \%$, drawing the attention of the scientific community.

Aims \& Methods: We visited in our unit of celiac disease and gluten-related conditions during 2016423 (F/M:312/111) new patients with clinical suspicion of $\mathrm{CD}$. Of these 113 they were not celiac but were investigated for suspected nonceliac gluten sensitivity. After in vitro tests for the exclusion of celiac disease, to verify the real prevalence of food allergy particularly to wheat protein, in non celiac patient referred our unit for symptoms after gluten ingestion, all these patients underwent allergologic workup consists on: skin prick tests for foods including wheat (Alk-abello), LTP (lipid transfer protein) (peach Alk abello), alpha amylase, wheat flour, barley, corn, rice, grass pollen and histamine. Also they all performed patch tests for suspected allergy to nickel, if they reported reactions after a few hours of ingestion of gluten. Molecular-based allergy (MA) diagnostics could overcome some limitations of sIgE in vitro assay using wheat flour extracts. We have used omega-5 gliding (TRI a 19) and nsLTP (TRIa 14), gli admin, wheat, gluten that are available in the immunoCap assay, whereas the Alfa -amylase/trypsin inhibitor (TRI a A/TI) is available only in the microarray ISAC assay. The sIgE to omega-5 gliadin assay is highly reliable and now widely used to identify the patient with WDEIA.

Results: In our overall population, $113(26.7 \%)$ non celiac patients had a history of immediate or not immediate reaction after ingesting gluten: the Allergologiic tests found wheat protein sensitization in 14 patients of these $(12.4 \%, 9 \mathrm{~F})$. In addition we also found $5(4.5 \%, 4 \mathrm{~F})$ patients with real allergy to wheat or wheat protein.

Conclusion: In our population $19 / 113(16.8 \%)$ non celiac patient had real reaction after ingesting gluten: $14(12.3 \%)$ had wheat protein sensitization and 5 $(4.5 \%)$ had WA. These percentages are different and very high than that reported in the literature.

Disclosure of Interest: All authors have declared no conflicts of interest.

\section{Reference}

Non- celiac wheat sensitivity as an allergic condition. personal experience and narrative review. A. Carroccio et al AmJ gastroent 2013.

\section{P0617 A QUESTIONNAIRE-BASED SYMPTOM EVALUATION STUDY IN 381 PATIENTS DIAGNOSED WITH BILE ACID MALABSORPTION BY SEHCAT FROM 2003 TO 2016}

H.R. Clausen, B. Damsgaard, J.F. Dahlerup, J. Agnholt, S.P. Jørgensen, K. Krogh

Department Of Hepatology And Gastroenterology, Aarhus University Hospital, Aarhus/Denmark

Contact E-mail Address: heleneraskclausen@studmed.au.dk

Introduction: Excessive amounts of bile acids entering the colon cause chronic diarrhoea (bile acid diarrhoea (BAD)). Diagnosis of BAD is possible by measuring the retention level of orally ingested ${ }^{75}$ Selenium homotaurocholic acid (SeHCAT). Standard treatment of BAD is bile acid sequestrants (BAS), such 
as colestyramine. Short-term outcome of having BAD is well-described, but longterm effects remain unclear. The aim of the present study was to describe longterm symptoms, adherence to treatment and quality of life in a well-defined group of patients with BAD

Aims \& Methods: Between 2003 and 2016, 559 patients referred to our hospital for diarrhoea had abnormally low seHCAT retention levels $(<15 \%$ at day 7$)$. Questionnaires about medical history, bowel function, use of medication, and quality of life were sent to all patients.

Results: Among 559 patients, $381(68.2 \%)$ responded ((242 women $(63.5 \%)$, median age: 53 years (range 22 to 89 ), median age at diagnosis 47 years (range 16 to 85$)$ ). In 123 respondents $(32.3 \%$ ) BAD was due to ileal dysfunction (type 1 ), $199(52.2 \%)$ had idiopathic BAD (type 2), and $59(15.5 \%)$ had BAD due to cholecystectomy (type 3 ). At follow-up, 272 patients (73.9\%) still reported bothersome diarrhoea and $244(65.1 \%)$ regularly used antidiarrheal medication. Treatment included BAS in $45.1 \%$ while $32.3 \%$ of patients used other treatment. 184 patients $(49.9 \%)$ reported that treatment had improved their symptoms, while $116(31.4 \%)$ reported that they were the same, and $69(18.7 \%)$ felt worse. Quality of life was still affected by diarrhoea in 242 patients $(63.5 \%)$. Conclusion: BAD must be considered as a chronic disease and despite correct diagnosis and treatment, most patients continue to have significant diarrhoea and reduced quality of life. This supports the need for further research in pathophysiology and new therapy principles.

Disclosure of Interest: All authors have declared no conflicts of interest.

\section{P0618 EFFICACY OF PERCUTANEOUS ENDOSCOPIC GASTRO- JEJUNOSTOMY (PEG-J) DECOMPRESSION THERAPY FOR PATIENTS WITH CHRONIC INTESTINAL PSEUDO-OBSTRUCTION (CIPO)}

H. Ohkubo ${ }^{1}$, T. Higurashi ${ }^{2}$, J. Arimoto ${ }^{1}$, A. Nakajima ${ }^{1}$

${ }^{1}$ Yokohama City Hospital Dept. of Gastroenterology, Yokohama/Japan

${ }^{2}$ Department Of Gastroenterology And Hepatology, Yokohama City University, Yokohama/Japan

Contact E-mail Address: ohkuboh@yokohama-cu.ac.jp

Introduction: Chronic intestinal pseudo-obstruction (CIPO) is an intractable rare digestive disease manifesting persistent small bowel distension without any mechanical cause. Intestinal decompression is a key treatment, but conventional method including a trans-nasal small intestinal tube is invasive and painful Therefore, a less invasive and tolerable new decompression method is urgently desired. We conducted a pilot study and assessed the efficacy and safety of percutaneous endoscopic gastro-jejunostomy (PEG-J) decompression therapy in CIPO patients.

Aims \& Methods: Eight definitive CIPO patients (2 males and 6 females) were enrolled. All patients received PEG-J decompression therapy. The number of days with any abdominal symptoms in a month (NODASIM), body mass index (BMI), serum albumin level (Alb), and small intestinal volume before and after PEG-J were compared in all patients.

Results: PEG-J was well tolerated and oral intake improved in all patients. NODASIM has significantly decreased (24.3 vs 9.3 days/months) and BMI/ Alb have significantly increased (14.9 vs $17.2 \mathrm{~kg} / \mathrm{m}^{2}$ and $2.6 \mathrm{vs} 3.8 \mathrm{~g} / \mathrm{dl}$, respectively), whereas total volume of the small intestine has not significantly reduced (4.05 vs $2.59 \mathrm{~L}, p=0.18$ ). Reflux esophagitis and chemical dermatitis were observed in one case but was successfully treated conservatively.

Conclusion: PEG-J decompression therapy can contribute greatly to improvement of abdominal symptoms and nutritional status in CIPO patients. Although sufficient attention should be paid to acid reflux symptoms, PEG-J has the potential to be a noninvasive novel decompression therapy for CIPO available at home. However, accumulation of more CIPO patients and longterm observation are needed.

Disclosure of Interest: All authors have declared no conflicts of interest.

\section{P0619 DIFFERENT ORAL GLUTEN LOADS INDUCE AN INFLAMMATORY AND ANTIOXIDANT RESPONSE IN HEALTHY VOLUNTEERS}

M. Di Stefano ${ }^{1}$, I. Benedetti ${ }^{2}$, E.V. Pesatori ${ }^{2}$, G.F. Manfredi ${ }^{2}$, M. Deamici ${ }^{2}$

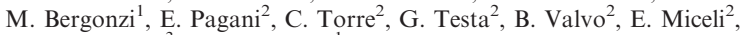
G.L. Marseglia ${ }^{3}$, G.R. Corazza ${ }^{1}$

${ }^{1}$ IRCCS Policlinico San Matteo, Pavia/Italy

${ }^{2}$ First Department Of Internal Medicine, IRCCS Policlinico San Matteo, pavia/ Italy

${ }^{3}$ Department Of Pediatrics, IRCCS Policlinico San Matteo, Pavia/Italy

Contact E-mail Address: irene.benedetti89@gmail.com

Introduction: In genetically susceptible subjects, gluten exposure determines celiac disease, but it has also been shown that gluten ingestion could alter intestinal absorption even in patients with non-intestinal diseases (Doherty, Lancet 1981) and in healthy subjects (Levine, NEJM 1966). However, whether gluten causes intestinal alterations in healthy volunteers (HV) is still a matter of debate: in HV we recently demonstrated that gallbladder motility and gastric emptying were not affected by the ingestion of gluten, whereas a slight increase of intestinal fermentation was evident (Di Stefano, $D L D$ 2015). A cytotoxic effect of gluten on intestinal cell lines was also previously shown (Rivabene, $B B A$ 1999). We recently demonstrated that in dyspeptic patients the ingestion of a standard meal induces both an inflammatory and an antioxidant response, correlated with symptom occurrence (Di Stefano, UEGW 2016). It is therefore possible that food-related alterations of the intestine could be responsible for post-prandial activation of inflammation and anti-oxidant systems.

Aims \& Methods: We therefore studied whether gluten intake induces alterations of post-prandial activation of inflammation and anti-oxidant systems. Twenty consecutive, non-smoking, normal-weight HV ( 8 females, mean age $27.9 \pm 3.6$ yrs) were enrolled. The presence of acute or chronic disorders was excluded in all of them. After an overnight fast and at the same time in the morning, all the subjects underwent evaluation of post-prandial modifications of serum levels of pro-inflammatory cytokines (IL-1 $\beta$, IL-6, TNF $\alpha$ ), endogenous antioxidant system (uric acid), glucose, insulin and serum lipopolysaccharide (LPS), measured as putative factors responsible for inflammatory response. Serum samples were collected at fasting and every 30 minutes for a 4-hour period after an oral gluten load of $2 \mathrm{gr}$ (in $10 \mathrm{HV}$ ) or $20 \mathrm{gr}$ (in the other $10 \mathrm{HV}$ ). The presence and severity of symptoms such as epigastric pain, epigastric burning, fullness, early satiety, abdominal pain, abdominal distention, bloating, flatulence, nausea, vomiting, belching, heartburn, regurgitation, diarrhea, and headache, were evaluated by visuo-analog score at fasting and every 30 minutes in the post-prandial period.

Results: In comparison with mean fasting values, none of the measured parameters showed a significant increase in the post-prandial period after the ingestion of $2 \mathrm{gr}$ of gluten. On the contrary, after the ingestion of $20 \mathrm{gr}$ of gluten mean post-prandial values of TNF $\alpha$ and IL- $\beta$ showed a significant increase $(2.45 \pm 1.75 \mathrm{pg} / \mathrm{mL}$ and $0.65 \pm 0.31 \mathrm{pg} / \mathrm{mL})$ as compared to mean fasting values $(1.17 \pm 1.49 \mathrm{pg} / \mathrm{mL}$ and $0.29 \pm 0.15 \mathrm{pg} / \mathrm{mL} ; \mathrm{p}<0.05)$. Mean post-prandial values of uric acid were also significantly higher $(74.98 \pm 33.02 \mathrm{nmol} / \mathrm{mL})$ than fasting values $(45.34 \pm 10.08 \mathrm{nmol} / \mathrm{mL} ; \mathrm{p}<0.05)$. No significant differences were detected in IL-6, glucose, insulin and LPS d after the ingestion of the $20 \mathrm{gr}$ gluten oral load. Symptoms were absent after both oral loads.

Conclusion: The ingestion of a high, but not a low, oral load of gluten induces a significant post-prandial inflammatory response causing the activation of the main endogenous anti-oxidant system. In HV, these activations are not accompanied by a symptomatic response. Further studies are needed to investigate the inflammatory and anti-oxidant post-prandial response in patients with glutenrelated disorders.

Disclosure of Interest: All authors have declared no conflicts of interest.

MONDAY, OCTOBER 30, 2017

09:00-17:00

NUTRITION I - HALL 7

P0620 THERAPEUTIC ACTION OF KETOGENIC ENTERAL NUTRITION IN OBESE AND OVERWEIGHT PATIENTS: A RETROSPECTIVE INTERVENTIONAL STUDY

C. Papadia ${ }^{1}$, P. Basset ${ }^{2}$, G. Cappello ${ }^{3}$, R. Shidrawi

${ }_{1}^{1}$ Gastroenterology, Homerton University Hospital, London/United Kingdom

${ }^{2}$ Statsconsultancy, London/United Kingdom

${ }^{3}$ Clinical Nutrition, Rome/Italy

Contact E-mail Address: cinzia.papadia@nhs.net

Introduction: Ketogenic Enteral Nutrition $\left(\mathrm{KEN}^{\mathrm{TM}}\right)$ is a modification of Blackburn's protein-sparing modified fast, using a hypocaloric, ketogenic liquid diet. The study is about Ketogenic enteral nutrition (KEN) in overweight and obese patients receiving short treatment of the nutritional solution as 24hour infusion. It is a retrospective analysis that examines safety, weight loss and body composition changes after three sequential 10-days cycles of KEN therapy. Aims \& Methods: Anthropometric and bio-impedance data from 629 patients who underwent KEN were collected before and after completing a ten-day cycle. The study focuses on the change in outcomes from the first cycle to the second cycle and from the first cycle to the third cycle. The following outcomes were explored: weight, waist circumference, BMI, fat mass, lean mass, dry lean mass, phase angle, wellness marker, water mass as a percentage of total body weight. The cycle 1, 2 and 3 outcomes were analyzed using descriptive statistics (mean, standard deviation, n) summarizing the outcome at each cycle. Statistical tests were used to test for significant differences between paired cycle 1 and cycle 2 outcomes and also between paired cycle 1 and cycle 3 outcomes. For normally distributed outcomes, the paired t-test was used. Whereas for skewed outcomes, the Wilcoxon signed-ranks test was used. Scatter plots were used to plot percentage of excess weight loss against phase angle. The Pearson's correlation coefficient was calculated. Regression analysis for the outcome percent change in weight from cycle 1 to cycle 2 for phase angle and basal metabolic rate (BMR)/Weight ratio as predictors was carried out.

Results: The results suggested significant changes for all analyzed parameters. There were significant decreases in weight, waist circumference, BMI, fat mass, lean mass, dry lean mass and phase angle. Quantitative changes in lean mass and dry lean mass were negligible with respect to changes in fat mass. There was also a statistically significant increase in water mass as a $\%$ of total body weight and wellness marker from cycle 1 to cycle 3 . The Pearson's correlation coefficients $r=0.18, p=0.004$ and $r=22, p=0.04$ indicated changes in cycle 1 and cycle 3 in percentage of weight excess to be significantly, positively correlated to phase angle. The multivariate linear regression model showed that for a 1 unit increase in $\mathrm{BMR} /$ weight there was a 3.3 percent decrease in percent change in weight. KEN treatment was overall well tolerated. $92 \%$ of patients on oral hypoglycaemics and $80 \%$ of patients on anti-hypertensive medication were able to discontinue their medication safely during KEN therapy.

Conclusion: KEN treatment is safe, well tolerated and results in rapid fat loss without detriment to dry lean mass.

Disclosure of Interest: R. Shidrawi: Director weight management system limited All other authors have declared no conflicts of interest. 


\section{P0621 ANALYSIS OF LONG-TERM WEIGHT REGAIN IN OBESE}

\section{PATIENTS TREATED WITH INTRAGASTRIC BALLOON}

B. Q. Sander ${ }^{1}$, M. Galvao Neto ${ }^{2}$, J. I.B. Scarparo ${ }^{3}$, E. Grecco ${ }^{4}$, T. F. Souza ${ }^{4}$, M. P. Sander ${ }^{1}$, D. S. Paiva ${ }^{1}$, L. R. Alberti ${ }^{5}$, V. N. Arantes ${ }^{5}$

${ }^{1}$ Dept. Of Bariatric Endoscopy, Sander Medical Center, Belo Horizonte/Brazil

${ }^{2}$ Bariatric Endoscopy, Gastro Obeso Center, São Paulo/Brazil

${ }^{3}$ Bariatric Endoscopy, Scarparo Scopia, São Paulo/Brazil

${ }^{4}$ Bariatric Endoscopy, Faculdade de Medicina do ABC - Hospital Mário Covas, São Paulo/Brazil

${ }^{5}$ Cirurgia, Faculdade de Medicina da UFMG, Belo Horizonte/Brazil

\section{Contact E-mail Address: brunosander@hotmail.com}

Introduction: Obesity is a global disease and its management includes pharmacological therapy, nonabsorptive surgery and, Intragastric Balloon (IGB). Any form of therapy, including surgery, is flawed by weight regain in the longterm. IGB has gained popularity recently; however, there is a lack of studies addressing the use of IGB and its effect on weight control in the long-term. This is the first study that aimed to assess the weight regain in a large cohort of patients treated with IGB, with a time span from two to five years after the removal of the device.

Aims \& Methods: All obese patients treated with IGB in a specialized obesity center, and that underwent balloon removal from June 2009 to June 2013 were invited to participate in the study. Exclusion criteria: pregnancy or bariatric surgery after balloon removal, impossibility to contact, and refusal to participate. Patients that agreed to participate were interviewed by a trained investigator in person and answered a questionnaire survey and had their body weight measured. Interviews started on July 2015 and ended on July 2016. Medical records of recruited patients were reviewed and the body weight at the moment of IGB introduction and removal registered. Patients were stratified by segment timeframe from balloon removal and interview date $(2,3,4$ and 5 years) and all intervening factors related to weight control, as well as behavior habits were analyzed and compared with logistic multivariate analysis.

Results: A total of 224 patients entered the study. Of these, $81.3 \%$ (182) were women. During the use of IGB, patients lost an average of $15 \%$ of their body weight; representing a mean loss of $66 \%$ of excessive weight. Between 2 and 5 years after removal of IGB, $67 \%$ (150) of the subjects had regained weight; the mean weight regain was $4 \mathrm{~kg}$ during this period. Most patients $(62 \%)$ regained $10 \%$ to $19 \%$ of weight lost during treatment. The mean weight regain increased during follow-up, but without significant difference among groups: 2 years $[\mathrm{n}=10]: 4.66 \pm 4.91 \mathrm{~kg} ; 3$ years $[\mathrm{n}=83]: 8.66 \pm 6.96 \mathrm{~kg} ; 4$ years $[\mathrm{n}=54]$ : $9.99 \pm 8.44 \mathrm{~kg}$ and 5 years $[\mathrm{n}=3]: 19,96 \pm 12,24 ;(\mathrm{p}=0,51)$. The lower the $\mathrm{BMI}$ at the beginning of treatment, the greater the weight regain after the IGB withdrawal. This correlation was inverse $(r=-0.20)$ and significant $(\mathrm{p}<0.01)$. The correlation was stronger and more significant with patients who had withdrawn the balloon at two years $(r=-.59, \mathrm{p}<0.01)$ followed by those in which balloon withdrawal was undertaken four years before $(\mathrm{r} .=-0.23, \mathrm{p}=0.03)$. Weight regain group contained more individuals who did not perform psychological and nutritional follow-up and who were also sedentary, during and after treatment. Each year, after removal of IGB, the chance of regaining weight increased 1.5 times. No follow-up with a nutritionist after the procedure increased chance of weight regain in 1.8 times. Lack of follow-up with a psychologist during treatment had a weight regain 1.9 times increased. Multivariate logistic analysis determined risk factors for weight regain according to time span after IGB removal. After 2 years of balloon removal, the significant risk factor was lack of follow-up with a psychologist during treatment; increasing the chance of weight regain 1.13 times compared with those subjects that received psychological follow-up. An independent and significant risk factor for weight regain after 3 years of IGB removal was the lack of follow-up with a nutritionist after the use of IGB. Chance of weight regain was 3.36 times higher in this group than in individuals who did the nutritional follow-up. After 4 years of IGB removal, sedentary behavior was an independent and significant risk factor, increasing the chance of weight regain 3.86 times compared with physically active behavior.

\begin{tabular}{lllll}
\hline$\%$ of weight regained $*$ & 2 years & 3 years & 4 years & 5 years \\
\hline$<10 \%$ & $20 \%(2)$ & $15.6 \%(13)$ & $18.5 \%(10)$ & $33.3 \%(1)$ \\
Between 10 and $19 \%$ & $70 \%(7)$ & $62.7 \%(52)$ & $59.3 \%(32)$ & $66.7 \%(2)$ \\
Between 20 and $29 \%$ & $10 \%(1)$ & $14.5 \%(12)$ & $14.8 \%(8)$ & 0 \\
Between 30 and $39 \%$ & 0 & $2.4 \%(2)$ & $1.9 \%(1)$ & 0 \\
Between 40 and $49 \%$ & 0 & $1.2 \%(1)$ & $5.6 \%(3)$ & 0 \\
Between 50 and $59 \%$ & 0 & $2.4 \%(2)$ & 0 & 0 \\
Between 90 and $99 \%$ & 0 & $1.2 \%(1)$ & 0 & 0 \\
\hline
\end{tabular}

Conclusion: IGB has a suboptimal long-term effect on body weight control after 2 to 5 years of balloon removal, with weight regain observed in up to two thirds of patients $(66 \%)$. The following variables adversely affect long-term body weight control after IGB removal: lack of psychological support and nutrition counseling and sedentary lifestyle. A multidisciplinary team approach is of paramount importance to assist obese patients treated with IGB in order to effectively maintain long-term body weight control.

Disclosure of Interest: All authors have declared no conflicts of interest.
P0622 LONG TERM EFFECT OF DUODENAL-JEUJENAL BYPASS LINEAR IMPLANTATION ON WEIGHT REDUCTION AND GLYCEMIC CONTROL

S. Fishman ${ }^{1}$, L. Mlynarsky ${ }^{1}$, L. Ben-Haim ${ }^{2}$, J. Rutenberg ${ }^{1}$, M. Shnell ${ }^{1}$, E. Santo ${ }^{1}$ ${ }^{1}$ Obesity Service, Dept. Of Gastroenterology, TASMC Dept. of Gastroenterology, Tel Aviv/Israel

${ }^{2}$ Dept. Of Endocrinology, TASMC Dept. of Gastroenterology, Tel Aviv/Israel

Contact E-mail Address: sigalf@tlvmc.gov.il

Introduction: The Duodenal-Jeujenal Bypass Linear (DJBL) is an endoscopic device which prevents ingested nutrients absorption in the duodenum and first part of jejunum. The resultant effects are weight reduction and improvement in glycemic control in patients with type 2 diabetes mellitus (T2DM).

Aims \& Methods: The aim of the current study is to assess weight and glycemic control changes resulted from the device implantation and a year after the device removal. Between February 2013 and September 2016, 51 diabetic patients were treated with DJBL in our center. This prospective observational study included 36 patients who completed at least 9 months of active treatment. However, adverse events and early removals were analyzed for the whole cohort. Blood tests, body weight and medications data were collected during scheduled visits and phone interviews. The primary end points were body weight and glycemic control changes a year after implantation. Secondary end points were the same parameters after device removal. The protocol was approved by the local ethic committee.

Results: Thirty six patients $(52.8 \%$ male) were treated for at least 9 months with the device, 10 of which completed a whole year follow-up after device removal. At the end of 12 months post implantation, the average body weight and BMI dropped from $109.5 \pm 19.1 \mathrm{~kg}$ and $37.4 \pm 5.0 \mathrm{Kg} / \mathrm{m}^{2}$ to $93.7 \pm 20.4 \mathrm{Kg}$ and $31.3 \pm 3.4 \mathrm{Kg} / \mathrm{m}^{2}$, respectively $(\mathrm{P} \leq 0.001)$. Although parameters increased up to $97.4 \pm 14.6 \mathrm{~kg}$ and $33.3 \pm 5.0 \mathrm{Kg} / \mathrm{m}^{2}$ at the end of a year follow-up, both parameters remained significantly lower than the baseline $(\mathrm{P}<0.05)$. HbAlc was reduced during the treatment from $7.6 \pm 1.6 \%$ to $6.6 \pm 1.2 \% \quad(\mathrm{P}=0.02)$ and increased to $6.8 \pm 1.0 \%$ after 12 month follow-up $(\mathrm{P}=\mathrm{NS})$. Among 15 insulin-dependent patients $(42 \%)$, insulin average dose was reduced by $65 \%$ $(\mathrm{p}=0.05)$, but the dose was doubled after a year follow-up. Interestingly, the glycemic control in this insulin-dependent population was difficult to maintain as evidenced by the increased $\mathrm{HbAlc}$ a year after the device removal $(6.9 \pm 1.1 \%$ to $7.3 \pm 1.1 \% \mathrm{p}=0.585)$. In contrast, among non-insulin dependent patients, there was a non-significant decrease in $\mathrm{HbA} 1 \mathrm{C}$ during the year post the device removal $(6.5 \pm 1.3$ to $6.4 \pm 0.8, \mathrm{P}=0.812)$. Ten patients $(19.6 \%)$ of the initial 51 treated suffered from adverse events, $4(7.8 \%)$ of which were categorized as severe ones (two major bleedings and 2 hepatic abscesses).

Conclusion: DJBL is an effective tool for weight reduction glycemic control among insulin and non-insulin dependent diabetic patients. Moreover, substantial metabolic achievements are preserved at least a year after device removal. Since DJBL bears a considerable amount of side effects, strategies to mitigate them are warranted.

Disclosure of Interest: All authors have declared no conflicts of interest.

P0623 SYNBIOTIC (INULIN, LACTOBACILLUS, BIFIDOBACTERIUM AND SACCHAROMYCES BOULARDII) IMPROVES FATTY LIVER DISEASE BY VIRTUE OF ITS ACTION ON LIPID PROFILES, LEPTIN, ADIPONECTIN, AND INFLAMMATORY BIOMARKERS

T.M. M. Falalyeyeva ${ }^{1}$, N. Kobyliak ${ }^{2}$, O. Tsyryuk ${ }^{1}$, I. Prybytko ${ }^{1}$, T. Beregova ${ }^{1}$, L. Ostapchenko

${ }^{1}$ Taras Shevchenko National University of Kyiv, Kyiv/Ukraine

${ }^{2}$ National Bohomolets Medical University, Ukraine, Kyiv/Ukraine

Contact E-mail Address: nazariikobyliak@gmail.com

Introduction: NAFLD is the most important cause of chronic liver disease and is considered the hepatic manifestation of the metabolic syndrome associated with type 2 diabetes. The prevalence of NAFLD in the general population reaches 15 $20 \%$ and it goes up to 76 to $90 \%$ in the obese population. The search of new nontoxic drugs for preventing the development of obesity is the most important challenge of modern science. The question about impact of probiotics and prebiotics on fat metabolism and obesity is being actively debated in the scientific literature. So the aim of the study was to investigate the effect of synbiotic (S) on development of experimental obesity in rats with NAFLD.

Aims \& Methods: The study was carried out on 60 white rats, that were divided into 6 groups (I-III - males, IV-VI - females). I and IV groups were intact control (4-month old). Newborn rats of groups II and III s.c. in volume $8 \mu \mathrm{l} / \mathrm{g}$ were administered a saline or monosodium glutamate (MSG) $(4 \mathrm{mg} / \mathrm{g})$ at $2-10$ days of life. Since the age of 1 month, rats of III and V group had been injected with water, rats III and VI groups - S (Inulin, Lactobacillus, Bifidobacterium and Saccharomyces boulardii) ("Opefera" World Medicine) in a dose of 1, 94 × 109 $\mathrm{KOE}+2,9 \mathrm{mg} /$ inulin. Introduction had been performed with 2-week course for 3 months courses alternated with two-week breaks) for 3 months. In 4-month rats anthropometrical parameters and visceral adipose (VAT) tissue mass were estimated, and adiponectin in serum and leptin in VAT were measured by ELISA. Results: In 4-month rats we diagnosed the changes of the anthropometrical parameters and significant increase of VAT mass that confirmed development of visceral obesity. In male rats, there were more pronounced changes. It was established that under condition of obesity caused by the introduction of MSG, the level of adiponectin in serum decreased but leptin in VAT was increased. S significantly improvement lipid profiles and histological state of liver: decrease of dystrophy, inflammation and necrosis and reduction of the NASH incidence. 
Also consumption of $\mathrm{S}$ led to reduction of pro-inflammatory cytokines and leptin and increased anti-inflammatory cytokines and adiponectin.

Conclusion: Thus, the introduction of $\mathrm{S}$ reduced the obesity, that shows the effectiveness of therapy for the prevention of obesity.

Disclosure of Interest: All authors have declared no conflicts of interest.

\section{P0624 IMPROVED EMPLOYEMENT OF INTRAGASTRIC} BALLOON FOR WEIGHT LOSS: A PRELIMINARY ANALYSIS

S.H. Kim, H.J. Chun, H.S. Choi, E.S. Kim, B. Keum, Y.T. Jeen, H.S. Lee, C.D. Kim

Department Of Internal Medicine, Division Of Gastroenterology And Hepatology, Korea University Anam Hospital, Seoul/Korea, Republic of

Contact E-mail Address: drchunhj@chol.com

Introduction: Endoscopic bariatric approaches are gaining traction as possible treatment modalities for obesity. Especially, intragastric balloon was demonstrated to be associated with a significant weight loss in obese patients. Despite many advances in the design and material of intragastric balloon devices, there still remains a need for improved devices which is safer, faster, and less expensive than before. In the present study, we evaluated feasibility of newly developed intragastric balloon.

Aims \& Methods: We used a newly developed intragstric balloon with improved employment for this study. The intragastric balloon was supplied as delicately rolled up inside a thin silicon sheath and mounted by surrounding the endoscope. Endoscopic intragastric balloon placement and positioning was simply performed with direct vision. 10 pigs were submitted to the novel intragastric balloon placement. We evaluated feasibility of the intragastric balloon and compared procedure time between the novel intragatric balloon and End-ball (Endalis, Brignais, France) intragastric balloon.

Results: In all cases, the novel intragastric balloons were successfully placed under usual sedation of diagnostic endoscopy. The procedures were simple and fast; the mean insertion time was 41.314 .3 and $153.8134 .8 \mathrm{sec}$ in novel intragastric balloon group and end ball group, respectively. The mean inflation time was 412.46 and $512.83 \mathrm{sec}$ in novel intragastric balloon group and end ball group, respectively

Conclusion: This preliminary data suggest that the procedure with the new intragastric balloon attain technical improvements in the placement without severe adverse events. The new intragastric balloon could offer constantly effective procedure regardless of the ability of the endoscope practitioner.

Disclosure of Interest: All authors have declared no conflicts of interest.

\section{References}

1. Imaz I, Martinez-Cervell C, Garcia-Alvarez EE, et al. Safety and effectiveness of the intragastric balloon for obesity. A metaanalysis. Obes Surg. 2008; 18:841-6.

2. Tsesmeli N, Cosimaros D. The future of bariatrics: endoscopy, endoluminal surgery, and natural orifice transluminal endoscopic surgery. Endoscopy. 2010;42:155-62.

\section{P0625 IS RE-IMPLANTATION OF THE DUODENAL-JEJUNAL BYPASS LINER VIABLE?}

S. Guenthert, A. Dienethal, J. Stein

Gastroenterology And Clinical Nutrition, DGD Clinics Sachsenhausen, Frankfurt Main/Germany

Contact E-mail Address: j.stein@em.uni-frankfurt.de

Introduction: The endoscopically implanted DJBL is a $60 \mathrm{~cm}$ long, impermeable fluoropolymer device which prevents food from making contact with the proximal intestine, thus inducing considerable weight loss and improvement of type 2 diabetes mellitus (T2DM). Both weight and HbA1c levels have been reported to increase post explantation. The question remains as to whether it is possible to reimplant the DJBL, and what the results would be in terms of BMI (Body Mass Index) change and T2DM control.

Aims \& Methods: The aim of this study was to investigate the safety, feasibility and effectiveness of DJBL re-implantation in patients who show a relapse in glucose levels after DJBL explantation. This prospective, observational study was conducted at the Department of Gastroenterology of DGD Clinics Sachsenhausen, Frankfurt (Germany) between 2014 and 2016. Five obese patients with T2DM and with a body mass index (BMI) ranging from 35$59 \mathrm{~kg} / \mathrm{m}^{2}$, who completed follow-up after their first implant and underwent removal of the DJBL after 12 months, were selected for re-implantation after an additional 4 months of follow-up. Weight loss, BMI, and HbAlc were analysed before reimplantation and twelve months thereafter.

Results: In all 5 patients, the DJBL was initially implanted and explanted without complications. Re-implantation and re-explantation were also performed without complications. Changes in body weight, BMI and glycated haemoglobin (HbAlc) are shown in Table 1
Table 1: Body weight, BMI and HbAlc changes at different timepoints

\begin{tabular}{llll}
\hline $\begin{array}{l}\text { Timepoint } \\
\text { (months) }\end{array}$ & $\begin{array}{l}\text { Mean weight } \\
\text { in kg }( \pm \text { SD, range })\end{array}$ & $\begin{array}{l}\text { Mean BMI } \\
( \pm \text { SD, range })\end{array}$ & $\begin{array}{l}\text { Mean HbA1c } \\
\text { in \% }( \pm \text { SD, range })\end{array}$ \\
\hline 0 & $115.8(45.4 ; 88-196)$ & $40.9(10.3 ; 35.3-59.2)$ & $9.1(1.3 ; 8-10.7)$ \\
6 & $97.4(39.8 ; 72-164)$ & $29.9(2.2 ; 26.4-51.2)$ & $7.6(0.8 ; 6.6-8.3)$ \\
12 & $95.0(38.8 ; 72-164)$ & $33.5(9.0 ; 29.549 .5)$ & $6.7(0.9 ; 5.9-7.8)$ \\
$16(0)$ & $97.1(37.8 ; 75-164)$ & $34.3(8.6 ; 29.3-49.5)$ & $7.7(1.6 ; 6.2-9.9)$ \\
$22(6)$ & $93.2(40.6 ; 63-164)$ & $32.8(9.7 ; 24.6-49.5)$ & $7.0(1.0 ; 5.7-7.7)$ \\
$28(12)$ & $92.5(43.6 ; 61-160)$ & $31.5(9.1 ; 23.8-48.6)$ & $7.0(0.7 ; 6.3-7.7)$ \\
\hline
\end{tabular}

Conclusion: The results of this observational study show that re-implantation of the DJBL is viable and safe even only 4 months after explantation. After reimplantation, weight and $\mathrm{HbAlc}$ levels decreased once more.

Disclosure of Interest: J. Stein: Jürgen Stein has received speakers' honoraria from GI Dynamics.

All other authors have declared no conflicts of interest.

\section{P0626 THE COMPARATIVE EFFICACY OF OBESITY TREATMENTS IN YOUNG PEOPLE - A SYSTEMATIC REVIEW AND META ANALYSIS}

S. S. Selvendran, N. Penney, N. Aggarwal, A. Darzi, S. Purkayastha Surgery And Cancer, Imperial College London, London/United Kingdom

\section{Contact E-mail Address: SS9612@ic.ac.uk}

Introduction: Obesity in the young population is becoming increasingly prevalent. It is associated with short- and long-term consequences. Early and effective interventions are paramount. Current treatment options include: lifestyle modifications, pharmacological therapies, endoscopic treatments and bariatric surgery. However, the relative effectiveness of these treatments in this cohort remains unclear.

Aims \& Methods: To systematically identify and meta-analyse studies evaluating treatments that reduce body mass index (BMI) in overweight and obese young people. A systematic literature review of EMBASE and MEDLINE databases was conducted. Studies were included/excluded based on pre-specified eligibility criteria. Included patients were 21 years or younger. Lifestyle modification and pharmacological therapy searches were restricted to randomised control trials. Results: 16,372 studies were identified with 80 studies complete with sufficient data for meta-analysis. Bariatric surgery caused the most weight loss in the shortand medium-term [pooled estimate of mean BMI loss: $13.77 \mathrm{~kg} / \mathrm{m}^{2}$ ]. Lifestyle modifications and pharmacological therapy had a more modest impact on weight [pooled estimate of mean BMI loss: $0.99 \mathrm{~kg} / \mathrm{m}^{2}$ and $0.94 \mathrm{~kg} / \mathrm{m}^{2}$ respectively]. Individual studies demonstrated that endoscopic treatment results in short-term BMI reduction, however insufficient data prevented meta-analysis. Conclusion: This is the first systematic review and meta-analysis to comprehensively summarise and quantify the comparative efficacy of BMI reducing treatment options in the obese, young population. Currently, bariatric surgery is rarely considered in this young cohort. However, due to its high efficacy, physicians and patients should have a lower threshold for considering bariatric surgery when lifestyle and pharmacological interventions have failed. The non-surgical interventions provide smaller but statistically significant impacts on BMI reduction. There should be effective communication discussing the relative efficacy of all treatment options and their associated complications between those involved. This knowledge will assist clinicians in determining a holistic, patient-centred treatment programme for obese, young patients.

Disclosure of Interest: All authors have declared no conflicts of interest.

\section{P0627 FOOD-DERIVED MICRORNA AND INFLUENCE ON THE}

\section{FECAL MICRORNA EXPRESSION}

J. Link ${ }^{1}$, C. Langner ${ }^{1}$, A. Canbay ${ }^{1}$, P. Malfertheiner ${ }^{1}$, A. Link ${ }^{1}$

${ }_{1}^{1}$ Department Of Gastroenterology, Hepatology And Infectious Diseases, Otto-vonGuericke University, Magdeburg/Germany

\section{Contact E-mail Address: alinkmail@gmail.com}

Introduction: Tumor development is a multistep process, which involves genetic and environmental factors. Diet is among the most important contributing factors and processed and red meat has been classified as carcinogenic for colorectal cancer. MicroRNAs (miRNAs) are functional, ubiquitously present molecules with great impact on tumor initiation and progression. Exogenous microRNA or xenomiRs have been identified in sera from different species suggesting an active cross-kingdom trespassing through biological barriers during digestive process. Aims \& Methods: In the present work, we evaluated whether miRNAs are present in various foods, and if miRNAs may be degraded through cooking and other forms of food processing. Furthermore, we tested if short-term vegetarian or meat-rich diet may influence human or plant-derived miRNA in feces and blood. For this purpose, six healthy subjects were asked to adhere to vegetarian or meat rich diet for 5 to 7 days and fecal and blood specimens were obtained at different time points. Plant-miRNAs were further investigated in gastric and colon mucosa. To evaluate the presence of miRNA in food, we selected several common foods prior and after cooking/processing. Quantitative real-time PCR was performed using TaqMan Assay. 
Results: All analyzed microRNAs were present in all studied foods with highest expression for miR-21 followed by miR-16 and miR-155. Especially, hash, beef and salmon showed the highest miRNA expression, while lowest expression was found in cheese and milk. Food procession led to only marginal changes (max. 1.5-fold) in miRNA expression and thus demonstrating its stability against degradation. Short-term changes in diet (from usual to vegetarian and to meatrich diet) in healthy subjects was not associated with variation in miR-21, miR155 and miR-16 expression. Interestingly, in comparison to several previous reports, we repeatedly failed to detect any plant miR-168 in sera. However, vegetarian-diet was associated with a significant increase in miR-168 level in feces (up to 8-fold), while meat-rich diet was associated with slight decrease if compared to the starting time point (mean \pm SD $0.0031 \pm 0.002$ for no diet vs $0.025 \pm 0.042$ for vegetarian vs. $0.0016 \pm 0.00096$ for meat-rich; $p=0.03$ KruskalWallis test, with $\mathrm{p}>0.05$ for Dunńs multiple comparison test for vegetarian vs. meat-rich).

Conclusion: The results of this study show that various foods provide a great source of miRNAs, which remains stable despite processing. We further demonstrate that minor short-term changes in diet do not impact on the miRNA expression pattern in feces and blood supporting its value as biomarkers. A functional role of diet-induced increase in plant-derived miRNA expression needs further evaluation.

Disclosure of Interest: All authors have declared no conflicts of interest.

\section{P0628 NEUROMEDIN U BLOCKS GASTRIC EMPTYING THROUGH VAGAL-DEPENDENT MECHANISMS AND IMPROVES ORAL GLUCOSE TOLERANCE}

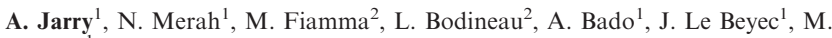
Le Gall ${ }^{1}$

${ }^{1}$ INSERM U1149, Paris/France

${ }^{2}$ INSERM U1158, Paris/France

Contact E-mail Address: annecharlottejarry@hotmail.fr

Introduction: The gut and brain peptide neuromedin $U$ (NMU) is reported to decrease food intake and body weight, and to improve oral glucose tolerance suggesting that NMU could exert an incretin effect. NMU is thus considered as a promising candidate for the treatment of obesity and diabetes. However, and in contradiction with previous observations, NMU was recently presented as a "decretin" hormone able to decrease insulin secretion. The pathways through which NMU controls glycemia are thus uncertain and we sought to clarify some of NMU mechanisms of action on glucose homeostasis.

Aims \& Methods: Oral (OGTT) and intraperitoneal (IPGTT) glucose tolerance tests were performed after an intraperitoneal injection of NMU or PBS in C57B16 mice that underwent a laparotomy or a troncular subdiaphragmatic vagotomy. During OGTT, blood was sampled to measure insulin secretion. [14]-C-glucose uptake was assessed in isolated intestinal loops in presence or absence of NMU. Gastric retention of a phenol red gavage and total intestinal transit time were evaluated after an intraperitoneal injection of NMU or PBS. Activation of vagus nerve neurons by intraperitoneal injection of NMU was assessed by $\mathrm{cFos}$ immunostaining on brainstem slides going through the nucleus of the solitary tract (NTS) and the dorsal nucleus of the vagus (DMV). Direct impact of NMU on pyloric contraction was assessed ex vivo in isometric chambers.

Results: A single intraperitoneal injection of NMU in C57B16 mice prevented the rise of glycemia following an oral but not an intraperitoneal load of glucose (OGTT versus IPGTT). Unexpectedly, during the OGTT, NMU injection prevented insulin secretion and only slightly improved peripheral insulin sensitivity. Furthermore intestinal [14]-C-glucose uptake in isolated intestinal loops was barely reduced by NMU addition $(-17 \% \mathrm{P}<0.05$ vs PBS). Actually NMU injection blocked gastric emptying (gastric retention of a phenol red gavage at 30 min: $+285 \% \mathrm{P}<0.0001$ vs PBS). This effect was partly prevented in vagotomized mice. In addition, injection of NMU induced c-fos expression in the nucleus of the solitary tract (NTS) of control but not vagotomized mice. In isotonic chambers, NMU directly induced pyloric contraction in a dose dependent manner (basal contraction $+21,7 \%$ at $10-6 \mathrm{M}$ ).

Conclusion: These data demonstrate that a single intraperitoneal injection of NMU blocks gastric emptying directly by inducing pylorus contraction and indirectly via afferent vagal fiber activation. Through the blockade of gastric emptying, NMU reduces intestinal nutrient absorption and thus improves oral glucose tolerance. The gastric emptying blockade induced by $\mathrm{NmU}$ could also contribute to its anorexigenic effect.

Disclosure of Interest: All authors have declared no conflicts of interest.

P0629 LOW FODMAP DIET \& PREBIOTIC

GALACTOOLIGOSACCHARIDES IMPROVE IRRITABLE BOWEL SYNDROME SYMPTOMS AND RESPONSE TO LOW FODMAP DIET IS PREDICTED BY URINE METABOLOME, STOOL SHORT-CHAIN FATTY ACIDS AND VOLATILE ORGANIC COMPOUNDS: A RANDOMISED CONTROLLED TRIAL

B. Wilson ${ }^{1}$, R. Hough ${ }^{2}$, T. Kanno ${ }^{1}$, M. Rossi ${ }^{1}$, G. Parkes $^{3}$, Q. Aziz ${ }^{3}$,

S. Anderson ${ }^{4}$, P. Irving ${ }^{5}$, M. Lomer ${ }^{1}$, A. J. Mason ${ }^{1}$, C. S.J. Probert ${ }^{2}$, K. Whelan

${ }^{1}$ King's College London, London/United Kingdom

${ }^{2}$ University of Liverpool, Liverpool/United Kingdom

${ }^{3}$ Barts Health NHS Trust, London/United Kingdom

${ }^{4}$ Guy's and St Thomas' NHS Foundation Trust, London/United Kingdom

${ }^{5}$ Guýs and St Thomaś Hospital Dept. of Gastroenterology - London/United

Kingdom
Contact E-mail Address: bridgette.wilson@kcl.ac.uk

Introduction: Dietary restriction of fermentable carbohydrates (low FODMAP diet, LFD) is effective at managing symptoms in 50-80 percent of patients with irritable bowel syndrome (IBS). Prebiotic B-galacto-oligosaccharide (B-GOS; HOST-G904) also reduce symptoms in IBS however the combination of the two therapies has not previously been investigated. What differentiates those who respond to the LFD from those who do not is unclear.

Aims \& Methods: This randomised controlled trial aimed to investigate whether: 1) addition of prebiotic B-GOS could improve symptoms of IBS alongside the LFD; and 2) if urinary metabolites, faecal short-chain fatty acids (SCFA) or volatile organic compounds (VOC) could identify factors at baseline that predict response. Sixty-nine adults fulfilling Rome criteria for IBS were recruited to a 3 arm RCT: control (sham diet/placebo), LFD (LFD/placebo) or LFD plus BGOS (LFD/B-GOS) for four weeks. Validated questionnaires (Global symptom question and gastrointestinal symptom rating scale) assessed GI symptoms (response) and urine and stool samples were collected for analysis at baseline and week 4 . To examine the relationship between responders and non-responders, urine metabolomics (700 MHz ${ }^{1} \mathrm{H}-\mathrm{NMR}$ ), stool SCFA (gas liquid chromatography (GLC)) and stool VOC (GC-mass spectrometry) were analysed on samples at baseline and 4-weeks. Urine metabolomics spectra and VOC profiles were analysed using unsupervised principal component analysis (PCA) and supervised pattern recognition methods (orthogonal partial least square discriminant analysis (OPLS-DA)) or PLS-DA respectively. Stool SCFA were compared using t-tests, models of prediction were tested using receiver operator characteristic (ROC) curves.

Results: There was a significant difference in response rates (adequate relief) between control $(30 \%)$, LFD $(50 \%)$ and LFD/B-GOS $(67 \%)(p=.046)$, with post-hoc differences specifically between control and LFD/B-GOS $(p=0.015)$. Individual IBS symptoms were more markedly improved in the LFD/B-GOS group compared to control. In the LFD group only, there was a significant difference in the urine metabolome between responders and non-responders at both baseline $\left(Q^{2}=0.296\right.$ vs randomised -0.175$)$ and at 4 -weeks $\left(Q^{2}=0.485\right.$ vs randomised -0.203 ). At baseline, there were significant greater stool isobutyrate between responders $(51.4 \mathrm{mg} / 100 \mathrm{~g})$ and non-responders $(31.9 \mathrm{mg} / 100 \mathrm{~g}, \mathrm{p}=0.04)$, with ROC curves supporting this as a predictor of response (AUC $=0.747$, $\mathrm{p}=0.063$ ). Finally, there was a significant difference in VOC profiles between responders and non-responders to the LFD at baseline $(\mathrm{p}=0.04)$. VOC profiles, modelled by PLS-DA, reveal significant separation of VOC profiles across the three treatment groups at 4-weeks $(\mathrm{p}=0.02)$

Conclusion: Addition of B-GOS to the LFD improves symptoms in IBS. Urine metabolomics, stool SCFA and VOC profiling are relatively low-cost and noninvasive techniques that may predict response to the LFD as well as helping to further understand the underlying mechanisms. Prospective clinical trials to test these algorithms are warranted and may lead to personalised therapy for patients with IBS.

Disclosure of Interest: B. Wilson: BW is funded by a PhD studentship provided by Clasado Biosciences

All other authors have declared no conflicts of interest.

\section{P0630 THE ANALYSIS OF PROTEIN CONSUMPTION PATTERNS} IN PATIENTS WITH SIBO

V. Pilipenko, V. Isakov, A. Vlasova

Gastroenterology \& Hepatology, Research Institute of Nutrition RAMS, Moscow/ Russian Federation

Contact E-mail Address: pilipenkowork@rambler.ru

Introduction: Small intestinal bacterial overgrowth (SIBO) is common in patients with gastrointestinal diseases. SIBO symptoms are improved with antimicrobial treatment, but recurrence rate is high (approximately $40 \%$ for 9 months). Dietary modification is essential for prevention of recurrence of SIBO however there are no detailed studies of nutrition in SIBO patients. Protein consumption is considered important for SIBO, as the proteins from animal source are essential for growth of $\mathrm{CH} 4 / \mathrm{H} 2$-producing microorganisms.

Aims \& Methods: The aim of the study was to assess the protein consumption patterns in patients with different types of SIBO. Three-day food diary was collected from 574 patients, undergoing $\mathrm{CH} 4 / \mathrm{H} 2$ lactulose breath test. The photographs used to estimate the size of the portions eaten. According to food composition and portion all dishes in food diary were converted into constituent products by food groups. Each food group were compared with the normal levels of a healthy diet pyramid based on daily calories (normal value $=1.0$ ). According to the breath test results the patients were divided into 4 subgroups of different SIBO types $(\mathrm{H} 2, \mathrm{CH} 4, \mathrm{H} 2$ and $\mathrm{CH} 4)$ and a subgroup with no signs of SIBO (Table 1).

Results: All types SIBO patients consumed less meat than control (no signs of SIBO), however patients with hyperproduction of $\mathrm{CH} 4$ only demonstrated highest consumption of fish, and it was a trend in patients with isolated H2-hyperproduction to consume more poultry than other groups. There were no differences between groups in consumption of processed meat foods or eggs. Conclusion: There are specific animal protein consumption patterns related to the type of the SIBO, which can be used for the planning of dietetic interventions in patients for prevention of SIBO recurrence

Disclosure of Interest: All authors have declared no conflicts of interest. 
P0631 LOW FODMAP DIET IMPROVES SYMPTOMS AND

QUALITY OF LIFE IN PATIENTS WITH RADIATION INDUCED

SMALL BOWEL DISEASE: A PILOT STUDY

T. Larsen ${ }^{1}$, T. Hausken ${ }^{2}$, S. O. Ystad ${ }^{2}$, N. Hovdenak ${ }^{1}$, B. Mueller ${ }^{3}$, G.A. Lied ${ }^{4}$ ${ }^{1}$ Department Of Clinical Medicine, University of Bergen, Bergen/Norway

${ }^{2}$ Haukeland University Hospital, National Centre for Functional Gastrointestinal Disorders, Bergen/Norway

${ }^{3}$ Hyperbaric Medical Unit, Haukeland University Hospital, Bergen/Norway

${ }^{4}$ Center For Nutrition, Clinical Medicine, University of Bergen, Bergen/Norway

Contact E-mail Address: trinelarsen92@gmail.com

Introduction: Patients suffering from chronic radiation-induced small bowel disease (RISBD) after cancer treatment have similar symptoms as patients with IBS (irritable bowel syndrome), despite dissimilar pathological origin. RISBD is a common late effect after pelvic radiation therapy for gastrointestinal (GI), gynecological and urological cancer. The delayed development of ischemia, fibrosis, dysmotility and malabsorbtion in GI tissue, leads to IBS-like symptoms like abdominal pain, diarrhea, constipation and bloating. The low FODMAP (fermentable oligo-, di-, monosaccharides and polyols) diet (LFD) is a widespread management strategy for IBS. The aim of the conducted study was to investigate the effects of LFD on symptoms and health related quality of life (HRQOL) for patients with chronic RISBD.

Aims \& Methods: In an open pilot study, 11 patients (mean age 46 years) with RISBD related IBS symptoms were instructed to follow LFD throughout a 4week intervention period. IBS Severity Scoring System (IBS-SSS) and IBS Symptom Questionnaire (IBS-SQ) were used to assess symptoms. An Ad hoc questionnaire measured grade of tissue damage and typical RISBD complaints (fecal incontinence, rectal mucus and rectal bleeding). Short Form Nepean Dyspepsia Index (SF-NDI) and 12-item Short Form Health Survey (SF-12) were used to evaluate HRQOL. A 3-day food record was used to estimate baseline intake of FODMAPs, to reveal dietary changes and to assess adherence to the diet. All schemes were completed at baseline and at 4 weeks. The study included no control group.

Results: FODMAP intake was successfully reduced, and main additional changes in the diet were reduced intake of energy, carbohydrates and fiber. The adherence to the diet was high (mean $94.8 \%$ ). IBS symptoms improved significantly based on mean total score of IBS-SSS and IBS-SQ, which changed from $310.2 \pm 60.7$ to $171.4 \pm 107.2(\mathrm{p}=0.001)$ and $27.4 \pm 4.1$ to $15.7 \pm 10.1(\mathrm{p}=0.002)$, respectively. The severity of abdominal pain, abdominal distension, belching/flatulence, constipation, diarrhea, early satiety, dissatisfaction with bowel habits and interference with life in general, improved significantly. Tendencies of improvement were also measured in comorbidity complaints (nausea, headache, backache, fatigue and muscle pain) and typical RISBD complaints. HRQOL improved based on SF-NDI total score, which changed from $30.5 \pm 9.4$ to $18.3 \pm 8.2(\mathrm{p}=0.001)$ and based on mental $(\mathrm{p}=0.047)$ and physical $(\mathrm{p}=0.134)$ component summary score of SF-12.

Conclusion: The low FODMAP diet seems effective in alleviating IBS symptoms, and improving HRQOL in patients with RISBD. High compliance to LFD is possible with adequate diet counseling and continuous guidance. Further controlled studies with larger sample size should be conducted to verify our results and hopefully enable the implementation of LFD as a future management strategy for chronic RISBD

Disclosure of Interest: All authors have declared no conflicts of interest.

\section{P0632 DIET SUPPLEMENTED WITH MEDIUM- AND LONG-CHAIN FATTY ACIDS DOES NOT AFFECT LOWER GI MOTILITY AND VISCERAL PAIN IN RATS}

P. Mosinska ${ }^{1}$, M. Martin-Ruiz ${ }^{2}$, R. Abalo ${ }^{2}$, J. Fichna

${ }^{1}$ Biochemistry, Medical University of Lodz, Lodz/Poland

${ }^{2}$ Universidad Rey Juan Carlos, Alcorcon, Madrid/Spain

Contact E-mail Address: paula.mosinska@gmail.com

Introduction: Dietary interventions are gaining popularity in terms of alleviating symptoms experienced by patients with functional GI disorders (FGID), especially with irritable bowel syndrome (IBS). Available strategies rely on low intake of foods high in short-chained carbohydrates, dairy products, wheat, spices etc. Although some studies indicate increased rectal sensitivity after duodenal lipid administration in patients with IBS and suggest delayed transit after high fat intake, the effects of dietary supplementation with different types of fatty acids in IBS have not been explored so far.

Aims \& Methods: The aim of the study was to evaluate the differences in GI motor function and visceral sensitivity in rats exposed to diet supplemented with either medium-chain- (MCFA) or long-chain fatty acids (LCFA). Male Wistar rats were fed with control diet (A), and diet supplemented with $3.5 \%$ coconut oil (B) (abundant with MCFA) or $3.5 \%$ evening primrose oil (C) (abundant with LCFAs) for 4 weeks. The effects of each diet on GI motility were measured radiographically after contrast administration (p.o; $X$ rays were taken $0-8 \mathrm{~h}$ before and after feeding, on day 0 and day 28 , respectively), and by performing the colon bead expulsion test. Temporal changes in the size of the stomach and caecum of each rat were analyzed based on digitalized X-rays, using an image processor. Visceral sensitivity was assessed with abdominal withdrawal reflex to colorectal distension. Body weight gain and food/water ingestion were measured throughout the experiment.

Results: Diet supplementation in neither group B nor group C affected the $\mathrm{G}$ motor function in comparison to control group (A). The number of contractions and the mean time of each contraction in response to colorectal distension measured during each $5 \mathrm{~min}$ for $40 \mathrm{~min}$ were higher in the control group (A), when compared to either B or C groups but the differences were not statistically significant. No changes in morphometric measurements of stomach and caecum, the body weight gain or food/water consumption were found.

Conclusion: Diets differing in MCFAs or LCFAs contents did not induce marked effects on GI motility and visceral pain in rats. Available data and the results obtained herein suggest that the amount of FAs intake, rather than the types of FAs may provoke IBS symptoms.

Disclosure of Interest: All authors have declared no conflicts of interest.

\section{References}

Simrén M1, Agerforz P, Björnsson ES, Abrahamsson H. Nutrient-dependent enhancement of rectal sensitivity in irritable bowel syndrome (IBS). 2007 Neurogastroenterology and Motility, 19(1):20-9.

Rao S, Kavelock R, Beaty J, AckersonK, Stumbo P. Effects of fat and carbohydrate meals on colonic motor response. 2000 Gut 46(2):205-211

Abalo R., Chen C., Vera G., Fichna J., et al. In vitro and non-invasive in vivo effects of the cannabonoid-1 receptor agonist AM841 on gastrointestinal motor function in the rat. 2015 Neurogastroenterology and Motility, 27:1553-1567. Vera G., Lopez-Perez AE., Martinez-Villaluenga M., Cabezos PA., Abalo R. Xray analysis of the effect of the 5-HT3 receptor antagonist granisetron on gastrointestinal motility in rats repeatedly treated with the antitumoral drug cisplatin. 2014. Experimental Brain Research, 232:2601-2612.

\section{P0633 INTESTINAL ADAPTATIONS AFTER SUBTOTAL PANCREATECTOMY IN MICE MAY CONTRIBUTE TO POSTPRANDIAL HYPERGLUCAGONEMIA}

A. Jarry ${ }^{1}$, G. Guillemain ${ }^{2}$, J. Le Beyec ${ }^{3}$, A. Bado ${ }^{3}$, J. Gauthier ${ }^{4}$, B. Blondeau ${ }^{2}$, J. Riveline ${ }^{5}$, M. Le Gall ${ }^{3}$

${ }^{1}$ Physiology, INSERM U1149, Paris/France

${ }^{2}$ INSERM UMR_S 938, Paris/France

${ }^{3}$ Physiology, U11 $\overline{4} 9$, Paris/France

${ }^{4}$ Centre Universitaire Du Diabète Et De Ses Complications, Service de

Diabétologie, Endocrinologie, Nutrition, Paris/France

${ }^{5}$ Service De Diabétologie, Endocrinologie, Nutrition, Centre Universitaire du

Diabète et de ses Complications, Paris/France

Contact E-mail Address: annecharlottejarry@hotmail.fr

Introduction: Glucagon and insulin are pancreatic peptides essential for the control of glucose homeostasis. Pancreatectomy in human leads to a loss of plasma insulin while glucagon is still detectable, suggesting the existence of an extrapancreatic source of glucagon. More specifically, in pancreatectomized patients, glucagon secretion is increased when glucose is administered orally, a release that

Abstract No: P0630

Table 1: Consumption of protein products in SIBO patients.

\begin{tabular}{|c|c|c|c|c|c|}
\hline Food & $\begin{array}{l}\text { No SIBO } \\
\mathrm{n}=65\end{array}$ & $\begin{array}{l}\text { H2 SIBO }(>20 \text { ppM }) \\
n=312\end{array}$ & $\begin{array}{l}\text { CH4 SIBO }(>12 \mathrm{ppm}) \\
\mathrm{n}=77\end{array}$ & $\begin{array}{l}\mathrm{H} 2 / \mathrm{CH} 4 \mathrm{SIBO} \\
\mathrm{n}=123\end{array}$ & Significance \\
\hline Meat & $0.58 \pm 0.71$ & $0.37 \pm 0.58$ & $0.42 \pm 0.67$ & $0.31 \pm 0.45$ & $\begin{aligned} & \mathrm{p}= 0.015 \text { no } \mathrm{SIBO} \text { vs H2 SIBO; } \\
& \mathrm{p}=0.017 \text { no SIBO vs H2 } / \\
& \text { CH4 SIBO; } \mathrm{p}=0.059 \text { no } \\
& \text { SIBO vs CH4 SIBO }\end{aligned}$ \\
\hline Poultry & $0.38 \pm 0.49$ & $0.52 \pm 0.63$ & $0.41 \pm 0.50$ & $0.39 \pm 0.48$ & $\begin{array}{l}\mathrm{p}=0.074 \mathrm{H} 2 \mathrm{SIBO} \text { vs } \mathrm{H} 2 / \mathrm{CH} 4 \\
\text { SIBO }\end{array}$ \\
\hline Eggs & $0.11 \pm 0.19$ & $0.14 \pm 0.22$ & $0.13 \pm 0.21$ & $0.10 \pm 0.17$ & NS \\
\hline Fish & $0.27 \pm 0.37$ & $0.22 \pm 0.43$ & $0.56 \pm 0.75$ & $0.33 \pm 0.77$ & $\begin{aligned} \mathrm{p}= & 0.001 \mathrm{CH} 4 \mathrm{SIBO} \text { vs } \mathrm{H} 2 \\
& \mathrm{SIBO} ; \mathrm{p}=0.004 \mathrm{CH} 4 \mathrm{SIBO} \\
& \text { vs H2/CH4 SIBO } \mathrm{p}=0.024 \\
& \text { CH4 SIBO vs No SIBO }\end{aligned}$ \\
\hline $\begin{array}{l}\text { Processed meat pro- } \\
\text { ducts (sausages, etc) }\end{array}$ & $0.08 \pm 0.11$ & $0.12 \pm 0.14$ & $0.07 \pm 0.11$ & $0.08 \pm 0.12$ & NS \\
\hline
\end{tabular}


is similar to the oral glucose-stimulated secretion of glucagon-like peptide 1 (GLP-1). GLP-1 is an incretin hormone secreted by enteroendocrine L cells (EEC) from the distal gut. GLP-1 and glucagon, both originate from the same proglucagon precursor, differentially processed by prohormone convertase 2 (PC2) into glucagon in pancreatic $\alpha$ cells and by prohormone convertase $1 / 3$ $(\mathrm{PCl} / 3)$ into GLP-1 in EEC

Aims \& Methods: We hypothesized that, after pancreatectomy, proglucagon can also be processed into glucagon in EEC. We developed a $75 \%$ subtotal pancreatectomy model in C57B16 mice. Control (Ct) mice underwent a laparotomy. Postsurgery, blood glucose levels were measured daily and oral glucose tolerance tests (OGTT) were performed after 1 week. Insulinemia and glucagonemia were also measured in fed and fasted mice and during OGTT. After 2 weeks, animals were sacrificed and the remnant pancreas was sampled for glucagon and insulin immunostaining and for alpha- versus beta-cell mass quantification. Proximal and distal intestinal segments were sampled for morphometric analyses as well as measurement of proconvertase and proglucagon mRNA levels. Colonic segments were incubated in a glucose-enriched medium for one hour and glucose-induced secretions of glucagon and GLP-1 were measured in the supernatant.

Results: As soon as one day post-surgery, pancreatectomized $(\mathrm{Px})$ mice developed a hyperglycemia that maintained for over a week $(351 \mathrm{mg} / \mathrm{dl}$ in Px mice vs $140 \mathrm{mg} / \mathrm{dl}$ in $\mathrm{Ct}$ mice, $\mathrm{P}<0.05,5$ days post-surgery). This hyperglycemic state was accompanied by an oral glucose intolerance (area under the curve $+278 \%$ in Px mice, $\mathrm{P}<0.01$ vs $\mathrm{Ct}$ mice, 1 week post-surgery). During, OGTT, intestinal glucose absorption increased (slope between 0 and $15 \mathrm{~min}+69.9 \%$ in Px mice $\mathrm{P}<0.01$ vs $\mathrm{Ct}$ mice, 1 week post-surgery). Glucagonemia increased in fasted pancreatectomized mice $(+146.6 \%$ in $\mathrm{Px}$ mice $\mathrm{P}<0.01$ vs $\mathrm{Ct}$ mice 1 week post-surgery). After sacrifice, alpha cell mass was decreased in the remaining pancreas $(-79.25 \%$ in $\mathrm{Px}$ mice $\mathrm{P}<0.05$ vs $\mathrm{Ct}$ mice, 2 weeks post-surgery). However an increased ability of the proximal colon to secrete glucagon ex vivo was observed $(+290.6 \%$ in Px mice $\mathrm{P}<0.05$ vs $\mathrm{Ct}$ mice, 2 weeks post-surgery). In pancreatectomized mice, an hypertrophy of the duodenum was associated with an increase in crypt depth $(+77.7 \%$, in Px mice $\mathrm{P}<0.05 \mathrm{vs}$ control mice, 2 weeks post-surgery) and villus height $(+53.8 \%$ in $\mathrm{Px}$ mice $\mathrm{P}<0.05$ vs control mice, 2 weeks post-surgery)

Conclusion: These data establish an ability of the whole gut to adapt in response to pancreatectomy. The upper intestine (duodenum) become hyperplasic and may contribute to the increased intestinal capacity to absorb glucose. The distal intestine (colon) is able to produce glucagon and may participate to the development of the reported hyperglucagonemia.

Disclosure of Interest: All authors have declared no conflicts of interest.

\section{P0634 QUALITY, VIABILITY AND COMPOSITION OF THE MULTISPECIES PROBIOTIC VSL\#3}

D. Mora ${ }^{1}$, S. Colombo ${ }^{1}$, G. Della Scala ${ }^{1}$, G. Gargari ${ }^{1}$, S. Arioli ${ }^{1}$, W. M. De Vos ${ }^{2}$ ${ }^{1}$ Department Of Food Environmental And Nutritional Sciences, University of Milan, Milan/Italy

${ }^{2}$ Rpu Immunobiology And 3 Dept Of Food Hygiene, University of Helsinki, Helsinki/Finland

\section{Contact E-mail Address: diego.mora@unimi.it}

Introduction: A probiotic formula to be functional and reliable should: i) contain viable cells, ii) have a reproducible composition, iii) to be taxonomically defined. Here we detail the consistency of the multispecies probiotic product VSL\#3, which has been produced for the last 20 years and is marketed globally for threating Inflammatory Bowel Disease, Pouchitis and other intestinal diseases (Mimura et al 2004; Reiff et al., 2009).

Aims \& Methods: To show consistency in the quality, viability and composition of the multispecies probiotic product VSL\#3 various batches of the multispecies probiotic VSL\#3 were analyzed in detail and derived from productions in the USA and Italy. The product batches have been tested using a series of microbiological, phylogenetic and genomic methods. The microbiological analysis included plating on selective media, cell counting and viability analysis by Flow Cytometry (FCM) using fluorescent dyes that allowed high throughput separation and quantification of live, dead and damaged cells (ISO 19344 IDF 232, 2015). A metagenetic approach, based on $16 \mathrm{~S} r R N A$ gene profiling, was used to define the bacterial community structure of different productions batches. In addition, Lactobacillus helveticus and L. acidophilus S-layer proteins, which are known to exert anti-inflammatory effects by reducing the activation of $\mathrm{NF}-\kappa \mathrm{B}$ on the intestinal epithelial Caco- 2 cell line, have been extracted and visualized on SDS-PAGE (Konstantinov et al., 2009; Taverniti et al., 2013). Moreover, urease activity of Streptococcus thermophilus, known to exert positive effect human health by competing with the undesired urease-positive bacteria of the human microbiota (Mora and Arioli, 2014), was quantified using a spectrophotometric-, and flow cytometry-based assay.

Results: The different test batches were all found to contain a common bacteria community structure based on the presence of the following species Streptococcus thermophilus, Lactobacillus acidophilus, Lactobacillus paracasei, Lactobacillus plantarum, Lactobacillus helveticus, Bifidobacterium breve and B. animalissubsp. lactis. The stability of the batches was confirmed by FCM and viable cells were always above the value of $2 \times 10^{10}$ event/g. The Lactobacillu helveticus and $L$. acidophilus S-layer protein SlpA was detected in each VSL\#3 batches tested thus highlighting that this relevant immunomodulatory factor was not subjected to degradation during the shelf-life of the product. Likewise, urease activity of $S$. thermophilus was stable in all VSL\#3 batches along the shelf-life.

Conclusion: In conclusion, stability, molecular and taxonomic comparative analysis shows that VSL\#3 is reliably and reproducibly produced in different parts of the world.

Disclosure of Interest: All authors have declared no conflicts of interest.

\section{References}

ISO 19344 IDF 232. 2015. Milk and milk products. Starter cultures, probiotics and fermented products; Quantification of lactic acid bacteria by flow cytometry; Konstantinov et al. 2008. S layer protein A of Lactobacillus acidophilus NCFM regulates immature dendritic cell and T cell functions. Proc Natl Acad Sci USA 105:19474-19479;

Mimura et al., 2004. Once Daily High Dose Probiotic Therapy (VSL\#3) for Maintaining Remission in Recurrent or Refractory Pouchitis. Gut 53:108-114; Mora and Arioli, 2014. Microbial urease in health and disease. Plos Pathogens 10(12): e1004472;

Reiff et al., 2009 Balancing Inflammatory, Lipid, and Xenobiotic Signaling Pathways by VSL\#3, a Biotherapeutic Agent, in the Treatment of Inflammatory Bowel Disease. Inflamm Bowel Dis 11:1721-1736;

Taverniti et al., 2013. S-Layer Protein Mediates the Stimulatory Effect of Lactobacillus helveticus MIMLh5 on Innate Immunity. Appl Environ Microbiol 79: 1221-1231;

\section{P0635 USE OF ALTERNATIVE LIPID EMULSION IN NON-} CRITICALLY ILL PATIENTS IN ACUTE HOSPITAL SETTING

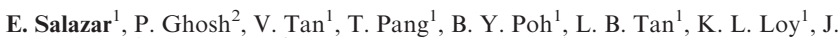
N.C. Chong ${ }^{1}$, D. H.L. $\mathrm{Ng}^{3}$, K. L. Ling ${ }^{1}$, Y.T. Wang ${ }^{1}$

${ }^{1}$ Gastroenterology And Hepatology, Singapore General hospital, Singapore/ Singapore

${ }^{2}$ Centre For Quantitative Medicine, Duke-National University of Singapore Medical School, Singapore/Singapore

${ }^{3}$ Gastroenterology And Hepatology, Tan Tock Seng Hospital, Singapore/ Singapore

Contact E-mail Address: ennaliza.salazar@sgh.com.sg

Introduction: Soybean oil intravenous lipid emulsion (IVLE) is also known as conventional lipid is rich in linoleic acid ( $\omega$-6 PUFA). $\omega$-6 PUFA may exaggerate inflammatory response and indirectly detrimental in the critically ill patients ${ }^{1,2}$ To overcome this, the use of alternative IVLEs such as medium chain triglycerides (MCT), fish oil and olive oil alone or in combination with soybean oil IVLE have been used to lower the content of $\omega-6$ PUFA. Most studies on alternative IVLEs have been conducted in the critically ill patients, elective surgical patients and cancer patients. No previous studies have evaluated the clinical outcomes of several different IVLEs in non-critically ill patients in acute hospital setting.

Aims \& Methods: The purpose of this study is to determine whether there is a difference in clinical outcome amongst patient who received conventional soybean oil IVLE versus alternative IVLEs in non-critically ill patients in acute hospital setting. All patients on parenteral nutrition (PN) were identified in a prospective compilation database from July 2007 to September 2010 and were analysed retrospectively. Patients were analysed based on the IVLE received, namely soybean oil based (Lipofundin-N 20\%, type 1), MCT oil based (Lipofundin MCT/LCT 20\%, type 2), olive oil based (ClinOleic, type 3) and fish oil containing (Lipidem, type 4). Patients must receive PN for at least 5 days in general ward or high dependency unit not requiring invasive/non-invasive ventilator support or inotropes support. Exclusion criteria included patients who received less than 5 days of $\mathrm{PN}$, intensive care unit (ICU) patients, PN started in ICU and continue in general ward or HDU, PN restarted in less than 3 days after receiving PN in ICU, patient on invasive/non-invasive ventilator support or inotropes support and home PN patients.

Results: 537 patients were started on PN and 388 patients were included in the study. 90 patients were on type 1 (soybean based) IVLE, 60 patients were on type 2 (MCT based) IVLE, 141 patients were on type 3 (olive oil based) IVLE and 97 patients were on type 4 (contain fish oil) IVLE. Baseline characteristic were similar in four groups of IVLEs. Majority of PN were initiated in patients admitted under surgical team. There were no difference in terms of mortality, readmission and infection rate between conventional and alternative IVLE as a group, odd ratio (OR) was 0.66 (CI $0.36-1.24 ; \mathrm{p}=0.16$ ), 1.71 (CI $0.84-3.73$; $\mathrm{p}=0.15)$ and $0.90(\mathrm{CI} 0.55-1.49 ; \mathrm{p}=0.73$ ) respectively (as shown in table 1 ). The length of stay in log-scale was significantly lower in alternative IVFE as a group $(\mathrm{p}=0.03)$. There were no difference in terms of mortality, readmission and infection rate between conventional IVLE versus each of the alternative IVLE. Length of stay was only statistically significantly lower for olive oil based IVLE (Type 3 ) when compared to conventional IVLE $(\mathrm{p}=0.05)$.

Table 1: Clinical outcomes with conventional IVLE versus alternative IVLE as a group

\begin{tabular}{lllll}
\hline & Conventional & $\begin{array}{l}\text { Alternative } \\
\text { (As a group) }\end{array}$ & OR (95\% CI) & P Value \\
\hline Mortality Yes (N) No (N) & 2170 & 49248 & $0.66(0.36-1.24)$ & 0.16 \\
Readmission Yes (N) No (N) & 1258 & 65183 & $1.71(0.84-3.73)$ & 0.15 \\
Infection Yes (N) No (N) & 4150 & 126171 & $0.90(0.55-1.49)$ & 0.72 \\
$\begin{array}{l}\text { Length of stay (Mean (SD) } \\
\quad \text { in log-scale) }\end{array}$ & $3.58(0.59)$ & $3.43(0.57)$ & & 0.03 \\
\hline
\end{tabular}

Conclusion: Length of stay was significantly lower in alternative IVLE compared with conventional IVLE. However, there were no clinical difference in terms of mortality, readmission and infection between conventional and alternative IVLE in non critically ill patients.

Disclosure of Interest: All authors have declared no conflicts of interest. 


\section{References}

1. Wanten GJA, Calder P. Immune modulation by parenteral lipid emulsions. AM J Clin Nutr 2007; 85: 1171-1184.

2. Furukawa K, Yamamori H, Takagi K, et al. Influences of soybean oil emulsion on stress response and cell-mediated immune function in moderately or severely stressed patients. Nutrition. 2002; 18:235-240.

3. Edmunds CE, Brody RA, Parrott JS, et al. the effects of different IV fat emulsions on clinical outcomes in critically ill patients. Crit Care Med 2014; 42:1168-1177.

\section{P0636 COMPLICATIONS AND EARLY MORTALITY IN PERCUTANEOUS ENDOSCOPIC GASTROSTOMY PLACEMENT IN LOMBARDY: A MULTICENTER PROSPECTIVE STUDY}

A. Anderloni ${ }^{1}$, F. Barzaghi ${ }^{2}$, G. Meucci ${ }^{3}$, R. Marino ${ }^{4}$, L. Amato ${ }^{5}$, M. Frigerio ${ }^{6}$, V. Saladino ${ }^{7}$, A. Toldi ${ }^{8}$, G. Manfredi ${ }^{9}$, A.E. Redaelli ${ }^{10}$, M. Feliziani ${ }^{11}$, G. De Roberto $^{12}$, F. Boni ${ }^{13}$, G. Scacchi ${ }^{14}$, D. Mosca ${ }^{15}$, M. Devani ${ }^{16}$, M. Arena ${ }^{17}$, M. Massidda ${ }^{18}$, P. Zanoni ${ }^{19}$, C. Ciscato ${ }^{20}$, V. Casini ${ }^{21}$, P. Beretta ${ }^{20}$, E. Forti ${ }^{22}$, R. Salerno ${ }^{23}$, V. Caramia ${ }^{24}$, G. Lollo ${ }^{1}$, M. Bianchetti ${ }^{25}$, C. Tomba ${ }^{26}$, M. Di Leo ${ }^{1}$, A. Repici ${ }^{1}$, M. Soncini ${ }^{27}$, G. Maconi ${ }^{28}$, G. Manes ${ }^{29}$, R. Gullotta ${ }^{30}$

${ }^{1}$ Humanitas Research Hospital, Milan, Italy., Rozzano/Italy

${ }^{2}$ Presidio Ospedaliero di Busto Arsizio, Busto Arsizio/Italy

${ }^{3}$ GI Unit, Ospedale S. Giuseppe, Milano, Milano/Italy

${ }^{4}$ ASL Lodi, Lodi/Italy

${ }^{5}$ Humanitas - Mater Domini, Castellanza/Italy

${ }^{6}$ Azienda Sanitaria Locale Della Provincia di Como, Como/Italy

${ }^{7}$ Ospedale di Legnano, Legnano/Italy

${ }^{8}$ Gastroenterology Unit, Valduce Hospital, Como/Italy

${ }^{9}$ Az Ospedaliera di Crema, Crema/Italy

${ }^{10}$ HS Gerarardo Monza, Monza/Italy

${ }^{11}$ Azienda Ospedaliera Sant'Antonio Abate, Gallarate/Italy

${ }^{12}$ Istituto Europeo di Oncologia, Milano/Italy

${ }^{13}$ Azienda Ospedaliera di Melegnano, Melegnano/Italy

${ }^{14}$ Ospedale Policlinico, Milano/Italy

${ }^{15}$ Azienda Ospedaliera Sant'Anna, Como/Italy

${ }^{16}$ AOG Salvini, Rho/Italy

${ }^{17}$ Ospedale San Paolo, Milano/Italy

${ }^{18}$ Istituto Clinico Humanitas Gavazzeni, Bergamo/Italy

${ }^{19}$ Casa di cura San Francesco, Bergamo/Italy

${ }^{20}$ ICCS istituto Clinico Città Studi, Milano/Italy

${ }^{21}$ Ospedale di Seriate, Seriate/Italy

${ }^{22}$ Endoscopy Unit, Niguarda-Ca' Granda Hospital, Milano/Italy

${ }^{23}$ Fatebenefratelli, Milano/Italy

${ }^{24}$ Medicina, Ospedale Maugeri, Pavia/Italy

${ }^{25}$ Istituto Clinico Humanitas Mater Domini, Castellanza/Italy

${ }^{26}$ Fondazione IRCCS Ca Granda Ospedale Maggiore Policlinico Milano, Cassina de' Pecchi/Italy

${ }^{27}$ San Carlo Borromeo, Milano/Italy

${ }^{28}$ Department Of Biomedical And Clinical Sciences - L.sacco University Hospital -

Milan Italy, Luigi Sacco University Hospital Gastroenterology Unit Biomed and Clinical Sciences, Milano/Italy

${ }^{29}$ Ospedale Salvini, Garbagnate/Italy

${ }^{30}$ Gastroenterology, Reckitt Benckiser Healthcare, Paderno Dugnano (mi)/Italy

Contact E-mail Address: andrea.anderloni@humanitas.it

Introduction: Percutaneous endoscopic gastrostomy (PEG) is currently the method of choice for medium- and long-term enteral feeding and is nowadays one of the most common endoscopic procedures performed worldwide. To date, data on complications and mortality rates are generally retrospective and only few prospective studies have been published on small number of patients.

Aims \& Methods: We aimed to prospectively evaluate the complication rates and the mortality of patients after PEG insertion or PEG replacement. This is a multicenter prospective cohort study. Between 15th September 2015 and 15th September 2016, all patients that underwent PEG insertion or replacement, were included. Details about patient's characteristics, ongoing therapies, comorbidities, indication for PEG placement/replacement, informed consent form signature were collected. Early and late (30 days) complications and mortality were assessed.

Results: 912 patients (426 [46.7\%] male) were enrolled in 34 centers. Patients mean age was $72 \mathrm{y}$ (SD16.01, range 18-99). ASA status was 1 in $4.0 \%, 2$ in $23.4 \%, 3$ in $58.4 \%, 4$ in $14.1 \%$. PEG 1 st placement was performed in 582 $(64 \%)$ patients. Indications were: dysphagia for cerebrovascular diseases in $33.3 \%$, degenerative neurological illness in $21.3 \%$, dementia in $14.6 \%$, head and neck cancer in $14.4 \%$, management of patient after the discharge in $8.9 \%$, nutritional support in oncological patients in 3.4\%, medication administration in $1.5 \%$, gastric decompression in $0.2 \%$. The informed consent form was signed by a relative (without legal guardianship) in $49.6 \%$, patient in $28,2 \%$, legal guardian in $16 \%$, medical director in $6,2 \%$ of patients. The majority of patients $(63.2 \%)$ had antiplatelet and/or anticoagulant concomitant therapy. Anesthetist was present in $47.7 \%$ of procedures and sedoanalgesia was the more common modality for sedation $(69.8 \%)$. The complication and mortality rate were $4.6 \%$ and $7.8 \%$, respectively. The most frequent complication was infection $(2.4 \%)$, followed by bleeding $(1.4 \%)$, tube dislodgment $(0.4 \%)$, buried bumper syndrome $(0.2 \%)$. PEG replacement was carried out in 330 $(36 \%)$ patients. The indications for PEG replacement were obstruction/malfunction/deterioration of the tube in $52.9 \%$, tube dislodgment in $26.4 \%$. Endoscopic procedure was necessary in $9.7 \%$ of cases. The informed consent was signed by relative (without legal guardianship) in $66.3 \%$, patient in $10.5 \%$, legal guardian in $16.25 \%$, medical director in $7 \%$ of patients. Thirty-days mortality was $2.4 \%$.

Conclusion: Our data confirm that PEG placement is a safe procedure with a mortality rate at 30 days of $8 \%$. To our knowledge this is the largest prospective study on the use of PEG. Surprisingly in more than $50 \%$ of patients the consent form was not properly signed, leading to possible medico-legal consequences. Moreover, in $9 \%$ of the cases PEG was placed for an early discharge (more than for real clinical indication).

Disclosure of Interest: All authors have declared no conflicts of interest.

\section{P0637 MEDICAL REGISTRAR REPORTING OF CHEST X-RAYS FOR NASOGASTRIC TUBE POSITION: HOW CAN IT BE MADE SAFER?}

M. Tierney, Z. Leach, C. Sibley, C. Rutter, C. Pither, T. Smith

Nutrition, University Hospitals Southampton, Southampton/United Kingdom

Contact E-mail Address: maeve.tierney@nhs.net

Introduction: Nasogastric tube (NG) feeding is an essential part of in-patient care. Tubes can be placed at the bedside with no need for specific equipment or sedation. However placement of NG tubes is not without risk and avoiding the introduction of substances into the respiratory tract through a misplaced NG tube was highlighted as a UK National Patient Safety Agency alert in 2005. In 2011 the NPSA made this a 'never event'. The only acceptable methods of checking the position of an NG tube are: $\mathrm{pH}<5.5$ on aspirate or confirmation on chest $\mathrm{x}$-ray (CXR) by competent medical staff. Reporting a CXR for NG tube position is a frequent request particularly for junior doctors out of hours. Practise vaires across the UK - some trusts require NG checking to be done only by senior clinicians (medical registrars or consultants) and some only allow reporting by a consultant radiologist. We assessed documentation of $\mathrm{NG}$ position on CXR by medical registrars from the region to find out if documentation was adequate, as would be expected of senior clinicians. NPSA guidance suggests four points should be documented in the medical notes to confirm NG position: 1. Does the tube follow the contours of the oesophagus and avoid those of the bronchi? 2. Does the tube clearly bisect the carina or the bronchi? 3. Does it cross the diaphragm in the midline? 4 . Is the tip clearly visible below the left hemi-diaphragm? All four criteria were met in only $17.6 \%$ of repsonses and answers were considered incorrect in $20.5 \%$. An aide-memoir sticker with an abbreviated version of the above four points, time, date, doctor signature and whether tube is safe to use or not with Yes/No answers, is used on some wards in Southampton and we assessed whether its use would improve quality of reporting both a correctly placed and misplaced NG tube.

Aims \& Methods: Medical registrars from first to final year of specialist training and from various specialties were presented with a CXR showing a correctly sited NG tube and were asked to complete a sticker answering yes or no, to check position and whether it was safe to use. Following this they were presented with a CXR showing an incorrectly sited NG tube and asked to use the sticker to assess position. The CXR was projected and anonymous responses collected after sufficient time for the group to complete both stickers.

Results: 31 complete responses were obtained for the correctly sited tube with $58 \%$ stating that it should be used and $42 \%$ that they would not use the tube without further review. 10 incomplete responses were obtained and therefore $86 \%$ of responses met NPSA guidance for reporting CXR for NG position. 28 complete responses were obtained for the incorrectly sited tube and $100 \%$ stated that the tube should not be used.

Conclusion: Use of the sticker increased compliance with NPSA guidance for CXR reporting for NG tube position from $17.6 \%$ to $86 \%$. The misplaced tube was correctly reported and not used in $100 \%$ of responses. The correctly sited tube was reported as safe to use in $58 \%$. The CXR used was of an anonymous real patient and was slightly rotated to reflect a real-life scenario which meant the tube was slightly off the midline. In this real-life scenario some trainees would be happy to make a judgement considering these factors and others may be cautious and follow the sticker statements exactly prompting further review by radiology or removal of the tube. Overall this increases patient safety and avoids use of a misplaced tube in accordance with UK guidance. We intend to roll out use of the sticker on all wards which use NG tubes to rapidly improve documentation and patient safety. The other option we may consider is developing a pathway for radiology consultants to report all these CXRs before the NG tube is used; however, this is likely to take considerable time and is unlikely to be available out of hours.

Disclosure of Interest: All authors have declared no conflicts of interest.

\section{References}

National Patient Safety Agency Alert 2005/PSA/05 Reducing the harm caused by misplaced nasogastric feeding tubes 2005

Lee $\mathrm{S}$ and Mason E. Competence in confirming correct placement of nasogastric feeding tubes amongst FY1 doctors BMJ Qual Improv Report 2013 2:u201014.w1198

Checking placement of nasogastric feeding tubes in adults (interpretation of $\mathrm{x}$ ray images): summary of a safety report from the National Patient Safety Agency BMJ 2011;342:d2586 
P0638 MICRONUTRIENT MONITORING IN HOME PARENTERAL NUTRITION PATIENTS: AN AUDIT OF PRACTICE IN A REGIONAL REFERRAL CENTRE

M. Tierney, L. Bakewell, E. Buse, C. Rutter, C. Pither, T. Smith

Nutrition, University Hospitals Southampton, Southampton/United Kingdom

Contact E-mail Address: maeve.tierney@nhs.net

Introduction: Intestinal failure patients by definition have reduced ability to absorb fluid and macronutrients through the gastrointestinal tract. Type two intestinal failure patients require months of intravenous nutrition (parenteral nutrition) for weeks or months either in hospital or at home. Type three intestinal failure patients generally require long-term parenteral nutrition (PN), which is given at home (HPN) and may be life-long. In addition to a reduction in the ability to absorb macronutrients, patients on long-term PN have a reduction in absorption of micronutrients (copper, zinc, selenium and manganese) and vitamins (A, B12, D and E) which are required for metabolism and enzymatic reactions at a cellular level. PN is routinely supplemented with micronutrients and should be sufficient. Toxicity can occur at high levels and deficiency can cause a variety of symptoms. ESPEN guidelines recommend that serum vitamin and trace element levels be checked at baseline and at least once per year. NICE guidelines specify more frequent monitoring for in-patients and that selenium; manganese and vitamin $\mathrm{D}$ should be checked three to six monthly in HPN patients. Some trace elements (copper and zinc in particular) are affected by acute illness. Current local practise is to avoid checking levels until there is evidence that inflammation or infection has resolved.

Aims \& Methods: Our aim was to audit the frequency of micronutrient screening in our cohort of HPN patients. All type two and three intestinal failure outpatients were included. Current in-patients were excluded due to the effect of acute illness on micronutrient levels. Patients on parenteral fluid rather than nutrition were excluded as current guidelines give recommendations for HPN patients and do not specify recommendations if fluid alone is required. A search of the blood results system was performed for all micronutrient results from one full year to the date of the search. Results were recorded in spreadsheet format and analysed. Many patients live out of the region; however, many local trusts do not have the laboratory facilities to check micronutrient levels so they tend to be done in Southampton. If no results were avaiable on the Southampton system then the local hospital was contacted for local results if available.

Results: 57 home parenteral nutrition patients were identified. $51(89.5 \%)$ of these patients had micronutrients checked at some point during their care. 44 of $49(89.7 \%)$ had micronutrients checked within one year (two of the 51 had only recently so did not have results within a year). 32 (61.5\%) of those who had micronutrients checked had them done within the last six months. 6 patients had never had micronutrients checked. One had them requested just prior to the time of audit but results were not yet available. Two were out of area and had not been to clinic within the past year. One of these commenced PN in 2015 and found it difficult to attend clinic. The other had not been seen in clinic due to an administrative error and has now been seen with micronutrients requested. Two further patients had never had micronutrients checked due to a persistently raised CRP.

Conclusion: Despite a lack of clarity between guidelines about the frequency of monitoring of micronutrients, it is recommended that HPN patients receiving long-term intravenous nutrition should have regular monitoring to reduce risk of deficiency or toxicity. The majority of our cohort of HPN patients had micronutrients checked annually and over half were checked six monthly. This is compliant with ESPEN guidelines; however, we need to aim for $100 \%$. We have introduced a template to use in clinic to trigger review of results and request micronutrients when required. Alongside this we have introduced a virtual ward round to remotely review all out-patients regularly and plan ahead to request blood tests when required. Following the introduction of these measures we will repeat the audit to find out if the situation has improved.

Disclosure of Interest: All authors have declared no conflicts of interest.

\section{References}

Pironi L et al. ESPEN guidelines on chronic intestinal failure in adults: Clinical Nutrition; 35 (2016) 247-307

Nutrition support for adults: oral nutrition support, enteral tube feeding and parenteral nutrition NICE Clinical guideline [CG32] Published date: February 2006

\section{P0639 CLINICAL NUTRITION - ARE WE IGNORANT OR NEGLIGENT?}

T.M.A. Gan, R. Asokkumar, E. Salazar

Gastroenterology And Hepatology, Singapore General Hospital, Singapore/

Singapore

Contact E-mail Address: aaron.gan2@mohh.com.sg

Introduction: Early recognition and delivery of nutritional care by physicians has been shown to improve outcomes in malnourished hospitalized patients. However, physicians encounter multiple barriers in providing appropriate nutritional care. While comprehensive international guidelines and nutritional training in medical education have been introduced to overcome these barriers, there appears to be a discrepancy in practice amongst physicians despite the availability of these resources.

Aims \& Methods: We aim to assess the knowledge and attitudes of physicians towards clinical nutrition in a large tertiary teaching hospital in Singapore. An anonymous questionnaire comprising 15 multiple-choice questions from standard nutrition textbooks was administered. The questionnaire was designed to assess (a) recognition of nutritional needs of hospitalized patients, (b) knowledge on principles of clinical nutrition, and (c) application of nutritional intervention in common clinical practice. We included consultants, fellows and residents working in units where nutritional problems were common. Finally, we conducted a separate 5-question opinion survey to assess each participant's nutritional training and exposure, based on a 5- point Likert scale ranging from "strongly agree" to strongly disagree".

Results: A total of 305 physicians volunteered to participate in this study. Forty $(13 \%)$ did not reveal their specialty or staff grade and were excluded from analysis. The remaining 265 responders comprised $77(29 \%)$ consultants, $58(22 \%)$ fellows, and $130(49 \%)$ residents. Amongst them, $232(87 \%)$ were from medical disciplines and $33(13 \%)$ from surgical disciplines. The median aggregate score (out of a maximum score of 15) obtained by consultants, fellows and residents was $6.0 \pm 2.2$ (range $2-12$ ), $7.0 \pm 1.8$ (range $3-11$ ), and $7.0 \pm 1.8$ (range $1-10$ ) respectively. All 3 grades of physicians achieved less than $50 \%$ of the maximum possible score. No significant difference in median aggregate score was observed between physicians from medical disciplines $(6.5 \pm 1.9)$ and those from surgical disciplines (7.0 \pm 1.8$)$. However, gastroenterologists performed significantly better than non-gastroenterologists (median aggregate score $9.0 \pm 2.2$ vs $6.0 \pm 1.8, \mathrm{p}<0.001)$. In the opinion survey, a majority of physicians $(63 \%)$ believed that nutrition-related teaching was inadequate during residency training and $44 \%$ felt that clinical nutrition was accorded insufficient attention during ward rounds. Only $33 \%$ of responders reported that they performed nutritional screening on admission, and a mere $10 \%$ were confident in providing nutrition counselling to malnourished patients. Interestingly, their overall performance was not different from that of other participants (see Table 1).

Table 1: Median aggregate scores by grade, specialty and response in opinion survey

\begin{tabular}{llll}
\hline & $\begin{array}{l}\text { Median } \\
\text { aggregate } \\
\text { score } \pm \text { SD }\end{array}$ & Range & P value \\
\hline Physician Grade & & & \\
Consultants $(\mathrm{n}=77)$ & $6.0 \pm 2.2$ & $2.0-12.0$ & 0.617 \\
Fellows $(\mathrm{n}=58)$ & $7.0 \pm 1.8$ & $3.0-11.0$ & \\
Residents $(\mathrm{n}=130)$ & $7.0 \pm 1.8$ & $1.0-11.0$ & \\
Specialty & & & \\
Medical disciplines $(\mathrm{n}=232)$ & $6.5 \pm 1.9$ & $1.0-12.0$ & 0.193 \\
Surgical disciplines $(\mathrm{n}=33)$ & $7.0 \pm 1.8$ & $2.0-10.0$ & \\
Gastroenterologists $(\mathrm{n}=25)$ & $9.0 \pm 2.2$ & $3.0-12.0$ & $<0.001$ \\
Non-gastroenterologists $(\mathrm{n}=240)$ & $6.0 \pm 1.8$ & $1.0-11.0$ & \\
Performed nutrition screening on admission & & & \\
Agreed ( $=81)$ & $7.0 \pm 2.0$ & $1.0-12.0$ & 0.321 \\
Disagreed ( $\mathrm{n}=99)$ & $7.0 \pm 2.2$ & $2.0-11.0$ & \\
Confident in providing nutrition counselling & & & \\
Agreed $(\mathrm{n}=23)$ & $7.0 \pm 2.0$ & $3.0-10.0$ & 0.467 \\
Disagreed (n=137) & $7.0 \pm 2.0$ & $2.0-11.0$ & \\
\hline
\end{tabular}

Conclusion: Our study highlights that knowledge on nutrition and its clinical application to hospitalized patients remains inadequate across all physician grades, especially amongst non-gastroenterologists. The current state of clinical nutrition-related teaching during residency training falls short of achieving its goals, and may need re-examination.

Disclosure of Interest: All authors have declared no conflicts of interest.

MONDAY, OCTOBER 30, 2017

09:00-17:00

\section{PAEDIATRIC: UPPER GI - HALL 7}

\section{P0640 OUTCOMES OF PER-ORAL ENDOSCOPIC MYOTOMY IN CHILDREN WITH ACHALASIA CARDIA}

Z. Nabi ${ }^{1}$, M. Ramchandani ${ }^{1}$, R. Chavan ${ }^{1}$, R. Kalapala ${ }^{1}$, D.N. Reddy ${ }^{2}$, S. Darisetty

${ }^{1}$ Gastroenterology, Asian Institute of Gastroenterology, Hyderabad/India

${ }^{2}$ Gastroenterology, Asian Inst. of Gastroenterology - Gastroenterology, Asian Inst. of Gastroenterology; Hyderabad/IN, Hyderabad/India

Contact E-mail Address: zaheernabi1978@gmail.com

Introduction: Per-oral endoscopic myotomy (POEM) is a novel treatment modality for achalasia cardia (AC). The studies are limited in paediatric population. Aims \& Methods: In this study our aim was to analyse the feasibility, safety and efficacy of per-oral endoscopic myotomy in children We retrospectively evaluated the data of all children ( $\leq 18$ years) who underwent POEM at our institution from September 2013 to February 2017. All POEM procedures were performed under general anaesthesia in an endoscopy suit. Technical feasibility, safety and efficacy were analysed. Clinical success was defined as Eckardt score $\leq 3$. Objective parameters including-timed barium swallow and high resolution manometry were assessed and compared before and after POEM.

Results: Thirty children (15-boys, 15-girls) with mean age of $14.1+3.32$ (4-18) years, underwent POEM during the specified period. The sub-types of AC weretype I (8), type II (19) and type III (1). Eight children had prior treatment with pneumatic balloon dilatation. POEM was successfully performed in all children. Anterior myotomy was performed in majority of children $23(76.7 \%)$. Mean total length of myotomy was $10.9 \pm 2.25 \mathrm{~cm}$, with $7.9 \pm 2.09 \mathrm{~cm}$ on esophageal and 
$3.03 \pm 0.67 \mathrm{~cm}$ on gastric side. Mean operating time was $76.7 \pm 45.5(30-240)$ minutes. Mild gas related adverse events requiring temporary discontinuation of procedure and or drainage procedure were encountered in eight $(26.7 \%)$ children (capno-peritoneum-4 and retro-peritoneal carbon dioxide -4). There was significant reduction in mean LES pressure after POEM $(36.25 \pm 16.5$ vs $11.47 \pm 5.06, p=0.0001)$. Significant improvement of esophageal emptying on timed barium esophagogram $(>50 \%)$ was documented in $94.4 \%$ children. At median follow up of 504 days $(30-1290)$ clinical success was noticed in 29 children $(96.7 \%)$. Mean Eckardt score before and after POEM were $6.8 \pm 1.63$ and $0.86 \pm 0.77$, respectively $(\mathrm{p}=0.0001)$. Gastroscopy revealed erosive gastroesophageal reflux in five children at 1 year $(27.8 \%)$.

Conclusion: Conclusion-POEM is safe and effective for children with achalasia cardia. POEM can be safely performed in an endoscopy suit in paediatric patients.

Disclosure of Interest: All authors have declared no conflicts of interest.

\section{References}

Khashab MA, El Zein M, Kumbhari V. Comprehensive analysis of efficacy and safety of per-oral endoscopic myotomy performed by a gastroenterologist in the endoscopy unit: a single-center experience. Gastrointest Endosc. 2016 Jan;83(1):117-25.

Nabi Z, Ramchandani M, Reddy DN, et al. Per Oral Endoscopic Myotomy in Children with Achalasia Cardia. J Neurogastroenterol Motil. 2016 Oct 30;22(4):613-619.

Chen WF, Li QL, Zhou PH, et al. Long-term outcomes of peroral endoscopic myotomy for achalasia in pediatric patients: a prospective, single-center study. Gastrointest Endosc. 2015 Jan;81(1):91-100.

\section{P0641 HIGH RATE OF HELICOBACTER PYLORI ANTIBIOTIC RESISTANCE IN ARMENIAN CHILDREN WITH GASTRODUODENAL DISEASE}

\section{T. Shahinyan ${ }^{1}$, G. Amaryan ${ }^{2}$, M. Hovsepyan ${ }^{2}$, C. Braegger ${ }^{3}$}

${ }^{1}$ Chair Of Pediatrics 2, Yerevan State Medical University, Yerevan/Armenia ${ }^{2}$ Pediatrics, Arabkir Joint Medical Center, Yerevan/Armenia

${ }^{3}$ Division Of Gastroenterology And Nutrition, University Children's Hospital

Zurich, Zurich/Switzerland

Contact E-mail Address: shahinyan_tatevik@yahoo.com

Introduction: Because of high prevalence of gastric malignancies in the adult population, high Helicobacter pylori $(\mathrm{Hp})$ prevalence in Armenia is suspected. Rising antibiotic resistance of $\mathrm{Hp}$ both in children and adults lead to decrease of effectiveness of standard eradication therapy $[1,2]$. The aim of this study is to determine frequency of $\mathrm{Hp}$ antibiotic resistance in Armenian children.

Aims \& Methods: 47 children with suspected gastroduodenal disease (GDD), hospitalized in Arabkir MC, were selected from April to December 2016 (23 boys and 24 girls, average age $8.98 \pm 4.10)$. Hp-associated GDD were diagnosed according to clinical, endoscopic and histological criteria. Antral biopsy was cultured on 5\% sheep blood Columbia agar and selective Hp media. Antibiotic susceptibility test was done by disk diffusion method.

Results: Hp-associated GDD was diagnosed in 40 patients out of $47: 37(92.5 \%)$ had gastritis and/or duodenitis, $3(7.5 \%)$ had peptic ulcer disease (PUD). Seven out of 47 children were excluded from the study due to both histology and culture negative for $\mathrm{Hp}$. Thirty-four $(85 \%)$ were treatment-naïve patients and $6(15 \%)$ had received eradication therapy previously. Main clinical symptoms were recurrent epigastric pain $34(85 \%)$, nausea $28(70 \%)$ and vomiting $13(32.5 \%)$. By endoscopy erosive gastritis and/or duodenitis was seen in $18(45 \%)$, non-erosive gastritis in $16(40 \%)$, PUD in $3(7.5 \%)$, normal mucosa in $3(7.5 \%)$. Rapid urease test was positive in all antral biopsies $(100 \%)$. Histology showed chronic gastritis and/or duodenitis in $28(70 \%)$, atrophic gastritis in $5(12.5 \%)$, gastric glandular dysplasia in $2(5 \%)$, gastric metaplasia of duodenal mucosa in $3(7.5 \%)$, normal mucosa in $2(5 \%)$. Hp was positive in $38(95 \%)$ and negative in $2(5 \%)$. Cultures were positive for $\mathrm{Hp}$ in 14 of 40 patients $(35 \%)$. Susceptibility test was possible in $12 \mathrm{Hp}$ strains from available14: all but to 2 were resistant to metronidazole $(83.3 \%), 4$ to clarithromycin $(33.3 \%), 3$ double resistant to both metronidazole and clarithromycin $(25 \%)$, and $66.6 \%$ to doxycycline. All strains were susceptible to amoxicillin and levofloxacine $(100 \%), 6$ strains were tested and found susceptible to nifuratel.

Conclusion: These data indicate a high rate of resistance to conventional triple therapy antibiotics: metronidazole $(83.3 \%)$ and clarithromycin $(33 \%)$. High resistance to doxycycline also was seen, despite limited use of this antibiotic in Armenian paediatric practice. High susceptibility to nifuratel might be useful for further development of specific eradication schemes for Armenia. High frequency of both erosive and non-erosive gastritis as well as high rate of gastric atrophy and dysplasia in these patients were noticed.

Disclosure of Interest: All authors have declared no conflicts of interest.

\section{References}

Prospective multicentre study on antibiotic resistance of Helicobacter pylori strains obtained from children living in Europe. Koletzko S et al. Gut 2006;55:1711-1716.

Recent Insights into Antibiotic Resistance in Helicobacter pylori Eradication. Gastroenterology Research and Practice, Volume 2012, Article ID 723183, 8 pages
P0642 GASTRIC MICROBIOTA OF CHILDREN WITH CHRONIC GASTRITIS

E. Kornienko, N. Parolova, P. Zykov

St. Petersburg State Paediatric Medical University, St. Petersburg/Russian

Federation

Contact E-mail Address: da@amamed.ru

Introduction: Children's gastric microbiota in the presence or absence of $H$. pylori (HP) has not been studied well.

Aims \& Methods: We aimed to study the composition of the microbiota in the biopsy material of the antral part of the stomach, according to the 16S-rRNA sequencing, of children with chronic gastritis, in the presence or absence of HP, and also to compare it with the histological data. Biopsy materials of mucous tunic from antral part of the stomach were taken from 16 children aged 10-17 with chronic gastritis and after the preliminary extraction the biopsy materials were examined using the method of sequencing with a pair of oligonucleotide primers, which are specific for the conservative regions of the 16S-rRNA gene, on the Life Technologies Ion Torrent sequencer using the 318v2 chip. Bioinformatic processing was conducted using the QIIME package. The results were compared with the data from the histological examination of the biopsy materials from the same part of the stomach as well as with the results of diagnosis using rapid urease test AMA RUT Expert with digital Reader.

Results: 8 out of 16 patients were identified as $\mathrm{HP}(+)$ positive, 2 of them had HP in small amounts, 6 of them - in significant amounts. The dominant types of bacteria in the stomach of all children were Proteobacteria, Bacteroidetes, Firmicutes; in a lesser extent - Actinobacteria, Cyanobacteria, Fusobacteria. $64.1 \%$ of $\mathrm{HP}(+)$ patients' microbiome was constituted of HP, among Proteobacteria it reached $75-99 \%$, the amount of other bacteria herewith shortened, and the species diversity decreased. Non-helicobacter microbiota of children with small amount of HP was almost identical in composition as HP(-) patients', the amount of other microbes was more numerous and diverse, also within Proteobacteria. The signs of inflammation in mucous coat of the stomach in case of HP presence were more pronounced than in HP absence, they correlated with the amount of HP.

Conclusion: Microbiome of the children's stomach is diverse, it is similar to adults'. The infection from HP inhibits another microbiota and it is accompanied with the signs of mucous coat inflammation, which correlates with the amount of HP

Disclosure of Interest: All authors have declared no conflicts of interest

\section{P0643 FEATURES OF CHRONIC GASTRITIS CAUSED BY CO- INFECTION OF HELICOBACTER PYLORI AND EPSTEIN-BARR VIRUS IN PEDIATRIC PATIENTS}

\section{E. Spivak, R. Levit}

Pediatric, Medical State University, Yaroslavl/Russian Federation

\section{Contact E-mail Address: spivak58@mail.ru}

Introduction: It is known that co-infection of the gastric mucosa with highly pathogenic Hp strains and the Epstein-Barr virus is a risk factor for the development of severe gastritis and gastric adenocarcinoma. However, the characteristics of such co-infection in children are not sufficiently studied

Aims \& Methods: The aim of this study is to estimate the role co-infection of highly pathogenic strains of Helicobacter pylori and Epstein-Barr virus in pediatric patients with chronic gastritis Patients and methods. 190 children aged 8-16 with chronic $\mathrm{Hp}$-associated gastritis were studied All the patients underwent clinical and laboratory examinations and endoscopy. Gastric biopsies were taken and inflammation graded according to the Sydney system. Polymerase chain reaction (PCR) was used to detect the presence of Epstein-Barr virus (EBV), Helicobacter pylori $(\mathrm{Hp})$ and its highly pathogenic strains in the gastric mucosa of the patients.

Results: Persistent EBV infection was found in 83 children $(43.7 \%)$ with chronic gastritis of the antral and (or) gastric body areas. Helicobacter pylori strains that possess the virulence factors (cytotoxin-associated gen A (CagA), vacuolating cytotoxin gen A (VacA), induced by contact with epithelium (IceA), and blood group antigen-binding adhesion (BabA) were detected in 49 patients $(25.8 \%)$. In most cases, the association of two or more virulence factors in one patient was observed. It was found that 39 pediatric patients had co-infection of the highly pathogenic strains of $\mathrm{Hp}$ and EBV. The study revealed no significant effect of the variant of the gastric mucosa infection on the clinical manifestations of gastritis the nature of intoxication, abdominal and dyspeptic syndromes. At the same time, the endoscopic and morphological data analysis has revealed a severe gastritis with the development of pangastritis and signs of gastric mucosa atrophy observed mainly in the antral region, in patients with co-infection (highly pathogenic strains of $\mathrm{Hp}+\mathrm{VEB}$ ). In addition, by correlation analysis, we found that the increase and development of the inflammatory process in the gastric mucosa was mostly influenced by the presence of CagA-positive strains of $H$. pylori in combination with EBV. We found that children infected by EBV without highly pathogenic Hp strains had mild mononuclear and polymorphonuclear cell infiltration without atrophy.

Conclusion: Co-infection with highly pathogenic Hp strains and the Epstein-Barr virus in pediatric patients is significantly associated with severe gastritis. Disclosure of Interest: All authors have declared no conflicts of interest. 
P0644 HELICOBACTER PYLORI INFECTION AND SPECIFIC IMMUNOGLOBULIN E ANTIBODIES TO FOOD ALLERGENS IN SYMPTOMATIC CHILDREN ADMITTED IN A DIGESTIVE ENDOSCOPY UNIT

V. Hurduc, B. Luiza, P. Doina

Pediatric Gastroenterology, Victor Gomoiu Clinical Children Hospital, "Carol

Davila" University of Medicine and Pharmacy, Bucharest/Romania

\section{Contact E-mail Address: v hurduc@yahoo.com}

Introduction: Helicobacter pylori is one of the most widespread bacterial infections worldwide, therefore nowadays its prevalence was decreasing, mostly in developed countries. There are some studies which support that $H$ pylori could favor the development of food allergy.

Aims \& Methods: To assess the relationship between $H$ pylori infection and specific immunoglobulin E (Ig E) antibodies to food allergens in symptomatic children. We conducted a prospective study of 394 symptomatic children (249 girls, age range 6 months- 18 years), mostly with uninvestigated dyspepsia requiring an endoscopic evaluation in our unit, from January 2015 to December 2016. All patients were evaluated for $H$ pylori infection by at least two standard invasive tests and for specific immunoglobulin $\mathrm{E}$ antibodies to major food allergens (R-biopharm, Germany). The nutritional status of patients was assessed in all cases by the new World Health Organization (WHO, 2007) growth charts. EPIINFO version 7 was used for statistical analysis. A two sided p-value less than 0.05 was considered statistically significant.

Results: Active $H$ pylori infection was documented in $246(62.3 \%)$ cases. The allergic sensitization to at least one of the food allergens was identified in 134 of 394 patients (34\%). The majority of Ig E positive children (109 of 134 cases; $81.3 \%$ ) were positive for cow's milk followed by egg $(17.9 \%)$, wheat $(7.46 \%)$, peanut $(4.5 \%)$, soybean $(3.73 \%)$. The allergic sensitization to food allergens was associated with abnormal levels of specific Ig E antibodies to common inhalatory allergens in 55 of 134 cases $(41.04 \%)$. Regarding the association of $\mathrm{H}$ pylori infection with an elevated serum Ig E level to at least one of the food allergens tested, there was no significant correlation $(\mathrm{p}=0.14) .77$ of $134(31.30 \%)$ patients positive for food specific Ig E antibodies were $H$ pylori infected and 57 of them $(38.55 \%)$ were $H$ pylori negative (Fisher exact test $=0.08$ ). The assessment of the patients nutritional profile in relationship with $H$ pylori infection and food allergy not revealed a statistically significant effect on the two ends of the poor nutritional status (undernutrition and overnutrition).

Conclusion: The recent decline of $H$ pylori infection is not evident in our study. There was no association between $H$ pylori infection and Ig E mediated food allergy. Undernutrition and overnutrition were not associated with the $H$ pylori infection and food allergy in our patients.

Disclosure of Interest: All authors have declared no conflicts of interest.

\section{P0645 CURRENT STATUS OF THE FIRST AND SECOND LINE THERAPY FOR HELICOBACTER PYLORI INFECTION IN SYMPTOMATIC CHILDREN: A SINGLE CENTER STUDY}

V. Hurduc, B. Luiza, P. Doina

Pediatric Gastroenterology, Victor Gomoiu Clinical Children Hospital, "Carol

Davila" University of Medicine and Pharmacy, Bucharest/Romania

Contact E-mail Address: v_hurduc@yahoo.com

Introduction: Current evidence suggests the decline of the eradication rates of $H$ pylori in children treated with standard first line therapy, partly determined by its antibiotic resistance.

Aims \& Methods: To evaluate the effectiveness of current first and second-line therapy recommendations for $H$ pylori eradication in children. We conducted a prospective open-label study of 158 symptomatic children (age range 6 months18 years; 106 girls) who required a first upper digestive endoscopy over the past year. Active $H$ pylori infection was documented in 122 of the 158 investigated children $(77.2 \%)$. Infected children were randomly assigned to receive either a $7-$ 14 days standard empiric triple therapy consisting of esomeprazole (ESO) plus amoxicillin (AMO) and clarithromycin (CLA) or metronidazole (MET), either a sequential therapy for 10-14 days. Bismuth salts are not easily available in our country. Eradication efficacy was assessed by follow-up endoscopy $4-8$ weeks after the end of anti- $\mathrm{H}$ pylori therapy by at least two different invasive tests. In patients failing to be cured through the first treatment a second alternative was applied (a triple therapy based on quinolones or metronidazole either sequential therapy) associated with antibiotic susceptibility testing. The primary and secondary outcomes were the rate of $H$ pylori eradication after the first and second line therapy, by intention to treat (ITT) and per-protocol (PP) analysis. Statistical analysis was performed with EPI INFO 7. The differences between eradication rates were analysed by $x^{2}$ test and the Odds Ratio $(\mathrm{p}<0.005$ was considered statistically significant).

Results: Patients with $H$ pylori infection were treated with an initial empiric first line standard therapy $(\mathrm{n}=52)$ or a sequential therapy $(\mathrm{n}=70)$. Of the 122 children, 9 patients were lost to follow-up $(7.38 \%)$. Overall $\mathrm{H}$ pylori was eradicated in $87 / 122$ children $(71.32 \%$ for ITT analysis versus $76.99 \%$ for PP analysis). The first eradication rates were significantly higher using the sequential therapy (55 70 cases; $78.57 \%$ for ITT analysis and $55 / 64$ cases; $85.93 \%$ for PP analysis) compared with standard first line triple therapy (32/52 cases; $61.53 \%$ for ITT analysis and $32 / 49$ cases; $65.30 \%$ for PP analysis). The ITT and the PP eradication rates were significantly higher with sequential treatment $(\mathrm{OR}=0.43 ; 95 \%$ CI: $0.19-0.97 ; p=0.04 ; x^{2}=3.43 ; p=0.06$ for ITT analysis and $\mathrm{OR}=0.30 ; 95 \%$ CI: $0.12-0.77 ; \mathrm{p}=0.013 ; \mathrm{x}^{2}=5.55 ; \mathrm{p}=0.01$ for PP analysis). A second-line therapy was recommended in 26 of cases with an overall eradication rate of $80.76 \%$ ( $21 / 26$ cases) for ITT analysis and respectively $87.5 \%$ (21/24 cases) for PP analysis. The choice of second line therapy was tailored by antimicrobial susceptibility only in some cases $(15 / 26$ cases; $57.7 \%)$. The eradication rates for PP analysis were: $90 \%$ ( $9 / 10$ cases) for triple therapy based on MET; $80 \%$ ( $8 / 9$ cases) for triple therapy based on quinolones and $80 \%$ ( $4 / 5$ cases) for sequential therapy.

Conclusion: This endoscopic series reveals a high rate of $H$ pylori infection $(77.2 \%)$. The sequential therapy achieved a significantly higher rate of eradication than the standard empiric triple regimens regardless of using ITT $(78.57 \%$ versus $61.53 \%)$ or PP $(85.93 \%$ versus $65.30 \%)$ analysis. The eradication rates for the second-line therapy was significantly higher $(87.5 \%$ for PP analysis) compared with the first-line empiric standard therapy (76.99\% for PP analysis). Disclosure of Interest: All authors have declared no conflicts of interest.

\section{P0646 GUT MICROBIOTA ALTERATIONS UNDER OLIGOFRUCTOSE-ENRICHED INULIN ADMINISTRATION IN PAEDIATRIC COELIAC DISEASE PATIENTS ON A GLUTEN-FREE DIET:A RANDOMIZED CONTROLLED TRIAL}

U. Krupa-Kozak ${ }^{1}$, N. Drabińska ${ }^{1}$, L. H. Markiewicz ${ }^{2}$, E. Jarocka-Cyrta ${ }^{3}$ ${ }^{1}$ Department Of Chemistry And Biodynamics Of Food, Institute of Animal Reproduction and Food Research, Polish Academy of Sciences, Olsztyn/Poland ${ }^{2}$ Department Of Immunology And Food Microbiology, Institute of Animal Reproduction and Food Research, Polish Academy of Sciences, Olsztyn/Poland ${ }^{3}$ Faculty Of Medical Science, Department Of Clinical Pediatrics, University of Warmia \& Mazury, Olsztyn/Poland

Contact E-mail Address: u.krupa-kozak@pan.olsztyn.pl

Introduction: Imbalanced gut microbiota is suggested to be involved in the pathogenesis of coeliac disease (CD). In many CD patients, despite a long-term treatment with a gluten-free diet (GFD), the intestinal dysbiosis is not completely restored. Prebiotics, substances of the unique ability to shape intestinal microbiota are promising, low-risk GFD supplement to remedy the intestinal dysbiosis in $\mathrm{CD}$ patients.

Aims \& Methods: The aim of the present study was to assess the effect of prebiotic oligofructose-enriched inulin (OEI) administration on the quantitative gut microbiota characteristics of CD children following a strict GFD for $\geq 1$ year. A randomized, placebo-controlled 12 -weeks dietary intervention was conducted on $34 \mathrm{CD}$ children ( $62 \%$ female, mean age 10 years) on GFD who were randomly assigned to prebiotic (OEI; $10 \mathrm{~g} /$ day) or placebo group (maltodextrin; $7 \mathrm{~g} /$ day). Before (baseline) and after the intervention, the anthropometric (weight, height) and biochemical blood parameters (C-reactive protein, creatinine, aspartate aminotransferase, alanine aminotransferase), quantitative gut microbiota characteristics (by real-time PCR) and concentration of short-chain fatty acids (by gas chromatography with a flame ionization detector) were assessed.

Results: Thirty CD patients completed the study. After 12-weeks intervention, the biochemical blood parameters reminded normative in all CD patients, and the gut microbiota counts within eachexperimental group did not differ from their counts at baseline. However, in comparison with placebo group, Bifidobacterium counts was significantly $(\mathrm{p}<0.01)$ higher in CD children consuming OEI-supplemented GFD. Moreover, the counts of Clostridium leptum group in children of prebiotic group did not show the decreasing tendency along with the time of GFD as it was observed in placebo group. These changes were reflected intotal bacteria number after the intervention that was constant in prebiotic group but tended to fall in placebo group. Microbiota counts corresponded well with microbial metabolic activity. In comparison with placebo group, the concentration of short-chain fatty acids increased significantly in prebiotic group $(50.27$ vs. $69.95 \mu \mathrm{mol} / \mathrm{g}, \mathrm{p}<0.05)$, mainly due to a significantly higher acetate formation $(28.82$ vs. $44.06 \mu \mathrm{mol} / \mathrm{g}, \mathrm{p}<0.05)$.

Conclusion: A long-term administration of OEI in GFD prevents from a gut dysbiosis observed along with the duration of the GFD, and maintains a constant quantity of beneficial bifidobacteria. Moreover, the administered of OEI in GFD stimulates activityof gut microbiota observed asthe increased SCFA formation.

Disclosure of Interest: All authors have declared no conflicts of interest.

Acknowledgment: The research was supported by statutory funds of the Department of Chemistry and Biodynamics of Food of the Institute of Animal Reproduction and Food Research PAS. Travel expenses were funded by KNOW (Leading National Research Centre) Scientific Consortium: "Healthy Animal Safe Food" (decision of Ministry of Science and Higher Education No. 05-1/ KNOW2/2015).

\section{P0648 EVALUATING GLUTEN IMMUNOGENIC PEPTIDES AS NON-INVASIVE MARKER OF GLUTEN-FREE DIET ADHERENCE IN PAEDIATRIC CELIAC DISEASE}

A. Rodriguez-Herrera ${ }^{1}$, I. Comino ${ }^{2}$, V. Segura ${ }^{2}$, J. Diaz $^{3}$, A. Muñoz-Suano ${ }^{4}$, A. Cebolla ${ }^{4}$, C. Sousa ${ }^{2}$, The Deliac Group ${ }^{2}$

${ }^{1}$ Gastroenterology And Nutrition, Instituto Hispalense Pediatria, Seville/Spain

${ }^{2}$ Departamento de Microbiología y Parasitología, Facultad de Farmacia, Universidad de Sevilla, Sevilla/Spain

${ }^{3}$ Servicio De Análisis Clínicos., Hospital Universitario INGESA de Ceuta, Ceuta/ Spain

${ }^{4}$ Biomedal, Sevilla/Spain

\section{Contact E-mail Address: alfonsorodriguez@ihppediatria.com}

Introduction: Treatment for celiac disease (CD) is a lifelong strict gluten-free diet (GFD). Patients should be followed-up with dietary interviews and serology as $\mathrm{CD}$ markers to ensure adherence to the diet. However, none of these methods 
offer an accurate measure of dietary compliance. Presence of gluten related substances in faeces proves that transit through gastrointestinal tract happened and confirms gluten ingestion.

Aims \& Methods: Detection of gluten immunogenic peptides (GIP) in stools as a marker of GFD adherence in CD paediatric patients was evaluated and compared against traditional methods of GFD monitoring.

A prospective, non-randomized, multi-centre follow-up study, 2 years long, including $64 \mathrm{CD}$ patients started on GFD when diagnosed was conducted (age range $0-18$ years)

Fecal GIP was quantified by enzyme-linked immunosorbent assay (ELISA). Anti-tissue transglutaminase (anti-tTG) IgA and anti-deamidated gliadin peptide (anti-DGP) IgA antibodies were measured simultaneously, during basal and follow-up visits at 0,6,12 and 24 months. Correlations between fecal GIP and serum antibodies were stablished by Cochran's and Friedman tests.

Results: 62 patients $(97 \%)$ had detectable GIP levels in stools, during basal visit, before initiation of the GFD, whereas $20.3 \%$ of the patients were found to have positive GIP after treated with a GFD. Dietary transgressions were more frequent in children over 8 years of age. $46.1 \%$ of them showed more than one detected transgression. Anti-tTG IgA remained in high concentrations in 48, 34 and $20 \%$ of the patients at 6,12 and 24 months of follow-up. Anti-DGP was positive in $13,4.5$ and $0 \%$ of cases when tested at 6,12 and 24 months follow-up. Both serological methods did not correlate with GIP in stools $(\mathrm{p}<0.05)$.

Conclusion: The GIP ELISA enabled direct and quantitative assessment of gluten exposure early after ingestion. Detection of GIP in stools revealed lack of accuracy of traditional serological methods to verify GFD compliance in CD paediatric patients. The antibodies can take several months or even years to decrease after initiation of the GFD and reduction (but incomplete suppression) of gluten intake appeared to be sufficient to decrease antibodies in sera. Faecal GIP could be a useful tool: 1) on the diagnosis of $\mathrm{CD}$, to ensure that a sufficient amount of gluten has been ingested to allow a correct CD diagnosis. 2) on treatment, for monitoring of short-term and long-term GFD compliance. 3) on the differential diagnosis of unresponsive $\mathrm{CD}$ versus dietary non-compliance.

Disclosure of Interest: A. Rodriguez-Herrera: Co-author Gluten detection in human body fluids. Spain P201400569

A. Cebolla: Co-author Gluten detection in human body fluids. Spain P201400569 C. Sousa: Co-author Gluten detection in human body fluids. Spain P201400569 All other authors have declared no conflicts of interest.

\section{References}

Comino I, Real A, Vivas S, Síglez MA, Caminero A, Nistal E, Casqueiro J, Rodríguez-Herrera A, Cebolla A, Sousa C. Monitoring of gluten-free diet compliance in celiac patients by assessment of gliadin 33-mer equivalent epitopes in feces. Am J Clin Nutr 2012;95:670-7.

Moreno ML, Rodriguez-Herrera A, Sousa C, Comino I. Biomarkers to Monitor Gluten-Free Diet Compliance in Celiac Patients. Nutrients 2017;9:06.PMID: 28067823

Shan L, Qiao SW, Arentz-Hansen H, Molberg Ø, Gray GM, Sollid LM, Khosla C. Identification and analysis of multivalent proteolytically resistant peptides from gluten: Implications for Celiac Sprue. J Proteome Res 2005;4:1732-41.

\section{P0649 INTUSSUSCEPTION IN CHILDREN WITH NEWLY DIAGNOSED CELIAC DISEASE: PREVALENCE AND NATURAL HISTORY}

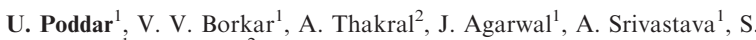
K. Yachha ${ }^{1}$, S. Kumar ${ }^{2}$

${ }^{1}$ Pediatric Gastroenterology, Sanjay Gandhi Post Graduate Institute of Medical Sciences, Lucknow/India

${ }^{2}$ Radiodiagnosis, Sanjay Gandhi Post Graduate Institute of Medical Sciences, Lucknow/India

\section{Contact E-mail Address: ujjalpoddar@hotmail.com}

Introduction: There are sporadic reports of occurrence of intussusception in celiac disease but no systematic study yet. The prevalence and natural history of intussusception in children with celiac disease is not known.

Aims \& Methods: We prospectively studied the prevalence and natural history of intussusception in newly diagnosed celiac disease in children. From January 2010 to October 2013, 150 children, diagnosed to have celiac disease on the basis of positive celiac serology (IgA tissue transglutaminase/anti-endomysial antibody) and duodenal biopsy showing villous atrophy, were prospectively recruited in this study. Abdominal ultrasonography was done before starting gluten-free diet and repeated in those who had intussusception, on day three and then weekly till the resolution of intussusception.

Results: A total 167 children were diagnosed to have new onset celiac disease during the study perios. Out of them 150 were recruited in the study and 17 were excluded; 15 did not get an ultrasonography before starting gluten-free diet and two children were consuming gluten-free diet prior to presentation to our hospital. The median age of 150 children with celiac disease was 72 (range, 16-204) months and $79(53 \%)$ were boys. Their median duration of symptoms was 18 months (range, 1-136 months). Diarrhea was presenting symptoms in $119(79 \%)$ of cases. A total 45 intussusceptions were detected in $37(24.7 \%)$ children. Majority $(86.5 \%)$ had single intussusception and the most common site was ileo-ileal $(46.7 \%)$, followed by jejuno-jejunal intussusception $(40 \%)$. Out of 45 intussusceptions, $43(95 \%)$ involved small bowel. All but one had asymptomatic intussusception. On gluten-free diet, intussusception resolved spontaneously within 7 days in $24(65 \%)$, within 14 days in $31(84 \%)$ and within 28 days in $34(92 \%)$ cases, and none required surgical or hydrostatic reduction. On univariate analysis, younger age, low weight $\mathrm{z}$ score, presentation with diarrhea, abdominal distension, rickets, lower serum albumin, more severe villous atrophy and refeeding syndrome were found to be significantly more common in children with intussusception as compared to children without intussusception. On multivariate analysis abdominal distension (Odds ratio: $3.9,95 \%$ Confidence Interval: 1.3-11.9) and hypoalbuminemia (Odds ratio: $1.8,95 \%$ Confidence Interval: 1.2 2.7) were found to be significantly associated with intussusception.

Conclusion: Intussusception is frequently $(25 \%)$ seen in children with newly diagnosed celiac disease, generally asymptomatic and resolves spontaneously on gluten-free diet. It is often associated with more severe disease. As spontaneous resolution is the rule for intussusception in the setting of pediatric celiac disease, attempt for reduction (either surgical or hydrostatic) should be deferred unless indicated otherwise.

Disclosure of Interest: All authors have declared no conflicts of interest.

\section{Reference}

Reilly NR, Aguilar KM, Green PH. Should intussusception in children prompt screening for celiac disease? J Pediatr Gastroenterol Nutr. 2013; 56: 56-59.

\section{P0651 META-ANALYSIS: PROTON PUMP INHIBITORS MODERATELY INCREASE THE RISK OF SMALL INTESTINAL BACTERIAL OVERGROWTH}

T. Su, S. Chen

Department Of Gastroenterology, Sir Run Run Shaw Hospital, Zhejiang

University, Institute of Gastroenterology, Zhejiang University, Hangzhou/China

Contact E-mail Address: 18770085122@163.com

Introduction: PPIs have become one of the most commonly prescribed classes of medications. ${ }^{1}$ Although PPIs are generally well tolerated, accumulating evidence suggests that PPIs have long-term risks. ${ }^{2}$ One potential risk of PPI use is development of small intestinal bacterial overgrowth (SIBO), which is defined as $>105$ bacteria colony-forming units (CFU) per $\mathrm{mL}$ upon culturing upper gut aspirates. However, this is controversial due to conflicting results from prior studies. ${ }^{3-8}$

Aims \& Methods: The aim of this meta-analysis was to evaluate the association between use of PPIs and the risk of SIBO. We systematically searched the online PubMed, Embase, Cochrane Library databases and Web of Science for relevant articles before November 2016. Two researchers identified and extracted data independently. The pooled analysis was performed using generic inverse-variance random-effects model. Subgroup and sensitivity analysis were conducted to assess the stability and heterogeneity of the pooled results. The risk of publication bias was evaluated by examining funnel plot asymmetry, Egger's test and Begg's test. All statistical analyses were performed using Stata software version 13.0

Results: A total of nineteen articles met the eligibility criteria for the meta-analysis. The pooled odds ratio (OR) showed a statistically significant association between increased risk of SIBO and PPI use (OR $=1.7195 \% \mathrm{CI}: 1.20-2.43)$. No statistically significant publication bias was found based on the Egger's test $(p=0.149)$ or Begg's test $(p=0.115)$. Furthermore, subgroup analyses demonstrated an association between SIBO and PPI use in studies that employed small bowel aspirates culture and glucose hydrogen breath tests (GHBT) as diagnostic tests for SIBO. However, when the pooled analysis was limited to studies recruiting irritable bowel syndrome (IBS) patients as study population, no statistically significant association between PPI use and SIBO was observed, which suggests that PPI use does not affect the risk of SIBO among IBS patients.

Conclusion: Our meta-analysis suggests that the use of PPI moderately increases the risk of SIBO, which highlights the need for appropriate prescription of PPIs. Disclosure of Interest: All authors have declared no conflicts of interest.

\section{References}

1. M, B. Top 200 prescription drugs of 2009. Pharmacy Times (2010).

2. Schnoll-Sussman, F. \& Katz, P. O. Clinical Implications of Emerging Data on the Safety of Proton Pump Inhibitors. Curr Treat Options Gastroenterol 15, 1-9, doi:10.1007/s11938-017-0115-5 (2017).

3. Giamarellos-Bourboulis, E. J., Pyleris, E., Barbatzas, C., Pistiki, A. \& Pimentel, M. Small intestinal bacterial overgrowth is associated with irritable bowel syndrome and is independent of proton pump inhibitor usage. $B M C$ Gastroenterology 16 (2016).

4. Enko, D. \& Kriegshauser, G. Functional 13C-urea and glucose hydrogen/ methane breath tests reveal significant association of small intestinal bacterial overgrowth in individuals with active Helicobacter pylori infection. Clinical biochemistry, doi:10.1016/j.clinbiochem.2016.08.017 (2016).

5. Schatz, R. A., Zhang, Q., Lodhia, N., Shuster, J., Toskes, P. P. \& Moshiree, B. Predisposing factors for positive D-Xylose breath test for evaluation of small intestinal bacterial overgrowth: a retrospective study of 932 patients. World J Gastroenterol 21, 4574-4582, doi:10.3748/wjg.v21.i15.4574 (2015).

6. Franco, D. L., Disbrow, M. B., Kahn, A. et al. Duodenal Aspirates for Small Intestine Bacterial Overgrowth: Yield, PPIs, and Outcomes after Treatment at a Tertiary Academic Medical Center. Gastroenterology research and practice 2015, 971582, doi:10.1155/2015/971582 (2015).

7. Jacobs, C., Coss Adame, E., Attaluri, A., Valestin, J. \& Rao, S. S. C. Dysmotility and proton pump inhibitor use are independent risk factors for small intestinal bacterial and/or fungal overgrowth. Alimentary Pharmacology and Therapeutics 37, 1103-1111 (2013). 
8. Ratuapli, S. K., Ellington, T. G., O'Neill, M. T. et al. Proton pump inhibitor therapy use does not predispose to small intestinal bacterial overgrowth. The American journal of gastroenterology 107, 730-735, doi:10.1038/ajg.2012.4 (2012).

\section{P0652 CLINICAL SIGNIFICANCE OF TRANSFORMING GROWTH FACTOR - B1 AND TUMOR NECROSIS FACTOR - A IN CHILDREN WITH FOOD PROTEIN INDUCED ENTEROCOLITIS SYNDROME}

O. T. Kamilova ${ }^{1}$, A. N. Aripov ${ }^{2}$, S. S. Sultankhodjaeva ${ }^{1}$, S. I. Geller ${ }^{1}$, D. X. Dustmuxamedova ${ }^{1}$, Z. S. Xudoyorova ${ }^{2}$, M. Rustamova ${ }^{3}$

${ }^{1}$ Gastroenterology, Republican Specialized Scientific-Practical Medical Center of Pediatrics, Tashkent/Uzbekistan

${ }^{2}$ Laboratory, Republican Specialized Scientific-Practical Medical Center of

Pediatrics, Tashkent/Uzbekistan

${ }^{3}$ Tashkent Medical Academy, Tashkent/Uzbekistan

Contact E-mail Address: okamilova@mail.ru

Introduction: Nowadays food allergy continue to increase, especially in westernized countries and is now recognized as a worldwide problem. Transforming growth factor- $\beta 1$ (TGF- $\beta 1$ ) is a profibrotic cytokine, which plays an important role in promoting the structural changes in food allergy. Also for patients with food protein - induced enterocolitis syndrome TNF- $\alpha$ appears to have an important role.

Aims \& Methods: The aim was to determine the significance of the Transforming Growth Factor - $\beta 1($ TGF $-\beta)$ and Tumor Necrosis Factor $-\alpha($ TNF $-\alpha)$ in children with food protein induced enterocolitis syndrome. It was examined 38 patients with FPIES at the age from 4 months to 3 years, the average age was $19 \pm 4$ months. The control group consisted of 11 healthy children of the same age. The determination of TGF- $\beta 1$ and TNF $-\alpha$ in serum was performed by an enzyme-linked immunosorbent assay kits from Bender Medsystem (Austria).

Results: The level of TGF- $\beta 1$ in patients with FPIES exceeded the norm and was respectively $33.5+1.6 \mathrm{ng} / \mathrm{ml}$, at norm $20.2+2.1 \mathrm{ng} / \mathrm{ml}, \mathrm{p}<0.001$. The indices of TNF $-\alpha$ were also increased and amounted to $8.8+1.3 \mathrm{ng} / \mathrm{ml}$ in comparison with the norm $4.2+0.67 \mathrm{ng} / \mathrm{ml} \mathrm{p}<0.001$. For our patients it was characterized by an increase in specific antibodies IgE to cow's milk in $18(47.3 \%)$ children. In these cases disease was more severely with vivid clinical manifestations (indomitable vomiting, stool with blood, expressed weight deficit). Also among of patients in this group TGF- $\beta 1$ level was significantly higher than in the control group, amounting to $42,01 \pm 7.5 \mathrm{ng} / \mathrm{ml}$. TNF - $\alpha$ indicators were also increased: $11 \pm 1.2 \mathrm{ng} / \mathrm{ml}$

Conclusion: It is believed that TGF - $\beta 1$ inhibits allergic inflammation, but in our observations, an increase in its values was established, especially in the group of children with high specific IgE levels to cow's milk. It is likely that an increase in TGF - $\beta 1$ stimulates the release of TNF - $\alpha$, which supports chronic inflammation in allergic diseases.

Disclosure of Interest: All authors have declared no conflicts of interest

\section{P0653 THE ROLE OF GTS1 \& GTM1 GENE POLYMORPHISMS IN NEWBORNS' OBESITY RISK}

C.O. Marginean ${ }^{1}$, C. Marginean ${ }^{2}$, L.E. Melit ${ }^{1}$, C. Banescu ${ }^{3}$, M.O. Marginean ${ }^{1}$ Pediatrics I, University of Medicine and Pharmacy Tirgu Mures, Targu Mures/ Romania

${ }^{2}$ Obstetrics \& Gynecology, University of Medicine and Pharmacy Tirgu Mures, Targu Mures/Romania

${ }^{3}$ Genetics, University of Medicine and Pharmacy Tirgu Mures, Targu Mures/ Romania

Contact E-mail Address: marginean.oana@gmail.com

Introduction: Newborns' birth weight is influenced by maternal factors (mother's weight at the onset of pregnancy, gestational weight gain - GWG), genetic, obstetrical and environmental factors, but also socio-economical ones. Excessive GWG, mother's increased BMI and the adipose tissue mass determine a bigger birth weight and increase the risk of newborns' obesity. Glutathione Stransferases (GSTs) is an oxidative stress-related gene which is associated with and ist complications.

Aims \& Methods: The aim of the study was to investigate the role of mother-child GTS1 and GTM1 polymorphisms as independent risk factors for newborn's weight, but also to establish correlations between these polymorphisms and anthropometrical parameters, and bioimpedance (BIA) ones, respectively. We assessed the anthropometrical parameters in both mothers and their newborns (BMI- body mass index, MUAC - medium upper arm circumference, TST tricipital skin thickness, weight - W), BIA parameters in mothers, but we also determined the clinical, paraclinical and genetic parameters in both mothers and newborns.

Methods: We performed a cross-sectional study on 202 mothers and their newborns in a Clinic of neonatology \& Gynecology and Obstetrics from Romania. Results: We noticed that in newborns with $\mathrm{W}>3000$ gr there was a significant statistical correlation between weight and mother's GST1 polymorphism $(\mathrm{p}=0.046)$, birth at term $(\mathrm{p}<0.001)$, with mother's percentage of fat mass assessed by BIA $(\mathrm{p}<0.001)$, and multi parity, respectively $(\mathrm{p}<0.001)$. We obtained a tendency towards correlations between $\mathrm{W}>3000 \mathrm{gr}$ and basal metabolism rate $(\mathrm{p}=0.063)$, and GWG seemed to be a protective factor for this $\mathrm{W}$ $(p=0.072)$. We also found that GSM1 in newborns was a risk factor with tendency towards statistical significance in newborns with increased birth weight. We did not find any interaction effect between newborns' and mothers' GST1 and GSM1 polymorphisms and anthropometrical parameters $(\mathrm{p}=0.545$ for MUAC, and 0.664 for TST), or paraclinical parameters.

Conclusion: Mother's GSTM1 is an independent risk factor for newborns' W $>3000 \mathrm{gr}$, while mother's GWG seems to be a protective factor for $\mathrm{W}>3000$ gr. Further studies are needed in order to determine the clear role of this polymorphisms in newborns' obesity risk. This research was supported by the Research Grants of the University of Medicine and Pharmacy Tîrgu Mures, Romania - "The role of genetic determinism of the mother in child's obesity correlated with measurements of bioimpedance and anthropometry" no.275/4 11.01.2017.

Disclosure of Interest: All authors have declared no conflicts of interest.

\section{References}

1. Bessa SS, Ali EMM, Hamdy SM. The role of glutathione S- transferase M1 and $\mathrm{T} 1$ gene polymorphisms and oxidative stress-related parameters in Egyptian patients with essential hypertension. Eur J Intern Med. 2009;20(6):625-630. doi:10.1016/j.ejim.2009.06.003.

2. Almoshabek HA, Mustafa M, Al-Asmari MM, Alajmi TK, Al-Asmari AK. Association of glutathione S-transferase GSTM1 and GSTT1 deletion polymorphisms with obesity and their relationship with body mass index, lipoprotein and hypertension among young age Saudis. JRSM Cardiovasc Dis. 2016:5:2048004016669645 doi:10.1177/2048004016669645.

3. Afrand M, Bashardoost $\mathrm{N}$, Sheikhha $\mathrm{MH}$, Afkhami-Ardekani M. Association between Glutathione S-Transferase GSTM1-T1 and P1 Polymorphisms with Metabolic Syndrome in Zoroastrians in Yazd, Iran. Iran J Public Health. 2015;44(5):673-682.

4. Wang G, Zhang L, Li Q. Genetic polymorphisms of GSTT1, GSTM1, and NQO1 genes and diabetes mellitus risk in Chinese population. Biochem Biophys Res Commun. 2006;341(2):310-313. doi:10.1016/j.bbrc.2005.12.195.

\section{P0654 SARCOPENIA IN CHILDREN WITH INTESTINAL TRANSPLANTATION}

R. Hegarty, J. Hind, D. Thangarajah

Paediatric Liver, GI And Nutrition Centre, King's College Hospital, London/ United Kingdom

Contact E-mail Address: anthiburtt@gmail.com

Introduction: Deficits in lean mass and muscle measures are well described in children with chronic disease e.g. childhood inflammatory bowel disease[1]. Sarcopenia is a poor prognostic biomarker in adults with advanced cancer, liver transplantation and in children with acute lymphoblastic leukaemia [2-4]. Psoas muscle cross sectional area (PCA) has been shown to correlate with whole body muscle mass and can be measured from axial imaging [5]. Little is known about sarcopenia in children with intestinal transplantation (IT). Sarcopenia is a poor prognostic biomarker in adults with advanced cancer, liver transplantation and in children with acute lymphoblastic leukaemia. Psoas muscle cross sectional area (PCA) has been shown to correlate with whole body muscle mass and can be measured from axial imaging [5]. Little is known about sarcopenia in children with intestinal transplantation (IT)

Aims \& Methods: The primary objective was to determine whether children who had IT show differences in total PCA when compared to healthy controls. The secondary objective was to investigate association of PCA and survival after IT. A retrospective, case note review of children who had IT at a single centre since inception in 2009 to May 2016. Controls were identified from abdominal trauma series. Total PCA $\left(\mathrm{mm}^{2}\right)$ was measured using direct techniques of magnetic resonance imaging or computed tomography at the level of the anterior superior iliac spine. To correct for body size, PCA index was derived for all subjects: PCA divided by height. PCA index was then described according to outcome. Statistical analysis was carried out using Social Sciences (SPSS) version 23. Results: 16 patients (9 male) underwent IT at our centre. Post-transplant axial imaging was available for 12 (6 males), median age 6.2[3.6 to 12.91 ] years patients in whom the diagnoses (n): Chronic intestinal pseudo-obstruction(3), gastroschisis(3), intestinal ischaemia(1), intestinal lymphangiectasia (1), volvulus (1), progressive familial intrahepatic cholestasis (1), Hirschsprung's disease (1) and intestinal failure of indeterminate aetiology(1). One patient was excluded from analysis as she was a bilateral amputee. Children who had IT had a significantly lower PCA and PCA index than controls; median PCA index $\left(\mathrm{x}_{10}^{-3} \mathrm{~mm}^{2}\right)[\mathrm{IQR}]$ in IT vs controls; $4.71[3.68$ to 6.10$]$ vs 9.55 [8.44 to 11.33$], \mathrm{p}<0.05$. There was a trend toward higher PCA index in those who survived $(n=9)$ compared to those who did not (2).

Conclusion: Children who underwent IT had sarcopenia of the psoas muscle in comparison to healthy controls. In IT patients who died there was a trend toward psoas muscle sarcopenia. This study adds to the evidence that body core muscle is consistently deficient in children with chronic disease and is the first to comment on children with IT. This small study provides the basis to develop PCA as a prognostic marker in children with transplantation.

Disclosure of Interest: All authors have declared no conflicts of interest.

\section{References}

1. Thangarajah, D., et al., Systematic review: Body composition in children with inflammatory bowel disease. Aliment Pharmacol Ther, 2015. 42(2): p. $142-57$.

2. Rayar, M., et al., Sarcopenia in children with acute lymphoblastic leukemia. J Pediatr Hematol Oncol, 2013. 35(2): p. 98-102.

3. Englesbe, M.J., et al., Sarcopenia and mortality after liver transplantation. $J$ Am Coll Surg, 2010. 211(2): p. 271-8. 
4. Fukushima, H., et al., Sarcopenia as a prognostic biomarker of advanced urothelial carcinoma. PLoS One, 2015. 10(1): p. e0115895.

5. Morrell, G.R., et al., Psoas Muscle Cross-sectional Area as a Measure of Whole-body Lean Muscle Mass in Maintenance Hemodialysis Patients. $J$ Ren Nutr, 2016. 26(4): p. 258-64.

\section{P0655 GENETIC PREDISPOSITION TO PRIMARY LACTOSE INTOLERANCE AND ITS INFLUENCE ON CHILDREN'S QUALITY OF LIFE AND DAIRY INTAKE}

C. Pienar ${ }^{1}$, E. Seclaman ${ }^{2}$, M. Lazarescu ${ }^{1}$, R. Costachescu ${ }^{1}$, S.A. Popescu ${ }^{3}$, I. Sporea ${ }^{4}$, I. Ciuca ${ }^{1}$, L. Pop ${ }^{1}$

${ }^{1}$ Pediatrics Department, "Victor Babes" University of Medicine and Pharmacy, Timisoara/Romania

${ }^{2}$ Biochemistry, "Victor Babes" University of Medicine and Pharmacy, Timisoara/ Romania

${ }^{3}$ Gastroenterology And Hepatology, "Victor Babes" University of Medicine, Timisoara/Romania

${ }^{4}$ Gastroenterology And Hepatology, "Victor Babes" University of Medicine and Pharmacy Timisoara, Timisoara/Romania

\section{Contact E-mail Address: cpienar@gmail.com}

Introduction: Primary lactose intolerance (PLI) is a frequent condition caused by a genetically programmed and progressive loss of lactase expression. It is considered that PLI is the ancestral variant, while lactase persistence is caused by 2 polymorphisms: the dominant C/T13910 and G/A22018. Homozygotes (CC or GG) have undetectable lactase levels. In clinical practice only half of people with PLI have symptoms. However, some studies showed that PLI subjects have lower dairy intake.

Aims \& Methods: To investigate whether genetic predisposition to PLI influences the quality of life and dairy intake in a group of Romanian children. We conducted a prospective study, recruiting consecutive children evaluated in our unit in May-August 2016. Our study population included 87 children aged 6-17 years (mean age $10.64 \pm 3.51$ years), $45(51.72 \%)$ girls. We used strip genotyping to identify genetic predisposition to IPL. Subjects were asked to complete a validated quality of life questionnaire and a dairy intake questionnaire. We used Spearman's test to evaluate the correlation between IPL and quality of life and dairy intake.

Results: $(51.7 \%)$ subjects had a CC genotype. $30(34.5 \%)$ subjects had a GG genotype. Our results were consistent with Hardy-Weinberg equilibrium. We found no correlation between homozygosity for PLI and dairy intake (CC: $\mathrm{r}=-0.06, \mathrm{p}=0.54 ; \mathrm{GG}: \mathrm{r}=-0.01, \mathrm{p}=0.86$ ). We found no correlation between either CC, or GG homozygosity and quality of life $(\mathrm{r}=-0.11, \mathrm{p}=0.3$ and $\mathrm{r}=-0.1, \mathrm{p}=0.34)$.

Conclusion: In our group genetic predisposition to IPL followed European trends. It did not influence quality of life and dairy intake.

Disclosure of Interest: C. Pienar: This work was supported by an internal grant of

"Victor Babes" University of Medicine and Pharmacy, PII-C4-TC-2016-08

All other authors have declared no conflicts of interest.

TUESDAY, OCTOBER 31, 2017

09:00-17:00

LIVER \& BILIARY II - HALL 7

\section{P0656 HYPOXIA CAUSES HEPATIC STELLATE CELLS \\ ACTIVATION IN THE ABSENCE OF CUX1}

E. Becker ${ }^{1}$, P. Di Fazio ${ }^{1}$, J. Hänze ${ }^{2}$, T.M. Gress ${ }^{3}$, D. Bartsch ${ }^{1}$, T

T. Wissniowski ${ }^{3}$

${ }^{1}$ Department Of Visceral Thoracic And Vascular Surgery, Philipps University

Marburg, Marburg/Germany

${ }^{2}$ Department Of Urology And Pediatric Urology, Philipps University Marburg, Marburg/Germany

${ }^{3}$ Klinik Für Gastroenterologie, Endokrinologie, Stoffwechel Und Infektiologie,

Philipps Universität Marburg, Marburg/Germany

\section{Contact E-mail Address: eva-mareike.becker@t-online.de}

Introduction: CUX1 (CUTL1) is a transcription factor belonging to homeobox proteins. It is responsible of driving the transcription of genes deputed to many cellular functions like proliferation, differentiation and cell death. It has been shown that its role can change and drive tumorigenesis. Up to now, its role is unknown in hepatic stellate cells. W

Aims \& Methods: We focused on CUX1 activity in hepatic stellate cells undergoing hypoxic stress. LX-2 cells were treated with $100 \mathrm{ng} / \mathrm{ml} \mathrm{CoCl} 2$ or kept at $37^{\circ}$ $\mathrm{C}$ at low oxygen $(<0.5 \%)$. Expression of hypoxia markers and activation markers was performed by RT-qPCR. Western blotting was performed to analyze the protein level of CUX1 and HIF-1alpha. Transfection with plasmid containing a promoter sequence for HIFlalpha was performed simultaneously with CUX1 knock-down.

Results: LX-2 cells treated for 6 hours with $\mathrm{CoCl} 2$ or low oxygen showed an overexpression or a restoration of COL1A1 and ACTA2 after knock down of CUX1. Additionally, CDKN1A, CDKN1B, VEGFA and HIF1alpha were up-regulated in LX.2 cells previously transfected with siCUX1. Protein level of CUX1 was significantly down-regulated, whereas HIFlalpha protein was strongly up-regulated by hypoxia condition. Transcriptional activity of HIF1alpha is not correlated with CUX1 expression.

Conclusion: CUX1 controls the activation of hepatic stellate cells. Its knock down promotes the hypoxia response. CUX1 could represent a key factor for controlling liver fibrogenesis. Its role in a liver fibrosis scenario needs to be further investigated.

Disclosure of Interest: All authors have declared no conflicts of interest.

P0657 HEPATIC FIBROBLAST GROWTH FACTOR-21 AND OMENTIN-1 MRNA LEVELS IN MORBIDLY OBESE WOMEN WITH NON-ALCOHOLIC FATTY LIVER DISEASE

M. Kukla ${ }^{1}$, M. Waluga ${ }^{1}$, M.Ż̈rniak ${ }^{2}$, M. Kajor ${ }^{1}$, t. Liszka ${ }^{1}$, M. Dyaczyński ${ }^{3}$, G. Kowalski ${ }^{4}$, R.J. Buldak ${ }^{1}$, M. Hartleb ${ }^{1}$

${ }^{1}$ Medical University of Silesia, Katowice/Poland

${ }^{2}$ Department Of Gastroenterology And Hepatology, Medical University of Silesia, Katowice/Poland

${ }^{3}$ Municipal Hospital, Siemianowice Ślaskie/Poland

${ }^{4}$ Medical University of Silesia, Bytom/Poland

Contact E-mail Address: mwaluga@poczta.onet.p

Introduction: Fibroblast growth factor-21 (FGF21) and omentin-1 have been recognized as potent antidiabetic agents, with potential hepatoprotective activity. Aims \& Methods: The aim of this study was to evaluate hepatic FGF21 and omentin-1 mRNA expression, and their serum levels as predictive markers of liver injury and insulin resistance in morbidly obese women with NAFLD. The study included 56 severely obese women who underwent intraoperative wedge liver biopsy during the bariatric surgery. Hepatic FGF21 and omentin-1 mRNA was assessed by quantitative real-time PCR, while their serum concentration with commercially available enzyme-linked immunosorbent assays.

Results: FGF21 serum level was significantly higher in patients with more extent steatosis (grade 2 and 3 ) compared to those without or with mild steatosis (grade 0 and 1$)(\mathrm{p}=0.049)$. However, ROC analysis showed poor discriminant power for FGF21 serum level in differentiation between more and less extensive steatosis with $\mathrm{AUC}=0.666$. There was evident tendency to higher levels of hepatic FGF21 mRNA in patients with lobular inflammation and fibrosis, and to lower levels in the case of ballooning degeneration and steatosis. There was positive mutual correlation between hepatic FGF21 and omentin-1 mRNA levels $(\mathrm{r}=0.73 ; \mathrm{p}<0.001)$. Fibrosis stage was associated with serum glucose and HOMA-IR ( $\mathrm{p}=0.03$ and $\mathrm{p}=0.02$, respectively). Serum omentin was not associated with histopathological features. Hepatic omentin-1 mRNA levels exerted the tendency to be lower in patients with advanced steatosis and hepatocyte ballooning.

Conclusion: In conclusion our study, which focused on hepatic FGF21 and omentin-1 mRNA expression, confirmed a marked expression of both molecules in the liver of morbidly obese patients with NAFLD. mRNA levels were affected by histopathological abnormalities. More extent steatosis was associated with evident change in serum FGF21 concentration in morbidly obese women with NAFLD. The vast amount of fat, both visceral and subcutaneous in severely obese patients may affect FGF21 and omentin-1 serum levels.

Disclosure of Interest: All authors have declared no conflicts of interest.

\section{References}

Kukla M, Mazur W, Bułdak RJ, Żvirska-Korczala K. Potential role of leptin, adiponectin and three novel adipokines - visfatin, chemerin and vaspin - in chronic hepatitis. Mol Med 2011; 17:1397-1410 [PMID: 21738955PMCID: PMC3321801 DOI: 10.2119/molmed.2010.00105]

Kukla M, Waluga M, Adamek B, et al. Omentin serum concentration and hepatic expression in chronic hepatitis C patients - together or apart? Pol J Pathol 2015; 66 (3): 231-238 [PMID: 26619101]

Kukla M, Berdowska A, Stygar D, et al. Serum FGF21 and RBP4 levels in patients with chronic hepatitis C. Scandinavian Journal of Gastroenterology 2012; 47: 1037-1047 [PMID: 22670657 DOI: 10.3109/00365521.2012.694901] Eisinger K, Krautbauer S, Wiest R, et al. Portal vein omentin is increased in patients with liver cirrhosis but is not associated with complications of portal hypertension. Eur J Clin Invest. 2013;43:926-932.

Dushay J, Chui PC, Gopalakrishnan GS, et al. Increased fibroblast growth factor 21 in obesity and nonalcoholic fatty liver disease. Gastroenterology 2010; 139 (2): 456-463 [PMID: 20451522 PMCID: PMC4862867 DOI: $10.1053 /$ j.gastro.2010.04.054]

Kukla M, Waluga M, Żrniak M, et al. Serum omentin and vaspin levels in cirrhotic patients with and without portal vein thrombosis. World J Gastroenterol 2017; 23(14): 2613-2624 DOI: 10.3748/wjg.v23.i14.2613

\section{P0658 LOUREIRIN B INHIBITS THE PROLIFERATION OF HEPATIC STELLATE CELLS VIA DOWN-REGULATING FRIZZLED- 4}

Z.

Dept. Of Gastroenterology, First Peoplés Hospital, Kunming/China

Contact E-mail Address: song4715@163.com

Introduction: Liver fibrosis is the result of repeated healing repair and interstitial reconstruction after chronic liver injury. Activation of hepatic stellate cells represents a critical event in fibrosis because these cells become the primary source of extracellular matrix in liver upon injury. Inhibiting HSCs' activation, proliferation, extracellular matrix production and promoting HSCs' apoptosis are the important therapeutic approaches of liver fibrosis.

Aims \& Methods: We aimed to investigate the anti-liver fibrosis ability of loureirin $\mathrm{B}$ and the molecular mechanisms involved it. After hepatic stellate cells 
(HSCs), which were separated from Sprague-Dawley rat, were treated with different concentrations of loureirin B, MTT assay was employed to determine HSCs proliferation, western blot were used to test the expressions of Frizzled-4 receptor protein and $\alpha$-SMA. In addition, enzyme-linked immunosorbent assay (ELISA) was performed to measure the content of $\alpha$-SMA, TGF- $\beta 1$ and VEGF in the cultured HSCs' supernatant, and reverse-transcription PCR (RT-PCR) were utilized to detect the expressions of Frizzled- 4 and $\alpha$-SMA genes.

Results: MTT test showed that the proliferation of HSCs was inhibited significantly with a time and dose dependent relationship by the treatment of loureirin $B$ with the half maximal inhibitory concentrations of $0.180 \mu \mathrm{g} / \mu \mathrm{L}$ $\left(\mathrm{IC}_{50}=0.180 \mu \mathrm{g} / \mu \mathrm{L}\right)$. Western blot analysis showed that the expressions of Wnt receptor Frizzled-4 protein and $\alpha$-SMA were obviously lower in the group of loureirin B treatment than that in the control group. Moreover, the Loureirin B also inhibited $\alpha$-SMA $(\mathrm{p}<0.01)$, TGF $\beta 1(\mathrm{p}<0.01)$ and VEGF $(\mathrm{p}<0.05)$ secretion in the cultured HSCs' supernatant in different degree by the ELISA assay, and RT-PCR results revealed that Loureirin B down-regulated the expressions of Frizzled-4 and $\alpha$-SMA genes in the level of mRNA

Conclusion: CONCLUSIONS: The Loureirin B mediated anti-hepatic fibrosis by inhibiting the proliferation of HSCs through restraining the Wnt signaling pathway.

Disclosure of Interest: All authors have declared no conflicts of interest.

\section{References}

1. Hernandez-Gea V, Friedman SL. Pathogenesis of liver fibrosis[J]. Annu Rev Pathol, 2011, 6:425-456.

2. Bataller R, Brenner D A. Liver fibrosis[J]. The Journal of Clinical Investigation, 2005, 115:208-218.

3. et al. Rosmarinic acid and baicalin epigenetically derepress peroxisomal proliferator-activated receptor $\gamma$ in hepatic stellate cells for their antifibrotic effect[J]. Hepatology, 2012, 55(4):1271-1281.

\section{P0659 MACROPHAGE CONTRIBUTES TO STEATOHEPATITIS THROUGH MEDIATING INFLAMMATORY CYTOKINES, AUTOPHAGY AND THE CROSSTALK WITH HEPATOCYTES}

J.K.C. Lau ${ }^{1}$, X. Zhang ${ }^{2}$, E.S. Chu ${ }^{2}$, J. Yu ${ }^{2}$

${ }^{1}$ Faculty Of Medicine, Shho College, the Chinese University of Hong Kong, Hong Kong/Hong Kong PRC

${ }^{2}$ Institute Of Digestive Disease And Department Of Medicine And Therapeutics,

The Chinese University Of Hong Kong, Hong Kong/Hong Kong PRC

Contact E-mail Address: jennie_lau@outlook.com

Introduction: Macrophages play a pivotal role in the pathogenesis of non-alcoholic steatohepatitis (NASH) and are a major component of inflammatory cells infiltrated in NASH. However, the precise mechanism of how macrophages contribute to the pathogenesis of NASH remains unexplored.

Aims \& Methods: We aimed to characterize the role and molecular regulators of macrophages in NASH and the therapeutic effects of macrophage depletion on NASH. C57BL/6 wildtype (WT) mice and transgenic LysM-Cre/DTR mice were fed with methionine-and-choline-deficient (MCD) diet for 5 weeks to induce steatohepatitis. Hepatic macrophages were depleted in WT mice by injecting liposomal clodronate (i.p. $50 \mathrm{mg} / \mathrm{kg} /$ week) and in $L y s M-C r e / D T R$ mice by injecting diphtheria-toxin (DTox) (i.p. $100 \mathrm{ng} / \mathrm{mice}$ ). Primary macrophages were isolated from bone marrow of WT mice. For the in vitro study, mouse immortalized hepatocytes AML-12 were cultured with primary macrophage conditioned medium and mouse primary macrophages were cultured with AML-12 hepatocytes conditioned medium to evaluate the interaction between macrophages and hepatocytes in steatohepatitis. A series of assays including cytokine profiling assay, DNA binding activity, flow cytometry and Western blot were performed. Results: Hepatic macrophage marker CD68 expression was significantly higher in human NASH patients compared with normal controls $(P<0.001)$ Steatohepatitis was established in WT mice and LysM-Cre/DTR mice fed MCD diet, concomitant with significantly enhanced hepatic macrophage infiltration as indicated by F4/80 staining. Macrophage depletion by liposomal clodronate or DTox attenuated steatohepatitis in both animal models. This was also associated with reduced hepatic necroinflammation, oxidative stress, hepatic triglyceride accumulation, and secretion of pro-inflammatory cytokines in both liposomal clodronate-treated WT mice and DTox-treated lys M-Cre/DTR mice as compared to the corresponding control mice. Macrophage depletion was also accompanied by the reduction of neutrophils, which together can reduce inflammation. Upregulated macrophages were associated with the increased expression of hepatic pro-inflammatory cytokines including interleukin (IL)-1a, IL-1b, IL12, IL-17, Granulocyte-macrophage colony-stimulating factor, Monocyte chemoattractant protein-1 (MCP-1) and macrophage inflammatory protein 1a (MIP-1a), and the activation of NF- $\kappa$ B and JNK signaling pathways in the liver. Macrophage-induced steatosis was mediated by increased hepatic lipogenesis through inducing liver $\mathrm{X}$ receptors $(\mathrm{LXR} \alpha$ and $\mathrm{LXR} \beta)$, sterol regulatory element binding protein-1c and carbohydrate-responsive element-binding protein Moreover, autophagy deficiency and endoplasmic reticulum (ER) stress were involved in macrophage-induced steatohepatitis as indicated by p62/SQSTM1 accumulation and increased GRP78 and p-IRE1a expression. For the in vitro study, macrophage conditioned medium significantly promoted lipid accumulation in AML-12 hepatocytes. On the other hand, MCD-cultured hepatocytes medium promoted primary macrophage polarization to M1 phenotype as well as the production of pro-inflammatory cytokines (TNF-a and MCP-1).

Conclusion: We demonstrated that macrophages contribute to the progression of NASH through promoting inflammation, lipogenesis, autophagy impairment, ER stress and also through a crosstalk with hepatocytes. Similarly, macrophage depletion has mitigation effects on NASH and may provide a potential treatment approach of steatohepatitis.

Disclosure of Interest: All authors have declared no conflicts of interest.

\section{P0660 NEWLY SYNTHESIZED BILE ACID DERIVATIVES PREVENT LIVER STEATOSIS IN VITRO THROUGH TARGETING OF NR1 SUBFAMILY NUCLEAR RECEPTORS}

H.M.D.S. Brito ${ }^{1}$, M. Batista ${ }^{2}$, M. Silva ${ }^{2}$, J. Salvador ${ }^{3}$, R. E. Castro ${ }^{1}$, C.M.P. Rodrigues

${ }^{1}$ Research Institute for Medicines (iMed.ULisboa), Faculty of Pharmacy, Universidade de Lisboa, Lisboa/Portugal

${ }^{2}$ Faculty of Pharmacy, University of Coimbra, Coimbra/Portugal

${ }^{3}$ Center for Neuroscience and Cell Biology, University of Coimbra, Coimbra/ Portugal

Contact E-mail Address: hugobrito1@gmail.com

Introduction: Non-alcoholic fatty liver disease (NAFLD) is considered the hepatic manifestation of metabolic syndrome, with simple liver steatosis being capable of gradually progressing to inflammation, fibrosis, cirrhosis and even hepatocellular carcinoma. Still, disease pathogenesis is complex and no targeted therapies have yet been approved for NAFLD. Bile acids (BAs) constitute a wide class of steroid molecules with pleiotropic functions, contributing to the homeostasis of lipids and glucose. In the liver, they specifically modulate nuclear receptors from the NR1 subfamily, such as Farnesoid X Receptor (FXR) and Liver X Receptor (LXR), thus tightly regulating bile acid synthesis and oxidation and storage of triglycerides.

Aims \& Methods: Our aim was to screen BA derivatives for their potential to selectively activate FXR, thus protecting liver cells against free fatty acid (FFA)induced lipid accumulation and lipotoxicity. Nineteen novel BA derivatives were analyzed by in silico molecular docking studies for FXR binding affinity, further evaluated in human cells using a FXR reporter assay. Assessment of FXR-dependent gene and protein expression was analyzed upon incubation of primary mouse hepatocytes and HepG2 cells with selected BA derivatives. In parallel, BA derivatives were co-incubated with oleic and palmitic acids (2:1) for assessment of cellular cytotoxicity and intracellular lipid accumulation.

Results: From the compound library, five BA derivatives showed stronger activation of FXR, comparing with their natural precursors. Incubation of HepG2 cells with FFAs led to a $\sim 25 \%$ reduction in cell viability and $\sim 35 \%$ increase in cell death, with a dose-dependent accumulation of lipid droplets. Pre-incubation of cells with selected derivatives efficiently prevented FFA-induced cell death and lipid accumulation. Finally, incubation of both HepG2 cells and primary mouse hepatocytes with BA derivatives strongly induced FXR, RXR, SHP, BSEP, FGF19 and VLDLR mRNA levels, and repressed PARP1, LXR, SREBP1-c and CYP7a1 mRNA expression. Molecular docking studies and FXR reporter assays confirmed ligand affinity to FXR. Furthermore, chenodeoxycholic acid (CDCA)-based derivatives were potent activators of FXR at lower concentrations comparing with the parent molecule.

Conclusion: In conclusion, we identified novel BA derivatives that directly modulate liver nuclear receptors, such as FXR and LXR, thus protecting liver cells against lipotoxicity and preventing FFA accumulation. These new molecules may be used as scaffolds for the development of targeted therapies for NAFLD.

Disclosure of Interest: All authors have declared no conflicts of interest.

(Supported by PTDC/BIM-MEC/0895/2014, SFRH/BD/110672/2015, and SFRH/BD/80975/2011, FCT, Portugal).

\section{P0661 DISSECTING AUTOPHAGY IN A NAFL/NASH MOUSE AND} HUMAN MODEL

S. C.H. Polte ${ }^{1}$, T. T. Wissniowski ${ }^{2}$, T.M. Gress ${ }^{2}$, D. Bartsch ${ }^{1}$, K. Okamoto ${ }^{3}$, T. Matono ${ }^{3}$, T. Stiewe ${ }^{4}$, M. Wanzel $^{4}$, P. Di Fazio ${ }^{1}$

${ }^{1}$ Department Of Visceral Thoracic And Vascular Surgery, Philipps University Marburg, Marburg/Germany

${ }^{2}$ Klinik Für Gastroenterologie, Endokrinologie, Stoffwechel Und Infektiologie, Philipps Universität Marburg, Marburg/Germany

${ }^{3}$ Second Internal Medicine, Tottori University, Yonago/Japan

${ }^{4}$ Center For Tumor And Immunobiology, Philipps University Marburg, Marburg/ Germany

Contact E-mail Address: sophia.polte@gmail.com

Introduction: NAFLD is currently classified in non-alcoholic fatty liver (NAFL) and non-alcoholic steatohepatitis (NASH). It is a liver disease related to metabolic syndrome with rising socio-economic impact worldwide. NAFLD is defined by significant lipid deposition in hepatocytes that is unrelated to alcohol consumption. This high prevalence of liver disease occurs after a protracted inflammatory status caused by insulin resistance derived from high consumption of fructose-rich goods as shown by the multi-parallel hit theory. Autophagy is a self-digesting mechanism that helps the cells to overcome stress conditions derived by nutrient deprivation and massive storage, e.g. lipid and proteins. Autophagy dysfunction has been implicated in lipid accumulation related diseases. Up to now, it in yet fully understood the role exerted by autophagy in liver diseases not related to alcohol.

Aims \& Methods: Here, autophagy has been analyzed in a mouse model of NAFL/NASH and in human in vitro model. Liver specimens were collected from 24 weeks old FLS and FLS-ob/ob mice. Liver tissue was snap frozen and kept at $-80^{\circ} \mathrm{C}$. RNA and proteins were isolated. RT-qPCR and western blotting was performed. HepG2 cells were incubated for $24 \mathrm{~h}$ with $2 \mathrm{mM}$ oleic acid (OA) 
alone and in combination with $100 \mathrm{pM}$ Bafilomycin and $2 \mathrm{mM}$ Caffeine. RTqPCR and western blotting was performed also. Real time fluorescence monitoring of HepG2 cells stably expressing ectopic MAP1LC3B-GFP-RFP-tag was performed with Incucyte.

Results: FLS-ob/ob mice, representing a NASH mouse model, showed an overexpression of the autophagy markers BECN1, MAP1LC3B, SQSTM, UVRAG, TFEB, PRKAA 1_1 and PRKAA 2_1 in comparison with FLS mice that represent the NAFL model. Furthermore, the protein level of BECN1 and MAP1LC3B was down-regulated in FLS-ob/ob mice, whereas SQSTM and UVRAG resulted over-expressed. Interestingly, the phosphorylated form of AMPK $\alpha$ was found up-regulated in FLS-ob/ob mice, while FLS mice did not show a quantifiable expression of $\mathrm{P}-\mathrm{AMPK} \alpha$. AMPK $\alpha$ was found stably expressed in both FLS-ob/ob and FLS mice. HepG2 cells treated with oleic acid showed a down-regulation of BECN1, MAP1LC3B, UVRAG and SQSTM proteins. AMPK $\alpha$ and its phosphorylated form resulted unvaried. Interestingly, Caffeine treatment caused a stronger reduction of autophagy markers, including the reduction of $\mathrm{P}-\mathrm{AMPK} \alpha$ also. The combination of Caffeine and OA had an additive effect compared to single compounds by causing a stronger reduction of autophagy markers protein level. Bafilomycin caused a reduction of MAP1LC3B and UVRAG only. Its combination with OA caused a stronger reduction of protein level. HepG2 MAP1LC3B-GFP-RFP showed an increase of red fluorescence after $48 \mathrm{~h}$ of treatment with $2 \mathrm{mM}$ OA

Conclusion: Autophagy mechanism is significantly activated in FLS-ob/ob mice, that lack of Leptin expression, and in HepG2 treated with OA, supporting that autophagy is strongly implicated in NASH. Targeting autophagy could represent a valid future therapeutic strategy for patients affected by NASH. Further studies are needed to better understand the role exerted by Leptin during autophagy in NASH.

Disclosure of Interest: All authors have declared no conflicts of interest.

\section{P0662 MODULATION OF MITOCHONDRIAL DYNAMICS BY}

\section{MIRNAS IN NAFLD}

A.L. Simão ${ }^{1}$, M. B. Afonso ${ }^{1}$, P. M. Rodrigues ${ }^{1}$, A. J. Amaral ${ }^{2}$, M. GamaCarvalho $^{2}$, A. Zorzano ${ }^{3}$, C.M.P. Rodrigues ${ }^{1}$, R. E. Castro

${ }^{1}$ Research Institute For Medicines (iMed. Ulisboa), Faculty of Pharmacy, Universidade de Lisboa, Lisboa/Portugal

${ }^{2}$ BioFIG - Centre for Functional and Integrative Genomics, Faculty of Sciences, Universidade de Lisboa, Lisbon/Portugal

${ }^{3}$ Institute for Research in Biomedicine (IRB Barcelona), Barcelona/Spain

Contact E-mail Address: adlsimao@ff.ul.pt

Introduction: Non-alcoholic fatty liver disease (NAFLD) pathogenesis associates with intracellular lipid accumulation in the liver. In addition, recent evidence supports a functional role for both mitochondrial dysfunction and microRNAs (miRNA/miRs) in NAFLD pathogenesis. In particular, deregulation of mitochondrial dynamics proteins, like mitofusin-2 (Mfn2) is frequently observed in obese and diabetic patients.

Aims \& Methods: Our aims were to profile global liver miRNA expression changes during NAFLD progression and correlate them with the development of mitochondrial dysfunction in both experimental and human NAFLD C57BL6 mice were fed either a standard or a fast food (FF) diet for 25 weeks; or a methionine- and choline-deficient (MCD) diet for 2 and 8 weeks. miRNA profiling was performed using liver RNA from 8 weeks MCD-fed mice, in TaqMan MicroRNA arrays. qPCR array data was analyzed using the HTqPCR package in Bioconductor. Liver biopsies were obtained from patients with simple steatosis or NASH. mRNA and protein expressions were analyzed by qRT-PCR and immunoblotting, respectively. miRNA targeting was evaluated by dual-luciferase reporter assays.

Results: Both FF- and MCD-fed mice developed NASH-like features, including liver steatosis, inflammation and insulin resistance; as well as progressive steatohepatitis, severe liver damage and fibrosis. Strikingly, liver Mfn2 protein levels significantly decreased in both models $(\mathrm{p}<0.05)$. Inversely, expression of Drp1, a mitochondrial fission protein, was found increased $(p<0.05)$. Other mitochondrial proteins, such as the voltage-dependent anion channel (VDAC), presented no expression changes. In human patients, Mfn2 protein levels decreased from steatosis to NASH $(\mathrm{p}<0.05)$. Microarray profiling indicated that liver miRNAs are significantly modulated during NAFLD progression. Specifically, 25 miRNAs were found significantly increased in the liver of MCD-fed mice, while, inversely, 27 miRNAs were decreased. Curiously, in silico analysis revealed that several of the up-regulated miRNAs could target Mfn2 in, at least, one Mfn2 3 UTR binding site. Binding of miRNAs to Mfn-2, including miR-34a, was validated in HepG2 cells using a dual-luciferase reporter vector containing the Mfn2 3UTR. Finally, overexpression of miR-34a in $\mathrm{C} 2 \mathrm{C} 12$ muscle cells lead to Mfn2 inhibition and insulin resistance $(\mathrm{p}<0.05)$.

Conclusion: In conclusion, mitochondrial dysfunction, particularly downregulation of Mfn2, plays a key role in human and experimental NAFLD and is targeted by miRNAs, like miR-34a. A better understanding of the network of miRNAs targeting mitochondrial proteins during NAFLD may help in the development of novel targeted therapies for metabolic diseases associated with mitochondrial dynamics dysfunction. (Supported by PTDC/BIM-MEC/0895/2014, SFRH/BD/104160/2014, FCT, PT and Gilead Sciences International Research Scholars Program 2015).

Disclosure of Interest: All authors have declared no conflicts of interest.
P0663 GRANULOCYTE COLONY STIMULATING FACTOR IN DECOMPENSATED LIVER DISEASE - OUR EXPERIENCE IN A TERTIARY HOSPITAL IN NEPAL

S. Thapaliya

Department Of Gastroenterology, Tribhuvan University Teaching Hospital, Institute of Medicine, Maharajgunj, Kathmandu/Nepal

Contact E-mail Address: sabinthapaliya@gmail.com

Introduction: Alcoholic Hepatitis, Decompensated Chronic Liver Disease and Acute-on-Chronic Liver Failure form a bulk of in-patients in Nepal. Mortality is quite high in these presentations despite all the medications currently available in our country. Prednisolone and Pentoxifylline are not up to the mark in terms of both short and long-term outcomes ${ }^{1}$. The only option that remains is liver transplant, which is not readily available in our country, and even if available in the near future, needs at least some months for planning and preparation and is costly. G-CSF (Granulocyte Colony Stimulating Factor) has shown both morbidity and mortality benefit in some studies in these groups of patients $2,3,4$. By comparing the outcomes in those receiving and not receiving G-CSF, we can suggest G-CSF therapy to reduce mortality and morbidity in these patients. Although most of the studies done in the role of G-CSF in ACLF (Acute-onChronic Liver failure) and decompensated CLD (Chronic Liver Disease) have used $5 \mu \mathrm{g} / \mathrm{Kg} /$ dose of G-CSF subcutaneously for $\geq 10$ doses $^{2}$, our study has used fixed dosage of $300 \mu \mathrm{g}$ of G-CSF subcutaneously twice a day for a total of 3 days (6 doses).

Aims \& Methods: We aimed to study the role of G-CSF in the treatment of alcoholic hepatitis, Decompensated CLD and ACLF. From January 2016 to December 26, a total 49 patients with alcoholic hepatitis, decompensated chronic liver disease and acute-on-chronic liver failure admitted in TUTH (Tribhuvan University Teaching Hospital) were studied. Patients were randomized (in a 1:1 ratio) to either the 'GCSF + SMT' (Standard Medical Therapy) group (cases) or the 'SMT-alone' (control) group according to computer-generated random numbers. Patients in G-CSF group received G-CSF $300 \mu \mathrm{g}$ twice daily for 3 days (total 6 doses). Mortality rates at 1 month and CTP (Child Turcotte Pugh) and MELD (Model for End stage Liver Disease) scores at enrollment and at Day 30 were compared in the two groups.

Results: A total of 49 patients [median age: 49 (range: $27-73$ ) years, $70 \%$ males) were included in the study, 24 of them received G-CSF along with SMT and 25 received SMT alone. Baseline characteristics were similar in both the groups. The 3-day GCSF therapy did not lead to any significant adverse effects. At one month, in GCSF + SMT group, 4 had died whereas in SMT alone group 15 had died. $83 \%$ survived in GCSF group whereas only $40 \%$ survived in control group $(\mathrm{P}=.002)$. Also significant improvement in CTP and MELD scores was seen in the group treated with GCSF at one month after therapy. Also, there were fewer complications of sepsis, hepatic encephalopathy and renal impairment in the GCSF group compared to the SMT alone group.

Conclusion: GCSF therapy improves survival and clinical outcome in patients with alcoholic hepatitis, decompensated chronic liver disease and acute-onchronic liver failure. It may be useful in patients who do not have access to transplant services and also to the patients awaiting transplantation to prevent worsening during the waiting period. Further studies are needed to explore whether lower doses (total 6 doses) of GCSF are as effective as higher doses (total 10 doses).

Disclosure of Interest: All authors have declared no conflicts of interest.

\section{References}

1. Thursz, M. R. et al. Prednisolone or pentoxifylline for alcoholic hepatitis. $N$. Engl. J. Med.372, 1619-1628 (2015).

2. Garg V1, Garg H, Khan A, Trehanpati N, Kumar A, Sharma BC, Sakhuja $P$, Sarin SK. Granulocyte colony-stimulating factor mobilizes CD34(+) cells and improves survival of patients with acute-on-chronic liver failure. Gastroenterology 2012;142:505-512

3. Duan X-Z, Liu F-F, Tong J-J, et al. Granulocyte-colony stimulating factor therapy improves survival in patients with hepatitis B virus-associated acuteon-chronic liver failure. World Journal of Gastroenterology: WJG. 2013;19(7):1104-1110.

4. Gaia S, Olivero A, Smedile A, Ruella M, Abate ML, Fadda M, et al. Multiple courses of G-CSF in patients with decompensated cirrhosis: consistent mobilization of immature cells expressing hepatocyte markers and exploratory clinical evaluation. Hepatol Int 2013; 7:1075-1083.

P0664 TITLE: STEM CELL TRANSPLANTATION IMPROVES SURVIVAL, QUALITY OF LIFE AND SYNTHETIC FUNCTIONS OF THE LIVER IN PATIENTS WITH END STAGE LIVER DISEASE

M. Albadry ${ }^{1}$, M. Amer ${ }^{2}$, W. A.E. Mostafa ${ }^{3}$, H. A. Bayomi ${ }^{2}$, A. El Shafie ${ }^{2}$, M. Alboraie ${ }^{4}$

${ }^{1}$ Department Of Tropical Diseases And Gastroenterology, Aswan University, Aswan/Egypt

${ }^{2}$ Department Of Tropical Medicine, Al Azhar University, Cairo/Egypt

${ }^{3}$ Microbiology \&immunology Department, Military Medical Academy, Cairo/ Egypt

${ }^{4}$ Department Of Internal Medicine, Al Azhar University, Cairo/Egypt

Contact E-mail Address: alboraie@azhar.edu.eg

Introduction: Cirrhosis and its complications are common causes of death among patients with end-stage liver disease. Liver transplantation as a definitive treatment is limited by shortage of donors and high cost. Autologous bone marrow 
mononuclear layer containing stem cells is a novel approach for regeneration of liver cell which can be a therapeutic option for those patients.

Aims \& Methods: To determine the outcome after intrasplenic or intrahepatic injection of autologous bone marrow stem cells (ABMSC) transplantation in patients with liver cell failure secondary to chronic hepatitis C infection. Sixty chronic hepatitis $\mathrm{C}$ patients with liver cell failure were prospectively enrolled. They were classified into 3 groups; group I: 20 patients underwent (ABMSC) injected intrasplenic. Group Ia: 10 patients underwent (ABMSC) injected intrasplenic after trans differentiation into hepatocytes with regular amount of growth factor. Group Ib: 10 patients underwent (ABMSC) injected intrasplenic after trans differentiation into hepatocytes with the double amount of growth factor. Group II: consisted of 20 patients underwent (ABMSC) injected intra hepatic (right portal branch). Group IIa: 10 patients underwent (ABMSC) injected intrahepatic after trans differentiation into hepatocytes using regular amount of growth factor. Group IIb: 10 patients underwent (ABMSC) injected intrahepatic; after trans differentiation into hepatocytes with the double amount of growth factor. Group III: (Control Group): consisted of 20 patients received traditional supportive treatment for chronic liver cell failure and symptomatic treatment of ascites and bleeding abnormalities. All groups of patients were followed regularly for nine months clinically, biochemically and ultrasonographically. Fatigue was assessed by the modified fatigue impact scale questionnaire before, during and at end of the study.

Results: Our study included 60 patients $(78.33 \%$ males) with mean age \pm SD (49.9 \pm 6 years). Patients who had ABMSC injection showed improvement in clinical parameters as bleeding tendency, ascites, lower limb edema and hepatic encephalopathy. There was statistically significant improvement in serum albumin level and prothrombin time in group I and group II in comparison group III. There was also statistically significant improvement in Child-Turcotte-Pugh in group I and group II in comparison group III at the third month and this was maintained till the end of the study. Fatigue improved in all patients who had ABMSC. There was improvement in serum albumin, ascites, lower limb edema, bleeding tendency and physical activity. Also there was improvement in MELD score and performance status.

Conclusion: Stem cell transplantation has a beneficial effect on synthetic functions of the liver and possibly improves survival and quality of life of patients with end stage liver disease. Autologous bone marrow transplantation is safe and beneficial technique in treatment of in patients with liver cell failure secondary to chronic hepatitis $\mathrm{C}$ infection.

Disclosure of Interest: All authors have declared no conflicts of interest.

\section{P0665 PERIPHERAL BLOOD MONONUCLEAR CELL TRANSPLANTATION STIMULATES HEPATOCYTES PROLIFERATION IN PATIENTS WITH ALCOHOLIC LIVER CIRRHOSIS}

G.R. Burganova ${ }^{1}$, S.R. Abdulkhakov ${ }^{1}$, I. M. Gazizov², A. A. Gumerova ${ }^{1}$, M. A. Titova ${ }^{1}$, R. Deev ${ }^{3}$, A. P. Kiyasov ${ }^{1}$

${ }^{1}$ Department Of Morphology And General Pathology, Kazan Federal University, Kazan/Russian Federation

${ }^{2}$ Department Of Normal Human Anatomy, Kazan State Medical University, Kazan/Russian Federation

${ }^{3}$ Department Of Patological Anatomy With Course Of Forensic Medicine, Ryazan State Medical University, Ryazan/Russian Federation

\section{Contact E-mail Address: guzel.burganova@gmail.com}

Introduction: It is well known that severe liver disease requires a liver transplantation to be treated. But liver transplantation is not available in many countries. An alternative treatment can be a transplantation of autologous bone marrow derived cells which can stimulate liver regeneration. Proliferating cell nuclear antigen (PCNA) shows nuclei of dividing cells and may be useful for calculating proliferation index.

Aims \& Methods: The aim of this study was to evaluate changes of proliferation intensity of liver cells in patients with alcoholic liver cirrhosis after autologous peripheral blood mononuclear cell (PBMC) transplantation. This uncontrolled open-labeled clinical trial was approved by Ethical committee of Ministry of Health of the Republic of Tatarstan, Russia. Eleven patients took part in the study, they received granulocyte colony-stimulating factor injections for 5 days for PBMC mobilization. On the 6th day PBMCs were collected and injected into the celiac trunk. Liver biopsies were obtained three times from each person on next time-points: before transplantation of PBMCs into the celiac trunk (initial), three and twelve months after the procedure. Liver biopsy specimens were embedded in paraffin and stained immunohistochemically with antibodies against PCNA. The PCNA labeling index was calculated as the number of PCNA-labeled nuclei for 1000 hepatocyte nuclei in each specimen and the results were expressed as percentage ratios. Statistical analysis was done by Wilcoxon signed-rank test using Statistica v.12 software. p value $<0.05$ was considered significant.

Results: Before the transplantation of PBMCs $28.3 \pm 18.3 \%$ of all the hepatocytes expressed PCNA without any topographic prevalence. Three months after the transplantation the proportion of proliferating hepatocytes increased up to $36.7 \pm 24.8 \%$. Twelve months after transplantation of PBMCs we found hepatocytes proliferation to be 2 times higher than before the procedure. Proportion of proliferating hepatocytes reached $50.2 \pm 17.0 \%(\mathrm{p}=0.04)$. Great increase in hepatocytes proliferation intensity coincided with biochemical improvements of serum bilirubin, ALT and alkaline phosphatase.

Conclusion: Our study showed that proposed treatment was safe and effective. We can conclude that after transplantation of autologous PBMC s proliferation of hepatocytes greatly contributes to the liver regeneration and improvement of blood biochemical data in patients with alcoholic liver cirrhosis. However, effect of our treatment is not constant and requires repeated PBMC transplantation. Disclosure of Interest: All authors have declared no conflicts of interest.

\section{P0666 ASSOCIATION BETWEEN NONALCOHOLIC FATTY LIVER DISEASE AND METABOLIC SYNDROME IN APPARENTLY HEALTHY KOREAN ADULTS}

K.M. Sohn ${ }^{1}$, Y.J. Jeon ${ }^{2}$

${ }^{1}$ Gastroenterology, Hansol Hospital, Seoul/Korea, Republic of

${ }^{2}$ MedGene Lab, Seoul/Korea, Republic of

\section{Contact E-mail Address: dr.sohnkm@gmail.com}

Introduction: The prevalence of non-alcoholic fatty liver disease (NAFLD) has increased and several studies have shown that there is an association between NAFLD and metabolic syndrome (MetS). The aim of this study was to determine how much impact the risk factors of metabolic syndrome has on ultrasonographic fatty liver, especially NAFLD.

Aims \& Methods: A total of 41,258 adults who underwent routine comprehensive health evaluations, including abdominal ultrasonography, were selected. We calculated the adjusted prevalence ratios (PRs) for components of MetS (high blood pressure (BP), impaired fasting glucose, low high-density lipoprotein cholesterol (HDL-C), and high triglycerides (TG) according to NAFLD.

Results: NAFLD was found in $13.8 \%$ of non-obese subjects and $52.3 \%$ of obese subjects. NAFLD was associated with most components of MetS in both obese and non-obese subjects. However, non-obese NAFLD patients had significantly higher PRs for certain components of MetS than did obese patients, especially among women. Body mass index, waist circumference, fasting blood glucose, triglyceride, HDL-C and aspartate aminotransferase, alanine aminotransferase, $\gamma$-glutamyl transpeptidase levels all affected NAFLD independently. The prevalence of metabolic syndrome was increased in mild $(40.8 \%)$ and moderate $(57.8 \%)$ NAFLD groups. When odd ratio $(95 \% \mathrm{CI})$ for NAFLD group was compared to the contrast group, there was an increased risk of metabolic syndrome with odd ratio of $12.8(95 \%$ CI, $9.1 \sim 17.0)$.

Conclusion: NAFLD and its severity has a close connection with MetS and also with each risk factors of MetS. Therefore, assessment for concurrent MetS among NAFLD patients is considered to be necessary.

Disclosure of Interest: All authors have declared no conflicts of interest.

\section{Reference}

1. Kwon YM, Oh SW, Hwang SS, et al. Association of nonalcoholic fatty liver disease with components of metabolic syndrome according to body mass index in Korean adults. Am J Gastroenterol. 2012 Dec;107(12):1852-8.

\section{P0667 LEAN-NAFLD IS THE STRONGEST PREDICTOR OF FUTURE OBESITY AMONG URBAN ADULT SRI LANKANS: RESULTS FROM A PROSPECTIVE, COMMUNITY COHORT FOLLOW-UP STUDY}

M. A. Niriella ${ }^{1}$, S. T. De Silva ${ }^{1}$, A. Kasthuriratna ${ }^{1}$, A. G. Ranasinghe ${ }^{1}$, A S. Dassanayake ${ }^{1}$, A. P. De Silva ${ }^{1}$, A. Pathmeswaran ${ }^{1}$, A. R. Wickramasinghe ${ }^{1}$, N. Kato ${ }^{2}$, H. J. De Silva ${ }^{1}$

${ }^{1}$ Faculty of Medicine, University of Kelaniya, Ragama/Sri Lanka

${ }^{2}$ National Center for Global Health and Medicine, Tokyo/Japan

Contact E-mail Address: maduniln@yahoo.co.uk

Introduction: Obesity is a global problem. Data from the South Asian region is limited.

Aims \& Methods: In a cohort follow-up study we investigated obesity among urban, adult, Sri Lankans [selected by age-stratified random sampling from Ragama-MOH area, Gampaha District; initial screening 2007 (aged 35-64 years); re-evaluation 2014 (aged 42-71 years)]. On both occasions structured interview, anthropometry, liver ultrasound, biochemical and serological tests were performed. Total body fat (TBF) and visceral fat percentage (VFP) were assessed by impedance in 2014. General-obesity (GO) was BMI $>25 \mathrm{~kg} / \mathrm{m}^{2}$. Central-obesity (CO) was waist circumference (WC) $>90 \mathrm{~cm}$ males and WC $>80 \mathrm{~cm}$ females. Non-alcoholic fatty liver disease (NAFLD) was diagnosed on ultrasound criteria, safe alcohol consumption and absence of hepatitis $\mathrm{B} / \mathrm{C}$ markers. Multinomial logistic regression was fitted to assess associations.

Results: In $2007(\mathrm{n}=2967), 614(20.7 \%)$ were overweight [51.9\%-women], $1161(39.1 \%)$ had GO [65.9\%-women] and $1584(53.4 \%)$ had CO [71\%-women]. Females $(\mathrm{p}<0.001)$, raised-TG $(\mathrm{p}<0.001)$, low-HDL $(\mathrm{p}<0.001)$, diabetes $(\mathrm{p}<0.001)$, hypertension $(\mathrm{p}<0.001)$, NAFLD $(\mathrm{p}<0.001)$, and low household income $(\mathrm{p}<0.001)$ were significantly associated with prevalent $\mathrm{GO}$ and $\mathrm{CO}$ respectively. Additionally, increased-age $(\mathrm{p}=0.05)$, low-educational level $(\mathrm{p}<0.001)$ and unhealthy eating $(\mathrm{p}<0.001)$ were associated with prevalent CO. Inadequate physical activity was not associated with either. $2137(72 \%)$ attended follow-up in 2014. Of those who were initially non-obese who attended follow-up, 189/1270(14.9\%) [64\% women] had developed GO (annual-incidence $2.13 \%$ ) and $206 / 947(21.9 \%$ ) [56.3\% women] had developed CO (annual incidence $3.12 \%$ ) after 7 years. TBF and VFP significantly correlated with incident GO and CO (p < 0.001). Females gender (OR-1.78, p $<0.001 ; 2.81, p<0.001)$ and NAFLD (OR-2.93, p < 0.001; OR-2.27, p < 0.001) independently predicted incident $\mathrm{GO}$ and $\mathrm{CO}$ respectively.

Conclusion: The prevalence and incidence of GO and $\mathrm{CO}$ were high in this cohort. Both incident $\mathrm{GO}$ and $\mathrm{CO}$ were associated with female gender and 
lean-NAFLD with lean-NAFLD being the strongest predictor of future development of GO.

Disclosure of Interest: All authors have declared no conflicts of interest.

\section{P0668 THE USE OF THE FATTY LIVER INDEX TO DETERMINE THE PREVALENCE OF FATTY LIVER DISEASE (HEPATIC STEATOSIS) IN AN IRISH POPULATION}

\author{
L. Reynolds, L. Rabbitt, C. Goulding \\ Acute Medical Unit, University Hospital Galway, Galway/Ireland
}

Contact E-mail Address: laura93reynolds@gmail.com

Introduction: Worldwide, the prevalence of fatty liver disease (FLD) is increasing, particularly in countries with rising obesity rates, such as Ireland. Studies suggest that up to $25 \%$ of those with FLD can progress to non alcoholic steatohepatitis (NASH) and be at risk of its sequelae, including cirrhosis and hepatocellular carcinoma. Indeed, NASH is now the second most common indication for liver transplantation in the US. Despite this alarming data, there is no prevalence data for Ireland in relation to FLD.

Aims \& Methods: We aimed to use a simple screening tool, the Fatty Liver Index (FLI) to identify those at risk of having fatty liver disease (FLD) amongst all comers presenting to an Acute Medical Unit (AMU) and to use this data as an indicator of prevalence of FLD in Ireland.

Methods: In this prospective cohort study, all patients attending the Acute Medical Unit (AMU) were invited to take part. Their height, weight and waist circumference were measured, and triglycerides (TG) were added to their 'routine AMU blood panel', which also included measurement of gamma glutamyl transferase (GGT). Exclusion criteria were as follows: known liver disease, excess alcohol intake ( $>17$ units per week for males, $>11$ units per week for females), age $<18$ years, pregnancy, active malignancy. The Fatty Liver Index (FLI), an algorithm based on Body Mass Index (BMI), waist circumference, TG and GGT was used to stratify patients into groups based on risk of having FLD. A FLI score of $>60$ is highly suggestive of having FLD, a score of 30-60 is indeterminate and a score of $<30$ is considered low risk for FLD. Ethical approval for this research was granted by the ethics committee of UHG.

Results: Data was completed on 316 participants; 58 were excluded, the majority due to either a history of alcohol excess or known liver disease. A total of 258 participants were therefore evaluated; $50 \%$ were male. One hundred and sixteen $(45 \%)$ participants scored $>60$ on the FLI; $57.3 \%$ of which were male. Only $16 \%$ of males had a FLI $<30$, compared with $44 \%$ of females. Males had a significantly higher FLI than females; 60.9 vs. $43.12(\mathrm{p}<0.0001)$. Those with $\mathrm{FLI}>60$ had a mean weight $=93.5 \mathrm{~kg}$ and $\mathrm{BMI}=31.5$, vs. $64.9 \mathrm{~kg}$ and 22.4 respectively for those with FLI $<30$ ( $\mathrm{P}>0.0001)$. There was a statistically significant difference in all parameters measured between all 3 groups $(p<0.0001)$, apart from height, although there was a trend toward lower height in the FLI $<30$ group, most likely due to the fact that it was $73 \%$ female. When only males were looked at, there was no height difference between the 3 groups. Those with a FLI $>60$ were older than those with FLI $<30,54.6$ vs. $48(\mathrm{p}=0.01)$.

Conclusion: In this study looking at prevalence of fatty liver in Ireland, $45 \%$ of participants were found to be at high risk, and $70 \%$ were at high or indeterminate risk. Worryingly, only $16 \%$ of males fell into the low-risk group. Apart from weight, GGT, TG and BMI this study also showed age and male sex to be significant risk factors for developing fatty liver. This group clearly needs follow up to further evaluate and manage their fatty liver.

Disclosure of Interest: All authors have declared no conflicts of interest.

\section{P0669 ROLE OF BISPHENOL A AS AN ENVIRONMENTAL FACTOR IN THE PROMOTION OF NON-ALCOHOLIC FATTY LIVER DISEASE: IN VITRO AND IN VIVO STUDY}

M. Dallio ${ }^{1}$, A. G. Gravina ${ }^{1}$, M. Masarone ${ }^{2}$, S. Errico ${ }^{3}$, C. Nicolucci ${ }^{3}$, R. Di

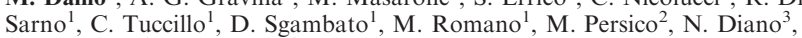
C. Loguercio ${ }^{1}$, A. Federico ${ }^{1}$

${ }^{1}$ Department Of Clinical And Experimental Medicine, University of Campania "Luigi Vanvitelli", Naples/Italy

${ }^{2}$ Department Of Internal Medicine And Hepatology, University of Salerno, Baronissi/Italy

${ }^{3}$ Department Of Experimental Medicine, University of Campania "Luigi Vanvitelli", Naples/Italy

\section{Contact E-mail Address: marcello.dallio@gmail.com}

Introduction: Bisphenol A (BPA) is an endocrine disrupting chemical, a heterogenic group of chemicals usually found in food packaging or insecticide residues on vegetable crops, associated with type 2 diabetes mellitus (T2DM), cardiovascular disease and liver enzyme abnormalities, and in general with the whole blood glucose homeostasis.

Aims \& Methods: We have evaluated BPA plasma and urine levels in non-alcoholic fatty liver disease (NAFLD) patients compared to healthy subjects and we evaluated the possibility to eliminate this environmental factor after a BPA-free diet regimen. Furthermore, we evaluated, in human HepG2 cells, the effects of exposure to different BPA concentrations on both oxidative stress induction and cell proliferation. We enrolled sixty patients with histologic diagnosis of NAFLD with or without T2DM, before a BPA-free diet, and healthy subjects, by subjecting them to evaluation of body composition using bioimpedance analysis. In vitro, the proliferation of BPA-exposed HepG2 cells at two different concentrations $(0.025$ and $0.05 \mu \mathrm{M})$ was evaluated, both at high $(\mathrm{H}-\mathrm{HepG})$, in order to simulate human hyperglycemia, and at low (L-HepG2) glucose concentrations, for $48 \mathrm{~h}$. Lipoperoxidation was also assessed by TBARS assay.

Results: BPA levels were significantly higher in 60 NAFLD subjects, both in urine and in plasma $(\mathrm{p}<0.0001)$ if compared to controls and, among this group, it appeared to be higher in 30 non-alcoholic steatohepatitis (NASH) patients compared to 30 simple steatosis $(\mathrm{NAFL})$ ones $(\mathrm{p}<0.05)$, independently from the presence of T2DM. After following a BPA-free diet for one month, NAFLD patients showed a significant reduction of BPA circulating levels $(\mathrm{p}<0.05)$ without a significant reduction of urine levels, which represents the only way to eliminate BPA amount released into circulation by the adipose BPA reservoir. In fact subjects with a higher fat percentage in body composition showed higher BPA levels in plasma and urine. In our population study, NASH patients showed a higher fat percentage in body composition in comparison to NAFL ones. Only H-HepG2 cells treated with BPA $(0.05 \mu \mathrm{M})$ increased proliferation compared to controls at $48 \mathrm{~h}(\mathrm{p}<0.0001)$. Moreover, BPA increased TBARS levels at $48 \mathrm{~h}$ in $\mathrm{H}-\mathrm{HepG} 2$ cells versus controls.

Conclusion: Our study reveals a possible role of BPA as an environmental factor involved in the progression of NAFLD, particularly in obese and/or T2DM patients.

Disclosure of Interest: All authors have declared no conflicts of interest.

\section{P0670 ASSESSMENT OF FATTY PANCREAS IN PATIENTS WITH} NON-ALCOHOLIC FATTY LIVER DISEASE

T. Alempijevic ${ }^{1}$, S. Dragasevic ${ }^{2}$, S. Zgradic ${ }^{3}$, M. Stojkovic Lalosevic ${ }^{4}$, B. Milicic ${ }^{4}$, D. Popovic ${ }^{1}$, T. Milosavljevic ${ }^{1}$

${ }^{1}$ Clinic For Gastroenterology And Hepatology, Clinical Center of Serbia, School of Medicine University of Belgrade, Belgrade/Serbia

${ }^{2}$ Clinic For Gastroenterohepatology, Clinical Center of Serbia, Belgrade/Serbia ${ }^{3}$ Gastroenterology, Clinical Center of Serbia, Belgrade/Serbia

${ }^{4}$ Institute for Medical Informatics, University of Belgrade, Faculty of Dentistry, Belgrade, Serbia, Belgrade/Serbia

Contact E-mail Address: tamara.alempijevic@med.bg.ac.rs

Introduction: The clinical implications of non-alcoholic fatty pancreas disease (NAFPD) are still the topic of debate in human studies. It has been shown that fatty infiltration in pancreas correlates with metabolic risk factors and may represent significant manifestation of metabolic syndrome (MeS) in association with nonalcoholic fatty liver disease (NAFLD). The aim of our study was to determine the association of fatty pancreas (FP) in NAFLD patients with features of MeSand to determine a simple new noninvasive scoring system for FP prediction in NAFLD patients.

Aims \& Methods: We conducted across-sectonal study that included 143 subjectswith NAFLD classified into two groups according to the severity grade of FP as follows: patients with non fatty pancreas and grade I light FP $(\mathrm{n}=59)$ and patients with grade II severly and grade III highly FP $(\mathrm{n}=84)$. Patients were analyzed for diagnostic criteria of $\mathrm{MeS}$, underwent sonographic examination with adiposity measurments and liver biopsy. Liver fibrosis was evaluated semiquantitative according to the METAVIR scoring system and using non-invasive markers of hepatic fibrosis (NAFLD fibrosis score (NFS), BARD score, Fibrosis-4 score (FIB-4), AST to Platelet Ratio Index (APRI)).

Results: Of these 143 patients with NAFLD, 84 had criteria for metabolic syndrom $(\mathrm{MeS})$, while there was no significant difference in frequency among FP groups. Out of all clinical and laboratory characteristics, no statistical differences were registred in demographic data, lifestyle factors, body mass index (BMI), systolic and distolic blood preasure, presence of hypertension and dyslipidemia, values of insulin, high density lipoprotein (HDL) and triglycerides. Waist circumference (WC) showed significant difference among groups, indicating WC as a posible marker for higher risk of FP in NAFLD patients $(P=0.018)$. Diabetes mellitus $(\mathrm{DM})$ was more frequent in patients with severly FP $(P=0.02)$, with greater portion of patients with HOMA-IR $>3$. Higher values of fasting plasma glucose, total cholesterol, serum amylase and lipase were associated with presence of highly fatty pancreas $(P=0.052, P=0.007, P=0.014 ; P=0.024$, retrospectively). Values of hemoglobin Alc $(\mathrm{HbAlc})>6 \%$ were significantly associated with NAFLD patients with severly FP, highlighting its impaired function in $\operatorname{MeS}(P=0.008)$. While no significant difference was found in the use of statins and hypertensives, higher number of patients with severly FP did not use antidiabetic agents and asociation was registred among NAFLD patients with use of metformin and glimeprid and the first FP group $(P=0.035)$. Out of all visceral fat amounts, only measures of mesenteric fat were associated with severly FP $(P=0.013)$. Results of our study determined highly significant association of NAFLD and NAFPD. Neither NAFLD non-invasive markers nor hystological reports of liver fibrosisshowed significant associationwith presence of fatty pancreas. In multivariate analysis of FP predictors in our study cohort, logistic regression approach was used. Model of predicting occurence of FP was designed from multivariate logistic regression analysis. The probability was estimated with the equation: $0.627+0.640 * 0.593 *$ glucose (fasting glucose level) - cholesterol level (total cholesterol) $+0.058+1.585 *$ serum lipase * ultrasonography level of liver steatosis. According to the score values for different cut off levels, best ability in the prediction of severly FP has shown the score value above 6.5.

Conclusion: Our study demonstrated that pancreatic fat infiltration due to its impaired function presents an additional factor that contributes to aggravation of $\mathrm{MeS}$ manifestations, affects glucose metabolism and severity of NAFLD. Interestingly, significant association was registred among NAFLD patients 
with use of antidiabetic agents and the absence of highly fatty pancreas, indicating its potential protective role. Simple new noninvasive scoring system was designed frommultivariate logistic regression analysis to estimate the occurence of severly FP in NAFLD with best ability in the prediction in score values above 6.5 .

Disclosure of Interest: All authors have declared no conflicts of interest.

\section{P0671 SERUM THYROID STIMULATING HORMONE IS INDEPENDENTLY ASSOCIATED WITH HEPATIC STEATOSIS AND STEATOHEPATITIS IN EUTHYROID SUBJECTS}

A. Eshraghian ${ }^{1}$, S. Nikeghbalian ${ }^{2}$, B. Geramizadeh ${ }^{2}$, S. Ali Malek-Hosseini ${ }^{2}$ ${ }^{1}$ Gastroenterology And Hepatology, Shiraz University of Medical Sciences, Shiraz Iran

${ }^{2}$ Transplant Research Center, Shiraz/Iran

Contact E-mail Address: eshraghiana@yahoo.com

Introduction: Non- alcoholic fatty liver disease (NAFLD) is a rapidly growing disease worldwide. The pathogenesis of NAFLD is not well recognized. Thyroid is totally involved in regulation of lipid and carbohydrate metabolism, body weight, and energy homeostasis. Therefore, the role of thyroid hormones in pathogenesis of hepatic steatosis is anticipated.

Aims \& Methods: This study aimed to investigate thyroid hormone abnormalities in euthyroid subjects with hepatic steatosis. A cross sectional study was conducted between September 2012 and September 2015 at Namazi hospital, Shiraz, Iran. Study subjects were healthy individuals who had undergone liver biopsy for evaluation of liver histology as a routine pre-transplant checkup before living related liver transplantation. Liver function tests, age, gender, weight, height, fasting plasma glucose, thyroid hormones, and lipid profile were recorded. Liver biopsy specimens were reviewed by an expert pathologist for hepatic steatosis and steatohepatitis. Individuals with a history of chronic liver disease, hepatitis B or C infection, hepato-billiary cancers, those with $>20$ grams/day alcohol consumption, and individuals receiving medications causing hepatic steatosis were excluded from the study.

Results: A total of 210 individuals (130 women and 80 men) were included. Seventy six individuals (36.19\%) had hepatic steatosis and 19 individuals had steatohepatitis $(9.04 \%)$ in liver histology. Mean age of individuals with and without hepatic steatosis were $32.9 \pm 6.69$ and $31.8 \pm 6.72$ years respectively $(\mathrm{P}=0.26)$. In univariate analysis higher weight, triglyceride, total cholesterol, alanine aminotransferase (ALT), alkaline phosphatase, fasting blood sugar (FBS) and thyroid stimulating hormone (TSH) were associated with hepatic steatosis $(\mathrm{P}<0.05)$. Serum T4 and T3 were not associated with hepatic steatosis $(\mathrm{P}>0.05)$. In regression analysis, higher FBS, higher alkaline phosphatase, higher ALT and higher TSH $(\mathrm{OR}=1.36$; $95 \% \mathrm{CI}$ : $1.02-1.80, \mathrm{P}=0.03)$ were independent predictors of hepatic steatosis. In regression analysis, higher serum TSH was independently associated with steatohepatitis compared to those without steatohepatitis $(6.83 \pm 6.04 \mathrm{mIU} / \mathrm{L}$ and $2.10 \pm 1.27 \mathrm{mIU} / \mathrm{L})$ $(\mathrm{OR}=2.11 ; 95 \% \mathrm{CI}: 1.45-3.07, \mathrm{p}<0.001)$. A cutoff value of $3.75 \mathrm{mIU} / \mathrm{L}$ for TSH was predictor of presence of steatohepatitis in liver biopsies (sensitivity $=75 \% ;$ specificity $=89 \% ; \mathrm{AUC}=0.754 ; \mathrm{P}=0.004)$

\begin{tabular}{lllc}
\hline & Odds Ratio (OR) & $95 \%$ Confidence Interval $(\mathrm{CI})$ & P-Value \\
\hline Weight & 1.05 & $0.96-1.16$ & 0.245 \\
Triglyceride & 1 & $0.98-1.01$ & 0.989 \\
ALT & 1.007 & $0.93-1.08$ & 0.867 \\
TSH & 2.11 & $1.45-3.07$ & $<0.001$ \\
\hline
\end{tabular}

Conclusion: Higher serum TSH is associated with hepatic steatosis and steatohepatitis in euthyroid subjects. Thyroid hormones may have crucial role in hepatic steatosis and may be targeted for treatment of NAFLD patients.

Disclosure of Interest: All authors have declared no conflicts of interest.

\section{Reference}

1. Bano A, Chaker L, Plompen EP, Hofman A, Dehghan A, Franco OH, Janssen HL, Darwish Murad S, Peeters RP. Thyroid Function and the Risk of Nonalcoholic Fatty Liver Disease: The Rotterdam Study. J Clin Endocrinol Metab 2016;101(8):3204-11

\section{P0672 IDENTIFICATION AND IN SILICO CHARACTERIZATION OF SIX NOVEL GANAB MUTATIONS IN POLYCYSTIC LIVER DISEASE}

L. F.m. Van De Laarschot ${ }^{1}$, R.H.m. Te Morsche ${ }^{1}$, A. Hoisschen ${ }^{2}$, W.R. Cnossen ${ }^{1}$, H. Venselaar ${ }^{3}$, J.M. Banales ${ }^{4}$, J.P.h. Drenth ${ }^{1}$ Gastroenterology \& Hepatology, Radboudumc, Nijmegen/Netherlands ${ }^{2}$ Department Of Human Genetics, Radboudumc, Nijmegen/Netherlands ${ }^{3}$ Centre For Molecular And Biomolecular Informatics, Radboudumc, Nijmegen/ Netherlands

${ }^{4}$ Liver And Gastrointestinal Diseases, Biodonostia Research Institute- Donostia University Hospital, San Sebastián/Spain
Contact E-mail Address: liyanne.vandelaarschot@radboudumc.nl

Introduction: Glucosidase II is part of the functional pathway of co-translational protein translocation and maturation in the endoplasmic reticulum. It is implicated in autosomal dominant polycystic liver disease (ADPLD) and autosomal dominant polycystic kidney disease (ADPKD). The $\beta$-subunit of glucosidase II, encoded by PRKCSH, has been identified as one of the causative genes of ADPLD. Recent data suggest that the $\alpha$-subunit of glucosidase II (GII $\alpha$ ), encoded by $G A N A B$, is associated with ADPKD and ADPLD. We aimed to identify $G A N A B$ mutations in an independent cohort of patients with the primary phenotype of polycystic liver disease (PLD) and to predict the influence of these mutations on glucosidase II function.

Aims \& Methods: We identified genetic mutations in $G A N A B$ using molecular inversion probe (MIP) analysis in a cohort of PLD patients. Both patients with ADPKD and ADPLD were included for analysis. Mutations identified with MIP analysis were validated using Sanger sequencing. Bioinformatics prediction tools (PolyPhen-2, Align GVGD, SIFT, MutationTaster) were used to predict the functional significance of the mutations. YASARA\&WHAT IF were used for analysis of the structural effects of the mutations. Primary cholangiocytes obtained from a patient with $G A N A B$ mutation (c.2515C $>$ T) were used to study loss of heterozygosity.

Results: We identified and validated 6 new bona fide $G A N A B$ mutations in 7 unrelated families. These are 2 frameshift (c.687delT and c.11_16delTAGCGG), 1 splicing (c.2691-28C > G), 2 nonsense (c.2509C $>\mathrm{T}$ and c.2656C $>\mathrm{T}$ ) and 1 missense (c.1835G $>$ C) mutation. In silico analysis showed c.687delT and c.11_16delTAGCGG are located in N-terminal domain of the protein. These mutations probably lead to a total defective protein. c. $1835 \mathrm{G}>\mathrm{C}$ is located in the active site of the protein. It is predicted to disrupt the composition of the active site and reduce enzymatic activity. The remaining mutations (c.2691$28 \mathrm{C}>\mathrm{G}, \mathrm{c} .2509 \mathrm{C}>\mathrm{T}$ and c. $2656 \mathrm{C}>\mathrm{T}$ ) are located in the distal C-terminal domain, which interacts with $P R K C S H$. The mutations could result in early termination of this domain. It is speculated this disrupts the ability of the two subunits to interact. Western Blot showed no differences in GII $\alpha$ expression in an ADPLD patient with $G A N A B$ mutation c. $2515 \mathrm{C}>\mathrm{T}$ compared to primary cholangiocytes obtained from a patient without PLD. This indicates in this patient no loss of heterozygosity occurs in cholangiocytes lining the hepatic cysts. Conclusion: We describe six novel GANAB mutations that can cause PLD in a mixed population of ADPKD and ADPLD patients. These mutations are found in functionally important domains of $\alpha$-subunit of glucosidase II, which may lead to impaired enzymatic activity of the complex. In contrast to other PLD related genes no loss of heterozygosity was found for $G A N A B$ in cyst epithelium.

Disclosure of Interest: All authors have declared no conflicts of interest.

\section{P0673 DIFFERENCES BETWEEN BY-PASS AND SLEEVE} GASTRECTOMY ON CLINICAL AND LABORATORY STATUS 6 AND 12 MONTHS AFTER INTERVENTION IN BARIATRIC SUBJECTS

D. Macor $^{1}$, S. Palmisano ${ }^{2}$, F. Masutti ${ }^{1}$, V. Lanzlotti ${ }^{3}$, R. Patti ${ }^{1}$, R. M. Buonocore ${ }^{4}$, C. Abazia ${ }^{4}$, N. De Manzini ${ }^{2}$, L. S. Croce', , C. Tiribelli

${ }^{1}$ Fondazione Italiana Fegato, Centro Studi Fegato, Basovizza/Italy ${ }^{2}$ Chirurgia Generale, ASUITS Ospedale Cattinara, Trieste/Italy ${ }^{3}$ Dipartimento Universitario Clinico Di Scienze Mediche Chirurgiche E Della Salute, Università degli Studi di Trieste, Trieste/Italy

${ }^{4}$ Clinica Patologie Del Fegato, ASUITS Ospedale Cattinara, Trieste/Italy

Contact E-mail Address: cpf@asuits.sanita.fvg.it

Introduction: In patients with morbid obesity, dietary treatment and physical activity are the first line of treatment, but if not responding, bariatric surgery is the only validated alternative. The main surgical procedures are sleeve gastrectomy (SG) and gastric bypass (GBP), and they are chooses in function of BMI, age and comorbidity. Both techniques have proven effective in weight loss. It is known that liver fibrosis evaluation with Point Shear Wave Elastography (pSWE) is difficult in these patients.

Aims \& Methods: To study the difference between SG and GB and their impact on main clinical and laboratory hepatic metabolic indicators and scores 6 and 12 months after the intervention and pSWE at 12 months. We studied 68 obese subject candidate to bariatric surgery ( 45 female, 23 male). 28 underwent GBP and $40 \mathrm{SG}$. Blood tests, physical examination were assessed before surgery, after 6 months (68 patients) and after 12 months (51 patients) and pSWE after 12 months.

Results: In the comparison between GBP vs SG there was a statistically significant difference in the reduction in Fatty Liver Index (61\% vs $37 \%, \mathrm{p}=0.015)$, waist circumference $(26 \%$ vs $18 \%, \mathrm{p}=0.045)$, BMI $(34 \%$ vs $28 \%, \mathrm{p}=0,016)$, total cholesterol $(23 \%$ vs $0,05 \%, p=0,001)$, ALT (increased by $15 \%$ in GBP decreased by $27 \%$ in $\mathrm{SG}, \mathrm{p}=0,023$ ) while no differences were observed in the other indicators considered. Ferritin level increased (52\%) in SG and decreased $(25 \%)$ in GBP $(p=0.02)$. No difference was observed for pSWE.

Conclusion: This study showed some significant differences in clinical and laboratory terms between the two types of intervention, in fact GBP seems to have a more powerful effect on weight loss and all related markers: all steatosis scores (FLI, HSI, LAP), BMI, waist circumference. This can be explained by better malabsorbitive effect of this intervention and by a lower BMI starting point for reasons related to the intervention technique.

Disclosure of Interest: All authors have declared no conflicts of interest.

\section{References}

Am J Gastroenterol. 2014 Sep;109(9):1404-14. doi: 10.1038/ajg.2014.155. Epub 2014 Jun 24. 
Development, external validation, and comparative assessment of a new diagnostic score for hepatic steatosis. Meffert PJ1, Baumeister SE1, Lerch MM2, Mayerle J2, Kratzer W3, Völzke H1 Clin GastroenterolHepatol. 2013 Sep;11(9):1201-4. doi: 10.1016/j.cgh.2012.12.031. Epub 2013 Jan 22.

External validation of the fatty liver index for identifying nonalcoholic fatty liver disease in a population-based study. Koehler EM1, Schouten JN, Hansen BE, Hofman A, Stricker BH, Janssen HL.

\section{P0674 AUTOSOMAL DOMINANT POLYCYSTIC LIVER DISEASE IS A RISK FACTOR TO HAVE A LARGER LIVER VOLUME COMPARED WITH PATIENTS WITH COMBINED POLYCYSTIC LIVER DISEASE AND AUTOSOMAL POLYCYSTIC KIDNEY DISEASE: RESULTS OF THE PLD REGISTRY}

R. Van Aerts ${ }^{1}$, M. De Jong ${ }^{1}$, W. Kievit ${ }^{1}$, F. Nevens ${ }^{2}$, H. Kim ${ }^{3}$, C. Ahn ${ }^{3}$, J.P.h. Drenth ${ }^{1}$

${ }^{1}$ Gastroenterology And Hepatology, Radboudumc Nijmegen, Nijmegen

Netherlands

${ }^{2}$ UZ Leuven, Leuven/Belgium

${ }^{3}$ Seoul National University Hospital, Seoul/Korea, Republic of

Contact E-mail Address: rene.vanaerts@radboudumc.nl

Introduction: Polycystic liver disease (PLD) occurs in the setting of 2 different genetic disorders: autosomal polycystic liver disease (ADPLD) and autosomal polycystic kidney disease (ADPKD). These patients may develop hepatomegaly as a result of multiple fluid-filled cysts. It is unclear whether PLD severity differs between ADPLD and ADPKD. Height adjusted liver volume (htTLV) reflects with symptomatic disease and diminished quality of life. We assessed hepatomegaly with htTLV, as an objective parameter, in a large cohort of ADPKD and ADPLD patients

Aims \& Methods: PLD patients, defined by $>10$ liver cysts on radiological imaging, were included in the international PLD registry. The cases were identified from clinical records at the University Leuven (Belgium), Seoul National University Hospital (South-Korea) and Radboud University Hospital Nijmegen (the Netherlands). For this cross-sectional analysis, we selected patients when height adjusted total liver volume was measured prior to liver reducing therapy. We performed univariate and multivariate analyses to explore risk factors associated with severity of disease.

Results: Out of a total 1674 patients in the PLD registry, 1222 patients $(1110$ ADPKD, 112 ADPLD) could be selected. In the ADPKD + PLD group height adjusted liver volume is significantly lower compared with ADPLD patients $(1050 \mathrm{ml} / \mathrm{m}$ vs $1922 \mathrm{ml} / \mathrm{m} ; \mathrm{p}=.000)$. Females have higher htTLV than men in both ADPLD and ADPKD. Severe disease (htTLV $>3.200 \mathrm{~mL} / \mathrm{m}$ ) is more prevalent in ADPLD. Diagnosis, gender and age are independent predictors for severity of disease.

Conclusion: In this cohort more ADPLD patients had moderate to severe PLD compared to those with ADPKD. Longitudinal studies are needed to further explore the differences in national course of PLD phenotype between ADPLD and ADPKD patients and to identify new risk factors.

Disclosure of Interest: All authors have declared no conflicts of interest.

P0675 CARDIOVASCULAR RISK DEVELOPMENT MODEL FOR THE (ATYPICAL) PATIENT WITH NON-ALCOHOLIC FATTY LIVER DISEASE

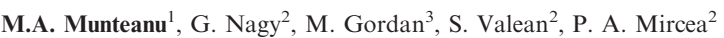

${ }^{1}$ Internal Medicine, University of Oradea, Faculty of Medicine and Pharmacy, Oradea/Romania

${ }^{2} 1$ st Medical Clinic, University of Medicine and Pharmacy "Iuliu Hatieganu" ClujNapoca, Cluj-Napoca/Romania

${ }^{3}$ The Techinal University of Cluj-Napoca, Cluj-Napoca/Romania

Contact E-mail Address: mihaimunteanual@yahoo.com

Introduction: Non-alcoholic fatty liver disease (NAFLD) affects about 1 billion people worldwide. Those with non-alcoholic steatohepatitis (NASH) among NAFLD patients have increased mortality rates compared to the general population, with cardiovascular diseases beeing the leading cause of death. Identifying patients at risk of developing cardiovascular events is of major importance, both in terms of prognosis, as in the terms of therapeutic attitude.

Aims \& Methods: Our aim was to cuantify the risk of developing atherosclerosis as a main cardiovascular risk factor, in NAFLD patients and to identify a screening strategy for those patients. We included patients with NAFLD and metabolic syndrome (MS) into 2 arms: with NAFLD and MS, and with NAFLD without MS. NAFLD diagnosis was based on clinical, biological and ultrasound determinations. We used Fibromax for evaluating the hepatic modifications (presence of NASH, the degree of steatosis and the stage of fibrosis; F3-F4 counted as advanced fibrosis). We performed ultrasound measurement of the carotid intimamedia thickness (in the common carotid artery, $1 \mathrm{~cm}$ before the bifurcation). Values above $0.07 \mathrm{~cm}$ were consider pathologic and values above $0.12 \mathrm{~cm}$ were considered as atherosclerotic (ats) plaques. Routine antropometric and laboratory determinations were performed.

Results: We included 95 patients with NAFLD, from which 53 were with MS and 43 were without MS. In all patients with NAFLD, with and without MS, there was a correlation between the value of intima-media thickness and the presence of NASH $(p=0.025)$, the degree of steatosis $(p=0.027)$ and the severity of fibrosis $(\mathrm{p}<0.001)$. Patients with NAFLD and MS had higher values pf the intima-media thickness than those with NAFLD, but without MS $(p=0.002)$
In all patients with NAFLD, there was a correlation between the presence of ats plaque and advanced fibrosis $(\mathrm{p}<0.001)$. The sex of the patients $(\mathrm{p}=0.638)$ and the level of total cholesterol $(p=0.438)$, LDL cholesterol $(p=0.505)$, HDL-cholesterol $(p=0.458)$, and triglycerides $(p=0.911)$ were not correlated with the presence of ats plaque, nor on the entire group of patients or on each arm. In the logistic model for the prediction of the risk of developing ats plaque, we included presence of NASH, the grade of steatosis, the stage of fibrosis, body mass index, abdominal circumference and creatinine clearence level. In this model, the stage of fibrosis has been shown to be a predictor with a positive effect on the presence of ats plaque $(\mathrm{p}=0.004$, adjusted $\mathrm{OR}=7.19$, CI $[1.86 ; 27.72])$. This model correctly classified $90.9 \%$ of patients with ats plaque and $91.7 \%$ of patients without ats plaque.

Conclusion: NAFLD patients were found to be atypical for the development of cardiovascular risk. Staging of fibrosis has proved to be an important prognostic factor. On the other hand, identifying NAFLD patients with ats plaques might select the patients in which liver biopsy might be indicated for better characterization of the liver disease. Screening and agressive treatment of cardiovascular risk factors should be done in every NAFLD patient.

Disclosure of Interest: All authors have declared no conflicts of interest.

\section{P0676 DEVELOPMENT AND VALIDATION OF AN AUTOMATED SYSTEM FOR ASSESSMENT OF LIVER STEATOSIS AND FIBROSIS IN ROUTINE HISTOLOGICAL IMAGES FROM PATIENTS WITH NAFLD}

R. Forlano ${ }^{1}$, B.H. Mullish ${ }^{1}$, J. Maurice ${ }^{1}$, N. Giannakeas ${ }^{2}$, N. Angkathunyakul ${ }^{3}$, J. Lloyd ${ }^{1}$, A. Tzallas ${ }^{1}$, M. Tsipouras ${ }^{4}$, M. Yee ${ }^{5}$, S. Taylor-Robinson ${ }^{1}$, R.D. Goldin ${ }^{3}$, M. Thursz ${ }^{1}$, P. Manousou

${ }^{1}$ Department Of Hepatology, Imperial College London, London/United Kingdom ${ }^{2}$ Technological Educational Institute, Epirus/Greece

${ }^{3}$ Imperial College London, Cellular Pathology, London/United Kingdom

${ }^{4}$ Informatics and Telecommunication Engeneering, Macedonia/Greece

${ }^{5}$ Endocrinology, London/United Kingdom

Contact E-mail Address: r.forlano@imperial.ac.uk

Introduction: Liver biopsy is the reference standard for diagnosing and staging non-alcoholic fatty liver disease (NAFLD). Steatosis grade and fibrosis stage are typically reported using semi-quantitative scores. Inter-and intra-observer variability in the current scoring systems may impact upon histological staging, and consequently upon the interpretation of responses to interventions in clinical trials.

Aims \& Methods: We developed an automated method for steatosis and fibrosis quantitation using biopsies of NAFLD patients. We further validated Liver Stiffness Measurements (LSM) and controlled attenuation parameter (CAP) in this group, using quantitative assessment as reference. 246 consecutive patients with biopsy-confirmed NAFLD and transient elastography within 3 months of the biopsy were evaluated. Biopsies were independently scored by two histopathologists and digitalised at 2x magnification. Areas of steatosis and fibrosis were annotated manually using the NDP.view2 to facilitate machine learning. Each image was then analysed by the automated software: fat percentage (fat $\%$ ) and Collagen ProportionateArea (CPA) computed by the software were compared with the manual annotation. They were also correlated with LSM and CAP.

Results: There was an excellent concordance between manual and automatic measurements, with inter-class correlation coefficient, ICC $=0.98$, $(95 \% \mathrm{CI}=0.96-0.99, \mathrm{p}=0.0001)$. There was good correlation between fat $\%$ and steatosis grade, but with significant overlap between groups. Results were similar between CPA and fibrosis stage.LSM was significantly associated with CPA $(\mathrm{Rho}=0.8, \mathrm{p}=0.001)$.CAP score correlated significantly with fat $\%$ (Rho $=0.45, p=0.002$ ) and effectively diagnosed steatosis $>5 \%$ (AUROC $0.82,95 \%$ sensitivity, $60 \%$ specificity), but could not distinguish between grades. Conclusion: We have developed an automated software, using low-resolution images to provide a rapid, easily performed, objective assessment of steatosis and fibrosis in NAFLD, with excellent correlation with experts' annotation. Objective measures would be helpful in the assessment of therapeutic response in clinical practice and in clinical trials for patients with non-alcoholic fatty liver disease.

Disclosure of Interest: All authors have declared no conflicts of interest.

\section{P0677 THE IMPORTANCE OF FIBROSIS SCORES AND} TRANSIENT ELASTOGRAPHY IN NAFLD EVOLUTION

D. Neagoe $^{1}$, A. Amzolini ${ }^{1}$, G. Ianosi ${ }^{2}$, M. Popescu ${ }^{3}$, A. Farmazon ${ }^{1}$, S. Ianosi ${ }^{1}$, A. Turculeanu ${ }^{4}$

${ }^{1}$ Internal Medicine, University of Medicine and Pharmacy, Craiova/Romania ${ }^{2}$ Surgical Department, University of Medicine and Pharmacy, Craiova/Romania ${ }^{3}$ Endocrinology, University of Medicine and Pharmacy, Craiova/Romania

${ }^{4}$ Clinical Laboratory, University of Medicine and Pharmacy, Craiova/Romania

Contact E-mail Address: dananeagoe2014@gmail.com

Introduction: Today, nonalcoholic fatty liver disease (NAFLD) is the most prevalent form of liver disease and it is an increasingly frequent cause of cirrhosis. Although several factors have been associated with the disease, the biological basis of the histological diversity of severity of NAFLD remains unknown. Several relatively noninvasive parameters have been identified as predictive for advanced fibrosis stage in patients with NAFLD, but none of them has sufficient sensitivity or specificity to replace liver biopsy. 
Aims \& Methods: Aim of our study was to compare two non-invasive methods: fibrosis scores based on serum markers and transient elastography Fibroscan (TE). We included 152 patients with NAFLD, 40 males $(26.31 \%)$ and 112 females with age from 23 to 79 years. 35 patients $(23.02 \%)$ were overweight, 9 patients had normal weight and $24(15.79 \%)$ had severe obesity. In all patients we calculated BMI and fibrosis scores: BARD, FIB-4 and NAFLD fibrosis score (NAFLD-FS). Blood samples were collected to determinate aminotransferases, glucose, albumin level, platelet count. The abdominal ultrasonography was performed by the same physician and steatosis was graded using a semi-quantitative scale of 1 (mild) to 3 (severe).TE was, also, performed by a single physician using conventional $\mathrm{M}$ probe or XL probe, with 10 valid acquisitions. We considered significant fibrosis (F2) when estimated cutoff of $\mathrm{F} 2$ was $7.1 \mathrm{kPa}$, severe fibrosis (F3) when cutoff value was $9.5 \mathrm{kPa}$, and cirrhosis (F4) with cutoff value $\geq 12.5 \mathrm{kPa}$

Results: $86.84 \%$ patients had metabolic syndrome and $51.31 \%$ had diabetes mellitus.40 patients had mild steatosis, 59 had moderate and 53 had severe steatosis. After we performed TE $69.07 \%$ of patients had no significant fibrosis, $14.47 \%$ had F2, $9.86 \%$ had F3 and $7.23 \%$ had F4. The area under the receiver-operating characteristic curve (AUROC) of TE was $0.823(95 \%$, $0.252-0.394)(\mathrm{p}<0.0001)$. Sensibility and specificity for cutoff $7.1 \mathrm{kPa}$ was 0.74 respectively 0.79 to exclude significant fibrosis. NAFLD-FS correlated statistic significant with TE $(\mathrm{p}<0.0001)$. BARD score did not correlate with TE and NAFLD-FS for significant fibrosis.FIB-4 correlated with TE for high degree fibrosis $(\mathrm{p}=0.004)$.

Conclusion: NAFLD-FS, FIB-4 and TE can be used together to evaluate the progression of fibrosis in NAFLD and to select the patients for liver biopsy. In our study BARD score was not useful in detection of high degree fibrosis. Disclosure of Interest: All authors have declared no conflicts of interest.

\section{P0678 EFFECTS OF UDCA AND STATIN COMBINATIONS ON LIPID PROFILE IN NAFLD PATIENTS}

S. S. Vyalov

Gastroenterology, Peoples Friendship University of Russia, Moscow/Russian Federation

Contact E-mail Address: svialov@mail.ru

Introduction: Dyslipidemia has an important role in NAFLD and inflammation progress and insulin resistance development. The studies suggested the role of UDCA in lipid profile correction. UDCA replaced bile acid balance and improved triglyceride and cholesterol levels in NAFLD patients, has immunomodulatory action.

Aims \& Methods: The aim of study was estimation of the efficacy of different dosage of ursodeoxycholic acid (UDCA) with statin combination treatment in patients with NASH and NAFLD. There are 180 patients with NAFLD. It was divided into two subgroups: fatty liver (90 subjects) - patients with normal level of ALT, and nonalcoholic steatohepatitis (NASH, 90 subjects) - patients with elevated level of ALT (the median is $78.5 \mathrm{U} / 1$ ). All patients was divided in 3 groups. The blood test, liver enzymes, lipid profile, HOMA-IR, Fibroscan and stool test was checked every 2 weeks of treatment. First group was taken UDCA $10 \mathrm{mg} / \mathrm{kg} /$ day + Statin. The patients of first group continue treatment for 3 months. Second group was taken UDCA $15 \mathrm{mg} / \mathrm{kg} / \mathrm{day}+$ Statin. Third group were fed the low-lipid and low-glycemic index diet only $(900 \mathrm{kcal} / \mathrm{day})$. After 3 months of treatment patients of third group (diet only) add UDCA $15 \mathrm{mg} / \mathrm{kg} / \mathrm{day}$ to treatment for extra 3 months.

Results: The subgroups did not show any difference in terms of initial total cholesterol (TC), low-density lipoprotein cholesterol (LDL-C), and triglycerides (TG). Analysis of the lipid spectrum showed a more intense dynamics in the group of patients taken UDCA combined with statin. After 3 months of follow-up there was a significant reduction in TC to $4.2 \mathrm{mmol} / 1$, LDL-C to $1.8 \mathrm{mmol} / 1$ and $\mathrm{TG}$ to $1.2 \mathrm{mmol} / 1$ in the fatty liver subgroup, and TC to $4.1 \mathrm{mmol} / 1$, LDL-C to $1.8 \mathrm{mmol} / 1$ and TG to $1.2 \mathrm{mmol} / 1$ in the NASH subgroup. Changes were similar in the subgroups. The level of total cholesterol, triglyceride and liver enzymes were decreased faster in group taken UDCA and statin independent from UDCA dosage. There are no side effects or liver enzyme elevation in group treated with UDCA and statins. In the NASH subgroup there was a significant decrease in ALT to $35 \mathrm{U} / 1$. At the end of the first month of statin therapy combined with UDCA in the NASH subgroup a significant positive dynamics of ALT was found in patients with NASH (initially $78.5 \mathrm{U} / 1$; after treatment decrease to $38.5 \mathrm{U} / 1)$. Stage of liver fibrosis was significant in patients taken UDCA and statin, as evidenced by the decrease in the index of fibrosis. Conclusion: 3-month statin therapy in combination with UDCA showed significant lipid-lowering effects in patients with NAFDL, as well as normalization of ALT in the NASH subgroup. Among the patients who previously had not taken statins in the NASH subgroup, higher levels of TC and TG were observed. The same subgroup showed more evident lipid-lowering effects than the fatty liver subgroup during the first month of the treatment. There are no differences between the dosages of UDCA.

Disclosure of Interest: All authors have declared no conflicts of interest.
P0679 EFFECT OF GRANULOCYTE COLONY-STIMULATING

FACTOR (G-CSF) ON MORTALITY AND COMPLICATIONS VIZ. SEPSIS, ENCEPHALOPATHY, HEPATORENAL SYNDROME, AND GASTROINTESTINAL BLEED IN SEVERE ALCOHOLIC HEPATITISA RANDOMIZED CONTROLLED STUDY

\section{A. Sharma}

Gastroenterology And Hepatology, Fortis Escorts Hospital Jaipur, Jaipur/India

Contact E-mail Address: abhinav11005@yahoo.com

Introduction: Severe alcoholic hepatitis has very high short-term mortality. Compared to standard medical therapy (SMT), GCSF improves clinical and biochemical profiles, morbidity and mortality in these patients. We evaluated efficacy of GCSF in modulating the disease course of severe alcoholic hepatitis over a period of 3 months in terms of mortality, morbidity by Discriminant function (mDF), Child-Turcotte-Pugh (CTP) and Model for End-Stage Liver Disease (MELD) scores and various complications viz. sepsis, GI bleed, encephalopathy, hepatorenal syndrome (HRS) in comparison to SMT. We also studied the mobilising effect of GCSF on bone marrow stem cells measured by counting CD34+ cells from peripheral blood.

Aims \& Methods: The present study was performed to evaluate the safety and efficacy of GCSF on mortality and complications viz. sepsis, encephalopathy, hepatorenal syndrome (HRS) and gastrointestinal bleed (GI Bleed) and also to investigate whether G-CSF therapy could improve the indices of severity of liver disease, such as Discriminant function (mDF), Child-Turcotte-Pugh (CTP), Model for End-Stage Liver Disease (MELD) score in patients with severe alcoholic hepatitis. 50 patients with severe alcoholic hepatitis were randomly assigned to groups A and B (25 in each). Both groups were given SMT, while in addition, patients in group A were given $5 \mu / \mathrm{kg}$ GCSF subcutaneous ( 10 doses for 5 days). We assessed survival, changes in CTP, MELD and mDF scores and the development of complications till 90 days.

Results: The baseline parameters in both groups were comparable. On day 6 group A had higher mean leukocyte and CD34 counts than group B $(\mathrm{P}<.001)$. On 90 days follow up 17 patients in group A $(68 \%)$ and 9 in group B $(36 \%)$ survived $(\mathrm{p}=.04)$. Mean changes for different scores were greater in group A then group B i.e. CTP $(-41.97 \%$ vs $-8.84 \%)$, MELD $(-50.89 \%$ vs $10.09 \%)$ and $\mathrm{mDF}(-74 \%$ vs $18 \%)(\mathrm{p}<.001)$. The percentages of patients who developed HRS, HE, or sepsis were lower in group A than in group B $(28 \%$ vs $64 \%, 32 \%$ vs $64 \%$, and $28 \%$ vs $68 \%$, respectively) $(\mathrm{p}<.001)$. There was no significant difference in GI bleed in both groups.

Conclusion: In severe alcoholic hepatitis, GCSF therapy significantly improves the survival. It also significantly reduces CTP, MELD, and $\mathrm{mDF}$ scores and prevents the development of complications.

Disclosure of Interest: All authors have declared no conflicts of interest.

\section{References}

1. Terai S, Ishikawa $\mathrm{T}$, Omori $\mathrm{K}$, Aoyama $\mathrm{K}$, Marumoto $\mathrm{Y}$, Urata $\mathrm{Y}$, Yokoyama Y, Uchida K, Yamasaki T, Fujii Y, OkitaK, Sakaida I. Improved liver function in patients with liver cirrhosis after autologous bone marrow cell infusion therapy. Stem Cells 2006; 24: 2292-2298

2. Tseleva T, Brenner DA. The phenotypic fate and functional role for bone marrow-derived stem cells in liver fibrosis. J Hepatol 2012; 56: 965-972

3. Spahr L, Lambert JF, Brandt LR et al. Granulocyte-colony stimulating factor induces proliferation of hepatic progenitors in alcoholic steatohepatitis: a randomized trial. Hepatology 2008; 48: 221-9.

4. Virendra Singh et al Granulocyte Colony-Stimulating Factor in Severe Alcoholic Hepatitis: A Randomized Pilot Stud Am J Gastroenterol 2014; 109:1417-1423

5. Theocharis SE, Papadimitriou LJ, Retsou ZP, et al. Granulocytecolony stimulating factor administration ameliorates liver regeneration in animal model of fulminant hepatic failure and encephalopathy. Dig Dis Sci 2003:48:1797-1803

6. Gaia S, Smedile A, Omede P, et al. Feasibility and safety of G-CSF administration to induce bone marrow derived cells mobilization in patients with end stage liver disease. $J$ Hepatol 2006;45:13-19.

7. Garg V, Garg H, Khan A et al. Granulocyte-colony stimulating factor mobilizes CD34+ cells and improves survival of patients with acute on chronic liver failure. Gastroenterology 2012; 142: 505-12

\section{P0680 ALCOHOLIC LIVER DISEASE/NON ALCOHOLIC FATTY LIVER DISEASE INDEX (ANI): HOW TO DISTINGUISH ALCOHOLIC FROM NON ALCOHOLIC LIVER DISEASE WITHOUT HISTOLOGY}

R. Gaspar $^{1}$, J. Santos-Antunes ${ }^{2}$, S. Rodrigues ${ }^{3}$, F. Carneiro ${ }^{4}$, G. Macedo ${ }^{5}$ ${ }^{1}$ Gastrenterology, Hospital São João, Porto/Portugal

${ }^{2}$ Gastroenterology Department, Centro Hospitalar S. João, Porto, Portugal, Porto/Portugal

${ }^{3}$ Centro Hospitalar De São João, Porto/Portugal

${ }^{4}$ Pathology Department Centro Hospitalar São João, Porto/Portugal

${ }^{5}$ Centro Hospitalar São João, Porto Medical School, Porto/Portugal

Contact E-mail Address: ruilopesgaspar@gmail.com

Introduction: Steatosis/steatohepatitis is one of the most common liver diseases with increasing prevalence and results from excessive alcohol consumption (alcoholic liver disease) or as nonalcoholic fatty liver disease (NAFLD). The differential diagnosis is of paramount importance as they have different management and therapeutic approaches, being liver biopsy the gold standard for establishing the diagnosis. The distinction between these two entities without biopsy is 
difficult due to the unreliable history of alcohol consumption and lack of sensibility and specificity of a single marker. In order to overcome these difficulties, ANI (alcoholic liver disease/nonalcoholic fatty liver disease index) was created for a non-invasive determination of fatty liver diagnosis.

Aims \& Methods: The aim of this study was to evaluate the reliability of ANI as a noninvasive method to distinguish NAFLD from ALD. A retrospective study between 2010 and 2015 in patients with definite diagnosis of NAFLD and ALD based on clinical, biochemical and histological criteria was performed. ANI scoring system in the differentiation of ALD and NAFLD was evaluated through the area under receiver-operating curve (AUROC). ANI score was calculated through Mayo Clinic formula.

Results: This study was carried out in 22 patients with ALD and 120 with NAFLD, 87 men $(61.3 \%)$ with a median age of $51 \pm 13$ years. NAFLD patients presented a body mass index (BMI) of $28.9 \pm 5.9$ vs $23.9 \pm 6$ in ALD. ANI showed a sensitivity of $81 \%$ and specificity of $79 \%$ for the diagnosis of ALD with a cut-off value of -1.96 [AUROC $0.806(0.715-0.898), \mathrm{p}<0.001$ ]. ANI greater than - 1.96 indicates a diagnosis of ALD whereas ANI less than -1.96 indicates a greater probability of NAFLD.

Conclusion: ANI scoring system is a non invasive diagnostic and reliable tool that may be used to distinguish NAFLD from ALD, decreasing the need for live biopsy. ANI greater than -1.96 suggests the diagnosis of ALD and ANI lesser than -1.96 suggest NAFLD

Disclosure of Interest: All authors have declared no conflicts of interest.

\section{P0681 TO DRINK OR NOT TO DRINK? A PROSPECTIVE COHORT STUDY ON THE EFFECTS OF ONE MONTH ALCOHOL ABSTINENCE IN MODERATE DRINKERS}

I. Munsterman ${ }^{1}$, M. Groefsema ${ }^{2}$, G. Weijers ${ }^{3}$, W. Klein ${ }^{4}$, D.w. Swinkels ${ }^{5}$, J.P.h. Drenth ${ }^{1}$, A. Schellekens ${ }^{6}$, E. Tjwa

${ }^{1}$ Gastroenterology \& Hepatology, Radboud University Medical Center, Nijmegen/ Netherlands

${ }^{2}$ Behavioural Science Institute, Radboud University, Nijmegen/Netherlands

${ }^{3}$ Medical Ultrasound Imaging Center, Radiology And Nuclear Medicine, Radboud university medical center, Nijmegen/Netherlands

${ }^{4}$ Radiology And Nuclear Medicine, Radboud university medical center, Nijmegen/ Netherlands

${ }^{5}$ Department Of Laboratory Medicine, Translational Metabolic Laboratory,

Radboud university medical center, Nijmegen/Netherlands

${ }^{6}$ Psychiatry, Radboud university medical center, Nijmegen/Netherlands

Contact E-mail Address: isabelle.munsterman@radboudumc.nl

Introduction: Alcohol consumption is an accepted phenomenon in Western society, although harmful effects of excessive alcohol intake on health have been widely shown. Alcohol has a direct toxic effect on the liver, potentially leading to steatohepatitis and eventually liver fibrosis and cirrhosis. Excessive use is furthermore associated with obesity, hypertension, hypercholesterolemia and diabetes. Cessation of alcohol may reverse these effects to some degree. The biological effects of short-term abstinence from moderate alcohol consumption are not known.

Aims \& Methods: A prospective interventional study in 16 healthy moderate drinkers ( $\leq 3$ units alcohol/day or 21 units/week) who discontinued alcohol use for one month. Nine complete abstainers served as a control group. In both groups an extensive health evaluation was performed at three time points: at baseline (T0), at end of cessation (T4) and after one month of resuming alcohol intake (T8). Outcomes consisted of morphometrics (blood pressure, body mass index and body impedance analysis), transient and shear wave elastography to assess liver stiffness and computer assisted ultrasonography to assess liver fat. A blood panel consisted of liver enzymes, cholesterol, glucose and iron metabolism and inflammatory parameters. Several quality of life components were assessed with VAS scales.

Results: The intervention and control group were comparable on baseline in terms of age, gender and morphometrics. Prior to intervention the participants consumed on average $10.4 \pm 4.5$ units alcohol/week and when they resumed alcohol use, an average of $12.4 \pm 5.1$ units/week. During the intervention period, complete cessation was ascertained by blood parameters. Liver stiffness and liver fat percentage did not differ between both groups nor change within participants at any time point. Compared to baseline, GGT decreased at T4 (28 to $22 \mathrm{IU} / \mathrm{L}, \mathrm{p}=.001)$ and increased at $\mathrm{T} 8$ to $26 \mathrm{IU} / \mathrm{L}(\mathrm{p}=.010)$. AST showed a similar trend (T4: $\mathrm{p}=.071$ resp.T8: 0.021). Cessation of alcohol also led to a significant drop in total cholesterol and LDL. Interestingly, subcutaneous fat decreased significantly at T4 $(19.5 \mathrm{~mm}$ to $18.1 \mathrm{~mm}, \mathrm{p}=.008)$. Ferritin was higher at baseline in drinkers compared to controls (median $107 \mu \mathrm{g} / \mathrm{L}$ (IQR $52-280)$ vs. $55 \mu \mathrm{g} / \mathrm{L}(\mathrm{IQR} 21-100), \mathrm{p}=.027)$ and decreased at $\mathrm{T} 4$ to $86 \mu \mathrm{g} / \mathrm{L}$ $(\mathrm{p}=.053)$. All parameters remained unaltered in the control group during all time points. Physical fitness improved significantly within drinkers after cessation of alcohol, other parameters of quality of life remained stable and were similar between groups.

Conclusion: Metabolic and biochemical changes within the liver, amelioration of lipid profile and improvement of physical fitness can be observed after one month of alcohol cessation in moderate drinkers. These pilot results should be further assessed in larger cohorts but already endorse the public message to subdue and possibly even altogether cease alcohol use.

Disclosure of Interest: All authors have declared no conflicts of interest.
P0682 PROBIOTICS REDUCE ETHANOL-INDUCED HEPATIC INJURY BY MODULATING GUT MICROBIOTA AND INTESTINAL BARRIER INTEGRITY IN MICE

Y. Zhang, G. Chen, J. Sun

Gastroenterology, Zhongshan Hospital, Fudan University, Shanghai/China

\section{Contact E-mail Address: xiaowanzizy@163.com}

Introduction: Gut-liver axis plays an important role in the pathogenesis of ALD. The metabolic products of intestine enter the blood through the damaged intestinal mucosal barrier and cause liver damage. Nowadays, probiotics have been used in the prevention and treatment of a variety of diseases, including ALD. It has become a hot spot for the study of regulating intestinal micro ecological balance and enhancing intestinal barrier function, to prevent alcoholic liver injury. In this study, ALD mice were given probiotics to observe the protective effects on ALD and explore the possible mechanism.

Aims \& Methods: Gut-liver axis plays an important role in the pathogenesis of ALD. The metabolic products of intestine enter the blood through the damaged intestinal mucosal barrier and cause liver damage. Nowadays, probiotics have been used in the prevention and treatment of a variety of diseases, including ALD. It has become a hot spot for the study of regulating intestinal micro ecological balance and enhancing intestinal barrier function, to prevent alcoholic liver injury. In this study, ALD mice were given probiotics to observe the protective effects on ALD and explore the possible mechanism.

Results: $\mathrm{HE}$ and oil red $\mathrm{O}$ staining of liver tissues in alcohol group showed significant steatosis and inflammatory response, and probiotics treatment can reduce liver injury. Compared with the control group, the LPS of alcohol group was significantly higher than that of the control group $(62.36 \pm 3.05 \mathrm{vs}$ $12.23 \pm 1.19 \mathrm{pg} / \mathrm{ml}, \mathrm{p}<0.05)$ and TNF- $\alpha(779.4 \pm 10.01$ vs $272.4 \pm 6.78 \mathrm{pg} / \mathrm{ml}$, $\mathrm{p}<<0.05)$ was significantly increased. Probiotics can effectively ease endotoxemia $(35.63 \pm 1.84$ vs $62.36 \pm 3.05 \mathrm{pg} / \mathrm{ml}, \mathrm{p}<0.05)$ and inflammatory factors TNF- $\alpha(526.6 \pm 25.04$ vs $779.4 \pm 10.01 \mathrm{pg} / \mathrm{ml}, \mathrm{p}<0.05)$ induced by alcohol. RT-PCR results showed that probiotics increased the expression of Reg $3 b$ and Reg3g $(\mathrm{P}<0.05)$. Compared with the alcohol group, the mRNA levels of intestinal tight junction protein Occludin and Claudin-1 were increased with probiotics treatment $(\mathrm{P}<0.05)$. However, compared with the alcohol group, the mRNA level of ZO-1 showed no significant difference $(\mathrm{P}>0.05)$. WB results showed that the expression of Occludin and Claudin-1 increased in probiotic treatment group $(\mathrm{P}<0.05)$.

In addition, Bacteroides $(\mathrm{p}<0.05)$ and Bacteroides $(\mathrm{p}<0.05)$ were significantly increased in the intestine of the alcohol group. While the Clostridium $(p<0.05)$, Lactobacillus $(\mathrm{p}<0.05)$ and Coccidia $(\mathrm{p}<0.05)$ were decreased. With the treatment of probiotics, the levels of Enterococcus $(\mathrm{p}<0.05)$ and Enterobacteriaceae $(p<0.05)$ decreased, and the levels of Lactobacillus $(p<0.05)$ and Bifidobacterium $(\mathrm{p}<0.05)$ increased.

Conclusion: Probiotics showed protective effects on ALD by reducing alcoholinduced endotoxemia and liver inflammatory injury as well as improving the intestinal mucosal barrier and restoring the intestinal micro ecological imbalance. Disclosure of Interest: All authors have declared no conflicts of interest.

\section{References}

Dugum, M. and A. McCullough, Diagnosis and Management of Alcoholic Liver Disease. J Clin Transl Hepatol, 2015. 3(2): p. 109-16.

Szabo, G., Gut-Liver Axis in Alcoholic Liver Disease. Gastroenterology, 2015. 148(1): p. $30-36$.

Wang, L., et al., Intestinal REG3 Lectins Protect against Alcoholic Steatohepatitis by Reducing Mucosa-Associated Microbiota and Preventing Bacterial Translocation. Cell Host Microbe, 2016. 19(2): p. 227-39.

Vassallo, G., et al., Review article: alcohol and gut microbiota - the possible role of gut microbiota modulation in the treatment of alcoholic liver disease. Alimentary Pharmacology \& Therapeutics, 2015. 41(10): p. 917-927

Llopis, M., et al., Intestinal microbiota contributes to individual susceptibility to alcoholic liver disease. Gut, 2016. 65(5): p. 830-839.

\section{P0683 THE OVERWEIGHT ROLE IN THE OCCURRENCE OF HEPATOTOXIC REACTIONS DURING CHEMOTHERAPY OF ACUTE LEUKEMIA}

I. Skrypnyk, G. Maslova

Internal Medicine \#1, Ukrainian Medical Stomatological Academy, Poltava/ Ukraine

Contact E-mail Address: scrin69@yandex.ru

Introduction: Chemotherapy drugs have a direct hepatotoxic effect, which leads to multiple increase in the risk of liver injury in patients with acute leukemias (AL). The development of hepatotoxicity limits the chemotherapy (CT) providing in full doses.

Aims \& Methods: We aimed to assess the overweight role in an increase of risk level of hepatotoxic reactions in AL chemotherapy dynamics. The study involved 84 patients with newly diagnosed AL (64 - acute myeloid leukemia (AML), 20 acute lymphoblastic leukemia (ALL)), ECOG I-II, aged 24-67 years, 41(48.8\%) women, $43(51.2 \%)$ - men. The body mass index (BMI) was determined, the liver function was assessed by the activity of alanine aminotransferase (ALT), aspartate (AST) aminotransferase, alkaline phosphatase (ALP), gamma-glutamyltranspeptidase (GGT), total bilirubin, total protein in the blood serum three times: at baseline and on the 28 th and $56^{\text {th }}$ day after starting induction of remission therapy, according to the ALL (prednisolone, doxorubicin, vincristin, asparaginase, cyclophosphamide, cytarabine, mercaptopurine) and AML (cytarabine, doxorubicin) treatment protocols. To assess the severity of hepatotoxic reactions 
the CTCAE scale was used. Overweight was detected in 40 patients: BMI $=25$ 29.9 - in $29(34.5 \%)$, BMI $>30$ - in $11(13.1 \%)$ patients. Depending on the overweight presence the patients were divided into 2 groups: I $(n=44)$ - patients with AL with normal body weight, II $(\mathrm{n}=40)$ - patients with AL and overweight. Results: In AL patients of group I before the start of chemotherapy functional liver state was not significantly different from healthy people. In group II there was an increase of ALT activity in 1.5 times, AST - in 1.2 times, ALP and GGT in 1.4 times compared to the norm $(\mathrm{p}<0.05)$ and reached grade I level, and no change in bilirubin and total protein levels. On the 28th day of treatment in 3 $(6.8 \%)$ patients of group I the violation of the functional liver state was revealed, which was characterized by the increased activity of ALT in 1.5 times, AST - in 1.2 times, ALP - in 1.3 times, GGT - in 1.5 times compared to normal levels, that consistent with grade I level, the bilirubin and total protein levels remained in the normal range. In group II hepatotoxicity was detected in $19(47.5 \%)$, which was characterized by the increased activity of ALT and AST in 2.3 and in 2.2 times respectively, GGT and ALP in 1.9 and 2.4 times respectively, the level of total bilirubin increased in 2.1 times $(\mathrm{p}<0.05)$, of which in $17(42.5 \%)$ patients hepatotoxic reactions were of grade I and in $2(5 \%)$ - of grade II level, with no statistically significant changes in protein synthesis liver function. On the 56th day of treatment in $7(15.9 \%)$ patients of group I the violation of the functional liver state was revealed, which was characterized by the increased activity of ALT in 1.8 times, AST - in 1.3 times, ALP - in 1.6 times, GGT - in 1.9 times compared to normal levels, the bilirubin and total protein levels remained in the normal range, that consistent with grade I. In group II hepatotoxicity was detected in $26(65 \%)$, which was characterized by the increased activity of ALT and AST in 2.6 and in 2.3 times respectively, GGT and ALP in 2.6 and 3.7 times respectively, the level of total bilirubin increased in 3.6 times $(\mathrm{p}<0.05)$, of which in $10(25 \%)$ patients hepatotoxic reactions were of grade I and in $16(40 \%)$ - of grade II level.

Conclusion: The presence of the overweight results in a significant increase in the frequency and degree of hepatotoxic reactions in patients with AL during chemotherapy.

Disclosure of Interest: All authors have declared no conflicts of interest.

\section{P0684 RESEARCH ON FERROPTOSIS IN HEPATOCYTE INJURY INDUCED BY ALCOHOLIC}

\section{Y. Wu, T. Liu, G. Zhang}

Xiangya Hospitol, Central South University, Changsha/China

\section{Contact E-mail Address: liuting818@126.com}

Introduction: Alcohol abuse and alcohol dependence have become the world's growing public health problems. With the improvement of living standards in China, alcohol consumption is growing and the incidence of alcoholic liver injury also increased year by year. However, the mechanism of alcohol-induced liver injury is not clear yet, and its pathogenesis is mainly related to oxidative stress, lipid peroxi-dation, cytokines and mitochondrial dysfunction. Ferroptosis is an iron-dependent cell death and its inhibitors can improve cell biochemical and morphological changes ${ }^{[1]}$. The presence of Ferroptosis in liver injury induced by alcohol has not yet been reported

Aims \& Methods: We aim to investigate the presence of Ferroptosis and the expression of glutathione peroxidase $4(\mathrm{GPX} 4)^{[2]}$ in alcoholic liver injury by establishing a cytological model of acute alcoholic liver injury and animal model of alcoholic liver injury, and further elucidating the pathogenesis of liver injury caused by ethanol. 1. By recovering human primary hepatic cell lines to establish a model of acute alcoholic liver injury, Fer-1 and erastin were used to intervention, DMSO control. 2. The biochemical indexes ALT, Fe, GSH and MDA were detected by the kit. The expression of GPX4 in liver cells was detected by qRT-PCR and Western Blot. 3. To establish an animal model of alcoholic liver injury, Fer-1 and erastin were used to intervention, DMSO control. HE staining and electron microscopy were used to observe the changes of liver tissue morphology.

Results: 1, Acute alcoholic liver injury cytology model showed that in the untreated group, the $\mathrm{Fe}$ content is $0.809 \pm 0.140 \mathrm{nmol} / \mathrm{mg}$, GSH $14.978 \pm 0.398 \mathrm{nmol} / \mathrm{mg}$, MDA $3.402 \pm 0.546 \mathrm{nmol} / \mathrm{mg}$; The content of $\mathrm{Fe}$ in the alcoholic model group was $1.059 \pm 0.005 \mathrm{nmol} / \mathrm{mg}$, GSH $6.826 \pm 0.013 \mathrm{nmol} / \mathrm{mg}$ and MDA $8.598 \pm 0.669 \mathrm{nmol} / \mathrm{mg}$, which was statistically significant compared with the untreated group $(\mathrm{P}<0.05)$. The content of $\mathrm{Fe}$ in Ferroptosis inhibitor Fer- 1 group was $0.889 \pm 0.007 \mathrm{nmol} / \mathrm{mg}$, GSH $11.11 \pm 0.413 \mathrm{nmol} / \mathrm{mg}$, MDA $5.465 \pm 0.317 \mathrm{nmol} / \mathrm{mg}$, compared significantly with alcoholic liver model group $(\mathrm{P}<0.05)$. The expression of GPX4 mRNA in the untreated group, alcohol model group and Ferroptosis inhibitor group was $2.041 \pm 0.236,1.420 \pm 0.219,1.693 \pm 0.294$ respectively. The protein level of GPX4 respectively were $9.65 \pm 1.453,1.36 \pm 0.770$ and $4.04 \pm 0.709$. The results of the above analysis showed that there was significant difference bitween alcoholic model group and Ferroptosis inhibitor group at both mRNA and protein level of GPX4 $(\mathrm{P}<0.05)$. 2, Liver microelectiography showed that the size of mitochondria in liver cells of untreated group was normal. The mitochondrial atrophy and membrane density were increased in the liver alcoholic model group, suggesting that Ferroptosis occurred in the liver injury induced by alcohol. The size of the mitochondria was normal and the mitochondrial ridge was seen in the liver of the Ferroptosis inhibitor group.

Conclusion: 1, Ferroptosis exists in alcohol-induced liver injury. 2, The acute alcohol liver injury cell model showed GSH content decreased, Fe and MDA content increased and the expression of GPX4 decreased in both protein and mRNA levels. Ferroptosis inhibitor Fer-1 could improve the above biochemical changes and the expression of GPX4. 3, In the animal model of alcoholic liver injury, electron microscopy showed that there was Ferroptosis in mouse liver cells, which showed that the mitochondria of the liver cells were smaller and the mitochondrial membrane density was increased. The Ferroptosis inhibitor Fer-1 could improve the above mitochondrial morphological changes. Disclosure of Interest: All authors have declared no conflicts of interest.

\section{References}

1. Dixon SJ, Lemberg KM, Lamprecht MR, Skouta R, Zaitsev EM, Gleason CE, Patel DN, Bauer AJ, Cantley AM, Yang WS, Morrison B 3rd, Stockwell BR. Ferroptosis: an iron-dependent form of nonapoptotic cell death. Cell. 2012 May 25;149(5):1060-72.

2. Yang WS, SriRamaratnam R, Welsch ME, Shimada K, Skouta R, Viswanathan VS, Cheah JH, Clemons PA, Shamji AF, Clish CB, Brown LM, Girotti AW, Cornish VW, Schreiber SL, Stockwell BR. Regulation of ferroptotic cancer cell death by GPX4. Cell. 2014 Jan 16;156(1-2):317-31.

\section{P0685 AMANITA PHALLOIDES HEPATOTOXICITY: 20-YEAR} EXPERIENCE IN A GASTROENTEROLOGY INTENSIVE CARE UNIT

C. Atalaia Martins, J. Carvalheiro, R. Ferreira, P. Amaro, M. Ferreira, L. Tomé Gastroenterology, Centro Hospitalar e Universitário de Coimbra, Coimbral Portugal

Contact E-mail Address: catarinatalaiamartins@gmail.com

Introduction: Ingestion of amatoxin-containing mushrooms is a rare medical emergency. Amatoxin can cause massive hepatocyte necrosis and acute liver failure. Liver transplantion can be life saving but liver transplantation criteria are complex and not consensual. Mushrooms poisoning is associated with a high mortality rate.

Aims \& Methods: We conducted a retrospective analysis of demographic, clinical, therapeutic and prognostic data of all patients with Amanita phalloides poisoning admitted to a Gastroenterology Intensive Care Unit (GICU) of a tertiary hospital between 1997 and 2017 .

Results: A total of 27 patients were included: $55.6 \%$ were male and the mean age was $53 \pm 15$ years old (range, 16-75). The most frequent initial symptoms were vomiting $(100 \%)$, diarrhea $(88.9 \%)$ and abdominal pain $(74.1 \%)$. The mean time between ingestion and onset of symptoms was 10.1 hours and GICU admission was 50.9 hours. At admission $33.3 \%$ presented hepatic encephalopathy $(25.9 \%$ grade $1 ; 3.7 \%$ grade 3 and $3.7 \%$ grade 4 ). Laboratory characterization at admission: mean INR was 4 , total bilirubin $3.5 \mathrm{mg} / \mathrm{dL}$, creatinine $1.9 \mathrm{mg} / \mathrm{dL}$ and factor V $25.8 \%$. The different criteria for emergent liver transplantation were assessed: $37 \%(\mathrm{n}=10)$ met Clichy's criteria, $59.3 \%(\mathrm{n}=16)$ met King's College criteria, $33.3 \%(n=9)$ met Ganzert criteria and 40.7\% $(n=11)$ met Escudié criteria. Overall, $59.3 \%$ fulfilled criteria for liver transplantation: $25.9 \%$ on admission and $33.3 \%$ during hospitalization. Regarding specific medical treatment: $92.6 \%$ received silibinine, $59.3 \%$ acetylcysteine and $48.1 \%$ penicillin. In the group of patients with emergent liver transplantation criteria, $63 \%$ were effectively transplanted. In the sub-group of patients who met criteria but were not transplanted $(n=6)$ : in $66.7 \%(n=4)$ the reason was absence of donor, in $16.7 \%(n=1)$ there was liver function recovery and in $16.7 \%(n=1)$ a contraindication was found (irreversible intestinal ischemia diagnosed intraoperatively). The mortality in this sub-group was $83.3 \%$. The mortality rate in transplanted patients was $60 \%$. All patients without emergent liver transplantation criteria survived.

Conclusion: Amanita phalloides poisoning has a severe and rapidly progressive presentation, often with indication for liver transplant. Patients are admitted late in GICU. Mortality rate remains high even in transplanted patients.

Disclosure of Interest: All authors have declared no conflicts of interest.

\section{References}

1. Bonanci M., Shetler K., Yu I. et al. Features of Patients With Severe Hepatitis Due to Mushroom Poisoning and Factors Associated With Outcome. Clin Gastroenterol Hepatol 2017;15(5):776-779

2. Ferreira R., Romãozinho J.M., Amaro P. et al. Assessment of emergency liver transplantation criteria in acute liver failure due to Amanita phalloides. Eur $J$ Gastreoenterol Hepatol 2011; 23 (12): 1226-32

3. Mas A. Mushrooms, amatoxins and the liver. J Hepatol 2005; 42: 144-146

4. Ganzert M, Felgenhauer N, Zilker T. Indication for liver transplantation following amatoxin intoxication. J Hepatol. 2005; 42:202-209

5. Escudié L, Francoz C., Vinel JP et al. Amanita phalloides poisoning: Reassessment of prognostic factors and indications for emergency liver transplantation. J Hepatol. 2007; 46: 466-73

P0686 BINGE DRINKING AMONG YOUNG STUDENTS IS A RISK FACTOR FOR THE DEVELOPMENT OF ALCOHOL USE DISORDER: RESULTS FROM A CROSS-SECTIONAL STUDY

G. Addolorato ${ }^{1}$, G. A. Vassallo ${ }^{1}$, G. Antonelli ${ }^{2}$, M. Antonelli ${ }^{1}$, C. Tarli ${ }^{1}$, A. Mirijello ${ }^{1}$, C. Mosoni ${ }^{1}$, M. M. Rando ${ }^{1}$, L. Sestito ${ }^{1}$, M. Barbara ${ }^{3}$,

M.F. Maida ${ }^{3}$, C. Cammà ${ }^{3}$, A. Gasbarrini ${ }^{4}$

${ }^{1}$ Catholic University of Rome, Rome/Italy

${ }^{2}$ Digestive And Liver Diseases Unit, Sapienza Università di Roma at Sant'Andrea University Hospital, Roma/Italy

${ }^{3}$ Biomedical Department Of Internal And Specialized Medicine, University of Palermo, Italy, Palermo/Italy

${ }^{4}$ Internal Medicine, Gastroenterology And Liver Diseases, Gemelli Hospital Dept. of Internal Medicine Dept. of Gastroenterology, Rome/Italy 
Contact E-mail Address: gabriele.vassallo86@libero.it

Introduction: Binge drinking is a common pattern of alcohol consumption among young population. At present few data are available on the possible relationship between binge drinking and alcohol use disorder (AUD) in adolescent. The aim of this study was to assess drinking habits, patterns of alcohol consumption, smoking habits, use of illicit drugs, the prevalence of binge drinking and AUD among young students. The correlation between binge drinking and AUD was also investigated.

Aims \& Methods: This study was performed on 2704 subjects attending high school. Questionnaires regarding socio-demographic data, anthropometric characteristics, pattern and amount of alcohol intake, smoking habits, use of illicit drugs, and physical activity were administered to students. Moreover Italian versions of AUDIT, STAI-Y1, STAY-Y2 and ZUNG scale were administered. Results: Alcohol intake was reported by 2126 students (79\%); among them 1278 $(60 \%)$ reported at least one episode of binge drinking in the last year and 715 $(34 \%)$ in the last month. According to AUDIT questionnaires, a diagnosis of AUD was made in $165(6 \%)$ subjects. The prevalence of AUD was higher in subjects that reported binge drinking behavior than in those that did not report binge drinking $(\mathrm{p}<0.0001)$.

Conclusion: Alcohol consumption and abuse among young students is alarming. Binge drinking behavior among young students seems to be very common and it seems a risk factor for the development of AUD.

Disclosure of Interest: All authors have declared no conflicts of interest.

\section{P0687 LOW LYSOPHOSPHATIDYLCHOLINE LEVELS MAY PREDICT SEVERE ALCOHOLIC HEPATITIS}

P. Fischer ${ }^{1}$, A. Horhat ${ }^{1}$, C. Hebristean ${ }^{2}$, B.D. Procopet $^{1}$, M. Tantau ${ }^{3}$, C. Socaciu ${ }^{2}$, H. Stefanescu ${ }^{4}$

${ }^{1}$ Gastroenterology, Dr. O. Fodor" Regional Institute of Gastroenterology and

Hepatology., Cluj-Napoca, Romania, Cluj-Napoca/Romania

${ }^{2}$ Research and Development Centre BIODIATECH for Applied Biotehnology in

Diagnostic and Molecular Therapy, Cluj-Napoca/Romania

${ }^{3}$ Endoscopy, Regional Institute of Gastroenterology and Hepatology, University of

Medicine and Pharmacy Iuliu Hatieganu Cluj-Napoca, Cluj-Napoca/Romania

${ }^{4} 3$ rd Medical Clinic, University of Medicine and Pharmacy "Iuliu Hatieganu",

Cluj-Napoca/Romania

Contact E-mail Address: adelinahorhat25@gmail.com

Introduction: Severe alcoholic hepatitis (SAH) remains a condition which bears high mortality and morbidity rates, as well as high healthcare costs. This is why adequate selection of patients who would benefit the most from corticotherapy is of utmost importance. Although serum biomarkers are available (Maddrey Discriminant Function - MDF), the diagnostic of SAH relies on liver biopsy. Previous metabolomic studies have shown a core metabolic phenotype represented by decreased serum lysophosphatidylcholines (LPC) and increased serum bile acids that occurs relatively early in liver diseases regardless of etiology, and remains stable in their evolution, including liver cirrhosis and hepato/cholangiocarcinoma (1). Our previous work also showed that decreased LPC levels are associated with alcoholic liver disease (ALD).

Aims \& Methods: The aim of the study was to assess the metabolic profile of patients with ALD and to identify potential new biomarkers associated with severity. Between December 2015 and September 2016, 64 patients with biopsy proven $\mathrm{AH}$ were included ( 38 with $\mathrm{SAH}-\mathrm{MDF}>32$ and 24 with non-severe $\mathrm{AH}$ - MDF < 32). Fasting serum was stored at -80 degrees after centrifugation at $5000 \mathrm{rpm}$ for 10 minutes. Specific purification protocol metabolomic analysis was performed using Thermo Scientific UHPLC UltiMate 3000 system, equipped with a Dionex quaternary pump delivery system and a Bruker Daltonics MaXis Impact MS detection equipment (version 2012). Biostatistical analysis The chromatograms obtained were processed using CompassDataAnalysis_4.2 software (Bruker, Germany) and about 3000-4000 molecular masses were identified. Those data were further processed using ProfileAnalysis (Bruker, Daltonics): time alignment, normalization by sum of bucket values in analysis, $80 \%$ bucket filter, internal recalibration, etc. The matrix obtained was further processed by MetaboAnalysis, to analyze samples through univariate and multivariate statistical analysis.

Results: Univariate and multivariate statistical analysis by MetaboAnalysis identified 10 potential biomarkers. Among them, LPC (18:0) showed good discrimination for SAH $(\mathrm{AUC}=0.804)$ with significantly lower values as compared with non-severe AH $\left(0.38\right.$ fold change, $\left.p=6 \times 10^{-11}\right)$.

Conclusion: SAH appears to have a different metabolic profile, mainly due to changes in lysophosphatidylcholine metabolism. Targeted metabolomic studies are required in order to confirm the results and to evaluate the possible applications in current clinical practice.

Disclosure of Interest: All authors have declared no conflicts of interest.

\section{Reference}

Krautbauer, Sabrina, et al. "Systemic saturated lysophosphatidylcholine is associated with hepatic function in patients with liver cirrhosis." Prostaglandins \& other lipid mediators 124 (2016): 27-33.

\section{Abstract No: P0685}

\begin{tabular}{|c|c|c|c|c|c|c|c|c|c|c|}
\hline & Gender & Age & $\begin{array}{l}\text { Hours from } \\
\text { ingestion to } \\
\text { GICU admission }\end{array}$ & $\begin{array}{l}\text { Clichy's } \\
\text { criteria } \\
\text { (Yes/No) }\end{array}$ & $\begin{array}{l}\text { King's } \\
\text { College } \\
\text { criteria } \\
\text { (Yes/No) }\end{array}$ & $\begin{array}{l}\text { Ganzert } \\
\text { criteria } \\
(\mathrm{Yes} / \mathrm{No})\end{array}$ & $\begin{array}{l}\text { Escudié } \\
\text { criteria } \\
(\mathrm{Yes} / \mathrm{No})\end{array}$ & $\begin{array}{l}\text { Listed } \\
\text { to ELT } \\
(\mathrm{Yes} / \mathrm{No})\end{array}$ & $\begin{array}{l}\text { ELT } \\
(\mathrm{Yes} / \mathrm{No})\end{array}$ & Outcome \\
\hline Patient 1 & Male & 48 & 72 & No & Yes & No & Yes & Yes & Yes & $\begin{array}{l}\text { Died from acute myo- } \\
\text { cardial infarction } \\
\text { after ELT }\end{array}$ \\
\hline Patient 2 & Female & 29 & 72 & Yes & Yes & No & Yes & Yes & Yes & Alive \\
\hline Patient 3 & Female & 52 & 48 & Yes & Yes & Yes & Yes & Yes & No & Died, absence of donor \\
\hline Patient 4 & Male & 67 & 24 & Yes & Yes & Yes & Yes & Yes & Yes & Alive \\
\hline Patient 5 & Female & 55 & 72 & No & Yes & No & No & Yes & No & $\begin{array}{l}\text { Alive, hepatic function } \\
\text { recovery }\end{array}$ \\
\hline Patient 6 & Male & 40 & 48 & Yes & Yes & Yes & Yes & Yes & Yes & $\begin{array}{l}\text { Died from cardiopul- } \\
\text { monary arrest after } \\
\text { ELT }\end{array}$ \\
\hline Patient 7 & Male & 16 & 48 & No & Yes & No & Yes & Yes & Yes & Alive \\
\hline Patient 8 & Female & 66 & 48 & Yes & Yes & Yes & Yes & Yes & Yes & $\begin{array}{l}\text { Died from cardiopul- } \\
\text { monary arrest after } \\
\text { ELT }\end{array}$ \\
\hline Patient 9 & Male & 43 & 96 & Yes & Yes & Yes & Yes & Yes & No & Died, absence of donor \\
\hline Patient 10 & Male & 55 & 72 & Yes & Yes & Yes & Yes & Yes & No & Died, absence of donor \\
\hline Patient 11 & Male & 48 & 16 & Yes & Yes & Yes & Yes & Yes & Yes & Alive \\
\hline Patient 12 & Female & 64 & 24 & Yes & Yes & Yes & Yes & Yes & No & Died, absence of donor \\
\hline Patient 13 & Male & 63 & 56 & No & Yes & No & No & Yes & Yes & $\begin{array}{l}\text { Died, peritonitis after } \\
\text { ELT }\end{array}$ \\
\hline Patient 14 & Male & 68 & 37 & No & Yes & No & No & Yes & Yes & Died \\
\hline Patient 15 & Female & 63 & 56 & No & Yes & Yes & No & Yes & Yes & $\begin{array}{l}\text { Died from acute myo- } \\
\text { cardial infarction } \\
\text { after ELT }\end{array}$ \\
\hline Patient 16 & Male & 70 & 57 & Yes & Yes & No & No & Yes & No & $\begin{array}{l}\text { Died, irreversible } \\
\quad \text { intestinal ischemia }\end{array}$ \\
\hline
\end{tabular}


P0688 APPLICATION OF THE ICA-AKI CRITERIA IN THE DIAGNOSIS OF ACUTE KIDNEY INJURY IN PATIENTS WITH ACUTE DECOMPENSATION OF CIRRHOSIS

R. Azevedo, J. Pinto, H. Ribeiro, F. Pereira, C. Leitão, A. Caldeira, E. Pereira, R. Sousa, A. Banhudo

Gastroenterology, Amato Lusitano Hospital, Castelo Branco/Portugal

Contact E-mail Address: richardazevedo13@gmail.com

Introduction: Acute kidney injury (AKI) is a common complication in patient with decompensated liver cirrhosis. Recently, the International Club of Ascites (ICA) defined new diagnostic criteria: the ICA-AKI criteria.

Aims \& Methods: This study aims to identify patients hospitalized for acute decompensation of cirrhosis with AKI, according to the ICA-AKI criteria, and to determine if its application leads to greater prognostic accuracy.

Methods: Retrospective analysis of hospitalized patients in a gastroenterology department for acute decompensation of cirrhosis, without acute-on-chronic liver failure, between January 2014 and December 2015. Identification of AKI patients according to ICA-AKI criteria. Compared the hospital length of stay, severity of liver disease and in-hospital and short-term mortality among patients with and without AKI. Compared the accuracy of the conventional criteria vs. ICA-AKI criteria in the prediction of mortality.

Results: 161 patients included, $85.7 \%$ male, mean age of $65 \pm 10.8$ years. Average length of stay of $11.6 \pm 9.5$ days. $39.8 \%$ of patients had AKI on admission or during hospitalization according to the ICA-AKI criteria $(60.9 \%$ in stage 1 , $20.3 \%$ in stage 2 and $18.8 \%$ in stage 3). Patients with AKI according to ICAAKI had longer hospitalizations (14.55 vs. 9.78 days, $p<0.05$ ), higher severity of hepatic disease quantified by the MELD and MELD-Na scores (17.62 vs. 12.83 $511, \mathrm{p}<0.05$ and 17.61 vs. $12.83 \mathrm{P}<0.05)$ and higher in-hospital, 28 and 90 -day mortality when compared to patients without AKI $(23.4$ vs. $6.2 \%, \mathrm{p}<0.05,31.3$ vs. $9.3 \%, p<0.05,42.9$ vs. $23.7 \%, p<0.05)$. There was a statistically significant association between the presence of infection and the development of AKI $(\mathrm{p}<0.05)$. The ICA-AKI area under the curve (AUC) to predict in-hospital, 28 and 90-day mortality was significantly higher than the AUC of conventional criteria $(0.682$ vs $0.533 ; 0.678$ vs. $0.588 ; 0.618$ vs. $0.509, p<0.05)$

Conclusion: The ICA-AKI criteria allow the identification of decompensated cirrhotic patients in whom a worse prognosis is predicted. Thus, they constitute a useful tool in daily clinical practice.

Disclosure of Interest: All authors have declared no conflicts of interest.

\section{References}

1. Angeli P, Gines P, Wong F, et al. Diagnosis and management of acute kidney injury in patients with cirrhosis: revised consensus recommendations of the International Club of Ascites. J Hepatol. 2015;62(4):968-974. doi:10.1016/ j.jhep.2014.12.029.

2. Wong F. The evolving concept of acute kidney injury in patients with cirrhosis. Nat Rev Gastroenterol Hepatol. 2015;12(12):711-719. doi:10.1038/ nrgastro.2015.174

3. Scott RA, Austin AS, Kolhe N V, McIntyre CW, Selby NM. Acute kidney injury is independently associated with death in patients with cirrhosis. Frontline Gastroenterol. 2013;4(3):191-197. doi:10.1136/flgastro-2012-100291.

\section{P0689 NESTED CASE-CONTROL STUDY FOR RISK FACTORS OF HEPATIC ENCEPHALOPATHY IN PATIENTS WITH LIVER CIRRHOSIS}

L.T. E. Yuan ${ }^{1}$, S. Chuah ${ }^{2}$, S. Yang ${ }^{2}$, C. Liang ${ }^{2}$, C. Wu ${ }^{2}$, W. Tai ${ }^{2}$, T. Hung ${ }^{3}$, S. Nguang ${ }^{4}$, J. Wang ${ }^{5}, \mathrm{~K}$. Tseng ${ }^{6}, \mathrm{M} . \mathrm{Ku}^{7}, \mathrm{P} . \mathrm{Hsu}^{8}, \mathrm{D} . \mathrm{Wu}^{5}, \mathrm{C} . \mathrm{Hsu}^{9}$

${ }^{1}$ Department Of Internal Medicine, Division Of Gastroenterology, Yuan's General Hospital, Kaohsiung/Taiwan

${ }^{2}$ Division Of Hepato-gastroenterology; Department Of Internal Medicine Kaohsiung Chang Gung Memorial Hospital, Taiwan, Kaohsiung/Taiwan

${ }^{3}$ Division Of Hepato-gastroenterology; Department Of Internal Medicine, Buddist Tzu Chi General Hospital, Dalin Branch, Taiwan, Dalin/Taiwan

${ }^{4}$ Division Of Gastroenterology, Pin-Tung Christian Hospital, Pin-Tung/Taiwan

${ }^{5}$ Division Of Gastroenterology, Department Of Internal Medicine, Kaohsiung

Medical University Hospital and Kaohsiung Medical University, Kaohsiung/ Taiwan

${ }^{6}$ Division Of Gastroenterology, Department Of Internal Medicine, Kaohsiung Medical University Hospital and Kaohsiung Medical University, Kaohsiung, Kaohsiung/Taiwan

${ }^{7}$ Division Of Gastroenterology, Foo Yin University Hospital, Pin-Tung/Taiwan ${ }^{8}$ Division Of Gastroenterology, Department Of Internal Medicine, Kaohsiung Veterans General Hospital, National Yang-Ming University, Kaohsiung/Taiwan ${ }^{9}$ Department Of Pharmacy, Kaohsiung Chang Gung Memorial Hospital, Kaohsiung/Taiwan

Contact E-mail Address: emyuan@hotmail.com

Introduction: The pathophysiology of hepatic encephalopathy is not fully understood. A nationwide nested case-control study was conducted to investigate risk factors in the development of hepatic encephalopathy (HE) among patients with liver cirrhosis (LC) in Taiwan.

Aims \& Methods: A total of 913 patients with incident HE and 3499 patients without HE (control) were identified from a cohort of liver cirrhosis $(n=14,428)$ using the population-based, Longitudinal Health Insurance Database 2000 in 1997-2012. Controls were matched to case patients on age at LC diagnosis $(+/$ -2 years), sex, Charlson Comorbid index score, year of LC and follow-up time at 1:1 ratio. A multivariate logistic regression model for $\mathrm{HE}$ was developed to explore the relative contribution of various risk factors, including patient demographics, infections, cirrhosis-related complications (hepatocellular carcinoma, decompensated cirrhosis), $H$. pylori therapy, and peptic ulcer bleeding. A Cox regression model for all-cause mortality was performed.

Results: 714 cases of HE and matched to 714 controls were enrolled in the analysis. Infections (adj. OR, 3.41, 95\% CI, 2.7-4.31, p <.0001) and frequency of infections yearly $(\geq 3$ :adj.OR $11.26,95 \% \mathrm{CI}, 5.7-22.22 ; 1-3$ : adj.OR 2.82 , $95 \%$ CI, 2.26-3.53) were significantly associated with increased risk of HE. $H$. pylori infection $(13.31 \%$ vs. $8.68 \%, p=0.0052)$ and sites of infections such as pneumonia $(14.99 \%$ vs. $10.50 \%, \mathrm{p}=0.0111)$, peritonitis $(14.29 \%$ vs $2.52 \%$, $\mathrm{p}=<0.0001)$, sepsis $(25.63 \%$ vs. $9.52 \%, \mathrm{p}=<0.0001)$, urinary tract infection $(18.77 \%$ vs. $11.20 \%, \mathrm{p}<0.0001)$, biliary tract infection $(7.14 \%$ vs. $3.22 \%$, $\mathrm{p}=0.0008)$ and cellulitis $(11.62 \%$ vs. $3.98 \%, p=0.0207)$ increased risk for HE. HE (adj HR, 0.90, 95\% CI, 0.76-1.06, p=0.02) and infections (adj. HR, 1.13, $95 \%$ CI $0.93-1.38, \mathrm{p}=0.23$ ) increased hazards of death but did not reach statistical significance.

Conclusion: This is the first reported case-control study of HE in Taiwan. The study provides further evidence that infections are strongly associated with HE development among patients with liver cirrhosis; risks for HE vary by relative frequencies and sites of infections. These data provides important information relevant to the prevention and management of cirrhotic patients at risk for HE. Disclosure of Interest: All authors have declared no conflicts of interest.

\section{References}

1. Schulz et al. Does H. pylori eradication therapy benefit patients with hepatic encephalopathy?:systematic review. J Clin Gastroenterol 2014 Jul;48(6):491-

2. Hung TH, Lay CJ, Chang CM, Tsai JJ, Tsai CC, Tsai CC. The effect of infections on the mortality of cirrhotic patients with hepatic encephalopathy. Epidemiol Infect. 2013 Dec;141(12):2671-8

3. Wong F, et al. Sepsis in cirrhosis: report on the 7 th meeting of the International Ascites Club. Gut 2005; 54: 718-725

4. Borzio $\mathrm{M}$, et al. Bacterial infection in patients with advanced cirrhosis: a multicentre prospective study. Digestive and Liver Disease 2001; 33: 41-48.

5. Strauss E, et al. Bacterial infections associated with hepatic encephalopathy: prevalence and outcome. Annual of Hepatology 2003; 2: 41-45.

6. Martin GS, et al. The epidemiology of sepsis in the United States from 1979 through 2000. New England Journal of Medicine 2003; 348: 1546-1554

7. Thuluvath PJ, et al. Spontaneous bacterial peritonitis - in-hospital mortality, predictors of survival, and health care costs from 1988 to 1998. American Journal of Gastroenterology 2001; 96: 1232-1236.

8. Guevara M, et al. Risk factors for hepatic encephalopathy in patients with cirrhosis and refractory ascites: relevance of serum sodium concentration. Liver International 2010; 30: 1137-1142.

9. Udayakumar N, et al. Predictors of mortality in hepatic encephalopathy in acute and chronic liver disease: a preliminary observation. Journal of Clinical Gastroenterology 2007; 41: 922-926.

10. Hamza RE, Villyoth MP, Peter G, Joseph D, Govindaraju C, Tank DC, Sreesh S, Narayanan P, Vinayakumar KR, Risk factors of cellulitis in Cirrhosis and antibiotic prophylaxis in preventing recurrence. Ann Gastroenterol 2014:27(4);374-37913. Zuo-Hua G, Chen-Chi

11. Arvaniti, V, D'Amico, G, and Fede, G (2010). Infections in patients with cirrhosis increase mortality four-fold and should be used in determining prognosis. Gastroenterology, 2010; 139: 1246-56

\section{P0690 SEPSIS-3 CRITERIA ARE MORE ACCURATE THAN SIRS CRITERIA IN PREDICTING IN-HOSPITAL MORTALITY IN PATIENTS WITH CIRRHOSIS AND BACTERIAL INFECTIONS}

S. Piano, M. Tonon, G. Chies, A. Romano, E. Vettore, M. Stanco, C. Pilutti, A. Brocca, P. Angeli

Department Of Medicine - Dimed, University of Padova, Padova/Italy

Contact E-mail Address: salvatorepiano@gmail.com

Introduction: Patients with cirrhosis have a high risk of sepsis, which confers a poor prognosis. Systemic inflammatory response syndrome (SIRS) criteria have several limitations in cirrhosis. Recently, new criteria for sepsis (Sepsis-3) have been suggested in the general population (increase of Sequential Organ Failure Assessment [SOFA] $\geq 2$ points from baseline). Outside the ICU, the qSOFA (at least 2 among: alteration in mental status, systolic blood pressure $<100 \mathrm{mmHg}$, or respiratory rate $\geq 22 / \mathrm{min}$ ) was suggested to screen sepsis. These criteria have never been evaluated in cirrhotic patients

Aims \& Methods: The aim of our study was to assess the ability of Sepsis-3 criteria in predicting in hospital mortality in patients with cirrhosis and bacterial infections. Consecutive patients with cirrhosis and bacterial/fungal infections were prospectively included. Demographic, laboratory and microbiological data were collected at diagnosis of infection. Preadmission SOFA was assessed using preadmission data as provided for acute kidney injury criteria. Patients were followed-up until death, liver transplantation or discharge.

Results: 259 patients were included (Age $=61 \pm 12$ years, MELD-score $=20 \pm 7$ ) The most common infections were urinary tract infection $(33 \%)$, spontaneous bacterial peritonitis $(23 \%)$ and pneumonia $(14 \%)$. SIRS, qSOFA and Sepsis-3 criteria were found in 34,23 and $64 \%$ of patients, respectively. During the hospitalization $19 \%$ of patients died. Sepsis-3 and qSOFA had significantly greater discrimination for in-hospital mortality $($ AUROC $=0.784$ and 0.732 , respectively) than SIRS (AUROC $=0.606 ; \mathrm{p}<0.01$ for both). In multivariate analysis, Sepsis-3 $(\mathrm{OR}=5.71 ; \mathrm{p}=0.007)$, qSOFA $(\mathrm{OR}=3.11 ; \mathrm{p}=0.004), \mathrm{CLIF}-$ $\mathrm{C}$-AD score $(\mathrm{OR}=1.05 ; \mathrm{p}=0.007), \mathrm{C}$-reactive protein $(\mathrm{OR}=1.62 ; \mathrm{p}=0.014)$ and nosocomial infections $(\mathrm{OR}=2.15 ; \mathrm{p}=0.058)$, were found to be independent 
predictors of in-hospital mortality. Patients with Sepsis-3 had higher incidence of acute-on-chronic liver failure (36 vs $11 \%$; p < 0.001 ), septic shock ( 15 vs $0 \%$; $\mathrm{p}<0.001)$ and transfer to the ICU (16 vs $2 \% ; \mathrm{p}=0.001)$ than those without Sepsis-3. Similar findings were found for qSOFA.

Conclusion: Sepsis-3 criteria are more accurate than SIRS criteria in predicting the severity of infections in patients with cirrhosis. qSOFA is a useful bedside tool to assess risk for worse outcomes in these patients. Patients with Sepsis-3 and positive qSOFA deserve more intensive management and strict surveillance.

Disclosure of Interest: All authors have declared no conflicts of interest.

\section{Reference}

Singer M, Deutschman CS, Seymour CW, et al. The Third International Consensus Definitions for Sepsis and Septic Shock (Sepsis-3). JAMA. 2016;315:801-810.

\section{P0691 A SUBCLINICAL HIGH TRICUSPID REGURGITATION PRESSURE GRADIENT IS A RISK FACTOR FOR SURVIVAL AFTER LIVING DONOR LIVER TRANSPLANTATION}

A. Ohyama, A. Takaki, Y. Umeda, T. Yasunaka, R. Yoshida, D. Nobuoka, F. Ikeda, H. Okada, H. Onishi, H. Shiraha, S. Nakamura, T. Yagi Gastroenterology And Hepatology, Okayama University Graduate School of Medicine, Dentistry and Pharmaceutical Sciences, Japan/Japan

\section{Contact E-mail Address: at841205@gmail.com}

Introduction: Portopulmonary hypertension (POPH) is characterized by pulmonary vasoconstriction, while hepatopulmonary syndrome (HPS) is characterized by vasodilatation. Given that HPS could be resolved after orthotopic liver transplantation (OLT) even when hypoxemia were severe $\left(\mathrm{PaO}_{2}<50 \mathrm{mmHg}\right)$, implications for OLT is accepted not only for deceased donor LT (DDLT), but also to living donor related LT (LDLT). However, the post-OLT course of POPH complicated patients are often unsatisfactory and severe (mPAP $>45$ to $50 \mathrm{mmHg}$ ) patients are considered as an absolute contraindication for OLT. The International Liver Transplant Society Practice Guidelines indicate that, unlike HPS, there are no data to support the concept that POPH (treated or untreated) should be an indication for OLT. Furthermore, the Practice Guidelines recommend that patients with mPAP $<35 \mathrm{mmHg}$ be indicated for OLT, and PA-targeted therapy should be initiated in patients with $\mathrm{mPAP} \geq 35 \mathrm{mmHg}$. However, almost all of the patients in these articles had received DDLT, and no such analyses have been for LDLT patients. Given that left or right lobe LDLT grafts are smaller than DDLT grafts, PH-induced hepatic venous pressure might result in a strong congestive impact on the LDLT grafted liver. Although patients with confirmed POPH and HPS are rare, relatively pulmonary hypertensive patients and mild HPS patients may be more common.

Aims \& Methods: The present objective is to investigate the clinical impact of subclinical PH on the inevitably small survival in grafted living donor OLT (LDLT). We recruited 84 OLT candidates for liver cirrhosis in a retrospective cohort study. Patients exhibiting a tricuspid regurgitation pressure gradient (TRPG) $\geq 25 \mathrm{mmHg}$ (median) on echocardiography were categorized as potentially having $\mathrm{PH}$ (subclinical $\mathrm{PH} ; \mathrm{n}=34$ ). The mean pulmonary artery pressure (mPAP) measured after general anesthesia with $\mathrm{FIO}_{2} 0.6$ (mPAP-FIO ${ }_{2} 0.6$ ) was also also included as advisory data. Patients exhibiting $\mathrm{pO}_{2}<80 \mathrm{mmHg}$ and an alveolar-arterial oxygen gradient $\left(\mathrm{AaDO}_{2}\right) \geq 15 \mathrm{mmHg}$ were categorized as potentially having HPS (subclinical HPS; $\mathrm{n}=29$ ). The clinical course after LDLT was investigated according to subclinical PH or HPS.

Results: Subclinical PH was correlated with a worse 1 -year survival $(\mathrm{p}=0.003)$. Subclinical PH $(p=0.012)$ and older donor age $(p=0.008)$ were correlated with a poor 40-month survival. Although a higher $\mathrm{mPAP}-\mathrm{FIO}_{2} 0.6$ was expected to correlate with a worse survival, a high $\mathrm{mPAP}-\mathrm{FIO}_{2} 0.6$ was not a significant risk factor for the post-LDLT survival. When the post-LDLT survival was investigated according to the TRPG and mPAP-FIO ${ }_{2} 0.6$ status, it was worst in patients who had a high TRPG and low mPAP-FIO 20.6 . The patients with a high $\mathrm{mPAP}-\mathrm{FIO}_{2} 0.6$ and low TRPG showed a good survival, probably because co-existing HPS released the regurgitation pressure.

Conclusion: In cirrhosis patients, $\mathrm{mPAP}-\mathrm{FIO}_{2} 0.6$ may not accurately reflect the congestive pressure to the liver, as the pressure might escape via a pulmonary shunt. Subclinical high TRPG is an important marker for predicting congestive pressure to a grafted small liver, resulting in a worse survival after LDLT.

Disclosure of Interest: All authors have declared no conflicts of interest.

\section{P0693 CARVEDILOL VERSUS PROPRANOLOL EFFECT IN THE PRIMARY PROPHYLAXIS OF VARICEAL BLEEDING IN CIRRHOTIC PATIENTS WITH PORTAL VEIN THROMBOSIS}

I. Girleanu ${ }^{1}$, A. $\operatorname{Trifan}^{1}$, C. Cojocariu ${ }^{1}$, A.M. Singeap ${ }^{1}$, C. Sfarti ${ }^{1}$, O. Stoica ${ }^{1}$, S. Chiriac ${ }^{1}$, T. Cuciureanu ${ }^{1}$, C. Stanciu ${ }^{2}$

${ }^{1}$ Gastroenterology, "Gr. T. Popa" University of Medicine and Pharmacy, Iasi/

Romania

${ }^{2}$ Institute of Gastroenterology and Hepatology, Iasi/Romania

\section{Contact E-mail Address: gilda iri25@yahoo.com}

Introduction: Portal vein thrombosis (PVT) is reconized as an independent factor of variceal bleeding. Beta blockers are the mainstay treatment to prevent variceal bleeding in cirrhotic patients. Carvedilol has been shown to be equal to propranolol in preventing first bleeding in cirrhotic patients, however, the efficacy of this policy in patients with PVT is unknown.
Aims \& Methods: The aim of this study was to evaluate the efficacy of carvedilol versus propranolol in primary prophylaxis of variceal bleeding in cirrhotic patients with occlusive portal vein thrombosis. Between January 2014 and December 2015, cirrhotic patients with occlusive non-malignant PVT were enrolled in a tertiary center. PVT was susspected by Doppler ultrasound and confirmed by computeted tomography. Cirrhotic patients with esophageal varices and no previous variceal bleeding were randomized to carvedilol $6.125 \mathrm{mg}$ daily or Propranolol $40 \mathrm{mg}$ daily. End points were esophageal variceal bleeding or death.

Results: During the study periode forty eight patients were evaluated. Twenty one and twenty seven patients were randomized in carvedilol and propranolol arms respectively. Mean age was $49 \pm 12.2$ years; $33(68.7 \%)$ were males; $60.4 \%$ had viral cirrhosis; mean Child-Pugh score was $7.2 \pm 2.6$ and mean follow up was $12.3 \pm 9.1$ months (range $1-29$ months). All the patients had occlusive non-malignant PVT, most of them involving only the trunk, and grade 2 or 3 esophageal varices. Both carvedilol and propranolol groups had comparable variceal bleeding rates $(14.2 \%$ vs. $14.8 \%, \mathrm{P}=0.002)$, bleed related mortality $(9.5 \%$ vs. $11.1 \%$, $\mathrm{P}=0.027)$ and overall mortality $(23.8 \%$ vs. $22.2 \%, \mathrm{P}=0.004)$ respectively. Adverse events in carvedilol group were hypotension $(n=2)$, requiring cessation of therapy, while and dyspnea $(n=3)$ resolved spontaneously. In the propranolol group there was 1 advers event that required discontinuation of treatment (grade 2 atrio-ventricular block).

Conclusion: Our study suggests that carvedilol is probably not superior to propranolol in preventing first variceal bleeding in cirrhotic patients with occlusive PVT, and they both can be used as primary prophylaxis.

Disclosure of Interest: All authors have declared no conflicts of interest.

P0694 ASSESSMENT OF PROGNOSTIC PERFORMANCE OF ALBI, CHILD-PUGH AND MELD SCORES IN PATIENTS WITH LIVER CIRRHOSIS COMPLICATED WITH ACUTE UPPER GASTROINTESTINAL BLEEDING

S. Xavier ${ }^{1}$, R. Villas-Boas ${ }^{1}$, P. Boal Carvalho ${ }^{2}$, J. Magalhães $^{2}$, C. Marinho ${ }^{3}$, J. Cotter ${ }^{4}$

${ }^{1}$ Life and Health Sciences Research Institute (ICVS), School of Medicine, University of Minho, Braga/Portugal

${ }^{2}$ Gastroenterology Department, Hospital da Senhora da Oliveira, Guimarães, Guimarães/Portugal

${ }^{3}$ School Of Medicine, University Of Minho, Life and Health Sciences Research Institute, Braga/Guimarães/Portugal

${ }^{4}$ Pt Government Associate Laboratory

${ }^{4}$ ICVS/3B's, Braga/Guimarães/Portugal

\section{Contact E-mail Address: smaxavier@gmail.com}

Introduction: The ALBI score was recently developed to assess the severity of liver dysfunction, taking into account albumin and bilirubin levels. We aimed to assess its prognostic performance in patients with liver cirrhosis complicated with upper gastrointestinal bleeding (UGIB) while comparing it with Child-Pugh (CP) and MELD scores.

Aims \& Methods: Retrospective unicentric study, including consecutive adult patients with cirrhosis admitted for UGIB between January 2011 and November 2015. Clinical, analytical and endoscopic variables were assessed and ALBI, CP and MELD scores at admission were calculated. Statistical analysis was performed using SPSS v21.0 and MedCalc v.16.4.3, and a two-tailed p value $<0.05$ was defined as indicating statistical significance.

Results: Included 111 patients with a mean age of $57 \pm 12$ years, $76.6 \%$ were males. Liver cirrhosis was most frequently alcoholic $(89.2 \%)$ and the most common etiology for UGIB was variceal hemorrhage, in $75.5 \%$ of patients. During the first 30 days of follow-up 12 patients $(10.8 \%)$ died, and during the 1 st year of follow-up another 10 patients died (1st year mortality of $19.8 \%$ ). When comparing the three scores, regarding in-stay and 30 days mortality, only ALBI score showed statistical significant results, with an area under the curve (AUC) of $0.82(p<0.01)$ for both outcomes. Regarding 1st year mortality, AUC for ALBI, CP and MELD scores, were $0.71(\mathrm{p}<0.01), 0.64(\mathrm{p}<0.05)$ and $0.66(\mathrm{p}=0.02)$, respectively, while for global mortality AUC were 0.75 $(\mathrm{p}<0.01), 0.72(\mathrm{p}<0.01)$ and $0.72(\mathrm{p}<0.01)$, respectively. When comparing the AUC of the three scores, no significant differences were found regarding 1 st year mortality and global mortality.

Conclusion: In our series, ALBI score accurately predicted both in-stay and 30 days mortality $(0.82(\mathrm{p}<0.01))$, while CP and MELD scores weren't able to predict these outcomes. All scores showed a fair prognostic prediction performance regarding 1st year and global mortality. These results suggest that ALBI score is particularly helpful in the assessment of short term outcomes, with a better performance than the most commonly used scores, and may assist the clinician in the stratification of care at admission and maybe even in the referral to liver transplant.

Disclosure of Interest: All authors have declared no conflicts of interest. 


\section{P0695 PROTON PUMP INHIBITORS IN CIRRHOTIC PATIENTS:} IT'S URGENT TO RETHINK THEIR USE!

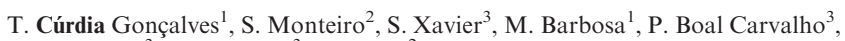
J. Magalhães ${ }^{3}$, C. Marinho ${ }^{3}$, J. Cotter ${ }^{2}$

${ }^{1}$ Gastroenterology Department, Hospital da Senhora da Oliveira, Guimarães/ Portugal

${ }^{2}$ Pt Government Associate Laboratory

${ }^{4}$ ICVS/3B's, Braga/Guimarães/Portugal

${ }^{3}$ Gastroenterology Department, Hospital da Senhora da Oliveira, Guimarães, Guimarães/Portugal

Contact E-mail Address: tiagomcg@hotmail.com

Introduction: Despite the progress in the treatment of cirrhosis, infections remain a common problem, being responsible for the great majority of morbidity and mortality in these patients.

Aims \& Methods: The aim of this study was to identify predictive factors for infection in the first hospitalization for decompensated cirrhosis (DC) Retrospective analysis of patients with the first hospitalization for DC between January of 2009 and March of 2016. Demographic, clinical and biochemical data was compared between patients with and without proven infection in the first hospitalization for DC.

Results: From the 179 patients with a first hospitalization for DC, $6 \%$ had ascites on admission, $45.8 \%$ had upper gastrointestinal bleeding, $38.5 \%$ had jaundice, and $28.5 \%$ had hepatic encephalopathy. Regarding medication, $29.6 \%$ of the patients were taking proton pump inhibitors (PPI), 22.3\% had beta-blockers prescribed, and $1.7 \%$ were on prophylactic antibiotic. In those 53 patients with proven infection, spontaneous bacterial peritonitis was the most common infection $(34 \%)$, followed by urinary tract infection $(30.2 \%)$ and pneumonia $(13.2 \%)$. Infected patients presented with jaundice $(\mathrm{p}=0.03)$, severe ascites $(\mathrm{p}=0.009)$, use of PPI $(p=0.003)$ and acute-on-chronic liver failure $(p=0.006)$ more frequently than those without infection. Additionally, infected patients presented with significantly increased values of C-reactive protein $(p<0.001)$, INR $(p=0.04)$, creatinine $(\mathrm{p}=0.013)$ and MELD scores $(\mathrm{p}=0.001)$. Mortality rates were higher in infected patients at 30 -day $(4.0 \%$ vs. $9.4 \%), 3$ months $(7.9 \%$ vs. $18.9 \%), 6$ months $(12.7 \%$ vs. $24.5 \%)$ and 1 year $(22.2 \%$ vs. $26.5 \%)$. In the multivariate analysis, the use of PPI was independently associated with an increased risk of infections $(\mathrm{OR}=2.3 ; 95 \%$ CI 1.052-5.173).

Conclusion: Almost a third of patients will develop infections right at the first hospital admission for decompensated cirrhosis, which are associated with higher short and long-term mortality rates. As PPI more than double the risk of infection, the indication for the use of these drugs should be strictly reviewed and their interruption considered in cirrhotic patients.

Disclosure of Interest: All authors have declared no conflicts of interest.

\section{P0697 CRITICAL FLICKER FREQUENCY TEST PREDICTS THE FIRST EPISODE OF OVERT HEPATIC ENCEPHALOPATHY IN PATIENTS WITH COMPENSATED LIVER CIRRHOSIS}

E. Shahini ${ }^{1}$, M. Barone ${ }^{2}$, A. Iannone ${ }^{1}$, M.T. Viggiani ${ }^{2}$, V. Corvace ${ }^{3}$, M. Principi ${ }^{2}$, A. Di $\mathrm{Leo}^{2}$

${ }^{1}$ Dept. Of Emergency And Organ Transplantation, Gastroenterology Unit, Bari Italy

${ }^{2}$ Emergency And Organ Transplantation, University of Bari, Bari/Italy

${ }^{3}$ San Camillo Hospital, Manfredonia (foggia), Italy., Gastroenterology Unit,

Foggia/Italy

Contact E-mail Address: vincistein00@gmail.com

Introduction: Critical flicker frequency (CFF) values $\leq 39 \mathrm{~Hz}$ identify cirrhotic patients with minimal hepatic encephalopathy $(\mathrm{mHE})$ and predict their risk of developing overt hepatic encephalopathy (oHE). However, these results have been obtained in cirrhotics with advanced liver disease suffering a previous episode of liver decompensation ( $74 \%$ of patients) or oHE (14\% of patients).

Aims \& Methods: Herein, we evaluated the effectiveness of CFF in predicting the first episode of oHE in compensated outpatients undergoing a long-term follow up. A total of 134 selected patients and 150 healthy subjects were evaluated using CFF. A CFF value was considered normal when $>39 \mathrm{~Hz}$ and pathological when $\leq 39 \mathrm{~Hz}$. At baseline, we evaluated demographic characteristics, laboratory tests, model for end-stage liver disease (MELD) score, and Child-Pugh class in all patients. Then, they were followed up for $31.5 \pm 18.9$ months and received clinical examinations and laboratory tests every six months.

Results: At baseline, all controls had a CFF $>39 \mathrm{~Hz}$ with a mean value significantly higher than that observed in 93 patients with CFF $>39 \mathrm{~Hz}(\mathrm{p}<0.001)$, while the remaining 41 patients showed a CFF $\leq 39 \mathrm{~Hz}$. Our analysis demonstrated a significant inverse correlation between CFF and MELD ( $\mathrm{r}$ : -0.33 , $p=0.0003)$, while the prevalence of CFF values $\leq 39 \mathrm{~Hz}$ significantly increased with the progression of the Child-Pugh class $(\mathrm{p}=0.003)$. Interestingly, the CFF value at baseline was predictive of the first episode of overt HE both by log-rank test $(\mathrm{p}<0.001)$ and $\mathrm{Cox}$ regression analysis $(\mathrm{HR}=5.623 ; 95 \% \mathrm{CI}=2.433-$ 12.991; $\mathrm{p}<0.001)$

Conclusion: We demonstrated, for the first time, that CFF predicts the first episode of oHE in a population of compensated cirrhotics that never experienced oHE. Cirrhotic patients should be routinely screened by CFF to identify patients at risk of oHE.

Disclosure of Interest: All authors have declared no conflicts of interest.

\section{References}

1. Romero-Gómez M, Cordoba J, Jover R, et al. Value of the critical flicker frequency in patients with minimal hepatic encephalopathy. Hepatology 2007;45:879-885.

2. Kircheis G, Hilger N, Häussinger D. Value of critical flicker frequency and psychometric hepatic encephalopathy score in diagnosis of low-grade hepatic encephalopathy. Gastroenterology 2014;146:961-969.

3. Romero-Gómez M, Ampuero J. Deciphering the spectrum of low-grade hepatic encephalopathy in clinical practice. Gastroenterology 2014;146:887890.

4. Vilstrup H, Amodio P, Bajaj J, et al. Hepatic Encephalopathy in Chronic Liver Disease: 2014 practice guideline by the American Association for the Study of Liver Disease and the European association for the Study of The Liver. AASLD practice guideline. Hepatology 2014;60:715-735.

P0698 OMNIPRESENCE OF LIVER FIBROSIS, BUT PORTAL HYPERTENSION ONLY IN SELECTED ADULT FONTAN PATIENTS

I. Munsterman ${ }^{1}$, T. Duijnhouwer ${ }^{2}$, R. Van Kimmenade ${ }^{2}$, C. Bronkhorst ${ }^{3}$, J.P.h. Drenth ${ }^{1}$, A. Van Dijk ${ }^{2}$, E. Tjwa ${ }^{1}$

${ }^{1}$ Gastroenterology \& Hepatology, Radboud University Medical Center, Nijmegen/ Netherlands

${ }^{2}$ Cardiology, Radboud university medical center, Nijmegen/Netherlands

${ }^{3}$ Pathology, Jeroen Bosch Hospital, 's-Hertogenbosch/Netherlands

Contact E-mail Address: isabelle.munsterman $@$ radboudumc.nl

Introduction: The Fontan circulation causes some degree of hepatic congestion by its nature of anatomical reconstruction. This may lead to liver fibrosis or even cirrhosis, but to what extend is unknown. A profound hepatic evaluation, incorporating several non-invasive and invasive modalities, in an asymptomatic Fontan patient cohort may further elucidate this.

Aims \& Methods: Consecutive patients with a Fontan circulation are prospectively included for screening of liver fibrosis. This screening consists of a blood panel including biomarkers, liver ultrasonography, transient elastography (Fibroscan), contrast-enhanced liver MRI or CT-scan and liver biopsy. Liver biopsies were systematically scored with the Fontan specific fibrosis score. Mild fibrosis was defined as a maximum score of 2 on sinusoidal and portal fibrosis component, severe fibrosis as score 3 or 4 on at least one component. Non-invasive markers for portal hypertension (PHT) such as presence of collaterals and splenomegaly on imaging, platelet count $\left(\mathrm{N} / \mu \mathrm{Lmm}^{3}\right) /$ spleen diameter $(\mathrm{mm})$ ratio (PSR) and von Willebrand factor (VWF) were measured.

Results: From November 2015 until March 2017, 52 Fontan patients were included (mean age $27 \pm 7.5$ years, $60 \%$ male). The majority of patients had one or more elevated liver enzymes (elevated GGT in $68 \%$, median $67 \mu \mathrm{mol} / \mathrm{L}$ IQR 51-107). Median platelets were $174 \times 10 \mathrm{e} 9 / 1$ (IQR 147-213) and mean VWF $104 \pm 23 \%$. Mean liver stiffness was $21.8 \pm 9.3 \mathrm{kPa}$. The majority $(77 \%)$ of ultrasonographies showed no sign of cirrhosis or splenomegaly (mean spleen size $11.9 \pm 1.8 \mathrm{~cm}$ ). However, $54 \%$ of advanced imaging (MRI or CT-scan) showed cirrhosis, $23 \%$ congestion and in $21 \%$ signs of PHT were observed. Of 33 patients with histology, none were without fibrosis or sinusoidal dilatation and cirrhosis was present in $21 \%$. Of these only $57 \%$ also showed signs of cirrhosis on imaging. Of all patients with signs of PHT on imaging only one $(13 \%)$ patient had histologically confirmed cirrhosis whereas five $(63 \%)$ had mild fibrosis. Liver stiffness was equal in patients with mild $(22.3 \pm 9.2)$ compared to severe fibrosis $(25.3 \pm 12.1)(\mathrm{p}=.458)$. Median PSR in patients with mild fibrosis was 1557 (IQR $1139-1910)$ and 1233 (IQR 1045-1451) in severe fibrosis $(\mathrm{p}=.114)$. VWF was comparable between groups $(99 \pm 12$ vs. $105 \pm 20 \%, \mathrm{p}=.241)$. Patients with severe fibrosis were not older (resp $28 \pm 7$ vs. $26 \pm 6$ years, $\mathrm{p}=.555$ ) nor had a longer duration of the Fontan circulation $(22 \pm 5$ vs. $21 \pm 4$ years, $p=.644)$

Conclusion: All Fontan patients are at risk of developing severe liver fibrosis, irrespective of age, duration of Fontan circulation and even when asymptomatic. Portal hypertension may occur in the absence of severe fibrosis. Assessment by solely non-invasive modalities may both under- and overestimate the incidence of fibrosis. For generalizability of the current findings, Fontan patients should be prospectively assessed with multimodality assessment in larger cohorts. Disclosure of Interest: All authors have declared no conflicts of interest.

P0699 A RANDOMIZED DOUBLE BLIND CONTROLLED TRIAL ON THE EFFECT OF LACTOBACILLUS RHAMNOSUS GG IN PATIENTS WITH MINIMAL HEPATIC ENCEPHALOPATHY

E. Shahini ${ }^{1}$, M. Barone ${ }^{1}$, A. Iannone ${ }^{2}$, M.T. Viggiani ${ }^{2}$, V. Corvace ${ }^{3}$, M. Principi ${ }^{2}$, A. Di Leo ${ }^{2}$

${ }_{1}^{1}$ Dept. Of Emergency And Organ Transplantation, Gastroenterology Unit, Bari Italy

${ }^{2}$ Emergency And Organ Transplantation, University of Bari, Bari/Italy

${ }^{3}$ San Camillo Hospital, Manfredonia (Foggia), Italy, Gastroenterology Unit, Foggia/Italy

Contact E-mail Address: vincistein00@gmail.com

Introduction: Probiotics has been recently used to treat cirrhotic patients with any grade of acute or chronic hepatic encephalopathy (HE). Herein, we evaluated the efficacy of Lactobacillus Rhamnosus GG (LRGG) on the treatment of minimal $\mathrm{HE}(\mathrm{mHE})$ in compensated cirrhotics.

Aims \& Methods: 134 patients were screened by critical flicker frequency (CFF) to diagnose mHE. Among them, 41 patients were $\mathrm{CFF}+(\leq 39 \mathrm{~Hz})$ and were 
randomized to placebo or LRGG treatment, for 2 months. In all intention to treat and per protocol patients, demographic characteristics, laboratory test, model for end-stage liver disease (MELD) score, and Child-Pugh class were evaluated.

Results: CFF value increased in both LRGG and placebo groups at the end of treatment. However, in the former group CFF value significantly increased as compared to placebo $(46.5 \pm 5.7$ vs. $41.1 \pm 4.6 \mathrm{~Hz}, \mathrm{p}=0.015)$, thus indicating a better therapeutic outcome on mHE. Moreover, mHE reversal was achieved in $75.0 \%$ vs. $35.7 \%$ of the patients from LRGG and placebo groups, respectively $(\mathrm{p}=0.047)$.

Conclusion: For the first time, we demonstrate that LRGG improves mHE (expressed by a significant CFF increase).

Disclosure of Interest: All authors have declared no conflicts of interest.

\section{References}

1. Romero-Gómez M, Cordoba J, Jover R, et al. Value of the critical flicker frequency in patients with minimal hepatic encephalopathy. Hepatology. 2007; 45(4): 879-85.

2. MK, Sharma BC, Sharma P, Sachdeva S, Srivastava S. Probiotics prevent hepatic encephalopathy in patients with cirrhosis: a randomized controlled trial. Clin Gastroenterol Hepatol. 2014; 12(6): 1003-8.

3. Dhiman RK, Rana B, Agrawal S, et al. Probiotic VSL\#3 reduces liver disease severity and hospitalization in patients with cirrhosis: a randomized, controlled trial. Gastroenterology. 2014; 147(6): 1327-37.

4. Saab S, Suraweera D, Au J, Saab EG, Alper TS, Tong MJ. Liver Int. 2016; 36(7): 986-93.

\section{P0700 ASSOCIATIONS OF GENETIC POLYMORPHISM OF TOLL- LIKE RECEPTOR 3(TLR-3) AND SEX- LINKED TOLL- LIKE RECEPTOR 7 (TLR-7) ALLELES WITH THE HEPATITIS C VIRUS INFECTION OUTCOME IN EGYPTIAN POPULATION: A MULTICENTRE FAMILY-BASED STUDY}

M. El-Bendary ${ }^{1}$, M. Neamatallah ${ }^{2}$, H. Elalfy ${ }^{1}$, T. Besheer ${ }^{1}$, A. Elkholy ${ }^{3}$, M. ElDiasty $^{1}$, M. Elsareef ${ }^{4}$, M. Zahran ${ }^{5}$, B. El-Aarag ${ }^{5}$, M. El-Setouhy ${ }^{6}$, A. Eladl ${ }^{7}$, G. Esmat ${ }^{8}$, T.R.W.F.B.S.\&.T.D.F.(Science \& Technology Development Foundation (Stdf), Project No.1784 (Tc/2/health/2009/hep-1.3) ${ }^{9}$

${ }^{1}$ Tropical Medicine And Hepatology, Mansoura Faculty of Medicine-Mansoura University, Mansoura/Egypt

${ }^{2}$ Medical Biochemistry, Mansoura Faculty of Medicine- Mansoura University,

Mansoura/Egypt

${ }^{3}$ Gastroenterology Department, Mansoura Health Insurance Hospital, Mansoura/ Egypt

${ }^{4}$ Biochemistry, Mansoura Faculty of Science, Mansoura/Egypt

${ }^{5}$ Chemistry, Menoufia University, Shebin El-kom/Egypt

${ }^{6}$ Ain Shams Faculty of Medicine, Cairo/Egypt

${ }^{7}$ Alazhar Faculty of Medicine -Assuit University, Assuit/Egypt

${ }^{8}$ Endemic Hepatogastroenterology And Infectious Diseases, Cairo university hospitals, Cairo/Egypt

${ }^{9}$ This research was funded by Science \& Technology Development Foundation (STDF), Cairo/Egypt

\section{Contact E-mail Address: mmelbendary@gmail.com}

Introduction: Hepatitis $\mathrm{C}$ virus infection is a pressing national problem in Egypt. $\mathrm{HCV}$ carries a variable clinical course in different patients. The human Toll like receptors (TLRs) family consists of ten receptors that are critically important for innate immunity. TLRs recognize and respond to diverse microbial molecules and enable the innate immune system to discriminate among groups of pathogens and to induce an appropriate cascade of effector responses. Toll-like receptors 3 and 7 are recognition receptors that allow the innate immune system binding to RNA materials. The genetic polymorphism of these receptors has a direct role on the outcome of many diseases.

Aims \& Methods: The aim of this study was to assess the association between genetic polymorphism of TLR 3 and Sex- linked TLR 7 alleles with HCV infection outcome in Egyptian families using high resolution techniques. A total of 135 Egyptian families (622 individuals) were recruited in this study from upper and lower Egypt (East and West delta). We compared the risk of allele carriage of selected markers in different groups. These groups included spontaneous virus clearance (SVC) (108 subject), chronic HCV (CHC) patients (216), and negative control (298) individuals. The rs3775291 (C/T) was genotyped for TLR3. While the rs3853839 (C/G) was genotyped for TLR7 by real time PCR using $\mathrm{Taq}^{\mathrm{Man}}$ allelic discrimination kit (Applied Biosystems) according to the manufacturer's protocol. The distribution of the TLR7 rs3853839 (C/G) polymorphism was assessed separately in female and male subpopulations of the study due to the X-linked nature of the TLR7 gene.

Results: As regard TLR3 the frequency of the C allele was $88.5 \%, 83.4 \%$ and $71.06 \%$ for the SVC, control and $\mathrm{CHC}$ groups respectively. While the allele frequency of the T allele was found to be $11.5 \%, 16.5 \%, 38.9 \%$ in SVC, control, and $\mathrm{CHC}$ groups respectively. When these alleles were compared between the 3 studied groups, The T allele of TLR 3 rs 3775291 was found to be significantly higher in chronic HCV group when compared to both spontaneous clearance (SVC) and control groups [OR $15.285(95 \%$ CI 2.04 to 114.08 , corrected P (Pc) $<0.02)$ and $\mathrm{OR} 5.17(95 \% \mathrm{CI} 2.30$ to $11.64, \mathrm{Pc}<0.0003)]$ respectively. As regard the sex- linked TLR-7 it was found that the frequency of the C allele was $69.5 \%$, $69.1 \%$ and $46.3 \%$ for the SVC, control and CHC groups respectively. While the frequency of the G allele was found to be $30.95 \%, 30.9 \%, 53.7 \%$ in SVC control and $\mathrm{CHC}$ groups respectively. When the association of these alleles with $\mathrm{HCV}$ infection outcome was studied in the female and male subpopulations it showed that: The C allele of TLR 7 rs3853839 in the female subpopulation was significantly higher in both HCV spontaneous clearance (SVC) and control groups when compared to chronic HCV group [OR 0.42 (95\% CI 0.21 to 0.82 , $\mathrm{Pc}<0.0372)$ and OR $0.40(95 \%$ CI 0.23 to $0.71, \mathrm{Pc}<0.0054)]$ respectively. The same results was also reported in the male subpopulation as the same allele was found to be significantly higher in both HCV spontaneous clearance (SVC) and control groups when compared to chronic HCV group [OR 0.289 $(95 \%$ CI 0.14 to $0.59, \mathrm{Pc}<0.0021)$ and OR $0.17(95 \%$ CI 0.1009 to 0.28 , $\mathrm{Pc}<0.0001)]$ respectively.

Table(1): Association of the allele T of TLR3 rs3775291 polymorphism among the studied groups.

\begin{tabular}{llll}
\hline & $\begin{array}{l}\text { control Vs } \\
\text { spontanous }\end{array}$ & $\begin{array}{l}\text { CHC vs } \\
\text { Spontaenous }\end{array}$ & CHC vs control \\
\hline Odds ratio (OR) & 2.9517 & 15.2857 & 5.1786 \\
$95 \%$ CI & 0.3648 to 23.8807 & 2.0481 to 114.0824 & 2.3038 to 11.6406 \\
z statistic & 1.015 & 2.659 & 3.979 \\
P & 0.3102 & 0.0078 & 0.0001 \\
Pc & 0.9306 & 0.0234 & 0.0003 \\
\hline
\end{tabular}

Conclusion: The risk of development of chronic HCV infection was associated with T allele carriage of TLR3rs3775291 SNP. While the carriage of C allele of TLR7 rs3853839C allele was associated with spontaneous HCV clearance in both male and female subpopulations in Egyptian families.

Disclosure of Interest: All authors have declared no conflicts of interest.

\section{References}

Yue, M., et al., Sex-specific association between X-linked Toll-like receptor 7 with the outcomes of hepatitis C virus infection. Gene, 2014. 548(2): p. 244-250. Schott, E., et al., A Toll-like receptor 7 single nucleotide polymorphism protects from advanced inflammation and fibrosis in male patients with chronic HCVinfection. Journal of hepatology, 2007. 47(2): p. 203-211.

Sá, K.S.G.d., et al., Toll-like receptor 3 gene polymorphisms are not associated with the risk of hepatitis B and hepatitis C virus infection. Revista da Sociedade Brasileira de Medicina Tropical, 2015. 48(2): p. 136-142.

\section{P0701 ACTIVATED HEPATIC STELLATE CELLS CAN DIRECTLY INDUCE PATHOGENIC TH17 CELLS IN CHRONIC HEPATITIS B VIRUS INFECTION}

Y. Liu ${ }^{1}$, W. Jiang ${ }^{2}$

${ }^{1}$ Zhongshan Hospital, Fudan University, Shanghai/China

${ }^{2}$ Gastroenterology \& Hepatology, Zhongshan Hospital, Fudan University,

Shanghai/China

Contact E-mail Address: liuyun2008294@126.com

Introduction: Th17 cells are involved in liver fibrosis by activating hepatic stellate cells (HSCs). We aimed to investigate whether HSCs could regulate the function of Th17 cells and the relevant mechanism.

Aims \& Methods: Sixty-five patients diagnosed with chronic hepatitis B (CHB) were enrolled in this study. To unravel the effect of HSCs on T cells, naïve $\mathrm{CD} 4{ }^{+} \mathrm{T}$ cells and $\mathrm{Th} 17$ cells were sorted from $\mathrm{CHB}$ patients and cultured with or without activated-HSCs, and cytokines expression and genes transcription were analyzed. In addition, the regulatory mechanism of HSCs was also investigated.

Results: ELISA and qRT-PCR showed that Th17 cells from CHB patients were more pathogenic via the expression of IL-17A, IL-23R, RORC, CCL20 and CCR6, and meanwhile, they could activate the primary HSCs. The co-culture experiment indicated that activated HSCs dramatically promoted the proliferation of $\mathrm{CD}^{+} \mathrm{T}$ cells in a time- and dose-dependent manner. In addition, they could also induce the naïve $\mathrm{CD}^{+} \mathrm{T}$ cells into $\mathrm{Th} 17$ cells which had a more pathogenic phenotype. Moreover, activated-HSCs-mediated induction of Th17 cells might depend on IL-1 $\beta$ and IL-6 release as well as COX-PGE2 pathway. Conclusion: Th17 cells cooperated with HSCs in a proinflammatory feedback loop provide us a more understanding of the pathogenic role of Th17 cells in the chronicity of HBV infection.

Disclosure of Interest: All authors have declared no conflicts of interest.

\section{P0702 EFFICACY AND SAFETY OF DIRECT ACTING ANTIVIRAL DRUGS IN EARLY TREATMENT OF HCV GENOTYPE 4 POST-} LIVING DONOR LIVER TRANSPLANTATION

\section{E. F. Fawzy ${ }^{1}$, A. M. Moussa ${ }^{2}$, I. M. Fawzy ${ }^{3}$, M. Elbadry ${ }^{2}$}

${ }^{1}$ Tropical Medicine And Gastroenterology Dep., Assiut University, Assiu/Egypt ${ }^{2}$ Tropical Medicine And Gastroenterology Dep., Aswan University, Aswan/Egypt ${ }^{3}$ Tropical Medicine And Gastroenterology Dep., Ain Shams University, Cairo/ Egypt

Contact E-mail Address: ehab_mostafa99@yahoo.com Introduction: Living donor liver transplantation (LDLT) has become the only life-saving treatment option for patients with end stage liver disease secondary to $\mathrm{HCV}$ infection in Egypt, unfortunately recurrence of infection is nearly universal, 
resulting in cirrhosis and graft failure within 5 years after transplant. Different studies have been published studying efficacy and safety of direct acting antiviral (DAA) drugs in treatment of HCV infection post-liver transplantation. We aimed to evaluate the efficacy and safety of treatment with DAA drugs early post-transplant period in LDLT recipients with HCV genotype 4

Aims \& Methods: The study was descriptive retrospective analysis on twenty-six LDLT patients with HCV genotype 4 treated with DAA drugs (fifteen patients received Sofosbuvir $400 \mathrm{mg}$ and weight based Ribavirin dose for 24 weeks, eight patients received Sofosbuvir $400 \mathrm{mg}$ and Daclatasvir $60 \mathrm{mg}$ for 24 weeks and three patients received Sofosbuvir $400 \mathrm{mg}$ and Simeprevir $150 \mathrm{mg}$ for 12 weeks) in the early post-transplant period who were transplanted during the period from January 2014 till December 2015 at Egyptian tertiary center for organ transplantation

Results: Twenty-six patients completed the treatment course. sustained virological response (SVR) at week 12 was achieved in $80.8 \%$ (21/26) of recipients, $100 \%$ (11/11) for Sofosbuvir- Daclatasvir and Sofosbuvir- Simeprevir group versus $66.7 \%(10 / 15)$ for Sofosbuvir-Ribavirin group $(\mathrm{p}<0.05)$. No major side effects had been reported, anemia developed in patients received ribavirin respond to treatment with erythropoietin and reduction of the ribavirin dose.

Conclusion: Use of Daclatasvir/Sofosbuvir or Simeprevir/Sofosbuvir in early treatment of $\mathrm{HCV}$ genotype 4 post living donor liver transplant recipient achieved higher rates SVR than Sofosbuvir/Ribavirin.

Disclosure of Interest: All authors have declared no conflicts of interest.

\section{P0703 OPTIMIZATION OF DAA TREATMENT SCHEDULE: FOCUS ON HCV GENOTYPE 3}

F. Morisco ${ }^{1}$, R. Granata ${ }^{1}$, S. Camera ${ }^{1}$, F. Morando ${ }^{1}$, A. Ippolito ${ }^{2}$, M. Milella $^{3}$,

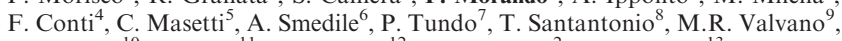
A. Termite ${ }^{10}$, P. Gatti ${ }^{11}$, V. Messina ${ }^{12}$, A. Iacobellis ${ }^{2}$, M. Librandi ${ }^{13}$,

N. Caporaso ${ }^{1}$, A. Andriulli ${ }^{13}$

${ }^{1}$ Gastroenterology Unit, Department of Clinical Medicine and Surgery, "Federico II' University, Napoli, Napoli/Italy

${ }^{2}$ Division of Gastroenterology, "Casa Sollievo della Sofferenza" Hospital, IRCCS

San Giovanni Rotondo, San Giovanni Rotondo/Italy

${ }^{3}$ Clinics of Infectious Diseases, University of Bari, Bari/Italy

${ }^{4}$ Centre For The Study Of Hepatitis, department Of Medical And Surgical Sciences (dimec), University of Bologna, Bologna/Italy

${ }^{5}$ Hepatology and Liver Transplantation Unit, University of Tor Vergata, Roma, Roma/Italy

${ }^{6}$ Department of Medical Sciences, University of Torino, Torino/Italy

${ }^{7}$ Division of Infectious Diseases, Hospital of Galatina, Galatina/Italy

${ }^{8}$ Clinics of Infectious Diseases, University of Foggia, Foggia/Italy

${ }^{9}$ Division of Gastroenterology, "Casa Sollievo della Sofferenza" Hospital, IRCCS,

San Giovanni Rotondo, San Giovanni Rotondo/Italy

${ }^{10}$ Liver Unit, Hospital of Castellaneta, Castellaneta/Italy

${ }^{11}$ Department Of Internal Medicine, Hospital of Ostuni, Ostuni/Italy

${ }^{12}$ Infectious and Tropical Diseases Unit, S. Anna and S. Sebastiano Hospital,

Caserta, Caserta/Italy

${ }^{13}$ Department Of Physiology, faculty Of Pharmacy, La Sapienza University, Roma/Italy

\section{Contact E-mail Address: federicamorando.fm@gmail.com}

Introduction: Direct antiviral agents (DAAs) have led to high sustained virological responses (SVR) in HCV patients. However, genotype 3 patients respond to treatment in a suboptimal way.

Aims \& Methods: This study aims to identify which of the several treatment schedules recommended for genotype 3 would constitute the best option. Twenty-four Italian centers were involved in this real-life study where HCV genotype 3 patients treated with DAAs. To expand the number of cases, we conducted a systematic review of literature on the outcome of genotype 3 patients treated with DAAs.

Results: A total of 233 patients with HCV genotype 3 were enrolled. Cirrhotic patients accounted for $83.7 \%$. Overall, the SVR 12 rate was achieved by 205 subjects $(88.0 \%)$; the SVR rates were $78.8 \%$ after sofosbuvir/ribavirin, $92.5 \%$ after sofosbuvir/daclatasvir \pm ribavirin, and $100 \%$ after sofosbuvir/ledipasvir (7patients). No difference of rate of SVR was observed in cirrhotic and non cirrhotic patients $(92.2 \mathrm{vs} 94.4)$ using a combination regimens of NS5A and NS5B inhibitors. The systematic review of literature provided data of 3311 patients: the mean weighted SVR12 rate was $84.4 \%$ (CI:80.4-87.8); the rates varied from $79.0 \%$ (CI:70.9-85.3) with sofosbuvir/ribavirin, to $83.7 \%$ (CI:66.293.1) with sofosbuvir/ledispavir, and to $88.2 \%$ (CI:83.3-91.7) with sofosbuvir/ daclatasvir.

Conclusion: Our results reinforce the concept that HCV genotype 3 should no more considered difficult to treat individuals. The optimal therapeutic regimen for these patients appears to be the combination sofosbuvir/daclatasvir, administered for 12 weeks without the use of RBV in non cirrhotic patients. In cirrhotics the meta-analytic approach suggests to extend therapy at 24 weeks.

Disclosure of Interest: All authors have declared no conflicts of interest.
P0704 IMPROVEMENT OF LIVER STIFFNESS VALUES

MEASURED BY TRANSITION ELASTOGRAPHY AFTER CHRONIC HEPATITIS C TREATMENT WITH DIRECT ACTION ANTIVIRALS AND EVOLUTIVE CORRELATION OF THROMBOCYTOPENIA AND PRESENCE OF ESOPHAGEAL VARICES

M. Perez Ferrer ${ }^{1}$, I. Maestro Prada ${ }^{1}$, A. Mcgee ${ }^{2}$, J.L. Castro Urda ${ }^{1}$, L.A. Castillo Hererra $^{1}$, C. Garcia Ramos

${ }^{1}$ Gastroenterology, Hospital Severo Ochoa, Leganes/Spain

${ }^{2}$ Epidemiology, Hospital Universitario Mostoles, Mostoles/Spain

Contact E-mail Address: miguelpferrer@gmail.com

Introduction: An improvement in liver stiffness (LS) measured by transient elastography (TE) has been observed in patients with chronic hepatitis $\mathrm{C}$ treated with direct action antivirals (DAA). ${ }^{(1,2)}$. The Baveno VI guidelines ${ }^{(3)}$ propose that patients with compensated advanced chronic liver disease (cACLD), LS measurement $<20 \mathrm{kPa}$ and a platelet count $>150000 / \mu \mathrm{L}$ can avoid screening endoscopy as their combination is highly specific for excluding clinically significant oesophageal varices $(\mathrm{EV})$. These criteria have been validated recently. $(4,5,6)$

Aims \& Methods: The aim of this study was to quantify LS regression both quantitatively (measured in Kilopascals) and qualitatively (Stages of F0-F4 fibrosis) in a stationary phase after the sustained virological response (SVR) in patients with cACLD (F4). The secondary objective was to assess whether this improvement in LF measurements has a clinical correlation with changes in platelet numbers and the presence of varices according to Baveno VI criteria. Results: 84 patients ( 49 men and 35 women) with cACLD were included in the study. Median TE on baseline (BL) prior to DAA treatment was [mean (range), $23.86(12.5-75) \mathrm{kPa}$ ] and decreased to [mean (range, $15.6(4.8-75) \mathrm{kPa}$ ] at SVR 24 and [mean Range), $16.19(3-62.7) \mathrm{kPa}$ ] at SVR $>54$. Both were statistically significant, showing a decrease in LS about $30 \%$ between BL and SVR24 and about $33 \%$ between BL and SVR $>54$. We did not find statistically significant differences between SVR 24 and SVR $>54$. Regarding the probability of qualitative improvement of the LS (improve from F4 to F3 or less) the AUC was 0.8 with $17.9 \mathrm{kPa}$ as the cut-off point which has a Sensitivity of 0.76 and Specificity of $0.81 . \mathrm{NPV}=78.12, \mathrm{PPV}=78.57 .32$ patients with highly suggestive cACLD $(\mathrm{LS}>15 \mathrm{kPa})$ underwent upper endoscopy (UE): $10(32 \%)$ had varices (5 small EV and 5 big EV). 17 (53\%) fulfilled the Baveno VI criteria (3 with small EV and 4 with small) EV. There were only 3 cases of EV misdiagnosed by Baveno VI Criteria. We did not find any significant differences in platelet levels or in the regression of varices.

\begin{tabular}{ll}
\hline & $\begin{array}{l}\text { Main Cohort } \\
\mathrm{n}=84\end{array}$ \\
\hline Age mean (st) & $60(9.8)$ \\
Male-sex (\%) & $49(58.3 \%)$ \\
Aetiology of liver disease (\%) & $\mathrm{HCV} \mathrm{100 \%}$ \\
Platelet count $\times 103 / \mathrm{mm} 3$ Baseline mean (st) & $149.21(59.62)$ \\
Platelet count $\times 103 / \mathrm{mm} 3$ SVR 54 mean (st) & $168.15(60.33)$ \\
Fibrosis Stage F0-1 in SVR 54 n (\%) & $8(12.7 \%)$ \\
Fibrosis Stage F2 in SVR 54 n (\%) & $12(19.5 \%)$ \\
Fibrosis Stage F3 in SVR 54 n (\%) & $13(20.63 \%)$ \\
Fibrosis Stage F4 in SVR 54 n (\%) & $30(47.62 \%)$ \\
Liver Stiffness (kPa) Baseline [n=84]mean (st) & $23.86(12.63)$ \\
Liver Stiffness (kPa) SVR 24 [n=59] mean (st) & $15.6(12.14)$ \\
Liver Stiffness (kPa) SVR 54 [n=63]mean (st) & $16.18(10.99)$ \\
Patients who underwent Upper Endoscopy n (\%) & $32(38.01 \%)$ \\
Patients with varices (VE) (any size) [n=32] n (\%) & $10(31.25 \%)$ \\
Patients fulfilling Baveno criteria [n=32]n (\%) & $17(53.15 \%)$ \\
Patients with high-risk VE [n=32] n (\%) & $5(15.6 \%)$ \\
Patients fulfilling Baveno criteria with & $4(12.5 \%)$ \\
$\quad$ hight-risk VE [n=32] n (\%) & \\
\hline
\end{tabular}

Conclusion: There is a significant improvement in LS data after treatment with DAA both at SVR24 and SVR > 54. This improvement seems to be more likely to happen in patients with lower TE values (in our study $17.9 \mathrm{kPa}$ ). We did not have enough available data in our study to support that this improvement in LS measured by TE has a relevant impact on the clinical management of the patient. The Baveno VI criteria are a useful tool in daily practice to avoid unnecessary UE. Further investigation with larger samples is needed.

Disclosure of Interest: All authors have declared no conflicts of interest.

\section{References}

1. Bachofner JA, Valli PV, Kröger A, Bergamin I, Künzler P, Baserga A, et al. Direct antiviral agent treatment of chronic hepatitis $C$ results in rapid regression of transient elastography and fibrosis markers fibrosis-4 score and aspartate aminotransferase-platelet ratio index. Liver Int. Mar 2017;37(3):369-76.

2. Knop V, Hoppe D, Welzel T, Vermehren J, Herrmann E, Vermehren A, et al. Regression of fibrosis and portal hypertension in $\mathrm{HCV}$-associated cirrhosis and sustained virologic response after interferon-free antiviral therapy. $J$ Viral Hepat. Dec 2016;23(12):994-1002.

3. de Franchis R, Baveno VI Faculty. Expanding consensus in portal hypertension: Report of the Baveno VI Consensus Workshop: Stratifying risk and 
individualizing care for portal hypertension. J Hepatol. Sept 2015;63(3):74352.

4. Llop E, Lopez M, de la Revilla J, Fernandez N, Trapero M, Hernandez M, et al. Validation of non invasive methods to predict the presence of gastroesophageal varices in a cohort of patients with compensated advanced chronic liver disease. J Gastroenterol Hepatol. Mar 2017 on press.

5. Jangouk P, Turco L, De Oliveira A, Schepis F, Villa E, Garcia-Tsao G. Validating, deconstructing and refining Baveno criteria for ruling out highrisk varices in patients with compensated cirrhosis. Liver Int. Feb 2017; 00:1-7.

6. Maurice JB, Brodkin E, Arnold F, Navaratnam A, Paine H, Khawar S, et al. Validation of the Baveno VI criteria to identify low risk cirrhotic patients not requiring endoscopic surveillance for varices. $J$ Hepatol. Noy 2016;65(5):899-905.

\section{P0705 8 VERSUS 12 WEEKS OF LEDIPASVIR/SOFOSBUVIR REGIMEN IN PATIENTS WITH CHRONIC HEPATITIS C GENOTYPE 1 INFECTION}

J.R. Carvalho, P. Alexandrino, F. Serejo, R.T. Marinho, F. Ramalho,

B. Rodrigues, P. Ferreira, M. S. Dias, H. Cortez-Pinto, C. Baldaia, A. J. Pedro, L. X. De Brito, L. Carrilho Ribeiro, N. Fatela, J. Velosa

Department Of Gastroenterology And Hepatology, North Lisbon Hospital Center, University of Lisbon, Portugal, Lisbon/Portugal

Contact E-mail Address: joana.rita.carvalho@gmail.com

Introduction: The therapeutic regimens for chronic hepatitis $\mathrm{C}$ are now tending to be shorter and ribavirin free, more cost-effective and with fewer adverse effects. Aims \& Methods: We aimed at comparing the 8 weeks versus the 12 weeks regimen of ledipasvir plus sofosbuvir in patients with hepatitis $\mathrm{C}$ virus (HCV) genotype 1 infection without cirrhosis, treatment naïve, HCV RNA $<6000000 \mathrm{UI} /$ $\mathrm{mL}$. We included 281 patients (pts) with genotype 1 and RNA $<6000000 \mathrm{UI} / \mathrm{mL}$ treated with ledipasvir plus sofosbuvir in the recommended dose: 120 pts - 8 weeks (group 1) and 161 pts - 12 weeks (group 2). The fibrosis stage was eval-

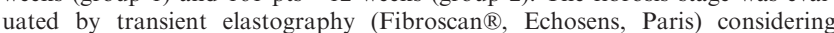
F4 $>12.5 \mathrm{kPa}$. Patients with undetectable RNA after 12 weeks of treatment were considered cured - sustained virologic response (SVR).

Results: No significant demographic and clinical differences were found between the two groups with the exception of the fibrosis stage (table). Two hundred and forty-four patients concluded the treatment; the SVR was $99 \%$ in group 1 and $100 \%$ in group 2 , without differences between the two groups $(\mathrm{p}=0.275)$. Thirty three patients are still in follow up: group $1-15$ pts and group $2-18$ pts. The reported adverse effects were mild in both groups (fatigue, insomnia, headache and pruritus) but more frequent in group $2(\mathrm{p}=0.046)$.

\begin{tabular}{|c|c|c|c|}
\hline Patients characteristics & 8 weeks $\mathrm{n}=120$ & 12 weeks $n=161$ & $\mathrm{p}$ \\
\hline Average age (years) & $50.4(20-81)$ & $53.6(19-86)$ & 0.473 \\
\hline Male sex, n (\%) & $62(51.7 \%)$ & $89(55.3 \%)$ & 0.548 \\
\hline \multicolumn{4}{|l|}{ Genotype (n) } \\
\hline 1a & 74 & 94 & 0.579 \\
\hline $1 \mathrm{~b}$ & 44 & 66 & 0.462 \\
\hline 1 and $1 \mathrm{a} / 1 \mathrm{~b}$ & $1+1$ & $1+0$ & \\
\hline Average $\mathrm{RNA} \pm \mathrm{SD}(\mathrm{UI} / \mathrm{mL})$ & $903275 \pm 1093067$ & $1826591 \pm 1611806$ & 0.348 \\
\hline \multicolumn{4}{|l|}{ Initial transient elastography (n) } \\
\hline $\mathrm{F} 0 / \mathrm{F} 1$ & 111 & 105 & $<0.001$ \\
\hline $\mathrm{F} 2 / \mathrm{F} 3$ & 8 & 56 & $<0.001$ \\
\hline $\mathrm{F} 4$ & 1 & 0 & 0.246 \\
\hline \multicolumn{4}{|c|}{ Previous treatment with $\mathrm{INF} \pm$ Ribavirin } \\
\hline Yes & 8 & 0 & 0.001 \\
\hline No & 112 & 161 & $<0.001$ \\
\hline HIV co-infection & 1 & 14 & 0.004 \\
\hline \multicolumn{4}{|l|}{ RNA - week $4, n^{\circ}(\%)$} \\
\hline Undetectable & $86(71.7 \%)$ & $124(77.0 \%)$ & 0.307 \\
\hline$<15 \mathrm{UI} / \mathrm{mL}$ & $13(10.8 \%)$ & $13(8.1 \%)$ & 0.430 \\
\hline \multicolumn{4}{|l|}{ Virologic response } \\
\hline SVR, $n^{\circ}(\%)$ & $103(99.0 \%)$ & $141(100.0 \%)$ & 0.275 \\
\hline No response (no compliance) $*$ & 1 & 0 & $*$ \\
\hline Awaiting response (in follow up) & 15 & 18 & 0.734 \\
\hline Lost for follow up & 1 & 2 & 0.742 \\
\hline Mild adverse effects, n (\%) & $7(6 \%)$ & $21(13 \%)$ & 0.046 \\
\hline
\end{tabular}

* Patient retreated with ledipasvir + sofosbuvir + ribavirine during 12 weeks with SVR

Conclusion: In patients with chronic HCV genotype 1 infection and RNA $<6000000 \mathrm{UI} / \mathrm{mL}$, the 8 weeks regimen of ledipasvir plus sofosbuvir without ribavirin has similar high cure rates with less adverse effects.

Disclosure of Interest: All authors have declared no conflicts of interest.

\section{References}

1. Backus LI, Belperio PS, Shahoumian TA, Loomis PT, Mole LA. Real-World effectiness of ledipasvir/sofosbuvir in 4, 365 treatment-naive, genotype 1 hepatitis C-infected patients. Hepatology 2016:64(2):405-414.

2. Ingiliz P, Christensen S, Kimhofer T, et al. Sofosbuvir and Ledipasvir for 8 weeks for the treatment of Chronic Hepatitis C Virus (HCV) infection in HCV-monoinfected and HIV-HCV-coinfected individuals: results from the german hepatitis C Cohort (GECCO-01). Clinical Infectious Disease 2016:63(10):1320-1324

\section{P0706 CLINICAL FEATURES OF PATIENTS DEVELOPING HCC AFTER ACHIEVING SVR WITH DAA AGAINST CHRONIC} HEPATITIS C

K. Ishida, T. Nakatani, Y. Motokawa, F. Tomooka, A. Shibamoto, Y. Fujimoto, Y. Aihara, S. Nagamatu, M. Uejima, H. Matuo, E. Kikuchi Gastroenterology, Nara Prefecture General Medical Center, Nara/Japan

Contact E-mail Address: k_ishida1549@yahoo.co.jp

Introduction: Although the conventional IFN-based therapy has made a significant achievement in treating patients with hepatitis $\mathrm{C}$ virus (HCV), including the preventive effect of hepatocarcinogenesis after achieving sustained virological response (SVR), patients intolerant of IFN, such as those with advanced age or liver cirrhosis (LC), could not savor its privilege. The appearance of directacting antivirals (DAAs) provided almost every patient with the chance to receive the treatment for HCV infection without any serious adverse effects (AEs). In addition, SVR could be highly expected in more than $95 \%$ of patients treated with DAAs. However, the preventive effect of DAAs for the future hepatocarcinogenesis following eradication of HCV remains unknown. In our facility, the administration of DAAs to patients with HCV infection led to $97 \%(153 / 157)$ of SVR, among which $5.9 \%$ (9/153) of patients developed hepatocellular carcinoma (HCC) within 2 years after achieving SVR.

Aims \& Methods: We evaluated the clinical features of patients developing $\mathrm{HCC}$ after confirming SVR with DAAs against HCV. One hundred and fifty-three patients achieving SVR defined as negative HCV-RNA 12 weeks after cessation of DAA (oral combination therapy with Daclatasvir/Asunaprevir, Ombitasvir/ Paritaprevir/Ritonavir, Sofosbuvir/Ledipasvir, or Sofosbuvir/Ribavirin) were enrolled in this study (Age 69.2 \pm 0.8 , male/female 71/82, genotype1/2 122/31,

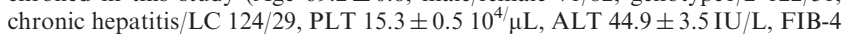
index $3.9 \pm 0.2$, APRI $1.3 \pm 0.1$, Wisteria floribunda agglutinin positive Mac-2binding protein (WFA(+)-M2BP) $3.3 \pm 0.5$ COI, AFP $12.1 \pm 2.4 \mathrm{ng} / \mathrm{mL}$, PIVKA-II $29.8 \pm 4.5 \mathrm{mAU} / \mathrm{mL}$ ). All patients were divided into 2 groups (A: 9 patients with HCC developing after SVR achievement, B: 144 without HCC after SVR achievement). Serum parameters (PLT, WFA(+)-M2BP, FIB-4 index, APRI, ALT, Alb, AFP, PIVKAII) and age were evaluated between 2 groups. Results: In group B, significant declining (pre-DAA treatment/the time of achieving SVR) was observed in $\operatorname{ALT}(44.8 \pm 3.6 / 19.5 \pm 1.1 \mathrm{IU} / \mathrm{L}), \operatorname{AFP}(11.9 \pm 3.1$ $4.1 \pm 0.3 \mathrm{ng} / \mathrm{mL}), \quad \mathrm{WFA}(+)-\mathrm{M} 2 \mathrm{BP}(3.2 \pm 0.5 / 1.9 \pm 0.3 \quad$ COI $) \quad$ FIB-4 index $(3.7 \pm 0.2 / 3.0 \pm 0.2)$ and APRI $(1.2 \pm 0.1 / 0.7 \pm 0.05)$, and significant increase in $\operatorname{Alb}(4.1 \pm 0.04 / 4.3 \pm 0.04 \mathrm{~g} / \mathrm{dL})$ and PLT $\left(15.4 \pm 0.5 / 16.1 \pm 0.5 \quad 10^{4 / \mu L}\right)$. In group A, significant declining was observed only in ALT $(45.1 \pm 10.9$ / $16.7 \pm 2.2 \mathrm{IU} / \mathrm{L})$. This result indicates that DAA treatment significantly ameliorates parameters related with hepatic fibrosis as well as hepatic inflammation in group B, however, it led to the significant amelioration only in those related with hepatic inflammatin in group A. Next, focusing on parameters after achieving SVR, WFA(+)-M2BP (A:3.4 $\pm 0.6 / \mathrm{B}: 1.7 \pm 0.2 \mathrm{COI})$ and FIB-4 index $(5.7 \pm 1.6$ $3.0 \pm 0.2)$ were significantly higher and Alb $(3.8 \pm 0.2 / 4.3 \pm 0.04 \mathrm{~g} / \mathrm{dL})$ was significantly lower in group A comparing with group B. When dividing group A into 2 groups $(\mathrm{C}$ : new occurrence/D: recurrence), $\operatorname{AFP}(3.0 \pm 1.3 / 6.2 \pm 1.8 \mathrm{ng}$ $\mathrm{mL})$, WFA(+)-M2BP $(2.0 \pm 0.8 / 3.8 \pm 0.7 \quad \mathrm{COI}), \quad$ FIB-4 index $(3.6 \pm 2.4 /$ $6.3 \pm 2.0)$ and APRI $(0.6 \pm 0.4 / 1.1 \pm 0.3)$ were higher and $\operatorname{Alb}(4.3 \pm 0.1$ $3.6 \pm 0.3 \mathrm{~g} / \mathrm{dL})$ and PLT $\left(20.9 \pm 13.2 / 13.4 \pm 3.410^{4} \mu \mathrm{L}\right)$ were lower in group D than group $\mathrm{C}$, although no significant difference was seen between 2 groups. This result suggests that there might be more patients with progressive hepatic fibrosis in group D comparing with group C. Finally, while univariate analysis showed WFA(+)-M2BP, FIB-4 index and Alb were significantly associated with the development of HCC after achieving SVR with DAA against HCV, multivariate analysis revealed only Alb was the significantly independent factor contributing to HCC development after SVR achievement.

Conclusion: Low level of serum albumin as well as the progression of hepatic fibrosis could be associated with the development of HCC after confirming SVR with DAA to HCV.

Disclosure of Interest: All authors have declared no conflicts of interest.

\section{P0707 EARLY OCCURRENCE OF HEPATOCELLULAR CARCINOMA IN PATIENTS WITH HEPATITIS C VIRUS TREATED WITH DIRECT-ACTING ANTIVIRALS}

S. Hai, E. Hatano, T. Okada, Y. Asano, N. Uyama, K. Suzumura, M. Tada, I. Nakamura, K. Iida, H. Sueoka, H. Iwama, A. Kurimoto, R. Ito, H. Nishida, J. Fujimoto

Department Of Surgery, Hyogo College of Medicine, Nishinomiya/Japan

Contact E-mail Address: shai1005@hyo-med.ac.jp

Introduction: Direct-acting antivirals (DAAs) are novel antiviral drugs for hepatitis $\mathrm{C}$ virus $(\mathrm{HCV})$ and have enabled the achievement of a high rate of sustained 
virological response (SVR) [1]. However, the impact of DAAs on the occurrence of hepatocellular carcinoma (HCC) and HCC recurrence after curative hepatic resection of HCC has been recently discussed [2,3], but remain unclear.

Aims \& Methods: The clinical data of 97 patients who underwent curative hepatic resection for primary HCC with HCV at our department between January 2012 and March 2017 were reviewed to clarify the impact of DAAs on HCC occurrence and recurrence. SVR was defined as no detection of HCV RNA in the serum at 24 weeks after the cessation of antiviral therapy.

Results: SVR was achieved in 21 patients treated with interferon (IFN)-based regimens and in 9 with DAAs at hepatectomy. Between the two groups, there were no significant differences in the clinical characteristics, including the age, prevalence of diabetes mellitus, drinking history, preoperative liver function, operative procedures, tumor size and presence of liver cirrhosis, but the median duration from the date of SVR to the date of HCC incidence was significantly shorter in patients treated with DAAs (14 days, range: -123 to 235 days) than in those treated with IFN-based regimens (324 days, range: 35 to 4190 days). In particular, HCC was detected within 24 weeks after the cessation of antiviral therapy in 3 patients treated with DAAs. After hepatectomy, SVR was achieved in 21 (DAAs: 16 patients, IFN-based regimens: 5 patients) of the 67 patients without SVR when hepatectomy was performed, and the 1- and 3-year disease-free survival (DFS) rates were $93.3 \%$ and $83.0 \%$ in patients after SVR treated with DAAs $(\mathrm{n}=25), 90.9 \%$ and $71.8 \%$ in patients with IFN-based regimens $(\mathrm{n}=26)$ and $57.8 \%$ and $19.7 \%$ in patients without SVR $(\mathrm{n}=46)$, respectively, regardless of the timing of hepatectomy, respectively. The DFS rate was significantly higher in patients with SVR than in those without SVR $(p<0.01)$, but was not markedly different according to the antiviral treatments $(\mathrm{p}=0.504)$. Conclusion: While DAAs were able to reduce the DFS rate, the early occurrence of HCC in patients after SVR treated with DAAs is more frequent than that among patients treated with IFN-based regimens. Therefore, careful follow-up with imaging series is needed even for patients with SVR treated with DAAs. Disclosure of Interest: All authors have declared no conflicts of interest.

\section{References}

1. Chung RT, Baumert TF. Curing chronic hepatitis C-the arc of a medical triumph. N Engl J Med. 2014;370:1576-8.

2. Conti F, Buonfiglioli F, Scuteri A, et al. Early occurrence and recurrence of hepatocellular carcinoma in HCV-related cirrhosis treated with direct-acting antivirals. J Hepatol. 2016;65:727-33.

3. Kozbial K, Moser S, Schwarzer R, et al. Unexpected high incidence of hepatocellular carcinoma in cirrhotic patients with sustained virologic response following interferon-free direct-acting antiviral treatment. $J$ Hepatol. 2016;65:856-8

\section{P0708 EFFICACY AND SAFETY OF SOFOSBUVIR AND RIBAVIRIN IN HCV POSITIVE PATIENTS WITH RENAL IMPAIRMENT}

\section{M.O. T. Butt}

Gastroenterology And Liver Transplant, Shifa International Hospital, Islamabad, Islamabad/Pakistan

\section{Contact E-mail Address: mosamabutt2016@gmail.com}

Introduction: Hepatitis C virus infection is a leading cause of chronic liver disease affecting more than 170 million people worldwide. HCV infection in the setting of renal impairment is not uncommon. Despite the major developments in the treatment of $\mathrm{HCV}$, treatment of this sub group of patient with impaired renal function is still a challenge.

Aims \& Methods: The aim of this study is determine the efficacy and safety of sofosbuvir and ribavirin in $\mathrm{HCV}$ positive patients with renal impairment. All consecutive patients of with HCV related liver disease with creatinine clearance less than $50 \mathrm{ml} / \mathrm{min}$ were included in the study. Data was collected for tolerability, efficacy and on treatment adverse events. All the patients received Sofosbuvir and dose adjusted Ribavirin according of $\mathrm{CrCl}$. Virological response was checked at 1 month (RVR), 3 months (EVR) and at the end of treatment.

Results: A total of 31 patients were included in the study were 31 out of which $17(54.8 \%)$ were male. Mean age was $52.23 \pm 17.6$ years while the mean BMI was $25.0 \pm 4.50 \mathrm{~kg} / \mathrm{m} 2.10(32.25 \%)$ patient were on regular hemodialysis. $26(83.9 \%)$ patients had CTP-A while $5(16.1 \%)$ had CTP-B disease. Majority of the patients $22(71 \%)$ were genotype 3 while $7(22.6 \%)$ were genotype $1.24(77.4 \%)$ patients were treatment naïve, while those who were treatment experienced, 3 patients received each Interferon and Peg interferon therapy. Treatment was stopped in 2 $(6.5 \%)$ patients because of disease decompensation while $3(9.7 \%)$ were lost to follow up. ETR was achieved in $25(96.1 \%)$ out of 26 patients who completed treatment. Similarly $12(80 \%)$ out of 15 patients have achieved SVR-12 so far. During the therapy $10(32.3 \%)$ patients had adverse events, $6(19.4 \%)$ suffered from depression while $4(12.9 \%)$ developed grade II anemia.

Conclusion: In resource constraint population where newer DAAs are not available, combination of sofosbovir and low-dose ribavirin in patients with renal impairment seems to be better tolerated and efficacious in terms of achieving the virological response.

Disclosure of Interest: All authors have declared no conflicts of interest.
P0709 CHRONIC HEPATITIS C A MAJOR HEALTH - RELATED QUALITY OF LIFE BURDEN IN COMPANSATED CIRRHOTIC PATIENTS

T. Cuciureanu ${ }^{1}$, A.M. Singeap ${ }^{2}$, I. Girleanu ${ }^{3}$, S. Chiriac ${ }^{2}$, A. Trifan ${ }^{2}$

${ }^{1}$ Institute Of Gastroenterology And Hepatology Iasi, UMF Gr T Popa Iasi, iasi Romania

${ }^{2}$ Gastroenterology, "Grigore T. Popa" University of Medicine and Pharmacy, iasi/ Romania

${ }^{3}$ Institute of Gastroenterology and Hepatology, Iasi/Romania

Contact E-mail Address: drcuciureanutudor@gmail.com

Introduction: Chronic hepatitis $\mathrm{C}$ infection is a systemic disease, one of the leading causes towards cirrhosis and hepatocellular cancer and it is to be considered nowadays a major health-related quality of life (HRQoL) burden.

Aims \& Methods: The aim of this study was to assess HRQoL impairment of hepatitis $\mathrm{C}$ virus $(\mathrm{HCV})$ infection among a broad sample of compensated $\mathrm{HCV}$ cirrhotic patients. We conducted a prospective study between January 1 st 2016 to January 31,2017 , in a tertiary center, in which we included 110 patients with compensated HCV cirrhosis, aged between 50 and 75, with no history of neuropsychiatric illness but associated comorbidities (diabetes type 2, hypertension, dyslipidemia). The patients were completely evaluated according to the national protocol. Health status and fatigue of our patients were evaluated using the FACIT- F (version 4) and SF-36 survey. Respondents with HCV compensated cirrhosis were compared with a control group matched for age and sex with no prior history of HCV infection on the Mental (MCS) and Physical (PCS) Component Summary scores.

Results: Unadjusted comparisons between subjects infected with HCV $(\mathrm{n}=110)$ and controls $(n=60)$ revealed that HCV patients had lower FACIT- F utility scores $(43.2 \pm 0.8$ vs $49.5 \pm 0.5, \mathrm{P}<0.05)$. Severe fatigue was present in $30 \%$ (33 patients) of the HCV group compared to $11.6 \%(7$ patients) in controls. Subgroup analyses of respondents age 60 years and older revealed lower MCS score in HCV patients compared to controls $(41.95$ vs. $49.72, \mathrm{p}<0.05)$. Control group registered higher PCS score $(53.30$ vs $45.2, \mathrm{P}<0.05)$ compared to the study group.

Conclusion: Although the results were obtained on a small group we observed that in untreated patients with chronic HCV infection, HRQoL is significantly impaired due to fatigue severity and age. Our result underline the need for effective antiviral treatment to decrease the burden of fatigue in this segment of population.

Disclosure of Interest: All authors have declared no conflicts of interest.

\section{P0710 SOFOSBUVIR IN COMBINATION WITH RIBAVIRIN IN GENOTYPE 3 HEPATIITS C PATIENTS WITH CIRRHOSIS. AN EXPERIENCE FROM TERTIARY CARE HOSPITAL}

\section{A. A. Langhah}

Gastroenterology Section, Medical Unit Iv, Jinnah Postgraduate Medical Centre, Karachi/Pakistan

Contact E-mail Address: draliakbar12@gmail.com

Introduction: Hepatitis C virus (HCV) is the most common cause of cirrhosis in this part of the world. Advent of Directly acting antivirals (DAAs) like Sofosbuvir (SOF) has dramatized the treatment and is the corner stone in treatment of $(\mathrm{HCV})$. Most trials have been conducted in HCV genotype 1 and data for Interferon free regimen in genotype $3(\mathrm{GT}-3)$ is limited especially in cirrhotics. Aims \& Methods: We aimed to evaluate the safety and efficacy of SOF plus Ribavirin (RIB) in patients with compensated and decompensated cirrhosis. This is a prospective real-world cohort study of HCV with compensated or decompensated cirrhosis. Efficacy was assessed by Sustained Viral Response after 06 months of completion of treatment. Adverse events were recorded on designed proforma on serial follow-up visits.

Results: The cohort consisted of 91 consecutive patients out of which 41 were compensated cirrhotics and 50 had decompensated cirrhosis. The mean age was $53.4 \pm 11$ years. Males were $47(51.6 \%)$ and females were $44(48.4 \%)$. Mean CTP and MELD score were 7.71 and 9.21 respectively. In compensated cirrhosis, SVR was achieved in $25(84 \%)$ treatment naïve patients compared to treatment experienced patients where $5(80 \%)$ achieved SVR. In decompensated cirrhosis SVR was achieved in $22(77.3 \%)$ treatment naïve patients, whereas $13(76.9 \%)$ patients achieved SVR in treatment experienced group. In $72 \%$ patients with cirrhosis, there were no side effects whereas most common adverse event was fatigue and drop of Hemoglobin by $1.0 \mathrm{gm} / \mathrm{dl}$. Furthermore, CTP and MELD scores decreased to 6.9 and 8.7 respectively after treatment.

Conclusion: Sofosbuvir in combination with Ribavirin in GT-3 HCV patients achieved good SVR in compensated cirrhosis than decompensated cirrhosis wheras fatigue and drop of $\mathrm{Hb}$ were the most common adverse effects. Disclosure of Interest: All authors have declared no conflicts of interest. 
P0711 RECURRENCE OF HEPATOCELLULAR CARCINOMA (HCC) IN PATIENTS WITH COMPENSATED LIVER CIRRHOSIS AND HCC TREATED WITH PARITAPREVIR/OMBITASVIR/RITONAVIR, DASABUVIR WITH RIBAVIRIN: A NATIONAL COHORT STUDY

D. Proca ${ }^{1}$, C.M. Preda ${ }^{2}$, C. Baicus ${ }^{2}$, C. P. Popescu ${ }^{2}$, M. Manuc ${ }^{2}$, C. S. Pop ${ }^{2}$, T. Voiosu ${ }^{2}$, E. Ceausu ${ }^{2}$, M. Diculescu ${ }^{2}$, A. Oproiu ${ }^{2}$

${ }^{1}$ Gastroenterology, Fundeni Clinica Institute, Bucharest/Romania

${ }^{2}$ Gastroenterology, University of Medicine and Pharmacy "Carol Davila",

Bucharest/Romania

Contact E-mail Address: doina.proca08@gmail.com

Introduction: New direct-acting antivirals (DAA) have changed the management of $\mathrm{HCV}$ infection by being effective in more than $90 \%$ of cases [1, 2]. Unfortunately, it has been reported an unexpected high rate of HCC early recurrence following DAA treatment, but more data are needed [3-5].

Aims \& Methods: From a national prospective cohort, enrolling 3717 Romanian patients with hepatitis $\mathrm{C}$ virus compensated liver cirrhosis who received reimbursed DAA with Paritaprevir/Ombitasvir/ritonavir, Dasabuvir and Ribavirin (OBV/PTV/r + DSV + RBV) for 12 weeks, from December 2015 to August 2016 we analyzed 21 patients with previous HCC. Most of them were treated through surgical resection $(9 / 21)$, followed by radiofrequency ablation (RFA) 6/21, transarterial chemoembolisation (TACE) $5 / 21$ and only one percutaneous ethanol injection (PEIT). The patients received DAA treatment only if they had no cancer relapse 6 months after their last therapy session for HCC. All these patients were evaluated through CT scan or MRI 3 to 6 months after having finished their DAA therapy. The median follow-up is 6 months $(3 \div 12)$. Data were obtained from the Romanian National Health Agency.

Results: Two female patients decompensated and died because of acute liver failure $(9.5 \%)$, the first one after having finished the 12 weeks therapy, and the other one in week 7 of DAA therapy. This cohort was $52 \%$ females, median age 64 years $(51-77), 76 \%$ IFN pre-treated, $50 \%$ associated NASH, $67 \%$ with severe necroinflammatory activity (severity score 3 - Fibromax), 30\% with co-morbidities, $24 \%$ with Child Pugh A6. The median MELD score was $9(6 \div 11)$. SVR was reported in $19 / 21$ as per protocol analysis $(90.5 \%)$. Recurrence rate of HCC was $29 \%$, higher in males $(40 \%)$ than females $(18 \%)$, higher in patients treated with TACE $(40 \%)$ than in those with hepatic resection $(33 \%)$, and the lowest risk of recurrence was encountered in RFA (17\%). These differences were not statistically significant because of the small sample size. The pattern of recurrence was: intrahepatic growth (1 patient), new intrahepatic lesion (1 nodule in 2 patients, up to 3 nodules less or equal to $3 \mathrm{~cm}$ in 1 case) and infiltrative ill-defined hepatocellular carcinoma in 2 patients. We found no correlation between the HCC-free interval of time and recurrence rate $(\mathrm{p}=0.62)$.

Conclusion: Early recurrence rate of HCC in treated patients with compensated liver cirrhosis that received DAA with OBV/PTV/r + DSV + RBV was $29 \%$, significantly increased compared to the natural recurrence rate. This rate is higher in males $(40 \%)$ and in patients treated with TACE $(40 \%)$.

Disclosure of Interest: All authors have declared no conflicts of interest.

\section{References}

1. Reig M, Boix L, Bruix J. The impact of direct antiviral agents on the development and recurrence of hepatocellular carcinoma. Liver Int. 2017; 37 Suppl 1:136-139.

2. ANRS collaborative study group on hepatocellular carcinoma. Lack of evidence of an effect of direct-acting antivirals on the recurrence of hepatocellular carcinoma: Data from three ANRS cohorts. J Hepatol. 2016; 65:73440 .

3. Conti F, Buonfiglioli F, Scuteri A, . Early occurrence and recurrence of hepatocellular carcinoma in HCV-related cirrhosis treated with directacting antivirals. J Hepatol. 2016; 65:727-33.

4. Reig M, Mariño Z, Perelló C, . Unexpected high rate of early tumor recurrence in patients with $\mathrm{HCV}$-related $\mathrm{HCC}$ undergoing interferon-free therapy. J Hepatol. 2016; 65(4):719-2.

5. Villani R, Facciorusso A, Bellanti F, . DAAs Rapidly Reduce Inflammation but Increase Serum VEGF Level: A Rationale for Tumor Risk during AntiHCV Treatment. PLoS One. 2016; 11:e0167934.

\section{P0712 SHEAR WAVE ELASTOGRAPHY FOR THE DIAGNOSIS OF ESOPHAGOGASTRIC VARICES}

H. Iijima ${ }^{1}$, T. Nishimura ${ }^{1}$, C. Nakano ${ }^{1}$, T. Aoki ${ }^{1}$, Y. Miyamoto ${ }^{2}$, N. Ishii ${ }^{2}$,

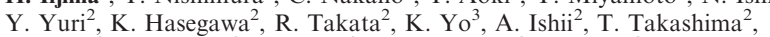

Y. Sakai ${ }^{2}$, N. Aizawa ${ }^{2}$, N. Ikeda ${ }^{2}$, H. Nishikawa ${ }^{2}$, Y. Iwata ${ }^{2}$, H. Enomoto ${ }^{2}$,

S. Nishiguchi ${ }^{3}$, J. Fujimoto ${ }^{3}$

${ }^{1}$ Ultrasound Imaging Center, Division Of Hepatobiliary And Pancreatic Diseases, Hyogo College of Medicine, Nishinomiya/Japan

${ }^{2}$ Division Of Hepatobiliary And Pancreatic Diseases, Hyogo College of Medicine, Nishinomiya/Japan

${ }^{3}$ Department Of Hepato-biliary-pancreatic Surgery, Hyogo College of Medicine, Nishinomiya/Japan

Contact E-mail Address: hiroko-i@hyo-med.ac.jp

Introduction: Shear wave elastography (SWE) has been used in clinical practice as a noninvasive method to diagnose liver fibrosis by measuring tissue stiffness.

Aims \& Methods: The usefulness of SWE in the diagnosis of esophagogastric varices (EGVs) associated with portal hypertension was evaluated. 550 patients who underwent measurements of liver stiffness (LS), spleen stiffness (SS) and EGV evaluation between January 2011 and July 2016 were included (no varices, $\mathrm{n}=340$; esophagus only, $\mathrm{n}=107$; stomach only, $\mathrm{n}=14$; esophagus and stomach, $\mathrm{n}=89$ ). Virtual Touch Quantification (VTQ) was used for measurements of LS and SS, and the spleen index (SI) was calculated. EGVs were evaluated according to forms and red colour sign (RC sign) based on the General Rules for Study of Hypertension in Japan.

Results: LS, SS and SI showed significant increase in accordance with the severity of forms $(\mathrm{p}<0.01)$. The area under the receiver operating characteristic curve (AUROC) of LS, SS and SI for detecting EGVs were 0.8526, 0.9048, 0.8199, with the cut off values $1.67 \mathrm{~m} / \mathrm{s}, 2.81 \mathrm{~m} / \mathrm{s}, 18.5 \mathrm{~cm}^{2}$, respectively, and SS showed usefulness in detecting EGVs. When LS, SS and SI were compared for their ability to detect EGVs which were $<$ F2 or with RC sign positive, LS, SS and SI were significantly higher for EGVs which require treatment $(\mathrm{p}<0.001)$. The AUROC of LS, SS and SI for detecting EGVs which require treatment were 0.8131, $0.8693,0.8270$, respectively. All modality showed good detecting ability, and SS particularly showed better performance. When each modality was compared in detection of gastric varices, LS and SS were significantly higher with the presence of gastric varices, but SI did not show a significant difference. The AUROC of LS, SS and SI for detecting gastric varices were 0.7359, 0.8611, 0.6470 , respectively, and SS showed a superior detecting ability while LS and SI showed decreased abilities.

Conclusion: LS, SS and SI were all useful in detecting EGVs and predicting the presence of EGVs which require treatments. SS particularly showed the highest diagnostic ability.

Disclosure of Interest: All authors have declared no conflicts of interest.

P0713 STAGING HEPATIC STEATOSIS IN NONALCOHOLIC FATTY LIVER DISEASE BY QUANTITATIVE CONVENTIONAL ULTRASOUND IMAGING, VALIDATED WITH HISTOPATHOLOGY

G. Weijers ${ }^{1}$, I. Munsterman ${ }^{2}$, A. Meel - Van Den Abeelen ${ }^{1}$, J. M. Thijssen ${ }^{1}$, J.P.h. Drenth ${ }^{2}$, E. Tjwa ${ }^{2}$, C. De Korte

${ }^{1}$ Medical Ultrasound Imaging Center, Radiology And Nuclear Medicine, Radboud university medical center, Nijmegen/Netherlands

${ }^{2}$ Gastroenterology \& Hepatology, Radboud university medical center, Nijmegen/ Netherlands

Contact E-mail Address: isabelle.munsterman@radboudumc.nl

Introduction: Nonalcoholic fatty liver disease (NAFLD) is the most common liver disorder in developed countries with a global prevalence of approximately $25 \%$. NAFLD represents a spectrum of disorders and starts with benign steatosis (NAFL, $\geq 5 \%$ hepatic steatosis but may lead to nonalcoholic steatohepatitis (NASH), inflammation with hepatocyte injury with or without fibrosis, which may lead to cirrhosis. Liver biopsy still is the gold standard for staging steatosis. The BRUNT score stages steatosis in 4 categories $(\leq 5 \%, 5-33 \%, 34-66 \%$, $\geq 66 \%$ ), which provides a limited sensitivity to detect changes in steatosis content. For evaluation of treatment and disease activity a quantitative and preferably non-invasive tool is needed urgently. Therefore we developed and tested a computer aided ultrasound (CAUS) protocol for the non-invasive assessment of hepatic steatosis using ultrasound (US) B-mode images, which showed high predictive values (area under the curve (AUC) up to 0.95) in cows and similar correlations in a human pilot study. The current study was conducted in order to assess the predictive values of CAUS in staging hepatic steatosis in-vivo.

Aims \& Methods: Consecutive patients indicated for a liver biopsy received a simultaneous transabdominal US examination (Siemens Acuson X150; Siemens Healthcare $\mathrm{GmbH}$, Erlangen, Germany, with $\mathrm{CH} 5-2$ transducer). Currently 205 patients with liver biopsies and QUS images are included. Biopsies were qualitatively scored using the Brunt-score for steatosis grading. Metavir, Ishak and/or Roenigk scores were used for disease specific fibrosis staging. All US images were further post-processed using the CAUS method. In CAUS corrections are applied for the: look up table (linearization); beam-profile; attenuation caused by the superficial tissue layers. Furthermore segmentation of large blood vessels and bile ducts is performed automatically. Finally echo-level and texture parameters are estimated on average and as a function of depth by estimation of slope and intercept of a linear fit.

Results: Best correlating CAUS parameter with BRUNT steatosis stage was found to be the residual attenuation coefficient (RAC, $\mathrm{R}=0.79, \mathrm{p}<0.01$ ). Parameters used in the multiple linear regression analysis using the leave one out method were: Residual attenuation coefficient $(\mathrm{RAC}=$ slope of the mean echo level (MU)); MU; slope of the Signal to noise ratio; and the lateral speckle size. AUC for HS was found to be $0.95(\mathrm{n}=71)$ with a sensitivity and specificity of $86 \%$ and $85 \%$ respectively, which are in line with previously performed studies. $87 \%$ of the included patients were found to have NASH with fibrosis. However, no significant correlations of the CAUS parameters to a pure fibrosis-group $(\mathrm{N}=106)$ was found.

Conclusion: CAUS is able to classify steatosis accurately and does not suffer from the presence of fibrosis, making CAUS a feasible tool for screening and evaluation of hepatic steatosis.

Disclosure of Interest: All authors have declared no conflicts of interest.

\section{P0714 UTILITY OF A NEW FUNCTION IN 3D SIM-NAVIGATOR: ELECTRIC FIELD, WHICH INDICATES THE PREDICTED} ABLATIVE AREA

A. Sakamoto, T. Kimura, T. Matsumae, G. Tanke, H. Yoshida, M. Taki, M. Fukuhara, S. Henmi, T. Yamashina, Y. Sawai, S. Saito, N. Nishijima, A. Nasu, H. Komekado, M. Asada, T. Tsumura, T. Maruo, R. Kita, Y. Osaki Gastroenterology And Hepatology, Osaka Red Cross Hospital, Osaka/Japan 
Contact E-mail Address: azusa-s@osaka-med.jrc.or.jp

Introduction: Recently, fusion imaging technology has been reported as useful for radiofrequency ablation (RFA) and various models of ultrasound equipment have been developed to include fusion imaging systems. The 3D SimNavigator (HITACHI) is a navigation system that can be used in real-time virtual sonography (RVS) by simulating and navigating the $3 \mathrm{D}$ positions of electrodes. As an addition, the 3D Sim-Navigator is now equipped with a new system that shows the predicted ablative area. This function indicates the electric field that occurs around the electrodes as the predicted ablative area on MPR images. The E-field Simulator (E-field) can manage various electrodes, such as monopolar and bipolar RF systems. E-field can be used to predict the ablative areas from not only a single electrode but also multiple electrodes, which was difficult to do in the past. We therefore examined the accuracy of E-field on 3D Sim-Navigator.

Aims \& Methods: We evaluated 10 nodules treated using a bipolar RF system (CelonPOWER; Olympus Medical Systems) with multiple electrodes and 72 nodules treated using a monopolar RF system (VIVARF system; STARmed) between April 2016 and March 2017 prospectively. We compared the major and minor axes of maximum axial ablative area for 72 nodules ablated by the monopolar RF system on both E-field and post-RFA contrast-enhanced computed tomography (CT). We then compared ablative volumes of 10 nodules ablated by the bipolar RF system on E-field and on post-RFA contrast-enhanced CT. Finally, we compared treatment efficacy using a grading system (J Gastroenterol 2013 Aug;48(8):951-65.) on E-field and on post-RFA contrastenhanced CT.

Results: On the evaluation of maximum axial ablative area by monopolar electrodes, major and minor axes were smaller on E-field than on CT, but these differences were not significant. On the evaluation of ablative volume by bipolar electrodes, the correlation coefficient for ablative volumes on E-field and CT was greater than 0.96 . On comparing treatment efficacy using the grading system, the degree of agreement between E-field and CT was 0.691 and the weighted kappa coefficient was 0.929 .

Conclusion: Ablative volume on E-field correlated well with that on post-RFA contrast-enhanced CT. Furthermore, good agreement was observed between the treatment efficacy based on E-field and post-RFA contrast-enhanced CT. E-field can be used to predict the ablative area from not only a single electrode, but also multiple electrodes, which was difficult to do in the past. The present results suggest that contrast-enhanced CT may become unnecessary for confirming therapeutic effects in the future.

Disclosure of Interest: All authors have declared no conflicts of interest.

\section{References}

Usefulness of a New Three-Dimensional Simulator System for Radiofrequency Ablation. Hirooka M, Koizumi Y, Imai Y, PLOS ONE. 2016 Feb 4.

Clinical utility of multipolar ablation with a three-dimensional simulator system for patients with liver cancer. Hirooka M, Koizumi Y, Imai Y, J Gastroenterol Hepatol. 2017 Feb 27. doi: 10.1111

Radiofrequency ablation for hepatocellular carcinoma: the relationship between a new grading system for the ablative margin and clinical outcomes. Nishikawa H, Osaki Y, Sakamoto A, J Gastroenterol. 2013 Aug;48(8):951-65.

\section{P0715 ATTENUATION COEFFICIENT MEASUREMENT (ACM) AS NOVEL REAL TIME ULTRASOUND ALTERNATIVE TO CAP (FIBROSCAN)}

O. Dynnyk ${ }^{1}$, N. Marunchyn ${ }^{2}$, N. Kobyliak ${ }^{2}$, O. Fedusenko ${ }^{3}$, V. Gurianov ${ }^{2}$ ${ }^{1}$ Bogomolets Institute of physiology of the Ukrainian National Academy of Sciences, Kyiv/Ukraine

${ }^{2}$ Endocrinology, Bogomolets National Medical University, Kyiv/Ukraine

${ }^{3}$ Shupyk National Medical Academy of Postgraduate Education, Kyiv/Ukraine

Contact E-mail Address: nazariikobyliak@gmail.com

Introduction: The presence of fat droplets in the hepatocytes (micro- or macrovesicular hepatic steatosis) under condition of chronic diffuse liver disease (CDLD) increases the attenuation of ultrasound (US). A group of Ukrainian scientists proposed an original algorithm for real-time US attenuation measurement (attenuation coefficient measurement - ACM - patent UA №2014 111234). Aims \& Methods: From total of 3274 patients who underwent to comprehensive abdominal US (2015-2016) in our clinic, 949 have been diagnosed with fatty liver according to Hamaguchi criteria. All these patient we provide ACM $(\mathrm{dB} / \mathrm{cm})$ measurement on SoneusP7 device (Ultrasign, Ukraine), with a $1-6 \mathrm{MHz}$ convex transducer in the right and left lobes. For diagnostic accuracy assessment (used $\mathrm{CT}$ as standard) and comparison with CAP measured by Fibroscan (Echosens, France) we included 142 patients for subanalysis. Evaluation of diagnostic accuracy of ACM performed using ROC-analysis.

Results: Depending on the stage of steatosis according to B-mode median, 25 and 75 quartiles for ACM were as follows: control group 1.57 (1, 32-1, 85); S1-1, 86 $(1,78-2,11)$; S2-2, $26(2,20-2,49)$ and respectively for $\mathrm{S} 3-2,7(2,40-2,82) \mathrm{dB} /$ $\mathrm{cm}$. ACM value increase parallel the hepatic steatosis progression $(\mathrm{p}<0.001)$, which was also accompanied with presence of very strong correlation between these parameters $(r=0.814, \mathrm{p}<0.001)$. In patient with NAFLD the association between maximum value of ACM and duration of T2DM and triglycerides (model 1, multiple correlation coefficient $=0.55 ; \mathrm{R}^{2}=0.26 ; \mathrm{p}=0.004$ ) and ALT (model 2, multiple correlation coefficient $=0.55 ; \mathrm{R}^{2}=0.25 ; \mathrm{p}=0.005$ ) were observed. After adjustment by the duration of T2DM the level of triglycerides $(\mathrm{r}=0.44, \mathrm{p}=0.012)$ and activity of ALT $(\mathrm{r}=0.44, \mathrm{p}=0.012)$ significantly correlated with ACM. The AUROC of ACM for steatosis diagnosis was $0.925(95 \%$ CI $0.877-0.973$ ). The optimal cutoff point was $>2.27 \mathrm{~dB} / \mathrm{cm}$, with sensitivity, specificity, PPV and NPV respectively $91.5,77.3,84.6$ and $83.8 \%$. ACM value also significantly correlated with CAP $(r=0.630, p<0.001)$.

Conclusion: The ACM as novel real-time ultrasound approach can be used for noninvasive hepatic steatosis diagnosis, allows clinicians to monitor disease progression and response to treatment.

Disclosure of Interest: All authors have declared no conflicts of interest.

\section{P0716 HOW DIFFERENT IS THE PERFORMANCE OF CEUS IN CIRRHOTIC VS. NON-CIRRHOTIC PATIENTS?}

T. Moga ${ }^{1}$, C. S. Ivascu ${ }^{1}$, C. G. Foncea ${ }^{1}$, D. Gherhardt ${ }^{1}$, R. Sirli ${ }^{1}$, S.A. Popescu ${ }^{1}$, M. Danila ${ }^{1}$, I. Sporea ${ }^{1}$

${ }^{1}$ Department Of Gastroenterology And Hepatology, Victor Babes University of Medicine, Timisoara/Romania

\section{Contact E-mail Address: moga.tudor@yahoo.com}

Introduction: The aim of this paper is to evaluate the performance of CEUS as a first step in the evaluation of focal liver lesions (FLLs) in cirrhotic and noncirrhotic patients.

Aims \& Methods: A retrospective study was performed on a cohort of 2904 FLLs evaluated by CEUS between September 2009-December 2016, according to EFSUMB guide lines (2) in a tertiary center. 979 (33.7\%) FLLs did not completely fulfill the EFSUMB-CEUS criteria thus needing to be confirmed by other imaging technique (contrast-enhanced CT, contrast-enhanced MRI) or histology. Lesions that were previously diagnosed have been excluded. Using CT, MRI and histology for the final diagnosis we calculated the specificity, sensibility and accuracy of CEUS for the difficult FLLs that challenged the examiner, in cirrhotic and non-cirrhotic patients.

Results: CEUS managed an overall correct diagnosis in $76.8 \%(752 / 979)$ of the lesions. From the 979 FLLs, 335 (34.3\%), (67.7\% HCC, 4.7\% Hemangioma, $3.8 \%$ Metastasis, $18.2 \%$ Other benign lesions and $5.3 \%$ Other malignant lesions), were detected in liver cirrhosis (LC) and $644(65.7 \%),(36.1 \%$ Metastasis, $19.5 \%$ Hemangioma, 6.8\% HCC, 31.3\% Other bening lesions and $6 \%$ Other malignant lesions) were in non-cirrhotic liver (NC). In LC CEUS performance for benign lesions was: $87.7 \% \mathrm{Se}, 93.6 \% \mathrm{Sp}$ and $90.4 \%$ Ac. For malignant lesions in LC, CEUS performed: $76.1 \%$ Se, $92.4 \% \mathrm{Sp}$ and $83.8 \%$ Ac. In the NC group, CEUS performance for benign lesions was: $82 \% \mathrm{Se}, 95.8 \% \mathrm{Sp}$ and $88.4 \%$ Ac. and for malignant lesions was: $90.16 \%$ Se, $90.13 \% \mathrm{Sp}$ and $89.4 \%$ Ac. CEUS performance on the most frequent lesions: Hepatocellular carcinoma (HCC) in LC: $65.2 \% \mathrm{Se}, 86 \% \mathrm{Sp}, 68.5 \%$ Ac. HCC in NC: $65.9 \% \mathrm{Se}, 94.7 \mathrm{Sp}, 92.1$ Ac. Metastasis in LC: $30.7 \mathrm{Se}, 97.3 \mathrm{Sp}, 92.9 \%$ Ac. Metastasis in NC: $78.5 \% \mathrm{Se}$, 91.2\% Sp, 85.5\% Ac. Hemangioma in LC: $68.7 \%$ Se, $95.6 \mathrm{Sp}, 93.4 \%$ Ac. Hemangioma in NC: $77.2 \% \mathrm{Se}, 96.1 \% \mathrm{Sp}$ and $91.3 \mathrm{Ac}$.

Conclusion: CEUS is an accurate and reliable method as a first step in the evaluation of FLLs. Liver cirrhosis does not influence significantly CEUS performance.

Disclosure of Interest: All authors have declared no conflicts of interest.

\section{References}

1. Strobel D, Seitz K, Blank W, Schuler A, Dietrich C, von Herbay A, Friedrich-Rust M, et al. Contrast-enhanced ultrasound for the characterization of focal liver lesions-diagnostic accuracy in clinical practice (DEGUM multicenter trial). Ultraschall Med 2008;29:499-505.

2. Albrecht T., Blomley M., Bolondy L., et al. for the EFSUMB study group. Guidelines for the use of contrast agents in ultrasound. Ultraschall 2014; 25: 249-256;

\section{P0717 CUX1 IN LIVER CANCER: EXPERIMENTAL STUDY IN} HYPOXIA MODEL

S. Blümel ${ }^{1}$, T. T. Wissniowski ${ }^{2}$, J. Hänze ${ }^{3}$, T.M. Gress ${ }^{2}$, D. Bartsch ${ }^{1}$, P. Di Fazio ${ }^{1}$

${ }^{1}$ Department Of Visceral Thoracic And Vascular Surgery, Philipps University Marburg, Marburg/Germany

${ }^{2}$ Klinik Für Gastroenterologie, Endokrinologie, Stoffwechel Und Infektiologie, Philipps Universität Marburg, Marburg/Germany

${ }^{3}$ Department Of Urology And Pediatric Urology, Philipps University Marburg, Marburg/Germany

Contact E-mail Address: sophie.bluemel@gmx.de

Introduction: CUX1 (CUTL1) is a transcription factor able to promote the expression of several genes implicated in cellular proliferation, differentiation and demise. In normal adult cells, it preferentially favors the expression of proapoptotic genes. Its aberrant expression in tumor turns its role as foe. It favors the expression of oncogenes and survival factors, especially in stress condition, thus supporting tumorigenesis.

Aims \& Methods: Here, we show CUX1 activity during hypoxia in liver cancer cells. CUX1was knocked down and its targets were analysed by RT-qPCR in HepG2 and Hep3B cells under hypoxic and/or normal culture condition. The hypoxia condition was established by $24 \mathrm{~h}$ treatment with $150 \mu \mathrm{M} \mathrm{CoCl} 2$ or with $0.5 \% \mathrm{O} 2$ atmosphere. Hypoxia markers and CUX1 were analysed by RT-qPCR. Transfection qith plasmid expressing a reporter sequence for HIF1alpha was performed in combination with CUX1 knock-down.

Results: Hypoxia determined the up-regulation of HIF1-alpha (Hypoxia inducible factorl-alpha) and a stable or up-regulated expression of its inhibitor FIH-1 (SLC2A1) up to $24 \mathrm{~h}$ prolonged hypoxia. VEGFA was significantly 
overexpressed. Knock-down of CUX1 determined a significant down-regulation of HIF-1alpha, FIH-1 and VEGFA. Interestingly, the expression of CDKN1A was only attenuated after CUX1 knock down and hypoxic stress. HIF1alpha transcriptional activity is dependent by CUX1 expression.

Conclusion: CUX1 exerts an oncogenic role in liver cancer by sustaining the survival mechanism beyond hypoxia. CUX1 silencing results in suppression of the hypoxia inducible factor and its target VEGFA causing a block of cell cycle in liver cancer cells modulated by the stable expression of CDKN1A.

Disclosure of Interest: All authors have declared no conflicts of interest.

\section{P0718 S-ADENOSYLMETHIONINE AFFECTS CELL CYCLE PATHWAYS AND SUPRESSES PROLIFERATION IN LIVER CELLS}

L. Yan, G. Zhang, Y. Chen

Department Of Gastroenterology, Xiangya Hospital, Central South University, Changsha/China

\section{Contact E-mail Address: yanlu_152@163.com}

Introduction: S-Adenosylmethionine (SAMe) is a kind of common liver-protecting medicine. Recent studies have shown that SAMe has the inhibitory effects on hepatocellular carcinoma(HCC). But the specific mechanism has not been elucidated

Aims \& Methods: Here, we examine the effects and relevant mechanism of SAMe on human hepatocellular carcinoma cell HepG2 and mouse hepatocyte AML12. RNA sequencing (RNA-Seq) was used to identify the differentially expressed genes between HepG2 cells which were treated with SAMe or not. And we used western blot and QRT-PCR to confirm some of these genes. MTS and flow cytometry-based assays were carried out in response to SAMe treatment. Results: A total of 472 SAMe-related genes were identified by RNA-Seq. We found that differentially expressed genes were enriched in cell cycle related signaling pathway significantly by the KEGG and GO Pathway enrichment analysis. Through the construction of protein-protein interaction network, we observed the module associated with cell cycle is in the middle of the whole network. All these results implied that cell cycle pathway may play a very important role in the regulation of SAMe effected on HepG2 cells. Then the RNA-Seqcharacterized genes involved in cell cycle (MCM3, MCM4, and E2F1) were confirmed by Western blot and QRT-PCR in HepG2 and AML12 cells. MTS analysis showed that SAMe could diminish cell proliferation. And flow cytometry-based assays indicated that treatment with SAMe altered cell cycle kinetic S phase cell cycle arrest.

Conclusion: Altogether, our data enforce the evidence of SAMe possessing of antiproliferative action in liver cells, capable of up-regulating MCM3, MCM4 and E2F1 expression leading to cell cycle inhibition, and provide an important theoretical basis for the clinical chemoprevention and treatment in HCC of SAMe.

Disclosure of Interest: All authors have declared no conflicts of interest.

\section{References}

1. Kim JU, Shariff MI, Crossey MM, Gomez-Romero M, Holmes E, Cox IJ, et al. Hepatocellular carcinoma: Review of disease and tumor biomarkers. World J Hepatol. 2016;8:471-84.

2. McGuire S. World Cancer Report 2014. Geneva, Switzerland: World Health Organization, International Agency for Research on Cancer, WHO Press, 2015. Adv Nutr. 2016;7:418-9.

3. Llovet JM, Bru C, Bruix J. Prognosis of hepatocellular carcinoma: the BCLC staging classification. Semin Liver Dis. 1999;19:329-38.

4. Finn RS. Development of molecularly targeted therapies in hepatocellular carcinoma: where do we go now? Clin Cancer Res. 2010;16:390-7.

5. Cho YK, Kim JK, Kim MY, Rhim H, Han JK. Systematic review of randomized trials for hepatocellular carcinoma treated with percutaneous ablation therapies. Hepatology. 2009;49:453-9.

6. Deng GL, Zeng S, Shen H. Chemotherapy and target therapy for hepatocellular carcinoma: New advances and challenges. World J Hepatol. 2015;7:78798.

7. Mato JM, Lu SC. Role of S-adenosyl-L-methionine in liver health and injury. Hepatology. 2007;45:1306-12.

8. Tabor CW, Tabor H. Methionine adenosyltransferase (S-adenosylmethionine synthetase) and S-adenosylmethionine decarboxylase. Adv Enzymol Relat Areas Mol Biol. 1984:56:251-82.

9. Martinez-Chantar ML, Corrales FJ, Martinez-Cruz LA, Garcia-Trevijano ER, Huang ZZ, Chen L, et al. Spontaneous oxidative stress and liver tumors in mice lacking methionine adenosyltransferase 1A. FASEB J. 2002; 16:1292-4

10. Lu SC, Alvarez L, Huang ZZ, Chen L, An W, Corrales FJ, et al. Methionine adenosyltransferase $1 \mathrm{~A}$ knockout mice are predisposed to liver injury and exhibit increased expression of genes involved in proliferation. Proc Nat Acad Sci U S A. 2001;98:5560-5.

11. Lu SC, Mato JM. S-adenosylmethionine in liver health, injury, and cancer. Physiol Rev. 2012;92:1515-42.

12. Cederbaum AI. Hepatoprotective effects of S-adenosyl-L-methionine against alcohol- and cytochrome P450 2E1-induced liver injury. World $J$ Gastroenterol. 2010;16:1366-76.

13. Yang H, Ramani K, Xia M, Ko KS, Li TW, Oh P, et al. Dysregulation of glutathione synthesis during cholestasis in mice: molecular mechanisms and therapeutic implications. Hepatology. 2009;49:1982-91.

14. Hardy ML, Coulter I, Morton SC, Favreau J, Venuturupalli S, Chiappelli F, et al. S-adenosyl-L-methionine for treatment of depression, osteoarthritis, and liver disease. Evid Rep Technol Assess (Summ). 2003:1-3.
15. Hussain Z, Khan MI, Shahid M, Almajhdi FN. S-adenosylmethionine, a methyl donor, up regulates tissue inhibitor of metalloproteinase-2 in colorectal cancer. Genet Mol Res. 2013;12:1106-18.

16. Li TW, Yang H, Peng H, Xia M, Mato JM, Lu SC. Effects of S-adenosylmethionine and methylthioadenosine on inflammation-induced colon cancer in mice. Carcinogenesis. 2012;33:427-35.

17. Chik F, Machnes Z, Szyf M. Synergistic anti-breast cancer effect of a combined treatment with the methyl donor S-adenosyl methionine and the DNA methylation inhibitor 5-aza-2'-deoxycytidine. Carcinogenesis. 2014;35:13844.

18. Ilisso CP, Sapio L, Delle Cave D, Illiano M, Spina A, Cacciapuoti G, et al. SAdenosylmethionine Affects ERK1/2 and Stat3 Pathways and Induces Apotosis in Osteosarcoma Cells. J Cell Physiol. 2016;231:428-35.

19. Yang H, Sadda MR, Li M, Zeng Y, Chen L, Bae W, et al. S-adenosylmethionine and its metabolite induce apoptosis in HepG2 cells: Role of protein phosphatase 1 and Bcl-x(S). Hepatology. 2004;40:221-31.

20. Lu SC, Ramani K, Ou X, Lin M, Yu V, Ko K, et al. S-adenosylmethionine in the chemoprevention and treatment of hepatocellular carcinoma in a rat model. Hepatology. 2009;50:462-71.

21. SC L. Where are we in the chemoprevention of hepatocellular carcinoma? Hepatology. 2010;51:734-6.

22. Trapnell C, Pachter L, Salzberg SL. TopHat: discovering splice junctions with RNA-Seq. Bioinformatics. 2009;25:1105-11.

23. Anders S, Huber W. Differential expression analysis for sequence count data. Genome Biol. 2010;11:R106.

24. Kanehisa M, Goto S, Sato Y, Furumichi M, Tanabe M. KEGG for integration and interpretation of large-scale molecular data sets. Nucleic Acids Res. 2012;40:D109-14.

25. Young MD, Wakefield MJ, Smyth GK, Oshlack A. Gene ontology analysis for RNA-seq: accounting for selection bias. Genome Biol. 2010;11:R14.

26. Forner A, Llovet JM, Bruix J. Hepatocellular carcinoma. Lancet. 2012;379:1245-55

27. Llovet JM, Bruix J. Systematic review of randomized trials for unresectable hepatocellular carcinoma: Chemoembolization improves survival. Hepatology. 2003;37:429-42.

28. Mato JM, Martinez-Chantar ML, Lu SC. S-adenosylmethionine metabolism and liver disease. Ann Hepatol. 2013;12:183-9.

29. Vendemiale G, Altomare E, Trizio T, Le Grazie C, Di Padova C, Salerno MT, et al. Effects of oral S-adenosyl-L-methionine on hepatic glutathione in patients with liver disease. Scand J Gastroenterol. 1989;24:407-15.

30. Frezza M, Surrenti C, Manzillo G, Fiaccadori F, Bortolini M, Di Padova C. Oral S-adenosylmethionine in the symptomatic treatment of intrahepatic cholestasis. A double-blind, placebo-controlled study. Gastroenterology. 1990;99:211-5.

31. Ansorena E, Garcia-Trevijano ER, Martinez-Chantar ML, Huang ZZ, Chen L, Mato JM, et al. S-adenosylmethionine and methylthioadenosine are antiapoptotic in cultured rat hepatocytes but proapoptotic in human hepatoma cells. Hepatology. 2002;35:274-80.

32. Ramani K, Yang H, Xia M, Ara AI, Mato JM, Lu SC. Leptin's mitogenic effect in human liver cancer cells requires induction of both methionine adenosyltransferase 2A and 2beta. Hepatology. 2008;47:521-31.

33. Latasa MU, Boukaba A, Garcia-Trevijano ER, Torres L, Rodriguez JL, Caballeria J, et al. Hepatocyte growth factor induces MAT2A expression and histone acetylation in rat hepatocytes: role in liver regeneration. FASEB J. 2001;15:1248-50.

34. Vermeulen K, Van Bockstaele DR, Berneman ZN. The cell cycle: a review of regulation, deregulation and therapeutic targets in cancer. Cell Prolif. 2003;36:131-49.

35. Parashar S, Cheishvili D, Arakelian A, Hussain Z, Tanvir I, Khan HA, et al. S-adenosylmethionine blocks osteosarcoma cells proliferation and invasion in vitro and tumor metastasis in vivo: therapeutic and diagnostic clinical applications. Cancer Med. 2015;4:732-44.

\section{P0719 BRG1 PROMOTES HEPATOCARCINOGENESIS BY MODULATING CYCLINB, D, E AND MATRIX METALLOPROTEINASE 7}

B. Kaufmann, B. Wang, M. Lu, N. Hüser, H. Friess, G. Von Figura,

D. Hartmann

Klinikum rechts der Isar/Technische Universität München, München/Germany

Contact E-mail Address: benedikt_kaufmann@hotmail.com

Introduction: The chromatin remodeler complex SWI/SNF plays an important role in physiological and pathological processes. The role of BRG1, a catalytic subunit of the SWI/SNF complex, that is known to be mutated in hepatocellular carcinoma (HCC) remains unclear. The aim of this work is to investigate the role of BRG1 on cell growth, cell invasion and its effect on the expression of target genes.

Aims \& Methods: We examined the expression of BRG1 in human tissue samples and HCC cell lines by qRT-PCR and Western Blot. We used siRNA to downregulate BRG1 in human $\mathrm{HCC}$ cell lines. Cell growth and cell invasion of siRNA-treated cells was analysed by growth curves, colony formation assay and invasion assay. The expression of target genes after BRG1 downregulation was investigated by qRT-PCR.

Results: BRG1 was found to be significantly increased in HCC samples compared to non-HCC samples. After BRG1 downregulation by siRNA, cell growth and cell invasion decreased in $\mathrm{HuH} 7$ and $\mathrm{HepG} 2$ cell lines. A positive modulating effect by BRG1 was shown for the expression of CyclinB, D, E and MMP7 in either HepG2 or $\mathrm{HuH} 7$ cell lines. 
Conclusion: Our results support the hypothesis that overexpression of BRG1 increases cell growth and cell invasion in HCC. Furthermore, the data highlight genes promoting proliferation and invasion that are being regulated by BRG1 during hepatocarcinogenesis. In particular, CyclinB, D, E and MMP7 appear to play a major role in this context and might be an important link between BRG1 expression and HCC development.

Disclosure of Interest: All authors have declared no conflicts of interest.

\section{P0720 PROGNOSTIC ROLE OF NEUTROPHIL-TO-LYMPHOCYTE RATIO IN HEPATOCELLULAR CARCINOMA (HCC)}

N. Elleuch, S. Jardak, A. Sabbek, A. Ben Slama, H. Jaziri, E. Hammami, B. Ahlem, K. Mehdi, J. Ali

Sahloul, Sousse/Tunisia

\section{Contact E-mail Address: Elleuchghorbel.nour@yahoo.fr}

Introduction: Inflammation may play an important role in progession, and a high neutrophil-to-lymphocyte ratio (NLR) has been reported as a poor prognostic indicator in several malignancies.

Aims \& Methods: This study was aimed to investigate the prognostic value of NLR in patients with HCC. We performed a retrospective study including patients with hepatocellular carcinoma admitted in the hepato-gastroenterology department of Sousse between January 2010 and December 2015.

Results: A total of 76 patients were included in this study. Mean age was 59.8 (33-87 years). The sex ratio was $3.22(\mathrm{M} / \mathrm{F}=58 / 18)$. Hepatocellular carcinoma occurred on a liver of cirrhosis in the majority of cases $(90.7 \%)$. The main causes of cirrhosis were hepatitis B virus infection (43 patients-62.3\%), hepatitis C virus infection (11 patients-16\%), non alcoholic steatohepatitis (6 patients-8.6\%) and alcohol consumption ( 5 patients-7.2\%). Our results showed that high NLR was associated with poor overall survival (OS) in HCC regardless of therapeutic choice $(\mathrm{P}<0.05)$. Otherwise, high NLR was significantly correlated with the presence of vascular invasion $(\mathrm{P}=0.002)$, lymph node mestasis $(\mathrm{P}=0.04)$, tumor multifocality $(P=0.01)$ and higher incidence of $A F P \geq 200 \mathrm{ng} / \mathrm{ml}$ $(\mathrm{P}=0.04)$.

Conclusion: Elevated NLR indicates a poor prognosis for patients with HCC. The NLR is a readily available and inexpensive biomarker, and its addition to established prognostic scores for clinical decision making warrants further investigation.

Disclosure of Interest: All authors have declared no conflicts of interest.

\section{P0721 REIC/DKK-3 PROTEIN CONCENTRATION INDUCE THE POSITIVE EFFECT TO THE MORTALITY OF HEPATOCELLULAR CARCINOMA}

A. Ohyama, D. Uchida, H. Shiraha, H. Sawahara, H. Kato, H. Okada Gastroenterology And Hepatology, Okayama University Graduate School of Medicine, Dentistry and Pharmaceutical Sciences, Okayama City/Japan

\section{Contact E-mail Address: at841205@gmail.com}

Introduction: The $\mathrm{Wnt} / \beta$-catenin plays essential roles in the growth of hepatocellular carcinoma (HCC). The Dickkopf (Dkk) protein family (Dkk1-4) is known as Wnt signal antagonists, and reduced expression in immortalized cells (REIC)/ Dkk-3 over-methylation is associated with poor prognosis of HCC patients. But the roles of REIC/Dkk-3 in inhibiting Wnt signaling remains still unclear.

Aims \& Methods: In our previous study, REIC/Dkk-3 protein induced significant production of interferon gamma from lymphocytes incubated with pancreatic cancer cells, indicating that REIC/Dkk-3 protein might activate cancer immunity in the tumor-bearing patients. ${ }^{1}$ We hypothesized that REIC/Dkk-3 expression was correlated with cancer immunity in HCC patients. Thus, we investigated the correlation between serum REIC/Dkk-3 protein level and the prognosis in HCC patient. We retrospectively studied $58 \mathrm{HCC}$ patients who underwent primary liver resection for HCC admitted to out unit from 2008 to 2017. Patient serum was gathered before resection. Serum REIC/Dkk-3 protein level was measured by an enzyme-linked immunosorbent assay.

Results: 58 HCC patients were divided into two groups, 41 REIC/Dkk-3 high concentration group (protein level $>800$ ) and 17 Dkk-3 low concentration group (protein level $<800$ ), according to the presence of REIC/Dkk-3 proteins in the blood, as detected by ELISA spectrometry. There was no significant difference in age, sex, Child-Pugh score and HCC stage in the patient groups. REIC/Dkk-3 Protein tended to be declining in liver cancer patients with poor prognosis. $(\mathrm{p}=0.186)$

Conclusion: Our results demonstrated that the serum Dkk-3 protein levels might be a prognosis marker in HCC patients. Further study is necessary with more number of HCC patients.

Disclosure of Interest: All authors have declared no conflicts of interest.

\section{Reference}

1. Uchida, D., H. Shiraha, H. Kato, et al., Synergistic anti-pancreatic cancer immunological effects by treatment with reduced expression in immortalized cells/dickkopf-3 protein and peripheral blood mononuclear cells. $J$ Gastroenterol Hepatol, 2016. 31(6): p. 1154-9

\section{P0722 SURGICAL OUTCOME OF PATIENTS WITH}

FIBROLAMELLAR HEPATOCELLULAR CARCINOMA. DOES IT DIFFERS FROM COMMON HEPATOCELLULAR CARCINOMA?

\section{E. EI-Hanafy}

Facultu Of Medicin, Mansoura University, Gastroenterology Surgical Center, Cairo/Egypt

Contact E-mail Address: dr_ehab_elhanafy@yahoo.com

Introduction: Fibrolamellar hepatocellular carcinoma (FL-HCC) has conventionally been considered to be a histologic variant of hepatocellular carcinoma (HCC), with distinct clinicopathologic features. It is a rare primary hepatic malignancy that was first described as a pathological variant of HCC by Edmondson in 1956 [1]. The etiology of FL-HCC remains unclear. It typically occurs in normal livers without underlying liver fibrosis or cirrhosis [2]. In contrast to HCC which usually found in the presence of cirrhosis or chronic hepatitis [3]. FL-HCC has been reported to occur in association with focal nodular hyperplasia (FNH) a type of benign liver lesion. Many series have mentioned that FLHCC is less aggressive than conventional HCC [4]. However, other studies have failed to confirm the observation of a better outcome in FL-HCC [5]. Other studies reported that the survival was similar between common HCC and FL$\mathrm{HCC}$, and that may be related to the higher resectability rate which improve the survival of patients with FL-HCC [6].

Aims \& Methods: The aim of this study was to evaluate the clinicopathological features and the surgical outcomes of patients with FL-HCC who were referred to our tertiary referral center over a 15 -year period. This is a retrospective study including 22 patients with a pathologic diagnosis of FL-HCC who underwent hepatectomy over a 15-year period. Tumor characteristics, survival and recurrence were evaluated

Results: There were 11 male and 11 female with a median age of 29 years (range from 21 to 58 years). Two $(9 \%)$ patients had hepatitis $\mathrm{C}$ viral infection and only 2 $(9 \%)$ patients had alpha-fetoprotein level $>200 \mathrm{ng} / \mathrm{mL}$. The median size of the tumors was $12 \mathrm{~cm}$ (range from $5-20 \mathrm{~cm}$ ). Vascular invasion was detected in 5 $(23 \%)$ patients. Four $(18 \%)$ patients had lymph node metastases. The median follow up period was $42 \mathrm{mo}$ and the 5-year survival was $65 \%$. Five $(23 \%)$ patients had a recurrent disease, 4 of them had a second surgery with 36 mo median time interval. Vascular invasion is the only significant negative prognostic factor.

FL-HCC $(\mathrm{n}=22)$

\begin{tabular}{ll}
\hline & FL-HCC $(\mathrm{n}=22)$ \\
\hline Number & $19(86 \%)$ \\
Single & $3(14 \%)$ \\
Multiple & Median $12 \mathrm{~cm}$ (range, 5-20) \\
Size $(\mathrm{cm})$ & 9 right, 10 left, 3 bilateral \\
Location & \\
Hepatic resection & $16(73 \%)$ \\
Hepatectomy & $4(18 \%)$ \\
Extended hepatectomy & $2(9 \%)$ \\
Localized resection & \\
Stage & $10(45 \%)$ \\
I & $5(23 \%)$ \\
II & $7(32 \%)$ \\
III & 0 \\
IV & $4(18 \%)$ \\
Nodal metastases & $5(23 \%)$ \\
Vascular invasion & $2(9 \%)$ \\
Positive safety margin & $4(18 \%)$ \\
Repeated hepatectomy & \\
\hline
\end{tabular}

\begin{tabular}{llll}
\hline Factor & No. $(\%)$ & $\begin{array}{l}\text { Overall } \\
\text { survival } \\
\text { (month) }\end{array}$ & p. value \\
\hline Age (year) & $16(73 \%)$ & 86 & \\
$<40$ & $6(27 \%)$ & 72 & 0.4 \\
$\geq 40$ & $11(50 \%)$ & 84 & 0.6 \\
Gender & $11(50 \%)$ & 79 & \\
Female & & 82 & 0.3 \\
Male & $8(36 \%)$ & 76 & \\
Tumor size (cm) & $14(64 \%)$ & & \\
$<10$ & $19(86 \%)$ & 89 & 0.2 \\
$\geq 10$ & $3(14 \%)$ & 77 & \\
Number & & & \\
1 & $16(73 \%)$ & 86 & \\
$>1$ & & & \\
Hepatic resection & & & \\
Hepatectomy & & & \\
\hline
\end{tabular}


Continued

\begin{tabular}{llll}
\hline Factor & No. $(\%)$ & $\begin{array}{l}\text { Overall } \\
\text { survival } \\
(\text { month })\end{array}$ & p. value \\
\hline $\begin{array}{l}\text { Extended hepatectomy } \\
\text { Localized resection }\end{array}$ & $4(18 \%)$ & 77 & 0.62 \\
$\begin{array}{l}\text { Nodal metastases } \\
\text { Negative }\end{array}$ & $2(9 \%)$ & 79 & \\
Positive & $18(82 \%)$ & 88 & 0.09 \\
Vascular invasion & $4(18 \%)$ & 78 & 0.03 \\
Absent & $17(77 \%)$ & 92 & \\
Present & $5(23 \%)$ & 58 & 0.08 \\
Safety margin & & & \\
Negative & $20(91 \%)$ & 87 & \\
Positive & $2(9 \%)$ & 72 & \\
\hline
\end{tabular}

Conclusion: FL-HCC has a favorable prognosis than common HCC and should be suspected in young patients with non cirrhotic liver. Aggressive surgical resection should be done for all patients. Repeated hepatectomy or excision of recurrent disease should be considered for these patients as it has a relatively indolent course.

Disclosure of Interest: All authors have declared no conflicts of interest.

\section{References}

1. Edmondson HA. Differential diagnosis of tumors and tumor-like lesions of liver in infancy and childhood. AMA J Dis Child. 1956 Feb. 91(2):168-86.

2. Collier NA, Weinbren K, Bloom SR, Lee YC, Hodgson HJ, Blumgart LH Neurotensin secretion by fibrolamellar carcinoma of the liver. Lancet. 1984;1:538-540.

3. McLarney JK, Rucker PT, Bender GN, Goodman ZD, Kashitani N, Ros PR. Fibrolamellar carcinoma of the liver: radiologic- pathologic correlation. Radiographics. 1999;19:453-471.

4. El-Serag HB, Davila JA. Is fibrolamellar carcinoma different from hepatocellular carcinoma? A US population- based study. Hepatology 2004;39:798-803.

5. Katzenstein HM, Krailo MD, Malogolowkin MH. Fibrolamellar hepatocellular carcinoma in children and adolescents. Cancer 2003;97:2006-2012.

6. Nagorney DM, Adson MA, Weiland LH, Knight CD Jr., Smalley SR, Zinsmeister AR. Fibrolamellar hepatoma. Am J Surg. 1985;149:113-119.

\section{P0724 MICROWAVE VERSUS RADIOFREQUENCY THERMAL ABLATION OF HEPATOCELLULAR ADENOMA: SAFETY AND EFFICACY}

H. El Alfy ${ }^{1}$, T. Besheer ${ }^{1}$, K. Farid ${ }^{1}$, A. Mohammed El Sayed Ahmed El Nakib ${ }^{1}$, A. El Shabrawi ${ }^{1}$, A. Elbadrawy ${ }^{2}$, A. El Morsy ${ }^{2}$, K. Zalata ${ }^{3}$, M. Abd El Aziz ${ }^{1}$

${ }^{1}$ Tropical Medicine, Mansoura University Hospitals, Mansoura/Egypt

${ }^{2}$ Diagnostic And Interventional Radiology, Mansoura Faculty of Medicine,

Mansoura/Egypt

${ }^{3}$ Pathology Department, Mansoura Faculty of Medicine, Mansoura/Egypt

Contact E-mail Address: el_naqueeb@yahoo.com

Introduction: Hepatocellular adenoma (HCA) is a rare benign tumor of the liver that typically presents in women within their reproductive years. The recent increase in the HCA prevalence is noticeably associated with the rising prevalence of obesity and the metabolic syndrome. Because of high risk of complications such as hemorrhage, rupture and malignant transformation, appropriate treatment strategy should be considered. Given the success of image-guided ablation in treating malignant hepatic tumors, there is increased interest in treating benign masses with percutaneous ablation.

Aims \& Methods: To investigate the efficacy and safety of Microwave versus Radiofrequency Ablation in management of HCA. Out of 320 Patients presented with hepatic focal lesions over 1 year, data of 15 patients diagnosed to have HCA were collected retrospectively. The diagnosis of HCA in those patients was based on radiological findings using triphasic pelvi-abdominal CT, dynamic MRI or cytopathological examination of FNAC for those whose radiological findings were not conclusive. The size of the all tumors was ranged between 2.5 to $3.4 \mathrm{~cm}$. Tumors were selected for treatment by percutaneous ablation after review at a multidisciplinary that included radiologists, hepatologists, hepatobiliary surgeons. Decisions to pursue percuteneous ablation therapy were based on a multidisciplinary discussion of multiple factors, including patient preference, number and location of tumors impacting the extent of a possible liver resection and hepatic reserve and the decreased morbidity compared with surgery. The patients were divided into 2 groups: Group(A):included 7 patients $(5$ females, 2 males) underwent Radiofrequency Ablation. Group(B):included 8 patients $(5$ females, 3 males)underwent Microwave Ablation. Follow up was perfomed every 3 months over a period of 1 year.

Results: Microwave and Radiofrequency thermal Ablation had the same safety and efficacy in management of HCA with no evidence of residual tumour, tumor recurrence nor complications but Microwave thermal ablation was superior in large size tumors and needed only single skin puncture while Radiofrequency ablation needed more than single puncture in the same session.
Conclusion: Percutaneous ablation of HCA using Microwave or Radiofrequency thermal Ablation is safe, feasible and able to eradicate the targeted hepatic focal lesion and prevent known complications of HCA. Of note Microwave ablation is much more efficient in treating larger lesions through single puncture in contrast to Radiofrequency which needs more than one puncture.

Disclosure of Interest: All authors have declared no conflicts of interest.

\section{References}

Agrawal S, Agarwal S, Arnason T, Saini S, Belghiti J. Management of hepatocellular adenoma: recent advances. Clin Gastroenterol Hepatol 2015; 13:12211230 .

Atwell TD, Brandhagen DJ, Charboneau JW, Nagorney DM, Callstrom MR, Farrell MA. Successful treatment of hepatocellular adenoma with percutaneous radiofrequency ablation. AJR Am J Roentgenol 2005; 184: 828-831.

Brace CL. Microwave tissue ablation: biophysics, technology, and applications. Crit Rev Biomed Eng 2010; 38:65-78.

Dokmak S, Paradis V, Vilgrain V, et al. A single-center surgical experience of 122 patients with single and multiple hepatocellular adenomas. Gastroenterology 2009; 137:1698-1705.

\section{P0725 METABOLIC DISORDERS ACROSS HEPATOCELLULAR} CARCINOMA IN ITALY

F. Morisco ${ }^{1}$, M. Guarino ${ }^{1}$, M.R. Valvano ${ }^{2}$, V. Cossiga ${ }^{1}$, F. Farinati ${ }^{3}$, E.G. Giannini ${ }^{4}$, F. Ciccarese ${ }^{5}$, F. Piscaglia ${ }^{6}$, G.L. Rapaccini ${ }^{7}$, M. Di Marco ${ }^{8}$, E. Caturelli ${ }^{9}$, M. Zoli ${ }^{10}$, F. Borzio ${ }^{11}$, R. Sacco ${ }^{12}$, C. Cammà ${ }^{13}$, M. Felder ${ }^{14}$, L. Benvengù ${ }^{15}$, A. Gasbarrini ${ }^{16}$, G. Svegliati Baroni ${ }^{17}$, F.G. Foschi ${ }^{18}$, G. Missale ${ }^{19}$, A. Masotto ${ }^{20}$, R. Virdone ${ }^{21}$, N. Caporaso' ${ }^{1}$, F. Trevisani ${ }^{10}$ ${ }^{1}$ Gastroenterology Unit, Department of Clinical Medicine and Surgery, "Federico II" University, Napoli, Napoli/Italy

${ }^{2}$ Division Of Gastroenterology, Casa Sollievo della Sofferenza" Hospital, IRCCS, San Giovanni Rotondo/Italy

${ }^{3}$ Department Of Surgery, Oncology And Gastroenterology, University of Padova, PadovalItaly

${ }^{4}$ Gastroenterology Unit, Department Internal Medicine, University of Genoa,

IRCSS-Azienda Ospedaliera Universitaria San Martino-IST, Genoa/Italy

${ }^{5}$ Surgery Unit, Policlinico San Marco, Zingonia/Italy

${ }^{6}$ Department of Medical and Surgery Sciences, University of Bologna, S. Orsola Malpighi Hospital, Bologna/Italy

${ }^{7}$ Division of Internal Medicine and Gastroenterology, Complesso Integrato

Columbus, Università Cattolica del Sacro Cuore, Rome/Italy

${ }^{8}$ Division of Medicine, Bolognini Hospital, Seriate/Italy

${ }^{9}$ Division of Gastroenterology, Belcolle Hospital, Viterbo/Italy

${ }^{10}$ Department of Medical and Surgery Sciences, Internal Medicine Unit, Alma-

Mater Studiorum, University of Bologna, Bologna/Italy

${ }^{11}$ Division of Radiology, Department of Medicine, Fatebenefratelli Hospital, Milan/Italy

${ }^{12}$ Division of Gastroenterology and Metabolic Disease, University Hospital of Pisa, Pisa/Italy

${ }^{13}$ Section of Gastroenterology, Biomedical Department of Internal and Specialized Medicine (DI.BI.M.I.S.), University of Palermo, Palermo/Italy

${ }^{14}$ Division of Gastroenterology, Bolzano Regional Hospital, Bolzano/Italy

${ }^{15}$ Department of Clinical and Experimental Medicine, Medical Unit, Univeristy of Padua, Padua/Italy

${ }^{16}$ Internal Medicine, Gastroenterology And Liver Diseases, Gemelli Hospital Dept. of Internal Medicine Dept. of Gastroenterology, Rome/Italy

${ }^{17}$ Division of Gastroenterology, Polytechinic University of Marche, Ancona/Italy ${ }^{18}$ Department of Internal Medicine, Ospedale per gli Infermi di Faenza, Faenzal Italy

${ }^{19}$ Division of Infectious Diseases and Hepatology, Parma University Hospital, Parma/Italy

${ }^{20}$ Gastroenterology Unit, Ospedale Sacro Cuore Don Calabria, Negrar/Italy ${ }^{21}$ Division of Internal Medicine 2, Ospedale Riuniti Villa Sofia Cervello, Palermo/ Italy

Contact E-mail Address: valentina.cossiga@gmail.com

Introduction: Metabolic disorders, such as obesity and diabetes, are well known risk factors for hepatocellular carcinoma (HCC). Conversely, their impact on the natural history of HCC patients is not established.

Aims \& Methods: This study aimed at evaluating the impact of metabolic disorders on clinical features, treatment and survival of HCC patients regardless of its etiology. We analyzed the Italian Liver Cancer (ITA.LI.CA) database regarding 839 HCC patients prospectively collected from 2009 to 2014. The following metabolic features were analyzed: BMI, diabetes, arterial hypertension, hypercholesterolemia and hypertriglyceridemia. According to these features, patients were divided into 3 groups: $0-1$ metabolic features, 2 metabolic features, 3-5 metabolic features.

Results: As compared with patients with $0-1$ metabolic features, patients with 3-5 features showed lower percentage of HCC diagnosis on surveillance (p 0.021) larger tumors ( $\mathrm{p}$ 0.038), better liver function (higher percentage of patients with Child-Pugh A [p 0.007] and MELD < 10 [p 0.003]), higher percentage of metastases $(p 0.024)$, and lower percentage of portal vein thrombosis $(p 0.010)$. The BCLC stage and treatment options were similar among the 3 groups, with the exception of a less frequent access to locoregional therapies for BCLC stage B patients with 3-5 features (p 0.012). Overall survival and survival according to BCLC stage and/or treatment did not significantly differ among the 3 groups. Diabetic patients showed a lower survival (p 0.046). MELD score, HCC 
morphology, nodule size, BCLC stage, portal vein thrombosis and metastasis were independent predictors of lead-time adjusted survival.

Conclusion: Our "real-world" study suggests that metabolic disorders shape the clinical presentation of HCC but do not seem to play a major role in setting the patient survival, except for diabetes.

Disclosure of Interest: All authors have declared no conflicts of interest.

\section{P0726 LIVER VOLUME AS A PREDICTOR OF RISK FOR HEPATOCELLULAR CARCINOMA IN CHRONIC HEPATITIS C PATIENTS}

N. Kang, J.W. Chung, J. Kim

Internal Medicine, Seoul National University Bundang hospital, Seong-Nam city/ Korea, Republic of

\section{Contact E-mail Address: namkyumd@gmail.com}

Introduction: Chronic hepatitis $\mathrm{C}$ virus (HCV) infection pose risk for development of hepatocellular carcinoma (HCC), even after viral eradication with effective antiviral therapy. Therefore, risk prediction is clinically important for effective surveillance of chronic hepatitis $\mathrm{C}(\mathrm{CHC})$ patients, but studies are insufficient compared to chronic hepatitis $\mathrm{B}$. The liver volume has been reported to correlate with the severity of liver cirrhosis, but it is not known whether decreased liver volume predicts the HCC risk in CHC.

Aims \& Methods: The aim of this study was to assess the significance of liver volume in the prediction of $\mathrm{HCC}$ risk in $\mathrm{CHC}$ patients. A retrospective cohort of 101 CHC patients who received 4-phase dynamic CT imaging studies during surveillance was analyzed for liver volume and outcome of surveillance. Liver volumes were measured on portal venous phase of CT image and corrected for body weight and height: liver volume index $(\mathrm{LVI})=$ ratio of the expected standard volume to the measured liver volume. Kaplan-Meier analysis with the logrank test used to compare HCC. Cox proportional hazard analysis was used to identify the independent predictors of HCC risk.

Results: The cumulative incidence of HCC was $2.1 \%, 16.2 \%$ and $46.1 \%$ at 1,4 and 8 years, respectively. The risk of $\mathrm{HCC}$ was significantly higher in patients with LVI $>1(\mathrm{P}<0.001)$. Presence of liver cirrhosis was also associated with higher risk for HCC. $(\mathrm{P}<0.001)$, whereas age, sex, alpha-fetoprotein and HCV RNA level were not significant predictors of HCC. Multivariate analysis show that LVI > 1 and presence of LC were independent predictors of HCC (HR: 63.53, CI: $3.24-1244.28, \quad \mathrm{P}<0.001 / \mathrm{HR}: \quad 3.10, \quad \mathrm{CI}: \quad 1.28-7.51, \quad \mathrm{P}=0.12$, respectively).

Conclusion: Decreased liver volume is an independent predictor of HCC in chronic hepatitis C. Liver volume index is useful in predicting risk of HCC in $\mathrm{CHC}$.

Disclosure of Interest: All authors have declared no conflicts of interest.

\section{P0727 HAND AND FOOT SYNDROME AS A PREDICTOR OF OUTCOME IN PATIENTS WITH HEPATOCELLULAR CARCINOMA TREATED WITH SORAFENIB}

M. Ochi, A. Okawara, N. Kakinoki, T. Kamoshida, S. Hirai

Department Of Gastroenterology, Hitachi General Hospital, Hitachi City/Japan

\section{Contact E-mail Address: masaochiphi@hotmail.co.jp}

Introduction: Sorafenib is a multi-thyrosine kinase inhibitor classified as a neovascularization inhibitor. A previous study indicated that the administration of a thyrosine kinase inhibitor, cetuximab, significantly prolonged the survival of patients with dermal disorder ${ }^{1}$. However, few studies have reported the efficacy of sorafenib administration in patients with hand and foot syndrome (HFS).

Aims \& Methods: In this study, we investigated the prognosis of sorafenib-treated patients with HFS. HFS grading was conducted according to the Common Terminology Criteria for Adverse Events (CTCAE) v.4.0. Patients with grade 1 or higher dermal disorder were regarded as having HFS, and grade 0 patients as not having HFS. For HFS evaluation, a double-check system was adopted: primary evaluation based on a specific evaluation sheet at the Pharmacists' Outpatient Clinic and final evaluation by physicians at the outpatient clinic. We examined the influence of HFS on the effects of treatment after the introduction of sorafenib in 42 patients with a history of multidisciplinary treatment, such as transcatheter arterial chemoembolization (TACE), between May 2009 and March 2017

Results: Grade 1 or higher HFS was observed in 22 patients $(53 \%)$, and it was absent in $20(47 \%)$. Overall, the median sorafenib administration period was 2.1 months. In the HFS-free and HFS groups, it was 0.9 and 2.7 months, respectively $(\mathrm{p}<0.001)$. Survival analysis was performed using the Kaplan-Meier method. Overall, the median survival was 5.2 months. In the HFS-free and HFS groups, it was 3.0 and 7.8 months, respectively $(\mathrm{p}<0.001)$. Multivariate analysis showed that the presence of HFS (hazard ratio, $0.41 ; 95 \% \mathrm{CI}, 0.19$ to $0.88 ; \mathrm{p}=0.023$ ) and administration period (hazard ratio, $0.45 ; 95 \% \mathrm{CI}, 0.20$ to $0.98 ; \mathrm{p}=0.045$ ) were significant predictive factors. The following were not significant predictive factors: age, BCLC staging, dosage, and tumor markers

Conclusion: The prognosis of hepatocellular carcinoma patients receiving sorafenib treatment was closely related to the presence of HFS and administration period. HFS was a predictor of outcome in patients with hepatocellular carcinoma treated with sorafenib. This study indicated that a multi-thyrosine kinase inhibitor, sorafenib, prolonged survival in patients with HFS, as demonstrated for cetuximab. HFS reduces the quality of life (QOL), and is a sorafenib administration-inhibiting factor. In our hospital, a system for patients to initially consult the Pharmacists' Outpatient Clinic, followed by the feed-back of grade-based
HFS control strategies to physicians at the outpatient clinic, was established. Skin control for sorafenib-treated patients with HFS may have prolonged the administration period, improving the prognosis

Disclosure of Interest: All authors have declared no conflicts of interest.

\section{Reference}

1. Jonker DJ, O'Callaghan CJ, Karapetis CS, et al: Cetuximab for the treatment of colorectal cancer. $N$ Engl J Med 2007; 357: 2040-2048.

\section{P0729 UNUSUAL METASTASIS OF HEPATOCELLULAR CARCINOMA}

I. Nakhcha ${ }^{1}$, I. Benelbarhdadi ${ }^{2}$, F.Z. Ajana ${ }^{1}$

${ }^{1}$ Service Medecine C Rabat Maroc, Hopital Ibn Sina Rabat, Rabat/Morocco

${ }^{2}$ Rabat, Ibn Sina Hospital, Rabat/Morocco

\section{Contact E-mail Address: Ibtissamnakhcha2@gmail.com}

Introduction: Hepatocellular carcinoma is the most common primary tumor of the liver and is estimated to cause more than a quarter of a million deaths each year throughout the world. Extrahepatic metastasis of HCC occurs in about 30 $50 \%$ of patients, and it depends on HCC stages. 1 The most frequent site is lung, followed by lymph node, bone, and adrenal gland.2 Extrahepatic metastases to unusually sites from HCC have been reported in a few case reports. We report cases of patients with unusual extrahepatic metastatic sites from HCC.

Aims \& Methods: We carried out a retrospective study of 16 patients with unusual extrahepatic metastasis of hepatocellular carcinoma out of 1047 cases of $\mathrm{HCC}$ treated at the hepatogastroenterology department "Medicine $\mathrm{C}$ " of the IBN SINA University Hospital during the past 22 years. The diagnosis was suspected based on clinical signs and imaging data, and confirmed by histology when the biopsy of the metastasis was possible, were excluded from this study, patients with lung metastasis, lymph node and portal thrombosis.

Results: Our study included 16 patients, 10 men and 6 women with a mean age of 58.5 years ranging from 37 years to 75 years. 13 patients had cirrhosis due to hepatitis $\mathrm{C}$ virus, 1 patient had a cirrhosis due to viral $\mathrm{B}$ infection and 2 patients had $\mathrm{HCC}$ within anoncirrhotic liver. All patients had one or more $\mathrm{HCC}$, ranging in size from 2 to $10 \mathrm{~cm}$. The AFP was normal in 11 cases and elevated in 4 cases $(>200 \mathrm{ng} / \mathrm{ml})$. We collected 4 cases of adrenal metastases, 3 costovertebral metastases, 2 gastric metastases, 2 brain metastases, 1 cranial metastasis, 1 clavicular metastasis, 1 ovarian metastasis, 1 nasopharyngeal metastasis, and a case of metastasis in the path of percutaneous biopsy of HCC. In 4 cases the diagnosis of $\mathrm{HCC}$ and metastasis was synchronous while in 12 cases median time from diagnosis of hepatocellular carcinoma andextrahepatic HCC was 15.5 months. Therapeutic abstention was decided in 14 patients for the advanced stage of the disease. cutaneous metastasis was resected surgically and HCC occurring in healthy liver was treated by lumpectomy and upper pole gastrectomy in gastric metastasis. The average survival was estimated at 14 months with a decline of 17.3 months, 6 cases were lost to follow and 6 deaths occurred in our series. Conclusion: The incidence of unusual and extrahepatic metastasis of HCC diagnosed during clinical course was not frequent. The diagnostic procedures for extrahepatic metastasis have not been standardized, however considering the substantial advances in treatment of HCC, the detection of extrahepatic HCC is crucial for patients to receive appropriate therapy, which ultimately determines patient survival.

Disclosure of Interest: All authors have declared no conflicts of interest.

\section{P0730 EPIDEMIOLOGICAL STUDY OF HISTOLOGICALLY} PROVEN ADVANCED HEPATOCHOLANGIOCARCINOMA: AN AGEO MULTICENTER RETROSPECTIVE STUDY IN FRANCE

M. Salimon ${ }^{1}$, C. Prieux-Klotz ${ }^{2}$, D. Tougeron ${ }^{3}$, T. Lecomte ${ }^{4}$, M. Caulet ${ }^{4}$, T. Matysiak Budnik ${ }^{1}$, V. Hautefeuille ${ }^{5}$, M. Tiako-Meyo ${ }^{6}$, A. Zaanan ${ }^{6}$, Y. Touchefeu ${ }^{1}$

${ }^{1}$ IMAD, Department of Gastroenterology, University Hospital, Nantes/France ${ }^{2}$ Department of Gastroenterology, Cochin University Hospital, Paris/France ${ }^{3}$ Gastroneterology, Poitiers unversity hospital, Poitiers/France

${ }^{4}$ Department of Gastroenterology, Tours University Hospital, Tours/France ${ }^{5}$ Department of Gastroenterology, Amiens University Hospital, Amiens/France ${ }^{6}$ Department of Gastroenterology, Hôpital Européen Georges Pompidou, Paris/ France

Contact E-mail Address: maeva.salimon@chu-nantes.fr

Introduction: Hepatocholangiocarcinoma is a rare primary hepatic tumor combining features of both, cholangiocarcinoma and hepatocellular carcinoma (cHCC-ICC). Few data concerning the epidemiology of cHCC-ICC have been reported, mainly from surgical series in Asian and American populations.

Aims \& Methods: The main objective of this retrospective multicenter study was to evaluate epidemiological features and overall survival of histologically proven advanced $\mathrm{CHCC}$ patients in a French population. Data from patients treated for histologically proven cHCC-ICC in six French university hospitals between 2008 and February 2017, were retrospectively collected. The main clinical, biological, therapeutic features and OS were reported. Statistical analysis was performed using Graph Pad Prism 6.

Results: Thirty patients were included $(76.6 \%$ of men, median age 64 years [extreme 37-88]. Cirrhosis was associated in 33.3\% of cases (Child-Pugh score A: $70 \%$ ). Positive serology for hepatitis $\mathrm{B}$ virus and $\mathrm{C}$ was found in respectively, 
$5(16.6 \%)$ and $2(6.6 \%)$ patients; and 1 co-infection was observed. Chronic alcoholism was noted in $33.3 \%$, diabetes and obesity (body mass index $\geq 30 \mathrm{~kg} / \mathrm{m}^{2}$ ) were both present in $26.6 \%$ of cases. Alpha-fetoprotein, Carbohydrate Antigen 19-9 and carcinoembyonnic antigen serum levels were above normal in respectively $39 \% \quad\left(\right.$ median $\left.=5.3 \mu \mathrm{g} / \mathrm{L} \quad\left[\begin{array}{lll}2-21 & 479\end{array}\right]\right), 50 \%$ $($ median $=21.8 \mathrm{IU} / \mathrm{mL}[4.5-20000])$ and $14 \% \quad($ median $=2.4 \mu \mathrm{g} / \mathrm{L}[2-88])$ of cases. Six patients $(20 \%)$ were initially treated by surgical resection. At the diagnosis of advanced non resectable disease, $66.6 \%$ of patients presented a multifocal hepatic lesions, $50 \%$ presented distant metastases (bones metastases $(23.3 \%)$, lung metastases $(20 \%)$ and peritoneal metastases $(13.3 \%)$. Twentyseven patients $(90 \%)$ received first line of systemic treatment. Twenty-four patients were treated by chemotherapy: Gemcitabine $(\mathrm{Gem})$ alone $(\mathrm{n}=1)$, Gem + oxaliplatine $\quad(n=12), \quad$ Gem + oxaliplatine + bevacizumab $\quad(n=9)$ Gem + cisplatine $(n=2)$. Two patients were treated by chemoembolization and 1 patient received sorafenib. Twenty-one $(70 \%)$ and $4(13.3 \%)$ patients received a second and third line of treatment, respectively. Median overall survival was 14.5 months.

Conclusion: Advanced cHCC-ICC appear to be aggressive tumors with a poor prognosis. Cirrhosis was associated in one third of patients. Systemic treatments are not standardized and must be evaluated in a dedicated study.

Disclosure of Interest: All authors have declared no conflicts of interest.

\section{P0731 A PROPOSAL FOR MODIFICATION OF BARCELONA CLINIC LIVER CANCER SYSTEM FOR HEPATOCELLULAR CARCINOMA STAGING BASED ON KOREAN MULTICENTER REGISTRY DATABASE}

J. Cheong, H. Cho

Gastroenterology, Ajou University Hospital, Suwon/Korea, Republic of

\section{Contact E-mail Address: jaeyoun620@gmail.com}

Introduction: Barcelona Clinic Liver Cancer (BCLC) C stage is defined as patients with preserved hepatic function and one of those adverse predictors including performance status (PS) 1-2, vascular invasion (VI), or extrahepatic spread (EHS). Therefore, BCLC C demonstrates extreme heterogeneity because patients with PS 1-2 are categorized to BCLC C regardless of tumor burden, VI or EHS. This study aimed to modify BCLC system based on PS to derive more relevant staging system.

Aims \& Methods: A total of 7501 subjects, who were registered in Korean Liver Cancer Study Group during the period 2008-2013, were analyzed. Kaplan-Meier analysis was used to compare overall survival (OS). The relative goodness-of-fit between staging system was compared by Akaike information criterion (AIC) and integrated area under the curve (iAUC).

Results: Two modified BCLC (mBCLC) systems (\#1 and \#2) were derived by reducing role of PS in BCLC system. The patients with PS 1 or 2 without VI or EHS were reassigned to stage 0, A, or B according to their tumor burden. Prognostic accuracy was compared between mBCLC systems and original BCLC by AIC and IAUC. As a result, mBCLC\#2 system identified as most explanatory and desirable model for HCC staging by showing smallest AIC value $(\mathrm{AIC}=70088.01)$ and largest IAUC (IAUC $=0.722)$, while original BCLC showed the biggest AIC value $(\mathrm{AIC}=70697.17)$ and smallest IAUC $(\mathrm{IAUC}=0.705)$. The mBCLC\#2 stage $\mathrm{C}$ was further sub-classified into $\mathrm{C} 1$, $\mathrm{C} 2, \mathrm{C} 3$ and $\mathrm{C} 4$ according to the variables which selected by statistical and clinical importance. The C1-C4 sub-groups showed significant different OS distribution between groups $(\mathrm{P}<0.001)$.

Conclusion: Modification of BCLC system based on PS derived accurate and relevant modified BCLC system for HCC patients.

Disclosure of Interest: All authors have declared no conflicts of interest.

\section{References}

1. Hsu CY, Lee YH, Hsia CY et al. Performance Status in Patients With Hepatocellular Carcinoma: Determinants, Prognostic Impact, and Ability to Improve the Barcelona Clinic Liver Cancer System. Hepatology. 2013, 57(1) $112-119$

2. Giannini EG, Moscatelli A, Pellegatta $G$ et al. Application of the Intermediate-Stage Subclassifi cation to Patients With Untreated Hepatocellular Carcinoma. Am J Gastroenterol 2016; 111:70-77

\section{P0732 HEPATIC LYMPHOMAS: A RARE ENTITY}

R. Gaspar ${ }^{1}$, A. Andrade ${ }^{1}$, J. Santos-Antunes ${ }^{1}$, R. Liberal ${ }^{1}$, F. Carneiro ${ }^{2}$, G. Macedo ${ }^{3}$

${ }^{1}$ Gastrenterology, Hospital São João, Porto/Portugal

${ }^{2}$ Pathology Department Centro Hospitalar São João, Porto/Portugal

${ }^{3}$ Centro Hospitalar São João, Porto Medical School, Porto/Portugal

\section{Contact E-mail Address: ruilopesgaspar@gmail.com}

Introduction: Primary tumors of the liver, beyond hepatocellular carcinoma, are difficult to characterize and are associated with poor prognosis. Hepatic involvement in the lymphomatous process is uncommon and primary hepatic lymphomas are rare. The etiology is not yet known but it is thought that hepatitis B and $\mathrm{C}$ may be risk factors. The therapeutic approach is not yet fully defined.
Aims \& Methods: The aim of this study was to evaluate the clinical manifestations, risk factors and prognosis in lymphomas with hepatic involvement. We conducted a retrospective study of patients diagnosed with hepatic lymphoma at our center between 2005 and 2016.

Results: During the 12 years, 36 hepatic lymphomas were identified, 27 primary hepatic lymphomas and 9 with secondary hepatic involvement. The mean age at diagnosis was $53.5 \pm 14.6$ years and $50 \%$ were males. Only one patient had hepatitis B and none had hepatitis C. The majority $(94.4 \%)$ had symptoms at the time of diagnosis, with fatigue $(83.3 \%)$, night sweats $(61.1 \%)$ and loss of weight $(61.1 \%)$ being the most common. The imaging presentation was of a single mass in $47.2 \%$ of cases, multiple masses in $30.6 \%$ and infiltrative mass in $22.2 \%$. The most common lymphoma subtypes were diffuse large B-cell lymphoma $(52.8 \%)$, MALT lymphoma $(11.1 \%)$ and Hodgkin's lymphoma $(11.1 \%)$. Survival at the end of one year was $63.8 \%$ and $27.8 \%$ at 3 years. Age $>60$ years $(\mathrm{p}=0.004)$ was the only factor that was significantly associated with higher mortality.

Conclusion: Hepatic lymphomas are rare entities that may occur in different ways, with diffuse large B-cell lymphoma being the most common subtype. They presented a 3 -year survival of only $27.8 \%$ and the age over 60 years was the only factor significantly associated with mortality.

Disclosure of Interest: All authors have declared no conflicts of interest.

\section{P0733 USE OF BILE ACIDS AS A REGULATOR OF GUT MICROBIOTA}

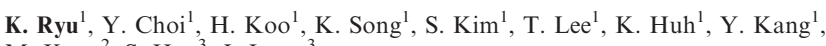
M. Kang ${ }^{2}, \mathrm{~S} . \mathrm{Han}^{3}$, J. Jeong ${ }^{3}$

${ }^{1}$ Gastroenterology, Konyang University College of Medicine, Daejeon/Korea, Republic of

${ }^{2}$ Dankook University Hospital, Cheonan-si/Korea, Republic of

${ }^{3}$ Anatomy \& Histology, Konyang University College of Medicine, Daejeon/Korea, Republic of

\section{Contact E-mail Address: medidrug@hotmail.com}

Introduction: Microbiotas, as part of our bodies, actually affect the body's metabolism, defense and digestion. Gut microbiotas are the typical examples and they are mainly regulated by bile acids. Each bile acid has different properties, ${ }^{1}$ and the inhibition or promotion effects of microbiotas are very diverse. ${ }^{2}$ Each individuals have a characteristic microbial distribution and composition within the intestinal tract, presumably related to the composition of their respective characteristic bile acids. ${ }^{3}$ The bile acids, released into the intestinal tract, are mostly reabsorbed (about 95\%) through the enterohepatic circulation and recycled. If a certain bile acid is continuously orally-supplied, the ratio of supplied bile acid to the total bile acid is gradually increased due to the continuous enterohepatic circulation. It has been reported that changes in the composition of intestinal bile acids cause dysbiosis, ${ }^{4}$ and it is presumed that an artificial change in the composition of bile acid can be an effective method of controlling the proliferation of target microbiotas.

Aims \& Methods: First, we wanted to identify the effects of certain bile acids on each microbiotas. We observed the effect of bile acids in human body (cholic acid, deoxycholic acid, chenodeoxycholic acid, ursodeoxycholic acid) on microorganisms by the disk diffusion method, used for antibiotic susceptibility test. Second, we intended to observe actual intestinal microbial changes through the supply of specific bile acids in animal models. Specific bile acid (UDCA), which is commercialized, was fed to rats, and real time polymerase chain reaction (RTPCR) was used to observe the changes of the colon microbiotas. Fermicutes and bacteroidetes, which consist more than $90 \%$ of total intestinal microbiotas, were analyzed. ${ }^{5}$ Next, to minimize the role of endogenous bile acid, we used bile duct ligation rat model. Next generation sequencing (NGS) method was used to analyze the changes of microorganisms according to the supplied amount of UDCA. Examinations were divided into three groups for 3 weeks, considering the capacity of commercial UDCA product: control group; $10 \mathrm{mg} / \mathrm{kg} / \mathrm{day}$ group; and $15 \mathrm{mg} / \mathrm{kg}$ /day group. Fecal contents were collected from cecum of sacrificed rats. Results: Each bile acid formed various inhibitory or promoting regions in target strains. Saccharomyces boulardii and Lactobacillus casei, which are commercially available as probiotics, were mainly inhibited by hydrophobic bile acids. Enterococcus faecalis and Klebsiella pneumoniae, which are frequently observed in bile during biliary infections, did not form a large inhibitory zone of bile acids. Escherichia coli, which is occasionally found in the lower intestinal tract, proliferated more around bile acid. Other microorganisms, which cause opportunistic infections in various organs in the body, showed various patterns. As a result of relative quantitative analysis(RT-PCR) based on the control group, the ratio of Fermicutes to Bacteroidetes was decreased in the group treated with UDCA for 3 weeks. In the cholestatic model, NGS showed changes in the proportion of intestinal microbiota and an increase in diversity after 3 weeks of UDCA supply. Conclusion: The effects of various kinds of bile acids on microbiotas are very diverse, and the oral administration of certain bile acids may cause changes in microbial environment in the intestinal tract. Finally, oral feeding of certain bile acids can be used as a therapeutic treatment for dysbiosis by controlling the microbial environment in the intestinal tract.

Disclosure of Interest: All authors have declared no conflicts of interest.

\section{References}

1. Begley M, et al. The interaction between bacteria and bile. FEMS Microbiol Rev. 2005; 29:625-51. 
2. Sandhya B. et al. Bile acid amphiphiles with tunable head groups as highly selective antitubercular agents. Med. Chem. Commun., 2014;5:1761-8.

3. Kakiyama G. et al. Modulation of the fecal bile acid profile by gut microbiota in cirrhosis. J Hepatol. 2013; 58(5):949-55.

4. Bajaj JS. et al. Linkage of gut microbiome with cognition in hepatic encephalopathy. Am J Physiol Gastrointest Liver Physiol. 2012;302:G168-75.

5. Turnbaugh PJ. et al. A core gut microbiome in obese and lean twins". Nature. 2009;457: 480-4

\section{P0734 MIRNA-21 IS OVEREXPRESSED IN PRIMARY BILIARY CHOLANGITIS AND MEDIATES LIVER INJURY AND NECROPTOSIS IN EXPERIMENTAL CHOLESTASIS}

M.B. B. Afonso ${ }^{1}$, P. M. Rodrigues ${ }^{1}$, A. L. Simão ${ }^{1}$, M. M. Gaspar ${ }^{1}$, T. Carvalho ${ }^{2}$, P. Borralho ${ }^{3}$, J.M. Banales ${ }^{4}$, R. E. Castro ${ }^{1}$, C.M.P. Rodrigues ${ }^{1}$ ${ }^{1}$ Research Institute for Medicines (iMed.ULisboa), Faculty of Pharmacy, Universidade de Lisboa, Lisbon/Portugal

${ }^{2}$ Histology and Comparative Pathology Laboratory, Instituto de Medicina Molecular, Lisbon/Portugal

${ }^{3}$ Escola Superior de Tecnologia da Saúde de Lisboa (ESTEsL); Instituto de Anatomia Patológica, Universidade de Lisboa; Hospital Cuf Descobertas, Lisbon Portugal

${ }^{4}$ Department Of Liver And Gastrointestinal Diseases, Biodonostia Research Institute - Donostia University Hospital - University of the Basque Country (UPV/EHU), CIBERehd, Ikerbasque, San Sebastian/Spain

Contact E-mail Address: adlsimao@ff.ulisboa.pt

Introduction: Inhibition of microRNA-21 (miR-21) prevents necroptosis in the mouse pancreas. In turn, we recently showed that necroptosis contribute to hepatic necro-inflammation in the common bile duct ligation (BDL) murine model.

Aims \& Methods: We aimed to evaluate the role of miR-21 in mediating deleterious processes associated with cholestasis. The functional crosstalk between miR21 and necroptosis was investigated in vitro. miR-21 expression was evaluated in the liver of primary biliary cholangitis (PBC) patients. C57BL/6 wild-type (WT) or miR-21-deficient ( $m i R-21^{-/}$) mice were subjected to BDL or sham surgeries, with biochemical, molecular and histological analysis of hepatic damage, fibrosis, necroptosis and bile acid metabolism, after either acute (3 days) or chronic (14 days) injury.

Results: Studies in $m i R-21^{-/-}$primary mouse hepatocytes established a functional link between miR-21 and necroptosis through cyclin dependent kinase 2 associated protein 1 (CDK2AP1). miR-21 expression increased in the liver of PBC patients and BDL WT mice at both 3 and 14 days. Notably, under BDL, $m i R-21^{-/-}$mice displayed decreased serum levels of liver injury markers compared with WT mice, accompanied by reduced hepatocellular degeneration, oxidative stress and pro-fibrotic gene expression. Hallmarks of necroptosis were decreased in the liver of BDL $m i R-21^{-1-}$ mice, via relieved repression of CDK2AP1. Further, $m i R-21^{-/-}$mice displayed improved adaptive response in the expression of bile acid homeostasis-associated genes.

Conclusion: miR-21 ablation ameliorates liver damage and necroptosis in BDL mice; as such, inhibition of miR-21 should arise as a promising approach to treat cholestatic liver diseases. Supported by FCT, Portugal through grants PTDC BIM-MEC/0895/2014 and UID/DTP/04138/2013, and fellowships SFRH/BD 91119/2012 (MBA), SFRH/BD/88212/2012 (PMR), and SFRH/BD/104160/201 (ALS).

Disclosure of Interest: All authors have declared no conflicts of interest.

\section{P0735 IS COMPLETE STONE REMOVAL FOR CHOLEDOCHOLITHIASIS ALWAYS NECESSARY IN EXTREMELY ELDERLY PATIENTS?}

N. Okuda, S. Sugimoto, H. Nakamura, H. Hisada, T. Temma, Y. Hashimoto, S. Hayashi, T. Ito, M. Takami, M. Yamamura, J. Oyamada, A. Kamei Gastroenterology, Ise Res Cross Hospital, Ise City/Japan

Contact E-mail Address: n-okuda@ise.jrc.or.jp

Introduction: Endoscopic sphincterotomy, endoscopic papillary balloon dilation, and endoscopic papillary large balloon dilation are widely recognized as safe and effective treatments of choledocholithiasis. However, endoscopic stone removal does have some complications. Although the life expectancy of elderly patients has increased dramatically worldwide, little information is available on the necessity of complete endoscopic stone removal in extremely elderly patients.

Aims \& Methods: The aim of this study was to evaluate the safety and efficacy of complete endoscopic stone removal in extremely elderly patients. We retrospectively evaluated all extremely elderly patients (older than 90 years) who had undergone endoscopic stone removal for choledocholithiasis at Ise Red Cross Hospital between January 2012 and December 2016. Included patients were divided into complete stone removal and incomplete stone removal (failure to achieve complete stone removal and insertion of a plastic stent) groups. We compared the complication rate, overall survival (OS), and disease-specific survival (DSS) rate between the two groups.

Results: In total, 67 patients were included this study; 36 (54\%) had complete stone removal and $31(46 \%)$ had incomplete stone removal. The median age of the patients was 92 years (range 90-100 years), median follow-up period was 462 days (range 6-1449 days) and the male-to-female ratio was 15:52. Baseline characteristics (age, body mass index, performance status, and comorbidities), rate of complications (perforation, bleeding, hypoxemia, or decreased blood pressure during the endoscopic procedure), and total number of endoscopic procedures did not significantly differ between the two groups. The median number of stones was one (range $0-5)$ and two (range $1-5)(P=0.013)$, while the median diameter of the largest stones was $9 \mathrm{~mm}$ (range $0-27)$ and $14 \mathrm{~mm}($ range $5-32)(P=0.001)$ in the complete and incomplete stone removal groups, respectively. During the follow-up period, OS was $33.3 \%$ and $41.9 \%$ and DSS was $5.56 \%$ and $3.23 \%$ in the complete and incomplete stone removal groups, respectively. Kaplan-Meier analysis found no significant difference in OS and DSS between the two groups $(\mathrm{P}=0.187$ and $\mathrm{P}=0.581$, respectively)

Conclusion: Patients in the incomplete stone removal group tended to have more numerous and larger stones. This single-centre retrospective study revealed no significant difference in OS and DSS between the two groups. Complete stone removal might not be always necessary in extremely elderly patients aged 90 years and older.

Disclosure of Interest: All authors have declared no conflicts of interest.

\section{P0736 THE EVALUATION OF TRANSPAPILLARY ENDOSCOPIC GALLBLADDER DRAINAGE WITH THE USE OF INTRADUCTAL ULTRASONOGRAPHY}

K. Hayasaka, T. Ujihara, R. Nakahara, D. Murakami, T. Shimizu, S. Suehiro, Y. Katsuyama, H. Harada Gastroenterology, New Tokyo Hospital, Chiba/Japan

Contact E-mail Address: dr25027@gk2.so-net.ne.jp Introduction: The number of indications for endoscopic transpapillary gallbladder drainage (ETGBD) to acute cholecystitis patients has been increasing with the aging of the population and prescribing antithrombotic agents. ETGBD is one of the challenging procedure because it is difficult to identify the cystic duct (CD) orifice. From November 2015, we performed with ETGBD using intraductal ultrasonography (IDUS) complementarily. The CD orifice can be identified using IDUS, resulting in cannulation in to the CD. We investigated the success rate and clinical outcomes of ETGBD in combination with using IDUS

Aims \& Methods: ERCP was performed in 1000 patients (1400 times) at New Tokyo Hospital between July 2012 and December 2016. Among them, a total of 97 patients underwent ETGBD; 58 patients with IDUS and 39 without IDUS. In this study, we investigated the success rate of ETGBD retrospectively. The success of ETGBD was defined as cannulation into the gallbladder within two trials. Results: The mean age and male proportion of patients in the group with IDUS was similar to that in the group without IDUS $(74.5 \pm 11.4$ vs $73.3 \pm 11.3$; $P=0.600$ and $18 / 40$ and $13 / 26 ; P=0.828)$. The procedure success rate was $78.4 \%(76 / 97)$ in total; $86.2 \%(50 / 58)$ in the group with IDUS and $66.7 \%(26$ 39 ) in the group without IDUS $(P=0.026)$. Using IDUS under fluoroscopic image allowed all patients in the group with IDUS to identify the CD orifice. ETGBD procedures in eight patients were unsuccessful because of a highly flexion of the $\mathrm{CD}$ in seven patients and an obstruction of the $\mathrm{CD}$ orifice caused by tumor invasion in one patient. Although both groups developed mild pancreatitis (one patient in the group with IDUS, and two patients in the group without IDUS), no significant difference was observed in the two groups $(P=0.563)$. All patients were successfully managed with conservative treatment. There were no any other complications in the two groups.

Conclusion: The success rate of ETGBD in the group with IDUS was significantly higher than that in the group without IDUS. IDUS is may be useful as a complementary option of endoscopic gallbladder drainage.

Disclosure of Interest: All authors have declared no conflicts of interest.

\section{P0737 THE RENDEZVOUS PROCEDURE FOR THE MANAGEMENT OF BILE DUCT INJURIES AFTER CHOLECYSTECTOMY: SHORT AND LONG-TERM OUTCOMES AND PREDICTORS FOR SUCCESS}

A.M. Schreuder ${ }^{1}$, K. A. Booij ${ }^{1}$, P.r. De Reuver ${ }^{1}$, O. M. Van Delden ${ }^{2}$, K. P. Van Lienden $^{2}$, M.G.h. Besselink ${ }^{1}$, O.R.c. Busch ${ }^{2}$, D.J. Gouma ${ }^{2}$, E. A.j. Rauws ${ }^{3}$, T. M. Van Gulik

${ }^{1}$ Department Of Surgery, Academic Medical Center, Amsterdam/Netherlands ${ }^{2}$ Interventional Radiology, Academic Medical Center, Amsterdam/Netherlands ${ }^{3}$ Gastroenterology, Academic Medical Center, Amsterdam/Netherlands

Contact E-mail Address: a.m.schreuder@amc.uva.nl

Introduction: Bile Duct Injury (BDI) following laparoscopic cholecystectomy is a persisting problem. The rendezvous procedure (RV) provides a combined endoscopic and percutaneous approach in order to re-establish bile duct continuity in complex BDI.

Aims \& Methods: The aim of this study is to assess short-term and long-term outcomes of the RV. All consecutive patients with BDI referred to our tertiary referral center between 1995 and 2016 were analyzed retrospectivally. RV procedure was performed when endoscopic stenting or PTC failed and when deemed feasible by a dedicated multidisciplinary team including a hepato-pancreato-biliary surgeon, gastroenterologist and interventional radiologist. Classification of $\mathrm{BDI}$, technical success of RV, procedure-related complications and outcomes were assessed.

Results: Among a total of 812 patients, RV was performed in $47(5.8 \%)$ patients, of which $31(66 \%)$ were diagnosed with complete transection of the bile duct (type D/Strasberg type E injury). Primary success rate of RV was $94 \%$ (44/47 patients). Reasons for failure $(\mathrm{N}=3)$ were inability to pass a stricture and inability to make contact between the two wires. In $26 / 47$ patients $(55 \%) \mathrm{RV}$ was the final successful treatment. In $17 / 47$ patients $(36 \%) \mathrm{RV}$ acted as a bridge to 
surgery; although the RV was initially successful, late complications (stenosis, stent dysfunction) required elective hepaticojejunostomy (HJ). Procedure-related adverse events occurred in 10 patients $(18 \%)$ with cholangitis being the most frequent complication $(\mathrm{N}=4.7 \%)$. No life-threatening adverse events and no 30-day mortality occurred.

Conclusion: In experienced hands, RV is safe with a final non-surgical success rate of $55 \%$. When endoscopic stenting fails in patients with complex BDI, RV can be considered as a viable treatment option before surgical repair.

Disclosure of Interest: All authors have declared no conflicts of interest.

\section{References}

1. Rystedt J, Lindell G et al. Bile Duct Injuries Associated With 55, 134 cholecystectomies: Treatment an d Outcome from a National Perspective. World $J$ Surg 2016

2. Booij KAC, de Reuver PR et al. Long-term Impact of Bile Duct Injury on Morbidity, Mortality, Quality of Life, and Work Related Limitations. 2017

3. Booij KAC, de Reuver PR et al, Morbidity and mortality after minor bile duct injury following laparoscopic cholecystectomy. Endoscopy 2014

4. de Reuver PR, Rauws EA, Bruno MJ et al; Survival in bile duct injury patients after laparoscopic cholecystectomy: a multidisciplinary approach of gastroenterologists, radiologists, and surgeons. Surgery 2007

5. De Reuver PR, Rauws EA, Vermeulen M, Dijkgraaf MGW, Gouma DJ, Bruno MJ, . Endoscopic treatment of post-surgical bile duct injuries. Gut 2007

6. Janssen JJ, van Delden OM, van Lienden KP et al. Percutaneous Balloon Dilatation and Long-Term Drainage as Treatment of Anastomotic and Nonanastomotic Benign Biliary Strictures. Cardiovasc Intervent Radiol. 2014 Jan 23

7. Calvo MM, Bujanda L, Heras I et al, The rendezvous technique for the treatment of choledocholithiasis. Gastrointestinal Endoscopy 2001

8. Nasr JY, Hashash JG, Orons P et al, Rendezvous procedure for the treatment of bile leaks and injury following segmental hepatectomy. Digestive and Liver Disease 2013

9. Donatelli G, Vergeau BM, Derhy S et al. Combined endoscopic and radiologic approach for complex bile duct injuries. Gastrointestinal Endoscopy 2014

10. Fiocca F, Salvatori FM et al, Complete transection of the main bile duct: minimally invasive treatment with an endoscopic-radiologic rendezvous. Gastrointestinal Endoscopy 2011

11. Tomoda $\mathrm{T}$, Kato $\mathrm{H}$ et al, Biliary Anastomotic stricture after adult living donor liver transplantation with duct-to-duct reconstruction: Outcome after endoscopic treatment including rendezvous procedure. Transplantation 2016

12. Ismael $\mathrm{HN}, \mathrm{Cox} \mathrm{S}$ et al, The morbidity and mortality of hepaticojejunostomies for complex bile duct injuries: a multi-institutional analysis of risk factors and outcomes using NSQIP. HPB 2017-2

13. Parlak E, Disibeyaz S et al. Endoscopic Treatment of Patients with Bile Duct Stricture After Cholecystectomy: Factors Predicting Recurrence in the Long Term. Dig dis sci 2015

\section{P0738 CHOLECYSTECTOMY AFTER ERCP CLEARANCE OF COMMON BILE DUCT STONES: DOES TIMING MATTERS? A PROSPECTIVE RANDOMIZED STUDY}

E. Atif, E. A. Elhanafy, A. Elnakeeb

Gastroenterological Surgical Center, Mansoura University, Mansoura/Egypt

\section{Contact E-mail Address: Dr.ehab.atif@gmail.com}

Introduction: The time interval between endoscopic retrograde cholangiopancreatography (ERCP) and laparoscopic cholecystectomy (LC) is a matter of debate. This study was planned to compare early LC versus late LC.

Aims \& Methods: This is a prospective randomized study on patients who are presented with concomitant gallbladder and common bile duct stone. The study population was divided into two groups; group (A) managed by early LC within three days after ERCP; and group (B) managed by late LC one month after ERCP.

Results: No significant difference between both groups as regards the conversion rate, the degree of adhesion, cystic duct diameter, and intraoperative common bile duct injury or bleeding. Recurrent biliary symptoms were significantly more in delayed LC group in $7(12.71 \%)$ patient versus 1 patient in early LC $(\mathrm{P}=0.03)$. Conclusion: No significant difference between both groups as regards the conversion rate. Recurrent biliary symptoms were significantly more in delayed LC while waiting LC. Morbidity was significantly more in delayed LC.

Disclosure of Interest: All authors have declared no conflicts of interest.
P0739 TRANSRECTAL GALLBLADDER PRESERVING CHOLECYSTOLITHOTOMY AND POLYPECTOMY BY PURE NOTES

B. Liu

Gastroenterology, The First Affiliated Hospital of Zhengzhou University, Zhengzhou/China

Contact E-mail Address: 3217265358@qq.com

Introduction: Transcolonic and transrectal NOTES in human cases was greatly restricted by the fact of fecal contamination. We developed a detachable intracolonic balloon to help keep the colon sterile by blocking the colonic lumen. Although cholecystectomy is widely used for treating gallbladder polyps and gallstones, there is still a controversy about whether or not the gallbladder should be preserved. However, postcholecystectomy syndromes, such as bile duct injury and the correlation with colon cancer, remind us of the importance of gallbladder preservation.

Aims \& Methods: Approved by the Independed Ethics Committee, we've completed 8 transrectal gallbladder preserving cholecyctolithotomy (TRGPC) and 3 transrectal gallbladder preserving polypectomy (TRGPP) and 2 combined cases by pure NOTES. Moreover, 1 case of TRGPC was done by hybrid NOTES. As the figures show, the balloon was placed in the transverse colon to block the colonic lumen, and the distal colon cavity was disinfected with povidone-iodine solution. An incision was made on the anterior rectal wall $12-17 \mathrm{~cm}$ from the anus. The endoscope was advanced into the peritoneal cavity with liver and gallbladder identified. The bile was aspirated before an incision on the gallbladder wall was made. Stones and/or polyps were found inside of the gallbladder. Stone extractor and biopsy forceps were used to take out the stones. The polyps were coagulated and removed by electric biopsy forceps. The muscular layer and the adventitial layer were successively closed with endoclips. Peritoneal cavity lavage was performed with sterile saline. The rectal incision was closed with endoclips and endoloops tightly. At the end of the procedure, the balloon was pulled out after being deflated.

Results: The mean operation time (from incision making till the last clipping) was $180.5 \mathrm{~min}$. (89-467 min.). 6 hours after anesthesia, the patients could drink water, and liquid diet was resumed 24 hours later. Postoperatively, 4 of the 14 patients felt mild abdominal distention which disappeared within 12 hours when they were able to get off the bed. For 1 patient with acute cholecystitis, a hybrid NOTES with laparoscopy was performed. Moreover, gallbladder drainage and peritoneal lavage were used, and the abdominal pain relieved soon. All the patients were discharged without any adverse events and all felt good during the follow-ups.

Conclusion: The usage of the detachable balloon can prevent the operative field from fecal contamination effectively. TRGPC and TRGPP by pure NOTES are suitable for both males and females. Transrectal route provides a novel alternative approach for the treatment of gallbladder polyps and gallstones. To our knowledge, this is the first human case series of transrectal gallbladder preserving cholecyslithotomy and polypectomy by pure NOTES. However, multicentered, prospective, controlled researches with more cases are needed in the future. Disclosure of Interest: All authors have declared no conflicts of interest.

\section{P0740 KML001, AN ORAL ARSENIC COMPOUND, AS PALLIATIVE CHEMOTHERAPY IN ADVANCED BILIARY TRACT CANCERS AFTER FAILURE OF GEMCITABINE BASED CHEMOTHERAPY}

J.H. Jo, I.R. Cho, H.S. Lee, M.J. Chung, J.Y. Park, S. Bang, S.W. Park, J. Chung, S.Y. Song

Department Of Internal Medicine And Institute Of Gastroenterology, Yonsei University Health System Dept. of Internal Medicine Dept. of Gastroenterology, Seoul/Korea, Republic of

Contact E-mail Address: woforce@naver.com

Introduction: Sodium metaarsenite (NaAs2O3: code name KML001) is an orally bioavailable arsenic compound with potential anti-cancer activity. However, the effect of KML001 has not been evaluated in patient with biliary tract cancers. We investigated the potential of KML001 as palliative chemotherapy in patients with advanced biliary tract cancers who non-respond to gemcitabine-based chemotherapy.

Aims \& Methods: The study was designed to evaluate safety, tolerability and effectiveness of KML001 as palliative chemotherapy in advanced biliary tract cancer. Inclusion criteria were 1) inoperable or metastatic cholangiocarcinoma and gallbladder cancer, and 2) previous history of failure to gemcitabine-based chemotherapy. Exclusion criteria were 1) naïve patient to chemotherapy, 2) ECOG PS $>3$, and 3) history of decompensated congestive heart failure, uncontrolled arrythmia, and QT prolongation (QTc $>480 \mathrm{~ms}$ ). KML001 (Kominox, Komipharm International Co., Ltd.) was administrated as $7.5 \mathrm{mg}$ daily to eligible subjects. Every two months, patient took response evaluation by biliary CT scan. Results: A total of 44 patients ( 21 females and 23 males) were enrolled prospectively between November 2011 and October 2014. Mean age of the patients was 
61.5 years old. There were 17 patients $(38.6 \%)$ with intrahepatic cholangiocarcinoma, 9 patients $(20.5 \%)$ with perihilar cholangiocarcinoma, 7 patients $(15.9 \%)$ with extrahepatic cholangiocarcinoma and 11 patients $(25.0 \%)$ with gallbladder cancer. All patients had stage IV disease and median number of prior anticancer treatments of patients was 2 (range 1-5). After an average of 1.5 months of treatment (range $0.5-10.0$ months), three patients $(6.8 \%)$ presented stable disease as best overall response, 23 patients $(52.3 \%)$ presented progression and 18 patients $(40.9 \%)$ could not survive until response evaluation. Median progression free survival was 1.7 months (interquartile range (IQR) $0.8-2.3$ months) and median overall survival from study-enroll was 2.5 months (IQR 1.4-4.9 months). During treatment, 25 patients $(55.6 \%)$ could maintain tolerable general condition without increasing ECOG PS, and 35 patients $(79.6 \%)$ could maintain or decrease the requirement for morphine as pain killer. During treatment, there were 12 cases $(12 / 44,27.3 \%)$ of severity grade 3 adverse events (AE) and no cases of grade 4 AE. Most common AE was ALT/AST elevation $(11 / 44,25 \%)$ followed by anemia $(10 / 44,22.7 \%)$. The major causes of the drop outs from study were due to disease progression or patient's death (30 cases, $66.7 \%$ ), and there were only 5 cases $(11.1 \%)$ who dropped out due to adverse drug reactions or severe AE.

Table 1: Treatment outcomes
Study period, mean, mo (range)

Progression free survival, mo (IQR)

Survival from study-enroll, mo (IQR)

Best response, n (\%)

SD

PD

Not evaluated

ECOG, n $(\%)$

Morphin requirement, n (\%)

Increased

Maintain

increased

maintain

decreased

Adverse event, n (\%)

Grade 1

Grade 2

\section{Grade 3}

Grade 4

Drop out cause, n (\%)
- drug reaction

Adverse event

Patient's death

Disease progression

Withdrawal consent

Loss of follow-up

Poor general condition
$1.5(0.5-10.0)$

$1.7(0.8-2.3)$

$2.5(1.4-4.9)$

$3(6.8 \%)$

$23(52.3 \%)$

$18(40.9 \%)$

$19(43.2 \%)$

$25(56.8 \%)$

$8(18.6 \%)$

$31(72.1 \%)$

$4(9.3 \%)$

$29(65.9 \%)$

$12(27.3 \%)$

$0(0 \%)$

$1(2.3 \%)$

$4(9.1 \%)$

$7(15.9 \%)$

$22(50.0 \%)$

$5(11.4 \%)$

$2(4.5 \%)$

$3(6.8 \%)$
$16(36.4 \%)$

Conclusion: KML001 was safe and well tolerated in respects of adverse events. KML001 was also shown promising result in disease control and pain control. KML001 can be another palliative treatment option for patients with advanced biliary tract cancers who non-respond to gemcitabine based chemotherapy. Disclosure of Interest: All authors have declared no conflicts of interest.

\section{P0741 ENDOSCOPIC DRAINAGE OF MALIGNANT STENOSIS OF THE BILIARY CONFLUENCE: HOW MANY SEGMENTS SHOULD BE DRAINED TO IMPROVE THE PATIENT SURVIVAL?}

F. Caillol ${ }^{1}$, E. Bories ${ }^{2}$, C. Zemmour ${ }^{3}$, C. Pesenti ${ }^{1}$, J.P. Ratone ${ }^{1}$, M. Giovannini ${ }^{1}$ ${ }^{1}$ Endoscopy, Paoli Calmettes Institute, Marseille/France

${ }^{2}$ Paoli Calmettes Institute, marseille/France

${ }^{3}$ Statistics, Paoli Calmettes Institute, Marseille/France

Contact E-mail Address: fcaillol@free.fr

Introduction: ESGE stated in 2012 that more than $50 \%$ of the liver had to be drained in case of unresectable hilary liver stenosis, however it remains unclear if unilateral or bilateral palliative drainage has to be perform for this kind of stenosis. Our policy is to try to drain the most possible segments of the liver in case of hilar stenosis. The aim of our study was to evaluate the efficiency of hilary drainage in function of the number of segments drained.

Aims \& Methods: The study is a retrospective analysis of a prospective registry of drainage of malignant stenosis of the hilum. Drainage were performed by 5 operators performing ERCP, EUS-drainage, and per-cutaneous drainage. The choice of the technique was left to the appreciation of the operators. All techniques could be associated. A Ct-scan or MRI was performed before and after drainage to decide the plan of the drainage and to evaluate efficiency and quality of the drainage. All drainages were performed under general anesthesia in a intubated patient. The quality of the drainage was evaluated by calculation of the percentage of drained segments. This percentage was calculated by dividing the number of liver segment drained with the number of liver segment. The number of liver segment was obtained by removing from the 8 classical liver segments with the segments resected in case of surgery, and/or the segments with invasion of more than $50 \%$ by tumor. The aim of the study was to evaluate the effect of the quality of the drainage on the patients survival. Quality of the drainage was defined by the percentage of liver segments drained.

Results: 60 (38 men) patients were included from from 01/2015 to 07/2016. Mean age $=69.84$ years old. The classification of the stenosis was type II for $17(29 \%)$ patients, type III for 20 patients $(34 \%)$, type IV for $22(37 \%)$. Histology corresponded to CCK from 26 patients $(43 \%)$, metastatic disease from colorectal cancer for 15 patients $(25 \%)$ and others cancers for $19(32 \%)$. Median followup was 8.5 months $(5.5-16.5)$. The median of survival was 5 months $(2.3-12.3)$. In unvaried and multi varied analysis there was a significant correlation between the percentage of segments drained $>80 \%(\mathrm{p}<0.05)$ and the survival. The other factor with impact on the survival was an invasion of the liver $>50 \%$ by tumor. There was no impact on the survival according to the different techniques used to drain the bile ducts. To confirm the efficiency of the quality of the drainage, a ROC curve was performed establishing a correlation between patients receiving chemotherapy and percentage of liver drained (area curve $=0.77(0.65-0.88)$. Conclusion: The survival of patient with a malignant stenosis of the biliary confluence is highly correlate with the rate of the liver segment drained.

Disclosure of Interest: All authors have declared no conflicts of interest.

\section{P0742 SYSTEMATIC REVIEW AND META-ANALYSIS OF TRANSABDOMINAL AND ENDOSCOPIC ULTRASOUND FOR GALLBLADDER POLYPS}

S. Z. Wennmacker ${ }^{1}$, M. P. Lamberts ${ }^{2}$, M. Di Martino ${ }^{3}$, J.P.h. Drenth ${ }^{2}$, K S. Gurusamy ${ }^{3}$, C. J.h.m. Van Laarhoven

${ }^{1}$ Dept. Of Surgery, Radboud University Medical Centre, Nijmegen/Netherlands

${ }^{2}$ Dept. Of Gastroenterology, Radboud Univers. Medisch Centrum, Nijmegen/ Netherlands

${ }^{3}$ Surgery, Royal Free Campus, UCL Medical School, London/United Kingdom

Contact E-mail Address: sarah.wennmacker@radboudumc.nl

Introduction: Approximately $0.6 \%$ to $4 \%$ of cholecystectomies are for gallbladder polyps. The decision to perform cholecystectomy is based on presence of gallbladder polyp on transabdominal ultrasound (TAUS) or endoscopic ultrasound (EUS), or both. This decision is also influenced by whether the polyp is a true or pseudo polyp. Pseudo polyps are non-neoplastic and do not need surgery. True polyps are neoplastic, either benign (adenoma) or (pre)malignant (dysplastic polyp/carcinoma). True polyps usually need surgery, as they are thought to have malignant potential through the adenoma-carcinoma sequence. (Pre)malignant lesions should be operated sooner than benign lesions. There has been no systematic review and meta-analysis on the accuracy of TAUS and EUS in the diagnosis of gallbladder polyps, true gallbladder polyps and (pre)malignant polyps.

Aims \& Methods: The aim was to determine and compare the accuracy of TAUS and EUS for diagnosis of gallbladder polyps, differentiating between true and pseudo polyps and differentiating between (pre)malignant and benign polyps. MEDLINE, EMBASE, Science Citation Index Expanded and the Cochrane library were searched. The search was not restricted based on language, publication status, or prospective or retrospective nature of the studies. Only studies with data on true positives, false positives, false negatives and true negatives for TAUS or EUS were included. Histopathology after cholecystectomy was the only accepted reference standard, except for studies on diagnosis of gallbladder polyp, for which follow-up by TAUS or EUS was also accepted as reference standard. Two authors independently screened abstracts, selected studies for inclusion and collected the data from each study. The quality of the studies was evaluated using the QUADAS-2 tool. If possible the bivariate model was used to obtain pooled estimates of sensitivity and specificity. Otherwise the principles of Takwiongi et al. for meta-analysis of sparse data were used. ${ }^{1}$ Hierarchical summary receiver operating characteristics curve (HSROC) were used to test whether TAUS or EUS was superior. Post-test probabilities were calculated using the median prevalence (as pre-test probabilities) and summary positive and negative likelihood ratios.

Results: A total of 17 studies were included in this review. For diagnosis of gallbladder polyps six studies on TAUS were included. The sensitivities and specificity of the studies ranged from 0.45 to 1.00 , and 0.91 to 0.98 respectively. There were no studies on EUS for this topic. For differentiating between true and pseudo polyps, seven studies were included. All seven studies retported on TAUS, four studies also reported on EUS. The sensitivities and specificities of the studies ranged from 0.47 to 1.00 and 0.51 to 0.98 for TAUS, and from 0.63 to 1.00 and 0.84 to 0.96 for EUS. For differentiating between (pre)malignant and benign polyps, five studies were included. Four studies reported on TAUS and three studies on EUS. The sensitivities and specificities of the studies ranged from 0.09 to 1.00 , and 0.46 and 1.00 for TAUS and from 0.69 and 0.92 , and 0.87 to 0.95 for EUS.

No studies were of high methodological quality. The results of the pooled sensitivities, specificities and post-test probabilities are shown in Table 1. HRSOC analysis showed no significant difference between the diagnostic accuracy of TAUS and EUS for differentiating between true and pseudo polyps and for 
differentiating between (pre)malignant and benign polyps $(\mathrm{p}=0.174$ and $\mathrm{p}=0.589$ respectively).

Conclusion: Diagnostic accuracy of TAUS for diagnosis gallbladder polyps is moderate and decreases further when differentiating between polyp types. TAUS would regularly provide false positive results, leading to unnecessary surgery. There was no evidence that diagnostic test accuracy of EUS was better than TAUS. Further studies of high methodological quality are needed to determine diagnostic accuracy of EUS and TAUS for differentiating between polyp types.

This abstract is based on a pre-peer review draft of a Cochrane Review

Disclosure of Interest: All authors have declared no conflicts of interest.

\section{Reference}

1. Takwoingi et al. Performance of methods for meta-analysis of diagnostic test accuracy with few studies or sparse data. Statistical Methods in Medical Research. Jun 2015.

\section{P0743 DIAGNOSTIC VALUE OF CONTRAST-ENHANCED ULTRASONOGRAPHY IN HIGH MECHANICAL INDEX CONTRAST MODE FOR POLYPOID LESIONS OF THE GALLBLADDER}

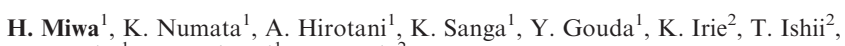
T. Kaneko ${ }^{1}$, K. Sugimori ${ }^{1}$, S. Maeda ${ }^{2}$

${ }^{1}$ Gastroenterological Center, Yokohama City University Medical Center, Yokohama/Japan

${ }^{2}$ Gastroenterology, Yokohama City University Graduate School of Medicine, Yokohama/Japan

Contact E-mail Address: miwa@yokohama-cu.ac.jp

Introduction: In its early stages, gallbladder cancer is an asymptomatic disease, and is associated with a poor prognosis if found in an inoperable condition. Several investigators have reported the utility of contrast-enhanced ultrasonography (CEUS) in low mechanical index (MI) contrast mode using a microbubble contrast agent for gallbladder lesions. However, CEUS images with low MI setting are influenced by the echogenicity of background B-mode and cannot depict precise vessel images, in contrast with high MI contrast mode.

Aims \& Methods: The aim of this study was to assess the diagnostic value of CEUS in high MI contrast mode for characterizing polypoid lesions of the gallbladder (PLG). Thirty-six patients with PLG, including 17 with gallbladder cancer and 19 with benign polyps, who underwent CEUS were enrolled. The institutional review board approved this study and informed consent was obtained. Perflubutane-based contrast agent and high MI contrast mode was used for CEUS. Two blinded readers retrospectively evaluated images obtained in B-mode and CEUS. Kappa values, which reflect inter-observer agreement. Subsequently, patients were stratified according to lesion size at the largest diameter, and the diagnostic accuracy for gallbladder cancer in B-mode and CEUS were assessed.

Results: Two patients with malignant PLG could not be evaluated in B-mode due to sludge. Kappa values for CEUS were graded as good or excellent, and were better than in B-mode. Age and size of malignant PLGs were significantly larger than benign lesions. In B-mode, $80 \%(12 / 15)$ of malignant PLGs exhibited heterogeneity $(p<0.01)$. On CEUS, malignant PLGs exhibited sessile-shape $(76 \%$ $[13 / 17])$, dilated vessels $(71 \%[12 / 17])$, irregular vessels $(82 \%$ [14/17]), and heterogeneous enhancement $(59 \%[10 / 17])(\mathrm{p}<0.01)$. Except for heterogeneous enhancement, all features remained significantly different after stratification according to size of PLG between $11 \mathrm{~mm}$ and $20 \mathrm{~mm}$ on CEUS. The sensitivity, specificity, and accuracy for diagnosis of gallbladder cancer was $80 \%(12 / 15)$, $79 \%(13 / 19)$, and $73 \%(25 / 34)$ in B-mode, $94 \%(16 / 17), 89 \%(17 / 19)$, and $92 \%$ $(33 / 36)$ on CEUS, and $88 \%(7 / 8), 91 \%(10 / 11)$, and $89 \%(17 / 19)$ on CEUS after stratification according to size, respectively.
Conclusion: CEUS in high mechanical index contrast mode was a useful modality for differentiating gallbladder cancer and benign PLGs.

Disclosure of Interest: All authors have declared no conflicts of interest.

\section{P0744 ASSOCIATION OF CIRCULATING ADIPONECTIN LEVELS AND TUMOR STAGE IN BILIARY TRACT CANCER}

A. Saray, B. Gogov, N. Zubcevic, D. Prohic, A. Mehmedović, A. Puhalović Department Of Gastroenterology And Hepatology, Clinical Center University of Sarajevo, Sarajevo/Bosnia And Herzegovina

\section{Contact E-mail Address: sarayaida19@gmail.com}

Introduction: Multiple recent studies have indicated that some of adipose tissuederived hormones may significantly influence the growth and proliferation of GI tumors including liver cancer $(1,2)$. However, the role of adipokines such as adiponectin and leptin in biliary tract cancer have not been well studied before. The aim of the study was to analyze plasma concentrations of adiponectin and leptin in cholangiocarcinoma (CC) patients and to compare these concentrations to clinicopathological parameters.

Aims \& Methods: Baseline levels of adiponectin and leptin were determined in 38 consecutive patients with newly diagnosed cholangiocarcinoma and 38 healthy control subjects. The association between adiponectin and leptin and tumor stage was evaluated using nonparametric Spearman's correlation test. Control subjects were matched to case patients by age, sex and BMI. Survival analysis used the Kaplan-Meier curve and the Cox proportional hazards model.

Results: Overall median adiponectin concentrations were lower in $\mathrm{CC}$ patients versus control subjects $(5.1 \mathrm{vs} 9.3 \mathrm{mg} / \mathrm{mL}, P=0.001)$. In CC patients with T stage 2-4 $(\mathrm{n}=22)$ median adiponectin concentrations were significantly lower than in CC patients with T stage $1(\mathrm{n}=16)(3.8$ vs $6.6 \mathrm{mg} / \mathrm{mL}, P=0.001)$. The mean leptin levels were not significantly decreased in CC patients $(P=0.45)$. Adiponectin concentrations were inversely correlated with tumor $\mathrm{T}$ stage $(\mathrm{r}=-0.811, P=0.01)$ of $\mathrm{CC}$ patients. Higher adiponectin levels at baseline were associated with increased overall survival in $\mathrm{T}$ stage $2-4$ patients (Cox $\mathrm{F}$ test $=2.139, \mathrm{P}<0.05$ ).

Conclusion: This study identified an association between adiponectin levels and tumor stage suggesting a potential role for adiponectin in progression of cholangiocarcinoma. Furthermore these results suggest, for the first time, that serum adiponectin levels might represent a prognostic indicator in patients with $\mathrm{CC}$. Our results support the hypothesis linking adipose-tissue derived hormons levels to growth of obesity-associated cancers (3). Adipokines appear to play an important role in risk prediction and management of cholangiocarcinoma patients.

Disclosure of Interest: All authors have declared no conflicts of interest.

\section{References}

1. Hillenbrand A, Fassler J, Huber N, et al. Changed adipocytokine concentrations in colorectal tumor patients and morbidly obese patients compared to healthy controls. BMC Cancer 2012, 12:545. doi:10.1186/1471-2407-12-545

2. Aleksandrova $\mathrm{K}$, Boeing $\mathrm{H}$, Nöthlings $\mathrm{U}$, et al Inflammatory and metabolic biomarkers and risk of liver and biliary tract cancer. Hepatology (Baltimore, Md). 2014;60(3):858-871. doi:10.1002/hep.27016.

3. Sun Y, Lodish HF. Adiponectin deficiency promotes tumor growth in mice by reducing macrophage infiltration. PLoS One. 2010;5(8):e11987

P0742

Table 1: Results of meta-analysis and post-test probabilities

\begin{tabular}{|c|c|c|c|c|c|c|c|}
\hline Index Test & Target condition & $\begin{array}{l}\text { Number of } \\
\text { studies (patients) }\end{array}$ & $\begin{array}{l}\text { Summary } \\
\text { sensitivity } \\
(95 \% \mathrm{CI})\end{array}$ & $\begin{array}{l}\text { Summary } \\
\text { specificity } \\
(95 \% \mathrm{CI})\end{array}$ & $\begin{array}{l}\text { Mininum, median } \\
\text { and maximum } \\
\text { prevalence of target } \\
\text { condition = pre-test } \\
\text { probability }\end{array}$ & $\begin{array}{l}\text { Positive post-test } \\
\text { probability }(95 \% \mathrm{CI})\end{array}$ & $\begin{array}{l}\text { Negative post-test } \\
\text { probability }(95 \% \mathrm{CI})\end{array}$ \\
\hline TAUS & Gallbladder polyp & $\begin{array}{l}6 \text { studies }(16260 \\
\text { patients) }\end{array}$ & $0.80(0.55-0.98)$ & $0.97(0.95-0.98)$ & $\begin{array}{l}\text { Minimum: } 0.4 \% \\
\text { Median: } 6.4 \% \\
\text { Maximum: } 53.3 \%\end{array}$ & $\begin{array}{l}0.10(0.07-0.11) \\
0.65(0.55-0.69) \\
0.97(0.95-0.97)\end{array}$ & $\begin{array}{l}0.00(0.00-0.00) \\
0.01(0.01-0.04) \\
0.19(0.08-0.38)\end{array}$ \\
\hline TAUS & True gallbladder polyp & $\begin{array}{l}7 \text { studies ( } 1272 \\
\text { patients) }\end{array}$ & $0.77(0.48-0.92)$ & $0.78(0.59-0.90)$ & $\begin{array}{l}\text { Minimum: } 9.1 \% \\
\text { Median: } 20.2 \% \\
\text { Maximum: } 60.0 \%\end{array}$ & $\begin{array}{l}0.26(0.16-0.39) \\
0.47(0.32-0.62) \\
0.84(0.74-0.91)\end{array}$ & $\begin{array}{l}0.03(0.01-0.07) \\
0.07(0.03-0.16) \\
0.31(0.15-0.53)\end{array}$ \\
\hline EUS & True gallbladder polyp & $\begin{array}{c}4 \text { studies }(267 \\
\text { patients) }\end{array}$ & $0.84(0.54-0.96)$ & $0.84(0.70-0.92)$ & $\begin{array}{l}\text { Minimum: } 9.1 \% \\
\text { Median: } 20.2 \% \\
\text { Maximum: } 60.0 \%\end{array}$ & $\begin{array}{l}0.35(0.20-0.53) \\
0.57(0.39-0.74) \\
0.88(0.79-0.95)\end{array}$ & $\begin{array}{l}0.02(0.01-0.07) \\
0.05(0.01-0.15) \\
0.22(0.07-0.51)\end{array}$ \\
\hline TAUS & $\begin{array}{l}\text { Dysplastic polyp/ } \\
\text { carcinoma }\end{array}$ & $\begin{array}{l}4 \text { studies ( } 1637 \\
\text { patients) }\end{array}$ & $0.60(0.22-0.89)$ & $0.89(0.76-0.96)$ & $\begin{array}{l}\text { Minimum: } 4.1 \% \\
\text { Median: } 20.1 \% \\
\text { Maximum: } 95.6 \%\end{array}$ & $\begin{array}{l}0.19(0.07-0.46) \\
0.59(0.30-0.83) \\
0.99(0.97-1.00)\end{array}$ & $\begin{array}{l}0.02(0.01-0.05) \\
0.10(0.04-0.24) \\
0.91(0.78-0.96)\end{array}$ \\
\hline EUS & $\begin{array}{l}\text { Dysplastic polyp/ } \\
\text { carcinoma }\end{array}$ & $\begin{array}{c}3 \text { studies ( } 350 \\
\text { patients) }\end{array}$ & $0.85(0.56-0.96)$ & $0.91(0.75-0.97)$ & $\begin{array}{l}\text { Minimum: } 4.1 \% \\
\text { Median: } 20.1 \% \\
\text { Maximum: } 95.6 \%\end{array}$ & $\begin{array}{l}0.28(0.12-0.54) \\
0.70(0.44-0.87) \\
0.99(0.99-1.00)\end{array}$ & $\begin{array}{l}0.01(0.00-0.02) \\
0.04(0.01-0.13) \\
0.78(0.51-0.92)\end{array}$ \\
\hline
\end{tabular}


P0745 PRETREATMENT BODY MASS INDEX AND WEIGHT CHANGE DURING INITIAL PERIOD OF CHEMOTHERAPY AFFECT SURVIVAL OUTCOME IN ADVANCED BILIARY TRACT CANCER PATIENTS

J. Kang, S.H. Lee, Y.H. Choi, J.H. Choi, J.K. Ryu, Y. Kim

Seoul National University College Of Medicine, Department of Internal Medicine and Liver Research Institute, Seoul/Korea, Republic of

\section{Contact E-mail Address: schmeiche180@ gmail.com}

Introduction: Recently many studies have been conducted to investigate the association between obesity and survival in cancer patients. Cancer has a significant influence on the nutrient status of patients and obesity can affect on the pharmacokinetics of anti-cancer drugs. The impact of obesity on survival is known to vary in different cancers. Biliary tract cancer was less frequently analyzed and most of the studies were on the relationship between obesity and cancer incidence.

Aims \& Methods: We performed this study to investigate the association between BMI and overall survival in advanced biliary tract cancer patients with chemotherapy. Between January 2005 and December 2015, two hundred and eighty-four patients who underwent chemotherapy for biliary tract cancer were retrospectively reviewed. The relationship between BMI $\left(\mathrm{kg} / \mathrm{m}^{2}\right)$ and overall survival (OS) was assessed. Based on World Health Organization BMI category and 2014 Clinical Practice Guidelines for Overweight and Obesity in Korea, BMI was classified as follows; underweight, $<18.5 \mathrm{~kg} / \mathrm{m}^{2}$; normal, $18.5-22.9 \mathrm{~kg} / \mathrm{m}^{2}$; overweight, $23-24.9 \mathrm{~kg} / \mathrm{m}^{2}$; obese, $\geqq 25 \mathrm{~kg} / \mathrm{m}^{2}$.

Results: Median OS was 12.1 months for underweight patients, 10.5 months for normal patients, 16.1 months for overweight group, 13.6 months for obese patients, respectively. $(p=0.047)$ Univariate analysis showed that BMI, local status of disease, operation, radiotherapy and ECOG performance were significantly associated with better survival. Compared with normal patients, overweight patients (BMI 23-24.9 $\mathrm{kg} / \mathrm{m}^{2}$ ) had a reduced risk of mortality in multivariate analysis (HR 0.491 , CI $0.334-0.721 ; 95 \% \mathrm{p}=0.036$ ). In the additional analysis for the effect of change in body weight and BMI to the overall survival, larger amount of change in body weight was associated with further decrease in overall survival.

Conclusion: Slightly overweight status and the maintenance of body weight during the initial period of chemotherapy is independent predictor of better overall survival in advanced biliary tract cancer patients with good performance status.

Disclosure of Interest: All authors have declared no conflicts of interest.

\section{P0746 THROUGH THE CATHETER BIOPSY METHOD FOR \\ BILIARY CARCINOMA}

\section{T. Okuzono}

Department Of Gastroenterology, Sendai Kousei hospital, Sendai/Japan

\section{Contact E-mail Address: okuzonotoru@gmail.com}

Introduction: To perform curative operation of biliary carcinoma, the pre-operative identification of exact proximal and distal margins is important. A biopsy forceps is conventionally inserted to common bile duct via duodenum ampulla guided with an antecedent guide wire. Cannulation of the bile duct with the biopsy forceps may sometimes be difficult in cases where no sphincterotomy is performed, placing the patient at risk of post-ERCP pancreatitis after multiple attempts to advance the forceps into the duct. Pancreatobiliary endoscopists have reported the biopsy methods.

Aims \& Methods: The aim of this study was to assess the feasibility and safety of the biopsy methods. This was a retrospective review of bile duct biopsies with this new method conducted in Sendai Kousei hospital from February 2015 to October 2016. All patients who had biliary stenosis were included. Patients' demographic data, technical success, adverse events and the diagnostic accuracy were evaluated.

Results: A total of 95 biopsy procedures were performed in 40 patients. The technical success rate was $95 \%(90 / 95)$. Post-ERCP pancreatitis occurred in 1 of 40 patients $(2.5 \%, 1$ grade 1 patient). There were no other adverse events like perforation or bleeding. The diagnostic yield of mapping biopsy procedures was $100 \%$ ( 7 of 7 patients).

Conclusion: The new biopsy methods to biliary stricture were feasible and safe. It opens up exciting possibilities for endoscopic preoperative diagnosis of the biliary carcinoma.

Disclosure of Interest: All authors have declared no conflicts of interest.

\section{Reference}

Hijioka S, Hara K, Mizuno N, Imaoka H, Mekky MA, Nagashio Y, et al. A novel technique for endoscopic transpapillary "mapping biopsy specimens" of superficial intraductal spread of bile duct carcinoma (with videos). Gastrointestinal endoscopy. 2014;79(6):1020-5.

\section{P0747 THE DEVELOPMENT OF A RISK SCORE TO PREDICT ADVERSE OUTCOMES OF EXPLORATORY SURGERY IN PERIHILAR CHOLANGIOCARCINOMA}

M. Gaspersz ${ }^{1}$, S. Buettner ${ }^{1}$, J. L.a. Van Vugt ${ }^{1}$, R.J. S. Coelen ${ }^{2}$, J. Vugts ${ }^{1}$, E. Belt ${ }^{3}$, J. De Jonge ${ }^{1}$, W. Polak ${ }^{1}$, F. E.j.a. Willemssen ${ }^{4}$, T. M. Van Gulik ${ }^{5}$, J.

N.m. Ijzermans ${ }^{1}$, B. Groot Koerkamp ${ }^{1}$
${ }^{1}$ Surgery, Erasmus University Medical Center, Rotterdam/Netherlands

${ }^{2}$ Surgery, Academic Medical Center, Amsterdam/Netherlands

${ }^{3}$ Surgery, Albert Schweitzer ziekenhuis, Dordrecht/Netherlands

${ }^{4}$ Radiology, Erasmus University Medical Center, Rotterdam/Netherlands

${ }^{5}$ Surgery, Academisch Medisch Centrum Dept. of Surgery, Amsterdam

Netherlands

Contact E-mail Address: m.gaspersz@erasmusmc.nl

Introduction: $\mathrm{Up}$ to half of all patients with resectable $\mathrm{PHC}$ on imaging are found unresectable during exploratory surgery. Moreover, mortality is high in patients who do undergo resection for PHC.

Aims \& Methods: The aim of this study was to identify patients at high risk for adverse outcomes of exploratory surgery and developing a preoperative risk score model to predict adverse outcomes in perihilar cholangiocarcinoma (PHC) patients undergoing exploratory surgery. Consecutive PHC patients who underwent exploratory laparotomy in two tertiary referral centers between 2002 and 2014 were identified. Baseline patient and tumor characteristics were collected from medical records. Adverse postoperative outcome was defined as unresectable disease at exploratory laparotomy, 90-day surgical mortality, or an R1 resection. A multivariable Cox proportional hazards analysis was performed to identify preoperatively available poor prognostic factors for an adverse outcome.

Results: In total, 331 patients underwent exploratory laparotomy with a median OS $(95 \%$ confidence interval) of 20.7 (17.8-23.6) months. Adverse outcomes were found in 229 patients $(69.2 \%)$ : 145 patients $(43.8 \%)$ had preoperative adverse outcomes of the exploratory surgery, $53(16 \%)$ patients had postoperative adverse outcomes and $31(9.4 \%)$ patients experienced both peri- and postoperative adverse outcomes. Median OS for patients with an adverse outcome was 13.1 months $(11.3-15.0)$ compared to 50.9 months $(39.6-62.2)$ in patients without adverse outcomes $(\mathrm{p}<0.001)$. Cholangitis at or before first presentation (hazard ratio (HR) 2.38, 95\% CI 1.16-4.99, $\mathrm{p}=0.0422$ ), CA 19-9 above $1000 \mathrm{U}$ $\mathrm{mL}$ (HR 3.26, 95\% CI 1.14-11.8, p=0.0195), and tumor involvement of the hepatic artery on imaging (HR 2.07, 95\% CI $1.01-4.33, \mathrm{p}=0.0492)$ were independent prognostic factors for an adverse outcome at exploratory laparotomy. A risk score based on these three factors identified patients with a substantial $(49 \%)$, high $(66 \%)$, and very high $(87 \%)$ risk of adverse outcome.

Table 1: Calculation of prognostic score

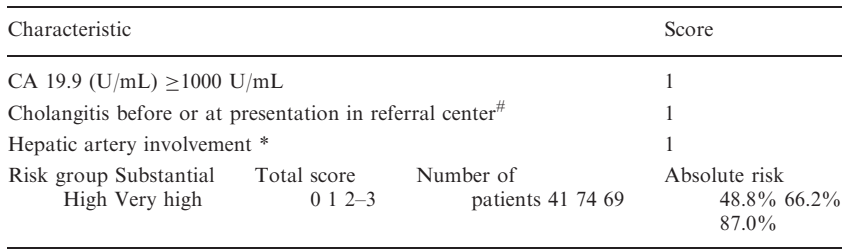

Conclusion: PHC patients undergoing exploratory laparotomy have a high risk of an adverse outcome. A preoperative risk score for adverse outcome may help clinicians to inform patients.

Disclosure of Interest: All authors have declared no conflicts of interest.

\section{P0748 A NEW PROGNOSTIC MODEL FOR PATIENTS WITH} PERIHILAR CHOLANGIOCARCINOMA

J. L.a. Van Vugt ${ }^{1}$, M. Gaspersz ${ }^{1}$, R.J. S. Coelen ${ }^{2}$, E. Roos ${ }^{2}$, J. De Jonge ${ }^{1}$, W. Polak ${ }^{1}$, O.R.c. Busch ${ }^{2}$, M.G.h. Besselink ${ }^{2}$, C.Y. Nio ${ }^{3}$, F. E.j.a. Willemssen ${ }^{4}$, J. N.m. Ijzermans ${ }^{1}$, D. Van Klaveren ${ }^{5}$, T. M. Van Gulik ${ }^{2}$, B. Groot Koerkamp ${ }^{1}$ ${ }^{1}$ Surgery, Erasmus University Medical Center, Rotterdam/Netherlands ${ }^{2}$ Surgery, Academic Medical Center, Amsterdam/Netherlands

${ }^{3}$ Academisch Medisch Centrum Dept. of Radiology, Amsterdam/Netherlands

${ }^{4}$ Radiology, Erasmus University Medical Cente, Rotterdam/Netherlands

${ }^{5}$ Department Of Public Health, Erasmus MC University Medical Center Rotterdam, Rotterdam/Netherlands

Contact E-mail Address: m.gaspersz@erasmusmc.nl

Introduction: Patients with perihilar cholangiocarcinoma (PHC) have few treatment options and a poor prognosis. Most staging models for patients with PHC have been developed for the minority of patients with potentially resectable disease and are not applicable to the vast majority of patients.

Aims \& Methods: The aim of this study was to develop a prognostic score for all PHC patients using variables available at presentation. All consecutive patients with PHC (regardless of tumor stage and treatment) in two tertiary referral centers between 2002 and 2014 were identified and included. Baseline patient and tumor characteristics were collected from medical records. Cox proportional hazards regression was used for multivariable analysis. Age, BMI, bilirubin, CA 19-9, and tumor size were modeled as continuous covariates.

Results: A total of 674 patients were included of whom $342(50.8 \%)$ had unresectable disease at presentation and $176(26.2 \%)$ underwent exploratory laparotomy. Multivariable analysis identified age (HR 1.41 (95\% CI 1.23-1.63)), BMI (HR 1.11 (95\% CI 1.05-1.17), serum bilirubin level (HR 1.45 (95\% CI 1.21 1.71), CA 19.9 serum level (HR 1.22 (95\% CI 1.07-1.38), tumor size (HR 1.33 (95\% CI 1.14-1.56), WHO performance status 3-4 (HR 1.48 (95\% CI 1.10-1.95), suspected distant metastases on imaging (HR 1.69 (95\% CI 1.29-2.20), unilateral HA involvement: HR 1.28 (95\% CI 1.05-1.57), and main/bilateral HA involvement: (HR 1.61 (95\% CI 1.21-2.14)) as independent prognostic parameters. Based on these factors, a prognostic score was created to predict survival for patients with PHC from the time of presentation. Discrimination using Kaplan- 
Meier curves, and calibration curves revealed good predictive abilities. The risk scored identified patients with a 1-year survival probability ranging from 15 $73 \%$.

Conclusion: We developed a prognostic score to predict overall survival for PHC patients using eight independent poor prognostic factors available at presentation. This score may help to inform patients and guide individualized treatment decision making.

Disclosure of Interest: All authors have declared no conflicts of interest.

\section{P0749 THE OUTCOMES OF ERCP FOR THE PALLIATION OF} MALIGNANT JAUNDICE IN ENGLAND BETWEEN 2001 AND 2015

P. Harvey ${ }^{1}$, S. Baldwin ${ }^{2}$, J. Mytton ${ }^{2}$, F. Evison ${ }^{2}$, P. Patel ${ }^{2}$, N. Trudgill ${ }^{1}$ ${ }_{1}^{1}$ Gastroenterology, Sandwell and West Birmingham NHS Trust, Birmingham United Kingdom

${ }^{2}$ Health Informatics, University Hospital Birmingham, Birmingham/United

Kingdom

Contact E-mail Address: Philipharvey@nhs.net

Introduction: Malignant biliary obstruction has a poor prognosis unless secondary to a resectable primary cancer. Recent data on PTC for the relief of malignant obstruction in a palliative setting demonstrated a high early mortality. We have therefore examined outcomes of ERCP in inoperable malignant obstruction.

Aims \& Methods: The Hospital Episode Statistics (HES) database contains diagnostic and procedural data for all hospital attendances in England. HES is linked to the Office for National Statistics (ONS) to provide mortality data. All subjects from April 2001 to April 2015 in England with an ICD10 code for cancer 2 years prior to ERCP or in the following 6 months were examined. Subjects undergoing a curative surgical procedure were excluded. Associations between demographics, co-morbidities, unit ERCP volume and mortality were examined by logistic regression.

Results: 49055 subjects were included in the study of whom $48.7 \%$ were male, median age 74.5 years (range 19-104). Pancreatic cancer was the most common aetiology $(63.5 \%)$ followed by liver and intrahepatic bile duct malignancy $(19.4 \%)$. Mortality was $4.16 \%, 10.9 \%$ and $19.6 \%$ for 7 day, in hospital and 30 day respectively. In multivariate analysis male gender (OR $1.14,(95 \%$ CI 1.08 $1.20) \mathrm{p}<0.001)$; increasing by age quintile $64-71(1.34,(1.23-1.47) \mathrm{p}<0.001)$, 72-77 (1.57, (1.44-1.72) p <0.001), 78-83 (1.83, (1.68-2.00) p < 0.001), >83 $(2.78, \quad(2.55-3.03), \mathrm{p}<0.001)$; most deprived quintile $(1.22, \quad(1.12-1.33)$, $\mathrm{p}<0.001)$; increasing co-morbidity score 1 to $5(1.09,(1.02-1.16), \mathrm{p}=0.012), 6$ to $10(1.23,(1.12-1.35), \mathrm{p}<0.001) 11$ to $15(1.49,(1.33-1.66), \mathrm{p}<0.001), 16$ to $20(1.97,(1.70-2.28), \mathrm{p}<0.001),>20(2.79(2.39-3.25), \mathrm{p}<0.001)$; advancing year of ERCP $2013 / 14(0.78,(0.68-0.90), p=0.001), 2014 / 15(0.85,(0.74-0.98)$ $\mathrm{p}=0.028)$; and previous renal failure $(1.92,(1.77-2.09), \mathrm{p}<0.001)$ were associated with increasing 30 day mortality. Asian ethnicity $(0.82$, (0.67-0.99), $\mathrm{p}=0.036)$, Cancer of extrahepatic and unspecified parts of biliary tree $(0.60$, $(0.55-0.65), \mathrm{p}<0.001)$ and upper tertile of unit ERCP activity $(>230)$ per annum $(0.86,(0.80-0.93), \mathrm{p}<0.001)$ were negatively associated with 30 day mortality.

Conclusion: Short-term mortality in subjects with malignant biliary obstruction following ERCP was high. A better prognosis was observed in; high-volume ERCP units, Asian ethnicity and extrahepatic primary cancers. Male gender, advancing age, increasing co-morbidity score, greater deprivation and previous renal failure predicted death at 30 days.

Disclosure of Interest: All authors have declared no conflicts of interest.

TUESDAY, OCTOBER 31, 2017

PAEDIATRIC: LIVER, BILIARY AND PANCREAS - HALL 7

P0750 EARLY DEVELOPMENT OF NONALCOHOLIC FATTY LIVER DISEASE IN GENETICALLY PREDISPOSED CHILDREN WITH OVERWEIGHT AND OBESITY DOES NOT COINCIDE WITH METABOLIC DERANGEMENTS

\section{K. Karnebeek ${ }^{1}$, J. Plat ${ }^{2}$, A. Vreugdenhil ${ }^{1}$}

${ }^{1}$ Department Of Pediatrics, Maastricht University Medical Centre, Maastricht/ Netherlands

${ }^{2}$ Department Of Human Biology, School Of Nutrition And Translational Research In Metabolism (nutrim), Maastricht University, Maastricht/Netherlands

\section{Contact E-mail Address: kylie.karnebeek@mumc.nl}

Introduction: Non-alcoholic fatty liver disease (NAFLD) is a common chronic liver disease and in particular a health threat in obese children. Single nucleotide polymorphisms in genes encoding PNPLA3 (rs738409) and TM6SF2 (rs58542926) contribute to the development of NAFLD. It is however unknown whether liver pathology and cardiometabolic disturbances coincide in carriers and non-carriers of these risk alleles in an at-risk obese pediatric population. Therefore, we assessed cardiometabolic derangements, genetic predisposition for NAFLD and liver transaminase levels in children with overweight and obesity

Aims \& Methods: One hundred and seventy-four children ( $49 \%$ boys) from the Centre for Overweight Adolescent and Children's Healthcare $(\mathrm{COACH})$ at the Maastricht University Medical Centre were genotyped for PNPLA3 I148M and TM6SF2 E167K. Anthropometric, cardiometabolic risk and liver-related parameters were determined.

Results: Anthropometric parameters did not differ significantly between carriers and non-carriers of the risk alleles. ALT and AST were significantly higher in
PNPLA3 G allele carriers as compared to the C allele carriers (ALT; CC 21, 00 $(17,00 ; 28,00) ;$ CG 26, $50(18,00 ; 34,50) ;$ GG 29, $00(23,00 ; 51,00)(\mathrm{p}=0.004)$ and AST; CC 24, 00 (19, 00;30, 00); CG 27, 50 (21, 00;40, 00); GG 30, $00(26,00 ; 36$, $00)(\mathrm{p}=0.004))$. The odds ratio for having ALT levels above the cut-off values increased for every PNPLA3 G allele, with an OR of $2,51(1,22 ; 5,18 ; p=0,013)$ for the PNPLA3 CG genotype and $5,54(1,53 ; 20,02 ; p=0,009)$ for the GG genotype, compared to the CC genotype. Carriers of the PNPLA3 risk allele did not show a deteriorated metabolic profile compared to non-carriers.

The TM6SF2 T allele carriers also showed a tendency towards increased transaminase levels, but a significantly healthier cardiometabolic profile, i.e. lower total cholesterol $(p=0.024)$, LDL cholesterol $(p=0.015)$ and triglyceride concentrations $(p=0.003)$ and a higher central retinal artery equivalent $(p=0.020)$ compared to the major allele carriers. Carrying the TM6SF2 $\mathrm{T}$ allele in addition to carrying the PNPLA3 'risk' allele, did not significantly increase the odds ratios for increased ALT concentrations. The prevalence of the metabolic syndrome did not differ between the different PNPLA3 genotype carriers nor between the TM6SF2 genotype carriers.

Conclusion: Despite significantly higher liver transaminase levels and a positive correlation between ALT levels and triglyceride and fasting insulin concentrations in PNPLA3 G allele carriers, these children did not have a more deteriorated cardiometabolic profile compared to non-carriers. The metabolic syndrome was not more prevalent in risk allele carriers. These results suggest that hepatic aberrations and metabolic disturbances apparently do not develop concordantly in this specific population. Furthermore, these children with a high liver health risk may not be identified by measuring cardiometabolic parameters.

Disclosure of Interest: All authors have declared no conflicts of interest.

\section{P0751 TWO-DIMENSIONAL SHEAR WAVE ELASTOGRAPHY IN} CHILDREN: WHAT IS THE NUMBER OF LIVER STIFFNESS MEASUREMENTS NEEDED FOR A HIGH QUALITY EVALUATION?

C. Pienar ${ }^{1}$, P. Velea ${ }^{1}$, D. Gherhardt ${ }^{2}$, I. Ciuca ${ }^{1}$, C. Paul ${ }^{1}$, O. Belei ${ }^{1}$,

S.A. Popescu ${ }^{2}$, I. Sporea ${ }^{2}$

${ }^{1}$ Pediatrics Department, "Victor Babes" University of Medicine and Pharmacy, Timisoara/Romania

${ }^{2}$ Gastroenterology And Hepatology, "Victor Babes" University of Medicine, Timisoara/Romania

Contact E-mail Address: cpienar@gmail.com

Introduction: Pediatric chronic liver diseases are becoming a public health issue. Ultrasound based elastographic techniques have emerged as non-invasive methods of pediatric liver fibrosis assessment. The most recent are two dimensional shear-wave elastographic (2D SWE) techniques. While they are proved to be highly reproducible in children, there is still no consensus regarding the number of measurements to be performed for a high-quality evaluation.

Aims \& Methods: We aimed to investigate the number of liver stiffness measurements (LSM) needed for a high-quality evaluation using a 2D SWE technique. We conducted a prospective study which included 73 children (age range: $3-17$ years, mean age $11.73 \pm 3.55$ years, $37 \%$ girls, mean body mass index (BMI) $25.12 \pm 7.38 \mathrm{~kg} / \mathrm{m} 2$ ). We used the 2D-SWE.GE (Logiq E9, GE Healthcare, Chalfont St Giles- UK), with a C1-6-D probe. One examiner performed 10 LSM for each child. We randomly extracted 1 LSM, 2 LSM, 3 LSM and 5 LSM from all 10 and calculated their respective medians. We employed the Friedman test to compare the medians of 1,2, 3, 5 and 10 LSMs. We used the interclass correlation coefficient (ICC) to assess the agreement between the medians of $1,2,3,5$ and 10 LSMs.

Results: Medians calculated from 1, 2, 3, 5 and 10 LSMs were similar $(4.21 \pm 1.05 \mathrm{kPa} \quad$ vs $4.22 \pm 0.91 \mathrm{kPa}, \quad 4.25 \pm 1.03 \quad$ vs $4.2 \pm 0.99 \mathrm{kPa}$ vs $4.19 \pm 0.99 \mathrm{kPa}, \mathrm{p}=0.94)$. Furthermore, the agreement between medians calculated from $1,2,3,5$ and $10 \mathrm{LSMs}$ was excellent $(\mathrm{ICC}=0.960,95 \%$ confidence interval: $0.944-0.974)$.

Conclusion: We suggest obtaining 5 LSM for a high-quality evaluation using this 2D SWE technique.

Disclosure of Interest: S.A. Popescu: I hereby confirm that I have received financial support (congress travel grants, speaker fee) from: Philips, General Electric, Abbvie, AstraZeneca, Zentiva

All other authors have declared no conflicts of interest.

I. Sporea: I hereby confirm that I have received financial support (congress travel grant or speaker fee) from Philips, Siemens, General Electric, Abbvie, Zentiva, Bristol Meyers Squibb

TUESDAY, OCTOBER 31, 2017

PANCREAS II - HALL 7

09:00-17:00

\section{P0752 PERCUTANEOUS EMBOLIZATION OF VISCERAL ARTERY} PSEUDO-ANEURYSMS - A TERTIARY CENTER EXPERIENCE

R. Chavan ${ }^{1}$, J. Singh ${ }^{1}$, Z. Nabi ${ }^{1}$, R. Kalapala ${ }^{1}$, D.N. Reddy ${ }^{1}$

${ }^{1}$ Gastroenterology, Asian Institute of gastroenterology, Hyderabad, Hyderabad/ India

Contact E-mail Address: drradhikachavan@gmail.com

Introduction: Visceral artery pseudo-aneurysms are rare, but potentially fatal if rupture. Pseudoaneurysm usually occurs most frequently after pancreatitis. Angioembolization with conventional trans-catheter approach is the standard treatment. Direct percutaneous embolization has been commonly used for treatment of peripheral artery pseudoaneurysm when trans-catheter approach is not feasible. However, very limited data is available regarding its safety and efficacy in visceral artery pseudoaneurysm. 
Aims \& Methods: We aimed to assess the technical feasibility, safety and effectiveness of direct percutaneous embolization as an alternative treatment option for visceral pseudo aneurysms. We retrospectively evaluated the data of patients who underwent percutaneous embolization at our institution from Feb 2007 to March 2017. All procedures were performed under ultrasound (US) guidance under local anaesthesia. Technical feasibility, safety and efficacy of percutaneous embolization were analysed. At 30 days follow up US with color Doppler/dual phase computed tomography was done to see for recurrence of pseudoaneurysm.

Results: 23 patients(18-male) with mean age of $34.47 \pm 21.28$ (7-72) years, underwent direct percutaneous embolization for visceral pseudoaneurysm. Most common aetiology for pseudoaneurysm was pancreatitis (16) followed by trauma (3), paracentesis (3) and surgery (1). The site of pseudoaneurysm wassplenic artery (13), left gastric artery (3), hepatic artery (3), inferior epigastric artery (3) and gastroduodenal artery (1). Mean size of pseudoaneurysm was $1.8 \pm 0.6(1-3.5) \mathrm{cm}$. Reasons for choosing percutaneous approach over transcatheter embolization included- technical difficulties in 11 patients, excess collaterals obstructing view of feeding artery in 5 patients, and recurrence after previous embolization in 6 patients. Agents used for embolization- glue with lipiodo (21), coil (1) and coil with glue (1). Mean procedural time was $11.3 \pm 2.11(8-16)$ minutes and fluoroscopy exposure time was $2.4 \pm 1.34$ (1-6) minutes. Percutaneous embolization was successfully performed in all patients (technical success-100\%). Mild adverse events included - local site pain in $19(80 \%)$ patients. Moderate adverse event included - splenic infarct in 5 patients, all of which responded to conservative management. There were no major adverse events and no occurrence of distant embolization. At median follow up of 910 days (30-3186) there was no recurrence of pseudoaneurysm (clinical success$100 \%$ ).

Conclusion: Percutaneous embolization is safe and effective for treatment of visceral artery pseudoaneurysm. Percutaneous technique may be considered as an alternative to trans-catheter embolization in cases of challenging anatomy, multiple collaterals and recurrence after previous embolization precluding transcatheter advancement.

Disclosure of Interest: All authors have declared no conflicts of interest.

\section{P0753 DEVELOPMENT OF AUTOIMMUNE PANCREATITIS IS INDEPENDENT OF P21 MEDIATED PANCREATIC INFLAMMATION}

G.M. Seleznik ${ }^{1}$, T. Reding ${ }^{1}$, L. Peter ${ }^{1}$, A. Gupta ${ }^{1}$, S. Sonda ${ }^{1}$, C. Verbeke ${ }^{2}$, E. Dejardin ${ }^{3}$, M. Heikenwälder ${ }^{4}$, R. Graf ${ }^{1}$

${ }^{1}$ Visceral \& Transplantation Surgery, University Hospital Zurich, Swiss HPB Centre, Zurich/Switzerland

${ }^{2}$ Department Of Pathology, University of Oslo, Oslo/Norway

${ }^{3}$ Laboratory Of Molecular Immunology And Signal Transduction, University of

Liège, Liege/Belgium

${ }^{4}$ Institute Of Virology, Technische Universität München, Helmholtz Center

Munich, Munich/Germany

Contact E-mail Address: anurag.gupta $@$ usz.ch

Introduction: Chronic (CP) and autoimmune pancreatitis (AIP) are characterized by different inflammatory processes. Whether CP is a prerequisite for autoimmunity is still unclear. AIP is considered mostly a T-cell mediated disease; however, in induction of chronic pancreatitis macrophages play a pivotal role. Cyclin dependent kinase (cdk) inhibitors are critical regulators in inflammatory disease as they can influence proliferation, activation and differentiation of inflammatory cells, and apoptosis. In particular, p21 has been described as a mediator of inflammation and various autoimmune diseases by regulating $\mathrm{T}$-cell activation and promoting macrophage development. We therefore examined the role of p21-mediated inflammation in AIP.

Aims \& Methods: Human pancreas samples from CP and AIP patients were evaluated for p21 expression. To investigate the effects of p21 in pancreatitis, we intercrossed lymphotoxin overexpressing mice $(\operatorname{Tg}($ Elal-LTa, $b))$ - a model to study CP and AIP - with p21 deficient ( $221-/-)$ mice. Infiltrating cells were visualized by immunohistochemistry, supported by gene expression analysis in an early and a progressive phase. Circulating autoantibodies and the presence of tertiary lymphoid organs (TLOs) were analysed to assess autoimmunity.

Results: p21 was upregulated in human $\mathrm{CP}$ patients but remained unchanged in AIP patients. p21 deficiency in LT mice $(L T p 21-/-)$ prevented early pancreatic injury. LTp21-/- mice had normal serum amylase, reduced inflammatory gene expression and cell influx. In acinar cells diminished proliferation and abrogated activation of non-canonical NF-kB pathway was observed. In contrast, 12 months old LT mice with and without p21 had similar inflammatory gene expression and T \& B cell infiltration. Interestingly, LT and LTp21-/- mice had comparable tertiary lymphoid organs (TLOs), autoantibodies and elevated $\mathrm{IgG}$ levels. However, acinar cell proliferation, acinar-to-ductal metaplasia and acinar non-canonical NF-kB pathway activation remained impaired in LTp21-/- pancreata.

Conclusion: Our findings indicate that $\mathrm{p} 21$ is crucial for pancreatitis in LT-driven pancreatic injury. p21 is involved in early acinar secretion of inflammatory mediators that attract innate immune cells. However, p21 is not essential for humoral immune response, accountable for autoimmunity and lack of p21 does not rescue AIP development. Remarkably, p21 interferes with acinar NF- $\kappa$ B signaling and renders acinar cells less susceptible to proliferation and transdifferentiation. We therefore suggest that chronic and autoimmune pancreatitis follow different inflammatory processes.

Disclosure of Interest: All authors have declared no conflicts of interest.

\section{P0754 GRANULOCYTES DRIVE PANCREATITIS IN A NOVEL MODEL OF INTERLEUKIN-17A-INDUCED PANCREATITIS VIA PEPTIDYL ARGININE DEIMINASE-DEPENDENT EXTRACELLULAR TRAP FORMATION}

M. Leppkes ${ }^{1}$, C. Maueröder ${ }^{2}$, S. Hirth ${ }^{3}$, C. Günther ${ }^{1}$, S. Nowecki ${ }^{1}$, U. Billmeier ${ }^{1}$, S. Paulus ${ }^{1}$, M. Biermann ${ }^{2}$, L. E. Munoz ${ }^{2}$, M. Hoffmann ${ }^{2}$, D. Wildner ${ }^{1}$, A. L. Croxford ${ }^{3}$, A. Waisman ${ }^{3}$, D. E. Jenne ${ }^{4}$, J. Mayerle ${ }^{5}$, M.M. Lerch ${ }^{6}$, G. Schett ${ }^{2}$, S. Wirtz ${ }^{1}$, M.F. Neurath ${ }^{1}$, M. Herrmann ${ }^{2}$, C. Becker ${ }^{1}$

${ }^{1}$ Medizin 1, Universitätskliniken Erlangen, Erlangen/Germany

${ }^{2}$ Medizin 3, Universitätskliniken Erlangen, Erlangen/Germany

${ }^{3}$ Institut Für Molekulare Medizin, Universitätsmedizin Mainz, Mainz/Germany

${ }^{4}$ Helmholtz Zentrum, Comprehensive Pneumology Center, Munich/Germany

${ }^{5}$ Medizinische Klinik Und Poliklinik Ii, Klinikum der LMU München-Grosshadern, München/Germany

${ }^{6}$ Medicine A, University Medicine Greifswald - Medicine A, University Medicine Greifswald; Greifswald/DE, Greifswald/Germany

\section{Contact E-mail Address: Moritz.leppkes@uk-Erlangen.de}

Introduction: Various forms of pancreatitis (e.g. severe acute pancreatitis, autoimmune pancreatitis type 2) are characterized by an infiltration of neutrophil granulocytes. Yet, despite sharing the feature of granulocytic infiltration, these diseases take opposing natural courses of disease. A novel function of granulocytes, the formation of aggregated neutrophil extracellular traps (aggNETs), has been described and called for a reevaluation of the specific role of neutrophils in pancreatitis. We were interested in the specific function of granulocytes in various models of pancreatic inflammation.

Aims \& Methods: Experimental models of pancreatic inflammation were employed including caerulein-induced pancreatitis and a novel model of IL$17 \mathrm{~A}$-induced pancreatitis. The outcome of disease was characterized by immunohistochemistry, RNA expression and flow cytometric analyses.

Results: Transgenic systemic delivery of IL-17A alone can induce granulocytosis and neutrophil infiltration to the pancreas. Interestingly, neutrophils do not remain in the interstitium, yet enter the pancreatic ducts and form aggregates in the ductal lumen. Our experimental models further indicate that peptidyl arginine deiminase 4 (PAD4) is critical for intraductal aggregate formation and that PAD4-deficiency abrogates disease progression. Mechanistically, we identify the pancreatic juice as a strong instigator of neutrophil chromatin extrusion. Characteristic single components of pancreatic juice, such as bicarbonate ions and calcium carbonate crystals, induce aggregated NET formation.

Conclusion: Granulocytes aim to contain an inflammatory focus and enter pancreatic ducts with potentially detrimental consequences to dependent areas of the organ.

Disclosure of Interest: M. Leppkes: M.L. has received a research scholarship from MSD Sharpe \& Dohme GmbH, Germany. No financial or non-financtial conflict of interest exists related to this study.

All other authors have declared no conflicts of interest.

\section{P0755 MITOCHONDRIAL FUNCTION AND DISTRIBUTION IN PANCREATIC DUCTAL EPITHELIAL CELLS}

E. Tóth ${ }^{1}$, J. Maléth $^{1}$, R. Erdô $s^{1}$, Z. Rázga ${ }^{2}$, L. Tretter ${ }^{3}$, G. Horváth ${ }^{3}$, Z. Rakonczay ${ }^{4}$, P. Hegyi ${ }^{5}$

${ }^{1}$ First Department Of Medicine, University of Szeged, Szeged/Hungary ${ }^{2}$ Department Of Pathology, University of Szeged, Szeged/Hungary

${ }^{3}$ Department Of Medical Biochemistry, Semmelweis University, Budapest Hungary

${ }^{4}$ Department Of Pathophysiology, University of Szeged, Szeged/Hungary ${ }^{5}$ Institute For Translational Medicine/1st Department Of Medicine, University of Pécs, Pécs/Hungary

\section{Contact E-mail Address: tothemesem $@$ gmail.com}

Introduction: Mitochondrial dysfunction is a hallmark of several disease pathogenesis including acute pancreatitis (AP). Our results suggest that mitochondrial damage is crucial in bile acid induced inhibition of pancreatic ductal $\mathrm{HCO}_{3}$ secretion, however the details of mitochondrial function and dysfunction in pancreatic ductal epithelial cells (PDEC) is not known yet.

Aims \& Methods: The aim of our study was to characterize the mitochondrial distribution and function in PDECs under physiological and pathophysiological conditions. Guinea pig and Cyclophilin D WT and knock out (KO) mouse pancreatic ducts were used. Mitochondrial distribution was studied by electron microscopy $(\mathrm{EM})$. Mitochondrial membrane potential $\left(\Delta \psi_{\mathrm{m}}\right)$ was measured by confocal microscopy and pancreatic ductal $\mathrm{HCO}_{3}{ }^{-}$secretion by microfluorometry

Results: EM measurements revealed that the mitochondrial density is significantly higher on the apical side of the guinea pig PDEC compared to the middle or the basal segment in HEPES solution. The apical mitochondrial density increased further in $\mathrm{CO}_{2} / \mathrm{HCO}_{3}{ }^{-}$buffered solution, or during the administration of $5 \mu \mathrm{M}$ forskolin. That was also confirmed by the $\Delta \psi_{\mathrm{m}}$ measurements as we detected increased TMRM fluorescence on the apical side of the PDEC during stimulation. The genetic $\mathrm{KO}$ of cyclophilin $\mathrm{D}$ significantly reduced the loss of $\Delta \psi_{\mathrm{m}}$ and protected pancreatic ductal $\mathrm{HCO}_{3}{ }^{-}$secretion during the administration of $500 \mu \mathrm{M}$ chenodeoxycholic acid

Conclusion: Our results revealed that mitochondrial function has a central role in the function of PDEC presumably by providing ATP for fluid and ion secretion. On the other hand the opening of MPTP seems to be crucial in the bile acid induced toxicity offering a potential therapeutic target in AP. Disclosure of Interest: All authors have declared no conflicts of interest. 
P0756 RELATIONSHIP BETWEEN NUCLEOTIDE-BINDING OLIGOMERIZATION DOMAIN-CONTAINING PROTEIN 2 VARIANTS AND SEVERITY OF ACUTE PANCREATITIS

M.M.M. M. Harputluoglu ${ }^{1}$, M. Ozbek ${ }^{2}$, B. Otlu ${ }^{3}$, U. Demirel ${ }^{4}$, M. Aladag ${ }^{1}$, M.

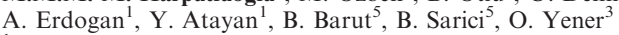

${ }^{1}$ Gastroenterology, Inonu University Medical Faculty, Malatya/Turkey

${ }^{2}$ Internal Medicine, Inonu University Medical Faculty, Malatya/Turkey

${ }^{3}$ Microbiology, Inonu University Medical Faculty, Malatya/Turkey

${ }^{4}$ Gastroenterology, Firat University Medical Faculty, Elazig/Turkey

${ }^{5}$ General Surgery, Inonu University Medical Faculty, Malatya/Turkey

\section{Contact E-mail Address: mharputluoglu@ hotmail.com}

Introduction: Infectious complications are main causes of mortality in severe acute pancreatitis (AP) (1). Most infections in AP are intestinal origin (2). The Nucleotide oligomerization domain 2 (NOD2) is a NOD-like receptor family member that senses and responds to bacterial wall peptides (3). Guenther et al. reported that $\mathrm{p} . \mathrm{R} 702 \mathrm{~W}$ mutation was found to be associated with multipl organ failure and mortality in patients with AP (4). We aimed to investigate whether there is a correlation between NOD2 variants and AP severity in this study.

Aims \& Methods: Group $1(\mathrm{n}=27)$ was healthy. Group $2(\mathrm{n}=36)$ and Group 3 $(n=32)$ were composed of mild and severe pancreatitis patients according to the Atlanta 2012 classification (5). Four NOD2 variants and serum interleukin-6 (IL6), Tumor Necrosis Factor- $\alpha(\mathrm{TNF}-\alpha)$ and lipopolysaccharide-binding protein (LBP) levels were studied.

Results: We detected p.R702W variant in 3 patients $(3 / 32,9.4 \%)$ in severe pancreatitis group, but this variant was not seen in the other two groups. 1007fs variant was found in 3,3 and 1 patient in mild $(3 / 36,8.3 \%)$ and severe pancreatitis $(3 / 32,9.4 \%)$ groups, and in healthy group $(1 / 27,3.7 \%)$, respectively. There was no significant difference the frequencies of NOD2 variants between groups. Serum IL-6, TNF- $\alpha$ and LBP levels were significantly higher in the severe pancreatitis group than in the healthy group and mild pancreatitis group (all $\mathrm{p}<0.001)$. However, there was no significant difference between these cytokine levels and NOD2 variants.

Conclusion: Our results suggest that there may be a relationship between the presence of p.R702W variant and severe pancreatitis.

Disclosure of Interest: All authors have declared no conflicts of interest.

\section{References}

1. Nathens AB, Curtis JR, Beale RJ, Cook DJ, Moreno RP, Romand JA, Skerrett SJ, Stapleton RD, Ware LB, Waldmann CS. Management of the critically ill patient with severe acute pancreatitis. Crit Care Med. 2004;32(12):2524-36

2. Li Q, Wang C, Tang C, He Q, Li N, Li J. Bacteremia in patients with acute pancreatitis as revealed by $16 \mathrm{~S}$ ribosomal RNA gene-based techniques*. Crit Care Med. 2013;41(8):1938-50

3. Strober W, Asano N, Fuss I, Kitani A, Watanabe T. Cellular and molecular mechanisms underlying NOD2 risk-associated polymorphisms in Crohn's disease. Immunol Rev. 2014;260(1):249-60

4. Guenther A, Nijmeijer RM, Nitsche C, Aghdassi A, Boemeester MA, Schulz HU, Papachristou GI, Mayerle J, van Santvoort HC, Zenker M, Simon P, Weber E, Whitcomb DC, Lerch MM, Weiss FU. Nucleotide-binding oligomerization domain protein 2 (NOD2/CARD15) mutation p.R702W predisposes to a fatal outcome of severe acute pancreatitis. Pancreatology 2013;13(3):S7-8

5. Banks PA, Bollen TL, Dervenis C, Gooszen HG, Johnson CD, Sarr MG, Tsiotos GG, Vege SS; Acute Pancreatitis Classification Working Group. Classification of acute pancreatitis-2012: revision of the Atlanta classification and definitions by international consensus. Gut. 2013;62(1):102-11.

\section{P0757 DETERMINANTS OF SEVERITY IN ACUTE PANCREATITIS - A NATION-WIDE PROSPECTIVE MULTICENTER STUDY}

H. Sternby ${ }^{1}$, F. Bolado ${ }^{2}$, H.J. Canaval-Zuleta ${ }^{3}$, C. Marra-López ${ }^{4}$, A. I. Hernando-Alonso ${ }^{5}$, A. Del-Val-Antonana ${ }^{6}$, G. García-Rayado ${ }^{7}$, R. Rivera Irigoin $^{8}$, F. J. Grau-Garcia ${ }^{9}$, L. Oms ${ }^{10}$, J. Millastre-Bocos ${ }^{11}$, I. PasqualMoreno $^{12}$, D. Martínez-Ares ${ }^{13}$, J. A. Rodriguez-Oballe ${ }^{14}$, A. Lopez-Serrano ${ }^{15}$ M. Ruiz-Rebollo ${ }^{16}$, A. Viejo-Almanzor ${ }^{17}$, P. Zapater ${ }^{18}$, E. De-Madaria ${ }^{19}$ ${ }^{1}$ Department Of Surgery, Institution of Clinical Sciences, Malmö/Sweden ${ }^{2}$ Gastroenterology, Complejo Universitario de Navarra, Pamplona/Spain ${ }^{3}$ Gastroenterology, Hospital son Llater, Palma de Mallorca/Spain ${ }^{4}$ Gastroenterology, Hospital Universitario Araba, Vitoria-Gasteiz/Spain ${ }^{5}$ Gastroenterology, Hospital Gregorio Maranon, Madrid/Spain

${ }^{6}$ Gastroenterology, Hospital Universitari i Politecnic la Fe, Valencia/Spain ${ }^{7}$ University Clinic Hospital Aragon Health Research Institute, Zaragoza/Spain ${ }^{8}$ Gastroenterology, Hospital Costa del Sol, Marbella/Spain

${ }^{9}$ Gastroenterology, Hospital Arnau de Vilanova, Valencia/Spain

${ }^{10}$ Surgery, Consorci Sanitari de Terrassa, Barcelona/Spain

${ }^{11}$ Gastroenterology, Hospital Universitario Miguel Servet, Zaragoza/Spain

${ }^{12}$ Gastroenterology, Hospital Clinico Universitario, Valencia/Spain

${ }^{13}$ Gastroenterology, Complexo Hospitalario Universitario de Vigo, Vigo/Spain

${ }^{14}$ Gastroenterology, Hospital Universitario Virgen Macarena, Seville/Spain

${ }^{15}$ Hospital Universitario Doctor Peset, Valencia/Spain

${ }^{16}$ Hospital Clinico Universitario, Valladolid/Spain

${ }^{17}$ Gastroenterology, Hospital Puerta del Mar, Cadiz/Spain

${ }^{18}$ (isabial-fundación Fisabio), Instituto de Investigación Sanitaria y Biomédica de

Alicante, Alicante/Spain
${ }^{19}$ Gastroenterology, Hospital General Universitario de Alicante - Gastroenterology, Hospital General Universitario de Ali, Alicante/Spain

Contact E-mail Address: hannasternby@gmail.com

Introduction: Acute pancreatitis (AP) has a wide clinical spectrum, ranging from mild cases to patients with high morbidity and a 3-5\% mortality. Severity classifications used local and systemic complications to define different subgroups of patients, but it is important to ascertain which of them are independent determinants for the clinical course of the disease.

Aims \& Methods: The aim of this study was to analyze which complications were independently associated to worse outcomes (time to oral refeeding, invasive treatment, ICU admission, hospital stay and mortality). Patients with AP were prospectively enrolled at 23 centers, $60 \%$ had biliary etiology, $17 \%$ necrotizing AP and $14 \%$ organ failure. Associations between possible determinants and outcome variables were assessed through binary logistic regression analysis (with adjustment for sex, etiology, Charlson comorbidity score (including age) and recurrent AP in the model).

Results: In total 1665 patients were included. Independent determinants of increased morbidity and mortality (vs category with lower risk) were: persistent organ failure (POF) vs transient organ failure (TOF), all local complications (especially combined peripancreatic and pancreatic necrosis) vs no local complications, infected necrosis vs sterile necrosis and multiple-organ POF vs singleorgan POF. We found no independent effect for late ( $>7$ th day) vs early POF nor POF associated to infected necrosis vs sterile POF.

Conclusion: Results of this study demonstrate that major determinants of severity to be included in a future classification of severity of AP are 1) local complications, where necrosis should be further categorized into infected vs sterile and 2) POF/TOF, with POF further categorized into multiple vs single POF.

Disclosure of Interest: All authors have declared no conflicts of interest.

\section{P0758 A LUMEN APPOSING METAL STENT WITH ANTI-REFLUX VALVE FOR ENDOSCOPIC ULTRASOUND-GUIDED DRAINAGE OF PSEUDOCYST AND WALLED-OFF NECROSIS}

I.R. Cho, M.J. Chung, J.H. Jo, H.S. Lee, J.Y. Park, S. Bang, S.W. Park, S.Y. Song, J. Chung

Department Of Internal Medicine, Yonsei University College of Medicine, Seoul/ Korea, Republic of

\section{Contact E-mail Address: inrae0428@yuhs.ac}

Introduction: Pancreatic pseudocyst (PC) and walled-off necrosis (WON) are frequent complications of acute pancreatitis. Drainage procedure is required when symptoms and complications such as pain, biliary obstruction, or infection occur. With technological advances, endoscopic ultrasound (EUS)-guided drainage replaced surgical treatment and has become the standard treatment. We developed a novel fully-covered lumen apposing metal stent (LAMS) with antireflux valve for the purpose of preventing complications such as stent migration or reflux of bowel contents while improving the stent patency. The aim of this study is to investigate the efficacy and the rate of complications of EUS guided drainage using novel LAMS with anti-reflux valve for PC and WON.

Aims \& Methods: We compared the treatment outcomes and the rate of complications of EUS-guided drainage using LAMS with EUS-guided drainage using plastic stents. Ten patients underwent EUS-guided drainage using the novel LAMS (LAMS group) and eighteen patients using conventional plastic stents (plastic stent group) from December 2013 to October 2016. A novel LAMS used in this study was designed to have bilateral flare ends, 4 anti-migration flaps (at each side) and a pair of 2 anti-reflux valves (inside the lumen). Technical success is defined as a successful placement of the stent without immediate complication and clinical success is defined as a resolution of the $\mathrm{PC} / \mathrm{WON}$ and disappearance of the symptoms.

Results: Among 10 patients treated with LAMS, 4 patients had complicated PC and 6 patients had WON. In plastic stent group, 15 patients had complicated PC and 3 patients had WON. The median size of fluid collection before treatment was $69.5 \mathrm{~mm}$ (range, $48-214 \mathrm{~mm}$ ) in LAMS group and $92.0 \mathrm{~mm}(56-253 \mathrm{~mm})$ in plastic stent group. Median duration of stent placement was 47 days (1-355 days) in LAMS group and 55 days (1-216 days) in plastic stent group. Treatment outcomes of the LAMS group were not inferior despite the significantly higher proportion of WON patients in the LAMS group compared to the plastic stent group. There were no statistically significant differences in the technical success rate $(90 \%$ versus $94.4 \% ; \mathrm{p}=0.595)$, clinical success rate $(70 \%$ vs. $77.8 \%$; $\mathrm{p}=0.491)$, resolution rate $(76.8 \%$ vs. $80.7 \% ; \mathrm{p}=0.705)$, complication rate $(40 \%$ vs. $50 \% ; \mathrm{p}=0.456)$. In LAMS group, 3 patients experienced mild fever and 1 patient showed peritonitis due to immediate stent migration. In plastic stent group, mild fever was developed in 4 patients, small amount of pneumoperitoneum was in 1 patients, self-migration of stent (after resolution of fluid collection) was in 2 patients. 3 patients showed serious adverse events - 1 patient in each group experienced peritonitis due to immediate stent migration and 1 patient in plastic stent group had active bleeding. Procedure time (30.7 minutes vs. 41.2 minutes; $\mathrm{p}=0.106$ ) and fasting period after treatment (3.1 days vs. 2.1 days; $p=0.344$ were also not different between two groups.

Conclusion: For EUS-guided drainage, LAMS showed acceptable treatment outcomes and the rate of complications even in relatively more severe cases of pancreatic fluid collection. And no additional fasting period was required in the LAMS group compared to the plastic stent group. Further well-designed prospective studies are needed to validate these findings.

Disclosure of Interest: All authors have declared no conflicts of interest. 
P0759 THE PREDICTORS OF STEP UP APPROACH USING ENDOSCOPIC ULTRASOUND-GUIDED TRANSMURAL DRAINAGE FOR WALLED-OFF NECROSIS

H. Shiomi ${ }^{1}$, A. Sakai ${ }^{1}$, T. Ezaki ${ }^{1}$, T. Nakagawa ${ }^{1}$, T. Kobayashi ${ }^{1}$, Y. Shiomi ${ }^{1}$, A. Masuda ${ }^{1}$, Y. Okabe ${ }^{2}$, T. Azuma ${ }^{1}$

${ }_{1}^{1}$ Gastroenterology, KobeUniversity Graduate School of Medicine, Kobe, Japan, Kobe/Japan

${ }^{2}$ Gastroenterology, Kakogawa Central City Hospital, Kakogawa/Japan

Contact E-mail Address: hshiomi@med.kobe-u.ac.jp

Introduction: Endoscopic ultrasound-guided transmural drainage (EUS-TD) has been shown to be a safe and effective minimally invasive treatment for walled-off necrosis (WON). However, in some cases, simple drainage is not sufficient to manage the symptoms of WON and step up approach such as direct endoscopic necrosectomy (DEN) and surgical necrosectomy may be required. The factors associated with the outcome of endoscopic treatment for WON remain unclear. Aims \& Methods: This study aimed to retrospectively correlate the clinical characteristics of WON with the outcome of endoscopic transmural drainage. 49 patients ( 38 males; mean age $60.79 \pm 13.44$ ) with symptomatic WON treated by an attempted EUS-TD initially were enrolled in this study. The relationship between the outcome of treatment and the clinical characteristics including morphological feature and expansion of WON was evaluated.

Results: The mean size of WON was $126.63+46.79 \mathrm{~mm}$. EUS-TD was technically successful in $48 / 49(97.9 \%)$ patients and $26(54.2 \%)$ improved with EUSTD alone while step up approach was needed in 19 patients. DEN, percutaneous drainage and surgery were done in 14,2 and 3 patients, respectively. All patients with step up approach had successful outcomes. Three patients died before step up approach was performed, but death was not related with the procedure itself. The patients who needed step up approach had multi-locular $(\mathrm{p}<0.05)$ and large size WON $(p=0.02)$ as compared to patients treated with EUS-TD alone. The extent of WON correlated significantly $(\mathrm{p}=0.001)$ with the type of treatment. Gas bubbles sign within necrotic tissue on the abdominal CT imaging early after EUS-TD indicated the necessity of necrosectomy during the treatment $(\mathrm{p}<0.01)$. Bleeding as adverse events was observed in 3 patients $(6.2 \%)$. Two patients improved with conservative therapy and 1 patient underwent transcatheter arterial embolization.

Conclusion: The step up approach is safe and effective for the treatment of WON. Multi-locular, large size and extensive WON were important predictors for performing a step up approach. Gas bubbles sign within necrotic tissue after EUSTD may help to perform necrocectomy.

Disclosure of Interest: All authors have declared no conflicts of interest.

P0760 THE ANALYSIS OF CLINICAL FEATURES AND RISK FACTOR OF RECURRENCE AFTER THE FIRST ATTACK OF ACUTE PANCREATITIS

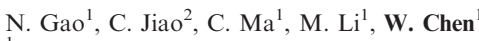

${ }^{1}$ The First Affliated Hospital of Soochow University, Suzhou/China

${ }^{2}$ the Affiliated Taizhou People's Hospital of Nantong University, taizhou/China

Contact E-mail Address: weichangchen@126.com

Introduction: Patients with a first episode of acute pancreatitis can develop recurrent or chronic pancreatitis (CP). However, little is known about the incidence or risk factors for these events.

Aims \& Methods: We performed a retrospective study of 489 patients with a first episode of acute pancreatitis from September 2012 - September 2015. We collected relevant information of disease course and follow up until June 2016. We classified these patients according to etiology, explore the relevant characteristics of recurrence episode and performed univariate and multivariate regression of possible risk factors.

Results: During a median follow-up of 30.4 months, 411 cases were followed up completely. The total recurrence rate was $27.5 \%(113 / 411)$, with a median recurrence interval of 6.5 (IQR 2.61-10.33) months. The recurrence rate and median recurrence interval of biliary group, hyperlipidemic group and other etiology group were $21.5 \%$ (57/265), 5.78 (IQR 2.52-9.92); 49.2\% (30/61), 8.28 (IQR 5.35-16.30); 30.6\% (26/85), 6.18 (IQR 2.60-9.07). To biliary AP, the recurrence rate of patients who underwent cholecystectomy less than two weeks after AP attack is lower than patients without cholecystectomy, and the difference was statistically significant. Logistic regression suggested that hyperlipidemic pancreatitis $(\mathrm{OR}=2.133 ; 95 \% \mathrm{CI}, 1.117-4.075 ; \mathrm{P}<0.001)$, pancreatic necrosis $(\mathrm{OR}=2.52 ; 95 \% \mathrm{CI}, 1.251-5.078 ; \mathrm{P}=0.01)$, smoking $(\mathrm{OR}=2.918 ; 95 \% \mathrm{CI}$, $1.728-4.928 ; \mathrm{P}=0.01)$, complicated with diabetes $(\mathrm{OR}=2.190 ; 95 \% \mathrm{CI}, 1.282$ 3.741; $\mathrm{P}=0.04)$ were independent risk factors for recurrence.

Conclusion: Quitting smoking, properly treating diabetes, hypertriglyceridemia and biliary disease, making close follow-up of patients complicated with pancreatic necrosis may reduce ARP attack.

Disclosure of Interest: All authors have declared no conflicts of interest.

\section{References}

1. Vipperla K, Papachristou GI, Easler J, et al. Risk of and factors associated with readmission after a sentinel attack of acute pancreatitis[J]. Clin Gastroenterol Hepatol, 2014, 12(11):1911-1919. DOI: 10.1016/ j.cgh.2014.04.035.

2. Bertilsson S, Swärd P, Kalaitzakis E. Factors That Affect Disease Progression After First Attack of Acute Pancreatitis[J]. Clin Gastroenterol Hepatol, 2015, 13(9):1662-1669.e3. DOI: 10.1016/j.cgh.2015.04.012.

3. Ahmed AU, Issa Y, Hagenaars JC, et al. Risk of Recurrent Pancreatitis and Progression to Chronic Pancreatitis After a First Episode of Acute
Pancreatitis[J]. Clin Gastroenterol Hepatol, 2016, 14(5):738-746. DOI: 10.1016/j.cgh.2015.12.040

4. Coté GA, Imperiale TF, Schmidt SE, et al. Similar efficacies of biliary, with or without pancreatic, sphincterotomy in treatment of idiopathic recurrent acute pancreatitis[J]. Gastroenterology, 2012, 143(6):1502-1509.el. DOI: 10.1053 j.gastro.2012.09.006

5. Tenner S, Baillie J, DeWitt J, et al. American College of Gastroenterology guideline: management of acute pancreatitis[J]. Am J Gastroenterol, 2013, 108(9):1400-1415; 1416. DOI: 10.1038/ajg.2013.218.

6. Testoni PA. Acute recurrent pancreatitis: Etiopathogenesis, diagnosis and treatment[J]. World J Gastroenterol, 2014, 20(45):16891-16901. DOI: 10.3748/wjg.v20.i45.16891

7. Wilcox CM, Seay T, Kim H, et al. Prospective Endoscopic Ultrasound-Based Approach to the Evaluation of Idiopathic Pancreatitis: Causes, Response to Therapy, and Long-term Outcome[J]. Am J Gastroenterol, 2016, 111(9):13391348. DOI: 10.1038 /ajg.2016.240.

8. Räty S, Pulkkinen J, Nordback I, et al. Can Laparoscopic Cholecystectomy Prevent Recurrent Idiopathic Acute Pancreatitis?: A Prospective Randomized Multicenter Trial[J]. Ann Surg, 2015, 262(5):736-741. DOI: 10.1097/ SLA. 0000000000001469 .

9. da CDW, Bouwense SA, Schepers NJ, et al. Same-admission versus interval cholecystectomy for mild gallstone pancreatitis (PONCHO): a multicentre randomised controlled trial[J]. Lancet, 2015, 386(10000):1261-1268. DOI: 10.1016/S0140-6736(15)00274-3.

[10] Munigala S, Conwell DL, Gelrud A, et al. Heavy Smoking Is Associated With Lower Age at First Episode of Acute Pancreatitis and a Higher Risk of Recurrence[J]. Pancreas, 2015, 44(6):876-881. DOI: 10.1097 MPA.0000000000000364

P0761 CROSSTALK BETWEEN INFLAMMATION AND COAGULATION IN PANCREATITIS-INDUCED ACUTE RENAL FAILURE

S. Chuklin ${ }^{1}$, S. Chooklin², B. Pidhirnyy ${ }^{2}$

${ }^{1}$ Biochemistry, Danylo Halytsky Lviv National Medical University, Lviv/Ukraine ${ }^{2}$ Regional Clinical Hospital, Lviv, Ukraine, Lviv/Ukraine

Contact E-mail Address: SChuklin@bigmir.net

Introduction: Clinical data has indicated that severe acute pancreatitis is a serious inflammatory disease with a systemic inflammatory response and multiple organ dysfunction Acute renal injury caused by acute pancreatitis is a common complication that is associated with a high rate of mortality. Although the pathogenesis of acute necrotizing is not completely clear, the activation of inflammatory cytokines and coagulation are keys in the etiology.

Aims \& Methods: We examined 155 patients with acute necrotizing pancreatitis According to the international classification in 98 patients we diagnosed the moderate severe AP, and in 57 patients the severe AP. Disorders of kidney function were in 48 patients. We determined the creatinine level, indicators of hemostasis and inflammation

Results: Analysis of the relationship of inflammation and hemostasis in patients with acute pancreatitis and renal dysfunction is accompanied by decreased of activated partial thrombin time $(\mathrm{F}=25.227, \mathrm{p}<0.00001)$, increased of thrombin time $(\mathrm{F}=19.428, \quad \mathrm{p}=0.00004)$ fibrinogen concentration $(\mathrm{F}=4.6046$ $\mathrm{p}=0.03568)$, D-dimers level $(\mathrm{F}=28.456, \mathrm{p}<0.00001)$, and level of soluble fibrin-monomer complexes $(\mathrm{F}=34.015, \mathrm{p}<0.00001)$, lack of activity of antithrombin III $(\mathrm{F}=42.123$, p $<0.00001)$, increased synthesis of $\mathrm{C}$-reactive protein $(\mathrm{F}=15.591, \mathrm{p}=0.00020)$, excessive production of proinflammatory cytokines IL-2 $(\mathrm{F}=29.997, \mathrm{p}<0.00001)$, IL-6 $(\mathrm{F}=21.076, \mathrm{p}=0.00002)$, and TNF- $\alpha$ $(\mathrm{F}=25.643, \mathrm{p}<0.00001)$. In acute pancreatitis patients with renal dysfunction was shown a direct correlation between severity of renal failure (SOFA score) and concentrations of IL-6 $(R=0.416484, p=0.000504), C R P(R=0.510742$, $\mathrm{p}=0.000012)$, D-dimers $(\mathrm{R}=0.321619, \mathrm{p}=0.008456)$, soluble fibrin-monomer complexes $(\mathrm{R}=0.290750, \mathrm{p}=0.017868)$, and duration of thrombin time $(\mathrm{R}=0.296007, \mathrm{p}=0.015814)$

Conclusion: The mechanism of the acute renal injury following acute necrotizing pancreatitis is complicated. The inflammatory cascades and hypercoagulative state are initiated this pathological process.

Disclosure of Interest: All authors have declared no conflicts of interest.

\section{P0762 A NEW IL13/IFN R RATIO PREDICTS SEVERITY IN ACUTE PANCREATITIS}

A. Rodriguez-Nicolas ${ }^{1}$, A. Martinez-Chamorro ${ }^{1}$, M.P. Jimenez-Gamiz ${ }^{1}$, A.M. Matas-Cobos ${ }^{2}$, F. Ruiz-Cabello ${ }^{1}$, E. Redondo-Cerezo ${ }^{2}$

${ }^{1}$ Immunology, "Virgen de las Nieves" University Hospital, Granada/Spain

${ }^{2}$ Gastroenterology And Hepatology, "Virgen de las Nieves" University Hospital, Granada/Spain

Contact E-mail Address: eredondoc@gmail.com

Introduction: Acute Pancreatitis (AP) may be severe in up to $20 \%$ of patients with substantial morbidity and mortality, which is related to a generalized inflammatory response. In some individuals, this severe inflammatory response is downregulated; in others it escapes control. Our group has previously described a TH1 profile associated with poor prognosis in AP, and a TH2 profile associated with a mild or moderate condition.

Aims \& Methods: Our aim was the development of an index for an early assessment of prognosis in AP. We analyzed 12 cytokines in 117 patients, upon 
admission to hospital. A receiver operating characteristic (ROC) analysis was built at day 0 for the prediction of severity. Later, a multiple discriminant analysis was performed, using the Wilks lambda test, to identify the variables that differ most between patients with mild AP and moderate/severe AP. A ratio calculated using the most discriminant cytokines was studied in relation to severity and mortality.

Results: ROC curves showed that TH1 cytokines IL6, IFN- $\gamma$ and TNF- $\alpha$ can be measured for the prediction of severe AP, while TH2 cytokines IL4, L13, GM$\mathrm{CSF}$, for the prediction of a mild or moderate condition. A stepwise analysis showed that IL13 and IFN- $\gamma$ were the biomarkers which contributed most to the discrimination between mild and moderate/severe AP (Wilks' lambda $=0.855$, $\mathrm{p}<0.0001 ;$ Wilks' lambda $=0.747, \mathrm{p}<0.0001$, respectively). We calculated the IL13/IFN $\gamma$ index. This ratio was significantly higher in patients with mild AP when we compared between groups $(\mathrm{p}=7.36 \times 10-8)$. This difference was also observed between severe AP and the rest of patients $(\mathrm{p}=0.007)$. The ROC curve was also modified, increasing the area under the curve (AUC), the sensibility and the specificity, in relation to AP severity.

Conclusion: We describe an IL13/IFN $\gamma$ ratio that could be of great interest in the assessment of prognosis in AP. A high value of the IL13/IFN $\gamma$ ratio at hospital admission is associated with a good prognosis of AP.

Disclosure of Interest: All authors have declared no conflicts of interest.

\section{P0763 CORRELATION BETWEEN POST-ERCP SERUM AMYLASE LEVELS AND CT FINDINGS IN ERCP-INDUCED PANCREATITIS: A PROSPECTIVE MULTICENTER OBSERVATIONAL STUDY}

O. Inatomi ${ }^{1}$, S. Bamba ${ }^{1}$, Y. Nakai ${ }^{2}$, K. Kusumoto ${ }^{2}$, B. Endoh ${ }^{3}$, A. Suzuki ${ }^{4}$, T. Suzuki ${ }^{5}$, T. Kawakami ${ }^{5}$, S. Oota ${ }^{6}$, A. Inoue ${ }^{6}$, Y. Itokawa ${ }^{2}$, K. Chikugo ${ }^{3}$, A. Andoh ${ }^{1}$, Y. Mizumoto ${ }^{3}$, K. Tanaka ${ }^{4}$

${ }^{1}$ Division Of Gastroenterology, Shiga Univ. of Medical Sciences, Otsu/Japan ${ }^{2}$ Digestive Disease Center, Department Of Gastroenterology And Hepatology, Kyoto Katsura Hospital, Kyoto/Japan

${ }^{3}$ Department Of Gastroenterology, Kyoto Medical Center, Kyoto Medical Center, Kyoto/Japan

${ }^{4}$ Department Of Gastroenterology, Kyoto Second Red Cross Hospital, Kyoto/ Japan

${ }^{5}$ Department Of Gastroenterology, Japanese Red Cross Kyoto Daiichi Hospital, Kyoto/Japan

${ }^{6}$ Department Of Radiology, Shiga University of Medical Science, Otsu/Japan

\section{Contact E-mail Address: osam@belle.shiga-med.ac.jp}

Introduction: According to the diagnostic criteria by Cotton et al. ${ }^{1)}$, post-ERCP acute pancreatitis is defined as the persistence of serum amylase levels three times or higher than the upper limit of the standard for $18 \pm 6 \mathrm{~h}$ after ERCP with persistent upper abdominal pain for $4 \mathrm{~h}$ or longer. However, the criterion of three times or higher than the upper limit was mostly based on retrospective studies, and has not been necessarily supported by imaging diagnosis. In this study, using CT findings as the gold standard of ERCP-induced pancreatitis, we investigated the cutoff serum amylase level that suggested ERCP-induced pancreatitis in a prospective multicenter study.

Aims \& Methods: At five high-volume centers, 2078 patients examined by ERCP between April 2015 and May 2016 were prospectively followed. CT was performed in patients whose serum amylase level exceeded the institutional upper limit on the day after ERCP (after 12-20 h) to investigate the presence or absence of pancreatitis findings. Two expert radiological diagnosticians assessed the images blinded and judged the presence or absence of pancreatitis based on the Balthazar grade. Patients with a preexisting high amylase level, clinically diagnosed pancreatitis, and had a difficult imaging evaluation due to the presence of cancer before ERCP were excluded from analysis. Correlations of the serum amylase level after $2 \mathrm{~h}$, that on the following day, abdominal pain under $4 \mathrm{~h}$, and that persisting longer than $4 \mathrm{~h}$ with the presence or absence of pancreatitis were investigated using ROC analysis and the chi-square test.

Results: Amylase levels increased on the following day in $402(21.5 \%)$ of the 1868 patients included, and 340 patients examined by CT were included in the analysis. ERCP-induced pancreatitis was diagnosed based on imaging in 204 patients $(10.9 \%)$. The cutoff amylase level for judging the presence or absence of pancreatitis on the following day was 2.75 times higher than the institutional upper limit (sensitivity: $73.3 \%$, specificity: $79.0 \%$, positive likelihood ratio: 3.48 , negative likelihood ratio: 0.34 ) with an AUC of 0.80 . The cutoff level after $2 \mathrm{~h}$ was 2.73 times higher than the institutional upper limit (sensitivity: $45.6 \%$, specificity: $79.7 \%$, positive likelihood ratio: 2.24 , negative likelihood ratio: 0.68 ) with an AUC of 0.63 . Abdominal pain under $4 \mathrm{~h}$ was noted in 36 of the 204 patients in the pancreatitis group, and 12 of 136 patients in the non-pancreatitis group with a sensitivity of $17.7 \%$; specificity, $91.1 \%$; positive likelihood ratio, 1.99 ; and negative likelihood ratio, 0.90. Abdominal pain that persisted longer than $4 \mathrm{~h}$ occurred in 75 patients in the pancreatitis group and 12 in the non-pancreatitis group with a sensitivity of $36.7 \%$; specificity, $91.1 \%$; positive likelihood ratio, 4.12; and negative likelihood ratio, 0.69 .

Conclusion: The appropriate cutoff serum amylase level for judging ERCPinduced pancreatitis on the day following ERCP is 2.75 times higher than the institutional upper limit. The diagnostic value of serum amylase levels $2 \mathrm{~h}$ after ERCP may be low. Regarding abdominal pain persisting for longer than $4 \mathrm{~h}$, the specificity was high but the sensitivity was low. Therefore, setting a cutoff serum amylase level on the day after ERCP is very useful to diagnose ERCP-induced pancreatitis.

Disclosure of Interest: All authors have declared no conflicts of interest.

\section{Reference}

1. Cotton PB, et al., Endoscopic sphincterotomy complications and their management: an attempt at consensus. Gastrointest Endosc 1991; 37: 383-393.

\section{P0764 CLINICAL EFFIACY AND SAFETY OF EUS-GUIDED} LUMEN-APPOSING METAL STENT ASSISTED PANCREATIC WALL-OFF NECROSIS DRAINAGE: A REAL-LIFE EXPERIENCE IN A TERTIARY HOSPITAL

S.T. Law ${ }^{1}$, C. De La Serna Higuera ${ }^{2}$, M. Perez-Miranda ${ }^{2}$, P. Gil ${ }^{2}$

${ }^{1}$ Department Of Medicine And Geriatrics, Tuen Mun Hospital, Hong Kong/Hong Kong PRC

${ }^{2}$ Gastroenterology And Hepatology, Rio Hortega, Valladolid/Spain

Contact E-mail Address: st1168@hotmail.com

Introduction: Recently, lumen apposing metal stent (LAMS) has been developed and employed in abscess drainage. However, its use is limited by its cost [1] and its safety rises concerns as apparently associated with more adverse events.[2] The aim of this study was to investigate the efficacy and safety of LAMS in endoscopic ultrasound (EUS) -guided pancreatic wall-off necrosis (WON) drainage. Aims \& Methods: Patients All consecutive patients with necrotizing pancreatitis with WON who underwent EUS-guided drainage using LAMS during the period of $1^{\text {st }}$ Jan 2012 and $30^{\text {th }}$ Jun 2016 were retrospectively retrieved from the institution database. Necrotizing pancreatitis and WON were defined as according to the revised 2012 Atlanta classification. Those encapsulated fluid collection outside the pancreas were excluded from this study. The following data were collected: patient demographics, EUS and microbiologic features of the necrosis, procedural characteristics and their outcomes. Procedures All procedures were performed by 2 endosonographers. Once the WON was identified, a 19-gauge needle was inserted with 0.035 -inch guidewire to allow for insertion of the AXIOS device, which was then advanced to create the fistula tract by using the electrocautery tip. Once the delivery catheter was inside the WON, the distal and proximal flange of the stent were deployed subsequently under EUS and endoscopic guidance respectively. Outcome measures Primary outcome measures were: 1.technical success defined as stent deployment without any difficulty nor reposition; 2.clinical success defined as symptom resolution with the abscess size $\leq 2 \mathrm{~cm}$ on computed tomography (CT); Secondary measures are: 1 . Stemt revision due to its migration/dislodgment in case of unresolved abscess; 2 adverse events; 3.WON recurrence Data analysis Continuous variables were expressed as median and IQR. Categorical data were expressed as absolute numbers and percentages.

Results: The clinical characteristics of the patients and their WONs are shown in Table 1. In the cohort, the deployment of LAMS (AXIOS: $15 \times 10 \mathrm{~mm}, \mathrm{n}=38$; $10 \times 10 \mathrm{~mm}, \mathrm{n}=8)$ was technically success in $45\left(97.8 \%, 3\right.$ cases required $2^{\text {nd }}$ attempt for proper deployment) cases while one case required to switch over to a fully-covered metal stent due to the lengthy insertion tract. $28(60.9 \%)$ cases were manageed with necrosectomy (median 1, IQR 2) in which $15(32.6 \%)$ of them had concurrent nasocystic drainage prior to each procedure. $43(93.5 \%)$ patients were treated successfully while two refractory cases required superimposed insertion of a fully-covered tubular metal stent coaxially and one case required to switch over to percutaneously drainage. $20(43.5 \%)$ cases reported to have adverse events. Six patients experienced immediate bleeding during insertion of LAMS in which two related to process of necrosectomy; among them, one case required the superimposed insertion of a tubular fully covered metal stent coaxially to control the bleeding while the other five had bleeding stopped spontaneously. Delayed bleeding was reported in 4 cases:three were managed by local administration of adrenalin, one managed by arterial embolisation to the bleeding site and one with uncontrolled bleeding due to disseminated intravascular coagulopathy and then died of multiple organ failure. Ten cases were reported to have stent migration during management in which two cases were dislodged during necrosectomy while the others were found during follow-up.

Table 1: Clinical Characteristics of the cohort and its management outcome measures

LAMS $(\mathrm{n}=46)$

Age, year

$70(26)$

Male sex

32(69.6)

Comorbidities

Diabetic mellitus ${ }^{1}$

Cardiovascular disease ${ }^{2}$

19(43.2)

Chronic obstructive airway disease

$3(6.5)$

Malignancy on chemotherapy ${ }^{3}$

$8(19.6)$

Cause of pancreatitis

Gallstones

$40(87.0)$

Miscellaneous ${ }^{4}$

2(4.5)

APACHE II score

10(7)

White cell count $(\mathrm{x} 1.000 / \mu \mathrm{L})$

13(7.20)

C-reactive protein $(\mathrm{mg} / \mathrm{L})$

150(129)

Total bilirubin $(\mathrm{mg} / \mathrm{dL})$

$0.7(0.57)$

$\operatorname{ALT}(\mathrm{U} / \mathrm{L})$

45(41.5)

Wall-off necrosis (WON) 
Table 1 Continued

LAMS $(\mathrm{n}=46)$

\begin{tabular}{|c|c|}
\hline Diameter $(\mathrm{cm})$ & $9(4.5)$ \\
\hline \multicolumn{2}{|l|}{ Location } \\
\hline Head & $13(28.3)$ \\
\hline Body/tail & $31(67.4)$ \\
\hline Whole & $2(4.3)$ \\
\hline \multicolumn{2}{|l|}{ Features } \\
\hline Poorly-defined wall & $4(8.7)$ \\
\hline Loculation & $11(23.9)$ \\
\hline Lumpy/mass content & $18(39.1)$ \\
\hline \multicolumn{2}{|l|}{ Organism } \\
\hline Gram positive & $7(15.2)^{5}$ \\
\hline Gram negative & $4(8.7)$ \\
\hline Mixed & $15(32.6)$ \\
\hline Unknown & $20(43.5)$ \\
\hline \multicolumn{2}{|l|}{ Site of cyst enterostomy ${ }^{6}$} \\
\hline Stomach & $36(78.3)$ \\
\hline Duodenal bulb & $10(21.7)$ \\
\hline \multicolumn{2}{|l|}{ Necrosectomy } \\
\hline Patient (n, \%) & $28(60.9)$ \\
\hline Number of Necrosectomy (n, IQR) & $1(2)$ \\
\hline Nasocystic drainage (n, \%) & $15(32.6)$ \\
\hline Technical success & $45(97.8)^{7}$ \\
\hline Stent revision & $12(26.1)$ \\
\hline Spontaneously migration & 8 \\
\hline Dislodged during necrosectomy & 2 \\
\hline Ineffective drainage & $2^{8}$ \\
\hline Adverse events & $20(43.5)$ \\
\hline Bleeding ${ }^{9}$ & 10 \\
\hline Migration & 10 \\
\hline \multicolumn{2}{|l|}{ Outcomes } \\
\hline Duration of hospital stay (days) & $64(33)$ \\
\hline Duration of stent insertion (week) & $10(4.5)$ \\
\hline Clinical success & $43(93.5)^{10}$ \\
\hline Recurrence & $3(6.5)$ \\
\hline
\end{tabular}

Data are either median (IQR) or no. $(\%)$ of patients, unless otherwise indicated. ${ }^{1}$ : coexisting dyslipidemia ${ }^{2}$ : coexisting congestive heart failure ${ }^{3}$ : myeloproliferative disease $(n=4))$, colon $(n=4)$, lung $(n=1)^{4}$ :alcoholism, hyperclcemia, post ERCP $(n=1)$; idiopathic $(n=3)^{5}$ : Enterococcus spp $(n=5)$, Methicillin-sensitive Staphylococcus aureus $(\mathrm{n}=2){ }^{6}$ : AXIOS $10 \times 10 \mathrm{~mm}: \mathrm{n}=6$ (transgastric); $\mathrm{n}=2$ (transduodenal) ${ }^{7}$ : mal-deployed stent: $\mathrm{n}=4$, all successfully placed in $2^{\text {nd }}$ attempt (among them, one changed into Wallflex stent $60 \times 10 \mathrm{~mm})^{8}$ : diverted to a tubular metal stent $(n=1)$; percutaneous drainage $(n=1)^{9}$ : immediate bleeding: $\mathrm{n}=6$, among them, one case managed by a fully-covered tubular metal stent placed co-axially within lumen of LAMS; early bleeding: within $48 \mathrm{hr}, \mathrm{n}=1$, embolisation required;delayed bleeding: all spontaneously subsided ${ }^{10}$ : refractory cases: managed by percutaneous drainage $(n=1)$, superimposed fully-covered tubular metal stent co-axially $(n=2) \mathrm{NB}$ : LAMS, lumen-apposing metal stent Conclusion: Pancreatic WON can be effectively treated by EUS- guided LAMS assisted drainage with high technical and clinical success rate. Bleeding and stent migration are the two major adverse events during the management that can be managed accordingly.

Disclosure of Interest: All authors have declared no conflicts of interest.

\section{References}

1. Kumar N, Conwell DL, Thompson CC. Direct endoscopic necrosectomy versus step-up approach for walled-off pancreatic necrosis: comparison of clinical outcome and health care utilization. Pancreas. 2014 Nov;43(8):1334 9.

2. Stecher SS, Simon P, Friesecke S, Glitsch A, Kühn JP, Lerch MM, Mayerle J. Delayed severe bleeding complications after treatment of pancreatic fluid collections with lumen-apposing metal stents. Gut. 2017 Jan 12. pii: gutjnl2016-313562.

P0765 RECTAL INDOMETHACIN IS PROTECTIVE AGAINST POST-ERCP PANCREATITIS IN HIGH-RISK AND AVERAGE-RISK POPULATION: A SYSTEMATIC REVIEW AND META-ANALYSIS

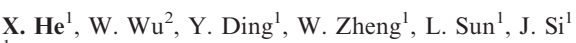

${ }^{1}$ Department Of Gastroenterology, Sir Run Run Shaw Hospital, hangzhou/China

${ }^{2}$ State Key Laboratory for Diagnosis and Treatment of Infectious Diseases, The

First Affiliated Hospital, School of Medicine, Zhejiang University, Hangzhou,

China, Hangzhou/China

Contact E-mail Address: hexingkang@zju.edu.cn
Introduction: Post-endoscopic retrograde cholangiopancreatography (ERCP) pancreatitis (PEP) is a common and serious adverse event following ERCP, with a reported incidence of $9.7 \%$ in unselected patients [1]. Given huge economic and clinical burden, effective approaches for post-ERCP pancreatitis prophylaxis remains a major priority for research. Nonsteroidal anti-inflammatory drugs (NSAIDs) have also been shown the potential efficacy in prophylaxis PEP across high-risk patients, especially for diclofenac or indomethacin [3-5]. Recently, a prospective, double-blind, controlled trial conducted by Levenick [6] and colleagues in the USA showed that the reduction in PEP using indomethacin was not as significant as previously reported. In fact, even more cases of pancreatitis occurred in indomethacin group compared with placebo group. Subsequently, a high-quality meta-analysis also concluded that there is no prophylaxis for the prevention of PEP among average-risk patients[7]. These findings raised the question that whether administration of rectal indomethacin should be recommended in average-risk patients.

Aims \& Methods: We aimed to determine the beneficial effect of rectal indomethacin in the prevention of post-ERCP pancreatitis in average-risk of patients. We systematically searched PubMed, EMBASE, Web of science and Cochrane library before October 2016. Studies that evaluated rectal administration of indomethacin in the prevention of post-ERCP pancreatitis were included in the analysis. We adopted a random-effects model to calculate overall relative risk (RR) and 95\% confidence interval (CI).

Results: We identified ten randomized clinical trials from initial search and finally included in the meta-analysis. Administration of rectal indomethacin significantly reduced the incidence of PEP in combined population (RR, 0.63; 95\% CI, 0.50-0.77). There was no significant heterogeneity across included studies $\left(\mathrm{I}^{2}=14.2 \%, \mathrm{P}=0.31\right)$. In subgroup analysis, rectal indomethacin was effective in both high-risk ( $\mathrm{RR}, 0.49 ; 95 \% \mathrm{CI}, 0.35-0.71)$ and average-risk (RR, 0.69; $95 \% \mathrm{CI}, 0.55-0.86$ ) patients and reduced the risk of mild and moderate to severe pancreatitis. The overall results remained unchanged and robust in sensitivity analyses. There was no evidence of significant publication bias among this meta-analysis.

Conclusion: Rectal administration of indomethacin is an effective approach to prevent the incidence of post-ERCP pancreatitis both in high-risk and averagerisk population undergoing ERCP. However, more high-quality randomized controlled trials are needed to further investigate the optimal timing for administration of indomethacin.

Disclosure of Interest: All authors have declared no conflicts of interest.

\section{References}

1. Kochar B, Akshintala VS, Afghani E, et al. Incidence, severity, and mortality of post-ERCP pancreatitis: a systematic review by using randomized, controlled trials. Gastrointest Endosc 2015;81:143-149 e9.

2. Dumonceau JM, Andriulli A, Deviere J, et al. European Society of Gastrointestinal Endoscopy (ESGE) Guideline: prophylaxis of post-ERCP pancreatitis. Endoscopy 2010;42:503-15.

3. Dai HF, Wang XW, Zhao K. Role of nonsteroidal anti-inflammatory drugs in the prevention of post-ERCP pancreatitis: a meta-analysis. Hepatobiliary Pancreat Dis Int 2009;8:11-6.

4. Elmunzer BJ, Waljee AK, Elta GH, et al. A meta-analysis of rectal NSAIDs in the prevention of post-ERCP pancreatitis. Gut 2008;57:1262-7.

5. Cheon YK, Cho KB, Watkins JL, et al. Efficacy of diclofenac in the prevention of post-ERCP pancreatitis in predominantly high-risk patients: a randomized double-blind prospective trial. Gastrointest Endosc 2007;66:1126-32.

6. Levenick JM, Gordon SR, Fadden LL, et al. Rectal Indomethacin Does Not Prevent Post-ERCP Pancreatitis in Consecutive Patients. Gastroenterology 2016;150:911-7; quiz e19

7. Inamdar S, Han D, Passi M, et al. Rectal indomethacin is protective against post-ERCP pancreatitis in high-risk patients but not average-risk patients: a systematic review and meta-analysis. Gastrointest Endosc 2016.

\section{P0766 THE IMPACT OF THE SPECIALISTIC}

GASTROENTEROLOGIC UNIT ON THE OUTCOME OF ACUTE PANCREATITIS (AP) IN THE VENETO REGION (NORTH-EAST OF ITALY)

L.G. G. Cavallaro ${ }^{1}$, B. Germaná ${ }^{1}$, A. Giacomin ${ }^{2}$, L. Frulloni ${ }^{3}$, A. Gabbrielli ${ }^{3}$, F. Farinati ${ }^{4}$, M. Saia ${ }^{5}$

${ }^{1}$ San Martino Hospital - Ulss1, Gastroenterologic Unit, Belluno/Italy

${ }^{2}$ San Bassiano Hospital - Ulss 7, Gastroenterologic Unit, Bassano del Grappa (VI)/ Italy

${ }^{3}$ Medicine, University of Verona Cattedra di Gastroenterologia Dept. of Medicine, Verona/Italy

${ }^{4}$ Dipartimento Di Scienze Chirurgiche E Gastroenterologiche, Policlinico

Universitario, Padova/Italy

${ }^{5}$ Direzione Medica, Sant'Antonio Hospital - ULSS16, Padova/Italy

Contact E-mail Address: cavallarolucas@libero.it

Introduction: Acute pancreatitis (AP) is one of the most common gastrointestinal diseases requiring hospitalization with an annual incidence of 13-50 cases per 100,000 persons. It is a potentially fatal disease with an overall mortality ranging from 2 to $8 \%$. Both epidemiology and outcomes are variable according to the different countries. Furthermore, few studies have considered the impact of hospital units on AP outcomes.

Aims \& Methods: To evaluate both the trend and outcomes of acute pancreatitis according to the admitting hospital units: Surgery, Internal/General Medicine, Gastrointestinal (GI) Unit, Intensive Care Unit (ICU). This is a retrospective 
cohort study based on the anonymous computerized database of hospital discharges in the Veneto Region (North-East of Italy). The principal diagnosis of AP according to the International Classification of Diseases 9th revision, Clinical Modification (ICD 9-CM, code 577.0) of the hospital discharges was selected. The period from January 2001 to December 2015 was analysed. Veneto population was considered as the reference population (in the period, it varied from $4,529,823$ to $4,927,527$ inhabitants, with $51 \%$ females). Hospitalization, Length of stay (LOS), in-hospital mortality, need for surgery (according to the DRG 191-194, 199-201 which identified bilio-pancreatic surgery) were reported according to the admitting hospital Units. Statistics: Chi squared for trend and Odds Ratio (OR) were applied.

Results: During the analysed period, 23,389 overall hospitalizations for AP, annual hospitalizations of 32 patients/100,000 inhabitants and in-hospital mortality of $3.2 \%$ were observed. Characteristics of the patients were: mean age: 62.2 $+/-19.3 y s, 54 \%$ Males (M); Female (F) mean age: $65 y$ s $+/-19.3 y s$, male mean age: $59.4+/-19.3 \mathrm{ys}(\mathrm{p}<0.05)$. Hospitalizations was higher in males $(\mathrm{M}: 35.4, \mathrm{~F}$ : 28.4, OR $1.24(95 \% \mathrm{CI}: 1.20-1.27, \mathrm{p}<0.05)$ and it increased in a stepwise progression from youngest to oldest patients (from 4.4 to $151.2 \mathrm{p}<0.05$ ); a similar trend was observed when considering in-hospital mortality (from 0.5 to $10.3 \%, \mathrm{p}<0.05$ ). From 2001 to 2015 , hospitalization ( 32.4 to $29.5, \mathrm{p}=0.04$ ), inhospital mortality $(1.41$ to $0.79, \mathrm{p}<0.05)$ and need for surgery (NFS: $5.6 \%$ to $3.0 \%, \mathrm{p}<0.05)$ trends decreased. Conversely, admission trends increased during the analysed period both in General Medicine (from 34 to $63, \mathrm{p}<0.05$ ) and Gastrointestinal (GI) units (from 14 to $29, \mathrm{p}<0.05$ ). The Overall in-hospital mortality was the lowest in GI Units with a NFS of $3.6 \%$ (see Table). In comparison to General Medicine Units, GI units were associated with a low inhospital mortality (OR: 0.37 , CI $95 \%$ : $0.28-0.49, \mathrm{p}<0.05$ ) and an high NFS (OR: 2.88, CI 95\%: 2.18-3.81, p < 0.05).

\begin{tabular}{lllll}
\hline $\begin{array}{l}\text { Admitting Hospital Unit } \\
(2001-2015)\end{array}$ & $\begin{array}{l}\text { Number of } \\
\text { cases (\%) }\end{array}$ & $\begin{array}{l}\text { Lengths of } \\
\text { stay (days) }\end{array}$ & $\begin{array}{l}\text { In-Hosp. } \\
\text { Mortality }\end{array}$ & NFS (\%) \\
\hline General Medicine (Non GI) & $6,960(30 \%)$ & $13.5 \pm 10.7$ & $4.3 \%$ & $88(1.3 \%)$ \\
Gastrointestinal (GI) Units & $3,797(16 \%)$ & $12 \pm 11.2$ & $1.7 \%$ & $135(3.6 \%)$ \\
Surgical Units & $12,112(52 \%)$ & $13+/-13.6$ & $2.7 \%$ & $443(3.7 \%)$ \\
Intensive Care Unit & $520(2 \%)$ & $17.2 \pm 21.8$ & $12.9 \%$ & $42(8.1 \%)$ \\
\hline
\end{tabular}

Conclusion: During the last 15 years in the Veneto Region, hospitalization rate, in-hospital mortality rate and need for surgery of acute pancreatitis significantly decreased. Conversely, admissions in both General Medicine and GI units increased. Management of AP in GI units seems to be related with a best outcome: lower in-hospital mortality and probably, more eligible patients for surgical treatment.

Disclosure of Interest: All authors have declared no conflicts of interest.

\section{References}

G. Uomo, R. Pezzilli, A. Gabbrielli et al. Diagnostic assessment and outcome of acute pancreatitis in Italy: Results of a prospective multicentre study ProInfAISP: Progetto informatizzato pancreatite acuta, Associazione Italiana Studio Pancreas, phase II. Digestive and Liver Disease 2007; 39: 829-837.

Anne F. Peery, Seth D. Crockett, Alfred S. Barritt et al. Burden of Gastrointestinal, Liver, and Pancreatic Diseases in the United States. Gastroenterology 2015;149:1731-1741

Stephen E. Roberts, Sian Morrison-Rees, Ann John et al. The incidence and aetiology of acute pancreatitis across Europe. Pancreatology 2017: 17: 155-165.

\section{P0767 COMPARISON OF CLINICAL COURSE AND OUTCOME OF ACUTE PANCREATITIS, RECURRENT ACUTE PANCREATITIS, ACUTE ON CHRONIC PANCREATITIS}

N. Berry, D. Sharma, J. Samanta, N. Dhaka, S. K. Sinha, R. Kochhar, R. Prasada, V. Gupta, T. Yaday

Gastroenterology, Postgraduate Institute of Medical Education and Research, Chandigarh/India

Contact E-mail Address: dibya.sharma@yahoo.com

Introduction: Recurrent acute pancreatitis (RAP) and acute on chronic pancreatitis (ACP) are likely to have less severe disease and local complications in comparison to acute pancreatitis (AP) possibly due to underlying chronic changes or fibrosis. However there is lack of literature regarding comparative studies between the natural course of disease and outcome of patients of AP vis-à-vis that of RAP and ACP.

Aims \& Methods: This study was conducted to compare the clinical course and outcomes of patients with AP, RAP and ACP. 248 consecutive patients with diagnosis of AP, RAP or ACP were included during study period. Outcome measures studied were severity, organ failure (OF), persistent organ failure (POF), need for ICU stay, ventilator and dialysis, hospital stay, need for percutaneous catheter drain (PCD), surgery and mortality.

Results: Out of $248,158(64 \%)$ patients had AP, $45(18 \%)$ patients had RAP and $45(18 \%)$ patients had ACP. $86(54 \%)$ of AP, $4(9 \%)$ of ACP and none of patients in RAP group had severe AP according to revised Atlanta classification ( $p<0.001)$. On comparison of OF, $101(63 \%$; of whom $54 \%$ had POF) of AP, 6 (13\%; of whom $9 \%$ had POF) of ACP and none of RAP patients developed OF $(\mathrm{p}<0.001)$. PCD and surgery requirement were seen in $89(56 \%) \& 9(6 \%)$ of
AP, $5(11 \%) \& 4(9 \%)$ of ACP and none of RAP patients respectively $(\mathrm{p}<0.001$ $\& \mathrm{p}=0.44$ ). Length of hospital stay (days) was significantly higher in AP and ACP as compared to RAP $(18.80 \pm 14.5 .8 .82 \pm 11.2 .3 .36 \pm 4.4, \quad \mathrm{P}<0.001)$ whereas ICU requirement was seen in significantly more patients in AP group $(59 ; 37 \%)$ than in $\operatorname{ACP}(5 ; 11 \%)$ and $\operatorname{RAP}(0 ; 0 \%)(\mathrm{p}<0.001) .41(26 \%)$ of AP, 2 $(4.4 \%)$ of ACP, but none of patient with RAP needed ventilatory support $(\mathrm{p}<0.001)$, while $13(8 \%)$ of AP patients, $1(2.2 \%)$ of ACP and none with RAP required dialysis $(\mathrm{p}<0.05)$. Mortality in AP, ACP and RAP was 29 $(18 \%), 2(4.4 \%), 0(0 \%)$ respectively $(\mathrm{p}<0.05)$.

Conclusion: Patients with first attack of AP had more severe disease course as compared to those with ACP, while those with RAP had the least severe course. Disclosure of Interest: All authors have declared no conflicts of interest.

\section{P0768 ACUTE PANCREATITIS IN PATIENTS WITH IPMNS: RETROSPECTIVE STUDY OF 346 PATIENTS OBSERVED FROM 2009 TO 2016}

L. Brozzi ${ }^{1}$, A. Amodio ${ }^{1}$, F. De Marchi ${ }^{1}$, R. Teresa Marzia ${ }^{1}$, I. Breoni ${ }^{1}$,

P. Campagnola ${ }^{2}$, S.F. Crinó ${ }^{1}$, A. Gabbrielli ${ }^{3}$, L. Frulloni ${ }^{1}$

${ }^{1}$ Medicine, AOUI Verona-Pancreas Center, verona/Italy

${ }^{2}$ Policlinico G.B. Rossi Dipt. di Gastroenterologia, Verona/Italy

${ }^{3}$ Gastroenterology, University of Verona, Verona/Italy

Contact E-mail Address: lorenzo.brozzi89@gmail.com

Introduction: In literature the frequency of acute pancreatitis (AP) in patients with IPMNs varies between 12 and $65 \%$, but most of studies are from surgical series and often pancreatitis occurred after surgery was included. Furthermore, most of the studies includes in the diction of "symptomatic IPMNs" the presence of less severe disorders, such dyspeptic symptoms, making series unclear.

Aims \& Methods: The aim of this study was to investigate the correlation between the presence of IPMN and acute pancreatitis to determine the frequency, evaluate the different characteristics from asymptomatic IPMNs patients, evaluate the possible differences between type and localization of IPMNs in occurrence of acute pancreatitis and his disease severity. A retrospective analysis was performed on all observed patients with IPMN-MD, IPMN-BD and mixed type at Gastroenterology Unit in the period between January 2009 and March 2016. In the study patients with an instrumental or histological diagnosis of IPMNs were included.

Results: We studied 346 patients (164 males and 182 females, mean age at the first report $61.6 \pm 12.2$ years). At the time of radiological diagnosis, $45 \%$ were asymptomatic, $51 \%$ had had symptoms, while $4 \%$ of the data were missing: the frequency of AP (excluding biliary etiology) of all 346 patients with IPMN was $26 \%$. AP was edematous in $85 \%$ of patients and necrotic in $15 \%$. We found increased frequency in patients with PA with IPMN of the main pancreatic duct (MD and mixed), and unifocal type. The localization to the body seems to be more correlated with the presence of AP. The number of cysts (for IPMN-BD and mixed type) was significantly lower in patients who have had AP.

Conclusion: Our medical extraction series confirms that the PA is an event that occurs in $26 \%$ of patients with IPMNs, with a prevalence of the male sex, it is associated with a IPMN central and mixed type, predominantly localized to the body. The pancreatitis is not associated with malignancy in resected patients

Disclosure of Interest: All authors have declared no conflicts of interest.

\section{P0769 APOLIPOPROTEIN B AND A-I RATIO PREDICTS SEVERE ACUTE PANCREATITIS}

J. Kim, K.J. Lee

Internal Medicine, Yonsei University Wonju College of Medicine Dept. of Internal Medicine, Wonju/Korea, Republic of

Contact E-mail Address: jawkim96@yonsei.ac.kr

Introduction: Severe acute pancreatitis (SAP) has a considerable mortality and morbidity rate. Although many indices have been developed to classify the severity of acute pancreatitis (AP), there is no ideal method for predicting SAP. The ratio of apolipoprotein B to A-I (apoB/A-I) is associated with metabolic syndrome and inflammatory status.

Aims \& Methods: This study aimed to investigate the association between severity of AP and serum apoB/A-I ratio. Patients with AP were prospectively enrolled at Yonsei University Wonju College of Medicine from March 2015 to August 2016. The severity of acute pancreatitis was assessed according to the revised Atlanta classification criteria (Atlanta 2012).

Results: Of 191 patients with AP, $134(70.2 \%)$ were classified as mild AP, 42 $(22 \%)$ as moderately severe AP, and $15(7.9 \%)$ as SAP. The apoB/A-I ratio was highest in patients with SAP compared with those with mild and moderately severe AP $(P=0.001)$. The apoB/A-I ratio positively correlated with Atlanta classification, computed tomography severity index, and Bedside index for severity of AP. The apoB/A-I ratio showed the highest ability to predict SAP in patients with AP compared with apolipoprotein B or apolipoprotein A-I alone. Conclusion: Serum apoB/A-I ratio appears to have value in predicting SAP in patients with AP.

Disclosure of Interest: All authors have declared no conflicts of interest. 


\section{References}

Walldius G, Jungner I. Apolipoprotein B and apolipoprotein A-I: risk indicators of coronary heart disease and targets for lipid-modifying therapy. $J$ Intern $\mathrm{Med}$ 2004; 255: 188-205.

Zhong L, Li Q, Jiang Y et al. The ApoB/ApoAl ratio is associated with metabolic syndrome and its components in a Chinese population. Inflammation 2010; 33: $353-8$.

Chenaud C, Merlani PG, Roux-Lombard P et al. Low apolipoprotein A-I level at intensive care unit admission and systemic inflammatory response syndrome exacerbation. Crit Care Med 2004; 32: 632-7.

Faraj M, Messier L, Bastard JP et al. Apolipoprotein B: a predictor of inflammatory status in postmenopausal overweight and obese women. Diabetologia 2006; 49: 1637-46.

Sierra-Johnson J, Somers VK, Kuniyoshi FH et al. Comparison of apolipoprotein-B/apolipoprotein-AI in subjects with versus without the metabolic syndrome. Am J Cardiol 2006; 98: 1369-73.

Enkhmaa B, Anuurad E, Zhang Z, Pearson TA, Berglund L. Usefulness of apolipoprotein B/apolipoprotein A-I ratio to predict coronary artery disease independent of the metabolic syndrome in African Americans. Am J Cardiol 2010; 106: 1264-9.

\section{P0770 IMAGING IN CHRONIC PANCREATITIS - DATA FROM THE SCANDINAVIAN BALTIC PANCREAS CLUB DATABASE}

T. Engjom ${ }^{1}$, F.G. Erchinger ${ }^{1}$, E. Tjora ${ }^{2}$, G. Dimcevski ${ }^{2}$

${ }^{1}$ Department Of Clinical Medicine, University of Bergen, Bergen/Norway

${ }^{2}$ Pediatric Department, Haukeland University Hospital, Bergen/Norway

Contact E-mail Address: trond.engjom@helse-bergen.no

Introduction: The Scandinavian-Baltic-Pancreatic-Club database collects data from patients with chronic pancreatitis (CP) in Nordic countries. Grading of structural changes is important in the description of a $\mathrm{CP}$ cohort

Aims \& Methods: We aimed to characterise structural changes of the pancreas in patients with CP. Subjects with definitive or probable CP according to the MANNHEIM diagnostic-criteria were included. Structural changes were graded according to the M-ANNHEIM-classification. A subgroup was also scored by the modified Cambridge score. Clinical data on disease-duration, nutrition, exocrine function, pain, alcohol/smoking habits and frequencies of malnutrition and diabetes were collected. A grouping of the M-ANNHEIM score (A: Normal $=0$, B: Minimal change $=1+2$ and $\mathrm{C}$ : Moderate $/$ marked $=3+4$ ) was performed for correaltion to the clinical data.

Results: The database contains 932 patients (623 men). The M-ANNHEIM-score was present from 446 subjects and both imaging scores from 93 subjects. According to M-ANNHEIM subjects were graded as 0: Normal $(8.1 \%), 1$ : Equivocal $(22.9 \%)$, 2: Mild $(12.1 \%)$, 3: Moderate $(17.9 \%)$ and 4: Marked $(39.0 \%)$. Correlation of the imaging scores: The imaging-scores demonstrated acceptable correlation $(\rho=0.75, \mathrm{p}<0.001)$ and good agreement $(\mathrm{ICC}=0.71$ $(0.59,0.8), \mathrm{p}<0.001)$. Agreement was best for groups with marked changes. The clinical parameters divided by M-ANNHEIM groups are presented in the table:

\begin{tabular}{llll}
\hline Clinical parameter & A: Normal & $\begin{array}{l}\text { B: Minimal } \\
\text { change }\end{array}$ & C: Marked \\
\hline Disease duration (Years) & $3.6(5.2)$ & $4.6(5.8)$ & $5.0(6.4)$ \\
Pain (VAS 0-100) & $38.3(39.2)$ & $39.4(36.5)$ & $35.5(35.3)$ \\
Nutrition (BMI kg/m²) & $24.2(5.0)$ & $24.7(5.5)$ & $23.7(4.7)$ \\
Malnutrition $(\%)$ & 4 & 10 & 11 \\
Faecal Elastase $(\mu \mathrm{g} / \mathrm{g})$ & $35[17-168]$ & $69[15-304]$ & $45[15-142]$ \\
Diabetes $(\%)$ & 37 & 43 & 39 \\
Smoke (Pack years) & $0[0-20]$ & $0[0-25]$ & $17[0-37]^{*}$ \\
Alcohol (Lifetime & $0[0-2.5]$ & $0[0-18]$ & $0[0-18]$ \\
$\quad$ years $>5$ units/day) & & & \\
\hline
\end{tabular}

$* \mathrm{p}<0.05$ (Kruskal-Wallis). No other differences reached significance. Values: mean (SD) or median [IQ-range]. Malnutrition: BMI $<18.5$.

Conclusion: Subjects with marked structural changes had the highest lifetime smoke-doses. There was poor correlation of structural changes to the clinical features. The two imaging scores demonstrated acceptable correlation and agreement. Poor agreement in normal/minimal-change groups may reduce the value of the scores where they are most needed. The results are presented on behalf of the SBPC study group

Disclosure of Interest: All authors have declared no conflicts of interest.
P0771 OSTEOPATHY IS COMMON IN PATIENTS WITH CHRONIC PANCREATITIS, BUT IS NOT RELATED WITH VITAMIN D AND FECAL ELASTASE LEVELS (P-BONE STUDY)

S. Stigliano ${ }^{1}$, A. Waldthaler ${ }^{2}$, E. Martínez-Moneo ${ }^{3}$, S. Robinson ${ }^{4}$, M. Malvik ${ }^{5}$, A. Hedstrom ${ }^{6}$, A. Kaczka ${ }^{7}$, M. Scholdei ${ }^{2}$, G. Delle Fave ${ }^{1}$, P. Simon ${ }^{8}$,

G. Capurso ${ }^{1}$

${ }^{1}$ Digestive And Liver Disease, Sant'Andrea Hospital, Roma/Italy

${ }^{2}$ Department For Internal Medicine I, University Hospital Regensburg,

Regensburg/Germany

${ }^{3}$ Hospital Universitario de Cruces, Bilbao/Spain

${ }^{4}$ Department of HPB Surgery, Newcastle upon Tyne, Newcastle/United Kingdom

${ }^{5}$ Tartu University Hospital, Tartu/Estonia

${ }^{6}$ Karolinska university hospital, stockholm/Sweden

${ }^{7}$ University Hospital Lodz, Lodz/Poland

${ }^{8}$ Ernst-Moritz-Arndt-University Greifswald, Greifswald/Germany

Contact E-mail Address: seri_stigliano@yahoo.it

Introduction: In patients with chronic pancreatitis (CP) malabsorption of vitamins $\mathrm{D}$ and $\mathrm{K}$, alcoholism, smoking and inflammatory status contribute to low bone mineral density (BMD). A recent meta-analysis estimated the prevalence of osteoporosis $(25 \%)$ and osteopenia $(40 \%)$ in CP and highlighted limitations of the reviewed studies.

Aims \& Methods: To evaluate the prevalence of osteoporosis and osteopenia in patients with $\mathrm{CP}$ and to investigate the correlation between BMD and $\mathrm{CP}$ features, and vitamin D and PEI. This is a multicentre cross-sectional study (PBONE, a Pancreas 2000 project) on prevalent CP patients. The Diagnosis and severity of the disease was defined according to the M-ANNHEIM classification. Clinical information and biochemical variables were recorded; PEI was assessed by fecal elastase. Standardized Osteodensitometry was performed by dual energy x-ray absorptiometry (DEXA). Categorical variables were analysed by means of Fisher's exact test, and continuous variables by t-test. A logistic regression analysis was performed to identify risk factors for osteoporosis or osteopenia. The relationship between continuous variables was assessed with Pearson correlation coefficient.

Results: 211 consecutive CP patients were enrolled at 6 Centres $(67 \% \mathrm{M}$; mean age $60 \pm 13$ years). Osteopenia was diagnosed in $42 \%$ and osteoporosis in $22 \%$ of cases. Aetiology was alcoholic in $43 \%$, and $18 \%$ had severe CP. $56 \%$ of patients had PEI. The mean value of vitamin D was $20 \mathrm{ng} / \mathrm{ml}$ and $56 \%$ of cases had vitamin D insufficiency. There was no correlation between vitamin D levels, or elastase levels and t-score at either spine or femur. Alcoholic aetiology was associated with higher risk of having low levels of fecal elastase (p 0.02) and with lower level of vitamin D (p 0.001) but not with osteopenia or osteoporosis. Female sex and older age seems to be associated with a higher risk of developing osteoporosis (OR 4.5 95\% CI 2-9.8 p 0.001; OR 1.09 95\% CI 1-1.3 p 0.01) while a higher $\mathrm{BMI}$ is associated with a reduced risk of its occurrence (OR $0.895 \% \mathrm{CI}$ $0.77-0.94$ p 0.001)

Conclusion: The present data confirm a high rate of osteopathy in CP patients. However, there was apparently no correlation between BMD, pancreatic exocrine function, severity of the disease or vitamin D levels. Other factors, such as vitamin $\mathrm{K}$ might deserve investigation for their possible relationship with bone mineral density in $\mathrm{CP}$ patients.

Disclosure of Interest: All authors have declared no conflicts of interest.

\section{P0772 NATURAL HISTORY OF PANCREATITIS ASSOCIATED} WITH SPINK1 MUTATIONS

N. Muller ${ }^{1}$, O. Hentic ${ }^{1}$, F. Maire ${ }^{1}$, P. Ruszniewski ${ }^{1}$, P. Lévy ${ }^{2}$, V. Rebours ${ }^{2}$ ${ }^{1}$ Beaujon Hospital, Clichy/France

${ }^{2}$ Service De Gastroenterologgie-pancreatologie, Université Denis Diderot Hopital Beaujon, Service de Pancreatologie, Clichy Cedex/France

Contact E-mail Address: nelly.muller@aphp.fr

Introduction: SPINK1 is a gene coding for the inhibitor of the cationic trypsinogen. Heterozygous mutations prevalence is estimated at $2 \%$. They are recognized as a risk factor for chronic pancreatitis. However few data are available regarding the natural history and the risk of complications in these patients.

Aims \& Methods: A prospective monocentric study was carried out from 2000 to 2016 to describe the natural history of SPINK 1 mutation related pancreatitis All patients referred for idiopathic acute and/or chronic pancreatitis with a SPINK1 mutation, were included and followed annually. Epidemiological, genetic, clinical and morphological data were collected.

Results: We included 158 patients. Mutations of SPINK1 were: heterozygous $(65 \%)$, homozygous $(8 \%)$ N34S, others $(27 \%)$. Median age at first symptoms and diagnosis was 20 [2-73] and 29 years [3-76]. During follow-up (median length:7.45 years), clinical manifestations were pancreatic pain $(73 \%)$, pseudocyst $(15 \%)$, acute pancreatitis $(77 \%)$, cholestasis $(6 \%)$, exocrine pancreatic insufficiency (EPI) $(33 \%)$, diabetes $(15 \%)$ and pancreatic adenocarcinoma $(n=6.4 \%)$. Calcifications and ductal abnormalities were found in $56 \%$ and $62 \%$. Endoscopic treatment and surgery were performed for $16 \%$ and $14 \%$ of the patients. Four patients died including 3 due to pancreatic cancer). The risk of pancreatic cancer at $55,60,70$ and 75 years was $9.4 \%, 14.7 \%, 28.9 \%$ and $46.7 \%$. Risk factors of cancer were calcifications $(\mathrm{p}=0.03)$ and EPI $(\mathrm{p}=0,04)$

Conclusion: SPINK1 mutations should be searched for in young patients with idiopathic pancreatitis. Risk of pancreatic cancer is probably underestimated. 
Cancer screening should be discussed especially in case of pancreatitis with calcifications.

Disclosure of Interest: All authors have declared no conflicts of interest.

P0773 EXOCRINE FUNCTION, NUTRITION AND ENZYME TREATMENT IN THE SCANDINAVIAN BALTIC PANCREAS CLUB DATABASE - PRELIMINARY DATA

F.G. Erchinger ${ }^{1}$, T. Engjom ${ }^{2}$, E. Tjora ${ }^{3}$, G. Dimcevski ${ }^{4}$

${ }_{1}^{1}$ Medical Department, Voss Hospital, Voss/Norway

${ }^{2}$ Medical Dpt, Haukeland University Hospital Medical Department, Bergen/ Norway

${ }^{3}$ Pediatric Department, Haukeland University Hospital, Bergen/Norway

${ }^{4}$ Department Of Clinical Medicine, University of Bergen, Bergen/Norway

Contact E-mail Address: friedemann.erchinger@helse-bergen.no

Introduction: The Scandinavian-Baltic-Pancreatic-club database collects patients with chronic pancreatitis (CP) from Nordic countries. Description of exocrine pancreatic insufficiency (EPI) and consequences is important in characterization of CP cohorts.

Aims \& Methods: Characterise EPI from CP in a Northern European cohort. Patients with definitive or probable CP (M-ANNHEIM diagnostic criteria) were included from nine centres. Demographic data, body-mass index (BMI), faecal elastase (FE), enzyme-doses and lab-parameters were collected. Values: Mean (SD) unless otherwise stated. EPI-classification grouped patients as follows: A: Normal, B-Mild: EPI not requiring enzymes, C-Proven: EPI requiring enzymes. Results: Included patients: 932 (623 men: 309 women). Missing EPI-classification: 46 patients. Included patients: 932 (623 men: 309 women). Missing EPIclassification: 46 patients.

\begin{tabular}{|c|c|c|c|}
\hline $\begin{array}{l}\text { Clinical } \\
\text { parameter }\end{array}$ & $\begin{array}{l}\text { (A) } \\
\text { Normal }\end{array}$ & $\begin{array}{l}\text { (B) Mild } \\
\text { insufficiency }\end{array}$ & $\begin{array}{l}\text { (C) Proven } \\
\text { insufficiency }\end{array}$ \\
\hline $\begin{array}{l}\text { Exocrine pancreatic function } \\
\qquad(\%)\end{array}$ & 33 & 16 & 51 \\
\hline $\begin{array}{l}\text { Faecal Elastase }(\mu \mathrm{g} / \mathrm{g}) \text { (mean } \\
\quad(\mathrm{SD})) \mathrm{p}<0.001\end{array}$ & $368(161)$ & 128 (144) & $51(69)$ \\
\hline $\begin{array}{l}\text { Nutrition: BMI }\left(\mathrm{kg} / \mathrm{m}^{2}\right) \text { (mean } \\
\quad(\mathrm{SD})) \mathrm{p}<0.005\end{array}$ & $24.6(4.9)$ & $23.7(4.3)$ & $22.6(4.3)$ \\
\hline $\begin{array}{l}\text { Frequency BMI }<18.5(\%) \\
\quad p<0.005\end{array}$ & 5 & & 16 \\
\hline $\begin{array}{l}\text { Vitamin D: Frequency } \\
\quad<25 \mathrm{nmol} / \mathrm{L}(\%) \text { (I vs II) } \\
\mathrm{p}<0.005\end{array}$ & 7.4 & 23.7 & 17.6 \\
\hline $\begin{array}{l}\text { Enzyme Treatment (lipase- } \\
\text { units/day) (median [IQ } \\
\text { range])* }\end{array}$ & \multicolumn{2}{|l|}{$0[0-75000]$} & $\begin{array}{l}120000 \\
{[75000-150000]}\end{array}$ \\
\hline $\begin{array}{l}\text { Hemoglobin: (median [IQ } \\
\text { range] } \mathrm{p}<0.05\end{array}$ & \multicolumn{2}{|l|}{$11.8(2.7-3.0)$} & $10.7(2.8)$ \\
\hline $\begin{array}{l}\text { Faecal Elastase and disease } \\
\text { duration (years)** }\end{array}$ & \multicolumn{2}{|l|}{$<10: 143(175)$} & $>10: 91(118)$ \\
\hline
\end{tabular}

${ }^{*} \mathrm{p}<0.001 .9 \%$ received $<50000$ lipase-units/day. 14 subjects having FE $>200$ received enzymes, 48 subjects with $\mathrm{FE}<100$ received no enzymes.

${ }^{* *}$ no age/sex differences in EPF

Conclusion: In our material frequency of EPI is higher than reported in the NAPS2 study (31\%). Consequences of EPI were lower BMI, more frequent underweight, higher enzyme-doses and lower haemoglobin. Need for vitamin D supplements was highest in the group with mild EPI not receiving enzymes. Exocrine function was correlated with disease duration, but neither with age nor gender.

Disclosure of Interest: All authors have declared no conflicts of interest.

\section{P0774 FLUID AND $\mathrm{HCO}_{3}{ }^{-}$SECRETION AND CFTR ACTIVITY IS INHIBITED BY CIGARETTE SMOKE EXTRACT IN GUINEA PIG PANCREATIC DUCTAL CELLS}

D. Talas ${ }^{1}$, P. Pallagi ${ }^{1}$, V. Venglovecz ${ }^{2}$, E. Gál ${ }^{1}$, K. Tóth ${ }^{1}$, A. Schnúr ${ }^{1}$, J. Maléth ${ }^{1}$, D. Csupor ${ }^{3}$, Z. Rakonczay ${ }^{4}$, P. Hegyi ${ }^{1}$

${ }^{1}$ First Department Of Medicine, University of Szeged, Szeged/Hungary

${ }^{2}$ Department Of Pharmacology And Pharmacotherapy, University of Szeged, Szeged/Hungary

${ }^{3}$ Department Of Pharmacognosy, University of Szeged, Szeged/Hungary

${ }^{4}$ Department Of Pathophysiology, University of Szeged, Szeged/Hungary

Contact E-mail Address: talasdave @icloud.com

Introduction: Smoking represents an independent risk factor for the development of chronic pancreatitis (CP). It is well documented that secretion of pancreatic ductal alkaline fluid (which is regulated mostly by the anion exchanger and CFTR) is diminished in CP.

Aims \& Methods: In this study we would like to understand whether smoking has any effects on pancreatic ductal fluid and $\mathrm{HCO}_{3}{ }^{-}$secretion. Guinea pigs were exposed to cigarette smoke four times a day for $30 \mathrm{~min}$ for 6 weeks. The CFTR expression was analysed by immunohistochemistry. Pancreatic ducts were isolated from guinea pig pancreas. Cigarette smoke extract (CSE) was prepared by smoking of 15 cigarettes into $10 \mathrm{ml}$ distilled water by a smoking machine. Intracellular $\mathrm{Ca}^{2+}$ concentration and $\mathrm{pH}$ were evaluated by microfluorometry. Fluid secretion was measured by video microscopy. CFTR currents were detected by whole cell configuration of patch clamp technique.

Results: Cigarette smoking significantly diminished the expression of CFTR and the fluid and $\mathrm{HCO}_{3}{ }^{-}$secretion in guinea pig pancreas. CSE dose dependently decreased fluid and $\mathrm{HCO}_{3}{ }^{-}$secretion in guinea pig pancreatic ducts via inhibition of anion exchanger, $\mathrm{Na}^{+} / \mathrm{H}^{+}$exchanger and $\mathrm{Na}^{+} / \mathrm{HCO}_{3}{ }^{-}$cotransporter and also forskolin-stimulated $\mathrm{Cl}^{-}$current of $\mathrm{CFTR} \mathrm{Cl}^{-}$channel. CSE incubation altered the pattern of carbachol-induced $\mathrm{Ca}^{2+}$ signal in pancreatic ducts suggesting that some of the inhibitory effects may be regulated by calcium signalling.

Conclusion: Cigarette smoking and CSE inhibits pancreatic ductal fluid and $\mathrm{HCO}_{3}{ }^{-}$secretion and the activity of the CFTR which may play role in the smoke-induced pancreatic damage. This study was supported by OTKA, MTA, SZTA and ÚNKP.

Disclosure of Interest: All authors have declared no conflicts of interest.

P0775 HISTOLOGICAL DIAGNOSIS WITH RAPID ON-SITE EVALUATION IN ENDOSCOPIC ULTRASOUND-GUIDED FINENEEDLE ASPIRATION OF PANCREATIC SOLID LESIONS

T. Hirayama ${ }^{1}$, K. Hara ${ }^{2}$, N. Mizuno ${ }^{2}$, S. Hijioka ${ }^{2}$, T. Kuwahara ${ }^{2}$, N. Okuno ${ }^{2}$, Y. Niwa ${ }^{3}$

${ }^{1}$ Department Of Gastroenterology, Yodogawa Christian Hospital, Osaka-shi/Japan ${ }^{2}$ Department Of Gastroenterology, Aichi Cancer Center Hospital, Nagoya/Japan ${ }^{3}$ Department Of Endoscopy, Aichi Cancer Center Hospital Dept. of Endoscopy, Nagoya/Japan

Contact E-mail Address: hirayamatakashijudye@yahoo.co.jp

Introduction: Rapid on-site cytologic evaluation (ROSE) for determining the suitability of a specimen often provides high efficacy of endoscopic ultrasoundguided fine needle aspiration (EUS-FNA). In our center, we propose an additional role of ROSE in histological diagnosis aimed at improving diagnostic accuracy.

Aims \& Methods: From January 2009 and December 2015, 215 patients were evaluated who underwent both EUS-FNA for pancreatic solid lesions and surgery. We retrospectively compared the diagnostic performance of ROSE during EUS-FNA with the final diagnosis confirmed by surgically resected specimens. Diagnosis by ROSE using Diff-Quik ${ }^{\mathrm{TM}}$ was carried out by both a cytopathologist and an endoscopist.

Results: The median of needle passes required for ROSE was 1 (range, 1-5). Final diagnoses for the 215 lesions were pancreatic ductal adenocarcinoma (PDAC; $\mathrm{n}=162$ ), pancreatic ductal adenosquamous carcinoma (PDASC; $\mathrm{n}=9$ ), pancreatic neuroendocrine tumor ( $\mathrm{pNET} ; \mathrm{n}=30$ ), solid pseudopapillary neoplasm (SPN; $\mathrm{n}=9)$, metastatic tumors $(\mathrm{n}=4)$, and acinar cell carcinoma $(\mathrm{ACC} ; \mathrm{n}=1)$. Primary lesions for metastatic tumors in the pancreas were renal cell cancer $(\mathrm{RCC} ; \mathrm{n}=2)$, small cell lung cancer (SCLC; $\mathrm{n}=1)$, and colon cancer $(\mathrm{n}=1)$. ROSE could not diagnose 14 cases. When adenocarcinoma (including suspicious) was suspected by ROSE, ROSE diagnosed 94.6\% (159/168) of adenocarcinomas. When special type tumor (pNET, SPN, RCC, SCLC) was suspected by ROSE, ROSE diagnosed $96.4 \%(27 / 28)$ of special type tumor.

Conclusion: All adenocarcinomas suspected by ROSE were malignant tumors When special type tumor (pNET, SPN, RCC, SCLC) was suspected by ROSE, Diagnostic accuracy of ROSE was $96.4 \%$. Diagnostic accuracy using ROSE is high agreement in final histological diagnosis. It is suggested that ROSE may also be useful for diagnosis of special type tumor.

Disclosure of Interest: All authors have declared no conflicts of interest.

\section{References}

1. Agarwal B, Krishna NB, Labundy JL, Safdar R, Akduman EI. EUS and/or EUS-guided FNA in patients with CT and/or magnetic resonance imaging findings of enlarged pancreatic head or dilated pancreatic duct with or without a dilated common bile duct. Gastrointest Endosc. 2008; 68: 237-42; quiz $334,5$.

2. Volmar KE, Vollmer RT, Jowell PS, Nelson RC, Xie HB. Pancreatic FNA in 1000 cases: a comparison of imaging modalities. Gastrointest.. 2005; 61: 854 61 .

3. DiMagno EP, Buxton JL, Regan PT, et al. Ultrasonic endoscope. Lancet (London, England). 1980; 1: 629-31.

4. Yoshinaga S, Suzuki H, Oda I, Saito Y. Role of endoscopic ultrasoundguided fine needle aspiration (EUS-FNA) for diagnosis of solid pancreatic masses. Dig. Endoscopy: official journal of the Japan Gastroenterological Endoscopy Society.. 2011; 23 Suppl 1: 29-33.

5. Klapman JB, Logrono R, Dye CE, Waxman I. Clinical impact of on-site cytopathology interpretation on endoscopic ultrasound-guided fine needle aspiration. Am J Gastroenterol 2003; 98: 1289-94.

6. Mansy SS, Abbas MA, Yehia HA, Abdelrazik SM, Ghanem LY, Amin TM. Value of the innovated technique agarose cell block in improving the 
sensitivity of urine cytology in cases of bladder carcinoma. Ultrastruct. Pathol 2006; 30: 379-85.

7. Nathan NA, Narayan E, Smith MM, Horn MJ. Cell block cytology. Improved preparation and its efficacy in diagnostic cytology. Am J Clin Pathol 2000; 114: 599-606.

\section{P0777 THE NOVEL ROLE OF GASTROKINE, A GASTRIC TUMOR SUPPRESSOR PROTEIN, IN PANCREATIC CARCINOGENESIS}

S. Steiner ${ }^{1}$, G.M. Seleznik ${ }^{1}$, T. Reding ${ }^{1}$, A. Gupta ${ }^{1}$, D. Lenggenhager ${ }^{2}$, M. Heikenwälder ${ }^{3}$, R. Graf ${ }^{1}$

${ }^{1}$ Visceral \& Transplantation Surgery, University Hospital Zurich, Swiss HPB Centre, Zurich/Switzerland

${ }^{2}$ Institute Of Pathology, University Hospital Zurich, Zurich/Switzerland

${ }^{3}$ Institute Of Virology, Technische Universität München, Helmholtz Center Munich, Munich/Germany

Contact E-mail Address: sabrina.steiner@usz.ch

Introduction: Pancreatic ductal adenocarcinoma (PDAC) has one of the most dismal prognoses of all cancer types. Diagnostic techniques for early malignant lesions are limited, which shows an evident need to understand the pathomechanism leading to PDAC and find a suitable marker for early detection. Initial processes in PDAC development involve acinar to ductal metaplasia (ADM) with further neoplastic progression into four pancreatic intraepithelial neoplastic (PanINs) stages. After accumulation of mutations, these lesions will further evolve into PDAC. Gastrokine $1 \& 2$ (GKN1 \& GKN2) are secreted proteins found almost exclusively in the stomach, where they are involved in gastric epithelial homeostasis. While current research focuses on the exploration of tumor-suppressive properties of GKN1 in gastric tumors, nothing is known about GKN function in other organs. A whole genome microarray of KrasG12D PtflaCre (KC) mice, a mouse model with predisposition to pancreatic cancer, revealed strikingly high gastrokine expression. We will further analyze the involvment of GKNs in the development and progression of PDAC and explore the possibility to use them as biomarkers.

Aims \& Methods: GKN1 \& GKN2 expression was confirmed by qPCR in human and mouse pancreas samples. The presence of GKN1 was verified by western blot and immunohistochemistry (IHC) in mouse pancreas. Mouse pancreatic juice and serum were analyzed by proteomic analysis. To investigate the role of GKNs in pancreatic carcinogenesis in vivo, we established mouse models by intercrossing $\mathrm{KC}$ mice with $\mathrm{Gkn}^{-/-}$and $\mathrm{Gkn}^{-/-}$mice respectively. The capacity of acinar cells lacking Gkn1 and Gkn2 to transdifferentiatie into ductal lesions in vitro was tested.

Results: GKNs were upregulated during early stages of pancreatic carcinogenesis in mouse and peri-tumoral human pancreas. GKNs were absent in healthy pancreas and tumor tissue. IHC showed specific GKN1 expression in premalignant PanIN lesions, while GKN2 positive cells were also localized in the stroma. ELISA and proteomic analysis in mice confirmed the secretion of GKNs into pancreatic juice. Preliminary results from the first timepoint of analysis showed accelerated tumor development in $\mathrm{GKN1}^{-/-} \mathrm{KC}$ mice. Wild type acinar cells transdifferentiated in vitro only in the presence of TGFa. On the contrary, $\mathrm{Gkn}^{-/-}$acinar cells transdifferentiated spontaneously, and resulted in a higher number of ADMs.

Conclusion: We identified for the first time specific gastrokine expression in preneoplastic lesions in human and mouse pancreatic tissue. The secretion into pancreatic juice during carcinogenesis could make gastrokine a potential biomarker for the detection of early pancreatic premalignant lesions. With our mouse models we will provide in vivo evidence on the role of GKNs as potential tumor supressors in the pancreas.

Disclosure of Interest: All authors have declared no conflicts of interest.

P0778 AUTOPHAGY IS ESSENTIAL FOR PANCREATIC CANCER DEVELOPMENT IN A NEW HUMANIZED GENETICALLYMODIFIED ADULT MOUSE MODEL

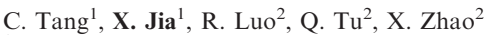

${ }^{1}$ Gastroenterology, West China Hospital, Sichuan University, Chengdu/China

${ }^{2}$ Kunming Institute of Zoology, Kunming/China

Contact E-mail Address: jiaxintong22@163.com

Introduction: Pancreatic cancer is one of the deadliest malignancies and there are no effective therapies for it. According to a search of The Cancer Genome Atlas (TCGA), alterations in Kirsten rat sarcoma (KRAS), Tumour protein (TP) 53, Cyclin-dependent kinase inhibitor (CDKN)2A and Cyclin-dependent kinase inhibitor $(\mathrm{CDKN}) 2 \mathrm{~B}$ are the most frequent genetic aberrations in human pancreatic cancer $(91 \%, 63 \%, 53 \%$ and $34 \%$ of cases, respectively). Macroautophagy (hereafter referred to as autophagy) has been implicated in some of human diseases, and it plays a complex role in pancreatic cancer.

Aims \& Methods: We aimed to investigate autophagy response in a new humanized genetically-modified adult mouse model of pancreatic cancer. To induce pancreatic cancer, lentiviruses expressing oncogenic $K R A S^{G 12 D}$ and shRNA targeting tumour suppressors $T p 53, C d k n 2 a$ and $C d k n 2 b$ (lentiviruses-KTCC) were injected into pancreas of 9 -week old adult mouse. Autophagy was detected by immunofluorescence staining for autophagic protein light chain-3 (LC-3) and Lysosomal-associated membrane protein 1(LAMP-1). Additionally, the expression of autophagic protein LC3, autophagy related protein 7 (ATG7), LAMP-1 and P62 were determined by western blot. In vitro, pancreatic duct epithelial cells of normal mice were primary isolated, LC3 was determined by immunofluorescence staining in the lentiviruses-KTCC infected primary cells.

Results: Mice developed pancreatic cancer ten weeks after lentiviruses-KTCC injection, both in macrography and histopathology analysed. The mRNA levels of autophagic genes Atg7 and Atg12 were up regulated. In addition, the LC3 and LAMP-1 positive areas were significantly increased. And co-localization of LC3 and LAMP-1 was found in pancreatic tumour sections. Moreover, the increased protein levels of ATG7, LC3, LAMP-1 and decreased P62 protein was observed in the pancreatic tumour tissues. In vitro, the protein level of LC3 in the lentiviruses-KTCC infected primary cells was increased by 6 times when compared with that in the control primary cells $(\mathrm{P}=0.0104)$.

Conclusion: An adult mouse model of pancreatic cancer can be generated by altering Kras, Tp53, Cdkn2a and Cdkn2b genes. Besides, increased autophagy was observed during the development of pancreatic cancer in our mouse model. These findings provide considerable insight into the role of autophagy in pancreatic cancer and autophagy inhibition might be a potential target in treating pancreatic cancer.

Disclosure of Interest: All authors have declared no conflicts of interest.

\section{P0779 HIPEC IN GI CANCERS. IS HYPERTHERMIA FRIEND OR}

FOE?

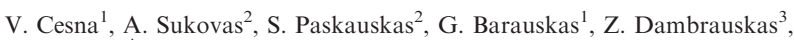
A. Gulbinas ${ }^{4}$

${ }^{1}$ Department Of Surgery, Lithuanian University of Health Sciences, Kaunas/ Lithuania

${ }^{2}$ Department Of Obstetrics And Gynaecology, Lithuanian University of Health Sciences, Kaunas/Lithuania

${ }^{3}$ Institute For Digestive Research, Lithuanian University of Health Sciences, Kaunas/Lithuania

Contact E-mail Address: gulbanta@gmail.com

Introduction: Hyperthermia as a positive additive to chemotherapy is described in multiple studies. Despite controversial results hyperthermal intraperitoneal chemotherapy (HIPEC) is a standard treatment option for some types of gastrointestinal cancer that invades peritoneum. However, the results of clinical data and basic research are uneven. Moreover, there is a lack of fundamental knowledge on additive cytotoxic effect of hyperthermia on cancer cells of different origin.

Aims \& Methods: Our aim was to analyse gastrointestinal cancer cell response to various hyperthermia levels, accompanied by chemotherapy, in a manner of cell cytotoxity, apoptosis and intracellular cisplatin concentration. Cancer cell lines of gastric (AGS), pancreatic (T3M4) and colorectal (CACO-2) origin were exposed to cisplatin and different temperature regimens $\left(37^{\circ} \mathrm{C}\right.$ to $\left.45^{\circ} \mathrm{C}\right)$ either in isolated manner, or in combination. Cells were treated for one hour, mimicking HIPEC timing in clinical setting. The intracellular concentration of cisplatin was measured immediately after experiment by mass spectrometry. 48 hours later changes of cell viability and apoptosis rates depending on temperature in addition to cisplatin treatment were evaluated by MTT and Annexin/7 AAD flow cytometry respectively.

Results: Response of AGS to hyperthermia was as implied. Viability of the cells was gradually decreasing by raising the temperature. CACO-2 cells had no significant response to temperature rise up to $42^{\circ} \mathrm{C}$, but at $43^{\circ} \mathrm{C}$ viability dropped by $14 \%$ constantly remaining at higher temperatures. T3M4 cells acted in unpredictable manner, whereas decreasing viability by $30 \%$ in the interval between $37^{\circ} \mathrm{C}$ to $42^{\circ} \mathrm{C}$ and $20 \%$ increase at $43^{\circ} \mathrm{C}$ was observed. Following simultaneous exposure to hyperthermia and cisplatin we observed no additive temperature affect in interval between $37^{\circ} \mathrm{C}$ to $41^{\circ} \mathrm{C}$. However, at particular temperature regimens, we observed temporary proliferation increase: AGS - at $42^{\circ} \mathrm{C}$ $(33 \%)$; T3M 4 - at $43^{\circ} \mathrm{C}(32 \%)$. Higher temperatures dramatically inhibited AGS - by $70 \%$, T3M 4 - by $76 \%$. There was the linear pattern of slight decrease (up to $26 \%$ at $45^{\circ} \mathrm{C}$ ) of viability in CACO-2 cells. Isobologram analysis of combined hyperthermia and cisplatin treatment revealed strong antagonism of hyperthermia and chemotherapy in all analyzed cell lines. Nevertheless, hyperthermia of $43^{\circ} \mathrm{C}$ in addition to cisplatin promoted apoptosis of AGS cells by $33 \%$, CACO-2 by $26 \%$, T3M 4 by $19 \%$. Moreover, application of hyperthermia $\left(43^{\circ} \mathrm{C}\right)$ could contribute to increase of intracellular cisplatin concentration by $30 \%, 20 \%$ and $18 \%$ AGS, CACO-2 and T3M4 cells respectively.

Conclusion: Our results indicate that there is no linear contribution of hyperthermia to chemotherapy in all analyzed cell lines. Therefore, in clinical setting it should be applied individually, regarding cancer type. Moreover, particular temperatures can worsen the treatment and increase cancer cell growth.

Disclosure of Interest: All authors have declared no conflicts of interest.

\section{P0780 CACHEXIA INVOLVEMENT IN THE LOCAL SPREAD OF PANCREATIC DUCTAL ADENOCARCINOMA}

L. Petrusel ${ }^{1}$, R. Suharoschi ${ }^{2}$, I. Rusu ${ }^{3}$, C. Pojoga ${ }^{1}$, R. Seicean ${ }^{4}$, A. Seicean ${ }^{1}$ ${ }^{1}$ Dept. Of Internal Medicine, Gastroenterology, Iuliu Hatieganu University of Medicine and Pharmacy, Cluj-Napoca/Romania

${ }^{2}$ Molecular Nutrition And Proteomics Laboratory, Institute of Life Sciences, University of Agricultural Sciences and Veterinary Medicine, Cluj-Napocal

Romania

${ }^{3}$ Dept. Of Pathology, Octavian Fodor Regional Institute of Gastroenterology and Hepatology, Cluj-Napoca/Romania 
${ }^{4}$ General Surgery, Clinical Emergency County Hospital Cluj-Napoca, ClujNapoca/Romania

Contact E-mail Address: andradaseicean@gmail.com

Introduction: Cachexia is a multifactorial syndrome, characterized by the loss of skeletal muscle mass which is not fully reversible by nutritional support. Activin play a dominant role in the development and progression of cachexia and also in tumor cell growth in pancreatic adenocarcinoma via non-SMAD (MAPK, PI3K Akt) pathways. Cachexia might be a keypoint in pancreatic ductal adenocarcinomas (PDAC) poor outcome.

Aims \& Methods: Our goal was to assess the significance of activin protein expression in PDAC related to the clinical stage and survival. There were included patients with histological proven of adenocarcinoma $(n=115)$ and a matched control group $(n=124)$. The plasma levels of activin was analyzed using western blot. The t test was used to determine the difference between the two groups, Kaplan-Meier curve and log-rank tests were used to determine the differences in survival curves of studied patients.

Results: The activin was overexpressed more frequently in PDAC compared to controls $(\mathrm{p}=0.001)$. and has been closely related to advanced clinical stage (stage III-IV), tumor size, location and with the presence of metastasis $(\mathrm{p}<0.05)$ Activin expression was higher in patients with type 2 diabetes $(p=0.04)$. No relationship between activin level and the patients age, sex or tumor size, was noted. Patients with activin high expression had a shorter survival time than PDAC patients with activin low expression $(\log -$ rank $=4.35, \mathrm{p}=0.03)$

Conclusion: Activin pathway is related to cachexia and the local spread of PDAC, metastasis, the presence of diabetes and survival.

Disclosure of Interest: All authors have declared no conflicts of interest.

\section{References}

1. Bachmann J, Büchler MW, Friess H, Martignoni ME. Cachexia in patients with chronic pancreatitis and pancreatic cancer: impact on survival and outcome. Nutr Cancer 2013; 65: 827-833

2. Bartosch-Haerlid. A., Andersson R. Cachexia in pancreatic cancer Mechanisms and potential intervention. e-SPEN, the European e-Journal of Clinical Nutrition and Metabolism 4, 2009 e337-e343

3. Zhou, X., Wang, J. L., Lu, J., Song, Y., Kwak, K. S. et al Reversal of cancer cachexia and muscle wasting by ActRIIB antagonism leads to prolonged survival. Cell 2010; 142, 531-543

4. Togashi Y, Akihiro K, Hiroki S et al. Activin signal promotes cancer progression and is involved in cachexia in a subset of pancreatic cancer. Cancer Letters 2015; $356819-827$

5. Chen J.L, K.L. Walton, C.E. Winbanks, K.T. Murphy, R.E. Thomson, Y Makanji, et al., Elevated expression of activins promotes muscle wasting and cachexia, FASEB J. 2014; 28: 1711-1723.

6. Lause P, van Maanen A, Thissen J. Role of Activin A and Myostatin in Human Cancer Cachexia. J Clin Endocrinol Metab, May 2015, 100 (5): 2030 2038

\section{P0781 THE INTRACELLULAR CYTOSKELETON MARKER IS} OVEREXPRESSED IN PANCREATIC DUCTAL ADENOCARCINOMA

L. Petrusel ${ }^{1}$, R. Suharoschi ${ }^{2}$, T. Zaharie $^{3}$, I. Rusu ${ }^{3}$, C. Pojoga ${ }^{4}$, R. Seicean ${ }^{5}$, A. Seicean ${ }^{4}$

${ }^{1}$ Dept. Of Internal Medicine, Gastroenterology, Iuliu Hatieganu University of Medicine and Pharmacy, Cluj-Napoca/Romania

${ }^{2}$ Molecular Nutrition And Proteomics Laboratory, Institute of Life Sciences, University of Agricultural Sciences and Veterinary Medicine, Cluj-Napoca/

Romania

${ }^{3}$ Dept. Of Pathology, Octavian Fodor Regional Institute of Gastroenterology and Hepatology, Cluj-Napoca/Romania

${ }^{4}$ Dept. Of Internal Medicine, Gastroenterology, Octavian Fodor Regional Institute of Gastroenterology and Hepatology, Cluj-Napoca/Romania

${ }^{5}$ General Surgery, Clinical Emergency County Hospital Cluj-Napoca, Cluj-

Napoca/Romania

Contact E-mail Address: andradaseicean@gmail.com

Introduction: Intracellular cytoskeleton in pancreatic ductal adenocarcinoma (PDAC) might be a key point in its poor outcome. Reliable biomarkers estimating the cytoskeleton involvement are lacking and their relationship with the cachexia is not known. Ezrin is involved in intracellular signaling and adhesion, by linking in the PI3K/Akt pathways.

Aims \& Methods: The goal is to assess the significance of ezrin protein expression in PDAC related to the clinical stage and survival. There were included patients with histological proven of adenocarcinoma $(n=51)$ and a matched control group $(\mathrm{n}=51)$. The plasma levels of ezrin were analyzed using western blot. The $t$ test was used to determine the difference between the two groups, Kaplan-Meier curve and log-rank tests were used to determine the differences in survival curves of studied patients.

Results: The ezrin was overexpressed more frequently in PDAC compared to controls $(p=0.009$ and $p=0.05)$. Ezrin expression has been closely related to advanced clinical stage $(\mathrm{p}=0.03)$, but not with the presence of metastasis No relationship between ezrin levels and the patients age, sex or tumor size and location of tumor was found. The survival of patients with high or low levels of ezrin expression was similar.

Conclusion: Ezrin pathway as a intracellular cytoskeleton biomarker is related to the local spread of PDAC, but not in metastasis or survival.

Disclosure of Interest: All authors have declared no conflicts of interest.

\section{References}

1. Capello M, Cappello P, Linty FC et al (2013) Autoantibodies to Ezrin are an early sign of pancreatic cancer in humans and in genetically engineered mouse models. J Hematol Oncol 6:67

2. Meng Y, Lu Z, Yu S, Zhang Q, Ma Y, Chen J: Ezrin promotes invasion and metastasis of pancreatic cancer cells. J Transl Med 2010, 8:61

3. Yeh TS, Tseng JH, Liu NJ et al. Significance of Cellular Distribution of Ezrin in Pancreatic Cystic Neoplasms and Ductal Adenocarcinoma, Arch Surg. 2005;140:1184-1190

4. Piao J, Liu S, Xu Y, et al. Ezrin protein overexpression predicts the poor prognosis of pancreatic ductal adenocarcinomas. Experimental and Molecular Pathology 98 (2015) 1-6.

5. Li J, Wei K, Yu H, Jin D, Wang G, Yu B (2015) Prognostic value of Ezrin in various cancers: a systematic review and updated meta-analysis. Sci Rep 5:17903

\section{P0782 QUALITY OF LIFE DURING CHEMOTHERAPY IN JAPANESE PATIENTS WITH UNRESECTABLE ADVANCED PANCREATIC CANCER}

K. Shibayama ${ }^{1}$, F. Koga ${ }^{2}$, S. Nakashita ${ }^{2}$, N. Oza ${ }^{2}$, T. Otsuka ${ }^{3}$, N. Ureshino ${ }^{3}$, Y. Kawaguchi ${ }^{2}$

${ }^{1}$ Department Of Nursing, Saga-Ken Medical Centre Koseikan, Saga/Japan

${ }^{2}$ Department Of Hepatobiliary And Pancreatology, Saga-Ken Medical Centre Koseikan, Saga/Japan

${ }^{3}$ Department Of Oncology, Saga-Ken Medical Centre Koseikan, Saga/Japan

Contact E-mail Address: kaoru_1238@yahoo.co.jp

Introduction: FOLFIRINOX (FFX) or nab-paclitaxel plus gemcitabine $(\mathrm{GnP})$ are standard regimens in patients with advanced pancreatic cancer. However, chemotherapy can impair quality of life $(\mathrm{QoL})$ due to adverse events. Because the life expectancy of this population is typically short, QoL is as important as the actual length of life.

Aims \& Methods: The aim of this study was to assess QoL during chemotherapy in patients with advanced pancreatic cancer. Twenty-one Japanese patients with unresectable advanced pancreatic cancer and performance status $0-1$ were included in this study. All patients were treated with FFX or GnP as first-line chemotherapy. QoL was assessed using the European Organization for Research and Treatment for Cancer Quality of Life Core Questionnaire (EORTC QLQC30), and anxiety and depression were measured using the Hospital Anxiety and Depression Scale (HADS) at baseline and 2 weeks and every month after initiation of chemotherapy. Changes between score at baseline and median score after chemotherapy were compared using Wilcoxon signed-rank test. Continuous variables are presented as median (range).

Results: Thirteen male and 8 female patients were included, with a median age of $65(50-75)$ years and BMI of $21.2(16.0-26.2) \mathrm{kg} / \mathrm{m}^{2}$. The chemotherapy regimens were FFX in 5 men and 2 women, modified-FFX in 4 men and 4 women, and $\mathrm{GnP}$ in 4 men and 2 women. Eight patients took opioids for pain, and 4 received celiac plexus neurolysis. Regarding global health status (GHS) and functional scales in QLQ-C30, baseline scores were: GHS, 50 (17-92)\%; physical, 87 (53$100) \%$; role, 83 (33-100)\%; emotional, 67 (33-100)\%; cognitive, $83(33-100) \%$; and social, $67(11-100) \%$. After chemotherapy, role function scale was decreased significantly $(p=0.04)$, and nausea $(p=0.02)$ and diarrhea $(p=0.049)$ were more frequently observed, while pain was relieved $(p=0.002)$. In analysis according to patients' background, a lot of evaluation in patients with BMI $<21 \mathrm{~kg} / \mathrm{m}^{2}$ tended to be worse than in those with BMI $\geq 21 \mathrm{~kg} / \mathrm{m}^{2}$ after chemotherapy. Regarding HADS at baseline, 5 patients reported depression, 7 borderline depression, 5 anxiety requiring intervention, and 5 anxiety requiring follow-up. There were no significant changes in HADS after chemotherapy.

Conclusion: In patients with advanced pancreatic cancer, GHS and mental status had already deteriorated at baseline. Although pain scale might be improved due to analgesic treatment, role function scale, nausea and diarrhea became worse during chemotherapy. In particular, QoL tended to deteriorate in lean patients. These results indicate that pain as well as QoL factors should always be considered to manage chemotherapy properly in patients with advanced pancreatic cancer.

Disclosure of Interest: All authors have declared no conflicts of interest.

\section{P0783 C-REACTIVE PROTEIN (CRP) LEVEL IS AN INDICATOR OF THE THERAPEUTIC EFFECT OF TREATMENT RESPONSE TO ENDOSCOPIC ULTRASOUND-GUIDED CELIAC NEUROLYSIS} (EUS-CPN) IN PATIENTS WITH PANCREATIC CANCER (PC) PAIN

G. Kimura, Y. Hashimoto, K. Umemoto, K. Watanabe, M. Sasaki, H. Takahashi, H. Imaoka, I. Oono, S. Mitsunaga, M. Ikeda Hepato-biliary-pancreatic Internal Medicine, National Cancer Center Hospital East, Kashiwa/Japan

Contact E-mail Address: gengen_cocacola@yahoo.co.jp

Introduction: Pain devastates the quality of life and is often difficult to control in unresectable PC. Opioid analgesics are used to alleviate the pain. However, pharmacologic intervention sometimes fails and the side effects could become major problems. Recently, EUS-CPN has been shown to have a therapeutic effect against PC pain in the short term.

Aims \& Methods: The objective of the study was to evaluate the efficacy and safety over the long term and to identify predictors of pain relief in patients 
treated by EUS-CPN. Clinical information was obtained retrospectively from the medical charts and endoscopy reporting system. The follow-up period was until the patient died or was lost to follow-up. Between November 2014 and March 2017, 70 patients with PC pain were enrolled. We performed EUS-CPN by injection of $5 \mathrm{ml}$ of bupivacaine mixed with $15 \mathrm{ml}$ of pure ethanol on the celiac plexus and superior mesenteric plexus using a $22 \mathrm{G}$ endoscopic ultrasound-guided fine needle aspiration needle. Treatment response was assessed by self-reported pain relief and change in the daily dose requirement of morphine. Treatment response was defined as to decrease or stay of the same amount of morphine consumption after EUS-CPN, or achieve morphine dosage level within 4 weeks after EUS-CPN if morphine consumption temporarily elevated because of the delayed response of EUS-CPN. Pain evaluation was conducted at 1 week, 4 weeks, 12 weeks after EUS-CPN and tumor disease progression.

Results: The overall response rate was $81.4 \%(57 / 70)$. The median duration of pain relief was 4.0 months. 10 patients required a second EUS-CPN due to pain relapse, and $90 \%(9 / 10)$ showed response to the repeat procedure. Of the 44 patients who showed response to the initial EUS-CPN during chemotherapy, the pain relief persisted after the diagnosis of tumor progression in 16 patients (16/44: 36.3\%). Between the response group and no-response groups, there were significant differences in the prevalences of liver metastasis (47 vs. $92 \%$, $P=0.003)$, lung metastasis (12 vs. $38 \%, P=0.02$ ) and lymph node metastasis (56 vs. $92 \%, P=0.015$ ), and in the serum levels of CEA (median: 6.7 vs. $17.1 \mathrm{ng}$ ml, $P=0.017$ ), CA19-9 (median: 617.2 vs. $3519 \mathrm{U} / \mathrm{ml}, P=0.009$ ), CRP (median: 0.61 vs. $1.91 \mathrm{mg} / \mathrm{dl}, P=0.029$ ) and albumin (median: 3.4 vs. $3.8 \mathrm{~g} / \mathrm{dl}, P=0.029$ ). Univariate analysis revealed a significantly smaller percentage of patients with CRP level elevation over $3.0 \mathrm{mg} / \mathrm{dl}$ from baseline within 4 weeks after EUS-CPN (11 vs. $38 \%, P=0.01$ ). The overall survival after EUS-CPN was also significantly longer in the response group as compared to the no-response group (median: 5.8 vs. 3.1 months, $P=0.01$ ).

Conclusion: Our study demonstrated that EUS-CPN had therapeutic effect on intractable pain in unresectable PC in the long follow-up. Repeat EUS-CPN was also effective in patients who showed a treatment response to the first EUS-CPN. Patients who did not benefit from EUS-CPN had strong cancer inflammation and aggressiveness, indicative of higher CRP, CA19-9 and lower albumin levels, and the higher frequencies of liver metastasis.

Disclosure of Interest: All authors have declared no conflicts of interest.

\section{P0784 PRESENTATION SYMPTOMS AND RISK FACTORS ARE ASSOCIATED WITH DIAGNOSTIC DELAY AND DISEASE STAGE BUT NOT WITH PROGNOSIS OF PANCREATIC CANCER}

G. Capurso ${ }^{1}$, C. Stornello ${ }^{1}$, L. Archibugi ${ }^{1}$, S. Stigliano ${ }^{1}$, M. Signoretti ${ }^{1}$, M. Milella ${ }^{2}$, V. Barucca ${ }^{3}$, C. Capalbo ${ }^{4}$, G. Delle Fave ${ }^{1}$

${ }^{1}$ Digestive \& Liver Disease Unit, Ii, Medical School Univesity "Sapienza", S. Andrea Hospital, Rome/Italy

${ }^{2}$ Oncology, IFO Cancer Institute, Rome/Italy

${ }^{3}$ Digestive Oncology Phd Program, Medical School University "Sapienza", S. Andrea Hospital, Rome/Italy

${ }^{4}$ Oncology Unit, Medical School Univesity "Sapienza”, S. Andrea Hospital, Rome/ Italy

\section{Contact E-mail Address: livia.archibugi@hotmail.it}

Introduction: Pancreatic ductal adenocarcinoma (PDAC) 5-year survival rate is only $6 \%$ as only $20 \%$ of patients are eligible for surgery at diagnosis, due to delayed diagnosis as symptoms are often unrecognized. Data on the association between presentation symptoms, diagnostic delay, disease stage and survival are however limited, with heterogeneous results. Furthermore, the association between risk factors for developing PDAC, such as smoking, obesity, alcohol intake, diabetes and PDAC family history and diagnostic delay has not been explored specifically.

Aims \& Methods: We aimed to investigate the association between presentation symptoms, diagnostic delay, risk factors for PDAC, stage at diagnosis and survival. This was a retrospective analysis of a single-centre cohort of prospectively evaluated PDAC patients with the above mentioned data recorded in a dedicated database. Fisher test for comparison of proportions for categorical variables and Student's t-test for continuous variables were employed. Multiple logistic regression analysis was employed to investigate factors associated with metastatic stage at diagnosis or death. Survival probability was calculated with the Kaplan-Meier curve and Cox analysis was employed to calculate hazard ratios (HR). Tests of statistical significance and confidence intervals were two-sided; a $\mathrm{p}<0.05$ was considered to be statistically significant.

Results: In 434 PDAC patients the mean diagnostic delay was 4 months $(95 \% \mathrm{CI}$ 3.6-4.4). Jaundice was the leading presentation symptom in $74(17 \%)$, weight loss in $164(37.7 \%)$, pain in $105(24 \%)$, new-onset diabetes in $43(9.9 \%)$. Diagnosis was incidental in 24 cases $(5.5 \%)$ or related with undetermined complaints in other $24(5.5 \%)$. The diagnostic delay was significantly shorter for patients with jaundice (mean 1.1 months) compared to those with pain (3.6), new-onset diabetes (5.46) and weight loss (5.96). PDAC family history, smoking and previous diabetes were not associated with presentation symptoms or delay, but obese patients ( 4.3 vs 3.4 months; $p=0.03)$ and those drinking alcohol (4.5 vs 3.6 months; $p=0.03$ ) had a longer delay. The mean diagnostic delay was of 4.5 months in patients with distant metastases, 4.1 in locally advanced and 3.4 in resectable disease $(\mathrm{p}=0.02)$. Patients presenting with jaundice had distant metastases at diagnosis in only $18 \%$ of cases compared to $45 \%$ of other presentations $(\mathrm{p}=0.00001)$. The mean survival was 11.8 months in the 395 patients with available follow-up. At Kaplan-Meier and Cox-proportional regression analysis age at diagnosis ( $\mathrm{HR}=1.02$ per year) and metastatic stage at diagnosis (HR 2.5), but not the different presentation symptoms, or the diagnostic delay were associated with worse survival.
Conclusion: Different presentation symptoms, the presence of obesity and alcohol intake are associated with the diagnostic delay, which is in turn related with disease stage at diagnosis. However, symptoms and delay do not seem to affect prognosis. These results suggest that diagnosis at the preclinical stage is necessary to change disease prognosis.

Disclosure of Interest: All authors have declared no conflicts of interest.

\section{P0785 PLASMA DNA GENOTYPING USING DIGITAL PCR FOR SURVEILLANCE OF PANCREATIC CANCER IN HIGH-RISK INDIVIDUALS}

Y. Mizukami ${ }^{1}$, Y. Ono ${ }^{2}$, H. Karasaki ${ }^{2}$, K. Koizumi ${ }^{3}$, T. Okada ${ }^{1}$, J. Sasajima ${ }^{1}$, M. Yamada ${ }^{4}$, S. Asahara ${ }^{5}$, K. Kawakubo ${ }^{6}$, H. Maguchi

${ }^{1}$ Department Of Medicine, Asahikawa Medical University, Asahikawa/Japan ${ }^{2}$ Center For Clinical And Biomedical Research, Sapporo Higashi Tokushukai Hospital, Sapporo/Japan

${ }^{3}$ Center For Gastroenterology, Shonan Kamakura General Hospital, Kanagawa/ Japan

${ }^{4}$ Department Of Gastroenterology, Shibetsu City Hospital, Shibetsu/Japan ${ }^{5}$ Department Of Gastroenterology, Chiba Tokushukai Hospital, Chiba/Japan ${ }^{6}$ Department Of Gastroenterology And Hepatology, Hokkaido University Graduate School of Medicine, Sapporo/Japan

Center For Gastroenterology, Teine-Keijinkai Hospital, Sapporo/Japan

Contact E-mail Address: mizu@asahikawa-med.ac.jp

Introduction: Cell-free DNA (cfDNA) shed from tumors into the general circulation offers opportunities to trace the genomic evolution of cancer. The level of cfDNA is generally higher in cancer patients than healthy individuals; however, detecting the rare fraction of circulating tumor-derived DNA (ctDNA) in patient plasma remains a technical challenge. Initial efforts have been made to quantify the ctDNA using conventional PCR, but the low sensitivity of this approach has limited its feasibility as a routine clinical test. New technologies for quantifying ctDNA are now sensitive enough for reliable application in the clinic (ref 1). Pancreatic ductal adenocarcinoma (PDA) is among the most lethal human malignancies. Intraductal papillary mucinous cystic neoplasias (IPMNs) are precursors of PDA and provide models of neoplastic progression from a benign intraductal tumor through increasing grades of dysplasia to PDA, and mutations in KRAS and/or GNAS mediate key signaling during early development of the tumors (ref 2 ). Better prediction of histological grades using non-invasive tools is urgently needed for IPMN patients to make appropriate management decisions.

Aims \& Methods: In the current study, we sought to determine if quantification of major driver mutations such as KRAS and GNAS in the plasma cfDNA could serve as biomarkers for diagnosis of localized PDAs and risk stratification of IPMNs. We first established protocols for absolute quantification of very low concentrations of the target mutant alleles using a Bio-Rad QX200 droplet digital PCR platform (ddPCR). Using this novel protocol, feasibility of the assay was tested. At present, 92 PDA and 145 IPMN patients with either benign or malignant disease have been recruited (UMIN000012810).

Results: Although ddPCR-based assays have rather high precision and sensitivity $(0.01 \%)$, limited plasma cfDNA yields in patients with resectable PDAs (Stage 0 II) resulted in significant intrinsic errors due to "subsampling". The issue associated with missing targets at very low abundance during compartmentalization could be effectively overcome by pre-amplification, and the sensitivity of mutant KRAS detection was greatly improved $(80.3 \%)$ relative to a standard protocol $(37.5 \%)$. Given the highly accurate assay, risk assessment of the IPMN cases was performed during follow-up. Although the concentration of plasma cfDNA in patient with IPMNs was not robustly higher than healthy subjects $(15.1+$ / $-5.8 \mathrm{ng} / \mathrm{mL}$ plasma vs $11.3+/-3.2 \mathrm{ng} / \mathrm{mL}$ plasma), mutant KRAS/GNAS alleles from patients, particularly in high-grade IPMNs, can be successfully quantified albeit at low levels. In the subset of patients who had no evidence of "high-risk stigmata" as well as "worrisome features" by imaging modalities (ref 3) and normal tumor markers (CA19-9 and CEA), oncogenic KRAS could be detected in plasma cfDNA earlier than the detection of invasive PDAs by imaging. Conclusion: By setting an appropriate protocol for ddPCR-based cfDNA assay, serial blood sampling allows physicians to conduct real-time detection and monitoring of tumor genomic alterations (ref 4). Although larger validation studies are required before introduction into the clinic, early results of the study indicate that our approach allows the detection of localized early-stage disease and offers an alternative tool to monitor IPMN progression non-invasively.

Disclosure of Interest: All authors have declared no conflicts of interest.

\section{References}

1. Wan JC, Massie C, Garcia-Corbacho J, et al. Liquid biopsies come of age: towards implementation of circulating tumour DNA. Nat Rev Cancer 2017; 17:223-238

2. Patra KC, Bardeesy N, Mizukami Y. Diversity of precursor lesions for pancreatic cancer: The genetics and biology of intraductal papillary mucinous neoplasm. Clin Transl Gastroenterol 2017;8:e86.

3. Tanaka M, Fernandez-del Castillo C, Adsay V, et al. International consensus guidelines 2012 for the management of IPMN and MCN of the pancreas. Pancreatology 2012;12:183-97.

4. Berger AW, Schwerdel D, Costa IG, et al. Detection of hot-spot mutations in circulating cell-free DNA from patients With intraductal papillary mucinous neoplasms of the pancreas. Gastroenterology 2016;151:267-270. 
P0786 TREATMENT EFFICACY AND ADVERSE EVENTS OF GEMCITABINE PLUS NAB-PACLITAXEL USED FOR METASTATIC PANCREATIC CANCER: A RETROSPECTIVE COHORT STUDY

I.R. Cho, J.H. Jo, H.S. Lee, M.J. Chung, J.Y. Park, S.W. Park, S.Y. Song, J. Chung, S. Bang

Department Of Internal Medicine, Yonsei University College of Medicine, Seoul Korea, Republic of

Contact E-mail Address: inrae0428@yuhs.ac

Introduction: Pancreatic cancer is a lethal disease and the fifth most common cause of cancer-related death in Korea. Pancreatic cancer patients show dismal prognosis with a 5 -year survival rate less than $10 \%$, because the majority of patients are diagnosed in advanced stage. Since the late 1990s, gemcitabinebased chemotherapy has been used as a mainstay of metastatic pancreatic cancer $(\mathrm{mPC}$ ) treatment and various combination therapies (such as combination with capecitabine or erlotinib) had been attempted to improve the patients survival, so far. Recently, MPACT trial, a randomized phase III trial showed that combination of gemcitabine and nab-paclitaxel had statistically significant survival benefit compared with gemcitabine monotherapy. Based on the results of this trial, gemcitabine with nab-paclitaxel combination therapy is currently being used as a standard therapy for pancreatic cancer patients. However, only $2 \%$ of the MPACT trial study population was Asian, and other researches on Asian population group are also lacking. Therefore, we investigated treatment efficacy and safety of gemcitabine plus nab-paclitaxel combination therapy for $\mathrm{mPC}$ treatment in Korean population.

Aims \& Methods: Total 66 metastatic pancreatic cancer patients treated with gemcitabine $\left(1000 \mathrm{mg} / \mathrm{m}^{2}\right)$ and nab-paclitaxel $\left(125 \mathrm{mg} / \mathrm{m}^{2}\right)$ regimen (on day 1,8 , 15 of a 28-day cycle) as the first line chemotherapy from February 2016 were identified using the Severance Hospital Pancreatic Cancer Cohort Registry. Treatment efficacy (overall survival (OS), progression-free survival (PFS), objective response rate) and treatment-related adverse events (AE) of patients (occurrence rate, severity grade and dose-intensity) were analyzed.

Results: The median follow-up period was 7.4 months (range 1.5-14.9 months); during this period, $21(31.8 \%)$ patients died. Median cumulative dose of gemcitabine and nab-paclitaxel were $13,000 \mathrm{mg} / \mathrm{m}^{2}$ and $1487.5 \mathrm{mg} / \mathrm{m}^{2}$. The median OS, PFS and objective response rate were 12.0 months $(95 \%$ confidence interval [CI] 9.515-14.485), 7.8 months ( $95 \%$ CI $5.021-10.579)$ and $48.5 \%$, respectively. The incidence of neurotoxicity was $54.5 \%$ and $12(18.2 \%)$ patients experienced grade $\geq 3$ neurotoxicity. $30(45.5 \%)$ patients showed grade $\geq 3$ neutropenia and $10(15.2 \%)$ patients had febrile neutropenia. Grade $>3$ gastrointestinal AE was occurred in $11(16.7 \%)$ patients and $28(42.4 \%)$ patients experienced dermatologic $\mathrm{AE}$ such as alopecia and skin eruption. About $59 \%$ of patients experienced treatment delays due to adverse events. Dose reduction was performed in 39 $(59.1 \%)$ patients and 14 patients experienced treatment cessation due to severe $\mathrm{AE}$

Table 1: Treatment efficacy and treatment-related adverse events of gemcitabine with nab-paclitaxel

\section{Variables}

Duration of chemotherapy

Cycles (28-day schedule)

Duration, days

$5(2-12)$

$141(32-435)$

Efficacy of Chemotherapy

Overall survival - months $(95 \% \mathrm{CI})$

$12.0(9.515-14.485)$

Progression-free survival - months $(95 \% \mathrm{CI})$

$7.8(5.021-10.579)$

Adverse events

Peripheral neuropathy

Grade $\geq 3$ neuropathy

$36(54.5 \%)$

Grade $\geq 3$ Neutropenia

$12(18.2 \%)$

Febrile neutropenia

$30(45.5 \%)$

$10(15.2 \%)$

$14(21.2 \%)$

$11(16.7 \%)$

Grade $\geq 3$ adverse event

$32(48.5 \%)$

General weakness

$28(42.4 \%)$

Dermatologic adverse event

$21(31.8 \%)$

Gemcitabine

$39(59.1 \%)$

nab-paclitaxel

$39(59.1 \%)$

n (\%)

Cessation of administration due to $A E$

n $(\%)$

$14(21.2 \%)$

Conclusion: These results suggest that gemcitabine and nab-paclitaxel combination therapy is effective for metastatic pancreatic cancer treatment in east-Asian population group. Similar to previous studies, this combination therapy showed remarkable neurotoxicity and myelosuppression. Careful monitoring and proper management during chemotherapy is required.

Disclosure of Interest: All authors have declared no conflicts of interest.
P0787 IMPACT OF PREOPERATIVE EUS-FNA ON PERITONEAL RECURRENCE AND SURVIVAL IN PATIENTS WITH PANCREATIC CANCER

S.H. Kim, S.J. Lee, K.H. Lee, J.K. Lee, K.T. Lee, J.K. Park

Gastroenterology, Samsung Medical Center, Seoul/Korea, Republic of

Contact E-mail Address: ilonaelona@hanmail.net

Introduction: Endoscopic ultrasound-guided fine needle aspiration (EUS-FNA) is a useful and safe method for tissue confirmation of malignancy. This method has the risk of tumor cell dissemination along the needle track or within the peritoneum by preoperative EUS-FNA.

Aims \& Methods: The aim of our study was to estimate the risk of peritoneal recurrence and the impact on long-term outcomes by preoperative EUS-FNA in resected pancreatic cancer. The records of patients diagnosed with pancreatic cancer who underwent curative resection between 2009 and 2013 were reviewed retrospectively. A total of 394 patients were included: 78 patients with preoperative EUS-FNA (EUS-FNA group) and 316 without preoperative EUS-FNA (Non-EUS-FNA group). Peritoneal recurrence was diagnosed based on image findings.

Results: Median length of follow-up was 23 months (range $1 \sim 94$ months). A total of 82 patients had peritoneal recurrence; $34.6 \%$ (27/78) in EUS-FNA group vs. $28.2 \%(89 / 316)$ in Non-EUS-FNA group $(P=0.26)$. Cancer-free survival and overall survival were not different between the groups: median cancer-free survival in EUS-FNA group was 10.8 months compared with 10.6 months in NonEUS-FNA group $(\mathrm{P}=0.83)$, and median overall survival in EUS-FNA group was 56.4 months compared with or 56.7 months in Non-EUS-FNA group $(\mathrm{P}=0.93)$.

Conclusion: Preoperative EUS-FNA for pancreatic cancer was not associated with an increased rate of peritoneal recurrence and mortality. Our study suggests that EUS-FNA is a safe method for obtaining tissues of pancreatic masses.

Disclosure of Interest: All authors have declared no conflicts of interest.

\section{References}

1. S. Ngamruengphong, C. Xu, T.A. Woodward, M. Raimondo, et al. Risk of gastric or peritoneal recurrence, and long-term outcomes, following pancreatic cancer resection with preoperative endosonographically guided fine needle aspiration. Endoscopy 2013;45:619-626

2. Kenji Ikezawa, Hiroyuki Uehara, Arata Sakai, et al. Risk of peritoneal carcinomatosis by endoscopic ultrasound-guided fine needle aspiration for pancreatic cancer. $J$ Gatroenterol (2013) 48:966-972

3. Abdul Hamid El Chafic, John Dewitt, Julia Kim LeBlanc, et al. Impact of preoperative endoscopic ultrasound-guided fine needle aspiration on postoperative recurrence and survival in cholangiocarcinoma patients. Endoscopy 2013;45:883-889

4. Akiko T, Akio K, Tomoaki M, Hjime Y, et al. Resected tumor seeding in stomach wall due to endoscopic ultrasonography-guided fine needle aspiration of pancreatic adenocarcinoma. World Gastroenterol 2015 July 21;21(27):8458-8461

5. Won Jae Yoon, Ebubekir S. Daglilar, Carlos Fernandez-del Castillo, et al. Peritoneal seeding in intraductal papillary mucinous neoplasm of the pancreas patients who underwent endoscopic ultrasound-guided fine-needle aspiration: The PIPI study. Endoscopy 2014;46:382-387

\section{P0788 HENT1 \& DPD EXPRESSION IN EUS-FNAB SAMPLES OF PANCREATIC DUCTAL ADENOCARCINOMA: TECHNICAL FEASIBILITY AND PROGNOSTIC SIGNIFICANCE OF GEMCITABINE+S1-BASED CHEMORADIOTHERAPY}

R. Yamada ${ }^{1}$, S. Isaji ${ }^{2}$, H. Inoue ${ }^{1}$, A. Hayasaki ${ }^{2}$, Y. Murata ${ }^{2}$, M. Kishiwada ${ }^{1}$,

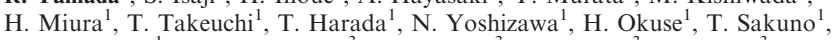
M. Nakamura ${ }^{1}$, M. Katsurahara ${ }^{3}$, Y. Hamada ${ }^{3}$, K. Tanaka ${ }^{3}$, N. Horiki ${ }^{3}$,

Y. Takei ${ }^{2}$

${ }^{1}$ Gastroenterology And Hepatology, Mie University Graduate School of Medicine, Tsu/Japan

${ }^{2}$ Hepatobiliary Pancreatic And Transplant Surgery, Mie University Graduate School of Medicine, Tsu/Japan

${ }^{3}$ Endoscopy, Mie University Graduate School of Medicine, Tsu/Japan

Contact E-mail Address: reiko-t@ @lin.medic.mie-u.ac.jp

Introduction: Gemcitabine (Gem) therapy had been the standard treatment for advanced pancreatic ductal adenocarcinoma (PDAC) for long time. Even though FOLFIRINOX, a combination of fluorouracil, oxaliplatin and irinotecan, and gemcitabine/nab-paclitaxel $(\mathrm{GnP})$ therapy are currently recommended as firstline drugs, Gem is still one of the important options. Efficient permeation of Gem into cells requires specialized integral membrane transporter proteins to cross plasma membranes. Among these transporters, the major mediators of Gem uptake into human cells are the human equilibrative nucleoside transporter 1 (hENT1). In the previous study started since 2005, we found hENT1 expression in endoscopic ultrasonography-guided fine-needle aspiration biopsy (EUSFNAB) samples to be a useful prognostic marker of Gem-based chemoradiotherapy (Gem-CRT) for pancreatic ductal adenocarcinoma (PDAC) (Pancreas 2016). Since November 2011, our institution has replaced Gem + tegafur/gimeracil/oteracil (S-1) (GS)-CRT with Gem-CRT in an attempt to improve clinical response and prognosis. Aggregate median survival time (MST: months) of GS-CRT was compared with previous Gem-CRT result, and the superior efficacy of GS-CRT was observed (MST: 16 versus 20, respectively), particularly in hENT1-negative patients (MST: 9 versus 14, respectively). However, there were no differences in 
hENT1-positive patients (MST: 25 versus 25, respectively). We suspect that genetic factors might play a role in this observed variability.

Aims \& Methods: In the present study, we evaluated hENT1 and dihydropyrimidine dehydrogenase (DPD: enzyme involved in the degradation of tegafur) expression in EUS-FNAB samples for evaluating and predicting the clinical efficacy of GS-CRT. EUS-FNAB samples were obtained prior to GS-CRT administration. In total, 95 formalin-embedded PDAC specimens were obtained. In the samples determined to have sufficient material remaining following cytological/histological diagnosis $(\mathrm{n}=76)$, hENT1 expression was evaluated via immunohistochemical (IHC) examination. Following IHC analysis of hENT1, a further assessment of DPD expression was carried out in those samples determined to have sufficient material remaining $(\mathrm{n}=58)$.

Results: By reusing the EUS-FNAB specimens after diagnosis of PDAC, hENT1 and DPD expression could be successfully assessed in $79.2 \%(76 / 95)$ and $61.1 \%$ (58/95) of these cases, respectively. In those sufficient for hENT1 testing, $67.1 \%$ (51/76) were found to be positive. And in those sufficient for DPD testing, $27.6 \%$ $(16 / 58)$ were found to be positive. MST was significantly longer in hENT1-positive patients ( 25 versus 14 negative; $\mathrm{P}=0.015$ ). As for $\mathrm{DPD}$, MST was significantly longer in DPD-negative patients (33 versus 14 positive; $\mathrm{P}<0.001$ ). In the multivariate model including pretreatment clinical factors (age, sex, tumor location, tumor size, UICC-T classification, hENT1 expression, and DPD expression) and the clinical response after GS-CRT (response of GS-CRT, reduction rate in serum CA19-9 level, and distant metastasis after GS-CRT), only hENT1 expression $(\mathrm{HR}=3.511 ; \quad 1.545-7.981, \quad \mathrm{P}=0.003)$ and $\mathrm{DPD}$ expression $(\mathrm{HR}=0.232 ; 0.108-0.496, \mathrm{p}<0.001)$ were found to be significant independent prognosis factors.

Conclusion: hENT1 and DPD expression observed in EUS-FNAB samples can be useful clinical predictors in PDAC cases treated with GS-CRT. Disclosure of Interest: All authors have declared no conflicts of interest.

\section{References}

Yamada R, Mizuno S, Uchida K, et al. Human Equilibrative Nucleoside Transporter 1 Expression in Endoscopic Ultrasonography-Guided Fine-Needle Aspiration Biopsy Samples Is a Strong Predictor of Clinical Response and Survival in the Patients With Pancreatic Ductal Adenocarcinoma Undergoing Gemcitabine-Based Chemoradiotherapy. Pancreas. 2016;45:761-71.

\section{P0789 EVALUATION OF THE EFFICACY OF ENDOSCOPIC SPHINCTEROTOMY IN THE TREATMENT OF SYMPTOMATIC IPMN WITHOUT WORRISOME FEATURES. A MULTICENTER FRENCH RESTROSPECTIVE STUDY}

J. Gonzalez ${ }^{1}$, D. Lorenzo ${ }^{1}$, J.P. Ratone ${ }^{2}$, A. Culetto ${ }^{3}$, P. Lévy ${ }^{4}$, M. Giovannini ${ }^{5}$, M. Barthet ${ }^{1}$

${ }^{1}$ Gastroenterology, AMU, AP-HM, Hôpital Nord, Marseille/France

${ }^{2}$ Endoscopy, Paoli Calmettes Institutes, Marseille/France

${ }^{3}$ Gastroenterology, CHU Rangueil, Toulouse/France

${ }^{4}$ Service De Gastroenterologgie-pancreatologie, Université Denis Diderot Hopital Beaujon, Service de Pancreatologie, Clichy Cedex/France

${ }^{5}$ Endoscopy, Institut paoli Calmettes, Marseille/France

Contact E-mail Address: jmgonza05@yahoo.fr

Introduction: Intraductal Papillary Mucinous Neoplasms (IPMN) require a close follow-up to early detect worrisome features, which imply a pejorative evolution towards dysplasia and surgery. However, some patients are symptomatic including post-prandial pancreas related abdominal pain (PPAP) or recurrent acute pancreatitis (RAP). We currently do not know if those symptoms are risk factor of cancer in IPMN, and there are no data about the efficacy of endoscopic sphincterotomy (ES) to manage them.

Aims \& Methods: This was a retrospective multi-centered observational study in 4 tertiary expert centers. The files of 27 patients that underwent ES for symptomatic IPMN without worrisome features nor surgical indication were considered. Six were excluded $(4=$ lack of data; $1=$ pseudocyst; $1=$ adenocarcinoma $)$, thus 21 were analyzed. Age, sex, medical history, time of follow-up, characteristics of IPMN and ERCP procedures, clinical success, evolution and need for surgery were recorded. The primary endpoint was to assess the efficacy of ES for improving the symptoms of pain related to IPMN. The efficacy was defined as the resolution or the decreasing $>50 \%$ of the symptoms' frequency. The secondary endpoints were to document, the need for a second ES, the occurrence of WF during follow-up, the need for surgery and its indications.

Results: There were 10 men and 11 women involved, with a mean age of 66 years old [45-87] and a mean total follow-up of 105 months [17-276]. Their symptoms were PPAP in 6 patients and RAP in the 15 others ( 5 being severe AP), with a mean of 3.6 episode/year [1-12]. The delay between diagnosis and ES was 41 months $[1-192]$ and the patients were followed by MRI $(81 \%)$, EUS $(95 \%)$ CTscan $(36 \%)$ or by alternating (MRI and EUS) for $45 \%$. The IPMN were involving the accessory branch duct in $71 \%$, or mixed type in $29 \%$, with a wirsung duct always $<7 \mathrm{~mm}$. The mean number of cysts was 3.5 [1-10], the larger measuring $12.7 \mathrm{~mm}$ [5-25] mean. They were located for $59 \%$ in the head, $17 \%$ in the body or tail, and they were diffuse in $24 \%$. None patient had worrisome features. Endoscopy: ERCP with pancreatic ES was performed in $100 \%$ of the cases ( 5 with pancreas divisum, 2 pre-cut required), associated with a biliary ES in $33 \%$ of the cases. A prophylaxis with NSAIDS was carried out in $38 \%$ of the cases and a plastic stent placement in $33 \%$. There were 4 postERC benign pancreatitis, and the mean follow-up after ES was 54 months [13167]. The clinical efficacy rate as defined after one session was $81 \%(17 / 21)$. Among them, one had a late recurrence (41 months) and underwent a second ES session with final success, whereas 4 have been operated ( 2 for initial pain, 2 for WF). Among the patients whom failed $(4 / 21)$, one had a second ES with final success and 3 finally had surgery. The two groups were comparable in terms of age, sex, mean follow-up time, and characteristics of IPMN. The final efficacy of ES was $86 \%(19 / 21)$. In total seven patients were operated after a mean of 19 months of follow-up, $24 \%$ if success of ERCP and in $75 \%$ if failure. The indications were WF $(n=4)$ or pain $(n=3)$. The histopathology showed four low-grade dysplasia, one high-grade dysplasia, and two no dysplasia (=surgery for pain). No patients evolved towards adenocarcinoma in the follow-up.

Conclusion: ES for symptomatic IPMN without WF is effective in $81 \%$ of the cases. A symptomatic IPMN (pain) does not seem to be a valuable indication for surgery, since no patients developed with cancer after a follow-up greater than 5 years.

Disclosure of Interest: M. Barthet: Consultant for Boston Scientific

All other authors have declared no conflicts of interest.

\section{P0790 STATIN USE DECREASES THE RISK OF PANCREATIC} CANCER OCCURRENCE: A META-ANALYSIS

L. Archibugi ${ }^{1}$, P.G. Arcidiacono ${ }^{2}$, G. Delle Fave ${ }^{1}$, G. Capurso ${ }^{1}$

${ }^{1}$ Digestive \& Liver Disease Unit, S. Andrea Hospital - University of Rome "La Sapienza", Rome/Italy

${ }^{2}$ Pancreato-biliary Endoscopy And Endosonography Division, Pancreas Translational \& Clinical Research Center, San Raffaele Scientific Institute IRCCS, Vita-Salute San Raffaele University, Milan/Italy

Contact E-mail Address: livia.archibugi@hotmail.it

Introduction: Statins are widely prescribed both for primary and secondary prevention of coronary artery disease and for the treatment of dyslipidemia. Several studies evaluated the association between statin use and the onset of pancreatic cancer (PDAC) in order to evaluate a possible chemopreventive effect, with heterogeneous results. Previous meta-analyses evaluating researches published up to 2012 did not find any association to the risk, but in the latest years new studies with interesting results have been published.

Aims \& Methods: The aim of our study was to conduct a new systematic review and meta-analysis to clarify this association. A comprehensive literature search of PUBMED for articles published up to November 2016 and abstracts presented between 2012-2016 at the DDW and ASCO conventions was carried out. Eligible studies were case-control studies (CC), cohort studies (C) and randomized controlled trials (RCTs) assessing the effect of statin use on the risk of PDAC, compared with placebo or no treatment. Studies had to report Odds Ratio (OR), Relative Risk (RR), or Hazard Ratio (HR), estimates with $95 \%$ confidence interval $(\mathrm{CI})$, or provide sufficient data for their calculation. Pooled adjusted ORs with corresponding $95 \%$ CIs were calculated using random effects model. Publication bias was assessed through Begg and Mazumdar test. Heterogeneity was assessed by means of the $\mathrm{I}^{2}$ value.

Results: A total of 21 studies $(12$ CC, 6 C, 3 RCTs) contributed to the analysis. A total of 11383 PDAC patients and 2991004 controls were included. The pooled incidence of PDAC was $0.27 \%(3161 / 1167130)$ among statin users and $0.44 \%$ (8144/1835153) among the non-users. The overall pooled result for all studies demonstrated a reduced risk of PDAC among statin users (OR 0.82; 95\% CI $0.69-0.96 ; p=0.019)$, compared to non-statin users. In a subgroup analysis the protective effect was limited to case-control studies (OR 0.72; 95\% CI $0.56-0.93$ ) and not to cohort (OR $0.93 ; 95 \%$ CI $0.73-1.19$ ) nor RCTs (OR $1.04 ; 95 \%$ CI $0.56-1.94)$. No publication bias was found.

Conclusion: This is the first meta-analysis showing that statins exert a protective effect on the incidence of PDAC. Further studies taking into account statin dose, duration and subgroups of patients are needed in order to clarify the association. Disclosure of Interest: All authors have declared no conflicts of interest.

\section{P0791 AN IMPROVED DIGITAL POLYMERASE CHAIN REACTION PROTOCOL TO CAPTURE LOW-COPY KRAS MUTATIONS IN PLASMA CELL-FREE DNA BY RESOLVING "SUBSAMPLING" ISSUES}

Y. Ono ${ }^{1}$, A. Sugitani ${ }^{2}$, H. Karasaki ${ }^{2}$, M. Ogata ${ }^{1}$, R. Nozaki ${ }^{2}$, J. Sasajima ${ }^{3}$, Y. Mizukami ${ }^{3}$

${ }^{1}$ Laboratory Of Clinical Bioinformatics, Center for Clinical and Biomedical Research, Sapporo Higashi Tokushukai Hospital, Sapporo/Japan

${ }^{2}$ Center for Clinical and Biomedical Research, Sapporo Higashi Tokushukai Hospital, Sapporo/Japan

${ }^{3}$ Department Of Medicine, Asahikawa Medical University, Asahikawa/Japan

Contact E-mail Address: y-ono_ccbr@tohtoku.jp

Introduction: Genetic alterations responsible for the initiation of cancer may serve as immediate biomarkers for early diagnosis. Plasma levels of circulating cell-free DNA (cfDNA) in cancer patients are higher than those in healthy individuals; however, the major technical challenge for the widespread implementation of cfDNA genotyping as a diagnostic tool is the insufficient sensitivity and specificity of detecting early-stage tumors that shed low amounts of cfDNA. Droplet Digital PCR (ddPCR) has rather high precision and sensitivity for absolute quantification $(0.01 \%)$. However, due to very low target concentrations of cfDNA in plasma, there may be an intrinsic error due to "subsampling" (Ref. 1). This is caused by limited cfDNA yield and missing targets at very low abundance during compartmentalization in ddPCR-based liquid biopsy assays. Such issues potentially result in large variations or errors in quantification, even when using highly accurate platform. Using ddPCR technology for early cancer diagnosis and risk stratification is still challenging. 
Aims \& Methods: In this study, we aimed to overcome the subsampling issue and to establish a more reliable framework for digital quantification of rare tumor cell-derived mutant alleles for non-invasive diagnosis of gastrointestinal cancer. To establish more reliable dd-PCR protocol for quantification of low-frequency alleles within a limited cfDNA pool, two-step multiplex ddPCR targeting eight clinically relevant mutant $K R A S$ variants was examined using a Bio-Rad QX200 droplet digital PCR platform. Plasma samples from patients with colorectal $(\mathrm{n}=10)$ and pancreatic cancer $(\mathrm{n}=9)$ were evaluated, and cfDNA from healthy volunteers $(\mathrm{n}=50)$ was utilized to calculate reference intervals.

Results: Limited cfDNA yields in patients with resectable colorectal and pancreatic cancers did not meet the requirement for efficient capture and quantification of rate mutant alleles by ddPCR. To overcome the the subsampling issues and achieve better assay specificity, we attempted pre-amplification of plasma cfDNA using primers flanking $K R A S$ exon 2 as the first-step PCR. Eight pre-amplification cycles followed by a second-run ddPCR were sufficient to obtain approximately 5000-10,000 target alleles/ng cfDNA, resolving the subsampling issue; furthermore, the signal-to-noise ratio for rare mutant alleles against the massive background presented by the wild-type allele was significantly enhanced. The cut-off limit of reference intervals for mutant KRAS was determined to be $\sim 0.09 \%$ based on samples from healthy individuals.

Conclusion: The modification introduced in the ddPCR protocol facilitated the quantification of low-copy alleles carrying driver mutations, such as oncogenic $K R A S$, in localized and early-stage cancers using small blood volumes, thus offering a minimally invasive modality for timely diagnosis.

Disclosure of Interest: All authors have declared no conflicts of interest

\section{Reference}

1. Lievens, A., et al., 2016. Measuring Digital PCR Quality: Performance Parameters and Their Optimization. PLoS One 11, e0153317.

\section{P0792 ANALYSIS OF CLINICAL PREDICTIVE FACTORS AFFECTING THE OUTCOME OF 2ND LINE CHEMOTHERAPY FOR THE PATIENT OF ADVANCED PANCREATIC CANCER}

J.E. Lee ${ }^{1}$, H.S. Lee ${ }^{1}$, M.J. Chung ${ }^{2}$, J.Y. Park ${ }^{2}$, S. Bang ${ }^{2}$, S.W. Park ${ }^{2}$, S.Y. Song ${ }^{2}$ ${ }^{1}$ Gastroenterology, Yonsei University, Seoul/Korea, Republic of

${ }^{2}$ Department Of Internal Medicine, Yonsei University College of Medicine, Seoul/ Korea, Republic of

\section{Contact E-mail Address: chelle884@yuhs.ac}

Introduction: Benefit of second line chemotherapy (SL) after failed first-line chemotherapy (FL) in advanced pancreatic cancer has not yet been established. We intend to identify prognostic factors and ultimately devise a model of clinical parameters for decision of SL versus basic supportive care (BSC) after failure of FL chemotherapy.

Aims \& Methods: 408 patients who received gemcitabine based-first-line chemotherapy for advanced pancreatic adenocarcinoma at Yonsei University Hospital between January 2010 and December 2014 were retrospectively reviewed. Significant clinical parameters regarding second line related survival were reviewed for analyzing predictive factors.

Results: 161 of $408(39.5 \%)$ received SL therapy. Median overall survival from the beginning of SL (OS2) was 20.0 weeks $(14.0 \sim 34.0)$. Significantly more SL patients presented higher body mass index $(\mathrm{BMI})(\mathrm{p}=0.001)$ and ECOG $0-1$ $(p=0.003)$ at diagnosis, lower rate of lung metastasis $(p<0.001)$ and longer duration of FL $(\mathrm{p}<0.001)$. More SL patients had received gemcitabine-based concurrent chemo-radiation therapy $(\mathrm{CCRT})(\mathrm{p}=0.029)$ compared to FL only patients. Prognostic factors significant to OS2 were BMI at diagnosis $(\mathrm{p}=0.019$ $\mathrm{HR}=0.870$ ), duration of FL therapy (median duration 16weeks (8.00-28.00) $\mathrm{p}=0.004, \mathrm{HR}=0.986)$, presence of peritoneal metastasis $(\mathrm{p}=0.002, \mathrm{HR} 1.732)$ at diagnosis, malignant thrombotic event during firstgline chemotherapy $(\mathrm{p}=0.001, \mathrm{HR}=0.428)$. Experience of CCRT was also a significant prognostic factor $(\mathrm{p}=0.001, \mathrm{HR}=2.245)$; initial staging of the CCRT group was TNM stage3, which might be the ultimate factor impacting OS2.

Conclusion: Study suggests that SL chemotherapy may be beneficial for patients with longer duration of FL chemotherapy, higher BMI at diagnosis, patients without peritoneal metastasis at diagnosis, no malignant thrombosis event during chemotherapy and patient initially TNM stage3, who received Gemcitabine based CCRT.

Disclosure of Interest: All authors have declared no conflicts of interest.

\section{References}

1. Berger AK, Abel U, Komander C, et al. Chemotherapy for advanced pancreatic adenocarcinoma in elderly patients $(>/=70$ years of age): a retrospective cohort study at the National Center for Tumor Diseases Heidelberg. Pancreatology 2014;14:211-5.

2. Chung KH, Ryu JK, Son JH, et al. Efficacy of Capecitabine Plus Oxaliplatin Combination Chemotherapy for Advanced Pancreatic Cancer after Failure of First-Line Gemcitabine-Based Therapy. Gut Liver 2017;11:298-305.

3. Yoo C, Kim BJ, Kim KP, et al. Efficacy of Chemotherapy in Patients with Unresectable or Metastatic Pancreatic Acinar Cell Carcinoma: Potentially Improved Efficacy with Oxaliplatin-containing Regimen. Cancer Res Treat 2016.

4. Viaud J, Brac C, Artru P, et al. Gemcitabine as second-line chemotherapy after Folfirinox failure in advanced pancreatic adenocarcinoma: A retrospective study. Dig Liver Dis 2017.
5. Vienot A, Beinse $G$, Louvet $C$, et al. Overall Survival Prediction and Usefulness of Second-Line Chemotherapy in Advanced Pancreatic Adenocarcinoma. J Natl Cancer Inst 2017.

6. Kadokura M, Ishida Y, Tatsumi A, et al. Performance status and neutrophillymphocyte ratio are important prognostic factors in elderly patients with unresectable pancreatic cancer. J Gastrointest Oncol 2016;7:982-988.

7. Fiore M, Ramella S, Valeri S, et al. Phase II study of induction chemotherapy followed by chemoradiotherapy in patients with borderline resectable and unresectable locally advanced pancreatic cancer. Sci Rep 2017:7:45845.

8. Kim ST, Choi YJ, Park KH, et al. A prognostic model to identify patients with advanced pancreas adenocarcinoma who could benefit from second-line chemotherapy. Clin Oncol ( $R$ Coll Radiol) 2012;24:105-11.

9. Rahma OE, Duffy A, Liewehr DJ, et al. Second-line treatment in advanced pancreatic cancer: a comprehensive analysis of published clinical trials. Ann Oncol 2013;24:1972-9.

10. Makris EA, MacBarb R, Harvey DJ, et al. Surrogate End Points for Overall Survival in Metastatic, Locally Advanced, or Unresectable Pancreatic Cancer: A Systematic Review and Meta-Analysis of 24 Randomized Controlled Trials. Ann Surg Oncol 2017.

11. Marianne S, Louise D, Jana S, et al. The role of second-line chemotherapy after gemcitabine failure in patients with advanced pancreatic cancer. Pancreas 2016;45: 601-605

P0793 RAPID ON SITE EVALUATION (ROSE): AN ESSENTIAL TOOL IN ECHO-ENDOSCOPIC (EUS) STUDY OF SOLID LESIONS OF THE PANCREAS

R. Gaspar ${ }^{1}$, P. Moutinho-Ribeiro ${ }^{2}$, V. Magno Pereira ${ }^{3}$, M. Silva $^{2}$, A. Peixoto ${ }^{3}$, S. Lopes ${ }^{4}$, F. Vilas-Boas ${ }^{2}$, H. Barroca ${ }^{4}$, J. Lopes ${ }^{5}$, F. Carneiro ${ }^{4}$, G. Macedo ${ }^{3}$ ${ }^{1}$ Gastrenterology, Hospital São João, Porto/Portugal

${ }^{2}$ Gastrenterologia Hospital Central do Funchal Funchal/Portugal

${ }^{3}$ Centro Hospitalar São João, Porto Medical School, Porto/Portugal

${ }^{4}$ Pathology Department Centro Hospitalar São João, Porto/Portugal

${ }^{5}$ Centro Hospitalar De São João, Porto/Portugal

Contact E-mail Address: ruilopesgaspar@gmail.com

Introduction: Rapid on site evaluation for endoscopic ultrasound-guided fineneedle aspiration (EUS-FNA) of the pancreas provides immediate information regarding cellular adequacy, avoiding repeated procedures.

Aims \& Methods: The aim of this study was to evaluate the impact of ROSE in EUS-FNA of solid pancreatic lesions. Retrospective study of consecutive EUSFNA of solid pancreatic lesions, in a tertiary center, between 2012 and 2016.

Results: A total of 259 EUS-FNA in 197 patients were included, $63.6 \%$ male, with mean age of $63.4(+/-12.8)$ years. The anatomical distribution of the lesions was: $56.4 \%$ in the head, $17 \%$ in the body, $10 \%$ in the uncinate process and $5.8 \%$ in the tail. The mean number of passes were $3.3(+/-1.4)$ and the needle size was $25 \mathrm{G}$ in $60.8 \%$ and $22 \mathrm{G}$ in $23.8 \%$. ROSE was performed in $34.7 \%$ of the punctures (23.6\% along with the initial EUS-FNA), with a mean number of passes 3.4 $(+/-1.9)$. The diagnostic yield of initial EUS-FNA without ROSE was $44.8 \%$ vs $83.6 \%$ when ROSE was performed $(\mathrm{p}<0.001)$. When not conclusive, there was no significant differences in the diagnostic yield of the repeated EUS-FNA (with and without ROSE). Beyond ROSE in the first puncture, higher levels of Ca 19.9 (199 vs $10 \mathrm{ng} / \mathrm{mL}, \mathrm{p}=0.001$ ), size of the lesion (36.1 vs. $29.8 \mathrm{~mm}, \mathrm{p}<0.001$ ), invasion of adjacent structures $(64.6 \%$ vs $47.9 \%, \mathrm{p}=0.009)$ and malignancy $(73.2 \%$ vs $25.4 \%, \mathrm{p}<0.001)$ were associated with EUS-FNA diagnostic accuracy. In multivariate analysis, ROSE $(\mathrm{p}=0.001)$ and the size of the lesion $(\mathrm{p}=0.023)$ were independent predictors of adequate diagnostic samples. In this series, the definitive diagnosis was of malignancy in $64.9 \%$ (ductal adenocarcinoma $54.7 \%$ ), benign in $25.8 \%$ and indeterminate in $9.3 \%$

Conclusion: In agreement with the reported evidence, ROSE along EUS-FNA improved the diagnostic yield in solid pancreatic lesions and should be considered whenever possible in the first procedure, until an overall adequate diagnostic yield $(>80 \%)$ is achieved.

Disclosure of Interest: All authors have declared no conflicts of interest.

\section{TUESDAY, OCTOBER 31, 2017}

09:00-17:00

ENDOSCOPY AND IMAGING II - HALL 7

\section{P0794 ESOPHAGEAL FISTULA HEALING BY MESENCHYMAL} STEM CELL-DERIVED EXTRACELLULAR VESICLES IN A THERMORESPONSIVE GEL: A PRE-CLINICAL PORCINE STUDY

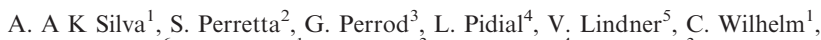
B. Dallemagne ${ }^{6}$, F. Gazeau ${ }^{1}$, C. Cellier ${ }^{3}$, O. Clément $^{4}$, G. Rahmi ${ }^{3}$

${ }^{1}$ Laboratoire Matières et Systèmes Complexes, Paris/France

${ }^{2}$ Nouvel Hopital Civil, Strasbourg/France

${ }^{3}$ Hôpital Européen Georges Pompidou Dept. de Gastroenterologie, Paris/France ${ }^{4}$ Inserm U970, Laboratoire Imagerie de l'Angiogenèse, Paris/France

${ }_{5}^{5}$ Anatomo-pathologie, Nouvel Hôpital Civil, Strasbourg/France

${ }^{6}$ IRCAD, Research Institute against Cancer of the Digestive System, Strasbourg/ France

Contact E-mail Address: gabriel.rahmi@egp.aphp.fr

Introduction: Postoperative digestive fistula remain a challenging condition associated with a high morbi-mortality, unsatisfactory healing rates and high refractoriness. The limitation of current approaches highlights the need for a better therapeutic strategy in terms of both long-lasting efficacy and safety. Mesenchymal stem cell (MSCs) are strongly involved in tissue injury repair. MSCs feature an immune-privileged status while displaying pro-angiogenic, 
and antifibrotic properties. Increasing evidences point out MSC action via subcellular entities known as extracellular vesicles (EVs). MSC EVs recapitulate the therapeutic properties of their cellular counterparts while offering remarkable advantages in terms of safety (no proliferation, no differentiation, no vascular occlusion following administration) and shelf life stability. Herein, we evaluated, in a porcine fistula model, the healing potential of allogenic MSC-EVs delivered through a thermoresponsive gel (Pluronic F127) allowing the administration in a sol state through a catheter and gelation in situ at body temperature to retain EVs at fistula site.

Aims \& Methods: Seventeen esophageal fistulas were surgically created by placing two plastic stents during 30 days into the neck of 9 pigs and randomized into control group $(n=6)$ and treated groups (gel alone $n=6$ and gel-EVs $n=5)$. In the gel-EVs group, Pluronic F127 gel contained allogenic EVs collected from the swine adipose stem cell conditioned medium. Clinical, endoscopic and radiological evaluation of fistula healing was performed at day 30 and day 45 , before histological assessment.

Results: All fistulas were successfully induced at day 30. At day 45, the control group featured open internal and external fistula orifices in all pigs. For this group, radiological evaluation showed open fistula tracts, which were confirmed by histology. In the gel group and gel-EVs groups, radiological examination showed a complete fistula closure in $67 \%(4 / 6)$ and $100 \%(5 / 5)$ of the animals, respectively. In the gel group, histological analysis confirmed a complete fistula for 3 from 6 cases while a partial closure was observed for 1 case from 6 . In the gel-EVs group, histological complete fistula closure was reported for 4 from 5 cases while a partial closure was evidenced in 1 from 5 cases. In comparison with control group, treated fistulas showed a reduced inflammatory infiltrate and fibrosis and an enhanced angiogenesis, especially in the gel-EVs group.

Conclusion: This study provides the first evidence in the literature that MSC-EVs may provide a therapeutic effect in a pre-clinical fistula model. EVs were successfully administered via a thermoresponsive Pluronic F127 hydrogel, gelling in situ to enable EV retention in the fistula tract. Besides, the gel further provided a proangiogenic and an anti-inflammatory effect. The combined action of MSC EVs and the gel enhanced fistula healing associated with an anti-fibrotic effect in this esophageal fistula model. This investigation paves the way towards a future subcellular localized fistula therapy merging safety and efficacy.

Disclosure of Interest: All authors have declared no conflicts of interest.

\section{P0795 REAL-TIME MULTIPHOTON MORPHOLOGICAL IMAGING FOR DIAGNOSING GASTRIC ATYPICAL HYPERPLASIA AND ADENOCARCINOMA}

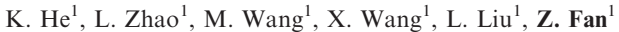

${ }^{1}$ The First Affiliated Hospital with Nanjing Medical Universtiy, Nanjing/China

\section{Contact E-mail Address: fanzhining $@$ njmu.edu.cn}

Introduction: Compared with histopathology, real-time histology or virtual biopsy is important for clinical diagnosis, especially for endoscopic examination. Based on two photon fluorescence (TPEF), multiphoton microscopy (MPM) imaging could demonstrate cell autofluorescence and second-harmonic generation (SHG) signal from collagen, which implied real-time information on tissue architecture and cellular morphology. More importantly, no contrast agent is needed for this live diagnosis. The aim of this study is to evaluate the feasibility of MPM to histologically diagnose gastric diseases, compared with other chromoendoscopy and H\&E histopathology.

Aims \& Methods: A pilot study was performed between March 2016 and August 2016. 30 gastric tissue slides (normal, low-grade dysplasia (LGD), high-grade dysplasia (HGD), and cancer) were examined under MPM. MPM and H\&E images were compared by the experienced pathologist. Cellular-to-nuclei ratio was analyzed to compare morphological features, while the major/minor axis ratio was calculated to reveal cellular asymmetry.

Results: Near-infrared light $(800 \mathrm{~nm})$ was optimized and applied for multiphoton autofluorescence imaging in gastric tissue. Under MPM, gastric dysplasia tissue demonstrated enlarged, while cancer cells were characterized by irregular size and shape, enlarged nuclei, and increased nuclear-to-cytoplasmic ratio. All these were confirmed by H\&E images. (Figure 1) The mean cellular/nuclei ratio for normal mucosa was $20.55 \pm 4.84$, LGD $34.60 \pm 3.90$, HGD $46.85 \pm 3.22$, and cancer $56.80 \pm 3.37(\mathrm{P}<0.05)$. The mean major/minor axis ratio for normal mucosa was $1.31 \pm 0.09$, LGD $2.02 \pm 0.16$, HGD $1.70 \pm 0.18$, and cancer $1.43 \pm 0.18$ $(\mathrm{P}<0.05)$.

Conclusion: MPM-based optical biopsy was feasible and efficient to clinically diagnose gastric cancer. With miniaturization and integration of endoscopy, MPM will be applied to provide real-time histological diagnosis without invasive biopsy for gastric cancer in the future.

Disclosure of Interest: All authors have declared no conflicts of interest.

\section{P0796 IMPROVEMENT IN HEPATIC TRANSAMINASES OVER 12 MONTHS AFTER SINGLE PROCEDURE DUODENAL MUCOSAL} RESURFACING IN TYPE 2 DIABETES PATIENTS

A. Van Baar ${ }^{1}$, M. Nieuwdorp ${ }^{2}$, L. Crenier ${ }^{3}$, R. Batterham ${ }^{4}$, F. Holleman ${ }^{5}$, P. Vignolo ${ }^{6}$, G. Costamagna ${ }^{7}$, J. Deviere $^{8}$, R. Haidry ${ }^{9}$, L. Rodríguez ${ }^{10}$, M. Galvao Neto ${ }^{11}$, J.J. G.h.m. Bergman ${ }^{1}$, G. Mingrone ${ }^{12}$

${ }^{1}$ Gastroenterology \& Hepatology, Academic Medical Center, Amsterdam/ Netherlands

${ }^{2}$ AMC-UvA Dept. of Vascular Medicine, Amsterdam/Netherlands
${ }^{3}$ Endocrinology, Erasme University Hospital, Brussels/Belgium

${ }^{4}$ Centre For Obesity Research, Department Of Medicine, University College Hospital, London/United Kingdom

${ }^{5}$ Internal Medicine, Academic Medical Center, Amsterdam/Netherlands ${ }^{6}$ CCO Clinical Center for Diabetes, Obesity and Reflux, Santiago/Chile ${ }^{7}$ Digestive Endoscopy Unit, Università Cattolica del Sacro Cuore, Rome/Italy ${ }^{8}$ Dept. Of Gastroenterology, Erasme University Hospital, Brussels/Belgium ${ }^{9}$ Gastroenterology, University College London Hospital, London/United Kingdom ${ }^{10}$ CCO Obesidad Diabetes y RGE Dept. of Surgery, Santiago/Chile

${ }^{11}$ Bariatric Endoscopy, Gastro Obeso Center, São Paulo/Brazil

${ }^{12}$ Internal Medicine, Catholic University, Rome/Italy

Contact E-mail Address: a.c.vanbaar@amc.nl

Introduction: Type 2 diabetes (T2D) and fatty liver disease are highly prevalent, often overlapping metabolic disorders where upstream insulin resistance is thought to be a common pathogenic driver. Simultaneous treatment of both conditions has been reported with insulin sensitizing interventions including weight loss, pharmacotherapy (GLP1 receptor agonists, TZDs), and bariatric surgery. Duodenal Mucosal Resurfacing (DMR) is a minimally invasive endoscopic procedure that has demonstrated glycemic efficacy in patients with T2D seemingly via an insulin sensitizing mechanism. ${ }^{1}$ We report data supporting 12 mo durability of improved metabolic indices in patients with T2D after a single DMR procedure in a single-arm, open-label, multicenter study.

Aims \& Methods: In this endoscopic DMR procedure, the duodenal mucosa was treated with hydrothermal ablation using a patented balloon catheter. Efficacy was analyzed in a modified intent-to-treat cohort (mITT, patients who received $\geq 1$ ablation) stratified into baseline alanine aminotransferase (ALT) level tertiles - lowest $($ ALT $<28 \mathrm{U} / \mathrm{L})$, middle $(28 \leq \mathrm{ALT}<37 \mathrm{U} / \mathrm{L})$, and upper $(\mathrm{ALT} \geq 37$ $\mathrm{U} / \mathrm{L}$ ). Change from baseline in ALT, aspartate aminotransferase (AST), body weight, homeostasis model assessment index (HOMA-IR) and HbAlc were analyzed using repeated measures ANOVA. Data are reported as mean(SD).

Results: Baseline ALT and HbAlc in the mITT cohort $(n=27$; age, 55(9) y; diabetes duration, $6(2.5) \mathrm{y}$; BMI, 32.5(4.2) $\left.\mathrm{kg} / \mathrm{m}^{2}\right)$ were $37.0(20.6) \mathrm{U} / \mathrm{L}$, and $8.7(1.0) \%$, respectively.12-mo reductions in $\mathrm{HbAlc}$, ALT, AST and weight were $-1.0(1.2) \%(\mathrm{P}=0.005),-8(14) \mathrm{U} / \mathrm{L}(\mathrm{P}=0.18),-6(7) \mathrm{U} / \mathrm{L}(\mathrm{P}=0.005)$, $-2.0(4.2) \mathrm{kg}(\mathrm{P}=0.83)$ and $-3.6(\mathrm{P}=0.037)$. In the upper tertile, ALT reductions from baseline $(59(23) \mathrm{U} / \mathrm{L})$ at 3 and 12 -mo were $-22(15)(\mathrm{P}=0.024)$, and $-16(20) \mathrm{U} / \mathrm{L} \quad(\mathrm{P}=0.72)$; respective body weight changes were $-3.8(2.9)$ $(\mathrm{P}=0.24)$, and $-1.9(5.9) \mathrm{kg}(\mathrm{P}=0.99) .12$-mo reductions in AST, HbAlc and HOMA-IR were: $-9(7) \mathrm{U} / \mathrm{L}(\mathrm{P}=0.13),-1.1(0.5) \%(\mathrm{P}=0.002)$; and $-4.7(5.2)$ $(\mathrm{P}=0.94)$. In the middle and lowest tertiles, 12-mo ALT reductions were $-4(11)$ and $-2(3) \mathrm{U} / \mathrm{L}$.

Conclusion: A single DMR procedure in patients with T2D produced significant reductions in $\mathrm{HbAlc}$ up to 12 months in the total cohort. In the highest baseline ALT subgroup, ALT was significantly lower at 3 months and remained lower than baseline at 12 months accompanied by significant lowering of hyperglycemia up to 12 months. Further studies are planned to quantify the efficacy, safety and durability of the hepatic and glycemic effects associated with DMR

Disclosure of Interest: R. Batterham: I consult for Novo Nordisk and I have participated in Speakers' Bureau's for Orexigen and Novo Nordisk.

J. Deviere: Research support and consulting fees, Fractyl

R. Haidry: "Educational Research grants from Cook Endoscopy and Pentax Medical Europe to support research infrastructure"

L. Rodríguez: Research suport from Fractyl Laboratories, Inc

M. Galvao Neto: Serves on the scientific advisory board of and receives research funding from GI Dynamics, Inc., and Fractyl Laboratories, Inc.

J.J.G.H.M. Bergman: Research support for IRB approved Revita-1 study: Fractyl Laboratories Inc.

G. Mingrone: Unknown at time of abstract submission.

All other authors have declared no conflicts of interest.

\section{Reference}

1. Rajagopalan H, Cherrington AD, Thompson CC, et al. Endoscopic Duodenal Mucosal Resurfacing for the Treatment of Type 2 Diabetes: 6 Month Interim Analysis From the First-in-Human Proof-of-Concept Study. Diabetes Care 2016.

\section{P0797 UPPER GASTROINTESTINAL ENDOSCOPIC FINDINGS IN ASYMPTOMATIC HEALTHY INDIVIDUALS WITH NORMAL AND DECREASED SERUM PEPSINOGENS FROM THE GISTAR PILOT STUDY}

I. Kikuste ${ }^{1}$, J. Young Park ${ }^{2}$, R. Murillo ${ }^{2}$, S. Parshutin ${ }^{3}$, I. Polaka ${ }^{3}$, A. Kirsners ${ }^{3}$, R. Herrero ${ }^{2}$, M. Leja ${ }^{3}$

${ }^{1}$ Digestive Diseases Centre GASTRO, LV/Latvia

${ }^{2}$ International Agency for Research on Cancer, Lyon/France

${ }^{3}$ Institute Of Clinical And Preventive Medicine \& Faculty Of Medicine, University of Latvia, LV/Latvia

Contact E-mail Address: ikikuste@gmail.com

Introduction: Limited data are available with regard to the prevalence of upper gastrointestinal endoscopic findings in asymptomatic healthy individuals as an endoscopy is an invasive and costly procedure.

Aims \& Methods: Individuals were recruited from general population in Latvia as part of the GISTAR pilot study. The study group has been referred for an upper endoscopy and was tested for serum pepsinogens and pepsinogen $\mathrm{I} / \mathrm{II}$ ratio $<3$ 
(Biohit, Plc., Helsinki, Finland) was considered decreased. Data were obtained from 1034 individuals (74\% response rate). Upper gastrointestinal endoscopy procedures were performed between February 2014 and June 2016. The study was approved by local ethics committees and the Ethics Committee of the International Agency for Research on Cancer.

Results: Altogether 1034 (45\% male) participants (average age 52; min-max 39$64)$ were enrolled and upper endoscopies performed in the study. Main endoscopic findings were not present only in $21 \%-219 / 1034$ of asymptomatic individuals. The majority of the participants $(\mathrm{n}=815,79 \%)$ showed abnormal endoscopic findings including the following: reflux esophagitis A- $18 \%$, reflux esophagitis B- $6 \%$, reflux esophagitis C-1, 3\% and histologically confirmed Barrett's esophagus $-1.3 \%$. The most common main endoscopic finding was noted gastric erosions $232 / 1034(22.4 \%)$. The prevalence of gastric peptic ulcer was $2.9 \%(30 / 1034)$ and gastric scar $0.9 \%(9 / 1034)$, but duodenal ulcer $2.3 \%(24 /$ $1034)$ and duodenal scar $5.9 \%(62 / 1034)$. In addition, three gastric adenocarcinoma cases $(0.29 \%)$ have been identified during the upper endoscopy. The study showed statistically significant difference between the groups of individuals with normal and decreased pepsinogen tests with gastric polyps $3.6 \%$ vs $9.2 \%$, $p=0.02$, gastric erosions $23.6 \%$ vs $11.2 \%, p=0.04$, duodenal ulcer (scar) $6.5 \%$ vs $1 \%, p=0.02$, duodenal lymphangiectasia $1.9 \%$ vs $6.1 \%, p=0.02$ and reflux esophagitis A $18.7 \%$ vs $9.2 \%, p=0.01$.

Conclusion: In this group of asymptomatic healthy individuals, only $20 \%$ showed normal endoscopic findings while the majority had clnically significant findings, some of which were related to the decreased pepsinogens levels. Futher studies are needed to confirm our observations.

Disclosure of Interest: All authors have declared no conflicts of interest.

\section{P0798 REBAMIPIDE SOLUTION AS A NOVEL SUBMUCOSAL INJECTION PROMOTES HEALING SPEED AND QUALITY OF ESD- INDUCED ULCER BY SUPRESSING FIBROSIS}

A. Fujimoto ${ }^{1}$, T. Nishizawa ${ }^{2}$, M. Shimoda ${ }^{3}$, O. Goto ${ }^{2}$, Y. Ochiai ${ }^{4}$, T. Maehata ${ }^{2}$, T. Akimoto ${ }^{2}$, Y. Mitsunaga $^{4}$, M. Kato $^{2}$, A. Nakayama ${ }^{2}$, M. Sasaki ${ }^{2}$, T. Uraoka ${ }^{2}$, N. Yahagi ${ }^{2}$

${ }^{1}$ Development Of Minimally, Keio University, Tokyo/Japan

${ }^{2}$ Department Of Pathology, Keio University, Tokyo/Japan

\section{Contact E-mail Address: ai-fujimoto@a8.keio.jp}

Introduction: Peroral administration of rebamipide in additional to proton pump inhibitor (PPI) was reported to be effective to promote ulcer healing after endoscopic submucosal dissection (ESD). In this pilot study, we assessed the efficacy and safety of a novel rebamipide solution as a submucosal injection agent for ESD using in vivo porcine models.

Aims \& Methods: The protocol was approved by the ethics review board of our animal experimental laboratory in advance (13055-(0)). ESDs of about $30 \mathrm{~mm}$ in diameter were performed at four sites in the stomachs of three pigs. An endoscopist blinded to the test agents performed the ESDs with a $2 \%$ rebamipide solution at two sites (rebamipide group) and with base solution alone at the other two sites (control group). The safety and the treatment results of rebamipide group were assessed. The gastric ulcer stages were evaluated by endoscopy once weekly up to week 4 after the ESD to determine a healing score based on ulcer staging using the classification of Sakita and Miwa. Al stage was defined as score 1. A2, H1, H2, S1, S2 were defined as healing score 2, 3, 4, 5, 6, respectively. The average scores of each week were compared in the rebamipide group and the control group. One pig was sacrificed at 1 week after the ESD and the other two were sacrificed at 4 weeks, for pathological evaluation of ESD-induced ulceration and ulcer scarring by HE. The number of neutrophils and width of the fibrosis were also compared between the rebamipide group and control group at 1 week and 4 weeks. Neutrophils were counted in five fields around the ESD-induced ulcer in HE stained sections under $\times 400$ magnification and the average counts of the five fields. The width of the fibrosis was defined as the maximum diameter of $\alpha$ SMA-positive area in the depth direction from the submucosa to the muscle layer at a site of ESD-induced ulceration or ulcer scarring.

Results: There were no adverse events related with the use of the rebamipide solution. The average healing score was significantly higher in the rebamipide group than in the control group at 2 weeks $(p=0.027)$. The folds into the centers of the ulcer scars were more regular in the rebamipide group than in the control group at 4 weeks after ESD. In the histopathological assessment of the ulcers at 4 weeks, mucosal healing was remarkably better in the rebamipide group than in the control group, with a thicker and more homogeneous mucosal layer. Fibrosis was significantly less extensive in the rebamipide group than in the control group at 4 weeks $(p=0.02)$. In the evaluation of inflammation, the average number of neutrophils was lower in the rebamipide group than in the control group at both 1 week and 4 weeks, but not to a significant extent.

Conclusion: The rebamipide solution appeared to be safe and effective as an injection material for promoting the healing of ESD-induced ulcers. It also seemed to smoothen the folds of the ulcer circumference after ESD by suppressing fibrosis.

Disclosure of Interest: All authors have declared no conflicts of interest.
P0799 EFFECT OF ILAPRAZOLE ON THE HEALING OF ENDOSCOPIC SUBMUCOSAL DISSECTION-INDUCED GASTRIC ULCER: INTERIM ANALYSIS OF RANDOMIZED, MULTICENTER STUDY

C.S. Bang ${ }^{1}$, W.G. Shin ${ }^{1}$, G.H. Baik ${ }^{1}$, H.Y. Kim ${ }^{1}$, S. Kae ${ }^{1}$, H.J. Jang ${ }^{2}$, M.H. Choi ${ }^{2}$

${ }^{1}$ Internal Medicine, Hallym University College of Medicine, Chuncheon/Korea, Republic of

${ }^{2}$ Gastroenterology, Hallym Univerisity Dongtan Sacred Hospital, Gyunggi-Do/ Korea, Republic of

Contact E-mail Address: sgun91@hallym.or.kr

Introduction: The optimal treatment regimen or duration of endoscopic submucosal dissection (ESD)-induced gastric ulcer has not been established. The aim of this study was to assess the efficacy of novel PPI, ilaprazole for the treatment of ESD-induced gastric ulcer.

Aims \& Methods: This was a prospective, open-label, randomized multicenter study. Between June 2015 and April 2017, a total of 88 patients who underwent ESD for gastric neoplasm were randomly allocated with ilaprazole $20 \mathrm{mg}$ or rabeprazole $20 \mathrm{mg}$ daily for 8 weeks. The primary outcome was ulcer healing rate at 4 and 8 weeks.

Results: In the intention-to-treat analysis, the ulcer healing rate of each treatment group was not significantly different at 4 or 8 weeks (ilaprazole vs. pantoprazole; $96.7 \%$ vs. $96.4 \%, P=0.80$ at 4 weeks, $99.7 \%$ vs. $99.0 \%, P=0.19$ at 8 weeks). There was no independent predictive factors for a high ulcer healing rate in the multivariate analysis.

Conclusion: According to this interim analysis of trial, ilaprazole and rabeprazole showed no significant difference in the healing of artificial gastric ulcer. Most of the ulcers achieved complete healing within 4 to 8 weeks.

Disclosure of Interest: All authors have declared no conflicts of interest.

\section{Reference}

Shin WG, Kim SJ, Choi MH, Kim KO, Jang HJ, Park CH, Baek IH, Kim KH, Baik GH, Kae SH, Kim JH, Kim HY. Gastrointest Endosc. 2012 Apr;75(4):73947. doi: 10.1016/j.gie.2011.11.004. Epub 2012 Jan 26.

\section{P0800 THE EFFECT OF VONOPRAZAN FOR ENDOSCOPIC SUBMUCOSAL DISSECTION-INDUCED ULCERATION AND POSTOPERATIVE BLEEDING}

G. Ikeda, N. Ueki, S. Futagami, S. Agawa, H. Noda, K. Higuchi, T. Akimoto, Y. Maruki, H. Yamawaki, Y. Kodaka, T. Kawagoe, K. Miyake, M. Kaise, K. Iwakiri

Department Of Gastroenterology, Nippon Medical School, Tokyo/Japan

Contact E-mail Address: gogogo.with.yxx@gmail.com

Introduction: ESD is the standard treatment for early gastric cancer and less invasive procedure compared with gastrectomy. Proton pump inhibitors (PPIs) have been widely used for the treatment of ESD-induced gastric ulcers. Many studies have reported that it is critical issues for ESD procedure to prevent postoperative bleeding. Vonoprazan, a potassium-competitive acid blocker (P-CAB), has a strong and continuous inhibition of gastric acid secretion, and is expected to improve effectively ESD-induced gastric ulcerations compared to the treatment with PPIs.

Aims \& Methods: To determine whether vonoprazan can ameliorate more effectively ESD-induced gastric ulcerations and can reduce the incidence of postoperative bleeding than PPIs, we compared healing rate of ulcerations and bleeding incidence in the patients treated with vonoprazan with those treated with PPI. 139 patients who underwent gastric ESD between January 2015 and December 2016 were enrolled in Nippon Medical School Hospital. 11 patients who were injected triamcinolone into mucosa preventing stricture of the prepylorus were excluded. 59 patients were treated with P-CAB for 4 weeks (P-CAB group) and 69 patients were treated with PPI (4 omeprazole, 22 esomerazole, 11 lansoprazole or 32 rabeprazole) for 4 weeks (PPI group), and subsequently underwent endoscopy for evaluation of ulcer size and intra gastric $\mathrm{pH}$ using gastric juice. The area of ulcerations was approximated by multiplying the length $(\mathrm{mm})$ by the width $(\mathrm{mm})$.

Results: The shrinking rate of ESD-induced ulcer at 4 weeks after ESD were not significantly $(95.4 \pm 8.1 \%$ and $94.5 \pm 6.4 \%, \mathrm{p}=0.4852)$ different between P-CAB and PPI groups. The post-ESD bleeding incidence $(6.8 \%)$ in the P-CAB group were not significantly $(\mathrm{p}=0.8189)$ different from that $(5.8 \%)$ in the PPI group. The intra gastric $\mathrm{pH}$ at 4 weeks after ESD in the P-CAB group was significantly higher than that in the PPI group $(6.9 \pm 1.0$ vs $6.1 \pm 1.9$, respectively, $\mathrm{p}=0.0028)$. Conclusion: Vonoprazan is superior to PPI in acid suppression, but there were no significant differences in ulcer healing and bleeding incidence between the two groups.

Disclosure of Interest: All authors have declared no conflicts of interest.

\section{P0801 INFLUENCE OF ANTITHROMBOTIC THERAPY ON DELAYED BLEEDING AFTER GASTRIC ESD: A RETROSPECTIVE ANALYSIS IN 665 CASES}

H. Noda, N. Ueki, T. Akimoto, G. Ikeda, K. Higuchi, S. Agawa, Y. Maruki, H. Yamawaki, Y. Kodaka, T. Kawagoe, K. Miyake, S. Futagami, K. Iwakiri Gastroenterology, Nippon Medical School Hospital, Bunkyo-ku, Tokyo/Japan 
Contact E-mail Address: stand.by.me.0106@gmail.com

Introduction: Endoscopic submucosal dissection (ESD) for superficial gastric neoplasms is gaining an acceptance as one of curative treatment options. However, delayed bleeding still exists as a major complication of ESD. On the other hands, the number of patients taking antithrombotic agents are increasing because the evidence of antithrombotic therapy for prevention of thrombotic events has been established and population aging advances, which is thought to be a serious problem related to increasing delayed bleeding after ESD.

Aims \& Methods: To assess the influence of antithrombotic therapy on delayed bleeding after ESD, we retrospectively investigated the delayed bleeding rate after ESD among the continuation of antithrombotic agents, the cessation of antithrombotic agents and heparin bridge therapy. 735 lesions in 665 patients were treated with ESD from January 2006 to December 2016. We compared the delayed bleeding rate in 153 patients receiving antithrombotic therapy with 512 patients without that. Furthermore, we compared the delayed bleeding rate in the patients continuing antithrombotic therapy with that in the patients with the cessation of antithrombotic therapy or with heparin bridge therapy. The patients who were taking two or more antithrombotic agents were treated with continuation aspirin or cilostazol. The cessation period of antithrombotic therapy before ESD followed the guidelines for therapeutic endoscopy in antithrombotic agentsusers from Japan Gastrointestinal Endoscopy Society. We defined delayed bleeding as a hematemesis, a melena, or a decrease of $\mathrm{Hb}>2 \mathrm{~g} / \mathrm{dl}$.

Results: The delayed bleeding rate in the patients receiving antithrombotic therapy was $14.4 \%(22 / 153)$, which was significantly higher than that in the patients without antithrombotic therapy $(5.7 \%: 29 / 512)(\mathrm{p}=0.0007)$. The median timing of delayed bleeding in patients receiving antithrombotic therapy and that in patients without antithrombotic therapy were $5.7 \pm 4.6$ days and $7.0 \pm 6.8$ days, respectively, without significant difference $(\mathrm{p}=0.48)$. Of 153 patients taking antithrombotic therapy, 38 continued a single antithrombotic agent (all of them were antiplatelet drugs) during ESD (continuation group), 38 discontinued antithrombotic therapy and resumed it after ESD (cessation group), and 30 switched to heparin therapy before ESD (heparin bridge group). One patient was excluded because of uncertain about the period of cessation. The delayed bleeding rate of continuation group, cessation group and heparin bridge group were $13.2 \%(5 / 38), 13.1 \%(11 / 84)$ and $20.0 \%(6 / 30)$, respectively, without significant difference $(\mathrm{p}=0.63)$. The delayed bleeding rate of continuation group was equal to that of cessation group $(\mathrm{p}=0.60)$. The delayed bleeding rate of heparin bridge group seemed to be high $(20.0 \%)$, but there was no significant diffrence compared to that of total number of continuation and cessation group $(13.1 \%(16 / 122), p=0.24)$. Deep vein thrombosis was observed in one patient in the cessation group.

Conclusion: Antithrombotic therapy increased the delayed bleeding rate However, the delayed bleeding rate in the patients continuing antithrombotic therapy during ESD was similar to that in the patients discontinuing antithrombotic therapy before ESD. Therefore, it is appropriate that the patients with high risk of thrombosis continue antithrombotic therapy on gastric ESD, but the heparin bridge therapy requires a further examination.

Disclosure of Interest: All authors have declared no conflicts of interest.

P0802 LOW-DOSE ASPIRIN DELAYS THE ULCER HEALING AND INCREASES THE RISK OF POSTOPERATIVE BLEEDING AFTER GASTRIC ENDOSCOPIC SUBMUCOSAL DISSECTION THROUGH THE DUODENOGASTRIC REFLUX

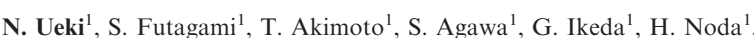
K. Higuchi ${ }^{1}$, Y. Maruki ${ }^{1}$, H. Yamawaki ${ }^{1}$, Y. Kodaka ${ }^{1}$, T. Kawagoe ${ }^{1}$, K. Miyake ${ }^{1}$, M. Kaise ${ }^{2}$, K. Iwakiri ${ }^{1}$

${ }^{1}$ Department Of Gastroenterology, Nippon Medical school, Tokyo/Japan

Contact E-mail Address: nobue@nms.ac.jp

Introduction: Endoscopic submucosal dissection (ESD) permits en bloc resection for larger lesions. The number of the patients taking anti-thrombotic agents including low-dose aspirin (LDA) has increased. The Japanese guidelines recommended endoscopic procedures without interruption of LDA therapy in patients at high risk of thrombotic events who use LDA alone. And, bile acid reflux is known to cause gastric mucosal damage though the exact mechanisms are still unclear

Aims \& Methods: In this study, we aimed to clarify whether LDA treatment and gastric bile acid contents synergistically affect on postoperative bleeding and healing of ulceration after gastric ESD procedure. A total 224 patients with gastric neoplasms were treated with ESD at Nippon Medical Hospital, between January 2013 and June 2016. To investigate whether anti-thrombotic agents affect the ESD procedure-induced ulceration and ESD postoperative bleeding rate, we compared ulceration reduction rate (one month after ESD), postoperative bleeding rate and gastric bile acid contents among the patients treated with low dose aspirin, other anti-thrombotic agents and non-antithrombotic agents On the day of ESD and one day after ESD, gastric juice was taken in endoscopy and total bile acids were measured spectrophotometrically after the enzyme reaction.

Results: Ulcer reduction rate in the patients treated with LDA was significantly $(\mathrm{p}=0.0036)$ higher compared to that in the patients with non-anti-thrombotic agents. ESD postoperative bleeding rate in the patients with LDA was significantly higher ( $p=0.028$ and $p<0.0001$, respectively) compared to those in the patients with other anti-thrombotic agents and the patients with non anti-thrombotic agents. The difference of gastric bile acid contents between pre- and postESD procedure in the patients with low dose aspirin was significantly $(\mathrm{p}=0.0426)$ higher compared to that in the patients with non anti-thromobotic agents.
Conclusion: LDA increased gastric bile acid contents, which delayed the ulcer healing and increased the bleeding after ESD.

Disclosure of Interest: All authors have declared no conflicts of interest.

P0803 NARROW-BAND IMAGING (NBI) ENDOSCOPY WITH DUAI FOCUS MAGNIFICATION FOR CHARACTERIZATION OF GASTRIC NON-NEOPLASTIC AND NEOPLASTIC LESIONS

R. Kuvaev ${ }^{1}$, S. Kashin ${ }^{1}$, A. Aleshicheva ${ }^{1}$, E. Kraynova ${ }^{2}$, A. Nadezhin ${ }^{2}$, E. Nikonov ${ }^{3}$

${ }^{1}$ Dept. Of Endoscopy, Yaroslavl Regional Cancer Hospital Dept. of Endoscopy, Yaroslavl/Russian Federation

${ }^{2}$ Pathology, Yaroslavl Regional Cancer Hospital, Yaroslavl/Russian Federation ${ }^{3}$ Gastroenterology, Pirogov Russian National Research Medical University, Moscow/Russian Federation

Contact E-mail Address: kuvaev roman@mail.ru

Introduction: Advanced endoscopic imaging has become an essential part of the diagnostic algorithm for characterization of gastric lesions. NBI endoscopy with magnification allows enhancing visualization of gastric mucosa and provides accurate pathology prediction. European Society of Gastrointestinal Endoscopy (ESGE) recommends the application of validated classification systems to support the use of optical diagnosis [1]. The improvement of current classifications and development of validated criteria or scales for diagnosis by advanced endoscopic imaging are a key areas of research for the endoscopic community

Aims \& Methods: The aim of this study was to evaluate the diagnostic accuracy of NBI features described in ESGE proposed NBI classification for gastric precancerous and neoplastic lesions [2]. 180 gastric lesions in 97 patients (mean age 59.7 years, $\mathrm{SD}=13.7,48 \%$ male, $52 \%$ female) were observed with NBI endoscopy and dual focus magnification (GIF- HQ190, Exera III, Olympus, Japan). Mucosal and vascular patterns were classified into type A (regular circular mucosal pattern with regular vesels), type B (regular tubulo-villous mucosal pattern with regular vesels) and type $\mathrm{C}$ (irregular mucosal and vascular pattern), presence of "light blue crests" - LBC (blue-whitish areas) and demarcation line were assessed. Forceps biopsy or endoscopic mucosal resection/endoscopic submucosal dissection were performed for a histological evaluation of lesions.

Results: From 180 gastric lesions (62 lesions with chronic gastritis, 67 - intestinal metaplasia (IM), 20 - hyperplastic polyp, 5 - low-grade dysplasia, 10 - highgrade dysplasia, 16 - adenocarcinoma) 70 lesions had pattern A, 62 lesions pattern B (36 lesions were LBC positive and 26 lesions were LBC negative) and 28 lesions - C pattern (table 1). 20 lesions of hyperplastic polyps had specific "coarse" pattern and were not classified due to the absence of appropriate category. Demarcation line was identified in $2 \%, 66 \%$ and $100 \%$ of lesions with pattern $\mathrm{A}, \mathrm{B}$, and $\mathrm{C}$, respectively.

Conclusion: ESGE proposed NBI classification for gastric precancerous and neoplastic lesions demonstrated high diagnostic accuracy in detection intestinal metaplasia and neoplasia. Combination of pattern B, LBC and demarcation line was found to be the most accurate criterion for intestinal metaplasia. Benign hyperplastic polyps were characterized by specific pattern that need to be validated in further studies.

Disclosure of Interest: All authors have declared no conflicts of interest.

\section{References}

1. East J.E. et al. Advanced endoscopic imaging: European Society of Gastrointestinal Endoscopy (ESGE) Technology Review. Endoscopy. 2016, Nov;48(11):1029-1045.

2. Pimentel-Nunes P. et al. A multicenter validation of an endoscopic classification with narrow band imaging for gastric precancerous and cancerous lesions. Endoscopy. 2012, Mar;44(3):236-46

\section{P0804 TRANSPLANTATION OF AUTOLOGOUS ESOPHAGUS MUCOSA TO PREVENT STRICTURE AFTER CIRCUMFERENTIAL ENDOSCOPIC SUBMUCOSAL DISSECTION OF EARLY SQUAMOUS CELL}

L. Zhongli, F. Chaoqiang, Y. Xin, P. Xue, Z. Xia, Y. Jin, X. Xia, B. Jianying Gastroenterology, Xinqiao Hospital, Third Military Medical University, Chongqing, Qing/China

Contact E-mail Address: 18253537@qq.com

Introduction: Esophageal endoscopic submucosal dissection (ESD) to remove superficial esophageal neoplasms is gradually becoming the standard treatment for superficial oesophageal cancer, but is associated with esophageal stenosis, particularly when ESD involves the entire circumference of the luminal. Many methods to prevent post-ESD stricture, such as repeated Endoscopic balloon dilatation (EBD), temporary stent insertion, and oral steroid and intralesional steroid injection, have been used in different institutions. In recent years, new technologies such as autologous oral mucosal sheets or extracellular matrix scaffold material have also been suggested to manage esophageal strictures. There are no standard guidelines to prevent stricture in a patient with circumferential mucosal defect after ESD. In this study, we aimed to assess the effectiveness and safety of endoscopic transplantation of autologous esophagus mucosa in preventing formation of strictures after ESD.

Aims \& Methods: We performed a single-arm, single-institute study. Nine patients who underwent wholly circumferential ESD for superficially extended 
Abstract No: P0803

Table 1: Results of the study

\begin{tabular}{|c|c|c|c|c|c|}
\hline Pattern or endoscopic feature & Pattern A & Pattern B & LBC & $\begin{array}{l}\text { Pattern } \mathrm{B}+\mathrm{LBC}+ \\
\text { demarcation line }\end{array}$ & Pattern C \\
\hline Expected outcome & $\begin{array}{l}\text { Absence of intestinal } \\
\text { metaplasia and neoplasia }\end{array}$ & $\begin{array}{l}\text { Intestinal } \\
\text { metaplasia }\end{array}$ & $\begin{array}{l}\text { Intestinal } \\
\text { metaplasia }\end{array}$ & $\begin{array}{l}\text { Intestinal } \\
\text { metaplasia }\end{array}$ & Neoplasia \\
\hline Sensitivity (CI 95\%) & $0.94(0.87-0.996)$ & $0.84(0.75-0.92)$ & $0.54(0.42-0.66)$ & $0.97(0.92-1.02)$ & $0.87(0.75-0.99)$ \\
\hline Specificity (CI 95\%) & $0.88(0.81-0.94)$ & $0.94(0.89-0.99)$ & $1.00(1.00-1.00)$ & $1.00(1.00-1.00)$ & $0.99(0.98-1)$ \\
\hline Accuracy (CI 95\%) & $0.9(0.85-0.95)$ & $0.89(0.85-0.94)$ & $0.8(0.75-0.87)$ & $0.99(0.98-1.0)$ & $0.97(0.94-1.00)$ \\
\hline Positive predictive value (CI 95\%) & $0.83(0.74-0.92)$ & $0.9(0.83-0.98)$ & $1.00(1.00-1.00)$ & $1.00(1.00-1.00)$ & $0.96(0.94-1.00)$ \\
\hline Negative predictive value (CI 95\%) & $0.96(0.91-0.998)$ & $0.89(0.83-0.95)$ & $0.75(0.67-0.83)$ & $0.99(0.98-1.00)$ & $0.97(0.94-1.00)$ \\
\hline
\end{tabular}

esophageal squamous cell carcinoma at the endoscopic center of Xinqiao Hospital, Third Military Medical University (Chongqing, China) from January 2015 to February 2017, were enrolled in this study. We collected specimens of autologous esophageal mucosal tissue from these patients. After undergone ESD, these mucosal patches were first attached to the "ulcer surface" by hemoclips and then fixed by means of a covered metal mesh stent. The stent was removed on post-procedure day 7. All patients were monitored by endoscopy.

Results: En bloc ESD was safely achieved in all cases. The overall longitudinal diameter of resected specimens was $117.8 \mathrm{~mm}$ (range, 70 to $150 \mathrm{~mm}$ ). Autologous esophagus mucosa were successfully transplanted to "ulcer surface" using an endoscope. The number of mucosal patchs ranged from 8 to 28. Complete reepithelialization occurred within a median time of 8.6 days with a graft survival rate at $93.06 \%$. Postprocedural stricture accompanied by dysphagia occurred in seven patients on post-procedure day 24.7 (range, 18-34 days). The median sessions of EBD and intralesional steroid injection was 3.3 (range 1-6). No other serious complications occurred in these patients, such as immediate bleeding and perforation. Eight patients were still alive during the mean follow-up period of 11.6 months (range, 2.5 to 21 months). One patient developed lung metastasis and died of the disease 15 months after ESD.

Conclusion: Transplantation of autologous esophagus mucosa appears to be a safe means of relieving the severity of esophageal stenoses following Circumferential ESD.

Disclosure of Interest: All authors have declared no conflicts of interest.

\section{References}

1. Bhatt A, Abe S, Kumaravel A, Vargo J, Saito Y. Indications and Techniques for Endoscopic Submucosal Dissection. Fully covered removable nitinol selfexpandable metal stents (SEMS) in malignant strictures of the esophagus: a multicenter analysis. Surg Endosc. 2012; 26(6):1664-9.

2. Hochberger J, Koehler P, Wedi E, Gluer S, Rothstein RI, Niemann H, 2012; 143(3):582-8.e1-2.

\section{P0805 ENDOSCOPIC CLOSURE OF POST-SURGICAL FISTULAS OR PERFORATIONS WITH OVER-THE-SCOPE-CLIP}

P. Santos ${ }^{1}$, D. Reis ${ }^{1}$, C. Noronha Ferreira ${ }^{1}$, J. Lopes ${ }^{1}$, L. Carrilho Ribeiro ${ }^{1}$, J. Velosa ${ }^{1}$

${ }^{1}$ Gastrenterologia E Hepatologia, Hospital de Santa Maria - Centro Hospitalar Lisboa Norte, Lisbon/Portugal

\section{Contact E-mail Address: patricia.sants@hotmail.com}

Introduction: Gastrointestinal perforations and post-surgical fistulas are feared complications with high morbidity and mortality. The over-the-scope-clip (OTSC) has been successfully used for its resolution.

Aims \& Methods: We aimed to evaluate the therapeutic efficacy of OTSC in the management of upper digestive tract post-surgical fistulas or perforations.

This was a retrospective review of consecutive patients at a single center from January 2011 to January 2017 who underwent upper endoscopy with attempt OTSC placement for the closure of post-surgical fistulas or perforations. Statistical analysis: IBM SPSS 23.0.

Results: A total of 18 patients ( $72 \%$ women), with a mean age of $48.9 \pm 18.1$ years were treated with an OTSC for the closure of post-surgical fistulas or perforations. Mean fistulas size was $5.8 \pm 3.5 \mathrm{~mm}$. Median follow-up time was 20.5 (5-84) months.

Etiology of fistulas: post-surgical $(n=16)$, perforation by foreign body $(n=1)$ and after endoscopic procedure $(\mathrm{n}=1)$. Location of the fistulas: cardia $(33 \%)$, esophagus $(28 \%)$, gastric body $(16 \%)$, antrum $(11 \%)$, esophagus-jejunal anastomosis $(6 \%)$ and duodenal bulb $(6 \%)$.

Overall clinical success rate was achieved in $72.2 \%(n=13)$. Fistulas were sucessfully closed in both non-surgical cases.

Regarding post-surgical fistulas $(n=16), 50 \%(n=8)$ were after bariatric surgery. There was a clinical success rate of $68.8 \%(n=11)$. The median time between surgery and the endoscopic intervention was 20.5 (2-550) days. There was no association between clinical success and the time between surgery and the endoscopic intervention $(p=0,624)$ or the location of the fistula $(p=0,334)$. In 1 case, endoscopic re-intervention with placement of OTSC was required due to persistence of fistula. In $5(31 \%)$ cases the OTSC was not effective, requiring endoscopic re-intervention with stent placement $(\mathrm{n}=2)$ or surgical intervention $(\mathrm{n}=3)$. The overall recurrence rate was $11 \%(\mathrm{n}=2)$.

Conclusion: OTSCs can be safely and effectively used in patients presenting with post-surgical fistulas or perforations and, when feasible, may be more advantageous and less costly than surgery. Further research is required to characterize the determinants of long-term success and risk factors for failure. Disclosure of Interest: All authors have declared no conflicts of interest.

\section{References}

1. Bège T, Emungania O, Vitton V, et al. An endoscopic strategy for management of anastomotic complications from bariatric surgery:a prospective study. Gastrointest Endosc. 2011;73:238-44.

2. Baron TH, Song LM, Ross A, Tokar JL, Irani S, Kozarek RA. Use of an overthe-scope clipping device: multicenter retrospective results of the first U.S. experience(withvideos). Gastrointest Endosc. 2012;76:202-8.

3. Wedi E, GonzalezS, Menke D, KruseE, Matthes K, Hochberger J. One hundred and one over-the-scope-clip applications for severe gastrointestinal bleeding, leaks and fistulas. World J Gastroenterol. 2016;22:1844-53.

4. Manta R, Manno M, Bertani H, et al. Endoscopic treatment of gastrointestinal fistulas using an over-the-scope clip (OTSC) device: case series from a tertiary referral center. Endoscopy. 2011;43:545-8.

\section{P0806 A PROSPECTIVE STUDY USING A NEW DEVICE FOR ENDOSCOPIC RESECTION OF EARLY NEOPLASIA IN BARRETT'S ESOPHAGUS}

R. E. Pouw ${ }^{1}$, T. Beyna ${ }^{2}$, K. Belghazi ${ }^{1}$, A.D. Koch ${ }^{3}$, E.J. Schoon ${ }^{4}$, R. Haidry ${ }^{5}$, B.L.a.m. Weusten ${ }^{6}$, R. Bisschops ${ }^{7}$, N.J. Shaheen ${ }^{8}$, M.B. Wallace ${ }^{9}$ N.E. Marcon ${ }^{10}$, K. Wang ${ }^{11}$, C. Leggett ${ }^{11}$, J. Ortiz Fernandez-Sordo ${ }^{12}$, K. Ragunath ${ }^{13}$, M. Di Pietro ${ }^{14}$, O. Pech ${ }^{15}$, H. Neuhaus ${ }^{2}$, J.J. G.h.m. Bergman ${ }^{1}$ ${ }^{1}$ Gastroenterology \& Hepatology, Academic Medical Center, Amsterdam/ Netherlands

${ }^{2}$ Department Of Internal Medicine, Evangel. Krankenhaus Düsseldorf, Düsseldorf Germany

${ }^{3}$ Erasmus MC - University Medical Center Rotterdam, Rotterdam/Netherlands

${ }^{4}$ Dept. Of Gastroenterology, Catharina Hospital Gastroenterology and Hepatology, Eindhoven/Netherlands

${ }^{5}$ Gastroenterology, University College London Hospital, London/United Kingdom ${ }^{6}$ Departement Of Gastroenterology And Hepatology, St Antonius Hospital, Nieuwegein/Netherlands

${ }^{7}$ Gastroenterology, Katholieke Universiteit Leuven, Leuven/Belgium

${ }^{8}$ Center For Esophageal Diseases Abd Swallowing, University of North Carolina School of Medicine, Chapel Hill/United States of America/NC

${ }^{9}$ Gastroenterology, Mayo Clinic Florida, Jacksonville, FL/United States of

America

${ }^{10}$ St Michael's Hospital, Toronto/Canada

${ }^{11}$ Mayo Clinic Rochester, Rochester/United States of America/MN

${ }^{12}$ Nottingham University Hospitals, Nottingham/United Kingdom

${ }^{13}$ Endoscopy \& Gastroenterologist Wolfson Digestive Diseases Centre, Queens

Medical Centre campus Nottingham University Hospitals, Nottingham/United Kingdom

${ }^{14}$ MRC Cancer Cell Unit, Cambridge University Hospitals MRC Cancer Cell Unit, Cambridge/United Kingdom

${ }^{15}$ Klinik Für Gastroenterologie Und, Krankenhaus Barmherzige Brüder Klinik für Gastroenterologie und Interventionelle Endoskopie - Klinik, Regensburg/Germany

Contact E-mail Address: roospouw@gmail.com

Introduction: Early neoplastic lesions in Barrett's Esophagus (BE) can be effectively and safely removed by endoscopic resection (ER) using multi-band mucosectomy (MBM). Recently a new MBM device became available, designed for improved visualization, easier passage of accessories, and better suction power compared to other marketed MBM devices.

Aims \& Methods: This study aims to document performance of the new MBM device for ER of early neoplastic lesions in BE.

This is a company sponsored, international, multicenter, single-arm, prospective registry study enrolling 300 subjects with early neoplasia in BE. Primary endpoint is successful ER defined as complete resection of the delineated target area in one procedure. Secondary outcomes: adverse events, procedure time.

Results: To date 259 subjects have been enrolled at 14 centers (Europe 10, US 3, Canada 1). Mean age was $67 \pm 9$ years, with $87 \%$ males. In these 259 subjects, a total of 301 lesions were removed using the new MBM device, with a mean of $2.5 \pm 1.92$ resections per procedure. Indication for ER was high-grade dysplasia (HGD) in $65 \%$, early adenocarcinoma in $20 \%$, visible lesion with low-grade dysplasia in $10 \%$, and a visible lesion suspicious for neoplasia without pre-treatment histology in $5 \%$. The primary endpoint of successful ER of a target lesion 
was reached in 290/301 (96\%) procedures. A perforation occurred in 3/301 ER procedures $(1 \%, 95 \%$ CI $0.21 \%-2.88 \%)$. Two perforations were closed with clips, all three patients received intra-venous antibiotics and were admitted to hospital for 2,3 and 9 days. Bleeding requiring intraprocedural hemostasis occurred during $15 \%$ of procedures. Significant post-procedural bleeding requiring an intervention occurred in 5 cases $(2 \%)$. Dysphagia requiring endoscopic dilatation occurred in 7 patients $(3 \%)$, after ER with a mean number of $4 \pm 2.9$ resected pieces. Mean total procedure time for ER using the new MBM device was $33 \pm 17.1$ minutes.

Conclusion: The new MBM device used in this study proved to be effective for resection of early neoplastic lesions in BE: successful ER was achieved in $96 \%$ of procedures. Perforations were seen in $1 \%$ and significant post-procedural bleeding in $2 \%$, complications were effectively managed endoscopically.

Disclosure of Interest: All authors have declared no conflicts of interest.

\section{P0807 COST-EFFECTIVE ANALYSIS COMPARING STANDARD BIOPSY VS. DIGITAL BIOPSY BY CONFOCAL ENDOMICROSCOPY}

C. Robles-Medranda, M. Valero, J. Ospina, M. Puga-Tejada, H. AlvaradoEscobar, M. Soria, H. Pitanga-Lukashok

Gastroenterology, Instituto Ecuatoriano de Enfermedades Digestivas, Guayaquil/ Ecuador

\section{Contact E-mail Address: carlosoakm@yahoo.es}

Introduction: Endoscopy has greatly influenced gastroenterological diagnosis. However, most lesions can be suspected but not certainly diagnosed only on the basis of endoscopic findings and therefore histology is needed. On the other hand the reliability of detecting lesions histologically depends on the site, number, and size of biopsy (Bx) specimens with a $20-30 \%$ probability of sampling mistakes. Probe based Confocal Laser Endomicroscopy (p-CLE) allows endoscopic in-vivo mucosal cellular evaluation of the gastrointestinal (GI) tract with a high $(90 \%)$ diagnostic accuracy. It allows to perform target $\mathrm{Bx}$. Moreover, the NPV is $>98 \%$. There is no information in the literature regarding the economic impact of performing digital biopsies (DBx) by p-CLE.

Aims \& Methods: The aim of this study is to perform a cost-effectiveness analysis comparing the diagnosis of upper GI tract pathologies using only standard Bx following the literature recommendations (LR) vs. the diagnosis with DBx using p-CLE. This was a retrospective study with prospective collection data of patients included from Jan 2014 to Nov 2016. The pathologies included for pCLE evaluation are summarized in Table 1. The diagnosis costs using standard $\mathrm{Bx}$ was calculated following the literature recommendations (Table 2). The standard $\mathrm{Bx}$ costs included the histological process and physician honoraria per $\mathrm{Bx}$ (USD 50.oo), and one biopsy forceps per patient (USD 38.oo). The DBx costs by p-CLE included the probe, the processor and the physician honoraria (USD 500.oo). Baseline characteristics, p-CLE indications, the diagnostic accuracy of p-CLE and costs were described.

Results: 78 patients were included, $51.2 \%$ were female. The mean age was 50.18 years old. p-CLE indications distribution was: esophagus $29(37.2 \%)$, stomach 46 $(59 \%)$ and duodenum $3(3.8 \%)$ subgroups. Biopsies were performed in $71 / 78$ cases (91.0). Table 1 shows the procedure cost reached with the different pathologies, by following the Bx LR for initial diagnosis and follow-up. The efficacy of p-CLE in our study was $91.7 \%$ sensitivity, $89.8 \%$ specificity, $64.7 \%$ PPV, $98.2 \%$ NPV and $90.1 \%$ accuracy for the diagnosis of the malignant upper GI tract pathology, by Digital Biopsy with p-CLE. Finally, the cost-effective analysis showed that when applying p-CLE a diminishing cost in \$ 188 USD and \$138 USD is detected if more than 10 biopsies are attempted by procedure.

Table 1: Cost analysis following the Literature Recommendations (LR) for initial diagnosis and follow-up

\begin{tabular}{|c|c|c|c|c|}
\hline Pathology & $\begin{array}{l}\text { No. of } \\
\text { Bx by LR }\end{array}$ & $\begin{array}{l}\text { No. of } \\
\text { Bx by } \\
\text { Updated } \\
\text { Sydney } \\
\text { System }\end{array}$ & $\begin{array}{l}\text { No. of } \\
\text { total } \mathrm{Bx}\end{array}$ & $\begin{array}{l}\text { Total } \\
\text { cost of } \\
\mathrm{Bx}^{\mathrm{c}} \text { (USD) }\end{array}$ \\
\hline Esophageal Tumor ${ }^{\mathrm{a}}$ & 8 & - & 8 & 438.00 \\
\hline Barrett's Esophagus 1 to $3.9 \mathrm{~cm}^{\mathrm{a}, \mathrm{b}}$ & 4 & - & 4 & 238.00 \\
\hline Barrett's Esophagus $\geq 4 \mathrm{~cm}^{\mathrm{a}, \mathrm{b}}$ & 8 & - & 8 & 438.00 \\
\hline Gastric Tumor $^{\mathrm{a}}$ & 8 & 5 & 13 & 688.00 \\
\hline $\begin{array}{l}\text { Gastric Atrophy and/or } \\
\text { Metaplasia a, b }\end{array}$ & 12 & - & 12 & 638.00 \\
\hline Gastric Ulcer $^{\mathrm{a}}$ & 8 & 5 & 13 & 688.00 \\
\hline
\end{tabular}

Bx: biopsies; LR: Literature Recommendations. a. For initial diagnosis. b. For follow-up. c. Cost includes histological process and physician honoraria per biopsy (USD 50.oo), and the Bx forceps per patient (USD 38.oo).

Conclusion: In our population, the digital biopsy by p-CLE proved to be more cost-effective, when $\geq 10$ biopsies were indicated, like in cases of a Barrett's Esophagus $\geq 4 \mathrm{~cm}$, a Gastric Tumor, or in the context of two or more suspected pathologies (e.g.: esophageal and gastric disease).

Disclosure of Interest: C. Robles-Medranda: KOL for Pentax Medical, Boston Scientific Consulting, US Endoscopy Consulting.

All other authors have declared no conflicts of interest.
P0808 GASTRIC PER-ORAL ENDOSCOPIC PYLOROMYOTOMY (G-POEM) IN THE TREATMENT OF REFRACTORY GASTROPARESIS: EXPERIENCE OF THE FIRST 9 CASES IN A MEXICO

O. V. Hernandez Mondragon, R. Palos Cuellar, G. Blanco Velasco, J.M. Blancas-Valencia, M. L. Hernandez Reyes

Endoscopy, IMSS, Mexico city/Mexico

Contact E-mail Address: mondragonmd@yahoo.co.uk

Introduction: Gastroparesis is a syndrome characterized by a delayed gastric emptying in absence of a mechanic obstruction. Reduction in QOL scores have been observed. Etiologies include: idiopathic, diabetic, post-surgical. Diagnosis is based on the combination of symptoms and a delayed gastric emptying scintigraphy(GES) of $>10 \%$ after $240 \mathrm{~min}$. Multiple treatments have been used with temporary results or high morbidity, so new treatment options have been explored. G-POEM is a new endoscopic treatment which is based in the POEM treatment for achalasic patients and consist in a creation of a submucosal tunnel in order to perform an endoscopic pyloromyotomy. Initial results have been promising.

Aims \& Methods: The aim of this study was to evaluate the safety and efficacy of G-POEM in a group of Mexican patients with refractory gastroparesis. This prospective study was carried out in a tertiary care center in Mexico city, between December 2016 and April 2017. We included patients with refractory gastroparesis defined as presence of symptoms such as: nausea, vomiting, early satiety with inability to finish a normal meal, bloating and upper gastrointestinal pain. These patients were on medical treatment and didńt respond and have a positive gastroparesis cardinal symptom index (GCSI) score combined with a $>10 \%$ of retention at $240 \mathrm{~min}$ in the GES study. Exclusion criteria were malignancy, peptic ulcer disease, normal GES and coagulation disorders. Procedure steps were based in POEM procedure, beginning $5 \mathrm{cms}$ before the pyloric arch with an longitudinal incision, then submucosal tunnel creation, myotomy of the piloric arch up to the serosa and $2 \mathrm{cms}$ before this point and finally closure with clips. Follow-up included GCSI, endoscopy and GES at 3 months after procedure. Characteristics of procedure, and patients were documented. Student paired ttest was used for comparisons between groups and $\mathrm{p}<0.05$ was considered as statistically significant.

Results: There were 9 patients included in this initial study, the mean age was $42.4 \pm 8.5$ years. 6 patients were female and 3 male. The most common etiology was postsurgical $4 / 9(44.4 \%)$, followed by diabetic $3 / 9(33.3 \%)$ and idiopathic $2 /$ $9(22.2 \%)$. The mean G-POEM time was $61.4 \pm 7.8 \mathrm{~min}$, and complications were self-limited and presented in only 4 patients, the GCSI score decreased $68 \%$ between the pre-procedure levels as well as the GES which decresed $67 \%$ compared with levels at 3 months after G-POEM $(34.3 \pm 5.8$ vs $13.1 \pm 3.2 \mathrm{p}=0.003$ / $20.74 \pm 5.3$ vs $6.83 \pm 1.78 \mathrm{p}=0.001$ respectively). $7 / 9(77.7 \%)$ normalized the GES $(<10 \%$ at $240 \mathrm{~min})$. Endoscopy at 3 months after procedure didńt show any complication (Table 1).

Conclusion: G-POEM is a safe and effective procedure in Mexican patients with refractory gastroparesis with a normalization of the GES in up to $77 \%$ of these patients.

Disclosure of Interest: All authors have declared no conflicts of interest.

\section{References}

1. Ahuja NK, Clarke JO. Pyloric Therapies for Gastroparesis. Curr Treat Options Gastroenterol. 2017 Mar;15(1):230-240.

2. Khashab MA, Ngamruengphong S, Carr-Locke D, Bapaye A, Benias PC, Serouya S, Dorwat S, Chaves DM, Artifon E, de Moura EG, Kumbhari V, Chavez YH, Bukhari M, Hajiyeva G, Ismail A, Chen YI, Chung H. Gastric per-oral endoscopic myotomy for refractory gastroparesis: results from the first multicenter study on endoscopic pyloromyotomy (with video). Gastrointest Endosc. 2017 Jan;85(1):123-128.

3. Mekaroonkamol P, Li LY, Dacha S, Xu Y, Keilin SD, Willingham FF, Cai Q. Gastric peroral endoscopic pyloromyotomy (G-POEM) as a salvage therapy for refractory gastroparesis: a case series of different subtypes. Neurogastroenterol Motil. 2016 Aug;28(8):1272-7.

4. Xue HB, Fan HZ, Meng XM, Cristofaro S, Mekaroonkamol P, Dacha S, Li LY, Fu XL, Zhan SH, Cai Q. Fluoroscopy-guided gastric peroral endoscopic pyloromyotomy (G-POEM): a more reliable and efficient method for treatment of refractory gastroparesis. Surg Endosc. 2017 Apr 13. doi: 10.1007/ s00464-017-5524-y. [Epub ahead of print]

5. Geyl S, Legros R, Charissou A, Mesturoux L, Couquet CY, Carrier P, Brayette A, El-Ouafi Z, Loustaud-Ratti V, Sautereau D, Monteil J, Jacques J. Peroral endoscopic pyloromyotomy accelerates gastric emptying in healthy pigs: proof of concept. Endosc Int Open. 2016 Jul;4(7):E796-9.

P0809 ENDOSCOPIC MANAGEMENT OF FOREIGN BODIES IN THE UPPER GASTROINTESTINAL TRACT: A RETROSPECTIVE STUDY OF 1294 CASES

C. Geng, X. Li, X. Lei, C. Wang

Department Of Gastroenterology, West China Hospital of Sichuan University

Chengdu/China

Contact E-mail Address: gengchong15scu@163.com

Introduction: Foreign body (FB) ingestion including food bolus impaction is frequently encountered in clinical practice. Few studies with large sample size 
Abstract No: P0808

Table 1: Characteristics of procedure and patients before and 3 months after G-POEM

\begin{tabular}{|c|c|c|c|c|c|c|c|}
\hline Patient & $\begin{array}{l}\text { Age/ } \\
\text { Gender }\end{array}$ & $\begin{array}{l}\text { GCSI pre } \\
\text { (score) }\end{array}$ & $\begin{array}{l}\text { GES pre } \\
(\%)\end{array}$ & $\begin{array}{l}\text { G-POEM } \\
\text { time (min) }\end{array}$ & $\begin{array}{l}\text { Adverse } \\
\text { events }\end{array}$ & $\begin{array}{l}\text { GCSI at } \\
3 \mathrm{~m} \text { (score) }\end{array}$ & $\begin{array}{l}\text { GES } \\
\text { at } 3 \mathrm{~m}(\%)\end{array}$ \\
\hline 1 & $25 / \mathrm{F}$ & 37 & 25.4 & 61 & Mild Abdominal pain & 16 & 8.5 \\
\hline 2 & $57 / \mathrm{F}$ & 41 & 25.4 & 65 & None & 25 & 9 \\
\hline 3 & $45 / \mathrm{F}$ & 31 & 22.4 & 60 & None & 15 & 5 \\
\hline 4 & $73 / \mathrm{F}$ & 36 & 16.7 & 97 & Mild abdominal pain & 12 & 3 \\
\hline 5 & $42 / \mathrm{M}$ & 25 & 20.9 & 55 & None & 10 & 5 \\
\hline 6 & $45 / \mathrm{M}$ & 35 & 22.4 & 45 & None & 14 & 11 \\
\hline 7 & $28 / \mathrm{M}$ & 29 & 26.4 & 55 & None & 5 & 12 \\
\hline 8 & $37 / \mathrm{F}$ & 35 & 12.5 & 60 & Mild abdominal pain & 10 & 4 \\
\hline 9 & $30 / \mathrm{F}$ & 40 & 15.5 & 55 & Mild Abdominal pain & 11 & 4 \\
\hline Total & $42.4 \pm 8.56 \mathrm{~F} 3 \mathrm{M}$ & $34.3 \pm 5.8$ & $20.74 \pm 5.3$ & $61.4 \pm 7.8$ & 5 None 4 Mild abdominal pain & $13.1 \pm 3.2$ & $6.83 \pm 1.78$ \\
\hline
\end{tabular}

towards endoscopic management of FBs had been reported. No direct evidence has demonstrated the relationship between duration of FB impaction and outcomes of endoscopic management. Moreover, it remained unclear whether endoscopic management of FBs under general anaesthesia could improve endoscopic outcomes when compared with topical pharyngeal anaesthesia.

Aims \& Methods: The aim of the present retrospective study is to analyze our endoscopic outcome and explores the best timing and anaesthesia methods of endoscopic intervention in population with FB ingestion. All consecutive patients suspected of FB ingestion were enrolled. The demographic, clinical and endoscopic data were collected and analyzed.

Results: Totally, 1294 cases were recruited in this retrospective research. The ages ranged from 7 months to 94 years, with a median age of 47.0 (31-63) years. The majority of patients (1191/1294 cases, 92.0\%) presented with some symptoms after FB ingestion, in order of frequency odynophagia (415 cases, $32.1 \%$ ), foreign body sensation ( 340 cases, $26.3 \%$ ) and sore throat ( 267 cases, $20.1 \%$ ). The duration of FB impaction ranged from 4 hours to more than 2 years with a median time of 1 (0.63-3) days. Bony FBs, jujube pit, food bolus and dental prosthesis were the most frequent FBs in population. Anatomically, FBs were mostly impacted in the oesophagus $(\mathrm{n}=1025,86.9 \%)$, especially in the upper oesophagus $(n=832,70.5 \%)$, followed by stomach $(n=95,8.1 \%)$, duodenum $(\mathrm{n}=36,3.0 \%)$ and pharynx $(\mathrm{n}=24,2.0 \%)$. Nearly half of the patients $(49.9 \%)$ developed FB-related complications, mainly including mucosal injures (356 cases, $27.5 \%$ ) and ulcers ( 210 cases, $16.2 \%)$. The most common underlying pathologies were oesophageal stricture (35 cases, 39.3\%) and oesophageal cancer (11 cases, $15.5 \%)$. As the duration of FB impaction increased, positive finding and successful removal of FB by endoscopy significantly decreased $(p<0.001)$. Furthermore, complication rate significantly increased with time $(\mathrm{p}<0.001)$. Age $(\mathrm{OR}=1.15,95 \% \mathrm{CI}$ : $1.20-1.91, \mathrm{p}<0.001)$, type and location of FBs $(\mathrm{OR}=4.51, \quad 95 \% \mathrm{CI}: \quad 2.95-6.90, \quad \mathrm{p}<0.001 ; \quad \mathrm{OR}=2.26, \quad 95 \% \mathrm{CI}: \quad 1.48-3.46$, $\mathrm{p}<0.001)$, anaesthesia methods $(\mathrm{OR}=1.35,95 \% \mathrm{CI}: 1.05-1.75, \mathrm{p}=0.02)$ and duration of $\mathrm{FB}$ impaction $(\mathrm{OR}=1.74,95 \% \mathrm{CI}: 1.50-2.00, \mathrm{p}<0.001)$ were verified as risk factors for development of FB-related complication by logistic regression analysis. General anaesthesia could not improve positive FB detection $(p=0.818)$ or success rate of endoscopic management of FBs $(p=0.135)$, as well as decrease the complication rate when compared with topical pharyngeal anaesthesia $(52.3 \%$ VS $47.5 \%, \mathrm{p}=0.033)$.

Conclusion: FB-related complication rate increased with time, endoscopic management under general anaesthesia could not improve therapeutic effects when compared with topical pharyngeal anaesthesia. Overall, Patients suspected of FB ingestion should receive endoscopic management as soon as possible.

Disclosure of Interest: All authors have declared no conflicts of interest.

\section{P0810 CLINICAL OUTCOMES AFTER ENDOSCOPIC RESECTION FOR ESOPHAGEAL SQUAMOUS CELL CARCINOMA COMPARING THE CASES WITH MM AND SM1 INVASION}

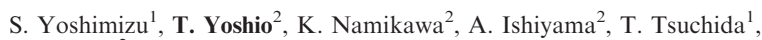
J. Fujisaki ${ }^{2}$

${ }^{1}$ Cancer Institute Hospital, Tokyo/Japan

${ }^{2}$ Gatroenterology, Cancer Institute Hospital, Tokyo/Japan

\section{Contact E-mail Address: toshiyuki.yoshio@jfcr.or.jp}

Introduction: Recent advances in endoscopic resection (ER) provide us increasing chances for resecting esophageal squamous cell carcinoma (ESCC) with muscularis mucosae (MM) and SM1 invasion. As MM/SM1 invasive cancer is reported to have $8-20 \%$ of metastatic risks and is defined as relative indication for ER in guideline by Japan Esophageal Society. For them, we perform additional therapy such as chemo radiotherapy (CRT) or operation considering the risk of metastasis and patients' condition.

Aims \& Methods: To know the difference of metastatic risk and long time outcomes, we retrospectively studied 121 cases of ESCC with pathological MM/SM1 invasion (MM/SM1:97/24) resected by ER from 2003 to 2013 in Cancer Institute Hospital. After pathological diagnosis of resected lesions, we performed additional therapy such as CRT, radiation therapy (RT) or operation, to the cases with lymphovascular invasion (LVI) or droplet infiltration (DI). Median observation period was 48 months.

Results: Enrolled cases included 112 males and 9 females and their median age was $66(39-86)$. We resected ESCC by ESD in 71 cases and by EMR-C in 50 cases and their median size was $27 \mathrm{~mm}$. Local recurrence was observed in 6 cases which were all after EMR $(12 \%)$. As for local recurrence 5 cases were treated by reEMR and 1 case by APC, resulted in no re-recurrence. Of 97 cases of MM, 15 cases $(15.5 \%)$ had LVI, 10 cases $(10.3 \%)$ had DI. We recommended additional therapy in 21 cases $(21.6 \%)$. Additional therapy was performed in 15 cases $(15.5 \%)($ ope/CRT/RT:9/5/1). No case died of ESCC and 22 cases $(22.7 \%)$ died of other diseases. Of 24 cases of SM1, 9 cases $(37.5 \%)$ had LVI, 5 cases $(20.8 \%)$ had DI. We recommended additional therapy in 12 cases $(50.0 \%)$. Additional therapy was performed in 9 cases $(37.5 \%$ ) (ope/CRT/RT/chemotherapy: 3/4/1/1). Three cases died of ESCC and 5 cases $(20.8 \%)$ died of other diseases. Comparing both groups, tumor size and local recurrence rate were not different each other. The frequency of LVI was significantly higher in SM1 than in MM $(p<0.05)$ and the frequency of DI was higher in SM1, although not significant $(\mathrm{p}=0.161)$. The metastatic recurrence was observed significantly frequent in SM1 than MM $(16.7 \%$ vs $2.1 \%$; p $<0.01)$. The 5 -year overall survival (OS)/disease specific survival (DSS)/relapse free survival (RFS) were $81.7 \%$ $100 \% / 94.1 \%$ for $\mathrm{MM}$ and $62.9 \% / 87.9 \% / 91.7 \%$ for SM1. OS and RFS were not different each other, however, CSS was superior in MM than in SM1 $(\mathrm{p}<0.01)$.

Conclusion: ESCC with MM invasion was superior in metastatic recurrence and CSS than ESCC with SM1 invasion, although we treat MM/SM1 in the same way. Additional therapy should be considered more positively in cases of SM1 than in cases of MM, considering metastatic risk and patients' conditions.

Disclosure of Interest: All authors have declared no conflicts of interest.

\section{P0811 GASTRIC ESD IN AN ANIMAL SURVIVAL MODEL USING} THE ANUBIS-SYSTEM

J. Bernhardt ${ }^{1}$, H. Steffen ${ }^{2}$, P. Koehler ${ }^{3}$, S. Schneider-Koriath ${ }^{2}$, K. Ludwig ${ }^{2}$ ${ }^{1}$ Dept. Of Endoscopy, Klinikum Suedstadt Rostock, Rostock/Germany

${ }^{2}$ Dept. Of Surgery, Klinikum Suedstadt Rostock, Rostock/Germany

${ }^{3}$ Friedrich Loeffler Institute, Neustadt/Germany

Contact E-mail Address: joern.bernhardt@kliniksued-rostock.de

Introduction: ESD in generally is still under evaluation. The one-piece resection of lesions larger then $2 \mathrm{~cm}$ has many advantages against piece meal resection. One problem in ESD is to lift and prepare the specimen simultaneously. We used the ANUBIS-system for intragastric ESD.

Aims \& Methods: The experimental study was conducted in a porcine model in general anesthesia. We started the study with 7 pigs in a survival model using the Anubisscope (Karl Storz, Germany). After insertion of the scope insufflations of the stomach was done using $\mathrm{CO}_{2}$. After the opening of the valves at the tip of the scope, an area of $5 \mathrm{~cm}$ in diameter was selected for resection. Injection was done through the working channel with a hyperosmolaric solution added with blue colour. After circle incision, preparation was done with the two arms of the scope using a grasper and a hook-knife. Also the grasper could use for coagulation. The specimen was removed with the scope after closing its valves.

Results: The procedure was successful in all animals with operation time ranging from 102 to 189 minutes with a learning curve. After weight gain in all cases, the animals were sacrificed after postoperative day 42 and the workup showed competent healing with a star-like scar.

Conclusion: The use of an operating platform like the Anubisscope has the advantage of flexible preparation in opposite position of the instruments in ESD. The disadvantages are the only two degrees of freedom of the flexible instruments and the rotation-like movements. Also, it is not possible to reach all regions of the stomach.

Disclosure of Interest: All authors have declared no conflicts of interest.

P0812 USEFULNESS OF NARROW BAND IMAGING WITH MAGNIFYING ENDOSCOPY AS A SCREENING TEST FOR GASTRIC CANCER

T. Morinushi

Gastroenterology, Kameda Medical Center, Chiba/Japan

Contact E-mail Address: moristatu@yahoo.co.jp 
Introduction: Narrow band imaging with magnifying endoscopy (NBI-ME) is useful for diagnosis of gastric cancer; however, whether NBI-ME is useful as a screening test for gastric cancers has not yet been determined. Additionally, it is important to consider the impact on the atrophy of the background gastric mucosa in gastric cancer screening because the incidence of gastric cancer depends largely on the degree of atrophy noted in the background gastric mucosa.

Aims \& Methods: We aimed to determine the usefulness of NBI-ME as a screening tool for gastric cancer. We retrospectively studied 3515 patients who had undergone screening upper gastrointestinal endoscopy between April 2013 and March 2014. We excluded patients with advanced gastric cancer and those who had undergone gastrectomy. Thus, we studied 1080 patients who received NBIME and 2435 patients who had undergone conventional endoscopy. We classified the degree of atrophy of the background gastric mucosa using the KimuraTakemoto classification. Severe atrophy was noted in 1620 patients (Group S), and mild atrophy in 1895 patients (Group M). We evaluated the biopsy rate, the detection rate of gastric neoplasms, and the accuracy of biopsy using NBI-ME compared to conventional endoscopy.

Results: The biopsy rate of NBI-ME and conventional endoscopy in Group M was 5.4 and $7.7 \%$, respectively, while in Group S it was 14.9 and $14.8 \%$, respectively. The biopsy rate did not differ significantly between those who received NBI-ME and those who had undergone conventional endoscopy. The detection rate of gastric neoplasms using NBI-ME and conventional endoscopy in Group $\mathrm{M}$ was 0 and $0.2 \%$, respectively, while in Group $\mathrm{S}$ it was noted to be 4.2 and $1.8 \%$, respectively. Thus, the detection rate of NBI-ME was significantly higher than that of conventional endoscopy in Group $\mathrm{S}(\mathrm{p}<0.01)$. The accuracy of biopsy with NBI-ME and conventional endoscopy in Group M was 0 and $3.2 \%$, respectively, but in Group S was noted to be 36.4 and $14.1 \%$, respectively. Thus, the accuracy of biopsy using NBI-ME is significantly superior to conventional endoscopy in Group $\mathrm{S}(\mathrm{p}<0.01)$.

Conclusion: NBI-ME as a screening test for gastric cancer is useful for patients with severe atrophy of the background gastric mucosa because this technique has shown a higher detection rate of gastric neoplasms and better accuracy of biopsy. Disclosure of Interest: All authors have declared no conflicts of interest.

\section{P0813 DIAGNOSTIC LIMITATIONS OF MAGNIFYING ENDOSCOPY WITH NARROW BAND IMAGING (ME-NBI) IN EARLY GASTRIC CANCER}

K. Matsumoto ${ }^{1}$, H. Ueyama ${ }^{1}$, K. Matsumoto ${ }^{1}$, Y. Akazawa ${ }^{1}$, H. Komori ${ }^{1}$, M. Hojo ${ }^{1}$, A. Nagahara ${ }^{2}$, T. $\mathrm{Yao}^{3}$, S. Watanabe

${ }^{1}$ Dept. Of Gastroenterology, Juntendo University, Bunkyo City/Japan

${ }^{2}$ Department Of Gastroenterology, Juntendo University Shizuoka Hospital, Shizuoka/Japan

${ }^{3}$ Department Of Human Pathology, Juntendo University School of Medicine, Tokyo/Japan

Contact E-mail Address: khmatsu@juntendo.ac.jp

Introduction: ME-NBI is an important modality to diagnose early gastric cancer. A unified diagnostic system, the magnifying endoscopy simple diagnostic algorithm for early gastric cancer (MESDA-G), was proposed in 2016 by Muto et al. ${ }^{[1]}$ and was thought to become a future mainstream system. Although the usefulness of ME-NBI has been often reported ${ }^{[2,3]}$, some cases are difficult to diagnose with ME-NBI.

Aims \& Methods: We aimed to clarify endoscopic and histological features of difficult-to-diagnose cases using MESDA-G. We retrospectively analyzed 362 cases of early gastric cancer that underwent endoscopic resection at our hospital from January 2013 to December 2016. Lesions for which ME-NBI images were not taken at maximum magnification or difficult to evaluate because of mucus or erosions were excluded. After exclusion, a total of 350 lesions were enrolled in this study. Two expert endoscopists reviewed ME-NBI images of all lesions. Lesions diagnosed as non-cancer by both experts were defined as ME-NBI incorrect diagnosis lesions (ME-NBI-IDL). ME-NBI and histopathological findings of ME-NBI-IDL were analyzed. ME-NBI findings were defined according to the presence (+) or absence (-) of a demarcation line (DL) and regular (R), irregular (I) or absent (A) of micro vascular (MV) and micro surface (MS) patterns. Results: Of 350 lesions, $32(9.1 \%)$ were ME-NBI-IDL. Macroscopic types were I/ $\mathrm{IIa} / \mathrm{IIa}+\mathrm{IIc} / \mathrm{IIb} / \mathrm{IIc}=3 / 13 / 2 / 7 / 7$, respectively. Twenty-seven of ME-NBI-IDL showed DL (-). Five of ME-NBI-IDL showed R or A of MS/MV patterns, despite of DL $(+)$. The details were as follows; In the DL (-) group, MV/MS were $\mathrm{R} / \mathrm{R}: 25, \mathrm{I} / \mathrm{R}: 1$, and $\mathrm{R} / \mathrm{A}: 1$. In the $\mathrm{DL}(+)$ group, $\mathrm{MV} / \mathrm{MS}$ were $\mathrm{R} / \mathrm{R}: 4$ and $\mathrm{A} / \mathrm{R}: 1$. Histological types of ME-NBI-IDL were gastric adenocarcinoma of fundic gland type (GAFG)/well-differentiated carcinoma (well)/well-to-moderately differentiated carcinoma (well to mod)/papillary carcinoma (pap)/signet ring cell carcinoma $(\operatorname{sig})=20 / 4 / 1 / 1 / 6$, respectively. Diagnostic difficulties were caused by two histological findings: 1) low-grade atypia type; surface structure of cancerous lesion was similar to surrounding mucosa due to very low-grade atypia (well/well to $\mathrm{mod} / \mathrm{pap}=4 / 1 / 1$, respectively.), 2) covered type; cancer was unexposed to the superficial mucosal layer (GAFG/sig $=20 / 6$, respectively).

Conclusion: Diagnostic limitations of ME-NBI (MESDA-G) were dependent on distinct histological characteristics. However, we suggest that the low-grade atypia type might be able to diagnose correctly by improving diagnostic accuracy of ME-NBI.

Disclosure of Interest: All authors have declared no conflicts of interest.

\section{References}

1. Muto M et al. Dig Endosc 2016

2. Nagahama T. Gastrointest Endosc 2011.

3. Maki S. Gastric Cancer 2013.

P0814 BEST PRACTICE IN PLACEMENT OF PERCUTANEOUS ENDOSCOPIC GASTROSTOMY WITH JEJUNAL EXTENSION TUBE FOR CONTINUOUS INFUSION OF LEVODOPA CARBIDOPA INTESTINAL GEL IN THE TREATMENT OF ADVANCED PARKINSON'S DISEASE: THE ROLE OF GASTROENTEROLOGIST

A.G. Gravina, I. Apicella, V. M. Ormando, M.R. Amato, M. I. Russo, M. Romano, P. Esposito

Medical And Surgical Department Of Clinical And Experimental Internistic " $f$ Magrassi", Hepatogastroenterology and Digestive Endoscopy Unit, University of Studies "L. Vanvitelli", Napoli/Italy

Contact E-mail Address: antonietta.gravina@yahoo.it

Introduction: Levodopa is the gold standard in pharmacological treatment of the advanced Parkinson's disease (APD) and its oral administration in advanced disease is associated with development of symptoms and motor and non-motor complications. Due to its short plasma half-life, Levodopa requires repeated dosing $(1,2)$. Impaired gastric emptying is common in APD and reduces the absorption of Levodopa, requiring progressively higher doses and more frequent administrations. Continuous duodenal administration of modulated Levodopa/ Carbidopa gel by Jejunal Extension Tube Placement through Percutaneous Endoscopic Gastrostomy (PEG-J), is a new therapeutic protocol in advanced APD (3).

Aims \& Methods: Aim of this work is to verify efficacy and safety of intestinal gel (LCIG) delivered continuously through an Intrajejunal percutaneous tube (PEGJ). 24 adult ( $14 \mathrm{M}, 10 \mathrm{~F}$, median age 71 years) with APD and preserved sensitivity to L-Dopa were enrolled. 7 days before PEG-J placement, to evaluate the possible presence of mucosal or anatomical gastric anomalies, each patient underwent a diagnostic esophagogastroduodenoscopy (EGD). Treatment with LCIG consists in a water-based suspension containing micronized levodopa $(20 \mathrm{mg} / \mathrm{mL})$ and carbidopa $(5 \mathrm{mg} / \mathrm{mL})$ in methylcellulose, administered by continuous jejunal infusion for $12 \mathrm{~h}$ /day using a portable pump by PEG-J. Clinical evalutations were performed at baseline(T0), before LCIG initiation, and after 3 (T3) and 6 (T6) months of therapy. To evaluate efficacy and safety outcomes it has been used Unified Parkinson's Disease Rating Scale (UPDRS) parts II, III and IV. For the analysis of the differences between the clinical variables and to exclude biases due to the small number of the sample in question, the non-parametric KruskalWallis $\mathrm{H}$ test was used for the comparison of three samples. A statistically significant value of $\mathrm{p}$ was less than 0.05 . The analyzes were carried out using SPSS version 13 (SPSS Inc., Chicago, IL, USA).

Results: 1) Success rate for PEG-J placement was $100 \%$; 2) Eight $/ 24$ patients $(33 \%)$ dropped-out LCIG at T3; 3) Sixteen/16 patients $(100 \%)$ showed statistically significant ( $\mathrm{p} 0.05$ ) higher performances in daily common activities and statistically significant (p 0.05) lower incidence and severity of motor fluctuations, as assessed by UPDRS part IV, compared to their best oral therapy; 4)During observational period, 6 patients experienced adverse events.

Conclusion: 1) This study demonstrates that continuous intrajejunal LCIG's infusion treatment is highly effective in decreasing motor fluctuations in advanced PD patients compared to oral administration of levodopa-carbidopa; 2) This therapeutic approach should be proposed in well selected APD patients with preserved sensitivity to L-dopa.

Disclosure of Interest: All authors have declared no conflicts of interest.

\section{References}

1. Lee A, Gilbert RM. Epidemiology of Parkinson Disease, Neurol Clin 2016 Nov; 34(4):955-65

2. Connolly BS, Lang AE. Pharmacological treatment of Parkinson disease: a review. JAMA 2014; 311(16):1670-83

3. Zulli C, Sica M, De MiccoR, Del Prete A, Amato MR, Tessitore A, Ferraro F, Esposito P. Continuous intra jejunal infusion of levodopa-carbidopa intestinal gel by jejunal extension tube placement through percutaneous endoscopic gastrostomy for patients with advanced Parkinson's disease: a preliminary study. Eur Med Pharmacol Sci 2016; 20(11):2413-17

\section{P0815 PREDICTIVE RISK MODEL FOR POST-ENDOSCOPIC} SUBMUCOSAL DISSECTION ULCER BLEEDING OF STOMACH

Y.H. Choe' ${ }^{1}$, S.K. Shin ${ }^{2}$, E.H. Kim ${ }^{1}$, Y.C. Lee ${ }^{1}$, S.K. Lee ${ }^{1}$, J. Park ${ }^{3}$

${ }^{1}$ Gastroenterology, Severance Hospital, Yonsei University College of Medicine, Seoul/Korea, Republic of

${ }^{2}$ Dept. Of Gastroenterology, Yonsei University College of Medicine Dept. of Internal Medicine, Seoul/Korea, Republic of

${ }^{3}$ Dept. Of Internal Medicine, Yonsei University, Seoul/Korea, Republic of

Contact E-mail Address: last_message@yuhs.ac

Introduction: Post-endoscopic submucosal dissection (ESD) bleeding is the most common complication of ESD. In previous studies, the post-ESD bleeding occurred from $1.8 \%$ to $15.6 \%$ of total procedures [1-4]. Recently, many patients who underwent ESD, have been prescribed antiplatelets or anticoagulants, because of various underlying diseases such as cerebrovascular accidents or 
cardiovascular diseases [5]. Thus, the verified risk prediction model of post-ESD bleeding may enable to determine preventive therapeutic options and restarting date of antiplatelet agents.

Aims \& Methods: The aim of this study is to develop the predictive risk model of post-ESD bleeding. A total of 5374 patients, who were taken ESD from January 2007 to November 2016, in a Korean tertiary hospital, were included in this retrospective study. To avoid overfitting of the prediction model, we divided the patients randomly into two groups, either a derivation group or a validation group. Preoperative and procedural-related variables were selected via univariate and multivariate analysis. A risk score was calculated to assess the bleeding prediction model of a patient in the derivation group and was discriminated in the validation group.

Results: Post-ESD bleeding occurred in 248 patients $(4.84 \%)$. In the derivation group, multivariate logistic regression revealed renal diseases (odds ratio [OR], $3.90 ; 95 \%$ confidence interval $[\mathrm{CI}], 1.48-9.09 ; P=0.0029)$, anti-platelet agent (OR, 3.07; 95\% CI, 1.44-6.05; $P=0.002$ ), and en-bloc resection (OR, 3.83; $95 \% \mathrm{CI}, 1.34-9.34 ; P=0.0059)$ as significant risk factors $(\mathrm{C}$-statistic $=0.607$; $95 \%$ CI, 0.552-0.661). In the validation set, the model also showed good discrimination power $(\mathrm{C}$-statistic $=0.580 ; 95 \%$ CI, 0.533-0.627). Based on the scoring system of odds ratio, bleeding risk was $4.1 \%$ in the low risk set (score $\leq 4$ ), $7.0 \%$ in the high risk set (score $>4, P=0.003$ ) (validation set).

Conclusion: Our study investigated a prediction scoring system of estimating the bleeding risk, including the patient, endoscopist factors. A risk score can be calculated before the procedure and the endoscopists can predict bleeding potency before the gastric ESD. Based on the scoring system, endoscopists may alter therapeutic plans such as prolongation of admission dates or medication schedules.

Disclosure of Interest: All authors have declared no conflicts of interest.

\section{References}

1. Goto O, Fujishiro M, Oda I, Kakushima N, Yamamoto Y, Tsuji Y, et al. A multicenter survey of the management after gastric endoscopic submucosal dissection related to postoperative bleeding. Dig Dis Sci 2012;57:435-9.

2. Koh R, Hirasawa K, Yahara S, Oka H, Sugimori K, Morimoto M, et al. Antithrombotic drugs are risk factors for delayed postoperative bleeding after endoscopic submucosal dissection for gastric neoplasms. Gastrointest Endosc 2013;78:476-83.

3. Yano T, Tanabe S, Ishido K, Suzuki M, Kawanishi N, Yamane S, et al. Different clinical characteristics associated with acute bleeding and delayed bleeding after endoscopic submucosal dissection in patients with early gastric cancer. Surg Endosc 2017. doi:10.1007/s00464-017-5513-1.

4. Park YM, Cho E, Kang HY, Kim JM. The effectiveness and safety of endoscopic submucosal dissection compared with endoscopic mucosal resection for early gastric cancer: a systematic review and metaanalysis. Surg Endosc 2011;25:2666-77.

5. Takeuchi T, Ota K, Harada S, Edogawa S, Kojima Y, Tokioka S, et al. The postoperative bleeding rate and its risk factors in patients on antithrombotic therapy who undergo gastric endoscopic submucosal dissection. $B M C$ Gastroenterol 2013;13:136.

\section{P0816 USEFULNESS OF DUAL RED IMAGING IN ESOPHAGEAL AND GASTRIC ENDOSCOPIC SUBMUCOSAL DISSECTION}

T. Yamasaki ${ }^{1}$, K. Yamamoto ${ }^{2}$, Y. Yasui ${ }^{2}$, Y. Sakata ${ }^{2}$, M. Suo ${ }^{3}$, T. Ochiai ${ }^{1}$, A. Kimura ${ }^{2}$, A. Nakata ${ }^{2}$, M. Yamamura ${ }^{2}$, N. Hirata ${ }^{1}$, T. Suekane ${ }^{2}$, E. Sasaki ${ }^{2}$, K. $\mathrm{Sano}^{2}$, K. Watanabe ${ }^{2}, \mathrm{H}$. Nebiki ${ }^{1}$

${ }^{1}$ Gastroenterology, Osaka City General Hospital Dept. of Gastroenterology, Osaka/Japan

${ }^{2}$ Hepatology, Osaka City General Hospital, Osaka/Japan

\section{Contact E-mail Address: tomorrow_aaa@yahoo.co.jp}

Introduction: Endoscopic submucosal dissection (ESD) remains difficult procedure due to bleeding and perforation. One of the reasons that these complications are occurred is unclear visibility of submucosal layer. Dual red imaging (DRI) is a novel endoscopic image enhanced technology that is focus on slightly deeper layer by using narrow long wavelength light than white light imaging(WLI). Therefore, DRI improves the visibility of vessels and demarcation line between the submucosal and muscle layers for colorectal ESD ${ }^{1}$. The aim of this study is to estimate efficacy of DRI for esophageal and gastric ESD.

Aims \& Methods: A retrospective analysis was performed on patients who underwent ESD for esophageal or gastric cancer in our institution. ESD was performed by circumferential incision using WLI at first. Submucosal dissection was performed using DRI in principle, and Ulcer bed was observed with DRI after resection. Total resection time (from precut to complete resection), perforation rate, and hemostasis rate after procedure were recorded. These parameters were compared with our matched previous procedures using propensity score (1:2). We recorded paired endoscopic images using DRI and WLI at the same point during the dissection, then estimated the visibility of vessels and submucosa by those images using visual analog scale $(0-100)$ by 5 endoscopists. We compared the visibility of DRI with WLI followed by the estimation.

Results: Twenty lesions (esophagus: 3 , stomach: 17) of 18 patients were enrolled for this study. Total resection time was $67.0 \pm 27.9 \mathrm{~min}$, and was shorter than matched data $(141.7 \pm 113.5 \mathrm{~min})$ significantly $(\mathrm{p}=0.0054))$. Hemostasis rate after resection and perforation rate were not different significantly. The score of vessels visibility using DRI and WLI were $68.6 \pm 17.5$ and $62.3 \pm 19.6$, respectively. The score of submucosal visibility using DRI and WLI were $70.0 \pm 18.0$ and $64.9 \pm 19.0$, respectively. DRI was superior to WLI in both scores significantly $(\mathrm{p}<0.0001)$.
Conclusion: Though our study is preliminary, DRI improves total resection time, visibility of vessels and demarcation line between the submucosal and muscle layers significantly as previous study showed on colorectal ESD. DRI uses narrow long wavelength light. The light reaches slightly deeper layer than WLI. Therefore, we can see not only clear vessels but intact submucosa with DRI, through submucosal surface is dirty and unclear by blood, fat, and fibrosis. We named this effect "noise cancelling effect" by DRI. This effect gets better the visibility better, therefore the esophageal and gastric ESD makes easier and safer. Disclosure of Interest: All authors have declared no conflicts of interest.

\section{Reference}

1. Ninomiya, Ther Adv Gastroenterol 2016, Vol. 9(5) 679-683

\section{P0817 EFFICACY OF BLUE LIGHT IMAGING USING LED LIGHT SOURCE FOR DIAGNOSIS OF EARLY GASTRIC CANCER}

O. Dohi ${ }^{1}$, Y. Naito ${ }^{1}$, S. Takayama ${ }^{1}$, K. Ogita ${ }^{1}, K$. Terasaki ${ }^{1}$, T. Nakano ${ }^{1}$, N. Iwai ${ }^{1}$, T. Ueda ${ }^{1}$, T. Okayama ${ }^{1}$, N. Yoshida ${ }^{1}$, K. Kamada ${ }^{1}$, K. Uchiyama ${ }^{1}$, T. Ishikawa ${ }^{1}$, T. Takagi ${ }^{1}$, O. Handa ${ }^{1}$, H. Konishi ${ }^{1}$, Y. Itoh ${ }^{1}$

${ }^{1}$ Gastroenterology And Hepatology, Kyoto Prefectural University of Medicine, Kyoto/Japan

Contact E-mail Address: osamu-d@koto.kpu-m.ac.jp

Introduction: Endoscopic diagnosis of early gastric cancer (EGC) is essential for ESD, which is a standard treatment for EGC. Magnifying endoscopy with blue laser imaging was useful for diagnosis of EGC prior to ESD [1]. A novel endoscopic system powered by unique four kinds of LED light sources sets a whitelight imaging, blue light imaging and linked color imaging.

Aims \& Methods: We prospectively evaluated whether blue light imaging with magnifying endoscopy (LED-BLI-ME) could be as efficient for diagnosis of EGC as blue laser imaging with magnifying endoscopy (BLI-ME). 43 patients with 45 tumorous lesions including 28 well-differentiated adenocarcinomas, two moderately differentiated adenocarcinoma, six poorly differentiated, and five adenomas that were diagnosed by biopsy specimens were enrolled in this study between Deccember 2016 and May 2017. The patients with EGC underwent LED-BLI-ME observation, following BLI-ME observation in the same day. At first, an endoscopist evaluated a demarcation line and micromorphology in the part of EGC using LED-BLI-ME according to vessel and surface classification system. Then, the same endoscopist evaluated adenmarcation line in the same part of EGC using BLI-ME. All of the EGCs were immidiately resected by ESD after the observation of two modalities. All of the EGCs could be evaluated one-to-one correspondence between the endoscopic image and pathological finding in detail. Primary end point was to analyze the diagnostic accuracy of LED-BLI-ME for EGC. Secondary end point was to compared the diagnostic accuracy and between LED-BLI-ME and BLI-ME. Our study was approved by the Ethical Review Committee of Kyoto Prefectural University of Medicine, and performed in accordance with the World Medical Association's Declaration of Helsinki. In addition, this study has been registered in the University Hospital Medical Information Network Clinical Trials Registry (UMIN-CTR; http://www.umin.ac.jp/ctr/) as trial UMIN 000025275.

Results: The clinicopathological features in the patients were as follows; mean age was $71.9 \pm 8.74$, gender (male:female) was $31: 14$, location(U:M:L) was 9:15:21, macroscopic type (elevated: flat: depressed) was 18:1:26, median tumor size was $18.1 \pm 0.298 \mathrm{~mm}$, and final pathological diagnosis (well-differentiated adenocarcinoma: moderately differentiated adenocarcinoma: poorly adenocarcinoma) was $37: 1: 7$, respectively. Diagnostic accuracy of demarcation line of EGC using LED-BLI-ME and BLI-ME were 91.1\% (41/45) and 91.1\% (41/45), respectively. The rate of high confidence for diagnosis of a demarcation line of EGC using LED-BLI-ME and BLI-ME were $84.4 \%(38 / 45)$ and $91.1 \%(41 / 45)$, respectively. There was no significant difference of diagnostic accuracy between two modalities. The consistency rate in the demarcation line, microvascular pattern, and microsurface pattern of EGC between LED-BLI-ME and BLI-ME was $80.0 \%(41 / 45), 97.8 \%(44 / 45)$, and $97.8 \%$ (44/45), respectively.

Conclusion: LED-BLI-ME were demonstrated the high diagnositc performance for diagnosis of EGC demarcation, similar to BLI-ME.

Disclosure of Interest: Y. Naito: Fujifilm Co. (collaboration research) Y. Itoh: Fujifilm Medical Co.

All other authors have declared no conflicts of interest.

\section{Reference}

1. Dohi O, Yagi N, Majima A, Horii Y, Kitaichi T, Onozawa Y, Suzuki K, Tomie A, Reiko Kimura-Tsuchiya, Tsuji T, Yamada N, Bito N, Okayama T, Yoshida N, Kamada K, Katada K, Uchiyama K, Ishikawa T, Takagi T, Handa O, Konishi H, Naito Y, Yanagisawa A, Itoh Y. Diagnostic ability of magnifying endoscopy with blue laser imaging for early gastric cancer: a prospective study. Gastric Cancer 2017;20:297-303. 
P0818 RISK FACTORS OF COMPLICATION RELATED TO ENDOSCOPIC MANAGEMENT OF FOREIGN BODIES IN THE ESOPHAGUS: A PROSPECTIVE STUDY IN 595 CASES FROM MULTIPLE CENTERS IN CHINA

L. Wang, L. Wang

The second Affiliated Hospital, School of Medicine, Zhejiang University, Hangzhou/China

Contact E-mail Address: wanglj76@hotmail.com

Introduction: Foreign bodies (FBs) ingestion is a common medical emergency accounting for $4 \%$ of all emergency endoscopies, secondary only to the GI bleeding. ${ }^{[1]} 70 \sim 75 \%$ of FBs are located in the esophagus ${ }^{[2]}$. The need of endoscopic management reached up to $63 \sim 76 \%{ }^{[3-5]}$ with $3 \% \sim 20 \%$ of incidence of complications ${ }^{[6]}$. According to the latest guidelines from ESGE, emergent endoscopy is recommended for the impaction of sharp-pointed objects within 24 hours ${ }^{[5]}$. However, there were still different opinions on the endoscopic methods with different FBs.

Aims \& Methods: The study was performed from October 2015 to August 2016 among 595 patients with clinical suspicion of foreign body ingestion from 18 general hospitals in China. The patient data including age, gender, clinical features, and data about endoscopic management including types and locations of foreign bodies, retrieval devices, outcomes and complications were collected and analyzed.

Results: 1) The most common types of foreign bodies were fish bones $(34.0 \%)$, chicken bones $(22.1 \%)$, fruit nucleus $(17.1 \%)$ and food bolus $(14.6 \%)$. The majority of them were short objects $(<2.5 \mathrm{~cm}, 74.0 \%)$, subsequently followed by middle objects $(2.5 \sim 6.0 \mathrm{~cm}, 24.5 \%)$ and long objects $(>6 \mathrm{~cm}, 1.5 \%)$. Most objects were lodged in the proximal esophagus $(75.9 \%)$, followed by the middle segment $(15.2 \%)$ and the distal segment $(8.9 \%)$ of esophagus. 2) $96.3 \%$ of all cases had obvious clinical symptoms. Clinical symptoms occurred more often in the proximal segment of the esophagus $(98.1 \%)$ than any other segments of the upper gastrointestinal tract $(92.6 \%)(\mathrm{P}<0.001)$. 3) The successful removal rate through endoscopy was $94.5 \%$. It was even higher with general anesthesia $(99.3 \%)$ than without it $(92.7 \%)(\mathrm{P}<0.01) .4)$ Complication rate was as high as $34.0 \%$, which was increased with long retention time and sharp objects $(\mathrm{P}<0.001)$. The rate was increased by $2.2-$ and 6.1 - folds after impacted for over 24 hours or 48 hours as compared to 12 hours. Logistic regression analysis indicated that sharp objects had obviously more complications than non-sharp ones (OR 3.36, 95\% CI: 1.97-5.74). In particular, the incidence of perforation was $5.6 \%$, which was strongly related with long retention time and sharp objects $(\mathrm{P}<0.01)$, but not with locations or lengths of the objects $(\mathrm{P}>0.05)$

Conclusion: General anesthesia could largely improve the retrieval rate through endoscopy. Foreign bodies, especially sharp ones, should be removed as soon as possible within 24 hours, to further decrease severe complications.

Disclosure of Interest: All authors have declared no conflicts of interest.

\section{References}

1. Mosca S, Manes G, Martino R et al. Endoscopic management of foreign bodies in the upper gastrointestinal tract: report on a series of 414 adult patients. Endoscopy. 2001; 33: 692-6.

2. Dray X, Cattan P. Foreign bodies and caustic lesions. Best Pract Res Clin Gastroenterol. 2013; 27: 679-89.

3. Ikenberry SO, Kue TL, Anderson MA et al. Management of ingested foreign bodies and food impactions. Gastrointest Endosc. 2011; 73: 1085-91.

4. Palta R, Sahota A, Bemarki A et al. Foreign-body ingestion: characteristics and outcomes in a lower socioeconomic population with predominantly intentional ingestion. Gastrointest Endosc. 2009; 69: 426-33.

5. Birk M, Bauerfeind P, Deprez PH, Hafner M, Hartmann D, Hassan C et al. Removal of foreign bodies in the upper gastrointestinal tract in adults: European Society of Gastrointestinal Endoscopy (ESGE) Clinical Guideline. Endoscopy. 2016;48:489-96.

6. Lai AT, Chow TF. Risk factors predicting the development of complications after foreign body ingestion. Br J Surg. 2003;90(12):1531-5.

\section{P0819 BIOPSY STRATEGIES FOR ENDOSCOPIC SCREENING OF PRE-MALIGNANT GASTRIC LESIONS}

\section{Z. Meng}

Department Of Gastroenterology, First Affiliated Hospital of Zhejiang Chinese Medical University, Hangzhou/China

Contact E-mail Address: zm1990morphine@163.com

Introduction: GA and IM are detectable precursor lesions of most gastric carcinomas. Early endoscopy examination may potentially result in early detection and treatment of advanced precursor lesions and cancer. The annual incidence of GC is quite low in central Europe, for GAwas $0.1 \%$ and for IM was $0.25 \%^{[1-2]}$. The histological assessment and biopsy sampling protocol has been standardized in the updated Sydney system and the recommended five gastric biopsies were widely applied in the staging system for gastric cancer risk stratification ${ }^{[3]}$. Some believe that the GA risk screening requires consideration of the cost, suffering of patients as well as rational utilization of medical resources, so adopt five biopsy may be presumably less appropriate in large-scare population gastric cancer risk screening. In this study, we evaluated the OLGA and OLGIM staging in a standardized five biopsy protocol through screening patients underwent endoscopy with abdominal pain and discomfort. In order to identify a more optimal biopsy strategy with quite high consistence with the standardized five biopsy protocol in OLGA and OLGIM staging and less number of biopsies during cancer risk assessment, we re-evaluated OLGA and OLGIM staging adopting different biopsy combinations (evaluated the appropriate biopsy locations and number of biopsies)

Aims \& Methods: Gastric atrophy (GA) and intestinal metaplastic (IM) are precursor lesions of gastric cancer (GC), the extent and severity of which was intimate correlated with the occurrence of GC. Operative Linkon Gastritis Assessment (OLGA) and Operative Linkon Gastric Intestinal Metaplasia Assessment (OLGIM) with five biopsy samples are superordinate stages system during Gastric risk stratification. We analysis the degree of GA and IM in these five locations and screen out an more representative, simple and convenient biopsy samples composition for gastric risk screening.

Methods: 368 patients with abdominal pain and other discomfort undergoing endoscopy were enrolled in the study. Five biopsy pieces (two from antrum, namely lesser and larger curvature, two from corpus, larger and lesser curvature and one from incisura angularis) were acquired and graded by senior gastrointestinal pathologists according to the updated Sydney system. Gastric risk stratification was calculated by adopting OLGA and OLGIM staging system.

Results: GA and IM are more frequently acquired in the incisura angularis mucosa than the both lesser and larger curve of antrum and corpus respectively $(\mathrm{P}<0.05)$, especially the moderate and severe degree. More GA and IM alternations happened in the lesser curve of the antrum and corpus than in the corpus $(\mathrm{P}<0.05)$. The composition three biopsy pieces (lesser curvature of the antrum and corpus, angulus biopsy) with quite accurate consistence with five biopsy pieces protocol using OLGA and OLGIM staging $(94.0 \%$ and $92.9 \%)$, together with a rather low omission diagnostic rate of OLGA and OLGIM III + IV $(3.8 \%$ and $7.2 \%$ )

Conclusion: The incisura angularis with more moderate and severe GA and IM should be recommended as conventional biopsy site during endoscopy. The three biopsy pieces (lesser curvature of the antrum and corpus, angulus biopsy) with quite high consistence, more convenience and less cost comparing with five biopsy pieces protocol using OLGA and OLGIM staging system, and thus could be recommend in the further gastric risk screening applying OLGA and OLGIM staging.

Disclosure of Interest: All authors have declared no conflicts of interest.

\section{References}

1. de Vries A C, van Grieken N C, Looman C W, el al. Gastric cancer risk in patients with premalignant gastric lesions: a nationwide cohort study in the Netherlands. Gastroenterology, 2008, 134(4): 945-952[PMID:18395075]

2. Dixon M F, Genta R M, Yardley J H, el al. Classification and grading of gastritis. The updated Sydney System. International Workshop on the Histopathology of Gastritis, Houston 1994. Am J Surg Pathol, 1996, 20(10): 1161-1181[PMID:8827022]

3. Isajevs S, Liepniece-Karele I, Janciauskas D, el al. Gastritis staging: interobserver agreement by applying OLGA and OLGIM systems. Virchows Arch, 2014, 464(4): 403-407[PMID:24477629]

\section{P0820 USEFULNESS OF LINKED COLOR IMAGING (LCI) FOR RECOGNITION OF EARLY GASTRIC CANCER AND GASTRIC ADENOMA}

N. Yagi, H. Kitae, A. Tomie, T. Yasuda, T. Omatsu, Y. Nakahata, T. Kurobe, A. Obora, T. Kojima

Gastroenterology, Murakami Memorial Hospital, Asahi University, Gifu/Japan

Contact E-mail Address: nyagi@koto.kpu-m.ac.jp

Introduction: LCI is a novel color enhancement feature available for LASEREO endoscopy systems (FUJIFILM CO., Tokyo, Japan), which can enhance the slight color difference on gastrointestinal mucosa. Recently we reported that color enhancement of the diffuse redness by LCI was useful for the diagnosis of $H$. pylori infection. Therefore, we expected that LCI facilitate the endoscopic recognition of early gastric cancer and gastric adenoma by enhancing its color difference between normal and atypical mucosa.

Aims \& Methods: The aim of this study was to evaluate the usefulness of LCI for recognition of early gastric cancer and gastric adenoma compared to conventional white light imaging (WLI), WLI with Indigo Carmine contrast staining (IC) and Blue Laser Imaging-bright (BLI-brt) imaging. We retrospectively analyzed 39 lesions in 33 patients who had endoscopic submucosal dissection (ESD) at Murakami Memorial Hospital from June 2014 to May 2016. All lesions were scanned with four imaging mode, WLI, IC, BLI-brt and LCI (LASEREO with EG-L590ZW scope), and assigned a recognition score from 3 (excellent visibility) to 0 (poor visibility) by three endoscopists.

Results: The overall mean recognition score of LCI was significantly higher than that of WLI/IC/BLI-brt ( $2.18 \pm 0.073$ vs. $1.56 \pm 0.080 / 1.86 \pm 0.099 / 1.91 \pm 0.073)$. For type 0-IIa lesions (18 lesions), the mean recognition score of LCI was significantly higher than WLI and BLI-brt $(2.26 \pm 0.12$ vs. $1.83 \pm 0.12 / 2.14 \pm 0.097)$, whereas no significant difference was seen between LCI and IC $(2.26 \pm 0.12$ vs. $2.35 \pm 0.12$ ). For type 0 -IIb or 0 -IIc lesions ( 21 lesions), the mean recognition score of LCI was significantly higher than WLI/IC/BLI-brt $(2.11 \pm 0.11$ vs. $1.33 \pm 0.10 / 1.44 \pm 0.13 / 1.73 \pm 0.10)$. And the mean recognition score of BLI-brt was also significantly higher than WLI/IC, but no significant difference was seen between WLI and IC. Regarding WLI, IC, and BLI-brt, the mean recognition score of type 0-IIa lesions in each mode was significantly higher than that of type 0 -IIb or 0 -IIc lesions. However, regarding LCI, there was no significant difference in the mean recognition score between type 0 -IIa lesions and type 0 -IIb or 0 IIc lesions. These results indicated that the detection of type 0 -IIb or 0-IIc lesion was difficult compared to 0-IIa in WLI, IC and BLI-brt mode. However, LCI mode enable us to detect these type 0 -IIb or 0-IIc lesion as easily as type 0-IIa. 
Conclusion: LCI mode was useful for recognition of early gastric cancer and gastric adenoma, especially for type 0-IIc and 0-IIb lesions that are frequently found in post- $H$. pylori eradication gastric mucosa.

Disclosure of Interest: All authors have declared no conflicts of interest.

\section{P0821 GASTROINTESTINAL STROMAL TUMORS SHOULD BE RESECTED EVEN IN A SMALL SIZE - A RETROSPECTIVE ANALYSIS IN 33 CASES}

O. Goto ${ }^{1}$, T. Akimoto ${ }^{1}$, Y. Mitsunaga ${ }^{1}$, A. Fujimoto ${ }^{1}$, Y. Ochiai ${ }^{1}$, T. Maehata ${ }^{1}$, H. Kawakubo ${ }^{2}$, H. Takeuchi ${ }^{2}$, Y. Kitagawa ${ }^{2}$, N. Yahagi

${ }_{1}^{1}$ Division Of Research And Development For Minimally Invasive Treatment,

Cancer Center, Keio University, School of Medicine, Tokyo/Japan

${ }^{2}$ Department Of Surgery, Keio University School of Medicine, Tokyo/Japan

\section{Contact E-mail Address: ogotou-gi@keio.jp}

Introduction: In gastrointestinal stromal tumors (GISTs) without a risk of metastasis to other organs, local resection is acceptable. In small GISTs, however, it is controversial whether surgical resection is necessary because a risk of recurrence/ metastasis in these tumors are considered to be quite low. Laparoscopic endoscopic cooperative surgery (LECS) is a promising surgical technique as one of minimally-invasive, function-preserving surgeries. By using this technique, we are aggressively resecting gastric SMTs including relatively small ones.

Aims \& Methods: To investigate necessity of surgical resection for small GISTs, we retrospectively assessed a malignant potential of these tumors which were resected nonexposed endoscopic wall-inversion surgery (NEWS) (nonexposure LECS technique) as well as feasibility and safety of this technique. Between August 2013 and October 2016, NEWS was conducted in 33 consecutive SMTs which met all of following conditions: possible GIST which was preoperatively diagnosed by histology or imaging modalities, less than $3 \mathrm{~cm}$ in size and intraluminally-/intramurally-growing type. In the NEWS procedure, a lesion was resected in a following manner: endoscopic mucosal markings, laparoscopic serosal markings just behind the mucosal markings, endoscopic submucosal injection, laparoscopic sero-muscular incision and suturing with the lesion inverted toward the inside of the stomach, endoscopic muco-submucosal incision and peroral retrieval. Short-term outcomes of NEWS and a potential risk of recurrence/metastasis in each tumor according the Fletcher's classification were assessed.

Results: Mean age and size of the lesion were $59.9 \pm 13.7$ years-old and $23.3 \pm 8.4 \mathrm{~mm}$, respectively. The procedure was successfully completed in all cases in a mean procedure duration of $206 \pm 43 \mathrm{~min}$. The patients were discharged without severe adverse events $7.3 \pm 1.5$ days after the procedure. The first endoscopy after the procedure was performed 5.8 months after discharge in 22 cases, which showed no residual food in the remnant stomach in all cases. Neither apparent impairment of food intake nor disease-related death occurred and a body weight loss was $0.9 \pm 2.3 \mathrm{~kg}$ during the mean observational period of 16 months. GIST was histologically diagnosed in 20 cases. A risk of recurrence/ metastasis in these GISTs was classified into high (2), intermediate (1), low (12) and very-low (5), respectively. In a comparison of two groups (high/intermediate and low/very-low), a mean tumor size and existence of delle formation were $31.7 \mathrm{~mm}$ and $21.6 \mathrm{~mm}(\mathrm{p}=0.036)$, and $67 \%$ and $12 \%(\mathrm{p}=0.028)$, respectively. Conclusion: Some small GISTs which could be retrieved transorally had a high malignant potential. NEWS was feasible, safe and therefore recommended for these tumors including ulcerated GISTs as a minimally-invasive surgical option to avoid an additional surgical scar for the retrieval of the specimen and a risk of iatrogenic tumor cell seeding into the peritoneum during the procedure. Disclosure of Interest: All authors have declared no conflicts of interest.

\section{Reference}

Goto O, Takeuchi H, Sasaki M, et al. Nonexposure technique of laparoscopyassisted endoscopic full-thickness resection for gastric subepithelial tumours. Endoscopy 2016; 48: 1010-5.

\section{P0822 LONG-TERM OUTCOMES OF PERORAL ENDOSCOPIC MYOTOMY FOR ACHALASIA IN PATIENTS OLDER THAN 70 YEARS}

J. Zhu, Q. Li, P. Zhou

Endoscopy Center, Zhongshan Hospital, Fudan University, Shanghai/China

\section{Contact E-mail Address: zjysjtu@yahoo.com}

Introduction: Achalasia is a rare esophageal motor disorder characterized by esophageal aperistalsis and incomplete relaxation of lower esophageal sphincter (LES). The Elderly patients have longer courses of disease, less perception of symptoms and similar manometric findings compared with younger patients. Peroral endoscopic myotomy (POEM) is a novel endoscopic therapy which recently has been described as an incisionless and less invasive scarless myotomy alternative for treating achalasia. However, very few studies concentrated on the long-term outcomes of POEM for achalasia patients, especially for elderly patients.

Aims \& Methods: Peroral endoscopic myotomy (POEM) is a minimally invasive endoscopic myotomy for achalasia. But the long-term outcomes of POEM for achalasia in elderly patients are unclear. We therefore aim to evaluate the efficacy and safety of POEM for achalasia in patients older than 70 years. A total of 33 consecutive patients aged $\geq 70$ years were enrolled in this study, who had undergone POEM for achalasia from May 2011 to March 2016 in the Endoscopy
Center of Zhongshan Hospital. We retrospectively assessed the short and long term outcomes of these patients including Eckardt score, lower esophageal sphincter pressure, adverse events and recurrence.

Results: All patients underwent POEM successfully. Mean procedure time was $50.7 \pm 22.0 \mathrm{~min}$ and postoperative hospital stay was $2.8 \pm 1.5$ days. No severe complications related to POEM were encountered. During a mean follow-up period of $40.5 \pm 19.1$ months (range from 12.2 to 70.9 months), the therapeutic success rate was $90.9 \%(30 / 33)$. Mean Eckardt score was $7.6 \pm 2.0$ and $1.4 \pm 1.3$ before and after POEM, respectively $(\mathrm{P}<0.001)$. Mean LES pressure decreased from a mean of $20.3 \pm 5.8 \mathrm{mmHg}$ to $3.9 \pm 2.9 \mathrm{mmHg}$ after POEM $(\mathrm{P}<0.001)$. Clinical gastroesophageal reflux occurred in 11 cases $(33.3 \%)$ after the procedure which was successfully resolved by proton pump inhibitors.

Conclusion: POEM can be a safe and effective treatment option for achalasia in elderly patients older than 70 years with significant improvement in symptoms. Disclosure of Interest: All authors have declared no conflicts of interest.

\section{References}

1. Campos GM, Vittinghoff E, Rabl C, et al. Endoscopic and surgical treatments for achalasia: a systematic review and meta-analysis. Ann Surg. 2009; 249(1): 45-57.

2. Gutschow CA, Leers JM, Schröder W, Prenzel KL, et al. Effect of aging on esophageal motility in patients with and without GERD. Ger Med Sci. 2011; 9: Doc22.

3. Schechter RB, Lemme EM, Novais P, et al. Achalasia in the elderly patient: a comparative study. Arq Gastroenterol. 2011; 48(1): 19-23.

4. Sonnenberg A. Hospitalization for achalasia in the United States 1997-2006. Dig Dis Sci. 2009; 54(8): 1680-1685.

5. Samo S, Carlson DA, Gregory DL, et al. Incidence and Prevalence of Achalasia in Central Chicago, 2004-2014, Since the Widespread use of High-Resolution Manometry. Clin Gastroenterol Hepatol. 2016; doi: 10.1016/j.cgh.2016.08.030.

6. Molena D, Mungo B, Stem M, et al. Hospitalization for esophageal achalasia in the United States. World J Gastrointest Endosc. 2015; 7(13): 1096 1102 .

7. Zaninotto G, Annese V, Costantini M, et al. Randomized control trial of Botox injection versus laparoscopic Heller myotomy for esophageal achalasia. Ann Surg. 2004; 239(3): 364-370

8. Leyden JE, Moss AC, MacMathuna P. Endoscopic pneumatic dilation versus botulinum toxin injection in the management of primary achalasia. Cochrane Database Syst Rev. 2006; 18;(4): CD005046.

9. Sheraz R. Markar, Giovanni Zaninotto. Laparoscopic Heller Myotomy for Achalasia: Does the Age of the Patient Affect the Outcome? World J Surg. 2015; 39(7): 1608-1613.

10. Boeckxstaens GE, Annese V, Bruley des Varammes S, et al. Pneumatic dilation versus laparoscopic Heller myotomy for idiopathic achalasia. $N$ Engl J Med. 2011; 364(19): 1807-1816.

11. Salvador R, Costantini M, Cavallin F, et al. Laparoscopic Heller myotomy can be used as primary therapy for esophageal achalasia regardless of age. $J$ Gastrointest Surg. 2014; 18(1): 1062112.

12. DE Low, AM Bodnar, SR Markar, et al. Surgical myotomy should be considered as primary treatment in elderly patients with achalasia. Gastroenterology. 2013; 144(5): S-1079.

\section{P0823 POST-POEM ESOPHAGITIS - REAL REFLUX ESOPHAGITIS OR JUST A HEALING PROCESS?}

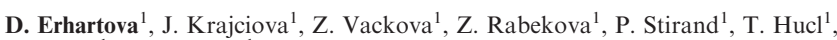
J. Spicak ${ }^{1}$, J. Martinek ${ }^{1}$

${ }^{1}$ Department Of Hepatogastroenterology, Institute for Clinical and Experimental Medicine, Prague/Czech Republic

Contact E-mail Address: denisaerhartova@gmail.com

Introduction: POEM has become a standard treatment of achalasia based on its excellent efficacy and safety. However, unlike in laparoscopic myotomy, POEM is not accompanied by an antireflux procedure and thus the rates of both clinically relevant post-POEM reflux (measured by symptoms and/or $24 \mathrm{~h} \mathrm{pH}$ monitoring) and post-POEM esophagitis are key questions of long-term POEM safety. Several studies have reported the rates of post-POEM reflux and postPOEM esophagitis being as high as $30-40 \%$. The aim of this retrospective analysis was to analyze whether esophageal erosions, detected endoscopically early after POEM, represent real reflux esophagitis or rather signs of still ongoing healing process after POEM.

Aims \& Methods: A single-center retrospective analysis of 192 patients who underwent POEM in our institution. Three months after POEM, 162 patients underwent both $\mathrm{pH}$ monitoring and endoscopy and treatment with PPIs was assessed. At 24-36 months, a control endoscopy was performed in 41 patients to screen for esophagitis. We reviewed all available video recordings of POEM procedures and subsequent control endoscopies to analyze whether the localization of erosions was limited to the site of submucosal tunneling (e.g. between 2-4 óclock in patients after anterior POEM) or also outside this area (real reflux esophagitis). We also analyzed evolution of esophagitis in time and correlated it with $\mathrm{pH}$ monitoring and intake of PPIs.

Results: At 3 months, esophagitis was present in 64 out of 162 patients $(39.6 \%)$. Of those, $21(33 \%)$ were on PPIs by the time of endoscopy and $43(67 \%)$ were not. Among 43 patients with esophagitis and available video recording, 29 $(67.4 \%)$ had erosion(s) only on the site of submucosal tunnel and $14(32.6 \%)$ had erosions also elsewhere. Among patients with erosion(s) only on site of the tunnel, $13(44.8 \%)$ had normal acid exposure time (assessed by $\mathrm{pH}$ monitoring 
performed off PPIs). At 24-36 months, esophagitis was present in 9 out of 41 examined patients $(21.9 \%), \mathrm{p}=0.04$ vs. 3 months. In 6 out of 10 patients with esophagitis present at 3 months, who underwent control endoscopy at 24-36 months, esophagitis resolved completely even though only 2 patients had been treated by a PPI.

Conclusion: After POEM, the majority of patients with esophagitis had erosions on site of submucosal tunnel and early esophagitis often occurred in patients without an abnormal acid exposure. These findings suggest that an ongoing healing process after POEM might interfere in findings of presumed postPOEM reflux esophagitis. Thus, the rate of real post-POEM reflux esophagitis may be lower than previously thought.

Disclosure of Interest: All authors have declared no conflicts of interest.

\section{P0824 ENDOSCOPIC SUBMUCOSAL TUNNEL DISSECTION FOR LESSER GASTRIC CURVATURE SUPERFICIAL NEOPLASMS}

D. Shi, R. Li, W. Chen, X. Wang, C. Ma, D. Zhang

Gastroenterology, the First Affiliated Hospital of Soochow University, Suzhou/ China

\section{Contact E-mail Address: 1rhcsz@163.com}

Introduction: Endoscopic submucosal dissection (ESD) has been widely used for resection of gastrointestinal neoplastic lesions, but there are still technical challenges in treating large ones especially located in lesser gastric curvature. In the tunnel technique, incisions at the lower and upper lesion edges are joined by a submucosal tunnel and then lateral incisions are made. The mucosa is thereby easily separated from the muscular layer. We report our experience of endoscopic submucosal tunnel dissection (ESTD) in lesser gastric curvature.

Aims \& Methods: To estimate the safety and efficiency of endoscopic submucosal tunnel dissection (ESTD) for lesser curvature superficial neoplasms.47 patients with lesser curvature superficial neoplasms undergoing endoscopic resection were analyzed retrospectively. 26 patients underwent ESTD and 21 received endoscopic submucosal dissection (ESD). Operation time, security, En bloc resection rate and complications were compared between the two groups. The major difference between ESTD and ESD is that, instead of pre-cutting circumferential mucosal, a submucosal tunnel was created by submucosal dissection from the oral incision to the anal incision. Bilateral resection was then performed to remove the lesion completely.

Results: The differences between the two groups in the age of the patients and the diameter of the lesions had no statistically significant $(P>0.05)$. En bloc resection rate was $100 \%$ in the study group and $90.5 \%$ in the control group $(19 / 21)$, and the difference was statistically significant $(P<0.05)$. The average operation time was 46 minutes ( $36 \sim 59$ minutes), the control group was 67 minutes ( $48 \sim 110$ minutes), the difference was statistically significant $(P<0.05)$. The intraoperative bleeding rate of the study group was $57.7 \%(15 / 26)$, the control group was $100 \%$, the difference was statistically significant $(P<0.05)$. The incidence of perforation was $0 \%$ in the study group and $9.5 \%$ in the control group $(2 / 21)$, and the difference was statistically significant $(P<0.05)$. There was 1 case of delayed bleeding after operation in the two groups, there were no postoperative perforation, and the difference was not statistically significant $(P>0.05)$. There were no recurrence and metastasis in the two groups after the operation. Conclusion: ESTD for lesser curvature superficial neoplasms can obviously shorten the operation time and have a higher security, compared with the traditional ESD operation.

Disclosure of Interest: All authors have declared no conflicts of interest.

\section{References}

1. Nicholas E, Haruhiro I, Haruo I, et al. Training in peroral endoscopic myotomy (POEM) for esophageal achalasia[J]. Endoscopy, 2012, 8(4):329.

2. Xu M D, Cai M Y, Zhou P H, et al. Submucosal tunneling endoscopic resection: a new technique for treating upper GI submucosal tumors originating from the muscularis propria layer (with videos).[J]. Gastrointestinal Endoscopy, 2012, 75(1):195-199.

3. Linghu E, Feng X, Wang X, et al. Endoscopic submucosal tunnel dissection for large esophageal neoplastic lesions.[J]. Endoscopy, 2013, 45(12):1032-4.

4. Von D S, Feussner H, Henke J, et al. Submucosal endoscopy: a novel approach to en bloc endoscopic mucosal resection (with videos)[J]. Gastrointestinal Endoscopy, 2007, 66(4):753.

5. Gan T, Yang J L, Zhu L L, et al. Endoscopic submucosal multi-tunnel dissection for circumferential superficial esophageal neoplastic lesions (with videos)[J]. Gastrointestinal Endoscopy, 2016, 84(1):143.

\section{P0825 PROPOFOL SEDATION DURING GASTROINTESTINAL ENDOSCOPY INDUCES EUPHORIA IN A LARGE SUBSET OF PATIENTS}

T. Brechmann ${ }^{1}$, C. Maier ${ }^{2}$, M. Kaisler ${ }^{2}$, J. Vollert ${ }^{2}$, W. Schmiegel ${ }^{1}$, S. Pak ${ }^{3}$,

N. Scherbaum ${ }^{4}$, F. Rist ${ }^{5}$, A. Riphaus ${ }^{3}$

${ }^{1}$ Department Of Gastroenterology And Hepatology, Berufsgenossenschaftiche Universitätsklinik Bergmannsheil gGmbH, Bochum/Germany

${ }^{2}$ Department Of Pain Medicine, Berufsgenossenschaftliche Universitätsklinik Bergmannsheil gGmbH, Bochum/Germany

${ }^{3}$ Department Of Internal Medicine And Gastroenterology, KRH Klinikum Agnes Karll Laatzen, Laatzen/Germany
${ }^{4}$ Department Of Psychiatry And Psychotherapy, LVR-Hospital Essen, Essen/ Germany

${ }^{5}$ Department Of Psychology, University of Münster, Münster/Germany

\section{Contact E-mail Address: thorsten.brechmann@rub.de}

Introduction: Propofol is recommended for sedation in gastrointestinal endoscopy (GE) [1], but preliminary data suggests addictive potentials in animal models [2, 3] and healthy volunteers [4]. There is an increasing number of reports of propofol addiction in physicians and nurses associated with high mortality up to $30 \%$ within the first year of abuse [5-7]. Some patients and medical laymen developed propofol addiction and feigned an indication for endoscopy to receive propofol $[8,9]$. Survivors after propofol abuse reported that the abiding memory of the intense relaxation many years ago was a major reason to abuse propofol years thereafter [10]. However, it is unknown how many patients develop a euphoric drug reaction after endoscopy.

Aims \& Methods: The primary objectives were to define the frequency of a euphoric reaction pattern under propofol sedation, to evaluate the reminiscence one week later and to investigate the superposition of negative feelings by that respective propofol-induced euphoria. Eighty-two patients undergoing elective GE under propofol-sedation in an ambulatory setting were enrolled in a prospective observational study. The grade of anxiety, expectation or relief about the examination's result and affective state in terms of cheerfulness, relaxation, activation, sedation and anxiety were surveyed using a numeric rating scale (1 to 10) immediately before (t1), after GE (t2) and seven days ( $\mathrm{t} 3$ ) later. Statistics: hierarchical cluster analysis, heat map, $\chi 2$ test and paired t-test.

Results: Mean propofol dosage was $264 \pm 120 \mathrm{mg}$. The average indices of cheerfulness, relaxation and activation increased significantly ( $\mathrm{t} 1$ to t2 to $\mathrm{t} 3$ ), whereas sedation and anxiety decreased. Two clusters of mood changes emerged (t1 vs. t2). One $(n=46.56 .1 \%)$ was characterized by an unease reaction pattern with equal values regarding cheerfulness, relaxation and anxiety, while relaxation decreased; the other cluster showed a euphoric reaction pattern $(n=36$, $43.9 \%$ ) with markedly increased cheerfulness, relaxation and decreased anxiety. These effects intensified at recall (t3). Despite similar endoscopy results, euphoric cluster patients rated these more positively. No association between cluster and personal traits, prior use of psychotropic drugs, alcohol consumption or smoking habits were found.

Conclusion: Propofol induces euphoria in nearly half the patients undergoing elective GE with persisting, even enhanced reminiscence. Consequently, endoscopists should be aware of a potential risk of addiction in vulnerable subjects. Disclosure of Interest: All authors have declared no conflicts of interest.

\section{References}

1. Dumonceau J-M, Riphaus A, Schreiber F, et al. Non-anesthesiologist administration of propofol for gastrointestinal endoscopy: European Society of Gastrointestinal Endoscopy, European Society of Gastroenterology and Endoscopy Nurses and Associates GuidelineUpdated June 2015. Endoscopy 2015;47:1175-89.

2. LeSage MG, Stafford D, Glowa JR. Abuse liability of the anesthetic propofol: self-administration of propofol in rats under fixed-ratio schedules of drug delivery. Psychopharmacology 2000;153:148-54.

3. Bonnet U. Einschatzung des Abhangigkeitsrisikos von Propofol. Fortschr Neurol Psychiatr 2011;79:442-52.

4. Zacny JP, Lichtor JL, Thompson W, et al. Propofol at a subanesthetic dose may have abuse potential in healthy volunteers. Anesth Analgesia 1993;77(3):544-52.

5. Monroe T, Hamza H, Stocks G, et al. The misuse and abuse of propofol. Subst Use Misuse 2011:46:1199-205.

6. Kranioti EF, Mavroforou A, Mylonakis P, et al. Lethal self administration of propofol (Diprivan). A case report and review of the literature. Forensic Sci Int 2007;167:56-8.

7. Maier C, Iwunna J, Tsokos M., et al. Deaths by propofol abuse. Results of questioning in legal-medical institutes in Germany, Austria and Switzerland. Anästhesist;2017.

8. Roh S, Park JM, Kim DJ A case of propofol dependence after repeated use for endoscopy. Endoscopy 2011;43 Suppl 2 UCTN:E362.

9. Koopmann A, Goltz C von der, Hermann D, et al. Propofol addiction initiated by anesthetic use. American J Psychiatr 2011;168:211-2.

10. Maier C, Leclerc-Springer J. Lebensbedrohliche Fentanyl- und Propofolabhangigkeit: Interview mit einer Uberlebenden. Der Anaesthesist 2012;61(7):601-7.

\section{P0826 ENDOSCOPIC SUBMUCOSAL DISSECTION FOR UPPER GI SUBMUCOSAL TUMOURS}

H. Awadie, D. J. Tate, A. Klein, L. Desomer, M. Ma, S. Heitman, N. J. Burgess, E. Y.t. Lee, V. Kwan, M.J. Bourke

Gastroenterology And Hepatology, Westmead Hospital, Sydney/Australia

Contact E-mail Address: halimawadie@gmail.com

Introduction: Submucosal tumours (SMT) in the upper gastrointestinal tract (UGI) impose diagnostic and therapeutic challenges. They may have malignant potential and endoscopic ultrasound (EUS) guided diagnosis is often inaccurate. A substantial proportion may not involve the muscularis propria (MP) and thus may be amenable to endoscopic excision. Snare-based techniques are usually unable to completely excise such lesions, though without complete excision ongoing endoscopic surveillance may be necessary. Endoscopic submucosal dissection (ESD) offers the possibility of complete resection and definitive 
histological diagnosis to guide management decisions. Western data on ESD for SMT is absent. Our aim was to analyse the data from our referral centre.

Aims \& Methods: A prospectively collected ESD database was analysed to identify patients with SMT of the UGI. All lesions underwent EUS assessment with the aim to exclude MP involvement prior to resection, and in the case of neuroendocrine tumours (NET) blood tests and Octreotate PET scan were performed.

Results: Over 42 months, 30 ESDs for SMT lesions were performed. The mean age was 62 years with 19 male patients $(63 \%)$. Mean lesion size was $18 \mathrm{~mm}$. Twenty five patients $(83.3 \%)$ had completely resected tumours. Four patients $(13 \%)$ had involvement of the MP which was identified during the resection, and one patient $(3 \%)$ had MP injury which precluded complete resection. Three of five lesions of the incompletely ESD procedures were in the proximal body of the stomach, however only two lesions of the completely resected lesions were in the proximal body (P-0.004). Otherwise, there were no significant differences between the patients and lesions characteristics. The histology of the SMT lesions were 9 NET, 6 leiomyoma, 5 Granular cell tumours, 4 inflammatory fibroid polyp, 2 GIST, 2 dysplastic Lipoma, one myofibroblastic tumour and one Warthin's like tumour. Nineteen patients had completed surveillance endoscopy (SE) without an endoscopic and histological recurrence (Median follow up 18 months). Six patients are pending SE. The four patients with deep MP involvement were referred for surgery.

Conclusion: ESD for selected UGI SMT is an effective treatment. Long-term endoscopic follow-up confirmed the absence of recurrence endoscopically and histologically. MP involvement cannot be reliably excluded by prior EUS. This technique should be considered for UGI SMT lesions without MP involvement in experienced centres.

Disclosure of Interest: All authors have declared no conflicts of interest.

\section{P0827 COMPARING APPROACHES TO SELF-EXPANDING} METALLIC STENTS INSERTION

L. Mooney ${ }^{1}$, N. Church ${ }^{2}$, R. Sinha ${ }^{1}$, C. Basquill ${ }^{1}$

${ }^{1}$ Centre For Liver And Digestive Disorders, NHS Lothian, Edinburgh/United

Kingdom

${ }^{2}$ Centre For Liver And Digestive Disordersl, NHS Lothian, Edinbrgh/United

Kingdom

Contact E-mail Address: leanne.mooney@nhs.net

Introduction: The incidence of oesophageal cancer has increased significantly over the past two decades. The majority of these cancers are incurable at diagnosis. Therefore, the management is aimed at maintaining quality of life by ensuring adequate nutrition and palliation of symptoms, mainly dysphagia. Self-expanding metallic stents (SEMS) have a well-recognised role in the palliative management of patients with oesophageal cancer. These stents are inserted endoscopically, under direct vision (EC) or with fluoroscopic assistance to endoscopy (FAE). There is little evidence to compare outcomes between these approaches.

Aims \& Methods: The objective of this study was to compare the outcomes, using various performance indicators, in patients who underwent SEMS for palliation in oesophageal cancer via different approaches (EC or FAE) at the Royal Infirmary of Edinburgh (RIE). A retrospective observational study was conducted between May 2014 to April 2016; a total of 62 SEMS. The approach to stent insertion was subject to operator choice and availability of fluoroscopic assistance, and as such can be akin to randomized study. We compared early and late complications associated with two techniques. Early complications included pain, vomiting, bleeding, perforation and tachyarrhythmia. Late complications included tumour overgrowth, oesophagitis, stricture and stent migration. We also compared morbidity, the need for repeat procedures and the number of additional stents required following each approach.

Results: Forty-seven stents where inserted by EC and fifteen by FAE. The median age among the two groups were comparable at 75 and 69 years respectively. There was male predominance in both the groups $(70 \%$ and $67 \%)$. Adenocarcinoma was the most common malignancy (56\%), followed by squamous cell carcinoma $(35 \%)$ among the study subjects. We observed a higher frequency of technical difficulties with EC placement $(13 \%)$ to FAE $(0 \%)$, however no malposition was observed in the EC group. Early complications were comparable in both groups, however chest pain $(21 \%)$ was more frequently associated with the EC group. Late complications such as tumour overgrowth, oesophagitis, oesophageal stricture and stent migration were comparable in both groups. Food bolus obstruction and dysphagia was more common in EC placement $(28 \%)$ to FAE $(0 \%)$ necessitating a higher number of endoscopies. However, a relatively higher number of re-stent procedures where performed in the FAE group. Median survival was comparable in both of the groups.

\begin{tabular}{lll}
\hline Variable & EC (47) & FAE (15) \\
\hline Male (\%) & $33(70)$ & $10(67)$ \\
Location of tumour Upper & $32(9)$ & $40(6)$ \\
$\quad$ Limit (IQR) Lower & $37(5.1)$ & $38(7)$ \\
$\quad$ Limit (IQR) & & $3(2.5)$ \\
Sedation and analgesia use- & $2(1.8)$ & $75(50)$ \\
$\quad$ median dose (IQR) & $50(50) 100(0)$ & $100(0)$ \\
$\quad$ Midazolam dose (mg) & & \\
\hline
\end{tabular}

(continued)
Continued

\begin{tabular}{|c|c|c|}
\hline Variable & $\mathrm{EC}(47)$ & FAE (15) \\
\hline \multicolumn{3}{|l|}{$\begin{array}{l}\text { Fentanyl (mcg) Local } \\
\text { Throat Spray (mg) }\end{array}$} \\
\hline Commonest Stent & $7 / 10$ ultraflex $(38)$ & $7 / 10$ ultraflex (33) \\
\hline Placement difficulties & $6(13)$ & $0(0)$ \\
\hline Previous failed attempts & $1(2)$ & $0(0)$ \\
\hline $\begin{array}{l}\text { Early complications: } \\
\text { Vomiting } \\
\text { Tachyarrhythmia } \\
\text { Apiration pneumonia } \\
\text { Chest pain }\end{array}$ & 3 (5) 2 (4) 3 (6) $10(21)$ & $1(7) 0(0) 1(7) 1(7)$ \\
\hline $\begin{array}{l}\text { Late complications Tumour } \\
\text { overgrowth } \\
\text { Oesophagitis Stricture } \\
\text { Stent migration: Mean } \\
\text { days }\end{array}$ & $\begin{array}{l}9(19) 2(4) \\
1(2) 9(20) 32\end{array}$ & $\begin{array}{l}5(33) 0(0) \\
1(7) 3(15) 29\end{array}$ \\
\hline Malposition & $0(0)$ & $1(7)$ \\
\hline Food bolus obstruction & $10(21)$ & $0(0)$ \\
\hline Second stent: Mean days & 8 (17) 96.1days & 5 (33) 171days \\
\hline Third stent: Mean days & 2 (4) 188.5 days & 2 (7) 286.5days \\
\hline Fourth Stent: Mean days & $0(0)$ & 2 (7) 377.5days \\
\hline $\begin{array}{l}\text { Median Survival days } \\
\text { (IQR) }\end{array}$ & $102(73)$ & $126(161)$ \\
\hline
\end{tabular}

Conclusion: Our results are comparable to findings of other authors $(1,2)$, in that neither approach is superior. Both FAE and EC techniques have similar early and late complications and comparable median survival. EC offers the advantage of stent placement with direct visualization, however a learning curve must be acknowledged. FAE insertion is a time-consuming procedure and exposes both patients and endoscopy staff to radiation. In conclusion, the approach used should be based on individual characteristics including operator experience, tumour characteristics and previous endoscopic interventions.

Disclosure of Interest: All authors have declared no conflicts of interest.

\section{References}

1. Ferreira F, et al. A comparative study between fluroscopic and endoscopic guidance in palliative oesophgeal stent placement. Diseases of Esophagus 2012;25:608-613

2. Chapman T et al. PTU-055.A comparsion of radiological and endoscopic oesophgeal stent placement in malignancy. Gut 2014 63:A62

\section{P0828 CAUSTIC INJURIES OF THE SUPERIOR} GASTROINTESTINAL TRACT: 15 YEARS OF EXPERIENCE

M. Rocha, T. Moreira, M. Salgado, L. Maia, S. Barrias, I. Pedroto Gastroenterology, Centro Hospitalar do Porto, Porto/Portugal

Contact E-mail Address: martalemosrocha@gmail.com

Introduction: Ingestion of caustic substances is relatively frequent and can cause serious lesions in the upper gastrointestinal tract, carrying important morbidity and even mortality.

Aims \& Methods: We aimed to characterize the population assisted for caustic ingestion, the therapeutic approach, complications and risk factors for severe oesophageal lesions.

Retrospective cohort of adults presenting due to caustic ingestion between 2000 and 2015. Demographic and clinical data were collected. The endoscopic Zargar classification was applied. We analysed risk factors for severe oesophageal lesions, defined as Zargar 2b-3. Statistical tests: Mann-Whitney; Spearman (significance level $5 \%$ ).

Results: Overall 72 patients were included, with a mean age $53 \pm 17$ years, $65.3 \%$ female. Ingestion was voluntary in $49.3 \%$ of the cases, $33.3 \%$ had previous suicide attempts. Alkaline substance in $90.4 \%$. Most common symptoms at admission: $60.3 \%$ odynophagia, $41.1 \%$ epigastric pain, $32.9 \%$ vomiting. Oropharyngeal lesions in $41.1 \%$. In $79.5 \%$ endoscopy was performed in the first 12 hours. Oesophageal lesions were present in $46.6 \%$ of patients (Zargar classification: I- $2.7 \%$, IIa- $23.3 \%$, IIb-5.5\%, IIIa-6.8\%, IIIb-8.2\%); gastric lesions in $58.9 \%$ and duodenal lesions in $13.7 \%$. $53.4 \%$ were hospitalized, $51.3 \%$ in intensive/intermediate care units. The mean length of hospital stay was 14.9 days. Medical treatment prescribed: $76.7 \%$ proton pump inhibitors, $15.1 \%$ corticoids, $15.1 \%$ prophylactic antibiotics. Parenteral feeding was initiated in $28.8 \%$ of patients. Eight patients required invasive ventilation and two were tracheotomised. Early complications: infections in 12 patients $(16.4 \%)$, perforations in $2(2.7 \%)$; late complications: stenosis in $7(9.6 \%)$ : dilation in 6 , surgery in 3 . One patient died from gastric perforation after voluntary ingestion of acid. Severe oesophageal lesions were associated with increased inflammatory parameters, tachycardia and/or hypotension at admission and motivated longer hospital stays, requirement of intensive care and further complications $(\mathrm{p} \leq 0.002)$. The ingestion of acidic substances $(100 \%$ of voluntary intake) was associated with severe oesophageal damage in $3 / 7(42.9 \%)$ patients, severe gastric lesion in $5 / 7(71.4 \%)$, acidemia in 5/7 (71.4\%), complications in 5/7 (71.4\%) and $100 \%$ hospitalization. 
Conclusion: Early changes in blood tests were seen in severe oesophageal lesions. Severe oesophageal lesions were associated with longer hospitalization and complications (infection/stenosis/perforation). Ingestion of acidic substances was a risk factor for severe digestive lesions.

Disclosure of Interest: All authors have declared no conflicts of interest.

\section{References}

Núnez O, et al. Estudio de los factores predictivos de lesiones digestivas graves tras la ingestión de cáusticos. Med Clin (Barc) 2004;123(16):611-4 Chirica M, et al. Caustic ingestion. Lancet 2016.

\section{P0829 NOVEL IMAGE ENHANCEMENT TECHNOLOGY USING LINKED COLOR IMAGING WITH ACETIC ACID INDIGOCARMINE MIXTURE FOR DIAGNOSIS OF EARLY GASTRIC NEOPLASM}

Y. Kawahara ${ }^{1}$, H. Kanzaki ${ }^{2}$, S. Kawano ${ }^{2}$, M. Iwamuro ${ }^{2}$, Y. Kono ${ }^{2}$, T. Gotoda ${ }^{2}$, H. Sakae ${ }^{2}$, Y. Baba ${ }^{2}$, Y. Obayashi ${ }^{2}$, Y. Okamoto ${ }^{2}$, H. Okada ${ }^{2}$

${ }^{1}$ Department Of Endoscopy, Okayama University Hospital Dept. of Endoscopy, Okayama/Japan

${ }^{2}$ Departments Of Gastroenterology And Hepatology, Okayama University Hospital, Okayama/Japan

\section{Contact E-mail Address: yoshirok@gmail.com}

Introduction: A value of the combination of magnifying endoscopy of and image enhancement endoscopy (IEE) technology (e.g. NBI, BLI) is reported in a diagnosis for the early gastric neoplasm. That method is useful, but in order to master it it is necessary to learn and familiarize complex classifications. Therefore, this diagnostic method is still more difficult for general endoscopists. Linked Color Imaging (LCI) was recently developed using a laser endoscopic system (Fujifilm Co., Tokyo, Japan). LCI acquires images by simultaneously using narrow-band short wavelength light and white light in an appropriate balance. This combination of light provides more information about the vasculature and architecture on the mucosal surface than that obtained with typical white-light imaging. When we use acetic acid indigocarmine mixture (AIM) with LCI mode, we reported that the magnifying images of early gastric cancer are very clear, three-dimensional and near to real histology. So, we examined the examined the utility of this method.

Aims \& Methods: This was a prospective observational study performed at a single tertiary referral center. The subjects are 120 lesions of 115 patients with gastric neoplasm. We are indicated of the endoscopic submucosal dissection (ESD), and were given preoperative endoscopy in our hospital from September 2014 to February 2017. Firstly we observed the lesions by magnifying endoscopy with the BLI mode and diagnosed using VS classification system. Secondly we observed the lesions by magnifying endoscopy with LCI + AIM method and diagnosed using VS classification system. Furthermore, we classified tumor differentiation into high differentiation, moderately differentiated, and poorly differentiated by its surface pattern. Finally, we classified the visualization ability of the surface fine structure in Clear, Visible, and Invisible and evaluated it. We carried out ESD and compared the image with the histopathology.

Results: By the pathology results, 92 lesions were gastric cancer and 28 lesions were gastric adenoma. The differentiation ability of a cancer and the non-cancer (adenoma) did not have the significant difference between the BLI mode and the LCI + AIM methods. Diagnosis of differentiation of gastric cancer was correct in 87 of 92 cases $(94.6 \%)$. In the classification of visualization ability, 32 lesions were Clear, 44 lesions were Visible, 44 lesions were Invisible by BLI mode, On the other hand, 45 lesions were Clear, 64 lesions were Visible, 11 lesions were Invisible by LCI + AIM method. In the visualization ability of the surface fine structure, LCI + AIM method is significantly clearer than BLI mode $(\mathrm{p}<0.001)$. Conclusion: When we use AIM, indigocarmine accumulates in pit of the duct, and duct structures become clear by the acetic acid, By LCI mode, we can observe the vascular pattern of the lesion clearly. So by the combination of AIM and LCI, we can observe the endoscopic images closer to actual histological images. By this method, we can compare histopathology with an endoscopic image intuitively, so we believe that a magnifying endoscopy diagnosis of the gastric cancer is enabled even if we do not use various confusing classifications.

Disclosure of Interest: All authors have declared no conflicts of interest.

P0830 THE SAFETY AND EFFECTIVENESS OF ENDOSCOPIC SUBMUCOSAL DISSECTION FOR EARLY GASTRIC NEOPLASMS IN PATIENTS AGED 85 YEARS OR OLDER

Y. Sumida ${ }^{1}$, T. Kuwai ${ }^{1}$, K. Tao ${ }^{1}$, S. Funaki ${ }^{1}$, Y. Miyasako ${ }^{1}$, T. Takasago ${ }^{1}$, T. Nishimura ${ }^{1}$, R. Miura ${ }^{1}$, H. Imagawa ${ }^{1}$, T. Yamaguchi ${ }^{1}$, A. Yamaguchi ${ }^{1}$, H. Kouno ${ }^{1}$, H. Kohno ${ }^{1}$, S. Ishaq ${ }^{2}$

${ }^{1}$ Gastroenterology, Kure MC \& Chugoku CC, Kure/Japan

${ }^{2}$ Gastroenterology, Dudley Group Hospitals NHS Foundation Trust, Dudley/

United Kingdom

Contact E-mail Address: sumiday@kure-nh.go.jp

Introduction: Endoscopic submucosal dissection (ESD) is one of the most useful methods for treating early gastric neoplasms. The advantages of ESD include the ability to control the size and shape of the resection, permitting en bloc resection of large and ulcerated lesions. The frequency of gastric ESD for patients aged 85 years or older has increased along with increases in the average population age. However, few studies have reported the short-term and long-term outcomes of gastric ESD in elderly patients.

Aims \& Methods: The aims of our study were to evaluate and compare the efficacy, safety, and clinical outcomes of gastric ESD in patients aged 85 years or older and in younger patients. The subjects were 705 patients who collectively presented with 876 gastric tumors (288 adenomas and 588 early gastric cancers). All patients underwent ESD at our hospital between June 2007 and December 2016. The patients were divided into two groups: elderly (Group A: aged $\geq 85$ years, consisting of 59 patients with a collective 71 lesions) and non-elderly (Group B: aged $<85$ years, consisting of 646 patients with a collective 805 lesions). We evaluated the clinical and pathological findings, resection rates, complications, and long-term outcomes, including the survival rate. The local and distant recurrence rates were analyzed in the cohort with curative resection and observationally managed with non-curative resection. The 3- and 5-year overall survival and tumor-specific survival rates were analyzed in the entire study cohort.

Results: The patients' mean ages were 87 (Group A) and 71 years (Group B), and the male-to-female ratios were 30/29 (Group A) and 646/805 (Group B). No significant differences were found in the mean tumor size for Group A $(15 \mathrm{~mm})$ and Group B $(20 \mathrm{~mm})$. Regarding histopathological findings, the prevalence rates of tubular adenoma were $28.3 \%$ (21/71; Group A) and 33.8\% (267/ 805; Group B); intramucosal carcinomas, 52.1\% (37/71; Group A) and 53.8\% (433/805; Group B); shallow submucosal invasive carcinomas $(<500 \mu \mathrm{m}), 7.0 \%$ (5/71; Group A) and 6.5\% (52/805; Group B); and deep submucosal invasive carcinomas $(>500 \mu \mathrm{m}), 11.3 \%(8 / 71$; Group A) and 6.6\% $(53 / 805$; Group B). Once again, the groups showed no significant differences. The en bloc resection rates were 100\% (71/71 lesions; Group A) and 97.1\% (782/805 lesions; Group B), histological complete resection rates were 94.4\% (67/71; Group A) and 92.9\% (748/805; Group B), and the curative resection rates were 78.8\% (56/71; Group A) and $86.3 \%$ (695/805; Group B). Among the non-curative cases, $13(86.6 \%)$ of the 15 patients in Group A and $41(46.3 \%)$ of the 110 patients in Group B received no additional treatment. These results were significantly higher for Group A than for Group B. Concerning complications, the postoperative hemorrhage rates were $2.8 \%(2 / 71$ patients; Group A) and 2.6\% (12/479 patients; Group B), and the perforation rates were $0 \%(0 / 71$; Group A) and $0.5 \%$ (4 805; Group B). Regarding long-term outcomes, analysis of recurrence revealed the local and distant recurrence rates to be $0 \%$ for Group A and $0.9 \%(7 / 746$; local) and $0.1 \%$ (1/746; distant) for Group B. Regarding survival analysis, the mean follow-up period in Group A and Group B was 839 and 1156 days, respectively. The 3 - and 5-year overall survival rates were $95.5 \%$ (3 year) and $74.7 \%$ (5 year) for Group A and $92.8 \%$ (3 year) and $87.7 \%$ (5 year) for Group B. The 3and 5-year tumor-specific survival rates were $100 \%$ for Group A and $99.2 \%$ (3 year) and $98.3 \%$ (5 year) for Group B (using the Kaplan-Meier method and long-rank test). There were no significant differences observed in the survival rates. $6(10.0 \%)$ of 59 patients in Group A and $54(9.3 \%)$ of 646 patients in Group B died, and disease-specific mortality rates in Groups A and B were $0 \%$ $(0 / 59)$ and $0.8 \%(5 / 646)$, respectively.

Conclusion: Gastric ESD in patients aged 85 years or older can be effectively and safely performed. According to the long-term outcomes, gastric ESD performed as a local resection (total biopsy) in elderly patients may be acceptable, even in non-curative cases.

Disclosure of Interest: All authors have declared no conflicts of interest.

\section{P0831 RESULTS FROM THE FIRST UK VIRTUAL COMPLEX}

\section{POLYP MDM}

A. Chattree, M. Rutter

Gastroenterology, University Hospital of North Tees, Stockton on Tees/United Kingdom

Contact E-mail Address: amitchattree $@$ nhs.net

Introduction: Data from the UK Bowel Cancer Screening Programme (BCSP) has established that the assessment and management of large non pedunculated colorectal polyps (LNPCPs) varies markedly, leading to variable and often suboptimal outcomes, especially for the most complex lesions ${ }^{1}$. A multicentre complex polyp multidisciplinary team meeting was created within the North East of England BCSP with the aim of ensuring more robust decision making and management of complex LNPCPs.

Aims \& Methods: A virtual multicentre MDM was conducted via audioteleconferencing within the North East of England between 2014-6 to discuss complex LNPCPs (LNPCPs with increased risk of malignancy or complexity associated with endotherapy, as defined in BSG/ACPGBI guidelines ${ }^{2}$ ). Non-complex LNPCPs were not discussed. Patient data was distributed securely via NHSmail. Outcomes were assessed prospectively using key performance indicators (KPIs) from the BSG/ACPGBI guidelines.

Results: 61 complex LNPCP cases were managed via the MDM with 8 excluded from analysis (7: managed prior to MDM referral, 1: MDM advice not followed), 27 lesions were managed with primary endotherapy, 23 with primary surgery and 3 cases conservatively. Of the endoscopic cases, 2 required surgery due to failed endotherapy and 2 due to a finding of malignancy. 12-month recurrence was $8.7 \%$ with no reported complications. The rate of surgical management using the BSG/ACPGBI KPI (including only surgically managed benign lesions or lesions subject to failed endotherapy) was $39.5 \%$. The en-bloc resection 
rate of complex LNPCPs with features suggestive of increased malignancy risk was $71.4 \%$. Breakdown of Outcomes $(\mathbf{n}=\mathbf{5 3})$ *Primary endoscopic therapy $(\mathbf{n}=\mathbf{2 7})-23$ excised with curative intent -21 cases with no recurrence at $1 \mathrm{y}-2$ cases with recurrence $(8.7 \%)$ both $<1 \mathrm{~cm}$ and managed endoscopically -4 required secondary surgery -2 failed endotherapy -2 proved to be malignant *Primary Surgical Management $(\mathbf{n}=23)-10 / 23$ malignant lesions $(43.5 \%)-9 / 23$ subject to transanal surgery $(39.1 \%)$ *Conservative management $(\mathbf{n}=\mathbf{3})$ Breakdown via High risk features of malignancy/SMSA4/SMSA3 $(n=53)$ *High risk features of malignancy (any SMSA score) $(\mathbf{n}=\mathbf{2 1})$-7 endoscopically managed -5 pEMR, 2 ESD $-2 / 7(28.6 \%)$ cases (both managed with pEMR) malignant -13 managed surgically $(4 / 13(30.8 \%)$ : transanal surgery) $-9 / 13$ malignant $(69.2 \%)-1$ managed conservatively *SMSA $4(\mathbf{n}=\mathbf{2 5})-12$ managed endoscopically -12 managed surgically (due to technical considerations) -3/12 malignant $(25 \%)-1$ managed conservatively ${ }^{*}$ SMSA3 $(\mathbf{n}=7)-4$ managed endoscopically -2 management surgically (both lesions originating from within appendix) -1 managed conservatively.

Conclusion: 61 complex LNPCP cases were managed via the MDM with 8 excluded from analysis (7: managed prior to MDM referral, 1: MDM advice not followed), 27 lesions were managed with primary endotherapy, 23 with primary surgery and 3 cases conservatively. Of the endoscopic cases, 2 required surgery due to failed endotherapy and 2 due to a finding of malignancy. 12 month recurrence was $8.7 \%$ with no reported complications. The rate of surgical management using the BSG/ACPGBI KPI (including only surgically managed benign lesions or lesions subject to failed endotherapy) was $39.5 \%$. The en-bloc resection rate of complex LNPCPs with features suggestive of increased malignancy risk was $71.4 \%$.

Disclosure of Interest: All authors have declared no conflicts of interest.

\section{References}

1. Chattree A, Nylander D, Silcock J, et al. UEG15-ABS-1622. Marked Variation in Endoscopic Mucosal Resection Outcomes Within the UK Bowel Cancer Screening Programme. United European Gastroenterology Journal 2015; 2015: (Suppl 1)

2. Rutter MD, Chattree A, Barbour JA, et al. British Society of Gastroenterology/Association of Coloproctology of Great Britain and Ireland Guidelines for the Management of Large Non-Pedunculated Colorectal Polyps. Gut 2015; 0:1-27.

\section{P0832 DIFFERENCES IN DISTRIBUTION OF SIZE, SHAPE AND SERRATED HISTOLOGY OF COLORECTAL ADENOMAS BETWEEN ENDOSCOPISTS WITH LOW ( $<20 \%)$ AND HIGH $(\geq 20 \%)$ ADENOMA DETECTION RATE}

D. Penz ${ }^{1}$, E. Waldmann ${ }^{1}$, A. Hinterberger ${ }^{2}$, B. Majcher ${ }^{1}$, A. Dokladanska ${ }^{1}$, A. Szymanska ${ }^{1}$, M. Trauner ${ }^{2}$, M. Ferlitsch ${ }^{3}$

${ }^{1}$ Department of Internal Medicine III, Division Of Gastroenterology \&

Hepatology, Medical University of Vienna, Vienna/Austria

${ }^{2}$ Department Of Medicine III, Head of Division of Gastroenterology and

Hepatology - Department of Medicine III, Head of Division o, Wien/Austria

${ }^{3}$ Quality Assurance Working Group, Austrian Society of Gastroenterology and

Hepatology (OEGGH), Vienna/Austria

Contact E-mail Address: daniela.penz@meduniwien.ac.at

Introduction: Patients of endoscopists with high $(\geq 20 \%)$ adenoma detection rate (ADR) have less risk for interval cancer than of those with an low ADR $(<20 \%)$. Lesion-related-factors, such as size, shape and histology influence these quality measure, as physicians with high ADR tend to detect more flat and serrated adenomas than those with low ADR.

Aims \& Methods: Our study aim is to investigate the differences of size, shape and serrated histology of adenomas between low- and high-ADR group in our screening cohort. We analyzed 221.534 screening colonoscopies performed by 261 endoscopists between 2007 and March 2017 within the austrian certificate of screening colonoscopy. T-Test was used to assess differences.

Results: $39.1 \%$ of endoscopists were categorized in the ADR low- and $60.9 \%$ in the high-ADR group. Overall, mean ADR was $23.06 \%$ (SD 0.55) with a minimum of $0.39 \%$ and a maximum of $48.72 \%$. In the low-ADR-group mean ADR was 14.56 (SD 0.42) and 28.51 (SD 0.50) in the high-ADR group. Relating to size, there was a significant difference $(\mathrm{p}=0.029)$ in detection of adenomas of 1 $2 \mathrm{~cm}$ with a mean of $8.44 \%$ (SD 6.02) in low- vs. $10.22 \%$ (SD 6.64) of all adenomas in high-ADR group but no differences between adenomas $<0.5 \mathrm{~cm}$, $0.5-1 \mathrm{~cm}$ and those bigger than $2 \mathrm{~cm}$. Regarding shape, proportion of pedunculated adenomas in low-group-ADR differ significantly higher $(\mathrm{p}=0.002)$, with a mean of $19.36 \%$ (SD 14.69) vs. $14.70 \%$ (SD 9.55) but there were no differences between flat and sessile adenomas. With a mean proportion of $4.43 \%$ (SD 5.61) vs. $6.64 \%$ (SD 5.97), the proportion of sessile serrated adenomas (SSA) differ significantly between low-ADR vs. high-ADR-group $(p<0.01)$. There was no significant difference regarding traditional serrated adenomas $(\mathrm{p}=0.800)$.

\begin{tabular}{lllll}
\hline size & ADR-Group & Mean & SD & p-value \\
\hline$<0.5 \mathrm{~cm}$ & $<20 \%$ & 53.49 & 20.58 & $\mathrm{p}=0.384$ \\
& $\geq 20 \%$ & 51.31 & 19.14 & \\
$0.5-1 \mathrm{~cm}$ & $<20 \%$ & 34.04 & 18.23 & $\mathrm{p}=0.980$ \\
\hline
\end{tabular}

\begin{tabular}{lllll} 
Continued & & & & \\
\hline size & ADR-Group & Mean & SD & p-value \\
\hline & $\geq 20 \%$ & 34.09 & 15.46 & \\
$1-2 \mathrm{~cm}$ & $<20 \%$ & 8.44 & 6.02 & $\mathrm{p}=0.029$ \\
& $\geq 20 \%$ & 10.22 & 6.64 & \\
$>2 \mathrm{~cm}$ & $<20 \%$ & 4.04 & 3.69 & $\mathrm{p}=0.470$ \\
& $\geq 20 \%$ & 4.39 & 3.81 & \\
shape & & & & \\
flat & $<20 \% \geq 20 \%$ & 26.6427 .11 & 25.1124 .23 & $\mathrm{p}=0.883$ \\
pedunculated & $<20 \% \geq 20 \%$ & 19.3614 .70 & 14.699 .55 & $\mathrm{p}<0.01$ \\
sessile & $<20 \% \geq 20 \%$ & 53.9958 .19 & 27.2326 .36 & $\mathrm{p}=0.216$ \\
SSA \& TSA & & & & \\
SSA & $<20 \% \geq 20 \%$ & 4.436 .64 & 5.615 .97 & $\mathrm{p}<0.01$ \\
TSA & $<20 \% \geq 20 \%$ & 0.880 .94 & 2.101 .44 & $\mathrm{p}=0.800$ \\
\hline
\end{tabular}

Conclusion: Endoscopists with higher ADR tend to detect significant more SSA and adenomas with $1-2 \mathrm{~cm}$ in size, but have lower proportions of pedunculated adenomas than those with a low ADR. In our study cohort no significant differences in flat shape or diminutive size was measurable.

Disclosure of Interest: All authors have declared no conflicts of interest.

\section{P0833 PREDICTIVE FACTORS FOR TECHNICALLY DIFFICULT ENDOSCOPIC SUBMUCOSAL DISSECTION (ESD). IMPLICATIONS FOR CASE SELECTION: A SPANISH PROSPECTIVE MULTICENTER COHORT STUDY}

J. C. Marín-Gabriel ${ }^{1}$, F. Ramos-Zabala ${ }^{2}$, F. Mugica ${ }^{3}$, A. Herreros De Tejada ${ }^{4}$, E. Albéniz Arbizu ${ }^{5}$, J. De La Peña Garcia ${ }^{6}$, O. Nogales Rincón ${ }^{7}$, A. Sanchez Yague $^{8}$, M.G. Fernández-Esparrach ${ }^{9}$, C. Dolz Abadia ${ }^{10}$, A. Alvarez Delgado ${ }^{11}$, H. Cortés-Pérez ${ }^{12}$, C. Guarner Argente ${ }^{13}$, A.J. Del Pozo-García ${ }^{1}$, J.B. Díaz Tasende $^{1}$

${ }^{1}$ Gastroenterology. Endoscopy Unit., University Hospital "12 de Octubre", Madrid/ Spain

${ }^{2}$ Gastroenterology, HM Montepríncipe University Hospital, Boadilla del Monte (Madrid)/Spain

${ }^{3}$ Gastroenterology, Donostia University Hospital, San Sebastian/Spain

${ }^{4}$ Gastroenterology, Puerta de Hierro University Hospital, Majadahonda/Spain

${ }^{5}$ Gastroenterology, Complejo Hospitalario de Navarra, Pamplona/Spain

${ }^{6}$ Gastroenterology, Marques de Valdecilla University Hospital, Santander/Spain

${ }^{7}$ Endoscopy Unit, Gregorio Marañon University Hospital, Madrid/Spain

${ }^{8}$ Gastroenterology, Hospital Costa del Sol, Marbella/Spain

${ }^{9}$ Inst. De Malaties Digestives, Hospital Clinic de Barcelona, Barcelona/Spain

${ }^{10}$ Gastroenterology, Hospital Son Llàtzer, Palma de Mallorca/Spain

${ }^{11}$ Endoscopy Unit, Salamanca University Hospital, Salamanca/Spain

${ }^{12}$ Gastroenterology, Cruces University Hospital, Baracaldo/Spain

${ }^{13}$ Servei De Patologia Digestiva, Hospital de la Santa Creu i Sant Pau, Barcelonal Spain

Contact E-mail Address: josecarlos.marin@salud.madrid.org

Introduction: ESD is a complex procedure, mainly in non-Asian countries where the learning process is not well established. Results may be improved in Western countries with a careful selection of lesions for ESD and avoiding those with a greater chance of technical difficulty. Factors predicting technically difficult ESD when it is performed by non-Asian endoscopists should be clarified.

Aims \& Methods: We aimed to identify the potential risk factors that are associated with a higher technical difficulty during ESD in a Western European setting where there are no available Asian experts. We prospectively recorded consecutive ESD cases performed by members of the ESD Working Group of the Spanish Society of Digestive Endoscopy. Demographic and clinical characteristics of the patients, location and morphology of the lesions, and technical factors were collected. We defined difficult ESD as those aborted procedures, time-consuming (duration $>180 \mathrm{~min}$.) or when changing the technique to piecemeal resection was needed to remove the tumor. Analyses were carried out using IBM SPSS software for Windows (IBM Corp., Armonk, NY, USA). Parametric continuous variables are reported as the mean \pm standard deviation (SD). A KolmogorovSmirnov test was used to evaluate normal distribution. Categorical variables are reported as either frequencies or percentages. Statistical differences between the groups were analyzed using a chi-squared method for categorical data. The meaningful variables with a $\mathrm{p}$ value $<0.1$ in the univariate analysis were included in the logistic regression model. Multivariate analysis was performed using binary logistic regression methods. Odds ratios (ORs) and $95 \%$ confidence intervals (CIs) were calculated to assess the strength of the influence of each individual variable.

Results: We included 265 lesions in 265 patients [mean age \pm SD: $69 \pm 10$ y; 150 males $(56.6 \%)]$. They were recruited in 15 Spanish University Hospitals between January 2016 and March 2017. Location of the lesions were: esophagus $(n=7$; $2.6 \%)$, cardia $(n=5 ; 1.8 \%)$; stomach $(n=48 ; 18.1 \%)$; duodenal bulb $(n=1$; $0.3 \%)$; colon $(\mathrm{n}=144 ; 54.3 \%)$ and rectum $(\mathrm{n}=60 ; 22.6 \%)$. Mean lesion size was $38.6 \pm 18.5 \mathrm{~mm}$. Median duration of the procedure was $105 \mathrm{~min}$. (8-375). In 73 cases $(27.5 \%)$ criteria for difficult ESD were fulfilled. Endoscopic resection was aborted in 7 cases $(2.6 \%)$. When endoscopic resection was achieved $(n=258$; $97.3 \%$ ) both situations, duration $>3 \mathrm{~h}$ and a piecemeal resection, were noted in $21(8.1 \%)$ patients. Duration $>3 \mathrm{~h}$ in 25 cases $(9.7 \%)$ and unsuccessful en bloc 
resection in $20(7.7 \%)$ were observed in isolation, respectively. Table 1 shows the univariate and multivariate analysis of factors regarding technically difficult ESD.

Table 1: Univariate and multivariate analysis of possible factors related to technically difficult ESD

\begin{tabular}{|c|c|c|c|c|}
\hline & \multicolumn{2}{|l|}{$\begin{array}{l}\text { UNIVARIATE } \\
\text { ANALYSIS }\end{array}$} & \multicolumn{2}{|c|}{$\begin{array}{l}\text { MULTIVARIATE } \\
\text { ANALYSIS }\end{array}$} \\
\hline Variables & OR (C.I. 95\%) & $\mathrm{p}$ & OR (C.I. 95\%) & $\mathrm{p}$ \\
\hline \multicolumn{5}{|l|}{ Procedure } \\
\hline Case load $\leq 10$ & $0.8(0.4-1.6)$ & 0.5 & & \\
\hline 2 endoscopists (vs. 1 operator) & $20.6(5.9-72.6)$ & $<0.0001$ & $9.7(0.9-94.9)$ & 0.06 \\
\hline \multicolumn{5}{|l|}{ Location } \\
\hline Colorectal & $2.6(1.2-5.7)$ & 0.01 & $2.2(0.6-8.3)$ & 0.2 \\
\hline Stomach & $0.5(0.2-1.0)$ & 0.06 & & \\
\hline \multicolumn{5}{|l|}{ Lesion } \\
\hline Size $>30 \mathrm{~mm}$ & $2.4(1.3-4.4)$ & 0.004 & $5.0(1.7-14.4)$ & 0.003 \\
\hline Recurrent tumor & $3.2(1.3-8.1)$ & 0.008 & $8.1(1.4-45.9)$ & 0.02 \\
\hline Protruded morphology & $0.9(0.5-1.9)$ & 0.9 & & \\
\hline Depressed component & $0.6(0.2-1.7)$ & 0.4 & & \\
\hline Poor manoeuvrability & $3.5(1.7-7.5)$ & 0.001 & $3.5(1.4-8.7)$ & 0.006 \\
\hline Previous biopsy & $1.0(0.6-1.8)$ & 0.9 & & \\
\hline Submucosal invasion & $1.3(0.5-3.7)$ & 0.6 & & \\
\hline $\begin{array}{l}\text { Severe submucosal } \\
\quad \text { fibrosis (F2 vs. F0/F1) }\end{array}$ & $3.3(1.7-6.4)$ & 0.0002 & $1.3(0.4-4.4)$ & 0.6 \\
\hline \multicolumn{5}{|l|}{ Complications } \\
\hline Intraprocedural bleeding & $4.1(1.9-8.7)$ & 0.0003 & $5.1(1.3-19.9)$ & 0.02 \\
\hline
\end{tabular}

Conclusion: The factors independently associated with technically difficult ESD (aborted procedures, time-consuming or finished with a piecemeal resection) were: lesion size $>30 \mathrm{~mm}$, poor manoeuvrability, recurrent lesions and intraprocedural bleeding. Except for the last one, the remaining factors can be identified during the first diagnostic endoscopy. Endoscopists who will start performing ESD should try to avoid these difficult procedures in the early part of their learning curves.

Disclosure of Interest: All authors have declared no conflicts of interest.

\section{References}

1. Takeuchi Y, Iishi H, Tanaka S, Saito Y, Ikematsu H, Kudo SE, et al. Factors associated with technical difficulties and adverse events of colorectal endoscopic submucosal dissection: retrospective exploratory factor analysis of a multicenter prospective cohort. Int J Colorectal Dis. 2014;29(10):1275-84.

2. Hori K, Uraoka T, Harada K, Higashi R, Kawahara Y, Okada H, et al Predictive factors for technically difficult endoscopic submucosal dissection in the colorectum. Endoscopy. 2014;46(10):862-70.

\section{P0834 EPOCH-MAKING TECHNIQUE OF FULL-THICKNESS RESECTION FOR THE COLORECTAL TUMOR BY USING LAPAROSCOPY ENDOSCOPY COOPERATIVE SURGERY (LECS)}

Y. Tamegai $^{1}$, Y. Fukunaga ${ }^{2}$, A. Chino ${ }^{1}$, S. Saito ${ }^{1}$, J. Fujisaki ${ }^{1}$, M. Ueno ${ }^{2}$ ${ }^{1}$ Dept. Of Endoscopy, Cancer Institute Hospital, Tokyo/Japan

${ }^{2}$ Dept. Of Surgery, Cancer Institute Hospital, Tokyo/Japan

Contact E-mail Address: yoshiro.tamegai@jfcr.or.jp

Introduction: We established the Laparoscopy Endoscopy Cooperative Surgery (LECS) procedure to overcome the limitation of colorectal endoscopic submucosal dissection (ESD). This procedure is a local full-thickness resection of the combined procedure of laparoscopy assited colectomy (LAC) and ESD procedure. Also, it is the method that is epoch-making for minimal invasive treatment that kept an intestinal function.

Aims \& Methods: The aim of this study was to investigate the feasibility and safety of LECS procedure applied with endoscopic submucosal dissection (ESD) technique obtained adequate surgical margin. We performed ESD on 1376 colorectal tumors in 1341 patients (male: female $=777: 564$; mean age, 66.1 years) Among these cases, six cases had perforation $(0.4 \%)$, and three of six cases required emergent surgery. We examined the cause of perforation and the limit of ESD from the view point of safety. We performed one-piece resection for 11 cases (male: female $=7: 4$; mean age, 63.5years) of colorectaltumors using LECS procedure. In the first, the indication of LECS is at high risk of the perforation by the treatment of ESD and EMR and is the lesion that safety cannot secure. In addition, the indication is the lesion which is curable by the local excision without lymph node dissection. Therefore, submucosal invasive (T1) cancer with the risk of lymph node metastases does not become the indication for this full-thickness resection technique. From the above-mentioned basic concept, indications of the LECS procedure for colorectal tumors were thought to be as follows: 1) Intramucosal carcinoma (Tis) and adenoma with high-grade atypia (Vienna Classification;Category 3,4) accompanied by wide and severe degree fibrosis in the submucosal layer (tumor recurrence after endoscopic and surgical resection); 2) submucosal tumors; 3) Intra-mucosal carcinoma (Tis) and adenoma with high-grade atypia involved appendix or diverticle. We examined the clinicopathological outcomes of the above-mentioned 11 cases.

Results: Four of six cases that caused perforation in ESD were cases with fibrosis in the submucosal layer. Three cases of those were moderate to severe degree fibrosis cases, and a limit of ESD seemed to exist in these lesions from the viewpoint of safety and curability. We accomplished full-thickness resection successfully for 11 cases using LECS procedure as follows: 5 cases of Tis cancer, 4 cases of adenoma, 1 case of schwannoma, and 1 case of GIST. The reasons that we judged as the indication of LECS procedure were as follows: three cases accompanied by severe degree fibrosis, 2 cases involved diverticle, 3 cases involved appendix, 2 cases of submucoal tumor, and 1 case of poor endoscopic operability. These cases were considered a limitation of ESD due to the high risk of perforation. An operative time was an average of 195.8 minutes (127 to 332), and the perioperative bleeding was an average of $8.9 \mathrm{~g} / \mathrm{dl}$ (3 to 20). We experienced no complications, and average post-operative hospital stay was 7.7(6 to 12) days. Histological examination of the resected specimens revealed negative lateral and deep margins. The postoperative follow-up was carried out first a half year later, and it was every one year subsequently. In the above-mentioned follow-up schedule, blood examination, colonoscopy, CT scan were performed for clinical evaluation. The residual/local recurrence case was absent for 31.6 months (range 10-60 months) for the mean follow-up period. Also, without complications such as postoperative anastomotic stricture or adhesive ileus, we followed favorable course.

Conclusion: We developed a LECS procedure to overcome the limit of ESD, and completed full-thickness one-piece resection of the tumors considered as high risk of perforation in the endoscopic treatment.

Disclosure of Interest: All authors have declared no conflicts of interest.

\section{Reference}

Fukunaga Y, Tamegai Y, Chino A, Ueno M, Nagayama S, Fujimoto Y, Konishi T, Igarashi M., New technique of en bloc resection of colorectal tumor using laparoscopy and endoscopy cooperatively (laparoscopy and endoscopy cooperative surgery - colorectal). Diseases of the Colon and Rectum 2014; 57, 2: 267-271

\section{P0836 HIGH INCIDENCE OF MINOR ADVERSE EVENTS AFTER ENDOSCOPIC MUCOSAL RESECTION OR SUBMUCOSAI DISSECTION FOR COLORECTAL NEOPLASMS}

Y. Song ${ }^{1}$, J. Byeon ${ }^{1}$, K. Kim ${ }^{2}$, D. Yang ${ }^{1}$, S.W. Hwang ${ }^{1}$, E.M. Song ${ }^{1}$, S.H. Park ${ }^{1}$ ${ }^{1}$ Gi, Asan Medical Center, Seoul/Korea, Republic of

${ }^{2}$ Internal Medicine, Konkuk University School of Medicine, Konkuk University Chungju Hospital, Chungju/Korea, Republic of

Contact E-mail Address: js092672@gmail.com

Introduction: Endoscopic mucosal resection (EMR) and submucosal dissection (ESD) are effective procedures for reducing the need for colorectal surgery. Many studies have focused on major adverse events related to EMR and ESD such as bleeding and perforation. However, data on structured assessment of minor post-procedural adverse events remain scarce. We therefore evaluated the incidence of major and minor adverse events within 30 days of EMR or ESD. Aims \& Methods: We prospectively performed a single-center study on consecutive patients who underwent colonoscopic EMR or ESD between April 2014 and April 2016. We asked for permission to interview the patients 1 day, 14 days, and 28 days after their procedure. Four gastroenterologists performed all procedures. The assigned nurse prospectively recorded the data and interviewed the patients in person on 1 day and $14 \pm 2$ days, and via phone on $28 \pm 2$ days after their respective EMR/ESD procedure. A standardized interview form was developed to assess the occurrence of (i) major adverse events (hospital visit required), (ii) minor adverse events, and (iii) working days lost due to adverse events. The primary outcome was the occurrence of all adverse events, which were classified as either "major" or "minor".

Results: We enrolled 630 patients who underwent EMR or ESD. $615(97.6 \%)$ patients (499 EMR, 116 ESD) were contacted. Male comprised 68.5\% $(\mathrm{n}=415)$, and the mean age was 57.9 years $( \pm 11.3)$. Major adverse events occurred in 31 $(5 \%), 16(2.5 \%)$, and $1(0.2 \%)$ patients on days 1,14 , and 28 , respectively. Minor adverse events occurred in $284(46.1 \%), 344(55.9 \%)$, and $25(4.1 \%)$ patients on days 1,14 , and 28 , respectively. Bleeding was the most common major event on days 1 and 14; abdominal bloating and bowel habit change were the most common minor events on days 1 and day 14 , respectively, $11(1.8 \%)$ and $2(0.3 \%)$ patients took leaves of absence due to adverse events on day 14 and 28 , respectively. The detailed events are listed in Table 1 .

Conclusion: Although the number of major adverse events was small, nearly half of the patients reported minor adverse events on 14 days following colon EMR ESD. Most minor adverse events subsided by the day 28 .

Disclosure of Interest: All authors have declared no conflicts of interest. 
Abstract No: P0836

Table 1: Number of Patients with Adverse events on Day 1, 14, and 28.

\begin{tabular}{|c|c|c|c|c|c|c|}
\hline & Day $1 \mathrm{~N}=3$ & $\%)$ & DAY $14 \pm 2$ & $0(58.5 \%)$ & DAY $28=$ & $6(4.2 \%)$ \\
\hline & Major 31(5. & & Major $16(2$. & & Major 1(0 & \\
\hline & $\begin{array}{l}\text { EMR } \\
19(3.8 \%)\end{array}$ & $\begin{array}{l}\text { ESD } \\
12(10.3 \%)\end{array}$ & $\begin{array}{l}\text { EMR } \\
11(2.2 \%)\end{array}$ & $\begin{array}{l}\text { ESD } \\
5(4.3 \%)\end{array}$ & $\begin{array}{l}\text { EMR } \\
1(0.2 \%)\end{array}$ & ESD \\
\hline Hematochezia & 15 & 8 & 9 & 4 & & \\
\hline Chest pain & & & 2 & & 1 & \\
\hline Microperforation & 1 & 2 & & & & \\
\hline Severe abdominal pain & 1 & 2 & & 1 & & \\
\hline Perforation & 2 & & & & & \\
\hline & Minor 284(4 & & Minor 344 & & Minor 25 & \\
\hline & EMR & ESD & EMR & ESD & EMR & ESD \\
\hline & $217(43.5 \%)$ & $67(57.8 \%)$ & $267(53.5 \%)$ & $77(66.4 \%)$ & $19(3.8 \%)$ & $6(5.2 \%)$ \\
\hline Bowel habit change & & & 156 & 41 & & \\
\hline Abdominal bloating & 112 & 28 & 30 & 14 & 5 & \\
\hline Minor bleeding & 36 & 25 & 45 & 22 & 2 & \\
\hline Mild abdominalpain & 42 & 16 & 54 & 24 & 2 & \\
\hline Dizziness & 33 & 13 & 25 & 10 & & 2 \\
\hline Headache & 26 & 7 & 19 & 6 & & \\
\hline Back pain & 17 & 3 & 16 & 3 & 1 & \\
\hline Easy fatigability & 10 & 1 & 65 & 21 & 4 & 2 \\
\hline General Myalgia & 4 & 2 & 26 & 8 & & \\
\hline Nausea/vomiting & 3 & 3 & 8 & 2 & & \\
\hline Tenesmus & 3 & 3 & 18 & 9 & 2 & 1 \\
\hline Febrile sesnse & 2 & 1 & 7 & & & \\
\hline Voiding difficulty & 1 & 1 & 5 & & & \\
\hline Dry mouth & 1 & 1 & & & & \\
\hline Epigastric pain & 2 & & 3 & & & \\
\hline Leg pain & & & 1 & & 1 & \\
\hline Urticaria & & 1 & 2 & 1 & 1 & \\
\hline Indigestion & & 1 & 23 & 5 & 3 & 1 \\
\hline Drowsiness & & 1 & & & & \\
\hline Herpes zoster & & & 2 & & & \\
\hline Flank pain/proctalgia & & & 1 & 3 & & \\
\hline Sleep disturbance & & & & & 1 & \\
\hline
\end{tabular}

P0837 DEVELOPING PATIENT-REPORTED EXPERIENCE MEASURES FOR GI ENDOSCOPY: RESULTS OF PATIENT INTERVIEWS

L.J. Neilson ${ }^{1}$, L. Sharp ${ }^{2}$, J. Patterson ${ }^{2}$, P. Hewitson ${ }^{3}$, C. Von Wagner ${ }^{4}$, C.J. Rees ${ }^{1}$ ${ }^{1}$ Dept. Of Gastroenterology, South Tyneside District Hospital, South Shields United Kingdom

${ }^{2}$ Institute Of Health And Society, Newcastle University, Newcastle upon Tyne/ United Kingdom

${ }^{3}$ University of Oxford, Oxford/United Kingdom

${ }^{4}$ Institute Of Epidemiology \& Health, University College London, London/United Kingdom

Contact E-mail Address: neilson.laurajane@hotmail.co.uk

Introduction: Patient experience is increasingly recognised as a key measure of quality of care. Ensuring positive experience is important to patients and fundamental in maximising participation in screening programmes and re-attendance for surveillance procedures. Current measures of patient experience of gastrointestinal (GI) endoscopy are clinician derived.(1) Patient Reported Experience Measures (PREMs) should be patient derived and incorporate pre-and postprocedure experience. We aimed to identify themes considered as important to patients undergoing GI procedures as a basis for developing PREMs.

Aims \& Methods: We aimed to identify themes important to patients undergoing GI investigations, to enable questionnaire development. Patients who had undergone upper or lower GI investigations (gastroscopy, colonoscopy and CT pneumocolon) were invited to attend for a semi-structured interview. 32 interviewees were purposefully sampled to ensure diversity. Interviews were conducted by a research fellow trained in qualitative methods and were audio recorded and transcribed verbatim. Recruitment continued until saturation was achieved. Analysis used qualitative thematic methods focusing on anticipated and emergent themes, using constant comparison to ensure that all perspectives were included in the explanation of the data.

Results: 168 patients were approached. 32 interviews were completed (12 gastroscopy, 10 colonoscopy and $10 \mathrm{CT}$ pneumocolon), with a male:female ratio of 18:14. The time interval from examination to procedure ranged from 5 to 44 days. Mean age was 63.1 years (SD 11.5)

The interviews provided an in-depth understanding of patient experience of GI procedures. 6 over-arching and inter-linking themes emerged across all procedures; anxiety, expectations, choice/control, communication/information, comfort and embarrassment/dignity. Relation of themes was seen e.g. if the procedure appointment was sooner than expected, patients were anxious about the potential outcome. Choice was important in terms of appointment, endoscopist and choice of pre-medication, however it was highly individualised. Communication prepared patients and managed expectations, with one patient describing poor endoscopist communication affecting the whole experience. Patients described embarrassment related to changing and waiting areas; sensitive nature of the test; exposure and physical reaction. Discomfort during the procedure was attributed to instrument and air insertion.

Conclusion: Despite heterogeneity between procedures consistent themes related to patient experience emerged. This work will be used to develop PREMs for Gastrointestinal Endoscopy.

Disclosure of Interest: L.J. Neilson: Research post previously funded by Aquilant endoscopy

C.J. Rees: Colin Rees has received research grants from ARC medical, Olympus Medical, Aquilant endoscopy, Norgine, travel grants from Boston scientific and Cook medical and speaking grants from Norgine and Olympus

All other authors have declared no conflicts of interest.

\section{Reference}

1. Brown S, Bevan R, Rubin G, Nixon C, Dunn S, Panter S, Rees CJ. Patientderived measures of GI endoscopy: a meta-narrative review of the literature. Gastrointest Endosc 2015;81(5):1130-40

\section{P0838 RANDOMIZED CONTROLLED TRIAL OF ABDOMINAL} VIBRATION STIMULATION AND WALKING EXERCISE FOR BOWEL CLEANSING PRIOR TO COLONOSCOPY

C. Noh, J.K. Kang, K.M. Lee, S.J. Shin, S.G. Lim, K.J. Lee Gastroenterology, Ajou university School of Medicine, Suwon/Korea, Republic of

Contact E-mail Address: cknoh23@gmail.com

Introduction: Adequate bowel preparation is important to perform colonoscopy for accurate mucosa examination, lesion detection and treatment. Walking exercise is known to be effective for colon cleansing. However, it is difficult for patients with uncomfortable walking to improve the status of bowel cleansing. Aims \& Methods: Therefore, we prospectively evaluated the clinical feasibility and clinical validity of the abdominal vibration stimulation for bowel 
preparation. In this randomized, prospective, investigator-blind study and single center study, 141 inpatients for elective colonoscopy were randomized to two groups. PEG solution was used for bowel cleaning in all patients. The one is walking over 3000 steps and the other is having abdominal vibrator more than 30 minutes before colonoscopy. After examination we recorded procedure results, sedation information, patient's satisfaction and adequacy of bowel preparation by using the Boston Bowel Preparations Scale (BBPS).

Results: There were no significant differences between vibrator group $(n=75)$ and walking group $(\mathrm{n}=66)$ in bowel preparation quality (Total BBPS 7.40 vs $7.23, \mathrm{p}=0.519)$, withdrawal time (30.40 vs $30.05 \mathrm{mins}, \mathrm{p}=0.829)$, number of polyps (4.09 vs $3.17, \mathrm{p}=0.085$ ), patient satisfaction (4.39 vs $4.12, \mathrm{p}=0.249$ ) and number of diarrhea after taking PEG $(11.49$ vs $11.42, p=0.503)$. Vibrator group was superior than walking group in time of first defecation after taking PEG (112. 89 vs 123.42 mins, $\mathrm{p}=0.005)$ and cecal intubation time (6.23 vs 8.52 mins, $\mathrm{p}=0.011$ )

Conclusion: Bowel preparation accompanied with abdominal vibration stimulation showed almost similar results to a walking group which was conventional methods for adequate bowel preparation. The patients with the conditions which cause uncomfortable gait such as old age, CVA, Parkinsons, or joint disease, bowel preparation with abdominal vibrator is expected to help in proper bowel cleansing for therapeutic colonoscopy.

Disclosure of Interest: All authors have declared no conflicts of interest.

\section{References}

1. Lieberman DA, Weiss DG, Bond JH, Ahnen DJ, Garewal H, Chejfec G. Use of colonoscopy to screen asymptomatic adults for colorectal cancer. Veterans Affairs Cooperative Study Group 380. N Engl J Med 2000;343:162-168.

2. Winawer SJ, Zauber AG, Ho MN, et al. Prevention of colorectal cancer by colonoscopic polypectomy. The National Polyp Study Workgroup. $N$ Engl J Med 1993;329:1977-1981.

3. Harewood GC, Wiersema MJ, Melton LJ 3rd. A prospective, controlled assessment of factors influencing acceptance of screening colonoscopy. Am J Gastroenterol 2002;97:3186-3194.

4. Tan JJ, Tjandra JJ. Which is the optimal bowel preparation for colonoscopy - a meta-analysis. Colorectal Dis 2006:8:247-258.

5. DiPalma JA, Brady CE 3rd. Colon cleansing for diagnostic and surgical procedures: polyethylene glycol-electrolyte lavage solution. $\mathrm{Am} J$ Gastroenterol 1989;84:1008-1016.

6. Huppertz-Hauss G, Bretthauer M, Sauar J, et al. Polyethylene glycol versus sodium phosphate in bowel cleansing for colonoscopy:a randomized trial Endoscopy 2005;37:537-541.

7. Calderwood AH, Jacobson BC. Comprehensive validation of the Boston Bowel Preparation Scale. Gastrointest Endosc 2010;72:686-92

\section{P0839 COMPARATIVE STUDY OF ELECTRICAL AND RHEOLOGICAL PROPERTIES OF DIFFERENT SOLUTIONS TO PERFORM SUBMUCOSAL INJECTION}

I. Bon ${ }^{1}$, V. Lorenzo-Zúñiga ${ }^{1}$, V. Moreno De Vega ${ }^{1}$, A. Rodríguez ${ }^{2}$, N. D. De La Ossa $^{3}$, I. Marín ${ }^{1}$, J. Boix ${ }^{1}$, R. Bartolí ${ }^{4}$

${ }^{1}$ Endoscopy/ter Group, Germans Trias/IGTP, Badalona/Spain

${ }^{2}$ University Hospital Germans Trias, Badalona/Spain

${ }^{3}$ Pathology Department, University Hospital Germans Trias, Badalona/Spain

${ }^{4}$ IGTP/CIBERehd, Badalona/Spain

Contact E-mail Address: ibon@igtp.cat

Introduction: Electrical and rhelogical properties of the submucosal cushing solutions are crucial to avoid complications secondary to endoscopic resections. Electrical resistance $(\mathrm{R})$ of a substance is a measure of the difficulty to pass an electric current through that solution. The higher the $\mathrm{R}$, the resection will be quicker, easier and safer, with less temperature increase. Our group has developed a new solution to perform submucosal injection (TriBio).

Aims \& Methods: To analize the electrical $(\mathrm{R})$ and rheological (temperature, viscosity, height and lasting of the cushing) properties of different submucosal solutions in an ex vivo model of porcine stomach. Tested solutions were: Saline (S), Gliceol ${ }^{\circledR}$ (GC), Hyaluronic acid (HA), Distilled water (DW), Platelet-rich plasma (PRP), Glucosated serum 10\% (GS), Gelaspan (GP), TriBio (TB) and PRP + TB. Measurements were done at time 0 and 30 minutes.

Results: The solutions that showed the best basal R were: PL, HA, GS, TB and $\mathrm{TB}+\mathrm{PRP}$. At 60 minutes, the best R were: PRP, TB, PRP + TB, HA and GS. The best durability at 60 minutes was for TB, PRP, TB + PRP and PL that maintained the height at around $80 \%$ of its original in comparison to the other substances with were at around $60 \%$. During the resection the solutions that underwent a lower temperature increase were: TB + PRP, PL, and TB.

\begin{tabular}{|c|c|c|c|c|}
\hline & $\begin{array}{l}\text { Viscosity } \\
\text { (pa) }\end{array}$ & $\begin{array}{l}\% \text { diminution } \\
\text { cushion ( } 60 \mathrm{~min})\end{array}$ & $\begin{array}{l}\text { Trans-epithelial } \\
\mathrm{R}(\mathrm{M} \Omega)\end{array}$ & $\begin{array}{l}\text { Increase in } \\
\mathrm{T}^{\mathrm{a}} \text { during } \\
\text { endoscopic } \\
\text { resection }\left({ }^{\circ} \mathrm{C}\right)\end{array}$ \\
\hline Basal & n.a. & n.a. & 10 & 83.5 \\
\hline Saline & 0.0043 & 39.6 & 9 & 49.1 \\
\hline Gelaspan & 0.009 & 45.5 & 9 & 116.6 \\
\hline Glyceol & 0.009 & 26.3 & 10 & 44.9 \\
\hline
\end{tabular}

Continued

\begin{tabular}{|c|c|c|c|c|}
\hline & $\begin{array}{l}\text { Viscosity } \\
\text { (pa) }\end{array}$ & $\begin{array}{l}\% \text { diminution } \\
\text { cushion }(60 \mathrm{~min})\end{array}$ & $\begin{array}{l}\text { Trans-epithelial } \\
\mathrm{R}(\mathrm{M} \Omega)\end{array}$ & $\begin{array}{l}\text { Increase in } \\
\mathrm{T}^{\mathrm{a}} \text { during } \\
\text { endoscopic } \\
\text { resection }\left({ }^{\circ} \mathrm{C}\right)\end{array}$ \\
\hline Glucosated saline $10 \%$ & 0.008 & 29 & 12 & 29.9 \\
\hline Pluronic $20 \%$ & $0.9 *$ & 0.52 & 14 & 12 \\
\hline Hyaluronic acid $5 \%$ & 0.04 & 24.4 & 13 & 12.5 \\
\hline TriBio (TB) & $0.9 *$ & 2.8 & 15 & 36.5 \\
\hline Platelet-rich Plasma (PRP) & $0.01 * *$ & 17 & 13 & 20.8 \\
\hline $\mathrm{TB}+\mathrm{PRP}$ & $0.45^{*}$ & 11.5 & 15 & 5.6 \\
\hline
\end{tabular}

n.a: not applicable $*$ gelification at $37^{\circ} \mathrm{C} * *$ viscoelastic solid when activated

Conclusion: Based on electrical and rheological properties, the best submucosal solutions to perform safer endoscopic resections are: TB + PRP, TB, PL and PRP.

Disclosure of Interest: V. Lorenzo-Zúñiga: Authorship of the patent

All other authors have declared no conflicts of interest.

J. Boix: authorship of the patent

R. Bartolí: Authorship of the patent

\section{P0840 PATIENT SATISFACTION RELATED TO QUALITY OF} INFORMATION GIVEN THROUGHOUT COLONOSCOPY

S. Sjöblom ${ }^{1}$, S. Jakobsson ${ }^{2}$, J. Ryhlander ${ }^{1}$, M. Simrén ${ }^{3}$, P. Stotzer ${ }^{3}$, G. Ringström ${ }^{1}$

${ }^{1}$ Department Of Gastroenterology, Sahlgrenska University Hospital, Gothenburg/ Sweden

${ }^{2}$ Centre For Person-centered Care, Institute of Health and Care Sciences

University of Gothenburg, Gothenburg/Sweden

${ }^{3}$ Institute Of Medicine, Sahlgrenska Academy, University of Gothenburg, Gothenburg/Sweden

Contact E-mail Address: sara.sjoblom@vgregion.se

Introduction: Patients with chronic diseases, such as inflammatory bowel disease, experience a lower degree of being involved in health care than others. Compared with other countries Swedish patients report lower possibility to be involved in their care and receive less information about care (1).

Aims \& Methods: To investigate the perception of written and oral information given before and after a colonoscopy, the perceived knowledge of planned follow-up and preference to be more involved in decisions/participate in their care in different patient groups. Outpatients ( $>18$ y) undergoing colonoscopy (all indications) were consecutively included $(n=862)$. Before the procedure patients completed questionnaires regarding sociodemographic data and the written information about bowel preparation and examination. After the procedure patients reported their perceptions about the information provided regarding the colonoscopy and the follow-up.

Results: Data from 862 patients were analyzed (447 females) (mean age 52; $18-90$ y) A large number of patients $(n=740,87 \%)$ rated the written information sent home before the colonoscopy as distinct, while a subset $(n=110,13 \%)$ rated it as indistinct/very indistinct. When questions of importance were asked to the medical staff during the colonoscopy most patients were content with the answers from the physician or nurse. A small proportion of patients $(n=57,7 \%)$ stated that they received too little information or that they did not understand the information about the colonoscopy results; these patients were mainly younger $(<50$ y) $(\mathrm{p}<0.001)$. The majority of the patients $(\mathrm{n}=602,74 \%)$ reported thorough knowledge about the follow-up, while $26 \%(n=207)$ lacked this knowledge. More than $1 / 3(n=275)$ of the patients wished to be more involved in decisions regarding their care and treatment. Desire for a higher degree of involvement were more pronounced in patients $<40 \mathrm{y}(\mathrm{p}<0.05)$ and in patients with IBD $(\mathrm{p}<0.05)$ compared to patients with other indications for colonoscopy. Patients referred from outpatient clinics in the hospital desires a higher degree of involvement than patients referred from primary care $(p<0.01)$.

Conclusion: The majority of the patients undergoing colonoscopy reported that they received satisfactory information about the procedure and preparation. However, there is room for improvement regarding follow-up information and patients' involvement in their care and treatment. Specifically, improvements seem warranted for younger patients and patients with chronic diseases, such as IBD.

Disclosure of Interest: All authors have declared no conflicts of interest.

\section{Reference}

1. 2016 Commonwealth Fund International Health Policy Survey of Adults. 


\section{P0841 THE INCIDENCE OF SYNCHRONOUS ADVANCED} NEOPLASIA OF RECTAL LATERALLY SPREADING TUMORS WITH A SKIRT

S. Osera, T. Shinohara, A. Kudo, T. Yamada, T. Momoi, H. Fukushima, M. Furutake, A. Tomori, T. Hisa

Department Of Gastroenterology, Saku Central Hospital Advanced Care Center, Saku, Nagano/Japan

Contact E-mail Address: shou0122@hotmail.com

Introduction: A "skirt" is a slightly elevated flat lesion with wide pits occasionally observed at the margin of laterally spreading tumors (LSTs), and rectal LSTs had significantly more skirt lesion in comparison to colonic LSTs. Although the clinicopathological, endoscopic, and molecular characteristics of LSTs with a skirt have been reported [1], there are no reports concerning the incidence of synchronous neoplastic lesions of rectal LSTs with a skirt.

Aims \& Methods: The aim of this retrospective study was to clarify the incidence of synchronous advanced neoplasia (AN) of rectal LSTs with a skirt. A total of 13,116 cases underwent colonoscopy in our hospital between January 2012 and June 2016. Of these, 101 consecutive rectal LSTs were examined to assess the incidence of synchronous AN detection rate and the number of $\mathrm{AN}$ according to the location of AN lesion; divided into the right colon, left colon and rectum. A skirt was defined on the basis of the following endoscopic findings: spreading across the margins of the LST, consisting of a slightly elevated flat lesion, and containing wide pits. AN was defined as the presence of any of the following features: adenomas larger than $10 \mathrm{~mm}$, adenomas with villous histology or highgrade dysplasia including intra-mucosal carcinoma and invasive cancer.

\begin{tabular}{llll}
\hline Synchronous advanced neoplasias of rectal LSTs with and without skirts \\
\hline & $\begin{array}{l}\text { Rectal LSTs } \\
\text { with skirts } \\
(\mathrm{n}=25)\end{array}$ & $\begin{array}{l}\text { Rectal LSTs } \\
\text { without skirts } \\
(\mathrm{n}=76)\end{array}$ & p value \\
& & & \\
\hline Patients with advanced neoplasia & & $36(47.4 \%)$ & 0.02 \\
Total & $5(20.0 \%)$ & $23(30.3 \%)$ & 0.03 \\
Right colon & $2(8.0 \%)$ & $21(27.6 \%)$ & 0.01 \\
Left colon & $1(4.0 \%)$ & $5(6.6 \%)$ & 0.41 \\
Rectum & $3(6.0 \%)$ & & 0.02 \\
Number of advanced neoplasia & & 74 & 0.03 \\
Total & 7 & 35 & 0.04 \\
Right colon & 2 & 34 & 0.44 \\
Left colon & 2 & 5 & \\
Rectum & 3 & &
\end{tabular}

Results: A skirt was observed in 25 of 101 rectal LSTs (24.8\%). Rectal LSTs with a skirt (median age 69 years, $52 \%$ female, mean size $51.7 \pm 27.1 \mathrm{~mm}$ ) had 22 highgrade dysplasia and 3 submucosal carcinomas, and rectal LSTs without a skirt (median age 72 years, $34 \%$ female, mean size $24.7 \pm 16.0 \mathrm{~mm}$ ) had 8 low-grade dysplasia, 45 high-grade dysplasia, and 23 submucosal carcinomas, respectively. The overall AN detection rate in rectal LSTs with a skirt $(20.0 \%)$ was significantly lower compared with rectal LSTs without a skirt $(46.8 \%, p=0.02)$. As for the analysis of AN detection rate according to the location, there were significant differences in the right colon $(8.0 \%$ vs $29.9 \%, p=0.03)$ and the left colon $(4.0 \%$ vs $27.3 \%, p=0.01$ ) between LSTs with and without a skirt. In contrast, there was no significant difference with respect to the rectum $(6.0 \%$ vs $6.5 \%, p=0.41)$. The total number of AN in rectal LSTs with a skirt ( $n=7$; right colon: 2 , left colon: 2 and rectum: 3 ) was significantly lower than in rectal LSTs without a skirt $(\mathrm{n}=74$; right colon: 35, left colon: 34 and rectum: 5). There were significant differences in the right colon $(p=0.03)$ and the left colon $(p=0.04)$, while, there was no significant difference between these groups with respect to the rectum.

Conclusion: The rectal LSTs with a skirt had significantly lower synchronous advanced neoplasia than rectal LSTs without a skirt, especially in the right and left colon. Our results may suggest that rectal LSTs with a skirt have different characteristics compared with rectal LSTs without a skirt in terms of the incidence of synchronous neoplastic lesion.

Disclosure of Interest: All authors have declared no conflicts of interest.

\section{Reference}

1. Osera S, Fujii S, Ikematsu H, et al. Clinicopathological, endoscopic, and molecular characteristics of the "skirt" - a new entity of lesions at the margin of laterally spreading tumors. Endoscopy 2016; 48: 448-455.
P0842 EVALUATION OF MUCOSAL HEALING WITH SHIELDS BASED ON DIFFERENT HYDROGELS IN A RAT MODEL OF THERMAL INJURY

I. Bon ${ }^{1}$, R. Bartolí ${ }^{2}$, V. Moreno De Vega ${ }^{1}$, J. Boix ${ }^{1}$, N. D. De La Ossa ${ }^{3}$, I. Marín ${ }^{1}$, V. Lorenzo-Zúñiga ${ }^{1}$

${ }^{1}$ Endoscopy/ter Group, Germans Trias/IGTP, Badalona/Spain

${ }^{2}$ IGTP/CIBERehd, Badalona/Spain

${ }^{3}$ Pathology Department, University Hospital Germans Trias, Badalona/Spain

Contact E-mail Address: ibon@igtp.cat

Introduction: Endoscopic resection of large lesions leads to extensive mucosal defects and submucosal exposure, with a substantial risk of adverse events. The prevention of these complications is inefficient with current methods. Endoscopic shielding, as a simple and safe technique, has been proposed to improve mucosal restoration, and therefore, the incidence of these events. Previous reports have confirmed the efficacy of the placement of hydrogels based on platelet-rich plasma (PRP) (1) or hialuronic acid with other substances (TriBio) (2), but never the combination of both hydrogels, in the prevention of delayed complications after mucosal damage.

Aims \& Methods: To assess the efficacy of endoscopic shielding with the combination of PRP and TriBio in a rat model of thermal injury. Thermal injury was obtained according to our rat model (3). Lesions were performed in male Sprague-Dawley rats $(400-450 \mathrm{~g})$ under general anesthesia. Animals were randomized to receive one of the following shields onto the lesions: PRP + TriBio, PRP and TriBio. Rats underwent endoscopic follow-up at 7 days and 2 weeks. Afterwards, animals were sacrificed and ulcers sites were macroscopically and histopathologically evaluated.

Results: Animals treated with PRP + TriBio obtained the best results in comparison with other hydrogels (PRP and TriBio). Mucosal healing rate (percentage of mucosal restoration) at 14 days was significantly higher with $\mathrm{PRP}+$ TriBio $(100 \%$ vs $82 \%$ and $90 \% ; p<0.05)$. Histological study confirmed these data, showing total restoration of mucosal layer with PRP + TriBio

Conclusion: The use of a combination of two covering agents (TriBio and PRP) is the best approach to obtain mucosal healing in a rodent model of endoscopic thermal injury in colon

Disclosure of Interest: R. Bartolí: Authorship of the patent

J. Boix: Authorship of the patent

V. Lorenzo-Zúñiga: Authorship of the patent

All other authors have declared no conflicts of interest.

\section{References}

1. Lorenzo-Zúñiga V, Boix J, Moreno de Vega V, Bon I, Marín I, Bartolí R. Efficacy of platelet-rich plasma as a shielding technique after endoscopic mucosal resection in rat and porcine models. Endosc Int Open. 2016 Aug;4(8):E859-64

2. Lorenzo-Zúñiga V, Boix J, Moreno de Vega V, Bon I, Marín I, Bartolí R. Endoscopic shielding technique with a newly developed hydrogel to prevent thermal injury in two experimental models. Dig Endosc. 2017 Mar 13.

3. Lorenzo-Zúñiga V, Boix J, Moreno de Vega V, de la Ossa ND, Òdena G, Bartoli R. Microperforation of the colon: animal model in rat to reproduce mucosal thermal damage. J Surg Res 2014; 188(2):415-8.

P0843 EFFICACY OF ENDOSCOPIC PLACEMENT OF A DRUGELUTING PLATFORM WITH DIFFERENT ANTITUMORAL AGENTS TO EVALUATE ACUTE NECROSIS IN AN AZOXYMETHANEINDUCED COLONIC TUMOURS IN RATS

R. Bartoli ${ }^{1}$, I. Bon ${ }^{2}$, V. Moreno De Vega ${ }^{2}$, J. Boix ${ }^{2}$, N. D. De La Ossa ${ }^{3}$, I. Marín ${ }^{2}$, V. Lorenzo-Zúñiga ${ }^{2}$

${ }^{1}$ IGTP/CIBERehd, Badalona/Spain

${ }^{2}$ Endoscopy/ter Group, Germans Trias/IGTP, Badalona/Spain

${ }^{3}$ Pathology Department, University Hospital Germans Trias, Badalona/Spain

Contact E-mail Address: rbartolisole@gmail.com

Introduction: Colonoscopic procedures have become massive in the last years since colorectal cancer (CRC) is becoming a prevalent disorder. The next frontier of this technique will be to provide an active substance in a precise site of the colon (targeted therapy). This has many advantages (targeted therapy, dose adjustment, limiting side effects, assessment of mucosal healing, etc.); however, this selective and direct administration of drugs is not possible nowadays, but it is a true challenge. Following these evidences we have developed drug eluting platform to locally treat CRC lesions

Aims \& Methods: To evaluate the efficacy of intratumoral injection of our drugeluting platform with different combinations of these antitumoral drugs (aflibercept $2 \mathrm{mg} / \mathrm{mL}$, cetuximab $16 \mathrm{mg} / \mathrm{mL}$, panitumumab $6 \mathrm{mg} / \mathrm{mL}$, irinotecan $3.5 \mathrm{mg}$ $\mathrm{mL}$ and bevacizumab $5 \mathrm{mg} / \mathrm{mL}$ ) in a rat model of azoxymethane-induced colorectal cancer. Rats underwent endoscopic follow-up at 1 and 2 weeks after endoscopic therapy. Afterwards, animals were sacrificed and tumors were excised and macroscopically and histopatologically evaluated. 
Results: Intratumoral injection was feasible in all animals with no adverse events. Basal mean size of tumors ranged from 6 to $8 \mathrm{~mm}$. Anti-VEGF in comparison with Anti-EGF obtained the best results (significantly reduction in size and cell necrosis). However, only aflibercept showed total acute tumoral necrosis.

Conclusion: Intratumoral injection of anti-VEGF in a drug-eluting platform is able to produce tumoral necrosis in an experimental model of CRC. This technique could open a new way to manage CRC.

Disclosure of Interest: R. Bartolí: Authorship of the patent

J. Boix: Authorship of the patent

V. Lorenzo-Zúñiga: Authorship of the patent

All other authors have declared no conflicts of interest.

\section{P0844 A THREE-DIMENSIONAL IMAGING SYSTEM IMPROVES THE ENDOSCOPIC VISIBILITY OF NON-POLYPOID COLORECTAL NEOPLASMS}

T. Matsumura, D. Maruoka, K. Okimoto, N. Akizue, T. Nakagawa,

H. Ishigami, M. Arai, N. Kato

Department Of Gastroenterology, Graduate School of Medicine, Chiba University, Chiba City/Japan

Contact E-mail Address: matsumura919@yahoo.co.jp

Introduction: Three-dimensional (3D) imaging techniques have been developed in the medical field. Previous research reports that simulated 3D colonoscopy improves the detection of colonic lesions [1]. A novel 3D imaging system has been recently developed, which can create $3 \mathrm{D}$ virtual video images from conventional two-dimensional (2D) endoscopic images [2]. However, actual cases have not been studied.

Aims \& Methods: This study aimed to investigate whether the 3D system can improve the visibility of colorectal neoplasms compared with conventional 2D endoscopy. We studied two non-polypoid colorectal neoplasms and recorded their videos using conventional 2D endoscopy and the 3D system. The movies were evaluated by 8 endoscopists ( 4 experts and 4 non-experts) and 4 medical students. Each neoplasm was assigned a visibility score between 4 (excellent visibility) and 1 (poor visibility).

Results: The mean visibility scores were $3.35 \pm 0.58$ for $2 \mathrm{D}$ endoscopy and $3.75 \pm 0.44$ for the $3 \mathrm{D}$ system. The score was significantly higher for the $3 \mathrm{D}$ system than for $2 \mathrm{D}$ endoscopy $(p<0.01)$. When comparing the evaluations by the experts, non-experts, and medical students, the differences in the scores by the non-experts and medical students were noted to be higher $(p<0.05)$. In contrast, the scores by the experts were also higher for the $3 \mathrm{D}$ system, but no statistical difference was observed $(3.50 \pm 0.53$ for $2 \mathrm{D}$ endoscopy and $3.87 \pm 0.35$ for the $3 \mathrm{D}$ system, $p=0.08$ ). As a result, 10 out of 12 observers noted that the $3 \mathrm{D}$ system had better visibility than conventional $2 \mathrm{D}$ colonoscopy, and none of the observers noted deterioration in visibility with the $3 \mathrm{D}$ system.

Conclusion: The present findings suggest that the 3D imaging system improves the visibility of non-polypoid colorectal neoplasms, and this is more effective for non-experts. Our findings would contribute to improvement in the detection of these neoplasms.

Disclosure of Interest: All authors have declared no conflicts of interest.

\section{References}

1. Sakata S, Grove PM, Stevenson AR, Hewett DG. Gut. 2016; 65: 730-731.

2. Matsumura T, Ishigami H, Okimoto K, et al. Three-dimensional imaging system for colonoscopy. Endoscopy 2017; 49: E1-E2

\section{P0845 PAIN DURING COLONOSCOPY: DIFFERENCES BETWEEN PATIENTS' EXPERIENCES AND CAREGIVERS' ASSESSMENT} J. Ryhlander $^{1}$, G. Ringström ${ }^{2}$, M. Simrén ${ }^{3}$, S. Sjöblom ${ }^{1}$, P. Stotzer ${ }^{3}$, S. Jakobsson ${ }^{4}$

${ }^{1}$ Gastro Endoscopy Unit, Sahlgrenska University Hospital, Gothenburg/Sweden ${ }^{2}$ Institute Of Medicine, Sahlgrenska Academy, University of Gothenburg. Gothenburg/Sweden

${ }^{3}$ Dept Of Internal Medicine, Sahlgrenska University Hospital - Dept of Internal Medicine, Sahlgrenska University Hospital; Gothe, Gothenburg/Sweden ${ }^{4}$ Centre For Person-centered Care, Institute of Health and Care Sciences, University of Gothenburg, Gothenburg/Sweden

\section{Contact E-mail Address: jessica.ryhlander@vgregion.se}

Introduction: Pain is a subjective perception, which contributes to difficulties to provide adequate pain relief according to every patient's needs. Colonoscopy is by many patients considered as a painful and strenuous procedure.

Aims \& Methods: To investigate congruence and differences between patients' and caregivers' report of pain during colonoscopy. Patients ( $\geq 18$ years) undergoing an outpatient colonoscopy (all indications) have consecutively been included $(\mathrm{n}=862)$. Before the procedure the patients completed questionnaires regarding sociodemographic information and anxiety. After the colonoscopy the patients registered their pain experience on a six-grade scale, ranging from "no pain" to "extremely severe pain". Caregivers (physicians and endoscopy nurses) estimated patient's pain using the same scale.
Results: Data from 785 patients has been collected, mean age 52 (18-90) years; 413 women. Approximately half of the patient's reported no or mild pain, $21 \%$ moderate pain and a subgroup of $14 \%$ were not adequately relieved. These subgroup reported severe, very severe or extremely severe pain. $90 \%$ of the patients were given analgesics and sedation during the investigation. For patients who reported, "severe, very severe or extremely severe pain" $(n=111)$, pain was underestimated by physicians and nurses in $58 \%$ of all assessments. This was most commonly seen among the youngest patients, $18-29$ years $(n=99)$, where pain was underestimated in $25.5 \%$ among the group. There was also a difference according to gender; physicians underestimated pain in $60 \%$ of men who reported "moderate pain" $(n=66)$ while the nurses underestimated pain in $27 \%$ among the same group of men. Women's pain was overestimated by caregivers in $26 \%(\mathrm{n}=188)$ of all cases with mild pain. Patients undergoing colonoscopy for the first time $(n=331)$, and reporting "moderate pain", were underestimated by physicians in $58 \%$ and by nurses in $25 \% .58 \%$ of the patient reports that they were anxious before the procedure. This group reported more pain than the group without anxiety $(\mathrm{p}<0.001)$. Presence of anxiety and a high level of pain among the group enhanced the agreement between the caregiver's and the patient's pain report. The agreement between pain reports from patients and caregivers were poor to fair, with slight differences between nurses $($ Kappa $=0.37 ; \mathrm{p}<0.000)$ and physicians (Kappa $=0.29 ; \mathrm{p}<0.000)$ In total, congruent pain reports between patients and caregivers were seen in $36 \%$ of all assessments.

Conclusion: Agreement between caregivers' and patients' pain reports is far from perfect, and the agreement is influenced by several factors such as the profession of the caregiver, as well as patient factors including pain severity, anxiety, age, gender and previous experience of colonoscopy. The goal for the future should be to individualize the use of analgesics based on every patient's needs, which seems to be of special importance in specific groups of patients.

Disclosure of Interest: All authors have declared no conflicts of interest.

\section{P0846 DEVELOPMENT OF A NEW ENDOSCOPIC} CLASSIFICATION AND FIRST INTERNATIONAL VALIDATION (FACILE GROUP) OF COLONIC LESIONS USING ADVANCED IMAGING MODALITIES IN IBD PATIENTS

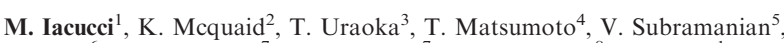
Y. Iwao ${ }^{6}$, M. Lowerison ${ }^{7}$, B.C. Lethebe ${ }^{7}$, S. Sanduleanu ${ }^{8}$, S. Ghosh ${ }^{1}$,

R. Kiesslich

${ }^{1}$ University Of Birmingham, Institute of Translational Medicine, Birmingham United Kingdom

${ }^{2}$ University of California San Francisco, USA, San Francisco/United States of America

${ }^{3}$ Gastroenterology, National Hospital Organization Tokyo Medical Center, Tokyo/ Japan

${ }^{4}$ Division Of Gastroenterology, Department Of Internal Medicine, Iwate Medical University, Morioka/Japan

${ }^{5}$ Gastroenterology, Leeds Teaching Hospitals NHS Trust, Leeds/United Kingdom ${ }^{6}$ Center For Preventive Medicine, Keio University School of Medicine Center for Preventive Medicine, Tokyo/Japan

${ }^{7}$ Clinical research Unit, Calgary/Canada

${ }^{8}$ Gastroenterology And Hepatology, Maastricht Hospital, Maastricht/Netherlands ${ }^{9}$ HSK, Dr. Horst-Schmidt-Kliniken, Wiesbaden/Germany

Contact E-mail Address: iacuccim@yahoo.it

Introduction: The SCENIC consensus proposed recommendations for optimal detection and management of dysplasia during colonoscopic surveillance for IBD $^{1}$. Characterization of colonic lesions in IBD remains challenging even by using advanced endoscopic imaging modalities (high definition [HD], virtual chromoendoscopy [VCE] dye chromoendoscopy [DCE]

Aims \& Methods: We aimed to develop a unified endoscopic classification of advanced imaging to predict histology of colonic lesions, and to validated by international experts (Frankfurt Advanced Chromoendoscopic Ibd LEsionsFACILE Group). We developed an endoscopic classification of IBD lesions, based on morphology, colour, demarcation, surface pattern, vessel pattern, signs of inflammation (table). A library of 60 colonic lesions, including dysplasia, sessile serrated adenomas/polyps, invasive cancer and pseudopolyps collected at surveillance colonoscopy by using HD, DCE and VCE with i-scan or NBI were assessed. The diagnostic performance of the score was tested based on the final histopathology and the inter-observer variability of the eight examiners. The examiners have had to perform a pre-test (45 minutes) before analyzing the colonic lesions. Multivariate analysis with bootstrapping, of characteristics of the classification was performed to determine the strength of endoscopic predictors of dysplasia.

Results: Of the 60 IBD lesions, 33 (55\%) were dysplasia, $6(10 \%)$ cancer, $9(15 \%)$ SSA/Ps and $12(20 \%)$ pseudopolyps. Across the experienced academic raters sensitivity, specificity, PPV, NPV and accuracy in predicting histology, were $72 \%, 72 \%, 91 \%, 40 \%, 72 \%$. Individual rater accuracy ranged from $66 \%$ to $77 \%$. Sensitivity, specificity, PPV, NPV, accuracy, for predictions made with high confidence were $72 \%, 90 \%, 97 \%, 46 \%, 76 \%$, which were significantly more accurate compared with a low confidence of diagnosis (76\% vs $65 \%$; $\mathrm{P}<0.001)$. Univariate analysis showed that the non polypoid lesions, irregular and vessel architecture and signs of inflammation within the lesion were predictive of dysplasia. Subsequent multivariate analysis confirmed that of these endoscopic findings non polypoid lesion OR 11.6 (95\% CI:6.71-20.2), surface pattern 
OR 0.31 (95\% CI:0.17-0.54), vessel architecture OR 5.1 (95\% CI: 2.7-10.2), sign of inflammation within the lesion OR 0.39 (95\% CI: 0.18-0.85) were independent predictors of dysplasia, with vessel architecture and morphology being the best predictors. The sensitivity, specificity, PPV, NPV and accuracy at the multivariate analysis stage were $94 \%(95 \%$ CI: $90-96 \%), 51 \%(95 \%$ CI: $43-58 \%), 88 \%$ (95\% CI: 82-92\%), 69\% (95\% CI:62-75\%), 85\% (95\% CI: $79-90 \%)$. Interobserver agreement of the raters improved from the pre-test $($ Kappa $=0.27 .95 \%$ CI: $0.19-0.38)$ to post test $(\mathrm{Kappa}=0.34 .95 \% \mathrm{CI}: 0.23-0.45 ; \mathrm{P}=0.02)$ but was moderate.

Conclusion: We developed and validated the first endoscopic classification using all imaging modalities (HD, VCE, DCE) to characterize and differentiate dysplastic from non-dysplastic lesions in IBD. Non polypoid lesions, irregular surface and vascular pattern as well as inflammation within the lesions were predictive of dysplasia. The inter-observer variability of the score was moderate. The classification will be further refined based on the multivariate analysis and a prospective study is ongoing.

Table 1: Advanced endoscopic classification of IBD lesion

Morphology (mm): Polypoid/non polypoid

Paris Classification (Ip, Is, IIa, IIb, IIc, III)

Endoscopic inflammatory activity (within the lesion)

No ulcerations

Ulceration

Endoscopic inflammatory activity (surrounding area)

No ulcerations

Ulcerations

Demarcation

Yes

No

Colour of the lesion (relative to the background)

Paler

Same intensity

Darker

Surface architecture (tissue)

Roundish

Villous -regular

Villous - irregular

Irregular/non-structural

Vessel architecture

Non visible

Regular

Irregular

Disclosure of Interest: All authors have declared no conflicts of interest.

\section{Reference}

1. Laine L, Kaltenbach T, Barkun A, et al. SCENIC international consensus statement on surveillance and management of dysplasia in inflammatory bowel disease. Gastroenterology. 2015; 148:639-651.

\section{P0847 GENDER DIFFERENCES IN ACCEPTANCE OF COLORECTAL CANCER SCREENING: PAIN AS THE \\ EXPLANATION}

B. Kirkøen ${ }^{1}$, P. Berstad ${ }^{2}$, E. Botteri ${ }^{2}$, E. Dalén ${ }^{3}$, J.A. Nilsen ${ }^{4}$, G. Hoff ${ }^{1}$, T. De Lange $^{4}$, T. Bernklev ${ }^{5}$

${ }^{1}$ Cancer Registry of Norway, Oslo/Norway

${ }^{2}$ Cancer Registry of Norway, Oslo, Norway, Oslo/Norway

${ }^{3}$ Østfold Hospital Trust, Grålum/Norway

${ }^{4}$ Vestre Viken Hospital Trust, Barum Hospital, Drammen/Norway

${ }^{5} R \& d$, Vestfold Hospital Trust, Sandefjord/Norway

Contact E-mail Address: benedicte.kirkoen@kreftregisteret.no
Introduction: Participants' experience with a screening test can influence adherence, and therefore the efficacy of a screening programme. We compared the satisfaction with decision and willingness to repeat colorectal cancer screening with flexible sigmoidoscopy(FS) and faecal immunochemical test (FIT).

Aims \& Methods: In a prospective, randomised trial 3257 individuals (50-74 years) were invited to either FS or FIT(1:1) of whom $1650(52.6 \%)$ attended. In total, 1497 screening participants completed at least one questionnaire measuring willingness to repeat screening, recommend screening and satisfaction with decision to attend, either before screening, and/or three times in the following year. There were 769 and 728 responders in the FS and FIT group respectively. Of these FS participants, 581 participants also completed a pain-questionnaire. Results: One year after screening, $10 \%$ of the FS participants were not willing to repeat screening, compared to $5 \%$ of FIT participants, adjusted OR $0.47(95 \%$ CI: $0.31-0.72)$. In the FS group, there was a higher percentage of women who would not repeat screening, compared to men, adjusted OR 2.52 (95\% CI: 1.48 4.28 ). Notably, $22 \%$ of women reported pain during the FS, compared to $5 \%$ of the men. Both men and women who self-reported pain were statistically significantly less willing to attend FS screening again compared to participants who reported no pain. When we added pain to the model, pain was significantly associated with the unwillingness to repeat FS OR 3.15 (95\%CI: 1.68-5.87), while gender was no longer OR 1.53 (95\% CI: 0.82-2.88).

Conclusion: Acceptance for FS and FIT were high among Norwegian screening participants, though FIT participants were more willing to repeat screening. Women were less willing to repeat screening with FS compared to men. This gender difference seemed partly due to pain, and therefore preventable.

Disclosure of Interest: All authors have declared no conflicts of interest.

\section{P0848 THE ROLE OF PROBE CONFOCAL LASER ENDOMICROSCOPY WITH IMAGE ENHANCED ENDOSCOPY IN CHARACTERISATION AND ENDOSCOPIC RESECTION OF DYSPLASTIC LESIONS IN INFLAMMATORY BOWEL DISEASE PATIENTS \\ M. Iacucci $^{1}$, X. Gui ${ }^{2}$, S. Ghosh ${ }^{1}$ \\ ${ }^{1}$ University Of Birmingham, Institute of Translational Medicine, Birmingham/ United Kingdom \\ ${ }^{2}$ Department Of Pathology, University of Calgary, Calgary/Canada}

Contact E-mail Address: iacuccim@yahoo.it

Introduction: Detection, characterization and therapeutic management of flat dysplastic lesions during surveillance colonoscopy in inflammatory bowel disease (IBD) can be a challenge. The recent SCENIC consensus has introduced a new terminology and concept "endoscopically resectable" when the distinct margins of a detected lesion could be identified. New endoscopic techniques and skills are required to recognize the margins reliably and assess the surrounding mucosa to plan endoscopic removal successfully and organ sparing. We report our experience of the use of probe confocal endomicroscopy (pCLE) combined with selective electronic virtual (VCE) and dye chromoendoscopy (DCE) for management of challenging dysplastic lesions during surveillance in IBD.

Aims \& Methods: IBD patients underwent surveillance colonoscopy using highdefinition (HD)-iSCAN (Pentax EC-3940Fi; Japan) VCE and DCE in combination with pCLE (Cellvizio, Paris, France). pCLE was applied following IV injection of fluorescein $5 \% 10 \mathrm{ml}$ to assess the histological features of the lesion, the margins and the mucosa surrounding the visible colonic lesion. Biopsies eventually proved dysplasia or SSA of the colonic lesions. The study was approved by the Calgary Conjoint Health Services Research Ethics Board of the University of Calgary. All patients gave informed consent.

Results: Seven patients with IBD and disease duration of $\geq 8$ years and in clinical remission $\{$ Eaden, $2001 \# 2\}$ (mean age 55 years; 6 male, $U C=4 \mathrm{CD}=3$ ) were prospectively included. They underwent surveillance colonoscopy using HDiSCAN (Pentax EC-3940Fi; Japan). When a colonic lesion was detected, selective iSCAN -VCE was performed with or without DCE (five out of seven had DCE)

\section{Abstract No: P0848}

\begin{tabular}{|c|c|c|c|c|c|c|}
\hline \multirow{3}{*}{$\frac{\mathrm{UC} / \mathrm{CD}}{\text { Left sided UC }}$} & \multicolumn{3}{|c|}{ Endoscopic Findings } & \multirow{3}{*}{$\begin{array}{l}\text { Endomicroscopy Findings } \\
\text { Villiform appearance of the } \\
\text { crypts with stellar open- } \\
\text { ing. The colonic mucosa } \\
\text { surrounding the lesion } \\
\text { was normal. }\end{array}$} & \multirow{3}{*}{$\begin{array}{l}\text { Histology } \\
\text { SSA }\end{array}$} & \multirow{3}{*}{$\begin{array}{l}\text { Outcome } \\
\text { En-block EMR }\end{array}$} \\
\hline & Kudo Par & rder & & & & \\
\hline & IIO/IV & IIb Size $>2.5 \mathrm{~cm}$ & distinct & & & \\
\hline Crohn's colitis & IIIS/IIIL & IIb Size $>2.5 \mathrm{~cm}$ & indistinct & $\begin{array}{l}\text { Villiform elongated appear- } \\
\text { ance of the crypts with } \\
\text { dark epithelium, } \\
\text { decreased number of the }\end{array}$ & LGD & Surgical resection \\
\hline
\end{tabular}


Abstract No: P0848 Continued

\begin{tabular}{|c|c|c|c|c|c|c|}
\hline \multirow[b]{2}{*}{$\mathrm{UC} / \mathrm{CD}$} & \multicolumn{3}{|c|}{ Endoscopic Findings } & \multirow[b]{2}{*}{ Endomicroscopy Findings } & \multirow[b]{2}{*}{ Histology } & \multirow[b]{2}{*}{ Outcome } \\
\hline & Kudo Pa & rder & & & & \\
\hline $\begin{array}{l}\text { Ulcerative } \\
\text { pancolitis }\end{array}$ & IIIL/IV & Is Size $>2.5 \mathrm{~cm}$ & distinct & $\begin{array}{l}\text { goblet cells with leakage } \\
\text { of fluorescein and dis- } \\
\text { tortion of the crypts } \\
\text { around the lesion. } \\
\text { Villiform appearance of the } \\
\text { crypts with stellar open- } \\
\text { ing of the lumen of the } \\
\text { crypts. Areas of dark } \\
\text { epithelium with } \\
\text { decreased number of } \\
\text { goblet cells. } \\
\text { Surrounding mucosa } \\
\text { was normal. }\end{array}$ & SSA with focal LGD & En-block EMR \\
\hline Colonic Crohn's & $\mathrm{II} 0 / \mathrm{IV}$ & Is Size $>2.5 \mathrm{~cm}$ & distinct & $\begin{array}{l}\text { Villiform -elongated } \\
\text { appearance of the crypts } \\
\text { with stellar opening of } \\
\text { the lumen. The mucosa } \\
\text { surrounding the lesion } \\
\text { was normal. }\end{array}$ & SSA & En-block EMR \\
\hline Colonic Crohn's & IIIL/IV & IIb Size $>2.5 \mathrm{~cm}$ & indistinct & $\begin{array}{l}\text { Villiform- elongated } \\
\text { appearance of the crypts } \\
\text { with dark epithelium } \\
\text { and decreased number } \\
\text { of goblet cells. The sur- } \\
\text { rounding mucosa } \\
\text { showed irregular archi- } \\
\text { tecture of the crypts and } \\
\text { leakage of fluorescein. }\end{array}$ & LGD & Surgical resection \\
\hline $\begin{array}{l}\text { Ulcerative } \\
\text { Pancolitis }\end{array}$ & $\mathrm{IIO}$ & IS Size $>5 \mathrm{~mm}$ & distinct & $\begin{array}{l}\text { In the midst of psudopolyp } \\
\text { villiform appearance of } \\
\text { the crypts with stellar } \\
\text { opening of the lumen }\end{array}$ & SSA & En-block EMR \\
\hline $\begin{array}{l}\text { Ulcerative } \\
\text { Pancolitis }\end{array}$ & IIIL/IV & IIb Size $>2.5 \mathrm{~cm}$ & indistinct & $\begin{array}{l}\text { Villiform appearance of the } \\
\text { crypts with dark epithe- } \\
\text { lium and absence of } \\
\text { goblet cells. The mucosa } \\
\text { surrounding the lesions } \\
\text { had irregular architec- } \\
\text { ture of the crypts }\end{array}$ & HGD & Surgical resection \\
\hline
\end{tabular}

with methylene blue $1 \%$ to characterize the surface, vascular pit pattern and the margins of the lesion. Each of the 7 patients had non polypoid colonic lesions, 4 were sessile (Paris Is) and 3 flat (IIa/IIb). Four of them were amenable to endoscopic therapy and were successfully removed using endoscopic mucosal resection (EMR) en-block or piecemeal technique. Interestingly, one patient with multiple scattered 'pseudopolyps' had a $8 \mathrm{~mm}$ sessile pseudopolypoid lesion with a suspicious areas of SSA in the midst that was confirmed by real pCLE.
The endoscopic, endomicroscopic and histological findings of all the lesions were described in Table 1.

Conclusion: This case series highlights the first successful use of pCLE in combination with VCE and DCE to predict, characterise and treat colonic neoplasia in IBD. pCLE may be an additional tool to aid the endoscopist in therapeutic management by deciding endoscopic resectability versus colectomy.

Disclosure of Interest: All authors have declared no conflicts of interest. 


\section{P0849 THE SAFETY AND EFFECTIVENESS OF COLORECTAL ENDOSCOPIC SUBMUCOSAL DISSECTION USING A SCISSORS- TYPE KNIFE IN ELDERLY PATIENTS}

T. Kuwai ${ }^{1}$, T. Yamaguchi ${ }^{1}$, H. Imagawa ${ }^{1}$, S. Funaki ${ }^{1}$, K. Tao ${ }^{1}$, R. Miura ${ }^{1}$, T. Takasago ${ }^{1}$, Y. Sumida ${ }^{1}$, Y. Miyasako ${ }^{1}$, A. Yamaguchi ${ }^{1}$, H. Kouno $^{1}$, H. Kohno , S. Ishaq ${ }^{2}$

${ }^{1}$ Gastroenterology, Kure MC \& Chugoku CC, Kure/Japan

${ }^{2}$ Gastroenterology, Dudley Group Hospitals, Birmingham City University,

Birmingham/United Kingdom

Contact E-mail Address: toshiokuwai@gmail.com

Introduction: Endoscopic submucosal dissection (ESD) is one of the most useful methods for treating early colorectal neoplasms and conventionally utilizes an IT, hook, or needle knife. However, because these devices are used without fixation to target, it confers a potential risk of complications due to unexpected incision. To reduce the risk of complications from ESD performed using a conventional knife, we used a scissors-type knife (SB Knife Jr: Akita Sumitomo Bakelite, Japan) that allows keeping an adequate dissection layer and preventing unexpected muscular layer injury. In the previous study, we reported that ESD performed using SB Knife Jr is a technically efficient and safe method for treating early colorectal neoplasms. However, the efficacy and safety of colorectal ESD using SB Knife Jr in elderly patients remain unclear.

Aims \& Methods: The aims of our study were to evaluate the efficacy, safety, and clinical outcomes of colorectal ESD using SB Knife $\mathrm{Jr}$ in patients aged $\geq 75$ years in comparison with those in younger patients. We evaluated 291 colorectal lesions in 271 patients (male-to-female ratio, 148:123; median age, 70 years) treated with ESD using SB Knife Jr between October 2010 to March 2017 at Kure Medical Center and Chugoku Cancer Center. The patients were divided into two groups, an elderly group (group A: age, $>75$ years; 95 patients, 97 lesions) and a non-elderly group (group B: age, $<75$ years; 176 patients, 194 lesions). We evaluated the en bloc resection rate, complete resection rate, curative resection rate, resected tumor size, procedural time, complications, and long-term outcomes, including survival rate. The 3 -year overall survival and tumor-specific survival rates were analyzed in the entire study cohort, and the local and distant recurrence rates were analyzed in the cohort with curative resection and observationally managed with non-curative resection.

Results: The mean age was 80.0 years in group A and 64.3 years in group B. The male-to-female ratios were 45:50 and 103:73 in groups A and B, respectively. Regarding histopathological findings, the prevalence rates of tubular adenoma were $37.1 \%(36 / 97)$ and $36.1 \%(70 / 194)$; Tis, 39.2\% (38/97) and $44.8 \%(87 / 194)$; T1a, $10.3 \%(10 / 97)$ and $10.3 \%(20 / 194)$; and $\mathrm{T} 1 \mathrm{~b}, 13.4 \%(13 / 97)$ and $8.8 \%(17)$ 194 ) in groups A and B, respectively, showing no significant difference. The mean resected tumor size was $33.9+16.6 \mathrm{~mm}$ in group $\mathrm{A}$ and $34.7+15.2 \mathrm{~mm}$ in group $\mathrm{B}$, and the median procedure time was $75.6 \mathrm{~min}$ (range, $10-420 \mathrm{~min}$ ) in group A and $75 \mathrm{~min}$ (range, 10-533 min) in group B, showing no significant difference. The en bloc resection rates were $96.9 \%(94 / 97)$ and $99.0 \%(192 / 194)$; the complete resection rates, $94.8 \%$ (92/97) and $94.8 \%$ (184/194); and the curative resection rates, $83.5 \%(81 / 97)$ and $88.1 \%(171 / 194)$ in groups A and B, respectively, showing no significant difference. Regarding complications, no perforation during the procedure occurred in any of the cases. The delayed bleeding rate was $1.0 \%(1 / 97)$ in group A and 2.6\% (5/194) in group B. Delayed perforation and rectal stricture occurred in one patient each in group $\mathrm{A}$ and were treated conservatively. Regarding long-term outcomes, the local recurrence rate was $1.0 \%(1 / 97)$ in group A and $0.5 \%(1 / 194)$ in group B, and no distant recurrence was observed in the recurrence analysis cohort. Regarding survival analysis (mean follow-up period: group A, $523 \pm 469$ days; group B, $628 \pm 582$ days), the 3 -year overall and disease-specific survival rates were respectively $98.8 \%$ and $100 \%$ in group A, and $93.3 \%$ and $98.3 \%$ in group B. One patient $(0.5 \%, 1 / 194)$ died of colorectal cancer and 5 patients $(2.6 \%, 5 / 194)$ died of other diseases in group B, while one patient $(1.1 \%, 1 / 95)$ died of other diseases in group A.

Conclusion: ESD performed with SB Knife Jr. is a technically efficient and safe method associated with favorable long-term outcomes in cases of early colorectal neoplasms both in elderly and non-elderly patients.

Disclosure of Interest: All authors have declared no conflicts of interest.

P0850 QUALITY IN COLONOSCOPY; HAVE YOU REALLY GOT TO THE CAECUM?

S. Budihal

Department Of Endoscopy, Nottingham University Hospitals, Nottingham/United Kingdom

Contact E-mail Address: shivbudihal@yahoo.co.uk Introduction: Poor quality and incomplete colonoscopy is associated with missed diagnosis and failure to prevent interval cancers. ${ }^{1}$ Caecal intubation rate is the most frequently used Quality Indicator of colonoscopy. British Society of Gastroenterology guidelines recommend obtaining "clear images of caecal landmarks or terminal ileum" while the European and American guidelines suggest "auditable photo documentation of completion preferably a panoramic view of the ileocecal valve and caecum". In this retrospective study we aimed to assess colonoscopists' practice in photo documentation of colonoscopy completion.

Aims \& Methods: Colonoscopy reports for colonoscopies performed at an endoscopy site in a University Hospital over a period of three months from $01 / 01 /$ 2014 to 31/03/2014 were retrieved from the Trust's Endoscopy database. Photo documentation from the reports were then analysed for caecal landmarks and terminal ileum images.

Results: A total of 292 colonoscopies were performed by 21 endoscopists $\{5$ Colorectal Surgeons (24\%), 3 Nurse Endoscopists (14\%), 3 Specialist Registrar Gastroenterology (14\%) and 10 Consultant Gastroenterologist $(48 \%)\}$. Caecal intubation was achieved in 248 cases $(85 \%)$. In $8(3 \%)$ cases the anastomosis was reached. The ileo-caecal valve was photographed in $172(70 \%)$ cases, the appendix in $102(41 \%)$ cases, the caecum in $83(33 \%)$ cases and a panoramic view was achieved only in $43(17 \%$ ) patients. Terminal Ileum was intubated in 53 cases $(21 \%)$ and images were recorded in 25 cases $(40 \%)$. In the case of anastomosis, 7 images $(88 \%)$ of the anastomosis were obtained. In some of the reports images were labelled and in a number of reports no photos were recorded at all.

Conclusion: This study shows that photo documentation is poor and needs to be improved in order to adhere to national and international guidelines. Evidence of caecal intubation is imperative as it can protect against medicolegal implications $^{2}$. Obtaining clear images of caecal landmarks will ensure definite caecal intubation and efforts towards recording them will prompt the endoscopist to attain adequate mucosal views. In order to achieve excellence we suggest colonoscopists obtain images of all caecal landmarks including the ileum (when intubated), label photographs and where possible record video clips of caecal intubation.

Disclosure of Interest: All authors have declared no conflicts of interest.

\section{References}

1. Bressler B, Paszat LF, Chen Z, et al. Rates of new or missed colorecta cancers after colonoscopy and their risk factors: a population-based analysis. Gastroenterol 2007;132:96-102

2. Rex DK, Bond JH, Feld AD. Medical-legal risks of incident cancers after clearing colonoscopy. Am J Gastroenterol 2001;96:952-7

\section{P0851 DETECTION AND CHARACTERIZATION OF SSA/PS DURING SURVEILLANCE COLONOSCOPY IN LONG STANDING IBD USING ADVANCED ENDOSCOPIC TECHNIQUES}

M. Iacucci ${ }^{1}$, O. Akinola ${ }^{2}$, R. Panaccione ${ }^{2}$, G.G. Kaplan ${ }^{2}$, X. Gui ${ }^{3}$, S. Urbanski ${ }^{4}$, S. Ghosh ${ }^{1}$

${ }^{1}$ University Of Birmingham, Institute of Translational Medicine, Birmingham United Kingdom

${ }^{2}$ IBD Clinic. Division Of Gastroenterology, University Of Calgary, Calgary/Canada ${ }^{3}$ Department Of Pathology, University of Calgary, Calgary/Canada

${ }^{4}$ Dept. Of Pathology, Alberta Health Services, Calgary/Canada

\section{Contact E-mail Address: iacuccim@yahoo.it}

Introduction: Sessile Serrated polyps (SSA/Ps) are pre-malignant lesions that may lead to colorectal cancer in accelerated manner. These lesions are easily missed by endoscopists as these are difficult to detect in IBD patients. We aimed to assess the prevalence, detection rate and endoscopic findings of SSA/Ps in long standing IBD patients prospectively undergoing surveillance colonoscopy using dye (DCE) or virtual electronic chromoendoscopy (VCE) or high definition white light (HD-WLE) colonoscopy alone.

Aims \& Methods: A total of 270 randomized patients (55\% men; age range $20-77$ years, median age 49 years) with long-standing IBD (median duration of the disease 14 years) undergoing surveillance colonoscopy were assessed by HDWLE $(n=90)$, VCE $(n=90)$ or DCE $(n=90)$. Surveillance colonoscopy with High Definition (HD) alone, or with iSCAN VCE or DCE was performed. Endoscopic features were recorded in each group with regard to location, morphology (polypoid/non polypoid), size and mucosal pit pattern, and these were characterised using the Kudo modified classification and Paris classification. The histology was reported by modified Vienna classification.

Results: Thirty -three SSA/Ps were detected in 20 (11UC; 9 CD; 11 female, age range $34-72 \mathrm{y}$, median age 61 years) patients out of the 270 patients with IBD enrolled $(12.2 \%)$. The endoscopic features of SSA/P lesions were: non-polypoid appearance $(51.5 \%)$, predominant localization in the proximal colon (vs distal) $(87 \%), \geq 5 \mathrm{~mm}$ in size $(48.4 \%)$, Kudo pit pattern modified type IIO (79\%). Kudo pit pattern modified type IIO had a sensitivity of $79 \%$ and specificity of $82 \%$ for diagnosing SSA/Ps at surveillance colonoscopy in IBD patients. There was no difference in detection rates of SSA/P using HD-WLE, DCE or VCE.

Conclusion: SSA/Ps are not an infrequent finding at surveillance colonoscopy in IBD. There are prevalent in the right colon location and these generally have Kudo pit pattern of IIO. SSA/Ps can be recognized endoscopically by Kudo pit

\begin{tabular}{|c|c|c|c|c|c|c|c|c|c|c|c|}
\hline & \multirow{2}{*}{$\mathrm{F}$} & \multirow{2}{*}{ Age mean } & \multirow{2}{*}{$\mathrm{UC} / \mathrm{CD}$} & \multicolumn{4}{|c|}{ Localization\&Size } & \multicolumn{2}{|c|}{ Paris classification } & \multicolumn{2}{|c|}{ Kudo pit pattern } \\
\hline & & & & Right & Left & $<5 \mathrm{~mm}$ & $\geq 5 \mathrm{~mm}$ & $\mathrm{Is} / \mathrm{p}$ & $\mathrm{IIb} / \mathrm{IIa}$ & $\mathrm{I} / \mathrm{II}$ & IIO III-IV \\
\hline Serrated adenoma $n=33$ & 11 & 61 & $11 / 9$ & 29 & 4 & 16 & 17 & 16 & 17 & 1 & 266 \\
\hline
\end{tabular}


pattern even in IBD patients. Further studies are needed to evaluate the natural history and outcome of the SSA/Ps pathway lesions in IBD patients.

Disclosure of Interest: All authors have declared no conflicts of interest.

\section{P0852 IN VIVO HISTOLOGICAL PREDICTION OF COLORECTAL POLYPS USING FICE TECHNOLOGY}

J. Cortez-Pinto ${ }^{1}$, J. Moleiro ${ }^{1}$, I. Marques ${ }^{1}$, R. Barosa ${ }^{2}$, J. Castela ${ }^{1}$, J. Pereira Da Silva $^{3}$, S. Faias ${ }^{3}$, S. Mão De Ferro ${ }^{1}$, P. Lage ${ }^{3}$, A. Dias Pereira

${ }^{1}$ Gastroenterology, IPOLFG, EPE, Lisboa/Portugal

${ }^{2}$ Gastroenterology, Hospital Garcia de Orta, Almada/Portugal

${ }^{3}$ Gastroenterology, Instituto Português de Oncologia Francisco Gentil, Lisboa/ Portugal

\section{Contact E-mail Address: joao_cpinto@hotmail.com}

Introduction: The histological characterization of colorectal polyps using FICE (Fujinon Intelligent Color Enhancement) technology presents high diagnostic acuity. However, the excellent results in histological prediction are a reflection of the clinical practice by trained endoscopists, and their application remains to be confirmed outside this context.

Aims \& Methods: To evaluate the in vivo histological prediction acuity of colorectal polyps $<10 \mathrm{~mm}$ (hyperplastic polyps vs adenomas) in WLE (White light endoscopy) and using FICE technology, comparing both modalities. Prospective evaluation, using WLE and FICE, of colorectal polyps $<10 \mathrm{~mm}$ in patients submitted to colonoscopy between $12 / 2016$ and $02 / 2017$ by four inexperienced endoscopists in FICE, except for a previous 20-minutes interactive session. Polyps were evaluated using the FICE classification [tubular or oval crypts (adenoma), round crypts or featureless appearance (hyperplastic polyps), indicating their confidence level (low $<90 \%$ vs high $>90 \%$ ). Statistics: SPSS v23

Results: 25 polyps were included, with a mean size of $4.5 \mathrm{~mm}, 14$ adenomas, 10 hyperplastic and 1 serrated adenoma. From the global assessment of all polyps and observations, the use of the FICE classification for prediction of adenomatous histology obtained values of sensitivity, specificity, positive and negative predictive value identical to WLE $(100 \%, 62.5 \%, 60 \%$ and $100 \%$, respectively). Overall, diagnostic acuity in histological prediction was identical in both modalities $(76 \%)$. The individual acuity of the endoscopists ranged from $66 \%$ to $100 \%$. Higher confidence scores were not associated with a higher probability of correct classification, both in WLE (77\% vs. $75 \%)$ and FICE $(75 \%$ vs. $80 \%)$, $\mathrm{p}>0.05)$.

Conclusion: The use of FICE technology by inexperienced endoscopists in the histological prediction of colorectal polyps has no advantage over WLE, having both suboptimal acuities. The lack of recourse to magnification may have contributed to these results.

Disclosure of Interest: All authors have declared no conflicts of interest.

\section{P0853 POLYP DETECTION RATES IN COLONOSCOPIES PERFORMED UNDER GENERAL ANAESTHETIC COMPARED TO CONVENTIONAL SEDATION}

B. Perren, A. S. Bancil, A.G. Lim, P. Youd, S. J. Moodie, P. Patel Medicine, Epsom and St Helier University Hospitals, London/United Kingdom

Contact E-mail Address: ben.perren@doctors.org.uk

Introduction: Colonoscopies are performed under general anaesthesia (GA) for various reasons, but mainly due to previous procedures being poorly tolerated with normal sedation. During the procedure, the endoscopist must be aware of the patient's comfort levels and reactions to endoscopic manoeuvres. Polyp detection rate is a recognised indicator of quality of colonoscopy. It has been demonstrated that use of sedation increases the quality of colonoscopy ${ }^{[1]}$. We hypothesised that complete removal of the patient factor may increase the detection rate of polyps further.

Aims \& Methods: The study was undertaken retrospectively at a district general hospital. Results were analysed for all colonoscopies performed under GA between February 2016 and February 2017 to identify whether polyps had been detected, the number of polyps detected, and the site of polyp detection. Studies performed for surveillance of previously diagnosed Inflammatory Bowel Diseases (IBD) were excluded. The age and gender of each group was also analysed to identify possible confounding factors.

Results: 40 colonoscopies were performed under propofol sedation within the defined criteria; 40 colonoscopies performed using conventional sedation were randomly selected to use for comparison. A significant increase in rate of polyp detection was found in GA studies ( $45 \%$ vs non-GA studies $30 \%, p=0.0384)$. GA studies identified a mean of 1.275 polyps per study, compared to a mean of 0.325 polyps where conventional sedation was used $(p<0.0001)$. There was an insignificant $(p=0.175)$ difference in age between the GA $(59.7 \pm 18.2)$ and nonGA $(64.7 \pm 14.2)$. There was a significant $(\mathrm{p}=0.0005)$ difference in gender between groups; there was an even distribution of gender in the conventional pool, while the GA pool of patients was $77 \%$ (31:9) female.

Conclusion: Use of propofol sedation during colonoscopy provides a statistically significant increase to both the rate of detection of polyps, and the number of polyps identified when compared to procedures performed for similar indications using conventional sedation. This may be due to elimination of the patient factor. The implications of this are unclear and thus further larger comparison studies are needed. It is not practical for all colonoscopies to be performed under GA due to capacity issues, anaesthetic requirements and time allocated to lists. However, it may be a clinically effective tool for high-risk patients, such as those being screened for hereditary colonic malignancies or IBD patients. Disclosure of Interest: All authors have declared no conflicts of interest.

\section{Reference}

1. Technical Performance of Colonoscopy: The Key Role of Sedation/ Analgesia and Other Quality Indicators. Radaelli, Franco, et al. 2008, The American Journal of Gastroenterology, pp. 1122-1130.

\section{P0854 PATIENT AND PHYSICIANS RELATED FACTORS ASSOCIATED WITH A HIGH ADENOMA DETECTION RATE IN ROUTINE COLONOSCOPY}

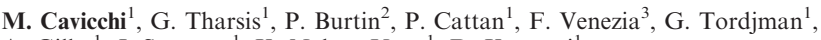
A. Gillet ${ }^{1}$, J. Samama ${ }^{1}$, K. Nahon-Uzan ${ }^{1}$, D. Karsenti ${ }^{1}$

${ }^{1}$ Pôle Digestif Paris Bercy, Clinique de Bercy, Charenton-le-Pont/France ${ }^{2} I G R$, Villejuif/France

${ }^{3}$ Digestive Endoscopy Unit, Clinique de Bercy, Charenton-le-Pont/France

Contact E-mail Address: m.cavicchi@wanadoo.fr

Introduction: Adenoma and polyp detection rates are correlated to the risk of interval colorectal cancer and is consequently considered as a quality benchmark for colonoscopy. We prospectively evaluated this variable in our daily practice, involving all the endoscopists of our endoscopy unit.

Aims \& Methods: 6027 colonoscopies were performed between 01/01/2016 and $31 / 12 / 2016$ by 30 physicians. Regarding patients, the following data were prospectively collected: age, gender, indication for colonoscopy, preparation procedure and quality of the preparation (assessed by the Boston Scale), number and size of polyps and polyp histopathology. Regarding physicians, age, gender, number of colonoscopies and mean withdrawal time (calculated from the normal colonoscopies) were studied. Neoplasia was defined as grade 4 or 5 of the Vienna classification (4: non-invasive high grade neoplasia (high grade adenoma/dysplasia, non-invasive carcinoma and suspicion of invasive carcinoma; 5 : invasive neoplasia (intramucosal carcinoma, submucosal carcinoma or beyond). Links between these data and polyp detection rate (PDR) were assessed by uniand multivariate analysis (stepwise logistic regression).

Results: We enrolled 2719 Male patients $(45.1 \%)$ and 3308 Female patients $(54.9 \%), 73.6 \%$ of them of 50 year-old or more. A sub-optimal preparation (defined by Boston scale score $<6$ or at least one sub-sore $<2$ ) was observed in $6.1 \%$ of the patients. Caecal intubation rate was $99 \% .21$ endoscopists were Male and 10 were under 50 year-old. The median number of colonoscopy per physician was 140 (range: 10-720). 2054 colonoscopies detected 3914 lesions or polyps: 2914 tubular/villous adenomas, 496 serrated adenomas, 242 hyperplastic polyps (hyperplastic polyps located in the rectum and sigmoid colon were not considered as at risk for cancer and were excluded), 212 other histology leading to a Mean Number of Polyps (MNP) of 0.65 and a Polyp Detection Rate (PDR) of $34.1 \% .1935$ coloscopies detected at least one adenoma (adenoma detection rate, $32.1 \%$ ). Large Polyp Detection Rate was $7.9 \%$ with detection of 538 polyps $>1(13.7 \%$ of polyps), in 477 patients. Neoplasia Detection Rate was 3.6\% (300 neoplasias in 220 patients). Among the endoscopists, the median PDR was $32.5 \%$ (range $14-62 \%$ ). Among them, 4 had a PDR $<20 \%, 17$ had a PDR between 20 and $39 \%$ and 9 had a PDR $>40 \%$. Mean withdrawal time was 490 seconds (range 228-831). 10 physicians had a mean withdrawal time $<420$ seconds with a mean ADR of $31 \%$ compared to $34.5 \%$ for physicians with a withdrawal time of more than 420 seconds $(p>0.05)$. In the univariate analysis, a high PDR was significantly associated with patient-dependant factors: age, Male gender, a familial history of polyp/cancer, screening or positive faecal immunochemical test (FIT+) and quality of preparation. Regarding physiciandependant factors, a high PDR was significantly associated with Male gender, high volume ( $>140$ colonoscopy per year) and withdrawal time. In the multivariate analysis, the only factors associated with a high PDR were: familial history of polyp/cancer, FIT+ and patient age.

Conclusion: In this large series of routine colonoscopies, we found medicallyrelevant polyps in more than one third of the patients, irrespectively of age and indications. In multivariate analysis, a high PDR was significantly associated with familial history of polyp/cancer, FIT + and age of the patient. This may suggest that the Male gender is no longer a risk factor for polyps. In addition, even if there are still discrepancies regarding PDR among physicians, we found no physician-dependant factor associated with a high PDR in the multivariate analysis.

Aknowledgment to all the endoscopists and nurses of the Clinique de Bercy. Disclosure of Interest: All authors have declared no conflicts of interest.

P0855 EFFICIENCY OF COLONOSCOPY IN CASE OF POSITIVE FECAL IMMUNOCHEMICAL TEST: ONE-YEAR EXPERIENCE AND RESULTS ON 391 PATIENTS IN ROUTINE PRACTICE IN FRANCE

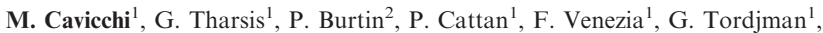
A. Gillet ${ }^{1}$, J. Samama ${ }^{1}$, K. Nahon-Uzan ${ }^{1}$, D. Karsenti ${ }^{1}$

${ }^{1}$ Pôle Digestif Paris Bercy, Clinique de Bercy, Charenton-le-Pont/France

${ }^{2} I G R$, Villejuif/France

Contact E-mail Address: m.cavicchi@wanadoo.fr

Introduction: Fecal immunochemical test (FIT) has progressively replaced the guaiac test for colorectal screening in average risk population in France since May 2015. With a high sensitivity and a good specificity, it is supposed to increase colonic cancer detection. However, its efficiency has not been described in routine colonoscopy.

Aims \& Methods: Among 6027 colonoscopies performed between 01/01/2016 and $31 / 12 / 2016$ in our endoscopy unit, 391 were performed for a positive FIT (FIT 
+ ). The following variables were prospectively collected: patient age and gender, quality of preparation (Boston scale), number, size and histopathology of polyps. Neoplasia was defined as categories 4 and 5 of the Vienna classification (highgrade dysplasia, in-situ carcinoma, intra-mucosal carcinoma, submucosal carcinoma or beyond). Comparison was made with the whole population of 3876 patients aged of 50 to 75 , referred for other reasons. Statistical analysis was performed in uni- and multivariate analysis using a stepwise logistic regression study.

Results: 391 patients with FIT + were included (6.4\% of the patients). 201 were Males and 190 were Female with a median age of 58 (range: 50-75). The preparation was considered as sub-optimal in $6.4 \%$ of cases (Boston scale score $<6$ or at least one sub-score $<2$ ). 498 lesions were detected in 230 patients. These lesions corresponded to 423 adenomas and adenocarcinomas, 41 serrated adenomas, 14 hyperplastic polyps (hyperplastic polyps located in the rectum and the sigmoid colon were not considered as lesions at risk and were therefore excluded from the analysis), 20 other pathology, leading to a mean number of polyps (MNP) of 1.27. Polyp detection rate (PDR) and adenoma detection rate (ADR) were $58.8 \%$ and $58 \%$, respectively. By comparison, PDR and ADR in the whole population were $39.1 \%$ and $37.0 \%$, respectively. From the 498 detected lesions, $122(24.5 \%)$ were $\geq 1 \mathrm{~cm}$ in 109 patients, resulting in a large polyp detection rate (LPDR) of $27.9 \%$ as compared to $9.4 \%$ in the whole population; 66 neoplastic lesions (13.2\% of the polyps) were detected in 57 patients, resulting in a neoplasia detection rate (NDR) of $14.6 \%$ as compared to $4.9 \%$ in the whole population. In Males, MNP, ADR and NDR were $1.52,67 \%$ and $18.9 \%$ respectively compared to $1,49.5 \%$ and $10 \%$ respectively in female patients. The multivariate analysis showed that FIT + was significantly associated with a high PDR (OR 2.8; 95\% CI 2.2-3.6), a high LPDR (OR 8.1; 95\% CI $5.4-12.3$ ) and a high NDR (OR $12.8 ; 95 \%$ CI 6.2-26.4).

Conclusion: In case of positive FIT, colonoscopy detects medically-relevant polyps in $59 \%$ of patients. Furthermore, the detection rates of large polyps and neoplastic lesions were very high, especially in Male patients, confirming the high predictive positive value of FIT compared to guaïac test. Our results are in agreement with the highest quality benchmarks recently proposed in case of positive FIT (et al., Am J Gastroenterol, 2016 Oct 11). Aknowledgment to all the endoscopists and nurses of the Clinique de Bercy.

Disclosure of Interest: All authors have declared no conflicts of interest.

\section{P0857 TWO LITERS OF POLYETHYLENE GLYCOL (PEG) WITH 15 MG OF BISACODYL VERSUS 4 LITERS OF PEG FOR BOWEL PREPARATION TO COLONOSCOPY. PROSPECTIVE RANDOMIZED STUDY. PRELIMINARY RESULTS}

E.M. Amine ${ }^{1}$, Z. Abdelkrim ${ }^{1}$, E.M. Aziz ${ }^{1}$, W. Khannoussi ${ }^{1}$, G. Kharrasse ${ }^{2}$, Z. Ismaili ${ }^{1}$

${ }^{1}$ Hepatogastroenterology, CHU Med VI Oujda, Oujda/Morocco

${ }^{2}$ Avicenne, Faculty of medicine Oujda, Oujda/Morocco

\section{Contact E-mail Address: mekamine@hotmail.com}

Introduction: Adequate bowel preparation is one of the most important quality factors of colonoscopy. Several formulations of bowel preparation have been evaluated for their ability to have a clean colon and be well tolerated by patients. Currently, PEG $4 \mathrm{~L}$ solution is the preferred method of bowel preparation to colonoscopy. This preparation has the disadvantage of being poorly tolerated by patients. Furthermore, recent studies have shown that a low -volume PEG solution (2L) associated with Bisacodyl for bowel cleansing is as effective and better tolerated as a large volume PEG (4L).

Aims \& Methods: This study aims to assess the efficacy and tolerance of the new regimen ( $2 \mathrm{~L}$ of PEG + Bisacodyl) compared to the classical regimen (4L of PEG). Materials and methods: A prospective comparative randomized study comparing the tolerance, acceptability and efficacy of a protocol A based on $4 \mathrm{~L}$ of PEG and a protocol $\mathrm{B}$ corresponding to $2 \mathrm{~L}$ of $\mathrm{PEG}+15 \mathrm{mg}$ of Bisacodyl. Using the Boston Bowel Préparation Scale (BBPS) by endoscopists, who did not know bowel preparation type, to evaluate the quality of preparation.

Results: Sixty-six patients were included (35 in group A and 31 in group B), with a sex ratio $=1$. The average age of patients was $52+/-15$ years [17-86 years] with a median of 51.5 years. Eight patients in the first group found the preparation difficult or moderately difficult vs 3 in group $B(p=N S)$ with mild to severe side effects $54 \%$ of group A and $29 \%$ of group B $(\mathrm{p}=0.03)$ and A mean side effect per patient was 2.26 vs. $1.89(\mathrm{p}=\mathrm{NS})$. Nausea and vomiting were respectively $(34 \%$ and $26 \%$ vs $19 \%$ and $13 \%)(\mathrm{p}=\mathrm{NS})$. However, 5 patients in group A had sleep disorders vs only one case in group B. Four patients who received 4 L of PEG had expressed their refusal to resume the same preparation if necessary while 2 patients in group B had refused. A score greater than or equal to 7 was recorded $60 \%$ versus $84 \%$ respectively $(\mathrm{p}=0.03)$. On average; the overall score obtained was 6.49 versus 7.32, respectively. This score (BBPS) tended to be better in protocol $\mathrm{B}$ than in protocol $\mathrm{A}$ in the right segment (2.55 vs 2.17), transverse colon (2.58 vs 2.43$)$ and left colon (2.19 vs 1.94$)$. This same trend was recorded in relation to the number of patients with a maximal score of $(9 / 9)$, with 4 patients in group B and no patient in group A. Seven patients refused to repeat the test even if indicated. This refusal was secondary to the preparation in 4 cases ( 2 cases of each group). Abdominal pain and post-endoscopic distension in both groups A and B were respectively $(62.8 \%$ and $65.7 \%$ vs $32 \%$ and $22.5 \%)(\mathrm{p}=0.016$ and $\mathrm{p}<0.001)$.

Conclusion: Preliminary results from our study suggest that the low-volume $2 \mathrm{~L}$ PEG with bisacodyl does improve patient tolerability with a tendency to be better for good Bowel preparation as compared to the traditional $4 \mathrm{~L}$ PEG. We continue our study to have a more significant number of patients view these encouraging results.

Disclosure of Interest: All authors have declared no conflicts of interest.

\section{P0858 COLONOSCOPY ON THE LEFT, RIGHT?}

I. Mocanu, A. Laranjo, S. Pires, N. Veloso, L. Gonçalves, R. Godinho, I. Medeiros

Gastroenterology, Hospital Espirito Santo de Évora, Évora/Portugal

Contact E-mail Address: irina.mocanu.24@gmail.com

Introduction: Colonoscopy is a cumbersome technique to train, with high variability of time spent to reach the cecum, depending on endoscopist experience, patient characteristics and type of colonoscope used. Recently, the ROLCOL study demonstrated an advantage in time and patient comfort during progression in right lateral position(RLP) when compared with traditional left lateral position (LLP).

Aims \& Methods: To compare time to reach cecum, patient and endoscopist comfort (using a visual analogue scale (VAS) in colonoscopies with progression on RLP and LLP. Prospective study, between January and April 2017, of colonoscopies under conscious sedation, randomly assigned to RLP and LLP. Olympus 190 series and Fuji EC-530 WI3 colonoscopes were used. Inclusion criteria: routine colonoscopies, patients $>18$ years of age, abdominal surgeries other than colonic, examinations done by residents in second and fifth year of training and specialists. Exclusion criteria: incomplete colonoscopies or prior history of colonic surgery.

Results: One hundred and eighty-eight colonoscopies (94 on each side) were included. There was no statistical difference in mean age (RLP:LLP $=61 \mathrm{Vs}$. 64 years; $p>0.05$ ), gender (49 Vs. 52 males; $p>0.05$ ), body mass index $(27.4$ Vs. 26.4; $p>0.05$ ), previous history of abdominal surgery (44 Vs. 34; $p>0.05$ ), endoscopists experience (residents: 53 Vs. 41, specialists: 41 Vs. 53 ; p >0.05), sedation dosage (midazolam 1.04 Vs. $1.09 \mathrm{mg}$, petidine 25 Vs. $25 \mathrm{mg}$; p >0.05) or colonic preparation (BBPS 6 Vs. 6,$01 ; \mathrm{p}>0.05$ ). Globally, there was no difference in "time to cecum" between the two positions (612 Vs. 633 seconds; $\mathrm{p}>0.05$ ), nor the patients comfort (VAS 3.92 Vs. 3.94; $p>0.05$ ). This did not change after exclusion of less experienced endoscopistś colonoscopies (581 Vs. 579 seconds, VAS 3, 71 Vs. 3.76; p > 0.05). Surgical and overweight patients did not benefit from any of the positions (time to cecum: 650 Vs. 702 and 570 vs 657; $\mathrm{p}>0.05$, comfort: 4.2 Vs. 4.12 and 3.83 Vs. 3.96; $p>0.05$, respectively). However, endoscopist́s comfort was higher in LLP (4.62 Vs. 3.78; $\mathrm{p}<0.05)$. Both groups required position change during progression or loop palpation by the nurse in equal percentage $(54 \%$ Vs. $46 \%$; p > 0.05 and $49.5 \%$ Vs. $50.5 \%$; $\mathrm{p}>0.05$ ). Additionally, there was no difference in time do cecum, patients or endoscopists comfort between the colonoscopies performed with Fuji or Olympus colonoscopes.

Conclusion: In our experience, progression in right lateral position did not show additional advantage over standard LLP in time to reach the cecum or patients comfort. We did not find any difference in time to progression or comfort between Olympus and Fuji colonoscopes.

Disclosure of Interest: All authors have declared no conflicts of interest.

\section{Reference}

"Right Or Left in COLonoscopy (ROLCOL)? A Randomized Controlled Trial of Right- versus Left-Sided Starting Position in Colonoscopy", 2015 Nov;110(11):1576-81. doi: 10.1038/ajg.2015.298. Epub 2015 Sep 29.

\section{P0859 NON-GRANULAR LATERALLY SPREADING COLONIC LESIONS $\geq 20 M M$ (LSL) ARE SMALLER AND MORE COMMONLY MULTIPLE THAN GRANULAR LSL; IMPLICATIONS OF A 'COLONIC MUCOSAL PHENOTYPE' FOR PRACTITIONERS OF ENDOSCOPIC RESECTION}

D. J. Tate ${ }^{1}$, H. Awadie ${ }^{1}$, L. Desomer ${ }^{1}$, M. Sidhu ${ }^{1}$, K. Goodrick ${ }^{1}$, N. G. Burgess ${ }^{1}$, M.J. Bourke ${ }^{2}$

${ }^{1}$ Department Of Gastroenterology And Hepatology, Westmead Hospital, Sydney/ Australia/NSW

${ }^{2}$ Westmead Clinical School, University of Sydney, Sydney/Australia/NSW

Contact E-mail Address: djtate@gmail.com

Introduction: The surface morphology of laterally spreading colonic lesions $\geq 20 \mathrm{~mm}$ (LSL) can be described as granular $(\mathrm{G})$ or non-granular (NG). NG lesions are more difficult to detect, harder to resect endoscopically and harbour a higher risk of submucosal invasive cancer (SMIC) than G lesions.

Aims \& Methods: We aimed to characterise the population of LSL in patients presenting for endoscopic mucosal resection (EMR). From 9/2008 until 03/2017 LSL were identified within a prospective observational cohort of patients undergoing endoscopic mucosal resection at a single academic tertiary centre. LSL were interrogated using high-definition white light (HDWL) at the time of EMR and the morphology of LSL were described along with patient, lesion and procedural factors. All LSL detected during the study period were eligible. LSL with mixed or indeterminate morphology were excluded. Serrated LSL were excluded. Lesions not attempted for EMR were excluded. Patients with multiple LSL were categorised according to the largest (dominant). 
Results: 1382 LSL in 1243 patients were analysed. 1155/1243 (92.9\%) patients had a solitary LSL. The majority of patients with multiple LSL had two $(77.3 \%)$ or three $(15.9 \%)$ lesions. $889 / 1382(64.3 \%)$ of LSL were G. G LSL were more likely to be solitary $(87.0 \%)$ than NG LSL $(77.5 \%, \mathrm{p}<.001)$. G LSL were more commonly large $(>40 \mathrm{~mm}$ in size) $(49.3 \%)$ than NG LSL $(26.0 \%, \mathrm{p}<.001)$ and were more commonly found in the right colon (proximal to transverse colon) $(54.2 \%$ versus $48.3 \%, \mathrm{p}=.034)$. In 88 patients with multiple LSL the dominant LSL was G (49/88 [55.7\%]). A dominant G LSL was associated with fewer other LSL than a dominant NG LSL, $\mathrm{p}=.029$.

Table 1; The morphology of the dominant (largest) laterally spreading lesion (LSL) predicts the presence and number of synchronous LSL. Morphology of the dominant lesion did not predict the others would be of the same morphology $(\mathrm{p}=.697)$. The dominant LSL was large in $43.2 \%$ of cases. Size of the dominant LSL predicted size of the other LSL $(\mathrm{p}<.001) .58 .0 \%$ of dominant LSL were located in the right colon. In $65.9 \%$ patients all LSL were in the same colonic segment; this was not predicted either by the location of the dominant LSL $(\mathrm{p}=.860)$ or its morphology $(\mathrm{p}=.228)$.

\begin{tabular}{|c|c|c|c|c|c|}
\hline Dominant LSL Morphology & \multicolumn{2}{|c|}{ Solitary $(n=1155)$} & \multicolumn{2}{|c|}{ Multiple $(\mathrm{n}=227)$} & $P$ \\
\hline Granular $(\mathrm{n}=889)$ & \multicolumn{2}{|l|}{$773(87.0)$} & \multicolumn{2}{|c|}{$116(13.0)$} & $<.001$ \\
\hline Non-granular $(\mathrm{n}=493)$ & \multicolumn{2}{|l|}{$382(77.5)$} & \multicolumn{2}{|c|}{$111(22.5)$} & \\
\hline \multirow[t]{2}{*}{ Dominant LSL Morphology } & \multicolumn{5}{|c|}{ Number of synchronous LSL } \\
\hline & 2 & 3 & 4 & 5 & $P$ \\
\hline Granular $(n=49)$ & $41(83.7)$ & $4(8.2)$ & $3(6.1)$ & $1(2.0)$ & .029 \\
\hline Non-granular $(\mathrm{n}=29)$ & $27(69.2)$ & $10(25.6)$ & 0 & $2(5.1)$ & \\
\hline
\end{tabular}

Conclusion: $7 \%$ of patients will have more than one LSL. In these patients the dominant lesion morphology predicts the presence and number of additional LSL (the Colonic Mucosal Phenotype). More than 20\% of NG LSL are associated with an additional LSL whereas G LSL are predominantly large and solitary. Practitioners of endoscopic resection should be aware that NG LSL may be multiple and ensure full examination of the colonic mucosal surface, particularly since they are higher risk for SMIC than G LSL.

Disclosure of Interest: All authors have declared no conflicts of interest.

\section{P0860 WIDE-FIELD PIECEMEAL COLD SNARE POLYPECTOMY OF LARGE SESSILE SERRATED POLYPS WITHOUT A SUBMUCOSAL INJECTION IS SAFE}

D. J. Tate, H. Awadie, F. Bahin, L. Desomer, R. Lee, S. Heitman, K. Goodrick, M.J. Bourke

Department Of Gastroenterology And Hepatology, Westmead Hospital, Sydney/ Australia/NSW

Contact E-mail Address: djtate@gmail.com

Introduction: Large series suggest endoscopic mucosal resection is safe and effective for the removal of large sessile serrated polyps $>10 \mathrm{~mm}$ (large SSP) but it exposes the patient to the risks of electrocautery, principally delayed bleeding. Aims \& Methods: We aimed to examine the feasibility and safety of piecemeal cold snare polypectomy (pCSP) for the resection of large SSP. Over 12 months sequential large SSP without endoscopic evidence of dysplasia referred to a tertiary endoscopy centre were considered for pCSP. The technique for pCSP was standardised. The lesion and its margins were assessed using high definition endoscopic imaging. Snare resection commenced at one margin including a 2 $3 \mathrm{~mm}$ rim of normal tissue. A thin-wire snare was used in all cases. Firm downward pressure and suction of luminal gas aided tissue capture. Subsequently the assistant closed the snare until resistance was felt, and then completely once the endoscopist was satisfied with the amount of captured tissue. If transection did not occur within five seconds gentle traction was exerted on the snare catheter against the tip of the colonoscope. If transection still did not occur, the snare placement was revised. The mucosal defect was then expanded with a flushing pump containing $0.9 \%$ saline. Further resections were then performed aligning the snare with the cut edge of the expanding mucosal defect. Once the resection was completed the mucosal defect was inspected for residual serrated tissue. If residual was detected further generous snare resection was performed. Oozing of blood from the resection site was common and was not actively treated. Submucosal injection was not performed. High-definition imaging of the defect margin was used to ensure the absence of residual serrated tissue. Adverse events were assessed at 2 weeks and surveillance was planned between 6 and 12 months. Results: 41 SSP were completely removed by pCSP in 34 patients. 7 patients had two lesions removed. The median size of SSP was $15 \mathrm{~mm}$ (IQR 14.5-20), range $10-35 \mathrm{~mm}$. The median duration of procedures was 4.5 minutes (IQR 1.4-6.3). There was no evidence of perforation or significant intra-procedural bleeding. There were no significant adverse events at 2 week follow up including delayed bleeding and post polypectomy syndrome. $8 / 41$ lesions underwent first follow-up at median 6 months with no evidence of recurrence.
Table 1: Baseline characteristics and outcomes of the 34 patients and 41 SSP that underwent piecemeal cold snare polypectomy (pCSP). IQR - interquartile range, $\mathrm{SCl}$ - first surveillance colonoscopy.

$\mathrm{pCSP}(\mathrm{n}=41)$

Patient

Age, mean (standard deviation)

$68.58(10.12)$

Sex, female $(\%)$

27 (79.4)

Lesion

Size, median (IQR)

$15(14.5-20)$

Location, proximal to transverse colon $(\%)$

$26(63.4)$

Paris classification $(\%)$

0 -IIa

40 (97.6)

0 -IIb

$1(2.4)$

Endoscopic evidence of dysplasia (\%)

Kudo, highest $(\%)$

II-O

Procedure

Duration, median minutes (IQR)

$4.5(1.4$ to 6.3$)$

Pieces, median (IQR)

$3.0(3-5)$

Protrusion within defect $(\%)$

$9(22.0)$

$0(0)$

Intra-procedural bleeding requiring intervention (\%)

$41(100)$

3 (7.3)

Low grade cytological dysplasia $(\%)$

$\mathrm{pCSP}(\mathrm{n}=41)$

Adverse events

Clinically significant post endoscopic bleeding (\%)

Delayed perforation $(\%)$

$0(0)$

Post procedural pain (\%)

Admission to hospital for related complication within 2 weeks $0(0)$

Follow up

Months to SC1, IQR

$6(5-7)$

Recurrence at $\mathrm{SC} 1,(\%), \mathrm{n}=8$

Histologic recurrence at $\mathrm{SC} 1,(\%), \mathrm{n}=5$

$0(0)$

Conclusion: There is potential for pCSP to become the standard of care for nondysplastic large SSP. This may reduce the burden on patients and healthcare systems of removing SSP, particularly by avoidance of delayed bleeding. Disclosure of Interest: All authors have declared no conflicts of interest.

\section{P0861 THE PROSPECTIVE OBSERVATION STUDY FOR OVER 10MM COLORECTAL LESIONS ENDOSCOPICALLY RESECTED USING BIPOLAR SNARE}

H. Nakamura, H. Ikematsu, N. Okamoto, R. Ito, D. Sato, T. Minamide, Y. Yamamoto, K. Takashima, T. Hombu, K. Nakajo, T. Kadota, Y. Yoda, K. Hori, Y. Oono, T. Yano

Gastroenterology And Endoscopy, National Cancer Center Hospital East, Kashiwa/Japan

Contact E-mail Address: hirosnak@east.ncc.go.jp

Introduction: Polypectomy of adenomas reduces death due to colorectal cancer; therefore colonoscopy is the gold standard to detect and treat adenomatous lesions. Most adenomatous lesions are less than $20 \mathrm{~mm}$ in size. Therefore, these are not indication for endoscopic submucosal dissection (ESD). Recently, there are some reports about cold snare polypectomy (CSP). CSP is effective and easy to remove lesions of less than $5-10 \mathrm{~mm}$ in size. On the other hand, in over $10 \mathrm{~mm}$ lesions, many endoscopists would remove it by endoscopic mucosal resection (EMR) with monopolar snare. It is expected that the bipolar snare would decrease the incidence of perforation because of electric current flow peculiar to bipolar snare that does not flow through the wall of colon. So, by using bipolar snare, hot snare polypectomy (HSP) that can be easily resected in a short time may be safely performed for over $10 \mathrm{~mm}$ colorectal lesions. However, there is no report about them.

Aims \& Methods: We aimed to clarify removal method, procedure time and complications for over $10 \mathrm{~mm}$ colorectal lesions endoscopically resected using bipolar snare. Consecutive patients with over $10 \mathrm{~mm}$ colorectal lesions endoscopically resected using bipolar snare in National Cancer Center Hospital East between September 2016 and March 2017 were enrolled in this study, prospectively. The removal method rate of these lesions, each procedure time, complete resection rate, bleeding rate and perforation rate, and pathological finding were assessed.

Results: A total 92 lesions in 67 patients were analyzed. 47 patients $(70 \%)$ were male, and the median age was 67 years (range: 44-88). The median lesion size was $13 \mathrm{~mm}$ (range: $10-30)$. The macroscopic type was $33(36 \%)$ polypoid lesions and $59(64 \%)$ flat lesions. The location was $55(60 \%)$ lesions in right colon, $31(34 \%)$ 
in left colon and $6(6 \%)$ in rectum. Pathological diagnosis was $22(24 \%)$ hyperplastic polyp or SSA/P, $42(46 \%)$ low grade dysplasia (LGD), $23(26 \%)$ high grade dysplasia (HGD), and $1(1 \%)$ submucosal invasive cancer. In the removal methods, HSP was $71(77 \%)$ lesions and EMR was $21(23 \%)$. The median procedure time of HSP and EMR was 37 seconds (range: 7-430) and 167 (range: $60-450)(\mathrm{p}<0.001)$. The median lesion size of was HSP and EMR was $12 \mathrm{~mm}$ (range: $10-30)$ and 20 (range: $10-28)(\mathrm{p}<0.001)$. The immediate bleeding of HSP and EMR occurred in $7(10 \%)$ lesions and $6(33 \%)(\mathrm{p}=0.009)$. The delayed bleeding of HSP and EMR occurred in $2(3 \%)$ lesions and 0 $(\mathrm{p}=0.437)$. Perforation was not occurred. No tumors were horizontal and vertical margin positive. In the pathological diagnosis, $86 \%$ of hyperplastic polyp or SSA $/ \mathrm{P}, 86 \%$ of LGD, and $57 \%$ of HGD was resected by HSP, and submucosal invasive cancer was resected by EMR.

Conclusion: Of over $10 \mathrm{~mm}$ colorectal lesions was resected by using bipolar snare, $77 \%$ were resected by HSP. The procedure time of HSP was significant shorter than EMR.

Disclosure of Interest: All authors have declared no conflicts of interest.

\section{P0862 THE SMSA POLYP SCORE RELIABLY PREDICTS ROBUST ENDPOINTS OF ENDOSCOPIC MUCOSAL RESECTION OF COLORECTAL LATERALLY SPREADING LESIONS}

M. Sidhu ${ }^{1}$, D. J. Tate ${ }^{1}$, L. Desomer ${ }^{2}$, G. Brown ${ }^{3}$, A. Moss ${ }^{4}$, S. Raftopoulos ${ }^{5}$, S. Zanati ${ }^{3}$, R. Singh ${ }^{6}$, S. Williams ${ }^{2}$, E. Lee ${ }^{1}$, M.J. Bourke ${ }^{7}$

${ }^{1}$ Westmead Hospital, Westmead/Australia

${ }^{2}$ Westmead Hospital, Sydney/Australia

${ }^{3}$ The Alfred Hospital, Melbourne/Australia

${ }^{4}$ Footscray Hospital, Melbourne/Australia

${ }^{5}$ Sir Charles Gairdner Hospital, Perth/Australia

${ }^{6}$ Lyell McEwin Hospital, Adelaide/Australia

${ }^{7}$ University of Sydney, Sydney/Australia

Contact E-mail Address: drmsidhu16@gmail.com

Introduction: The 'SMSA' polyp scoring system is a method of risk stratifying the difficulty of polypectomy based on expert consensus opinion. The score is simple, intuitive and has previously not been evaluated in a large multicentre setting.

Aims \& Methods: We aimed to determine the ability of the SMSA polyp score to predict robust endpoints after endoscopic mucosal resection (EMR) of colonic laterally spreading lesions (LSL). The SMSA polyp score was applied to a prospectively collected multicentre database of LSL resected by EMR over eight years. This score describes the complexity of polypectomy with respect to four major domains (table 2) and is subsequently divided into four levels. Standard inject and resect EMR procedures were performed with detailed patient, procedural and outcome data recorded prospectively over the study period including all features of the SMSA. In patients who had multiple lesions resected the largest lesion was retained for analysis. The primary endpoints were correlation of SMSA score with completion rate, adverse event rate and adenoma recurrence. Results: 2305 lesions in 2305 patients $(47.4 \% \mathrm{M}, 45,2 \%$ right colon) underwent EMR. The majority of lesions were SMSA $4(50.2 \%)$ with a median lesion size of $30 \mathrm{~mm}$ (range $20-160 \mathrm{~mm}$ ). Failed single session EMR occurred in $97(4.2 \%)$ and this was predicted by increasing SMSA $(\mathrm{p}<.001)$. Intra-procedural bleeding was significantly more common with increasing SMSA (SMSA 2, 19/229 (8.3\%) versus SMSA $4291 / 1158(25.1 \%), p<.001)$. Clinically significant post EMR bleeding (CSPEB) was more common as SMSA increased with $4(1.7 \%)$ in the SMSA 2 group and $90(7.8 \%)$ in the SMSA 4 group, $p=0.001$. Intra-procedural perforation and delayed perforation were no different between the groups. After EMR surgery at two weeks was more common in the SMSA 4 group $(\mathrm{p}<.001)$. Of those patients that underwent their first surveillance colonoscopy (SC1), endoscopic recurrence (EDR) was more common in the SMSA 4 group than the SMSA 2 group, 206 (23.7) as compared to $9(5.4 \%), \mathrm{p}<.001$. This was also the case for histologic recurrence $(\mathrm{p}<.001)$. The difference in EDR persisted to the second surveillance colonoscopy (SC2) with no recurrences in the SMSA 2 group versus $9.1 \%$ in the SMSA 4 group.

Table 1: Outcomes after endoscopic mucosal resection at the initial procedure, 2 weeks and subsequent surveillance procedures.

\begin{tabular}{lllll}
\hline & SMSA 2 & SMSA 3 & SMSA 4 & p value \\
\hline Total & $229(9.9)$ & $918(39.8)$ & $1158(50.2)$ & \\
Successful EMR (\%) & $226(98.7)$ & $894(97.4)$ & $1088(94.0)$ & $<.001$ \\
Duration - min (median IQR) & $10(5-15)$ & $15(10-20)$ & $30(20-45)$ & $<.001$ \\
IPB (\%) & $19(8.3)$ & $115(12.5)$ & $291(25.1)$ & $<.001$ \\
Deep injury * (\%) & $11(4.8)$ & $33(3.6)$ & $54(4.7)$ & .448 \\
CSPEB (\%) & $4(1.7)$ & $46(5.0)$ & $90(7.8)$ & .001 \\
Delayed Perforation (\%) & $0(0)$ & $2(0.2)$ & $5(0.4)$ & .640 \\
Surgery at 2w (\%) & $20(8.7)$ & $50(5.4)$ & $117(10.1)$ & .001 \\
Underwent SC1 (n) & 166 & 685 & 871 & \\
EDR SC1 (\%) & $9(5.4)$ & $71(10.4)$ & $206(23.7)$ & $<.001$ \\
HDR SC1 (\%) & $3(6.4)$ & $36(13.4)$ & $120(28.7)$ & $<.001$ \\
Surgery SC1 (\%) & $1(0.6)$ & $7(1.0)$ & $12(1.4)$ & .747 \\
Underwent SC2 (n) & 88 & 326 & 462 & \\
EDR SC2 (\%) & $0(0)$ & $21(6.4)$ & $42(9.1)$ & .008 \\
Surgery SC2 (\%) & $0(0)$ & $1(0.3)$ & $3(0.6)$ & .766 \\
\hline
\end{tabular}

EMR - endoscopic mucosal resection, IPB - intra-procedural bleeding, IPP intraprocedural perforation, CSPEB - clinically significant post endoscopic bleeding (bleeding after EMR requiring admission to hospital or re-intervention), $2 \mathrm{w}$ - two weeks, SC1/2 - surveillance colonoscopy $1 / 2$. * target sign or actual hole corresponding to DMI type III/IV - Sydney Classification (1)

\begin{tabular}{llllllll}
\hline \multicolumn{1}{l}{ SMSA score (2) } & \multicolumn{1}{l}{$l$} & & & \\
\hline Size & Points & Morphology & Points & Access & Points & Site & Points \\
$<1 \mathrm{~cm}$ & 1 & Pedunculated & 1 & Easy & 1 & Left & 1 \\
$1-1.9 \mathrm{~cm}$ & 3 & Sessile & 2 & Difficult & 3 & Right & 2 \\
$2-2.9 \mathrm{~cm}$ & 5 & Flat & 3 & & & & \\
$3-3.9 \mathrm{~cm}$ & 7 & & & & & & \\
$>4 \mathrm{~cm}$ & 9 & & & & & & \\
SMSA Level & & Points & & & & & \\
1 & & $4-5$ & & & & \\
2 & & $6-9$ & & & & \\
3 & & $9-12$ & & & & \\
4 & & & & & & & \\
\hline
\end{tabular}

Conclusion: SMSA is a simple readily applicable clinical score that identifies a subgroup of patients who are at increased risk of EMR related complications including CSPEB and recurrence. This information is useful for planning EMR lists with respect to time and resource allocation. Moreover SMSA could have a major impact on training, both in identifying appropriate training cases and providing an objective benchmark against which to assess the progress of trainees in EMR.

Disclosure of Interest: All authors have declared no conflicts of interest.

\section{References}

1. Burgess NG, Bassan MS, McLeod D, Williams SJ, Byth K, Bourke MJ. Deep mural injury and perforation after colonic endoscopic mucosal resection: a new classification and analysis of risk factors. Gut. 2016 Jul 27;: gutjnl-2015-309848.

2. Gupta S, Miskovic D, Bhandari P, Dolwani S, McKaig B, Pullan R, et al. A novel method for determining the difficulty of colonoscopic polypectomy. Frontline Gastroenterol. BMJ Publishing Group Ltd; 2013 Oct 1;4(4):244-8.

\section{P0863 USE OF ACETIC ACID FOR EVALUATING SESSILE SERRATED ADENOMA/POLYP: A PILOT STUDY}

S. Yamamoto, M. Shafazand

Gastroenterology, Sahlgrenska University Hospital Östra, Göteborg/Sweden

Contact E-mail Address: shun0515suke@gmail.com

Introduction: Sessile serrated adenoma/polyp (SSA/P) has been accumulated increasing attention since its risk for developing to cancer had been clarified. These polyps are difficult not only to detect but also to determine their precise margin after detection especially in right side colon. Such a difficulty leads high recurrence rate after endoscopic resection. Magnifying function and narrow band imaging (NBI) is reported to be useful for evaluation of SSA/P but it needs special equipment, extra time, and expertise. Easier, uncomplicated, and non time consuming method is desired. The use of acetic acid or acetic acid-indigo carmine mixture have been introduced into endoscopic diagnosis in Barrett's esophagus, early gastric cancer, and colorectal early cancer. However, there have been no reports on using this agent as aid for the optimal diagnosis of the margin of SSA/P. If this rather cheap agent were helpful for realizing the precise margin of SSA/P, it could decrease insufficient removal of the polyp and recurrence after that.

Aims \& Methods: The aim of this pilot study is to assess whether the acetic acid could facilitate the recognition of the margin of SSA/P. We used acetic acid as a mixture with indigo carmine and compared it to conventional evaluating methods; narrow band imaging (NBI) and indigo carmine. From December 2016 to February 2017, patients in whom SSA/P more than $10 \mathrm{~mm}$ were found in right side colon in daily practical colonoscopy by single endoscopist in our institute were included. We used the standard scope without magnifying function. First we observed lesions with conventional white light and NBI. Second, we recorded pictures with indigo carmine (IC) spray alone on it. Finally, we sprayed the mixture of acetic acid and indigo carmine mixture (AIC) directly through the endoscopic working channel without using catheter onto lesion. Using recorded pictures during these procedures, ability for recognizing the margin of polyps were compared between IC and AIC, or NBI and AIC by 3 endoscopists, and the concordance rate of diagnosis among these three were assessed as Kappa statistics.

Results: $9 \mathrm{SSA} / \mathrm{P}$ lesions in 7 patients were investigated. In all cases, AIC was helpful to recognize the margin of polyps without causing any obstructive effect. We also observed disappearance of mucous on the surface, dilated crypts caused by acid contact, and aceto-whitish reaction on the surficial glands in all lesions. In comparing the ability for recognizing the margin of the lesions, 6.3 in 9 lesions (mean among 3 practitioners) were thought to be as AIC better than IC, and 
similarly 7.3 in 9 were better than NBI. Kappa value among participants was 4.8; moderate agreement $(\mathrm{p}=0.0016)$. All polyps were removed endoscopically after evaluation. All lesions were histologically diagnosed as SSA/P without dysplasia. Conclusion: Acetic acid was useful and promising to facilitate the endoscopic recognition of the precise margin of SSA/P in right side colon. Strength of this method is that it is very simple and needs no special equipment nor skill.

Disclosure of Interest: All authors have declared no conflicts of interest.

\section{References}

1. Heiko Pohl, et al. Incomplete polyp resection during colonoscopy-Results of the complete adenoma resection study. Gastroenterology. 2013, 144, 74-80.

2. Ijspeert JE, et al. Prevalence, distribution and risk of sessile serrated adenomas/polyps at a center with a high adenoma detection rate and experienced pathologists. Endoscopy. 2016, 48, 740-6.

\section{P0864 AUTOLOGOUS BLOOD, A NOVEL AGENT FOR PREOPERATIVE COLONIC LOCALIZATION: A SAFETY AND EFFICACY COMPARISON STUDY}

E.J. Kim ${ }^{1}$, J. Chung ${ }^{1}$, S. Kim ${ }^{1}$, J.H. Kim ${ }^{1}$, Y.J. Kim ${ }^{1}$, K.O. Kim ${ }^{1}$, K.A. Kwon ${ }^{1}$, D.K. Park ${ }^{1}$, S.W. Park ${ }^{2}$, J. Baek ${ }^{2}$

${ }^{1}$ Department Of Internal Medicine, Gachon University Gil Medical Center,

Incheon/Korea, Republic of

${ }^{2}$ Department Of Surgery, Gachon University Gil Medical Center, Incheon/Korea, Republic of

\section{Contact E-mail Address: kkoimge $@$ naver.com}

Introduction: Preoperative localization or tattooing is essential for minimally invasive surgery. Although preoperative endoscopic tattooing using India ink or indocyanine green is widely used, clinical evidence and safety profile supporting the use of these agents is lacking.

Aims \& Methods: We assessed the efficacy and safety of preoperative endoscopic tattooing using autologous blood. A total of 80 patients who underwent endoscopic tattooing using either India ink or autologous blood were included in this study. From February 2016, all patients who required localization of a target lesion before colorectal surgery underwent endoscopic tattooing using autologous blood at a single tertiary medical center, and the outcomes were collected prospectively. As a comparison, we retrospectively reviewed the medical records of a further 40 consecutive patients who underwent endoscopic tattooing using India ink before February 2016. The primary outcomes were the visibility of the tattooing in the peritoneal cavity and related adverse events.

Results: Endoscopic tattoos produced using India ink were visible in 38 (95\%) patients, and tattoos created using autologous blood were visible in $36(90 \%)$ patients. In the autologous blood group, the tattoo could not be identified in four patients due to excessive peritoneal fat, bleeding tendency, congenital anomaly, and tattooing to an inadequate depth. Eight $(20 \%)$ patients in the India ink group and four patients $(10 \%)$ in the autologous blood group experienced endoscopic tattooing-related adverse events.

Conclusion: Preoperative endoscopic tattooing using autologous blood is a feasible and safe modality for the preoperative localization of colorectal lesions. Disclosure of Interest: All authors have declared no conflicts of interest.

\section{P0865 SUBMUCOSAL INVASION IN COLORECTAL LATERALLY SPREADING TUMORS (LST) AND ABILITY OF THE ENDOSCOPIST FOR CANCER DETECTION}

H. Soliman ${ }^{1}$, B. Brieau ${ }^{2}$, M. Guillaumot ${ }^{2}$, S. Leblanc ${ }^{2}$, R. Coriat ${ }^{2}$, F. Prat ${ }^{3}$, S. Chaussade ${ }^{4}$

${ }^{1}$ Gastro-enterology, Hopital Saint Antoine, Paris/France

${ }^{2}$ Department Of Gastroenterology, Cochin Hospital, Assistance Publique-Hôpitaux de Paris, Paris/France

${ }^{3}$ Hopital Cochin Dept. of Gastroenterology, Paris/France

${ }^{4}$ Service De Gastro-entérologie, CHU Cochin Dept. de Gastroenterologie, Paris/ France

Contact E-mail Address: heithemsoliman90@hotmail.com

Introduction: Laterally spreading tumors (LSTs) are defined as lesions $>10 \mathrm{~mm}$ with a low vertical axis and lateral extension. They are separated in 2 groups with 2 subclasses for each of them: granular LST (LST-G) with, or without large nodule; and non-granular LST (LST-NG), separated into flat lesions (IIa) and depressed lesions (IIa + IIc). Every subclass have been associated with a proper risk of cancer and submucosal invasion $(\mathrm{T} 1 \mathrm{sm})^{1,2}$. Knowing this aspect could help for the decision of the resection technique (endoscopic mucosectomy EMR, endoscopic submucosal dissection ESD, or surgery). The aim of our study was to determine the rate of cancer (submucosal and mucosal adenocarcinoma) in a western series of LST treated by endoscopic resection, and to evaluate the ability of the endoscopist to predict the depth of cancer invasion.

Aims \& Methods: The entire patients with a LST $\geq 20 \mathrm{~mm}$ treated between January 2012 and December 2016 in our single center were included. Endoscopic data were collected (size, location, LST classification, analysis of pit pattern, endoscopic suspicion of cancer). We also reported the resection technique, histological results, and the follow-up at 1 year.

Results: 377 LST were included in our study. The average age was 67.7 years old. The mean size of lesion was $40.6 \mathrm{~mm}$. LST were located in the right colon, the rectum, the left colon and the transverse colon in $44.5 \%, 32.6 \%, 14.0 \%$ and $8.8 \%$, respectively. The resection technique used was a monobloc EMR in
$15.4 \%$, piecemeal EMR (pEMR) in $42.9 \%$, ESD in $27.3 \%$ and assisted ESD in $14.5 \%$. ESD was associated with a significant lower risk of recurrence after 1 year $(4.9 \%$ against $18.1 \%)$. Considering the LST classification, there were $27.0 \%$ LST-G without large nodule, $28.4 \%$ LST-G with large nodule, 35.5\% flat LST-NG IIa, and 9.0\% LST-NG with depression IIa + IIc. The overall rate of adenocarcinoma was $19.7 \%$, and $9.0 \%$ with submucosal invasion. The rates of adenocarcinoma and the rates of submucosal invasion in every subtype of LST are reported in table 1 . They were higher for LST-G with large nodule $(34.5 \%$ and $15.9 \%$ respectively) and for LST-NG with depression $(35.3 \%$ and $20.6 \%)$. Regarding the prediction of submucosal cancer by the endoscopist, we report a low sensitivity, and positive predictive value (respectively $64.7 \%$ and $32.8 \%$ ). However, we had a good specificity and negative predictive value $(86.0 \%$ and $96.0 \%)$. Endoscopic predictors of submucosal cancer were invasive pit pattern $($ HR 33.0; $\mathrm{p}=5.78 \mathrm{e}-07)$ and depression $(\mathrm{HR}=11.86 ; \mathrm{p}=0.049)$.

\begin{tabular}{lllll}
\hline Rate of adenocarcinoma and submucosal invasion for each type of LST \\
\hline & LST G & $\begin{array}{l}\text { LST-G with } \\
\text { large nodule }\end{array}$ & $\begin{array}{l}\text { LST-NG } \\
\text { type IIa }\end{array}$ & $\begin{array}{l}\text { LST-NG } \\
\text { IIa + IIc }\end{array}$ \\
\hline Number (n/\%) & $102 / 27,0$ & $107 / 28,4$ & $134 / 35,5$ & $34 / 9,0$ \\
Cancer $(\mathrm{n} / \%)$ & $12 / 11,8$ & $37 / 34,5$ & $11 / 8,2$ & $12 / 35,3$ \\
T1sm (n/\%) & $5 / 4,9$ & $17 / 15,9$ & $4 / 3,0$ & $8 / 20,6$ \\
\hline
\end{tabular}

Conclusion: Our western series confirm similar rates of submucosal adenocarcinoma according to the type of LST as compared to Asian series. LST-G with large nodule, and LST-NG with depression were associated with a higher risk of submucosal invasion and invasive pit pattern was the stronger predictor of malignancy. Endoscopic submucosal dissection should be systematically performed in these cases.

Disclosure of Interest: All authors have declared no conflicts of interest.

\section{References}

1. Kudo and al., Gastrointestinal endoscopy, 2008

2. Facciorusso and al., World Journal of Gastroenterology, 2015

\section{P0866 ETHNIC VARIATION OF COLONIC POLYPS: FINDINGS FROM AN INTERNATIONAL HOSPITAL FOR MEDICAL TOURISM IN THAILAND}

S. Techapaitoon ${ }^{1}$, V. Permpoon ${ }^{1}$, K. Pongpirul ${ }^{2}$

${ }_{1}^{1}$ Digestive Disease Center, Bumrungrad International Hospital, Bangkok/Thailand ${ }^{2}$ Department Of International Health, Johns Hopkins Bloomberg School of Public Health, Baltimore/United States of America/MD

Contact E-mail Address: kpongpi1@jh.edu

Introduction: Evidence on an international variation of pathological types and anatomical distribution of colonic polyps is beneficial for early detection and management but limited.

Aims \& Methods: To characterize differences in colonoscopy findings by ethnicity, a random sample of patients aged at least 50 years without colonic symptoms or history of colonic diseases who underwent colonoscopy were reviewed. Of 26,508 subjects, 2651 were randomly selected. Of 1300 subjects who met the inclusion criteria, abnormal findings were identified in 878 cases $(67.54 \%)$, of which 452 cases had 940 polyps and 7 cancer lesions were found in 6 cases. Of 452 patients with polyps, half had only one polyp (53.76\%) and were Asian (54.65\%), followed by Caucasian $(26.99 \%)$, Middle Eastern $(15.71 \%)$, and other ethnic origins $(2.65 \%)$ (Figure). Ethnicity-specific polyp prevalence were $36.26 \%$, $38.05 \%, 27.24 \%$, and $34.15 \%$, respectively. Polyps of Caucasian subjects tended to be smaller $(4.52 \mathrm{~mm})$ and locate on the left side of the colon $(65.63 \%)$ than that of other ethnicities $(44.44 \%-60.53 \%)$. The majority of the polyps $(84.04 \%)$ were small $(<=5 \mathrm{~mm})$ whereas $8.19 \%$ were large $(>=10 \mathrm{~mm})$; hyperplastic polyp, tubular adenoma (TA), and tubulovillous (TVA) adenoma were identified in $43.19 \%, 53.83 \%$, and $2.34 \%$, respectively. Premalignant (TA + TVA) polyps were found in $56.08 \%, 50.19 \%$, and $64.23 \%$ of the polyps of Asian, Caucasian, and Middle Eastern patients, respectively. Premalignant lesions were found in $52.91 \%$ of small polyps.

Conclusion: The findings suggested that number, size, distribution, and pathological type of colonic polyps vary across ethnic groups. As more than half of small polyps were a tubular adenoma, we propose that polyps of all sizes should be removed when feasible.

Disclosure of Interest: All authors have declared no conflicts of interest. 
P0867 ARE WE READY FOR COLONIC ESD IN FRANCE?

J. Jacques ${ }^{1}$, J. Albouys ${ }^{1}$, A. Charissoux ${ }^{2}$, J. Rivory ${ }^{3}$, D. Sautereau ${ }^{1}$, T. Ponchon ${ }^{4}$, R. Legros ${ }^{1}$, M. Pioche ${ }^{5}$

${ }^{1}$ Hepato-gastro-enterology, CHU Limoges - Hepato-Gastro-Enterology, $\mathrm{CHU}$

Limoges; Limoges/FR, Limoges/France

${ }^{2}$ Pathology, CHU Limoges, Limoges/France

${ }^{3}$ Hepatogastroenterology, Hopital Edouard Herriot, Lyon/France

${ }^{4}$ Dept. Of Digestive Diseases, Edouard Herriot University Hospital, Lyon/France ${ }^{5}$ Hepatogastreoenterology, Hopital Edouard Herriot, Lyon/France

Contact E-mail Address: jeremiejacques@gmail.com

Introduction: Endoscopic submucosal dissection represents the standard of care for large superficial colo-rectal neoplasms in Japan. In Europe, only few studies reported promising results, essentially in the rectal location. Colonic ESD is more technically challenging because of the colonic loops, intestinal motility, the folded anatomy, problems caused by inconstant gravity, and submucosal fibrosis. Colonic ESD is although more risky because perforations are most often non clinically significant in the rectal location (under peritoneal reflection) contrary to the colonic location. Here we reported our results of two years of colonic ESD performed by two French expert teams that began colonic ESD after a strong animal training and a strong experience in rectal and upper-digestive tract ESD. Aims \& Methods: Retrospective bicentre study of all cases of colonic ESD performed between 01/2016 and 03/2017 for superficial pre-cancerous or cancerous neoplasms. Primary Endpoint was to evaluate the En bloc, R0, curative resection rate and extended curative resection rate (Curative resection + non-curative due to positive horizontal margins and without recurrent disease on endoscopic control). Secondary endpoints were to compare these results with results of rectal ESDs performed during the same period.

Results: 87 colonic ESD were performed in two French centers between 01/2015 and $03 / 2017$ for superficial pre-cancerous or cancerous neoplasms. During the same period 93 rectal ESDs were performed for superficial pre-cancerous or cancerous neoplasms. Descriptive results: male $54(61 \%)$, mean size of the specimen: $49 \mathrm{~mm}$, mean duration of procedure $125.1 \mathrm{~min}$, mean speed of ESD: 18.9 $\mathrm{mm}^{2} /$ min, perforation rate: $9(10.3 \%)$, post procedural bleeding rate: $2.3 \%$, secondary surgery $13(15.3 \%)(3(23 \%)$ for a perforation; $10(77 \%)$ for a failure or pathological risk factor or lymph node metastasis) Colonic location: $26(30 \%)$ sigmoid, $8(9.2 \%)$ left colon, $6(6.9 \%)$ splenic flexure, $9(10.3 \%)$ transverse colon, $10(11.5 \%)$ hepatic flexure, $14(16.1 \%)$ right colon, $14(16.1 \%)$ caecum Pathological analysis: low grade dysplasia in $24.7 \%$, high grade dysplasia in $35.8 \%$, intra-mucosal carcinoma in $19.8 \%$, superficial submucosal carcinoma in $7.5 \%$, deep submucosal carcinoma in $8.6 \%$ and $\mathrm{T} 2$ adenocarcinoma in $3.7 \%$. Only 2 patients $(3.1 \%)$ had a recurrent disease during endoscopic follow up. Primary Endpoint: En bloc resection: $87.4 \%$, R0 resection: $73.5 \%$, curative resection: $63.8 \%$, extended curative resection: $85.7 \%$ Secondary Endpoint: No statistically significant parameters have been found between rectal and colonic ESD (Table 1).

\begin{tabular}{llll}
\hline & RECTUM $\mathrm{n}=87$ & COLON $\mathrm{n}=93$ & $\mathrm{p}$ \\
\hline Age $(\mathrm{Y})$ & 66.9 & 66.9 & 0.99 \\
Duration (min) & 124 & 125 & 0.93 \\
Large diameter (mm) & 54.6 & 49 & 0.03 \\
Small diameter (mm) & 41.6 & 38 & 0.17 \\
Surface (mm2) & 1948 & 1937.5 & 0.95 \\
Speed (mm2/min) & 20.1 & 18.9 & 0.6 \\
Exclusive ESD & $96 \%$ & $92 \%$ & 0.09 \\
Monobloc resection & $94.40 \%$ & $87.40 \%$ & 0.09 \\
R0 resection & $74.10 \%$ & $73.50 \%$ & 0.73 \\
Curative resection & $68.50 \%$ & $63.90 \%$ & 0.87 \\
Extended curative resection & $91.40 \%$ & $85.80 \%$ & 0.3 \\
Perforation & $7.70 \%$ & $10.30 \%$ & 0.6 \\
Post ESD bleeding & $6.60 \%$ & $2.30 \%$ & 0.28 \\
Pathological analysis & & & 0.76 \\
$\quad$ DBG & $24.80 \%$ & $24.70 \%$ & \\
DHG & $33.70 \%$ & $35.80 \%$ & \\
Intramucosal ADK & $27 \%$ & $19.80 \%$ & \\
sm < 1000 ADK & $3.40 \%$ & $7.50 \%$ & \\
sm $>1000$ ADK & $9 \%$ & $8.60 \%$ & \\
T2 ADK & $2.20 \%$ & $3.70 \%$ & \\
secondary surgery & $8(8.8 \%)$ & $13(15.3 \%)$ & 0.16 \\
Perforation & $0(0 \%)$ & $3(23 \%)$ & \\
failure & $2(25 \%)$ & $6(46.1 \%)$ & \\
Pathological reason & $6(75 \%)$ & $4(30.8 \%)$ & \\
Recurrence & $0(0 \%)$ & $2(3.1 \%)$ & 0.7 \\
\hline
\end{tabular}

Conclusion: Colonic ESD could be perfomed with similar results than rectal ESD in French expert teams with prior strong experience in animal ESDs and rectal ESDs. It allows en bloc resection of large superficial colorectal neoplasms with a very low risk of recurrence. RCTs comparing colonic ESD to piece-meal EMR are needed to determinate the appropriate place of each technique in Europe. Disclosure of Interest: All authors have declared no conflicts of interest.
P0868 RISK OF COLORECTAL NEOPLASM IN PATIENTS WITH ACROMEGALY -A CASE-CONTROL STUDY

Y. Ochiai ${ }^{1}$, T. Iizuka ${ }^{1}$, N. Inoshita ${ }^{1}$, S. Yamada ${ }^{2}$, S. Hoteya ${ }^{1}$

${ }^{1}$ Dept. Of Gastroenterology, Toranomon Hospital Dept. of Gastroenterology, Tokyo/Japan

${ }^{2}$ Dept. Of Hypothalamic And Pituitary Surgery, Toranomon Hospital Dept. of Gastroenterology, Tokyo/Japan

Contact E-mail Address: y.ochiai.1987@gmail.com

Introduction: It is well known that acromegalics have been at an increased risk of colorectal neoplasm. However, there has been few reports of them in Japanese patients with acromegaly. In this study, we attempted to elucidate the risk of colorectal neoplasm in Japanese patients with acromegaly comparing with healthy controlled patients.

Aims \& Methods: Between April 2008 and September 2016, a total of 745 patients were underwent Hardy operation in our institute. Among them, a total of 178 patients were performed colonoscopy in our hospital during perioperative period and were enrolled in the case group. In contrast, a total of 356 patients were selected randomly from those who were performed colonoscopy in our medical check-up in the same period by means of being matched to cases of the age and gender. The incidence, size, location and histology of colorectal neoplasm were investigated.

Results: As background, 84 patients $(47.2 \%)$ were men and 94 patients were women, and the median age was 47.5 years old (18 to 75$)$ in the case group. The median height/weight was $165.5 \mathrm{~cm} / 65.1 \mathrm{~kg}$ in the case group and it was significantly larger than the control group $(163.4 \mathrm{~cm} / 60.1 \mathrm{~kg})(p=0.038$, $p<0.001$ respectively). The median Body Mass Index (BMI) were 23.4 in the case group and 22.5 in the control group. There was a significant difference between two groups $(p<0.001)$. The frequency of colorectal neoplasm was $66.8 \%$ (119/178 patients) in the case group and was significantly higher than control group of $24.2 \%(86 / 356$ patients $)(p<0.001)$. The average number and size of neoplasm were 2.44 and $4.74 \mathrm{~mm}$ in the case group and 1.77 and $3.89 \mathrm{~mm}$ in the control group. There was a significant difference between two groups $(p=0.001)$. The distribution of neoplasm in the case group vs in the control group was shown $12.6 \%$ vs $12.8 \%$ in cecum, $30.3 \%$ vs $36 \%$ in ascending colon, $23.5 \%$ vs $30.2 \%$ in transverse colon, $15.1 \%$ vs $10.5 \%$ in descending colon, $66.4 \%$ vs $40.7 \%$ in sigmoid solon, and $31.9 \%$ vs $19.8 \%$ in rectum. The incidence of neoplasm in sigmorectal region had a significant difference in two groups (the case group $80.7 \%$ vs the control group $53.5 \%)(p<0.001)$. In terms of neoplasm larger than $5 \mathrm{~mm}$, the frequency was $34.3 \%$ (61/178 patients) in the case group and $7.6 \%(27 / 356$ patients $)$ in the control group $(p<0.001)$. In addition, as for neoplasm larger than $10 \mathrm{~mm}$, that was $15.2 \%(27 / 178$ patients $)$ and $2.2 \%(8 / 356$ patients) respectively $(p<0.001)$. The number of neoplasm resected by endoscopy was 71 lesions $(24.5 \%$ of all) in the case group and 25 lesions $(16.4 \%)$ in the control group. The pathological examination in the case and control group showed eight and one had hyperplastic polyps, 2 and zero had sessile serrated adenoma/polyp, 3 and zero had inflammatory polyp, 48 and 19 had low to moderate grade adenomas, 4 and 3 had High grade adenomas, one and zero had carcinoid, and 5 and 2 had carcinoma respectively. There were no significant differences between two groups in the limited cases of high grade adenoma and carcinoma.

Conclusion: This study showed the acromegalic patients had an increased risk of colorectal neoplasm especially, in the sigmorectal region. It is important to be performed colonoscopy at the diagnosis of acromegaly.

Disclosure of Interest: All authors have declared no conflicts of interest.

\section{P0869 COMPARISON OF WHITE LIGHT COLONOSCOPY AND A NOVEL ROBOTIC COLONOSCOPE IN THE ASSESSMENT OF ULCERATIVE COLITIS}

S. Pallotta ${ }^{1}$, E. Tumino ${ }^{2}$, G. Manes ${ }^{3}$, S. Ardizzone ${ }^{4}$, R. Sacco ${ }^{5}$, F. Iannuzzi ${ }^{1}$, R. De Franchis ${ }^{6}$

${ }^{1}$ Digestive Endoscopy, Luigi Sacco Hospital, Milan/Italy

${ }^{2}$ A.O.U. Pisana UO Gastroenterologia, Pisa/Italy

${ }^{3}$ Asst Rhodense, Garbagnate Milanese/Italy

${ }^{4}$ Department Of Biomedical And Clinical Sciences, Università degli Studi di Milano, Milano/Italy

${ }^{5}$ Division of Gastroenterology and Metabolic Disease, University Hospital of Pisa, PisalItaly

${ }^{6}$ Medicine, Istituto di Ricovero e Cura a Carattere Scientifico, Milano/Italy

Contact E-mail Address: rasmusklump1974@gmail.com

Introduction: Colonoscopy in ulcerative colitis (UC) is performed as first diagnosis and during screening for dysplasia and disease flares. It is an invasive procedure with a burden of discomfort and possible complications. To overcome discomfort and complications due to colonoscopy an Italian high-tech start-up (Endotics, Peccioli, Italy) developed a soft, self-propelled, disposable robotic colonoscope (R), approved with CE mark.

Aims \& Methods: We wanted to compare diagnostic performance and tolerability of $\mathrm{R}$ with those of standard white light colonscope (S) in patients with ulcerative colitis. Consecutive patients referred for colonoscopy to our endoscopy departments with clinically mild to moderate ulcerative colitis that signed the informed consent for both procedures were studied first with $\mathrm{R}$ and then with $\mathrm{S}$ (Olympus $\mathrm{CF}-145$ ), by two different operators, blinded to previous observations. $\mathrm{R}$ had the following technical specifications: $17 \mathrm{~mm}$ outer diameter, rest position length probe $30 \mathrm{~cm}$; maximum length of probe $54 \mathrm{~cm}$; working length $210 \mathrm{~cm}$; NTSC CMOS analog camera. Conscious sedation with midazolam was administered as 
needed. We recorded endoscopic activity according to Baron criteria, time to reach the caecum, sedation, patient's pain/discomfort and operator's difficulty. Results: We studied 12 patients $(7 \mathrm{M} / 5 \mathrm{~F})$, mean age 41 yrs and disease duration 5.33 yrs. 53 colonic segments out of the 54 evaluated had the same assessment of disease activity (absent $=0$ points, mild $=1 \mathrm{pt}$, moderate $=2 \mathrm{pts}$ and severe $=3$ pts) with $\mathrm{R}$ and $\mathrm{S}$. Mean activity score with $\mathrm{R}$ was $0.35 \mathrm{pts}$ (SD 0.60) and $0.33 \mathrm{pts}$ (SD 0.60) with S, without significant difference. The caecum was reached in $11 / 12$ cases by $\mathrm{S}$ in an average of $29.42 \mathrm{~min}$ (SD 28.94), and in $10 / 12$ cases by $\mathrm{R}$, in an average of $46.67 \mathrm{~min}$ (SD $24.98 \mathrm{~min}$ ), with a mean difference of $17,25 \mathrm{~min}$., not statistically significant. Incomplete colon explorations with R clustered in Milan, probably because of smaller experience. An average of 1.45 (SD 0.79) mg of midazolam were used during S while 0.41 (SD 0.38) mg during R. Mean pain/ discomfort on a $0-10$ scale was 2.08 (SD 1.67) for R and 4.17 (SD 1.74) for S, with a statistically significant difference $(\mathrm{p}=0.066)$ favouring $\mathrm{R}$. Mean perceived operator's difficulty on a $0-10$ scale was 4.44 (SD 1.78) for R, and 4.08 (SD 1.44) for $\mathrm{S}$, with a mean difference of 0.42 pts favouring $\mathrm{S}$, not statistically significant. Conclusion: $\mathrm{R}$ appears to be a promising method for disease staging in patients with ulcerative colitis, because of comparable accuracy and reduction in pain and discomfort. A tool channel to obtain biopsies and perform therapeutic endoscopy, together with images of higher definition (CMOS digital Camera HD ready) and virtual chromo-endoscopy, useful for follow-up and screening for dysplasia in patients with long duration of disease are now available in the latest version of R. The "column" connected to the latest version of the robot is the size of a portable suitcase, and suitable for remote operation. Further studies with newer versions of $\mathrm{R}$ are needed to assess the role of this technology from an economic point of view and in special settings like failed screening colonoscopies, dysplastic lesions in UC, bed-side colonoscopy, colonoscopy in rural areas.

Disclosure of Interest: All authors have declared no conflicts of interest.

\section{Reference}

Cosentino F, Tumino E, Passoni GR, Morandi E, Capria A. Int J Artif Organs. 2009 Aug;32(8):517-27.

\section{P0870 WHAT IS THE CONCORDANCE FOR THE DIAGNOSIS OF LATERALLY SPREADING-TYPE LESIONS (LST) AMONGST WESTERN AND JAPANESE EXPERT ENDOSCOPISTS?}

B. Manorahan ${ }^{1}$, A. Jimenez ${ }^{2}$, S. Sansone ${ }^{1}$, M. Yamada ${ }^{3}$, M. San Juan ${ }^{1}$, N. Gonzalez ${ }^{4}$, E. Albéniz Arbizu ${ }^{5}$, M. A. Bianco 6 , R. Bisschops ${ }^{7}$, K. Homma ${ }^{8}$, H. Ikematsu' ${ }^{9}$, Y. Morita ${ }^{10}$, T. Uraoka ${ }^{11}$, K. Ragunath ${ }^{12}$, A. Parra-Blanco ${ }^{1}$ ${ }^{1}$ Gastroenterology, NIHR Nottingham Digestive Diseases Biomedical Research Unit, Nottingham University Hospitals NHS Trust, Nottingham, Nottingham/ United Kingdom

${ }^{2}$ Research Unit, University Hospital of the Canary Islands, Tenerife/Spain ${ }^{3}$ Endoscopy Division, National Cancer Center Hospital, Tokyo, Tokyo/Japan ${ }^{4}$ Gastroenterology "Henry Cohen", Montevideo Clinic Hospital, Montevideo/ Uruguay

${ }^{5}$ Servicio Aparato Digestivo, Complejo Hospitalario de Navarra, Pamplona/Spain ${ }^{6}$ Gastroenterology, Hospital Maresca, Torre del Greco/Italy

${ }^{7}$ Gastroenterology, Katholieke Universiteit Leuven, Leuven/Belgium

${ }^{8}$ Therapeutic Endoscopy, Nihonkai General Hospital, Sakata/Japan

${ }^{9}$ Endoscopy Division, National Cancer Center Hospital East, Kashiwa/Japan

${ }^{10}$ Division Of Gastroenterology, Department Of Internal Medicine, Graduate School of Medicine, Kobe University, Kobe/Japan

${ }^{11}$ Gastroenterology, National Hospital Organization Tokyo Medical Center, Tokyo/Japan

${ }^{12}$ Endoscopy \& Gastroenterologist Wolfson Digestive Diseases Centre, Oueens Medical Centre Campus Nottingham University Hospitals, Nottingham/United Kingdom

Contact E-mail Address: mzybm3@nottingham.ac.uk

Introduction: The LST classification and Paris classification systems are internationally used to describe polyp morphology. Differences between Japanese and Western endoscopists in the use of classification systems, have been observed. We aimed to evaluate the inter-observer agreement of LST classification amongst Western \& Japanese experts.

Aims \& Methods: A total of 40 endoscopic video clips depicting LSTs $(10 \%$ malignant) were by 6 expert endoscopists; 3 from Japan and 3 from the West. Assessments included LST classification (LST-G homogeneous, LST-G mixed, LST-NG flat, LST-NG pseudodepressed), Paris classification, invasiveness, treatment suggestion and mean size of lesion. We calculated the interobserver agreement with weighted kappa and Chi square.

Results: Japanese endoscopists diagnosed more lesions as LST-G than Western $(62.7$ vs. $45.4 \%)$, Western diagnosed more LST-NG than Japanese (54.6 vs $37.3 \% ; \mathrm{p}=0.007)$. The interobserver agreement of the LST classification among the six experts was good with a weighted Kappa of 0.61 (IC 95\% 0.430.78 ) for Japanese, and moderate at 0.45 (IC95\% $0.27-0.64$ ) for Western. Difference in concordance between the two cohorts was not statistically significant $(\mathrm{p}=0.22)$. When only two categories were considered (LST-G vs NG), agreement was very good for Japanese (weighted Kappa of $0.81 ; 95 \%$ CI $0.65-0.97)$ and good for Western endoscopists $(0.65$; 95\% CI $0.46-0.85)$ Again, difference in concordance was not statistically significant $(\mathrm{p}=0.22)$. Piecemeal Endoscopic Resection was suggested in 34, 7\% cases by Western, but never by Japanese endoscopists, whereas Endoscopic Submucosal Dissection was recommended in $50.4 \%$ and $16.1 \%$ cases by Japanese and Western experts respectively $(\mathrm{p}<0.0001)$.
Conclusion: This study is the first to validate the LST classification system comparing East and West. There were significant differences in the types of LST diagnosed, and concordance was good in the Japanese cohort and moderate in the Western, but not significantly different. The recommendations for treatment were also different. We suggest a modification of the classification system to enable a more unanimous diagnosis and therapeutic strategy.

Disclosure of Interest: All authors have declared no conflicts of interest.

\section{References}

Kudo, S., Kashida, H., Tamura, T., Kogure, E., Imai, Y., Yamano, H. \& Hart, A. R. 2000. Colonoscopic diagnosis and management of nonpolypoid early colorectal cancer. World J Surg, 24, 1081-90.

Uraoka, T., Saito, Y., Matsuda, T., Ikehara, H., Gotoda, T., Saito, D. \& Fujii, T. 2006. Endoscopic indications for endoscopic mucosal resection of laterally spreading tumours in the colorectum. Gut, 55, 1592-7.

Yamada, M., Saito, Y., Sakamoto, T., Nakajima, T., Kushima, R., ParraBlanco, A. \& Matsuda, T. 2016. Endoscopic predictors of deep submucosal invasion in colorectal laterally spreading tumors. Endoscopy, 48, 456-64.

Van Doorn, S. C., Hazewinkel, Y., East, J. E., Van Leerdam, M. E., Rastogi, A., Pellisé, M., Sanduleanu-Dascalescu, S., Bastiaansen, B. A., Fockens, P. \& Dekker, E. 2015. Polyp morphology: an interobserver evaluation for the Paris classification among international experts. Am J Gastroenterol, 110, 180-7.

Shigita, K., Oka, S., Tanaka, S., Sumimoto, K., Hirano, D., Tamaru, Y., Ninomiya, Y., Asayama, N., Hayashi, N., Nagata, S., Arihiro, K. \& Chayama, K. 2016. Clinical significance and validity of the subclassification for colorectal laterally spreading tumor granular type. J Gastroenterol Hepatol, $31,973-9$.

P0871 CLINICAL USEFULNESS OF THE SMSA DIFFICULTY SCORE AND COMPARISON WITH A SUBJECTIVE SCORE FOR THE MANAGEMENT OF LARGE NON-PEDUNCULATED COLORECTAL LESIONS. A MULTICENTER STUDY FROM THE SPANISH ENDOSCOPY SOCIETY ENDOSCOPIC RESECTION GROUP

E. Albéniz Arbizu ${ }^{1}$, M. Fraile González², C. Guarner Argente ${ }^{3}$, D. MartínezAres $^{4}$, B. Ibañez Beroiz ${ }^{5}$, A. Herreros De Tejada ${ }^{6}$, J. Santiago ${ }^{6}$, M.A. Alvarez ${ }^{7}$ X. Bessa Casserras ${ }^{7}$, M. Rullan ${ }^{8}$, O. Nogales Rincón ${ }^{9}$, F. Ramos-Zabala ${ }^{10}$, E. Valdivielso Cortazar ${ }^{11}$, R. Pardeiro Pertega ${ }^{11}$, P. Alonso ${ }^{11}, J_{\text {. Cobian }}{ }^{12}$, F. Mugica ${ }^{12}$, C.J. Gargallo Puyuelo ${ }^{13}$, A. Elosua ${ }^{8}$, J. De La Peña ${ }^{14}$, J.G. Martinez-Cara ${ }^{15}$, E. Redondo Cerezo ${ }^{16}$, M. Pellisé Urquiza ${ }^{17}$, L. Rivero Sánchez $^{18}$, A. Huerta ${ }^{19}$, J. Gonzalez Santiago ${ }^{20}$, A. Alvarez Delgado ${ }^{21}$, J. Espinós ${ }^{22}$, A. Repiso Ortega ${ }^{23}$, F.J. Navajas ${ }^{23}$, J. Carbo Perseguer ${ }^{24}$, J. Merlo ${ }^{25}$, M. Rodriguez Tellez ${ }^{26}$, V.A. Jimenez-Garcia ${ }^{26}$, F. Sabado ${ }^{27}$,

E. Saperas Franch ${ }^{28}$, P. Huelin Alvarez ${ }^{3}$, M. Concepcion-Martin ${ }^{3}$, E. Spanish Endoscopy Society Endoscopic Resection Group Members ${ }^{29}$

${ }^{1}$ Servicio Aparato Digestivo, Complejo Hospitalario de Navarra, Pamplona/Spain ${ }^{2}$ Hospital San Pedro, Logroño/Spain

${ }^{3}$ Servei De Patologia Digestiva, Hospital de la Santa Creu i Sant Pau, Barcelona/ Spain

${ }^{4}$ Complejo Hospitalario Universitario de Vigo, Vigo/Spain

${ }^{5}$ Navarrabiomed, Pamplona/Spain

${ }^{6}$ Hospital Puerta de Hierro, Madrid/Spain

${ }^{7}$ Hospital del Mar, Barcelona/Spain

${ }^{8}$ Complejo Hospitalario de Navarra, Pamplona/Spain

${ }^{9}$ Hospital Gregorio Marañon Dept. de Endoscopia Digestiva, Madrid/Spain

${ }^{10}$ Servicio De Aparato Digestivo, Hospital Universitario HM Montepríncipe, Madrid/Spain

${ }^{11}$ H Juan Canalejo, Coruña/Spain

${ }^{12}$ Hospital Universitario Donostia, San Sebastian/Spain

${ }^{13}$ Hospital Lozano Blesa, Zaragoza/Spain

${ }^{14} \mathrm{H} M$. de Valdecilla, Santander/Spain

${ }^{15} \mathrm{HU}$ Virgen de las Nieves, Granada/Spain

${ }^{16}$ Digestive Diseases, Hospital Virgen de las Nieves, Granada/Spain

${ }^{17}$ Hospital Clinic, Barcelona/Spain

${ }^{18}$ Gastroenterology, Hospital Clinic Barcelona, Barcelona/Spain

${ }^{19} \mathrm{H}$ Galdakao Usansolo, Galdakao/Spain

${ }^{20}$ CAU de Salamanca.IBSAL, Salamanca/Spain

${ }^{21}$ Endoscopy Unit, CAU de Salamanca, Salamanca/Spain

${ }^{22}$ Gastroenterology, Hospital Mutua Terrassa, Barcelona/Spain

${ }^{23} \mathrm{H}$ Virgen de la Salud, Toledo/Spain

${ }^{24} \mathrm{H}$ la Paz-Carlos III, Madrid/Spain

${ }^{25}$ Centro Médico Quirúrgico Servidigest SL, Barcelona/Spain

${ }^{26}$ Hospital Universitario Virgen de la Macarena, Sevilla/Spain

${ }^{27} \mathrm{CH}$ Provincial Castellon, Castellon/Spain

${ }^{28}$ Hospital General de Catalunya, Barcelona/Spain

${ }^{29}$ Spanish Endoscopy Society, Madrid/Spain

Contact E-mail Address: edualbeniz@hotmail.com

Introduction: A scoring system based on size, morphology, site and access (SMSA score) was established for determining the complexity of polypectomy or endoscopic mucosal resection (EMR) and it could predict outcomes.

Aims \& Methods: The aim of this study was to assess the usefulness of the SMSA scoring system relating the difficulty level with outcomes and complications of EMR and to compare it with a subjective classification of difficulty. We conducted a prospective multi-center study with 1997 consecutive patients with large $(\geq 20 \mathrm{~mm}$ ) non-pedunculated colorectal lesions (LNPCL) treated by EMR $(\mathrm{n}=2198)$ at 23 hospitals belonging to the Spanish Endoscopy Society Endoscopic Resection Group, from January 2013 to October 2016. We 
calculated the SMSA score of difficulty and assessed the ability of SMSA to identify 5 outcomes: 3 -months recurrence, 1-year recurrence, global recurrence (endoscopy not effective after 2 or more treatments), delayed bleeding and perforation. We compared results with those obtained using a subjective classification of difficulty: easy or medium vs difficult. Comparisons were conducted using chi-squared tests and complemented with logistic regression models.

Results: The SMSA scoring system classified 690 polyps $(39 \%)$ as level 4 and $1098(61 \%)$ as level 3, whereas the subjective classification system classified 399 $(22 \%)$ as difficult and $1389(78 \%)$ as easy or medium. The agreement between measures of difficulty was weak $(\mathrm{k}=0.33) .255$ patients $(19.9 \%)$ had recurrence 3 months after EMR, $84(11.6 \%)$ had recurrence at 1 year, $78(3.5 \%)$ suffered delayed bleeding and $35(1.8 \%)$ perforation. The level 4 polyps had significantly higher rates of recurrence at 3 months and 1 year $(\mathrm{p}<0.001$ in both cases) and delayed bleeding $(\mathrm{p}=0.012)$. We did not found differences for global recurrence and perforation. Using the subjective indicator of difficulty, recurrence was also more frequent in the higher difficulty category at 3-months, 1-year and the global recurrence $(\mathrm{p}<0.001$ in all three cases) and for delayed bleeding too $(\mathrm{p}=0.006)$, but not for perforation.

Conclusion: The SMSA grading tool is a predictor of outcomes or recurrences and bleeding following resection of LNPCL. However, in our multi-center sample, it does not appear to overcome the ability of a subjective indicator of difficulty made by the endoscopist during the EMR. It seems that this score can be used to facilitate planning, training or competency assessment, but efforts should be focused on validating the scoring system in a real situation, adjusting the score of variables or including new ones.

Disclosure of Interest: All authors have declared no conflicts of interest.

\section{Reference}

Gupta S, Miskovic D, Bhandari P, et al. A novel method for determining the difficulty of colonoscopic polypectomy. Dis Colon Rectum 2013; 56: 960-966 Sansone S, et al. Clinical utility of the SMSA grading tool for the management of colonic neoplastic lesions. Dig Liver Dis (2017), http://dx.doi.org/10.1016/ j.dld.2016.12.030

\section{P0872 NARROW BAND IMAGING GUIDED BIOPSY IMPROVES THE YIELD OF HISTOLOGY FOR THE DIAGNOSIS OF GASTROINTESTINAL TUBERCULOSIS (GITB)}

N. Berry, S. K. Sinha, S. Malik, R. Kochhar, K. Vaiphei, K. Sharma, N. Dhaka, A. Koshi

Gastroenterology, Postgraduate Institute of Medical Education and Research. Chandigarh/India

\section{Contact E-mail Address: sarojksinha@hotmail.com}

Introduction: Accurate diagnosis of gastrointestinal tuberculosis (GITB) is challenging due to pauci-bacillary nature of disease and poor sensitivity of histopathology. Role of tissue acquisition using narrow band imaging magnification (NBI-M) to improve yield of histopathology over high-definition white light endoscopy (HD-WLE) has not been assessed.

Aims \& Methods: To compare the utility of narrow band imaging with magnification versus high-definition white light imaging guided endoscopic biopsy for diagnosis of gastrointestinal tuberculosis. In this prospective study from July 2015 to November 2016, adult cases of clinically suspected GITB were recruited. All patients underwent mantoux test, contrast enhanced computed tomography of abdomen, esophagogastroduodenoscopy and/or colonoscopy using both HDWLE and NBI-M and guided biopsies using both were taken. Histopathological examination was done by two independent pathologists. A final diagnosis of GITB was made if acid-fast bacilli were seen in tissue or grown in culture, histopathology showed caseous necrosis with granulomatous inflammation or clinical/radiological and endoscopic features were suggestive of tuberculosis and a good clinical response to antitubercular therapy.

Results: A total of 55 cases of clinically suspected GITB were recruited. A final diagnosis of tuberculosis was made in 32 cases (duodenal $n=4$, ileocolic $n=28$ ). Concomitant evidence of active or healed pulmonary tuberculosis was seen $21 \%$ of cases. The mean age, haemoglobin and erythrocyte sedimentation rate of patients with tuberculosis were $36.41+14.6$ years, $10.2+2.4 \mathrm{~g} / \mathrm{dl}$, $37.8+15.3 \mathrm{~mm} /$ hour respectively. The mean duration of symptoms was 10.9 months. The most common symptoms were pain abdomen $(78 \%)$, weight loss $(62.5 \%)$, and loss of appetite $(40.6 \%)$, fever $(37.5 \%)$, vomiting $(34 \%)$ and diarrhoea $(22 \%)$. Mantoux test was positive in $40.6 \%$ cases. The most common endoscopic findings were ulcerations $(75 \%)$, nodularity $(46.8)$, distorted ileocecal valve $(28 \%)$ and strictures $(21.8 \%)$ (Table 1$)$. The most common radiological findings were mural wall thickening $(65.6 \%)$, mesenteric lymphadenopathy $(56 \%)$ and strictures $(40 \%)$ (Table 1$)$. NBI-M guided biopsy confirmed the diagnosis of GITB in $46.88 \%$, while HD WLE guided biopsy confirmed diagnosis of GITB in $28.12 \%(\mathrm{P}, 0.04)$. The two sets of biopsies together confirmed diagnosis of GITB in $53.1 \%$. The area under curve for NBI-M plus HDWLE, NBI-M alone and HDWLE alone were $0.77(0.63-0.87) 0.73(0.60-0.85)$ and $0.64(0.50$ 0.77 ) respectively. Patients were started on anti-tubercular therapy for nine months. Four patients underwent surgery for intestinal obstruction while on antitubercular therapy. Twenty eight completed full therapy and improved. Conclusion: NBI-M guided biopsy improved the yield of histology for diagnosis of GITB.

\begin{tabular}{llll}
\hline Endoscopic findings & \multicolumn{3}{c}{ Radiological findings } \\
\hline Parameter & $\mathrm{N}(\%)$ & Parameter & $\mathrm{N}(\%)$ \\
Ulcerations & $24(75)$ & Mesenteric lymphadenopathy & $18(56)$ \\
Nodularity & $15(46.8)$ & Mural thickening & $21(65.6)$ \\
Stricture & $7(21.8)$ & Stricture & $13(40)$ \\
Distorted Ileocecal valve & $9(28.1)$ & Ascites & $5(15.6)$ \\
Hyperemia & $3(9.3)$ & Peritoneal thickening & $1(3.1)$ \\
Pseudopolypii & $2(6.2)$ & Omental nodularity & $1(3.1)$ \\
Intussception & $1(3.1)$ & Psoas abscess & $1(3.1)$ \\
\hline
\end{tabular}

Disclosure of Interest: All authors have declared no conflicts of interest.

\section{P0873 EFFICACY AND SAFETY OF ENDOSCOPIC RESECTION OF LARGE COLORECTAL ADENOMAS - CLINICAL EXPERIENCE OF A TERTIARY REFERRAL CENTER}

L. Mlynarsky ${ }^{1}$, S. Zelber-Sagi ${ }^{2}$, E. Miller ${ }^{3}$, R. Kariv ${ }^{3}$

${ }^{1}$ Sackler Faculty Of Medicine, Tel-Aviv University, Tel Aviv/Israel

${ }^{2}$ School Of Public Health, Faculty Of Social Welfare And Health Sciences, University of Haifa, Haifa/Israel

${ }^{3}$ Gasteroenterology And Hepatology, Tel Aviv Medical center, Tel-aviv/Israel

\section{Contact E-mail Address: revitalk@tlvmc.gov.il}

Introduction: Colorectal cancer is a leading cause for cancer related mortality. Adenomatous polyp, the precursor, can usually be endoscopically resected to prevent cancer. Currently, there are no criteria for surgical vs. endoscopic resection and decision is individually made by the treating physician.

Aims \& Methods: We aimed to evaluate factors associated with short-term efficacy and safety of endoscopic resection of large $(\geq 20 \mathrm{~mm})$ and giant $(\geq 40 \mathrm{~mm})$ adenomas. Consecutive cases that underwent endoscopic resection of adenomas larger than $20 \mathrm{~mm}$ were included. Endoscopic, clinical and histological details of polyps and of the endoscopic procedure were recorded as well as the need for surgical resection.

Results: Total of 351 resections were included. Average diameter was $30.34 \pm 10.66 \mathrm{~mm}$. Surgery was indicated in $21(5.98 \%)$ cases. In a multivariate analysis for efficacy, two variables were independent risk factors for surgery: adenoma size (OR 95\% CI $1.08(1.04-1.12)$ ) and cecal location (OR 95\% CI 5.97 (1.60-22.33)). Post-polypectomy complications were documented in 85 cases $(24.2 \%)$ : bleeding - $69(19.7 \%, 54 / 69$ managed during procedure), perforations $-8(2.3 \%)$ and significant discomfort up to early termination of procedure $15(4.3 \%)$. Only $21(6.0 \%)$ developed serious complications requiring further hospitalization. In multivariate analysis for safety, independent risk factors for post-polypectomy complications were: adenoma size (OR 95\%CI 1.04 (1.011.06 ), polyp morphology (sessile OR 95\%CI $2.55(1.45-4.51)$, flat OR $95 \% \mathrm{CI}$ $2.40(1.04-5.52))$ and submucosal adrenaline injection (OR 95\% CI 1.87 (1.11$3.20)$ ). Every increment of $1 \mathrm{~mm}$ in adenoma diameter above $20 \mathrm{~mm}$, increased the need for surgery by $8 \%$ and the risk for complications by $4 \%$.

Conclusion: Resection of large or giant adenomas is generally a safe procedure when performed by an experienced endoscopist. Although adenoma size is the most significantly related to efficacy and safety, each case of giant adenoma should be evaluated in a referral center for feasibility of endoscopic resection. Disclosure of Interest: All authors have declared no conflicts of interest.

Abstract: P0871. Outcome by SMSA grade. Odds ratios and 95\% CI ROC Curves by SMSA using the score in the continuous format.

\begin{tabular}{|c|c|c|c|c|c|c|c|c|c|}
\hline & $\begin{array}{l}\text { SMSA score } \\
\text { Level } 3\end{array}$ & Level 4 & p-value & $\begin{array}{l}\text { Subjective difficulty score } \\
\text { Not difficult }\end{array}$ & Difficult & p-value & $\begin{array}{l}\text { ROC Curves by SMSA } \\
\text { ROC curve }\end{array}$ & $\mathrm{OR}(95 \% \mathrm{CI})$ & p-value \\
\hline 3-months recurrence & $73(12.2 \%)$ & $125(27.6 \%)$ & $<0.001$ & $125(15.2 \%)$ & $72(30 \%)$ & $<0.001$ & $0.64(0.60,0.69)$ & $1.28(1.18,1.38)$ & $<0.001$ \\
\hline 1-year recurrence & $29(7.7 \%)$ & $46(17.2 \%)$ & $<0.001$ & $46(9.0 \%)$ & $28(20.9 \%)$ & $<0.001$ & $0.66(0.59,0.72)$ & $1.33(1.18,1.50)$ & $<0.001$ \\
\hline Global recurrence & $25(5.8 \%)$ & $27(8.9 \%)$ & 0.106 & $27(4.7 \%)$ & $24(14.7 \%)$ & $<0.001$ & $0.54(0.46,0.63)$ & $1.08(0.94,1.24)$ & 0.318 \\
\hline Delayed bleeding & $29(3.1 \%)$ & $35(5.6 \%)$ & 0.012 & $41(3.4 \%)$ & $24(6.6 \%)$ & 0.006 & $0.61(0.53,0.68)$ & $1.23(1.09,1.39)$ & 0.001 \\
\hline Perforation & $18(1.9 \%)$ & $10(1.6 \%)$ & 0.631 & $18(1.5 \%)$ & $10(2.8 \%)$ & 0.105 & $0.55(0.46,0.64)$ & $1.06(0.88,1.27)$ & 0.521 \\
\hline
\end{tabular}


P0874 COLORECTAL MUCOSAL DEFECT CLOSURE FOLLOWING ENDOSCOPIC MUCOSAL RESECTION: A SYSTEMATIC REVIEW AND META-ANALYSIS

\section{Palmela ${ }^{1}$, P. Marques Da Costa ${ }^{2}$, A. O. Ferreira}

${ }^{1}$ Gastroenterology Department, Hospital Beatriz Ângelo, Loures/Portugal

${ }^{2}$ Gastroenterology And Hepatology Department, Hospital de Santa Maria, Lisboa/

Portugal

Contact E-mail Address: palmela.carolina@gmail.com

Introduction: Clinically meaningful delayed bleeding is the most frequent adverse event following endoscopic colorectal mucosal resection. Observational and interventional studies on the efficacy of prophylactic closure (PC) following endoscopic mucosal resection (EMR) showed conflicting results.

Aims \& Methods: The primary objective of this review is to evaluate the effectiveness in preventing bleeding and post-polypectomy syndrome (PPS) or perforation of PC of colonic mucosal defects following endoscopic resection. We performed a systematic review and meta-analysis of randomized controlled trials (RCTs) from MEDLINE. We included studies with humans submitted to colonoscopy and in whom mucosal flat or sessile (Paris classification 0-II or Is) lesions with an estimated size $>10 \mathrm{~mm}$ were found and removed.

Results: 269 articles were initially screened: 5 were RCTs, 4 of them were pooled in the quantitative analysis. A total of 555 patients and 557 resected lesions (proximal colon: 220; distal: 337) were included. Endoscopic procedures: 459 loop polypectomies and 98 submucosal dissections. A total of 298 lesions were randomized to PC versus 259 to non-closure (NC). Number of events on PC group: delayed bleeding $(n=3)$, PPS and perforation $(n=6)$. Number of events on NC group: delayed bleeding $(n=13)$, PPS and perforation $(n=14)$. Prophylactic mucosal defect closure was effective in reducing delayed bleeding risk (OR 0.206, 95\% CI 0.054-0.779; $\mathrm{p}=0.020 ; \mathrm{I}^{2}=0,0 \% ; 2$ RCT and 452 lesions included). There was a non-significant trend for PPS/perforation risk reduction after PC (OR 0.349, 95\% CI 0.114-1.070, $\mathrm{p}=0.066 ; \mathrm{I}^{2}=0,0 \% ; 2$ RCT and 374 lesions included).

Conclusion: Prophylactic closure of mucosal defects after EMR of flat or sessile colorectal lesions $\geq 10 \mathrm{~mm}$ reduces de risk of delayed bleeding. Further studies are needed to evaluate the effect on PPS/perforation prevention.

Disclosure of Interest: All authors have declared no conflicts of interest.

P0875 RECTAL ESD IN VERY OLD PATIENTS ( $>80$ YEARS): A FRENCH MULTICENTER RETROSPECTIVE STUDY

R. Legros ${ }^{1}$, J. Albouys ${ }^{1}$, M. Guillaumot ${ }^{2}$, V. Lepilliez ${ }^{3}$, S. Chaussade ${ }^{2}$, M. Pioche ${ }^{4}$, S. Leblanc ${ }^{2}$, M. Barret ${ }^{2}$, F. Prat $^{2}$, J. Rivory $^{4}$, D. Sautereau ${ }^{1}$, T. Ponchon ${ }^{4}$, J. Jacques

${ }^{1}$ Hepato-gastro-enterology, CHU Limoges - Hepato-Gastro-Enterology, CHU

Limoges; Limoges/FR, Limoges/France

${ }^{2}$ Gastroenterology, Cochin Hospital, Paris/France

${ }^{3}$ Hopital prive Jean Mermoz, Lyon/France

${ }^{4}$ Hepatogastreoenterology, Hopital Edouard Herriot, Lyon/France

Contact E-mail Address: rom1.legros@gmail.com

Introduction: Endoscopic Submucosal Dissection has become the standard of care for large superficial rectal precancerous and cancerous lesions. It allows an en-bloc resection of large superficial lesions that increase the quality of pathological analysis and considerably decrease the risk of recurrence. Risk of having large superficial lesions increase with age and no study have focused specifically on ESD in very old people ( $>80$ years). Indeed in this population alternative surgery had a high morbidity and is often refused or contraindicated for these patients

Aims \& Methods: Retrospective bicentre study of all cases of rectal ESDs performed between $06 / 2010$ and 12/2016 for superficial pre-cancerous or cancerous neoplasms in patients older than 80 years four French teams that performed more than 150 ESDs in the last 5 years. Primary Endpoint was to evaluate the En bloc, R0, curative resection rate and complications in patients older than 80 years. Secondary endpoints were to compare these results with results of rectal ESDs performed during the same period for patients younger than 80 years.

Results: 58 rectal ESDs were performed in four French centers between 06/2010 and $12 / 2016$ for superficial pre-cancerous or cancerous neoplasms in patients older than 80 years. Descriptive results: male $28(48 \%)$, mean size of the specimen: $56.4 \mathrm{~mm}$, mean duration of procedure 143.7 min, mean speed of ESD: 19.8 $\mathrm{mm}^{2} / \mathrm{min}$, perforation rate: $3(5.2 \%)$, post procedural bleeding: $7(12 \%)$, secondary surgery $2(3.4 \%)$ (All for pathological reason) Pathological analysis: lowgrade dysplasia in $19 \%$, high-grade dysplasia in $28 \%$, intra-mucosal carcinoma in $5 \%$, superficial submucosal carcinoma in 5\%, deep submucosal carcinoma in $5 \%, \mathrm{~T} 2$ adenocarcinoma in $3.4 \%$ and anal SCC in $6.9 \%$. Only 1 patients $(1.7 \%)$ had a recurrent disease during endoscopic follow up. Primary Endpoint: En bloc resection: $86.2 \%$, R0 resection: $62.5 \%$, curative resection: $62.5 \%$, Secondary Endpoint: Duration of procedure was longer in older people. Speed of ESD was lower. En bloc and R0 resection were lower in very old patients. Cancer was more frequent in very old patients. All comparative data between very old patients $(>80$ years) and younger patients $(<80$ years $)$ are resumed in table 1 .

\begin{tabular}{llll}
\hline & $>80(\mathrm{n}=58)$ & $<80(\mathrm{n}=275)$ & $\mathrm{p}$ \\
\hline Duration & 143.69 & 113.78 & 0.053 \\
Large diameter $(\mathrm{mm})$ & 56.43 & 56.36 & 0.98 \\
\hline
\end{tabular}

: Continued

\begin{tabular}{llll}
\hline & $>80(\mathrm{n}=58)$ & $<80(\mathrm{n}=275)$ & $\mathrm{p}$ \\
\hline small diameter $(\mathrm{mm})$ & 43.31 & 41.42 & 0.54 \\
Surface $(\mathrm{mm} 2)$ & 2289.67 & 2154.64 & 0.68 \\
Speed $(\mathrm{mm} 2 / \mathrm{min})$ & 19.76 & 24.72 & 0.01 \\
Perforation & $5.20 \%$ & $6.30 \%$ & 1 \\
post ESD bleeding & $12 \%$ & $5.50 \%$ & 0.07 \\
Exclusive ESD & $86.20 \%$ & $95.50 \%$ & 0.007 \\
$\quad$ En bloc & $86.20 \%$ & $95.50 \%$ & 0.007 \\
R0 & $62.50 \%$ & $74.20 \%$ & 0.08 \\
Curative & $62.50 \%$ & $69.30 \%$ & 0.31 \\
Pathological analysis & & & 0.009 \\
$\quad$ LGD & $19 \%$ & $23.6 \%$ & \\
$\quad$ HGD & $28 \%$ & $34 \%$ & \\
im ADK & $31 \%$ & $29 \%$ & \\
sm < 1000 ADK & $5 \%$ & $6.9 \%$ & \\
sm $>1000$ ADK & $5 \%$ & $3.6 \%$ & \\
T2 ADK & $3.4 \%$ & $2.1 \%$ & \\
anal SCC & $6.9 \%$ & $0.3 \%$ & 0.39 \\
Secondary surgery & $3.40 \%$ & $7.30 \%$ & 0.84 \\
Recurrence & $1.70 \%$ & $1.40 \%$ & \\
\hline
\end{tabular}

Conclusion: ESD is feasible and efficient in very old patients. However, En bloc resection and $\mathrm{R} 0$ resection are less frequent than in younger patients probably due to more challenging lesions (more frequent cancer on the pathological analysis). ESD should be the treatment of choice for large rectal superficial neoplasms of the rectum in very old patients in view of its oncological efficiency and its safety in comparison to the surgical alternative.

Disclosure of Interest: All authors have declared no conflicts of interest.

Reference

Dong J, Wang W, Yu K, Gao Y, Cheng X, Liu P, et al. Outcomes of laparoscopic surgery for rectal cancer in elderly patients. J BUON. 2016 Jan;21(1):80-6.

\section{P0876 COLONOSCOPY SPLIT-DOSE PROTOCOL} IMPLEMENTATION: A SINGLE-CENTRE EXPERIENCE

C. Palmela, C. Gomes, M.P. Costa Santos, M. Rocha, S. Pereira, D. Tavares, R. Ribeiro, A. O. Ferreira, E. Barjas, M. Cravo

Gastroenterology Department, Hospital Beatriz Ângelo, Loures/Portugal

Contact E-mail Address: palmela.carolina@gmail.com

Introduction: Split-dose bowel preparation (SD) is more effective in bowel cleansing than previous day preparation (PD).

Aims \& Methods: The main objective of the study was to explore the impact of a SD protocol in bowel cleansing quality in patients from a district hospital. This was an exploratory observational study of patients who underwent total colonoscopy between jun/2016-mar/2017 with polyethylenoglycol bowel preparation before and after SD protocol implementation. Bowel cleansing quality was assessed prospectively (using Boston Bowel Preparation Score) and compared between SD and PD groups. Tolerance was assessed using a patient questionnaire.

Results: A total of 344 patients were included, $53 \%$ were male, mean age of $61.8 \pm 13.6$ years. Bowel preparation: $66 \%$ SD and $34 \%$ PD. Overall, $72 \%$ of colonoscopies occurred in morning shifts. Mean interval between finishing bowel preparation and colonoscopy was $4 \mathrm{~h} 50$ (SD) and 8h09 (PD). Adequate bowel cleansing was found in $81 \%$ of patients (SD $83 \%$ vs. PD $79 \% ; p=0.34$ ). There was an association between adequate bowel cleansing and a shorter interval between finishing preparation and colonoscopy ( $5 \mathrm{~h} 40$ vs. $7 \mathrm{~h} 15 ; \mathrm{p}=0.010)$. Split-dose preparation was associated with a better cleansing in the right colon $(2.17 \pm 0.69$ vs. $2.03 \pm 0.65 ; p=0.047)$ and a trend for better overall cleansing $(6.70 \pm 1.87$ vs. $6.32 \pm 1.90 ; \mathrm{p}=0.067)$. On morning shifts, there was a significant association between SD prep and better overall cleansing $(p=0.030)$ and also right colon cleansing $(\mathrm{p}=0.034)$. After adjusting for morning shifts, we found an association between SD preparation and better bowel cleansing (risk difference $0.406 ; 95 \% \mathrm{CI}-0.023-0.834 ; \mathrm{p}=0.063)$. There was no difference between groups on bowel urgency (SD 2.6\% vs. PD $1.7 \% ; \mathrm{p}=0.718$ ). SD preparation was associated with worse sleep quality (SD $25 \%$ vs. DA $7 \% ; \mathrm{p}=0.004$ ).

Conclusion: The implementation of a split-dose bowel preparation protocol in our hospital was associated with better bowel cleansing, especially on the right colon. Split-dose preparation was not associated with higher bowel urgency, although there was a worse sleep quality.

Disclosure of Interest: All authors have declared no conflicts of interest. 
P0877 EQUAL ADENOMA DETECTION RATE IN COLONOSCOPIES OF PATIENTS WITH SPINAL CORD INJURY AND CONTROLS - A CASE-CONTROL STUDY

A. Blanco Belver ${ }^{1}$, M. Aach ${ }^{2}$, W. Schmiegel ${ }^{1}$, T. A. Schildhauer ${ }^{2}$, R. Meindl ${ }^{2}$, T. Brechmann ${ }^{1}$

${ }^{1}$ Department Of Gastroenterology And Hepatology, Berufsgenossenschaftliches University Hospital Bergmannsheil Gmbh, Bochum/Germany

${ }^{2}$ Department Of General And Trauma Surgery, Spinal Cord Injury Unit,

Berufsgenossenschaftliches Universitätsklinikum Bergmannsheil gGmbH, Bochum/ Germany

\section{Contact E-mail Address: thorsten.brechmann@rub.de}

Introduction: Spinal cord injury (SCI) is a devastating event that occurs with a discharge incidence of 109 per million, which results in an estimated prevalence of up to 2525 per one million [1]. Cancer is a major cause of death in patients with spinal cord injury (SCI) [2]. Preventive strategies claim increasing attention, but must deal with special problems. Most SCI patients suffer from neurogenic bowel alterations, characterized by loss of bowel control, diarrhea, reduced bowel motility, constipation or evacuation difficulties. Therefore, bowel preparation requires modified and intensified regimens and more intensive care to prevent pressure ulcers and optimum skin management $[3,4,5]$. Colonoscopy itself might be associated with reduced efficacy in SCI patients, since a poorer quality of bowel cleansing or failure to reach the caecum could diminish adenoma detection rates (ADR) [6, 7], while complication rates could increase due to severe morbidity.

Aims \& Methods: The primary objective was to determine the adenoma detection rate (ADR) in general, right and left hemicolon. Secondary objectives were polyp, advanced adenoma and carcinoma detection rates, size of adenomas and polyps in either localization, intensity and effect of bowel preparation, rate of complete colonoscopies, need for re-endoscopy, duration of colonoscopic procedures, deepest point of insertion and complication rates. We reviewed retrospectively consecutive SCI patients who underwent colonoscopy from 2003 to 2014 and assigned an age-, gender- and year of performance-matched control group

Results: In $236 \mathrm{SCI}$, compared to 414 control patients, bowel preparation lasted longer $(3.57 \pm 1.5$ vs. $1.15 \pm 0.6$ days, $\mathrm{p}=0.001)$, achieved insufficient cleansing results more often $(23.7$ vs. $3.6 \%)$ and caused more adverse events. Colonoscopy needed longer time ( 36.9 vs. $25.0 \mathrm{~min}, \mathrm{p}=0.001)$, remained incomplete more often $(24.6$ vs. $4.6 \%)$, resulting in more re-colonoscopies (14.8 vs. $4.3 \%)$ Endoscopy- and sedation-related adverse events were equal. However, neither overall nor size-dependent polyp (30.9 vs. $34.8 \%$ ), adenoma ( 21.2 vs. $21.0 \%$ ), advanced adenoma $(6.8 \%$ vs. $7.2 \%)$ or cancer $(1.7$ vs. $2.0 \%)$ detection rate differed.

Conclusion: Despite intensified protocols, bowel preparation shows inferior results in SCI patients and colonoscopy needs more efforts to succeed, but achieves a comparable quality.

Disclosure of Interest: All authors have declared no conflicts of interest.

\section{References}

1. Noonan VK, Fingas M, Farry A, Baxter D, Singh A, Fehlings MG, Dvorak MF. Incidence and prevalence of spinal cord injury in Canada: a national perspective. Neuroepidemiology 2012;38:219-26.

2. Middleton JW, Dayton A, Walsh J, Rutkowski SB, Leong G, Duong S. Life expectancy after spinal cord injury: a 50-year study. Spinal Cord 2012;50:803-11.

3. Frisbie JH, Chopra S, Foo D, Sarkarati M. Colorectal Carcinoma and myelopathy. J Am Paraplegia Soc 1984;7:33-6.

4. Ancha HR, Spungen AM, Bauman WA, Rosman AS, Shaw S, Hunt KK, Post JB, Galea M, Korsten MA. Clinical trial: the efficacy and safety of routine bowel cleansing agents for elective colonoscopy in persons with spinal cord injury - a randomized prospective single-blind study. Aliment Pharmacol Ther. 2009;30:1110-7

5. Stratton MD, McKirgan LW, Wade TP, Vernava AM, Virgo KS, Johnson FE, Longo WE. Colorectal cancer in patients with previous spinal cord injury. Dis Colon Rectum 1996;39:965-8

6. Burke CA, Church JM. Enhancing the quality of colonoscopy: the importance of bowel purgatives. Gastrointest Endosc 2007;66:565-73.

7. Gupta N. How to Improve Your Adenoma Detection Rate During Colonoscopy. Gastroenterology. 2016;151:1054-1057.

\section{P0878 RISK FACTORS AND PRACTICAL CONSEQUENCES OF COLORECTAL ESD CONVERSION TO EMR AT A WESTERN REFERRAL CENTER IN DAILY PRACTICE}

E. Perez-Cuadrado-Robles, H. Piessevaux, T. Moreels, P.H. Deprez Gastroenterology Department, Cliniques Universitaires Saint-Luc, Brussels/ Belgium

Contact E-mail Address: kikemurcia@gmail.com

Introduction: There are limited data concerning risk factors and consequences of colorectal endoscopic submucosal dissection (ESD) conversion to mucosal resection (EMR) in western centers.

Aims \& Methods: Hospital-based frequency-matched case-control retrospective study. All patients were identified from a database of 225 consecutive dissections between 2013 and 2017. The cases were those with ESD conversion to EMR for a $>20 \mathrm{~mm}$ colonic lateral spreading tumor (LST). The controls were randomly selected by frequency 1:2 matching for tumor size $( \pm 50 \mathrm{~mm})$ and location (rectum/non-rectum). The presences of different factors were evaluated to determine whether they predicted ESD conversion.

Results: One-hundred and seventeen patients (39 cases, 78 controls) were included (mean age: $68 \pm 10.9$ years, $52.1 \%$ male). Mean tumor size was $38.5 \pm 14.4 \mathrm{~mm}$ and the most common location was the right colon/transverse $(\mathrm{n}=71,60.7 \%)$. By multivariable analysis using backward stepwise method, nonlifting [OR: 0.324 (CI95\%: $0.117-0.896), p=0.03$ ], duration $>120 \mathrm{~min}$ [OR: 3.127 (CI95\%: $1.305-7.493, p=0.011$ ) and LST-G type [OR: 2.881 (CI95\%: $1.074-7.727), p=0.036]$ were independent factors associated to ESD conversion. Duration was also significant when adjusted by fibrosis $\left(\mathrm{OR}^{\mathrm{a}}: 21.212, p=0.028\right)$ and related to en-bloc resection $(62.8 \%$ vs. $91.9 \%, p<0.001)$. Fibrosis $(p=0.032)$ and non-retroview $(p=0.025)$ were significant factors only in univariate analysis. Previous biopsy/polypectomy, referral status, presence of a fellow, circumferential $(>75 \%)$ tumor, intra-procedure complications $(14.5 \%)$ and start time were not associated to ESD conversion. R0 was achieved in $64.7 \%(n=11$ ) 17) cases with en-bloc EMR resection and in $91 \%$ controls $(p<0.001)$. There were two recurrences $(13.3 \%)$ at 3 -months within the 15 cases with piecemeal resection, treated endoscopically. No recurrences were noted in en-bloc (ESD or EMR) resection patients $(p=0.044)$. Three $(7.7 \%)$ and one $(1.3 \%)$ patients had an indication for surgery in case-control groups $(p=0.107)$.

Conclusion: The presence of certain factors should be assessed during the procedure to identify patients who are high-risk for ESD conversion regardless of size or location, particularly the duration of the dissection.

Disclosure of Interest: All authors have declared no conflicts of interest.

\section{P0879 CONTRIBUTION OF COLONOSCOPY IN ELDERLY} PATIENTS OLDER THAN 70 YEARS

A. El Mekkaoui ${ }^{1}$, A. Taiymi ${ }^{1}$, A. Zazour ${ }^{1}$, W. Khannoussi ${ }^{2}$, G. Kharrasse ${ }^{3}$, Z. Ismaili ${ }^{4}$

${ }^{1}$ Hepato Gastro Enterology, University hospital Mohammed VI, Oujda/Morocco ${ }^{2}$ Hepato Gastroenterology, Farabi Hospital, Oujda/Morocco

${ }^{3}$ Avicenne, Faculty of medicine Oujda, Oujda/Morocco

${ }^{4}$ Medical School, Casablanca/Morocco

Contact E-mail Address: mekamine@hotmail.com

Introduction: The elderly patients are considered as a particular population. Colonoscopy has an important place at this population because of the limited number of normal paraclinic examinations and the high incidence of tumor disease, specially the colo rectal cancer.

Aims \& Methods: The aim of this study was to determine the indications and results of colonoscopy in people older than 70 years We performed a retrospective descriptive study over a period of 2 years from the beginning of the endoscopic unit until February 2017, conducted in the department of hepatogastroenterology of our university hospital. All patients over the age of 70 years who have underwent colonoscopy have been included.

Results: A total of 1059 colonoscopies were performed, $10.3 \%$ were indicated for people older than 70 years. The mean age was 74.15 years with a median age of 75 years and a maximum age of 91 years. The prevalence of males was $51 \%$. $10.5 \%$ of cases $(\mathrm{n}=10)$ were diabetic, $12.6 \%(\mathrm{n}=12)$ hypertensive, $12.6 \%$ $(\mathrm{n}=12)$ with ischemic heart disease. $8.4 \%(\mathrm{n}=8)$ had cirrhosis and $9 \%(\mathrm{n}=9)$ had a digestive neoplasia. Colonoscopy was indicated for hematocheziain $40 \%$ $(\mathrm{n}=38)$, transit disorders in $33.6 \%(\mathrm{n}=32)$, abdominal pain in $14.7 \%(\mathrm{n}=14)$, IBD in $3.1 \%(\mathrm{~N}=3)$, radiographic abnormalities in $13.6 \%(\mathrm{n}=13)$, iron deficiency anemia in $4.2 \%$, and for patients with a family history of colorectal cancer in $1 \%$ of cases. Colonoscopy was abnormal in $83 \%(\mathrm{n}=93)$, with polyps in $48.3 \%(n=45)$, suspected lesions of malignancy in $16.1 \%(n=15)$, Diarrhea in $19.3 \%(\mathrm{n}=18)$, IBD in $3.2 \%(\mathrm{n}=3)$, arteriovenous malformations in $12 \%$ $(\mathrm{n}=11)$ and rectal erosions in $2.1 \%(\mathrm{n}=2)$. The rate of malignant lesions diagnosed by colonoscopy was $53.3 \%$ of females $(n=8)$ versus $46.7 \%$ of males $(\mathrm{n}=7)(\mathrm{p}=0.26), 13.3 \%(\mathrm{~N}=2)$ with hematochezia versus $86.7 \%(\mathrm{n}=13)$ without hematochezia $(\mathrm{p}=0.07)$, and $26.7 \%$ with altered general status $(n=4)$ versus $73.3 \%$ with conservated general status $(n=11)(p=0.07)$. The evolution of $46.6 \%$ of patientsdiagnosed with malignant tumor pathology showed that $33.3 \%$ of patients underwent surgery and $13.3 \%$ of patients had died.

Conclusion: In our study, suspected lesions of malignancy were diagnosed in $16.1 \%$ of cases. Colonoscopy in this age group is intended not only to detect possible polyps which could degenerate in the future but rather to diagnose the cancer already installed, especially with the presence of clinical signs.

Disclosure of Interest: All authors have declared no conflicts of interest.

\section{P0880 ENDOSCOPIC CLOSURE OF ACUTE IATROGENIC PERFORATIONS OF THE GASTROINTESTINAL TRACT AND PREDICTORS OF NEED FOR EARLY SURGERY: A SYSTEMATIC REVIEW AND META-ANALYSIS}

A. Gabr ${ }^{1}$, N. Ammar ${ }^{2}$, M. El Houssine ${ }^{2}$, M. Rutter ${ }^{3}$

${ }^{1}$ East Kent University Hosptials NHS, FD/United Kingdom

${ }^{2}$ Public Health Department, Ain Shams University, Cairo/Egypt

${ }^{3}$ Gastroenterology, University Hospital of North Tees, Stockton on Tees/United Kingdom

Contact E-mail Address: ahmedgabr@hotmail.co.uk

Introduction: Acute iatrogenic perforations are one of the recognized complications of both diagnostic and therapeutic gastrointestinal endoscopy. For decades, surgical treatment has been the standard of care, but endoscopic closure has become a more popular approach, due to feasibility and the reduction of the 
burden of surgery, combined with the availability of various endoscopic closure devices.

Aims \& Methods: To assess the technical and clinical success and safety of endoscopic closure, in total, and for each endoscopic device used. Also, to identify factors predicting surgery as a first line treatment, and failure of endoscopic treatment. Medical literature (Cochrane library, EMBASE, MEDLINE) from 1966 till September 2016 was searched. A systematic review and meta-analysis were performed on studies reporting technical and clinical success of endoscopic closure of acute iatrogenic perforations, according to PRISMA (Preferred Reporting Items for Systematic Reviews and Metaanalyses) guidelines.

Results: 764 studies were identified. 28 studies, in human, met our inclusion criteria and were analysed. A total of 474 endoscopic closures were attempted in these studies. The overall technical success rate was $93.1 \%(\mathrm{n}=451 / 474,95 \%$ CI: $89.8 \%-96.4 \%)$, clinical success was $89.7 \% \quad(n=431 / 474,95 \%$ CI: $85.5 \%-93.9 \%)$, and complication rate was $1.3 \%(\mathrm{n}=7 / 474,95 \%$ CI: $0.3 \%-2.3 \%)$. Technical success for endoclip closure was $96.6 \%(95 \% \mathrm{CI}$ : $94.2 \%-98.2 \%)$, and clinical success was $93 \%(95 \% \mathrm{CI}: 87.1 \%-97.2 \%)$, and complication rate was $1.5 \%$ (95\% CI: $93.75 \%-96.25 \%)$. For OTSC (Over the scope clip device), technical success was $83.8 \%(95 \%$ CI: $63.9 \%-96.6 \%)$, clinical success was $77.9 \%(95 \%$ CI: $56.8 \%-93.3 \%)$, and complication rate was $4.1 \%$ (95\% CI: $90.3 \%-99.1 \%)$. The technical success rate for Self-expanding metal stent (SEMS) is $100 \%(95 \% \mathrm{CI}: 71.5 \%-100 \%)$, clinical success is $91 \%(95 \% \mathrm{CI}$. $74.1 \%-108 \%)$, and complication rate of $9.1 \%$ (95\% CI: $78 \%-112 \%)$. Only one study for endosuturing met our criteria, with technical and clinical success rate of $100 \%$, and without any complication. Factors predicting failure of endoscopic treatment and need for early surgical intervention included large perforation size, leucocytosis, fever, severe abdominal pain, large amount of peritoneal free air, necrotic or soft inflammatory margins, unfavourable anatomical site, stool contamination, diffuse peritonitis and failure of endoscopic closure.

Conclusion: Our study suggests that endoscopic closure is a suitable treatment option for acute iatrogenic gastrointestinal perforations. Several factors have been suggested as predictors of need for surgery as a first line treatment. The study is limited by the low methodological quality of most studies included, indicating the need for further research.

Disclosure of Interest: All authors have declared no conflicts of interest.

\section{References}

1. Chavez, Y.H.et al (2013). A large international multicentre experience with an over-the-scope clipping device for endoscopic management of Gastrointestinal perforations, fistulae, and leaks in 188 patients Citation: Gastrointestinal Endoscopy, May 2013, vol./is. 77/5 SUPPL. 1(AB148AB149), 0016-5107.

2. Voemarns, R., Moine, O., and Von Rentlen, D. (2012). Efficacy of endoscopic closure of acute Perforations of the Gastrointestinal Tract. Clinical gastroenterology and hepatology 2012; 10:603-608.

3. Jin, Young-Joo. et al (2013).ERCP related duodenal perforation. Endoscopy 2013; 45: 806-812.

4. Fujishiro, M. et al (2006). Nonsurgical management of post-ESD perforation. Endoscopy 2006; 38: $1001 \pm 1006$.

\section{P0881 REAL-TIME HISTOLOGICAL CHARACTERIZATION OF COLORECTAL POLYPS - THE IMPACT OF TRAINING}

J. Castela, J. Cortez-Pinto, S. Mão De Ferro, I. Rosa, S. Ferreira, J. Pereira Da Silva, I. Claro, S. Faias, P. Lage, A. Dias Pereira

Núcleo Regional do Sul da Liga Portuguesa Contra o Cancro, Lisboa/Portugal

\section{Contact E-mail Address: joanarocastela@gmail.com}

Introduction: Narrow-band imaging (NBI) allows, after training, "in-vivo" classification of colorectal polyps. Recent guidelines propose a "do not resect" strategy for rectosigmoid hyperplastic polyps $\leq 5 \mathrm{~mm}$, characterized with a high degree of confidence, with $\geq 90 \%$ negative predictive value (NPV) for adenomatous histology.

Aims \& Methods: We aimed to evaluate the impact of training for the simultaneous application of NICE (International Colorectal Endoscopic Classification) and WASP (Workgroup Serrated Polyps and Polyposis) classifications. Prospective, single-center study of patients undergoing elective colonoscopy (colonoscopy-CF-H190®, Olympus), in two periods: P1: January-February/ 2016 and P2: January-February/2017. Endoscopists had no prior experience in NBI in P1, except for an interactive session of 20 minutes, and after P1 applied the technique during 2016 at their discretion. Polyps were assessed for its location, size and morphology (Paris classification); NICE/WASP: 1p (hyperplastic), 1 s (sessile serrated), 2 (adenoma), 3 (deep submucosal invasive cancer); confidence level (low: $<90 \%$ vs high: $>90 \%$ ) of the predictions. NICE/WASP classifications were compared with histology. Learning impact evaluated by P1 and P2 comparison. Statistics: Software-R (version 3.1.2I).

Results: 290 polyps of 135 patients were analysed (P1: $\mathrm{n}=192 ; \mathrm{P} 2: \mathrm{n}=98)$, average size $6.4 \mathrm{~mm}(2-35 \mathrm{~mm}), 60 \%$ located in the left colon, $71.0 \% 0$-Is. No difference in size, location, morphology and histology between P1 and P2 groups. An improvement of global acuity in the application of NICE/WASP classification in P2 was observed: $74.5 \%(95 \% \mathrm{CI}: 67.7 \%-80.5 \%)$ vs $81.6 \%(95 \% \mathrm{CI}$ : $72.5 \%-88.7(\mathrm{p}=0,222)$, statistically signficant for high confidence level predictions (P1: $77.6 \%, \mathrm{P} 2: 75.5 \%$ of the predictions): $78.5 \%$ (95\% CI: $71.1 \%-84$, $8 \%)$ vs $90.5 \%(95 \%$ CI: $81.5 \%-96.1 \%)(\mathrm{p}=0.044)$. In the P2 data subanalysis, NICE/WASP classification for adenoma showed sensitivity, specificity, positive predictive value and NPV of $82 \%(70-99 \%), 94 \%(80-99 \%), 96 \%(87-100 \%)$ and $72 \%(56-85 \%)$, respectively; for rectosigmoid polyps $\leq 5 \mathrm{~mm}$, with high confidence level $(\mathrm{n}=35)$, NPV for adenoma was $80 \%(59-93 \%)$.
Conclusion: The overall performance of endoscopists significantly improved with one year of training, but did not meet the standards for the implementation of the "do not resect" strategy. However, the results may have been affected by the addition of an additional category (1s) to the NICE classification.

Disclosure of Interest: All authors have declared no conflicts of interest.

\section{References}

1. Hayashi N, Tanaka S, Hewett DG, et al. Endoscopic prediction of deep submucosal invasive carcinoma: validation of the narrow-band imaging international colorectal endoscopic (NICE) classification. Gastrointest Endosc. 2013 Oct;78(4):625-32.

2. IJspeert JE, Bastiaansen BA, van Leerdam ME, et al. Development and validation of the WASP classification system for optical diagnosis of adenomas, hyperplastic polyps and sessile serrated adenomas/polyps. Gut. 2015 Mar 9. pii: gutjnl-2014-308411.

3. Rex DK, Kahi C, O'Brien M, et al. The American Society for Gastrointestinal Endoscopy PIVI (Preservation and Incorporation of Valuable Endoscopic Innovations) on real-time endoscopic assessment of the histology of diminutive colorectal polyps. Gastrointest Endosc. 2011 Mar;73(3):419-22.

4. Patel SG, Schoenfeld P, Kim HM, et al. Real-Time Characterization of Diminutive Colorectal Polyp Histology Using Narrow-Band Imaging: Implications for the Resect and Discard Strategy Gastroenterology. 2016 Feb;150(2):406-18.

\section{P0882 “O-RING SIGN" AS A NOVEL COLONOSCOPIC FINDING WITH NARROW-BAND IMAGING FOR DETECTING DEPRESSED- TYPE COLORECTAL LESIONS}

\section{T. Fujii}

Dept. Of Gastroenterology, TF Clinic Dept. of Gastroenterology, Tokyo/Japan

Contact E-mail Address: tfclinic@khaki.plala.or.jp

Introduction: In recent years, post-colonoscopy colorectal cancer (PCCRC) has become a focus of attention as likely representing "missed" or "rapidly-growing" lesions in colonoscopic screening for colorectal cancer (CRC). Currently, lesions thought responsible for PCCRC include sessile serrated adenomas/polyps or flat and depressed-type lesions occurring on the right side of the colon, and there is an increasing need for endoscopic modalities to prevent overlooking these lesions. Colorectal screening using narrow-band imaging (NBI) during colonoscope withdrawal from the cecum, which was started at our clinic since November 2008, suggested that NBI colonoscopy was superior to white-light imaging (WLI) colonoscopy in detecting flat and depressed-type lesions (1). With NBI, the depressed area of a lesion is recognized as "whitish" and the surrounding ring-like mucosa as "brownish", which constitutes the "O-ring sign".

Aims \& Methods: We aimed to evaluate the incidence and characteristics of the "O-ring sign" in depressed-type colorectal lesions. A total of 227 endoscopically resected and histologically confirmed depressed lesions (IIa + IIc, 156; IIc, 71) were included for analysis. The colonoscopic images of these lesions were retrospectively examined for "O-ring sign" positivity and intensity (grade 0 , negative; grade 1 , mildly to moderately positive; and grade 2 , highly positive). Of these, 16 were excluded as unevaluable and a total of 211 evaluable lesions were analyzed. Results: Of the 211 lesions (IIa + IIc, 141; IIc, 70) analyzed, 84 (IIa + IIc, 60; IIc; 24), 105 (IIa + IIc, 69; IIc, 36), and 22 (IIa + IIc, 12; IIc, 10) were found to be in grades 0,1 , and 2 , respectively, with $60.2 \%$ of these shown to be "O-ring sign"positive $(127 / 211)$, with IIa + IIc and IIc accounting for $57.4 \%(81 / 141)$ and $65.7 \%(46 / 70)$, respectively, of these lesions. While an examination by tumor size and location revealed no clear tendency in "O-ring sign" positivity, an examination by grade revealed a higher "O-ring sign" positivity rate among those with high-grade dysplasia $(84.6 \% ; 11 / 13)$ than those with low-grade dysplasia $(59.2 \%$; 116/196)

Conclusion: NBI colonoscopy screening for the "O-ring sign" as an index appears to improve the detection of depressed-type colorectal lesions.

Disclosure of Interest: All authors have declared no conflicts of interest.

\section{Reference}

1. Fujii T. Gastrointest Endosc 2010; W1480

\section{P0883 THE LEARNING CURVE FOR COLORECTAL ENDOSCOPIC SUBMUCOSAL DISSECTION (ESD) BETWEEN EXPERT AND TRAINEE ENDOSCOPIST}

N. Akimoto ${ }^{1}$, K. Mitsui ${ }^{1}$, T. Teramoto ${ }^{2}$, S. Fujimori ${ }^{1}$, K. Iwakiri ${ }^{1}$

${ }^{1}$ Gastroenterology, Nippon Medical School, Graduate School of Medicine, Tokyo/ Japan

${ }^{2}$ Gastroenterology, Machida Icho Hospital, Tokyo/Japan

Contact E-mail Address: naohiko.akimoto07@gmail.com

Introduction: Endoscopic submucosal dissection (ESD) has been acceptable as a minimally invasive therapy and providing en-bloc resection for early malignant and pre-malignant lesions of gastrointestinal cancer. Colorectal ESD has some difficulties such as a risk of perforation and its severity compare to gastric ESD. Hence, Colorectal ESD is more challenging than gastric ESD in endoscopic technique. In Japan, where has high incidence of gastric cancer, endoscopists 
could have many experiences of gastric ESD that may be beneficial for the introduction of colorectal ESD. However there is little knowledge about the learning curves of the young endoscopists who perform the colorectal ESD first. Aims \& Methods: We conducted multi-center retrospective observational study to elucidate the safety and learning curve of the trainee who perform the colorectal ESD first. The study subjects were consecutive 261 cases of colorectal ESD performed by three endoscopists in Nippon Medical School Hospital and Machida Icho Hospital from 2010 to August 2016. The ESD devices were Flush knife BT (Fujifilm), Dual knife (Olympus), Hook knife (Olympus) or a combination of these selected by operators demand. The endoscopist A and B, who had over 10000 examinations of colonoscopy and experiences of gastric ESD (as expert group), and endoscopist $\mathrm{C}$ had about 1000 colonoscopy and stared colorectal ESD first (as trainee group). The completion rate of operation, which is defined as en-block resection rate without changing operator, operation time, speed (time $/ \mathrm{mm} 2$ ) and complications were analyzed in each endoscopist. Furthermore, we divided these procedures in three periods equally such as early, middle and late.

Results: The median age was 70 (range 26-91) years old, and genders were 158 males and 103 females. Tumor locations were proximal colon, distal colon and rectum in $143(54.8 \%), 59(22.6 \%)$, and $59(22.6 \%)$, respectively. The histological types were well and moderately differentiated tubular adenocarcinoma, adenoma, hyperplastic polyp, SSA $/ \mathrm{P}$, and neuroendocrine tumor in $139(60.4 \%)$, $68(29.6 \%), 5(2.1 \%), 10(4.3 \%)$ and $8(3.8 \%)$, respectively. There is no statistical significance in age, gender, tumor location, tumor size and histological type. The average operation time was 68.0 minutes, the median 80.1 (range 13-353) minutes. The completion rates of endoscopist $\mathrm{A}$ and $\mathrm{B}$ reached $100 \%$ in all three time periods and endoscopist $\mathrm{C}$ showed an increase: $41 \%, 67 \%$, and $100 \%$ in the initial, middle, late period, respectively. In the operation time, there was a significant difference between endoscopist A $(73.1 \pm 38.9$, median 67.5) and $\mathrm{C}$ $(100 \pm 60.8$, median 85$), \mathrm{B}(62.9 \pm 45.1$, median 55.5$)$ and $\mathrm{C}(\mathrm{P}<0.001)$. In addition, there was difference in the operation speed of endoscopist $\mathrm{C}$ among each periods. The complications were perforation and delayed bleeding in 8 cases $(3.1 \%)$ and 2 cases $(0.8 \%)$. All cases recovered with medical and/or endoscopic treatment alone.

Conclusion: Trainee endoscopist may have a good learning curve in completion rate and increasing experience reflects in a remarkable success in colorectal ESD. We concluded that the training of colorectal ESD first was acceptable by the trainee endoscopist who had no experience of gastric ESD.

Disclosure of Interest: All authors have declared no conflicts of interest.

\section{P0884 LARGE BALLOON DILATATION VERSUS MECHANICAL LITHOTRIPSY AFTER ENDOSCOPIC SPHINCTEROTOMY IN MANAGEMENT OF LARGE COMMON BILE DUCT STONES AMONG CIRRHOTIC PATIENTS}

M. I. Radwan ${ }^{1}$, M.H. Emara Elzanan ${ }^{2}$, I. M. Ibrahim ${ }^{1}$, M. E. Morsy ${ }^{3}$ ${ }^{1}$ Tropical Medicine, Zagazig University Dept. of Tropical Medicine Faculty of Medicine, Zagazig/Egypt

${ }^{2}$ Tropical Medicine, Kafrelsheikh University, Kafr Elshikh/Egypt

${ }^{3}$ Medicine, Alahrar Teaching Hospital, Al-ahrar/Egypt

\section{Contact E-mail Address: emara_20007@yahoo.com}

Introduction: Removal of large common bile duct (CBD) stones is one of the challenges during ERCP and it seems more difficult in cirrhotic patients due to suspected higher rates of adverse events especially bleeding diathesis. Patients with liver cirrhosis can tolerate ERCP to treat their biliary tract or pancreatic diseases. Patients with liver cirrhosis are three times more susceptible to cholelithiasis, than the non-cirrhotic population plus the biliary and pancreatic cancer and other diseases and that is why ERCP is increasingly performed for patients with cirrhosis. Endoscopic sphincterotomy (EST) has become a standard step in mangement of CBD stones and introduction of both mechanical lithotripsy (ML) and large balloon dilation (LBD) facilitated extraction of the large stones. Despite the increasing use of both techniques, a head-to-head comparison between EST followed by LBD and EST followed by ML is lacking in the literature, and to the best of our knowledge none were done on cirrhotic patients Aims \& Methods: This study aimed at comparing the therapeutic benefits and complications between mechanical lithotripsy and large balloon dilation after sphincterotomy in patients with liver cirrhosis. Ninety eight cirrhotic patients with calcular obstructive jaundice were included and randomly divided into 2 groups: Group A; 49 patients treated by large balloon dilation (LBD), Group B; 49 patients treated by mechanical lithotripsy. All patients underwent sphincterotomy initially. Inclusion criteria for this study included: Age of 18-60 years, any sex, liver cirrhosis patients Child A or B with clinical and laboratory proved obstructive jaundice, presence of large bile duct stones and deep selective cannulation of the bile duct. Exclusion criteria included: Known allergy to the used contrast material, Child $\mathrm{C}$ cirrhosis, the Need for needle knife precutting in order to achieve bile duct cannulation, Selective bile duct cannulation achieved after more than five accidental pancreatograms and severe Coagulopathy All patients were subjected to thorough history taking, complete clinical examination. Pancreatic enzymes concentrations were measured 4 hours before and 24 hours after the procedure, complete blood cell count and liver function tests were performed before and the morning after the procedure. Before and during ERCP, stone size and number were verified. Diagnosis of common bile duct stones was confirmed either by pre-ERCP investigations including abdominal ultrasonography (US), CT, MRCP or at the time of ERCP Large CBD stone: Is defined before ERCP by a bile duct stone $12 \mathrm{~mm}$ or more (transverse diameter of the largest stone) by US, CT or MRCP and during cholangiography showed large filling defect more than $12 \mathrm{~mm}$ that could not be extracted using a standard balloon catheter
Results: There were no dropouts and all subjects remained in the study till the end. The demographic and biochemical data of both groups at base line were comparable. At base line, there was no significant difference between both groups regarding CBD diameter $(18.96 \pm 2.5$ vs $18.8 \pm 1.5$, p value 0.8$)$, stones size $(17.1 \pm 2.2$ vs $17.2 \pm 1.53$, p value 0.71$)$ and stone number $(1.49 \pm 0.65$ vs $1.41 \pm 0.49, \mathrm{p}$ value 0.61$)$ The overall success rate for stone extraction in this study is $95.6 \%(94 / 98)$. The success rate of ERCP was also comparable between the two groups. The success rate for CBD clearance was $98 \%$ and $93.8 \%$ for large balloon dilation and mechanical lithotripsy respectively. Although, patients in balloon dilation group showed more success in stone retrieval rate, the difference was not statistically significant ( $P$ value 0.61$)$. The rate of adverse events in this study was $10.2 \%(10 / 98)$ and bleeding was the commonest reported complication $50 \%(5 / 10)$; group B that was treated by ML developed more $(16.3 \%)$ adverse events than group A treated by LBD (4.1\%) and the difference was satstically significant $(\mathrm{P}$ value 0.04$)$

The reported complications in both groups

\begin{tabular}{llll}
\hline Complications & Group A & Group B & P value \\
\hline No & $47(95.9 \%)$ & $41(83.7 \%)$ & 0.04 \\
Bleeding & $1(2 \%)$ & $4(8 \%)$ & \\
Pancreatitis & $1(2 \%)$ & $2(4 \%)$ & \\
Cholangitis & $0(0 \%)$ & $2(4 \%)$ & \\
\hline
\end{tabular}

Conclusion: In conclusion endoscopic sphincterotomy plus LBD is a safe and effective treatment for endoscopic removal of large common bile duct stones in cirrhotic patients when compared with endoscopic sphincterotomy plus ML. Bleeding is the most common adverse event reported in cirrhotic patients undergoing these procedures

Disclosure of Interest: All authors have declared no conflicts of interest.

\section{P0885 SAFETY AND EFFICACY OF ENDOSCOPIST-DIRECTED BALANCED PROPOFOL SEDATION (BPS) DURING ENDOSCOPIC RETROGRADE CHOLANGIO-PANCREATOGRAPHY (ERCP)}

A. Lapidus ${ }^{1}$, I. M. Gralnek ${ }^{2}$, A. Suissa ${ }^{3}$, A. Klein ${ }^{3}$, K.A. Yassin ${ }^{3}$, I. Khamaysi ${ }^{1}$ ${ }^{1}$ The Ruth And Bruce Rappaport Faculty Of Medicine, Technion - Israel Institute of Technology, Haifa/Israel

${ }^{2}$ Institute Of Gastroenterology And Hepatology, Ha'Emek Medical Center, Afula/ Israel

${ }^{3}$ Department Of Gastroenterology, Rambam Health Care Campus, Haifa/Israel

\section{Contact E-mail Address: alon.lap@gmail.com}

Introduction: Endoscopist-directed balanced Propofol sedation (BPS), defined as a fixed dose of an opioid and benzodiazepine combined with incremental doses of Propofol, has been shown to be a safe and effective moderate sedation regimen for gastroscopy and colonoscopy. However, there are very limited data on the safety and efficacy of endoscopist-directed BPS in ERCP.

Aims \& Methods: We aimed to evaluate the safety and efficacy of endoscopistdirected BPS, as well as to compare patient outcomes with anesthesiologistadministered BPS, for both in-patients and out-patients undergoing ERCP. We performed a retrospective cohort study using prospectively collected endoscopy data from a tertiary care, university-affiliated medical center where endoscopistdirected BPS during ERCP is routine practice amongst the ERCPists, all of whom have up-to-date advanced cardiac life support (ACLS) certification. ERCP nurses also maintain up-to-date certification in ACLS and cardiopulmonary resuscitation equipment and medications are available within the advanced endoscopy suite. During ERCP, the endoscopist and the endoscopy nurse as a team were responsible for monitoring patient vital signs (e.g., pulse, blood pressure, oxygen saturation levels). Each endoscopist was responsible for directing the provision and dosing of the BPS. Patient-level demographics and pre/post procedure vital signs were collected along with BPS drug dosages, American Society of Anesthesiologists score (ASA) and measured "hard endpoint" patient outcomes, including: need for bag-mask ventilation or endotracheal intubation, aborted ERCP due to sedation effects, need for hospital admission post-ERCP (out-patients only) or need for change in level of hospital care (in-patients only), and mortality within 24 hours of ERCP.

Results: Over the course of 17 months (October 2015 - March 2017), 501 patients underwent ERCP and received endoscopist-directed BPS (Cohort 1: $380(76 \%)$ inpatient, mean age 64.1 years, $46 \%$ males, $24 \%$ ASA I, $65 \%$ ASA II, $11 \%$ ASA III). During this same time period, 24 patients received anesthesiologist-administered BPS (Cohort 2: $19(79 \%)$ inpatient, mean age 65.0 years, $67 \%$ males, $12 \%$ ASA I, $25 \%$ ASA II, 38\% ASA III, 25\% ASA IV). In Cohort 1 , the indications for ERCP were: $231(46 \%)$ suspected choledocholithiasis, $68(13 \%)$ stent replacement, $62(12 \%)$ evaluation of know/suspected malignancy, $48(10 \%)$ jaundice, $40(8 \%)$ post-hepatobiliary intervention complications, $8(2 \%)$ abdominal pain, and $44(9 \%)$ other/unspecified. BPS dosages (mean \pm SD: range) were: Fentanyl $0.06 \mathrm{mg} \pm 0.02 \mathrm{mg}$ : $0.05-0.10 \mathrm{mg}$; Midazolam $1.7 \mathrm{mg} \pm 0.7 \mathrm{mg}: 1.0$ $2.5 \mathrm{mg}$; and Propofol $178 \mathrm{mg} \pm 103 \mathrm{mg}: 10-640 \mathrm{mg}$. Propofol dose inversely correlated with patient age $(\mathrm{r}=-0.42, \mathrm{p}<0.001)$, ASA score $(\mathrm{r}=-0.19, \mathrm{p}<0.001)$ and Mallampati score $(\mathrm{r}=-0.24, \mathrm{p}<0.001)$. No clinically meaningful differences were found in patient vital signs pre and post ERCP. Moreover, no patient required bag-mask ventilation, endotracheal intubation nor hospital admission change in level of in-hospital care following ERCP. One patient in Cohort 2 who 
received anesthesiologist-directed BPS, required bag-mask ventilation and the ERCP was aborted due to sedation effects. There was no mortality from any cause within 24 hours of ERCP. All patients were discharged from the advanced endoscopy suite without adverse events.

Conclusion: Endoscopist-directed BPS appears feasible, safe and efficacious for ASA I-III patients undergoing inpatient or outpatient ERCP.

Disclosure of Interest: All authors have declared no conflicts of interest.

\section{P0886 A NOVEL METHOD OF PREVENTING DUODENOBILIARY REFLUX BY MEANS OF SUSPENDED OVERLENGTH BILIARY STENTS IN PATIENTS WITH BILIARY STRICTURE}

\section{Y. Huang, X. Yan, H. Chang, Y. Zhang, W. Yao, K. Li \\ Gastroenterology And Hepatology, Peking University Third Hospital, Beijing China}

\section{Contact E-mail Address: 13911765322@163.com}

Introduction: Endoscopic insertion of plastic or metal stents is a well-established treatment for malignant or part of benign biliary obstruction. The major limitation of this technique is stent occlusion. Duodenobiliary reflux has been considered as a key contributor to stent occlusion. No appropriate method can so far prevent duodenobiliary reflux. Different strategies to prolong the patency of plastic stents included changing stent size, stent design, but stent sluge due to duodenal biliary reflux remains an unsolved problem. We have been using a novel suspended overlength biliary stents (reformed with nasobiliary tube) as substitution for ordinary biliary plastic stent to prevent the reflux from January 1, 2016 to December 31, 2016

Aims \& Methods: The aim of the study is to evaluate the efficacy and patency of the suspended overlength biliary stents. The suspended overlength biliary stents (SOBS) were placed in introhepatic bile duct in 61 patients with extrahepatic bile ducts stricture who were followed up at least three months from January 1, 2016 to December 31,2016 . Nasobiliary tube of $7.5 \mathrm{Fr}$ or $8.5 \mathrm{Fr}$ with multiple side holes were cut $30 \mathrm{~cm}$ with operation knife from the top on sterile operating table. The purpose of the set of $30 \mathrm{~cm}$ length is to ensure the tail reaches the duodenal horizontal part. The SOBS were placed in the introhepatic biliary duct by using conveyer under the fluoroscopic guidance at the end of ERCP. Radiography of the Meglumine Diatrizoate was performed in each patient of SOBS group to evaluate the existence of duodenal biliary reflux. 74 patients who were performed at least two or more ERCP with extrahepatic bile ducts stricture treated with ordinary plastic stents (OBS group) from last ten years were compared with SOBS group.

Results: (1) The mean age of SOBS and OBS were $68.8 \pm 15.8 \mathrm{yrs}$ and $60.4 \pm 14.7$ yrs $(P=0.002)$, respectively. (2) $35(57.4 \%)$ and $34(45.9 \%)$ patients were malignant biliary obstruction in SOBS and OBS group, respectively $(P=0.227)$. Malignant obstruction included bile duct cancer and biliary duct invasion of pancreatic cancer. Benign obstruction included autoimmune pancreatits, chronic pancreatits, post operation stenosis, inflammatory stenosis due to cholelithiasis. (3) The mean first and second patency was 4.5 months and 5.6 months in OBS groups. All the patients in OBS group experienced at least three ERCP to exchange plastic stents. The mean first and second patency were 3.9 months and 4.4 months in OBS patients with malignant biliary obstruction. Yet the mean patency was 5.1 and 6.5 months in OBS patients with benign biliary obstruction. (4) The occlusion rates of SOBS and OBS group after 3, 4, 5, 6 months of first ERCP were $13.1 \%$ and $36.5 \%, 27.1 \%$ and $55.4 \%, 42.4 \%$ and $67.6 \%, 55.9 \%$ and $77.0 \%$, respectively $(P=0.003,0.001,0.005,0.015)$. Multiple factors with logistic regression analysis showed that gender, age, location of biliary stricture were not related with the patency except the character of stricture. (5) SOBS were occluded in only 11 patients $(11 / 61,18 \%)$ with the patency from 80 days to 12 months (mean patency was 5.2 months). The mean follow-up period of the other 50 patients in SOBS groups was 6.5 months. (range from 3 months to 15 months). No evidence of duodenal biliary reflux were detected in SOBS group.

Conclusion: Suspended overlength biliary stents can prolong the patency and reduce the occlusion rate effectively due to duodenobiliary reflux in both malignant and benign biliary stricuture.

Disclosure of Interest: All authors have declared no conflicts of interest.

\section{References}

1. Dumonceau JM, Tringali A, Blero D, et al. Biliary stenting: indications, choice of stents and results: European Society of Gastrointestinal Endoscopy (ESGE) clinical guideline. Endoscopy 2012;44: 277-98

2. Dua KS, Reddy ND, Rao VG, et al. Impact of reducing duodenobiliary reflux on biliary stent patency: an in vitro evaluation and a prospective randomized clinical trial that used a biliary stent with an antireflux valve. Gastrointest Endosc 2007;65:819-28.

3. Donelli G, Guaglianone E, Di Rosa R, et al. Plastic biliary stent occlusion: factors involved and possible preventive approaches. Clin Med Res 2007;5:53-60.
P0887 DUODENOSCOPES AND LINEAR ECHOENDOSCOPES ARE

CONTAMINATED WITH DIGESTIVE TRACT BACTERIA: NATIONWIDE PERSISTENT HIGH PREVALENCE IN THE NETHERLANDS

A. W. Rauwers' ${ }^{1}$, A. F. Voor In 'T Holt ${ }^{2}$, W. De Groot ${ }^{2}$, J. G. Buijs ${ }^{3}$, B. E. Hansen ${ }^{1}$, M. C. Vos ${ }^{2}$, M.J. Bruno ${ }^{1}$

${ }^{1}$ Gastroenterology \& Hepatology, Erasmus MC University Medical Center,

Rotterdam/Netherlands

${ }^{2}$ Medical Microbiology And Infectious Diseases, Erasmus MC University Medical Center, Rotterdam/Netherlands

${ }^{3}$ Staff Office Medical Devices, Erasmus MC University Medical Center,

Rotterdam, the Netherlands, Rotterdam/Netherlands

Contact E-mail Address: a.rauwers@erasmusmc.nl

Introduction: Recent studies describe multiple outbreaks of multi-drug resistant organisms caused by contaminated duodenoscopes, used for endoscopic retrograde cholangiopancreatography (ERCP) procedures. Contamination of duodenoscopes is attributed to their complex design, which includes a side viewing tip, forceps elevator and elevator wire channel. Linear echoendoscopes (LEs), used for endoscopic ultrasound (EUS) procedures, have a similar design with an additional balloon channel. Previously, we found that contamination of duodenoscopes was widespread in the Netherlands. It is unclear if the increased awareness of contamination and associated outbreaks has improved reprocessing outcomes of duodenoscopes and linear echoendoscopes (DLEs).

Aims \& Methods: This cross-sectional study was conducted to determine the prevalence of bacterial contamination of all reprocessed DLEs in The Netherlands. All 75 Dutch ERCP/EUS centres were invited to sample all DLEs using centrally distributed kits, according to uniform sampling methods explained by video instructions. Local staff sampled four to six sites per DLE depending on endoscope type, including swabs (protection cap, forceps elevator), flushes (biopsy, suction, air/water and forceps elevator channel) and brushes (biopsy, air/water and balloon channel). Samples were centrally cultured. Sampling procedure, culture processing and culture interpretation were consistent with Dutch guidelines. Contamination was defined as AM20: any microorganism with $\geq 20$ colony forming units (CFU) $/ 20 \mathrm{~mL}$, and MGO: presence of microorganisms with gastrointestinal or oral origin, independent of CFU count. Results: Between October 2016 and April 2017, 62 (85\%) Dutch ERCP/EUS centres responded with 1130 samples of 215 DLEs (158 duodenoscopes and 57 LEs). Fifteen different DLE types from three distinct manufacturers were sampled. In total 29/62 (47\%) centres had at least one AM20 or MGO contaminated DLE, similar to the prevalence (31/66 centres; $47 \%$ ) in our previous study $(p=0.98)$. Twenty-eight $(13 \%)$ DLEs from $19(31 \%)$ centres were AM20 contaminated. Thirty-two $(15 \%)$ DLEs from $23(37 \%)$ centres were contaminated with MGO, including Enterobacteriaceae, Pseudomonas aeruginosa and Candida spp. AM20 contamination was identified in eight different DLE types, MGO contamination in all nine duodenoscope types and three LE types. For both definitions, contamination was not duodenoscope or LE dependent (Pvalues $>0.72)$, nor type $(\mathrm{P}$-values $>0.14)$ or microbial surveillance dependent $(\mathrm{P}$-values $>0.45)$.

Conclusion: Similar to our previous findings, in $47 \%$ of all Dutch ERCP/EUS centres at least one patient-ready DLE was AM20 or MGO contaminated. Of all DLEs, $15 \%$ was contaminated with digestive tract bacteria, indicating inadequate reprocessing. These results suggest that any additional awareness of contamination has had no lasting effect or that the current reprocessing technique is not suitable for current DLE designs. This highlights the need for new preventive measures to minimize the risk of interpatient microbial transmission by DLE. Disclosure of Interest: B.E. Hansen: dr. Bettina E. Hansen received grants and personal fees from Intercept Pharmaceuticals Inc., personal fees from Novartis, personal fees from Albiero and grants from Roche International outside the submitted work.

M.J. Bruno: Prof. dr. Marco J. Bruno received personal fees from Boston Scientific, personal fees from Cook Medical and grants from Pentax Medical outside the submitted work.

All other authors have declared no conflicts of interest.

\section{P0888 TECHNICAL EVOLUTION OF A NEWLY-DEVELOPED DIGITAL CHOLANGIO/PANCREATO-SCOPY (SPYGLASS DS) FOR PREOPERATIVE EVALUATION OF PANCREATOBILIARY NEOPLASM}

K. Ito, S. Koshita, T. Ogawa, Y. Kanno, K. Masu, H. Kusunose, T. Sakai, T. Murabayashi, S. Hasegawa, F. Kozakai, Y. Noda

Gastroenterology, Sendai City Medical Center, Sendai/Japan

Contact E-mail Address: keiito@openhp.or.jp

Introduction: Although a newly digital cholangio/pancreato-scopy (SpyDS) has been reported to be useful for therapeutic purpose in patients with biliary diseases, clinical application for diagnostic purpose of pancreatobiliary neoplasm remains unclarified.

Aims \& Methods: To evaluate the usefulness and safety of cholangio/pancreatoscopy using a SpyDS for preoperative evaluation of pancreatobiliary neoplasm. Patients and methods: Between Oct/2015 and Feb/2017, consecutive 26 patients (19, cholangiocarcinoma; 7, IPMN) who underwent cholangio/pancreasto-scopy using a SpyDS for preoperative evaluation were included in this study. Diagnostic accuracy of malignancy and tumor extent evaluation by cholangio/ pancreato-scopic guided biopsy/cytology and adverse event after the procedure were retrospectively investigated.

Results: Of 19 patients with cholangiocarcinoma, success rate of SpyDS guided mapping biopsy was $83 \%$ (103/124 sites). Diagnostic accuracy of longitude 
Abstract: P0890. Table 1: Learning curves and competence in ERCP

\begin{tabular}{|c|c|c|c|c|}
\hline Study Endpoint & $\begin{array}{l}\text { No. of AETs meeting } \\
\text { inclusion criteria* }\end{array}$ & $\begin{array}{l}\text { No. of } \\
\text { evaluations }\end{array}$ & $\begin{array}{l}\text { No. of AETs achieving } \\
\text { competence }(\%)\end{array}$ & $\begin{array}{l}\text { No. of AETs achieving } \\
\text { competence }(\%)\end{array}$ \\
\hline & & & Primary Analysis* & Sensitivity Analysis** \\
\hline \multicolumn{5}{|l|}{ Basic Technique } \\
\hline - Intubation & 20 & 2239 & $20(100)$ & $19(95)$ \\
\hline - Achieving short position & 20 & 2226 & $19(95)$ & $15(75)$ \\
\hline - Identifying the papilla & 20 & 2223 & $19(95)$ & $18(90)$ \\
\hline \multicolumn{5}{|l|}{ Technical Aspects } \\
\hline - Overall cannulation & 19 & 2075 & $13(68.4)$ & $6(31.5)$ \\
\hline - Cannulation: native papilla & 17 & 1041 & $3(17.6)$ & $0(0)$ \\
\hline - Stent removal & 14 & 737 & $13(92.8)$ & $9(64.2)$ \\
\hline - Wire placement in biliary duct & 18 & 1815 & $16(88.8)$ & $8(44.4)$ \\
\hline - Sphincterotomy & 15 & 731 & $10(66.6)$ & $0(0)$ \\
\hline - Balloon sweep & 19 & 1602 & $18(94.7)$ & $10(52.6)$ \\
\hline - Stone clearance & 14 & 697 & $12(85.7)$ & $6(42.8)$ \\
\hline - Stricture dilation & 10 & 432 & $9(90)$ & $3(30)$ \\
\hline - Stent insertion & 17 & 1029 & $14(82.3)$ & $3(17.6)$ \\
\hline \multicolumn{5}{|l|}{ Cognitive Aspects } \\
\hline - Demonstrated clear understanding of indiciation & 20 & 2264 & $20(100)$ & $14(70)$ \\
\hline - Appropriate use of fluoroscopy & 20 & 2169 & $18(90)$ & $7(35)$ \\
\hline - Proficient use of real time & 20 & 2219 & $19(95)$ & $9(45)$ \\
\hline - Logical plan based on cholangiogram & 20 & 2220 & $19(95)$ & $10(50)$ \\
\hline - Demonstrated understanding of use of indomethacin & 19 & 1630 & $19(100)$ & $16(84.2)$ \\
\hline \multicolumn{5}{|l|}{ Overall Technical Success } \\
\hline Overall Cognitive Success & 20 & 2268 & $12(60)$ & $5(25)$ \\
\hline
\end{tabular}

* Primary analysis: success defined as score of 1 or 2 (no assistance/minimal verbal cues), Acceptable failure rate $-\mathrm{p} 0=0.1$ and unacceptable failure rate $-\mathrm{p} 1=0.3 * *$ Sensitivity analysis: success defined as score of 1 (stringent definition of success)

tumor extent using SpyDS plus mapping biopsy was 92\%. One patient developed mild cholangitis after the procedure. As for IPMN, pancreatoscopy using a SpyDS could visualize intraductal papillary tumors in all patients, and SpyDS guided biopsy/cytology was successfully performed. Diagnostic accuracy of malignancy was $100 \%$ without any adverse event after the procedure.

Conclusion: Preoperative evaluation using a SpyDS plus histological evaluation for pancreatobiliary neoplasm was found to be useful and safety. Further study is needed to establish evidence about the usefulness of this techdnique.

Disclosure of Interest: All authors have declared no conflicts of interest.

\section{P0889 ACUTE PANCREATITIS AND HYPERAMYLASAEMY DEVELOPMENT AFTER ENDOSCOPIC RETROGRADE CHOLANGIOPANCREATOGRAPY - CHALLENGES AND PREVENTION}

P. Petrov ${ }^{1}$, B. Petrov ${ }^{1}$, T. Vukova ${ }^{2}$

${ }^{1}$ University hospital "Sofiamed"- Sofia, Sofia/Bulgaria

${ }^{2}$ Excitable Structures, Institute of Biophysics and Biomedical Engineering -

Bulgarian Academy of Sciences, Sofia/Bulgaria

\section{Contact E-mail Address: dr.p.petrov@gmail.com}

Introduction: Endoscopic retrograde cholangiopancreatography (ERCP) is one of the most technically complex procedures performed by gastroenterologists. After a significant increase in the indications for implementing ERCP, gastroenterologists began to pay greater attention to complications identification and prevention. Despite the widespread improvement of endoscopic techniques and increased experience of endoscopists, the rate of complications has not declined significantly.

Aims \& Methods: To analyze the frequency of probable causes of asymptomatic hyperamylasaemy and acute pancreatitis after ERCP and their prevention. Two groups of patients were covered: a retrospective (340) and prospective (154) group. Patients had evidence of bile ducts impaired passability of varying etiology. In these cases ERCP is the final stage in the diagnostic and therapeutic algorithm. All ERCPs were carried out by one expert endoscopist. Patients from the prospective group were administered intramuscularly with Diclofenac $(75 \mathrm{mg})$ before and after the manipulation. The methods used in the study were: demographic data; history and physical examination; laboratory data; imaging methods; ERCP; clinical course and statistical methods for processing data received.

Results: The most common indication for ERCP in all patients was cholestasis constellation $(88.1 \%)$. In a minority of patients ERCP was purely diagnostic $(6.1 \%)$, while at $93.9 \%$ it was also therapeutic. Of all patients at 47 of cases $(9.5 \%)$ hyperamylasaemy was observed, and at 12 or $2.4 \%$ - acute pancreatitis was observed. Patients who developed acute pancreatitis often have acute calculous cholecystitis. Due to the small number of patients with acute pancreatitis, however, these results should be carefully commented. Patients who developed acute pancreatitis were found to have a lower level of alkaline phosphatase and ESR and higher values of leukocytes on the 72nd hour. The univariant logistic regression analysis identified the following risk factors for developing hyperamylasaemy: cholelithiasis; sclerosing papilooditis of ERCP; normal values of serum total bilirubin and elevated CRP levels in receiving. In order to establish a predictive model a multiple logistic regression analysis was performed. The model includes: average total bilirubin at entry and cannulation of the pancreatic duct more than 3 times. The estimated true percentage for predicting lack of hyperamylasaemy with this predictive model is very good - $97 \%$. Univariant logistic regression analysis identified the following risk factors for the development of acute pancreatitis: cannulation of the pancreatic duct and the presence of calculous cholecystitis when entering.

Conclusion: We detected a low incidence of asymptomatic hyperamylasaemy $(9.5 \%)$ and acute pancreatitis $(2.4 \%)$ in the group of patients which were subjected to ERCP. Clinical and laboratory parameters characterizing the patients who developed these complications, and risk factors for acute pancreatitis and asymptomatic hyperamylasaemy were determined. The effect of intramuscular Diclofenac administered before and after ERCP has no effect.

Disclosure of Interest: All authors have declared no conflicts of interest.

\section{P0890 MOST ADVANCED ENDOSCOPY TRAINEES DO NOT MEET COMPETENCE FOR NATIVE PAPILLAE CANNULATION IN ERCP: RESULTS FROM A PROSPECTIVE MULTICENTER STUDY}

S. Wani ${ }^{1}$, M. Hall ${ }^{1}$, V. Simon ${ }^{1}$, S. Han ${ }^{1}$, D. Early ${ }^{2}$, R. Keswani ${ }^{3}$

${ }^{1}$ University of Colorado Anschutz Medical Center, Aurora/United States of America

${ }^{2}$ Washington University in St. Louis, St. Louis/United States of America

${ }^{3}$ Northwestern University, Chicago/United States of America

Contact E-mail Address: sachin.wani@ucdenver.edu

Introduction: Advanced endoscopy trainees (AETs) achieve ERCP competency at variable rates and specific case volumes do not ensure competence. However, training and credentialing guidelines continue to utilize an absolute procedure volume to determine competence. There are limited data on whether current training composition and volumes ensure ERCP competence in the US.

Aims \& Methods: (i) To define ERCP learning curves, utilizing a centralized database, with a focus on cannulation rates using a large national sample of AET programs (AETPs). (ii) To critically examine the composition of current ERCP training in AETPs. ASGE-recognized AETPs were invited to participate and AETs were graded on every ERCP after completion of 25 hands-on ERCP exams. Grading was performed using our previously developed and validated tool [The EUS and ERCP Skills Assessment Tool (TEESAT)] which assesses technical and cognitive competence in a continuous fashion. Grading for each skill was done using a 4-point scoring system: 1-no assistance, 2-minimal verbal cues, 3-multiple verbal cues or hands-on assistance and 4-unable to complete. A comprehensive data collection and reporting system was built using REDCap, a web-based data collection software, and SAS to create learning curves using cumulative sum (CUSUM) analysis for overall and individual technical and cognitive components of ERCP. Individual results and comparison to peers were sent to AETs and trainers quarterly. Acceptable and unacceptable failure rates were set a priori. AETs with $<20$ evaluations were excluded and success was defined as a skill score of 1 or 2 . Individual and combined graphs to assess change in cannulation success rates were constructed and the Cochran-Armitage trend test was used to assess improvement in success rates. 
Abstract: P0891. Table 1.

\begin{tabular}{|c|c|c|c|c|}
\hline & E1 & Notes & A1 & Notes \\
\hline Procedure numbers & 132 & & 118 & \\
\hline \multicolumn{5}{|l|}{ Complications } \\
\hline Pancreatitis (PERCPP) & $3 ; 2.3 \%$ & One death within 30 days & $2 ; 1.7 \%$ & \\
\hline Perforation & $1 ; 0.8 \%$ & Retroduodenal & 0 & \\
\hline Hyperamylasaemia & $1 ; 0.8 \%$ & Metal stent for cholangiocarcinoma & $1 ; 0.8 \%$ & Plastic stent for bile leak \\
\hline Immediate bleeding & $1 ; 0.8 \%$ & Controlled with metal stent & $2 ; 1.7 \%$ & Haemostasis with adrenaline injection \\
\hline Delayed bleeding & 0 & & 0 & \\
\hline Intramural duodenal haematoma & 0 & & $1 ; 0.8 \%$ & On CT \\
\hline Post-ERCP cholangitis & 0 & & 0 & \\
\hline \multicolumn{5}{|l|}{ Patient \& procedural characteristics } \\
\hline Female & $71 ; 53.8 \%$ & & $79 ; 66.9 \%$ & \\
\hline Median age & 63.5 & & 63 & \\
\hline History of Sphicter of Oddi dysfunction & 0 & & 0 & \\
\hline History of PERCPP & 0 & & 0 & \\
\hline History of chronic pancreatitis & $1 ; 0.8 \%$ & & $1 ; 0.8 \%$ & \\
\hline Trainee involvement & 0 & & 0 & \\
\hline Normal LFTs & $21 ; 16.5 \%$ & No data for 5 patients & $11 ; 10 \%$ & No data for 8 patients \\
\hline Non-dilated bile ducts & $39 ; 29.5 \%$ & & $18 ; 15.3 \%$ & \\
\hline Pancreatic duct wire/contrast & $19 ; 15 \%$ & Excludes 5 unsuccessful cannulations & $30 ; 25.9 \%$ & Excludes 2 unsuccessful cannulations \\
\hline Precut sphincterotomy & $4 ; 3 \%$ & & $9 ; 7.6 \%$ & \\
\hline
\end{tabular}

Results: Of the 62 programs invited, 20 AETPs participated and 20 AETs were included in the final analysis. At the end of training, median number of ERCPs performed/AET was 350 (15-500). Overall, 2649 ERCP exams were graded; the majority were ASGE biliary grade $1(77 \%)$ and only $14 \%$ for pancreatic indication. Among biliary ERCP cases, AETs attempted native papilla cannulation and sphincterotomy in $1199(53 \%)$ and 901 (40\%) cases, respectively. The mean time allowed for cannulation was: overall - $4.0 \mathrm{~min}$ (SD 4.3), native papilla - $5.7 \mathrm{~min}$ (SD 4.8), and AET failed cannulation cases - $6.2 \mathrm{~min}$ (SD 5). There was no change in the time allowed for native papilla cannulation during the 1-year period $(\mathrm{p}=0.28)$. AETs were involved in a small proportion of cases requiring advanced cannulation techniques such as double-wire technique, placement of pancreatic duct stent and precut sphincterotomy $(6 \%)$. Learning curves for individual endpoints, overall technical and cognitive aspects noted substantial variability. Majority of AETs achieved overall technical (60\%) and cognitive (100\%) competence at the end of training. While there was a statistically significant improvement in overall and native papilla cannulation rates (both $\mathrm{p}<0.001$ ), only $18 \%$ of AETs achieved competence for native papilla cannulation (Table 1). Conclusion: The results of this study confirm the substantial variability in learning curves and competence among AETs in ERCP validating the shift away from threshold numbers to determine competence. We report the feasibility of establishing a centralized national database to report individualized ERCP learning curves using a novel web-based comprehensive data collection and reporting system. Using strict definitions, a minority of AETs achieved competency in native papilla cannulation which may, in part, be due to limited cannulation time provided to AETs. Selective native papilla deep cannulation needs to be an important benchmark for assessing competence. Methods to improve native papilla cannulation rates and strategies to increase AET exposure to advanced ERCP techniques are required.

Disclosure of Interest: S. Wani: Consultant for Boston Scientific, Medtronic All other authors have declared no conflicts of interest.

\section{P0891 SIMILAR POST-ERCP PANCREATITIS RATES IN ENDOSCOPIST- VS ASSISTANT-CONTROLLED WIRE-GUIDED BILE DUCT CANNULATION: A SINGLE CENTRE OBSERVATIONAL STUDY}

M. Hu, A. Bhagwat, M. Hayat

Dept Of Gastroenterology, Northumbria Healthcare NHS Foundation Trust, North Shields/United Kingdom

Contact E-mail Address: maxworth.hu@gmail.com

Introduction: A recent randomised study by Buxbaum et $\mathrm{al}^{1}$ demonstrated a significantly lower rate of post-endoscopic retrograde cholangiopancreatography (ERCP) pancreatitis (PERCPP) in endoscopist- versus assistant-controlled bile duct cannulation. We set out to audit the rates of PERCPP at our centre based on this finding.

Aims \& Methods: All ERCPs performed by two endoscopists between April 2015 and March 2016 were audited retrospectively. The two endoscopists practiced endoscopist- (E1) and assistant-controlled (A1) wire-guided cannulation exclusively. Both E1 \& A1 had access to the same medications, sphincterotomes, equipment, and teams. Data was obtained and anonymised from electronic patient records and endoscopy reporting software. PERCPP was defined as abdominal pain with hyperamylasaemia at least three times the upper limit of normal (ULN) 24-48 hours after ERCP with or without corresponding imaging findings within 7 days; or abdominal pain with a further rise in pre-existing hyperamylasaemia to at least three times ULN 24-48 hours after ERCP with or without corresponding imaging findings within 7 days. Additional data was collected for CT evidence of post-ERCP complications, hyperamylasaemia without pancreatitis, bleeding, and post-ERCP cholangitis. Patient and procedural characteristics predisposing to pancreatitis were also recorded

Results: A total of 250 procedures were audited. E1 performed 132 procedures (54\% female; median age 63.5$)$ and Al performed 118 procedures $(67 \%$ female; median age 63). The rates of PERCPP in E1 and A1 were 2.3\% (3 patients) and $1.7 \%$ ( 2 patients) respectively. One 84 -year-old patient of $\mathrm{E} 1$ with a presumed malignant common bile duct (CBD) stricture had PERCPP and died 17 days after ERCP, having opted for palliation. The remaining 4 patients had uneventful conservative management of PERCPP. E1 had one patient with immediate bleeding post-sphincterotomy controlled with a CBD stent whilst A1 had 2 patients requiring adrenaline injection for haemostasis. In addition, E1 had one patient with retroduodenal perforation managed conservatively and Al had one patient with CT evidence of intramural duodenal haematoma which was uneventful. Both E1 and A1 had one case each of uncomplicated hyperamylasaemia. A summary of complications, with patient and procedural characteristics, is listed in Table 1 .

Conclusion: In our observational study, which was not intended or powered for statistical analysis, there was no overt difference in the rates of PERCPP when comparing between endoscopist- or assistant-controlled wire-guided CBD cannulation. The overall complication rate was similar and although there were some differences in procedural characteristics between the two endoscopists, there were no characteristics predisposing to PERCPP overtly skewed towards either endoscopist's case load. Further randomised trials, or a crossover study; provided the endoscopists and assistants are equally competent in both methods of wire cannulation, are needed to clarify the safety profile of either technique.

Disclosure of Interest: All authors have declared no conflicts of interest.

\section{Reference}

1. Buxbaum et al. Randomized Trial of Endoscopist-Controlled vs. AssistantControlled Wire-Guided Cannulation of the Bile Duct. 2016. Am J Gastroenterol 111 (12), 1841-1847.

\section{P0892 CONFIRMATION OF THE EFFECT OF AN ANTAGONIST TO CONSCIOUS SEDATION ON THE PREVENTION OF ASPIRATION PNEUMONIA AFTER ENDOSCOPIC RETROGRADE CHOLANGIOPANCREATOGRAPHY}

Y. Taya ${ }^{1}$, M. Kuwatani ${ }^{2}$, K. Sakurai ${ }^{1}$, R. Sasaki ${ }^{1}$, S. Tsunematsu ${ }^{1}$, M. Matsumoto', U. Baba ${ }^{1}$, Y. Tsukamoto ${ }^{1}$, S. Muto ${ }^{1}$, T. Kimura ${ }^{1}$

${ }_{1}^{1}$ Gastroenterology \& Hepatology, Hokkaido Medical Center, Sapporo/Japan ${ }^{2}$ Gastroenterology And Hepatology, Hokkaido University Hospital, Sapporo/Japan

Contact E-mail Address: abe yoko_0922@yahoo.co.jp

Introduction: Most endoscopic retrograde cholangiopancreatography (ERCP) related procedures are performed under "conscious sedation", a drug-induced depression of consciousness during which patients are comfortable and able to maintain purposeful responses to verbal or tactile stimulation, and cardiorespiratory function generally remains intact ${ }^{1)}$. Meanwhile, we sometimes observe adverse events related to conscious sedation after ERCP such as aspiration pneumonia ${ }^{2)}$. So far, it is unknown whether immediate recovery from conscious sedation with antagonists is necessary or not.

Aims \& Methods: We aimed to reveal the efficacy of flumazenil, an antagonist to benzodiazepines, on the prevention of adverse events, especially, aspiration pneumonia related to conscious sedation which is most frequent after ERCP. One hundred ninety patients who underwent ERCP between January to December 
2014 in a general hospital in Japan were included. The patients were divided to two groups: the group with flumazenil (F group) and the group without flumazenil (non-F group) just after ERCP and they were compared and analyzed. Examination items were 1) patient characteristics, 2) procedure characteristics, and 3) occurrence rates of post-ERCP aspiration pneumonia. Pearson's Chisquared test and Fisher's exact test were used for statistical analysis of categorical data.

Results: 1) One hundred fifteen patients $(60.5 \%)$ were administered flumazenil just after ERCP (F group) and 75 patients $(39.5 \%)$ were not (non-F group). The median age, 76 (47-94) in the F group and 78 (46-94) in the non-F group; male/ female ratio, $57 / 58$ in the $F$ group and $34 / 41$ in the non-F group. The distributions of the basic disease (CBD stone/malignant biliary disease/malignant pancreatic disease/others) were $64 / 8 / 17 / 26$ in the $\mathrm{F}$ group and $30 / 11 / 16 / 18$ in the non-F group. The distributions of used benzodiazepines (midazolam/diazepam/ none) and the patient number who got aspiration pneumonia were $102 / 13 / 0$ and 1 in the $\mathrm{F}$ group and 59/13/2 and 1 in the non-F group, respectively (Fisher's test: $\mathrm{p}$-value $=0.074$ ). There were no significant differences of patient characteristics between both groups. 2) The mean procedure times were 36.7 minutes in the $F$ group and 30.3 minutes in the non-F group, respectively. The details of procedures for the major papilla (endoscopic sphincterotomy/endoscopic papillary balloon dilation/others/none) were $40 / 13 / 5 / 56$ in the $\mathrm{F}$ group and $25 / 5 / 3 / 41$ in the non-F group. The distributions of endoscopic biliary drainage or stenting/ none were 94/21 in the F group and 59/16 in the non-F group. (Fisher's test: pvalue $=0.85$ ) There were no significant differences of procedure characteristics between both groups. 3) Two patients $(1.05 \%)$ developed aspiration pneumonia after ERCP. One (94 years old, male) was in the F group and the other ( 81 years old, female) was in the non-F group (Pearson's Chi-squared test p-value $=1$ ). Both of them were over 80 -year old. The patients who were given oxygen during ERCP were $86(45.2 \%)$. Of them, one patient developed pneumonia (Pearson's Chi-squared test $\mathrm{p}$-value $=1$ ).

Conclusion: Flumazenil did not have no preventive effect on the occurrence of aspiration pneumonia related to conscious sedation after ERCP.

Disclosure of Interest: All authors have declared no conflicts of interest.

\section{References}

1. Nekisa Zekeri, Sergio Coda, Shelby Webster et al. Risk factors for endoscopic sedation reversal events: a five-year retrospective study. Frontline Gastroenterology 2015;6:270-277

2. Andrews PJ, Wright DJ, Lamont MC, Flumazenil in the outpatient. A study following midazolam as sedation for upper gastrointestinal endoscopy. Anaesthesia 1990;45:445-458

\section{P0893 PREVENTION OF POST-ERCP PANCREATITIS: RESULTS OF AN AGGRESSIVE HYDRATION PROTOCOL}

P. Marcos, C. Atalaia-Martins, S. Barbeiro, I. Cotrim, C. Gonçalves, L. Eliseu, H. Vasconcelos

Gastroenterology, Centro Hospitalar de Leiria ("Leiria Hospital Center"), Leiria/ Portugal

\section{Contact E-mail Address: pedromarcos1ster@gmail.com}

Introduction: Pancreatitis is the leading complication of endoscopic retrograde cholangiopancreatography (ERCP). Some studies have shown that aggressive intravenous hydration may reduce the incidence of this serious adverse event, the post-endoscopic retrograde cholangiopancreatography pancreatitis (PEP). In our department we implemented an aggressive hydration protocol (AHP) for patients undergoing ERCP in order to prevent PEP.

Aims \& Methods: The aim of this study was to evaluate the impact of this protocol on the incidence and severity of PEP. Patients and methods Retrospective analysis of all patients submitted to ERCP in one center during 16 months, including patients hospitalized in the gastroenterology department who were managed according to the AHP and patients hospitalized in other departments who underwent standard hydration $(\mathrm{SH})$. Patients who underwent AHP received intravenous sodium lactate solution (RL) at $200 \mathrm{~mL} /$ hour starting 1 hour before, during the procedure and the anesthetic recovery; in those who remained asymptomatic after the ERCP, the RL was maintained at $100 \mathrm{~mL} /$ hour for 8 hours and after it was changed to a balanced salt solution with glucose (PG) at $80 \mathrm{~mL} /$ hour; in those patients who had abdominal pain or amylase $>3$ times normal limit after ERCP, the RL was maintained at $200 \mathrm{~mL} /$ hour during 8 hours and after it was changed to PG at $120 \mathrm{~mL} / \mathrm{h}$. We evaluated the incidence and severity of PEP, the established patient and procedure-related risk factors for PEP (except the difficulty of cannulation) and the occurrence of complications. Data were analyzed with SPSS statistical software.

Results: We analyzed 192 patients, 290 ERCP (AHP $\mathrm{n}=168$, SH $\mathrm{n}=122)$. The incidence of PEP was $3.8 \%(11 / 290)$, slightly lower in the $\mathrm{SH}$ group $(4 / 11$ versus $7 / 11 ; p=0.766$ ). In the $\mathrm{SH}$ subset, only $1 \mathrm{PEP}$ was moderate and the remaining 3 were mild. In the AHP group, 3 PEP were moderate and 4 were mild. There were no complications related to AHP. We didn't find any patient or procedure-related variable significantly associated with the development of PEP.

Conclusion: Our AHP didn't reduce the incidence of PEP or its severity. Indeed, the AHP group presented more PEPs than the SH group, although the difference between both PEP incidences was not significant. Despite our study didńt show any advantage related to the use of an AHP, intravenous aggressive hydration may have a role in PEP prophylaxis. Further studies are needed to establish its true value.

Disclosure of Interest: All authors have declared no conflicts of interest.

\section{References}

1. Committee ASoP, Chandrasekhara V, Khashab MA, Muthusamy VR, Acosta RD, Agrawal D, Bruining DH, Eloubeidi MA, Fanelli RD, Faulx AL, Gurudu SR, Kothari S, Lightdale JR, Qumseya BJ, Shaukat A, Wang A, Wani SB, Yang J, DeWitt JM. Adverse events associated with ERCP. Gastrointest Endosc. 2017;85(1):32-47.

2. Buxbaum J, Yan A, Yeh K, Lane C, Nguyen N, Laine L. Aggressive hydration with lactated Ringer's solution reduces pancreatitis after endoscopic retrograde cholangiopancreatography. Clin Gastroenterol Hepatol. 2014;12(2):303-307 e301.

3. Sagi SV, Schmidt S, Fogel E, Lehman GA, McHenry L, Sherman S, Watkins J, Cote GA. Association of greater intravenous volume infusion with shorter hospitalization for patients with post-ERCP pancreatitis. J Gastroenterol Hepatol. 2014;29(6):1316-1320.

4. Dumonceau JM, Andriulli A, Elmunzer BJ, Mariani A, Meister T, Deviere J, Marek T, Baron TH, Hassan C, Testoni PA, Kapral C, European Society of Gastrointestinal E. Prophylaxis of post-ERCP pancreatitis: European Society of Gastrointestinal Endoscopy (ESGE) Guideline - updated June 2014. Endoscopy. 2014;46(9):799-815.

5. Smeets XJ, da Costa DW, Besselink MG, Bruno MJ, Fockens P, Mulder CJ, van der Hulst RW, Vleggaar FP, Timmer R, Drenth JP, van Geenen EJ. Systematic review: periprocedural hydration in the prevention of post-ERCP pancreatitis. Aliment Pharmacol Ther. 2016;44(6):541-553.

\section{P0894 ERCP CYTOLOGY YIELD - DOES THE BRUSH MATTER?}

I. Mocanu, A. Laranjo, S. Pires, N. Veloso, L. Gonçalves, R. Godinho, I. Medeiros

Gastroenterology, Hospital Espirito Santo de Évora, Évora/Portugal

Contact E-mail Address: irina.mocanu.24@gmail.com

Introduction: Extra-hepatic biliary tree strictures are caused by a variety of malignant and benign diseases. The brushing of such stenosis during ERCP is safe and easy to perform, however, it has a low sensibility, ranging from 30 to $60 \%$. Efforts to improve citology yield include dilation of the stricture prior to brushing and multiple brush passages, both without significant success. In 2011, US Endoscopy introduced the Infinity® cytology brush - a 14Fr $(4,75 \mathrm{~mm})$ device that combines soft and stiff bristles in order to improve acquisition of cytology samples.

Aims \& Methods: We aimed to determine if a new-design brush can improve the diagnostic yield of biliary cytology. From February 2015 until December 2016, the new Infinity ${ }^{\circledR}$ brush was used in all cases of ERCP cytology. These were compared with historical controls, where a classical 8 Fr biliary cytology brush was used. In both groups, at least two passages were made, with transfer to a thin prep solution, as per prior protocol. Follow-up data, namely clinical course, radiological data or other histological results were collected for a definitive diagnosis.

Results: Thirty-five new brush cases were compared with 52 historical controls. There was no significant difference between gender $(57 \%$ Vs. $52 \%$ male; $\mathrm{p}>0.05$ ), age (mean 70.5 Vs. 74 years; $p>0.05$ ), location of stricture (common bile duct $77 \%$ Vs. $84 \% ; p>0.05$ ), length of stricture (between $1-$ $3 \mathrm{~cm}$ in $80 \%$ Vs. $84 \% ; \mathrm{p}>0.05)$ or dilation prior to sampling $(71 \%$ Vs. $57.7 \% ; \mathrm{p}>0.05$ ) between the two groups. Physicians' impression of malignant stricture during ERCP was more frequent in new brush cases, with near statistical significance $(91 \%$ Vs. $75 \% ; p=0.052)$. Sufficient sample size for cytological analysis was more frequently obtained in cases when compared to controls $(86 \%$ vs. $100 \%, p<0.05)$. Definitive diagnosis were as follows (cases Vs. controls): pancreatic neoplasia $57 \%$ Vs. $48 \%$, cholangiocarcinoma $25.7 \%$ Vs. $23 \%$, ampuloma 0 Vs. $1.9 \%$, benign conditions $17.3 \%$ Vs. $21 \%$, no definitive diagnosis 0 Vs. $6.1 \%$. In malignant cases, there was no difference between sensibilities of the cases $(31 \%)$ and controls $(27 \%)$, either for pancreatic cancer $(20 \%$ Vs. $24 \%$; $\mathrm{p}>0.05)$ nor cholangiocarcinoma $(55.6 \%$ Vs. $50 \%$; p > 0.05$)$. Specificity, positive predictive value and negative predictive value of Infinity ${ }^{\circledR}$ brush and classical 8Fr brush were as follows: $100 \%$ Vs. $100 \% ; 100 \%$ Vs. $100 \%, 23 \%$ vs. $35.7 \%$. Of the 16 strictures with benign features on ERCP ( 3 cases Vs. 13 controls), 14 had negative cytology and 2 were inconclusive (both controls).

Conclusion: There were significantly more samples with adequate cellularity for cytological analysis with the new Infinity $($ brush, however, the global sensibility of the new brush was not superior to the standard devices. With both brushes, the yield is twice as better for cholangiocarcinoma than for pancreatic cancer. One disadvantage we noted with the Infinity ${ }^{\circledR}$ brush is the increased difficulty in passing very tight, fibrotic strictures, even after dilation, due to its width and rigidity. Further ways to improve results would be the routine production of a smear sample, cell block analysis or intra-ductal biopsies. The future era of cholangioscopy could dramatically increase our ability to sample biliary strictures. Disclosure of Interest: All authors have declared no conflicts of interest. 
P0895 PROSPECTIVE STUDY OF EARLY PRECUT VS. UTMOST PRECUT WITH PANCREATIC STENT IN INITIAL PANCREATIC DUCT CANNULATION

E.T. Park, B.C. Yun, S.U. Lee, B.H. Han

Internal Medicine, Kosin University, Gospel Hospital, Busan/Korea, Republic of

Contact E-mail Address: euntpark@hanmail.net

Introduction: In biliary access, repeated biliary cannulation attempts are a risk factor for post ERCP pancreatitis (PEP). Early precut is an effective technique for successful biliary cannulation and can significantly reduce the incidence of PEP. The aim of this study was prospectively to evaluate clinical efficacy the performance of utmost early precut with pancreatic stent in the patients in whom pancreatic duct cannulation was performed initially.

Aims \& Methods: When guidewire was placed in the pancreatic duct initially by chance, the patients were randomized into early precut (Group A) or utmost early precut sphincterotomy with pancreatic stent (Group B). In Group A, pancreatic duct cannulation within 5 times and attempted precut papillotomy without pancreatic stent. In Group B, from the first, pancreatic stent was inserted and then precut with an incision over a pancreatic stent was done. Main outcome measurements were frequency of successful CBD cannulation and post-procedure related complications

Results: From January 2015 to August 2016, the two groups were similar with regard to patient demographics. A total of 50 patients were enrolled. 26 patients were assigned to the Group A and 24 to the Group B. Successful CBD cannulation was achieved in 23 of $26(88.5 \%)$ patients in the Group A and 23 of 24 $(95.8 \%)$ patients in the Group B. The mean cannulation time was 16.9 minutes in the Group A and 14.8 minutes in the Group B. Post-procedure hyperamylasemia was significantly higher in Group A. The overall incidence of post-procedure pancreatitis was $11.5 \%(3 / 26)$ in the Group A and $4.2 \%(1 / 24)$ in the Group B $(\mathrm{P}<0.001)$.

Conclusion: In patients with pancreatic duct cannulation initially by chance, compare to early precut group, utmost early precut with pancreatic stent over the guidewire group not only facilitate biliary cannulation and the success rates but also promise low incidence of post-ERCP pancreatitis. In experienced hands, utmost early precut technique can dramatically reduce the trauma of ampulla and risk of PEP compared with conventionally persistent cannulation attempts. Disclosure of Interest: All authors have declared no conflicts of interest.

\section{References}

1. Rrakalathan S., Philip M, Michael J. B. Early Precut Sphincterotomy Does Not Increase Risk During Endoscopic Retrograde Cholangiopancreatography in Patients With Difficult Biliary Access: A Meta-analysis of Randomized Controlled Trials. Clinical Gastroenterology and Hepatology 2015;13:1722-1729.

2. Alberto M., Milena D., Nicola G., et al. Early precut sphincterotomy for difficult biliary access to reduce post-ERCP pancreatitis: a randomized trial. Endoscopy 2016;48: 530-535.

3. David Z., Martín G., Cecilia C., et al. Early precut is as efficient as pancreatic stent in preventing post-ERCP pancreatitis in high-risk subjects - A randomized study. Rev. Esp Enferm Dig. 2016;108: 558-562.

4. Tiing L., Andrew B., Mingjun S., et al. A comparative study of strategies for prevention of post-ERCP pancreatitis after early pre-cut sphincterotomy for biliary access. Gastrointest Endosc 2015; 81 (Suppl): AB358.

\section{P0896 POST-ERCP BLEEDING IN THE ERA OF MULTIPLE \\ ANTIPLATELET AGENTS}

H. Oh ${ }^{1}$, I. El Hajj ${ }^{2}$, J. Easler ${ }^{2}$, E. Fogel ${ }^{2}$, J. Watkins ${ }^{2}$, L. Mchenry ${ }^{2}$, G. Lehman ${ }^{2}$, J.S. Choi ${ }^{3}$, H. Kang ${ }^{4}$, S. Sherman ${ }^{2}$

${ }^{1}$ College Of Medicine, Chung-Ang University, Seoul/Korea, Republic of

${ }^{2}$ Gastroenterology And Hepatology, Indiana University, Indianapolis/United States

of America/IN

${ }^{3}$ Inje University, Busan/Korea, Republic of

${ }^{4}$ Anesthesiology, Chung-Ang University, Seoul/Korea, Republic of

Contact E-mail Address: ohcgi@cau.ac.kr

Introduction: Antithrombotic therapy with antiplatelet agents (APA) has been increasingly utilized during the last few decades. This study aimed to determine the risk of post-ERCP bleeding among those patients who are taking APAs, especially in the era of multiple agents.

Aims \& Methods: From July 2012 to May 2016, the patients who underwent 1st therapeutic ERCP were identified from the ERCP database of 3 institutions in USA and Korea. The primary outcomes were the frequency, type, and severity of ERCP-related bleeding according to the use of APA.

Results: The frequencies of post-ERCP bleeding among the four different groups were 16 of $2083(0.8 \%)$ in No drug group, 12 of $256(4.7 \%)$ in Aspirin group, 3 of $48(6.3 \%)$ in Single APA group, and 4 of $48(8.3 \%)$ in Multiple APA group $(\mathrm{p}<0.001)$. Most cases of post-ERCP bleeding were mild $(29 / 35,88 \%)$. In the univariate analysis, post-ERCP bleeding was associated with age, pull-type sphincterotomy, and APA, and inversely associated with balloon dilation of the biliary orifice. In the multivariate analysis, pull-type sphincterotomy (odds ratio [OR] 7.829, 95\% confidence interval [CI] 1.411-43.453, $\mathrm{p}=0.019$ ) and country (Korea; OR $0.124,95 \%$ CI $0.042-0.361, \mathrm{p}<0.001$ ) were associated with post-ERCP bleeding.

Conclusion: The frequency of post-ERCP bleeding was statistically higher in patients on any APAs within 6 days prior to ERCP. However, in the multivariate analysis, APA use was not associated with post-ERCP bleeding. Until a large study adequately powered to detect differences in bleeding rates, caution is recommended when considering invasive procedures during ERCP in patients on APAs.

Disclosure of Interest: All authors have declared no conflicts of interest.

P0897 PROSPECTIVE COMPARISON OF DIGITAL SPYGLASS DIRECT VISUALIZATION SYSTEM VS DIRECT PERORAL CHOLANGIOSCOPY USING A MULTIBENDING ENDOSCOPE AS A SINGLE-OPERATOR CHOLANGIOSCOPY FOR MANAGING BILIARY LESIONS

Y.N. Lee ${ }^{1}$, J.H. Moon ${ }^{1}$, H.J. Choi ${ }^{1}$, H.K. Kim ${ }^{2}$, T.H. Lee ${ }^{1}$, H.W. Lee ${ }^{1}$, M.H. Choi ${ }^{1}$, S. Cha ${ }^{1}$, Y.D. Cho ${ }^{1}$, S. Park ${ }^{1}$

${ }^{1}$ Digestive Disease Center And Research Institute, Department Of Internal Medicine, SoonChunHyang University School of Medicine, Bucheon and Seoul/ Korea, Republic of

${ }^{2}$ Department Of Pathology, SoonChunHyang University School of Medicine, Bucheon/Korea, Republic of

Contact E-mail Address: yunnah $@$ schmc.ac.kr

Introduction: In a recent, a digital version of single-operator cholangioscope (SpyGlass DS) and direct POC (DPOC) using a mulibending ultraslim endoscope were introduced as improved forms of each POC, especially in image quality and technical difficulty, respectively.

Aims \& Methods: In this study, we prospectively compared the procedure success rate of SpyGlass DS and DPOC for diagnosis and treatment of BD lesions. A total of 15 patients with $\mathrm{BD}$ lesions (diameter of $\mathrm{CBD} \geq 8 \mathrm{~mm}$ ) requiring evaluation or treatment using POC were enrolled prospectively. All patients received POCs using SpyGlass DS and mulibending ultraslim endoscope for DPOC. According to the presence of obstructive lesion, all patients were classified as obstructive type or non-obstructive type, respectively. Procedural success defined as an ability to advance the cholangioscope to the desired target and get adequate cholangioscopic visualization for the targeted lesion.

Results: The overall technical success rates of SpyGlass DS and DPOC were $100 \%$ and $93.3 \%$, respectively $(P=0.5)$. In DPOC, 1 patient was failed to insert the endoscope into the CBD. In SpyGlass DS, 2 patients were failed to visualize the targeted lesion. The procedural success rates of SpyGlass DS and DPOC according to the type of lesion were not different in 9 obstructive type $(100 \%$ vs. $88.9 \%, P=0.5)$ and 6 non-obstructive type $(66.7 \%$ vs. $100 \%$, $P=0.227)$. The successful diagnostic and therapeutic procedures by DPOC and SpyGlass DS were observed in 8 of 8 patients $(100 \%)$ and 7 of 9 patients $(77.8 \%)(P=0.265)$, respectively.

Conclusion: Both advanced image quality of SpyGlass DS and improved technical difficulty of DPOC by a mulibending ultraslim endoscope showed comparable and high procedure success rates in patients with dilated BD. Future prospective studies focused on overall cost savings and long-term clinical outcomes are seems to be required for deciding adequate indications of each POC systems.

Disclosure of Interest: All authors have declared no conflicts of interest.

P0899 THE DILEMMA OF MANAGMENT BORDERLINE COMMON BILE DUCT STONE. DOES STONE SIZE MATTER?: A PROSPECTIVE RANDOMIZED STUDY

E. Abdellatif, E. Elhanafy, A. Elnakeeb

Surgery, Gastroenterology Surgical Center, Mansoura/Egypt

Contact E-mail Address: dr.ehab.atif@icloud.com

Introduction: Management of common bile duct stones (CBDS) in patients with borderline CBD presents a surgical challenge. The aim of this study was to compare conservative treatment with endoscopic stone extraction for the treatment of borderline CBD with stones.

Aims \& Methods: This prospective randomized controlled trial includes patients with $\mathrm{CBDS}$ in borderline $\mathrm{CBD}(\mathrm{CBD}<10 \mathrm{~mm})$ associated with gallbladder stones who were treated with conservative treatment or endoscopic stone extraction followed by laparoscopic cholecystectomy (LC) and intraoperative cholangiogram (IOC). The primary outcome was successful CBD clearance. The secondary outcomes were the overall complications, cost, and hospital stay. Results: LC and IOC revealed complete clearance of CBDS in $48(96 \%)$ cases in the endoscopic retrograde cholangiopancreatography (ERCP) group (52\% of patients by ERCP, and $44 \%$ of patient passed the stone spontaneously), and in the remaining two patients, the CBDS was removed by transcystic exploration. In the conservative group, LC and IOC revealed complete clearance of CBDS in $90 \%$ of cases, and in the remaining $10 \%$ of patients, the CBDS was removed by transcystic exploration. Post-ERCP pancreatitis (PEP) is noticed significantly in the ERCP group $(2[4 \%]$ versus $8[16 \%] ; \mathrm{P}=.04)$. The average net cost was significantly higher in the ERCP group. Recurrent biliary symptoms developed significantly in the ERCP group after 1 year $(10 \%$ versus $0 \% ; \mathrm{P}=.02)$ in the form of recurrent cholangititis and recurrent CBDS.

Conclusion: Management of CBDS in patients with borderline CBD represents a surgical challenge. Borderline CBD increases the technical difficulty of ERCP and increases the risk of PEP. Conservative management of CBDS in borderline CBD not only avoids the risks inherent in ERCP and unnecessary preoperative ERCP, but it is also effective in clearing CBDS. The hepatobiliary surgeon 
should consider a conservative line of treatment in CBDS in borderline CBD in order to decrease the cost and avoid unnecessary ERCP.

Disclosure of Interest: All authors have declared no conflicts of interest.

\section{P0900 PROSPECTIVE STUDY ON METHODS AND SUCCESS OF BILIARY CANNULATION OF 458 VIRGIN PAPILLAS - QUALITY ASSURANCE OF ERCP AT OUR DEPARTMENT}

Á. Orbán-Szilágyi, K. Lorinczy, K. Rábai, M. Horváth, F. Zsigmond, T. Gyökeres

Gastroenterology, Medical Centre, Hungarian Defence Forces, Budapest/Hungary

Contact E-mail Address: tiborgyokeres@freemail.hu

Introduction: The cannulation of a virgin papilla is the most difficult and highrisk step in ERCP and it requires significant experience to maximize the success and to minimize poor outcomes. Cannulation rate is one of the accepted quality indicators of ERCP. It is mandatory to regularly assess quality indicators of endoscopic procedures to maintain and improve endoscopic service.

Aims \& Methods: We prospectively collected data about cannulation details of all patients with virgine papillas and post-ERCP complications from April 2016 to April 2017.

Results: During this 12-months period we have performed 1102 ERCPs, in 458 of them we had virgin papilla. All ERCPs had therapeutic intentions and all of the patients were followed up. In 13 patients papillas were not accessible due to duodenal stenosis $(10 / 13)$ or postoperative situations $(3 / 10)$. In two of them the indication was ceased (because the biliary obstruction resolved spontaneously), 11 patients got percutaneous transhepatic drainage (PTD). The primary cannulation success rate of accessible papillas was $88.5 \%(394 / 445)$ while the overall cannulation success was $96.6 \%(430 / 445) .56 .1 \%$ of primary successful cannulations were achieved by conventional method, in $14.2 \%$ we used pancreatic guidewire assisted technique, in $20.1 \%$ we used early precut sphincterotomy, and in the rest cases we used combined techniques. In 51 primary unsuccessful cases we repeated ERCP attempt in 4 days on average and successfully cannulated $70.5 \%(36 / 51)$ of them at the second or third attempts. 27 of them were achieved by conventional method, 7 of them after extending the precut, one case was cannulated using pancreatic guidewire technique, and we used profilactic pancreatic stent in one patient, as well. Out of the 15 patients with finally unsuccessful cannulation, we performed precut without deep cannulation in 10 cases. 7 of them resolved after precut, 2 of the 10 patients got PTD and one patient refused further investigation. In 4 patients out of the 15 unsuccessful cannulations the obstruction resolved without any further intervention and one patient got PTD. We had in sum $3.4 \%(15 / 445)$ post-ERCP pancreatitis, 7 of them were mild, and 8 moderate, we had no severe one. We observed endoscopic signs of bleeding during ERCP in 34 cases that needed some endoscopic interventions (infiltration/coagulation/stenting), 7 of them (1.6\%) required blood transfusion. Three patients suffered perforation during ERCP. One of them got biliary stent and was discharged uneventfully on the 8th day. We had 2 sphincterotomy related perforations, 1 had early surgery - he died on the 14th day, another patient had delayed surgery, he recovered.

Conclusion: Quality assessment of ERCP performance is essential. Our overall cannulation rate was acceptable. We used pancreatic guidewire technique just after first guidewire passage of pancreatic duct and switched on to precut at early phase of process to avoid long lasting traumatisation of the papilla. Our complication rate of post-ERCP pancreatitis was good while the post-sphincterotomy bleeding rate should be considered higher than in the literature, therefore we changed the settings of electrosurgical current.

Disclosure of Interest: All authors have declared no conflicts of interest.

\section{P0901 RISK FACTORS FOR POST-ENDOSCOPIC RETROGRADE CHOLANGIOPANCREATOGRAPHY PANCREATITIS: A PROSPECTIVE MULTICENTER STUDY}

A. Suzuki ${ }^{1}$, B. Endoh ${ }^{2}$, K. Chikugo ${ }^{3}$, T. Kawakami ${ }^{4}$, T. Suzuki ${ }^{4}$, N. Yoshitaka ${ }^{5}$, K. Kusumoto ${ }^{5}$, Y. Itokawa ${ }^{5}$, O. Inatomi ${ }^{6}, \mathrm{~S}$. Bamba $^{6}, \mathrm{~K}$. Uno ${ }^{1}$, Y. Mizumoto ${ }^{2}$, K. Tanaka ${ }^{1}$

${ }^{1}$ Department Of Gastroenterology, Kyoto Second Red Cross Hospital, Kyoto/ Japan

${ }^{2}$ Department Of Gastroenterology, Kyoto Medical Center, Kyoto/Japan

${ }^{3}$ Department Of Gastroenterology, National Hospital Organization Kyoto Medical Center, Kyoto/Japan

${ }^{4}$ Department Of Gastroenterology, Japanese Red Cross Kyoto Daiichi Hospital, Kyoto/Japan

${ }^{5}$ Digestive Disease Center, Department Of Gastroenterology And Hepatology, Kyoto Katsura Hospital, Kyoto/Japan

${ }^{6}$ Division Of Gastroenterology, Shiga University of Medical Science, Shiga/Japan

Contact E-mail Address: azmaked@gmail.com

Introduction: The procedures related to endoscopic retrograde cholangiopancreatography (ERCP) play a major role in the diagnosis and treatment of pancreatobiliary disease. However, post-ERCP pancreatitis (PEP) remains the most common and severe complication of ERCP. The incidence rates of and risk factors for PEP have mainly been reported from retrospective studies.

Aims \& Methods: This study aimed to identify the incidence rate of and risk factors for PEP in a prospective large cohort study. This is a prospective cohort study of all patients who underwent ERCP-related procedures at 5 high-volume centers between February 2015 and May 2016. Patients who presented with acute pancreatitis, post biliary reconstruction, and failure to reach the papilla were excluded. The incidence rates of PEP and its severity were examined. Multivariate analysis was used to identify the risk factors for the disease. Patients who presented with at least two of the following three manifestations were diagnosed with PEP: 1) elevated levels of serum amylase, 2) abdominal pain lasting more than 24 hours, and 3) abnormal findings of acute pancreatitis on computed tomography. An increase in serum amylase level of at least three times greater than the normal upper limit at approximately $18 \mathrm{~h}$ after the procedure (the next morning) was regarded as significant. The severity of PEP was graded according to the severity assessment of the Japanese Ministry of Health, Labour, and Welfare.

Results: A total of 1932 patients were finally analyzed. PEP occurred in 142 patients $(7.3 \%)$; it was mild in 117 patients $(6.0 \%)$ and severe in 25 patients $(1.3 \%)$. Univariate analysis showed that female gender, naive papilla, surgically altered gastrointestinal anatomy, no coexistence of acute cholangitis, diagnostic ERCP, elective ERCP, procedure time after reaching the papilla, number of cannulation attempts, precut sphincterotomy, intraductal ultrasonography, pancreatic duct injection, insertion of guidewire into the pancreatic duct, and placement of prophylactic pancreatic stent were significant risk factors. In the multivariate analysis, female gender (OR 2.273; 95\%CI 1.570-3.291), naïve papilla (OR 3.024; 95\% CI 1.805-5.066), surgically altered gastrointestinal anatomy (OR 2.607; 95\% CI $1.378-4.931$ ), procedure time after reaching the papilla (OR 1.009; 95\%CI 1.001-1.017), pancreatic duct injection (OR 2.297; 95\% CI $1.493-3.534$ ), and intraductal ultrasonography (OR 1.620; 95\% CI 1.015-2.585) were independent risk factors.

Conclusion: The incidence of PEP was similar to those reported in previous studies. These risk factors are important as predictors of PEP. A shorter procedure time and avoidance of unnecessary pancreatic duct injection are important for prevention of PEP.

Disclosure of Interest: All authors have declared no conflicts of interest.

\section{P0902 USEFULNESS OF AMY MEASUREMENTS AT 2 HOURS AFTER ENDOSCOPIC RETROGRADE CHOLANGIOPANCREATOGRAPHY IN PREDICTING THE SEVERITY OF POST-ERCP PANCREATITIS, : MULTICENTER PROSPECTIVE STUDY, SOSUI}

K. Takumi ${ }^{1}$, T. Suzuki ${ }^{1}$, H. Sato ${ }^{1}$, N. Yoshida ${ }^{1}$, Y. Nakai ${ }^{2}$, K. Kusumoto ${ }^{2}$, Y. Itokawa ${ }^{2}$, A. Suzuki ${ }^{1}$, B. Endoh ${ }^{3}$, K. Chikugo ${ }^{3}$, O. Inatomi ${ }^{4}$, S. Bamba ${ }^{4}$ Y. Mizumoto ${ }^{3}, \mathrm{~K}$. Tanaka

${ }^{1}$ Gastoroenterology, Japanese Red Cross kyoto Daiichi Hospital, Kyoto/Japan ${ }^{2}$ Digestive Disease Center, Department Of Gastroenterology And Hepatology, Kyoto Katsura Hospital, Kyoto/Japan

${ }^{3}$ Department Of Gastroenterology, Kyoto Medical Center, Kyoto/Japan

${ }^{4}$ Division Of Gastroenterology, Shiga University of Medical Science, Shiga/Japan

Contact E-mail Address: mbyqm671@yahoo.co.jp

Introduction: The severity of post-ERCP pancreatitis (PEP) is a major problem because of occasional lethality. However, no predictor of the severity has been identified. In the present multicenter prospective study, SOSUI, we investigated the potential predictors of severity.

Aims \& Methods: Of 2078 subjects who underwent ERCP between February 2015 and May 2016 at five high-volume centers, 1932, excluding those who had been complicated by pancreatitis, undergone biliary tract reconstruction, or had papilla not reached, were included. Of the 1932 patients, 163 who developed PEP were compared between the mild and severe cases to examine potential predictors of severity. PEP was diagnosed based on two or more of the following three conditions: (1) serum amylase elevation (above the upper limits of each center) on the following day, (2) abdominal pain lasting for longer than 24 hours, and (3) pancreatitis on CT. Severity was assessed based on the severity criteria of the Ministry of Health, Labour and Welfare. For continuous variables, the Mann-Whitney U test was employed. For binomial comparison, univariate analysis was conducted using a chi-square test.

Results: Twenty-five severe $(1.3 \%)$ and 138 mild (7.1\%) PEP patients were included. Patient and procedural factors were examined, demonstrating that the AMY values at 2 hours after ERCP were significantly higher in the severe cases than in the mild ones $(\mathrm{P}=0.005)$. ROC analysis was conducted on the AMY values at 2 hours as a predictor of severe PEP, demonstrating that the cutoff value was 3.7 times higher than the upper limits of each center (sensitivity: $64 \%$; specificity: $70 \%$; and AUC: 0.65 ). In multivariate analysis using logistic regression by dividing the AMY values at 2 hours after ERCP into higher or lower than 3.7 times the reference values of each center, abdominal pain immediately after ERCP and AMY values at 2 hours after ECRP were identified as independent factors.

Conclusion: Severe PEP should be diagnosed as early as possible for intervention. However, it may take a long time to assess clinical courses or examine images after examination. In the present study, abdominal pain immediately after ERCP and AMY values at 2 hours were identified as predictors of severity, being useful for facilitating early therapeutic intervention.

Disclosure of Interest: All authors have declared no conflicts of interest.

\section{References}

Cotton PB, Lehman G, Vennes J, et al. Endoscoppic sphincterotomy complications and their management:an attempt at consensus, Gastorintest Endosc 1991:37:383-393

Freeman ML, DiSario JA, Nelson DB, et al. Risk factors for post-ERCP pancreatitis: A prospective multicenter study. Gastorointest Endosc 2001;54:425-434

Cheng CL, Sherman S, Watkins JL, et al. Risk Factors for Post-ERCP pancreatitis: A prospective multicenter study. AM J Gastroenterology 2006;101:139-147 
Young KC, Kwang BC, James L, et al. Frequency and Severity of post-ERCP pancreatitis correlated with of pancreatic ductal opacification. Gastrointest Endosc 2007;65:385-393

\section{P0903 ENDOSCOPIC ULTRASOUND-BASED TRANSDUODENAL CHOLECYSTOLITHOTOMY VIA DOUBLE-FLANGED FULLY COVERED METAL STENT WITH HOT STENT DELIVERY}

\author{
S. Sun \\ Endoscopy Center, Shengjing Hospital of China Medical University, Shenyang \\ China
}

Contact E-mail Address: sunsiyucmu@aliyun.com

Introduction: Laparoscopic cholecystectomy (LC) has become the 'gold standard' for the treatment of symptomatic gallstones. However, before clinical implementation, instruments still need modification, and a more convenient treatment is still needed.

Aims \& Methods: The aim of this study was to evaluate the transduodenal tractcholecystoscopy technique in the treatment of gallbladder disease without cholecystectomy. The patient with cholesterol gallstones and high surgical risks were enrolled between January 2015 and March 2017. Endoscopic ultrasound (EUS)-guided cholecystoduodenostomy by deploying a double-flanged fully covered metal stent with hot stent delivery was performed and endoscopic sphincterotomy (EST) was also performed during this procedure for those patients with accompanying common bile duct stones. One or two weeks later an forwardviewing endoscope was advanced into the gallbladder via the stent, and cholecystolithotomy or polypectomy was performed. After the stents were removed, a pigtail-type naso-cholecystic drainage catheter was inserted into the gallbladder over the guide wire and removed 2 days later. Four weeks later gallbladder was assessed by abdominal ultrasound.

Results: EUS-guided cholecystoduodenostomy with double flanged mental stent deployment was successfully performed in all of 26 patients (Male/Female, 11/15; mean age, $61 \pm 16.19 \mathrm{yrs})$. After the procedure, fistulas had formed in each of thepatients and the stones of 7 patients expelled themselves completely. Endoscopic cholecystolithotomy (19) and polyps resection (2) were successfully performed through the stents, and then the stents were removed. Common bile duct stones were also successfully removed in 6 patients. EGD showed all the fistula closed completely after 3 days. The ultrasound examination of the gallbladder 4 weeks later showed no stones remaining and also showed satisfactory functioning of the gallbladder. The mean follow-up period was 11 months (range: $1-27$ months). Cholesterol gallstones recurrence were not detected in any patient during follow-up.

Conclusion: The EUS-guided placement of a novel metal stent with hot stent delivery is a safe and simple approach for performing an endoscopic cholecystoduodenostomy, which can subsequently allow procedures tobe performed for treating biliary disease, including cholecystolithotomy.

Disclosure of Interest: All authors have declared no conflicts of interest.

\section{References}

1. Strain M, Chisevescu D, Blaj S, et al. The first NOTE (Natural Orifice Translumenal Endoscopic) cholecystectomy in Romania: An experimental model in pig. Chirurgia (Bucur) 2009;104:173-9.

2. Cuadrado-Garcia A, Noguera JF, Olea-Martinez JM, et al. Hybrid natural orifice transluminal endoscopic cholecystectomy: Prospective human series. Surg Endosc 2011;25:19-22.

3. Cho YB, Park CM, Chun HK, et al. Transvaginal endoscopic cholecystectomy using a simple magnetic traction system. Minim Invasive Ther Allied Technol 2011;20:174-8.

4. Ogredici O, Linke GR, Lamm S, et al. Routine cholangiography during rigid-hybrid transvaginal natural orifice transluminal endoscopic cholecystectomy. Surg Endosc 2014;28:910-7.

5. Hall RC. Is natural orifi ce transluminal endoscopic cholecystectomy as safe as laparoscopic cholecystectomy? Arch Surg 2008;143:604.

6. Parra V, Kedia P, Zerbo S, et al. Drainage of infected pancreatic necrosis by using 2 lumen-apposing metal stents, a nasocystic drain, and hydrogen peroxide. Gastrointest Endosc 2015;81:1261.

\section{P0904 QUANTITATIVE ENDOSCOPIC ULTRASOUND ELASTOGRAPHY IN THE DIFFERENTIAL DIAGNOSIS OF PANCREATIC SOLID TUMORS}

H. Ribeiro ${ }^{1}$, C. Leitão ${ }^{1}$, J. Pinto ${ }^{2}$, R. Azevedo ${ }^{1}$, F. Pereira ${ }^{1}$, R. Sousa ${ }^{1}$, A.I.L. Pires Caldeira ${ }^{1}$, E. Pereira ${ }^{1}$, A. Banhudo ${ }^{1}$

${ }^{1}$ Gastroenterology, Amato Lusitano Hospital, Castelo Branco/Portugal

${ }^{2}$ Gastrenterologia, Unidade Local de Saúde de Castelo Branco, Castelo Branco/ Portugal

Contact E-mail Address: helena.britoribeiro@gmail.com

Introduction: Quantitative second generation endoscopic ultrasound (EUS) elastography allows the quantitative analysis of tissue stiffness and can be a useful auxiliary tool in the differential diagnosis of pancreatic solid tumors (1)(2).

Aims \& Methods: The aim of this study was to evaluate the accuracy of the quantitative EUS elastography in the differential diagnosis of pancreatic solid masses, discriminating malignant from benign masses, using strain ratio (SR) analysis. A prospective study was performed for 15 months and included 29 consecutive patients who underwent EUS for the evaluation of solid pancreatic masses. EUS elastography was performed by 2 operators, using a linear echoendoscope. The mean of 3 measures was considered as the SR final result for each lesion. EUS-fine-needle aspiration of the lesions was performed after SR assessment and the final diagnosis was based on the cytology or histology results. Accuracy of the elastography was obtained by the analysis of ROC curves.

Results: Included 29 patients in a total of 30 lesions with conclusive histological/ cytologic diagnosis ( 8 inflammatory masses, 19 adenocarcinomas, 2 neuroendocrine tumors and 1 undifferentiated carcinoma). The mean SR value was significantly higher in the malignant tumors comparing with the benign tumors $(55.56$ vs $23.93, \mathrm{p}=0.001$ ). The sensitivity and specificity of SR for detection of pancreatic malignancy for a cut of 15.89 were, respectively, $95.45 \%$ and $87.5 \%$ (area under the curve of $0.89,95 \% \mathrm{CI}$ ). The overall accuracy of the EUS elastography using the SR for the detection of pancreatic malignancy was $93 \%$.

Conclusion: Quantitative EUS elastography presents good accuracy in the differentiation between malignant and benign pancreatic masses. It is a promising EUS technique in the diagnostic approach of solid pancreatic lesions, which may complement the study and characterization of the tumors, aiding in the diagnostic and follow-up of this patients.

Disclosure of Interest: All authors have declared no conflicts of interest.

\section{References}

1. Garcia JI, Noia JL, Abdulkader I, Forteza J, Munoz. Gastroenterology.; 2010;139(4):1172-80.

2. Cosgrove D, Piscaglia F, Bamber J, Bojunga J, Gilja OH, Klauser AS, et al. EFSUMB Guidelines and Recommendations on the Clinical Use of Ultrasound Elastography. Part 2: Clinical Applications.: 238-53.

\section{P0905 IS ENDOSCOPIC ULTRASONOGRAPHY EXAMINATION USEFUL BEFORE ENDOSCOPIC SUBMUCOSAL DISSECTION IN PATIENTS OF SUPERFICIAL ESOPHAGEAL SQUAMOUS CELL CARCINOMA?}

Y.Y. Chu ${ }^{1}$, S.C. $\mathrm{Ng}^{2}$, J.M. Lien ${ }^{1}$, C.T. Chiu ${ }^{1}$

${ }^{1}$ Gastroenterology And Hepatology, Chang Gung Memorial Hospital, Taoyuan Taiwan

${ }^{2}$ Internal Medicine, Chang Gung Memorial Hospital, Keelung/Taiwan

Contact E-mail Address: chu2235@yahoo.com

Introduction: Esophageal squamous cell carcinoma (ESCC) ranked the seventh most frequent cancers, and is the fifth leading cause of cancer death in Taiwan. The overall 5-year survival rate of ESCC is $12-15 \%$. It is import to diagnose ESCC in early stage and receive curative treatment. Endoscopic submucosal dissection (ESD) is a well-developed skill to complete resect gastrointestinal (GI) tract early cancer. The limitation of ESD is cancer invasion to submucosal layer and increasing the risk of lymph node metastasis $(10-45 \%)$ of ESCC.Endoscopic ultrasonography (EUS) is an examination for detecting the invasion depth (T stage) of GI tract cancer, the reported accuracy of T1 stage is around $85 \%$ of ESCC, however, the result of detail discrimination of EUS among each subdivision of mucosa (T1a or $\mathrm{m}$ ) or submucosa (T1b or sm) is unsatisfied.

Aims \& Methods: The aim of this study is to evaluate the accuracy of T1 stage subdivision of ESCC by higher frequency EUS probe prior to ESD treatment. From 2013 to 2016, there are 825 patients diagnosed with ESCC, further stage with EUS, computered tomography and PET-CT. The EUS examination is performed with miniprobe (UM-2R, $12 \mathrm{MHz}, \mathrm{UM}-3 \mathrm{R}, 20 \mathrm{MHz}$; Olympus Optical Co. Ltd., Tokyo, Japan) and water immersion method.

The enrolled criteria of this study: 1. ESCC was proved by biopsy result. 2. T1a or T1b stage diagnosed by EUS. 3. No lymph nodes or distant metastasis. 4. Treatment with ESD. All EUS-T stage results were correlated with final pathological $\mathrm{T}$ stage, including $\mathrm{T} 1 \mathrm{a} / \mathrm{T} 1 \mathrm{~b}$.

Results: Total 90 patients enrolled in this study and there were 83 patients with T1a and 7 were T1b stage of ESCC by EUS. After ESD treatment, all specimens were confirmed as T1 on pathology, EUS stage of T1a in 83 patients and 66 $(80 \%)$ were confirmed as T1a, $17(20 \%)$ were T1b on pathology; of $7 \mathrm{~T} 1 \mathrm{~b}$ (patients refuse surgery) on EUS, $2(29 \%)$ were confirmed as T1a and $5(71 \%)$ were T1b on pathology, further subdivision of pathological T1a: $68(\mathrm{~m} 1: 33, \mathrm{~m} 2$ : 15, m3: 20), and T1b: $22(<\mathrm{sm} 1: 6,>\mathrm{sm} 2: 16)$. The sensitivity, specificity, positive predictive value and negative predictive value of EUS T1a is $97 \%$, $23 \%, 80 \%$ and $71 \%$, respectively. All the patients with pathological T1b stage received further esophagectomy or concurrent chemoradiotherapy if surgery unfit.

Conclusion: EUS demonstrates excellent accuracy for T1a diagnosis, but low accuracy of distinguishing between stages T1a and T1b of ESCC. Even with high-frequency miniprobe $(20 \mathrm{MHz})$, the main cause of understage of $\mathrm{T} 1 \mathrm{~b}$ is difficult to detect the cancer micro-infiltration into submucosal layers, and peritumoral inflammation or submucosal fibrosis causes some difficulties in differentiating mucosal from submucosal lesions is the reason of overstage of T1a. To improve the diagnostic accuracy, developing advanced EUS device and more experience endoscopist are needed. Comparing with other locoregional staging method, EUS remains a valuable tool of detecting ESCC Tla stage when ESD treatment is considered.

Disclosure of Interest: All authors have declared no conflicts of interest. 


\section{P0906 COMPARISON OF DIAGNOSTIC PERFORMANCES FOR}

\section{THE EVALUATION OF SUSPECTED MAIGNANT BILIARY} STRICTURE AMONG SAME SESSION EUS-AND ERCP-GUIDED TISSUE SAMPLING

S.J. Yeo' ${ }^{1}$, C.M. Cho ${ }^{2}$, M.K. Jung ${ }^{1}$, A.N. Seo ${ }^{3}$, H.I. Bae ${ }^{3}$

${ }_{1}^{1}$ Division Of Gastroenterology And Hepatology, Dept. Of Internal Medicine, Kyungpook National University Medical Center, Daegu/Korea, Republic of

${ }^{2}$ Kyungpook National University School of Medicine, Daegu/Korea, Republic of ${ }^{3}$ Department Of Pathology, Kyungpook National University School of Medicine, Daegu/Korea, Republic of

\section{Contact E-mail Address: sekj319@hanmail.net}

Introduction: Determining the cause of suspected biliary strictures is always challenging in clinical practice. Although EUS-guided tissue sampling (EUS-TS) revealed a better diagnostic yield in suspected malignant biliary obstructive lesions comparing to ERCP-guided tissue sampling (ERCP-TS), there was few studies for which techniques are better dependent on primary tumor.

Aims \& Methods: The aim of our study is to compare the diagnostic yields between EUS-TS and ERCP-TS in patients with suspected malignant biliary obstructive lesion according to primary tumor sites. By reviewing medical records, we enrolled patients who underwent same-session examination of EUS and ERCP for the evaluation of suspected pancreatobiliary obstructive lesion. For cytopathologic diagnosis, endoscopic ultrasound-guided fine needle aspiration (EUS-FNA) or biopsy (EUS-FNB) and ERCP-TS using brush cytology and/or forceps biopsy were performed. The diagnostic performances were compared between two techniques according to primary tumor sites.

Results: From January 2011 to September 2016, we enrolled 125 patients and 32 patients were excluded due to the following reasons: loss of follow up in 8 , ERCP-TS from pancreatic duct in 23, and ERCP-TS from periampullary biopsy in 4. Among the enrolled patients (93 patients; 62 males, mean age 65.8 years), $86(92.5 \%)$ had malignant tumor such as cholangiocarcinoma in 39 , pancreatic cancer in 37 , and other malignant tumors in 10 patients. And 7 $(7.5 \%)$ patients had benign lesions. EUS-TS revealed higher rate of overall diagnostic accuracy comparing to ERCP-TS $(82.8 \%$ vs. $60.2 \%, \mathrm{p}=0.001)$. Depending on primary lesions, the diagnostic accuracy for pancreatic lesions was statistically higher in EUS-TS than ERCP-TS $(84.4 \%$ vs. $51.1 \%, p=0.003)$. Conclusion: EUS-TS is superior to ERCP-TS for the evaluation of suspected malignant pancreatobiliary obstructive lesions. Especially, if the biliary obstruction was caused by pancreatic lesions, EUS-TS would need to be a priority for cytopathologic diagnosis

Disclosure of Interest: All authors have declared no conflicts of interest.

\section{P0907 PREVALENCE OF POSTERIOR MEDIASTINAL LYMPHADENOPATHIES IN PATIENTS UNDERGOING ENDOSCOPIC ULTRASONOGRAPHY FOR NON-MALIGNANT INDICATIONS: A PORTUGUESE SINGLE-CENTRE PROSPECTIVE STUDY}

J. Veloso Do Carmo, S. C. Marques, M. Bispo, C. Chagas

Gastroenterology, Hospital Egas Moniz - Centro Hospitalar de Lisboa Ocidental, Lisboa/Portugal

\section{Contact E-mail Address: joanavcarmo@gmail.com}

Introduction: Significant heterogeneity in geographic distribution in the prevalence and features of mediastinal lymphadenopathies have been documented in CT studies. Awareness of the geographic prevalence and characteristics of lymphadenopathies will be relevant when performing endoscopic ultrasonography (EUS) for nodal staging of malignant neoplasia.

Aims \& Methods: 1. To document the prevalence and characteristics of mediastinal lymphadenopathies in patients submitted to EUS for non-malignant extrathoracic disease. 2. To identify predictive factors for the presence of mediastinal lymphadenopathies. A prospective, unicentric study was performed between July and December 2016. Mediastinal stations 9, 8, 7, 6, 5, 4L and 2 were systematically evaluated using a linear echoendoscope in all patients undergoing EUS due to benign extra-thoracic pathology, without history of oncologic disease. Demographic, clinical and EUS features were analysed, including location, number, shape, dimensions and echogenicity of the lymphadenopathies.

Results: We analysed 75 patients: M/F, 32/43; Mean age, 63 years. The majority $(72 \%)$ of the patients presented lymphadenopathies in at least one mediastinal station and $88 \%$ of these were found in stations 7 or $4 \mathrm{~L}$. Only $6 \%$ of these had short axis diameter $>10 \mathrm{~mm}$, most were oval $(59 \%)$ or triangular $(37 \%)$ and $40 \%$ had a hyperechogenic center. The prevalence of lymphadenopathies was higher in smoker patients $(83 \%$ vs $64 \%, \mathrm{p}=0.024)$, with a higher average number of lymphadenopathies per patient in this group $(2.1$ vs 1.6 ; $\mathrm{p}=0.017$ ). Similar findings were documented in patients with relevant occupational or environmental respiratory exposure (prevalence $83 \%$ vs $71 \%$; average number 3 vs 1.7). By logistic regression analysis, none of the variables analysed was independently associated with the presence of mediastinal lymphadenopathies.

Conclusion: This prospective Portuguese study documented a higher prevalence of mediastinal lymphadenopathies than previously reported in northern Europe ${ }^{1}$, in patients with no evidence of oncologic disease. This higher prevalence, mostly in smokers or patients with relevant occupational exposure, may negatively influence the specificity and positive predictive value for malignancy of mediastinal lymph node $(\mathrm{N})$ staging by EUS, with particular relevance in esophageal and pulmonary cancer staging.

Disclosure of Interest: All authors have declared no conflicts of interest.

\section{Reference}

1. Kalaitzakis E, Sadik R, Doig L, Meenan J. Defining the lymph node burden in a Northern European population without malignancy: the potential effect of geography in determining a need for FNA? Diseases of the Esophagus 2009;22:409-417.

\section{P0908 ACCURACY OF ENDOSCOPIC ULTRASOUND IN GASTRIC ADENOCARCINOMA PATIENT SELECTION FOR NEOADJUVANT THERAPY}

J. M. Costa ${ }^{1}$, J. B. Soares ${ }^{1}$, B. Gonçalves ${ }^{1}$, S. Carvalho ${ }^{2}$, R. Gonçalves ${ }^{1}$

${ }^{1}$ Gastroenterology, Hospital de Braga, Braga/Portugal

${ }^{2}$ Pathology, Hospital de Braga, Braga/Portugal

Contact E-mail Address: julianamcosta87@gmail.com

Introduction: Recent studies demonstrated the positive impact of neoadjuvant treatment for gastric adenocarcinoma $\mathrm{T} \geq 2$ and/or $\mathrm{N}+$.

Aims \& Methods: We aimed to assess the accuracy of endoscopic ultrasound in the selection of patient with gastric adenocarcinoma for neoadjuvant therapy. A unicentric retrospective analysis of patients with the anatomopathological diagnosis of gastric adenocarcinoma between 2011 and 2016, who performed endoscopic ultrasound for staging and underwent surgery without prior neoadjuvant treatment. The concordance (kappa) and accuracy [sensitivity (S) and specificity (E)] of the endoscopic ultrasound for $\mathrm{T} \geq 2$ and/or $\mathrm{N}+$ (criteria for neoadjuvant treatment) were assessed using the anatomopathological staging of the resected surgical specimen.

Results: The final sample included 144 patients $(64.6 \%$ male) with a median age of $68.5 \pm 12.2$ years. In most cases $(80.6 \%$ ), the neoplasia was distal (antrum, incisura angularis and body). The neoplasia was of the intestinal type, diffuse and mixed in $65.3 \%, 18.8 \%$ and $16 \%$ of the cases, respectively. After examination of the resected surgical specimen, $53.5 \%$ of patients had criteria for neoadjuvant treatment $(\mathrm{T} \geq 2$ and/or $\mathrm{N}+$ ). The overall kappa, sensitivity and specificity of the endoscopic ultrasound for $\mathrm{T} \geq 2$ and/or $\mathrm{N}+$ were $0.720(\mathrm{p}<0.001), 85.2 \%(95 \%$ CI: $75.6-92.1 \%)$ and $87.3 \%(95 \%$ CI: $76.5-94.4 \%)$, respectively. The overall kappa, sensitivity and specificity of the endoscopic ultrasound for $\mathrm{T} \geq 2$ and/or $\mathrm{N}+$ were higher in proximal lesions (cardia and JEG) $(\mathrm{k}=0.924, \mathrm{~S}-94.4 \%$ and $\mathrm{E}-100 \%)$ compared with distal lesions $(\mathrm{k}=0.671, \mathrm{~S}-82.5 \%$ and $\mathrm{E}-84.9 \%)$ and in intestinal type lesions $(\mathrm{k}=0.765, \mathrm{~S}-84.9 \%$ and $\mathrm{E}-92.7 \%)$ compared with diffuse type lesions $(\mathrm{k}=0.682, \mathrm{~S}-88.4 \%$ and $\mathrm{E}-80 \%)$ or mixed $(\mathrm{k}=0.566, \mathrm{~S}-81.8 \%$ and $\mathrm{E}-75 \%$ ).

Conclusion: In one of the largest series of patients, we showed that endoscopic ultrasound has an overall high agreement and accuracy in the selection of gastric adenocarcinoma patients for neoadjuvant therapy, although they higher for proximal and intestinal lesions.

Disclosure of Interest: All authors have declared no conflicts of interest.

\section{References}

1. Smyth EC, Verheij M, Allum W, Cunningham D, Cervantes A \& Arnold D on behalf of the ESMO Guidelines Committee*. Gastric cancer: ESMO Clinical Practice Guidelines for diagnosis, treatment and follow-up. Annals of Oncology, 2016. (Supplement 5): v38-v49, 2016.

2. Cunningham D, Allum W, Stenning S, et al. Perioperative Chemotherapy versus Surgery Alone for Resectable Gastroesophageal Cancer. The New England Journal of Medicine, 2006. 6;355(1):11-20.

3. Bhandari S, Shim CS, Kim JH, et al. Usefulness of three-dimensional, multidetector row CT (virtual gastroscopy and multiplanar reconstruction) in the evaluation of gastric cancer: a comparison with conventional endoscopy, EUS, and histopathology. Gastrointestinal Endoscopy, 2004. 59(6):619-26.

4. Kwee RM, Kwee TC. The accuracy of endoscopic ultrasonography in differentiating mucosal from deeper gastric cancer. American Journal of Gastrenterology, 2008. 103, 1801-1809.

\section{P0909 IS IT USEFULL TO REPEAT ENDOSCOPIC ULTRASOUND WITH FINE NEEDLE ASPIRATION OF PANCREATIC CYSTIC LESIONS? A RETROSPECTIVE STUDY}

S. Faias ${ }^{1}$, J. Pereira Silva ${ }^{1}$, I. Marques ${ }^{2}$, R. Fonseca ${ }^{3}$, A. Dias Pereira ${ }^{2}$ ${ }^{1}$ Gastroenterology, Instituto Português de Oncologia Francisco Gentil, Lisboa Portugal

${ }^{2}$ Gastroenterology, IPO Lisboa, Lisboa/Portugal

${ }^{3}$ Pathology, IPO Lisboa, Lisboa/Portugal

Contact E-mail Address: sandrarfaias@hotmail.com

Introduction: Pancreatic cystic neoplasms (PCNs) require initial imaging characterization and frequently follow-up. Endoscopic ultrasound with fine-needle aspiration (EUS-FNA) for CEA measurement and cytology of cystic fluid is the most accurate diagnostic method in these lesions. The role of repeated EUS-FNA with cystic fluid analysis in follow-up of PCNs is not clear.

Aims \& Methods: To determine if patients with pancreatic cysts with a second repeated EUS-FNA for cystic fluid analysis for CEA and cytology had a change in cyst classification or on clinical decision. Retrospective analysis of a EUS database, with 284 patients who had EUS-FNA for pancreatic cyst evaluation from 2007-16, of which 35 had 2 EUS procedures, and of these, 22 had 2 consecutive EUS-FNA procedures. 
Results: In our series: $16 / 22$ females $(73 \%)$, mean age $=58 \pm 13$ years old $(29-77)$. Mean follow-up time $=60 \pm 34$ months $(6-116)$. Cyst location: head $/$ body $/$ tail: 11 8/3. Mean size in $1^{\text {st }}$ EUS-FNA: $3 \pm 1,5 \mathrm{~cm}(1,2-7 \mathrm{~cm})$ vs 2 nd EUS-FNA: 3 , $1 \pm 1,9 \mathrm{~cm}(1,2-10 \mathrm{~cm})$; with both EUS-FNAs with $36 \%$ cysts $>3 \mathrm{~cm}$. Mass/ mural nodule present:7/22 vs $4 / 22$ on $2^{\text {nd }}$ EUS-FNA. Repetition of EUS-FNA due to: nodule, imagiologic change or increasing size. Mean interval between the two subsequent EUS-FNAs: 35 months (3-117). Cysts with CEA level $>192 \mathrm{~g} /$ $\mathrm{mL}$ (7 vs 10 patients) and acellular cystic fluid samples (62\% vs $59 \%$ ), between the $1^{\text {st }}$ and the $2^{\text {nd }}$ EUS-FNA, not statistical significant. There were 4 patients operated that had previously a repeated EUS-FNA ( $2^{\text {nd }}$ EUS-FNA 3, 4,7 and 10 months after the $1^{\text {st }}$ EUS-FNA). Surgical pathology (respectively): Intraductal papillary mucinous neoplasm, mucinous cystic neoplasm, solid pseudopapillary neoplasm and a neuroendocrine tumour. Comparing the group of patients who had pancreatic cyst surgery with patients on imagiological follow up, there was a statistical difference in cystic size $>3 \mathrm{~cm}$ and the presence of a mural nodule or mass in the surgery group, but no significant differences in age, CEA value or a diagnostic cytology between the two groups. The mean time between the two EUS-FNA procedures was significantly shorter in the surgical patients group. Conclusion: Clinical follow-up of pancreatic cysts with successive EUS-FNAs can be useful in larger cysts and if worrisome features (mural nodule or mass) are present, and should be performed early on follow-up. If these features are absent, most cysts have an identical CEA (increase to $>192 \mathrm{mg} / \mathrm{mL}$ in only $14 \%$ of patients, without statistical significance) ant the EUS-FNA doesn't change clinical decision making.

Disclosure of Interest: All authors have declared no conflicts of interest.

\section{P0910 DETERMINATION OF INTRACYSTIC GLUCOSE CONCENTRATIONS IN THE DIFFERENTIAL DIAGNOSIS OF PANCREATIC CYSTS: A PROSPECTIVE STUDY}

A. Cantamessa, M. Bruno, S. Gaia, G. Saracco, C. De Angelis Gastroenterology Unit, A.O. Città della Salute e della Scienza di Torino Università of Turin, Turin/Italy

\section{Contact E-mail Address: eusdeang $@$ hotmail.com}

Introduction: Despite advances in imaging techniques, differential diagnosis of pancreatic cysts still remains challenging. There has been an increasing interest in new pancreatic cyst biomarkers as a way to differentiate different cyst subtypes and avoid unnecessary surgery. Recently intracystic fluid glucose has been proposed as a promising marker. The aim of this prospective study was to verify this early finding.

Aims \& Methods: We enrolled in the study all the patients who underwent Endoscopic Ultrasound (EUS) guided Fine Needle Aspiration of a pancreatic cyst at our Institution from October 2015 to February 2017. The cyst fluid was sent for cytology, mucin staining and determination of amylase, Carbohydrate Antigen 19-9 (CA 19-9), Carcinoembryonic Antigen (CEA) and glucose. When deemed necessary by the endoscopist, needle-based confocal laser endomicroscopy (nCLE) of the cyst wall and/or contrast-enhanced EUS was performed. A definitive diagnosis of the nature of the cyst was reached relying on surgery, citology or mucin staining, a typical pattern of nCLE or by consensus (on EUS and radiological features) by three expert endosonographers, blinded to cyst markers concentrations.

Results: Twenty-nine patients (13 males, median age 72 years, range: $30-83$ ) entered the study. Nineteen $(66 \%)$ pancreatic cysts were unilocular while 10 $(34 \%)$ were multilocular, the median largest diameter was $45 \mathrm{~mm}$ (range: 20 $70 \mathrm{~mm})$. Sixteen $(55 \%)$ cysts were located in the pancreatic head, $10(35 \%)$ in the body and $3(10 \%)$ in the tail. CE-EUS was performed in $14(48.3 \%)$ patients, nCLE in $12(42.8 \%)$ subjects. Eighteen $(62.1 \%)$ cysts were finally classified as mucinous ( 6 mucinous cystadenomas; 12 intraductal papillary mucinous neoplasm) and $11(37.9 \%)$ as non-mucinous (6 serous cystadenomas; 5 pseudocysts). The final diagnosis was reached relying on surgery in 9 patients $(31 \%)$, on citology in $4(13.8 \%)$, on nCLE in $8(27.6 \%)$ and on consensus in $8(27.6 \%)$. Mean glucose concentrations in mucinous cyst were significantly lower than in non-mucinous cysts $(7.7 \mathrm{mg} / \mathrm{dl}$ vs $95.7 \mathrm{mg} / \mathrm{dl}, \mathrm{p}<0.0001)$. In the diagnosis of mucinous cysts, sensitivity of CA 19-9 (cutoff more than $5000 \mathrm{U} / \mathrm{ml}$ ), CEA (cutoff more than $192 \mathrm{mg} / \mathrm{ml}$ ) and glucose (cutoff less than $50 \mathrm{mg} / \mathrm{dl}$ ) was respectively $22.2 \%, 66.7 \%$ and $94.4 \%$. Specificity was respectively $72.7 \%, 100 \%$ and $100 \%$. Accuracy was respectively $41.4 \%, 79.3 \%$ and $96.6 \%$. Only two subjects in this cohort were affected by diabetes, this condition did not impact on intracystic glucose concentration.

Conclusion: Although limited by the small sample size, this study confirms the utility of intracystic glucose levels in differentiating mucinous from non-mucinous pancreatic cysts. This cheap, new marker outperformed CA 19-9 and CEA in sensitivity, specificity and accuracy.

Disclosure of Interest: All authors have declared no conflicts of interest.

\section{P0912 UTILITY OF LIQUID BASED CYTOLOGY IN EUS-FNA SAMPLES FOR THE PANCREATIC LESIONS}

Y. Ishida ${ }^{1}$, Y. Okabe ${ }^{1}$, T. Taira ${ }^{2}$, T. Sakaue ${ }^{1}$, M. Fukahori $^{1}$, M. Yasumoto ${ }^{1}$, T. Ushijima ${ }^{1}$, Y. Naito ${ }^{3}$, O. Tsuruta ${ }^{1}$, T. Torimura

${ }^{1}$ Division Of Gastroenterology, Department Of Medicine, Kurume University School of Medicine, Kurume/Japan

${ }^{2}$ Diagnostic Pathology, Kurume University Hospital, Kurume/Japan

${ }^{3}$ Department Of Pathology, Kurume University School of Medicine, Kurume/Japan

Contact E-mail Address: ishida_yuusuke@kurume-u.ac.jp
Introduction: Liquid-based cytology (LBC) preparation method is one of the preparation methods of cytology samples, which is being increasingly used in gynecological and non-gynecological cytology samples, due to its ability to decrease screening time, insufficient sample rate, and air-drying artifacts compared to a conventional smear method. Additionally, immunocytochemistry (ICC) can be performed after LBC.

Aims \& Methods: The aim of this study is to show the actual method of LBC and to evaluate the utility of LBC in EUS-FNA samples of the pancreatic lesions. 292 specimens obtained by EUS-FNA from patients with pancreatic disease were enrolled in this study. Clinical diagnosis was pancreatic cancer (PC) in 210 cases, acinar cell carcinoma in three cases, adenosquamous cell carcinoma in one case, invasive ductal carcinoma derived from IPMN in three cases, metastatic pancreatic tumor in eight cases, pancreatic neuroendocrine tumor (PNET) in 20 cases, solid pseudopapillary neoplasm in one case, serous cystic neoplasm (SCN) in five cases, mass-forming pancreatitis in four cases, and autoimmune pancreatitis (AIP) in 40 cases. Just after EUS-FNA, tissue sample in the FNA needle was flushed out into petri dish with saline. The specimen was carefully examined to divide visual tissue core from liquid specimen. Divided visual tissue core was formalin-fixed and processed for pathological evaluation. All residual liquid specimen in whole was immediately immersed in liquid-based fixation medium (CytoRich ${ }^{\mathrm{TM}}$ Red) at the bedside. The liquid was centrifugalized and processed for cytological evaluation in the laboratory. Sufficient material rate of cytology was retrospectively evaluated in all cases, and diagnostic yield between cytology and histology in patients with malignant diseases was compared. In addition, availability of immunohistochemistry (IHC) and ICC was assessed retrospectively.

Results: Sufficient material rate in cytological evaluation was $96.6 \%$ in all enrolled specimens (282/292). 10 insufficient materials were obtained from PC in two, SCN in four, PNET in one, and AIP in three. In seven of the 10 cases with insufficient materials, sampling error occurred because sufficient material was not confirmed using rapid onsite evaluation. Accuracy of cytology, histology, and combination of cytology and histology were $84.4 \%, 72.9 \%$, and $90.0 \%$, respectively. In the study of 255 specimens with malignant diseases, sufficient material rate of cytology and histology are $99.1 \%(223 / 225)$ and $89.3 \%(201 / 255)$. Positive predictive values of cytology and histology are $81.7 \%(184 / 225)$ and $67.6 \%(152 / 225) .65 .8 \%(48 / 73)$ of pathologically non-diagnosed cases could be diagnosed as malignancy owing to cytology. In 26 specimens where immunochemistry was needed, IHC was available in 23 of the 26 specimens $(88.5 \%)$ and ICC was available in all specimens. In the three specimens, IHC was not available owing to pathological insufficiency.

Conclusion: LBC in pancreatic cell samples obtained by EUS-FNA may be useful for reducing insufficient material rate and conducting ICC as well as in samples in other medical fields.

Disclosure of Interest: All authors have declared no conflicts of interest.

\section{P0913 ANTIBIOTIC PROPHYLAXIS AFTER PANCREATIC CYST PUNCTURE - LESS IS MORE? ONE-TIME VERSUS EXTENDED CIPROFLOXACIN PROTOCOL}

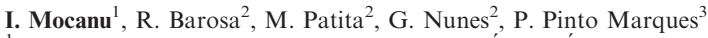
${ }_{1}^{1}$ Gastroenterology, Hospital Espirito Santo de Évora, Évora/Portugal ${ }^{2}$ Gastroenterology, Hospital Garcia de Orta, Almada/Portugal

${ }^{3}$ Gastroenterology, Hospital da Luz, Lisbon/Portugal

\section{Contact E-mail Address: irina.mocanu.24@gmail.com}

Introduction: Echoendoscopy with fine needle aspiration (EUS-FNA) is a useful tool for the characterization of pancreatic cystic lesions (PCL) due to its ability to provide samples for biochemical and cytological examination. This is a safe procedure, with low complications rate $(0-2,5 \%)$. Nevertheless, peri-procedural and even several days after EUS-FNA antibiotic prophylaxis has been the standard practice due to the possible risk of pancreatic infection.

Aims \& Methods: We aimed to compare adverse outcomes after EUS FNA of PCL with two protocols: group 1: Ciprofloxacin $200 \mathrm{mg}$ iv, one-dose, immediately before FNA, and group 2: Ciprofloxacin $200 \mathrm{mg}$ iv, one-dose, immediately before FNA plus three days of oral Ciprofloxacin, $500 \mathrm{mg}$, bid. Retrospective study of single-operator EUS-FNA of CLP in two centers with different antibiotic prophylaxis protocols, between January 2014 and December 2016. A telephonic questionnaire regarding post-procedural complications was applied to all patients that agreed to enter the study.

Results: Two hundred and four EUS-FNA were analyzed: $51.5 \%(\mathrm{n}=105)$ in group $2.60 \%(\mathrm{n}=123)$ women, mean age $63.4+-12.8$ years. We were able to contact $86.9 \%(n=86)$ patients in group 1 and $94.3 \%(n=99)$ in group 2 $(\mathrm{p}>0.05)$. The mean time between EUS-FNA and questionnaire application was 14, 7 months for group 1 and 17 months for group $2(p>0.05)$. There was no statistical difference between the two protocol groups regarding the morphological features of the PCLs (size, morphology, location) or procedure (needle size, location of puncture, number of passages or percentage of cysts with complete collapse after aspiration). Five patients had intra-cystic limited bleeding after puncture (two in group 1 and three in group 2). In group $1,6.2 \%(n=6)$ reported complications: 1 major (mild acute pancreatitis) and 5 minor (epigastric pain, vomiting). In group $2,9.1 \%(\mathrm{n}=9)$ reported 1 major (mild acute pancreatitis) and 8 minor (epigastric pain, transient fever, nausea) complications, with no difference between the two groups $(p>0.05)$. The patients with intra-cystic bleeding did not have a worse outcome. Additionally, five patients $(1.9 \%)$ reported any type of adverse reaction related to antibiotic use (75\% in group 2). Conclusion: EUS-FNA is a safe procedure, with $1 \%$ rate for major complications in our series and none of them with evidence of infection. We did not find any additional benefit from extension of antibiotic prophylaxis beyond the one-time administration during EUS-FNA. It has even been questioned whether antibiotic 
prophylaxis is mandatory during EUS-FNA of PCLs, since it does not seem to have a protective effect. Moreover, the raise in antibiotic resistance and possible adverse effects related to their use should be balanced against the very low infectious complication rate of EUS-FNA. One limitation of our study is its retrospective nature, with a significant delay between the EUS-FNA and the time to inquiry, which could have biased the patientś answers.

Disclosure of Interest: All authors have declared no conflicts of interest.

P0914 EUS-GUIDED FNA IN THE STUDY OF THE ADRENAL GLAND: NATIONAL RETROSPECTIVE MULTICENTER STUDY

A. Martin-Cardona ${ }^{1}$, G. Fernández-Esparrach ${ }^{2}$, J.C. Súbtil Íñigo ${ }^{3}$, J. Iglesias Garcia $^{4}$, M. Garcia-Guix ${ }^{1}$, A. Barturen Barroso ${ }^{5}$, A.Z. Gimeno García ${ }^{6}$, J. Miguel Esteban ${ }^{7}$, A. Pardo Balteiro ${ }^{8}$, A. J. Velasco Guardado ${ }^{9}$, E. Vazquez Sequeiros $^{10}$, C. Loras Alaustrey ${ }^{11}$, B. Martinez Moreno ${ }^{12}$, A. Castellot ${ }^{13}$, C. Huertas Nadal ${ }^{14}$, M. Martínez Lapiedra ${ }^{15}$, A. Sanchez Yague ${ }^{16}$, Á. Terán Lantarón $^{17}$, V. Jair Morales ${ }^{2}$, M. T. Betes Ibáñez ${ }^{3}$, D. De La Iglesia Garcia ${ }^{4}$ C. Sánchez-Montes ${ }^{2}$, M. D. Lozano Escario ${ }^{3}$, J. Lariño Noia ${ }^{4}$, A. Gines ${ }^{18}$, J. B. Gornals ${ }^{1}$

${ }^{1}$ Endoscopy Unit-digestive Diseases Department, Hospital Universitari de Bellvitge, Hospitalet de Llobregat (Barcelona)/Spain

${ }^{2}$ Endoscopy Unit, Hospital Clínic, University of Barcelona, Barcelona/Spain

${ }^{3}$ Clínica Universitaria de Navarra, Navarra/Spain

${ }^{4}$ Hospital Clínico Universitario de Santiago de Compostela, Santiago de Compostela/Spain

${ }_{6}^{5}$ Hospital Universitario Las Cruces, Bilbao/Spain

${ }^{6}$ Hospital Universitario de Canarias, Santa Cruz de Tenerife/Spain

${ }^{7}$ Hospital Clinico San Carlos, Madrid/Spain

${ }^{8}$ Hospital Universitario Joan XXIII, Tarragona/Spain

${ }^{9}$ Hospital Universitario de Salamanca, Salamanca/Spain

${ }^{10} \mathrm{MD}$ Anderson, Madrid/Spain

${ }^{11}$ Hospital Universitari de Terrassa, Terrassa/Spain

${ }^{12}$ Hospital General Universitario de Alicante, Alicante/Spain

${ }^{13}$ Hospital Insular de Gran Canaria, Las Palmas de Gran Canaria/Spain

${ }^{14}$ Hospital Dr. Josep Trueta, Girona/Spain

${ }^{15}$ Instituto Oncológico Valenciano, Valencia/Spain

${ }^{16}$ Hospital Costa del Sol, Marbella/Spain

${ }^{17}$ Hospital Universitario Marqués de Valdecilla, Santander/Spain

${ }^{18}$ Dept. Of Gastroenterology, Hospital Clínic. Barcelona., Barcelona/Spain

\section{Contact E-mail Address: martincardona $@$ gmail.com}

Introduction: The endoscopic ultrasound (EUS) has proven useful in the study and evaluation of the adrenal gland (AG) by endoscopic ultrasound-guided fineneedle aspiration (EUS-FNA), in both the left and right glands.

Aims \& Methods: To analyze the diagnostic performance, safety, impact on clinical management, predictors of malignancy and cyto-pathological correlation of the EUS-FNA with adrenal gland.

National multicenter retrospective study. Mailing-SEED (Spanish Society of Digestive Endoscopy) partners. Participation: 17 Spanish centers. Inclusion period: April/2003 to April/2016. Inclusion criteria: All adrenal gland (Left/ Right) punctured by endoscopic ultrasound. Variables reviewed: demographic characteristics, clinical, extension studies, EUS and pathological data. Comparison between variables; and estimation of multivariate logistic model for cytology, mortality and therapeutic change.

Results: A review of 205 EUS-FNA of adrenal gland in 200 patients (154 males). Average age: 65.3 (DE 9.6). Primary tumor: lung 69\%, unknown $10 \%$, another $20 \%$. Adrenal gland left (191); adrenal gland right (14). Main features: CT scanpathological $(71.2 \%)$, PET scan-high uptake $(82 \%)$, hypoechoic $(88 \%)$ and suspected by endoscopist $(69 \%)$. Puncture technique most commonly used: cytological needle $(75 \%), 22-\mathrm{G}(64 \%)$, suction-syringe $(66.3 \%)$, passes 2.17 (DE 1.38 ). $92 \%$ of samples allowed a cytological diagnosis, $60 \%$ malignant. Variables associated with malignancy: CT scan pathological morphology (OR: 2.99 IC 95\% 1.41-6.44), heterogeneous pattern EUS (OR: 2.11 CI 95\% 1.13-3.97), morphology EUS 'Variegated' (OR: 2.46 CI 95\% $1.00-6.24)$ and suspected by endoscopist (OR 17.46 CI $95 \% 6.2$ to 58.5 ). A size $<30 \mathrm{~mm}$ was associated with lower mortality (OR: 2.27 CI 95\% 1.17 to 4.48 ). Pathological suspicion of the endoscopist was associated with a greater therapeutic change (OR: 4.48 CI 95\% 2.38 to 8.62). No description of adverse events.

Conclusion: Adrenal gland by endoscopic ultrasound-guided fine-needle aspiration is a safe and high diagnostic yield method. The variables most associated with malignancy are the suspicious echo endoscopic images objectified by expert endoscopists, a heterogeneous pattern and variegated morphology. The results suggest the possibility of developing a predictive malignancy model preprocedure.

Disclosure of Interest: All authors have declared no conflicts of interest.

\section{References}

1. Schuurbiers OC, Tournoy KG, Schoppers HJ, et al. EUS-FNA for the detection of left adrenal metastasis in patients with lung cancer. Lung Cancer 2011;73:310-315.

2. Eloubeidi MA, Black KR, Tamhane A, et al. A large single-center experience of EUS-guided FNA of the left and right adrenal glands: diagnostic utility and impact on patient management. Gastrointest Endosc 2010;71:745-53.

3. DeWitt J, Alsatie M, LeBlanc J, et al. Endoscopic ultrasound-guided fineneedle aspiration of left adrenal gland masses. Endoscopy 2007;39: 65-71.

\section{P0915 RELIABILITY OF GIST'S PRE-TREATMENT RISK ASSESSMENT CLASSIFICATION WITH EUS-FNB BY USING DESIGNED CORE NEEDLES}

R. Grassia, P. Capone, F. Cereatti, E. Iiritano, VjeroK., M. Martinotti, G. Tanzi, F. Buffoli

Gastroenterology, ASST Cremona, Cremona/Italy

Contact E-mail Address: robertograssia@yahoo.it

Introduction: The current classifications of non-metastatic GIST are based on post-operative pathologic criteria and are usefully employed to estimate the potential risk of postoperative recurrence and determine the value of adjuvant Imatinib. The proposed pre-treatment classification currently recognized risk factors as tumor diameter, mitotic rate and site (gastric vs non-gastric). EUSguided tissue samples remains mainstay for pathological diagnosis of GIST, but previous studies showed that EUS-FNA with standard 19 or 22 gauge needles does not reliably reflect GIST's proliferation and size.

Aims \& Methods: We aimed to investigate the EUS-FNB diagnostic yield for GIST and to evaluate whether EUS-FNB samples reflect prognostic criteria obtained from resected GISTs.

A prospectively maintained database was retrospectively reviewed to identify consecutive patients with surgically resected subepithelial lesions who received a diagnosis of GIST at a previous EUS-FNB with a 19 or 22 gauge core-needle (EchoTip ${ }^{\circledR}$ ProCore ${ }^{\mathrm{TM}}$, Cook Medical). Size from EUS examinaton and mitotic/ proliferative indexes obtained from EUS-FNB samples were compared with surgical specimens.

Results: Between November 2012 and December 201618 patients were studied ( 11 males; mean age 71.6 years, range $44-88$ yo). The tumour site was the stomach in 15 out of 18 patients and the duodenum in 3 out of 18 patients. Agreement between EUS-FNB and surgical pathology was $100 \%$ with respect to the diagnosis of GIST (18/18). Proliferative indexes (Ki67/MIB1) were determinable in $14 / 18(77.7 \%)$ of biopsies versus all cases (as expected) in resected specimens. In our series Ki67/MIB1 were generally underestimated. We found only 2 patients with the required number of $50 \mathrm{HPFs}$ for mitotic count examination. They showed a mitotic index $<5 / 50$ HPFs comparable to surgical specimens. No mitotic figures were seen in core biopsy specimen from any of the remaining 16 patients. In these patients the number of HPFs for mitotic count examination ranged from 1 to 22 . In their corresponding surgical specimen we found mitoses in 16/16 patients, ranging from 1 to 5 per 50 consecutive HPFs. Tumour size of the surgical specimen exceeded $(>5 \mathrm{~mm})$ tumor EUS-size in 12 out of the 18 investigated cases $(66.6 \%)$ and was equal $( \pm 5 \mathrm{~mm})$ in 6 cases $(33.3 \%)$.

Conclusion: In our experience, EUS-guided tissue core biopsy have an extremely high diagnostic accuracy for GIST diagnosis, but underestimates the proliferation indexes and rarely allows for a reliable mitotic count. The main reason responsible for these results is the uneven distribution of the mitotic figures throughout the lesion, which can cause the biopsy to miss the most mitotically active areas. Furthermore, EUS examination generally underestimates the size of the lesions; this limit is fundamentally linked to the "bidimensional" evaluation of lesions obtained by ultrasound. In addition, the underestimation of the size is greater for large lesions because of the low depth of field evaluated by high frequencies used in EUS. Our data obtained with EUS-FNB are similar to previous studies with FNA and constitute a major limitation for developing a possible pre-treatment and biopsy-based risk classification of GIST. Alternative parameters (genotype profiling) must be validated on pre-surgical biopsy samples from GISTs for prognostication purposes.

Disclosure of Interest: All authors have declared no conflicts of interest.

P0916 TECHNICAL FEASIBILITY AND SAFETY OF ENDOSCOPIC ULTRASOUND (EUS)-GUIDED FIDUCIAL MARKER PLACEMENT USING A NOVEL SYSTEM WITH PRE-LOADED 22-GAUGE NEEDLES IN PANCREATIC CANCER PATIENTS

E.J. Shin ${ }^{1}$, S. Kim ${ }^{2}$, M. A. Khashab ${ }^{1}$, M.I. Canto

${ }^{1}$ Gastroenterology And Hepatology, Johns Hopkins University School of Medicine, Baltimore/United States of America/MD

${ }^{2}$ Department Of Internal Medicine, Research Institute Of Clinical Medicine, Chonbuk National University Medical School, Jeonju/Korea, Republic of

Contact E-mail Address: eshin3@jhmi.edu

Introduction: Pancreatic cancer (PC) remains a disease with overall poor prognosis, despite significant advances over the past decade. Stereotactic body radiation therapy (SBRT) is able to deliver higher biological effective dose to the tumor over a shorter period of time with reduced local toxicity compared to conventional external beam radiation therapy. EUS-guided fiducial placement has been shown to improve the accuracy and localization during SBRT. 
Conventional EUS-guided fiducial placement requires back-loading each fiducial through the tip of the FNA needle. Thus, delivery of multiple fiducials can be cumbersome and time-consuming.

Aims \& Methods: We aimed to evaluate the feasibility, safety, and performance characteristics of fiducial deployment in PC patients using a novel exchangeable FNA system with pre-loaded 22-gauge EUS fiducial needles. This was a single-center pilot study of 10 consecutive PC patients undergoing EUS-guided fiducial placement for SBRT. The fiducial delivery system contains a 22-gauge EUS needle pre-loaded with 2 gold markers with knurled design. After the 2 markers were deployed, the EUS fiducial needle insert was exchanged out for a second pre-loaded EUS fiducial needle insert through the exchangeable FNA system for total deployment of 4 markers in each patient. All patients underwent CT after fiducial placement as part of SBRT to evaluate successful deployment and complications. The primary endpoint was procedure success, defined as deployment of at least 3 fiducials into the desired target area. Secondary endpoints were total procedure time, fiducial delivery time, and safety.

Results: Fiducial placement was attempted in 10 consecutive patients with PC (mean age 61.7 years, males $60 \%)$. The tumor was located in the head $(n=6)$, neck $(\mathrm{n}=2)$, and the body $(\mathrm{n}=2)$ of the pancreas. Mean size of the tumor was $2.7 \mathrm{~cm}$ (range 1.6-5.3). Procedure success was achieved in all $10(100 \%)$ patients. All 10 patients successfully received 4 fiducials. Mean total procedure time was 12.2 minutes (range 5-18). By comparison, using historic controls of the first 10 patients who underwent conventional EUS-guided fiducial placement, the mean total procedure time was 26 minutes (range 16-44, $\mathrm{p}=0.002$ ). Mean fiducial delivery time was 4.2 minutes (range 1-8). There were no immediate or delayed (7 days) complications.

Conclusion: EUS-guided fiducial placement with a novel exchangeable FNA system with pre-loaded 22-gauge EUS fiducial needle is quick, technically feasible and safe. This system may theoretically decrease the risk to the clinical staff by eliminating the need for back-loading fiducials through exposed needle tip and handling of potentially dirty needles. Given the potential safety and time advantages, further prospective studies are warranted for validation

Disclosure of Interest: E.J. Shin: Consultant, C2 Therapeutics No conflict of interest relevant to the abstract.

M.A. Khashab: Consultant, Boston Scientific No conflict of interest relevant to the abstract.

M.I. Canto: No conflict of interest relevant to the abstract.

All other authors have declared no conflicts of interest.

\section{P0917 DEVELOPMENT AND VALIDATION OF A HIGHLY SENSITIVE AND SPECIFIC AUTOMATED ALGORITHM TO EVALUATE THE ABUNDANCE OF BUBBLES IN SMALL BOWEL CAPSULE ENDOSCOPY}

O. Pietri ${ }^{1}$, G. Rezgui ${ }^{2}$, A. Histace ${ }^{2}$, M. Camus ${ }^{3}$, I. Nion-Larmurier ${ }^{1}$, E. Abou

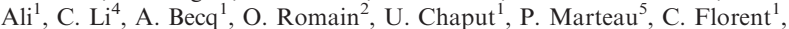
X. Dray ${ }^{1}$

${ }^{1}$ Department Of Digestive Diseases, APHP Saint Antoine Hospital, Paris/France ${ }^{2}$ ETIS, ENSEA, Cergy Pontoise/France

${ }^{3}$ Department Of Gastroenterology, Cochin Hospital, Assistance Publique-Hôpitaux de Paris, Paris/France

${ }^{4}$ College Of Arts And Sciences, Drexter University, Philadelphia/United States of America/PA

${ }^{5}$ UPMC, Paris University, Sorbonne University, Paris/France

Contact E-mail Address: olivia.pietri@hotmail.fr

Introduction: Bubbles can impair the visualization of the small bowel (SB) mucosa during capsule endoscopy (CE).

Aims \& Methods: Our aim was to develop and to validate a computed algorithm, which would evaluate theabundance of bubbles in SB-CE. Two sets of $200 \mathrm{SB}$ CE normal still frames were extracted from 45 complete third-generation SB-CE videos. Two experienced SB-CE readers analyzed both sets of images twice, in a random order. Each still frame was categorized as "scarce in" or "abundant in" bubbles $(<10 \%$ or $\geq 10 \%$ of bubbles covering the frame, respectively). Reproducibility ( $\kappa$ coefficient), sensitivity (Se), specificity (Sp), Receiver Operating Characteristic [ROC] curve, and calculation times were measured for different algorithms (Grey-level of co-occurrence matrix [GLCM], fractal dimension, Hough transform, and Speeded-Up Robust Features [SURF]) using the experts' reading as reference. Algorithms with highest reproducibility,
Se and Sp were then selected for a validation step on the second set of frames. Criteria for validation were $\kappa=1, \mathrm{Se} \geq 90 \%, \mathrm{Sp} \geq 85 \%$, and a low calculation time.

Results: Both SURF and GLCM algorithms had high operating points (Se and Sp over $90 \%)$ and a perfect reproducibility $(\kappa=1)$. At the validation step, the GLCM detector strategy had the best diagnostic capabilities, with $\mathrm{Se}=95.79 \%$, $\mathrm{Sp}=95.19 \%$, and a mean calculation time of $0.037 \mathrm{~s}$ per frame. Table 1 : Sensitivity (Se), specificity (Sp), negative predictive value (NPV), positive predictive value (PPV) and area under receiver operating characteristic curve (AUROCC) of four algorithms for evaluation of bubble abundance in small bowel capsule endoscopy still frames (development step).

Conclusion: A GLCM detector strategy has high diagnostic performances to categorize "scarce in" or "abundant in bubbles" SB-CE frames. This algorithm is of interest for clinical use (i.e. quality in CE reporting) and for research (providing an objective comparison tool of different preparations, including antibubble agents)

Disclosure of Interest: X. Dray: Xavier Dray has received consultance fees from Covidien GI solutions

All other authors have declared no conflicts of interest.

\section{P0918 AGE AND GASTRIC EMPTYING TIME ARE PREDICTIVE FACTORS FOR INCOMPLETE CAPSULE ENDOSCOPY: RESULTS OF A MULTIVARIATE ANALYSIS IN A LARGE STUDY} POPULATION

E. Macías ${ }^{1}$, I. Fernández-Urién Sainz ${ }^{1}$, J.F. Juanmartiñena ${ }^{1}$, I. Elizalde ${ }^{1}$, A. Borda ${ }^{1}$, G. Ezpeleta ${ }^{2}$, J.J. Vila ${ }^{1}$

${ }^{1}$ Gastroenterology, CHN, Pamplona/Spain

${ }^{2}$ Preventive Medicine, CHN, Pamplona/Spain

Contact E-mail Address: ifurien $@$ yahoo.es

Introduction: Capsule endoscopy has been demonstrated to be a first-line tool for small bowel visualization. However, it has some limitations such as incomplete examinations - i.e: the capsule does not reach the cecum - leading to missing lesions.

Aims \& Methods: To evaluate those factors that can predict incomplete examinations, to identify those patients at risk for incomplete procedures and to define those approachs that may improve the efficiency of the examination reducing the time of the diagnostic process as well as the need to repeat procedures. A total of 1918 patients who underwent capsule enteroscopy at our center between 2008 and 2015 were retrospectively analyzed. We evaluated variables such as age, sex, anthropometric parameters, comorbidity, drugs, outpatient care, analytical parameters, indication of the test and transit times. Initiallly, a univariate analysis and then, a multivariate analysis using a logistic regression model were carried out. Results: In the univariate analysis, the following variables showed a statistically significant association with the rate of incomplete examinations: age, gender, indication of procedure, outpatient care, history of abdominal surgery, heart disease, capsule ingestion posture, hemoglobin levels, renal failure and both gastric and small bowel transit times. These variables were included in the multivariate analysis were age $>65$ years $(\mathrm{OR}=1.99,95 \% \mathrm{CI}: 1.34-2.95)$, gastric transit time $>41$ minutes $(\mathrm{OR}=2.60,95 \% \mathrm{CI},: 1.72-3.93)$ and small bowel transit time $>$ than 286 minutes $(\mathrm{OR}=3.5295 \% \mathrm{CI}: 2.26-5.48)$ showed a statistically significant association with the risk of incomplete examination.

Conclusion: Incomplete capsule endoscopy is predictable. Patients older than 65 years and/or a gastric emptying time greater than 42 minutes are independent predictive factors for incomplete procedures. In these clinical scenarios, pharmacological preventive measures or endoscopic introduction should be taken into account to avoid incomplete examinations.

Disclosure of Interest: All authors have declared no conflicts of interest.

\section{P0919 A NOVEL CAPSULE TECHNOLOGY PLATFORM FOR} SPECIFIC LOCALIZED COLON DRUG DELIVERY

N. Arber ${ }^{1}$, H. Elizabeth ${ }^{2}$, S. Doron ${ }^{3}$, G. Nathan ${ }^{1}$, J. Maayan ${ }^{1}$, L. Jesse ${ }^{2}$, M. Alaa ${ }^{1}$, S. Benny ${ }^{4}$, G. Ian ${ }^{5}$, R. Tova ${ }^{6}$, L. Evgenya

${ }^{1}$ Health Promotion Center And Integrated Cancer Prevention Center, Tel Aviv Medical Center, Tel Aviv/Israel

${ }^{2}$ Rambam Health Care Campus, Haifa/Israel

${ }^{3}$ Soroka Medical Center, Beer Sheva/Israel

\begin{tabular}{|c|c|c|c|c|c|c|}
\hline & Sensitivity $(\%)$ & Specificity $(\%)$ & $\begin{array}{l}\text { Negative predictive } \\
\text { value }(\%)\end{array}$ & $\begin{array}{l}\text { Positive predictive } \\
\text { value }(\%)\end{array}$ & $\begin{array}{l}\text { Area under } \\
\text { Receiver operating } \\
\text { characteristic curve }\end{array}$ & $\begin{array}{l}\text { Calculation time }(\mathrm{s}) \\
\text { by frame }(\text { mean } \pm \mathrm{SD})\end{array}$ \\
\hline \multicolumn{7}{|l|}{ Development step } \\
\hline Algorithm 1: GLCM & 94.38 & 93.58 & 95.32 & 92.31 & 0.9852 & $0.040 \pm 0.003$ \\
\hline Algorithm 2: Fractal dimension & 84.27 & 82.57 & 86.54 & 79.78 & 0.9269 & $10.1 \pm 0.7$ \\
\hline Algorithm 3: Hough transform & 85.39 & 81.65 & 87.25 & 79.17 & 0.9252 & $1.45 \pm 1.2$ \\
\hline Algorithm 4: SURF & 94.38 & 97.24 & 95.45 & 96.55 & 0.9897 & $11.47 \pm 7.11$ \\
\hline \multicolumn{7}{|l|}{ Validation step } \\
\hline Algorithm1: GLMC & 95.79 & 95.19 & 96.12 & 94.79 & & $0.037 \pm 0.005$ \\
\hline Algorithm 4: SURF & 94.74 & 94.23 & 95.15 & 93.75 & & $1.45 \pm 1.78$ \\
\hline
\end{tabular}


${ }^{4}$ Laniado Hospital, Netanya/Israel

${ }^{5} \mathrm{Ha}$ 'Emek Medical Center, Afula/Israel

${ }^{6}$ Bnai Zion Medical Center, Haifa/Israel

${ }^{7}$ Medisanto Medical Center, Tel Aviv/Israel

Contact E-mail Address: nadira@tlvmc.gov.il

Introduction: A many variety of pharmaceuticals for the treatment of colon disease can be more effective and have less side effects if targeted for precise delivery in the colon. Over the years, many types of delivery vehicles have been developed with the aim of targeting the colon, such as $\mathrm{Ph}$ based delivery technologies, time dependent drug release mechanisms, pressure based mechanisms, flora sensitive mechanisms and others. These technologies have performed with variable degrees of success due to the wide distribution of motility and other physiological variability between patients. We describe a novel capsule technology which incorporates a diffused gas sensor that allows for an accurate sensing of colon entrance; as well as a $3 \mathrm{D}$ real time positioning system that allows for an accurate, programmable, localized, and in colon drug delivery system.

Aims \& Methods: Data was collected from 14 patients that swallowed capsules in a multi-center clinical trial using an x-ray imaging capsule (GUT 2016). The patients were sent home to continue their normal life routine while the capsule naturally traveled in the gastrointestinal tract until excretion. (Subjects signed informed consent forms and the study was performed after local IRB approval). The capsules contained electronics and software that allowed for live communication between the capsule and a recording device that is placed directly on the patient's back. This device tracks the position of the capsule and communicates with it, receiving diffused gas pressure from the capsule sensor and fusing this information with $3 \mathrm{D}$ position information from the capsule. The capsule system exhibited position accuracy of $\pm 1 \mathrm{~cm}$ and the ability to detect movements in real time, as well as potential of $\sim 1 \mathrm{ml}$ of payload for drug containment.

Results: The average total transit time of the capsule was 43 hours (range: 15-68 hours). The average transit time to cecum was 13.8 hours, and the average time across the colon was 12.8 hours (range $6-25$ ). The position tracking and the RF communication between the capsule and the recorder showed $>90 \%$ coverage in all cases, even in obese patients. No adverse events were reported. Figure 1 illustrates the recorder placement on the patient back. Figure 2 is a typical averaged capsule position trace in the colon.

Conclusion: A capsule with accurate position tracking, 2-way communication, and on line algorithms can determine colonic entrance and identify exact locations in the colon. A wide variety of drugs can accurately be delivered to their exact target in the colon. It enables for a more effective (high dose) and less toxic (no systemic delivery) therapy for IBD and cancer.

Disclosure of Interest: N. Arber: Bayer Bio-view Gi-View Micro-medic Checkcap

All other authors have declared no conflicts of interest.

\section{P0920 COLON CAPSULE ENDOSCOPY: HOW DOES INADEQUATE PROCEDURE CLEANSING CORRELATE WITH PROCEDURE ACCURACY?}

S. Perek ${ }^{1}$, S. Farkash ${ }^{2}$, N. Schwartz ${ }^{3}$

${ }^{1}$ Medicine A, Rambam Health Care Campus, Haifa/Israel

${ }^{2}$ Pathology, HaEmek Medical Center, Afula/Israel

${ }^{3}$ HaEmek Medical Center, Afula/Israel

Contact E-mail Address: shayperek@gmail.com

Introduction: Colon Capsule Endoscopy (CCE) procedure requires a preparation regimen, which provides a clean colon, clear capsule images and promotes capsule propulsion through the entire colon. A four-point cleansing grading scale system (poor, fair, good and excellent) has been used to describe the quality of colon preparation. "Poor" and "fair" are considered inadequate cleansing, while "good" and "excellent" - adequate for polyp detection. Adequate cleansing has been associated with significantly higher diagnostic accuracy, when compared with inadequate cleansing ( $72 \%$ vs. $28 \%$ respectively).[1]

Aims \& Methods: Evaluate the relation between CCE procedure cleansing and accuracy - with an emphasis on inadequate cleansing. This is an additional post study analysis[2], including 767 screening population participants, from 17 sites in the United States and Israel, who underwent CCE procedure followed by a blinded colonoscopy. Capsule preparation included $12 \mathrm{mg}$ Senna, 4 liters split dose of sulfate-free polyethehylene glycol (PEG), with 6 and $3 \mathrm{oz}$ of oral sulfate solution - for capsule propulsion. The CCE video was reviewed by 1 of 5 highly experienced CCE gastroenterologists. These physicians assessed colon cleansing on a "poor" to "excellent" scale. Incomplete studies were included in the analysis. Polyps were considered a match, if the size measured by the capsule $( \pm 50 \%)$ range overlapped the size measured by the colonoscopy $( \pm 50 \%)$ range, and polyp location estimates by the 2 methods were in the same or adjacent segments[2]. Results: The mean age of the analyzed cohort was 57.1 (SD 5.8) with 342 males $(44.6 \%)$.

Conclusion: This analysis indicates, that when utilizing 4 liter PEG and oral sulfate solutions for CCE procedure preparation, current cleansing assessment scale and methodology may need to be re-evaluated - to better correlate with polyp detection. Currently, "fair" cleansing may not indicate inadequate cleansing for polyp detection.

Disclosure of Interest: S. Perek: Employee of Medtronic

S. Farkash: Employee of Medtronic

N. Schwartz: Employee of Medtronic

\section{References}

1. Van Gossum A et al. Capsule Endoscopy versus Colonoscopy for the Detection of Polyps and Cancer. New England Journal of Medicine 2009; 361:264-270.

2. Rex DK et al. Accuracy of capsule colonoscopy in detecting colorectal polyps in a screening population. Gastroenterology 2015; 148: 948-957.

\section{P0921 COLON CAPSULE ENDOSCOPY MAY REDUCE} COLONOSCOPY MISS RATE - A MULTICENTER STUDY

\section{S. Perek ${ }^{1}$, N. Schwartz ${ }^{2}$}

${ }^{1}$ Medicine A, Rambam Health Care Campus, Haifa/Israel

${ }^{2}$ HaEmek Medical Center, Afula/Israel

\section{Contact E-mail Address: shayperek@gmail.com}

Introduction: Colonoscopy miss rate is an area of intense focus, as it directly correlates with colorectal cancer incidence rate. Previous studies reported a colonoscopy miss rate of $2 \%-22 \%$, depending on polyp size and histology [1]. Colon Capsule Endoscopy (CCE) is a visualization diagnostic modality of the colon mucosa, which has demonstrated high sensitivity for polyps and adenomas [2]. Determining the nature of polyps detected by CCE but missed by the imperfect gold standard (colonoscopy), may facilitate both optimization of CCE application (potential CCE additive value) and increase colonoscopy polyp detection

Aims \& Methods: Characterize polyps detected by CCE, which were missed by colonoscopy. 695 screening population participants, from 17 sites in the United States and Israel, underwent CCE procedure followed by a blinded colonoscopy. The overall colonoscopy adenoma detection rate in this study was very high $-39 \%$ [2]. Following the blinded colonoscopy, the patient's $\mathrm{CCE}$ report was assessed. Based on the findings in this report, the colonoscopy performing physician decided whether or not to immediately follow up with a second colonoscopy. 70 of the CCE findings were detected by the second colonoscopy. These 70 polyps were compared with 683 polyps detected by blinded colonoscopy, using logistic regression model. Adjusted Odds Ratios (Adj.OR) and corresponding Confidence Intervals (CI) were estimated.

Results: Of the 70 polyps missed by first colonoscopy and detected by second colonoscopy, $20(29 \%)$ were $6 \mathrm{~mm}$ or larger (based on colonoscopy size estimation), $19(27 \%)$ were either adenomatous or sessile serrated lesions and $16(23 \%)$ were described as either flat or sessile-flat by colonoscopy performing physician. Stratification of polyps based on location:

\begin{tabular}{lll}
\hline & $\begin{array}{l}\text { Detected by } \\
\text { blinded colonoscopy } \\
(\mathrm{n}=683)\end{array}$ & $\begin{array}{l}\text { Detected after } \\
\text { CCE and } \\
\text { unblinding }(\mathrm{n}=70)\end{array}$ \\
\hline Cecum & $64(84 \%)$ & $12(16 \%)$ \\
Ascending & $181(94 \%)$ & $11(6 \%)$ \\
Transverse & $98(96 \%)$ & $4(4 \%)$ \\
Descending-Sigma & $243(90 \%)$ & $26(10 \%)$ \\
Rectum & $97(85 \%)$ & $17(15 \%)$ \\
\hline
\end{tabular}

Abstract: P0920

\begin{tabular}{lll}
\hline Colon cleansing & Sensitivity $(\geq 6 \mathrm{~mm}) ; \mathrm{n}=272$ & Specificity $(\geq 6$ mm $) ; \mathrm{n}=495$ \\
\hline Adequate cleansing & $153 / 195=78.5 \%(72.2 \%-83.7 \%)$ & $308 / 350=88.0 \%(84.2 \%-91.0 \%)$ \\
Inadequate cleansing & $54 / 77=70.1 \%(59.1 \%-79.2 \%)$ & $130 / 145=89.7 \%(83.5 \%-93.7 \%)$ \\
P-value & 0.147 & 0.600 \\
Poor cleansing & $3 / 9=33.3 \%(11.7 \%-64.9 \%)$ & $15 / 16=93.8 \%(69.7 \%-100 \%)$ \\
Fair, good and excellent cleansing & $204 / 263=77.6 \%(72.1 \%-82.2 \%)$ & $423 / 479=88.3 \%(85.1 \%-90.9 \%)$ \\
P-value & 0.007 & 1 \\
\hline
\end{tabular}

Poor cases had significantly lower CCE sensitivity compared with fair, good and excellent cases, for 6 mm polyps (P-value $=0.007)$. When stratifying cases based on the current adequacy cutoff, sensitivity for $6 \mathrm{~mm}$ polyps is similar in inadequate ("poor" + "fair") cases compared to adequate ("good" + "excellent") cases (70.1\%, $78.5 \%$ respectively; P-value $=0.147$ ). 
Multivariate logistic regression revealed that after adjusting to polyp's size, cecal and rectal segments were associated with increased chance of CCE additive value to colonoscopy (cecum vs. ascending or transverse colon: Adj.OR $=3.2[95 \% \mathrm{CI}$ 1.3-7.6] and Adj.OR $=4.5$ [95\% CI: $1.4-14.6]$ respectively; rectum vs. ascending or transverse colon: Adj.OR =2.6 [1.1-5.8] and Adj.OR=3.6 [95\% CI: 1.2-11.4] respectively). There were 59 patients $(8.49 \%$ of study population), with at least one CCE additive value to colonoscopy event.

Conclusion: CCE has the ability to detect polyps missed by traditional colonoscopy, especially lesions in the cecum and rectum.

Disclosure of Interest: S. Perek: Employee of Medtronic

N. Schwartz: Employee of Medtronic

\section{References}

1. Van Rijn JC et al. Polyp miss rate determined by tandem colonoscopy: a systemic review. Am J Gastroenterology 2006; 101(2): 343-50.

2. Rex DK et al. Accuracy of capsule colonoscopy in detecting colorectal polyps in a screening population. Gastroenterology 2015; 148: 948-957.

\section{P0922 ENDOSCOPIC MANAGEMENT OF POSTOPERATIVE PANCREATIC FISTULAS AFTER DISTAL PANCREATECTOMY OR ENUCLEATION}

M. Camus ${ }^{1}$, S. Chaouch ${ }^{1}$, S. Leblanc ${ }^{1}$, S. Gaujoux ${ }^{1}$, F. Paye ${ }^{2}$, P. Balladur ${ }^{2}$, J.C. Vaillant ${ }^{3}$, F. Menegaux ${ }^{3}$, P.P. Massault ${ }^{4}$, M. Barret ${ }^{1}$, R. Coriat ${ }^{1}$, S. Chaussade ${ }^{1}$, B. Dousset ${ }^{4}$, F. Prat ${ }^{1}$

${ }^{1}$ Gastroenterology, University Paris 5 APHP Hopital Cochin, Paris/France

${ }^{2}$ Surgery, University Paris 6 APHP Saint Antoine Hospital, paris/France

${ }^{3}$ Surgery, University Paris 6 APHP Pitie Salpetriere Hospital, Paris/France

${ }^{4}$ Surgery, University Paris 5 APHP Cochin hospital, Paris/France

Contact E-mail Address: marine.camus@gmail.com

Introduction: Only small series ( $<10$ patients) have described endoscopic management of postoperative pancreatic fistulas (POPF). The purpose of this retrospective study was to describe the indications, technique and results of endoscopic treatment of POPF.

Aims \& Methods: From a prospective database of an endoscopic unit of a tertiary center, patients with POPF who underwent pancreatic endoscopic treatment during a retrograde endoscopic cholangiopancreatography (ERCP) were identified between January 2010 and June 2016. POPF was classified according to the definition of the International Study Group of Pancreatic Fistula Working Group. The indications, the techniques and results of endoscopic drainage and the patients' outcomes were registered.

Results: Among 6473 ERCPs performed during the inclusion period, 31 patients had POPF treated endoscopically ( 14 men, 7 women, mean age \pm standard deviation [SD]: 63 years $[ \pm 15])$. Surgeries at the origin of the fistula were: distal pancreatectomy with spleen resection $(n=19)$; spleen preserving distal pancreatectomy $(n=3)$; central pancreatectomy $(n=2)$; enucleation $(n=4)$; partial pancreatectomy $(\mathrm{n}=1)$; left nephrectomy $(\mathrm{n}=2)$, for the following indications: IPMN $(\mathrm{n}=7)$, pancreatic adenocarcinoma $(\mathrm{n}=5)$, neuroendocrine tumors $(\mathrm{n}=4)$, insulinoma $(\mathrm{n}=3)$, adrenocortical carcinoma $(\mathrm{n}=6)$, metastasis $(\mathrm{n}=2)$, pheochromocytoma $(\mathrm{n}=1)$, cystadenomas $(\mathrm{n}=2)$, spleen artery aneurysm $(\mathrm{n}=1)$. The mean time between surgery and first ERCP $( \pm \mathrm{SD}$, [range] $)$ was 70 days $( \pm 145,[6-806])$. POPF was grade B in 19 patients and grade C in 12 patients. All patients had somatostatin analogs and $13(41.9 \%)$ had an external radiological drainage prior to endoscopic drainage. In 9 patients $(29 \%)$ a pancreatic cystogastrostomy was performed during follow-up (FU) in addition to the transpapillary drainage. The pancreatic cannulation rate was $96.8 \%$ at the first ERCP and $100 \%$ after 2 ERCPs: $90.3 \%$ by the major papilla, $9.7 \%$ by the minor papilla. All patients had a pancreatic sphincterotomy and the placement of a pancreatic plastic stent. 3 early complications $(<72 \mathrm{~h})$ occurred: 2 post-sphincterotomy bleeding and 1 perforation, all medically treated. The mean FU $( \pm \mathrm{SD}$, [range]) was 378 days ( \pm 497 [2-2227]) after the first ERCP. During FU, there were 3 stent migrations and 2 stent obstructions requiring a subsequent ERCP for stent replacement. Eight patients $(25.8 \%)$ died after surgery complications. Among the 23 remaining patients, the closure rate of POPF was $100 \%$ within an average $( \pm \mathrm{SD}$ [range]) of 153 days $( \pm 81$ [16-326]) after the first ERCP, after a mean number of ERCP performed per patient $( \pm$ SD) $2.5( \pm 1.0)$. No late reopening fistula occurred after removal of the pancreatic stent.

Conclusion: This retrospective study, the most important one reported to date, shows that the endoscopic treatment of POPF resistant to medical therapy is an effective option.

Disclosure of Interest: All authors have declared no conflicts of interest.

\section{P0923 EFFICIENCY OF PANCREATIC STENTS IN DIFFICULT CANNULATION - A RETROSPECTIVE SINGLE - CENTER STUDY}

V. Sandru ${ }^{1}$, M. Ilie ${ }^{1}$, O.M. Plotogea ${ }^{1}$, E. Rinja ${ }^{1}$, I. Moroi ${ }^{1}$, B. Ungureanu ${ }^{2}$, G. Constantinescu ${ }^{1}$

${ }^{1}$ Dept. Of Gastroenterology, Clinical Emergency Hospital Bucharest, Bucharest/ Romania

${ }^{2}$ Department Of Gastroenterology, Emergency County Hospital, Craiova, Craiova Romania
Contact E-mail Address: drsandruvasile@gmail.com

Introduction: Difficult biliary cannulation is defined by the presence of one or more of the following: more than 5 contacts with the papilla while attempting to cannulate; more than 5 minutes spent attempting to cannulate following visualization of the papilla; more than one unintended, pancreatic duct cannulation or opacification. In these situations, pancreatic stent insertion might prove to be very useful for prophylactic and tactical purposes.

Aims \& Methods: We are proposing in this paper to present the experience of Clinical Emergency Hospital Bucharest regarding difficult biliary cannulation and how pancreatic stent insertion proves to be efficient in these situations. This paper is a retrospective study of the patients who presented difficult cannulation and to whom pancreatic stents were inserted for prophylactic and tactical purpose. The study included patients with ERCP (Endoscopic Retrograde Cholangiopancreatography) performed in Clinical Emergency Hospital Bucharest between December 2014 and December 2016. The patients were evaluated in terms of pancreatic stents efficiency regarding successful cannulation and post ERCP pancreatitis rate. The stents used were $5 \mathrm{Fr}, 3 \mathrm{~cm}$ and $5 \mathrm{~cm}$.

Results: We introduced in the study 158 patients with ERCP and difficult biliary cannulation, who required pancreatic stent insertion for prophylactic and tactical purpose. Patients' mean age was 60 years, while the sex ratio was $2: 1$ in favor of the female gender. Pancreatic stents proved their efficiency in $98 \%$ cases, in only 3 patients deep cannulation being unsuccessful. On average, patients required 1 , 15 ERCP procedures in order to obtain biliary access. ERCP indication for benign pathology was predominant $(60 \%)$. The stents used were $5 \mathrm{Fr}, 5 \mathrm{~cm}$ (102 patients) and $5 \mathrm{Fr}, 3 \mathrm{~cm}$ (56 patients). Precut sphincterotomy was performed in 82 cases ( 37 before stent insertion and 45 after stent insertion). From all patients included, only 19 patients $(12 \%)$ presented post procedure elevation of serum amylase 3 times higher than normal value associated with abdominal pain. Conclusion: Pancreatic stents proved to be efficient in obtaining biliary cannulation in difficult situations. Regardless of their length, $5 \mathrm{Fr}(3 \mathrm{~cm}, 5 \mathrm{~cm})$ stents ensure the same success rate for cannulation and offers protection against post ERCP pancreatitis, as long as they are correctly inserted.

Disclosure of Interest: All authors have declared no conflicts of interest.

\section{Reference}

1. Testoni Pier Alberto et al. Papillary cannulation and sphincterotomy techniques at ERCP: European Society of Gastrointestinal Endoscopy (ESGE) Clinical Guideline. Endoscopy. 2016 Jul;48(7):657-83.

\section{P0924 ENDOSCOPIC ULTRASONOGRAPHY-GUIDED BILIARY DRAINAGE WITHOUT DILATION DEVICE USING A THIN DELIVERY-SYSTEM STENT: A PRECLINICAL STUDY}

I. Masahiro ${ }^{1}$, M. Kitano ${ }^{1}$, Y. Kawaji ${ }^{1}$, H. Abe ${ }^{1}$, T. Tamura $^{1}$, N. Junya ${ }^{1}$,

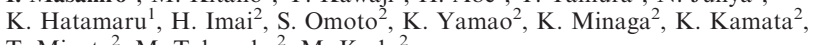
T. Miyata ${ }^{2}$, M. Takenaka ${ }^{2}$, M. Kudo 2

${ }^{1}$ Second Department Of Internal Medicine, Wakayama Medical University,

Wakayama/Japan

${ }^{2}$ Gastroenterology And Hepatology, Kindai University, Osakasayama/Japan

Contact E-mail Address: itonaga@wakayama-med.ac.jp

Introduction: Endoscopic ultrasonography (EUS)- guided biliary drainage (EUS$\mathrm{BD}$ ) is increasingly used in the treatment of malignant biliary obstruction after failed ERCP. However, Multi-step process for EUS-BD is closely related to adverse events.

Aims \& Methods: The present study was designed to determine feasibility and safety of stent placement using a thin delivery-system stent without dilation step during EUS-BD. Three types of the new designed partially covered laser-cut metal stents (6-mm-wide and 60-mm-long) with $7 \mathrm{Fr}$ delivery catheter with hard tip (7Fr hard tip), 7.5Fr delivery catheter with hard tip (7.5Fr hard tip) and 7Fr delivery catheter with soft tip (7Fr soft tip) were prepared respectively. A phantom model with a silicon plate was created. The plate was punctured with s 19-G needle and a guidewire was passed the plate. The delivery system was advanced over the guidewire to pass the plate and the resistance force was measured. A biliary obstruction model was created by clipping the papilla in 10pigs, EUS-BD (choledochoduoduodenostomy) using the thin delivery system stents was attempted following 19-G needle puncture without the use of dilation devices. The technical success and adverse events within 2 weeks after EUS-BD were analyzed for three types of stents.

Results: Among the three types of stents, $7 \mathrm{Fr}$ soft tip had the least resistance in the phantom model. In the animal model, the median common bile duct diameter before puncture measured on EUS and the median procedure time was $7.66 \mathrm{~mm}$ (4.05-9.5) and 29.3 minutes (16-47) respectively. In all pigs, EUS-BD using the three types of stents were technically successful. Dilation was unnecessary in $25 \%$ $(1 / 4), 0 \%(0 / 2)$ and $100 \%(4 / 4)$ for the 7Fr hard tip, 7.5Fr hard tip and 7Fr soft tip, respectively. Even in the cases requiring dilation, stent placement was successful, immediately after dilation only with a thin catheter $(5.5 \mathrm{Fr})$. Neither cautery dilation nor balloon dilation was needed. There were no procedurerelated complications occurring during and 2 week after EUS-BD. All stents remained in place without migration. At necropsy, fistulas were created between the bile duct and duodenum in all pigs and the growth of fibrous tissue was observed in the microscopic findings. 
Conclusion: Among the three types of stents, the 7Fr soft tip was suitable for EUS-BD without dilation in the phantom and animal models. The thin deliverysystem stent may be technically feasible and safety for EUS-BD and possibly reduce adverse events.

Disclosure of Interest: All authors have declared no conflicts of interest.

\section{P0925 TREATMENT WITH FULLY-COVERED METAL STENTS OF POST-SPHINCTEROTOMY EARLY AND LATE BLEEDING}

P. Gambitta ${ }^{1}$, S. Pallotta ${ }^{2}$, A. D’Alessandro ${ }^{3}$, F. Iannuzzi ${ }^{2}$, E. Bareggi²,

A. Ballerini

${ }^{1}$ Digestive Endoscopy, ASST Fatebenefratelli- Sacco, Milan/Italy

${ }^{2}$ Digestive Endoscopy, Luigi Sacco Hospital, Milan/Italy

${ }^{3}$ Clinical Medicine And Surgery, University of Naples Federico II Clinical and

Experimental Medicine, Naples/Italy

\section{Contact E-mail Address: alex balle $@$ libero.it}

Introduction: Post-endoscopic sphincterotomy bleeding is treated endoscopically with pharmacologic injection, electrosurgical cohagulation, balloon tamponade or clipping, but severe cases may require angiographic or surgical approach. An alternative long-acting tamponade treatment with fully-covered metal stents (FCMS) has been advocated.

Aims \& Methods: We report here on the use of FCMS in post-sphincterotomy early and late bleeding. Patients referred for in- and out-patient ERCP were informed of the potential off-label treatment with FCMS of post-sphincterotomy bleeding, and of treatment approval by the local ethical committee. We treated post-spincterotomy bleeding first with adrenaline and/or sclerosing agent injection. When this first line hemostasis failed, we placed short FCMS in the distal choledochus. Endoscopy was rescheduled after 1 month to remove the FCMS. During the early post-procedural period the patients were treated with blood transfusions if needed, and antiplatelet drugs as well as oral anticohagulants were avoided.

Results: 17 Patients $(10 \mathrm{M} / 7 \mathrm{~F})$, aged on an everage 70 years (range 38-90) received 18 FCMS $(10 \mathrm{~mm} \times 40 \mathrm{~mm}$, Boston Scientific) for failed hemostasis since 2015. Technical success was $100 \%$. Hemostasis was obtained in 16 patients, with an everage loss of $1 \mathrm{~g} / \mathrm{dl}$ of $\mathrm{Hb}$ and 9 packed red cells units transfused. In our cases we had 1 outlayer, a patient that had received needle-knife sphincterotomy without early bleeding but developed delayed bleeding with hypovolemic shock He received a second ERCP and a FCMS after 3 days. After further 3 days the patient was in the intensive care unit because of persistent bleeding and we performed a third ERCP. The FCMS was in place, but we removed it, retreated the bleeding by injective therapy and placed a second FCMS, obtaining a stable hemostasis. This single patient received $8 / 9$ of the packed red cells unit used in the study. The FCMS spontaneously migrated in $4 / 18$ cases, one had been removed for incomplete bleeding control and substituted after 3 days, and 13 were easily removed as per protocol. In two cases FCMS were removed and a plastic double pig-tail stent was put in place, for persistent choledochal distal stenosis. Conclusion: These cases represent a large collection of evidence showing that treating post-sphincterotomy early bleeding with FCMS is feasible, safe and effective. Late bleeding associated with needle-knife pre-cut was much harder to control and required endoscopic revision, intensive care unit support and restenting. Our results are consistent with and support previous research in the field.

Disclosure of Interest: All authors have declared no conflicts of interest.

\section{References}

1. DeBenedet T. et al "Post Sphincterotomy bleeding: fully covered metal stents for hemostasis" F1000 research 2013, 2:171

2. Wang Y. Et al "Clinical research for delayed hemorrhage after endoscopic sphincterotomy" Int J Clin Exp Med 2015; 8(4): 5753-5759.

\section{P0926 EFFICACY OF SELF-EXPANDABLE METALLIC STENT PLACEMENT IN THE MANAGEMENT OF ANASTOMOTIC STRICTURE AFTER ORTHOTOPIC LIVER TRANSPLANTATION}

S. Jang, M. Parsi, R. Lopez, A. Bhatt, J. J. Vargo

Gastroenterology, Cleveland Clinic, Cleveland/United States of America/OH

Contact E-mail Address: jangs@ccf.org

Introduction: Anastomotic bile duct stricture (ABS) remains as one of the most common complications after orthotopic liver transplantation (OLT). Current standard of practice of endoscopic retrograde cholangiopancreatography (ERCP) with insertion of multiple plastic stents (PS) often requires multiple procedures before achieving satisfactory stricture resolution. In recent years, studies utilizing covered self-expandable metallic stent (cSEMS) in refractory ABS management reported varying degree of success.

Aims \& Methods: The aim of this study was to analyze efficacy of SEMS in resolution of anastomotic stricture in patients with orthotopic liver transplantation. In addition, we sought to identify factor(s) influencing the likelihood of stricture resolution, the rate of adverse outcome(s) A retrospective cohort study was conducted using a registry of consecutive patients who underwent ERCP with biliary SEMS placement from January of 2010 to November of 2016 for the management of refractory ABS. Demographic variables including age, gender, and clinical variables including body max index (BMI), number or prior ERCP with PS insertion, stent brand and dimensions and duration of SEMS insertion period were collected. The rates of stricture resolution, adverse outcomes including post ERCP pancreatitis, cholangitis and stent dysfunctions (occlusion, and) migration) were calculated. This study was approved by the Institutional Review Board of the Cleveland Clinic.

Results: There were 47 OLT patients who underwent ERCP-cSEMS insertion for refractory ABS during the study period. Of 47 patients, 37 patients $(78.8 \%)$ achieved stricture resolution after single SEMS treatment. Longer duration of SEMS insertion was the only variable associated with increasing probability of stricture resolution as there was $20 \%$ increase in odds of stricture resolution for every additional week SEMS was in place. Among those who achieved initial stricture resolution, 27 patients $(73 \%)$ maintained bile duct patency throughout the follow up period. The most common adverse outcome was internal migration of cSEMS which occurred in 11 patients $(23.4 \%)$. Post-ERCP pancreatitis was observed in $3(6.4 \%)$ patients

Conclusion: The efficacy rate observed in resolving refractory ABS with cSEMS placement appears to be comparable to that of multiple ERCPs with PS placement method. Furthermore, durability of ABS resolution with cSEMS use further supports its potential long-term efficacy. Hence, cSEMS should be considered as a viable alternative treatment of refractory ABS. Longer stent insertion period is associated with higher likelihood of ABS resolution. The high rate of internal migration observed with SEMS warrants further endeavor in stent design improvements.

Disclosure of Interest: All authors have declared no conflicts of interest.

\section{References}

1. Pascher A, Neuhaus P. Biliary complications after deceased-donor orthotopic liver transplantation. J Hepatobiliary Pancreat Surg 2006;13:487-496

2. Sharma S, Gurakar A. Biliary strictures following liver transplantation: past, present and preventive strategies. Liver Transpl. 2008;14:759-69.

3. Chaput U, Scatton O, et al. Temporary placement of partially covered selfexpandable metal stents for anastomotic biliary strictures after liver transplantation: a prospective, multicenter study. Gastrointest Endosc. 2010;72:1167-1174

4. Albert JG, Filmann N, et al. Long-term follow-up of endoscopic therapy for stenosis of the biliobiliary anastomosis associated with orthotopic liver transplantation. Liver Transpl. 2013;19:586-93

5. Poley J, Lekkerkerker $\mathrm{M}$, et al. Clinical outcome of progressive stenting in patients with anastomotic strictures after orthotopic liver transplantation. Endoscopy. 2013; 45:567-70

6. Devière $J$, Nageshwar Reddy D, et al. Successful management of benign biliary strictures with fully covered self-expanding metal stents. Gastroenterology 2014; 147:385-95

7. Tarantino I, Traina, M, et al, Fully covered metallic stents in biliary stenosis after orthotopic liver transplantation. Endoscopy. 2012;44:246-250.

8. Kao D, Zepeda-Gomez S, et al. Managing the post-liver transplantation anastomotic biliary stricture: multiple plastic versus metal stents: a systematic review. Gastrointest Endosc 2013; 77: 679-691

\section{P0927 "DISCONNECTED PANCREATIC DUCT" FOLLOWING EUS GUIDED DRAINAGE OF PANCREATIC FLUID COLLECTION - IS IT CLINICALLY RELEVANT? LONG-TERM FOLLOW UP FROM A LARGE VOLUME TERTIARY CARE CENTRE}

S. Lakhtakia, J. Basha, P. Pal, R. Gupta, M. Ramchandani, S. Kumar, R. Kalapala, M. Tandan, Z. Nabi, P.M. Reddy, G.V. Rao, D.N. Reddy Gastroenterology, Asian Institute of Gastroenterology, Hyderabad/India

Contact E-mail Address: drsundeeplakhtakia@gmail.com

Introduction: Disconnected pancreatic duct (DPD) can occur in patients after drainage of pancreatic fluid collections (PFC). Concerns have been raised about clinical relevance of DPDS.

Aims \& Methods: To assess the frequency of DPD and its clinical significance after EUS guided drainage of PFC. Patients of acute or chronic pancreatitis with symptomatic PFC, who underwent Endoscopic Ultrasound (EUS) guided drainage between January 2011 to December 2016 were included, after an informed consent. Stents used for drainage procedure were either bi-flanged metal stent (BFMS) or double pigtail plastic stent. All these patients underwent MRCP between 4 to 8 weeks after drainage to evaluate pancreatic duct (PD) anatomy and confirm resolution of PFC. Subsequently, they had Endoscopic Retrograde Pancreatography (ERP) and, if required, stent removal. BFMS was removed in all patients. Plastic stents were retained indefinitely, if DPD was confirmed. All patients were systematically followed at 3-6 monthly intervals for any recurrence of PFC or new onset clinical event.

Results: A total of 407 patients (346 males, mean age 33.5 years, range 5-69 years) were followed up after EUS guided drainage for PFC. Of these 260 underwent BFMS and 147 underwent plastic stents placement. 319 patients had pancreatogram (ERP and MRCP) after resolution of PFC. The pancreatic duct abnormalities observed were as follows: DPD in $197(61.7 \%)$, PD leak in 36 $(11.3 \%)$, PD stricture in $20(6.3 \%)$ patients. Normal PD was seen in 43 $(13.5 \%)$ patients. Calcific pancreatitis was noted in $23(7.2 \%)$. Among the patients with DPD, the location of PD "cut off' was in head $(32,16.2 \%)$, genu $(59,29.9 \%)$, body $(90,45.6 \%)$ and tail $(16,8.1 \%)$. Overall, the follow-up after stent removal ranged from 3-72 months, with a median follow up of 12 months. Pseudocyst recurred in 29 patients $(8.5 \%)$ at a mean follow up of 3.5 month (range 1-30 months), which included DPDS 27, PD stricture 1, and CCP 1. 20/29 had pain (symptomatic) and rest 9 were asymptomatic. 15/20 underwent intervention (EUS drainage with plastic stent in 12, surgery in 3 ) and 5 resolved spontaneously. 
Conclusion: Disconnected Pancreatic Duct is observed in $60 \%$ patients following EUS guided drainage of PFC. However; only a small proportion $(<10 \%)$ of DPD had symptomatic recurrent fluid collection.

Disclosure of Interest: All authors have declared no conflicts of interest

\section{P0928 FULLY COVERED SELF-EXPANDABLE METAL STENT IN THE MANAGEMENT OF DUODENAL RETROPERITONEAL PERFORATIONS DURING ERCP: A SINGLE CENTER EXPERIENCE}

M. Pizzicannella ${ }^{1}$, G. Andrisani ${ }^{1}$, M. Martino ${ }^{1}$, R. Rea ${ }^{1}$, M. Pandolfi ${ }^{1}$, F.M. Di Matteo ${ }^{1}$

${ }^{1}$ Digestive Endoscopy Unit, Campus Bio Medico, Rome/Italy

\section{Contact E-mail Address: f.dimatteo@unicampus.it}

Introduction: ERCP-related perforation is rare $(0.39 \%)$, but it is associated with an overall mortality of $7.8 \%$. Duodenal retroperitoneal perforation (Type II) is the most frequent, among the ERCP-related perforations. The management of this complication has not been standardized yet: traditionally surgery was considered the only rescue therapy, but in the last years the majority of cases has been managed conservatively. The endoscopic treatment included biliary stent and/or nasobiliary drainage. In our institution, from 2010 we has been using fully covered self-expandable metallic stent (FCSEMS) with nasobiliary drainage always after resolution of the initial indication for ERCP. These stents have the advantage of covering the laceration and allowing free flow of bile into the duodenum instead of into the retroperitoneal space. The aim of this study was to evaluate in our cohort of patients, the benefits of FCSEMS in type II perforations

Aims \& Methods: We experienced six type II perforations associated with ERCP. We retrospectively evaluated the clinical findings, the length of hospital stay, the need for surgery and death.

Results: Of the 3250 ERCP procedures performed from March 2010 to November 2016 , only six $(0.18 \%)$ resulted in perforations (male/female, 2/4; median age: 69 years; age range: $54-80$ years). ERCP procedures were performed with carbon dioxide insufflation. Five patients underwent ERCP for biliary stones. In all cases standard sphynterotomy was performed and perforation was immediately detected. Successful closure of persistent sphincterotomyrelated duodenal perforation using FCSEMS was obtained in all patients. One patient developed ERCP-related pacreatitis, successfully treated with medical therapy. Three FCSEMS were successfully removed after a median of 18 days, the remaining three fell out spontaneously. The median length of hospital stay was 8.5 days (range 4-20 days). There were no deaths or need for surgery.

Conclusion: The placement of FCSEMS is easy, safe and quick. In our cohort of patients, FCSEMS is the effective endoscopic approach for management of type II perforations recognized during ERCP.

Disclosure of Interest: All authors have declared no conflicts of interest.

\section{References}

1. Weiser R, Pencovich N, Mlynarsky L, Berliner-Senderey A, Lahat G, Santo E, Klausner JM, Nachmany I.Management of endoscopic retrograde cholangiopancreatography-related perforations: Experience of a tertiary center. Surgery. 2017 Apr;161(4):920-929

2. Canena J, Liberato M, Horta D, Romão C, Coutinho A. Short-term stenting using fully covered self-expandable metal stents for treatment of refractory biliary leaks, postsphincterotomy bleeding, and perforations. Surg Endosc. 2013 Jan;27(1):313-24

3. Odemis B1, Oztas E, Kuzu UB, Parlak E, Disibeyaz S, Torun S, Kayacetin E. Can a Fully Covered Self-Expandable Metallic Stent be Used Temporarily for the Management of Duodenal Retroperitoneal Perforation During ERCP as a Part of Conservative Therapy? Canena J, Surg Laparosc Endosc Percutan Tech. 2016 Feb;26(1):e9-e17

\section{P0929 EFFICACY AND ONCOLOGIC SAFTY OF ENDOSCOPIC DUODENAL STENTING IN PATIENTS WITH ADVANCED PANCREATIC CANCER}

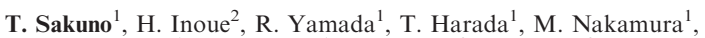

M. Katsurahara ${ }^{1}$, Y. Hamada ${ }^{1}$, K. Tanaka ${ }^{3}$, N. Horiki ${ }^{3}$, Y. Takei ${ }^{1}$

${ }^{1}$ Gastroenterology And Hepatology, Mie University Hospital, Tsu, Mie/Japan

${ }^{2}$ Gastroenterology \& Hepatology, Mie University Graduate School of Medicine

Tsu, Mie/Japan

${ }^{3}$ Endoscopy, Mie University Hospital, Tsu, Mie/Japan

\section{Contact E-mail Address: dpi5tuag@yahoo.co.jp}

Introduction: Duodenal obstruction is often seen in patients with advanced pancreatic cancer (APC). Endoscopic duodenal stenting (DS) has become increasingly popular due to its less invasiveness compared with surgical gastrojejunostomy. With recent new advances in chemotherapy and radiation therapy, conversion surgery after neoadjuvant therapy are performed in some cases with APC.

Aims \& Methods: To evaluate the efficacy and safety of DS as palliative treatrnent and bridge to sugery in patients with APC. We retrospectively analyzed patients who received DS placement between March 2012 and March 2017. Twenty-three patients (11 men/12 women median age, 65.9; 46-82 years) with APC. GOO scoring system (GOOSS) was used as an index of clinical success. Results: A total of 23 Patients consisting of 6 patients treated with chemotherapy and 11 patients treated with neoadjuvant chemoradiotherapy were included in this study. Obstructed parts of the duodenum were D1 in 2, D2 in 5, D3 in 12, and D4 in 4 . Technically success of DS was achieved in $100 \%$ of patients. The mean GOOSS before DS and after DS were 0.5 and 2.6 respectively $(\mathrm{p}<0.001)$. The median time to restart soft solid meal after DS was 2.9 days. Biliary obstruction was seen in 13 patients and managed with endoscopic biliary stenting. Adverse events occurred in 6 patients, including 1 with cholangitis, 1 with bleeding and 3 with stent occlusion. The reintervention technical and clinical success rates were $100 \%(5 / 5)$ and $80 \%(4 / 5)$, respectively. The median survival time after DS was 159 days. In neoadjuvant chemoradiotherapy patients, 4 patients were performed surgery for APC.

Conclusion: DS in patients with APC was effective and safe. The findings of this study suggest that DS is worth considering as the bridge to surgery in patients receiving neoadjuvant therapy.

Disclosure of Interest: All authors have declared no conflicts of interest.

\section{P0930 CLINICAL FAILURE IN COLONIC STENTING FOR MALIGNANT LARGE BOWEL OBSTRUCTION: OUTCOMES AND RISK FACTORS IN 172 PROCEDURES}

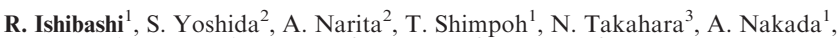

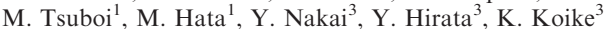

${ }^{1}$ Department Of Gastroenterology, Graduate School Of Medicine, The University of Tokyo, Tokyo/Japan

${ }^{2}$ Department Of Endoscopy And Endoscopic Surgery, Graduate School of

Medicine, The University of Tokyo, Tokyo/Japan

${ }^{3}$ Dept. Of Gastroenterology, University of Tokyo, Tokyo/Japan

Contact E-mail Address: rei1484@gmail.com

Introduction: Endoscopic stenting with self-expandable metallic stents (SEMS) has become widely accepted for an alternative to emergency surgery in the management of malignant large bowel obstruction (LBO) after the initial introduction of SEMS in 1991. Recent years, advance in procedural equipment and accumulation of experience have improved technical success rate in colonic stenting for malignant LBO. However, there is still a divergence in the success rate of technical and clinical.

Aims \& Methods: The aim of this study is to clarify the factors that reduce clinical success rate of stent placement. This study was conducted by a retrospective chart review at a single center. The endoscopy database and clinical records from the University of Tokyo Hospital, Tokyo, Japan, were reviewed retrospectively between May 2007 and February 2017. Patient's symptoms, characteristics, clinical data were obtained from electric medical record. The obstructions were diagnosed clinically and radiologically and were evaluated by the Colorectal Obstruction Scoring System (CROSS). Technical success was defined as deployment of a stent across the entire length of the stricture on the first attempt. Clinical success was defined as resolution of symptoms and radiological relief of the obstruction within 24 hours, as confirmed by radiographic observations. Results: A total of 172 patients (91 males, 81 females; mean age 66.9 years) underwent colonic stenting for malignant LBO. This procedure was performed to 80 colorectal cancer (CRC) patients $(48 \%)$ and to 92 patients $(52 \%)$ with cancer of other etiology. Stenting was performed as a bridge to surgery (BTS) therapy in 33 patients $(19.2 \%)$, whereas stenting was planned palliative procedure in 139 patients $(80.8 \%)$. Sixty-seven patients $(39 \%)$ had a tumor causing obstruction in the proximal colon, and 105 patients $(61.0 \%)$ in the distal colon or rectum. The mean length of obstruction was $6 \mathrm{~cm}$, and obstruction of 60 patients $(34.9 \%)$ longer than $6 \mathrm{~cm}$. The technical and clinical success rate was $98.8 \%$ and $89.5 \%$, respectively. Clinical success rate dropped from $98.9 \%(91 / 92)$ to $78.8 \%$ $(63 / 80)$ when the obstruction caused by extra-colonic malignancy (ECM) $(\mathrm{p}<0.05)$. There was also reduction of clinical success rate from $99.1 \%(111$ $112)$ to $71.7 \%(43 / 60)$ in all patients $(\mathrm{p}<0.05)$ when attempting to stent lesions of longer than $6 \mathrm{~cm}$. Clinical success rate was $85.7 \%(90 / 105)$ and $95.5 \%(64 / 67)$ $(\mathrm{p}<0.05)$ when the patient had obstruction of distal colon or rectum vs in proximal colon. In multivariate analysis, placement in distal colon or rectum, obstruction by ECM, and obstruction longer than $6 \mathrm{~cm}$ were independent risk factors associated with lower clinical success rate (odds ratio [OR] 3.19; 95\% CI 0.96-10.6, [OR] 24.5; 95\%CI 3.19-189.3, [OR] 31.7; 95\% CI 4.32-232.7).

Conclusion: The placement of SEMS for malignant LBO is safe and effective, but clinical success rate drops in the cases with obstructions of distal colon or rectum, longer than $6 \mathrm{~cm}$, or caused by ECM. Considering the indication of colonic stenting for malignant $\mathrm{LBO}$, these factors should be evaluated before procedure. Disclosure of Interest: All authors have declared no conflicts of interest.

\section{P0931 THE CLINICAL EFFICACY OF MITOMYCIN-C IN.JECTION THERAPY IN REFRACTORY BENIGN ESOPHAGEAL STENOSIS: A PRELIMINARY STUDY}

S.G. Lim $^{1}$, J. Kim ${ }^{1}$, K.M. Lee ${ }^{1}$

${ }^{1}$ Gastroenterology, Ajou University School of Medicine, Suwon/Korea, Republic of

Contact E-mail Address: mdlsk75@gmail.com

Introduction: Refractory Esophageal Stenosis (RES) definition differs among studies. Unresolved benign esophageal stenosis even after 5 or more sessions of endoscopic dilation therapy in most studies. Until now, there have been no treatment showing the satisfactory results. Mitomycin C (MMC) inhibits DNA synthesis/reduces fibroblastic collagen formation. Tried in RES in several studies. The meta-analysis about treatment of refractory gastrointestinal stenosis with MMC in total 24 studies. The most commonly reported site esophagus $(79 \%)$ Only 9 recruited adult patients $(n=38)$. Of these, 23 patients were RES. 
Complete/partial response of MMC for RES patients: $59.5 \% / 40.5 \%$, respectively.

Aims \& Methods: The aim of this study was to evaluate the clinical efficacy of MMC injection therapy in patients who had refractory benign esophageal stenosis. Between October 2013 and February 2016 Enrollment criteria: Patients with refractory benign esophageal stenosis who did not respond even after five or more endoscopic dilation therapy. The patients should be more than eighteen year old. Exclusion criteria: Malignant esophageal stenosis, Pregnant or breast feeding women, Deteriorated patients who could not tolerate endoscopic procedures. The method of MMC application -Via 23 gauge injection needle (Optimos injector, Taewoong Medical, Korea) $-4 \mathrm{~mL}$ of saline-diluted MMC was prepared, with concentration of $0.5 \mathrm{mg} / \mathrm{mL}$-Injection of $0.5 \mathrm{ml} \mathrm{MMC}$ at eight points of the stenotic site immediately after bougination. Follow-up -Interview After 1 week and every four weeks after MMC injection upto 52 weeks. Endoscopy Four weeks after MMC injection and when the obstructive symptoms appeared. Primary end-point -The rate of clinical success. Secondary end-points -The mean score of GOOSS before and after MMC injection therapy, Complication rates. Definition -Clinical success: Improvement of GOOSS score more than one point after MMC injection therapy, compared before MMC injection

Results: Ten patients with refractory benign esophageal stenosis were initially enrolled. Two patients were excluded due to death from hypovolemic shock due to persistent bleeding and esophagectomy as patient's wish. Finally, Eight patients were analyzed. The rate of clinical success of MMC injection therapy in patients with refractory benign esophagea stenosis was $87.5 \%$. Mean scores of GOOSS were significantly reduced after MMC injection therapy, from 2.5 to 0.29 . In all patients, MMC injection therapy below two sessions was needed to improve the symptoms of enrolled patients. Major complications did not occur in any patients.

Table 1: Outcomes of MMC injection therapy

\begin{tabular}{ll}
\hline Variables & values \\
\hline Number of Bougie dilation before MMC injection 5/6/7/8/9 & $5 / 1 / 0 / 1 / 1$ \\
The number of session of MMC injection 1/2 & $3 / 5$ \\
Mean GOO score before MMC injection & 2.5 \\
Mean score of GOOSS after final MMC injection & 0.29 \\
Mean diameter of stenosis before MMC injection, mm & 5.2 \\
Mean diameter of stenosis 3 month after final & 8.9 \\
$\quad$ MMC injection, mm & 87.5 \\
Clinical success rate (\%) & $0(0) 0(0) 0(0)$ \\
Complications (N, \%) perforation bleeding requiring &
\end{tabular}

Conclusion: In our study, the mitomycin injection therapy was effective in patients who had retractable benign esophageal stenosis. The mitomycin injection therapy could be considered as an alternative for retractable benign esophageal stenosis. A large-scale prospective studies are required in future.

Disclosure of Interest: All authors have declared no conflicts of interest.

\section{References}

1. Ferguson DD. Evaluation and management of benign esophageal strictures. Dis Esophagus 2005;18:359-64.

2. Spechler SJ. AGA technical review on treatment of patients with dysphagia caused by benign disorders of the distal esophagus. Gastroenterology 1999; 117:233-54.

3. Shah JN. Benign refractory esophageal strictures: widening the endoscopist's role. Gastrointest Endosc 2006;63:164-7.

4. Siersema PD, de Wijkerslooth LR. Dilation of refractory benign esophageal strictures. Gastrointest Endosc 2009;70:1000-12.

5. Siersema PD. Treatment options for esophageal strictures. Nat Clin Pract Gastroenterol Hepatol 2008;5:142-52.

6. Pereira-Lima JC, Ramires RP, Zamin I, Jr., et al. Endoscopic dilation of benign esophageal strictures: report on 1043 procedures. Am J Gastroenterol 1999;94:1497-501.

7. Lew RJ, Kochman ML. A review of endoscopic methods of esophageal dilation. J Clin Gastroenterol 2002;35:117-26.

8. Rahbar R, Jones DT, Nuss RC, et al. The role of mitomycin in the prevention and treatment of scar formation in the pediatric aerodigestive tract: friend or foe? Arch Otolaryngol Head Neck Surg 2002;128:401-6.

\section{P0932 TREATMENT WITH MULTIPLE REABSORBABLE STENTS OF COMPLETE AND PARTIAL LOWER GI ANASTOMOSIS DEHISCENCE AND STENOSIS}

P. Gambitta ${ }^{1}$, S. Pallotta ${ }^{1}$, F. Iannuzzi ${ }^{1}$, E. Bareggi ${ }^{1}$, A. Ballerini ${ }^{1}$, A. D'Alessandro ${ }^{2}$

${ }^{1}$ Digestive Endoscopy, ASST Fatebenefratelli-Sacco, Milan/Italy

${ }^{2}$ Clinical Medicine And Surgery, University of Naples Federico II Clinical and

Experimental Medicine, Naples/Italy

Contact E-mail Address: rasmusklump1974@gmail.com

Introduction: Anastomotic stenosis or dehiscence are serious complications of colorectal surgery. Colostomy or ileostomy and later redo anastomosis or
Hartmann's procedure are the mainstay of therapy, but edema and inflammation due to abdominal sepsis or later adherences can prevent the use of standard management, and therefore alternative salvage repair methods may be required. Reabsorbable polydioxanone stents are a proposed method but with still debatable results in terms of correct placement, clinical success, early and late stenosis, stent migration, perforation, bleeding, fecal incontinence and local pain.

Aims \& Methods: We report here on the use of reabsorbable polydioxanone stents (RPS) in colorectal anastomotic stenosis or dehiscence. We treated with RPS placement, endoscopic dilation and daily mesalazine enemas and/or suppositories 5 complex cases since 2015. Endoscopy was repeated 1 month after and then every two months or earlier if needed. Endoscopic dilation and progressive apposition of partially overlapping stents were repeated until a stable lumen was observed. During follow-up the patients were treated with local mesalazine.

Results: 5 Patients ( $3 \mathrm{M} / 2 \mathrm{~F}$ ), age (49 years mean; range 23-70) were informed of the off-label use of stents and treatment approval by the local ethical committee. See table 1 . Technical initial success was $100 \%$. We did not dilate patients with dehiscence prior to stent placement. After the first RPS we could deploy a 2 nd (100\%, average 3.5 - range $1.5-6$ months) and a 3 rd stent $(40 \%$, average 11 , range $7.5-14.5$ months). Later, after $2.5-15.5$ months, lumen was stable and restored. Granulation tissue in-between stent fibers produced early stenosis, treated by re-stenting. 1 stent prolapsed partially from the anus and was shortened with scissors. Bleeding was not present. Pelvic pain in all the patients needed NSAIDs and reduced over time.

Conclusion: These cases show that stenting with RPS of anastomotic dehiscences is feasible, safe and effective. Due to restenosis associated with dehiescence multiple stents were needed. Distal migration was easily managed. Distal anastomoses might explain the presence of pelvic pain. 3 patients have been subjected to recanalization without complaints.

Disclosure of Interest: All authors have declared no conflicts of interest.

\section{References}

1. Biodegradable polydioxanone stents: a new option for therapy-resistant anastomotic strictures of the colon. Janik V et al. Eur Radiol (2011) 21: 1956-1961

2. Stents for colorectal obstruction: past, present and future. Kim EJ et al World J Gastroenterol 2016 January 14; 22(2): 842-852

\section{P0933 EVALUATION OF FACTORS ASSOCIATED TO A}

\section{SUCCESSFUL DILATION IN POST-ESD STRICTURES}

\section{E. Perez-Cuadrado-Robles ${ }^{1}$, H. Piessevaux ${ }^{1}$, T. Moreels ${ }^{1}$, R.C.P. Yeung ${ }^{1}$,} E. Danse ${ }^{2}$, P.H. Deprez

${ }^{1}$ Department Of Gastroenterology, Cliniques Universitaires Saint-Luc, Brussels/ Belgium

${ }^{2}$ Radiology, Cliniques Universitaires Saint-Luc; Université Catholique de Louvain, Brussels/Belgium

\section{Contact E-mail Address: kikemurcia@gmail.com}

Introduction: The prevalence of post-ESD esophageal strictures is non-negligible, with a critical impact on the patients' quality of life. Balloon-dilation may be the first-line therapy. However, factors associated to a successful dilation in postESD strictures remain unclear.

Aims \& Methods: This is an observational and analytical retrospective study. Sixty-eight consecutive patients (mean age: $65 \pm 11 \mathrm{y}, 76.5 \%$ men) who underwent endoscopic dilation because of post-ESD symptomatic esophageal strictures between 2006 and 2016 were included. They had Barrett's esophagus $(n=46$, $67.6 \%)$, epidermoid carcinoma $(n=21,30.9 \%)$ and other $(n=1)$. Patients with previous endoscopic dilations or resections, esophageal surgery, radiotherapy or motor disorders were excluded. Clinical, morphological and technical features were collected and analysed to determine the factors associated with a positive outcome, defined as the absence of dysphagia during at least twelve months following last dilation. The need of surgery, gastrostomy or prosthesis was considered as negative.

Results: Resected lesions (95.6\% en-bloc) presented a median size of $52.5 \mathrm{~mm}$ (range: $22-110$ ) and achieved $\geq 75 \%$ of the circumference in $50 \%$ of cases $(p=0.435)$. They were mostly located in inferior esophagus $(70.6 \%, p=0.231)$ and required two endoscopic sessions in 8 patients $(11.8 \%)$. Oral and injected corticoids were used in $11(16.2 \%)$ and $12(17.6 \%)$ cases, with no effect in outcome $(p=0.181 / 0.282)$. No specific factor could be associated to a better outcome (median follow-up: 23 months): early dilation ( $\leq 15$ days after ESD), number of dilations $(p=0.345)$, short $(\leq 2 \mathrm{~cm})$ stenosis $(p=0.319)$, balloon diameter $(p=0.475)$ and Barrett's vs. squamous cell carcinoma $(\mathrm{p}=0.458)$. The overall positive outcome rate was $92.6 \%$ after a median of 3 (range:1-27) dilations and 5 months of treatment ( 1 dilation $/ 6.7$ weeks). A prosthesis was placed in 4 patients with clinical improvement in two of them. There were 3 complications (4.4\%, all perforations).

Conclusion: Most of patients presenting with post-ESD strictures and dysphagia improve at long-term buy they need a median of 3 sessions. No specific factor was associated to a better outcome.

Disclosure of Interest: All authors have declared no conflicts of interest. 
Abstract: P0932

\begin{tabular}{|c|c|c|c|c|c|c|}
\hline Patient Age (Y) M/F & Primary disease & Primary treatment & Complication & $\begin{array}{l}\text { Secondary treat- } \\
\text { ment and result }\end{array}$ & $\begin{array}{l}\text { N. Stents N } \\
\text { dilations }\end{array}$ & $\begin{array}{l}\text { Follow-Up } \\
\text { (months) }\end{array}$ \\
\hline $\mathrm{CD} 70 \mathrm{~F}$ & sigmoid adeno $\mathrm{K}$ & $\begin{array}{l}\text { Sigmoid resection } \\
\text { and colorectal } \\
\text { anastomosis } \\
\text { with colostomy } \\
\text { and } \\
\text { radiotherapy }\end{array}$ & $\begin{array}{c}\text { anastomotic } \\
\text { stenosis }\end{array}$ & $\begin{array}{l}\text { Endoscopic dilation } \\
\text { Sub-total } \\
\text { dehiscence }\end{array}$ & 34 & 8.5 \\
\hline RS $52 \mathrm{M}$ & rectal adeno $\mathrm{K}$ & $\begin{array}{l}\text { Knight-Griffen rec- } \\
\text { tosigmoid } \\
\text { resection }\end{array}$ & $\begin{array}{l}\text { Perianal fistulas an } \\
\text { anastomotic } \\
\text { dehiescence }\end{array}$ & $\begin{array}{l}\text { Ileostomy }+3 \text { re-do } \\
\text { low colorectal } \\
\text { anastomosis }+1 \\
\text { FC-SEMS pla- } \\
\text { cement and } \\
\text { removal Sub- } \\
\text { total } \\
\text { dehiescence }\end{array}$ & $\begin{array}{l}34+1 \text { FC-SEMS } \\
\text { placement and } \\
\text { removal }\end{array}$ & 15.5 \\
\hline AI $23 \mathrm{M}$ & $\begin{array}{l}\text { Occlusion due to } \\
\text { Hirsch prung's } \\
\text { disease }\end{array}$ & $\begin{array}{l}\text { Ileostomy, left emi- } \\
\text { colectomy, } \\
\text { coloanal anasto- } \\
\text { mosis, closure of } \\
\text { ileostomy }\end{array}$ & $\begin{array}{l}\text { Abscess and } \\
\text { dehiescence }\end{array}$ & $\begin{array}{l}\text { Re-do ileostomy } \\
\text { and anastomo- } \\
\text { sis, } 1 \text { FC-SEMS } \\
\text { placement and } \\
\text { removal Abscess } \\
\text { and total } \\
\text { dehiescence }\end{array}$ & 23 & 2.3 \\
\hline UN $53 \mathrm{M}$ & sigmoid adeno $\mathrm{K}$ & $\begin{array}{l}\text { Sigmoid resection } \\
\text { and colorectal } \\
\text { anastomosis }\end{array}$ & $\begin{array}{l}\text { anastomotic steno- } \\
\text { sis and } \\
\text { dehiescence }\end{array}$ & $\begin{array}{l}\text { Endoscopic dilation } \\
\text { and } 1 \text { FC-SEMS } \\
\text { placement and } \\
\text { removal Sub- } \\
\text { total dehiscence }\end{array}$ & 23 & 15 \\
\hline DN $54 \mathrm{~F}$ & sigmoid adeno $\mathrm{K}$ & $\begin{array}{l}\text { Sigmoid resection } \\
\text { and colorectal } \\
\text { anastomosis }\end{array}$ & $\begin{array}{l}\text { anastomotic steno- } \\
\text { sis and } \\
\text { dehiescence }\end{array}$ & $\begin{array}{c}\text { Endoscopic dilation } \\
\text { and } 1 \text { FC-SEMS } \\
\text { placement and } \\
\text { removal Sub- } \\
\text { total dehiscence }\end{array}$ & 23 & 10 \\
\hline
\end{tabular}

P0934 EFFECTIVENESS OF REPEATED DILATIONS IN THE MANAGEMENT OF ESOPHAGEAL BENIGN STRICTURES

A. Benezech, A. Debourdeau, J. Gonzalez, M. Barthet

Service De Gastroentérologie, Assistance Publique - Hôpitaux de Marseille, CHU Nord, Marseille/France

Contact E-mail Address: alban.benezech@ap-hm.fr

Introduction: Refractory or recurrent esophageal begnign strictures (REBS) are frequent, and defined as the impossibility to reach or maintain a diameter of $14 \mathrm{~mm}$ after 5 sessions of endoscopic dilation (ED). Because of a lack of guidelines, their management remains challenging, sometimes leading to radical surgical procedure.

Aims \& Methods: The aim of this study was to define the efficacy of long-term and repeated ED in the management of REBS. This was a monocentric retrospective study involving patients managed in our tertiary center between January 2002 and April 2017 for REBS. All the endoscopic dilations were performed using Savary bougies or hydraulic balloons, depending on the operator's choice. Demographical and clinical data were recorded for each patient. The endoscopic management was detailed with the number of procedures, the endoscopic device used, the diameter of dilation, and potential concomitant treatment (as self-expanding metal stent, steroid injection or incisional therapy). The primary endpoint was the efficacy of sustained and recurrent ED, defined as the absence of further dilation within 3 months of the last procedure or an interval between the 2 last ED greater than 3 months. A failure was considered in case of death, need for surgery, permanent enteral feeding tube or an interval between the last 2 procedures lower than 3 months. The secondary endpoints were to document the characteristics of dilation procedures and concomitant treatments, the decreasing of the number of dilations per trimester, and to elucidate potential predictive factors for success of ED.

Results: A total of 39 patients ( 23 men) with a mean age of $47.5 \pm 20.7$ years were included. The etiologies of strictures were anastomotic $(46.1 \%)$, caustic $(28.2 \%)$, peptic $(10.3 \%)$ or other etiologies (radiation injuries, esophageal diverticulectomy, severe viral esophagitis, $15.4 \%$ ). A clinical success of repeated ED was acheived in 27 patients $(69.2 \%)$. Twelve patients $(30.8 \%)$ experienced failure, among them seven $(17.9 \%)$ required frequent dilatations, two $(5.1 \%)$ underwent surgery, two $(5.1 \%)$ maintained an enteral feeding tube, and one patient $(2.6 \%)$ died consecutively to inhalation pneumonia. A mean of $9.8 \pm 4$ ED sessions were performed per patient, with a mean treatment duration of $22.6 \pm 20.1$ months. Regarding concomitant treatments, 16 patients $(41 \%)$ had at least one fullycovered metallic stent placement, incisional therapy was performed in 11 patients $(28.2 \%)$, and 3 patients $(7.7 \%)$ received corticosteroid injections. The number of dilations per trimester gradually decreased over time. No significant predictive factor of success was found, such as etiology of stricture or the use of concomitant treatment, particularly. Nevertheless, an greater number of dilations during the first trimester could promote the success of the management $(3.2 \pm 2.2$ dilations in the success group vs $2.2 \pm 0.8$ in failure group, $\mathrm{p}=0.056$ )

Conclusion: Repeated and maintained endoscopic dilations are effective $(70 \%)$ in the management of REBS, regardless of the etiology of stricture. A prolonged management up to 2 years, and the initial rhythm of endoscopic procedures may favor the final success. A systematic schedule for ED would improve the efficacy of this management.

Disclosure of Interest: All authors have declared no conflicts of interest.

\section{P0935 EFFICACY AND SAFETY OF NEWLY DEVELOPED ENDOSCOPIC COLONIC STENTS WITH AN INCREASED EXPANDABLE FORCE: A RETROSPECTIVE COMPARISON WITH CONVENTIONAL COLONIC STENTS}

T. Nishimura ${ }^{1}$, T. Kuwai ${ }^{1}$, H. Kohno ${ }^{1}$, H. Kouno ${ }^{1}$, A. Yamaguchi ${ }^{1}$, T. Yamaguchi ${ }^{1}$, H. Imagawa ${ }^{1}$, Y. Miyasako' ${ }^{1}$, Y. Sumida ${ }^{1}$, T. Takasago ${ }^{1}$, R. Miura ${ }^{1}$, K. Tao ${ }^{1}$, S. Funaki ${ }^{1}$, S. Ishaq ${ }^{2}$

${ }^{1}$ Gastroenterology, Kure Medical Center and Chugoku Cancer Center, kure/Japan ${ }^{2}$ Gastroenterology, Dudley Group Hospitals NHS Foundation Trust, Dudley/ United Kingdom

Contact E-mail Address: tomoyukin@kure-nh.go.jp

Introduction: Endoscopic stenting with self-expandable metallic stents (SEMSs) is a widely accepted procedure for treating malignant colonic obstruction. This procedure was covered by the National Health Insurance of Japan in January 2012, and the WallFlex colonic stent and Niti-S colonic stent can currently be used in Japan. In the previous study, we reported that the WallFlex colonic stent has more expanded force than the Niti-S colonic stent. On the other hand, the risk of stent-related perforation was lower when using the Niti-S stent due to its structure. Currently, we newly developed an SEMS (Niti-S structure, with 18-mm diameter with increased expanded force compared with the conventional type), which comprised the benefits of both WallFlex and Niti-S. In this study, we compared the efficacy and safety of the newly developed colonic stent with the conventional colonic stents.

Aims \& Methods: This study aimed to compare the efficacy and safety of the newly developed colonic stent with the conventional colonic stents (the WallFlex colonic stent and the Niti-S colonic stent). Overall, 91 patients (96 lesions, male/ female: 48/43, average age: 73.2 years) underwent endoscopic SEMS placement between November 2011 and March 2017 at Kure Medical Center and Chugoku Cancer Center. The WallFlex colonic stent was used in 36 patients (38 lesions: Group W), the Niti-S colonic stent in 51 patients (53 lesions: Group N), and the newly developed colonic stent in 5 patients ( 5 lesions: Group D). Stratified analysis of the clinical background, technical success rate, procedure time, clinical success rate, and complications was performed to compare Group W, Group N, and Group D

Results: Endoscopic SEMS placement was attempted in 96 lesions as a bridge to surgery (BTS) in 52 lesions (54\%) and as palliative therapy (PAL) in 44 lesions $(46 \%)$. In Group W, SEMS was placed in 19 lesions (50\%) as BTS and in 19 lesions $(50 \%)$ as PAL; in Group N, SEMS was placed in 32 lesions $(60 \%)$ as BTS and in 21 lesions $(40 \%)$ as PAL; and in Group D, SEMS was placed in 1 lesion $(20 \%)$ as BTS and in 4 lesions $(80 \%)$ as PAL. The technical success rate was $100 \%$ in all groups. The overall clinical success rate was $93.7 \%(90 / 96): 89.5 \%$ $(34 / 38)$ in Group W, 96.2\% (51/53) in Group N, and 100\% $(5 / 5)$ in Group D. Complications within 7 days included abdominal pain $(3 / 38,8 \%)$, poor 
expansion $(1 / 38,3 \%)$, and fever $(1 / 38,3 \%)$ in Group $\mathrm{W}$ and perforation due to obstructive colitis $(2 / 53,4 \%)$ in Group N. Complications after 8 days included stent-related perforations $(4 / 38,11 \%)$ and stent occlusion $(1 / 38,3 \%)$ in Group $\mathrm{W}$ and stent occlusion $(2 / 53,4 \%)$ in Group N. All 4 patients with stent-related perforations had undergone palliative stenting with the WallFlex colonic stent, and the stent-related perforation rate in Group W was significantly higher than that in Group N $(\mathrm{P}<0.05)$. In Group $\mathrm{D}$, there were no complications and no stent occlusion.

Conclusion: The technical and clinical success rates were extremely high in all groups. The risk of stent-related perforation was higher when using the WallFlex stent, while there was also perforation due to obstructive colitis when using the Niti-S stent. On the other hand, there were no complications when using the newly developed endoscopic colonic stent with an increased expanded force. Although these results were obtained for a limited sample size, this new stent is expected to exhibit more efficacy and safety than conventional colonic stents. Disclosure of Interest: All authors have declared no conflicts of interest.

TUESDAY, OCTOBER 31, 2017

09:00-17:00

\section{SURGERY II - HALL 7}

\section{P0936 PERORAL ENDOSCOPIC MYOTOMY FOR THE CASES IN WHICH HELLER-DOR OPERATION WERE INEFFECTIVE}

H. Shiwaku ${ }^{1}$, K. Yamashita ${ }^{1}$, N. Kore-Eda ${ }^{1}$, T. Ohmiya ${ }^{1}$, H. Okada $^{1}$,

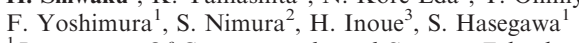

${ }^{1}$ Department Of Gastroenterological Surgery, Fukuoka University Faculty of

Medicine, Fukuoka/Japan

${ }^{2}$ Department Of Pathology, Faculty Of Medicine, Fukuoka University, Fukuokal Japan

${ }^{3}$ Digestive Disease Center, Showa University Koto-Toyosu Hospital, Tokyo/Japan

Contact E-mail Address: hiro.shiwaku@gmail.com

Introduction: Heller-Dor operation is a radical treatment for cases of esophageal achalasia. However, postoperative recurrence of symptoms is observed in some cases. Conventionally, repeat surgery or endoscopic balloon dilatation is additionally performed on such cases. Repeat surgery is associated with issues related to adheision, whereas endoscopic balloon dilatation is associated with issues related to effect reliability and constancy. Peroral endoscopic myotomy (POEM) is a new endoscopic treatment that was first reported by Inoue et al. in 2008. POEM can be accomplished normally without being affected by the previous Heller Dor operation, if POEM is performed at the contralateral site of the previous myotomy. Moreover, POEM enables making a long myotomy that cannot be made in laparotomy. At our hospital, we have performed POEM on 12 cases that previously underwent the Heller-Dor operation. Herein, we report on the treatment outcomes.

Aims \& Methods: We performed POEM on 210 cases between September 2011 and April 2017. Of those cases, 12 cases $(5.7 \%)$ had previously undergone HellerDor operation. The treatment outcomes were retrospectively investigated. The reasons for the relapse of the symptoms were investigated based on the results of medical interview, endoscopy, esophagography, esophageal manometry, and 24hour PH monitoring test.

Results: The POEM cases that previously underwent Heller-Dor operation included 8 men and 4 women with the mean age of $54.8 \pm 15.4$ years. As regards the cause for the recurrence of symptoms, the lower esophageal sphincter was the responsible lesion in 10 patients, the esophageal body in 1 patient, and another reason in 1 patient. POEM was performed by incising the posterior wall in all cases, and the treatment was not prematurely discontinued for any cases. The mean length of the muscular layer incision was $14.3 \pm 6.1 \mathrm{~cm}$ on the esophageal side and $2.8 \pm 1.0 \mathrm{~cm}$ on the gastric side. The mean surgical duration was $136.5 \pm 35.6$ minutes. No accidental symptom associated with POEM was observed. The integrated relaxation pressure was preoperatively $22.1 \pm 8.6 \mathrm{mmHg}$ and $13.6 \pm 5.4 \mathrm{mmHg}$. The Eckardt score was $4.1 \pm 2.5$ preoperatively, $0.67 \pm 1.1$ at 3 months after the surgery, and $1.0 \pm 1.2$ at 12 months after the surgery $(\mathrm{P}<0.05)$. The symptoms improved in all cases.

Conclusion: The treatment outcomes of the POEM performed on cases that previously underwent Heller-Dor operation were favorable. POEM differs from repeat surgery by laparotomy as it can be used to perform a long muscular layer incision without adhesion if an appropriate route is used. The major advantages of POEM are the reliability and constancy of the effectiveness compared to endoscopic balloon dilatation. POEM is a very effective treatment method for cases for which Heller-Dor operation has not been sufficiently effective. Disclosure of Interest: All authors have declared no conflicts of interest.

\section{P0937 PREDICTORS OF POSTOPERATIVE LYMPHOPAENIA AFTER SURGERY FOR ESOPHAGEAL AND ESOPHAGO-GASTRIC JUNCTION CANCER}

O. De Simoni ${ }^{1}$, S. Mocellin ${ }^{1}$, P. Moroni ${ }^{2}$, L.M. Saadeh ${ }^{3}$, F. Cavallin ${ }^{4}$, R. Alfieri ${ }^{4}$, M. Cagol ${ }^{4}$, C. Castoro ${ }^{4}$, M. Scarpa ${ }^{4}$

${ }^{1}$ Surgical Oncology Unit, Veneto Institute of Oncology, Padova/Italy

${ }^{2}$ University of Padova, Padova/Italy

${ }^{3}$ Oncological Surgery Unit, Veneto Institute of Oncology, Padova/Italy

${ }^{4}$ Esophageal And Digestive Tract Surgical Unit, Regional Centre For Esophageal Disease, Veneto Institute of Oncology (IOV-IRCCS), Padova/Italy

Contact E-mail Address: ottavia.desimoni@gmail.com

Introduction: Postoperative immunosuppression in transthoracic esophagectomy for patients with esophageal cancer is a common finding [1]. In fact, both transhiatal and transthoracic esophagectomy induce severely depressed monocyte and
T-lymphocyte cytokine production [2]. Remarkably, lymphopenia is an independent predictive factor for long-term survival in patients with esophageal cancer [3].

Aims \& Methods: The aim of this study was to assess the predictors of postoperative immune suppression in esophageal or esophago-gastric junction cancer. One hundred ninety three consecutive patients with esophageal or esophago-gastric junction cancer were enrolled in this retrospective study. White blood cells count, polymorph nucleate cells and lymphocytes counts were observed at preoperative time, post-operative day (POD) 1, 3 and 7. Post-operative outcome details were also retrieved. Multiple regression models were fitted to the data to test the association between potential predictors and preoperative lymphopaenia and lymphopenia on POD1 as parameters of immune suppression. Results: The preoperative lymphocyte count was 1, 240/ml (IQR: 0.895-1.700) while the lymphocyte count on POD1 was $0.670 / \mathrm{ml}$ (IQR: $0.500-0.982$ ), $\mathrm{p}<0.001$; on POD3, it was $0.800 / \mathrm{ml}$ (IQR: $0.580-1.070$ ), $\mathrm{p}<0.001$; and on POD7, it was $0.825 / \mathrm{ml}$ (IQR: $0.550-1.180$ ), $\mathrm{p}<0.001$. In a modeltha also included the interval between the end of neoadjuvant therapyand the esophagectomy and the preoperative whiteblood cells count, only the final dose of radiotherapy resulted to be an independent predictor of preoperative lymphocyte count. Similarly, in a model that also included the intervalbetween the end of the neoadjuvant therapy and the esophagectomy, the number of surgical accesses (laparotomy, thoracotomy and cervicotomy) and the number of nodal metastasis, only the final dose of radiotherapy resulted to be an independent predictor of lymphocytes count on POD1

Conclusion: Patients with esophageal and esophago-gastric junction cancer present a significant post operative immunosuppression that lasts at least for the first postoperative week. The total amount of radiation received by the mediastinum is the only predictor of the preoperative and postoperative lymphocyte count. Disclosure of Interest: All authors have declared no conflicts of interest.

\section{References}

1. Yamaguchi Y(1), Hihara J, Hironaka K, Ohshita A, Okita R, Okawaki M, Matsuura K, Nagamine I, Ikeda T, Ohara M, Hamai Y. Postoperative immunosuppression cascade and immunotherapy using lymphokine-activated killer cells for patients with esophageal cancer: possible application for compensatory anti-inflammatory response syndrome. Oncol Rep. 2006 Apr;15(4):895-901.

2. van Sandick JW(1), Gisbertz SS, ten Berge IJ, Boermeester MA, van der Pouw Kraan TC, Out TA, Obertop H, van Lanschot JJ. Immune responses and prediction of major infection in patients undergoing transhiatal or transthoracic esophagectomy for cancer. Ann Surg. 2003 Jan;237(1):35-43.

3. Feng JF(1), Liu JS, Huang Y. Lymphopenia predicts poor prognosis in patients with esophageal squamous cell carcinoma. Medicine (Baltimore). 2014 Dec;93(27):e257. doi: 10.1097/MD.0000000000000257.

\section{P0938 FLEXIBLE ENDOSCOPIC TREATMENT FOR ZENKER'S DIVERTICULUM OF HIGH ANESTHETIC RISK PATIENTS WITH THE SB KNIFE. TERTIARY CENTRE CENTRE EXPERIENCE}

S. Ishaq ${ }^{1}$, A. Antonello ${ }^{2}$, A. Aziz ${ }^{3}$, L. Priestnall ${ }^{4}$, T. Kuwai ${ }^{5}$, S. Kong ${ }^{6}$, F. Walji ${ }^{4}$, K. $\mathrm{Siau}^{7}$, A. Gait ${ }^{8}$, C.J.j. Mulder ${ }^{9}$

${ }^{1}$ Gastroenterology, Dudley Group Hospitals, Birmingham City University, Birmingham/United Kingdom

${ }^{2}$ Peterborough City Hospital, Peterborough/United Kingdom

${ }^{3}$ Radiology, Dudley Group Hospitals, Dudley/United Kingdom

${ }^{4}$ Dudley Group Hospitals, Dudley/United Kingdom

${ }^{5}$ Department Of Gastroenterology, Kure Medical Center and Chugoku Cancer

Center, Kure/Japan

${ }^{6} S G U$, Grenada/Grenada

${ }^{7}$ Royal College Of Physicians, JAG Clinical Fellow, London/United Kingdom

${ }^{8}$ Dept. Of Anaesthesiology, Russell Hall Hospital, Birmingham/United Kingdom

${ }^{9}$ Dept. Of Gastroenterology, VU University Medical Center, Amsterdam/

Netherlands

Contact E-mail Address: Sauid.Ishaq@dgh.nhs.uk

Introduction: Surgery and endoscopic stapling have been the mainstay of treatment modalities for Zenker's diverticulum. The need for general anesthesia (GA) is however a limiting factor in high-risk patients, as patients with ZD are often elderly with cardiorespiratory co-morbidities. In recent years, flexible endoscopic septum division (FESD) has gained popularity as a less invasive treatment modality for $\mathrm{ZD}$, that can be performed under deep sedation, and has the potential to be performed safely in patients otherwise deemed unfit for full GA.

Aims \& Methods: The aim of this pilot study was to assess the efficacy and safety of FESD for patients deemed unfit for GA. Patients had been referred to our tertiary centre for FESD. Data on 14 consecutive day case patients (median age 81, range 67-95, 7 females) with an ASA score of 3 that underwent FESD between August 2015 and November 2016 were collected retrospectively. All patients underwent FESD under deep sedation with propofol and remifentanyl, and did not require ventilatory support. The amount of propofol used was recorded. Demographic data as well as information of the nature of symptoms and recurrences was retrieved. The severity of dysphagia was measured with the Dakkak scale. Data on procedural and 30-day post-procedural complications, including sedation-related side effects, were collected

Results: All patients complained of dysphagia (median Dakkak score 2, range 13 ) and regurgitation before the procedure. There were no procedure-related nor sedation related adverse events in any of the patients. There were no 30-day postprocedural adverse events. All patients but one experienced complete symptom relief after one treatment session. Median propofol used was $173 \mathrm{mg}$ (range 59- 
$330 \mathrm{mg}$ ). One patient required an additional treatment session due to persisting dysphagia (score 1) to achieve symptom remission. This was carried out without complications.

Conclusion: Our results suggest that FESD is a safe, feasible and effective treatment in high risk patient who are otherwise not suitable for GA, and can be performed as a day case in an endoscopy unit. It is less invasive hence should be considered as a preferred option over endoscopic stapling. Close interaction and team working with anesthesiologist is important to ensure a safe outcome

Disclosure of Interest: All authors have declared no conflicts of interest.

\section{P0939 INCREASING LYMPH NODE RETRIEVAL IN ESOPHAGECTOMY, OUTCOMES OF THE DUTCH UPPER GASTROINTESTINAL CANCER AUDIT}

L. R. Van Der Werf ${ }^{1}$, J. Dikken ${ }^{2}$, M.I. Van Berge Henegouwen ${ }^{3}$, V. E.p.p. Lemmens ${ }^{4}$, G. A.p. Nieuwenhuijzen ${ }^{5}$, B.P.1. Wijnhoven ${ }^{6}$, On Behalf Of The Dutch Upper Gi Cancer Audit Group ${ }^{7}$

${ }^{1}$ Oncological Surgery, Erasmus Medical Center, Rotterdam/Netherlands ${ }^{2}$ Surgery, LUMC, Leiden/Netherlands

${ }^{3}$ Surgical Department, Academic Medical Centre, Amsterdam/Netherlands

${ }^{4}$ Epidemiology, Erasmus Medical Centre, Rotterdam/Netherlands

${ }^{5}$ Oncological Surgery, Catharina ziekenhuis, Eindhoven/Netherlands

${ }^{6}$ Surgery, Erasmus MC Rotterdam Dept. of Surgery, Rotterdam/Netherlands

${ }^{7}$ Dutch Institute for Clinical Auditing, Leiden/Netherlands

\section{Contact E-mail Address: L.vanderwerf@dica.nl}

Introduction: To improve the quality of esophageal cancer surgery in the Netherlands, the number of retrieved lymph nodes (LNs) is evaluated in one of the quality indicators in the Dutch Upper gastrointestinal Cancer Audit (DUCA).(1) All hospitals in the Netherlands can use the outcomes of the DUCA indicators it to monitor their own results in relation to the national average. The aim of this study was to analyze the outcomes of the quality indicator: 'a minimal number of 15 retrieved LNs' in the past years and to determine factors associated with this outcome.

Aims \& Methods: Patients with an esophageal carcinoma who underwent an esophagectomy with curative intention and who were registered in the Dutch Upper gastrointestinal Cancer Audit between 2011-2016 were included in this retrospective national cohort study. The primary outcome: 'percentage of patients with $\geq 15$ LNs' was analyzed by year and hospital. Factors tested with univariable and multivariable analysis for the association with $\geq 15$ LNs were: age, Charlson score, weight loss, BMI, cT-, cN-, cM-stage, tumor location, neoadjuvant therapy, type of procedure, intraoperative complications, hospital volume and year of resection. The postoperative outcomes: radicality, intraoperative complications, morbidity, and mortality were tested on association with $>15$ LNs.

Results: The overall percentage of patients in the Netherlands with $\geq 15$ LNs increased between 2011 and 2016 from $51 \%$ to $81 \%$. The variation between hospitals decreased. Multivariable analysis showed an independent association with $>15$ LNs for the factors: cN2-stage (OR $[95 \%$ confidence interval]: $1.37[1.05-1.79]$ ), resection in a hospital with $26-50$ or $>50$ resections/year (reference: $0-25$ resections, $2.03[1.57-2.63]$ and 3, 21[2.43-4.23]) and resection between 2012-2016 (reference: 2011, ORs: 1.55, 1.81, 2.43, 2.20, 2.64). Factors independent associated with $<15$ LNs are: neo-adjuvant chemo-radiation-therapy (reference: no neoadjuvant therapy, $0.66[0.47-0.93]$ ), intraoperative complications $(0.55[0.39-0.80])$ and open and minimally invasive transhiatal resections (reference: open transthoracic, $0.24[0.18-0.32]$ ) and $0.38[0.26-0.55])$. Postoperative morbidity and mortality were not associated with $\geq 15$ LNs.

Conclusion: The outcomes of the quality indicator 'a minimal number of 15 retrieved LNs' is increased between 2011 and 2016. The variation between hospitals is decreased. The $\mathrm{cN}$-stage, neoadjuvant therapy, type of procedure, intraoperative complications, year of resection and hospital volume seem to be associated with the outcomes.

Disclosure of Interest: All authors have declared no conflicts of interest.

\section{Reference}

Busweiler LA, Wijnhoven BP, van Berge Henegouwen MI, et al. Early outcomes from the Dutch Upper Gastrointestinal Cancer Audit. Br J Surg 2016; 103(13): $1855-63$.

\section{P0940 COLONIC INTERPOSITION AFTER OESOPHAGECTOMY IN ADULTS: A SYSTEMATIC REVIEW AND META-ANALYSIS OF THE INFLUENCE OF CONDUIT CHOICE AND ROUTE ON OUTCOME \\ J. Brown ${ }^{1}$, D. Chan ${ }^{2}$, W. Lewis ${ }^{2}$, G. Clark ${ }^{2}$, G. Blackshaw ${ }^{2}$, A. Foliaki ${ }^{2}$ ${ }^{1}$ Cardiff Medical School, Cardiff/United Kingdom \\ ${ }^{2}$ General Surgery, University Hospital of Wales, Cardiff/United Kingdom}

Contact E-mail Address: HawkinsJ12@cardiff.ac.uk

Introduction: Colonic interposition was first described as a method to create a neo-oesophagus in 1911 by Kelling and Vuillet, and in contemporary practice is usually only considered if the stomach is not a viable neo-conduit because of the associated high morbidity and mortality. There is no consensus on the optimum choice of colonic conduit (right vs. left) or the route of placement (posterior mediastinal, retrosternal or subcutaneous).
Aims \& Methods: The aim of this review was to determine the optimum choice and route of conduit following colonic interposition after oesophagectomy in adults. PubMed, MEDLINE and the Cochrane Library (January 1985 to January 2017) were systematically searched for studies which reported outcomes of colonic interposition after oesophagectomy in adults. The primary outcome measure was overall morbidity and secondary outcome measure was operative mortality.

Results: Twenty-seven studies, involving 1849 patients (median age 60 years, 1177 males, 697 malignant disease) who underwent colonic interposition were analysed. The overall pooled morbidity rate of left vs. right colonic conduit was $9.6 \%$ [95\% CI $(6.24-12.87), \mathrm{p}<0.00001]$ vs.16.5\% [95\% CI (11.07-22.02), $\mathrm{p}<0.00001]$ respectively. The overall pooled mortality rate of left vs. right colonic conduit was $5.6 \%$ [95\% CI $(3.50-7.60)$, p $<0.0001]$ vs. $10.3 \%$ [95\% CI (7.23$13.27), \mathrm{p}<0.0001]$ respectively. Retrosternal route placement was associated with the lowest overall pooled morbidity of $9.2 \%$ [95\% CI $(6.48-11.99)$, $\mathrm{p}<0.00001]$, and lowest overall pooled mortality of $4.8 \%$ [95\% CI $(3.74$ $5.89), \mathrm{p}<0.0001]$

Conclusion: Left colon is the conduit of choice for colonic interposition after oesophagectomy in adults and the retrosternal route should be favoured.

Disclosure of Interest: All authors have declared no conflicts of interest.

\section{References}

1. Bothereau H, Munoz-Bongrand N, Lambert B, Montemagno S, Cattan P, Sarfati E. Esophageal reconstruction after caustic injury: is there still a place for right coloplasty?. The American Journal of Surgery. 2007;193(6):660-664.

2. Mansour K, Bryan F, Carlson G. Bowel Interposition for Esophageal Replacement: Twenty-Five-Year Experience. The Annals of Thoracic Surgery. 1997;64(3):752-756.

3. Doki Y, Okada K, Miyata H, Yamasaki M, Fujiwara Y, Takiguchi S et al. Long-term and short-term evaluation of esophageal reconstruction using the colon or the jejunum in esophageal cancer patients after gastrectomy. Diseases of the Esophagus. 2008;21(2):132-138

4. Fürst H, Hartl W, Löhe F, Schildberg F. Colon Interposition for Esophageal Replacement. Annals of Surgery. 2000;231(2):173-178.

5. Hamai Y, Hihara J, Emi M, Aoki Y, Okada M. Esophageal reconstruction using the terminal ileum and right colon in esophageal cancer surgery. Surgery Today. 2011;42(4):342-350.

6. Motoyama S, Kitamura M, Saito R, Maruyama K, Sato Y, Hayashi K et al. Surgical Outcome of Colon Interposition by the Posterior Mediastinal Route for Thoracic Esophageal Cancer. The Annals of Thoracic Surgery. 2007;83(4):1273-1278

7. Renzulli P, Joeris A, Strobel O, Hilt A, Maurer C, Uhl W et al. Colon interposition for esophageal replacement: a single-center experience. Langenbeck's Archives of Surgery. 2004;389(2):128-133.

8. Saeki H, Morita M, Harada N, Egashira A, Oki E, Uchiyama $\mathrm{H}$ et al Esophageal replacement by colon interposition with microvascular surgery for patients with thoracic esophageal cancer: the utility of superdrainage. Diseases of the Esophagus. 2012;26(1):50-56.

9. Boukerrouche A. Isoperistaltic left colic graft interposition via a retrosternal approach for esophageal reconstruction in patients with a caustic stricture: mortality, morbidity, and functional results. Surgery Today. 2013;44(5):827-833.

10. Demeester T, Johansson K, Franze I, Eypasch E, Lu C, Mcgill J et al Indications, Surgical Technique, and Long-Term Functional Results of Colon Interposition or Bypass. Annals of Surgery. 1988;208(4):460-474.

11. Gvalani A, Deolekar S, Gandhi J, Dalvi A. Antesternal Colonic Interposition for Corrosive Esophageal Stricture. Indian Journal of Surgery. 2012;76(1):56-60.

\section{P0941 THE ROLE OF LIVER RESECTION AFTER CHEMOTHERAPY FOR SYNCHRONOUS COLORECTAL LIVER METASTASIS}

A. Ouchi, Y. Shimizu, K. Komori, Y. Senda, T. Kinoshita, S. Natsume, T. Ooshiro

Department Of Gastroenterological Surgery, Aichi Cancer Center Hospital, Aichi/ Japan

Contact E-mail Address: akira.ouc@gmail.com

Introduction: The combination of chemotherapy and molecular targeted therapy has improved the outcome of patients with colorectal liver metastasis (CRLM); however, treatment outcomes of liver resection after preceding chemotherapy for CRLM, especially synchronous CRLM (sCRLM), remain unclear. For patients with technically resectable and oncologically suitable sCRLM, we conduct hepatic resection for the metastases at an interval of 3 months after colorectal resection, whereas for those with technically unresectable or oncologically unsuitable SCRLM, we perform preceding chemotherapy. The aim of this study was to retrospectively evaluate treatment outcomes of our strategy for sCRLM and explore prognostic factors for determining the indication for liver resection, particularly in patients receiving chemotherapy for SCRLM.

Aims \& Methods: Between April 2006 and March 2016, 157 consecutive patients underwent primary tumor resection for SCRLM at our department; of them, 35 patients (S group) underwent subsequent staged liver resection, 109 patients received preceding chemotherapy, and remaining 13 patients received other treatments. Among those who received preceding chemotherapy, 25 patients (CS group) underwent liver resection after chemotherapy, but 84 patients (C group) could not undergo liver resection and continued ongoing chemotherapy. In the 
CS group, 16 patients had unresectable disease (due to lack of remnant liver volume, invasion to hepatic hilar area, or invasion to three hepatic veins) and 9 initially had unsuitable disease (progressive primary disease or suspicion of other distant metastasis); therefore, upfront chemotherapy was selected.

Results: The frequency of adverse prognostic factors tended to be higher in the $\mathrm{CS}$ group than in the $\mathrm{S}$ group, e.g., tumor number (mean $\pm \mathrm{SD}: 6.0 \pm 3.5 \mathrm{vs}$ $2.7 \pm 1.7)$ and number of metastatic lymph nodes $(4.2 \pm 6.3$ vs $2.7 \pm 2.3)$. Nevertheless, overall survival (OS) in the CS and S groups since primary tumor resection was equivalent (3-year survival rate: $86.9 \%$ vs $93.4 \%$, Logrank $\mathrm{P}=0.34$ ) and much better than that in the $\mathrm{C}$ group (3-year survival rate: $40.2 \%$ ). Although liver-limited relapse-free survival (RFS) since hepatectomy tended to be worse in the CS group than in the $\mathrm{S}$ group (3-year survival rate: $45.0 \%$ vs $62.7 \%$, Log-rank $\mathrm{P}=0.14)$, RFS after hepatectomy was equivalent in the CS and S groups (3-year survival rate: $33.3 \%$ vs $21.6 \%$, Log-rank $\mathrm{P}=0.97$ ). Early tumor shrinkage (ETS) was found to be a stronger poor prognostic factor for liver resection after chemotherapy than existing prognostic factors in univariate and multivariate analyses, and RFS was much better in patients with ETS than in those with non-ETS (3-year survival rate: $62.5 \%$ vs $7.7 \%$, Log-rank $\mathrm{P}=0.05)$.

Conclusion: OS and RFS in the CS group compared favorably with those in the S group despite the high frequency of poor prognostic factors; patients with ETS had a better prognosis after liver resection. Liver resection after chemotherapy revealed comparatively favorable prognosis in well-selected patients with sCRLM, and early responsiveness to chemotherapy was useful in determining the indication for liver resection in patients receiving chemotherapy for SCRLM. Disclosure of Interest: All authors have declared no conflicts of interest.

\section{P0942 SURGICAL OUTCOME OF HEPATOPANCREATODUODENECTOMY ASSOCIATED WITH HEMIHEPATECTOMY OR MORE EXTENDED LIVER RESECTION FOR BILIARY CANCERS}

T. Sano ${ }^{1}$, K. Komaya ${ }^{1}$, T. Arikawa ${ }^{1}$, T. Saito ${ }^{1}$, S. Ishiguro ${ }^{1}$, S. Komatsu ${ }^{1}$,

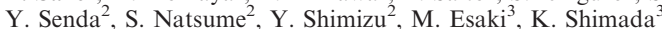

${ }^{1}$ Gastroenterological Surgery, Aichi Medical University, Nagakute/Japan

${ }^{2}$ Department Of Gastroenterological Surgery, Aichi Cancer Center Hospital, Aichi/ Japan

${ }^{3}$ Hepatobiliary And Pancreatic Surgery, National Cancer Center Hospital, Tokyo/ Japan

Contact E-mail Address: sano.tsuyoshi.508@mail.aichi-med-u.ac.jp

Introduction: Hepatopancreatoduodenectomy associated with hemihepatectomy or more extended liver resection (HPD) is occasionally indicated in patients with advanced gallbladder or bile duct cancers, and is still a highly invasive surgical procedure. Feasibility of this invasive procedure for biliary cancers has not fully invastigated.

Aims \& Methods: The aim of this study is to review surgical outcome of HPD in terms of case series of a single surgeon at three Japanese tertiary referral centers. Consecutive one hundred one hepatobiliary resections with caudate lobectomy were carried out by a single surgeon (TS) at National Cancer Center, Tokyo, Aichi Cancer Center, Nagoya, and Aichi Medical University, Nagakute since 2002 and 2016. Of these 14 included 13 patients with bile duct cancer and one with gallbladder cancer underwent HPD were analyzed. Essentially, liver transection was performed in terms of Pean clamp crushing method under intermittent inflow occlusion and pancreatojujunostomy was carried out in terms of duct to mucosa anastomosis.

Results: There were 9 men and 5 women. The median age was 66 years old (3481). All patients underwent preoperative biliary drainage and 12 patients underwent preoperative portal vein embolization. As to the extent of liver resection, left hemihepatectomy was performed in 2 , right hemihepatectomy in 11 , and right trisectionectomy in 1 patient, respectively. Combined portal vein resection and reconstruction was carried out in $6(42.9 \%)$, and hepatic arterial resection and reconstruction in one patients $(7.1 \%)$. The median operation time was 822 minutes (518-992), and the median intraoperative blood loss was $1716 \mathrm{ml}(730$ 2930), respectively. Although one patient underwent left trisectionectomy with segmental portal vein resection and reconstruction of 101 hepatobiliary resections resulted in the postoperative mortality (mortality rate $1.0 \%$ ), HPD was accomplished with zero mortality in this studied period. Histologically, the proximal bile duct margins were confirmed positive for cancer cells in two patients with bile duct cancer (R1 resection). Four patients with bile duct cancer survived more than 5 years without evidence of tumor recurrence, on the other hand one patient with gallbladder cancer died from recurrence 7 months after the surgery. Conclusion: HPD may become a safe and potentially feasible surgical procedure for bile duct cancer, the indication of HPD for gallbladder cancer should be elucidated for further investigations in terms of large case series.

Disclosure of Interest: All authors have declared no conflicts of interest.

\section{References}

1. Sano T, Shimada K, Sakamoto Y, Yamamoto J, Yamasaki S, Kosuge T. One hundred two consecutive hepatobiliary resections for perihilar cholangiocarcinoma with zero mortality. Ann Surg. 2006;244:240-247. PMID: 16858186

2. Sano T, Shimada K, Sakamoto Y, Ojima H, Esaki M, Kosuge T. Prognosis of perihilar cholangiocarcinoma; hilar bile duct cancer versus intrahepatic cholangiocarcinoma involving the hepatic hilus. Ann Surg Oncol. 2008;15:590-599. PMID: 18057991
3. Sano T, Shimizu Y, Senda Y, Komori K, Ito S, Abe T, Kinoshita T, Nimura Y. Isolated caudate lobectomy with pancreatoduodenectomy for a bile duct cancer. Langenbeck's Arch Surg. 2013;398:1145-1150. PMID: 24026222

P0943 PERIOPERATIVE ADMINISTRATION OF BROADSPECTRUM ANTIBIOTICS COVERING ENTEROBACTERIACEAE DECREASE THE INCIDENCE OF SURGICAL SITE INFECTION FOLLOWING PANCREATODUODENECTOMY

K. Tanaka, Y. Nakanishi, T. Asano, T. Noji, T. Nakamura, T. Tsuchikawa, K. Okamura, T. Shichinohe, S. Hirano

Department Of Gastroenterological Surgery 2, Hokkaido University Graduate School of Medicine, Sapporo/Japan

Contact E-mail Address: kimitaka.t@gmail.com

Introduction: Pancreatoduodenectomy (PD) is one of the operations associated with high rate of surgical site infection (SSI). The one of the reasons is said that SSI is associated with the preoperative biliary infection caused by preoperative examinations or drainage of biliary tract. In our clinical trial [1], SSI after pancreatobiliary surgery could be decreased by using the perioperative selective antibiotics based on preoperative bile culture. The bacteria cultured from SSI were Enterobacteriaceae such as Enterococcus and Enterobacter species with high frequency.

Recently, however, most of the patients who had planned to undergo PD received internal biliary drainage preoperatively. It means preoperative collection of the bile juice is hard for the patient with internal biliary drainage. In order to solve this problem, we compared the conventional perioperative prophylactic antibiotics (Cefmetazole: CMZ) and the broad-spectrum antibiotics covering the Enterobacteriaceae (Piperacillin/Tazobactam +Vancomycin: PIPC/ $\mathrm{TAZ}+\mathrm{VCM})$, retrospectively. The aim of this study is to assess the impact of two types of perioperative antibiotics usage in patients undergoing PD.

Aims \& Methods: Sixty-nine patients underwent PD at Hokkaido University Hospital (Japan) between April 2015 and March 2016, when prospective surgical site infection surveillance was performed. Thirty-eight patients were administered CMZ as perioperative prophylactic antibiotics from April 2015 to March 2016, and 31 were PIPC/TAZ + VCM from April 2016 to March 2017. CMZ was injected intravenously every three hours from the start of operation, and once after the operation. PIPC/TAZ was injected intravenously every three hours from the start of operation and three times on the next day of the operation, whereas VCM was injected intravenously in the morning of operation $(20 \mathrm{mg} / \mathrm{kg})$ and just after the operation $(15 \mathrm{mg} / \mathrm{kg})$.

Comparison between CMZ group and PIPC/TAZ + VCM group was performed to variables from clinical features (gender, age, body mass index, performance of neoadjuvant chemotherapy and preoperative biliary stent), operative outcomes (operation time and blood loss) and postoperative course (the incidence of SSI, the duration of postoperative antibiotics and postoperative stay). The side effects of VCM or PIPC/TAZ, and the occurrence of multidrug resistant bacteria were investigated, as well.

Results: There were no significant differences in clinical features and operative outcomes between the two groups. The patients with PIPC/TAZ + VCM received significantly shorter duration of postoperative antibiotics administration than the patients with $C M Z(5.9 \pm 8.5$ vs. $13.0 \pm 18.2$ days; $p=0.048)$. Significantly lower incidence of SSI was revealed in patients with PIPC/TAZ+VCM $(9 / 31(29.0 \%))$ than in those with CMZ $(20 / 38(52.6 \%))(p=0.048)$. Especially, significantly lower incidence of incisional SSI was revealed in patients with PIPC/ TAZ + VCM (2/31(6.5\%)) than in those with CMZ $(14 / 38(36.8 \%))(p=0.004)$. The redneck syndrome as one of the side effects of VCM was observed in 5/31 patients $(16.1 \%)$. No multidrug resistant bacteria were identified.

Conclusion: The broad-spectrum perioperative antibiotics covering Enterobacteriaceae such as Enterococcus and Enterobacter species could decrease the incidence of SSI and duration of postoperative antibiotics administration compared to CMZ. More strict indication to select the patients who should be administered VCM, because the occurrence of side effects of VCM was relatively high

Disclosure of Interest: All authors have declared no conflicts of interest.

\section{Reference}

1. Okamura K, Tanaka K, Miura T, Nakanishi Y, Noji T, Nakamura T, Tsuchikawa T, Okamura K, Shichinohe T, Hirano S. Randomized controlled trial of perioperative antimicrobial therapy based on the results of preoperative bile cultures in patients undergoing biliary reconstruction. $J$ Hepatobiliary Pancreat Sci. 2017 Mar 29. doi: 10.1002/jhbp.453. [Epub ahead of print] PubMed PMID: 28371248.

\section{P0944 PARADIGM SHIFT? SHOULD A LYMPHADENECTOMY ROUTINELY BE PERFORMED ALSO AMONG GALLBLADDER CANCER PATIENTS WITH T1A DISEASE?}

N. Köhn ${ }^{1}$, R. Warschkow ${ }^{2}$, D. Nussbaum ${ }^{3}$, D. Candinas ${ }^{4}$, B. Gloor ${ }^{1}$, B. Schmied ${ }^{2}$, D. Blazer ${ }^{3}$, M. Worni ${ }^{1}$

${ }^{1}$ Visceral Surgery And Medicine, Inselspital, University Clinic, Bern/Switzerland ${ }^{2}$ Surgery, Kantonsspital St. Gallen, St. Gallen/Switzerland

${ }^{3}$ Surgery, Duke University Medical Center, Durham/United States of America/NC ${ }^{4}$ Inselspital, Universität Bern Inselspital Visceral und Transplant.-Chirurgie, Bern Switzerland 
Contact E-mail Address: mathias.worni@insel.ch

Introduction: Gallbladder cancer is the fifth most common gastrointestinal malignancy and the most common of all biliary tract cancers. Overall, it is associated with a poor prognosis. Consensus guidelines suggest that patients with Tla cancers can be observed with cholecystectomy alone while patients with $\mathrm{T} 1 \mathrm{~b}$ or greater lesions should undergo lymphadenectomy in the hepatoduodenal ligament. Extent of surgery is especially important for those patients given efficient systemic therapy is lacking.

Aims \& Methods: The aim of this study was to critically explore whether those suggestions hold true on a population-based level given that current evidence is mainly based on small patient series. Especially we assessed the rate of T-stage specific lymph node positivity and the impact of lymphadenectomy on long-term outcomes. The National Cancer Data Base of the United States was reviewed from 2004 to 2012 to identify non-metastatic gallbladder cancer patients with T1a, T1b, or T2 stage primary tumors. Patients were grouped by whether a dedicated lymphadenectomy was performed or not while those with missing information were excluded. Groups were compared for baseline characteristics. Predictors of dedicated lymphadenectomy were assessed by univariate and multivariable adjusted logistic regression with adjustment for important patient- and tumor characteristics. Overall survival was assessed using Cox proportional hazard regression analyses before and after full bipartite pairwise propensity score matching.

Results: Of the 3879 patients included, $287(7.4 \%)$ had T1a, $661(17.0 \%) \mathrm{T} 1 \mathrm{~b}$ and $2931(75.6 \%)$ T2 gallbladder cancer. Most patients were female $(\mathrm{n}=2751$, $70.9 \%$ ), median age was 72 years (range $21-90$ ). Among patients with T1a, T1b, and T2 disease, $102(35.5 \%), 278(42.1 \%)$, and 1526, (52.1\%) underwent a dedicated lymphadenectomy, respectively. Over the study period, the rate of lymph node excision increased from $43 \%$ to $58 \%$ ( $\mathrm{p}$ for trend $=0.005$ ). The rates of positive lymph nodes were $11.8 \%, 16.2 \%$, and $42.5 \%$ for T1a, T1b, and T2-stage, respectively. 5-year overall survival rate was $31.6 \%$ for patients without and $44.6 \%$ for patients with a dedicated lymphadenectomy and $58.6 \%, 43.9 \%$, and $34.5 \%$ for T1a, T1b, and T2-stage, respectively. After multivariable adjustment, the odds of undergoing a lymphadenectomy increased with tumor stage compared to T1a disease (vs. T1b: OR: 1.37; CI: 1.01-1.86, vs. T2: OR: 1.95; CI: 1.48-2.57). Compared to their counterparts, patients who underwent lymphadenectomy for gallbladder cancer were more likely to have had an R0 resection status, underwent chemo- or radiation therapy, were diagnosed in later years, were younger, had a lower Charlson-Deyo-comorbidity score, were operated in high volume centers and traveled a longer distance to the treatment facility. In univariate analysis, no survival benefit of lymphadenectomy was found for T1a disease (HR 1.04, 95\% CI 0.70-1.54) while lymphadenectomy improved overall survival in T1b (HR $0.72,95 \% \mathrm{CI} 0.58-0.90$ ) and T2 stage (HR $0.59,95 \% \mathrm{CI}$ 0.53-0.65). Given significant bias of undergoing lymphadenectomy, full bipartite, pairwise propensity-score matching was performed. A trend towards overall survival improvement was then also found for T1a disease (HR $0.65,95 \%$ CI 0.39 1.09). Overall survival benefit remained for T1b (HR $0.68,95 \% \mathrm{CI} 0.51-0.91)$ and T2-stage (HR $0.63,95 \%$ CI $0.55-0.71$ ).

Conclusion: Our results support current consensus guidelines that T1b and T2 gallbladder cancer patients should undergo LA. However, based on the high rate of nodal positivity among patients with T1a disease and the trend towards overall survival improvement among T1a patients who underwent lymphadenectomy, we suggest to rethink this dogma and advocate to perform lymphadenectomy also in surgically fit patients with T1a disease.

Disclosure of Interest: All authors have declared no conflicts of interest.

\section{P0945 RECTAL SWAB CULTURE CAN DIRECT ANTIMICROBIAL PROPHYLAXIS AND PREDICT THE RISK OF INFECTIOUS COMPLICATIONS AFTER PANCREATICODUODENECTOMY}

M. De Pastena ${ }^{1}$, S. Paiella ${ }^{1}$, D. Ciprani ${ }^{1}$, G. Marchegiani ${ }^{1}$, A.M. Azzini ${ }^{2}$, E. Concia ${ }^{2}$, R. Salvia ${ }^{1}$, C. Bassi ${ }^{1}$

${ }^{1}$ General And Pancreatic Surgery Department, Pancreas Institute, University of Verona, Verona/Italy

${ }^{2}$ Infectious Diseases Division, University of Verona Hospital, Verona/Italy

\section{Contact E-mail Address: salvatore.paiella $a$ univr.it}

Introduction: Despite improvements in the perioperative care, the morbidity rate after Pancreaticoduodenectomy (PD) is still higher than $50 \%$. In particular, infectious complications (ICs) occur in about one-third of cases. This study aims to examine the correlation between preoperative rectal swab (RS) and intraoperative bile cultures, and its impact on postoperative course of patients submitted to PD.

Aims \& Methods: The institutional electronic database was queried for all consecutive PD performed from January 2015 to July 2016. Data were retrospectively analyzed. Based on the positivity/negativity of preoperative surveillance RS for multi-antibiotic resistant Gram-negative and Gram-positive enteric rods, the population was divided into two groups consequently compared (RS+ vs. RS-).

Results: Three hundred thirty-eight patients were considered for the analysis. Overall 50 patients $(14.8 \%)$ showed a RS+. Preoperative biliary drain (PBD) was the only independent risk factor associated to $\mathrm{RS}+(\mathrm{p}=0.021, \mathrm{OR} 2.6)$. The groups statistically differed in the overall morbidity, ICs, sepsis, pulmonary complications, reoperation and mortality $(\mathrm{p}<0.05)$. At multivariate analysis, ICs and mortality remained independently associated to $\mathrm{RS}+(\mathrm{p}=0.013 \mathrm{OR}$ $2.9, \mathrm{p}=0.009$ OR 3.4, respectively). Bile-culture showed a perfect correlation (species and antibiotic susceptibility) with RS culture in 157 patients $(86.7 \%)$ ). The most common microorganisms found were E. Coli ESBL $(6.6 \%)$ and Klebsiella pneumoniae carbapenemase-resistant (2.2\%).
Conclusion: Preoperative RS-culture's positivity strictly correlates to biliary colonization with enteric rods that occurs after PBD. Infectious complications and mortality after PD are independently associated with a positive RS culture. Our study suggests that preoperative RS can direct antibiotic prophylaxis in order to reduce the burden of ICs and deadly events after PD.

Disclosure of Interest: All authors have declared no conflicts of interest.

\section{References}

Di Carlo P, Gulotta G, Casuccio A, Pantuso G, Raineri M, Farulla CA, et al. KPC - 3 Klebsiella pneumoniae ST258 clone infection in postoperative abdominal surgery patients in an intensive care setting: analysis of a case series of 30 patients. BMC Anesthesiol. 2013;13(1):13.

Tzouvelekis LS, Markogiannakis A, Psichogiou M, Tassios PT, Daikos GL. Carbapenemases in Klebsiella pneumoniae and other Enterobacteriaceae: an evolving crisis of global dimensions. Clin Microbiol Rev. 2012;25(4):682-707.

Grundmann H, Glasner C, Albiger B, Aanensen DM, Tomlinson CT, Andrasevic AT, et al. Occurrence of carbapenemase-producing Klebsiella pneumoniae and Escherichia coli in the European survey of carbapenemase-producing Enterobacteriaceae (EuSCAPE): a prospective, multinational study. Lancet Infect Dis. 2017;17(2):153-63.

Fong ZV, McMillan MT, Marchegiani G, Sahora K, Malleo G, De Pastena M, et al. Discordance Between Perioperative Antibiotic Prophylaxis and Wound Infection Cultures in Patients Undergoing Pancreaticoduodenectomy. JAMA Surg. 2016;151(5):432-9

\section{P0946 EFFECT OF TRICLOSAN-COATED SUTURE USE ON THE INCIDENCE OF SUPERFICIAL INCISIONAL SURGICAL SITE INFECTIONS AFTER GASTROENTEROLOGIC SURGERY: A PROPENSITY SCORE MATCHING RETROSPECTIVE STUDY}

K. Yamashita, S. Takeno, S. Hoshino, H. Shiwaku, S. Hasegawa Department Of Gastroenterological Surgery, Fukuoka University Faculty of Medicine, Fukuoka/Japan

Contact E-mail Address: kanefumi0519@yahoo.co.jp

Introduction: Surgical site infections (SSIs) after gastroenterological surgery cause significant morbidity, prolong hospitalisation and increase health care costs. Thus, SSI prevention is critical. To prevent bacterial colonisation in suture material, which disables local mechanisms of wound decontamination, triclosancoated sutures were developed. We retrospectively analysed the efficacy of triclosan-coated polydioxanone sutures in abdominal fascia and skin closure using a propensity score matching analysis. We further analysed the surgery types for which these sutures are best suited.

Aims \& Methods: The study protocol followed the principles of the Declaration of Helsinki and received ethical approval from the Ethics Committee of the Fukuoka University (approval no. 12-7-06). At our department, we used conventional abdominal closure methods during gastroenterological surgery before August 2012. Thus, we retrospectively collected surveillance data over a 1.5-year period for the control group. From September 2012, we began using triclosancoated sutures for closure. Hence, we collected data for the study group from September 2012 to September 2013. In total, we included 1768 patients (control group, $n=640$; study group, $n=1128$ ) who underwent gastroenterological surgery. Baseline differences and selection bias were adjusted using propensity score matching.

Results: Before matching, the SSI incidence differed significantly between the control and study groups for all gastroenterological surgeries [12.4\% (140/ $1128)$ vs. $5.5 \%(35 / 640) ; \mathrm{p}<0.001]$. The SSI incidence according to the surgery type was as follows in the control and study groups: $12.7 \%(26 / 204)$ vs. $10.5 \%$ $(12 / 114)(\mathrm{p}=0.347)$ for upper gastrointestinal (GI) surgery, $14.6 \%(43 / 294)$ vs. $5.3 \%(10 / 190)(\mathrm{p}=0.001)$ for lower GI surgery, $8.8 \%(24 / 274)$ vs. $4.1 \%(7 / 169)$ $(\mathrm{p}=0.045)$ for hepato-biliary-pancreatic surgery, $18.2 \%(40 / 220)$ vs. $7.8 \%(5 / 64)$ $(\mathrm{p}=0.030)$ for emergency surgery and $5.1 \%(7 / 136)$ vs. $1.0 \%(1 / 103)(\mathrm{p}=0.074)$ for others. Of 1768 cases, 483 pairs were matched using propensity score matching. No parameter used for the propensity score differed between the groups. After matching, we found a significant difference in the SSI incidence between the control and study groups for all gastroenterological surgeries $[9.7 \%(47 / 436)$ vs. $5.7 \%(28 / 455) ; \mathrm{p}<0.001]$. We found a significant difference in the SSI incidence between the control and study groups for lower GI $[17.0 \%(16 / 78)$ vs. $4.3 \%(4 / 90) ; p=0.008]$ and hepato-biliary-pancreatic $[16.4 \%(11 / 56)$ vs. $4.3 \%$ $(4 / 63) ; p=0.049]$ surgeries. No significant difference was found between the groups for upper GI surgery, emergency surgery and others. Multivariable logistic regression analysis showed that triclosan-coated suture use for lower G surgery was the independent factor affecting the SSI incidence $(p=0.017)$. The sutures demonstrated a significant efficacy in lower GI surgery.

Conclusion: Few studies have focussed on the types of surgery best suited for triclosan-coated sutures. Our findings suggest that abdominal fascia and skin closure using these sutures reduces the SSI risk, particularly after lower GI surgery.

Disclosure of Interest: All authors have declared no conflicts of interest. 
P0947 COMPARISON OF POSTOPERATIVE CONDITIONS BETWEEN ESOPHAGOGASTROSTOMY WITH THE DOUBLE-FLAP TECHNIQUE AND THAT WITH A CIRCULAR STAPLER IN LAPAROSCOPIC PROXIMAL GASTRECTOMY

M. Hayashi, H. Kawakubo, S. Mayanagi, K. Fukuda, R. Nakamura, K. Suda, N. Wada, Y. Kitagawa

Department Of Surgery, Keio University School of Medicine, Tokyo/Japan

\section{Contact E-mail Address: sas.jtf@gmail.com}

Introduction: In recent years, laparoscopic proximal gastrectomy (LPG) has been actively performed in our institution to reduce invasiveness. However, proximal gastrectomy is sometimes followed by reflux. Until February 2015, we performed esophagogastrostomy with a circular stapler (CS) accompanied by fundoplication in LPG. Since March 2015, to avoid the postoperative complication, we have been using esophagogastrostomy with the double-flap technique (DFT) in LPG for gastric cancer.

Aims \& Methods: We conducted this study to examine whether DFT can reduce the incidence of reflux and influence postoperative conditions. From January 2012 to February 2017, 47 LPGs were performed at Keio University Hospital. First, surgical time, bleeding, incidence of postoperative complication, postoperative hospital stay, and incidence of anastomotic stenosis were examined as surgical factors and compared between the DFT and CS groups. Second, gastroesophageal reflux finding on endoscopy, condition of the remnant stomach according to residue, gastritis, bile (RGB) classification at postoperative 6 months and 1 year, and proton pump inhibitor (PPI) intake were examined as postoperative factors. Finally, albumin and hemoglobin levels at postoperative 6 months and 1 year were examined as nutrient factors. Gastroesophageal reflux was assessed with scores of $0-5$ in accordance with the Los Angeles (LA) classification.

Results: Twenty-three LPGs with DFT and 24 LPGs with CS were performed during the period. Compared with the CS group, the DFT group had a significantly longer surgical time $(272.3 \pm 35.5$ vs $241.1 \pm 26.7 \mathrm{~min}, \mathrm{p}<0.01)$. Other surgical factors showed no statistically significant differences between the two groups. As for postoperative factors, although no significant differences in PPI intake, LA classification, and RGB classification were found, the DFT group showed a significantly lower score than the CS group $(\mathrm{p}<0.01)$. Postoperative nutrient factors did not show any significant difference between the groups.

Conclusion: Although LPG with DFT required a longer surgical time than LPG with CS, DFT is thought to be a safe reconstruction method in LPG. In addition to its safety, DFT can reduce postoperative reflux in patients who undergo LPG. Disclosure of Interest: All authors have declared no conflicts of interest.

\section{References}

1. Hayami M, Hiki N, Nunobe S, et al: Clinical Outcomes and Evaluation of Laparoscopic Proximal Gastrectomy with Double-FlapTechnique for Early Gastric Cancer in the Upper Third of the Stomach. Ann Surg Oncol. 2017 Jan 27.

2. Yoshitake U, Hidehumi S, Tsuyoshi E, et al: Laparoscopic proximal gastrectomy for early gastric cancer. Surg Today

\section{P0948 NOVEL ENDOSCOPIC REPAIR TECHNIQUE FOR GASTROINTESTINAL LEAKS AND PERFORATIONS USING NEGATIVE PRESSURE THERAPY WITH OPEN-PORE POLYURETHANE-FOAM AND FILM DRAINAGE}

G. Loske ${ }^{1}$, F. Rucktaeschel ${ }^{2}$, T. Schorsch ${ }^{1}$, C. T. Mueller ${ }^{1}$

${ }^{1}$ Department For General, Abdominal, Thoracic And Vascular Surgery, katho-

lisches Marienkrankenhaus gGmbH, Hamburg/Germany

${ }^{2}$ Medical Clinic, Westküstenkliniken, Heide/Germany

\section{Contact E-mail Address: loske.chir@marienkrankenhaus.org}

Introduction: Gastrointestinal (GI) leaks and perforations are difficult to manage and often mandate laparotomy and extensive surgical interventions for their repair. Endoscopic Negative Pressure Therapy (ENPT) has been developed to treat GI defects such as leaks, fistulae and perforations. However, ENPT has only been utilized in the management of rectal and esophageal leakages. By modifying the delivery catheter we were able to adapt ENPT to treat duodenal defects, that otherwise would have required surgery or more invasive methods to be repaired.

Aims \& Methods: Herein, we report ENPT using open-pore Polyurethane-foam and Film Drainage in a series of 10 patients with duodenal leakages. This is an open-label, retrospective, single-center study. Open-pore polyurethane-foam drainage (OPD) devices were constructed out of a piece $(1.5 \mathrm{~cm} \times 1.5 \mathrm{~cm} \times 3 \mathrm{~cm})$ of open-pore polyurethane-foam which was fixed surrounding the tip of a nasogastric drainage tube. Small bore open-pore film drainage (OFD) device was constructed with a strip of a very thin fragment open-pore double layered film $\left(2 \times 2^{-10} \mathrm{~cm}\right)$ which was wrapped surround the tip of a nasogastric drainage tube. The open- film consists out of two permeable membranes with a small interspace. Fluids are drained along the interspace and through the membranes. Diameter of small-bore OFD is $4-6 \mathrm{~mm}$, depending on the diameter of the drainage tube. OPD is inserted transorally. The foam is grasped with endoscopic forceps and guided to the duodenal lumen. After correct placement into the duodenal lumen for intraluminal ENPT, the tube is transferred out nasally, to become a nasoduodenal tube. Due to its smaller outer diameter OFD insertion is similar to placing a naso-gastric or naso-intestinal feeding tube (i.e. through the nose). After nasal insertion into the esophagus OFD is grasped with a forceps and advanced into the stomach, and guided into the duodenal lumen for intraluminal ENPT. In case of a duodeno- cutanous fistula the pull-through technique has been used for duodenal placement. In one case rendezvous technique was used in combination with operative procedure. OPD and OFD are connected to an electronic vacuum device and negative pressure is applied. We use standard negative pressure of $125 \mathrm{mmHg}$, continuous suction, and high intensity, which results in collapse of the duodenal lumen around the open-pore foam or film with subsequent closure of the defect zone. Furthermore, duodenal secretions are actively removed through the tubes.

Results: We treated 10 patients with ENPT because of a duodenal leakage. Reason of duodenal defects were: rupture of operative suture $(n=8)$, iatrogenic perforation during $\operatorname{ERCP}(\mathrm{n}=1)$, perforation due to an operative drain $(\mathrm{n}=1)$. Nine patients were treated with intraluminal and one patient with intracavitary variant of ENPT. In 7 patients we used the OPD device, in one patient OFD, and in two patients OPD and OFD. All leakages $(100 \%)$ were successfully closed after a treatment period of 11 days in median (range 7-19 days).

Conclusion: ENPT using small diameter tube with open-pore film was effective to treat duodenal leakages. Advantage of OFD is the small diameter which allows easy endoscopic placement through small openings and nasally insertion. OPD and OFD devices can be positioned accurately at the site of duodenal leaks and perforations using common endoscopic techniques and thus represent a potential addition to the armamentarium to treat these difficult lesions.

Disclosure of Interest: G. Loske: Gunnar Loske is a consultant for Lohmann \& Rauscher.

All other authors have declared no conflicts of interest.

\section{P0949 INFLUENCE OF THE HYBRID METHOD OF DETOXICATION ON BLOOD CLARIFICATION EFFECTIVENESS AT PATIENTS WITH THE MULTIORGAN FAILURE}

K. G. Khalikulov, B. A. Saidkhanov, D. B. Saidkhanova

Extracorporeal Detoxication, National Center of Surgery named after acad.

V.Vakhidov, Tashkent City/Uzbekistan

Contact E-mail Address: drhgh@hotmail.com

Introduction: The main releaser for development of multiorgan failure syndrome (MOFS) in most cases are aggressive mediators of inflammation which are very often occur after surgical complications and more than in $70 \%$ of cases leads to lethal outcomes. Increase of an endotoxemia leads to development of the expressed pathological processes and to a fast decompensation of bodies of natural detoxication with the subsequent development of a multiorgan failure. Increase in detoxication ability of sorbents can happen due to change of chemical composition, or due to collimating of padding properties to them by their modification by various agents by means of an immobilization on their surface of organic and inorganic compounds. In this plan especially important role is got by the researches directed to development of sorbents with oxidizing activity.

Aims \& Methods: Aim of study is to estimate effectiveness of the modified haemosorbent application for patients about the MOFS. The experimental part of work was conducted on 14 not purebred dogs with the acute liver failure modelled by bandaging of distal department of the CBD. After development of pathological process animals were divided into 2 groups. To the first group of animals the procedure of a haemosorbtion was carried out by a reference technique with use of a haemosorbent of SKN-2K. To the second group of animals the haemosorbtion was carried out by the developed technique with the same sorbent, but the solution of a neutral anolyte subjected to oxidizing modification. For this purpose, in the flowing mode carried out a half-hour incubation of a sorbent from 21 of solution of a neutral anolyte.

Results: Results showed that at animals of the 2nd group in comparison with group of comparison improvement of a condition of an organism was expressed in much larger degree. After performing detoxication therapy by the developed technique a normalization of all studied parameters is registered. The same tendency is revealed also concerning nontoxical components. It is necessary to pay special attention to dynamics of a ratio of the common protein and an index average molecules/the common protein, pointing to synthesis process activation. The carried-out all-clinical blood test revealed the considerable improvement of indexes of white blood. In group of comparison it was not succeeded to achieve the complete normalization of the studied indexes. On the basis of what the conclusion was drawn on high effectiveness of the developed technique, and expediency of its introduction in clinical practice. Under our observation there were 45 patients needing carrying out getter detoxicating in the postoperative period. The control group (25) was created by a random sample of case histories from set of the patients with a similar syndrome who were earlier on treatment in our clinic and receiving a course of haemo perfused therapy by a reference technique. The analysis of results of treatment of patients of a basic group showed that the positive dynamics of clinical indexes expressed in decrease of manifestations of an intoxication syndrome, improvement of health and laboratory indexes is noted in earlier terms, and degree of expressiveness of positive changes at them was much higher. Dynamics of decrease in endogenic intoxication is reflected by data of laboratory researches.

Conclusion: Thus, the received results showed that it is possible to increase quality of detoxicating by a pretreatment of a haemo sorbent solution of a neutral anolyte. As a result of it the sorbent gains padding, oxidizing properties. At such modification there is an inclusion of oxygen-containing and acid groups in structure of a haemo sorbent. Besides, on a surface of a sorbent protogenic groups of carboxylic and phenolic types thanks to which the oxidized coals gain the expressed cation-exchange ability are formed. Therefore, besides actually getter, such sorbent follow-up gains oxidizing properties. Modification of a sorbent solution of a neutral anolyte incidentally allows to solve also other problem connected about need of use of anticoagulants for prevention of a thrombogenesis in a column. Use it in this quality provides decrease in risk of postoperative violations from system of a hemostasis. 
Disclosure of Interest: All authors have declared no conflicts of interest.

\section{References}

Medvedinskiy I.D., Zislin B.D., Yurchenko L.N. Multiorgan failure development concept at hestosis model//Anesthesiology and ICU.- 2000.- №3.- p.48-51. Moroz V.V., Lukach V.N., Shifman E.M., Dolgih V.T., Yakovleva I.I. Sepsis. Clinico-pathophysiological aspects of intensive therapy. Manual for Doctors. Petrozavodsk: Intellect, 2004.- p. 290.

Musllomhi M.C. Holigan A.W:, Williamson K. Recent development in the pathophysiology and management of pre-eclampsia//Br. J. Anaesth. -1996. $76(1)-131-148$

Salyukov Yu. L., Shutov V.Yu, Revzin A.I. et al. Combined usage of intravenous natrium hypochloride with combined haemocorrection at multiorgan failure treatment/VIII international congress of anesthesiology and intensive care. 2002. P.176.

Macedonckaya T.P. Combined use of gluthamin and pektine at multiorgan failure after peritonitis. Autoreferat of dissertation. M, 2003

Peritonitis and multiorgan failure treatment results using methods of extracorporeal haemocorrection/Fillipov S.I., Arestovich R.A., Kulamihin et al. VIII international congress of anesthesiology and intensive care. 2002. P.280.

Various methods anticoagulation influence to coagulopathy development on patients with multiorgan failure./Loginov S.P., Pavlova T.A., Evdokimov E.A. et al.//VII international congress «Actual aspects of extracorporeal blood purification», M., 2010.-p.53

\section{P0950 CARDIAC SEPTAL DEFFECT OCCLUDER DEVICE FOR ENDOSCOPIC TREATMENT OF GASTRO-CUTANEOUS AND GASTRO-PULMONARY LEAKS AFTER BARIATRIC SURGERY}

A.J. Baptista ${ }^{1}$, W. Bandel ${ }^{2}$, A. Gelrud ${ }^{1}$, A. Salinas ${ }^{1}$, L.C. Sabagh ${ }^{3}$, A. Ospina ${ }^{3}$, M.A. Guzman ${ }^{1}$, J.F. Piñerúa Gonsálvez ${ }^{1}$, H. Rass ${ }^{1}$, A. Oropeza ${ }^{1}$, M. Antor ${ }^{1}$

${ }^{1}$ Hospital de Clínicas Caracas, Caracas/Venezuela

${ }^{2}$ Kids Cardiac Center, Miami/United States of America

${ }^{3}$ Clinica Reina Sofia, Bogota/Colombia

\section{Contact E-mail Address: albertogebaptista@hotmail.com}

Introduction: Gastric leaks are severe complications of Bariatric Surgery (BS). Surgical re-intervention may be indicated but is associated with high morbidity and mortality. The use of self-expanding esophageal metallic stents (SEMS) has become an effective alternative. Over the scope clips (OTSC) have also been used. Nevertheless some patients develop a refractory fistulae after stent removal or other failed endoscopic treatments. Cardiac Septal Defect Closure Devices (CSDCD), used in interventional cardiology have been described to treat postsurgical digestive fistulae in non-bariatric cases.

Aims \& Methods: We aim to present the experience using CSDCD for gastric leaks secondary to BS. In this retrospective study, patients with leaks secondary to gastric bypass (GBP) or sleeve gastrectomy (SG) from 4 centers were included Data collected from november 2012 to january 2016 included sex, age, type of surgery, previous treatment, tract path, size of the leak opening and defect closure. Leaks were grouped according to the International Sleeve Gastrectomy Expert Panel Consensus in acute (post-operative days 1-7), early (1-6 weeks), late (after 6 weeks) and chronic (>12 weeks). Biliary catheters were adapted to introduce the CSDCD through the gastroscopes working channels. Clinical success was defined as complete and permanent resolution of abdominal or thoracic drainage with imaging documentation of closure (CT with contrast or upper GI contrast study) after 2 months.

Results: 42 patients with leaks were included (31 SG, 11 GBP). Three acute leaks, 5 early, 22 late and 12 chronic. Prior failed therapies included: SEMS and enteral tube feeding (ETF), SEMS alone, SEMS and gastrostomy of excluded stomach, OTSC, ETF alone, jejunostomy and none ( 3 acute leaks had CSDCD as the primary treatment). Tract path was grouped as gastric-cutaneous (37), gastricpleural (3) and gastric-bronchial (2). Median follow-up was 34, 8 weeks. All 3 patients with acute leak failed to close the defect. The CSDCD were removed within 7 days and SEMS were placed instead leading to defect closure. The 5 patients with early leaks had initial good response but within 30 days drainage recurred. The CSDCD were removed and replaced for a larger diameter device leading to permanent defect closure. Clinical success was achieved on 38 patients $(90,5 \%)$. In one patient with late leak the tract path was connected to an undrained cavity. Evolution was unsatisfactory and total gastrectomy accomplished.

Conclusion: CSDCD are effective to treat post bariatric surgery late and chronic leaks despite of the failed previous endoscopic treatment. Although early leaks finally healed it seems advisable to maintain conservative treatment and wait for the leak to become late or chronic before CSDCD placement. Acute leaks must be managed with a combination of SEMS, ETF, OTSC as first step.

Disclosure of Interest: All authors have declared no conflicts of interest.

P0951 COMPARATIVE EVALUATION OF LAPAROSCOPIC SINGLE-PORT (SILS) CHOLECYSTECTOMY AND LAPAROSCOPIC FOUR-PORT CHOLECYSTECTOMY

A. Alekberzade, N. Krylov, E. Lipnitsky

Surgery, Sechenov First Moscow State Medical University, Ministry of Health of

the Russian Federation, Moscow/Russian Federation
Introduction: Laparoscopic cholecystectomy is the gold-standard for the treatment of gallbladder stone disease. Single-incision laparoscopic (SILS) cholecystectomy was introduced with the aim of reducing the invasiveness of classic laparoscopic surgery. Despite satisfactory cosmetic results of SILS cholecystectomy and its repute of a painless procedure, there are few published studies comparing early and long-term postoperative period of laparoscopic SILS cholecystectomy versus laparoscopic four-port cholecystectomy.

Aims \& Methods: The aim of this study is the comparative evaluation of SILS cholecystectomy and laparoscopic four-port cholecystectomy. Early and longterm postoperative period has been analyzed in 240 patients who underwent laparoscopic cholecystectomy including 120 cases of single-port technique and 120 cases of four-port technique. Both groups were compared in surgical time, pain syndrome severity (visual analog scale), need for analgesics, postoperative complications, hospital-stay, daily activity recovery and return to physical work, patients' satisfaction of surgical results and their aesthetic effect.

Results: It was revealed that SILS cholecystectomy is associated with lower severity of postoperative pain, quick recovery of daily activity and return to physical work, high satisfaction of surgical results and their aesthetic effect compared with four-port cholecystectomy. Disadvantages of SILS cholecystectomy include longer duration of surgery, high incidence of postoperative umbilical hernia. However, hernia was predominantly observed during the period of surgical technique development.

Conclusion: Further studies to standardize, evaluate the safety and benefits of SILS cholecystectomy are necessary.

Disclosure of Interest: All authors have declared no conflicts of interest.

\section{References}

1. Bucher P., Pugin F., Buchs N.C. et al. Randomized clinical trial of laparoendoscopic single-site versus conventional laparoscopic cholecystectomy. Br J Surg. 2011;98(12):1695-1702. doi: 10.1002/bjs.7689.

2. Lai E.C., Yang G.P., Tang C.N. et al. Prospective randomized comparative study of single incision laparoscopic cholecystectomy versus conventional four-port laparoscopic cholecystectomy. Am J Surg. 2011;202(3):254-258. doi: 10.1016/j.amjsurg.2010.12.009.

3. Marks J., Tacchino R., Roberts K. et al. Prospective randomized controlled trial of traditional laparoscopic cholecystectomy versus single-incision laparoscopic cholecystectomy: report of preliminary data. Am J Surg. 2011;201(3):369-372; discussion 372-373. doi: 10.1016/ j.amjsurg.2010.09.012.

4. Ma J., Cassera M.A., Spaun G.O. et al. Randomized controlled trial comparing single-port laparoscopic cholecystectomy and four-port laparoscopic cholecystectomy. Ann Surg. 2011;254(1):22-27. doi: 10.1097/ SLA.0b013e3182192f89.

5. Sinan H., Demirbas S., Ozer M.T. et al. Single-incision laparoscopic cholecystectomy versus laparoscopic cholecystectomy: a prospective randomized study. Surg Laparosc Endosc Percutan Tech. 2012;22(1):12-16. doi: 10.1097/ SLE.0b013e3182402448.

\section{P0952 LAPAROSCOPIC SURGERY'S 100 MOST INFLUENTIAL} MANUSCRIPTS: A BIBLIOMETRIC ANALYSIS

K. Mellor ${ }^{1}$, A. Powell ${ }^{2}$, W. Lewis ${ }^{3}$

${ }^{1}$ General Surgery, Morriston Hospital, Swansea/United Kingdom

${ }^{2}$ Division Of Cancer And Genetics, Cardiff University, Cardiff/United Kingdom

${ }^{3}$ General Surgery, University Hospital of Wales, Cardiff/United Kingdom

Contact E-mail Address: kt.mellor@hotmail.co.uk

Introduction: Bibliometric analysis highlights key topics and publications, which have shaped the development of laparoscopic surgery (LS). Here the 100 most cited manuscripts in the field of LS are analyzed.

Aims \& Methods: The Thomson Reuters Web of Science database was used to identify all English language full manuscripts for the study with the search term 'laparoscop*'and 'surg*'. The 100 most cited papers were further analyzed by topic, journal, author, year and institution.

Results: 71,524 eligible papers were returned and the median (range) citation number was 585.76 (range 1635 to 368 ). The two most cited papers received 1635 citations each. The first $\left(\right.$ Nelson $\left.{ }^{1}\right)$ compared laparoscopic and open colectomy for malignancy, and the second $\left(\right.$ Clavien $\left.^{2}\right)$ described the classification of post-operative morbidity. Annals of Surgery published the highest number of papers $(n=22)$ and received most citations $(n=12,356)$. The country with the highest number of publications was the USA $(n=55)$, and the year with the greatest number of publications was $2004(\mathrm{n}=13)$. The most ubiquitous topic was the treatment of cancer $(n=35)$, followed by surgical technique $(n=24)$, and comparison of laparoscopic with open surgery $(n=22)$.

Conclusion: The most cited manuscripts highlighted laparoscopic surgical technique and compared laparoscopic with open surgery, and this review provides the most influential references in this arena, serving as a guide as to a citable paper. Disclosure of Interest: All authors have declared no conflicts of interest.

\section{References}

1. Nelson H, Sargent D, Wieand HS, Fleshman J, Anvari M, Stryker SJ, Beart RW, Hellinger M, Flanagan R, Peters W, Ota D, (2004) A comparison of laparoscopically assisted and open colectomy for colon cancer. NEJM 350: 2050-2059 
2. Clavien PA, Barkrun J, de Oliveira ML, Vauthey JN, Dindo D, Schulick RD, de Santibanes E, Pekolj J, Slankamenac K, Bassi C, Graf R, Vonlanthen R, Padbury R, Cameron JL, Makuuchi M (2009) The Clavien-Dindo Classification of Surgical Complications Five Year Experience. Annals of Surgery 250: 187-196

\section{P0953 BASELINE CHARACTERISTICS IN LAPAROSCOPIC SIMULATOR PERFORMANCE}

N. Oussi ${ }^{1}$, P. Renman ${ }^{2}$, E. Georgiou ${ }^{3}$, L. Enochsson ${ }^{4}$

${ }^{1}$ Division Of Surgery, Karolinska Institutet, Huddinge/Sweden

${ }^{2}$ Division Of Surgery, Medical School, Umeå University, Umeå/Sweden

${ }^{3}$ Medical Physics Dept., Athens University Medical School, MPLSC, Medical

School, National and Kapodistrian University of Athens, Athens/Greece

${ }^{4}$ Department Of Surgical And Perioperative Sciences, Umeå University, Division of Surgery, Luleå/Sweden

\section{Contact E-mail Address: ninos.oussi@ki.se}

Introduction: Laparoscopic technique is the first choice for multiple surgical procedures today. Laparoscopic surgery differs from traditional open surgery in several aspects, for example two-dimensional view of a three-dimensional interior, higher demands on eye-hand coordination and lack of tactile feedback. Laparoscopic surgical skills can be substantially improved by simulator training. Learning via simulators are under constant development and it is important to understand the value of baseline characteristics and abilities to further optimize simulators and training curricula within surgical education. In this study, focus, will be on the impact of PC-gaming experience and visuospatial skill.

Aims \& Methods: The aim of the study is to further analyse different factors to laparoscopic simulator training. 48 medical students completed three tasks in a laparoscopic virtual reality simulator, a validated Minimally Invasive Surgical Trainer (MIST, Mentice, Gothenburg, Sweden). Prior to the task, they performed a visuospatial test and answered questions regarding baseline characteristics (e.g. PC-gaming experience, age, gender, previous simulator experience). The data where analysed regarding different parts of the simulation (time, economy of movement, error and total score).

Results: The group with high PC-gaming experience performed significantly better in total time (Mean differerence $=85.49, \mathrm{p}=0.021$ ) and economy of movement $(\mathrm{M}=25.30, \mathrm{p}=0.018)$ in task 1 and 2 . There were no differences between either of the groups in task number 3 . A high visuospatial score correlated with a better result in time to completion $(\mathrm{M}=68.89, \mathrm{p}=0.026)$ and total score $(\mathrm{M}=80.16, \mathrm{p}=0.036)$. The group with both low PC-gaming experience and low visuospatial score performed worst in the simulator exercises.

Conclusion: Both PC-gaming experience and visuospatial abilities have an impact on laparoscopic simulator performance. No remaining significant differences by the third simulation exercise indicates a learning effect that could be more important than baseline skills. It could be valuable to identify individuals with both low PC-gaming experience and low visuospatial score to offer them additional simulator training.

Disclosure of Interest: All authors have declared no conflicts of interest.

\section{References}

1. van Empel PJ et al. Mapping the Maze of Minimally Invasive Surgery Simulators. Journal of Laparoendoscopic \& Advanced Surgical Techniques. 2012;22(1):51.

2. Grantcharov TP et al. Randomized clinical trial of virtual reality simulation for laparoscopic skills training. British Journal of Surgery. 2004;91(2):146.

3. Seymour NE et al. Virtual reality training improves operation room performance: results of randomized, double-blinded study. Annals of surgery. 2002;236(4):458-63.

4. Larsen CR et al. Effect of virtual reality training on laparoscopic surgery: randomised controlled trial. BMJ: British Medical Journal (Overseas \& Retired Doctors Edition). 2009:338(7705):1253.

5. Chaudhry A et al. Learning rate for laparoscopic surgical skills on MIST VR, a virtual reality simulator: quality of human-computer interface. Annals of Royal College of Surgeons of England. 1999;81(4):281-6.

6. Jalink MB et al. The effects of video games on laparoscopic simulator skills American Journal of Surgery. 2014;208(1):151-6.

7. Glassman D et al. Effect of playing video games on laparoscopic skills performance: A systematic review. Journal of Endourology/Endourological Society. 2016;30(2):146-52.

8. Nomura T et al. Can interview prior to laparoscopic simulator traning predict a trainee's skills? Journal of Surgical Education. 2008;65(5):335-9.

\section{P0954 PERITONEAL TUBERCULOSIS: EPIDEMIOLOGICAL DATA, CLINICAL AND EVOLUTIVE ASPECTS ACCORDING TO THE EXPERIENCE OF A TUNISIAN CENTER}

W. Ben Ameur, J. Hanen, E. Hammami, E. Nour, M. Kssia, B. Ahlem, A. Salem, A. Ben Slama, J. Ali

Gastroenterology Service, Sahloul, Sousse/Tunisia

Contact E-mail Address: wafabenameur1@hotmail.fr

Introduction: Tuberculosis is a major cause of morbidity and mortality worldwide. Its incidence is continually increasing. Peritoneal localization is a particular entity, even less well known, because of its atypical and confusing symptomatology, which in most cases imposes a malignant condition.
Aims \& Methods: We collected all patients hospitalized for peritoneal tuberculosis between 2005 and 2015. The aim of this retrospective study was to study the epidemiological, clinical, paraclinical, diagnostic, therapeutic and evolutionary specificities of peritoneal tuberculosis in its various presentations.

Results: The total number of patients was 65 . It was 15 men $(23.1 \%)$ and 50 women $(76.9 \%)$. The sex ratio was 0.3 . The mean age at diagnosis was 40 years (15-79 years). No personal history of tuberculosis has been found in our series. A personal history of tuberculosis was found in 3 patients $(4.6 \%)$. The general signs of tuberculin impregnation were frequently found $(91 \%)$. The digestive functional signs that brought the patients to consult are: abdominal pain $(87.7 \%)$, abdominal distension $(87.5 \%)$, diarrhea $(16.9 \%)$ and sub occlusive syndromes $(4.6 \%)$. An abdominal mass was observed in only 4 patients $(6.1 \%)$. Hepatomegaly and splenomegaly were noted in 2 cases for each. The intradermal reaction was positive in only $24 \%$ of patients. The research of BK in the ascites fluid was systematically performed in all patients but returned negative in all cases. The quantiferon-TB Gold was performed in 3 patients only and returned positive. The mean level of CA 125 was $250.3 \mathrm{IU} / \mathrm{ml}$. Confirmation of diagnosis was obtained on the histological analysis of peritoneal biopsies or adnectomy pieces. The main operative findings (in patients with coelioscopy or exploratory laparotomy) were: Whitish granulations (98\%), adhesions $(43.1 \%)$ and agglutinated loops $(1.5 \%)$. The presence of tuberculous granuloma was observed in 52 patients $(81 \%)$. The course of treatment was as follows: cure in 50 patients $(80.6 \%)$, recurrence in 6 patients $(9.6 \%)$, relapse in 2 patients and 3 patients were lost to follow-up. The mortality in our series was $0 \%$

Conclusion: Peritoneal tuberculosis raises diagnostic problems in the first place, because of its polymorphic and non-evocative clinical expression; Hence the value of carrying out radiological, endoscopic and histo-bacteriological investigations to confirm the diagnosis before the evolution towards serious or even fatal forms.

Disclosure of Interest: All authors have declared no conflicts of interest.

\section{P0955 ABDOMINAL TUBERCULOSIS: A RETROSPECTIVE SERIES}

\section{OF 150 CASES}

S. Jardak, H. Kchir, N. Maamouri, H. Chaabouni, N. Ben Mami

Tunis, Rabta b, Tunis/Tunisia

Contact E-mail Address: sondajardak1@gmail.com

Introduction: Tuberculosis is still a problem of public health in Tunisia, which is a country of endemicity. The epidemiological situation of the disease is marked by the rise of the extrapulmonaires forms especially abdominal tuberculosis.

Aims \& Methods: The aim of this study was to analyze the epidemiologic, clinical, diagnostic, therapeutic and evolutive features of abdominal tuberculosis in a series of 150 patients. This was a retrospective and descriptive monocentric study of 150 cases of abdominal tuberculosis conducted from 2004 to 2014 in a tunisian center. Diagnosis of tuberculosis was based on histological evidence or otherwise on a beam of arguments.

Results: There were 150 patients enrolled. The mean age was 37.2 (17-72 years). Ninety seven $(64.6 \%)$ were females. Symptoms were ascites $107(71.3 \%)$, abdominal pain $28(18.6 \%)$, weight loss and reduced appetite $80(53.3 \%)$. Un tableau pseudochirurgical a révélé la maladie dans $9.3 \%$ des cas (14 cas). Sub-acute and acute intestinal obstruction was seen in $10(6.6 \%)$ and $4(2.6 \%)$ respectively. 105 patients $(70 \%)$ had peritoneal tuberculosis, $34(22.6 \%)$ had intestinal tuberculosis. The association of the two forms was found in one case. 10 patients $(6.6 \%)$ had a Lymph node involvement. Basis of diagnosis of abdominal tuberculosis were histo-pathology (tissue obtained during surgery, colonoscopy, CT or ultrasound guided biopsy, laparoscopy and upper gastro intestinal endoscopy) in 122 $(81.3 \%)$ patients. Response to medical treatment was found in $141(94 \%)$ patients.

Conclusion: Abdominal tuberculosis is one of the most common site of extrapulmonary tuberculosis. No single test is adequate for diagnosis of abdominal tuberculosis in all patients. Abdominal TB remains an ongoing diagnostic dilemma requiring a high index of clinical suspicion.

Disclosure of Interest: All authors have declared no conflicts of interest.

TUESDAY, OCTOBER 31, 2017

09:00-17:00

IBD II - HALL 7.

P0956 PATHOBIONT-FREE MICROBIOTA PROTECTS AGAINST GUT INFLAMMATION INDUCED BY AN INNATE IMMUNE DEFICIENCY OR DIETARY PERTURBANT OF THE MICROBIOTA

B. Chassaing ${ }^{1}$, A. T. Gewirtz ${ }^{2}$

${ }^{1}$ Institute For Biomedical Sciences, Georgia State University, Atlanta/United States of America

${ }^{2}$ Institute For Biomedical Sciences, Georgia State University, Atlanta/United States of America/GA

Contact E-mail Address: bchassaing@gsu.edu

Introduction: Inability to maintain a stable and beneficial microbiota is associated with chronic gut inflammation, which classically manifests as colitis but may more commonly exist as low-grade inflammation that promotes metabolic syndrome. Alterations in microbiota and associated inflammation can originate from dysfunction in host proteins that manage microbiota, such as the flagellin receptor TLR5, and/or be promoted by exogenous factors that disturb hostmicrobiota interactions, such as the detergent-like dietary emulsifiers carboxymethylcellulose $(\mathrm{CMC})$ and polysorbate $80[1,2,3]$. That the complete absence of a microbiota (i.e. germ-free conditions) eliminates all evidence of inflammation in 
TLR5-deficient and emulsifier-treated mice demonstrates that these models of gut inflammation are microbiota dependent. We hypothesize that such microbiota dependency of inflammation reflects an inability to manage pathobiont bacteria, such as Adherent-Invasive E. coli (AIEC).

Aims \& Methods: Our goal was to examine extent to which microbiota mismanagement and associated inflammation in TLR5-deficient and emulsifier-treated mice would manifest in a limited pathobiont-free microbiota. WT and TLR5deficient mice were maintained in gnotobiotic isolators containing altered Schaedler flora (ASF), a community of eight bacterial species. Mice were treated with either CMC or P80, or inoculated with AIEC reference strain LF82 [4]. Feces were assayed for bacterial loads, microbiota composition, and inflammatory marker lipocalin-2. Fecal LPS and flagellin bioactivity were measured via a cell-based reporter assay, and morphologic and metabolic parameters were determined.

Results: Neither CMC nor P80 induced evidence of intestinal inflammation nor metabolic syndrome in WT ASF animals. Analogously, relative to similarly maintained WT mice, loss of TLR5 did not result in low-grade intestinal inflammation nor metabolic syndrome under ASF conditions. Concomitantly, the ASF microbial community was not disturbed by CMC nor P80 and, moreover, was similar between WT and T5KO animals. Inoculation with AIEC strain LF82 resulted in profound alteration of the ASF community in T5KO mice compared to WT control animals. AIEC LF82 inoculation in ASF T5KO mice correlated with microbiota exhibiting elevated levels of bioactive LPS and flagellin, and was associated with modest level of low-grade inflammation and increased adiposity. Conclusion: In a limited-complexity pathobiont-free microbiota, loss of the flagellin receptor TLR5 or emulsifier consumption does not impact microbiota composition nor its ability to promote inflammation. Addition of AIEC to this ecosystem perturbs microbiota composition, increases levels of lipopolysaccharide and flagellin, but only modestly promotes gut inflammation and adiposity, suggesting that the phenotypes previously observed require disruption of complex microbiota.

Disclosure of Interest: All authors have declared no conflicts of interest.

\section{References}

1. Chassaing B, Koren O, Goodrich JK, Poole AC, Srinivasan S, Ley RE, et al. Dietary emulsifiers impact the mouse gut microbiota promoting colitis and metabolic syndrome. Nature 2015;519:92-6.

2. Chassaing B, Ley RE, Gewirtz AT. Intestinal epithelial cell toll-like receptor 5 regulates the intestinal microbiota to prevent low-grade inflammation and metabolic syndrome in mice. Gastroenterology 2014;147:1363-77 e17.

3. Vijay-Kumar M, Aitken JD, Carvalho FA, Cullender TC, Mwangi S, Srinivasan S, et al. Metabolic syndrome and altered gut microbiota in mice lacking Toll-like receptor 5. Science 2010;328:228-31.

4. Darfeuille-Michaud A, Boudeau J, Bulois P, Neut C, Glasser AL, Barnich N, et al. High prevalence of adherent-invasive Escherichia coli associated with ileal mucosa in Crohn's disease. Gastroenterology 2004;127:412-21.

\section{P0958 ISOLATION AND CHARACTERIZATION OF LAMINA PROPRIA MONONUCLEAR CELLS FROM HUMAN COLONIC MUCOSA}

S. Musladin ${ }^{1}$, I. Zlatar ${ }^{1}$, L. Pribolšan ${ }^{1}$, K. Ilić ${ }^{1}$, P. Nikolić ${ }^{1}$, M. Antolić ${ }^{2}$, S. Cužić ${ }^{2}$, G. Aralica ${ }^{3}$, L. Patrlj ${ }^{3}$, R. Kliček ${ }^{3}$, M. Bosnar ${ }^{1}$, V. Eraković Haber ${ }^{1}$ ${ }^{1}$ In Vitro Pharmacology, Fidelta d.o.o., Zagreb/Croatia

${ }^{2}$ In Vivo Pharmacology, Fidelta d.o.o., Zagreb/Croatia

${ }^{3}$ Clinical Hospital Dubrava, Zagreb/Croatia

\section{Contact E-mail Address: sanja.musladin $@$ glpg.com}

Introduction: Lamina propria represents a thin layer of connective tissue rich in lymphoid cells and macrophages, underlying the epithelium of mucous membranes. Lamina propria mononuclear cells (LPMCs) are immune system's first line of defense in the intestine characterized by the ability of differentiating invading pathogens from beneficial intestinal flora and swiftly removing them. Disrupted regulation of LPMCs is implicated in pathology of a group of disorders causing chronic intestinal inflammation, termed Inflammatory bowel disease (IBD). Two major types of IBD are ulcerative colitis, limited to the colon and Chron's disease affecting any segment of the gastrointestinal tract. Chron's disease and ulcerative colitis were shown to be mediated by Th1-polarized helper (CD4+) T-cells and Th2-polarized CD4+ and NKT cells, respectively, while Th17-polarized cells are involved in pathogenesis of both diseases ${ }^{1}$. Therefore, research into biology and regulation of LPMCs is of essential importance for development of treatments for IBD symptoms.

Aims \& Methods: The aim of this study was development of a robust method for isolation and characterization of LPMCs from human colonic mucosa, compatible with further ex vivo cell culturing and research. Mucosal tissue samples contained the tissue not affected by tumor, removed during tumor surgery of patients that consented to donation of tumor and the surrounding resected tissue. The tissue may be considered as healthy in terms of IBD. We have employed an isolation method consisting of disruption of epithelial cells by incubation of mucosa in a DTT and EDTA containing solution, followed by enzymatic and mechanical digestion of tissue and purification of mononuclear cells by density gradient centrifugation. Isolated cells were characterized by flow cytometry (FACS) detection of cell-type specific surface antigens and cytological analysis. To examine the plasticity of isolated cells in terms of polarization towards IBDassociated phenotypes, the cells were seeded at conditions mediating Th1, Th2 and Th17 differentiation of CD4+ T-cells and analyzed for activation of differentiation-specific gene expression and cytokine production by qPCR and ELISA assays. Aliquots of cells were cryopreserved and further analyzed for the effect of cryopreservation on distribution of surface antigens and CD4+ T-cell responses. Results: Approximately 95\% cell viability and 90\% leukocyte (CD45+) cell purity was determined by FACS analysis of isolated LPMCs. According to cytological analysis, CD45-negative cells may represent CD45-negative population of plasma cells; no contamination with epithelial cells was detected. Within CD45+ cell population, 26-47\% T-lymphocytes, 17-24\% B-lymphocytes, 8-17\% macrophages and $21-46 \%$ monocytes were detected by FACS and cytological analyses $(\mathrm{N}=3)$. Cryopreservation did not significantly affect cell viability and surfaceantigen distribution. Isolated cells were successfully polarized towards Th1, Th2 and Th17 CD4+ T-cell phenotypes, as confirmed by IFNY gene expression and cytokine production for Th1, IL13 gene expression and IL5 cytokine production for Th2 and IL17 cytokine production for Th17 differentiated cells. Differentiation was confirmed also in cryopreserved cells, with lower level of phenotype-specific cytokine production.

Conclusion: Method for LPMC isolation from human colonic mucosa tissue samples was successfully established with approximately $95 \%$ viability of isolated cells, $90 \%$ purity of CD $45+$ cells and no detectable epithelial cell contamination. Within CD $45+$ cells, $26-47 \%$ of T-lymphocytes, $17-24 \%$ B-lymphocytes, $8-17 \%$ macrophages and $21-46 \%$ of monocytes were detected. Isolated cells were successfully polarized towards IBD-associated Th1, Th2 and Th17 CD4+ T-cell phenotypes, as confirmed by activation of phenotype-specific gene expression and cytokine production. Cryopreservation of isolated LPMCs did not significantly affect cell viability, distribution of cell-type specific surface antigens or polarization towards Th1, Th2 and Th17 CD4+ T-cell phenotypes.

Disclosure of Interest: All authors have declared no conflicts of interest.

\section{Reference}

Nemoto, Y. \& Watanabe, M. in Crohn's Disease and Ulcerative Colitis 183-194 (Springer US, 2012). doi:10.1007/978-1-4614-0998-4_15

\section{P0959 FACTORS ASSOCIATED WITH DISABILITY IN INFLAMMATORY BOWEL DISEASE: OUTPATIENT CROSS- SECTIONAL STUDY}

J. M. $\operatorname{Costa}^{1}$, D. Matos ${ }^{2}$, J. B. Soares ${ }^{1}$, R. $\operatorname{Costa}^{1}$, B. Arroja ${ }^{1}$, R. Gonçalves ${ }^{1}$ ${ }^{1}$ Gastroenterology, Hospital de Braga, Braga/Portugal

${ }^{2}$ School Of Health Sciences, University of Minho, Braga/Portugal

Contact E-mail Address: julianamcosta87@gmail.com

Introduction: The Inflammatory Bowel Disease-Disability Index (IBD-DI) has recently been validated to measure disability in IBD.

Aims \& Methods: We aimed to assess disability in IBD outpatients using IBD-DI and to determine the sociodemographic, clinical and psychological factors that are associated with greater disability. Between August and October of 2016, patients with an established diagnosis of Crohn's Disease (CD) or Ulcerative Colitis (UC) for at least 3 months and followed up at our outpatient clinic were invited to participate. Socio-demographic and clinical data were collected from electronic health record and interview. Optimism and disability were evaluated by applying, personally or by phone, the validated Portuguese versions of the Revised Life Orientation Test (LOT-R) and IBD-DI, respectively. The association between sociodemographic, clinical and psychological variables (optimism) and IBD-DI (scale $0-100$, proportional to the reported disability) was determined by univariate and multivariate analysis.

Results: A total of 143 patients (70 DC and 73 UC; $50.3 \%$ females) with a mean age of $38 \pm 13$ years were included. Most $(85.3 \%)$ was in clinical remission. The median IBD-DI-PT score was $17.9 \pm 10.7$, with no significant difference between DC and CU $(p=0.944)$. In univariate analysis, female gender, high level education, number of days off from work, articular manifestations, number of comorbidities, use of psychotropic drugs and pessimism (low LOT-R score) were significantly associated with higher disability (IBD-DI-PT score). In multivariate analysis, only female gender $(B=0.150)$, number of comorbidities $(B=0.186)$ and pessimism $(B=0.370)$ were significantly associated with higher disability. Clinical activity was associated with higher disability only for $C D$ patients $(B=0.321)$. Conclusion: IBD outpatients reported low levels disability associated with their disease, which can be explained by the high percentage of patients in clinical remission. Comorbidities and psychological factors (optimism) emerged as the main predictive factors of greater disability, reinforcing the importance of multidisciplinary approach to these patients. Clinical activity seems more important to $\mathrm{CD}$ than UC patients in terms of disability.

Disclosure of Interest: All authors have declared no conflicts of interest.

\section{References}

1. Moradkhani A, Beckman LJ, Tabibian JH. Health-related quality of life in inflammatory bowel disease: psychosocial, clinical, socioeconomic, and demographic predictors. Journal of Crohn's and Colitis. 2013; 7(6):467-73.

2. Gower-Rousseau C, Sarter H, Savoye G, et al. Validation of the Inflammatory Bowel Disability Index in a population-based cohort. Gut. 2015; 0:1-9.

3. Soares JB, Pereira R, Costa JM, et al. The Inflammatory Bowel Disease Disability Index: validation of the Portuguese version according to the COSMIN checklist. European journal of gastroenterology \& hepatology. 2016; 28:1151-60. 


\section{P0960 BIOLOGIC THERAPY REDUCES T CELL ACTIVATION IN}

\section{PATIENTS WITH CROHN'S DISEASE}

L. D. Garcia, A.E. Z. Carvalho, E. A. Rosseto, C. C.V. Nora, F. S. Flaquer, C.L. P. Mangueira, L. V. Rizzo, K. I.L. De Carvalho

Experimental Research, Hospital Israelita Albert Einstein, São Paulo/Brazil

\section{Contact E-mail Address: dargeniole@gmail.com}

Introduction: Imbalance of immune bowel environment is associated with development of many diseases, such as Crohn's disease (CD). Although the pathogenesis of $C D$ remains unclear, a key feature of $C D$ is dysregulation of immune system (Zhang and $\mathrm{Li}, 2014$ ). Human immune cells are the front line of inflammatory responses and include, in addition to other cells, CD4+ and CD8+ T cells, which comprises adaptive phase and require proper inflammatory cues for their effector function. After activation, CD4+ and CD8+ T cells express several markers that give them powerful functional abilities (Abbas et al., 1996). CD38 and HLA-DR besides being classically markers of cellular activation, are also known as markers of diseases progression (Lovelace et al., 2017). Some studies shows that CD4+ T cells play a key role in the immune inflammatory response leading to CD but this cells are poorly characterized in the blood of the patients. Aims \& Methods: This study aimed to characterized CD4+ and CD8+ T cells in the blood of patients with CD.The study was performed in individuals with $\mathrm{CD}$ $(n=46)$ and healthy controls $(n=38)$. Blood of healthy donors and patients with CD was collected in clinical laboratory from Hospital Albert Einstein. CD4+ and CD $8+T$ cells was quantified by multiparametric flow cytometry. Dosage of calprotectin and ASCA was performed by commercial Elisa kit. The groups were compared for numerical measures using Student's t-tests, ANOVA, MannWhitney or Kruskal-Wallis, depending on the suitability of the data and the number of groups to be compared. The computational package used for the analyzes was R 3.0.3 and GraphPad Prisma 6.

Results: The highest prevalence in both group was female, aged between 19 and 66 years with a median of 37.5 years. Among clinical exams, $61 \%$ of cases present a value greater than 200 in the dosage of Calprotectin, whereas in the contro group the rate was only $18.4 \%(p<0.001)$. ASCA $\operatorname{IgG}(p=0.001)$ and $\operatorname{IgA}$ $(\mathrm{p}<0.001)$ measures were also higher in group of cases when compared to control group. The population of CD3 + cells in peripheral blood of healthy controls and patients with $\mathrm{CD}$ was evaluated both in biological and corticoid treatment. A significant increase in this population was observed in patients receiving corticosteroids $(\mathrm{p}=0.02)$ compared to healthy controls. In the population of CD4+ a significant increase was observed in patients undergoing treatment with corticosteroids in relation to patients undergoing biological treatment $(\mathrm{p}=0.0027)$. In CD8+ population, no statistical difference was observed between the groups. The markers CD38, CD62L and HLADR were also evaluated. In relation to CD38 $(\mathrm{p}=0.0022)$ and CD62L $(\mathrm{p}=0.015)$ in subpopulation of CD4+ T cells, a significant increase in the expressions was observed in the group of patients receiving corticosteroids in relation to the group receiving biological therapy. Regarding HLADR, statistical difference $(\mathrm{p}=0.01)$ was observed between the group undergoing biological treatment and the group of healthy controls. In the subpopulation of CD8 $+\mathrm{T}$ cells, a significant increase $(\mathrm{p}=0.02)$ was observed in the CD38 marker when compared patients on corticoid treatment with patients undergoing biological treatment. Regarding the CD62L marker, only a statistical difference $(p=0.04)$ was observed when compared patients on corticosteroid treatment with healthy controls. Finally, a statistical difference $(p=0.02)$ was also observed in relation to the HLADR marker when comparing the group of patients receiving corticoid with the group of patients in use of biological therapy.

Conclusion: We conclude that the use of biological therapy suppresses activated state of $\mathrm{CD} 4+$ and $\mathrm{CD} 8+\mathrm{T}$ cells by regulation of $\mathrm{CD} 38, \mathrm{CD} 62 \mathrm{~L}$ and HLADR in relation to the use of corticosteroids.

Disclosure of Interest: All authors have declared no conflicts of interest.

\section{References}

1. Abbas AK, Murphy KM, Sher A. Functional diversity of helper T lymphocytes. Nature 1996; 383, 787-793.

2. Lovelace ES, Maurice NJ, Miller HW, Slichter CK, Harrington R, Margare A, Prlic M, De Rosa S, Polyak SJ. Silymarin suppresses basal and stimulus induced activation, exhaustion, differentiation, and inflammatory markers in primary human immune cells. PLoS One. 2017; 12(2):e0171139.

3. Zhang Y, Li Y. Inflammatory bowel disease: pathogenesis. World $J$ Gastroenterol 2014; 20: 91-99.

\section{P0961 CHANGES OF THE MUCOSAL COLON MICROFLORA ARE CAUSE OF INFLAMMATION AT THE PATIENTS WITH ULCERATIVE COLITIS}

A. $\operatorname{Kasianov}^{1}$, A. Fedotova ${ }^{1}$, M. Logacheva ${ }^{1}$, K. Klimina ${ }^{2}$, A. Zhurov ${ }^{3}$, I. Ruchkina ${ }^{4}$, A. Parfenov ${ }^{4}$, O. Knyazev ${ }^{4}$, N. Fadeeva ${ }^{4}$

${ }^{1}$ Laboratory Of Evolutionary Genomics, The A.N. Belozersky Institute Of PhysicoChemical Biology, Moscow/Russian Federation

${ }^{2}$ Laborotory Of System Biology And Computational Genetics, Vavilov institute of general genetics, Moscow/Russian Federation

${ }^{3}$ Moscow Institute of Physics and Technology, Dolgoprudny/Russian Federation

${ }^{4}$ Ibd, Moscow Clinical Research Center, Moscow/Russian Federation

Contact E-mail Address: artem.kasianov@gmail.com

Introduction: Advancing of sequencing technologies in the first decade of the XXI century gives the opportunity to realization of large scale projects such as the 1000 human genomes project. This project provides enormous amount of the data for H.sapiens population genetics studies and different GWAS studies aimed to investigate connection of the human genetics variations with different diseases. It is especially interested to study the connection between the human genetics, the microbiome metagenomics of mucosa and different pathological conditions. Previously revealed, the size of the summary gut microbiome metagenome is on order more than the size of a human genome. The most of works devoted to the study of the connection of metagenome changes with different pathological conditions based on the investigation of fecal samples. On our opinion, in the case of ulcerative colitis it is more effective to study the mucosal microbiome of the affected regions.

Aims \& Methods: The aim of our work was to study the composition of mucosal microbiomes in the colon mucosa biopsies from patients with ulcerative colitis by using the target high throughput sequencing of bacterial 16S rRNA genes. Biopsies from four caucasoid race patients with left-sided ulcerative colitis in the abating relapse phase (Meyo endoscopical index - 3, Rachmelevich clinical index -4) and two patients from control group with irritable bowel syndrome were collected. The DNA was extracted from mucosal biopsies and 16S rRNA genes from it's were target sequenced by using Illumina Miseq sequencer. Sequencing reads were quality checked by the FastQC software and trimmed by using the trimmomatic software. To characterize the composition of the microbiota, trimmed reads were analyzed by the QIIME software. The obtained results were compared with the earlier published data: SRA Project - ERP001780 (96 samples from patients of control group, 44 samples from patients with ulcerative colitis)[1] and SRA Project - SRP056002 (703 samples from patients with ulcerative colitis).

Results: More than 124 bacterial genuses were founded in biopsies of four patients with ulcerative colitis. The analyzed samples of patients with ulcerative colitis were split in two groups by using the PCoA analysis. The first group was characterized by decreasing the concentration of the Firmicutes type bacteria (pvalue $<0.005)$ and increasing the concentration of Bacteroidetes type bacterias (p-value $<0.05$ ). For the second group, It was founded decreasing of the concentration of the Actinobacteria type bacterias (p-value $<0.05$ ), 70 -fold excess of Bacteroides vulgatus species bacterias concentration was revealed for one sample of the first group (normalized number of reads in control samples was less than 0.003 and reached 0.21 in samples of patients with ulcerative colitis). Additionally, the concentration of Escherichia coli species bacterias was increased in the 40 times for that sample (normalized number of reads in control samples was less than 0.0008 and reached 0.03 in samples of patients with ulcerative colitis). Although, the predominance of Proteobacteria genus bacteria was not founded. The concentration of Faecalibacterium prausnitzii species bacteria was decreased by three orders of the magnitude for samples from the first group (normalized number of reads in control samples reached 0,2 and was less than 0,06 in samples of patients with ulcerative colitis).

Conclusion: The concentration increase of the conditional-pathogenic mucosal microflora (a mostly Bacteroidetes type bacteria) was discovered, which playing the important role in the development of ulcerative colitis and lead to a mucosa damage. Also, the deficiency of Faecalibacterium prausnitzii species bacteria was discovered, which decrease resistance of mucosa to the conditional-pathogenic microflora.

Disclosure of Interest: All authors have declared no conflicts of interest.

\section{Reference}

1. Maomeng Tong, Xiaoxiao Li, Laura Wegener Parfrey, Bennett Roth, Andrew Ippoliti, Bo Wei, James Borneman, Dermot P. B. McGovern, Daniel N. Frank, Ellen Li, Steve Horvath, Rob Knight, and Jonathan Braun. A Modular Organization of the Human Intestinal Mucosal Microbiota and Its Association with Inflammatory Bowel Disease. PLoS One. 2013; 8(11): e80702

\section{P0962 COLONIC INFLAMMATION DELAYS DEVELOPMENT OF STREPTOZOTOCIN-INDUCED DIABETES IN MICE THROUGH MODULATION OF ENDOGENOUS INCRETIN SYSTEM}

H. Zatorski, M. Sałaga, M. Zielińska, J. Fichna

Department Of Biochemistry, Medical University of Lodz, Lodz/Poland

Contact E-mail Address: zatorski.h@gmail.com

Introduction: The role of incretin hormone, GLP-1, in inflammatory bowel diseases (IBD) development and exacerbations is still poorly understood. GLP-1 decreases blood glucose level and is co-secreted by intestinal L cells with GLP-2, which may be responsible for intestinal growth and enhancement of intestinal function. The prevalence of type 2 diabetes among IBD patients is low, even though IBD sufferers may be at a greater risk of development of T2D due to overproduction of pro-inflammatory cytokines and excessive administration of diabetogenic drugs. We hypothesize that the possible mechanism underlying this phenomenon is related to changes in the levels of incretin hormones.

Aims \& Methods: The primary aim of this study was to investigate, in the mouse model, whether coexisting colitis postpones development of experimental diabetes. The secondary goal was to investigate the potential involvement of incretin hormones as an underlying factor. Experimental diabetes was induced by administration of streptozotocin for 5 consecutive days $(50 \mathrm{mg} / \mathrm{kg}$, i.p.). Mice with blood glucose levels above $200 \mathrm{mg} / \mathrm{dL}$ were considered as diabetic. To develop a chronic and relapsing colonic inflammation, mouse model of colitis induced by intracolonic administration of TNBS (first dose: $150 \mathrm{mg} / \mathrm{kg}$ at day 1 , booster dose: $75 \mathrm{mg} / \mathrm{kg}$ at days $12,23,34,45,56)$ was used. On day 60 mice were sacrificed and macroscopic score, ulcer score, colon length and bowel thickness were recorded. In all experiments the levels of inflammation markers: myeloperoxidase (MPO) activity, TNF- $\alpha$ and IL-1 $\beta$ expression were determined. The 
effect of colitis on T2D development was studied by assessing fasting glucose levels, as well as the incretin hormone levels, GLP-1 and GLP-2 in the blood.

Results: The development of hyperglycemia in mice treated with TNBS was delayed compared to a non-inflamed group, what was associated with significantly higher level of GLP-1 in blood. Surprisingly, the levels of GLP-2 were significantly reduced in diabetic mice with colitis, suggesting that two distinct mechanisms are involved in the regulation of the incretin hormones in response to intestinal inflammation. There were no significant differences in macroscopic score, colon length, and bowel thickness in diabetic mice with or without colitis. Notably, ulcer score was significantly increased in diabetic mice with colitis compared to diabetic mice with no inflammation. No changes in MPO, TNF$\alpha$, IL-1 $\beta$ were observed between these groups.

Conclusion: We propose that GLP-1 production may be stimulated in response to colonic inflammation and thus postpone development of diabetes. This observation is the first to reveal possible connections between T2D and IBD and may lead to better understanding of the pathophysiology of these two diseases. Furthermore, our data suggest that the incretin hormones may become a potential new therapeutic option in treatment of IBD.

Disclosure of Interest: All authors have declared no conflicts of interest.

\section{P0963 EOSINOPHILS-ASSOCIATED CYTOKINES AS \\ INFLAMMATORY BOWEL DISEASE BIOMARKERS}

K. Neubauer ${ }^{1}$, M. Matusiewicz ${ }^{2}$, I. Bednarz-Misa ${ }^{2}$, S. Górska ${ }^{3}$, A. Gamian²,

M. Krzystek-Korpacka ${ }^{2}$

${ }^{1}$ Gastroenterology And Hepatology, Wroclaw Medical University, Wroclaw/ Poland

${ }^{2}$ Medical Biochemistry, Wroclaw Medical University, Wroclaw/Poland

${ }^{3}$ Laboratory Of Medical Microbiology, Ludwik Hirszfeld Institute of Immunology and Experimental Therapy, Polish Academy of Sciences, Wroclaw/Poland

\section{Contact E-mail Address: kasianeu@gmail.com}

Introduction: Pathogenesis of inflammatory bowel disease (IBD) is multifactorial and establishing diagnosis requires a performance of series of variable tests. The alternative, non-invasive, markers of IBD are intensively searched for. Eosinophils are acidophilic multifunctional granulocytes that remain outside the mainstream research on IBD. However, they are a rich source of cytotoxic proteins, pro- and anti-inflammatory cytokines, chemokines and growth factors and are likely to contribute to both inflammatory and regenerative phases of the disease. Accordingly, peripheral eosinophils of IBD patients are primed and preactivated. They display increased responsiveness, adhesiveness, migration, and degranulation and are characterized by up-regulated secretion of their mediators.Locally, increased number and activation of eosinophils have been repeatedly observed in areas of active inflammation. Despite the acknowledged contribution of eosinophils to the disease pathogenesis, available data on cytokines closely related to the development and activity of peripheral eosinophils in IBD patients are either scattered or non-existent.

Aims \& Methods: Aim of the study was assessment of the circulating eosinophilassociated cytokines and growth factors as differential markers and indicators of mucosal healing in inflammatory bowel disease.

Study population consisted of 277 individuals: 101 patients with Crohn's disease (CD), 77 with ulcerative colitis, 16 with irritable bowel syndrome (IBS) and 83 healthy controls. The disease severity was assessed using the Crohn's Disease Activity Index (CDAI) for CD and the Mayo Disease activity index (MDAI) for UC. Additionally, Mayo endoscopic score was applied to evaluate the severity of bowel inflammation in UC patients. The concentrations of eosinophilassociated cytokines and growth factors: eotaxin, GM-CSF, IFN $\gamma$, IL4, IL5, IL8, IL12(p70), IL13, RANTES and TNF $\alpha$ were measured simultaneously in patient's sera using Luminex $\mathrm{xMAP}{ }^{\circledR}$ technology and referred to IBD activity and the levels of hsCRP. The suitability of eosinophil-associated cytokines and growth factors as differential markers and potential indicators of mucosal healing, individually and in multi-marker panels, was evaluated using ROC analysis. Results: As compared to IBS patients or healthy controls, patients with CD had significantly higher levels of IL5, IL8, IL12(p70), GM-CSF, and TNF $\alpha$ and patients with UC the levels of eotaxin, IL4, IL5, IL8, IL12(p70), IL13, GM$\mathrm{CSF}$, and TNF $\alpha$. As compared to CD patients, patients with UC had significantly higher levels of eotaxin, IL4, IL5, IL8, and IL1. In turn, the concentrations of hsCRP were significantly higher in CD than UC. Except for IL13, all cytokines and hsCRP positively correlated with CDAI but solely IL12(p70) and hsCRP were significantly higher in patients with active than inactive CD. In UC, a positive correlation with MDAI was observed for hsCRP, GM-CSF, IL12(p70), and IFN $\gamma$ and a negative one for IL8. The concentrations of hsCRP, GM-CSF, IFN $\gamma$, IL12(p70) and RANTES were higher in UC patients with active than inactive disease whereas those of IL8 and TNF $\alpha$ were significantly lower. As differential individual markers, eotaxin displayed superior accuracy as an indicator of active UC $(71 \%)$, followed by hsCRP as an indicator of active CD (66\%). The combined assessment of eotaxin, hsCRP and IFN $\gamma$ had slightly higher accuracy $(78 \%)$ and allowed for a correct classification of $72 \%$ patients. The concentrations of hsCRP, GM-CSF, IFNg, and IL12(p70) were significantly and positively correlated with the degree of bowel inflammation, expressed as Mayo endoscopic subscore. Of these, a drop in GM-CSF had superior accuracy as an indicator of mucosal healing $(91 \%)$, allowing for a correct classification of $87 \%$ of patients. IL5, IL8, IL12(p70), TNF $\alpha$, and GM-CSF were significantly higher in both CD and UC than in IBS. Of these, IL8 had superior accuracy in differentiating IBS and IBD (91\%), allowing for a correct classification of $93 \%$ of patients.
Conclusion: Eosinophil-associated cytokines are elevated in IBD, more pronouncedly in UC, and may support the differential diagnosis of IBD and aid in monitoring of mucosal healing.

Disclosure of Interest: All authors have declared no conflicts of interest.

\section{P0964 MACROPAHGE IL10 SIGNALING IS REQUIRED FOR THE THERAPEUTIC EFFECT OF ANTI-TNF THERAPY IN INFLAMMATORY BOWEL DISEASE}

P. J. Koelink ${ }^{1}$, F. M. Bloemendaal ${ }^{1}$, L. Westera ${ }^{1}$, A. B. Van 'T Wout $^{2}$, A K. Gloudemans ${ }^{2}$, B. Li ${ }^{3}$, T. L. Geiger ${ }^{3}$, M. E. Wildenberg ${ }^{4}$, G.R. Van Den Brink ${ }^{5}$ ${ }_{2}^{1}$ AMC Amsterdam, Amsterdam/Netherlands

${ }^{2}$ Janssen Prevention Center, Janssen Pharmaceutical Companies of Johnson \& Johnson, Leiden/Netherlands

${ }^{3}$ Department Of Pathology, St. Jude Chlidren's Research Hospital, Memphis/ United States of America

${ }^{4}$ Tytgat Institute, AMC Amsterdam, Amsterdam/Netherlands

${ }^{5}$ Dept. Of Gastroenterology, Academisch Medisch Centrum, Amsterdam/ Netherlands

Contact E-mail Address: p.j.koelink@amc.nl

Introduction: Interleukin(IL) 10 is an important anti-inflammatory cytokine for the maintainance of gut homeostasis. Defects in the IL10 signalling pathway in macrophages leads to disregulation of regulatory (M2) type macrophages and subsequent inflammatory bowel disease (IBD). IBD patients are frequently succesfully treated with anti-TNF $\alpha$ antibody therapy, although not all patients are responsive.

Aims \& Methods: We determined the effect of anti-TNF $\alpha$ therapy in both IL10 knock-out $(\mathrm{KO})$ mice and in the CD4 + CD45Rb high T-cell transfer model of colitis. Macrophage populations were quantified using qPCR analysis for CD206 and iNOS and flow cytometry for CD206. IL10 mRNA and protein levels were analysed with qPCR and ELISA.

Results: Colitis in the IL10 KO mice was completely resistant to anti-TNF $\alpha$ therapy, in sharp contrast to the colitis in SCID or Rag1 KO mice upon transfer of $\mathrm{CD} 4+\mathrm{CD} 45 \mathrm{Rb}$ high T-cells, which was significantly reduced by anti-TNF $\alpha$ therapy. Succesfull anti-TNF $\alpha$ therapy was accompanied by an increase of IL10 levels and an increase of regulatory (M2) type macrophages in the intestine. Blocking IL10 signaling, with an IL10 Receptor blocking antibody, diminshed the therapeutic efficacy of anti-TNF $\alpha$ therapy. Anti-TNF $\alpha$ therapy was also effective in RAG1 KO mice that received a transfer with IL10 Receptor KO CD $4+C D 45 \mathrm{Rb}$ high T-cells, indicating that IL10 signaling in T-cells was not important for the therapeutic efficacy of anti-TNF $\alpha$. In contrast, LysMCre IL10Ra flox/flox Rag1 KO, mice defective in macrophage IL10 signaling, were unresponsive to anti-TNF $\alpha$ therapy upon receiving CD $4+$ CD 45 Rb high T-cells. In these mice there was also no increase of intestinal M2 macrophages.

Conclusion: IL10 signaling in macrophages is pivotal for the therapeutic efficacy of anti-TNF $\alpha$ therapy in animal models for IBD. Defects in the IL10 pathway may also play a role in anti-TNF $\alpha$ non-responders which is subject of further investigation.

Disclosure of Interest: All authors have declared no conflicts of interest.

\section{P0965 LONG-TERM CONSEQUENCES OF ANTIBIOTIC THERAPY:} ROLE OF SCFAS AND INTESTINAL BARRIER INTEGRITY

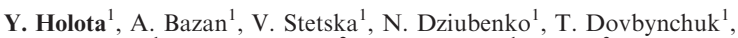
T. Chervinska ${ }^{1}$, L. Zakordonets ${ }^{2}$, T. Serhiychuk ${ }^{1}$, I. Kaji ${ }^{3}$, G. Tolstanova ${ }^{1}$ Educational And Scientific Centre "institute Of Biology And Medicine", Taras Shevchenko National University of Kyiv, Kyiv/Ukraine

${ }^{2}$ Bogomolets National Medical University, Kyiv/Ukraine

${ }^{3}$ UCLA School Of Medicine/Cure, West LA VA Medical Center, Los Angeles/ United States of America

Contact E-mail Address: juliagolota@gmail.com

Introduction: Epidemiological studies revealed that antibiotics exposure increases the risk of inflammatory bowel diseases (IBD) development (Hviid, 2011, Shaw, 2011, Kronman, 2012). However, mechanisms of this association are not fully understood. Recent studies revealed sustained alterations in gut microbiota after antibiotic treatment (Rashid, 2015, Dethlefsen, 2011), the full consequences of which remain unknown.

Aims \& Methods: Here we investigated long-term effects of antibiotic treatment on gut microbiota, short-chain fatty acids (SCFAs) production, transport \& sensing, colon mucosa functional state, surface mucus layer and epithelial barrier integrity. Male Wistar rats $(n=178,140-160 \mathrm{~g})$ were treated for 14 days with broad-spectrum antibiotic ceftriaxone (Cf) $(300 \mathrm{mg} / \mathrm{kg}$, i.m.) or vehicle; euthanized in 1,14 or 56 days after $\mathrm{Cf}$ withdrawal. The fecal microbiota was analyzed by bacteriological culture methods; fecal SCFAs- by gas chromatography; colonic localization and levels of FFA2 \& FFA3 receptors and MCT1, MCT4 \& SMCT1 transporters of SCFAs - by immunohistochemistry; levels of FFA2, FFA3, ERK1/2, p38, HIF1 $\alpha$ proteins in colon mucosa - by Western blot analysis; superoxide dismutase (SOD) and matrix metalloproteinases (MMP) activity - by zymography method; catalase activity, level of thiobarbituric acid (TBA) reactive substances, mucus glycoproteins and its carbohydrate composition colorimetrically; epithelial permeability was evaluated by Evans blue permeation and bacterial translocation.

Results: Cf injection leads to compositional changes of fecal microbiota which progress over time. In 56 days, we found increased level of Clostridium spp, E. coli, conditionally pathogenic and hemolytic bacteria. Levels of Bifidobacterium 
\& Lactobacillus were unchanged during the study. Moreover, the concentrations of acetic, propionic, butyric acids and total SCFAs were decreased 2.9-, 13.8-, 8.5-, 4.8-fold $(\mathrm{P}<0.05)$, respectively, the first day after $\mathrm{Cf}$ withdrawal. These changes were accompanied by decreased immunoreactivity for the FFA2, FFA3 receptors, SMCT1 and increase MCT1 \& MCT4 transporters of SCFAs in colon mucosa. In 56 days, concentration of SCFAs and level of FFA3 was still below control values. These changes evoked a significant shift in colonic mucosal homeostasis. We revealed increased level of TBA-active substances, decreased the activity of SOD and catalase antioxidant enzymes with increased HIFl $\alpha$, activation ERK1/2 and decreased p38 MAP kinases activity after Cf injection. Hypoxia and oxidative stress development were associated with increased permeability of colon epithelium and bacterial translocation to blood in 56 days after Cf withdrawal. Moreover, the levels of mucus glycoproteins, hexoses and fucose decreased while sialation of mucus glycoproteins increased which is typical for IBD development. These changes were accompanied by increased activity of MMP-9 and decrease - MMP-2 in rats colon mucosa.

Conclusion: We showed for the first time that antibiotic treatment induced longterm changes in SCFAs composition, levels of their receptors and transporters in colonic mucosa. It was associated with alterations in mucus composition, increased epithelial permeability that might increase susceptibility to IBD development.

Disclosure of Interest: All authors have declared no conflicts of interest.

\section{References}

Hviid A et al. Antibiotic use and inflammatory bowel diseases in childhood. Gut. 2011;60(1):49-54.

Shaw SY et al. Association between the use of antibiotics and new diagnoses of Crohn's disease and ulcerative colitis. Am J Gastroenterol. 2011;106(12):2133-42. Kronman MP et al. Antibiotic exposure and IBD development among children: a population-based cohort study. Pediatrics. 2012;130(4):e794-803.

Rashid $\mathrm{M}$ et al. Determining the long-term effect of antibiotic administration on the human normal intestinal microbiota using culture and pyrosequencing methods. Clin Infect Dis. 2015;60 Suppl 2:S77-84.

Dethlefsen L et al. Incomplete recovery and individualized responses of the human distal gut microbiota to repeated antibiotic perturbation. Proc Natl Acad Sci USA. 2011;108 Suppl 1:4554-61.

\section{P0966 THE ROLE OF TLR2-MEDIATED TREG/TH17 IMBALANCE IN THE PATHOGENESIS OF ULCERATIVE COLITIS}

Y.F. Xie ${ }^{1}$, J.Y. $\mathrm{Hao}^{2}$, Y.H. Pang ${ }^{2}$, Y.H. Ma ${ }^{3}$, Z.R. $\mathrm{Li}^{2}$, X.Q. $\mathrm{He}^{2}$, X.J. $\mathrm{Liu}^{2}$ ${ }^{1}$ Gastroenterology Department, Beijing Chaoyang Hospital, Beijing/China ${ }^{2}$ Gastroenterology Department, Beijing Chaoyang Hospital, Capital Medical University, Beijing/China

${ }^{3}$ Department Of Medicine, Beijing Haidian Maternal and Child Health Hospital, Beijing/China

\section{Contact E-mail Address: 1xjw2012@126.com}

Introduction: According to our previous report, the imbalance of Treg/Th17 cells in the DSS induced UC mice is related with the reduction of CD45RA ${ }^{-}$FoxP $3{ }^{\text {high }}$ activated Treg(FrII) cells, which has the real function of immunosuppression, and with the elevation of CD45RA ${ }^{-}$FoxP $3^{\text {low }}$ Treg(FrIII) cells, which provide $\mathrm{FoxP}^{+}{ }^{+} \mathrm{L} 17 \mathrm{a}^{+}$trait but lack of immunosuppressive capacity.Activation of Tolllike receptor 2(TLR2) leads to elevation of FrIII, which show FoxP3 ${ }^{+} \mathrm{IL}_{17 \mathrm{a}^{+}}$ trait by secrecting IL-17a.

Aims \& Methods: To investigate the influence of TLR2 on imbalanced Treg/Th17 in DSS induced ulcerative mice .18 mice were divided into three groups, including healthy control mice (group A), ulcerative colitis mice (group B) and TLR2mAb intervened-UC mice (group C).Weight and DAI (disease activity index) of every mice were recorded once a day. After being sacrificed, pathological examination of the rectum of all the mice were carried out. Treg cells with their subsets, Th17 cells and $\mathrm{CD} 3{ }^{+} \mathrm{CD} 8{ }^{-} \mathrm{CD} 25^{+} \mathrm{FoxP} 3^{+} \mathrm{IL}-17 \mathrm{a}^{+}$cells from the lamina propria of colon (LPC) and other tissues of mice were evaluated by flow cytometry.

Results: (1) Declined vitality, anorexia, weight lost and bloody purulent stool were the major symptoms of UC mice. The symptoms of TLR2mAb intervenedUC mice were alleviated, and the DAIs of these mice showed difference from UC mice $(\mathrm{P}<0.05)$. The inflammation of colonic mucosa in TLR $2 \mathrm{mAb}$ intervened$\mathrm{UC}$ mice were also attenuated compared with UC mice. (2) Compared with group A, the Treg cells of group B were increased in PBMC, MLN and LPC $(1.275 \pm 0.063$ vs $1.168 \pm 0.028,6.067 \pm 0.152$ vs $3.652 \pm 0.639,5.657 \pm 0.211$ vs $1.173 \pm 0.150, \mathrm{P}<0.05$ respectively). The level of Treg cells in PBMC of group $\mathrm{C}$ was declined compared with group $\mathrm{B}(1.153 \pm 0.028$ vs $1.275 \pm 0.063, \mathrm{P}<0.05)$ (3) The levels of Th17 cells were elevated in PBMC and LPC of group B compared with group A $(0.488 \pm 0.015$ vs $0.328 \pm 0.023,4.300 \pm 0.137$ vs $1.333 \pm 0.126, \mathrm{P}<0.05)$, but Th17 cells were decreased in PBMC of group $\mathrm{C}$ compared with group $\mathrm{B} \quad(0.273 \pm 0.061$ vs $0.488 \pm 0.015, \quad \mathrm{P}<0.05)$. (4) Descended FrII cells were detected in PBMC, MLN and LPC of group B compared with group $\mathrm{A}(0.016 \pm 0.005$ vs $0.030 \pm 0.009, \quad 0.123 \pm 0.012$ vs $0.465 \pm 0.024,0.233 \pm 0.022$ vs $0.367 \pm 0.018, \mathrm{P}<0.05$ respectively). Otherwise, increased FrII cells were in spleen, PBMC, MLN and LPC of group C compared with group B $(0.577 \pm 0.016$ vs $0.220 \pm 0.309,0.030 \pm 0.009$ vs $0.016 \pm 0.005$, $0.467 \pm 0.016$ vs $0.123 \pm 0.012,0.365 \pm 0.019$ vs $0.233 \pm 0.022, \mathrm{P}<0.05$ respectively). FrIII cells were elevated in PBMC, MLN and LPC of group B compared with group $\mathrm{A}(4.215 \pm 0.490$ vs $3.128 \pm 0.171,14.463 \pm 0.463$ vs $8.740 \pm 1.140$, $9.487 \pm 0.604$ vs $3.760 \pm 0.104, \mathrm{P}<0.05$ respectively). Moreover, declined FrIII cells were observed in spleen and PBMC of group C compared with group B $(8.967 \pm 0.354$ vs $14.122 \pm 0.438,3.070 \pm 0.763$ vs $4.215 \pm 0.490, \mathrm{P}<0.05)$. (5) Compared with group $\mathrm{A}, \mathrm{CD}^{+} \mathrm{CD}^{-} \mathrm{CD} 25^{+} \mathrm{FoxP}^{+}{ }^{+} \mathrm{IL} 17 \mathrm{a}^{+}$cells were increased in PBMC, MLN and LPC in group $\mathrm{B}(3.915 \pm 0.264$ vs $2.533 \pm 0.076$, $1.672+0.158$ vs $0.898+0.044,5.967 \pm 0.100$ vs $2.308+0.252, \mathrm{P}<0.05$ respectively). But the level of $\mathrm{CD}^{+} \mathrm{CD} 8^{-} \mathrm{CD} 25^{+} \mathrm{FoxP} 3^{+} \mathrm{IL} 17 \mathrm{a}^{+}$cells were decreased in spleen, PBMC, MLN and LPC compared with group $\mathrm{B}(0.538 \pm 0.021$ vs $1.238 \pm 0.017,2.523 \pm 0.066$ vs $3.915 \pm 0.264,0.897 \pm 0.040$ vs $1.672 \pm 0.158$, $2.443 \pm 0.052$ vs $5.967 \pm 0.100, \mathrm{P}<0.05$ respectively).

Conclusion: By blocking TLR2, TLR2mAb could improve the level of FrII cells in PBMC, MLN and LPC, but reduce the levels of FrIII and FoxP $3^{+} \mathrm{IL}_{17 \mathrm{a}^{+} \text {cells }}$ in DSS induced UC mice. Furthermore, TLR2mAb could alleviate the DAI index as well as the inflammation of colitis in DSS induced UC mice. Disclosure of Interest: All authors have declared no conflicts of interest.

\section{References}

1. Immunity 2009; 30(6): 899-911.

2. World journal of gastroenterology 2016; 22(42): 9356-9367.

\section{P0967 FIRST ANALYSIS FROM UK IBD TWIN BIOBANK; 16S RRNA GENE SEQUENCING IDENTIFIES REDUCED DIVERSITY IN ACTIVE IBD AND TAXA ASSOCIATED WITH ACTIVE DISEASE PHENOTYPE TO LEVEL OF SPECIES}

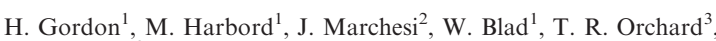
J. Mcdonald ${ }^{2}$

${ }^{1}$ Gastroenterology, Chelsea and Westminster Hospital, London/United Kingdom ${ }^{2}$ Imperial College London, London/United Kingdom

${ }^{3}$ Gastroenterology, Imperial College Healthcare NHS Trust, London/United Kingdom

Contact E-mail Address: marcus.harbord@chelwest.nhs.uk

Introduction: Previous studies have shown that the gut microbiota plays an important role in IBD, however there is not a consensus on which bacteria are responsible for the disease. $16 \mathrm{~S}$ gene profiling studies generate large amounts of information, however they can be confounded by genetic and environmental factors. Twin studies are instrumental in controlling for some of these variabilities, and in this study we investigated the microbiota of twin pairs discordant for Crohn's disease (CD) and Ulcerative colitis (UC) using 16S rRNA gene sequencing, with the aim of identifying taxa associated with disease.

Aims \& Methods: Participants were recruited via the UK IBD Twin Registry. Stool samples were collected and frozen using standard methods. Participants who had received antibiotics within 3 months were excluded. Harvey Bradshaw Index and Simple Clinical Colitis Activity Index were recorded. Full medical history was available from the UK IBD Twin Registry. Samples underwent 16S rRNA sequencing using the Illumina MiSeq platform and analysed using our data analysis pipeline. PERMANOVA was used to evaluate associations with clinical metadata, which included matching of twin pairs for analysis, and STAMP was used to identify taxonomic differences between groups.

Results: 20 twin pairs discordant for CD (5MZ:15DZ mean age 52 years) and 17 discordant for UC (6MZ:11DZ mean age 59.7 years) were recruited. 7 subjects with CD had active disease as did 4 with UC.

Gut microbiota from active CD patients had lower bacterial diversity compared to remission CD patients and healthy twins (Shannon diversity index, $\mathrm{p}<0.001$ healthy vs active $C D$, active vs remission $C D, 1$-way ANOVA post-hoc $=$ Tukey). Active UC patients also had lower bacterial diversity compared to remission UC patients and heathy twins (Shannon diversity index, $\mathrm{p}<0.01$ healthy vs active $\mathrm{UC}, \mathrm{p}<0.05$ active vs remission).

We found that active CD patients had a higher proportion of Clostridium hylemonae and Lactobacillus delbrueckii compared to healthy twins, and a lower proportion of Bacteroides uniformis, Bacteroides vulgatus, and Faecalibacterium prausnitzii $(\mathrm{p}<0.05)$. We found that active UC patients had a lower proportion of Alistipes spp. compared to their healthy twins and UC patients in remission $(\mathrm{p}<0.05)$

Conclusion: This study confirms previous findings showing decreased diversity in IBD patients and changes in some bacterial taxa, however our study is the first to show decreases in Alistipes spp. in active UC.

Disclosure of Interest: All authors have declared no conflicts of interest.

\section{P0968 $\mathrm{CD4}^{+}$T CELLS OF IBD PATIENTS ARE CHARACTERIZED BY AN INCREASED EXPRESSION OF THE NUCLEOTIDE RECEPTOR P2Y2, WHICH IMPACTS RELEVANTLY ON PRO- INFLAMMATORY SIGNALING CASCADES}

J. Panteleev-Ivlev ${ }^{1}$, S. Zundler ${ }^{1}$, R. Atreya ${ }^{1}$, M. F. Neurath ${ }^{1}$, I. Atreya ${ }^{1}$

${ }^{1}$ Medical Clinic 1, University of Erlangen-Nuremberg, Erlangen/Germany

Contact E-mail Address: imke.atreya@uk-erlangen.de

Introduction: Chronic and acute inflammation is often associated with an upregulation of extracellular UTP and ATP nucleotides, which are able to interact with various cell types via purinergic $G$ protein-coupled P2 receptors. Interestingly, former studies already described an increased expression of the ATP/UTP receptor subtype P2Y2 (P2Y2R) in the colonic tissue of IBD patients [1]. However, it remained unknown in how far immune cells of IBD patients are also characterized by an altered P2Y2R expression and whether this might impact on their pro-inflammatory capacity.

Aims \& Methods: P2Y2R mRNA and protein expression on primary $\mathrm{CD}^{+} \mathrm{T}$ cells from the blood of IBD patients or healthy donors was analyzed via qPCR or western blot, respectively. Furthermore, primary human $\mathrm{CD}^{+} \mathrm{T}$ cells were stimulated in the presence of UTP or the selective P2Y2R agonist 2-thio-UTP and 
subsequently analyzed for STAT3 signaling (western blot), NFkB signaling (NFkB Transcription Factor Assay, western blot) and cytokine secretion (ELISA).

Results: Peripheral blood $\mathrm{CD}^{+} \mathrm{T}$ cells from IBD patients could be characterized by a significantly increased P2Y2R expression compared to healthy controls, while expression levels of the P2Y4 receptor subtype turned out to be comparable between both groups. Further subdividing the group of included IBD patients into Crohn's disease and ulcerative colitis patients, we could not observe a significant difference in the $\mathrm{P} 2 \mathrm{Y} 2 \mathrm{R}$ levels between both disease entities. Interestingly, the increased P2Y2R expression in the lymphocyte compartment of IBD patients seemed to be limited to $\mathrm{CD} 4^{+} \mathrm{T}$ cells, as $\mathrm{CD} 8^{+} \mathrm{T}$ cells of those patients even showed decreased P2Y2R levels. Regarding potential regulators of P2Y2R expression in the context of IBD, our data identified IL-6 and TGF-beta as potent inducers of $\mathrm{P} 2 \mathrm{Y} 2 \mathrm{R}$ expression in human $\mathrm{CD}^{+} \mathrm{T}$ cells. Interestingly high extracellular UTP levels resulted in a decreased expression of the TGF-betal receptor on $\mathrm{CD}^{+} \mathrm{T}$ cells, implicating a potential negative feedback loop in which P2Y2R signaling might inhibit TGF-beta induced P2Y2R expression over time. Regarding the impact of P2Y2R signaling on the pro-inflammatory capacity of human lymphocytes, our data indicate that the selective P2Y2R agonist 2-Thio-UTP is able mediate NFkB as well as STAT3 activation and to induce secretion of the pro-inflammatory cytokines IL-6 and IL-17 in stimulated human $\mathrm{CD} 4^{+} \mathrm{T}$ cells.

Conclusion: The observed increased expression of $\mathrm{P} 2 \mathrm{Y} 2 \mathrm{R}$ in $\mathrm{CD}^{+} \mathrm{T}$ cells of IBD patients together with the demonstrated pro-inflammatory effects of P2Y2R signaling in human $\mathrm{T}$ cells markedly strengthen the role of $\mathrm{P} 2 \mathrm{Y} 2 \mathrm{R}$ as a promising molecular target in IBD.

Disclosure of Interest: All authors have declared no conflicts of interest.

\section{Reference}

1. Grbic DM, Degagné E, Langlois C, Dupuis AA, Gendron FP. Intestinal inflammation increases the expression of the P2Y6 receptor on epithelial cells and the release of CXC chemokine ligand 8 by UDP. J Immunol. 2008 Feb 15;180(4):2659-68.

\section{P0969 HIF-1A STABILIZATION THROUGH HYDROXYLASE INHIBITION AMELIORATES DSS-INDUCED COLITIS AND INDUCES AUTOPHAGY}

J. Cosin Roger ${ }^{1}$, S. Simmen ${ }^{1}$, H. Melhem ${ }^{1}$, K. Atrott ${ }^{1}$, I. Frey-Wagner ${ }^{1}$ M. Hausmann ${ }^{1}$, C. De Valliere ${ }^{1}$, M. R. Spalinger ${ }^{1}$, P. Spielmann ${ }^{2}$, R.H. Wenger ${ }^{2}$, J. Zeitz ${ }^{1}$, S. Vavricka ${ }^{1}$, G. Rogler ${ }^{1}$, P.A. Ruiz

${ }^{1}$ Gastroenterology And Hepatology, University of Zurich, University Hospital Zurich, Zurich/Switzerland

${ }^{2}$ Institute Of Physiology, University of Zurich, Zurich/Switzerland

Contact E-mail Address: jesus.cosin@uv.es

Introduction: Environmental hypoxia has been increasingly recognized as an important environmental factor associated with Inflammatory Bowel Disease (IBD). Hypoxia allows the stabilization of hypoxia inducible factor (HIF) complexes and has been linked to the activation of autophagy. HIF $1 \alpha$ is induced in the inflamed mucosa from IBD patients and mouse models of colitis, but its role in intestinal inflammation is still controversial since both, positive and negative effects have been reported.

Aims \& Methods: We aim to elucidate the effects of HIF $1 \alpha$ stabilization in autophagy and the development of intestinal inflammation in a murine model of colitis. Female C57BL/6 J mice between 8-10 weeks of age were exposed to $2 \%$ DSS in drinking water for 7 days, and received $8 \mathrm{mg}$ of the hydroxylase inhibitor dimethyloxaloylglycine (DMOG) intraperitoneally every second day. Mice were killed at day 9 , mucosal damage was assessed by colonoscopy and murine endoscopic score of colitis severity (MEICS) was calculated. H\&E staining was performed to analyze the histological damage. Expression of TNF, IL-6, IL-1 $\beta$ and Nlrp3 was analyzed by $q \mathrm{PCR}$ and protein expression of $\mathrm{p}-\mathrm{NF} \kappa \mathrm{B}, \mathrm{NF} \kappa \mathrm{B}, \mathrm{p}-$ mTOR, mTOR, N1rp3, p62, LC3 was assessed by Western Blot. HT-29 cells were subjected to normoxia $(21 \% \mathrm{O} 2)$ or hypoxia $(0.2 \% \mathrm{O} 2)$ for $24 \mathrm{~h}$ in the presence or absence of $40 \mu \mathrm{M}$ chloroquine. Cells were stained with DAPI and LysoTracker Yellow-HCK-123 to monitor lysosomal accumulation. Chromatin immunoprecipitation (ChIP) analysis was performed using an antibody against HIF $1 \alpha$ and PCR was performed using the promoter-specific primers for the p62 promoter binding sites of HIF $1 \alpha$.

Results: DMOG administration induced a significant lower reduction of body weight in DSS-treated mice compared to DSS-treated mice administered with vehicle. Furthermore, mice administered with DMOG presented less reduction in the colon length, a significant reduction in the MEICS and histological scores showing intact crypts in large areas without extensive infiltration or thickening of the mucosa. The mRNA expression of the pro-inflammatory factors TNF, IL-6, IL-1 $\beta$ and Nlrp3 was significantly reduced in mice treated with DMOG compared to vehicle-treated mice. At a protein level, DMOG administration reduced $\mathrm{p}-\mathrm{NF} \kappa \mathrm{B}$ and NLRP3 expression. DMOG-treated mice also showed activation of autophagy, as evidenced by a decrease in p-mTOR and p62 expression and increase of LC3II. In vitro, hypoxia induced a significant accumulation of lysosomes in HT-29 cells and a reduction of p-NF $\kappa$ B. The late-stage autophagy inhibitor chloroquine reverted this effect indicating that was autophagymediated. Finally, ChIP analysis revealed that hypoxia induced the binding of HIF $1 \alpha$ to the promoter of $\mathrm{p} 62$

Conclusion: Our results indicate that DMOG-mediated HIF $1 \alpha$ stabilization ameliorates DSS-induced colitis, activates autophagy and significantly reduces inflammatory gene expression and signaling. In cultured intestinal epithelial cells hypoxia triggers lysosomal formation and HIF $1 \alpha$ binds to the promoter of p62, thereby promoting autophagy.

Disclosure of Interest: All authors have declared no conflicts of interest.

\section{P0970 PROTECTIVE EFFECT AND ACTION MECHANISM OF APOCYNIN IN IBD MOUSE MODEL}

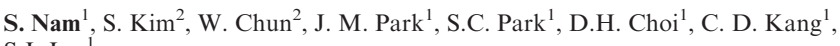
S.J. Lee ${ }^{1}$

${ }^{1}$ Department Of Internal Medicine, Kangwon National University School of

Medicine, Chuncheon-si, Gangwon-do/Korea, Republic of

${ }^{2}$ Department Of Pharmacology, College Of Medicine, Kangwon National

University, Chuncheon-si/Korea, Republic of

Contact E-mail Address: pinetrees@daum.net

Introduction: There are several medical treatment options for inflammatory bowel disease (IBD), but all have drawback due to their significant adverse effects. Many new drugs are being developed for more safe and effective treatment. Apocynin is a chemical 4-hydroxy-3-methoxyacetophenone which is an inhibitor of NADPH oxidase and has showed promising effect in various chronic inflammatory diseases such as asthma and atherosclerosis. Due to its anti-inflammatory effect and safety profile, apocynin can be new candidate for the treatment of IBD.

Aims \& Methods: In this study, we aimed to investigate effect of apocynin on colonic inflammation and the action mechanism using chemically-induced colitis mouse model. We used dextran sulfate sodium (DSS)-induced colitis model. 8 weeks old male BALB/c mice were divided into four groups (each group, $n=6$ ): control, DSS only, DSS with apocynin, and DSS with sulfasalazine. Water (control and DSS group), apocynin $(400 \mathrm{mg} / \mathrm{kg})$ and sulfasalazine $(150 \mathrm{mg} / \mathrm{kg}$ ) were administrated by oral route using sonde during 7 days. For western blot anaylsis, mouse colon was lysed and protein was extracted. The following antibodies were used; iNOS (BD Biosciences), COX-2, Nrf2 (Santa Cruz Biotechnology Inc), MCP-1, TNF- $\alpha$, p-Nrf2, HO-1 (Abcam), and $\beta$-actin (Sigma).

Results: Protective effect of apocynin was evident by weight change and colon length. Histologic analysis also showed improved erosion and decreased neutrophilic infiltration in apocynin group compared to DSS group. In colon tissue, several pro-inflammatory enzymes and cytokines are decreased by apocynin. Apocynin also activated anti-inflammatory pathway by inducing activation of Nrf2 and production of heme oxygenase-1 (HO-1).

Conclusion: Apocynin, a NADPH-oxidase inhibitor, showed significant antiinflammatory effect in DSS induced colitis model. Considering its good safety profile, this molecule can be a new candidate for the treatment of IBD. Disclosure of Interest: All authors have declared no conflicts of interest.

\section{P0971 THE USE OF RAPID EVAPORATIVE IONISATION MASS SPECTROMETRY (REIMS) IN FAECAL SAMPLES TO IDENTIFY INFLAMMATORY BOWEL DISEASE}

S.T.R. Powles ${ }^{1}$, L. W.L. Chong ${ }^{1}$, S. J. Cameron ${ }^{2}$, L. C. Hicks ${ }^{3}$, Z. Takats ${ }^{2}$, H. R.T. Williams ${ }^{1}$, T. R. Orchard

${ }^{1}$ Division Of Digestive Diseases, Department Of Surgery And Cancer, Imperial College London, London/United Kingdom

${ }^{2}$ Division Of Computational And Systems Medicine. Department Of Surgery And Cancer, Imperial College London, London/United Kingdom

${ }^{3}$ Gastroenterology, Imperial College Healthcare NHS Trust, London/United Kingdom

Contact E-mail Address: s.powles@imperial.ac.uk

Introduction: Faecal metabolic profiling has been shown to distinguish Inflammatory Bowel Disease (IBD) from healthy controls (HC), specifically with depletion of gut-associated short chained fatty acids (SCFA) as the predominant feature separating these groups (1). Previous and current studies have used proton nuclear magnetic resonance (1H NMR) spectroscopy or mass spectroscopy (MS) to measure faecal metabolites to examine the metabolic, microbiomic and clinical response of IBD patients to different treatments. Both techniques require a significant amount of sample pre-processing.

Rapid Evaporative Ionization Mass Spectrometry (REIMS) is a relatively new technology which applies a laser to a biological sample, and the resulting vapour, containing gas phase ions of metabolites and structural lipids, is analysed by a mass spectrometer (2). Unprocessed faecal samples can be rapidly assessed using this technique to obtain lipidomic spectral profiles (2). To our knowledge this is the first study that has used REIMS to investigate whether IBD patients can be distinguished from healthy controls using faecal samples.

Aims \& Methods: Unprocessed faecal samples from 109 IBD patients and 46 healthy controls were analysed using Rapid Evaporative Ionization Mass Spectrometry. Clinical and dietary data were collected, and patients with significant other co-morbidities were excluded. Partial least squares discriminative analysis (PLSDA) was performed to examine whether there was differences in the metabolic data between patients with Inflammatory Bowel Disease and healthy controls. Further subgroup analysis was then carried out including examining whether ulcerative colitis could be distinguished from Crohn's disease. 
Participant characteristics

\begin{tabular}{lll}
\hline & IBD & Healthy Control \\
\hline Number $(\%)$ & 109 & 46 \\
Age (mean) & 48 & 55 \\
Male & $51(47 \%)$ & $19(41 \%)$ \\
Caucasian & $63(58 \%)$ & $21(46 \%)$ \\
Crohn's disease & $62(57 \%)$ & - \\
High IBD $\left(>30 \mathrm{~kg} / \mathrm{m}^{2}\right)$ & $14(13 \%)$ & $10(21 \%)$ \\
\hline
\end{tabular}

Results: Supervised multivariate analysis (PLSDA) was able to separate faecal samples of IBD patients from healthy controls, and sub-analysis showed a stronger separation of Crohn's disease patients from healthy controls (Q2 quality assurance of 0.2 ) compared to UC from healthy controls. A model comparing Crohn's disease from UC was able to separate the two groups.

Conclusion: This early preliminary analysis shows that there is a degree of separation between samples of IBD patients compared to healthy control. This suggests that REIMS analysis has good potential as a research tool in IBD metabonomic studies, and importantly in the current ongoing longitudinal IBD research. Further analysis will include identifying the principal metabolites that separate these groups.

Disclosure of Interest: All authors have declared no conflicts of interest.

\section{References}

1. Marchesi J.R. et al. J. Proteome Res. 2007, 6, 546-551

2. Balog J et al. Anal. Chem. 2010, 82, 7343-7350

\section{P0972 THE PATHOGENIC MECHANISM OF ARYL HYDROCARBON RECEPTOR MEDIATED ABNORMAL DIFFERENTIATION OF INTESTINAL ILC3/ILC1 IN CROHN'S DISEASE}

X. Zhao, H. Zhang

Department Of Gastroenterology, Jiangsu Province Hospital and Nanjing Medical University, Nanjing/China

Contact E-mail Address: zhaoxj91718@163.com

Introduction: The abnormal differentiation of intestinal innate lymphoid cells ILC3 3 and ILC1 exist in autoimmune disease. ILC3 decreased and ILC1 increased in Crohn's disease (CD) patients, suggesting that $C D$ patients have abnormal intestinal ILC3/ILC1 alteration.

Aims \& Methods: The present study investigated the aberrant colonic mucosal ILC3/ILC1 in active CD patients and 2, 4, 6-trinitrobenzene sulphonic acid (TNBS)-induced colitis mice. The expressions of aryl hydrocarbon receptor $(\mathrm{AhR})$ in colon of active and quiescent $\mathrm{CD}$ patients were detected by western blot and immunofluorescence. The ILC3/ILC1 were investigated in CD patients and 2, 4, 6-trinitrobenzene sulphonic acid (TNBS)-induced colitis mice (AhR ${ }^{-1-}$, $\left.\mathrm{AhR}^{+/+}\right)$.

Results: Compared to quiescent CD patients, the expression of aryl hydrocarbon receptor (AhR) in the intestinal tissue in active $\mathrm{CD}$ patients was decreased. Meanwhile, the number of ILC3 in active CD patients and AhR knockout mice was decreased while ILC1 increased. The intestinal inflammation in AhR knockout mice given TNBS was more severe than wild-type mice.

Conclusion: These findings suggest that AhR may mediate abnormal differentiation of ILC3/ILC1, and the production of inflammatory cytokines, finally, promotes the pathogenesis of CD

Disclosure of Interest: All authors have declared no conflicts of interest.

\section{References}

1. Kaser A, Zeissig S, Blumberg RS. Inflammatory bowel disease. Annu Rev Immunol. 2010;28:573-621.

2. Peters CP, Mjösberg JM, Bernink JH, Spits H. Innate lymphoid cells in inflammatory bowel diseases. Immunol Lett. 2016 Apr;172:124-131.

3. Artis D, Spits H. The biology of innate lymphoid cells. Nature. 2015 Jan;517(7534):293-301.

4. Giacomin PR, Moy RH, Noti M, et al. Epithelial-intrinsic IKK $\alpha$ expression regulates group 3 innate lymphoid cell responses and antibacterial immunity. $J$ Exp Med. 2015 Sep;212(10):1513-1528.

5. Hepworth MR, Monticelli LA, Fung TC, et al. Innate lymphoid cells regulate CD4+ T-cell responses to intestinal commensal bacteria. Nature. 2013 Jun;498(7452):113-117.

6. Lahoti TS, Hughes JM, Kusnadi A, et al. Aryl hydrocarbon receptor antagonism attenuates growth factor expression, proliferation, and migration in fibroblast-like synoviocytes from patients with rheumatoid arthritis. $J$ Pharmacol Exp Ther. 2014 Feb;348(2):236-245.

7. Monteleone I, Rizzo A, Sarra M, et al. Aryl hydrocarbon receptor-induced signals up-regulate IL-22 production and inhibit inflammation in the gastrointestinal tract. Gastroenterology. 2011 Jul;141(1):237-248.
P0973 PREVALENCE AND GENETIC DIFFERENCES IN

ADHESION-RELATED GENES AMONG COMMENSAL AND ADHERENT-INVASIVE $E$. COLI STRAINS

C. Camprubí-Font, A. Segú-Roig, N. Maeso-Sánchez, M. López-Siles, M. Martinez-Medina

Biology Department, Universitat de Girona, Girona/Spain

Contact E-mail Address: c.camprubi4@gmail.com

Introduction: Long polar fimbriae ( $\mathrm{LpfA}$ ), FimH adhesin and ChiA chitinase have been related with adherent-invasive E. coli (AIEC) pathogenesis. Controversial results have been found regarding the prevalence of LpfA in AIEC vs non-AIEC $(1,2)$. Some FimH amino acid variants were reported to be specific for AIEC (3) whereas other variants were associated with phylogroup and disease origin of the strains (4). Differences in the ChiA sequence were reported between LF82 and K-12 strains but this gene has not been studied in other AIEC yet (5).

Aims \& Methods: The prevalence of $1 p f \mathrm{~A}$ and the distribution of fimH and chiA variants in a collection of AIEC and non-AIEC from different disease origins Crohn's disease (CD), ulcerative colitis (UC) and colorectal cancer (CRC)- were studied with the purpose to determine if these genes could be used as molecular markers for AIEC identification and disease diagnostics. In a collection of 37 AIEC and 29 non-AIEC isolated from CD, UC, CRC patients and controls, $\operatorname{lpf} A$ gene was PCR-amplified to assess its presence and fim $H$ and chiA genes were sequenced to identify point mutations. For comparison of FimH and ChiA protein sequences, UPGMA phylogenetic tree and allele identification was performed using MEGA5. The genetic differences were annotated using as reference the K-12 strain. Then, they were analysed statistically according to AIEC pathotype, phylogroup, and disease origin by the $\chi 2$ test and non-parametric tests were used to evaluate amino acid variability regarding the adhesion and invasion indices.

Results: Low gene frequency for $\operatorname{lpf} A 141$ and $\operatorname{lpf} A 154$ was reported $(11.7 \%$ and $16.7 \%$ respectively). Lpf A154 was only found in strains from A $(22 \%)$ and B1 phylogroup $(86 \%)$ and no relation with AIEC phenotype or disease was observed. Two main clusters of FimH were obtained by phylogenetic analysis, classifying the strains according to the presence of S78N mutation. N70S and $\mathrm{S} 78 \mathrm{~N}$ variants were characteristic from strains of $\mathrm{B} 2$ and $\mathrm{D}$ phylogroups as none of the A or B1 strains presented it. Despite statistical significance was not reached, the strains with $\mathrm{N} 70 \mathrm{~S}, \mathrm{~S} 78 \mathrm{~N}, \mathrm{~V} 163 \mathrm{~A}, \mathrm{R} 166 \mathrm{H}$ mutations showed the highest adhesiveness. Regarding ChiA, two main clusters defined by the presence or absence of an insertion in 312-314 residues were obtained. None of the five previously mutations found in LF82 strain were associated with AIEC strains, whereas the V415A variant was found specifically in AIEC $(20 \%)(p=0.049)$. Of note, among the strains harbouring the 312-314 insertion, a subcluster that shared identical amino acid sequence included the LF82 strain and the $44 \%$ of AIEC strains but only the $10 \%$ of the non-AIEC $(\mathrm{p}=0.019)$. No differences between FimH/ChiA variants and origin of isolation was observed.

Conclusion: In contrast with other studies, no relation of $1 p f A$ presence nor in FimH mutations with AIEC pathotype or disease was observed. Nonetheless, a variant in ChiA sequence more frequently found in AIEC isolates was reported, being an interesting signature sequence for the detection of at least a subgroup of AIEC strains. Further confirmation in a wider strain collection would be required.

Disclosure of Interest: All authors have declared no conflicts of interest.

\section{References}

1. Dogan et al. 2014. IBD.20(11):1919-32

2. Chassaing et al. 2011. J Clin Invest. 121(3):966-75.

3. Dreux et al. 2013. PLoS Pathog 9(1): e1003141

4. Iebba et al.2012. Infect Immun.80(4):1408-1417.

5. Low et al.2013 Gastroenterology 145(3):602-612

\section{P0974 DISTINCT GUT MICROBIOTA PROFILES IN PATIENTS WITH PRIMARY SCLEROSING CHOLANGITIS AND ULCERATIVE COLITIS}

L. Bajer ${ }^{1}$, M. Kverka ${ }^{2}$, M. Kostovcik ${ }^{3}$, P. Macinga ${ }^{1}$, J. Dvorak ${ }^{2}$, Z. Stehlikova ${ }^{2}$, J. Brezina ${ }^{4}$, J. Spicak ${ }^{1}$, P. Drastich ${ }^{1}$

${ }^{1}$ Department Of Hepatogastroenterology, Institute for Clinical and Experimental Medicine, Prague/Czech Republic

${ }^{2}$ Institute of Microbiology of the Czech Academy of Sciences, Prague/Czech Republic

${ }^{3}$ Department Of Genetics And Microbiology, Charles University in Prague, Faculty of Science, Prague/Czech Republic

${ }^{4}$ Hepatogastroenterology, Institute for Clinical and Experimental Medicine. Prague/Czech Republic

Contact E-mail Address: lukasbajer1@gmail.com

Introduction: Primary sclerosing cholangitis (PSC) is a progressive disorder of biliary tree which can lead to end-stage liver disease, liver transplantation or even death ${ }^{1,2}$. Colitis accompanying PSC is considered to be a phenotype of IBD (inflammatory bowel disease) distinct from ulcerative colitis (UC) and is often referred to as "PSC-IBD" ${ }^{3}$.

Aims \& Methods: Our aim was to compare the gut bacterial microbiota of patients with PSC and UC. Stool samples were prospectively collected and relevant clinical data obtained from 106 study participants, 43 PSC patients with $(\mathrm{n}=32)$ or without $(\mathrm{n}=11)$ concomitant IBD, 32 UC patients, and 31 healthy controls (HC). Sequencing of the 16S rRNA gene including the V3 and V4 regions was performed on Illumina MiSeq platform to cover low taxonomic 
levels. Data were further processed in QIIME employing MaAsLin and LEfSe tools for analysis of the output data.

Results: Microbial profiles in both PSC and UC were characterized by low bacterial diversity and significant change in global microbial composition. Rothia, Enterococcus, Streptococcus, Veillonella, and three other genera were markedly overrepresented in PSC regardless of concomitant IBD. Rothia, Veillonella and Streptococcus were tracked to the species level to identify Rothia mucilaginosa, Streptococcus infantus, $S$. alactolyticus, and $S$. equi along with Veillonella parvula and $V$. dispar. PSC was further characterized by decreased abundance of Adlercreutzia equolifaciens and Prevotella copri. Decrease in genus Phascolarctobacterium was linked to presence of colonic inflammation regardless of IBD phenotype. Akkermansia muciniphila, Butyricicoccus pullicaecorum and Clostridium colinum were decreased in UC along with genus Roseburia. Unclassified Actinomyces species were markedly increased in overlap syndrome of autoimmune hepatitis (AIH) and PSC. Low levels of serum albumin were significantly correlated with enrichment of order Actinomycetales.

Conclusion: PSC was characterized by microbial features independent of concomitant IBD. Several bacterial taxa clearly distinguished IBD phenotypes (PSCIBD and UC) as well as PSC from PSC/AIH.

Disclosure of Interest: All authors have declared no conflicts of interest.

\section{References}

1. Gow PJ, Chapman RW. Liver transplantation for primary sclerosing cholangitis. Liver 2000; 20(2): 97-103

2. Chapman RW, Arborgh BA, Rhodes JM, Summerfield JA, Dick R, Scheuer PJ, Sherlock S. Primary sclerosing cholangitis: a review of its clinical features, cholangiography, and hepatic histology. Gut 1980; 21(10): 870-877

3. Loftus EV, Jr., Harewood GC, Loftus CG, Tremaine WJ, Harmsen WS, Zinsmeister AR, Jewell DA, Sandborn WJ. PSC-IBD: a unique form of inflammatory bowel disease associated with primary sclerosing cholangitis. Gut 2005; 54(1): 91-96

\section{P0976 HYPOXIA INDUCIBLE FACTOR (HIF)-1 ACCELERATES EPITHELIAL WOUND HEALING THROUGH INTEGRIN REGULATION}

B. J. Goggins ${ }^{1}$, K. Minahan ${ }^{2}$, D. Knight ${ }^{2}$, J. Horvat ${ }^{2}$, M. M. Walker ${ }^{3}$, S. Keely ${ }^{2}$ ${ }^{1}$ School Of Biomedical Sciences And Pharmacy, University of Newcastle, Callaghan/Australia/NSW

${ }^{2}$ University of Newcastle School of Biomedical Science and Pharmacy, Callaghan/ Australia

${ }^{3}$ School Of Medicine And Public Health, The University of Newcastle Australia, Callaghan/Australia/NSW

\section{Contact E-mail Address: simon.keely@newcastle.edu.au}

Introduction: The characteristic inflammation associated with IBD contributes to repeated cycles of epithelial wounding and repair in the intestine. The epithelium functions as a selective barrier, critical for mucosal protection. During intestinal inflammation, damage to the vasculature leads to reduced oxygen availability (hypoxia) at the mucosa. Epithelial wound healing processes occur in this hypoxic environment and are critical to restore barrier integrity and gut homeostasis. A key factor in the co-ordination of mucosal wound healing is the transcription factor Hypoxia inducible factor (HIF)-1. HIF-1 mediates an array of protective mechanisms for cell survival and repair. Previous work has shown that pharmacological stabilisation of HIF- $1 \alpha$ by prolyl hydroxylase inhibitors (PHDi) is protective in murine models of colitis. Importantly, our work has identified HIF-1-mediated induction of integrin- $\beta 1$ at the mucosa as critical to this process. Aims \& Methods: We aimed to examine the functional role and post-translational activity of epithelial $\alpha$-integrin subunits dimerizing with integrin- $\beta 1$ to promote HIF-1-mediated wound healing by PHDi. Cell migration and inhibition scratch assays were performed on T84 monolayers $\left(\sim 1 \% \mathrm{O}_{2}\right.$ and /or PHDi (AKB-4924), wound closure was monitored over 24 hours. Monolayers were stained by immunofluorescence for $\alpha$-integrin subunits at 12 hours post-wound. Novel biopsywound models were used to extend the study of PHDi-mediated wound healing in vivo.

Results: PHDi treatment significantly accelerated wound closure in T84 monolayers, which was inhibited by antibodies functionally targeting integrin- $\alpha 3$. Immunofluorescent imaging of cell monolayers showed both hypoxia and PHDi treatments increased expression of integrin- $\alpha 3$, localized at the leading edge of the wound. PHDi treatment also significantly accelerated mucosal wound closure in the colons of mice following biopsy wounds with elevated expression of integrin- $\alpha 3$ associated with the wound epithelium.

Conclusion: These data suggest that PHDi-mediated HIF-1 stabilisation promotes mucosal healing through regulation of epithelial integrins, in particular $\alpha 3 \beta 1$. Integrin- $\alpha 3 \beta 1$ is a key regulator of epithelial cytoskeletal organization therefore PHDi compounds may enhance wound closure through integrin$\alpha 3 \beta 1$-mediated re-organisation of the cytoskeleton.

Disclosure of Interest: All authors have declared no conflicts of interest

\section{References}

1. Keely, S., et al., FASEB J, 2009. 23(5): p. 1338-46.

2. Goggins, B.J., et al., Front Immunol, 2013. 4: p. 272

3. Keely, S., et al., Mucosal Immunol, 2014. 7(1): p. 114-23.
P0977 EFFECTS OF TIME ON URINARY METABOLIC

SIGNATURES IN INFLAMMATORY BOWEL DISEASE

L. C. Hicks ${ }^{1}$, S.T.R. Powles ${ }^{2}$, L. W.L. Chong ${ }^{2}$, J. Swann ${ }^{3}$, E. Holmes ${ }^{3}$, H. R.T. Williams ${ }^{2}$, T. R. Orchard ${ }^{2}$

${ }^{1}$ Gastroenterology, Imperial College Healthcare NHS Trust, London/United Kingdom

${ }^{2}$ Division Of Digestive Diseases. Department Of Surgery And Cancer, Imperial College London, London/United Kingdom

${ }^{3}$ Division Of Computational And Systems Medicine. Department Of Surgery And Cancer, Imperial College London, London/United Kingdom

Contact E-mail Address: 1.hicks@imperial.ac.uk

Introduction: Metabolic profiling (metabonomics) has been proposed as a novel clinical tool in IBD to predict development of complex disease, or for longitudinal non-invasive monitoring of activity and/or response to drug treatment. Urinary metabonomics can distinguish IBD from healthy controls(1) but no studies to date have assessed the stability of these discriminatory profiles over time. Studies in healthy adults show metabolic signatures are largely unchanged over periods of upto 3 years(2), but signals are influenced by multiple external factors including medication and surgery, so how these change in IBD is unknown. The aim of this study was to compare baseline urinary metabolic profiles of IBD patients with a repeated sample several years later to assess similarity, and also to test if any clinical outcomes could be retrospectively predicted from the baseline sample.

Aims \& Methods: Two urine samples from 39 IBD patients (22 Crohn's disease (CD) and 17 ulcerative colitis (UC)) were collected - one at baseline and one several years later (range 7-9 yrs). These were analysed by $1 \mathrm{H}$ NMR spectroscopy. Disease progression was defined as initiation of immunosuppression or biologics, progression of disease location or phenotype, or surgery. Principal components analysis was used to visualise the variance between the two timepoints within the cohort. Orthogonal partial least squares discriminant analysis (OPLSDA) was used to establish if the metabolic signatures could be used to predict adverse clinical outcomes in the patients studied.

Results: $57 \%$ of CD patients and $17 \%$ of UC patients had clinical progression at follow-up sampling. PCA showed clustering of sample pairs from the baseline and several years later in most individuals, suggesting intra-individual similarity across time. OPLSDA showed no statistical models could be built to predict combined poor outcome based on the initial urinary metabolic profile $(\mathrm{p}=0.26)$. However, the small subgroup who went on to require surgical intervention could be separated from the cohort in a model $(\mathrm{Q} 2=0.015 ; \mathrm{p}=0.03)$ constructed on their baseline profiles.

Conclusion: The metabolic profile of IBD in an individual appears relatively stable over a significant time period despite a variety of clinical outcomes and interventions. Variations in longitudinal measurements appear to be subtle, and therefore application of this technique for disease monitoring and risk stratification could prove difficult. These results may suggest that metabolic profiling could be exploited to predict a higher risk of requiring future surgery.

Disclosure of Interest: All authors have declared no conflicts of interest.

\section{References}

1. Williams H.R.T. et al., (2009). Am J Gastroenterol

2. Bernini, P., et al., (2009). J Proteome Res

\section{P0978 BACTERIAL TRANSLOCATION CONTINUES IN ULCERATIVE COLITIS DESPITE MUCOSAL HEALING AND CONTINUED CLINICAL REMISSION}

\section{A. Steel}

Gastroenterology, Royal Liverpool \& Broadgreen University Hospital, Liverpool/ United Kingdom

Contact E-mail Address: alan.steel $@$ rlbuht.nhs.uk

Introduction: Mucosal healing is considered to be the hallmark of successful therapy in Ulcerative Colitis, and has become increaingly used as a target of therapeutci interventions. Loss of mucosal integrity and associated translocation of bacterial components across the mucosal epithelial barrier occurs in Ulcerative Colitis and persistance of a structural mucosal defecit is a characteristic of chronic inflammatory bowel disease. Mucosal healing can be defined by clinical, endoscopic and histological means but no clear definition of the required extent of mucosal healing exists, nor is there agreeent on how functional mucosal healing is defined.

Aims \& Methods: To define the extent and associations of mucosal healing in patients with Ulcerative Colitis and the relationship with bacterial translocation and clinical remission. Patients with established diagnosis of Ulcerative Colitis undergoing endoscopic evaluation were recruited to the study (Ethics: South West London REC2 10/H0706/26). Clinical history and long-term follow-up data were recorded. Blood and mucosal samples were prepared as mononuclear cells. Healthy controls recruited from cohort undergoing routine lower gastrointestinal investigations without positive findings. Flow cytometry characterisation of cells by cell surface CD45RO, CD27, CD3, CD14, CD8 and CD161 and cytokine expression after staphylococcal enterotoxin B stimulation by IL-2, IL$17 \mathrm{a}$, IL-22, TNF, IL-17f and IFN $\gamma$. Immunohistochemistry to define tight junction apical epithelial expression (Claudin 1, Claudin 4 and Occludin) and lipopolysaccharide within the lamina propria. Peripheral blood markers of bacterial translocation: bacterial DNA (16srDNA), lipopolysaccharide Binding protein (LBP), soluble CD14 and plasma lipopolysaccharide. Statistical analysis by Mann Whitney or Kruskall Wallis analysis with Dunn's post test correction, or by Spearman rho correlation. 
Results: 28 patients with Ulcerative Colitis, duration of disease 4 months to 31 years (median 6.3 years) and 22 Healthy Controls were recruited to the study. Half of the patinets had active disease as assessed by Ulcerative Colitis Severity Score. Disease severity positively correlated with frequency of mucosal Th17 $(\mathrm{CD} 4+\mathrm{IL}-17 \mathrm{a}+)$ and IL-17f. Breaches in tight junction protein expresion were greater in UC than controls: Claudin $1(\mathrm{p}<0.0001)$, Claudin $4(\mathrm{p}=0.0016)$ and occludin $(\mathrm{p}=0.03)$. The serum marker of bacterial translocation, lipopolysaccharide binding protein (LBP) was elevated in UC compared to controls $(\mathrm{p}=0.0078)$ and was positively correlated with breaches of Claudin 1 and Occludin ( $\mathrm{p}=0.018$ and $\mathrm{p}=0.012$ respectively) Staining colon biopsies for the presence of lipopolysaccharide in the lamina propria demonstrated positive findings in healthy controls, supported by data from 16s rDNA analysis of blood from healhty controls. In the Ulcerative Colitis cohort in clinical remission the abscence of lipopolysaccharide in the lamina propria was associated with elevated levels of LBP and increased breaches of Occludin $(\mathrm{p}=0.0022)$.

Conclusion: Breaches of tight junction proteins in the colon of patients with stable clinical remission can be detected and are assocaited with perturbations of mucosal immunological function and markers of bacterial translocation. Lipopolysaccaride presence in the lamina propria of patients in remission appears to be assocated with less tight junction breaches and reduced local and systemic evidence of bacterial translocation. These findings require further study, specifically to examine the role of mucosal immune tolerance to lipopolysaccharide and other bacterial cell products that may be present in the healed mucosa of ulcerative colitis.

Disclosure of Interest: All authors have declared no conflicts of interest.

\section{References}

Salim SY Inflam Bowel Dis 2011 17:362-81

Ignacio A Front Immunol 2016 7:54

Heller F Gastroenterology 2005; 129:550-564

\section{P0979 EFFECT OF FIBER AND FAT CONSUMPTION ON DISEASE ACTIVITY AND QUALITY OF LIFE IN PATIENTS WITH INFLAMMATORY BOWEL DISEASE}

I. Pefkianaki, I. Mouzas, I. Koutroubakis

School Of Medicine, University of Crete, Heraklion/Greece

Contact E-mail Address: medp2011775@med.uoc.gr

Introduction: Diet may influence intestinal inflammation via various pathways but the evidence regarding the role of fiber or fat intake in patients with inflammatory bowel disease (IBD) is controversial.

Aims \& Methods: The aim of this study was to investigate the association between dietary fiber or total fat intake and disease activity or quality of life in Greek IBD patients. We prospectively collected food frequency questionnaires (FFQ) from consecutive IBD patients at outpatient visits. The European Prospective Investigation into Cancer and Nutrition (EPIC) Study FFQ for Greek population with the MAFF photographic food atlas were used in order to collect information for dietary habits of IBD patients. Moreover, disease activity using the simple colitis activity index (SCCAI) (for ulcerative colitis (UC)) and Harvey-Bradshaw index (HBI) for Crohn's disease (CD)) as well as quality of life using the short inflammatory bowel disease questionnaire (SIBDQ) were evaluated. Patients' demographic, clinical characteristics, nutritional status, laboratory data $[\mathrm{C}$ reactive protein $(\mathrm{CRP})$, haemoglobin, erythrocyte sedimentation rate (ESR), platelets and albumin] and treatment data were recorded and analysed for all participants.

Results: A total of 141 consecutive IBD patients (53 UC, $88 \mathrm{CD}$, mean age $47.2 \pm 16.1$ years, 84 males 57 females, mean BMI 26.7 +5.3$)$ were included. Patients' mean daily fiber intake was 21.8 gr (IQR 13.8-34.6) and mean daily total fat was $161.4 \mathrm{gr}(121.2-237)$ with $43.5 \%$ percent energy from fat. Regarding disease activity $34(24.1 \%)$ patients had active disease with HBI or SCCAI score $>5$ whereas $45(31.9 \%)$ patients had poor quality of life with SIBDQ score $<50$ There was no difference in daily fiber or fat consumption between patients with UC and CD [median daily fiber intake 23.5 (IQR 10.8-36.5) gr vs 21.7 (14.6$34.2)$ gr $(\mathrm{p}=0.86)$, median fat intake $148.7(112.4-242.8)$ gr vs 169.4 (127.1$236.7)$ gr $(\mathrm{p}=0.30)]$. There was a significant negative correlation between fiber intake and disease activity in both $\mathrm{UC}(\mathrm{r}=-0.37 \mathrm{p}=0.007)$ and $\mathrm{CD}(\mathrm{r}=-0.32$, $\mathrm{p}=0.002$ ) while there was not significant correlation between fat intake and disease activity (in both diseases $\mathrm{p}>0.05$ ). No association of fiber or fat consumption with abnormal CRP, increased ESR, presence of anemia, thrombocytosis or low albumin was found (all with $p>0.05$ ). SIBDQ score had a significant correlation only with fiber intake $(r=0.31 \mathrm{p}=0.0002)$ but not with fat intake $(r=0.07 \mathrm{p}=0.40)$

Conclusion: Higher dietary fiber intake is associated with lower disease activity and better quality of life in patients with IBD. Fat consumption does not seem to influence the disease activity and patients' quality of life. Based on these results it could be suggested that the amount of fiber consumption by IBD patients may play an important role in the disease course.

Disclosure of Interest: All authors have declared no conflicts of interest.
P0980 PROGRESSION IN CROHN'S DISEASE BEHAVIOUR IN A PROSPECTIVE EUROPEAN POPULATION-BASED INCEPTION COHORT - THE ECCO-EPICOM COHORT

J. Burisch $^{1}$, A. Goldis ${ }^{2}$, F. Magro ${ }^{3}$, S. Turcan ${ }^{4}$, R. D’Inca ${ }^{5}$, S. Čuković-Čavka ${ }^{6}$,

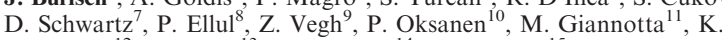
R. Nielsen ${ }^{12}$, N. Arebi ${ }^{13}$, R. Salupere ${ }^{14}$, M. Fumery ${ }^{15}$, J.F. Dahlerup ${ }^{16}$, D. Christodoulou ${ }^{17}$, L. Kievit ${ }^{18}$, D. Duricova ${ }^{19}$, N. Pedersen ${ }^{20}$, D. Valpiani ${ }^{21}$, I. Kaimakliotis ${ }^{22}$, V. Hernández ${ }^{23}$, L. Kupcinskas ${ }^{24}$, J. Halfvarson ${ }^{25}$, E. Langholz ${ }^{26}$, P.L. Lakatos ${ }^{9}$, P. Munkholm ${ }^{27}$

${ }^{1}$ Department Of Gastroenterology, North Zealand University Hospital,

Frederikssund/Denmark

${ }^{2}$ Dept. Of Gastroenterology, County Hospital Timisoara Gastroenterology,

Timisoara/Romania

${ }^{3}$ Centro Hospitalar São João, Amadora/Portugal

${ }^{4}$ Gastroenterology, The State University of Medicine and Pharmacy

"N.Testemitanu», Chisinau/Moldova

${ }^{5}$ Department Of Surgery, Oncology And Gastroenterology, Azienda Ospedaliera di

Padova, Padova/Italy

${ }^{6}$ Division Of Gastroenterology And Hepatology, University Hospital Center

Zagreb, University of Zagreb School of Medicine, Zagreb/Croatia

${ }^{7}$ Soroka Hospital, Beer ShevalIsrael

${ }^{8}$ Medicine, Mater Dei Hospital, Msida/Malta

${ }^{9} 1$ st Department Of Medicine, Semmelweis University Faculty of Medicine 1st Dept. of Medicine - 1st Department of Medicine, Semmel, Budapest/Hungary

${ }^{10}$ Department Of Gastroenterology And Alimentary Tract Surgery, Tampere University Hospital, Tampere/Finland

${ }^{11}$ Gastroenterology Department, OU Careggi Regional Referral Center for Inflammatory Bowel Disease, Florence/Italy

${ }^{12}$ Medical Department, The National Hospital of the Faroe Islands, Torshavn Faroer Islands

${ }^{13}$ St. Mark's Hospital, Imperial College London, London/United Kingdom ${ }^{14}$ Department Of Gastroenterology, Tartu University Hospital, Tartu/Estonia ${ }^{15}$ Gastroenterology Unit, Epimad Registry, CHU Amiens Sud, Avenue LaennecSalouel, Amiens University Hospital, Amiens/France

${ }^{16}$ Department Of Hepatology And Gastroenterology, Aarhus University Hospital, Aarhus/Denmark

${ }^{17} 1$ st Division Of Internal Medicine And Hepato-gastroenterology Unit, University Hospital, Ioannina/Greece

${ }^{18}$ Department Of Medicine, Herning Central Hospital, Herning/Denmark

${ }^{19}$ IBD Clinical And Research Center, ISCARE I.V.F. a.s., Prague/Czech Republic ${ }^{20}$ Gastroenterology Department, Slagelse Hospital, Slagelse/Denmark

${ }^{21}$ Department Of Gastroenterology And Digestive Endoscopy, Morgagni Hospital, Forli/Italy

${ }_{22}^{22}$ American Gastroenterology Center, Nicosia/Cyprus

${ }^{23}$ Instituto de Investigación Biomédica, Xerencia de Gestión Integrada de Vigo,

Vigo/Spain

${ }^{24}$ Lithuanian University of Health Sciences Inst. for Digestive Research, Kaunas/ Lithuania

${ }^{25}$ Dept. Of Gastroenterology, Faculty Of Medicine And Health, Örebro University, Örebro/Sweden

${ }^{26}$ Herlev University Hospital, Herlev/Denmark

${ }^{27}$ Gastroenterology, Hepatology, University of Copenhagen, North Zealand Hospital - Gastroenterology, Hepatology, University of Copen, Frederikssund Denmark

Contact E-mail Address: burisch@gmail.com

Introduction: Crohn's disease (CD) is a progressive disease that over time can lead to the development of complications such as strictures or internal penetrating disease that will ultimately lead to surgery. Only few population-based studies from the biological era and widespread use of immunodulators have investigated the change in disease behaviour and subsequent risk of surgery in CD.

Aims \& Methods: The EpiCom-cohort is a population-based cohort of unselected patients with inflammatory bowel disease diagnosed in 2010 in Eastern and Western European centres. Patients were followed prospectively for five years and clinical data were captured throughout the follow-up period. Disease behaviour was defined according the Montreal classification as B1: non-stricturing, non-penetrating, B2: stricturing; B3: penetrating based on endoscopy, cross-sectional imaging or surgery. The risk of progressing from $\mathrm{B} 1$ to $\mathrm{B} 2 / \mathrm{B} 3$ as well as the risk of surgical resection was analysed by Cox regression analyses using the proportional hazard assumption including multiple covariates (age, gender, disease location, diagnostic delay, smoking status, change in behaviour, geographic region and treatment with biologics within 6 months from diagnosis).

Results: A total of 488 incident CD patients were included in the study, of which $347(71 \%)$ had B1. A total of $141(29 \%)$ patients had complicated CD at diagnosis. After 5 years' follow-up, this number increased to $190(39 \%)$ (Table 1). Of patients diagnosed with $\mathrm{B} 1,35(10 \%)$ progressed to $\mathrm{B} 2$ while $14(4 \%)$ progressed to B3 after a median of 21 months (range: 0-62). The proportion of B1 patients changing behaviour was highest during the $1^{\text {st }}$ year of disease $(5 \%)$ but stable during the remaining follow-up period (approx. 2\%/year). Colonic location (L2 vs L4 HR: 0.3 CI95\%: 0.1-0.8), extra-intestinal manifestations at diagnosis (HR: 0.2 CI95\%: $0.1-0.8$ ), and the need for early biologics (HR: 2.5 CI95\%: $1.2-5.1$ ) were associated with progression in behaviour. During follow-up, a total of 107 $(22 \%)$ patients had a resection. Of patients with $\mathrm{B} 1$ as initial behaviour a total of $37(11 \%)$ patients had a resection. A change in behaviour from B1 to B2/B3 (HR 6.8 CI95\%: 3.0-15.6) and early biologics (HR 0.5 CI95\%: $0.2-0.5$ ) was 
associated with the risk for resection. No difference in the results was found between Eastern and Western European patients.

Table 1: Disease behaviour in Crohn's disease at diagnosis and follow-up

\begin{tabular}{lllll}
\hline & \multicolumn{3}{c}{ At follow-up } & \\
\cline { 2 - 4 } & B1 & B2 & B3 & $\begin{array}{l}\text { Total } \\
\text { (diagnosis) }\end{array}$ \\
\hline $\begin{array}{l}\text { Bt diagnosis } \\
\text { non-penetrating }\end{array}$ & $298(61 \%)$ & $35(7 \%)$ & $14(3 \%)$ & $347(71 \%)$ \\
$\begin{array}{l}\text { B2, strictuing } \\
\text { B3, penetrating } \\
\text { Total (follow-up) }\end{array}$ & - & $89(18 \%)$ & $11(3 \%)$ & $100(21 \%)$ \\
\hline
\end{tabular}

Conclusion: In this European population-based inception cohort of unselected CD patients $14 \%$ of patients with B1 progressed to B2 or B3 after five years of follow-up. The risk of surgery was increased in patients with B1 who progressed to B2/B3. No clinical predictors for progression in behaviour including smoking and treatment with biological therapy could be identified.

Disclosure of Interest: All authors have declared no conflicts of interest.

P0981 CHANGES IN GUT MICROBIOTA COMPOSITION CORRELATE WITH SHORT TERM CHANGES IN FACEAL CALPROTECTIN AND CRP IN PATIENTS WITH CROHN'S DISEASE

D. Chan $^{1}$, D. Kumar ${ }^{1}$, M. Mendall ${ }^{2}$

${ }^{1}$ Department Of Gastroenterology And Colorectal Surgery, St. George's Hospital, London/United Kingdom

${ }^{2}$ Deparment Of Gastroenterology, Croydon University Hospitall, Croydon/United Kingdom

Contact E-mail Address: derekchan65@hotmail.com

Introduction: Introduction: Faecal microbiota are believed to play an important role in the pathogenesis of Crohn's disease (CD). It is not known for certain whether gut microbiota composition (GMC) changes in response to inflammation or whether inflammation in the gut wall is a response to changes in GMC. Dynamic changes in bowel wall inflammation and short term changes in GMC have not previously been studied, but would give a clue as to the aetiological importance of GMC.

Aims \& Methods: We aimed to assess the correlation between short changes in GMC and a faecal marker of gut inflammation faecal calprotectin (FC) and CRP a marker of systemic inflammation.

We conducted a blinded sham controlled cross-over study of 32 subjects assigned to IgG4 guided exclusion diets. Each subject was randomised to one month of true or sham diet followed by a one month washout period followed by a further cross-over trial diet. Stool and blood specimens were obtained at time 0,1 month (completion of first trial diet), 2 months (completion of washout) and 3 months (end of cross-over trial diet). Taxonomic profiling was performed using 16s RNA next generation sequencing. Copy counts were made for firmicutes and bacteroidetes as a phylum; Roseburia and Bifidobacterium as a genus and Faecalibacterium prausnitzii as a species. Shannon diversity was calculated.

Results: 26 people fully completed the study. For each participant this provided six timepoints for comparison: timepoint $1-2,2-3,3-4,1-3,1-4$ and $2-4$. The relative abundance of bacteria groups were calculated. Kendall Tau correlation coefficient was used to assess correlation between change in FC and CRP to change in relative abundance of various bacteria. We found significant negative correlation with change in FC and CRP with Bifidobacterium genus and change in CRP with Roseburia genus. There were poor correlation and inconsistent changes in other species and firmicutes and bacteroidetes and Shannon diversity.
Conclusion: Bifidobacterium relative abundance correlate closely with short term changes in FC suggesting a possible causal association. Other genera and species had poor and inconsistent correlations with short term changes in FC suggesting that they may not be causally related to short term changes in gut inflammatory activity.

Disclosure of Interest: All authors have declared no conflicts of interest.

P0982 COLORECTAL CANCER IN INFLAMMATORY BOWEL DISEASE: RISK FACTORS IN A PROSPECTIVE MULTICENTER NESTED CASE-CONTROL IG-IBD STUDY

L. Biancone ${ }^{1}$, A. Armuzzi ${ }^{2}$, M.L. Scribano ${ }^{3}$, R. D'Inca ${ }^{4}$, C. Papi ${ }^{3}$, L. Spina ${ }^{5}$,

L. Guidi ${ }^{6}$, F. Moccisro ${ }^{7}$, P. Alvisi ${ }^{8}$, A. Ruffa ${ }^{9}$, B. Neri ${ }^{9}$, W. Fries ${ }^{10}$, G. Riegler ${ }^{11}$ M. Daperno ${ }^{12}$, F. Castiglione ${ }^{13}$, S. Renna ${ }^{14}$, S. Festa ${ }^{15}$, E. Calabrese ${ }^{9}$,

G. Meucci ${ }^{16}$, F. Rogai ${ }^{17}$, S. Ardizzone ${ }^{18}$, A. Rossi ${ }^{1}$, A. Orlando ${ }^{19}$, F. Pallone ${ }^{1}$ ${ }^{1}$ Systems Medicine, University of Rome "Tor Vergata", GI Unit, Roma/Italy

${ }^{2}$ Presidio Columbus, Fondazione Policlinico Gemelli Università Cattolica, Rome Italy

${ }^{3}$ GI Unit, AO S. Camillo Forlanini, Rome, Rome/Italy

${ }^{4} \mathrm{DDr}$., division of gastroenterology, Padova/Italy

${ }^{5}$ GI Unit, Università S. Donato, Milano, Mlan/Italy

${ }^{6}$ Gastroenterology \& Internal Medicine-celiac Cisease Unit, A.gemelli Foundation, Catholic University of Sacred Heart, Rome/Italy

${ }^{7}$ GI Unit, ARNAS Civico-DI Cristina Benfratelli, Palermo, Palermo/Italy

${ }^{8}$ GI Unit, Ospedale Maggiore, Bologna, Bologna/Italy

${ }^{9}$ Systems Medicine, Università Tor Vergata Internal Medicine, Roma/Italy

${ }^{10}$ GI Unit, Università di Messina, Messina, Messina/Italy

${ }^{11}$ GI Unit, SUN, Seconda Università, Napoli, Naples/Italy

${ }^{12} 12$ GI Unit, Ospedale Mauriziano, Torino, Turin/Italy

${ }^{13}$ GI Unit, Università Federico II, Napoli, Naples/Italy

${ }^{14} 14$ GI Unit, Ospedale Cervello, Palermo/Italy

${ }^{15}$ GI Unit, AO S.Filippo Neri, Rome, Rome/Italy

${ }^{16}$ GI Unit, Ospedale S. Giuseppe, Milano, Mlan/Italy

${ }^{17}$ GI Unit, AOU Careggi, Firenze, Florence/Italy

${ }^{18}$ GI Unit, Ospedale Fatebenefratelli, Milano, Mlan/Italy

${ }^{19}$ GI Unit, Ospedale Cervello, Palermo/Italy

Contact E-mail Address: biancone $@$ med.uniroma2.it

Introduction: Risk factors for colorectal cancer (CRC) in Inflammatory Bowel Disease (IBD) are still debated (1).

Aims \& Methods: In a prospective multicenter, nested case-control IG-IBD study at 4 years, we aimed to assess the frequency and risk factors for incident CRC in IBD. The role of IBD phenotype vs thiopurines (IS) and/or anti-TNFs use as risk factors for CRC was also evaluated. From Jan. 2012 to March 2017, all incident cases of CRC in IBD pts referring to 16 IG-IBD Units were recorded. Each IBD pt with CRC (IBD-CRC) was matched with 2 IBD pts with no cancer (IBD-C) for: IBD type (Crohn's Disease, CD vs Ulcerative Colitis, UC), gender, age $( \pm 5$ yrs). Cases of CRC derive from a larger cohort of IBD pts referring to the same Units, with incidence of any cancer separately reported at 4 years (follow up at 3 yrs reported for cancer overall)(2). Statistical analysis: data expressed as median (range), Wilcoxon test, Chi-squared test, Fisher exact test; multivariate logistic regression analysis.

Results: Incident cases of CRC occurred in 66 IBD pts: 41 UC (UC-CRC), 25 CD (CD-CRC). IBD group therefore included 198 pts (66 IBD-CRC, 132 IBD-C), UC group included 123 pts (41 UC-CRC, 82 UC-C) and CD group included 75 $\mathrm{CD}$ pts $(25 \mathrm{CD}-\mathrm{CRC}, 50 \mathrm{CD}-\mathrm{C})$. The frequency of incident $\mathrm{CRC}$ was higher in the tested UC versus CD population $(62.1 \%$ vs $37.9 \% ; \mathrm{p}=0.009)$. Gender was equally distributed in IBD groups (UC: $14 \mathrm{~F}[34 \%]$; CD:12 F [48\%]; $=1$ ). The median age was comparable between IBD-CRC and IBD-C (UC-CRC vs UC-C 62 [37-86] vs 59 [35-86]; CD-CRC vs CD-C:51 [23-76] vs 55 [22-76]; $\mathrm{p}=1)$. UC duration was longer in pts with vs without CRC $(20[0-37]$ vs $10[0-35] ;<10 \mathrm{yrs}$ :

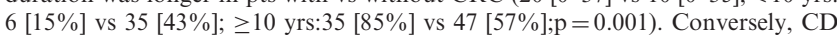
duration was comparable between pts with vs without CRC (20 [1-47] vs 13 [0$35] ;<10$ yrs:6 [24\%] vs $15[30 \%] ; \geq 10$ yrs:19 [76\%] vs $35[70 \%] ; \mathrm{p}=0.58)$. IBD

Abstract: P0981

\begin{tabular}{|c|c|c|c|c|c|c|c|c|c|c|c|c|c|}
\hline & \multirow[b]{2}{*}{ Timepoint } & \multicolumn{2}{|c|}{ Firmicutes } & \multicolumn{2}{|c|}{ Bacteroidetes } & \multicolumn{2}{|c|}{ Roseburia } & \multicolumn{2}{|c|}{ Bifidobacterium } & \multicolumn{2}{|c|}{ Faecalibacterium prausnitzii } & \multicolumn{2}{|c|}{ Shannon Diversity } \\
\hline & & $\begin{array}{l}\text { Kendal } \\
\text { Tau }\end{array}$ & $\mathrm{p}$ value & $\begin{array}{l}\text { Kendal } \\
\text { Tau }\end{array}$ & $\mathrm{p}$ value & $\begin{array}{l}\text { Kendal } \\
\text { Tau }\end{array}$ & $\mathrm{p}$ value & $\begin{array}{l}\text { Kendal } \\
\text { Tau }\end{array}$ & $\mathrm{p}$ value & $\begin{array}{l}\text { Kendal } \\
\text { Tau }\end{array}$ & $\mathrm{p}$ value & $\begin{array}{l}\text { Kendal } \\
\text { Tau }\end{array}$ & $\mathrm{p}$ value \\
\hline FCP & $1-2$ & 0.352 & 0.015 & -0.211 & 0.147 & 0.056 & 0.731 & -0.265 & 0.068 & -0.179 & 0.233 & 0.131 & 0.374 \\
\hline FCP & $2-3$ & -0.073 & 0.624 & -0.173 & 0.234 & 0.043 & 0.785 & -0.320 & 0.027 & -0.239 & 0.107 & -0.220 & 0.129 \\
\hline FCP & $3-4$ & -0.067 & 0.657 & 0.227 & 0.117 & -0.027 & 0.870 & -0.147 & 0.315 & 0.190 & 0.214 & 0.114 & 0.441 \\
\hline FCP & $1-3$ & 0.048 & 0.739 & -0.123 & 0.381 & -0.031 & 0.835 & -0.009 & 0.967 & 0.014 & 0.934 & -0.048 & 0.739 \\
\hline FCP & $1-4$ & 0.048 & 0.739 & 0.031 & 0.835 & -0.054 & 0.707 & -0.453 & 0.001 & 0.043 & 0.770 & -0.060 & 0.677 \\
\hline FCP & $2-4$ & 0.062 & 0.675 & 0.006 & 0.982 & 0.203 & 0.152 & -0.431 & 0.002 & -0.080 & 0.582 & -0.043 & 0.774 \\
\hline CRP & $1-2$ & -0.117 & 0.427 & 0.040 & 0.797 & -0.130 & 0.398 & 0.183 & 0.207 & -0.083 & 0.585 & 0.143 & 0.327 \\
\hline CRP & $2-3$ & 0.114 & 0.440 & 0.207 & 0.154 & 0.004 & 1 & -0.124 & 0.400 & -0.040 & 0.804 & 0.134 & 0.362 \\
\hline CRP & $3-4$ & -0.201 & 0.168 & 0.020 & 0.907 & -0.490 & 0.001 & -0.262 & 0.072 & 0.028 & 0.874 & -0.111 & 0.454 \\
\hline CRP & $1-3$ & 0.242 & 0.080 & -0.054 & 0.707 & -0.003 & 1.000 & 0.185 & 0.182 & 0.151 & 0.278 & 0.017 & 0.917 \\
\hline CRP & $1-4$ & 0.111 & 0.428 & 0.091 & 0.518 & 0.009 & 0.967 & -0.060 & 0.677 & 0.060 & 0.677 & 0.031 & 0.835 \\
\hline CRP & $2-4$ & -0.103 & 0.465 & 0.126 & 0.369 & -0.158 & 0.260 & 0.080 & 0.573 & 0.089 & 0.531 & 0.215 & 0.122 \\
\hline
\end{tabular}


pts with CRC were younger at diagnosis of IBD than their IBD-C (UC-CRC vs UC-C: median yrs:39 [15-76] vs $46[20-81] ;<40$ yrs, $\mathrm{n}=: 21[51 \%]$ vs 27 $[33 \%] ; \geq 40$ yrs: $20[49 \%]$ vs $55[67 \%] ; \mathrm{p}=0.04 ; \mathrm{CD}-\mathrm{CRC}$ vs CD-C: median yrs $27[6-67]$ vs $37[10-67] ;<40 \mathrm{yrs}, \mathrm{n}=19$ [76\%] vs $26[52 \%] ; \geq 40 \mathrm{yrs:} 6[24 \%]$ vs 24 $[48 \%] ; \mathrm{p}=0.04)$. The median age at diagnosis of CRC was comparable in UC vs $\mathrm{CD}(60$ [35-83] vs 51 [20-76]; $\mathrm{p}=0.19)$. The frequency of CRC was comparable between UC pts using or not IS and/or anti-TNFs (CRC-UC vs CRC-UC: IS monotherapy $6[15 \%]$ vs 17 [21\%]; Anti-TNFs monotherapy: no CRC; Combotherapy: $5[12 \%]$ vs $6[7 \%]$; no IS/no anti-TNFs: $11[27 \%]$ vs $23[28 \%]$; $\mathrm{p}=0.88$ ). No differences were also observed between CD pts treated or not with IS and/or anti-TNFs (CRC-CD vs CRC-C: IS monotherapy: 4 [16\%] vs 9 [18\%]; Anti-TNFs monotherapy:2 [8\%] vs $3[6 \%]$; Combotherapy: $10[40 \%]$ vs $23[46 \%]$; no IS/no anti-TNFs: 16 [64\%] vs 35 [70\%]; $\mathrm{p}=0.59$ ). CD pts with $\mathrm{CRC}$ showed a higher frequency of pattern B1 (B1 vs B2 vs B3 (n [\%]): 14 [56\%] vs $3[12 \%]$ vs 8 $[32 \%] ; \mathrm{p}=0.019)$. Risk factors for CRC considered In multivariate analysis included: age $(<40$ vs $\geq 40 \mathrm{yrs})$, IBD duration $(<10$ vs $\geq 10$ yrs $)$, smoking habits $(\mathrm{Yes} / \mathrm{No} ; \mathrm{Y} / \mathrm{N})$, ISS-anti-TNFs $(\mathrm{Y} / \mathrm{N})$, IBD-related surgery, UC extent (extensive vs distal; subtotal vs distal), CD pattern (B3 vs B1, B2 vs B1), perianal $\mathrm{CD}$. In UC, the only significant risk factor was UC duration (OR [95\% CI]: OR $3.33[1.44-9.1 \mathrm{I}]$, as the other risk factors were not significant: OR 0.94 [0.36$2.98] ; 1.28$ [0.48-3.04];0.96 [0.36-3.08];1.78 [0.60-4.66];1.36 [0.66-2.89];0.38 [0.08$1.23]$, respectively). In $\mathrm{CD}$, perianal disease was the only significant risk factor for CRC (OR 3.11 [1.16-8.33I, as no other significant risk factors were identified (OR 2.21 [0.70-9.87]; 1.66 [0.62-4.96]; 0.56 [0.19-1.41]; 0.99 [0.41-2.52], 0.54 [0.2-1.43], 0.83 [0.26-2.55], 0.23 [0.04-0.83], respectively)

Conclusion: In a prospective, multicenter, nested-case control IG-IBD study, incident cases of $\mathrm{CRC}$ were more frequent in $\mathrm{UC}$ than in $\mathrm{CD}$. In our cohort, $\mathrm{UC}$ duration and perianal CD, but not immunomodulators use, were identified as risk factors for incident cases of CRC in IBD

Disclosure of Interest: L. Biancone: The study was not supported by any grant nor funded and any of the below reported disclosures are related to the study. LB. Lecture fees or Advisory Board: Zambon, MS\&D, Takeda, Abbvie, Sofar, Ferring, Wassermann;

A. Armuzzi: The study was not supported by grants nor funded.Disclosures are not related to the study: Consulting, lecture fees:Abbvie, Astra, Biogen, Celltrion, Ferring, Hospira, Janssen, Lilly, MSD, Mundipharma, Pfizer, Samsung, Sofar, Takeda, Zambon.Research grant: MSD

M.L. Scribano: The study was not supported by grants nor funded. The reported disclosures are not related to the study:. Lecture fees: Zambon; Advisory Board: Abbvie, Biogen Idec, Mundipharma, Pfizer, Takeda.

R. D'inca: The study was not supported by grants nor funded. The reported disclosures are not related to the study: Speaker and advisory board member: Abbvie, MSD, Zambon, Mundipharma, Hospira, Biogen, Takeda, Holstone

C. Papi: The study was not supported by grants nor funded. The reported disclosures are not related to the study: Consultant or educational grants: Takeda, Abbvie, MSD Chiesi. Sofar, alfa Wassermann

L. Guidi: The study was not supported by grants nor funded. All disclosures are not related to the study: lecture fees:Abbvie, MSD, Takeda, Zambon, consultant: AbbVie, MSD Mundipharma, support: for research not related to this study:AbbVie, MSD

W. Fries: The study was not supported by grants nor funded. The reported disclosures are not related to the study: Advisory boards: Abbvie, Mundipharma, speaker fees: Takeda, Zambon, educational grant: MSD

G. Riegler: The study was not supported by grants nor funded. The reported disclosures are not related to the study: Lecture fees: Zambon, Abbvie.

M. Daperno: The study was not supported by grants nor funded. The reported disclosures are not related to the study: Lectures, board/congress fees: Abbvie, Pfizer, Takeda, Mundipharma, Janssen, MS\&D, SOFAR, Ferring, Chiesi F. Castiglione: The study was not supported by grants nor funded. The reported disclosures are not related to the study: Consultant to: MS\&D, Takeda, AbbVie; Lecture fees: Abbvie, MSD, Takeda, Chiesi; Unrestricted research grants: AbbVie, Sofar, MS\&D, Ferring.

E. Calabrese: The study was not supported by grants nor funded. The reported disclosures are not related to the study: lecture fees from Abbvie, Takeda, MSD G. Meucci: The study was not supported by grants nor funded. The reported disclosures are not related to the study: Lecture fees or board for: Chiesi, MS\&D, Mundipharma, Takeda, Zambon.

S. Ardizzone: The study was not supported by grants nor funded. These disclosures are not related to the study: Consultant to: Abbvie, Mundipharma, MS\&D, Takeda, Recordati, Zambon;Unconditioned grants:MS\&D, Abbvie, Takeda, Mundipharma, Ferring, Sofar, Zambon

A. Orlando: The study was not supported by grants nor funded. The reported disclosures are not related to the study: Lecture fees: Abbvie, Chiesi, MSD, Otsuka, Takeda, Sofar, Mundipharma: Consultant: Abbvie, MSD, Sofar, Takeda.

F. Pallone: The study was not supported by grants nor funded. The reported disclosures are not related to the study: lecture fees from Zambon, Takeda. All other authors have declared no conflicts of interest.

\section{References}

1. Beaugerie L.N Engl J Med 2015;372:1441;

2. Biancone L.JCC 2016;10(8):913
P0983 EPIDEMIOLOGY OF INFLAMMATORY BOWEL DISEASE IN SPAIN: INTERIM ANALYSIS OF THE NATIONWIDE EPIDEMIBD STUDY OF GETECCU

M. Chaparro ${ }^{1}$, J.L. Cabriada ${ }^{2}$, M.J. Casanova ${ }^{1}$, D. Ceballos ${ }^{3}$, M. Esteve ${ }^{4}$, H. Fernández Rosaenz ${ }^{5}$, M. Barreiro-De Acosta ${ }^{6}$, V. García-Sánchez ${ }^{7}$, D. Ginard ${ }^{8}$, F. Gomollon ${ }^{9}$, R. Lorente Poyatos ${ }^{10}$, P. Nos ${ }^{11}$, S. Riestra Menéndez $^{12}$, M. Rivero Tirado ${ }^{13}$, P. Robledo Andrés ${ }^{14}$, C. Rodríguez ${ }^{15}$ B. Sicilia Aladrén ${ }^{16}$, E.A. Torrella Cortes ${ }^{17}$, F. Rodríguez-Artalejo ${ }^{18}$, J P. Gisbert ${ }^{3}$, O. N Behalf Of The Epidemibd Group ${ }^{1}$

${ }^{1}$ Hospital Universitario de La Princesa, IIS-IP and CIBEREehd, Madrid/Spain

${ }^{2}$ Gastroenterology, Hospital de Galdakao-Usansolo, Galdakao/Spain

${ }^{3}$ Gastroenterology, Hospital Universitario Dr. Negrin, Las Palmas/Spain

${ }^{4}$ Gastroenterology, Hospital Universitario Mútua de Terrassa, Terrassa/Spain

${ }^{5}$ Gastroenterology, Hospital de San Pedro, Logroño/Spain

${ }^{6}$ Gastroenterology, University Hospital of Santiago, Santiago de Compostela/Spain ${ }^{7}$ Gastroenterology, Hospital Universitario Reina Sofía and IMIBIC, Córdobal Spain

${ }^{8}$ Gastroenterology, Hospital Son Espases, Palma de Mallorca/Spain

${ }^{9}$ Gastroenterology, Hospital Clínico Universitario Lozano Blesa, IIS Aragón and CIBERehd, Zaragoza/Spain

${ }^{10}$ Gastroenterology, Hospital General de Ciudad Real, Ciudad real/Spain

${ }^{11}$ Gastroenterology, Hospital Universitario y Politécnico de La Fe and CIBERehd, Valencia/Spain

${ }^{12}$ Gastroenterology, Hospital Universitario Central de Asturias, Gijón/Spain

${ }^{13}$ Gastroenterology, Hospital Universitario de Valdecilla, Santander/Spain

${ }^{14}$ Gastroenterology, Hospital San Pedro de Alcantara, Cáceres/Spain

${ }^{15}$ Gastroenterology, Complejo Hospitalario de Navarra, Pamplona/Spain

${ }^{16}$ Gastroenterology, Hospital Universitario de Burgos, Burgos/Spain

${ }^{17}$ Gastroenterology, Hospital General Universitario Morales Meseguer, Murcia/ Spain

${ }^{8}$ Preventive Medicine And Public Health, Universidad Autónoma de Madrid IdiPaz and CIBERESP, Madrid/Spain

Contact E-mail Address: mariachs2005@gmail.com

Aims \& Methods: Aims: Primary: to evaluate the incidence of inflammatory bowel disease (IBD) in Spain. Secondary: to describe the characteristics of patients at diagnosis; to evaluate the need for immunomodulators (IMM), biologics, surgery and hospital admissions in the first year of diagnosis and to assess the time from the onset of symptoms to the diagnosis of the disease.

Methods: Prospective and population-based nationwide study. Adult patients diagnosed with IBD $3 / 4$ Crohn's disease (CD), ulcerative colitis (UC) or indeterminate colitis (IC) $3 / 4$ during 2017 in the 17 Spanish regions are being included. Each case is also being followed-up for 12 months after diagnosis to describe changes in phenotype or location, to evaluate the requirement of IMM and biologics and to determine the need for hospitalization or surgery during the first year from the diagnosis. Data was captured in a web-based database (AEG-REDCap).

Results: An updated interim analysis of the incidence of IBD is planned 6 months after the beginning of the study (June 2017). Up to April $1^{\text {st }} 2017,557$ patients from 156 centres have been included: $53 \%$ males, mean age 40 years, $27 \%$ smokers. $51 \%$ had UC, $44 \%$ CD, and $5 \%$ IC. $13 \%$ of patients had a family history of IBD. The mean time to IBD diagnosis from symptoms onset was 3 months (range $0-198$ ). At the time of diagnosis, $10 \%$ of the patients had extraintestinal manifestations (the most frequent being rheumatologic manifestations, in $6 \%$ of the cases). In CD patients, $53 \%$ had ileal location, $20 \%$ colonic, $26 \%$ ileocolonic, $2.4 \%$ upper gastrointestinal tract involvement, and $13 \%$ perianal disease; $8 \%$ of patients had stenosing and $7 \%$ fistulising behaviour at the time of diagnosis. In UC patients, $30 \%$ had extensive colitis and $36 \%$ left-sided colitis at diagnosis. The therapeutic requirements were: $28 \%$ oral steroids, $13 \%$ intravenous steroids, $9 \%$ thiopurines, $0.9 \%$ methotrexate, $0.4 \%$ cyclosporin, and $3.2 \%$ anti-TNFs. Twelve patients $(2.2 \%)$ were operated $(5$ abdominal and 7 perianal surgery), and 133 patients $(24 \%)$ required hospital admission within the first 3 months following diagnosis.

Conclusion: In this large nationwide epidemiologic study we describe how the treatment requirement and the consumption of health resources was already high from the time of diagnosis as, indeed, a relevant proportion of IBD patients required surgery or hospital admission within the first 3 months after the diagnosis.

Disclosure of Interest: J.L. Cabriada: Jose Luis Cabriada served as consultant or received research funding to MSD, Takeda, Janssen, Otsuka Ph and Kern Ph. R. Lorente Poyatos: Speaker consultant: MSD, Abbvie and Takeda

P. Nos: Educational activities, research projects, scientific meetings and advisory boards sponsored by Abbvie, MSD, Tillots, Janssen, Otsuka, Kern, Pfizer, Gebro, Zambon and Takeda.

S. Riestra Menéndez: Research grants from MSD and Abbvie and payments for scientific collaborations from MSD, Abbvie, Takeda, shire, ferring, faes J.P. Gisbert: Speaker, consultant, advisory member for or research funding from MSD, Abbvie, Hospira, Pfizer, Kern Pharma, Biogen, Takeda, Janssen, Roche, Ferring, Faes Farma, Shire Ph., Dr. Falk Ph., Tillotts Ph., Chiesi, Casen Fleet, Gebro Ph., Otsuka Ph., Vifor Ph.

All other authors have declared no conflicts of interest. 
P0984 IMPACT OF ANORECTAL COMPLAINTS ON QUALITY OF LIFE IN PATIENTS WITH INFLAMMATORY BOWEL DISEASE - A SURVEY OF THE DUTCH NATIONAL CROHN'S AND COLITIS ORGANISATION

P. F. Vollebregt ${ }^{1}$, A. A. Van Bodegraven ${ }^{1}$, M.l. Markus-De Kwaadsteniet ${ }^{2}$, D. Van Der Horst ${ }^{2}$, R. J. Felt-Bersma ${ }^{1}$

${ }^{1}$ Gastroenterology And Hepatology, VU University Medical Centre Amsterdam, Amsterdam/Netherlands

${ }^{2}$ Dutch Crohn's and Colitis organisation, Woerden/Netherlands

Contact E-mail Address: p.vollebregt@vumc.nl

Introduction: Anorectal complaints occur in a considerable group of patients with IBD. There is a dearth of evidence relating to the impact of these complaints on IBD patients' lives. We aimed to survey the effect of anorectal complaints on quality of life in a large cohort of patients who engaged in the Dutch Crohn's and Colitis organisation (CCUVN)

Aims \& Methods: In October 2016, the CCUVN had a membership database of 10,047 patients. A comprehensive study questionnaire was sent out online by the CCUVN in January 2015 and October 2016 to a voluntary panel, which consisted of 1710 CCUVN patients. The panel is represented by patients who voluntary participate in online surveys with regard to disease related subjects. Inclusion criteria were age over 18 years old and a self-reported diagnosis of Crohn's disease (CD), ulcerative colitis (UC) or IBD-unclassified (IBD-U). The survey included the St. Marks incontinence score, perianal disease activity index, faecal incontinence quality of life (FI-QOL) questionnaire and the SF-36 questionnaire. Multiple imputation was used for covariates with missing data. Multivariate regression analysis was performed.

Results: A total of 1094 patients (64\%) responded the online survey. Mean age was 47 years (range 18-87), CD diagnosis was predominant (621 CD patients $(57 \%), 431$ UC patients $(39 \%)$ and 42 IBD-U patients $(4 \%))$ and diagnosis was established for a mean period of 13 years (interquartile range 3-19 years). Active perianal disease was present in $243 \mathrm{CD}$ patients $(39 \%)$ and perianal surgery (abscess- or fistula-related) was previously performed in $153(25 \%)$. Faecal incontinence ( $\geq 1$ episode per month) was reported in $305 \mathrm{CD}(58 \%), 230 \mathrm{UC}$ $(56 \%)$ and 20 IBD-U $(51 \%)$ patients. Weekly episodes occurred in $41 \mathrm{CD}(8 \%)$, 22 UC ( $5 \%$ ) and 3 IBD-U (8\%) patients (mean St. Marks incontinence score 14). FI-QOL scores were not different between the different diagnoses. Multivariate regression analysis (adjusted for gender, diagnosis and previously performed abdominal operations) showed a reduced total SF-36 score in patients with faecal incontinence $(\beta-8.57[-11.33 ;-5.81] ; p<0.0001)$ and active perianal disease $(\beta-4.13[-7.35 ;-0.91] ; p=0.01)$. A better score was reported in UC patients compared to CD patients ( $\beta 3.53[0.48-6.58] ; p=0.02)$. Previously performed perianal surgery was not associated with SF-36 score in the multivariate analysis.

Conclusion: Anorectal complaints have a substantial impact on the quality of life in patients with IBD, with a (usually unmet and) clear need to treat. More awareness for this highly distressing and most commonly cumbersome treatable disease manifestation is needed.

Disclosure of Interest: All authors have declared no conflicts of interest.

\section{P0985 CURRENT UNDERSTANDING OF POUCH MICROBIOTA IN HEALTH AND DISEASE; A SYSTEMATIC REVIEW}

J. Segal ${ }^{1}$, S. Oke ${ }^{1}$, G. Hold ${ }^{2}$, A. Hart ${ }^{3}$

${ }^{1}$ St Marks Hospital, Harrow/United Kingdom

${ }^{2}$ School Of Medicine, University of Aberdeen, Aberdeen/United Kingdom

${ }^{3}$ Gastroenterology, St Mark's Hospital and Academic Institue, London/United Kingdom

Contact E-mail Address: jonathansegal@doctors.org.uk

Introduction: The human gut microbiome is made up predominately of four major bacterial phyla, Firmicutes, Bacteroidetes, Proteobacteria and Actinobacteria. Changes or imbalance of these phyla is termed dysbiosis. Specifically, in inflammatory bowel disease (IBD), key changes have been identified such as a reduction in beneficial bacterial species including Faecalibacterium prausnitzii and increases in more pathogenic species including members of the Enterobacteriacae. Currently it is not understood if dysbiosis is the cause of, or the effect of, intestinal inflammation. It is difficult to chronologically assess the microbiota changes prior to developing IBD as currently we are unable to predict those individuals who will develop the disease. The pouch is a potential model to study pathogenesis of inflammation as $40 \%$ of those that develop pouchitis do so within 12 months. The relative short time from pouch formation to inflammation allows the longitudinal study of the microbiota which gives insight into potential microbiota patterns occurring both in disease and non-diseased states. Interestingly, inflammation within the pouch is rarely seen in patients who have this operation for Familial Adenomatous Polyposis (FAP), thus raising the possibility that pouchitis shares a similar pathogenesis to the inflammation that is seen in ulcerative colitis.

Aims \& Methods: 1. To understand changes in pouch microbota over time. 2. To understand pouch microbiota that is associated with pouch inflammation A computer assisted search of the on-line bibliographic databases MEDLINE and EMBASE was carried between 1966 and February 2016. Randomised controlled trials, cohort studies and observational studies were included. Inclusion criteria: Studies which reported microbiota analysis on either faecal samples or tissue from the ileo-pouch anal anastomosis. Studies that provided information on specific bacterial taxa. Exclusion criteria: Studies which did not report on patterns of individual bacterial taxa differences in the pouch. Studies on the microbiota of Crohn's disease or UC in isolation without any data on pouch patients. Studies with less than ten patients.

Results: The search strategy found 844 . There were a total of 27 papers included in the analysis. Microbiota in pouchitis: Bacteroidetes, Enterococcaceae, Lachnospiraceae, Faecalibacterium, Ruminococcaceae, Streptococci Alcaligenaceae and Bifidobacterium were reduced in patients with pouchitis. Whereas Enterobacteriaceae, including E. coli Fusobacterium and Clostridia were increased in patients with pouchitis. One study highlighted bacteria that were exclusively found in pouchitis which included Leptospira, Pseudoalteromonas, Desulfosporosinus, Microcystis, Methylobacter. Chronic pouchitis was associated with a significant increase in Staphylocococus aureus and it has been suggested that this may be a responsible pathogen for chronic pouchitis. Furthermore, Enterococcus, F. prausnitzii, Lachnospiraceae and Insertae Sedis $X I V$ and have been shown to be significantly reduced in chronic pouchitis patients. These differences were largely due to a decrease in sequences from members of the genera Ruminococcus, Dorea, Clostridium, and Eubacterium.

Conclusion: The microbiota undoubtedly plays an important role in both the inflamed and the healthy pouch. However, a direct causal relationship has not yet been established between individual microbiota changes and inflammation. There are many studies that highlight changes in bacterial composition, but studies are limited by heterogeneity of and in particular, analysis techniques and sampling strategies. Studies used a variety of methods to define microbial diversity which can be broadly split into culture vs culture-independent approaches. Culture-based studies are likely to have a bias towards culturing more aerobically friendly microbes than exist in a true pouch environment, thus over-representing aerobic bacteria whilst possibly under-representing anaerobic bacteria. The use of $16 \mathrm{~S}$ rRNA analysis methods will negate this effect and represents the future in accurately determining the microbiota.

Disclosure of Interest: All authors have declared no conflicts of interest.

\section{P0986 LIVE-VACCINES AND BREASTFEEDING IN NEWBORN EXPOSED IN UTERO TO ANTI TNF: A MULTICENTER FRENCH STUDY IN INFLAMMATORY BOWEL DISEASE}

S. Bendaoud ${ }^{1}$, S. Nahon ${ }^{2}$, L. Beaugerie ${ }^{3}$, J. Gornet ${ }^{4}$, B. Pariente ${ }^{5}$, L. Peyrin-

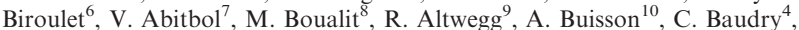
L. Plastaras ${ }^{11}$, F. Heluwaert ${ }^{12}$, M. Amil ${ }^{13}$, M. Antoni ${ }^{14}$, K. Bideau ${ }^{15}$, V. Quentin ${ }^{16}$, G. Pineton De Chambrun ${ }^{17}$, R. Faroux ${ }^{18}$, F. Skinazi ${ }^{19}$, C. Locher $^{20}$, E. Cuillerier ${ }^{21}$, A. Boureille ${ }^{22}$, A. Aubourg ${ }^{23}$, I. Rosa ${ }^{24}$, M. Simon ${ }^{1}$ ${ }^{1}$ Institut Mutualiste Montsouris, Paris/France

${ }^{2}$ Hopial Montfermeuil, ParisMontfermeil/France

${ }^{3}$ Hopital Saint Antoine, Paris/France

${ }^{4}$ Hopital Saint Louis, paris/France

${ }^{5}$ Hopital Claude Huriez, lille/France

${ }^{6} \mathrm{CHU}$ Nancy, Nancy/France

Abstract: P0985. Table 1: Evolution of pouch microbiota over-time

\begin{tabular}{|c|c|c|c|c|c|}
\hline Study & Year & Analysis method & UC or FAP & Comparator & Key findings in UC \\
\hline \multicolumn{6}{|c|}{ Two-three months } \\
\hline Almeida & 2008 & Mucus- culture & $\mathrm{UC}$ & $\begin{array}{l}\text { Two vs eight months after } \\
\text { ileostomy closure }\end{array}$ & Enterobacter Klebsiella \\
\hline Kohyama & 2009 & $\begin{array}{l}\text { Faeces-PCR Terminal restriction } \\
\text { fragment length polymorphism } \\
\text { amplification }\end{array}$ & $\mathrm{UC}$ & Uc vs healthy controls & C. coccides \\
\hline Hinata & 2012 & Faeces-16S RNA & $\mathrm{UC}$ & UC vs healthy & $\begin{array}{l}\text { C. coccides group C. leptumsubgroup } \\
\text { B. fragilis group Atopobium }\end{array}$ \\
\hline \multicolumn{6}{|c|}{ Six-eight months } \\
\hline Almeida & 2008 & Mucus- culture & $\mathrm{UC}$ & Two vs eight months post ileostomy & $\begin{array}{l}\text { Most prevalent: E coliVeillonella } \\
\text { Enterobacter, Klebsiella Peptococcus }\end{array}$ \\
\hline Bednarz & 2015 & Swab-culture & $\mathrm{UC}$ & UC longitudinal & Enterobacteriaceae most common \\
\hline \multicolumn{6}{|l|}{1 year } \\
\hline Luukkonen & 1988 & Faeces-culture & $\mathrm{UC}$ & Kock ileostomy and ileostomy & Transformation to a "colonic" microbiota \\
\hline Hinata & 2012 & Faeces-PCR & $\mathrm{UC}$ & Healthy volunteers & Enterococcus Lactobacillus \\
\hline
\end{tabular}


${ }^{7}$ Hopital Cochin, paris/France

${ }^{8}$ Centre hospitalier Lille, Lille/France

${ }^{9}$ Centre hospitalier Montpellier, montpellier/France

${ }^{10}$ Dept. Of Gastroenterology, CHU Estaing Clermont-Ferrand, Clermont-ferrand/ France

${ }^{11}$ Hopital Colmar, colmar/France

${ }^{12}$ Centre Hospitalier Annecy Gennois, Epagny Metz-Tessy/France

${ }^{13}$ Centre Hospitalier-Site la Roche/Yon, La Roche-sur-Yon/France

${ }^{14}$ Centre Hospitalier Orange, Orange/France

${ }^{15}$ Centre Hospitalier de Cornouaille, Quimper/France

${ }^{16}$ Centre Hospitalier de Saint Brieuc, Saint Brieuc/France

${ }^{17}$ Centre hospitalier de Montpellier, Montpellier/France

${ }^{18}$ Centre Hospitalier la Vendée, La Roche-sur-Yon/France

${ }^{19}$ Hopital Delafontaine, saint Denis/France

${ }^{20}$ Centre hospitalier de meaux, Meaux/France

${ }^{21}$ Hopital de Dreux, Dreux/France

${ }^{22} \mathrm{CHU}$ Nantes, Nantes/France

${ }^{23} \mathrm{CHRU}$ Tours, Tours/France

${ }^{24}$ Centre hospitalier Intercommunal de Créteil, creteil/France

Contact E-mail Address: sihem_bend@yahoo.fr

Introduction: Anti TNF cross placenta during pregnancy and are detectable in the newborn in the first 6 months at least. Along this, European consensus recommends to avoid live vaccines within 6 months of live in newborn exposed in utero to anti TNF treatments. Moreover, anti-TNF treatments are of low risk for breastfed infants but data are scarce. The aim of our study was to evaluate the rate and tolerance 1) of live-vaccines before and after 6 months of life in newborn exposed in utero to anti TNF and 2) of breastfeeding under anti TNF.

Aims \& Methods: We performed an observational and retrospective study in 22 French departments of gastroenterology from February 2016 to April 2017. Included patients were inflammatory bowel disease (IBD) women, pregnant under anti TNF, giving birth to alive newborn and agree to answer a questionnaire concerning 1) live-vaccines (BCG, rotavirus, MMR) in their child during first year, 2) breastfeeding and complication and 3) information given during pregnancy.

Results: 124 pregnant women treated with antiTNF were included. The mean age of women was 31 year (IQR 5). 96 (77\%) women received anti TNF for Crohn's disease and $28(23 \%)$ for ulcerative colitis or undetermined colitis. AntiTNF used were Infliximab in $76(61 \%)$ patients, Adalimumab in $46(37 \%)$ patients, Golimumab in one patient $(1 \%)$ and Certolizumab in one $(1 \%)$ patient. Thiopurine was associated in $29(23 \%)$ patients. $31(25 \%)$ patients experienced flare of IBD during pregnancy, $18(15 \%)$ women were treated with steroids. AntiTNF was discontinued before 26 gestational weeks in $66(53 \%)$ women and resumed after delivery in $112(90 \%)$ patients. 55 women $(45 \%)$ breastfed their infant and no complication was noted. Among 69 women who did not perform lactation, $42(63 \%)$ did not for personal choice and and $25(37 \%)$ because not recommended by the gastroenterologist. Concerning vaccination questionnaires, 96 responses were obtained. BCG was performed in 29 children $(30 \%)$ and was administered before 6 months in 15 children $(14 \%)$. One local abscess was reported with favorable evolution. Rotavirus vaccination was performed in 7 children $(7 \%)$ and before 6 months in $5(\%)$ cases. One case of fever was reported. MMR vaccination (Measles, Mumps and Rubella) was performed in 62 children $(65 \%)$; before 6 months in 5 cases. Information concerning fetal exposure to antiTNF and vaccination recommendation was given to $111(91 \%)$ IBD women during pregnancy, by at least the gastroenterologist in $89 \%$ of cases, the obstetrician in $25 \%$ of cases and the pediatrician in $16 \%$ of cases. Information was at least oral in $105(86 \%)$ cases and written in $39(32 \%)$ cases. Conclusion: Half of women breastfed their child with no reported complication. BCG was administered in $30 \%$ of the newborn and performed before 6 months in $50 \%$ of cases, with only one case of local abscess. Rotavirus vaccination is rare but often performed before 6 months. Information to pregnant IBD women is only given by gastroenterologist in the majority of cases. Information in maternity by obstetrician and pediatrician should be improved.

Disclosure of Interest: All authors have declared no conflicts of interest.

\section{P0987 OUTCOME OF ENDOSCOPIC ALLY RESECTED}

\section{DYSPLASIC LESIONS IN ULCERATIVE COLITIS}

H. Saoula, A. F. Boutaleb, H. Mahiou, M. Aissaoui, Y. Zmiri, N. Hamidouche, R. Osmane, Y. Aissat, A. Mitiche, L. Gamar, K. Belhocine, N. Bounab, T. Boucekkine, L. Kecili, S. Berkane, Z. Amir, M. Nakmouche Gastroenterology, CHU Bab El Oued, Algiers/Algeria

Contact E-mail Address: houriasaoula@yahoo.fr

Introduction: For a long time, dysplasic lesions in ulcerative colitis were only treated by surgery. Recent guidelines recommend the complete endoscopic resection of dysplasic lesions in ulcerative colitis.

Aims \& Methods: The aim of this study was to determine the outcome of dysplasic lesions resected endoscopic ally in ulcerative colitis. In this prospective study between January 2008 and January 2015, dysplasic lesions detected in patients with longstanding ulcerative colitis were assessed for their resecability, then when it was possible were resected. The patients were followed, and an endoscopic control was done at 6 month than every one year.

Results: 36 dysplasic lesions were identified in 25 patients; 5 lesions were judged not resectable and reffered to surgery. 31 lesions were resected in 21 patients: 22 low-grade dysplasia, 7 lesions indefinite for dysplasia, and 2 high-grade dysplasia. 18 patients $(85.7 \%$ ) had endoscopic control: mean 2.8 (maximum: 5 minimum: 1). 2 patients refused next colonoscopy, one patient was not controlled because of a bad bowel preparation for 4 times. In 13 patients $(72.2 \%)$ no dysplasia was detected after a mean follow up of 30.16 months (range: 7.56$62.5)$. Neoplasic lesions were found in 5 patients $(27.7 \%)$ : one adenocarcinoma of the sigmoid detected in a women that have had a high-grade dysplasia resected in the sigmoid; in 3 patients new dysplasic lesions localized in other segments of the colon than those initially resected. In one patient a serrated rectal adenoma was found in the same place where was resected a serrated adenoma, reflecting an incomplete resection.

Conclusion: Our results confirm that a complete endoscopic resection may be sufficient in dysplasic lesions occurred in ulcerative colitis. Nevertheless a closer follow-up is necessary because these patients may develop newer neoplasic lesions.

Disclosure of Interest: All authors have declared no conflicts of interest.

\section{P0988 CYTOMEGALOVIRUS INFECTION IS ASSOCIATED WITH A POOR OUTCOME IN PATIENTS WITH ULCERATIVE COLITIS TREATED BY VEDOLIZUMAB}

X. Roblin ${ }^{1}$, L. Brichet ${ }^{1}$, S. Pillet ${ }^{2}$, E. Del Tedesco ${ }^{1}$, J.M. Phelip ${ }^{1}$, B. Pozzetto ${ }^{2}$, N. Williet ${ }^{1}$

${ }^{1}$ University of St. Etienne Dept. de Gastroenterologie, Saint Etienne/France

${ }^{2}$ Virology, Saint Etienne university, Saint Etienne/France

Contact E-mail Address: xavier.roblin@chu-st-etienne.fr

Introduction: Cytomegalovirus (CMV) infection has been associated to resistance to several immunomodulatory therapies in Ulcerative Colitis (UC) patients. The impact of CMV infection in UC patients treated with Vedolizumab is unknown. The aim of this study was to analyze the outcome of UC patients treated with Vedolizumab and to analyze the risk factors for CMV disease associated to Vedolizumab therapy.

Aims \& Methods: We performed a retrospective case-control study of all patients with UC treated with Vedolizumab from June 2014 through November 2016 at our IBD center. All eligible patients had presented latent CMV infection with presence of IgG against CMV and undetectable CMV DNA load determined by real time PCR (qPCR) in colonic biopsies before treatment. During the followup, a colonoscopy or rectoscopy with sampling of 2 biopsies in inflamed tissue was performed for each patient in case of loss of clinical response under Vedolizumab. Patients with primary non response to Vedolizumab were excluded. All patients with high mucosal CMV DNA load ( $>250$ copies/mg of tissue) were treated with valganciclovir. Patients with undetectable CMV DNA load disease were considered as control group.

Results: Thirty two patients were eligible (sex ratio M/F: 3/1, mean age: 43.6 years, mean disease duration: 7.5 years, E3 phenotype according to the Montreal classification: $90 \%$ ). Six patients $(19 \%)$ exhibited high colonic CMV DNA load (median load: 734 copies/mg of tissue) during the first year of the follow-up and were treated with valganciclovir for 6 weeks in association with continued Vedolizumab treatment; the 26 others patients with a undetectable CMV DNA load were included in the control group. After antiviral treatment, the CMV DNA load was found undetectable for all 6 patients. Treatment change was more frequent in the CMV disease group (HR $=3.15$ [1.02-9.7], $\mathrm{p}=0.03507$ ) with only $16.7 \%$ patients who continued Vedolizumab treatment versus $65.4 \%$ in the control group $(\mathrm{p}=0.052)$. Colectomy was also more frequent in the CMV group $(33.3 \%$ versus $7.7 \% \mathrm{p}=0.064)$. By multivariate analysis, the only factor associated with the occurrence of CMV disease was a fecal calprotectin less than $600 \mathrm{mcg} / \mathrm{g}$ stools at the beginning of the vedolizumab treatment. A previous CMV colic infection also was more frequent but not statistically significant. There was no association between CMV infection and Vedolizumab levels in the sera.

Conclusion: The occurrence of CMV disease, documented with high CMV DNA load on colonic biopsy samples, in UC treated with Vedolizumab is responsible for a negative impact on the natural evolution of UC, with more therapeutic failure and surgical treatment, even after an efficient antiviral treatment. Disclosure of Interest: All authors have declared no conflicts of interest.

\section{P0989 F-CALPROTECTIN USE IN INFLAMMATORY BOWEL DISEASE IS CHARACTERISED BY IMPROVED DIAGNOSTIC ACCURACY, LESS PATIENT HARM AND DECREASED COSTS, COMPARED WITH CONVENTIONAL SEROLOGICAL MARKERS AND COLONOSCOPY. THE SPANISH SCENARIO}

B. Mascialino ${ }^{1}$, A. A. Vora ${ }^{2}$

${ }_{1}^{1}$ Immunodiagnostics, Thermo Fisher Scientific, Uppsala/Sweden

${ }^{2}$ Immunodiagnostics, Thermo Fisher Scientific, Portage/United States of America/ $M N$

Contact E-mail Address: barbara.mascialino2@thermofisher.com

Introduction: Gastrointestinal disorders may exhibit overlapping symptoms making diagnosis difficult in the primary and specialty care settings. Inflammatory bowel disease (IBD), with a prevalence of $<0.5 \%$ in the general population[1], is characterized by chronic inflammation of the gastrointestinal tract, non-specific elevation of conventional inflammatory markers such as ESR and CRP and may present with extra-intestinal manifestations. Irritable bowel syndrome (IBS), in contrast, is a functional disorder without gastrointestinal inflammation and with an estimated prevalence of 10-20\% [2]. Endoscopy is the gold standard for detecting and quantifying IBD vs. IBS, but due to the low prevalence of IBD, is negative in the majority of cases. Furthermore, it is invasive, expensive, and uncomfortable for the patient and not without risks. Moreover, inadequate bowel preparation prior to colonoscopy is known to 
increase the burden of disease from both the clinical and the economic perspective: shorter intervals between repeated procedures, higher missed rates, patient inconvenience, and increased risk of complications are reported in the scientific literature. F-Calprotectin (FC) is a fecal marker of intestinal inflammation; IBD patients exhibit FC levels significantly higher than the general population; IBS patients have FC levels higher than healthy controls, but significantly lower than IBD patients [3]. Therefore, FC can be used as a pre-endoscopic test to differentiate between IBD and IBS. The present study aims at evaluating the costeffectiveness of FC compared to the combined usage of CRP and ESR, and the gold standard to distinguish IBD from IBS in Spain.

Aims \& Methods: An 18-week Markov model was developed for each diagnostic strategy, simulating 1000 patients presenting to a primary care physician with non-specific gastrointestinal symptoms. In the model, $1.6 \%$ of the colonoscopies brought about complications [4], which may result in Emergency Room visits and surgery. Inadequate colon preparation $(23 \%)[5]$ and consequent repeated colonoscopies $(30.3 \%)[6]$ were also included in the calculations. Outcomes include cost savings, cost per corrected IBD diagnosed, and colonoscopy reduction. Uncertainty was addressed with sensitivity analysis.

Results: FC is cost-effective when compared to CRP + ESR, and to colonoscopy (Table 1): It results in more correctly IBD diagnoses at a lower price; It reduces the number of unnecessary endoscopies, increasing the number of correctly diagnosed IBD $(\mathrm{N}=63)$ and IBS $(\mathrm{N}=26)$ patients.

\begin{tabular}{llll}
\hline Clinical and health economics results & & \\
\hline & F-Calprotectin & CRP + ESR & Colonoscopy \\
\hline N correctly diagnosed IBS & 683 & 657 & 900 \\
N correctly diagnosed IBD & 98 & 35 & 100 \\
Total costs (EUR) & 290527 & 477787 & 582106 \\
Average cost/patient (EUR) & 290.5 & 477.8 & 582.1 \\
Colonoscopy complication & 1978 & 2269 & 6313 \\
$\quad$ costs (EUR) & & & \\
N colonoscopies avoided & 706.3 & 640.6 & 0 \\
Savings ascribable to the & 336338 & 305051 & 0 \\
$\quad$ avoided colonoscopies & & &
\end{tabular}

Conclusion: Results show that the usage of FC as pre-endoscopic diagnostic tool is associated with fewer colonoscopies and correctly identifies more disease while decreasing costs compared to the alternatives. Consequently, FC demonstrates superior value both from patient and payer perspective, while simultaneously increasing diagnostic efficacy.

Disclosure of Interest: B. Mascialino: Employee of Thermo Fisher Scientific A.A. Vora: Employee of Thermo Fisher Scientific

\section{References}

1. Molodecky N.A., et al, Gastroenterology (2012), 142: 46-54.

2. Canavan C., et al, Clinical Epidemiology (2014), 4 (6): 71-80.

3. von Roon et al, American Journal of Gastroenterology (2007), 102; 803-813.

4. Ranasinghe I. et al, Gastroenterology (2016), 150: 103-113.

5. KilgoreT.W. et al, Gastrointestinal endoscopy (2011), 73 (6): 1240-1245.

6. Hendry P.O. et al, Colorectal disease (2006), 9: 745-748.

\section{P0990 RISK OF SERIOUS INFECTION IN HEALTHCARE WORKER WITH INFLAMMATORY BOWEL DISEASE: A CASE-CONTROL STUDY OF THE GETAID}

C. Gagniere ${ }^{1}$, A. Bourrier ${ }^{2}$, P. Seksik ${ }^{3}$, J. Gornet ${ }^{4}$, O. Dewit ${ }^{5}$, S. Nancey ${ }^{6}$, R. Altwegg ${ }^{7}$, V. Abitbol ${ }^{8}$, D. Laharie ${ }^{9}$, C. Reenaers ${ }^{10}$, A. Buisson ${ }^{11}$, M. Nachury ${ }^{12}$, S. Viennot ${ }^{13}$, L. Vuitton ${ }^{14}$, C. Stefanescu ${ }^{15}$, P. Marteau ${ }^{16}$, G. Bouguen ${ }^{17}$, J. Cosnes ${ }^{18}$, A. Amiot ${ }^{1}$

${ }^{1}$ Dept. Of Gastroenterology, Henri Mondor Hospital, APHP Dept. of Gastroenterology, Creteil/France

${ }^{2}$ Gastroenterology, Saint Antoine Hospital, Paris/France

${ }^{3}$ Gastroenterology And Nutrition Unit, Gastroenterology \& Nutrition Department, Paris/France

${ }^{4}$ Hôpital Saint-Louis Gastroentérologie, Paris Cedex/France

${ }^{5}$ UCL Saint Luc, Brussels/Belgium

${ }^{6}$ Dept. De Gastroenterologie, Hospices civils de Lyon, Pierre Benite/France

${ }^{7}$ Hopital Saint Eloi Hepatologie Gastro enterologie, Montpellier cedex/France

${ }^{8}$ Hopital Cochin Gastroentérologie, Paris Cedex/France

${ }^{9} \mathrm{CHU}$ de Bordeaux Hopital Haut-Leveque Dept. de Gastroenterologie, Pessac cedex/France

${ }^{10}$ Chu Sart Tilman, Assistants en formation gastro, réseau Ulg - CHU Sart Tilman, Assistants en formation gastro, réseau, Lantremange/Belgium

${ }^{11}$ Dept. Of Gastroenterology, CHU Estaing Clermont-Ferrand, Clermont-ferrand/ France

${ }^{12} \mathrm{CHU}$ Lille, Lille/France

${ }^{13}$ Caen Hospital, Caen/France
${ }^{14}$ Dept. Of Gastroenterology, Besançon university hospital gastroenterology, Courbevoie/France

${ }^{15}$ Service De Gastroenterologie Et Assistance Nutritive, Beujon Hospital, Clichy, Clichy/France

${ }^{16}$ Gastroenterology \& Digestive Endoscopy Unit, Saint-Antoine Hospital, Paris/ France

${ }^{17} \mathrm{CHU}$ Rennes, Rennes/France

${ }^{18}$ Gastroenterology, hopital St Antoine Gastroenterology GETAID, Paris/France

Contact E-mail Address: charlotte.gagniere@aphp.f

Introduction: The increased use of immunomodulators and biological agents for the treatment of patients with inflammatory bowel disease (IBD) is associated with a key safety concern considering potential serious infection. Healthcare workers were thought to be at substantial risk for acquiring such infection due to daily and close interactions with infected patients and asymptomatic carriers of pathogens.

Aims \& Methods: We performed a retrospective observational study, collecting data from the Groupe d'Etude Thérapeutique des Affections Inflammatoires du tube Digestif (GETAID) from January 2015 to June 2016, on all 482 consecutive patients with IBD $(68.5 \%$ with rohn's disease, $28.4 \%$ with ulcerative colitis and $3.1 \%$ with IBD undetermined) who work as healthcare workers $(27.2 \%$ of physicians, $33.0 \%$ of nurses; $13.1 \%$ of nurses' aides and $26.7 \%$ of other healthcare personnel working in interaction with in-hospital patients), in 17 tertiary centers in France and Belgium. We selected a control group of patients with IBD who do not work as healthcare personnel from the monocentric MICISTA database. Controls were matched on age $( \pm 2.5$ years), sex, IBD type and date of IBD diagnosis $( \pm 2.5$ years). Serious infection was defined as (1) Clostridium difficile infection (2) community-acquired pneumonia (3) Mycobacterium tuberculosis infection (4) any community-acquired infection that required hospitalization. Serious infection-free survival was studied with Kaplan-Meier method, logrank test and Cox regression model. In each patient, the duration of IBD was divided into semesters which were independently analyzed regarding the occurrence of serious infection too take into account the influence of various treatments.

Results: 482 patients (126 male; median age: 24.0 [IQR 19.9-32.1] years) were included in the present study. The median follow-up period was 9.3 [4.6-16.2] years. A total of 74 serious infection was recorded in healthcare workers including 14 Clostridium difficile infection, $19 \mathrm{EBV}$ or CMV-related serious viral infection, 8 tuberculosis infection including 4 tuberculosis and 4 tuberculous primoinfection, 8 community-acquired pneumonia and 25 miscellaneous serious infection. The probabilities of serious infection-free survival were $1.0 \%, 6.1 \%, 10.8 \%$ and $14.1 \%$ at $1,5,10$ and 15 years. No difference was found between healthcare workers and control patients regarding the occurrence of serious infection in time-dependent analysis and in independent semester analysis. However, a increased risk of tuberculosis infection was found in healthcare workers $(0.07$ infections for 100 patient-years vs. $0.009, \mathrm{p}=0.02$ ). In multivariate analysis, serious infection was decreased in patients with Crohn's disease $(\mathrm{OR}=0.63$, IC95\% $\% 0.43-0.91], p=0.01)$ and increased in patients treated with corticosteroids $(\mathrm{OR}=3.05$, IC95\%[2.06-4.52], $\mathrm{p}<0.001)$, immunosuppressant $(\mathrm{OR}=1.98$, IC95\%[1.38-2.84], $\mathrm{p}<0.001)$ and anti-TNF agents $(\mathrm{OR}=2.93, \mathrm{IC} 95 \%[2.02$ 4.27], $\mathrm{p}<0.001)$

Conclusion: Although there is an higher exposure to potential pathogens in healthcare worrkers, this is not associated with an higher risk of serious infection as compared with controls with the exception of tuberculosis infection. Prospective studies are needed to confirm that the level of occupational exposure to potential pathogens should not be taken into account when discussing the introduction of immunomodulator or biological agents with the exception of the risk of tuberculosis infection.

Disclosure of Interest: P. SEKSIK: Philippe Seksik received consulting fees from Abbvie, Merck-MSD and Biocodex, grants from Biocodex, sponsored travel from Merck-MSD and Takeda

J. Gornet: Jean-Marc Gornet has received fees from Sanofi, Merck Serono, Roche, Novartis, Amgen and travel accommodation from Abbvie and MSD. O. Dewit: Dewit O: lecture fees from MSD

S. Nancey: Stephane Nancey has received consulting fees from Merck, Abbvie, Takeda, Ferring, Norgine, Vifor Pharma, Novartis, Janssen Cilag, Hospira, Takeda and HAC-Pharma.

V. Abitbol: Vered Abitbol has received lecture fees from Ferring, MSD, Vifor Pharma and Abbvie.

D. Laharie: David Laharie has received consulting and lecture fees from AbbVie, Ferring, Janssen Cilag, MSD, Pfizer, and Takeda

C. Reenaers: Reenaers C: consulting fees from Abbvie, MSD, Janssen; lectures fees from Abbvie, MSD, Roche, Takeda, Falk. EL; consulting and lecture fees from Abbvie and MSD.

A. Buisson: Anthony Buisson has received lecture fees from Abbvie, MSD Ferring, Takeda, Hospira and Vifor Pharma. This author has also received a consulting fee from Abbvie.

M. Nachury: Nachury M declares lecture fees from Abbvie, MSD, Takeda and Hospira.

S. Viennot: Stephanie Viennot has received consulting fees from Abbvie, MSD, Takeda, Vifor Pharma and Ferring.

L. Vuitton: Lucine Vuitton has received lecture fees from Abbvie, MSD, Takeda, Norgine, and Ferring. This author has also received consulting fees from Abbvie and Takeda.

C. Stefanescu: Stefanescu M: consulting fee from MSD and sponsored travel from Abbvie, Msd, Takeda, Mayoli. 
P. Marteau: Philippe Marteau:has received payments for lectures/speakers bureau participationfrom Abbvie, Ferring, Hospira, Pfizer.

G. Bouguen: Guillaume Bouguen has received consulting fees from MSD and Abbvie. This author has also received lecture fees from MSD, Abbvie, Takeda, and Ferring.

J. Cosnes: Jacques Cosnes has served as a speaker for Abbvie and Falk Foundation and is an advisory board member for VIFOR PHARMA.

A. Amiot: Abbvie, Hospira, Takeda, Gilead, Biocodex, MSD, Janssen, Ferring and Takeda.

All other authors have declared no conflicts of interest.

P0991 CORRELATION BETWEEN INFLAMMATORY BIOMARKERS AND ENDOSCOPIC SCORES IN ULCERATIVE COLITIS: THUS EXTENSION MAKES THE DIFFERENCE?

C. $\operatorname{Arieira}^{1}$, F. Dias De Castro ${ }^{1}$, M.J. Moreira ${ }^{2}$, J. Cotter ${ }^{2}$

${ }^{1}$ Gastroenterology Department, Hospital da Senhora da Oliveira, Guimarães,

Guimarães/Portugal

${ }^{2}$ Pt Government Associate Laboratory

${ }^{4}$ ICVS/3B's, Braga/Guimarães/Portugal

Contact E-mail Address: catia_arieira@hotmail.com

Introduction: Several endoscopic scores have been used to assess the severity of inflammatory activity in Ulcerative Colitis (UC), however, few consider the extension of the disease. Scores such as the Dublin Score (DS) and the Modified Mayo Endoscopic Score (MMES) combine the severity of inflammation with the extent of the disease.

Aims \& Methods: We aimed to calculate the correlation between the endoscopic scores -Mayo Endoscopic Score (MES), DUBLIN, MMES and the biomarkers of inflammation - erythrocyte sedimentation rate (ESR), C-reactive protein (CRP) and Calprotectin - and to compare the ability of these scores to predict Calprotectin $>100 \mu \mathrm{g} / \mathrm{g}$. This was a retrospective study, including patients with diagnosis of left or extensive UC who underwent colonoscopy between 2015 and 2016. The biomarkers were obtained with a maximum interval of one week in relation to colonoscopy and without introduction of new therapy. The Spearman test calculated the correlation between scores and biomarkers. ROC curves (AUC) were obtained for each score to predict Calprotectin $>100 \mu \mathrm{g} / \mathrm{g}$.

Results: 60 patients were included, $46.7 \%$ female patients with mean age $45.3 \pm 12.8$ years with mean values of ESR $4.4 \pm 12.8 \mathrm{~mm}$, CRP $5.12 \pm 6.00 \mathrm{mg}$ 1 and Calprotectin $354 \pm 430 \mu \mathrm{g} / \mathrm{g}$. The correlation between Calprotectin and MES was $r s=0.623 \mathrm{p}<0.001$, for DS $r s=0.548 \mathrm{p}<0.001$ and for MMES $\mathrm{rs}=0.588 \mathrm{p}<0.001$. Regarding CRP, a correlation with the MES was $\mathrm{rs}=0.415 \mathrm{p}=0.001$ and with the MMES was $\mathrm{rs}=0.404 \mathrm{p}=0.001$, but no correlation was found with the DS. There was no significant correlation between ESR and endoscopic scores. To predict values of Calprotectin $>100 \mu \mathrm{g} / \mathrm{g}$ the AUC for the MES was 0.848 , for the DS 0.801 and for the MMES 0.815 , and there was no statistically difference between the curves.

Conclusion: Although there is a good correlation between endoscopic scores and Calprotectin, the correlation between scores that take into account the extension were not superior to Mayo Endoscopic Score.

\section{P0992 IBD - IS IT A RISK FACTOR FOR THE DIAGNOSIS OF HEPATIC STEATOSIS?}

C. Arieira ${ }^{1}$, S. Monteiro ${ }^{1}$, S. Xavier ${ }^{1}$, F. Dias De Castro ${ }^{1}$, J. Magalhães ${ }^{1}$, M.J. Moreira ${ }^{2}$, J. Cotter ${ }^{2}$

${ }^{1}$ Gastroenterology Department, Hospital da Senhora da Oliveira, Guimarães, Guimarães/Portugal

${ }^{2}$ Pt Government Associate Laboratory

${ }^{4}$ ICVS/3B's, Braga/Guimarães/Portugal

Contact E-mail Address: catia_arieira@hotmail.com

Introduction: Although is not yet established, recent studies suggest an increase prevalence of hepatic steatosis (HS) in patients with inflammatory bowel disease (IBD). Factors such as chronic inflammation, previous surgeries, drug-induced hepatotoxicity, malnutrition and intestinal dysbiosis seem to be involved in the pathogenesis of this disease.

Aims \& Methods: We aimed to assess the frequency of HS in IBD patients quantified by CAP (controlled attenuation parameter) and by clinical-analytical methods: Hepatic Steatosis Index (HSI) and Fatty Liver Index (FLI). A secondary aim is to investigate risk factors associated with HS in IBD patients. This was a cross-sectional study that included consecutive outpatients that were observed in our department between January and March 2017. Patients with known liver disease or alcohol habits were excluded. HS was defined as HSI $\geq 36$ or FLI $\geq 60$ or CAP $>248$.

Results: 149 patients included with mean age $40.7 \pm 13$ years, 83 female $(55.7 \%)$, $59.7 \%$ with Crohn's disease (CD). 62 patients $(41.7 \%)$ had CAP $>248.20$ $(13.4 \%)$ FLI $>60$ and $40(26.8 \%)$ HSI $>36$. There were no differences in the mean CAP value $(244 \pm 54.2)$, HSI $(33.3 \pm 5.18)$, and FLI $(31.5 \pm 25.3)$ among patients with CD and Ulcerative Colitis. We found that patients with CAP $>248$ were more frequently obese $(27.4 \%$ vs $0 \%$ p $<0.001)$, males $(54.8 \%$ vs. $36.8 \%$ $\mathrm{p}=0.029)$ and presented more frequently metabolic syndrome $(25 \%$ vs $4.6 \%$ $\mathrm{p}<0.001)$. Regarding the IBD factors, patients with HS had a higher frequency of previous surgeries $(30.6 \%$ vs $16.1 \% \mathrm{p}=0.035)$. There were no differences between hospitalization, duration of the disease, use of corticosteroids or other IBD treatments.
Conclusion: In our cohort the frequency of HS varied between $13.4 \%$ and $41.7 \%$ defined by non-invasive methods. We found that the presence of metabolic syndrome and obesity were more frequent in patients with HS. Regarding factors related to IBD, patients with previous history of surgery were more frequently diagnosed with HS.

Disclosure of Interest: All authors have declared no conflicts of interest.

\section{P0993 BONE HEALTH IN CROHN'S DISEASE IN THE ERA OF TNF- ALPHA INHIBITORS}

S. Hakimian ${ }^{1}$, J. Kheder ${ }^{2}$, D. Cave ${ }^{2}$, B. Hyatt ${ }^{2}$

${ }_{1}^{1}$ Internal Medicine, University of Massachussetts, Worcester/United States of America/MA

${ }^{2}$ Gastroenterology, UMass Medical Center, Worcester/United States of America

Contact E-mail Address: shahrad.hakimian@umassmemorial.org

Introduction: Osteoporosis and fractures are common in Crohn's disease (CD). Recently, several inflammatory cytokines, including tumor necrosis factor (TNF)-alpha have been linked to increased bone resorption. Therefore, it is hypothesized that anti-TNF therapy may influence osteoporosis and fracture risk. However, few studies have evaluated osteoporosis and fracture risk in the CD population.

Aims \& Methods: The aim of this study is to gain a better understanding of the epidemiologic risk factors for osteoporosis and vitamin D deficiency in the era of TNF-alpha inhibitors. We conducted a retrospective review of 714 consecutive patients with CD in our GI clinic between 2008 and 2015 to identify 464 patients who met the inclusion criteria for the study comprising of all adults older than 18 years with confirmed CD based on labs and endoscopic findings. Data extracted for analysis included demographics data, disease phenotype, duration of disease, measures of disease activity, imaging and endoscopic data. Statistical analysis was performed using student t-test and chi-square test.

Results: We reviewed the charts of 290 patients with CD treated with TNF-alpha inhibitors (TNF) and 174 patients who are anti-TNF naïve (NB). There were 207 $(45 \%)$ males and $257(55 \%)$ females in this cohort. TNF patients tended to be younger (average age of $43+/-15$ and $54+/-18$ years in TNF and NB groups respectively). Mean duration of disease was $14.9+/-10.2$ for TNF and $18.6+/$ -19.2 for NB group. Approximately half of the patients had a smoking history. Average BMI was $27.6+/-6.6$. Rates of vitamin D deficiency, insufficiency and normal vitamin D-25-OH levels were not significantly different between TNF and NB groups. Vitamin D level was not associated with age, duration of disease, or inflammatory markers (ESR). However, there was a weak positive correlation between nutritional status (lowest albumin) and vitamin D level (Pearson's $\mathrm{R}=0.19$ ). DXA scans were available for 168 patients which included 100 patients in the TNF group and 68 patients in the NB group. There was similar rate of osteoporosis ( $16 \%$ vs $18 \%$ ), osteopenia ( $53 \%$ vs $57 \%$ ) and normal bone density ( $31 \%$ vs $25 \%$ ) between the TNF and NB groups respectively. Furthermore, there was no statistically significant difference in T-scores at the hip $(-1.2 \mathrm{vs}-1.3)$, the spine $(-1.0$ vs -0.95$)$, or the lowest T-scores $(-1.5$ vs -1.4$)$ between TNF and NB patients. However, Z-scores at the spine $(-0.47 \mathrm{vs}-0.05)$, the hip $(-0.55 \mathrm{vs}$ $-0.40)$ and the lowest Z-scores $(-0.91$ vs -0.67$)$ were lower in the TNF group, but only reached significance at the spine $(\mathrm{P}=0.03)$. Interestingly, a significantly higher proportion of TNF patients under 60 years of age met the criteria for osteoporosis (T-score $<-2.5$ below the mean) as compared to NB patients $(15 \%$ vs $3.6 \%$ ). Additionally, rates of osteoporosis in the NB group were very different before and after age $60(3.6 \%$ vs $30 \%)$ [table 1]. There was no correlation with bone density and vitamin $\mathrm{D}$ level, nutritional status (based on lowest albumin level), or degree of inflammation (highest ESR or CRP levels). However, there was a moderate positive correlation with BMI and bone density (Pearson $\mathrm{R}=0.39)$ and a negative correlation with age $(\mathrm{R}=-0.25)$.

Table 1): Osteoporosis rates in patients on anti-TNF therapy (TNF) and those naïve to biologic medications (NB) before and after age 60 .

\begin{tabular}{llc}
\hline Group & Age $<60$ & Age $>60$ \\
\hline TNF & $15.4 \%$ & $18.2 \%$ \\
NB & $3.6 \%$ & $30.0 \%$
\end{tabular}

Conclusion: Rates of vitamin D deficiency, and osteoporosis were similar among patients on anti-TNF medications to those on no biologics. TNF group patients were diagnosed with osteoporosis at an earlier age compared to NB group. Patients on anti-TNFs also had statistically lower Z-scores at the spine. Prospective studies are necessary to further determine the role of anti-TNF medications in osteoporosis.

Disclosure of Interest: All authors have declared no conflicts of interest.

\section{P0994 THE AVAILABILITY OF INFLIXIMAB TROUGH LEVELS IN IBD PATIENTS ON MAINTENANCE THERAPY DEEPLY IMPACTS THERAPEUTIC DECISION-MAKING}

T. Lobaton ${ }^{1}$, F. Cañete ${ }^{1}$, A. Teniente ${ }^{2}$, E. Cabré ${ }^{1}$, M. Mañosa ${ }^{1}$, E. Martínez ${ }^{2}$, E. Domènech ${ }^{1}$

${ }^{1}$ Gastroenterology, Hospital Universitari Germans Trias i Pujol, Badalona/Spain

${ }^{2}$ Immunology, Hospital Universitari Germans Trias i Pujol, Badalona/Spain

Contact E-mail Address: trianany2010@gmail.com 
Introduction: Infliximab (IFX) trough levels (ITLs) have emerged as a promising tool for the management of inflammatory bowel disease (IBD) patients and they correlate with clinical response and endoscopic remission. However, its use in clinical practice is still under debate, particularly in clinically stable patients

Aims \& Methods: 1) to describe real-life ITLs in clinically stable IBD patients; 2 ) to identify factors associated with infratherapeutic ITLs; and 3) to evaluate the impact of ITLs availability by comparing the CCD with TLGD. The decisions between experts were also compared. Both comparisons were calculated by the linear Cohen's Kappa $(\kappa)$ index. IBD patients on maintenance IFX therapy were prospectively included from June 2015 to June 2016. Demographic, clinical and biological data including C-reactive protein (CRP) levels from the same infusion day were collected. At each IFX infusion, patients were visited by their physician; a "current clinical decision" (CCD) was taken regarding clinical data and CRP. ITLs were measured just before the IFX infusion and were considered as infratherapeutic if $<2 \mu \mathrm{g} / \mathrm{ml}$. Once ITLs were known, 3 experts took a hypothetical decision on treatment based on the same clinical and biological data plus ITLs (ITL-guided decision -TLGD-).

Results: A total of 224 IFX infusions from 74 patients $(76 \%$ Crohn's disease) were analyzed. Median (IQR) disease and IFX therapy duration was 10 years (518 ) and 23 months (7-61), respectively; $87 \%$ received concomitant immunosuppressant therapy; $70 \%$ were on standard dosing, whereas $10 \%$ were scheduled every $4-6$ weeks and $20 \%$ every $10-12$ weeks. $60 \%$ of the patients were in clinical and biological remission. Median (IQR) CRP levels were $3.1 \mathrm{mg} / \mathrm{mL}(1.5-6.1)$. Median (IQR) ITLs were $1.79 \mu \mathrm{g} / \mathrm{ml}(0.35-3.74)$, with $52 \%$ of patients having infratherapeutic ITLs. In the multivariate analysis, the only risk factor for infratherapeutic ITLs was the presence of biological activity. Concordance between CCD and TLGD was poor $(\kappa=0.10[95 \% \mathrm{CI}: 0.01-0.20] / \kappa=0.11$ [95\% CI:0.01$0.21] / \kappa=0.10[95 \% \mathrm{CI}: 0-0.21])$ for experts $\mathrm{A} / \mathrm{B} / \mathrm{C}$, respectively. This "disagreement" was mainly due to a higher proportion of dose-escalations according to the TLGD as compared to the CCD. Among the 203 infusions in which no action was taken according to the CCD, $93(40 \%), 48(20 \%)$ and $65(30 \%)$ would have been dose-escalated according to the TLGD for experts A, B and C, respectively. Moreover, concordance between experts was moderate $(\kappa=0.55$ [95\% CI:0.41-0.71] $/ \kappa=0.40$ [95\%CI:0.26-0.55] $/ \kappa=0.30$ [95\%CI:0.21-0.40]) for experts A-B/B-C/A-C respectively.

Conclusion: Our results highlight the impact of the inflammatory burden on ITLs and the need of re-defining their therapeutic range in patients clinically stable. Both the clinical and economical impact of ITL-assisted decision-making in IBD patients should be evaluated in prospective cohorts.

Disclosure of Interest: E. Domènech: Fees for advisory, lectures and research grants from MSD, AbbVie, Takeda, Kern and Pfizer.

All other authors have declared no conflicts of interest.

\section{P0995 THE DIAGNOSTIC UTILITY OF LINKED-COLOR IMAGING IN THE EVALUATION OF MUCOSAL INFLAMMATION IN PATIENTS WITH ULCERATIVE COLITIS}

H. Hamamoto, S. Kanmura, S. Arima, Y. Nasu, S. Tanoue, F. Sasaki,

S. Hashimoto, A. Ido

Digestive And Lifestyle Diseases, Kagoshima University Graduate School of Medical and Dental Sciences, Kagoshima/Japan

Contact E-mail Address: h-hitomi@m3.kufm.kagoshima-u.ac.jp

Introduction: Recent studies recommend the histological mucosal healing of the intestinal tissue as a treatment goal in ulcerative colitis (UC). The intestinal activity in $\mathrm{UC}$ is usually evaluated based on conventional endoscopic findings by white-light imaging (WLI). However, these findings are not always correlated with the histological findings, and often tend to underestimate the inflammatory activity in comparison to the histological findings. Linked-color imaging (LCI) is a new endoscopy system that enhances the color differences of the gastrointestinal mucosa. We investigated the efficiency of LCI in the evaluation of the intestinal activity, including the histological activity, in patients with UC.

Aims \& Methods: Thirty-one UC patients underwent colonoscopy from August to December 2016. Twenty-one UC patients, who were evaluated using an ECL600ZP endoscope with the LASEREO system (FUJIFILM Co., Tokyo, Japan), were enrolled. All of the target lesions were observed by WLI and LCI, and biopsy specimens were obtained from the lesions with the reddest tones in each view. A total of 96 lesions were biopsied. We quantified the color tones on LCI based on the $\mathrm{L}^{*} \mathrm{a} \mathrm{b}^{*}$ color values (LCI-L, LCI-a, LCI-b), where L* a* and b* represent lightness, red color, and yellow color, respectively. We also quantified the color tones on WLI and LCI (WLI-L, WLI-a, WLI-b) based on the L*a*b* color values. The endoscopic images were classified according to the Mayo endoscopic score (MES), and biopsied specimens were classified according to the Geboes score. The endpoint of this study was to measure the correlation between the L*a*b* color values and the (1) MES, (2) Geboes score, (3) mucosal healing, and (4) histological healing. Furthermore, we defined MES 0 as inactive endoscopic disease, mucosal healing, and a Geboes score of $\leq 2$ as inactive histological disease, histological healing.

Results: (1) A moderate correlation was observed between the LCI-a and MES values $(r=0.56, p<0.001)$. (2) The Geboes score was moderately correlated with the LCI-a value $(\mathrm{r}=0.45, \mathrm{p}<0.001)$ and weakly correlated with WLI-a $(\mathrm{r}=0.24$, $\mathrm{p}<0.001)$, and WLI-b values $(\mathrm{r}=0.28, \mathrm{p}=0.006)$. (3) The LCI-a and WLI-a values were significantly higher in patients with active endoscopic disease than they were in those with mucosal healing $(\mathrm{p}<0.001)$. The area under the curve (AUC) of the receiver operating characteristic (ROC) curve of LCI-a for mucosa healing was 0.82 , while the ROC-AUC of WLI-a was 0.71 . (4) The LCI-a values of was patients with active histological disease were significantly higher, in comparison to those with histological healing $(\mathrm{p}=0.001)$. The ROC-AUC of LCI-a for histological healing was 0.69 . Moreover, WLI was not correlated with histological activity.
Conclusion: LCI was superior to WLI for predicting mucosal healing and histological healing. The findings suggest the utility of LCI in evaluating mucosal inflammation in patients with UC.

Disclosure of Interest: All authors have declared no conflicts of interest

\section{P0996 WHAT SITUATIONS PRODUCE PSYCHOLOGICAL MALAISE IN PATIENTS WITH INFLAMMATORY BOWEL DISEASE? PERCEPTIONS FROM PHYSICIANS AND PATIENTS. THE ENMENTE PROJECT}

M. Barreiro-De Acosta ${ }^{1}$, A. Panadero ${ }^{2}$, M. Cañas ${ }^{3}$, L. Carmona ${ }^{4}$, Y. Modino ${ }^{5}$, J. Guardiola ${ }^{6}$, L. Cea-Calvo ${ }^{7}$, B. Juliá De Páramo ${ }^{7}$, C. Romero ${ }^{7}$, M. Gobbo ${ }^{8}$, I. Marín-Jiménez

${ }^{1}$ Gastroenterologist, IBD Unit, Gastroenterology Department. Complexo Universitario de Santiago de Compostela, Santiago/Spain

${ }^{2}$ Psychologist specialized in Inflammatory Bowel Disease from Confederation of Associations of Crohn's Disease and Ulcerative Colitis of Spain (ACCU), Madrid/ Spain

${ }^{3}$ Nurse, IBD Unit at Gastroenterology Department, Hospital Clínico San Carlos, Madrid/Spain

${ }^{4}$ Clinical epidemiologist, Instituto de Salud Musculoesquelética (InMusc), Madrid/ Spain

${ }^{5}$ Patient representative from the Confederation of Associations of Crohn's Disease and Ulcerative Colitis of Spain (ACCU España), Madrid/Spain

${ }^{6}$ Gastroenterologist, IBD Unit, Gastroenterology Department; Hospital

Universitari de Bellvitge-IDIBELL, L'Hospitalet de Llobregat, Barcelona/Spain

${ }^{7}$ Medical Affairs, Merck Sharp \& Dohme De España, Madrid/Spain

${ }^{8}$ Psychologist, Positivamente, Centro de Psicología, Madrid/Spain

${ }^{9}$ Gastroenterologist, Gastroenterology Department and Institute of Medical Research Gregorio Marañón (IiSGM); Hospital General Universitario Gregorio Marañón, Madrid/Spain

\section{Contact E-mail Address: manubarreiro@hotmail.com}

Introduction: Inflammatory Bowel Disease (IBD) patients live situations that may trigger negative feelings and psychological malaise. ENMENTE Project aims globally to improve identification and early management of psychological impact in IBD patients followed in Spanish hospital gastroenterology clinics. The aim of the study was to describe possible differences among perceptions from physicians and patients about the clinical situations triggering anxiety in patients with IBD

Aims \& Methods: During April 2016 two surveys were available on-line, one for IBD patients, in the ACCU Spain website (Confederation of IBD Spanish Patients' Associations) and another one for physicians members of GETECCU (Spanish Group for IBD treatment). Both invited their members to participate by email and the patients' survey was announced in social networks. The scientific committee (3 gastroenterologists, 2 psychologists, 1 nurse and 1 patient) decided which potentially stressful clinical situations were considered. Physicians and patients rated these situations on a scale from 1 to 10 as potential triggers of anxiety for the patient. A Mann-Whitney test was used to compare perceptions from patients and physicians taking 151 valid questionnaires from physicians and a randomized sample of 155 patients' questionnaires.

Results: The survey was completed by 912 patients (mean age $39( \pm 10)$ years, $67 \%$ women) and 170 physicians (mean age $44( \pm 10)$ years, $58 \%$ women). Having an ostomy, fecal incontinence in public or surgery are important triggers of anxiety agreed between physicians and patients (table). Patients, however experience anxiety from a possible new flare or from being fatigued, whereas physicians are more concerned about anxiety due to telling about a new IBD diagnosis and about pregnancy in IBD patients (table).

Mean scores from physicians and patients about clinical situations triggers anxiety or depression

\begin{tabular}{llll}
\hline $\begin{array}{l}\text { To what extent these clinical situations } \\
\text { may trigger anxiety or depression? }\end{array}$ & $\begin{array}{l}\text { Physicians } \\
(\mathrm{n}=151)\end{array}$ & $\begin{array}{l}\text { Patients } \\
(\mathrm{n}=155)\end{array}$ & $\mathrm{p}$-value \\
\hline The lack of diagnosis & 6.3 & 6.0 & $\mathrm{~ns}$ \\
The diagnosis of IBD & 6.2 & 5.6 & $\mathrm{p}<0.05$ \\
The performance of an endoscopy & 5.6 & 5.7 & $\mathrm{~ns}$ \\
The explanation of an ostomy & 6.6 & 5.9 & $\mathrm{~ns}$ \\
A new oral treatment & 4.8 & 4.8 & $\mathrm{~ns}$ \\
A new auto-injectable treatment & 5.6 & 5.3 & $\mathrm{~ns}$ \\
A new intra-venous treatment & 5.9 & 5.3 & $\mathrm{~ns}$ \\
A surgery & 6.7 & 6.5 & $\mathrm{~ns}$ \\
Having an ostomy & 6.9 & 6.6 & $\mathrm{p}<0.05$ \\
A pregnancy & 5.9 & 4.0 & $\mathrm{p}<0.05$ \\
The pain & 6.3 & 6.1 & $\mathrm{~ns}$ \\
An episode of public incontinence & 6.8 & 6.6 & $\mathrm{~ns}$ \\
A new flare & 6.2 & 6.5 & $\mathrm{p}<0.05$ \\
Changes in the body image & 6.3 & 5.9 & $\mathrm{~ns}$ \\
Tiredness, fatigue, reduction in performance & 6.0 & 6.3 & $\mathrm{p}<0.05$ \\
\hline
\end{tabular}

Conclusion: The main anxiety triggers in patients were having an ostomy, fecal incontinence in public, a surgery, a new flare and the feeling of fatigue. These last 
two situations were scored higher by patients than by physicians. Teaching the patient to identify and early manage a new flare and the treatment of fatigue are aspects that would help to reduce the anxiety feeling and should be taken into account in clinical practice Acknowledgements. Funded by Merck Sharp \& Dohme of Spain and endorsed by ACCU España and by GETECCU

Disclosure of Interest: All authors have declared no conflicts of interest.

\section{P0997 EVALUATION OF LISA-TRACKER IMMUNOASSAY INFLIXIMAB AND ANTI-INFLIXIMAB FOR THE THERAPEUTIC DRUG MONITORING OF SB2}

A. Berger ${ }^{1}$, A. Haccourt ${ }^{1}$, J. Salameh ${ }^{2}$, X. Roblin ${ }^{3}$, S. Paul ${ }^{4}$

${ }^{1}$ Laboratoire D'immunologie Cic1408, CHU Saint-Etienne, Saint-Etienne/France ${ }^{2}$ Biogen France, Nanterre/France

${ }^{3}$ University of St. Etienne Dept. de Gastroenterologie, Saint Etienne/France

${ }^{4}$ Immunology, Hôpital Nord, Saint Etienne/France

\section{Contact E-mail Address: stephane.paul@ chu-st-etienne.fr}

Introduction: Flixabi, an infliximab biosimilar referencing Remicade $\Re$, was developed by Samsung Bioepis, the joint venture between Samsung BioLogics and Biogen. SB2 received approval in EU for all approved indications of the reference infliximab. Many decision algorithms based on the measure of Infliximab (IFX) trough levels and antibodies to infliximab (ATI) have been increasingly used to optimize infliximab in Crohn's disease and ulcerative colitis. The aim of our study was to appreciate if the biosimilar SB2 could be efficiently monitored using the Lisa-Tracker infliximab and anti-infliximab immunoassays developed by Theradiag (France).

Aims \& Methods: During this evaluation, standard curves of Infliximab and two different batches of SB2 were compared and then accuracy of the Lisa-Tracker IFX kit in detecting the spiked concentration of SB2 was measured using the Lisa-tracker assay. Levels of infliximab (from 5 spiked samples with known amount of SB2 and 10 clinical samples from patients treated with infliximab) were calculated according to each of the 3 standard curves (infliximab, SB2 batch1 and SB2 batch2). All samples and standards were tested in duplicate. $\mathrm{R}^{2}$ must be $>0.95$ and the slope must be comprised between 0.9 and 1.1 . Intra-run and inter-run precision were also measured with spiked samples of different known SB2 (from 2 to $12 \mu \mathrm{g} / \mathrm{ml}$ ) amounts. Capacity of polyclonal antibodies directed against infliximab to block the detection of SB2 using the Lisa-Tracker infliximab assay and the capacity of SB2 to block the detection of anti-infliximab antibodies using the Lisa-Tracker anti-infliximab assay were tested.

Results: We demonstrated the perfect equivalence of infliximab standard curve to the SB2 standard curve and that the Lisa-Tracker assay is suitable for the quantification of SB2 in human serum samples $\left(\mathrm{R}^{2}=0.99\right.$; the levels of infliximab of the 20 samples were calculated according to the 3 standard curves infliximab, SB2 batch 1 and SB2 batch 2 with CV ranged from 2.1 to $12.6 \%$ ). Quantification of SB2 is not affected by serum matrix and the $\%$ of recovery were comprised between $82 \%$ and $113 \%$. High intra-run and inter-run precision were obtained with the Lisa-Tracker infliximab assay for the quantification of SB2 (CV ranged from 3.3 to $17.9 \%$ ). Finally, the capacity of polyclonal antibodies to infliximab to block the detection of SB2 in 5 spiked samples was demonstrated with percentages of inhibition comprised between $80 \%$ and $97 \%$. The capacity of SB2 to block the detection of anti-infliximab antibodies in 5 positive clinical samples was also confirmed with high percentages of inhibition between $84 \%$ and $95 \%$. Conclusion: In conclusion, Lisa-Tracker Infliximab and anti-Infliximab assays are suitable for the monitoring of patients treated with SB2. Acknowledgements: Biogen provided the SB2 drug for this study. Biogen reviewed the abstract and provided feedback to the authors.

Disclosure of Interest: All authors have declared no conflicts of interest.

\section{P0998 THE MEASURE OF TROUGH LEVELS OF INFLIXIMAB IS LINKED TO THERAPEUTIC RESPONSE IN IBD PATIENTS}

X. Roblin ${ }^{1}$, A. Berger ${ }^{2}$, G. Boschetti ${ }^{3}$, B. Flourie ${ }^{3}$, S. Nancey ${ }^{4}$, S. Paul ${ }^{2}$ ${ }_{1}^{1}$ Gastroenterology, CHU Saint-Etienne, Saint-Etienne/France

${ }^{2}$ Laboratoire D'immunologie Cic1408, CHU Saint-Etienne, Saint-Etienne/France ${ }^{3}$ Gastroenterology, Lyon-Sud University Hospital, Pierre Benite/France ${ }^{4}$ Gastroenterologie, CH Lyon Sud Gastro secteur Jules Courmont, Pierre Benite Cedex/France

\section{Contact E-mail Address: xavier.roblin@ chu-st-etienne.fr}

Introduction: If the association between trough levels of infliximab (TLI) and clinical remission or mucosal healing is demonstrated, we don't really know the cause and effect between TLI and target value to obtain this association. So, the aim of our study was to evaluate the causality or the association between TLI and clinical remission.

Aims \& Methods: We prospectively included all IBD patients treated in our IBD unit and in clinical remission (CDAI $<150$ for Crohn's Disease $(\mathrm{CD})$ or partial mayo score $<3$ for ulcerative colitis (UC) with biomarker normalization (fecal calprotectin $<250 \mu \mathrm{g} / \mathrm{g}$ stools) or in deep remission (clinical remission with fecal calprotectin $<50 \mu \mathrm{g} / \mathrm{g}$ stools). We analyzed median of TLI and fecal calprotectin at the inclusion (M0) and 6 months before eligibility (M-6). We excluded patients with deep remission at M-6.

Results: 111 patients were included $(60 \mathrm{CD}$, sex ratio $\mathrm{M} / \mathrm{F}: 0.8,51$ patients in deep remission at M0). All these 111 patients were in clinical remission at M-6. Median fecal calprotectin at M-6 were similar in the two groups of patients $(210 \mu \mathrm{g} / \mathrm{g}$ in the group of patients who achieved deep remission at M0 vs
$220 \mu \mathrm{g} / \mathrm{g}$ in the group of patients who achieved only biomarker remission respectively; $p=0.40$ ). Conversely, median TLI at M-6 was significantly lower in patients who did not achieved deep remission at M0 $(2.8 v s 4.1 \mu \mathrm{g} / \mathrm{L}$ respectively; $\mathrm{p}=0.01$ ). A ROC curve analysis was not able to isolate a cut-off value associated to deep remission achievement. (AUROC $=0.61$ ). Next, we analyzed separately median of TLI and fecal calprotectin levels 6 months before eligibility (M-6) of patients in deep remission at M0 (51 patients). The median TLI was significantly lower at M-6 than at M0 $(4.1 \mu \mathrm{g} / \mathrm{mL}$ os $5.9 \mu \mathrm{g} / \mathrm{mL}$ respectively; $\mathrm{p}=0.03)$. Conversely, median fecal calprotectin was significantly higher at M-6 in comparison to M0 (190 vs $35 \mu \mathrm{g} / \mathrm{g}$ stool; $\mathrm{p}=0.01)$. A negative and weak significant correlation between fecal calprotectin and TLI wad observed (Spearman's rank correlation coefficient $(\mathrm{q})=-0.25 ; \mathrm{p}=0.045$ ).

Conclusion: Although TLIs may increase with decreased drug clearance due to deep remission, we show for the first time that the residual rate is the causal element for achieving clinical remission.

Disclosure of Interest: All authors have declared no conflicts of interest.

\section{P0999 SMOKING STATUS INFLUENCES FECAL VOLATILE ORGANIC COMPOUNDS COMPOSITION}

J. De Swart ${ }^{1}$, N. Van Gaal ${ }^{1}$, D. J.C. Berkhout ${ }^{2}$, S. Bosch ${ }^{1}$, T.G. J. De Meij ${ }^{2}$, K. H.N. De Boer ${ }^{1}$

${ }^{1}$ Gastroenterology And Hepatology, VU University Medical Center, Amsterdam Netherlands

${ }^{2}$ Department Of Pediatric Gastroenterology, VU University Medical Center, Amsterdam/Netherlands

Contact E-mail Address: bosch.sofie@gmail.com

Introduction: Fecal volatile organic compounds (VOCs) are gaseous carbonbound metabolic products considered to reflect intestinal microbiota composition. There is increasing evidence that VOCs may serve as non-invasive biomarkers for a broad range of gastrointestinal diseases. As smoking leads to a substantial shift in intestinal microbial composition in healthy and diseased persons, the aim of this study was to assess the effect of smoking status on fecal VOC pattern.

Aims \& Methods: In this cross-sectional pilot-study adult smokers, non-smokers and former smokers scheduled for colonoscopy at the VU University medical center were instructed to collect a fecal sample prior to bowel cleansing. Patients were included if no abnormalities were found during colonoscopy. Exclusion criterium was use of antibiotics three months prior to participation. All participants completed a questionnaire on standard demographics, BMI, diet and smoking habits. Fecal VOC profiles were measured using an electronic nose device (Cyranose 320®)

Results: Fecal samples from 56 subjects (11 smokers, 21 non-smokers, 24 former smokers) were analyzed. Median age was 62 years (27-82 years). Furthermore, there were no significant differences between groups for the variables age, sex, BMI, diet, sample weight, chronic diseases and medication and supplement use. Fecal VOC profiles differed between smokers and non-smokers (PC1: $p$ value $=0.003$ ). Smokers could be distinguished from non-smokers based on fecal VOC profiles was possible with an overall accuracy of $75 \%$ and corresponding sensitivity and specificity values of $72.7 \%$ and $76.2 \%$. No significant differences between the fecal VOC profiles from smokers and former smokers (PC1: $p$ value $=0.083)$ and between profiles from non-smokers and former smokers (PC1: $p$-value $=0.081)$ were observed.

Conclusion: This study showed that smoking status has a significant influence on fecal VOC profiles. This implicates that the smoking status should be taken in account when performing (fecal) VOC analysis. The finding that VOC profiles did not differ between smokers and former-smokers could be due to the wide divergence (6 month - 43 years) in smoke-free time in former smokers group. Disclosure of Interest: All authors have declared no conflicts of interest.

\section{References}

1. de Meij TG, de Boer NK, Benninga MA, Lentferink YE, de Groot EF, van de Velde ME, et al. Faecal gas analysis by electronic nose as novel, noninvasive method for assessment of active and quiescent paediatric inflammatory bowel disease: Proof of principle study. J Crohns Colitis. 2014.

2. Ahmed I, Greenwood R, Costello B, Ratcliffe N, Probert CS. Investigation of faecal volatile organic metabolites as novel diagnostic biomarkers in inflammatory bowel disease. Aliment Pharmacol Ther. 2016;43(5):596-611.

3. Buijck M, Berkhout DJ, de Groot EF, Benninga MA, van der Schee MP, Kneepkens CM, et al. Sniffing Out Paediatric Gastro-intestinal Diseases: the Potential of Volatile Organic Compounds as Biomarkers for Disease. J Pediatr Gastroenterol Nutr. 2016.

4. Opstelten JL, Plassais J, van Mil SW, Achouri E, Pichaud M, Siersema PD, et al. Gut Microbial Diversity Is Reduced in Smokers with Crohn's Disease. Inflamm Bowel Dis. 2016;22(9):2070-7.

5. Biedermann L, Zeitz J, Mwinyi J, Sutter-Minder E, Rehman A, Ott SJ, et al. Smoking cessation induces profound changes in the composition of the intestinal microbiota in humans. PLoS One. 2013;8(3):e59260.

6. Biedermann L, Rogler G. Environmental factors and their impact on the intestinal microbiota: a role for human disease? Dig Dis. 2012;30 Suppl 3:207. 
P1000 CLOSTRIDIUM DIFFICILE INFECTION AND IBD PATIENTS IN ONE CLINICAL CENTER

\author{
A. Atanassova ${ }^{1}$, A. Georgieva ${ }^{2}$ \\ ${ }^{1}$ Gastroenterology, Medical University of Varna, Varna/Bulgaria
}

${ }^{2}$ Clinict Of Gastroenterology, Medical University, Varna, UMHAT "S. Marina" Varna, Varna/Bulgaria

\section{Contact E-mail Address: aniatanassova@abv.bg}

Introduction: The prevalence of Clostridium difficile infection (CDI) in patients suffering from inflammatory bowel disease (IBD) has increased rapidly over the past several decades. However, the exact global epidemiology remains unclear because of insufficient data from developing countries.

Aims \& Methods: The goal of our study is to examine the incidence of CDI in patients with IBD. A retrospective, observational study evaluating IBD patients in a referral center was performed to evaluate the incidence of Clostridium difficile. Diagnosis was confirmed with stool toxin analysis. Demographic information, diagnosis, anatomic location, IBD therapy, antibiotic exposure, hospitalizations, and surgeries were recorded. For a period of 3 years 202 IBD patients were studied, 105 of which have UC and 97 - Chron's disease (CD). We used the Clostridium difficile Glutamat Dehydrogenase + Toxin A + B based on the principle of quantitative immunochromatographic assay for the determination of Clostridium difficile Glutamat Dehydrogenase, Toxin A and Toxin B in stool samples.

Results: The results show that all patients with a positive CDI test have a clinical picture, which resembles a relapse of the disease $(p<0.05)$. There's a tendency towards growth in the incidence of IBD patients who are CDI positive. Their number in 2016 is significantly higher than that in 2014 . In 2014 it was $-5.90 \%$ with CD and $12.50 \%$ with UC, whereas in 2016- $12.20 \%$ with CD and $27.80 \%$ with UC $(\mathrm{p}<0.05)$. The results show that the incidence of CDI patients with UC is significantly higher than in patients with $\mathrm{CD}$, respectively $18.1 \%$ to $9.30 \%$ $(\mathrm{p}<0.05)$. There is a stong correlation between CDI incidence in patients with IBD and the severity of their disease. Patients positive for CDI have a much more severe course of the disease, UC $(46.40 \%)$ and CD $(24.20 \%)(\mathrm{p}<0,05)$.

Conclusion: There is an increase in incidence of CDI, and patients with UC are more affected by it. The results of our study are confirmed by other authors as well. A significant part of patients with CDI have a severe disease that needs extra prospective researches to determine the incidence and influence of the infection amongst patients with IBD, who receive different therapy regimes and also to understand how the CDI affects the evolution of the disease.

Disclosure of Interest: All authors have declared no conflicts of interest.

\section{References}

1. Ben-Horis S, Margalit M, Bossuyt P, Maul J, Shapira Y, Bojic D, et al. Combination immunomodulator and antibiotic treatment in patients with inflammatory bowel disease and clostridium difficile infection. Clin Gastroenterolo Hepatol 2009;7:981-7

2. Ben-Horin S, Margalit M, Bossuyt P, Maul J, shaprira Y, Bojic D, et al. Prevalence and clinical impact of endoscopic pseudomembranes in patients with inflammatory bowel disease and Clostridium difficile infection, $J$ Crohns Colitis 2010;4:194-8

3. Das R, Feuerstadt P, Brandt LJ. Glucocorticoids are associated with increased risk of short-term mortality in hospitalized patients with clostridium difficile-associated disease. Am J Gastroentrol 2010; 105;2040-9

4. Elliot B, Chang BJ, Colledge CL, Riley TV, Clostridium difficile - associated diarrhea. Intern Med $j$ 2007;37:561-8

5. Issa M, Vajayapal A, Graham MB, Beaulieu DB, Otterson MF, Lundeen S, et al. Impact of clostridium difficile on inflammatory bowel disease. Clin Gastroenterolo Hepatol 2007 Mar;5(3):345-51.

6. Lamontagne F, Labble AC, Haeck O, Lesur O, Lalalncete M, Patino C, et al. Impact of emergency colectomy on survival of patients with fulminant Clostridium difficile colitis during an epidemic caused by a hypervirulent strain. Ann Surg 2007;245: 267-727.

7. Schneeweiss S, Korzenik J, Solomon DH, Cannning C, Lee J, Bressler B. Infliximab and other immunomodulating drugs in patients with inflammatory bowel disease and the risk of serious bacterial infections. Aliment Pharmacol Ther 2009 Aug;30(3):253-64.

\section{P1001 DEVELOPMENT OF A NEW SCORE PREDICTIVE OF SUSTAINED CLINICAL REMISSION IN PATIENTS WITH INFLAMMATORY BOWEL DISEASE UNDER INFLIXIMAB- AZATHIOPRINE COMBOTHERAPY}

B. Sivard ${ }^{1}$, N. Williet ${ }^{1}$, J. Phelip ${ }^{1}$, E. Del Tedesco ${ }^{1}$, S. Paul ${ }^{1}$, X. Roblin ${ }^{1}$ ${ }^{1}$ Hepato-gastroenterology, university hospital of Saint-Etiennne, Saint-Priest en Jarez/France

Contact E-mail Address: xavier.roblin@chu-st-etienne.fr

Introduction: There is no blood test predictive of sustained clinical remission in patients with Crohn's Disease (CD) or Ulcerative colitis (UC) under Infliximab (IFX) - azathioprine (AZA) combotherapy.

Aims \& Methods: All patients with CD or UC, consecutively treated by the combination of IFX-AZA between August 2015 and March 2017, were included in this monocentric study. Clinical, biological (blood cells count, liver function enzymes, C-reactive protein (PCR)) were retrospectively collected at baseline, at week 14 (W14) and at 6 months (W24) from the start of combination therapy.
Trough level of IFX (TLI) at W14 was also recorded. Sustained clinical remission was defined as clinical remission for 6 months under combotherapy with no need of AZA dose modification, nor therapeutic switch or need for surgery. A predictive score before combotherapy was developed basing on receiver operating characteristic (ROC) curves and logistic regression analyses.

Results: Of 71 patients enrolled (median age: 36.3 yrs; women: $52.5 \%$; CD: $61.3 \%$; current tobacco: $28.7 \%), 58(81.7 \%)$ experienced sustained clinical remission. The clinical biological score was calculated at baseline by adding attributed points for these variables as follows: alkaline phosphatase $\leq 58 \mathrm{UI} / \mathrm{L}$ (11 pts), lymphocytes $\leq 1.2 \times 10^{9} / \mathrm{L}(6 \mathrm{pts})$, red blood cell count $<4.4 \times 10^{12} / \mathrm{L}(5 \mathrm{pts})$, mean corpuscular volume $>87 \mathrm{fl}(4 \mathrm{pts})$, white blood cell count $<9.3 \times 10^{9}$ $\mathrm{L}(4.5 \mathrm{pts})$, neutrophils $<5.0 \times 10^{9} / \mathrm{L}$ (4 pts), body mass index $>22 \mathrm{~kg} / \mathrm{m}^{2}$ (3 pts), platelets count $<330 \times 10^{9} / \mathrm{L} \quad(3 \mathrm{pts}), \quad \mathrm{PCR}>3.7 \mathrm{mg} / \mathrm{L} \quad(3 \mathrm{pts})$ and Gamma-glutamyl transpeptidase $<30 \mathrm{UI} / \mathrm{L}$ (3 points). A total score $\geq 17$ was predictive of sustained clinical remission with good performance (Area under the curve (AUC): $90.1 \%$ [95\% CI: 81.8\% - 98.5\%]; sensitivity (Se): $81.3 \%$, specificity (Sp): 90.9\%; Positive predictive value (PPV): $97.7 \%$; Negative predictive value (NPV): 50\%); especially in UC patients (AUC: 98.2\% [95\% CI: 93.9\%-100\%], Se: $90.5 \%$, Sp and PPV: $100 \%$; NPV: $66.7 \%)$. Corresponding outcomes for CD were also satisfying (AUC: $83.9 \%$ [95\%CI: $70 \%-97.8 \%]$, Se: $75 \%$, Sp: $85.7 \%$, PPV: $96 \%$; NPV: $42.9 \%$ ). In contrast, performance of TLI at $\mathrm{W} 14(>2.87 \mu \mathrm{g} / \mathrm{mL})$ for the prediction of sustained clinical remission were moderately interesting (AUC: $60.9 \%$ [95\%CI: $38.7 \%-83.1 \%$ ]; Se: $79.3 \%$, Sp: $61.3 \%$; PPV: $90.2 \%$; NPV: $40 \%$ ). These results were not significantly different between patients who had received an AZA monotherapy before combotherapy compared to those who did not.

Conclusion: This new score is a promising tool for the prediction at baseline of sustained clinical remission in inflammatory bowel disease patients who start combotherapy. It may help to identify easily patients who should benefit optimization of IFX rather than early switch treatment in this setting. However, a prospective validation is needed before recommending its use in clinical practice. Disclosure of Interest: All authors have declared no conflicts of interest.

\section{P1002 LOWER GI SYMPTOMS IN YOUNG PATIENTS: CAN SYMPTOMS AND NON-INVASIVE TESTS BE USED SYSTEMATICALLY TO AVOID UNNECESSARY COLONOSCOPIES?}

A. Alakkari, B. Ryan

Gastroenterology Department, Adelaide and Meath Hospital, Dublin/Ireland

Contact E-mail Address: alakkar@yahoo.com

Introduction: Young patients commonly present with lower gastrointestinal symptoms to gastroenterology clinics nationwide. Most colonoscopies in such patients are normal, but risk potentially serious complications. There is an over reliance on endoscopy in clinical practice leading to increasing demand on limited resources. As a result, some patients with potentially life-threatening conditions (e.g. colon cancer) may have a significant delay in diagnosis that may result in a worsened prognosis. Many endoscopic procedures are absolutely necessary, but many might be avoided if other, non-invasive forms of investigations were available which could reliably exclude significant pathology. This in turn could lead to reduced waiting times and improved access for those who really need them.

Aims \& Methods: We aimed to assess colonoscopies, relevant faecal and blood tests in young patients with lower gastrointestinal symptoms. Colonoscopies performed over a 1-year period were retrospectively identified from the Endoscopy Reporting System. Patient charts, faecal calprotectin (FC), C Reactive Protein (CRP), and Coeliac serology were reviewed. A raised $\mathrm{CRP}>5 \mathrm{mg} / \mathrm{L}$ or $\mathrm{FC}$ of $\geq 50 \mathrm{ug} / \mathrm{g}$ was considered abnormal. A Colonoscopy with mucosal inflammation confirmed on histology was considered abnormal. Inclusion and exclusion criteria are in table 1

Table 1: Study criteria

\begin{tabular}{ll}
\hline Inclusion criteria & Exclusion criteria \\
\hline Age $\leq 45$ years & Known Iron deficiency anaemia \\
$\begin{array}{l}\text { Presenting complaint: diarrhoea, } \\
\text { constipation and abdominal } \\
\text { pain/bloating }\end{array}$ & Overt or obscure GI bleeding \\
& \\
& Known Inflammatory \\
& Bowel Disease \\
\hline
\end{tabular}

Results: 2155 medical GI outpatient colonoscopies performed over 12 months were identified. 242 met inclusion criteria for the study. Median age of the patient cohort was 34 years (range 16-45), with $141(58 \%)$ females. The cohort was stratified according to indications; Group A; $132(55 \%)$ patients with diarrhoea predominant symptoms, and Group B; 110 (45\%) patients with constipation, abdominal pain and bloating. Colonoscopy was normal in $104(79 \%)$ of Group A and $102(93 \%)$ of Group B $(\mathrm{p}=0.002) .36(15 \%)$ Colonoscopies were abnormal; 7 patients had active ileitis, 22 had colonic inflammation (12 IBD, 2 lymphocytic colitis, 8 non-specific inflammation), and 7 had ileocolonic inflammation (all diagnosed with IBD). 28 of $36(78 \%)$ patients with mucosal inflammation confirmed on histology had diarrhoea $(p=0.0001)$. FC was available in 36 patients, and CRP in 171 patients. In group A the negative predictive value, positive predictive value and specificity of CRP/FC were $88 \%, 43 \%$ and 
$86 \%$ respectively. In group B these figures were $95 \%, 38 \%$ and $95 \%$ respectively. 21 patients had incidental early adenomatous polyps. $180(74 \%)$ patients were investigated for Coeliac Disease. 4 had positive findings: 3 in Group A and 1 in Group B. One patient in Group A had both coeliac disease and lymphocytic colitis.

Conclusion: Colonoscopy has low yield in young symptomatic patients, especially those with non-diarrhoeal symptoms. Non-invasive tests should be used systematically to better identify patients requiring colonoscopy. We are conducting a prospective study to explore non-invasive diagnostic paradigms. Implementation of these strategies will help reduce colonoscopy waiting times.

Disclosure of Interest: All authors have declared no conflicts of interest.

\section{P1003 FAECAL CALPROTECTIN AND MAGNETIC RESONANCE IMAGING ARE RELIABLE TOOL TO DETECT ENDOSCOPIC POSTOPERATIVE RECURRENCE IN CROHN'S DISEASE}

A. Buisson ${ }^{1}$, P. Baillet ${ }^{1}$, G. Cadiot $^{2}$, M. Goutte ${ }^{1}$, F. Goutorbe ${ }^{3}$, H. Brixi ${ }^{2}$, C. Hoeffel ${ }^{2}$, C. Allimant ${ }^{1}$, M. Reymond ${ }^{1}$, H. Obritin-Guilhen ${ }^{1}$, G. Bommelaer ${ }^{1}$, B. Pereira ${ }^{4}$, C. Hordonneau ${ }^{5}$

${ }^{1}$ Dept. Of Gastroenterology, CHU Estaing Clermont-Ferrand, Clermont-ferrand/ France

${ }^{2}$ Hépato-gastroenterologiee, Hôpital Robert Debré, Reims Cedex/France ${ }^{3} \mathrm{CH}$ Bayonne, Bayonne/France

${ }^{4}$ Dept. Of Statistics, CHU Clermont-Ferrand, Clermont-ferrand/France

${ }^{5}$ Dept. Of Radiology, CHU Estaing Clermont-Ferrand, Clermont-ferrand/France

Contact E-mail Address: a_buisson@hotmail.fr

Introduction: As surgical resection is not curative in Crohn's disease (CD), postoperative recurrence (POR) remains a crucial issue. The POCER trial (1) has recently confirmed that the therapeutic management has to be adapted according to endoscopic findings within the first year following surgery to prevent POR. However, as colonoscopy is a burdensome procedure, alternative tools have been developed such as faecal biomarkers and magnetic resonance imaging (MRI). Aims \& Methods: We aimed to assess the performances of MRI and faecal calprotectin to detect endoscopic POR in CD patients. Adult CD patients from two tertiary centers who underwent ileal or ileocolonic resection were consecutively included in this prospective study. All the patients underwent magnetic resonance enterography including diffusion-weighted sequences with apparent diffusion coefficient (ADC) calculation $\left(\mathrm{mm}^{2} / \mathrm{s}\right)$ and evaluation of Clermont score (2), MaRIA (3) and MR score (4) within the first year after surgery or restoration of intestinal continuity (median $=6$ months IQR [5.0-9.3]) Colonoscopy and MRI were performed within a median time of 14 days IQR [6.5-31] and stools were collected for faecal calprotectin measurement the day before the colonoscopy.

Results: Overall, $30 \mathrm{CD}$ patients were enrolled in the study. Among them, 15 $(50.0 \%)$ were female and $7(23.3 \%)$ were active smokers. Disease location was ileal, colonic or ileocolonic in $15(50.0 \%), 1(3.3 \%)$ and 14 patients $(46.7 \%)$, respectively. The patients included in this study were treated with no medication $(20.0 \%)$, 5-ASA $(6.7 \%)$, thiopurines $(56.7 \%)$ or anti-TNF agents $(20.0 \%)$ in preventing endoscopic POR. Within the first year after surgery, endoscopic findings according to Rutgeerts index were i0 for 8 patients $(26.7 \%)$, il for 5 patients( $16.7 \%)$, i2a for 4 patients $(13.3 \%)$, i2b for 6 patients $(20.0 \%)$, i3 for 5 patients $(16.7 \%)$ and i4 for 2 patients $(6.7 \%)$. In defining endoscopic POR as Rutgeerts index $\geq \mathrm{i} 2$, ADC value $(2.22$ vs $2.12 ; \mathrm{p}=0.35)$, related contrast enhancement (RCE) $(77 \%$ vs $119 \%, 0.056)$, Clermont score (9.3 vs 7.9 $\mathrm{p}=0.11)$, MRI score $(\mathrm{p}=0.57)$ and MaRIA (5.3 vs $6.4 ; \mathrm{p}=0.21)$ were not significantly different in patients with endoscopic POR. Using Rutgeerts index $\geq \mathrm{i} 2 \mathrm{~b}$ as cut-off value to define endoscopic POR, ADC mean value was lower in patients with endoscopic POR $(\geq \mathrm{i} 2 \mathrm{~b})(2.03$ vs $2.27, \mathrm{p}=0.032)$. Clermont score (7.4 vs $10.4, p=0.038)$ and related contrast enhancement (RCE) $(75 \%$ vs $132 \%$, $0.01)$ were higher in patients with endoscopic POR ( $\geq \mathrm{i} 2 \mathrm{~b})$. In contrast, MaRIA $(5.0$ vs $7.3 ; \mathrm{p}=0.15)$ and MRI scores $(\mathrm{p}=0.17)$ were not significantly higher in patients with endoscopic POR ( $\geq \mathrm{i} 2 \mathrm{~b})$. In case of severe endoscopic POR $(\geq i 3)$, RCE ( $87 \%$ vs $135 \% ; p=0.046)$ was increased while none of the following MRI parameters was significantly different: ADC (2.05 vs $2.20 ; \mathrm{p}=0.18)$, Clermont score ( 8.0 vs $11.0 ; \mathrm{p}=0.14$ ), MaRIA (5.3 vs $7.8 ; \mathrm{p}=0.25$ ) and MRI score $(\mathrm{p}=0.11)$. Faecal calprotectin values were significantly higher in patients with endoscopic POR defined as $\geq \mathrm{i} 2(87.8$ vs $314.9 \mu \mathrm{g} / \mathrm{g} ; \mathrm{p}=0.0064)$, $\geq \mathrm{i} 2 \mathrm{~b}(92.6$ vs $366.1 \mu \mathrm{g} / \mathrm{g} ; \mathrm{p}=0.0075)$ or in patients presented with severe endoscopic $P O R \geq \mathrm{i} 3$ (98.8 vs $544.4 \mu \mathrm{g} / \mathrm{g} ; \mathrm{p}=0.0003)$. CRP value was not significantly higher in patients with endoscopic POR defined as $\geq \mathrm{i} 2(2.5$ vs $5.9 ; \mathrm{p}=0.10)$ and $\geq \mathrm{i} 2 \mathrm{~b}$ ( 3.2 vs $6.2 ; p=0.43)$, but was increased in patients with severe endoscopic POR ( 3.0 vs $9.8 ; \mathrm{p}=0.02$ ). Using ROC curves, we determined the best thresholds and their performances to detect endoscopic POR (Table 1).
Table 1: Performances of MRI parameters and faecal calprotectin to detect endoscopic postoperative recurrence in Crohn's disease

\begin{tabular}{llllll}
\hline & AUC & Se & Spe & NPV & PPV \\
\hline Detection of Rutgeerts $\geq \mathrm{i} 2$ & & & & & \\
Clermont score $>8.4$ & 0.70 & $36 \%$ & $77 \%$ & $47.6 \%$ & $66.7 \%$ \\
MaRIA $>7$ & 0.67 & $29 \%$ & $77 \%$ & $45.5 \%$ & $62.5 \%$ \\
Faecal calprotectin $>100 \mu \mathrm{g} / \mathrm{g}$ & 0.84 & $60 \%$ & $100 \%$ & $64.7 \%$ & $100 \%$ \\
Detetection of Rutgeerts $\geq \mathrm{i} 2 \mathrm{~b}$ & & & & & \\
Clermont score $>8.4$ & 0.73 & $47 \%$ & $82.4 \%$ & $66.7 \%$ & $66.7 \%$ \\
MaRIA $>7$ & 0.70 & $38.5 \%$ & $82.4 \%$ & $62.5 \%$ & $63.6 \%$ \\
Faecal calprotectin $>100 \mu \mathrm{g} / \mathrm{g}$ & 0.79 & $66.7 \%$ & $92.9 \%$ & $76.5 \%$ & $88.9 \%$ \\
Detection of Rutgeerts $\geq \mathrm{i} 3$ & & & & & \\
Clermont score $>8.4$ & 0.67 & $42.9 \%$ & $73.9 \%$ & $81 \%$ & $33.3 \%$ \\
MaRIA $>7$ & 0.63 & $42.9 \%$ & $78.3 \%$ & $81.8 \%$ & $37.5 \%$ \\
Faecal calprotectin $>100 \mu \mathrm{g} / \mathrm{g}$ & 0.96 & $100 \%$ & $89.5 \%$ & $100 \%$ & $77.8 \%$ \\
\hline
\end{tabular}

Conclusion: Faecal calprotectin and MRI are reliable tools to detect endoscopic $\mathrm{POR}$ in $\mathrm{CD}$ patients and could be used as non-invasive alternative options to colonoscopy.

Disclosure of Interest: A. Buisson: None related to this work. Lecture fees for Abbvie, Hospira, Takeda, MSD, Ferring, Vifor Pharma, Sanofi-Aventis. Consulting fees for Abbvie, Hospira, Takeda.

All other authors have declared no conflicts of interest.

\section{References}

1. De Cruz et al. Lancet 2015

2. Hordonneau et al. Am J Gastroenterol 2014

3. Rimola et al. Gut 2009

4. Koilakou et al. Inflamm Bowel Dis 2010

\section{P1004 SMALL BOWEL PREPARATION IN PATIENTS WITH CROHN'S DISEASE: THE POLYETHYLENE-GLYCOL IS NO LONGER USEFUL}

L. Lagin ${ }^{1}$, A. Bourreille ${ }^{2}$, M. Flamant ${ }^{3}$, M. Freyssinet ${ }^{4}$, C. Le Berre ${ }^{2}$, S. Bruley Des Varannes ${ }^{5}$, C. Trang 6

${ }^{1}$ Loire Atlantique, CHU Nantes, nantes/France

${ }^{2}$ Hépato-gastro-entérologie, $\mathrm{CHU}$ de Nantes Hôtel Dieu, Nantes CEDEX/France ${ }^{3}$ Clinique Jules Verne, Nantes/France

${ }^{4}$ Lappareil Digestif, Imad, CHU Nantes, Nantes/France

${ }^{5}$ Institut Des Maladies De L'appareil Digestif, Hopital Hotel Dieu, Nantes/France ${ }^{6}$ Hepato-gastroenterology Unit, hospital, Nantes/France

Contact E-mail Address: ludovic.lagin@gmail.com

Introduction: PEG preparations reduce the acceptability of endoscopic explorations but remains recommended before performing a small bowel videocapsule (SBVC). No study has evaluated its impact on the small bowel cleanness compared to other modalities in patients with Crohn's disease (CD). The objective of this study was to compare three methods of preparation, one using polyethyleneglycol and two simplified methods in adult patients with CD.

Aims \& Methods: Three methods of preparation i.e. low-residue diet the evening before more 1 L PEG before the capsule (PEG), liquid diet the evening before the capsule (LD) and $1.5 \mathrm{~L}$ of water at the time of the capsule (Water) were compared. All patients were prospectively included and had an SB3 (Medtronic) capsule after the ingestion of a Patency Agile ${ }^{\mathrm{TM}}$ capsule. The small bowel was identified between the 1st duodenal and the 1st cecal images or the last recorded image and was separated into 3 equal segments. A standardized quantitative cleanliness score was calculated rating the luminosity, the presence of bubbles, turbid liquids, food residues and the percentage of visible mucosal surface in the entire small bowel and in each tertiles. The quantitative score ranged from 0 (worse) to 10 (best) and a qualitative score was obtained rating the cleanness in excellent, good, fair and poor. After anonymization of the films, the recordings were randomized and re-read by 4 investigators blinded to the mode of preparation. The demographic and clinical characteristics of the patients were collected at the time of the capsule. The cleanness scores were compared on the whole small bowel and on each segment.

Results: Between January 2015 and August 2016, 97 patients (38 men, mean age $40.8 \mathrm{yrs})$ were included and performed 105 capsules with 35 capsules in each group of preparations. The groups were comparable concerning the clinical and demographic characteristics (sex ratio, disease duration, history of intestinal resection, phenotype, treatment, indication of the capsule). The mean duration of the small bowel transit time was comparable between the three groups (198 min (PEG), $245 \mathrm{~min}$ (LD), $226 \mathrm{~min}$ (Water) $(\mathrm{P}=0.102)$. The endoscopic activity of the disease was comparable between the 3 groups $(P=0.358)$. The cecal intubation rate was significantly lower in the PEG group $66 \%$ versus $91 \%$ (LD) and $94 \%$ (Water) $(\mathrm{P}=0.04)$. No capsule impaction was observed. The mean quantitative cleanliness score for the whole small bowel was not significantly better for the PEG group (5.7) compared to the other modalities LD (6.3) and Water (6.5) $(\mathrm{P}=0.262)$. In the first tertile, the quantitative score was significantly better in the LD (7.9) and Water (7.6) groups compared to the PEG group (6.8) $(\mathrm{P}=0.043)$. The preparation by water was considered qualitatively better compared to the two other modalities $(\mathrm{P}=0.04)$. 
Conclusion: This is the first study evaluating the relevance of PEG preparation in a large population of adult patients with CD. Our study has demonstrated that there is no benefit in using PEG for the preparation of the small bowel before the capsule in patients with CD. Quantitatively, the two simplified preparation methods were more efficient than the preparation with PEG and qualitatively, the preparation using water was considered as the most efficient.

Disclosure of Interest: A. Bourreille: Advisory Boards: Medtronic Cours, formations: Medtronic Aids for research: Medtronic

All other authors have declared no conflicts of interest.

\section{P1005 VALUE OF 75SEHCAT IN THE DIAGNOSIS OF BILE ACID MALABSORPTION IN CROHŃS DISEASE WITH CHRONIC DIARRHOEA}

B. Gros $^{1}$, L. Mena ${ }^{2}$, J.M. Benítez-Cantero ${ }^{3}$, E. Carmona ${ }^{2}$, E. Iglesias-Flores ${ }^{1}$, F.R. Maza ${ }^{2}$, R. Medina ${ }^{4}$, V. García-Sánchez ${ }^{3}$ ${ }_{1}^{1}$ Gastroenterology, Hospital Reina Sofía, Córdoba/Spain

${ }^{2}$ Nuclear Medicine, Hospital Reina Sofía, Córdoba/Spain

${ }^{3}$ Gastroenterology, Hospital Universitario Reina Sofía, Córdoba/Spain

${ }^{4}$ Gastroenterology, Instituto Maimónides de Investigación Biomédica, Córdoba/

Spain

\section{Contact E-mail Address: begrosal@gmail.com}

Introduction: Bile acid malabsorption (BAM) is a well-known disorder associated to inflammatory bowel disease patients, however, it is underdiagnosed in clinical practice. ${ }^{75} \mathrm{SeHCAT}$ test is the current clinical gold standard for diagnosing bile acid diarrhoea widely used in Europe.

Aims \& Methods: We aimed to analyze the incidence of BAM in Crohn's disease (CD) patients with chronic diarrhoea through ${ }^{75} \mathrm{SeHCAT}$ and to assess whether there is a relationship between the malabsorption degree and the presence of ilea resection. We analyzed, retrospectively, 30 patients with $\mathrm{CD}$ and chronic diarrhoea with and without ileal resection during the period between August 2015April 2016. In all patients, an inflammatory activity was previously discarded through biomarkers and endoscopy/MRI. No patients had received treatment with bile acid sequestrants (Cholestyramine) before the study. We perform the test after the ingestion of a ${ }^{75} \mathrm{SeHCAT}$ capsule $(0,37 \mathrm{MBq})$ prior fasting the night before. Seven-day measurements were compared with 3 hours activity to calculate the abdominal retention percentage. The interpretation of the retention results was made considering $>10 \% \mathrm{BA}$ is consistent with a normal result. Mild BAM is considered $7-10 \%$, moderate $4-7 \%$ and severe $<4 \%$ retention at seventh day measurements. Epidemiological and clinical data were collected from the local database ENEIDA.

Results: We studied 15 women and 15 men with mean age of 46.5 years old (2658 ). The median duration of the disease was 13 years (2-27). At diagnosis, ileal location was present in $50 \%$ of the patients, ileocolonic $40 \%$ and colonic in $10 \%$. According to the behavior of the disease, $50 \%$ presented non-stricturing nopenetrating, $23 \%$ stricturing and $27 \%$ penetrating pattern, and $43 \%$ patients had perianal disease. In relation with smoking status, $34 \%$ were active smokers. Regarding actual treatment, $40 \%$ of the patients are controlled with only immunosuppressive drugs, $10 \%$ with biologics, while $20 \%$ used combotherapy. $83.3 \%$ of the patients had undergone ileal resection. Median length of intestinal resection was $24 \mathrm{~cm}(2-80)$. Six of them needed more than one intestinal resection during the evolution of the disease. Median time from CD diagnosis to surgery was 24 months ( $0-192$ months). In the total number of patients, the median of depositions was 6 per day (2-10). After performing ${ }^{75} \mathrm{SeHCAT}$ scan, $96.8 \%$ of patients demonstrated abnormal values defined as $<10 \%$ abdominal retention of ${ }^{75}$ SeHCAT after seven days. All patients with ileal resection had BAM but also the majority of patients without resection $(86 \%) .92 \%$ of patients with ileal resection had severe malabsorption and $8 \%$ moderate malabsorption. $72 \%$ of patients without resection had severe malabsorption, 14\% moderate malabsorption and $14 \%$ had normal retention. There was no statistical association $(\mathrm{p}=0.253)$ between ${ }^{75} \mathrm{SeHCAT}$ retention and intestinal resection.

Conclusion: ${ }^{75} \mathrm{SeHCAT}$ is an useful procedure for diagnosing BAM in patients with $\mathrm{CD}$ and chronic diarrhea. High incidence of BAM is present in CD patients and there was no significant association between ${ }^{75} \mathrm{SeHCAT}$ retention and performing surgery.

Disclosure of Interest: All authors have declared no conflicts of interest.

\section{P1006 CLINICAL USEFULNESS OF ENDOSCOPIC ASSESSMENT IN ULCERATIVE COLITIS USING LINKED COLOR IMAGING: A PRELIMINARY PILOT STUDY WITH 10 PATIENTS}

K. Yamanouchi ${ }^{1}$, R. Iwakiri ${ }^{2}$, R. Shimoda ${ }^{2}$, Y. Sakata ${ }^{2}$, N. Tsuruoka ${ }^{2}$, S. Shirai ${ }^{2}$, K. Yamamoto ${ }^{2}$, K. Akutagawa ${ }^{2}$, T. Noda ${ }^{3}, H$. Endo ${ }^{4}$, K. Fujimoto ${ }^{5}$

${ }_{1}^{1}$ Internal Medicine \& Gastrointestinal Endoscopy, Takagi hospital, Okawa/Japan ${ }^{2}$ Dept. Of Gastrointestinal Endoscopy, Saga Medical School Dept. of Gastroenterology, Saga/Japan

${ }^{3}$ Internal Medicine And Endoscopy, Karatsu Red Cross Hospital, Karatsu/Japan ${ }^{4}$ Internal Medicine And Endoscopy, Saiseikai Karatsu Hospital, Karatsu/Japan ${ }^{5}$ Dept. Of Internal Medicine, Saga Medical School, Saga/Japan

Contact E-mail Address: kymstd@yahoo.co.jp

Introduction: Linked Color Imaging (LCI) is a novel image-enhanced endoscopy technique which increases distinctiveness of the mucosal color of endoscopic images. The aim of this study is to investigate the usefulness of LCI to evaluate the activity of mucosal inflammation in ulcerative colitis (UC) patients
Aims \& Methods: Totally, 10 consecutive patients with inactive or mildly active UC were enrolled, and fifty-three areas were assessed by LCI. All examinations were conducted with a LASEREO endoscopic system (FUJIFILM CO., Tokyo, Japan). During the colonoscopy, each region of interest (ROI) of terminal ileum, cecum, ascending colon, transverse colon, descending colon, sigmoid colon, and rectum was observed by both white light imaging (WLI), and LCI. The Commission international de l'éclairage (CIE) LAB color differences $(\Delta C)^{1)}$ were calculated among WLI and LCI in each ROI. After ROI was observed by colonoscopy, the biopsy specimen was taken in each ROI. Inflammatory cell infiltration, erosion, crypt abscesses, and goblet cell depletion were assessed as the histologic findings of acute inflammation. For evaluation of chronic inflammation, crypt atrophy, crypt distortion, and basal plasmacytosis were assessed. The correlation between $\Delta \mathrm{C}$ and Mayo endoscopic sub-score was assessed, indicating that the higher $\Delta \mathrm{C}$ mean easier color difference for recognition.

Results: The mean age of patients who were enrolled in the present study was $41.6 \pm 17.7$ years. The sex ratio (men/women) was $4: 6$. The type of extent of UC (ulcerative proctitis/left-sided UC/extensive UC) was 0:3:7. 1.Correlation between CIELAB color differences and histology. The mean $\triangle \mathrm{C}$ of ROI without inflammatory cell infiltration was significantly higher than that of ROI with inflammatory cell infiltration $(15.9 \pm 4.9$ vs. $12.5 \pm 6.7, p=0.046)$. The mean $\Delta \mathrm{C}$ was not affected by histological findings of erosions, crypt abscesses, goblet cell depletion, crypt atrophy, crypt distortion, and basal plasmacytosis. LCI distinguished colon mucosal white color compared to WLI with use of threedimensional color space, indicating the remission-colon mucosa of UC with no inflammatory cell infiltration in ROI was easily detected by LCI. 2.Correlation between CIELAB color differences and Mayo endoscopic subscore. Low Mayo endoscopic sub-scores tended to be inversely proportional to high $\Delta \mathrm{C}(\Delta \mathrm{C}$ : $15.5 \pm 4.9,11.8 \pm 5.1,13.0 \pm, 9.1 \pm 5.3$ ), but not significant in the present evaluation. The colon mucosa with the low Mayo endoscopic sub-scores were relatively easily detected by LCI compared to WLI.

Conclusion: The present pilot trial indicated that the inactive UC mucosa could be easily detected as the white area by the LCI mode compared to WLI, suggesting that LCI might be one of novel approaches for evaluation of disease activity of UC.

Disclosure of Interest: All authors have declared no conflicts of interest.

\section{Reference}

1. Kuehni RG. Color-tolerance data and the tentative CIE $1976 \mathrm{~L}$ a b formula. J Opt Soc Am. 1976;66:497-500.

\section{P1007 THE ROLE OF SENSE OF COHERENCE IN DETERMINING HEALTH RELATED QUALITY OF LIFE AND DISABILITY IN} INFLAMMATORY BOWEL DISEASE

G. Wild, M. Vitagliano, I. Albanese, K. Shirlow

Gastroenterology, Mcgill University Health Centre, Montreal/Canada

Contact E-mail Address: gary.wild@mcgill.ca

Introduction: There is an ever growing body of evidence that indicates that Health Related Quality of Life (HRQoL) is significantly impaired in IBD. While clinical variables and disease activity status influence HRQoL, a host of psychosocial and personality variables also play an important role. This contention is supported by evidence of impaired HRQoL and disability in IBD patients in the absence of demonstrable disease activity. Moreover, individual differences may play a key role in the psychological adaptation to living with IBD and coping with the psychological distress associated with the disorder. Sense of coherence (SOC) is an emerging theoretical and dynamic construct that seeks to explain why some individuals in the face of adversity experience illness while others do not. Antonovsky's theory of SOC suggests that individuals with strong SOC exhibit effective and flexible coping strtaegies when faced with stressors (e.g. chronic medical condition) whereas those with weak SOC are less likely to adapt to health stressors and have less motivation when confonted with challenges to their health. While a number of studies have shown that SOC appears to have an impact on HRQoL, data regarding this association in IBD are limited. Aims \& Methods: The goal of the current study was to examine the associations between an individual's sense of coherence, and their overall health-related quality of life. The first part of this analysis looked at whether sense of coherence accounted for more of the variance in disability level, and an individual's illness perception, as compared to other reported psychosocial factors. The second part of this study evaluated whether sense of coherence, as well as illness perception, were associated with an individual's self-reported quality of life, and if this interaction was moderated by the individual's level of anxiety. Additionally, the correlations between sense of coherence, and self-efficacy, as well as self-reported emotional intelligence were also were further evaluated. This is a cross-sectional observational cohort of IBD patients attending MUHC (McGill University Health Center) IBD outpatient clinics. The patient population $(\mathrm{N}=95)$ demographics are as follows: mean age $(42.4 \pm 12.44)$, gender $(40.6 \%$ male $)$, disease type $(58.4 \% \mathrm{CD}, 33.7 \% \mathrm{UC})$, disease activity $(27.7 \%$ active, $65.3 \%$ inactive). Patients completed multiple validated questionnaires pertaining to a variety of psychosocial parameters. Data was analyzed by multiple linear regression using statistics software (SPSS version 17.0).

Results: Preliminary analyses of this patient population reveal that $40.2 \%$ of the variance in level of disability is explained by sense of coherence. This model suggests a significant negative correlation between sense of coherence and level of disability $(B=-0.64, p<0.05)$. A smaller, albeit significant contribution of sense of coherence with illness perception was additionally found $(14.1 \%$ of the variance, and $B=-0.39, p<0.05)$. Furthermore, $32.3 \%$ of the variance in self- 
reported quality of life was explained by sense of coherence $(B=0.43, p<0.05)$ and illness perception $(B=-0.28, p<0.05)$. However, the individual's report of anxiety did not moderate this relationship. Significant positive correlations between sense of coherence and self-efficacy were also seen in this patient population $(\mathrm{r}=0.61, \mathrm{p}<0.01)$, as well as with self-reported emotional intelligence $(\mathrm{r}=0.52, \mathrm{p}<0.01)$.

Conclusion: These data indicate that Sense of Coherence is an important psychological construct that is associated with HRQoL and disability in IBD. Screening for these factors, in addition to the physiological markers, should be considered in order to provide the most holistic treatment strategies.

Disclosure of Interest: All authors have declared no conflicts of interest

\section{P1008 FAECAL AMINO ACID PROFILES AS NOVEL NON-} INVASIVE BIOMARKERS FOR THE DETECTION OF PAEDIATRIC INFLAMMATORY BOWEL DISEASE: A METABOLOMIC APROACH

S. Bosch ${ }^{1}$, N. Van Gaal ${ }^{1}$, R. Zuurbier ${ }^{1}$, E. Struijs ${ }^{2}$, A. Bakkali², K.h.n. (. De Boer $^{1}$, T.G. J. De Meij ${ }^{3}$

${ }^{1}$ Gastroenterology And Hepatology, VU University medical centre, Amsterdam/ Netherlands

${ }^{2}$ Department Of Clinical Chemistry, VU University Medical Center, Amsterdam/ Netherlands

${ }^{3}$ VU University Medical Centre Dept. of Pediatric Gastroenterology, Amsterdam/ Netherlands

\section{Contact E-mail Address: s.bosch1@vumc.nl}

Introduction: Inflammatory bowel disease (IBD) is primarily assessed by endoscopy, which is a costly and invasive procedure with serious risk of complication, underlining the need for novel non-invasive diagnostic biomarkers. In previous studies, plasma amino acid analysis has revealed significant differences between IBD subjects and controls. This 'aminogram' has not yet been studied in faecal samples of IBD patients. The aim of this explorative study was to compare faecal amino acid composition between paediatric de novo IBD patients and healthy controls, and between the phenotypes ulcerative colitis (UC) and Crohn's disease (CD).

Aims \& Methods: In this cross-sectional case-control study, paediatric treatment naïve IBD patients from a tertiary centre were included, before bowel cleansing and colonoscopy. Control patients were recruited from schools in the province North Holland, the Netherlands. All participants collected a faecal sample on which amino acid analysis was performed by means of high performance liquid chromatography (HPLC, Biochrom 30). To correct for the influence of faecal consistency, the samples were freeze dried for 24 hours before the analysis was performed. To prevent bias by peak overlap of different amino acids, outcomes of $5 \mathrm{nmol} / \mathrm{mg}$ or lower were excluded from further analysis.

Results: Faecal samples from 15 subjects ( 5 healthy, 5 UC, 5 CD) were analysed. Median age was 14 (8-17) years. Subjects and controls were matched on age and sex. A total of 42 different amino acids were analysed, of which 30 were excluded due to quantities of $\leq 5 \mathrm{nmol} / \mathrm{mg}$. In particular, alanine, glycine, phenylalanine, leucine, isoleucine, valine and lysine differed between IBD patients and healthy controls with ratios up to 5:1 (table 1). In addition, $\mathrm{UC}$ and $\mathrm{CD}$ patients differed remarkably based on levels of glycine, phenylalanine and serine with ratios up to 4:1 (table 1).

Table 1: Levels of amino acids in patients with Crohn's disease, ulcerative colitis and healthy controls

\begin{tabular}{llll}
\hline & $\begin{array}{l}\text { healthy } \\
\text { controls } \\
\text { median }\end{array}$ & $\begin{array}{l}\text { ulcerative } \\
\text { colitis } \\
\text { median }\end{array}$ & $\begin{array}{l}\text { Crohn's } \\
\text { disease } \\
\text { median }\end{array}$ \\
\hline $\begin{array}{l}\text { Alanine } \\
\text { Phenylalanine }\end{array}$ & $\begin{array}{l}2.07(1.88-4.39) \\
\text { Glycine }\end{array}$ & $5.28(3.31-10.38)$ & $8.21(4.59-13.05)$ \\
Leucine & $1.06(0.91-2.65)$ & $1.91(1.10-3.58)$ & $5.28(2.17-5.97)$ \\
Isoleucine & $1.00(0.86-2.88)$ & $3.04(2.34-5.32)$ & $4.13(3.80-7.45)$ \\
Valine & $0.76(0.09-2.15)$ & $1.69(0.88-2.01)$ & $3.07(1.68-4.56)$ \\
Lysine & $0.96(0.76-2.61)$ & $2.43(2.35-5.19)$ & $4.41(3.29-6.64)$ \\
Serine & $1.72(1.21-4.03)$ & $2.63(1.27-5.64)$ & $4.62(2.27-8.04)$ \\
\hline
\end{tabular}

${ }^{\mathrm{a}} 11$ levels are displayed in $\mathrm{nmol} / \mathrm{mg}$

Conclusion: This was the first pilot study to assess the potential of the faecal aminogram as non-invasive biomarker for disease activity of paediatric IBD. We observed remarkable differences in faecal amino acid composition between IBD patients and healthy controls, and between the IBD phenotypes. Whether these differences reflect decreased absorption or increased loss by inflamed intestines needs to be elucidated. Currently, we are awaiting the results of a larger proof-ofconcept study on these faecal amino acid profiles.

Disclosure of Interest: All authors have declared no conflicts of interest.

\section{References}

1. Levy I, Gralnek IM Complications of diagnostic colonoscopy, upper endoscopy, and enteroscopy. Best Pract Res Clin Gastroenterol 2016;30(5):705-18.

2. Patti GJ, Yanes O, Siuzdak G Innovation: Metabolomics: the apogee of the omics trilogy. Nat Rev Mol Cell Biol 2012;13(4):263-9.
3. Yau YY, Leong RW, Shin S, et al. Bimodal plasma metabolomics strategy identifies novel inflammatory metabolites in inflammatory bowel diseases. Discov Med 2014;18(98):113-24.

4. Hisamatsu T, Okamoto S, Hashimoto M, et al. Novel, objective, multivariate biomarkers composed of plasma amino acid profiles for the diagnosis and assessment of inflammatory bowel disease. PLoS One 2012;7(1):e31131.

\section{P1009 INFLIXIMAB TROUGH LEVELS AND ANTIBODIES TO INFLIXIMAB IN ASSOCIATION WITH DISEASE ACTIVITY AND MUCOSAL HEALING IN PATIENTS WITH INFLAMMATORY BOWEL DISEASE}

E. Orfanoudaki ${ }^{1}$, M. Gazouli ${ }^{2}$, K. Foteinogiannopoulou ${ }^{1}$, E. Theodoraki ${ }^{1}$, E. Legaki ${ }^{2}$, I. Koutroubakis ${ }^{1}$

${ }^{1}$ Gastrenterology, University Hospital of Heraklion, Heraklion/Greece

${ }^{2}$ Medical School, Basic Medical Sciences, Laboratory Of Biology, National and Kapodistrian University of Athens, Athens/Greece

Contact E-mail Address: elina_or@yahoo.gr

Introduction: Measurement of infliximab trough levels (IFX-TLs) and antibodies to infliximab (ATIs) has been suggested as an important parameter for the optimization of IFX treatment of patients with inflammatory bowel disease (IBD). Aims \& Methods: We aimed to cross-sectionally investigate the correlation between IFX-TLs or ATIs and clinical, biochemical as well as endoscopic activity in Greek IBD patients. Consecutive IBD patients on maintenance treatment with IFX, were included. IFX-TLs and ATIs were measured using ELISA (Eagle BioSciences, Nashua, NH, USA) on serum samples drawn before infusion. At the same time quality of life using short IBD questionnaire (SIBDQ) and clinical disease activity using Harvey-Bradshaw Index (HBI) for Crohn's disease (CD) or simple colitis activity index (SCAI) for ulcerative colitis (UC) were assessed. Moreover, biomarkers (hemoglobin, ESR, CRP, platelets, albumin) were measured and latest colonoscopies (within 6 months) were reviewed and evaluated for presence or not of mucosal healing.

Results: A total of 74 patients receiving IFX maintenance therapy [55 CD, 19 UC, 49 males, mean age 42.3 years, 45 on combination therapy with immunomodulators (IMMs), 10 under intensified dose (either $10 \mathrm{mg} / \mathrm{kg} / 8 \mathrm{w}$ or $5 \mathrm{mg} / \mathrm{kg} / 4$ $6 \mathrm{w})]$ were studied. Median time since IFX initiation was 26 (13-71) months and median value of serum IFX-TL was $4.83 \mu \mathrm{g} / \mathrm{ml}(0.03-30.7)$. Seven out $74(9.5 \%)$ were positive for ATIs $(>10 \mathrm{tAU} / \mathrm{ml})$. Patients on combination treatment had significantly higher IFX-TLs $(6.98 \mu \mathrm{g} / \mathrm{ml}, 0.34-30.7)$ compared to those on IFX monotherapy $(1.85 \mu \mathrm{g} / \mathrm{ml}, 0.09-25.8)(\mathrm{p}=0.01)$. Patients with positive ATIs had median IFX-TLs $0.99 \mu \mathrm{g} / \mathrm{ml}(0.09-1.40)$ statistically lower compared to those with negative ATIs $(6.01 \mu \mathrm{g} / \mathrm{ml}, 0.03-30.69)(\mathrm{p}=0.0005)$. The correlations of IFX-TLs and ATIs with clinical, biochemical and endoscopic indices of disease activity in IBD patients are presented in Table 1. No other significant correlations of IFX-TL or ATIs with other disease characteristics were observed. In the logistic regression analysis only IFX-TLs (OR 0.86, 95\% CI $0.76-0.97 \mathrm{p}=0.017$ ) and duration of IFX treatment (OR $0.97,95 \%$ CI $0.95-0.99 \mathrm{p}=0.04$ ) were independently correlated with the presence of mucosa healing.

Table 1: Correlations of infliximab trough levels and antibodies to infliximab with clinical, biochemical and endoscopic indices of disease activity in patients with inflammatory bowel disease

\begin{tabular}{|c|c|c|c|c|}
\hline \multirow{2}{*}{$\mathrm{N}=74$} & \multicolumn{2}{|c|}{$\begin{array}{l}\text { Infliximab trough } \\
\text { levels (IFX-TLs) }\end{array}$} & \multicolumn{2}{|c|}{$\begin{array}{l}\text { Antibodies to } \\
\text { Infliximab (ATIs) }\end{array}$} \\
\hline & $r$ & $p$ & $r$ & $p$ \\
\hline $\mathrm{HBI}(\mathrm{CD})$ & 0.11 & 0.41 & -0.31 & 0.02 \\
\hline SCAI (UC) & 0.18 & 0.48 & 0.17 & 0.47 \\
\hline SIBDQ & 0.09 & 0.44 & -0.06 & 0.63 \\
\hline $\mathrm{CRP}(\mathrm{mg} / \mathrm{dl})$ & -0.27 & 0.02 & 0.24 & 0.04 \\
\hline $\mathrm{Hgb}(\mathrm{mg} / \mathrm{dl})$ & 0.08 & 0.48 & -0.19 & 0.10 \\
\hline ESR 1st hour (mm) & 0.09 & 0.42 & -0.05 & 0.65 \\
\hline $\operatorname{PLT}\left(\times 10^{9} / \mu \mathrm{L}\right)$ & 0.19 & 0.09 & -0.17 & 0.88 \\
\hline Alb (mg/dl) & 0.003 & 0.77 & 0.009 & 0.94 \\
\hline ATIs & -0.34 & 0.01 & - & - \\
\hline Combined with IMMs & 0.25 & $\mathbf{0 . 0 3}$ & -0.26 & 0.02 \\
\hline Mucosal healing $(\mathrm{N}=53)$ & 0.38 & 0.006 & -0.34 & 0.01 \\
\hline
\end{tabular}

Conclusion: Therapeutic drug monitoring is valuable in IBD-patients on maintenance IFX-treatment. Combination treatment with IFX and IMMs is associated with higher IFX-TLs compared to IFX monotherapy. Higher IFX-TLs are independently associated with the presence of mucosal healing.

Disclosure of Interest: All authors have declared no conflicts of interest.

\section{References}

1. Vande Casteele et al, The relationship between infliximab concentrations, antibodies to infliximab and disease activity in Crohn's disease, Gut 2014;0:1-7 
2. Vande Casteele et al, Trough concentrations of infliximab guide dosing for patients with inflammatory bowel disease, Gastroenterology 2015 Jun;148(7):1320-9.e3

3. Reinisch et al, Factors Associated With Short- and Long-Term Outcomes of Therapy for Crohn's Disease, Clinical Gastroenterology and Hepatology 2015;13:539-547

\section{P1010 COMPARATIVE INVESTIGATION OF ENTEROBACTERIA BUSH IN ULCERATIVE COLITIS PATIENTS, AND THEIR CONSANGUINEOUS, AND NON-CONSANGUINEOUS RELATIVES}

\section{K. Fukuda}

St Luke's International Hospital, Tokyo/Japan

Contact E-mail Address: fukukats@1uke.ac.jp

Introduction: In recent years, the gut microbiota has been recognized as a relevant fingerprint to predict the development of inflammatory bowel disease (IBD) like ulcerative colitis (UC). Accordingly, inter-individual variation in the gut microbial community may reflect inter-individual variation in the risk of developing IBD or other diseases. Further recently, the Next-Generation Sequencing (NGS) has been validated for determining bacterial species in faecal samples. Essentially, NGS is a molecular biology sequencing technique for the precise identification and assessment of bacterial species.

Aims \& Methods: With the major focus of our study being to establish a relevant biomarker of disease activity in UC patients based on the intestinal microbiota, 82 UC patients together with 61 healthy relatives as controls were included for investigations of gut microbiota in faecal samples. Twenty-five patients had active UC (group I) and 57 had quiescent UC; 29 with mild inflammation in the large intestine (group II), and 28 without inflammation (group III). The patients' relatives were consanguineous (group IV, $\mathrm{n}=33$ ), and non-consanguineous (group $\mathrm{V}, \mathrm{n}=28$ ). The subjects' age ranged from 15 to 69 years. Faecal bacteria between groups I to $\mathrm{V}$ were compared by the t-test. The Discriminant analysis in all five groups was done for each Phylum, Class, Order, Family, Genus and Species. The Canonical Discriminant Function Coefficient (Df) for each bacterial community was calculated. The quantity of each bacteria was multiplied by the Df value, and the sum was termed the Discriminant Score (Ds). Next, we tried to analyze the quantity and the diversity of the bacteria which had significant difference.

Results: We obtained 1011 varieties of bacteria as Phyla, Class, Order, Family, Genera and Species. Any individual bacterial quantity with 0 value $\geq 95 \%$ of group I and group $\mathrm{V}$, the mean of the individual quantity of bacteria $\leq 0.05 \%$ cases (648) were excluded. The t-statics was done on 363 bacteria between groups I to V. Significant difference was calculated in 18 Species, 10 Genera, and 4 Families. The Discriminant analysis was done on these 18 Species from all groups. The Ds value showed an increasing tendency in this order: group I $<$ group II $<$ group III $<$ group IV $<$ group V. Significant difference was calculated for group I vs group II, vs group III, vs group IV, and vs group V $(\mathrm{P}<0.05)$. Likewise, group $\mathrm{V}$ vs group I, vs group II, vs group III $(\mathrm{P}<0.05)$, indicating a relevant association between gut microbial species and the development of UC. In bacteria having significant difference, especially Bacteroidaceae Family and Bacteroides Genus were numerous clearly, and both were higher in group I, active UC. It's odd that the diversity of Bacteroides Genus was higher in Group V, non-consanguineous relatives, but the quantity of Bacteroides Genus was higher in group I. And Bacteroides fragilis was increased in group I, and the others of Bacteroides were increased in group V, it depends on the amount of Bacteroides fragilis. In active UC, the amount of Bacteroides fragilis was increased, but the diversity of Bacteroides Genus was decreased. It's very interesting, and the balance can be key point between Bacteroides fragilis and the diversity of Bacteroides in UC activity. And about Genus Anaerococcus, Finegoldia and Peptoniphilus, about Species Anaerococcus vaginalis, Finegoldia magna and Peptoniphilus gorbachii were increased in group I significantly. All these bacteria belong to Peptoniphilaceae Family.

Conclusion: In this study, we compared 363 bacteria between active UC patient to control, significant difference was calculated in 18 Species, 10 Genera, and 4 Families. To our knowledge, this is the first report on so many bacteria being related to UC activity. Additionally, the Ds related to UC, or otherwise absence of UC in the five groups. Potentially, Ds might be a clinically relevant biomarker of disease activity in UC. This is the first application of the Ds to the study of microbiota in UC patients, consanguineous and non-consanguineous relatives by using NGS. Moreover we could obtain a lot of interesting results about the quantity and the diversity of the bacteria, especially Bacteroides and Peptoniphilaceae. Clinical trial No: UMIN000017103

Disclosure of Interest: All authors have declared no conflicts of interest.
P1011 EVALUATION OF PET-MRI AND FECAL BIOMARKERS TO MONITOR DISEASE ACTIVITY IN PATIENTS WITH ULCERATIVE COLITIS

J. Langhorst ${ }^{1}$, J. H. Boone ${ }^{2}$, A. K. Koch ${ }^{1}$, A. Rueffer ${ }^{2}$, G. Dobos ${ }^{3}$

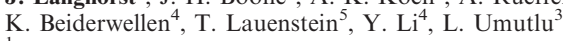

${ }^{1}$ Department For Internal And Integrative Medicine, Kliniken Essen-Mitte Integrative Gastroenterologie, Essen/Germany

${ }^{2}$ Techlab Inc, Blacksburg/United States of America/VA

${ }^{3}$ Enterosan, Labor L $+S$, Bad-Bocklet-Grossenbrach/Germany

${ }^{4}$ Diagnostic And Interventional Radiology And Neuroradiology, University

Hospital of Essen, Essen/Germany

${ }^{5}$ Radiology, Evangelisches Krankenhaus, Duesseldorf/Germany

Contact E-mail Address: j.langhorst@kliniken-essen-mitte.de

Introduction: Endoscopy is the gold standard diagnostic tool in ulcerative colitis (UC). However noninvasive methods like cross-sectional imaging and fecal biomarkers are needed for interval clinical assessments and assessing response to medical treatment. The combination of positron emission tomography (PET) with $18 \mathrm{~F}$-fluorodeoxyglucose (18F-FDG) with magnetic resonance imaging (MRI) as integrated PET/MRI in one examination is a new cutting-edge technology for the non-invasive assessment of the inflammatory activity in UC. In addition a panel of noninvasive biomarkers like Lactoferrin and Calprotectin are increasingly popular and used in all-day patient care.

Aims \& Methods: To compare the performance of non-invasive biomarkers to PET/MRI and colonoscopy in patient with UC. In every patient a PET/MRI including the maximum standardized uptake value ratio gut/liver (SUVQuot) and a colonoscopy including an endoscopy index (EI) was performed within 48 hours and the Disease Activity Index Mayo score (DAI) was calculated. Fecal Lactoferrin (LF), CalprotectinIMUN (CalI), CalprotectinCALPREST (CalP), PMN-elastasis (PMN-E), S100A12, Eosinophil-derived Neurotoxin (EDN) as well as CRP were correlated to the SUVQuot, the DAI and the EI using correlation analyses. Sensitivity, specificity and diagnostic accuracy were calculated using optimized cut-offs. All analyses were performed using SPSS (IBM SPSS Statistics for Windows, release 22.0).

Results: 32 patients ( 21 female), mean age $44.4 \pm 10.63$ years (range $23-67$ ) with diagnosed UC were included in the study. Mean time since diagnosis was 11.41 years $(\mathrm{SD}=6.42)$. EI and SUVQuot $(\mathrm{r}(32)=.45 ; \mathrm{p}=.009)$, EI and DAI $(\mathrm{r}$ $(32)=.87 ; \mathrm{p}=.000)$ as well as DAI and SUVQuot correlated significantly ( $\mathrm{r}$ $(32)=.40 ; \mathrm{p}=.022)$. SUVQuot was correlated significantly with LF (r $(32)=.36 ; \mathrm{p}=.046), \operatorname{EDN}(\mathrm{r}(32)=.49 ; \mathrm{p}=.005)$, and CRP $(\mathrm{r}(32)=.36$; $\mathrm{p}=.043$ ), but not with PMN-e, CalP, S100A12, and Call (all $\mathrm{p}>.05$ ). DAI was correlated significantly with PMN-e (r $(32)=.55 ; \mathrm{p}=.001, \mathrm{LF}(\mathrm{r}(32)=.55$; $\mathrm{p}=.001), \operatorname{EDN}(\mathrm{r}(32)=.70 ; \mathrm{p}=.000), \operatorname{CalP}(\mathrm{r}(32)=.46 ; \mathrm{p}=.008)$, and CRP ( $(32)=.56 ; \mathrm{p}=.001$ ), but not with $\mathrm{S} 100 \mathrm{~A} 12$ and Call (all $\mathrm{p}>.05$ ). EI was correlated significantly with LF $(\mathrm{r}(32)=.61 ; \mathrm{p}=.000)$, EDN $(\mathrm{r}(32)=.68$; $\mathrm{p}=.000)$, PMN-e $(\mathrm{r}(32)=.51 ; \mathrm{p}=.003)$, S100A12 ( $\mathrm{r}(32)=.41 ; \mathrm{p}=.021)$, CalP (r $(32)=.52 ; \mathrm{p}=.002), \mathrm{CRP}(\mathrm{r}(32)=.44 ; \mathrm{p}=.012)$, but not with Call $(\mathrm{p}>.05)$. The median levels (inactive/active) were: LF: $1.75 / 20.13 \mu \mathrm{g} / \mathrm{g}$, CalI:

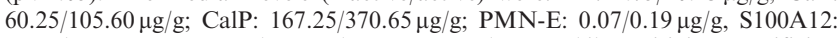
18.82/88.96; EDN: $0.39 / 2.35 \mu \mathrm{g} / \mathrm{g}$; CRP: $0.15 / 0.75 \mathrm{mg} / \mathrm{dl}$.Sensitivity, specificity, diagnostic accuracy (confidence interval) and optimized cut-off for LF was $87.5 \% / 87.5 \% / 87.5 \%$ (CI $72.1 \%-100 \%$ ); $4,27 \mu \mathrm{g} / \mathrm{g}$; CALP: $62.5 \% / 62.5 \% \% /$ $62.5 \% \quad$ (CI $38.9 \%-97.5 \%) ; 361 \mu \mathrm{g} / \mathrm{g}$; CALI: $62.5 \% / 62, \quad 5 \% / 62.5 \% \quad$ (CI $36.7 \%-86.2 \%), \quad 68, \quad 43 \mu \mathrm{g} / \mathrm{g} ; \quad \mathrm{PMN}-\mathrm{e}: \quad 83.3 \% / 75.0 \% / 81.25 \% \quad$ (CI $60.5 \%-99.9 \%$ ); $0.085 \mu \mathrm{g} / \mathrm{g}$; S100A12: $75.0 \% / 75.0 \% / 75 \%$ (CI $56.9 \%-94.1 \%$ ); 60.40; EDN: $75.5 \% / 75.0 \% / 75 \%$ (CI $80.5 \%-100 \%$ ); $1,30 \mu \mathrm{g} / \mathrm{g}$; CRP: $70.8 \%$ / $75.0 \% / 71.9 \%$ (CI $53.5 \%-94.9 \%$ ); $0,35 \mathrm{mg} / \mathrm{dl}$.

Conclusion: Using EI as gold standard reference we found that fecal biomarkers LF, EDN, PMN-E and S100A12 can reliably distinguish between active and inactive UC. However, Calprotectin did not perform well. LF, EDN and CRP were significantly correlated to the SUVQuot which was significantly correlated with EI and DAI. In conclusion, Lactoferrin and Eosinophilderived Neurotoxin performed best using endoscopy and PET/MRI as reference.

Disclosure of Interest: J. Langhorst: Research grant by Techlab Inc.

J.H. Boone: Employee of Techlab Inc.

All other authors have declared no conflicts of interest.

\section{References}

Beiderwellen K, Kinner S, Gomez B, Lenga L, Bellendorf A, Heusch P, Umutlu L, Langhorst J, Ruenzi M, Gerken G, Bockisch A, Lauenstein TC. Hybrid imaging of the bowel using PET/MR enterography: Feasibility and first results. EUR J RADIOL. 2016; Feb;85(2):414-21

Langhorst J, Boone J, Lauche R, Rueffer A, Dobos GJ. Fecal Lactoferrin, Calprotectin, PMN-Elastase, CRP and White Blood Cell Count as an Indicator for Mucosal Healing and Clinical Course of Disease in Patients with Mild to Moderate Ulcerative Colitis: Post Hoc Analysis of a Prospective Clinical Trial. JOURNAL OF CROHNS AND COLITIS 2016; Feb 13

\section{P1012 MMP3 (METALLOPROTEINASE 3): A NEW MARKER FOR ASSESSING LOSS OF RESPONSE TO INFLIXIMAB IN IBD}

B. Barberio, R. D'Inca, L. Digiglio, S. Facchin, M. Piciocchi, R. Cardin, M.G. Vettorato, M. Dalla Gasperina, A. Rigo, G.C. Sturniolo Discog, Azienda Ospedaliera di Padova, Padova/Italy

Contact E-mail Address: brigida.barberio@gmail.com 
Introduction: At present, drug response to infliximab is monitored by trough levels (TL) and anti-drug antibody levels (ATI). Recently, another pathway of drug degradation has been hypothesized since MMP3 and MMP9 were found to be able to cleave IgG, like infliximab, in both animal and human experimental studies (1).

Aims \& Methods: We collected serum samples in 102 patients (27 Crohn's Disease and 75 Ulcerative Colitis) treated with stable doses of infliximab for at least 6 months $\left(\mathrm{t}_{0}\right)$ and 6 months thereafter $\left(\mathrm{t}_{1}\right)$. In each patient TL, ATI values and MMP3 levels were assessed at $t_{0}$ and $t_{1}$ by ELISA. In addition, MMP3 levels were also determined in 28 healthy subjects as controls. Clinical (HBI or Mayo score) and biochemical (CRP, fecal calprotectin) markers were assessed to define disease remission/activity. TL were considered therapeutic if $>3.8 \mathrm{mcg} / \mathrm{ml}$, ATI were considered positive if $>10 \mathrm{mcg} / \mathrm{ml}$. Data are presented as mean \pm Standard Error Mean (SEM). Comparison among groups was performed by non-parametric tests.

Results: MMP3 levels were similar at $\mathrm{t} 0$ and $\mathrm{t} 1$ in patients which maintained therapeutic TL $(14.5 \pm 1.7 \mathrm{pg} / \mathrm{ml}$ and $15.0 \pm 1.6 \mathrm{pg} / \mathrm{ml}$, respectively) and in healthy controls $(13.1 \pm 0.8 \mathrm{pg} / \mathrm{ml})$. Patients with low TL but ATI negative had significantly higher MMP3 levels compared to the group with low TL and ATI positive $(33.2 \pm 3.0$ and $20.0 \pm 2.7$ respectively, $\mathrm{p}=0.0003)$, showing another pathway of drug degradation. 21 patients lost response between t0 and $t 1: 15$ out of 21 patients demonstrated high levels of MMP3 $(22.0 \pm 2.1 \mathrm{pg} / \mathrm{ml})$ already at $\mathrm{t} 0$; in addition, 17 of these 21 patients were in clinical remission at $\mathrm{t} 0$, while at t1 all patients had disease activity.

Conclusion: Serum MMP3 levels are useful in predicting loss of response to anti TNFa in patients with low TL but without ATI. High MMP3 levels predict with $90.5 \%$ accuracy loss of response over the next 6 months.

Disclosure of Interest: All authors have declared no conflicts of interest.

\section{Reference}

1. Biancheri, P., Brezski, R. J., Di Sabatino, A., Greenplate, A. R., Soring, K. L., Corazza, G. R., .. \& \& Snoek, S. A. (2015). Proteolytic cleavage and loss of function of biologic agents that neutralize tumor necrosis factor in the mucosa of patients with inflammatory bowel disease. Gastroenterology, 149(6), 1564-1574.

\section{P1013 USEFULNESS OF A MULTIDISCIPLINARY APPROACH COMBINING BOTH RHEUMATOLOGY AND GASTROENTEROLOGY FOR THE ASSESSMENT AND TREATMENT OF INFLAMMATORY BOWEL DISEASE PATIENTS}

M. Barreiro-De Acosta ${ }^{1}$, E. Pérez-Pampín ${ }^{2}$, R. Ferreiro Iglesias ${ }^{1}$, R. Mejuto ${ }^{1}$, A. Mera ${ }^{2}$ J.E. Dominguez-Muñoz ${ }^{1}$

${ }^{1}$ Gastroenterology, University Hospital of Santiago, Santiago de Compostela/Spain ${ }^{2}$ Rheumathology, University of Santiago de Compostela, Santiago de Compostela/ Spain

Contact E-mail Address: manubarreiro@hotmail.com

Introduction: More than one third of inflammatory bowel disease patients (IBD) present extraintestinal manifestations, with articular manifestations being the more common, clearly the more incapacitating and which more alter the quality of life of IBD patients. These patients could benefit from a multidisciplinary approach for quicker diagnosis and for optimizing treatments.

Aims \& Methods: The aim of the study was to evaluate the impact of a multidisciplinary approach carried out by both a rheumatologist and a gastroenterologist in the management of these patients. Therapeutic changes after the combined assessment were also evaluated. Methods From April 2015 to April 2017 , all IBD patients reporting articular pain to the IBD-dedicated gastroenterologist were referred to an experienced rheumatologist. The day of the consultation a multidisciplinary committee with a rheumatologist and a gastroenterologist evaluated and discussed in all patients their possible diagnosis and potential changes in their treatment. Assessment was made according to current guidelines and data recorded in a common database regarding the reasons why patients were remitted from IBD, their rheumatologic diagnosis and all changes implemented in their treatments. Results are shown in percentages.

Results: 112 consecutive IBD patients were remitted from the IBD Unit and analyzed by the committee. Mean age 38 years (ranging from 18 to 73). Most patients were women $(67 \%), 19 \%$ were smokers and $23 \%$ former smokers. $51 \%$ of patients analyzed had Crohn's disease and $49 \%$ ulcerative colitis. The main causes for derivation from IBD were a suspicion of inflammatory arthropathies in $43 \%$ and of arthromyalgias in $40 \%$. The more frequent diagnosis after the rheumatology consultation and the committee meeting were inflammatory arthropathies associated with IBD in $41 \%(51.5 \%$ presented axial arthropathies and $48.5 \%$ presented peripheral arthropathies) and fibromyalgia in $15 \%$. Regarding treatment changes, after the multidisciplinary committee with a rheumatologist and a gastroenterologist, changes were made in $28 \%$ of patients. Of those, in $35 \%$ of patients methotrexate was added in patients with biologic treatment (in some of them patients were in monotherapy, but in others the drug was introduced for replacing thiopurines). In $24 \%$ of patients sulfasalazine was introduced instead of mesalamine. In the other patients either other biologics like ustekinumab were introduced or the doses of anti-TNF were optimized in accordance with rheumatologic schedules.

Conclusion: A multidisciplinary consultation combining inflammatory bowel disease and rheumatology allows both an earlier detection of inflammatory arthropathies associated with IBD and earlier changes in treatment, thereby helping to optimize the hospitality resources. Fibromyalgia is common among IBD patients, though it is important that it is detected it should not be confused with inflammatory arthropathies.

Disclosure of Interest: All authors have declared no conflicts of interest.

\section{P1014 PERFORMANCE OF THE BÜHLMANN QUANTUM BLUE® ADALIMUMAB RAPID TEST DEDICATED FOR THERAPEUTIC DRUG MONITORING OF SERUM ADALIMUMAB TROUGH LEVELS}

T. Schuster ${ }^{1}$, M. Wieser ${ }^{1}$, S. Kräuchi ${ }^{1}$, R. Sokoll ${ }^{1}$, F. Bantleon ${ }^{1}$, J. Weber ${ }^{1}$, M. Schneider ${ }^{1}$, A.P. Abel ${ }^{2}$

${ }^{1}$ Development, BÜHLMANN Laboratories AG, Schönenbuch/Switzerland

${ }^{2} B \ddot{U} H L M A N N$ Laboratories AG, Schönenbuch/Switzerland

\section{Contact E-mail Address: ms@buhlmannlabs.ch}

Introduction: Adalimumab is a human monoclonal antibody used for the treatment of inflammatory diseases like Crohn's Disease (CD) and Ulcerative Colitis (UC). For efficient treatment trough levels of adalimumab need to be adjusted within a therapeutic window between 5 to $10 \mu \mathrm{g} / \mathrm{mL}^{1}$. A rapid test allows a much faster analysis of trough levels, providing a great advantage over test formats that need samples to be sent to a central laboratory. Here, we present the analytical performance characteristics as part of the validation of the Quantum Blue ${ }^{\circledR}$ Adalimumab test from BÜHLMANN.

Aims \& Methods: The sandwich lateral flow immunoassay uses a tumor necrosis factor alpha coated label and a highly specific monoclonal antibody to detect adalimumab in a diluted human serum sample. Evaluation was performed according to CLSI guidelines. The linearity study included multiple concentration levels covering and exceeding the expected measuring interval, obtained by blending two sample pools, low and high. The within laboratory precision was performed with five (5) samples within the measuring range (20 days - two runs per day in two replicates). Method comparison was performed with a commercial available ELISA (RIDASCREEN ${ }^{\circledR}$ ADM Monitoring, Art. No. G09043, RBiopharm, Darmstadt, Germany) and is based on 106 serum samples. For recovery analysis six clinical samples over the measuring interval of the test were spiked with $6 \mu \mathrm{g} / \mathrm{mL}$ adalimumab in negative serum and compared with the expected values. The high dose hook effect was evaluated with spiked pooled human serum in two runs, the second run with an additional dilution of the samples into the measuring interval of the test.

Results: The BÜHLMANN Quantum Blue ${ }^{\circledR}$ Adalimumab test exhibits a linear range from 0.93 to $34.61 \mu \mathrm{g} / \mathrm{mL}$. The recovery of clinical samples ranged from $80 \%$ to $89 \%$. No high dose hook effect was observed up to $1002 \mu \mathrm{g} / \mathrm{mL}$ of adalimumab. The applied additional dilution into the measuring interval yielded a linear relationship. The mean total within-laboratory precision of the device was $23.9 \%$ (20.7 to $28.9 \%$ ) with a repeatability of $21.2 \%(16.4$ to $27.8 \%)$. The method comparison revealed a slope of 1.18 and a regression coefficient (r) of 0.92 (Passing Bablok) suggesting that the new Quantum Blue ${ }^{\circledR}$ Adalimumab test showed an excellent correlation compared to the ELISA method.

Conclusion: The BÜHLMANN Quantum Blue ${ }^{\circledR}$ Adalimumab lateral flow test enables the quantitative determination of adalimumab trough level in serum with time to result of only 15 minutes. The developed test allows to measure adalimumab over a wide range, well beyond the therapeutic window. Hence, it represents a valuable tool for the clinician to assess the adalimumab trough level. Disclosure of Interest: All authors have declared no conflicts of interest.

\section{Reference}

1. Moss, A. C. Optimizing the use of biological therapy in patients with inflammatory bowel disease. Gastroenterol. Rep. 3, 63-8 (2015).

P1015 ADALIMUMAB TROUGH LEVELS AND ANTIADALIMUMAB ANTIBODIES CORRELATE WITH CLINICAL AND ENDOSCOPIC ACTIVITY IN CROHN'S DISEASE PATIENTS

G. Pellegatta ${ }^{1}$, S. Lo Pumo ${ }^{1}$, G. Bodini ${ }^{1}$, I. Baldissarro ${ }^{1}$, E.G. Giannini ${ }^{1}$, V. Savarino ${ }^{1}$, A. Jain ${ }^{2}$, E. Savarino ${ }^{3}$

${ }^{1}$ Division Of Gastroenterology, Department Of Internal Medicine, University of Genoa, Genova/Italy

${ }^{2}$ Prometheus Laboratories Inc., San Diego/United States of America

${ }^{3}$ Department Of Surgery, Oncology And Gastroenterology, University of Padua, Padua/Italy

Contact E-mail Address: gaia.pellegatta@gmail.com

Introduction: Adalimumab (ADA) is an anti-TNF $\alpha$ drug approved for patients with refractory luminal Crohn's disease (CD). Recently, mucosal healing (MH) emerged as a major therapeutic goal in inflammatory bowel disease. Few data are available on ADA trough levels (TL), anti-ADA antibodies (AAA) during long term follow-up of $\mathrm{CD}$ patients, and their potential association with $\mathrm{MH}$ and disease outcome.

Aims \& Methods: The aim of our prospective study was to evaluate a possible association between achievement of MH, ADA TL, and AAA in CD patients. Moreover, we assessed the presence of a possible association between clinical outcome and $\mathrm{MH}$. We prospectively enrolled moderate to severe $\mathrm{CD}$ patients who were primary responders to ADA treatment. Blood samples were withdrawn at standardized time points during treatment $(0-, 2-, 6$-week and every 8 weeks thereafter), before ADA administration. ADA TL were measured using an homogenous mobility shift assay (HMSA; Prometheus Lab, San Diego, United States). Disease activity was assessed by means of Harvey-Bradshaw Index (HBI remission defined by $\mathrm{HBI}<5$ ). As to endoscopic activity, we defined $\mathrm{MH}$ in case 
of a value of Crohn's Disease Endoscopic Index of Severity below 8, so far we included patients with complete $\mathrm{MH}$ and those with a minimal residual endoscopic activity. Endoscopic evaluation was performed within two weeks of blood sampling, and at least after 6 months of ADA treatment.

Results: In our prospective study we enrolled $22 \mathrm{CD}$ patients primary responders to ADA therapy ( 13 males, median age $=43$ years, range $23-67$ years $)$ who had a median treatment duration of 52 weeks (range 24-121 weeks). ADA TL were significantly higher $(P=0.0002)$ in patients who achieved $\mathrm{MH}(12.1 \mathrm{mcg} / \mathrm{mL}$, range $6.8-17.2 \mathrm{mcg} / \mathrm{mL})$ as compared to patients without $\mathrm{MH}(4.50 \mathrm{mcg} / \mathrm{mL}$, range $0.0-9.9 \mathrm{mcg} / \mathrm{mL}$ ). Receiver Operating Characteristic curve identified an ADA TL cut-off of $6.43 \mathrm{mcg} / \mathrm{mL}$ as the threshold with the highest accuracy for identification of patients who achieve MH (AUROC 0.934, sensitivity $100 \%$, specificity $81.8 \%$, PPV 84.6 , PNV 100). Moreover, achievement of $\mathrm{MH}$ was associated with absence of AAA $(P=0.012)$. Lastly, HBI was significantly lower $(P=0.0002)$ in patients with $\mathrm{MH}(4$, range $3-8)$ than in patients without (11, range $4-17)$

Conclusion: In our cohort of $\mathrm{CD}$ patients, we observed a clear association between ADA TL, AAA development, and MH. In particular, an ADA TL of $6.43 \mathrm{mcg} / \mathrm{mL}$ has been identified as the best cut-off to obtain endoscopic remission or at least a minimal residual endoscopic activity. Moreover, we observed that CD patients on ADA therapy who achieved MH had a lower disease clinical activity. Thus, our data support the use of therapeutic ADA monitoring for the management of $\mathrm{CD}$ patients in order to obtain clinical and endoscopic remission of the disease

Disclosure of Interest: All authors have declared no conflicts of interest

\section{References}

Roblin X et al. Association between pharmacokinetics od Adalimumab and mucosal healing in Patients with Inflammatory Bowel Diseases. Clinical Gastroenterology and Hepatology 2014;12:80-8

Karmiris K et al. Influence of trough serum levels and immugenity on long-term outcome of adalimumab therapy in Crohn's disease. Gastroenterology 2009; $137: 1628-40$

Bodini $\mathrm{G}$ et al. Adalimumab trough serum levels and anti-adalimumab antibodies in the long-term clinical outcome of patients with Crohn's disease. Scandinavian Journal of Gastroenterology 2016.

\section{P1016 ULTRASONOGRAPHIC RESPONSE TO ANTI-TNFS IS ASSOCIATED WITH BETTER OUTCOMES IN CROHN'S DISEASE}

E. Calabrese ${ }^{1}$, F. Zorzi ${ }^{1}$, E. Lolli ${ }^{1}$, S. Onali ${ }^{1}$, M. C. Fantini ${ }^{1}$, L. Biancone ${ }^{1}$, G. Monteleone

${ }^{1}$ Gastroenterology, Department Of Systems Medicine, University of Rome Tor Vergata, Rome/Italy

Contact E-mail Address: emma.calabrese@uniroma2.it

Introduction: Crohn's disease (CD) management targets mucosal healing on ileocolonoscopy as a treatment goal.

Aims \& Methods: We hypothesized that ultrasonographic response to anti-TNFs is also associated with better long-term outcomes. Patients with CD treated with anti-TNFs who had serial small intestine contrast ultrasonography (SICUS) between January 2011 and April 2017 were identified. Disease site (based on bowel wall thickness), extent of lesions, and presence of complications (stenosis, prestenotic dilation, abscess, or fistulas) were evaluated using SICUS. Inclusion required pre-therapy SICUS with follow-up SICUS after 12 months, or 2 SICUS $\geq 12$ months apart while on maintenance therapy. At second SICUS, complete responders had improved lesions, non-responders had worsening or new lesions, and partial responders had other scenarios. CD-related outcomes of corticosteroid need, hospitalization, and surgery were assessed at one year from the second SICUS.

Results: Seventy-nine CD patients treated with anti-TNFs (37\% with Infliximab, $63 \%$ with Adalimumab) were identified. Most patients had ileal disease $(67 \%$ ) and stricturing phenotype $(52 \%)$. Based on SICUS, thirty-six patients $(46 \%)$ were complete sonographic responders, 30 partial (38\%), and 13 non-responders $(16 \%)$. Complete and partial responders at SICUS had a reduced risk for surgery in comparison with non responders $[\mathrm{p}=0.003(\mathrm{OR}: 34.6, \mathrm{CI}: 1.7-700), \mathrm{p}=0.003$ (OR 12, CI:0.6-2.40)]. Complete responders at SICUS had a reduced number of hospitalizations in comparison with non responders $[\mathrm{p}=0.04$ (OR 4.2, CI:117.3)]. Complete and partial responders at SICUS had a reduced risk for need for rescue corticosteroids in comparison with non responders $[\mathrm{p}=0.005$ (OR:7.8, CI:1.9-32.4), $\mathrm{p}=0.002$ (OR 5.2, CI:1.7-15.3)].

Conclusion: Ultrasonographic response to medical therapy is associated with significant reductions in long-term risk of surgery, hospitalizations and steroid usage among CD patients. These findings suggest the significance of response assessed by ultrasonography as a treatment target.

Disclosure of Interest: All authors have declared no conflicts of interest.

\section{P1017 CONTINUOUS MONITORING WITH THE TELEMEDICINE TOOL MYIBDCOACH SHOWS AN ASSOCIATION BETWEEN} NOVEL STRESS AND INFLAMMATORY BOWEL DISEASE FLARES

D. S.j. Wintjens ${ }^{1}$, M. De Jong ${ }^{1}$, A. E. Van Der Meulen - De Jong ${ }^{2}$, M. Romberg Camps $^{3}$, M. Becx ${ }^{4}$, M. H. Verwey ${ }^{2}$, B. Winkens ${ }^{5}$, A. A.m. Masclee ${ }^{1}$,

D.M. Jonkers ${ }^{1}$, M.J. Pierik ${ }^{1}$
${ }^{1}$ Department Of Internal Medicine, Division Of Gastroenterology And Hepatology, Maastricht University Medical Centre+, Maastricht/Netherlands

${ }^{2}$ Department Of Gastroenterology And Hepatology, Leiden University Medical Center, Leiden/Netherlands

${ }^{3}$ Department Of Internal Medicine And Gastroenterology, Zuyderland Medical Center, Sittard-Geleen/Netherlands

${ }^{4}$ Department Of Gastroenterology, Antonius Medical Centre, Nieuwegein/ Netherlands

${ }^{5}$ Department Of Methodology And Statistics, School for Public Health and Primary Care (CAPHRI), Maastricht/Netherlands

Contact E-mail Address: d.wintjens@maastrichtuniversity.nl Introduction: Inflammatory bowel disease (IBD) is characterized by recurrent flares of disease activity, which may lead to hospitalisations, surgery, and eventually disease progression. The exact role of psychosocial factors as triggers remains controversial and current literature focuses on the global presence of psychosocial symptoms preceding a flare instead of distinguishing pre-existing from novel symptoms. In this prospective study, we aim to explore the impact of newly developed symptoms of anxiety, depression, fatigue, psychological stress, and life events on IBD flares.

Aims \& Methods: IBD patients were recruited from the MyIBDCoach study cohort (de Jong et al., Lancet 2017, in Press). MyIBDCoach is a telemedicine tool to monitor IBD patients at home. During the 12-month study period, participants reported on disease activity and psychosocial parameters (including psychological stress, anxiety, depression, fatigue, and life events) through MyIBDCoach every 1-3 months. Flares were defined as clinical disease activity in combination with one of the following: faecal calprotectin $>250 \mu \mathrm{g} / \mathrm{g}$, disease activity on endoscopy or other imaging techniques, or dose escalation or initiation of a new drug to induce remission. For all psychosocial parameters, a binary variable was created to indicate whether symptoms were newly developed or preexisting with reference to the previous measurement, thereby correcting for invariability. A generalized estimating equation model was used to separately determine which psychosocial parameters were associated with flares in the three preceding months, correcting for immortal time bias after a flare and adjusting for gender, disease phenotype, smoking status and disease duration. Results: In total, 2748 measurements from 381 IBD patients were included. Fiftyone patients $(13 \%)$ flared after clinical remission was achieved. Newly developed psychological stress was associated with a flare in the following three months (odds ratio $[\mathrm{OR}]=3.01 ; 95 \% \mathrm{CI}=1.48,6.12$ ). Newly reported symptoms of depression $(\mathrm{OR}=1.29 ; 95 \% \quad \mathrm{CI}=0.53,3.14)$, anxiety $(\mathrm{OR}=1.06 ; 95 \%$ $\mathrm{CI}=0.46,2.43)$, fatigue $(\mathrm{OR}=1.59 ; 95 \% \mathrm{CI}=0.79,3.22)$, or the occurrence of a life event $(\mathrm{OR}=2.07 ; 95 \% \mathrm{CI}=0.94,4.55)$ were not significantly associated, although the latter did occur more frequently before flares.

Conclusion: Newly developed psychological stress is associated with disease flares in IBD patients. Therefore continuous monitoring of patients' stress levels and interventions such as mindfulness and coaching might be interesting to prevent flares and eventually improve disease course.

Disclosure of Interest: All authors have declared no conflicts of interest.

\section{P1018 STRAIN ELASTOGRAPHY AND DIFFERENTIAL} DIAGNOSIS OF INFLAMMATORY AND FIBROTIC STRICTURES IN CROHN'S DISEASE

L. P. Orlova, T. V. Samsonova, I. Khalif, M. Shapina, P. Evgrafov

Department Of Ultrasound, State Scientific Centre of Coloproctology named after A.N. Ryzhih of the Russian Ministry of Healthcare, Moscow, Russian Federation, Moscow/Russian Federation

Contact E-mail Address: lporlova2013@yandex.ru

Introduction: Strain elastography has become a new emerging technique in ultrasonic diagnostics of gastrointestinal pathology. Currently there is few published data on the use of elastography for making the diagnosis and following the course of inflammatory bowel diseases.

Aims \& Methods: Objective. To assess the accuracy of strain elastography concerning stricture detection in Crohn's disease (CD).

Materials and Methods. 24 patients aged from 18 to $43 \mathrm{y}$ were included into the study, 1 of them having a colonic stricture, 1 patient having a stricture of ileotransverse anastomosis and 22 patients having a stricture in the small intestine. Surgical treatment was carried out in 22 patients, in each case histopathological evaluation of surgery specimens was conducted. We performed transcutaneus ultrasonic examination of the bowel using $7.5 \mathrm{MHz}$ linear and $3.5 \mathrm{MHz}$ convex probes with power Doppler mode and colonoscopy in all 24 patients. Strain elastography was used during each US-examination to differentiate inflammatory and fibrotic strictures.

Results: Ultrasonic examination invariably showed local narrowing of the intestinal lumen in stricture sites. Inflammatory stricture length was $29 \mathrm{~mm}(21.1-$ 55.5), (median (2.5th - 97.5th percentile)) with intestinal wall thickening of $6 \mathrm{~mm}(4.23-9.00)$ and the presence of ulcers. The lesion length in fibrotic strictures was $30 \mathrm{~mm}(20-60)$, wall thickness $-6 \mathrm{~mm}(4.16-8.27)$, ulcers were visualized either. In $22.7 \%$ of cases we observed the signs of partial bowel obstruction. Strain ratio (SR) values for inflammatory strictures were $1.53(0.43-3.17)$, for fibrotic strictures $-4.19(1.57-6.42)$, the difference being statistically significant (Mann-Whitney test, $\mathrm{p}<0.05$ ). According to morphologic studies inflammatory strictures were characterized by transmural inflammatory infiltration. In fibrotic strictures we found fibrosis in submucosal layer with loci of muscularis propria involvement. No significant differencies were found between ultrasonic and morphologic data, $\mathrm{p}>0.05$. 
Conclusion: The proposed ultrasonic and elastographic signs of stricturizing CD facilitate preoperative detection and differential diagnosis of fibrotic and inflammatory strictures, helping to choose appropriate surgical treatment.

Disclosure of Interest: All authors have declared no conflicts of interest.

\section{References}

1. Migaleddu V, Quaia E, Scanu D, Carla S, Bertolotto M, Campisi G, Sirigu D, Virgilio G. Abdom Imaging. 2011 Apr;36(2):142-8.

2. Dillman JR, Stidham RW, Higgins PD, Moons DS, Johnson LA, Keshavarzi NR, Rubin JM. J Ultrasound Med. 2014 Dec;33(12):2115-23.

3. Fufezan O, Asavoaie C, Tamas A, Farcau D, Serban D. Med Ultrason. 2015 Dec;17(4):422-30.

4. Fraquelli M, Branchi F, Cribiù FM, Orlando S, Casazza G, Magarotto A, Massironi S, Botti F, Contessini-Avesani E, Conte D, Basilisco G, Caprioli F. Inflamm Bowel Dis. 2015 Nov;21(11):2605-12.

\section{P1019 THE INFLAMMATORY BOWEL DISEASE DISABILITY INDEX IN INFLAMMATORY BOWEL DISEASE: RELATIONSHIP WITH DISEASE CHARACTERISTICS AND QUALITY OF LIFE IN A COHORT OF SICILIAN PATIENTS}

G. C. Morreale $^{1}$, E. Gianquinto ${ }^{2}$, V. Calvaruso ${ }^{1}$, M.G. Cilluffo ${ }^{1}$, B. Scrivo ${ }^{1}$, M. Cappello

${ }^{1}$ Di.bi.mis., Gastroenterology and Hepatology Unit, Palermo/Italy

${ }^{2}$ Di.bi.mis., Policlinico Paolo Giaccone, Palermo/Italy

\section{Contact E-mail Address: dottgaetanocmorreale@gmail.com}

Introduction: IBDs are disabling conditions that negatively affect physical, psychological, familial and social dimensions of life. The concept of quantifying disability has become established for the evaluation of many other chronic diseases. Thus, specific tools have been used to assess the impact of disease and its treatment options on relevant end-points such as health-related quality of life (HRQL), measured by the IBD-Questionnaire (IBD-Q). Recently, the IBDDisability Index (IBD-DI) has been developed to evaluate the entire spectrum of limitations in functioning in patients with IBD. This index is inspired to the International Classification of Functioning, Disability and Health (ICF). The aim of the present study was to assess the relationship between the IBD-DI, clinical characteristics and HRQoL in a cohort of Sicilian patents with ulcerative colitis(UC) and Crohn's disease (CD) followed up in a referral center.

Aims \& Methods: IBD-Q and IBD-DI questionnaires were administered to consecutive UC and CD adults outpatients from July 2016 to April 2017. The IBDDI consists of 28 items that evaluate the 4 domains of body functions, activity and participation, body structures and environmental factors. IBD-Q consists of 32 questions grouped into 4 dimensions: bowel, systemic, social, emotional. Scores range from 1 (poorest QoL) to 7 (best QoL) with higher scores indicating better QoL. Disease activity was assessed by partial Mayo score for UC and by Harvey-Brandshaw Index for CD. The mean differences of DI score in relation to dichotomic clinical variables were performed by Student's t test. By linear regression analysis we assessed also the relationship between DI and IBD-Q. Differences were reported as statistically significant if the $\mathrm{p}$ value was $<0.05$. Results: Data from UC and CD patients were analysed separately. 100 UC patients (59\% males, median age 49 years) were enrolled; $17 \%$ were smokers. $83 \%$ had inactive or mild disease, $17 \%$ moderate disease. None of the recruited patients had severe disease. Concomitant medications at the time of the interview were conventional therapy (5-aminosalicylic acid, oral steroids) in 72 patients $(72 \%)$ or immunosuppressive therapy (immunosuppressant or anti-TNF-a) in 28 patients $(28 \%)$. The mean IBD-DI score was $23.15 \pm 17.492 ; 62 \%$ of patients had low DI $\leq 25(62 / 100)$ while $7 \%$ had high DI $(>50)$. No correlations were found between IBD-DI and gender, disease duration, disease extension (Montreal Classification) and immunosuppressive therapy. IBD-DI was related to clinical disease activity $(\mathrm{p}<0.001)$ and extraintestinal manifestations $(P=0.005)$ By linear regression analysis, IBD-DI was significantly associated with IBD-Q $\left(\mathrm{R}^{2} 0,573 ; \mathrm{p}<0.001\right)$. Interestingly, $5 \%(\mathrm{n}=5)$ of patients with inactive or mild disease had severe disability $(\geq 50)$ and $5 \%(n=5)$ with active disease had low disability $(\leq 25) .54$ CD patients $(59 \%$ males, median age 41 years) were enrolled; $22 \%$ were smokers. $94 \%$ had mild disease, $17(6 \%)$ severe disease. Concomitant medications at the time of the interview were conventional therapy (5-aminosalicylic acid, oral steroids) in 22 patients $(40 \%)$ or immunosuppressive therapy (immunosuppressant or anti-TNF-a) in 32 patients $(60 \%)$. The mean IBD-DI score was $20.17 \pm 16.24 ; 72 \%$ of patients had low $\mathrm{DI} \leq 25(39 / 54)$ while only 2 patients had high DI $(>50)$. No correlations were found between IBD-DI and disease characteristic (gender, disease duration, disease extension, extraintestinal manifestations. By linear regression analysis, IBDDI was significantly associated with IBD-Q $\left(\mathrm{R}^{2} 0,604 ; \mathrm{p}<0.001\right)$.

Conclusion: Our preliminary results show that the IBD-DI is significantly related to HR-QoL both in UC and CD. In UC IBD-DI is also related to disease activity and presence of extraintestinal manifestations. However, most of our patients were in clinical remission. A larger sample with different grades of disease activity could provide a more accurate evaluation of the reliability of this tool in measuring functional status and disability in IBD. IBD-DI could become a major endpoint in RCTs targeting the course of IBD

Disclosure of Interest: All authors have declared no conflicts of interest.

\section{Reference}

1. Development of the first disability index for inflammatory bowel disease based on the international classification of functioning, disability and health. 2017 Apr;66(4):588-596. doi: 10.1136/gutjnl-2015-310151. Epub 2015 Dec 8.

\section{P1020 DIPEPTIDYL PEPTIDASE 4 (DPP-4): A BIOMARKER OF DISEASE ACTIVITY AND PROGNOSIS IN INFLAMMATORY} BOWEL DISEASE

P. Pinto-Lopes ${ }^{1}$, J. Afonso ${ }^{1}$, G. Macedo ${ }^{2}$, F. Magro ${ }^{1}$

${ }^{1}$ Department Of Biomedicine - Pharmacology And Therapeutics Unit. Internal Medicine Department, Faculty of Medicine of Porto University. Hospital São João, Porto, Porto/Portugal

${ }^{2}$ Department Of Gastroenterology, Hospital São João, Porto, Porto/Portugal

Contact E-mail Address: pedro.lopes.md@gmail.com

Introduction: DPP-4 is a membrane-bound glycoprotein expressed on the cell surface of enterocytes and lymphocytes. It is released in plasma, maintaining its proteolytic activity and inactivating cytokines, chemokines and neuropeptides. Aims \& Methods: We aimed to investigate the diagnostic and prognostic value of DPP-4 in patients with inflammatory bowel disease (IBD). A total of 203 adult patients $[\mathrm{n}=149$ IBD patients; $\mathrm{n}=42$ healthy controls; $\mathrm{n}=12$ immune controls systemic lupus erythematosus (SLE) in remission] were prospectively recruited. Plasma DPP-4 was analysed in all groups; faecal samples from IBD patients were collected for DPP-4 and calprotectin analysis. Disease activity was assessed using the Harvey-Bradshaw Index (HBI) for Crohn's disease (CD), the partial Mayo Score (pMS) for Ulcerative colitis (UC) and the Systemic Lupus Erythematosus Disease Activity Index for SLE. A multi-biomarker model was derived using logistic regression to evaluate predictors of disease activity and Cox regression to evaluate predictors of treatment escalation (disease outcome). Treatment escalation was defined as the need for escalation to immunomodulatory/biologic therapies or intestinal resection surgery, as a consequence of a disease flare.

Results: Median plasma DPP-4 values were lower in active CD vs CD in remission [1043ng/mL, interquartile range (IQR): $824-1345$ vs $1685 \mathrm{ng} / \mathrm{mL}$, IQR: $1519-2237 ; P<0.001]$; as well as in active UC vs UC in remission (1323 ng/ mL, IQR: $1064-1766$ vs $2149 \mathrm{ng} / \mathrm{mL}$, IQR: $1616-2478 ; P<0.001)$. IBD patients in remission showed plasma DPP-4 values significantly lower than healthy subjects; no differences were found between immune and healthy controls. Median faecal DPP-4 values were much lower in active UC vs UC in remission [1213ng/ $\mathrm{mL}$, IQR: $559-1682$ vs $7814 \mathrm{ng} / \mathrm{mL}$, IQR: 2555-7985; $P<0.001]$, but no differences were found between active $C D$ and $C D$ in remission. In CD, plasma DPP-4 correlated strongly with faecal calprotectin (FC) $(\mathrm{r}=-0.61, P<0.001)$, C-reactive protein $(\mathrm{CRP})(\mathrm{r}=-0.60, P<0.001)$ and $\mathrm{HBI}(\mathrm{r}=-0.56, P<0.001)$, while no statistically significant correlation was found with faecal DPP-4. In UC, plasma DPP-4 correlated moderately with FC $(\mathrm{r}=-0.31, P<0.05)$ and CRP $(\mathrm{r}=-0.32, P<0.05)$, strongly with pMS $(\mathrm{r}=-0.52, P<0.001)$ and with faecal DPP-4 $(r=0.52, P<0.001)$. The multivariable logistic regression model showed that plasma DPP-4 and CRP are independent predictors of CD activity [odds ratio (OR): $26.95 ; 95 \%$ confidence interval $(\mathrm{CI}): 3.01-240.96 ; P=0.003$ and $\mathrm{OR}$ : 38.02; $95 \%$ CI: $4.25-340.48 ; P=0.001$, respectively]. In UC patients, plasma DPP-4, FC and CRP showed to be independent predictors of disease activity (OR: 62.10; $95 \%$ CI: $2.93-1315.62 ; P=0.008$ and OR: $65.90 ; 95 \%$ CI: $4.96-$ $875.53 ; P=0.002$ and OR: 48.19 ; $95 \% \mathrm{CI}: 3.35-692.76 ; P=0.004$, respectively). At follow-up (median 578 days; IQR: 426-688), plasma DPP-4 and CRP independently predicted treatment escalation in CD [hazard ratio (HR) $9.09 ; 95 \% \mathrm{CI}$ : $1.77-46.57 ; P=0.008$ and HR 5.90; $95 \%$ CI: $1.53-22.78 ; P=0.010$; respectively], as well as in UC (HR 7.80; 95\% CI: $1.60-38.04 ; P=0.011$ and HR $12.23 ; 95 \%$ CI: $2.30-64.98 ; P=0.003$; respectively). At 1 year, the proportion of patients who needed treatment escalation was $66 \%$ in $\mathrm{CD}$ and $41 \%$ in UC, if $\geq 2$ biomarkers criteria were met.

Conclusion: An activity assessment model and a prognostic model that combines plasma DPP-4 and other biomarkers accurately predicts IBD activity and an aggressive disease course. Faecal DPP-4 correlates well with plasma DPP-4 in Ulcerative colitis, but not in Crohn's disease.

Disclosure of Interest: F. Magro: Has received fees for speaking engagements from ScheringPlough/MSD, Abbvie, Lab Vitória, and Dr Falk Pharma Portugal, and fees for consultancies and honoraria from MSD.

All other authors have declared no conflicts of interest.

\section{P1021 ROLE OF PET-CT TO ASSESS DISEASE ACTIVITY IN ULCERATIVE COLITIS AND ITS CORRELATION WITH CLINICAL CRITERIA, ENDOSCOPY AND FECAL BIOMARKERS}

N. Berry, S. K. Sinha, A. Bhattacharya, K. Prasad, C. Vaishnavi, K. Vaiphei, J. Samanta, R. Prasada, N. Dhaka, R. Kochhar

Gastroenterology, Postgraduate Institute of Medical Education and Research, Chandigarh/India

Contact E-mail Address: nehaberry86@hotmail.com

Introduction: Disease activity in ulcerative colitis (UC) is best assessed clinically by Mayo score and endoscopy. Positron emission tomography -computerized tomography (PET-CT) is a non-invasive imaging technique to assess disease activity, extent, treatment response in UC, specially in pediatric population, sick patients and those unwilling for endoscopy. 
Aims \& Methods: We conducted a prospective observational study at our tertiary care centre with the aim of assessing and correlating UC disease activity by clinical criteria, endoscopy, histology, serum and fecal biomarkers and PETCT. 60 eligible patients of UC were enrolled into 3 groups ( 26 remission, 24 moderate and 10 severe activity) as per Mayo score and ${ }^{18} \mathrm{~F}$ FDG PET-CT was performed within 72 hours of endoscopy. ESR, CRP and fecal calprotectin levels were determined for all patients.

Results: Of 60 enrolled patients, $10 \%$ patients had proctitis, $43.3 \%$ had left-sided colitis and $46.7 \%$ had extensive colitis. ESR, CRP, fecal calprotectin levels and rectal PET activity were significantly higher in patients with moderate and severe disease activity as compared to those in remission. Rectal PET activity showed a significant correlation with the Mayo score $(\mathrm{k}=0.465, \mathrm{p}<0.001)$, endoscopic sub-score $(\mathrm{k}=0.526, \mathrm{p}<0.001)$, histological score $(\mathrm{k}=0.496, \mathrm{p}<0.001)$, and fecal calprotectin levels $(\mathrm{k}=0.279, \mathrm{p}=0.031)$. Extent evaluation by PET-CT and colonoscopy also showed a significant correlation $(\mathrm{k}=0.582, \mathrm{p}<0.001)$ with each other. We found that CRP at a cut-off level of $<12 \mathrm{mg} / \mathrm{L}$ had a sensitivity of $70.59 \%$ and specificity of $92.3 \%$, and fecal calprotectin at a cutoff of $<143 \mathrm{ug} / \mathrm{g}$ had a sensitivity of $82.35 \%$ and specificity of $88.46 \%$ to predict remission. Besides, PET-CT identified sacroilitis in 1, mesenteric lymphadenopathy in 5 , mesenteric stranding in 4 , and adenocarcinoma in 1 patient.

Conclusion: PET-CT is a reliable non-invasive tool for assessing disease activity in UC with good correlation with the Mayo score, endoscopic score, histology and fecal calprotectin. It is an accurate measure to determine disease extent, and a good predictor of remission. Thus, with a better patient compliance, it holds promise in replacing colonoscopy where it is refused or difficult to perform.

Biomarkers and rectal PET activity of patients

\begin{tabular}{|c|c|c|c|c|c|}
\hline Variables & $\begin{array}{l}\text { Remission } \\
(\mathrm{n}=26)\end{array}$ & $\begin{array}{l}\text { Moderate } \\
\text { activity } \\
(\mathrm{n}=24)\end{array}$ & $\begin{array}{l}\text { Severe } \\
\text { activity } \\
(\mathrm{n}=10)\end{array}$ & $\begin{array}{l}\text { Total } \\
(\mathrm{n}=60)\end{array}$ & $\begin{array}{l}\mathrm{P*} \text { value } \\
\text { between } \\
\text { groups }\end{array}$ \\
\hline $\operatorname{ESR}(\mathrm{mm})($ mean $\pm \mathrm{SD})$ & $23.3 \pm 18.7$ & $35.1 \pm 22.3$ & $48.9 \pm 9.7$ & $32.3 \pm 21.0$ & 0.002 \\
\hline $\mathrm{CRP} \mathrm{mg} / \mathrm{L}($ mean $\pm \mathrm{SE})$ & $5.6 \pm 1.2$ & $25.3 \pm 4.3$ & $93.3 \pm 23.4$ & $28.1 \pm 5.7$ & 0.000 \\
\hline $\begin{array}{l}\text { Fecal calprotectin }(\mu \mathrm{g} / \mathrm{g}) \\
\quad(\text { mean } \pm \mathrm{SE})\end{array}$ & $72.27 \pm 13.65$ & $276.29 \pm 30.43$ & $426.6 \pm 64.95$ & $212.93 \pm 24.23$ & 0.001 \\
\hline $\begin{array}{l}\text { Rectal PET-CT activity } \\
\quad\left(\mathrm{SUV}_{\max }\right)(\text { Mean } \pm \mathrm{SD})\end{array}$ & $6.04 \pm 5.50$ & $10.79 \pm 3.88$ & $12.70 \pm 10.50$ & $9.05 \pm 6.56$ & 0.004 \\
\hline
\end{tabular}

Disclosure of Interest: All authors have declared no conflicts of interest.

\section{P1022 CLINICAL PERSPECTIVE ON DRUG MONITORING WITH \\ AN INFLIXIMAB RAPID QUANTIFICATION ASSAY}

F. Magro ${ }^{1}$, J. Afonso ${ }^{2}$, S. Lopes ${ }^{1}$, R. Coelho ${ }^{1}$, R. Gonçalves ${ }^{3}$, P. Caldeira ${ }^{4}$

P. Lago ${ }^{5}$, M.H. Tavares Sousa ${ }^{6}$, J. Ramos ${ }^{7}$, A.R. Gonçalves ${ }^{8}$, P. Ministro9,

I. Rosa ${ }^{10}$, T. Meira ${ }^{11}$, A. Andrade ${ }^{12}$, J. B. Soares ${ }^{4}$, D. Carvalho ${ }^{13}$, P. Sousa ${ }^{9}$,

A. Vieira ${ }^{11}$, C. Camila Dias ${ }^{14}$

${ }^{1}$ Gastroenterology, Centro Hospitalar São João, Porto/Portugal

${ }^{2}$ Biomedicine - Unity Pharmacology And Therapeutics, Faculty of Medicine of

Porto, Porto/Portugal

${ }^{3}$ Gastroenterology, Hospital de Braga, Braga/Portugal

${ }^{4}$ Gastroenterology, Centro Hospitalar do Algarve, Faro/Portugal

${ }^{5}$ Gastroenterology, Centro Hospitalar do Porto, Porto/Portugal

${ }^{6}$ Centro Hospitalar do Algarve, Portimão/Portugal

${ }^{7}$ Gastroenterology, Hospital De Sto António Dos Capuchos, Lisbon, Portugal,

Lisbon/Portugal

${ }^{8}$ Gastroenterology And Hepatology, Hospital de Santa Maria, Centro Hospitalar

Lisboa Norte-EPE, Lisboa, Portugal, Lisbon/Portugal

${ }^{9}$ S. Teotónio Hospital, Viseu/Portugal

${ }^{10}$ Gastroenterology, IPOLFG, EPE, Lisboa/Portugal

${ }^{11}$ Hospital Garcia Orta, Almada/Portugal

${ }^{12}$ Gastroenterology, Hospital São João, Porto/Portugal

${ }^{13}$ Serviço De Gastrenterologia, Centro Hospitalar de Lisboa Central EPE, Lisboa/ Portugal

${ }^{14}$ Department of Community Medicine, Information and Health Decision Sciences, Faculty of Medicine of the University of Porto, Porto/Portugal

Contact E-mail Address: joanavafonso@gmail.com

Introduction: The importance of therapeutic drug monitoring (TDM)-based algorithms to guide infliximab (IFX) adjustments in inflammatory bowel disease (IBD) patients is unquestionable. Currently, most of the methods commercially available for the quantification of IFX are ELISA-based delaying the target dosage adjustment to the following infusion. A new rapid assay is now available in the market.

Aims \& Methods: This study aimed to explore a recently-launched IFX quantification rapid test from a clinical perspective. A prospective cohort involving 110 ulcerative colitis (UC) patients under IFX maintenance therapy was study. IFX trough levels were quantified using a rapid quantification assay and a commonly used reference kit.

Results: IFX values were statistically different when comparing patients with or without endoscopic remission (Mayo endoscopic score $=0$ ) and stratified by their faecal calprotectin (FC) levels, regardless the methodology used. Though the two methods correlated well with each other (Spearman correlation coefficient $=0.843, \mathrm{p}<0.001$; intraclass correlation coefficients $=0.857,95 \%$ CI: $0.791-0.903$ ), values obtained with the reference kit were on average 2.62 units higher than those obtained with the rapid system. Nevertheless, $3 \mathrm{ug} / \mathrm{ml}$ was shown to be an appropriate cut-off to assess endoscopic remission and inflammatory burden levels with both assays. In fact, eighty eight percent of patients that had a positive outcome showed IFX values, with the rapid assay, above $3 \mathrm{ug} /$ $\mathrm{mL}$, Mayo endoscopic score $\leq 1$ and for an FC concentration $<250 \mathrm{ug} / \mathrm{g}$.

Conclusion: Our results show that the rapid IFX assessment system is a reliable alternative to the time-consuming ELISAs in patients on the maintenance phase of IFX operating a threshold of $3 \mathrm{ug} / \mathrm{ml}$.

Disclosure of Interest: F. Magro: Prof. Magro has received fees for speaking engagements from ScheringPlough/MSD, Abbvie, Lab Vitoria, and Dr.Falk Pharma Portugal, and fees for consultancies and honoraria from MSD.

All other authors have declared no conflicts of interest.

\section{P1023 THE IMPACT OF AN INFLAMMATORY BOWEL DISEASE EDUCATION CLINIC ON PATIENT OUTCOMES AND RESOURCE UTILISATION}

W. On, B. Collins, H. Stanley, A. Westhoff, S. Tynan, D. Mcclements, V. Theis, J. Mclindon, R. Chandy, A. Bassi

Gastroenterology, St Helens \& Knowsley Teaching Hospitals NHS Trust,

Liverpool/United Kingdom

\section{Contact E-mail Address: weiheng.on@gmail.com}

Introduction: Patient education and awareness in those who have inflammatory bowel disease (IBD) and is regarded by the United Kingdom (UK) IBD standards group as a key standard in the provision of care towards patients with IBD. ${ }^{1}$ Our district general hospital implemented an IBD education clinic in 2015 which is run quarterly per annum by a multidisciplinary panel of doctors, specialist nurses, pharmacists and dietitians. Patients who have been newly diagnosed with IBD are invited to attend this clinic. Clinic attendees are given an overview of their disease, treatment modalities, options of non-medical support (e.g: psychology) and are made aware of our telephone helpline open access service. To date, there has not been any published data on the impact of an IBD education clinic on resource use or patient outcomes.

Aims \& Methods: We aimed to evaluate the impact of the education clinic on resource use in patients who attended the clinic compared to patients who did not. A retrospective analysis was done of patients who were diagnosed with IBD between January 2013 and May 2015. 40 patients were identified and divided equally (20 patients each) into clinic attenders (CA) and non-attenders (NA). Resource use was determined at 12 months from diagnosis in the NA group and 12 months from attendance in the CA group. The median time from diagnosis to clinic attendance in the CA group was 7 months. Data was obtained from our hospital's electronic database system. Statistical analysis was carried out with the student's t-test.

Results: The median age was 37 in the CA group and 33 in the NA group. In the CA group, 10 patients had ulcerative colitis (UC), 9 patients had Crohn's Disease (CD) and 1 patient had unclassified IBD. In the NA group, 13 patients had UC and 7 patients had $C D$. The median of resource use and patient outcomes over a 12 month period (from diagnosis in the NA group and from clinic attendance in the CA group) is detailed in the table below.

\begin{tabular}{llll}
\hline Resource use and patient outcomes & NA & CA & p-value \\
\hline Steroid courses & 0.47 & 0.51 & 1 \\
Unplanned hospital admissions & 0.19 & 0.21 & 1 \\
IBD telephone helpline consultations & 0.24 & 2.58 & 0.001 \\
Clinic appointments & 3.71 & 2.84 & 0.08 \\
Blood tests (excluding essential monitoring blood tests) & 5.81 & 3.68 & 0.14 \\
Endoscopies & 0.33 & 0.26 & 0.77 \\
Radiological imaging & 0.24 & 0.16 & 0.5 \\
Therapy escalation & 1.4 & 1.15 & 0.62 \\
\hline
\end{tabular}

Conclusion: In our cohort of patients, patients who attended the IBD education clinic were more likely to utilise our open access IBD telephone helpline service. There was a trend towards increased frequency of outpatient clinic appointments and blood tests in patients who did not attend the IBD education clinic although this was not statistically significant. There were no differences in the rates of hospital admissions or steroid courses in either group. The limitations of our data include the small sample size and short follow-up period.

Disclosure of Interest: All authors have declared no conflicts of interest.

\section{Reference}

1. THE IBD STANDARDS GROUP. http://s3-eu-west-1.amazonaws.com/files.crohnsandcolitis.org.uk/Publications/PPR/ibd_standards_13.pdf [online] 
P1024 EMERGING ROLE OF IL-33/ST2 LEVELS IN PREDICTING MUCOSAL RESPONSE TO ANTI-TNF THERAPY IN ULCERATIVE COLITIS

L.R. Lopetuso ${ }^{1}$, V. Petito ${ }^{1}$, C. Graziani ${ }^{1}$, T. T. Pizarro ${ }^{2}$, F. Scaldaferri ${ }^{1}$, A. Gasbarrini ${ }^{1}$

${ }^{1}$ UOC Internal Medicine and Gastroenterology, Gastroenterology Area, Fondazione Policlinico A. Gemelli, Catholic University of Sacred Heart, Rome/ Italy

${ }^{2}$ Pathology, Case Western Reserve University, Cleveland/United States of America/OH

\section{Contact E-mail Address: lopetusoloris@libero.it}

Introduction: Tumor necrosis factor (TNF) inhibitors (anti-TNF) are considered to be effective in inducing mucosal healing in patients with moderate-to-severe Ulcerative Colitis (UC). The role of IL-33 and its receptor, ST2, in intestinal inflammation is incompletely understood, with both pro-inflammatory and regulatory properties described. Recent evidence has shown that anti-TNF is able to modulate the IL-33/ST2 axis in inflammatory conditions.

Aims \& Methods: The aim of our study was to explore the potential role of the IL-33/ST2 axis in the mucosal healing process mediated by anti-TNF therapy in UC. Endoscopic MAYO score was calculated before the first anti-TNF infusion (T0) and after 6 weeks (T2). 24 UC patients (MAYO score at T0 $\geq 2$ ), grouped into 12 responders with mucosal healing (MAYO score $\leq 1)$ and 12 non-responders to anti-TNF at T2 (MAYO score $\geq 2$ ) were enrolled. 10 healthy controls undergoing routine colonoscopy for tumor screening were also enrolled. At each time point, serum samples were collected and ELISA performed to assess IL-33 ST2 protein levels. Intestinal biopsies were also taken from the rectum and IHC was done to evaluate mucosal IL-33/ST2 expression and localization.

Results: IL-33 protein levels were significantly increased in responders vs. nonresponders, both at T0 and T2. Among responders, IL-33 protein was slightly reduced at T2 vs. T0, while unchanged in non-responders. Interestingly, significantly higher levels of ST2 were found in responders vs. not responders at T0, while no differences between groups were found at T2. Among responders, ST2 levels were dramatically reduced at T2 vs. T0. No significant differences were found in non-responders at both time points. Healthy controls showed significantly lower levels of both IL-33 and ST2 compared with other groups. IHC confirmed these observations. In particular, IL-33 and ST2 staining was more intense within the inflamed and ulcerated mucosa of responders compared to non-responders at T0. After 6 weeks, ST2 staining was even more evident in responders, notably localized to the healed mucosa and in close proximity to areas of re-epithelialization. Little to no staining for both IL-33 and ST2 was present in healthy controls.

Conclusion: Our results suggest a possible role for IL-33/ST2 in predicting gut mucosal wound healing in patients with moderate-to-severe UC treated with anti-TNF. Further studies are underway to determine mechanisms of action that support these findings.

Disclosure of Interest: All authors have declared no conflicts of interest.

\section{P1025 INFLIXIMAB TROUGH LEVELS IN THE INDUCTION PHASE ARE ASSOCIATED WITH PROLONGED REMISSION IN CROHŃS DISEASE PATIENTS}

M.D. Martín-Arranz ${ }^{1}$, M. Jaquotot Herranz ${ }^{2}$, E. Martín-Arranz ${ }^{2}$, A. Martínez Feito $^{3}$, M. Díaz-Almirón ${ }^{4}$, C. Suárez Ferrer ${ }^{2}$, J. Poza Cordon ${ }^{2}$, S. Gómez Senent $^{2}$, L. García Ramirez ${ }^{1}$, M.P. Aracelia ${ }^{5}$, D. Pascual-Salcedo ${ }^{3}$

${ }^{1}$ Gastroenterology, La Paz, Madrid/Spain

${ }^{2}$ Gastroenterology, University Hospital La Paz, Madrid/Spain

${ }^{3}$ Inmunology, IdiPaz, MADRID/Spain

${ }^{4}$ Bioestadistics, University Hospital La Paz, Madrid/Spain

${ }^{5}$ Inmunology, University Hospital La Paz, Madrid/Spain

\section{Contact E-mail Address: mmartinarranz@salud.madrid.org}

Introduction: Higher infliximab (IFX) serum concentrations have been associated with higher rates of clinical remission in inflammatory bowel disease patients ${ }^{1,2}$. However, the correlation between IFX trough levels (TL) during the induction phase and in medium or long-term remission in Crohn's disease (CD) patients is less clear ${ }^{3}$.

Aims \& Methods: We aimed to analyse whether the IFX-TL were able to predict medium ( 22 weeks) and long-term (54 weeks) remission in CD patients. Consecutive CD patients receiving IFX $(5 \mathrm{mg} / \mathrm{kg}$ at weeks $0,2,6$ and every eight weeks at least until 22 week) were included retrospectively. IFX-TL were measured by capture ELISA and anti-Infliximab antibodies (ATI) by a bridging ELISA before each infusion ${ }^{4}$. Clinical response and biological parameters were also measured. The investigator defined remission based on clinical (HarveyBradshaw score), biological and endoscopic or imaging technics; partial or no response, as well as intensification decided by investigators were considered as non-remission patients. U-Mann Whitney test was used to assess association between IFX-TL and remission. ROC curves were included to obtain cut points for clinical use.

Results: 32 CD patients were screened, but finally 29 analysed, because 3 patients had IFX discontinued at week 14. Baseline characteristics included: 16 female: $55.2 \%, 31 \%$ smoker, $31 \%$ ex-smoker, $79.3 \%$ ileal or ileocolonic involvement, $44.8 \%$ inflammatory behavior, with $6.9 \%$ previous abdominal surgery and $86.2 \%$ on concomitant immunosuppressant, $34.5 \%$ non-naive to biological therapy. On week $22,44.8 \%$ patients were in remission; on week $54.40 \%$ were in remission.

\begin{tabular}{llllll}
\hline \multicolumn{7}{c}{ Week } & $\begin{array}{l}\text { REMISSION } \\
\text { Median }(\mathrm{IQR}) \\
\text { IFX-TL }(\mu \mathrm{g} / \mathrm{mL})\end{array}$ & $\begin{array}{l}\text { NO REMISSION } \\
\text { Median }(\mathrm{IQR}) \\
\text { IFX-TL }(\mu \mathrm{g} / \mathrm{mL})\end{array}$ & $\mathrm{Z}$ & $\mathrm{p}$ \\
\hline Week 6 & 22 & $24.6(18.4-41.3)$ & $12.7(5.2-22.2)$ & -2.982 & 0.002 \\
& 54 & $28.2(18.3-40)$ & $15.1(5.6-21.3)$ & -2.441 & 0.014 \\
Week 14 & 22 & $9.5(6.8-14)$ & $1.8(0.9-9.2)$ & -2.647 & 0.007 \\
& 54 & $8.4(5.2-13.3)$ & $3.8(1.3-9.5)$ & -1.580 & 0.123 \\
\hline
\end{tabular}

On week 6 , IFX-TL $>24 \mu \mathrm{g} / \mathrm{mL}$ was associated with remission at week 22 (ROC 0.83 ; S: $61.5 \%$, Sp: $81.3 \%$ ) and at week 54 (ROC 0.79; S 60\%, E $80 \%$ ). On week 14, IFX-TL $>11 \mu \mathrm{g} / \mathrm{mL}$ was associated with remission at week 22 (ROC 0.80; S: $41.7 \%$, Sp: $93.8 \%$ ) and at week 54 (ROC 0.70; S: $44.4 \%$, Sp: $86.7 \%$ ).

Conclusion: IFX-TL during the induction period are associated with remission at 6 and 12 months. Optimal TL ranges associated to remission can be calculated to guide clinical decisions. can be calculated to guide clinical decisions.

Disclosure of Interest: All authors have declared no conflicts of interest.

\section{References}

1. Steenholdt C, Bendtzen K, Brynskov J, Thomsen OØ, Ainsworth MA. Cutoff levels and diagnostic accuracy of infliximab trough levels and anti-infliximab antibodies in Crohn's disease. Scand J Gastroenterol 2011; 46: 310-318.

2. Bortlik M, Duricova D, Malickova K, Machkova N, Bouzkova E, Hrdlicka $\mathrm{L}$ et al. Infliximab trough levels may predict sustained response to infliximab in patients with Crohn's disease. J Crohns Colitis 2013; 7: 736-743.

3. Cornillie F, Hanauer SB, Diamond RH, Wang J, Tang KL, $\mathrm{Xu} \mathrm{Z}$ et al. Postinduction serum infliximab trough level and decrease of C-reactive protein level are associated with durable sustained response to infliximab: a retrospective analysis of the ACCENT I trial. Gut. 2014 Nov;63(11):1721-7.

4. Pascual-Salcedo D, Plasencia C, Ramiro S, Nuño L, Bonilla G, Nagore D, et al. Influence of immunogenicity on the efficacy of long-term treatment with infliximab in rheumatoid arthritis. Rheumatology (Oxford). 2011 Aug;50(8):1445-52.

\section{P1026 CORRELATION BETWEEN CLINICAL, ENDOSCOPIC AND HISTOLOGICAL ACTIVITY IN INFLAMMATORY BOWEL DISEASE: A PROSPECTIVE STUDY}

B. Neri $^{1}$, A. Ruffa ${ }^{1}$, S. Romeo ${ }^{1}$, E. Grasso ${ }^{1}$, F. Zorzi ${ }^{1}$, G. Palmieri ${ }^{2}$, L. Biancone ${ }^{1}$ ${ }^{1}$ Medicina Dei Sistemi, Università degli studi di Roma Tor Vergata, Roma/Italy ${ }^{2}$ Anatomia Patologica, Universita degli studi di Roma Tor Vergata, Roma/Italy

Contact E-mail Address: benedettoneri@gmail.com

Introduction: Several histological scores of activity have been developed in Inflammatory Bowel Disease (IBD). However, their usefulness in clinical practice and the correlation between clinical, endoscopic and histological scores is undefined.

Aims \& Methods: To assess, in a prospective study, the correlation between clinical, endoscopic and histological activity scores in a cohort of IBD patients (pts) undergoing colonoscopy. Secondary end-point was to assess the role of histological scores in clinical practice. From Feb. to Dec. 2016, IBD pts undergoing colonoscopy according to clinical indication were enrolled. Inclusion criteria: 1. Diagnosis of IBD; 2. Age $>18$ and $<80$ yrs; 3. Regular follow up; 4. Indication for colonoscopy; 5. No severe comorbidities. During colonoscopy, $\geq 2$ biopsies were taken from $\geq 1$ macroscopically involved area and, possibly, from $\geq 1$ uninvolved area. Clinical activity was assessed by the Mayo score $($ activity $\geq 3)(2)$ for Ulcerative Colitis (UC) and the CDAI (activity $\geq 150)(1)$ for Crohn's Disease (CD) pts. All colonoscopies were performed by one IBDdedicated gastroenterologist. Endoscopic activity was assessed in UC pts by the Mayo score (activity $\geq 2)(2)$, and in $\mathrm{CD}$ by the Rutgeerts' score (recurrence $\geq 2)(3)$ or the SES-CD (activity $\geq 3)(4)$, according to previous surgery. Histological activity was assessed by one pathologist using the Global Histologic Activity Score (GHAS) for CD (5) or the Geboes simplified score for UC (activity $\geq 3.1)(6)$. Each of the 3 investigators was blind regarding the other activity scores. Statistical analysis. Data expressed as median [range], correlations assessed by the Spearman correlation test.

Results: IBD group included 107 pts: 67 (62.6\%) UC (M 36 [53.7\%], age 50 [2480], UC duration 14 yrs [1-48]), 40 (37.4\%) CD (M 24 [60\%], age 46 [19-75], CD duration 14 yrs [1-36]). UC extent included: pancolitis in $34(51 \%)$, left sided in $17(25 \%)$, proctitis in $16(24 \%)$ pts.CD involved $(n=[\%])$ : the ileum $(21[52 \%])$, colon $(6[15 \%])$, ileum-colon $(12[30 \%])$, upper GI $(1[3 \%])$, previous surgery in $18(45 \%)$. CD behavior was fibrostricturing $(\mathrm{n}=15 ; 37 \%)$, penetrating $(\mathrm{n}=11 ; 28 \%)$, non-stricturing non-penetrating $(\mathrm{n}=14 ; 35 \%)$, No complications occurred. Clinical Activity: UC. The day of colonoscopy, UC was clinically active in $14(21 \%)$, in remission in $53(79 \%)$ pts. Endoscopic activity: UC. Endoscopic activity was observed in $31(46 \%)$, remission in $36(54 \%)$ pts. (Mayo score: $0[\mathrm{n}=14] ; 1[\mathrm{n}=22] ; 2[\mathrm{n}=13], 3[\mathrm{n}=18])$. Histologic activity: $U C$. Microscopic activity was observed in 24/67 (36\%) pts: 4/24 pts showed endoscopic remission. Correlations: UC. In UC, a significant correlation was observed between: clinical and endoscopic scores $(r=0.52 ; p<0.0001)$; clinical and histologic scores $(\mathrm{r}=0.28 ; \mathrm{p}=0.02)$; endoscopic and histological scores $(\mathrm{r}=0.56 ; \mathrm{p}<0.0001)$. Clinical Activity: $C D$. At colonoscopy, $\mathrm{CD}$ was clinically 
active in $5(12 \%)$, in remission in $35(88 \%)$ pts. Endoscopic activity: $C D$ Colonoscopy was incomplete in $2 / 40$ pts. In the 24 pts with no previous surgery, SES-CD was: $0(\mathrm{n}=4), 3(\mathrm{n}=1) 4(\mathrm{n}=3), 6(\mathrm{n}=2) ; 8(\mathrm{n}=3) ; 9(\mathrm{n}=2) ; 10(\mathrm{n}=1)$; $11(\mathrm{n}=1) ; 12(\mathrm{n}=1) ; 13(\mathrm{n}=1) ; 14(\mathrm{n}=1) ; 17(\mathrm{n}=1) ; 19(\mathrm{n}=1)$ pts (median $4[0$ 19]; activity: $20 / 24$ [80\%], remission: $4 / 24$ [20\%]). In the 16 pts with previous surgery, the Rutgeerts' score was: $0 \quad(n=3) ; 1 \quad(n=1), 2 \quad(n=6) ; 3 \quad(n=2) 4$ $(\mathrm{n}=4)($ recurrence: $12 / 16[75 \%])$. Histologic activity: $C D$. The GHAS was:0 $(\mathrm{n}=5) ; 1 \quad(\mathrm{n}=11) ; 2(\mathrm{n}=3) ; 4(\mathrm{n}=1) ; 6(\mathrm{n}=2) ; 7(\mathrm{n}=1) ; 9(\mathrm{n}=1)$ in pts without previous surgery, and $0(\mathrm{n}=3) ; 1(\mathrm{n}=9) ; 3(\mathrm{n}=1) ; 10(\mathrm{n}=1)$ in pts with previous surgery.In $\mathrm{CD}$, the histological score showed a slightly significant correlation with SES-CD $(\mathrm{r}=0.41 ; \mathrm{p}=0.046)$ and no correlation with the Rutgeerts' score $(\mathrm{r}=0.31 ; \mathrm{p}=0.247)$.

Conclusion: In a prospective study, a significant correlation was observed between clinical, endoscopic and histological activity in UC. Histological activity may be observed in UC patients in endoscopic remission, thus suggesting that this finding may represent a predictive marker of clinical relapse.

Disclosure of Interest: L. Biancone: The study was not supported by grants nor funded. The reported disclosures are not related to the study: L. Biancone Lecture fee(s): MSD, Takeda, Abbvie, Zambon, A. Armuzzi Lecture fee(s): Abbvie,

All other authors have declared no conflicts of interest.

\section{References}

1. Best WR Gastroenterology 1976;70:439

2. Schroeder KW. NEJM 1987;317:1625

3. Rutgeerts P. Gastroenterology 1990;99:956

4. Daperno M. GIE 2004;60:505

5. D'Haens GR. Gastroenterology 1998;114:262

6. Jauregui-Amezaga A. JCC 2017;11:305

\section{P1027 THE RELATIONSHIP BETWEEN SERUM INFLIXIMAB AND ADALIMUMAB CONCENTRATIONS AND THEIR CORRESPONDING ANTI-DRUG ANTIBODY LEVELS: ANALYSIS OF OVER 50, 000 PATIENT RESULTS USING LAB-DEVELOPED CHEMILUMINESCENT IMMUNOASSAYS}

\section{K. Y. Chun ${ }^{1}$, J. Yang ${ }^{2}$}

${ }^{1}$ Esoterix Specialty Medicine, Labcorp, Calabasas/United States of America/CA ${ }^{2}$ Medical Affairs, LabCorp, Calabasas/United States of America/CA

\section{Contact E-mail Address: chunk@1abcorp.com}

Introduction: Assays to measure TNF inhibitors and anti-drug antibodies $(A D A b)$ in patient serum are being utilized to manage failure to respond and loss of response. These monitoring assays may also be used to proactively titrate doses and dosing intervals. Here, 36,477infliximab (IFX) and 21,284 adalimumab patient results from the past 4 years were analyzed.

Aims \& Methods: Measurements of drug and ADAb levels were performed by lab-developed chemiluminescent immunoassays.[1] Lower limits of quantitation are $0.4 \mathrm{ug} / \mathrm{mL}$ for IFX and $0.6 \mathrm{ug} / \mathrm{mL}$ for adalimumab. ADAb assays are drug tolerant in the presence of up to $100 \mathrm{ug} / \mathrm{mL}$ of drug. Clinical histories and blood collection timing are unknown.

Results: Of 36,477 measured IFX samples, 56\% $(20,353)$ were ADAb-free and $44 \%(16,224)$ had measureable ADAb ranging from 22 to 3.5 million ng/mL. In the absence of ADAb, IFX concentrations were from 0.4 to $>100 \mathrm{ug} / \mathrm{mL}$, with the majority $(>60 \%$ ) between 3.0 to $20 \mathrm{ug} / \mathrm{mL}$. In the presence of $\mathrm{ADAb}$, an inverse relationship between IFX concentrations and anti-IFX antibodies is clearly evident (Table 1). As a reference, an ADAb-free mean drug level was determined from samples with IFX between 0.4-30 ug/mL. Decreasing mean IFX concentrations (column 3) with increasing ADAb (column 1) are demonstrated where low ADAb $(<200 \mathrm{ng} / \mathrm{mL})$ appears to have minimal impact on mean drug levels while high ADAb $(>1000 \mathrm{ng} / \mathrm{mL})$ is invariably associated with severely diminished IFX $(10 \%$ or less than the mean). A similar drug $\mathrm{ADAb}$ relationship was observed with adalimumab (Table 2). Interestingly, even low titer anti-adalimumab antibodies are associated with a diminished mean adalimumab $(3.4-5.6 \mathrm{ug} / \mathrm{mL})$ where the baseline mean adalimumab (6.1 $\mathrm{ug} / \mathrm{mL}$ ) was calculated from 10, 784 ADAb-free samples with adalimumab from $0.6-15 \mathrm{ug} / \mathrm{mL}$. Anti-adalimumab antibody levels $>300 \mathrm{ng} / \mathrm{mL}$ are associated with free adalimumab concentrations $<30 \%$ of the mean. Of all adalimumab patient samples, $60 \%(12,873)$ were free of ADAb, and $40 \%$ (8411) were ADAb positive, ranging from 25 to 2.8 million $\mathrm{ng} / \mathrm{mL}$. For adalimumab in Table 2, as ADAb levels increase (column 1), their corresponding mean drug concentrations diminish (column 3), similar to IFX results in Table 1.

Table 1: Anti-Infliximab Antibody Distribution and Corresponding Mean Free Drug Levels

\begin{tabular}{|c|c|c|c|}
\hline $\begin{array}{l}\text { Anti-Infliximab } \\
\text { Antibody } \\
\text { Ranges }(\mathrm{ng} / \mathrm{mL}) \\
\text { undetected }\end{array}$ & $\begin{array}{l}\mathrm{n} \\
15692\end{array}$ & $\begin{array}{l}\text { Mean Drug } \\
\text { Concentration } \\
(\mathrm{ug} / \mathrm{mL}) \\
10\end{array}$ & $\begin{array}{l}\% \text { with } \\
\text { Undetectable } \\
\text { Drug }(<0.4 \mathrm{ug} / \mathrm{mL}) \\
0 \%\end{array}$ \\
\hline $22-100$ & 6785 & 9.5 & $11 \%$ \\
\hline $101-200$ & 2684 & 8.6 & $19 \%$ \\
\hline $201-300$ & 1344 & 6.3 & $27 \%$ \\
\hline $301-500$ & 1427 & 5.2 & $38 \%$ \\
\hline $501-700$ & 789 & 3.7 & $53 \%$ \\
\hline
\end{tabular}

\begin{tabular}{llll} 
Table 1: Continued & & & \\
\hline Anti-Infliximab & & Mean Drug & $\%$ with \\
Antibody & & Concentration & Undetectable \\
Ranges $(\mathrm{ng} / \mathrm{mL})$ & $\mathrm{n}$ & $(\mathrm{ug} / \mathrm{mL})$ & $\begin{array}{l}\text { Drug }(<0.4 \mathrm{ug} / \mathrm{mL}) \\
701-1000\end{array}$ \\
802 & 3.0 & $79 \%$ \\
$1001-2000$ & 1246 & 1.0 & $86 \%$ \\
$2001-4000$ & 575 & $<0.4$ & $100 \%$ \\
$4001-3.5$ million & 572 & $<0.4$ & \\
\hline
\end{tabular}

Table 2: Anti-Adalimumab Antibody Distribution and Corresponding Mean Free Drug Levels

\begin{tabular}{|c|c|c|c|c|}
\hline \multirow{3}{*}{$\begin{array}{l}\text { Anti-Adalimumab } \\
\text { Antibody } \\
\text { Ranges (ng/mL) } \\
\text { undetected }\end{array}$} & \multirow[b]{3}{*}{$\begin{array}{l}\mathrm{n} \\
10784\end{array}$} & \multirow{3}{*}{$\begin{array}{l}\text { Mean Drug } \\
\text { Concentration } \\
\text { (ug/mL) } \\
6.1\end{array}$} & \multirow{3}{*}{$\begin{array}{l}\% \text { with } \\
\text { Undetectable } \\
\text { Drug }(<0.6 \mathrm{ug} / \mathrm{mL}) \\
0 \%\end{array}$} & - \\
\hline & & & & \\
\hline & & & & \\
\hline $25-100$ & 3904 & 5.6 & $5 \%$ & \\
\hline $101-200$ & 1144 & 3.4 & $12 \%$ & \\
\hline $201-300$ & 570 & 2.4 & $24 \%$ & \\
\hline $301-500$ & 655 & 1.8 & $39 \%$ & \\
\hline $501-700$ & 330 & 1.1 & $57 \%$ & \\
\hline $701-1000$ & 303 & 0.7 & $75 \%$ & \\
\hline $1001-2000$ & 496 & $<0.6$ & $93 \%$ & \\
\hline $2001-4000$ & 394 & $<0.6$ & $97 \%$ & \\
\hline $4001-2.8$ million & 610 & $<0.6$ & $99 \%$ & \\
\hline
\end{tabular}

Conclusion: Upon analysis of 57,861 infliximab and adalimumab patient samples from 2012-2016, 43\% exhibited anti-drug antibodies. We found that low-titer antibodies do not appear to impact drug levels. Our findings are consistent with American Gastroenterological Association Critical Care Pathways for Crohn's Disease and Ulcerative Colitis where low and high antibody scenarios are managed very differently (increase drug/consider immunomodulator vs. switch drug within class). High resolution antibody assays may be helpful in dosing TNF inhibitors and in other treatment and management decisions.

Disclosure of Interest: All authors have declared no conflicts of interest.

\section{Reference}

1. Marini et al. AAPS Journal 2016 DOI:10.1208/s12248-016-9981-3.

\section{P1028 REAL-WORLD USE OF IMMUNOSUPPRESSIVES AMONG PATIENTS WITH INFLAMMATORY BOWEL DISEASE TREATED WITH VEDOLIZUMAB}

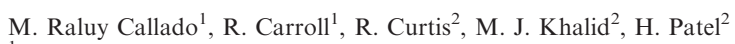

${ }^{1}$ Evidera, London/United Kingdom

${ }^{2}$ Takeda Development Centre Europe Ltd, London/United Kingdom

Contact E-mail Address: hari.patel@takeda.com

Introduction: Immunosuppressives (IM) are often used as maintenance therapy to reduce the risk of relapse among patients with inflammatory bowel disease (IBD). Vedolizumab (VDZ), a humanized monoclonal antibody that targets the $\alpha 4 \beta 7$ integrin, has been approved for the treatment of moderately-to-severely active Crohn's disease (CD) and ulcerative colitis (UC).

Aims \& Methods: The aim was to assess the real-world use of IM therapy and compare outcomes in IBD patients with, versus without a history of IM use, who initiated treatment on VDZ in the United States. The Explorys Universe database was used to identify all IBD patients $>18$ years of age who: (1) initiated VDZ therapy between May 20, 2014, and February 22, 2016 (the date of VDZ initiation was the assigned index date); and (2) had 365 days of available data pre- and post- index date (follow-up). Patients were stratified based on the use of IM at any point in their treatment history before the index date. Key outcomes in the follow-up period included the use of IM; and the incidence of IBD-related surgeries, hospitalizations, and flares (defined as the use of intravenous corticosteroids, IBD-related surgeries, or hospitalizations).

Results: A total of 567 patients were included, of which $68.4 \%$ had CD, and $31.6 \%$ had UC. Mean (standard deviation [SD]) age at index was 44 (15.0) years; $58.6 \%$ were female. Overall, $54.6 \%$ had a history of prior IM use, and $64.6 \%$ received anti-TNFs before starting VDZ therapy. On average, patients initiated VDZ 4.5 (3.6) years following their initial diagnosis. Of the $54.6 \%$ of patients with a history of IM therapy, $61.0 \%$ did not use IM during maintenance treatment with VDZ during follow-up. Of the $45.4 \%$ of patients without a history of IM use, $87.0 \%$ did not initiate IM therapy during follow-up. Amongst VDZ patients with a history of IM vs. without history of IM use, there was a trend of increased flares $(38.7 \%$ vs. $30.0 \%, p=0.034)$, hospitalizations $(21.9 \%$ vs. $19.8 \%, \mathrm{p}=0.605)$, and surgeries $(10.6 \%$ vs. $6.6 \%, \mathrm{p}=0.103)$. Findings for UC and $C D$ patients are presented in Table 1.

Conclusion: The majority of patients with a history of IM use did not use IM therapy after initiating VDZ in a real-world clinical practice. The use of IM after initiating VDZ was also low amongst patients without a history of IM use. Lower rates of healthcare resource utilization were observed among patients without a history of IM use. Further research is needed to better understand the degree to 
which IM therapy is used concomitantly with VDZ and potential impact on outcomes in real-world clinical practice.

Disclosure of Interest: M. Raluy Callado: Miriea Raluy Callado is a full-time employee of Evidera.

R. Carroll: Robert Carroll is a full-time employee of Evidera.

R. Curtis: Employee of Takeda Development Centre Ltd.

M.J. Khalid: Employee of Takeda Development Centre Ltd.

H. Patel: I am currently an employee of Immensity Consulting Inc., which received funding from Takeda Development Centre Ltd.

\section{P1029 MOLECULAR SURROGATES OF HISTOLOGIC ACTIVITY IN CROHN'S DISEASE}

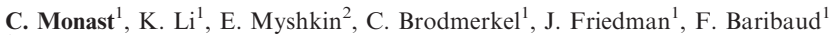
${ }^{1}$ Janssen Research \& Development, LLC, Spring House/United States of America/ $P A$

${ }^{2}$ Clarivate Analytics, Boston/United States of America/MA

\section{Contact E-mail Address: cmonast1@ITS.JNJ.com}

Introduction: Biomarkers of inflammatory bowel disease activity have been researched for decades but objective markers of disease severity that support clinical decision-making are still needed. Well-established markers include serum C-reactive protein and fecal calprotectin, but their use as a standalone surrogate for disease activity has been controversial. We hypothesize that novel objective markers of tissue inflammation are best identified at the site of disease with a tissue-level assessment of disease activity.

Aims \& Methods: Biopsy samples were obtained from participants in the UNITI trials of ustekinumab in moderate-to-severe Crohn's disease. The UNITI induction trials included two cohorts, patients who failed $\geq 1$ TNF antagonists (UNITI-1) or patients who failed conventional therapies (UNITI-2). Pairs of adjacent biopsies were taken from the rectum, splenic flexure, and ileum. One biopsy from each pair was assessed by Global Histology Disease Activity Score (GHAS) while the other was submitted to microarray analysis. Partial least squares regression and random forest were used to identify biomarkers associated with histological severity in the UNITI-1 cohort. Robustness of the resulting models was assessed using cross-validation within the training set and multiple external validation sets (defined within the UNITI-1 and UNITI-2 cohorts).

Results: In UNITI-1, a single multivariate model comprising 16 genes was identified that predicted histological activities in rectum or splenic flexure biopsies. This model was characterized by $\mathrm{R}^{2}=0.78$ for the training set, and $\mathrm{R}^{2}=0.59$, 0.54 , and 0.32 on external validation sets also from UNITI-1. A separate 14-gene model capturing histological activity in ileal biopsies was characterized by $\mathrm{R}^{2}=0.5$ for the training set and $\mathrm{R}^{2}=0.45$ in the external validation set. In general, both models contained genes related to tissue degradation, barrier function, and immune regulation, including CXCL11 (I-TAC). Both models retained performance in external validation datasets from UNITI-2 but exhibited lower performance. De novo models generated from UNITI-2 also exhibited lower performance. Indeed, weighted gene co-expression network analysis indicated weaker associations between gene expression and histology scores for UNITI-2 compared to UNITI-1 subjects.

Conclusion: Our analysis supports the ability of biopsy transcriptomics combined with machine learning approaches to capture disease-relevant variability in Crohn's disease and, more importantly, supports the use of similar approaches to identify additional surrogate markers. Interestingly, this approach was more successful in the TNF antagonist failure cohort compared to the conventional therapy failure cohort. We hypothesize that this is related to increased strength of the transcriptional signal in the TNF antagonist failure cohort. We identified specific genes that could be used together as surrogates for histologic measurement, which may not be susceptible to the subjectivity inherent in GHAS scoring. Finally, the specific genes identified by our analysis provide insight into the molecular processes driving histological disease activity in Crohn's disease. Disclosure of Interest: C. Monast: Janssen Research \& Development, LLC employee

K. Li: Janssen Research \& Development, LLC employee

E. Myshkin: Consultant to Janssen Research \& Development, LLC

C. Brodmerkel: Janssen Research \& Development, LLC employee

J. Friedman: Janssen Research \& Development, LLC employee

F. Baribaud: Janssen Research \& Development, LLC employee

\section{Reference}

1. Pacou M, Mesana L, Gauthier A, Naessens D, Sloan S, Danese S, Bonovas $\mathrm{S}$, Abrams K."Indirect Treatment Comparison of Ustekinumab Versus Other Biologics In Moderate To Severe Crohn's Disease: A 1-Year Treatment Sequence Analysis." Value in Health 19,no. 7 (2016):A576

\section{P1030 INDIRECT TREATMENT COMPARISON OF USTEKINUMAB VERSUS OTHER BIOLOGICS IN MODERATE-TO-SEVERE CROHN'S DISEASE PATIENTS HAVING FAILED ANTI-TNF THERAPY - A 1-YEAR TREATMENT SEQUENCE ANALYSIS INCLUDING DELAYED RESPONDERS}

L. Mesana ${ }^{1}$, M. Pacou ${ }^{2}$, D. Naessens ${ }^{3}$, S. Sloan ${ }^{4}$, A. Gauthier ${ }^{1}$

${ }^{1}$ Amaris, London/United Kingdom

${ }^{2}$ Amaris, Paris/France

${ }^{3}$ Janssen Pharmaceutica, Beerse/Belgium

${ }^{4}$ Janssen Global Services, Horsham/United States of America/PA

Contact E-mail Address: laura.mesana@amaris.com

Introduction: Indirect evidence is needed to inform the clinical efficacy of ustekinumab in Crohn's disease (CD). Indirect treatment comparisons in CD are challenged by withdrawal trial designs limiting placebo arm transitivity. This treatment sequence analysis builds on previous work ${ }^{1}$ proposing a solution to challenges inherent to $\mathrm{CD}$ datato compare one year efficacy of biologics in $\mathrm{CD}$ patients having failed anti-TNF therapy. Analyses accounted for delayed responders (induction non-responders attaining response after additional doses) to generate more comprehensive estimates of biologics' relative efficacies.

Aims \& Methods: A systematic literature review identified randomized controlled trials in CD patients having failed anti-TNF therapy for induction and maintenance of ustekinumab (UST), adalimumab (ADA), or vedolizumab (VDZ). Clinical response (CDAI-100 point reduction) and remission (CDAI $\leq 150)$ were assessed. The probability of achieving response after induction was multiplied by the conditional probability of maintaining response/achieving remission at one year. Separate calculations were conducted for early and delayed responders. Their respective treatment sequence rates were summed to obtain overall response and remission rates. Placebo rates were imputed using data from patients induced and maintained on placebo from the IM-UNITI study, adjusted for responder and remitter induction rates. Bayesian analyses generated relative

Abstract: P1028. Table 1: Characteristics and outcomes among patients newly started on vedolizumab stratified by IBD type and history of immunosuppressive therapy

\begin{tabular}{|c|c|c|c|c|c|c|}
\hline & \multicolumn{3}{|l|}{$\mathrm{CD}(\mathrm{N}=388)$} & \multicolumn{3}{|l|}{$\mathrm{UC}(\mathrm{N}=179)$} \\
\hline & $\begin{array}{l}\text { With history of } \\
\text { IM use }(\mathrm{N}=225)\end{array}$ & $\begin{array}{l}\text { Without history of } \\
\text { IM use }(N=163)\end{array}$ & P-value & $\begin{array}{l}\text { With history of } \\
\text { IM use }(\mathrm{N}=85)\end{array}$ & $\begin{array}{l}\text { Without history of } \\
\text { IM use }(N=94)\end{array}$ & P-value \\
\hline Mean (SD) age, years & $43(14.8)$ & $47(14.7)$ & 0.009 & $41(14)$ & $44(16)$ & 0.193 \\
\hline Female, $\%$ & $64.9 \%$ & $59.5 \%$ & 0.290 & $51.8 \%$ & $47.9 \%$ & 0.655 \\
\hline $\begin{array}{c}\text { Mean (SD) time from } \\
\text { diagnosis to VDZ } \\
\text { initiation, years }\end{array}$ & $6.0(3.9)$ & $4.2(3.4)$ & $<0.001$ & $3.6(2.7)$ & $2.6(2.6)$ & $<0.001$ \\
\hline $\begin{array}{l}\text { Pre-index exposure to } \\
\text { anti-TNF therapy, \% }\end{array}$ & $78.2 \%$ & $55.2 \%$ & $<0.001$ & $68.2 \%$ & $44.7 \%$ & 0.002 \\
\hline \multicolumn{7}{|l|}{$\begin{array}{l}\text { IBD-related measures } \\
\text { in the } 365 \text { days pre-index }\end{array}$} \\
\hline Hospitalisations & $42.2 \%$ & $28.8 \%$ & 0.0076 & $28.2 \%$ & $33.0 \%$ & 0.520 \\
\hline Surgeries & $18.7 \%$ & $6.7 \%$ & 0.0008 & $3.5 \%$ & $2.1 \%$ & 0.669 \\
\hline Flares & $56.9 \%$ & $43.6 \%$ & 0.0103 & $37.6 \%$ & $43.6 \%$ & 0.449 \\
\hline \multicolumn{7}{|l|}{$\begin{array}{l}\text { IBD-related measures in the } \\
365 \text { day follow-up period }\end{array}$} \\
\hline Hospitalisations & $24.9 \%$ & $20.2 \%$ & 0.328 & $14.1 \%$ & $19.1 \%$ & 0.426 \\
\hline Surgeries & $12.4 \%$ & $7.4 \%$ & 0.128 & $5.9 \%$ & $5.3 \%$ & $>0.999$ \\
\hline Flares & $43.6 \%$ & $32.5 \%$ & 0.035 & $25.9 \%$ & $25.5 \%$ & $>0.999$ \\
\hline
\end{tabular}

Note: IM therapy included use of azathioprine, 6-mercaptopurine, methotrexate, mycophenolate mofetil, cyclosporine, and Tacrolimus 
odds ratios (OR), credible intervals $(\mathrm{CrI})$, and posterior distribution probabilities for superiority of UST.

Results: Accounting for delayed responders, the absolute proportions of patients having maintained response and being in remission at one year were $30 \%$ of patients receiving UST every 8 weeks, $19 \%$ of those receiving VDZ every 4 weeks, and $23 \%$ of patients receiving ADA every other week or weekly. Based on a one-year treatment sequence analysis, probabilities for UST to be better than VDZ for achieving and maintaining response and remission were $99 \%(\mathrm{OR}[\mathrm{CrI}]: 1.94[1.07 ; 3.48])$ and $98 \%(\mathrm{OR}[\mathrm{CrI}]: 1.82[1.00 ; 3.28])$, respectively. UST had higher likelihoods of remission than ADA given weekly $(83 \%$, OR[CrI]:1.36[0.72;2.58) or every other week $(85 \%, \mathrm{OR}[\mathrm{CrI}]: 1.41[0.74 ; 2.68])$.

Conclusion: This approach deals with methodological issues inherent to CD trial data. In CD patients having failed anti-TNF therapy, ustekinumab had higher likelihoods of response or remission than adalimumab and vedolizumab over a one-year treatment sequence. Additional induction doses and continued maintenance therapy with ustekinumab have demonstrated benefits in delayed responders compared to other biologics. Previous research is limited to indirect treatment comparisons in early responders only. Including delayed responders provides a more accurate picture of CD patients' response to biologics and better informs clinical practice.

Disclosure of Interest: L. Mesana: Consultant to Janssen Scientific Affairs, LLC M. Pacou: Consultant to Janssen Scientific Affairs, LLC

D. Naessens: Janssen Scientific Affairs, LLC employee

S. Sloan: Janssen Scientific Affairs, LLC employee

A. Gauthier: Janssen Scientific Affairs, LLC investigator

\section{Reference}

1. Pacou M, Mesana L, Gauthier A, Naessens D, Sloan S, Danese S, Bonovas S, Abrams K."Indirect Treatment Comparison of Ustekinumab Versus Other Biologics In Moderate To Severe Crohn's Disease: A 1-Year Treatment Sequence Analysis." Value in Health 19, no. 7 (2016):A576.

\section{P1031 INDIRECT TREATMENT COMPARISON OF USTEKINUMAB VERSUS OTHER BIOLOGICS IN MODERATE-TO-SEVERE CROHN'S DISEASE PATIENTS HAVING FAILED CONVENTIONAL THERAPY - A 1-YEAR TREATMENT SEQUENCE ANALYSIS INCLUDING DELAYED RESPONDERS}

\author{
L. Mesana ${ }^{1}$, M. Pacou ${ }^{2}$, D. Naessens ${ }^{3}$, S. Sloan ${ }^{4}$, A. Gauthier ${ }^{1}$ \\ ${ }^{1}$ Amaris, London/United Kingdom \\ ${ }^{2}$ Amaris, Paris/France \\ ${ }^{3}$ Janssen Pharmaceutica, Beerse/Belgium \\ ${ }^{4}$ Janssen Global Services, Horsham/United States of America/PA
}

Contact E-mail Address: laura.mesana $@$ amaris.com

Introduction: Indirect evidence is needed to inform the clinical efficacy of ustekinumab in Crohn's disease (CD). Indirect treatment comparisons in CD are challenged by withdrawal trial designs limiting placebo arm transitivity. This treatment sequence analysis builds on previous work ${ }^{1}$ proposing a solution to challenges inherent to $\mathrm{CD}$ data to compare one year efficacy of biologics in $\mathrm{CD}$ patients having failed conventional therapy. Analyses accounted for delayed responders (induction non-responders attaining response after additional dosing) to generate more comprehensive estimates of biologics' relative efficacies. Aims \& Methods: A systematic literature review identified randomized controlled trials in CD patients having failed conventional therapy for induction and maintenance of ustekinumab (UST), adalimumab (ADA), or vedolizumab (VDZ). Clinical response (CDAI-100 point reduction) and remission $(\mathrm{CDAI} \leq 150)$ were assessed. The probability of achieving response after induction was multiplied by the conditional probability of maintaining response/achieving remission at one year. Separate calculations were conducted for early and delayed responders. Their respective treatment sequence rates were then summed to obtain overall response and remission proportions. Placebo rates were imputed using data from patients induced and maintained on placebo from the IM-UNITI study and adjusted for responder and remitter induction rates. Bayesian analyses generated relative odds ratios $(\mathrm{OR})$, credible intervals $(\mathrm{CrI})$, and posterior distribution probabilities for superiority of ustekinumab.

Results: When accounting for delayed responders, the absolute proportions of patients having maintained response and being in remission at one year were of $50 \%$ in patients receiving UST, $39 \%$ in those receiving VDZ, and 33 or $36 \%$ in patients receiving ADA every other week or weekly, respectively. Based on a oneyear treatment sequence analysis, probabilities for UST to be better than VDZ for achieving and maintaining response and remission were $84 \%$ (OR[CrI]:1.39[0.73;2.62]) and 69\%(OR[CrI]:1.19[0.59;2.38]), respectively. UST had higher likelihoods of remission than ADA given weekly $(75 \%$, OR[CrI]:1.33[0.58;3.02]) or every other week ( $82 \%$, OR[CrI]:1.47[0.64;3.33]) Conclusion: This approach deals with methodological issues inherent to $\mathrm{CD}$ trial data. In CD patients having failed conventional therapy, higher likelihoods of response or remission were observed for ustekinumab versus adalimumab and vedolizumab over a one-year treatment sequence. Additional induction doses and continued maintenance therapy with ustekinumab in delayed responders have demonstrated benefits compared to other biologics. Previous research' is limited to indirect treatment comparisons in early responders only. Including delayed responders provides a more accurate picture of $\mathrm{CD}$ patients' response to biologics and better informs clinical practice.
Disclosure of Interest: L. Mesana: Consultant for Janssen Scientific Affairs, LLC M. Pacou: Consultant for Janssen Scientific Affairs, LLC

D. Naessens: Janssen Scientific Affairs, LLC employee

S. Sloan: Janssen Scientific Affairs, LLC employee

A. Gauthier: Consultant for Janssen Scientific Affairs, LLC

\section{Reference}

Pacou, M., L. Mesana, A. Gauthier, D. Naessens, S. Sloan, S. Danese, S. Bonovas, and K. Abrams. "Indirect Treatment Comparison of Ustekinumab Versus Other Biologics In Moderate To Severe Crohn's Disease: A 1-Year Treatment Sequence Analysis." Value in Health 19, no. 7 (2016): A576.

\section{P1032 EFFICACY AND TOLERABILITY OF INITIATING, OR SWITCHING TO, INFLIXIMAB BIOSIMILAR CT-P13 IN INFLAMMATORY BOWEL DISEASE (IBD): A LARGE SINGLE- CENTRE EXPERIENCE}

R.P. Ratnakumaran, N. To, D. Gracie, C. Selinger, T. Clark, N. Carey,

G. Dowson, K. Leigh, L. Bourner, A.C. Ford, P. J. Hamlin

Gastroenterology, Leeds Teaching Hospital, Leeds/United Kingdom

Contact E-mail Address: raguprakash.ratnakumaran@nhs.net

Introduction: Anti-TNF therapies have revolutionised the management of IBD. Recently, the infliximab (IFX) biosimilar (CT-P13) received market authorisation for IBD allowing cost benefits with switches to CT-P13 with annual savings of $£ 5400 /$ patient $(70 \mathrm{~kg}$ patient receiving $5 \mathrm{mg} / \mathrm{kg} 8$ weekly). We present our experience of switching patients from the originator IFX to CT-P13 for new and existing patients.

Aims \& Methods: Recorded baseline characteristics included indication, age, sex, disease duration, treatment duration, concomitant immunomodulators, baseline CRP and HBI/Mayo scores. Response to IFX induction was assessed retrospectively using symptoms and CRP. Treatment response and remission rates, primary and secondary loss of response rates, and adverse events in patients who initiated IFX in the 12 months pre-Feb 2016 were compared with those who initiated CT-P13 in the 12 months post-Feb 2016. Sustained response was compared for existing IFX patients who switched to CT-P13 in Feb 2016 against those who continued with the original IFX. Drug and antibody levels were measured before switch and at 3,6, and 12 months post.

Results: 53 patients commenced IFX in the 12 months pre-Feb 2016 (26 Crohn's Disease (CD), 13 fistulating CD, 13 Ulcerative Colitis (UC), 1 IBD-Unclassified (IBD-U) compared with 69 patients who commenced CT-P13 in the subsequent 12 months (22CD, 9 fistulating CD, 35UC, 3IBD-U). Baseline characteristics did differ, with a greater proportion of UC patients in the CT-P13 cohort $(51 \% \mathrm{v}$ $24.5 \%(p=0.003))$. This group had a higher mean CRP $(20.2$ v10.6 $(p=0.008))$ although a lower median Mayo score $(5 \mathrm{v} 11(p=0.007))$. There was no difference in response $(12(23 \%) \vee 15(21.74 \%)(p=0.905))$, remission $(14(26 \%) \vee 29(42 \%)$ $(p=0.074))$, primary non-response $(8(15 \%) \vee 4(5.8 \%)(p=0.087))$, secondary loss of response $(12(23 \%)$ v $15(21.74 \%)(p=0.905))$, or adverse events $(6(11 \%)$ v $6(8.7 \%)(p=0.629))$ in those who initiated originator IFX compared with CTP13. 191 patients switched to CT-P13 post-Feb 2016, while 19 patients continued on originator IFX. There was no difference in the response $(2(11 \%)$ v $24(12.6 \%)$ $(p=0.797))$, remission $(9(47 \%) \vee 111(58.1 \%)(p=0.367))$, secondary loss of response $(8(42 \%) \vee 47(24.6 \%)(p=0.098))$, or adverse events $(0 \% \vee 9(4.7 \%)$ $(p=1))$ between the originator IFX and CT-P13 respectively. Drug and antibody levels were available pre-switch to CT-P13 for 134 patients. Therapeutic drug levels with no significant antibodies were associated with clinical remission 12 months post-switch compared with low/undetectable drug levels and significant antibodies $(60(67 \%) \vee 0 \%(p=0.0008))$. Loss of response or discontinuation of therapy at 12 months post-switch was significantly lower in those with therapeutic drug levels and no significant drug antibodies compared with patients low/ undetectable drug levels and significant antibodies $(18(20 \%) \vee 7(100 \%)$ $(p<0.0001)$ and $6(7 \%)$ $6(86 \%)(p<0.0001)$ respectively $)$

Conclusion: There was no significant difference in response and remission rates, primary and secondary loss of response, or adverse events between originator IFX and CT-P13 during the first 12 months after switching. The presence of low undetectable drug levels and significant antibodies pre-switch was associated with loss of response and discontinuation of treatment. Switching to CT-P13 in 191 patients in our unit lead to $>£ 1$ million in savings.

Disclosure of Interest: All authors have declared no conflicts of interest.

\section{P1033 SAFETY AND EFFICACY OF HELICOBACTER PYLORI ERADICATION IN PATIENTS WITH INFLAMMATORY BOWEL} DISEASE

S. Shinzaki ${ }^{1}$, T. Fujii ${ }^{2}$, S. Bamba ${ }^{3}$, T. Kobayashi ${ }^{4}$, H. Tanaka ${ }^{5}$, T. Yoshino ${ }^{6}$, A. Yamada ${ }^{7}$, N. Kamata ${ }^{8}$, T. Hibi ${ }^{4}$

${ }^{1}$ Department Of Gastroenterology And Hepatology, Osaka University Graduate School of Medicine, Suita/Japan

${ }^{2}$ Department Of Gastroenterology And Hepatology, Tokyo Medical and Dental University, Tokyo/Japan

${ }^{3}$ Division Of Gastroenterology, Shiga University of medical science, Shiga/Japan ${ }^{4}$ Center For Advanced Ibd Research And Treatment, Kitasato University Kitasato Institute Hospital, Tokyo/Japan

${ }^{5}$ IBD Center, Sapporo Kosei General Hospital, Sapporo/Japan 
${ }^{6}$ IBD Center, Tazuke Kofukai Medical Research Institute, Kitano Hospital, Osaka/Japan

${ }^{7}$ Department Of Internal Medicine, Toho University Sakura Medical Center, Sakura/Japan

${ }^{8}$ Department Of Gastroenterology, Osaka City University Graduate School of Medicine, Osaka/Japan

Contact E-mail Address: shinzaki@gh.med.osaka-u.ac.jp

Introduction: Low prevalence of Helicobacter pylori (HP) infection has been reported in patients with inflammatory bowel disease (IBD), however, it is unclear whether the eradication therapy for HP can exacerbate disease activity of IBD. We then aimed to clarify the safety and efficacy of HP eradication in patients with IBD.

Aims \& Methods: This was a multicenter, retrospective cohort study in 26 institutions. Patients who eradicated HP by proton pump inhibitor and amoxicillinbased triple therapy after the diagnosis of IBD (ulcerative colitis (UC) or Crohn's disease (CD)) from March 2005 to July 2015 were enrolled. Two IBD patients without HP eradication whose gender, age, disease, location, severity, and observation period were matched with each HP-eradicated patient were enrolled in the same institution. Disease activity of IBD at baseline, 2 and 6 months after observation (eradication) was investigated. Exacerbation of IBD was defined as increase/addition of IBD drug. IBD-associated hospitalization or surgery; and physicians' assessment was also analyzed. Factors associated with exacerbation of IBD were assessed by univariate and multivariate logistic regression analysis.

Results: A total of 429 IBD (378 UC and 51 CD) patients, including 144 patients who eradicated HP (eradication group) and 285 control patients (non-eradication group), were enrolled. IBD exacerbation rates in 2 and 6 months of observation were $8.3 \%(12 / 144)$ and $11.8 \%(17 / 144)$ in eradication group, which showed no significant differences compared with those of $4.9 \%(14 / 285)$ and $7.7 \%(22 / 285)$ in non-eradication group. Physicians' assessment showed similar results in terms of disease exacerbation, but in 2 months of observation no patient was improved in eradication group whereas $3.2 \%(9 / 285)$ of patients was improved in noneradication group $(P=0.019)$. Multivariate analysis revealed that the independent factor of IBD exacerbation after HP eradication was active disease at baseline (OR 5.3 (95\% CI: $1.5-16.9), P=0.011)$. HP was eradicated in $82.9 \%(102 /$ 123) of patients using clarithromycin as first-line treatment and $90.4 \%(19 / 21)$ using metronidozole as second-line, both of which were comparable with previous reports in non-IBD patients.

Conclusion: HP eradication therapy does not exacerbate disease activity of IBD without affecting eradication rate, but may not improve disease activity, suggesting that careful observation is neccesary after eradication, especially for patients with active disease.

Disclosure of Interest: S. Shinzaki: I have received lecture fees from Mitsubishi Tanabe Pharma, AbbVie, EA Pharma, and Eisai.

T. Fujii: T. Fujii has received a research grant from Eisai, and lecture fees from Mitsubishi Tanabe Pharma, AbbVie, EA Pharma, and Eisai.

S. Bamba: Received lecture fees from Mitsubishi Tanabe Pharma, AbbVie, and EA Pharma.

T. Kobayashi: Received research grants and lecture fees from Mitsubishi Tanabe Pharma and Eisai; research grant from Otsuka Pharmaceutical; lecture fees from AbbVie, Zeria Pharmaceutical, JIMRO, and Ajinomoto Pharmaceuticals; and consulting fees from Nippon Kayaku.

H. Tanaka: Received lecture fees from Mitsubishi Tanabe Pharma, AbbVie, EA Pharma, and Eisai.

A. Yamada: Received lecture fees from AbbVie, and EA Pharma.

T. Hibi: Received advisory and lecture fees from Zeria Pharmaceutical; advisory fees from Eisai, consulting fees from AbbVie, AstraZeneca Pharmaceuticals, EA Pharma, and Takeda Pharmaceutical; and lecture fees from JIMRO and Mitsubishi Tanabe Pharma.

All other authors have declared no conflicts of interest.

\section{P1034 EFFECTIVENESS OF IMMUNOSUPPRESSORS IN CROHN'S DISEASE}

L. Kecili ${ }^{1}$, N. Kaddache ${ }^{1}$ L Si Salah ${ }^{1}$, A Rebiha ${ }^{1}$, S. Khouitar ${ }^{1}$, K. Soualah ${ }^{1}$ H. Bellimi ${ }^{1}$, F. Ihaddadene ${ }^{1}$, R. Benbaya ${ }^{1}$, K. Bouchaoui ${ }^{2}$, A. Tata ${ }^{2}$, B. Bahaz ${ }^{1}$, T. Boucekkine ${ }^{1}, \mathrm{~S}$. Berkane ${ }^{1}$

${ }^{1}$ Gastroenterology, CHU Mustapha, algiers/Algeria

${ }^{2}$ EPH Blida, Blida/Algeria

Contact E-mail Address: keli_002000@yahoo.fr

Introduction: The use of immunosuppressors, including Azathioprine (AZT) in inflammatory bowel disease (IBD) is considered reference treatment. They are indicated especially in the case of corticodependence, corticoresistance and in postoperative reccurence. Their efficacy is primarily judged on clinical evaluation and relapses. Few data are available on long-term healing during Crohn's disease (CD). The aim of this study is to evaluate the long-term clinical effectiveness of AZT in luminal Crohn's disease and in prevention of postoperative recurrence with endoscopic evaluation.

Aims \& Methods: In this retrospective study, 133 consecutive patients followed for intestinal MC with or without anoperineal involvement were included between $1 / 1 / 2016$ and $31 / 03 / 2016$. All patients with Rutgeerts score greater than or equal to 2 received AZT. All patients were under AZT for at least 12 months, maintaining remission for luminal involvement or preventing postoperative recurrence. All our patients have received clinical (HBI), biological (CRP) and endoscopic evaluation (and MR enterography in some cases). They were checked at different times (during the first 2 years $=\mathrm{G} 1$, between 2 and 5 years $=\mathrm{G} 2$ and beyond 5 years $=\mathrm{G} 3$ ). Endoscopic remission was defined by the absence of ulceration. Statistical analysis used the Student Fisher and U tests of Mann Whitney.

Results: AZT was prescribed in $46.6 \%(62 / 133)$ cases of luminal involvement and $53.4 \%$ of cases to prevent post-operative recurrence. In the luminal disease group, the sex ratio $\mathrm{M} / \mathrm{F}$ was 1 , the age at diagnosis was 27 years \pm 9 ; A delay in diagnosis was estimated at 1.5 years \pm 2.5 ( $0-13$ years). Smoking was found in $26 \%$ of cases $(16 / 61)$. It was ileocolonic or colonic in $16.4 \%$ and $49.2 \%$ of cases, phenotype $\mathrm{B} 1$ or $\mathrm{B} 2$ in $32.8 \%$ and $44.6 \%$ respectively. $47.5 \%$ had more than 2 corticosteroid treatments (CTC), the average time to start AZT was $4 \pm 6$ years. The mean duration of treatment was $3.5 \pm 5.2$ years. The persistence of endoscopic lesions was observed in $46 \%, 20 \%$ and $25 \%$ of cases in G1, G2 and G3 respectively. Loss of response was observed in patient with more than 2 cures of CTC $(48 \%$ versus $25 \%, \mathrm{p}=0.042)$, and the B2B3 phenotype of the disease $[47.2 \%$ versus $26 \%(\mathrm{~B} 1), \mathrm{p}=0.037]$ There was no correlation between HBI and endoscopic healing. For postoperative recurrence, sex ratio $2.3 \mathrm{~F} / 1 \mathrm{M}$, age at diagnosis was 32 years $\pm 11(17-70)$; The duration of the disease before surgery was 34 months $\pm 44(0-156)$, the time between surgery and AZT was estimated at 3 years $\pm 6(0-20) .26 \%$ of patients were smokers. $63 / 72(87.5 \%)$ underwent surgery for stenosis and 5.5\% after severe flare. $61 \%$ had intestinal resection and $20.8 \%$ a right hemi colectomy. $6 / 72(8.3 \%)$ had more than 2 resections. $60 / 72$ had a colonoscopy before and after AZT. The persistence of i2-i4 lesions was observed in $44 \%, 21 \%$ and $19 \%$ in G1, G2 and G3 respectively.

Conclusion: In our work, the existence of endoscopic lesions and the absence of symptoms is not uncommon. The prediction after one year of clinical relapse in case of endoscopic lesions is uncertain. The aim of this study, which is still ongoing, is to determine the rate and predictive factors of failure, as well as the modalities of endoscopic control especially concerning timing.

Disclosure of Interest: All authors have declared no conflicts of interest.

\section{P1035 EVALUATION OF PHARMACOKINETIC PROFILES OF SB2 AS A BIOSIMILAR OF REFERENCE INFLIXIMAB}

D. Shin, J.W. Kang, S. Park, Y. Lee, S. Lee

Samsung Bioepis Co., Ltd., Incheon/Korea, Republic of

Contact E-mail Address: sr0427.lee@samsung.com

Introduction: Based on the totality of evidence with similar analytical, pharmacokinetic (PK) and clinical results, SB2 was approved by European Medicines Agency and U.S. Food and Drug Administration as a biosimilar of the reference infliximab (INF) for all indications for which INF has been approved. Here we report the PK profiles of SB2 compared to that of INF in two animal models, healthy subjects and patients with rheumatoid arthritis (RA).

Aims \& Methods: The pre-clinical PK profiles were evaluated in single and repeated dose studies $(1,3$, and $10 \mathrm{mg} / \mathrm{kg}$ of SB2, European Union sourced INF [EU-INF] or United States sourced INF [US-INF]) in two animal models (Sprague Dawley [SD] rat and transgenic Tg197 mouse). The clinical Phase I pivotal study for PK was conducted in healthy subjects ${ }^{1}$. The subjects received a single $5 \mathrm{mg} / \mathrm{kg}$ intravenous infusion of study drugs (SB2, EU-INF or US-INF) and were observed for 10 weeks. PK equivalence was to be concluded if the $90 \%$ confidence interval (CI) for the ratio geometric least squares means (LSMeans) of the primary PK endpoints (area under the concentrationtime curve [AUC] from time zero to infinity $\left[\mathrm{AUC}_{\mathrm{inf}}\right]$, AUC from time zero to the last quantifiable concentration $\left[\mathrm{AUC}_{\text {last }}\right]$ and maximum concentration $\left[\mathrm{C}_{\max }\right]$ ) were within the standard equivalence margin of 0.8 to 1.25 . The steady state $\mathrm{PK}$ profile was assessed in a Phase III study in RA patients ${ }^{2}$. In this study, the patients received $3 \mathrm{mg} / \mathrm{kg}$ of SB2 or EU-INF at weeks $0,2,6$ and then every 8 weeks up to 46 weeks. PK analyses were performed up to week 30 . Infliximab serum concentration was measured through two different enzyme-linked immunosorbent assays for pre-clinical and clinical studies.

Results: In pre-clinical studies, available PK profiles from animal studies showed no significant differences in $\mathrm{C}_{\max }$ and $\mathrm{AUC}_{\text {last }}$ between SB2, EU-INF and USINF. In healthy subjects, the $90 \%$ CIs for the primary PK parameters were within the pre-defined equivalence margin of 0.8 to 1.25 between SB2 and reference products (SB2 vs. EU-INF and SB2 vs. US-INF). In RA patients, the mean trough level was comparable between SB2 (ranging from 1.915 to 17.965 to $\mu \mathrm{g}$ / $\mathrm{mL}$ ) and EU-INF (ranging from 2.224 to 16.954 to $\mu \mathrm{g} / \mathrm{mL}$ ) from week 2 to week 30. The PK profiles were also comparable between SB2 and INF when analysed by the presence of ADA in both Phase I and Phase III clinical studies.

Conclusion: Similar PK profiles of SB2 and reference products were confirmed in pre-clinical and clinical studies. Altogether, the previously demonstrated analytical similarity and the data presented here indicate that similar PK are expected in all indications approved for SB2.

Disclosure of Interest: D. Shin: Employee of Samsung Bioepis

J.W. Kang: Employee of Samsung Bioepis

S. Park: Employee of Samsung Bioepis

Y. Lee: Employee of Samsung Bioepis

S. Lee: Employee of Samsung Bioepis 


\section{References}

1. Shin D et al., (2015), A Randomized, Phase I Pharmacokinetic Study Comparing SB2 and Infliximab Reference Product (Remicade((®)) in Healthy Subjects.

2. Choe J-Y et al., (2015), A randomised, double-blind, phase III study comparing SB2, an infliximab biosimilar, to the infliximab reference product Remicade in patients with moderate to severe rheumatoid arthritis despite methotrexate therapy.

\section{P1036 INFLIXIMAB DOSE BANDING SHORTENS LENGTH OF STAY OF INFLAMMATORY BOWEL DISEASE PATIENTS}

J. Ollivier $^{1}$, F. Poullenot ${ }^{2}$, A. Berroneau ${ }^{1}$, S. Djabarouti ${ }^{1}$, F. Xuereb ${ }^{1}$, F. Zerbib ${ }^{2}$, D. Laharie ${ }^{2}$, D. Breilh ${ }^{1}$

${ }^{1}$ Pharmacy, CHU de Bordeaux, Pessac/France

${ }^{2}$ Gastroenterology, CHU de Bordeaux, Pessac/France

Contact E-mail Address: julien.ollivier@chu-bordeaux.fr

Introduction: According to infliximab (IFX) license in inflammatory bowel diseases (IBD), infusion doses are based on patient weight. In daily practice, treatment is prepared by pharmacist after clinical patient assessment, leading to an increased duration of hospital stay and consequently costs. A pharmacokinetic study (1) has shown that a weight-based dose (WBD) strategy does not reduce interindividual variability of IFX trough levels when compared to fixed doses. According to these findings, our hospital implemented dose banding (DB) of IFX infusions, defined by doses rounded up or down according to one of eight predetermined standard doses with a maximum theoretical deviation of $+/-5 \%$, that allowed to prepare infusions at the pharmacy before patient admission Aims \& Methods: The aim of the study was to compare hospitalisation length of stay in patients receiving IFX DB as compared to those treated with IFX WBD. From February to March 2017, we conducted a prospective, case-control study in our unit, including all IBD patients admitted for an IFX infusion. Patients who should receive an IFX dose between 250 and $800 \mathrm{mg}$ were included in the DB group (treatment pre-prepared at the pharmacy, sent to the hospital unit before patient admission and administered just after the clinical validation). Patients who should receive an IFX dose below $250 \mathrm{mg}$ or above $800 \mathrm{mg}$ were included in the WBD group (treatment prepared after clinical validation including weight, and then sent to the hospital unit). Patients were analysed only when precise length of stay could be obtained and measured in minutes. Primary objective was to compare the length of stay at hospital in both groups. Secondary objective was to compare the proportion of IFX doses cancelled, reattributed and wasted infusions and the saved or wasted price associated (reimbursement price of one $100 \mathrm{mg}$ IFX vial: $382.28 €$ )

Results: Among the 373 IBD patients treated by IFX during the study period, 116 $(31 \%)$ patients $(51 \mathrm{M} / 65 \mathrm{~F}$; median age: 41 years) were included in the study ( 75 in the DB group and 41 in the WBD group) corresponding to 128 infusions ( 84 in DB and 44 in WBD groups). Mean length of hospitalisation stay were $238 \pm 21 \mathrm{~min}$ in the DB group and $308 \pm 32 \mathrm{~min}$ in the DBW group, respectively $(p<0.001)$. DB was associated with a mean reduction of length of stay of $23 \%$, corresponding to 70 minutes per patient. DB reduced significantly the mean duration of stay by decreasing the waiting time between clinical assessment and start of the infusion: $16 \mathrm{~min}$ vs. $84 \mathrm{~min}$ with WBD $(\mathrm{p}<0.001)$. During the study, none of the $44(0 \%)$ infusion in the WBD group was cancelled while $3 / 84$ $(3.5 \%)$ were cancelled in the DB group $(p=0.55)$. Two out these three infusions could be reattributed to other patients, saving $2801 €$

Conclusion: When used routinely in IBD, IFX DB is associated with a shortened length of stay as compared to WBD, with a mean reduction of 70 minutes per patient. As IFX DB seems having similar efficacy to weight-based doses, it may improve functioning of daily hospitalisation units.

Disclosure of Interest: All authors have declared no conflicts of interest.

\section{Reference}

1. Fasanmade, A. A., Adedokun, O. J., Blank, M., Zhou, H., Davis, H. M. Pharmacokinetic properties of infliximab in children and adults with Crohn's disease: a retrospective analysis of data from 2 phase III clinical trials. Clinical therapeutics, 2011;33(7): 946-964

\section{P1037 RAPIDITY OF ONSET OF RESPONSE TO ADALIMUMAB (ADA) IN LUMINAL CROHŃS DISEASE (CD). DATA FROM RAPIDA TRIAL}

I. Marín-Jiménez ${ }^{1}$, F. Casellas ${ }^{2}$, M. Esteve ${ }^{3}$, L. Castro-Laria ${ }^{4}$, S. García-López ${ }^{5}$, D. Ceballos ${ }^{6}$, A. Echarri ${ }^{7}$, M. Martín-Arranz ${ }^{8}$, D. Busquets ${ }^{9}$, J. Llaó $^{10}$, M. Navarro-Llavat ${ }^{11}$, J. Huguet ${ }^{12}$, F. Argüelles-Arias ${ }^{4}$, R. Vicente ${ }^{5}$, L. Rodríguez-San Pedro ${ }^{13}$, G. Díaz ${ }^{13}$, R. Casado ${ }^{13}$, M. Barreiro-De Acosta ${ }^{14}$ ${ }^{1}$ Departamento De Gastroenterología, Hospital Gregorio Marañón, Madrid/Spain ${ }^{2}$ Crohn-colitis Care Unit, Hospital Universitari Vall d'Hebron, Barcelona/Spain ${ }^{3}$ Departmento De Gastroenterología, Hospital Universitario MútuaTerrassa, Terrassa/Spain

${ }^{4}$ Fea Aparato Digestivo, Hospital Virgen Macarena, Sevilla/Spain

${ }^{5}$ Department Of Gastroenterology, Hospital Universitario Miguel Servet, Zaragoza/Spain
${ }^{6}$ Departamento De Gastroenterología, Hospital Universitario Dr. Negrin, Las Palmas/Spain

${ }^{7}$ Departamento De Gastroenterología, Complejo Hospitalario Universitario de Ferrol, Ferrol ( A Coruña)/Spain

${ }^{8}$ Departamento De Gastroenterología, Hospital Universitario La Paz, Madrid/ Spain

${ }^{9}$ Departamento De Gastroenterología, Hospital Universitario Dr. Josep Trueta, Girona/Spain

${ }^{10}$ Hospital de la Santa Creu i Sant Pau, Universitat Autónoma de Barcelona, Barcelona/Spain

${ }^{11}$ Departamento De Gastroenterología, Hospital de Sant Joan Despí Moisès

Broggi, Sant Joan Despi - Barcelona/Spain

${ }^{12}$ Hospital General Universitario de Valencia, Valencia/Spain

${ }_{13}^{13}$ Abbvie SLU, Madrid/Spain

${ }^{14}$ Hospital Clínico Universitario de Santiago, Santiago de Compostela, Santiago de Compostela/Spain

Contact E-mail Address: drnachomarin@hotmail.com

Introduction: Rapidity of response to treatment in $\mathrm{CD}$ is now considered a field of major interest, due to the importance of achieving the highest benefit in the shortest possible time. There are no studies specifically designed to evaluate the rapidity of response to ADA neither other antiTNF therapies. The aim of this trial was to evaluate the rapidity of onset of clinical response to ADA therapy.

Aims \& Methods: Adult antiTNF naïve patients with active luminal (HarveyBradshaw Index $(\mathrm{HBI})>8$ ) moderate-to-severe CD (excluding penetrating and stricturing disease), with no response to a full and adequate course of therapy with corticosteroids and/or immunosuppressants, were enrolled in this interventional, prospective, open label, single arm and multicenter clinical trial. Patients received standardized ADA treatment $(160 \mathrm{mg}-80 \mathrm{mg}-40 \mathrm{mg}$ eow). The HBI was evaluated to determine the response at day 4 and week 1 ; and clinical remission at weeks 2, 4 and 12. Response was defined as a decrease of, at least, 3 points in the HBI global score and remission was defined as HBI global score $<5$. CRP (C Reactive Protein) and fecal calprotectin (FC) were analyzed at baseline, day 4 , week $1,2,4,12$. The modified intention to treat (mITT) population was the primary population for efficacy analysis and consisted of those patients enrolled in the study who had received at least one dose of ADA. Treatment-emergent serious adverse events (AEs) were recorded to assess safety throughout the study until 70 days after last treatment dose. All patients who received at least one dose of ADA were included in the safety population. Statistical analyses were performed by the t-test or the Wilcoxon signed rank test, as applicable. Time to clinical response was analyzed using a Kaplan-Meier survival analysis model.

Results: 86 antiTNF naïve patients were analyzed. A response at day 4 and week 1 , was experienced by $60.5 \%$ and $74.4 \%$ of patients, respectively. Remission was achieved by $53.5 \%$ of patients at week $2,61.6 \%$ at week 4 and $54.7 \%$ at week 12 . The median time to obtain response was 4.0 days $(95 \%$ confidence interval (CI): $1.0,4.0)$ and the median time to remission was 7.0 days $(95 \% \mathrm{CI}: 4.0,14.0)$.

\begin{tabular}{llll}
\hline & $\begin{array}{l}\text { Median CRP } \\
\text { levels }(\mathrm{mg} / \mathrm{L})\end{array}$ & $\begin{array}{l}\text { Median FC } \\
\text { levels }(\mu \mathrm{g} / \mathrm{g})\end{array}$ & $\begin{array}{l}\mathrm{p} \text {-value vs baseline } \\
\text { for CRP and FC }\end{array}$ \\
\hline Baseline & 4.87 & 795 & $\mathrm{p}<0.0001$ \\
Day 4 & 1.70 & 430 & $\mathrm{p}<0.0001$ \\
Week 1 & 1.50 & 438 & $\mathrm{p}<0.0001$ \\
Week 2 & 1.56 & 419 & $\mathrm{p}<0.0001$ \\
Week 4 & 1.98 & 296 & $\mathrm{p}<0.0001$ \\
Week 12 & 1.68 & 300 & $\mathrm{p}<0.0001$ \\
\hline
\end{tabular}

During the study, $42.5 \%$ of the patients suffered from any adverse event (AE). Only 3 patients $(3.5 \%)$ showed a serious AE

Conclusion: ADA produces rapid clinical remission and response since day 4 in patients with moderate-to-severe CD unresponsive to therapy with corticosteroids and/or immunosuppressants.

Disclosure of Interest: F. Casellas: Dr. Francesc Casellas has received research funding from AbbVie, MSD, Shire, Ferring and Zambon.

M. Esteve: Dr Esteve has served as a consultant for Abbvie, MSD, Takeda and Tillots Pharma and has received speaker fees from MSD and Abbvie

S. García-López: Dr. Santiago García has received research and funding from AbbVie, MSD, Shire, FAES and Ferring and has served occasionally as a consultant for Abbvie and MSD.

A. Echarri: Dr Ana Echarri has received research funding from Abbvie and Shire, and speaker fees from Abbvie, Takeda, MSD, Shire, Pfizer

M. Martín-Arranz: Dra. Martín Arranz has served as consultant for Abbvie, MSD, Ferring and has received speaker fees from Abbvie, MSD, Ferring, Chiesi, Tillots.

M. Navarro-Llavat: Dr. Mercè Navarro-Llavat has received research funding from AbbVie and speaker fees from AbbVie, MSD, Takeda, Ferring, Shire Pharmaceuticals, Zambon and Allergan.

J. Huguet: Dr. Jose María Huguet Malaves has received research funding from AbbVie and MSD and speaker fees from Abbvie, MSD, Ferring and Takeda. F. Argüelles-Arias: Dr. FAA has served as a consultant for AbbVie, MSD, Kern Pharma, Celltrion, and Takeda Also, has received research funding fromMSD, Kern Pharma, Celltrion, and Takeda, and speaker fees from MSD, Kern Pharma, Celltrion, and Takeda. 
R. Vicente: Dr. Raquel Vicente has received medical education funding and speaker fees from Abbvie, MSD, Shire y Ferring.

L. Rodríguez-San Pedro: Lucía Rodríguez San Pedro Baselga is employee of AbbVie.

G. Díaz: Gema Díaz is employee of AbbVie.

R. Casado: Rocío Casado is employee of AbbVie.

M. Barreiro-de Acosta: Consultant and advisory member for or has received research funding from MSD, Abbvie, Hospira, Kern Pharma, Takeda, Pfizer, Ferring, FaesFarma, Shire Pharmaceuticals, Dr. Falk Pharma, Chiesi, GebroPharma, Otsuka Pharmaceutical, and ViforPharma.

All other authors have declared no conflicts of interest.

\section{P1038 HIGH-DOSE INTRAVENOUS IRON ISOMALTOSIDE IN PATIENTS WITH GASTROINTESTINAL DISEASES}

\section{R. Derman ${ }^{1}$, J.F. Dahlerup ${ }^{2}$, W. Reinisch ${ }^{3}$}

${ }^{1}$ Thomas Jefferson University, Philadelphia/United States of America/PA

${ }^{2}$ Department Of Hepatology And Gastroenterology, Aarhus University Hospital, Aarhus/Denmark

${ }^{3}$ Department Of Internal Medicine III, Medical University of Vienna, Vienna/ Austria

Contact E-mail Address: walter.reinisch@meduniwien.ac.at

Introduction: Patients with gastrointestinal diseases such as inflammatory bowel disease (IBD) often suffer from iron deficiency anaemia (IDA) and have a high annual iron need. Intravenous administration of high-dose iron is the most efficient approach to replenish iron stores. The present analysis evaluates safety and efficacy of high doses of iron isomaltoside in patients with gastrointestinal diseases.

Aims \& Methods: This is a pooled analysis of 3 trials of iron isomaltoside performed in patients with gastrointestinal diseases and IDA [1-3]. Outcome measures were adverse drug reactions (ADRs) and haemoglobin $(\mathrm{Hb})$ measurements. Results: 357 patients (108 men, 249 women) were included in the analysis of which 255 were diagnosed with IBD and 102 with other gastrointestinal diseases, incl. bariatric surgery, gastrointestinal bleeding etc. A cumulative dose of $\leq 1000 \mathrm{mg}$ and $>1000 \mathrm{mg}$ iron isomaltoside was administered in 199 and 158 patients, respectively. ADRs were observed in $13.6 \%$ (36 events in 27 patients) and $12.0 \%$ (30 events in 19 patients) of the patients dosed with $\leq 1000 \mathrm{mg}$ and $>1000 \mathrm{mg}$ iron isomaltoside, respectively $(p=0.8)$. Similar frequencies was observed in the IBD subgroup $(14.3 \%$ versus $12.1 \%, p=0.8) .0 .5 \%$ of the patients experienced a serious ADR ( 2 events in 2 patients; grand mal convulsion and syncope). ADRs with a patient frequency $>1 \%$ are shown in the table below:

\begin{tabular}{lll}
\hline & Patients $(\%)$ & \\
\cline { 2 - 3 } & $\begin{array}{l}\leq 1000 \mathrm{mg} \text { iron } \\
\text { ADomaltoside } \mathrm{N}=199\end{array}$ & $\begin{array}{l}>1000 \mathrm{mg} \text { iron } \\
\text { isomaltoside } \mathrm{N}=158\end{array}$ \\
\hline Flushing & 3.0 & 0 \\
Constipation & 0 & 1.9 \\
Increased hepatic enzyme & 1.5 & 0.6 \\
Diarrhoea & 0.5 & 1.3 \\
Dyspepsia & 0 & 1.3 \\
Headache & 1.0 & 1.3 \\
Hypersensitivity & 1.0 & 1.3 \\
Abdominal pain & 1.0 & 0.6 \\
Hypertension & 1.0 & 0 \\
Hypotension & 1.0 & 0.6 \\
Nausea & 1.0 & 0 \\
Urticaria & 1.0 & 0.6 \\
\hline
\end{tabular}

No ADRs of hypophosphatemia were reported. In patients dosed with $\leq 1000 \mathrm{mg}$ iron isomaltoside, $\mathrm{Hb}$ increased with a mean of $1.72(95 \%$ confidence interval (CI): 0.12$) \mathrm{g} / \mathrm{dL}$ from baseline to week $3,2.00(0.12) \mathrm{g} / \mathrm{dL}$ to week 4 , and 2.32 $(0.13) \mathrm{g} / \mathrm{dL}$ to week 8 . In patients dosed with $>1000 \mathrm{mg}$ iron isomaltoside, $\mathrm{Hb}$ increased with a mean of $2.04(0.10) \mathrm{g} / \mathrm{dL}$ from baseline to week $3,2.51(0.09) \mathrm{g} /$ $\mathrm{dL}$ to week 4 , and $3.01(0.12) \mathrm{g} / \mathrm{dL}$ to week 8 . The observed increase in $\mathrm{Hb}$ was statistically significantly higher in patients dosed with $>1000 \mathrm{mg}$ iron isomaltoside $(p<0.04)$. In the IBD subgroup, a similar dose-depended statistically increase in $\mathrm{Hb}$ was observed at week 3 and onwards $(p<0.02)$.

Conclusion: No dose-response for ADRs was observed with administration of high cumulative doses of iron isomaltoside whereas $\mathrm{Hb}$ increased more after 3 weeks with doses $>1000 \mathrm{mg}$. Thus, high doses $(>1000 \mathrm{mg}$ ) of iron isomaltoside can be administered without additional safety concerns including concerns of hypophosphatemia and with efficacious increases in $\mathrm{Hb}$ in patients with gastrointestinal diseases.

Disclosure of Interest: R. Derman: Richard Derman has been a consultant for Pharmacosmos $\mathrm{A} / \mathrm{S}$, and the investigator/institution received a fee per patient J.F. Dahlerup: The investigator/institution received a fee per patient. W. Reinisch: The investigator/institution received a fee per patient.

\section{References}

1. Reinisch W et al. Am J Gastroenterol 2013;108:1877-88

2. Dahlerup JF et al. Scand J Gastroenterol 2016;51:1332-8

3. Derman R et al. Am J Hematol. 2017;92:286-91

\section{P1039 EFFICACY AND SAFETY OF GOLIMUMAB IN ULCERATIVE COLITIS. PRELIMINARY DATA FROM A MULTICENTER ITALIAN STUDY}

F. Bossa ${ }^{1}$, M.R. Valvano ${ }^{2}$, C. Giuseppe ${ }^{3}$, A. Lauria ${ }^{4}$, N. Buccianti ${ }^{5}$, E. Vinci ${ }^{6}$ E. Shaini ${ }^{7}$, M. Mendolaro ${ }^{8}$, L. Grossi ${ }^{9}$, S. Mazzuoli ${ }^{10}$, A. Rispo ${ }^{11}$, G. Pranzo $^{12}$, L. Sebkova ${ }^{13}$, A. Tursi ${ }^{14}$, A. Miranda ${ }^{15}$, M. Patturelli ${ }^{16}$, R. Spagnuolo ${ }^{17}$,

C. Ricciardelli ${ }^{18}$, C. Sgarro ${ }^{19}$, P. Paese ${ }^{20}$, G. Inserra ${ }^{21}$, A. Azzarone ${ }^{22}$, W. Fries ${ }^{23}$ A.C. Privitera ${ }^{24}$, M. Principi ${ }^{25}$, M. Cappello ${ }^{26}$, A. Andriulli ${ }^{2}$

${ }^{1}$ Division Of Gastroenterology, Casa Sollievo della Sofferenza Hospital, San

Giovanni Rotondo/Italy

${ }^{2}$ Division Of Gastroenterology, Casa Sollievo della Sofferenza" Hospital, IRCCS, San Giovanni Rotondo/Italy

${ }^{3}$ Medicina Clinica E Sperimentale, IBD Unit, Messina/Italy

${ }^{4}$ Uoc Di Gastroenterologia, Ospedale Bianchi Melacrino Borrelli, Reggio Calabria Italy

${ }^{5}$ IBD Unituoc Di Medicina Generale, Ospedale Madonna delle Grazie, Matera/

Italy

${ }^{6}$ IBD Unit, Azienda Ospedaliera per l'Emergenza Cannizzaro, Catania/Italy

${ }^{7}$ Emergency And Organ Transplantation, Section Of Gastroenterology, AOU

Policlinico, Bari, Bari/Italy

${ }^{8}$ Gastroenterology And Hepatology Section, University of Palermo School of Medicine, Palermo/Italy

${ }^{9} G$ d́Annunzio University of Chieti-Pescara, School of Gastroenterology c/o

Digestive Physiopathology, Pescara/Italy

${ }^{10}$ Gastroenterology \& Artificial Nutrition, San Nicola Pellegrino" Hospital, Trani/ Italy

${ }^{11}$ Dept. Clinical Medicine And Surgery, University'Federico IÍof Naples, Naples/

Italy

${ }_{12}^{12}$ Presidio valle d'itria, Martina Franca/Italy

${ }^{13}$ Ciaccio, Ospedale Pugliese, Catanzaro/Italy

${ }^{14}$ Gastroenterology Sevice, ASL BAT, Andria (BT), Andria/Italy

${ }^{15}$ Internistic Medical Department "magrassi -lanzara", University of Campania

"L.Vanvitelli", Naples/Italy

${ }^{16}$ Gastroenterology And Endoscopy Unit, University of Campania "L. Vanvitelli",

Napoli/Italy

${ }^{17}$ Università, Catanzaro/Italy

${ }^{18}$ Gastroenterology Unit, Poggiardo Hospital, Poggiardo/Italy

${ }^{19}$ Gastroenterology And Digestive Endoscopy, OORR Foggia, Foggia/Italy

${ }^{20}$ Azienda Ospedaliera di Cosenza U.O. Gastroenterologia, Cosenza/Italy

${ }^{21}$ Policlinico, Catania, Catania/Italy

${ }^{22}$ Gastroenterologia, Ospedale San Paolo di Bari, Bari/Italy

${ }^{23}$ GI Unit, Università di Messina, Messina, Messina/Italy

${ }^{24}$ Sofar ospedale Cannizzaro di Ct, Catania/Italy

${ }^{25}$ Emergency And Organ Transplantation, University of Bari, Bari/Italy

${ }^{26}$ Di.bi.mis., Gastroenterology and Hepatology Unit, Palermo/Italy

Contact E-mail Address: f.bossa@operapadrepio.it

Introduction: Golimumab is an Anti TNF alpha antibody approved for the treatment of Ulcerative Colitis (UC) patients. Its efficacy and safety were studied in randomized, double blind trials ${ }^{1,2}$, but its effectiveness and safety in daily clinical practice are still little known ${ }^{3}$.

Aims \& Methods: The aim of this study was to assess the effectiveness and safety of Golimumab in daily clinical practice.

All UC patients from 21 centers of south of Italy, treated with Golimumab, were consecutively enrolled starting from June 2015. Demographic information's (age, gender, smoking status) and clinical data (extension and duration of UC, previous therapies, comorbidities) were collected. Clinical, laboratory and endoscopic data during the treatment with Golimumab were collected every three months.

Results: A total of 190 patients (120 males) were enrolled. The mean age at diagnosis and mean duration of disease were respectively $38.8 \pm 14.6$ years, and $9.1 \pm 7.0$ years. Only 21 patients were active smokers $(11 \%)$. About the extension, 111 were pancolitis $(58 \%), 72$ had a distal colitis $(38 \%)$ and 7 a proctitis $(4 \%)$. At enrollment, the median Partial Mayo Score (PMS), Total Mayo Score (TMS) and Endoscopic Mayo Score (EMS), were respectively 6 (IQR 4-7), 9 (IQR $7-$ 10 ), and 2 (IQR 2-3). The median values of ESR, C Reactive Protein and faecal calprotectine were respectively $25 \mathrm{~mm} / 1^{\text {st }}$ hour (IQR $15-38$ ), $3 \mathrm{mg} / \mathrm{dl}$ (IQR $1-9$ ), and $250 \mathrm{mg} / \mathrm{kg}$ (IQR $174-500)$. One hundred twenty five patients $(66 \%)$ were naïve to anti TNF alpha, while 65 have been treated with Infliximab $(n=42)$, Adalimumab $(\mathrm{n}=5)$ or both $(\mathrm{n}=19)$. The indications for Golimumab were: steroid-resistance in 37 patients $(20 \%)$, steroid-dependence in $130(68 \%)$, extraintestinal manifestations in $6(3 \%)$, and Anti TNF alpha failure in $17(9 \%)$. Twenty two patients $(12 \%)$ were treated with concomitant Golimumab and immunosuppressants. A total of 142 patients have been completed at least 3 months of therapy. Of these patients, a significant reduction of mean PMS $(\mathrm{n}=142 ; \mathrm{p}<0.001)$, TMS $(\mathrm{n}=45 ; \mathrm{p}<0.001)$, EMS $(\mathrm{n}=45 ; \mathrm{p}<0.001)$, ESR $(\mathrm{n}=125 ; \mathrm{p}<0.001)$, and $\operatorname{CRP}(\mathrm{n}=134 ; \mathrm{p}<0.001)$ were observed after 3 months. The rate of responders (reduction of $>2$ points of PMS) was $60 \%$, while the rate of clinical remission (PMS $<2$ ) was $39 \%$, and the rate of mucosal healing $(\mathrm{EMS} \leq 1)$ was $53 \%$. Among the 85 responder patients, $67(79 \%)$ have also completely discontinuated the steroids. At univariate analysis for predictive factors of response (gender, duration of disease, smoking status, previous Anti 
TNF, combo therapy, PMS, EMS, TMS, ESR, CRP, calprotectin, and indication to therapy), the naïve status to anti TNF alpha $(\mathrm{p}=0.0001)$, higher values of CRP $(p=0.0001)$, PMS $(p=0.0001)$, TMS $(p=0.0001)$ and EMS $(p=0.006)$ were associated with better response. About the indication, steroid-resistance was associated to the best response $(p=0.002)$, while Anti TNF resistance with the worse response $(\mathrm{p}=0.002)$. At multivariate analysis only TMS $(\mathrm{p}<0.001$, OR 1.5 - CI95\% 1.2-1.8) and Naïve to Anti TNF alpha $(\mathrm{p}=0.015$, OR 3.0 - CI $1.2-7.5)$ were confirmed associated to better response. To date, only 33 patients have discontinuated Golimumab (17\%). A total of 15 adverse events ( 3 serious) were observed. Ten non-responder patients underwent to colectomy ( 7 of them were refractory to other anti TNF alpha).

Conclusion: Golimumab was effective and safe in induction of response in UC patients in daily clinical practice.

Disclosure of Interest: All authors have declared no conflicts of interest

\section{References}

1. Sandborn WJ, Feagan BG, Marano C, et al. Subcutaneous golimumab induces clinical response and remission in patients with moderate-to-severe ulcerative colitis. Gastroenterology 2014;146:85-95

2. Sandborn WJ, Feagan BG, Marano C, et al. Subcutaneous golimumab maintains clinical response in patients with moderate-to-severe ulcerative colitis. Gastroenterology 2014;146: 96-109.

3. Bosca-Watts MM, Cortes X, Iborra M, et al. Short term effectiveness of golimumab for ulcerative colitis: observational multicenter study. World $J$ Gastroenterol. 2016;22: 10432-10439.

\section{P1040 OUTCOMES OF PATIENTS IN REMISSION WITH INFLAMMATORY BOWEL DISEASE WITH UNDETECTABLE INFLIXIMAB TROUGH LEVELS AND POSITIVE ANTIBODIES TO INFLIXIMAB}

G.S.Z. Tun ${ }^{1}$, R. W. Downey ${ }^{1}$, K. Robinson ${ }^{1}$, A. Wright ${ }^{1}$, L. Marshall ${ }^{1}$, M. F. Hale ${ }^{1}$, A. J. Lobo ${ }^{1}$

${ }^{1}$ Gastroenterology, Sheffield Teaching Hospitals NHS Foundation Trust, jf/United Kingdom

Contact E-mail Address: gloria.tun@sth.nhs.uk

Introduction: The formation of antibodies to infliximab (ATI) is associated with increased drug clearance. Patients with undetectable infliximab (IFX) levels and positive ATI may indicate a group who may no longer be benefitting from the drug. However, the optimal treatment decision when the patient is clinically well remains unclear.

Aims \& Methods: The aim was to assess the course of disease of patients in remission, with undetectable IFX levels and positive antibodies. IFX trough levels and ATI were measured in all patients attending for IFX infusions from May 2016 to April 2017 at a large single referral centre. Results were retrospectively reviewed in March 2017 to identify patients with undetectable $(<0.8 \mathrm{mg} / \mathrm{L})$ IFX trough levels and positive ATI $(>10 \mathrm{mg} / \mathrm{L})$. A local guideline suggested that in in well patients of this cohort, patients should be switched to an alternative biologic if duration of IFX treatment was $<12$ months, or if the duration of therapy was $>12$ months to consider withdrawal of IFX or to assess disease activity - with withdrawal of IFX in inactive disease or a switch to an alternative biologic for active disease. Trough levels for IFX and ATI were measured using drug tolerant enzyme-linked immunosorbent assays (Biohit, UK). Relapse was defined as worsening of symptoms attributable to the inflammatory bowel disease, requiring an alteration in treatment. Kaplan-Meier with Tarone-Ware test was used to compare survival curves for relapse and Cox regression model used to analyse the impact of the different treatment decision on the rate of relapse. Results: 47/223 patients had undetectable IFX drug levels with positive ATI Follow-up data was available in 45 patients. 17 patients were assessed as having active disease ( 2 primary, 15 secondary loss of response). Of the 28 in remission (at time of most recent TDM, median Harvey Bradshaw Index (HBI) 3.5; median Simple Clinical Colitis Activity Index (SCCAI) 2.5). 79\% had a HBI/ SSCAI $<5$ but all were deemed to be in remission at time of review. $6 / 28$ patients had received prior anti-tumour necrosis factor- $\alpha$ antagonists and 14/28 were receiving concomitant immunosuppression (thiopurines 12; methotrexate 2). Of these patients in remission, 7 continued on IFX (1 dose escalated), 13 had IFX withdrawn and 8 were switched to an alternative biologic (adalimumab 4; vedolizumab 3; ustekinumab 1). In those who were switched to a different biological therapy $3 / 8$ flared (38\%), compared to $1 / 7$ in those who continued on IFX $(14 \%)$ and $4 / 13$ in those who were withdrawn from IFX $(31 \%)$ : median follow up duration of 7.5 months (range $2-10$ months). There was no significant difference in the survival curves regarding rate of relapse based on the different management decisions taken $(\mathrm{p}=0.81$; Figure 1). Patients withdrawn from IFX were not at an increased risk of relapse compared to those who continued on treatment (hazard ratio $1.62, \mathrm{p}=0.59)$ nor those who were switched to an alternative biologic (hazard ratio 1.42, $\mathrm{p}=0.65$ ). Figure 1. Kaplan Meier of the rate of relapse during follow-up of patients in clinical response with undetectable IFX levels with positive ATI who were continued on $\operatorname{IFX}(n=7)$, switched to an alternative biologic $(n=8)$ and withdrawn from IFX $n=13(p=0.81)$.

Conclusion: Initial early experience in our centre suggests that withdrawal of IFX in selected patients with undetectable IFX levels and positive ATI is not associated with an increased risk of flare. Although not statistically significant, the rate of relapses in those who continued on infliximab was lower compared to those who were switched or withdrawn from therapy, however further follow-up and greater numbers are required in this group.

Disclosure of Interest: All authors have declared no conflicts of interest.

\section{P1041 PHARMACOKINETIC SIMILARITY OF ABP 710 TO INFLIXIMAB: RESULTS FROM A RANDOMISED, SINGLE-BLIND, SINGLE-DOSE, PARALLEL-GROUP STUDY IN HEALTHY SUBJECTS}

V. Chow, N. Zhang, P. Kaur, A. Kaliyaperumal, E. Krishnan Amgen, Thousand Oaks/United States of America/CA

Contact E-mail Address: vchow@amgen.com

Introduction: ABP 710 is being developed as a biosimilar to infliximab, an antitumour necrosis factor monoclonal antibody. Analytical and functional comparisons between ABP 710 and infliximab have been completed. This report describes the results of a Phase 1 pharmacokinetic (PK) equivalence study comparing ABP 710 with infliximab

Aims \& Methods: This was a single-blind, single-dose, 3-arm, parallel-group study in healthy adults, 18 to 45 years of age and with a body mass index of 18 to $30 \mathrm{~kg} / \mathrm{m}^{2}$. Subjects were randomised to receive a $5 \mathrm{mg} / \mathrm{kg}$ intravenous (IV) infusion of ABP 710 or infliximab sourced from the EU and the US after pretreatment with an antihistamine and acetaminophen 30 minutes prior to start of infusion. The primary objective was demonstration of PK similarity of ABP 710 with infliximab EU and with infliximab US based on area under the serum concentration-time curve from time 0 to infinity $\left(\mathrm{AUC}_{\mathrm{inf}}\right)$ as the primary endpoint. The criteria to achieve PK equivalence were for geometric mean (GM) ratio and its $90 \%$ confidence interval $(\mathrm{CI})$ to be within the range of 0.80 to 1.25 . Secondary endpoints included maximum observed serum concentration $\left(\mathrm{C}_{\max }\right)$, safety, and immunogenicity.

Results: A total of 148 subjects received study treatment (ABP 710: $n=49$; infliximab EU: $n=49$; infliximab US: $n=50$ ). After a single dose, the adjusted least square (LS) GM of $\mathrm{AUC}_{\mathrm{inf}}$ and $\mathrm{C}_{\max }$ were as follows: $\mathrm{ABP} 710,33559 \mu \mathrm{gh} / \mathrm{mL}$ and $123 \mu \mathrm{g} / \mathrm{mL}$; infliximab EU, $33706 \mu \mathrm{gh} / \mathrm{mL}$ and $121 \mu \mathrm{g} / \mathrm{mL}$; infliximab US, $37523 \mu \mathrm{gh} / \mathrm{mL}$ and $127 \mu \mathrm{g} / \mathrm{mL}$. Ratios of adjusted LS GM (90\% CIs) for $\mathrm{AUC}_{\text {in }}$ and $\mathrm{C}_{\max }$ between ABP 710 and infliximab EU were $0.996(0.9042,1.0963)$ and $1.021(0.9624,1.0827)$ and that between ABP 710 and infliximab US were 0.894 $(0.8122,0.9848)$ and $0.972(0.9167,1.0301)$. The ratios of adjusted LS GM $(90 \%$ CIs) of $\mathrm{AUC}_{\text {inf }}$ and $\mathrm{C}_{\max }$ between infliximab US and infliximab EU were 1.113 $(1.0115,1.2252)$ and $1.05(0.9906,1.1138)$. The $90 \%$ CIs of these ratios were fully contained within the 0.80 to 1.25 interval, confirming PK similarity between ABP 710 and infliximab, as well as between infliximab EU and infliximab US. There were no deaths, serious adverse events, or treatment-emergent adverse events (TEAEs) leading to discontinuation from the study; 1 subject in the infliximab EU group developed polyarthritis that resolved with treatment and the subject completed the study. The incidence of TEAEs was similar in the 3 groups (ABP 710: $83.7 \%$; infliximab EU: $83.7 \%$; infliximab US: $86.0 \%$ ); the majority was mild or moderate. The most frequently reported TEAEs were somnolence, headache, nasopharyngitis, upper respiratory tract infection, nausea, and lethargy. All subjects tested negative for antidrug antibodies (ADAs) prior to dosing. At the end of study (Day 57), $40 \%$ subjects on ABP $710,27 \%$ on infliximab EU, and $32 \%$ on infliximab US were positive for binding ADAs; $13 \%$ on ABP $710,19 \%$ on infliximab EU and $10 \%$ on infliximab US were positive for neutralising ADAs. Conclusion: Results of this study demonstrate PK similarity between ABP 710 and infliximab sourced from the EU and the US, as well as between infliximab EU and infliximab US following a single $5 \mathrm{mg} / \mathrm{kg}$ IV infusion in healthy subjects. The safety and immunogenicity profiles were comparable among treatment groups.

Disclosure of Interest: V. Chow: I am a full time employee and stockholder of Amgen Inc

N. Zhang: I am a full time employee and stockholder of Amgen Inc

P. Kaur: I am a full time employee and stockholder of Amgen Inc

A. Kaliyaperumal: I am a full time employee and stockholder of Amgen Inc

E. Krishnan: I am a full time employee and stockholder of Amgen Inc

P1042 EPIDEMIOLOGY AND BURDEN OF COMPLEX PERIANAL FISTULAS IN PATIENTS WITH CROHN DISEASE- A SYSTEMATIC LITERATURE REVIEW

J. Panés ${ }^{1}$, H. Patel ${ }^{2}$, E. Rupniewska ${ }^{3}$, S. Khan ${ }^{4}$, D. Bojic ${ }^{5}$, M. J. Khalid ${ }^{2}$, W. Reinisch ${ }^{6}$

${ }^{1}$ Dept. Of Gastroenterology, Hospital Clinic Barcelona Dept. of Gastroenterology Dept. of Gastroenterology, Hospital Clínic Bar, Barcelona/Spain

${ }^{2}$ Takeda Development Centre Europe Ltd, London/United Kingdom

${ }^{3}$ RTI Health Solutions, Manchester/United Kingdom

${ }^{4}$ RTI Health Solutions, RTP/United States of America

${ }^{5}$ Europe And Canada Medical Affairs, Takeda Pharmaceuticals International, Zurich/Switzerland

${ }^{6}$ Medical University of Vienna, Vienna/Austria

Contact E-mail Address: hari.patel@takeda.com

Introduction: Complex perianal fistulas (CPF) are common among Crohn's disease $(C D)$ patients and are associated with substantial morbidity. The burden and management of CPF are poorly studied.

Aims \& Methods: To systematically review the literature on epidemiology, global disease burden, and treatment outcomes for CPF in CD patients. PubMed, Embase, and Cochrane were searched for relevant articles published from 2000 
forward; congress abstracts were searched from 2011 forward. CPFs were defined as fistulas with intersphincteric, transsphincteric, suprasphincteric, extrasphincteric, or horseshoe tracts.

Results: 535 records were reviewed by 2 independent researchers, and 63 relevant articles and abstracts were selected for inclusion (including 3 epidemiology and 3 burden; the rest were treatment guidelines/patterns or treatment outcome studies). The estimated cumulative incidence of $\mathrm{CPF}$ in $\mathrm{CD}$, based mostly on studies conducted in referral centres, ranges from $12 \%-14 \%$ ( 2 studies). CPF can result in significant morbidity and greatly diminished quality of life; up to $59 \%$ of patients ( 1 study) are at risk of fecal incontinence. Treatment options include a combination of medical and surgical interventions. However, across all options identified, a high proportion of patients experience treatment failure (lack of or inadequate response) and relapse (Table). Only 4 identified studies were conducted specifically in patients refractory to anti-tumor necrosis factor (TNF)$\alpha$ agents - a population with high unmet needs (one study of perifistular injections of infliximab, and three studies of surgical interventions). Available data suggest that anti-TNF- $\alpha$ dose escalation or switching between different antiTNF- $\alpha$ agents is of limited value ( 2 studies). Table - Rates of treatment failure and relapse or reoccurrence among patients withcomplex perianal fistulain Crohn's Disease ${ }^{\mathrm{a}}$

Conclusion: CPFs in CD pose substantial clinical burden. There is a high unmet need for effective treatment options for CPF in CD patients, especially those refractory to anti-TNF- $\alpha$ agents, as evidenced by high treatment failure and relapse rates.

Disclosure of Interest: All authors have declared no conflicts of interest

\section{P1043 VITAMIN D IS RELATED TO THE EFFECTS OF ANTI-TNF TREATMENT IN CROHŃS DISEASE PATIENTS}

M. Bafutto ${ }^{1}$, M. B.G. $\operatorname{Costa}^{1}$, K. T.P.E. Silva ${ }^{1}$, J. P.V. Costa ${ }^{1}$, C. C.S.O. Reviglio ${ }^{1}$, E.C. Oliveira ${ }^{2}$, J. R. Filho

${ }^{1}$ Gastroenterology, Federal University of Goiás, Goiânia/Brazil

${ }^{2}$ Surgery, Organización Panamericana de Gastroenterología (OPGE), Goiânial Brazil

Contact E-mail Address: maurobafutto@yahoo.com.br

Introduction: Vitamin D deficiency is common in patients with Crohn's disease (CD). It is believed that this deficiency is related to the CD activity. Vitamin D supplementation have many effects, including imunomodulation. However, the role of Vitamin D (VD) in severe CD patients using Anti-TNF is still unclear. Aims \& Methods: To evaluate the results of the VD replacement at different doses; check possible immunomodulatory action of vitamin D in CD patients with Anti- TNF. We conducted a double-blind, randomized, prospective study. 42 patients were selected with history of moderate to severe CD in use of antiTNF, of both sexes, between 18 to 60 years, with dosage of 25 -hydroxyvitamin D $<75 \mathrm{nmol} / \mathrm{L}(30 \mathrm{ng} / \mathrm{ml})$ who signed the informed consent. Were excluded patients with less than 18 or over 70 years, pregnant women, chronic kidney or liver disease, sarcoidosis, tuberculosis, hyper- or hypoparathyroidism, neoplasias, use of anticonvulsants; and patients who received calcium supplements or VD in the last 6 months. 30 patients were randomized. Patients were submitted to a questionnaire of sun exposure, quality of life (IBDQ), clinical examination, VD dosage, C-reactive protein (CRP), fecal calprotectin (FC) and were divided into three groups: 1 Group $(\mathrm{G} 1)$ : 10 patients receiving 2,000 U/VD, $\mathrm{VO} /$ week for 8 weeks, 2 Group 2 (G2): 10 patients receiving 10,000 U/VD, VO/week for 8 weeks; 3 Group (G3): 10 patients receiving $50,000 \mathrm{U} / \mathrm{VD}, \mathrm{V} . \mathrm{O} /$ week for 8 weeks. At the end of 8 weeks the patients answered IBDQ and were submitted to $\mathrm{VD}, \mathrm{FC}$ and $\mathrm{CRP}$ dosage. All patients were followed for $52 \mathrm{w}$ and checked for disease activity recurrence (CDAI $>150, \mathrm{FC}>300, \mathrm{CT}$ scan), FC, CRP and VD levels.

Results: IBDQ improvement was observed in all groups with statistically significant results in $\mathrm{G} 2(\mathrm{p}=0.04)$ and $\mathrm{G} 3(\mathrm{p}=0.01)$. Increased VD were observed in all groups $($ mean $\pm \mathrm{SD} \times$ mean $\pm \mathrm{SD})$ : $\mathrm{G} 1-(19.5 \pm 5.1 \times 26 \pm 6.7) \mathrm{p}=0.07 ; \mathrm{G} 2$ $(19.1 \pm 4.1 \times 26 \pm 5.8) \mathrm{p}=0.04 ; \mathrm{G} 3-19.5 \pm 6.4 \times 46.4 \pm 12.7) \mathrm{p}<0.0001$. CRP dosage were reduced, although not statistically significant, at G2 and G3 $(5.8 \pm 4 \times 39 \pm 2.8) \mathrm{p}=0.18(5.2 \pm 7.3 \times 2.4 \pm 3.6) \mathrm{p}=0.2 ;$ and increased in G1
$(8.1 \pm 10,3 \times 13,4 \pm 19.9) \mathrm{p}=0.3$. There was a significant decrease in $\mathrm{FC}$ in G3 $(1014 \pm 850 \times 483 \pm 564) \quad \mathrm{p}=0.04$, no significant decrease in G2 $(767 \pm 751 \pm 823 \times 535) \mathrm{p}=0.2$, and increase in G1 $(1101 \pm 744 \times 1357 \pm 819)$ $\mathrm{p}=0.4$. $52 \mathrm{w}$ follow period showed that recurrent disease activity were predominant in patients with VD $<30(\mathrm{p}=0.0004)$ and statically significant results were observed in disease activity recurrence rate $(\mathrm{p}=0.006), \mathrm{FC}(\mathrm{p}=0.02)$ and CRP $(\mathrm{p}=0.01)$ when compared patients with $\mathrm{VD}>30$ and $\mathrm{VD}<30$.

Conclusion: 50,000 U/week was the best dosage for VD replacement and is related to immunomodulation. Most of patients with CD in Anti-TNF therapy have recurrent disease when $\mathrm{VD}<30$ and a hight remission rate with $\mathrm{VD}>30$. VD levels are related to the effects of Anti-TNF therapy in CD patients. Disclosure of Interest: All authors have declared no conflicts of interest.

\section{P1045 CLINCAL EFFECTIVENESS OF GOLIMUMAB IN CROHN'S DISEASE - AN OBSERVATIONAL STUDY BASED ON THE SWEDISH NATIONAL QUALITY REGISTRY FOR INFLAMMATORY BOWEL DISEASE}

S. Rundquist ${ }^{1}$, C. Eriksson ${ }^{1}$, L. Nilsson ${ }^{2}$, L. Angelison ${ }^{3}$, S. Jäghult ${ }^{4}$, J. Björk ${ }^{5}$, O. Grip ${ }^{6}$, H. Hjortswang ${ }^{7}$, H. Strid ${ }^{8}$, P. Karlén ${ }^{2}$, S. Montgomery ${ }^{9}$, J. Halfvarson ${ }^{1}$ Dept. Of Gastroenterology, Faculty Of Medicine And Health, Örebro University, Örebro/Sweden

${ }^{2}$ Dept. Of Internal Medicine, Danderyd Hospital, Stockholm/Sweden

${ }^{3}$ Dept. Of Internal Medicine, Helsingborg Hospital, Helsingborg/Sweden

${ }^{4}$ Stockholm Gastro Center, Karolinska Institutet Danderyd Hospital, Stockholm/ Sweden

${ }^{5}$ Dept. Of Medicine, Center For Digestive Diseases, Karolinska University Hospital, Stockholm/Sweden

${ }^{6}$ Dept. Of Gastroenterology, Skåne University Hospital Malmö, Malmö/Sweden

${ }^{7}$ Dept. Of Gastroenterology, Linköping University, Linköping/Sweden

${ }^{8}$ Dept. Of Internal Medicine, Södra Älvsborgs Sjukhus, Borås/Sweden ${ }^{9}$ Clinical Epidemiology And Biostatistics, School Of Medical Sciences, Örebro University, Örebro/Sweden

Contact E-mail Address: sara.rundquist@regionorebrolan.se

Introduction: Golimumab is approved for the treatment of moderate to severe ulcerative colitis, but not Crohn's disease (CD). Therefore, its potential efficacy in $\mathrm{CD}$ remains largely unknown. Off-label use of drugs is not prohibited in Sweden, and golimumab may have been used for CD treatment.

Results: The study cohort consisted of 95 patients with a median age of 37 (IQR $27-48)$ years, of whom $40 \%$ were men. The majority of the patients $(90.5 \%)$ had previously experienced treatment failure for at least one anti-TNF agent. At the start of golimumab, $41 \%$ were on a concomitant immunomodulator and $16 \%$ on corticosteroids. After a median follow-up time of 21 (IQR 10-36) months, 60 $(63 \%)$ patients had stopped treatment with golimumab. Reasons for discontinuation were inadequate response; $\mathrm{n}=45(75 \%)$, intolerance; $\mathrm{n}=11(18 \%)$ and other reasons; $n=4(7 \%)$. Estimated drug continuation rates were $73 \%$ at 12 weeks and $42 \%$ at 52 weeks. Concomitant treatment with corticosteroids at baseline seemed to be associated with a higher risk of discontinuation of golimumab (unadjusted HR: $1.97 ; 95 \% \mathrm{CI}: 1.04-3.73 ; \mathrm{p}=0.04$ ), although the assocation did not remain significant after adjusting for potential confounding factors (adjusted HR: $1.76 ; 95 \% \mathrm{CI}: 0.84-3.67 ; \mathrm{p}=0.13$ ).

Aims \& Methods: We aimed to describe the CD population that is treated with golimumab in Sweden and to assess the long-term effectiveness, defined as drug continuation rate, as well as identify predictors of drug discontinuation. Patients with $\mathrm{CD}$ who received at least one injection of golimumab were identified through the Swedish national quality registry for inflammatory bowel disease (SWIBREG). Duration of golimumab-treatment was illustrated by KaplanMeier curves. Univariate and multivariate Cox proportional hazard regression models were used to identify predictors of golimumab discontinuation. The variables sex, age, duration of disease, location, perianal disease, smoking status, previous surgery, concomitant treatment with corticosteroids or immunomodulators at baseline, prior anti-TNF therapy and CRP at baseline were included in the models.

Abstract: P1042

\begin{tabular}{|c|c|c|c|c|c|c|}
\hline \multirow{2}{*}{ Treatment } & \multicolumn{3}{|c|}{ Treatment failure $^{\mathrm{b}}$} & \multicolumn{3}{|c|}{ Relapse/recurrence } \\
\hline & Rates, \% & $\begin{array}{l}\text { Number of } \\
\text { studies }\end{array}$ & $\begin{array}{l}\text { Number of patients } \\
\text { per study (range) }\end{array}$ & Rates, \% & $\begin{array}{l}\text { Number } \\
\text { of studies }\end{array}$ & $\begin{array}{l}\text { Number of patients } \\
\text { per study (range) }\end{array}$ \\
\hline $\begin{array}{l}\text { Anti-TNF- } \alpha \text { agents } \\
\quad(\text { agent unspecified })\end{array}$ & $46-60$ & 3 & $39-66$ & 27 & 1 & 66 \\
\hline Infliximab & $12-58$ & 4 & $6-52$ & 41 & 1 & 52 \\
\hline Adalimumab & $22-73$ & 4 & $9-38$ & 0 & 1 & 9 \\
\hline Surgical interventions & $0-69$ & 10 & $5-40$ & $13-20$ & 3 & $5-10$ \\
\hline $\begin{array}{l}\text { Combined medical and } \\
\text { surgical management }\end{array}$ & $0-80$ & 15 & $9-212$ & $0-41$ & 7 & $9-71$ \\
\hline Standard of care ${ }^{\mathrm{c}}$ & $30-68$ & 4 & $15-250$ & $23-66$ & 3 & $79-250$ \\
\hline
\end{tabular}

${ }^{a} 44$ studies reported treatment outcomes for CPF in CD patients. Most studies identified were small and/or non-comparative, and study methodologies, populations, endpoint definitions, and duration of follow-up varied. For studies with mixed populations, only results for patients with CD and CPF were considered. ${ }^{b}$ Defined as lack of or inadequate response to therapy (i.e. lack of complete response). ${ }^{c}$ Defined as standard of care used at each centre excluding anti-TNFs and surgery in 2 studies and as standard medical care at each centre including anti-TNF and surgery in 2 studies. 
Conclusion: Golimumab-treated patients with $\mathrm{CD}$ are a treatment-refractory group. Despite this, more than $40 \%$ of the patients seemed to have sustained clinical benefit after one year, since they were receiving continued golimumab treatment. Co-concomitant corticosteroid treatment at start of golimumab appears to be associated with worse outcome.

Disclosure of Interest: C. Eriksson: Research grant from the Swedish government's agreement on medical training and research and speaker's fee from Takeda.

O. Grip: OG has received Consulting fees during the last 5 years from Ferring, Takeda, Viphor Pharma, Abbvie and Jansen-Cilag.

H. Hjortswang: Consultant/advisory board for AbbVie, Takeda, Janssen, Tillotts. Lectures for AbbVie, Takeda, Ferring, Tillotts, Falk pharma, Shire.

P. Karlén: Consultant/advisory board for Abbvie, Ferring, Otsuka, Takeda. Lectures for Abbvie, Ferring, Hospira, Otsuka, Takeda, Vifor. Principal Investigator for Abbvie, Amgen, Chemo-centryx, Celgene, Ferring, GSK, Jansen, MSD, Otsuka, Pfizer, Roche, Takeda.

J. Halfvarson: Consultant/lecture fees from Abbvie, Hospira, Medivir, Pfizer, RenapharmaVifor, Tillotts Pharma, Janssen, MSD and Takeda. Grant support from Janssen, MSD and Takeda. Outside the submitted work.

All other authors have declared no conflicts of interest.

P1046 EARLY IMPROVEMENT IN QUALITY OF LIFE IN PATIENTS WITH LUMINAL CROHNSS DISEASE TREATED WITH ADALIMUMAB. DATA FROM RAPIDA TRIAL

F. Casellas ${ }^{1}$, M. Barreiro-De Acosta ${ }^{2}$, M. Esteve ${ }^{3}$, L. Castro-Laria $^{4}$, R. Vicente ${ }^{5}$, D. Ceballos ${ }^{6}$, A. Echarri ${ }^{7}$, M. Martín-Arranz ${ }^{8}$, D. Busquets ${ }^{9}$, J. Llaó $^{10}$ M. Navarro-Llavat ${ }^{11}$, J. Huguet ${ }^{12}$, F. Argüelles-Arias ${ }^{4}$, J. Boudet ${ }^{5}$, L. Rodríguez-San Pedro ${ }^{13}$, G. Díaz ${ }^{13}$, R. Casado ${ }^{13}$, I. Marín-Jiménez $^{14}$ ${ }^{1}$ Crohn-colitis Care Unit, Hospital Universitari Vall d'Hebron, Barcelona/Spain ${ }^{2}$ Departamento De Gastroenterología, Hospital Clinico Universitario de Santiago, Santiago de Compostela, Santiago de Compostela/Spain

${ }^{3}$ Departmento De Gastroenterología, Hospital Universitario MútuaTerrassa, Terrassa/Spain

${ }^{4}$ Fea Aparato Digestivo, Hospital Virgen Macarena, Sevilla/Spain

${ }^{5}$ Department Of Gastroenterology, Hospital Universitario Miguel Servet, Zaragoza/Spain

${ }^{6}$ Departamento De Gastroenterología, Hospital Universitario Dr. Negrin, Las Palmas/Spain

${ }^{7}$ Departamento De Gastroenterología, Complejo Hospitalario Universitario de Ferrol, Ferrol (A Coruña)/Spain

${ }^{8}$ Departamento De Gastroenterología, Hospital Universitario La Paz, Madrid/ Spain

${ }^{9}$ Departamento De Gastroenterología, Hospital Universitario Dr. Josep Trueta, Girona/Spain

${ }^{10}$ Hospital de la Santa Creu i Sant Pau, Universitat Autónoma de Barcelona, Barcelona/Spain

${ }^{11}$ Departamento De Gastroenterología, Hospital de Sant Joan Despí Moisès

Broggi, Sant Joan Despi - Barcelona/Spain

${ }^{12}$ Hospital General Universitario de Valencia, Valencia/Spain

${ }^{13}$ Abbvie SLU, Madrid/Spain

${ }^{14}$ Departamento De Gastroenterología, Hospital Gregorio Marañón, Madrid/Spain

Contact E-mail Address: 1.garcia@ms-c.es

Introduction: Clinical response and patient's quality of life improve as a result of the direct benefit of Crohn's disease (CD) effective treatment. Rapidity of response to treatment in $\mathrm{CD}$ is a field of major interest, due to the importance of achieving the highest benefit in the shortest possible time. There are no studies specifically designed for early evaluation of the quality of life in patients with active CD receiving adalimumab therapy. The aim of this study was to evaluate the rapidity of improvement of quality of life in response to adalimumab therapy in adult antiTNF naïve patients with active luminal (Harvey-Bradshaw Index $\geq 8$ ) moderate-to-severe $\mathrm{CD}$, and with no response to a full and adequate course of therapy with corticosteroids and/or immunosuppressants.

Aims \& Methods: To this purpose we designed an interventional, prospective, open label, single arm and multicenter clinical trial. Quality of life was evaluated by using the validated questionnaires EuroQol-5D (EQ-5D) and the 36 items version of the Inflammatory Bowel Disease Questionnaire (IBDQ-36). Questionnaires were administered at baseline, day 4 and weeks 1, 24 and 12 with standardized adalimumab treatment $(160 \mathrm{mg}-80 \mathrm{mg}-40 \mathrm{mg}$ eow). The modified intention to treat (mITT) population was the primary population for analysis and consisted of those patients enrolled in the study who had received at least one dose of adalimumab. Statistical analyses were performed by the t-test or the Wilcoxon signed rank test, as applicable.

Results: Eighty-six patients were included. At baseline, the median EQ-5D index score was 0.68 . EQ-5D scores improved significantly versus baseline, at day 4 and weeks $1,2,4$ and 12, with median changes of $0.05(p=0.0005), 0.05(p<0.0001)$, $0.11(\mathrm{p}<0.0001), 0.10(\mathrm{p}<0.0001)$ and $0.11(\mathrm{p}<0.0001)$, respectively. Similarly, EQ-5D VAS median scores also improved significantly, compared to baseline (median score at baseline: 55.00), at day 4 and thereafter, with median changes of $5.00,5.50,9.50,10.00$ and 12.00 , respectively $(\mathrm{p} \leq 0.0001$ at all timepoints). The comparison, versus baseline, of the IBDQ-36 overall score (median score at baseline: 142.50 ) at day 4 and weeks $1,2,4$ and 12 , also yielded statistically significant differences, with median improvements of 14.0, 18.0, 29.0, 42.0 and 35.5 respectively ( $p<0.0001$ at all time-points). Restoration of normal health (IBDQ-36 score > 209) was obtained in $9 \%$ of patients at day 4 and increased to $35 \%$ at week 12 .

Conclusion: Adalimumab produces rapid improvement of quality of life since day 4 in patients with moderate-to-severe Crohńs disease.
Disclosure of Interest: M. Barreiro-de Acosta: Speaker, consultant and advisory member or research funding from MSD, Abbvie, Hospira, Kern Pharma, Takeda, Pfizer, Ferring, FaesFarma, Shire Pharmaceuticals, Dr. Falk Pharma, Chiesi, GebroPharma, Otsuka Pharmaceutical, and ViforPharma.

M. Esteve: Dr Esteve has served as a consultant for Abbvie, MSD, Takeda and Tillots Pharma and has received speaker fees from MSD and Abbvie

R. Vicente: Dr. Raquel Vicente has received medical education funding and speaker fees from Abbvie, MSD, Shire y Ferring.

A. Echarri: Dr Ana Echarri has received research funding from Abbvie, Shire and speaker fees from Abbvie, Takeda, MSD, Shire, Pfizer

M. Martín-Arranz: Dra. Martín Arranz has served as consultant for Abbvie, MSD, Ferring and speaker fees for Abbvie, MSD, Ferring, Chiesi, Tillots.

M. Navarro-Llavat: Dr. Mercè Navarro-Llavat has received research funding from AbbVie and speaker fees from AbbVie, MSD, Takeda, Ferring, Shire Pharmaceuticals, Zambon and Allergan.

J. Huguet: Dr. Jose María Huguet Malaves has received research funding from AbbVie and MSD and speaker fees from Abbvie, MSD, Ferring and Takeda.

F. Argüelles-Arias: Dr. FAA has served as a consultant for AbbVie, MSD, Kern Pharma, Celltrion, and Takeda Also, has received research funding fromMSD, Kern Pharma, Celltrion, and Takeda, and speaker fees from MSD, Kern Pharma, Celltrion, and Takeda.

L. Rodríguez-San Pedro: Lucía Rodríguez San Pedro Baselga is employee of AbbVie.

G. Díaz: Gema Díaz is employee of AbbVie.

R. Casado: Rocío Casado is employee of AbbVie.

I. Marín-Jiménez: Speaker, consultant, advisory member or research funding from MSD, Abbvie, Hospira, Takeda, Janssen, Ferring, Faes Farma, Shire Pharmaceuticals, Dr. Falk Pharma, Chiesi, Gebro Pharma, Otsuka Pharmaceuticals, Astrazeneca and Tillotts Pharma.

All other authors have declared no conflicts of interest.

\section{P1047 EVALUATION OF QUALITY OF LIFE IN IBD PATIENTS TREATED WITH ANTI-TNFA THERAPY}

G. Lorenzon, R. Caccaro, G. Girardin, B. Barberio, A. Rigo, E. Savarino, G.C. Sturniolo, R. D'Inca

Surgery, Oncology And Gastroenterology, Gastroenterology, Padua/Italy

\section{Contact E-mail Address: gretalorenzon90@gmail.com}

Introduction: Anti-tumor necrosis factor- $\alpha$ (anti-TNF $\alpha)$ agents are commonly used treatment options for moderate to severe Crohn's Disease (CD) and Ulcerative Colitis (UC). However, despite their clinical effectiveness, few data regarding the role on quality of life (QoL) are available.

Aims \& Methods: To prospectively evaluate disease activity and QoL in a singlecentre cohort of CD and UC patients, after introduction of anti-TNF $\alpha$ agents (infliximab or adalimumab). All consecutive adult CD and UC patients who started Infliximab (IFX) or Adalimumab (ADA) from 2014 to 2015 at Padua University Hospital were enrolled. Disease severity was evaluated through laboratory tests (Haemoglobin, C-reactive protein (CRP) and Fecal calprotectin) and commonly used scores (Harvey Bradshow Index (HBI) for CD and Modified Truelove and Witts Severity Index (MTWSI) for UC) for each patient, at anti$\mathrm{TNF} \alpha$ introduction and 12 months thereafter; QoL was assessed through the Short-Inflammatory Bowel Disease Questionnaire (S-IBDQ).

Results: A total of 115 patients were consecutively evaluated, 33 patients were excluded due to non-compliance $(11.3 \%)$ or drop-out for adverse events or primary non response $(17.3 \%)$ within 12 months. Eighty-two $(71.5 \%)$ were included in the statistical analysis (M/F 53/29, median age 43 years, CD/UC 42/40). Forty and 42 patients started IFX and ADA, respectively. QoL was significantly higher in $\mathrm{CD}$ than UC at baseline (median S-IBDQ 49 vs $32, \mathrm{p}=0.004$ ). In CD patients, anti-TNF $\alpha$ determined significant reduction of HBI (median 3 vs $1 ; p<0.01$ ), CRP (median 5 vs $2.9 \mathrm{mg} / \mathrm{L} ; \mathrm{p}=0.004$ ), fecal calprotectin (median $429 \mathrm{vs} 119 \mu \mathrm{g}$ / $\mathrm{g} ; \mathrm{p}<0.001$ ) but not haemoglobin (median 13.6 vs $13.2 \mathrm{~g} / \mathrm{dL}, \mathrm{p}=0.25$ ). QoL significantly improved (median S-IBDQ 49 vs $59 ; \mathrm{p}<0.001$ ), both in IFX and ADA groups (IFX: $\mathrm{p}<0.001$; ADA: $\mathrm{p}=0.02$ ). In UC patients, anti-TNF $\alpha$ therapy improved disease activity (median MTWSI 7 vs $4, p=0.03$ ), haemoglobin levels (median $11.6 \mathrm{vs} 13.2 \mathrm{~g} / \mathrm{dL} ; \mathrm{p}=0.006$ ), fecal calprotectin (median $1600 \mathrm{vs}$ $108 \mathrm{ug} / \mathrm{g} ; \mathrm{p}=0.004$ ), but not CRP (median 5 vs $2.9 \mathrm{mg} / \mathrm{L}, \mathrm{p}=0.08$ ). QoL improved at 12 months (median S-IBDQ: 32 vs $56, \mathrm{p}=0.001$ ) both in patients treated with $\operatorname{IFX}(\mathrm{p}=0.003)$ and $\operatorname{ADA}(\mathrm{p}=0.005)$. No adverse events were reported during the study period.

Conclusion: Anti-TNF $\alpha$ therapy is safe and improves disease activity and quality of life of UC and CD patients.

Disclosure of Interest: All authors have declared no conflicts of interest.

\section{P1048 ENDOSCOPIC AND HISTOLOGIC FINDINGS CORRELATE WITH FREE INFLIXIMAB FOUND IN UNINFLAMED TISSUE IN IBD PATIENTS}

H. Bar-Yoseph ${ }^{1}$, A. Blatt ${ }^{1}$, S. Pressman ${ }^{1}$, S. Gerassy ${ }^{1}$, B. Ungar ${ }^{2}$, S. Ben-Horin ${ }^{2}$, Y. Chowers

${ }^{1}$ Gastroenterology, Rambam Health Care Campus, Haifa/Israel

${ }^{2}$ Gastroenterology, Chaim Sheba Medical Center Ramat Gan Israel, Ramat Gan/ Israel

Contact E-mail Address: haggaiby@gmail.com

Introduction: Anti-TNF agents are widely used in the treatment of inflammatory bowel diseases (IBD). Despite the fact that the intestine is the main therapeutic 
target, little or no information is available regarding the ratios of free and TNFbound infliximab in the intestinal tissue.

Aims \& Methods: We aimed to assess the presence of free versus TNF-bound infliximab in the intestinal tissue of IBD patients and its possible association with clinical outcomes. Protein was extracted from frozen intestinal tissues of infliximab-treated patients. Free infliximab and TNF-bound infliximab were detected using ELISA and normalized to tissue protein concentration. Concurrent serum drug levels (SDL), anti-drug antibodies (ADA), serum TNF-bound infliximab levels, patient's pharmacotherapy, clinical response based on physician global assessment, endoscopic appearance (severity determined according to mayo scoring in ulcerative colitis and endoscopist's assessment of ulceration severity, extent of disease and affected area in Crohn's disease) and pathological results (severity determined by observing pathologist graded as normal, mild, moderate and severe disease) at the time of colonoscopy were determined. Correlation were performed using Spearman's rank correlation test.

Results: Twenty four biopsies from 13 patients (11 Crohn's disease and 2 ulcerative colitis patients) were tested. Non-inflamed tissue infliximab levels, but not inflamed tissue levels, correlated with SDL $(R=0.8499, \quad p=0.0037$, $\mathrm{FDR}=0.0185)$ and were negatively correlated with the endoscopic appearance $(\mathrm{R}=-0.7214, \mathrm{p}=0.0185)$ and pathological severity $(\mathrm{R}=-0.7959, \mathrm{p}=0.0059)$. TNF-bound infliximab was measured in both inflamed and non-inflamed specimens and did not correlate with drug levels in the serum or tissue. ADA was only detected in a single patient, precluding statistical analysis. Notably, no TNFbound infliximab was measured in the serum.

Conclusion: These findings show that pharmacokinetic-pharmacodynamics interaction, as measured by SDL, better reflects drug levels in healthy mucosa rather than the inflamed one, and suggest a more complex drug/target interaction in inflamed tissue, which cannot be explained by target binding only. Future studies assessing SDL dynamic changes during the process of mucosal healing may allow their use as surrogate markers for this purpose.

Disclosure of Interest: B. Ungar: Bella Ungar has received consultancy fees from Abbvie and Jannsen.

S. Ben-Horin: SBH has received consultancy and/or advisory board fees from Schering-Plough, AbbVie, Celltrion, Pfizer, Ferring, Janssen and Takeda; and has received research support from Celltrion, AbbVie \& Takeda

Y. Chowers: YC declare Abbvie grant support, lecture and advisory fees, Janssen lecture and advisory fees, Takeda grant support lecture and advisory fees, Medtronics advisory fees

All other authors have declared no conflicts of interest.

\section{P1050 LONG-TERM OUTCOMES OF MAINTENANCE ANTIBIOTIC} THERAPY IN CHRONIC ANTIBIOTIC-DEPENDENT POUCHITIS

S. Poo ${ }^{1}$, J. Segal ${ }^{2}$, S. Mclaughlin ${ }^{3}$, O. Faiz ${ }^{4}$, S. Clark ${ }^{2}$, A. Hart ${ }^{5}$

${ }^{1}$ Imperial NHS Trust, London/United Kingdom

${ }^{2}$ Surgery And Cancer, Imperial College London, London/United Kingdom

${ }^{3}$ Royal Bournemouth and Christchurch Hospitals, Bournemouth/United Kingdom

${ }^{4}$ St. Marks Hospital, Harrow/United Kingdom

${ }^{5}$ IBD Unit, St Mark's Hospital, Harrow/United Kingdom

Contact E-mail Address: stephanie.pooxw@gmail.com

Introduction: Restorative proctocolectomy (RPC) is considered the treatment of choice in patients with ulcerative colitis (UC) who have failed medical therapy and in some patients with familial adenomatous polyposis (FAP). After RPC, approximately $40 \%$ of UC patients develop acute pouchitis at 5 years, for which the mainstay treatment is empirical antibiotics. $10-15 \%$ of these patients subsequently develop chronic antibiotic-dependent pouchitis (CADP), and are maintained on long-term antibiotics to maintain clinical remission. Clostridium difficile and tendon rupture are recognised significant complications associated with long-term ciprofloxacin. We aimed to evaluate the effectiveness and safety of long-term antibiotic use in patients with chronic pouchitis.

Aims \& Methods: A retrospective analysis of patients started on antibiotic therapy between Jan 2007 - Jan 2009 for CADP was performed. 39 patients (30 males) who underwent RPC for UC $(n=37)$ and FAP $(n=2)$ with a diagnosis of CADP (defined as the need for antibiotics for at least one month and with a Pouch Disease Activity Index (PDAI) of greater than 7) were identified. Antibiotic-associated complications and functional outcomes were evaluated. Patients were followed-up at a tertiary referral centre until the last clinic appointment or the development of pouch failure, defined as the need for an ileostomy. Results: The median follow-up was 102 months (range 9-125); 27 (69\%) patients with CADP remained on antibiotics at last follow-up. The most common antibiotics used were ciprofloxacin $(30 \%)$, metronidazole $(19 \%)$ or a combination of both $(11 \%)$. Median $24 \mathrm{~h}$ stool frequency was 10 (range 4-25) at last follow-up. This was unchanged from baseline $(\mathrm{p}>0.05)$. Reported complications included: $7(18 \%)$ perianal fistula, $12(31 \%)$ anastomotic strictures requiring dilatation, 6 $(15 \%)$ perianal sepsis, $5(13 \%)$ incontinence and $11(28 \%)$ antibiotic-related side effects. Of 39 patients, $7(18 \%)$ achieved remission while $8(21 \%)$ developed pouch failure requiring an ileostomy or pouch revision, over a median duration of 6 years (range 3-9). Antibiotic resistance was identified from at least one stool sample in $28 / 36(78 \%)$ patients, however Clostridium difficile toxin was not detected in any stool samples. No patient developed tendon rupture. 36 pouchrelated hospital admissions occurred in 11/39 (28\%) patients. Reasons for admission included: 23 obstructive symptoms, 1 incontinence, 1 bleeding from pouch, 11 elective examinations under anaesthetic.

Conclusion: The management of chronic pouchitis remains challenging despite long term antibiotic therapy. This is the first study to report the long-term outcomes of long term antibiotics on patients with CADP. $69 \%$ of patients remained on antibiotics at last follow up. Although the use of antibiotics in chronic pouchitis is justified, the complication rates remain high and pouch failure is an inevitable consequence and occurs in $21 \%$ of our patient cohort. Antibiotic resistance was reported in $78 \%$ of patients. The utility of long term antibiotics must therefore be weighed against potential complications associated with pouchitis and antibiotics.

Disclosure of Interest: S. McLaughlin: Simon McLaughlin sits on the advisory board honoraria and sponsorship to attend medical conferences received from Abbvie.

All other authors have declared no conflicts of interest.

\section{P1051 ANTIBODIES TO INFLIXIMAB OCCUR THROUGHOUT TREATMENT BUT DEVELOPMENT IS DELAYED BY IMMUNOSUPPRESSION}

R. W. Downey, G.S.Z. Tun, K. Swallow, G. Wild, A. Wright, K. Robinson, L. Marshall, R. Sargur, M. F. Hale, A. J. Lobo

Gastroenterology, Sheffield Teaching Hospitals NHS Foundation Trust, jf/United Kingdom

Contact E-mail Address: rdowney1@sheffield.ac.uk

Introduction: Infliximab (IFX) is an important agent in the treatment of inflammatory bowel disease (IBD). However, its use may be limited by the development of antibodies to infliximab (ATI). The aim of this study was to assess the timing and significance of ATI development in clinical practice.

Aims \& Methods: Since May 2016, all IBD patients receiving intravenous IFX for maintenance treatment, at a large, single, referral centre, have undergone therapeutic drug monitoring (TDM). Serum IFX trough levels and ATI were both measured by drug-tolerant enzymelinked immunosorbent assays (Biohit, UK) from 199 patients at a variable interval following the start of IFX treatment. IFX trough levels were considered undetectable $(<0.8 \mathrm{mg} / \mathrm{L})$, low $(0.8-2.9 \mathrm{mg} /$ $\mathrm{L})$, therapeutic $(3-7 \mathrm{mg} / \mathrm{L})$ and supratherapeutic $(>7 \mathrm{mg} / \mathrm{L})$. ATI were considered positive if $>10 \mathrm{mg} / \mathrm{L}$. Clinical remission was defined as a Harvey Bradshaw Index or Simple Colitis Activity Index $\leq 4$. Risk factors for ATI development were assessed by binary logistic regression model, using 522 sera taken from patients on maintenance therapy. Time to develop ATI, undetectable IFX levels and loss of response to treatment was assessed by Cox regression and Kaplan-Meier analysis.

Results: Male sex $(\mathrm{OR}=2.1 ; \mathrm{p}<0.001)$, week of treatment (OR for each extra week of treatment $=0.999 ; \mathrm{p}<0.038$ ) and use of concomitant immunosuppression (IS) $(\mathrm{OR}=0.373 ; \mathrm{p}<0.001)$ were associated with ATI formation. During the period of observation, those not taking IS were more likely to develop ATI at any time throughout their IFX treatment (Hazard Ratio $=2.4 ; p=0.03$ ). 59/199 patients were ATI positive at their first TDM, of whom 2 were in their first 6 months of IFX treatment. 140/199 patients were ATI negative at their first TDM. During follow-up, we observed 32 patients develop ATI (19 patients developed antibodies within 6 months of starting IFX treatment, 5 between 6-12 months treatment and 8 after 12 months). At any given time, patients with positive ATI are 3.4 times as likely $(\mathrm{p}=0.002)$ to develop undetectable levels than ATI negative patients. Transient ATI formation was seen in only 5/162 patients. Figure 1 . Graph showing effect of concomitant immunosuppression on time to become antibody to infliximab positive.

Conclusion: Although IS therapy protects against ATI formation, the risk of antibody development continues throughout treatment. If concomitant IS is aimed at reducing ATI formation, then this also needs to be continued for the duration of IFX therapy.

Disclosure of Interest: All authors have declared no conflicts of interest.

\section{P1052 A DRUG-RESISTANT ASSAY CAN BETTER DIRECT THE NEED FOR ADALIMUMAB DOSE-ESCALATION AFTER INDUCTION THERAPY IN ANTI-TNF NAÏVE PATIENTS WITH CROHN'S DISEASE}

G. Moors ${ }^{1}$, B. Verstockt ${ }^{1}$, S. Bian ${ }^{2}$, S. Tops ${ }^{2}$, V. Ballet ${ }^{1}$, G. Van Assche ${ }^{1}$, S. Vermeire ${ }^{1}$, A. Gils ${ }^{2}$, M. Ferrante ${ }^{1}$

${ }^{1}$ Department Of Gastroenterology And Hepatology, University Hospitals Leuven, Leuven/Belgium

${ }^{2}$ Laboratory For Therapeutic And Diagnostic Antibodies, Department Of Pharmaceutical And Pharmacological Sciences, KU Leuven, Leuven/Belgium

Contact E-mail Address: gitte.moors@uzleuven.be

Introduction: Antidrug antibodies (ADA) may develop in up to $35 \%$ of patients with Crohn's disease (CD) receiving adalimumab (ADM). Although ADA to ADM are predominantly neutralizing, standard drug-sensitive assays do not allow ADA measurement in patients with detectable trough levels (TL) and drug-tolerant assays are suboptimal in case of high TL.

Aims \& Methods: We aimed to identify ADA using a drug-resistant assay, instead of the standard drug-sensitive assay, and to correlate their presence with clinical outcome. Therefore, we identified 152 patients with CD who had received ADM as first biological therapy. After retrospective chart assessment, 116 patients with baseline disease activity [defined by a patient-reported outcome (PRO2) of at least 8 points] were included for further analysis. Clinical remission was defined as an average daily stool frequency $\leq 2.8$ and an average abdominal pain score $\leq 1$. Serum samples were available in $70 / 116$ at week 12 after ADM initiation. ADA presence was determined via both a monoclonal drug-sensitive assay in case of undetectable TL, and via an in-house developed drug-resistant assay regardless of TL [1].

Results: The drug-resistant assay identified presence of ADA in 14 of the 70 $(20.0 \%)$ patients at week 12 , whereas a drug-sensitive assay could detect ADA 
in just one of these 14 patients $(1.4 \%)(\mathrm{p}<0.001)$. Median (IQR) TL were significantly higher in the ADA negative group compared to the ADA positive group [9.21 (7.00-12.99) vs. $3.45(1.72-5.44) \mu \mathrm{g} / \mathrm{mL}, \mathrm{p}<0.001]$. A significant correlation between TL and ADA levels could be found (Spearman' rho $-0.562, \mathrm{p}<0.001)$. Although the presence of these ADA was not significantly associated with lack of clinical remission at week 12, a clear tendency was observed $(\mathrm{p}=0.136)$. During median (IQR) follow-up of $1.46(0.32-3.48)$ years, 43 out of 116 patients $(37.1 \%)$ needed ADM dose-escalation. Importantly, escalation-free-survival significantly differed between ADA positive and negative patients $(\mathrm{p}=0.001)$. Univariate analyse could not identify any more factors (weight, BMI, gender, disease behaviour, disease location, CRP, serum albumin, PRO2, concomitant therapy, smoking) associated with ADA presence at week 12 . Interestingly, $50 \%$ of the ADA positive patients had TL above $4 \mu \mathrm{g} /$ $\mathrm{mL}$ and would not have been dose optimized proactively according to current practice. Though, 3 out of these 7 patients needed dose-escalation afterwards which could have been expected based on the ADA positivity.

Conclusion: A drug-resistant assay can identify ADA to ADM before all drug has been neutralised and TL become undetectable. As these ADA at week 12 are significantly associated with need for dose-escalation and can appear before TL drops below the threshold of $4 \mu \mathrm{g} / \mathrm{mL}$, they may be better to identify those patients who could benefit from dose-escalation. Moreover, the differences in TL between patients at week 12 can finally be explained by the presence of ADA measured with a drug-resistant assay.

Disclosure of Interest: B. Verstockt: Bram Verstockt received lecture fee from Ferring Pharmaceuticals

G. Van Assche: Financial support from Abbott, Ferring, Janssen, MSD and Abbott, PDL BioPharma, UCB Pharma, Sanofi-Aventis, Abbott, Abbvie, Ferring, Novartis, Biogen Idec, Janssen Biologics, NovoNordisk, Zealand Pharma A/S, Takeda, Shire, Novartis and BMS

S. Vermeire: Financial support from MSD, Abbvie and UCB Pharma; lecture fees from Abbott, Abbvie, MSD, Ferring Pharmaceuticals and UCB Pharma; consultancy fees from Pfizer, Ferring Pharmaceuticals, Shire Pharmaceuticals Group, MSD, and AstraZeneca Pharmaceuticals.

A. Gils: Speaker for MSD, Janssen Biologicals, Abbvie, Pfizer, and Takeda. Consultant for UCB and Takeda. License of (anti-)infliximab, (anti-)adalimumab, and vedolizumab ELISA to apDia and infliximab, adalimumab lateral flow to R-Biopharm AG.

M. Ferrante: Financial support from Takeda; lecture fees from Ferring, Boehringer-Ingelheim, Chiesi, MSD, Tillotts, Janssen Biologics, AbbvieTakeda, Mitsubishi Tanabe, Zeria; consultancy fees from Abbvie, Boehringer-Ingelheim, Ferring, MSD, and Janssen Biologics.

All other authors have declared no conflicts of interest.

\section{Reference}

1. Bian S, Ferrante M, Gils A. Validation of a Drug-Resistant AntiAdalimumab Antibody Assay to Monitor Immunogenicity in the Presence of High Concentrations of Adalimumab. AAPS J 2017 Mar;19(2):468-74.

\section{P1053 ADHERENCE TO MAINTENANCE THERAPY IN PATIENTS WITH INFLAMMATORY BOWEL DISEASE BEFORE AND AFTER THE INTRODUCTION OF THE SHARED MEDICATION RECORD}

\section{K. Roelsgaard}

Department Of Internal Medicine, Randers Regional Hospital, Randers/Denmark

Contact E-mail Address: klaroe@rm.dk

Introduction: Compliance is a significant problem in the medication of patients with chronic diseases, especially during periods where patients are completely unaware of their disease and just take their drugs to prevent disease recurrence. (1) During the last years Shared Medication Record (SMR) was introduced in Denmark (2). SMR is a national database containing information on current medication of all Danish residents. SMR include information on where, when, and how much medicine the patients by at the pharmacies. Therefore with SMR it becomes possible for doctors to see if patients retrieve the prescribed medicine at the pharmacy. Patients with chronic inflammatory bowel diseases (IBD), ulcerative colitis (UC) or Crohns Disease (CD), have periods of flares of the disease but in many cases also long periods when the disease is in remission. The majority of patients need medication to reduce the risk of recurrence of disease activity. This means that they need to take medicine even if they have no symptoms of disease. Previous American studies have shown that a number of patients in this situation do not take their medication and thus are at increased risk for relapse of the disease (1). There are no corresponding data for Danish patients. We wanted to find out the proportion of Danish IBD patients in remission who buy the prophylactic treatment as prescribed, and whether this proportion will change when the patients are informed about that the doctor can see if they pick up the medicine at the pharmacy.

Aims \& Methods: The purpose of this study was to investigate whether Danish patients with IBD in remission buy the prophylactic treatment as prescribed, if these patients buy a larger part of their medicine when they know that the doctor can see which medicines they buy at the pharmacy. 100 consecutive patients with $\mathrm{UC}$ or CD in remission for at least six months and treated with a fixed dose of Mesalazine, azathioprine, or Mercaptupurine during the preceding six months were enrolled from Randers Regional Hospital Adult Gastroenterological Outpatient Clinic. Patients were randomized 1:1 either to receive information that the doctor could follow their pharmacy refills, or not to get information on this. The patients were not informed that they participated in a study. All patients had a second visit six to 12 months later. Patients who had flares in disease activity during the study period was excluded. Adherence to the treatment was defined as pharmacy refills according to the prescribed dose for at least $80 \%$ of the period of the preceeding six months. Fisher's exact test was used as test of indenpendence between groups

Results: $67 \%$ of the patients in the study were adherent to their medical treatment during the first study period decreasing to $48 \%$ during the second study period $(\mathrm{P}<0.01)$. There was no difference in the decrease in adherence between patients informed about SMR and those who were not informed. Younger patiens were less prone to adherence compared to older patients at the first study visit (Age groups: $19-39 / 40-59 / 60+$ Years, adherence $48 / 71 / 81 \%)(\mathrm{P}<0.05)$. We found no differences related to disease (UC/CD), sex, 5-ASA/antipurins, or administration route (oral/rectal).

Conclusion: Adherence to treatment fell from the first visit when the disease had been in remission for at least six months, to the second study visit when the disease had been in remission for at least 12 months. This was indenpendent of whether the patients were aware that the physician could follow their medication refills or not. This might indicate that adherence to medical treatment of IBD decreases over time when the disease is in remission.

Disclosure of Interest: All authors have declared no conflicts of interest.

\section{References}

1. Kane S. Medication Nonadherence and the Outcomes of Patients with Ulcerative Colitis Quiescent. Am J Med. 2003; 114: 39-43.

2. http://sundhedsdatastyrelsen.dk/da/registre-og-services/om-faellesmedicinkort

\section{P1054 CLINICAL EFFICACY AND SAFETY OF GOLIMUMAB IN BIOLOGIC NAÏVE AND EXPERIENCED PATIENTS WITH ACTIVE ULCERATIVE COLITIS NON-RESPONDER OR INTOLERANT TO CONVENTIONAL THERAPIES}

B. Orlandini ${ }^{1}$, E. Capoferro ${ }^{2}$, S. Bagnoli ${ }^{1}$, G. Dragoni ${ }^{1}$, A. Geccherle ${ }^{2}$, F. Rogai ${ }^{1}$ ${ }^{1}$ Gastroenterology, IBD Unit, Careggi University Hospital, Florence/Italy

${ }^{2}$ Ospedale sacro cuore Negrar Unita IBD Unit Colon Proctolo, Verona/Italy

Contact E-mail Address: beatrice.orlandini2@gmail.com

Introduction: Golimumab (GOL) is a fully human monoclonal antibody to TNF $\alpha$ approved for the treatment of patients with moderate to severe ulcerative colitis (UC) with inadequate response or intolerance to steroids or immunosuppressive therapies. The aim of this study is to evaluate the efficacy and safety of GOL in both biologic naïve $(\mathrm{BN})$ and biologic experienced (BE) patients.

Aims \& Methods: Data were prospectively collected from a cohort of UC patients treated with GOL from March 2015 to March 2017 at two centers. Data were analyzed from two patient cohorts, namely BN patients and patients who have already undergone treatment with infliximab or adalimumab (BE). Patients received GOL $200 \mathrm{mg}$ sc. at week 0, GOL $100 \mathrm{mg}$ sc. at week 2 , then $50 \mathrm{mg}$ or $100 \mathrm{mg}$ sc. every 4 weeks depending on body weight. The primary outcomes were clinical response rate and incidence of adverse events (AEs).

\begin{tabular}{|c|c|c|c|c|}
\hline & Total & $\begin{array}{l}\text { Biologic } \\
\text { Naïve (BN) }\end{array}$ & $\begin{array}{l}\text { Biologic } \\
\text { Experienced } \\
\text { (BE) }\end{array}$ & $\mathrm{p}$ value \\
\hline \multicolumn{5}{|l|}{ PATIENT CHARACTERISTICS } \\
\hline Number of patients (n, \%) & $59(100)$ & $27(46)$ & $32(54)$ & \\
\hline $\operatorname{Sex}(n, \%)$ & & & & 0.9 \\
\hline Male & $28(47)$ & $13(48)$ & $15(47)$ & \\
\hline Female & $31(53)$ & $14(52)$ & $17(53)$ & \\
\hline Charlson Comorbidity index (n, \%) & & & & 0.7 \\
\hline 0 & $56(95)$ & $26(96)$ & $30(94)$ & \\
\hline$\geq 1$ & $3(6)$ & $1(4)$ & $2(6)$ & \\
\hline Charlson Global index (n, \%) & & & & 0.4 \\
\hline $0-1$ & $55(77)$ & $18(67)$ & $27(85)$ & \\
\hline$>1$ & $14(23)$ & $9(33)$ & $5(15)$ & \\
\hline Age at diagnosis (years) (median, IQR) & $33(23-45)$ & $33(25-45)$ & $32(23-45)$ & 0.5 \\
\hline Disease duration (years) (median, IQR) & $8(4-14)$ & $8(4-15)$ & $9(3-14)$ & 0.7 \\
\hline $\begin{array}{l}\text { Disease extension } \\
\quad \text { (Montreal classification) (n, \%) }\end{array}$ & & & & 0.3 \\
\hline E1 & $2(3)$ & $0(0)$ & $2(6)$ & \\
\hline E2 & $33(56)$ & $17(63)$ & $16(50)$ & \\
\hline E3 & $24(41)$ & $10(37)$ & $14(44)$ & \\
\hline $\begin{array}{l}\text { Endoscopic disease activity } \\
\text { (Mayo score) }(\mathrm{n}, \%)\end{array}$ & & & & 0.3 \\
\hline 1 & $2(3)$ & $2(7)$ & $0(0)$ & \\
\hline 2 & $12(20)$ & $5(18)$ & $7(22)$ & \\
\hline 3 & $45(77)$ & $20(74)$ & $25(78)$ & \\
\hline Previous ICS use (n, \%) & & & & 0.7 \\
\hline Steroid dependence & $49(83)$ & $23(85)$ & $26(81)$ & \\
\hline Steroid resistance & $10(17)$ & $4(15)$ & $6(19)$ & \\
\hline Previous IMS (n, \%) & & & & 0.7 \\
\hline Yes & $41(69)$ & $18(67)$ & $23(72)$ & \\
\hline Concomitant combination therapy $(\mathrm{n}, \%)$ & $4(7)$ & $1(4)$ & $3(9)$ & \\
\hline STUDY RESULTS & & & & \\
\hline
\end{tabular}


: Continued

\begin{tabular}{|c|c|c|c|c|}
\hline & Total & $\begin{array}{l}\text { Biologic } \\
\text { Naïve (BN) }\end{array}$ & $\begin{array}{l}\text { Biologic } \\
\text { Experienced } \\
\text { (BE) }\end{array}$ & $\mathrm{p}$ value \\
\hline Surgery required after treatment $(\mathrm{n}, \%)$ & $13(22)$ & $3(11)$ & $10(31)$ & 0.2 \\
\hline Adverse events (n, \%) & & & & 0.8 \\
\hline Yes & $10(17)$ & $4(15)$ & $6(19)$ & \\
\hline Discontinuation due to adverse events (n, \%) & $5(50)$ & $3(75)$ & $2(33)$ & \\
\hline $\begin{array}{l}\text { Time to discontinuation due to adverse } \\
\text { events (months) (median, IQR or mead, SD) }\end{array}$ & $4(1-4)$ & $4(2-4)$ & $2(1-4)$ & \\
\hline Patients eligible for analysis ( $\mathrm{n}, \%)$ & $54(92)$ & $24(89)$ & $30(94)$ & \\
\hline Duration of therapy (months) (median, IQR) & $7(4-14)$ & $7(4-13)$ & $7(4-14)$ & 0.8 \\
\hline \multicolumn{5}{|l|}{ Follow-up data at 3 months (n, \%) } \\
\hline Clinical response & $26(48)$ & $14(58)$ & $12(40)$ & 0.8 \\
\hline Treatment failure & $28(52)$ & $10(42)$ & $18(60)$ & 0.8 \\
\hline \multicolumn{5}{|l|}{ Follow-up at March 2017 (n, \%) } \\
\hline Clinical response & $17(31)$ & $8(33)$ & $9(30)$ & 0.5 \\
\hline Treatment failure & $37(69)$ & $16(67)$ & $21(70)$ & 0.5 \\
\hline
\end{tabular}

Results: Overall, data from 59 patients were analyzed. Of these, $27(46 \%)$ were $\mathrm{BN}$ and $32(54 \%)$ BE. Baseline patient's characteristics and main study results are shown in Table 1. BN and BE patients were comparable in terms of comorbidity profile, age at diagnosis, disease duration, pattern of previous and concomitant conventional therapies, as well as of disease extension and severity. Overall, surgical intervention after GOL therapy was performed in $13(22 \%)$ cases: $3(11 \%)$ belonging to the $\mathrm{BN}$ and $10(31 \%)$ and $\mathrm{BE}$ group, respectively $(\mathrm{p}=0.2)$. In $10(17 \%)$ patients AEs were recorded, most of which were genitourinary or herpes simplex infections. Of note, two cases of basal cell carcinoma were registered. The rate of AEs did not significantly differ among the BN and BE groups $(\mathrm{p}=0.8)$. In $5(50 \%)$ patients AEs were responsible for therapy discontinuation after a median (IQR) period of $4(1-4)$ months. Of the $54(92 \%)$ patients who continued therapy, median (IQR) duration of GOL treatment was 7 (4-14) months. At 3 months follow-up, $26(48 \%)$ patients showed clinica response to GOL. Clinical response rate was similar in the BN and BE cohorts $(\mathrm{p}=0.8) .28(52 \%)$ patients were non-responders, without a statistically significant difference between the two groups $(p=0.8)$. At March 2017, $17(31 \%)$ patients maintained clinical response, whereas $37(69 \%)$ failed the treatment. No statistically significant differences were noticed between the $\mathrm{BN}$ and $\mathrm{BE}$ cohorts concerning the clinical response or the treatment failure $(p=0.5)$. Conclusion: In our cohort, clinical response at 3 months follow-up was obtained in almost half of patients while at the last follow-up in one third. BN and BE patients had similar results in terms of clinical response, even though there seems to be a better early response in BN patients. Even if not statistically significant, surgery was required most frequently in the BE group. Rate of AEs was acceptable and similar in both $\mathrm{BN}$ and $\mathrm{BE}$ patients. Although our findings need to be confirmed in larger series, GOL therapy appears to be safe and to achieve an acceptable disease control in both the biologic naïve and experienced setting.

Disclosure of Interest: All authors have declared no conflicts of interest.

\section{P1055 ENDOSCOPIC FOLLOW-UP AND THERAPEUTIC ATTITUDE AFTER ILEOCOLONIC RESECTION IN A NATIONWIDE SPANISH COHORT OF CROHN'S DISEASE PATIENTS. PRACTICROHN STUDY}

M. Barreiro-De Acosta ${ }^{1}$, V. García-Sánchez ${ }^{2}$, A. Gutiérrez ${ }^{3}$, M.D. Martín Arranz $^{4}$, L. Cea-Calvo ${ }^{5}$, C. Romero ${ }^{5}$, B. Juliá De Páramo ${ }^{5}$, E. Domènech ${ }^{6}$ ${ }^{1}$ Unidad de Gastroenterología Complejo Hospitalario Universitario de Santiago, Santiago de Compostela/Spain

${ }^{2}$ Unidad Clínica de Aparato Digestivo Hospital Universitario Reina Sofía, IMIBIC, Córdoba/Spain

${ }^{3}$ Unidad de Gastroenterología Hospital General Universitario de Alicante, CIBEREHD Madrid, Alicante/Spain

${ }^{4}$ Servicio de Digestivo Hospital Universitario La Paz, Madrid/Spain

${ }^{5}$ Medical Affairs, Merck Sharp \& Dohme de España, Madrid/Spain

${ }^{6}$ Servicio de Gastroenterología, Hospital Universitari Germans Trias i Pujol and CIBERehd, Barcelona/Spain

Contact E-mail Address: manubarreiro@hotmail.com

Introduction: Endoscopic recurrence precedes clinical recurrence after ileocolonic resection for Crohn's disease (CD). Guidelines recommend an ileocolonoscopy within the first year after surgery where timely treatment decisions may be affected. The aim of our study was to describe the endoscopic monitoring and associated treatment decisions within the first year after surgery in a cohort of CD patients operated on between 2007 and 2010

Aims \& Methods: PRACTICROHN was a study that included patients aged $\geq 18$ years-old from 26 Spanish hospitals who underwent CD-related ileocolonic resection with ileocolonic or ileorectal anastomosis between January 2007 and December 2010. Clinical data was retrospectively collected from clinical charts during 5 years follow-up after surgery. Categorical variables were compared with the $\chi 2$ test or Fisher's exact test.

Results: Three hundred and fourteen patients were analyzed (mean age 40 years [SD 13], 48\% men). Throughout the observational period, 262 patients $(83.4 \%)$ underwent colonoscopy and 52 patients $(16.5 \%)$ lacked endoscopic follow-up. First endoscopy was performed mainly within the first year after surgery $(45.5 \%$, $\mathrm{n}=143)$ and the leading reason was a planned monitoring $(66.4 \%, \mathrm{n}=95)$. In total, $30.3 \%$ of the patients underwent planned endoscopy within first year after surgery. By year, it was found a positive trend in the number of both total and "planned" endoscopies carried out within this period $(\mathrm{p}=0.017$ and $\mathrm{p}=0.027$, respectively) (table). Table

\begin{tabular}{llllll}
\hline \multirow{2}{*}{ Patients underwent surgery } & $\begin{array}{l}2007 \\
\mathrm{n}=75\end{array}$ & $\begin{array}{l}2008 \\
\mathrm{n}=83\end{array}$ & $\begin{array}{l}2009 \\
\mathrm{n}=77\end{array}$ & $\begin{array}{l}2010 \\
\mathrm{n}=79\end{array}$ & p-value
\end{tabular}

Patients with endoscopy within first year after surgery, $\mathrm{n}(\%)$

Patients with "planned" endoscopy within first year after surgery, $\mathrm{n}(\%)$

$24(33.80) \quad 36(43.90) \quad 36(46.75) \quad 47(59.49) \quad 0.017$

$15(20.00) \quad 25(30.12) \quad 21(27.27) \quad 34(43.04) \quad 0.027$

Treatments received by patients at discharge were Immunomodulators $(30.8 \%)$, antibiotics $(21.3 \%)$, corticoesteroids $(19.1 \%)$, mesalazine $(12.1 \%)$ and antiTNFa $(0.9 \%)$. A total of 235 patients $(74.8 \%)$ had a medication change throughout the follow-up period, mainly within the first year after surgery $(47.1 \%, \mathrm{n}=148)$. Median time to first medication change was 10 (IQR 4-22) months. More often therapeutic decision was the introduction or dose escalation of thiopurines or change to a more potent agent $(68.1 \%, \mathrm{n}=160)$ followed by the introduction of an antiTNFa drug or dose escalation $(51.4 \%, \mathrm{n}=121)$. Ninety-two out of 95 patients with planned endoscopy within first year after surgery had Rutgeerst scoring (RS) available, $37(40.2 \%)$ and $55(59.7 \%)$ showed RS $\geq \mathrm{i} 2$ and $<\mathrm{i} 2$ respectively. More patients with a $\mathrm{RS} \geq \mathrm{i} 2$ had a medication change as compared to patients with a RS $<\mathrm{i} 2$, but the differences didn't reach statistical significance $(45.95 \%$ vs $36.36 \%)$. Reasons for medication change were "endoscopic without clinical recurrence" $(52.9 \% \mathrm{RS}>\mathrm{i} 2$ vs $10.0 \% \mathrm{RS}<\mathrm{i} 2, \mathrm{p}=0.010)$, "clinical recurrence" (29.4\% RS $\geq \mathrm{i} 2$ vs $25.0 \% \mathrm{RS}<\mathrm{i} 2)$ and "others" (23.5\% RS $\geq \mathrm{i} 2$ vs $60.0 \%$ $\mathrm{RS}<\mathrm{i} 2, \mathrm{p}=0.045)$.

Conclusion: The number of planned endoscopies carried out within the first year after surgery increased significantly from 2007 to 2010 showing a steadily implementation of guidelines recommendations. Changes in medication within this period were more frequent in the setting of endoscopic recurrence. Acknowledgements. Funded by Merck Sharp \& Dohme of Spain

Disclosure of Interest: All authors have declared no conflicts of interest.

P1056 ETROLIZUMAB TREATMENT IMPROVES HISTOLOGICAL ACTIVITY AS ASSESSED BY BOTH THE ROBARTS HISTOPATHOLOGY AND NANCY HISTOLOGICAL INDICES

L. Peyrin-Biroulet ${ }^{1}$, B. G. Feagan ${ }^{2}$, G. De Hertogh ${ }^{3}$, D. T. Rubin ${ }^{4}$, R. Maciuca ${ }^{5}$, J. Mcbride ${ }^{5}$, U. Arulmani ${ }^{5}$, A. Scherl ${ }^{5}$

${ }^{1}$ Nancy Univ Hospital, Lorraine Univ, Lorraine/France

${ }^{2}$ Univ of Western Ontario, London/Canada/ON

${ }^{3}$ Univ of Leuven, Leuven/Belgium

${ }^{4}$ Univ of Chicago Medicine, Chicago/United States of America/IL

${ }^{5}$ Genentech, Inc., South San Francisco/United States of America/CA

Contact E-mail Address: arulmani.udayasankar@gene.com

Introduction: Etrolizumab, an anti- $\beta 7$ monoclonal antibody targeting $\alpha 4 \beta 7$ and $\alpha \mathrm{E} \beta 7$ integrins, showed efficacy and safety versus placebo (PBO) during 10 weeks (wk) of induction in patients with moderate-to-severe ulcerative colitis in the Phase 2 EUCALYPTUS trial (Vermeire S. Lancet. 2014;384:309-18). Since a reduction in histologic inflammation has been linked with improved long-term clinical outcome (Bryant RV. Gut. 2016;65:408-14), and the FDA recommends using both histologic and endoscopic assessments for efficacy evaluation, the effect of etrolizumab on histologic inflammation was evaluated in mucosal biopsies from EUCALYPTUS patients using the Robarts histopathology index (RHI; Mosli MH. Gut. 2017;66:50-8) and Nancy histological index (NHI; Marchal-Bressenot A. Gut. 2017;66:43-9).

Aims \& Methods: 124 patients were randomly assigned (1:1:1) to receive subcutaneous etrolizumab $(100 \mathrm{mg}$ at wk 0 , 4, and 8 , with PBO at wk 2 , or $420 \mathrm{mg}$ loading dose at wk 0 , followed by $300 \mathrm{mg}$ at wk 2, 4, and 8) or matching PBO. Biopsies were taken using flexible sigmoidoscopy/full colonoscopy from the most inflamed colonic area within $10-40 \mathrm{~cm}$ from the anal verge at baseline (BL) and at wk 10.62 patients provided consent for long-term sample storage for research; batched H\&E-stained slides were scored by a single pathologist using the Geboes scale (later converted to RHI) and NHI. At wk 10, mean changes in RHI and NHI scores for pooled etrolizumab or PBO were calculated. Subanalyses explored histologic response (reductions of $\geq 6$ or 10 points or $\geq 50 \%$ improvement from BL RHI and $>1$ or 2 points reduction from BL NHI), remission (no neutrophils, $\mathrm{RHI} \leq 4$ and $\mathrm{NHI}=0, \leq 1$ or 2 ) and correlation with endoscopic improvement.

Results: Analysis included 56 patients with BL data and BL NHI $>1$. At wk 10 , RHI and NHI scores decreased by a greater extent with etrolizumab compared with PBO, regardless of anti-tumor necrosis factor $\alpha$ (aTNF) experience (RHI -8.4 vs $-1.6 ; \mathrm{P}=0.032$ and $\mathrm{NHI}-1.2$ vs $-0.2 ; \mathrm{P}=0.011$ for all comers). $\mathrm{A}$ greater proportion of etrolizumab-treated patients achieved categorical histologic improvement and remission. Of patients with an endoscopic subscore $(\mathrm{ES}) \leq 1$ at wk $10(\mathrm{n}=6), 100 \%$ experienced histologic response as assessed by RHI $(5 / 5$ with RHI non-missing at wk 10), and $83 \%(5 / 6)$ by NHI. Mean (SD) RHI changes were $-19.2(10.0)$ in patients with an $\mathrm{ES} \leq 1$ at wk 10 versus $-4.4(10.1)$ in patients with an ES $>1$. Mean (SD) NHI changes were $-2.5(1.5)$ in patients with an $\mathrm{ES} \leq 1$ at wk 10 versus $-0.6(1.3)$ in patients with an $\mathrm{ES}>1$. Spearman's 
correlation coefficients between RHI and NHI were 0.82 at BL and 0.91 at wk 10 , while both histologic scores were similarly correlated with ES $(0.25-0.28$ at BL and $0.38-0.40$ at wk 10).

Table 1: Percentage of Patients Achieving Histologic Response and Remission at Week 10

\begin{tabular}{|c|c|c|c|c|c|c|}
\hline & \multicolumn{2}{|l|}{$\begin{array}{l}\text { aTNF-naive } \\
(\mathrm{n}=16)\end{array}$} & \multicolumn{2}{|c|}{$\begin{array}{l}\text { aTNF-experienced } \\
(\mathrm{n}=34)\end{array}$} & \multicolumn{2}{|l|}{$\begin{array}{l}\text { All comers } \\
(\mathrm{n}=50)\end{array}$} \\
\hline & Etrolizumab & Placebo & Etrolizumab & Placebo & Etrolizumab & Placebo \\
\hline \multicolumn{7}{|c|}{ RESPONSE (decrease from baseline) } \\
\hline $\mathrm{RHI} \geq 6$ & $55 \%$ & $17 \%$ & $46 \%$ & $36 \%$ & $49 \%$ & $30 \%$ \\
\hline$\geq 10$ & $55 \%$ & $0 \%$ & $33 \%$ & $21 \%$ & $40 \%$ & $15 \%$ \\
\hline$\geq 50 \%$ & $36 \%$ & $0 \%$ & $25 \%$ & $7 \%$ & $29 \%$ & $5 \%$ \\
\hline $\mathrm{NHI} \geq 1$ & $73 \%$ & $17 \%$ & $52 \%$ & $14 \%$ & $58 \%$ & $14 \%$ \\
\hline$\geq 2$ & $55 \%$ & $0 \%$ & $32 \%$ & $14 \%$ & $39 \%$ & $10 \%$ \\
\hline \multicolumn{7}{|c|}{ REMISSION (absolute score) } \\
\hline $\mathrm{RHI} \leq 4$ & $36 \%$ & $0 \%$ & $8 \%$ & $7 \%$ & $17 \%$ & $5 \%$ \\
\hline No neutrophils & $36 \%$ & $0 \%$ & $8 \%$ & $7 \%$ & $17 \%$ & $5 \%$ \\
\hline $\mathrm{NHI}=0$ & $36 \%$ & $0 \%$ & $4 \%$ & $14 \%$ & $14 \%$ & $10 \%$ \\
\hline$\leq 1$ & $45 \%$ & $0 \%$ & $20 \%$ & $14 \%$ & $28 \%$ & $10 \%$ \\
\hline$\leq 2 *$ & $73 \%$ & $33 \%$ & $56 \%$ & $21 \%$ & $61 \%$ & $25 \%$ \\
\hline
\end{tabular}

*Must have achieved $\geq 1$ point improvement

NHI $0=$ no histologically significant disease

NHI $1=$ chronic inflammatory infiltrate with no acute inflammatory infiltrate NHI $2=$ mildly active disease

Conclusion: Histologic activity assessment using RHI or NHI demonstrates improvement after wk 10 with etrolizumab treatment and was greater in aTNF-naive patients. Importantly, RHI or NHI reductions were associated with improved ES at wk 10

Disclosure of Interest: L. Peyrin-Biroulet: Consultant/Advisor for Merck, Abbvie, Janssen, Genentech, Mitsubishi, Ferring, Norgine, Tillots, Vifor, Therakos, Pharmacosmos, Pilège, BMS, UCB-pharma, Hospira, Celltrion, Takeda, Biogaran, Boerhinger-Ingelheim, Lilly, Pfizer [rest of discl. on request] B.G. Feagan: Research grants from AbbVie Inc., Amgen Inc., AstraZeneca MedImmune Ltd., Atlantic Pharmaceuticals Ltd., Boehringer-Ingelheim, Celgene Corporation, Celltech, Genentech Inc/Hoffmann-La Roche Ltd., Gilead Sciences Inc. [rest of discl. on request]

G. de Hertogh: Fee to intitution from Janssen R\&D, LLC for central pathology review

D.T. Rubin: Research grants from Prometheus Laboratories, AbbVie, UCB Pharma, Janssen, Takeda, Genentech. Consultant/Advisor for AbbVie, UCB Pharma, Janssen, Takeda, Emmi, Genentech, Amgen, Celgene, Pfizer R. Maciuca: Employee of Genentech. Roche stock-holder J. McBride: Employee of Genentech. Roche stock-holder

U. Arulmani: Employee of Genentech. Roche stock-holder.

A. Scherl: Employee of Genentech

P1057 GO-COLITIS: EFFICACY AND QUALITY OF LIFE DURING GOLIMUMAB MAINTENANCE IN UK PATIENTS WITH MODERATE TO SEVERE ULCERATIVE COLITIS

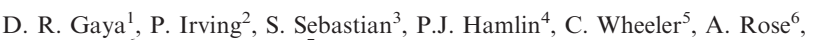
G. Gillespie ${ }^{6}$, C. S.J. Probert ${ }^{7}$

${ }^{1}$ Gastroenterology Unit, Glasgow Royal Infirmary, Glasgow/United Kingdom

${ }^{2}$ Guýs and St Thomaś Hospital Dept. of Gastroenterology - Guy's and St Thomas Hospital Dept. of Gas, London/United Kingdom

${ }^{3}$ Hull Royal Infirmary, Hull/United Kingdom

${ }^{4}$ Leeds Gastroenterology Institute, St. James's University Hospital, Leeds/United Kingdom

${ }^{5}$ Medical Affairs, MSD, Hoddesdon/United Kingdom

${ }^{6}$ MSD Sharp \& Dohme Division of Immunology, Hoddesdon/United Kingdom

${ }^{7}$ Gastroenterology Research Unit, Cellular And Molecular Physiology, University

of Liverpool, Liverpool/United Kingdom

Contact E-mail Address: chris.probert@liverpool.ac.uk

Introduction: GO-COLITIS (NCT02092285; 2013-004583-56) is a phase 4, multicentre, open-label, single-arm trial in the UK assessing efficacy of golimumab (GLM) in induction and maintenance of clinical response in patients with moderate to severe ulcerative colitis (UC) resistant to conventional treatment. Results of the maintenance phase are presented here.

Aims \& Methods: Anti-TNF naive adults with UC $>3$ months who responded to induction therapy with subcutaneous GLM at week 6, according to partial Mayo score (PMS), continued to receive a maintenance dose of $50 \mathrm{mg}$ or $100 \mathrm{mg}$ GLM (dependent on the patient's weight) every 4 weeks as per the Summary of Product Characteristics for a total of 54 weeks. Measurements were taken at week 6, week 30 and week 54 . The primary endpoint was the proportion of patients meeting PMS response criteria at week 54 (defined as decrease in PMS of $\geq 2$ points and $\geq 30 \%$ from baseline, plus a decrease in rectal bleeding subscore of $\geq 1$ point or absolute rectal bleeding score $\leq 1$ ). Secondary endpoints included proportion of patients meeting PMS remission criteria at week 54 (defined as PMS $\leq 2$ and no individual Mayo subscore $>1$ ), change from baseline in IBDQ and EQ-5D at each visit and normalization of CRP.
Results: Overall, 205 patients enrolled in GO-COLITIS and received at least one dose of GLM. Of these, 140 patients responded in the induction phase and received GLM in the maintenance phase. Clinical response was maintained through week 54 in $52 / 140$ patients $(37.1 \% ; 95 \%$ CI, $29.1 \%$ to $45.7 \%)$ and $42 /$ 140 patients were in remission at week $54(30.0 \%$; $95 \%$ CI, $22.6 \%$ to $38.3 \%)$. Improvements in PMS subscores from baseline to week 54 were noted in stool frequency (mean change, $-1.9 ; \mathrm{SD}, 1.1[\mathrm{n}=59]$ ), rectal bleeding (mean change, -1.5 ; SD, $0.8[\mathrm{n}=59]$ ), and physician's global assessment (mean change, -1.8 ; SD, $0.8[\mathrm{n}=57])$. Normal CRP levels at week 54 were seen in $50 / 59$ patients (response rate, $84.7 \% ; 95 \%$ CI, $73.0 \%$ to $92.8 \%$ ). IBDQ and EQ-5D results are summarised in the Table. Serious adverse events (SAEs) occurred in 49/205 patients $(23.9 \%)$, with 3 SAEs considered treatment-related

Table: Mean (SD) Change from baseline to week 54 in IBDQ and EQ-5D.

\begin{tabular}{lllllll}
\hline & $\mathrm{n}$ & Baseline & $\mathrm{n}$ & Week 54 & $\mathrm{n}$ & $\begin{array}{l}\text { Change From } \\
\text { Baseline }\end{array}$ \\
\hline IBDQ total score & 138 & $116.4(32.7)$ & 59 & $186.2(27.1)$ & 59 & $66.8(36.7)$ \\
EQ-5D index score & 136 & $0.7(0.2)$ & 60 & $0.9(0.2)$ & 58 & $0.2(0.3)$ \\
\hline
\end{tabular}

Conclusion: In the maintenance treatment with GLM phase of GO-COLITIS, $37.1 \%$ and $30.0 \%$ of patients with moderate to severe UC in the UK demonstrated clinical response and remission at week 54, respectively. Improvements in patient-reported quality of life measures (IBDQ/EQ-5D) were seen; the degree of improvement in IBDQ total score exceeded the IBDQ increase cutoff (i.e. $>20$ ) for patient defined-remission previously identified as representative of a patientdefined improvement in an assessment of UC clinical endpoints. ${ }^{1}$ Adverse events were consistent with previous observations.

Disclosure of Interest: D.R. Gaya: Daniel R. Gaya: speaker for Abbvie, Dr Falk Pharma, Ferring, MSD, Shire, Takeda, Vifor.

P. Irving: Peter Irving: research funding from and is a speaker or advisory board member for Abbvie, Dr Falk Pharma, Ferring, Genentech, Hospira, Janssen, Johnson and Johnson, MSD, Pharmacosmos, Shire, Takeda, Tillotts, Topivert, Vifor Pharma \& Warner Chilcott.

S. Sebastian: Shaji Sebastian: research funding from and is a speaker or advisory board member for Abbvie, Dr Falk Pharma, Ferring, MSD, Takeda, Warner Chilcott and Vifor Pharma.

P.J. Hamlin: P. John Hamlin: speaker for Abbvie, Ferring, Janssen, MSD, Takeda, Tillotts, Warner Chilcott.

C. Wheeler: Colin Wheeler: consultant for Aegerion, Amryt Pharma, Astrazeneca, Daiichi Sankyo, GSK, MSD UK, Takeda, Sanofi and Shionogi; was an employee of MSD UK and owns stocks and shares in Clovis Oncology, Novelion, Shield Therapeutics and Tesaro.

A. Rose: Anita Rose: employee of MSD UK and owns stocks and shares in Merck \& Co., Inc., Kenilworth, NJ USA.

G. Gillespie: Gillian Gillespie: employee of Merck \& Co., Inc., Kenilworth, NJ USA and owns stocks and shares in Merck \& Co., Inc., Kenilworth, NJ USA. C.S.J. Probert: Christopher S.J. Probert: speaker and a consultant for Abbvie, Aventis, Dr Falk Pharma, Ferring, Janssen, MSD, Napp, Pfizer, Shield, Shire, Takeda and Vifor.

\section{Reference}

1. Higgins PDR, et al. Gut. 2005;54:782-788.

\section{P1058 A UNITED STATES CLAIMS DATABASE ANALYSIS COMPARING SAFETY, MEDICAL RESOURCE UTILIZATION, AND TREATMENT COSTS ASSOCIATED WITH THE MANAGEMENT OF INFLAMMATORY BOWEL DISEASE BY DRUG CLASS}

G. H. Long ${ }^{1}$, A. Tatro ${ }^{2}$, Y. $\mathrm{Oh}^{2}$, S. R. Reddy ${ }^{3}$, A. N. Ananthakrishnan ${ }^{4}$

${ }^{1}$ Roche Pharmaceuticals Ltd., Welwyn Garden City/United Kingdom

${ }^{2}$ Genentech, Inc. South San Francisco/United States of America/CA

${ }^{3}$ Partnership for Health Analytic Research, Beverly Hills/United States of America/CA

${ }^{4}$ Massachusetts General Hospital and Harvard Medical School, Boston/United States of America/MA

Contact E-mail Address: oh.young@gene.com

Introduction: Only a third of patients (pts) with inflammatory bowel disease (IBD) treated with current pharmacological options achieve clinical remission at 1 year, and most experience drug-related adverse events (AEs). Herein we characterise the clinical and economic burden of IBD treatment limitations in terms of AEs of interest, medical resource utilisation (MRU) and associated medical costs.

Aims \& Methods: Using pts taking only aminosalicylates (ASA) as a reference, we compared AE incidence, MRU and medical costs in pts with ulcerative colitis (UC) or Crohn's disease (Crohn's) who initiated treatment with oral corticosteroids (OCS), immunosuppressants (IS), anti-tumor necrosis factor agents (aTNF) or with combinations thereof. Eligible pts (aged $>18$ years) in the IMS PharMetrics insurance claims database between $7 / 1 / 10$ and $6 / 30 / 15$ had $\geq 2$ medical claims ( $\geq 7$ days apart) and a diagnosis of UC (ICD-9-CM: 556.x) or Crohn's (ICD-9-CM: 555.x), with >1 qualifying claim in the year preceding treatment. Univariate comparisons included statistical tests of significance $\left(\chi^{2}, \mathrm{~F}\right.$ test, or Kruskal Wallis). Multivariate analyses were based on Cox proportional hazards regression, negative binomial regression, logistic regression or linear regression 
analysis adjusted by significant socio-demographic, clinical and disease severity covariates.

Results: This analysis included 30,676 (Crohn's: $N=14,528$; UC: $N=16,148$ ). OCS monotherapy was the strongest predictor of any AE occurring for pts with Crohn's or UC (Crohn's: HR, 1.62 [1.51-1.73]; UC: HR, 1.57 [1.49-1.66]). A similar pattern was observed for severe infection (Crohn's: HR, 2.43 [2.07-2.85]; UC: HR, 2.37 [2.07-2.72]) and bone-related conditions (Crohn's: HR, 1.88 [1.74 2.03]; UC: HR, 1.77 [1.67-1.89]). The strongest predictors for serious hepatic events were IS + OCS (Crohn's: HR, 2.38 [1.72-3.31]; UC: HR, 2.36 [1.753.18]) and OCS (Crohn's: HR, 2.09 [1.65-2.66]; UC: HR, 1.92 [1.57-2.34]). Pts with UC or Crohn's receiving OCS or IS + OCS were more likely to have emergency department visits; IBD-related hospitalisation, visits or procedures; and gastrointestinal surgery compared with pts receiving other therapies. Annualised total medical costs were greatest for aTNF + IS or aTNF therapy in both Crohn's and UC. However, annualised medical service costs (that exclude IBD drug costs) were highest for pts initiating OCS-containing therapies (Crohn's: OCS, $\$ 27,041$ and OCS + IS, \$23,332; UC: OCS, \$19,659) followed by other index therapies (Crohn's: ASA, $\$ 10,823$ and aTNF + IS, $\$ 19,151[\mathrm{P}<0.001]$ UC: ASA, \$7,980 and aTNF + IS, \$18,771 [P < 0.001]).

Conclusion: Chronic OCS use was associated with increased risk of severe infection, bone conditions, and serious hepatic events compared with other therapies. Consistent with an increased AE risk, OCS regimens were associated with higher rates of MRU and medical service costs compared with other therapies. Although limiting the use of OCS regimens may be more costly initially, treatment decisions should consider downstream benefits of alternate options.

Disclosure of Interest: G.H. Long: Employee of Roche. Roche stock-holder

A. Tatro: Employee of Genentech. Roche stock-holder

Y. Oh: Employee of Genentech. Roche stock-holder

S.R. Reddy: Employee of PHAR, LLC, paid by Genentech/Roche to conduct this research

A.N. Ananthakrishnan: Consultant/Advisor for Abbvie, Takeda, and Merck

\section{P1059 CAN EARLY DRUG AND ANTI-INFLIXIMAB-ANTIBODY LEVELS PREDICT PRIMARY NON RESPONSE TO INFLIXIMAB THERAPY?}

H. Bar-Yoseph ${ }^{1}$, Y. Chowers ${ }^{1}$, N. Levhar ${ }^{2}$, L. Selinger ${ }^{2}$, U. Manor ${ }^{2}$, M. Yavzori ${ }^{2}$, E. Fudim², O. Picard ${ }^{2}$, U. Kopylov², R. Eliakim², S. Ben-Horin ${ }^{2}$, B. Ungar ${ }^{2}$

${ }^{1}$ Gastroenterology, Rambam Health Care Campus, Haifa/Israel

${ }^{2}$ Gastroenterology Institute, - Gastroenterology institute/IL, Ramat Gan/Israel

Contact E-mail Address: bellageyshis@gmail.com

Introduction: Infliximab has been shown to induce and maintain long-term clinical remission in inflammatory bowel disease (IBD) patients. However, $10-30 \%$ of patients show no clinical benefit by the end of induction (week 14) and are considered primary non-responders. The mechanisms underlying primary nonresponse have not yet been clearly defined.

Aims \& Methods: In this study we aimed to evaluate to which extent pharmacokinetics (early induction infliximab and anti-infliximab-antibody (ATI) levels) are associated with primary non response. A retrospective observational casecontrol study of patients with IBD attending the Gastroenterology Department of Sheba medical center and treated with infliximab between 2009 and 2016 was performed. Clinical scores were determined and sera were collected prospectively before infusions. Infliximab and ATI levels were measured by our previously described drug-tolerant ELISA assay.

Results: Thirty five primary non responders have been identified and matched at $1: 3$ ratio with 105 primary responders for a total of 140 patients. Both week 2 and week 6 infliximab levels were significantly lower among primary non-responders compared to responders (week 2: median level $7.2 \mu \mathrm{g} / \mathrm{ml}$ vs. $13.5 \mu \mathrm{g} / \mathrm{ml}$, $\mathrm{p}=0.0019$, week 6: median level $2.2 \mu \mathrm{g} / \mathrm{ml}$ vs. $9.5 \mu \mathrm{g} / \mathrm{ml}, \mathrm{p}<0.0001$, respectively). ATI appeared more frequently (either week 2 or $6, \mathrm{OR}=4.6$, CI 2 $10.8, \mathrm{p}=0.0004)$ and at higher levels in non-responders compared to responders (week 2: median ATI $7.3 \mu \mathrm{g} / \mathrm{ml}$-eq vs. $3.8 \mu \mathrm{g} / \mathrm{ml}$-eq, $\mathrm{p}=0.005$, week $6: 10.8 \mu \mathrm{g} / \mathrm{ml}$ eq vs. $4.4 \mu \mathrm{g} / \mathrm{ml}$-eq, $\mathrm{p}=0.008$, respectively). Moreover, week 2 ATI levels $>4.3 \mu \mathrm{g} / \mathrm{ml}$-eq $(\mathrm{AUC}=0.78, \mathrm{p}=0.0004$, sensitivity $77 \%$, specificity $71 \%)$ and infliximab levels $<6.8 \mu \mathrm{g} / \mathrm{ml}$ (AUC $=0.68, \mathrm{p}=0.002$, sensitivity $50 \%$, specificity $86 \%$ ) were predictive of primary non-response. In analyses of various demographic and clinical factors correlated with primary non-response the only independent factors found significant on multivariable analyses were lower infliximab and higher ATI levels at either week 2 or 6, infliximab monotherapy and previous IBD-related surgeries. Sub-analyses according to IBD type demonstrated that in addition to lower drug and higher ATI levels, older age and previous surgeries increased the risk for primary non-response among Crohn's disease patients. Monotherapy was the only other factor significant among ulcerative colitis patients, although multivariate analysis was not performed due to limited sample size $(\mathrm{n}=32)$.

Conclusion: Although direction of causality cannot be ascertained, infliximab levels below $6.8 \mu \mathrm{g} / \mathrm{ml}$ and ATI levels above $4.33 \mu \mathrm{g} / \mathrm{ml}$-eq before the second infusion (week 2) are predictive of primary non response, in addition to previous CD-related surgeries and infliximab monotherapy. These findings suggest that preemptive interventions in a subset of patients might minimize this phenomenon.

Disclosure of Interest: Y. Chowers: Abbvie - grant support, lecture and advisory fees Janssen - lecture and advisory fees Takeda - grant support lecture and advisory fees Medtronics - advisory fees

U. Kopylov: Speaker fees - abbvie Research support, speaker and advisory fees jannsen
S. Ben-Horin: SBH has received consultancy and/or advisory board fees from Schering-Plough, AbbVie, Celltrion, Pfizer, Janssen and Takeda; and has received research support from Celltrion, AbbVie \& Takeda B. Ungar: I received consultation fees from Abbvie and Jannsen. All other authors have declared no conflicts of interest.

\section{P1060 TREATMENT EXPERIENCE WITH TOPICAL PRODUCTS FOR ULCERATIVE COLITIS-THE PATIENT́S PERSPECTIVE IN EUROPE AND THE USA}

T. Bayrhoffer ${ }^{1}$, A. Thompson ${ }^{2}$, T. Knittel ${ }^{3}$

${ }^{1}$ DBA Effimed Research LLC, San Francisco/United States of America/CA

${ }^{2}$ Brera Life Sciences, Guildford/United Kingdom

${ }^{3}$ InDex Pharmaceuticals, Stockholm/Sweden

Contact E-mail Address: thomas.knittel@indexpharma.com

Introduction: Topical therapies for ulcerative colitis have shown evidence of good efficacy and can induce better responses and earlier improvement in distal ulcerative colitis (UC) when compared with oral therapies. Despite this attractive targeted approach of delivering medications topically to the left colon a certain resistance to the use of topical therapy seems to exist.

Aims \& Methods: The goal of this market research was to assess the familiarity with and perceptions of patients towards topical products. A qualitative market research study was performed in the USA and 3 European countries (Germany, UK and Italy). The primary patient recruitment sources were online web portals, e-mail campaigns and social networking sites. Informal feedback gathered from outspoken patient activists to identify the right sources was also used. In order to select UC patients with more advanced disease and/or a longer disease history current or past steroid medication was mandatory as a qualification for inclusion in the market research. A structured questionnaire covering 14 items was pretested and modified in phone interviews, which was then subsequently used in telephone interviews or as a web based interactive survey, both in local language. A total of 148 patient responses were obtained via 10 phone interviews and 138 web-survey, 60 patients came from the US, 27, 25 and 36 from Germany, UK and Italy, respectively.

Results: In this survey cohort patients had been diagnosed with UC for $>5$ years on average, $2 / 3$ of patients had left-sided disease and less than a third had extensive disease. The majority of patients experienced at least 1-2 flare-ups each year and less than $18 \%$ of patients had them only rarely. ASA and steroids were the most commonly used medications in all countries, biological treatments were reported as highest in $35 \%$ (US) to the lowest $16 \%$ (UK) as stated by the patients. The vast majority of patients stated that they had treatment experience with rectal products at some point during their treatment journey with only a slightly lower number in the US $(83 \%)$ compared to the EU countries (Range 89 $92 \%$ ). Rectal enemas were the most common formulation delivery for topical ASA products in all markets $(79 \%)$ followed by suppository $(25 \%)$ and foam $(13 \%)$. A total of $53 \%$ of patients were not concerned about the rectal mode of administration, while $47 \%$ reported some concerns. These mainly comprised the need to hold the enema in place, a generally uncomfortable feeling with rectal medications and painful administration.

Conclusion: Despite a certain resistance to use topical therapy almost all patients stated to have used rectally administered products at some point during their disease journey and even patients in the USA were very familiar with these medications. Although physicians see patients as the primary driver for the resistance to use topical products in UC, less than $50 \%$ of the patients were actually concerned about the use of topical therapy in this study, thereby calling for better physician-patient communication.

Disclosure of Interest: T. Bayrhoffer: Consultancy for Index Pharmaceuticals A. Thompson: Consultancy for Index Pharmaceuticals

T. Knittel: Consultancy, CMO position and share holding of Index Pharmaceuticals

\section{P1061 SELF-MANAGEMENT IN INFLAMMATORY BOWEL} DISEASE: A PERSPECTIVE OF NURSES AND PHYSICIANS

S. P. Van Den Burg, N. M.s. Peek, A. E. Van Der Meulen - De Jong, J.P.w. Maljaars

Gastroenterology And Hepatology, LUMC, Leiden/Netherlands

Contact E-mail Address: s.p.van_den_burg@1umc.nl

Introduction: Over the last years, self-management (SM) has been advocated as an instrument to empower and enable patients, increase patient involvement and reduce healthcare costs in chronic diseases. Although many SM programs have been developed for inflammatory bowel disease (IBD) patients, little research has been performed on needs and wishes of IBD physicians, nurses and patients. Nurses and physicians play an important role in providing and stimulating $\mathrm{SM}^{1-3}$

Aims \& Methods: This study aimed to gain insight in what caregivers consider good SM-options, and which patient- or disease-related factors they consider of influence on the degree of SM a patient is willing to apply. During a nurses IBD day at our hospital, a survey regarding SM was distributed among 46 nurses. Also, 50 IBD-interested gastroenterologists were invited to respond to the same survey by email with a link to the survey. The survey contained questions regarding the caregivers' views on ways for patients to apply SM in an outpatient setting (12 options were given). Caregivers were asked to state whether they thought these options would be valuable to patients or not, and to name their top three options. Also, caregivers were asked their views on factors that could 
influence the degree of SM a patient is willing to apply, such as: disease duration, activity and perception, health literacy, self-efficacy, patients' age, and level of trust between patient's and their IBD team. Caregivers were asked per item whether they thought this factor would be of influence and to name the three most important factors.

Results: 38 nurses (mean age 42 years) and 32 physicians (mean age 44 years) responded to the survey. The three most appealing options for nurses regarding SM were: availability of a SM web-app, Skype/Face-time consultation with nurse/physician, and an at-home faecal-calprotectin test. Physicians preferred the availability of a SM web-app, an at-home faecal-calprotectin test, and making patients in charge of their patient records. When comparing the value of each of the 12 possible choices in which patients could apply SM, only one option was valued differently between nurses and physicians. $56 \%$ of physicians favoured patients being in charge of patients records compared to $18 \%$ of nurses $(\mathrm{p}=0.001)$. Physicians thought that the 3 most important factors influencing SM in patients were: level of trust between physician and patient, self-efficacy, and disease perception. Also, $41 \%$ of the physicians found health literacy to be an important factor. Nurses suggested that self-efficacy, disease perception and disease activity were most important. One factor was valued differently between nurses and physicians: $78 \%$ of nurses thought that patients' age was an important factor in patient's SM, compared to $34 \%$ of physicians $(\mathrm{p}=0.000)$. Conclusion: Overall, nurses and physicians agree on many aspects of SM in IBDcare. However, more physicians are in favour of making patients in charge of their patient records. Nurses and physicians agree that patient characteristics, in contrast to disease characteristics, influence SM, with self-efficacy being the most important. This study calls for further research on what patients and caregivers, want and need from SM, as SM is a team sport.

Disclosure of Interest: All authors have declared no conflicts of interest.

\section{References}

1. Bagnasco A, Di Giacomo P, Da Rin Della Mora, Roberta, Catania G, Turci $\mathrm{C}$, Rocco $\mathrm{G}$ et al. Factors influencing self-management in patients with type 2 diabetes: a quantitative systematic review protocol. $J$ Adv Nurs 2014;70(1):187-200.

2. Bos Touwen I, Schuurmans M, Monninkhof E, Korpershoek Y, Spruit Bentvelzen L, Ertugrul-van der Graaf I et al. Patient and disease characteristics associated with activation for self-management in patients with diabetes, chronic obstructive pulmonary disease, chronic heart failure and chronic renal disease: a cross-sectional survey study. PLOS ONE 2015;10(5):e0126400-.

3. Matthias M, Bair M, Nyland K, Huffman M, Stubbs D, Damush T et al. Selfmanagement support and communication from nurse care managers compared with primary care physicians: a focus group study of patients with chronic musculoskeletal pain. Pain Manag Nurs 2010;11(1):26-34.

\section{P1062 DISTINCT PATTERNS OF SHORT-CHAIN FATTY ACIDS IN PATIENTS WITH ULCERATIVE COLITIS EXPERIENCING A FLARE DURING TREATMENT WITH MESALAMINE OR A HERBAL COMBINATION OF MYRRH, CHAMOMILE FLOWERS AND COFFEE CHARCOAL}

J. Langhorst ${ }^{1}$, A. K. Koch ${ }^{1}$, A. Rueffer ${ }^{2}$, G. Dobos ${ }^{3}$

${ }_{1}^{1}$ Department For Internal And Integrative Medicine, Kliniken Essen-Mitte Integrative Gastroenterologie, Essen/Germany

${ }^{2}$ Enterosan, Labor L + S, Bad-Bocklet-Grossenbrach/Germany

${ }^{3}$ Internal And Integrative Medicine, Kliniken Essen-Mitte, Essen/Germany

\section{Contact E-mail Address: j.langhorst@kliniken-essen-mitte.de}

Introduction: The combination of myrrh, chamomile flowers, and coffee charcoal has shown first evidence for potential efficacy in maintaining remission in ulcerative colitis (UC). SCFA are end products of the microbial fermentation of dietary fibers in the gut. They are involved in the regulation of the gut immune system, promote mineral absorption, lipid metabolism, mucin production and expression of antimicrobial peptides. UC patients often show reduced occurrence of SCFA especially during flare. This might lead to unfavorable health impairments including higher risk of inflammation and heightened cancer risk.

Aims \& Methods: The purpose of the present study was to evaluate the influence of mesalazine and of the herbal preparation on SCFA in ulcerative colitis Analyses was proceeded as sub-study of a randomized double-blind, doubledummy, controlled clinical trial that has been published previously. Patients were treated with the herbal preparation consisted of $100 \mathrm{mg}$ myrrh, $70 \mathrm{mg}$ chamomile extract and $50 \mathrm{mg}$ coffee charcoal (Myrrhinil-Intest $\AA$; Repha $\mathrm{GmbH}$, Hannover, Germany) 4tblx $3 / \mathrm{d}$ or the gold standard treatment with mesalamine $500 \mathrm{mg} 3 \mathrm{xd}$. Clinical activity was monitored with the CAI (Rachmilewitz) with a CAI $>4$ indicating a clinical flare. Fecal samples were collected, gas chromatography was performed and SCFA (butyrate, acetate, propionate, iso-valerate and iso-butyrate) were measured at baseline, in the event of a clinical flare or at the end of the 12-month time interval.

Results: A total of $\mathrm{n}=89$ patients were included in the study. $\mathrm{N}=43$ pts developed a clinical flare (descriptive statistics are shown in table 1). Patients who were treated with mesalamine showed a significant decline of the SCFA (M basline $=66.12 ; \mathrm{SD}=39.59 ; \mathrm{M}$ flare $=29.83 ; \mathrm{SD}=15.05 ; 95 \%$ BCI [18.8658.60]) in the event of a flare. In contrast, patients who were treated with the herbal preparation showed no significant decline of the SCFA (M basline $=64.80 ; \quad \mathrm{SD}=53.74 ; \quad \mathrm{M}$ flare $=48.09 ; \mathrm{SD}=35.90 ; 95 \%$ BCI $\quad[-.71-$ 42.28]) in the event of a flare. There was no significant decline in SCFA in the patients in remission neither for the herbal preparation $(\mathrm{n}=19$; $\mathrm{M}$ baseline $=65.77 ; \mathrm{SD}=43.38 ; \mathrm{M} 12 \mathrm{month}=48.63 ; \mathrm{SD}=23.23 ; 95 \%$ BCI [-7.02-
39.95]) nor for mesalamine ( $\mathrm{n}=27$; $\mathrm{M}$ basline $=57.01$; $\mathrm{SD}=34.40$; $\mathrm{M} 12$ month$=42.01 ; \mathrm{SD}=19.84 ; 95 \%$ BCI $[-.34-29.54])$.

Conclusion: Findings show that the herbal preparation might induce different effects on the SCFA of patients with UC compared to mesalamine and therefore might exhibit different modes of action in treating UC. Since a decline of SCFA might lead to unfavorable health impairments like higher cancer risk, treatment options like the herbal preparation might yield additional beneficial effects in the treatment of UC. A combination of the two treatment modalities might be useful and should be investigated in further studies.

Disclosure of Interest: J. Langhorst: Has served as a Speaker for Repha; Research grant from Repha GmbH

All other authors have declared no conflicts of interest.

\section{References}

1. Langhorst J, Varnhagen I, Schneider SB, et al. Randomised clinical trial: a herbal preparation of myrrh, chamomile and coffee charcoal compared with mesalazine in maintaining remission in ulcerative colitis-a double-blind, double-dummy study. ALIMENTARY PHARMACOLOGY \& THERAPEUTICS. Sep 2013;38(5):490-500.

2. Langhorst J, Frede A, Knott M et al. Distinct Kinetics in the Frequency of Peripheral CD4+ T Cells in Patients with Ulcerative Colitis Experiencing a Flare during Treatment with Mesalazine or with a Herbal Preparation of Myrrh, Chamomile, and Coffee Charcoal. PLOS ONE. (2014) Aug 21;9(8):e104257.

\section{P1063 GRANULOCYTE-MONOCYTE APHERESIS (GMA) IN DIFFICULT- TO-TREAT INFLAMMATORY BOWEL DISEASE (IBD). A SINGLE-CENTER REAL-LIFE EXPERIENCE}

A. Caroli, F. Lamboglia, I. Franceschet, C. Pozzan, D. Checchin, F. Bortoluzzi, R. Cappuccio, A. Vitalba

UOC di Gastroenterologia, Ospedale dell'Angelo, Mestre; Ospedale SS Giovanni e Paolo, Venezia, Mestre-Venezia/Italy

Contact E-mail Address: alessandro.caroli@ulss12.ve.it

Introduction: Selective GMA using Adacolumn ${ }^{\circledR}$ device is a non-pharmacological therapeutic option for patients affected by IBD, but its precise role among the various treatments available and its true effectiveness are still debated. In particular, steroid-dependent patients, refractory or intolerant to immunosuppressant and biologics, represent a sub-group of patients with limited options of treatment. Recently, a multicentric open-label trial [the ART trial *] showed, for the first time, a clinical benefit of GMA in these problematic patients.

Aims \& Methods: The aim of this study was to further evaluate, in our real-life clinical experience, the efficacy and safety of GMA in these difficult-to-treat patients. We retrospectively reviewed the clinical data of patients treated with GMA-Adacolumn ${ }^{\circledR}$ in our center between $1 / 1 / 2008$ and $31 / 12 / 2016$. Only steroid-dependent and/or AZA/IFX/ADA-resistant or intolerant cases were considered. GMA was performed once a week for a minimum of five consecutive weeks. Occasionally, one or two additional sessions were performed. A clinical response was defined as $a \geq 3$ points reduction of the clinical activity index (CAI) for ulcerative colitis ( $\overline{\mathrm{UC}}$ ) and $\mathrm{a} \geq 100$ points reduction of the Crohn disease activity index (CDAI) for Crohn disease (CD) after 12 weeks from the beginning of the treatment.

Results: The study population included a total of 30 patients (17 males, 13 females, mean age 49 years, range $21-73$ ) affected by UC (20 patients) and CD (10 patients). Eight patients ( 5 UC, $3 \mathrm{CD}$ ) were excluded from the final analysis for incomplete data. In the remaining 22 pts a clinical response, at week 12, was observed in $15(68 \%)$. The response was better in UC $(11 / 15=73 \%)$ than in CD $(4 / 7=57 \%)$. A steroid-sparing effect was observed in all responsive patients. GMA was generally well tolerated, as only 4 patients $(13 \%)$ reported mild adverse events (headache in two, hypotension in one, vascular procedure complication in one) and no patients discontinued the apheresis due to the adverse events.

Conclusion: In our real-life single-center experience, focused to a selected group of difficult-to-treat patients affected by IBD, GMA with Adacolumn ${ }^{\circledR}$ seems to be, in a short-time evaluation, a useful and safe option of treatment, supporting the recent data from the ART trial. Prospective randomized trials in larger sample of patients and with more extended follow-up are needed to confirm these results.

Disclosure of Interest: All authors have declared no conflicts of interest.

\section{Reference}

*Dignass A et al., JCC 2016,10: 812-820.

P1064 SYNERGISTIC AND ADDITIVE EFFECTS OF MYRRH, CHAMOMILE AND COFFEE CHARCOAL - COMPONENTS OF A TRADITIONAL HERBAL MEDICINE ENHANCE ANTISPASMODIC AND CYTOKINE/CHEMOKINE-INHIBITING ACTIVITY OF THE HERBAL COMBINATION

C. Vissiennon ${ }^{1}$, S. Paulo ${ }^{2}$, D. Hammoud ${ }^{3}$, K. Goos ${ }^{1}$, K. Nieber ${ }^{4}$, J. Arnhold ${ }^{2}$ ${ }^{1}$ Repha GmbH Biologische Arzneimittel, Langenhagen/Germany

${ }^{2}$ Medizin. Fakultät, University Hospital, Universität Leipzig, Leipzig/Germany 
${ }^{3}$ Inter-Regional University of Industrial Engineering, Biotechnologies and Applied Sciences (IRGIB Africa University), Cotonou/Benin

${ }^{4}$ Institute Of Pharmacy, University of Leipzig, Leipzig/Germany

\section{Contact E-mail Address: cica.vissiennon@uni-leipzig.de}

Introduction: The herbal medicinal product Myrrhinil-Intest $\AA$, a combination of myrrh (Commiphora molmol E.), chamomile flower (Matricaria chamomilla L.) and coffee charcoal (Coffea Arabica L.) is used for the treatment of gastrointestinal complaints. Clinical data suggest its use for the maintenance therapy of inflammatory bowel disease [1]. Despite existing clinical data, pharmacological data supporting the observed efficacy remain insufficient.

Aims \& Methods: The present study aims to investigate the influence of the single and combined herbal extracts with regard to its antispasmodic and immunemodulating activities. Thus, the effect of myrrh, chamomile flower, coffee charcoal extract on intestinal motility was determined using isometric tension measurement in isolated rat small intestinal preparations. Furthermore chemokine (IL8; CXCL10; CXCL13) release from cytokine-(10 ng/mL TNF $\alpha ; 5 \mathrm{ng} / \mathrm{mL}$ IL $1 \beta ; 10 \mathrm{ng} / \mathrm{mL} \mathrm{IFN} \gamma$ )-challenged intestinal epithelial cells (Caco2) and LPSchallenged human macrophages (THP-1) was investigated respectively using an ELISA test system. Budesonid served as positive control. To characterize the combined effect, concentration-response relations of single components and the herbal combination were compared and $\mathrm{IC}_{50}$ values derived. Interpretation of the data was based on a dose reduction index (DRI; $\left.=\mathrm{IC}_{50 \text {-single }} / \mathrm{IC}_{50 \text {-comb. }}\right)$ which estimates the extent to which the dose of one or more components in the combination can be reduced to achieve effect levels that are comparable with those achieved with single component.

Results: Myrrh and chamomile flower extract exerted spasmolytic effects by inhibiting acetylcholine-induced contractions in rat small intestinal preparations $\left(\mathrm{IC}_{50}: \operatorname{myrrh}=144 \mu \mathrm{g} / \mathrm{mL}\right.$; chamomile flower $\left.=383 \mu \mathrm{g} / \mathrm{mL}\right)$. In combination, chamomile flower and myrrh interacted additively $\left(\mathrm{IC}_{50}:\right.$ myrrh $=74 \mu \mathrm{g} / \mathrm{mL}$; chamomile flower $=196 \mu \mathrm{g} / \mathrm{mL}$ ) resulting in a DRI of 1.9. All three plant components inhibited CXCL13 release from LPS-stimulated human macrophages $\left(\mathrm{IC}_{50}\right.$ : myrrh $=19 \mu \mathrm{g} / \mathrm{mL}$; chamomile flower $=82 \mu \mathrm{g} / \mathrm{mL}$; coffee charcoal $=106 \mu \mathrm{g} / \mathrm{mL}$ ). Synergistic effects exerted by the herbal combination in inhibiting CXCL13 release significantly reduced $\mathrm{IC}_{50}$ values $\left(\mathrm{IC}_{50}\right.$ : myrrh $=5 \mu \mathrm{g} / \mathrm{mL}$; chamomile flower $=22 \mu \mathrm{g} / \mathrm{mL}$; coffee charcoal $=29 \mu \mathrm{g} / \mathrm{mL}$ ) resulting in a DRI of 3.7 . Cytokine induced CXCL10 release from $\mathrm{Caco} 2$ cells was reduced by all herbal components $\left(\mathrm{IC}_{50}:\right.$ myrrh $=41 \mu \mathrm{g} / \mathrm{mL}$; chamomile flower $=364 \mu \mathrm{g} / \mathrm{mL}$; coffee charcoal $=447 \mu \mathrm{g} / \mathrm{mL}$ ) with comparably high $\mathrm{IC}_{50}$ values. However, application of the herbal combination, significantly reduced the $\mathrm{IC}_{50}$ of the plant extracts (myrrh- $\mathrm{IC}_{50}=25 \mu \mathrm{g} / \mathrm{mL}, \quad \mathrm{DRI}=1.7$; chamomile flower- $\mathrm{IC}_{50}=124 \mu \mathrm{g} / \mathrm{mL}$, $\mathrm{DRI}=2.9$; coffee charcoal-IC $50=124 \mu \mathrm{g} / \mathrm{mL}, \mathrm{DRI}=3.6)$. IL8 release from cytokine-challenged $\mathrm{Caco} 2$ cells was inhibited after myrrh $\left(\mathrm{IC}_{50}=3 \mu \mathrm{g} / \mathrm{mL} ; 28 \% \max \right.$ inhib.) and coffee charcoal ( $\mathrm{IC}_{50}=218 \mu \mathrm{g} / \mathrm{mL} ; 75 \%$ max inhib.) but increased after chamomile flower treatment $\left(\mathrm{EC}_{50}=39 \mu \mathrm{g} / \mathrm{mL} ; 29 \% \max\right.$ stimul.). Treatment with all three plant extracts resulted in a moderate IL8 inhibition with an inverted U-shape concentration-response curve $\left(\mathrm{IC}_{50}\right.$ : myrrh $=56 \mu \mathrm{g}$ $\mathrm{ml}$; coffee charcoal $=281 \mu \mathrm{g} / \mathrm{ml} ; 77 \%$ max. inhib.).

Conclusion: The herbal components myrrh, chamomile flower and coffee charcoal influenced chemokine signalling of stimulated intestinal epithelial cells and activated macrophages. Myrrh and chamomile flower additionally exerted antispasmodic effects. Synergistic and additive effects between the plant extracts justify the combinatory composition of the traditional herbal medicinal product (Myrrhinil-Intest $\mathbb{R}$ ) and its application for the treatment of inflammatory intestinal disorders.

Disclosure of Interest: C. Vissiennon: Author Cica Vissiennon is employed by Repha GmbH Biologische Arzneimittel

K. Goos: Co-Author Karl-Heinz 30 Goos is shareholder of Repha $\mathrm{GmbH}$ Biologische Arzneimittel

All other authors have declared no conflicts of interest.

\section{Reference}

1. Langhorst $\mathbf{J}$ et al. Alimentary Pharmacology \& Therapeutics 2013; 38: 490500

\section{P1065 ARE TROUGH LEVELS OF ANTI-TNF DRUGS RELATED WITH TREATMENT FAILURE AND DURATION OF TREATMENT?}

J. González-López1, M. Barreiro-De Acosta ${ }^{2}$, C. Llamas ${ }^{3}$, J.E. DominguezMuñoz ${ }^{2}$, J.M. Giráldez-Montero ${ }^{1}$, R. Ferreiro Iglesias ${ }^{2}$, M.J. Lamas-Díaz ${ }^{1}$ ${ }^{1}$ Pharmacy, University Hospital of Santiago, Santiago de Compostela/Spain ${ }^{2}$ Gastroenterology, University Hospital of Santiago, Santiago de Compostela/Spain ${ }^{3}$ University of Santiago de Compostela, Santiago de Compostela/Spain

\section{Contact E-mail Address: manubarreiro@hotmail.com}

Introduction: Therapeutic drug monitoring of infliximab (IFX) and adalimumab (ADA) in inflammatory bowel disease are commonly used if patients show loss of response, but there are scarce data about the influence of trough levels on prognosis.

Aims \& Methods: The aim of the study was to evaluate the relationship between drug concentration of anti-TNF and treatment failure and duration of treatment in a real life environment. METHODS A prospective observational study was performed in a cohort of patients with inflammatory bowel disease. Patients were included consecutively through visits to the hospital to receive IFX or ADA. Inclusion criteria were all age $>18$ year patients, being treated with one of the study drugs at standard dose as maintenance therapy and giving consent to participate in the study. In the first visit, blood samples were extracted for drug concentration determination. Patients were followed-up for the next 2 years. Trough drug concentrations and drug antibodies were measured by an ELISA technique (Promonitor). Patients with IFX levels $<3 \mathrm{mcg} / \mathrm{mL}$ and ADA $<5 \mathrm{mcg} / \mathrm{mL}$ were considered under lower limit of therapeutic range (LLTR). After the follow-up period, the number of patients that continued (success) or discontinued (failures) the treatment were analyzed. Any change in dosage (intensification) or in the type of drug was considered as discontinuation of the treatment. Chi square test was used to analyze the dependence between the categorical variables and t-student for continuous variables with normal distribution.

Results: 134 patients were consecutively included, 53 male $(40 \%), 92(68 \%)$ patients with Crohn's disease (CD) and $42(32 \%)$ with ulcerative colitis (UC), $82(61 \%)$ received maintenance treatment with IFX and $52(39 \%)$ with ADA. Mean age was 43 [19-71] years. After finishing the follow-up, $107(79 \%)$ patients continued the same treatment regimen (success). A total of $59 \%$ of this group of patients were with drug concentrations above LLTR. The remaining 27 patients changed their treatment (failures). In the failure group, only $41 \%$ of the patients were above LLTR, $18.2 \%$ less with respect to success treatment group. These results did not reach statistical significance $(p=0.09)$, but are in agreement with current evidence. When type of inflammatory disease was analyzed, a greater percentage of patients below the LLTR were found in the UC group $(41.3 \%$ vs $52.4 \%, \mathrm{CD}$ and UC respectively), but the difference did not reach statistical significance. Regarding the duration of the treatments, the duration was similar in the group of patients with low concentrations compared to the group with high drug concentrations (46.6 vs 42.6 months). However, when the duration of treatment was analyzed in patients who discontinued, significant differences were observed (43.8 vs 20.2 months in the <LLTR and > LLTR groups respectively). Therefore, there are patients who relapse rapidly even with drug levels above LLTR. We observed 14 patients with positive antibodies, all of them with drug concentrations below the LLTR.

Conclusion: The percentage of patients who continue treatment was higher when drug concentrations were above LLTR. However, these high levels did not prevent the early relapse of some of them.

Disclosure of Interest: All authors have declared no conflicts of interest.

\section{P1066 IMMUNOSUPPRESSIVE CO-TREATMENT WITH INFLIXIMAB AND ADALIMUMAB IS NOT SUPERIOR TO ANTI-TNF MONOTHERAPY TO PREVENT TREATMENT FAILURE AND TREATMENT DISCONTINUATION IN ULCERATIVE COLITIS}

S. Vieujean ${ }^{1}$, E. Louis ${ }^{2}$, C. Reenaers ${ }^{1}$

${ }^{1}$ Gastroenterology, CHU Liège, Liège/Belgium

${ }^{2}$ University Hospital CHU of Liège, Liège/Belgium

Contact E-mail Address: catherine.reenaers@chu.ulg.ac.be

Introduction: In Crohn's disease there is clear benefit from combination therapy with infliximab (IFX) and immunosuppressive drugs (IS), while the benefit seems more limited for adalimumab (ADA). Although some studies suggest a benefit of combination therapy with IFX in ulcerative colitis (UC) few data are available [1].

Aims \& Methods: Our aim was to compare real life efficacy of anti-TNF monotherapy (IFX and ADA) and anti-TNF + IS for UC maintenance. This was a retrospective study of patients with UC treated with IFX or ADA in 2 Belgian academic and regional Hospitals. Treatment periods were divided into 6-month semesters. A combination therapy semester was defined as anti-TNF + IS for at least 3 months, a failure semester as anti-TNF withdrawal for secondary loss of response, intolerance or surgery, a treatment optimisation semester as anti-TNF dose escalation or steroids start. Semesters with and without failure and with or without optimisation were compared through univariate and multivariate analysis. Patients receiving 6 months anti-TNF + IS during the first semesters were separately analysed.

Results: 478 semesters in 60 patients with IFX and 175 semesters in 33 patients with ADA were included. The mean IFX and ADA treatment duration were respectively $49( \pm 33)$ months and $38( \pm 19)$ months. Within patients treated with IFX, 32/60 patients received IFX + IS during the first semester. IFX was administrated as monotherapy in $361 / 478$ semesters $(76 \%)$. Respectively $218 / 478(46 \%)$ and $78 / 478$ semesters $(16 \%)$ with IFX required dose escalation and corticosteroids course. IFX + IS was associated with more semesters with failure $(5 \%$ vs $3 \%, \mathrm{p}=0.02)$ and numerically more semesters with dose escalation $(64 \%$ vs $28 \%$, $\mathrm{p}=0.06)$. There was no difference in corticosteroids use $(\mathrm{p}=0.63)$. IS during the first semester was not associated with lower risk of IFX failure $(p=0.41)$ nor with a longer survival without IFX withdrawal $(\mathrm{p}=0.20)$. Continuing the IS treatment beyond the first semester was not associated with fewer semesters with failure $(\mathrm{p}=0.18)$. Within patients treated with ADA, 19/33 patients received IFX + IS during the first semester. ADA was administrated as monotherapy in 93/175 semesters (53\%). Respectively $84 / 175(48 \%)$ and $42 / 175(24 \%)$ semesters with ADA required dose escalation and corticosteroids course. ADA + IS was not associated with less semesters with failure $(7 \%$ vs $5 \%, p=0.58)$, less semesters with corticosteroids use $(\mathrm{p}=0.63)$. More semesters with ADA + IS required ADA dose escalation $(61 \%$ vs $30 \%, p=0.01)$. IS during the first semester was not associated with lower risk of ADA failure $(\mathrm{p}=0.84)$ nor with a longer survival without ADA withdrawal $(\mathrm{p}=0.78)$. Continuing the IS treatment beyond the first semester was not associated with fewer semesters with failure $(p=0.20)$. Conclusion: In this real-life experience, combination therapy of IFX or ADA with IS during the first semester or prolonged after the first semester was not associated with less dose escalations, steroid courses or treatment failures. 
Disclosure of Interest: All authors have declared no conflicts of interest.

\section{Reference}

1. Panaccione R et al. Gastroenterology. 2014 Feb;146(2):392-400

\section{P1067 EFFICACY AND SAFETY OF ADALIMUMAB AFTER INFLIXIMAB FAILURE IN PEDIATRIC ULCERATIVE COLITIS: A REAL-LIFE EXPERIENCE FROM THE SIGENP-IBD REGISTRY}

M. Aloi ${ }^{1}$, M. Bramuzzo ${ }^{2}$, S. Arrigo ${ }^{3}$, C. Romano ${ }^{4}$, D. Iacorte ${ }^{5}$, S. Gatti ${ }^{6}$, M.T. Illiceto ${ }^{7}$, F. Zucconi ${ }^{1}$, D. Dilillo ${ }^{8}$, G. Zuin ${ }^{8}$, D. Knafelz ${ }^{9}$, A. Ravelli ${ }^{10}$, G. D'Arcangelo ${ }^{1}$, P. Alvisi ${ }^{11}$

${ }^{1}$ Pediatric Gastroenterology And Liver Unit, Sapienza University of Rome Dept. of Pediatric Gastroenterology SIGENP IBD Group, Rome/Italy

${ }^{2}$ Institute For Maternal And Child Health, Irccs "burlo Garofalo,", Pediatric

Department, Gastroenterology and Nutrition Unit, Trieste/Italy

${ }^{3}$ Pediatric Gastroenterology Unit, Institute "Giannina Gaslini”, Genova/Italy

${ }^{4}$ Pediatric Gastroenterology And Endoscopy, Pediatric Department, Pediatric

Department, Messina/Italy

${ }^{5}$ Pediatric Gastroenterology Unit, Maggiore Hospital, Bologna/Italy

${ }^{6}$ Pediatric Gastroenterology Unit, Salesi Children Hospital, Ancona/Italy

${ }^{7}$ Pediatric Gastroenterology And Endoscopy Unit, Spirito Santo Hospital, Pescara Italy

${ }^{8}$ Pediatric Department, Gastroenterology Unit, Buzzi Children Hospital, Milan/ Italy

${ }^{9}$ Gastroenterology And Nutrition Unit, "Bambino Gesü" Children Hospital, Rome Italy

${ }^{10}$ University Department Of Pediatrics, A.C.S. International Pharma Group SRL, Brescia/Italy

${ }^{11}$ GI Unit, Ospedale Maggiore, Bologna, Bologna/Italy

Contact E-mail Address: marina.aloi@uniroma1.it

Introduction: The objective of the present study was to evaluate the effectiveness and safety of adalimumab (ADA) in children with ulcerative colitis (UC) who experienced previous infliximab (IFX) failure or intolerance.

Aims \& Methods: This retrospective study included all children with UC from a national pediatric registry who received ADA therapy. The primary endpoint was the rate of corticosteroid (CS) free remission (PUCAI $<10$ ) at week 52. Secondary outcomes were: the rate of continuous clinical response and remission, primary non-response and loss of response at Weeks 12, 30, and 52 and rate of mucosal healing $(\mathrm{MH})$ at week 52 .

Results: A total of 32 children with UC received ADA (median age $10 \pm 4$ years). Median disease duration before ADA therapy was 27 months. All patients received previous IFX therapy $(43 \%$ intolerant, $50 \%$ not-responders, $7 \%$ positive anti-IFX antibodies). Fifty-two weeks after ADA initiation 13 patients $(41 \%)$ were in CS-free remission. $\mathrm{MH}$ occurred in 9 patients $(28 \%)$ at 52 week. The cumulative probability of clinical relapse-free course was $69 \%, 59 \%$ and $53 \%$ at 12,30 and 52 weeks, respectively. Ten patients $(31 \%)$ had a primary failure and $5(15 \%)$ loss of response to ADA. No significant differences in terms of efficacy were reported between not-responders and intolerant to IFX $(\mathrm{p}=1.0)$. Overall, 19 patient $(59 \%)$ maintained ADA therapy during 52-week follow-up. Seven patients $(22 \%)$ experienced a an adverse event. No serious side effects were observed and none resulted in ADA discontinuation.

Conclusion: In this cohort of children with UC ADA had a favorable short- and long-term efficacy, allowing to recover a significant percentage of patients intolerant or not-responding to IFX. The efficacy was not related to the cause of IFX discontinuation (intolerance/failure). Overall, the safety profile was good. Larger, prospective, controlled trial with longer follow-up should be suggested to better clarify the role of ADA in pediatric UC.

Disclosure of Interest: All authors have declared no conflicts of interest.

P1068 LOW FODMAP DIET IMPROVE DISEASE ACTIVITY AND QUALITY OF LIFE IN PATIENTS WITH INFLAMMATORY BOWEL DISEASE

G. Bodini ${ }^{1}$, E.G. Giannini ${ }^{1}$, V. Savarino ${ }^{1}$, M. Crespi ${ }^{1}$, S. Lo Pumo ${ }^{1}$, C. Zanella ${ }^{1}$, I. Baldissarro ${ }^{1}$, E. Savarino ${ }^{2}$

${ }^{1}$ Department Of Internal Medicine, IRCCS San Martino DIMI, Genova/Italy ${ }^{2}$ Department Of Surgery, Oncology And Gastroenterology, University of Padua, Padua/Italy

Contact E-mail Address: giorgia.bodini $@$ gmail.com

Introduction: Inflammatory Bowel Diseases (IBD), Crohn Disease (CD) and Ulcerative Colitis (UC), are idiopathic autoimmune conditions whose pathogenesis is still unknown. It has been hypothesized that, in genetic predispose subjects, a deregulated immune response associated to an increase of intestinal permeability may lead to bowel damage and clinical manifestations. Thus, environmental factors and, in particular, food intake may play a pivotal role in IBD pathogenesis.

Aims \& Methods: The aim of this prospective study was to evaluate the effects of a 6-week low fermentable Oligosaccharides, disaccharides, monosaccharides and polyols (FODMAP) diet on disease activity and quality of life in patients with IBD. At first visit (T0), patients were clinically evaluated by a gastroenterologist and a nutritionist, filled a questionnaire on quality of life (the IBD-Q) and underwent blood tests as well as fecal calprotectin assessment. Disease activity was defined using the Mayo score and the Harvey Bradshaw Index (HBI) for UC and $\mathrm{CD}$, respectively. After the baseline visit, patients were randomized into two groups: A) patients underwent a low FODMAP diet; B) patients underwent a diet with normal FODMAP amount. A food diary was used to assess patients' adherence to the different diets. After six weeks (T1), patients had a second visit to assess disease activity, complete the IBD-Q, and repeat blood tests as well as fecal calprotectin assessment.

Results: In this prospective, interventional, cohort study, we enrolled 55 consecutive IBD patients who agreed to participate from an initial cohort of 127 IBD patients. Twenty-six patients were randomised to a low FODMAP diet (group A), while 29 patients to a standard FODMAP diet (group B). Among CD patients $(\mathrm{n}=35,63.6 \%)$, median HBI values significantly decreased during the study, in the whole population and in group $\mathrm{A}$, whereas no change was recorded in group $\mathrm{B}$ (respectively $P=0.02 ; P=0.02 ; P=0.3$ ). Among $\mathrm{UC}$ patients $(\mathrm{n}=20$, $36.4 \%$ ), median Mayo scores did not significantly decrease during the study, both considering the whole population and the two groups $(P=0.3, P=0.3$, and $P=0.8$, respectively). Moreover, despite no statistically significant difference in quality of life in both groups at T0, in group A quality of life improved after the diet compared to group B (respectively, $P=0.06, P=0.05$ and $P=1$ ).

Conclusion: We demonstrated that a low FODMAP diet, for a limited period of 6 weeks, is able to improve both disease activity, at least for CD, and quality of life in IBD patients. Further, larger multicentre studies are needed to confirm these preliminary data.

Disclosure of Interest: All authors have declared no conflicts of interest.

\section{TUESDAY, OCTOBER 31, 2017}

PAEDIATRIC: LOWER GI - HALL 7

09:00-17:00

\section{P1069 SUBCUTANEOUS USTEKINUMAB PROVIDED CLINICAL AND BIOLOGICAL BENEFIT FOR 9/12 REFRACTORY PEDIATRIC CROHN'S DISEASE}

C. Martinez-Vinson, J. Hugot, M. Bellaiche, J. Viala

Gastroentérologie Pédiatrique, Hôpital Robert Debré, Paris/France

Contact E-mail Address: christine.martinez-vinson@aphp.fr

Introduction: Ustekinumab has shown a good safety profile and efficacy to induce and maintain remission in adult patients with refractory Crohn's Disease (CD). Data are lacking in children.

Aims \& Methods: All CD patients under 18 years who received ustekinumab were included in this retrospective observational study performed in a single tertiary pediatric centre.

Results: See table.

Conclusion: Subcutaneous ustekinumab is effective to induce and maintain remission in severe pediatric CD refractory to anti-TNF antibodies.

Disclosure of Interest: All authors have declared no conflicts of interest.

\section{References}

Sandborn WJ, Feagan BG, Fedorak RN, Scherl E, Fleisher MR, Katz S, Johanns J, Blank M, Rutgeerts P; Ustekinumab Crohn's Disease Study Group. A randomized trial of Ustekinumab, a human interleukin-12/23 monoclonal antibody, in patients with moderate-to-severe Crohn's disease. Gastroenterology. 2008 Oct;135(4):113041.

Sandborn WJ, Gasink C, Gao L-L, Blank MA, Johanns J, Guzzo C, Sands BE, Hanauer SB, Targan S, Rutgeerts P, Ghosh S, de Villiers WJ, Panaccione R, Greenberg G, Schreiber S, Lichtiger S, Feagan BG; CERTIFI Study Group. Ustekinumab induction and maintenance therapy in refractory Crohn's disease. $N$ Engl J Med. 2012 Oct 18;367(16):1519-28.

Kopylov U, Afif W, Cohen A, Bitton A, Wild G, Bessissow T, Wyse J, Al-Taweel T, Szilagyi A, Seidman E. Subcutaneous ustekinumab for the treatment of antiTNF resistant Crohn's disease-the McGill experience. J Crohns Colitis. 2014 Nov;8(11):151622.

Wils P, Bouhnik Y, Michetti P, Flourie B, Brixi H, Bourrier A, Allez M, Duclos B, Grimaud JC, Buisson A, Amiot A, Fumery M, Roblin X, Peyrin-Biroulet L, Filippi J, Bouguen G, Abitbol V, Coffin B, Simon M, Laharie D, Pariente B; Groupe D"Etude Thérapeutique des Affections Inflammatoires du Tube Digestif. Subcutaneous Ustekinumab Provides Clinical Benefit for Two-Thirds of Patients With Crohn's Disease Refractory to Anti-Tumor Necrosis Factor Agents. Clin Gastroenterol Hepatol. 2016 Feb;14(2):242-50.

Rinawi F, Rosenbach Y, Assa A, Shamir R. Ustekinumab for Resistant Pediatric Crohn's Disease. J Pediatr Gastroenterol Nutr. 2016 Apr;62(4):e34-5.

Cameron FL, Garrick V, Russell RK. Ustekinumab in Treatment of Refractory Paediatric Crohn Disease. J Pediatr Gastroenterol Nutr. 2016 Mar;62(3):e30.

Bishop C, Simon H, Suskind D, Lee D, Wahbeh G. Ustekinumab in Pediatric Crohn's Disease Patients: Case Review. J Pediatr Gastroenterol Nutr. 2016 Feb 4. 
P1070 TROUGH LEVELS TO INFLIXIMAB AT W6 ARE PREDICTIVE OF REMISSION AT W14, IN PEDIATRIC CROHN DISEASE

C. Martinez-Vinson, O. Courbette, C. Aupiais, J. Viala, J. Hugot Gastroentérologie Pédiatrique, Hôpital Robert Debré, Paris/France

Contact E-mail Address: christine.martinez-vinson@aphp.fr

Introduction: Loss of response to anti-tumor necrosis factor (TNF) agents is a common clinical problem. $40 \%$ of patients lose response within 12 month of therapy initiation. This retrospective study aimed to analyse factors associated with remission after 14 weeks of induction treatment by IFX in children with CD.

Aims \& Methods: All patients aged from 2 to 18 years old with CD meeting European Crohn's and Colitis Organisation criteria and treated for the first time by IFX between January 2002 and March 2014 at a single tertiary pediatric center were considered for inclusion in this retrospective study. The following baseline characteristics were anonymously recorded for each patient: gender, age at $\mathrm{CD}$ onset, age at inclusion, duration of disease, $\mathrm{CD}$ classification according to Paris classification, prior exposure to $\mathrm{CD}$ treatments, previous intestinal resections and reasons for anti-TNF initiation. At each infusion visit (week 0, 2, 6 and 14) disease activity was determined using Pediatric Crohn's disease Activity Index (PCDAI). Four blood samples were obtained for each patient (at W0, W2, W6 and W14). The following laboratory tests were recorded: ESR, CRP, hemoglobin, hematocrit, albumin, blood levels of lymphocytes, Trough levels to IFX (TRI) and antibodies to IFX (ATIs). Children were classified in three groups according to response at week 14: (1) remission was defined by a Crohn's Disease Activity Index under or equal to 10; (2) partial response was defined by a PCDAI decreased by more 12.5 points since inclusion without a remission as defined above; (3) absence of partial response or remission.

Results: We analyzed 107 patients with CD, with a total of 428 visits until W14. The principal reason to start infliximab was failure of immunosuppressive therapy $(60 \%)$. Infliximab proved to be an effective treatment in our cohort since $75.7 \%(n=81)$ patients were responders to infliximab and $40 \%(n=42)$ were in clinical remission whereas $24.3 \%(n=26)$ were non respondents at W14. At week 14,107 patients were divided in three groups related to the clinical activity of their disease: lack of clinical response, partial clinical response, clinical remission. It concerns respectively 26, 39 and 42 patients. Major baseline characteristics were not associated with clinical remission: sex, age at diagnosis, disease location, time between diagnosis and induction, age at induction. Drugs associated with infliximab at W0, W2, W6 or W14, whether it was immunosuppressive agents or corticoids were not associated with remission. Patients with low albumin levels had a worse response at induction Activity score at induction was also statistically associated with clinical remission: each decreasing of 10 points of activity score at induction increase of 0.48 times the risk to obtain clinical remission. Trough residual of infliximab $>8.5 \mu \mathrm{g} / \mathrm{ml}$ at w6 increase of 11.3 times the risk to obtain clinical remission at w14. Lack of growth retardation at induction increased of 3.98 times the risk to obtain clinical remission at w14.

Conclusion: Infliximab measurement in combination with evaluation of clinical severity (low body weight, growth retardation, hypoalbuminemia, severe disease) appears to be a reasonable strategy for predicting both short- and long-term treatment outcomes with IFX in the initial stage of treatment. Early detection of response to IFX is critical for the management of CD, especially in acute severe patients: it seems that the infliximab trough level at week 6 (more than 8.5 ) is predictive of a remission at week 14 . Second, some patients, especially patients with low body weight, growth retardation, hypoalbuminemia and severe disease may require higher doses than standart doses.

Disclosure of Interest: All authors have declared no conflicts of interest.

\section{References}

Hyams J, Crandall W, Kugathasan S, et al. Induction and maintenance infliximab therapy for the treatment of moderate-to-severe Crohn's disease in children. Gastroenterology 2007; 132:863-73.

Gisbert JP, Panés J. Loss of response and requirement of infliximab dose intensification in Crohn's disease: a review. Am J Gastroenterol 2009;104:760-7.

Hyams JS, Lerer T, Griffiths A, et al. Long-term outcome of maintenance infliximab therapy in children with Crohn's disease. Inflamm Bowel Dis 2009;15:81622.

De Bie CI, Hummel TZ, Kindermann A, et al. The duration of effect of infliximab maintenance treatment in paediatric Crohn's disease is limited. Aliment Pharmacol Ther 2011;33:243-50.

Crombé V, Salleron J, Savoye G, et al. Long-term outcome of treatment with infliximab in pediatric-onset Crohn's disease: a population-based study. Inflamm Bowel Dis 2011;17:2144-52.

Assa A, Hartman C, Weiss B, et al. Long-term outcome of tumor necrosis factor alpha antagonist's treatment in pediatric Crohn's disease. $J$ Crohns Colitis 2013;7:369-76.

Steenholdt C, Bendtzen K, Brynskov J et al. Cut-off levels and diagnostic accuracy of infliximab trough levels and anti-infliximab antibodies in Crohn's disease. Scand J Gastroenterol 2011;46:310-8.

Maser EA, Villela R, Silverberg MS, et al. Association of trough serum infliximab to clinical outcome after scheduled maintenance treatment for Crohn's disease. Clin Gastroenterol Hepatol 2006;4:1248-54.

Bortlik M, Duricova D, Malickova K, et al. Infliximab trough levels may predict sustained response to infliximab in patients with Crohn's disease. J Crohns Colitis 2013;7:736-43.

Baert F, Drobne D, Gils A, et al. Early trough levels and antibodies to infliximab predict safety and success of reinitiation of infliximab therapy. Clin Gastroenterol Hepatol 2014;12:1474-81. 
Paul S, Del Tedesco E, Marotte H, et al. Therapeutic drug monitoring of infliximab and mucosal healing in inflammatory bowel disease: a prospective study. Inflamm Bowel Dis 2013;19:2568-76.

Cornillie F, Hanauer SB, Diamond RH, et al. Postinduction serum infliximab trough level and decrease of C-reactive protein level are associated with durable sustained response to infliximab: a retrospective analysis of the ACCENT I trial. Gut 2014;63:1721-7.

Hämäläinen A, Sipponen T, Kolho KL, et al. Serum infliximab concentrations in pediatric inflammatory bowel disease. Scand J Gastroenterol 2013:48:35-41.

Hyams JS, Ferry GD, Mandel FS, et al. Development and validation of a pediatric Crohn's disease activity index. J Pediatr Gastroenterol Nutr 1991;12:439-47. Singh N, Rosenthal CJ, Melmed GY, et al. Early infliximab trough levels are associated with persistent remission in pediatric patients with inflammatory bowel disease. Inflamm Bowel Dis 2014;20:1708-13.

Fasanmade AA, Adedokun OJ, Blank M, et al. Pharmacokinetic properties of infliximab in children and adults with Crohn's diseasae:retrospective analysis of data from 2 phase III clinical trials. Clin Ther 2011;33:946-64.

Dotan I, Ron Y, Yanai H, et al. Patient factors that increase infliximab clearance and shorten half-life in inflammatory bowel disease: a population pharmacokinetic study. Inflamm Bowel 2014:20:2247-59.

\section{P1071 INFLIXIMAB INDUCED PSORIASIS IN A COHORT OF CHILDREN WITH INFLAMMATORY BOWEL DISEASE: A 12 YEARS FOLLOW-UP STUDY}

C. Martinez-Vinson, O. Courbette, C. Aupiais, J. Viala, J. Hugot Gastroentérologie Pédiatrique, Hôpital Robert Debré, Paris/France

Contact E-mail Address: christine.martinez-vinson@aphp.fr

Introduction: In adult Inflammatory bowel disease (IBD), skin adverse reactions have an estimated prevalence of 1.6 to $22 \%$. This side effect occurs more frequently in patients treated with infliximab (IFX) for IBD. Datas in the pediatric population are lacking so far.

Aims \& Methods: All patients aged 2 to 18 years, with Crohn's disease (CD) or Ulcerative colitis (UC) and treated for the first time by IFX between January 2002 and March 2014, were considered for inclusion in this monocentric retrospective study.

Results: Basline Patients: 115 patients were treated with IFX for CD and 23 for UC. IFX treatment was initiated at the age of 14 , about 2 years after diagnosis. The indication for treatment was in $61.6 \%(\mathrm{n}=85)$ resistance to conventional therapy, in $26.8 \%(n=37)$ a perianal fistulizing disease and in $11.6 \%(n=16)$ a severe colitis. At the first injection, the median PCDAI was $35(25 ; 45)$ for CD and the median PUCAI $35(25 ; 45)$ for UC. The duration of treatment with IFX ranged from 45 days to 8 years and median was 23.9 months $(11.6 ; 36.5)$. Psoriasis: 20 patients (14\% of the cohort) had an IFX-induced psoriasis. $70 \%$ of them $(n=14)$ of patients were in remission when the psoriasis was diagnosed. Psoriasis was diagnosed at the 8th injection $(6 ; 15)$, though $355(239 ; 532)$ days after the start of biotherapy. $20 \%$ of patients had a combo therapy: $50 \%$ of them were treated by 6 -mercaptopurine, $25 \%$ by azathioprine and $25 \%$ by methotrexate. The median IFX trough levels (TRI) when psoriasis occured was $4.7 \mathrm{mcg} / \mathrm{mL}$ $(1.8 ; 9.6)$ and $4.1 \mathrm{mcg} / \mathrm{mL}(2.1,8.8)$ at the previous visit. Median Antibodies to IFX (ATI) rate was $0 \%$. All were supported by local treatments. No patients discontinued biotherapy following the psoriasis. Personal or family history of psoriasis, and the smoking status have not been collected. We compared the population of patients with psoriasis $(n=20)$ and without psoriasis $(n=127)$ with an univariate model. All children in the psoriasis group were followed for a $\mathrm{CD}$. There was more perineal location of $\mathrm{CD}$ in psoriasis group with a significant difference $(\mathrm{p}=0.033)$.

Conclusion: $14 \%$ of our IBD patients treated with IFX developed psoriasis during follow-up. All were $\mathrm{CD}$, more frequently it occurred for $\mathrm{CD}$ with perineal lesions, at the 8th injection in median, with no ATI.

Disclosure of Interest: All authors have declared no conflicts of interest.

\section{References}

Kerbleski JF, Gottlieb AB. Dermatological complications and safety of antiTNF treatments. Gut 2009;58:1033-9.

Davet N, Ritchlin C. Paradoxical adverse events associated with anti-TNF alpha agents. Seneschal J, Milpied B, Vergier B et al. Cytokine imbalance with increased production of interferon-alpha in psoriasiform eruptions associated with antitumour necrosis factor-alpha treatments. $\mathrm{Br} J$ Dermatol 2009;161:1081-8

Collamer A N, Battafarano D F. Psoriatic skin lesions induced by tumor necrosis factor antagonist therapy: clinical features and possible immunopathogenesis. Semin Arthritis Rheum 2010;40:233-40.

De Gannes G C, Ghoreishi M, Pope J et al. Psoriasis and pustular dermatitis triggered by TNF-alpha inhibitors in patients with rheumatologic conditions. Arch Dermatol 2007;143:223-31.

Tillack C, E hmann L M, F riedrich M et al. Anti-TNF antibody-induced psoriasiform skin lesions in patients with infl ammatory bowel disease are characterised by interferon- $\gamma$-expressing Th 1 cells and IL-17 A/IL-22-expressing Th 17 cells and respond to anti-IL-12/IL-23 antibody treatment. Gut 2014; 63: 567-77. Rahier J -F, Buche S, Peyrin-Biroulet L et al. Severe skin lesions cause patients with inflammatory bowel disease to discontinue anti-tumor necrosis factor therapy. Clin Gastroenterol Hepatol Off Clin Pract J Am Gastroenterol Assoc 2010; 8: 1048-55.

Broge T, Nguyen N, Sacks A et al. Infl iximab-associated psoriasis in children with Crohn's disease may require withdrawal of anti-tumor necrosis factor therapy. Infl amm Bowel Dis 2013; 19: 75-7.

Collamer A N, Guerrero K T, Henning J S et al. Psoriatic skin lesions induced by tumor necrosis factor antagonist therapy: a literature review and potential mechanisms of action. Arthritis Rheum 2 008; 5 9: 9 96- 1001.

Guerra I, Algaba A, Pérez-Calle J L et al. Induction of psoriasis with antiTNF agents in patients with infl ammatory bowel disease: a report of 21 cases. $J$ Crohns Colitis 2012; 6: 518- 23.

Fiorino G, Allez M, Malesci A et al. Review article: anti TNF-alpha induced psoriasis in patients with inflammatory bowel disease. Aliment Pharmacol Ther 2009; 29: 921-7.

Fidder H, Schnitzler F, Ferrante $M$ et al. Long-term safety of infliximab for the treatment of inflammatory bowel disease: a single-centre cohort study. Gut 2009; 58: $501-8$.

Afzali A, W heat C L, Hu J K et al. The association of psoriasiform rash with anti-tumor necrosis factor (anti-TNF) therapy in inflammatory bowel disease: a single academic center case series. J Crohns Colitis 2014; 8: 480-8.

Cleynen I, Vermeire S. Paradoxical infl ammation induced by anti-TNF agents in patients with IBD. Nat Rev Gastroenterol Hepatol 2 012; 9: 496-503.

\section{P1072 PLATELET ABNORMALITIES AND ANEMIA IN PAEDIATRIC IBD: ARE THEY LINKED?}

M. Votto ${ }^{1}$, F. Viviano ${ }^{2}$, F. Viola ${ }^{2}$

${ }^{1}$ Pediatria, IRCCS Policlinico San Matteo, PAVIA/Italy

${ }^{2}$ Pediatria E Neuropsichiatria Infantile, Università La Sapienza, Roma/Italy

Contact E-mail Address: martinavotto@gmail.com

Introduction: Crohn's Disease (CD) and Ulcerative Colitis (UC) are two mayor forms of Inflammatory Bowel Disease (IBD). In children with IBD anemia is common and is a combination of iron deficiency and anemia of chronic disease (ACD). IBD are associated with several alterations of platelets, including number, shape, and function ${ }^{1}$. In clinical practice, the most common platelet alteration is thrombocytosis. In IBD, thrombocytosis is associated with iron deficiency anemia and chronic inflammation ${ }^{2}$. The importance of platelet function is due to the substantially increased incidence of thromboembolic phenomena in IBD

Aims \& Methods: The aim of the study is to demonstrate the link between anemia, thrombocytosis and platelet aggregation in pediatric IBD patients. This study includes 51 children and adolescents recruited from the Pediatric Gastroenterology Unit of Policlinico Umberto I in Rome. Patients younger 6 years, with inherited platelet defects, hemoglobinopathies, and receiving therapies that alter platelet function, are excluded. We collect disease activity scores (Pediatric Crohn's Disease Activity Index [PCDAI], Pediatric Ulcerative Colitis Activity Index [PUCAI]). The laboratory investigations include: complete blood count, mean corpuscular volume (MCV), mean platelet volume (MPV), mean corpuscular haemoglobin concentration (MCHC), levels of hemoglobin ( $\mathrm{Hb})$ and levels of serum ferritin (SF), transferrin and iron. Diagnostic criteria for anemia are based on ECCO guidelines. Platelet aggregation is evaluated on platelet-rich plasma in an AggRAM aggregometer with Born's Method. The results were reported as the maximal percentage of aggregation observed after 4 min stimulation in response to collagen $(1 \mu \mathrm{g} / \mathrm{mL})$ and adenosine diphosphate (ADP $0.8 \mu \mathrm{M}$ and ADP $2 \mu \mathrm{M}$ )

Results: The study include 51 children and adolescents, 24 with UC and 27 with $\mathrm{CD}$ (median age is 15.3 years \pm 3.5 ). Iron deficiency anemia combined to ACD is the most common type $(58.3 \%$ in UC and $50 \%$ in CD). Hemoglobin levels are significantly lower in patients with UC compared to CD patients $(\mathrm{p}=0.0320)$. No significant differences are observed between mean values of red cells, MCV, $\mathrm{MCHC}$, RDW, iron, transferrin and serum ferritin both in CD and UC. Thrombocytosis prevails in UC compared to CD patients, but no significant correlation was found. No differences are oserved between mean values of PDW and MPV in both groups.In patients with UC, a negative correlation was found between mean values of hemoglobin and platelet count $(p=0.0134)$. Moreover in patients with $\mathrm{CD}$, disease activity was positively correlated with platelet count $(\mathrm{p}=0.0040)$. Platelet aggregation results higher in anemic patients. In anemic children, mean baseline platelet aggregations - induced by

ADP $0.8 \mu \mathrm{M}$ and collagen $1 \mu \mathrm{g} / \mathrm{mL}$ - are significantly higher in UC compared to $\mathrm{CD}(\mathrm{p}=0.001$ and $\mathrm{p}=0.030$ respectively). Another significant correlation is observed between platelet aggregation - induced by ADP $0.8 \mu \mathrm{M}$ and ADP $2 \mu \mathrm{M}$ - in anemic UC patients compared to non-anemic UC patients $(\mathrm{p}=0.002$ and $\mathrm{p}=0.040$ respectively). Platelet aggregation-induced by ADP $0.8 \mu \mathrm{M}$-is significantly higher in anemic UC patients with active disease (PUCAI $>20$ ) compared to same patients whose disease is in remission $(\mathrm{p}=0.042)$ and compared to patients with active $\mathrm{CD}(\mathrm{p}=0.054)$

Conclusion: In our cohort, mixed anemia (iron deficiency anemia combined to anemia of chronic disease) is the most common type of anemia. Thrombocytosis is a condition more frequent in anemic IBD patients, specially in UC. In UC, anemia and disease activity are significant correlated with platelet hyperaggregation. Therefore UC patients-specially with active disease and anemia-might present major risk of thrombosis, independently from acquired or inherited hemostasis defects. 
Disclosure of Interest: All authors have declared no conflicts of interest.

\section{References}

1. Voudoukis E. et al; Multipotent role of platelets in inflammatory bowel diseases: a clinical approach. World J Gastroenterol 2014;20:3180-90.

2. Voudoukisa Eet al; Association between thrombocytosis and iron deficiency anemia in inflammatory bowel disease. Eur $J$ Gastroenterol Hepatol 2013;25:1212-6.

3. Danese $\mathrm{S}$ et al; Platelets in inflammatory bowel disease: clinical, pathogenic, and therapeutic implications. AJ Gastroenterol 2004;99:938-45.

\section{P1073 RELATIONSHIP BETWEEN CLINICAL COURSE OF ULCERATIVE COLITIS (UC) DURING PREGNANCY AND OUTCOMES OF PREGNANCY: A RETROSPECTIVE EVALUATION STUDY}

A. Ito, O. Teppei, T. Katutishi

Dept. Medicine, Inst. Gastroentelorgy, Tokyou Womens Medical University, Tokyo/Japan

Contact E-mail Address: itoayumi@ige.twmu.ac.jp

Introduction: Ulcerative colitis (UC) is a chronic, intractable disease with a long clinical course. UC has a marked influence on the lifestyle of patients, and its effects on pregnancy and childbirth can especially become a problem for women in their child-bearing years. Various studies have suggested that it is desirable for pregnant women with UC to give birth while remaining in a state of remission. Aims \& Methods: The present study evaluated pregnant women with UC attending our hospital who became pregnant during remission, in order to examine the factors that contributed to recurrence of UC during pregnancy. We investigated 40 pregnant patients in remission (44 cases) attending our hospital between January 2008 and July 2016 who had remained in remission for one year prior to pregnancy. After becoming pregnant while in remission, patients who stayed remission until delivery were classified into the ongoing remission group (35 cases) and patients with recurrence during pregnancy were classified into the recurrence group (9 cases). Remission was defined as a Lichtiger clinical activity index (CAI) of less than 4 . Relapse was defined as a CAI $\geq 5$ with the need for initiation or dose escalation of steroids or administration of biological agents during pregnancy. Items examined: Clinical characteristics (age at onset, disease duration, age of becoming pregnant, disease type, and treatment), the CAI in the first, second, and third trimesters, and whether or not patients continued treatment during pregnancy were examined and compared between the two groups. The reasons for discontinuation of treatment were also investigated

Results: There were significant differences between the two groups with respect to the age of becoming pregnant $(32.9 \pm 4.6$ years in the ongoing remission group vs. $28.3 \pm 7.0$ years in the recurrence group), the CAI in the second trimester $(2.9 \pm 4.6$ vs. $3.5 \pm 1.6)$, the CAI in the third trimester $(2.9 \pm 0.7$ vs. $5.4 \pm 2.0$ ), and whether oral treatment was continued (continuation of treatment [yes:no]; 30:5 in the ongoing remission group vs. 5:4 in the recurrence group). Regarding the discontinuation of oral treatment, two patients in the ongoing remission group and one patient in the recurrence group discontinued it on their own judgment, while two patients in the recurrence group discontinued it due to hyperemesis. Discussion: The present study revealed that factors influencing the recurrence of UC during pregnancy were the age of becoming pregnant and the continuation of oral treatment. Our results showed that younger women were more susceptible to recurrence. As expected, discontinuing oral treatment was a factor that contributed to recurrence. However, the reasons for discontinuing treatment during pregnancy differed from those for non-pregnant women. Some patients discontinued treatment on their own judgment because they were concerned about adverse effects on the fetus, while others had difficulty with continuing treatment due to hyperemesis. With regard to the effects of medications on the fetus, medical staff should provide an explanation about the safety of treatment and should be aware that patients may have various concerns about drug therapy. If patients have difficulty continuing oral treatment due to severe hyperemesis, administration of local therapy should be considered.

Conclusion: During pregnancy, it is important to continue treatment for UC so that patients can give birth while remaining in remission. Accordingly, intervention by medical staff is particularly necessary in order to provide pregnant women with information and explanations regarding treatment.

Disclosure of Interest: All authors have declared no conflicts of interest.

P1074 USE OF STEROIDS IN ADULTS AND ADOLESCENTS WITH INFLAMMATORY BOWEL DISEASE IN THE BIOLOGICS ERA

I. Parisi ${ }^{1}$, L. Whitley ${ }^{1}$, C. Murray ${ }^{2}$, F. Kiparissi ${ }^{3}$, F. Rahman ${ }^{1}$, S. Mehta ${ }^{1}$, E. Seward ${ }^{1}$, D. Graham ${ }^{1}$, H. Kwok ${ }^{1}$, S. Mccartney ${ }^{1}$

${ }^{1}$ GI Medicine, University College London Hospitals, London/United Kingdom

${ }^{2}$ Gastroenterology, Royal Free Hospital, London/United Kingdom

${ }^{3}$ Great Ormond Street Hospital, London/United Kingdom

Contact E-mail Address: ioannaparis@hotmail.com
Introduction: Corticosteroids have been effectively used for decades for the rapid induction of clinical remission in patients with Inflammatory Bowel Disease (IBD). However their adverse event profile is well-known, particularly with long-term use and in younger populations, and they have no role in maintenance of remission. Steroid-sparing agents and more recently biological therapy have reduced systemic steroid use but the true incidence of steroid prescriptions especially in the outpatient setting is not known.

Aims \& Methods: The aim of this study was to capture real-life steroid use in both adult and adolescent populations older than 13 years old who attend a dedicated IBD clinic in a tertiary referral centre. We tried to identify risk factors associated with appropriate or excessive steroid use in the whole cohort and in the two subgroups separately. All consecutive IBD patients who were followed up for at least one year in the adult and adolescent clinic in UCLH and attended their IBD appointment during February and March 2017 were included in the study. A steroid assessment questionnaire was completed by the clinician during the visit for eligible patients. Use and type of steroids prescribed during the past year was recorded, as well as appropriate bone protection and disease activity based on Physician Global Assessment.

Results: 60 adolescents and 59 adults were included in the study. Two thirds of adolescents had Crohn's disease while in the adult population Crohn's and ulcerative colitis (UC) were equally distributed. 57 (95\%) adolescents had been exposed to thiopurines and $47(78 \%)$ to anti-TNFs as opposed to $69 \%$ $(\mathrm{p}=0.002)$ and $39 \%(\mathrm{p}<0.001)$ of adults respectively. Vedolizumab exposure was similar in adults and adolescents. The percentage of patients with moderate to severe disease at last visit was comparable between two groups $(18 / 60,30 \%$ adolescents vs $18 / 59,31 \%$ adults, $\mathrm{p}=\mathrm{NS}) .27 / 119(23 \%)$ of patients were offered steroids during the past year and the incidence was the same in the adult and adolescent populations. $4 / 27(15 \%)$ received excessive steroids, ie $>2$ courses within the preceding 12 months. Of the 27, 17 (62\%) had Prednisolone and the rest received Budesonide, while the choice of steroid was associated with a diagnosis of Crohn's disease and was not different between the two groups. One third of patients did not receive bone protection with the steroid prescription, however this was mainly observed with Budesonide rather than Prednisolone ( $7 / 10$ vs 2 $17, \mathrm{p}=0.003)$. Steroid use overall was not associated with the age group or initial diagnosis but was expectedly associated with severity of disease (sever$\mathrm{e}>$ moderate $>$ mild, $\mathrm{p}<0.001$ ). The use of thiopurines or anti-TNFs did not affect steroid prescriptions, but interestingly the majority of Vedolizumab users received steroids in contrast to Vedolizumab naïve patients $(7 / 9,78 \%$ vs $20 / 109$, $18 \%, \mathrm{p}<0.001)$. This association remained significant even after adjustment for disease severity and other co-factors, suggesting possibly the higher need for 'bridging' steroids due to the slow-acting effect of Vedolizumab therapy (binary logistic regression showed naïve to Vedolizumab patients were protected against steroid use, OR 0.043 CI 95\% 0.005-0.382, p=0.005)

Conclusion: The use of steroids in an outpatient population with IBD is still common regardless of age group and use of anti-TNFs, as almost 1 in 4 patients received some type of oral steroid during a period of one year. Newer biologics, such as Vedolizumab, may predispose patients to higher steroid use, possibly due to slow induction of remission.

Disclosure of Interest: All authors have declared no conflicts of interest.

\section{Reference}

Raine T. Excess steroid use in IBD: too much, how much, and why? Results from a UK nationwide audit. 2017 Mar 31.

P1075 REMISSION INDUCTION IN CORTICOSTEROID NAÏVE CHILDREN AND ADOLESCENTS WITH ULCERATIVE COLITIS BY ADSORPTIVE LEUCOCYTAPHERESIS AS MONOTHERAPY OR IN COMBINATION WITH A LOW DOSE PREDNISOLONE AFTER FAILURE OF FIRST-LINE MEDICATIONS

T. Tanaka ${ }^{1}$, M. Akagi ${ }^{2}$, T. Iiboshi ${ }^{2}$, H. Goishi ${ }^{2}$, T. Kajihara ${ }^{2}$, A. R. Saniabadi ${ }^{3}$, T. Miura

${ }^{1}$ Dept. Of Gastroenterology, Akitsu Prefectural Hospital, Hiroshima/Japan

${ }^{2}$ Akitsu Hospital, Hiroshima/Japan

${ }^{3}$ JIMRO, Takasaki/Japan

Contact E-mail Address: tomotaka@c.do-up.com

Introduction: In patients with active ulcerative colitis (UC), myeloid lineage leucocytes are known to be elevated with activation behaviour, including the $\mathrm{CD} 14+\mathrm{CD} 16+\mathrm{DR}++$ monocyte phenotype, which is a major source of tumour necrosis factor- $\alpha .^{2}$ Therefore, selective depletion of myeloid leucocytes by adsorptive granulocyte/monocyte apheresis (GMA) with an Adacolumn is expected to promote remission, or enhance drug efficacy. Potentially, GMA should be a relevant treatment option in patients in whom drug therapy has limitations.

Aims \& Methods: Our major objective was to apply GMA as remission induction therapy in paediatrics and adolescents UC setting when first-line 5-aminosalicylates had failed. Thirty consecutive patients with active ulcerative colitis (UC), age 11-19 years, body weight $33-55.5 \mathrm{~kg}$ were given mesalazine $(\mathrm{n}=23)$ or sulphasalazine $(\mathrm{n}=7)$ as the first-line medication. Twenty patients relapsed while receiving the first-line medications or did not respond, and received GMA with the Adacolumn, at 2 sessions in the first week, then weekly, up to 11 sessions. Patients who achieved $\geq 5$ decrease in the clinical activity index (CAI) after 5 
GMA sessions continued with GMA while non-responders received GMA in combination with a low dose prednisolone, 0.5 to $1.0 \mathrm{mg} / \mathrm{kg}$ bodyweight. At entry and week 12 , patients were clinically and endoscopically evaluated with each patient serving as her or his own control.

Results: At entry, all 30 patients were corticosteroid naïve and none had deep colonic lesions or extensive loss of the mucosal tissue at the affected sites (GMA non-responder features). Ten patients achieved stable remission with the first-line medications and did not receive GMA. Six patients did not respond well to the first 5 GMA sessions and received prednisolone together with GMA, while 12 patients responded well to GMA, and achieved stable remission, but 2 patients withdrew to receive high dose prednisolone (up to $2 \mathrm{mg} / \mathrm{kg}$ bodyweight). At entry, the average CAI was $14 \pm 0.4$. Prednisolone was tapered to $0 \mathrm{mg}$ within 3 months in those who received. Therefore, at week 12, all 30 patients were in remission, majority with mucosal healing.

Conclusion: In this treatment design, GMA in young corticosteroid naïve patients with active UC refractory to the first-line 5-aminosalicylates was associated with clinical remission and mucosal healing, while in non-responders to GMA monotherapy, addition of a low dose prednisolone enhanced the efficacy of GMA and tapering of the prednisolone dose was not associated with relapse. Therefore, the majority of young steroid naïve UC patients who fail to respond to first-line 5aminosalicylates should respond well to GMA and avoid pharmacologicals. Additionally, GMA has a good safety profile, which is a very favourable feature in growing patients.

Disclosure of Interest: A.R. Saniabadi: Dr. Saniabadi has a non-regular employment position at JIMRO.

All other authors have declared no conflicts of interest.

\section{References}

1. Saniabadi AR, et al. Therapeutic leukocytapheresis for inflammatory bowel disease. Transfus Apher Sci 2007;37:191-200

2. Belge KU, et al. The proinflammatory $\mathrm{CD} 14+\mathrm{CD} 16+\mathrm{DR}++$ monocytes are a major source of TNF. J Immunol 2002;168:3536-42.

\section{P1076 EARLY SCREENING FOR INFLAMMATORY BOWEL}

\section{DISEASE IN CHILDREN WITH AUTOIMMUNE LIVER DISEASE}

D. Thangarajah, E. Cernat, B. Vadamalayan, D. Hadzic, M. Samyn Paediatric Liver, GI And Nutrition Centre, King's College Hospital, London/ United Kingdom

\section{Contact E-mail Address: anthiburtt@gmail.com}

Introduction: Autoimmune liver disease (AILD), in particular autoimmune sclerosing cholangitis (ASC) is associated with inflammatory bowel disease (IBD). Little is known about the effect of IBD on the outcome of AILD. Faecal calprotectin (FC), a stool biomarker, screens for bowel inflammation without the need for invasive endoscopic evaluation and is easy to collect.

Aims \& Methods: The aim of this study was to review the investigations and outcomes of IBD and AILD in children with a primary diagnosis of AILD and to identify possible risk factors for development of IBD in children with AILD. Children with AILD were identified from electronic case notes between 2007 and 2010 and those with a diagnosis of IBD prior to AILD excluded. AILD was diagnosed and treated as per centre protocol. Diagnostic endoscopy for IBD was performed, based on GI symptoms and/or elevated FC ( $>60 \mathrm{U} / \mathrm{g})$. Data were documented at time of liver diagnosis; endoscopy and last liver follow up. Patients were classified as AILD-IBD or AILD.Mann Whitney and Chi squared test were used to analyse data where appropriate, significance $\mathrm{p}<0.05$.

Results: Out of 37 (12 male) children, diagnosed with AILD (ASC 11), 23 underwent diagnostic endoscopy after a median time from diagnosis of 27.6 [20.1 to 53.9] weeks. $20 / 23$ reported GI symptoms and FC was elevated in 13/18 tested. At endoscopy $57 \%(13 / 23)$ a diagnosis of IBD (AILD-IBD group) (UC $(n=12)$, IBD-U $(\mathrm{n}=1)$ ) was made, $10 / 23$ were within normal limits (AILD group). There was no difference in gender or diagnosis of ASC between the 2 groups. At presentation of AILD, children in the AILD-IBD group were significantly leaner in terms of weight and BMI, had lower haemoglobin, with a trend for younger age at presentation (table 1). GI symptoms and $\mathrm{FC}>60 \mathrm{U} / \mathrm{g}$ were significantly more prevalent in the AILD-IBD group. At the time of endoscopy, 22 were on treatment for AILD with prednisolone and 13 with an additional agent (azathioprine or mycophenolate mofetil). Biochemical remission for AILD was achieved in $45 \%$ at endoscopy and in $74 \%$ at last liver follow-up (median 4.1 [3.5 to 5.0] years) with no difference for both groups. All patients are alive; however, in the AILD-IBD group 1 underwent an isolated liver transplantation (LT) and 1 required a subtotal colectomy. One girl underwent LT combined with subtotal colectomy after decompensation of her liver disease.

Table 1: Diagnostic parameters of AILD at presentation median [IQR]/*p value $<0.05$

\begin{tabular}{lll}
\hline & AILD-IBD & AILD \\
\hline $\mathrm{n}$ (male) & $13(5)$ & $10(3)$ \\
Age (years) & $12.5[10.3$ to 14.6$]$ & $14.4[12.0$ to 15.5$]$ \\
ASC $(\mathrm{n})$ & 6 & 2 \\
Weight $(\mathrm{kg})$ & $48.8[38.8$ to 55.9$]$ & $61.5[52.9 \text { to } 75.4]^{*}$ \\
Height $(\mathrm{cm})$ & $160.7[152.3$ to 168.0$]$ & $164.0[154.4$ to 168.8$]$ \\
BMI $\left(\mathrm{kg} / \mathrm{m}^{2}\right)$ & $19.2[18.2$ to 20.2$]$ & $22.5[19.5 \text { to } 27.3]^{*}$ \\
Haemoglobin $(\mathrm{g} / \mathrm{l})$ & $113.0[90.5$ to 122.0$]$ & $127.5[118.5 \text { to } 146.5]^{*}$ \\
\hline & & (continued)
\end{tabular}

Table 1: Continued

\begin{tabular}{lll}
\hline & AILD-IBD & AILD \\
\hline AST(IU/1) & $235.0[113.5$ to 470.5$]$ & $461.0[241.0$ to 1039.0$]$ \\
IgG $(\mathrm{g} / \mathrm{l})$ & $25.3[16.2$ to 37.8$]$ & $25.1[19.2$ to 30.1$]$ \\
Faecal calprotectin $(\mathrm{U} / \mathrm{g})$ & $298.5[114.5$ to 439.8$]$ & $42.5[34.6 \text { to } 66.3]^{*}$ \\
GI symptoms (n) & 12 & 8 \\
Faecal protectun $>60 \mathrm{U} / \mathrm{g}(\mathrm{n} /$ total $)$ & $11 / 12$ & $2 / 6$ \\
\hline
\end{tabular}

Conclusion: In our cohort $35 \%$ of children presenting with AILD were subsequently diagnosed with IBD. Possible risk factors for development of IBD in AILD are low haemoglobin, being leaner and younger at diagnosis. An elevated FC and the presence of GI symptoms are useful to assess the need for diagnostic endoscopy when considering diagnosis of IBD in the context of AILD. As concurrent immunosuppression may mask mild symptoms and signs of IBD a lower threshold for endoscopy should be considered in these patients.

Disclosure of Interest: All authors have declared no conflicts of interest.

P1077 CLINICAL AND LABORATORY VARIABLES THAT PREDICT CLINICAL AND ENDOSCOPIC REMISSION IN CHILDREN WITH CROHN'S DISEASE TREATED WITH INFLIXIMAB

G. D'Arcangelo, S. Oliva, F. Tarani, F. Viola, F. Civitelli, S. Cucchiara, M. Aloi Pediatric Gastroenterology And Liver Unit, Sapienza University of Rome, ROME/ Italy

Contact E-mail Address: giuliadarcangelo87@gmail.com

Introduction: We aimed to identify early clinical and laboratory predictors of sustained clinical and endoscopic remission in children with Crohn's disease (CD) under infliximab (IFX)

Aims \& Methods: Prospective study conducted in children with moderate-tosevere CD starting IFX treatment. All patients underwent endoscopy, weighted pediatric CD activity index (wPCDAI) assessment, C-reactive protein (CRP) at week 0 and 52. wPCDAI and CRP were also evaluated at 14 weeks. The primary outcome was to determine the ability of 14-week wPCDAI and CRP to predict steroid-free sustained remission and mucosal healing at 1 year. As a secondary outcome we sought to evaluate the concordance between wPCDAI and Simple Endoscopic Score for CD (SES-CD) at week 52.

Results: Forty-one children were enrolled (median age 13.5 2.7 , females $41.5 \%$ ). At 1 year, $21(51 \%)$ and $16(39 \%)$ were in clinical (wPCDAI $<12.5$ ) and endoscopic $($ SES-CD $<3)$ remission, respectively. Fourteen-week wPCDAI didn't differ between patients who achieved both clinical and endoscopic remission at 1 year $(p=0.21$ and $p=0.35$, respectively). By using a multivariable logistic regression model, neither week-14 wPCDAI nor CRP were predictors of 1-year clinical $(\mathrm{p}=0.83$ and $\mathrm{p}=0.30$, respectively) and endoscopic remission $(\mathrm{p}=0.22$ and $\mathrm{p}=0.48)$. wPCDAI resulted significantly correlated with 1 -year SES-CD $(r=0.38, p=0.01)$. The concordance between wPCDAI and SES-CD was excellent and good for severe disease and remission ( $\mathrm{k}$ cohen 0.87 and 0.76 ), moderate and absent for mild and moderate disease, respectively.

Conclusion: Based on our results, 14-week post induction wPCDAI and CRP are not predictors of 1-year sustained steroid-free clinical and endoscopic remission in children with CD under biologic therapy. Continuous wPCDAI shows a good correlation with SES-CD, particularly for patients in remission and with severe disease.

Disclosure of Interest: All authors have declared no conflicts of interest.

\section{TUESDAY, OCTOBER 31, 2017} OTHER LOWER GI DISORDERS II - HALL $7_{-}$

09:00-17:00

\section{P1078 THALIDOMIDE FOR THE TREATMENT OF REFRACTORY GASTROINTESTINAL BLEEDING CAUSED BY ANGIODYSPLASIAS}

X. Wang ${ }^{1}$, Q. Wang ${ }^{2}$, H. Huang ${ }^{3}$, W. Lu ${ }^{1}$

${ }^{1}$ Gastroenterology Department, the First People's Hospital, Hangzhou/China

${ }^{2}$ Gastroenterology, The First People's Hospital, Hangzhou/China

${ }^{3}$ Department, Institute name, Hangzhou/China

Contact E-mail Address: 15968807972@163.com

Introduction: Gastrointestinal angiodysplasia, either inherited or acquired, is an important and challenging cause of acute gastrointestinal haemorrhage, particularly in the elderly, sometimes refractory to treatment. Although multiple treatment modalities, both medical and surgical, are being used, there is no established medical treatment available for these patients. Thalidomide is a potent inhibitor of angiogenesis in experimental models. As angiodysplasias are a result of unregulated vessel growth, antiangiogenic treatment may inhibit growth of angiodysplasias. But its benefits and risks nevertheless remain unclear. Aims \& Methods: This retrospective study evaluates the efficacy, safety, and sideeffect of thalidomide in the treatment of patients with refractory gastrointestinal bleeding from angiodysplasias. Patients with recurrent gastrointestinal bleeding of angiodysplasia who were from Hangzhou First people's Hospital from October 2012 to July 2015 were collected for this open and nonrandomized study. Thalidomide was started with $50 \mathrm{mg} /$ day and then increased incrementally by $100 \mathrm{mg} /$ day, if tolerated, and continued for 6 months. Adverse events, hemoglobin, blood chemistry, the changes of coagulation and blood transfusion were monitored during the treatment and for 6-months post-treatment. 
Results: Twenty-one patients with chronic refractory angiodysplasis bleeding were recruited in this study, included 10 women, aged between 40-85;11 cases of male, aged between 31-70. One patient with colic vascular malformation died of massive hemorrhage due to self withdrawal. Among the remaining 20 patients who were given thalidomide regularly for 6 months. (1). Eight patients come across constipation, sleepiness and dry mouth. There were no skin rashes, peripheral neuropathy and any other adverse reactions during the treatment. All side-effects resolved when thalidomide was discontinued. (2). The red blood cell after treatment $(3.76 \pm 0.56) * 10^{\wedge} 9 / \mathrm{L}$ compared with before treatment $(3.38 \pm 0.60)^{*} 10^{\wedge} 9 / \mathrm{L}$; hemoglobin after treatment $(94.7 \pm 13.15 \mathrm{~g} / \mathrm{L})$ compared with before treatment $(83.2 \pm 19.76 \mathrm{~g} / \mathrm{L})$; HCT after treatment $(0.32 \pm 0.05)$ compared with before treatment $(0.29 \pm 0.06)$; the difference was statistically significant $(\mathrm{P}<0.05)$. (3). The ALT after treatment $(32.9 \pm 18.5 \mathrm{U} / \mathrm{L})$ compared with before treatment $(30.6 \pm 12.8 \mathrm{U} / \mathrm{L})$; AST after treatment $(28.1 \pm 8.56 \mathrm{U} / \mathrm{L})$ compared with before $(28.0 \pm 12.4 \mathrm{U} / \mathrm{L}) ; \gamma$-GT after treatment $(34.4 \pm 8.4 \mathrm{U} / \mathrm{L})$ compared with before $(35.6 \pm 12.7 \mathrm{U} / \mathrm{L})$; AKP after treatment $(85.5 \pm 19.8 \mathrm{U} / \mathrm{L})$ compared with before $(83.0 \pm 20.8 \mathrm{U} / \mathrm{L})$; ALB after treatment $(36.2 \pm 3.1 \mathrm{~g} / \mathrm{L})$ compared with before $(36.3 \pm 4.3 \mathrm{~g} / \mathrm{L})$; there was no statistically significant difference $(\mathrm{P}>0.05)$. (4). Prothrombin time $(\mathrm{PT})$ after treatment $(12.1 \pm 1.3 \mathrm{~s}) \mathrm{com}-$ pared with before $(11.8 \pm 1.4 \mathrm{~s})$; APTT after treatment $(30.2 \pm 3.7 \mathrm{~s})$, compared with before $(31.0 \pm 6.2 \mathrm{~s})$; the difference was not statistically significant $(\mathrm{P}>0.05)$. (5). 6 cases of colonic capillary malformation review colonoscope, and the vascular malformation improved significantly after treatment.

Conclusion: Thalidomide, with its antiangiogenic mechanism of action, seems to be a promising drug in bleeding angiodysplasias as a treatment option for patients unable to benefit from other available modalities of treatment. the study drug was well tolerated.

Disclosure of Interest: All authors have declared no conflicts of interest.

\section{References}

1. Diggs NG, Holub JL, Lieberman DA, et al. Factors that contribute to blood loss in patients with colonic angiodysplasia from a population-based study. Clin Gastroenterol Hepatol, 2011,9(5):425-420.

2. Varma P, Misra M, Radhakrishnan VV, et al. Fetal post-operative gastro intestinal hemorrhage because of angio-dysplasia of small intestine in aortic regurgitation. Interact Cardiovasc Thorac Surg, 2004,3(1):118-120.

3. Luckraz H, Hashim S, Ashraf S. Aortic stenosis and angiodysplasia in the elderly:common things occur commonly? Interact Cardiovasc Thorac Surg, 2003,2(4):526-528

4. Dabak V, Kuriakose P, Kamboj G, et al. A pilot study of thalidomide in recurrent GI bleeding due to angiodysplasias. Dig Dis Sci, 2008, 3(6):16321635 .

5. Kirkham SE, Lindley KJ, Elawad MA, et al. Treatment of multiple small bowel angiodysplasias causing severe life-threatening bleeding with thalidomide. J Pediatr Gastroenterol Nutr, 2006,42(5):585-587.

\section{P1079 NEWLY DEVELOPED ENDOSCOPIC DETACHABLE SNARE LIGATION THERAPY FOR COLONIC DEVERTICULAR HEMORRHAGE: A MULTICENTER PHASE II TRIAL}

K. Kobayashi ${ }^{1}$, T. Narasaka ${ }^{2}$, D. Akutsu ${ }^{3}$, M. Nishi ${ }^{4}$, M. Wakayama ${ }^{5}$, Y. Hiroshima ${ }^{6}$, S. Endo ${ }^{7}$, T. Mamiya $^{8}$, S. Hirai ${ }^{9}$, K. Ikezawa ${ }^{10}$, H. Ishida ${ }^{11}$, M. Hirose ${ }^{12}$, T. Yamada ${ }^{3}$, H. Suzuki ${ }^{2}$, T. Kaneko ${ }^{3}$, Y. Mizokami ${ }^{2}$, I. Hyodo ${ }^{1}$ Internal Medicine, Tokyo Metropolitan Bokutoh Hospital, Tokyo/Japan ${ }^{2}$ Endoscopic Center, University of Tsukuba Hospital, Ibaraki/Japan ${ }^{3}$ Gastroenterology, University of Tsukuba, Ibaraki/Japan

${ }^{4}$ Gastroenterology, Tsukuba Gakuen Hospital, Ibaraki/Japan

${ }^{5}$ Gastroenterology, Koyama Memorial Hospital, Ibaraki/Japan

${ }^{6}$ Gastroenterology, Hitachinaka General Hospital, Ibaraki/Japan

${ }^{7}$ Gastroenterology, Shinmatsudo Central General Hospital, Chiba/Japan

${ }^{8}$ Gastroenterology, Ryugasaki Saiseikai Hospital, Ibaraki/Japan

${ }^{9}$ Gastroenterology, Hitachi General Hospital, Ibaraki/Japan

${ }^{10}$ Gastroenterology, Tsukuba Memorial Hospital, Ibaraki/Japan

${ }^{11}$ Gastroenterology, Mito Medical Center, Ibaraki/Japan

${ }^{12}$ Gastroenterology, Kasumigaura Medical Center, Ibaraki/Japan

\section{Contact E-mail Address: kats0625@hotmail.co.jp}

Introduction: Colonic diverticular bleeding is the most common cause of lower gastrointestinal bleeding. We have reported the preliminary safety results of endoscopic detachable snare ligation (EDSL), a new method for diverticular hemorrhage ${ }^{1)}$. The bleeding diverticulum was ligated with a detachable snare. Unlike the endoscopic band ligation, removal of the scope to attach a ligation device and reinsertion for treatment are not needed in this method. We performed a clinical trial to evaluate the effecy and safety of EDSL.

Aims \& Methods: This multicenter single arm phase II study was conducted in 12 Japanese institutions. Patients suspected of diverticular bleeding were enrolled from June 2015 to March 2017. Patients with serious heart, renal, or liver failure, sepsis, disseminated intravascular coagulation, and high-dose steroid use (prednisolone dosage $>10 \mathrm{mg}$ /day) were excluded. The primary endpoint was the early (within 1 month) rebleeding rate in patients who were treated with EDSL.
The secondary endpoints were overall early rebleeding rate in patients who had confirmed diverticular bleeding (intention to treat: ITT), success rate of EDSL, total procedure time, EDSL procedure time, identification rate of bleeding diverticula, and adverse events. This study was approved by the ethics committee of each participating hospital and conformed to the Helsinki Declaration and the Japanese Clinical Research Guidelines.

Results: Of 123 patients with diverticular hemorrhage, 101 were treated with EDSL and the early rebleeding rate was $5 \%(5 / 101)$. The rebleeding rate in ITT population was $9 \%(11 / 123)$. Success rate of EDSL was $78 \%(96 / 123)$. The median total procedure time and EDSL procedure time were $40(15-150)$ and $4(1-57) \mathrm{min}$, respectively. The identification rate of bleeding diverticula was $60 \%$ (123 in 205 enrolled patients). Two mild adverse events occurred; colonic diverticulitis cured with antibiotics and temporary abdominal pain during EDSL. Conclusion: EDSL is an effective, safe, and convenient treatment method for colonic diverticular hemorrhage.

Disclosure of Interest: All authors have declared no conflicts of interest.

\section{Reference}

1. Akutsu D, Narasaka T, Wakayama M et al. Endoscopic detachable snare ligation: a new treatment method for colonic diverticular hemorrhage. Endoscopy. 2015; 47: 1039-42.

\section{P1080 RISK FACTORS FOR EARLY AND LATE RE-BLEEDING IN PATIENTS WITH COLONIC DIVERTICULAR BLEEDING}

Y. Sato, H. Yasuda, S. Nakatsu, M. Yamashita, Y. Matsuo, H. Yamamoto, F. Itoh

Gastroenterology And Hepatology, St. Marianna Medical University, Kawasaki/ Japan

Contact E-mail Address: y2satou@marianna-u.ac.jp

Introduction: Incidence of colonic diverticular bleeding has increased in recent years. Colonic diverticular bleeding is problematic because of the following reasons: the low detection rate of the bleeding source by endoscopy and frequent rebleeding. At our hospital, we have a policy of performing emergency lower gastrointestinal endoscopy for all patients with colonic diverticular bleeding within $24 \mathrm{~h}$ of their admission. We have reported that the following factors can contribute to the successful identification of the bleeding source: extravasation revealed by abdominal contrast computed tomography (CT), and mounting of a hood to the tip of an endoscope during lower gastrointestinal endoscopy. However, risk factors for re-bleeding in patients with colonic diverticular bleeding were still unknown

Aims \& Methods: In this study, we examined the risk factors for early and late rebleeding in patients with colonic diverticular bleeding. From January 2004 to April 2016, we admitted 432 patients (285men and 147 women, mean age: $71 \pm 13$ years) to our hospital for treatment following a diagnosis of colonic diverticular bleeding based on abdominal CT and endoscopy findings. Early and late rebleeding was defined as macroscopically bloody stools as a result of colonic diverticular bleeding during hospitalization and after discharge, respectively. Risk factors for early and late re-bleeding were retrospectively examined using univariate and multivariate analysis.

Results: Early re-bleeding occurred in 112 patients $(26 \%$; 86 men and 26 women, mean age: $71 \pm 12$ years). The mean duration until re-bleeding was $3.9 \pm 2.4$ days, and on average, early re-bleeding recurred $1.7 \pm 1.2$ times. On average, lower gastrointestinal endoscopy was performed $2.7 \pm 1.2$ times and endoscopic hemostatic treatment was performed $1.0 \pm 1.0$ times. In the univariate analysis, significant differences were seen in males $(P=0.005)$, in the use of oral antiplatelet agents $(\mathrm{P}=0.012)$, and in patients not undergoing endoscopic hemostasis $(P=0.004)$. In the multivariate analysis, male gender $(P=0.006$; odds ratio $2.06,95 \% \mathrm{CI} 1.23-3.44)$, the use of oral antiplatelet agents $(\mathrm{P}=0.008$; odds ratio $1.85,95 \% \mathrm{CI} 1.17-2.93)$, and patients not undergoing endoscopic hemostasis $(\mathrm{P}=0.005$; odds ratio $1.5,95 \% \mathrm{CI} 0.31-0.81)$ were independent risk factors for early re-bleeding. Late re-bleeding was seen in 72 of 345 patients who were able to follow up $(21 \% ; 46$ men and 26 women, mean age: $73 \pm 12$ years). The mean duration until late re-bleeding was $41 \pm 40$ months, and on average, late re-bleeding recurred $1.5 \pm 1.2$ times. Only the use of oral antiplatelet agents $(P=0.05$; odds ratio $1.72,95 \% \mathrm{CI} 0.98-2.98$ ) was identified as an independent risk factor for late re-bleeding in the univariate and multivariate analysis.

Conclusion: Not undergoing endoscopic hemostasis and male gender were identified as risk factors for early re-bleeding, indicating the importance of choosing measures and hemostatic treatments to improve the detection rate of bleeding sources during endoscopy. The use of oral antiplatelet agents was a risk factor for both early and late re-bleeding, suggesting the need for patient management through multi-departmental cooperation.

Disclosure of Interest: All authors have declared no conflicts of interest.

\section{Reference}

Nakatsu $\mathrm{S}$ et al, Urgent computed tomography for determining the optimal timing of colonoscopy in patients with acute lower gastrointestinal bleeding. Intern Med. 2015;54(6): 553-558. 


\section{P1081 ACUTE LOWER GASTROINTESTINAL BLEEDING-IS}

\section{NOBLADS THE ANSWER?}

S. Xavier ${ }^{1}$, J. Magalhães ${ }^{2}$, J. Cotter ${ }^{3}$

${ }^{1}$ Life and Health Sciences Research Institute (ICVS), School of Medicine, University of Minho, Braga/Portugal

${ }^{2}$ Gastroenterology Department, Hospital da Senhora da Oliveira, Guimarães, Guimarães/Portugal

${ }^{3}$ Pt Government Associate Laboratory, 4 ICVS/3B's, Braga/Guimarães/Portugal

Contact E-mail Address: smaxavier@gmail.com

Introduction: Acute lower gastrointestinal bleeding (ALGIB) constitutes an important gastroenterological emergency. A new score (NOBLADS) that intends to determine the risk of severe ALGIB was recently developed. We aimed to assess the validity of this score in a cohort of patients with ALGIB.

Aims \& Methods: Retrospective study. Emergency consecutive admissions for ALGIB were reviewed. Severe ALGIB was defined as transfusion of $\geq 2$ units of packed red blood cells (PRBC) and/or hematocrit decrease of $\geq 20 \%$ within the first $24 \mathrm{~h}$ and/or recurrent bleeding after $24 \mathrm{~h}$ of stability. NOBLADS score was calculated and its discriminative capacity for severe ALGIB as well as for other outcomes was assessed.

Results: Included 118 patients with a mean age of $73 \pm 14.4$ years and $52.5 \%$ males. Most frequent etiologies for ALGIB were diverticular bleeding $(23.7 \%)$ and post-polipectomy $(21.2 \%)$. ALGIB was severe in $38.1 \%$ of patients. NOBLADS score showed a weak discriminative capacity to determine severe ALGIB (AUC $=0.68, \mathrm{p}<0.01$ ). However, when comparing patients with NOBLADS $<4$ and $\geq 4$, patients with higher scores were significantly older $(69.2 \pm 15.7$ years vs $78.6 \pm 10.0$ years, $p<0.01)$, had lower hemoglobin levels at admission $(11.8 \pm 2.6 \mathrm{~g} / \mathrm{dL}$ vs $10.2 \pm 2.5 \mathrm{~g} / \mathrm{dL}, \mathrm{p}<0.01)$, were transfused with more units of PRBCs during the first 24 hours and during hospital in-stay $(0.4 \pm 0.9$ vs $1.1 \pm 1.3, \mathrm{p}<0.01$ and $1.0 \pm 2.2$ vs $3.0 \pm 3.3, \mathrm{p}<0.01$, respectively) and were more frequently admitted to intermediate care units $(35.2 \%$ vs $59.6 \%$, $\mathrm{p}<0.01$ ). No differences were found between the two groups regarding in-stay length, rebleeding rate, need for surgery or death.

Conclusion: NOBLADS score showed a weak discriminative capacity to determine severe ALGIB however, patients with NOBLADS $\geq 4$ had greater PRBCs transfusion need and were more frequently admitted to intermediate care units. New or improved scores that can predict severe ALGIB are needed to determine more precisely appropriate care and to allow for a standardized approach. Disclosure of Interest: All authors have declared no conflicts of interest.

\section{P1082 COLORECTAL CANCERS (CRCS) DEPENDING ON THE SCREENING INTERVAL IN IBARAKI, JAPAN}

\section{Y. Saitoh ${ }^{1}$, H. H. Suzuki ${ }^{2}$, I. Hyodo}

${ }^{1}$ Dept. Of Gastroenterology, Ibarakiken Medical Center, Ibaraki/Japan

${ }^{2}$ University of Tsukuba, Tsukuba/Japan

${ }^{3}$ Gastroenterology, University of Tsukuba, Ibaraki/Japan

Contact E-mail Address: saitoitsyo@yahoo.co.jp

Introduction: In Japan, CRC screening was launched as a national policy for all people aged over 40 years in 1992. 2-day FIT has been widely accepted, and has been recommended performing in every year.

Aims \& Methods: The aim of this study is to verify the validity of an annual 2-day FIT. This analysis was performed by the OC-SENSOR DIANA (EIKEN, JAPAN) automated analyzer with cut-off value of $20 \mu \mathrm{g} \mathrm{Hb} / \mathrm{g}$ stool. Between 2011 and 2014, 957 CRCs and 12,290 polyps were detected. 957 CRCs were analyzed by screening history about sizes and Dukes' stage. The person who had undergone 2-day FIT 1 year before was defined as repeated group. And hadn't undergone more than 5 years before was defined as first-time group.

Results: The number of FIT positive participants was 9,370 for the first time group and 25,595 for the repeated group. With regard to CRCs and benign polyps, 457 CRCs and 3,010 benign polyps were detected for the first time group, and 500 CRCs and 9,280 benign polyps were detected for the repeated screening group. The positive predictive value (PPV) for CRCs was higher by the first time group than by the repeated group. The PPV for benign polyps was higher by the repeated group than by the first time group. 393 cases in the first time group and 438 cases in the repeated group were reported for sizes. In the first time group, 231 cases were 1-24 mm, 110cases were $25-49 \mathrm{~mm}$, and 52 cases were over $50 \mathrm{~mm}$. In the repeated group, 288 cases were $1-24 \mathrm{~mm}, 117$ were 25 $49 \mathrm{~mm}$, and 33 cases were over $50 \mathrm{~mm}$. The size of detected cancers by the repeated group was significantly smaller than by the first time group. In the first time group, 187 cases were intra-mucosal Dukes A, 109 cases were Dukes A, 65 cases were Dukes B, 61 cases were Dukes C, 21 cases were Dukes D. In the repeated group, 258 cases were intra-mucosal Dukes A, 127 cases were Dukes A, 36 cases were Dukes B, 54 cases were Dukes C, and 7 cases were Dukes D. As a result, Dukes' stage was higher in the first group, and was lower in the repeated group.

Table 1: Dukes

\begin{tabular}{rllllll}
\hline & Intra-mucosal A & $\mathrm{A}$ & $\mathrm{B}$ & $\mathrm{C}$ & $\mathrm{D}$ & Unknown \\
\hline $\begin{array}{r}\text { First-time } \\
\text { group }\end{array}$ & 187 & 109 & 65 & 61 & 21 & 14 \\
Repeated & 258 & $(23.9 \%)$ & $(14.2 \%)$ & $(13.3 \%)$ & $(4.6 \%)$ & $(3.1 \%)$ \\
group & $(51.7 \%)$ & 127 & 36 & 54 & 7 & 16 \\
$\begin{array}{r}\text { P value } \\
\text { valu) }\end{array}$ & 0.0008 & $(25.5 \%)$ & $(7.2 \%)$ & $(10.8 \%)$ & $(1.4 \%)$ & $(1.5 \%)$ \\
\hline
\end{tabular}

Conclusion: Repeated FIT screening might be more efficient to detect benign polyps, smaller CRCs, and CRCs of intra-mucosal Dukes A. We'll go on researching CRCs locations about screening history.

Disclosure of Interest: All authors have declared no conflicts of interest.

\section{References}

1. Mandel JS, Church TR, Ederer F, et al: Colorectal Cancer Mortality:Effectiveness of biennial screening for fecal occult blood, Jnatl Cancer Inst 1999;91(5):434-7.

2. Jorgensen OD, Kronborg O, Fenger C: A randomized study of screening for colorectal cancer using faecal occult blood testing:result afret 13 years and seven biennial screening rounds. Gut 2002;50:29-32

\section{P1083 ANALYSIS OF THE CONCENTRATION OF FIT FOR COLORECTAL CANCERS FROM THE SCREENING (2000-2014) IN IBARAKI, JAPAN}

Y. Saitoh, I. Hyodo, H. Suzuki, M. Kaneko

Dept. of Gastroenterology, Ibarakiken Medical Center, Ibaraki, Japan, Mito/ Japan

Contact E-mail Address: saitoitsyo@yahoo.co.jp

Introduction: In Japan, CRC screening has been launched as a national policy for all people aged over 40 years since 1992. 2-day FIT has been widely accepted, and has been recommended performing in every year. It is demanded that CRC screening is performed effectively depending on the capacity of colonoscopies. We will show so much data on fecal hemoglobin concentration (referred as concentration) and progress of colorectal cancer among them.

Aims \& Methods: The aim of this study is to analyze the concentration of FIT for colorectal cancers (CRCs) from the screening. The cut off value is adapted $20 \mu \mathrm{g}$ $\mathrm{Hb} / \mathrm{g}$ stool and the rate of further examination is around $75 \%$ for many years. In Ibaraki prefecture, CRCs were detected 3,421 cases from the screening (20002014) with 2-day FIT. The concentration of FIT was grouped in 20-80, 80-140, $140-200$ and over $200 \mu \mathrm{g} \mathrm{Hb} / \mathrm{g}$ stool. Screening have been performed with the OC-SENSOR DIANA (EIKEN, JAPAN) automated analyzer. CRCs were analyzed with age group $(40-49,50-59,60-69$, over 70 year-old $)$, size(1-24, 25-49, over $50 \mathrm{~mm}$ ), location(proximal, distal), Dukes' classification(Dukes A-m, Ainvasive, $\mathrm{B}, \mathrm{C}, \mathrm{D})$ depending on the concentration. The chi-squared test was used to compare of each group.

Results: There was no difference in gender and age group for concentration. The concentration of CRCs in the distal colon was significantly higher in the proximal colon [distal 39\% $(861 / 2,200)$ and proximal $32 \%(337 / 1,053)$ with over $200 \mu \mathrm{g} \mathrm{Hb} / \mathrm{g}$ stool]. The concentration of CRCs with larger size was significantly higher than smaller size [1-24 mm 27\% (533/1,961), 25-49 mm 54\% (439/818) and over $50 \mathrm{~mm} 64 \%(169 / 263)$ with over $200 \mu \mathrm{g} \mathrm{Hb} / \mathrm{g}$ stool]. The concentration of invasive CRCs was significantly higher than intra-mucosal CRCs [intra-mucosal $23 \%(370 / 1,617)$ and invasive $50 \%(888 / 1,793)$ with over $200 \mu \mathrm{g} \mathrm{Hb} / \mathrm{g}$ stool]. The concentration of Dukes B, C and D were significantly higher than Dukes A except for intra-mucosal. There was no difference between Dukes B and D [Dukes A except for intra-mucosal 36\% (325/910), B 68\% (247/363), C 60\% $(232 / 385)$ and D $69 \%(61 / 89)$ with over $200 \mu \mathrm{g} \mathrm{Hb} / \mathrm{g}$ stool].

Table 1: Fecal $\mathrm{Hb}$ concentration and progress of colorectal cancer

\begin{tabular}{llllllllllllllllll}
\hline \multicolumn{3}{c}{ Age } & \multicolumn{1}{c}{ Size $(\mathrm{mm})$} & \multicolumn{3}{c}{ Location } & \multicolumn{1}{c}{ Dukes } \\
\hline $\begin{array}{l}\text { conc. } \\
(\mu \mathrm{g} / \mathrm{g})\end{array}$ & $40-49$ & $50-59$ & $60-69$ & $70-$ & $1-24$ & $25-49$ & $50-$ & proximal distal A-m & A & B & C & D \\
$20-80$ & 48 & 190 & 588 & 605 & 993 & 224 & 53 & 495 & 871 & 885 & 375 & 61 & 82 & 15 \\
$80-140$ & 14 & 51 & 185 & 199 & 268 & 107 & 16 & 146 & 286 & 230 & 140 & 32 & 38 & 6 \\
$140-200$ & 17 & 33 & 117 & 100 & 167 & 48 & 25 & 75 & 182 & 132 & 70 & 23 & 33 & 7 \\
$200-$ & 55 & 177 & 529 & 497 & 533 & 439 & 169 & 337 & 861 & 370 & 325 & 247 & 232 & 61 \\
total & 134 & 451 & 1,419 & 1,401 & 1,961 & 818 & 264 & 1,053 & 2,200 & 1,617 & 910 & 363 & 385 & 89 \\
\hline
\end{tabular}

Conclusion: In 20-80 $\mathrm{g} \mathrm{Hb} / \mathrm{g}$ stool, there were CRCs with smaller size, no invasion, in the proximal colon, Dukes A except for intra mucosal CRCs and so on. When the cut off value is raised over $80 \mu \mathrm{g} \mathrm{Hb} / \mathrm{g}$ stool, the detection of early stage CRCs and proximal CRCs may be lost. There were many advanced CRCs with concentration over $200 \mu \mathrm{g} \mathrm{Hb} / \mathrm{g}$ stool. Therefore, when the participants, who are positive with high concentration of FIT, need to take a further examination as soon as possible. Why concentration of CRCs in the distal colon were higher than in the proximal colon? It may be related to the fact that the number of detectable CRCs in the distal colon are more than in the distal colon. We will go on researching mechanism about this.

Disclosure of Interest: All authors have declared no conflicts of interest.

\section{References}

1. Mandel JS, Church TR, Ederer F, et al: Colorectal Cancer Mortality:Effectiveness of biennial screening for fecal occult blood, Jnatl Cancer Inst 1999;91(5):434-7.

2. Jorgensen OD, Kronborg O, Fenger C: A randomized study of screening for colorectal cancer using faecal occult blood testing:result afret 13 years and seven biennial screening rounds. Gut 2002;50:29-32. 
P1084 COLONOSCOPY SURVEILLANCE DETECTS A HIGH PREVALENCE OF ADVANCED COLORECTAL NEOPLASIA AND SERRATED POLYPOSIS SYNDROME IN HODGKIN LYMPHOMA SURVIVORS

L. S. Rigter ${ }^{1}$, M.C.w. Spaander ${ }^{2}$, B. M. Aleman ${ }^{3}$, T.M. Bisseling ${ }^{4}$, L.M.g. Moons ${ }^{5}$, A. Cats ${ }^{1}$, P. J. Lugtenburg ${ }^{6}$, C. P. Janus ${ }^{7}$, E. J. Petersen ${ }^{8}$, J. M. Roesink ${ }^{9}$, R. W. Van Der Maazen ${ }^{10}$, P. Snaebjornsson ${ }^{11}$, E.J. Kuipers ${ }^{12}$ M.J. Bruno ${ }^{13}$, E. Dekker ${ }^{14}$, G. A. Meijer ${ }^{11}$, J. P. De Boer ${ }^{15}$, F. E. Van Leeuwen ${ }^{16}$ M. E. Van Leerdam ${ }^{1}$

${ }^{1}$ Gastroenterology, Netherlands Cancer Institute, Amsterdam/Netherlands

${ }^{2}$ Gastroenterology \& Hepatology, Erasmus Medical Center Rotterdam, Rotterdam Netherlands

${ }^{3}$ Radiation Oncology, Netherlands Cancer Institute, Amsterdam/Netherlands

${ }^{4}$ Dept. Of Gastrotenterology \& Hepatology, Radboud University Medical Centre,

Nijmegen/Netherlands

${ }^{5}$ Gastroenterology And Hepatology, University Medical Center Utrecht, Utrecht Netherlands

${ }^{6}$ Hematology, Erasmus MC Cancer Institute, Rotterdam/Netherlands

${ }^{7}$ Radiation Oncology, Erasmus MC Cancer Institute, Rotterdam/Netherlands ${ }^{8}$ Hematology, University Medical Center Utrecht, Utrecht/Netherlands

${ }^{9}$ Radiation Oncology, University Medical Center Utrecht, Utrecht/Netherlands

${ }^{10}$ Radiation Oncology, Radboud University Medical Center, Nijmegen/Netherlands

${ }^{11}$ Pathology, Netherlands Cancer Institute, Amsterdam/Netherlands

${ }^{12}$ Department Of Gastroenterology And Hepatology, Erasmus MC University

Medical Center, Rotterdam/Netherlands

${ }^{13}$ Department Of Gastroenterology \& Hepatology, University Medical Center

Rotterdam - Department of Gastroenterology \& Hepatology, University Medica,

Rotterdam/Netherlands

${ }^{14}$ Gastroenterology \& Hepatology, AMC - Gastroenterology \& Hepatology, AMC, Amsterdam/NL, Amsterdam/Netherlands

${ }^{15}$ Medical Oncology, Netherlands Cancer Institute, Amsterdam/Netherlands

${ }^{16}$ Epidemiology, Netherlands Cancer Institute, Amsterdam/Netherlands

Contact E-mail Address: 1.rigter@nki.nl

Introduction: Hodgkin lymphoma (HL) survivors treated with abdominal radiotherapy and/or alkylating chemotherapy have an increased risk of colorectal cancer. This study evaluated the prevalence of colorectal neoplasia in HL survivors.

Aims \& Methods: The primary aim of this multicenter cohort study was to assess the diagnostic yield of advanced colorectal neoplasia detected by a first surveillance colonoscopy among HL survivors treated with abdominal radiotherapy and/or procarbazine. Advanced colorectal neoplasia was defined as an advanced adenoma (high-grade dysplasia, $\geq 25 \%$ villous component, or $\geq 10 \mathrm{~mm}$ diameter), an advanced serrated lesion (dysplasia or $\geq 10 \mathrm{~mm}$ diameter), or CRC. Results were compared with a Dutch general population cohort that underwent a primary screening colonoscopy $(n=1276$ asymptomatic individuals between $50-75$ years of age). This study demonstrates the results of a predefined interim analysis.

Results: A colonoscopy was performed in $101 \mathrm{HL}$ survivors, who were significantly younger than general population controls (median 51 years (interquartile range $45-57$ ) vs. 60 years (interquartile range 55-65), $\mathrm{p}<0.001$ ). A mean of 3.5 neoplastic lesions was detected per HL survivor (standard deviation 4.9) vs. 1.1 per control (standard deviation 1.8, p < 0.001). Despite their young age, the prevalence of advanced neoplasia was higher in HL survivors than in controls $(25 \% \quad(95 \%$ confidence interval $16-33 \%)$ vs. $12 \%(10-14 \%), p<0.001)$ Advanced adenomas were detected in $14 \%(8-21 \%)$ of HL survivors and $9 \%$ of controls $(7-10 \%, \mathrm{p}=0.08)$. The prevalence of advanced serrated lesions was higher in HL survivors than in controls $(12 \% \quad(6-18 \%)$ vs. $4 \% \quad(3-5 \%$, $\mathrm{p}<0.001)$. Serrated polyposis syndrome was present in $6 \%(2-11 \%)$ of HL survivors and absent in controls $(\mathrm{p}<0.001)$

Conclusion: HL survivors treated with abdominal radiotherapy and/or procarbazine have a high prevalence of advanced colorectal neoplasia. Colonoscopy surveillance should therefore be implemented as standard of care.

Disclosure of Interest: M.E. van Leerdam: M.E. van Leerdam obtained funding from the Dutch Society of Gastroenterology and Hepatology (Maag Lever Darm Stichting (MLDS) funding project FP14-04).

All other authors have declared no conflicts of interest.

\section{P1085 INCIDNCE OF PROSTATE CANCER IN COLORECTAL CANCER PATIENTS: NATIONWIDE RETROSPECTIVE COHORT STUDY}

H.S. Kim ${ }^{1}$, D.H. Lee ${ }^{1}$, K.D. Han ${ }^{2}$, D.W. Shin ${ }^{1}$, Y.J. Choi ${ }^{1}$, H. Yoon ${ }^{1}$, N. Kim ${ }^{1}$ Department Of Internal Medicine, Seoul National University Bundang Hospital, Seongnam/Korea, Republic of

${ }^{2}$ Department Of Biostatistics, The Catholic University of Korea, Seoul/Korea, Republic of

Contact E-mail Address: sincere24@naver.com

Introduction: Colorectal cancer is one of the most common cancers diagnosed worldwide, and prostate cancer is also the most common malignancy in men and a major cause of cancer deaths. Both colorectal cancer and prostate cancer also tend to occur more frequently in developed countries. There have been severa reports on the association between colorectal cancer and prostate cancer, but the conclusions are inconsistent

Aims \& Methods: We aim to investigate the incidence of prostate cancer as a second primary malignancy among patients with prior primary colorectal cancer (CRC) using a nationwide population-based dataset. This study is a nationwide population-based retrospective cohort study. We followed up with patients registered in the Republic of Korea National Health Insurance Corporation who were diagnosed with colon cancer between 2007 and 2014 and investigated the incidence of prostate cancer (one year lag period). The incidence of prostate cancer was also evaluated in age and gender-matched controls using a cohort of patients diagnosed with colorectal cancer during the same period. The incidence rate (IR) was defined as the number of newly diagnosed prostate cancer patients per 1000 person-years. To assess the role of detection bias-related to the followup of CRC, follow-up started at the date of CRC diagnosis and continued until the earliest date of prostate cancer diagnosis, death, loss to follow-up, or the 2015 year-end. We used Cox proportional hazards models to identify prostate cancer occurrences among CRC patients. We also performed the multivariable analysis. Multivariable models included the variables of age, sex, body mass index, hypertension, diabetes mellitus, dyslipidemia, and income.

Results: We analyzed a total of 85,462 first primary CRC survivors. During the follow-up period of 494,222 person-years, $2005(2.3 \%)$ developed prostate cancer (incidence rate 4.06/1,000 person-years). The median duration of follow-up was 5.78 years. Compared with the general population, CRC patients had a significantly increased risk of secondary prostate cancer $(\mathrm{HR}=2.30,95 \%$ $\mathrm{CI}=2.182-2.426 ; \mathrm{P}<0.001$ ). Multivariate analysis (including age, sex, body mass index, hypertension, diabetes mellitus, dyslipidemia, and income) showed that age $<55$ years $(\mathrm{HR}=20.85,95 \% \mathrm{CI}=11.88-36.59 ; \mathrm{P}<0.001)$ is a significant independent predictor of prostate cancer development.

Conclusion: Men who develop colorectal cancer are at an increased risk of prostate cancer, with the greatest risk in men under the age of 55. This data suggests that CRC patients under 55 years old require regular screening for prostate cancer.

Disclosure of Interest: All authors have declared no conflicts of interest.

\section{P1086 SITE AND STAGE DISTRIBUTION OF SCREEN DETECTED AND CLINICALLY DETECTED COLORECTAL CANCERS IN THE NETHERLANDS}

E. Toes-Zoutendijk ${ }^{1}$, A. Kooyker ${ }^{1}$, M. A. Elferink ${ }^{2}$, V. Lemmens ${ }^{2}$,

M. E. Van Leerdam ${ }^{3}$, I. Lansdorp-Vogelaar ${ }^{4}$

${ }^{1}$ University Medical Center Rotterdam, Rotterdam/Netherlands

${ }^{2}$ Netherlands Comprehensive Cancer Organization, Utrecht/Netherlands

${ }^{3}$ Dept. Of Gastroenterology, Netherlands Cancer Institute, Amstelveen

Netherlands

${ }^{4}$ Department Of Public Health, Erasmus University Medical Center, Rotterdam/ Netherlands

Contact E-mail Address: e.toes-zoutendijk@erasmusmc.nl

Introduction: To reduce the burden of colorectal cancer (CRC) mortality, screening has been introduced. Screening can be beneficial if cancers are detected in an earlier stage or in a pre-malignant stage, as survival rates of these patients will improve.

Aims \& Methods: In this study stage distribution of screen detected CRCs were compared with clinically detected CRCs in the Netherlands. All CRCs detected in men and women aged 55 to 75 years in the Netherlands in 2015 were included in the analysis. Data were gathered from the Dutch Cancer Registry. The current analysis is based on $70 \%$ of these cancers that had staging information available at initial data retrieval. Data will be updated in May 2017. Proportions of site and stage distribution of screen detected and clinically detected CRCs were compared.

Results: A total of $6,517 \mathrm{CRCs}$ in 2015 with staging information were available for the preliminary analysis. Of those, $2.591(39.8 \%)$ were diagnosed as a result of CRC screening (screen detected), 3,463 (53.1\%) presented with symptoms (clinically detected), $118(1.8 \%)$ were detected during surveillance colonoscopy, $202(3.1 \%)$ as coincidental finding and of $143(2.2 \%)$ the method of detection was unknown. Screen detected cancers were more often diagnosed in an earlier stage (stage I and II) compared with clinically detected cancers, 1,687 $(66.5 \%)$ and $1,355(39.6 \%)$ respectively $(p<0.001)$. Screen detected cancers were more often diagnosed in the left side of the colon compared with clinically detected cancers, $46.2 \%$ vs $31.5 \% \quad(p<0.001)$. Comparison of stage distribution by location showed that left sided cancers were most often diagnosed in an early disease stage with $59.0 \%$ of the CRCs in stage I or II, followed by the right sided cancers with $52.8 \%$ of the CRCs in stage I or II. The CRCs of the rectum were most often diagnosed in a late disease stage, only $39.4 \%$ in stage I or II. Table 1 shows the comparison of stage distribution by location and method of detection. With screening, $68.8 \%$ of the right sided cancers, $71.0 \%$ of the left sided cancers, and $56.5 \%$ of the rectum CRCs were diagnosed in an early disease stage (stage I and II) which was all higher than clinically detected cancers $(p<0.001)$.

Table 1: Stage distribution of screen detected and clinically detected colorectal cancers by location and method of detection

\begin{tabular}{llllllll}
\hline & \multicolumn{2}{l}{ Screen detected } & & & \multicolumn{2}{l}{ Clinically detected } \\
\cline { 2 - 3 } \cline { 7 - 8 } & Right sided & Left sided & Rectum & & Right sided & Left sided & \multicolumn{2}{l}{ Rectum } \\
\hline Stage & $\mathrm{n}(\%)$ & $\mathrm{n}(\%)$ & $\mathrm{n}(\%)$ & $\mathrm{n}(\%)$ & $\mathrm{n}(\%)$ & $\mathrm{n}(\%)$ \\
I & $254(38.3 \%)$ & $637(54.5 \%)$ & $316(45.7 \%)$ & & $147(12.2 \%)$ & $217(20.2 \%)$ & $188(17.0 \%)$ \\
II & $203(30.6 \%)$ & $193(16.5 \%)$ & $75(10.8 \%)$ & $381(31.7 \%)$ & $277(25.7 \%)$ & $130(11.8 \%)$ \\
III & $166(25.0 \%)$ & $262(22.4 \%)$ & $257(37.1 \%)$ & & $344(28.6 \%)$ & $310(28.8 \%)$ & $543(49.1 \%)$ \\
IV & $41(6.2 \%)$ & $77(6.6 \%)$ & $44(6.4 \%)$ & $329(27.4 \%)$ & $272(25.3 \%)$ & $245(22.2 \%)$ \\
\hline
\end{tabular}

Conclusion: Screen detected CRCs show a more favourable stage distribution compared with clinically detected cancers, with two third of the cancers 
diagnosed in stage I or II. As those patients will have better survival rates, it is expected that screening will decrease CRC mortality rates.

Disclosure of Interest: All authors have declared no conflicts of interest.

\section{P1087 LOCATION AND SEX PREDOMINANCE OF MISMATCH REPAIR DEFICIENT COLORECTAL CANCER IN IVORY COAST DIFFER FROM ITS EUROPEAN COUNTERPART}

L. Bienfait ${ }^{1}$, B. Doukoure ${ }^{2}$, N. D’Haene ${ }^{1}$, I. Salmon ${ }^{1}$, P. Demetter ${ }^{1}$, L. Verset ${ }^{1}$ ${ }^{1}$ Department Of Pathology, Erasme Hospital, Université Libre de Bruxelles, ULB, Brussels/Belgium

${ }^{2}$ Pathology, Université Félix Houphouet-Boigny, Abidjan/Côte D'ivoire

Contact E-mail Address: lucie.bienfait@erasme.ulb.ac.be

Introduction: According to European and American series, ${ }^{1,2}$ up to $20 \%$ of colorectal cancers are characterised by instability at microsatellite sites and have deleterious mutations in mismatch repair (MMR) genes (MLH1, MSH2, MSH6 and PMS2) or hypermethylation of the MLH1 promotor gene. MMR deficient colorectal cancers are predominantly found in the right colon. Although an increasing rate of colorectal cancer has been observed in many low- and middle-income countries including in West-Africa, ${ }^{3}$ data on epidemiology and biology of colorectal cancer in native Africans from this region are scarce.

Aims \& Methods: We aimed to study the incidence of MMR deficiency in Ivory Coast and to compare the data with those from a tertiary center in Belgium. Immunohistochemistry for MLH-1, MSH-2, MSH-6 and PMS-2 was performed on paraffin-embedded tissue samples from 83 colorectal cancers (54\% males) operated in Abidjan and from 343 colorectal cancers (48\% males) from the Erasme University Hospital in Brussels. Immunohistochemical staining was interpreted as normal or loss of expression.

Results: Colorectal cancer is occurring at a younger age in Ivory Coast compared to Belgium (median age: 53 vs. 66). In both populations, MMR deficiency was detected in $13 \%$ of cases (11 and 43 cases, respectively). Whereas MMR deficient cancers in Brussels were mainly found in women (26/43 i.e. $61 \%$ ), only $3 / 11$ $(27 \%)$ of the MMR deficient cancers from the Abidjan series occurred in female patients. Moreover, the predominant location of MMR deficient tumours was different between both series: in the Brussels patients group, MMR deficient tumours were mainly located in the right colon $(33 / 43$ i.e. $77 \%$ ) whereas in the Abidjan group they were predominant (10/11 i.e. 91\%) in the left colon. With regard to the involved proteins, $6 / 11(55 \%)$ of the MMR deficient cases from Ivory Coast were characterised by loss of expression of MSH2 and MSH6 whereas this immunohistochemical staining pattern was observed in only $9 / 43$ $(20 \%)$ cases from Belgium.

Conclusion: Our pilot study reveals marked differences in presentation of MMR deficient colorectal cancer between the two geographic regions. In contrast to Europe, MMR deficient colorectal cancer in Ivory Coast is mainly found in male patients and in the left colon. Moreover, there are differences with regard to the involved mismatch repair proteins. Together with the younger age at presentation, these data suggest differences in epidemiology and biology of colorectal cancer in native Africans from West Africa compared to the European population.

Disclosure of Interest: All authors have declared no conflicts of interest

\section{References}

1. Mohan HM, Ryan E, Balasubramanian I et al. Microsatellite instability is associated with reduced disease specific survival in stage III colon cancer. Eur J Surg Oncol 2016; 42: 1680-1686.

2. Poulogiannis G, Frayling IM, Arends MJ. DNA mismatch repair deficiency in sporadic colorectal cancer and Lynch syndrome. Histopathology 2010; 56: $167-179$.

3. Dakubo JC, Naaeder SB, Tettey Y, Gyasi RK. Colorectal carcinoma: an update of current trends in Accra. West Afr J Med 2010; 29: 178-183.

\section{P1088 ROUTINE MOLECULAR ANALYSIS FOR LYNCH SYNDROME IN PATIENTS WITH ADVANCED ADENOMA OR COLORECTAL CANCER WITHIN A NATIONAL SCREENING PROGRAM FOR COLORECTAL CANCER}

A. Goverde ${ }^{1}$, W. Nm Dinjens ${ }^{2}$, M.J. Bruno ${ }^{3}$, R.M.W. Hofstra ${ }^{1}$, M. Doukas ${ }^{2}$, M. M. Van Der Weiden ${ }^{2}$, H.j. Dubbink ${ }^{2}$, A. Wagner ${ }^{1}$, M.C.w. Spaander ${ }^{3}$ ${ }^{1}$ Clinical Genetics, Erasmus MC, University Medical Center Rotterdam, Rotterdam/Netherlands

${ }^{2}$ Pathology, Erasmus MC, University Medical Center, Rotterdam, Netherlands, Rotterdam/Netherlands

${ }^{3}$ Gastroenterology \& Hepatology, Erasmus MC, University Medical Center Rotterdam, Rotterdam/Netherlands

\section{Contact E-mail Address: a.goverde.1@erasmusmc.nl}

Introduction: Lynch syndrome (LS) is the most common hereditary cause of colorectal cancer (CRC). Identifying LS carriers and their affected family members is of great importance for prevention of CRC. Routine screening for LS by immunohistochemical staining (IHC) in CRC patients $\leq 70$ years of age is recommended. LS screening in adenoma patients could yield more benefit, since CRC can still be prevented in these patients. A small number of participants of the national CRC screening program is expected to have LS. We aimed to assess the diagnostic yield of IHC for LS in patients with advanced and multiple adenomas or CRC within the Dutch national fecal immunochemical test (FIT)-based CRC screening program.

Aims \& Methods: We included participants of the national CRC screening program, referred to our center after a positive FIT from December 2013 to December 2016. IHC for MLH1, MSH2, MSH6 and PMS2 protein was performed on advanced adenomas and CRCs found at colonoscopy. Adenomas were considered advanced if they had a villous component, high-grade dysplasia or were $\geq 10 \mathrm{~mm}$ in size. Also, in cases with $\geq 4$ non-advanced adenomas, IHC was performed on the largest adenoma. $M L H 1$ hypermethylation analysis was used to distinguish sporadic $M L H 1$ deficiency from $M L H 1$ deficiency suspect for LS. Patients with IHC suspect for LS were offered germline mutation analysis. If no pathogenic mutation was found, we performed somatic mutation analysis. Results: A total of 1006 patients (54\% male; mean age of 67 years $( \pm 6$ years)) with positive FIT were included in the study. At colonoscopy, $352(35 \%)$ patients (63\% male; mean age of 67 years ( \pm 6 years) had a CRC and/or adenoma eligible for IHC. A total of 322 adenoma patients were analyzed. None had aberrant IHC. Of the examined adenomas, $151(47 \%)$ had a villous component and/or high grade dysplasia $(132(41 \%)$ with villous component and $37(11 \%)$ with high grade dysplasia). Out of 48 CRC patients, 7 (15\%) showed loss of protein expression. All seven cases had loss of MLH1 and PMS2 protein. Five cases had $M L H 1$ promoter hypermethylation. The two patients without $M L H 1$ promoter hypermethylation were referred for genetic counselling. Both patients had no family history suspect for LS. In both cases no germline $M L H 1$ mutation was found and somatic mutation analysis showed that both had a likely sporadic tumor.

Conclusion: Our results indicate that routine LS screening by IHC and $M L H 1$ hypermethylation in patients with advanced and multiple adenoma within a national FIT-based screening CRC program is not an effective strategy. The diagnostic yield of LS screening in younger adenoma patients should be assessed. Also, our results imply that $M L H 1$ promoter hypermethylation may be a late event in oncogenesis, since none of the adenomas had aberrant IHC.

Disclosure of Interest: All authors have declared no conflicts of interest.

P1089 COLORECTAL CANCER SCREENING PROGRAMS AND THE RATE OF SURGICAL ONCOLOGY PROCEDURES IN THE VENETO REGION (ITALY): ARE FOLLOW-UP COLONOSCOPIES REALLY NEEDED?

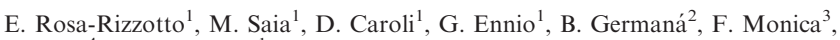
P. Pilati ${ }^{4}$, F. De Lazzari ${ }^{1}$

${ }^{1}$ Dipto. Di Gastroenterologia, St. Anthony Hospital Gastroenterology Unit Dept. of Medicine, Padova/Italy

${ }^{2}$ San Martino Hospital - Ulss1, Gastroenterologic Unit, Belluno/Italy

${ }^{3}$ Department Of Gastroenterolgy, Cattinara Hospital, Trieste/Italy

${ }^{4}$ Surgery Unit, Padova/Italy

Contact E-mail Address: d.caroli@libero.it

Introduction: Colorectal cancer (CRC) is a leading cause of cancer mortality in the Veneto Region (North-eastern part of Italy). Population screening of adults between 50 and 75 for CRC was begun in 2002, and it became standard practice in all 21 local health units (LHU) of the region in 2008, 14 LHU provided also follow-up colonosopies and 7 LHU no. The current retrospective cohort study was carried out to evaluate the impact of CRC screening on the rate of surgical oncology procedures to treat colon and rectal cancer.

Aims \& Methods: Data from hospital discharge records (HDR) regarding CRC patients hospitalized between 2000 and 2015 were collected. All CRC patients whose principal diagnosis was colon and/or rectal cancer were included in the study. The number of patients studied rose approximately $18 \%$ reaching 1,547,097 for the last year (2015). The Standardized Hospitalization Ratio (SHR) using five-year age groupings was calculated and expressed per 10,000 population.

Results: During the study period, 30,399 surgical procedures for colorectal cancer were performed (colon $63 \%$, rectum $36 \%$, secondary malignant neoplasm $1 \%$ ) with a SHR of 139.1 ; the number was higher in males (1.69 vs. 1.02; OR: 1.66; CI $95 \%$ : $1.62-1.7 ; \mathrm{p}<0.05)$. An analysis of the annual SHR distribution uncovered two distinct phases: during the first phase there was a rising tendency that reached a maximum value in 2007 (166.9; X2 trend: 46.731; $p<0.05)$ and during the second there was a falling tendency that reached its minimum value in $2015(102.3$; X2 trend: $429.791 ; \mathrm{p}<0.05)$. When the cancer sites were analyzed, it was seen that despite the peak in 2007, the rate of surgical procedures of the proximal colon during the last year was the same as the 2000 value (41.5); there was, instead, a relevant decrease in the rate of procedures on the distal colon and rectum which fell from 94.4 to $59.2(-37.3 \%)$. The study also shows that there was no significant difference in the reduction in surgical procedures for CCR in LHU in which the screening program included a follow-up colonoscopy (SHR 2015: 139.8; -29\%) with respect to those centers wehere it was not forseen (SHR 2015: 138.5; -28\%) 
Conclusion: Study findings confirmed that CRC screening was effective in reducing the number of oncological surgical oncology procedures particularly with regard to the distal colon and rectum. Data analysis showed that the screening seemed to accelerate reaching the peak rate in surgical procedures that took place in 2007. After that time point the number of operations began to fall as far as the distal colon was concerned (it fell by $37.3 \%$ ). Finally our data suggest that the real benefit in reduction of oncological surgery procedures is due to the first screening colonoscopy.

Disclosure of Interest: All authors have declared no conflicts of interest.

\section{P1090 ETHNIC VARIATION IN ADENOMA DETECTION IN THE UK FLEXIBLE SIGMOIDOSCOPY BOWEL CANCER SCREENING PROGRAMME}

D. N.F. Lim, S. Mellor, S. Szarvas-Jones, D. Bools, H. Whitsey, B. Miri, A. Mapiye, P. Wurm, R. J. Robinson

Digestive Diseases Centre, University Hospital of Leicester NHS Trust, Leicester/ United Kingdom

Contact E-mail Address: dennis.lim@uhl-tr.nhs.uk

Introduction: The NHS bowel scope screening programme was introduced in 2013 with all adults aged 55 invited for a 'one-off' flexible sigmoidoscopy followed by a colonoscopy if significant adenomas are detected. University Hospitals of Leicester Bowel Cancer Screening Centre serves an ethnically diverse community with approximately $25 \%$ of the population eligible for sigmoidoscopy screening being British Asian Indians and $45 \%$ being British Whites ${ }^{1}$. Within the faecal occult blood based bowel cancer screening programme we have previously reported a lower polyp detection rate (PDR) and adenoma detection rate (ADR) in Asians undergoing colonoscopy compared to White British $^{2}$. This study aims to evaluate PDR, ADR and cancer detection (CDR) in British Asian Indians taking part in the bowel scope screening programme. Aims \& Methods: Patients who underwent screening sigmoidoscopy between February $1^{\text {st, }} 2015$ to $28^{\text {th }}$ Feb 2017 were included. All individuals participating in screening sigmoidoscopy routinely report their self-selected ethnic origin. This database was cross referenced with the endoscopic and histology findings from the 'Exeter' online database. The findings in British Asian Indians were compared with British Whites.

Results: A total of 4287 patients underwent screening sigmoidoscopy over the 2-year period. 1169 individuals had polyps (500 adenomas). Overall polyp detection rate (PDR) was $1169 / 4287(27.3 \%)$, adenoma detection rate (ADR) was $500 / 4287(11.7 \%)$, hyperplastic polyps were 642/4287 (15\%), sessile serrated lesions were $13 / 4287(0.3 \%)$, and other polyps were $14 / 4287(0.3 \%)$ (Inflammatory polyps 13 , Juvenile polyp 1). Cancer detection rate was $6 / 4287$ $(0.14 \%)$. During the period studied, 3509 British white individuals $(82 \%)$ and 778 British Asian Indians screened. Six cancers were detected in British white $(\mathrm{CDR}=0.17 \%)$ but none in British Asian Indians.

\begin{tabular}{llll}
\hline & British White & British Asian Indians & P value \\
\hline PDR & $30.8 \%$ & $11.4 \%$ & $<0.001$ \\
& $(95 \%$ CI $26.7-34.9 \%)$ & $(95 \%$ CI $10.1-12.4 \%)$ & \\
ADR & $12.0 \%$ & $9.0 \%$ & $<0.01$ \\
& $(95 \%$ CI $8.0-16.0 \%)$ & $(95 \%$ CI $6.7-11.2 \%)$ & \\
Conversion & $4.0 \%$ & $1.8 \%$ & $<0.001$ \\
$\quad$ to colonoscopy & $(95 \%$ CI $2.8-5.2 \%)$ & $(95 \%$ CI $0.9-3.2 \%)$ & \\
\hline
\end{tabular}

Conclusion: This study found no cancers and significantly lower PDR and ADR in British Asian Indians compared to British White participants in the bowel scope screening programme. Further long term evaluation of these differences is needed and may shed light on factors contributing to the development of bowel cancer.

Disclosure of Interest: All authors have declared no conflicts of interest.

\section{References}

1. 2011 Census programme. Key statistics for Local Authorities in England and Wales.

2. Verma A.M, Lewin V, Chilton A.P, de Caestecker J, Dixon A, Jameson J, Wurm P, Robinson R.J. Variations In Adenoma Detection Rate And Cancer Detection Rate In Individuals From Different Ethnic Groups Undergoing Bowel Cancer Screening Colonoscopy. Gut 2014;63:A122

\section{P1091 DEAD BOX POLYPEPTIDE 27 PROMOTES TUMORIGENICITY IN COLORECTAL CANCER THROUGH ACTIVATING NUCLEAR FACTOR KAPPA B PATHWAY AND ITS EXPRESSION IS ASSOCIATED WITH POOR SURVIVAL IN PATIENTS}

H. Chen ${ }^{1}$, J. Tang ${ }^{2}$, C.C. Wong ${ }^{1}$, J. Fang ${ }^{2}$, J.J.Y. Sung ${ }^{1}$, J. Yu

${ }^{1}$ Institute Of Digestive Disease And Department Of Medicine And Therapeutics, State Key Laboratory Of Digestive Disease, The Chinese University of Hong Kong, Hong Kong/Hong Kong Prc

${ }^{2}$ Division Of Gastroenterology And Hepatology, Shanghai Institute Of Digestive

Disease, Ren Ji Hospital, Shanghai Jiao Tong University, Shanghai/China
${ }^{3}$ Institute Of Digestive Disease, Partner State Key Laboratory Of Digestive

Disease, Chinese University of Hong Kong, Hong Kong/Hong Kong Prc

Contact E-mail Address: inflyings@gmail.com

Introduction: We identified for the first time that DDX27 (DEAD box polypeptide 27) gene was amplified in colorectal cancer (CRC) by whole genome sequencing. Amplification of DDX27 was detected in $47 \%(47 / 100)$ of primary CRC tumors and positively correlated with its mRNA overexpression. DDX27 plays a pivotal oncogenic role in colorectal carcinogenesis by promoting cell proliferation and inhibiting apoptosis. In this study, we investigate its function, mechanism of action and clinical implication in CRC.

Aims \& Methods: Downstream effectors and pathways of DDX27 were identified by promoter luciferase reporter assay, $\mathrm{RT}^{2}$ Profiler PCR array and western blot. The interacting partners of DDX27 were screened by BioID method and further validated using immunoprecipitation assay and immunofluorescence staining method. Clinical implication of DDX27 was assessed in two human CRC cohorts by quantitative PCR method and immunohistochemical staining of tissue microarrays.

Results: Promoter luciferase reporter assays revealed that DDX27 mainly activated nuclear factor kappa B (NF- $\kappa$ B) pathway in CRC cell lines (HCT116 and $\mathrm{SW} 480)$. Ectopic expression of DDX27 promoted transcription of NF- $\kappa \mathrm{B}$ signaling targets including BCL2A1, BIRC3, CCL20, CXCL3, NFKBIA, TNF and TNFAIP3. Conversely, silencing of DDX27 showed an opposite effect on NF- $\kappa$ B signaling. Treatment of NF- $\kappa$ B inhibitors CAPE and JSH-23 abrogated the promoting effect of DDX27 on CRC cells growth. We reveled that DDX27 enhanced and prolonged $\mathrm{NF}-\kappa \mathrm{B}$ signaling via reducing the accumulation of nuclear $\mathrm{I} \kappa \mathrm{B} \alpha$, which negatively regulates transcriptional activities of $\mathrm{NF}-\kappa \mathrm{B}$ and transport NF- $\kappa$ B proteins back to the cytoplasm. DDX27 overexpression markedly increased the recruitment of NF- $\kappa \mathrm{B}$ p65 inside nuclear and promoted $\mathrm{NF}-\kappa \mathrm{B}$ activity in $\mathrm{CRC}$ cells under TNF- $\alpha$ stimulation. NPM1 was identified as a potential binding partner of DDX27 by BioID method to screen for proteinprotein interactions. The interaction of NPM1 and DDX27 inside nucleus was further validated by endougenous immunoprecipitation assay and immunofluorescence staining. Knockdown of NPM1 abrogated DDX27-activating NF- $\kappa$ B signaling, as well as its tumor-promoting function. Kaplan-Meier curves showed that higher DDX27 expression was significantly associated with shortened survival in patients with CRC of two independent cohorts $(\mathrm{N}=199$ for Beijing cohort using quantitative PCR method, and $N=275$ for Shanghai cohort using immunohistochemical staining of tissue microarrays; both $\mathrm{P}<0.05$ ).

Conclusion: DDX27 plays an important oncogenic role in promoting CRC tumorigenicity via activation of NF- $\kappa \mathrm{B}$ pathway. Higher expression of DDX27 is correlated with poor prognosis in CRC patients.

Disclosure of Interest: All authors have declared no conflicts of interest.

\section{P1092 CLINICOPATHOLOGICAL STUDY OF SERRATED LESIONS} OF THE COLORECTUM

M. Sugimura, M. Iwabuchi, K. Ukai

Gastroenterology, Sendai Medical Center Dept. of Gastroenterology, Sendai/Japan

Contact E-mail Address: msugimura@snh.go.jp

Introduction: Serrated lesions of the colorectum are the precursors of microsatellite unstable carcinomas. However, their clinical and pathologic features are still unclear and need further exploration.

Aims \& Methods: The aims of this study was to clarify the clinicopathological features of colorectal serrated lesions. We reviewed clinical charts and pathology files of 5352 endoscopically resected specimens performed during January 2007 and December 2016 in our hospital. A total of 463 serrated lesions $(8.7 \%)$ resected were classified into three categories: HP (hyperplastic polyp), SSA/P (sessile serrated adenomas/polyps), and TSA (traditional serrated adenoma), according to the WHO criteria. We examined the features of these cases and evaluate the morphologic characteristics by using immunochemical staining for Ki-67 and the expression of MUCs (MUC2, MUC5AC and MUC6) in differentiating serrated lesions.

Results: Of these 463 lesions, a total of 241 (52.1\%) were HP, $102(22.0 \%)$ SSA $/ \mathrm{P}$, and $120(25.9 \%)$ TSA. Male to female ratio $(\mathrm{M} / \mathrm{F})$ was 2.38 for HP, 0.98 for SSA/P, and 2.45 for TSA. Mean size of SSA/Ps $(13.1 \mathrm{~mm})$ and TSAs $(10.4 \mathrm{~mm})$ were significantly larger than that of $\mathrm{HP}(8.1 \mathrm{~mm})(\mathrm{p}<0.005$, respectively). SSA/Ps were located predominantly in the proximal colon, whereas HP and TSA were mainly located in the sigmoid colon and rectum. $84 \%$ of SSA/Ps were flat in macroscopic appearance. SSA/Ps and HPs were whitish or almost the same as adjacent mucosa in color, whereas TSAs had a tendency to be reddish. Magnified colonoscopy showed Type II open pit pattern as characteristic of SSA/Ps, whereas pinecone-shaped pit pattern as that of TSAs. Incidences of concomitant carcinomas in HP, SSA/P, and TSA were $0 \%$ ( 0 out of 241 ), $2.9 \%$ (3 out of 102 ), and $4.2 \%$ ( 5 out of 120 ), respectively. Ki-67 positive cells in HP showed regular, symmetric distribution, and those in SSA/P did irregular asymmetrical pattern, whereas most of those cells in TSA distributed in the socalled ectopic crypts. Expression levels of MUC2, MUC5AC and MUC6 were 
significantly different between serrated lesions, SSA/Ps and HPs were positive for MUC5AC in comparison with TSAs.

Conclusion: Our studies showed the three types of serrated lesions have their own distinct features and could be helpful to distinguish between them. SSA/P and TSA are premalignant lesions of colorectum and we should detect these lesions and completely remove endoscopically.

Disclosure of Interest: All authors have declared no conflicts of interest

\section{P1093 IN SITU DETECTION OF MIGRATING COLORECTAL CANCER CELL-RELEASED LARGE EXTRACELLULAR EXOSOME CLUSTERS}

G. Valcz ${ }^{1}$, E. I. Buzás ${ }^{2}$, T. Krenács ${ }^{3}$, A.V. Patai ${ }^{1}$, Á. Nagy ${ }^{1}$, L. Homolya ${ }^{4}$, G. Török ${ }^{4}$, Z. Szállási ${ }^{5}$, K. Dede ${ }^{6}$, S. Spisák ${ }^{7}$, Z. Tulassay ${ }^{1}$, P. Igaz $^{1}$, B. Molnar ${ }^{1}$ ${ }^{1}$ 2nd Dept. Of Internal Medicine, Semmelweis University, Budapest/Hungary ${ }^{2}$ Department Of Genetics, Cell- And Immunobiology, Semmelweis University, Budapest/Hungary

${ }^{3} 1$ st Department Of Pathology And Experimental Cancer Research, Semmelweis University, Budapest/Hungary

${ }^{4}$ Institute Of Enzymology, Research Centre For Natural Sciences, Hungarian Academy of Sciences, Budapest/Hungary

${ }^{5}$ Computational Health Informatics Program (chip), Boston Children's Hospital, Boston/United States of America/MA

${ }^{6}$ Department Of General Surgery And Surgical Oncology, Uzsoki Teaching Hospital, Budapest/Hungary

${ }^{7}$ Department Of Medical Oncology, Dana-Farber Cancer Institute, Boston/United States of America/MA

Contact E-mail Address: arpad.patai@gmail.com

Introduction: It is generally accepted that exosomes, small, membrane-bounded vesicles are formed in multivesicular bodies (MVBs) which fuse with plasma membrane resulting in the release of individual exosomes into the extracellular space. Recently, sporadic in vitro observations of a novel, unconventional mechanism have been reported in which the exosome-like vesicles remain in one block during their secretion.

Aims \& Methods: Our aim was to examine this phenomenon in migrating colorectal cancer (CRC) cells in situ. Immunohistochemistry (IHC) examination of migrating, individual cancer cells was performed in surgically removed, metastatic CRC samples $(\mathrm{n}=38)$. We used epithelial specific cytoplasmic (cytokeratin/CK) and cell membrane (E-cadherin) markers for the identification of migrating CRC cells as well as ALIX and CD63 proteins for the detection of exosomal transport. Samples were analyzed with confocal and stimulated emission depletion (STED) microscopy-based 3D reconstructions.

Results: 3D reconstructions showed ALIX-positive and CD63-positive exosome clusters (ECs) with 0.62 to $1.94 \mu \mathrm{m}$ diameter (mean \pm SD: $1.17 \pm 0.34 \mu \mathrm{m}$ ) localized partially inside, and/or outside the cytoplasm in $85.96 \%(n=98 / 114)$ of migrating CRC cells. E-cadherin IHC showed that ECs were not only captured during their exit from the cytoplasm and localized among plasma membrane proteins, but they were also detected extracellularly, in the plasma membrane-stroma interface. STED-microscopic images showed that released ECs were composed of smaller, distinguishable ALIX-positive spheroids of 98 to $150 \mathrm{~nm}$ diameter (mean $\pm \mathrm{SD}: 128.96 \pm 16.73 \mathrm{~nm}$ ), which fall into the size ranges of exosomes.

Conclusion: Our study demonstrates in situ for the first time that besides conventional exosome release, migrating $\mathrm{CRC}$ cells also secrete large, extracellular ECs. These structures might fundamentally contribute to the autocrine/paracrine regulation of cancer development, which effect may differ from that mediated by conventionally secreted exosomes.

Disclosure of Interest: All authors have declared no conflicts of interest.

P1094 ALTERED ARGININE METABOLISM IN HYPERPROLIFERATIVE INTESTINAL EPITHELIAL CELLS: A POTENTIAL ROLE IN TUMORIGENESIS AND WOUND HEALING

J. H.m. Van Der Meer ${ }^{1}$, W. L. Smit ${ }^{1}$, B. J. Meijer ${ }^{1}$, C. N. Spaan ${ }^{1}$, T. B.m. Hakvoort ${ }^{1}$, M.A. Boermeester ${ }^{2}$, G.R. Van Den Brink ${ }^{3}$, V. Muncan ${ }^{1}$ Tytgat Institute For Liver And Intestinal Research, Academic Medical Center, Amsterdam/Netherlands

${ }^{2}$ Dept. Of Surgery, Academic Medical Center Amsterdam, Amsterdam/ Netherlands

${ }^{3}$ Dept. Of Gastroenterology, Academisch Medisch Centrum, Amsterdam/ Netherlands

Contact E-mail Address: j.h.vandermeer@amc.nl

Introduction: The semi-essential amino acid arginine is important for intestinal epithelial cell proliferation and is an essential dietary component delivered by solid food. In homeostatic proliferating adult intestinal enterocytes arginine is catabolized, by the enzyme arginase 2. During embryonic development, the demand for arginine increases, due to rapid growth. Arginine is lacking from milk and arginine therefore has to be synthesized de novo to sustain epithelial expansion in the rapidly growing intestinal tube. Neonatal enterocytes are capable of de novo synthesis of arginine from citrulline. The rate-limiting enzyme in this process is argininosuccinate synthetase 1 (ASS1). Similarly to postnata development, hyperproliferative epithelium is a hallmark of tumorigenesis and wound healing.

Aims \& Methods: The aim of this study is, to investigate, whether de novo arginine synthesis via ASS1 plays a role in intestinal carcinogenesis and repair.
We performed immunohistochemistry on intestines from $\mathrm{APC}^{\mathrm{f} / \mathrm{fl}}$ mice, and $\mathrm{C} 57 \mathrm{BL} / 6$ mice that either underwent colonic biopsies or irradiation of the intestine. Intestinal organoids from APC ${ }^{\mathrm{fl} / \mathrm{fl}}$ mice were transduced with a shRNA for ASS1. RNA and protein levels of ASS1 were measured by qPCR and western blot. Arginine concentrations were determined by HPLC. Protein synthesis was measured by [35S]-methionine labelling.

Results: ASS1 expression is highly increased in adenomas and hyperpoliferative crypts during epithelial repair after mechanical wounding and irradiation wounds, compared to homeostasis. Variable expression of ASS1 is seen in different colorectal cancer cell lines, and expression correlates with resistance against arginine deprivation. In $\mathrm{APC}^{-/-}$organoids generated from the $\mathrm{APC}^{\mathrm{fl} / \mathrm{fl}}$ genotype, ASS1 RNA and protein are highly expressed, with concomitant increase of intracellular arginine. Upon knockdown of ASS1 in $\mathrm{APC}^{\mathrm{fl} / \mathrm{fl}}$ organoids, protein sysnthesis is reduced and organoid growth is compromised. Furthermore, knockdown of ASS1 decreases overall protein synthesis.

Conclusion: In a hyperproliferative state, intestinal epithelial cells synthesize arginine via ASS1. Our data suggests this plays a functional role to support growth and protein synthesis during intestinal carcinogenesis and repair.

Disclosure of Interest: G.R. van den Brink: G.R. van den Brink is an employee of GlaxoSmithKline

All other authors have declared no conflicts of interest.

\section{P1095 ENHANCED COLONIC TUMORIGENESIS IN TLR4-DEFICIENT MICE IS ASSOCIATED TO IMPAIRMENT OF ANTIGEN PRESENTATION BY EPITHELIAL CELLS}

M. Scarpa ${ }^{1}$, I. Castagliuolo ${ }^{2}$, C. Marchiori ${ }^{2}$, S. Morgan ${ }^{3}$, A. Kotsafti ${ }^{1}$, P. Brun ${ }^{2}$, A. Porzionato ${ }^{2}$, I. Angriman ${ }^{4}$, C. Castoro ${ }^{1}$, M. Scarpa ${ }^{1}$

${ }^{1}$ Esophageal And Digestive Tract Surgical Unit, Regional Centre For Esophageal Disease, Veneto Institute of Oncology (IOV-IRCCS), Padova/Italy

${ }^{2}$ Dept. Of Molecular Medicine, University of Padova, Padova/Italy

${ }^{3}$ Barnsley Hospital NHS Foundation Trust, Barnsley/United Kingdom

${ }^{4}$ Dept. Of General Surgery, University of Padova, Padova/Italy

Contact E-mail Address: melania.scarpa@iov.veneto.i

Introduction: Toll-like receptors have a pivotal role in natural immunity but their role is indefinite and, sometimes controversial, in tumor development. TLR4 signalling through MyD88 has been linked to tumour growth and progression in mice models, whereas in humans TLR4/MyD88 signalling seems to correlate with colorectal cancer poor prognosis [1]. On the other hand, TLR4 signalling seem to be correlated to adaptive immunosurveillance in inflammatory related colonic carcinogenesis [2].

Aims \& Methods: The aim of this study was to define the role of TLR4 in the immune surveillance mechanisms in a non-inflammatory model of colonic carcinogenesis. The azoxymethane (AOM) induced colon carcinogenesis mouse model was used. Colonic mucosal samples were collected from wild type (WT) C57bl/6 and TLR 4 knock out (KO) mice before AOM administration (T0), at 4 and at 8 months after the first AOM injection. Colons were removed and examined for occurrence of adenoma and inflammatory infiltrate. Macroscopic tumor load was assessed counting the number of polyps. Flow cytometry on colonic mucosa single cell suspensions for CD8+ lymphocytes expressing CD28, CD38 or CD69, for CD4+ lymphocytes expressing CD25 or CD25 and FoxP3 and for epithelial cells expressing CD80 or MHC-I or MHC-II were performed. Non parametric statistics was used.

Results: In TLR4KO mice sacrificed at 8 months, 1.4 polyps/mouse were observed while none was observed at $\mathrm{T} 0$ or at 4 months $(p=0.006)$. In TLR4KO mice at 8 months, the rates of epithelial cells expressing CD80, CD86, MHC-I and MHC-II 8 months were significantly lower than in those at $\mathrm{T} 0$ or at 4 months $(\mathrm{p}=0.01, \mathrm{p}=0.003, \mathrm{p}=0.003, \mathrm{p}=0.001$, respectively $)$. Moreover, at 8 months, 5/7 TLR4KO mice compared to 0/7 WT ones had at least a colonic adenocarcinoma $(\mathrm{p}=0.02)$. At this time point, CD4+CD25+, $\mathrm{CD} 4+\mathrm{Cd} 25+\mathrm{FoxP} 3+, \mathrm{CD} 8+\mathrm{CD} 28+, \mathrm{CD} 8+\mathrm{CD} 38+$ cells rate was significantly lower in TLR4KO mice than in WT ones $(p<0.001, p<0.001, p=0.01$, $\mathrm{p}=0.002$, respectively). Similarly, at 8 months the rate of epithelial cells expressing MHC-I and MHC-II were significantly lower in TLR4KO than in WT mice $(\mathrm{p}=0.02, \mathrm{p}<0.001$, respectively).

Conclusion: TLR4 deficiency significantly accelerate the progression of colonic carcinogenesis through a progressive decline of antigen presentation and lack of co-stimulation at later stages. These impairments are associated to a decline of $\mathrm{T}$ cell response in all its form (Treg, $\mathrm{T}$ helper and cytotoxic). All these findings are coherent with a pivotal role of TLR4 in the immune surveillance mechanism. Disclosure of Interest: All authors have declared no conflicts of interest.

References

1. Wang EL, Qian ZR, Nakasono M, Tanahashi T, Yoshimoto K, Bando Y, Kudo E, Shimada M, Sano T. High expression of Toll-like receptor 4/myeloid differentiation factor 88 signals correlates with poor prognosis in colorectal cancer Br J Cancer 2010; 102, 908-915

2. Scarpa M, Cardin R, Bortolami M, Kotsafti A, Scarpa MC, Pozza A, Maran G, Picciocchi M, Ruffolo C, D'Incà R, Sturniolo GC, Castagliuolo I, Castoro C, Angriman I. Mucosal immune environment in colonic carcinogenesis: CD80 expression is associated to oxidative DNA damage and TLR4$\mathrm{NF}_{\kappa} \mathrm{B}$ signalling. Eur $J$ Cancer 2013 Jan;49(1):254-63. 
P1096 ENDOSCOPIC FOLLOW UP CAN SELECT PATIENTS FOR

MULTI-GENE TESTING IN ATTENUATED ADENOMATOUS POLYPOSIS WITH NO APC OR MUTYH IDENTIFIED MUTATIONS

R. Cannizzaro ${ }^{1}$, R. Magris ${ }^{1}$, G. Cini $^{2}$, M. Quaia ${ }^{3}$, S. Maiero ${ }^{1}$, E. Canton ${ }^{4}$, A. Viel ${ }^{3}$, M. Fornasarig ${ }^{1}$

${ }^{1}$ Oncological Gastroenterology, Centro di Riferimento Oncologico di Aviano S.O.C. di Gastroenterologia, Aviano/Italy

${ }^{2}$ Experimental Oncology ${ }^{1}$ Centro di Riferimento Oncologico di Aviano, Aviano/ Italy

${ }^{3}$ Funcional Onco-genomics And Genetics, Centro di Riferimento Oncologico di Aviano, Aviano/Italy

${ }^{4}$ Clinical Trials Office, Centro di Riferimento Oncologico di Aviano, Aviano/Italy

Contact E-mail Address: rcannizzaro@cro.it

Introduction: Less than a hundred polyps defines attenuated familial adenomatous polyposis (AFAP). APC or MUTYH involvement has been described in $60 \%$ of the cases. The natural history of AFAP without identified genetic defects is not enough evaluated. In our study we compare clinical and endoscopic features of polyposis in patients carrying APC or MUTYH mutation and wild type patients.

Aims \& Methods: 102 cases (35 F, 67 M; mean age 51; range 28-79) of AFAPs were registered at our Institution between 1996 and 2014. They had no cancer family history and presented more than 10 adenomas at index colonoscopy. Genetic testing for APC and MUTYH genes was performed. Patients were put in a program, after having cleaned the colon, consisting in colonoscopy after one year and then the colonoscopic interval was based on the number of polyps from 1 to 3 years. Odds Ratio test was used to compare APC or MUTYH mutated and wild-type patients.

Results: Out of 102 patients with AFAP we identified a genetic defect in 36 patients $(35.3 \%$; 12 with APC and $24 \mathrm{MUTYH})$ and $66(64.7 \%)$ were wildtype. The mean endoscopic follow up was 10 years $(2-31)$ in the mutated group and 9.7 years (2-23) in the wild-type group. Table 1 describes endoscopic and clinical features between the two groups. We observed some statistically differences between groups: the mutated group was younger than 50 years of age with a higher number of polyps, right colon was mainly involved and endoscopic follow-up was mostly every year. Patients of wild-type group never underwent to colectomy during follow-up and they displayed few adenoma recurrences in $24 \%$ of cases. On the other hand $14 \%$ of mutated patients underwent colectomy for dense polyposis and $28 \%$ had more polyps than at index colonoscopy and $11 \%$ no polyps at all. These $11 \%$ displayed mutation in MUTYH Regarding extra-colonic manifestations we observed duodenal adenomas in 5 $(13.8 \%)$ patients and desmoids tumor in one patient of mutated group. Two gastric cancers and one melanoma were diagnosed in the wild type group.

Table 1: Comparison of clinical and endoscopical feature between APC or MUTYH carriers versus wild type patients.

\begin{tabular}{|c|c|c|c|c|c|}
\hline & \multicolumn{2}{|c|}{$\begin{array}{l}\text { APC or MUTYH } \\
\text { mutation }=36\end{array}$} & \multicolumn{2}{|l|}{$\begin{array}{l}\text { Wild } \\
\text { type }=66\end{array}$} & \multirow{2}{*}{ Statistics } \\
\hline & Number & $\%$ & Number & $\%$ & \\
\hline \multicolumn{6}{|l|}{ Gender } \\
\hline male & 19 & 52,78 & 48 & 72,73 & $\begin{array}{l}\text { OR } 2.3860 \text {, } \\
95 \% \text { CI: } 1.0203 \\
\text { to } 5.5794\end{array}$ \\
\hline female & 17 & 47,22 & 18 & 27,27 & $\mathrm{P}=0.0448$ \\
\hline \multicolumn{6}{|l|}{ Age at onset } \\
\hline$<50$ & 25 & 69,44 & 13 & 19,70 & $\begin{array}{l}\text { OR } 9.265795 \% \\
\text { CI: } 3.6441 \\
\text { to } 23.5597\end{array}$ \\
\hline$\geq 50$ & 11 & 30,56 & 53 & 80,30 & $\mathrm{P}<0.0001$ \\
\hline \multicolumn{6}{|l|}{$\begin{array}{l}\text { Number of polyps } \\
\text { at index colonoscopy }\end{array}$} \\
\hline$<20$ & 17 & 47,22 & 47 & 71,21 & $\begin{array}{l}\text { OR } 0.361795 \% \\
\text { CI: } 0.1555 \\
\text { to } 0.8411\end{array}$ \\
\hline$>20$ & 19 & 52,78 & 19 & 28,79 & $\mathrm{P}=0.0182$ \\
\hline \multicolumn{6}{|l|}{ Polyps site } \\
\hline right & 29 & 80,56 & 18 & 27,27 & $\begin{array}{l}\text { OR } 11.047695 \% \\
\text { CI: } 4.1162 \\
\text { to } 29.6510\end{array}$ \\
\hline left & 7 & 19,44 & 48 & 72,73 & $\mathrm{P}<0.0001$ \\
\hline \multicolumn{6}{|l|}{ Synchron colorectal cancer } \\
\hline yes & 10 & 27,78 & 21 & 31,82 & $\begin{array}{l}\text { OR } 0.824295 \% \\
\text { CI: } 0.3369 \\
\text { to } 2.0161\end{array}$ \\
\hline no & 26 & 72,22 & 45 & 68,18 & $P=0.6718$ \\
\hline \multicolumn{6}{|l|}{ Treatment polyposis } \\
\hline colectomy & 13 & 36,11 & 14 & 21,21 & $\begin{array}{l}\text { OR } 2.099495 \% \\
\text { CI: } 0.8532 \\
\text { to } 5.1660\end{array}$ \\
\hline endoscopic polypectomy & 23 & 63,89 & 52 & 78,79 & $P=0.1065$ \\
\hline \multicolumn{6}{|l|}{ Colonoscopic interval } \\
\hline 1 year & 19 & 52,78 & 0 & 0,00 & $\begin{array}{l}\text { OR } 455.000095 \% \\
\text { CI: } 23.3975 \\
\text { to } 8848.1863\end{array}$ \\
\hline $2-3$ year & 4 & 11,11 & 52 & 78,79 & $\mathrm{P}=0.0001$ \\
\hline Burden polyps in follow up & & & & & \\
\hline
\end{tabular}

Table 1: Continued

\begin{tabular}{|c|c|c|c|c|c|}
\hline & \multicolumn{2}{|c|}{$\begin{array}{l}\text { APC or MUTYH } \\
\text { mutation }=36\end{array}$} & \multicolumn{2}{|l|}{$\begin{array}{l}\text { Wild } \\
\text { type }=66\end{array}$} & \multirow{2}{*}{ Statistics } \\
\hline & Number & $\%$ & Number & $\%$ & \\
\hline no & 4 & 11,11 & 36 & 54,55 & \\
\hline $\begin{array}{l}\text { less than at index } \\
\text { colonoscopy }\end{array}$ & 4 & 11,11 & 16 & 24,24 & $\begin{array}{l}\text { OR } 0.093695 \% \\
\text { CI: } 0.0274 \\
\text { to } 0.3197\end{array}$ \\
\hline $\begin{array}{l}\text { more than at index } \\
\text { colonoscopy and } \\
\text { dense polyposis }\end{array}$ & 15 & 41,67 & 0 & & $\mathrm{P}=0.0002$ \\
\hline Tot & 36 & 100,00 & 66 & 100,00 & \\
\hline
\end{tabular}

Conclusion: We observed a different behavior between mutated and wild-type patients. Patients with genetic involvement still developed adenomas during the follow-up and some needed colectomy. Instead, wild-type patients had mostly no recurrence. Constitutional genetic background could be suspected in wild-type patients when a continuous development of new polyps has observed and further genetic investigation should be offered by multi-gene testing.

Disclosure of Interest: All authors have declared no conflicts of interest.

P1097 GLOBAL DNA HYPOMETHYLATION ALONG THE COLORECTAL NORMAL-ADENOMA-CANCER SEQUENCE

K. Szigeti ${ }^{1}$, O. Galamb ${ }^{1}$, A. Kalmár ${ }^{1}$, G. Valcz ${ }^{1}$, E. Markus ${ }^{1}$, Z. Tulassay ${ }^{2}$, B. Wichmann ${ }^{3}$, P. Igaz ${ }^{1}$, B. Molnar ${ }^{1}$

${ }^{1}$ 2nd Department Of Internal Medicine, Semmelweis University, Budapest/

Hungary

${ }^{2}$ 2nd Department Of Medicine, SE II. Belgy. Klinika Belgyogyaszat, Budapest/ Hungary

${ }^{3}$ 2nd Medicine, Molecular Gastroscopy, Semmelweis University, Budapest/

Hungary

Contact E-mail Address: kri.szigeti@gmail.com

Introduction: Besides local hypermethylation on promoters of certain tumor suppressor genes, global DNA hypomethylation is characteristic in various types of cancers including colorectal cancer (CRC). The DNA methylation level of long interspersed nuclear element-1 (LINE-1) repetitive retrotransposon sequences constituting $17 \%$ of the human genome can be used to estimate global methylation level.

Aims \& Methods: We aimed to analyze the alterations of the global DNA methylation levels along the colorectal normal-adenoma-carcinoma sequence progression on the basis of LINE-1 methylation and to study the methyl-cytosine pattern in tissue samples. Genomic DNA was isolated from 10 colorectal adenoma, 10 CRC and 30 normal colonic biopsy samples. Bisulfite conversion of DNA samples was performed using EZ DNA Methylation-Direct Kit (Zymo). For methylation level quantification of the LINE-1 retrotransposable element, bisulfitespecific PCR (BS- PCR) was applied, and $146 \mathrm{bp}$ long PCR products were sequenced on Pyromark Q24 system (Qiagen). Tissue localization of 5-methylcytosine (5-mC) in normal, adenoma and CRC tissues was analyzed by immunohistochemistry using mouse monoclonal anti-5mC antibody (GeneTex).

Results: According to the LINE-1 bisulfite sequencing results, significant $(\mathrm{p}<0.01)$ global DNA hypomethylation was detected both in CRC $(63 \pm 8.7 \%)$ and adenoma samples $(67 \pm 5.1 \%)$ compared to normal tissue $(72 \pm 1.4 \%)$. 5-mC labeling of both the epithelial and stromal components of normal samples was strong (scoring values: +2 and +3 ) with diffuse and nuclear staining. In adenomas, decreased nuclear 5 -mC staining (scoring value: +2 ) was detectable in the epithelium and the stroma compared to normal epithelium. In CRC samples significantly lower 5-mC levels could be observed than in normal tissue samples $(\mathrm{p}<0.05)$

Conclusion: Global DNA hypomethylation could be shown in CRC compared to healthy normal tissue samples both by LINE-1 bisulfite-sequencing and by 5 -mC immunohistochemistry. Genome-wide DNA methylation decrease occurs already in adenoma stage of colorectal carcinogenesis.

Disclosure of Interest: All authors have declared no conflicts of interest.

\section{P1098 CIRCULATING MIRNA CHANGES IN HUMAN} COLORECTAL CANCER DEVELOPMENT AND IN ANIMAL MODEL

Z.B. Nagy $^{1}$, B.K. Bartak ${ }^{1}$, A. Kalmár ${ }^{1}$, B. Wichmann ${ }^{2}$, O. Galamb ${ }^{2}$, E. Markus ${ }^{2}$, K. Szigeti ${ }^{2}$, Á. Nagy ${ }^{2}$, P. Igaz ${ }^{2}$, Z. Tulassay ${ }^{1}$, B. Molnar ${ }^{1}$

${ }^{1}$ 2nd Dept. Of Internal Medicine, Semmelweis University, Budapest/Hungary

${ }^{2}$ Hungarian Academy of Sciences, Budapest/Hungary

Contact E-mail Address: nagyzsofiab@gmail.com

Introduction: MiRNAs have a critical relevance in regulation during tumorigenesis. The expression profiles of miRNAs alter along tumor progression moreover; these miRNA may spread into tumor macro- and microenvironment. 
Extracellular miRNAs are stable and its expression is less characterized in plasma. Altered and overlapped miRNA profiles between tissue and plasma are less explored.

Aims \& Methods: The present study was designed to characterize the tissue and circulating miRNA profile through colorectal adenoma-carcinoma sequence in human specimens and also in mice peripheral blood samples. Furthermore, the purpose of our study was to determine the origin of detected miRNAs in tumoradherent $\mathrm{C} 38 / \mathrm{C} 57 \mathrm{BL} / 6$ and non-adherent CBAJ mice tumor models. To achieve that goal, human peripheral blood and biopsy of normal (N), tubular (AT), tubulovillous adenoma (ATV) and colorectal cancer (CRC) were selected and plasma were also collected two times a week over 45 days from C57BL/6-C38, CBAJ mice. MiRNAs were isolated and Affymetrix GeneChip miRNA array analysis was performed for screening of the altered miRNA profile. RT-qPCR method was used for validation.

Results: In the case of human samples out of 1733 detectable miRNAs, 306 miRNAs were expressed in normal, 334 in adenoma and 321 in CRC Characteristic miRNA expression alteration was observed in the comparison of AD vs. CRC (miR-149*, miR-3196, miR-4687) in plasma. In the case of $\mathrm{N}$ vs. CRC, overexpression of miR-612, miR-1296, miR-933, miR-937 and miR-1207 was validated by RT-PCR $(p<0.05)$. Partial co-expression of these miRNAs was observed in tissue pairs as well. We identified high plasma levels of 94 miRNAs in healthy animals and 176 miRNAs in late tumor stages. Based on CBAJ-C38 mice model experiment where the injected tumorous cells could not adhere miR-676 found to be a host originated while miR-92a was a tumorderived miRNA. MiR-676 and miR-92a shown significant overexpression (388x and $37 \mathrm{x} \mathrm{p}<0.05)$ in plasma samples based on real-time PCR and microarray results.

Conclusion: Circulating miRNAs alteration could observe in animal models and in the human circulatory system. Cancer-associated miRNAs in the circulation may originate from the immunologic system or from other metastatic regions far from the primer tumor location.

Disclosure of Interest: All authors have declared no conflicts of interest

\section{P1099 RHOA: THE KEY SIGNALING PATHWAY OF MICRORNA- 126 IN SUPPRESSING THE EPITHELIAL-MESENCHYMAL TRANSITION, PROLIFERATION, MIGRATION AND INVASION OF COLORECTAL CANCER CELLS}

M. Huang, W. Yuan, S. Wu, Z. Shen, Z. Yang, Y. Quan, C. Zhu, K. Tang, X. Wang

Department Of Gastroenterology, The Third Xiangya Hospital Of Central South University, Changsha, Hunan, China, Hunan Key Laboratory of Nonresolving Inflammation and Cancer, Changsha, Hunan, China, Changsha/China

\section{Contact E-mail Address: hmy02420@126.com}

Introduction: The mechanism of colorectal cancer (CRC) invasion and metastasis is still unclear. Epithelial-mesenchymal transition (EMT) is one of the key molecular steps in the process of distant metastasis. EMT is referred to conversion of cells with an epithelial phenotype into cells with a mesenchymal phenotype, which lead to loss of cell polarity, with acquisition of migratory and invasive properties of cancer cells. ${ }^{1}$ MicroRNAs (miRNAs) are small non-coding RNAs that negatively regulate gene expression at post-transcriptional phase. ${ }^{2}$ MicroRNA-126 (miR-126) originates from a common precursor structure located within the egfl7 gene, which acts as a tumor suppressive miRNA in various carcinomas. ${ }^{3}$ Our previous studies found that miR-126 was significantly down-regulated in human CRC tissues and negatively related with patient's prognosis. ${ }^{4}$ MiR-126 was also low-expressed in high metastatic cell lines, and inhibited proliferation, invasion and metastasis of CRC in vitro. ${ }^{5}$ However, whether miR-126 can regulate the process of EMT in CRC is still unclear. Ras homologue A (RhoA) is one of most characterized members of Rho GTPases which belong to Ras superfamily. RhoA, as molecular switch, cycles between an active GTP-bound state and inactive GDP-bound state. RhoA and its components of signaling pathway are known to participate in a diverse array of cellular events related to invasion and metastasis of cancer cells. ${ }^{6}$ Our previous study found that miR-126 down-regulated RhoA and ROCK activity in CRC cells. ${ }^{5}$ Whether RhoA activity and RhoA signaling pathway play an important role in miR-126 regulating EMT process, cell proliferation, migration and invasion of CRC remains unclear.

Aims \& Methods: To identify RhoA signaling pathway associated with the functions of miR-126 in EMT, proliferation, migration and invasion of CRC cells. Constructed CRC cell lines of miR-126 over-expression or knockdown. Performed MTT, colony formation, wound-healing, migration, invasion assays and RT PCR, western blot analysis to study the functions of miR-126 in EMT, proliferation, migration, invasion and expression RhoA signaling pathway of CRC cells. Constructed pDsRed2-V14RhoA (constitutively active RhoA, V14RhoA) and pDsRed2-N19RhoA (domain-negative, N19RhoA) mutants, then transfected them into the CRC cell lines of miR-126 over-expression or knockdown to restore RhoA activity. Pulldown assay detected RhoA activity after transfected. Then repeated the experiments above to investigate the biological behavior changes of CRC cells.

Results: MiR-126 promoted the expression of E-cadherin and suppressed the expression of SLUG, Snail, Vimentin, Fibronectin of CRC cells. MiR-126 also inhibited proliferation, migration and invasion of CRC cells, and negatively regulating RhoA signaling pathway. V14RhoA mutant effectively increased the activity of RhoA and reversed the role of miR-126 by promoting EMT, proliferation, migration and invasion in miR-126 overexpressing HCT116 cells. Conversely, N19RhoA mutant effectively decreased the activity of RhoA and suppressed EMT, proliferation, migration and invasion in miR-126-silenced SW480 cells
Conclusion: RhoA signaling pathway was the key signaling pathway of miR-126 in suppressing the EMT, proliferation, migration and invasion of CRC cells. Disclosure of Interest: All authors have declared no conflicts of interest.

\section{References}

1. Thiery JP, Acloque H, Huang RY, Nieto MA. Epithelial-mesenchymal transitions in development and disease. Cell. 2009;139(5):871-890.

2. Carrington JC, Ambros V. Role of microRNAs in plant and animal development. Science. 2003;301(5631):336-338.

3. Bu J, Li H, Li XY, Liu LH, Sun W, Xiao T. Prognostic Role of MicroRNA126 for Survival in Malignant Tumors: A Systematic Review and MetaAnalysis. Dis Markers. 2015:739469.

4. Yuan W, Guo YQ, Li XY, et al. MicroRNA-126 inhibits colon cancer cell proliferation and invasion by targeting the chemokine (C-X-C motif) receptor 4 and Ras homolog gene family, member A, signaling pathway. Oncotarget. 2016;7(37):60230-60244.

5. Hall A. Rho GTPases and the actin cytoskeleton. Science. 1998;279(5350): 509-514.

6. Li N, Tang A, Huang S, et al. MiR-126 suppresses colon cancer cell proliferation and invasion via inhibiting RhoA/ROCK signaling pathway. Mol Cell Biochem. 2013;380(1-2):107-119.

\section{P1100 DNA METHYLATION CHANGES PRECEDE AND CONTRIBUTE TO SPORADIC MUTATIONS IN COLORECTAL ADENOMA AND CANCER DEVELOPMENT THROUGH INDUCED GENOMIC INSTABILITY}

B. Molnar, O. Galamb, B. Péterfia, B. Wichmann, A. Kalmár, Z.B. Nagy, B.K. Bartak, K. Szigeti, E. Markus, Z. Tulassay

2nd Dept. Of Medicine, Semmelweis University, Budapest/Hungary

Contact E-mail Address: mb@bel2.sote.hu

Introduction: Colorectal cancer development is characterised by sporadic mutations and epigenetic alterations. DNA mutations occur randomly and sporadically in growth-related genes, mostly on cytosine nucleotides. Active demethylation of cytosines in relation to RNA expression alterations may lead to genetic instability and DNA mutations. Whole genomic DNA methylation and mutation analysis with RNA expression profiling could demonstrate the primary and secondary order of the epigenetic and genetic changes and their relation to the malignant phenotype development.

Aims \& Methods: In this study we aimed the whole genome methylation analysis and targeted mutation analysis of colorectal cancer (CRC)-related genes (12) with upstream whole genomic mRNA expression evaluation. Special focus was set on the p53 pathway and the involved genes. Methyl capture sequencing (Illumina) was performed on normal (N: 6), adenomatous (Ad: 15) and colorectal cancer (CRC: 9) biopsy specimens. Methylation results were confirmed by in silico methylation studies' results (TCGA) and by methylation array-PCR (Qiagen Methylation array). Specimens were further evaluated for 32 mutations of 12 CRC-related genes (APC, BRAF, CTNNB1, EGFR, FBXW7, KRAS, NRAS, MSH6, PIK3CA, SMAD2, SMAD4, TP53) by new generation sequencing (Roche 454 Junior). mRNA expression evaluation was performed by whole genomic expression analysis (HGU 133, Affymetrix). Tageted pathway analysis was performed for the p53 pathway. Bioinformatic analysis included overall quantitative methylation analysis, selection of top hyper/hypomethylated genes, methylation changes on the tumor mutation regions and related pathway gene promoters were evaluated by targeted analysis.

Results: Overall hypomethylation was observed on the N-Ad-CRC sequence in the gene body and non coding genomic regions. In Ad-N comparison e.g. p73, NGFR, PDGFRA genes were hypermethylated for their promoters, FMN1, SLC16A7 genes were hypomethylated, respectively. In CRC-N comparison DKK2, SDC2, SOX1 genes showed hypermethylation, while ERBB4, CREB5, CNTN1 genes were hypomethylated in the promoter regions. In silico analysis on the TCGA database yielded confirmatory results. The common hyper- and hypomethylated genes were also in correlation with methylation array results yielded by methylation specific PCR. A significant negative correlation of the top methylated genes could be demonstrated to the RNA expressions. In the certain mutation hot spot, genebody regions significant DNA methylation alterations (mainly hypomethylation) were detected. APC, P53 and KRAS mutations were found in $30 \%, 15 \%, 21 \%$ of adenomas, and in $29 \%, 53 \%, 29 \%$ of CRCs, respectively. RNA expressions profiling could not detect any significant quantitatve changes related to these DNA sequence alterations. The p53 gene body was addressed by hypermethylations in adenomas. mRNA expression changes were observed in most of the 553 pathway genes showing promoter methylation alterations.

Conclusion: DNA methylation with consecutive phenotypic effect can be observed in a high number of gene promoters and gene body regions through CRC development. The mutation hot spot areas of the most relevant colorectal cancer mutation genes (APC, p53,KRAS, NRAS) were involved in methylation alterations without detectble quantitative RNA expression changes. p53 cancepathway genes are highly methylated in promoters and gene body with corresponding expression changes. The tumor mutation hot spot areas in the p53 gene are hypomethylated and thus genetically instabile and therefore mutated. Disclosure of Interest: All authors have declared no conflicts of interest. 
P1101 MIR-126 REGULATES TUMOR GROWTH AND METASTASIS IN COLORECTAL CANCER BY RECRUITING TUMOR ASSOCIATED MACROPHAGES THROUGH PARACRINE SIGNALING OF CXCL12

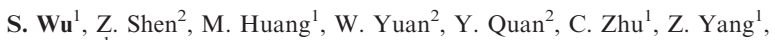

X. Wang ${ }^{1}$

${ }^{1}$ Department Of Gastroenterology, The Third Xiangya Hospital of Central South University, Changsha/China

${ }^{2}$ Department Of Gastroenterology, The Third Xiangya Hospital Of Central South University, Changsha, Hunan, China, Hunan Key Laboratory of Nonresolving Inflammation and Cancer, Changsha, Hunan, China, Changsha/China

Contact E-mail Address: echoecho0428@163.com

Introduction: Colorectal carcinoma is one of the leading causes of cancer-related mortality worldwide. Tumor associated macrophages (TAMs) are critical stromal components intimately involved with the progression, invasion, and metastasis of cancer cells. Recently, increasing studies have demonstrated that microRNA-126 (miR-126) had an important role in colorectal cancer. The expression of miR-126 decreased significantly in colorectal cancer, particularly in highly metastatic cell lines, indicating that miR-126 may inhibit tumor development and metastasis. However, the mechanism underlying miR126 inhibiting cancer is uncertain, and its function in cross-talk between colorectal cancer cells and TAMs are still in its infancy.

Aims \& Methods: In this study, we investigate the cross-talk between cancer cells and TAMs in colorectal cancer microenvironment, and find out what role the miR-126-CXCL12-IL6 axis plays in it. Methods: (1)The effect of miR-126 on CXCL12 expression was assessed in the CRC cell line Caco2 transferred with a miR-126 mimic or inhibitor to increase or decrease miR-126 expression; (2) We build a co-culture system of TAMs and transferred cancer cells, and use AMD3100(100 ng/ml) to block CXCL12/CXCR4 axis, then detect the recruitment and inflammation factors secretion of TAMs; (3) Furthermore, the TAMs co-cultured before were taken away from the previous system and put into a new co-culture system with untreated colorectal cancer cells, and IL6 neutralizing antibodies were added in. We detect the expression of EMT associated factors and STAT3 pathway activation by western blot, cell growth by CCK8, metastasis by Transwell. The definition of statistical significance was defined as $\mathrm{P}<0.05$ (two-tailed).

Results: (1)miR-126 negatively regulate CXCL12 expression in post-transcript level; (2)Inhibiting miR-126 of colorectal cancer cells could promote TAMs recruitment and up-regulate inflammation factors IL1 $\beta$ and IL6 expression. However, blocking CXCL12/CXCR4 axis by AMD3100 could reverse this effect, vice versa;(3) Inhibiting miR-126 of colorectal cancer cells could recruiting TAMs, therefore down-regulate E-cadherin protein, up-regulate slug protein, and activate STAT3 pathway of untreated cancer cells. It could also promote cancer cells growth and metastasis. In addition, IL6 neutralizing antibodies could block this effect, vice versa.

Conclusion: Our results reveal a novel mechanism by that miR-126 repress recruitment and inflammatory factor secretion of TAMs through controlling secretion and paracrine signaling of $\mathrm{CXCL} 12$ to inhibit colorectal cancer growth and metastasis.

Disclosure of Interest: All authors have declared no conflicts of interest.

P1102 THE EFFICACY OF NEXT-GENERATION OF IMAGE ENHANCED COLONOSCOPY (BLUE LASER IMAGING) IN THE DETECTION OF COLONIC LESIONS: A PILOT STUDY

R. Shimoda ${ }^{1}$, Y. Sakata ${ }^{1}$, N. Tsuruoka ${ }^{1}$, S. Shirai ${ }^{1}$, T. Noda ${ }^{2}$, H. Endo ${ }^{3}$, K. Akutagawa ${ }^{4}$, R. Iwakiri ${ }^{5}$, K. Fujimoto ${ }^{6}$

${ }^{1}$ Internal Medicine And Endoscopy, Saga Medical School, Saga/Japan

${ }^{2}$ Internal Medicine And Endoscopy, Karatsu Red Cross Hospital, Karatsu/Japan

${ }^{3}$ Internal Medicine And Endoscopy, Saiseikai Karatsu Hospital, Karatsu/Japan

${ }^{4}$ Internal Medicine, Taku City Hospital, Taku/Japan

${ }^{5}$ Dept. Of Gastrointestinal Endoscopy, Saga Medical School Dept. of

Gastroenterology, Saga/Japan

${ }^{6}$ Dept. Of Internal Medicine, Saga Medical School, Saga/Japan

Contact E-mail Address: shimodar@cc.saga-u.ac.jp

Introduction: Narrow Band Imaging (NBI) enable detection for vascular-rich small, flat lesion and recognition of mucosal surface compared with normal colonic mucosa and vascular pattern in colonoscopy. However, recent studies reported no significant difference in overall adenoma detection rate with the use of NBI compared with white light imaging (WLI). Blue Laser Imaging (BLI) is next-generation of image enhanced endoscopy technique using LASER light source that were realized with lighting and image processing suitable for visualization of microvessels and structures in the superficial portion of the mucous membrane.

Aims \& Methods: Our aim was to determine whether the use of BLI enhances the adenoma detection rate (ADR) and miss rate compared with WLI. A total of 130 patients who underwent screening or surveillance colonoscopy in Saga University Hospital were included. Three patients were excluded because of poor bowel preparation. One hundred and twenty-seven patients were randomized to tandem colonoscopy with BLI followed by WLI (BLI-WLI group) or WLI followed by WLI (WLI-WLI group). Polyp (adenoma) detection rates, miss rates and the number of polyps (adenoma) detected per person (mean adenoma detection rate) were examined between the two groups.

Results: The BLI-WL group and WL-WL group comprised 64 and 63 patients, respectively. The proportion of patients with polyps (adenoma) was $62.5 \%$ in BLI-WL group compared with $63.5 \%$ in WL-WL group. There was no significant difference between two groups regarding ADR (). The number of adenomas detected per person (ADR) of BLI-WL group and WL-WL group were 2.84 and
1.90 , respectively. However, the polyp miss rate of BLI-WL group was less than that of WL-WL group (1.6\% vs $10.0 \%: P=0.0014)$.

Conclusion: There were no significant difference in the overall polyp (adenoma) detection rate with BLI-WLI group or WL-WL group. However, miss rate was higher in WL-WL group compared with BLI-WL group $(10.0 \%$ vs $1.6 \%$, $P=0.0014)$. Further, BLI detected more polyps per patient compared with WL group (2.84 vs 1.90). BLI may improve polyp miss rate and the number of polyps per subject (mean adenoma detection rate) in the colonoscopy.

Disclosure of Interest: All authors have declared no conflicts of interest.

\section{P1103 COLORECTAL CANCER SCREENING COLONOSCOPY -} ABSENT DISTAL POLYPS IN ADVANCED PROXIMAL NEOPLASIA

R. N. Patel ${ }^{1}$, M. Hussein ${ }^{1}$, L. Polak ${ }^{1}$, A. Kumar ${ }^{2}$, S. Samanta ${ }^{1}$

${ }^{1}$ Gastroenterology, Barking, Havering and Redbridge University Trust, Essex/United Kingdom

${ }^{2}$ Colorectal Surgery, Barking, Havering and Redbridge University Trust, Essex/United Kingdom

\section{Contact E-mail Address: rajan.patel $@$ nhs.net}

Introduction: The National Health Service Bowel Cancer Screening Programme (NHS BCSP) offers colonoscopy to people testing positive for Faecal Occult Blood Test (FOBT) after the age of 60. In addition, the 'Bowel scope screening' test offers once-only flexible sigmoidoscopy (FS) to people in the UK after the age of 55. The Norwegian (NORCCAP) and Italian (SCORE) trials evaluated the effectiveness of FS screening and reported a non-statistically significant decrease in colorectal cancer (CRC) specific mortality at follow-up. It is unclear if significant proximal neoplasia is being missed in people undergoing flexible sigmoidoscopy alone.

Aims \& Methods: We aim to investigate the distributions of pathology within the BCSP at a busy district general hospital in London. In 2015, 22,539 FOBT kits were returned out of the $43,884(51.4 \%)$ sent out in the boroughs of Barking, Havering and Redbridge (BHR). Of those returned, $398(1.8 \%)$ tested positive. We collected data for the 326 patients who attended for colonoscopy at BHR University Hospitals ( $81.9 \%$ ). Subgroup analyses included age, sex, histology, location of polyps, number of polyps, polyp size and therapies.

Results: Mean age 67, Male $60.4 \%$. Polyps were found in 199 patients $(61 \%), 488$ polyps found in total, mean number of polyps 2.5 (Range 1-14), mean size $7 \mathrm{~mm}$ (Range $1 \mathrm{~mm}-60 \mathrm{~mm}) ; 49(15 \%)$ hyperplastic, $156(47.9 \%)$ adenomas and 16 $(4.9 \%)$ adenocarcinoma. Patients with adenoma/carcinoma were older $(67.6$ vs. $66.5, \mathrm{p}=0.02$ ) but there was no difference in sex (Male $61.4 \%$ vs. $59.4 \%$, $\mathrm{p}=0.49$ ) when compared to those without adenoma/carcinoma. Of 172 patients with adenoma/carcinoma, $111(64.5 \%)$ were proximal to the splenic flexure (SF). 5 out of $16(31.2 \%)$ adenocarcinomas were proximal to SF and $2(40 \%)$ of these patients had no polyps distal to the SF

\begin{tabular}{llll}
\hline Adenoma/Carcinoma according to location in colon & \\
\hline $\begin{array}{l}\text { Adenoma/ } \\
\text { Carcinoma }\end{array}$ & $\begin{array}{l}\text { SF + Distal } \\
(\mathrm{n}=61)\end{array}$ & $\begin{array}{l}\text { Proximal to } \\
\text { SF }(\mathrm{n}=111)\end{array}$ & *p value \\
\hline Age & 67.4 & 67.7 & 0.67 \\
Male & $41(67.2 \%)$ & $67(60.4 \%)$ & 0.32 \\
Number of polyps & 1.7 & 3 & $<0.01$ \\
Size of polyps $(\mathrm{mm})$ & 8.6 & 6.0 & $<0.01$ \\
Carcinoma & 11 & 5 & 0.71 \\
\hline
\end{tabular}

Conclusion: Patients with adenomas/carcinomas are older and those with proximal adenomas/carcinomas have more polyps but are smaller in size. One in three adenocarcinomas picked up during colonoscopy would be out of reach of a flexible sigmoidoscopy. Furthermore, over one third of the proximal cancers did not have distal polyps.

Disclosure of Interest: All authors have declared no conflicts of interest.

\section{References}

1. Atkin W, Edwards R, Kralj-Hans I, Wooldrage K, Hart A, Northover J et al. Once-only flexible sigmoidoscopy screening in prevention of colorectal cancer: a multicentre randomised controlled trial. The Lancet. 2010;375(9726):1624-1633.

2. Hoff G, Grotmol T, Skovlund E, Bretthauer M. Norwegian Colorectal Cancer Prevention Study Group. Risk of colorectal cancer seven years after flexible sigmoidoscopy screening: randomised controlled trial. BMJ. 2009; (338):B1846. Results of a randomized trial of FS in Norway.

3. Segnan N, Armaroli P, Bonelli L, et al. Once-only sigmoidoscopy in colorectal cancer screening: follow-up findings of the Italian Randomized Controlled Trial-SCORE. J Natl Cancer Inst. 2011; 103(17):1310-1322. Results of a randomized trial of FS in Italy. 
P1104 STUDIES ON CLINICOPATHOLOGICAL CHARACTERISTICS AND THE LONG-TERM PROGNOSIS OF DEPRESSED-TYPE COLORECTAL CARCINOMAS

S. Kudo ${ }^{1}$, T. Kurata ${ }^{1}$, K. Ichimasa ${ }^{1}$, Y. Kouyama ${ }^{1}$, S. Matsudaira ${ }^{1}$ N. Toyoshima ${ }^{1}$, Y. Mori ${ }^{1}$, M. Misawa ${ }^{1}$, N. Ogata ${ }^{1}$, T. Kudo ${ }^{1}$, T. Hisayuki ${ }^{1}$, T. Hayashi ${ }^{1}$, K. Wakamura $^{1}$, E. Hidaka ${ }^{1}$, T. Baba ${ }^{1}$, F. Ishida ${ }^{1}$

${ }^{1}$ Digestive Disease Center, Showa University Northern Yokohama Hospital, Yokohama/Japan

Contact E-mail Address: kudos@med.showa-u.ac.jp Introduction: Colorectal cancers have two development theories. One of the development theories is "adenoma-adenocarcinoma sequence" developing from protruded-tyepes "polypes" we know generally. The other is considered to emerge directly from nomal epithelium, not through the adenomatous stage. Recently, it is revealed most of this type are depressed-type carcinomas. This theory is called "de novo" pathway. We studied clinicopathological characteristics and long-term prognosis mainly on depressed-type colorectal carcinomas. Aims \& Methods: The aim is to clarify the pathological characteristics of depressed-type colorectal carcinomas compared with flat- and protruded-type. A total of 29030 colorectal neoplasms excluding advanced carcinomas were resected endoscopically or surgically in our Center from April 2001 to December 2016. Of these, 1127 lesions were T1 carcinomas. According to the morphological/development classification, 244 lesions $(21.7 \%)$ were depressedtype, 385 lesions $(34.2 \%)$ were flat-type and 498 lesions $(44.1 \%)$ were protrudedtype. We analyzed the pathological features of these lesions.

Results: The rate of distant metastasis or recurrence was $0.9 \%(10 / 1127)$. Among these 10 cases, 5 cases were developed from depressed-type lesions and one showed a para-aortic lymph node metastasis and four showed a lung metastases. The rate of submucosal invasion in all the lesions was $72.4 \%$ in depressed-type, $3.2 \%$ in flat-type and $2.9 \%$ in protruded-type. Within less than $5 \mathrm{~mm}$ in diameter, that was $10.6 \%, 0 \%$ and $0 \%$ respectively. Among T1 carcinomas, the rate of vessel invasion was $64.3 \%$ in depressed-type, $34.3 \%$ in flat-type and $38.4 \%$ in protruded-type, that of poorly differentiated or mucinous adenocarcinoma was $17.2 \%, 10.4 \%$ and $13.5 \%$, that of massively submucosal invasion was $94.7 \%$, $71.7 \%$ and $69.7 \%$, and that of tumor budding was $36.5 \%, 16.1 \%$ and $17.3 \%$, respectively. The rates of these pathological factors were significantly higher in depressed-type lesions. On the other hand, the rate of adenomatous componen was $4.9 \%, 52.2 \%$ and $50.8 \%$,respectively. It was significantly lower in depressedtype lesions, suggesting that they emerge directly from normal epithelium without going through the adenoma stage. The rate of lymph node metastasis was $11.6 \%$, $6.8 \%$ and $13.0 \%$, respectively. And 2 depressed-type lesions had synchronous liver metastasis

Conclusion: Depressed-type colorectal carcinomas invade massively even when they are small. They had higher risks of vascular invasion, poorly differentiated or mucinous adenocarcinoma, massive invasion, and tumor budding than flat- or protruded-types. For their rapid growth and malignant potential, whether the lesion is depressed-type or not is very important in the diagnosis of colorectal carcinomas.

Disclosure of Interest: All authors have declared no conflicts of interest

\section{P1105 ENDOSCOPIC OBSERVATIONS OF MICROSURFACE STRUCTURES MAY REFLECT MOLECULAR SUBCLONES IN COLORECTAL TUMORS}

E. Yamamoto ${ }^{1}$, H. Aoki ${ }^{2}$, H. Yamano ${ }^{1}$, H. Suzuki ${ }^{2}$, H. Nakase ${ }^{1}$

${ }^{1}$ Department Of Gastroenterology And Hepatology, Sapporo medical university, Sapporo/Japan

${ }^{2}$ Dept. Of Molecular Biology, Sapporo Medical University, Sapporo/Japan

\section{Contact E-mail Address: eiichiro@xa3.so-net.ne.jp}

Introduction: Recent cancer genome analyses identified extensive genetic intratumoral heterogeneity (ITH), suggesting a Big Bang model in which tumors grow as an expansion producing numerous subclones. As subclone mixing is indicative of an early malignant potential, ITH may be a hallmark to identify precursor lesions at high-risk of developing CRC.

Aims \& Methods: We aim to clarify the association between ITH of driver mutations and clinicopathological characteristic in colorectal tumors. A total of 711 specimens from 477 colorectal tumors were analyzed in this study. Subcomponents of the tumors were endoscopically defined using the microsurface structures according to Kudo's pit pattern classification, and biopsy specimens were obtained from respective portions. BRAF, KRAS and TP53 mutations were analyzed by pyrosequencing and direct sequencing. Hot spot mutations of 50 cancer related genes were analyzed using the Ion PGM sequencer.

Results: ITH of KRAS and TP53 mutations was frequently observed in colorectal tumors with multiple subcomponents. Heterogeneity of KRAS or TP53 mutations between early and advanced subcomponents (e.g., KRAS G12D in early portion and $K R A S$ G13D in advanced portion) progressively increased during the steps of carcinogenesis: high grade adenomas (KRAS, 8\%; TP53, $0 \%$ ), noninvasive cancers $(K R A S, 14 \% ; T P 53,12 \%)$ and submucosal cancers (KRAS, $25 \% ; T P 53,38 \%)$. These results suggest that subcomponents defined by microsurface structure may represent genetically distinct subclones. By contrast, only 4 of $41 B R A F$-mutant lesions exhibited ITH of BRAF mutation. Pathological findings of the 4 lesion were low grade adenoma $(n=1)$, non-invasive CRCs with adenoma $(\mathrm{n}=2)$ and serrated lesions $(\mathrm{n}=1)$, whereas majority of the $B R A F$-mutant lesions without ITH $(\mathrm{n}=37)$ were serrated lesions or CRC with serrated lesions. Sequencing of 50 cancer-related genes in 8 tumors with KRAS/ TP53 ITH revealed that both subcomponents exhibited identical mutations in genes including $F B X W 7, H R A S$ and $S K T 11$, suggesting that these were founder mutations.
Conclusion: Our results demonstrate that degree of ITH of $K R A S / T P 53$ mutations increases during the progression of colorectal tumorigenesis. Intratumoral variations in the microsurface structure may represent molecular subclones in early colorectal lesions and may be predictive of the malignant progression. Disclosure of Interest: All authors have declared no conflicts of interest.

\section{P1106 THE DIAGNOSTIC VALUE OF HYPOXIA INDUCED EXOCELLULAR VESICLES IN COLORECTAL CANCER PATIENT PLASMA}

A. Abols ${ }^{1}$, D. Santare ${ }^{2}$, R. Toleikiene ${ }^{1}$, E. Zandberga ${ }^{1}$, R. Vangravs ${ }^{2}$, M. Takatalo-Laine ${ }^{3}$, M. Palviainen ${ }^{3}$, P. Siljander ${ }^{3}$, M. Leja ${ }^{2}$, A. Line

${ }^{1}$ Latvian Biomedical Research and Study Centre, Riga/Latvia

${ }^{2}$ Department Of Science, Riga East University Hospital, Riga/Latvia

${ }^{3}$ University of Helsinki, Helsinki/Finland

Contact E-mail Address: daiga.santare@lu.lv

Introduction: Hypoxia signalling has been found to enhance cancer cell survival, chemoresistance, motility, tumour angiogenesis as well as self-renewal capacity and proliferation of putative cancer stem cells. One of the key player in hypoxia is carbonic anhydrase IX (CAIX) which is a hypoxia-inducible enzyme. CAIX is overexpressed in a variety of cancers including colon cancer and plays a crucial role in maintaining favourable intracellular $\mathrm{pH}$ in hypoxia. There is also evidence that extracellular vesicle (EV) production is increased in response to hypoxia and promotes adaptive response of cancer cells and we have previously demonstrated, that CAIX positive EVs secretion is increased in response to hypoxia in colorectal cancer cell lines SW480 and SW620.

Aims \& Methods: Within this study, we explored a possibility to use CAIX for the isolation of hypoxic EVs from colorectal cancer (CRC) patients' plasma. EVs were isolated from plasma samples of $27 \mathrm{CRC}$ patients and 25 healthy donors (HD) by using sequential centrifugation, filtration and size-exclusion chromatography steps. EVs where quantified by Nanoparticle tracking analysis (NTA) and CAIX positive EVs where determined by ApogeeA50.

Results: Statistically significant increase in the amount and size of EVs was observed between CRC and HD cohorts based on NTA data. ApogeeA50 data revealed that there is a tendency for the percentage of CAIX-positive EVs to increase with the stage of CRC and is higher in stage III-IV CRC patients than in HD. In addition, it is higher in patients with metastasis than without distant metastases.

Conclusion: There is an increased total EV number, EV size and CAIX positive EV amount in CRC patient plasma compared to HD plasma, that might have diagnostic and prognostic value. (Financed by Latvian Council of Science Collaboration project No: 625/2014).

Disclosure of Interest: All authors have declared no conflicts of interest.

\section{P1107 THE GENESIS STUDY: GENETIC BIOPSY FOR PREDICTION OF SURVEILLANCE INTERVALS AFTER ENDOSCOPIC RESECTION OF COLONIC POLYPS}

A. W. Berger ${ }^{1}$, K. Raedler ${ }^{2}$, C. Langner ${ }^{3}$, L. Ludwig ${ }^{4}$, N. Dikopoulos ${ }^{4}$

K.F. Becker ${ }^{5}$, J. Slotta-Huspenina ${ }^{5}$, M. Quante ${ }^{6}$, D. Schwerdel ${ }^{1}$, L. Perkhofer ${ }^{1}$, A. Kleger ${ }^{1}$, E. Zizer ${ }^{1}$, F. Oswald ${ }^{2}$, T. Seufferlein ${ }^{7}$, A. Meining ${ }^{1}$ ${ }^{1}$ Department Of Internal Medicine I, Ulm University, Ulm/Germany

${ }^{2}$ Clinic For Internal Medicine I, Ulm University, Ulm/Germany

${ }^{3}$ Institute For Pathology, Medical University Graz, Graz/Austria

${ }^{4}$ Gastroenterologische Schwerpunktpraxis, Dornstadt/Germany

${ }^{5}$ Institute For General Pathology And Pathological Anatomy, Technical University Munich, München/Germany

${ }^{6}$ II Department Of Internal Medicine, Technical University Munich, München/ Germany

${ }^{7}$ Klinik Für Innere Medizin I, Universitätsklinikum Ulm - Klinik für Innere Medizin I, Universitätsklinikum Ulm; Ulm/DE, Ulm/Germany

Contact E-mail Address: Alexander.Meining@uniklinik-ulm.de Introduction: Colorectal cancer (CRC) is an important contributor to cancer mortality and morbidity worldwide. $80 \%$ of CRCs arise via the adenoma-carcinoma sequence, 10-20\% CRCs by sessile serrated adenomas (SSA). Hyperplastic polyps are regarded harmless. Current surveillance strategies for CRC following polypectomy are determined by endoscopic and histopathological factors. Such a distinction has also been challenged.

Aims \& Methods: The study was aimed for molecular characterization of colonic polyps in patients who underwent screening colonoscopy. Correlation of the genetic analysis with endoscopic, clinical and histopathological data was attempted to potentially better define relevant risk marker or sub-groups at risk for prediction of surveillance intervals. 100 Patients were enrolled in this multicenter study (NCT02595645; Median age: 62.9 y, 50 males, 50 female). Up to 6 representative polyp biopsies were collected and stored in a formalin-free medium and finally embedded in paraffin-blocks, followed by histopathological assessment. Targeted Next Generation Sequencing (tNGS) was performed from identical tissue specimens (38 CRC-related genes; GeneRead DNAseq Targeted Panels V2, Qiagen () ) on a MiSeq platform (Illumina $\left.{ }^{\circledR}\right)$. Genetic and histopathological analysis was done blinded to the endoscopic and clinical data.

Results: In 100 patients, 224 polyps were removed. 121 polyps $(54.0 \%)$ are sized $<10 \mathrm{~mm}, 71(31.7 \%)$ were $\geq 10 \mathrm{~mm}$. For 32 polyps $(14.3 \%)$ no size was available. 90 polyps $(40.2 \%)$ were located in the left, 126 polyps $(56.3 \%)$ in the right colon, for 8 polyps $(3.6 \%)$ no location was noted. 112 polyps $(50.0 \%)$ were adenomatous and 110 polyps $(49.1 \%)$ non-adenomatous lesions. No data were available 
for 2 polyps $(0,9 \%)$. Clinical, endoscopic and histopathological data were correlated to molecular characteristics. Significant associations of genetic alterations with endoscopic or histopathological polyp characteristics were observed for BRAF, KRAS, TCF7L2, FBXW7 and CTNNB1 mutations. Multivariate analysis revealed that polyps $\geq 10 \mathrm{~mm}$ have a significant higher relative risk (RR) for harboring oncogene mutations (RR 3.467 (1.742-6.933)). Adenomas and rightsided polyps are independent risk factors for CTNNB1 mutations (RR 18.559 $(2.371-145.245)$ and $12.987(1.637-100.00))$.

Conclusion: We were able to show that assessment of the mutational landscape of resected polyps/poly biopsy can easily be integrated in the workflow of current colonoscopy practice. There are distinct genetic patterns related to size and location of polyps and the clinician can appreciate this additional information to better estimate a patient's individual risk.

Disclosure of Interest: All authors have declared no conflicts of interest.

\section{P1108 QUANTITY, FRAGMENT LENGTH AND GLOBAL DNA METHYLATION LEVEL ALTERATIONS OF CIRCULATING CELL-FREE DNA IN COLORECTAL ADENOMA, CANCER AND INFLAMMATORY BOWEL DISEASES}

E. Markus ${ }^{1}$, A. Kalmár ${ }^{1}$, O. Galamb ${ }^{2}$, K. Szigeti ${ }^{1}$, B.K. Bartak ${ }^{1}$, Z.B. Nagy ${ }^{1}$, B. Wichmann ${ }^{2}$, Z. Tulassay ${ }^{1}$, P. Igaz $^{1}$, B. Molnar ${ }^{1}$

${ }^{1}$ 2nd Department Of Internal Medicine, Semmelweis University, Budapest/ Hungary

${ }^{2}$ Hungarian Academy of Sciences, Budapest/Hungary

\section{Contact E-mail Address: eszter1991@gmail.com}

Introduction: Cell-free DNA (cfDNA) is circulating in human plasma and its amount is different in certain physical conditions. It is well known, that in healthy people the quantity of cfDNA is very low, but it rises in chronic disorders such as cancer. At the same time, very high cfDNA level can be measured in healthy people during physical exercise.

Aims \& Methods: We aimed to analyze cfDNA changes (quantity, fragment length, global DNA methylation level) in physiological conditions (during physical exercise) and its alterations in neoplastic and inflammatory colorectal diseases. Plasma was separated from 64 patients (16 colorectal carcinomas (CRC), 13 colonic adenomas (AD), 19 inflammatory bowel disease (IBD), and 16 normal (N) donors without evidence of disease). Plasma samples were also collected from 6 healthy athletes before, during and after physical training. DNA was isolated with High Pure Viral Large Volume NA isolation Kit (Roche). cfDNA was quantified with Qubit fluorometry (Invitrogen). CfDNA fragment length distribution was assessed by Bioanalyzer 2100 using High Sensitivity DNA assay (Agilent). Global DNA methylation was analysed by bisulfite pyrosequencing of long interspersed nuclear element-1 (LINE-1) (Qiagen).

Results: High increase of cfDNA amounts was observed in plasma samples of patients with colonic adenoma $(20.61 \pm 10.70 \mathrm{ng} / \mathrm{ml})$, colorectal cancer $(24.13 \pm 20.02 \mathrm{ng} / \mathrm{ml})$ and IBD $(22.27 \pm 14.60 \mathrm{ng} / \mathrm{ml})$ compared to healthy subjects $(10.33 \pm 3.22 \mathrm{ng} / \mathrm{ml})$. Highly elevated cfDNA amounts were found in plasma samples of athletes during physical exercise $(66.17 \pm 29.00 \mathrm{ng} / \mathrm{ml})$, while the cfDNA amount decreased after physical activity $(51.87 \pm 39.80 \mathrm{ng} / \mathrm{ml})$. Characteristic cfDNA fragment length distribution pattern (with different peak heights at $180 \mathrm{bp}, 360 \mathrm{bp}, 550 \mathrm{bp}$ ) was observed in each patient group. Global DNA hypomethylation was shown in CRC plasma samples with advanced tumor stage (N: $79 \% \pm 0.80 \%$, AD: $79 \% \pm 1.70 \%$, advanced CRC: $70 \% \pm 0.03 \%$ ). Conclusion: Remarkable increase was found in plasma samples of colorectal AD, $\mathrm{CRC}$, IBD patients and also in healthy athletes during physical exercise. CfDNA fragment length analysis showed differences between each group. Global DNA hypomethylation could be observed only in CRC patients with advanced tumor stage. Based on our results, the above DNA analysis methods might contribute to non-invasive detection for colorectal diseases

Disclosure of Interest: All authors have declared no conflicts of interest.

\section{P1109 ANALYSIS OF SFRP1, SFRP2, SDC2 AND PRIMA1 PROMOTER METHYLATION IN CELL-FREE PLASMA DNA FOR NON-INVASIVE DETECTION OF COLORECTAL ADENOMA AND CANCER}

B.K. Bartak ${ }^{1}$, A. Kalmár ${ }^{1}$, B. Péterfia ${ }^{1}$, A.V. Patai ${ }^{1}$, O. Galamb ${ }^{2}$, G. Valcz ${ }^{2}$, B. Wichmann ${ }^{2}$, Z.B. Nagy ${ }^{1}$, E. Markus ${ }^{1}$, K. Szigeti ${ }^{1}$, A. Nagy ${ }^{1}$, Z. Tulassay ${ }^{2}$, P. $\operatorname{Igaz}^{1}$, B. Molnar ${ }^{2}$

${ }^{1}$ 2nd Department Of Internal Medicine, Semmelweis University, Budapest/ Hungary

${ }^{2}$ Hungarian Academy of Sciences, Budapest/Hungary

Contact E-mail Address: bartak.barbara@gmail.com

Introduction: Epigenetic instability, primarily aberrant DNA methylation seems to be a common phenomenon in colorectal cancer (CRC) influencing gene expression and contributing to tumor formation. In recent years, blood-based assays came into focus as potential screening tools for colorectal adenoma and cancer cases, as the analysis of the methylation status of circulating cell-free DNA (cfDNA) in plasma samples provides a good opportunity for cancer detection.

Aims \& Methods: Our aim was to analyse the methylation pattern of four selected genes in biopsy and plasma samples of healthy, colorectal adenoma and CRC patients. Moreover, we aimed to examine the effect of methylation alterations on protein expression. MethyLight (ML) PCR was used after bisulfite-conversion to study certain DNA sequences of the promoter regions of SFRP1, SFRP2, SDC2 and PRIMA1 in 32 biopsy-plasma pairs and in 121 additional plasma samples. For validation, DNA methylation status ( $\beta$-values) of the 4 markers was determined using methyl capture sequencing data from our research group. Furthermore, the methylation status of the four candidates' whole promoter regions was examined in silico using Illumina Infinium HumanMethylation450 BeadChip methylation array data downloaded from The Cancer Genome Atlas (TCGA) database and from NCBI Gene Expression Omnibus Database (GEO accession number: GSE48684). In order to examine the effect of promoter methylation on the protein expression, immunohistochemistry analysis was performed. Results: Using ML assays, methylation of SFRP1, SFRP2, SDC2 and PRIMA1 was observed in $85.1 \%, 72.3 \%, 89.4 \%$ and $80.9 \%$ in the plasma fraction of patients with CRC and $89.2 \%, 83.8 \%, 81.1 \%$ and $70.3 \%$ of adenoma patients, respectively. In multiple logistic regression analysis, the four markers together were able to distinguish CRC patients from healthy individuals with $91.5 \%$ sensitivity and $97.3 \%$ specificity (AUC $=0.978$ ) and could differentiate adenoma samples from healthy controls with $89.2 \%$ sensitivity and $86.5 \%$ specificity $(A U C=0.937)$. In silico analyses confirmed our results on the altered methylation pattern of the four markers in tissue samples. Immunohistochemistry analysis indicated decreasing protein levels of the four markers along the colorectal adenoma-carcinoma sequence.

Conclusion: Our findings suggest that SFRP1, SFRP2, SDC2 and PRIMA1 can be promising epigenetic biomarker candidates for colorectal adenoma and cancer diagnosis with high sensitivity and specificity.

Disclosure of Interest: All authors have declared no conflicts of interest.

\section{Reference}

Galamb O, Kalmár A, Péterfia B, Csabai I, Bodor A, Ribli D, Krenács T, Patai ÁV, Wichmann B, Barták BK et al. Aberrant DNA methylation of WNT pathway genes in the development and progression of CIMP-negative colorectal cancer. Epigenetics. 2016 Aug 2;11(8):588-602.

\section{P1110 URINE-NMR METABOLOMICS FOR SCREENING OF ADVANCED COLORECTAL ADENOMA AND EARLY STAGE COLORECTAL CANCER}

E. Kim ${ }^{1}$, H.N. Kwon ${ }^{2}$, S.H. Kim ${ }^{1}$, N.Y. Paik ${ }^{1}$, S. Hong ${ }^{3}$, T.J. Kim ${ }^{1}$, D.K. Chang ${ }^{1}$, J.J. Kim ${ }^{4}$, S.H. Park ${ }^{2}$, Y. Kim ${ }^{3}$

${ }^{1}$ Samsung Medical Center Dept. of Gastroenterology, Seoul/Korea, Republic of ${ }^{2} 2$ College of Pharmacy, Natural Product Research Institute, Seoul National University, Seoul/Korea, Republic of

${ }^{3}$ Gastroenterolgy, Samsung Medical Center, Seoul/Korea, Republic of

${ }^{4}$ Department Of Medicine, Samsung Medical Center, Sungkyunkwan University School of Medicine, Seoul/Korea, Republic of

Contact E-mail Address: er.kim@samsung.com

Introduction: Metabolomics, a dynamic portrait of the metabolic status of living systems, has demonstrated its great potential for use in the diagnosis of various cancers by applying advanced analytic techniques and bioinformatics tools. Recently, very few metabolic markers in CRC have been consistently discovered, but metabolic profiles of patients with CRC including precancerous lesion in colorectum remains poorly understood and warrants investigation due to its non-invasive sampling method. In the last decade, several metabolomic approaches have been applied toward identifying metabolic alterations in CRC using variety of sample types including urine, tissue, serum, and feces. However there are only few urinary metabolomic studies and especially nuclear magnetic resonance (NMR) spectroscopy, which has several advantages including relatively high degree of reproducibility, easy-to-identify metabolites, high throughoutput, and non-destructive sample treatment, has not been applied to urine samples.

Aims \& Methods: In this study, we investigate the differences in urine metabolic profiles of patients with colorectal neoplasia (CRN) including CRC and precancerous lesion, and healthy volunteers using a NMR-based urine metabolomic study. In addition, we evaluate applicability as diagnostic tool of urine metabolomics for early detection of precancerous colorectal lesion with high sensitivity and specificity. Urine metabolomic profiles from patients with colorectal neoplasia (CRN; 36 advanced adenomas and 56 various stages CRC) and healthy controls $(\mathrm{n}=156)$ were analyzed by NMR spectroscopy. Healthy and CRN groups were statistically discriminated using orthogonal projections to latent structure discriminant analysis (OPLS-DA). The class prediction model was validated by three-fold cross-validation. The advanced adenoma and stage 0 CRC were grouped as pre-invasive CRN

Results: After patients underwent endoscopic resection or surgical resection for CRN, advanced adenoma has been diagnosed in 36 patients, stage O CRC in 24 patients, stage I CRC in 8 patients, stage II CRC in 7 patients, stage III CRC in 13 patients and stage IV CRC in 4 patients. CEA and CA 19-9 levels for patient with stage I to IV CRC and healthy control were also assessed. Among patients with stage I to IV CRC, CEA and CA 19-9 were increased only in two patients, respectively. The sensitivity and specificity of CEA and CA $19-9$ were $6.2 \%$ and 99.3\% The OPLS-DA score plot showed statistically significant discrimination between pre-invasive CRN as well as advanced CRC and normal with a $\mathrm{Q} 2$ value of 0.746 . In the prediction validation study, the sensitivity and specificity for diagnosing pre-invasive CRN was $96.2 \%$ and $95 \%$, respectively. The grades predicted by the PLS-DA model showed that area under the curve was 0.823 for taurine, 0.783 for alanine and 0.842 for 3 -aminoisobutyrate. In multiple receiver operating characteristics curve analyses, taurine, alanine, and 3-aminoisobutyrate were good discriminator for CRC patients.

Conclusion: NMR-based urine metabolomic profiles significantly and accurately discriminate between patients with pre-invasive CRN as well as 
advanced $\mathrm{CRC}$, and healthy control with high accuracy. It demonstrates an applicability of urine-NMR metabolomics as screening tool for accurate diagnosis of pre-invasive CRN

Disclosure of Interest: All authors have declared no conflicts of interest.

\section{References}

Lin Y, Ma C, Liu C, et al. NMR-based fecal metabolomics fingerprinting as predictors of earlier diagnosis in patients with colorectal cancer. Oncotarget 2016;7:29454-64

Ma Y, Zhang P, Wang F, et al. An integrated proteomics and metabolomics approach for defining oncofetal biomarkers in the colorectal cancer. Ann Surg 2012;255:720-30.

Wang H, Wang L, Zhang H, et al. (1)H NMR-based metabolic profiling of human rectal cancer tissue. Mol Cancer 2013;12:121.

P1111 VALIDATION OF THE UTILITY OF A FAECAL IMMUNOCHEMICAL TEST FOR HAEMOGLOBIN (FIT) IN PATIENTS PRESENTING TO PRIMARY CARE WITH NEW BOWEL SYMPTOMS

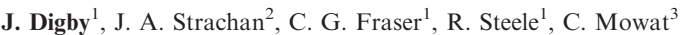

${ }^{1}$ University of Dundee, Dundee/United Kingdom

${ }^{2}$ Blood Sciences, Ninewells Hospital \& Medical School, Dundee/United Kingdom ${ }^{3}$ Gastroenterology, Ninewells Hospital \& Medical School, Dundee/United Kingdom

Contact E-mail Address: jaynedigby@nhs.net

Introduction: Symptoms alone are poor predictors of underlying colonic pathology. ${ }^{1}$ Only $14 \%$ of patients referred for colonoscopy from primary care have significant bowel disease (SBD), [colorectal cancer (CRC), high risk adenoma (HRA, defined as $\geq 3$ or any $\geq 1 \mathrm{~cm}$ ) and inflammatory bowel disease (IBD)]. ${ }^{2} \mathrm{We}$ have reported that undetectable faecal haemoglobin ( $\mathrm{f}-\mathrm{Hb}$ ), measured by a faecal immunochemical test (FIT) is a good rule-out test for SBD. ${ }^{2,3}$ Since December 2015, GPs in Tayside have been encouraged to use FIT test as an adjunct to history, examination and mandatory blood tests in patients referred with bowe symptoms. Referrals are vetted by a Consultant and triaged to test or clinic. We have examined the impact of the introduction of the FIT test on referral rates and colonoscopy yield.

Aims \& Methods: Patients in primary care with new bowel symptoms were encouraged to complete a FIT in addition to blood count and renal function check. We prospectively recorded FIT tests received, referrals to secondary care and colonoscopy findings over 1 year from December 2015 to December 2016. FIT tests were analysed by HMJACKarc (Kyowa Medex Co. Ltd., Japan) with an analytic range of $<10$ to $>400 \mu \mathrm{g} \mathrm{Hb} / \mathrm{g}$ faeces. Referral rates with FIT were examined along with clinical findings at colonoscopy.

Results: 5,655 FIT tests were received. $76.2 \%$ had undetectable $\mathrm{fHb}$, and 152 $(2.7 \%)$ were untestable. 4,108 patients were referred of whom $2,338(57 \%)$ returned a FIT. In 1,378 patients with a FIT result vetted to colonoscopy, 284 had SBD $(20.6 \%)$; 86 CRC, 124 HRA and 74 IBD. $44 \%$ of patients scoped had undetectable $\mathrm{f}-\mathrm{Hb}$ in whom prevalence of SBD was $6.6 \%$. Only $13 \%$ of SBD was in this cohort; 7/7 CRC patients had co-existing iron deficiency anaemia, 14/25 HRA and 3/6 IBD had anaemia or diarrhoea. $32.2 \%$ of those with $\mathrm{f}-\mathrm{Hb}>10 \mu \mathrm{g} \mathrm{Hb} / \mathrm{g}$ faeces had SBD rising to $54.9 \%$ in those with $\mathrm{f}-\mathrm{Hb}$ $>400 \mu \mathrm{g} \mathrm{Hb} / \mathrm{g}$ faeces. 2,677 patients completed a FIT but were not referred, in whom $94.1 \%$ had undetectable $\mathrm{f}-\mathrm{Hb}$. Referrals to secondary care were $14 \%$ down on the previous year.

Conclusion: A FIT test is an essential adjunct to the history, examination and blood tests in the assessment of bowel symptoms. Undetectable $\mathrm{f}-\mathrm{Hb}$ is reassuring Primary Care that SBD is unlikely and referrals to secondary care have reduced. At colonoscopy, yield of SBD has increased and is high in those with detectable $\mathrm{f}-\mathrm{Hb}$. Excluding patients with co-existing anaemia or diarrhoea, an undetectable $\mathrm{f}-\mathrm{Hb}$ is a good rule-out test and will miss only $5 \%$ of all SBD. Furthermore, $\mathrm{f}-\mathrm{Hb}$ concentration could aid triage irrespective of symptoms.

Disclosure of Interest: C.G. Fraser: Prof. Callum Fraser has undertaken consultancy with Immunostics, Ocean, New Jersey, USA; Mode Diagnostics, Glasgow, Scotland; and Kyowa-Medex Co., Tokyo, Japan: and has received travel support from Alpha Labs, Eastleigh, UK.

All other authors have declared no conflicts of interest.

\section{References}

1. Jellema P, van der Windt DAWM, Bruinvels DA et al. Value of symptoms and additional diagnostic tests for colorectal cancer in primary care; systematic review and meta-analysis. BMJ 2010;340:c1269.

2. Mowat C, Digby J, Strachan JA et al. Faecal haemoglobin and faecal calprotectin as indicators of bowel disease in patients presenting to primary care with bowel symptoms. Gut 2016;65(9):1463-9.

3. McDonald PJ, Digby J, Innes $\mathrm{C}$ et al. Low faecal haemoglobin concentration potentially rules out significant colorectal disease. Colorectal Dis 2013;15:e151-9.
P1112 NEW FECAL IMMUNOASSAY TEST (FIT) FOR THE COLORECTAL CANCER SCREENING IN ILE DE FRANCE: IMPACT OF AGE, GENDER AND HEMOGLOBIN LEVEL

S. Bercier ${ }^{1}$, A. Liautaud ${ }^{2}$, A. Kaufmanis ${ }^{3}$, C. Vincelet ${ }^{4}$, A. Bernoux ${ }^{5}$,

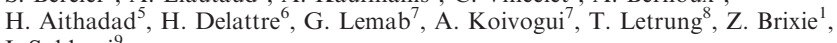
I. Sobhani ${ }^{9}$

${ }^{1}$ ADOC94, Joinville Le Pont/France

${ }^{2}$ ADECA75, Paris/France

${ }^{3} A D C 77, A D C /$ France

${ }^{4} A D M Y 78, A D M Y /$ France

${ }^{5} A D M C 91$, ADMC/France

${ }^{6}$ ADK92, ADK/France

${ }^{7} \mathrm{CDC} 93, \mathrm{CDC} /$ France

${ }^{8}$ APSVO 95, APSVO/France

${ }^{9}$ Dept. De Hepato-gastroenterologie, Universite Paris 12 Hopital H. Mondor Dept. of Hepato-Gastroenterology, Créteil/France

Contact E-mail Address: iradj.sobhani@aphp.fr

Introduction: The immunological screening test (FIT) for colorectal cancer (CCR) was introduced in January 2015 in Ile de France (15 million habitants) after Hemocult was abandoned due to its low sensitivity. The Hemocult (HC) launched in 2007 had reached less than 30\% participation rate.

Aims \& Methods: We report one-year on FIT in CRC screening in Ile de France and compare results to those with $\mathrm{HC}$ test for speculating on adjustment actions. The raw data were extracted by request from the registry of the screening structure (in various areas $75,77,78,91,92,94$ and 95 ) covering a target population of 3026366 habitants. Rates of participation were calculated, and after one-year experience period, profiles of individual with positive tests, rates of those with normal colonoscopy, with polyps (all stages combined) and with cancer + highgrade dysplasia (HGD) were described. Results were compared to those with HC from the launch to the end of the last campaign (Dec 2014) normalized for mean one-year output. The comparisons were made by an X2 test (qualitative variable) or a student $\mathrm{t}$ (quantitative variable) and multivariate stepwise analysis was performed for identifying predictive factors for cancer diagnosis.

Results: At the end of the HC-based screening campaigns 2014, 2.5 million individuals were annually invited and the participation rates since 2009 , ranged from $28.5 \%$ to $24.6 \%$, with females showing higher rates for participation $(30.7$ to $26.1 \%$ ) than men ( 26 to $21.8 \%, \mathrm{p}<0.0001$ ). During the first (2015) year FIT experience, the O Sensor device was used by 450120 (34\% estimated participation rate) individuals (versus 294603 participants with $\mathrm{HC}$ in $2014 \mathrm{p}<0.00001 ; 24 \%$ estimated participation rate), and the rate of positivity was $4.6 \%$ (versus $1.9 \%$ with HC; $\mathrm{p}<0.00001)$. Among all positive FIT individuals, 93\% underwent colonoscopies: $35 \%$ were normal (vs $41 \%$; $p<0.01$ ), $13.2 \%$ presenting with cancer or HGD and $52 \%$ with polyps (vs. $37.7 \% ; \mathrm{p}=0.00051$ ). More women $(54.5 \%)$ than men $(45.5 \%)$ responded to invitations while positivity rate in women $(3.7 \%)$ was lower than in men $(5.5 \% ; \mathrm{p}<0.00001)$. More advanced polyps and cancer were found in men $(14.5 \%)$ than in women $(11.3 \%$; $\mathrm{p}<0.00001)$ with normal colonoscopy rates lower in men $(24.2 \%)$ than in women $(40.7 \% ; \mathrm{p}<0.0001)$. The levels (mean $\mathrm{ng} / \mathrm{mL} ; \mathrm{SD})$ of FIT were significantly higher $(\mathrm{p}<0.001)$ in individuals with cancer +HGD $(667 ; 9)$ or with polyps $(374 ; 4)$ than in those with normal colonoscopy $(440 ; 4)$. In multivariate analysis, risk factors for abnormal colonoscopies with cancer + HGD were male gender, FIT $>340 \mathrm{ng} / \mathrm{mL}$ and age $>63.5$ yrs. More men than women with positive FIT test did not yet undergo colonoscopy

Conclusion: FIT test has gained a better adhesion in Ile de France probably because to its simplicity and a wider distribution. The lower rate of normal colonoscopies and a higher positive predictive value for cancer leads to more early lesions detected indicating its higher specificity. Men of 63.5 yrs old or more with Hemoglobin levels higher than $340 \mathrm{ng} / \mathrm{mL}$ are of very high risk of cancer and should be absolutely conducted to the colonoscopy in case positive FIT test. Disclosure of Interest: All authors have declared no conflicts of interest.

\section{P1113 ARTIFICIAL INTELLIGENCE CAN PREDICT THE PRESENCE OF LYMPH NODE METASTASIS IN T1 COLORECTAL CANCER}

K. Ichimasa ${ }^{1}$, S. Kudo ${ }^{1}$, Y. Mori ${ }^{1}$, M. Misawa ${ }^{1}$, S. Matsudaira ${ }^{1}$, Y. Kouyama ${ }^{1}$, T. Baba ${ }^{2}$, K. Wakamura ${ }^{1}$, T. Hayashi ${ }^{1}$, T. Hisayuki ${ }^{1}$, T. Kudo ${ }^{1}$, N. Toyoshima ${ }^{1}$, H. Nakamura ${ }^{1}$, T. Ishigaki ${ }^{1}$, Y. Yagawa ${ }^{1}$, K. Takeda ${ }^{1}$, Y. Maeda ${ }^{1}$, Y. Ogawa ${ }^{1}$, S. Hamatani ${ }^{3}$, H. Miyachi ${ }^{1}$, F. Ishida ${ }^{1}$

${ }^{1}$ Digestive Disease Center, Showa University Northern Yokohama Hospital

Digestive Disease Center, Yokohama/Japan

${ }^{2}$ Showa University Yokohama Northern Hospital, Yokohama/Japan

${ }^{3}$ Department Of Pathology, Jikei University School of Medicine, Tokyo/Japan

Contact E-mail Address: ichitommy14@yahoo.co.jp

Introduction: Most T1 colorectal cancers (CRCs) undergo surgical colectomy in accordance with established clinical guidelines despite the low incidence (approximately $10 \%$ ) of lymph node metastasis (LNM). Therefore, many patients without LNM undergo unnecessary surgeries.

Aims \& Methods: To reduce unnecessary surgeries, we aimed to predict the risk of LNM in T1 CRCs by using artificial intelligence (AI). Data on 690 consecutive patients with T1 CRCs who had undergone colectomy between April 2001 and March 2016 were retrospectively analyzed. Data of a randomly selected 590 patients were used for machine learning for the AI model, which analyzed five clinicopathological factors: tumor location, lymphatic invasion, vascular invasion, tumor budding and histological grade. The remaining 100 patients served as a test set for validating the AI model and output the predicted LNM as positive or negative. To validate the AI model, sensitivity, specificity and 
accuracy were calculated and compared with American, European and Japanese guidelines.(1-5)

Results: Sensitivity was $100 \%(95 \%$ CI, 56\%-100\%) in all models. Specificity and accuracy of the AI model, American, European and Japanese guidelines were $68 \%(58 \%-78 \%)$ vs. $45 \%(35 \%-56 \%)$ vs. $12 \%(6 \%-21 \%)$ vs. $8 \%(3 \%-15 \%)$, and $71 \%(61 \%-80 \%)$ vs. $50 \%(40 \%-60 \%)$ vs. $20 \%(13 \%-29 \%)$ vs. $16 \%(9 \%-25 \%)$, respectively. The rate of unnecessary surgeries of the AI model was calculated as $29 \%$ in comparison with American $50 \%(\mathrm{P}=0.004$, odds ratio [OR] 2), European $80 \%(\mathrm{P}<0.001$, OR 10), and Japanese $84 \%(\mathrm{P}<0.001$, OR 13).

Conclusion: AI can significantly reduce unnecessary surgeries compared with current guidelines while providing high sensitivity. AI will help in making decisions as to whether additional surgery is indicated after endoscopic resection of T1 CRCs. Grant support: Grants-in-Aid for Scientific Research (Number 17K 15972) from the Japan Society for the Promotion of Science.

Disclosure of Interest: All authors have declared no conflicts of interest.

\section{References}

1. Network NCC. NCCN Clinical Practice Guidelines in Oncology (NCCN Guidelines $\left.{ }^{\circledR}\right)$ Rectal Cancer (Version 1.2017). 2017.

2. Network NCC. NCCN Clinical Practice Guidelines in Oncology (NCCN Guidelines $($ ) Colon Cancer (Version 1.2017). 2017.

3. Labianca R, Nordlinger B, Beretta GD, et al. Early colon cancer: ESMO Clinical Practice Guidelines for diagnosis, treatment and follow-up. Ann Oncol 2013;24 Suppl 6:vi64-72.

4. Glimelius B, Tiret E, Cervantes A, et al. Rectal cancer: ESMO Clinical Practice Guidelines for diagnosis, treatment and follow-up. Ann Oncol 2013;24 Suppl 6:vi81-8.

5. Watanabe T, Muro K, Ajioka Y, et al. Japanese Society for Cancer of the Colon and Rectum (JSCCR) guidelines 2016 for the treatment of colorectal cancer. Int J Clin Oncol 2017.

\section{P1114 RISK FACTORS OF ADVANCED METACHRONOUS NEOPLASM IN COLONOSCOPIC SURVEILLANCE AFTER COLON CANCER RESECTION}

K. Nam ${ }^{1}$, J.E. Shin ${ }^{1}$, K.B. Bang ${ }^{1}$, H.D. Shin ${ }^{1}$, H. Namgung ${ }^{2}$, D.G. Park ${ }^{2}$ ${ }^{1}$ Gastroenterology, Dankook University Hospital, Cheonan/Korea, Republic of ${ }^{2}$ Surgery, Dankook University Hospital, Cheonan/Korea, Republic of

\section{Contact E-mail Address: nambag1108@gmail.com}

Introduction: Regular surveillance colonoscopy after colon cancer resection is recommended to detect metachronous adenoma and cancer. However, risk factors of advanced metachronous neoplasm during postoperative surveillance have not been fully evaluated yet.

Aims \& Methods: This study aimed to assess the risk of advanced metachronous neoplasm during surveillance colonoscopy in patients who underwent curative colon cancer resection. The patients who underwent curative colonic resection for non-metastatic colon cancer between January 2002 and December 2012 in a single tertiary center were retrospectively reviewed.

Results: A total of 298 patients were enrolled in this study. Surveillance colonoscopy was performed after perioperative clearing colonoscopy. Among the patients, $182(61.6 \%)$ were male, and the median age was 65 years. On perioperative clearing colonoscopy, accompaning high-risk adenomas $(\geq 3$, size $\geq 10 \mathrm{~mm}$, with high-grade dysplasia and villous histology) were detected in 95 patients $(31.9 \%)$ and were significantly associated with old age ( $\geq 65$ years), male sex, alcohol use, smoking, and stage 3 colon cancer $(P<0.05)$. During the postoperative follow-up periods (median, 5.35 years), advanced metachronous neoplasm was found in 45 patients $(15.1 \%)$ during surveillance colonoscopy, including colon cancer in 4 patients $(1.3 \%)$. In the multivariate analysis, distal colon cancer (distal to splenic flexure; odds ratio $[\mathrm{OR}]=4.463 ; \mathrm{P}=0.002$ ), accompaning high-risk adenomas on perioperative clearing colonoscopy $(\mathrm{OR}=3.414 ; \mathrm{P}=0.001)$, and hypertension $(\mathrm{OR}=2.344 ; \mathrm{P}=0.026)$ were significant risk factors of advanced metachronous neoplasm during surveillance colonoscopy.

Conclusion: Patients who had distal colon cancer, accompaning high-risk adenoma on perioperative clearing colonoscopy, and hypertension may need a shorter colonoscopic surveillance interval. A more tailored surveillance strategy is needed to improve overall outcome in patients who undergo curative colon cancer resection.

Disclosure of Interest: All authors have declared no conflicts of interest.

\section{References}

1. Kahi CJ, Boland CR, Dominitz JA, Giardiello FM, Johnson DA, Kaltenbach $\mathrm{T}$, et al. Colonoscopy Surveillance After Colorectal Cancer Resection: Recommendations of the US Multi-Society Task Force on Colorectal Cancer. Gastroenterology 2016;150:758-68.e11.

2. Fuccio L, Spada C, Frazzoni L, Paggi S, Vitale G, Laterza L, et al. Higher adenoma recurrence rate after left- versus right-sided colectomy for colon cancer. Gastrointest Endosc 2015;82:337-43.
P1115 PROSPECTIVE COMPARISON OF THE NOVEL FULL

SPECTRUM ENDOSCOPY (FUSE) AND ADVANCED HIGH DEFINITION-WHITE LIGHT ENDOSCOPY FOR DETECTION OF POLYPS IN ROUTINE PRACTICE

A. Martinez- Alcala García ${ }^{1}$, F. J. Martínez-Alcala ${ }^{1}$, C. Martínez- Alcalá ${ }^{1}$, K. Thakar ${ }^{1}$, R. Madero ${ }^{2}$, K. Mönkemüller ${ }^{3}$, F. Martínez Alcalá ${ }^{1}$ ${ }^{1}$ Centro de Innovaciones Digestivas Martínez-Alcalá, Seville/Spain

${ }^{2}$ Bioestadistica, Complejo Hospitalario La Paz, Madrid/Spain

${ }^{3}$ Gastroenterology, Frankenwald Klinikum, Kronach/Germany

Contact E-mail Address: alvaromalcala@gmail.com

Introduction: Despite major advances in white light endoscopy detection of colon polyps remains challenging with significant polyp miss rates. The novel second generation full spectrum endoscopy (FUSE) is a new scope with two additional cameras in the sides that provides a panoramic $330^{\circ}$ field of view.

Aims \& Methods: The aim of this study is to identify the role of the FUSE in improving polyp detection. This was a single-center, prospective, randomized, open label study in patients that presented for routine colonoscopy at an outpatient unit during a six months period. Patients were randomized to either FUSE (FUSE colonoscope CDVL slim c38) or standard frontal view (SFV) colonoscopy (Olympus Evis Exera III 190). The primary outcomes were polyp detection rates (PDR), diverticulum detection rate (DDR) and complete colonoscopy. Secondary outcomes were procedure time, adverse event rates, size and characteristics of the polyps and success of endoscopic treatment (R0 resection). All procedures were performed by experienced endoscopists, who had carried out $>5000$ colonoscopies and had cecal intubation rates of $>95 \%$.

Results: A total of 197 patients $(49.2 \%$ female, $50.8 \%$ male, median age 60 years, range \pm 16 years) were studied. No significant difference was seen between the 2 groups for the primary endpoints of polyps detection rate (PDR), diverticulum detection rate (DDR) or complete colonoscopy (table 1). About secondary endpoints: R0 endoscopic resection was achieved in $95 \%$ in both groups $(\mathrm{p}=0.68)$. The median procedure time in minutes was higher with SFV $(26.1 \pm 13 \mathrm{~min})$ than FUSE $21.5+/-10.7 \mathrm{~min}(-4.6$, IC95\%: -8 a -1.2$)$ $(\mathrm{p}=0.008)$. There were no significant differences regarding adverse events, determination of colon cleanliness, or others epidemiologic factors. 2 case were excluded of the statistical analysis due to surveillance of polyposis syndrome, to avoid skewing of results.

Conclusion: In expert hands, PDR and DDR exceed 50\% with advanced white light and FUSE systems. FUSE was not superior to advanced white light endoscopy for the PDR and DDR. However, with FUSE we can reduce procedure duration without any additional adverse events or increased discomfort. These data further demonstrate the safety and feasibility of the new FUSE system.

Disclosure of Interest: All authors have declared no conflicts of interest.

\section{P116 UNDERWATER ENDOSCOPIC MUCOSAL RESECTION FOR NON-PENDULATED COLORECTAL LESIONS. IS THE DISTAL CAP REALLY NECESSARY?}

L.H. Lenz Tolentino ${ }^{1}$, C. Pennacchi ${ }^{2}$, J. F. De Oliveira ${ }^{2}$, E. Q. Mendonça ${ }^{2}$, M. Minata ${ }^{3}$, E. Gonzalez ${ }^{2}$, G. De Paulo ${ }^{2}$, S. Geiger ${ }^{2}$, M. Sorbello ${ }^{2}$, M. Simas De Lima ${ }^{2}$, F. Kawaguti ${ }^{2}$, B. D.C. Martins ${ }^{2}$, E. R. Baba ${ }^{2}$,

A. V. Safatle-Ribeiro ${ }^{2}$, C. C. Gusmon ${ }^{2}$, R. Uemura ${ }^{2}$, A. Tellian ${ }^{4}$, F. Rolim ${ }^{4}$, C. Hashimoto ${ }^{4}$, U. Ribeiro Jr. ${ }^{2}$, F. Maluf-Filho ${ }^{2}$

${ }_{1}^{1}$ Endoscopia, Fleury Medicina e saúde, Sao Paulo/Brazil

${ }^{2}$ Gastrointestinal Endoscopy Unity, Cancer Institute of University of São Paulo, São Paulo/Brazil

${ }^{3}$ Endoscopy, Cancer Institute of Sao Paulo State, Sao Paulo/Brazil

${ }^{4}$ Gastroenterology, University of Sao Paulo, Sao Paulo/Brazil

Contact E-mail Address: ktpennacchi@yahoo.com.br

Introduction: Underwater Endoscopic Mucosal Resection (UEMR) has been demonstrated as a safe and effective technique for removal of flat and sessile colorectal lesions. Until now, only UEMR with cap has been described in literature. In addition there is no consensus about the electrosurgical settings.

Aims \& Methods: This prospective study was conducted between January and November 2016 in two university tertiary referral centers. UEMR was performed using a standard colonoscope without the distal cap. The lesions were marked with snare tip prior to resection. Insufflation was switched off. Then, the colon lumen was entirely deflated. Water at room temperature was infused using an irrigation pump until complete filling of the lumen was achieved. All gas pockets in the operative field were evacuated. Two sizes of polypectomy snare $(13$ and $25 \mathrm{~mm})$ were used according to the preference of the endoscopist. No submucosal injection was performed. One of these three different electrosurgical settings (DRYCUT, AUTOCUT and ENDOCUT) was selected. All resection wounds were carefully inspected after UEMR. Endoclips were employed for the management of bleeding and suspected perforation.

Results: Between January and November 2016, 45 patients (27 female, mean age 67 years, range 53-87) with 55 non-pedulated colorectal lesions (mean size $16 \mathrm{~mm}$, range $10-40 \mathrm{~mm}$ ) were included. Six lesions were located in cecum 21 in the ascending colon, nine in transverse, eight in descending colon, five in sigmoid, and six in rectum. In five patients, we selected DRYCUT mode; in fifteen AUTOCUT; and in twenty-five the ENDOCUT mode. All lesions were successfully and completely removed by UEMR. The procedure time was recorded in 24 resections (mean 13 minutes, range 4-40). Thirty-three of them were removed en bloc $(60 \%)$. Histology revealed the following: $40 \%$ tubular adenomas; $20 \%$ - tubulovillous adenomas; $25.45 \%$ - sessile serrated adenomas; $3.65 \%$ - traditional serrated polyps; $7.25 \%$ intramucosal adenocarcinomas and 
$3.65 \%$ were superficial submucosal carcinomas $(<1000 \mu \mathrm{m})$. During UEMR, two cases (both using AUTOCUT mode) of spurting bleeding were observed $(4.45 \%)$. Hemostasis was easily achieved in both cases by clipping. No patient requires blood transfusion. One patient had abdominal pain on the day after resection without signs of pneumoperitoneum on CT scan. There was no perforation or delayed bleeding.

Conclusion: This study supports the existing data indicating acceptable rates of technical success and low incidence of adverse events with UEMR. The results of this study without cap were similar with the previous ones using cap. Further comparative studies with and without cap, using different settings and especially between UEMR and traditional EMR are needed.

Disclosure of Interest: All authors have declared no conflicts of interest.

\section{References}

1. Bourke MJ. Endoscopic mucosal resection in the colon: A practical guide. Techniques in Gastrointestinal Endoscopy. 2011;13:35-49.

2. Binmoeller KF, Weilert F, Shah J, Bhat Y, Kane S. "Underwater" EMR without submucosal injection for large sessile colorectal polyps (with video). Gastrointest Endosc. 2012 May;75(5):1086-91.

3. Binmoeller KF. Underwater endoscopic mucosal resection. $J$ Interv Gastroenterol. 2014;4(4):113-6.

4. Curcio G, Granata A, Ligresti D, Tarantino I, Barresi L, Liotta R, et al. Underwater colorectal EMR: remodeling endoscopic mucosal resection. Gastrointest Endosc. 2015 May;81(5):1238-42.

5. Binmoeller KF, Hamerski CM, Shah JN, Bhat YM, Kane SD, GarciaKennedy R. Attempted underwater en bloc resection for large $(2-4 \mathrm{~cm}) \mathrm{col}$ orectal laterally spreading tumors (with video). Gastrointest Endosc. 2015 Mar;81(3):713-8.

\section{P1117 ADENOMA DETECTION RATE INFLUENCES RISK PREDICTION OF METACHRONOUS ADVANCED COLORECTAL NEOPLASIA IN LOW-RISK PATIENTS}

S.H. Kim, S.J. Lee, T.J. Kim, E.R. Kim, S.N. Hong, D.K. Chang, Y. Kim Gastroenterology, Samsung Medical Center, Seoul/Korea, Republic of

\section{Contact E-mail Address: ilonaelona $@$ hanmail.net}

Introduction: Current guidelines recommend surveillance colonoscopy after 10 years or surveillance in 5-10 years in individuals with no or 1-2 non-advanced adenomas.

Aims \& Methods: We hypothesized that risk of metachronous advanced colorectal neoplasia (AN) differs based on clinical characteristics and colonoscopy quality. We identified 7,171 participants with no or non-advanced adenomas at first-time screening colonoscopy. The risk of metachronous AN at surveillance colonoscopy 3-5 years later was investigated according to clinical characteristics and endoscopist adenoma detection rate (ADR).

Results: In multivariate analyses, strong associations between increasing age, male sex, current smoking, family history of colorectal cancer, follow-up interval, increasing number of adenoma, and low ADR and risk of any metachronous colorectal neoplasia were observed. For metachronous AN, increasing age, male sex, increasing number of adenoma, and low ADR were independent risk factors. Among patients with $1-2$ small adenomas, women with age $\geq 60$ years or men comprised a hidden-risk group, which had $5.3 \%$ risk of metachronous AN at surveillance. Women $<60$ years old with $1-2$ low-risk adenomas had very low risk $(1.2 \%)$ of metachronous $\mathrm{AN}$ as individuals with no adenoma. Furthermore, incidence of metachronous AN was significantly higher in individuals who were colonoscopy screened by endoscopists with a lower ADR $(<32 \%)$ than in those screened by endoscopists with a higher ADR $(\geq 32 \%)(3.2 \%$ vs. $0.6 \%$, respectively; $P=0.001$ )

Conclusion: According to patient and adenoma characteristics, and ADR of the endoscopist, the risk of metachronous $\mathrm{AN}$ varies among low-risk patients. In recommending surveillance colonoscopy, these factors should be taken into consideration.

Disclosure of Interest: All authors have declared no conflicts of interest.

\section{References}

1. Brenner H, Chang-Claude J, Seiler CM, et al. Protection from colorectal cancer after colonoscopy: a population-based, case-control study. Ann Intern Med 2011;154:22-30.

2. Kahi CJ, Imperiale TF, Juliar BE, et al. Effect of screening colonoscopy on colorectal cancer incidence and mortality. Clin Gastroenterol Hepatol 2009; 7:770-5; quiz 11.

3. Nishihara R, Wu K, Lochhead P, et al. Long-term colorectal-cancer incidence and mortality after lower endoscopy. $N$ Engl J Med 2013;369:1095-105.

4. Shaukat A, Mongin SJ, Geisser MS, et al. Long-term mortality after screening for colorectal cancer. $N$ Engl J Med 2013;369:1106-14.

5. Edwards BK, Ward E, Kohler BA, et al. Annual report to the nation on the status of cancer, 1975-2006, featuring colorectal cancer trends and impact of interventions (risk factors, screening, and treatment) to reduce future rates. Cancer 2010;116:544-73

6. Atkin WS, Edwards R, Kralj-Hans I, et al. Once-only flexible sigmoidoscopy screening in prevention of colorectal cancer: a multicentre randomised controlled trial. Lancet 2010;375:1624-33.

7. Brenner H, Chang-Claude J, Seiler CM, et al. Long-term risk of colorectal cancer after negative colonoscopy. J Clin Oncol 2011;29:3761-7.
8. Holme O, Loberg M, Kalager M, et al. Effect of flexible sigmoidoscopy screening on colorectal cancer incidence and mortality: a randomized clinical trial. JAMA 2014;312:606-15.

9. Imperiale TF, Glowinski EA, Lin-Cooper C, et al. Five-year risk of colorectal neoplasia after negative screening colonoscopy. $N$ Engl $\mathrm{J} \mathrm{Med}$ 2008:359:1218-24.

10. Schoen RE, Pinsky PF, Weissfeld JL, et al. Colorectal-cancer incidence and mortality with screening flexible sigmoidoscopy. $N$ Engl $\mathrm{J} \mathrm{Med}$ 2012;366:2345-57.

\section{P1118 EXPERIENCE OF PER ANAL ENDOSCOPIC MYECTOMY (PAEM)}

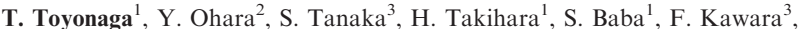
T. Ishida ${ }^{3}$, Y. Morita ${ }^{3}$, E. Umegaki ${ }^{3}$

${ }^{1}$ Endoscopy, Kishiwada Tokushukai Hospital, Kishiwada/Japan

${ }^{2}$ Dept. Of Endoscopy, Kobe University Hospital, Kobe/Japan

${ }^{3}$ Division Of Gastroenterology, Department Of Internal Medicine, Graduate School of Medicine, Kobe University, Kobe/Japan

\section{Contact E-mail Address: toyonaga $@$ med.kobe-u.ac.jp}

Introduction: The technique of endoscopic submucosal dissection has recently been improved, and large and complexed lesions such as those invading ileocecal valve and appendix orifice can be resected en bloc. However, lesions accompanying severe fibrosis in the submucosal layer and exhibiting the muscle retraction (MR) sign are often difficult to be resected completely ${ }^{1)}$. We devised a new method called 'Per Anal Endoscopic Myectomy' for rectal lesions involving severe fibrosis, in which dissection is done between the inner circular and outer longitudinal muscles instead of between submucosal layer and muscle layer ${ }^{2}$. Aims \& Methods: The aim of this study is to examine the usefulness and safety of PAEM. The PAEM cases performed in our hospital and an affiliated hospital were retrospectively reviewed. When fibrosis in the submucosal layer was suspected, pocket creation method ${ }^{3)}$ was applied and if severe fibrosis with MR sign was found, PAEM was selected. In PAEM procedure, after dissecting circumferentially around the fibrotic area with a double tunneling method, the inner circular muscle is cut in a circular manner, which makes the outer longitudinal muscle clearly visible. The space between the inner circular and outer longitudinal muscles is sparse and suitable traction with the tunneling method makes it easier to dissect this space. PAEM was performed only for rectal lesions, and no clip closure was carried out after the procedure in most cases.

Results: Ten rectal lesions were treated with PAEM between July 2015 and March 2017. Among them, 7 cases including 2 cases with mucosal cancer, 4 cases with deep submucosal invasion and 1 case with muscle invasion were resected en bloc with negative margin. The other 3 cases showed tumor invasion to the muscle layer and the vertical margin was positive. The clinical course after PAEM was preferable in all cases. Three cases which achieved resection with negative margin but found lymphovascular invasion of the tumor underwent additional surgery or adjuvant chemoradiation. In surgical cases, they could permit anus-preservation.

Conclusion: PAEM for lesions exhibiting MR sign with severe fibrosis will enable complete en bloc resection and lead to accurate pathological diagnosis. No complications were recorded in our experiences. Further investigation into the significance of PAEM would be needed.

Disclosure of Interest: T. Toyonaga: Dr. Toyonaga invented the Flush knife-BT in conjunction with Fujifilm and receives royalties from its sale.

All other authors have declared no conflicts of interest.

\section{References}

1. Toyonaga T, Tanaka S, Man IM, et al. Clinical significance of the muscleretracting sign during colorectal endoscopic submucosal dissection. Endosc Int Open 2015;3:E246-251.

2. Rahni DO, Toyonaga T, Ohara Y, et al. First reported case of per anal endoscopic myectomy (PAEM): A novel endoscopic technique for resection of lesions with severe fibrosis in the rectum. Endosc Int Open. 2017;5(3):E146-E150. 3. Hayashi Y, Sunada K, Takahashi H, et al. Pocket-creation method of endoscopic submucosal dissection to achieve en bloc resection of giant colorectal subpedunculated neoplastic lesions. Endoscopy 2014;46 Suppl 1 UCTN:E421-422.

\section{P1119 LOCAL RECURRENCE AFTER ENDOSCOPIC MUCOSAL RESECTION FOR HIGH-RISK LESIONS: MAY WE BETTER PLAN THE ENDOSCOPIC FOLLOW-UP ACCORDING TO PROCEDURAL, MORPHOLOGICAL AND HISTOLOGICAL CHARACTERISTICS?}

C. Quondamcarlo, C. Lucidi, R. Lapenta

Gastroenterology, Regina Elena Cancer Institute, Rome/Italy

Contact E-mail Address: qcinzia@ymail.com

Introduction: Endoscopic mucosal resection (EMR) is an increasingly used technique for the removal of large sessile and flat-laterally-spreading colorectal lesions. At present, surveillance colonoscopies are ever performed to ensure detection and adequate treatment of residual or recurrent adenoma (RRA), which, occurring in $10-40 \%$ of non-pedunculated lesions, currently represents the main limitation of this technique. Fortunately, endoscopic detection of RRA in the post EMR scar is currently highly accurate using HD-WL (high definitionwhite light) and NBI (narrow band imaging). Anyway, indications for follow-up 
colonoscopies and optimal time intervals are currently unclear. An adequate comprehension of the predicting factors of RRA would be very relevant to try to prevent it, better plan surveillance intervals, establish role and timing of surgery and reduce costs.

Aims \& Methods: Our aims were to assess the frequency of local recurrence after EMR for high-risk lesions, to identify risk factors for recurrence, and to provide follow-up suggestions. We considered all consecutive patients undergone EMR of flat or sessile adenomas $\geq 10 \mathrm{~mm}$ containing high-grade dysplasia or adenocarcinoma until pT1 from May 2015 to April 2016. EMR procedure was performed by 2 endoscopists with extensive EMR "inject and cut" experience and only lesions with a positive lifting sign were endoscopically treated. An endoscopic follow-up was performed at a planned interval of 3,6 and 12 months by using high-definition instruments white light (HD-WL) and narrow-band imaging (NBI) allowing an appropriate scare assessment with the execution of biopsies when needed. Data collection included: (a) lesion size, morphology, Paris classification, pit pattern (kudo), technique of removal (en bloc vs piecemeal) and histology for all primary lesions and RRA; (b) Information about budding, grading, microinvasion, margins, submucosal extension for all pT1 removed "en bloc".

Results: 50 patients were included (mean age $63 \pm 12$ years, 54\% females). The mean size of lesions was $21 \mathrm{~mm}$ (range $10-50 \mathrm{~mm}$ ), $40 \%$ were sessile, $35 \%$ granular LST and the reimaging $25 \%$ non-granular LST, the most frequent sites of location were sigmoidal colon $(40 \%)$, ascending colon $(25 \%)$ and cecum $(12 \%)$. According to the morphological characteristics, $60 \%$ of lesions were removed "en bloc" and $40 \%$ "piecemeal". Metallic clips were used as prophylaxis in $35 \%$ of patients and only in 1 for intraprocedural bleeding. No postprocedural bleeding or perforation occurred. APC has never been used. During the endoscopic follow-up a suspect early (3-months) RRA was documented and immediately treated in $16 \%$ and histologically confirmed only in $8 \%$. At 6 -month controls a RRA was again detected only in 2 of these patients. New cases of RRA were not found both at 6 and 12 month controls. Only 1 case of RRA was documented for lesions treated "en bloc" but this was the only one with histological characteristics strongly suggesting high risk of RRA (pT1, > $2 \mathrm{~cm}$, grading G2, free-disease margins $<1 \mathrm{~mm}$ ). As concerned lesions removed "piecemeal", RRA ( $70 \%$ high grade dysplasia and 30\% low grade dysplasia) was higher in pT1, lesions $>2 \mathrm{~cm}$, non-granular LST. No correlation was found with other factors.

Conclusion: EMR results a technique safe and effective particularly for lesions removable "en bloc"; in this cases in fact the rate of RRA seems to be low and easily histologically predictable. Although factors related to RRA in "piecemeal" EMR appear less clear more attention would be applied in relation to determined morphological pattern and presence of in situ carcinoma. Moreover, RRA seems to be ever early suggesting a close follow up only in the first period.

Disclosure of Interest: All authors have declared no conflicts of interest.

\section{Reference}

Tim D. G. Belderbos, Max Leenders, Leon M. G. Moons, Peter D. Siersema. Local recurrence after endoscopic mucosal resection of nonpedunculated colorectal lesions: systematic review and meta-analysis. Endoscopy 2014; 46: 388-400.

\section{P1120 TREATMENT STRATEGY FOR LOCAL RECURRENCES}

\section{AFTER ENDOSCOPIC RESECTION OF COLORECTAL NEOPLASMS}

S. Ito, K. Hotta, K. Imai, Y. Yamaguchi, Y. Kishida, K. Takizawa,

N. Kakushima, M. Tanaka, N. Kawata, M. Yoshida, H. Ishiwatari,

H. Matsubayashi, H. Ono

Division Of Endoscopy, Shizuoka Cancer Center, Shizuoka/Japan

\section{Contact E-mail Address: sa.ito@scchr.jp}

Introduction: Local recurrences after endoscopic resection (ER) frequently occur, especially after piecemeal resection. Recently, the efficacy of endoscopic submucosal dissection (ESD) for local recurrences has been reported. However, an appropriate treatment strategy for these lesions including ESD remains unclear.

Aims \& Methods: This study aimed to clarify the appropriate treatment strategy for local recurrences after ER. A total of 81 patients ( 81 lesions) who received treatment for local recurrences after ER for colorectal neoplasms between January 2010 and December 2016 were enrolled. Patients with pathological diagnosis of hyperplastic polyp, sessile serrated adenoma/polyp, and submucosal invasive cancer in their first ER were excluded. Seven patients who underwent surgery because of submucosal invasion or technically difficult locations were also excluded. Procedural outcomes, recurrence rate and disease control rate (DCR) were evaluated according to preoperative endoscopic diagnosis of recurrent lesions (adenomatous or cancerous). The DCR was defined as proportion of patients who were diagnosed with curative resection after ER or received additional surgery based on pathological diagnosis after ER.

Results: Seventy-four patients were included in the final analysis. Forty-nine patients diagnosed with adenomatous recurrences were treated by cold polypectomy in 15 , by endoscopic mucosal resection (EMR) in 26, and by ESD in 8 patients. Cold polypectomy was applied only to diminutive $(<5 \mathrm{~mm})$ lesions and there was no local recurrence. The en bloc resection rates of EMR and ESD were $53.8 \%$ and $100 \%$, respectively $(P=0.030)$. Two cases $(7.7 \%)$ in the EMR group developed local recurrences, but additional ER achieved curative resection. The DCR of three methods were all 100\%. Meanwhile, 25 patients diagnosed with cancerous recurrences were treated by EMR in 7 and by ESD in 18 patients. The en bloc resection rates of EMR and ESD were $28.6 \%$ and $83.3 \%$, respectively $(P=0.017)$. Three cases $(42.9 \%)$ in the EMR group developed local recurrences. One case required surgery because of invasive local recurrence, the second case required chemotherapy because of distant metastasis, and the third case was followed-up because of severe comorbidities. Therefore, the DCR in the EMR group was significantly lower than that of in the ESD group $(28.6 \%$ vs. $83.3 \%, P=0.017)$.

Conclusion: The selection of ER for local adenomatous recurrences could be based on lesion size. On the other hand, ESD is desirable for local cancerous recurrences to achieve complete disease control.

Disclosure of Interest: All authors have declared no conflicts of interest.

\section{P1121 A COMPARATIVE STUDY ON EFFICACY OF CHEMOTHERAPY AFTER ENDOSCOPIC COLONIC STENTING VS. THAT AFTER COLONIC SURGERY IN THE MANAGEMENT OF OBSTRUCTIVE COLORECTAL CANCER}

N. Hanabata ${ }^{1}$, Y. Sasaki ${ }^{2}$, K. Kanazawa ${ }^{1}$, S. Igarashi ${ }^{1}$, K. Hasui ${ }^{1}$, K. Shimaya ${ }^{1}$, H. Numao ${ }^{1}$, M. Munakata' ${ }^{1}$, S. Fukuda ${ }^{3}$

${ }_{1}^{1}$ Gastroenterology, Aomori Prefectural Central Hospital, Aomori/Japan

${ }^{2}$ Medical Informatics, Hirosaki University Graduate School of Medicine, Hirosaki/ Japan

${ }^{3}$ Gastroenterology And Hematology, Hirosaki University Graduate School of Medicine, Hirosaki/Japan

Contact E-mail Address: ohana@pastel.ocn.ne.jp

Introduction: Endoscopic stent placement in acute large-bowel obstruction due to colorectal cancer has been established as a palliative therapy or bridge to surgery with good outcomes in Japan. While, efficacy of chemotherapy after endoscopic colonic stenting has not been evaluated.

Aims \& Methods: The aim of this study was to evaluate efficacy of chemotherapy after endoscopic colonic stenting by comparing with that after surgery. Sixty five patients with colorectal cancer of stage IV presenting obstructive symptom visiting Aomori Prefectural Central Hospital from January 2012 to December 2016 were classified into SC group who have underwent chemotherapy after endoscopic colonic stenting (32 patients) and OC group who have underwent chemotherapy after surgery ( 23 patients). The patient's background, adverse effects and prognosis were compared with two groups.

Results: There have not been any significant differences in patient age $(65.5 \pm 9.3$ in $\mathrm{SC}$ vs. $61.5 \pm 14.1$ in $\mathrm{OC}, \mathrm{p}=0.21)$, male to female ratio $(25: 7$ in $\mathrm{SC}$ vs. 14:9 $\mathrm{p}=0.16)$ and performance status $(0.6 \pm 0.7$ in $\mathrm{SC}$ vs. $0.7 \pm 0.8$ in $\mathrm{OC}, \mathrm{p}=0.66)$. The averaged colorectal obstruction scoring system $(0$, Requiring continuous decompressive procedure; 1, No oral intake; 2, Liquid or enteral nutrient; 3, Soft solids, low-residue, and full diet with symptoms of stricture; 4, Soft solids, low-residue, and full diet without symptoms of stricture) score was significantly severe in OC $(1.7 \pm 1.1)$ than in SC $(0.7 \pm 0.9, \mathrm{p}<0.01)$. Adverse effects included perforation (1), stent slippage (6), re-obstruction by tumor progression (3) in SC, and ileus (1), abdominal abscess (1), renal insufficiency (1) in OC. The frequency of combined use of molecular target drugs (Bevacizumab/Panitumumab/ Cetuximab) at first chemotherapy has significantly differed $(\mathrm{p}<0.01)$ between SC $(0 / 11 / 9)$ and OC $(12 / 4 / 0)$. The median survival day was not significantly different between SC (595) and OC $(459, \mathrm{p}=0.93)$. In SC, survival was found significantly longer with additional surgery $(913, \mathrm{n}=13$ ) than without $(325$, $\mathrm{n}=19, \mathrm{p}<0.01)$. In OC, survival did not significantly differed between with resection of the primary tumor $(666, \mathrm{n}=16)$ and without $(595, \mathrm{n}=7, \mathrm{p}=0.93)$. Conclusion: This study has demonstrated that the survival of SC was identical to that of OC, and additional surgery was found to significantly improve the prognosis in SC. Chemotherapy after endoscopic colonic stenting has been considered tolerable as a palliative therapy or bridge to surgery for obstructive colorectal cancer.

Disclosure of Interest: All authors have declared no conflicts of interest.

\section{P1122 A NEW MANEUVER TO PLACE A THROUGH-THE-SCOPE STENT IN A MALIGNANT COLONIC STRICTURE INACCESSIBLE WITH A STANDARD-CALIBER COLONOSCOPE: "OVER-THE- CATHETER" COLONOSCOPE REPLACEMENT TECHNIQUE}

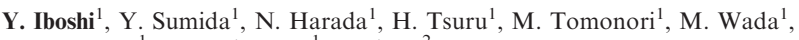
T. Osoegawa ${ }^{1}$, M. Nakamuta ${ }^{1}$, E. Ihara ${ }^{2}$

${ }^{1}$ Gastroenterology, Clinical Research Institute, National Kyushu Medical Center, Fukuoka/Japan

${ }^{2}$ Medicine And Bioregulatory Science, Kyushu University, Fukuoka/Japan

\section{Contact E-mail Address: yoichiroiboshi@gmail.com}

Introduction: A self-expandable metallic stent (SEMS) placement is potentially a colostomy-sparing option to manage a malignant colonic obstruction (MCO). However, in patients with coexisting peritoneal dissemination (carcinomatous adhesion), for example, insertion of a standard caliber colonoscope (SCC) is challenging, whereas such an endoscope equipped with a large working channel is suitable for through-the-scope (TTS) SEMS placement. Failure in stenting necessitates continuous tube drainage, stoma formation, or other surgical procedures and decreases quality of life (QOL).

Aims \& Methods: We examined feasibility and efficacy of, "Over-the-Catheter" Colonoscope Replacement technique (OTC-CR) detailed below, in palliative (not preoperative) SEMS placement for MCO. From Oct 2012 to Dec 2016, MCO patients were consecutively considered for decompression by SEMS placement unless stoma formation was preferred. When a conventional TTS procedure was unsuccessful, specifically, when the MCO site was inaccessible with an SCC (CF-H260AI, Olympus Medical Systems, Tokyo, Japan) with a $13.2 \mathrm{~mm}$ tip diameter and $3.7 \mathrm{~mm}$ working channel, needed for a $22 \mathrm{~mm}$ (not $18 \mathrm{~mm}$ ) SEMS 


\section{Abstract No: P1122}

List of 6 cases with malignant colonic obstruction in whom "Over-the-Catheter" colonoscope replacement technique was tried.

\begin{tabular}{|c|c|c|c|c|c|c|c|c|}
\hline $\begin{array}{l}\text { Case } \\
\text { No. }\end{array}$ & $\begin{array}{l}\text { Age, } \\
\text { Sex }\end{array}$ & $\begin{array}{l}\text { Primary } \\
\text { cancer site }\end{array}$ & $\begin{array}{l}\text { Site of } \\
\text { stricture }\end{array}$ & $\begin{array}{l}\text { Nature of } \\
\text { stricture }\end{array}$ & $\begin{array}{l}\text { Alternative } \\
\text { scope }\end{array}$ & $\begin{array}{l}\text { Distance from } \\
\text { Reinserted SCC } \\
\text { to stricture }\end{array}$ & $\begin{array}{l}\text { Technical/Clinical } \\
\text { outcome }\end{array}$ & $\begin{array}{l}\text { CROSS } \\
\text { score* change }\end{array}$ \\
\hline 1 & $71, \mathrm{~F}$ & Peritoneum & Splenic flexure & Extrinsic & PCF-PQ260L & Away (SCJ) & Success/Success & $1 \rightarrow 4$ \\
\hline 2 & $66, \mathrm{~F}$ & Sigmoid colon & Sigmoid colon & Intrinsic & PCF-PQ260L & Close & Success/Success & $2 \rightarrow 4$ \\
\hline 3 & $76, \mathrm{M}$ & Pancreas & Splenic flexure & Extrinsic & PCF-PQ260L & Away (SCJ) & Success/Success & $1 \rightarrow 4$ \\
\hline 4 & $41, \mathrm{~F}$ & $\begin{array}{l}\text { Transverse } \\
\quad \text { colon (resected) }\end{array}$ & Sigmoid colon & Extrinsic & GIF-Q260J & Close & Success/Success & $1 \rightarrow 4$ \\
\hline 5 & $55, \mathrm{~F}$ & Ovary & Sigmoid colon & Extrinsic & EG-580NW & $\begin{array}{l}\text { Away } \\
\quad \text { (Sigmoid colon) }\end{array}$ & Success/Success & $0 \rightarrow 3$ \\
\hline 6 & $49, \mathrm{~F}$ & Stomach & Transvers colon & Extrinsic & PCF-PQ260L & Close & Success/Success & $0 \rightarrow 3$ \\
\hline
\end{tabular}

*CROSS, ColoRectal Obstruction Scoring System (Reference 1)

Among 63 palliative MCO cases, initial attempt to place a $22 \mathrm{~mm}$ SEMS by TTS procedure was unsuccessful in 6 cases (Table), all of whom had peritoneal dissemination. The reasons for technical failures were; impossible insertion of an SCC to the stricture due to carcinomatous adhesions or narrowing in 5 cases and failure in passing GW through the stenosis due to a limited viewing angle to the stricture in one case. With OTC-CR, approach to the main stenosis with a thinner (alternative) scope, GW traverse, "over-the-catheter" replacement to the SCC, and a $22 \mathrm{~mm}$ SEMS placement were successful in all of the 6 cases with adequate clinical improvements (CROSS score change). Notably, ultimate TTS procedures were possible from reinserted SCC distant from the stricture in 3 cases. No adverse events occurred during the procedures.

delivery system passage (e.g. Niti-S Colonic Stent, Taewoong-Medical, Seoul, South Korea) or when a guidewire was unable to be passed through the stricture because of limited maneuverability of the endoscope, procedures below were followed. (1) A thinner scope, PCF-PQ260I/L, with a $9.2 \mathrm{~mm}$ tip and a $2.8 \mathrm{~mm}$ channel (Olympus Medical Systems, Tokyo, Japan) is inserted and advanced to the stricture, facilitated by its "passive bending" feature. An ultrathin endoscope (for transnasal use) or a gastroscope are alternatives. (2) A guidewire ( $\mathrm{GW}$ ), 0.035 inch Jagwire) is traversed through the stricture by coordinating manipulation of an ERCP-catheter (Article-No.0130211; MTW Endoskopie, Wesel, Germany). (3) Fluorescent examination through the catheter to delineate the lesion. (4) For colonoscope replacement, withdraw the scope and the catheter, leaving only GW. (5) Before reinsertion of the SCC, insert the catheter from the channel until it sticks out of the scope tip. (6) Pass GW backward into the catheter tip. Then move the catheter over GW past the anus and further advance it proximally until it reaches the stricture site. (7) Gently move the SCC "over the catheter" into the rectum and advance it in the more straightened (less winding) intestine. (8) After the colonoscope is advanced only as proximally as possible, shift to a standard TTS maneuver (withdraw only the catheter, leaving GW and insert the SEMS delivery along GW past the stricture where it is deployed). Results: See table.

Conclusion: "Over-the-Catheter" colonoscope replacement (OTC-CR) technique can be a salvage maneuver which facilitates a successful SEMS placement in MRO cases with peritoneal disseminations that preclude a conventional TTS procedure. Reachability of thin endoscopes and appropriate rigidity and force transmissibility of an ERCP-catheter that enable advancement of an SCC with maximum safety are thought to be linked to reduced chances of technical failures. Disclosure of Interest: All authors have declared no conflicts of interest.

\section{Reference}

1. Japan Colonic Stent Safe Procedure Research Group (http://colon-stent.com/001_mainpage_en.html)

\section{P1123 RESULT OF THE FIRST ROUND OF THE COLORECTAL CANCER SCREENING PROGRAMME IN THE BALEARIC} ISLANDS (SPAIN)

M. Florido ${ }^{1}$, M. Novella ${ }^{2}$, M. Heredia ${ }^{3}$, E. Miro ${ }^{4}$, C. Sanchez-Contador ${ }^{4}$, J. Gelabert ${ }^{3}$, S.P. Ortega ${ }^{1}$, E.Y. Iyo ${ }^{1}$, J. Reyes ${ }^{1}$, P. Garcia-Cortes ${ }^{3}$, J.A. Lucero ${ }^{2}$, Z. Salman ${ }^{2}$, C. Rodriguez ${ }^{2}$, A. Ruiz ${ }^{2}$, M.J. Garcia ${ }^{2}$

${ }_{1}^{1}$ Gastroenterology, Hospital Comarcal de Inca, Inca/Spain

${ }^{2}$ Gastroenterology, Hospital Can Misses, Ibiza/Spain

${ }^{3}$ Gastroenterology, Hospital Mateu Orfila, Mahon/Spain

${ }^{4}$ Direcció General De Salut Pública I Participació, Conselleria de Sanitat,

Palma/Spain

Contact E-mail Address: monica.florido@hcin.es

Introduction: Colorectal cancer (CRC) is the most common cause of cancer in western countries. In Balearic Islands 700 new cases per year are diagnosed. The cost-effectiveness of CRC screening programmes are clearly demonstrated in the studies and the important public health problem of CRC justifies the development of control strategies. The aim of this study is to present the results and impact during the first round of the program in Balearic Islands.

Aims \& Methods: The first round includes the period from January 2015 through December 2016. The program has been developed in the areas of Menorca, Ibiza, Formentera and Tramuntana (Mallorca), including 30\% of the Balearic Islands population. The target population (people who reside in these areas aged between 50 and 69 years old) was 75,575 individuals. Exclusion criteria. Colonoscopy performed in the previous 5 years, previous diagnosis of $\mathrm{CRC}$, follow-up colonoscopies because colon disease and severe illness-contraindication for the participation. People received the invitation by letter. Quantitative immunochemical fecal occult blood testing (i-FOBT/OC-Sensor) was the screening method. The kit was delivered at pharmacies joined the program. The samples were deposited in urns placed in health centres. Participants who tested positive $(\geq 100 \mathrm{ng} / \mathrm{ml})$ were referred to pre-endoscopy evaluation and follow-up colonoscopy. The colonoscopies were performed according to the quality criteria of guidelines.

Results: Overall participation rate (number of people who provide their i-FOBT sample) was $36.5 \%(n=21,555)$. Positive rate of $\mathrm{i}$-FOBT was $7 \%(1438)$ and $94.3 \%$ of those positive tests underwent a colonoscopy $(5,7 \%$ of exclusions in pre-endoscopy evaluation). 996 colonoscopies were performed. 47 adenomas with high grade dysplasia, 24 carcinomas in situ and 60 adenocarcinomas were found. Only $19 \%$ of these adenocarcinomas were T3 or T4 lesions while the rest presented earlier stages. $26 \%$ of colonoscopies were classified as high risk $(\geq 5$ adenomas or at least one $\geq 20 \mathrm{~mm}$ ). They have been reported 2 cases of colon perforations, both resolved by endoscopic treatment.

Conclusion: We obseved an acceptable participation rate in the first round of the colorectal cancer screening programme of the Balearic Islands. The index of positivity rate of i-FOBT and the results of the endoscopic explorations are according with the observed in other colorectal cancer screening programmes. We can conclude a successful development of the first round of the programme in our area.

Disclosure of Interest: All authors have declared no conflicts of interest.

\section{References}

Segnan N, Patnick J, von Karsa L, et al., editors. European guidelines for quality assurance in colorectal cancer screening and diagnosis. First edition. Luxembourg: European Commission, Publications Office of the European Union; 2010

Kaminski MF, Wieszczy P, Rupinski M, Wojciechowska U, Didkowska J, Kraszewska E, Kobiela J, Franczyk R, Rupinska M, Kocot B, ChaberCiopinska A, Pachlewski J, Polkowski M, Regula J. Gastroenterology. 2017 Apr 17. pii: S0016-5085(17)35441-0. doi: 10.1053/j.gastro.2017.04.006.

\section{P1125 ENTEROENDOCRINE, MUSASHI 1, AND NEUROGENIN 3 CELLS IN THE LARGE INTESTINE OF THAI PATIENTS WITH IRRITABLE BOWEL SYNDROME}

M. El-Salhy ${ }^{1}$, T. Patcharatrakul ${ }^{2}$, J.G. Hatlebakk ${ }^{3}$, T. Hausken ${ }^{4}$, O.H. Gilja ${ }^{3}$, S. Gonlachanvit ${ }^{2}$

${ }^{1}$ University Of Bergen, Clinical Medicine, bergen/Norway

${ }^{2}$ Medicine

${ }^{6}$ King Chulalongkorn Memorial Hospital, Bangkok/Thailand

${ }^{3}$ Medicine, National Centre for Functional Gastrointestinal Disorders, Bergen/

Norway

${ }^{4}$ Haukeland University Hospital, National Centre for Ultrasound in

Gastroenterology, Bergen/Norway

Contact E-mail Address: magdy.elsalhy@sklbb.no

Introduction: The prevalence, gender distribution, and clinical presentation of irritable bowel syndrome (IBS) differ between Asian and Western countries. The densities of enteroendocrine cells are abnormal in Western IBS patients. This study aimed at studying large-intestine enteroendocrine, Musashi 1(Msi 1; a marker for both intestinal stem cells and their early progeny), and neurogenin 3 (neurog 3; a marker for early intestinal endocrine cell progenitors) cells in Thai and IBS patients. 
Aims \& Methods: Thirty Thai IBS patients, and age and sex matched 20 Thai controls were included. Four biopsy samples were taken from each of the sigmoid colon and the rectum during a standard colonoscopy. Sections from these biopsy samples were immunostained for serotonin, peptide YY, oxyntomodulin (enteroglucagon), pancreatic polypeptide, somatostatin, Msi 1, neurog 3. The densities of immunoreactive cells were determined with computerized image analysis (1). Results: In both the colon, and rectum the density of serotonin cells was lower in IBS patients than controls. Whereas the density of PYY cells was increased in both the colon and rectum of IBS-D, it was reduced in IBS-M and IBS-C. The density of oxyntomodulin cells was reduced in both the colon and rectum of all IBS subtypes. While the density of PP cells was unaffected in the colon, it was reduced in the rectum. Somatostatin cell density was unaffected in both the colon and rectum. The densities of Msi 1 and neuro 3 were unchanged in both the colon and rectum.

Conclusion: The present findings of abnormal densities of the large-intestine enteroendocrine cells in Thai patients combined with previously reported changes in Western IBS patients (2) support the notion that intestinal enteroendocrine cells are involved in the pathophysiology of IBS. However, the changes in the enteroendocrine cells differed from those in Western patients. The present observations highlight that IBS differs in Asian and Western countries, and show that the changes in large-intestine enteroendocrine cells in Asian and Western IBS patients might be caused by different mechanisms.

Disclosure of Interest: All authors have declared no conflicts of interest.

\section{References}

1. El-Salhy M, Sandstrom O, Nasstrom E, Mustajbasic M, Zachrisson S. Application of computer image analysis in endocrine cell quantification. Histochem J 1997; 29: 249-256.

2. El-Salhy M, Hausken T, Gilja OH, Hatlebakk JG. The possible role of gastrointestinal endocrine cells in the pathophysiology of irritable bowel syndrome. Expert Rev Gastroenterol Hepatol 2017; 11: 139-148.

\section{P1126 SUBJECT GLOBAL SATISFACTION SCORE TO ASSESS OVERALL EFFECT OF NALDEMEDINE COMPARED WITH PLACEBO ON CONSTIPATION AND ABDOMINAL SYMPTOMS IN SUBJECTS WITH CHRONIC NON-CANCER PAIN AND OPIOID- INDUCED CONSTIPATION}

J. Tack ${ }^{1}$, M. Camilleri ${ }^{2}$, B. Cai ${ }^{3}$, T. Yamada ${ }^{3}$, J.C. Arjona Ferreira ${ }^{3}$ ${ }^{1}$ Gastroenterology, University of Leuven, University Hospital Gasthuisberg, Leuven/Belgium

${ }^{2}$ Mayo Clinic, Mayo Clinic Dept. of Gastroenterology Charlton Bldg., Rm. 8-110, Rochester, MN/United States of America

${ }^{3}$ Shionogi Inc, New Jersey/United States of America

Contact E-mail Address: jan.tack@med.kuleuven.ac.be

Introduction: Opioid-induced constipation (OIC) is a common side effect of opioid therapy that significantly affects multiple aspects of a patient's life. Naldemedine (NAL) is a peripherally-acting mu-opioid receptor antagonist developed for the treatment of OIC. In Phase 3 studies, NAL improved the frequency of spontaneous bowel movements, straining, consistency of stools, and patient assessment of constipation symptoms (PAC-SYM) and quality of life (PAC-QOL), measures of patient's quality of life, compared with placebo (PBO). The aim of this analysis is to assess the impact of NAL on overall satisfaction and to show if a simple score can assess the impact of treatment of OIC with NAL $0.2 \mathrm{mg}$ once daily on patient's satisfaction with constipation and abdominal symptoms in patients with OIC associated with non-cancer pain.

Aims \& Methods: In three Phase 3 randomized, double-blind, PBO-controlled trials of NAL (2 of 12-week duration [COMPOSE 1 and COMPOSE 2] and 1 of 52-week duration [COMPOSE 3]), a 7-grade scale ( $1=$ markedly, $2=$ moderately, or 3 =slightly worsened; 4 = unchanged; 5 =slightly, $6=$ moderately, or $7=$ markedly improved) was used to assess overall satisfaction with constipation and abdominal symptoms at the last study visit. The number and proportion of subjects in each grade were calculated and the overall difference between groups was assessed by Wilcoxon rank sum test. The mean subject global satisfaction score (SGSS) was also compared between groups. For SGSS, scores from 1 to 7 were replaced with scores from -3 to +3 , with 4 (unchanged) replaced with 0 .
This proposed new approach provides a single score that may be easier to interpret than the 7 -grade scale.

Results: There were 547 subjects in COMPOSE 1, 550 in COMPOSE 2, and 1246 in COMPOSE 3 (all $\geq 18$ years of age) randomized (1:1) to NAL $0.2 \mathrm{mg}$ once daily or PBO. The baseline characteristics of the study population were consistent between groups in each trial and between trials. Overall satisfaction assessment was completed in 372 subjects in COMPOSE 1, 296 in COMPOSE 2, and 1101 in COMPOSE 3. There were greater improvements in satisfaction with constipation and abdominal symptoms in the NAL group compared with the PBO group in all three studies (all $\mathrm{P} \leq 0.0005$; Table). The mean SGSS were 1.5 and 0.9 with NAL and PBO, respectively, in the two 12-week studies pooled, and 1.7 and 1.0 , respectively, in the 52 -week study.

\begin{tabular}{|c|c|c|c|c|c|c|}
\hline & \multicolumn{2}{|c|}{ COMPOSE 1} & \multicolumn{2}{|c|}{ COMPOSE 2} & \multicolumn{2}{|c|}{ COMPOSE 3} \\
\hline & NAL & PBO & NAL & $\mathrm{PBO}$ & NAL & PBO \\
\hline Total subjects assessed & 185 & 187 & 204 & 192 & 497 & 504 \\
\hline \multicolumn{7}{|c|}{ Global satisfaction category, n (\%) } \\
\hline Markedly worsened (1) & $\begin{array}{l}3 \\
(1.6)\end{array}$ & $\begin{array}{l}7 \\
(3.7)\end{array}$ & $\begin{array}{l}6 \\
(2.9)\end{array}$ & $\begin{array}{l}6 \\
(3.1)\end{array}$ & $\begin{array}{l}12 \\
(2.4)\end{array}$ & $\begin{array}{l}10 \\
(2.0)\end{array}$ \\
\hline $\begin{array}{l}\text { Moderately } \\
\quad \text { worsened (2) }\end{array}$ & $\begin{array}{l}11 \\
(5.9)\end{array}$ & $\begin{array}{l}8 \\
(4.3)\end{array}$ & $\begin{array}{l}5 \\
(2.5)\end{array}$ & $\begin{array}{l}10 \\
(5.2)\end{array}$ & $\begin{array}{l}3 \\
(1.1)\end{array}$ & $\begin{array}{l}14 \\
(2.8)\end{array}$ \\
\hline Slightly worsened (3) & $\begin{array}{l}4 \\
(2.2)\end{array}$ & $\begin{array}{l}7 \\
(3.7)\end{array}$ & $\begin{array}{l}3 \\
(1.5)\end{array}$ & $\begin{array}{l}12 \\
(6.3)\end{array}$ & $\begin{array}{l}5 \\
(1.4)\end{array}$ & $\begin{array}{l}11 \\
(2.4)\end{array}$ \\
\hline Unchanged (4) & $\begin{array}{l}27 \\
(14.6)\end{array}$ & $\begin{array}{l}58 \\
(31.0)\end{array}$ & $\begin{array}{l}28 \\
(13.7)\end{array}$ & $\begin{array}{l}54 \\
(28.1)\end{array}$ & $\begin{array}{l}74 \\
(14.9)\end{array}$ & $\begin{array}{l}169 \\
(33.5)\end{array}$ \\
\hline Slightly improved (5) & $\begin{array}{l}49 \\
(26.5)\end{array}$ & $\begin{array}{l}38 \\
(20.3)\end{array}$ & $\begin{array}{l}37 \\
(18.1)\end{array}$ & $\begin{array}{l}39 \\
(20.3)\end{array}$ & $\begin{array}{l}100 \\
(20.1)\end{array}$ & $\begin{array}{l}116 \\
(23.0)\end{array}$ \\
\hline $\begin{array}{l}\text { Moderately } \\
\text { improved (6) }\end{array}$ & $\begin{array}{l}39 \\
(21.1)\end{array}$ & $\begin{array}{l}41 \\
(21.9)\end{array}$ & $\begin{array}{l}53 \\
(26.0)\end{array}$ & $\begin{array}{l}39 \\
(20.3)\end{array}$ & $\begin{array}{l}129 \\
(26.0)\end{array}$ & $\begin{array}{l}89 \\
(17.7)\end{array}$ \\
\hline $\begin{array}{l}\text { Markedly } \\
\quad \text { improved (7) }\end{array}$ & $\begin{array}{l}52 \\
(28.1)\end{array}$ & $\begin{array}{l}28 \\
(15.0)\end{array}$ & $\begin{array}{l}72 \\
(35.3)\end{array}$ & $\begin{array}{l}32 \\
(16.7)\end{array}$ & $\begin{array}{l}174 \\
(35.0)\end{array}$ & $\begin{array}{l}95 \\
(18.8)\end{array}$ \\
\hline $\begin{array}{l}\text { P-value between } \\
\text { groups* }\end{array}$ & \multicolumn{2}{|l|}{0.0005} & \multicolumn{2}{|c|}{$<0.0001$} & \multicolumn{2}{|c|}{$<0.0001$} \\
\hline
\end{tabular}

${ }^{*}$ Wilcoxon rank sum test

Conclusion: Treatment of OIC with NAL $0.2 \mathrm{mg}$ once daily for 12 or 52 weeks led to greater satisfaction with constipation and abdominal symptoms compared with PBO, consistent with previously-reported improvements of PAC-SYM and PAC-QOL with NAL compared with PBO. The proposed SGSS appears to be a simple way to assess the impact on quality of life of OIC treatment. Disclosure of Interest: J. Tack: advisory board to Shionogi

M. Camilleri: advisory board to Shionogi INC 2 years ago

B. Cai: Employee of Shionogi Inc.

T. Yamada: Employee of Shionogi INC

J.C. Arjona Ferreira: Employee of Shionogi INC

\section{P1127 IBEROGAST PREVENTS CHANGES IN INTESTINAL}

\section{PERMEABILITY INDUCED BY PSYCHOLOGICAL STRESS IN MICE}

P. Aubert ${ }^{1}$, J. Chevalier ${ }^{1}$, T. Durand ${ }^{1}$, A. Bessard ${ }^{1}$, O. Kelber $^{2}$, H. Abdel-Aziz ${ }^{2}$, M. Neunlist ${ }^{1}$

${ }^{1}$ Inserm 1235 "tens", INSERM, Nantes/France

${ }^{2}$ Innovation \& Development, Bayer Consumer Health, Darmstadt/Germany

Contact E-mail Address: michel.neunlist@univ-nantes.fr

Introduction: The herbal preparation STW 5 has been reported to increase intestinal chloride secretion. However, the ability of STW 5 to modulate paracellular and transcellular permeability remains currently unknown. Therefore, we aimed

Abstract No: P1125

Table 1: Densities of enteroendocrine, Msi 1, and neurog 3 cells in the colon of Thai and Norwegian controls and IBS patients.

\begin{tabular}{|c|c|c|c|c|c|c|c|c|c|c|}
\hline \multirow[b]{2}{*}{ Cell type } & \multicolumn{5}{|l|}{ Colon } & \multicolumn{5}{|l|}{ Rectum } \\
\hline & Controls & IBS-total & IBS-D & IBS-M & IBS-C & Controls & IBS-total & IBS-D & IBS-M & IBS-C \\
\hline Serotonin & $202 \pm 20$ & $119 \pm 10^{* * *}$ & $104 \pm 9 * *$ & $93 \pm 8 * *$ & $144 \pm 21$ & $333 \pm 25$ & $176 \pm 12 * * *$ & $163 \pm 14 * * *$ & $166 \pm 16^{* *}$ & $191 \pm 23^{* *}$ \\
\hline PYY & $79 \pm 8$ & $95 \pm 10$ & $143 \pm 20 * *$ & $68 \pm 7$ & $67 \pm 7$ & $177 \pm 19$ & $184 \pm 23$ & $315 \pm 27 * * *$ & $102 \pm 9 *$ & $112 \pm 20^{*}$ \\
\hline Oxyntomodulin & $70 \pm 7$ & $40 \pm 4 * * *$ & $42 \pm 7 *$ & $39 \pm 7 * *$ & $39 \pm 6 * *$ & $224 \pm 30$ & $93 \pm 10 * * *$ & $78 \pm 13^{* *}$ & $72 \pm 10^{* *}$ & $86 \pm 15^{* *}$ \\
\hline PP & $46 \pm 5$ & $54 \pm 4$ & $56 \pm 8$ & $51 \pm 11$ & $55 \pm 6$ & $177 \pm 17$ & $100 \pm 13^{*}$ & $117 \pm 21^{*}$ & $73 \pm 17^{*}$ & $61 \pm 8^{* * *}$ \\
\hline Somatostatin & $91 \pm 12$ & $77 \pm 7$ & $86 \pm 13$ & $60 \pm 9$ & $78 \pm 9$ & $106 \pm 12$ & $102 \pm 8$ & $118 \pm 15$ & $116 \pm 16$ & $83 \pm 11$ \\
\hline Msi 1 & $5.0 \pm 0.4$ & $5.0 \pm 0.3$ & $5.0 \pm 0.6$ & $5.0 \pm 0.8$ & $5.0 \pm 0.5$ & $7.0 \pm 0.5$ & $8.0 \pm 0.5$ & $8.0 \pm 1.0$ & $7.0 \pm 1.0$ & $8.0 \pm 0.8$ \\
\hline Neurog 3 & $130 \pm 10$ & $129 \pm 11$ & $131 \pm 19$ & $105 \pm 16$ & $138 \pm 18$ & $274 \pm 31$ & $258 \pm 28$ & $287 \pm 54$ & $182 \pm 35$ & $268 \pm 42$ \\
\hline
\end{tabular}

Data was expressed as mean \pm SEM. *,$P<0.05 ; * *, P<0.001 ; * * *, P<0.0001$ 
to study the ability of STW 5 to modulate intestinal permeability under basal and repeated acute stress conditions.

Aims \& Methods: C57 bl6 mice were gavaged for 14 days with STW $5(3 \mathrm{~mL} / \mathrm{kg})$. After 10 days of treatment, mice were subjected to water avoidance stress (WAS) during 4 consecutive days. In vivo permeability to FITC -Sulfonic Acid (FSA, $400 \mathrm{Da}$ ) and Horse Radish Peroxydase (HRP, 44KDa), total transit time and colonic transit (fecal pellet output - FPO) were assessed at Day 0 (D0), D10 and D14 of IB treatment. Ex vivo permeability to FSA and HRP was assessed on jejunum, ileum, proximal colon and distal colon at D14 using Ussing chambers. Corticosterone blood level was measured at D11 and D14.

Results: In vivo permeability to FSA and HRP as well as total transit time were not modified by STW 5 in basal and WAS conditions. However, STW5 prevented the increase in permeability to FSA induced by WAS in the distal colon ex vivo. Conversely, STW 5 prevented the increase in permeability to HRP induced by WAS in the jejunum and proximal colon. Furthermore, while STW 5 tended to increase colonic transit as compared to control in basal conditions, it prevented the increase in colonic transit induced by WAS. Finally, STW 5 did not modify the changes in corticosterone induced by WAS.

Conclusion: Our study suggest that STW 5 can prevent WAS induced changes in paracellular and transcellular permeability in specific regions of the gastrointestinal tract. Such effects could contribute to the therapeutic effects of STW 5 in irritable bowel syndrome and support novel therapeutic indications for pathologies in which barrier functions are altered.

Disclosure of Interest: O. Kelber: Olaf Kelber is employed by Bayer

H. Abdel-Aziz: Heba Abdel aziz is employed by Bayer

M. Neunlist: This work was supported by a research grant to MN by Bayer

All other authors have declared no conflicts of interest.

\section{P1129 ALTERING SPHINGOSINE-1-PHOSPHATE WITH AGING INDUCES MOTILITY DYSFUNCTION OF COLON SMOOTH MUSCLE BY BKCA UPREGULATION IN RATS}

\section{S. Xiaoxue}

The First Affiliated Hospital Of Nanjing Medical University, Nanjing Medical University, Nanjing/China

\section{Contact E-mail Address: shenxiaoxue0066@163.com}

Introduction: Large conductance $\mathrm{Ca}^{2+}$ - activated $\mathrm{K}+$ channel (BKCa channel) was shown to play critical roles in regulating smooth muscle contractility by modulating membrane potential, at the same time, age-associated changes in $\mathrm{BKCa}$ expression may contribute to the development of motility disorders of the gastrointestinal tract. Sphingosine-1-Phosphate (S1P), component of Sphingolipids in the cell membranes, may affect BKCa expression. Thus, in this study, we investigated whether altered S1P due to aging may affect the motility of colon smooth muscle (CSM) in rats.

Aims \& Methods: Thus, in this study, we investigated whether altered S1P due to aging may affect the motility of colon smooth muscle (CSM)in rats. Forty Sprague-Dawley rats at the same age were randomly divided into five groups. After different times of administration, finally they were divided into differentage-group: 10-week group, 20-week group, 40-week group, 60-week group and 80 -week group. Colonic motility function and contractility of circular muscle strips were measured. The expression of $\mathrm{BKCa}$ and phosphorylated myosin light chain (P-MLC) level were tested in colonic tissues of rats with varying ages by immunohistochemical, RT-PCR and western blot. S1P levels in colonic tissues were tested by LC-MS/MS analysis. Primary cultured colonic smooth muscle cells (SMCs) from normal adult rats were used in complementary in vitro studies. In the absence and presence of S1P with different concentrations, the expression of BKCa, P-MLC level, single-channel activity, intracellular $\mathrm{Ca}^{2+}$ mobilization were tested. At the same time, in the presence and absence of S1P, SMCs were transfected with anti-S1P antibody. BKCa siRNA transfection was used to investigate whether P-MLC expression and intracellular $\mathrm{Ca}^{2+}$ mobilization were affected by BKCa expression in SMCs. The expression and phosphorylation of Akt, JNK, ERK, NF- $\kappa \mathrm{B}$, and PKC were examind by western blot analysis to investigate the effect between S1P and BKCa.

Results: Aged rats showed prolonged colonic transit time and weakness of circular muscle contraction compared with the young (10 weeks old) SD rats.LC-MS/MS analysis exhibited that the levels of S1P were significantly greater in the CSM from aged rats, demonstrating that S1P varies depending on age. BKCa ( $\alpha$-subunit and $\beta$-subunit) levels in CSM were shown to increase in an age-dependent manner from 10- to 80 -week-old rats by mRNA ,protein and immunohistochemical, but P-MLC expression decreased. In colon SMCs , by BKCa siRNA transfection, we found P-MLC levels increased. Exogenously added S1P upregulated $\mathrm{BKCa}$ in colon SMCs in a concentration-dependent manner, impaired intracellular $\mathrm{Ca}^{2+}$ mobilization though inhibiting $\mathrm{Ca}^{2+}$ influx and induced the decline of P-MLC.Our results also provedt that S1P upregulated $\mathrm{BKCa}$ through the $\mathrm{Akt} / \mathrm{ERK} / \mathrm{JNK}$ pathways. The expression of $\mathrm{BKCa}$ decreased by treatment with inhibitor of $\mathrm{Akt} / \mathrm{ERK} / \mathrm{JNK}$ pathways or siRNA.

Conclusion: The results of our study show that altered S1P due to aging upregulates BKCa via the Akt/ERK/JNK mediated pathway in CSM.BKCa upregulation inhibits $\mathrm{Ca}^{2+}$ influx and MLC phosphorylation and thereby reduces the contractility of CSM to evoke contractile dysfunction. These findings represent the first evidence for the mechanism of the contractile dysfunction of CSM observed in older individuals, which may be implicated in age-associated gastrointestinal motility disorders.

Disclosure of Interest: All authors have declared no conflicts of interest.

\section{References}

Maczis M, Milstien S, Spiegel S. Sphingosine-1-phosphate and estrogen signaling in breast cancer. Advances in Biological Regulation. 2016;60:160-165. Prossnitz ER, Barton M. Estrogen Biology: New Insights into GPER Function and Clinical Opportunities. Mol Cell Endocrinol. 2014;389(1-2):71-83.

Liu J, Jin X, Zhao N, et al. Bisphenol A promotes X-linked inhibitor of apoptosis protein-dependent angiogenesis via $\mathrm{G}$ protein-coupled estrogen receptor pathway. J Appl Toxicol. 2015;35(11):1309-1317.

Choi S, Kim JA, Kim TH, et al. Altering sphingolipid composition with aging induces contractile dysfunction of gastric smooth muscle via KCa1.1 upregulation. Aging Cell. 2015;14(6):982-994.

\section{P1130 DIABETES-RELATED ALTERATIONS IN THE EXPRESSION OF THE INFLAMMATORY CYTOKINES, TUMOR NECROSIS FACTOR ALPHA AND INTERLEUKIN 6 IN THE MYENTERIC GANGLIA AND ITS MICROENVIRONMENT OF DIFFERENT INTESTINAL SEGMENTS}

L. Chandrakumar, D. Mezei, B. P. Barta, Z. Szalai, N. Bódi, M. Bagyánszki Department Of Physiology, Anatomy And Neuroscience, University of Szeged, Szeged/Hungary

Contact E-mail Address: lalitha.biochem87@gmail.com

Introduction: Growing amount of evidence has indicated that increase of the hyperglycaemia-induced oxidative stress and decreased effectiveness of the endogenous antioxidant protection play the major role in the initiation of diabetesrelated neuronal damage ${ }^{1,2}$. Using a streptozotocin-induced diabetic rat model we recently demonstrated that nitrergic myenteric neurons, which are key regulators of peristalsis, display different susceptibilities to diabetic damage and also to insulin treatment in the different gut segments ${ }^{3}$. Based on these results we suggested the importance of the molecular differences in the neuronal microenvironment in the pathogenesis of diabetic nitrergic neuropathy.

Aims \& Methods: Aim to reveal the quantitative differences in the expression of the pro-inflammatory cytokines like tumor necrosis factor alpha (TNF $\alpha$ ) and interleukin 6 (IL6) in the myenteric ganglia and its microenvironment of the different intestinal segments, quantitative immunogold electron microscopy was used. Ten weeks after the onset of diabetes, segments from the duodenum, ileum and colon of streptozotocin-induced diabetic, insulin-treated diabetic, and control rats were processed for post-embedding immunohistochemistry.

Results: The density of $\mathrm{TNF} \alpha$ - and IL6-labelling gold particles was strictly region-dependent, with increasing to the distal part of the gastrointestinal tract of controls. In diabetic rats, the number of TNF $\alpha$ gold particles was significantly increased in the duodenal, decreased in the colonic myenteric ganglia, while did not show any significant differences in the ileal ganglia. The number of IL6 gold particles was not affected by diabetes in the myenteric ganglia of different gut regions. The diabetes-related alterations of TNF $\alpha$ - and IL6 expression were not protected by the immediate insulin replacement in any of the investigated intestinal segments. The differences in TNF $\alpha$ - and IL6 density were not significant in the capillary endothelium under different experimental conditions.

Conclusion: Based on these findings we presume that regionally alterations in the $\mathrm{TNF} \alpha$ and IL6 expression are correlated with the diabetes-related region-specific nitrergic myenteric neuropathy.

Disclosure of Interest: All authors have declared no conflicts of interest.

\section{References}

1. Bagyánszki M, Bódi N (2012) Diabetes-related alterations in the enteric nervous system and its microenvironment. World Journal of Diabetes 3(5):80-93.

2. Jancsó Zs, Bódi N, Borsos B, Fekete É, Hermesz E (2015) Gut region-specific accumulation of reactive oxygen species leads to regionally distinct activation of antioxidant and apoptotic marker molecules in rats with STZ-induced diabetes. The International Journal of Biochemistry \& Cell Biology 62:125-131.

3. Izbéki F, Wittman T, Rosztóczy A, Linke N, Bódi N, Fekete E, Bagyánszki M (2008) Immediate insulin treatment prevents gut motility alterations and loss of nitrergic neurons in the ileum and colon of rats with streptozotocininduced diabetes. Diabetes Res Clin Pract 80(2):192-198.

\section{P1131 ROLE OF SEMAPHORIN 3A IN THE POSTNATAL} DEVELOPMENT OF THE ENTERIC NERVOUS SYSTEM

J. Gonzales, C. Le Berre, E. Dikkongue, M. Neunlist, H. Boudin TENS INSERM U1235, Nantes/France

Contact E-mail Address: jacques.gonzales@univ-nantes.fr

Introduction: Critical developmental stages of the enteric nervous system (ENS) occur during the postnatal period leading to the formation of a mature neuroglial network characterized by the assembly of enteric neurons into ganglia and the formation of a highly organized pattern of neuronal connectivity. However, the mechanisms underlying these maturation processes are poorly understood. Semaphorin 3A (SEMA3A) is a secreted protein playing key roles in the neuronal circuitry formation of the central nervous system. Here, we studied the expression, the cellular distribution and the role of SEMA3A and its receptor neuropilin 1 (NRP1) in the maturation of enteric neurons. 
Aims \& Methods: Gene and protein expression of SEMA3A and its receptor NRP1 were analyzed in rat distal colon from postnatal day 1 (PN1) to adulthood by qRT-PCR and Western blot respectively. The cellular distribution of SEMA3A and NRP1 was performed at PN7 and PN36 in whole mount distal colon tissue by double immunofluorescence for SEMA3A or NRP1 with specific markers of glia (S100b), neurons (Hu, Tuj-1), and muscle cells (a-SMA). The impact of SEMA3A on neuronal outgrowth was assessed in cultures of enteric neurons cocultured with SEMA3A-transfected COS-7 cells.

Results: A peak of mRNA expression for SEMA3A and NRP1 was observed in distal colon at P7, corresponding to a stage of intense neural circuit remodeling. At the protein level, NRP1 was also found to be predominantly expressed during the early postnatal period. Immunohistofluorescence of colon tissue indicated that SEMA3A immunoreactivity was not associated with any specific cellular profile, but was distributed in small clusters disseminated throughout the tissue, a pattern consistent for a secreted protein. NRP1 was found in neurons, mainly associated with axonal processes, and was not detected in glial or muscle cells. Enteric neurons cultured in the presence of SEMA3A-expressing COS cells showed a strong reduction in axon length and complexity, while the ganglion size was unaffected.

Conclusion: This study shows the expression of SEMA3A and its receptor NRP1 in the ENS during early postnatal period. By controlling axonal outgrowth, SEMA3A might be an important factor to restrict the axonal trajectories in the appropriate paths between ganglia.

Disclosure of Interest: All authors have declared no conflicts of interest.

\section{P1132 A POPULATION-BASED STUDY ON BOWEL HABITS IN A PORTUGUESE COMMUNITY: PREVALENCE OF CONSTIPATION}

M. Silva ${ }^{1}$, S. Gomes ${ }^{2}$, J. Silva ${ }^{3}$, M. Fazendeiro ${ }^{4}$, M. Ribeiro ${ }^{5}$, A. Peixoto ${ }^{6}$, R. Gaspar ${ }^{6}$, R. Morais ${ }^{6}$, J. A. Sarmento ${ }^{6}$, G. Macedo ${ }^{6}$

${ }^{1}$ Gastroenterology, Centro Hospitalar São João, Porto Medical School, Porto/Portugal

${ }^{2}$ General Practice, USF Alfena, Alfena/Portugal

${ }^{3}$ General Practice, USF Casa dos Pescadores, Povoa de Varzim/Portugal

${ }^{4}$ General Practice, UCSP S. Miguel, Castelo Branco/Portugal

${ }^{5}$ USF A Ribeirinha, Guarda/Portugal

${ }^{6}$ Centro Hospitalar São João, Porto Medical School, Porto/Portugal

Contact E-mail Address: marcocostasilva87@gmail.com

Introduction: Constipation is a chronic disorder with an estimated prevalence of $17 \%$ in Europe. Epidemiological studies on bowel habits in the Portuguese general population have not been done previously, as in many other western countries. The aim of this population-based study was to describe bowel habits and the prevalence of self-reported constipation in a Portuguese community.

Aims \& Methods: We aimed to describe bowel habits and the prevalence of selfreported constipation in a Portuguese community. Methods: Cross-sectional study with convenience sampling between November 2015 and November 2016. The physician applied a questionnaire, to adult patients at primary health care consultation. The questionnaires were anonymous, and the only personal information the participants were required to give was their age and sex. The questionnaire contained objective questions on possible causes and constipation-associated conditions and medications (according to the criteria defined by the World Gastroenterology Organization), daily water and fiber intake, physical activity, bowel habits and Bristol stool scale (BSS). Descriptive statistics and uni and multivariate analysis were performed using IBM SPSS Statistics 22 with $\mathrm{p}<0.05$ deemed to be statistically significant.

Results: A total of 814 questionnaires were performed to individuals from 35 different municipalities ( $54 \%$ women; mean age $46 \pm 18$ years). Concerning possible causes of constipation, $43 \%$ subjects had a history of constipation-associated condition and $36 \%$ were taking constipation-associated drugs. Regarding bowel habits, $35 \%$ subjects had $<1$ bowel movement per day and $2 \%$ had $<1$ bowel movement per week. Using BSS, $66 \%$ of the cases reported type III or type IV stool consistency. Among women, $19 \%$ reported a change in bowel movements according to the phase of the mentrual cycle. In total, $22 \%$ of subjects considered to be constipated, and $78 \%$ of these, complied the Roma III criteria for functional constipation. Noteworthy, $6 \%$ of subjects with daily bowel movements and $38 \%$ of those with $<1$ weekly bowel movement considered to have constipation. Complaints of excessive straining, tenesmus, feeling of incomplete evacuation and abdominal pain were associated with the presence of constipation ( 64 vs. $12 \%$, 56 vs. $20 \%, 54$ vs. $19 \%$ and 37 vs. $18 \%$, respectively; $\mathrm{p}<0.0001$ ). No associations were found between constipation and physical activity, daily water or fiber intake.

Conclusion: This study represents the first assessment of bowel habits in the Portuguese population, depicting the high prevalence and related risk factors of a disorder that decreases the health-related quality of life and has major economic consequences. The prevalence of constipation $(22 \%)$ was higher than that reported in other European countries.

Disclosure of Interest: All authors have declared no conflicts of interest.

\section{References}

1. Walter S, Hallböök O, Gotthard R, Bergmark M, Sjödahl R. a populationbased study on bowel habits in a Swedish community: prevalence of faecal incontinence and constipation. Scand J Gastroenterol. 2002;37:911-6.

2. Lindberg $\mathrm{G}$ et al; World Gastroenterology Organisation. World Gastroenterology Organisation global guideline: Constipation - a global perspective. J Clin Gastroenterol. 2011;45:483-7.

\section{P1133 DIAGNOSTIC DISCORDANCE BETWEEN TESTS OF EVACUATION: A PROSPECTIVE STUDY}

\section{Carter $^{1}$, E. Bardan ${ }^{2}$, M. Beer-Gabel ${ }^{2}$}

${ }^{1}$ Sackler Faculty Of Medicine, Tel Aviv University, Tel Aviv/Israel

${ }^{2}$ Gastroenterology, Sheba Medical Center Dept. of Gastroenterology, Ramat Gan/ Israel

\section{Contact E-mail Address: dr.dancarter@gmail.com}

Introduction: Objective means of evaluation of the defecatory process include high resolution ano-rectal manometry (HRM), balloon expulsion test (BET) and imaging of the defecatory process (X-ray defecography, dynamic transpelvic ultrasound (DT-PUS) or MR defecography). These tests have a place in the evaluation of suspected evacuatory dysfunction (ED), fecal incontinence (FI) and chronic pelvic pain (CPP). Test choice may influence subsequent patient management; however, there is only limited information regarding the agreement between HRM, DT-PUS and BET.

Aims \& Methods: The aims of this study were to compare the diagnostic yield and agreement between different tests of evacuation and to define the relation between the diagnoses of evacuation dysfunction to objective evacuatory failure. 63 consecutive patients ( 60 females, mean age 51ys) were prospectively evaluated with HRM, BET and PUS. Inter test agreement for the diagnosis of anismus was assessed using the Kappa statistic. Correlation between anismus to evacuatory failure (assessed by PUS) was also assessed.

Results: 36 patients were assessed for ED, 6 for CPP and 21 for FI. Anismus was diagnosed in 26 patients by HRM and 45 patients by DT-PUS. All cases of anismus diagnosed by HRM or DT-PUS had a positive BET. The Kappa agreement for the diagnosis of anismus between HRM and DT-PUS was poor $(0.143 \pm 0.01)$. 9 patients had significant pelvic floor anatomic pathology (4 rectal prolapse, 6 pathological pelvic descent, 4 enterocele and 3 rectocele $>3.5 \mathrm{~cm}$ ). There was a moderate correlation between diagnosis of anisums on DT-PUS to failure to evacuate the rectum $(\mathrm{r}=0.636)$. The correlation between rectal evacuation on DT-PUS to the diagnosis of anismus on manometry was weak $(r=0.296)$

Conclusion: There is considerable disagreement between the results of various evacuatory tests, and between the diagnoses of evacuatory dysregulation to failure of rectal evacuation. Therefore, more than one test should be applied in order to evaluate the defecatory dysfunction.

Disclosure of Interest: All authors have declared no conflicts of interest.

\section{P1134 DIAGNOSTIC USE OF ENDOSCOPIC FULL-THICKNESS WALL RESECTION (EFTR) IN PATIENTS WITH SYMPTOMS OF CHRONIC INTESTINAL PSEUDO-OBSTRUCTION (CIPO)}

P.V. Valli ${ }^{1}$, D. Pohl ${ }^{1}$, M. Fried ${ }^{1}$, R. Caduff ${ }^{2}$, P. Bauerfeind

${ }^{1}$ University Hospital Zurich, Zürich/Switzerland

${ }^{2}$ Institute Of Surgical Pathology And Molecular Pathology, University Hospital Zurich, Zürich/Switzerland

Contact E-mail Address: piero.valli@usz.ch

Introduction: Complex gastrointestinal (GI) motility disorders such as chronic intestinal pseudo-obstruction (CIPO) or Hirschsprung's disease (HD) are challenging to diagnose and treat appropriately. Thorough assessment of patient history, radiographic exams, endoscopy and motility measurements aid in diagnostic workup, yet underlying histology is the cornerstone to enable a more distinct diagnosis of neuromuscular GI disorders. Traditionally, surgical procedures have been performed to obtain specimen suitable for accurate histologic analysis.

Aims \& Methods: We performed endoscopic full-thickness resection (eFTR) using a full-thickness-resection device (FTRD) under moderate propofol sedation in four patients with suspected severe neuromuscular gut disorders including CIPO.

Results: Patient 1: A 21-year-old male patient with cerebral palsy suffering from acute small bowel ileus with a history of laparotomy, detorquation and appendectomy after cecal volvulus at the age of 15 . Histologic analysis revealed irregular configuration of the myenteric plexus, but primary neuro- or myopathic disorder such as HD were ruled out. Thus, GI dysmotility due to cerebral palsy syndrome was suspected. Patient 2: After a life-long history of recurrent obstipation, colonic dilatation, ileus symptoms and various colonic segment resections, diagnostic eFTR was performed in a 55-year-old female patient. The diagnosis of hypoganglionosis was established by LDH histochemical and by immunohistochemical reactions with Calretinin and Map2-Kinase. Patient 3: A 19-year-old male patient with a history of a sigmoid volvulus and massively dilated large bowel segments. Enzyme histochemistry excluded HD upon histopathological analysis. The inner muscle layer showed fibrosis, eosinophilic leiomyositis and lymphocytic ganglionitis. Congenital CIPO was diagnosed due to degenerative leiomyopathy. Patient 4: A 56-year-old male patient with acute ileus and a yearlong history of constipation and abdominal pain. Histopathological analysis 
revealed hypoganglionosis, severe fibrosis of the inner muscle layer and reduced ICC networks $(<50 \%)$. HD was excluded and acquired CIPO was diagnosed due to hypoganglionosis. Technical as well as histological success providing large fullthickness specimen of excellent quality was achieved in all four patients (success rate $100 \%$ ). The mean procedure time was 12 minutes (range 5-20 min). The mean diameter of the resected specimen was $21 \mathrm{~mm}$ (range 20-22 mm). No adverse events connected to the procedure itself occurred during the follow up.

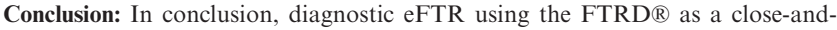
resect device is a well feasible and effective technique to provide full-thickness gut specimens of excellent quality and size, allowing detailed routine and sophisticated histologic, histochemical and immunohistochemical analysis for the diagnosis of neuromuscular gut disorders.

Disclosure of Interest: All authors have declared no conflicts of interest.

\section{P1135 TRANS-ABDOMINAL INTERFERENTIAL ELECTRICAL STIMULATION IS EFFECTIVE IN MANAGING REFRACTORY LOWER GASTROINTESTINAL DYSMOTILITY DISORDERS}

J. Moore, P.R. Gibson, R. E. Burgell

Gastroenterology, Monash University, Melbourne/Australia/VIC

Contact E-mail Address: judith.moore $@$ monash.edu

Introduction: There is emerging interest in non-pharmacological management of gastrointestinal dysmotility via neuromodulatory techniques. A new method of non-invasive neuromodulation - transabdominal interferential electrical stimulation - has apparent efficacy in a paediatric population with difficult constipation.

Aims \& Methods: We report our experience of its use in adult patients with functional constipation who are refractory to conventional management.

This is a descriptive case series of consecutive adult patients presenting to a tertiary referral functional gastrointestinal disorders clinic with refractory constipation that were taught and used home-based interferential stimulation for an hour a day over a three-month period by a functional gut nursespecialist between October 2015 and Feb 2017. The validated PAC-SYM and PAC-QOL questionnaires were given at commencement of stimulation and after three months of use. Overall symptom severity was assessed by $10 \mathrm{~cm}$ VAS An intention to treat analysis was conducted for any patients who commenced stimulation.

Results: Seven patients with refractory constipation (3 with slow-transit and 4 with idiopathic constipation) responding inadequately to pharmacological and non-pharmacological therapies underwent stimulation. Mean (range) age was 47 (26-73) y and 2 were male. All 7 patients completed the stimulation period. There was a reduction in PAC-SYM in all patients (median IQR 24 [18-36] vs 14 [10$21])$ and VAS median IQR 8 [7-9] vs 4 [2-5] $(\mathrm{p}=0.004)$. PAC-QOL was assessed in 4 patients where this also fell from 75 [69-85] to 38 [19-52]. Four were able to cease previously heavy daily laxative use and 2 were able to halve their use, one currently weaning off prucalopride. One remained on daily laxative use despite soft, formed stool. All reported satisfaction with stool type. Ongoing benefit remains in 2 after 4 and 12 months since ceasing its use, where the rest tend to use the stimulator intermittently.

Conclusion: Interferential electrical stimulation improved symptoms in patients with functional constipation. Randomized placebo controlled trials are justified. Disclosure of Interest: All authors have declared no conflicts of interest.

\section{Reference}

1. Hutson, J., Dughetti, L., Stathopoulos, L. \& Southwell, B. (2015) Transabdominal electrical stimulation (TES) for the treatment of slow transit constipation (STC). Pediatr Surg Int DOI 10.1007/s00383-015- 368 1-4

\section{P1136 YH12852, A NOVEL AND HIGHLY SELECTIVE 5-HYDROXYTRYPTAMINE 4 RECEPTOR AGONIST, INCREASES STOOL FREQUENCY IN HEALTHY VOLUNTEERS AND PATIENTS WITH FUNCTIONAL CONSTIPATION: RESULTS OF A RANDOMIZED DOUBLE-BLIND PLACEBO/ ACTIVE-CONTROLLED PHASE 1/2A TRIAL}

S.J. Moon ${ }^{1}$, H.G. Yoo ${ }^{1}$, S. Lee' 2 , S.B. Jang 2 , M.K. Kim², H. Na ${ }^{2}$, H. Lee ${ }^{1}$ ${ }^{1}$ Department Of Clinical Pharmacology And Therapeutics, Seoul National University College of Medicine and Hospital, Seoul/Korea, Republic of ${ }^{2}$ Yuhan R\&d Institute, Yuhan Corporation, Seoul/Korea, Republic of

\section{Contact E-mail Address: andrew90@yuhan.co.kr}

Introduction: The $5-\mathrm{HT}_{4}$ receptor is an attractive drug target that can stimulate gastrointestinal motility. YH12852 is a novel, potent and highly selective 5-HT receptor agonist currently under clinical development for the treatment for gastrointestinal motility disorders (GIMDs).

Aims \& Methods: The study aim was to evaluate the safety, tolerability, pharmacokinetic and pharmacodynamic profiles for YH12852 in healthy volunteers with $\leq 3$ spontaneous bowel movements per week (SBMs/week) and patients with functional constipation (FC). A randomized, double-blind, placebo- and active-controlled phase $1 / 2 \mathrm{a}$ study was performed in healthy subjects and FC patients, who were administered YH12852 $(0.3,0.5,1,2$ or $3 \mathrm{mg})$, prucalopride $2 \mathrm{mg}$ or placebo once daily after breakfast for 2 weeks. Subjects recorded bowel habits throughout the study period. Intensive pharmacokinetic blood samples were also collected (ClinicalTrials.gov identifier NCT02538367)

Results: Twenty-nine healthy subjects and 27 FC patients were enrolled. Treatment-emergent adverse events (TEAEs) were mostly mild and no serious adverse event was reported. The most frequently reported AE in the YH12852 and prucalopride group was headache. TEAEs in the YH12852 groups were similar to those in the prucalopride group. The change from baseline in weekly SBM frequencies averaged over the 2-week treatment period were $3.5,3.8,6.3$, 4.6 and 3.9 for YH12852 $0.3,0.5,1,2$ and $3 \mathrm{mg}$, respectively, 4.0 prucalopride and 2.6 placebo. The proportion of responders (defined as those with a $>1$ increase in SBM/week over 2 weeks from baseline) was higher in the YH12852 treatment groups $(87.5 \%, 100.0 \%, 100.0 \%$, and $100.0 \%$ in $0.3,0.5,1$ and $2 \mathrm{mg}$, respectively), with the exception of the $3 \mathrm{mg}$ group $(80.0 \%)$, compared with that in the prucalopride $(83.3 \%)$ and placebo groups $(28.6 \%)$. The mean stool consistencies assessed by the Bristol Stool Form Scale were comparable between the YH12852 and prucalopride groups. The time to reach the peak concentration of YH12852 was 4 hours. Steady state was achieved approximately 5 days after the first administration of YH12852 with a half-life of 23-28 hours. YH12852 showed a linear pharmacokinetic profile over $0.3-3 \mathrm{mg}$.

Conclusion: YH12852 was well tolerated and its safety profile was comparable to that of prucalopride. YH12852 appeared to increase bowel movement greater than prucalopride, particularly at $0.5-2 \mathrm{mg}$. YH12852 may have a significant potential for the treatment of lower GIMDs.

Disclosure of Interest: S. Lee: The affiliates of Yuhan Corporation are stockholders and/or employees

S.B. Jang: The affiliates of Yuhan Corporation are stockholders and/or employees

M.K. Kim: The affiliates of Yuhan Corporation are stockholders and/or employees

H. Na: The affiliates of Yuhan Corporation are stockholders and/or employees All other authors have declared no conflicts of interest.

P1137 HEALTHCARE PROFESSIONALS FAIL TO PROVIDE ADEQUATE COUNSELLING AND SUPPORT ABOUT OPIOIDINDUCED CONSTIPATION TO STRONG-OPIOID USERS

V. Andresen ${ }^{1}$, V. Banerji ${ }^{2}$, G. Hall ${ }^{2}$, A. Lass $^{3}$, A. V. Emmanuel ${ }^{4}$

${ }^{1}$ Gastroenterology, Israelitic Hospital, Hamburg/Germany

${ }^{2}$ InsightDojo, London/United Kingdom

${ }^{3}$ Medical, Shionogi Limited, London/United Kingdom

${ }^{4}$ Neuro-gastroenterology, National Hospital for Neurology and Neurosurgery,

London/United Kingdom

Contact E-mail Address: V.Andresen@ik-h.de

Introduction: Constipation is a common side effect of opioid use. Available laxative therapies for opioid-induced constipation (OIC) leave the patient with significant residual symptoms, which may lead them to adjust or stop their opioid intake in order to have a bowel movement, unless effectively counselled. ${ }^{1}$

Aims \& Methods: This subgroup analysis of an international survey investigated counselling resources, information-seeking, and sources of support in subjects with constipation caused by the use of strong opioids (e.g. buprenorphine, fentanyl). This was a quantitative, questionnaire-based, online survey conducted in France, Germany, Italy, Spain and the UK among respondents aged $\geq 40$ years with largely non-cancer-related chronic pain, treated long-term with strong opioids and having constipation $(\mathrm{N}=2016)$. The survey assessed past medical history, opioid use, treatment and treatment-seeking behaviour, symptoms, burden of disease, and effects on quality of life of constipation.

Results: In general, responders find it difficult to combine pain management relief and constipation and dislike having to balance between them (36\%). Approximately one-fifth $(22 \%)$ of respondents were very or somewhat dissatisfied with the effectiveness of their current constipation treatment and only $43 \%$ strictly adhered to prescribed treatment regimens, with $32 \%$ researching other treatment options. A significant number of responders (44\%) admitted that their constipation becomes so bothersome that they have to combine different methods to relieve it, and $40 \%$ often cut down their opioid medication or even skip it entirely $(9 \%)$ to relieve constipation. To manage their constipation, respondents regularly used a variety of approaches, including dietary measures $(48 \%)$, exercise $(23 \%)$ and single $(32 \%)$ or multiple $(15 \%)$ laxative treatments. Only $45 \%$ of respondents reported that their healthcare professionals (HCPs) had warned them about constipation as a potential side effect of opioid use. Almost twothirds of respondents $(63 \%)$ reported that their HCP was the main information source on opioid-induced constipation. Although $58 \%$ of respondents stated that they would have liked their HCP to provide more information about OIC, $48 \%$ preferred to deal with constipation on their own, rather than discuss it with their HCP. Other common sources of information were online search engines $(44 \%)$ and online health forums $(29 \%)$. Less than half of respondents $(43 \%)$ strictly adhered to prescribed treatment regimens, with $32 \%$ researching other treatments.

Conclusion: A proportion of patients are not satisfied with their current constipation treatment and they sometimes find balancing the need for adequate pain relief with constipation side effects challenging; consequently, many fail to adhere to their prescribed treatment regimens, or resort to using suboptimal strategies, such as reducing their opioid intake, to relieve constipation. Despite this dissatisfaction, many HCPs are not counselling patients adequately about constipation as a common potential side effect of opioid use. While most patients would like to have more support from their HCP, nearly half prefer to deal with constipation on their own, perhaps due to embarrassment or resignation. Disclosure of Interest: A. Lass: Contractor to Shionogi Ltd.

All other authors have declared no conflicts of interest.

\section{Reference}

1. LoCasale RJ et al. J Manag Care Spec Pharm 2016;22:246-53. 
P1138 THREE-DIMENSIONAL HIGH-RESOLUTION ANORECTAL MANOMETRY IN CHILDREN AFTER SURGERY FOR ANORECTAL DISORDERS

M. Banasiuk ${ }^{1}$, A. Banaszkiewicz ${ }^{1}$, M. Dziekiewicz ${ }^{1}$, A. Kamiński ${ }^{2}$, P. Albrecht ${ }^{1}$ ${ }^{1}$ Dept. Of Pediatric Gastroenterology, Medical University of Warsaw, Warsaw/ Poland

${ }^{2}$ Dept. Of Pediatric Surgery, Medical University of Warsaw, Warsaw/Poland

Contact E-mail Address: mbanasiu@tlen.pl

Introduction: Three-dimensional high-resolution anorectal manometry (3DHRAM) is the most precise tool to assess function of the anal canal and may be useful in evaluation of children after surgery on lower gastrointestinal tract that may present wide spectrum of symptoms from gastrointestinal tract. Our aim was to evaluate children after surgery for anorectal disorders using 3DHRAM.

Aims \& Methods: We performed a prospective study of 43 children (30 male, mean age, 7 years) after surgery for anorectal disorders at the Departments of Pediatric Gastroenterology and Nutrition, Medical University of Warsaw, Poland. The group consisted of 24 children after surgery for Hirschsprung's disease (HD), 12 children after surgery for anal atresia (AA) and 7 children after proctocolectomy for other reasons (PC). In all children conventional manometric parammeters were obtained. Pressures of the anal canal was divided into 8 segments and the resting and squeeze pressures of puborectalis muscle (PRM) were recorded in segments covering its anatomical localization. These data were compared to raw data obtained in our laboratory from healthy children published previously ( $\mathrm{HC}$ group). To assess correlation between manometry and symptoms, all children (after surgery and $\mathrm{HC}$ group) were divided into groups with respect to symptoms, as follows: asymptomatic (A), nonretentive fecal incontinent (NFRI), constipated (C) and retentive fecal incontinent (RFI).

Results: The lowest values of resting, squeeze and the pressure of PRM were observed in AA $(55.6 \mathrm{mmHg}, 121.7 \mathrm{mmHg}$ and $44.17 \mathrm{mmHg}$, respectively). As compared to asymptomatic children, the lowest mean and maximum resting pressures were observed in NRFI $(69.6 \mathrm{mmHg}$ and $61.3 \mathrm{mmHg}$, respectively; $\mathrm{p}<0.000$ ). Significantly lower maximum squeeze pressure were recorded in both, NRFI and RFI (168.1 $\mathrm{mmHg}$ and $103.8 \mathrm{mmHg}$, respectively; $\mathrm{p}=0.03$ ). ROC cut-off value for mean resting pressure between asymptomatic children and patient with fecal incontinence was $68,5 \mathrm{mmHg}$. Significantly lower PRM resting pressure were observed in NRFI group and lower PRM squeeze pressure in RFI (45.6 $\mathrm{mmHg}$ and $63.6 \mathrm{mmHg}$, respectively). Threshold of urge were significantly higher in group $\mathrm{C}$ as compared to A group $\left(87.5 \mathrm{~cm}^{3}\right.$ and $30 \mathrm{~cm}^{3}$, respectively; $\mathrm{p}=0.003$ ).

Conclusion: Our study demonstrated lower pressure parameters in children after surgery with the lowest values in patients suffering from anal atresia, which was correlated with incontinence. 3DHRAM may be useful tool for assessing the function of the anorectum of children after surgery.

Disclosure of Interest: M. Banasiuk: Equipment support from manufacturer of the equipment (Covidien AG)

All other authors have declared no conflicts of interest.

\section{P1139 UK CLINICAL EXPERIENCE AT 52 WEEKS WITH LINACLOTIDE FOR IRRITABLE BOWEL SYNDROME WITH CONSTIPATION}

A. V. Emmanuel ${ }^{1}$, J. Mclaughlin ${ }^{2}$, Y. Yiannakou ${ }^{3}$, S. Mclain-Smith ${ }^{4}$

${ }^{1}$ University College London, London/United Kingdom

${ }^{2}$ Salford Royal, Salford/United Kingdom

${ }^{3}$ NHS Foundation Trust, County Durham and Darlington, Durham/United Kingdom

${ }^{4} \mathrm{pH}$ Associates Ltd, Marlow/United Kingdom

Contact E-mail Address: a.emmanuel@ucl.ac.uk

Introduction: Linaclotide, a guanylate cyclase $\mathrm{C}$ agonist, has been shown in clinical trials to relieve constipation and improve abdominal pain and discomfort in patients with irritable bowel syndrome with constipation (IBS-C), but there are limited UK-specific real-world data to support this.

Aims \& Methods: A multi-centre, observational, prospective 52-week study was conducted in eight specialist hospitals in England and Scotland. The primary objective was to describe the change in IBS-Symptom Severity Scale (IBS-SSS) score from baseline at 12 weeks after linaclotide initiation. Consenting patients aged $\geq 18$ years initiated on linaclotide $(290 \mathrm{mcg})$ for IBS-C were recruited. Data on patient demographic and clinical characteristics, concomitant medications, patient-reported outcomes, including IBS-SSS score, and adverse events were collected. Results at 12 weeks (primary endpoint) have been presented previously; here we report analysis of real-world clinical experience 52 weeks post-linaclotide initiation.

Results: 202 patients were recruited; $185(92 \%)$ were female. At baseline, median age was 44.9 (range $18-77$ ) years; $84(42 \%)$ reported concomitant laxative use. Mean baseline IBS-SSS score was 339 (standard deviation $[\mathrm{SD}] \pm 92 ; \mathrm{n}=193$ ); $129(67 \%)$ patients had IBS-C classified as severe (score $\geq 300), 54(28 \%)$ moderate $(175-300)$, nine $(5 \%)$ mild $(75-175)$ and one $(0.5 \%)$ normal $(<75)$. At 52 weeks, mean IBS-SSS score was $256(\mathrm{SD} \pm 116 ; \mathrm{n}=78) ; 31(40 \%)$ patients had severe IBS-C, 27 (35\%) moderate, 14 (18\%) mild and six $(8 \%)$ normal. IBS-SSS scores improved significantly between baseline and 52 weeks, with a mean decrease of $71(\mathrm{SD} \pm 106)$ points overall (t-test $\mathrm{p}<0.001 ; \mathrm{n}=76$ with paired data) and $94(\mathrm{SD} \pm 102)$ points in the patients remaining on linaclotide $(\mathrm{p}<0.001 ; \mathrm{n}=34)$. Of the 76 patients with paired data, $41(54 \%)$ reported responding to treatment (ie reduction in IBS-SSS score of $>50$ points, or score fell below 150 [if baseline score $>150])$ [Table]. At 52 weeks, $41(20 \% ; n=202)$ patients remained on linaclotide, $87(43 \%)$ had stopped ( $<4$ doses in past week), most commonly due to side effects $(n=51)$ or lack of efficacy $(n=18)$, and 74 $(37 \%)$ were lost to follow-up/not known. Overall, 174 adverse events possibly related to linaclotide were reported in $77(38 \%)$ patients, most commonly diarrhoea $(25 \% ; \mathrm{n}=51)$, abdominal pain $(9 \% ; \mathrm{n}=18)$ and abdominal distension $(6 \% ; n=13)$

Table: Change in IBS-SSS score at 52 weeks from start of linaclotide

\begin{tabular}{lll}
\hline Change in IBS-SSS & Patients, $\mathrm{n}$ & Patients, \% \\
\hline$-350<-300$ & 1 & 1 \\
$-300<-250$ & 5 & 7 \\
$-250<-200$ & 5 & 7 \\
$-200<-150$ & 4 & 5 \\
$-150<-100$ & 11 & 14 \\
$-100<-50$ & 14 & 18 \\
$-50<0$ & 15 & 20 \\
No change & 1 & 1 \\
$>0<50$ & 10 & 13 \\
$50<100$ & 7 & 9 \\
$100<150$ & 3 & 4 \\
$150<200$ & 0 & 0 \\
$200<250$ & 0 & 0 \\
$250<300$ & 0 & 0 \\
$300<350$ & 0 & 0 \\
Total & 76 & 100 \\
\hline
\end{tabular}

Conclusion: Linaclotide was associated with a significant improvement in IBSSSS score at 52 weeks and was reasonably well tolerated. These results provide valuable insights into the longer-term outcomes of linaclotide treatment in patients with IBS-C in real-world clinical practice.

Disclosure of Interest: A.V. Emmanuel: Served on advisory boards for Allergan, Almirall, Shire, Takeda

Y. Yiannakou: Educational grant and speaker fees from allergan

S. McLain-Smith: SMS is an employee of $\mathrm{pH}$ Associates, an independent research consultancy which was commissioned by the sponsor to provide support with the design and conduct of the study, data analysis and medical writing All other authors have declared no conflicts of interest.

\section{P1140 EFFECT OF FAECAL MICROBIOTA TRANSPLANTATION ON GUT BACTERIAL FERMENTATION PRODUCTS IN PATIENTS WITH IRRITABLE BOWEL SYNDROME}

T. Mazzawi ${ }^{1}$, J. Valeur ${ }^{2}$, T. Hausken ${ }^{3}$, M. El-Salhy ${ }^{4}$, J.G. Hatlebakk ${ }^{3}$,

G.A. Lied $^{5}$

${ }^{1}$ Gastroenterology-medicine, Haukeland University Hospital, Bergen/Norway

${ }^{2}$ Unger-Vetlesen Institute, Lovisenberg Diaconal Hospital, Oslo/Norway

${ }^{3}$ Haukeland University Hospital, National Centre for Functional Gastrointestinal Disorders, Bergen/Norway

${ }^{4}$ Gastroenterology-medicine, Stord Hospital Helse-Fonna, Stord/Norway

${ }^{5}$ Center For Nutrition, Clinical Medicine, University of Bergen, Bergen/Norway

Contact E-mail Address: tarek.mazzawi@med.uib.no

Introduction: Irritable bowel syndrome (IBS) may be associated with disturbances of gut microbiota composition and functions, such as altered bacterial fermentation.

Aims \& Methods: The aim was to study the effect of faecal microbiota transplantation (FMT) on gut bacterial fermentation products: short-chain fatty acids (SCFAs). Patients diagnosed with IBS according to Rome III criteria $(n=13)$ were included. They received freshly donated faeces from relatives, instilled into the descending part of the duodenum via gastroscope. Faecal samples were collected from the donors and the patients before FMT and from the patients after FMT at weeks $1,3,12$ and $20 / 28$. All the samples were stored at $-80^{\circ} \mathrm{C}$ until analysis. Faecal concentrations of major SCFAs (acetic, propionic and n-butyric acids) and minor SCFAs (iso-butyric, n-valeric, iso-valeric, n-caproic and isocaproic acids) were analysed by vacuum distillation followed by gas chromatography. The patients completed IBS symptom questionnaire (IBS-SQ) before and after FMT at weeks $1,3,12$, and 20/28, assessing the following domains: nausea, bloating, abdominal pain, diarrhea, constipation and anorexia.

Results: Before FMT, concentrations of several SCFAs were significantly lower in IBS patients compared to donors (Table 1). After FMT, concentrations of SCFAs increased within the first 3 weeks, and the increment lasted up to 28 weeks (Table 1). Symptom scores as assessed by IBS-SQ improved from before FMT until week $20 / 28$ after FMT as follows: nausea $(P=0.0013)$, bloating $(P<0.0001)$, abdominal pain $(P=0.0005)$, diarrhea $(P<0.0001)$, constipation $(P=0.03)$, and anorexia $(P=0.09)$. Correlations were found between abdominal pain and both acetic acid $(\mathrm{r}=0.69, P=0.04)$ and total SCFAs $(\mathrm{r}=0.69$, $P=0.044)$ in IBS patients before FMT. Inverse correlations were found 3 weeks after FMT between nausea and iso-valeric acid $(\mathrm{r}=-0.65, P=0.014)$, and between constipation and propionic $(\mathrm{r}=-0.74, P<0.0001)$, iso-butyric $(\mathrm{r}=-0.79, P<0.0001)$, n-valeric acids $(\mathrm{r}=-0.79, P<0.0001)$ and iso-valeric $(\mathrm{r}=-0.72, P<0.0001)$ 
Abstract No: P1140

Table 1: Concentrations ( $\mathrm{mmol} / \mathrm{kg}$ ) of short-chain fatty acids (SCFAs) in faecal samples collected from donors and patients with irritable bowel syndrome (IBS) before and after faecal microbiota transplantation (FMT).

\begin{tabular}{|c|c|c|c|c|c|c|c|c|c|c|c|}
\hline \multirow[b]{2}{*}{ SCFAs } & \multirow[b]{2}{*}{$\begin{array}{l}\text { Donor, } \\
(n=13)\end{array}$} & \multirow{2}{*}{$\begin{array}{l}\text { Patients } \\
\text { before FMT, } \\
(n=9)\end{array}$} & \multicolumn{4}{|c|}{ Patients after FMT } & \multirow[b]{2}{*}{$* \mathrm{P}$} & \multirow[b]{2}{*}{$* * \mathrm{P}$} & \multirow[b]{2}{*}{$* * * \mathrm{P}$} & \multirow[b]{2}{*}{$* * * * \mathrm{P}$} & \multirow[b]{2}{*}{$* * * * * \mathrm{P}$} \\
\hline & & & $\begin{array}{l}\text { Week 1, } \\
(n=12)\end{array}$ & $\begin{array}{l}\text { Week 3, } \\
(n=10)\end{array}$ & $\begin{array}{l}\text { Week } 12 \text {, } \\
(n=13)\end{array}$ & $\begin{array}{l}\text { Week } 20 / 28 \\
(n=12)\end{array}$ & & & & & \\
\hline Acetic acid & $/ 33.9 \pm 2.8$ & $23.6 \pm 6$ & $31.1 \pm 4.9$ & $35.5 \pm 3.9$ & $25.8 \pm 4.4$ & $28.5 \pm 2.4$ & 0.77 & $>0.9$ & $>0.9$ & 0.3 & $>0.9$ \\
\hline Propionic acid & $9.5 \pm 1$ & $6.2 \pm 1.6$ & $7.9 \pm 1.5$ & $8.2 \pm 1.5$ & $7.3 \pm 1.9$ & $8.1 \pm 1.2$ & 0.18 & $>0.9$ & $>0.9$ & 0.2 & $>0.9$ \\
\hline n-butyric acid & $10.4 \pm 1.6$ & $4.7 \pm 1.2$ & $7.7 \pm 1.8$ & $8.4 \pm 1.5$ & $5.8 \pm 1.4$ & $5.96 \pm 1.11$ & 0.049 & 0.78 & $>0.9$ & 0.095 & 0.25 \\
\hline Iso-butyric acid & $1.27 \pm 0.17$ & $0.67 \pm 0.11$ & $0.77 \pm 0.12$ & $0.92 \pm 0.13$ & $0.7 \pm 0.12$ & $0.98 \pm 0.2$ & 0.03 & 0.089 & $>0.9$ & 0.025 & 0.96 \\
\hline n-valeric acid & $1.4 \pm 0.18$ & $0.68 \pm 0.008$ & $1.05 \pm 0.2$ & $1.06 \pm 0.15$ & $0.77 \pm 0.13$ & $0.93 \pm 0.093$ & 0.013 & 0.67 & $>0.9$ & 0.042 & 0.47 \\
\hline Iso-valeric acid & $1.6 \pm 0.2$ & $0.8 \pm 0.2$ & $0.9 \pm 0.15$ & $1.16 \pm 0.2$ & $0.8 \pm 0.14$ & $1.27 \pm 0.2$ & 0.014 & 0.046 & $>0.9$ & 0.011 & $>0.9$ \\
\hline n-caproic & $0.8 \pm 0.02$ & $0.3 \pm 0.1$ & $0.5 \pm 0.2$ & $0.5 \pm 0.1$ & $0.2 \pm 0.08$ & $0.3 \pm 0.09$ & 0.2 & $>0.9$ & $>0.9$ & 0.059 & 0.17 \\
\hline Iso-caproic & $0.01 \pm 0.005$ & $0.02 \pm 0.02$ & $0.008 \pm 0.006$ & $0.013 \pm 0.01$ & $0.01 \pm 0.005$ & $0 \pm 0$ & $>0.9$ & $>0.9$ & $>0.9$ & $>0.9$ & 0.6 \\
\hline Total SCFAs & $58.8 \pm 5.4$ & $37 \pm 8$ & $49.9 \pm 8$ & $55.7 \pm 6.2$ & $41.4 \pm 7.1$ & $46 \pm 4.7$ & 0.17 & $>0.9$ & $>0.9$ & 0.15 & 0.6 \\
\hline
\end{tabular}

Data are presented as mean \pm SEM. Comparison: Kruskal-Wallis multiple comparisons test with Dunn's post test. *Donors at the beginning of the study vs. patients on FMT day before faecal installation, **Donors at the beginning of the study vs. patients 1 week after FMT, *** Donors at the beginning of the study vs. patients 3 weeks after FMT, ****Donors at the beginning of the study vs. patients 12 weeks after FMT, *****Donors at the beginning of the study vs. patients $20 / 28$ weeks after FMT. FMT: faecal microbiota transplantation. SCFAs: short-chain fatty acids

Conclusion: Our results reveal differences in faecal fermentation products between patients with IBS and healthy donors, and suggest that FMT may act to normalise such alterations of gut microbial functions.

Disclosure of Interest: All authors have declared no conflicts of interest.

P1141 HEALTHCARE RESOURCE USE IN PATIENTS WITH IRRITABLE BOWEL SYNDROME WITH DIARRHOEA BASED ON A SURVEY OF PHYSICIANS IN THE UNITED KINGDOM

\author{
A. Marciniak ${ }^{1}$, D. Collomb ${ }^{1}$, S. Baker ${ }^{1}$, R. Goosey ${ }^{2}$ \\ ${ }^{1}$ Allergan plc, Marlow/United Kingdom \\ ${ }^{2}$ Kantar Health, Epsom/United Kingdom
}

Contact E-mail Address: Anne.Marciniak@Allergan.com

Introduction: Irritable bowel syndrome with diarrhoea (IBS-D) is a chronic gastrointestinal disorder associated with significantly increased healthcare resource use (HCRU) and a substantial economic burden. In clinical practice, adequate relief (AR) of symptoms is an important measure of treatment effectiveness. ${ }^{1,2}$ However, the difference in HCRU related to IBS-D between patients with AR and those with inadequate relief (IR) has not yet been assessed.

Aims \& Methods: This objective of this study was to quantify the HCRU in patients with AR of IBS-D symptoms compared to patients with IR. An online survey assessing HCRU was distributed to general practitioners (GPs) recruited from market research panels in the UK in August 2016. GPs optedin to complete the survey via an email link and were screened before being invited to complete the main survey. Screening criteria included having seen patients with a chronic gastrointestinal condition in the past three months, having seen patients with IBS-D in the past 3 months and having decided on what treatments were prescribed for patients with IBS. The survey was a 15-minute web-based survey, including 12 questions collecting information on the use of medical services and procedures amongst patients with IBS-D for the first year following diagnosis and for subsequent years. Respondents were required to answer the survey considering patients who had AR of IBS-D symptoms and those who did not (IR), based on the respondents' own assessments. Statistical analyses included t-tests for two independent samples comparing mean scores for those with IR vs those with AR during the first year after diagnosis at a 5\% risk level, with $\mathrm{p}<0.05$ denoting significance.

Results: The online survey was completed by $50 \mathrm{GPs}$, with responses from $46 \mathrm{GPs}$ included in this analysis (four responses were excluded due to data quality reasons). The reported total number of medical visits was significantly higher for patients with IR vs patients with AR during the first year after diagnosis (mean 10.11 vs $5.20 ; \mathrm{p}<0.001$ ), with similar results seen for subsequent years (mean 8.20 vs 4.38). Significantly higher numbers of GP office visits, outpatient visits and emergency room visits were reported for patients with IR, with the greatest difference seen for GP office visits (2.37 more visits/year on average; incremental 4-week increase of $0.182 ; \mathrm{p}=0.003$ ) [Table]. Similarly, the total reported mean number of procedures was significantly higher for patients with IR vs patients with $\mathrm{AR}(8.11$ vs $4.52 ; \mathrm{p}=0.046)$, with number of colonoscopies having the greatest difference between the two groups $(2.17$ vs $0.91 ; 1.26$ more procedures/year on average; incremental 4-week increase of $0.097 ; \mathrm{p}=0.008$ ). Similar results were seen for the subsequent years after diagnosis, with patients with IR having more medical procedures compared to patients with AR (4.08 vs 2.56)

Table

\begin{tabular}{|c|c|c|c|c|}
\hline $\begin{array}{l}\text { Mean resource use } \\
\text { (standard deviation) }\end{array}$ & $\begin{array}{l}\text { Adequate } \\
\text { relief } \\
\left(1^{\text {st }} \text { year }\right. \\
\text { after } \\
\text { diagnosis })\end{array}$ & $\begin{array}{l}\text { Inadequate } \\
\text { relief } \\
\left(1^{\text {st }} \text { year }\right. \\
\text { after } \\
\text { diagnosis })\end{array}$ & $\begin{array}{l}\text { Difference } \\
\text { per year }\end{array}$ & $\begin{array}{l}\text { Incremental } \\
\text { mean } \\
\text { quantity } \\
\text { per } 4 \text { weeks }\end{array}$ \\
\hline \multicolumn{5}{|l|}{ Medical visits } \\
\hline Total & $5.20(4.87)$ & $10.11(5.30)^{*}$ & 4.91 & 0.378 \\
\hline GP office & $3.33(2.87)$ & $5.70(3.12)^{*}$ & 2.37 & 0.182 \\
\hline Outpatient & $0.96(1.53)$ & $2.30(1.68)^{*}$ & 1.35 & 0.104 \\
\hline Emergency room & $0.52(1.24)$ & $1.17(1.36)^{*}$ & 0.65 & 0.050 \\
\hline Hospitalisations & $0.39(1.00)$ & $0.93(1.10)$ & 0.54 & 0.042 \\
\hline \multicolumn{5}{|l|}{ Procedures } \\
\hline Total & $4.52(6.96)$ & $8.11(7.58)^{*}$ & 3.59 & 0.276 \\
\hline Colonoscopy & $0.91(1.47)$ & $2.17(1.61)^{*}$ & 1.26 & 0.097 \\
\hline Sigmoidoscopy & $0.70(1.43)$ & $1.37(1.56)$ & 0.67 & 0.052 \\
\hline Ultrasound & $1.13(2.06)$ & $1.76(2.26)$ & 0.63 & 0.048 \\
\hline Endoscopy & $0.67(1.28)$ & $1.22(1.37)$ & 0.54 & 0.042 \\
\hline $\begin{array}{l}\text { Computerised } \\
\text { tomography scan }\end{array}$ & $0.48(1.01)$ & $0.85(1.10)$ & 0.37 & 0.028 \\
\hline X-ray & $0.63(1.53)$ & $0.74(1.68)$ & 0.11 & 0.008 \\
\hline
\end{tabular}

" $\mathrm{p}<0.05$ compared to patients with adequate relief

Conclusion: GPs reported that patients with IBS-D considered as having IR of symptoms had increased HCRU, including more GP office visits and more colonoscopies, compared to patients with AR. These results highlight that IR is potentially an important driver of increased HCRU in patients with IBS-D, emphasising that effective treatments that provide AR may reduce HCRU and the associated economic burden.

Disclosure of Interest: A. Marciniak: Anne Marciniak is an employee of Allergan plc and shareholder in Pfizer, Amgen, and Allergan plc.

D. Collomb: David Collomb is an employee of Allergan plc.

S. Baker: Stephen Baker is an employee of Allergan plc.

R. Goosey: Richard Goosey is an employee of Kantar Health, paid consultants to Allergan plc.

\section{References}

1. Jan Irvine E et al. Gastroenterology 2006; 130: 1538-1551.

2. Passos MCF et al. Am J Gastroenterol 2009; 104: 912-919. 
P1142 RANDOMISED PLACEBO CONTROLLED ESCITALOPRAM INTERVENTION IN IBS WITH PANIC DISORDER: EVALUATION BY GSRS AND BY EXPERIENCE SAMPLING METHOD

L. Vork ${ }^{1}$, M. Drukker ${ }^{2}$, Z. Mujagic ${ }^{1}$, D. Keszthelyi ${ }^{1}$, J. Van $\mathrm{Os}^{2}$, A. A.m. Masclee ${ }^{1}$, C. Leue ${ }^{2}$, J. Kruimel ${ }^{1}$

${ }^{1}$ Division Of Gastroenterology-hepatology, Department Of Internal Medicine, Maastricht University Medical Center +, Maastricht/Netherlands ${ }^{2}$ Department Of Psychiatry And Psychology, Maastricht University Medical Center+, Maastricht/Netherlands

Contact E-mail Address: 1.vork@maastrichtuniversity.nl Introduction: Selective Serotonin Reuptake Inhibitors (SSRI's) have shown efficacy in reducing symptoms but less so on pain in irritable bowel syndrome (IBS). Comorbid anxiety frequently occurs in IBS. We hypothesized that SSRI's will particularly be effective in reducing abdominal pain in IBS patients with pronounced comorbid anxiety. As methods for symptom evaluation were used 1) gastrointestinal symptom rating scale (GSRS) as primary parameter and 2) a new method called the Experience Sampling Method (ESM). With ESM digital assessments are completed randomly and repeatedly during daily life, therewith capturing fluctuating symptom patterns more accurately than retrospective questionnaire methods

Aims \& Methods: IBS patients with comorbid panic disorder were included in a randomized controlled trial on escitalopram versus placebo. Measurements were completed at baseline $(\mathrm{t}=0)$ and after $3(\mathrm{t}=3)$ and 6 months $(\mathrm{t}=6)$. At each time point, the gastrointestinal symptom rating scale (GSRS) and a 7-day ESM period were completed. Subjects completed ESM assessments on a palmtop computer at 10 random moments each day during 7 consecutive days. ESM periods were analysed when at least $1 / 3$ (i.e. 23) of the assessments were completed. Mixed linear modelling was used with the GSRS-abdominal pain domain (GSRS-AP) as the dependent and treatment group as the independent variable, as well as with ESM- abdominal pain scores as the dependent and treatment group and ESManxiety scores as the independent variables.

Results: In total, 29 subjects (15 escitalopram and 14 placebo; 21 female; $37 \pm 14.8$ years; equal abdominal pain and anxiety scores at baseline) were included. Average GSRS-AP scores were not significantly different between escitalopram and placebo at $\mathrm{t}=3$ (B: 0.265 , SE: $0.451, \mathrm{p}=0.557)$ or $\mathrm{t}=6(\mathrm{~B}: 0.229$ SE: $0.539, p=0.670)$. For the ESM analyses, at $t=6$, average abdominal pain scores were significantly lower $(\mathrm{B}: 1.30$, SE: $0.623, \mathrm{p}=0.037)$ (on a $1-7$ scale) in the escitalopram group compared to placebo. With increasing anxiety levels (scores 2, 3 and 4) this difference further increased to 1.57, 1.84 and 2.11, respectively. A sensitivity analysis on subjects that completed all 3 valid ESM periods (i.e. at least $1 / 3$ of total number of assessments at $t=0, t=3$ and $t=6$ ) showed similar results.

Conclusion: Using GSRS as primary outcome, no significant effect of escitalopram over placebo on abdominal pain was found over a 6-month period. However, using ESM, a significant improvement in abdominal pain was observed, related to anxiety scores. These data 1) challenge the value of traditional retrospective methods with end-of-period symptom recording and 2) are in favour of novel more accurate momentary symptom registrations such as the Experience Sampling Method.

Disclosure of Interest: All authors have declared no conflicts of interest.

\section{P1143 LINACLOTIDE ACCELERATES COLONIC TRANSIT AND IMPROVES COLONIC CONTRACTILITY IN IBS WITH CONSTIPATION}

A. D. Farmer $^{1}$, A. R. Hobson ${ }^{2}$

${ }^{1}$ Gastroenterology, University Hospitals of North Midlands, Stoke on Trent/United Kingdom

${ }^{2}$ Functional Gut Clinic, London/United Kingdom

\section{Contact E-mail Address: a.farmer@qmul.ac.uk}

Introduction: Linaclotide, a guanylate cylase- $\mathrm{C}$ agonist, stimulates intestinal fluid secretion and decreases visceral hypersensitivity and is licensed for use in irritable bowel syndrome with constipation (IBS-C). There is a relative paucity of data concerning its effect on gastrointestinal (GI) motility.

Aims \& Methods: We aimed to compare the effect of linaclotide on segmental and pan-enteric motility in IBS-C. 14 patients with Rome III defined IBS-C (3 male, mean age 37 years, range 20-64) underwent a wireless motility capsule (WMC) using a standardized protocol. Segmental transit was derived from measures around known anatomical landmarks as identified by compartmental $\mathrm{pH}$ changes. Ileal and colonic motility measures are presented as area under the curve (AUC) derived from contraction amplitude and frequency. Validated questionnaires assessing GI (verbal descriptor anchored visual analog scale (VDVAS) assessing sensory intensity (VDVAS-I) and unpleasantness (VDVAS-U)) and somatic symptoms (Personal Health Questionnaire (PHQ) as well as quality of life (EQ-5D) were administered. The WMC and questionnaires were repeated after 28 days of linaclotide $290 \mathrm{mcg}$ po od.

Results: Changes in GI motility are shown in Table 1. Linaclotide improved VDVAS-I and VDVAS-U $(130.7 \pm 20.8$ vs. $106.5 \pm 33, p=0.03$ and $113 \pm 22$ vs. $85.8 \pm 33, p=0.01)$ and quality of life $(58.4 \pm 21.2$ vs. $68 \pm 17.6, p=0.02)$. Linaclotide reduced somatic symptoms $(8.3 \pm 2.2$ vs. $5.9 \pm 1.4, p=0.007)$.
Change in $\mathrm{pH}$ across the ileocaecal junction correlated with improvement in VDVAS-I and $\mathrm{U}(\mathrm{r}=0.6, p=0.03$ and $\mathrm{r}=0.64, p=0.02)$.

Table 1: Changes in GI physiology following linaclotide.

\begin{tabular}{llll}
\hline & $\begin{array}{l}\text { Baseline } \\
\text { (mean and } \\
\text { standard } \\
\text { deviation) }\end{array}$ & $\begin{array}{l}\text { Post treatment } \\
\text { (mean and } \\
\text { standard } \\
\text { deviation) }\end{array}$ & $\begin{array}{l}\text { P } \\
\text { Value }\end{array}$ \\
\hline Gastric emptying time (minutes) & $154 \pm 64$ & $177 \pm 57$ & 0.4 \\
Small bowel transit time (minutes) & $353 \pm 152$ & $299 \pm 139$ & 0.3 \\
Colonic transit time (minutes) & $3017 \pm 1305$ & $1983 \pm 1216$ & 0.04 \\
Whole gut transit time (minutes) & $3517 \pm 1375$ & $2432 \pm 1180$ & 0.04 \\
Ileal contractility (AUC) & $262 \pm 142.4$ & $221 \pm 113.5$ & 0.5 \\
Colonic contractility (AUC) & $90.9 \pm 78.3$ & $134.6 \pm 93$ & 0.006 \\
Changein pH across & $-2.4 \pm 0.2$ & $-2.1 \pm 0.4$ & 0.03 \\
\multicolumn{1}{c}{ the lleocaecal junction } & & & \\
\hline
\end{tabular}

Linaclotide improved VDVAS-I and VDVAS-U (130.7 \pm 20.8 vs. $106.5 \pm 33$, $p=0.03$ and $113 \pm 22$ vs. $85.8 \pm 33, p=0.01)$ and quality of life $(58.4 \pm 21.2$ vs. $68 \pm 17.6, p=0.02)$. Linaclotide reduced somatic symptoms $(8.3 \pm 2.2$ vs. $5.9 \pm 1.4, p=0.007)$. Change in $\mathrm{pH}$ across the ileocaecal junction correlated with improvement in VDVAS-I and $\mathrm{U}(\mathrm{r}=0.6, p=0.03$ and $\mathrm{r}=0.64, p=0.02)$. Conclusion: Linaclotide reduced colonic transit and enhances contractility. These changes in GI motility are accompanied by improvements in symptoms and quality of life and are associated with a reduction in extra-gastrointestinal symptoms. Change in $\mathrm{pH}$ across the ileocaecal junction has been proposed as a surrogate marker of caecal fermentation. Thus the beneficial effect of linaclotide on symptoms may therefore be related to a reduction in fermentation. This potential biomarker warrants further exploration.

Disclosure of Interest: A.D. Farmer: Speaker Bureau - Allergan Advisory boardAllergen

All other authors have declared no conflicts of interest.

\section{P1144 RELATIONSHIP BETWEEN RIFAXIMIN THERAPY AND SEHCAT TEST IN PATIENTS WITH DIARRHEA-PREDOMINANT IRRITABLE BOWEL SYNDROME OR FUNCTIONAL DIARRHEA}

A. Ruiz Cerulla, S. Maisterra, C. Arajol, P. Gilabert, K. Serra, O. Puig, P. Notta, F. Rodriguez-Moranta, L. Rodriguez-Alonso, E. Sanchez-Pastor, J. Guardiola Gastroenterology, Hospital Universitari de Bellvitge, Hospitalet de Llobregat/ Spain

Contact E-mail Address: alexandraruizcerulla@gmail.com

Introduction: Bile acids (BAs) and gut microbiota have been involved in IBS pathophysiology. BA diarrhea (BAD) is often found in patients with irritable bowel syndrome with diarrhea (IBS-D) or functional diarrhea (FD) ${ }^{1}$. Colestyramine and rifaximin have both been shown to improve symptoms in these patients $\mathbf{2}^{2,3}$. It is unknown whether a SeHCAT test may help to predict response to rifaximin or whether rifaximin treatment affects SeHCAT test result. Aims \& Methods: a) To determine if a SeHCAT test may be used to predict response to rifaximin in patients with IBS-D or FD. b) To assess if rifaximin modifies SeHCAT result.

Consecutive patients diagnosed with IBS-D or FD were prospectively included in the study. All patients received rifaximin ( $400 \mathrm{mg}$ TID for $2 \mathrm{w}$ ). A SeHCAT test was performed to evaluate presence of BAD before and 1 month after rifaximin treatment. BAD was defined as SeHCAT retention $<10 \%$. Number of daily stools, number of daily watery stools, Bristol stool scale, abdominal pain, distension and presence of urgency were recorded before and after treatment. IBS severity score (IBS-SS) was also calculated.

Results: Forty-one patients were included. BAD was present in 23 patients $(56 \%)$. No clinical differences were found between BAD or non-BAD patients at study entry. Rifaximin resulted in a significant improvement in the number of daily stools $(\Delta-1.5 ; \mathrm{P}<0.01)$, daily watery stools $(\Delta-2.1 ; \mathrm{P}<0.01)$, Bristol scale $(\Delta-1.1 ; \mathrm{P}<0.01)$, abdominal pain $(\Delta-0.5 ; \mathrm{P}<0.01)$, distension $(\Delta-0.3$; $\mathrm{P}<0.01)$, urgency $(\Delta-0.7 ; \mathrm{P}<0.01)$ and in the IBS-SS $(\Delta-78 ; \mathrm{P}<0.01)$. No differences were found between BAD and non-BAD patients in the improvement of any item. Rifaximin treatment did not modify SeHCAT value $(9.5 \%$ before treatment and $10.7 \%$ after treatment; $\mathrm{P}=0.4$ ).

Conclusion: Half of the patients diagnosed with IBS-D or FD present BAD according the SeHCAT test. Rifaximin treatment confers significant clinical improvement irrespective of the presence of BAD. Rifaximin treatment does not affect SeHCAT test.

Disclosure of Interest: All authors have declared no conflicts of interest.

\section{References}

1. Wedlake L, A'Hern R, Russell D, Thomas K, Walters JR, Anfreyev HJ Systematic review: the prevalence of idiopathic bile acid malabsorption as 
diagnosed by SeHCAT scanning in patients with diarrhoea-predominant irritable bowel syndrome. Aliment Pharmacol Ther. 2009 Oct; 30(7): 707-17.

2. Lembo A, Pimentel M, Rao S, Schoenfeld P, Cash B, et al. Repeat Treatment With Rifaximin Is Safe and Effective in Patients With Diarrhea-Predominant Irritable Bowel Syndrome. Gastroenterology. 2016; 151: 1113-1121.

3. Fernández-Bañares F, Rosinach M, Piqueras M, Ruiz-Cerulla A, Modolell I, Zabana Y, Guardiola J, Esteve M. Randomised clinical trial: colestyramine vs. hydroxypropyl cellulose in patients with functional chronic watery diarrhoea. Aliment Pharmacol Ther. 2015 Jun; 41(11): 1132-40.

\section{P1145 FECAL MICROBIOTA TRANSPLANTATION FOR PATIENTS WITH POST-INFECTIOUS OR ANTIBIOTIC-INDUCED IRRITABLE BOWEL SYNDROME: RESULTS FROM A PROSPECTIVE PILOT STUDY}

Y. H. Van Beurden ${ }^{1}$, A. E. Budding ${ }^{2}$, E. M. Terveer ${ }^{3}$, J. J. Keller ${ }^{4}$, E. J. Kuijper ${ }^{5}$, C. M.j.e. Vandenbroucke-Grauls ${ }^{2}$, C.J.j. Mulder ${ }^{1}$

${ }^{1}$ Gastroenterology And Hepatology, VU university medical center, Amsterdam/ Netherlands

${ }^{2}$ Medical Microbiology And Infection Control, VU University Medical Center, Amsterdam/Netherlands

${ }^{3}$ Medical Microbiology, Leiden University Medical Center, Leiden/Netherlands ${ }^{4}$ Gastroenterology And Hepatology, Haaglanden Medical Center. The Hague Netherlands

${ }^{5}$ Afd. Medische Microbiologie, Leids Univers. Medisch Centrum, Leiden Netherlands

Contact E-mail Address: y.vanbeurden $@$ vumc.nl

Introduction: In $10-15 \%$ of patients with irritable bowel syndrome (IBS), the onset of symptoms follows an episode of gastrointestinal infection or use of antibiotics. Both antibiotic-induced and post-infectious IBS may be caused by alterations of the gut microbiota, which provides a rationale for treatment with fecal microbiota transplantation (FMT).

Aims \& Methods: We performed an open label pilot study in the VU University medical center in Amsterdam from August 2016 through January 2017, to evaluate the effect of FMT in patients with post-infectious IBS $(n=5)$ and patients with antibiotic-induced IBS $(\mathrm{n}=5)$. Participants with clinical symptoms lasting over six months, an IBS-symptom severity score (IBS-SSS) of at least 175 points, and a negative screening for gastrointestinal pathology were eligible. Donor feces $(198 \mathrm{ml})$ was provided by the Netherlands Donor Feces Bank (NDFB) and administered via a duodenal tube. Participants did not receive pre-treatment or bowel cleansing. Participants were followed for eight weeks via two validated questionnaires: IBS-SSS (range 100-500), and IBS-Quality of Life score (IBS-QOL; range $0-100 \%$ ). Fecal samples were obtained before and after FMT for microbiota analysis. Following the recommendation of the European Agency Medicines, FMT was considered clinically effective if participants reported an IBS-SSS improvement of at least 30\% compared to baseline, eight weeks after FMT.

Results: The mean IBS-SSS of all patients had improved significantly from $328( \pm 74)$ points at baseline to $245( \pm 117)$ at eight weeks after FMT $(\mathrm{p}=0.008)$; the median IBS-SSS improved with $40 \%$ from 340 (range $230-480$ ) to 205 (range $80-470$ ). Also, a significant improvement was seen in abdominal pain $(\mathrm{p}=0.039)$, satisfaction about bowel habit $(\mathrm{p}=0.018)$, and influence of IBS symptoms on daily life $(\mathrm{p}=0.011)$. There was no difference in bloating, or number of days with abdominal pain. The mean IBS-QOL improved from $51 \%$ to $66 \%(\mathrm{p}=0.008)$. FMT was considered effective in three patients with antibiotic-induced IBS, and one patient with post-infectious IBS ( $\geq 30 \%$ improvement). In general, fecal microbiota analysis showed that the microbiota composition of responders shifted to that of their corresponding donors. Nonresponders initially also did (which was associated with IBS-SSS improvement), however, eight weeks after FMT, the microbiota composition had shifted back towards baseline.

Conclusion: Treatment with FMT appears promising for antibiotic-induced and post-infectious IBS. Based on these results, a randomized placebo controlled tria is warranted.

Disclosure of Interest: A.E. Budding: A.E. Budding has proprietary rights to the IS-pro technique, and is co-owner of the spin-off company IS-diagnostics. All other authors have declared no conflicts of interest.

P1146 CONSECUTIVE WEEKS OF INADEQUATE RELIEF: A POST HOC ANALYSIS OF THE POOLED ELUXADOLINE PHASE 3 STUDIES IN PATIENTS WITH IRRITABLE BOWEL SYNDROME

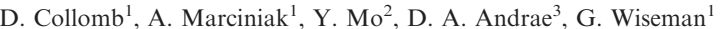

${ }^{1}$ Allergan plc, Marlow/United Kingdom

${ }^{2}$ Allergan plc, Jersey City/United States of America/NJ

${ }^{3}$ Allergan plc, Austin/United States of America/TX

Contact E-mail Address: gwen.wiseman@allergan.com

Introduction: Adequate relief (AR) of irritable bowel syndrome (IBS) symptoms is a commonly used global outcome measure to assess treatment benefit in both clinical trials and real-life clinical practice. Conversely, inadequate relief (IR) is considered an important driver for patients to use healthcare resources such as general practitioner or gastroenterologist consultations. Eluxadoline (ELX) is a mixed $\mu$-opioid receptor (OR) and $\kappa$-OR agonist and $\delta$-OR antagonist approved for the treatment of IBS with diarrhoea (IBS-D) in the US and Europe.
Aims \& Methods: To evaluate IR in patients treated with ELX in a post hoc analysis from two randomised, double-blind, placebo (PBO)-controlled Phase 3 trials (IBS-3001, IBS-3002). Patients meeting Rome III criteria for IBS-D were randomised 1:1:1 to twice-daily (BID) ELX (75 or $100 \mathrm{mg}$ ) or PBO. Efficacy was evaluated through Week 26. For evaluation of AR, patients were asked "In the last 7 days, have you had adequate [or satisfactory] relief of your IBS symptoms?" (Yes/No) on a weekly basis, via an electronic diary. As previously described, patients answering "Yes" for $\geq 50 \%$ of the total weeks during the target time interval were considered AR responders.,2 Patients answering "No" were considered to have IR. This analysis evaluated the number of consecutive weeks that patients reported IR over Weeks 1-12 and 13-24 of treatment. Patients without AR analysis were ineligible for intention-to-treat (ITT) analysis; missing data were not imputed.

Results: Overall, 2428 patients with IBS-D were enrolled across the two Phase 3 trials and 2423 were included in the ITT analysis. In the pooled dataset, a significantly greater proportion of patients were AR responders ${ }^{2}$ with either ELX 100 or $75 \mathrm{mg}$ BID vs PBO at 12 weeks $(56.1 \%$ [p $<0.001]$ and $56.3 \%[\mathrm{p}=0.001]$ vs $46.4 \%$, respectively) and at 26 weeks $(51.5 \%[\mathrm{p}<0.001]$ and $49.0 \%[\mathrm{p}=0.004]$ vs $41.8 \%$, respectively). ${ }^{3}$ Over the first 12 weeks of treatment, a greater proportion of patients reported no IR of IBS-D symptoms with ELX 100 or $75 \mathrm{mg}$ BID vs PBO (Table). Greater proportions of patients reported only $1-<5$ consecutive weeks of IR with ELX 100 or $75 \mathrm{mg}$ BID vs PBO. A significantly lower proportion of patients reported IR for $>8$ consecutive weeks with ELX 100 or $75 \mathrm{mg}$ BID vs PBO $(13.2 \%[\mathrm{p}<0.0001]$ and $15.6 \%[\mathrm{p}=0.004]$ vs $22.6 \%$, respectively). In contrast, a greater proportion of patients reported IR for the full 12 consecutive weeks with PBO vs ELX 100 or $75 \mathrm{mg}$ BID (Table). Similar results were observed in Weeks 13-24 of treatment, with ELX-treated patients generally having fewer consecutive weeks of IR compared to PBO-treated patients (Table).

Table: Consecutive weeks of inadequate relief

\begin{tabular}{|c|c|c|c|c|c|c|}
\hline \multirow[b]{2}{*}{$\begin{array}{l}\text { Inadequate } \\
\text { relief rate, \% } \\
\text { Number of } \\
\text { consecutive } \\
\text { weeks with } \\
\text { inadequate relief }\end{array}$} & \multicolumn{3}{|c|}{ Weeks $1-12$} & \multicolumn{3}{|c|}{ Weeks $13-24$} \\
\hline & $\begin{array}{l}\mathrm{PBO} \\
(\mathrm{n}=809)\end{array}$ & $\begin{array}{l}\text { ELX } \\
75 \mathrm{mg} \\
\text { BID } \\
(\mathrm{n}=808)\end{array}$ & $\begin{array}{l}\text { ELX } \\
100 \mathrm{mg} \\
\text { BID } \\
(\mathrm{n}=806)\end{array}$ & $\begin{array}{l}\mathrm{PBO} \\
(\mathrm{n}=809)\end{array}$ & $\begin{array}{l}\text { ELX } \\
75 \mathrm{mg} \\
\text { BID } \\
(\mathrm{n}=808)\end{array}$ & $\begin{array}{l}\text { ELX } \\
100 \mathrm{mg} \\
\text { BID } \\
(\mathrm{n}=806)\end{array}$ \\
\hline 0 & 14.4 & 23.6 & 24.8 & 26.8 & 39.5 & 37.1 \\
\hline $1-<5$ & 46.7 & 50.2 & 49.2 & 35.9 & 33.8 & 37.6 \\
\hline $5-\leq 8$ & 16.3 & 10.7 & 12.7 & 13.1 & 8.6 & 8.5 \\
\hline$>8-<12$ & 5.3 & 3.8 & 2.9 & 4.7 & 3.9 & 3.7 \\
\hline 12 & 17.3 & 11.8 & 10.3 & 19.4 & 14.1 & 13.1 \\
\hline
\end{tabular}

Conclusion: In this post hoc analysis of the pooled ELX Phase 3 studies, ELXtreated patients experienced fewer consecutive weeks of IR compared to those receiving PBO, within both Weeks 1-12 and 13-24 of treatment. As IR is thought to drive increased healthcare provider visits, these data suggest that ELX could potentially reduce healthcare resource use and subsequent healthcare costs associated with IBS. Further prospective study of the impact of ELX on AR and any subsequent reduction in healthcare costs is required, including the relationship between the number of consecutive weeks of IR and patients' behaviour towards healthcare resource use.

Disclosure of Interest: D. Collomb: David Collomb is an employee of Allergan plc.

A. Marciniak: Anne Marciniak is an employee of Allergan plc and shareholder in Pfizer, Amgen, and Allergan plc.

Y. Mo: Yifan Mo is an employee of Allergan plc.

D.A. Andrae: David A. Andrae is an employee of Allergan plc and shareholder in Allergan plc.

G. Wiseman: Gwen Wiseman is an employee of Allergan plc.

\section{References}

1. Lembo AJ et al. $N$ Engl J Med 2016; 374: 242-253

2. Chey WD et al. Aliment Pharmacol Ther 2017; doi: 10.1111/apt.14031 [Epub]

3. Covington PS. Poster 55 presented at ACCP Global Conference on Clinical Pharmacy, USA, 2015

P1147 CLINICAL RESPONSE AND DISCONTINUATION OVER TIME IN PHASE 3 TRIALS OF ELUXADOLINE FOR IRRITABLE BOWEL SYNDROME WITH DIARRHOEA

A. Marciniak ${ }^{1}$, R. T. Carson ${ }^{2}$, J. L. Abel ${ }^{2}$, D. A. Andrae ${ }^{3}$, K. Fust ${ }^{4}$, D. Becker ${ }^{5}$ ${ }^{1}$ Allergan plc, Marlow/United Kingdom

${ }^{2}$ Allergan plc, Jersey City/United States of America/NJ

${ }^{3}$ Allergan plc, Austin/United States of America/TX

${ }^{4}$ Optum, Plymouth/United States of America/MA

${ }^{5}$ Optum, Burlington/Canada/ON

Contact E-mail Address: Anne.Marciniak@Allergan.com

Introduction: Irritable bowel syndrome with diarrhoea (IBS-D) is a chronic gastrointestinal disorder characterised by recurrent abdominal pain associated with diarrhoea. Eluxadoline, a mixed $\mu$ - and $\kappa$-opioid receptor agonist and $\delta$-opioid 
receptor antagonist, is approved for the treatment of IBS-D in adults, based on two large Phase 3 clinical trials where eluxadoline met the primary composite response endpoint of simultaneous improvement in abdominal pain and stool consistency over 26 weeks.

Aims \& Methods: These post hoc analyses of two double-blind, placebocontrolled, Phase 3 clinical trials of eluxadoline (IBS-3001 and IBS-3002) aimed to assess responder and non-responder rates over 4-week intervals for eluxadoline vs placebo, and the proportion of responders/non-responders who continued or discontinued treatment. Patients meeting Rome III criteria for IBS$\mathrm{D}$ were randomised to twice-daily treatment with eluxadoline $(75 \mathrm{or} 100 \mathrm{mg})$ or placebo. Patients rated IBS symptoms daily, including worst abdominal pain (WAP; $0-10$ scale, with $0=$ no pain and $10=$ worst imaginable pain), stool consistency (Bristol Stool Form Scale [BSFS]), and Global Symptom Score (GSS; $0-4$ scale, where $0=$ no symptoms and $4=$ very severe symptoms). A responder for a 4-week interval was defined as a patient with a simultaneous daily improvement of $\geq 30 \%$ in WAP score vs baseline and BSFS score $<5$ with $\geq 50 \%$ of days demonstrating a response and a minimum of 20 days of diary data. The intention-to-treat (ITT) analysis set included all patients randomised to a treatment group. For these analyses, patients with baseline GSS $\geq 3$ (severe or very severe symptoms) and self-reported inadequate symptom control with loperamide were classified as having severe IBS-D.

Results: Of the 2423 patients in the pooled Phase 3 ITT population, 249 were classified as having severe IBS-D. Over Weeks $1-4,26.8 \%$ and $30.3 \%$ of patients with severe IBS-D were responders with eluxadoline 75 and $100 \mathrm{mg}$, respectively, vs $8.1 \%$ of patients on placebo (Table). Higher proportions of patients were responders with eluxadoline vs placebo over each subsequent 4-week interval, with response rates observed at Weeks 1-4 consistently maintained across all subsequent 4 -week time intervals. With both eluxadoline and placebo, proportions of responders discontinuing were $<2 \%$ across each 4 -week interval, and discontinuation rates in non-responders were higher than in responders (Table). Similar findings were observed in the ITT analysis set: over Weeks $1-4,22.7 \%$ and $24.6 \%$ of patients were responders with eluxadoline 75 and $100 \mathrm{mg}$, respectively, vs $12.5 \%$ of patients on placebo. Over Weeks $21-24,28.1 \%$ and $30.2 \%$ of patients were responders with eluxadoline 75 and $100 \mathrm{mg}$, respectively, vs $20.1 \%$ on placebo. Discontinuation rates among responders with both eluxadoline and placebo remained $<1 \%$ across all 4 -week time intervals.

Table

\begin{tabular}{|c|c|c|c|c|c|c|c|c|c|c|c|c|}
\hline \multirow[b]{3}{*}{ Weeks } & \multicolumn{4}{|c|}{ Placebo $(\mathrm{n}=74)$} & \multicolumn{4}{|c|}{$\begin{array}{l}\text { Eluxadoline } \\
75 \mathrm{mg}(\mathrm{n}=86)\end{array}$} & \multicolumn{4}{|c|}{$\begin{array}{l}\text { Eluxadoline } \\
100 \mathrm{mg}(\mathrm{n}=89)\end{array}$} \\
\hline & \multicolumn{2}{|c|}{$\begin{array}{l}\text { Responder, } \\
\%\end{array}$} & \multicolumn{2}{|c|}{$\begin{array}{l}\text { Non- } \\
\text { responder, } \\
\%\end{array}$} & \multicolumn{2}{|c|}{$\begin{array}{l}\text { Responder, } \\
\%\end{array}$} & \multicolumn{2}{|c|}{$\begin{array}{l}\text { Non- } \\
\text { responder, } \\
\%\end{array}$} & \multicolumn{2}{|c|}{$\begin{array}{l}\text { Responder, } \\
\%\end{array}$} & \multicolumn{2}{|c|}{$\begin{array}{l}\text { Non- } \\
\text { responder, } \\
\%\end{array}$} \\
\hline & $\mathrm{C}$ & DC & $\mathrm{C}$ & DC & $\mathrm{C}$ & DC & $\mathrm{C}$ & $\mathrm{DC}$ & $\mathrm{C}$ & $\mathrm{DC}$ & $\mathrm{C}$ & DC \\
\hline $1-4$ & 8.1 & 0.0 & 89.2 & 2.7 & 25.6 & 1.2 & 68.6 & 4.7 & 29.2 & 1.1 & 58.4 & 11.2 \\
\hline $5-8$ & 18.9 & 1.4 & 73.0 & 6.8 & 30.2 & 0.0 & 55.8 & 14.0 & 31.5 & 1.1 & 49.4 & 18.0 \\
\hline $9-12$ & 18.9 & 0.0 & 63.5 & 17.6 & 31.4 & 0.0 & 52.3 & 16.3 & 30.3 & 1.1 & 47.2 & 21.3 \\
\hline $13-16$ & 16.2 & 1.4 & 63.5 & 18.9 & 30.2 & 0.0 & 52.3 & 17.4 & 27.0 & 0.0 & 48.3 & 24.7 \\
\hline $17-20$ & 24.3 & 0.0 & 50.0 & 25.7 & 33.7 & 0.0 & 40.7 & 25.6 & 37.1 & 0.0 & 37.1 & 25.8 \\
\hline $21-24$ & 14.9 & 1.4 & 54.1 & 29.7 & 30.2 & 1.2 & 39.5 & 29.1 & 29.2 & 1.1 & 38.2 & 31.5 \\
\hline
\end{tabular}

$\mathrm{C}$, patients continuing on treatment; DC, patients discontinuing from treatment

Conclusion: Proportions of responders with eluxadoline 75 and $100 \mathrm{mg}$ were consistently higher vs placebo across all 4-week intervals in the treatment period in patients defined as having severe IBS-D. Furthermore, discontinuation rates among patients showing a treatment response remained consistently low compared to non-responders. However, as these analyses were conducted in a clinical trial setting, the relatively high continuation rates in non-responders may not reflect the real-world situation. These findings suggest that eluxadoline has sustained efficacy in treating the diarrhoea and abdominal pain associated with IBS-D, including in patients with severe and inadequately managed symptoms. Disclosure of Interest: A. Marciniak: Anne Marciniak is an employee of Allergan plc and shareholder in Pfizer, Amgen, and Allergan plc.

R.T. Carson: Robyn T. Carson is an employee of Allergan ple and shareholder in Allergan plc.

J.L. Abel: Jessica L. Abel is an employee of Allergan ple and shareholder in Allergan plc.

D.A. Andrae: David A. Andrae is an employee of Allergan ple and shareholder in Allergan plc.

K. Fust: Kelly Fust is an employee of Optum, paid consultants to Allergan plc. D. Becker: Debbie Becker is an employee of Optum, paid consultants to Allergan plc.

\section{P1148 THE LOW FODMAP DIET REDUCES CAECAL FERMENTATION COMPARED TO TRADITIONAL DIETARY ADVICE: A RANDOMISED CONTROLLED TRIAL}

A. D. Farmer ${ }^{1}$, N. Saini ${ }^{2}$, A. R. Hobson ${ }^{2}$

${ }^{1}$ Gastroenterology, University Hospitals of North Midlands, Stoke on Trent/United Kingdom

${ }^{2}$ Functional Gut Clinic, London/United Kingdom
Contact E-mail Address: a.farmer@qmul.ac.uk

Introduction: Diets reducing the content of fermentable short chain carbohydrates (fermentable oligo-, di-, monosaccharides, and polyols (FODMAPs)) as well as the National Institute of Health Care Excellence (NICE) diet have been reported to be effective in the treatment of patients with irritable bowel syndrome (IBS) $(1,2)$. The mechanisms by which this efficacy is achieved are incompletely understood but it has been proposed that such diets reduce fermentation, mediated by changes in the microbiota (3). Change in $\mathrm{pH}$ around the ileocaecal junction is considered to be a surrogate biomarker of caecal fermentation $(4,5)$. Aims \& Methods: We aimed to compare the effect of a low FODMAP diet vs. the NICE diet on change in ileocaecal $\mathrm{pH}$. We performed a single centre, randomized controlled trial of adult patients with Rome III defined IBS-mixed bowel habit (IBS-M) comparing the two dietary interventions. At baseline, patients ingested a wireless motility capsule (WMC) using a standardized protocol. Segmental transit times were derived from measures around known anatomical landmarks as identified by compartmental $\mathrm{pH}$ changes. Ileal and colonic motility measures are presented as area under the curve (AUC) derived from contraction amplitude and frequency. Validated questionnaires evaluating GI (verbal descriptor anchored visual analog scale (VDVAS) assessing sensory intensity (VDVAS-I) and unpleasantness (VDVAS-U)) and somatic symptoms (Personal Health Questionnaire (PHQ)) as well as quality of life (EQ-5D) were administered. The WMC and questionnaires were repeated after 28 days of dietary interventions. The primary endpoint was change in ileocaecal $\mathrm{pH}$ after the intervention. Secondary outcomes included changes in transit times, contractility and symptom scores.

Results: After screening, 32 patients ( 23 female, median age 37 years, range 18-65) were randomized. Baseline symptom severity and demographics were similar between the two groups. Relative to baseline, there was a reduction in the change in ileocaecal $\mathrm{pH}$ with the low FODMAP diet group compared to the $\mathrm{m}-\mathrm{NICE}$ group $(3.37 \pm 0.3$ vs $0.0003 \pm 0.4, p=0.047)$ suggesting reduced fermentation. Changes in GI motility are shown in Table 1.

Table 1: Changes in segmental/whole gut transit times and ileal/colonic motility, relative to baseline between the low FODMAP and m-NICE diets. Both the low FODMAP and NICE diets improved VDVAS-I and VDVAS-U $(-18 \pm 17$ vs. $-26 \pm 27, p=0.4$ and $-13.6 \pm 26$ vs. $-29.7 \pm 29, p=0.2$, respectively). Similarly, both diets reduced somatic symptoms $(-2 \pm 1.4$ vs. $-0.8 \pm 1.8, p=0.07)$ and improved quality of life $(9.5 \pm 10.2$ vs. $4.4 \pm 9.8, p=0.23)$.

\begin{tabular}{llll}
\hline & $\begin{array}{l}\text { Low } \\
\text { FODMAP }\end{array}$ & NICE & P value \\
\hline $\begin{array}{l}\text { Change in Gastric emptying } \\
\text { time (minutes) } \\
\begin{array}{c}\text { Change in Small bowel } \\
\text { transit time (minutes) }\end{array}\end{array}$ & $122 \pm 249$ & $7 \pm 370$ & 0.5 \\
$\begin{array}{c}\text { Change in Colonic transit } \\
\text { time (minutes) }\end{array}$ & $512 \pm 1425$ & $53 \pm 1728$ & 0.53 \\
$\begin{array}{c}\text { Change in Whole Gut } \\
\text { Transit time (minutes) }\end{array}$ & $710 \pm 1486$ & $-35 \pm 1588$ & 0.3 \\
$\begin{array}{c}\text { Change in Ileal contractility } \\
\text { (AUC) }\end{array}$ & $64 \pm 139$ & $114 \pm 175$ & 0.5 \\
$\begin{array}{l}\text { Change in Colonic contrac- } \\
\text { tility (AUC) }\end{array}$ & $5 \pm 58$ & $26 \pm 72$ & 0.5 \\
\hline
\end{tabular}

Conclusion: The low FODMAP diet reduces caecal fermentation in comparison to the NICE diet as indexed by a reduction in the change in $\mathrm{pH}$ across the ileocaecal junction. Both diets improved GI and extra-GI symptoms as well as quality of life. Neither diet has a demonstrable differential effect on ileal/colonic contractility or segmental/whole gut transit times. It is therefore plausible that the efficacy of the low FODMAP diet in IBS-M is via mediated by alterations in the microbiota.

Disclosure of Interest: A.D. Farmer: Speaker Bureau and Advisory Boards for Allergan

All other authors have declared no conflicts of interest.

\section{References}

1. Bohn et al. Gastro 2015 .

2. Eswaran et al. Am J Gastro 2016.

3. McIntosh et al. Gut 2016.

4. Farmer et al. World J Gastro 2014

5. Ringel-Kulka et al. Am J Gastro 2015.

\section{P1149 ORAL A-GALACTOSIDASE IMPROVES} GASTROINTESTINAL TOLERANCE TO A DIET HIGH IN GALACTOOLIGOSACCHARIDES: ADJUNCT THERAPY TO A LOW FODMAP DIET IN IRRITABLE BOWEL SYNDROME

C. J. Tuck ${ }^{1}$, K. M. Taylor ${ }^{2}$, J. S. Barrett ${ }^{1}$, P.R. Gibson ${ }^{1}$, J. G. Muir ${ }^{1}$

${ }_{1}^{1}$ Dept Gastroenterology, Monash University, Melbourne/Australia/VIC

${ }^{2}$ Dept Gastroenterology, Monash University and Alfred Hospital, Melbourne Australia/VIC

Contact E-mail Address: caroline.tuck@monash.edu

Introduction: Galacto-oligosaccharides (GOS) are indigestible short-chain carbohydrates (FODMAPs, fermentable, oligo-, di-, mono-saccharides and polyols) 
associated with triggering gastrointestinal symptoms in irritable bowel syndrome (IBS).

Aims \& Methods: This study aimed to assess whether oral $\alpha$-galactosidase coingestion with foods high in GOS and low in other FODMAPs would reduce symptoms and breath hydrogen production in a double-blind, placebo-controlled, cross-over trial approved by Monash University Ethics Committee. Patients meeting the Rome III criteria for IBS who produced $>10$ ppm hydrogen on two consecutive breath samples following $10 \mathrm{~g}$ fructan were recruited. Participants were randomly assigned to full-dose enzyme (300 GALU $\alpha$-galactosidase), half-dose (150 GALU $\alpha$-galactosidase) and placebo (glucose). Following a 3-day low FODMAP run-in period, participants consumed provided diets high in GOS for a further 3-days. Gastrointestinal symptoms were measured daily using a $100 \mathrm{~mm}$ visual-analogue-scale. Breath samples were taken hourly on the second last day and analysed as area-under-the-curve, faecal samples were taken on the final day.

Results: Thirty-one participants with IBS (20 IBS-D, 4 IBS-C, 7 IBS-M) completed the study. The addition of high GOS foods resulted in a significant increase in overall symptoms (median 13.0 [IQR 1.5-22.0] to 35.5 [12.8-54.0] $\mathrm{mm} ; \mathrm{p}=0.000$, Wilcoxon signed-rank test). No significant increase in overal symptoms was seen with the full-dose enzyme (14.0 [3.5-24.0] vs 14.7 [2.3-32.7] $\mathrm{mm} ; \mathrm{p}=0.422)$. Twenty-one participants exhibited GOS-sensitivity $(>10 \mathrm{~mm}$ increase for overall symptoms). Of those, full-dose enzyme reduced overall symptoms $(24.5[17.5-35.8] \mathrm{mm}$ vs $5.5(1.5-15.0) \mathrm{mm} ; \mathrm{p}=0.006)$ and bloating $(20.5$ [9.5-42.0] vs $6.5[2.0-15.8] ; \mathrm{p}=0.017)$. Breath hydrogen production was minimal with no differences seen between placebo (mean $5248 \pm$ SD $3339 \mathrm{ppm} .12 \mathrm{~h}$ ) and full-dose $(5585 \pm 3205 ; \mathrm{p}=0.597$, paired samples t-test $)$

Conclusion: An oral $\alpha$-galactosidase supplement taken with high GOS foods provides a clinically significant reduction in symptoms in GOS-sensitive individuals with IBS. The lack of change in breath hydrogen suggests the mechanism may not be related to reduced gas and distention, rather suggesting a role of alterations to the microbiota. Future analysis of the faecal microbiota may provide insight for the mechanism of action. This strategy can be easily translated into practice to improve tolerance specifically to high GOS foods for patients with IBS as an adjunct therapy to the low FODMAP diet.

Disclosure of Interest: J.S. Barrett: The Department of Gastroenterology financially benefits from the sales of a digital application and booklets on the low FODMAP diet.

P.R. Gibson: The Department of Gastroenterology financially benefits from the sales of a digital application and booklets on the low FODMAP diet. P Gibson has published an educational/recipe book on the low FODMAP diet.

J.G. Muir: The Department of Gastroenterology financially benefits from the sales of a digital application and booklets on the low FODMAP diet. All other authors have declared no conflicts of interest.

P1150 A SYSTEMATIC REVIEW AND META-ANALYSIS TO DETERMINE WHETHER BALLOON EXPULSION TESTING MIGHT BE AN APPROPRIATE INITIAL OFFICE-BASED TEST FOR DYSSYNERGIC DEFECATION

E. D. Shah ${ }^{1}$, J. Farida ${ }^{1}$, W.D. Chey ${ }^{2}$

${ }^{1}$ Division Of Gastroenterology, University of Michigan, Ann Arbor/United States of America

${ }^{2}$ Gastroenterology, Internal Medicine, University of Michigan, Ann Arbor, MI United States of America

Contact E-mail Address: ershah@med.umich.edu

Introduction: Balloon expulsion testing (BET) is a recommended means of identifying dyssynergic defecation (DD) in patients with chronic constipation (CC) but remains poorly standardized and underutilized outside of specialized centers. We aimed to assess the clinical utility of BET as an initial test for DD and to determine appropriate testing parameters.

Aims \& Methods: We performed a literature search (PubMED, EMBASE, conference abstracts from 1950-2016) to identify (1) case-control studies of DD or unselected CC subjects and healthy controls and (2) cohort studies of unselected subjects with CC. Eligible studies reported BET test parameters and results as well as presence of DD defined by constipation symptoms and a positive reference test (anorectal manometry[ARM], defecography, or electromyography[EMG]). Study quality was assessed using QUADAS criteria. We extracted age, sex, enrollment criteria, BET test parameters (subject position, stool surrogate, volume instilled, allowed expulsion time), and DD diagnostic criteria. Data were independently extracted by two authors. Meta-analysis was performed using a bivariate mixed-effects regression model. Meta-regression was performed to evaluate effects of individual test parameters. Between-study heterogeneity of summary results was assessed using an $\mathrm{I}^{2}$ statistic. Publication bias was assessed using Deeks' funnel plot assymetry test.

Results: We identified 15 eligible studies comprising 2,090 individual assessments of balloon expulsion, of which 14 studies of 1,760 subjects were eligible for cohort analysis and 15 studies of 1,988 subjects were eligible for case-control analysis. Among cohort studies, the AUC was 0.80 (95\% CI $0.61-0.91)$ with $70 \%$ sensitivity $(95 \%$ CI $52 \%-83 \%)$ and $77 \%$ specificity $(95 \%$ CI $70 \%-82 \%)$. Among pooled cohort and case-control studies, the AUC was $0.84(95 \%$ CI $0.68-0.93)$ with $70 \%$ sensitivity $(95 \%$ CI $53 \%-82 \%)$ and $81 \%$ specificity $(95 \%$ CI $75 \%$ $86 \%$ ). Further test performance characteristics stratified by subject position are reported in Table 1. Subject positioning (seated vs. left lateral decubitus) did not significantly affect AUC in cohort $(\mathrm{p}=0.82)$ or case-control $(\mathrm{p}=0.43)$ analysis. Pooled sensitivity $(\mathrm{p}=0.50)$ and specificity $(\mathrm{p}=0.66)$ were similar between seated and left lateral BET in analysis of unselected CC cohort studies, though specificity was higher with left lateral BET in pooled analysis of all studies $(p=0.03)$. The allowable time for balloon expulsion did not significantly affect summary sensitivity $(\mathrm{p}=0.92)$ or specificity $(\mathrm{p}=0.96)$ in meta-regression within the evaluated range of 1 and 5 minutes. While there were not enough studies to warrant meta-analysis of balloon distension characteristics, 13 of 17 studies instilled 50 $60 \mathrm{~mL}$ of water. When pooling cohort and case-control studies, both age $(\mathrm{p}<0.01)$ and gender $(\mathrm{p}<0.01)$ appeared to influence test performance. Choice of reference test did not significant affect test performance (ARM $[p=0.35]$, defecography $[\mathrm{p}=0.43]$, or $\mathrm{EMG}[\mathrm{p}=0.08]$ ). Continent of origin $(\mathrm{p}=0.34)$ and year of study $(\mathrm{p}=0.29)$ did not appear to significantly influence test performance. There was no evidence of publication bias $(\mathrm{p}>0.5)$.

Table 1: Sensitivity and specificity of balloon expulsion testing in diagnosing dyssynergic defecation (stratified by subject positioning with $95 \%$ confidence intervals)

\begin{tabular}{lll}
\hline $\begin{array}{l}\text { Summary test } \\
\text { characteristic }\end{array}$ & Seated position & $\begin{array}{l}\text { Left lateral } \\
\text { position }\end{array}$ \\
\hline $\begin{array}{l}\text { Case-control and cohort studies (optimal estimates) } \\
\text { Sensitivity } \\
69 \%(54 \% \text { to } 85 \%)\end{array}$ & $54 \%(7 \%$ to $100 \%)$ \\
$\begin{array}{l}\text { Specificity } \\
\text { Only cohort studies evaluating unselected subjects with }\end{array} \quad \begin{array}{c}\text { constipation } \\
\quad \text { real world estimates })\end{array}$ & $76 \%(70 \%$ to $83 \%)$ \\
$\begin{array}{l}\text { Sensitivity } \\
\text { Specificity }\end{array}$ & $69 \%(53 \%$ to $86 \%)$ & $76 \%(51 \%$ to $100 \%)$ \\
\hline
\end{tabular}

Conclusion: The performance characteristics of balloon expulsion could support use as an office-based point-of-care test to screen for dyssynergic defecation in chronically constipated subjects.

Disclosure of Interest: W.D. Chey: Dr. Chey is a consultant for Ironwood Pharmaceuticals and Allergan. He is co-CMO of My Total Health and holds a patent on My GI Health

All other authors have declared no conflicts of interest.

\section{P1151 PREVALENCE OF ANAL SQUAMOUS INTRAEPITHELIAL LESIONS IN LIVER TRANSPLANTED PATIENTS}

A. Albuquerque ${ }^{1}$, H. Pessegueiro Miranda ${ }^{2}$, J. Lopes $^{3}$, J. Gandara ${ }^{2}$, S. Rodrigues ${ }^{4}$, R. Gaspar ${ }^{4}$, R. Morais ${ }^{5}$, R. Ramalho ${ }^{1}$, E. Rodrigues-Pinto ${ }^{1}$, H. Cardoso ${ }^{4}$, H. Barroca ${ }^{3}$, C. Camila Dias ${ }^{5}$, F. Carneiro ${ }^{3}$, G. Macedo ${ }^{1}$ ${ }_{1}^{1}$ Gastroenterology Department, Hospital São João, Gastroenterology Department, Porto/Portugal

${ }^{2}$ Pancreatic and Liver Transplant Unit Centro Hospitalar Porto, Porto/Portugal ${ }^{3}$ Pathology Department Centro Hospitalar São João, Porto/Portugal

${ }^{4}$ Gastroenterology, Centro Hospitalar São João, Porto/Portugal

${ }^{5}$ Department of Community Medicine, Information and Health Decision Sciences, Faculty of Medicine of the University of Porto, Porto/Portugal

Contact E-mail Address: a.albuquerque.dias@gmail.com

Introduction: Anal squamous intraepithelial lesions are precancerous lesions of anal squamous cell carcinoma and are largely related to human papillomavirus infection. Immunosuppressed patients have a higher prevalence of these lesions. There are some studies in renal transplant recipients, but no information exists regarding prevalence in liver transplantation.

Aims \& Methods: Our aim was to evaluate the prevalence of anal squamous intraepithelial lesions in liver transplant recipients compared with healthy subjects. This was a prospective case-control study involving liver transplant recipients that were compared with a healthy control group. All patients were submitted to anal cytology. Those with abnormal cytological results, namely high-grade squamous intraepithelial lesions (HSIL), atypical squamous cells which cannot exclude high-grade squamous intraepithelial lesions (ASC-H), low-grade squamous intraepithelial lesions (LSIL) and atypical squamous cells of undetermined significance (ASC-US), were submitted to high-resolution anoscopy with biopsies of any suspicious lesion.

Results: A total of 59 liver transplant recipients and 57 controls underwent anal cytology. In the liver transplant group, $37(63 \%)$ were men, with a mean age of $54 \pm 10$ years. The most common indication for transplantation was alcoholic cirrhosis in 26 patients $(44 \%)$, the majority of the patients were only on tacrolimus $(\mathrm{n}=23,39 \%)$ and had been transplanted a mean of $8 \pm 5$ years ago. In the healthy control group, $36(63 \%)$ were men, with a mean age $59 \pm 11$ years. Regarding anal cytology, $10(17 \%)$ of liver transplant recipients had abnormal results, 7 patients had ASC-US, 1 patient ASC-H and 2 patients HSIL. In the control group only 1 patient $(2 \%)$ had an ASC-US result $(\mathrm{p}=0.005)$. Anal squamous intraepithelial lesions were confirmed in $7 / 10$ of liver transplant patients and $0 / 1$ in control group $(\mathrm{p}=0.013)$ by high-resolution anoscopy with biopsies. Smoking was the only risk factor for abnormal anal cytology $(\mathrm{OR}=5.9$, $95 \% \mathrm{CI}=1.224-28.121, \mathrm{p}=0.027)$.

Conclusion: Liver transplant patients have a higher risk of anal squamous intraepithelial lesions and screening should be considered especially in smokers. Disclosure of Interest: All authors have declared no conflicts of interest. 
TUESDAY, OCTOBER 31, 201709:00-17:00

OESOPHAGEAL, GASTRIC AND DUODENAL

\section{DISORDERS II - HALL 7}

\section{P1152 IMMUNOHISTOCHEMICAL EXPRESSION OF DIAMINE OXIDASE IN THE UPPER GASTROINTESTINAL TRACT OF PATIENTS WITH GASTROINTESTINALLY MEDIATED FOOD ALLERGY}

T. Vasilakis ${ }^{1}$, R. J. Rieker ${ }^{2}$, J. Heinrich ${ }^{3}$, A. Missbichler ${ }^{4}$, A. F. Hagel ${ }^{1}$, J. Kressel ${ }^{1}$, M. Raithel ${ }^{3}$

${ }^{1}$ University Clinical Center Erlangen, Erlangen/Germany

${ }^{2}$ Pathologisches Institut, University Clinical Center Erlangen, Erlangen/Germany ${ }^{3}$ St. Marien Waldkrankenhaus, Erlangen/Germany

${ }^{4}$ SCIOTEC Diagnostic Technologies GmbH, Tulln/Austria

\section{Contact E-mail Address: thomas.vasilakis@gmx.de}

Introduction: Gastrointestinally mediated food allergy (GMA) is a common disease, whose prevalence has risen in recent years. The scientific research on this disease has increased, however its diagnosis still remains difficult to date. Diamine oxidase (DAO) is an enzyme that deactivates histamine, the main mediator in allergic reactions, through oxidative deamination. It has been shown that the enzymatic activity of DAO is diminished in the colonic mucosa of patients with GMA. Up to now no studies concerning the expression of DAO in the upper gastrointestinal tract (GIT) of patients with GMA have been published. Therefore, the objective of this study was to analyse the immunohistochemical staining of DAO and its localisation in the upper GIT of patients with or without GMA.

Aims \& Methods: The study was retrospective. The GMA group consisted of 21 patients with diagnosed food allergy. The control group (CG) included 17 patients with neither food allergy nor food intolerance. Tissue samples from esophagus, cardia (subdivided in esophageal and gastric region), corpus, antrum and duodenum already obtained during endoscopy were immunohistochemically stained for DAO. The expression of DAO was semi-quantitatively analysed with the following scale based on the staining intensity of DAO (SIDAO): $0=$ none, $1=$ low, $2=$ medium, $3=$ high intensity. The localisation of DAO was also examined vertically from the epithelium to the submucosa in all tissues. The analysis was performed twice by the same examiner in two separate points of time. Furthermore, the tissue samples were immunohistochemically stained for MBP and CD117 in order to count the number of eosinophils and mast cells respectively. Two measurements were performed inside an area of $100 \mu \mathrm{m}^{2}$ in the lamina propria (LP); one in the subepithelial superficial lamina propria (SLP) and one in the deeper lamina propria (DLP). The average of these two measurements constituted the number of these cells for each tissue.

Results: Immunohistochemical analysis found DAO in all segments of the upper GIT, but the SI-DAO was overall low. Across the upper GIT the median SIDAO in the CG was 1.1 (range 0.9-1.3). The SI-DAO did not differ between the examined tissues. The SI-DAO was also low in the GMA group (median 1.0; range $0.8-1.3$ ) and it was statistically significantly lower compared to the controls (median: 0.8 vs. $1.1 ; \mathrm{p}=0.04$ ) only in duodenum. In the GMA group the SI-DAO in the esophageal region of the cardia and in corpus was significantly higher than in duodenum (see Table 1). The strongest DAO-staining was detected in the subepithelial lamina propria (SLP) of all tissues. A strong inverse correlation of SI-DAO with the number of eosinophils across the upper GIT was observed only in the GMA group $(r=-0,89, p=0,02)$. An inverse correlation of the SIDAO with the number of mast cells was also observed in both groups, but this result was not statistical significant (GMA: $r=-0.71, p=0.11 ; \mathrm{CG}: \mathrm{r}=-0.6$, $\mathrm{p}=0.14)$.

Table 1: SI-DAO in the upper GIT. P1-Value: comparison between GMA and control group. P2-Value: comparison of the SI-DAO in duodenum with the other segments of the upper GIT in the GMA-group. $\mathrm{N}=$ number of tissue samples

\begin{tabular}{clllllll}
\hline \multirow{2}{*}{ Group } & Parameter & Esophagus & $\begin{array}{l}\text { Cardia } \\
\text { - regophageal } \\
\text { region }\end{array}$ & $\begin{array}{l}\text { gastric } \\
\text { Cardia }\end{array}$ & Corpus & Antrum & Duodenum \\
\hline GMA & $\mathrm{N}$ & 9 & 10 & 8 & 18 & 18 & 18 \\
Group & Median & 1.3 & 1.1 & 1.1 & 1.2 & 1.1 & 0.8 \\
& 25th Percentile & 0.8 & 0.8 & 0.8 & 0.7 & 0.7 & 0.4 \\
& 75th Percentile & 1.5 & 1.3 & 1.1 & 1.5 & 1.4 & 1.3 \\
Control & N & 8 & 9 & 8 & 15 & 14 & 11 \\
Group & Median & 1.3 & 1.2 & 1.3 & 0.9 & 0.9 & 1.1 \\
& 25th Percentile & 1.1 & 1.0 & 0.9 & 0.8 & 0.7 & 1.0 \\
& 75th Percentile & 1.7 & 1.4 & 1.6 & 1.2 & 1.1 & 1.5 \\
& P1 - Value & 0.33 & 0.41 & 0.46 & 0.29 & 0.28 & $\mathbf{0 . 0 4} *$ \\
& P2 - Value & 0.07 & $\mathbf{0 . 0 1 *}$ & 0.06 & $\mathbf{0 . 0 2} *$ & 0.16 & x \\
\hline
\end{tabular}

Conclusion: The above findings indicate that DAO is present in low amounts in all segments of the upper GIT. But only in the duodenum a significant difference was found between GMA and CG, thus indicating that histamine-mediated symptoms most likely arise in duodenum. Therefore, regarding the upper GIT, the immunohistochemical staining for DAO only in duodenum could serve as an additional diagnostic parameter for detecting patients with GMA and possibly other histamine-mediated diseases. The above mentioned distribution pattern of DAO strengthens the theory that DAO acts extracellularly and is responsible for the elimination of the transepithelially absorbed exogenous histamine as well as of the endogenous histamine, as its highest staining intensity is found at the SLP throughout the upper GIT.

Disclosure of Interest: All authors have declared no conflicts of interest.

\section{Reference}

1. Raithel, M. et al., 1999. The involvement of the histamine degradation pathway by diamine oxidase in manifest gastrointestinal allergies. Inflamm Res, 48 Suppl 1, S75-6.

P1153 PREVALENCE OF ATROPHIC GASTRITIS IN GENERAL POPULATION: A GASTROPANEL $®$ - BASED STUDY, COMPARED WITH OLGA HISTOLOGICAL CLASSIFICATION

F. Di Mario ${ }^{1}$, S. Grillo ${ }^{1}$, S. Cesario ${ }^{1}$, C. Miraglia ${ }^{1}$, S. Scida $^{1}$, S. Landi ${ }^{1}$, P. Crafa $^{1}$, R. Cannizzaro ${ }^{2}$, G. Grande ${ }^{3}$, V. Corrente ${ }^{4}$, M. Franceschi ${ }^{5}$, M. Riccó ${ }^{6}$, L. Franzoni ${ }^{1}$, C. Scarpignato ${ }^{1}$, M. Rugge ${ }^{7}$

${ }^{1}$ Department Of Medicine And Surgery, University Of Parma, Italy, University of Parma, Parma/Italy

${ }^{2}$ Oncological Gastroenterology, Centro di Riferimento Oncologico di Aviano

S.O.C. di Gastroenterologia, Aviano/Italy

${ }_{3}^{3}$ Aou Modena, Gastroenterology and Digestive Endoscopy Unit, Modena/Italy

${ }^{4}$ University Hospital, Parma (Italy), Parma/Italy

${ }^{5}$ Endoscopic Unit, Department Of Surgery, Ulss4, Hospital ULSS4 Alto Vicentino, Santorso/Italy

${ }^{6}$ Local Health Unit Of Parma, Public Health Department, Occupational Health And Safety Service, Parma, Italy, University Hospital, Parma (Italy), Parmal Italy

${ }^{7}$ Pathology, Medical School of the Padova University, Padova/Italy

Contact E-mail Address: francesco.dimario@unipr.it

Introduction: Chronic atrophic gastritis (CAG) is claimed to be a pre-cancerous condition for gastric cancer, being serological markers like serum pepsinogens and gastrin-17 (G17) proposed as non-invasive useful tools. OLGA histological classification has been proposed as of prognostic value. Aim of the study was to assess, by using serological markers, the prevalence of CAG in general population and to correlate the results with OLGA classification.

Aims \& Methods: One thousand and nine hundred consecutive patients $(M=915$ mean age $=53.5$, range $=27-82$ ) showing upper-gastrointestinal $(\mathrm{GI})$ troubles entered the study. Exclusion criteria were: previous history of upper-GI neoplasms, previous surgery, concomitant Proton Pumps Inhibitor (PPI) therapy. All patients underwent a blood sample for GASTROPANEL ${ }^{\circledR}$ (BioHit Oyj, Finland) based on: pepsinogen I (PGI), pepsinogen II (PGII), gastrin-17 (G-17) and $\mathrm{IgG}$ against Helicobacter pylori. (Hp-IgG). The normal values are: PGI: $30-120 \mu \mathrm{g} / \mathrm{L}$, PGII: 2-15 $\mu \mathrm{g} / \mathrm{L}, \mathrm{G}-17$ : 1-9 pmol/L, Hp-IgG: $<30$ EIU, respectively. Low levels of PGI (PGI $<30 \mu \mathrm{g} / \mathrm{L}$ ) as well as high levels of G-17 $(\mathrm{G}-17>15 \mathrm{pmol} / \mathrm{L})$ were considered diagnostic for CAG. Eighty-three patients with serology suggestive for CAG underwent upper endoscopy and histology according with OLGA staging. The relationship between PGI, G-17 and the different OLGA stages was statistically analyzed.

Results: Eighty-three $(M=44$, mean age $=61$, range $=49-82)$ out of 1900 investigated patients showed CAG $(4 \%)$. Out of the 83 CAG patients, $19.2 \%$ was classified as OLGA $2,56.6 \%$ as OLGA 3 and $24.2 \%$ as OLGA 4 . The relationship between PGI levels and OLGA stage shows a statistically significant difference between the stages OLGA $0,1,2$ and the stages 3 and 4 (PGI mean values: OLGA 0: $72.45 \mu \mathrm{g} / \mathrm{L}$, OLGA 1: $85.95 \mu \mathrm{g} / \mathrm{L}$, OLGA 2: $47.38 \mu \mathrm{g} / \mathrm{L}$, OLGA 3: $16.00 \mu \mathrm{g} / \mathrm{L}$, OLGA 4: $10.10 \mu \mathrm{g} / \mathrm{L} ; \mathrm{p}<0.001)$. The relationship between OLGA stages and G-17 serum levels shows a statistically significant difference (G-17 mean values: OLGA 0: $4.6 \mathrm{pmol} / \mathrm{L}$, OLGA 1: $5.2 \mathrm{pmol} / \mathrm{L}, \mathrm{OLGA} 2: 26.5 \mathrm{pmol} / \mathrm{L}$, OLGA 3: $44.6 \mathrm{pmol} / \mathrm{L}$, OLGA 4: $38.5 \mathrm{pmol} / \mathrm{L} ; \mathrm{p}<0.01$ by comparing OLGA $0-1$ against OLGA $2-4)(\mathrm{p}<0.02)$.

Conclusion: The prevalence of CAG assessed by serological markers is low in the studied population $(4 \%)$. The majority of patients $(56.6 \%)$ were classified in the OLGA 2 stage. Both PG1 and G-17 showed a statistical significant relationship with the more severe degrees of CAG.

Disclosure of Interest: All authors have declared no conflicts of interest.

\section{P1154 IMPROVEMENT OF SYMPTOMS IN PATIENTS AFFECTED BY CHRONIC ATROPHIC GASTRITIS: A TWO-YEAR PROSPECTIVE STUDY BY USING L-CYSTEINE}

G. Masciangelo ${ }^{1}$, L. Franzoni ${ }^{1}$, C. Miraglia ${ }^{1}$, P. Crafa ${ }^{1}$, S. Scida ${ }^{1}$ M. Franceschi ${ }^{2}$, R. Cannizzaro ${ }^{3}$, N. Dal Bò ${ }^{4}$, C. Scarpignato ${ }^{1}$, M. Rugge ${ }^{5}$, F. Di Mario

${ }^{1}$ Department Of Medicine And Surgery, University of Parma, Parma/Italy ${ }^{2}$ Endoscopic Unit, Department Of Surgery, Ulss4, Hospital ULSS4 Alto Vicentino, Santorso/Italy

${ }^{3}$ Oncological Gastroenterology, Centro di Riferimento Oncologico di Aviano S.O.C. di Gastroenterologia, Aviano/Italy

${ }^{4}$ Gastroenterological Unit, Treviso Hospita, Treviso/Italy

${ }^{5}$ Pathology, Medical School of the Padova University, Padova/Italy

Contact E-mail Address: francesco.dimario@unipr.it

Introduction: Chronic atrophic gastritis (CAG) is considered to be a precancerous condition of gastric cancer; it is either autoimmune in origin or caused by infection with Helicobacter pylori. The majority of CAG patients are asymptomatic or experience aspecific manifestations like epigastric fullness, early satiation, nausea, bloating. The drugs currently used for upper gastrointestinal (GI) diseases, mainly proton pump inhibitors (PPIs), appear inappropriate in such patients, mainly because acid production is virtually lacking. The use of 
L-cysteine has been proposed as adjuvant therapy in CAG; the amino acid binds covalently to acetaldehyde (a Group I human carcinogen), removing it from the stomach. The aim of present study was to use L-cysteine to improve the symptoms in patients with diagnosis of CAG.

Aims \& Methods: One hundred fourteen consecutive patients $(M=43$, mean age $=52.5 \mathrm{yr}$, range $=31-69$ years) (with histological diagnosis of CAG by means of both gastric histology (moderate to severe chronic, atrophic, body gastritis according to the OLGA staging system) and serology (pepsinogen 1 $<25 \mu \mathrm{g} / \mathrm{l}$; gastrin-17 >14 pmol/1 - GastroPanel ${ }^{\circledR}$, Biohit Oyj, Finland) entered the study. Forty-one patients (11 M, mean age $49.4 \mathrm{yr}$, range $27-71$ years) were treated with L-cysteine (100 mg 3 times daily, with meals) for 24 months (Group 1). As a control group we enrolled $73 \mathrm{CAG}$ patients $(\mathrm{M}=32$, mean age $=55.3 \mathrm{yr}$, range $=32-77$ yrs) followed up for 24 months without any related therapy (Group 2). Symptoms (epigastric fullness, early satiation, nausea, bloating) were recorded at baseline and after 3, 6, 12, 24 months, according to severity score $(0-3$ for each symptom, min. $0=$ no symptoms; $\max .12=$ full symptomatic).

Results: The global symptomatic score results as follows, lasting the 24 months follow-up. Group 1: baseline 4.93; 3 months 3.36; 6 months 2.96; 24 months 2.64. Group 2: baseline 5.9, 3 months 6.2, 6 months 5.6, 24 months $5.8(\mathrm{p}<0.01)$. Subdividing the CAG patients according to the etiology (autoimmune gastritis or previous Helicobacter pylori infection) no differences were found in improving symptoms. No relevant side effects were observed during the study.

Conclusion: The administration of L-cysteine to subjects affected by moderatesevere chronic atrophic gastritis seems able to improve the symptoms in a twoyear follow-up.

Disclosure of Interest: All authors have declared no conflicts of interest.

\section{P1155 PROGNOSTIC SIGNIFICANCE OF SERUM INFLAMMATORY MARKERS IN GASTRIC CANCER}

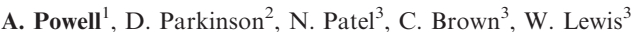

${ }^{1}$ Division Of Cancer And Genetics, Cardiff University, Cardiff/United Kingdom

${ }^{2}$ Cardiff \& Vale University Health Board, Cardiff/United Kingdom

${ }^{3}$ General Surgery, University Hospital of Wales, Cardiff/United Kingdom

Contact E-mail Address: powella16@cardiff.ac.uk

Introduction: Despite undergoing potentially curative resection a significant proportion of patients develop cancer recurrence. Several cellular and humoral components of systemic inflammatory response have been reported and associated with poor outcome. To date, no study has comprehensively examined the relationship between readily available markers of inflammation and survival in gastric cancer.

Aims \& Methods: Patients undergoing surgery for stage I-III gastric cancer between 2004-2016 at a regional unit were identified. Measurements of various systemic inflammation markers were recorded pre-operatively. Pathological factors were recorded from reports issued at the time of resection. The modified Glasgow Prognostic Score (based on CRP and Albumin), NeutrophilLymphocyte Ratio, Platelet-Lymphocyte Ratio and Neutrophil-Platelet score were calculated. Pathological variables including TNM stage, differentiation and vascular invasion were also recorded. Survival endpoints of overall survival (OS) and disease-free survival (DFS) were used.

Results: 331 patients were identified and 291 patients underwent potentially curative resection for gastric cancer. On univariable DFS analysis, female gender, proximal location, T-stage, N-stage, TNM, vascular invasion, poor differentiation, lymph node ratio, R1 status, platelet count and mGPS were significantly associated with poor survival. On multivariable DFS analysis, mGPS (Hazard Ratio (HR) $2.5195 \%$ Confidence Interval (CI) $(1.35-4.65) ; \mathrm{p}=0.011$ ) was the only inflammatory marker to retain independent significance. This was also observed in OS with mGPS (HR 2.75 (95\% CI 1.65-4.59); $p<0.001)$. The mGPS was associated with advanced T-stage $(\mathrm{p}=0.001)$, TNM stage $(\mathrm{p}=0.013)$ and poor differentiation $(\mathrm{p}=0.030)$.

Conclusion: The mGPS was the only inflammatory based marker to independently predict poor DFS and OS and may represent the optimum method for systemic inflammatory response quantification.

Disclosure of Interest: All authors have declared no conflicts of interest.

\section{P1156 ANALYSIS OF REBLEEDING PATIENTS IN UPPER}

\section{GASTROINTESTINAL BLEEDING IN A SINGLE CENTER SERIES}

R. Jiménez-Rosales ${ }^{1}$, E. Ortega-Suazo ${ }^{1}$, P. Abellán-Alfocea ${ }^{1}$, F. Valverde López $^{2}$, J.G. Martinez-Cara ${ }^{1}$, E. Redondo Cerezo ${ }^{1}$

${ }^{1}$ Digestive Diseases, Hospital Virgen de las Nieves, Granada/Spain

${ }^{2}$ Gastroenterology, "Virgen de las Nieves" University Hospital, Granada/Spain

\section{Contact E-mail Address: eredondoc@gmail.com}

Introduction: Upper gastrointestinal bleeding (UGIB) is one of the main causes of hospital admission and urgent endoscopy in Gastroenterology departments. In-hospital mortality from UGIB has decreased throughout the past 2 decades with a corresponding increase in the performance of endoscopy and endoscopic therapies. This may suggest that improvements in the therapeutic procedures for patients with UGIB could be responsible of the mortality decline. Despite this, UGIB represents a true emergency, associated with significant morbidity, mortality and healthcare costs. Furthermore, rebleeding after initial endoscopic therapy is observed in $10-20 \%$, and it has been associated with a higher mortality rate. Therefore, the definition of predictive factors for rebleeding is of outstanding importance.
Aims \& Methods: The aim of our study is to analyze risk factors and outcomes in a population of patients who suffered rebleed. We present a retrospective study on a prospectively built database of patients with GI bleeding admitted to the Emergency Room of "Virgen de las Nieves" University Hospital over 42 months, from January 2013 to July 2016. All patients underwent upper endoscopy, and information regarding patients' demographic data, comorbidities, current medications (including antiplatelet drugs, NSAIDS and oral anticoagulants), clinical presentations, hemodynamics, admission laboratory test results, and endoscopic findings was collected. Interventions were documented, including the need for blood transfusion and the number of packed red cells units per patient, endoscopic therapy, interventional radiology procedures, and surgery. Clinical outcomes documented were in-hospital and delayed 6-months mortality, rebleeding and delayed 6-months bleeding and cardiovascular events.

Results: 507 patients were included (339 males; aged 64.21 \pm 16.4 ). The incidence of rebleeding was $17.3 \%(\mathrm{n}=88)$. In the univariate analysis, factors related with rebleeding were creatinine levels $(1.52$ vs. $1.15 ; \mathrm{p}<0.001)$, tachycardia $(96.28$ vs. $88.24 ; \mathrm{p}<0.001)$, low levels of albumin $(2.80$ vs. $3.238 ; \mathrm{p}<0.001)$ and low systolic blood pressure $(103$ vs. $113 ; \mathrm{p}<0.001)$. In a logistic regression analysis tachycardia and high creatinine were independent risk factors for rebleeding, and albumin showed as an independent protective factor (Table 1). Rebleeding was associated with in-hospital mortality $(\mathrm{p}<0.0001)$; by contrast, it was not related nor with delayed 6-months mortality, neither with delayed cardiovascular and hemorrhagic events. The UGIB risk scores AIMS 65 and Rockall showed poor predictive ability for acute mortality in the rebleeding patients' group and was similar for Blatchford score (based on AUROc).

\begin{tabular}{llll}
\hline & Exp (B) & IC 95\% & P \\
\hline PULSE & 1.021 & $1.007-1,034$ & 0.003 \\
ALBUMIN & 0.406 & $0.267-0.618$ & 0.000 \\
CREATININ & 1.403 & $1.079-1.824$ & 0.012 \\
\hline
\end{tabular}

Conclusion: Rebleeding in UGIB is associated with increased in-hospital mortality; nevertheless, it is not related with delayed 6-months mortality, hemorrhagic and cardiovascular events. High creatitinine and low albumin levels were independent risk factors for rebleeding, suggesting a potential predictive role of these parameter. AIMS65, Rockall and Blatchford were predictors for in-hospital mortality but worked poorly in the patients who suffered rebleeding. Disclosure of Interest: All authors have declared no conflicts of interest.

\section{P1157 A CASE-CONTROL STUDY ON THE RISK OF UPPER GASTROINTESTINAL MUCOSAL INJURIES IN SUBJECTS PRESCRIBED NSAIDS AND ANTI-THROMBOTIC DRUGS USING THE LARGE ORGANIZED DATABASE OF CLAIMS IN JAPAN (APPROXIMATELY 3.7 MILLION OF POPULATION ON AN ACCUMULATED BASIS)}

N. Sugisaki ${ }^{1}$, R. Iwakiri ${ }^{2}$, K. Fujimoto ${ }^{3}$

${ }^{1}$ Graduate School Of Medical Science, Saga University, Saga/Japan

${ }^{2}$ Dept. Of Gastrointestinal Endoscopy, Saga Medical School, Saga/Japan

${ }^{3}$ Dept. Of Internal Medicine, Saga Medical School, Saga/Japan

Contact E-mail Address: nobuyuki_sugisaki@eapharma.co.jp

Introduction: Upper gastrointestinal (GI) adverse effects induced by NSAIDs and anti-thrombotic drugs are increasing along with progressive aging of society. Recently it is essential to perform pharmaco-epidemiological studies to identify adverse effects in the real-world setting using a large-scale medical database. We conducted a case-control study on the risk of upper GI mucosal injuries in subjects prescribed NSAIDs and anti-thrombotic drugs using the large organized database of claims in Japan.

Aims \& Methods: The medical claims database developed by Japan Medical Data Center (JMDC) Co., Ltd. was selected as data source in the present retrospective observational study. The JMDC claims database comprised of integrated medical and pharmacy claims, and includes both hospital and outpatient care from over 90 payers (approximately 3.7 million of population on an accumulated basis). Eligible subjects were aged 20 to 74 and registered for at least 3 months in the database from January 2009 to December 2014. The evaluated upper GI mucosal injuries were peptic ulcers (143,271 cases), upper GI bleeding (10,545 cases) and gastroesophageal reflux disease (GERD: 154,755 cases) with diagnosis by ICD10 codes and implementation of the upper GI endoscopy. For the each test-case, ten controls who matched age, sex and diagnosis month were identified from the database. Multivariate logistic regression analysis was used to calculate odds ratios of occurrence of each upper GI mucosal injuries caused by NSAIDs, COX-2 selective inhibitors, low-dose aspirin, antiplatelet drugs (except lowdose aspirin) and anticoagulants.

Results: The odds ratios of peptic ulcers were $1.45,1.31,1.50,1.53$ and 1.62 for NSAIDs, COX-2 selective inhibitors, low-dose aspirin, antiplatelet drugs, and anticoagulants, respectively. All the medicines significantly affected the occurrence of peptic ulcers ( $\mathrm{p}<0.0001$ in each). The odds ratios of upper GI bleeding were 1.76, 1.62, 1.96, 1.82 and 2.38, and those of GERD were 1.54, 1.41, 1.89, 1.67 and 1.91 , and these odds ratios were statistically significant in each medicine with GI bleeding and GERD ( $p<0.0001$ in each). The odds ratios of all the upper GI mucosal injuries were the highest in the patients with anticoagulants, and the ratios were relatively low in those with NSAIDs and COX-2 selective inhibitors. The odds ratios tended to increase with the number of prescribed 
medicines ( 1 agent $<2$ agents $<3$ agents, peptic ulcers: $1.38<2.49<4.52$, upper GI bleeding: $1.74<3.95<7.77$, GERD: $1.61<2.96<5.88$, respectively). The upper GI mucosal injuries were exacerbated in complication of lifestyle-related diseases, including hyperlipemia and diabetes mellitus.

Conclusion: Prescribing NSAIDs and anti-thrombotic medicines was associated with increased risks of developing upper GI injury. The present case-control study utilizing the large organized database of claims in Japan provided precise clinical evidence for safety management of medical drugs in the clinical settings in Japan.

Disclosure of Interest: All authors have declared no conflicts of interest

\section{Reference}

Kimura S, Sato T, Ikeda S, Noda M, Nakayama T. Development of a database of health insurance claims: standardization of disease classifications and anonymous record linkage. $J$ Epidemiol 20: 413-419, 2010.

P1158 GASTROINTESTINAL BLEEDING UNDER ANTICOAGULATION THERAPY: SYSTEMATIC REVIEW OF THE REBLEEDING RISK, ITS REVERSIBILITY PROFILE AND RISK STRATIFICATION TO SELECT PATIENTS FOR LEFT ATRIAL APPENDAGE OCCLUSION

\author{
A. Hadefi ${ }^{1}$, A. Aminian ${ }^{2}$, A. Bondue ${ }^{3}$, J. Devière $^{4}$, A. Lemmers ${ }^{4}$ \\ ${ }^{1}$ Gastroenterology, Hepatopancreatology And Digestive, Erasme Hospital, \\ Université Libre de Bruxelles, Brussels/Belgium \\ ${ }^{2}$ Cardiology Department, Hôpital civil Marie Curie, Charleroi/Belgium \\ ${ }^{3}$ Cardiology Department, Erasme Hospital, Université Libre de Bruxelles, \\ Brussels/Belgium \\ ${ }^{4}$ Gastroenterology, Hepatopancreatology And Digestive Oncology, Erasme \\ Hospital, Université libre de Bruxelles ULB, Brussels/Belgium
}

\section{Contact E-mail Address: alia.hadefi@gmail.com}

Introduction: Percutaneous left atrial appendage occlusion (LAAO) is increasingly recognized as valid alternative therapy to reduce thrombo-embolic risk in patients with non valvular atrial fibrillation (AF) and contraindications for long term oral anticoagulation (OAC) therapy ${ }^{1,2}$. Patients at high thromboembolic risk with previous gastrointestinal bleeding (GIB) might be at risk of bleeding recurrence in case of resuming anticoagulation. They could be selected for alternative therapies like LAAO. Up to now, there is no scientific consensus for patient selection for LAAO based on recurrent GIB risk.

Aims \& Methods: We aimed to review the literature on gastrointestinal (GI) bleeding recurrence and proposed to define the reversibility profile of each lesion in an organ by organ and lesion by lesion approach to stratify the risk of bleeding individually. We systematically collected data from both prospective and retrospective studies from pubmed in order to extract rebleeding risk by etiology. The reversibility profile was defined by type of treatment needed to cure the lesion. Low reversibility (LR) profile was defined as a need for heavy treatment (surgery, radiotherapy, embolisation) to cure the lesion or as diffuse lesions.

Results: The most frequent reported causes of bleeding are peptic gastroduodenal ulcer $(60 \%)$ for upper GI, diverticulosis $(40 \%)$, colitis $(20 \%)$ and anorectal diseases $(20 \%)$ for lower GI and angiodysplasia $(23 \%)$ for the midgut, these latters being responsible for $5 \%$ of all GI bleeding causes. The rate of reccurent GI bleeding under OAC therapy is $5-7 \%$ with vitamin $\mathrm{K}$ antagonists (VKA), an incidence that might increase with direct OAC. Recurrent bleeding rates for upper GIB, lower GIB and obscure GIB are respectively $5-7 \%, 16 \%$, and $40.3 \%$.

In the upper GI tract, lesions at high risk of bleeding reccurence are Dieulafoy lesions and angiodysplasia with reported rates up to $40 \%$ in some series. In the lower GI tract, lesions at highest risk are diverticular disease, angiodysplasia, colitis and radiation rectitis with bleeding recurrence rates reaching $60 \%, 20 \%$, $40 \%$ and $20 \%$ respectively. For the midgut, angiodysplasia $(20 \%)$ and bleeding of unknown origin $(20 \%)$ are associated to the highest risk of reccurent bleeding. LR profile lesions with high rebleeding risk are present for diffuse angiodysplasia, colonic diverticulosis and Dieulafoy lesions.

Conclusion: In conclusion, GI lesions at high risk of reccurent bleeding with low reversibility profile are infrequent and include in particular: diffuse angiodysplasia, colonic diverticulosis and Dieulafoy lesions. Patients with AF having those lesions with GIB under anticoagulation might be the best candidates for alternative therapies like LAAO. Larger studies are needed to assess the long term outcome of patients treated by LAAO for GIB under current oral anticoagulant therapies.

Disclosure of Interest: All authors have declared no conflicts of interest.

\section{References}

1. Holmes DR, Reddy VY, Turi ZG, et al. Percutaneous closure of the left atrial appendage versus warfarin therapy for prevention of stroke in patients with atrial fibrillation: a randomised non-inferiority trial. Lancet 2009;374:534-42.

2. Reddy VY, Doshi SK, Sievert H, et al. Percutaneous left atrial appendage closure for stroke prophylaxis in patients with atrial fibrillation: 2.3-Year Follow-up of the PROTECT AF (Watch-man Left Atrial Appendage System for Embolic Protection in Patients with Atrial Fibrillation) Trial. Circulation 2013;127:720-9.
P1159 AN AUDIT INTO THE MANAGEMENT OF BLEEDING

PEPTIC ULCER DISEASE

K. Siau ${ }^{1}$, A. Vijayasingam ${ }^{2}$

${ }^{1}$ Royal College Of Physicians, JAG Clinical Fellow, London/United Kingdom

${ }^{2}$ Postgraduate School of Medicine, Birmingham/United Kingdom

Contact E-mail Address: keith@siau.org

Introduction: Peptic ulcer disease (PUD) accounts for $25-56 \%$ of acute upper gastrointestinal bleeding (AUGIB) and is associated with high mortality.

Aims \& Methods: In line with international guidelines, we aimed to audit our practice of bleeding PUD at a district general hospital within the West Midlands, UK. We retrospectively identified all patients with AUGIB who had inpatient endoscopic confirmation of PUD between November 2012-2014. We scrutinised all endoscopy and case records to assess management related to PUD, in addition to follow-up records with the general practitioner.

Results: We identified 91 patients (median age 78.4,65.9\% male), of whom $63.7 \%$ were admitted with AUGIB, whereas $36.3 \%$ developed bleeding during their inpatient stay. The majority were duodenal $(74.7 \%)$ vs. gastric $(25.3 \%)$. $31.8 \%$ were related to aspirin/non-steroidal anti-inflammatory drug use. $48(52.7 \%)$ had high risk (Forrest $1 \mathrm{a}-2 \mathrm{~b}$ ) lesions, of whom 38/48 (79.2\%) received dual endoscopic therapy and $6 / 48(12.5 \%)$ received adrenaline monotherapy. $18.8 \%$ received the recommended adrenaline volume of $13 \mathrm{ml}$. Of $90 \%$ prescribed intravenous proton pump inhibitor infusion, $85 \%$ did not complete the full 72 -hour duration. Rebleeding occurred in 12 patients $(13.2 \%)$ after a median of 3-days post endoscopy. $10(83.3 \%)$ underwent repeat OGD, $2(16.7 \%)$ underwent CT embolisation, whilst $3(25.0 \%)$ underwent surgery. Aspirin resumption was observed in $4 / 20(20.0 \%)$ after haemostasis for PUD, whilst rebleed planning was documented on the endoscopy report in $33.0 \%$. Regarding H. pylori, $51 \%$ underwent assessment with serology/biopsy. $10(36 \%)$ had positive results, of whom $7(70 \%)$ received eradication. $12 / 23(52.2 \%)$ patients underwent followup endoscopy following gastric ulcer. The median transfusion requirement per patient was 2 units. Despite this, rates of anaemia at discharge and at 6 months were high at $83.5 \%$ and $62.9 \%$ respectively, with iron therapy initiated in $12.1 \%$. Overall, our 30 -day mortality rate was $12.1 \%$.

Conclusion: In this single-centre study, the management of PUD could be improved in multiple areas in line with international guidelines. Audits in other centres are required to quality assure the management of PUD. Interestingly, rates of anaemia at discharge and on follow-up are high. Such patients may benefit from iron replacement at discharge.

Disclosure of Interest: All authors have declared no conflicts of interest.

P1160 COMPARISON OF SCORING SYSTEMS FOR NONVARICEAL UPPER GI BLEEDING; FOCUS ON THE SIMPLE PREDICTION SYSTEM, AIMS65

M.S. Kim ${ }^{1}$, J. Choi ${ }^{2}$, W.C. Shin ${ }^{2}$

${ }^{1}$ Internal Medicine, Seoul National University Hospital, Seoul/Korea, Republic of ${ }^{2}$ Internal Medicine, Sanggye Paik Hospital, Inje University College of Medicine, Seoul/Korea, Republic of

Contact E-mail Address: kingtta21@naver.com

Introduction: In nonvariceal upper GI bleeding (NVUGIB) patients, it is important to predict the risk early and to take appropriate medical treatment. Recently, the AIMS65 score has been used to make simple predictions.

Aims \& Methods: The aim of this study is to compare the risk stratification of each predictor by comparing AIMS65 score with Glasgow-Blatchford score (GBS), full Rockall score and pre-endoscopic Rockall score. We retrospectively studied patients who visited the Sanggye Paik Hospital with NVUGIB between 2013 and 2016. Among these, AIMS65, GBS, and Rockall scores were measured. The primary outcome was inpatient mortality, and secondary outcomes were rebleeding, needs of endoscopic intervention, requirement of transfusion, intensive care unit (ICU) admission. Each scoring system and prognostic factors were compared using the ROC curve.

Results: In this study, 17 of the 512 selected patients died, and re-bleeding was confirmed in 30 patients. AUROC between AIMS 65 and mortality was measured at 0.844 higher than 0.721 and $0.743 / 0.749$ for GBS and pre-endoscopic full Rockall score. $(\mathrm{p}<0.001)$ For AUROC for secondary outcomes, AIMS 65 was superior to other scores in predicting the ICU admission $(0.728, \mathrm{p}<0.001)$. There was no significant difference in the superiority of each scoring system by pairwise comparison. GBS was superior to other scores for predicting blood transfusion requirement. $(\mathrm{p}<0.0001)$

Conclusion: The AIMS65 score in NVUGIB patients was not inferior to other scoring systems in predicting the prognosis, such as mortality and ICU admission rate. At the emergent department, we recommend using AIMS65 which is easier to access than other scoring systems.

Disclosure of Interest: All authors have declared no conflicts of interest.

\section{References}

1. Stanley AJ, Laine L, Dalton HR, Ngu JH, Schultz M, Abazi R, et al Comparison of risk scoring systems for patients presenting with upper gastrointestinal bleeding: international multicentre prospective study. BMJ (Clinical research ed). 2017;356:i6432.

2. Zhong M, Chen WJ, Lu XY, Qian J, Zhu CQ. Comparison of three scoring systems in predicting clinical outcomes in patients with acute upper gastrointestinal bleeding: a prospective observational study. Journal of digestive diseases. 2016;17(12):820-8.

3. Robertson M, Majumdar A, Boyapati R, Chung W, Worland T, Terbah R, et al. Risk stratification in acute upper GI bleeding: comparison of the 
AIMS65 score with the Glasgow-Blatchford and Rockall scoring systems. Gastrointestinal endoscopy. 2016:83(6):1151-60.

4. Park SM, Yeum SC, Kim BW, Kim JS, Kim JH, Sim EH, et al. Comparison of AIMS65 Score and Other Scoring Systems for Predicting Clinical Outcomes in Koreans with Nonvariceal Upper Gastrointestinal Bleeding. Gut and liver. 2016;10(4):526-31.

5. Hyett BH, Abougergi MS, Charpentier JP, Kumar NL, Brozovic S, Claggett BL, et al. The AIMS65 score compared with the Glasgow-Blatchford score in predicting outcomes in upper GI bleeding. Gastrointestinal endoscopy. 2013;77(4):551-7.

6. Chandra S. AIMS65 score predicts short-term mortality but not the need for intervention in acute upper GI bleeding. Gastrointestinal endoscopy. 2013;78(2):381-2.

7. Saltzman JR, Tabak YP, Hyett BH, Sun X, Travis AC, Johannes RS. A simple risk score accurately predicts in-hospital mortality, length of stay, and cost in acute upper GI bleeding. Gastrointestinal endoscopy. 2011;74(6):1215-24

P1161 COMPARISON OF RISK-SCORING SYSTEMS IN PREDICTING NEEDS OF INTERVENTION AND CLINICAI OUTCOMES OF UPPER GASTROINTESTINAL BLEEDING

M. Ooi, W. Low, R. Bryant, N. Q. Nguyen

Department Of Gastroenterology And Hepatology, Royal Adelaide Hospital, Adelaide/Australia/SA

\section{Contact E-mail Address: marieooi_al@yahoo.com}

Introduction: There are several risk-scoring systems are available to assist the management of upper gastrointestinal bleeding (UGIB). The aim of this study is to compare the performance of pre-endoscopy (pre-RS), post-endoscopy Rockall score (post-RS), GBS and AIMS65 scores in predicting the need for interventions and outcomes in patients admitted to hospital for UGIB.

Aims \& Methods: Data related to the three scoring systems were collected prospectively and scores calculated in consecutive patients who were admitted with acute UGIH to the Royal Adelaide Hospital over 24 months. The performance of these scores, using operating characteristic (ROC) curves, in predicting the following outcomes was assessed:the need for endotherapy, rebleeding risk, transfusion requirement, surgical intervention and death. All patients received high dose acid suppression therapy.

Results: Of the 777 patients (491M; $66.4 \pm 0.6 \mathrm{yrs})$ who presented with UGIB, 622 $(402 \mathrm{M} ; 65.7 \pm 0.6 \mathrm{yrs})$ underwent endoscopy with $123(38 \%)$ required endoscopic therapy, $42(13 \%)$ repeated endo-therapy and $12(3.7 \%)$ surgery for haemostasis. 155 (89M; 68.8 $\pm 0.6 \mathrm{yrs})$ patients were treated conservatively due to medically unfit $(\mathrm{n}=50)$, low suspicion $(\mathrm{n}=25)$, known diagnosis $(\mathrm{n}=17)$, and suspected MWT $(n=28)$. Death occurred in $33(8 \%)$ patients, of which $10(2.4 \%)$ were related to bleeding. Patients who required endoscopic or surgical intervention had higher GBS, pre-ERS, post-ERS and AIMS65 scores $(\mathrm{P}<0.001)$. On ROC analyses, GBS and post-RS were superior to pre-RS and AIMS65 in predicting the need for (1) endotherapy (AUC: 0.75 and 0.78 vs. 0.65 and $0.59, \mathrm{P}<0.01$ ), (2) repeated endoscopy due to re-bleed (AUC: 0.66 and 0.65 vs. 0.56 and 0.60 , $\mathrm{P}=0.03$ ), and (3) blood transfusion (AUC: 0.83 vs. 0.72 and $0.70, \mathrm{P}<0.01$ ). The scoring systems performed equally well in predicting the need for surgery (GBS vs. Rockall vs. GBS AUC: 0.72 vs. 0.67 vs. 0.71 ) and death (AUC: 0.75 and 0.72 vs. 0.69 ).

Conclusion: Although GBS performs equally well for predicting need of surgery and death, it is superior to both Rockall and AIMS65 scores in predicting the need for endotherapy, repeated endoscopy, and blood transfusion in patients with acute UGIH. These findings suggest that GBS is the best performed risk scoring system in the evaluation of patients with UGIB.

Disclosure of Interest: All authors have declared no conflicts of interest.

\section{P1162 STEROID ADMINISTRATION IS AN INDEPENDENT RISK FACTOR FOR REBLEEDING IN HEMORRHAGIC DUODENAL ULCER WITH A DOSE-RESPONSE RELATION}

W. Hatta, Y. Kondo, T. Koike, T. Kanno, N. Ara, K. Asanuma, N. Asano, A. Imatani, T. Shimosegawa

Division Of Gastroenterology, Tohoku University Graduate School of Medicine, Sendai/Japan

\section{Contact E-mail Address: waku-style@festa.ocn.ne.jp}

Introduction: Hemorrhagic gastroduodenal ulcer is commonly seen in routine clinical practice, and there have been many studies investigating risk factors for rebleeding. However, few studies have evaluated hemorrhagic gastric ulcers (HGUs) and hemorrhagic duodenal ulcer (HDUs) separately. Furthermore, the relation between steroid administration and rebleeding in hemorrhagic gastroduodenal ulcer remains unclear.

Aims \& Methods: The aim of this study was to clarify the difference of rebleeding between HGU and HDU, and associated factors for rebleeding of $\mathrm{HGU}$ and HDU. Between March 2005 and September 2016, 176 consecutive patients with hemorrhagic gastroduodenal ulcer (106 with HGU and 70 with HDU), who underwent endoscopic hemostasis, were enrolled in this study. Regular dose proton pump inhibitor was administrated to all patients after the diagnosis of hemorrhagic gastroduodenal ulcers. Rebleeding was defined as hematemesis or melena with ulcers confirmed by endoscopy or a decrease in the hemoglobin level $>2 \mathrm{~g} / \mathrm{dl}$ in the presence of endoscopically proven ulcers. First, we compared the rebleeding rate between $\mathrm{HGU}$ and HDU. Subsequently, associated factors for rebleeding of HGU and HDU were calculated by logistic regression analysis individually. The estimated factors were age $(<65 / \geq 65$ years $)$, gender, location of ulcer (upper third/middle or lower third in $\mathrm{HGU}$ and $2^{\text {nd }}$ portion/bulbs in HDU), underlying comorbidities (ischemic heart disease, liver cirrhosis, hypertension, diabetes mellitus, and hyperlipidemia), number of ulcers (multiple/ single), hemostasis method (pure ethanol injection therapy/other therapies), antiplatelet therapy, anticoagulant therapy, NSAIDs administration, steroid administration, antacid administration in the initial ulcer bleeding, hypoalbuminemia (serum albumin level $\leq 2.5 \mathrm{~g} / \mathrm{dl}$ ), and hemodialysis. We further investigated the detailed association between steroid administration and rebleeding in HDU, including dose-response relation.

Results: The rebleeding rate of HGU and HDU were $5.7 \%$ and $22.9 \%$, respectively, which was statistically significant $(P=0.001)$. There was no missing data in the estimated factors. Although no factor was associated with rebleeding in HGU, multivariate logistic regression analysis revealed that the independent risk factors for rebleeding in HDU were multiple ulcers [odds ratio $(95 \%$ confidence interval $)=24.2(2.76-213), P=0.004]$, steroid administration [14.0 (1.73-113), $P=0.013$ ], and hemodialysis [9.53 (1.00-90.7), $P=0.049$ ]. Regarding the detail of steroid administration, multivariate analysis showed that middle or high steroid administration ( $>20 \mathrm{mg}$ in prednisolone) (52.7 [3.19-871], $P=0.006$ ) was a significant risk factor for rebleeding of HDU, with a dose-response relation $(P$ for trend $=0.015$ )

Conclusion: HDU developed significantly higher rebleeding after endoscopic hemostasis, compared with HGU. In addition to multiple ulcers and hemodialysis, we firstly demonstrated by multivariate analysis that steroid administration is an independent risk factor for rebleeding of HDU after endoscopic hemostasis, with a dose-response relation.

Disclosure of Interest: All authors have declared no conflicts of interest.

\section{P1163 EFFICACY AND SAFETY OF BIO-INSERT MINERAL} SMECTITE IN CONTROLLING GASTROINTESTINAL HEMORRHAGE: AN ANIMAL PILOT STUDY

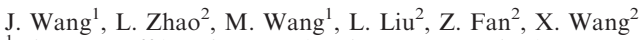

${ }^{1}$ The First Affiliated Hospital with Nanjing Medical Universtiy, Nanjing/China

${ }^{2}$ Digestive Endoscopy Center, the First Affiliated Hospital of Nanjing Medical University, Nanjing/China

\section{Contact E-mail Address: kit9178@sina.com}

Introduction: Gastrointestinal bleeding is common in clinics, especially after endoscopic operation. Besides from hemoclips, APC or electrocoagulation, more novel hemostasis approaches should be developed to improve endoscopic bleeding management. Granular smectite is bioinert mineral and efficient for curing diarrhea. Inspired by its dehydration and tissue-covering effect, this pilot study was to investigate its efficacy and safety for controlling hemorrhage in rats.

Aims \& Methods: 32 rats were divided into four equal groups. For hemorrhage model, a horizontal 10-mm incision was made on the lower part of the left hepatic lobe. Commercial hemostatic powder, smectite, starch and normal saline were respectively applied. Bleeding duration and blood loss were recorded. 1 week later, rats were sacrificed and liver tissue was collected for histopathology. Results: Smectite demonstrated the best hemostasis effect, and its mean coagulation time was $1.45 \pm 0.026 \mathrm{~min}$. Commercial hemostatic chitosan styptic powder need $2.5 \pm 0.04 \mathrm{~min}$ for complete clotting, while Starch group was $4.275 \pm 0.056 \mathrm{~min}$ and normal saline group was $4.925 \pm 0.108 \mathrm{~min}(\mathrm{p}<0.05)$. Similarly, smectite led to less loss of blood $(0.6188 \pm 0.034 \mathrm{~g})$, while rats lost $2.3288 \pm 0.123 \mathrm{~g}$ blood $(\mathrm{p}<0.05)$ under normal saline treatment. For starch and commercial chitosan, the blood loss was respectively $2.0862 \pm 0.061 \mathrm{~g}$ and $1.2325 \pm 0.028 \mathrm{~g}$. Histopathologic results confirmed that smectite was biocompatible to tissue.

Conclusion: The mineral smectite powder was the superior candidate for hemostasis treatment in vivo. Compared with common polysaccharide agents, smectite could induce faster coagulation and reduce blood loss. More importantly, bioinert smectite was biocompatible and even promoted the wound healing. For gastrointestinal application, smectite powder could be delivered through endoscopic spray tube, while its inspiring efficacy required more endoluminal hemostasis tests.

Disclosure of Interest: All authors have declared no conflicts of interest.

\section{P1164 PREDICTORS OF LIFE THREATENING MUCOSAL ULCERATION AFTER VARICEAL SCLEROTHERAPY}

\section{A. S. Hanafy}

Internal Medicine, Zagazig University, Zagazig/Egypt

\section{Contact E-mail Address: dr_amr_hanafy@yahoo.com}

Introduction: Life-threatening bleeding could occur early after variceal sclerotherapy in cirrhotic patients.

Aims \& Methods: We aimed to determine simple predictive factors of this complication in cirrhotic patients. Among 750 patients treated with variceal sclerotherapy (esophageal varices $(\mathrm{EV}), \mathrm{n}=655,87.3 \%$ ) and (gastric varices $(\mathrm{GV}$ ), $\mathrm{n}=95,12.7 \%$ ) Zagazig University hospital-endoscopy unit- Internal medicine department, in the period from October 2014 till July 2016. 150 patients $(20 \%$, mean age $46.2 \pm 9.4$ years) $(\mathrm{EV}=129, \mathrm{GV}=21)$ developed bleeding due to sclerotherapy induced ulcers confirmed by endoscopy $6.4 \pm 2.1$ days after the procedure. Cirrhosis was post viral hepatitis C $(89 \%)$, hepatitis B $(10 \%)$ and cryptogenic in $(1 \%)$. A case-control study was performed comparing these 
patients with 150 patients who underwent endoscopic variceal sclerotherapy without the development of bleeding due sclerosant ulceration.

Results: Bleeding occurred $6.4 \pm 2.1$ days (2-10) following sclerotherapy. Twentythree patients died following the bleeding $(15.3 \%)$. Using a multivariate analysis; pre-procedural factors as serum albumin $\leq 2 \mathrm{~g} / \mathrm{dl}$ [OR 1.3], total bilirubin $>1.6 \mathrm{mg} / \mathrm{dl}$ [OR 1.5], high platelet ratio index (APRI) $>1$ [OR 1.2], low prothrombin concentration $<50 \%$ [OR 1.5]. Intra-procedural factors as amount of ethanolamine $>15.5 \mathrm{ml}$ [OR 2.6], amacryate $>3.5 \mathrm{ml}$ [OR 2.9]. Post- procedural factors within 24 hours after endoscopy: leucocytosis $>12,000$ cell/ul [OR 1.9], drop of hemoglobin $>10 \%$ of the pre-endoscopic value [OR 3.2], prolonged INR $>1.55$ [OR 1.2].

Conclusion: Bleeding related to sclerosant ulcers is not uncommon, but may be life threatening. The proposed predictive factors should be watched and minimized before and during variceal sclerotherapy

Disclosure of Interest: All authors have declared no conflicts of interest.

\section{References}

1. Carbonell N, Pauwels A, Serfaty L, Fourdan O, Lévy VG, Poupon R. Improved survival after variceal bleeding in patients with cirrhosis over the past decades. Hepatology 2004; 40: 652-9.

2. De Franchis R. Evolving consensus in portal hypertension: Report of the Baveno IV consensus workshop on methodology of diagnosis and therapy in portal hypertension. J Hepatol 2005; 43: 167-76.

3. Consensus conference: complications of portal hypertension in adults. Gastroenterol Clin Biol 2004; 28: B318-23.

\section{P1165 IMPACT OF SLEEP DISORDER IN PATIENTS WITH FUNCTIONAL DYSPEPSIA}

\section{K. Huh ${ }^{1}$, J.K. Park ${ }^{2}$, K.W. Jung ${ }^{3}$, C.M. Shin ${ }^{4}$, J.W. Kim ${ }^{5}$}

${ }^{1}$ Gastroenterology, Konyang University Hospital, Daejeon/Korea, Republic of

${ }^{2}$ Department Of Internal Medicine, GangNeung Asan Hospital, Gangneung/Korea, Republic of

${ }^{3}$ Gastroenterology, Asan Medical Center, Seoul/Korea, Republic of

${ }^{4}$ Gastroenterology, Bundang Seoul University Hospital, Bundang/Korea, Republic of

${ }^{5}$ Gastroenterology, Ilsan Paik Hospital, Goyang/Korea, Republic of

\section{Contact E-mail Address: kchuh2020@hanmail.net}

Introduction: Few studies were reported on the association between sleep disorders and Rome III-based functional dyspepsia (FD).

Aims \& Methods: The aim of this study is to investigate the prevalence of sleep disorders in FD patients and the risk factors associated with sleep disorders. This multicenter, cross-sectional study had been conducted from August 2014 to December 2016 at 6 hospitals in Korea. Inclusion criteria were FD patients ( $\geq 18$ years) met the Rome III criteria among the patients visited the gastroenterology department for dyspepsia. Exclusion criteria were prior surgery to the upper gastrointestinal tract, history of ulcer disease, erosive GERD, history of malignancy, and severe comorbidity. Healthy control group who had no clinical history of gastroduodenal related disorder and no abnormal finding on endoscopy recruited from health examination center for screening. The Pittsburgh Sleep Quality Index was used to assess sleep disturbance. Hospital anxiety and depression scale was used to identify anxiety and depression.

Results: This study included 160 FD patients and 223 healthy control groups The total Pittsburgh Sleep Quality Index score was higher in FD patients than health controls $(7.8 \pm 4.3$ vs $5.6 \pm 3.1, \mathrm{p}=0.000)$ The prevalence of sleep disorder was significantly higher in FD patients than healthy control $(41.2 \%$ vs $18.4 \%$, $\mathrm{p}=0.000)$. In univariate analysis, FD was significant risk factor for sleep disorder (OR 3.12, p < 0.0001). The independent risk factors for sleep disorder in multivariate analysis were FD (OR 1.80, $\mathrm{p}=0.026)$, female $(\mathrm{OR} 1.78, \mathrm{p}=0.028)$ and depression (OR 2.91, p $<0.001)$.

Conclusion: FD significantly impacted on sleep disorder. FD was independent risk factor in sleep disorder.

Disclosure of Interest: All authors have declared no conflicts of interest

\section{P1166 PREVALENCE OF DYSPEPSIA IN INDIVIDUALS WITH GASTRO-OESOPHAGEAL REFLUX-TYPE SYMPTOMS IN THE COMMUNITY: A META-ANALYSIS}

L.H. Eusebi ${ }^{1}$, R.P. Ratnakumaran ${ }^{2}$, M.L. Artesiani ${ }^{1}$, F. Bazzoli ${ }^{1}$, A.C. Ford ${ }^{3}$

${ }^{1}$ Department of Medical and Surgical Sciences, University of Bologna, Bologna/Italy

${ }^{2}$ Gastroenterology, Leeds Teaching Hospital, Leeds/United Kingdom

${ }^{3}$ Libacs, University of Leeds, Leeds/United Kingdom

\section{Contact E-mail Address: raguprakash.ratnakumaran@nhs.net}

Introduction: Dyspepsia and gastro-oesophageal reflux are highly prevalent in the general population, but the two conditions are felt to be separate entities. However, There are numerous mechanisms implicated in the pathogenesis of functional dyspepsia, some of which are common to gastro-oesophageal reflux symptoms (GORD), including visceral hypersensitivity and delayed gastric emptying. To inform future research on potential shared pathophysiological mechanisms, it is important to estimate the strength of association between the two conditions, and whether this association remains stable depending on the criteria used to define these conditions, as well as geographic location.
Aims \& Methods: We conducted a systematic review and meta-analysis to estimate the prevalence of dyspepsia in individuals with gastro-oesophageal reflux symptoms, and to quantify the overlap between the two disorders. MEDLINE, EMBASE, and EMBASE Classic were searched (up until September 2016) to identify population-based studies reporting the prevalence of dyspepsia and GORS in adults ( $\geq 15$ years), defined using specific symptom-based criteria or a questionnaire. The prevalence of dyspepsia and weekly GORS were extracted for all studies. Pooled prevalence, according to study location and criteria used to define weekly GORS or dyspepsia, as well as odds ratios (OR), with $95 \%$ confidence intervals (CIs) were calculated. The degree of overlap between the two was examined.

Results: Of 14,132 papers evaluated, 79 reported prevalence of weekly GORS. Nineteen of these study populations, containing 111,459 participants, also reported the proportion of individuals with dyspepsia. The prevalence of dyspepsia in those with weekly GORS was $43.9 \%(95 \% \mathrm{CI}, 35.1-52.9 \%)$. The pooled OR for dyspepsia in individuals with weekly GORS, compared with those without, was 6.94 (95\% CI 4.33 to 11.12$)$. The OR for dyspepsia in weekly GORS remained significantly higher in all geographical regions studied, and across all diagnostic criteria used. The pooled degree of overlap between the two conditions was $25.9 \%(95 \%$ CI $19.9-32.4 \%)$, varying from $22 \%$ when the Bowel Disease Questionnaire was used to define weekly GORS, to $42.6 \%$ with the Mayo Reflux Questionnaire.

Conclusion: The OR of dyspepsia in individuals with weekly GORS was sevenfold that of individuals without GORS, and that there is overlap between the two conditions in up to one-quarter of individuals. Reasons for this remain speculative, but may include shared pathophysiological mechanisms or residual confounding.

Disclosure of Interest: All authors have declared no conflicts of interest.

\section{References}

1. Eusebi LH, Ratnakumaran R, Yuan Y, et al. Global Prevalence of, and Risk Factors for, Gastro-oesophageal Reflux Symptoms: A Meta-analysis. GUT 2017.

2. Ford AC, Marwaha A, Sood R, et al. Global prevalence of, and risk factors for, uninvestigated dyspepsia: a meta-analysis. Gut 2015.

3. Choung RS, Locke GR, 3rd, Schleck CD, et al. Overlap of dyspepsia and gastroesophageal reflux in the general population: one disease or distinct entities? Neurogastroenterol Motil 2012.

4. Hsu CS, Wen SH, Hung JS, et al. Overlap of Dyspepsia in Patients with Gastroesophageal Reflux Disease: Impact of Clinical, Metabolic, and Psychosocial Characteristics. Dig Dis Sci 2017.

\section{P1167 PSYCHOSOCIAL PREDICTORS OF LATER} GASTROINTESTINAL SYMPTOMS

M.P. P. Jones ${ }^{1}$, N. Koloski ${ }^{2}$, L. Van Oudenhove ${ }^{3}$, A. Ejova ${ }^{1}$, N.J. Talley ${ }^{4}$ ${ }^{1}$ Psychology, Macquarie University Psychology, North Ryde/Australia ${ }^{2}$ Faculty Of Health And Medicine And Priority Research Center For Digestive Health And Neurogastroenterology, The University of Newcastle Australia, Callaghan/Australia/NSW

${ }^{3}$ Translational Research Center For Gastrointestinal Disorders (targid), Katholieke Universiteit Leuven, Leuven/Belgium

${ }^{4}$ University of Newcastle Faculty of Health PVC Office, Callaghan/Australia

Contact E-mail Address: mike.jones@mq.edu.au

Introduction: Functional gastrointestinal (GI) disorders (FGIDs) have been theorized to be a disorder of the brain-gut axis with both somatic markers (e.g., abdominal pain) and psychological ones. Exactly how abdominal and psychological disorders are related remains poorly understood. While there have been a small number of methodologically rigorous longitudinal studies that examine this question $(1,2)$, even these have typically only studied a small number of psychological traits and only quite general GI symptoms. There remains a need for further longitudinal studies of how psychological traits predict subsequent FGID symptoms.

Aims \& Methods: The present study is a longitudinal examination of the influence of a broad range of psychosocial predictors on later bowel-related and epigastric discomfort in a community sample. A sample of 188 individuals randomly sampled from the Australian electoral roll were surveyed, and, of them, 123 met Rome criteria for irritable bowel syndrome (IBS) or functional dyspepsia (FD) while 65 did not meet criteria for FGIDs. Subjects were of mean age 49 $(\mathrm{SD}=15$, range $20-87)$ and $73 \%$ were female. A broad range of psychological constructs (24 in total) were measured at baseline. Among them were anxiety, depression, somatization, childhood abuse, neuroticism, hypochondriasis, somatic vs psychological symptom attribution, coping, and social support. GI symptoms were measured 18 months later using two Likert-type scales: one concerning epigastric pain and the other concerning bowel symptoms. Exploratory factor analysis (EFA) was used as a data-reduction technique to create a smaller number of composite predictors. Statistical analysis was based on ordinal regression.

Results: Ten composite scores were derived via EFA to be examined as predictors. In the full sample $(N=188)$ a number of psychological traits (including worry, non-sexual childhood abuse, psychological symptom attribution and childhood sexual abuse; see Table 1) were individually related to subsequent bowel symptom severity 18 months later. All four of these were independently associated with epigastric pain severity 18 months later (Table 1). There was evidence of variation in psychological predictors of gastrointestinal symptoms between individuals who met and did not meet criteria for IBS or FD. With respect to bowel symptoms, no psychological trait reached statistical significance 
as a predictor among individuals with FGIDs (Table 1). Epigastric symptom severity was predicted by worry and psychological attribution of symptoms among FGID individuals but no psychological trait predicted symptom severity among non-FGID individuals (Table 1).

Conclusion: A range of psychosocial factors predict later gastrointestinal symptom burden. For bowel symptoms, associations between psychological traits and symptom burden appear to be most clearly driven by the non-FGID subgroup, among whom psychological attributions for symptoms and problem-focused coping are positively related to later symbptom burden. For epigastric symptoms, a range of psychological traits were relevant, with the predictive patterns being most clearly driven by individuals who qualified for FGIDs. In light of these results, studies of the brain-gut axis need to consider a greater array of psychological traits, particularly outside of anxiety and depression. Further, the commonly reported associations between psychological traits and gastrointestinal symptoms may be moderated by symptom level but are relatively similar across upper and lower gastrointestinal symptoms.

Disclosure of Interest: All authors have declared no conflicts of interest.

\section{References}

1. Koloski et al. 2012 Gut p1284

2. Koloski et al. 2016 Aliment Pharmacol Ther p1

\section{P1168 AN INCREASED PREVALANCE OF NEURODEGENERATIVE/DEMYELINATING PROCESS IN PATIENTS WITH ESOPHAGEAL ACHALASIA-A PROSPECTIVE STUDY}

M. Jerie ${ }^{1}$, Z. Vackova ${ }^{1}$, H. Svecová ${ }^{2}$, E. Meluzinova ${ }^{3}$, J. Krajciova ${ }^{1}$, Z. Vojtech ${ }^{1}$, J. Spicak ${ }^{1}$, J. Vymazal ${ }^{4}$, J. Martinek

${ }^{1}$ Department Of Neurology, Hospital Na Homolce, Prague/Czech Republic

${ }^{2}$ Institute for Clinical and Experimental Medicine, Prague/Czech Republic

${ }^{3}$ Charles University University Hospital Motol, Prague/Czech Republic

${ }^{4}$ Hospital Na Homolce, Prague/Czech Republic

Contact E-mail Address: martin.jerie@ homolka.cz

Introduction: In the recent years, there has been an increasing recognition of the presence of gastrointestinal (GI) dysfunction in patients with neurologic diseases. There are no studies examining a relationship between achalasia and neurodegenerative/demyelinating diseases of central nervous system, although these diseases might have common features. For example, a number of genetic variations have been shown to increase the risk of both conditions (e.g. HLADQB1-insertion on chromosome 6 may be strongly associated with both achalasia and multiple sclerosis suggesting that autoimmune processes are involved in their etiopathogenesis). Several other findings (e.g. inflammatory infiltrates, Lewy's bodies, geospatial gradient etc.) are also present in both patients with achalasia and with neurodegenerative/demyelinating diseases.

Aims \& Methods: The aim of our prospective study is to examine a prevalence of neurodegenerative/demyelinating diseases in a cohort of consecutive patients with confirmed esophageal achalasia. Achalasia was diagnosed by highresolution manometry, endoscopy and esophagogram. A total of 140 consecutive patients with esophageal achalasia have been questioned about the occurrence of neurological diseases and symptoms in their personal and family history. Those with a suspicion of a neurological disease were referred for a detailed clinical neurological examination, MR imaging of the brain and electrophysiological study. A total of 106 patients with a neurodegenerative/demyelinating disease have been questioned by using a questionnaire about the occurrence of symptoms typical for achalasia (dysphagia, regurgitation, chest pain).

Results: A total of 51 out of 140 patients $(36.4 \%)$ exhibited some neurological symptoms-most often visual disturbances in 17 patients $(33.3 \%)$ and limbs paresthesia in 12 patients $(23.5 \%)$. Among patients with a presence of neurological symptoms, 5 patients $(3.6 \%)$ had definitely been diagnosed with a neurodegeneration/demyelinating disease (multiple sclerosis - 2 patients, Lebeŕs optic neuropathy - 1 patient, Parkinsońs disease - 1 patient and Allgrove syndrome - 1 patient). Furthermore, 7 patients with a positive questionnaire had been diagnosed with other neurological diseases (tetany $n=2$, carpal tunnel syndrome $n=3$, epilepsy $n=2)$. Fourteen patients $(27.4 \%)$ among those with neurological symptoms (vs. 0 out of 89 patients without neurological symptoms) had a positive family history of a neurodegenerative or a demyelinating disease. Among 106 patients with a neurodegenerative/demyelinating disease, 30 of them $(28 \%)$ described dysphagia in their personal history. These patients will be examined by esophageal manometry.

Conclusion: Our results imply an increased prevalence of neurodegenerative demyelinating diseases in patients with achalasia $(3.6 \%$ vs. approx. $1.4 \%$ in the Czech controls). Also, a high prevalence of dysphagia was found among patients with a neurodegenerative/demyelinating disease. These results warrant further confirmation in a large population-based study.

Disclosure of Interest: All authors have declared no conflicts of interest.

\section{P1169 HIGH RESTING PARASYMPATHETIC CARDIAC VAGAL TONE CONFERS A UNIQUE FUNCTIONAL BRAIN NETWORK DURING ACUTE OESOPHAGEAL PAIN}

J. K. Ruffle ${ }^{1}$, S. J. Coen ${ }^{2}$, V. Giampietro ${ }^{3}$, S. C.r. Williams ${ }^{3}$, A. D. Farmer ${ }^{4}$ Q. Aziz ${ }^{1}$

${ }^{1}$ Centre For Neuroscience And Trauma, Blizard Institute, Wingate Institute Of Neurogastroenterology, Barts and the London School of Medicine \& Dentistry, Oueen Mary University of London, London/United Kingdom

${ }^{2}$ Research Department Of Clinical, Educational And Health Psychology, University College London, London/United Kingdom

${ }^{3}$ Institute Of Psychiatry, Psychology \& Neuroscience, Department Of

Neuroimaging, King's College London, London/United Kingdom

${ }^{4}$ Gastroenterology, University Hospitals of North Midlands, Stoke on Trent/United Kingdom

Contact E-mail Address: j.ruffle@qmul.ac.uk

Introduction: Visceral pain is a complex percept influenced by numerous factors. Of these, differences in the autonomic nervous system (ANS)-in particular, parasympathetic cardiac vagal tone (CVT)-has been suggested to have a physiological role in the regulation and modulation of painful sensory signalling, to the extent of vagal nerve stimulation (to raise subject CVT) being tested as a possible antinociceptive.

Aims \& Methods: To date, no studies have explored the brain functional connectivity or network properties of CVT in relation to a painful stimulus, and thus this was our aim. In 21 healthy participants ( 10 male; mean age 30 years (range 21-53 years)), we quantified resting CVT using a Neuroscope. For all subjects, functional MRI data were acquired using a 3T MRI scanner during painful oesophageal balloon distention, as described elsewhere(1). The effect of resting CVT on brain networks during acute oesophageal pain were determined by means of network based statistics(2). Brain nodes were selected a priori of previous autonomic/visceral pain literature and included the following: bilateral nucleus accumbens (NAc), amygdala, insula, pallidum, thalamus and single hypothalamus $\left(\sum^{\text {Nodes }}=11\right)(3)$. Blood oxygen level dependant (BOLD) signal during oesophageal balloon distention to pain tolerance threshold was extracted from each of these regions and cross-correlated to produce nodal correlation matrices of $55 r$ values. Participants were dichotomised by means of median split based upon resting CVT value, and a two-tailed $t$-test of was undertaken. A primary threshold was applied $(t=1.65 ; \mathrm{p}<0.05)$ and permutation tested 50,000 times to ensure statistical stringency applied.

Results: We identified a unique subcortical brain connectivity network in the high resting CVT individuals when exposed to acute oesophageal pain. This complex symmetrical network comprised all 11 nodes with a total of 18 edges (significant

Abstract No: P1167

Table 1: Associations between individual psychological traits and symptom severity. Numeric entries are odds ratios (SE $E_{\text {odds ratio }}$ ), *** indicates $p<.001$, ** indicates $p<0.01, *$ indicates $p<0.05$, and ${ }^{\wedge}$ indicates $0.05 \leq p \leq 0.1$.

\begin{tabular}{|c|c|c|c|c|c|c|}
\hline Predictor & $\begin{array}{l}\text { Bowel } \\
\text { Non-FGID }\end{array}$ & $\begin{array}{l}\text { Symptom } \\
\text { FGID }\end{array}$ & $\begin{array}{l}\text { Severity } \\
\text { Combined }\end{array}$ & $\begin{array}{l}\text { Epigastric } \\
\text { Non-FGID }\end{array}$ & $\begin{array}{l}\text { Symptom } \\
\text { FGID }\end{array}$ & $\begin{array}{l}\text { Severity } \\
\text { Combined }\end{array}$ \\
\hline Problem-focused coping & $2.92(1.23)^{*}$ & $0.93(0.14)$ & $1.16(0.15)$ & $1.00(0.44)$ & $1.03(0.15)$ & $1.12(0.15)$ \\
\hline Worry & $1.44(0.51)$ & $1.35(0.22)^{\wedge}$ & $1.60(0.22) * * *$ & $1.60(0.65)$ & $1.55(0.26)^{* *}$ & $1.71(0.24)^{* * *}$ \\
\hline Avoidant coping & $0.41(0.21)^{\wedge}$ & $0.89(0.14)$ & $0.91(0.12)$ & $0.97(0.39)$ & $0.93(0.15)$ & $0.99(0.13)$ \\
\hline Doctor relationship & $2.96(1.77)^{\wedge}$ & $1.09(0.16)$ & $1.06(0.14)$ & $1.00(0.54)$ & $1.03(0.16)$ & $0.97(0.13)$ \\
\hline Childhood non-sexual abuse & $1.65(0.68)$ & $1.06(0.15)$ & $1.33(0.17)$ & $1.94(0.88)$ & $1.05(0.15)$ & $1.32(0.17)^{*}$ \\
\hline Social support & $0.53(0.22)$ & $0.85(0.12)$ & $0.88(0.11)$ & $0.68(0.32)$ & $0.82(0.12)$ & $0.87(0.11)$ \\
\hline $\begin{array}{l}\text { Somatic rather than } \\
\text { psychological attribution }\end{array}$ & $0.27(0.11) * * *$ & $0.93(0.15)$ & $0.72(0.10)^{*}$ & $0.83(0.36)$ & $0.64(0.11)^{* *}$ & $0.63(0.09)^{* * *}$ \\
\hline Doctor reassurance & $0.99(0.31)$ & $1.16(0.26)$ & $0.94(0.14)$ & $1.06(0.34)$ & $1.49(0.33)^{\wedge}$ & $1.01(0.16)$ \\
\hline Somatisation & $2.92(1.23)^{*}$ & $0.93(0.14)$ & $1.16(0.15)$ & $0.30(0.30)$ & $1.27(0.18)^{\wedge}$ & $1.54(0.20)^{* * *}$ \\
\hline Childhood sexual abuse & $1.44(0.51)$ & $1.35(0.22)^{\wedge}$ & $1.60(0.22) * * *$ & $2.16(0.88)^{\wedge}$ & $1.11(0.18)$ & $1.16(0.16)$ \\
\hline
\end{tabular}

When considered jointly with other predictors, psychological attribution of symptoms was significantly positively related to both bowel symptom severity (non-FGID: $\mathrm{OR}=0.31, \mathrm{SE}=0.13$; Full: $\mathrm{OR}=0.74, \mathrm{SE}=0.11$ ) and epigastric symptom severity (FGID: $\mathrm{OR}=0.63, \mathrm{SE}=0.09$; Full: $\mathrm{OR}=0.63, \mathrm{SE}=0.11$ ). The same was the case for worry (Bowel: Full: $\mathrm{OR}=1.40, \mathrm{SE}=0.21$; Epigastric: FGID: $\mathrm{OR}=1.58, \mathrm{SE}=0.27$; Full: $\mathrm{OR}=1.54$, $\mathrm{SE}=0.23$ ). For bowel symptoms, problem-focused coping $(\mathrm{OR}=2.30, \mathrm{SE}=0.98)$ was an additional independent positive (notably, not negative) predictor among participants without FGIDs. 
functional connections). These interconnections included the following: thalamus-amygdala, thalamus-hypothalamus, hypothalamus-NAc, amygdala-putamen amygdala-NAc and insula-putamen. No significant network was identified for the low CVT group.

Conclusion: During acute oesophageal pain, resting cardiac vagal tone yields a unique functional brain network comprising numerous complex subcortical brain regions, many of which have been previously associated with either visceral pain or modulation of baseline autonomics either at the physiological or neuroanatomical level (3). Previous research has suggested that a high resting CVT may be protective of nociceptive signalling, and furthermore studies investigating vagal nerve stimulation have included and report that of anti-nociception (4). Given the well-established role of these subcortical regions in pain processing, we suggest that this network identified may be of significance as to the neurophysiological process of parasympathetic modulation of painful sensory signalling. Lastly, to date, no studies have undertaken real-time assessment of the ANS (including CVT) during functional brain imaging and acute visceral pain. Future studies should investigate for this.

Disclosure of Interest: All authors have declared no conflicts of interest

\section{References}

1. Ruffle, JK et al. The influence of extraversion on brain activity at baseline and during the experience and expectation of visceral pain. Pers. Individ. Dif. 2015;74:248-253.

2. Zalesky A et al. Connectivity differences in brain networks. Neuroimage. 2012;60(2):1055-62.

3. Ruffle JK et al. Brain morphological differences in gastrointestinal disorders may be in part due to alteration in resting autonomic function. Gastroenterology. 2017;152(5):S918-S9.

4. Farmer, AD et al. Electrical vagal nerve stimulation prevents the development of acid induced esophageal hyperalgesia. NGM. 2016;28:48.

\section{P1170 RAPID DRINK CHALLENGE (RDC) TEST DURING} OESOPHAGEAL HIGH RESOLUTION MANOMETRY (HRM) IN PATIENTS WITH OESOPHAGO-GASTRIC JUNCTION OUTFLOW OBSTRUCTION

D. Biasutto, F. Mion, A. Garros, S. Roman

Digestive Physiology, Lyon I University and Hospices Civils de Lyon, Lyon/France

\section{Contact E-mail Address: d.biasutto@unicampus.it}

Introduction: Oesophago-gastric junction outflow obstruction (OGJOO) is of unclear significance. It may be secondary to an incomplete form of achalasia, a mechanical obstruction or be idiopathic. Rapid drink challenge (RDC) test is easy to perform during oesophageal HRM.

Aims \& Methods: We aimed to assess the yield of RDC in patients with OGJOO. From a database of 3522 consecutive oesophageal HRM performed from 01/ 2012 to $03 / 2017$, we extracted patients with OGJOO according to the Chicago Classification v3.0. HRM protocol consisted of $105-\mathrm{ml}$ water swallows in supine position and RDC test (200-ml free drinking) in sitting position. Distal contractile integral (DCI) integrated relaxation pressure (IRP), distal and pan-oesophageal pressurization (POP; homogeneous oesophageal pressurization $>30 \mathrm{mmHg}$ ) were reported for 5-ml swallows. POP and oesophageal shortening (OS) were analysed during RDC. Symptom severity was assessed with Eckardt score. Causes of OGJOO were determined by reviewing patients' chart for previous history, complementary work up and treatment. Quantitative data were expressed as median (range) and qualitative data as percentage. They were compared using non parametric and Chi square tests.

Results: 75 patients $(2 \%)$ ( 29 males, mean age 62 years $(25-92))$ were included. The dominant symptom was dysphagia $(69 \%)$, regurgitation $(9 \%)$, chest pain $(5 \%)$, other $(13 \%)$, no symptom $(3 \%)$. The causes of EGJOO were previous oesophago-gastric surgery $(43 \%)$, incomplete achalasia $(7 \%)$, mediastinal neoplasia $(7 \%)$, miscellaneous $(19 \%)$ and unknown $(25 \%)$. RDC test was successfully performed in 70 patients $(93 \%)$ and associated with POP and OS in $41 \%$ and $13 \%$ respectively. Dysphagia as dominant symptom was more frequent $(79 \%$ vs $59 \%, \mathrm{p}=0.057)$ and more severe (Eckardt score $5(1-11)$ vs $3(0-10), \mathrm{p}=0.01)$ in patients with POP during RDC compared to those without. The same observation was achieved in patients with OS vs those without (dysphagia $100 \%$ vs 62 , $\mathrm{p}=0.02$ and Eckardt score $6(2-10)$ vs $1(0-11), \mathrm{p}=0.02)$. Manometric parameters are reported in the table. The causes of OGJOO were similar in patients with and without POP during RDC (previous oesophago-gastric surgery $34 \%$ and $51 \%$ respectively, achalasia $14 \%$ and $2 \%$, mediastinal neoplasia $3 \%$ and $7 \%$, miscellaneous $10 \%$ and $22 \%$, unknown $38 \%$ and $17 \%$ ). OS was not observed in patients with unknown cause of EGJOO vs $20 \%$ of patients with an identified cause $(\mathrm{p}=0.09)$.

\begin{tabular}{|c|c|c|c|c|}
\hline $\begin{array}{l}\text { Manometry } \\
\text { parameters } \\
\text { for 5-ml swallows }\end{array}$ & $\begin{array}{l}\text { POP during } \\
\text { RDC } \\
n=29\end{array}$ & $\begin{array}{l}\text { No POP } \\
\text { during RDC } \\
\mathrm{n}=41\end{array}$ & OS $n=9$ & No OS $n=61$ \\
\hline $\begin{array}{c}\text { Mean IRP }(\mathrm{mmHg}) \\
\text { Maximal IRP } \\
\text { (mmHg) } \\
\text { Mean DCI } \\
\text { (mmHg.s.cm) } \\
\text { Maximal DCI }\end{array}$ & $\begin{array}{l}19(15-35)^{*} \\
29(18-57)^{*} \\
2700 \\
(496-14492)^{*} \\
4536 \\
(639-42139)^{*}\end{array}$ & $\begin{array}{l}18(15-29) \\
24(16-78) \\
1384 \\
(109-16108) \\
2146\end{array}$ & $\begin{array}{l}24(16-35) \\
31(23-78) \$ \\
1503(593-4095) \\
2264(1036-10127) \\
20(0-70) 0(0-40)\end{array}$ & $\begin{array}{l}18(15-29) \\
25(16-57) \\
1671(109-16108) \\
3016(156-42139) \\
0(0-100) 0(0-100)\end{array}$ \\
\hline
\end{tabular}

\begin{tabular}{llll} 
: Continued & & & \\
\hline $\begin{array}{l}\text { Manometry } \\
\text { parameters } \\
\text { for 5-ml swallows }\end{array}$ & $\begin{array}{l}\text { POP during } \\
\text { RDC } \\
\mathrm{n}=29\end{array}$ & $\begin{array}{l}\text { No POP } \\
\text { during RDC } \\
\mathrm{n}=41\end{array}$ & OS $\mathrm{n}=9$ \\
\hline $\begin{array}{l}\text { (mmHg.s.cm) } \\
\text { \%distal }\end{array}$ & $20(0-100)^{*}$ & $(156-38846)$ & \\
$\begin{array}{l}\text { pressurization } \\
\text { \%POP }\end{array}$ & $0(0-100)$ & $0(0-100) 0(0-60)$ & \\
\hline
\end{tabular}

${ }^{*} \mathrm{p}<0.05$ vs patients without POP; $\mathrm{t} \mathrm{p}<0.05$ vs patients without OS

Conclusion: While RDC test cannot be used to determine EGJOO cause, patients with POP or OS during RDC had more severe dysphagia than those without. In patients with POP, the highest DCI might be secondary to obstruction. Further prospective studies should determine if RDC test could help to select patients who might benefit from treatment.

Disclosure of Interest: F. Mion: consulting for Medtronic

S. Roman: consulting for Medtronic; research support from Sandhill and Crospon

All other authors have declared no conflicts of interest.

\section{P1171 THE NORMATIVE VALUES OF A NEW 36 CHANNELS} WATER PERFUSION ESOPHAGEAL MOTILITY CATHETER

S. Bor, S. Kipcak

Gastroenrology Sec., Ege Reflux Study Group, Ege University, izmir/Turkey

Contact E-mail Address: kipcaksezgi@gmail.com

Introduction: High resolution manomety (HRM) is performed with solid-state catheters (SS) in many centers. However according to Chicago classification, very limited data from healthy volunteers are available for some catheters and starting from IRP, numerical values are crucial for the diagnosis. Because of the cost of the SS-HRM catheters many centers especially from developing countries use water perfusion HRM (W-HRM) catheters up to 24 channels and normal values are even more limited.

Aims \& Methods: We evaluated a prototype 36 channels W-HRM reused catheter allowing to measure 3 -D pressure vector volume analysis of lower esophageal sphincter in healthy volunteers and compared to 36 channels SS-HRM catheters (LaborieMMS Canada). We included 43 healthy volunteers without any upper gastrointestinal complaint. Upper gastrointestinal endoscopy and $24 \mathrm{~h}$ impedance-pH monitoring performed in all subjects. Four subjects were excluded because of silent GERD. 39 subjects were analysed $(25$ males, W-HRM $(n=39)$, SS-HRM $(\mathrm{n}=33))$. Thirty-three patients underwent two esophageal manometry studies within two consecutive days with a random order. Procedures were performed in supine position with receiving ten times $5 \mathrm{ml}$ water, five times solid food and multiple water swallow with $200 \mathrm{ml}$ of water. 36 channel water- perfused 3-D HRM catheter and 36 channel solid state HRM catheter were used (Laborie-MMS Canada).

Results: There was significant differences between two catheters in terms of Integrated Relaxation Pressure (IRP), Distal Contractile Integrale (DCI) and DCI- expanded, LES resting pressure, \% of ineffective peristalsis, and esophageal length both with water and solid food swallows (Table). No difference has been shown with distal latency (DL), LES length, breaks size (Table).

\begin{tabular}{|c|c|c|c|}
\hline & & $\begin{array}{l}36 \mathrm{ch} \text { solid } \\
\text { statemean } \pm \mathrm{SD}\end{array}$ & $\begin{array}{l}36 \text { ch water } \\
\text { perfusion } \\
(\text { mean } \pm \mathrm{SD})\end{array}$ \\
\hline & $\begin{array}{l}\text { Esophageal length } \\
\quad \text { (inspirium) }(\mathrm{cm})\end{array}$ & $23.1 \pm 2$ & $22.5 \pm 2$ \\
\hline \multirow[t]{4}{*}{$5 \mathrm{ml}$} & LES resting pressure $(\mathrm{mmHg})$ & $34.8 \pm 13$ & $17.4 \pm 7$ \\
\hline & IRP $(\mathrm{mmHg})$ & $17.6 \pm 5(7-25)$ & $6.5 \pm 4(0-18)$ \\
\hline & DCI $\left(\mathrm{mmHg}^{*} \mathrm{~s}^{*} \mathrm{~cm}\right)$ & $1281 \pm 759$ & $701 \pm 432$ \\
\hline & Mean $\%$ ineffective peristalsis & $15 \%$ & $28 \%$ \\
\hline \multirow{5}{*}{$\begin{array}{l}\text { solid } \\
\text { food }\end{array}$} & LES resting pressure $(\mathrm{mmHg})$ & $33.5 \pm 11$ & $17.6 \pm 7$ \\
\hline & IRP (mmHg) & $17.6 \pm 5$ & $6.4 \pm 4$ \\
\hline & DCI (mmHg*s*cm) & $1969.1 \pm 1051$ & $1159.9 \pm 836$ \\
\hline & DCI expanded $\left(\mathrm{mmHg}^{*} \mathrm{~s} * \mathrm{~cm}\right)$ & $2122.9 \pm 1117$ & $1171.1 \pm 895$ \\
\hline & Mean $\%$ ineffective peristalsis & $12 \%$ & $23 \%$ \\
\hline
\end{tabular}

Conclusion: Water perfusion HRM catheter exhibits significantly lower values especially for IRP, DCI, LES resting pressure. Centers which are working with water perfusion catheters should not accept universal normal established with solid state catheters and need to work on their normative values. The ineffective peristalsis pattern is common with water perfusion catheters. Disclosure of Interest: All authors have declared no conflicts of interest. 
P1172 LOW-VOLUME MULTIPLE RAPID SWALLOW BETTER DISTINGUISH PERISTALTIC ESOPHAGEAL RESERVE COMPARED TO HIGH-VOLUME RAPID DRINKING TEST

I. Martinucci $^{1}$, N. De Bortoli ${ }^{1}$, S. Tolone ${ }^{2}$, M. Furnari ${ }^{3}$, M. Frazzoni ${ }^{4}$, L. Frazzoni ${ }^{5}$, L. Fuccio ${ }^{5}$, M. Bellini ${ }^{1}$, V. Savarino ${ }^{3}$, R. Penagini ${ }^{6}$, S. Marchi ${ }^{1}$, E. Savarino

${ }^{1}$ Gastroenterology Unit, University of Pisa, Pisa/Italy

${ }^{2}$ Surgery, Second University of Naples, Naples/Italy

${ }^{3}$ Di.m.i., Gastroenterology Unit, DiMI, Gastroenterology Unit, University of Genoa., Genoa/Italy

${ }^{4}$ Digestive Pathophysiology Unit, Baggiovara Hospital, Modena/Italy

${ }^{5}$ Department Of Medical And Surgical Sciences, S. Orsola-Malpighi Hospital, Bologna/Italy

${ }^{6}$ Dipartimento Di Scienze Mediche, Università degli Studi di Milano Dipto. di Gastroenterologia, Milan/Italy

${ }^{7}$ Department Of Surgery, Oncology And Gastroenterology, University of Padua, Padua/Italy

Contact E-mail Address: martinucci.irene@gmail.com Introduction: The Chicago Classification V3.0 (CCv3) defined ineffective esophageal motility (IEM) by the presence of $50 \%$ or more of weak or failed peristaltic waves during high resolution manometry $(\mathrm{HRM})$. Both low-volume $(10 \mathrm{ml}) \mathrm{mul}$ tiple rapid swallow (MRS) and high-volume $(200 \mathrm{ml})$ rapid drinking test (RDT) have been suggested as test to recognize the esophageal peristaltic reserve. Which test might better represent the esophageal peristaltic reserve is still a matter of discussion.

Aims \& Methods: The aim of this study was to compare the diagnostic value of MRS and RDT in patients with IEM. From a larger group of patients evaluated for heartburn (twice/week with poor response to standard dose proton pump inhibitors), we enrolled consecutive patients with IEM and with functional heartburn $(\mathrm{FH})$. FH were enrolled as controls. IEM was defined according to the $\mathrm{CCv} 3$, and $\mathrm{FH}$ were defined according to the Rome IV criteria and with normal peristalsis. HRM was performed according to the Italian Guidelines. All patients underwent 3 MRS $(10 \mathrm{ml}$ of water in 5 swallows in less then $10 \mathrm{~s}$ ) and 1 RDT $(200 \mathrm{ml}$ of water freely drunk). All patients underwent 24-h impedance and $\mathrm{pH}$ recording (MII-pH). Mean DCI of MRS and DCI of RDT were compared with mean DCI of 10 single swallows (SS). The MRS/SS and RDT/SS ratio were calculated.

Results: We evaluated 30 patients with IEM (18 males and 12 females; mean age $45.7 \pm 11.4 \mathrm{yrs})$ and 30 patients with $\mathrm{FH}$ ( 13 males and 17 females; mean age $41.2 \pm 13.6$ ). The MII-pH showed higher acid exposure time (AET) and number of reflux events in IEM than in FH $(\mathrm{p}<0.05)$. Mean DCI of SS resulted lower in patients with IEM compared to FH $(\mathrm{p}<0.05)$. One-hundred and eighty MRS and 60 RDT were evaluated. DCI of MRS was lower than $450 \mathrm{mmHg}$-s-cm in $39 \%(35 / 90)$ of IEM patients, and in 7\% (6/90) of FH $(\mathrm{p}<0.05)$. DCI of RDT was lower than $450 \mathrm{mmHg}$-s-cm in $77 \%(23 / 30)$ of IEM patients, and in $50 \%(15 /$ $30)$ of FH $(p<0.05)$. The MRS/RDT ratio resulted $>1$ in both groups. All results are reported in Table 1.

Table 1: Results of SS, MRS and RDT in patients with IEM and FH

\begin{tabular}{llll}
\hline & IEM group & FH group & $p$ \\
\hline Mean DCI (mmHg-s-cm) & $508.7 \pm 318.6$ & $1493.7 \pm 799.2$ & 0.001 \\
Mean DCI MRS & $614.2 \pm 437.1$ & $1982.6 \pm 974.4$ & 0.001 \\
Mean DCI RDT & $506.9 \pm 311.5$ & $1269.2 \pm 1027.6$ & 0.001 \\
MRS/SS ratio & $1.2 \pm 0.4$ & $1.6 \pm 0.7$ & 0.008 \\
RDT/SS ratio & $0.9 \pm 0.3$ & $1 \pm 0.5$ & 0.351 \\
MRS/RDT & $1.3 \pm 0.4$ & $1.4 \pm 0.7$ & 0.499 \\
\hline
\end{tabular}

Conclusion: MRS is a more reliable test to detect peristaltic esophageal reserve when compared to RDT both in patients with IEM or those with normal esophageal peristalsis.

Disclosure of Interest: All authors have declared no conflicts of interest.

\section{P1173 HIGH-VOLUME RAPID DRINKING TEST BETTER DISTINGUSH ESOPHAGEAL BODY INHIBITION COMPARED TO LOW-VOLUME MULTIPLE RAPID SWALLOWS}

I. Martinucci $^{1}$, N. De Bortoli ${ }^{1}$, S. Tolone ${ }^{2}$, M. Furnari ${ }^{3}$, M. Frazzoni ${ }^{4}$,

L. Frazzoni ${ }^{5}$, L. Fuccio ${ }^{5}$, M. Bellini ${ }^{1}$, V. Savarino ${ }^{3}$, R. Penagini ${ }^{6}$, S. Marchi ${ }^{1}$, E. Savarino

${ }^{1}$ Gastroenterology Unit, University of Pisa, Pisa/Italy

${ }^{2}$ Surgery, Second University of Naples, Naples/Italy

${ }^{3}$ Di.m.i., Gastroenterology Unit, DiMI, Gastroenterology Unit, University of Genoa., Genoa/Italy

${ }^{4}$ Digestive Pathophysiology Unit, Baggiovara Hospital, Modena/Italy

${ }^{5}$ Department Of Medical And Surgical Sciences, S. Orsola-Malpighi Hospital, Bologna/Italy

${ }^{6}$ Dipartimento Di Scienze Mediche, Università degli Studi di Milano Dipto. di Gastroenterologia, Milan/Italy

${ }^{7}$ Department Of Surgery, Oncology And Gastroenterology, University of Padua, Padua/Italy

Contact E-mail Address: martinucci.irene@gmail.com
Introduction: The Chicago Classification (CC V3.0) defined the presence of esophago-gastric outflow obstruction (EGJ-OO) when the value of integrated releasing pressure (IRP) is higher than $15 \mathrm{mmHg}$ during high-resolution manometry (HRM). Both low-volume $(10 \mathrm{ml})$ multiple rapid swallow (MRS) and highvolume $(200 \mathrm{ml})$ rapid drinking test (RDT) were never evaluated to detect the inhibition of esophageal body in patients with EGJ-OO.

Aims \& Methods: The aim of this study was to compare the efficacy of MRS and RDT in evaluating the esophageal body inhibition during repetitive swallow in patients with EGJ-OO and in patients with functional heartburn $(\mathrm{FH})$ considered as control group. From a larger group of patients evaluated for dysphagia with negative upper endoscopy, we enrolled consecutive patients with EGJ-OO, and as control group, patients with functional heartburn $(\mathrm{FH})$ defined according to the Rome IV criteria. EGJ-OO was defined according to CC V3.0. HRM performed according to Italian guidelines. All patients underwent 3 MRS $(10 \mathrm{ml}$ of water in 5 swallow in less then $10 \mathrm{~s}$ ) and 1 RDT ( $200 \mathrm{ml}$ of water freely drunk). The mean DCI of MRS and the DCI of RDT were compared with DCI of 10 single swallows (SS) mean value. The MRS/SS and RDT/SS ratio were calculated. The lack of esophageal body inhibition during multiple swallow tests were defined if contraction was identified measuring $>3 \mathrm{~cm}$ using the $30 \mathrm{mmHg}$ isobaric contour tool.

Results: We evaluated 30 patients with EGJ-OO (18 females; mean age $49.5 \pm 12.4 \mathrm{yrs})$ and 30 patients with FH (17 females; mean age $41.2 \pm 13.6)$ Impedance and $\mathrm{pH}$ 24-h analysis was performed to select patients with $\mathrm{FH}$ (normal AET and number of reflux and lack of reflux-symptom correlation). During HRM the mean DCI resulted similar in patients with EGJ-OO compared to FH $(\mathrm{p}<0.839)$. One-hundred and eighty MRS and 60 RDT were evaluated. The lack of body inhibition was found in $11 \%(20 / 180)$ during MRS and in 53\% $(16 / 30)$ during RDT in EGJ-OO. No patients in FH showed lack of body inhibition during both MRS and RDT. All results are reported in Table 1.

Table 1: Results of SS, MRS and RDT in patients with EGJ-OO and FH

\begin{tabular}{llll}
\hline & EGJ-OO group & FH group & $p$ \\
\hline Mean DCI MRS & $1455 \pm 1436.1$ & $1982.6 \pm 974.4$ & 0.101 \\
Mean DCI RDT & $817.3 \pm 665.4$ & $1269.2 \pm 1027.6$ & 0.047 \\
MRS weak/failed (90) & $24 / 30$ & $2 / 4$ & N/A \\
RDT weak/failed (30) & $6 / 20$ & $3 / 12$ & N/A \\
MRS/SS ratio & $0.9 \pm 0.3$ & $1.6 \pm 0.7$ & 0.001 \\
$\begin{array}{l}\text { RDT/SS ratio } \\
\text { MRS complete } \\
\quad \text { body inhibition (\%) }\end{array}$ & $0.5 \pm 0.4$ & $1 \pm 0.5$ & 0.001 \\
$\begin{array}{l}\text { RDT complete } \\
\text { body inhibition (\%) }\end{array}$ & 89 & 100 & 0.001 \\
\hline
\end{tabular}

Conclusion: Patients with EGJ-OO showed less peristaltic reserve during MRS and RDT. The RDT evidenced more frequently lack of body inhibition in patients with EGJ-OO.

Disclosure of Interest: All authors have declared no conflicts of interest.

P1174 ESOPHAGEAL BOLUS FLOW METRICS ON PRESSURE FLOW ANALYSIS (PFA) OF ESOPHAGEAL HIGH RESOLUTION IMPEDANCE MANOMETRY (HRIM) IN GASTROESOPHAGEAL REFLUX DISEASE (GERD)

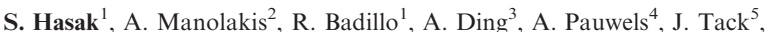
N. Rommel ${ }^{6}$, T. Omari ${ }^{7}$, C.P. Gyawali

${ }^{1}$ GI, Washington University in St. Louis, St. Louis/United States of America/MO ${ }^{2}$ Department Of Gastroenterology, School Of Medicine, University Of Thessaly, Larissa, Greece, University of Thessaly, Larissa/Greece

${ }^{3}$ University of California in San Diego, San Diego/United States of America/CA

${ }^{4}$ Targid, Katholieke Universiteit Leuven, Leuven/Belgium

${ }^{5}$ Gastroenterology, University of Leuven, University Hospital Gasthuisberg,

Leuven/Belgium

${ }^{6}$ University of Leuven, Leuven/Belgium

${ }^{7}$ University of Adelaide, Adelaide/Australia

Contact E-mail Address: hasak.s@wustl.edu

Introduction: Esophageal motor dysfunction and abnormal bolus transit are often encountered on high resolution impedance manometry (HRIM) performed prior to anti-reflux surgery (ARS) in gastroesophageal reflux disease (GERD). Esophageal motor dysfunction can be characterized using established software tools embedded in HRIM software, while new paradigms of interrogation of bolus presence and flow have been introduced with automated pressure flow analysis (PFA). The prevalence, and clinical relevance of these motor and bolus flow abnormalities to symptom presentation or symptom outcome from ARS are incompletely understood.

Aims \& Methods: Our aim was to evaluate the interrelationships between esophageal motor function and bolus transit using varying bolus consistencies, in health and in the context of symptomatic GERD prior to ARS. HRIM studies (Medtronic, Duluth, GA) from 18 controls $(28.4 \pm 1$ yr, 50\% F) and 86 GERD patients $(56.2 \pm 2 \mathrm{yr}, 58 \% \mathrm{~F})$ were reviewed, all of which were performed using $5 \mathrm{~mL}$ water (10 swallows), $5 \mathrm{~mL}$ viscous ( 5 swallows) and $1 \mathrm{~cm}^{3}$ bread boluses ( 2 swallows). All controls were asymptomatic; all GERD patients completed symptom questionnaires evaluating dominant and secondary symptoms using 5 point Likert scales for symptom frequency and severity $(0=$ none, $4=$ frequent 
or severe); the product of frequency and severity constituted the symptom score, assessed at baseline and after ARS. Automated PFA was performed for each test swallow by blinded investigators using purpose built software (Esophageal AIMplot, copyright T Omari) programmed in MatLab (The MathWorks Inc, Natick, MA, USA). The nadir impedance point (NI) indicating peak distension, and the contractile deceleration point (CDP) were determined. Latency was measured from NI to the contraction front at the CDP (distension to contraction latency, DCL). Intrabolus pressures (IBP) were measured during accommodation (IBP-A), compartmentalized transport (IBP-CT) and esophageal emptying (IBPEE). Bolus clearance in the esophageal body was assessed using the impedance ratio (IR) based on the relationship between NI and impedance at the contractile peak. Bolus flow at the esophagogastric junction (EGJ) was assessed using bolus flow time (BFT) in seconds when both pressure and impedance traces indicated a flow permissive paradigm. Univariate and multivariate analyses were performed to assess the relationships between bolus flow metrics and motor function, taking baseline symptom scores and symptom change following ARS into consideration. Results: Of 86 patients, $72.1 \%$ had typical symptoms. The mean baseline symptom score was $158.8 \pm 15.8$, with a mean decline of $139.9 \pm 15.8$ following ARS. Peak pressures in the proximal esophagus were lower in GERD patients compared to controls with all bolus consistencies $(\mathrm{p} \leq 0.007)$. Concurrently, metrics assessing esophageal bolus presence (NI) and clearance (IR) were consistently abnormal in GERD patients (Table, $\mathrm{p}=0.001$ for all bolus consistencies for each comparison to controls). Trans-EGJ bolus flow metrics were similarly abnormal in GERD (BFT, $\mathrm{p} \leq 0.001$ for all bolus consistencies compared to controls). IBP metrics were highest with solid swallows, especially in GERD patients compared to controls (Table). Within GERD, a gradient of increasing dysfunction in bolus presence and bolus clearance was noted, with least abnormalities with water swallows and the worst metrics with bread swallows $(\mathrm{p} \leq 0.02$ across groups for each comparison). IBP-EE $(\mathrm{p} \leq 0.05)$ and trans-EGJ BFT $(\mathrm{p} \leq 0.004)$ were higher during water and viscous swallows with atypical symptoms compared to typical symptoms. NI was consistently lower in patients reporting $\leq 75 \%$ symptom improvement following ARS with all bolus consistencies $(\mathrm{p} \leq 0.046)$.

Pressure Flow Analysis Comparisons Using Water, Viscous and Bread Swallows Between Controls and GERD Patients

\begin{tabular}{|c|c|c|c|c|c|c|}
\hline & \multicolumn{2}{|c|}{ water swallows } & \multicolumn{2}{|c|}{ viscous swallows } & \multicolumn{2}{|c|}{ bread swallows } \\
\hline & controls & GERD & controls & GERD & controls & GERD \\
\hline $\begin{array}{l}\text { Proximal peak } \\
\quad \text { pressure }(\mathrm{mmHg})\end{array}$ & $98.5 \pm 6$ & $75.2 \pm 3^{*}$ & $105.5 \pm 5$ & $84.7 \pm 2.5^{*}$ & $109.1 \pm 5$ & $91.4 \pm 3^{*}$ \\
\hline $\begin{array}{l}\text { Distal peak } \\
\quad \text { pressure }(\mathrm{mmHg})\end{array}$ & $72 \pm 9$ & $63.9 \pm 3$ & $75.0 \pm 8$ & $59.4 \pm 3^{*}$ & $68.9 \pm 7$ & $60.9 \pm 3$ \\
\hline $\begin{array}{l}\text { Nadir impedance } \\
\quad \text { (ohms) }\end{array}$ & $309 \pm 27$ & $882 \pm 59^{*}$ & $300 \pm 13$ & $633 \pm 45^{*}$ & $302 \pm 23$ & $650 \pm 40^{*}$ \\
\hline $\begin{array}{l}\text { Distension to } \\
\quad \text { contraction } \\
\text { latency (s) }\end{array}$ & $3.2 \pm 0.1$ & $3.4 \pm 0.3$ & $1.7 \pm 0.3$ & $2.0 \pm 1.0$ & $1.5 \pm 0.2$ & $1.6 \pm 0.2$ \\
\hline Impedance ratio & $0.3 \pm 0$ & $0.5 \pm 0$ * & $0.3 \pm 0$ & $0.4 \pm 0^{*}$ & $0.4 \pm 0$ & $0.5 \pm 0^{*}$ \\
\hline Distal IBP (mmHg) & $6.6 \pm 1.1$ & $8 \pm 0.5$ & $10.5 \pm 1.0$ & $13.4 \pm 0.6^{*}$ & $11.4 \pm 1.0$ & $15.3 \pm 0.9^{*}$ \\
\hline IBP-A $(\mathrm{mmHg})$ & $5.2 \pm 1.0$ & $5.1 \pm 0.5$ & $8.5 \pm 0.9$ & $8.6 \pm 0.5$ & $8.9 \pm 1.1$ & $12 \pm 1.0^{*}$ \\
\hline IBP-CT (mmHg) & $6.4 \pm 1.0$ & $8.7 \pm 0.5^{*}$ & $8.5 \pm 0.8$ & $9.5 \pm 0.5$ & $9.1 \pm 0.9$ & $11.6 \pm 0.6^{*}$ \\
\hline IBP-EE $(\mathrm{mmHg})$ & $10.3 \pm 0.8$ & $11.7 \pm 0.5$ & $12.5 \pm 0.8$ & $16.1 \pm 0.6^{*}$ & $13.1 \pm 0.8$ & $17.7 \pm 0.7 *$ \\
\hline Bolus flow time (s) & $4.1 \pm 0.5$ & $2.0 \pm 0.2 *$ & $3.8 \pm 0.5$ & $2.1 \pm 0.1^{*}$ & $3.3 \pm 0.4$ & $1.8 \pm 0.2 *$ \\
\hline
\end{tabular}

${ }^{*} \mathrm{p} \leq 0.04$ compared to controls. IBP: intrabolus pressure; IBP-A: IPB-accomodation; IBP-CT: IBP-compartmentalized transport; IBP-EE:IBP-esophageal emptying

Conclusion: Automated pressure flow analysis of HRIM studies demonstrates abnormalities of esophageal bolus transit and esophageal emptying in conjunction with lower proximal smooth muscle contractile function with all bolus consistencies in GERD compared to controls. Metrics assessing esophageal bolus presence may have prognostic value in GERD patients undergoing ARS.

Disclosure of Interest: N. Rommel: Holder of patent on AIM technology with Taher Omari

T. Omari: Holder of patent on AIM technology with Nathalie Rommel

C.P. Gyawali: Medtronic (research, speaker); Ironwood (consulting); Torax (consulting); Quintiles (consulting); Allergan (speaker)

All other authors have declared no conflicts of interest.

\section{References}

Carlson DA, et al. Neurogastroenterol Motil. 2017

Rommel N, et al. Neurogastroenterol Motil. 2014 May;26(5):636-45.

\section{P1175 PROVOCATIVE TESTING INCREASES THE DIAGNOSTIC YIELD OF HIGH RESOLUTION OESOPHAGEAL MANOMETRY IN PATIENTS WITH OESOPHAGEAL DIVERTICULA}

F. Wuestenberghs ${ }^{1}$, C.A. Melchior ${ }^{2}$, A.M. Leroi $^{2}$, G. Gourcerol ${ }^{2}$

${ }^{1}$ Hepato-gastroenterology, CHU UCL Namur, Yvoir/Belgium

${ }^{2}$ Digestive Physiology Unit, Rouen University Hospital, Rouen Cedex/France

Contact E-mail Address: fabien.wuestenberghs@uclouvain.be
Introduction: Oesophageal diverticula are rare diverticula of the gastrointestinal tract known to be associated with oesophageal motor disorders.

Aims \& Methods: The aim was to study manometric abnormalities associated with oesophageal diverticula, using both wet and solid swallows. Patients underwent high resolution oesophageal manometry (HRM) in the upright position. 18 patients with oesophageal diverticula were found and were free of previous surgery. Traction diverticulum was excluded in all patients. We also included 10 healthy controls. HRM was performed using wet $(5 \mathrm{~mL}$ of water) swallows in both groups, followed by solid (meat) swallows in patients. Mean age of the controls was 50 years old while it was 70 years old for patients.

Results: The main reported symptom was dysphagia $(76 \%)$. HRM found 11 $(61 \%)$ patients with an oesophageal motor disorder, including 2 oesophagogastric junction outflow obstruction [OGJOO], 4 achalasia (subtype 2: $n=2$; subtype $3: n=2)$, 4 distal oesophageal spasms [DES] and 1 jackhammer oesophagus, and was normal in $7(39 \%)$ patients. In those patients with normal findings, solid swallows identified $4(57 \%)$ additional motor disorders, including 2 OGJOO, 1 jackhammer oesophagus and 1 DES. Provocative testing using solid swallows increased the diagnostic yield by $22 \%$ in overall patients and by $57 \%$ in patients with normal manometry using wet swallows only. Mean pressure slopes at midoesophagus and distal oesophagus were greater in patients than healthy controls $\left(\mathrm{p}<0.05\right.$ for wet swallows), as previously reported ${ }^{1}$. Other metrics are summarized in the table.

HRM metrics with comparisons between wet swallows in controls and patients, and between wet and solid swallows among patients. * $\mathrm{p}<0.05 * * \mathrm{p}<0.01 * * * \mathrm{p}<0.00$

\begin{tabular}{llll}
\hline & $\begin{array}{l}\text { Controls } \\
\text { (liquids) }\end{array}$ & $\begin{array}{l}\text { Patients } \\
\text { (liquids) }\end{array}$ & $\begin{array}{l}\text { Patients } \\
\text { (solids) }\end{array}$ \\
\hline Number of swallows & 9.30 & 9.94 & 9.41 \\
EGJ resting pressure (mmHg) & 29.30 & 28.76 & 34.66 \\
Mean IRP 4s (mmHg) & 11.50 & 14.32 & $18.25^{*}$ \\
Mean DCI (mmHg.s.cm) & 1315.10 & 2877.99 & $7343.07^{* * *}$ \\
Distal latency (s) & 6.70 & 6.05 & $7.11^{*}$ \\
$\begin{array}{l}\text { Intrabolus pressure (mmHg) } \\
\text { Mean pressure slope }\end{array} \quad 8.10$ & $11.88^{*}$ & 15.19 \\
$\quad$ mid-oesophagus (mmHg/s) & -0.65 & $2.27^{* * *}$ & 2.59 \\
$\begin{array}{c}\text { Mean pressure slope distal } \\
\text { oesophagus (mmHg/s) }\end{array}$ & -0.36 & $1.41^{* *}$ & 0.46 \\
\hline
\end{tabular}

Conclusion: While more than one-third of HRM using wet swallows were normal, provocative testing using solid increased the diagnostic yield of the procedure by more than $50 \%$ in these patients. Analysis of pressure slopes confirmed the association of propagating peristalsis with the disease.

Disclosure of Interest: All authors have declared no conflicts of interest.

\section{Reference}

1. Carlson DA, Gluskin AB, Mogni B, et al. Esophageal diverticula are associated with propagating peristalsis: a study utilizing high-resolution manometry. Neurogastroenterol Motil 2016; 28: 392-398.

\section{P1176 EVALUATION OF ESOPHAGOGASTRIC JUNCTION CONTRACTILITY AFTER DIFFERENT TREATMENTS FOR ACHALASIA}

S. Tolone ${ }^{1}$, E. Savarino ${ }^{2}$, N. De Bortoli ${ }^{3}$, M. Frazzoni ${ }^{4}$, M. Furnari ${ }^{5}$, L. Frazzoni ${ }^{6}$, V. Savarino ${ }^{7}$, L. Docimo ${ }^{1}$, L. Swanström ${ }^{8}$

${ }^{1}$ Surgery, University of Campania "Luigi Vanvitelli",Naples, Naples/Italy

${ }^{2}$ Department Of Surgery, Oncology And Gastroenterology, University of Padua, Padua/Italy

${ }^{3}$ Division Of Gastroenterology, Department Of Internal Medicine, University of Pisa, Pisa/Italy

${ }^{4}$ Digestive Pathophysiology Unit, Baggiovara Hospital, Modena/Italy

${ }^{5}$ Di.m.i., Gastroenterology Unit, DiMI, Gastroenterology Unit, University of Genoa., Genoa/Italy

${ }^{6}$ Department Of Medical And Surgical Sciences, S. Orsola-Malpighi Hospital, Bologna/Italy

${ }^{7}$ Dept Internal Medecine, Universita di Genova, Genova/Italy

${ }^{8}$ University of Chicago, Chicago/United States of America

Contact E-mail Address: salvatore.tolone@unicampania.it

Introduction: The management of achalasia targets relieving the obstruction at the esophagogastric junction (EGJ) by pneumatic dilation (PD), laparoscopic Heller myotomy (LHM) plus a fundoplication variant (Dor, Toupet and more rarely Nissen/Nissen-Rossetti). However, effective ablation of the LES barrier can induce gastroesophageal reflux disease (GERD). Recently, new metrics to evaluate EGJ function with high resolution manometry (HRM) have been introduced, such as EGJ contractile integral (EGJ-CI). Currently there are few data investigating how achalasia treatments impact EGJ function based on these metrics.

Aims \& Methods: We aimed to assess the EGJ-CI metric in achalasia before and after different treatments, to verify if post-operative changes in this metric correlate to symptom relief and iatrogenic GERD following surgical treatments. Methods Between 2014 and 2015, we enrolled consecutive achalasia patients. All patients underwent clinical evaluation with Eckardt and GERDQ score, as 
well as upper endoscopy, barium esophagogram and HRM before and 6 months after treatment. Achalasia was classified according to the Chicago Classification V3.0. The EGJ-CI was calculated using the distal contractile integral tool-box during three consecutive respiratory cycles. Patients underwent to pneumatic dilatation (PD), or LHM plus a Dor (LHM-D), Toupet (LHM-T) or a NissenRossetti (LHM-NR) fundoplication. Ethical approval for the study was obtained.

Results: We enrolled 35 achalasia patients (14 Type I, 16 Type II and 5 Type III). Ten patients underwent PD, 11 LHMD, 8 LHM-T and 6 LHM-NR. At baseline, no differences among treatment groups regarding age, sex, pre-operative mean Eckardt score, GERDQ score, integral relaxation pressure (IRP) and EGJ-CI were recorded. All Type III subjects underwent LHM-D (3) and LHM-T (2). After all the procedures, in all the patients there was a significant decrease in Eckardt score, IRP and EGJ-CI ( $\mathrm{p}<0.001,<0.001$ and $<0.05$, respectively). $\mathrm{PD}$ and LHM-NR showed higher EGJ-CI $(20 \pm 9.3$ and $25.3 \pm 11.1 \mathrm{mmHg} * \mathrm{~cm}$, respectively) and IRP $(12.2 \pm 3.4$ and $13 \pm 4.5$, respectively) than LHM-D and LHM-T $(18.4 \pm 5.9, \mathrm{p}<0.05$ and $9.3 \pm 4.1 \mathrm{p}<0.05 \mathrm{mmHg} * \mathrm{~cm}$, respectively for EGJ-CI; $5.2 \pm 2.5, \mathrm{p}<0.05$ and $2.3 \pm 3.7 \mathrm{p}<0.001 \mathrm{mmHg} * \mathrm{~cm}$, respectively for IRP). Post-operative Eckardt score was lower in LHM-D and LHM-T $(2.1 \pm 0.5$ and $2.0 \pm 0.6$, respectively) than PD and LHM-NR $(4.2 \pm 1.0, \mathrm{p}<0.01$ and $3.7 \pm 1.5, \mathrm{p}<0.05)$. Post-operative GERDQ score was significant higher in LHM-T $(3.0 \pm 1.7$ vs. $8.2 \pm 3.9, \mathrm{p}<0.05)$. Low post-operative EGJ-CI values correlated with an increased risk of higher post-operative GERDQ score ( $\mathrm{p}<0.05$, odds ratio $4.223,95 \%$ CI $0.964-2.123$ ).

Conclusion: All procedures performed to treat achalasia produced an adequate relief of dysphagia. LHM-D and LHMT seem to result in a stronger alteration of the EGJ, with LHM-T resulting in an increased risk of post-operative reflux. Disclosure of Interest: All authors have declared no conflicts of interest.

\section{P1177 MULTIPLE RAPID SWALLOWING IN JACKHAMMER ESOPHAGUS PATIENTS: EVIDENCE FOR ALTERED NEURAI CONTROL}

A. Mauro ${ }^{1}$, S. Tolone ${ }^{2}$, E. Savarino ${ }^{3}$, N. De Bortoli ${ }^{4}$, M. Franchina ${ }^{1}$,

R. Penagini ${ }^{1}$

${ }^{1}$ Department Of Pathophysiology And Transplantation; Università Degli Studi Di Milano-Italy, Gastroenterology and Endoscopy Unit, Fondazione IRCCS Cà

Granda, Ospedale Maggiore Policlinico, Milano/Italy

${ }^{2}$ Division of General and Bariatric Surgery, Department of Surgery, Second University of Naples, Naples-Italy, Naples/Italy

${ }^{3}$ Division of Gastroenterology, Department of Surgery, Oncology and

Gastroenterology, University of Padua, Padua-Italy., Padua/Italy

${ }^{4}$ Division of Gastroenterology, Department of Translational Research and New

Technology in Medicine and Surgery, University of Pisa, Cisanello Hospital, Pisa/ Italy

\section{Contact E-mail Address: aURELIO.MAURO88@gmail.com}

Introduction: Jackhammer esophagus is a rare esophageal motility disorder. Little is known about its physiopathology; however, an excess of cholinergic drive has been suggested as an important etiologic factor. ${ }^{1}$ Multiple rapid swallowing (MRS) is an adjunctive test in order to evaluate integrity of inhibitory and excitatory neural pathways. In healthy subjects body motor inhibition is observed during MRS and a contraction stronger than single swallows (SS) occurs after MRS, the so-called peristaltic reserve (MRS/SS DCI ratio >1). In patients with nutcracker esophagus preservation of motor inhibition during MRS has been described with traditional manometry ${ }^{2}$. No study has evaluated peristaltic reserve and motor inhibition with high-resolution manometry (HRM) in patients with jackhammer esophagus.

Aims \& Methods: To evaluate MRS in a consecutive multicenter series of 42 Jackhammer esophagus patients (18 Male; 63 years; 55-71) according to Chicago 3 classification. 18 healthy subjects (HS) (seven male; 28 years; 23-33) from a published series were used as a control group. ${ }^{3}$ All patients underwent solid state HRM with ten $5 \mathrm{ml} \mathrm{SS}$ and one to three $10 \mathrm{ml}$ MRS (30 patients performed at least two MRS). Standard HRM parameters during SS were evaluated. During MRS presence/absence of motor inhibition and 4 second integrated relaxation pressure ( $4 \mathrm{sec}$ IRP) were evaluated. After MRS distal contractile integral (DCI) was evaluated and DCI ratio between MRS and SS was measured. Mann Whitney, Wilcoxon and chi-squared tests were used when appropriate; data are shown as median-IQ range.

Results: Descriptive data in jackhammer patients are shown in table 1. Twelve patients did not have motor inhibition during at least one MRS $(28 \%$ vs $5 \%$ in HS, $p<0.05)$. There was a trend toward a lower $4 \mathrm{~s}$ IRP during MRS compared to SS (see table 1); however, values were higher than those of 4s IRP MRS in HS $(5.1 \mathrm{mmHg} ; 2.2-11$ vs $1.6 \mathrm{mmHg} ; 0.3-2, \mathrm{p}<0.0001)$. MRS DCI was significantly lower than SS DCI; interestingly 26 patients had a MRS/SS DCI ratio $<1(62 \%$ vs $22 \%$ in HS, p < 0.005 ) and it was lower than the MRS/SS DCI ratio of HS
$(0.8 ; 0.4-1.1$ vs $1.9 ; 1.1-2, \mathrm{p}<0.0001)$ suggesting a reduction of the hypercontractile activity in the esophageal body.

HRM parameters during single and multiple rapid swallows in jackhammer patients. Median; interquartile range.

\begin{tabular}{llll}
\hline & SS & MRS & $\mathrm{p}$ value \\
\hline 4 s IRP, mmHg & $7.5 ; 4.9-13.1$ & $5.1 ; 2.2-10.1$ & $\mathrm{p}=0.06$ \\
DCI, mmHg.sec.cm & $6506 ; 5605-8582$ & $5537 ; 3568-8572$ & $\mathrm{p}<0.05$ \\
CFV, $\mathrm{cm} / \mathrm{s}$ & $3.9 ; 2.9-5.5$ & $4.4 ; 2.9-6.4$ & $\mathrm{p}=0.10$ \\
DL, sec & $6.8 ; 6.2-7.6$ & $6.8 ; 5.5-7.4$ & $\mathrm{p}=0.09$ \\
\hline
\end{tabular}

SS, single swallows; MRS, multiple rapid swallows; $4 \mathrm{sec}$ IRP, 4 second integrated relaxation pressure DCI, distal contractile integral; CFV, contractile front velocity; DL, distal latency.

Conclusion: Contrary to what occurs in healthy subjects, MRS reduce DCI value compared to SS in jackhammer esophagus patients, suggesting altered neural control of peristalsis. Differently to what previously observed with traditional manometry, motor inhibition during MRS is altered in a quarter of patients. Studies are needed in order to evaluate if reduction of DCI during MRS can improve dysphagia and chest pain in these patients.

Disclosure of Interest: All authors have declared no conflicts of interest.

\section{References}

1. Roman S, Neurogastroenterol Motil, 2012 mar;24 suppl 1: 32-9;

2. Brito EM, Am J Gastroeterol, 2003; 98 (1):40-5.

3. Elvevi A, 2015 Feb;47(2):103-7.

\section{P1178 GASTRIC PERORAL ENDOSCOPIC MYOTOMY (G-POEM) AS TREATMENT FOR FUNCTIONAL DELAYED GASTRIC EMPTYING: INITIAL ASIAN EXPERIENCE}

M. Cai ${ }^{1}$, J. Xu ${ }^{1}$, P. Zhou ${ }^{1}$

${ }^{1}$ Endoscopy Center, Zhongshan Hospital, Fudan University, Shanghai/China

Contact E-mail Address: cai.mingyan@zs-hospital.sh.cn

Introduction: Functional delayed gastric emptying is a difficult-to-treat disorder, which is often expressed clinically as nausea/vomiting, fullness/early satiety, bloating and weight loss. Gastric peroral endoscopic myotomy (G-POEM) has been regarded as a novel and minimally-invasive therapy for functional delayed gastric emptying refractory to medical therapy. We herein report our initial experience of G-POEM in an Asian population with focus on technique in addition to safety and efficacy of this promising endoscopic therapy.

Aims \& Methods: The data of consecutive patients who underwent G-POEM by a single expert endoscopist from October 2015 to November 2016 was collected. Procedures were performed, similar to POEM for achalasia, including initial mucosal incision, creating a submucosal tunnel, full-thickness (pyloro)myotomy, and closure of the mucosal entry. Patient demographics, etiology, Gastroparesis Cardinal Symptoms Index (GCSI) and gastric emptying scintigraphy (GES) were recorded before and after the procedure. Treatment outcomes and procedure related adverse events were also evaluated.

Results: A total of fourteen patients with refractory functional delayed gastric emptying, including eleven post-surgical $(78.6 \%)$ and three diabetic $(21.4 \%)$, were enrolled. The median age was 60 (range, 26-82) years. All patients were suffering from nausea, vomiting, bloating and weight loss. They all failed medical therapy including proton pump inhibitor, metoclopramide, mosapride, or domperidone. All fourteen patients underwent G-POEM successfully $(100 \%)$ with the mean procedure time of $43.71 \pm 13.08$ mins. Gastric emptying scintigraphy (GES) was performed in five patients with improvement of mean half empty time $(191.88 \pm 83.19 \mathrm{mins}$ vs. $91.44 \pm 32.92 \mathrm{mins})$, and retention at 2 hours $(70.02 \pm 12.68 \%$ vs. $33.48 \pm 20.32 \%)$. The median hospital stay after procedure was 6 days (range, $4-10)$. No procedure related adverse event $(0 \%)$ was observed. During a median follow-up of eight months (range, 3-17.5 months), one patient (post-surgical) had symptom recurrence after 45 days after the procedure because of stenosis related to scan formation. One of the two diabetic patients with severe diabetes and diagnosis as diabetic peripheral neuropathy showed little response to G-POEM, while the GCSI was 34 before and 28 after. The other showed initial improvement with GCSI score 22 before and 10 after during the first follow-up. However, his symptoms reoccurred seven months after G-POEM and the latest GCSI score was 19. Overall GCSI after G-POEM (mean, $5.00 \pm 2.57$ ) decreased significantly comparing with the one before (mean, 22.55 \pm 3.42 ).

Conclusion: G-POEM is a promising new endoscopic treatment option for functional delayed gastric emptying. Our initial data suggest it is safe and effective. Large studies are needed to determine safety, efficacy, long-term outcomes and determine which patient population benefits the most from this treatment option. Disclosure of Interest: All authors have declared no conflicts of interest. 


\section{P1179 EFFECTIVENESS OF CAP-ASSISTED DEVICE IN THE} ENDOSCOPIC MANAGEMENT OF FOOD BOLUS OBSTRUCTION IN THE UPPER ESOPHAGUS

M. Ooi, E. J. Young, N. Q. Nguyen

Department Of Gastroenterology And Hepatology, Royal Adelaide Hospital, Adelaide/Australia/SA

\section{Contact E-mail Address: marieooi_al@yahoo.com}

Introduction: Although cap-assisted technique has been shown to be effective in retrieving foreign bodies from the upper gastrointestinal tract, there is no data in food bolus obstruction (FBO). This study aimed to assess the performance of cap-assisted technique in the management of esophageal FBO, as compared to conventional endoscopic methods.

Aims \& Methods: Outcomes of all patients who presents with FBO requiring emergency endoscopic management between 2011 and 2016 were prospectively collected. The main measured outcomes were procedural success, procedural time, complications and length of hospital stay.

Results: Of the 315 patients (214M:102F; $58.3 \pm 18.3$ years) with FBO, $267(84.7 \%)$ had evidence of food bolus in the esophagus on endoscopy and $48(15.2 \%)$ patients had spontaneous passage of food bolus. Out of the 199 patients who had impacted FBO, 93 had cap-assisted technique and 106 had conventional approach, with no differences in the type and location of FB. The success rate of cap-assisted technique $(100 \%)$ was comparable to that of conventional techniques $(97.2 \%, \mathrm{P}=0.10)$. Patients who had failed extraction by conventional techniques $(n=3)$ were successfully treated when switched to cap-assisted approach. Cap-assisted approach was associated with a shorter total procedural time $(34 \pm 8$ and $43 \pm 22 \mathrm{~min}, \mathrm{P}=0.006)$, a shorter length of hospital stay $(0.95 \pm 0.56$ and $1.58 \pm 1.36$ days, $\mathrm{P}=0.0017)$ and more en-bloc removal $(87.3 \%$ vs $22.8 \%, \mathrm{P}<0.001)$. There were more complications in the conventional than the cap-assisted group $(7 / 106$ vs. $0 / 93 ; \mathrm{P}=0.01)$

Conclusion: Cap-assisted technique is $100 \%$ effective in the management of impacted $\mathrm{FBO}$ in the esophagus, with a significantly shorter procedural time and no complication. Although the findings suggest that cap-assisted technique should be the first line technique in the management esophageal FBO, further evaluation with a randomized multicenter trial is warranted.

Disclosure of Interest: All authors have declared no conflicts of interest.

\section{P1180 DO THE ENDOSCOPIC FINDINGS OF GASTRITIS BRING}

\section{THE FD SYMPTOMS}

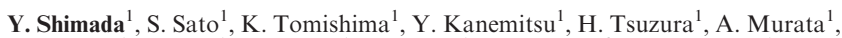
N. Amano ${ }^{1}$, S. Sato ${ }^{1}$, T. Genda ${ }^{1}$, K. Iijima ${ }^{1}$, M. Kusano ${ }^{2}$, A. Nagahara ${ }^{1}$ ${ }^{1}$ Gastroenterology And Hepatology, Juntendo University Shizuoka Hospital, Shizuoka/Japan

${ }^{2}$ Gastroenterology And Hepatology, Gunma University Hospital, Gunma/Japan

\section{Contact E-mail Address: yshimada@juntendo.ac.jp}

Introduction: Functional dyspepsia (FD) is defined that there is no evidence of organic disease which is likely to explain the symptoms. However, there was no obvious definition about "organic disease". Therefore, during the endoscopic examination, it happens that we can't judge whether obvious mucosal change like gastric erosion fulfill the exclusion criteria or not. Indeed, this issue was pointed out that there is notable inconsistencies in the exclusion criteria of FD (1). Recently, the Kyoto classification of gastritis has been published and used widely for the management of gastritis in Japan (2). In this classification, endoscopic findings are defined precisely. However, it is not discussed whether we can consider such findings as the "organic disease" or not. If these findings have no relation to FD symptoms, we can diagnose patients group as FD even if they have such endoscopic findings.

Aims \& Methods: The aim of this study is to explore which endoscopic findings defined in the Kyoto classification of gastritis bring FD symptoms. To assess the symptoms of patients, we employed the modified-Frequency of Scale for the Symptoms of GERD questionnaire (m-FSSG) (3), which was developed for evaluation of dyspeptic symptoms in patients. It comprised 14 symptoms and was rated on a scale of zero to four in each question. This questionnaire is useful to distinguish between NERD and FD because the m-FSSG contains seven question about reflux symptoms (question 1, 4, 6, 7, 9, 10 and 12) and seven questions about dyspeptic symptoms (question $2,3,5,8,11,13$ and 14). We investigated 19 endoscopic findings (atrophy, intestinal metaplasia, diffuse redness, spotty redness, mucosal swelling, enlarger fold, nodularity, foveolar-hyperplastic polyp, xanthoma, depressive erosion, regular arrangement of collecting venules: RAC, fundic gland polyp, red streak, raised erosion, hematin, erosion, patchy redness, map-like redness, multiple white and flat elevated lesions) were classified based on the definition in the Kyoto classification of gastritis. Data were extracted from the records of subjects who underwent upper gastrointestinal endoscopy at our department between April 2015 and March 2017. Of the 2,079 subjects analyzed, we focused on 255 subjects who answered m-FSSG at the time of endoscopy after excluding subjects diagnosed with organic disease (GERD, peptic ulcers and cancers) endoscopically. We also excluded subjects who answered had symptoms related acid reflux (score 3 or 4 ) in m-FSSG. We divided 255 subjects into FD group and Control group. FD group was defined as the subjects who had epigastralgia (score 2, 3 or 4), postprandial fullness (score 3 or 4) or early satiation (score 3 or 4 ) in m-FSSG. After that, we compared the rate of FD group of patients with or without findings in each 19 finding.

Results: This study included 55 subjects in FD group and 200 subjects in Control group. The ratio was statistically lower in men than women in FD group than in Control group $(43.6 \%$ vs. $61.0 \%, \mathrm{p}=0.02)$. Atrophy $(16.6 \%$ vs. $30.4 \%$, $\mathrm{p}=0.01)$, spotty redness $(9.1 \%$ vs. $23.0 \%, \mathrm{p}=0.02)$, hyperplastic polyp $(9.0 \%$ vs. $0 \%, \mathrm{p}=0.016)$, were frequently seen in Control group than in FD group.
RAC $(43.0 \%$ vs. $67.3 \%, p=0.002)$ were frequently seen in Control group than in FD group. Multivariate logistic regression (stepwise selection) showed that low age (under 65 years old) (OR 2.427, 95\% CI $0.209-0.812, \mathrm{p}=0.01$ ) and RAC (OR $2.123,95 \%$ CI $0.206-0.821, \mathrm{p}=0.012)$ were related to FD. None of the findings of gastritis examined were related to FD.

Conclusion: It is revealed that endoscopic gastritis is NOT related to FD symptoms.

Disclosure of Interest: All authors have declared no conflicts of interest.

\section{References}

1. Sugano K. Should we still subcategorize helicobacter pylori-associated dyspepsia as functional disease? J Neurogastroenterol Motil. 17: 366-371. 2011

2. Haruma K, et al. Kyoto classification of gastritis. Nihon Medical Center. Tokyo. 2014 (written in Japanese)

3. Kusano M, et al. Development and evaluation of a modified Frequency Scale for the Symptoms of Gastroesophageal Reflux Disease to distinguish functional dyspepsia from non-erosive reflux disease. J. Gastroenterol Hepatol. 27: 1187-91. 2012

\section{P1181 IMPACT OF EPIGASTRIC PAIN SYNDROME} ACCOMPANYING PANCREATIC ENZYME ABNORMALITIES EXHIBITED RAPID EARLY PHASE OF GASTRIC EMPTYING AND EARLY CHRONIC PANCREATITIS USING ENDOSONOGRAPHY

K. Kirita ${ }^{1}$, S. Futagami ${ }^{1}$, S. Agawa ${ }^{1}$, G. Ikeda ${ }^{1}$, H. Noda ${ }^{1}$, K. Higuchi ${ }^{1}$, T. Akimoto ${ }^{1}$, Y. Maruki $^{1}$, H. Yamawaki ${ }^{1}$, Y. Kodaka ${ }^{1}$, N. Ueki ${ }^{1}$, T. Kawagoe ${ }^{1}$, K. Iwakiri ${ }^{1}$

${ }^{1}$ Department Of Gastroenterology, Nippon Medical School, Tokyo/Japan

Contact E-mail Address: kumiko-k-21@nms.ac.jp

Introduction: There was not available data about the overlap between functional dyspepsia (FD) and pancreatic diseases.

Aims \& Methods: We aimed to determine whether epigastric pain syndrome (EPS) accompanying with pancreatic enzyme abnormalities were associated with early chronic pancreatitis proposed by Japan Pancreas Society (JPS) using endosonography. We enrolled 99 consecutive patients presenting with typical symptoms of FD, including patients with postprandial distress syndrome (PDS) $(n=59)$, EPS with pancreatic enzyme abnormalities $(n=41)$ and EPS without pancreatic enzyme abnormalities $(n=42)$ based on Rome III criteria. Gastric motility was evaluated using the ${ }^{13} \mathrm{C}$-acetate breath test. Early chronic pancreatitis was detected by endosonography and graded from 0 to 7 .

Results: The ratio of female patients among EPS patients $(34 / 41)$ with pancreatic enzyme abnormalities was significantly $(\mathrm{p}=0.0018)$ higher than the ratio of female EPS patients $(20 / 42)$ without it. Postprandial abdominal distention and physical component summary (PCS) scores in EPS patients with pancreatic enzyme abnormalities were significantly disturbed compared to those in EPS patients without it. Interestingly, $\mathrm{AUC}_{5}$ and $\mathrm{AUC}_{15}$ values $(24.85 \pm 1.31$ and $56.11 \pm 2.51$, respectively) in EPS patients with pancreatic enzyme abnormalities were also significantly $(\mathrm{p}=0.002$ and $\mathrm{p}=0.001$, respectively) increased compared to those $(19.75 \pm 1.01$ and $47.02 \pm 1.99$, respectively) in EPS patients without it. Overall, $64 \%$ of EPS patients with pancreatic enzyme abnormalities were diagnosed by endosonography as having concomitant early chronic pancreatitis proposed by JPS.

Conclusion: Further studies are warranted to clarify how EPS patients with pancreatic enzyme abnormalities were associated with early chronic pancreatitis proposed by JPS.

Disclosure of Interest: All authors have declared no conflicts of interest.

\section{P1182 ENDOSCOPIC AUTOLOGOUS TRANSPLANTATION OF ESOPHAGEAL MUCOSA FOR TREATING THE REFRACTORY CAUSTIC ESOPHAGEAL STRICTURE}

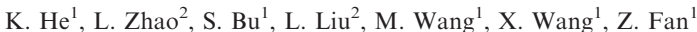

${ }^{1}$ The First Affiliated Hospital with Nanjing Medical Universtiy, nanjing/China ${ }^{2}$ Digestive Endoscopy Center, the First Affiliated Hospital of Nanjing Medical University, Nanjing/China

Contact E-mail Address: fanzhining@njmu.edu.cn

Introduction: Caustic esophageal injury is corrosive burns of esophagus and mostly caused by ingestion of chemical caustic substances such as strong acid or alkali. The caustic esophageal stricture is refractory and patients suffered from multiple dilation. Clinical ESD results revealed that benign stricture would be usually caused by more $75 \%$ mucosa loss of circumferential esophagus. Cultured skin tissue was effective for wound healing and preventing undesired fibrosis. We hypothesized that autologous esophageal mucosa transplantation might inhibit refractory fibroplasia and delay the recurrence of esophageal strictures.

Aims \& Methods: A man presented with the caustic esophageal stricture (32$40 \mathrm{~cm}$ from the incisors), which was caused by accidental exposure of anhydrous acetic acid. Under X-ray, routine endoscopic dilation was firstly applied to a diameter of $1.28 \mathrm{~cm}$. Three days later, with the informed consent, ESD operation was utilized to dissect $5^{*} 0.5 \mathrm{~cm}$ normal esophageal mucosa at the $18-23 \mathrm{~cm}$ location from the incisors. Mucosal defect was cured by fibrin glue after Argon plasma coagulation. Under endoscopic surveillance, the excised mucosa was transplanted to the surface of the narrowed segment and fixed with 3 titanium clips. 
Results: The endoscopic follow-up was planned every month. After 1 months, $18-23 \mathrm{~cm}$ esophageal region has healed to be normal. Within 6 months, the stricture process was excitingly delayed as expected. The patient stated his symptom was remarkably improved. Gastroscopy revealed the esophageal implanted lesion was covered with an epithelium and the luminal surface was flat, without ulceration.

Conclusion: Autologous esophageal mucosa transplantation might facilitate tissue re-epithelialization, reduce pathological fibroplasia, and be helpful for managing or preventing esophageal strictures. More clinical controlled trials are required to provide evidenced-based recommendation and promote its clinical application.

Disclosure of Interest: All authors have declared no conflicts of interest.

\section{P1183 FUNCTIONAL DYSPEPSIA IS STRONGLY ASSOCIATED WITH OBSTRUCTIVE SLEEP APNOEA-A CASE CONTROL STUDY}

R. N. Lui ${ }^{1}$, P. Cheong ${ }^{1}$, C. K. Cheung ${ }^{1}$, Y. Chan ${ }^{2}$, K. Yuen ${ }^{1}$, F. Fang ${ }^{1}$, J. C. Wu ${ }^{1}$ Department Of Medicine And Therapeutics, Chinese University of Hong Kong, Hong Kong/Hong Kong PRC

${ }^{2}$ Hong Kong Institute Of Integrative Medicine, Chinese University of Hong Kong, Hong Kong/Hong Kong PRC

\section{Contact E-mail Address: rashidlui@gmail.com}

Introduction: Functional dyspepsia (FD) is commonly associated with sleep disturbance, which has been attributed to comorbid anxiety, depression and bothersome gastrointestinal symptoms. However, it is unclear whether obstructive sleep apnoea (OSA) is specifically associated with FD.

Aims \& Methods: We aimed to compare the prevalence of FD in patients with OSA and healthy volunteers. A total of 60 consecutive OSA patients (defined as Epworth sleepiness scale $>=10$, and apnea-hypopnea index $>=10$ /hour during polysomnography; mean age: 47.6 years, male: $83.3 \%$ ) and 60 healthy age-andsex-matched volunteers were recruited in a prospective case-control study. Questionnaires were applied for the diagnosis of functional gastrointestinal disorders (FGIDs) according to Rome III criteria. Anxiety and depression were evaluated using the Hospital Anxiety and Depression Scale (HADS), sleep quality was evaluated by the Pittsburgh Sleep Quality Index, and fatigue was assessed by the Multidimensional Fatigue Inventory (MFI-20) Chinese version.

Results: The prevalence of FD was $28.3 \%$ and $8.3 \%$ (17 vs $5, \mathrm{P}=0.005)$, and the prevalence of symptomatic gastro-oesophageal reflux disease was $18.3 \%$ and $5 \%$ (11 vs $3, P=0.023$ ) in the OSA group and healthy volunteer group, respectively. OSA patients had higher anxiety and depression symptom scores, worse sleep quality, and more fatigue compared with healthy volunteers. In the multivariate logistic regression adjusting for age, sex and body mass index, OSA (odds ratio $4.93,95 \%$ CI $1.01-24.1, \mathrm{P}=0.049$ ) and depression (odds ratio $4.91,95 \% \mathrm{CI}$ $1.44-16.71, \mathrm{P}=0.011$ ) were independently associated with FD.

Conclusion: To the best of our knowledge, this is the first study showing that OSA is independently associated with functional dyspepsia. Sleep disturbances previously attributed to psychological comorbidities in FGID may in fact arise from undiagnosed OSA. We recommend screening for OSA as part of the management of FD.

Disclosure of Interest: All authors have declared no conflicts of interest.

\section{P1184 INDIVIDUAL ASSESSMENT OF GASTRIC ACID PRODUCTION BY MEANS OF A NON-INVASIVE TEST: RELATIONSHIP BETWEEN MAXIMAL ACID OUTPUT AND PEPSINOGEN I LEVELS}

F. Di Mario ${ }^{1}$, S. Scida ${ }^{2}$, C. Miraglia ${ }^{2}$, L. Franzoni ${ }^{2}$, M. Franceschi ${ }^{3}$, A. Bertelè ${ }^{4}$, R. Cannizzaro ${ }^{5}$, N. Dal Bò ${ }^{6}$, E. Savarino ${ }^{7}$, N. De Bortoli ${ }^{8}$, M. Rugge ${ }^{9}$,

C. Scarpignato ${ }^{2}$

${ }^{1}$ University Of Parma, Department of Clinical and Experimental Medicine, section of Gastroenterology, Parma/Italy

${ }^{2}$ Department Of Medicine And Surgery, University Of Parma, Italy, University of Parma, Parma/Italy

${ }^{3}$ Endoscopic Unit, Department Of Surgery, Ulss4, Hospital ULSS4 Alto Vicentino, Santorso/Italy

${ }^{4}$ Department Of Clinical \& Experimental Medicine, Clinical Pharmacology \&

Digestive Pathophysiology Unit, University of Parma, Parma/Italy

${ }^{5}$ Oncological Gastroenterology, Centro di Riferimento Oncologico di Aviano

S.O.C. di Gastroenterologia, Aviano/Italy

${ }^{6}$ Gastroenterological Unit, Treviso Hospita, Treviso/Italy

${ }^{7}$ Uo Gastroenterology Department Of Surgical, Oncological And

Gastroenterological Sciences, University Of Padova, Padova Italy, University of

Padova, Padova/Italy

${ }^{8}$ Division Of Gastroenterology, Department Of Internal Medicine, University of Pisa, Pisa/Italy

${ }^{9}$ Pathology, Medical School of the Padova University, Padova/Italy

\section{Contact E-mail Address: chiara.miraglia@studenti.unipr.it}

Introduction: The assessment of acid secretion is important in order to prescribe PPIs. The gold-standard to measure the maximal acid output (M.A.O.) is the collection of gastric after an i.m. injection of pentagastrin. However, this method is not currently used in clinical practice. Serum pepsinogen I (PGI) has been proposed as a non-invasive surrogate. Aim of this study was to compare in a group of patients with different acid related diseases serum levels of PGI and M.A.O.
Aims \& Methods: We enrolled 124 patients $(M=84$, mean age $=45.3+/-13.05$ range $=17-80)$ affected by chronic atrophic gastritis $(28, \mathrm{M}=17$, mean age 51.2 $+/-13.7$ ys range $20-73$ ) characterized by achlorhydria or low levels of acid production (Group 1), or by duodenal ulcer $(50, \mathrm{M}=42$, mean age $=43.5+/-$ 11.5 range $=17-65$ ) in which an hypersecretory status is claimed (Group 2). As control group, we studied 46 patients $(\mathrm{M}=44$ mean age $=44.0+/-13.4$ range $=25-80$ ) with normal upper GI endoscopy and gastric histology, without previous history of neoplasms or upper gastrointestinal surgery (Group 3). In all patients we measured M.A.O. by means of two hours collection of gastric juice (basal 30', followed by an i.m. injection of pentagastrin at the dosage of $6 \mu \mathrm{g} / \mathrm{kg}$ ) (M.A.O. normal values: 5-25 mEq/h). All patients underwent blood sample for determination of serum PGI (BioHit Ojy, Finland; normal values: 30-120 $\mu \mathrm{g} / 1$ ). All determinations, both for M.A.O. and PGI were made off medication.

Results: The mean M.A.O. value in Group 1 was $2.15 \mathrm{mEq} / \mathrm{h}$, in Group 252.49 $\mathrm{mEq} / \mathrm{h}$, in Group $317.48 \mathrm{mEq} / \mathrm{h}$. A statistically significant difference was found between the 3 groups (Group 1 vs. Group 2 p $<0.000001$; Group 1 vs. Group 2 $\mathrm{p}<0.0001$; Group 2 vs. Group $3 \mathrm{p}<0.0001)$. The PGI mean values in Group 1 was $11.39 \mu \mathrm{g} / 1$, in Group $2107.72 \mu \mathrm{g} / 1$, in Group $384.28 \mu \mathrm{g} / 1$ (Group 1 vs Group 2: $\mathrm{p}<0.000001 ;$ Group 1 vs Group 2: $\mathrm{p}<0.00001$; Group 2 vs Group 3 $\mathrm{p}<0.05)$. The relationship between M.A.O. and PGI showed a Pearson $\mathrm{R}=0.683(\mathrm{p}=0.0001)$. No statistically significant difference was found comparing M.A.O. and PGI in the single groups $(\mathrm{p}=\mathrm{ns})$.

Conclusion: Serum PGI levels are fitting with M.A.O. both in hypo- and hyperacid secretory conditions like chronic atrophic gastritis and duodenal ulcer, as well as in control subjects, suggesting that PGI could be adopted in clinical practice to assess gastric acid production in individual subjects for a proper management of acid related diseases.

Disclosure of Interest: All authors have declared no conflicts of interest.

\section{P1185 THE DISCREPANCY BETWEEN THE ACID REGURGITATION SYMPTOMS AND ENDOSCOPIC GERD GRADE IN DIABETES MELLITUS PATIENTS}

K. Sakitani, N. Suzuki

The Institute for Adult Life Diseases, Asahi Life Foundation, Tokyo/Japan

Contact E-mail Address: sakitani-tky@umin.ac.jp

Introduction: Gastroesophageal reflux disease (GERD) is caused by reflux of gastric acid into esophagus. It is reported that chronic inflammation caused by GERD can lead to precancerous changes; Barrett's esophagus. Thus, it could be critical to assess the GERD. Diagnosis of GERD is based on the subjective symptoms such as acid regurgitation. GERD is also diagnosed by endoscopy, and the Los Angeles (LA) classification is commonly used to grade reflux esophagitis endoscopically. It is well known that diabetes mellitus (DM) patients can develop neuropathy and their ability to feel pain can decrease. However, it is not known whether there is a discrepancy between the symptoms and endoscopic GERD grade in DM patients.

Aims \& Methods: From May 2015 to September 2016, patients who were taken esophagogastroduodenoscopy by experienced endoscopists at our institution were consecutively enrolled. All the patients completed the Gastrointestinal Symptom Rating Scale (GSRS); an interview-based rating scale consisting of 15 items including the question on acid regurgitation (Dig Dis Sci 1988). The GSRS has a seven-graded Likert type scale (score 1 represents absence of symptom, and score 7 represents very bothersome symptom). Gastric atrophy, reflux esophagitis, and esophageal hiatal hernia were diagnosed endoscopically. The study patients were categorized according to the grade of LA classification of GERD. The prevalence of patients with positive GSRS score (i.e. 2 or more) in the DM group and non-DM group were compared using the Pearson's chisquare test.

Results: A total of 2438 patients (647 females and 1791 males, mean age 62.4 years) were examined. Of these, 1040 patients $(42.7 \%)$ were DM, and 1398 patients $(57.3 \%)$ were non-DM patients. The prevalence of gastric atrophy, reflux esophagitis, and esophageal hiatal hernia were not different in DM group and non-DM group. Mild GERD (LA-A) was found in 251 patients $(10.6 \%, 92 \mathrm{DM}$ patients and 159 non-DM patients), and severe GERD (LA-B and more severe) was found in 76 patients $(3.12 \%, 40 \mathrm{DM}$ patients and 36 nonDM patients). Of the 36 non-DM patients with severe endoscopic GERD, 30 patients $(83.3 \%)$ had positive GSRS score of acid regurgitation; however, of the $40 \mathrm{DM}$ patients with severe endoscopic GERD, 19 patients $(47.5 \%)$ had positive GSRS score of acid regurgitation. The prevalence ratio of patients with positive GSRS score in severe endoscopic GERD was statistically lower in DM patients than in non-DM patients $(\mathrm{p}=0.0016)$.

Conclusion: There is a discrepancy between the subjective symptoms and endoscopic GERD grade in DM patients. DM patients' ability to feel acid regurgitation could decrease.

Disclosure of Interest: All authors have declared no conflicts of interest.

\section{Reference}

Dig Dis Sci. 1988 Feb;33(2):129-34. 
P1186 SARCOPENIA AS A LEADING RISK FACTOR FOR EROSIVE ESOPHAGITIS: A LARGE-SCALE CROSS-SECTIONAL STUDY

T. Kim, T.J. Kim, H. Lee, J.J. Kim

Department Of Medicine, Samsung Medical Center, Sungkyunkwan University School of Medicine, Seoul/Korea, Republic of

Contact E-mail Address: attack836@naver.com

Introduction: Obesity is an established risk factor for reflux esophagitis. Yet, the association of sarcopenia and obesity status with reflux esophagitis remains unclear. We studied the association between obesity, sarcopenia, and sarcopenic obesity and risk of reflux esophagitis in a large number of asymptomatic men and women.

Aims \& Methods: We conducted a cross-sectional study of 32,762 asymptomatic adults who underwent routine health check-ups including screening endoscopy from August 2006 to December 2011. Sarcopenia was defined as appendicular skeletal muscle mass (ASM)/body weight ( $\%$ ) value beyond two standard deviations below the mean for healthy young adults. Participants were categorized into four groups according to obese and sarcopenic status: normal, obese, sarcopenic, and sarcopenic obese.

Results: In a multivariate model adjusted for age, sex, smoking status, alcohol intake, regular exercise, and metabolic variables, risk of reflux esophagitis was higher in obese [adjusted odds ratio(aOR), 1.38; 95\% confidence interval (CI), 1.26-1.52], sarcopenic (aOR, 2.20; 95\% CI, 1.48-3.29), and sarcopenic obese participants (aOR, 1.68; 95\% CI, 1.39-2.03) than in normal participants. The ORs comparing sarcopenic and sarcopenic obese participants to obese participants were $1.59(95 \%$ CI, $1.06-2.38)$ and 1.22 (95\% CI, 1.02-1.47), respectively. In addition, the risk of reflux esophagitis according to sarcopenic and obese status was observed similarly in all subgroups that were evaluated. Conclusion: Our findings suggest that sarcopenia, regardless of obesity, is more harmful condition for reflux esophagitis than obesity without sarcopenia. Disclosure of Interest: All authors have declared no conflicts of interest.

\section{P1187 FOOD RATE (FREQUENCY AND TIME SPENT IN EATING) IN PATIENTS WITH MORBID OBESITY. DIFFERENCES AND SIMILARITIES BETWEEN MEN AND WOMEN}

J. Perez De La Serna ${ }^{1}$, A. Ruiz De León ${ }^{1}$, S. Ayllón Cano ${ }^{1}$, C. Sevilla Mantilla ${ }^{1}$, A. Sánchez Pernaute ${ }^{2}$, E. Rey ${ }^{1}$, B. Merchan Gomez ${ }^{1}$

${ }^{1}$ Gastroenterology, Hospital Clínico San Carlos, Madrid/Spain

${ }^{2}$ Surgery, Hospital Clínico San Carlos, Madrid/Spain

Contact E-mail Address: ayllonsonia@hotmail.com

Introduction: Obesity is considered one of the 21 st century plagues in the Western world. Although its etiology is multifactorial, eating habits represent an important factor in its development. (1). The caloric load, the proportion of its components and its patterns have been the object of multiple studies (1-5). One of the most controversial aspects is the relationship between the frequency of meals and body weight.

Aims \& Methods: The aim of the study was to determine the food rhythm (frequency and time spent in eating) in patients with morbid obesity (MO), based on data from the diary of the $24-\mathrm{H}$ esophageal $\mathrm{pH}$-monitoring studies.

This was a retrospective study, including 100 patients ( 77 women), with MO in whom bariatric surgery was indicated and 118 non-obese subjects from the control group of the Spanish Digestive Motility Group.

All were submitted to esophageal manometry and $24-\mathrm{H}$ esophageal $\mathrm{pH}$-monitoring according to the usual technique. During pHmetry it was recommended that the diet be similar as usual.

The relationship of demographic aspects with the number of meals and the time invested in them, based on data from the $\mathrm{pH}$ meter was analyzed.

Statistical analysis was performed with the STATGRAPHICS Centurion program. The limit of statistical significance was set at $\mathrm{p}<0.05$.

The study was approved by the ethics committee of our hospital. The informed consent of the participants was obtained for the performance of the tests.

Results: The mean age of patients with MO was 43.1 years (range 19-68) with no difference between men and women. The mean BMI was 41.55 (M 43.3; F 41, $\mathrm{P}<0.05)$

In the 118 subjects of the control group ( 63 women), the mean age was 28.3 years (range: 18-72 years), BMI 23.2, (M 24.2; F 22.3, P < 0.05). Age and BMI differences between controls and obese were statistically significant.

The number of meals is higher in the obese patients than in the controls. $76.3 \%$ of the controls performed $\leq 4$ meals. In obese patients this percentage was $56 \%$. The mean time spent at each meal showed statistically significant differences between controls $(20.5 \pm 7.7 \mathrm{~min})$ and obese $(17.7 \pm 7.25 \mathrm{~min})$ and between controls and obese women $(17.8 \pm 7.2 \mathrm{~min})$.

In MO, the relationship between BMI and the number of meals shows a higher BMI in those who do less number of meals, with significant differences between those who perform $\leq 3$ meals and those that perform $\geq 4$. This difference is set at the expense of men and not achieved statistical significance in women.

Conclusion: - MO patients eat more times a day than controls, this behavior is more evident in women.

- MO patients eat faster than controls without differences between men and women.

- Our results show an inverse relationship between the number of meals and body mass index. This behavior is more evident in men.

Disclosure of Interest: All authors have declared no conflicts of interest.

\section{References}

1. Reséndiz Barragán AM, Hernández Altamirano SV, Sierra Murguía MA, Torres Tamayo M. Hábitos de alimentación de pacientes con obesidad severa. Nutr Hosp. 2015;31(2):672-681.

2. Varela Moreiras G, Serrano Iglesias M, Alonso Aperte E, García González A, Achón y Tuñón M. Alimentación y Sociedad en la España del siglo XXI. 2015, 20-22 FUNDACIÓN MAPFRE. https://www.fundacionmapfre.org fundacion/es es/salud-prevencion/publicaciones-y-estudios/estudios/salud informe-alimentacion-sociedad-espana-siglo-xxi.jsp.

3. Alegre AP, Centurión DA, Larzabal MT, Schafer JY. SOBREPESO Y OBESIDAD, RELACIÓN CON LA FRECUENCIA DE COMIDAS TRABAJO FINAL DE INVESTIGACION. http://www.barcelo.edu.ar/ uploads/contenido/b4bdfb934aa61de11a57d63e47e75c13.pdf

4. Colombo P, Mangana M, Bianchi PA, Penagini R. Effect of calories and fat on postprandial gastro-oesophageal reflux. Scand $J$ Gastroenterol 2002;37(1):3-5

5. Fox M, Barr C, Nolan S, Lomer M, Anggiansah A, Wong T. The effects of dietary fat and calorie density on esophageal acid exposure and reflux symptoms. Clin Gastroenterol Hepatol 2007;5(4):439-44.

\section{P1188 ONE DRINK CAN INCREASE A RISK FOR ESOPHAGEAL, STOMACH AND COLORECTAL CANCER IN A COHORT OF 23,323,730 KOREAN ADULTS}

Y.J. Choi, D.H. Lee

Internal Medicine, Seoul National University Bundang Hospital, Seongnam/Korea, Republic of

Contact E-mail Address: oliv00e@naver.com

Introduction: Epidemiologic findings of low-volume alcohol consumption in relation to gastrointestinal cancers including gastric cancer are inconsistent.

Aims \& Methods: The association between alcohol intake and esophageal, gastric and colorectal cancer risk was examined in a population-based prospective cohort of 23,323,730 adults in Korea who had undergone a biennial evaluation provided by the National Health Insurance Corporation between the years 2009 and 2012.

Results: After median 5.4 years of follow-up, 9171 esophageal, 135,382 gastric and 154,970 colorectal cancer cases were identified. Cox proportional hazards regression models were used to estimate hazard ratios (HR) and corresponding $95 \%$ confidence intervals $(95 \% \mathrm{CI})$. Light drinking as well as moderate to heavy alcohol consumption significantly increased the risks of the three gastrointestinal cancers (HR 1.51; 95\% CI, 1.43-1.60; HR 1.08; 95\% CI, 1.06-1.09; HR 1.12; $95 \%$ CI, 1.11-1.14) compared with non-drinkers after adjusting for age, sex, smoking, exercise, income, body mass index, and diabetes. For esophageal cancer, there was a dose-dependent linear relationship. However, no association was observed between prediagnostic alcohol consumption and all causemortality.

Conclusion: Light drinking including even one alcoholic drink a day is associated with increased risks of esophageal, gastric and colorectal cancer.

Disclosure of Interest: All authors have declared no conflicts of interest.

\section{P1189 THE INFLUENCES OF VISCERAL FAT AREA ON THE SITES OF ESOPHAGEAL MUCOSAL BREAKS AND SYMPTOM SEVERITIES IN SUBJECTS WITH GASTROESOPHAGEAL REFLUX DISEASES}

E. Cho, J.H. Kim

Internal Medicine, Dongguk University College of Medicine, Goyang/Korea, Republic of

Contact E-mail Address: kimjaehak@dumc.or.kr

Introduction: Some studies have suggested the central obesity as a risk factor for gastroesophageal reflux diseases (GERD). However, the associations between visceral adipose tissue (VAT) and the sites of esophageal erosions or the symptom severities of GERD have not been studied yet.

Aims \& Methods: The aim of this study was to evaluate the influences of visceral fat area on the locations of erosions and symptoms of GERD. The subjects who underwent abdomen computerized tomography and esophagogastroduodenoscopy for routine checkup at the same day were collected from January 2007 to October 2016. 177 subjects who had erosive esophagitis (LA class A to D) were enrolled. Questionnaires including gastrointestinal symptoms were written before examinations. The abdominal obesity was evaluated by measuring visceral adipose tissue (VAT), subcutaneous adipose tissue (SAT), ratio of VAT to SAT, total adipose tissue (TAT), body mass index (BMI) and waist circumference (WC).

Results: Lesser curvature (LC) side of esophagogastric junction (EGJ) was the most frequent site of mucosal breaks (103 cases, $58.2 \%)$ followed by posterior wall side ( 71 cases, $40.1 \%$ ), anterior wall side ( 25 cases, $14.1 \%$ ) and fundus side $(16$ cases, $9.0 \%)$. Mucosal breaks in LC side were frequently observed in male subjects $(61.3 \%$ vs. $36.4 \%, \mathrm{p}=0.04)$. BMI $(25.6 \pm 4.5$ vs. $24.2 \pm 3.1, \mathrm{p}=0.019)$ and WC $(89.0 \pm 11.8$ vs. $85.0 \pm 9.1, \mathrm{p}=0.01)$ were significantly higher in LC group. Moreover, VAT, ratio of VAT to SAT, and TAT were significantly higher in LC group. In the multivariate analysis, a higher VAT area (odds ratio (OR) $3.47,95 \%$ confidence interval 1.38 to $8.73,1^{\text {st }}$ quartile vs. $4^{\text {th }}$ quartile, $\mathrm{p}<0.01$ ) and ratio of VAT to SAT (OR $2.99,95 \%$ CI 1.15 to $6.70,1^{\text {st }}$ quartile vs. $4^{\text {th }}$ quartile, $\mathrm{p}=0.02$ ) were strongly associated with the mucosal breaks in LC 
side. However, TAT was not significant in the multivariate analysis. Lower HDL-cholesterol levels (OR $0.28,95 \%$ CI 0.11 to $0.67, \mathrm{p}<0.01$ ) and much coffee consumption (OR 2.50, 95\% CI 1.06 to $5.86, \mathrm{p}=0.035$ ) were associated with the severities of GERD.

Conclusion: Mucosal breaks in LC side of EGJ were associated with visceral obesity measured by VAT, ratio of VAT to SAT, BMI and WC. Life style modification such as in left decubitus sleeping position might be emphasized in the subjects with visceral obesity.

Disclosure of Interest: All authors have declared no conflicts of interest.

\section{P1190 A LESS COMPETENT OESOPHAGO-GASTRIC JUNCTION IS ASSOCIATED WITH OESOPHAGEAL ACID HYPERSENSITIVITY EVEN IN HEALTHY CONTROLS}

C. Lottrup ${ }^{1}$, A. L. Krarup ${ }^{1}$, P. Ejstrud ${ }^{2}$, B. P. Mcmahon $^{3}$, A. M. Drewes ${ }^{1}$ ${ }^{1}$ Mech-sense, Department Of Gastroenterology And Hepatology, Aalborg University Hospital, Aalborg/Denmark

${ }^{2}$ Surgery A, Aalborg University Hospital, Aalborg/Denmark

${ }^{3}$ TAGG Research Centre, Tallaght Hospital and Trinity College, Dublin/Ireland

Contact E-mail Address: chlo@rn.dk

Introduction: In normal subjects, the oesophago-gastric junction (OGJ) sphincter complex maintains a tight barrier between the oesophagus and stomach acid However, gastro-oesophageal reflux disease (GORD) caused by acidic reflux has a prevalence of up to $26 \%$ [1]. One major factor determining whether gastrooesophageal reflux occurs and eventually generates symptoms is the competency of the OGJ, which can be studied using distensibility testing. This way, we have previously shown in patients with Barrett's oesophagus and healthy controls that an incompetent sphincter function was associated with more frequent reflux symptoms [2]. In the same patient groups, we also found greater oesophageal acid exposure and lower mucosal baseline impedance to be associated with impaired sphincter function. The latter probably represents a proxy for mucosal damage [3]. Other factors known to increase the perception of gastrooesophageal reflux episodes are greater acidity, larger volume, and more proximal extent of the reflux content along with impaired mucosal integrity and sensitisation (peripheral and central) [1]. All of this said no studies of our knowledge have specifically addressed the possible association between sphincter function of the OGJ and oesophageal sensitivity.

Aims \& Methods: We aimed to characterize oesophageal sensitivity in relation to OGJ competence, hypothesizing that sensitivity increases with impaired sphincter function. Twenty-three patients with Barrett's oesophagus (mean age: $64.2 \pm 7.7$ years) and 12 healthy controls (mean age: $54.9 \pm 10.8$ years) were examined. A standard upper endoscopy to locate the OGJ was followed by distensibility testing of the OGJ using the EndoFLIP probe. At a later visit, experimental oesophageal sensitivity was assessed using a multimodal stimulation probe. After placement in the oesophagus just above the OGJ, the probe allows the filling and emptying of an attached polyurethane bag with water, stimulation with electrical current, and infusion of acid. Using this probe, mechanical distension of the bag, thermal stimulation at increasing temperature, electrical stimulation, and acid perfusion with $0.1 \mathrm{M}$ hydrochloric acid (a Bernstein test) were performed. All stimulations were stopped when the subject felt moderate pain, equal to seven on a $0-10$ visual analogue scale validated for visceral pain. Data were analysed using multi-level, mixed-effects regression analysis in Stata 12.

Results: Oesophageal acid sensitivity increased with a more incompetent sphincter function: A lower tolerated acid volume was associated with greater distensibility index $(P=0.03)$ and with lower pressure $(P=0.03)$ in the OGJ in all subjects analysed together and separately in healthy controls $(P=0.006$ and 0.01 respectively). Performing separate analyses in patients with $\mathrm{BO}$, these associations were not present (all $P>0.7$ ). Sphincter function was associated with neither oesophageal sensitivity to mechanical, heat, nor electrical stimulation (all $P>0.13$ ).

Conclusion: Oesophageal acid sensitivity increased with a more incompetent OGJ. Based on this and previous findings, we suggest that even in some healthy controls, a modest degree of OGJ incompetence allows gastric acid to reflux. This may again lead to low-grade oesophageal inflammation and mucosal damage, thus evoking acid hypersensitivity. The latter mechanism probably constitutes a reflex protective mechanism towards acid reflux.

Disclosure of Interest: B.P. McMahon: Barry P McMahon holds a minor share in Crospon Inc., Galway, Ireland who manufactures the EndoFLIP probe.

All other authors have declared no conflicts of interest.

\section{References}

1. Herregods TVK, Bredenoord AJ, Smout AJPM. Pathophysiology of gastroesophageal reflux disease: new understanding in a new era. Neurogastroenterol Motil. 2015 Sep;27(9):1202-13.

2. Lottrup C, McMahon BP, Ejstrud P, et al. Esophagogastric junction distensibility in hiatus hernia. Dis Esophagus. 2016 Jul;29(5):463-71.

3. Lottrup C. Barrett's oesophagus - functional properties, acid clearance, and sensitivity. $\mathrm{PhD}$ thesis, Arhus University; 2016.
P1191 THE MUCOSAL INTEGRITY IN PHENOTYPES OF GASTROESOPHAGEAL REFLUX DISEASE AND FUNCTIONAL HEARTBURN

P. Ergun, S. Kipcak, S. Bor

Gastroenterology Sec., Ege Reflux Study Group, Ege University, Izmir/Turkey

Contact E-mail Address: pelinergun@yahoo.com

Introduction: Three different phenotypes of gastroesophageal reflux disease (GERD) such as erosive reflux (ERD), nonerosive reflux (NERD), esophageal hypersensitivity $(\mathrm{EH})$ and functional heartburn $(\mathrm{FH})$ might have different pathophysiological changes within the esophageal epithelium and the data is limited. Aims \& Methods: We aim to investigate the electrophysiological differences and diffusion characteristics as a reflection of tissue integrity using Ussing chamber system. Distal esophageal mucosal biopsies from 14 healthy controls $(5$ men, $40.6 \pm 11.2$ years) and 62 patients with GERD (40 men, $42.9 \pm 12.3$ years, $\mathrm{n}=26 \mathrm{LA}$ grade $\mathrm{A} / \mathrm{B}, \mathrm{n}=8 \mathrm{LA}$ grade $\mathrm{C} / \mathrm{D}, \mathrm{n}=22 \mathrm{NERD}, \mathrm{n}=6 \mathrm{EH})$ and 11 patients with FH were studied from November 2015 until March 2017. GERD and quality of life questionnaires, high-resolution esophageal manometry, $24 \mathrm{~h}$ impedance-pH monitoring, upper gastrointestinal endoscopy with esophageal biopsies were performed in all patients. Biopsies were put into the chambers to measure the transepithelial resistance (TEER), potential difference (PD) and tissue permeability via fluorescein diffusion within two hours as well as evaluation of dilated intercellular spaces with light microscopy.

Results: Esophageal biopsies of healthy volunteers $(163.6 \pm 41.1 \mathrm{ohms})$ had significantly higher TEER when compared to total GERD patients $(132.5 \pm 38.7$ ohms). Although the TEER results of whole GERD subtypes were decreased compared to healthy controls, only ERD groups were significantly lower $(123.3 \pm 29.8 \mathrm{ohms})$ (Table 1). There was also no significant difference in any parameters between NERD, FH and EH groups. The mucosal permeability of GERD subtypes was significantly higher than the healthy controls. The PPIunresponsive subjects $(\mathrm{n}=10,94.8 \pm 36.5$ pmols $)$ were much more permeable to fluorescein compared to PPI-responsive subjects $(n=52,56.0 \pm 32.4$ pmols $)$ within all GERD patients $(\mathrm{p}=0.009)$.

Table 1

\begin{tabular}{llll}
\hline GROUPS & TEER (Ohms) & PD (V) & $\begin{array}{l}\text { PERMEABILITY } \\
\text { (pmols) }\end{array}$ \\
\hline Healthy Controls & $163.6 \pm 41.1$ & $2.2 \pm 0,9$ & $43.9 \pm 16.6$ \\
GERD (total) & $132.5 \pm 38.7^{* *}$ & $2.6 \pm 1.5$ & $62.2 \pm 35.8^{*}$ \\
Esophageal & $150.6 \pm 23.9$ & $2.2 \pm 1.0$ & $71.8 \pm 34,5$ \\
$\quad$ Hypersensitivity & & & \\
NERD & $139.6 \pm 50.2$ & $2.2 \pm 1.3$ & $65.6 \pm 39,2^{*}$ \\
ERD (total) & $123.3 \pm 29.8^{*}$ & $3.0 \pm 1.6^{* * \varnothing}$ & $58.3 \pm 34.7^{* *}$ \\
ERD grade A/B & $130.5 \pm 27.8^{*}$ & $2.9 \pm 1.6$ & $54.0 \pm 30.5$ \\
ERD grade C/D & $105.8 \pm 32.7^{*}$ & $3.2 \pm 1.6$ & $72.6 \pm 43.6$ \\
Functional Heartburn & $145.3 \pm 42.7$ & $1.9 \pm 0.9$ & $67.0 \pm 35.2$
\end{tabular}

Conclusion: The TEER and permeability results imply that ERD and NERD groups showed a barrier disruption. However, epithelial permeability was not different in EH and FH groups. The dilatation of intercellular spaces may contribute to increased mucosal permeability in true-NERD and ERD patients. EH and FH patients might have different pathophysiology than others.

Disclosure of Interest: All authors have declared no conflicts of interest.

\section{P1192 PEPSIN AND PH LEVELS OF HUMAN GASTRIC JUICES IN GASTROESOPHAGEAL REFLUX DISEASE SUBGROUPS AND} FUNCTIONAL HEARTBURN

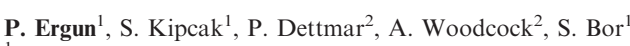

${ }^{1}$ Gastroenterology Sec., Ege Reflux Study Group, Ege University, Izmir/Turkey ${ }^{2}$ RD Biomed Limited, Hull/United Kingdom

Contact E-mail Address: pelinergun@yahoo.com

Introduction: The major noxious agents of gastroesophageal reflux disease (GERD) on the esophageal epithelium are gastric acid and pepsin. Nevertheless, there is no precise information about pepsin concentrations in gastric juice.

Aims \& Methods: We aim to address the pepsin values and $\mathrm{pH}$ results among subtypes of GERD and functional heartburn. 46 patients with GERD (23 erosive reflux disease LA grade A/B (ERD-A/B), 5 ERD-C/D, 14 nonerosive reflux disease-NERD, 4 esophageal hypersensitivity-EH), 8 functional heartburn (FH) and 17 healthy controls (HC) were included into the study. Upper gastrointestinal endoscopies were performed off PPI. Patients were instructed not to aspirate the local anaesthetic solution and biopsy channel of the endoscope was dried before the suction. The gastric juices from the subjects were aspirated during endoscopy into a special beaker and their $\mathrm{pH}$ values were measured immediately. The specimens were analysed using the Peptest lateral flow device (RD Biomed Ltd UK), a colorimetric assay containing two unique human monoclonal antibodies that capture and detect pepsin protein.

Results: There were no significance between pepsin levels in any GERD phenotypes, FH and healthy controls (Table 1). The $\mathrm{pH}$ results of patients with ERD $(1.8 \pm 0.6)$ were significantly lower versus $\mathrm{HC}(2.6 \pm 1.5)$. The $\mathrm{pH}$ levels of the 
esophageal hypersensitivity $(1.5 \pm 0.2)$ were significantly decreased when compared to HC $(2.6 \pm 1.6)$ and also true NERD $(4.0 \pm 2.0)$.

Table 1

\begin{tabular}{lll}
\hline & Pepsin $(\mathrm{ng} / \mathrm{ml})$ & $\mathrm{pH}$ \\
\hline ERD (total) & $514.7 \pm 282.1$ & $1.8 \pm 0.6^{\prime \prime}$ \\
ERD-A/B & $521.0 \pm 284.9$ & $1.8 \pm 0.6$ \\
ERD-C/D & $485.5 \pm 299.2$ & $2.1 \pm 1.0$ \\
Total NERD & $456.9 \pm 322.1$ & $3.5 \pm 2.1$ \\
True NERD & $428.1 \pm 293.0$ & $4.0 \pm 2.0$ \\
EH & $536.0 \pm 432.1$ & $1.5 \pm 0.2^{* \alpha}$ \\
GERD (total) & $494.5 \pm 294.1$ & $2.4 \pm 1.6$ \\
FH & $654.2 \pm 300.4$ & $2.1 \pm 1.1^{¥}$ \\
HC & $596.2 \pm 302.8$ & $2.6 \pm 1.5$ \\
\hline
\end{tabular}

Conclusion: Pepsin may be considered a damaging factor in pathophysiology of GERD, but we could not find any difference between GERD phenotypes and unaffected controls. NERD group had less gastric acid versus other groups but this finding needs more studies to confirm.

Disclosure of Interest: All authors have declared no conflicts of interest.

\section{P1193 NON INVASIVE DIAGNOSIS OF UPPER GI DISEASES IN A PRIMARY CARE SETTING: A STUDY ON 1,900 PATIENTS}

F. Di Mario ${ }^{1}$, S. Cesario ${ }^{1}$, S. Grillo ${ }^{1}$, S. Landi ${ }^{1}$, G. Baldassarre ${ }^{2}$, R. Cannizzaro ${ }^{3}$, P. Crafa ${ }^{1}$, M. Riccó ${ }^{4}$, L. Franzoni ${ }^{1}$, C. Miraglia ${ }^{1}$, S. Scida ${ }^{1}$, C. Scarpignato ${ }^{1}$, M. Rugge ${ }^{5}$, M.F. Roberti ${ }^{1}$

${ }^{1}$ Department Of Medicine And Surgery, University Of Parma, Italy, University of Parma, Parma/Italy

${ }^{2}$ Digestive Endoscopy Unit, ULSS4 Alto Vicentino, Santorso/Italy

${ }^{3}$ Oncological Gastroenterology, Centro di Riferimento Oncologico di Aviano

S.O.C. di Gastroenterologia, Aviano/Italy

${ }^{4}$ Local Health Unit Of Parma, Public Health Department, Occupational Health And Safety Service, Parma, Italy, University Hospital, Parma (Italy), Parma/ Italy

${ }^{5}$ Pathology, Medical School of the Padova University, Padova/Italy

Contact E-mail Address: simone.grillo89@gmail.com

Introduction: Gastropanel ${ }^{\circledR}$ is a non invasive test suggested able to perform a kind of "serological biopsy" of gastric mucosa. Aim of the study was to assess a proper diagnosis of different upper gastrointestinal (GI) diseases in a population suffering from upper GI disturbances in a primary care settings by means of serology.

Aims \& Methods: We enrolled 1900 consecutive patients $(M=769$; mean age $=56.4$ ys, range $=29-78$ ys) showing upper GI troubles. Exclusion criteria: upper GI surgery, alarm symptoms. All patients underwent a blood sample for Gastropanel® (BioHit Oyj, Finland): pepsinogen I (PGI), pepsinogen II (PGII), gastrin-17 (G-17) and IgG against Helicobacter Pylori (Hp-IgG). The normal values: PGI: $30-120 \mu \mathrm{g} / \mathrm{L}$, PGII: $2-15 \mu \mathrm{g} / \mathrm{L}, \mathrm{G}-17$ : 1-9 pmol/L, Hp-IgG: <30 $\mathrm{U} / \mathrm{L}$. The diagnosis of $\mathrm{Hp}$-related non-atrophic gastritis was made by means of the levels of PGII $>10 \mu \mathrm{g} / \mathrm{L}$ and $\mathrm{Hp}-\mathrm{IgG}>30 \mathrm{U} / \mathrm{L}$; the diagnosis of gastroesophageal reflux disease (GERD) was made when G-17 was low: $<2 \mathrm{pmol} / \mathrm{L}$; the diagnosis of chronic atrophic gastritis (CAG) was made when PGI was $<30 \mu \mathrm{g} / \mathrm{L}$ and $\mathrm{G}-17>14 \mathrm{pmol} / \mathrm{L}$. Finally when all the four parameters were normal, the subjects were classified as normal. All patients underwent upper GI endoscopy to support the serological diagnosis, with appropriate gastric biopsy according with OLGA classification.

Results: Four hundred and eighty eight patients were classified as affected by $\mathrm{Hp}$ related non-atrophic gastritis $(26 \%) ; 782$ patients were classified as GERD patients $(41 \%) ; 547$ patients were classified as normal $(29 \%) .83$ patients were classified as CAG (4\%). In $96 \%$ out of the 488 patients with Hp-related nonatrophic gastritis the features was confirmed by gastric histology (OLGA 0, 1, 2); in $91 \%$ out of the 782 patients diagnosed as GERD subjects, the diagnosis was confirmed by oesophagitis at endoscopy in 313 patients, positive Demeester score in 170 out of 221 patients at 24 hours pH-metry or presence of typical symptoms (heartburn and/or regurgitation) in 547 patients. The great majority of 83 patients in which the diagnosis was CAG showed a picture of OLGA 3 $(56.6 \%)$ or OLGA $4(24.2 \%)$

Conclusion: Non-invasive diagnosis of upper-GI diseases when alarm symptoms are absent seems to be promising to improve the appropriateness of endoscopy, as well as to address subjects affected by $\mathrm{Hp}$ infection to the cure and GERD patients to the therapy for reflux. The diagnosis of a precancerous condition like CAG before performing endoscopy could address to a better bioptic sampling Disclosure of Interest: All authors have declared no conflicts of interest.
P1194 CHANGES IN ANTHROPOMETRIC AND METABOLIC PARAMETERS RELATED TO GASTROESOPHAGEAL REFLUX DISEASE IN NON-OBESE CASES

S. Yavuz ${ }^{1}$, Ö. Gül Utku ${ }^{1}$, A. Gungunes ${ }^{2}$, N. Dindar Badem ${ }^{3}$, B. Ergul ${ }^{1}$, D. Oğuz ${ }^{1}$ ${ }_{1}^{1}$ Gastroenterology, Kır1kkale Universty, k1rkkale/Turkey

${ }^{2}$ Endocrinology, Kır1kkale Universty, kır1kkale/Turkey

${ }^{3}$ Biochemistry, Kirlkkale Universty, kır1kkale/Turkey

Contact E-mail Address: ozlemgulx@yahoo.com

Introduction: Prevalence of obesity and the obesity-related diseases have been increasing in recent years(1). In the studies conducted there are views claiming that visceral fat-related central obesity causes digestion system diseases and increase in the number of gastroesophageal reflux symptoms in particular (1-6). Aims \& Methods: In this study, in the cases of non-obese (Body mass index $(\mathrm{BMI})<30$ ), it is aimed to assess the effects of insulin resistance and the changes observed in the anthropometric and bioelectrical impedance measurements upon the reflux symptoms. Our study $120 \mathrm{BMI}<30$ dyspeptic non-obese, gastroesophageal reflux disease (GERD) diagnosed according to FSSG scale (Frequency scale for the symptoms of gastroesophageal reflux disease) and 50 BMI $<30$ non-dyspeptic, non-obese and not (GERD) diagnosed according to FSSG cases have been included. The cases included in the study concerned have been surveyed by means of the questionnaire including the demographic data and the extra esophageal reflux symptom. Serum biochemistry analyses (fasting glucose, insulin, lipid panel, uric acid, TSH, ALT) have been checked. Waist circumference has been measured. Body compositions and anthropometric measurements have been assessed through the bioelectrical impedance method (TANITA).

Results: In this study a statistically significant difference $(\mathrm{p}<0.05)$ has been found when GERH-diagnosed group is compared with the healthy control group in regard to waist circumference, BMI; LDL, Fat, Fat Mass, Total Body Water(TBW), obesity level, reflux score, acid reflux score and total score measurements. Fat free mass (FFM), muscle mass, bone mass, bone mineral density (BMR) measurments in between the both groups have not been found stattistically significant difference $(\mathrm{p}>0.05)$ (Table 1$)$. Considering the extra esophageal reflux symptoms a significant difference $(p<0.05)$ between the group suffering from sore throat, apnea, teeth grinding and GORH and the healthy control group has been found. Within the patients group a positive correlation between acid reflux score and BMI $(r=0.298)(\mathrm{p}<0.001)$, LDL $(r=0.387)(p<0.001)$, visceral fat $(r=0.180)(p<0.049)$ has been determined. A negative correlation between acid reflux score and TBW $(r=-0.273)$ $(\mathrm{p}<0.003)$ has been determined.

Table 1: Metabolic parameters and bioelectrical impedans findings

\begin{tabular}{|c|c|c|c|c|}
\hline & $\begin{array}{l}\text { Control Group } \\
(\mathrm{n}=50) \\
\text { Median } \\
(\text { Min.-Max.) }\end{array}$ & $\begin{array}{l}\text { Patient Group } \\
(\mathrm{n}=120) \\
\text { Median } \\
\text { (Min.-Max.) }\end{array}$ & $\begin{array}{l}\text { Total Number } \\
(\mathrm{N}=170) \\
\text { Median } \\
\text { (Min.-Max.) }\end{array}$ & P Value \\
\hline Glucose & $90.50(77-165)$ & $92(53-165)$ & $91.50(53-165)$ & 0.508 \\
\hline Insulin & $8.05(1.90-93)$ & $8.35(1.32-108)$ & $8.20(1.32-108)$ & 0.553 \\
\hline HDL & $51.50(3.10-99)$ & $47.50(3.10-90)$ & $48.50(3.10-99)$ & 0.066 \\
\hline LDL & $74.50(42-129)$ & $87(11-243)$ & $80(1-243)$ & 0.035 \\
\hline Triglyceride & $81(33-350)$ & $97(28-404)$ & $95(28-404)$ & 0.184 \\
\hline Total Cholesterol & $146.50(73-222)$ & $161(19-310)$ & $159(19-310)$ & 0.216 \\
\hline Uric Acid & $4.05(1.20-41)$ & $4.30(1.20-59)$ & $4.20(1.20-59)$ & 0.471 \\
\hline TSH & $1.68(0.07-7.56)$ & $1.54(0.02-10)$ & $1.58(0.02-10)$ & 0.476 \\
\hline ALT & $12.50(5-39)$ & $13(5-65)$ & $13(5-65)$ & 0.213 \\
\hline Fat & $19.30(3-41.80)$ & $24.75(3-95.20)$ & $22.55(3-95.20)$ & 0.016 \\
\hline Fat Mass & $12.80(1.10-36.20)$ & $15.15(1.10-43.40)$ & $14.20(1.10-43.40)$ & 0.012 \\
\hline FFM & $45.95(19-75.10)$ & $46.05(36.80-74.20)$ & $45.95(19-75.10)$ & 0.613 \\
\hline Muscle Mass & $43.95(35-70.50)$ & $43.90(34.90-516)$ & $43.90(34.90-516)$ & 0.520 \\
\hline TBW & $32.70(25.10-52.60)$ & $32.50(25.10-52.60)$ & $32.55(25.10-52.60)$ & 0.341 \\
\hline TBW Yüzde & $55.80(41.60-80.90)$ & $52.50(41.60-80.90)$ & $53.80(41.60-80.90)$ & 0.018 \\
\hline Bone Mass & $2.40(1.90-3.70)$ & $2.40(1.90-4)$ & $2.40(1.90-4)$ & 0.442 \\
\hline BMR & $5.858(55.94-9.138)$ & $5.851 .50(78.12-8.996)$ & $5.858(55.94-9.138)$ & 0.586 \\
\hline Metabolic Age & $16(12-64)$ & $27(12-66)$ & $22(12-66)$ & 0.002 \\
\hline Visceral Fat & $1(1-12)$ & $3(1-10)$ & $3(1-12)$ & $<0.001$ \\
\hline Degree of Obesity & $0.70(-29.90-35.90)$ & $10.95(-29.90-35.90)$ & $8.80(-29.90-35.90)$ & 0.015 \\
\hline Homa-IR (mg/dl) & $1.90(0.42-19.06)$ & $1.84(0.27-22.93)$ & $1.88(0.27-22.93)$ & 0.495 \\
\hline Acid Reflux Score & $13.50(5-23)$ & $15(8-23)$ & $14(5-23)$ & 0.020 \\
\hline Reflux Score & $7(7-7)$ & $19(9-35)$ & $15(7-35)$ & $<0.001$ \\
\hline BMI & $22.43 \pm 3.43$ & $23.79 \pm 3.60$ & $23.39 \pm 3.59$ & $<0.022$ \\
\hline $\begin{array}{l}\text { Waist } \\
\quad \text { Circumference }\end{array}$ & $74.63 \pm 5.62$ & $79.94 \pm 10.60$ & $78.38 \pm 9.71$ & $<0.001$ \\
\hline Total Score & $20.50(12-30)$ & $33(17-53)$ & $29(12-53)$ & $<0.001$ \\
\hline
\end{tabular}

Mann Whitney U Test(Monte Carlo) - Min.:Minimum - Max.:Maximum

Conclusion: The frequency and severity of gastroesophageal reflux symptoms in the non-obese is closely related with body fat composition as those in the obese. Increase in abdominal and visceral fat composition may cause high risk of gastroesophageal reflux disease in individuals irrespective of their obesity.

Disclosure of Interest: All authors have declared no conflicts of interest. 


\section{References}

1. Sogabe, M., et al., Influence of metabolic syndrome on upper gastrointestinal disease. Clin J Gastroenterol, 2016. 9(4): p. 191-202.

2. El-Serag, H.B., et al., Obesity is an independent risk factor for GERD symptoms and erosive esophagitis. The American journal of gastroenterology, 2005. 100(6): p. 1243-1250.

3. Sakaguchi, M., et al., Obesity as a risk factor for GERD in Japan. Journal of gastroenterology, 2008. 43(1): p. 57-62.

4. Corley, D.A. and A. Kubo, Body mass index and gastroesophageal reflux disease: a systematic review and meta-analysis. The American journal of gastroenterology, 2006. 101(11): p. 2619-2628.

5. El-Serag, H.B., et al., Obesity increases oesophageal acid exposure. Gut, 2007. 56(6): p. 749-755.

6. El-Serag, H.B., et al., Anthropometric correlates of intragastric pressure. Scandinavian journal of gastroenterology, 2006. 41(8): p. 887-891.

\section{P1195 PROXIMAL ESOPHAGEAL BASELINE IMPEDANCE LEVELS ARE ABLE TO DISCRIMINATE BETWEEN SCLERODERMA PATIENTS WITH AND WITHOUT ESOPHAGEAL INVOLVEMENT}

M. Della Coletta ${ }^{1}$, P. Zentilin ${ }^{2}$, E. Marabotto ${ }^{3}$, S. Tolone ${ }^{4}$, N. De Bortoli ${ }^{5}$, G. Bodini ${ }^{6}$, V. Savarino ${ }^{2}$, E. Savarino ${ }^{1}$

${ }^{1}$ Division Of Gastroenterology, Department Of Surgery, Oncology And

Gastroenterology, University of Padua, Padua/Italy

${ }^{2}$ Dept. Of Internal Medicine, University of Genoa, Genova/Italy

${ }^{3}$ University of Genoa, Genoa, Italy, Genoa/Italy

${ }^{4}$ Surgery, Second University of Naples, Naples/Italy

${ }^{5}$ Division Of Gastroenterology, Department Of Internal Medicine, University of Pisa, Pisa/Italy

${ }^{6}$ Department Of Internal Medicine, IRCCS San Martino DIMI, Genova/Italy

Contact E-mail Address: marcodellacoletta@gmail.com

Introduction: Esophageal baseline impedance (BI) levels have been recently proposed as a marker of mucosal integrity. Indeed, patients with non-erosive reflux disease (NERD) showed lower distal esophageal BI levels compared to healthy controls (HCs) due to the presence of abnormal distal esophageal acid exposure time (AET). On the other hand, no differences were found between NERD and HCs at proximal esophagus due to the limited proximal migration of the refluxate. Systemic sclerosis $(\mathrm{SSc})$ is a systemic disease characterized by the deposition of collagen and matrix proteins in the connective tissue of the skin and visceral organs, such as the gastrointestinal tract. This event could potentially affect the conductivity of the oesophageal wall and consequently reduce BI levels, also at proximal level, but data in this regard are limited.

Aims \& Methods: We aimed to prospectively compare BI levels between a group of NERD patients and two groups of SSc patients, one with a clear manometric picture of scleroderma esophagus (i.e. hypotensive esophago-gastric junction pressure and absent peristalsis) and one without esophageal involvement Consecutive patients with heartburn and those with a definite diagnosis of SSc underwent upper endoscopy in order to assess the presence of esophageal mucosal lesions. Further, a group of healthy subjects was used as controls (HCs) Thereafter, all endoscopy-negative and SSc patients underwent esophagea high-resolution manometry and impedance- $\mathrm{pH}$ testing off-therapy. Impedance$\mathrm{pH}$ tracings were blindly and manually reviewed, and we measured distal AET and $\mathrm{BI}$ values at $3,5,7,9,15$ and $17 \mathrm{~cm}$ above the lower esophageal sphincter, during the overnight rest, for at least 30 minutes after excluding swallows and reflux induced changes. NERD was diagnosed in case of reflux symptoms and abnormal AET.

Results: Fifty patients [38F; mean age $51 \mathrm{yrs}$ ] with NERD, $50 \mathrm{SSc}$ patients [44F; mean age $48 \mathrm{yrs}$ ] with esophageal involvement, $30 \mathrm{SSc}$ patients [28F; mean age $49 \mathrm{yrs}$ ] without esophageal involvement and $50 \mathrm{HCs}$ [37F; mean age $49 \mathrm{yrs}$ ] were enrolled. All 50/50 (100\%) NERD patients [median 7.2] and 44/50 (88\%) SSc patients with esophageal involvement [8.3] showed an abnormal AET compared to $8 / 30(26 \%)$ SSc patients without esophageal involvement [3.1] and $0 / 50(0 \%)$ HCs [0.8] $(\mathrm{p}<0.01)$. BI details are shown in the Table. Proximal median BI levels (i.e. at 9, 15 and 17) were similar between NERD, SSc patients without esophageal involvement and HCs $(\mathrm{p}=\mathrm{ns})$, whereas they were much lower in SSc patients with esophageal involvement $(\mathrm{p}<0.05)$

\begin{tabular}{lllll}
\hline $\begin{array}{l}\text { Median } \\
\text { BI Values }\end{array}$ & NERD & $\begin{array}{l}\text { SSc patients } \\
\text { with esophageal } \\
\text { involvement }\end{array}$ & $\begin{array}{l}\text { SSc patients } \\
\text { without esophageal } \\
\text { involvement }\end{array}$ & HCs \\
\hline at $3 \mathrm{~cm}, \Omega$ & 1180 & 961 & 1890 & 2965 \\
at $5 \mathrm{~cm}, \Omega$ & 1250 & 1114 & 2310 & 2688 \\
at $7 \mathrm{~cm}, \Omega$ & 1684 & 1230 & 2670 & 3110 \\
at $9 \mathrm{~cm}, \Omega$ & 2480 & 1640 & 2540 & 2890 \\
at $15 \mathrm{~cm}, \Omega$ & 2770 & 1520 & 2960 & 3320 \\
at $17 \mathrm{~cm}, \Omega$ & 2840 & 1980 & 3100 & 3250 \\
\hline
\end{tabular}

Conclusion: Proximal esophageal BI levels are able to segregate between scleroderma patients with and without esophageal involvement. The advent of nove and poorly invasive methods for the assessment of esophageal mucosal impedance will allow us to perform this measurement without the need of prolonged probe insertion.

Disclosure of Interest: V. Savarino: Consulting fee from Malesci, Reckitt, AlfaWasserman, Abbvie

E. Savarino: Consulting fee from Medtronic, Sofar, Takeda, Abbvie, MSD

All other authors have declared no conflicts of interest.

\section{P1196 GASTRIN-17 AS A NON-INVASIVE MARKER OF EARLY}

\section{GERD RELAPSE: A PROSPECTIVE ONE-YEAR STUDY}

F. Di Mario ${ }^{1}$, S. Speroni ${ }^{1}$, S. Scida ${ }^{1}$, C. Miraglia ${ }^{1}$, V. Corrente ${ }^{1}$, M. Franceschi ${ }^{2}$, E. Savarino ${ }^{3}$, N. De Bortoli ${ }^{4}$, A. Tursi ${ }^{5}$, G. Brandimarte ${ }^{6}$, L. Franzoni ${ }^{1}$,

\section{Scarpignato}

${ }^{1}$ Department Of Medicine And Surgery, University Of Parma, Italy, University of Parma, Parma/Italy

Endoscopic Unit, Department Of Surgery, Ulss4, Hospital ULSS4 Alto Vicentino, Santorso/Italy

${ }^{3}$ Uo Gastroenterology Department Of Surgical, Oncological And

Gastroenterological Sciences, University Of Padova, Padova Italy, University of

Padova, Padova/Italy

${ }^{4}$ Division Of Gastroenterology, Department Of Internal Medicine, University of Pisa, Pisa/Italy

${ }^{5}$ Gastroenterology Sevice, ASL BAT, Andria (BT), Andria/Italy

${ }^{6}$ Division of Internal Medicine and Gastroenterology, "Cristo Re" Hospital, Rome, Italy, Roma/Italy

Contact E-mail Address: francesco.dimario@unipr.it

Introduction: Gastroesophageal reflux disease (GERD), is characterized by frequent relapses after withdrawal of therapy and no prognostic markers of relapse are available to predict the outcome of the patients. Gastrin-17 (G-17) has been proposed as a non-invasive marker of reflux disease as well as a good marker of response to the therapy. Pepsinogen I (PG I) and Gastrin-17 (G-17) are claimed to increase in a statistically significant manner after proton pump inhibitors (PPIs) therapy. Aim of the study was to assess the prognostic value of G-17 serum levels in order to single out GERD patients more prone to develop an early reflux relapse in a prospective open study.

Aims \& Methods: We prospectively enrolled 221 consecutive GERD patients (F 113 , mean age 52.5 years; range $28-74$ years) with endoscopically proved diagnosis of esophagitis, according to the L.A. classification, all symptomatic (heartburn and/or regurgitation). All patients were treated with rabeprazole $20 \mathrm{mg}$ once a day for 6-8 weeks, assessing at the end of the therapy the symptoms' modifications by means of a questionnaire. In the group of asymptomatic patients, we performed a one-year follow-up, recording the GERD relapse episodes; only ondemand antacids were permitted. All patients underwent at baseline a blood sample and after the acute course of PPI therapy.

Results: One hundred eighty five patients were asymptomatic after the 6-8 weeks of PPI therapy and entered in the prospective evaluation for 12 months. 19 subjects were lost lasting the follow-up and finally 166 patients were available for the study analysis. 72 patients experienced at least one GERD relapse episode (first group) against 94 ones free of symptoms for one year (second group). The mean values of both PG I and G-17 after the 6-8 weeks of PPI therapy in comparison with the baseline levels, were higher in first group than in the second one (first group: baseline PG I $96 \mu \mathrm{g} / \mathrm{L}, \mathrm{G}-172.6 \mathrm{pmol} / \mathrm{L}$; after therapy: PG I $164 \mu \mathrm{g} / \mathrm{L}, \mathrm{G}-1719 \mathrm{pmol} / \mathrm{L}$; p < 0.0001; second group: baseline PG I $98 \mu \mathrm{g} /$ L, G-17 $2.9 \mathrm{pmol} / \mathrm{L}$; after therapy: PG I $116 \mu \mathrm{g} / \mathrm{L}, \mathrm{G}-176.3 \mathrm{pmol} / \mathrm{L}$; p: ns). The good response to full dose of PPI, assessed by an increase of both PG I and G-17, seems to be the pathophysiological background to explain the prognostic value of such markers.

Conclusion: Gastrin-17 and pepsinogen I increase after full-dose of PPI in GERD acute phase seems to be a simple non-invasive marker to predict early GERD relapse in one-year follow-up.

Disclosure of Interest: All authors have declared no conflicts of interest.

P1197 THE DIAGNOSTIC VALUE OF ESOPHAGEAL MUCOSAL AND BASELINE IMPEDANCE MEASUREMENTS IN PATIENTS WITH GASTROESOPHAGEAL REFLUX DISEASE

S. Kipcak ${ }^{1}$, P. Ergun ${ }^{1}$, S. Bor ${ }^{1}$

${ }^{1}$ Gastroenrology Sec., Ege Reflux Study Group, Ege University, Izmir/Turkey

Contact E-mail Address: kipcaksezgi@gmail.com

Introduction: Various biomarkers have been studied to evaluate the integrity of esophageal epithelium in distinguishing phenotypes of gastroesophageal reflux disease (GERD). Baseline impedance (BI) measurement is likely to be one of these and can be measured during the 24-hour ambulatory intra-esophageal impedance-pH study. Mucosal impedance (MI) measurement is a technique introduced in recent years and is a practical method that can be applied during endoscopy, but the validation studies are insufficient. BI \& MI measured with the same regular impedance catheter and data from 118 patients with different reflux phenotypes and controls were evaluated.

Aims \& Methods: Patients were divided into five groups: mild (ERD A-B, $\mathrm{n}=31$ ), severe erosive esophagitis (ERD $\mathrm{C}$ - $\mathrm{D}, \mathrm{n}=11$ ), non-erosive reflux disease (NERD, $\mathrm{n}=26$ ), functional heartburn-esophageal hypersensitivity (FH-EH, $\mathrm{n}=17$ ), healthy controls. High resolution manometry, 24-h MII-pH, upper gastrointestinal endoscopy were performed. BI values were taken at the sleeping period at night where reflux and swallowing did not occur. MI measured during endoscopy, a regular impedance-pH catheter passed through the biopsy 
channel of the scope. Distal two rings were contacted to the distal and proximal parts of the esophagus approximately 20-30 seconds. MMS Omega ambulatory recorder and Greenfield ( $6 \mathrm{imp}, 1 \mathrm{pH}$ ) impedance catheter were used.

Results: MI can differentiate ERD from non-erosive groups but do not have a diagnostic value to discriminate NERD from FH-EH or controls. However, BI can segregate NERD from ERD addition to controls (Table 1).

Table 1

\begin{tabular}{llll}
\hline & $\begin{array}{l}\text { Baseline } \\
\text { impedance }\end{array}$ & $\begin{array}{l}\text { Distal } \\
\text { mucosal } \\
\text { impedance }\end{array}$ & $\begin{array}{l}\text { Mucosal } \\
\text { impedance } \\
\text { (proximal } \\
\text { esophagus) }\end{array}$ \\
\hline Groups & $2267 \pm 393^{\#}$ & $2673 \pm 547^{\#}$ & $3190 \pm 515$ \\
Control $(\mathrm{n}=15 / \mathrm{n}=18)$ & $1906 \pm 716^{\#}$ & $2654 \pm 721^{\#}$ & $3350 \pm 880$ \\
FH and EH $(\mathrm{n}=17)$ & $1305 \pm 759^{* \#}$ & $2423 \pm 852^{\#}$ & $3407 \pm 1074$ \\
NERD $(\mathrm{n}=26)$ & $868 \pm 481^{*}$ & $1538 \pm 646^{*}$ & $3096 \pm 928$ \\
ERD A-B $(\mathrm{n}=31)$ & $441 \pm 301^{*}$ & $1355 \pm 672^{*}$ & $3236 \pm 1653$ \\
ERD C-D $(\mathrm{n}=11)$ & & & \\
\hline
\end{tabular}

Conclusion: As a new diagnostic tool, MI needs validation studies and our results failed to show additional diagnostic value in non-erosive patients compared to healthy controls. Since regular catheters are failed, new baloon-shaped catheters should be validated. BI might be a better tool to discriminate NERD from controls. This implicates that the esophageal epithelial resistance is impaired in this particular group compared to controls.

Disclosure of Interest: All authors have declared no conflicts of interest.

\section{P1198 ENDOSCOPIC-HISTOPATHOLOGIC ESOPHAGEAL FINDINGS IN ATROPHIC BODY GASTRITIS PATIENTS WITH GASTRO-ESOPHAGEAL REFLUX SYMPTOMS}

M. Carabotti ${ }^{1}$, G. Esposito ${ }^{2}$, E. Lahner ${ }^{2}$, E. Pilozzi ${ }^{2}$, G. Galli ${ }^{3}$, G. Ranazzi ${ }^{2}$, E. Di Giulio ${ }^{2}$, B. Annibale ${ }^{2}$

${ }^{1}$ University Sapienza, Rome/Italy

${ }^{2}$ Department Of Medical-surgical Sciences And Translational Medicine,

Sant'Andrea Hospital, University Sapienza, Rome/Italy

${ }^{3}$ Medical-surgical Department Of Clinical Sciences And Translational Medicine,

Sant'Andrea Hospital, School of Medicine, University Sapienza, Rome/Italy

Contact E-mail Address: mcarabotti@yahoo.it

Introduction: Atrophic body gastritis (ABG) is characterized by loss of oxyntic glands with consequent reduced acid secretion, hypergastrinaemia and, in a later stage, pernicious anemia (PA). Up to $40 \%$ of ABG patients complain of dyspepsia. Despite hypochlorhydria, in $21 \%$ of autoimmune gastritis (AG) patients gastro-esophageal reflux (GER) symptoms have been reported and in one third of them (mostly non acidic) reflux has been documented at $\mathrm{pH}$-monitoring. At now, data regarding endoscopic and histopathologic GER-related esophageal findings in this setting are lacking.

Aims \& Methods: Aim of this study was to assess the occurrence of GER symptoms and endoscopic-histopathologic esophageal findings in ABG patients. During 12 -months, 35 consecutive ABG patients $[80 \%$ female; median age 60 yrs (27-81); BMI $25.7 \mathrm{~kg} / \mathrm{m}^{2}(18.2-32.3)$; fasting gastrinaemia $329 \mathrm{pg} / \mathrm{ml}$ (215-1476); pepsinogen I $10 \mathrm{ng} / \mathrm{l}(0-44)$; positive Ab against parietal cells $74.3 \%$ ], undergoing scheduled gastroscopy for malignancy surveillance irrespective to gastrointestinal (GI) symptoms, were included. The presence of GER typical symptoms (heartburn and regurgitation), atypical (cough, no cardiac chest pain and dysphagia) and dyspepsia [postprandial distress syndrome (PDS) and epigastric pain syndrome (EPS)] was assessed by a standardized questionnaire. Hiatal hernia and erosive esophagitis were evaluated. Biopsies from distal esophageal (at least 2) and gastric mucosa (at least 5) were collected. Histopathological evaluation, performed by experienced pathologist who was unware of clinical data, assessed: i) dilatation of intercellular spaces (DIS); ii) basal cell layer hyperplasia; iii) intraepithelial eosinophils; iv) neutrophils and iv) mononuclear cells. Gastric biopsies were evaluated according to updated Sydney System. Moderate-severe body gastric atrophy was confirmed in all patients. No patient was in treatment with anti-secretory drugs.

Results: $74.3 \%(26 / 35)$ of ABG patients complained at least one GI symptom: $45.7 \%(16 / 35)$ reported GER symptoms [typical $(2 / 14)$, atypical $(9 / 14)$ and both typical and atypical symptoms (5/14)], 48.6\% (17/35) PDS and $11.4 \%(4 / 35)$ EPS dyspepsia. One GER patient had erosive esophagitis (LA-C according to Los Angeles classification) with concomitant hiatal hernia and another one presented a short tongue of columnar lined mucosa at distal esophagus with an area of intestinal metaplasia (Barrett's esophagus, C2M2 according to Prague Classification). Among ABG symptomatic patients, 34.6\% (9/26) presented GER-related microscopic esophagitis, whereas the remaining symptomatic patients were free of microscopic esophagitis. Esophageal findings, both endoscopic and microscopic esophagitis, were exclusively present in symptomatic ABG patients (Fisher-t: $\mathrm{p}=0.00027$ ). Evaluating only autoimmune gastritis patients $(n=26)$, the occurrence of esophageal findings was not related to the presence symptoms (Fisher-t: $\mathrm{p}=0.1915$ ).

Conclusion: A third of ABG symptomatic patients, investigated by specific questionnaire and esophageal biopsies, presented pathological findings of esophageal mucosa ranging from GER-related microscopic esophagitis to Barrett's esophagus. These findings show that, even in hypochlorhydric patients, esophageal mucosal damage may occur, suggesting the opportunity to accurately investigate GI symptoms, including GER symptoms, in ABG patients.

Disclosure of Interest: All authors have declared no conflicts of interest.

P1199 CLINICAL PRESENTATION RISK FACTORS AND ENDOSCOPICAL FEATURES IN GERD: A COMPARATIVE STUDY BETWEEN GERD AND DYSPEPTIC SUBJECTS ON A SAMPLE OF 2300 PEOPLE IN A PRIMARY CARE SETTING

F. Di Mario ${ }^{1}$, T.B. Tene Fokam ${ }^{2}$, S. Landi ${ }^{2}$, S. Cesario ${ }^{2}$, S. Grillo ${ }^{2}$, S. Scida ${ }^{2}$, C. Miraglia ${ }^{2}$, E. Savarino ${ }^{3}$, N. De Bortoli ${ }^{4}$, L. Franzoni ${ }^{2}$, G. Grande ${ }^{5}$, C. Scarpignato ${ }^{2}$

${ }^{1}$ University Of Parma, Department of Clinical and Experimental Medicine, section of Gastroenterology, Parma/Italy

${ }_{2}^{2}$ Department Of Medicine And Surgery, University of Parma, Parma/Italy

${ }^{3}$ Division Of Gastroenterology, Department Of Surgery, Oncology And

Gastroenterology, University of Padua, Padua/Italy

${ }^{4}$ Division Of Gastroenterology, Department Of Internal Medicine, University of Pisa, Pisa/Italy

${ }^{5}$ Aou Modena, Gastroenterology and Digestive Endoscopy Unit, Modena/Italy

Contact E-mail Address: francesco.dimario@unipr.it

Introduction: Gastroesophageal reflux disease (GERD) is considered a multi-factorial disease characterized by the presence of both typical and extra-oesophageal symptoms, as well as related risks factors, such as smoking habits. In primary care setting, dyspeptic symptoms could overlap GERD symptoms, being the differential diagnosis sometimes difficult, almost because when we performed in such patients upper-gastrointestinal (GI) endoscopy, a picture of esophagitis is observed in no more than $30-40 \%$ of the subjects. Aim of the study was to search in a primary care settings possible differences in clinical presentations in a group of patients with GERD, in comparison with a group of dyspeptic patients.

Aims \& Methods: One thousand and six hundred consecutive dyspeptic patients $(\mathrm{M}=766$; mean age $=51.5$ years; range $=27-79 \mathrm{yr})$ were enrolled in the study, according with presence of upper-GI troubles like epigastric pain, fullness, nausea, vomiting but not heartburn or regurgitation. All patients showed a negative upper-GI endoscopy and were helicobacter Pylori (H.p.) negative (Urea Breath Test and HpSA). Patients with history of peptic ulcer, neoplasms or upper-GI surgery were excluded. Seven hundred and one patients showing heartburn and/or regurgitation, a picture of esophagitis according with L.A. classification or a positive DeMeester score $(>14)$ at 24 hours $\mathrm{pH}$-metry were enrolled in GERD group. By using a questionnaire, we collected data on both alcohol and smoking habits.

Results: No differences were found for sex and age between the two groups (GERD: $\mathrm{M}: \mathrm{F}=1: 1.2$, mean age: 56 years; dyspeptics: $\mathrm{M}: \mathrm{F}=0.9$, mean age: 53 years; $p=n s$ ); heavy smokers was $29 \%$ in GERD group, $26 \%$ in dyspeptics; $\mathrm{p}=\mathrm{ns}$. Heavy drinkers were $23 \%$ in GERD group, $22 \%$ in dyspeptics; $\mathrm{p}=\mathrm{ns}$. In GERD group, typical symptoms were detected in 547 out of 701 patients, being heartburn the prevalent symptom in 479 subjects and regurgitation in 68 ones. Atypical symptoms, mainly chronic cough were present in 303 patients. 313 patients showed grade A esophagitis, 32 grade B, 4 grade C. One hundred and seventy out of 221 patients showed a positive DeMeester score for acid reflux.

Conclusion: Sex, age, smoking habits and alcohol consumption seem no differ in the two studied population. In GERD cohort, the majority of patients experienced Non Erosive reflux Disease (NERD). The majority of GERD patients suffered by typical symptoms; chronic cough represented the most frequent manifestation among the atypical and extra-oesophagial ones.

Disclosure of Interest: All authors have declared no conflicts of interest.

\section{P1200 REAL-WORLD RESPONSE OF PATIENTS WITH} GASTROESOPHAGEAL REFLUX DISEASE TO EMPIRICAL TREATMENT WITH PROTON PUMP INHIBITORS: A MULTICENTER, PROSPECTIVE, OBSERVATIONAL STUDY IN CHINA

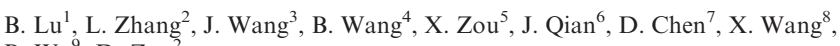
B. $\mathrm{Wu}^{9}$, D. Zou

${ }^{1}$ Gastroenterology, The First Affiliated Hospital of Zhejiang Chinese Medical University, Hangzhou/China

${ }^{2}$ Gastroenterology, Changhai Hospital, Shanghai/China

${ }^{3}$ Gastroenterology, China-Japan Union Hospital of Jilin University, Changchun/ China

${ }^{4}$ Gastroenterology, Tianjin Medical University General Hospital, Tianjin/China ${ }_{5}^{5}$ Department Of Gastroenterology, Nanjing Drum Tower Hospital, Nanjing/China ${ }^{6}$ Gastroenterology, Peking Union Medical College, Beijing/China

${ }^{7}$ Gastroenterology, Daping Hospital, Third Millitary Medical University, Chongqing/China

${ }^{8}$ Gastroenterology, Oinghai University Affiliated Hospital, Xining/China ${ }^{9}$ Gastroenterology, The Third Affiliated Hospital, Sun Yat-sen University, Guangzhou/China

Contact E-mail Address: zdw_2017@outlook.com

Introduction: In China, $13.6 \%$ of gastrointestinal outpatients suffer from gastroesophageal reflux disease (GERD), among which only $36.9 \%$ undergo endoscopy [1]. For patients with symptoms of GERD, empirical proton pump inhibitor (PPI) treatment is recommended as a diagnostic test of GERD and as a therapeutic trial to control symptoms by Chinese GERD consensus guidelines [2] 
Currently, there are no real-world data assessing the efficacy of short-term empirical treatment with PPIs in GERD patients in China.

Aims \& Methods: This was a multicenter, prospective, observational study carried out in a real-world setting. The primary objective was to determine the overall responder rate in patients with typical GERD symptoms after 4 weeks of empirical treatment with PPIs. Responders were defined as having heartburn/regurgitation on $\leq 1$ day during the prior 7 days, assessed by the Gerd-Q questionnaire. Outpatients aged between 18 and 65 years with a Gerd-Q score $\geq 8$ were enrolled if they were prescribed standard-dose PPIs as empirical treatment and were not planned to have an endoscopy within 4 weeks of enrolment. The PPI regimen prescribed was decided completely at the physicians' discretion. Patient demographics, diagnosis, prescribed PPI regimens, Gerd-Q score and symptom frequency were recorded. Data were collected at baseline, 2 weeks and 4 weeks after initiating PPI treatment. Results from the full analysis set (FAS) are presented. Results: A total of 1,000 patients from 10 centers were screened for this study, of which 987 met the inclusion criteria and were included in the FAS. The mean age was $45.2 \pm 11.6$ years, the mean body mass index was $23.4 \pm 3.3 \mathrm{~kg} / \mathrm{m}^{2}$, and $50.3 \%$ of the patients were male. The mean duration of GERD was $0.8 \pm 2.6$ years, with a mean baseline Gerd-Q score for the week before screening of $10.5 \pm 1.9$. During the 4 weeks' treatment, the proportion of patients receiving at least one dose of PPI was $99.5 \%$. Esomeprazole was the most frequently received PPI ( $57.1 \%$ of patients). Other PPIs (rabeprazole, lansoprazole, pantoprazole and omeprazole) were received by $50.1 \%$ of patients and $7.2 \%$ of the patients sequentially received $\geq 2$ PPIs in the duration of the study. A total of $787(79.7 \%)$ patients either completed the 4-week PPI treatment or withdrew after response, of which the responder rate was $74.0 \%[95 \%$ CI, $70.7 \%$ $77.0 \%$ ] (Table 1). Among the 818 patients who completed 2 weeks' treatment, the responder rate was $57.0 \%[95 \% \mathrm{CI}, 53.5 \%-60.4 \%]$. The overall median time to response was 13 days $[95 \% \mathrm{CI}, 12-15]$. Over the study duration, patients' Gerd-Q score demonstrated a decreasing trend. The proportion of patients with a Gerd-Q score $\geq 8$ reduced from $100 \%$ at baseline to $29.5 \%$ and $17.4 \%$ at 2 and 4 weeks, respectively.

Table 1: Responder rate ${ }^{[1]}$ and median time to response for different PPIs

\begin{tabular}{llll}
\hline & Esomeprazole & Other PPIs & Total \\
\hline 4-week responder & $75.2(342 / 455)$ & $72.3(240 / 332)$ & $74.0(582 / 787)$ \\
$\quad$ rate, \% [95\% CI] & {$[70.9-79.1]$} & {$[67.1-77.0]$} & {$[70.7-77.0]$} \\
2-week responder & $57.1(268 / 469)$ & $56.7(198 / 349)$ & $57.0(466 / 818)$ \\
$\quad$ rate, \% [95\% CI] & {$[52.5-61.7]$} & {$[51.4-62.0]$} & {$[53.5-60.4]$} \\
$\begin{array}{c}\text { Median time to response }{ }^{[2]}, \\
\quad 12[11-14]\end{array}$ & $14[12-17]$ & $13[12-15]$ \\
$\quad$ days [95\% CI] & & &
\end{tabular}

${ }^{[1]}$ Responder rate $=$ number of responders/number of patients who completed the corresponding treatment or withdrew after response. ${ }^{2]}$ Response was defined as heartburn/regurgitation frequency $\leq 1$ days during the prior 7 days.

Conclusion: In Chinese clinical practice, short-term PPI empirical treatment effectively improves symptom control in GERD patients and gains a satisfactory overall responder rate.

Disclosure of Interest: All authors have declared no conflicts of interest.

\section{References}

1. Lin Lin et al. Chin J Intern Med, 2015,53:517-520.

2. Chinese Society of Digestive Diseases. Chin J Dig, 2014,34:649-661.

\section{P1201 WIRELESS ELECTRICAL STIMULATION FOR MANAGING GASTROESOPHAGEAL REFLUX DISEASE IN THE RABBIT MODEL}

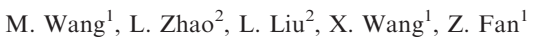

${ }^{1}$ The First Affiliated Hospital with Nanjing Medical Universtiy, Nanjing/China ${ }^{2}$ Digestive Endoscopy Center, the First Affiliated Hospital of Nanjing Medical University, Nanjing/China

\section{Contact E-mail Address: fanzhining@njmu.edu.cn}

Introduction: Electrical stimulation of lower esophageal sphincter (LES) has been approved to increase LES pressure (LESP) and commercially treat gastroesophageal reflux disease (GERD). Endostim ${ }^{\mathrm{TM}}$-like active appliance was efficient but need reoperation for replacing the battery part. Therefore, one novel passive LES stimulation system was invented and evaluated here in rabbits.

Aims \& Methods: Different from Endostim ${ }^{\mathrm{TM}}$, wireless power technology was firstly applied in the implanted part. The LES stimulation could be easily selfcontrolled and powered by external system. No reoperation or implantation replacement need. In order to evaluating its efficacy and safety, GERD rabbit model was created by balloon dilation and applied to optimizing stimulating parameters. Body weight, food intake and LESP were recorded in the experimental course.

Results: A total of 12 rabbits (male, $3 \sim 4 \mathrm{~kg}$ ) were successfully applied for equipment implantation. LES stimulation was delivered at $20 \mathrm{~Hz}, 215 \mu \mathrm{s}, 3-8 \mathrm{~mA}$ in multiple 30-minute sessions. The muscle contraction phenomenon was obviously observed during the surgery in the stimulation group(6 rabbits). The mean LESP was increased significantly in stimulation group than sham group (stimulation group: $9.25 \pm 1.24 \mathrm{mmHg}$ vs $13.99 \pm 1.28 \mathrm{mmHg}, \quad \mathrm{P}<0.05$; sham control group: $9.00 \pm 1.22 \mathrm{mmHg}$ vs $9.23 \pm 1.27 \mathrm{mmHg}, \mathrm{P}=0.267)$. After operation, the body weight initially decreased but recovered after one week. No serious implantation or stimulation-related adverse effects were reported in the twomonth follow-up.

Conclusion: Electric stimulation represents a prospective approach for treating GERD in clinics. This novel wireless LES stimulation system was also safe and effective for treating GERD rabbits. After implantation, its passive medical strategy will benefit patients and keep the long-term efficacy for clinical GERD management.

Disclosure of Interest: All authors have declared no conflicts of interest.

\section{P1202 SYSTEMATIC REVIEW AND META-ANALYSIS OF OUTCOMES AFTER LAPAROSCOPIC ANTI-REFLUX SURGERY RELATED TO OBESITY}

T. Abdelrahman ${ }^{1}$, A. Latif ${ }^{2}$, D. Chan ${ }^{1}$, N. Patel ${ }^{1}$, C. Brown ${ }^{1}$, W. Lewis ${ }^{1}$, T. Havard ${ }^{2}$, X. Escofet ${ }^{2}$

${ }^{1}$ General Surgery, University Hospital of Wales, Cardiff/United Kingdom

${ }^{2}$ General Surgery, Royal Glamorgan Hospital, Llantrisant/United Kingdom

Contact E-mail Address: tarig007@yahoo.com

Introduction: Laparoscopic Anti-Reflux Surgery (LARS) is an established alternative treatment to pharmacological therapy for patients with Gastro Oesophageal Reflux Disease (GORD), yet its safety and efficacy in obese patients is controversial. A systematic review and meta-analysis was performed to compare LARS related to obesity.

Aims \& Methods: The primary outcome measure was the relative incidence of recurrent reflux related to BMI. Secondary outcome measures were relative incidence of perioperative complications, re-intervention rates in the form of endoscopic dilatation or surgery, conversion to open surgery, and early return to theatre. Embase, MEDLINE and the Cochrane Library (January 1970 to November 2016) were searched for studies reporting clinical outcomes of LARS in patient cohorts stratified by Body Mass Index (BMI). Data was grouped according to BMI, $<30 \mathrm{~kg} / \mathrm{m}^{2}$ (non-obese) and $\geq 30 \mathrm{~kg} / \mathrm{m}^{2}$ (obese). Results were pooled in meta-analyses as Odds Ratios (OR).

Results: Eleven eligible observational studies comparing LARS in non-obese $(n=6003)$ and obese $(n=1620)$ patients were identified. Recurrence of reflux was significantly lower in the non-obese cohort (OR $0.34,95 \%$ CI 0.19 to $0.60, \mathrm{p}<0.001)$, however no significant differences were observed in rates of operative morbidity (OR $0.87,0.65$ to $1.18, \mathrm{p}=0.38$ ), redo surgery (OR 1.08, 0.68 to $1.72, p=0.73$ ), endoscopic dilatation (OR $1.06,0.49$ to $2.33, p=0.88$ ), conversion to open surgery (OR $1.17,0.55$ to $2.48, \mathrm{p}=0.68$ ), or early return to theatre (OR 0.77, 0.44 to $1.37, \mathrm{p}=0.38$ ).

Conclusion: LARS can be performed safely in obese patients, but risks higher GORD recurrence. Clinicians and patients should be aware that obesity may adversely affect LARS outcome and careful consideration be given in the consent process inherent within the optimal management of GORD.

Disclosure of Interest: All authors have declared no conflicts of interest.

\section{P1203 GASTROESOPHAGEAL REFLUX DISEASE REFRACTORY TO PROTON PUMP INHIBITOR THERAPY. INCOMPLETE ACID INHIBITION OR DIAGNOSTIC ERROR?}

J. Perez De La Serna, A. Ruiz De León, C. Sevilla Mantilla, A. Zataraín Valles, O. N. López García, E. Rey, B. Merchan Gomez

Gastroenterology, Hospital Clínico San Carlos, Madrid/Spain

Contact E-mail Address: jperezsernab@gmail.com

Introduction: The use of PPIs represents the main treatment in gastroesophageal reflux disease (GERD), having demonstrated its effectiveness both in the control of inflammation and symptomatology. However, between $10-20 \%$ of patients present persistent symptoms or lesions despite the treatment.

Aims \& Methods: The aim of the study was to assess the presence of acid reflux in patients submitted to our department with the diagnosis of refractory GERD, due to low or no response to PPIs. This was a retrospective study including 190 patients ( 55 men, 135 women) referred to our service with the diagnosis of GERD from January 2008 to December 2015. Based on the diagnostic criteria, two groups were made. Group 1: included 63 patients $(33.2 \%)$ diagnosed of GERD due to typical symptomatology and at least one positive complementary test (radiology, pHmetry or endoscopy). All of them underwent a $24-\mathrm{H}$ pHmetry study with a dual channel, esophageal and gastric, on-PPI treatment. In 17 patients the pHmetry was completed with multichannel intraluminal impedance (15 cases) or Bilitec ( 2 cases). Group 2: included 127 patients $(66.8 \%)$ who had been diagnosed of GERD only on the basis of typical symptoms; all of them underwent esophageal double channel $24-\mathrm{H}$ pH-metry off-PPI. All of the studies (24-hour pHmonitoring or multichannel intraluminal impedance-pH studies) (MARK III, Delta and Digitrapper pH-Z; Synectics, Gyven, Medtronic) were performed according to standard technique.

Results: Pathological reflux was present in 91 patients (47.9\%), 24 from group 1 and 67 from group 2. Pathological acid reflux was therefore ruled out as a cause of symptoms in $52.1 \%$ of all cases studied: 60 patients $(47.2 \%)$ from group 2 , and 39 patients $(61.9 \%)$ from group 1 . In addition, out of the 24 patients with pathological reflux in group 1 (true refractory patients), 9 had an incomplete response, with a percentage of time with $\mathrm{pH}<4$ less than $7.5 \%$ (mild reflux), which probably was not the cause of the symptomatology.

Conclusion: Proton pump inhibitors (PPIs) are the drugs of choice in the treatment of GERD. However, its efficacy may be compromised for a variety of reasons including: non-compliance, bioavailability, episodes of nocturnal acid break-through, poor gastric emptying, etc. In most of the patients referred for 
studying with the diagnosis of refractory reflux to PPIs, this diagnosis had only been based on GERD-compatible symptoms. When the diagnosis is exclusively clinical, about a half $(47.2 \%)$ of the patients with persistent symptoms on double doses of PPIs, considered as GERD patients refractory to PPIs, have an incorrect diagnosis (patients do not have pathological reflux). More than half of the patients $(61.9 \%)$ who have a diagnosis of GERD confirmed by complementary tests that do not respond to treatment with PPIs, acid reflux is not the cause of their symptoms.

Disclosure of Interest: All authors have declared no conflicts of interest.

\section{P1204 LOW-FODMAP DIET RESULTED EFFECTIVE IN REDUCING SYMPTOM PERCEPTION IN PATIENTS WITH FUNCTIONAL HEARTBURN}

I. Martinucci ${ }^{1}$, E. Savarino ${ }^{2}$, G. Guidi ${ }^{1}$, K. Nardi ${ }^{1}$, S. Tolone ${ }^{3}$, M. Frazzoni ${ }^{4}$, L. Fuccio ${ }^{5}$, L. Frazzoni ${ }^{5}$, M. Bellini ${ }^{1}$, V. Savarino ${ }^{6}$, S. Marchi ${ }^{1}$, N. De Bortoli ${ }^{1}$ ${ }^{1}$ Gastroenterology Unit, University of Pisa, Pisa/Italy

${ }^{2}$ Department Of Surgery, Oncology And Gastroenterology, University of Padua, Padua/Italy

${ }^{3}$ Surgery, Second University of Naples, Naples/Italy

${ }^{4}$ Digestive Pathophysiology Unit, Baggiovara Hospital, Modena/Italy

${ }^{5}$ Deprtment Of Medical And Surgical Sciences, S.Orsola-Malpighi University

Hospital, Bologna/Italy

${ }^{6}$ Dept Internal Medecine, Universita di Genova, Genova/Italy

Contact E-mail Address: martinucci.irene@gmail.com

Introduction: Recently, low-FODMAP diet has been proposed as potential treatment in patients with irritable bowel syndrome (IBS) given its high efficacy in symptoms relief. Recent data showed that IBS frequently overlap with functional heartburn $(\mathrm{FH})$ and functional dyspepsia.

Aims \& Methods: The aim of this study was to evaluate the efficacy of lowFODMAP diet in reducing heartburn in patients with $\mathrm{FH}$ and no pathophysiological evidence of gastroesophageal reflux (GERD) compared to patients with non-erosive reflux disease (NERD). As secondary aim we investigated the reduction of lower gastrointestinal symptoms in both groups. We enrolled patients with heartburn and negative upper endoscopy who were scheduled for upper pathophysiological tests (esophageal manometry and impedance and $\mathrm{pH}$ monitoring, MII-pH) at Gastroenterology Unit in University of Pisa. We excluded patients older than 75 and younger than 18, those with primary esophageal motor disorders and with previous abdominal surgery. Medical history, voluptuary habits and response to proton pump inhibitor (PPI) treatment were recorded. By means of MII-pH we splitted patients in two populations: NERD group (abnormal esophageal acid exposure or number of refluxes) and FH group (normal esophageal acid exposure and number of reflux, no symptom-reflux correlation and no heartburn relief during PPI treatment). All enrolled patients were evaluated with validated questionnaires (Likert and VAS) to evaluate heartburn occurrence pre- and post a nutritional approach with low-FODMAP diet for 6 weeks.

Results: We included 31 patients (20 female; mean age 49.1 yrs; mean BMI 24.4 ) into the study. NERD group was composed of 13 patients ( 6 female; mean age 48.7 yrs; mean BMI 25.7). FH group was composed by 18 patients (11 female; mean age 50.9 yrs; mean BMI 23.9). All patients showed symptom improvement regarding bloating, abdominal pain and stools composition $(\mathrm{p}<0.001)$ after low-FODMAP diet (see Table 1). Moreover, we observed a very important improvement of heartburn in the FH group (from $8.4 \pm 2.5$ to $2.3 \pm 1.1$; $\mathrm{p}<0.001$ on VAS scale) compared to the NERD group $(7.2 \pm 2.2$ a $6.9 \pm 1.9$; $\mathrm{p}=0.624$ on VAS).

Table 1: abdominal symptoms perception pre- and post- low-FODMAP diet in NERD and FH groups

\begin{tabular}{llllllll}
\hline & \multicolumn{2}{l}{ NERD group } & & \multicolumn{2}{l}{ FH group } & \\
\cline { 2 - 3 } & Pre-diet & Post-diet & P value & Pre-diet & Post-diet & P value \\
\hline Abdominal pain & $3.6 \pm 1.8$ & $2.7 \pm 0.9$ & $0.041^{*}$ & $5.8 \pm 2.1$ & $1.7 \pm 1.3$ & $0.0001^{*}$ \\
Bloathing & $4.3 \pm 2.6$ & $3.1 \pm 1.7$ & 0.187 & $6.3 \pm 1.5$ & $2.1 \pm 0.9$ & $0.0001^{*}$ \\
Wind & $4.9 \pm 2.3$ & $3.3 \pm 1.7$ & 0.055 & $6.1 \pm 1.9$ & $2.2 \pm 1.15$ & $0.0001^{*}$ \\
BSC & $3 / 13$ & $7 / 13$ & 0.226 & $2 / 18$ & $13 / 18$ & $0.005^{*}$ \\
$\quad$ (type 3-5) & & & & & & \\
\hline
\end{tabular}

Legend: $\mathrm{BSC}=$ Bristol Stool Classification; ${ }^{*}=$ statistically significant $(\mathrm{p}<0.05)$

Conclusion: This pilot study showed that a low-FODMAP diet was able to reduce heartburn perception in patients with $\mathrm{FH}$ and who did not obtain any symptom relief after PPI treatment. Larger prospective randomized controlled trial is mandatory to further explore these findings

Disclosure of Interest: All authors have declared no conflicts of interest.
P1205 GENDER DIFFERENCES IN NEOPLASTIC PROGRESSION IN BARRETT'S ESOPHAGUS: A MULTICENTER PROSPECTIVE COHORT STUDY

C. A.m. Roumans ${ }^{1}$, A. Treling ${ }^{2}$, D. Rizopoulos ${ }^{3}$, E. W. Steyerberg ${ }^{4}$, M.J. Bruno ${ }^{2}$, M.C.w. Spaander ${ }^{2}$

${ }^{1}$ Department Of Public Health, Erasmus University Medical Center, Rotterdam Netherlands

${ }^{2}$ Department Of Gastroenterology \& Hepatology, Erasmus University Medical Center, Rotterdam/Netherlands

${ }^{3}$ Department Of Biostatistics, Erasmus University Medical Center, Rotterdam/ Netherlands

${ }^{4}$ Department Of Medical Statistics And Bioinformatics, Leiden University Medical Center, Leiden/Netherlands

Contact E-mail Address: c.roumans@erasmusmc.nl

Introduction: Because of a higher prevalence of $\mathrm{BE}$ in males, recommendations in current guidelines are mainly based on male BE patients and make no difference in surveillance according to gender. Nevertheless, it is unknown whether female $\mathrm{BE}$ patients have the same neoplastic progression and acceleration rate as male patients.

Aims \& Methods: The aims of this study were (1) to evaluate the difference between males and females in probability of and (2) time to neoplastic progression, as well as (3) gender differences in stage distribution of neoplastic progression in surveilled BE patients. In this multicenter prospective cohort study we included 729 patients with BE who met the inclusion criteria of a segment of $>2 \mathrm{~cm}$ and confirmed intestinal metaplasia. Endoscopic surveillance was performed according to the American College of Gastroenterology guidelines. Cox regression modelling as well as accelerated failure time modelling were used to estimate differences in probability of and time to neoplastic progression to HGD, EAC and both HGD and EAC between sexes, respectively. All models were adjusted for age, presence of esophagitis and length of BE. In case of a limited number of events, descriptive statistics were used.

Results: 532 males (73\%; median age 58 years, IQR 51-67) and 197 females (median age 64 years, IQR 57-70) were included with a median follow-up of 8.2 years (IQR 5.3-10.3). High-grade dysplasia (HGD) was detected in 35 males versus 4 females, EAC in 12 males versus 5 females. The total number of patients with neoplastic progression was $56(8 \%)$, which was twice as high among males compared to females (HR $1.90,95 \%$ CI $0.92-3.92$ ). Especially the risk of HGD was higher in males than in females (HR 3.34, 95\% CI 1.17-9.50). The ratio HGD/EAC in males was 2.92, in females 0.80. Apparently in females proportionally more EAC was identified compared to males. Though these data might suggest accelerated neoplastic progression rates in females, time to event was significantly shorter for males in HGD (AR 0.45 , 95\% CI $0.22-0.94$ ). There was no difference for overall neoplastic progression (AR $0.59,95 \% \mathrm{CI}$ $0.32-1.09)$. Stage distribution is as shown in Table 1, females tend to have a higher stage of neoplastic progression than males.

Conclusion: The risk of HGD and overall neoplastic progression and acceleration rate of HGD development is higher in male BE patients compared to females. On the other hand descriptive statistics show proportionally more EAC in females as well as an advanced stage of EAC at diagnosis. Further research into the differential aspects of neoplastic progression in BE between men and women, may have future consequences for gender specific guideline recommendations, including the timing of follow-up.

Table 1: Stage distribution of neoplastic progression between males and females

\begin{tabular}{llllllll}
\hline & Stage 0 & & Stage 1 & & Stage 2 & & Total \\
\hline Male & 40 & $85 \%$ & 4 & $8.5 \%$ & 3 & $6.4 \%$ & 47 \\
Female & 5 & $56 \%$ & 3 & $33 \%$ & 1 & $11 \%$ & 9 \\
Total & 45 & $80 \%$ & 7 & $13 \%$ & 4 & $7.1 \%$ & 56 \\
\hline
\end{tabular}

Disclosure of Interest: All authors have declared no conflicts of interest.

\section{P1206 SINGLE SESSION FOCAL CRYOBALLOON ABLATION THERAPY IS SAFE AND EFFECTIVE IN THE TREATMENT OF DYSPLASTIC BARRETT'S ESOPHAGUS}

S. Van Munster ${ }^{1}$, A. Overwater ${ }^{2}$, J.J. G.h.m. Bergman ${ }^{1}$, B.L.a.m. Weusten ${ }^{2}$ ${ }^{1}$ Dpt. Of Gastroenterology And Hepatology, Academic Medical Center, Amsterdam, Amsterdam/Netherlands

${ }^{2}$ Dpt. Of Gastroenterology And Hepatology, Universy Medical Center Utrecht, Utrecht/Netherlands

Contact E-mail Address: s.n.vanmunster@amc.uva.nl

Introduction: Given its proven safety and efficacy, RadioFrequency Ablation (RFA) is the preferred ablation modality for dysplastic Barrett's Esophagus (BE). However, RFA is associated with significant drawbacks, such as the need for large controller units, multiple deployment steps and capital investment. The Focal CryoBalloon Ablation system (FCBA; C2 Therapeutics Inc. Redwood City, $C A, U S A)$ is another ablation method -based on the application of extreme cold- that has been recently developed to overcome these RFA drawbacks. Additionally, FCBA might be better tolerated. FCBA comprises a handheld, through-the-scope system with a conformable balloon that is simultaneously inflated and cooled using nitrous oxide, resulting in ice patches of approximately $2 \mathrm{~cm}^{2}$ on the targeted mucosa. Previous studies applying FCBA to limited areas of $\mathrm{BE}$ (1 to 2 small BE islands per patient) have shown promising results. Data on 
efficacy and safety of FCBA in the treatment of larger BE segments, however, are lacking. Therefore we aimed to assess the safety and efficacy of a single treatment with FCBA for dysplastic BE.

Aims \& Methods: Patients were seen between March and December 2016 at two tertiary referral centers in the Netherlands. Patients with a $\mathrm{BE}<6 \mathrm{~cm}$ in length and with a confirmed diagnosis of low-grade (LGD) or high-grade dysplasia (HGD) or after endoscopic resection for visible lesions, were included. Exclusion criteria included previous focal ablation therapy and strictures. At baseline, all visible BE was treated with side by side ablations of 10 seconds, including circumferential treatment of the gastroesophageal junction (GEJ). Pain scores were assessed directly post-treatment and at days 2 and 7. Follow-up endoscopy with biopsy and photo documentation was scheduled after 3 months. Primary outcomes included dysplasia regression rate and incidence of esophageal stricture or other adverse events.

Results: We enrolled 20 patients with dysplastic BE $(85 \%$ male, mean age $66( \pm 8)$ years), with a median BE length of C0M2 (IQR $0-0 ; 1-3)$ and with a baseline diagnosis of LGD $(10 ; 50 \%)$, HGD $(1 ; 5 \%)$, or mucosal adenocarcinoma $(9 ; 45 \%)$. Ten $(50 \%)$ had undergone endoscopic resection of a visible lesion before cryoablation and $8(40 \%)$ had undergone previous circumferential RFA. During a median ablation time of 16 minutes (IQR 11-19), all BE, including circumferential ablation of GEJ was successfully ablated in all patients. No adverse events occurred, and median pain directly post-treatment was 4 out of 10 (IQR $0-5$ ), whereas this was 1 (IQR $0-2$ ) and 0 (IQR $0-1$ ) at days 2 and 7. At the 3-month follow-up endoscopy, median endoscopic regression of initial BE was found to be $95 \%$ (IQR 83-98), this included 3 patients (15\%) with a complete $100 \%$ regression. All biopsies confirmed squamous regeneration without evidence for subsquamous BE. No significant esophageal strictures or other complications were noted.

Conclusion: Our multicenter, prospective trial shows that a single treatment with Focal CryoBalloon ablation therapy is safe, well-tolerated and effective for eradication of dysplastic BE.

Disclosure of Interest: All authors have declared no conflicts of interest.

\section{P1207 CRYOBALLOON ABLATION OF DYSPLASTIC BARRETT'S ESOPHAGUS CAUSES SHORTER DURATION AND LESS SEVERE POST-PROCEDURAL PAIN AS COMPARED TO RADIOFREQUENCY ABLATION}

S. Van Munster ${ }^{1}$, A. Overwater ${ }^{2}$, J.J. G.h.m. Bergman ${ }^{1}$, B.L.a.m. Weusten ${ }^{2}$ ${ }^{1}$ Gastroenterology And Hepatology, AMC, Amsterdam/Netherlands ${ }^{2}$ Dpt. Of Gastroenterology And Hepatology, Universy Medical Center Utrecht, Utrecht/Netherlands

Contact E-mail Address: s.n.vanmunster@amc.uva.nl

Introduction: Radiofrequency ablation (RFA) is safe and effective for eradication of dysplastic Barrett's Esophagus (BE), but may be associated with significant post-procedural pain. As an alternative, cryoablation using the Focal CryoBalloon Ablation system (FCBA) has recently been developed, which ablates $\mathrm{BE}$ by freezing it using nitrous oxide. Early uncontrolled studies suggest comparable safety and efficacy of FCBA and RFA in eradicating dysplastic BE. Therefore, secondary endpoints like pain might play a determining role in selecting the best treatment modality. In contrast to heat-based ablation, FCBA preserves the extracellular matrix which might be associated with less pain while maintaining sufficient depth of ablation. In this study, we aimed to compare post-procedural pain between focal RFA and FCBA.

Aims \& Methods: Between January 2016 and March 2017 all patients undergoing focal ablation therapy of BE, either with RFA or FCBA performed in two tertiary referral centers in the Netherlands, were approached to complete a digital diary. A short questionnaire was daily sent to patients for 14 days post- treatment, to assess (1) odynophagia, (2) chest pain in rest (both were assessed using VAS score ranging from 0 to 10), (3) dysphagia (assessed using a score ranging from 0 to 4 ) and (4) use of analgesics. Primary outcome included maximum VAS score (maximum score for either item 1 or 2), secondary outcomes included area under the curves (AUCs) for all items assessed, maximum reported VAS score at any time, time to VAS 0 and analgesics use. According to national guidelines, all visible BE was ablated, including circumferential treatment of the gastro-esophageal junction (GEJ). In a standardized way, all patients were advised to use paracetamol (up to 4 times $500 \mathrm{mg}$ daily) as necessary with additional ibuprofen, if needed.

Results: Fifty-five patients were included ( 35 with focal RFA; 20 with CBA) and median BE length was similar for the two groups (FCBA: C0M2, RFA: C0M1, p0.72). All other baseline characteristics were similarly comparable for both groups (table 1A). Maximum VAS score was lower after FCBA compared with RFA at all days, reaching statistical significance at 13/14 days (table 1B). All AUC curves were significantly smaller after FCBA compared to RFA: for maximum VAS score $(12.3$ vs $26.7, \mathrm{p}<0.01)$, for odynophagia $(11.6$ vs 26.7 , $\mathrm{p}<0.01)$, for pain in rest (7.8 vs 20.5, p < 0.01), for use of analgesics $(0.9$ vs 3.1, $\mathrm{p}<0.01)$ and for dysphagia $(2.6$ vs $8.2, \mathrm{p}<0.01)$. The maximum median VAS score reported on any of the 14 days was 2 (IQR 0-4) after FCBA and 4 (IQR $3-$ 7) after RFA $(\mathrm{p}<0.01)$. After 4 (IQR 1-10) days, half of the FCBA patients reported a pain score of 0 , whereas this was 13 (IQR 10-15) days for RFA $(\mathrm{p}<0.01)$. FCBA patients used analgesics during median 1 (IQR 1-1) days, compared to 4 (IQR $1-11)$ days for RFA patients $(\mathrm{p}<0.01)$.

Table 1: Baseline characteristics and maximum pain scores

\begin{tabular}{|c|c|c|c|c|}
\hline & & $\begin{array}{l}\text { FCBA } \\
(\mathrm{N}=20)\end{array}$ & $\begin{array}{l}\text { RFA } \\
(\mathrm{N}=35)\end{array}$ & P-value \\
\hline \multicolumn{5}{|c|}{ 1A. Baseline characteristics } \\
\hline \multicolumn{2}{|c|}{ Male gender, n $(\%)$} & $17(85 \%)$ & $29(83 \%)$ & 0.84 \\
\hline \multicolumn{2}{|l|}{ Age, mean (SD) years } & $65( \pm 8)$ & $66( \pm 8)$ & 0.66 \\
\hline \multirow{3}{*}{$\begin{array}{l}\text { Worst diagnosis } \\
\text { prior to FCBA }\end{array}$} & LGD, n & $10(50 \%)$ & $19(54 \%)$ & 0.54 \\
\hline & HGD, n & $1(5 \%)$ & $6(17 \%)$ & \\
\hline & $\mathrm{EAC}, \mathrm{n}$ & $9(45 \%)$ & $10(29 \%)$ & \\
\hline \multirow[t]{2}{*}{ Prior treatment } & $\mathrm{ER}, \mathrm{n}$ & $10(50 \%)$ & $17(52 \%)$ & 0.92 \\
\hline & $\begin{array}{l}\text { Circumferential } \\
\text { RFA, n }\end{array}$ & $10(50 \%)$ & $23(66 \%)$ & 0.33 \\
\hline \multirow{2}{*}{$\begin{array}{l}\text { BE length } \\
\text { prior to FCBA }\end{array}$} & Circumferential, cm & $0(0-0)$ & $0(0-0)$ & 0.64 \\
\hline & Maximum, $\mathrm{cm}$ & $2(1-2)$ & $1(1-3)$ & 0.72 \\
\hline \multicolumn{5}{|c|}{ 1B. Maximum pain scores on day 1 to 14 post treatment } \\
\hline \multirow{8}{*}{$\begin{array}{l}\text { Maximum VAS, } \\
\text { median (IQR) }\end{array}$} & Day 1 & $2(0-4)$ & $3(1-7)$ & 0.06 \\
\hline & Day 2 & $1(0-4)$ & $3(1-6)$ & 0.02 \\
\hline & Day 3 & $1(0-3)$ & $3(1-4)$ & 0.03 \\
\hline & Day 4 & $1(0-2)$ & $2(1-4)$ & 0.05 \\
\hline & Day 5 & $0(0-2)$ & $2(1-5)$ & 0.02 \\
\hline & Day 6 & $0(0-1)$ & $2(1-5)$ & $<0.01$ \\
\hline & Days 7 to 9 & $0(0-1)$ & $2(0-4)$ & $<0.01$ \\
\hline & Days 10 to 14 & $0(0-0)$ & $1(0-2 / 3)$ & $<0.01$ \\
\hline
\end{tabular}

Conclusion: In this multicenter, non-randomized, open prospective cohort study, patients reported less post-procedural pain and dysphagia after FCBA as compared with RFA and, moreover, FCBA patients used less analgesics. Although a randomized trial should provide definitive evidence for differences in post-procedural tolerability, our results strongly suggest a significantly different postprocedural course, thus favoring FCBA over RFA.

Disclosure of Interest: All authors have declared no conflicts of interest.

P1208 COMPARATIVE OUTCOMES OF RADIOFREQUENCY ABLATION FOR BARRETT'S OESOPHAGUS WITH DIFFERENT BASELINE HISTOLOGY

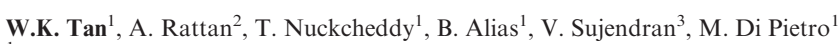
${ }^{1} M R C$ Cancer Unit, University of Cambridge, Cambridge/United Kingdom ${ }^{2}$ Department Of Gastroenterology, Addenbrookes Hospital, Cambridge/United Kingdom

${ }^{3}$ Department Of General Surgery, Addenbrookes Hospital, Cambridge/United Kingdom

Contact E-mail Address: weikeithtan@gmail.com

Introduction: Radiofrequency ablation (RFA) with endoscopic mucosal resection is recommended for Barrett's Oesophagus (BO) related neoplasia. In this study, we evaluated RFA treatment outcomes for BO stratified according to baseline histology, i.e. low-grade dysplasia (LGD), high-grade dysplasia (HGD) and intramucosal carcinoma (IMC).

Aims \& Methods: The aim of this study was to compare RFA outcomes for BO with low-grade dysplasia (LGD), high-grade dysplasia (HGD) and intramucosal carcinoma (IMC). We retrospectively reviewed the treatment outcomes of patients with dysplastic BO between January 2007-2017. Patients received 3monthly RFA until endoscopic and histologic remissions were achieved. Outcomes measured were: 1) complete remission of dysplasia (CRD) and intestinal metaplasia (CRIM), 2) stricture rate, and 3) durability of CRD and CRIM. Patients on active treatment protocol were excluded.

Results: We identified 113 patients who completed RFA treatment (21 LGD, 46 HGD and 46 IMC). There were no significant difference between the groups in the age, gender, circumferential and maximum length of $\mathrm{BO}$, and stricture rate. CRD and CRIM were achieved in $94.7 \%$ and $78.8 \%$ of patients, respectively. When stratified according to baseline histology, there was no significant difference in CRD rate among LGD $(95.2 \%)$, HGD $(95.7 \%)$ and IMC $(93.5 \%)$ $(\mathrm{p}=0.89)$. Similarly, there was no significant difference in CRIM rate among LGD $(71.4 \%)$, HGD $(76.1 \%)$ and IMC $(84.8 \%)(\mathrm{p}=0.31)$. CRD durability at 12 and 36 months $(\mathrm{n}=107)$ were $99.0 \%$ and $97.0 \%$, respectively. CRIM durability $(\mathrm{n}=89)$ at 12 and 36 months were $98.5 \%$ and $92.7 \%$, respectively. When stratified according to baseline histology, CRD durability at 12 and 36 months for LGD and IMC were $100 \%$ at both time points, and $97.7 \%$ and $93.6 \%$ for HGD, respectively $(\log$ rank $\mathrm{p}=0.31)$. CRIM durability at 12 and 36 months for LGD, HGD and IMC were $100 \%, 96.4 \%, 100 \%$, and $100 \%, 88.5 \%, 95.5 \%$, respectively $(\log$ rank $\mathrm{p}=0.60)$

Conclusion: The treatment outcomes for BO were similar in patients with different baseline histology. Our results showed that once CRD and CRIM were achieved, these were durable over time.

Disclosure of Interest: All authors have declared no conflicts of interest. 
P1209 IS IT REASONABLE TO PROPOSE AN ENDOSCOPIC MUCOSAL RESECTION FOR BARRET'S OESOPHAGUS WITH HIGH-GRADE DYSPLASIA ON THE BIOPSIES?

J. Gonzalez ${ }^{1}$, J. Casanova ${ }^{1}$, A. Benezech ${ }^{1}$, E. Bories ${ }^{2}$, S. Garcia ${ }^{3}$, M. Giovannini ${ }^{4}$, M. Barthet ${ }^{1}$

${ }^{1}$ Gastroenterology, AMU, AP-HM, Hôpital Nord, Marseille/France ${ }^{2}$ Institut Paoli Calmettes, Marseille/France

${ }^{3}$ Cyto-pathology, AMU, AP-HM, Hôpital Nord, Marseille/France

${ }^{4}$ Dept. Of Endoscopy, Paoli-Calmettes, Marseille Cedex/France

Contact E-mail Address: jmgonza05@yahoo.fr

Introduction: Endoscopic mucosal resection (EMR) is currently the first-line treatment for high-grade dysplasia (HGD) in Barret's esophagus (BE). Despite improvements in the characterization of dysplasia, the management depend on the pathological analysis of biopsies previously performed. However, the level of concordance between cytologists in such situation remains discussed. The aim of this study was to evaluate the discordance between biopsy sample (BS) and EMR specimen in case of HGD, as well as cytologists' inter and intra-observatory variability.

Aims \& Methods: This was a retrospective study including a prospective histological relecture (BS and specimen) in two expert centers. The inclusion criteria were: BE with HGD on pre-operative biopsies resected by EMR. The initial biopsies from other centers were collected and re-exanimated by our cytologists. The BS discordant with EMR specimens were recorded in a numeric file (Teleslide) and a second lecture was carried out by 2 experts and 2 fellows (1 of each per center). Five diagnoses were considered: no metaplasia (no BE), metaplasia without dysplasia, LGD, HGD, Adenocarcinoma. Concordance statistical tests were performed to assess the variability between BS and EMR specimen, and among the cytologists.

Results: Between January 2005 and December 2015, 87 patients have undergone EMR for HGD on biopsies, in both centers. Among them, $41(47 \%)$ had a discordant result between biopsies and resection specimen. The histological diagnosis was LGD in 23 cases $(16.4 \%)$, metaplasia in 13 cases $(14.9 \%)$, adenocarcinoma in 4 cases $(4.6 \%)$ and one patient had no metaplasia. Finally, 33 patients could be analyzed, 29 men and 4 women, with a mean age of 63 years old. The mean length of $\mathrm{BE}$ according to Prague classification was $\mathrm{C} 3-\mathrm{M} 5$, with relief abnormalities in $18.2 \%$ of the cases. A mean number of 1.4 endoscopic session was performed, with a mean of 2.7 resected pieces per EMR, which was macroscopically complete in $63.6 \%$ of the cases. The mean follow-up was 38 months. After histological relecture, the Kappa coefficient for the diagnosis of HGD was low on the initial BS, and ranged between 0 and 0.6 for the EMR specimen. The inter-observatory concordance was 0.2 (for both BS and EMR specimen) for the diagnosis of HGD. For other diagnoses, it was ranged between 0 and 0.5 for biopsies and between 0 and 0.6 for EMR specimen. The kappa coefficient regarding HGD between experts was 0.5 for biopsies and 0 for EMR, whereas it was 0.4 and 0.5 for the fellows, respectively. The intra-observatory (between BS and EMR specimen) after relecture was ranged between 0 and 0.6 .

Conclusion: The discordance rate between initial diagnosis of HGD on BS and final results on EMR specimen is high, around $47 \%$. The intra and inter observatory concordance is insufficient, even in expert tertiary centers. Thus, the question about performing EMR based on random biopsies rather than endoscopic assessment has to be asked, and clearly evaluated in further studies. This could have a direct impact on the management of BE.

Disclosure of Interest: M. Barthet: Consultant for Boston Scientific

All other authors have declared no conflicts of interest.

P1210 CD4+ AND CD8+ LYMPHOCYTE RATE AND PDL-1 LEUKOCYTE EXPRESSION ARE PREDICTIVE OF CLINICAL COMPLETE RESPONSE AFTER NEOADJUVANT CHEMORADIOTHERAPY FOR SQUAMOUS CELL CANCER OF THE THORACIC OESOPHAGUS

M. Fassan ${ }^{1}$, F. Cavallin ${ }^{2}$, V. Guzzardo ${ }^{1}$, A. Kotsafti ${ }^{3}$, R. Alfieri ${ }^{3}$, M. $\mathrm{Cagol}^{3}$, M. Scarpa ${ }^{3}$, V. Chiarion Sileni ${ }^{2}$, I. Castagliuolo ${ }^{4}$, M. Rugge ${ }^{5}$, C. Castoro ${ }^{3}$, M. Scarpa ${ }^{3}$

${ }^{1}$ University of Padova, Padova/Italy

${ }^{2}$ Veneto Institute of Oncology, Padova/Italy

${ }^{3}$ Esophageal And Digestive Tract Surgical Unit, Regional Centre For Esophageal Disease, Veneto Institute of Oncology (IOV-IRCCS), Padova/Italy

${ }^{4}$ Dept. Of Molecular Medicine, University of Padova, Padova/Italy

${ }^{5}$ Pathology, Medical School of the Padova University, Padova/Italy

Contact E-mail Address: machikots@hotmail.com

Introduction: Neoadjuvant chemoradiotherapy (CT-RT) before esophagectomy is standard management for locally advanced squamous cell carcinoma (SCC) of the esophagus and in several cases, it can lead to complete response (CR)

Aims \& Methods: The aims of this study were to identify possible immunological predictors of CR after neoadjuvant CT-RT and of cancer persistence/recurrence in patients who had a clinical CR. Eighty-eight consecutive patients with SCC of the thoracic oesophagus who underwent to neoadjuvant CT-RT were included in this retrospective study. Patients were included in the study if they had had neoadjuvant CTRT for locally advanced SCC and having had histologicla samples taken at diagnosis available in our Pathology Unit archive. Immunohistochemistry for PD1, PDL1, CD80, CD4 and CD8 was performed. Outcome details were retrieved and clinical CR and recurrence/relapse rate was compared. Non parametric comparison tests and non paramentric combination test were used for small sample size comparison.

Results: After neoadjuvant CT-RT, 23 patients had CR, while 65 had partia response, stable disease or progression. CD $8+$ and CD4+ lymphocyte rate and
PDL1 leukocyte expression were significantly higher in patients who had CR compared to those who had not $(p<0.0014, p<0.001$ and $p=0.004$ respectively). The accuracy of leukocyte expression of PDL1 and CD8+ and CD4+ lymphocyte rate was $0.76(\mathrm{p}=0.001), 0.81(\mathrm{p}=0.0001)$ and $0.75(\mathrm{p}=0.0001)$, respectively. Within the CR group, all patients with high infiltration of CD4+ $\mathrm{T}$ cell recurred/relapsed while only the $38.9 \%$ of those with low CD4 $+\mathrm{T}$ cell infiltration did the same $(\mathrm{p}=0.058)$.

Conclusion: In our group of patients, CD4+ and CD8+ lymphocyte rate and PDL-1 leukocyte expression were predictive of clinical complete response after neoadjuvant chemoradiotherapy for squamous cell cancer of the thoracic oesophagus with adequate accuracy. Moreover, high infiltration level of CD4+ T cell was associated to recurrence/relapse. These preliminary observations might be used to plan further study aimed to identify reliable predictors of response to chemoradiation in oesophageal SCC.

Disclosure of Interest: All authors have declared no conflicts of interest.

\section{P1211 IDENTIFICATION OF THREE DISTINCT BIOLOGICAL SUBTYPES IN ESOPHAGEAL AND JUNCTIONAL} ADENOCARCINOMA BY RNA SEQUENCING

S. Hoefnagel ${ }^{1}$, B. Scicluna ${ }^{1}$, S. Calpe ${ }^{1}$, C. Del Sancho-Serra ${ }^{1}$, K.K. Krishnadath ${ }^{2}$ ${ }^{1}$ CEMM, AMC, Amsterdam/Netherlands

${ }^{2}$ Gastroenterology And Hepatology, AMC, Amsterdam/Netherlands

Contact E-mail Address: s.j.hoefnagel@amc.uva.nl

Introduction: Esophageal adenocarcinoma (EAC) is a highly aggressive malignancy with poor prognosis. Advances in therapy have achieved incremental improvements in overall outcome in EAC but over- and undertreatment of undefined subgroups of patients might undermine these benefits (Courrech Staal et al. 2010). The biological diversity of EAC complicates patient selection and treatment stratification and impedes the development of new targeted agents. Further insight into the heterogeneous molecular pathology of EAC and a possible relation to outcomes and response to current treatment strategies is urgent. Aims \& Methods: We included $\sim 75$ patients with EAC and junctional adenocarcinomas between 2012 and 2017. Most patients received neoadjuvant chemo-radiotherapy (nCRT) with carboplatin and paclitaxel. Pre-treatment tissue samples of the tumor and healthy adjacent mucosa were collected during upper gastrointestinal endoscopy. RNA was extracted from all tumor biopsies and a subset of matched healthy biopsies. Samples were sequenced using Illumina sequencing technology. Count files were obtained, which served as input into the $\mathrm{R}$ language and environment for statistical computing for further analyses.

We performed unsupervised hierarchical clustering on the tumor RNA profiles to identify distinct clusters associated with clinical outcome.

Results: We could identify three distinct subtypes with a metabolic, immune and cell cycle regulating signature respectively. The subtype with the immune signature was associated with tendency to poorer response to therapy. We will develop a subtype classifier to perform subtype prediction in an independent patient cohort from the TCGA database (The Cancer Genome Atlas Research 2017). Conclusion: Our studies support the existence of three distinct EAC/junctional subtypes associated with different response to therapy. Based on these subgroups, we will develop an EAC subtype classifier that might improve stratification of patients for (targeted) therapies and subsequently improve outcomes. Disclosure of Interest: All authors have declared no conflicts of interest.

\section{References}

Courrech Staal, E. F., B. M. Aleman, H. Boot, M. L. van Velthuysen, H. van Tinteren, and J. W. van Sandick. 2010. 'Systematic review of the benefits and risks of neoadjuvant chemoradiation for oesophageal cancer', $\mathrm{Br} J$ Surg, 97: 1482-96.

The Cancer Genome Atlas Research, Network. 2017. 'Integrated genomic characterization of oesophageal carcinoma', Nature, 541: 169-75.

\section{P1212 CLINICAL VALUE OF LONG NONCODING RNA TRPM2-AS EXPRESSION IN ESOPHAGEAL SQUAMOUS CELL CARCINOMA}

H. Xü ${ }^{1}$, G. $\mathrm{Yao}^{2}$, L. Lin

${ }^{1}$ Gastroenterology, The First Affiliated Hospital of Nanjing Medical University, Nanjing/China

${ }^{2}$ Thoracic Surgery, The First Affiliated Hospital of Nanjing Medical University Nanjing/China

Contact E-mail Address: xuhairong0519@163.com

Introduction: Esophageal squamous cell cancer (ESCC) is one of the most common carcinomas worldwide. Long noncoding RNAs (lncRNAs) have been reported to play an importance role in the progression of many diseases especially in cancers. Recent studies reveal that lncRNA TRPM2-AS is upregulated in many cancers and may function in tumor development and metastasis. This study aimed to explore the expression of TRPM2-AS and its clinical value in ESCC.

Aims \& Methods: To investigate the function of long noncoding RNA TRPM2AS in ESCC, RT-qPCR was used to monitor the expression level of long noncoding RNA TRPM2-AS in 50 paired ESCC tissues and ESCC cell lines. Moreover, the associations between long non-coding RNA TRPM2-AS expression level and clinical characteristics was analyzed. In addition, over-expression and RNA interference (RNAi) approaches were used to study the biological functions of TRPM2-AS in ESCC cells. Cell growth and proliferation was 
analyzed by cell counting kit- 8 assay. Cell cycle and apoptosis were evaluated by flow cytometric analysis. Protein levels of p53 were determined by western blot analysis. Differences between groups were tested for significance using Student'st-test (two-tailed)

Results: ESCC tissues examined in this study showed an obvious increment in TRPM2-AS expression when compared to normal tissues. Meanwhile, TRPM2AS expression was positively related to lymph nodes metastasis, TNM stage and clinical stage. And upregulated TRPM2-AS expression was turned to be remarkably correlated with the shorter survival of ESCC patients which could act as an independent prognostic factor for both overall survival time and disease-free survival. In addition, overexpression of TRPM2-AS could promote the proliferation and inhibit the apoptosis of ESSC cells, while knockdown of TRPM2-AS had a reverse function. Furthermore, downregulation of TRPM2-AS enhanced the expression of p53 in ESCC cells.

Conclusion: This study suggested that long non-coding RNA TRPM2-AS could be a potential oncogene of ESCC. TRPM2-AS expression might be served as another potential therapeutic target and prognostic biomarker. In addition, our study presents the first evidences that TRPM2-AS contributes a lot to inhibiting apoptosis of ESCC by regulating the expressions of p53 in vitro, which may be a potential oncogene and therapeutic target for ESCC.

Disclosure of Interest: All authors have declared no conflicts of interest

\section{P1213 ROLE OF CD80 EXPRESSION IN INFLAMMATORY- RELATED ESOPHAGEAL CARCINOGENESIS}

M. Scarpa ${ }^{1}$, L. Dall'Olmo ${ }^{2}$, M. Fassan ${ }^{3}$, A. Porzionato ${ }^{4}$, A. Kotsafti ${ }^{1}$,

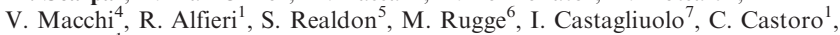
M. Scarpa ${ }^{1}$

${ }^{1}$ Esophageal And Digestive Tract Surgical Unit, Regional Centre For Esophageal Disease, Veneto Institute of Oncology (IOV-IRCCS), Padova/Italy

${ }^{2}$ Ospedale Civile di Venezia, Venezia/Italy

${ }^{3}$ University of Padova, Padova/Italy

${ }^{4}$ Dept. Of Molecular Medicine, University of Padova Dept. of Molecular Medicine, Padova/Italy

${ }^{5}$ Istituto Oncologico Veneto Endoscopy Unit, Padova/Italy

${ }^{6}$ Pathology, Medical School of the Padova University, Padova/Italy

${ }^{7}$ Molecular Medicine, University of Padua, Padova/Italy

Contact E-mail Address: melania.scarpa@iov.veneto.it

Introduction: Esophageal adenocarcinoma (EAC) is an increasingly common cancer with a poor prognosis. EAC is the final step of a pathway starting with esophageal reflux disease, intestinal metaplasia, and dysplasia. The expression of costimulatory molecules such as CD80 and CD86 in the esophageal cancer tissue is significantly lower than in the normal mucosa of healthy patients. This may be one of the mechanisms of immune escape of cancer cells in the esophageal cancer. Aims \& Methods: The aim of this study was to investigate the role of CD80 in the inflammatory esophageal carcinogenesis and to characterize the immune environment of EAC. Mucosa samples from cancer and from healthy esophagus were obtained during esophagectomy from patients affected by EAC. Fresh biopsies were obtained from patients who underwent endoscopy for screening or follow-up. Immunohistochemistry for CD80 was performed. Fresh biopsies were analyzed by flow cytometry to quantify the expression of CD80, its receptor CD28 and the lymphocytes activation marker CD38 on esophageal epithelial cells and CD8 infiltrating lymphocytes, respectively. A model of reflux induced esophageal carcinogenesis was created with a esophago-gastro-jejunostomy: $\mathrm{C} 57 \mathrm{bl} / 6$ mice were randomized to receive or not intraperitoneal injections of anti-CD80 antibody. The esophago-gastric specimens were collected $32 \pm 2$ weeks after randomization and analyzed in a blinded fashion. Non parametric statistics was used.

Results: Flow cytometric analysis of esophageal biopsies from healthy controls, Barrett esophagus, dysplastic esophagus and esophageal adenocarcinoma reveals that the expression of the costimulatory molecule CD 80 by epithelial cells peaks during metaplasia in the inflammatory esophageal carcinogenesis. In the mice that received antiCD80 antibodies the rate of dysplasia in the fore stomach was significantly higher $(5 / 7)$ compared to that observed in vehicle treated wild type mice $(1 / 6)$

Conclusion: The human and the in vivo data that we obtained suggest that in inflammation-driven esophageal carcinogenesis there is evidence of an active immune surveillance process mediated by the overexpression of CD80 costimulatory molecule on esophageal epithelial cells.

Disclosure of Interest: All authors have declared no conflicts of interest.

\section{P1214 TUMOR MICROENVIRONMENT IN ESOPHAGEAL ADENOCARCINOMA: NUCLEAR P53 OVER-EXPRESSION IS ASSOCIATED TO WORSE DISEASE-FREE SURVIVAL}

A. Kotsafti ${ }^{1}$, M. Fassan ${ }^{2}$, L.M. Saadeh ${ }^{3}$, V. Guzzardo ${ }^{2}$, M. Scarpa ${ }^{1}$, F. Cavallin ${ }^{1}$, M. Agostini ${ }^{2}$, M. Cagol $^{1}$, R. Alfieri ${ }^{1}$, M. Rugge ${ }^{4}$, I. Castagliuolo ${ }^{2}$, C. Castoro ${ }^{1}$, M. Scarpa ${ }^{1}$

${ }^{1}$ Esophageal And Digestive Tract Surgical Unit, Regional Centre For Esophageal Disease, Veneto Institute of Oncology (IOV-IRCCS), Padova/Italy

${ }^{2}$ University of Padova, Padova/Italy

${ }^{3}$ Oncological Surgery Unit, Veneto Institute of Oncology, Padova/Italy

${ }^{4}$ Pathology, Medical School of the Padova University, Padova/Italy

Contact E-mail Address: machikots@hotmail.com
Introduction: Esophageal adenocarcinoma (EAC) microenvironment is characterized by several oncogenes activation and oncosuppressor down regulation within the tumor cells, by lack of cytokines with anti-cancer effect and by high expression of immuno-suppressive factors. The interplay between oncogenes' disregulation and immune microenvironment and its effect on patients' prognosis is still largely unknown.

Aims \& Methods: The aim of this study was to evaluate the effect of the interplay between dysregulation of oncogenes and oncosuppressor genes within the tumor cells and immune microenvironment on EAC prognosis. Mucosa samples from EAC tissue were obtained during esophagectomy from 169 consecutive patientsoperated. Immunohistochemistry for MLH1, MSH2, MSH6, PMN2, cMyc, p16, HER2 and nuclear p53 expression was performed. CD8 infiltration, CD8 and NK cells cytolytic activity (CD107) of tumor infiltrating lymphocytes and antigen presenting cells activity within the tumor (CD80) were analysed with immunohistochemistry. Mutational analysis for BRAF was performed. Cox proportional hazard models were created to investigate the role of each marker adjusted for cancer stage. The association between each marker and the presence of nodal metastasis was assessd with Fisher exact test.

Results: In our EAC patients' series, one patients had BRAF mutation (V600E) and 5 of them had microsatellite instability. CD107 overexpression within the cancer was associated to the presence of nodal metastasis $(\mathrm{p}=0.029)$. In patients with EAC, nuclear p53 overexpression revealed to be an independent predictor of early recurrence with $[\mathrm{HR}=2.995(95 \% \mathrm{CI}=1.1224-7.9896) \mathrm{p}=0.029]$ as well as cancer stage $[\mathrm{HR}=2.993(95 \% \mathrm{CI}=1.6693$ to 5.3666$) \mathrm{p}<0.001]$. On the other hand, nuclear p53 overexpression also tended to be an independent predictor of overall survival $[\mathrm{HR}=2.9359(95 \% \mathrm{CI}=0.9613$ to 8.9663$) \mathrm{p}=0.06]$ while cancer stage confirmed to be the main survival predictor $[\mathrm{HR}=1.8689(95 \%$ $\mathrm{CI}=1.0810$ to 3.2314$) \mathrm{p}=0.025]$

Conclusion: In EAC, CD8 and NK cytolyic activity within the tumor was associated to nodal metastasis and CD107 expression might be used as a marker of it. Moreover, nuclear p53 overexpression within the tumor might be used as a marker of early recurrence after esophagectomy and then used to plan follow up strategies. No apparent relation between prognosis and mismatch repair genes expression or BRAF mutation was observed.

Disclosure of Interest: All authors have declared no conflicts of interest.

\section{P1215 PHARMACOLOGICAL INHIBITION OF MONOCARBOXYLATE TRANSPORTER 1 INDUCES APOPTOSIS IN METASTATIC ESOPHAGEAL ADENOCARCINOMA CELLS}

E. Chueca Lapuente ${ }^{1}$, A. Valero Torres ${ }^{2}$, S. Arechavaleta Tabuenca ${ }^{3}$,

M.Á. Sáenz ${ }^{4}$, M.A. García-González ${ }^{5}$, C. Hörndler ${ }^{6}$, A. Lanas ${ }^{4}$, E. Piazuelo ${ }^{1}$

${ }^{1}$ CIBER Enfermedades Hepáticas y Digestivas, Madrid/Spain

${ }^{2}$ Hospital Clinico Universitario Lozano Blesa, Zaragoza/Spain

${ }^{3}$ Instituto de Salud Carlos III, Madrid/Spain

${ }^{4}$ Universidad de Zaragoza, Zaragoza/Spain

${ }^{5}$ Instituto Aragonés de Ciencias de la Salud (IACS), Zaragoza/Spain

${ }^{6}$ Hospital Universitario Miguel Servet, Zaragoza/Spain

Contact E-mail Address: educhuec@gmail.com

Introduction: Altered glucose metabolism has become a recognised feature of tumor cells, which is characterized by an increased glucose uptake and preferential dependence on glycolysis for energy production. As a consequence, cancer cells produce large amounts of lactate, which is pumped out the cytosol by monocarboxylate transporters (MCTs), mainly MCT 1 and 4. MCT inhibition has previously been related to increase apoptosis in cancer cells, but this aspect has not been investigated in esophageal adenocarcinoma (EAC) yet.

Aims \& Methods: We aimed to analyze the expression of MCT1 and 4 in human samples of EAC and to evaluate in vitro the effect of extracellular glucose concentration and pharmacological inhibition of MCT1 on lactate concentration, intracellular $\mathrm{pH}(\mathrm{pHi})$, and cell apoptosis. MCT1 and MCT4 expression was assessed by immunohistochemistry in 29 human samples of EAC. For the in vitro study, two different EAC cell lines were used: OE33 (ECACC), established from an EAC of the lower esophagus and OACM5.1C (ECACC), obtained from a lymph node metastasis derived from an EAC. MCT1 and MCT4 expression and location were assessed by immunocytochemistry in both cell lines. The MCT1 selective inhibitor AZD3965 (0,10 and 100 nM) was added to the culture medium under a normoxic and hypoxic atmosphere in standard $(11 \mathrm{mM})$ or high $(30 \mathrm{mM})$ glucose content in the media. Apoptosis was determined by flow cytometry (Annexin V-FITC and propidium iodide). Intracellular lactate concentration was evaluated in a colorimetric assay. pHi was evaluated by flow cytometry with the probe SNARF-1 AM. Experiments were performed at least in triplicate. Statistical analysis was performed by student-t test.

Results: MCT1 and MCT4 expression was found in all the EAC samples evaluated. MCT1 expression was confined to tumor cells, with $62 \%$ of the biopsies showing moderate or intense staining, whereas MCT4 was expressed in both tumor and stromal cells, with $40 \%$ of moderate/intense stained samples. Both cell lines expressed MCT1 transporter, whereas MCT4 expression was present in OE33 cells and only in a small population of the metastatic cell line. High extracellular glucose concentration increased intracellular lactate levels in OE33 cells but not in the metastatic line, and did not have any effect either in $\mathrm{pHi}$ or in apoptosis under any of the conditions evaluated (normoxia and hypoxia) in both cell lines. Treatment with AZD3965 (10 and $100 \mathrm{nM})$ increased intracellular lactate concentration in OE33 and OACM $5.1 \mathrm{C}$ cells, but this increase was higher $(600-700 \%)$ in the metastatic cell line than in OE33 cells $(50-70 \%)$. Under normoxic conditions AZD3965 significantly increased pHi of both cell lines whereas under hypoxic atmosphere had no effect on metastatic cells. The highest concentration $(100 \mathrm{nM})$ of the MCT1 inhibitor significantly 
increased apoptosis of OACM5.1C cells whereas did not affect apoptosis of OE33 cells.

Conclusion: Metastatic and non-metastatic esophageal adenocarcinoma cells exhibit different glycolitic metabolism and response to pharmacological inhibition of MCT1, which increases apoptosis in metastatic cells. Further preclinical studies are necessary in order to define the potential of blocking lactate transporters on the treatment of metastatic EAC.

Disclosure of Interest: All authors have declared no conflicts of interest.

\section{P1216 THE PREDICTIVE FACTOR FOR PERFORATION IN \\ ESOPHAGEAL ESD}

Y. Nagami, M. Ominami, M. Shiba, S. Fukunaga, F. Tanaka, N. Kamata, H. Yamagami, T. Tanigawa, T. Watanabe, Y. Fujiwara

Graduate School Of Medicine, Osaka City University Graduate School of Medicine Dept. of Gastroenterology, Osaka/Japan

\section{Contact E-mail Address: yasuaki1975@hotmail.com}

Introduction: Although endoscopic submucosal dissection (ESD) is accepted as a standard treatment for early stage esophageal neoplasia, esophageal perforation is sometimes experienced as main adverse event. Esophageal perforation causes mediastinal emphysema, mediastinitis, and pneumothorax, those sometimes require emergency surgery.

Aims \& Methods: We evaluated the predictive factors for esophageal perforation in patients who received esophageal ESD. This was a retrospective observational study in a single institution. Between May 2004 and March 2016, 549 consecutive patients with 927 esophageal lesions were treated with ESD. Exclusion criteria was cases with lacking data. The primary outcome was determination of the predictive factors for esophageal perforation in patients who received esophageal ESD. The secondary outcome was clinical outcomes. Perforation was defined as a visual hole in the esophageal wall exposing the mediastinal cavity. Logistic regression multivariate regression analysis with generalized estimating equations were used to analyze repeated measures data.

Results: A total of 549 cases with 927 lesions were evaluated. Of those, perforation occurred in 15 cases $(2.7 \%)$ with 15 lesions $(1.6 \%)$. A lesion diameter (Odds ratio; $\mathrm{OR}=1.05,95 \%$ confidence intervals; CI: $1.02-1.07, p<0.001$ ) and the proximity of the tumor to a previous ESD scar (OR $=6.66,95 \%$ CI: $1.80-24.6, p=0.004)$ were both associated with perforation using crude logistic regression analysis. Multivariate logistic regression analysis also showed that a lesion diameter $(\mathrm{OR}=1.05,95 \% \mathrm{CI}: 1.03-1.07, p<0.001)$ and the proximity of the tumor to a previous ESD scar (OR $=13.0,95 \%$ CI: $2.48-67.9, p=0.002)$ were independent predictive factors for perforation.

Conclusion: Larger lesion and the proximity of the tumor to a previous ESD scar increased the likelihood of perforation in patients who received esophageal ESD. Disclosure of Interest: T. Tanigawa: Faculty member of a course sponsored by EA pharma Co., Ltd

T. Watanabe: Faculty member of a course sponsored by EA pharma Co., Ltd. Y. Fujiwara: Faculty member of a course sponsored by EA pharma Co., Ltd. Research grants from Ono.

All other authors have declared no conflicts of interest.

\section{P1217 SAFETY AND EFFICACY OF CHEMORADIOTHERAPY AFTER ENDOSCOPIC RESECTION IN PATIENTS WITH SUPERFICIAL ESOPHAGEAL SQUAMOUS-CELL CARCINOMA}

T. Wada ${ }^{1}$, C. Katada ${ }^{1}$, M. Azuma ${ }^{1}$, T. Yano ${ }^{1}$, S. Tanabe ${ }^{2}$, W. Koizumi ${ }^{1}$, H. Moriya ${ }^{3}$, K. Yamashita ${ }^{3}$, S. Komori ${ }^{4}$, K. Ishido ${ }^{1}$

${ }^{1}$ Gastroenterology, Kitasato University, School of Medicine, Sagamihara/Japan

${ }^{2}$ Research And Development Center For New Frontier, Kitasato University School of Medicine, Sagamihara/Japan

${ }^{3}$ Department Of General Surgery, Kitasato University, School of Medicine, Sagamihara/Japan

${ }^{4}$ Department Of Radiology And Radiation Oncology, Kitasato University,

School of Medicine, Sagamihara/Japan

Contact E-mail Address: t.wada@kitasato-u.ac.jp

Introduction: According to the current Japanese guidelines for the diagnosis and treatment of esophageal cancer, endoscopic resection is indicated for pathological T1a (epithelium/lamina propria mucosae) and relatively indicated for Tla(muscularis mucosae) and T1b(a tumor invading the submucosa to a depth of $200 \mu \mathrm{m}$ or less). In accordance with the guidelines, we have actively performed endoscopic resection for esophageal squamous-cell carcinoma(ESCC). Evidence of submucosal or lymphovascular invasion on histopathological examination of the resected specimens was considered to indicate non-curative resection, chemoradiotherapy (CRT) was additionally administered, taking into account the risk of lymph-node metastasis. In principle, CRT comprised 2 courses of cisplatin plus 5-fluorouracil. Patients who were 76 years or older or who had mild renal dysfunction received 2 courses of nedaplatin plus 5-fluorouracil. The radiation dose was 50.4 Gy in patients who had a tumor-positive vertical margin and $41.4 \mathrm{~Gy}$ in those who had a tumor-negative vertical margin. The field of radiation was determined according to the recommendations of the Japan Clinical Oncology Group Study JCOG0508(UMIN-CTR: identification number UMIN000000553).

Aims \& Methods: We retrospectively studied patients who underwent endoscopic resection in our hospital to evaluate the clinical utility of additional CRT in patients who had evidence of submucosal or lymphovascular invasion on histopathological examination of the endoscopically resected specimens. Among
364 patients with ESCC (425 lesions) who underwent endoscopic resection from January 2000 through July 2015, a total of 93 patients (93 lesions) had a histopathological diagnosis with submucosal or lymphovascular invasion. Among these 93 patients, 41 received additional CRT (CRT group), and 52 were followed without CRT(follow-up group). CRT comprised cisplatin plus 5-fluorouracil and radiation in 37 patients, nedaplatin plus 5-fluorouracil and radiation in 2, docetaxel plus cisplatin, 5-fluorouracil, and radiation in 1, and radiation alone in 1 .

Results: The median age was 68 years (53 to 79 ) in the CRT group and 72 (59 to $89)$ in the follow-up group $(\mathrm{p}=0.01)$. The median tumor diameter was $22 \mathrm{~mm}$ ( 6 to 55$)$ in the CRT group and 25 ( 3 to 47 ) in the follow-up group $(p=0.63)$. The tumor invades the MM in 9 patients, the SM1 in 3, and the submucosa to a

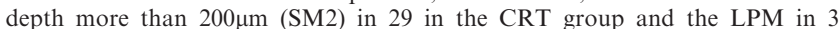
patients, the MM in 16, the SM1 in 18 , and the SM2 in 15 in the follow-up group $(\mathrm{p}=0.91)$. Lymphatic invasion was positive in 21 patients in the CRT group and 12 in the follow-up group $(\mathrm{p}<0.01)$. Vascular invasion was positive in 27 patients in the CRT group and 29 in the follow-up group $(\mathrm{p}=0.32)$. Involvement of the submucosal vertical margin was found in 7 patients in the CRT group and 9 in the follow-up group $(\mathrm{p}=0.97)$. CRT-related grade 3 or 4 early adverse events were leukopenia $24.3 \%$ (10 patients), neutropenia $29.3 \%$ (12), febrile neutropenia $4.9 \%$ (2), diarrhea $2.4 \%$ (1), anorexia $17.0 \%$ (7). In the CRT group, 38 of 40 patients received chemotherapy as scheduled. Treatment was discontinued in the second course in 2 patients, and 7 required dose reduction. Lymph-node metastasis were found in 2 patients in the CRT group and 7 in the follow-up group $(\mathrm{p}=0.15)$. In 2 patients with recurrence in the CRT group, lymph-node metastases were seen in the irradiated field 46 and 49 months after treatment, respectively. 1 patient in the CRT group and 3 in the follow-up group died of esophageal cancer $(\mathrm{p}=0.43)$. The overall survival $(\mathrm{OS})$ rate at 2 years was $97.1 \%$ in the CRT group and $93.8 \%$ in the follow-up group $(\mathrm{p}=0.02)$. The relapse-free survival (RFS) rate at 2 years was $97.1 \%$ in the CRT group and $83.4 \%$ in the follow-up group $(\mathrm{p}=0.02)$

Conclusion: Additional CRT after endoscopic resection in patients with esophageal squamous-cell carcinoma who have submucosal invasion, lymphovascular involvement, or vertical-margin invasion can become an effective organ-preservation strategy.

Disclosure of Interest: All authors have declared no conflicts of interest.

\section{Reference}

Muto M, Minashi K, Nihei K, et al. Efficacy of combined endoscopic resection and chemoradiotherapy for clinical stage I esophageal squamous-cell carcinoma (ESCC): A single-arm confirmatory study (JCOG0508). J Clin Oncol 34, 2016 (suppl; abstr 4013)

\section{P1218 SAFETY, EFFICACY AND OUTCOME OF ENDOSCOPIC SUBMUCOSAL DISSECTION FOR THE TREATMENT OF EARLY BARRETT'S NEOPLASIA}

M. Omae, M. Konradsson, P. Elbe, M. Nilsson, M. Lindblad, I. Rouvelas, F. Baldaque-Silva

Gastrocentrum, Karolinska University Hospital, stockholm/Sweden

\section{Contact E-mail Address: masami.nego@jfcr.or.jp}

Introduction: Endoscopic submucosal dissection (ESD) was developed in Japan for en bloc resection of large gastrointestinal neoplasias and has progressively been adopted in the West. Currently, early Barrett's neoplasia is mainly treated with endoscopic mucosal resection (EMR) and/or radiofrequency ablation, being the role of ESD in this context not well-established yet. Our aim is to evaluate the safety, efficacy and outcome of ESD for the treatment of early Barrett's neoplasia.

Aims \& Methods: Fifty consecutive ESD cases of early Barrett neoplasia were performed in 42 patients in our center between 2011 and 2016. All ESDs were performed under full narcosis after multidisciplinary team conference discussion and patient's consent. The primary endpoint was the rate of en bloc resection. Secondary endpoints included rate of R0 and curative resection, a comparison of pre- and post- ESD histology, procedure time, procedure-related adverse events, and rate of remission at follow-up. This study was approved by the Stockholm Regional Ethical Committee.

Results: Mean age was 67 years-old (range $46-84$ ), being $74 \%$ male and $72 \%$ long segment BE. The mean specimen size was $52 \mathrm{~mm}$ (range $16-150 \mathrm{~mm}$ ). ESD resections included $<25 \%, 25-50 \%, 50-75 \%$ and $75-100 \%$ of the lumen circumference in $4 / 31 / 12 / 3$ of cases, respectively. En bloc, R0 and curative resection were obtained in $96 \%(48 / 50), 80 \%(40 / 50)$ and $70 \%(35 / 50)$ of cases, respectively. The pre-ESD histology corresponded to low-grade dysplasia $(n=5)$, high-grade dysplasia $(n=30)$ and adenocarcinoma $(n=15)$. One case of LGD was upstaged to intramucosal AC, 10/30 cases of HGD were upstaged to adenocarcinoma. In 8/ 15 cases of AC, there was submucosal invasion on the ESD specimen. In 14/50 of resected specimens there was multifocal neoplasia. The mean procedure time was 120 minutes. There were 2 perforations $(4 \%)$ treated endoscopically and $2(4.0 \%)$ postoperative bleedings treated conservatively. Six patients $(12 \%)$ developed esophageal strictures that were managed endoscopically. The 30 days mortality was $0 \%$. In the 15 non-curative cases, 10 went through esophagectomy, 2 patients to further ESD, 1 received chemoradiotherapy and 2 patients are under surveillance. In the 10 esophagectomy cases, 4 patients had AC in the remnant Barrett's esophagus and 2 patients had lymph node metastasis. Complete remission was 
found in $100 \%(35 / 35)$ of patients with curative resection at median follow-up of 21 months (4-60 months).

Conclusion: In the proper setting, ESD is safe and effective for the treatment of early Barrett's neoplasia with high en bloc and complete resection rates and good curative rate. ESD enables full pathological assessment in lesions not suitable for en bloc resection with EMR. There were no recurrences in the curative cases, which increases the role of ESD for the management of early Barrett's neoplasia. Disclosure of Interest: All authors have declared no conflicts of interest.

\section{P1219 ENDOSCOPIC EVALUATION AT THE PRIMARY SITE OF CT1 ESOPHAGEAL CANCER AFTER PROTON BEAM THERAPY AND CLINICAL RESULTS OF SALVAGE ENDOSCOPIC THERAPY FOR LOCAL RECURRENCE}

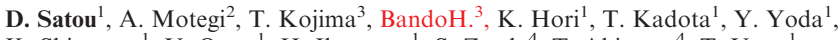
K. Shinmura ${ }^{1}$, Y. Oono ${ }^{1}$, H. Ikematsu ${ }^{1}$, S. Zenda ${ }^{4}$, T. Akimoto ${ }^{4}$, T. Yano ${ }^{1}$ ${ }^{1}$ Dept. Of Gastroenterology, Endoscopy Division, National Cancer Center Hospital East, Kashiwa, Japan., kashiwa/Japan

${ }^{2}$ National Cancer Center Hospital East, Kashiwa/Japan

${ }^{3}$ Gastroenterology And Oncology, National Cancer Center Hospital East, kashiwa Japan

${ }^{4}$ Division Of Radiation Oncology, National Cancer Center Hospital East, Kashiwa/ Japan

Contact E-mail Address: dsatou@ncc.go.jp

Introduction: Recently, it has been reported that proton beam therapy (PBT) is the effective treatment for patients with esophageal squamous cell carcinoma (ESCC). However, there are few reports regarding the endoscopic evaluation of efficacy after PBT at the primary site.

Aims \& Methods: The aim of this study is to clarify the adequate endoscopic evaluation for primary lesion of ESCC after PBT, and the clinical results of salvage endoscopic treatment for local recurrence. Patients with clinical T1 ESCC, and who had been treated with PBT between April 2013 and June 2016 at the National Cancer Center Hospital East were investigated. The total dose of PBT was 60 Gray-Equivalent (GyE). The efficacy of PBT at the primary site was evaluated with endoscopy, and the definition of complete response (CR) was used according to the same criteria as that of conventional chemoradiotherapy (CRT) as follows; disappearance of tumor lesion and ulcer, and absence of cancer cells with biopsy was verified. The endoscopic evaluation was performed within 2 months after the completion of PBT, and we repeatedly evaluated every month if the lesion did not achieve CR. The treatment for local recurrence after PBT was chosen based on the depth of the tumor as follows; endoscopic resection (ER) for cT1a, esophagectomy or photodynamic therapy (PDT) for cT1b or deeper depending on patient s condition.

Results: Among 44 patients who underwent PBT, the median age was 70 years (range, 41-79). The number of patients with clinical stage I was $23(52 \%)$, and those with stage II, III, and IV were $16(36 \%), 2(5 \%)$, and $3(7 \%)$, respectively. All patients underwent concurrent systemic chemotherapy. 43 patients $(98 \%)$ could achieve a CR at the primary site and only one patient $(2 \%)$ did not show a CR (non-CR) at the primary site. The median time to CR from the start of PBT at the primary site was 85 days (range, 70-554 days) and 6 months or longer period was required to confirm CR due to the remaining PBT induced erosion or ulceration in 7 patients $(15 \%)$. One patients whose primary site did not reached to CR showed prolonged ulceration for 385 days. Of the 43 patients, 6 patients $(14 \%)$ developed local recurrence without regional lymph node recurrence or distant metastasis, and the median time to local recurrence from CR was 257 days (range, 111-722 days). The endoscopic finding of local recurrence was resembling submucosal tumors (SMT) in 3 and flat lesion in other 3 tumors., All 6 patients with local recurrence were indicated for endoscopic treatment (ER:4, PDT:2). No complications, such as major bleeding or perforation, were observed. And, 6 patients $(100 \%)$ were alive without any recurrence at the median follow up period of 11 months (range, 1-32 months).

Conclusion: Longer period was required to confirm CR after PBT with chemotherapy in some cases comparing with the historical reports of that of conventional CRT. Careful closed endoscopic follow-up according to the way of conventional CRT enabled early detection of local recurrence and preferable local control with salvage endoscopic treatment.

Disclosure of Interest: All authors have declared no conflicts of interest.

\section{P1220 CLINICAL OUTCOMES OF ENDOSCOPIC SUBMUCOSAL DISSECTION FOR SUPERFICIAL ESOPHAGEAL NEOPLASMS OF PATIENTS WITH HEAD AND NECK CANCER}

J. F.D. Oliveira ${ }^{1}$, E. Q. Mendonça ${ }^{1}$, B. D.C. Martins ${ }^{1}$, F. Kawaguti ${ }^{1}$, M. S. De Lima ${ }^{1}$, S. Geiger ${ }^{1}$, C. Pennacchi ${ }^{1}$, C. C. Gusmon ${ }^{1}$, R. Uemura ${ }^{1}$ E. R. Baba ${ }^{1}$, A. V. Safatle-Ribeiro', L.H. Lenz Tolentino ${ }^{1}$, G. De Paulo', M. Sorbello ${ }^{1}$, M. Minata ${ }^{1}$, R. N. Moura ${ }^{1}$, U. Ribeiro Jr. ${ }^{1}$, F. Maluf-Filho ${ }^{1}$ ${ }^{1}$ Gastrointestinal Endoscopy Unity, Cancer Institute of University of São Paulo, Sâo Paulo/Brazil

Contact E-mail Address: ktpennacchi@yahoo.com.br

Introduction: Surveillance programs of gastrointestinal endoscopy for detection of a second primary cancer in patients with head and neck squamous cell carcinoma (HNSCC) are very important since it can detect synchronous or metachronous esophageal squamous cell carcinoma (ESCC) in up to $15 \%-25 \% 1$. The detection of ESCC in an early phase has paramount importance, since superficial lesions are amenable to endoscopic submucosal dissection (ESD) 2.
Aims \& Methods: The aim of this study was to investigate the clinical outcomes of ESD in superficial esophageal neoplasm (SEN) of HNSCC patients in an oncology tertiary center. From 2010 to 2016, 3280 endoscopies were performed in patients with HNSCC and in 1887 chromoscopy with Lugol and NBI were performed. A total of $26 \mathrm{SENs}$, submitted to ESD, in 25 patients were retrospectively analyzed.

Results: The median tumor size was $4,37 \mathrm{~cm}( \pm 1.83)$. The en bloc resection were $100 \%$ and free margin (R0) were $92.3 \%$. The two patients with positive margins had a depressed component in endoscopic evaluation. Recurrence occurred in $11,5 \%(3 / 26)$ and one of these patients was successfully treated with mucosectomy. The circumferential extension, number of patients e stenosis rate are show in table 1

Table 1: The circumferential extension, number of patients and stenosis rate

\begin{tabular}{lll}
\hline $\begin{array}{l}\text { Circumferential } \\
\text { Extension }\end{array}$ & $\begin{array}{l}\text { Number } \\
\text { of lesions }\end{array}$ & $\begin{array}{l}\text { Stenosis } \\
\text { Rate }\end{array}$ \\
\hline $25-49 \%$ & $1(2.56 \%)$ & $0(0 \%)$ \\
$50-74 \%$ & $14(53.84 \%)$ & $5(35.71 \%)$ \\
$75-99 \%$ & $6(23.07 \%)$ & $2(33.33 \%)$ \\
$100 \%$ & $5(19.23 \%)$ & $5(100 \%)$ \\
$75-100 \%$ & $11(42.30 \%)$ & $7(63.63 \%)$ \\
\hline
\end{tabular}

The circumference of the resection $\geq 75 \%$ was significantly associated with postoperative stricture $(\mathrm{OR}=3.5 ; \mathrm{P}<0.05)$. The average number of endoscopic dilatations for resolution of stenosis was $9.16( \pm 7.62)$. No procedure-related mortality occurred. Follow-up data median was 11 months.

Conclusion: Endoscopic surveillance of HNSCC is very important for SENs detecting. Esophageal ESD of these lesions are feasible and safe with acceptable complication risks despite the high rates of stenosis in resections $>75 \%$ of the circumference.

Disclosure of Interest: All authors have declared no conflicts of interest.

\section{References}

1. Sturgis EM, Miller RH. Second primary malignancies in the head and neck cancer patient. Ann Otol Rhinol Laryngol 1995;104: 946-954

2. Toyanaga T, Man IM, East JE et al (2013) 1,635 endoscopic submucosal dissection cases in the esophagus, stomach, and colorectum: complication rates and long-term outcomes. Surg Endosc 27:1000-1008

P1221 ENDOSCOPIC CRYOBALOON ABLATION IS SAFE, WELL-TOLERATED AND HIGHLY EFFECTIVE IN THE ERADICATION OF ESOPHAGEAL SQUAMOUS CELL NEOPLASIA

K. Yan ${ }^{1}$, S. Van Munster ${ }^{2}$, C. $\mathrm{Jian}^{3}$, L. Fugang ${ }^{4}$, Z. Deli ${ }^{3}, \mathrm{~W} . \mathrm{Li}^{1}, \mathrm{~S} . \mathrm{He}^{5}$, Y. Zhang ${ }^{6}$, D. Lizhou ${ }^{1}$, Y. Liu ${ }^{5}$, L. Xudong ${ }^{6}$, L. Xue ${ }^{7}$, L. Ning ${ }^{8}$, S. Dawsey ${ }^{9}$, J.J. G.h.m. Bergman ${ }^{2}$, B.L.a.m. Weusten ${ }^{9}$, G. Wang ${ }^{6}$

${ }^{1}$ Dpt. Of Endoscopy, Cancer Institute and hospital, Chinese Academy of Medical Sciences, Beijing/China

${ }^{2}$ Gastroenterology And Hepatology, AMC, Amsterdam/Netherlands

${ }^{3}$ Dpt. Of Endoscopy, Feicheng's People's hospital, Feicheng/China

${ }^{4}$ Dpt. Of Endoscopy, Donping's People's hospital, Donping/China

${ }^{5}$ Cancer Institute and Hospital, Chinese Academy of Medical Sciences, Beijing/ China

${ }^{6}$ Cancer Institute and Hospital, Chinese Academy of Medical Sciences \& Peking Union Medical College, Beijing/China

${ }^{7}$ Dpt. Of Pathology, Cancer Institute and Hospital, Chinese Academy of Medical Sciences, Beijing/China

${ }^{8}$ National Cancer Institute, Bethesda/United States of America/MD

${ }^{9}$ Dpt. Of Gastroenterology And Hepatology, Universy Medical Center Utrecht, Utrecht/Netherlands

Contact E-mail Address: keyan@cicams.ac.cn

Introduction: Globally, $80 \%$ of all esophageal cancer cases are esophageal squamous cell cancer (ESCC), arising from esophageal squamous cell neoplasia (ESCN). Patients with ESCC have poor prognosis, but when diagnosed at the stage of ESCN, curative endoscopic treatment can be performed. ESCN mainly occurs in developing countries, often with limited endoscopic expertise and resources, like Central and Eastern Asia and Eastern and Southern Africa. Hence, an easy-to-use, low-cost treatment for ESCN would be of great value. Focal Cryoballoon Ablation therapy (FCBA) (C2 Therapeutics Inc. Redwood City, $C A, U S A)$ is a new endoscopic ablation therapy that comprises a through-the-scope catheter with a conformable balloon that obviates the need for sizing, a handle, and a small disposable cryogen cartridge. The balloon is simultaneously inflated and cooled with nitrous oxide from the cartridge, resulting in ice patches of approximately $2 \mathrm{~cm}^{2}$. FCBA is easy to use and requires no capital equipment. Early studies for FCBA of Barrett's esophagus have shown promising results, however, limited data are available for FCBA of ESCN. In this study we aimed to assess the safety, tolerability and efficacy of FCBA in the eradication of ESCN.

Aims \& Methods: In this ongoing multi-center prospective trial in China, patients with one flat type (Paris 0-IIb) unstained lesion (USL) on Lugol's chromoscopy, $<6 \mathrm{~cm}$ in length and $<50 \%$ of circumference, with a confirmed diagnosis of Moderate or High Grade Intraepithelial Neoplasia (MGIN/HGIN) were 
enrolled. At baseline, side-by-side ablations of 10 seconds were performed on USLs. Treatment was repeated every 3-months until eradication of ESCN was confirmed. Outcomes: safety and tolerability (11-point visual analog scale (VAS) for pain), complete response (CR) rates (absence of MGIN or worse in biopsies), neoplastic progression and adverse events.

Results: We enrolled 80 patients (63 MGIN, 17 HGIN) with a median lesion of 2 (IQR $2-3$ ) cm in length. Of these, 79 patients $(99 \%$ ) were successfully treated; 3 developed superficial, self-limited mucosal lacerations upon balloon inflation and 2 of them were successfully re-ablated 3 months later. A median of 5 (IQR 4-7) side-by-side ablations were performed per patient, in a median ablation time of 8 (IQR 5-10) minutes. As of April 2017, 77/79 (97\%) patients completed a 3-month follow up endoscopy and $69 / 77$ patients $(89 \%)$ exhibited endoscopic and histologic CR. Eight patients had residual USL and were again treated; 5 had a CR 3 months later and results for other 3 pts are pending. To date, 4 patients have undergone a 12 month endoscopy and all continue to exhibit endoscopic and histologic CR. No significant strictures have been noted on follow-up. Three patients developed fever shortly after treatment which was treated with aspirin. Post-procedure median VAS was 1 (IQR $0-2$ ) at day 2, and 0 (IQR $0-0)$ at days 7 and 30 .

Conclusion: Preliminary results of our multicenter open prospective cohort study suggest that FCBA of ESCN is safe, well-tolerated, and highly effective in inducing endoscopic and histologic remission. Longer term (12 month) follow-up data is pending.

Disclosure of Interest: All authors have declared no conflicts of interest

\section{P1222 THE ENDOSCOPIC TREATMENT STRATEGY FOR SUPERFICIAL ESOPHAGEAL CANCER}

\section{N. Yamaguchi}

Department Of Gastroenterology And Hepatology, Nagasaki University Hospital, Nagasaki/Japan

Contact E-mail Address: naoyuki3435@nagasaki-u.ac.jp

Introduction: Endoscopic submucosal dissection (ESD) allows en bloc removal of superficial esophageal squamous cell carcinoma (SCC). However, esophageal stricture often occurs after ESD when the lesion involves more than three-fourth of the circumference of the lumen. Frequent balloon dilatation via endoscopy is required in such situation, thus causing health economic problem. In this study, we investigated the clinical outcomes, and prevention of post-ESD stenosis.

Aims \& Methods: A total of 667 cases in 516 consecutive patients were treated by ESD in our department from April 2006 to December 2016. We investigated the following 2 items. 1. Clinical outcomes and complications. 2. Usefulness of oral steroids administration, the local steroids injection, endoscopic transplantation of tissue-engineered autologous oral mucosal epithelial cell sheets, or steroid oral + local injection combination therapy for the prevention of post-ESD stenosis.

Results: 1. Clinical outcomes: En bloc resection rate was $99.8 \%$ and en bloc curative resection rate was $90.0 \%$. The rate of perforation, post-ESD bleeding, and post-ESD stenosis was $0.2 \%, 0.8 \%$ and $6.1 \%$, respectively. 2. Prevention of post-ESD stenosis: (1) Oral steroid vs Steroid injection vs Cell sheet transplantation: In oral steroid group, the stenosis rate was $14.9 \%$, and the ulcer healing period was 59.5 days. In steroid injection group, the stenosis rate was $12.9 \%$, and the ulcer healing period was 66.0 days. In cell sheet transplantation group, the stenosis rate was $40.0 \%$ and the ulcer healing period was 36.0 days. There was no significant difference between these 3 therapies, and these therapies prevent postESD stenosis to significant extent. However, ulcer healing period of the cell sheet transplantation was significantly shorter compared with the other 2 therapies. (2) The usefulness of SH oral + local injection combination therapy. We investigated limitations of steroid administration, and cell sheet transplantation in order to prevent post-ESD stenosis. The following 4 factors (more than $9 / 10$ of circumferential resection, more than $5 \mathrm{~cm}$ of longitudinal resection, cervical esophagus, post history of chemo-radiation therapy or endoscopic resection) were the stenosis prevention treatment-resistance factors. Therefore, we examined the stenosis rate according to the number of these 4 factors. The stenosis rate of the cases which have 0 or 1 factor, the case which has more than 2 factors in semicircular cases, and the complete circular cases is $4.9 \%, 30.3 \%$, and $44.8 \%$, respectively. The stenosis rate of the cases which have more than 2 factors and complete circular cases are significantly higher, compared to the cases which have 0 or 1 factor. As a result, the cases which have more than 2 factors and complete circular cases were regarded as the stenosis prevention treatment-resistant cases. In contrast, in $\mathrm{SH}$ oral + local injection combination therapy, the stenosis rate of the cases which have more than 2 factors and the complete circular cases is $12.5 \%$ and $14.3 \%$, respectively. Taken together, the stenosis rate of $\mathrm{SH}$ oral + local injection combination therapy is significantly lower, compared to the other 3 therapies.

Conclusion: Esophageal ESD achieved high en bloc resection rate and curability with low rates of complications. Oral steroid, steroid injection therapy and cell sheet transplantation may be effective treatment strategy for reducing post-ESD stenosis. However, the above-mentioned 4 factors are the stenosis prevention treatment-resistant factors in these 3 therapy cases. Furthermore, the cases which have more than 2 factors and complete circular cases were regarded as the stenosis prevention treatment-resistant cases. SH oral + local injection combination therapy is very useful for prevention of post-ESD stenosis and has a potential to overcome treatment-resistant stenosis.

Disclosure of Interest: All authors have declared no conflicts of interest.
P1223 DIAGNOSIS OF TUMORS IN THE CERVICAL AND UPPER

THORACIC ESOPHAGUS: EFFICACY OF ENDOSCOPIC ULTRASONOGRAPHY USING A JELLY-FILLING METHOD WITH WATER-SOLUBLE LUBRICATING JELLY

N. Matsuura ${ }^{1}$, N. Hanaoka ${ }^{1}$, R. Ishihara ${ }^{1}$, S. Yamamoto ${ }^{1}$, T. Akasaka ${ }^{1}$, Y. Takeuchi ${ }^{1}$, K. Higashino ${ }^{1}$, N. Uedo ${ }^{1}$, H. Iishi $^{1}$

'Gastrointestinal Oncology, Osaka international Cancer Institute, Osaka/Japan

Contact E-mail Address: ibura9@yahoo.co.jp

Introduction: Endoscopic ultrasonography (EUS) is the standard modality for qualitative diagnosis of submucosal tumors (SMTs) and determining the depth of invasion of esophageal cancer. Standard EUS, however, comprises a continuous water-filling or water-filled balloon method, which creates some problems (e.g., difficulty filling the water, aspirating water, poor image quality because of balloon compression). Aspiration of water is especially problematic during the diagnosis of lesions in the cervical or upper thoracic esophagus. To resolve such disadvantages, we recently reported a method that includes probe EUS with a jelly-filling technique (EUS-J) for evaluating superficial esophageal squamous cell carcinoma (SCC). The procedure is characterized by filling the esophageal lumen with a water-soluble lubricating jelly (K-Y lubricating jelly; Johnson \& Johnson, Tokyo, Japan) that is used for routine endoscopy and is harmless to humans. In the current study, we retrospectively evaluated the usefulness of EUS-J with water-soluble lubricating jelly for lesions located in the cervical and upper thoracic esophagus.

Aims \& Methods: Patients with an esophageal SCC or SMT in the cervical or upper thoracic esophagus were included. EUS-J with water-soluble lubricating jelly was performed using a high-resolution probe. Before examination, several 5-mL syringes containing the Water Soluble Lubricating Jelly were prepared. With the patient under conscious sedation with midazolam, an endoscope (GIF-2TQ260M; Olympus, Tokyo, Japan) was inserted into the targeted area in the esophagus. A $30-$ or $20-\mathrm{MHz}$ miniature probe was then inserted through the left channel of the endoscope, and $40 \mathrm{~mL}$ of jelly was instilled through the right channel until the esophageal lumen was filled.

Results: From December 2010 to March 2017, we used EUS-J to evaluate 61 patients with esophageal SCCs and 10 with SMTs in the cervical or upper thoracic esophagus. There were 10 lesions in the cervical esophagus and 61 in the upper thoracic esophagus. Among the 61 SCCs, 13 were recurrences following chemoradiotherapy. The other 48 SCCs did not undergo chemoradiotherapy. Of these 61 patients with esophageal SCC, 60 lesions $(98.3 \%)$ could be detected with EUS-J. 44 lesions treated either by esophagectomy $(n=7)$ or endoscopic resection $(\mathrm{n}=37)$. Histologic diagnosis was T1a in 27 lesions, T1b in 17 lesions. The overall accuracy of diagnosing invasion depth was $70.5 \%$ ( $31 / 44$ lesions) by EUS. Among the 10 SMTs, we diagnosed seven leiomyomas derived from muscularis mucosa and one lesion due to vertebral body compression. The remaining two lesions were, respectively, a diminutive SMT $(<3 \mathrm{~mm})$ and a small lesion in the cervical esophagus adjacent to the hypopharynx. Neither was detectable using EUS-J because of their small size and difficulty with instrumental maneuvering. There were no adverse events during EUS-J, including aspiration pneumonia. Conclusion: EUS-J with water-soluble lubricating jelly is useful and safe for diagnosing lesions in the cervical or upper thoracic esophagus. To our knowledge, this is the first report of using EUS with lubricating jelly for lesions located in this anatomic region.

Disclosure of Interest: All authors have declared no conflicts of interest.

\section{Reference}

Hanaoka N, Ishihara R, Matsuura M, et al. Esophageal EUS by filling watersoluble lubricating jelly for diagnosis of depth of invasion in superficial esophageal cancer. Gastrointest Endosc. 2015;82:164-5.

\section{P1224 OPTIMAL SURGERY TIME AFTER ENDOSCOPIC RESECTION IN EARLY GASTRIC CANCER: LONG-TERM FOLLOW- UP STUDY FOR SURGICAL AND ONCOLOGICAL SAFETY}

J.H. Cha ${ }^{1}$, J. Kim ${ }^{1}$, S.H. Choi ${ }^{2}$, D.H. Jung ${ }^{1}$, J.J. Park ${ }^{1}$, Y.H. Youn ${ }^{1}$, H. Park ${ }^{1}$, H. Kim ${ }^{3}$, J. Cheong ${ }^{3}$, W.J. Hyung ${ }^{3}$, S.H. Noh ${ }^{3}$

${ }^{1}$ Department Of Internal Medicine, Gangnam Severance Hospital, Yonsei University College of Medicine, Seoul/Korea, Republic of

${ }^{2}$ Department Of Surgery, Gangnam Severance Hospital, Yonsei University College of Medicine, Seoul/Korea, Republic of

${ }^{3}$ Department Of Surgery, Severance Hospital, Yonsei University College of Medicine, Seoul/Korea, Republic of

Contact E-mail Address: TRISTAN82@yuhs.ac

Introduction: The patients with early gastric cancer (EGC) who have undergone non-curative endoscopic resection (ER) need additional surgery. Our previous study reported short-term data about 29 days were optimal time when considering better surgical outcome. (Ann Surg Oncol. 2014 Jan;21(1):232-9.). This study is long-term follow-up study to evaluate the impact of previously proposed optimal time interval from ER to additive surgery by on the surgical and oncological outcomes.

Aims \& Methods: A total of 2850 patients who were diagnosed with EGC underwent ER at the Severance and Gangnam Severance Hospitals, Seoul, Korea, between January 2007 and December 2014 . We analyzed totally $302(10.6 \%)$ patients who underwent additive gastrectomy after non-curative ER. The patients were divided into 2 groups according to the time interval point, as the earlier operation group (group A) and the later operation group (group B). The time interval point, at which operative time and estimated intraoperative blood loss (EBL) of the earlier operation group and the later operation group 
showed the greatest disparities, was evaluated. We retrospectively evaluated longterm follow-up study for oncological outcomes about follow-up duration, locoregional recurrence, distant recurrence.

Results: The median follow-up duration is $40.36 \pm 20.74$ months in all patients Based on the previous our study, we divided patients two groups who underwent operation before and after 29 days. Of the 302 patients, 133 were in Group A ( $\leq 29$ days) and 169 in Group B (>29days). There were more differences between two groups about ASA score, ER Specimen size, intra-op. transfusion, POD\#1 Hemovac ${ }^{\circledR}$ discharge, Maximal postoperative CRP in the clinicopathological characteristics. Like previous our study the operative time, EBL, tumor size was significantly longer and more in group A compared with group B. There were totally 7 patients locoregional and distance recurrence during follow-up period. There were no differences in oncological outcomes between two groups.

\begin{tabular}{llll}
\hline Oncological recurrence for Each Group & & \\
\hline & $\begin{array}{l}\text { Group A } \\
(\leq 29 \text { days }) \\
\mathrm{n}=133\end{array}$ & $\begin{array}{l}\text { Group B } \\
(>29 \text { days }) \\
\mathrm{n}=169\end{array}$ & p-value \\
\hline $\begin{array}{l}\text { Characteristics } \\
\begin{array}{c}\text { F/u duration (months, } \\
\text { mean } \pm \text { SD) }\end{array}\end{array}$ & $37.02 \pm 20.54$ & $44.18 \pm 19.49$ & 0.002 \\
$\begin{array}{c}\text { Locoregional } \\
\text { recurrence (n, \%) }\end{array}$ & $1(0.8)$ & $1(0.6)$ & 0.757 \\
\begin{tabular}{l} 
Distant recurrence (n, \%) \\
\hline
\end{tabular} & $3(2.3)$ & $2(1.2)$ & \\
\hline
\end{tabular}

Conclusion: Based on long-term follow-up data, surgery time after ER in EGC does not affect oncological outcome. These long-term follow-up results suggest that additive surgery at about 1 month after ER is optimal for better surgical outcomes without affecting the oncological outcomes.

Disclosure of Interest: All authors have declared no conflicts of interest.

\section{Reference}

1. Kim MJ, Kim JH, Lee YC, et al. Is there an optimal surgery time after endoscopic resection in early gastric cancer? Ann Surg Oncol 2014;21:232-239.

\section{P1225 THE POINT TO DISTINGUISH EARLY GASTRIC CANCER FROM DEPRESSION TYPE OF GASTRIC INTESTINAL METAPLASIA}

T. Wakatsuki, S. Furutachi, H. Yamashita

Department Of Gastroenterology \& Hepatology, Okayama Medical Center. Okayama/Japan

Contact E-mail Address: t_wakatsuki0530@yahoo.co.jp

Introduction: This study discusses two endoscopic findings which improve the accuracy of the diagnosis of early gastric cancers (EGC). After successful Helicobacter pylori eradication, we often observe multiple reddish depressed lesions called "patchy redness" or "map-like redness" in the gastric mucosa. Even though most are intestinal metaplasia (IM), EGC is found among these lesions. A light blue crest (LBC) has been a highly accurate sign of the IM. There are, now, additional two endoscopic findings that should improve the accuracy of diagnosis of EGC. They are 1) "intraepithelial microinvation (IEMI)", and 2) "Over flow". Over flow is that the endoscopic finding that the structure of the depressed lesion spreads to the outside of the depression.

Aims \& Methods: The aim of this study is to clarify the usefulness of two endoscopic findings in order to detect the EGC in the group thought to be an IM. This is a retrospective control study. There were 234 cases of EGC performed endoscopic submucosal dissection (ESD) between November 2011 and August 2016 in Okayama Medical Center. We included 57 cases in this study. As EGC group, we included 45 cases that were 0 -IIc, $10 \mathrm{~mm}$ or less, pT1a (M), a differentiation type cancer. As IM group, we included 12 cases identified as IM by biopsy in the same period. The EGC group is then divided into 2 groups; 1 ) eradication group (13 cases) who had a history of eradication and, 2) a small EGC group (32 cases) without a history of eradication. We evaluated 1) the ratio of $L B C, 2$ ) the ratio of irregular microsurface pattern 3) the ratio of irregular microvascular pattern, 4) the ratio of IEMI and 5) the ratio of Over flow.

Results: The ratio of LBC was $75 \%$ in IM group, which was significantly higher than eradication group $(7.7 \%)$, and small EGC group $(9.4 \%)$. The ratio of irregular microsurface pattern was $33 \%$ in IM group, which was significantly lower than eradication group $(92 \%)$, and small EGC groups $(84 \%)$. The ratio of irregular microvascular pattern was $0 \%$ in IM group, which was significantly lower than eradication group $(92 \%)$, and small EGC group $(81 \%)$. The ratios of IEMI were $0 \%$ in IM group, $39 \%$ in eradication group and $41 \%$ in small EGC group. The ratios of Over flow were $0 \%$ in IM group, $46 \%$ in eradication group and $50 \%$ in small EGC group. Furthermore, the ratios to have either in two endoscopic findings were $0 \%$ in IM, $77 \%$ in eradication group and $69 \%$ in small EGC group.

Conclusion: IEMI and Over flow leads to the diagnosis of ECG in addition to other endoscopic findings.

Disclosure of Interest: All authors have declared no conflicts of interest.
P1226 IRON DEFICIENCY ANEMIA-ARE THERE ANY

\section{PREDICTORS OF GASTROINTESTINAL MALIGNANCY?}

\section{S. Xavier ${ }^{1}$, J. Magalhães ${ }^{2}$, J. Cotter ${ }^{3}$}

${ }^{1}$ Life and Health Sciences Research Institute (ICVS), School of Medicine,

University of Minho, Braga/Portugal

${ }^{2}$ Gastroenterology Department, Hospital da Senhora da Oliveira, Guimarães, Guimarães/Portugal

${ }^{3}$ Pt Government Associate Laboratory, 4 ICVS/3B's, Braga/Guimarães/Portugal

Contact E-mail Address: smaxavier@gmail.com

Introduction: Iron deficiency anemia (IDA) may be the only sign of gastrointestinal (GI) malignancy. The identification of predictive factors of GI malignancy in patients with IDA could help the physician to establish patients' priority to endoscopic assessment, contributing to an earlier diagnosis.

Aims \& Methods: Retrospective study of 344 patients submitted to endoscopic assessment for IDA. Included adult patients with IDA and excluded patients with GI or extra-GI bleeding, total gastrectomy, exclusively vegetarian diet or insufficient medical records.

Results: Included 121 patients with mean age of $68.5 \pm 17.0$ years and $54.5 \%$ females. GI malignancy was identified in $14.9 \%$ of patients (gastric in 12 , colonic in 6 patients). A statistically significant association was found between the presence of GI malignancy and age $(75.2 \pm 12.9$ vs $67.2 \pm 17.4, p<0.05)$, male gender $(72.2 \%$ vs $40.8 \%, p=0.01)$, GI symptoms $(61.1 \%$ vs $11.7 \%, p<0.01)$, weight loss $(61.1 \%$ vs $5.8 \%, \mathrm{p}<0.01)$, need for hospitalization $(88.9 \%$ vs $49.5 \%$, $\mathrm{p}<0.01)$, iron seric levels and transferrin saturation $(19.7 \pm 10.1 \mathrm{mg} / \mathrm{L}$ vs $30.4 \pm 18.9 \mathrm{mg} / \mathrm{L}, \mathrm{p}<0.01$ and $6.1 \pm 4.1 \%$ vs $9.2 \pm 6.3 \%, \mathrm{p}<0.03$, respectively). At logistic regression analysis only weight loss $(\mathrm{p}<0.01)$, GI symptoms $(\mathrm{p}<0.01)$, transferrin saturation $(\mathrm{p}<0.01)$ and need for hospitalization $(\mathrm{p}=0.02)$ showed a significant relation with the diagnosis of GI malignancy. Transferrin saturation showed a weak discriminative capacity (AUC $=0.67$, $\mathrm{p}=0.01$ ) however, values of transferrin saturation $\leq 11 \%$ had a sensibility of $94.4 \%$ and a negative predictive value of $97.1 \%$ for GI malignancy (CI $95 \%$ 85.1-99.9).

Conclusion: In patients with IDA the diagnosis of GI malignancy is established in a significant percentage of patients and patients with GI symptoms, weight loss or with need for hospitalization should be given priority in the performance of endoscopic examinations. Transferrin saturation may help the physicians in establishing the urgency of endoscopic assessment, since patients with values over $11 \%$ have a very low probability to have GI malignancy.

Disclosure of Interest: All authors have declared no conflicts of interest.

\section{P1227 IMPROVEMENT OF DETECTION RATE OF EARLY GASTRIC CANCER BY A TRAIN-THE-TRAINER (TTT) COURSE IN CHINA: A PROSPECTIVE CONTROLLED STUDY}

J. Hu ${ }^{1}$, M. Cai ${ }^{1}$, Q. Shi ${ }^{1}$, B. Zhu ${ }^{1}$, Y. Huang ${ }^{1}$, W. Qin ${ }^{2}$, Z. Ren ${ }^{1}$, J. Liu ${ }^{1}$, J. Lian ${ }^{1}$, Z. Zhang ${ }^{1}$, P. Zhou ${ }^{1}$, K. Yao ${ }^{3}$

${ }_{1}^{1}$ Endoscopy Center And Endoscopy Research Institute, Endoscopy Center and Endoscopy Research Institute, Zhongshan Hospital, Fudan University, Shanghai/ China

${ }^{2}$ Endoscopy Center And Endoscopy Research Institute, Fudan University

Zhongshan Hospital, Shanghai/China

${ }^{3}$ Dept. Of Endoscopy, Fukuoka University Chikushi Hsopital - Dept. of Endoscopy, Fukuoka University Chikushi Hsopital; Chi, Chikushino/Japan

Contact E-mail Address: hu.jianwei@zs-hospital.sh.cn

Introduction: Detection of gastric cancer in its early stage is pertinent in reducing disease-specific mortality. However, this has not been achieved in many countries where the incidence of gastric cancer is high. In order to overcome this problem, we developed an e-learning system for international endoscopists to improve endoscopic diagnosis of early gastric cancer. However, it has not been investigated whether such learning system is useful in clinical practice.

Aims \& Methods: The objective of this trial is to investigate whether the intensive on-site TTT course is useful for increasing early detection rate of gastric cancer in Chinese high-volume endoscopy center. Five Chinese doctors (the TTT group) who were invited to the TTT course and the other five age and experiece-matched Chinese doctors (the non-TTT group) in the same facility who did not attend the TTT course were included in this trial. Endoscopists of the TTT group attended 2-day learning course 4 times from March 20, 2015 to March 25, 2016. Endoscopists of the non-TTT group did not attend any learning program during the same period. Lectures of the TTT course included the detection of early gastric cancer by screening endoscopy using white-light endoscopy alone and the feature of the detected subtle gastric mucosal lesion using white-light endoscopy or magnifying endoscopy with narrow-band imaging. Contents used in the lecture had been reported to be useful by an e-learning trial [1, 2]. All the instructions were given by an experienced Japanese endoscopist (K. Yao) who constructed the e-learning system $[1,2]$. Endoscopists also received on-site handson instructions in order to check whether they obtained enough knowledge and technique. Furthermore, we held case conferences in order to share common experiences. During the period, the number of both newly detected early gastric cancers and screening gastrosocpy procedures was recorded. The primary endpoint was to compare the early detection rate between the TTT and the non-TTT group. (Early detection rate $=$ the number of newly detected early gastric cancers/ the number of screening endoscopy procedures)

Results: The data obtained from the 275 consecutive cases of screening gastroscopy procedures by the TTT group endoscopists and from the 323 consecutive cases of screening endoscopy procedures by the non-TTT group endoscopists were analyzed. In the TTT group, four cases with early gastric cancers were detected and no early gastric cancer was detected. The early detection rates of 
the TTT and the non-TTT group were $1.5 \%$ and $0 \%$, respectively. There was a significant difference in early detection rate between the TTT and the non-TTT group (Fisher's exact test, $\mathrm{P}=0.046$ ).

Conclusion: This clinical trial clearly showed that the systematic intensive TTT course is useful for improving early detection rate of gastric cancer in clinical practice at Chinese high-volume endoscopy center. (NCT02385578)

Disclosure of Interest: All authors have declared no conflicts of interest.

\section{References}

1. Yao K, et al. Development of an e-learning system for the endoscopic diagnosis of early gastric cancer: an international multicenter randomized controlled trial. EBioMedicine 2016;9:140-147

2. Yao K, et al. Development of an e-learning system for teaching endoscopists how to diagnose early gastric cancer: basic principles for improving early detection. Gastric Cancer 2017;20(Suppl 1): S28-S31

\section{P1228 COMPARISON OF ENDOSCOPIC SUBMUCOSAL DISSECTION AND SURGERY FOR THE TREATMENT OF EARLY GASTRIC CANCER: SINGLE-CENTER LONG-TERM OUTCOME STUDY}

H.I. Jung ${ }^{1}$, S.J. Kim ${ }^{1}$, I.T. So ${ }^{1}$, J.H. Choi ${ }^{1}$, Y.J. Lee ${ }^{1}$, H.J. Lee ${ }^{1}$, K.B. Cho ${ }^{1}$, K.S. Park ${ }^{1}$, S.W. Ryu ${ }^{2}$, Y.G. Son ${ }^{2}$, J.Y. Lee

${ }^{1}$ Department Of Internal Medicine, Keimyung University School of Medicine, Daegu/Korea, Republic of

${ }^{2}$ Department Of Surgery, Keimyung University School of Medicine, Daegu/Korea, Republic of

\section{Contact E-mail Address: hdwjhi@naver.com}

Introduction: Endoscopic submucosal dissection (ESD) is believed to be a possible modality for early gastric cancer. But there is little report about long-term outcomes of the ESD directly compare with the surgery. The purpose of this study is the comparison between the two treatment modalities about the outcomes.

Aims \& Methods: We performed a retrospective analysis of 1243 patients with stage I early gastric cancer without lymph node involvement. 551 patients were treated with ESD, and 692 patients were treated with subtotal or total gastrectomy. Long-term overall and disease-specific survival rates, development of new lesions, and complications were analyzed.

Results: The mean age was higher in the ESD group ( $64.9 \pm 9.5$ vs. $58.5 \pm 11.7$, $\mathrm{P}=0.001)$ and female distribution was higher in surgery group $(30.5 \% \mathrm{vs} .38 .9 \%$, $\mathrm{P}=0.001)$. In ESD group, diabetes was more frequent $(12.9 \%$ vs. $7.1 \%$, $\mathrm{P}<0.001)$. The overall survival rate was similar $(96.2 \%$ vs. $96.7 \%, \mathrm{P}=0.136)$, but disease-specific survival rate was significantly higher in ESD group $(99.8 \%$ vs. $98.7 \%, P=0.037$, log-rank test). During 10 year follow up period, new lesions were observed in $3.6 \%$ of ESD group and in $1.3 \%$ of surgery group $(\mathrm{P}<0.001)$. ESD group showed less complications $(4.5 \%$ vs. $16.3 \%, \mathrm{P}<0.001)$ and shorter hospital day than surgery group $(5.27$ days vs. 12.09 days, $\mathrm{P}<0.001)$.

Conclusion: Although the development of new lesions were more frequent than surgery, ESD has similar overall survival rate and even higher disease-specific survival rate than surgery. Also, ESD has less complications and shorter hospital day than surgery. Therefore, ESD is an effective therapeutic method in early gastric cancer as well as surgery.

Disclosure of Interest: All authors have declared no conflicts of interest

\section{P1229 HEMATOLOGISTS SHOULD ORDER ENDOSCOPIC EXAMINATION TO EXPERTS OF ENDOSCOPY IN CASE OF ENDOSCOPIC CHECK-UP OF GASTROINTESTINAL MALIGNANT LYMPHOMA}

T. Yamasaki, T. Sakurai, J. Mitobe, M. Mitsunaga, M. Saruta Department Of Gastroenterology And Hepatology, The Jikei University, Tokyo/ Japan

Contact E-mail Address: takusan.yamasan@gmail.com

Introduction: Gastric malignant lymphoma (ML) is most popular lymphoma of the gastrointestinal tract. Especially we often see gastric MALT lymphoma in cases of $H$. pylori (HP) infection positive, and we also sometimes find out HP negative gastric MALT lymphoma. Since gastric carcinoma (GC) is more common rather than gastric MALT lymphoma, typical endoscopic diagnostic characteristics of GC are established on usual endoscopic examination for most of gastroenterologists. On the other hand, endoscopic characteristics of gastric MALT lymphoma on usual endoscopy have not been established for most of gastroenterologists.

Aims \& Methods: Our aim of this study is to estimate of the difficulty on endoscopic diagnosis of gastric MALT lymphoma for 24 gastroenterologists (12 experts and 12 trainees of endoscopy) on usual endoscopic examination. We investigated a total of 72 gastric MALT lymphoma cases in our hospital and other 7 hospitals. We estimated total number of endoscopic examinations to achieve diagnosis of gastric MALT lymphoma endoscopically and histologically. In addition, we had analyzed the difference of abilities to diagnose of gastric MALT lymphoma between experts and trainees on endoscopic examinations using past endoscopic profiles (245 profiles).

Results: The average number of endoscopic examinations up-to diagnose gastric MALT lymphoma was 3.4 times (from 1 to 7 times) on whole endoscopies after first appearance of lymphoma. Though average total number of endoscopic examinations of experts was only 1.2 times, on the other hand average total number of endoscopic examinations of trainees was 5.4 times. There was a significant difference between experts and trainees of endoscopy on average total number of endoscopic examinations $(p=0.022)$. Major reason of misdiagnosis was insufficient recognition of endoscopic appearances of ML on endoscopic trainees. Typical representative appearances of gastric MALT lymphoma were erosions, ulcers and surface irregularities. Since these lesions are also appeared as typical appearances of GC and gastritis, most of non-expert of endoscopy cannot distinguish the difference of GC, ML and gastritis due to those similarities. For example, even if typical ML cases, non-expert could not diagnose repeatedly, but expert could diagnose minimal lesions of ML at first endoscopy. Especially HP positive gastric MALT lymphoma was similar to gastritis and GC on endoscopic findings, most of non-expert could not diagnose exactly.

Conclusion: There was significant difference of ability to diagnose gastric MALT lymphoma between specialists and trainees. Therefore, hematologists should order endoscopic examination to experts of endoscopy knowledgeable of ML in case of endoscopic check-up of gastrointestinal malignant lymphoma. Disclosure of Interest: All authors have declared no conflicts of interest.

\section{P1230 SURVIVAL BENEFITS OF GASTRECTOMY IN PATIENTS WITH STAGE IV GASTRIC CANCER: A POPULATION-BASED STUDY}

X. $\mathrm{He}^{1}, \mathrm{~W} . \mathrm{Wu}^{2}, \mathrm{Y} . \mathrm{Ding}^{1}$, Z. $\mathrm{Jin}^{1}, \mathrm{~L} . \mathrm{Sun}^{1}, \mathrm{~J} \cdot \mathrm{Si}^{1}$

${ }^{1}$ Department Of Gastroenterology, Sir Run Run Shaw Hospital, hangzhou/China ${ }^{2}$ State Key Laboratory for Diagnosis and Treatment of Infectious Diseases, The First Affiliated Hospital, School of Medicine, Zhejiang University, Hangzhou, China, Hangzhou/China

Contact E-mail Address: hexingkang@zju.edu.cn

Introduction: Gastric cancer is the fifth most common cancer worldwide and the third leading cause of cancer-related death globally [1]. With the widespread eradication of $H$. pylori and early detection, the incidence and mortality of gastric cancer have been declined rapidly in the past few decades [2-3]. However, the prognosis of patients with metastatic diseases was still dismal and most cases survived less than one year [4]. Current primary and standard therapies for metastatic gastric cancer mainly included chemotherapy, consisting of fluoropyrimidine/cisplatin-based combination regimens[5]. Although radical gastrectomy was the first-line treatment for early gastric cancer[6], the value of gastrectomy in stage IV was still a great controversy.

Aims \& Methods: We aimed to investigate the impact of primary tumor resection on survival outcomes among patients with metastatic cancer. We identified an eligible cohort of the population with stage IV gastric cancer from the Surveillance, Epidemiology and End Results (SEER) database between 2004 to 2012. The overall prognosis of advanced patients with or without gastrectomy was assessed by Kaplan-Meier and log-rank analysis. Multivariate Cox proportional hazards regression models was performed to analyze the effect of primary tumor resection on overall and cancer-specific mortality. To further reduce potential baseline bias in patient selection between two groups, we adopted 1:1 propensity score matching to re-examine the effect of resection.

Results: We finally identified 12984 eligible patients with metastatic GC between 2004 and 2012, including 1977 patients with gastrectomy and 11007 without resection. The median survival time for patients with or without surgery were 9.0, 4.0 months respectively. Patients who received surgery had a significantly better survival outcome compared with those without gastrectomy $(P<0.05)$. In multivariate Cox analysis, gastrectomy was associated with decreased overall mortality (HR, $0.55,95 \%$ CI $0.52-0.58, P<0.001)$ and cancer-specific mortality (HR, $0.55,95 \%$ CI $0.52-0.58, P<0.001$ ). In the propensity score matched model analysis, gastrectomy was associated with increased overall (HR, $0.54,95 \% \mathrm{CI}$, $0.51-0.58$ ) and cancer-specific survival (HR, $0.54,95 \% \mathrm{CI}, 0.50-0.55$ ).

Conclusion: Based on population-based studies, we demonstrated that there was a survival advantage of gastrectomy in patients with stage IV gastric cancer. Further prospective studies are needed to verify our findings.

Disclosure of Interest: All authors have declared no conflicts of interest.

\section{References}

1. Siegel RL, Miller KD and Jemal A. Cancer statistics, 2015. CA Cancer J Clin. 2015; 65(1):5-29.

2. Munoz N and Asvall J. Time trends of intestinal and diffuse types of gastric cancer in Norway. Int J Cancer. 1971; 8(1):144-157.

3. Zhu AL and Sonnenberg A. Is gastric cancer again rising? $J$ Clin Gastroenterol. 2012; 46(9):804-806.

4. Fujitani K, Yang HK, Mizusawa J, Kim YW, Terashima M, Han SU, Iwasaki Y, Hyung WJ, Takagane A, Park do J, Yoshikawa T, Hahn S, Nakamura K, Park CH, Kurokawa Y, Bang YJ, et al. Gastrectomy plus chemotherapy versus chemotherapy alone for advanced gastric cancer with a single non-curable factor (REGATTA): a phase 3, randomised controlled trial. Lancet Oncol. 2016; 17(3):309-318.

5. Wagner AD, Unverzagt S, Grothe W, Kleber G, Grothey A, Haerting J and Fleig WE. Chemotherapy for advanced gastric cancer. Cochrane Database Syst Rev. 2010; (3):CD004064.

6. Dikken JL, van de Velde CJ, Coit DG, Shah MA, Verheij M and Cats A. Treatment of resectable gastric cancer. Therap Adv Gastroenterol. 2012; $5(1): 49-69$. 


\section{P1231 DEVELOPMENT OF AND EXPERIENCE WITH AN} INSULATED SCISSORS-TYPE KNIFE (SB KNIFE)

\section{Kobayashi}

Yokkaichi Municipal Hospital Dept. of Gastroenterology, Yokkaichi/Japan

Contact E-mail Address: makoto-kobayashi@aroma.ocn.ne.jp

Introduction: Endoscopic submucosal dissection (ESD) is technically difficult and is associated with risks of perforation and bleeding. Although knife-type instruments are primarily used to make incisions during ESD, it is necessary to be proficient in endoscopic procedures and be able to perform them simultaneously with electrification and incision. Scissors-type knives are fairly easy to manipulate in colorectal ESD. We have fabricated SB knife Jr type (SBJr), short scissorstype knife with outer insulated layer, in collaboration with SUMITOMO BAKELITE CO.

Aims \& Methods: SBJr is short length (electrode length: $3.5 \mathrm{~mm}$ ) to be easy to handle in narrow colorectal lumen. The surface of the rotatable monopolar scissors is coated with insulating material in order to enhance the cutting power and prevent electric effects in the surrounding tissue. The shearing structure make sharp cutting quality and very small round tips prevent to grasp the muscular layer. SBJr was used in circumference incision, submucosal dissection and hemostasis. After infected hyaluronic acid in submucosal layer, grasped the tissue, confirming safety, make incision. SBJr was used not only used in incision but also in hemostasis. At sites containing blood vessels or bleeding, they were grasped and induceed coagulation using SBJr. It has been used on 180 colorectal lesions from January 2008

Results: The circumference incision and submucosal dissection were basically performed with High-frequency cutting wave. There were 3 cases of perforation during ESD and 1 case of post-operative bleeding. The procedure itself was fairly easy and was particularly effective at fibrosis sites, sites difficult to stable the endoscope and sites containing blood vessels, where conventional devices would encounter difficulties. Due to the very small round tips of the instrument, detailed operation become simply. For coagulation of blood vessels or bleeding, it is not required to replace SBJr which is used cutting and coagulation.

Conclusion: This short insulated scissors-type knife (SBJr) made it easier to perform colorectal ESD, we conclude that it is an effective device and easy for a beginner in colorectal ESD.

Disclosure of Interest: All authors have declared no conflicts of interest.

\section{P1232 GASTROINTESTINAL LYMPHOMAS}

R. Gaspar ${ }^{1}$, A. Andrade ${ }^{1}$, J. Santos-Antunes ${ }^{1}$, R. Liberal ${ }^{1}$, F. Carneiro ${ }^{2}$, G. Macedo

${ }^{1}$ Gastrenterology, Hospital São João, Porto/Portugal

${ }^{2}$ Diagnostics, IPATIMUP - Diagnostics, IPATIMUP; Porto/PT, Porto/Portugal

${ }^{3}$ Centro Hospitalar São João, Porto Medical School, Porto/Portugal

\section{Contact E-mail Address: ruilopesgaspar@gmail.com}

Introduction: The gastrointestinal tract (GIT) is the most commonly extranodal site affected in lymphomatous pathology. Infection with Helicobacter pylori, human immunodeficiency virus and Epstein Barr and immunosuppression have been studied as possible risk factors. The diagnosis is often late, presenting at an advanced stage, with limited therapeutic options.

Aims \& Methods: The objective of this study was to characterize the anatomical distribution, clinical manifestations, risk factors and prognosis of GI lymphomas. Retrospective study of patients diagnosed with GI lymphomas between 1997 and 2016.

Results: During the 20 years, 127 GI lymphomas were identified. The mean age at diagnosis was $58.2 \pm 16.5$ years, $53 \%$ of males. The majority $(92.9 \%)$ had symptoms at the time of diagnosis, with fatigue $(54.3 \%)$ being the most common. The most commonly affected organ was the stomach $(65.3 \%)$ and the most common subtypes were diffuse large B-cell $(48.8 \%)$ and MALT $(27.6 \%)$. Most were diagnosed through upper endoscopy $(68.5 \%)$ and $10.2 \%$ after exploratory laparotomy. Sixty-five percent of patients underwent chemotherapy and $26 \%$ underwent surgery. In the univariate analysis, lymphomas $>4 \mathrm{~cm}(\mathrm{p}=0.037)$, anorexia $(\mathrm{p}=0.006)$, tobacco use $(\mathrm{p}=0.038)$, alcohol consumption $(\mathrm{p}=0.006)$, lymphadenopathy $(\mathrm{p}=0.014)$, high $\mathrm{b} 2$-microglobulin $<0.01), \quad$ ECOG $>2$ $(\mathrm{p}<0.001)$ and IPI index $>2(\mathrm{p}<0.001)$ were associated with lower survival. Conclusion: The stomach is the main organ affected by lymphomatous disease but, unlike the literature in which MALT lymphoma predominates, the most common subtype was diffuse large B-cell lymphoma. Upper endoscopy was the main form of diagnosis. Alcohol and tobacco consumption, lymphadenopathy, lymphoma $>4 \mathrm{~cm}$, b2 high microglobulin, ECOG $>2$ and IPI index $>2$ are poor prognostic factors.

Disclosure of Interest: All authors have declared no conflicts of interest.

\section{P1233 THE EFFECT OF OBESITY ON EARLY GASTRIC CANCER IN PATIENTS UNDERGOING ENDOSCOPIC TREATMENT}

S. Jin ${ }^{1}$, S.Y. Nam ${ }^{1}$, S.W. Jeon ${ }^{2}$, Y. Kwon ${ }^{1}$, H.S. Lee ${ }^{1}$

${ }^{1}$ Gastroenterology, Kyungpook National University Medical Center, Daegu/Korea, Republic of

${ }^{2}$ Dept. Of Internal Medicine, Kyungpook National University, Daegu/Korea, Republic of

Contact E-mail Address: ga2001@hanmail.net

Introduction: Previous studies have shown that non-cardiac gastric cancer had no associations with the obesity even if cardiac or gastroesophageal junctional cancer was related with the obesity. These studies have included high portion of advanced gastric cancer. Patients with most advanced cancer already experienced weight loss.

Aims \& Methods: We evaluated the effect of body mass index (BMI) on early gastric cancer in patients undergoing endoscopic treatment for early gastric cancer. A total of 748 patients with early gastric cancer undergoing endoscopic treatment (endoscopic submucosal dissection) including age and sex matched healthy controls consist of this case-control study. Body mass index was classified into underweight (BMI < 18.5), normal (BMI 18.5-23), overweight (BMI 23-25), and obese (BMI $\geqq 25$ ) by Asian-Pacific guideline. Adjusted analysis using odds ratio (OR) and $95 \%$ confidence interval (CI) was performed to evaluate the effect of BMI on early gastric cancer.

Results: The mean age was 57 years and male sex was $60 \%(n=447)$. BMI was higher in gastric cancer comparing to healthy control ( 24 vs $23, P<0.001)$. The OR of gastric cancer was increased according to the BMI increase; 1.57 $(95 \%$ CI $, 0.89-2.79, P=0.12)$ in normal BMI, 1.88 (95\% CI, $1.06-3.35$, $\mathrm{P}=0.03)$ in overweight, and $2.28(95 \% \mathrm{CI}, 1.29-4.06, P=0.005)$ in obese persons comparing to underweight $(P$ value for trend $<0,001)$.

Conclusion: The early gastric cancer was strongly associated with the increased BMI and its effect has dose-dependent pattern.

Disclosure of Interest: All authors have declared no conflicts of interest.

P1234 COMPARATIVE STUDY OF THE ENDOSCOPIC ULTRASONOGRAPHY-GUIDED FINE-NEEDLE ASPIRATION VS MUCOSAL-INCISION ASSISTED BIOPSY FOR THE HISTOLOGICAL DIAGNOSIS OF GASTROINTESTINAL SUBEPITHELIAL TUMORS

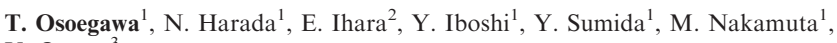
Y. Ogawa

${ }^{1}$ Gastroenterology, Clinical Research Institute, National Kyushu Medical Center, Fukuoka/Japan

${ }^{2}$ Department Of Medicine And Bioregulatory Science, Graduate School Of Medical Sciences, Kyushu university, Fukuoka city/Japan

${ }^{3}$ Medicine And Bioregulatory Science, Kyushu University, Fukuoka/Japan

Contact E-mail Address: t.osoegawa@gmail.com

Introduction: Gastrointestinal subepithelial tumors include potentially malignant tumors. When considering the diagnostic yield for subepithelial tumors, it is important to evaluate whether the samples obtained are adequate for histological analysis, as immunohistological analysis is indispensable for a definitive diagnosis. However, it may be difficult to make a correct histological diagnosis with only by the endoscopic ultrasonography-guided fine-needle aspiration (EUSFNA). Therefore, there has been an interest in exploring an alternative modality for tissue sampling as mucosal-incision assisted biopsy (MIAB) based on the endoscopic submucosal dissection.

Aims \& Methods: The aim of this study was to compare the usefulness of EUSFNA and MIAB in the histological diagnosis of gastrointestinal subepithelial tumors (SET). We performed the retrospective study comparing 37 patients who underwent either EUS-FNA $(n=18)$ or MIAB $(n=19)$. Diagnostic yield feasibility and safety of those EUS-FNA and MIAB were compared.

Results: The location of the SET was esophagus $(n=6)$, stomach $(n=29)$, and duodenum $(\mathrm{n}=2)$. The histological diagnosis were gastrointestinal stromal tumors $(n=10)$, leiomyoma $(n=17)$, aberrant pancreas $(n=3)$, poorly differentiated adenocarcinoma $(n=2)$, metastatic carcinoma (renal cell carcinoma, $\mathrm{n}=1$ ), and no-diagnosis $(\mathrm{n}=4)$. There were no significant differences in the clinical characteristics-including sex and age-of the patients in the EUS-FNA and MIAB. In EUS-FNAs/MIABs, the morphological findings of the SET by EUS were as follows. The mean diameter of tumor was $29.16 \pm 15.6$, $17.77 \pm 7.16 \mathrm{~mm}(\mathrm{p}=0.0034)$. The rate of intraluminal growth was $55.6 \%$ $94.7 \%(\mathrm{p}=0.0078)$. The mean procedure time was $40.89 \pm 10.79 \mathrm{~min}$ in EUS-FNA and $55.15 \pm 27.42 \mathrm{~min}$ in MIAB $(\mathrm{p}=0.0234)$. Histological diagnosis was made in $72.2 \%$ of the EUS-FNAs and $94.7 \%$ of the MIABs $(\mathrm{p}=0.0897)$. No complications were found in either method.

Conclusion: The mean procedure time was significantly longer in MIAB than in EUS-FNA. However, the mean diameter of the tumor was significantly smaller in MIAB than in EUS-FNA the higher diagnostic yield in MIAB than in EUSFNA was borderline significant.

Disclosure of Interest: All authors have declared no conflicts of interest.

\section{P1235 CLINICAL TRENDS AND BURDEN OF DEATH IN GASTRIC} CANCER: A SIX-YEARS SURVEY

P. Crafa ${ }^{1}$, S. Landi ${ }^{1}$, S. Grillo ${ }^{1}$, M. Franceschi ${ }^{2}$, A. Noto ${ }^{1}$, S. Scida ${ }^{1}$,

C. Miraglia ${ }^{1}$, L. Franzoni ${ }^{1}$, V. Corrente ${ }^{1}$, R. Cannizzaro ${ }^{3}$, G.M. Cavestro ${ }^{4}$ M. Rugge ${ }^{5}$,F. Di Mario ${ }^{1}$, P. Caruana ${ }^{6}$, C. Mancini ${ }^{6}$

${ }^{1}$ Department Of Medicine And Surgery, University of Parma, Parma/Italy

${ }^{2}$ Endoscopic Unit, Department Of Surgery, Ulss4, Hospital ULSS4 Alto Vicentino, Santorso/Italy

${ }^{3}$ Oncological Gastroenterology, Centro di Riferimento Oncologico di Aviano

S.O.C. di Gastroenterologia, Aviano/Italy

${ }^{4}$ Dept. of Gastroenterology, Università Vita-Salute San Raffaele, Milano, Italy,

Milano/Italy

${ }^{5}$ Pathology, Medical School of the Padova University, Padova/Italy

${ }^{6}$ Department of Pathology, Parma Hospital, Parma, Italy, Parma/Italy

Contact E-mail Address: pellegrino.crafa@unipr.it 
Introduction: In 2012 the reported incidence of gastric cancer in both sexes was $12.3 / 100,000$ but the mortality was still high $(8.7 / 100,000)$, being early detection strictly related to a better survival. Parma area is considered at medium-low incidence of gastric cancer. For early diagnosis, the detection of a precancerous condition like atrophic gastritis seems crucial, but the majority of such patient is asymptomatic and the use of non invasive diagnosis like serology (Pepsinogens and Gastrin 17) as suggested in the guidelines of Kyoto and Maastricht V is up to now limited in clinical practice. Aim of the study, therefore was to establish the burden of gastric cancer in the diagnosis of the last six years, focusing on the detection of early gastric cancer.

Aims \& Methods: Six years (from July 2010 to July 2016) were considered in search for diagnosis of gastric cancer as reported in the archives of the Pathology Department of Parma University. Overall, 816 cases of gastric cancer were found but we decide to consider only the surgically removed cases, therefore the available sample is based on 584 cases. For every cases we classified the cancer in early, following the Kodama classification, and advanced. The presence of atrophic gastritis nearby the neoplasia was assessed according with OLGA classification. In both early and advanced cancer the node status was investigated. Results: Overall, 584 cases of gastric cancer was detected in the six years considered interval $(\mathrm{M}=318, \mathrm{~F}=223$, mean age 78ys, range $36-105 \mathrm{ys})$ The diagnosis of early gastric cancer was made in $44 / 584(7.53 \%)(\mathrm{M}=24, \mathrm{~F}=20$ mean age $75.68 \mathrm{ys}$, range $47-92 \mathrm{ys})$. A diagnosis of advanced gastric cancer was established in $540 \mathrm{pts}(\mathrm{M}=318, \mathrm{~F}=222$, mean age $78.20 \mathrm{ys}$, range $36-105 \mathrm{ys})$. The picture of chronic atrophic gastritis was found in more than $95 \%$ of the cases, both in early and advanced ones. The node status was also recorded. Early cancers showed a $25 \%$ of node metastasis compared with $84.65 \%$ in advanced ones. As regards the number of involved nodes, in early presentation of neoplasia we found $98 \%$ of pN1 staging whereas in advanced the pN1 cases were only $9.1 \%$.

Conclusion: Early gastric cancer diagnosis is still confirmed as very infrequent and this could account for the high mortality rate for the gastric neoplasia. The search for precancerous condition like chronic atrophic gastritis is therefore mandatory.

Disclosure of Interest: All authors have declared no conflicts of interest.

\section{P1236 GASTRIC ADENOCARCINOMA OF FUNDIC GLAND TYPE: CANCER GENOMIC DATA}

H. Ueyama ${ }^{1}$, T. Hayashi ${ }^{2}$, T. Saito ${ }^{2}$, Y. Akazawa ${ }^{1}$, H. Komori ${ }^{1}$, K. Matsumoto ${ }^{1}$ K. Matsumoto ${ }^{1}, \mathrm{M}$. Hojo ${ }^{1}, \mathrm{~A} . \mathrm{Nagahara}^{3}, \mathrm{~T} . \mathrm{Yao}^{2}, \mathrm{~S}$. Watanabe ${ }^{1}$

${ }^{1}$ Department Of Gastroenterology, Juntendo University School of Medicine, Tokyo/Japan

${ }^{2}$ Department Of Human Pathology, Juntendo University School of Medicine, Tokyo/Japan

${ }^{3}$ Department Of Gastroenterology, Juntendo University Shizuoka Hospital,

Shizuoka/Japan

\section{Contact E-mail Address: psyro@juntendo.ac.jp}

Introduction: Gastric adenocarcinoma of fundic gland type (GAFG) is an uncommon variant of gastric adenocarcinoma which has a distinct clinicopathological, immunohistochemical, and endoscopic features 1-3). However, the molecular biological features of GAFG have not been well elucidated.

Aims \& Methods: We evaluated clinical and molecular characteristics of GAFG in comparison with conventional gastric adenocarcinoma. Among 831 patients with gastric adenocarcinoma who underwent surgery or endoscopic resection in Juntendo hospital between January 2010 and December 2016, we enrolled 13 cases of GAFG, defined as an extremely well differentiated adenocarcinoma with expression of pepsinogen-I and/or $\mathrm{H}^{+} / \mathrm{K}^{+}$-ATPase 1). To clarify concurrent mutations of GAFG, next generation sequencing (NGS) was perfumed for all cases of the tumor and normal tissue formalin-fixed paraffin-embedded samples, using Ion PGM ${ }^{\mathrm{TM}}$ system with the Hotspot Cancer Panel v2 targeting 50 genes (Thermo Fisher Scientific). We also carried out immunohistochemical staining including MUC2, MUC5AC, MUC6, and CD10, and analyzed association among genetic alterations and mucin phenotype.

Results: Clinicopathologically, all patients [62.9 y (range 43-80), 8 male, 5 female] had submucosal invasive adenocarcinomas with no $H$. pylori infection; the mean depth of the submucosal invasion was $288.4(50-700) \mu \mathrm{m}$. Neither local recurrence nor metastases were detected at a median of 6.7 months (1-47) follow-up after treatment. NGS analysis revealed that nine of 13 patients $(69.2 \%)$ had oncogenic driver mutations including GNAS $(5 / 13,38.5 \%), K R A S(2 / 13$, $15.4 \%), \operatorname{STK} 11(1 / 13,7.7 \%)$, and $\operatorname{TP} 53(1 / 13,7.7 \%)$, and these alterations were mutually exclusive. Mutations of $A P C$ and $C T N N B 1$, members of the $\mathrm{Wnt} / \beta$-catenin pathway, were absent in all cases examined. Compared with public data sets of conventional gastric adenocarcinoma 4), the frequency of GNAS mutation was significantly higher $(38.5 \%$ vs. $6.2 \%, \mathrm{p}<0.01)$ in GAFG. Furthermore, the frequency of TP53 mutation was significantly lower $(7.7 \%$ vs. $47.8 \%, p<0.05)$ in GAFG. However, there were no significant differences in the frequencies of KRAS and STK11 mutations. In addition, all lesions showed gastric phenotype (i.e. immunopositive for MUC5AC and/or MUC6), and there were no significant differences between genetic alterations and mucin phenotype.

Conclusion: The present clinical and comprehensive genetic analysis identified that GAFG is a neoplasm with low-grade malignancy, and the frequent presence of GNAS mutation is a characteristic genetic feature of GAFG.

Disclosure of Interest: All authors have declared no conflicts of interest.

\section{References}

1. Ueyama $\mathrm{H}$ et al. Gastric adenocarcinoma of fundic gland type (chief cell predominant type): proposal for a new entity of gastric adenocarcinoma. Am. J. Surg. Pathol. 2010; 34: 609-19.

2. Ueyama $\mathrm{H}$ et al. Gastric adenocarcinoma of the fundic gland type (chief cell predominant type). Endoscopy. 2014; 46(02): 153-157.

3. Ueyama $\mathrm{H}$ et al. Establishment of endoscopic diagnosis for gastric adenocarcinoma of fundic gland type (chief cell predominant type) using magnifying endoscopy with narrow-band imaging. Stomach and intestine. 2015; 50(12): 1533-1547.

4. Cancer Genome Atlas Research Network. Nature. 2014.

\section{P1237 LNCRNA-HOTAIR INDUCES THE UBIQUITINATION OF RUNX3 IN GASTRIC CANCER}

S. Chen ${ }^{1}$, M. Xue ${ }^{2}$, L. Chen ${ }^{1}$, L. Wang ${ }^{2}$

${ }^{1}$ Gastroenterology, Sir Runrun Shaw Hospital, School of Medicine, Zhejiang University, Hangzhou/China

${ }^{2}$ The second Affiliated Hospital, School of Medicine, Zhejiang University, Hangzhou/China

\section{Contact E-mail Address: 4094108@qq.com}

Introduction: Runt-related transcription factor 3 (Runx3) is a transcription factor playing an inhibitory role in the malignant behavior of gastric cancer. Long noncoding RNAs (LncRNAs) exert their functions mainly by binding with corresponding proteins, among which, transcription factors are the most common ones. However, the LncRNAs that could bind with and affect the expression or activity of Runx3 are still unclear.

Aims \& Methods: Potential Runx3-binding LncRNAs were screened by an online prediction software RPISeq, and then validated by RNA Immunoprecipitation. Specific HOTIAR binding site with Runx3 was confirmed further by RNA Pull down. The E3 ubiquitin ligases being involved in the ubiquitin-proteasome degradation of Runx3 were recognized through co-immunoprecipitation assay. Besides, the expressions of HOTIAR and Runx 3 were measured in human gastric cancer tissues and correlated with each other.

Results: A total of 11 lncRNAs that might bind with Runx3 were selected by in silico analysis, among which, HOTIAR, MALAT1, PVT1 and LET were significantly amplified in RNAs precipitated by Flag antibody. Ectopic expression of HOTIAR inhibits the protein expression of Runx3, while addition of exogenous MG132 could partially recover this. RNA Pull down assay with a stepwise deletion of Runx3 shows that HOT-D2 (1951-2100) is a major Runx3 binding site along the sequence of HOTIAR. Mex3b was markedly enriched by Runx3 antibody, and siRNAs targeting either HOTIAR or Mex3b could enhance the stability of Runx3 by impairing its ubiquitination. in addition, HOTIAR was negatively associated with the protein level of Runx3 in gastric cancer tissue. Conclusion: HOTAIR induces the ubiquitin-proteasome degradation of Runx 3 by enhancing its interaction with a E3 ubiquitin ligase Mex3b in gastric cancer. Disclosure of Interest: All authors have declared no conflicts of interest.

\section{P1238 CAB39L FUNCTIONS AS A TUMOUR SUPPRESSOR IN GASTRIC CANCER THROUGH LKB1-MEDIATED AMPK ACTIVATION}

W. Li ${ }^{1}$, C.C. Wong ${ }^{2}$, Y. Dong ${ }^{1}$, J. Xu ${ }^{3}$, Y. Qian ${ }^{4}$, W. Kang ${ }^{5}$, P.W.Y. Chiu ${ }^{6}$, E. $\mathrm{Ng}^{6}, \mathrm{~J} . \mathrm{Yu}^{2}$

${ }^{1}$ Department Of Surgery, Institute of Digestive Disease, The Chinese University of Hong Kong, Hong kong/Hong Kong PRC

${ }^{2}$ Institute Of Digestive Disease, Partner State Key Laboratory Of Digestive

Disease, Chinese University of Hong Kong, Hong Kong/Hong Kong PRC

${ }^{3}$ Chinese University of Hong Kong, Hong Kong/Hong Kong PRC

${ }^{4}$ Department Of Gastroenterology, Sir Run Run Shaw Hospital, School of Medicine, Zhejiang University, Hangzhou/China

${ }^{5}$ Anatomical And Cellular Pathology, Chinese University of Hong Kong, Hong Kong/Hong Kong PRC

${ }^{6}$ Prince Of Wales Hospital, Chinese University of Hong Kong Dept. of Surgery Prince of Wales Hospital, Hong Kong/Hong Kong PRC

Contact E-mail Address: liweilin@surgery.cuhk.edu.hk

Introduction: Promoter hypermethylation plays a vital role in cancer development through transcriptional silencing of tumour suppressive genes. Using Infinium Human Methylation 450 BeadChip $(450 \mathrm{~K})$ array, we first identified calcium binding protein 39-like (CAB39L) to be preferentially methylated in gastric cancer (GC) and it may function as a potential tumour suppressor gene.

Aims \& Methods: In this study, we aim to investigate the biological function, molecular mechanism and clinical implications of CAB39L in GC. Clinical relevance was validated by bisulfite genomic sequencing (BGS), western blot and immunohistochemistry (IHC). In vitro functional assays were carried out by cell viability, colony formation, apoptosis, cell cycle, cell invasion and migration assays in GC cell lines. In vivo tumorigenicity was evaluated in an orthotopic mice model. Pathway analysis was performed using RNAseq and Phosphokinase Antibody Array. The interaction of CAB39L with its protein partners was determined by co-immunoprecipitation.

Results: CAB39L mRNA was down-regulated in 13 out of $14 \mathrm{GC}$ cell lines. Silencing of CAB39L was associated with promoter hypermethylation, and demethylation $5^{\prime}$ - treatment using Azadeoxycytidine (5-Aza) restored the expression of CAB39L. In human GC, CAB39L mRNA and protein level $(p<0.0001)$ 
were significantly decreased in GC tissues comparing to adjacent normal tissues both in Chinese cohort $(n=48$ pairs $)$ and TCGA cohort $(n=450)$. CAB39L hypermethylation was correlated with poor overall survival in Chinese cohort $(\mathrm{n}=87, \mathrm{p}<0.005)$ and validated in TCGA cohort $(\mathrm{n}=354, \mathrm{p}<0.005)$, which suggesting that CAB39L might function as a tumour suppressor. The functional importance of CAB39L in GC was therefore examined. Ectopic expression of CAB39L in three GC cell lines (AGS, BGC823, MKN45) suppressed cell proliferation in MTT $(p<0.01)$ and colony formation assays $(p<0.0001)$. CAB39L induced apoptosis and G1 cell cycle arrest in GC cells, concomitant with the enhanced expression of cleaved caspase-8, caspase-3, p21 and decreased cyclin D3 expression. Cell migration and invasion abilities were inhibited by CAB39L in wound healing and gel invasion assays, respectively. Conversely, CAB39L knockdown in MKN28 demonstrated opposite effects. Orthotopic mice model also showed inhibited tumorigenicity with CAB39L-overexpressing BGC823 cells. Mechanistically, RNAseq and gene set enrichment analysis (GSEA) revealed that AMPK and ERBB2/ERBB4 signaling were involved in the tumour suppressive role of CAB39L in GC. Consistent with our RNAseq data, Phospho-Kinase Antibody Array identified AMPK as the top activated kinase; whilst ERK1/2 was the most strongly down-regulated in CAB39L overexpressing GC cells, suggesting that CAB39L up-regulates AMPK concomitant with down-regulation of ERBB2/4-ERK signalling. Moreover, co-immunoprecipitation identified a direct interaction between CAB39L and LKB1, a bona-fide tumour suppressor that functions to activate AMPK to suppress tumorigenesis. Western blot confirmed activation of LKB1-AMPK $\alpha / \beta$ cascade in GC cells expressing CAB39L, while an opposite effect was observed in CAB39L-silenced MKN28 cells. Administration of an AMPK activator, AICAR, inhibited growth of control cells but not CAB39L-expressing (thus AMPK activated) cells, suggesting that AMPK activation by CAB39L contributes to tumour suppression. Conclusion: CAB39L is a novel tumour suppressor silenced by promoter methylation in GC. CAB39L inhibits gastric tumorigenesis via LKB1-mediated activation of $\mathrm{AMPK} \alpha / \beta$. CAB39L methylation may serve as an independent prognostic biomarker for GC patients.

Disclosure of Interest: W. LI: No conflicts of interest.

C.C. Wong: No conflicts of interest.

Y. Dong: No conflicts of interest.

J. Xu: No conflicts of interest.

Y. Qian: No conflicts of interest.

W. Kang: No conflicts of interest.

P.W.Y. Chiu: No conflicts of interest.

E. Ng: No conflicts of interest.

J. Yu: No conflicts of interest.

P1239 FUNCTIONAL CHARACTERIZATION AND CLINICAL SIGNIFICANCE OF PKNOX2, A NOVEL TUMOR SUPPRESSOR IN GASTRIC CANCER
L. Zhang ${ }^{1}$, C.C. Wong ${ }^{1}$, W. $\mathrm{Li}^{1}$, J. Xu ${ }^{1}$, L. Cao ${ }^{1}$, J. Yu ${ }^{2}$
${ }^{1}$ Chinese University of Hong Kong, Hong Kong/Hong Kong PRC
${ }^{2}$ Institute Of Digestive Disease, Partner State Key Laboratory Of Digestive
Disease, Chinese University of Hong Kong, Hong Kong/Hong Kong PRC

\section{Contact E-mail Address: zhangliforever@126.com}

Introduction: Using $450 \mathrm{~K}$ DNA methylation array, we identified PBX/Knotted 1 Homeobox 2 (PKNOX2) as a novel gene differentially methylated in gastric cancer (GC). PKNOX2 belongs to the TALE (three-amino acid loop extension) family of homedomain transcription factors, which play fundamental roles in cell proliferation, differentiation and death. However, the role of PKNOX2 in GC remains largely unknown.

Aims \& Methods: We aimed to investigate the function, molecular mechanism and clinical implication of PKNOX2 in GC. PKNOX2 promoter methylation was evaluated by bisulfite sequencing. PKNOX2 expression was determined by RT-PCR and qPCR. Biological functions of PKNOX2 were determined in GC cells by MTT, colony formation, apoptosis, cell cycle, cell migration/invasion assays, and in vivo using nude mice tumorigenicity assay. Pathways targeted by PKNOX2 were identified by Cancer pathway PCR array and dual luciferase reporter assay.

Results: PKNOX2 was silenced in 15 out of 18 GC cell lines through promoter methylation, and 5'-Azadeoxycytidine treatment restored PKNOX2 expression. PKNOX2 mRNA expression was down-regulated in GC compared to adjacent normal tissues $(P<0.001)$. Moreover, promoter methylation of PKNOX2 was associated with poor survival in GC patients $(P<0.05)$, suggesting that PKNOX2 may function as a tumor suppressor in GC. We therefore examined its functional effect in GC. Ectopic expression of PKNOX2 in GC cell lines suppressed cell proliferation, induced cell apoptosis and cell cycle arrest, concomitant with increased expression of apoptosis markers cleaved caspase-9,-7,-3 and PARP and cell cycle inhibitors p53, p21 and p27. PKNOX2 also attenuated cell migration and invasion by inhibiting epithelial-mesenchymal transition. PKNOX2 knockdown in HGC27 cells resulted in opposite effects. Tumorigenicity assay in nude mice showed that stable PKNOX2 expression significantly suppressed tumor growth in vivo. To probe the mechanism of action of PKNOX2 in GC, we performed Cancer pathway PCR array, which unveiled a profound up-regulation ( $>6$-fold) of Insulin like Growth Factor Binding Protein 5 (IGFBP5) in PKNOX2-overexpressing GC cells. IGFBP5 knockdown abolished the growth inhibitory effect of PKNOX2 in GC cells, indicating that IGFBP5 mediated the tumor suppressive function of PKNOX2. Chromatin immunoprecipitation (ChIP) assay showed that PKNOX2 bind directly to IGFBP5 promoter to mediate transcription. Consistent with our data, PKNOX2 expression was positively correlated with IGFBP5 expression in the TCGA GC dataset. IGFBP5 mediated the tumor suppressive effect of
PKNOX2 via activating p53 signaling pathway, as determined by western blot and luciferase reporter assays. Consequently, p 53 transcription target genes were coordinately up-regulated in PKNOX2 overexpressing cells, leading to tumor suppression.

Conclusion: PKNOX2 functions as a novel tumor suppressor silenced in GC by promoter methylation. Its tumor suppressive effect is mediated via IGFBP5 and the activation of $\mathrm{p} 53$ signaling pathway. Promoter methylation of PKNOX2 may be a useful biomarker for predicting patient prognosis.

Disclosure of Interest: All authors have declared no conflicts of interest.

\section{P1240 HOXC10 IS UPREGULATED IN HUMAN GASTRIC CANCER AND PROMOTES CELL G1-S TRANSFORMATION AND PROLIFERATION THROUGH DIRECT TRANSCRIPTIONAL REPRESSION OF P21}

L. Wang ${ }^{1}$, Z. Jin ${ }^{2}, \mathrm{~S} . \mathrm{Chen}^{2}, \mathrm{~S} . \mathrm{Lai}^{2}, \mathrm{X} . \mathrm{He}^{2}, \mathrm{~J}^{\mathrm{Si}}{ }^{2}$

${ }^{1}$ The Second Affiliated Hospital, School of Medicine, Zhejiang University, Hangzhou/China

${ }^{2}$ Institute of Gastroenterology, Zhejiang University, Hangzhou/China

\section{Contact E-mail Address: wanglj76@hotmail.com}

Introduction: Gastric cancer is one of the most common malignancies worldwide. A multitude of factors contribute to the progression of gastric cancer, including activation of oncogenes and inactivation of tumor suppressors. To find the key molecules and their mutual relations in gastric carcinogenesis is of great significance for the diagnosis and treatment of gastric cancer. The homeobox (Hox) superfamily genes encode transcription factors and usually regulate the expression of target genes at transcription level. Dysregulation of Hox genes will cause the abnormality in individual development and tissue formation, and may also lead to malignant transformation. Our group have previously screened by gastric cancer tissue microarray and found that the expression of HoxC10 was remarkably upregulated in gastric cancer. At the present time, the function and molecular mechanisms of HoxC10 in gastric cancer remains poorly understood. Aims \& Methods: To investigate the expression of HoxC10 in gastric cancer, tissue samples from two clinical cohorts were used. We analyzed HoxC10 expression in gastric cancer tissues and the corresponding adjacint non-tumor tissues, as well as its correlation with clinical pathological parameters. In addition, we also verified the results by utilizing bioinformatics analysis. We studied the effects of HoxC10 on gastric cancer cell cycle control and proliferation through combination of in vitro cell function tests and in vivo nude mice model. Then, we screened HoxC10 potential downstream targets by using cDNA microarray and verified the results by RT-qPCR. We studied the effects of HoxC10 on p21 expression and its downstream cell cycle-related proteins and validated the correlation of HoxC10 and p21 expression in vitro and in vivo. By application of dual-luciferase reporter assay and chromatin immunoprecipitation, we explored the role of $\mathrm{HoxC} 10$ in p21 transcription repression.

Results: HoxC10 mRNA expression were significantly higher in fresh frozen gastric cancer tissues than in matching adjacent non-tumor tissues $(91.43 \%$, $64 / 70, \mathrm{P}<0.01)$. The expression of $\mathrm{HoxC} 10$ was related to the depth of tumor invasion, lymph node metastasis and tumor stage $(\mathrm{P}<0.01)$. Using tissue microarray, HoxC10 protein expression was also found widely upregulated in gastric cancer tissues $(91.3 \%, 137 / 150, \mathrm{P}<0.01)$ and was closely correlated with patient survival $(\mathrm{HR}=2.8 ; 95 \% \mathrm{CI} 2.0-7.2)$. Besides, in TCGA database of gastric cancer, HoxC10 was upregulated by 122 times $(\mathrm{n}=33, \mathrm{P}<0.01)$, and in Kaplan-Meier Plotter database, high HoxC10 expression was associated with poor prognosis of patients with gastric cancer ( $\mathrm{HR}=1.8 ; 95 \%$ CI $1.5-2.16$ ). HoxC10-overexpressing gastric cancer cells showed accelerated G1-S phase transformation and proliferation, whereas HoxC10 knocking-down induced cell cycle arrest in G1 phase and repressed cell proliferation. Moreover, overexpression of HoxC10 accelerated gastric tumor growth in a mouse xenograft model, while knocking-down of HoxC10 inhibited gastric tumor growth. cDNA microarray showed that HoxC10 regulates multiple downstream genes including p21, a potent cell cycle regulator. A significantly negative correlation between HoxC10 and p21 were detected in gastric cancer cells and tissues. Knockingdown of HoxC10 also altered the expression of some p21 downstream cell cycle-related proteins, such as CDK2 and CDK4. Furthermore, we found that HoxC10 binds to the $\mathrm{p} 21$ promoter directly and could inhibit p21 transcription. Conclusion: Taken together, our results suggest that HoxC10 functions as a tumor promoting gene in gastric cancer and may be an important regulator of cell cycle control through $\mathrm{p} 21$ transcription regulation.

Disclosure of Interest: All authors have declared no conflicts of interest.

\section{References}

1. Shah N, Sukumar S. The Hox genes and their roles in oncogenesis. Nat Rev Cancer. 2010; 10(5): 361-71.

2. Ladam F, Sagerstrom CG. Hox regulation of transcription: more complex(es). Dev dyn. 2014; 243(1): 4-15.

3. Harper JW, Adami GR, Wei N, Keyomarsi K, Elledge SJ. The p21 Cdkinteracting protein Cip1 is a potent inhibitor of G1 cyclin-dependent kinases. Cell. 1993; 75(4): 805-16. 


\section{P1241 RECOVERY OF GASTRIC FUNCTION IN CHRONIC} ATROPHIC GASTRITIS BY USING L-CYSTEINE: A 3 YEARS STUDY

F. Di Mario ${ }^{1}$, L. Franzoni ${ }^{2}$, S. Grillo ${ }^{3}$, S. Landi ${ }^{4}$, C. Miraglia ${ }^{2}$, S. Scida ${ }^{2}$, M. Riccò ${ }^{5}$, G. Grande $^{6}$, M. Franceschi ${ }^{7}$, M.P. Panozzo ${ }^{8}$, A. Antico ${ }^{8}$,

R. Cannizzaro ${ }^{9}$, G.M. Cavestro ${ }^{10}$, M. Rugge ${ }^{11}$, C. Scarpignato ${ }^{2}$

${ }^{1}$ University Of Parma, Department of Clinical and Experimental Medicine, section of Gastroenterology, Parma/Italy

${ }^{2}$ Department Of Medicine And Surgery, University Of Parma, Italy, University of Parma, Parma/Italy

${ }^{3}$ Gastroenterology, University of Parma, Italy, PARMA/Italy

${ }^{4}$ University of Parma, Parma/Italy

${ }^{5}$ Local Health Unit Of Parma, Public Health Department, Occupational Health And Safety Service, Parma, Italy, University Hospital, Parma (Italy), Parma/ Italy

${ }^{6}$ Aou Modena, Gastroenterology and Digestive Endoscopy Unit, Modena/Italy ${ }^{7}$ Endoscopic Unit, Department Of Surgery, Ulss4, Hospital ULSS4 Alto Vicentino, Santorso/Italy

${ }^{8}$ Department Of Clinical Pathology, Ulss 4 Alto Vicentino, Santorso, Italy, Hospital ULSS4 Alto Vicentino, Santorso/Italy

${ }^{9}$ Oncological Gastroenterology, Centro di Riferimento Oncologico di Aviano

S.O.C. di Gastroenterologia, Aviano/Italy

${ }^{10}$ Dept. of Gastroenterology, Università Vita-Salute San Raffaele, Milano, Italy, Milano/Italy

${ }^{11}$ Pathology, Medical School of the Padova University, Padova/Italy

\section{Contact E-mail Address: francesco.dimario@unipr.it}

Introduction: The relationship between Helicobacter pylori (H.p.) eradication and atrophic changes is debated. Although some studies report a partial restoration of serum pepsinogen I (PGI) levels after eradication, it is not clear whether this finding reflects gastric mucosal healing. L-cysteine, reducing acetaldehyde production after food intake, has been proposed for prevention of gastric carcinogenesis in patients with chronic atrophic gastritis (CAG). To assess modifications in gastric function after L-cysteine administration in CAG by means of PGI and gastrin 17 (G17) serum levels

Aims \& Methods: 62 patients (18 men, mean age $47.2 \mathrm{yrs}$ ), with histological diagnosis of moderate to severe chronic, atrophic, body gastritis (according to the O.L.G.A. staging system) and PGI serum levels $<25 \mu \mathrm{g} / \mathrm{L}$, underwent upper gastrointestinal endoscopy with gastric biopsy samplings and PGI and G-17 measurement by means of Gastropanel ${ }^{\circledR} .22$ out of 62 patients had autoimmune gastritis while 40 of them reported previous H.p. infection. All patients, Helicobacter pylori negative at baseline, were treated with L-cysteine $(100 \mathrm{mg}$ three times daily), up to now 24 out of 62 reached 36 months-treatment. Serum PGI and G-17 were measured at baseline and after 3, 6, 12, 24, 36 months after starting therapy.

Results: The PGI showed an increased serum level after the starting of L-cysteine administration, as it follows: PGI mean value at baseline was $8.42 \mu \mathrm{g} / 1$, but after 3 months therapy was 10.58 , after 6 months 11.65 , after 12 months 12.19 , after 24 months 13.88, and after 36 months was 14.21 (p: 0.0001). The G-17 serum level resulted gradually decreased over the 36 months therapy, as it follows: G-17 mean value was $51.33 \mathrm{pmol} / 1$ at baseline, 43.13 after 3 months therapy, 38.66 after 6 months, 28. 34 after 24 months and 26.03 after 36 months (p: 0.0041 )

Conclusion: After L-cysteine administration, patients with chronic, atrophic, body gastritis showed long-lasting improvements of physiological gastric function, reflected by a significant increase of PGI levels and a parallel decrease of G-17 serum levels over a 36 months follow-up period.

Disclosure of Interest: All authors have declared no conflicts of interest

\section{P1242 ANDROGEN RECEPTOR DIRECTLY REGULATES CELL CYCLE-RELATED KINASE TO PROMOTE GASTROCARCINOGENESIS}

\section{G. Zhao ${ }^{1}$, X. $\mathrm{Li}^{2}$, L. Xu ${ }^{3}$, N. Zhang ${ }^{3}$}

${ }^{1}$ Inner Mongolia People's Hospital, Hohhot/China

${ }^{2}$ State Key Laboratory Of Oncology In South China, Sun Yat-sen University

Cancer Center, Guangzhou/China

${ }^{3}$ Sun Yat-sen University, Guangzhou/China

\section{Contact E-mail Address: cnzhaoguijun@hotmail.com}

Introduction: Signaling pathway mediated by androgen receptor (AR) plays an important role in the development of gender-related tumors, such as hepatocellular carcinoma, prostate cancer. Gastric cancer (GC) is the third cause of cancer related death all over the world, and its incidence in male is also much higher than female. However, the molecular mechanisms of AR in gastric cancer are still poorly characterized.

Aims \& Methods: To investigate the role of AR in gastric cancer, we identify the transcriptional downstream targets of $\mathrm{AR}$ by chromatin immunoprecipitation. We detected mRNA and protein expression level of AR and its target in paired GC samples and GC cell lines by RT-PCR and western blot. The biological functions of AR signaling pathway in GC cell lines were determined by colony formation and cell migration/invasion assay.

Results: CCRK was demonstrated as the direct target of AR by chromatin immunoprecipitation assay. AR expression was elevated in most (6/7) GC cell lines compared with the immortalized gastric cell line GES1. CCRK was upregulated in all (7/7) tested GC cell lines. The correlation of AR and CCRK expression was statistically significant. Higher mRNA level of both AR and CCRK were detected in GC tissues compared with the adjacent normal tissues $(\mathrm{P}<0.01)$. Ectopic re-expression of $\mathrm{AR}$ or CCRK by stable transfection promoted colony formation and invasiveness $(\mathrm{P}<0.05)$. Consistently, the numbers of colony formation, migrated cells and invasive cell were reduced by knockdown of $\mathrm{AR}$ or $\mathrm{CCRK}$ in $\mathrm{GC}$ cell lines $(\mathrm{P}<0.01)$

Conclusion: Our results demonstrate that AR directly regulates CCRK expression in GC. AR and CCRK gene may act as a potential oncogene in gastrocarcinogenesis by playing an important role in promoting of cell proliferation, migration and invasion, which may partially explain the higher prevalence of gastric cancer among males.

Disclosure of Interest: All authors have declared no conflicts of interest.

P1243 FLYING OFF COURSE WITH A 2WW DIRECT ACCESS TO TEST PILOT: NOTTINGHAM'S EXPERIENCE OF THE SUSPECTED UPPER GASTRO-INTESTINAL CANCER PATHWAY CHANGE WITH GP VETTING AND OGD BOOKING

N. R. Lewis, J. Catton, E. James

Trent Oesophago-gastric Unit, Nottingham University Hospitals NHS Trust, Nottingham/United Kingdom

Contact E-mail Address: nina.lewis@nuh.nhs.uk

Introduction: Timely progress through diagnostic pathways is a leading quality measure for NHS cancer services. A hypothesis of sooner diagnosis being achievable with direct access to hospital tests from primary care is a core part of CRUK ACE program (1), and in the context of UGI cancer pathway, there is known wide variation of direct access (DA) OGD (2). This pilot evaluates the efficacy and utility of DA OGD or clinic (DA OPD) for GP concerns a patient may have OG cancer. Comparison was made with thestandard 2WW pathway, where allocation to OGD v OPD first is performed by OG consultant.

Aims \& Methods: Pilot and UGI standard 2WW referrals 01/01/16-01/08/16 were identified from Cancer Centre records.

Results: 192 patients were in the pilot pathway, 430 via the standard 2 WW. GPs were more likely to allocate patients to DA OGD (52\%) compared to $32 \%$ having DTT OGD allocated by the hospital. Despite under-utilisation of protected slots for DA OGD, time to DA OGD compared to DTT did not differ $(11.0$ days $[95 \%$ CI $10.5,11.2]$ versus 12.4 days [ $95 \%$ CI $11.0,13.9])$. The same was seen for DA OPD. The total time on pathway was not improved in the pilot group at 16.8 days [ $95 \%$ CI $4.9,28.6$ ] compared to 17.9 days [ $95 \%$ CI $16.9,18.9]$. The subgroup of patients allocated in the pilot to DA OGD did have a quicker exit from the pathway, at 12.4 days [95\% CI 6.5,18.3] compared to 14.8 days [95\% CI $12.9,16.6]$ on the DTT OGD group. The pilot overall detected 8 cancers $(4.2 \%)$; the standard $2 \mathrm{WW}$ path detected $55(12.8 \%)$. OG cancers were in 4 of the DA OGD $(4 \%)$ and 14 of the DTT OGD $(10.2 \%)$. A further 10 non-OG cancers were detected in the DTT group after clinicians requested further investigations to determine the cause of their symptoms. Those patients allocated to OPD first by either GP or hospital were as likely to have cancer as those having OGD, with $4.3 \%$ of those in the pilot having acancer detected this way, but none OG cancer, and $10.3 \%$ found to have cancers in the standard $2 \mathrm{WW}$ group following investigation directed after clinic visit. Of these $65 \%$ were cancers other than OG cancers and would not be detected on OGD alone.

Conclusion: OGD as a sole investigation for symptoms has its utility in excluding or detecting OG cancer. A high proportion of cancers detected via $2 \mathrm{WW}$ criteria on OG pathway are outside of OG tract and require other tests to diagnose them. In this pilot no additional utility of opening direct access OGD for GP concerned a patient may have an OG cancer was not demonstrated unless exclusion of OG cancer is viewed as the major purpose of the pathway. Our data demonstrates that such a view would be detrimental and lead to missed opportunity to detect other cancers that cause symptoms overlapping with those often ascribed to OG tumours.

Disclosure of Interest: All authors have declared no conflicts of interest.

Reference

1.www.cancerresearchuk.org 2. PLoS ONE 11(7):e0159725

\section{P1244 WHAT IS THE YIELD OF ROUTINE D2 BIOPSIES IN THOSE} PRESENTING WITH WEIGHT LOSS AT GASTROSCOPY?

M. Yalchin, S. Zonoozi, M. Hussein, N. Jayasooriya, K. Besherdas Gastroenterology, Barnet Hospital, DJ/United Kingdom

Contact E-mail Address: m.yalchin@nhs.net

Introduction: Coeliac disease is a common cause of malabsorption in Western countries. The gold standard method of diagnosing coeliac disease is by way of duodenal biopsy. Weight loss is a symptom of malabsorption. Patients referred for upper gastrointestinal endoscopy with symptoms of a weight loss commonly undergo duodenal biopsy to assess for presence of coeliac disease. We hypothesise that those patients with weight loss and who routinely have duodenal biopsies very rarely have coeliac disease unless there are other pointers towards malabsorption.

Aims \& Methods: A single-centre, retrospective analysis of consecutive patients undergoing upper gastrointestinal endoscopy for the sole indication of weight loss was undertaken within a large associate teaching hospital within North London from 2005-2016. Of these patients, we reviewed those that had duodenal (D2) biopsies and the results. If they proved abnormal, we looked back for additional markers of malabsorption, clinically and biochemically.

Results: 142 consecutive patients, 65 were Male, 77 were female, underwent OGD for weight loss. Out of this cohort, $62 \%(\mathrm{n}=88)$ had a duodenal biopsy. $89 \%$ $(\mathrm{n}=78)$ of these had a normal biopsy. $11 \%(\mathrm{n}=10)$ had an abnormal biopsy, 
and 6 of these patients had coeliac, whilst 4 had other pathology such as granulomas or duodenitis.

For all the patients who had abnormal D2 biopsies, they had other clinical markers of malabsorption, such as abdominal pain and diarrhoea, or biochemical indices such as anaemia or elevated TTG antibodies.

Conclusion: From this study we conclude that the yield of routine duodenal biopsies in patients endoscoped for the sole indication of weight loss is poor. In patients with weight loss in whom coeliac disease is identified on biopsy is always associated with additional symptoms or abnormalities in blood indices. We conclude that there is no need to take biopsies of the duodenum on a routine basis for weight loss alone unless there are other signs of malabsorption. This will save time (both from taking the biopsy and sampling in the lab), lower the cost (forceps and pot) and improve the safety (potential perforation and bleeding risk) of the procedure.

Disclosure of Interest: All authors have declared no conflicts of interest.

P1245 FUNDING DISPARITIES IN DIGESTIVE CANCER

\section{RESEARCH IN THE UNITED STATES}

\section{P. S. Liang ${ }^{1}$, K. O'Connell ${ }^{2}, \mathrm{M} . \mathrm{Du}^{2}$}

${ }^{1}$ Medicine, New York University, New York/United States of America

${ }^{2}$ Memorial Sloan Kettering Cancer Center, New York/United States of America

\section{Contact E-mail Address: petersliang $@$ gmail.com}

Introduction: In 2015, the five most common digestive cancers (colorectal, pancreatic, liver, gastric, and esophageal) accounted for $16 \%$ of incident cancer cases and $24 \%$ of all cancer deaths. It is unclear whether the amount and recent trends in US federal funding for digestive cancer research corresponds to the burden of disease.

Aims \& Methods: We obtained the total annual funding for cancer (including the five most common digestive cancers) from 2008 to 2015 using a public database of research funded by US federal agencies. We calculated funding in 2015 constant USD using the Consumer Price Index. Cases and deaths estimated by the American Cancer Society were used to calculate funding per death or case for each cancer. For comparison, we also extracted data for the three most common cancers (breast, lung, prostate) and all cancers combined. As funding for research in the United States was boosted by the American Recovery \& Reinvestment Act in 2009-2010 and declined thereafter, we analyzed trends in funding and disease burden from 2010-2015 using the Spearman correlation.

Results: In 2015, 8 billion USD in federal funding was issued to all cancer research and 658 million USD to the five common digestive cancers. The five digestive cancers accounted for 23-24\% of all cancer deaths from 2010-2015, but only received $7 \%$ of cancer funding in 2010 , and $8 \%$ of funding in 2015. The ratio of the proportion of all cancer death to the proportion of all cancer funding, a marker of funding disparity, was 2.9. In comparison, breast cancer accounted for $7 \%$ of cancer deaths in all years, $11 \%$ of funding in both 2010 and 2015 , and had lower proportional death than proportional funding (ratio $=0.6$ ). Prostate cancer likewise had lower proportional death than funding (ratio $=0.8$ ). Funding disparity, measured by proportional death and funding, was highest for esophageal and gastric cancer among digestive cancers and for lung cancer overall. The estimated number of deaths and cases decreased for colorectal cancer but increased for the other digestive cancers during the study period. Funding per death among digestive cancers in 2015 was highest for liver cancer and lowest for esophageal cancer. Funding per death for breast cancer was more than 3 -fold that of liver cancer and nearly 7-fold that of esophageal cancer. From 2010-2015, funding per death for digestive cancers decreased by $20 \%$ for esophageal cancer, $24 \%$ for colorectal and gastric cancer, and $28 \%$ for liver cancer; funding per death increased by $10 \%$ for pancreatic cancer. Statistically significant trends were observed for colorectal and liver cancer ( $\mathrm{p}<.05$ for both). Over the same period, funding per incident case decreased by $15 \%$ for esophageal cancer, $21 \%$ for colorectal cancer, $34 \%$ for gastric cancer, and $37 \%$ for liver cancer; funding increased for pancreatic cancer by $6 \%$. Statistically significant trends were observed for liver $(\mathrm{p}<.01)$, colorectal, and gastric $(\mathrm{p}<.05)$ cancer. Despite the larger relative funding decrease for liver cancer, it remained the bestfunded digestive cancer relative to both incident case and death. Liver cancer received more than 2.5 -fold funding per incident case than colorectal and gastric cancer in 2015. Gastric cancer was the only digestive cancer to measure in the bottom two for both funding per death and per incident case.

Conclusion: From 2010 to 2015 in the US, federal research funding relative to the burden of disease has decreased for all digestive cancers except pancreatic cancer. Although a similar trend was observed for all cancer research, there appears to be a funding disparity for digestive cancers - especially gastric cancer - compared to breast cancer and all cancers combined. Greater investment and more equitable funding allocation may improve digestive cancer outcomes.

Disclosure of Interest: All authors have declared no conflicts of interest.

\section{TUESDAY, OCTOBER 31, 201709:00-17:00}

\section{H. PYLORI II - HALL 7 .}

\section{P1246 HELICOBACTER PYLORI ERADICATION MODULATES ABERRANT CPG ISLAND HYPERMETHYLATION IN GASTRIC CARCINOGENESIS}

J. Choi ${ }^{1}$, S.G. Kim ${ }^{2}$, B.G. Kim ${ }^{3}$, S. Koh ${ }^{3}$, J.W. Kim ${ }^{3}$, K.L. Lee ${ }^{3}$

${ }^{1}$ Internal Medicine, Inje University of College of Medicine, Sanngye Paik Hospital, Seoul/Korea, Republic of

${ }^{2}$ Internal Medicin And Liver Research Institute, Seoul University College of

Medicine, Seoul/Korea, Republic of
${ }^{3}$ Department Of Internal Medicine, SMG-SNU Boramae Medical Center, Seoul/ Korea, Republic of

Contact E-mail Address: doct00@hanmail.net

Introduction: Helicobacter pylori infection induces aberrant DNA methylation in gastric mucosa. We evaluated the long-term effect of $H$. pylori eradication on promotor $\mathrm{CpG}$ island hypermethylation in gastric carcinogenesis.

Aims \& Methods: $H$. pylori-positive patients with gastric adenoma or early gastric cancer who underwent endoscopic resection were enrolled. According to $H$. pylori eradication after endoscopic resection, the participants were randomly assigned to H. Pylori eradication or non-eradication group. H. pylori-negative gastric mucosa from normal participants provided the normal control. $\mathrm{CpG}$ island hypermethylation of tumor-related genes (p16, CDH1, and RUNX-3) was evaluated by quantitative MethyLight assay in non-tumorous gastric mucosa. The gene methylation rate and median values of hypermethylation were compared after one year by $H$. pylori status.

Results: In $H$. pylori-positive patients, hypermethylation of p16 was found in $80.6 \%$, of CDH1 in $80.6 \%$, and of RUNX-3 in $48.4 \%$. This is significantly higher than normal control (p16, 10\%; CDH1, 44\%; RUNX-3, 16\%) $(\mathrm{p}<0.05)$. In the $H$. pylori eradication group, methylation rates of $\mathrm{pl} 6$ and $\mathrm{CDH} 1$ decreased in $58.1 \%$ and $61.3 \%$ of the patients, and the median values of hypermethylation were significantly lower at one year compared with the noneradication group. However, RUNX-3 hypermethylation did not differ significantly at one year after $H$. pylori eradication. The non-eradication group hypermethylation did not change after one year.

Conclusion: $H$. pylori infection was associated with promotor hypermethylation of genes in gastric carcinogenesis, and $H$. pylori eradication might reverse p16 and $\mathrm{CDH} 1$ hypermethylation

Disclosure of Interest: All authors have declared no conflicts of interest.

\section{P1247 CURCUMIN DOWNREGULATES INTERLEUKIN (IL)-17 BY INCREASING THE EXPRESSION OF INDOLEAMINE 2,3- DIOXYGENASE (IDO) IN HELICOBACTER PYLORI-INFECTED HUMAN GASTRIC MUCOSA}

T. Larussa, R. Liparoti, S. Gervasi, E. Suraci, R. Marasco, M. Imeneo, F. Luzza Department Of Health Sciences, University of Catanzaro, Catanzaro/Italy

Contact E-mail Address: tiziana.larussa@gmail.com

Introduction: IDO promotes the effector T-cells apoptosis by catalyzing the ratelimiting first step in tryptophan (Trp) catabolism. We demonstrated that the high expression of IDO in $H$. pylori-infected human gastric mucosa attenuates Thl and Th17 immune responses. The anti-inflammatory and anti-microbial properties of the nutraceutical compound curcumin suggest its use as an anti- $H$. pylori agent, but mechanisms that underlie its helpful activity are still not clear.

Aims \& Methods: Five antral biopsies were taken from 22 patients $(10 \mathrm{M}$, median age 47.5 yrs, range 20-74) who underwent gastroscopy for dyspeptic symptoms: 1 for urease quick test (Eurospital, Trieste, Italy), 2 for histology (Giemsa staining for $H$. pylori), and 2 for organ culture. ${ }^{13} \mathrm{~A} \mathrm{C}$-urea breath test was also performed (at least two tests positive and all the three tests negative to be considered $H$. pylori-infected or uninfected). Biopsy samples were immediately placed in an organ culture chamber, treated with and without curcumin $200 \mu \mathrm{M}$ (Sigma, St. Louis, MO, USA) for 20 hours and evaluated for the expression of IDO and IL-17 by Western blotting. Levels of IL-17 were also measured in culture supernatant by ELISA. Further antral biopsies from a subgroup of 14 patients were treated with curcumin in addition with the IDO inhibitor 1-methyl-L-Trp (1-MT, Sigma, St. Louis, MO, USA) and the expression of IL-17 was assessed in total protein extracts by Western blotting. The ratio of IDO and IL-17 with $\beta$-actin was calculated by densitometry, while results from ELISA were normalized on protein content. Values were given as means \pm SD arbitrary units (a.u.) and $\mathrm{pg} / \mathrm{mL}$, respectively. Data were analysed using the Mann-Whitney U test. Results: In gastric biopsies cultures from $H$. pylori-infected patients $(\mathrm{n}=13)$, IDO significantly increased in curcumin-treated compared with untreated samples $(1.26 \pm 0.64$ a.u. vs $0.87 \pm 0.61, p=0.02)$. Levels of IL-17 were significantly lower in curcumin-treated compared with untreated samples, both in gastric biopsies $(0.53 \pm 0.26$ a.u. vs $0.85 \pm 0.31$ a.u., $\mathrm{p}=0.02)$ and culture supernatant $(22.91 \pm 13.72 \mathrm{pg} / \mathrm{mL}$ vs. $40.46 \pm 21.69 \mathrm{pg} / \mathrm{mL}, \mathrm{p}=0.04)$.$) . In the subgroup of$ $H$. pylori-infected patients $(\mathrm{n}=9)$, samples treated with curcumin in addition to IDO inhibitor 1-MT, IL-17 expression was significantly higher compared with untreated samples as well as with those treated with curcumin alone $(1.44 \pm 0.52$ a.u. vs $0.91 \pm 0.34$ a.u. and vs $0.78 \pm 0.21$ a.u., $p=0.04$ and $\mathrm{p}=0.01$, respectively). Both IDO and IL-17 expression were very low in untreated $H$. pylori-uninfected samples and did not significantly change when treated with curcumin alone or in addition to IDO inhibitor 1-MT.

Conclusion: Curcumin is capable of down-regulating IL-17 production in H. pylori-infected human gastric mucosa. This effect is, at least in part, mediated by increasing IDO expression. This endorses the potential role of curcumin in dampening $H$. pylori-induced immune-mediated inflammatory changes. Disclosure of Interest: All authors have declared no conflicts of interest.

\section{Reference}

Larussa T, Leone I, Suraci E, Nazionale I, Procopio T, Conforti F, Abenavoli L, Hribal ML, Imeneo M, Luzza F. Enhanced expression of indoleamine 2,3-dioxygenase in Helicobacter pylori-infected human gastric mucosa modulates Thl/ Th2 pathway and interleukin 17 production. Helicobacter. 2015 Feb;20(1):41-8. 
P1248 ALTERATIONS IN SALIVARY MICROBIOTA IN SUBJECTS WITH HELICOBACTER PYLORI-ASSOCIATED GASTRITIS

W.K. Leung ${ }^{1}$, K.S. Lau ${ }^{1}$, T. S. Tong ${ }^{1}$, K. Chu ${ }^{2}$, P. C. Sham ${ }^{3}$ ${ }^{1}$ Department Of Medicine, University of Hong Kong, Hong Kong/Hong Kong PRC ${ }^{2}$ Department Of Surgery, The University of Hong Kong, Hong Kong/Hong Kong PRC

${ }^{3}$ Centre For Genomic Sciences, The University of Hong Kong, Hong Kong/Hong Kong PRC

Contact E-mail Address: waikleung@hku.hk

Introduction: Alterations in salivary microbiota have been linked to elevated inflammatory responses and has been reported in patients with inflammatory bowel disease and pancreatic cancer. As yet, the potential association between salivary microbiota and patients with gastric pathology has not be determined. Aims \& Methods: In this study, we characterized the salivary microbiota in patients with $H$. pylori (HP)-associated gastritis and the potential changes of salivary microbiota after receiving HP eradication. We enrolled subjects who were scheduled for diagnostic upper GI endoscopy. We excluded patients with peptic ulcer or cancer found on endoscopy, who have received prior HP eradication therapy, and who have recent exposure to antibiotics or acid suppressive therapies. Unstimulated saliva samples were obtained from subjects during fasting state prior to endoscopy. During endoscopy, gastric biopsies were obtained for determination of HP statuses by rapid urease test and histology. Another gastric biopsy was obtained for characterization of gastric microbiota. Serial salivary samples were obtained from HP-infected subjects 8-week after completing HP eradication therapy. Bacterial DNA was extracted for 16s rRNA sequencing by using the MiSeq Platform (Illumina). OTU clustering was performed and taxonomy assigned to the Greengene and HOMD database. Alpha and beta diversity were determined. Linear Discriminant Analysis Effect Size (LEfSe) was used to identify differentially expressed bacterial DNA in different groups. Results: We enrolled 16 subjects with confirmed HP-gastritis and 14 HP-negative subjects. Baseline salivary samples of all subjects were found to have significantly higher microbial diversity than corresponding gastric samples. The predominant microbial family identified in the stomach is Helicobacteraceae $(55.2 \%)$ whereas Helicobacteracece constitutes only $0.1 \%$ of salivary microbiota. In contrast, the predominant families in saliva microbiota are Prevotellaceae $(23.9 \%)$ and Neisseriaceae $(20.3 \%)$. When compared to HP-negative subjects, salivary microbiota in HP-positive patients showed a significant increase in the Bacteroidetes and Spirochaetaceae, and a decrease in Flavobacteriaeceae families. HP eradication therapy resulted in a significant reduction in the relative abundance of families Bacteriodetes and Spirochaetaceae, and an increase in Flavobacteriaceae. Conclusion: There was a significant difference in the microbial diversity and compositions between gastric and salivary microbiota in HP-infected subjects, with Helicobacteracece dominating the gastric microbiota. HP-infected subjects have distinctive microbiota in the saliva which is reversed by HP eradication therapy. The significance of these microbial alterations in the saliva of HPinfected subjects and its correlation with gastric diseases deserves further investigation.

Disclosure of Interest: All authors have declared no conflicts of interest

\section{P1249 EFFECT OF ALCOHOL DRINKING ON SEROCONVSERSION IN HELICOBACTER PYLORI-SERONEGATIVE KOREANS}

J.H. Kim, S. Lee, H.G. Kwon, S.P. Lee, J.H. Kim, I. Sung, H.S. Park, C.S. Shim Internal Medicine, Gastroenterology, Konkuk University Hospital, Seoul/Korea, Republic of

\section{Contact E-mail Address: kjhcess@gmail.com}

Introduction: Seroconversion of Helicobacter pylori occurs more often in seronegative adults living with a $H$. pylori-infected family member or in those with a history of upper gastrointestinal endoscopy. Nonetheless, there are controversies on alcohol drinking and seroconversion; a positive correlation was found in Korea, but an inverse correlation was found in Japan.

Aims \& Methods: In this study, we tried to elucidate the risk factors for seroconversion including alcohol drinking. Korean adults who showed negative finding on the serum anti-H. pylori $\operatorname{IgG}$ assay were analyzed. Subjects were included if they underwent serum anti- $H$. pylori IgG assay, serum pepsinogen assay, and upper gastrointestinal endoscopy annually at our center. Subjects were excluded if they had a history of $H$. pylori eradication or gastrectomy.

Results: Of the 267 included subjects, $26(9.7 \%)$ showed seropositive findings at the mean follow-up of $39.0 \pm 19.1$ months. Subjects with seroconversion showed a higher body mass index $(\mathrm{p}=0.033)$, heavier alcohol drinking $(\mathrm{p}=0.001)$, more intake of nonsteroidal anti-inflammatory drug $(\mathrm{p}=0.015)$, and longer follow-up period $(\mathrm{p}=0.038)$. On multivariate analysis, heavy alcohol drinking [odds ratio $(\mathrm{OR})=6.867,95 \%$ confidence interval $(\mathrm{CI})=2.089-22.577, \mathrm{p}=0.002)]$ and social drinking $(\mathrm{OR}=5.026,95 \% \mathrm{CI}=1.410-17.913, \mathrm{p}=0.013)$ were independent risk factors for seroconversion.

Conclusion: In Korean adults, the amount of alcohol drinking positively correlates with seroconversion in $H$. pylori-seronegative subjects. Although seroconversion does not indicate an active $H$. pylori infection, our study findings suggests that drinking is a significant risk factor for new $H$. pylori infection in Korean adults.

Disclosure of Interest: All authors have declared no conflicts of interest

\section{References}

1. Lee SP, Lee SY, Kim JH, Sung IK, Park HS, Shim CS, Jin CJ. Correlation between Helicobacter pylori infection, IgE hypersensitivity, and allergic disease in Korean adults. Helicobacter 2015;20:49-55.
2. Kim JH, Kim HY, Kim NY, Kim SW, Kim JG, Kim JJ, Roe IH, Seo JK, Sim JG, Ahn H, Yoon BC, Lee SW, Lee YC, Chung IS, Jung HY, Hong WS, Choi KW; Korea H. pylori Study Group, South Korea. Seroepidemiological study of Helicobacter pylori infection in asymptomatic people in South Korea. J Gastroenterol Hepatol 2001;16:969-975.

3. Kim N, Lim SH, Lee KH, Kim JM, Cho SI, Jung HC, Song IS. Seroconversion of Helicobacter pylori in Korean male employees. Scand $J$ Gastroenterol 2005;40:1021-1027.

4. Brown LM. Helicobacter pylori: Epidemiology and routes of transmission. Epidemiol Rev 2000;22:283-297.

5. De Schryver A, Van Winckel M, Cornelis K, Moens G, Devlies G, De Backer G. Helicobacter pylori infection: further evidence for the role of feco-oral transmission. Helicobacter 2006;11:523-528.

6. Osaki T, Okuda M, Ueda J, Konno M, Yonezawa H, Hojo F, Yagyu K, Lin Y, Fukuda Y, Kikuchi S, Kamiya S. Multilocus sequence typing of DNA from faecal specimens for the analysis of intra-familial transmission of Helicobacter pylori. J Med Microbiol 2013;62:761-765.

7. Jung JH, Choi KD, Han S, Jung HY, Do MY, Chang HS, Choe JW, Lee GH, Song HJ, Kim DH, Choi KS, Lee JH, Ahn JY, Kim MY, Bae SE, Kim JH. Seroconversion rates of Helicobacter pylori infection in Korean adults. Helicobacter 2013;18:299-308.

8. Kikuchi S, Ohgihara A, Hasegawa A, Miki K, Kaneko E, Mizukoshi H. Seroconversion and seroreversion of Helicobacter pylori antibodies over a 9 -year period and related factors in Japanese adults. Helicobacter 2004;9:335-341.

9. Sugiyama T, Naka H, Yachi A, Asaka M. Direct evidence by DNA fingerprinting that endoscopic cross-infection of Helicobacter pylori is a cause of postendoscopic acute gastritis. J Clin Microbiol 2000;6:2381-2382.

10. Kamada T, Hata J, Manabe M, Kusunoki H, Fujii M, Hashimoto H, Haruma K. Can dental treatment be the infection route of $\mathrm{H}$. pylori transmission in adults? Three cases of acute gastric mucosal lesions after dental treatment. Dig Endosc 2007;19:32-35.

\section{P1250 AUTOIMMUNE GASTRITIS WITH PREVIOUS O CONCURRENT H. PYLORI INFECTION PRESENTS DISTINCT FUNCTIONAL AND MORPHOLOGICAL FEATURES}

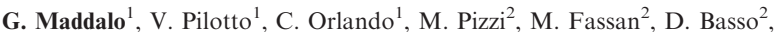

M. Rugge ${ }^{2}$, F. Farinati ${ }^{1}$

${ }^{1}$ Surgery, Oncology And Gastroenterology, Gastroenterology Unit, University of Padua, Padua/Italy

${ }^{2}$ Medicine, University of Padua, Padua/Italy

Contact E-mail Address: gemma.maddalo@gmail.com

Introduction: Autoimmune gastritis (AIG) results in hypo/achlorhydria due to parietal cells destruction ${ }^{1}$. It is characterized by lower levels of serum pespinogen (Pg) I and PgI/PgII ratio and increased levels of gastrin ${ }^{2}$. Some Authors support an association between AIG and Helicobacter pylori (HP) infection ${ }^{3}$.

Aims \& Methods: The aim of our study was to assess epidemiologic, serologic and pathologic features of AIG patients with and without previous HP infection. Two hundred and eleven consecutive patients with AIG, undergoing endoscopy were included. Serum gastrin, PgI, PgII and Cromogranin A levels were determined in all patients. Multiple gastric biopsies were obtained for histology, OLGA staging and HP detection. Previous or current HP infection (HP+) was confirmed in patients by anamnestic and/or pathologic and/or serologic data. Statistics was performed using non parametric tests.

Results: Present or previous HP infection was confirmed in 50/211 patients while 161 were negative (HP-). When we compared HP+ vs HP- AIG, no differences were found for age and gender distribution, antral and fundic/body atrophy, OLGA staging, PgI, PgII, PgI/PgII ratio and Cromogranin A levels. Gastrin levels and Gastrin/PgI ratio, a global marker of gastric damage we previously identified, were higher in HP- vs HP+ $(\mathrm{p}<0.02)$. Interestingly, $15 \% \mathrm{HP}$ - presented antrum atrophy. Severity of ECL hyperplasia was higher in HP- $(p=0.02)$ with a $3.5 \mathrm{RR}$ of developing nodular or carcinoid lesions when compared with $\mathrm{HP}+$. Serum PgI, PgII PgI/PgII and gastrin levels correlated with disease severity in HP- $(\mathrm{p}<0.01)$ but not in HP+

Conclusion: HP+ AIG have/have had a mild infection without differences in OLGA staging when compared with HP-. HP + are characterized by lower gastrin and gastrin/PGI levels that, considered the lack of differences in OLGA staging, seem to suggest an impairment of gastrin release and could have a selective G Cell damage leading to lower levels of gastrin and Gastrin/PgI ratio. Consequently, they have lower degrees of ECL hyperplasia and a lower risk of developing carcinoids when compared with HP-. The presence of antrum atrophy in HP- could be explained with the supposed autoimmune antrumdamage hypothesis in $\mathrm{AIG}^{4-5}$. In conclusion, HP+ and HP- AIG have some differences in serological phenotype and HP infection may correlate in AIG with lower ECL hyperplasia and lower risk of neuroendocrine tumor.

Disclosure of Interest: All authors have declared no conflicts of interest.

\section{References}

1. Toh BH. Diagnosis and classification of autoimmune gastritis. Autoimmun Rev. 2014;13(4-5):459-462. doi:10.1016/j.autrev.2014.01.048 [doi].

2. Agreus L, Kuipers EJ, Kupcinskas L, et al. Rationale in diagnosis and screening of atrophic gastritis with stomach-specific plasma biomarkers. Scand $J$ Gastroenterol. 2012;47(2):136-147. doi:10.3109/00365521.2011.645501.

3. Amedei A, Bergman MP, Appelmelk BJ, et al. Molecular mimicry between Helicobacter pylori antigens and $\mathrm{H}+, \mathrm{K}+-$ adenosine triphosphatase in human 
gastric autoimmunity. $J$ Exp Med. 2003;198(8):1147-1156. doi:10.1084/ jem.20030530.

4. Venerito M, Varbanova M, Rohl F-W, et al. Oxyntic gastric atrophy in Helicobacter pylori gastritis is distinct from autoimmune gastritis. J Clin Pathol. 2016;69(8):677-685. doi:10.1136/jclinpath-2015-203405

5. Jevremovic D, Torbenson M, Murray JA, Burgart LJ, Abraham SC. Atrophic autoimmune pangastritis: A distinctive form of antral and fundic gastritis associated with systemic autoimmune disease. Am J Surg Pathol. 2006;30(11):14121419. doi:10.1097/01.pas.0000213337.25111.37.

\section{P1251 THE INVESTIGATION OF MIR-155, MIR-21, MIR-146A AND MIR-223 EXPRESSIONS IN HELICOBACTER PYLORI POSITIVE AND NEGATIVE INDIVIDUALS}

N. Uyar Alpaslan ${ }^{1}$, E. Ucbilek ${ }^{2}$, I. Ö. Barlas ${ }^{1}$, O. Sezgin ${ }^{2}$, S. Yaras, ${ }^{2}$ ${ }^{1}$ Medical Biology And Genetics, Mersin University Faculty of Medicine, Mersin/Turkey

${ }^{2}$ Gastroenterology, Mersin University Faculty of Medicine, Mersin/Turkey

\section{Contact E-mail Address: enucbilek@hotmail.com}

Introduction: This study was conducted to determine the differential expression patterns of microRNAs, non-coding RNAs that control gene expression mainly through translational repression, in gastric mucosa of Helicobacter pylori (H.pylori) positive patients. Several miRNA have been associated with promoting the inflammatory response initiated by the $H$. pylori infection, increasing the malignant progression of the gastric epithelium, and enhancing the invasiveness and migratory capacity of cancer cells.Using serum specimens, expression patterns of hsa-miR-155, hsa-miR-21, hsa-miR-146-a and hsa-mir-223 were determined by Real-Time Polymerase Chain Reaction (Real-Time PCR).

Aims \& Methods: Patients who underwent upper gastrointestinal endoscopy, in Mersin University Faculty of Medicine, Department of Gastroenterology and diagnosed H.pylori positive and negative were recruited. H. pylori status was assessed by the rapid urease test. Serum specimens of patients, were taken for miRNA isolation. hsa-miR-155, hsa-miR-21, hsa-miR-146-a and hsa-mir-223 expression levels were determined using comparative $2^{-\Delta \Delta C T}$ analysis by using Real-Time PCR Systems, SDS 2.0.3 software programme. Statistical analysis of miRNAs between H.pylori positive and negative groups were compared with the Mann-Whitney U test. $\mathrm{p}<0.05$ was considered statistically significant. The relationship between categorical variables were tested using Pearson's chi-square test.

Table 1

\begin{tabular}{llllllll}
\hline & \multicolumn{2}{l}{ H.pylori positive $\mathrm{N}=46$} & & \multicolumn{2}{l}{ H.pylori negative $\mathrm{N}=49$} & \\
\cline { 2 - 3 } & Average & Median & & Average & Median & p value \\
\hline hsa_miR_155 & $0,990199 \pm 2,006536$ & 0,393990 & & $0,749915 \pm 1,276566$ & 0,295334 & 0,48 \\
hsa_miR_223 & $6,929772 \pm 19,222485$ & 2,031475 & & $7,064999 \pm 18,103347$ & 2,108900 & 0,97 \\
hsa_miR_146a & $4,730933 \pm 8,343877$ & 2,138397 & & $9,332742 \pm 28,023032$ & 1,447617 & 0,28 \\
hsa_miR_21 & $4,412535 \pm 5,519648$ & 2,030767 & & $4,669136 \pm 8,019229$ & 1,858662 & 0,85 \\
\hline
\end{tabular}

Results: H.pylori positive $(\mathrm{n}=46)$ and negative $(\mathrm{n}=49)$ were included. H.pylori was not able to effective the hsa-miR-155, hsa-miR-21, hsa-miR-146-a and hsamiR-223 expressions in serum specimens and nuclear factor-kB (NF-kB) and Transforming growth factor, beta (TGFB) pathways. There was no statistically difference between $H$. pylori positive and negative individuals in the analysis of hsa-miR-155, hsa-miR-21, hsa-miR-146-a and hsa-miR-223 miRNA expressions levels (Table 1). In surveys, there is no statistically difference between in each groups, the level of education, intake of smoking-alcohol, hypertension, diabetes, cardiovascular disease, family history of gastroduodenal disease, type of gastroduodenal disease.

Conclusion: This study may contribute to the literature in terms of preventing pro-cancerous progression in the cases of cancer resulting from $H$. pylori infection before the onset of the cancer process.

Disclosure of Interest: All authors have declared no conflicts of interest.

\section{References}

1 Johannes G., Arnoud H., Ernst J. Pathogenesis of Helicobacter pylori Infection. Clinical Microbiology. July 2006, p. 449-490.

2 Cadamuro A., Rossi A., Maniezzo N., Silva A. Helicobacter pylori infection: Host immune response, implications on gene expression and microRNAs. World J Gastroenterol 2014 February 14; 20(6): 1424-1437.

3 Matsushima K., Isomoto H., Inoue N., Nakayama T. MicroRNA signatures in Helicobacter pylori-infected gastric mucosa.Int. J. Cancer: 2011,128;361in $\mathrm{He}$.
370 .

4 Zabaleta J. MicroRNA: a bridge from $H$. pylori infection to gastritis and gastric cancer development. Frontiers in genetics. 2012;3, 294. 2010, 854-863.

5 Zhai R., Wei Y., Su L., Liu G., Mathew H., John C. Whole-miRNome profiling identifies prognostic serum miRNAs in esophageal adenocarcinoma: the influence of Helicobacter pylori infection status. Carcinogenesis. 2015: 8793.

6 Xiao B., Liu Z. Induction of microRNA-155 during Helicobacter pylori Infection and Its Negative Regulatory Role in the Inflammatory Response.JID. 2009:200.
7 Li B., Zhao Y., Guo G., Li W. Plasma microRNAs, miR-223, miR-21 and miR-218, as Novel Potential Biomarkers for Gastric Cancer Detection. Plos One.July 2012,7:7.

8 Zhang Z., Li Z., Gao C., Chen P., Chen J., Liu W., Xiao S., Lu H. miR-21 plays a pivotal role in gastric cancer pathogenesis and progression. Lab Invest 2008; 88: 1358-1366.

9 Wang H., Wang L., Wu Z. Three dysregulated microRNAs in serum as novel biomarkers for gastric cancer screening. Med Oncol. (2014) 31:298.

\section{P1252 INTERLEUKIN LEVEL IN PATIENTS INFECTED WITH} CAGA(+) AND CAGA(-) STRAINS OF HELICOBACTER PYLORI

N.V. Baryshnikova ${ }^{1}$, Y.P. Uspenskiy ${ }^{2}$, A. Suvorov ${ }^{1}$

${ }^{1}$ Science Research Institute, St-Petersburg/Russian Federation

${ }^{2}$ First Pavlov Saint-Petersburg State University, St-Petersburg/Russian Federation

Contact E-mail Address: baryshnikova_nv@mail.ru

Introduction: Change of interleukin level can be in patients with of chronic infections, in particular at Helicobacter pylori invasion. Change of interleukin level in $H$. pylori-infected patients is intensively studied. For example, increase in level of interleukin- 8 according to a number of works is accompanied by infection with virulent strains of a microorganism. CagA gene coding synthesis of the cytotoxin (CagA) of the same name capable, in addition, to exert impact on development of interleukin, in particular interleukin-8 is considered a marker of presence of pathogenecity island of $H$. pylori

Aims \& Methods: The aim was to define features of change of level of interleukin$1 \beta$ and interleukin- 8 (pro-inflammatory) and interleukin-4 (anti-inflammatory) at patients with the chronic gastritis associated with $H$. pylori infection depending on existence or lack of a gene of cagA in a microorganism genome. 40 patients with the chronic gastritis associated with $H$. pylori have been examined. Endoscopy was made for all patient for specification of a condition of stomach mucosa and taking biopsy from stomach antrum ( 2 bioptates) for the purpose of verification of $H$. pylori infection. Detection of a microorganism was made by rapid urease test, a histologic method and molecular-genetic research-the polymerase chain reaction (PCR) with definition of genes of urease (ureC, ureI). Besides, the PSR method presence of a cagA gene in a genome of $\mathrm{H}$. pylori was detected. All patients were divided on two groups: with cagA $(+)$ strains and with cagA $(-)$ strains. Levels of interleukins $1-\beta,-4,-8$ decided by immunefermental analysis (the Vektor-Best sets, Russia).

Results: cagA gene was detected in 30 patients $(\operatorname{cagA}(+)$ group) and absence in 10 patients (cagA $(-)$ group). In cagA $(+)$ patients mean level of interleukin-1- $\beta$ was $395.6 \mathrm{pg} / \mathrm{ml}$, but in cagA $(-)$ patients $-311.2 \mathrm{pg} / \mathrm{ml}(\mathrm{p}<0.05)$. Level of interleukin- 8 in cagA( + ) patients was $2.4 \mathrm{pg} / \mathrm{ml}$ but in cagA $(-)$ patients $-0.32 \mathrm{pg} / \mathrm{ml}$ $(\mathrm{p}<0.05)$.Level of interleukin-4 y cagA $(+)$ patients was $21.6 \mathrm{pg} / \mathrm{ml}$, but in cagA $(-)$ patients $-83.4 \mathrm{pg} / \mathrm{ml}(\mathrm{p}<0.05)$

Conclusion: presence in a genome of $H$. pylori cagA gene is accompanied by reliable increase in level of pro-inflammatory interleukins $(1-\beta, 8)$ and decrease in level anti-inflammatory interleukin- 4 that can be an additional factor of development of an inflammation during $H$. pylori invasion.

Disclosure of Interest: All authors have declared no conflicts of interest.

\section{P1253 THE TRANSITION OF HELICOBACTER PYLORI} ERADICATION IN OUR INSTITUTION

M. Ono, T. Kitagataya, Y. Shimoda, J. Ito, T. Kudo, K. Hatanaka, Y. Yamamoto, H. Naruse

Gastroenterology, Hakodate Municipal Hospital, Hakodate/Japan

Contact E-mail Address: cdf41180@par.odn.ne.jp

Introduction: The incidence and mortality rate of gastric cancer are high in Japan. The International Agency for Research of Cancer (IARC) reported that $80 \%$ of gastric cancer is caused by Helicobacter pylori $(H$. pylori) infection and that the incidence of gastric cancer can be reduced by $30 \%-40 \%$ through $H$. pylori eradication. H. pylori eradication therapy for chronic gastritis was approved for national health insurance in February 2013 in Japan. However, the success rate of $H$. pylori eradication by conventional primary triple therapy has been decreased by resistance to clarithromycin. Vonoprazan, which is a potassium ioncompetitive acid blocker (P-CAB), became available in Japan in February 2015, before its release on the world market. There have been some reports on the usefulness of vonoprazan for $H$. pylori eradication.

Aims \& Methods: The aim of this study was to investigate the transition of $H$. pylori eradication therapy including therapy with vonoprazan in our institution. This study was a retrospective single center study between January 2010 and December 2016. The subjects were patients who had received H. pylori eradiation therapy. The subjects were divided into a conventional triple therapy group (PPI: omeprazole lansoprazole, esomeprazole, and rabeprazole) and a group who received triple therapy with vonoprazan instead of a PPI. Regimens with amoxicillin, clarithromycin and a PPI or vonoprazan were regarded as $1^{\text {st }}$ line treatment, and a regimen with metronidazole instead of clarithromycin was regarded as $2^{\text {nd }}$ line treatment. Success rates of $1^{\text {st }}$ line treatment and $2^{\text {nd }}$ line treatment with a PPI or vonoprazan were compared.

Results: There were 1278 patients who underwent H.pylori eradication therapy. The mean age of the patients was 61.5 years (13-92), and the patients included 722 males and 556 females. The patients who received H.pylori eradication therapy included 857 patients with chronic gastritis, 300 patients with stomach duodenal ulcers, 82 patients with gastric cancer, 27 patients with polyps, and 12 other patients. The number of patients with chronic gastritis undergoing 
eradication therapy of $H$. pylori increased remarkably from $48.4 \%$ in 2012 to $73.8 \%$ in 2016 . The total success rate was $76.5 \%$. The success rate of the conventional PPI regimen was $75.8 \%(846 / 1116)$, and that of the vonoprazan regimen was $85.1 \%(74 / 87)$. The success rate of vonoprazan regimen was significantly higher than that of the PPI regimen (Fisher's exact test: $\mathrm{p}<0.05$ ). The success rate of H.pylori eradication in 2 nd line treatment was $84.7 \%(210 /$ 248). The success rate of H.pylori eradication in patients who received the conventional PPI regimen was $84.4 \%(35 / 224)$, and that in patients who received the vonoprazan regimen was $87.5 \%(21 / 24)$.

Conclusion: Vonoprazan is considered to be useful for H.pylori eradication instead of a PPI in first line treatment.

Disclosure of Interest: All authors have declared no conflicts of interest.

\section{References}

Shinya $\mathrm{Y}$ et al. Usefulness of vonoprazan, a potassium ion-competitive acid blocker, for primary eradication of Helicobacter pylori. World J Gastrointest Pharmacol Ther 2016; 7(4): 550-555.

Herrero R et al. The fight against gastric cancer - the IARC Working Group report. Best Pract Res Clin Gastroenterol 2014; 28: 1107-1114

\section{P1254 ERADICATION OF $H$. PYLORI INFECTION IN PATIENTS NAÏVE TO TREATMENT USING CONCOMITANT THERAPY OR BISMUTH QUADRUPLE THERAPY (THREE-IN-ONE PILL): A REAL- LIFE OBSERVATIONAL STUDY}

A.G. Gravina ${ }^{1}$, G. Nardone ${ }^{2}$, A. Miranda ${ }^{1}$, D. Sgambato ${ }^{1}$, A. Rocco ${ }^{2}$, G. Visciola ${ }^{1}$, D. Compare $^{2}$, M. Dallio ${ }^{1}$, L. Granata ${ }^{1}$, M.R. Romito ${ }^{2}$,

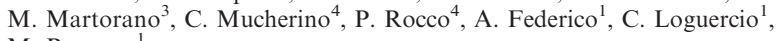
M. Romano ${ }^{1}$

${ }^{1}$ Dipartimento Di Internistica Clinica E Sperimentale "f Magrassi", UOC Epatogastroenterologia, Università degli Studi della Campania "L Vanvitelli", Napoli/Italy

${ }^{2}$ Dipartimento Di Medicina Clinica E Sperimentale, UOC Gastroenterologia, Università Federico II, Napoli, Napoli/Italy

${ }^{3}$ Uo Gastroenterologia, Sapri, P.O. dell'Immacolata, Sapri/Italy

${ }^{4}$ Uoc Gastroenterologia, Azienda Ospedaliera "S.Anna e S.Sebastiano",

Caserta/Italy

\section{Contact E-mail Address: antonietta.gravina@yahoo.it}

Introduction: Clarithromycin (CLA)-containing quadruple therapy, i.e. concomitant therapy (CT), and bismuth-containing quadruple therapy (BQT) have been suggested as first line therapies for eradication of $H$. pylori infection. Both treatments are reported to have an eradication rate higher than $90 \%$. International guidelines recommend that chosing one regimen vs the other should be based on the regional prevalence of antimicrobial resistance, knowledge of patient's previous antimicrobial exposure or allergy to amoxicillin, and patient's wish. However, this therapeutic approach has never been tested systematically.

Aims \& Methods: The primary endpoint of this study was to evaluate the eradication rate in $H$. pylori-infected subjects naive to treatment in an area of high $(>15 \%)$ CLA resistance using CT or BQT, based on knowledge of patient's previous antimicrobial exposure or allergy to amoxicillin, and patient's wish. Secondary endpoint was to assess any difference between CT and BQT in terms of efficacy, safety and compliance. Because in Italy bismuth compounds are not available, BQT was by using the new three-in-one pill (TiOP) (metronidazole $125 \mathrm{mg}+$ tetracycline $125 \mathrm{mg}+$ bismuth $140 \mathrm{mg}$ ). Patients and Methods: 1) Observational study on 144 consecutive dyspeptic patients $(57 \mathrm{M}$ and $87 \mathrm{~F}$, median age $51 \mathrm{yrs})$; 2) Diagnosis of $H$ pylori infection was through ${ }^{13} \mathrm{C}$ Urea Breath Test (UBT), or rapid urease test and histology in patients who performed endoscopy; 3) Groups of treatment: a) $84 \mathrm{CT}$ (36 M and $48 \mathrm{~F}$, median age $52 \mathrm{yrs}$ ) and $60 \mathrm{BQT}(21 \mathrm{M}$ and $39 \mathrm{~F}$, median age $50 \mathrm{yrs})$ patients; 4) Regimens: a) CT: esomeprazole $40 \mathrm{mg}$ bid + amoxicillin $1 \mathrm{~g}$ bid + clarithromycin $500 \mathrm{mg}$ bid + tinidazole $500 \mathrm{mg}$ bid; b) BQT: esomeprazole $40 \mathrm{mg}$ bid + TiOP 3 tablets qid. Both for 10 days; 5) Evaluation of eradication was performed at least 45 days after the end of therapy by UBT; 6) Compliance to and side effects of both regimens were assessed by a questionnaire at the end of therapy; 6) Statistics: ITT and PP analyses; significance of differences by chi square test $(\mathrm{p}<0.05)$.

Results: 1$)$ Eleven/144 patients $(7.6 \%, 95 \%$ CI $4.3-13.5)[6 / 84(7.1 \%, 95 \%$ CI $3.3-$ $15.4)$ in the CT group and $5 / 60(8.3 \%, 95 \%$ CI $3.6-19.3)$ in the BQT group] discontinued therapy because of severe side effects (p: ns); 2) Overall eradication rate was $122 / 133(91.7 \%, 95 \% \mathrm{CI} 87.2-96.5)$ in the PP analysis and $122 / 144$ $(84.7 \%, 95 \%$ CI $79-90.1)$ in the ITT analysis; 3) PP eradication rate was $69 / 78$ $(88.5 \%, 95 \%$ CI $81.6-95.8)$ in the CT group and $53 / 55(96.4 \%, 95 \%$ CI 91.6 $101.4)$ in the BQT group $(\mathrm{p}=\mathrm{ns})$; 3) ITT eradication rate was $69 / 84(82.1 \%$, $95 \%$ CI $74.3-90.8)$ in the CT group and $53 / 60(88.3 \%, 95 \%$ CI $80.6-96.8)$ in the BQT group $(\mathrm{p}=\mathrm{ns})$; 4) Among patients who completed therapy, no significant differences between the two groups were found as to the incidence of side effects $(29.4 \%$ in the CT group and $35.8 \%$ in the BQT group) or compliance to treatment.

Conclusion: 1) In an area of high CLA resistance, use of CT or BQT as first-line therapy, based on patient's previous antimicrobial exposure, allergy to amoxicillin and wish, is a highly effective strategy with an eradication rate $>90 \% ; 2$ ) BQT was more effective but with more side effects compared to CT, however differences were not statistically significant.

Disclosure of Interest: All authors have declared no conflicts of interest.
P1255 CYP3A5 GENOTYPE STATUS AFFECTS OUTCOME OF FIRST-LINE VONOPRAZAN-CONTAINED HELICOBACTER PYLORI ERADICATION THERAPY

M. Sugimoto ${ }^{1}$, H. Ban ${ }^{1}$, A. Otsuki ${ }^{2}$, H. Itoi ${ }^{2}$, O. Inatomi ${ }^{3}$, A. Andoh ${ }^{2}$ ${ }^{1}$ Division Of Digestive Endoscopy, Shiga University of Medical Science Hospital, Otsu/Japan

${ }^{2}$ Department Of Gastroenterology, Shiga University of Medical Science Hospital, Otsu/Japan

${ }^{3}$ Division Of Gastroenterology, Shiga University School of Medicine Dept. of Gastroenterology, Otsu/Japan

Contact E-mail Address: sugimo@belle.shiga-med.ac.jp

Introduction: Potent acid inhibition with acid inhibitory drugs is crucial to the success of eradication for Helicobacter pylori infection. Vonoprazan competitively inhibits the binding of potassium ion to $\mathrm{H}^{+} / \mathrm{K}^{+}$-ATPase in gastric parietal cells and inhibits $\mathrm{H}^{+} / \mathrm{K}^{+}$-ATPase activity at 400 -fold more potent than that of lansoprazole, proton pump inhibitor (PPI). Therefore, the eradication regimen contained vonoprazan has advantage for increasing the eradication rate compared with a PPI-based therapy. Although vonoprazan is mainly metabolized by CYP3A $4 / 5$, it is unclear whether its acid inhibitory effect and outcome of $H$. pylori eradication differ among CYP3A $4 / 5$ genotypes. Our aim was to clarify the influence of CYP3A4/5 genotypes on outcome of $H$. pylori eradication including vonoprazan in Japanese.

Aims \& Methods: We investigated the influence of CYP3A4/5 and CYP2C19 genotypes and susceptibility to antimicrobial agents for outcome of vonoprazan-contained eradication regimen for 7 days in 105 Japanese: (1) with amoxicillin $750 \mathrm{mg}$ and clarithromycin $20 \mathrm{mg}$ twice daily (bid) as the first-line treatment $(\mathrm{n}=76)$; (2) with amoxicillin $750 \mathrm{mg}$ and metronidazole $250 \mathrm{mg}$ bid as the second-line $(\mathrm{n}=29)$. Eradication status was assessed at eight weeks via a ${ }^{13} \mathrm{C}$-urea breath test. CYP3A $4 * 22$, CYP $3 A 5 * 3$ and CYP 2 C19*2/*3 were genotyped for all patients.

Results: Eradication rate on intention-to-treat analysis was $82.9 \%(95 \%$ confidence interval: $72.5 \%-90.6 \%, 63 / 76)$ in the first-line treatment and $93.1 \%$ $(77.2 \%-99.1 \%, 27 / 29)$ in the second-line. None with CYP3A $4 * 22$ was observed. $38.3 \%$ of patients $(46 / 120)$ were CYP 3 A $5 * 1 / * 3$ type and $55.0 \%$ were $* 3 / * 3$ type. In naive patients, the prevalence of clarithromycin-resistant strain was $42.4 \%$ (14/33). Eradication rate in patients with CYP3A5*1 carrier type (CYP3A5 $* 1 /{ }^{*} 1$ and $* 1 / * 3$ types) was $72.7 \%(54.5 \%-86.7 \%, 24 / 33)$, which was significantly lower than that in the *3/*3-type $(90.7 \%, 77.9 \%-97.4 \%, 39 / 43, \mathrm{p}=0.039)$ in the first-line treatment. However, no significant differences of clinical outcome in the second-line therapy were seen among CYP3A4/5 genotypes.

Conclusion: Eradication rates of vonoprazan-based eradication therapy can be achieved high compared with PPI-based therapy. However, because CYP 3 A $5 * 3$ genotype may be one of determinate for outcome of eradication regimen including vonoprazan, genotyping of CYP3A $5 * 3$ will be required to be paid attention for clinical outcome before treatment.

Disclosure of Interest: All authors have declared no conflicts of interest.

\section{P1256 COMPARISON OF HELICOBACTER PYLORI ERADICATION RATES: VONOPRAZAN VS. PROTON PUMP INHIBITOR}

N. Kozawa, T. Mochida, A. Igarashi, C. Kamitsuma, J. Tsuchiya, M. Ike, K. Yasuda, M. Kaneshiro, H. Ikemiyagi, Y. Fukami, K. Yoshino, S. Sakita Gastroenterology, Yokohama-city Red Cross Hostpital, Yokohama/Japan

Contact E-mail Address: naokooo122@gmail.com

Introduction: Proton pump inhibitor (PPI) based therapy is one of most popular H.pylori eradication in the world. On the other hand, eradication rates have been decreasing because of resistance to the clarithromycin (CAM). It is known that most antibiotics are not effective under strong acid secretion. So in order to improve the eradication rate, gastric acid must be reduced more rapidly and strongly. Vonoprazan(VPZ) is a new potassium competitive acid blocker and the usefulness is expected in Japan.

Aims \& Methods: Our aim was to investigate the efficacy of the VPZ-based eradication therapy. The subjects were 999 patients who were diagnosed as H.pylori infection in our institution from June 2014 to December 2016. The patients were grouped into three: VPZ group and conventional PPI (Lamsoprazole or Rabeprazole) group. We evaluated the first-, the second-line eradication rate and statistical analysis. Each regimen of VPZ group was first-line eradication (VPZ $20 \mathrm{mg}$ bid+ amoxicillin $750 \mathrm{mg}$ bid+ CAM $200 \mathrm{mg}$ bid for 7 days), second-line eradication (VPZ $20 \mathrm{mg}$ bid + amoxicillin (AMPC) $750 \mathrm{mg}$ bid+ Metronidazole $250 \mathrm{mg}$ bid for 7 days). Each regimen of PPI group was first-line eradication (PPI bid+ AMPC $750 \mathrm{mg}$ bid+ CAM $200 \mathrm{mg}$ bid for 7 days), second-line eradication (PPI bid + amoxicillin $750 \mathrm{mg}$ bid+ Metronidazole $250 \mathrm{mg}$ bid for 7 days). One of the following PPI was used: Lamsoprazole (LPZ) $30 \mathrm{mg}$, Rabeprazole (RPZ) $20 \mathrm{mg}$. After several months, eradication status was examined by urea breath test, stool antigen testing and blood antibody test.

Results: The number of first-line regimen of VPZ patients was 109, and the eradication was achieved in 97 patients $(87 \%)$. RPZ patients were 308 , and the eradication was achieved in 228 patients $(74 \%)$, and LPZ patients were 377 , and the eradication was achieved in 300 patients $(80 \%)$ respectively. The eradication rate of VPZ was statistically higher than RPZ and LPZ $(P=0.0006$, and $=0.019$ respectively). The number of second-line regimen of VPZ patients was 24 , and the eradication was achieved in 19 patients $(79 \%)$. RPZ patients were 68 , and the eradication was achieved in 60 patients $(88.2 \%)$, and LPZ patients were 68 , and the eradication was achieved in 60 patients $(88.2 \%)$ respectively. There were statically no significant differences in second-line regimens. Adverse events 
such as eruption and diarrhea were reported in $6.6 \%(9 / 136)$ of patients in VPZ, in $15.5 \%(58 / 375)$ in RPZ and in $6.1 \%(28 / 456)$ in $\mathrm{LPZ}$.

Conclusion: The first-line regimen with VPZ was superior to conventional PPI regimen, and was a result not to be inferior in the safety either.

Disclosure of Interest: All authors have declared no conflicts of interest.

\section{P1257 CAN TWO WEEK BISMUTH BASED QUADRUPLE THERAPY FOR RESISTANT H. PYLORI INFECTION STILL \\ BE USED IN THE UK?}

H. Logan-Ellis, L. Bensaid, R. P.H. Logan

Gastroenterology, King's College Hospital, London/United Kingdom

\section{Contact E-mail Address: hughloganellis $@$ nhs.net}

Introduction: Eradication of $H$. pylori infection cures peptic ulcer disease (PUD); however, current treatment strategies are far from ideal and many patients require repeated courses of treatment. We, and others, have recently documented that currently, within the UK, less than $30 \%$ of patients with with proven PUD, are subsequently documented to have been cured by $H$. pylori eradication (1)' Currently there is no consensus regarding 2nd line treatments, but the British National Formulary recommends a two regimen containing bismuth subcitrate, omeprazole, tetracycline and metronidazole. However since 2016, bismuth subcitrate has not been available in the UK, but is thought to be an important in treating persistent $H$. pylori infection ${ }^{(2)}$

Aims \& Methods: The aim of this observational cohort study was to evaluate the effectiveness of a 2 week bismuth based quadruple therapy in patients who had persistent infection following previous failed eradication therapy. Patients were identified from electronic hospital records using endoscopy data set, patient administration records, and pathology data sets from Jan 2011 to Dec 2016. Initial failed H.pylori eradication was defined by either by a positive ${ }^{13} \mathrm{C}$-Urea Breat Test $\left({ }^{13} \mathrm{C}\right.$-UBT) or positive HpStool Antigen test (HpSA) following $H$. pylori treatment in either primary or secondary care. All patients were seen, assessed and warned about the importance of compliance with treatment and of possible side effects by the specialist (RL) or the dyspepsia nurse specialist (LB). After treatment with omeprazole $20 \mathrm{mg}$ bd, tetracycline $500 \mathrm{mg}$ qds, metronidazole $400 \mathrm{mg}$ qds and bismuth subcitrate qds for two weeks, eradication was assessed at least 4 weeks after finishing treatment by ${ }^{13} \mathrm{C}$-UBT (or HpSA in those patients unable to attend hospital appointment). Endoscopy was also performed in those patients whom had failled mutliple previous treatments to ascertain the clinical need for further treatment by assessing any underlying ulcer diathesis and for $H$. pylori culture and sensitivity testing.

Results: Within the inclusion period, (and from $>560$ patient records), 41 patients ( 22 female), mean age $44 \mathrm{yr}$, (range 17-84 yr) were identifed as having persistent H.pylori infection by $+\mathrm{ve}^{13} \mathrm{C}$-UBT $(\mathrm{n}=25)$, HpSA $(\mathrm{n}=10)$ or histology/CLOtest $(n=6)$. All had failed at least one treatment, but $14 / 41(34 \%)$ had failed 2 or more. Most patients were non or light drinkers $(90 \%)$ and nonsmokers $(74 \%)$. OGD was performed in 19 patients to clarify indication for further attempts at $H$. pylori eradication and showed evidence of ulcer disease (ulcer, scarring or erosions) in 13/19 (68\%). Culture and antibiotic sensitivity testing was unsuccessful in $5 / 5$ patients. At least 6 weeks after the end of treatment a repeat ${ }^{13} \mathrm{C}$-UBT or HpSA was negative in $34 / 36$ (94\% $(95 \%$ CI $82-98 \%)$. 5 patients failed to attend for assessment of eradication. During the follow up period from 2011 onwards, 11 patients underwent repeat ${ }^{13} \mathrm{C}$-UBT or HpSA testing, and all remained cured of infection.

Conclusion: Two week standard bismuth based quadruple therapy remains a highly effective treatment for persistent $H$. pylori infection in those patients in whom eradication of infection is mandated by the an underlying ulcer diathesis. The high eradication rate is likely due to the use of bismuth/high doses of antibiotics, but also by specialists ensuring patients complied with their medication. These data, together with the poor outcomes of $H$. pylori eradication when undertaken by general physicians, also highlight the need for $H$. pylori eradication to be only undertaken by specialists who have access to alternative sources of colloidal bismuth subcitrate.

Disclosure of Interest: All authors have declared no conflicts of interest.

\section{References}

1. Porter B, Lim L, Logan RPH. Should General Physicians/Internists or GI specialists manage Peptic Ulcer Disease. UEG 2016, A1563.

2. Dore MP, Lu H, Graham DY; Role of bismuth in improving Helicobacter pylori eradication with triple therapy. Gut 2016; 65:870-8.

\section{P1258 MAY PROBIOTICS MONOTHERAPY ERADICATE HELICOBACTER PYLORI? A SYSTEMATIC REVIEW WITH POOLED-DATA ANALYSIS}

G. Losurdo, R. Cubisino, M. Barone, M. Principi, E. Ierardi, A. Di Leo Emergency And Organ Transplantation, University of Bari, Bari/Italy

Contact E-mail Address: giuseppelos@alice.it

Introduction: Despite several evidences in literature have demonstrated a role for probiotics as adjunctive treatment for Helicobacter pylori ( $\mathrm{H}$. pylori) eradication, national and international guidelines as well as meta-analyses suggest that only co-administration of probiotics may have a beneficial effect on the prevention of side effects and eradication rates. Herein, we performed a systematic review with pooled-data analysis aimed to clarify whether probiotics alone may eradicate the bacterium
Aims \& Methods: Methods of analysis and inclusion criteria were based on PRISMA recommendations. Relevant publications were identified by a research in PubMed, MEDLINE, Science Direct and EMBASE. The end-point was to estimate the mean eradication rate and variations of delta value at urea breath test across all studies and, overall, with a pooled data analysis. The data have been expressed ad proportions/percentages, and 95\% confidence intervals $(\mathrm{CI})$ were calculated. For continuous variables, we calculated the weighted mean difference. Odd ratios (OR) were calculated, where available, based on the Mantel-Haenszel method. Data were entered into the RevMan 5.3 software.

Results: Ten studies (both randomized clinical trials and open label pilot studies) were selected. In one study patients with peptic ulcers were selected, while in the remaining 9 only dyspeptic patients were recruited. Probiotics eradicated $H$. pylori in 50 out of 391 cases. The mean weighted eradication rate was $14 \%$, with a $95 \%$ CI of $2-25 \%(\mathrm{p}=0.02)$. Most of studies investigated a probiotic formulation based on a single lactobacilli strain. Lactobacilli eradicated the bacterium in 30 out of 235 patients, with a mean weighted rate of $16 \%(95 \%$ CI 1 $31 \%$ ). Multistrain combinations were effective in 14 out of 105 patients, with a pooled eradication rate of $14 \%(95 \% \mathrm{CI}-16-43 \%)$. In the comparison probiotics versus placebo, we found an $\mathrm{OR}=9.65$ in favor of probiotics, with a $95 \% \mathrm{CI}$ of $1.97-47.36(\mathrm{p}=0.005)$. Finally, probiotics induced a mean reduction in delta values of $8.61 \%$ o $(95 \% 5.88-11.34, \mathrm{p}<0.00001)$. No study provided data about adverse events.

Conclusion: Probiotics alone show a minimal effect on the eradication of $\mathrm{H}$. pylori, thus suggesting a presumable direct effect. However, they cannot be indicated as a therapeutic regimen for the low eradication rate.

Disclosure of Interest: All authors have declared no conflicts of interest.

\section{P1259 EFFECTIVENESS AND SAFETY OF PYLERA ${ }^{\circledR}$ IN PATIENTS} INFECTED BY HELICOBACTER PYLORI: A LARGE, PROSPECTIVE, REAL-LIFE STUDY

A. Tursi ${ }^{1}$, M. Franceschi ${ }^{2}$, L. Allegretta ${ }^{3}$, E. Savarino ${ }^{4}$, R. De Bastiani ${ }^{5}$, W. Elise ${ }^{6}$, G. Baldassarre ${ }^{2}$, A. Ferronato ${ }^{2}$, S. Scida ${ }^{7}$, C. Miraglia ${ }^{7}$, A. Penna ${ }^{8}$ C. Licci ${ }^{9}$, G.L. Rizzo ${ }^{10}$, P. Giuseppe ${ }^{11}$, C. Cassieri ${ }^{12}$, G. Brandimarte ${ }^{12}$, M. Picchio ${ }^{13}$, F. Di Mario ${ }^{14}$

${ }^{1}$ Gastroenterology Service, ASL BAT Gastroenterology Service, Andria/Italy ${ }^{2}$ Digestive Endoscopy Unit, ULSS4 Alto Vicentino, Santorso/Italy

${ }^{3}$ Division of Gastroenterology, "S. Caterina Novella" Hospital, galatina Italy

${ }^{4}$ Department Of Surgery, Oncology And Gastroenterology, University of Padua, PadualItaly

${ }^{5}$ Italian Association for Gastroenterology in Primary Care (GICA-CP), Feltre/ Italy

${ }^{6}$ Division of Gastroenterology, ASL RM6, Albano Laziale/Italy

${ }^{7}$ Department Of Medicine And Surgery, University Of Parma, Italy, University of Parma, Parma/Italy

${ }^{8}$ Division of Gastroenterology, "S. Paolo" Hospital, Bari/Italy

${ }^{9}$ Private Practice Gastroenterologist, Monopoli/Italy

"P" "Salus" Home Care, Brindisi/Italy"

${ }^{11}$ Digestive Endoscopy Service, "Valle d'Itria" Hospital, Martina Franca/Italy ${ }^{12}$ Division of Internal Medicine and Gastroenterology, "Cristo Re" Hospital, Rome/Italy

${ }^{13}$ Division of Surgery, "P. Colombo" Hospital, ASL RM6, Velletri/Italy

${ }^{14}$ University Of Parma, Department of Clinical and Experimental Medicine, section of Gastroenterology, Parma/Italy

Contact E-mail Address: francesco.dimario@unipr.it

Introduction: The new bismuth-containing quadruple therapy is currently advised due to emergence of $H$. pylori strains resistant to several antibiotics. Our aims was to assess the real-life effectiveness and safety of this therapeutic regimen in a large population of patients who were infected by $H$. pylori.

Aims \& Methods: Consecutive dyspeptic $H$. pylori-positive patients were enrolled, both naïve for treatment and already unsuccessfully treated. Patients were treated with Pylera ${ }^{\circledR}$ (3-in-1 capsules containing bismuth subcitrate potassium $140 \mathrm{mg}$, metronidazole $125 \mathrm{mg}$ and tetracycline $125 \mathrm{mg}$ ) 3 capsules four times a day plus omeprazole $20 \mathrm{mg}$ or esomeprazole $40 \mathrm{mg}$ two times a day for 10 days. Eradication was confirmed using a urea-breath test (at least 30 days after the end of treatment). Efficacy and safety were assessed.

Results: Three hundred and twenty patients were included in the study: 131 $(40.9 \%)$ patients were naïve, and $189(59.1 \%)$ patients with previous failure treatment. $H$. pylori eradication was achieved in $299(93.4 \%, 95 \%$ confidence intervals (CIs) 83.1 to 1.0 ) patients. No difference in eradication rate was found either between naïve and previously treated patients $(95.4 \%$ vs $92.1 \%, \mathrm{P}=0.336)$. Treatment-emergent adverse events occurred in 61 patients $(19.1 \%, 95 \%$ CIs 14.6 to 24.549). They were mild in all cases except in ten, who discontinued the study due to diarrhea (four patients) and diffuse urticarial rash (6 patients).

Conclusion: This bismuth-containing quadruple therapy achieved a remarkable eradication rate in real life, irrespective as first treatment or as a salvage therapy, despite the frequent occurrence of mild adverse events.

Disclosure of Interest: All authors have declared no conflicts of interest. 
P1260 COMPARISON OF CLARITHROMYCIN- AND LEVOFLOXACIN-CONTAINING TRIPLE THERAPIES FOR FIRSTLINE HELICOBACTER PYLORI ERADICATION IN IRAN

H. Fakheri ${ }^{1}$, A. Sadoogh ${ }^{2}$, S. Tirgar Fakheri ${ }^{2}$

${ }^{1}$ Gut And Liver Research Center, Mazandaran University of Medical Sciences, Sari/Iran

${ }^{2}$ Mazandaran University of Medical Sciences, Sari/Iran

Contact E-mail Address: septirgar74@gmail.com

Introduction: According to the Maastricht IV consensus report, Clarithromycincontaining triple therapy can be considered as a suitable option for first-line Helicobacter pylori (H. pylori) eradication in areas with less than $20 \%$ resistance rates to Clarithromycin. On the other hand, resistance to Clarithromycin is increasing in Iran, influencing the efficacy of standard triple therapy in this country. Therefore, regimens containing other antibiotics have to be considered in Iran.

Aims \& Methods: One hundred and forty patients with peptic ulcer disease and naïve $H$. pylori infection were randomly divided into two groups to receive either 10-day standard triple therapy (Pantoprazole 40 mg, Amoxicillin $1 \mathrm{gr}$ and Clarithromycin $500 \mathrm{mg}$, all given twice daily) or 10-day Levofloxacin-containing triple therapy (Pantoprazole $40 \mathrm{mg}$ BD, Amoxicillin $1000 \mathrm{mg}$ BD and Levofloxacin $500 \mathrm{mg} /$ daily). Eight weeks after the treatment, H. pylori eradication was assessed by ${ }^{14} \mathrm{C}$ - urea breath test.

Results: One hundred and thirty three patients completed the study. According to intention to treat analysis, H. pylori eradication rates were $75.7 \%(95 \%$ confidence interval $(\mathrm{CI}): 65.7 \%-85.7 \%)$ and $58.5 \%(95 \% \mathrm{CI}=47.1 \%-70 \%)$ in standard and Levofloxacin-containing therapies, respectively. Also, per-protocol eradication rates were $83 \%(95 \% \mathrm{CI}: 74 \%-92 \%)$ and $61 \%(95 \% \mathrm{CI}=49 \%$ $-73 \%$ ), respectively. The rates of severe adverse effects of therapy were $7.1 \%$ and $2.9 \%$ in the mentioned groups, respectively.

Conclusion: Both Clarithromycin-containing triple therapy and Levofloxacincontaining triple regimen do not seem to be suitable options for first-line $H$. pylori eradication in Iran. We suggest using Clarithromycin in quadruple regimens such as hybrid or concomitant therapies and reserve Levofloxacin to be used in second-line eradication regimens, as it is recommended by Maastricht $\mathrm{V}$ Consensus Report.

Disclosure of Interest: All authors have declared no conflicts of interest.

\section{P1261 EFFICACY AND TOLERABILITY OF REBAMIPIDE IN TRIPLE THERAPY FOR ERADICATION OF HELICOBACTER PYLORI: A RANDOMIZED CLINICAL TRIAL}

\section{S. S. Vyalov}

Gastroenterology, Peoples Friendship University of Russia, Moscow/Russian

Federation

Contact E-mail Address: svialov@mail.ru

Introduction: Rebamipide is an orally prostaglandin $\mathrm{E}_{2}$ and $\mathrm{I}_{2}$ synthesis inductor. A latest clinical trial showed that the adhesion of $H$. pylori to stomach wall was reduced by rebamipide. This could improve eradication rates by increasing the availibility of Helicobacter to antimicrobial.

Aims \& Methods: We aimed to determine eradication rate, the effectiveness and advantage of rebamipide in triple eradication therapy of $H$. pylori infection. Subjects comprised patients undergoing eradication therapy for $H$. pylori infection in our clinics. Patients with a history of eradication therapy, gastrectomy, or allergy to medications in triple therapy were excluded. Written informed consent was obtained for each patient. This trial was performed as a randomised openlabelled clinical study with the permission of an institutional review board. All patients was tested on $H$. pylory infection. The number of cases was 160 ( 80 cases in each group). Each patient was randomly enrolled for rebamipide therapy group (RBD) (esomeprazol $40 \mathrm{mg}$, amoxicillin $1000 \mathrm{mg}$, clarithromycin $500 \mathrm{mg}$, rebamipide $100 \mathrm{mg}$ twice a day for 14 days) or standart triple therapy group (STD) (esomeprazol $40 \mathrm{mg}$, amoxicillin $1000 \mathrm{mg}$, clarithromycin $500 \mathrm{mg}$, twice a day for 14 days). Before starting therapy, we checked the background characteristics of each patient (age; gender; weight; height; drinking habit; smoking habit; use of probiotics, bismuth, PPI; and endoscopic findings). After the therapy, we asked physicians and patients about medication compliance and side effects. The primary endpoint was the eradication rate. The secondary endpoints were the rates of side effects.

Results: For RBD therapy group and STD therapy group, the eradication rates were $94.0 \%(95 \%$ confidence interval, $85 \%-100 \%)$ and $82 \%(95 \%$ confidence interval, $74 \%-95 \%)$ respectively, and the rates of side effects were $8.5 \%(95 \%$ confidence interval, $7.0 \%-26.5 \%)$ and $29 \%$ (95\% confidence interval, $17.5 \%$ $41.0 \%)$ respectively. For each group, statistically significant difference in eradication rate was seen. The RBD group revealed trends of high eradication rates and low rates of side effects.

Conclusion: The findings suggest that rebamipide is effective in eradication of H. pylori infection, significantly improve eradication rate in triple therapy. The advantage of rebamipide has efficacy and good tolerability.

Disclosure of Interest: All authors have declared no conflicts of interest.

\section{References}

1. Nebiki H, Higuchi K, Arakawa T, Ando K, Uchida T, Ito H, Harihara S, Kuroki T, Kobayashi K. Effect of rebamipide on Helicobacter pylori infection in patients with peptic ulcer. Dig Dis Sci. 1998 Sep;43(9 Suppl):203S206S. PubMed PMID: 9753251.

2. Kato M, Asaka M, Sugiyama T, Kudo M, Nishikawa K, Sukegawa M, Hokari K, Katagiri M, Sato F, Kagaya H, Takeda H. Effects of rebamipide in combination with lansoprazole and amoxicillin on Helicobacter pyloriinfected gastric ulcer patients. Dig Dis Sci. 1998 Sep;43(9 Suppl):198S-202S. PubMed PMID: 9753250.

3. Hayashi S, Sugiyama T, Amano K, Isogai H, Isogai E, Aihara M, Kikuchi M, Asaka M, Yokota K, Oguma K, Fujii N, Hirai Y. Effect of rebamipide, a novel antiulcer agent, on Helicobacter pylori adhesion to gastric epithelial cells. Antimicrob Agents Chemother. 1998 Aug;42(8):1895-9. PubMed PMID: 9687380; PubMed Central PMCID: PMC105706.

4. Kim CD, Kim HH, Hong KW. Inhibitory effect of rebamipide on the neutrophil adherence stimulated by conditioned media from Helicobacter pyloriinfected gastric epithelial cells. J Pharmacol Exp Ther. 1999 Jan;288(1):1338. PubMed PMID: 9862763.

5. Song KH, Lee YC, Fan DM, Ge ZZ, Ji F, Chen MH, Jung HC, Bo J, Lee SW, Kim JH. Healing effects of rebamipide and omeprazole in Helicobacter pylori-positive gastric ulcer patients after eradication therapy: a randomized double-blind, multinational, multi-institutional comparative study. Digestion. 2011;84(3):221-9. doi: 10.1159/000329353. Epub 2011 Jul 8. PubMed PMID: 21757914.

\section{TUESDAY, OCTOBER 31, 201709:00-17:00 \\ SMALL INTESTINAL II - HALL 7}

\section{P1262 OBSERVER AGREEMENT FOR DIAGNOSIS OF ACUTE GRAFT-VERSUS-HOST DISEASE AFTER ALLOGENEIC STEM CELL TRANSPLANTATION WITH MICROVILLI ATROPHY IN THE TERMINAL ILEUM}

Y. Sugihara ${ }^{1}$, S. Hiraoka ${ }^{1}$, N. Fujii ${ }^{2}$, S. Takashima ${ }^{1}$, Y. Yamasaki ${ }^{1}$, D. Takei ${ }^{3}$, T. Inokuchi ${ }^{1}$, M. Takahara ${ }^{1}$, Y. Morito ${ }^{4}$, S. Takahashi ${ }^{5}$, K. Kuwaki ${ }^{6}$,

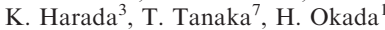

${ }^{1}$ Department Of Gastroenterology And Hepatology, Okayama University Graduate School of Medicine, Okayama/Japan

${ }^{2}$ Department Of Hematology And Oncology, kayama University Graduate School of Medicine, Okayama/Japan

${ }^{3}$ Division Of Endoscopy, Okayama University Graduate School of Medicine, Okayama/Japan

${ }^{4}$ Department Of Gastroenterology And Hepatology, Mitoyo General Hospital, Mitoyo/Japan

${ }^{5}$ Department Of Gastroenterology And Hepatology, Kagawa Prefectural Central Hospital, Kagawa/Japan

${ }^{6}$ Center For Innovative Clinical Medicine, Okayama University Graduate School of Medicine, Okayama/Japan

${ }^{7}$ Department Of Diagnostic Pathology, Okayama University Graduate School of Medicine, Okayama/Japan

Contact E-mail Address: y.sugi117@gmail.com

Introduction: Intestinal graft-versus-host disease (GVHD) represents one of the most serious complications of allogeneic stem cell transplantation (allo-SCT) Almost $10 \%-40 \%$ of patients who undergo allo-SCT develop significant clinical acute graft-versus-host disease (GVHD) [1]. Endoscopic and histological proofs are required because of the number of differential diagnoses manifesting as diarrhoea. Previous studies reported that endoscopic features, such atrophied terminal ileum [2,3], are useful in diagnosing acute GVHD. However, these previous studies did not address details of the random sequence, and were performed in a single centre.

Aims \& Methods: The present study investigated the incidence of the characteristic finding, particularly microvilli atrophy of the terminal ileum, of colonoscopy, and inter-observer agreement of the finding among experienced endoscopists in multiple centres. Consecutive patients who underwent alloHSCT were referred to the Okayama University Graduate School of Medicine between May 2008 and September 2015. Fifty-four patients underwent colonoscopy after allo-SCT as intestinal acute GVHD was suspected. We recorded the colonoscopic imaging. Subsequently, they were checked by three observers, from different institutions, to determine whether microvilli atrophy in the terminal ileum is present. Demographic information, disease, symptoms, and histological finding were obtained from the patients' medical records retrospectively. All study participants provided informed consent. The local ethics review committee granted ethical approval (approval 1610-013), and was registered in the University Hospital Medical Network Clinical Trials Registry (UMIN-CTR) as number UMIN 000025390.

Results: Definitive pathological and non-pathological GVHD were found in 22 patients and 32 patients, respectively. In the analysis of all 54 lesions, for three observer (A, B, and C), sensitivity of the microvilli atrophy in the terminal ileum was $86.4 \%, 77.3 \%$, and $79.2 \%$, whereas specificity of the appearance were $62.5 \%, 62.5 \%$, and $86.2 \%$. In addition, the positive predictive value of the appearance was $61.3 \%, 58.6 \%$, and $82.6 \%$, and negative predictive value (NPV) was $87.2 \%, 87.2 \%$, and $83.9 \%$, respectively. The kappa coefficient of the inter-rater reliability was $0.85,0.63$, and 0.63 in observers $\mathrm{AB}, \mathrm{AC}$, and $\mathrm{BC}$. Conclusion: Conclusions: Microvilli atrophy in the terminal ileum is an effective colonoscopy finding for real-time predictive histological diagnosis of acute intestinal GVHD. We achieved substantial inter-observer agreement for the analysis of microvilli atrophy in the terminal ileum and excellent agreement for predictive histological diagnosis.

Disclosure of Interest: All authors have declared no conflicts of interest.

\section{References}

1. Iqbal N, Salzman D, Lazenby AJ et al. Diagnosis of gastrointestinal graftversus-host disease. Am J Gastroenterol 2000; 95: 3034-3038 
2. Kreisel W, Dahlberg M, Bertz $\mathrm{H}$ et al. Endoscopic diagnosis of acute intestinal GVHD following allogeneic hematopoietic SCT: a retrospective analysis in 175 patients. Bone Marrow Transplant 2012; 47: 430-438

3. Mashiba T, Usami A, Soga Y et al. Endoscopic findings of intestinal graftversus-host disease following allogenic bone marrow and peripheral blood stem cell transplantation. Gastroenterol Endosc 2003; 45: 929-934

\section{P1263 EPIGALLOCATECHIN-3-GALLATE FROM GREEN TEA AMELIORATE 5-FLUROURACIL-INDUCED INTESTINAL MUCOSITIS}

J.M. Lee ${ }^{1}$, G. Min ${ }^{2}$, W. Kim ${ }^{3}$, S.J. Choi ${ }^{2}$, I.K. Yoo ${ }^{2}$, S.H. Kim ${ }^{2}$, J.M. Lee ${ }^{4}$, H.S. Choi ${ }^{2}$, E.S. Kim ${ }^{2}$, B. Keum ${ }^{2}$, H.J. Chun ${ }^{2}$, H.S. Lee ${ }^{2}$, Y.T. Jeen ${ }^{2}$, C.D. Kim², K.C. Yoon ${ }^{3}$

${ }_{1}^{1}$ Gastroenterology, Korea University Medical Center, Seoul/Korea, Republic of ${ }^{2}$ Division of Gastroenterology and Hepatology, Department of Internal Medicine, Korea University College of Medicine, Seoul/Korea, Republic of

${ }^{3}$ Division Of Gastroenterology And Hepatology, Department Of Internal Medicine, Institute of Gastrointestinal Medical Instrument Research, Korea University

College of Medicine, Seoul/Korea, Republic of

${ }^{4}$ Gastroenterology, Korea Univ. medical center, Seoul/Korea, Republic of

\section{Contact E-mail Address: ytjeen@korea.ac.kr}

Introduction: Chemotherapy-induced mucositis is a common complication during anticancer treatment. Epigallocatechin-3-gallate (EGCG), derived from green tea, has been shown to have antioxidant effects and immunomodulatory activities. However, studies on EGCG for chemotherapy-induced mucositis have been scarce.

Aims \& Methods: In this study, we aimed to prove the protective effect of EGCG in murine chemotherapy-induced mucositis model.Twenty-four 8-wk-old male C57BL/6 mice were randomized to 4 groups: control, EGCG, 5-Flurouracil (5-FU), EGCG plus 5-FU. Mucositis was induced by intraperitoneal injection of $5-\mathrm{FU}(400 \mathrm{mg} / \mathrm{kg})$. EGCG $(50 \mathrm{mg} / \mathrm{kg})$ was administered orally for 5 days from the day before administration of 5-FU. After 6 days of 5-FU injection, the mice were sacrificed and intestinal tissue was obtained. WBC count was performed with whole blood from Inferior vena cava of mice. The end points were villus height, villus/crypt ratio, histologic characteristics, and mRNA expression of tumor necrosis factor $(T N F)-\alpha$, and interleukin $(I L)-6$.

Results: In 5-FU group, neutropenia was confirmed by laboratory test (5-FU, $0.650 \mathrm{~K} / \mu \mathrm{L}$; Control, $5.317 \mathrm{~K} / \mu \mathrm{L}$ ), indicating sufficient 5-FU effect. Histologic findings showed that crypt dilatation, villus stunting, and villus atrophy were reduced in EGCG plus 5-FU group than in 5-FU group (Figure 1). Quantitatively, mean villus height (EGCG plus 5-FU, $352 \mu \mathrm{m} ; 5-\mathrm{FU}, 319 \mu \mathrm{m}$ ) and villus/crypt ratio (EGCG plus 5-FU, 3.28; 5-FU, 2.31) in EGCG plus 5-FU group, compared with 5-FU treated group, were significantly higher. mRNA expression of TNF- $\alpha$ was significantly lower in EGCG plus 5-FU group compared with 5-FU group $(\mathrm{P}<0.05)$ (Figure 2). Figure 1. Effects of EGCG administration on chemotherapy-induced mucositis in mice jejunum (A) control (B) 5-Fluorouracil (5-FU) group with significant villus atrophy and crypt dilatation (c) EGCG group (d) EGCG plus 5-FU group with mild villi destruction and less crypt dilatation Figure 2. mRNA expression of TNF- $\alpha$. 5-FU plus EGCG group showed significantly lower $2^{\wedge} \mathrm{ddCT}$ than 5 -FU only group. $(* * * \mathrm{P}<0.05)$

Conclusion: EGCG derived from green tea reduced 5 -FU induced intestinal mucositis, suggesting a possibility for novel treatment of chemotherapy-induced mucositis.

Disclosure of Interest: All authors have declared no conflicts of interest.

\section{P1264 INCREASED SUSCEPTIBILITY TO ENTEROPATHOGENIC BACTERIA BY PROTON PUMP INHIBITORS IN THE MURINE MODEL OF FOOD POISONING}

E. Yasutomi ${ }^{1}$, N. Hoshi ${ }^{1}$, J. Inoue ${ }^{1}$, S. Adachi ${ }^{1}$, T. Otsuka ${ }^{1}$, D. Watanabe ${ }^{1}$, Y. Ku ${ }^{1}$, H. Yamairi ${ }^{1}$, M. Ooi ${ }^{1}$, S. Fukuda ${ }^{2}$, M. Yoshida ${ }^{1}$, T. Azuma ${ }^{1}$

${ }^{1}$ Division Of Gastroenterology, Department Of Internal Medicine, Kobe University Graduate School of Medicine, Kobe/Japan

${ }^{2}$ Institute For Advanced Biosciences, Keio University, Yamagata/Japan

Contact E-mail Address: eiichiro_yasutomi@yahoo.co.jp

Introduction: Proton pump inhibitors (PPIs) have become one of the most commonly prescribed medicine due to their strong effects of suppressing gastric acid and high curative effects for acid related diseases. On the other hand, their side effects have been attracting more attention. One of them is the increased incidence of infectious intestinal diseases. Those are mostly reported by clinical publications, and the exact mechanism has not been clarified. To investigate whether PPIs can increase the susceptibility to peroral enteropathogenic bacterial infection, we used Citrobacter rodentium (C. rodentium), a well-known attaching/ effacing-associated pathogen, which is used for the model of human enterohemorrhagic Escherichia coli (EHEC) and enteropathogenic Escherichia coli (EPEC) infection: the major causes of food poisoning.

Aims \& Methods: To investigate whether LAZ can influence the steady-state intestinal environment, C57BL/6 J mice were divided into two groups, and $8 \mathrm{mg} / \mathrm{kg} /$ day of lansoprazole (LAZ group) or saline (control group) were administrated intraperitoneally for two weeks. The ileal contents and feces were collected before and after LAZ administration. Genomic DNA of the gut microflora was analyzed by $16 \mathrm{~S}$ ribosomal RNA (16SrRNA) gene sequencing, and the metabolites were analyzed by a CE-TOFMS platform. To examine the changes of immune cell distribution by LAZ, hematopoietic cells in the lamina propria were analyzed by flow cytometry. The changes of gene expressions of the ileum were comprehensively analyzed by microarray. Finally, mice were orally inoculated with $\mathrm{C}$. rodentium after LAZ administration, and the establishment of enteritis was evaluated by body weight loss, histology and inflammatory cytokine expressions examined by real-time PCR.

Results: At steady-state, no prominent change of metabolites and gut microflora were observed in the feces of LAZ group. On the other hand, the concentrations of short chain fatty acids, such as butyrate and propionate, were decreased in the ileum of LAZ group. The result of $16 \mathrm{SrRNA}$ analysis also showed that the composition of ileal microflora were different between LAZ and control group. However, it did not show the change of immune cell distribution in the intestine. The gene expression levels in the ileum were not altered either. Interestingly, the ileal microflora of LAZ group became similar to that of the feces. As mice have a habit of coprophagia, it was assumed that perorally invaded bacteria could survive and pass through the stomach due to suppression of gastric acid by LAZ. Accordingly, in the LAZ group, infectious inflammation was established by less numbers of $\mathrm{C}$. rodentium inoculation, indicating that PPIs could raise the susceptibility to peroral enteropathogenic bacterial infection.

Conclusion: Our data showed that administration of PPIs could alter the intestinal environment such as microflora and luminal metabolites. However, neither the gene expressions nor distribution of immune cells in the intestinal tissue were affected. It was assumed that the increased risk of peroral enteropathogenic bacterial infection was not because of the immunological modification by PPIs, but it was mainly because of the increased number of pathogenic bacteria passing through the stomach.

Disclosure of Interest: All authors have declared no conflicts of interest.

\section{P1265 EXPRESSION OF AMINO ACID TRANSPORTERS IN ANTITUMOR AGENT-INDUCED GASTROINTESTINAL MUCOSAL INJURIES}

M. Kodo ${ }^{1}$, R. Kawashima ${ }^{2}$, W. Koizumi ${ }^{3}$, T. Ichikawa $^{2}$

${ }^{1}$ Gastroenterology, Graduate school of Medical Sciences, Kitasato University, Sagamihara/Japan

${ }^{2}$ Laboratory Of Biochemistry, Graduate school of Medical Sciences, Kitasato University, Sagamihara/Japan

${ }^{3}$ Gastroenterology, Kitasato University School of Medicine, Sagamihara/Japan

Contact E-mail Address: m.s.s.r.t.m.m.f@gmail.com

Introduction: Because recent studies have demonstrated that amino acid transporters, which transport amino acids into cells, are correlated to various cancers and are major transporters that supply essential amino acids to tumor cells, these transporters are considered as novel biological tumor markers. On the other hand, because anticancer agents are frequently used clinically, the number of patients suffering from anticancer agent-induced intestinal mucosal injuries is increasing; hence, it is important to take appropriate measures for reducing these side effects. Because the L-type amino acid transporter (LAT) transports a wide range of nonselective amino acids, including essential amino acids, it is considered to be a gastrointestinal transporter that is important for nutrient absorption. Furthermore, the involvement of other amino acid transporters in inflammation has been reported in several studies.

Aims \& Methods: We aimed to clarify the pathophysiological role of an amino acid transporter in gastrointestinal tract inflammation caused by an antitumor agent in this study. The antitumor agent fluorouracil (5-FU) was orally administered to mice. The severity of mucositis was assessed based on the length, villus height, mucus production, cell infiltration, and immune response of the intestinal tract. We measured the mRNA expressions of LATs in the tissues of the small intestines. In addition, we compared the protein expressions among the small intestines using anti-LAT antibodies.

Results: After the administration of 5-FU, the body weight, food intake, water consumption, and fecal volume decreased; thus, a systemic influence was observed. The length and villus height of the intestinal tract decreased because of the administration of 5-FU, and mucosal damage with histological change was observed. The number of PAS-positive cells decreased in the small intestinal mucosa, and it was assumed that the defensive function of the epithelial cells had decreased. In addition, an increase in the mRNA expression of IL-1 $\beta$, IL-6, and TNF- $\alpha$ in the Peyer's patch along with an increase in the cell infiltration after the administration of 5-FU significantly enhanced the immune response associated with the inflammatory cytokine production. Furthermore, on investigating the mRNA and protein expressions of LAT1 and LAT2 in the tissues of the small intestines, we observed that LAT1 expression significantly increased and LAT2 expression decreased after the administration of 5-FU.

Conclusion: It was considered that the uptake capacity of amino acids, such as Gly, Ala, Ser, Thr, Cys, Asn, and Gln, that transported through LAT may be decreased in case of small intestinal mucosal injuries. On the other hand, LAT1 expression associated with the production of inflammatory cytokines suggested that LAT1 is a gastrointestinal inflammatory marker.

Disclosure of Interest: All authors have declared no conflicts of interest. 
P1266 A MUCOUS DEPENDENT MECHANISM OF ACETYL SALICYLIC ACID- INDUCED SMALL INTESTINAL MUCOSAL INJURY IN RATS

Y. Suyama ${ }^{1}$, O. Handa ${ }^{1}$, S. Takayama ${ }^{1}$, R. Mukai ${ }^{1}$, A. Majima ${ }^{1}$, Y. Onozawa ${ }^{1}$, A. Fukui ${ }^{1}$, T. Okayama ${ }^{1}$, K. Kamada ${ }^{1}$, K. Katada ${ }^{1}$, K. Uchiyama ${ }^{1}$, T. Ishikawa ${ }^{1}$, T. Takagi ${ }^{2}$, Y. Naito ${ }^{1}$, Y. Itoh ${ }^{1}$

${ }^{1}$ Molecular Gastroenterology And Hepatology, Kyoto Prefectural University of Medicine, Kyoto/Japan

${ }^{2}$ Kyoto Prefectural University of Medicine Molecular Gastroenterology and Hepatology, Kyoto/Japan

Contact E-mail Address: yosuke-s@koto.kpu-m.ac.jp

Introduction: Acetyl salicylic acid (ASA) has been used for the secondary prevention of cardiovascular diseases. Especially, the enteric coatedASA is widely used to prevent ASA-induced gastric mucosal injury. Recent technology such as video capsule endoscopy and balloon endoscopy enabled us to look inside the smal intestine in more detail. Consequently, not a few cases of ASA-induced small intestinal mucosal injury have been reported. However, the effective prophylaxis and treatment is not clear yet. Previously, we reported direct detrimental effect of ASA on small intestinal epithelial cells using an in vitro model [1]. However, there are the thick mucus layer between intestinal lumen and epithelial cells. The mucous has been reported to prevent foreign objects such as bacteria, medicine and food from epithelial cells.

Aims \& Methods: This study was conducted to clarify the role of mucus on ASAinduced small intestinal mucosal injury using a rat model. Male Sprague-Dawley rats, 9 weeks old was used. These rats were divided into four groups; group 1 : sham (carboxy methyl cellulose: CMC alone), group2: polysorbate-80 (P80) alone, group 3: ASA alone, and group 4: P80 plus ASA. CMC and/or $50-200 \mathrm{mg} / \mathrm{kg}$ ASA was injected into the proximal duodenum of fed rats. P80, an emulsifier, which has been reported to reduce mucous thickness [2], was administered via drinking water for 2 weeks before ASA treatment. Indeed, P80 also reduced the thickness of mucous layer in our analyses. One hour after ASA treatment, $1 \%$ Evans blue was injected into a vein of rats to visualize small intestinal lesions. Ninety minutes after ASA treatment, the entire small intestine was removed for histological assessment. To further investigate the importance of mucus, rebamipide (Reb, $300 \mathrm{mg} / \mathrm{kg}$ ) or saline were orally administered for one week prior to P80 and ASA treatment. Reb is a gastric muco-protective drug widely used for the treatment of gastric ulcer, and increases mucous secretion by small intestinal goblet cell

Results: Evans blue method suggested that high-dose ASA $(200 \mathrm{mg} / \mathrm{kg})$ induced severe jejunal mucosal lesions, which was further confirmed by the histological examination. Although lower doses of ASA (50 and $100 \mathrm{mg} / \mathrm{kg}$ ) did not cause mucosal damage, P80 significantly induced Evans blue exudate and severe mucosal lesions in jejunum at these concentrations, suggesting the pivotal role of mucus in these phenomena. Moreover, orally administered Reb significantly suppressed reducing small intestinal mucus and the exacerbation of ASA-induced mucosal lesions by $\mathrm{P} 80$, indicating that mucus is inevitable in the protection of ASA-induced small intestinal mucosal injury.

Conclusion: A mucous secretion increasing therapy might be a useful for the prevention of ASA-induced small intestinal mucosal injury.

Disclosure of Interest: Y. Naito: Contribution and lecture fee from Otsuka Pharmaceutical Co.

Y. Itoh: Endowed chair and research support from Otsuka Pharmaceutical Co. All other authors have declared no conflicts of interest.

\section{References}

1. Akifumi Fukui, et al: Acetyl salicylic acid induces damage to intestinal epithelial cells by oxidation-related modifications of ZO-1. Am J Physiol Gastrointest Liver Physio 2012.

2. Benoit Chassaing, et al: Dietary emulsifiers impact the mouse gut microbiota promoting colitis and metabolic syndrome. Nature 2015.

\section{P1267 ANTIBIOTIC-INDUCED DYSBIOSIS IN THE MOUSE SMALL INTESTINE PROMOTES ALLERGIC SENSITISATION}

G. Burns ${ }^{1}$, B. J. Goggins ${ }^{1}$, K. Minahan ${ }^{2}$, P. Foster ${ }^{3}$, N.J. Talley ${ }^{4}$, M M. Walker ${ }^{3}$, J. Horvat ${ }^{2}$, S. Keely ${ }^{1}$

${ }^{1}$ School Of Biomedical Sciences And Pharmacy, University of Newcastle, Callaghan/Australia/NSW

${ }^{2}$ University of Newcastle School of Biomedical Science and Pharmacy, Callaghan Australia

${ }^{3}$ School Of Medicine And Public Health. The University of Newcastle Australia, Callaghan/Australia/NSW

${ }^{4}$ Faculty Of Health And Medicine And Priority Research Center For Digestive Health And Neurogastroenterology. The University of Newcastle Australia, Callaghan/Australia/NSW

Contact E-mail Address: simon.keely@newcastle.edu.au

Introduction: Food allergy is characterised by a T helper type- 2 immune response against a food antigen, manifesting as symptoms including nausea, diarrhoea, vomiting or anaphylactic events. It is estimated that $10 \%$ of the Australian population have a food allergy, and common allergens include cow's milk, shellfish and peanuts. Epidemiological studies have identified antibiotics as a significant risk factor for food allergy in infants.

Aims \& Methods: We examined how the broad spectrum antibiotic amoxicillin influenced mucosal immune responses to peanut proteins and the development of peanut allergy in mice. Balb $/ \mathrm{C}$ mice were treated daily with $5 \mathrm{mg} / \mathrm{kg}$ amoxicillin or PBS for 5 days (days $0-4$ ). On days 5 and 6 animals received $0.2 \mathrm{mg}$ peanut extract or PBS vehicle by oral gavage. Animals were rechallenged with peanut or vehicle by oral gavage on days 11 and 13 and sacrificed on day 16 and immune responses to peanut challenge in blood and intestinal tissues were assessed by protein, mRNA and histological analysis.

Results: The proportion of circulating eosinophils was increased in the blood of mice treated with both antibiotics and peanut. Histological examination revealed an increase in small intestinal eosinophils, predominantly at the villous tips, indicating recruitment to the mucosa. RNA and protein analysis revealed an increase in IL-5 associated with increased Nod-Like Receptor Protein 3 (NLRP3) inflammasome activation.

Conclusion: These studies demonstrate that antibiotic treatment prior to food antigen challenge can lead to altered mucosal immune homeostasis, facilitating IL-5-mediated eosinophil recruitment, characteristic of allergic responses. Importantly, we have demonstrated an adjuvant-free model of food sensitisation and small intestinal eosinophilia. These findings contribute to a better understanding of how disruption of mucosal homeostasis by antibiotics contributes to the development of allergic sensitisation and reaction.

Disclosure of Interest: All authors have declared no conflicts of interest.

\section{P1268 PREBIOTIC EFFECTS ON HEALTHY AND CHEMOTHERAPY-INDUCED SMALL BOWEL INJURY IN RATS}

R. Yazbeck ${ }^{1}$, R. J. Lindsay ${ }^{2}$, M. S. Geier ${ }^{2}$, R. N. Butler ${ }^{3}$, S. Jaenisch ${ }^{3}$, G S. Howarth ${ }^{2}$

${ }^{1}$ Flinders Centre for Innovation in Cancer, Adelaide/Australia

${ }^{2}$ School Of Animal And Veterinary Science, University of Adelaide, Adelaide

Australia

${ }^{3}$ Surgery, Flinders University, Adealaide/Australia

Contact E-mail Address: roger.yazbek@flinders.edu.au

Introduction: Intestinal mucositis is a severe side-effect of chemotherapy with a current deficiency in effective treatments.

Aims \& Methods: This study investigated three prebiotics, galacto-oligosaccaride (GOS), mannan-oligosaccharide (MOS) and fructo-oligosaccharide (FOS) for their potential to reduce the severity of 5-Fluorouracil (5-FU)-induced intestinal mucositis in rats. Female Dark Agouti rats $(n=8 /$ group) were orally gavaged with either 5\% FOS, GOS, MOS or water (controls) for 16 days, and received an intraperitoneal injection of 5-Fluorouracil (5-FU: $150 \mathrm{mg} / \mathrm{kg}$ ) or saline (controls), on day 13. Rats were housed in metabolic cages for the duration of the study, and metabolic data was recorded daily. Rats were killed on day 16 and visceral organ weights and lengths were analyzed post mortem. Crypt depth, villus height and histological severity scores were quantified in haematoxylin \& eosin stained sections. Sucrase and myeloperoxidase [MPO] activity were quantified by biochemical assay. White and red blood cell types were quantified by whole blood analysis. Fecal volatile fatty acids (VFAs), acetic, propionic, isobutyic, butyric, isovaleric and valeric acid were also measured. Statistical analysis was by oneway ANOVA or Kruskal Wallis and Mann Whitney U test, where $\mathrm{p}<0.05$ was considered statistically significant. Data are expressed as mean \pm standard error of the mean

Results: \%Bodyweight loss was significantly decreased in all treatment groups following 5-FU injection. Total fecal output was increased in rats treated with GOS or FOS, pre and post 5-FU compared to saline treated controls $(\mathrm{p}<0.05)$. Ileal villus height was significantly higher in GOS treated rats pre 5-FU $(284.16 \pm 11.95 \mu \mathrm{m})$ compared to respective water controls $(240.40 \pm 8.83 \mu \mathrm{m}$; $\mathrm{p}<0.05$ ). Villus height and crypt depth was significantly decreased in all treatment groups after 5 -FU injection $(\mathrm{p}<0.05)$ and prebiotic treatment did not significantly modify this parameter. Similarly, jejunal and ileal sucrase activity was decreased in all groups after 5-FU injection $(\mathrm{p}<0.05)$, correlating with histological measurements. Tissue MPO activity was significantly increased post 5-FU injection, reflecting increased neutrophil activation, and was unchanged by prebiotic treatment. Interestingly, MOS and GOS both lowered $\%$ circulating neutrophils pre 5 -FU compared to water controls $(\mathrm{p}<0.05)$. Pre 5 FU treatment with GOS significantly increased the fecal VFAs acetic acid $(16.76 \pm 1.22 \mathrm{mM} / \mathrm{L})$ and propionic acid $(4.60 \pm 0.99 \mathrm{mM} / \mathrm{L})$ compared to saline treated controls $(7.73 \pm 0.92 \mathrm{mM} / \mathrm{L}$ and $3.05 \pm 0.28 \mathrm{mM} / \mathrm{L}$ respectively; $\mathrm{p}<0.05)$. MOS and GOS treatment also significantly increased fecal acetic and propionic acid post 5 -FU compared to water control $(\mathrm{p}<0.05)$

Conclusion: Our study has found that prebiotics, MOS, GOS and FOS modified some parameters of intestinal health and immune regulation in healthy rats; however, these prebiotics were not protective against 5-FU induced intestinal damage. Furthermore, our findings have demonstrated that prebiotic treatment significantly increases VFA production, suggesting functional changes to the intestinal microbiome. Further studies are indicated to investigate prebiotics, both alone and in combination, during the repair phase of intestinal mucositis, and to determine their effect on gut microbial composition.

Disclosure of Interest: All authors have declared no conflicts of interest. 
P1269 THE RELATION OF CHEMOKINE RECEPTOR CXCR3 AND GUT-HOMING MARKERS ON SMALL INTESTINAL LAMINA PROPRIA T-LYMPHOCYTES IN CROHN'S DISEASE PATIENTS

A. T. Do ${ }^{1}$, A. Shah ${ }^{2}$, E. Shanahan ${ }^{2}$, P. Gashemi ${ }^{2}$, T. Hansen ${ }^{2}$, N. Koloski ${ }^{2}$, S. Keelly ${ }^{3}$, N.J. Talley ${ }^{4}$, G. Holtmann ${ }^{2}$

${ }^{1}$ Faculty Of Medicine, The University Of Queensland, Woolloongabba/Australia ${ }^{2}$ Princess Alexandra Hospital, Department of Gastro and Hepatology,

Woolloongabba/Australia/QLD

${ }^{3}$ University Of Newcastle, Faculty of Health \& Medicine, New Castle/Australia/ NSW

${ }^{4}$ Faculty Of Health And Medicine And Priority Research Center For Digestive Health And Neurogastroenterology, The University of Newcastle Australia, Callaghan/Australia/NSW

Contact E-mail Address: a.do@uq.edu.au

Introduction: Crohn's disease has been thought to be caused by abnormal immune responses affecting many parts of digestive tract in which Th1 cells are particularly dominant. The recruitment of Th1 cells is regulated by interaction of their expression of chemokine receptor CXCR3 and its ligands. There have been many IBD murine models showing the increase of CXCR3 expression and its roles on the disease promotion. However, there are limited evidences in the roles of CXCR3 in human IBD. In fact, a small study in large bowel in a cohort of 10 Crohn's disease patients showed lower expression of CXCR3 on T lymphocytes, compared to colon cancer patients. In terms of inhibition of Tlymphocyte migration into intestine in IBD patients, anti a4b7 (Vedolizumab) therapy is currently shown to be effective.

Aims \& Methods: Our study aimed to assess expression of CXCR3 by different subsets of small intestinal lamina propria T-cells and its association with a4 and b7 integrin in Crohn's disease (CD) patients. Total of 56 duodenal biopsies were obtained from $\mathrm{CD}(\mathrm{n}=15)$, functional dyspepsia (FD)/irritable bowel syndrome (IBS) $(\mathrm{n}=24)$ or iron deficiency patients $(\mathrm{n}=17)$ with ethical approval. Lamina propria (LP) cells were isolated from biopsies using EDTA, collagenase and gradient density centrifugation with Ficoll. Expression of CXCR3, a4, and b7 on isolated T-lymphocytes was examined by flow cytometry. Statistical significance was assessed using T-test or Spearman correlation.

Results: The expression of CXCR3 on CD4 lymphocytes was significantly lower $(35.6 \%)$ in $\mathrm{CD}$ patients, compared to $61.6 \%$ in control group (FD/IBS/iron deficiency). Although the expression of CXCR3 on CD8 lymphocytes was higher than CD4 lymphocytes, it was not different between CD and other group $(75.8 \%$ in $\mathrm{CD}$ patients vs $82.2 \%$ in controls). Similar observation was obtained on the double positive CD4 and CD8 lymphocytes. Interestingly, only expression of CXCR3 on CD4 lymphocytes positively correlated with expression of the gut-homing integrins, a 4 and b7.

Conclusion: These observations showed significant expression of CXCR3 across different patients with GI-related disease, with consistently higher expression seen in $\mathrm{CD}^{+}$lymphocytes compared to CD4 lymphocytes. An unexpected reduction of CXCR3 expression was seen in small intestinal of CD patients, which associated with gut-homing integrins. This result showed CXCR3 expression may play a role in migration of CD4 lymphocytes but not CD8 lymphocytes into duodenum in relation with integrins, a 4 and b7. However, CXCR3 expression on CD4 lymphocytes in CD patients' small intestine may have protective role. This propose further study to clarify.

Disclosure of Interest: All authors have declared no conflicts of interest.

\section{P1270 METHODOLOGICAL QUALITY OF CLINICAL PRACTICE GUIDELINES ON PROBIOTICS IN ACUTE GASTROENTRITIS IN CHILDREN USING THE APPRAISAL OF GUIDELINES FOR RESEARCH \& EVALUATION II INSTRUMENT (AGREEII)}

A. Maruy Saito ${ }^{1}$, J.C. Herrera Rodriguez ${ }^{1}$, R. Rojas Galarza ${ }^{2}$

${ }^{1}$ Dept. Of Pediatrics, U Peruana Cayetano Heredia, Lima/Peru

\section{Contact E-mail Address: aldo.maruy@gmail.com}

Introduction: Acute Gastroenteritis (AGE) is one of the diseases that most frequently affects paediatric population. Successful treatment in AGE has been mainly based on prevention and treatment of its complications; every day, we find more publications on the use of adjuvants to decrease its duration. Probiotics have gained greater importance because some of them report benefits. We look for Clinical Practice Guidelines (CPG) that recommend their use in AGE in children. The AGREE II instrument was developed to address the issue of variability in guideline quality, so it is a tool that assesses the methodological rigour and transparency in which a guideline is developed.

Aims \& Methods: To assess the methodological quality of clinical practice guidelines $(\mathrm{CPG})$ on the use of probiotics in infant diarrhoea. The search was conducted in December 2016, of CPG based on the evidence, the last 10 years and unrestricted language, PubMed, ScienceDirect, and Lilacs. In PubMed, the search terms were: probiotics AND infants; limit: 'guideline'. In ScienceDirect: Probiotics AND infants; limit: Medicine and Dentistry and LILACS: 'probiotics' AND 'infants' and 'probióticos' AND 'guía'.The CPG selected were evaluated with the instrument AGREE II by two evaluators who were independent one from the other and by a third evaluator, when there were discrepancies. The concordance of the evaluations was measured with the index of kappa.

Results: Ten documents were recovered only in PubMed, and selected only 03 that fulfilled the criteria about probiotics. The other two were located in a direct way. The 5 CPG were: 1 . Diarrhea and vomiting caused by GE - NICE; 2. CPG on the diagnosis and treatment of the AID in Pediatrics - Peru; 3. ESPGHANESPID evidence-based guidelines for the management of AGE in children in Europe. Update (2014); 4. Clinical practice guideline ILA on the management of GEA in children under 5 years and 5. Use of probiotics for management of
AGE. A position paper by the ESPGHAN Working Group for Probiotics and Prebiotics. All of the CPG consider that the administration of Lactobacillus rhamnosus GG and Saccharomyces boulardii should be considered in the management of children with AGE as an adjunct to rehydration therapy with different level of evidence. The concordance between evaluators was $>0.9$ and $<1.0$ for all guidelines. Four CPG had one rating higher than $60 \%$ in 4 or more domains and one in only one domain. Only a CPG was greater than $60 \%$ in all domains. Four had an overall score greater than or equal to 5 and were 'recommended', and one with modifications, according to the instrument AGREE II. The CPG 5 is a Position Paper of a Working Group, not a Guideline, and its the reason for the lower rates obtained.

\begin{tabular}{|c|c|c|c|c|c|}
\hline Domain & $\begin{array}{l}\text { CPG1 } \\
\text { NICE }\end{array}$ & $\begin{array}{l}\text { CPG2 } \\
\text { Gonzales } \\
\text { et al }\end{array}$ & $\begin{array}{l}\text { CPG3 } \\
\text { Guarino } \\
\text { et al }\end{array}$ & $\begin{array}{l}\text { CPG4 } \\
\text { Polanco } \\
\text { et al. }\end{array}$ & $\begin{array}{l}\text { CPG5 } \\
\text { Swajeska } \\
\text { et al. }\end{array}$ \\
\hline 1.Scope and purpose & $97.22 \%$ & $100 \%$ & $83.33 \%$ & $88.89 \%$ & $19.44 \%$ \\
\hline $\begin{array}{l}\text { 2.Stakeholder } \\
\text { Involment }\end{array}$ & $88.89 \%$ & $80.56 \%$ & $77.78 \%$ & $69.44 \%$ & $30.56 \%$ \\
\hline $\begin{array}{l}\text { 3.Rigour and } \\
\text { Development }\end{array}$ & $79.17 \%$ & $75.00 \%$ & $73.96 \%$ & $67.71 \%$ & $54.16 \%$ \\
\hline $\begin{array}{l}\text { 4. Clarity of } \\
\text { Presentation }\end{array}$ & $97.22 \%$ & $100 \%$ & $80.56 \%$ & $69.44 \%$ & $61.11 \%$ \\
\hline 5.Applicability & $68.75 \%$ & $58.33 \%$ & $54.17 \%$ & $41.67 \%$ & $27.08 \%$ \\
\hline $\begin{array}{l}\text { 6.Editorial } \\
\text { Independence }\end{array}$ & $68.75 \%$ & $75.00 \%$ & $62.5 \%$ & $62.50 \%$ & $58.33 \%$ \\
\hline Rate Overall & 7 & 6 & 5 & 5 & 3 \\
\hline Recommendation & Yes & Yes & Yes & Yes & $\begin{array}{l}\text { Yes with } \\
\text { modifications }\end{array}$ \\
\hline
\end{tabular}

Conclusion: Conclusions: The selected CPG have good methodological quality, but are not specific to probiotics. Despite, they should be spread for better decision making.

Disclosure of Interest: A. Maruy Saito: CONSULTANT BIOCODEX SPEAKER MEAD JOHNSON NUTRITION

All other authors have declared no conflicts of interest.

\section{References}

1. AGREE Next Steps Consortium (2009) Update 2013. The AGREE II Instrument [Electronic version]. Retrieved: March, 10, 2017, from http:// www.agreetrust.org.

2. National Collaborating Centre for Women's and Children's Health (UK). Diarrhoea and vomiting caused by gastroenteritis: diagnosis, assessment and management in children younger than 5 years. NICE Clinical Guidelines, No. 84. London: RCOG Press; 2009. Available from: https://www.nice.org.uk/guidance/cg84.

3. Gonzales C, Bada C et al. Guía de Práctica Clínica sobre el Diagnóstico y Tratamiento de la Diarrea Aguda Infecciosa en Pediatría Perú-2011. Rev. Gastroenterol. Perú; 2011; 31-3: 258-277.

4. Guarino A, Ashkenazi S, et al. ESPGHAN/ESPID evidence-based guidelines for the management of acute gastroenteritis in children in Europe: update 2014. JPGN 2014 Jul; 59(1):132-52.

5. Polanco Allué I, Salazar-Lindo E, Gutiérrez-Castrellón P; Grupo IberoLatinoamericano sobre el Manejo de la Diarrea Aguda (GILA). [IberoLatin American clinical practice guide on the management of acute gastroenteritis in children under 5 years: diagnostic and health care criteria]. $A n$ Pediatr (Barc). 2014;80 Suppl 1:5-8.

6. Szajewska H, Guarino A, et al. Use of probiotics for management of acute gastroenteritis: a position paper by the ESPGHAN Working Group for Probiotics and Prebiotics. JPGN 2014 Apr;58(4):531-9.

P1271 COMBINATION OF TWO IN VITRO MODELS TO STUDY THE IMPACT OF CHRONIC CO-EXPOSURE OF A PESTICIDE WITH A PREBIOTIC ON THE INTESTINAL ENVIRONMENT

M. Requile ${ }^{1}$, D.O. Gonzalez Alvarez ${ }^{1}$, S. Delanaud ${ }^{1}$, L. Rhazi ${ }^{2}$, V. Bach ${ }^{1}$, F. Depeint ${ }^{2}$, H. Khorsi-Cauet ${ }^{1}$

${ }^{1}$ PERITOX UMR-I101 INERIS; Université Picardie Jules Verne, Amiens/France ${ }^{2}$ Unité Transformation et Agroressources; Institut Polytechnique UniLaSalle,

Beauvais/France

Contact E-mail Address: marina.requile@u-picardie.fr

Introduction: The excessive use of pesticides, often found as residues in our diet and as contaminants in drinking water, has become a public health problem. Most of these substances are considered as endocrine disruptors and their daily exposure is likely to have severe and irreversible consequences. Indeed, preliminary studies have shown that chronic exposure to low doses of chlorpyrifos (CPF) causes intestinal imbalance (dysbiosis) in vitro.

Aims \& Methods: The objective of this study is to evaluate the preventive potential of a prebiotic (inulin) in co-exposure with the CPF on the intestinal dysbiosis, the bacterial translocation and the integrity of the intestinal mucosa. For this we

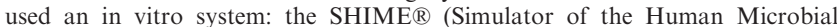


Intestinal Ecosystem). The SHIME® consists of series of fermenters, mimicking the human intestinal environment from the stomach to the colon.

The SHIME® was exposed to a daily dose of $3.5 \mathrm{mg}$ of CPF, combined with $10 \mathrm{~g}$ of inulin for 30 days. The samples were collected at day 0 (baseline, without CPF or inulin), D15 and D30 to determine the profile and microbial metabolism.

Results: Contrary to the previous results with CPF alone showing dysbiosis, prebiotic supplementation seems to reestablish CPF-induced imbalance, particularly in the potentially pathogenic microflora (Staphylococcus), which an increase in short term (D15: $p<0.001$ ), and a recovery at D30. (difference vs D0: NS). The same happened for the beneficial flora (Bifidobacteria).

Inulin also beneficially influences the fermentation profile, with higher production of volatile fatty acids (VFA), especially propionic acid and butyric acid.

Conclusion: The $\mathrm{CPF}$ /inulin co-exposure therefore had a positive impact on bacterial profile and metabolism, suggesting that prebiotic supplementation could reduce some intestinal damages caused by an exposure to CPF.

Perspective: In a second step, the Caco-2/TC7 cell line, which mimics a functional human intestinal epithelium, will be exposed to samples from the colic fermenters of the SHIME $(\mathrm{D} 0, \mathrm{CPF} \pm$ inulin at D15 and D30) in porder to mesure the impact on the epithelial barrier.

Disclosure of Interest: All authors have declared no conflicts of interest.

\section{References}

Joly Condette C, Elion Dzon B, Hamdad F, Biendo M, Bach V, Khorsi-Cauet H. Use of molecular typing to investigate bacterial translocation from the intestinal tract of chlorpyrifos-exposed rats. Gut Pathog. 2016;8:50

Joly Condette C, Bach V, Mayeur C, Gay-Quéheillard J, Khorsi-Cauet H. Chlorpyrifos Exposure During Perinatal Period Affects Intestinal Microbiota Associated With Delay of Maturation of Digestive Tract in Rats. J Pediatr Gastroenterol Nutr. 2015 Jul;61(1):30-40.

Joly C, Gay-Quéheillard J, Léké A, Chardon K, Delanaud S, Bach V, KhorsiCauet H. Impact of chronic exposure to low doses of chlorpyrifos on the intestinal microbiota in the Simulator of the Human Intestinal Microbial Ecosystem (SHIME®) and in the rat. Environ Sci Pollut Res. 2013 May 1;20(5):2726-34 Reygner J, Lichtenberger L, Elmhiri G, Dou S, Bahi-Jaber N, Rhazi L, KhorsiCauet H. Inulin Supplementation Lowered the Metabolic Defects of Prolonged Exposure to Chlorpyrifos from Gestation to Young Adult Stage in Offspring Rats

\section{P1272 THE DIET FEATURES OF DIARRHEA PREDOMINANT IRRITABLE BOWEL SYNDROME PATIENTS WITH SMALL INTESTINAL BOWEL OVERGROWTH}

H. Wei, Z. Liu, K. Wang, W. Zheng, L. Duan

Gastroenterology, Peking University Third Hospital, Beijing/China

Contact E-mail Address: weihui2012@bjmu.edu.cn

Introduction: Lactulose methane and hydrogen breath test(LMHBT) is now widely used in assessing small bowel bacterial overgrowth(SIBO) and irritable bowel syndrome(IBS). SIBO and IBS have similar clinical symptoms such as floating, diarrhea and abdominal pain. Although there is no clear relationship between IBS and SIBO yet, they are both in relationship with dysbiosis. Diet is associated with disease closely, especially for gastrointestinal diseases. Different dietary structures have different effects on the structure, metabolism and function of intestinal microbiota. Reduce fermentable oligosaccharides, disaccharides, monosaccharides and polyols (FODMAPs) diet can release parts of IBS symptoms obviously. There were rare reports for the effects of diet on LMHBT, especially for IBS-D with SIBO patients, nor sufficient researches of the effects of diet on intestinal microbiota profiles in IBS-D or SIBO patients. High fat diet may change the intestinal microbiota pofiles and SCFAs was reported in the past researches, most of which were animal experiments, lacking of effects diet on the clinical manifestations and intestinal microbiota profiles in patients with IBS-D, especially for high-fat diet.

Aims \& Methods: We aimed to analyze the characteristics of diet and lactulose methane and hydrogen breath test (LMHBT) in patients of diarrhea predominant irritable bowel syndrome (IBS-D) with small intestinal bacterial overgrowth (SIBO), and comparing with health controls. IBS-D patients (1865 years old), who met Rome III criteria were enrolled in Gastrointestinal outpatient clinic of Peking University Third Hospital from. Health volunteers were enrolled as controls (HC). All subjects underwent colonoscopy to excluded colonic organic disorders. All subjects completed the case report form (CRF), IBS symptom severity scale (IBS-SSS), the MOS item short form health survey (SF36). The patients were divided into IBS-D with SIBO (IBS-P) group and IBS-D without SIBO (IBS-N) group according to the LMHBT.Diet information was collected by food frequency questionnaire (FFQ) and three days recalls. The high fat diet is defined as the daily total calories supplying from fat is more than $50 \%$. Statistical analysis were performed by SPSS 20.0 software, $\mathrm{P}<0.05$ was considered statistically significant.

Results: Eighty-eight IBS-D patients and $32 \mathrm{HC}$ were enrolled. The positive rate of LMHBT in IBS-D was significantly higher than that of HC $[39.8 \%(35 / 88)$ vs. $12.5 \%(4 / 28), \mathrm{P}=0.005]$. The $28 \mathrm{HC}$ with negative LMHBT were enrolled in the follow-up analysis. (1) There were no significant differences in age and gender ratio among IBS-P, IBS-N and HC. The BMI of IBS-P was significantly lower than IBS-N [(21.57 \pm 0.54$)$ vs. $\left.(23.30 \pm 0.53) \mathrm{kg} / \mathrm{m}^{2}, \mathrm{P}=0.032\right]$. High fat diet IBS-D patients and along with IBS-P had higher abdominal pain scores. (2)The proportion of dietary protein and carbohydrate in IBS-D was significantly higher than that of $\mathrm{HC}(14.39 \%$ vs. $12.22 \%, \mathrm{P}=0.001 ; 53.94 \%$ vs. $46.25 \%$, $\mathrm{P}=0.003$, respectively), while the intake of fiber was a little lower than $\mathrm{HC}$ $[(16.85 \pm 1.54)$ vs. $(21.29 \pm 3.07) \mathrm{g} / \mathrm{d}, \mathrm{P}=0.169]$. The proportion of diet fat was significantly higher in IBS-P than IBS-N $[(47.19 \pm 2.62) \%$ vs. $(40.74 \pm 1.66) \%$, $\mathrm{P}=0.038]$. IBS-P had more high fat diet individuals than IBS-N $[37.1 \%(13 / 35)$ vs. $20.8 \%(11 / 53), \mathrm{P}=0.088]$. (3) The positive rate of LMHBT in IBS-D was significantly higher than that of $\mathrm{HC}[39.8 \%$ vs. $12.5 \%, \mathrm{P}=0.005]$. IBS-D patients with high fat diet had higher LMHBT positive rate than that of non-high fat diet patients $[54.2 \%(13 / 24)$ vs. $17.2 \%(11 / 64), \mathrm{P}=0.001)]$. The baseline of breath methane in IBS-P was significantly higher than that of in IBS-N $[(8.69 \pm 0.39)$ vs. $(6.39 \pm 0.47) \mathrm{ppm}, \mathrm{P}=0.002]$. Breath methane peak value was positively related with the fat proportion of diet $(r=0.413, P=0.022)$.

Conclusion: About $39.8 \%$ IBS-D patients existed SIBO. IBS-D patients with high fat diet had higher morbidity of SIBO, and the proportion of diet fat was positively correlated with breath methane baseline of LMHBT. SIBO can worsen nutritional status for IBS-D patients. High-fat diet might be one of the risk factors for IBS-D with SIBO.

Disclosure of Interest: All authors have declared no conflicts of interest.

\section{P1273 SYSTEMATIC REVIEW AND META-ANALYSIS PREVALENCE OF SMALL INTESTINAL BACTERIAL OVERGROWTH IN CHRONIC LIVER DISEASE}

A. Shah ${ }^{1}$, P. Ghasemi ${ }^{2}$, E. Shanahan ${ }^{1}$, G. Macdonald ${ }^{1}$, L. Fletcher ${ }^{1}$, M. Morrison ${ }^{3}$, M.P. P. Jones ${ }^{4}$, G. Holtmann

${ }^{1}$ Department Of Gastroenterology \& Hepatology, University of Queensland, Faculty of Medicine and Biomedical Sciences, Australia \& Metro South Health Service \& Translational Research Institute, Brisbane/Australia

${ }^{2}$ Department Of Gastroenterology And Hepatology, Princess Alexandra Hospital, Oueensland Health, Brisbane/Australia

${ }^{3}$ University of Queensland, Diamantina Institute, Microbial Biology and Metagenomics, Brisbane/Australia

${ }^{4}$ Psychology, Macquarie University Psychology, North Ryde/Australia

Contact E-mail Address: ayesha17@gmail.com

Introduction: Alterations to the gut microbiota and bacterial translocation have been implicated as relevant factors for the progression of chronic liver disease (CLD). While the sequence of events leading to translocation remains unclear, deficiencies in local host immune defences, increased permeability of the intestinal mucosal barrier and dysbiosis of the gut microbiota are suggested to play a role. Small intestinal bacterial overgrowth (SIBO), in which an excessive and/or abnormal type of bacteria is present in the small bowel has been implicated as a potential factor in translocation. However, systematic assessments of the extent of SIBO in CLD remain limited. We therefore aimed to compare the prevalence of small intestinal bacterial overgrowth (SIBO) in patients with chronic liver disease (CLD) and controls.

Aims \& Methods: Using the search terms 'small intestinal bacterial overgrowth (SIBO)' and 'chronic liver disease (CLD)' or 'small intestinal bacterial overgrowth (SIBO)' and 'cirrhosis', 19 case-control studies that met inclusion criteria were identified. Data were extracted to calculate prevalence rates and $95 \%$ confidence intervals $(\mathrm{CI})$.

Results: The final dataset included 1,000 adult patients with CLD and 488 controls. Nine studies employed glucose breath tests (GBT), four lactulose breath tests (LBT), with one each of glycine-1-14 ${ }^{\mathrm{C}}$-labeled glycocholate and ${ }^{14} \mathrm{C}-\mathrm{D}$ xylose breath test. Five studies utilised culture methods and one quantitative PCR. Across all testing methods, the prevalence of SIBO in patients with CLD was $38.9 \%(95 \%$ CI $36.9-42.0)$ versus $9.8 \%$ (95\% CI $7.5-12.8)$ in controls. Prevalence of SIBO in CLD was increased as compared to controls ( $\mathrm{RR}=7.15,95 \%$ CI 4.9-10.4). In patients with cirrhosis the prevalence of SIBO was $40.1 \%$ (95\% CI 36.6-43.8) compared to $7.3 \%$ (95\% CI $4.9-10.8$ ) in the respective controls. When the method of detection was limited to breath tests, the prevalence of SIBO in CLD was $35.8 \%$ (95\% CI 32.6-39.1) compared to $8.0 \%$ (95 CI 5.7-11.0) in controls. In contrast, based upon culture techniques, the prevalence of SIBO in CLD was $68.3 \%(95 \%$ CI $59.62-76.0)$ vs $7.94 \%(95 \%$ CI 3.44-12.73) in controls.

Conclusion: Regardless of the diagnostic modality, prevalence of SIBO is significantly increased in patients with CLD when compared to controls. It is notable that culture-based detection leads to a higher prevalence in CLD, suggesting breath tests may underestimate overall prevalence. Given the levels of SIBO detected, further studies need to explore the role of intestinal dysbiosis for the progression of CLD.

Disclosure of Interest: All authors have declared no conflicts of interest.

\section{P1274 IS THERE AN ASSOCIATION BETWEEN ENTERIC METHANE (CH4) PRODUCTION AND SYMPTOMS IN PATIENTS WITH UNEXPLAINED GI SYMPTOMS?}

A. Shah ${ }^{1}$, P. Ghasemi ${ }^{2}$, M. Morrison ${ }^{3}$, L. Fletcher ${ }^{1}$, G. Holtmann ${ }^{1}$, T. Hansen ${ }^{4}$ ${ }^{1}$ Department Of Gastroenterology \& Hepatology, University of Queensland, Faculty of Medicine and Biomedical Sciences, Australia \& Metro South Health Service \& Translational Research Institute, Brisbane/Australia

${ }^{2}$ Department Of Gastroenterology And Hepatology, Princess Alexandra Hospital, Queensland Health, Brisbane/Australia

${ }^{3}$ University of Queensland, Diamantina Institute, Microbial Biology and

Metagenomics, Brisbane/Australia

${ }^{4}$ Princess Alexandra Hospital, Department of Gastro and Hepatology,

Woolloongabba/Australia/QLD

Contact E-mail Address: ayesha17@gmail.com 
Introduction: In humans, enteric methane $(\mathrm{CH} 4)$ production is highly variable and related to the gastrointestinal microbiome and diet. Previous work suggests that $\mathrm{CH} 4$ production is more common in patients with 'constipating' conditions such as encopresis and diverticulosis. We aimed to explore the link between gastrointestinal symptoms breath $\mathrm{CH} 4$ exhalation in patients with unexplained GI symptoms.

Aims \& Methods: Consecutive patients $(\mathrm{n}=100)$ with unexplained GI symptoms underwent a combined $\mathrm{H} 2 / \mathrm{CH} 4$ breath test after ingestion of $75 \mathrm{~g}$ of glucose. $\mathrm{H} 2$ and $\mathrm{CH} 4$ were measured by Breathtreacker microlyser (Quintron, USA) Gastrointestinal symptoms were assessed utilising the (Structured Assessment of Gastrointestinal Symptoms Instrument (SAGIS). The association between methane exhalation and symptoms during the 2 weeks prior the test were evaluated using non parametric test.

Results: 100 consecutive patients (55f), aged $52.2 \pm 15.7$ yrs (mean \pm SD) were included. Of these, 14 with positive GBT and 19 without SAGIS data were excluded, resulting in 67 data-sets available for analysis. Methane peak and methane baseline values were highly correlated $(\mathrm{r}=0.96, \mathrm{p}<0.001)$. Methane peak (and baseline) were inversely correlated with the SAGIS diarrhoea score $(-0.35, \mathrm{p}<0.01$, Figure 1$)$. Contrary to current opinion, $\mathrm{CH} 4$ exhalation was not associated with constipation $(\mathrm{r}<0.1, \mathrm{P}>0.4)$. In addition, excessive belching and acid eructation were significantly associated with the baseline and peak $\mathrm{CH} 4$ exhalation $(\mathrm{r}$ all $\geq 0.3$, $\mathrm{p}$ all $<0.04$ ).

Conclusion: There is an inverse association between $\mathrm{CH} 4$ exhalation and diarrhoea symptoms. At the same time, $\mathrm{CH} 4$ is associated with bloating and acid eructation. These data suggest that $\mathrm{CH} 4$ or metabolic products from $\mathrm{CH} 4$ producing microbes modulate human gut function.

Disclosure of Interest: All authors have declared no conflicts of interest.

\section{P1275 CELIAC DISEASE AND POSITIVE IGA TISSUE}

TRANSGLUTAMINASE IN PATIENTS WITH DISTAL

\section{RADIUS AND ANKLE FRACTURE: A CASE-CONTROL STUDY}

A. M. Hjelle ${ }^{1}$, P. F. Mielnik ${ }^{1}$, G. S. Tell ${ }^{2}$, K.E.a. Lundin ${ }^{3}$, R. M. Nilsen ${ }^{4}$, E. Apalset ${ }^{5}$

${ }^{1}$ Departement Of Rheumatology, District General Hospital of Foerde,

FØRDE/Norway

${ }^{2}$ Departement Of Global Public Health And Primary Care, University of Bergen, Bergen/Norway

${ }^{3}$ Gastroenterology, Oslo University Hospital, Oslo/Norway

${ }^{4}$ Department Of Health And Social Sciences, Western Norway University of Applied Sciences, Bergen/Norway

${ }^{5}$ Departement Of Rheumatology, Haukeland University Hospital, Bergen/Norway

Contact E-mail Address: myhreanja@hotmail.com

Introduction: The prevalence of osteoporosis is higher among patients suffering a distal radius fracture than in healthy controls [1]. Celiac disease (CD) is associated with low bone mineral density [2], and overall findings indicate an increased risk of fracture in CD patients [3, 4]. This study is to our knowledge the first case control study investigating whether there is a higher prevalence of $\mathrm{CD}$ in adult patients suffering a peripheral fracture (distal radius or ankle) than in healthy age- and sex matched controls.

Aims \& Methods: Main objective was to investigate if patients with a recent fracture of the distal radius or ankle have a higher risk of having CD than healthy controls. 400 consecutive patients over the age of 40 with acute distal radius fracture $(\mathrm{n}=293)$ or ankle fracture $(\mathrm{n}=107)$ were included in a case control study by referral from the orthopedic department at Forde General Hospital, Norway. The controls were 197 age- and sex-matched subjects from Sogn and Fjordane County identified from the National Population Registry, with no previous fracture history. BMD of the hips and spine was measured and history of previous fracture, comorbidities, medication, life-style factors, body mass index (BMI) and nutritional factors were registered. Serum analysis to detect secondary osteoporosis including IgA tissue transglutaminase was performed.

Results: See table.

\begin{tabular}{lllll}
\hline & $\begin{array}{l}\text { Ankle } \\
\text { fracture }\end{array}$ & $\begin{array}{l}\text { Distal } \\
\text { radius } \\
\text { fracture }\end{array}$ & $\begin{array}{l}\text { All } \\
\text { fractures }\end{array}$ & Controls \\
\hline $\mathrm{N}$ & 107 & 293 & 400 & 197 \\
Fracture cases \% & 26.8 & 73.3 & & \\
Female gender \% & 69.1 & 80.2 & 77.3 & 82.7 \\
T-score $<-2,5 \%$ & 23.4 & 44.7 & 39.0 & 22.3 \\
T-score $-1.0-2,5 \%$ & 36.4 & 33.8 & 34.5 & 34.0 \\
Mean age & 62.9 & 57.1 & 61.4 & 60.3 \\
Mean BMI & 26.5 & 28.6 & 27.1 & 27.2 \\
Known CD $\%$ & $0(\mathrm{n}=0)$ & $1.4(\mathrm{n}=4)$ & $1.0(\mathrm{n}=4)$ & $1.5(\mathrm{n}=3)$ \\
Positive TTG $\%$ & $2.8(\mathrm{n}=3)$ & $2.4(\mathrm{n}=7)$ & $2.5(\mathrm{n}=10)$ & $1.3(\mathrm{n}=2)$ \\
Known CD or & $2.8(\mathrm{~N}=3)$ & $3.8(\mathrm{n}=11)$ & $3.5(\mathrm{n}=14)$ & $2.8(\mathrm{n}=5)$ \\
$\quad$ positive TTG & & & & 5.6 \\
Vitamin D & 6.6 & 2.4 & 3.6 & \\
$\quad$ deficiency & & & \\
\hline
\end{tabular}

${ }^{1}$ body mass index ${ }^{2}$ celiac disease ${ }^{3} \mathrm{IgA}$ tissue transglutaminase ${ }^{4} \mathrm{Vit} \mathrm{D}<37 \mathrm{nmol} / 1$
Conclusion: The prevalence of celiac disease was in preliminary analyses not significantly higher among persons suffering a distal fracture $(3.5 \%)$ than among healthy age- and sex- matched controls $(2.8 \%)$. Osteoporosis and low Vitamin D levels are significant risk factors for distal radius fracture, but this is not the case with ankle fractures.

Disclosure of Interest: All authors have declared no conflicts of interest.

\section{References}

1. Oyen, J., et al., Osteoporosis as a risk factor for distal radial fractures: a casecontrol study. J Bone Joint Surg Am, 2011. 93(4): p. 348-56.

2. Bianchi, M.L. and M.T. Bardella, Bone in celiac disease. Osteoporos Int, 2008. 19(12): p. 1705-16.

3. Olmos, M., et al., Systematic review and meta-analysis of observational studies on the prevalence of fractures in coeliac disease. Dig Liver Dis, 2008. 40(1): p. 46-53.

4. Hjelle, A.M., et al., Celiac disease and risk of fracture in adults-a review. Osteoporos Int, 2014. 25(6): p. 1667-76.

\section{P1276 INTERLEUKIN 1BETA, CLAUDIN AND OCCLUDIN} CONCENTRATIONS IN SERUM AND AUTONOMIC NERVOUS SYSTEM ACTIVITY AMONG NEUROLOGICAL ASYMPTOMATIC CELIAC DISEASE PATIENTS-PILOT STUDY

M. Zwolinska-Wcislo ${ }^{1}$, M. Przybylska-Feluś ${ }^{1}$, A. Furgała ${ }^{1}$, B. Brzozowski ${ }^{1}$, O. Kaczmarczyk ${ }^{2}$, K. Gil ${ }^{1}$, T. Mach ${ }^{1}$

${ }^{1}$ Department of Clinical Dietetics, Chair of Gastroenterology, Hepatology and Infectious Diseases, Jagiellonian University Medical College, Krakow, Poland, Krakow/Poland

${ }^{2}$ University Hospital in Krakow, Poland, Krakow/Poland

Contact E-mail Address: mzwcislo@su.krakow.pl

Introduction: Celiac disease (CeD) is an immune disorder, triggered by gluten intolerance in genetically susceptible patients. Clinical outcome includes several extraintestinal manifestations, including neurologic complications in about $10 \%$ of patients. Previous studies confirmed the subclinical changes of autonomic nervous system (ANS) activity resulting to impaired gastric myoelectric activity. In $\mathrm{CeD}$ disturbances of parasympathetic-sympathetic balance of the ANS activity with sympathetic dominance is observed. Tight junctions impairment is one of postulated pathomechanism in $\mathrm{CeD}$ and its complications. Transmembrane proteins of tight junctions include claudin and occludin. There is lack of publications referring to soluble claudin and occludin concentrations in CeD. Interleukin-1 beta (IL-1 beta) is an important mediator of the, and there are no data concerning on its influence on gastric myoelectric activity and ANS activity in CeD.

Aims \& Methods: The study was aimed to evaluate occludin, claudin and IL1 beta concentrations in serum in neurologically asymptomatic patients with $\mathrm{CeD}$ and its correlation with selected parameters of ANS activity markers (heart rate variability, electogastrography $\mathrm{HRV}$ ) and gastric myoelectric activity (EGG). Thirty four patients with $\mathrm{CeD}(70 \%$ females, mean age 34 years, $41.2 \pm 16.6)$ without neurological symptoms were tested for occludin, claudin, IL-1beta concentrations in serum using ELISA, and HRV and EGG.

Results: Biochemical parameters. Patients with $\mathrm{CeD}$ presented with lower average level of occludin $(1.41(0-2.9) \mathrm{ng} / \mathrm{ML})$ than healthy subjects $(1.68(0.39-4.8) \mathrm{ng} /$ ML) $(P=0.07$, the Mann-Whitney test). No significant impact of CeD on the average results of IL- 1 beta concentrations was observed $(P=0.44$, the MannWhitney test). The rest HRV. In the celiac group the assessment of HRV revealed a negative statistically significant correlation of claudin concentration and very low frequencies (VLF-RRI; Spearman's rank correlation coefficient: $r=-0.51$, $P=0.018)$ as well as positive correlation between IL-1 beta and $\mathrm{LF} / \mathrm{HF}$ was demonstrated $(\mathrm{r}=0.51, P=0.032)$. Statistically significant, negative and strong correlation of IL-1 beta concentration and DP (Dominant Power of EGG) $(r=-$ $0.58, P=0.038$ ) was shown.

Conclusion: ANS activity measured by EGG and HRV seems to be correlated to presence of IL-1beta. In celiac group the serum concentration of claudin and occludin do not correlate to ANS activity. Due to former research on autonomic imbalance in $\mathrm{CeD}$ with sympathetic overdrive, the hypothesis of influence of IL-1 beta on ANS activity should be considered.

Disclosure of Interest: All authors have declared no conflicts of interest.

\section{P1277 WIDE HETEROGENEITY AND HIGH MORTALITY IN UNDEFINED AND NON-COELIAC REFRACTORY SPRUE: A RETROSPECTIVE EVALUATION OF 7 CASES}

A. Schiepatti, F. Biagi, M. Zuffada, G. Maiorano, G.R. Corazza University of Pavia, Pavia/Italy

\section{Contact E-mail Address: salinana $@$ hotmail.it}

Introduction: Small bowel villous atrophy (VA) is mainly related to coeliac disease (CD) that develops in HLA DQ2/DQ8 positive patients and improves on a gluten-free diet. Other forms of VA unrelated to CD are common variable immune-deficiency, autoimmune enteropathy, small bowel malignancies, medication-related enteropathies, HIV, tropical sprue, and giardiasis [1-3]. However, there are also forms of VA in which CD can be neither confirmed nor excluded and there are forms of VA in which, although $\mathrm{CD}$ is excluded, a definitive diagnosis cannot be made. Some years ago, we coined the terms undefined sprue (US) and non-coeliac refractory sprue (NCRS) to define these two 
conditions [4]. Although other Authors described similar patients [2,3], it is still unclear whether US and NCRS are two independent conditions or whether they are two faces of the same coin. Alternatively, they could be umbrella terms covering one or more atrophic enteropathies still awaiting to be identified, as was the case of olmesartan-associated enteropathy until a few years ago.

Aims \& Methods: Our aim was to clarify the nature of these forms of VA. We retrospectively re-evaluated the clinical notes of patients referred to our Clinic from January 1999 to October 2016 with a previous diagnosis of refractory CD. Patients with VA in whom CD could not be confirmed and in whom other causes of VA were ruled out were selected.

Results: 7 patients $(2 \mathrm{~F}$, age at diagnosis of VA $46 \pm 26$ years, all with severe malabsorption) were identified. 3 were DQ2 positive and so were diagnosed as US: case 1 (F, 32 years) died of ulcerative jejunitis 8 years later; case $2(\mathrm{M}, 38)$ died of pulmonary embolism complicating systemic candidiasis 1 year later and case $3(\mathrm{M}, 71)$ died of intestinal lymphoma after 4.5 years. The remaining 4 patients were HLA DQ2/DQ8 negative, so a diagnosis of NCRS was made. 3 were DQ7.5 positive: case 4, (F, 61) died of EATL type 2 after 4.5 years; case 5 $(\mathrm{M}, 25)$ is still alive 10 years after diagnosis of VA and in good clinical conditions; case $6(\mathrm{M}, 69)$ is still alive but could be affected by a form of enteropathy due to candesartan. He is in good clinical condition and has been refusing followup. The last patient, case $7(\mathrm{M}, 29)$ is DQ7.3 positive. He is still alive 11 years after the diagnosis of VA, although complains of diarrhoea.

Conclusion: We described 7 patients with VA unrelated to CD or other known enteropathies. Although overall mortality among them is very high $(57 \%)$ and mainly due to lymphoproliferative disorders, 3 of the 4 DQ2/DQ8 negative patients are alive many years after the diagnosis of VA with no evidence of malignancy. This suggest that these 7 patients are not affected by the same condition. We speculate that DQ2 positive patients who died of lymphoma could be affected by a form of $\mathrm{CD}$ who escaped diagnosis and get complicated. However, we feel that the 3 DQ2/8 negative patients with long survival are affected by a still unidentified form of VA.

Disclosure of Interest: All authors have declared no conflicts of interest

\section{References}

1. Schiepatti A et al. Eur J Gastroenterol Hepatol 2017;29:572-6

2. Aziz I et al. Gut 2016 doi: 10.1136/gutjnl-2016-312271

3. DeGaetani M et al. Am J Gastroenterol 2013;108:647-53

4. Biagi F et al. Eur J Gastroenterol Hepatol 2001;13:561-5

\section{P1278 EVERYDAY LIFE RESTRICTIONS CAUSED BY LONG-TERM TREATED CELIAC DISEASE: PREVALENCE AND ASSOCIATED FACTORS}

L. Kivelä ${ }^{1}$, S. Alin ${ }^{1}$, S. Kröger ${ }^{1}$, K. Kaukinen ${ }^{2}$, K. Kurppa

${ }^{1}$ Tampere Center For Child Health Research, University of Tampere, University of Tampere/Finland

${ }^{2}$ School Of Medicine, University of Tampere, Tampere/Finland

Contact E-mail Address: laura.kivela@ fimnet.fi

Introduction: Strict gluten-free diet (GFD) in celiac disease is burdensome and difficult to maintain, which might predispose to poor dietary adherence and impaired quality of life. We aimed to evaluate adult patients' experience of living with celiac disease diagnosed in childhood, and identify factors associated with possible everyday life restrictions caused by the disease.

Aims \& Methods: 232 adults (women 69\%, median age $27.0 \mathrm{yr}$ ) with a childhood diagnosis of celiac disease fulfilled a questionnaire evaluating their experiences about general health and lifestyle, possible co-morbidities, adherence and attitudes towards GFD and long-term follow-up of celiac disease. In addition, they completed validated Gastrointestinal Symptom Rating Scale (GSRS) and Psychological General Well-Being (PGWB) surveys for gastrointestinal symptoms and quality of life. Patient records were used to confirm clinical and histological presentation at diagnosis and other relevant medical data.

Results: Altogether $108(47 \%)$ out of the 232 responders felt that celiac disease restricts their daily life. This was experienced especially when eating in a restaurant $(72 \%)$, traveling abroad $(38 \%)$ and visiting a friend $(30 \%)$. Patients reporting restrictions had more often anemia $(38 \%$ vs $22 \%, \mathrm{p}=0.013)$ and severe symptoms $(16 \%$ vs $6 \%, \mathrm{p}=0.047)$ at diagnosis, whereas the groups did not differ in age, gender or other clinical and histological presentation. Current age (median 26.3 vs $27.7 \mathrm{yr}, \mathrm{p}=0.315$ ) and time from the diagnosis (18.6 vs $17.9 \mathrm{yr}$, $\mathrm{p}=0.468$ ) were also comparable, as well as were self-experienced general health and concern about health, presence of co-morbidities and complications, smoking, physical exercise, socioeconomic status, membership of celiac society and presence of celiac disease in relatives. There was also no difference in specific gastrointestinal symptoms as measured by GSRS scores, but patients considering the disease restrictive reported more overall symptoms possibly related to celiac disease than those without restrictions $(32 \%$ vs $17 \%, \mathrm{p}=0.007)$. Furthermore, despite similar dietary adherence (strict GFD $78 \%$ vs $82 \%, p=0.770$ ) they experienced adhering to the diet more challenging (somewhat difficult $33 \%$ vs $7 \%, \mathrm{p}<0.001$ ) and had significantly lower PGWB vitality scores (median 17 vs $18, \mathrm{p}=0.023)$.

Conclusion: Almost half of the patients diagnosed in childhood experienced celiac disease to cause marked restrictions in adulthood. This was associated with current symptoms, lower vitality scores and difficulties to maintain GFD. Patients with severe symptoms and anemia at diagnosis might require special attention and tailored follow-up in these circumstances.

Disclosure of Interest: All authors have declared no conflicts of interest.
P1279 SYSTEMATIC REVIEW OF THE ECONOMIC BURDEN OF COELIAC DISEASE

T. Boulanger ${ }^{1}$, A. J. Taylor ${ }^{2}$, D. A. Leffler ${ }^{3}$, E. S. Mearns ${ }^{1}$, M. Gerber ${ }^{3}$

${ }^{1}$ IBM Watson Health, Boston/United States of America/MA

${ }^{2}$ Takeda, London/United Kingdom

${ }^{3}$ Takeda, Cambridge/United States of America/MA

Contact E-mail Address: aliki.taylor@takeda.com

Introduction: The prevalence of diagnosed coeliac disease (CD) has rapidly increased in developed countries over the last 30 years. The economic burden of diagnosing, managing, and monitoring $\mathrm{CD}$ can be substantial but is poorly understood. To assess the economic burden of $\mathrm{CD}$, we systematically reviewed current evidence quantifying economic costs and health resource utilization (HRU) for CD in North America and Europe.

Aims \& Methods: Searches of Medline, Embase, EconLit, the Cochrane Library, and conference abstracts systematically identified literature published in English during the last 10 years assessing direct and indirect costs, cost-effectiveness analyses, and HRU for CD.

Results: Of 33 studies meeting criteria for inclusion, most (20) were from Europe, and most (18) reported or modeled costs associated with screening and diagnosis. Cost per positive $C D$ diagnosis of screening patients already undergoing upper gastrointestinal biopsy for other indications, such as anaemia or irritable bowel syndrome, ranged from approximately $\$ 1,300$ in Canada to more than $€ 44,000$ in the Netherlands (costing year not reported). In these populations, screening was judged to be cost-effective with various strategies combining diagnostic modalities, including serology then biopsy, compared to no screening. Strategies using either endoscopy/biopsy or serology alone were not considered cost-effective. Direct annual excess costs to a US payer per diagnosed CD patient totaled $\$ 4,000$ (\$US 2007) to $\$ 6,000$ (\$US 2013) more than for a person without $\mathrm{CD}$, chiefly due to outpatient care, with higher costs among patients with poor disease control. High use of outpatient care is also reflected in studies of HRU, although hospitalization, emergency visits and medication use are also more common in individuals with CD than in controls. After initiation of a glutenfree (GF) diet, patients visit primary care providers less often, but use more medications. Patients often pay out of pocket for gluten-free (GF) foods, which cost $240-518 \%$ more than gluten-containing equivalents. Three studies on absenteeism from Scandinavia found fewer days missed from school and work following diagnosis and initiation of a GF diet.

Conclusion: Most economic studies of CD have focused on the cost of screening and diagnosis, especially in Europe. Methods of screening generally are considered cost-effective when they combine diagnostic modalities, such as serology then biopsy, in people being evaluated for symptoms. Much of the cost to a payer of managing $\mathrm{CD}$ derives from outpatient care, especially for patients with poorly controlled disease. Patients on a GF diet lose fewer days from work and school but pay high costs for GF foods.

Disclosure of Interest: A.J. Taylor: Aliki Taylor is employed by Takeda Development Centre Europe, London, UK

D.A. Leffler: Daniel Leffler is employed by Takeda Pharmaceuticals International Co, Cambridge, USA

M. Gerber: Michele Gerber is employed by Takeda Pharmaceuticals International $\mathrm{Co}$, Cambridge, USA

All other authors have declared no conflicts of interest.

P1280 PNPLA3 RS738409 POLYMORPHISM PREDICTS THE DEVELOPMENT AND THE SEVERITY OF HEPATIC STEATOSIS, BUT NOT METABOLIC SYNDROME, IN PATIENTS WITH CELIAC DISEASE

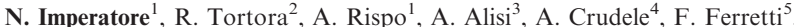
V. Nobili ${ }^{4}$, L. Miele ${ }^{5}$, N. Gerbino ${ }^{1}$, V. Di Martino ${ }^{1}$, N. Caporaso ${ }^{1}$, F. Morisco ${ }^{1}$ ${ }^{1}$ Gastroenterology, Department of Clinical Medicine and Surgery, School of Medicine Federico II of Naples, Naples/Italy

${ }^{2}$ Hepatology Unit, Cardarelli Hospital, NAPLES/Italy

${ }^{3}$ 2Liver Research Unit - Bambino Gesù Children's Hospital, IRCCS, Rome, Rome/Italy

${ }^{4}$ Hepato-metabolic Unit - Bambino Gesù Children's Hospital, IRCCS, Rome, Rome/Italy

${ }^{5}$ Catholic University, Rome, Rome/Italy

Contact E-mail Address: nicola.imperatore $@$ alice.it

Introduction: Metabolic syndrome (MS) and hepatic steatosis (HS) are frequent in patients with celiac disease (CD) after commencing gluten-free diet (GFD), but data about predictive factors for such a condition are still scarce.

Aims \& Methods: We aimed to evaluate the role of PNPLA3 rs738409 in the development of MS and HS in CD patients after starting GFD. From June 2014 to September 2016 we consecutively enrolled all patients referred to academic gastroenterological centre, suffering from $\mathrm{CD}$, with our without HS. All patients underwent anthropometrics and serological investigations, ultrasonography (US) evaluation to assess the degree and severity of HS and genotyping of PNPLA3 rs738409 polymorphism

Results: Finally, 370 subjects were enrolled (136 with HS and 234 without HS). At genotyping assays, CC genotype was found in 194 subjects $(52.4 \%)$, CG genotype in 138 subjects $(37.3 \%)$, while 38 individuals $(10.2 \%)$ showed the GG genotype. At binary logistic regression, only $\mathrm{G}$ and $\mathrm{GG}$ alleles were predictive for the development of HS (OR 1.97; p $<0.01$ for G carriers and OR 6.9; $p<0.001$ for GG carriers), while BMI (OR 3.8; p < 0.001) and waist circumference (OR 2.8; $\mathrm{p}=0.03$ ) at $\mathrm{CD}$ diagnosis were the only independent factors for the development of MS. The intergroup comparison among HS patients showed that 
the severe grade of HS was more frequently observed in GG than in CC carriers (74\% vs $11.3 \%, \mathrm{p}<0.001$, OR 21.8$)$.

Conclusion: PNPLA3 G and GG carriers with CD have higher susceptibility to hepatic steatosis, but not to metabolic syndrome. Moreover, patients with GG alleles display a more relevant disease in terms of severity of HS based on US evaluation.

Disclosure of Interest: All authors have declared no conflicts of interest

\section{P1281 PROTON PUMP INHIBITORS AS RISK FACTOR FOR METABOLIC SYNDROME AND HEPATIC STEATOSIS IN PATIENTS WITH CELIAC DISEASE ON GLUTEN-FREE DIET}

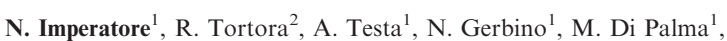
V. Di Martino ${ }^{1}$, N. Caporaso ${ }^{3}$, A. Rispo ${ }^{3}$

${ }^{1}$ Gastroenterology, Department of Clinical Medicine and Surgery, School of Medicine Federico II of Naples, Naples/Italy

${ }^{2}$ Hepatology Unit, Cardarelli Hospital, Naples/Italy

${ }^{3}$ Department Of Clinical Medicine And Surgery, Gastroenterology Unit AOU Federico II, Naples/Italy

Contact E-mail Address: nicola.imperatore@alice.it

Introduction: Recent research has shown that patients with celiac disease (CD) are at-risk of developing metabolic syndrome (MS) and hepatic steatosis (HS) after commencing gluten-free diet.

Aims \& Methods: We aimed to evaluate the predictive factors for MS and HS in $\mathrm{CD}$ after one year of GFD. All consecutive newly diagnosed CD patients were enrolled. We prospectively collected data about: BMI; waist circumference; blood pressure; cholesterol; triglycerides, glucose and insulin blood levels; insulin resistance (through the homeostatic model assessment HOMA-IR); treatment with proton pump inhibitors (PPI). Diagnosis of MS was made in accordance with current guidelines and HS was diagnosed by ultrasonography. The prevalence of MS and HS was re-assessed after 1 year of GFD. A logistic regression analysis was performed to identify risk factors for MS and HS occurrence after 1 year of GFD

Results: Of 301 patients with newly diagnosed CD, $13(4.3 \%)$ met criteria for diagnosis of MS and $25.9 \%$ presented HS at the time of CD diagnosis; 99 subjects $(32.8 \%)$ had long-term exposure to PPI during the study period. After 1 year, $72(23.9 \%)$ patients had developed MS $(4.3 \%$ vs $23.9 \% ; p<0.001$, OR 6.9$)$ and $112(37.2 \%)$ had developed HS $(25.9 \%$ vs $37.2 \% ; p<0.01$, OR 1.69). At multivariate analysis, high BMI at diagnosis (OR 10.8; $\mathrm{p}<0.001)$ and PPI exposure (OR 22.9; $\mathrm{p}<0.001)$ were the only factors associated with the occurrence of MS; HOMA-IR (OR 9.7; p < 0.001) and PPI exposure (OR 9.2; $\mathrm{p}<0.001)$ were the only factors associated with the occurrence of $\mathrm{HS}$

Conclusion: PPI exposure adds further risk of occurrence of MS and HS for patients with $\mathrm{CD}$ on GFD. The use of PPI in patients with CD on GFD should be limited to strict indications.

Disclosure of Interest: All authors have declared no conflicts of interest.

P1282 HOW MANY HOURS DO STABLE COELIAC PATIENTS TAKE UP IN GASTROENTEROLOGY OUT-PATIENT CLINICS?

M. Yalchin, R. Eckersley, T. Coysh, S. Mann

Gastroenterology, Barnet Hospital, DJ/United Kingdom

Contact E-mail Address: m.yalchin@nhs.net

Introduction: Patients with coeliac disease $(C D)$ are usually stable clinically and biochemically at their annual review. Due to pressures on out-patient services we reviewed data to assess which patients actually require specialist gastrointestinal (GI) input, and raise the question on who ought to see CD patients in clinics.

Aims \& Methods: Barnet Hospital is a District Hospital serving a population of 500,000 just outside North West London. This was a single-centre, retrospective study, of confirmed CD patients, under the care of three different gastroenterologists. Data was obtained from each consultant from their database. We reviewed the electronic records relating to each patient's last three clinic appointments, and assessed whether or not they were stable clinically and biochemically. We excluded patients with other significant GI co-morbidities and who were newly diagnosed within the last two years.

Results: There were 192 patients with CD of whom, 160 fulfilled the inclusion criteria. Female $=107 ; 67 \%$. In each of the last 3 clinic reviews $83 \%(n=133)$ were stable. Only $17 \%(\mathrm{n}=27)$ required specialist GI input; including $18 \%$ $(n=7)$ due to poor adherence to a gluten-free diet, $28 \%(n=11)$ due to GI symptoms, $18 \%(\mathrm{n}=7)$ due to weight loss, $18 \%(\mathrm{n}=7)$ due to anaemia, $8 \%$ $(\mathrm{n}=3)$ due to elevated TTG and $10 \%(\mathrm{n}=4)$ other. Annual reviews by a gastroenterologist occurred in $83 \%(n=133)$. Dieticians saw patients less than once per year in $62.5 \%(n=100)$ of cases, and $37.5 \%(n=60)$ were actually never referred to a dietician.

Conclusion: BSG guidelines recommend that patients with CD are seen annually by a dietician and/or clinician for a clinical review, biochemical checks and for dietary adherence and advice ${ }^{1}$. NICE guidelines also state that patients should be seen annually for clinical review, height and weights check, bloods and to assess the need for nutritional advice ${ }^{2}$. We have demonstrated that in a hospital that does not have a dietician-led coeliac service, gastroenterologists typically see straight forward and stable patients annually $83 \%$ of the time. Assuming a 10 minute consultation, this equates to 22 hours per anum of specialist time, equivalent to 6.3 clinics a year. Given the need to access our services for urgent referrals or unwell patients with other conditions, this study forms the basis for a business case to set up a dietician-led coeliac service, with access to gastroenterologists where needed.

Disclosure of Interest: All authors have declared no conflicts of interest.

\section{References}

1. Coeliac disease: recognition, assessment and management. NICE guideline [NG20]. Sept 2015

2. Ludvigsson JF, Bai JC, Biagi F, Card TR, Ciacci C, Ciclitira PJ, Green PH, Hadjivassiliou M, Holdoway A, van Heel DA, Kaukinen K, Leffler DA, Leonard JN, Lundin KE, McGough N, Davidson M, Murray JA, Swift GL, Walker MM, Zingone F, Sanders DS. Diagnosis and management of adult coeliac disease: guidelines from the British Society of Gastroenterology. Gut 2014;63:1210-28.

\section{P1283 MONITORING PATIENTS WITH COELIAC DISEASE: WHO} ACTUALLY NEEDS A DEXA SCAN?

M. Yalchin, R. Eckersley, T. Coysh, S. Mann

Gastroenterology, Barnet Hospital, DJ/United Kingdom

Contact E-mail Address: m.yalchin@nhs.net

Introduction: Patients with coeliac disease (CD) should be seen annually for a clinical review, blood tests and a DEXA scan if needed ${ }^{1,2}$. The indication for a DEXA scan is unclear due to conflicting recommendations in current guidelines $^{2,3}$. The aim of our study was to audit our practice, with a focus on requests for DEXA scans.

Aims \& Methods: This was a single-centre, retrospective study of CD patients under the care of 3 consultants. We accessed the electronic records to identify if haematological and biochemical profiles were being monitored annually. We also identified when patients had their first DEXA scans and whether or not they were indicated ${ }^{2,3}$.

Results: Data were collected on 160 patients $($ Female $=107[67 \%])$. Annual checks of FBC occurred in $94 \%$ of patients, vitamin B12 in $74 \%$, folate in $77 \%$, ferritin in $88 \%$, calcium in $85 \%$ and vitamin $\mathrm{D}$ in $69 \%$. DEXA scans occurred in $74 \%$ of patients $(n=119)$, including $65 \%(n=77)$ who were screened around the time of diagnosis. However, only $24 \%(n=28)$ actually warranted the scan according to guidelines 2,3 , and $68 \%(\mathrm{n}=81)$ did not fulfil criteria for a DEXA. In $8 \%$ of patients $(n=10)$, there was inadequate data. Of the 81 patients who did not warrant a DEXA scan, 77 results were available: normal in $48 \%$ $(n=37)$, osteopenia in $43 \%(n=33)$ and osteoporosis in $9 \%(n=7)$. Of the 7 patients that had osteoporosis, 4 patients were under 50 years old $(57 \%)$. Of the appropriate DEXA requests, $25 \%(\mathrm{n}=7)$ were normal, $39 \%(\mathrm{n}=11)$ had osteopenia and $36 \%(\mathrm{n}=10)$ had osteoporosis.

Conclusion: Most CD patients require very little clinical input at their routine appointments. Annual blood checks and adherence to a gluten-free diet are standard enquiries. However, there is a cohort of patients who are not getting their regular blood tests: $33 \%$ for bone profile and $25 \%$ for haematinics. Clinicians tend to order a DEXA in most CD patients because it is easier than attempting to judge an individual's risk in the setting of conflicting guidance $^{2,3}$. The pick-up rate of osteoporosis in $36 \%$ of appropriately screened patients (vs $9 \%$ in inappropriate scan requests) suggests that targeted screening allows for a more rational and cost-effective use of a limited resource. We hope that the guidelines can now be updated with more clarity for the practitioners who request DEXA scans in CD patients.

Disclosure of Interest: All authors have declared no conflicts of interest.

\section{References}

1. Coeliac disease: recognition, assessment and management. NICE guideline [NG20]. Sept 2015

2. Ludvigsson JF, Bai JC, Biagi F, Card TR, Ciacci C, Ciclitira PJ, Green PH, Hadjivassiliou M, Holdoway A, van Heel DA, Kaukinen K, Leffler DA, Leonard JN, Lundin KE, McGough N, Davidson M, Murray JA, Swift GL, Walker MM, Zingone F, Sanders DS. Diagnosis and management of adult coeliac disease: guidelines from the British Society of Gastroenterology. Gut 2014;63:1210-28.

3. Osteoporosis: assessing the risk of fragility fracture. NICE Guideline (CG146). Aug 2012.

\section{P1284 CELIAC DISEASE AND ADHERENCE TO THE} GLUTEN-FREE DIET: A 30-YEAR FOLLOW-UP STUDY

\section{F. Branchi ${ }^{1}$, M. Bravo ${ }^{1}$, L. Norsa ${ }^{1}$, F. Ferretti ${ }^{2}$, M.T. Bardella ${ }^{3}$, D. Conte ${ }^{4}$,} L. Elli ${ }^{3}$

${ }^{1}$ Center For The Prevention And Diagnosis Of Celiac Disease, Gastroenterology And Endoscopy Unit, Fondazione IRCCS Ca' Granda Ospedale Maggiore

Policlinico, Milan/Italy

${ }^{2}$ Department Of Pathophysiology And Transplantation, Università degli Studi di Milano, Milan/Italy

${ }^{3}$ Gastroenterology And Endoscopy Unit, Fondazione Irccs Cà Granda Ospedale Maggiore Policlinico, Center for the Diagnosis and Prevention of Celiac Disease, Milan/Italy

${ }^{4}$ Department Of Pathophysiology And Transplantation, Università Degli Studi Di Milano, Milan- Italy, Gastroenterology and Endoscopy Unit, Fondazione IRCCS Ca' Granda Ospedale Maggiore Policlinico, Milan/Italy 
Contact E-mail Address: federica.branchi@gmail.com

Introduction: A life-long gluten-free diet (GFD) is the only available treatment for patients with celiac disease (CD). Adherence to the GFD is associated with symptoms remission, reversal of mucosal atrophy and, possibly, prevention of CD-related complications. However, data on the long-term effects of a good or poor adherence to the GFD are limited.

Aims \& Methods: The aim of this study was to assess the rate and accuracy of compliance to a strict GFD in patients with a CD history of more than 30 years and to compare endpoints such as complications, symptoms and histology between patients following a strict GFD and patients not compliant to the diet. Between 2015 and 2016, data from all patients diagnosed with CD at the Fondazione IRCCS Ca' Granda before 1985 were retrieved. Patients not undergoing regular follow-up at the clinic were contacted in order to collect recent clinical updates as regards symptoms, adherence to the GFD, laboratory data and complications occurrence. All patients were asked to fill in a questionnaire investigating their knowledge on gluten free products and behavioral rules; in case of patients with $\mathrm{CD}$ diagnosis in childhood, the same questionnaire was administered to their parents.

Results: Clinical data from 196 patients were collected and analyzed. Patients were divided into 3 groups according to their adherence to GFD: 133 patients reporting a lifelong strict GFD, 29 patients on GFD at the time of follow-up but with a history of at least 5 years of gluten-containing diet (GCD), and 35 patients who reported to be on a GCD. No significant differences were found between groups regarding symptoms and histology at diagnosis, onset of associated autoimmune disorders, family history of $\mathrm{CD}$ and compliance to follow up. The onset of complications at follow-up did not significantly differ in the three groups as well. Follow-up histology was available in 63 patients $(32.1 \%)$. Persistence of villous atrophy was as expected more frequent in patients on GCD as on GFD $(41 \%$ vs $7 \%, p=0.008)$; however 20 patients showed normal histology during long-term GCD. The questionnaire was returned by 90 patients and 66 parents: a slightly better knowledge about the GFD and its behavioral rules was found between patients on lifelong GFD and patients with ongoing or past GCD $(\mathrm{p}=0.03)$.

Conclusion: Poor adherence to the GFD is reported by almost one-third of patients with a long-term history of $\mathrm{CD}$, confirming the high rate of poor compliance to such a strict diet among patients. Poor adherence to the GFD was confirmed as a major predictor of persistence of villous atrophy, but this does not necessarily imply the development of CD complications. Moreover, results from follow up biopsies showed that a GCD does not imply recurrence of villous atrophy in all patients, attesting the possibility that some $\mathrm{CD}$ patients may maintain a gluten tolerance over time.

Disclosure of Interest: All authors have declared no conflicts of interest.

\section{References}

1. Ciacci C, Cirillo M, Cavallaro R, Mazzacca G. Long-term follow-up of celiac adults on gluten-free diet: prevalence and correlates of intestinal damage. Digestion 2002; 66(3): 178-185

2. Errichiello S, Esposito O, Di Mase R, Camarca ME, Natale C, Limongelli MG, Marano C, Coruzzo A, Lombardo M, Strisciuglio P, Greco L. Celiac disease: predictors of compliance with a gluten-free diet in adolescents and young adults. J Pediatr Gastroenterol Nutr 2010; 50(1): 54-60

3. Matysiak-Budnik T, Malamut G, de Serre NP, Grosdidier E, Seguier S, Brousse N, Caillat-Zucman S, Cerf-Bensussan N, Schmitz J, Cellier C. Long-term follow-up of 61 coeliac patients diagnosed in childhood: evolution toward latency is possible on a normal diet. Gut 2007; 56(10): 1379-1386

\section{P1285 PATIENTS WITH SHORT BOWEL SYNDROME STRATIFIED BY BASELINE PARENTERAL SUPPORT VOLUME: POST HOC ANALYSIS OF THE CLINICAL EFFECT OF TEDUGLUTIDE}

P.B. Jeppesen ${ }^{1}$, S. M. Gabe ${ }^{2}$, D. L. Seidner ${ }^{3}$, H. Lee ${ }^{4}$, C. Olivier ${ }^{5}$ ${ }_{1}^{1}$ Rigshospitalet, Copenhagen/Denmark

${ }^{2}$ St Mark's Hospital, Northwick Park, London/United Kingdom

${ }^{3}$ Vanderbilt University Medical Center, Nashville/United States of America/TN

${ }^{4}$ Shire Human Genetic Therapies, Inc., Lexington/United States of America/MA ${ }^{5}$ Shire International GmbH, Zug/Switzerland

Contact E-mail Address: Palle.Bekker.Jeppesen @ regionh.dk

Introduction: Parenteral support (PS) volume needs vary depending on disease severity in patients with intestinal failure associated with short bowel syndrome (SBS-IF). Patient classification has focused on the diagnosis that led to resection and the remnant bowel anatomy.

Aims \& Methods: Recently, the idea that grading severity of SBS-IF is based on magnitude of PS volume needs led to this clinical trial data post hoc analysis of patients with SBS-IF based on their baseline PS volume. STEPS (NCT00798967; EudraCT2008-006193-15) was a 24-week, placebo-controlled study of teduglutide (TED) $0.05 \mathrm{mg} / \mathrm{kg} /$ day in patients with SBS-IF. Three baseline PS volume groups were evaluated: Group I, $\leq 9 \mathrm{~L} /$ week; Group II, $>9$ to $\leq 18 \mathrm{~L} /$ week; and Group III, >18 L/week

Results: The predominant diagnosis leading to SBS-IF in Group I $(12 / 28 ; 43 \%)$ and Group II $(15 / 41 ; 37 \%)$ was vascular gut complications; in Group III $(8 / 16$; $50 \%$ ), Crohn's disease. Baseline PS volume and TED-induced volume reduction (\% change) at Week 24 was highest in Group III (Table). Evaluation of individual patient response showed a close, linear, and significant correlation between absolute PS volume reduction at Week 24 in relation to TED treatment and daily volume at baseline $\left(\mathrm{y}=-0.387 \mathrm{x}+90.03, \mathrm{R}^{2}=0.61 ; P<0.0001\right)$; no significant correlation was observed in the placebo group $\left(y=-0.06 x-220.15, R^{2}=0.02\right.$;
$P=0.36$ ). Adverse events were reported by $93 \%$ (Group I), $80 \%$ (Group II), and $71 \%$ (Group III) of TED patients.

Table: Baseline PS Volume and Percent Change at Week 24

\begin{tabular}{|c|c|c|c|c|c|c|}
\hline \multirow{3}{*}{$\begin{array}{l}\text { Mean (SD) } \\
\text { PS Volume, } \\
\text { Actual Based }\end{array}$} & \multicolumn{6}{|c|}{ Baseline PS Volume } \\
\hline & \multicolumn{2}{|c|}{$\leq 9 \mathrm{~L} /$ week } & \multicolumn{2}{|c|}{$>9-\leq 18 \mathrm{~L} /$ week } & \multicolumn{2}{|c|}{$>18 \mathrm{~L} /$ week } \\
\hline & $\begin{array}{l}\text { TED } \\
(\mathrm{n}=15)\end{array}$ & $\begin{array}{l}\mathrm{PBO} \\
(\mathrm{n}=13)\end{array}$ & $\begin{array}{l}\text { TED } \\
(\mathrm{n}=20)\end{array}$ & $\begin{array}{l}\mathrm{PBO} \\
(\mathrm{n}=21)\end{array}$ & $\begin{array}{l}\text { TED } \\
(\mathrm{n}=7)\end{array}$ & $\begin{array}{l}\mathrm{PBO} \\
(\mathrm{n}=9)\end{array}$ \\
\hline Baseline, $\mathrm{mL} /$ day & $\begin{array}{l}806.9 \\
(328.11)\end{array}$ & $\begin{array}{l}856.1 \\
(310.24)\end{array}$ & $\begin{array}{l}1791.8 \\
(338.33)\end{array}$ & $\begin{array}{l}1870.2 \\
(408.27)\end{array}$ & $\begin{array}{l}3826.1 \\
(715.49)\end{array}$ & $\begin{array}{l}3527.4 \\
(749.04)\end{array}$ \\
\hline $\begin{array}{l}\text { Change at } \\
\text { Week 24, \% }\end{array}$ & $\begin{array}{l}-28.5 \\
(18.45)^{*}\end{array}$ & $\begin{array}{l}-29.2 \\
(35.49)^{\dagger}\end{array}$ & $\begin{array}{l}-33.5 \\
(20.97)^{*}\end{array}$ & $\begin{array}{l}-19.1 \\
(20.40)^{3}\end{array}$ & $\begin{array}{l}-36.7 \\
(13.97)\end{array}$ & $\begin{array}{l}-14.9 \\
(17.07)^{\S}\end{array}$ \\
\hline
\end{tabular}

$\mathrm{PBO}=$ placebo. ${ }^{*} \mathrm{n}=13 ;{ }^{\dagger} \mathrm{n}=12 ;{ }^{\star} \mathrm{n}=19,{ }^{\S} \mathrm{n}=8$.

Conclusion: Higher baseline PS volume in patients with SBS-IF correlates with greater absolute reduction in PS volume with TED treatment. This research was funded by Shire International GmbH, Zug, Switzerland.

Disclosure of Interest: P.B. Jeppesen: I have served as a consultant and on the speaker bureau for Shire.

S.M. Gabe: I have served as a consultant for Shire.

D.L. Seidner: I have served as a consultant for Shire.

H. Lee: I am an employee for Shire.

C. Olivier: I am an employee for Shire.

P1286 POST HOC ANALYSIS OF THE RELATIONSHIP BETWEEN PLASMA CITRULLINE AND PARENTERAL SUPPORT NEEDS IN PATIENTS WITH SHORT BOWEL SYNDROME WITH INTESTINAL FAILURE RECEIVING TEDUGLUTIDE

P.B. Jeppesen ${ }^{1}$, S. M. Gabe ${ }^{2}$, D. L. Seidner ${ }^{3}$, H. Lee ${ }^{4}$, C. Olivier ${ }^{5}$

${ }^{1}$ Rigshospitalet, Copenhagen/Denmark

${ }^{2}$ St Mark's Hospital, Northwick Park, London/United Kingdom

${ }^{3}$ Vanderbilt University Medical Center, Nashville/United States of America/TN

${ }^{4}$ Shire Human Genetic Therapies, Inc., Lexington/United States of America/MA

${ }^{5}$ Shire International $\mathrm{GmbH}, \mathrm{Zug} /$ Switzerland

Contact E-mail Address: Palle.Bekker.Jeppesen@regionh.dk

Introduction: Plasma citrulline has been proposed as a biomarker for remnant intestinal length, but it is unclear if plasma citrulline levels reflect intestinal absorptive function.

Aims \& Methods: This post hoc analysis investigated the relationship between reductions in parenteral support (PS) volume and changes in plasma citrulline levels with teduglutide (TED) in patients with intestinal failure associated with short bowel syndrome (SBS-IF). STEPS (NCT00798967; EudraCT2008006193-15) was a 24-week, placebo-controlled study of TED $0.05 \mathrm{mg} / \mathrm{kg} / \mathrm{day}$ in patients with SBS-IF. Plasma citrulline levels were assessed at baseline and Week 24 in all patients randomised to TED and in patients stratified by bowel anatomy.

Results: In the TED arm $(n=42)$, plasma citrulline levels at baseline were significantly correlated with remnant small bowel length $\left(\mathrm{R}^{2}=0.14 ; P=0.02\right.$; $\mathrm{n}=36)$ but not with baseline PS volume $\left(\mathrm{R}^{2}=0.03 ; P=0.30 ; \mathrm{n}=39\right)$. The correlation between baseline plasma citrulline and plasma citrulline change at Week 24 was significant $\left(\mathrm{R}^{2}=0.80 ; P=<0.0001 ; \mathrm{n}=39\right)$. No correlation was found between change in plasma citrulline levels and change in PS volume at Week 24 with TED $\left(\mathrm{R}^{2}=0.05 ; P=0.16 ; \mathrm{n}=39\right)$. When patients were analysed by bowel anatomy subgroups, significant increases in plasma citrulline were seen with TED but not placebo (Table).

Table: Mean (SD) Change From Baseline at Week 24 in PS Volume and Plasma Citrulline Stratified by Bowel Anatomy

\begin{tabular}{|c|c|c|c|c|c|c|}
\hline \multirow[b]{2}{*}{$\begin{array}{l}\text { Mean (SD) } \\
\text { Change at } \\
\text { Week } 24\end{array}$} & \multicolumn{2}{|c|}{$\begin{array}{l}\text { No Colon, } \\
\text { Stoma Present, } \\
\text { No Colon-in- } \\
\text { Continuity }\end{array}$} & \multicolumn{2}{|c|}{$\begin{array}{l}\geq 50 \% \text { Colon, } \\
\text { No Stoma, } \\
\text { Colon-in- } \\
\text { Continuity }\end{array}$} & \multicolumn{2}{|l|}{$\begin{array}{l}\text { Other } \\
\text { Bowel } \\
\text { Anatomies }\end{array}$} \\
\hline & $\begin{array}{l}\text { TED } \\
\left(\mathrm{n}=17^{*}\right)\end{array}$ & $\begin{array}{l}\mathrm{PBO} \\
\left(\mathrm{n}=16^{\dagger}\right)\end{array}$ & $\begin{array}{l}\text { TED } \\
(\mathrm{n}=18)\end{array}$ & $\begin{array}{l}\mathrm{PBO} \\
(\mathrm{n}=20)\end{array}$ & $\begin{array}{l}\text { TED } \\
(\mathrm{n}=7)\end{array}$ & $\begin{array}{l}\mathrm{PBO} \\
\left(\mathrm{n}=7^{\star}\right)\end{array}$ \\
\hline $\begin{array}{l}\text { PS Volume } \\
\mathrm{mL} / \text { day }\end{array}$ & $-919(644)^{\S}$ & $-340(436)$ & $-355(306)$ & $-327(349)$ & $-728(532)$ & $-297(498)$ \\
\hline$\%$ & $-40(18)^{\|}$ & -19 (29) & $-23(16)$ & $-24(22)$ & $-40(19)$ & $-19(31)$ \\
\hline $\begin{array}{l}\text { Citrulline } \\
\mu \mathrm{M}\end{array}$ & $30.1(18.9)^{\S}$ & $2.2(6.9)$ & $11.8(9.9)^{\S}$ & $0.8(5.3)$ & $24.0(20.9)^{\S}$ & $-2.7(7.6)$ \\
\hline$\%$ & $138(50)^{\S}$ & $8(31)$ & $87(63)^{\S}$ & $21(71)$ & $116(85)^{\S}$ & $-1(40)$ \\
\hline
\end{tabular}

$\mathrm{PBO}=$ placebo.

${ }^{*} \mathrm{n}=14$ with data; ${ }^{\dagger} \mathrm{n}=13$ with data; ${ }^{*} \mathrm{n}=6$ with data; ${ }^{\S} P \leq 0.01 ;{ }^{\prime \prime} P<0.05$.

Conclusion: Plasma citrulline may serve as a marker of remnant small bowel length in patients with SBS-IF, but plasma citrulline does not predict PS 
volume reductions with TED. Plasma citrulline changes with TED may reflect increased enterocyte mass. This research was funded by Shire International $\mathrm{GmbH}$, Zug, Switzerland.

Disclosure of Interest: P.B. Jeppesen: I have served as a consultant and on the speaker bureau for Shire.

S.M. Gabe: I have served as a consultant for Shire.

D.L. Seidner: I have served as a consultant for Shire.

H. Lee: I am an employee for Shire.

C. Olivier: I am an employee for Shire.

P1287 LACTULOSE, LACTOSE AND FRUCTOSE INGESTION

INDUCES SPECIFIC PATTERNS OF GASTROINTESTINAL SYMPTOMS IN CHINESE SUBJECTS WITH FUNCTIONAL DYSPEPSIA AND IRRITABLE BOWEL SYMPTOMS

V. Tan $^{1}$, N. H.S. Chu ${ }^{1}$, C. K. Yao ${ }^{1}$, P.R. Gibson ${ }^{2}$

${ }^{1}$ The University of Hong Kong, Hong Kong/Hong Kong PRC

${ }^{2}$ Dept. Of Gastroenterology, Alfred Hospital Dept. of Gastroenterology,

Melbourne/Australia

\section{Contact E-mail Address: vpytan@hku.hk}

Introduction: Prevalence rates of Functional Dyspepsia (FD) in East Asia are three times higher than Irritable Bowel Syndrome (IBS) rates. Many researchers have suggested that IBS subjects in the region experience their pain and discomfort in the upper abdomen, leading to misdiagnosis as FD.

Aims \& Methods: We aimed to compare patterns of gastrointestinal (GI) symptom genesis in the Chinese population with FD or IBS during provocative hydrogen breath testing (HBT) with lactulose, lactose and fructose. Subjects fulfilling the ROME III classification of FD and IBS, and control subjects with no known GI disorder/symptoms were recruited. All subjects underwent HBT $+/-$ methane assessment with lactulose $(10 \mathrm{ml})$, lactose $(25 \mathrm{~g})$ and fructose $(25 \mathrm{~g})$. Subsequent breath tests were performed after a washout period of at least one week. Breath tests were performed after an overnight fast, with the patient sedentary. Breath samples taken every 15 minutes for $3 \mathrm{~h}$. GI symptoms were recorded during these $3 \mathrm{~h}$ and at a telephone follow-up 24 hours later.

Results: A total of 353 subjects completed at least one breath test examination and 313 subjects completed all three breath tests. $16 \%, 55 \%$ and $29 \%$ were control, FD and IBS subjects. All study subjects were ethnic Chinese, the mean age was 53 [Range; $18-76$ ) y and $27 \%$ [95\% CI; 23-32\%] were male. $85 \%[95 \% \mathrm{CI} ; 82-89 \%]$ of subjects were hydrogen-producers and $100 \%$ methane-producers. Symptoms were induced in a relatively low proportion of healthy controls. Both FD and IBS subjects experienced similar proportions of epigastric pain on consumption of lactulose, lactose and fructose. See Table 1. Subjects with FD experience more belching than subjects with IBS when lactulose [58 vs. $42 \%, p=0.011]$ and lactose $[62$ vs. $46 \%, p=0.014]$ were ingested, respectively. Subjects with IBS experience significantly more "lower GI" symptoms of abdominal pain and development of loose stools when lactulose was ingested when compared with subjects with FD. In general subjects with IBS experienced both epigastric pain and abdominal pain when any of the three carbohydrate solutions were ingested. Healthy controls experienced minimal symptoms.

Conclusion: Chinese subjects commonly co-produced hydrogen and methane. Ingestion of poorly absorbed sugars induces symptom patterns in patients with FD in similar proportions. Chinese IBS subjects commonly experienced epigastric and abdominal pain. Reducing poorly absorbed short-chain carbohydrates (FODMAPs) might be efficacious in FD as it is in IBS

Disclosure of Interest: All authors have declared no conflicts of interest.

Table 1: Induction of Gastrointestinal Symptoms with Lactulose, Lactose and Fructose Hydrogen Breath Testing in Functional Dyspepsia and Irritable Bowel Syndrome Subjects

\begin{tabular}{lccc}
\hline & $\begin{array}{l}\text { Functional } \\
\text { Dyspepsia }\end{array}$ & $\begin{array}{l}\text { Irritable Bowel } \\
\text { Syndrome }\end{array}$ & P-value \\
\hline Lactulose \% [95\% Confidence Interval] & & \\
Epigastric Pain & $40 \%[33-47 \%]$ & $29 \%[20-38 \%]$ & 0.056 \\
Belching & $58 \%[51-65 \%]$ & $42 \%[32-52 \%]$ & 0.011 \\
Abdominal Pain & $4 \%[1-7 \%]$ & $37 \%[28-47 \%]$ & $<0.0001$ \\
Flatulence & $34 \%[27-40 \%]$ & $27 \%[18-36 \%]$ & 0.246 \\
Loose Stools & $19 \%[14-25 \%]$ & $30 \%[21-39 \%]$ & 0.045 \\
Lactose \% [95\% Confidence Interval] & & \\
Epigastric Pain & $44 \%[36-52 \%]$ & $41 \%[31-52 \%]$ & 0.692 \\
Belching & $62 \%[55-69 \%]$ & $46 \%[36-56 \%]$ & 0.014 \\
Abdominal Pain & $5 \%[2-8 \%]$ & $35 \%[25-44 \%]$ & $<0.0001$ \\
Flatulence & $50 \%[42-58 \%]$ & $50 \%[40-60 \%]$ & 1.00 \\
Loose stools & $28 \%[21-35 \%]$ & $38 \%[27-48 \%]$ & 0.109 \\
Fructose \% $[95 \%$ Confidence Interval] & & \\
Epigastric Pain & $37 \%[30-45 \%]$ & $26 \%[16-35 \%]$ & 0.060 \\
Belching & $52 \%[44-59 \%]$ & $31 \%[35-56 \%]$ & 0.331 \\
Abdominal Pain & $23 \%[1-7 \%]$ & $77 \%[18-37 \%]$ & $<0.001$ \\
Flatulence & $27 \%[20-34 \%]$ & $23 \%[14-32 \%]$ & 0.507 \\
Loose Stools & $19 \%[13-25 \%]$ & $29 \%[20-39 \%]$ & 0.082 \\
\hline
\end{tabular}


were evaluated: Group 1 (no colon/stoma present/no colon-in-continuity), Group $2(\geq 50 \%$ colon/no stoma/colon-in-continuity), and Group 3 (other bowel anatomies). Clinical response was defined as $\geq 20 \%$ reduction from baseline in weekly parenteral support (PS) volume at Weeks 20-24. Data presented as mean (SD).

Results: The predominant diagnosis in Group 1 was Crohn's disease, whereas the predominant diagnosis in Group 2 was vascular complications (Table). Group 1 patients required the highest baseline PS volumes compared with Group 2 or Group 3. TED-induced PS volume reduction (change in $\mathrm{L} /$ week) took longer to be realised in Group 2 (Week 12, -0.9 [1.2]; Week 24, -2.5 [2.1]) compared with Group 1 (Week 12, -5.5 [3.8]; Week 24, -6.4 [4.5]) or Group 3 (Week 12, -2.7 [1.2]; Week 24, -5.1 [3.7]). Response rates were higher with TED versus placebo in all groups, but the difference was significant only in Group $1(76 \%$ vs $19 \%$, $P=0.001$; Group 2, 56\% vs $40 \%, P=0.36$; Group 3, $57 \%$ vs $29 \%, P=0.33$ ). Adverse events were reported by $94 \%, 72 \%$, and $86 \%$ of Group 1, Group 2, and Group 3 patients receiving TED, respectively.

Table: Baseline Patient Characteristics

\begin{tabular}{|c|c|c|c|c|c|c|}
\hline \multirow[b]{2}{*}{ Parameter } & \multicolumn{2}{|c|}{$\begin{array}{l}\text { Group 1: No Colon, } \\
\text { Stoma Present, } \\
\text { No Colon-in- } \\
\text { Continuity }\end{array}$} & \multicolumn{2}{|c|}{$\begin{array}{l}\text { Group 2: } \\
\geq 50 \% \text { Colon, } \\
\text { No Stoma, } \\
\text { Colon-in- } \\
\text { Continuity }\end{array}$} & \multicolumn{2}{|c|}{$\begin{array}{l}\text { Group 3: } \\
\text { Other Bowel } \\
\text { Anatomies }\end{array}$} \\
\hline & $\begin{array}{l}\text { TED } \\
(\mathrm{n}=17)\end{array}$ & $\begin{array}{l}\text { PBO } \\
(\mathrm{n}=16)\end{array}$ & $\begin{array}{l}\text { TED } \\
(\mathrm{n}=18)\end{array}$ & $\begin{array}{l}\text { PBO } \\
(\mathrm{n}=20)\end{array}$ & $\begin{array}{l}\text { TED } \\
(\mathrm{n}=7)\end{array}$ & $\begin{array}{l}\mathrm{PBO} \\
(\mathrm{n}=7)\end{array}$ \\
\hline $\begin{array}{l}\text { Mean (SD) } \\
\text { age, year }\end{array}$ & $\begin{array}{l}52.1 \\
(10.3)\end{array}$ & $\begin{array}{l}50.7 \\
(12.9)\end{array}$ & $\begin{array}{l}51.3 \\
(14.9)\end{array}$ & $\begin{array}{l}50.3 \\
(18.7)\end{array}$ & $\begin{array}{l}49.6 \\
(11.7)\end{array}$ & $\begin{array}{l}46.0 \\
(13.2)\end{array}$ \\
\hline \multicolumn{7}{|l|}{ Sex, n $(\%)$} \\
\hline Men & $8(47)$ & $7(44)$ & $9(50)$ & $10(50)$ & $3(43)$ & $2(29)$ \\
\hline Women & $9(53)$ & $9(56)$ & $9(50)$ & $10(50)$ & $4(57)$ & $5(71)$ \\
\hline $\begin{array}{l}\text { Mean (SD) } \\
\text { BMI, } \mathrm{kg} / \mathrm{m}^{2}\end{array}$ & $\begin{array}{l}23.6 \\
(3.6)\end{array}$ & $\begin{array}{l}22.4 \\
(3.2)\end{array}$ & $\begin{array}{l}22.1 \\
(2.9)\end{array}$ & $\begin{array}{l}22.3 \\
(3.0)\end{array}$ & $\begin{array}{l}20.8 \\
(2.1)\end{array}$ & $\begin{array}{l}22.0 \\
(3.6)\end{array}$ \\
\hline \multicolumn{7}{|l|}{$\begin{array}{l}\text { Cause of SBS-IF, n } \\
(\%)\end{array}$} \\
\hline Crohn's disease & $9(53)$ & $7(44)$ & 0 & 0 & $1(14)$ & $1(14)$ \\
\hline $\begin{array}{l}\text { Vascular } \\
\quad \text { complications }\end{array}$ & $2(12)$ & $2(13)$ & $9(50)$ & $13(65)$ & $2(29)$ & $1(14)$ \\
\hline Injury & $1(6)$ & $3(19)$ & $3(17)$ & $1(5)$ & 0 & 0 \\
\hline Volvulus & 0 & 0 & $2(11)$ & $4(20)$ & $1(14)$ & $2(29)$ \\
\hline Cancer & $1(6)$ & 0 & 0 & $1(5)$ & 0 & $1(14)$ \\
\hline Other & $4(24)$ & $4(25)$ & $4(22)$ & $1(5)$ & $3(43)$ & $2(29)$ \\
\hline $\begin{array}{l}\text { Colon-in- } \\
\quad \text { continuity, n (\%) }\end{array}$ & $0(0)$ & $0(0)$ & $18(100)$ & $20(100)$ & $7(100)$ & $7(100)$ \\
\hline \multicolumn{7}{|l|}{ Stoma present, n (\%) } \\
\hline Ileostomy & $6(35)$ & $9(56)$ & 0 & 0 & 0 & 0 \\
\hline Colostomy & 0 & 0 & 0 & 0 & $4(57)$ & $1(14)$ \\
\hline Other & 0 & $2(13)$ & 0 & 0 & 0 & 0 \\
\hline $\begin{array}{l}\text { Ileocaecal valve } \\
\text { present, } \mathrm{n}(\%)\end{array}$ & 0 & 0 & $3(17)$ & $8(40)$ & 0 & $1(14)$ \\
\hline $\begin{array}{l}\text { Mean }(\mathrm{SD}) \\
\text { estimated } \\
\text { remaining small } \\
\text { bowel length, cm }\end{array}$ & $\begin{array}{l}137.7 \\
(70.9)^{*}\end{array}$ & $\begin{array}{l}113.7 \\
(79.8)^{*}\end{array}$ & $\begin{array}{l}52.2 \\
(27.4)\end{array}$ & $\begin{array}{l}39.2 \\
(30.4)^{\dagger}\end{array}$ & $\begin{array}{l}59.3 \\
(44.4)^{*}\end{array}$ & $\begin{array}{l}49.3 \\
(30.0)^{*}\end{array}$ \\
\hline $\begin{array}{l}\text { Mean (SD) PS } \\
\text { duration, year }\end{array}$ & $7.1(6.7)$ & $5.1(3.1)$ & $6.2(6.5)$ & $6.1(6.4)$ & $6.4(5.6)$ & $7.2(8.2)$ \\
\hline $\begin{array}{l}\text { Mean (SD) PS } \\
\text { volume, L/week }\end{array}$ & $14.5(9.6)$ & $18.8(7.9)$ & $10.6(5.8)$ & $10.5(5.3)$ & $12.4(7.1)$ & $9.1(3.9)$ \\
\hline
\end{tabular}

$\mathrm{BMI}=$ body $\quad$ mass $\quad$ index; $\quad \mathrm{PBO}=$ placebo; $\quad \mathrm{PS}=$ parenteral $\quad$ support; TED $=$ teduglutide. ${ }^{*} \mathrm{n}=15 ;{ }^{\dagger} \mathrm{n}=19 ;{ }^{\star} \mathrm{n}=6$.

Conclusion: Patients with SBS-IF in Group 1 had the highest baseline PS volume needs and responded most and fastest to TED with PS volume reductions, compared with patients in Group 2 or Group 3. This research was funded by Shire International GmbH, Zug, Switzerland.

Disclosure of Interest: P.B. Jeppesen: I have served as a consultant and on the speaker bureau for Shire.

S.M. Gabe: I have served as a consultant for Shire.

D.L. Seidner: I have served as a consultant for Shire.

H. Lee: I am an employee for Shire.

C. Olivier: I am an employee for Shire.

P1290 GENE EXPRESSION PROFILING OF SUPERFICIAL SPORADIC NON-AMPULLARY DUODENAL EPITHELIAL TUMORS

Y. Sakaguchi ${ }^{1}$, N. Yamamichi ${ }^{1}$, S. Tomida ${ }^{2}$, C. Takeuchi ${ }^{1}$, Y. Takahashi ${ }^{1}$, N. Kageyama-Yahara ${ }^{1}$, M. Fujishiro, ${ }^{1}$, K. Koike ${ }^{1}$

${ }^{1}$ Dept. Of Gastroenterology, University of Tokyo, Tokyo/Japan

${ }^{2}$ Graduate School Of Medicine, Dentistry And Pharmaceutical Sciences, Okayama University, Okayamashi/Japan

Contact E-mail Address: sakaguchi-tky@umin.ac.jp

Introduction: Sporadic non-ampullary duodenal epithelial tumors (SNADETs) are rare, accounting for less than $1 \%$ of gastrointestinal neoplasms, and thus the mechanism behind the pathogenesis and carcinogenesis of these neoplasms is still poorly understood. However, with the overall increase of small bowel cancer in recent years, there is an increasing need to clarify the morphology of SNADETs. This study was conducted with the objective of identifying genetic markers and pathways specific to superficial SNADETs through gene-expression analysis.

Aims \& Methods: This was a prospective pilot study on patients with a diagnosis of superficial SNADETs who were treated at the Department of Gastroenterology, Graduate School of Medicine, The University of Tokyo Hospital, Tokyo, Japan. Before enrollment, all patients had undergone endoscopic evaluation to preclude ampullary lesions, and had a preoperative histologic diagnosis of either adenoma or adenocarcinoma. Patients with familial polyposis were excluded. Immediately before resection of the target lesions, a single biopsy sample from the duodenal tumor and a paired sample from the surrounding normal duodenal mucosa were endoscopically obtained from each patient, followed by RNA extraction. Gene expression profiling with an oligonucleotide microarray was performed in a training set of 4 matched tumornormal tissue superficial SNADET pairs. Genes and pathways with consistent differences between pairs were identified, followed by a set-level gene enrichment analysis with a pre-valuated curated gene set. Results were confirmed with rtPCR in all other independent SNADET pairs.

Results: From Nov 2014 to Jan 2016, a total of 12 consecutive patients were enrolled in this study. One patient was excluded due to a post-treatment diagnosis of familial polyposis. In a training set of 4 tumor-mucosa pairs, 626 probes (168 up-regulated, 458 down-regulated) which consistently demonstrated over a 2-fold expression difference between tumor and normal mucosa in all matched pairs were identified. Rt-PCR of genes most highly differentially expressed between the tumors and normal mucosa was performed in the 4 pairs in the training set as well as 7 independent pairs. Consistent gene expression patterns concurrent with microarray results were demonstrated in all pairs, confirming the results of this study. Gene set enrichment analysis of the training set using a curated data set demonstrated a strong association between SNADETS with colorectal adenomas $(\mathrm{p}<0.00001)$ and APC down-regulation $(p<0.00001)$. No other significant associations were demonstrated.

Conclusion: Superficial SNADETs demonstrate gene expression characteristics with a strong resemblance to colorectal adenomas. Gene expression characterization of these lesions has also demonstrated the significant role of APC down-regulation in the pathogenesis of SNADETs, suggesting that an adenoma-carcinoma sequence similar to colorectal adenomas may be seen in SNADETs. Further analysis of genes which may play a key role in the carcinogenesis of these neoplasms is required.

Disclosure of Interest: All authors have declared no conflicts of interest.

\section{P1292 USE OF 3D COMPUTED TOMOGRAPHIC ENTEROCLYSIS TO OBTAIN INFORMATION ON THE LENGTH OF THE SMALI INTESTINE AND ON THE SIZE, SHAPE, LOCATION OF INTESTINAL NEOPLASIAS}

T. Yoshikawa, K. Ohno, T. Kurokami

Dept. Of Gastroenterology, Shizuoka General Hospital, Shizuoka/Japan

Contact E-mail Address: t-yoshi@med.nagoya-u.ac.jp

Introduction: We established a new imaging technique, 3D computed tomographic (CT) enteroclysis, to explore the small bowel (1). In our hospital, this examination is performed routinely to detect gross lesions in the small intestine. In our study, we analysed the clinical performance of 3D CT enteroclysis to evaluate its safety, feasibility, and usefulness for small intestinal neoplasias.

Aims \& Methods: Data on 3D CT enteroclysis performed in our hospital from January 2010 to March 2017 were reviewed. In 3D CT enteroclysis, the small bowel was inflated with air using a nasoduodenal tube, CT images were taken, and then $3 \mathrm{D}$ overviews, virtual endoscopy views, and virtual dissection views were generated using a virtual colonoscopy system. Total volume of injected air, intraintestinal pressure, and length of the depicted small bowel were recorded. The images of small intestinal neoplasias were collected and compared to intraoperative findings.

Results: One-hundred thirty-nine 3D CT enteroclysis were performed for 93 males and 46 females. The mean age was 49.2 y (17-87). Examinations were performed for definite/suspected Crohn's disease in 55, intestinal obstruction in 54 , small intestinal neoplasias in 25 , and others in 5 patients. Vomiting/belching and abdominal pain (requiring pain medication) were noted in 10 and 5 patients, respectively, but no additional treatments were necessary. The volume of air and intraintestinal pressure were recorded in 77 examinations for patients without previous resection of the small bowel. The mean total volume of injected air was $1872 \pm 656 \mathrm{ml}$, the mean maximum intraintestinal pressure was $2.7 \pm 0.8 \mathrm{kPa}$, the mean length of the depicted small bowel was $517 \pm 102 \mathrm{~cm}$, and whole small bowel tracing was achieved in $71.4 \%$ of these 77 examinations. Twenty small intestinal neoplasias were depicted in 4 cases of submucosal tumors, 3 cases of gastrointestinal stromal tumors (GISTs), in 3 cases of neuroendocrine tumors, in 2 cases of cancers, in 2 cases of lipomas, 2 cases of malignant lymphomas, 1 case of metastatic cancer, 1 case of Peutz-Jeghers syndrome, 1 case of Peutz-Jeghers type polyp, and 1 case of pyogenic granuloma. Surgery was performed for 12 cases; the total length of the small intestine and estimated location of the lesion by 3D CT enteroclysis were comparable to intraoperative findings in 9 cases (no measurement in 3 cases). In a case of multiple GISTs, some of the lesions of $6-8 \mathrm{~mm}$ in size were missed and no lesion smaller than $4 \mathrm{~mm}$ was depicted.

Conclusion: 3D CT enteroclysis can be performed safely, and the whole small bowel could be examined in most cases. 3D CT enteroclysis can depict stenosis and lesions bigger than $1 \mathrm{~cm}$, measure the length of the small intestine, measure 
the size and locate the position of the lesions, and present objective information for surgery and follow-up of small intestinal neoplasias. Therefore, 3D CT enteroclysis is a powerful new tool for diagnosis, pre-surgical evaluation, and followup for small intestinal neoplasias.

Disclosure of Interest: All authors have declared no conflicts of interest.

\section{Reference}

1. Yoshikawa T, Takehara Y, Kikuyama M, et al. Computed tomographic enteroclysis with air and virtual enteroscopy: protocol and feasibility for small bowel evaluation. Dig Liver Dis. 2012; 44: 297-302.

\section{P1293 INK TATTOOING FOR BALLOON-ASSISTED} ENTEROSCOPY-TIME WELL SPENT?

\author{
C. Römmele ${ }^{1}$, A. Ebigbo ${ }^{1}$, H. Messmann ${ }^{1}$, S. Gölder ${ }^{1}$ \\ ${ }^{1}$ Department Of Internal Medicine Iii, Klinikum Augsburg, Augsburg/Germany
}

Contact E-mail Address: christoph.roemmele@klinikum-augsburg.de Introduction: Balloon-assisted enteroscopy is a well-established tool in the diagnosis and therapy of small bowel diseases. Ink tattooing of the small bowel is used in some centers to mark pathologic lesions or the depth of small bowel insertion.

Aims \& Methods: The purpose of this study was to determine the safety, the detection rate within a surgical operation or video capsule endoscopy and the clinical relevance of ink tattooing during balloon-assisted enteroscopy (BAE). Between 01.01.2010 to 31.12.2015 229 BAE were performed in 156 patients (pts) at the endoscopy unit of Klinikum Augsburg. We performed a retrospective analysis of all $81(52 \%)$ patients who received an ink tattooing during BAE. Results: Main indications for BAE were known angiodysplasia (37 pts), suspected bleeding of the small intestine (32 pts) and anemia (19 pts). Other indications were known or suspected tumor of the small intestine (17 pts) and Crohn's disease ( 3 pts). In 27 patients no pathologic findings were found. In 41 patients an active bleeding, angiodysplasia or hemangioma were found and further therapeutic interventions were performed. Tumor/polyps (11 pts), inflammatory lesions (7 pts) and ulcerative diverticula (1 pts) were other findings. In all 81 patients ink tattooing of the small intestine was performed with no complications. $46(57 \%)$ of 81 patients received a follow-up mainly due to re-bleeding. 5 Patients underwent surgery directly after enteroscopy with ink-tattooing and therefore received no follow-up. In total $26(32 \%)$ patients received a capsule endoscopy after BAE at our hospital. The ink tattooing could be detected via capsule endoscopy in 19 of these 26 patients $(73 \%)$. The ink tattooing of the previous antegrade BAE could be detected via retrograde BAE only in 2 of 11 $(18 \%)$ patients. Nine patients received a second ink-tattooing of the small intestine within these examinations without any complications. Ink tattooing had no clinical relevance or therapeutic consequence in 62 of the $81(72 \%)$ patients within the observation period. 5 of these 62 Patients received no further diagnostic or therapeutic steps due to their clinical situation. In 9 patients ink tattooing influenced the choice of approach (antegrade versus retrograde) for reenteroscopy after a video capsule endoscopy. In 7 patients the ink tattooing was used for intraoperative localization and in 3 patients for intraoperative localization as well as for enteroscopy. The intraoperative detection rate of the ink tattooing was $100 \%$.

Conclusion: Ink tattooing of the small intestine is a minimally invasive and safe endoscopic procedure to mark the depth of scope insertion or a pathologic lesion during balloon-assisted enteroscopy. It is a useful tool to avoid unnecessary examinations and aids the intraoperative localization of pathologic lesions. A complete enteroscopy of the small intestine via BAE from retrograde and antegrade is achieved rarely in our setting.

Disclosure of Interest: All authors have declared no conflicts of interest.

\section{P1294 THE ROLE OF CAPSULE ENDOSCOPY IN THE DETECTION OF SMALL BOWEL TUMOURS IN A LOW-RISK POPULATION; A SINGLE-CENTRE EXPERIENCE}

M. Hussey ${ }^{1}$, G. Holleran ${ }^{2}$, T. Nuzum ${ }^{1}$, S. Crowther ${ }^{3}$, D. Mcnamara ${ }^{1}$ ${ }^{1}$ Gastroenterology, Tallaght Hospital, Dublin/Ireland

${ }^{2}$ Trinity Academic Gastroenterology Group, Trinity College, Dublin/Ireland ${ }^{3}$ Pathology, Tallaght Hospital, Dublin/Ireland

\section{Contact E-mail Address: husseyma@tcd.ie}

Introduction: Small bowel tumours (SBT) are very rare and generally grow insidiously, with alarm symptoms such as obstruction and overt GIB often occurring at a later stage of disease. The introduction of small bowel capsule endoscopy (SBCE) has enabled earlier detection of SBT and has been shown to affect the therapeutic course. However, the reported frequency of small bowel malignancies diagnosed by SBCE is highly variable.

Aims \& Methods: We aimed to review the frequency of small bowel tumours diagnosed by SBCE in a high-volume tertiary centre, and to assess the clinical presentation and outcome of patients. A retrospective review of the database in our institution was undertaken. All patients who had undergone SBCE from 2011-2016 were identified. All patients had undergone SBCE using the standard protocol. The Given SB3 Pillcam was used for the majority of procedures from 2014 onwards and the SB2 was used prior to 2014. All videos were analysed by experienced gastroenterologists and reported using the Rapid reader software system (Given Imaging, Covidien, Medtronic, Yoqneam, Israel). SBCE reports for all indications were reviewed and all patients with reports suggestive of a small bowel tumour were included initially. A chart review was then performed and information was obtained on patient demographics, clinical presentation/ indication for SBCE, laboratory markers, previous endoscopic or radiological procedures, further endoscopic or surgical interventions carried out, and final histological diagnosis. Only patients who went on to have a histological diagnosis of a small bowel malignancy were included in the final analysis.

Results: A total of 1670 SBCEs were carried out.The overall indications for SBCE were as follows: suspected small bowel Crohn's disease 34\% $(n=568)$, iron deficiency anaemia/obscure occult GIB $41 \%(n=685)$, obscure overt GI bleeding $16 \%(\mathrm{n}=267)$, non-specific abdominal pain $6 \%(\mathrm{n}=100)$, known or suspected polyposis syndrome $2 \%(\mathrm{n}=33)$ or Coeliac disease $1 \%(\mathrm{n}=17)$. In total $1 \%(n=19)$ of SBCE reports identified a possible small bowel tumour. Of these, a final histological diagnosis of a small bowel malignancy was made in 14 patients. Of the 5 patients with a benign diagnosis, one patient was diagnosed with myoepithelial hamartoma, two patients an adenomatous polyp, one a lymphangioma, and one ganglion cells only. Of the 14 patients diagnosed with a small bowel malignancy, the majority, $71 \%(\mathrm{n}=10)$ were female and the mean age was 58 years (18-90). The indication for referral in $12(86 \%)$ patients was obscure gastrointestinal bleeding, with occult bleeding in $57 \%(\mathrm{n}=8)$ and overt bleeding in $29 \%(n=4)$. Only one patient was referred for SBCE due to abnormal imaging and the other patient had persistent diarrhoea. The mean length of time from presentation to diagnosis of their small bowel tumour by SBCE was 18.8 months (16-24). Patient's mean Haemoglobin at diagnosis was $10.6 \mathrm{~g} / \mathrm{dL}$ (7.4-12.4). Only 3 patients had a recognised risk factor, 2 with coeliac disease and 1 patient had previous colorectal cance. In $79 \%(n=11)$ of all patients, SBCE was the first investigation suggestive of a small bowel tumour. In all, $7(50 \%)$ patients underwent cross sectional imaging prior to SBCE, of which $3(42 \%)$ had a reported no small bowel abnormalities. All patients had undergone at least one negative upper and lower endoscopy prior to referral, and SBCE was the third investigation in $50 \%(n=7)$ of patients. After SBCE, $60 \%(n=8)$ of patients underwent a double balloon enteroscopy (DBE) for further evaluation of findings and to obtain tissue samples. In terms of histological diagnoses, adenocarcinomas were found in $43 \%(n=6)$, Neurendocrine tumours in $29 \%(n=4)$, Gastrointestinal stromal tumours (GISTs) in $14 \%(\mathrm{n}=2)$, and lymphoma in $14 \%(n=2)$. All patients eventually underwent surgical resection of their small bowel tumours and $80 \%(\mathrm{n}=11)$ patients remain well at present, after a mean follow up time of 15.2 months $(2-36)$.

Conclusion: SBT are rare but accounted for $1 \%$ of significant SBCE findings in our cohort. The lack of concordance between SBCE and cross sectional imaging suggests this modality should remain an early diagnostic test in OGIB.

Disclosure of Interest: All authors have declared no conflicts of interest.

\section{TUESDAY, OCTOBER 31, 201709:00-17:00}

\section{NUTRITION II - HALL 7}

\section{P1295 VITAMIN D PREVENTS HEPATIC STEATOSIS AND CARDIOVASCULAR DAMAGE IN A RAT MODEL OF FATTY WESTERN DIET}

G. Mazzone ${ }^{1}$, C. Morisco ${ }^{2}$, V. Lembo ${ }^{1}$, G. D’Argenio ${ }^{1}$, M. D’Armiento ${ }^{2}$, A. Rossi ${ }^{1}$, C. Del Giudice ${ }^{2}$, N. Caporaso ${ }^{1}$, F. Morisco

${ }^{1}$ Department Of Clinical Medicine And Surgery, University of Naples Federico II, Napoli/Italy

${ }^{2}$ Department Of Advanced Biomedical Sciences, University of Naples Federico II, Naples/Italy

Contact E-mail Address: v.lembo@hotmail.it

Introduction: The western diet (WD) high in fat and fructose is considered one of the most relevant cause of metabolic disorders and cardiovascular diseases (CVD). The aim of this study was to evaluate whether daily vitamin $\mathrm{D}_{3}$ supplementation is able to modulate hepatic steatosis, or restore insulin resistance and the metabolic alterations contributing to CVD and hearth failure (HF) caused by a westernized diet, in a rat model without specific vitamin D deficiency.

Aims \& Methods: Eighteen adult male Wistar rats were divided into 3 groups, each of 6 rats, fed with: Group 1: Standard Diet, $3.3 \mathrm{kcal} / \mathrm{g}$ (SD); Group 2: Western Diet, $5.6 \mathrm{kcal} / \mathrm{g}$, (WD) containing $13 \mathrm{IU} /$ day $/$ rat of vitamin $\mathrm{D}_{3}$; Group 3: Western Diet + Vit D (WD Vit D) containing $23 \mathrm{IU} /$ day/rat of vitamin $\mathrm{D}_{3}$. The experiment was conducted for 6 months. Standardized tail-cuff blood pressure (BP) measurements of conscious rats and transthoracic echocardiography were performed in basal condition (Time 0), and after 3 and 6 months of diet. Hepatic steatosis and collagen myocardial fibrosis were assessed using standard methods. Serum insulin and $25(\mathrm{OH}) \mathrm{D}_{3}$ concentrations were determined using rat-specific ELISA kits. Insulin resistance was determined according to the Homeostasis Model of Assessment (HOMA-IR) method.

Results: In WD rats the percentage of hepatocytes with steatotic vacuoles was $61 \%$, while in WDVitD group was only $27 \%$. In WD group HOMA-IR was significantly higher than in SD $(41.9 \pm 8.9$ vs $6.17 \pm 1.3, \mathrm{p}<0.01)$ and it was reduced by vitamin $D$ supplementation in WDVitD group $(41.9 \pm 8.9$ vs $19.4 \pm 5.2, \mathrm{p}<0.05)$. At baseline, no differences in systolic blood pressure (SBP) were detected among the three groups showing normal systolic blood pressure. SD did not increase SBP, significantly, during the study period. On the contrary, WD, enhanced SBP by $27 \pm 12 \% \mathrm{p}<0.001$ at 3 months, and by $47 \pm 11 \%, p<0.001$ at 6 months. At the end of the study, SBP resulted to be higher in WD group $(117 \pm 3 \mathrm{mmHg})$ compared with both $\mathrm{SD}(84 \pm 3 \mathrm{mmHg}$, $\mathrm{p}<0.001)$ and with WDVit.D $(101 \pm 4 \mathrm{mmHg}, \mathrm{p}<0.01)$. During the study period, WD group showed a significant increase of left ventricular mass (LVM) $(52 \pm 25 \%$ at 3 months $\mathrm{p}<0.05$, and $123 \pm 43 \%$ at 6 months $\mathrm{p}<0.001$, vs basal conditions,). Supplementation of VitD abolished the WDinduced increase of LVM $(25 \pm 19 \%$ at 3 months, and $34 \pm 20 \%$ at 6 months, $p<0.05$ vs baseline respectively). At the end of the study LVM resulted to be higher in the WD group in comparison to both SD and WDVitD groups, while 
no difference was detected between SD and WDVit.D. However, some collagen staining resulted still present in WDVitD rats.

Conclusion: These results suggest that a daily supplementation of vitamin $\mathrm{D}_{3}$ is able to improve insulin sensitivity, and to prevent diabetes. Vitamin $\mathrm{D}_{3}$ also reduced hepatic steatosis and prevent cardiac alterations such as the increase of systolic blood pressure and left ventricular hypertrophy in WD rats. Disclosure of Interest: All authors have declared no conflicts of interest.

\section{P1297 ELIPSE BALLOON SYSTEM: OUR EXPERIENCE. A PRELIMINARY STUDY}

E. V. Avallone, M. A. Vitale, A. De Cesare, G. Villotti, R. Urgesi, M. C. Di Paolo, L. Pallotta, M. G. Graziani

Gastroenterology And Digestive Endoscopy, Azienda Ospedaliera San Giovanni Addolorata, rome/Italy

\section{Contact E-mail Address: veronicaele@hotmail.it}

Introduction: Gastric balloons (IGB) are an emerging option for overweight and obese patients with a body mass index (BMI) greater than $31 \mathrm{~kg} / \mathrm{m} 2$ and they provide greater efficacy with lower risks than do conventional surgical procedures. The balloon treatment is based on gastric space-occupying effects that increase the feeling of satiety and may also effect gut neuroendocrine signaling. However, widespread use of current generation IGBs has been limited by several factors: placement and removal endoscopies require sedation, special training and equipment; patients lost to follow-up are susceptible to IGB deflation and unplanned passage into the gastrointestinal tract. The ElipseTM is the world's first procedureless gastric balloon that not require endoscopy or anesthesia.

Aims \& Methods: We conducted a study to prospectively analyze the safety and effectiveness of IGB ELIPSE in overweight adults. Six patients, 1 male and 5 female (average age 40 , mean $\mathrm{BMI}=40 \mathrm{~kg} / \mathrm{m} 2$ ), were included in this study. Each patient swallowed Elipse ${ }^{\mathrm{TM}}$ balloon intended to remain in the stomach for 16 weeks. Each balloon was filled with $560 \mathrm{~mL}$ of filling fluid. Patients returned every 2 weeks for abdominal ultrasound which documented the correct positioning of the device. All patients were followed up by a nutritionist with a specific semiliquid diet.

Results: All 6/6 patients successfully swallowed the device. There were no major adverse effects. All 6 patients had a significant weight loss (about $16 \mathrm{Kg}$ ). In all of the patients, the balloon remained full throughout 16 weeks, self-emptied, and were passed spontaneously without needing endoscopic removal.

Conclusion: This study demonstrates the efficiency, security and simplicity of the Elipse ${ }^{\text {TM }}$ system. Moreover, we highlighted the non necessity of deep sedation and use of invasive endoscopic examinations for positioning and removal of the IGB.

Disclosure of Interest: All authors have declared no conflicts of interest

\section{References}

Roman S, Napoleon B, Mion F et al. Intragastric balloon for "non-morbid" obesity: A retrospective evaluation of tolerance and efficacy. ObesSurg 2004; 14 : 539-544

Machytka E1, Gaur S2, Chuttani R3, Bojkova M1, Kupka T1, Buzga M4, Giannakou A5, Ioannis K6, Mathus-Vliegen E7, Levy S2, Raftopoulos I5.Elipse, the first procedureless gastric balloon for weight loss: a prospective, observational, open-label, multicenter study. Endoscopy. 2017 Feb;49(2):154160. doi: 10.1055/s-0042-119296. Epub 2016 Dec 12.

El Kareh I, Genser L, Siksik JM et al. Small-bowel obstruction secondary to migration of an intragastric balloon. J Gastrointest Surg 2016; 20: 1409-1410. DOI: $10.1007 / \mathrm{s} 11605-016-311$

\section{P1298 NUTRITIONAL STATUS EVALUATION TOOL IS NOT USED AND NUTRITIONAL SUPPORT CONSULTATION IS RARELY REQUESTED BY THE TREATING PHYSICIAN IN A TERTIARY GREEK HOSPITAL}

G. Vlachonikolou ${ }^{1}$, L. Bounou ${ }^{1}$, S. Kalousios ${ }^{1}$, A. Theodorou-Kanakari ${ }^{1}$, E.A. Mavroeidi ${ }^{1}$, M. Roma ${ }^{1}$, A. Kasti ${ }^{2}$, G. D. Dimitriadis ${ }^{1}$, K. Triantafyllou ${ }^{1}$ 2nd Dept Of Internal Medicine And Research Institute, National and Kapodistrian University of Athens, Medical School, Athens/Greece

${ }^{2}$ Department Of Nutrition, Attikon University General Hospital, Athens/Greece

\section{Contact E-mail Address: ktriant $@$ med.uoa.gr}

Introduction: There is evidence that the level of nutrition is a major determinant for the outcome of hospitalized patients. The Malnutrition Universal Screening Tool (MUST) is a five-step screening tool using the Body Mass Index (BMI), unintentional weight loss before admission and the effect of acute disease on subject's nutritional status to identify adults who are malnourished, at risk of malnutrition or obese. It also includes management guidelines which can be used to develop a care plan. Although it is simple and quick, its use varies widely among different health care settings.

Aims \& Methods: We aimed to evaluate the need of nutritional support consultation, the rate of MUST tool use by the treating physicians and the rate of nutritional support services utilization in a tertiary Greek hospital. This was a prospective, cross-sectional, nutritional service-utilization, chart-review study of hospitalized patients with follow-up until discharge, in a tertiary 500 beds hospital in Athens, Greece. We reviewed data of patients admitted during three consecutive hospital on-call days in March 2017 regarding the number of patients at need for nutritional consultation during hospitalization and at discharge. Intensive care, psychiatric, pediatric, obstetrics and day clinic admissions were excluded. We used data from patient's MUST tool (in case of absence of these data, we calculated the MUST score using subject's data) to identify patients at high risk of malnutrition and we used BMI to identify overweight and obese subjects. We measured the utilization of the nutrition department services according to the actual consultations.

Results: We included data from 461 patients (50\% males, aged $67 \pm 19 \mathrm{yrs}$ ). MUST evaluation for malnutrition was not available in any of the reviewed charts. We therefore calculated the MUST score using the available data in the charts. There were $73(15.8 \%)$ overweight-obese and $85(18.4 \%)$ at high risk of malnutrition patients admitted at the internal medicine $(\mathrm{n}=127)$ and surgery $(\mathrm{n}=31)$ sections of the hospital. Nutritional consultation was requested in $4(3.1 \%)$ and $1(3.2 \%)$ internal medicine and surgery patients, respectively by the treating physician during hospitalization. Among the 383 patients discharged at the end of our observation, there were $72(18.8 \%)$ overweight-obese and $46(12 \%)$ at high risk of malnutrition patients. Nutritional consultation was recommended in 24 (6 obese, 15 undernourished and 3 patients with low MUST score) of them.

Conclusion: MUST -a simple screening tool for malnutrition- is not used by the medical staff and nutritional support services are significantly underutilized in a tertiary Greek hospital.

Disclosure of Interest: All authors have declared no conflicts of interest.

\section{P1299 CHANGE OF VITAMIN D AND BONE MINERAL DENSITY AFTER BARIATRIC SURGERY IN CHINESE POPULATION}

M. Han ${ }^{1}$, W. Lee ${ }^{2}$

${ }^{1}$ Internal Medicine, Min-Sheng General Hospital, Taoyuan/Taiwan

${ }^{2}$ Surgery, Min-Sheng General Hospital, Taoyuan/Taiwan

\section{Contact E-mail Address: minglun @ms18.hinet.net}

Introduction: Bariatric surgery is an effective treatment for morbid obesity. In Taiwan, the numbers of patient who received bariatric surgery increased gradually. However, for long-term follow-up, nutritional deficiency may develop in post-bariatric (metabolic) surgery patients, especially in patients who received mal-absorptive or combination procedure. Deficiency of nutrition may cause anemia, peripheral neuropathy, secondary parathyroidism and osteoporosis. Follow-up of the nutritional status for patients after bariatric surgery is an important issue.

Aims \& Methods: The aim of our study is to evaluate the change of Vitamin D and bone Mineral Density after bariatric surgery in Chinese population. This prospective cohort study included 50 patients (ranged from 20 to 65 years old) who received bariatric surgery at one teaching hospital in Taoyuan, Taiwan. Patient with osteoporosis before surgery were excluded in this study. Baseline (2012-2014) and one year after bariatric surgery (2013-2015), venous blood was collected from each patients for assessment of the Calcium, Vitamin D and parathyroid hormone (PTH) levels. BMD $(\mathrm{g} / \mathrm{cm} 2)$ was also measured at lumbar spine (L2-L4) by dual energy x-ray absorptiometry (DEXA).

Results: Among 50 patients, 15 patients received laparoscopic sleeve gastrectomy, 24 patients received laparoscopic mini-gastric bypass (MGB), 5 patients received laparoscopic Roux-en-Y gastric bypass (RYGB) and 6 patients received laparoscopic duodeno-jejunal bypass with sleeve gastrectomy (DJB-SG). The characteristic of the study population was shown as table 1 . The differences of mean for calcium, vitamin $\mathrm{D}, \mathrm{PTH}$ and $\mathrm{BMD}$ after bariatric surgery were $-0.16 \mathrm{mg} / \mathrm{dl} \quad(\mathrm{P}=0.03), 4.2 \mathrm{ng} / \mathrm{ml}(\mathrm{P}<0.01), 8.6 \mathrm{pg} / \mathrm{ml} \quad(\mathrm{P}=0.06)$ and $-0.04 \mathrm{~g} / \mathrm{cm}^{2}(\mathrm{P}=0.14)$ respectively.

Table 1: Characteristics of study population one year after bariatric surgery

\begin{tabular}{|c|c|c|c|c|c|}
\hline & LSG & MGB & RYGB & DJBSG & OVER ALL \\
\hline $\mathrm{N}$ & 15 & 24 & 5 & 6 & 50 \\
\hline Age, years & $34.7(7.4)$ & $37.4(11.7)$ & $41.4(14.1)$ & $44(10)$ & $37.8(10.5)$ \\
\hline $\operatorname{Sex}(M: F)$ & $7: 8$ & $5: 19$ & $2: 3$ & $3: 4$ & $17: 33$ \\
\hline $\operatorname{BMI}\left(\mathrm{kg} / \mathrm{m}^{2}\right)$ & $29.8(4.7)$ & $27.6(4.4)$ & $27.9(2.9)$ & $24(2.4)$ & \\
\hline $\mathrm{CA}(\mathrm{mg} / \mathrm{dl})$ & $9.6(0.3)$ & $9.1(0.3)$ & $9.2(0.4)$ & $9.3(0.4)$ & $9.5(0.4)$ \\
\hline PTH-I(pg/mL) & $63.8(21.3)$ & $70.4(25.9)$ & $73.1(4.2)$ & $50(14)$ & $62(29.4)$ \\
\hline VIT.D (ng/ml) & $19.4(7.7)$ & $14(6.9)$ & $12.9(8.6)$ & $16.9(5.3)$ & $15.6(7.5)$ \\
\hline $\begin{array}{l}\text { VIT.D insufficiency } \\
\qquad(<10 \mathrm{ng} / \mathrm{ml})\end{array}$ & $1(6.7 \%)$ & $8(33.3 \%)$ & $3(60 \%)$ & 0 & $12(23.5)$ \\
\hline $\begin{array}{l}\text { VIT.D deficiency } \\
\qquad(<5.3 \mathrm{ng} / \mathrm{ml})\end{array}$ & 0 & 0 & $1(20 \%)$ & 0 & $1(2 \%)$ \\
\hline $\operatorname{BMD}\left(\mathrm{g} / \mathrm{c} \mathrm{m}^{2}\right)$ & $1.11(0.25)$ & $1.15(0.23)$ & $1.18(0.26)$ & $1.11(0.19)$ & $1.15(0.17)$ \\
\hline
\end{tabular}

Conclusion: One year after bariatric surgery, the prevalence of osteoporosis and osteopenia was low. The serum Vitamin D level increased significantly but no significant change of BMD was noted. Further longitudinal studies are warranted to clarify the long-term effect of bariatric surgery on BMD in Chinese population.

Disclosure of Interest: All authors have declared no conflicts of interest. 
P1300 INTRAGASTRIC BALLOON: A LARGE BRAZILIAN MULTICENTRIC STUDY OVER 10,000 CASES AND 20 YEARS OF EXPERIENCE

B. Q. Sander ${ }^{1}$, C. Marchesini ${ }^{2}$, D. S. Paiva ${ }^{1}$, J. Schemberk Jr. ${ }^{3}$, G. Baretta ${ }^{4}$ G. Cairo Nunes ${ }^{5}$, J. I.B. Scarparo ${ }^{6}$, E. Grecco 7 , T. F. Souza ${ }^{7}$, M. Galvao Neto ${ }^{8}$ ${ }^{1}$ Dept. Of Bariatric Endoscopy, Sander Medical Center, Belo Horizonte/Brazil ${ }^{2}$ Clínica Caetano Marchesini, CuritibalBrazil

${ }^{3}$ Clinica ObesoGastro, Curitiba/Brazil

${ }^{4}$ Clinica Dr. Giorgio Baretta, Curitiba/Brazil

${ }^{5}$ Clínica Dr. Gabriel Cairo Nunes, São Paulo/Brazil

${ }^{6}$ Bariatric Endoscopy, Scarparo Scopia, São Paulo/Brazil

${ }^{7}$ Bariatric Endoscopy, Faculdade de Medicina do ABC - Hospital Mário Covas, São Paulo/Brazil

${ }^{8}$ Florida International University, Miami/United States of America/FL

\section{Contact E-mail Address: brunosander@hotmail.com}

Introduction: The intragastric balloon has been used for more than 20 years in Brazil as an endoscopic method for assisting weight loss, and some intercurrences were observed during more than 10,000 procedures performed. With the assistance of a multidisciplinary team the results have been satisfactory.

Aims \& Methods: To assess the efficacy and complications of the weight loss with IGB in patients seen at the 07 private centers. A total of 10,255 patients with IGB implanted from 1997 to 2017 were analyzed from a prospective fed databank. A liquid filled IGB with a volume in-between 620 to $700 \mathrm{ml}$ was used. Initial BMI started at $27 \mathrm{~kg} / \mathrm{m} 2$ (as approved by Brazilian health authorities) and were followed up by a multidisciplinary team during implant. IGB maximum period implant was 09 months. Statistical analysis was performed according to sex and degree of excess weight (overweight and grade I, II and III). Data were analyzed using Student t-test, and and Tukey post-test. The level of significance was set at $\mathrm{p}<0.05$.

Results: 492 patients $(4,8 \%)$ were excluded from the final analysis associated with weight loss: $226(2,2 \%)$ due to early removal-These were analyzed in relation to the previous consultation with psychologist and $94 \%$ of them did not make a psychological evaluation before the procedure, $158(1.54 \%)$ due fail on weight loss or weight gain-These were analyzed in relation to follow-up with nutritionist and $88 \%$ did not undergo nutritional monitoring during the use of IGB, 108 $(1.05 \%)$ due to incomplete data. There were also spontaneous hyperinflation on $0.99 \%(\mathrm{n}=101)$ and balloon spontaneous deflation or leakage in $0.82 \%(\mathrm{n}=84)$. Incidence of complications not leading to removal were $6.65 \% \quad(n=682)$. Complications other happened as fungal contamination in in $7.9 \%(\mathrm{n}=810)$ Wernick Korsakoff syndrome $0.01 \%(\mathrm{n}=2)$, pregnancy during implant period was $1.2 \%(n=123)$ and Dieulafoy lesion $0.009 \%(n=1)$. The incidence of complications with IGB removal was $0.05 \%(n=6)$ : gastric perforation. On the 9,763 remaining patients, $7.615(78 \%)$ were women and $2.148(22 \%)$ were men. Mean age was 31.13 years. The patients showed a significant weight loss, with a significantly lower final BMI $(27.16 \pm 8.42 \mathrm{~kg} / \mathrm{m} 2$; range: $15.71-34)$ than the initial BMI $(33.42 \pm 6.62 \mathrm{~kg} / \mathrm{m} 2$; range: $27-79.35)$. The mean Weight Loss was $-16.98 \mathrm{~kg}(+/-16.8 \mathrm{~kg})$. Percent EW L was higher in the overweight group $(129.92 \% \mathrm{EWL})$, followed by obesities grades I $(73.64 \%)$, II $(57.85 \%)$ and III $(41.33 \%)$ sequentially. A total of $5.2 \%(66 / 1,268)$ of the patients followed up for 18 months after withdrawal of the IGB were submitted to bariatric surgery.

Conclusion: The intragastric balloon has been established as an valid endoscopic therapeutic option for weight loss, especially in patients with overweight and obesity grades I and II and multidisciplinary team follow-up is mandatory for successful treatment.

Disclosure of Interest: All authors have declared no conflicts of interest.

\section{References}

Moura D, Oliveira J, De Moura EG, et al. Effectiveness of intragastric balloon for obesity: A systematic review and meta-analysis based on randomized control trials. Surg Obes Relat Dis 2016;12:420-9.

Genco A, Cipriano M, Bacci V, et al. Intragastric balloon followed by diet vs intragastric balloon followed by another balloon: a prospective study on 100 patients. Obes Surg 2010;20:1496-500. Genco A, Maselli R, Frangella F, et al. Intragastric balloon for obesity treatment: results of a multicentric evaluation for balloons left in place for more than 6 months. Surg Endosc 2015;29:2339-43. Crea N, Pata G, Della Casa D, et al. Improvement of metabolic syndrome following intragastric balloon: 1 year follow-up analysis. Obes Surg 2009;19:1084-8

Mazure RA, Salgado G, Villarreal P, et al. Intragastric balloon and multidisciplinary team. Nutr Hosp 2009;24:282-7.

Sallet JA, Marchesini JB, Paiva DS, et al. Brazilian multicenter study of the intragastric balloon. Obes Surg 2004;14:991-8.

\section{P1301 WEIGHT REGAIN AFTER BARIATRIC SURGERY - ARGON PLASMA COAGULATION FOR GASTROJEJUNAL ANASTOMOSIS DECREASE}

B. Q. Sander ${ }^{1}$, D. S. Paiva ${ }^{1}$, M. P. Sander ${ }^{1}$, M. Galvao Neto ${ }^{2}$, G. Baretta ${ }^{3}$,

E. Grecco ${ }^{4}$, T. F. Souza ${ }^{4}$, J. I.B. Scarparo ${ }^{5}$

${ }^{1}$ Dept. Of Bariatric Endoscopy, Sander Medical Center, Belo Horizonte/Brazil

${ }^{2}$ Florida International University, Miami/United States of America/FL

${ }^{3}$ Clínica Dr. Giorgio Baretta, Curitiba/Brazil

${ }^{4}$ Bariatric Endoscopy, Faculdade de Medicina do ABC - Hospital Mário Covas, São Paulo/Brazil

${ }_{5}^{5}$ Bariatric Endoscopy, Scarparo Scopia, São Paulo/Brazil

Contact E-mail Address: brunosander@hotmail.com
Introduction: The weight regained has been a described growing problem in patients after bariatric surgery. This weight regained is multifactorial and associated with dilation of Gastrojejunostomy (GJ). For the patients with significant weight regain some revisional procedures had be attempted and more recently endoscopic revisional procedures had being described.

Aims \& Methods: To evaluate the safety and effectiveness of argon plasma coagulation (APC) decreasing the diameter of the gastroenteric anastomosis in patients who have undergone RYGB for morbid obesity and regained weight associated to dilation of the GJ.From Jan-2014 to April-2017 554 RYGB subjects with weight regain a dilated anastomosis $(>18 \mathrm{~mm})$ and at least 2 years from procedure were submitted APC application. In relation to the anastomotic diameter, the majority of studies use a diameter of more than $20 \mathrm{~mm}$ to define anastomosis dilation, although some studies use smaller diameters such as $12 \mathrm{~mm}$, similar to that created manually in the gastrojejunal anastomosis using a 36 Fr Fouchet bougie. In the patients in the present study, the minimum crosssection diameter was $18 \mathrm{~mm}$ and the maximum measured in the first session $40 \mathrm{~mm}$. This anastomotic diameter was measured using a 33-mm long Olympus ${ }^{\circledR}$ articulated device. Interval between an APC session was 60 days with a maximum of 03 applications. APC set was at $2-3 \mathrm{~L} / \mathrm{m}$ with $65-85 \mathrm{~W}$. GJ diameter target was $8-12 \mathrm{~mm}$ estimated with pre-measured grasper. At first APC session, pre-op weight and BMI, post-op weight nadir, actual weight and BMI and estimated diameter of GJ were the variables collected. Complications during treatment were also collected. In the present study, psychological and nutritional evaluations were performed before APC and during treatment and physical activity was strongly recommended. Data were analyzed with descriptive statistics, student's t test and Spearman correlation.

Results: Of the 554 patients, $79.06 \%$ were women and $20.94 \%$ were men. Average time between bariatric surgery and the first APC was 96.35 months $( \pm 42.17)$ and average weight regained in this interval was $22.08 \mathrm{~kg}( \pm 11.05)$. The mean diameter of the anastomosis was $24.78 \mathrm{~mm}( \pm 6.04)$ and the average number of APC sessions were 1.78 times $( \pm 0.61)$. The average reduction of anastomotic diameter was $14.86 \mathrm{~mm}( \pm 7.24)$ and the final average diameter was $11.79 \mathrm{~mm}( \pm 3.89)$. The average weight loss between the first and last APC was $13.37 \mathrm{~kg}( \pm 7.82)$ and the average decrease of BMI was $4.59 \mathrm{~kg} / \mathrm{m} 2( \pm 2.78)$. 122 patients $(22.02 \%)$ did not achieve the target GJ diameter and 05 patient $(0.9 \%)$ did not lose weight even with the desired GJ diameter. From the 146 subjects $(26.36 \%)$ followed up to 12 months, the weight regain was less than $20 \%$ of the weight loss. Of the 554 patients APC, $51(9.2 \%)$ required dilatation balloon due to symptomatic stenosis at least once. No further complications were reported.

Conclusion: Argon Plasma Coagulation (APC) has been shown to be an effective and safe endoscopic technique for the reduction of gastro enteric anastomosis in patients undergoing bariatric surgery who have regained weight with dilation of the anastomosis. The reintroduction of the patient to the multisciplinary team is mandatory in cases of weight regain and loss to postoperative follow-up. A psychological and/or psychiatric evaluation is mandatory, as well as nutritional therapy and encouragement of physical activity. The monitoring of food intake and body weight, closer follow-up of the operated patients, appropriate choice of technique according to the patient and the experience of the surgeon, and a good learning curve are all factors that can reduce the failure rate of bariatric surgery. The reintroduction of the patient to the multidisciplinary team is mandatory if better results and sustainable weight loss and comorbidity control are to be obtained.

Disclosure of Interest: All authors have declared no conflicts of interest.

\section{References}

Galvão Neto M, Rodriguez L, Zundel N. Endoscopic revision of Roux-en-Y gastric bypass stomal dilation with a suturing device: preliminary results of a first out-of-United States series. Bariatric Times. 2011; 8: 1-34.

Baretta GAP, Alhinho HCAW, Matias JEF et al. Argon Plasma Coagulation of Gastrojejunal Anastomosis for Weight Regain After Gastric Bypass. Obes Surg 2015; 25: 72-79.

\section{P1302 EXCESS WEIGHT IN THE ELDERLY: A BRAZILIAN} EXPERIENCE WITH THE INTRAGASTRIC BALLOON TREATMENT

R.J.F. Fernandez ${ }^{1}$, E. Usuy ${ }^{2}$, M. Galvao Neto ${ }^{3}$, C.F. Diestel ${ }^{1}$, S. Barrichello ${ }^{1}$, A. F. Teixeira ${ }^{1}$

${ }^{1}$ Dept. Of Digestive Endoscopy, Endogastro Med Service, Rio de Janeiro/Brazil

${ }^{2}$ Bariatric Endoscopy, Usuy Clinic, rio de janeiro/Brazil

${ }^{3}$ Bariatric Endoscopy, Gastro Obeso Center, São Paulo/Brazil

Contact E-mail Address: ricfittipaldi@hotmail.com

Introduction: With the aging of the population, the incidence of obesity has also increased among the elderly. However, there is a higher incidence of severe comorbidities in this population comparing to adults, which often makes bariatric surgery unfeasible. In this scenario, treatment with the intragastric balloon (IGB) may be an interesting option.

Aims \& Methods: We aimed to assess the efficacy and complications of obesity treatment in the elderly using a non adjustable IGB. A total of 77 patients were analyzed. The minimal initial body mass index (BMI) was $28 \mathrm{~kg} / \mathrm{m} 2$. The level of significance was set at $\mathrm{p}<0.05$.

Results: 58 patients were women $(75.3 \%)$. Mean age was $64.26(60-80)$ years. Ten patients had no comorbidities, 52 had hypertension, 45 had dyslipidemia, 32 had insulin resistance, 12 had tipe II diabetes, and 10 had isquemic heart desease. There was no major complications. Results are shown on table 1. The treatment success rates according to the following criteria: $\geq \mathbf{1 0} \%$ total body weight loss (TBWL) and $\geq \mathbf{2 5 \%}$ excess weight loss (EWL) were $96.11 \%$ (74 patients) and 
98.7\% (76 patients) respectively. 30 patients reached a normal body mass index (BMI) $\left(23-28 \mathrm{~kg} / \mathrm{m}^{2}\right)$ according to the Pan American Health Organization (OPAS). Elderly shows a higher BMI reduction $(\mathrm{p}=0.0002)$ and \%TBWL $(\mathrm{p}=0.0003)$ than adults.

\section{Table 1}

$\mathrm{n}=77$

\section{Body weight $(\mathrm{kg})$}

Baseline

Final

Reduction

$\%$ TBWL

BMI $(\mathrm{kg} / \mathrm{m} 2)$

Baseline

Final

Reduction

Excess weight $(\mathrm{kg})$

Baseline

Final

$\%$ EWL

$103.37 \pm 17.14$

$81.66 \pm 15.71$

$21.71 \pm 7.58$

$21.07 \pm 6.07$

$37.89 \pm 5.41$

$29.86 \pm 4.76$

$8.03 \pm 2.88$

$35.53 \pm 16.98$

$13.82 \pm 15.49$

$69.27 \pm 30.01$

${ }^{*} \mathrm{p}<0.0001$ for all comparisons between values at baseline and at the end of the study. IGB(intragastric balloon); TBWL(total body weight loss); EWL(excess weight loss)

Conclusion: Endoscopic treatment of obesity with an IGB shows to be an excellent therapeutic option for the elderly.

Disclosure of Interest: All authors have declared no conflicts of interest.

\section{P1303 THE EFFECT OF A CONTROLLED GLUTEN CHALLENGE IN PATIENTS WITH SUSPECTED NON-COELIAC GLUTEN SENSITIVITY: A RANDOMIZED, DOUBLE-BLIND PLACEBO- CONTROLLED CHALLENGE}

H. F. Dale ${ }^{1}$, J.G. Hatlebakk ${ }^{2}$, N. Hovdenak ${ }^{3}$, S. O. Ystad ${ }^{4}$, G.A. Lied ${ }^{5}$ ${ }^{1}$ Centre For Nutrition, Department Of Clinical Medicine, University of Bergen, Bergen/Norway

${ }^{2}$ Medicine, Gastroenterology, National Center for Functional Gastrointestinal Disorders, Haukeland University Hospital, Bergen/Norway

${ }^{3}$ Department Of Clinical Medicine, University of Bergen, Bergen/Norway

${ }^{4}$ Haukeland University Hospital, National Centre for Functional Gastrointestinal Disorders, Bergen/Norway

${ }^{5}$ Center For Nutrition, Clinical Medicine, University of Bergen, Bergen/Norway

Contact E-mail Address: hanna.dale@outlook.com

Introduction: Non-coeliac gluten sensitivity (NCGS) is a new entity with unknown prevalence and mechanisms, and there is a need for a standardized procedure to confirm the diagnosis. The objective of this study was to characterize the response to a gluten challenge, when performed according to the updated Salerno criteria.

Aims \& Methods: Twenty patients (14F/6M, age range: $21-62$ y) with suspected NCGS, without coeliac disease and wheat allergy, were included while on a gluten-free diet. All patients went through four periods of double-blinded provocation with gluten and placebo containing muffins. They were instructed to eat two muffins a day (11 g gluten) for four days, followed by a three days' wash-out. Gastrointestinal symptoms were recorded with questionnaires at baseline and after each provocation, while fatigue and quality of life were registered at baseline and end of the trial.

Results: Four out of twenty patients $(20 \%)$ correctly identified the two periods when they received muffins containing gluten, hence were diagnosed with NCGS. The diagnosed group tended to show higher symptom scores than the not-diagnosed group both at baseline, after gluten exposure and after placebo, but no clear difference was seen in symptom change after provocation with gluten and placebo. The not-diagnosed group showed more severe symptoms with placebo than with gluten $(\mathrm{p}=0.029)$. Symptom severity at baseline was significantly correlated with fatigue $(r=0.63, p=0.003)$ and reduced quality of life $(r=0.76$, $\mathrm{p}=0.0001$ )

Conclusion: This randomized, double-blind placebo-controlled challenge with gluten diagnosed four patients with NCGS according to the Salerno criteria. However, according to the symptom registrations there are no clear difference between the diagnosed and the not-diagnosed group, or between symptoms after gluten provocation and placebo, indicating no specific effect of gluten in a group of patients with suspected NCGS.

Disclosure of Interest: All authors have declared no conflicts of interest.
P1304 THE ROLE OF BILE ACIDS AND GUT MICROBIOTA IN CORONARY ARTERY DISEASE: RESULTS OF THE MABAC STUDY IN HUMAN (MICROBIOTA ATHEROMA AND BILE ACID IN CORONARY DISEASE)

H. Duboc ${ }^{1}$, C. Chong Nguyen ${ }^{2}$, D. Rainteau ${ }^{3}$, H. Sokol $^{3}$, L. Humbert ${ }^{3}$, P. Seksik ${ }^{3}$, B. Coffin ${ }^{4}$, O. Varenne ${ }^{2}$, A. Bado ${ }^{4}$, D. Duboc ${ }^{2}$

${ }^{1}$ Department of Hepato Gastro Enterology and University Paris Diderot, Sorbonne Paris Cité, INSERM UMR 1149, Louis Mourier Hospital, APHP, Colombes, France, Colombes/France

${ }^{2}$ Department of Cardiology and University Paris Descartes, Cochin Hospital, APHP, Paris, France, Paris/France

${ }^{3}$ U1157 INSERM, LABEX Transimmunom, DHU I2B 27 University Pierre et Marie Curie, France, Paris/France

${ }^{4}$ Department of Hepato Gastro Enterology and University Paris Diderot, Sorbionne Paris Cité, INSERM UMR 1149, Louis Mourier Hospital, APHP. Colombes, France, Colombes/France

Contact E-mail Address: henri.duboc@gmail.com

Introduction: By targeting specific receptors into the vascular system, bile acids (BA) are cholesterol derivatives that are now considered as hormones. BA regulates the basal energy expenditure and gluco-lipidic metabolism. In animal models of atheroma developement (ApoE -/- and Ldl -/- mouse model) a powerful anti-atherosclerotic effect of circulating BA has been evidenced: BA are metabolites of the gut microbiota, suspected to play a role in the development of atherosclerosis. This study examined whether variations in BA or in the gut microbiota composition can be describe in the human Coronary Artery Disease pathophysiology.

Aims \& Methods: Consecutive patients undergoing coronary angiography between February and May 2015 were enrolled. To avoid physiological or induced variations in circulating BA or in the gut microbiota, highly restrictive exclusion criteria were applied. Circulating and fecal BA were quantified by high pressure liquid chromatography and tandem mass spectrometry. The fecal microbiota composition was assessed by 454 pyrosequencing of the total fecal bacterial DNA.

Results: 80 patients were prospectively included of 406 screened, and divided in two groups: with $(n=45)$ and a group without $(n=35)$ CAD. The mean serum concentration of total BA was $1.02 \pm 0.16 \mu \mathrm{mol} / 1$ in patients with, versus $2.16 \pm 0.38 \mu \mathrm{mol} / 1$ in patients without $\mathrm{CAD}(\mathrm{P}=0.005)$. This decrease, (adjusted for gender and age) was an independent predictor of CAD (odd ratio $=0.51$; $95 \%$ confidence interval $0.31,0.85 ; \mathrm{P}=0.01$ ). The BA concentrations in feces were similar in both groups. There was no group-specific pattern in the fecal microbiota. In a subgroup of 17 patients, one month of statin therapy increased the serum BA concentration from $0.68 \pm 0.08$ to $1.37 \pm 0.213 \mu \mathrm{mol} / \mathrm{l} ; \mathrm{P}=0.01$ ). Conclusion: There was no specific microbiota signature associated with CAD. However, the decreased serum BA concentration was a strong predictor of CAD in humans. With respect to the powerful anti-atherosclerotic effect of BA in animal models, and their role in human lipid metabolism and diabetes, this study unraveled the existence of a new metabolic disturbance associated with CAD.

Disclosure of Interest: All authors have declared no conflicts of interest.

\section{P1305 LOW FODMAP DIET: REINTRODUCTION PHASE DOES NOT MODIFY EFFICACY, BUT BEWARE OF REAL TRIGGER FOODS!}

M. Bellini ${ }^{1}$, A. Rossi ${ }^{2}$, D. Gambaccini ${ }^{1}$, D. Menicucci ${ }^{3}$, L. Bazzichi ${ }^{2}$, B. Fani ${ }^{4}$, R. Sellitri ${ }^{4}$, M.G. Mumolo ${ }^{4}$, N. De Bortoli ${ }^{4}$, L. Ceccarelli ${ }^{4}$, M. Mosca ${ }^{2}$, S. Marchi ${ }^{4}$

${ }^{1}$ Ospedale Di Cisanello Pisa, Pisa/Italy

${ }^{2}$ U.O. Reumathology, Ospedale Di Cisanello Pisa, Pisa/Italy

${ }^{3}$ Department Of Translational Research And Of New Surgical And Medical

Technologies, University of Pisa., Pisa/Italy

${ }^{4}$ U.O. Gastroenterology, Ospedale Di Cisanello Pisa, Pisa/Italy

Contact E-mail Address: mbellini@med.unipi.it

Introduction: The low-FODMAP diet (LFD) is used to treat patients with irritable bowel syndrome (IBS) even if some nutritional concerns have been raised. It starts with an elimination phase and is followed by a reintroduction phase to clearly detect the "symptom trigger" foods in order to suggest a definitive and less restrictive diet tailored to the patient.

Aims \& Methods: The aims of this study were to evaluate: 1) the effects of FODMAP reintroduction on a) body composition and nutritional status, using Bioelectrical Impedance Vector Analyses (BIVA), b) abdominal symptoms, c) quality of life, d) anxiety/depression, e) sleep quality. 2) if the patients' perception of the "trigger" foods was accurate.

66 IBS patients (54F, $12 \mathrm{M} ; 44.8 \pm 13.0$ yrs.) started (T0) a LFD for 8 weeks (T1) and followed a 9-14 week reintroduction period (T2). They underwent blood tests at T0 and T1. BIVA, anthropometric data, IBS-Symptom Severity Score, Bristol Stool Chart (BSC), SF36, Hospital Anxiety and Depression Scale and Pittsburgh Sleep Quality Index were performed at T0, T1 and T2. The patients were monitored by a nutritionist to verify their compliance.

Results: Neither change of blood tests at T1 nor variations of anthropometric data and BIVA were reported at T1 and T2 in comparison with T0. A significant improvement in abdominal symptoms (IBS-SSS), anxiety and quality of life, was recorded at $\mathrm{T} 1$, this remaining unchanged also at $\mathrm{T} 2(\mathrm{p}<0.0001)$. Depression improved at T2 ( $<<0.01$ vs. T0). Sleep quality improved at T1 ( $<0.05$ vs. T0) and at T2 ( $<<0.001$ vs.T0). Normal BSC feces were reported by 38 patients at T0 to 60 patients both at T1 and T2. The degree of symptom relief with the diet was $1.5 \pm 0.6$ at $\mathrm{T} 1$ and $1.6 \pm 0.7$ at $\mathrm{T} 2$ and the degree of satisfaction was high 
both at $\mathrm{T} 1(8.4 \pm 1.6)$ and $\mathrm{T} 2(8.2 \pm 1.7)$. When starting, LFD patients considered as trigger foods lactose $(67 \%)$, fructans $(27 \%)$, fructose $(17 \%)$, galacto-oligosaccharides (GOS) (17\%) and polyols (3\%); the reintroduction phase (T2) enabled us to detect lactose in $70 \%$, fructans in $30 \%$, fructose in $37 \%$, GOS in $33 \%$ and polyols in $27 \%$, as real triggers. The agreement (Cohen's kappa) was moderate for lactose (k: 0.50$)$, fair for fructans $(\mathrm{k}: 0.39)$ and fructose $(\mathrm{k}: 0.32)$ and poor for polyols (k: 0.16$)$ and GOS (k: 0.01).

Conclusion: Not only did reintroduction not affect the improvements achieved during the elimination phase, but it also precisely identified the foods responsible for the clinical manifestations. This enabled us to suggest a personalized diet for the patients. The real role played by FODMAPs in generating symptoms was abundantly underestimated and misunderstood by our patients. This underlines the fact that LFD has to be administered and carried out under the guide of an expert nutritionist.

Disclosure of Interest: All authors have declared no conflicts of interest.

\section{P1306 EXPRESSION OF THE FRUCTOSE TRANSPORTER GLUT5 IN PATIENTS WITH FRUCTOSE MALABSORPTION}

P. Staubach ${ }^{1}$, S. Krüger ${ }^{1}$, A. K. Koch ${ }^{2}$, J. Langhorst ${ }^{3}$, C. Röcken ${ }^{1}$, U. Helwig ${ }^{4}$ ${ }^{1}$ Department Of Pathology, University of Kiel, Kiel/Germany

${ }^{2}$ Department Of Integrative Gastroenterology, University of Duisburg-Essen, Essen/Germany

${ }^{3}$ Department For Internal And Integrative Medicine, Kliniken Essen-Mitte Integrative Gastroenterologie, Essen/Germany

${ }^{4}$ Medical Practice For Internal Medicine Oldenburg, University of Kiel, Oldenburg/ Germany

Contact E-mail Address: piastaubach@gmx.de

Introduction: Fructose malabsorption (FM) is a frequent finding in patients with abdominal symptoms due to high levels of fructose in Western diets. The role of monosaccharide transporter dysfunction in the small intestine is incompletely understood. The aim of this study was to investigate the histoanatomical distribution of the main fructose transporter GLUT5

Aims \& Methods: The study included 257 patients with FM diagnosed by hydrogen breath test and grouped according to the response to a fructose-free diet. 42 healthy individuals and 31 patients with coeliac disease (CD) served as control. The fructose breath test was done with $50 \mathrm{~g}$ fructose. Fructose malabsorption was defined as an increase of $20 \mathrm{ppm}$ of endexpiratory hydrogen. Formalin-fixed and paraffin-embedded duodenal biopsy specimens were obtained in all cases. Histology was assessed using hematoxylin and eosin stained tissue sections. Expression of GLUT5 was studied by immunohistochemistry. Expression patterns of GLUT5 were correlated with clinico-pathological patient characteristics. Results: The expression of GLUT5 did not differ significantly between patients with FM complete diet responders $(n=183)$ and healthy controls $(n=42)$. Also patients with FM responding to a fructose free diet did not differ in GLUT5 expression or in max. H2 increase and AUC measured in fructose breath testing from patients not responding to the diet $(n=40)$. However, in patients with CD $(\mathrm{n}=29)$ significant differences in GLUT5 expression were found compared to patients with FM and healthy controls $(p=0.009)$. The severity of $C D$ assessed by the Marsh score significantly correlated with the GLUT5 expression $\left(r_{\mathrm{s}}=-\right.$ $0.563, \mathrm{p}=0.001$ )

Conclusion: Changes in GLUT5 expression may not cause symptoms in adult patients with FM. The symptoms induced by FM could be associated with mechanisms known in the pathology of irritable bowel syndrome. However, in secondary malabsorption decreased GLUT5 expression was detected. Further investigation is needed to understand the essential factors in FM and the influence on functional gastrointestinal disorders.

Disclosure of Interest: All authors have declared no conflicts of interest.

\section{P1307 BETTER RESPONSE TO LOW FODMAP DIET IN JH NEGATIVE PATIENTS WITH DISORDERS OF GUT-BRAIN INTERACTION}

V. Schindler ${ }^{1}$, A. Zweig ${ }^{1}$, A. Becker ${ }^{2}$, J. Zeitz ${ }^{1}$, D. Runggaldier ${ }^{1}$, M. Fried ${ }^{1}$, D. Pohl ${ }^{1}$

${ }^{1}$ Gastroenterology And Hepatology, University of Zurich, University Hospital

Zurich, Zurich/Switzerland

${ }^{2}$ UniversitätsSpital Zürich, Zürich/Switzerland

\section{Contact E-mail Address: valeria.schindler@usz.ch}

Introduction: Previous studies have shown a reduction of gastrointestinal symptoms in patients with disorders of gut-brain interaction (FGID) when following a diet low in FODMAPs. Symptom relief due to a low FODMAP diet has especially been investigated in patients suffering from irritable bowel syndrome (IBS) and has proven to reduce gastrointestinal symptoms in up to $86 \%$ of patients with IBS. In addition, there is evidence for an association between gastrointestinal symptoms and joint hypermobility $(\mathrm{JH})$. However, there is no clear data regarding response rates to a diet low in FODMAPs in patients suffering from $\mathrm{JH}$. In this study we aimed to assess and compare the response to a diet low in FODMAPs in JH positive and JH negative patients with FGIDs.

Aims \& Methods: Data of patients presenting with FGID at the tertiary ambulatory functional bowel clinic between January 2015 and July 2016 were analyzed. FGIDs were diagnosed according to Rome III criteria. JH was assessed by physicians using Beighton score and rated positive for scores $\geq 4 / 9$ points. Patients received professional nutritional counseling on a diet low in FODMAPs. A global symptom response was assessed by a professional nutritionist after 4 to 6 weeks following a low FODMAP diet.

Results: Of all 84 patients screened for JH, $62(73.8 \%)$ were female and 22 $(26.2 \%)$ were male. Median age was 35 [range 18-79] years. Females were more likely to exhibit JH compared to males $(38 / 62$ [61.3\%] vs. 6/22 [27.3\%]; $\mathrm{p}=0.006$ ). Global symptom response rate to a diet low in FODMAPs was $64 / 84$ $(76.2 \%)$. Our data showed significantly better response to a low FODMAP diet in $\mathrm{JH}$ negative patients than in $\mathrm{JH}$ positive patients $(36 / 40$ [90.0\%] vs. $28 / 44$ $[63.6 \%], p=0.005$, ITT). Response of 7 patients was unknown because of early therapy discontinuation before nutritional re-counseling. When excluding 7 patients with therapy discontinuation from our calculations, the difference in diet response between $\mathrm{JH}$ negative and $\mathrm{JH}$ positive patients remained significant $(36 / 39[92.3 \%]$ vs. $28 / 38$ [73.7\%]; $p=0.036)$.

Conclusion: Our data indicate an association between global symptom response to a diet low in FODMAPs and joint hypermobility status in FGID patients. An underlying structural pathology or different pathophysiological factors (motility, intestinal permeability) causing gastrointestinal symptoms in JH positive patients and limiting response to low FODMAP diet should be considered. Our findings represent a further step towards pathophysiological features in FGIDs and might help to select patients for individually appropriate therapies.

Disclosure of Interest: M. Fried: Allergan, MSD, Astra, Vifor, Abbvie, UCB

D. Pohl: Allergan, Vifor, Astra, Permamed

All other authors have declared no conflicts of interest.

\section{P1308 CHANGES IN GASTROINTESTINAL SYMPTOMS, SMALL INTESTINAL BACTERIA, AND DUODENAL PHYSIOLOGY FOLLOWING A LOW-FIBER, HIGH-SUGAR DIET}

G. Saffouri ${ }^{1}$, B. Schmidt ${ }^{1}$, E. J. Battaglioli ${ }^{1}$, Y. Bhattarai ${ }^{1}$, J. Cho ${ }^{2}$, H. Lekatz $^{1}$, M. Grover ${ }^{1}$, G. Farrugia ${ }^{1}$, P. Kashyap ${ }^{1}$

${ }^{1}$ Gastroenterology And Hepatology, Mayo Clinic, Rochester/United States of America/MN

${ }^{2}$ Gastroenterology And Hematology, Mayo Clinic, Rochester/United States of America/MN

Contact E-mail Address: saffouri.george $@$ mayo.edu

Introduction: Gastrointestinal symptoms are often associated with dietary intolerances and are common in the developed world consuming a western diet low in fiber.

Aims \& Methods: To determine the effect of a high-sugar, low-fiber diet on GI symptoms, small intestinal bacteria, and duodenal physiology. This is a prospective single-center study. Healthy adults with baseline fiber intake $\geq 14 \mathrm{~g} / 1000$ calories/day; $<10 \%$ daily calories from added sugar; $>5$ servings of fruits and vegetables/day; and $\leq 13 \%$ daily calories from saturated fat were recruited. Exclusion criteria included known GI disease or symptoms, antibiotic/probiotic use within 4 weeks of the study, pregnancy, and vulnerable adults. At baseline visit, participants completed a symptom and demographic questionnaire and underwent esophagogastroduodenoscopy (EGD) with duodenal biopsies and aspirates. Symptoms assessed included: constipation, straining, incomplete evacuation, hard stools, abdominal pain associated with bowel movements, diarrhea, bloating, nausea/vomiting, heartburn, fatigue, and appetite. All participants consumed a 7-day standardized diet with typical United States macronutrient calorie distribution: $50 \%$ carbohydrates, $35 \%$ fat, $15 \%$ protein. The diet was low in fiber $(<10 \mathrm{~g} / 1000$ calories/day $)$ and high in simple sugar ( $\geq 50 \%$ daily carbohydrates). After dietary intervention, participants filled out follow up questionnaires and underwent repeat EGD with duodenal biopsies and aspirates. Before and after the diet, quantitative aerobic and anaerobic cultures were performed on duodenal aspirates. Duodenal biopsies were mounted in an Ussing chamber. Intestinal permeability was evaluated using transepithelial electrical resistance (TEER) and FITC flux (4KDa; a measure of paracellular transport). Secretory responses were quantified in voltage clamp mode by measuring baseline short circuit current (Isc) and change in Isc ( $\Delta$ Isc) in response to increasing concentrations $(0.003-300 \mu \mathrm{M})$ of serotonin $(5-\mathrm{HT})$ on the submucosal side. These measurements were repeated after the dietary intervention. Data are presented as mean \pm SEM. Data were analyzed using paired-t test unless specified and $\mathrm{p}<0.05$ was considered significant.

Results: A total of 10 participants (5 female; median age $26 ; 70 \%$ Caucasian) were recruited. Average BMI was $23.1 \mathrm{~kg} / \mathrm{m}^{2}$. At baseline, all participants were asymptomatic. After dietary intervention, all patients endorsed at least one new symptom and $9 / 10$ participants endorsed multiple $(\geq 2)$ new symptoms. At baseline $4 / 10$ participants had positive duodenal cultures $(>100,000 \mathrm{CFU} / \mathrm{mL}$, anaerobic) despite having no symptoms. Of the 6 who had no growth initially, 1 developed bacterial overgrowth following intervention. There was no significant difference in TEER $\left(26.45 \pm 1.98\right.$ vs $\left.26.18 \pm 2.45 \mathrm{Ohms} / \mathrm{cm}^{2}\right)$, FITC flux $(217 \pm 34.72$ vs $217.6 \pm 42.57 \mathrm{ng} / \mathrm{ml})$ or baseline Isc $(48.27 \pm 6.39$ vs $51.58 \pm$ $6.83 \mu \mathrm{A} / \mathrm{cm} 2$ ) before and after dietary intervention. Interestingly there was a significantly lower $\Delta$ Isc response to increasing concentrations of 5-HT after dietary intervention $(\mathrm{P}<0.05$, two-way ANOVA).

Conclusion: A low-fiber, high simple sugar diet led to gastrointestinal symptoms in participants who normally consume a high-fiber diet. This was associated with a significant decrease in 5-HT evoked secretory response in the duodenum, suggesting a potential role for dietary modulation of host serotonergic pathway. There was no correlation with quantitative bacterial cultures and there was no overall significant change in intestinal permeability. Diet may mediate these 
effects via a qualitative change in gut bacteria but this still needs to be determined.

Disclosure of Interest: All authors have declared no conflicts of interest.

\section{P1309 STRESS AND STRESS-RELATED PEPTIDE AMPLIFY THE ANOREXIC ACTIONS OF CHOLECYSTOKININ}

N. Yamaguchi, E. Hosomi, M. Ochiai, S. Ro, K. Chieko, K. Maezawa, S. Nagoshi, K. Takayama, K. Yakabi

Gastroenterology And Hepatology, Saitama medical University, kawagoe/Japan

Contact E-mail Address: yamaguoota810@yahoo.co.jp

Introduction: Recently roles of gut hormons on appetite control have been known. Among then, CCK is well known to suppress appetite and gastric motirity. On the other hand, patients of functional dispepisia (FD) have hyper sensitivity to CCK. And revels of CCK inbrad was shown to be high in FD patients. In FD patients, stress have important roles of pathogenesis of the disease.

Aims \& Methods: We undertook to clarify whether stress influences the actions of cholecystokinin $(\mathrm{CCK})$ on appetite and gastric emptying. As stress, we gave restraint stress, corticotropin-releasing factor (CRF) or urocortin 1 (UCN1) injection intraperitneally (IP). We also examined the effects of CCK and restraint stress on c-Fos expression in the neurons of appetite center of the brain. In the gastric emptying study, SD rats were fasted overnight. The amounts of the mixture (food and glass beads) left in the stomach were measured at 2 hours after the perorally injection of mixed food, and gastric emptying rate was calculated. In the study on appetite, CCK was IP injected and the amounts of food was measured at 1 and 2 hours after the injection. In some experiments, CRF or UCN1 was IP injected and the interaction with CCK on food intake was examined. In another study, restraint stress was given to rats and the interaction with CCK was evaluated. To study the involvement of brain in the interaction between CCK and stress, c-Fos expression in the neurons was examined and evaluated. Results: CCKdose-dependently inhibited gastric emptying. CCK dose-dependently inhibited food intake during $1 \mathrm{hr}$ and $2 \mathrm{hr}$. CRF $(10 \mu \mathrm{g} / \mathrm{kg}$ rat) significantly inhibited food intake. However, there was no interactive action between CCK and CRF on food intake. UCN1 $(3 \mathrm{nmol} / \mathrm{kg}$ rat) inhibited food intake at 1 and 2 hours. There was an synergistic action between CCK and UCN1 on food intake. Restraint stress amplified suppressive effect of CCK on gastric emptying and food intake. C-fos expression of the neurons in the nucleus of soritary tract (NTS) and paraventricular nucleus of hypothalamus (PVN) by CCK was amplified by the addition of restraint stress.

Conclusion: The result suggests that stress might amplify anorexic effects of CCK through the activation of satiety center of the brain that might be the possible pathogenesis for postprandial distress syndromes of FD.

Disclosure of Interest: All authors have declared no conflicts of interest.

\section{P1310 PEPTIDE TYROSINE-TYROSINE (PYY) ENHANCES EFFECTS OF CHOLECYSTOKININ (CCK) ON GASTRIC MOTILITY AND FOOD INTAKE IN RATS}

E. Hosomi ${ }^{1}$, N. Yamaguchi ${ }^{1}$, K. Maezawa ${ }^{1}$, M. Ochiai ${ }^{1}$, S. Ro ${ }^{1}$, K. Chieko ${ }^{1}$, K. Takayama ${ }^{1}$, K. Yakabi ${ }^{1}$

${ }^{1}$ Gastroenterology And Hepatology, Saitama medical University, Kawagoe/Japan

Contact E-mail Address: hosomi@saitama-med.ac.jp

Introduction: Cholecystokinin (CCK) and peptide tyrosine-tyrosine (PYY) have been known to suppress appetite and gastric emptying. Both peptides raise in blood by feeding, and maintain high levels simultaneously for 1-2 hours. Therefore there might be possible to cause interactive actions between two peptides, inducing satiation to finish food intake.

Aims \& Methods: In this study, we undertook to elucidate whether CCK and PYY have the interaction to decrease food intake. Study on gastric emptying. Male SD rats were fasted overnight, and $1 \mathrm{~mL}$ of mixture of food and glass beads was given into the stomach and then PYY or CCK or CCK followed by PYY was IP injected. The mixed food left in the stomach $1 \mathrm{hr}$ after the injection was measured and gastric emptying rate was calculated. Study on appetite. PYY or CCK was IP injected to the rats just before setting food to eat. The amounts of food were measured at 1 and 2 hrs after the injection. To clarify the involvement of the brain in the interaction between CCK and PYY, c-Fos expression was examined.

Results: CCK $(0.5-10 \mathrm{nmol} / \mathrm{kg})$ dose-dependently inhibited gastric emptying $(\mathrm{p}<0.001)$. CCK $10 \mathrm{nmol} / \mathrm{kg}$ maximally inhibited food intake $(\mathrm{p}<0.01)$ PYY $25-2500 \mathrm{pmol} / \mathrm{kg}$ significantly inhibited gastric emptying for 1 or $2 \mathrm{hrs}$ after the injection $(\mathrm{p}<0.01)$. PYY $250 \mathrm{pmol} / \mathrm{kg}$ significantly inhibited food intake for $1 \mathrm{hr}$ after the injection $(\mathrm{p}<0.01)$. The combination of CCK $10 \mathrm{nmol} / \mathrm{kg}$ and PYY $250 \mathrm{pmol} / \mathrm{kg}$ inhibited gastric emptying more than CCK alone (significant, $\mathrm{p}<0.01$ ) or PYY alone (not significant). PYY and CCK additively inhibited food intake when PYY was injected 20 minutes later from CCK injection. PYY significantly amplified c-Fos expression induced by CCK in the nucleus of soritary tract (NTS) and paraventricular nucleus of hypothalamus (PVN) in the brain.
Conclusion: The combination of PYY with CCK amplified the suppression of gastric emptying and food intake. The result suggests that the sequencing secretion of CCK and PYY might strengthen the inhibition of food intake through the activation of satiety center in the brain, that is important for terminating food intake and adjusting energy intake.

Disclosure of Interest: All authors have declared no conflicts of interest.

\section{WEDNESDAY, NOVEMBER 01, 201709:00-14:00} LIVER AND BILIARY III - HALL 7

\section{P1311 REGULATION OF MICRORNAS BY P53 FAMILY MEMBERS IN HEPATOCELLULAR CARCINOMA}

R. Hermelink ${ }^{1}$, M. Bender ${ }^{2}$, L. Kaser ${ }^{1}$, E. Aschenbrenner ${ }^{1}$, K. Pollinger ${ }^{1}$, C. Kunst ${ }^{1}$, M. Müller-Schilling ${ }^{1}$

${ }^{1}$ Department Of Internal Medicine I, University Hospital Regensburg,

Regensburg/Germany

${ }^{2}$ Department Of Internal Medicine IV, Hepatology And Gastroenterology, University Hospital Heidelberg, Heidelberg/Germany

Contact E-mail Address: rafaelhermelink@web.de

Introduction: Transcriptions factors belonging to the p53 family (p53, p63, p73) respond to cellular stress signals by inducing an accurately defined set of genes. In a number of tumors, also in hepatocellular carcinoma (HCC), p53 proteins can exert cancerogenic or tumorsuppressive functions. MicroRNAs are small, non-coding RNA molecules which play an important role in gene regulation. It is known that expression patterns of microRNAs can be controlled by the p53 family. Depending on disease and cellular origin different sets of p53-induced microRNAs have been identified.

Aims \& Methods: Little is known about p53-dependent microRNA signatures in HCC. The aim of the study was therefore to identify p53-family-regulated microRNAs in HCC. Hep3B cells were transfected with rAd-p53 and -p73. Microarray analyses were performed to identify p53- and p73-regulated microRNAs. Verification of p53- and p73-dependent microRNA expression was performed by qPCR. To evaluate the effect of HCC-relevant therapeutics on p53-dependent regulation of microRNAs transfected Hep3B cells were incubated with Doxorubicin, Sorafenib, Tivantinib, and Regorafenib in vitro.

Results: Overexpression of $\mathrm{p} 53$ and $\mathrm{p} 73$ induced a rash of microRNAs. p53 induced miR-34a by 2.4 -fold, miR-145 by 2.7 -fold and led to a slight reduction of miR-149. In the presence of p73 miR-34a was induced by 5.4 -fold, miR145 by 3.2 -fold, and miR-149 by 55 -fold. p53-dependent expression of miR-34a was further increased in the presence of Doxorubicin (5.7-fold), Regorafenib (2.5-fold) and Tivantinib (1.9-fold) compared to controls. Moreover, incubation with Regorafenib resulted in an up to 5.4-fold increase of p53-dependent expression of miR-149 and miR-192.

Conclusion: p53 proteins affect the microRNA signature in HCC. Beside the already known induction of miR-34a we demonstrate for the first time a regulation of miR-145 and -149 by $p 53$ und $p 73$. We hypothesize that regulation of tumorsuppressive microRNAs represents an effector mechanism by which p53 family members exert their role in tumor development and treatment response. The observed synergistic effect of p53 and HCC-relevant therapeutics on microRNA expression might provide new options for the development of therapeutic and prognostic measures in HCC

Disclosure of Interest: All authors have declared no conflicts of interest.

\section{P1312 IGFBP2 IS REGULATED BY THE P53 FAMILY OF TRANSCRIPTION FACTORS IN HEPATOCELLULAR CARCINOMA}

D. Gschwind ${ }^{1}$, M. Lohse ${ }^{1}$, E. Aschenbrenner ${ }^{1}$, K. Pollinger ${ }^{1}$, S. Schlosser ${ }^{1}$, C. Kunst ${ }^{1}$, M. Müller-Schilling ${ }^{1}$

${ }^{1}$ Department For Internal Medicine I, Universitiy Hospital Regensburg,

Regensburg/Germany

Contact E-mail Address: dana.gschwind@stud.uni-regensburg.de

Introduction: $\mathrm{p} 53$ transcription factors (p53, p63, p 73) respond to cellular stress by transcriptional regulation of specific sets of genes. In hepatocellular carcinoma (HCC) and other tumors p53 family members exert cancerogenic or tumorsuppressive effects. Depending on their splice variants-with transactivation domain (TA)/dominant negative (DN)-and the characteristics of the particular binding site (BS) p53 proteins activate or inhibit specific target genes. We previously identified the IGFBP2 gene (Insulin-Like Growth Factor Binding Protein 2 ) as one out of 7 putative target genes for p53 proteins with prognostic relevance in $\mathrm{HCC}$.

Aims \& Methods: The aim of this study was to characterize the so far unknown regulation of the IGFBP2 gene by 533 family members in HCC. Hep3B cells were transfected with rAd-p53 and -TAp73 and transcriptional regulation of IFGBP2 was determined by qPCR. Intra- and extracellular IGFBP2 protein levels were analyzed by Western Blot and ELISA. Transfac database analyses were performed to identify potential BS for p53 and p73 in the IGFBP2 locus. These sequences were cloned, mutated and analyzed for $\mathrm{p} 53$ family binding in luciferase reporter assays. Binding of p53 and p 73 to the identified BS was confirmed by CHIP experiments.

Results: TAp73 transfection increased IGFBP2 expression by up to 60-fold and resulted in elevated intra- and extracellular IGFBP2 protein levels, whereas IGFBP2 protein was not detected in controls. p53 transfection induced IGFBP2 expression by up to 7-fold. Two potential p53 and p73 BS are located in the promoter region, another 5 potential p73 and one p53 BS were identified in intron 1 of the IGFBP2 gene. Intron 1-dependent luciferase activity was increased by up to 110-fold after TAp73 transfection and up to 20-fold after p53 transfection. Mutation and deletion of the identified p53 BS in intron 1 resulted in a reduction of luciferase activity by up to $85 \%$. Deletion of one 
potential p73 BS reduced luciferase activity by $85 \%$. CHIP analyses verified binding of p53 and TAp73 to the particular BS.

Conclusion: These results clearly identify IGFBP2 as novel target gene for TAp73 and $\mathrm{p} 53$ in HCC. We demonstrate for the first time an interaction of TAp73 with IGFBP2 and therefore an important, so far unknown link between the $\mathrm{p} 53$ family network and IGF pathways. p53 proteins exert predominantly tumor-inhibiting effects, whereas the IGF axis is of critical importance for proliferative events. The particular ratio of both important signaling seems to be decisive for tumor characteristics and treatment response. Our results therefore not only expand our knowledge of cancerogenic processes, but also indicate that finetuning of these signalling pathways provides new therapeutic options in clinical management of $\mathrm{HCC}$

Disclosure of Interest: All authors have declared no conflicts of interest.

\section{P1313 HOW TO IMPROVE THE RELIABILITY OF LIVER FIBROSIS EVALUATION USING 2D-SWE.GE}

F. Bende, I. Sporea, R. Sirli, M. Danila, S.A. Popescu

Gastroenterology And Hepatology, University of Medicine and Pharmacy "Victor Babes" Timisoara, Timisoara/Romania

Contact E-mail Address: bendefelix@gmail.com

Introduction: Liver stiffness (LS) evaluation as a marker of fibrosis is usually considered reliable when it fulfills some quality criteria. Classic criteria used for Transient Elastography (TE) are: $\geq 10$ valid measurements, $\geq 60 \%$ success rate, and interquartile range/median ratio $(\mathrm{IQR} / \mathrm{M}) \leq 0.30$ [1]. However, new quality criteria were proposed using the IQR/M ratio, therefore the LS measurements can be classified into three categories: very reliable (IQR $/ \mathrm{M}<0.10$ ), reliable $(0.10<\mathrm{IQR} / \mathrm{M} \leq 0.30)$, poorly reliable (IQR/M $>0.30)$ [2].

Aims \& Methods: The aim of this study was to assess the impact of using quality criteria for liver stiffness (LS) evaluation by means of 2D Shear Wave Elastography from General Electrics (2D-SWE.GE), while using Transient Elastography (TE) as the reference. We included 226 subjects in our study, with or without chronic liver disease, in whom LS was assessed using 2DSWE.GE (LOGIQ E9, GE Healthcare) and TE (FibroScan, EchoSens). Reliable LS measurements were defined for TE as the median value of 10 measurements with a success rate of $\geq 60 \%$ and an interquartile range (IQR) $<30 \%$ of the median LS values. For 2D-SWE.GE 10 LS measurements were acquired in a homogenous area and the IQR and the IQR/M were calculated in each case. We divided our subjects into 3 groups according to the 2D-SWE.GE IQR/M: IQR/M < 0.10: $41(18.1 \%)$ cases; $0.10<$ IQR/M $\leq 0.30: 155(68.6 \%)$ cases; IQR $\mathrm{M}>0.30: 30(13.3 \%)$ cases. We calculated the correlation coefficient between TE and 2D-SWE.GE in each group.

Results: All $226(100 \%)$ subjects included had 10 valid measurements by means of 2D-SWE.GE and reliable results by TE. A strong positive correlation was found between LS values obtained by means of 2D-SWE.GE and TE in the IQR/ $\mathrm{M}<0.10$ group $(\mathrm{r}=0.84, \mathrm{p}<0.0001)$. A strong positive correlation was found between LS values obtained by means of 2D-SWE.GE and TE in the $0.10<\mathrm{IQR} / \mathrm{M} \leq 0.30$ group $(\mathrm{r}=0.80, \mathrm{p}<0.0001)$. A weak positive correlation was found between LS values obtained by means of 2D-SWE.GE and TE in the IQR $/ M>0.30$ group $(r=0.41, p=0.02)$. The correlations were significantly stronger in the IQR/M $<0.10$ and $0.10<\mathrm{IQR} / \mathrm{M} \leq 0.30$ groups as compared to the IQR $/ \mathrm{M}>0.30$ group (both $\mathrm{p}=0.0013$ ). No statistical differences were found between the correlations in the IQR $/ \mathrm{M}<0.10$ and $0.10<\mathrm{IQR} / \mathrm{M} \leq 0.30$ groups $(\mathrm{p}=0.43)$.

Conclusion: Using the IQR $/ \mathrm{M}<0.30$ as quality criteria significantly increase the reliability of LS measurements by means of 2D-SWE.GE. Using IQR/M $<0.10$ criteria does not significantly improve the reliability of 2D-SWE.GE LS measurements as compared to $0.10<\mathrm{IQR} / \mathrm{M}<0.30$ criteria.

Disclosure of Interest: I. Sporea: I hereby confirm that I have received financial support (congress travel grant or speaker fee) from Philips, Siemens, General Electric, Abbvie, Zentiva, Bristol Meyers Squibb

R. Sirli: I hereby confirm that I have received financial support (congress travel grant or speaker fee) from Philips, Abbvie, Zentiva

S.A. Popescu: I hereby confirm that I have received financial support (congress travel grants, speaker fee) from: Philips, General Electric, Abbvie, AstraZeneca, Zentiva

All other authors have declared no conflicts of interest.

\section{References}

1. Castera L, Foucher J, Bernard PH, et al. Pitfalls of liver stiffness measurement: a 5-year prospective study of 13,369 examinations. Hepatology 2010; 51: $828-835 \mathrm{a}$

2. Boursier J, Zarski JP, de Ledinghen V, et al; Multicentric Group from ANRS/HC/EP23 FIBROSTAR Studies. Determination of reliability criteria for liver stiffness evaluation by transient elastography. Hepatology 2013; 57 . $1182-1191$.

\section{P1314 MONITORING OF LIVER FUNCTION IN PATIENTS WITH NONALCOHOLIC FATTY LIVER DISEASE IN COMBINATION WITH METABOLIC SYNDROME}

K. Aksentiychuk, E. Sklyarov, N. Kurlyak, A. Bochar

Therapy \#1 And Medical Diagnostics, LNMU by Danylo Galytskyi Dept. of

Therapy, Lviv/Ukraine
Contact E-mail Address: akskris@mail.ru

Introduction: $13 \mathrm{C}$ - methacetin breath test (13C-MBT) is used to specify the detoxification function of the liver by determination its metabolic capacity and degree of hepatocytes recovery.

Aims \& Methods: The study involved 113 patients with MS aged from 37 to 82 years, the average age was $55.8 \pm 3.5$. There were 75 men and 88 women. The criteria, which has been examined for the functional state of the liver was 13CMBT. The control group included 25 patients with a BMI of $25-30 \mathrm{~kg} / \mathrm{m} 2$ without severe concomitant diseases. Indicators of $13 \mathrm{C}$-MBT were metabolic rate, cumulative dose of $13 \mathrm{C}$-methacetin on 40 and 120 minutes. Also evaluation was carried by mathematical deduction which measured the liver dysfunction stage. Results: The data showed normal detoxication liver function in patients without MS $(20.11 \% \pm 0.55)$. The results were below normal in patients with BMI higher than $25 \mathrm{~kg} / \mathrm{m} 2$, which indicated that there were initial changes in the functional state of liver. In patients with steatosis - cumulative dose on 120 minute was $15.12 \% \pm 0.49$, which corresponded to a moderate reduction of detoxification function with the mass of function hepatocytes $50-100 \%$. The data of $13 \mathrm{C}-\mathrm{MBT}$ in patients with steatohepatitis showed pronounced changes of the liver detoxification function $(8.88 \pm 0.64 \%)$. All indicators at steatosis group have indicated the moderate decline detoxification function with the level of function hepatocytes $50-100 \%($ Mvmax $40=0.66$, Cum.Dose $40=0.7$, Cum.Dose $120=0.54)$. The data of patients with steatohepatitis indicated the detoxification function with declining of the mass of function hepatocytes to $20-50 \%$ (Mvmax $40=0.46$, Cum. Dose $40=0.5$, Cum. Dose $120=0.32$ ). The results of the control group showed its compliance with norm, but one of the indicators (Cum. Dose 120) was related to the moderate decline detoxification function. Despite it, most of the criteria have been accorded to the norm. However, the results of data suggest the opportunity of early violation of detoxification liver function in patients with a BMI over $25 \mathrm{~kg} / \mathrm{m} 2$.

Conclusion: In assessing to the data of $13 \mathrm{C}$ - MBT, main attention is paid to mathematical calculation of $\mathrm{CO} 2$ labeled methacetin. It allows to identify the early stages of the liver detoxification function violation.

Disclosure of Interest: All authors have declared no conflicts of interest.

\section{P1315 IDENTIFICATION OF P73 AS A NOVEL TRANSACTIVATOR OF IGFBP4 GENE EXPRESSION IN HEPATOCELLULAR CARCINOMA}

D. Gschwind ${ }^{1}$, S. Heckel ${ }^{1}$, E. Aschenbrenner ${ }^{1}$, K. Pollinger ${ }^{1}$, S. Schlosser ${ }^{1}$, C. Kunst ${ }^{2}$, M. Müller-Schilling

${ }^{1}$ Department For Internal Medicine I, Universitiy Hospital Regensburg,

Regensburg/Germany

Contact E-mail Address: sebastian-heckel@outlook.de

Introduction: Members of the p53-family, including p53, p63 and p73, are known for their involvement in the regulation of cell cycle, cell senescence and apoptosis. In their role as transcription factors and depending on their splice variants-with transactivation domain (TA) or dominant negative (DN) - p53 and its siblings are capable of activating or inhibiting the transcription of specific target genes. We previously identified the gene for Insulin-like Growth Factor Protein 4 (IGFBP4) as potential p53-family target gene with prognostic relevance in hepatocellular carcinoma (HCC). In contrast to p53, the IGF system takes part in tissue growth and cell survival. IGFBP4 acts as inhibitor limiting IGF effects suggesting a possible interaction with p53 affairs.

Aims \& Methods: The aim of the study was to characterize the regulatory influence of $\mathrm{p} 53$ family members on the IGFBP4 gene. Hep3B cells were transfected with rAd-p53, -TAp63, -TAp73, -DNp63, and -DNp73. Transcriptional regulation of IGFBP4 was determined by real time qPCR. Intra- and extracellular IGFBP4 protein levels were examined by Western Blotting and ELISA. TRANSFAC database analysis was performed to identify potential p53-family binding sites in the IGFBP4 locus. Identified sequences were cloned, deleted and analyzed by luciferase reporter assays to evaluate binding of p53-family members.

Results: IGFBP4 expression was increased by more than 30 -fold in TAp73transfected Hep3B cells, by more than 15-fold in DNp63- and by 3-fold in p53-transfected cells. Induction of intracellular IGFBP4 protein was detected in all transfected Hep3B cells, whereas extracellular IGFBP4 levels were only measurable after TAp73 and DNp63 transfection. Database analysis identified 2 putative p 73 binding sites within intron 1 of the IGFBP4 gene. Intron 1-dependent luciferase activity was increased by up to 20-fold in TAp73-transfected cells. This induction was reduced by up to $70 \%$ when one of the putative binding sites was deleted.

Conclusion: These results identify the IGF inhibitor IGFBP4 as novel target gene for TAp73 in HCC. By demonstrating for the first time the interaction of TAp73 and IGFBP4 we enhance our knowledge in a so far unknown association of p53family network and IGF signaling. Since in an independent study we identified IGFBP2 as a novel p53-family target gene, these results highlight the link between p53-family-mediated tumor-inhibiting mechanisms and IGF-dependent cell proliferation. We therefore suppose that the particular balance of these pathways decides on growth, cancerogenesis and treatment response.

Disclosure of Interest: All authors have declared no conflicts of interest. 
P1316 NONALCOHOLIC FATTY LIVER DISEASE IN PATIENTS

WITH 2 TYPE DIABETES MELLITUS AND CORONARY HEART

DISEASE AGAINST THE BACKGROUND OF METABOLIC

SYNDROME. HOW TO DIAGNOSE?

K. Aksentiychuk, E. Sklyarov, N. Kurlyak, A. Bochar

Therapy \#1 And Medical Diagnostics, LNMU by Danylo Galytskyi Dept. of

Therapy, Lviv/Ukraine

Contact E-mail Address: akskris@mail.ru

Introduction: It is known that to determine nonalcoholic fatty liver disease (NAFLD), which develops in progress of body mass index (BMI) from $19 \%$ to $35 \%$, using instrumental and laboratory methods, which include an ultrasound, the determination of the transaminase levels, steatotest, 13C-methacetine test. However, the above research methods do not allow to clearly differentiate steatosis from the steatohepatitis, that reduces their credibility.

Aims \& Methods: 163 patients ( 75 men, 88 women) with 2 type diabetes mellitus and coronary heart disease with metabolic syndrome, were examined. The average age was $55.82 \pm 75$. On the above criteria of ultrasound, 72 patients were diagnosed as steatosis group, 66 - steatohepatitis group. In 25 patients liver pathology was not found, which identified as a control group.

For verification of steatosis and steatohepatitis diagnosis the level of ALT, diameter vena portae and data $13 \mathrm{C}$-methacetin breath test were evaluated.

Results: The rate of liver metabolism based on ${ }^{13} \mathrm{C}$-methacetine test results in patients without NAFLD was $22.07 \pm 0.66 \%$, in patients with steatosis $17.1 \pm 0.84 \%$, steatohepatitis $-14.3 \pm 0.62 \%$. Cumulative dose of methacetine on 120 minute was $20.25 \pm 0.46 \%$ in patients without pathology of liver, $16.1 \pm 0.49 \%$ in patients with steatosis, $11.4 \pm 0.36$ in patients with steatohepatitis. ALT level in control group was $0.4 \pm 0.05 \mathrm{mmol} / \mathrm{l}$, with steatosis $0.6 \pm 0.06 \mathrm{mmol} / 1$, steatohepatitis $0.9 \pm 0.1 \mathrm{mmol} / 1$. The diameter of vena portae in control group was $11.2 \pm 0.26 \mathrm{~mm}$, in group of steatosis $11.9 \pm 0.21 \mathrm{~mm}$, in patients with steatohepatitis $13.7 \pm 0.15 \mathrm{~mm}$. There were investigated, that the rate of metabolism and its cumulative dose on 120 minute has decreased in steatosis group in comparison of the control group, with simultaneous significant increasing of ALT level and diameter of the portal vein. However, a significant reduction of the metabolism rate of methacetin and the cumulative dose ${ }^{13} \mathrm{CO}_{2}$ in the background of the increase of a diameter of vena portae compared with a group of steatosis, were examined in steatohepatitis group.The investigation found that in ALT and the diameter of the portal vein negatively correlated with cumulative dose of ${ }^{13} \mathrm{CO}_{2}$ on 120 minute in patients with steatohepatitis. Therefore, a decrease in the metabolic capacity from 15 to $10 \%$ accompanied by an increase in ALT levels (more $0,68 \mathrm{mmol} / \mathrm{l}$ ) and diameter of the portal vein $(13 \mathrm{~mm})$

Conclusion: The differentiation between steatosis and steatohepatitis should be moderate with the speed of a metabolism, a cumulative dose of methacetine on 120 minutes via $13 \mathrm{C}-\mathrm{MBT}$ and vena portae diameter measurement. The sensitivity of the method is based on the definition of the three proposed indicators - ALT level, total concentration of ${ }^{13} \mathrm{C}$-methacetin on 120 minute and the portal vein size, which is $75 \%$ for steatosis, and $76 \%$ for steatohepatitis. Disclosure of Interest: All authors have declared no conflicts of interest.

\section{P1317 BMP1-3 IN LIVER FIBROSIS}

I. Grgurevic ${ }^{1}$, L. Grgurevic ${ }^{2}$, S. Vukicevic ${ }^{2}$, I. Erjavec ${ }^{2}$, I. Dumic Cule ${ }^{2}$ ${ }^{1}$ Gastroenterology, Dubrava, Zagreb/Croatia

${ }^{2}$ Medical School University of Zagreb, Zagreb/Croatia

Contact E-mail Address: lgrgurev@mef.hr

Introduction: Liver fibrosis (LF) is a progressive pathological process resulting in accumulation of excess extracellular matrix proteins. A metalloprotease BMP1 cleaves various matrix proteins and procollagen type I. We have recently discovered that BMP1-3 isoform circulates in the plasma and its neutralization decreases the progression of chronic kidney disease. The role of BMP1 in liver diseases associated with fibrosis has not been investigated. Bone Morphogenetic Protein 1 (BMP1) and mammalian tolloid (mTLD) are alternatively spliced products of the Bmpl gene that are essential for tissue pattering and ECM assembly by biosynthetic processing of a wide range of ECM precursors specifically procollagen type I undergoing enzymatic cleavage by BMP1 at the C-terminal end of the procollagen finally leading to liver cirrhosis, portal hypertension and liver failure.

Aims \& Methods: We used the model of $\mathrm{CCl}_{4}$-inducedLF in rats. Animals were treated with BMP1-3 antibody at two different concentrations $(20$ and $50 \mu \mathrm{g} / \mathrm{kg})$ for 3 and 8 weeks. Extent of LF was assessed by histology, morphometric analyses, shear wave elastography and hydroxyproline (HP) content measurement. Gene expression analyses were performed by qRT-PCR, and protein localization by immunohistochemistry. BMP7 protein $(150 \mu \mathrm{g} / \mathrm{kg})$ was used as a comparative therapy.

Results: In our experiments the presence of BMP1-3 was immunohistochemically demonstrated in both healthy and cirrhotic liver suggesting that at least a part of circulating BMP1-3 is produced in the liver. Administration of BMP1-3 Ab resulted in an effective inhibition of the fibrosis progression. Administration of BMP1-3 Ab at a dose $20 \mu \mathrm{g} / \mathrm{kg}$ and $50 \mu \mathrm{g} / \mathrm{kg}$ exerted antifibrotic activity comparable to that of BMP7. Apart from blocking the BMP1-3 activity, which is important for collagen maturation, administration of BMP1-3Ab was accompanied by the reduced expression of $\operatorname{Tg} f \beta, \operatorname{Itg} \beta 6$ and $\alpha$-Sma gene expression whereas expression of Decorin mRNA was enhanced. Decorin has evident anti-fibrotic activity in the liver after injury with $\mathrm{CCl}_{4}$ and silencing caused an increased activation of HSCs both in vivo and in vitro. Rats treated with both BMP1-3 antibody concentrations had significantly lower amount of collagen type I when compared to the $\mathrm{CCl}_{4}$-treated group. Direct proportionality was found between the degree of fibrosis and liver stiffness. We showed for the first time a distinct correlation between the HP level in liver, morphometric analysis and elastography of the liver.

Conclusion: Our results suggest that neutralization of BMP1-3 is a promising therapeutic aproach in preventing the liver fibrosis progression.

Disclosure of Interest: All authors have declared no conflicts of interest.

\section{P1318 LIVER TRANSIENT ELASTOGRAPHY IN NON-ALCOHOLIC FATTY LIVER DISEASE: IS THERE ANY PREDICTIVE ROLE IN THE DEVELOPMENT OF COLORECTAL POLYPS?}

M. Gravito-Soares ${ }^{1}$, E. Gravito-Soares ${ }^{2}$, D. Gomes $^{2}$, A. Simão ${ }^{3}$, L. Tomé ${ }^{1}$ Centro Hospitalar e Universitário Coimbra, Coimbra, Portugal, Coimbral Portugal

${ }^{2}$ Gastroenterology, Centro Hospitalar e Universitário Coimbra, Coimbra, Portugal, Coimbra/Portugal

${ }^{3}$ Internal Medicine, Centro Hospitalar e Universitário Coimbra, Coimbra,

Portugal, Coimbra/Portugal

Contact E-mail Address: ms18498@gmail.com

Introduction: Recent studies have demonstrated an association between decreased glucose tolerance, dyslipidemia and metabolic syndrome; and increased risk of colorectal polyps. Patients with non-alcoholic fatty liver disease (NAFLD) often have these risk factors. The association between NAFLD and colorectal polyps has been poorly studied.

Aims \& Methods: We aimed to evaluate the prevalence and risk factors of colorectal polyps in patients with NAFLD. This was a retrospective observational cohort study of 237 patients with NAFLD submitted to transient elastography by Fibroscan ${ }^{\circledR}$, between $01 / 2015-02 / 2017$. Exclusion criteria: age $<18$ years, absence of total colonoscopy with good preparation $<3$ years, inflammatory bowel disease, hereditary polyposis syndromes and personal/family history of colorectal polyps/neoplasia. Compared patients with colorectal polyps(cases) and without colorectal polyps(controls). Demographic variables, cardiovascular/metabolic risk factors, comorbidities, laboratory parameters and Fibroscan $^{(B)}$ scores of steatosis $(\mathrm{CAP}>300 \mathrm{~dB} / \mathrm{m})$ and fibrosis(F4: $\left.>10 \mathrm{KPa}\right)$ were evaluated.

Results: Of the 237 NAFLD patients who performed Fibroscan ${ }^{\circledR}, 103$ underwent total colonoscopy. The prevalence of colorectal polyps was $28.2 \%(\mathrm{n}=29)$ : $19.4 \%(20 / 103)$ hyperplastic, $16.5 \%(17 / 103)$ adenoma and $4.8 \%(5 / 103)$ advanced adenoma/adenocarcinoma. The mean age was $58.32 \pm 51$ years (vs57.09 $\pm 10.53 ; \mathrm{p}=0.089$ ), with men predominant $(51.7 \% \mathrm{vs} 63.5 \% ; \mathrm{p}=0.272)$, mostly located in the left colon $(55,2 \% \mathrm{vs} 44.8 \% ; \mathrm{p}=0.314)$ and number and mean size of $1.46 \pm 0.88$ and $6.89 \pm 6.56 \mathrm{~mm}$, respectively. After multivariate analysis, colorectal polyps were associated with F4 liver fibrosis (34.5\%vs $14.9 \% ; \mathrm{p}=0.026 ; \mathrm{OR}=3.01) \quad$ and obesity $\quad\left(\mathrm{BMI}>30 \mathrm{Kg} / \mathrm{m}^{2}: \quad 55.2 \% \mathrm{vs}\right.$ $29,7 \% ; \mathrm{p}=0.016 ; \mathrm{OR}=2.91)$; hyperplastic polyps were associated with liver fibrosis for a cut-off value of $6.9 \mathrm{Kpa}$ (AUROC:0.689; $=0.008 ; \mathrm{S}=85.7 \%$; $\mathrm{Sp}=51.2 \%)$, mainly $\mathrm{F} 4(42.8 \% \mathrm{vs} 14.6 \% ; \mathrm{p}=0.004 ; \mathrm{OR}=4.38)$, hyperuricemia gout $(23.8 \% \mathrm{vs} 8.5 \% ; \mathrm{p}=0.042 ; \mathrm{OR}=3.35)$ and peptic ulcer disease $(9.5 \% \mathrm{vs}$ $1.2 \% ; \mathrm{p}=0.043 ; \mathrm{OR}=8.53) ;$ adenoma was associated with liver steatosis $(88.2 \% \mathrm{vs} 83.7 \% ; \mathrm{p}=0.024 ; \mathrm{OR}=3.50), \quad \mathrm{F} 4$ liver fibrosis $(41.2 \% \mathrm{vs} 16.2 \%$; $\mathrm{p}=0.041 ; \mathrm{OR}=3.24) \quad$ and obesity $\quad(58.8 \% \mathrm{vs} 32.6 \% ; \mathrm{p}=0.040 ; \mathrm{OR}=2.96)$; advanced adenoma/adenocarcinoma was associated with F4 fibrosis $(20.0 \% \mathrm{vs} 2.1 \% ; \mathrm{p}=0.021 ; \mathrm{OR}=1.224), \quad$ hyperuricemia $/$ gout $\quad(40.0 \% \mathrm{vs} 10.3 \%$; $\mathrm{p}=0.044 ; \mathrm{OR}=1.50)$ and dilated cardiomyopathy $(20.0 \% \mathrm{vs} 1.0 \% ; \mathrm{p}=0.003$; $\mathrm{OR}=1.24)$.

Conclusion: More than $1 / 4$ of the patients with NAFLD have colorectal polyps, being $16.5 \%$ adenoma and $4.8 \%$ advanced adenoma/adenocarcinoma. Obesity and liver steatosis are independent risk factors for colorectal adenoma. Liver fibrosis, especially F4 is an independent risk factor for all types of colorectal polyps.

Disclosure of Interest: All authors have declared no conflicts of interest.

\section{P1319 "SUBTRACTED ADULTHOOD MASS INDEX" (SAMI)- A NEW INDEX TO PREDICT NAFLD RISK IN NON-OBESE INDIVIDUALS}

A. Kıyak ${ }^{1}$, S. Elibol ${ }^{1}$, M. Saruc ${ }^{2}$, Ö. Saygılı ${ }^{3}$, N. Tözün ${ }^{4}$

${ }^{1}$ School Of Medicine, Acrbadem University, Istanbul/Turkey

${ }^{2}$ Gastroenterology, Acibadem University Faculty of Medicine Acibadem Bakirkoy Hospital, Istanbul/Turkey

${ }^{3}$ Radiology, Ac1badem University, Istanbul/Turkey

${ }^{4}$ School Of Medicine, Acibadem University, Istanbul/Turkey

Contact E-mail Address: atakiyak@gmail.com

Introduction: Non-alcoholic fatty liver disease (NAFLD) is a common clinicopathological condition which may progress from simple steatosis to NASH, cirrhosis and hepatocellular carcinoma(HCC). Although obesity is accepted as the main risk factor for NAFLD, non-obese individuals are often diagnosed with NAFLD suggesting that high BMI may not be "a sine qua non" for the presence of NAFLD. Recent studies suggested that there might be a correlation between weight gain and metabolic diseases.

Aims \& Methods: In our research; the relationship between NAFLD in non-obese individuals and the amount of weight gain during adulthood was investigated and a new index that is different from BMI was proposed. 362 individuals were included in the survey. The subjects were selected among patients who had abdominal ultrasonography(USG) in our clinic, during the last 6 months. A $5 \%$ increase in echogenicity detected in the USG was defined as the diagnostic 
limit for hepatosteatosis. The beginning of adulthood was taken as 20 years old. Exclusion criteria were; HBsAg or anti-HCV positivity, alcohol use, liver disease, pregnancy, cancer treatment, renal insufficiency, being younger than 25 years old. Patients were evaluated for the presence of diabetes, hypertension or cardiovascular disease and ALT, AST, fasting plasma glucose and cholesterol values were recorded. Statistical evaluations were done with IBM SPSS.

NAFLD evaluation with SAMI 3 cut-off value

\begin{tabular}{lll}
\hline & NAFLD $(+)$ & NAFLD $(-)$ \\
\hline SAMI $>3$ & 144 & 38 \\
SAMI $<3$ & 25 & 77 \\
& & \\
Sensitivity & $85,2 \%$ & \\
Specificity & $66,95 \%$ & \\
PPV & $79,12 \%$ & \\
NPV & $75,49 \%$ & \\
\hline
\end{tabular}

Results: Among 362 participants $169(46.7 \%)$ were men with an average age of $44.81 \pm 10.73 .78(21.6 \%)$ participants were obese, the average age of the group being $46.78 \pm 9.12$. Out of 78 obese individuals $73(93.5 \%)$ were NAFLD $(+)$. The average age of the 284(78.4\%) non-obese subjects was $44 \pm 11.05$. Among nonobese people $169(59.5 \%)$ were $\mathrm{NAFLD}(+)$ and average age was $48.07 \pm 10.13$ while amongst NAFLD $(-)$ people was $38.74 \pm 10.04$. Non-obese NAFLD $(+)$ patients reported they had gained significant amount of weight during their adulthood. This information led us to create, a new index named "Subtracted Adulthood Mass Index" (SAMI) to estimate the risk of NAFLD development in non-obese individuals. SAMI is calculated by dividing the difference between the subject's current weight and his/her weight at the age of 20 years to his/her height squared $(\mathrm{kg} / \mathrm{m} 2)$. SAMI values for non-obese attendants were calculated. When the cut-off value was set as SAMI $4 \mathrm{~kg} / \mathrm{m} 2$, sensitivity was $76.3 \%$, specificity was 79.1, positive predictive value(PPV) was $84.3 \%$ and negative predictive value(NPV) was $69.4 \%$. At a cut-off of SAMI $3 \mathrm{~kg} / \mathrm{m} 2$ sensitivity was $85.2 \%$, specificity was $66.9 \%$, PPV was $79.1 \%$, NPV was $75.4 \%$.

Conclusion: In this pilot study, we found that weight gain in adulthood is an important predictor of NAFLD development in non-obese individuals. The new index named SAMI can correctly identify non-obese people under the risk of developing NAFLD. Cut-off value of SAMI has been set as $3 \mathrm{~kg} / \mathrm{m}^{2}$. We also observed that NAFLD prevalence increases as SAMI value goes up. We propose that SAMI is appropriate for clinical use to estimate the risk of NAFLD in onobese individuals.

Disclosure of Interest: All authors have declared no conflicts of interest.

\section{References}

1. Clinical and metabolic factors associated with development and regression of nonalcoholic fatty liver disease in nonobese subjects. Kim NH, Kim JH, Kim YJ, Yoo HJ, Kim HY, Seo JA, Kim NH, Choi KM, Baik SH, Choi DS, Kim SG - Liver Int. - April 1, 2014; 34 (4); 604-11

2. Lean NAFLD: An Underrecognized Outlier. Wattacheril J, Sanyal AJ - Curr Hepatol Rep - June 1, 2016; 15 (2); 134-139

3. Epidemiology and risk factors of nonalcoholic fatty liver disease (NAFLD). Duseja A, Chalasani N - Hepatol Int - December 1, 2013; 7 Suppl 2()$; 755-64$

\section{P1320 CHARACTERIZATION OF CISPLATIN RESISTANCE IN HEPATOMA CELL LINES}

S. Guttmann ${ }^{1}$, G. Chandhok ${ }^{1}$, S. Reinartz Groba ${ }^{1}$, C. Niemietz ${ }^{1}$, V. Sauer ${ }^{1}$, G. Ciarimboli ${ }^{2}$, U. Karst ${ }^{3}$, A. Zibert ${ }^{1}$, H. Schmidt ${ }^{1}$

${ }^{1}$ Transplantationsklinik, Universitätsklinikum Münster, Münster/Germany ${ }^{2}$ Experimentelle Nephrologie, Medizinische Klinik D, Universitätsklinikum Münster, Münster/Germany

${ }^{3}$ Institute Of Inorganic And Analytical Chemistry, University Münster Münster/Germany

Contact E-mail Address: guttmann.sarah@ukmuenster.de

Introduction: Cisplatin-treated cancer patients often face therapy failure caused by acquired cisplatin (cp) resistance. Development of resistance was previously associated with modulation of transporters mediating cellular copper metabolism. Overexpression of the Wilson disease protein ATP7B, a TGN copper transporter, was proposed to increase cellular $\mathrm{cp}$ efflux.

Aims \& Methods: The human hepatoma cell line HepG2 was compared with a HepG2-variant lacking functional ATP7B expression (KO) in regard to cp sensitivity. Hepatoma cell lines were generated that displayed cp resistance by stepwise increasing $\mathrm{cp}$ concentrations. Cells were examined via growth, cell viability assay (MTT) and analysed for apoptosis (Annexin V staining). Inductively coupled plasma mass spectrometry was used to determine intracellular cp level. Gene expression analysis (RT-qPCR) was carried out to determine the impact of various transporters. Overexpression of individual transporter genes and siRNA treatment were used for confirmation of the data.

Results: Treatment of $\mathrm{HepG} 2$ and $\mathrm{KO}$ cells with various cp concentrations revealed no significant differences in cell viability and intracellular cp accumulation. To examine how KO cells that lack ATP7B can adapt to high cp levels, a cp resistant subline $(\mathrm{CpR})$ was generated. $\mathrm{Cp}$ resistance was confirmed by viability assays, cell growth, apoptosis and intracellular $\mathrm{cp}$ load. Gene expression analysis of more than 16 transporters demonstrated an upregulation of metallothionein 1 $(M T 1: 8.91 \pm 4)$ and a downregulation of organic cation transporter 3 (OCT3: $-5.17 \pm 2$ ) compared to control cell lines. Weaning and regrowth of the CpR cell line in the presence of $\mathrm{cp}$ revealed a stable phenotype of resistance in the cells. Downregulation of OCT3 was identified to be permanent while MT1 upregulation was transient and rapidly induced by $\mathrm{cp}$. Overexpression of OCT3 in CpR cells resulted in loss of $\mathrm{cp}$ resistance to a level of untreated cells indicating that downregulation of OCT3 is responsible for acquired cp resistance.

Conclusion: We suggest that ATP7B does not seem to be involved in cp resistance, at least in hepatic cells. OCT3 represents a novel marker of $\mathrm{cp}$ resistance. OCT3 expression could be a valuable tool for improved prognosis of cisplatin therapy.

Disclosure of Interest: All authors have declared no conflicts of interest.

\section{P1321 AUTOMATED RAPID DETECTION SYSTEM USING THE QUENCHING PROBE METHOD FOR DETECTING RS738409 POLYMORPHISM IN PNPLA3 IN NONALCOHOLIC FATTY LIVER DISEASE}

Y. Kawaguchi ${ }^{1}$, A. Nakamura ${ }^{2}$, K. Shibayama ${ }^{3}$, F. Koga ${ }^{1}$, S. Nakashita ${ }^{1}$, D. Mori ${ }^{2}$

${ }^{1}$ Department Of Hepatobiliary And Pancreatology, Saga-Ken Medical Centre Koseikan, Saga/Japan

${ }^{2}$ Department Of Clinical Laboratory, Saga-ken Medical Centre Koseikan, Saga/ Japan

${ }^{3}$ Department Of Nursing, Saga-Ken Medical Centre Koseikan, Saga/Japan

Contact E-mail Address: kawaguy222@gmail.com

Introduction: Recent studies have shown that the single nucleotide polymorphism (SNP) rs738409 in the PNPLA3 gene is strongly associated with severity of nonalcoholic fatty liver disease (NAFLD). ${ }^{1,2}$ However, the traditional direct sequencing (DS) method is time-consuming and labor-intensive. The idensy $^{\mathrm{TM}}$ (ARKRAY, Inc.), which is based on the quenching probe (QP) method, automatically detects target genes in blood samples by fluorescence quenching within $90 \mathrm{~min} .^{3}$

Aims \& Methods: The current study compared the QP and DS methods for detecting SNPs in the PNPLA3 gene, and established the impact of the genotype on prognosis of NAFLD. We enrolled 107 patients with fatty liver irrespective of etiology. We used the i-densy fully automated genotyping system with QP. The requisite number of tips, reaction tubes, reagent packs and blood samples were set in their designated places. The forward and reverse polymerase chain reaction (PCR) primers and guanine QP were 5'-cttctctctcetttgctttcacag-3', 5'-gtgtgagcacacttcagagg-3' and 5'-ggcatgaagcaggaacatac-3', respectively. PCR consisted of initial denaturation for $1 \mathrm{~min}$ at $95^{\circ} \mathrm{C}$, and 60 cycles of denaturation at $95^{\circ} \mathrm{C}$ for $1 \mathrm{~s}$ and annealing at $61^{\circ} \mathrm{C}$ for $30 \mathrm{~s}$. After completion of the PCR, we analyzed melting temperatures. The SNP genotypes were determined by monitoring the change in fluorescence intensity with increasing temperature. The results obtained with the QP method were compared with those obtained with the conventional DS method. Then, we analyzed 73 patients with NAFLD according to PNPLA3 genotype in terms of alanine aminotransferase (ALT), aspartate aminotransferase to platelet ratio index (APRI), Fibroscan value, and cumulative hepatocellular carcinoma (HCC) development rate.

Results: The genotypes obtained with the QP method were identical to those obtained with the conventional DS method. In 73 patients with NAFLD, the frequency of the PNPLA3 genotypes CC, CG and GG was $21(28.8 \%)$, $24(32.9 \%)$ and $28(38.4 \%)$, respectively. Serum ALT, APRI and Fibroscan value according to PNPLA3 genotypes CC, CG and GG were 26 (14-59), $33(11-113)$ and $46(17-175) \mathrm{U} / \mathrm{L}, 0.3(0.1-1.0), 0.5(0.2-6.8)$ and $0.7(0.2-3.1)$, and $4.4(2.7-25.1), 5.6(2.5-26.5)$ and $6.6(3.6-38.6) \mathrm{kPa}$, respectively $(p=0.001$, $p=0.001$ and $p=0.033$ by Jonckheere-Terpstra test). HCC developed in none of the patients with $\mathrm{CC}$ genotype, one $(4.2 \%)$ with $\mathrm{CG}$ and four $(14.3 \%)$ with GG. The cumulative HCC development rate in patients with GG genotype was significantly higher than that in those with $C C+C G$ genotype $(p=0.043$ by logrank test).

Conclusion: The i-densy using the QP method can automatically, quickly and easily identify PNPLA3 genotypes in real-world clinical settings. These findings indicate the feasibility of personalized medicine for NAFLD.

Disclosure of Interest: All authors have declared no conflicts of interest.

\section{References}

1. Romeo S, Kozlitina J, Xing C, et al. Genetic variation in PNPLA3 confers susceptibility to nonalcoholic fatty liver disease. Nat Genet 2008; 40: 1461-5

2. Kawaguchi T, Sumida Y, Umemura A, et al. Genetic polymorphisms of the human PNPLA3 gene are strongly associated with severity of non-alcoholic fatty liver disease in Japanese. PLoS One 2012; 7: e38322

3. S. Suzuki, M. Komori, M. Hirai, et al. Development of a Novel, FullyAutomated Genotyping System: Principle and Applications. Sensors 2012; 12: $16614-27$ 
P1322 EFFECT OF ORLISTAT ON TOTAL LIVER FAT

QUANTIFICATION BY NOVEL MAGNETIC RESONANCE IMAGING IN OBESE PATIENTS WITH NON-ALCOHOLIC

STEATOHEPATITIS: INTERIM ANALYSIS OF A PROSPECTIVE, RANDOMIZED, SINGLE-CENTER, OPEN-LABEL TRIAL

J. Ye, W. Yanqin, H. Xuan, Z. Bihui

Gastroenterology, The First Affiliated Hospital Of Sun Yat-Sen University,

Guangzhou/China

Contact E-mail Address: sophiazhong@hotmail.com

Introduction: Orlistat is an effective pharmacologic weight loss treatment by inhibiting intestinal lipase to reduce dietary fat absorption. Previous studies have suggested that it lowers liver fat by ultrasound or semi quantitative histological score in nonalcoholic fatty liver diseases (NAFLD).

Aims \& Methods: We aimed to examine the efficacy of orlistat versus placebo in reducing liver fat content by the magnetic resonance imaging (MRI) based on chemical shift imaging. A total of 51 NAFLD patients diagnosed by MRI were randomized and to receive trice-daily $120 \mathrm{mg}$ oral Orlistat or placebo for 6 months, among them 30 (14 in the Orlistat group and 16 in the placebo group) were included in the interim analysis. Both groups received. Clinical parameters, laboratory tests and liver fat content were measured at baseline and 6 months including body mass index (BMI), waist hip ratio (WHR), liver enzymes, haemoglobin Alc, total cholesterol (CHOL), serum triglycerides (TG), fasting plasma insulin (FPI), homeostasis model assessment IR (HOMA-IR). The primary outcome was a change in liver fat quantified by MRI which is based on Dixon technique with two-point chemical shift-based fat-water separation method ${ }^{[1]}$. The chi-squared test and paired $t$ test were used to compare mean differences between fat fractions between two groups.

Results: At baseline, BMI was higher in Orlistat group $(31.38 \pm 3.19)$ than that of the placebo group $(26.78 \pm 3.02, \mathrm{p}<0.001)$, while the other baseline characteristics including liver fat fraction and the proportion of hypertension, hyperlipidaemia, diabetes mellitus type 2 , and hyperlipidemia were similar. Compared to baseline, end-of-treatment liver fat content was significantly lower in the Orlistat arm $(19.38 \% \pm 9.52 \%$ to $11.56 \% \pm 7.49 \%$, change was $7.72 \pm 6.39 \%, P=0.001)$ but not in the placebo $(16.05 \% \pm 8.7 \%$ to $14.17 \% \pm 9.58 \%$, change was $1.43 \pm 9.84 \%, \mathrm{P}=0.640)$ arm. Change of BMI was the only independent factors correlated with reduction of liver fat $\operatorname{content}(\beta=0.522, \mathrm{p}=0.006)$.

Conclusion: Orlistat did significantly decreased liver fat in NAFLD patients via its effect of lowering weight.

Disclosure of Interest: All authors have declared no conflicts of interest.

\section{Reference}

1. Zhi Dong, Shi-Ting Feng. MR quantification of total liver fat in patients with impaired glucose tolerance and healthy subjects. PLOS ONE, 2014,9(10):e111283

\section{P1323 LONG-TERM COPPER EXPOSURE OF HEPATIC CELLS LACKING FUNCTIONAL ATP7B}

S. Guttmann, S. Reinartz Groba, C. Niemietz, P. Ballmaier, R. Muhmann, V. Sauer, A. Zibert, H. Schmidt

Transplantationsklinik, Universitätsklinikum Münster, Münster/Germany

Contact E-mail Address: guttmann.sarah@ukmuenster.de

Introduction: Copper transporter ATP7B is essential for hepatic Cu homeostasis and loss of function results in the inherited autosomal recessive disorder Wilson Disease (WD). Symptoms of WD are i.e. elevated $\mathrm{Cu}$ accumulation in liver and brain. Understanding of molecular mechanisms involved in $\mathrm{Cu}$ homeostasis is essential to improve therapeutic options. The molecular impact following longterm elevated $\mathrm{Cu}$ in hepatic cells lacking functional ATP7B has not been explored.

Aims \& Methods: HepG2 cells lacking functional ATP7B (KO) were used for generation of a copper resistant subline $(\mathrm{CuR})$. Cell growth, cell viability (MTT) and intracellular $\mathrm{Cu}$ load (atomic absorption spectroscopy) was assessed. RTqPCR was performed to quantify the expression of genes related to $\mathrm{Cu}$ homeostasis genes. Functional analysis of candidate genes was assessed via siRNA transfection. Additional measurements of CTR1 expression was performed by flow cytometry (FACS) and Western Blot.

Results: KO cells can survive for many months in the presence of high $\mathrm{Cu}$ and gain resistance $(\mathrm{CuR})$. Characterization of $\mathrm{CuR}$ cells revealed increased survival up to 12 -fold compared to control, whereas a high intracellular $\mathrm{Cu}$ accumulation was noticed. Once $\mathrm{Cu}$ resistance was established, the termination of $\mathrm{Cu}$ exposure did not result in the loss of resistance indicating a stable modification of the cells. Two genes involved in $\mathrm{Cu}$ homeostasis displayed an altered expression pattern (upregulation of MT1 and downregulation of CTR 1 by $28.1 \pm 2.5$ and $-3.2 \pm 0.4$ fold, respectively) which was however transient and dependent on $\mathrm{Cu}$ exposure. The role of MDR1 which presented a stable modification was further investigated by siRNA and drug activation (verapamil). Notably, cell viability and intracellular $\mathrm{Cu}$ load was significantly affected by siRNA and verapamil indicating that MDR1 is involved in $\mathrm{Cu}$ homeostasis. In addition, hepatic cells derived from a WD patient and from the rat animal model confirmed our observations. Conclusion: Our analysis of long-term $\mathrm{Cu}$ exposure presents new insights in copper metabolism and suggests a new role of MDR1 in the pathogenesis of Wilson disease.

Disclosure of Interest: All authors have declared no conflicts of interest.
P1324 THE NONALCOHOLIC FAT LIVER DISEASE FIBROSIS

SCORE IN PREDICTING FIBROSIS IN MORBIDLY OBESE PATIENTS BEFORE BARIATRIC SURGERYTHE NONALCOHOLIC FAT LIVER DISEASE FIBROSIS SCORE IN PREDICTING FIBROSIS IN MORBIDLY OBESE PATIENTS BEFORE BARIATRIC SURGERY

R. G. Silva Junior ${ }^{1}$, T.F.A. Cavalcante ${ }^{1}$, W. R. Freitas Júnior ${ }^{2}$, A. Vieira ${ }^{1}$, L. A. Szutan ${ }^{2}$

${ }^{1}$ Medicine Department, Santa Casa School of Medical Sciences, São Paulo/Brazil ${ }^{2}$ Surgery Department, Santa Casa School of Medical Sciences, São Paulo/Brazil

Contact E-mail Address: thicianie@hotmail.com

Introduction: Non-alcoholic fatty liver disease (NAFLD) and non-alcoholic steatohepatitis (NASH) are increasingly common cause of chronic liver disease worldwide. Most patients with severe obesity who undergo bariatric surgery have NAFLD, which is associated insulin resistance, type 2 diabetes mellitus, hypertension, and obesity-related dyslipidemia. Identifying significant fibrosis in patients is crucial to evaluating prognosis and possible therapeutic intervention. Currently, liver biopsy is the gold standard for diagnosis of liver fibrosis.

Aims \& Methods: We aimed to evaluate the NAFLD fibrosis score for the assessment of significant fibrosis in patients with morbid obesity before undergoing Roux-en-Y gastric bypass. A total of 69 NAFLD patients (median BMI $47 \mathrm{~kg}$ m2) were prospectively enrolled from June 2015 to November 2016 at one Brazilian university hospital. All patients were evaluated with routine laboratory before bariatric surgery. Age, body mass index, hyperglycemia, platelet count, albumin and AST/ALT ratio were applied to the score formula. Biopsies were interpreted by a single experienced pathologist. NAFLD and fibrosis were classified according to the NASH Clinical Research Network NAFLD activity score. These scores were compared to liver biopsy findings and the area under the receiver operating characteristic curve (AUROC) was calculated for the diagnostic test.

Results: On liver biopsy, 29 patients $(42 \%)$ had some degree of fibrosis, with 13 patients $(18.8 \%)$ having significant fibrosis (F2-4). With standard thresholds, the specificity for the NAFLD fibrosis score for identification of significant fibrosis was $58.9 \%$. Using modified thresholds, the specificity could increase. For predicting significant fibrosis, for a cut-off of 1.05 , the score had $46.15 \%$ sensitivity and $96.43 \%$ specificity with AUROC of 0.74

Conclusion: The nonalcoholic fat liver disease fibrosis score has good accuracy to identify and significant liver fibrosis in morbidly obese patients subjected to bariatric surgery.

Disclosure of Interest: All authors have declared no conflicts of interest.

\section{References}

1. Brunt EM, Kleiner DE, Wilson LA, et al. Nonalcoholic fatty liver disease (NAFLD) activity score and the histopathologic diagnosis in NAFLD: distinct clinicopathologic meanings. Hepatology. 2010;53(3):810-20.

2. Ooi GJ, Burton PR, Doyle L, et al. Modified thresholds for fibrosis risk scores in nonalcoholic fatty liver disease are necessary in the obese. Obes Surg. 2016; doi: 10.1007/s11695-016-2246-5.

3. Machado MV, Cortez-Pinto H. Non-invasive diagnosis of nonalcoholic fatty liver disease. A critical appraisal. J Hepatol. 2013;58:1007-19.

\section{P1325 COMPARISON OF FIBROSCAN ${ }^{\circledR}$ AND FATTY LIVER INDEX} (FLI) TO SCREEN FOR FATTY LIVER DISEASE IN A LARGE COHORT OF EMPLOYEES: WHERE IS THE OPTIMAL CUT OFF?

M. Teufelhart, C. Rabitsch, B. Mehl, R. Winker, B. Meyer, T. Scherzer Health And Prevention Center, Sanatorium Hera, Vienna/Austria

Contact E-mail Address: manuela.teufelhart@hera.co.at

Introduction: FLI was developed as a simple predictor of steatosis (Bedogni et al.,2006) and a cut-off value $>60$ ruled in (positive likelihood ratio [LR+] 4.3) steatosis in Italians diagnosed with ultrasound. Fibroscan ${ }^{\circledR C A P}$ is more sensitive for the diagnosis of steatosis than ultrasound. Aim of this study is to evaulate the use of Fibroscan ${ }^{\circledR}$ in comparison with FLI to screen for fatty liver disease in Austrian bank employees and to recalculate optimal FLI cut-off values. Beside weight reduction and diet EASL guidelines recommend physical activity $>150 \mathrm{~min}$. per week for prevention and treatment of steatosis.

Aims \& Methods: More than 1000 Austrian bank employees will be screened for liver diseases with Fibroscan ${ }^{\circledR}$. A $\mathrm{kPa}$ value $\geq 7.9$ (M-probe) and $\geq 7.2$ (XLprobe) is categorized as fibrosis. Additionally Fibroscan $₫$ CAP with a cut-off value $\geq 248 \mathrm{~dB} / \mathrm{m}$ is defined as steatosis, $\geq 300 \mathrm{~dB} / \mathrm{m}$ as high-grade steatosis. Heavy drinkers are identified with AUDIT and SIAC questionnaires. Weekly physical activity is classified into "none", " $\leq 150 \mathrm{~min}$ " and " $>150 \mathrm{~min} "$

Results: To date 482 employees [age $45.9 \pm 8.7$ (mean \pm SD); m:207, f:275; BMI $25.4 \pm 4.4)]$ have been included in this analysis. 14/482 $(2,9 \%)$ employees had signs of liver fibrosis and received further investigations. Fibroscan ${ }^{\circledR C A P}$ values $\geq 248 \mathrm{~dB} / \mathrm{m}$ are shown in 156/482 (32,4\%) employees (m:91 [58\%], f:65 $[42 \%]), 56 / 482(11,6 \%)$ had CAP values $\geq 300 \mathrm{~dB} / \mathrm{m}$. Furthermore $15 / 156(9,6 \%)$ patients with steatosis are heavy drinkers. FLI significantly predicts Fibroscan ${ }^{\circledR} C A P$ values $(\operatorname{adj} . R 2=0.551, t(424)=22.9, \mathrm{p}<0.001)$, which indicates a large effect size. For this study population, a FLI cut-off value $\geq 40$ $(\mathrm{CAP} \geq 248 \mathrm{~dB} / \mathrm{m})$ and $\geq 50(\mathrm{CAP} \geq 300 \mathrm{~dB} / \mathrm{m})$ rules in fatty liver disease with a LR+ of 4.7 (SN:84\%; SP:75\%) and 4.8 (SN:83\%; SP:83\%), respectively. An additional consideration of physical activity level in the current cohort revealed that inactivity increases the LR+ of FLI values to predict NAFLD (trendwise significant, $\mathrm{p}=0.051$ ), indicating lower cut-off values. 
Conclusion: We conclude that Fibroscan ${ }^{\circledR}$ represents an eligible tool to diagnose liver diseases in Austrian bank employees. Compared to the previous work of Bedogni et al FLI predicts fatty liver at a lower cut-off level, at least for the examined population. This difference might be due to the fact that FibroScan ${ }^{\circledR C A P}$ is more sensitive than ultrasound.

Disclosure of Interest: All authors have declared no conflicts of interest.

\section{Reference}

Bedogni et al. (2006): The Fatty Liver Index: a simple and accurate predictor of hepatic steatosis in the general population. BMC Gastroenterology, 6:33

\section{P1326 METABOLOMICS IDENTIFIES PROGRESSIVE NAFLD}

D. Crisan ${ }^{1}$, M. Grigorescu ${ }^{1}$, A. Suciu ${ }^{2}$, H. Stefanescu ${ }^{2}$, F. Romanciuc ${ }^{3}$,

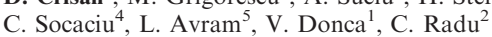

${ }^{1}$ University of Medicine and Pharmacy Cluj-Napoca, Cluj-Napoca/Romania ${ }^{2}$ Hepatology, Cluj-Napoca University of Medicine and Pharmacy $3 \mathrm{rd}$ Medical Clinic, Cluj-Napoca/Romania

${ }^{3}$ Faculty of Food Science and Technology, University of Agricultural Sciences and Veterinary Medicine, Cluj-Napoca, cluj-napoca/Romania

${ }^{4}$ Research and Development Centre BIODIATECH for Applied Biotehnology in

Diagnostic and Molecular Therapy, Cluj-Napoca/Romania

${ }^{5}$ Clinical Municipal Hospital Cluj-Napoca, Cluj-Napoca/Romania

Contact E-mail Address: crisan.dc@gmail.com

Introduction: Nonalcoholic fatty liver disease (NAFLD) is an affection with increasingly prevalence worldwide, having an important impact on morbidity and mortality, especially when it associated severe fibrosis.

Aims \& Methods: We aimed to assess the metabolites that are associated with fibrosis stages in NAFLD, using metabolomic method. A total of 40 patients were included in the study, 30 diagnosed with nonalcoholic fatty liver disease (NAFLD) and 10 controls. Steatosis and fibrosis were assessed using Fibromax elaborated by Biopredictive (R) (Paris, France). New metabolomic techniques (high performance liquid chromatography coupled with mass spectrometry (HPLC-MS) and principal component analysis (PCA) were used to identify final products of various metabolic pathways correlated with liver fibrosis.

Results: Of the 30 patients with NAFLD included in the study, 6 patients $(20 \%)$ had severe fibrosis. The metabolomic profile identified four metabolites that are associated with severe fibrosis: $1,25(\mathrm{OH})$ vitamin $\mathrm{D},(\mathrm{p}=0.03)$, lisophosphatidyletanolamine LPE 0:0:22:6 (p=0.05), Lysophosphatidylcholine LPC 18:2 $(\mathrm{p}=0.003)$, and high levels of butenyl carnitine $(\mathrm{p}=0.04)$. Of these, LPE was the strongest predictor of severe fibrosis (AUROC-0.795, Sensitivity $(\mathrm{Se})=88.33 \%$, specificity $(\mathrm{Sp})=78.79 \%)$, but the others molecules were also significantly associated with severe fibrosis: vitamin $\mathrm{D}(\mathrm{AUROC}=0.776)$, butenyl carnitine (AUROC $=0.737)$, LPC 18:2 (AUROC $=0.768)$. As the metabolomics permits the evaluation of all these molecules the same time, we can use them combined in order to increase the diagnostic accuracy. In our case, the combined use of the four metabolites determined an AUROC of 0.839 , with Se of $100 \%$ and $\mathrm{Sp}$ of $68.5 \%$.

Conclusion: Using metabolomics, we can identify patients with fatty liver and severe fibrosis who are significantly exposed to a progressive disease and a higher mortality.

Disclosure of Interest: All authors have declared no conflicts of interest

\section{P1327 CHRONIC RENAL FAILURE IS ASSOCIATED WITH THE DEVELOPMENT OF NAFLD/NASH}

S. Gehring ${ }^{1}$, P. Di Fazio ${ }^{2}$, T.M. Gress ${ }^{3}$, T.T. Wissniowski ${ }^{1}$

${ }^{1}$ Gastroenterology, Philipps University, Marburg/Germany

${ }^{2}$ Department Of Visceral Thoracic And Vascular Surgery, Philipps University

Marburg, Marburg/Germany

${ }^{3}$ Klinik Für Gastroenterologie, Endokrinologie, Stoffwechel Und Infektiologie,

Philipps Universität Marburg, Marburg/Germany

Contact E-mail Address: sonjagehring@gmx.de

Introduction: Chronic renal failure (CRF) is frequently associated bone metabolism and osteoporosis with lowered levels of Vitamine D and/or hyperparathyreodism particular in case of hemodialysis. Younger studies suggest an association of low vitamin D levels with non-alcoholic fatty liver disease (NAFLD) and nonalcoholic steatohepatitis (NASH), as well as metabolic syndrome, and diabetes mellitus. Unfortunately, a causality could not yet be proven.

Aims \& Methods: Our aim was to identify patients on higher risk to develop NAFLD/NASH in a selected patient cohort being admitted for renal disorders
176 patients, admitted to the department of nephrology of the University Hospital Marburg for renal disorders whose plasma vitamin D concentration, phosphate and parathormone levels and liver enzyme levels had been quantified beforehand, were enrolled and a retrospective investigation of laboratory parameters (including electrolytes, hormones, and vitamins) and pre-existing medical conditions (imcluding high blood pressure, diabetes, hyperlipoproteinaemia, and more) followed. Appropriat statistical test were used to characterise the cohort (ANOVA; MANN-Whitney-U; FISHER-EXACT) using SPSS TM . Other hepatopathies were excluded. Steatosis was assessed by ultrasonography.

Results: Patients were divided into 4 groups according to plasma vitamin D levels (normal $>25 \mathrm{ng} / \mathrm{ml}$; low $<25 \mathrm{ng} / \mathrm{ml}$ ) and transaminase levels (AST/ALT $/ \gamma$-GT $>30 \mathrm{U} / 1$; normal: AST/ALT/ $\gamma$-GT $<30 \mathrm{U} / 1$ ). Low 1,25-hydroxyvitamin D levels correlated signifactly with high kreatinine, urea, and LDL levels, while low 25-hydroxyvitamin D levels correlated with high cholesterol and trigylceride levels, suggesting a relationship between low vitamin D levels and fat metabolism disorders. Interestingly end stage renal failure (chronic hemodialysis) was significantly correlated with the development of NAFLD/NASH with significantly higher levels of AST/ALT and gGT, hyperparathyreodism and hyperphosphatemia Transaminases were significantly lower if Vitamin D was supplemented Conclusion: Vitamin D deficiency is often present in patients with kidney diseases such as chronic renal failure. Vitamin D levels are correlated to age and sex of the patient. Patients suffering from renal failure are on high risk developing NAFLD/NASH if diminished vitamin D levels are present Supplement of Vitamin D saves from NAFLD/NASH The correlation of hyperparathyreodism and NAFLD/NASH has to be further investigated in larger patient groups Disclosure of Interest: All authors have declared no conflicts of interest.

\section{P1328 NONINVASIVE DIAGNOSICS OF NONALCOHOLIC FATTY LIVER IN PATIENTS WITH TYPE 2 DIABETES MELLITUS}

K. Aksentiychuk ${ }^{1}$, E. Sklyarov ${ }^{1}$, A. Bochar ${ }^{2}$

${ }^{1}$ Therapy \#1 And Medical Diagnostics, LNMU by Danylo Galytskyi Dept. of Therapy, Lviv/Ukraine

${ }^{2}$ Lviv National Medical University, Lviv/Ukraine

Contact E-mail Address: akskris@mail.ru

Introduction: Usually for the determination of nonalcoholic fatty liver disease (NAFLD) there are instrumental and laboratory techniques, including ultrasound diagnosis, determination of aminotransferases, steatotest, 13C-methacetin breath test (13C-MBT). These methods in the diagnosis of NAFLD clinical forms is not specific and do not allow make difference between steatosis and steatohepatitis. The determination of NAFLD clinical forms is a priority in the prediction of further disease and choice of treatment. Steatohepatitis is the active form of NAFLD and progresses to fibrosis oftenly with subsequent liver parenchyma degeneration into cirrhosis. Simultaneously, steatosis could be possibly treated in the early stages of disease.

Aims \& Methods: The study involved 65 patients with type 2 diabetes and coronary heart disease with metabolic syndrome, aged 37 to 82 years (mean age $55.82 \pm 3.46), 29$ men, 36 women. According to the ultrasound, the stage of fatty infiltration were differentiated by such criteria for steatosis as diffuse liver parenchyma echogenicity intensification against the background of a slight increase in its size (liver echogenicity was significantly higher than normal kidney or lumbar muscle echogenicity); for steatohepatitis - hyperechogenicity of liver parenchyma and expansion of portal vein $(13 \mathrm{~mm}$ or more in diameter).

Results: For steatosis and steatohepatitis determination the ALT monitoring was used, where the level exceeding $0.68 \mathrm{mmol} / 1$ signed to steatohepatitis, and below $0.68 \mathrm{mmol} / 1$ - to steatosis. Portal vein diameter size above $13 \mathrm{~mm}$ subscribed steatohepatitis, and below 13 - steatosis. 13C-MBT data, which assesed the liver antitoxic function was used, where the result $13 \mathrm{CO} 2$ on 120 minute from 10 till $15 \%$ was classificated as liver detoxification function violation like steatohepatitis, and from 15 till 20 - steatosis. The present study found that indicators of ALT and portal vein diameter negatively correlated with cumulative dose $13 \mathrm{CO} 2$ for 120 minute in case of steatohepatitis. Thus, reduction cumulative dose $13 \mathrm{CO} 2$ on 120 minute from $15 \%$ to $10 \%$ was accompanied by ALT level increasing (more than $0.68 \mathrm{mmol} / \mathrm{l}$ ) and portal vein diameter enlargement (over $13 \mathrm{~mm})$.

Conclusion: Differentiation between steatosis and steatohepatitis should be perform by the cumulative dose $13 \mathrm{CO} 2$ on 120 minute evaluation and ALT levels and portal vein diameter assessment.

Disclosure of Interest: All authors have declared no conflicts of interest. 
P1329 NON-OBESE FATTY LIVER DISEASE IN TYPE 2 DIABETES: NOVEL CONDITION OR SIMILAR TO TYPICAL NAFLD STATE?

N. Kobyliak ${ }^{1}$, N. Bosak ${ }^{2}$, G. Mykhalchyshyn ${ }^{1}$, D. Kyriienko ${ }^{3}$, O. Dynnyk ${ }^{1}$ Endocrinology, Bogomolets National Medical University, Kyiv/Ukraine

${ }^{2}$ Bogomolets National Medical University, Kyiv/Ukraine

${ }^{3}$ Kyiv City Clinical Endocrinology Center, Kyiv/Ukraine

${ }^{4}$ Bogomolets Institute of physiology of the Ukrainian National Academy of

Sciences, Kyiv/Ukraine

Contact E-mail Address: natusiabosak@gmail.com

Introduction: Non-alcoholic fatty liver disease (NAFLD), the hepatic counterpart of the metabolic syndrome in close relation to obesity and encompasses a disease spectrum spanning simple steatosis through nonalcoholic steatohepatitis (NASH) with/without cirrhosis, and hepatocellular carcinoma [1]. Clearly, not all obese subjects develop NAFLD and NAFLD can also be found in non-obese patients. Globally, the reported prevalence of non-obese NAFLD varies widely, ranging from $3 \%$ to $30 \%$ [2]. Today remains unclear how patients without obesity develop NAFLD, therefore it is important to understand the clinical and pathological conditions of non-obese NAFLD.

Aims \& Methods: In this study, we investigated the liver stiffness and liver fat content in addition to other clinical and metabolic parameters in type 2 diabetes patient with non-obese and obese NAFLD detected on ultrasonography (US). In this cross-sectional study, 245 T2D patients with age of 40-80 years from the Kyiv City Clinical Endocrinology Center were selected. Inclusion criteria were: age over 18 years, presence of T2D in association with fatty liver disease. The diagnosis of fatty liver was based on the results of abdominal ultrasonography, which was done by trained technicians with Ultima PA (Radmir Co., Kharkiv, Ukraine). Of 4 known criteria (hepatorenal echo contrast, liver brightness, deep attenuation, and vascular blurring), the participants were required to have hepatorenal contrast and liver brightness to be given a diagnosis. According to body mass index (BMI) value patients were assigned else to NAFLD group $(\mathrm{n}=157$, $\left.\mathrm{BMI} \geq 30.0 \mathrm{~kg} / \mathrm{m}^{2}\right)$ or to non-obese NAFLD $\left(\mathrm{n}=88, \mathrm{BMI}<30.0 \mathrm{~kg} / \mathrm{m}^{2}\right)$ group We performed 10 valid measurements of liver stiffness (LS) measured by Shear Wave Elastography (SWE) in every patient, and a median value was calculated, the result being measured in $\mathrm{kPa}$. Also in all patient we calculated fatty liver index (FLI). FLI a validated prediction score for hepatic steatosis severity designed Bedogni et al [3]. Changes in transaminases activity, serum lipids and cytokines (TNF- $\alpha$, IL-1 $\beta$, IL-6, IL-8, INF- $\gamma$ ) levels were evaluated.

Results: Non-obese NAFLD patient had higher LS $(7.52 \pm 0.2$ vs $6.87 \pm 0.09$, $\mathrm{p}=0.001)$ values measured by SWE, which were accompanied with increased transaminase activity: for ALT $-42.1 \pm 3.17$ vs $35.8 \pm 1.28(\mathrm{p}=0.028)$ and for AST $-40.81 \pm 2.68$ vs $34.31 \pm 1.33(\mathrm{p}=0.016)$ respectively. In contrast to nonobese group patient in NAFLD group had significantly higher FLI $(86.59 \pm 1.09$ vs $68.06+1.98, p<0.001)$. Markers of chronic systemic inflammatory state were also significantly higher in NAFLD obese as compared to nonobese patient: IL- $1 \beta-44.64 \pm 2.0$ vs $31.02 \pm 1.78(\mathrm{p}<0.001)$; TNF- $\alpha-54.11 \pm 2.20$ vs $42.28 \pm 1.81(\mathrm{p}<0.001)$; IL-8 $-29.18 \pm 1.27$ vs $22.05 \pm 0.99(\mathrm{p}<0.001)$ and INF- $\gamma-195.60 \pm 9.47$ vs $132.47 \pm 7.54(p=0.016)$ respectively. Changes of IL-6 between groups were insignificant.

Conclusion: At present, the clinical-laboratory characteristics of non-obese NAFLD are not fully understood. Therefore, we found that non-obese NAFLD associated with higher liver stiffness values and transaminases activities. On the other hand, patient with obese NAFLD are characterized with more pronounced liver fat content and elevation of markers of chronic systemic inflammatory state.

Disclosure of Interest: All authors have declared no conflicts of interest.

\section{References}

1. Mykhalchyshyn G, Kobyliak N, Bodnar P. Diagnostic accuracy of acylghrelin and it association with non-alcoholic fatty liver disease in type 2 diabetic patients. J Diabetes Metab Disord 2015; 14: 44

2. Kim D, Kim WR. Nonobese Fatty Liver Disease. Clin Gastroenterol Hepatol. 2017;15:474-485.

3. Bedogni G, Bellentani S, Miglioli L, Masutti F, Passalacqua M, Castiglione A et al. The Fatty Liver Index: a simple and accurate predictor of hepatic steatosis in the general population. BMC Gastroenterol. 2006; 6: 33 .

\section{P1330 CHARACTERIZATION OF LEAN INDIVIDUALS WITH NON-ALCOHOLIC FATTY LIVER DISEASE: RESULTS OF A COHORT STUDY}

A. Eshraghian ${ }^{1}$, M.R. Fattahi ${ }^{2}$, R. Niknam ${ }^{2}$, S.A. Taghavi ${ }^{3}$

${ }^{1}$ Gastroenterohepatology Research Center, Shiraz University of Medical Sciences, Shiraz/Iran

${ }^{2}$ Endoscopy Ward, Namazi Hospital, Fars-Shiraz/Iran

${ }^{3}$ Gastroenterology, Shiraz University of Medical Sciencez, shiraz/Iran

Contact E-mail Address: eshraghiana@yahoo.com

Introduction: Non-alcoholic fatty liver disease (NAFLD) is usually considered as the hepatic manifestation of metabolic syndrome and obesity. However, a subgroup of NAFLD patients are lean. Some studies have reported severe liver fibrosis in lean NAFLD and these patients are still at risk for development of liver cirrhosis.

Aims \& Methods: This study aimed to investigate the prevalence and risk factors of lean NAFLD in a cluster of Iranian population. Study population was recruited from Kavar cohort study which has been started from 2006 in Kavar town, a small town near Shiraz. The study sampling was performed between September 2011 and September 2015 among adult subjects (age $>18$ years) who underwent voluntary hepatobiliary ultrasound. NAFLD was diagnosed using ultrasound and absence of chronic liver diseases such as autoimmune hepatitis, hepatitis $\mathrm{B}$ or $\mathrm{C}$ viruses induced hepatitis, hepato-billiary cancers, Wilson's disease, $>10 \mathrm{~g} /$ day alcohol consumption, and receiving some specific medications known to cause hepatic steatosis (like amiodarone, valporic acid, etc). Lean individuals were defined as those with body mass index (BMI) $<25 \mathrm{~kg} / \mathrm{m}^{2}$. Student's t-test was used for comparisons of continuous variables and Chi-square test was used for comparison of categorical variables. Receiver operating characteristics (ROC) curve analysis using area under curve (AUC) was used for analysis of optimal cutoff values for BMI and waist circumference in association with lean NAFLD.

Results: 1343 individuals were included. 165 individuals $(12.3 \%)$ was diagnosed to have NAFLD. 129 individuals $(9.6 \%)$ had mild NAFLD and 36 individuals $(2.7 \%)$ had moderate NAFLD. None of the participants had severe NAFLD. In univariate analysis, history of diabetes mellitus $(\mathrm{DM})(\mathrm{OR}=2.25 ; 95 \% \mathrm{CI}$ : $1.15-4.40, \mathrm{P}=0.015)$ and metabolic syndrome $(\mathrm{OR}=2.80 ; 95 \% \mathrm{CI}: 1.74-4.48$, $\mathrm{P}<0.001)$ were associated with NAFLD. Higher BMI and waist circumference, higher systolic and diastolic blood pressure, higher serum triglyceride, total cholesterol, fasting plasma glucose (FPG) and alanine aminotransferase (ALT) were associated with NAFLD $(\mathrm{P}<0.05)$. In multivariate regression analysis, higher BMI and waist circumference, higher serum ALT, FPG and cholesterol were independent predictors of NAFLD in our study population (Table). A cutoff value of $22.3 \mathrm{~kg} / \mathrm{m}^{2}$ for BMI was predictor of NAFLD (sensitivity $=72 \%$; specificity $=60 \% ;$ AUC $=0.728, P<0.001$ ). A cutoff value of $79.5 \mathrm{~cm}$ for waist circumference was predictor of NAFLD in our study population (sensitivity $=80 \% ; \quad$ specificity $=68 \% ; \quad$ AUC $=0.753, \quad \mathrm{P}<0.001) . \quad$ Table: Multivariate regression analysis showing independent risk factors for lean NAFLD

\begin{tabular}{llll}
\hline & $\begin{array}{l}\text { Odds } \\
\text { Ratio (OR) }\end{array}$ & $\begin{array}{l}95 \% \text { Confidence } \\
\text { Interval (CI) }\end{array}$ & P-Value \\
\hline BMI & 1.27 & $1.106-1.459$ & 0.001 \\
Waist circumference & 1.078 & $1.047-1.112$ & $<0.001$ \\
ALT & 1.018 & $1.004-1.033$ & 0.015 \\
Cholesterol & 1.008 & $1.003-1.013$ & 0.003 \\
FPG & 1.010 & $1.003-1.017$ & 0.004 \\
\hline
\end{tabular}

Conclusion: Lean NAFLD was prevalent in our study population and was associated with metabolic risk factors. BMI and waist circumference can be used for prediction of NAFLD in lean individuals.

Disclosure of Interest: All authors have declared no conflicts of interest.

\section{Reference}

1. Akyuz U, Yesil A, Yilmaz Y. Characterization of lean patients with nonalcoholic fatty liver disease: potential role of high hemoglobin levels. Scand J Gastroenterol. 2015; 50(3): 341-346. P1331 ASSESSING BAVENO VI CRITERIA WITH A NEW POINT-
SHEAR WAVE ELASTOGRAPHY TECHNIQUE: THE BAVELASTPQ STUDY

M. Garcovich ${ }^{1}$, G. Gibiino ${ }^{1}$, E. Di Stasio $^{2}$, B.E. Annicchiarico ${ }^{1}$, E. Carli ${ }^{1}$, M.E. Ainora ${ }^{1}$, L. Riccardi ${ }^{1}$, M.E. Riccioni ${ }^{1}$, M. Pompili ${ }^{1}$, M. Siciliano ${ }^{1}$, A. Gasbarrini ${ }^{1}$, M.A. Zocco

${ }^{1}$ Internal Medicine, Gastroenterology And Hepatology, Catholic University of Sacred Heart - Policlinico "A.Gemelli", Rome/Italy

${ }^{2}$ Catholic University of Sacred Heart - Policlinico "A.Gemelli", Rome/Italy

Contact E-mail Address: matteogarcovich@yahoo.it

Introduction: While some studies have evaluated the ability of new "real-time" elastography devices such as 2-D Shear Wave Elastography (SWE) and Virtual Touch Quantification (ARFI) in predicting the presence of high-risk gastroesophageal varices, no study has explored the potential role of another pointSWE technique, ElastPQ, in the assessment of clinically significant portal hypertension.

Aims \& Methods: The aim of our study was to identify a liver stiffness cut-off value measured by ElastPQ and/or laboratory parameters that could help identify those patients who can safely avoid screening endoscopy, similarly to the recently proposed Baveno VI criteria which recommends a liver stiffness value $<20 \mathrm{kPa}$ measured by transient elastography in combination to a platelet count $>150,000 / \mu \mathrm{l}$. Data were collected on 1385 patients who underwent ElastPQ measurement from January 2013 to January 2016 in our Department. Inclusion criteria were a liver stiffness value of $\geq 7 \mathrm{kPa}$ and an upper gastrointestinal endoscopy within 12 months, with a diagnosis of compensated chronic liver disease. We choose this specific liver stiffness cut-off value in order to reasonably rule-in all patients with advanced fibrosis and cirrhosis, based on the limited literature available on this specific elastographic technique. Exclusion criteria were history of decompensated liver disease, evidence of porto-spleno-mesenteric vein thrombosis and non-cirrhotic portal hypertension. Varices were graded as low risk (grade $<2$ ) or high risk $($ grade $\geq 2$ )

Results: The study included 184 patients $(114[62 \%]$ hepatitis C, and $160[87 \%]$ Child-Pugh A). Varices were present in $36 \%$ cases, with $10 \%$ prevalence of highrisk varices. According to ROC curve analysis liver stiffness measurement and 
platelet count were evaluated as predictors of high-risk varices. Overall 74/184 $(40 \%)$ met the new "BAVElastPQ" criteria (that is, liver stiffness $<12 \mathrm{kPa}$ and platelet count $>150,000 / \mu \mathrm{l})$. Within this group $11 / 63(17 \%)$ had any grade of varices and only $1 / 73(1 \%)$ had high-risk varices. The BAVElastPQ criteria gave sensitivity of 0.95 , specificity of 0.44 , a positive predictive value of 0.16 and a negative predictive value of 0.98 . The AUROC for liver stiffness and platelet count was 0.81 and 0.76 , respectively.

Conclusion: The BAVElastPQ criteria correctly identified $99 \%$ of patients without high-risk varices. By applying such criteria we could have potentially avoided $40 \%$ surveillance endoscopies in our cohort. To our knowledge this is the first study that evaluated the potential role of a new p-SWE technique such as ElastPQ in the non-invasive assessment of clinically significant portal hypertension, similarly to the recently proposed Baveno VI criteria though using ElastPQ as an alternative to transient elastography.

Disclosure of Interest: All authors have declared no conflicts of interest.

\section{P1332 PROTON PUMP INHIBITORS INTAKE NOT ASSOCIATED WITH HEPATIC ENCEPHALOPATHY IN CIRRHOTIC PATIENTS}

C. Teixeira ${ }^{1}$, A. Antunes ${ }^{2}$, E. Dantas ${ }^{1}$, A. M. Vaz ${ }^{2}$, P. Queirós ${ }^{2}$, A. L. Alves ${ }^{1}$, B. Peixe ${ }^{2}$, I. Cremers ${ }^{1}$, H. Guerreiro ${ }^{2}$, A. P. Oliveira

${ }^{1}$ Gastroenterology, Centro Hospitalar de Setubal, Setubal/Portugal

${ }^{2}$ Gastroenterology, Centro Hospitalar do Algarve, Faro/Portugal

Contact E-mail Address: ac.corda.teixeira@gmail.com

Introduction: Proton pump inhibitors (PPI) are commonly prescribed and predispose to small bowel bacterial overgrowth. Hepatic encephalopathy is a frequent complication of cirrhosis and is associated with intestinal dysbiosis.

Aims \& Methods: This study aimed to identify a possible association between PPI intake and hepatic encephalopathy development in cirrhotic patients. Retrospective analysis of consecutive cirrhotic patients hospitalized in two Gastroenterology Departments over 3.5 years. Collection of clinical data, PPI intake, infection and hepatic encephalopathy at hospitalization. Statistical analysis was performed with SPSS 21, considering statistical significance $\mathrm{p}<0.05$. Results: 386 patients, 321 males $(83.2 \%)$, mean age $60.3 \pm 12.1$ years. Main etiologies of cirrhosis were alcohol $(67.4 \%)$, alcohol plus hepatitis C Virus $(16.3 \%)$ and hepatitis $\mathrm{C}$ virus $(5.2 \%)$. Hepatic encephalopathy was present in $222(57.5 \%)$ of the patients and $26.9 \%$ had PPI intake. In univariate analysis hepatic encephalopathy was associated with infection $(\mathrm{p}<0.001)$, gastrointestinal bleeding $(\mathrm{p}<0.001)$ and Model for End-Stage Liver Disease (MELD) $(\mathrm{p}<0.001)$ There was no association between hepatic encephalopathy and PPI intake $(p=0.057)$, gender $(p=0.228)$ or age $(p=0.352)$. In multivariate analysis, hepatic encephalopathy maintained association with infection $(p<0.001)$, gastrointestinal bleeding $(\mathrm{p}<0.001)$ and MELD score $(\mathrm{p}=0.001)$.

Conclusion: In our series, PPI intake was not associated with hepatic encephalopathy development in cirrhotic patients.

Disclosure of Interest: All authors have declared no conflicts of interest.

\section{Reference}

Dam G, Vilstrup H, Watson H, Jepsen P. Proton Pump Inhibitors as a Risk Factor for Hepatic Encephalopathy and Spontaneous Bacterial Peritonitis in Cirrhosis Patients With Ascites. Hepatology. 2016;64(4):1265-72.

\section{P1333 CLINICAL IMPACT OF MULTIDRUG-RESISTANT BACTERIAL INFECTIONS IN LIVER CIRRHOSIS}

A.C. Cunha ${ }^{1}$, A. G. Antunes ${ }^{1}$, A.M. Vaz ${ }^{1}$, P. Queirós ${ }^{1}$, T. Gago ${ }^{1}$, J. Roseira ${ }^{1}$, A.R. Clara ${ }^{1}$, M.S. Eusébio ${ }^{1}$, B. Peixe ${ }^{1}$, H. Guerreiro

${ }^{1}$ Gastroenterology Department, Algarve Hospital Center, Faro/Portugal

Contact E-mail Address: anacdacunha@gmail.com

Introduction: The incidence of bacterial infections in cirrhotic patients is significantly higher than that observed in general population, being one of the most important causes of decompensation. In theory, the final result of an infectious disease depends of three major factors: the antibiotic resistance of the bacteria and the prompt administration of treatment; the intrinsic virulence or pathogenicity of the bacteria; and finally the patient status in relation to risk factors such as immune status, age, diet and stress. There are conflicting results regarding the clinical outcome of multidrug-resistant (MDR) bacterial infections in cirrhotic patients.

Aims \& Methods: Our aims within this study were to 1) analyze the incidence of MDR bacteria in patients with decompensated cirrhosis at admission (less than 24 hours after hospitalization), 2) to study its impact on 30 and 90-day mortality, and 3) to identify independent risk factors for 30 and 90-day mortality. We conducted a retrospective cohort study which evaluated all admissions due to decompensated cirrhosis in one center between 2010 and 2015. MDR bacteria infections, its acquisition site and the antibiotic resistance patterns were defined according with the European Centre for Disease Prevention and Control (ECDC) criteria.

Results: A total of 681 hospitalizations were evaluated and $41 \%$ had a bacterial infection at admission. The 30 and 90 -day mortality rate was $14.7 \%$ and $38.1 \%$, respectively. The most common infection was spontaneous bacterial peritonitis (SBP; $40.5 \%$ ), followed by urinary tract infection (UTI; $25 \%$ ). About $55.6 \%$ of the patients had a microbiological documented infection (MDI). MDR bacteria were identified in $18.6 \%$ of all bacterial infections, matching $34.5 \%$ of the nosocomial acquired infections and $8.3 \%$ of the community-acquired (CA) infections. In $40.6 \%$ of the UTI there was a MDI by a MDR bacteria. In patients with documented infections, no difference was noticed between non-MDI, non-MDR bacteria or MDR bacteria regarding the $30(p=0.801)$ and the 90 -day $(p=0.525)$ mortality rate. In the multivariate analysis, elevated BUN and bilirubin, presence of bacterial infection and lower albumin, sodium and $\mathrm{SpO} 2$ were independently associated with 30 and 90-day mortality. Higher INR and age were independently associated with 90-day mortality.

Conclusion: The presence of bacterial infection, independently of the antibiotic resistance profile, was associated with a worse prognosis in cirrhotic patients. We identified a high incidence of MDR bacteria in patients with UTI. Given the described risk factors for MDR bacteria development, we hypothesize that our results are probably the consequence of a bad antibiotic politics in our district, especially when managing CA UTIs.

Disclosure of Interest: All authors have declared no conflicts of interest.

\section{P1334 STATIN THERAPY IN THE REDUCTION OF PORTAL HYPERTENSION IN PATIENTS WITH LIVER CIRRHOSIS: A META- ANALYSIS OF RANDOMIZED CONTROLLED TRIALS}

\section{J.G. E. Cataluna, J. G. Cervantes}

Institute Of Digestive And Liver Diseases, St. Luke's Medical Center - Quezon City, Quezon City/Philippines

Contact E-mail Address: doycataluna@yahoo.com

Introduction: Statins have been shown to decrease intrahepatic vascular resistance and improve vasodilation of the liver vasculature via enhanced nitric oxide production. This meta-analysis of randomized controlled trials (RCTs) was conducted to determine if statin therapy reduces portal hypertension as measured by the hepatic venous pressure gradient (HVPG) among adult patients with liver cirrhosis.

Aims \& Methods: The objective of this research was to perform a meta-analysis of randomized control trials (RCTs) and determine if statin therapy reduces portal hypertension as measured by the hepatic venous pressure gradient (HVPG) among adult patients with liver cirrhosis. Specifically, this meta-analysis determined the pooled/standardized mean difference with $95 \%$ confidence interval using the random effects models. A comprehensive literature search from the PubMed- Medline, EMBASE, Cochrane Library, and Ovid databases was performed using the following terms: cirrhosis, portal hypertension, HVPG, and statin. Three studies were selected. The quality of the included studies was independently appraised by four authors using the Jadad scale. All disagreements and discrepancies of the reviewers were discussed. Trial results were combined under a random-effects model. Continuous data were analyzed by calculating the standard mean difference with $95 \%$ confidence interval $(\mathrm{CI})$ and a significant $\mathrm{p}$ value of 0.05. Cochrane Review Manager Software version 5.0 statistical software was used for all analyses.

Results: Three trials comprising of 98 patients met the inclusion criteria. In the random-effect model, the weighted mean difference was $0.76 \mathrm{mmHg}$, favoring statin therapy over placebo. There was no evidence of significant heterogeneity $\left(\mathrm{P}=0.51 ; \mathrm{I}^{2}, 0 \%\right)$

Conclusion: Statin therapy reduces portal hypertension as measured by the HVPG among adult patients with liver cirrhosis. The findings of this study reinforce the promising role of statins in decreasing portal hypertension. Further RCTs with larger population and with longer duration of follow-up as well as the use of different statin drugs to explore further on the class effect are recommended.

Disclosure of Interest: All authors have declared no conflicts of interest.

\section{References}

Tsochatzis EA, Bosch J, Burroughs AK. New therapeutic paradigm for patients with cirrhosis. Hepatology 2012; 56(5): 1983-1992.

Finegold JA, Manisty CH, Goldacre B, Barron AJ, Francis DP. What proportion of symptomatic side effects in patients taking statins are genuinely caused by the drug? Systematic review of randomized placebo-controlled trials to aid individual patient choice. Eur J Prev Cardiol 2014; 21(4): 464-474.

Abraldes JG, Rodriguez-Vilarrupla A, Graupera M, et al. Simvasta- tin treatment improves liver sinusoidal endothelial dysfunction in CCl4 cirrhotic rats. $J$ Hepatol 2007;46:1040-1046.

Pollo-Flores $\mathrm{P}$, et al. Three months of simvastatin therapy vs placebo for severe portal hypertension in cirrhosis: a randomized controlled trial. Digestive and Liver Disease. November 2015.

Abraldes, JG, et al. Simvastatin lowers portal pressure in patients with cirrhosis and portal hypertension: a randomized controlled trial. Gastroenterology. 2009; 136: 1651-1658.

Zafra C, et al. Simvastatin enhances hepatic nitric oxide production and decreases the hepatic vascular tone in patients with cirrhosis. Gastroenterology. 2004; 126: 749-755.

D'Amico G, Garcia-Pagan JC, Luca A, et al. Hepatic vein pressure gradient reduction and prevention of variceal bleeding in cirrhosis: a systematic review. Gastroenterology 2006;131:1611-1624.

Juan G. Abraldes, et al. for the BLEPS Study Group. Addition of Simvastatin to Standard Therapy for the Prevention of Variceal Rebleeding Does Not Reduce Rebleeding but Increases Survival in Patients With Cirrhosis. Gastroenterology 2016;150:1160-1170

Gupta TK, Toruner M, Chung MK, Groszmann RJ. Endothelial dysfunction and decreased production of nitric oxide in the intra- hepatic microcirculation of cirrhotic rats. Hepatology 1998;28: 926-931. 


\section{P1335 IN HOW MANY PATIENTS WE WILL MISDIAGNOSE}

\section{ESOFAGEAL VARICES BY USING THE BAVENO VI CRITERIA?}

S.A. Popescu ${ }^{1}$, R. Lupusoru ${ }^{1}$, R. Sirli $^{1}$, M. Danila ${ }^{1}$, L. Gheorghe ${ }^{2}$, A. Seicean ${ }^{3}$, A. Trifan ${ }^{4}$, M. Curescu ${ }^{5}$, A. Goldis ${ }^{1}$, L. D. Sandulescu ${ }^{6}$, C. Cijevschi Prelipcean ${ }^{7}$, C. Stanciu ${ }^{4}$, C. Brisc ${ }^{7}$, S. Iacob ${ }^{2}$, I. Sporea ${ }^{1}$

${ }^{1}$ Department Of Gastroenterology And Hepatology, Victor Babes University of

Medicine and Pharmacy Timisoara, Timisoara/Romania

${ }^{2}$ Gastroenterology Department, Carol Davila University of Medicine and

Pharmacy, Bucharest, Bucharest/Romania

${ }^{3}$ Dept. Of Internal Medicine, Gastroenterology, Iuliu Hatieganu University of Medicine and Pharmacy, Cluj-Napoca/Romania

${ }^{4}$ Institute Of Gastroenterology And Hepatology, "Grigore T. Popa" University of Medicine and Pharmacy, Iasi/Romania

${ }^{5}$ Department Of Infectious Diseases, Victor Babes University of Medicine and Pharmacy Timisoara, Timisoara/Romania

${ }^{6}$ Gastroenterology, University of Medicine and Pharmacy of Craiova, Craiova/ Romania

${ }^{7}$ Department Of Gastroenterology, Oradea University of Medicine and Pharmacy, Oradea, Oradea/Romania

Contact E-mail Address: alinamircea.popescu@gmail.com

Introduction: The place of noninvasive techniques for the prediction of presence of portal hypertension in patients with liver cirrhosis is one of the current research topics.

Aims \& Methods: The aim of this study was to evaluate the applicability of the Baveno VI criteria in a cohort of known compensated HCV liver cirrhosis patients, to see how often we misclassify the presence of esophageal varices (EV). Material and method We did a prospective multicentre study, from September 2015 to December 2016, which included all patients with perfectly compensated $\mathrm{HCV}$ liver cirrhosis, diagnosed by means of elastography, ultrasound, endoscopic and biological criteria prior to interferon-free treatment. All patients were evaluated by upper gastrointestinal endoscopy, transient elastography (TE) and biological tests. By using Baveno VI criteria we classified the patients in: probably without EV (liver stiffness - LS $<20 \mathrm{kPa}$ and thrombocites $>150.000 / \mathrm{mm}^{3}$ ), probably with EV $\left(\mathrm{LS}^{3} 25 \mathrm{kPa}\right)$ and the "gray zone" in between these criteria.

Results: Out of 403 patients, $127(30.7 \%)$ had LS $<20 \mathrm{kPa}, 89(22 \%)$ had LS between $20-25 \mathrm{kPa}, 190(47.3 \%)$ had LS $>25 \mathrm{kPa}, 120(29.7 \%)$ had thrombocytes $>150.000 / \mathrm{mm}^{3}$, while $283(70.3 \%)$ had thrombocytes $<150.000 / \mathrm{mm}^{3}$. For the subgroup probably with EV, the Baveno VI criteria had PPV $=84.6 \%$ $(\mathrm{Se}=40.7 \%, \mathrm{Sp}=74.6 \%, \mathrm{NPV}=26.8 \%)$ for predicting the presence of esophageal varices, while for the subgroup probably without EV had NPV $=80.3 \%$ $(\mathrm{Se}=50.2 \%, \mathrm{Sp}=58.6 \%, \mathrm{PPV}=75.6)$. The subgroup that had LS $<20 \mathrm{kPa}$ and thrombocytes $>150.000 / \mathrm{mm}^{3}$, was compound of 60 patients. Using these criteria we correctly classified $80 \%$ patients, with a $\mathrm{Se}=80 \%, \mathrm{Sp}=28.3 \%$, $\mathrm{PPV}=50 \%, \mathrm{NPV}=61.2 \%, \mathrm{AUROC}=0.70, \mathrm{CI}=(68.2-71.3)$. The best cut-off value for TE for predicting the presence of $\mathrm{EV}$ of any grade in our group was $>23 \mathrm{kPa}, \quad$ AUROC $0.79 \quad(\mathrm{Se}=68.8 \%, \quad \mathrm{Sp}=56.9 \%, \quad \mathrm{PPV}=44.7 \%$, $\mathrm{NPV}=78.4 \%$ ).

Conclusion: By using the Baveno VI criteria in patients with liver cirrhosis for the prediction of presence of esophageal varices, we can misclassify only $20 \%$ of patients.

Disclosure of Interest: S.A. Popescu: I hereby confirm that I have received financial support (congress travel grants, speaker fee) from: Philips, General Electric, Abbvie, AstraZeneca, Zentiva

R. Sirli: I hereby confirm that I have received financial support (congress travel grant or speaker fee) from Philips, Abbvie, Zentiva

I. Sporea: I hereby confirm that I have received financial support (congress travel grant or speaker fee) from Philips, Siemens, General Electric, Abbvie, Zentiva, Bristol Meyers Squibb

All other authors have declared no conflicts of interest.

\section{P1336 COMBINED RADIOLOGIC-BLOOD PARAMETERS AND BLOOD DERIVED NON-INVASIVE FIBROSIS SCORES IN PREDICTING OUTCOMES IN CHRONIC HEPATITIS C}

R.B. Thandassery ${ }^{1}, \mathrm{H}$. Wani ${ }^{1}$, S. Al Kaabi ${ }^{1}$, M. Khanna ${ }^{2}$

${ }^{1}$ Gastroenterolgy, Hamad General Hospital, Doha/Qatar

${ }^{2}$ Radiodiagnosis, Hamad General Hospital, Doha/Qatar

Contact E-mail Address: doc.ragesh@gmail.com

Introduction: Non-invasive fibrosis scores (NIFs) are increasingly replacing liver biopsy (LB) for estimation of liver fibrosis. Only limited studies have evaluated the role of NIFs in predicting development of esophageal varices (EV) and decompensation in cirrhotic patients. Similarrly there are limited studies evaluating combination of radiologic and blood parameters in predicting fibrosis and outcomes of cirrhosis.

Aims \& Methods: We aimed to compare combined radiologic-blood parameters (CRBP) and blood derived non-invasive fibrosis scores (NIF) for predicting pre treatment cirrhosis, development of esophageal varices (EV) and liver decompensation post antiviral treatment (AVT). 1605 patients (Jan 2002 to June 2015) with CHC underwent liver biopsy (LB) and received AVT with pegylated interferon and ribavirin. Three CRBPs (platelet count-bisplenic diameter index [PSI], platelet count-bisplenicdiameterportal vein index [PSPVI], platelet count-bisplenic diameter-portal vein-caudate lobe length index [PSCLI]) and nineteen blood derived NIFs were calculated from routine blood tests and abdominal ultrasound done prior to starting AVT. AUROCs were calculated for each of these parameters for predicting cirrhosis on pre treatment LB and development of EV and decompensation on follow-up after AVT.

Results: Mean age was $41.9 \pm 9.7$ years ( $85 \%$ males), predominantly genotypes 4 $(65 \%)$ and $1(11 \%)$. Pretreatment LB (Scheuer criteria) showed stage-0 fibrosis in $1.9 \%$, stage- 1 in $32.9 \%$, stage- 2 in $39.5 \%$, stage- 3 in $19 \%$, and stage- 4 (cirrhosis) in $6.6 \%$ of the patients. After AVT, there were $1,089(67.8 \%)$ responders, 482 $(30 \%)$ nonresponders and $34(2.1 \%)$ relapsers. After median follow-up of 6580.5 patient-years post AVT; 39 (2.4\%) developed EV (2 patients had both esophageal and gastric varices and one had only gastric varices) and $52(3.2 \%)$ had decompensation (bleed-9, ascites-39, jaundice-22, hepatic encephalopathy-7, hepatorenal syndrome-4). CRBPs had higher accuracy for prediction of cirrhosis and EV, while NIFs had higher accuracy for predicting decompensation (Table 1). The highest AUROCs were seen with PSI for predicting cirrhosis (AUROC $=0.882$ ) and EV (AUROC $=0.885$ ) and with FIB-4 score for decompensation (AUROC $=0.854$ ). At a cut of 1200 PSI had sensitivity of $87.2 \%$ and specificity of $75 \%$ for predicting cirrhosis and at a cut off of 1100 PSI had sensitivity of $89.4 \%$ and specificity of 85.7 for predicting EV. At a cut off of 2.5 , FIB-4 score had a sensitivity of $82.5 \%$ and specificity of $80.1 \%$ in predicting decompensation. Conclusion: CRBPs predict cirrhosis and development of esophageal varices with high accuracy. Some of the blood derived NIFs have high accuracy in predicting decompensation post antiviral treatment. Application of these simple scores may help in non-invasive screening of patients at high risk for development of esophageal varices and decompensation after antiviral treatment.

Disclosure of Interest: All authors have declared no conflicts of interest.

\section{P1337 A SMART APPROACH TO THE DIAGNOSIS OF MINIMAL HEPATIC ENCEPHALOPATHY}

T. Nuzum ${ }^{1}$, M. Hussey ${ }^{1}$, T. Khanna ${ }^{2}$, E. Macgregor ${ }^{2}$, J. Olaniyi ${ }^{2}$, C. Tersaruolo ${ }^{3}$, N. Breslin ${ }^{1}$, D. Menamara

${ }^{1}$ Gastroenterology, AMNCH, Tallaght, Dublin/Ireland

${ }^{2}$ Trinity College, Dublin/Ireland

${ }^{3}$ Gastroenterology Trinity Academic Gastroenterology Group (TAGG), AMNCH Tallaght, Dublin/Ireland

\section{Contact E-mail Address: tatiana.nuzum@amnch.ie}

Introduction: Minimal Hepatic Encephalopathy (MHE) is present in more than $30 \%$ of patients with chronic liver disease (CLD), and is associated with a poor prognosis including a higher incidence of falls, RTAs and overall mortality. Detection of MHE is often difficult due to time constraints associated with the current gold standard, the psychometric hepatic encephalopathy score (PHES), which includes five paper-based tests. A smartphone application (EncephalApp Stroop Test) has been suggested as a viable alternative.

Aims \& Methods: We aimed to validate the use of the EncephalApp Stroop Test as a screening tool for MHE in an Irish population. CLD patients and healthy controls were recruited from outpatients. CLD patients were identified based on clinical and radiological evidence. Written consent was obtained. Exclusion criteria: cognitive impairment from any other cause, colour-blindness or dyslexia. Baseline demographics, level of education and medical history were obtained, and a Child-Pugh score was calculated where relevant. Each patient performed the PHES test and the Stroop test. Outcomes included the time taken to perform each PHES test as well as the total PHES, the Stroop on + off time and time taken to complete 5 correct runs on the Stroop App. Results were analysed using a Stroop cut-off defined by a previous publication: on + off time $>190$ seconds. Both tests were compared and correlation coefficients calculated. Results were compared amongst patient groups using a student t-test, where $\mathrm{p}<0.05$ was considered significant. ROC analysis was used to establish a standard local cut-off for a positive Stroop test.

Results: A total of 96 patients (51 males) were recruited. Overall, the mean age was $51.7 \pm 16.6$ years; mean years spent in education $13.8 \pm 4.2$. In all there were 35 CLD and 61 healthy controls. While there was no difference in age or years spent in education, there were more men in the CLD group compared to the controls, $23 / 35(66 \%)$ vs. $28 / 61(46 \%), p=0.06$. Within the CLD cohort, 30 $(86 \%)$ patients were Child-Pugh class A, $4(11 \%)$ were class B and $1(3 \%)$ was class C. As expected, more CLD patients had a positive PHES, $4 / 35(11 \%)$ vs. $3 /$ $61(7 \%)$. Overall, correlation between the two tests was poor. $7(7 \%)$ participants had a positive PHES, while $47(49 \%)$ had a positive Stroop test $(\kappa=0.1516)$. Among controls, $27(44 \%)$ had a positive Stroop test, while $3(5 \%)$ had a positive PHES $(\kappa=0.1223)$. Similarly, $20(57 \%)$ CLD patients had a positive Stroop test and $4(11 \%)$ had a positive PHES $(\kappa=0.1765)$. Regarding the Stroop test, the mean time taken to complete 5 correct runs was significantly higher in CLD patients, mean $117.9 \mathrm{~s}[58.3-217.8 \mathrm{~s}]$ vs. $101.2 \mathrm{~s}[55.3-218.6 \mathrm{~s}], \quad \mathrm{p}=0.02$. However, mean Stroop on + off times were similar, $198.4 \mathrm{~s}[102.6-307.7 \mathrm{~s}]$ in the CLD cohort versus $187.9 \mathrm{~s}$ [101.2-420.9 s] in the control group. Of note, CLD patients with a positive PHES had a significantly higher Stroop on + off time compared to those with a negative PHES, mean $260 \mathrm{~s}$ vs. $190 \mathrm{~s}, \mathrm{p}=0.02$. ROC analysis gave a Stroop on + off time of $>187 \mathrm{~s}$ as a positive cut-off for our cohort, sensitivity $=100 \%$, specificity $=59 \%$. In total, 39 patients had an on + off time $>187 \mathrm{~s}, 18(51 \%), 2$ fewer false positives, in the CLD group, and a slightly better correlation $(\kappa=0.2404)$. On average, PHES took 20 minutes to complete, and the Stroop test 5 minutes. Older age and limited years of education correlated with a poorer performance on the Stroop test $(r=0.62, p<0.0001$ and $r=-0.322, p<0.0001$, respectively). However, neither age nor years of education correlated with performance on the PHES $(r=0.087, \quad r=0.12$, respectively). 
Abstract: P1336. Table 1: Predictive Accuracy of Different Combined Radiological and Blood Parameters and Non-invasive Fibrosis Scores for Predicting Cirrhosis, Development of Esophageal Varices and Decompensation

\begin{tabular}{|c|c|c|c|}
\hline NIFs/CRBPs & $\begin{array}{l}\text { Cirrhosis on liver biopsy } \\
\text { (pre treatment)* }\end{array}$ & $\begin{array}{l}\text { Esophageal varices } \\
\text { (post antiviral treatment)* }\end{array}$ & $\begin{array}{l}\text { Decompensation } \\
\text { (post antiviral treatment) }\end{array}$ \\
\hline AST-platelet ratio index (APRI) & $0.842(0.800-0.884), 0.021$ & $0.769(0.607-0.932), 0.083$ & $0.538(0.063-0.783), 0.120$ \\
\hline Fibrosis-4 (FIB-4) & $0.827(0.781-0.874), 0.024$ & $0.841(0.716-0.886), 0.018$ & $0.854(0.738-0.956), 0.041$ \\
\hline Lok score & $0.800(0.754-0.846), 0.024$ & $0.820(0.775-0.876), 0.023$ & $0.803(.858-0.993), 0.127$ \\
\hline GUCI score & 0.847 (0.805-0.889), 0.021 & $0.817(0.667-0.967), 0.077$ & $0.604(0.062-0.775), 0.144$ \\
\hline King score & $0.851(0.811-0.892), 0.021$ & $0.816(0.742-0.869), 0.032$ & $0.641(0.016-0.898), 0.150$ \\
\hline Fibrosis index & $0.820(0.771-0.870), 0.021$ & $0.819(0.752-0.869), 0.032$ & $0.767(0.529-0.873), 0.079$ \\
\hline Fibrosis cirrhosis index & $0.794(0.745-0.843), 0.025$ & $0.801(0.658-0.945), 0.073$ & $0.646(0.603-0.998), 0.143$ \\
\hline Cirrhosis discrimination score & $0.793(0.747-0.839), 0.023$ & $0.766(0.883-0.849), 0.042$ & $0.804(0.610-0.988), 0.089$ \\
\hline Age platelet index & $0.819(0.789-0.858) 0.020$ & $0.757(0.686-0.827), 0.036$ & $0.816(0.663-0.948), 0.078$ \\
\hline Fibro-index & $0.799(0.753-0.845), 0.023$ & $0.558(0.308-0.809), 0.128$ & $0.678(0.445-0.873), 0.114$ \\
\hline Globulin platelet index & $0.817(0.793-0.881), 0.021$ & $0.500(0.243-0.757), 0.131$ & $0.500(0.297-0.800), 0.146$ \\
\hline Doha score & $0.838(0.797-0.880), 0.021$ & $0.796(0.733-0.858), 0.032$ & $0.685(0.586-0.842), 0.044$ \\
\hline Fibro alpha & $0.636(0.568-0.704), 0.035$ & $0.622(0.371-0.874), 0.128$ & $0.644(0.362-0.999), 0.146$ \\
\hline AST-ALT ratio & $0.605(0.545-0.665), 0.031$ & $0.565(0.356-0.774), 0.107$ & $0.671(0.405-0.999), 0.137$ \\
\hline Pohl score & $0.586(0.524-0.649), 0.032$ & $0.588(0.485-0.691), 0.052$ & $0.619(0.392-0.836), 0.126$ \\
\hline Globulin albumin ratio & $0.762(0.708-0.816), 0.027$ & 0.507 (0.407-0.607), 0.051 & $0.452(0.225-0.661), 0.110$ \\
\hline Mean platelet volume & $0.689(0.563-0.816), 0.064$ & $0.461(0.289-0.633), 0.088$ & $0.648(0.478-0.756), 0.067$ \\
\hline RDW to platelet count ratio & $0.485(0.426-0.545), 0.030$ & $0.829(0.816-0.943), 0.032$ & $0.839(0.742-0.959), 0.060$ \\
\hline $\mathrm{PSI}^{\wedge}$ & $0.882(0.700-0.963), 0.042$ & 0.885 (0.794-0.977), 0.047 & $0.596(0.319-0.874), 0.142$ \\
\hline PSPV & $0.872(0.709-0.936), 0.033$ & $0.845(0.703-0.983), 0.071$ & $0.551(0.300-0.803), 0.128$ \\
\hline PSPVCLI & $0.850(0.737-0.922), 0.047$ & $0.852(0.738-0.962), 0.057$ & $0.495(0.248-0.743), 0.126$ \\
\hline
\end{tabular}

*AUROC (95\% Confidence interval), standard error

\begin{tabular}{llllll}
\hline Test & PHES & PHES - & Stroop+ & Stroop - & Kappa \\
\hline All patients & 7 & 89 & 39 & 57 & 0.107 \\
CLD & 4 & 31 & 18 & 17 & 0.2404 \\
\hline
\end{tabular}

Results: A community of 37 operational taxonomic units (OTUs) was sufficient to pinpoint characteristic features of the microbiome before and after the intervention. Within this predictive community, three OTUs were found to be differentially abundant: Lactobacillus brevis and Lactococcus lactis increased significantly and Enterococcus durans decreased significantly in the probiotic group. Zonulin normalized in $20 \%$ of patients in the probiotic group. Predicted metagenome functions (assessed by PICRUSt) and calprotectin did not show any differences during intervention.

Conclusion: In conclusion, a six months intervention with a multispecies probiotic enriched the microbiome of cirrhotic patients with probiotic bacteria. Additionally, the abundance of Enterococcus durans was reduced and the gut barrier was strengthened.

Conclusion: This is the first study investigating the efficacy of the Stroop te Ireland. It was quicker and easier to perform compared to PHES test. Age and years in education had a greater impact on the Stroop test, which may affect its application and interpretation. While our ROC analysis suggests a similar cut off to previously published values, there is significant variability and local validation is likely to be required. Overall the comparison with the gold standard PHES was poor. However, there were no false negative Stroop tests suggesting it may be a convenient filter test for MHE

Disclosure of Interest: All authors have declared no conflicts of interest.

\section{P1338 MULTISPECIES PROBIOTIC ENRICHES THE MICROBIOME WITH LACTOBACILLUS AND LACTOCOCCUS AND REDUCES ENTEROCOCCUS ABUNDANCE IN PATIENTS WITH LIVER CIRRHOSIS: RESULTS OF A RANDOMIZED, DOUBLE- BLIND, PLACEBO-CONTROLLED TRIAL}

A. Horvath ${ }^{1}$, M. Durdevic ${ }^{2}$, B. Leber ${ }^{3}$, B. Schmerboeck ${ }^{3}$, F. Rainer ${ }^{1}$

P. Douschan ${ }^{1}$, E. Krones ${ }^{1}$, W. Spindelboeck ${ }^{1}$, F. Durchschein ${ }^{1}$, G. Zollner ${ }^{1}$, R. Stauber ${ }^{1}$, P. Fickert ${ }^{1}$, P. Stiegler ${ }^{3}$, V. Stadlbauer ${ }^{1}$

${ }^{1}$ Department Of Gastroenterology And Hepatology, Medical University of Graz, Graz/Austria

${ }^{2}$ Centre For Medical Research, Medical University of Graz, Graz/Austria

${ }^{3}$ Department Of Transplantation Surgery, Medical University of Graz, Graz/ Austria

Contact E-mail Address: angela.horvath@medunigraz.at

Introduction: Cirrhosis is accompanied by significant changes of the intestinal microbiome including the overgrowth of the intestine with potential pathogens that can translocate through a weakened gut barrier and cause severe infections. We hypothesized that probiotic bacteria repress intestinal pathogen growth and strengthen the gut barrier.

Aims \& Methods: Therefore, we conducted a randomized, double-blind, placebocontrolled study to test the effects of the multispecies probiotic Ecologic Barrier (Winclove, Amsterdam, The Netherlands)/Omnibiotic Hetox (Allergosan, Graz, Austria) on microbiome composition, predicted metagenome functions, and tight junction function in cirrhosis patients. A once daily dose of the probiotic mixture $\left(1.5^{*} 10^{10} \mathrm{CFU}\right)$ or placebo was administered to 58 patients with Child's A cirrhosis. We analysed the stool microbiome prior, immediately after the intervention and six months following end of treatment. Hypervariable region 1-2 of the bacterial $16 \mathrm{~S}$ rDNA was sequenced and predictive communities were identified using Ada Boost Classifier. Functional predictions were analysed by Phylogenetic Investigations of Communities by Reconstruction of unobserved States (PICRUSt). Zonulin and calprotectin were assessed in stool as markers for gut permeability and intestinal inflammation, respectively.

\section{P1339 THE IMPACT OF DIABETES MELLITUS ON SHORT-TERM AND LONG-TERM OUTCOMES AFTER LIVER TRANSPLANTATION}

A. Eshraghian ${ }^{1}$, S. Nikeghbalian ${ }^{2}$, M.R. Fattahi ${ }^{3}$, S. Ali Malek-Hosseini ${ }^{2}$

${ }^{1}$ Gastroenterohepatology Research Center, Shiraz University of Medical Sciences, Shiraz/Iran

${ }^{2}$ Transplant Research Center, Shiraz/Iran

${ }^{3}$ Endoscopy Ward, Namazi Hospital, Fars-Shiraz/Iran

Contact E-mail Address: eshraghiana@yahoo.com

Introduction: Diabetes mellitus (DM) is a growing disease worldwide. Some previous studies have reported negative impact of DM in patients with chronic liver disease.

Aims \& Methods: This study aimed to investigate the prevalence of DM in patients with liver cirrhosis and its impact on post-liver transplant short-term and long-term outcomes. In a cross-sectional study patients with liver cirrhosis on liver transplant waiting list who had undergone liver transplantation between March 2012 and March 2015 at Shiraz Transplant Center, Shiraz, Iran were included. Clinical and laboratory data of patients were recorded and patients were followed during post-liver transplant period. DM was diagnosed if the patient had fasting plasma glucose (FPG) $\geq 126 \mathrm{mg} / \mathrm{dL}$ or random plasma glucose $\geq 200 \mathrm{mg} / \mathrm{dL}$ in 2 different checkings or receiving anti-diabetic medications. The impact of DM on post-transplant outcomes was investigated using student t-test and chi-square test. Multivariate logistic regression was used for analysis of independent risk factors of mortality after liver transplantation. Kaplan-Meier method was used for analysis of survival.

Results: 1014 patients were included in the study. 259 patients $(25.5 \%)$ found to have DM. Prevalence of DM was significantly higher among patients with cirrhosis due to non-alcoholic steatohepatitis $(\mathrm{NASH})(\mathrm{P}<0.001)$. Portal vein thrombosis (PVT) was significantly higher among cirrhotic patients with DM $(\mathrm{OR}=1.79 ; 95 \% \mathrm{CI}: 1.18-2.70 ; \mathrm{P}=0.005)$. Mean model for end stage liver disease (MELD) score, body mass index (BMI) and duration of liver disease were not different in patients with and without DM $(\mathrm{P}>0.05)$. Mean blood urea nitrogen (BUN) in 30 days post-transplant period was $16.17 \pm 17.4 \mathrm{mg} / \mathrm{dL}$ in patients without DM compared to $18.39 \pm 11.95 \mathrm{mg} / \mathrm{dL}$ in patients with $\mathrm{DM}$ $(P<0.001)$. Mean serum creatinine level was $1.46 \pm 0.95 \mathrm{mg} / \mathrm{dL}$ in patients without DM compared to $1.78 \pm 1.92 \mathrm{mg} / \mathrm{dL}$ in patients with $\mathrm{DM}(\mathrm{P}=0.001)$. 
Rejection episodes $(\mathrm{OR}=1.38 ; 95 \% \mathrm{CI}: 0.47-1.1, \mathrm{P}=0.129)$ and acute kidney injury $(\mathrm{OR}=1.33: 95 \% \mathrm{CI}: 0.96-1.84, \mathrm{P}=0.07)$ after liver transplantation were not statistically different in patients with and without DM. Using Kaplan-Meier method, mean post-liver transplant survival was $50.94 \pm 0.67$ months in cirrhotic patients without DM and $45.26 \pm 1.46$ months in cirrhotic patients with DM $(\mathrm{P}<0.001)$. Post-liver transplant survivals at 6 months, 1 year and 4 years were outlined in Table. In patients with DM, presence of hepatocellular carcinoma $(\mathrm{HCC})(\mathrm{OR}=4.01 ; 95 \% \mathrm{CI}: 1.64-9.78, \mathrm{P}=0.002)$, acute kidney injury within 30 days after transplant $(\mathrm{OR}=2.05 ; 95 \% \mathrm{CI}: 1.02-4.10, \mathrm{P}=0.042)$ and pre-transplant PVT $(\mathrm{OR}=2.51 ; 95 \% \mathrm{CI}: 1.16-5.14, \mathrm{P}=0.019)$ were independent predictors of mortality after liver transplantation.

Table: Post-liver transplant survival at 6 months, 1 year and 4 years in patients with and without DM

\begin{tabular}{llll}
\hline & 6 month survival & 1 year survival & 4 year survival \\
\hline With diabetes & $81 \% \pm 2.5 \%$ & $79.1 \% \pm 2.6 \%$ & $75.9 \% \pm 2.8 \%$ \\
Without diabetes & $90.6 \% \pm 1.1 \%$ & $88.7 \% \pm 1.2 \%$ & $85.4 \% \pm 1.3 \%$ \\
\hline
\end{tabular}

Conclusion: Diabetes mellitus is prevalent in patients with liver cirrhosis especially among those with NASH. Patients with DM may have lower post-transplant survival and need more intense follow-up.

Disclosure of Interest: All authors have declared no conflicts of interest.

\section{References}

1. Wlazlo N, Beijers HJ, Schoon EJ, Sauerwein HP, Stehouwer CD, Bravenboer B. High prevalence of diabetes mellitus in patients with liver cirrhosis. Diabet Med 2010; 27(11):1308-11.

2. Aguilar M, Liu B, Holt EW, Bhuket T, Wong RJ. Impact of obesity and diabetes on waitlist survival, probability of liver transplantation and post transplant survival among chronic hepatitis C virus patients. Liver Int 2016; 36(8):1167-75

\section{P1340 VALIDATION OF THE BAVENO VI CRITERIA ON A COHORT OF CIRRHOTIC PATIENTS}

I.M. Ratiu ${ }^{1}$, R. Lupusoru ${ }^{1}$, N. Baltes ${ }^{1}$, C. Pienar ${ }^{2}$, I. Sporea ${ }^{1}$

${ }^{1}$ Department Of Gastroenterology And Hepatology, University of Medicine and

Pharmacy "Victor Babes" Timisoara, Timisoara/Romania

${ }^{2}$ Gastroenterology, Pediatrics, University of Medicine and Pharmacy "Victor

Babes" Timisoara, Timisoara/Romania

Contact E-mail Address: ratiu_iulia@yahoo.com

Introduction: The Baveno VI guidelines propose that cirrhotic patients with a liver stiffness measurement (LS) $<20 \mathrm{kPa}$ and a platelet count $>150000 / \mu \mathrm{L}$ can avoid screening endoscopy as their combination is highly specific for excluding clinically significant varices.

Aims \& Methods: The aim of the study was to validate the Baveno VI criteria. We did a retrospentive study, from 2009-2014. We took all the patients with transient elastography data. Inclusion criteria were a LS $>12 \mathrm{kPa}$ and an upper gastrointestinal endoscopy within 12 months, with a diagnosis of chronic liver disease. Varices were graded as low risk $($ grade $<2)$ or high risk $(>$ grade 2$)$.

Results: The study included 774 patients (hepatitis C virus $40.5 \%$, hepatitis B virus $16.1 \%, 31.6 \%$ etanolics, $11.8 \%$ other etiology, and $47.5 \%$ were Child Pugh A). Varices were present in $561 / 774(2.4 \%)$ cases, with $8 \%$ prevalence of high risk varices. $306 / 774(39.6 \%)$ were at low risk and $468 / 774(60.4 \%)$ had high risk varices. 59/774 (7.6\%) met the Baveno VI criteria. The Baveno VI criteria gave a $\mathrm{Se}=62.2 \%, \mathrm{Sp}=80.6 \%, \mathrm{NPV}=44.6 \%, \mathrm{PPV}=89.5 \%$, positive likelihood ratio $=3.4$, negative likelihood ratio $=0.47$. If we combined the $\mathrm{LS}<20 \mathrm{kPa}$ and platelet count $>150,000$, the AUROC was 0.73 , CI $(0.68-0.74), \mathrm{p}<0.0001$. Conclusion: The Baveno VI criteria has correctly appoint $85.3 \%$ of patients who could safely avoid endoscopy.

Disclosure of Interest: I. Sporea: I hereby confirm that I have received financial support (congress travel grant or speaker fee) from Philips, Siemens, General Electric, Abbvie, Zentiva, Bristol Meyers Squibb

All other authors have declared no conflicts of interest.

\section{Reference}

1. Roberto de Franchis, Expanding consensus in portal hypertension Report of the Baveno VI Consensus Workshop: Stratifying risk and individualizing care for portal hypertension, 2015 Sep;63(3):743-52. doi: 10.1016/ j.jhep.2015.05.022

\section{P1341 MORTALITY PREDICTING MODEL IN LIVER CIRRHOSIS PATIENTS}

R. Lupusoru ${ }^{1}$, I. Sporea ${ }^{1}$, S.A. Popescu ${ }^{1}$, A. Stepan ${ }^{1}$, A. Pascaru ${ }^{1}$, M. Danila ${ }^{1}$, A. Barbulescu ${ }^{1}$, I.M. Ratiu $^{1}$, B. Miutescu ${ }^{1}$, R. Sirli $^{1}$

${ }^{1}$ Department Of Gastroenterology And Hepatology, Victor Babes University of

Medicine and Pharmacy Timisoara, Timisoara/Romania

Contact E-mail Address: raluca_lupusoru@yahoo.ro
Introduction: Cirrhotic patients very often need to be hospitalized and it is known that they have a higher mortality rate.

Aims \& Methods: The aim of the study was to assess the factors associated with mortality among cirrhotic patients and to create a new score for predicting mortality. The study was retrospective, and we included all hospitalized patients with the final diagnosis of liver cirrhosis on a period of 7 years. We divided them in two cohorts, an initial group, which was analysed; and a control group, in which we validated the score. We performed univariate and multivariate analysis in order to determine a prediction model for mortality.

Results: A total of 1163 cirrhotic patients were included. In-hospital mortality rate was $10 \%$. The initial cohort included 899 patients. Regarding cirrhosis etiology: $384 / 899(42 \%)$ had hepatitis C, $158 / 899(17.5 \%)$ had hepatitis B, 293/899 (32.5\%) were alcoholic, 6/899 (0.6\%) were autoimmune, $7 / 899$ $(0.7 \%)$ were cardiac, $13 / 899(1.4 \%)$ were primary biliary cirrhosis and in $5 \%$ of cases the etiology was unknown. In univariate analysis, hyponatremia $(\mathrm{p}<0.0001)$, hyperpotasemia $(\mathrm{p}<0.0001)$, hypoalbuminemia $(\mathrm{p}<0.0001)$, high values of bilirubin $(p<0.0001)$, high values of creatinine $(p<0.0001)$ were strongly associate with in hospital mortality. In multivariate analysis, the model including albumin, sodium, potassium, creatinine and bilirubin (all p-values $<0.05)$ had an AUROC $=0.78$, CI $(0.75-0.81), p<0.0001$. Using this factors as predictors, by multiple regression analysis we obtained in the initial group the following score: ABCPS score $=0.04+0.03 *$ Albumin $+0.05+0.02 *$ Creatinine $+0.04+0.04 *$ Bilirubin + $0.05+0.28 *$ Potassium $+0.04 * 0.07 *$ Sodium.

Conclusion: Prevention and prompt treatment of kidney injury, hyponatremia, hyperpotassemia, can improve survival. ABCPS score can be an usefull score to rule out patients with high mortality rate.

Disclosure of Interest: I. Sporea: I hereby confirm that I have received financial support (congress travel grant or speaker fee) from Philips, Siemens, General Electric, Abbvie, Zentiva, Bristol Meyers Squibb

S.A. Popescu: I hereby confirm that I have received financial support (congress travel grants, speaker fee) from:Philips, General Electric, Abbvie, AstraZeneca, Zentiva

R. Sirli: I hereby confirm that I have received financial support (congress travel grant or speaker fee) from Philips, Abbvie, Zentiva

All other authors have declared no conflicts of interest.

\section{P1342 BARIATRIC SURGERY IS ASSOCIATED WITH INCREASED RISK OF DEVELOPING ACUTE LIVER INJURY: A NATIONWIDE ANALYSIS}

P.T. Kroener ${ }^{1}$, P. K. Wander ${ }^{1}$, V. Popov ${ }^{2}$, C. C. Thompson ${ }^{3}$

${ }^{1}$ Mt. Sinai St. Luke's/West, New York/United States of America

${ }^{2}$ New York University School of Medicine, New York/United States of America

${ }^{3}$ Division Of Gastroenterology, Hepatology, And Endoscopy, Brigham and

Women's Hospital, Boston/United States of America

\section{Contact E-mail Address: thomaskroner@gmail.com}

Introduction: Bariatric surgery provides a durable method of weight loss but is associated with serious adverse events. Some studies report an increase in druginduced acute liver injury following bariatric surgery.

Aims \& Methods: We aimed to assess if bariatric procedures increase the risk of acute liver failure in a large inpatient cohort. We retrospectively analyzed discharge data on patients who developed acute liver injury (ALI) using the Nationwide Inpatient Sample (NIS) database from 2010-2013. Discharges with an ICD-9 code indicating ALI were included. The primary outcome was ALI in patients with a history of bariatric surgery compared to all other patients with an inpatient diagnosis of ALI. Secondary outcomes were mortality in the two cohorts and independent socio-demographic and medical risk factors for mortality in each cohort. Variables tested include age, gender, race, income, Charlson criteria, hospital factors and medical comorbidities including Malnutrition, HTN, Anemia, CKD, Diabetes, CHF, Coagulopathy, Alcoholism, HBV and $\mathrm{HCV}$. Univariate and multivariate logistic regression analyses were performed to identify independent predictors.

Results: During the study period, a total of 437,390 patients were diagnosed with acute liver injury and were included in the study, of which 3,799 had previously undergone bariatric surgery. In the post-bariatric cohort, mean age was 58.7 years and $77 \%$ were women. The prevalence of acute liver injury in all inpatient admissions for that time period was higher in patients with history of bariatric surgery $(0.85 \%)$ than in non-bariatric patients $(0.75 \%), p<0.01$. Patients with history of bariatric surgery displayed odds ratio of 1.52 of developing ALI when compared to patients with no history of bariatric surgery $(95 \% \mathrm{CI}: 1.43-1.61$, $\mathrm{p}<0.01)$. The rate of overall inpatient mortality was higher in non-bariatric cohort $(15.9 \%$ versus $9.3 \%)$. Post-bariatric patients admitted for ALI were more likely to be younger, female, Caucasian and residing in more affluent areas. Post-bariatric patients were also more likely to have higher rates of malnutrition, anemia, alcoholism, and significantly lower prevalence of hepatitis $\mathrm{B}$ and $\mathrm{C}, \mathrm{CHF}$, diabetes and kidney disease. In a multivariate regression model, the presence of CHF and coagulopathy increased mortality risk, and diagnosis of alcoholism was associated with lower mortality risk from ALI in patients with prior history of bariatric surgery (Table). 


\begin{tabular}{llll}
\hline Comorbidity & Adjusted Odds Ratio & $95 \% \mathrm{CI}$ & p-value \\
\hline Malnutrition & 1.86 & $0.89-3.88$ & 0.10 \\
HTN & 0.64 & $0.34-1.18$ & 0.16 \\
Anemia & 0.63 & $0.36-1.11$ & 0.12 \\
CKD & 1.66 & $0.79-3.54$ & 0.18 \\
Diabetes & 1.04 & $0.55-1.94$ & 0.91 \\
CHF & 3.92 & $1.77-8.71$ & $<\mathbf{0 . 0 1}$ \\
Coagulopathy & 1.75 & $1.03-2.96$ & $\mathbf{0 . 0 4}$ \\
Alcoholism & 0.48 & $0.25-0.95$ & $\mathbf{0 . 0 4}$ \\
HCV & 0.86 & $0.27-2.72$ & 0.80 \\
\hline
\end{tabular}

Conclusion: Bariatric surgery increases the risk of subsequent acute liver injury. Post-bariatric surgery patients admitted for ALI are more likely to have anemia, malnutrition, and alcoholism, supporting the hypothesis that baseline nutritional status may predispose to drug-induced ALI. Addressing these potentially modifiable risk factors may decrease the significant morbidity and mortality of ALI. Disclosure of Interest: All authors have declared no conflicts of interest.

\section{P1344 LONGITUDINAL MONITORING OF LIVER STIFFNESS BY ACOUSTIC RADIATION FORCE IMPULSE IMAGING IN PATIENTS WITH CHRONIC HEPATITIS B RECEIVING ENTECAVIR}

S. Wu ${ }^{1}$, H. Ding ${ }^{2}$, L. Liu ${ }^{1}$, N. Xu ${ }^{3}$, W. Jiang ${ }^{1}$

${ }^{1}$ Gastroenterology \& Hepatology, Zhongshan Hospital, Fudan University,

Shanghai/China

${ }^{2}$ Ultrasound, Zhongshan Hospital, Fudan University, Shanghai/China

${ }^{3}$ Zhongshan Hospital, Fudan University, Shanghai/China

Contact E-mail Address: wu.shengdi@zs-hospital.sh.cn

Introduction: Acoustic radiation force impulse (ARFI) imaging measures liver stiffness (LS), which significantly correlates with the stage of liver fibrosis in treatment-naive patients with chronic hepatitis B (CHB). So far, the use of ARFI elastography to monitor change in liver fibrosis has not been properly evaluated during antiviral therapy in CHB patients.

Aims \& Methods: We aimed to prospectively assess the clinical usefulness of ARFI during long-term antiviral therapy in CHB patients. Seventy-one CHB patients were consecutively recruited and received antiviral therapy with entecavir. Paired liver biopsies were performed in 27 patients at baseline and week 78 of entecavir therapy. LS was assessed by ARFI at multiple follow-up sessions.

Results: LS significantly decreased with treatment and continued to decrease after normalization of alanine aminotransaminase. Overall, 97.2\% patients achieved improvement of LS, whereas $19.7 \%$ patients had more than $30 \%$ reduction in LS values between baseline and week 104. Multivariate linear regression analysis showed that the degree of LS reduction significantly correlated with the baseline levels of LS value, platelet and cholinesterase. In the 27 patients who received paired liver biopsies, LS significantly correlated with stage of fibrosis and inflammatory grade at baseline. LS values decreased more significantly in patients with fibrosis regression than those with static histological fibrosis. Changes in LS value (change threshold $=15 \%$ ) was significantly correlated with the changes in histological fibrosis staging $(r=0.63, P<0.001)$.

Conclusion: In CHB patients, LS assessed by ARFI was significantly reduced during antiviral therapy. Longitudinal monitoring of LS might be a promising noninvasive assessment of fibrosis regression during long-term antiviral therapy in CHB. Further studies on large populations are warranted.

Disclosure of Interest: All authors have declared no conflicts of interest

\section{References}

1. Chang TT, Liaw YF, Wu SS, et al. Long-term entecavir therapy results in the reversal of fibrosis/cirrhosis and continued histological improvement in patients with chronic hepatitis B. Hepatology 2010;52(3):886-93.

2. Friedrich-Rust M, Poynard T, Castera L. Critical comparison of elastography methods to assess chronic liver disease. Nature reviews. Gastroenterology \& hepatology 2016;13(7):402-11.

3. Friedrich-Rust M, Buggisch P, de Knegt RJ, et al. Acoustic radiation force impulse imaging for non-invasive assessment of liver fibrosis in chronic hepatitis B. Journal of viral hepatitis 2013;20(4):240-7.

4. European Association for Study of L, Asociacion Latinoamericana para el Estudio del H. EASL-ALEH Clinical Practice Guidelines: Non-invasive tests for evaluation of liver disease severity and prognosis. Journal of hepatology 2015;63(1):237-64

5. Wong GL, Wong VW, Choi PC, et al. On-treatment monitoring of liver fibrosis with transient elastography in chronic hepatitis $\mathrm{B}$ patients. Antiviral therapy 2011;16(2):165-72.

6. Osakabe K, Ichino N, Nishikawa T, et al. Reduction of liver stiffness by antiviral therapy in chronic hepatitis B. Journal of gastroenterology 2011;46(11):1324-34.
P1345 CHRONIC HEPATITIS B VIRUS INACTIVE CARRIERSIMPACT OF METABOLIC DISORDERS IN STEATOSIS ASSESSED BY FIBROSCAN

S. Xavier ${ }^{1}$, S. Monteiro ${ }^{2}$, C. Arieira ${ }^{2}$, J. Magalhães ${ }^{2}$, C. Marinho ${ }^{3}$, J. Cotter ${ }^{4}$ ${ }^{1}$ Life and Health Sciences Research Institute (ICVS), School of Medicine, University of Minho, Braga/Portugal

${ }^{2}$ Gastroenterology Department, Hospital da Senhora da Oliveira, Guimarães, Guimarães/Portugal

${ }^{3}$ School Of Medicine, University Of Minho, Life and Health Sciences Research Institute, Braga/Guimarães/Portugal

${ }^{4}$ Pt Government Associate Laboratory, 4 ICVS/3B's, Braga/Guimarães/Portugal

\section{Contact E-mail Address: smaxavier@gmail.com}

Introduction: In chronic infection with Hepatitis B virus (HBV) hepatic steatosis is mainly attributable to metabolic risk factors, rather than virologic factors. We aimed to assess the presence of hepatic steatosis in chronic HBV inactive carriers using non-invasive methods, namely controlled attenuation parameter (CAP) determined with fibroscan. We also aimed to identifiy risk factors associated to hepatic steatosis and their impact in the values of fibrosis determined with fibroscan.

Aims \& Methods: Fibroscan was performed in chronic HBV inactive carriers, with assessment of hepatic transient elastography and CAP, with simultaneous assessment of anthropometric, clinical and analytical parameters. CAP values of 248,268 and $280 \mathrm{~dB} / \mathrm{m}$ defined cut-offs of steatosis grade I, II and III, respectively.

Results: Included 49 patients with a mean transient elastography of $5.1 \pm 1.5 \mathrm{Kpa}$ and a mean CAP of $248.9 \pm 49.3 \mathrm{~dB} / \mathrm{m}$. A significant association was found between the value of CAP and the presence of steatosis in the last ultrasound $(234.4 \pm 46.0 \mathrm{~dB} / \mathrm{m}$ vs $289.4 \pm 34.0 \mathrm{~dB} / \mathrm{m}, \mathrm{p}<0.01)$, the presence of elevated triglycerides $(239.9 \pm 49.8 \mathrm{~dB} / \mathrm{m}$ vs $284.1 \pm 28.1 \mathrm{~dB} / \mathrm{m}, \mathrm{p}=0.01)$ and obesity $(244.0 \pm 46.7 \mathrm{~dB} / \mathrm{m}$ vs $290.7 \pm 46.6 \mathrm{~dB} / \mathrm{m}, \mathrm{p}=0.01)$. When comparing patients with $\mathrm{CAP}>$ and $\leq 268 \mathrm{~dB} / \mathrm{m}$, patients with higher CAP values more frequently were overweight $($ BMI $\geq 25 \mathrm{~kg} / \mathrm{m} 2)(45.8 \%$ vs $84.0 \%, \mathrm{p}<0.01)$ and had metabolic syndrome (MS) $(12.5 \%$ vs $40 \%, \mathrm{p}<0.03)$, and also presented with higher values of BMI $(24.6 \pm 2.6 \mathrm{~kg} / \mathrm{m} 2$ vs $29.2 \pm 6.4 \mathrm{~kg} / \mathrm{m} 2, \mathrm{p}<0.02)$, waist circumference $(85.0 \pm 9.0 \mathrm{~cm}$ vs $97.9 \pm 11.3 \mathrm{~cm}, \mathrm{p}<0.01)$ and triglycerides $(95.6 \pm 31.4 \mathrm{mg}$ $\mathrm{dL}$ vs $136.1 \pm 62.9 \mathrm{mg} / \mathrm{dL}, \mathrm{p}=0.01)$ and lower values of HDL cholesterol $(58.9 \pm 14.3 \mathrm{mg} / \mathrm{dL}$ vs $50.4 \pm 14.4 \mathrm{mg} / \mathrm{dL}, \mathrm{p}<0.01)$. A significant association was also found between the value of elastography and female gender (females $4.6 \pm 1.3 \mathrm{KPa}$ vs males $5.8 \pm 1.5 \mathrm{KPa}, \mathrm{p}<0.01)$, elevated triglycerides $(4.9 \pm 1.5 \mathrm{KPa}$ vs $6.0 \pm 1.1 \mathrm{KPa}, \mathrm{p}=0.03)$ and obesity $(4.9 \pm 1.4 \mathrm{KPa}$ vs $6.5 \pm 1.1 \mathrm{KPa}, \mathrm{p}<0.01)$.

Conclusion: Different components of MS seem to contribute both to fibrosis and steatosis in chronic HBV inactive carriers. In this subset of patients, the interpretation of fibrosis values assessed by fibroscan should be made with caution since it may be influenced by metabolic parameters.

Disclosure of Interest: All authors have declared no conflicts of interest.

\section{P1346 ANTIVIRAL EFFICACY OF UNANI HERBS (SAUSSUREA}

\section{LAPPA AND ARTEMISIA ABSINTHIUM) IN CHRONIC HEPATITIS B}

S. Ansari ${ }^{1}$, M. A. Siddiqui ${ }^{1}$, S. Malhotra ${ }^{2}$, M. Maaz

${ }^{1}$ Department Of Moalajat (medicine), School of Unani Medicine, Jamia Hamdard, New Delhi, India, New Delhi/India

${ }^{2}$ Department Of Gastroenterology, Batra Hospital and Research Centre, New Delhi/India

Contact E-mail Address: drshabnamansari.md@gmail.com

Introduction: Chronic hepatitis B (CHB) is a serious health concern in terms of its high prevalence, as well as restricted and costly health care resources in India. ${ }^{1}$ Unani herbal drugs such as root of Saussurea lappa and whole plant of Artemisia absinthium have been used successfully in the treatment of hepatitis in Unani Medicine for centuries. These drugs have exhibited potential anti-hepatitis B, hepatoprotective, immuno-modulatory, anti-inflammatory and anti-oxidant activities in various animal models. ${ }^{2}$ With this background, present prospective clinical trial was tried to be designed according to the guidelines of American association for the study of liver diseases (AASLD) and implemented after Institutional ethical clearance.

Aims \& Methods: Objective was to evaluate the antiviral effect of herbal drugs, Saussurea lappa and Artemisia absinthium against hepatitis B virus (HBV) in the management of $\mathrm{CHB}$ and to collect data to warrant further clinical trials. In an open prospective single-arm study, we assigned 30 patients with $\mathrm{HBeAg-negative}$ or $\mathrm{HBeAg}$-positive $\mathrm{CHB}$ to receive decoction of root of Saussurea lappa, $15 \mathrm{~mL}$ (containing approx. $1 \mathrm{~g}$ of dried aquoues extract) in the morning and decoction of Artemisia absinthium, $15 \mathrm{~mL}$ (containing approx. $1 \mathrm{~g}$ of dried aquoues extract) in the evening once daily empty stomach for 12 weeks. Physiochemical standardization and TLC and HPTLC fingerprinting of decoctions were also done. Test drug was evaluated for its efficacy in loss of HBsAg (miniVIDAS $($ ) and $\mathrm{HBeAg}$ (CLIA tech.), a plasma HBV DNA level (RT PCR) of less than $200 \mathrm{IU} / \mathrm{mL}$ at week 12 (after treatment). Normalization of alanine aminotransferase levels [ALT (enzymatic essay)] without any significant adverse effects (clinical parameters, haemogram, kidney function test, and blood sugar fasting and post prandial) at week 6 (mid-treatment) and 12 (after treatment) would be suggestive of resolution of inflammation of liver.

Results: 1 . HBsAg loss was observed in $5(35.71 \%)$ patients in HBeAg-positive group $(\mathrm{n}=14)(\mathrm{p}<0.05)$ and $4(25 \%)$ patients in HBeAg-negative group $(\mathrm{n}=16)$ at week 12 (after treatment) $(\mathrm{p}=0.10)$. These $9 \mathrm{HBsAg}$ negative patients were further assessed on 26th week of treatment, all 9 maintained their negative HBsAg status. 2. HBeAg loss was observed in $71.42 \%$ patients at week 12 
$(\mathrm{p}<0.001)$. cVR was achieved in $57.14 \%$ patients in HBeAg-positive group $(\mathrm{p}<0.01)$ and $37.5 \%$ of patients in $\mathrm{HBeAg}$-negative group at week 12 $(\mathrm{p}<0.05)$. 3. Normalization of ALT was observed in $85.71 \%$ and $81.25 \%$ patients in HBeAg-positive and HBeAg-negative group respectively at week 12 $(\mathrm{p}<0.001)$ without any significant adverse effects in clinical and laboratory parameters $(\mathrm{p}>0.05)$ at mid and after treatment.

Conclusion: 1. Our study has substantiated that Saussurea lappa and Artemisia absinthium have antiviral effect against HBV in chronic hepatitis B. 2. Test drug effectively suppressed HBV DNA, HBeAg and HBsAg in study patients. 3 . Normalization of liver function test showed that the test drug can improve functions of liver without any significant adverse effects for a treatment duration of 12 weeks. 4. Limitation include small sample size, small duration and without control. Unlike other studies on CHB evaluating effect of conventional antivirals, we have enrolled only selected clinically stable CHB patients who were not diabetic and hypertensive, without liver fibrosis, and not have other neurological, kidney and heart disease, being a pilot study. 4. Future recommendation of double blind randomized clinical trial with satndard control with large sample size, longer duration and including cirrhotic patients.

Disclosure of Interest: All authors have declared no conflicts of interest.

\section{References}

1. WHO. Hepatitis B. In section Global Alert and Response (GAR) [Internet] 2014 [Cited 2014 Jan 7]. Available from:://www.who.int/mediacentre/factsheets.fs204/en/print.html.

2. Chen HC, Chou CK, Lee SD, Wang JC, Yeh SF. Active compounds from Saussurea lappa Clarks thatsuppress hepatitis B virus surface antigen gene expression in human hepatoma cells. Antiviral Res. 1995;27(1-2):99-109.

\section{P1347 QUANTIFICATION OF SERUM HBSAG IS A HELPFUL MARKER TO OPTIMIZE THE MANAGEMENT OF ANTIVIRAL NUC THERAPY IN CHRONIC HBEAG-NEGATIVE HEPATITIS B}

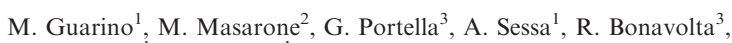
N. Caporaso ${ }^{1}$, F. Morisco ${ }^{1}$

${ }^{1}$ Gastroenterology Unit, Department of Clinical Medicine and Surgery, "Federico II' University, Napoli, Napoli/Italy

${ }^{2}$ Internal Medicine and Hepatology Unit, San Giovanni e RuggiD'Aragona,

University of Salerno, Salerno/Italy

${ }^{3}$ Department of Translational Medical Science, University of Naples "Federico II", Napoli/Italy

Contact E-mail Address: asessa1990@gmail.com

Introduction: Serum HBsAg loss is the recommended stopping rule in nucleo(t)side-analogues (NUC) responders, even if this event occurs rarely.

Aims \& Methods: We aimed to investigate in patients with chronic $\mathrm{HBeAb}+$ hepatitis the kinetics of HBsAg levels during the NUC therapy to evaluate the predictive parameters of $\mathrm{HBsAg}$ seroclearance. Patients with $\mathrm{CHB}$, receiving NUC antiviral therapy with stable viral suppression (HBV-DNA $<20 \mathrm{IU} / \mathrm{ml}$ ), were recruited at the Gastroenterology Unit of the University of Naples "Federico II". Sequential serum samples from these patients were tested for HBsAg quantification with the ElecsysHBsAg II Quant immunoassay (Roche Diagnostics, Indianapolis, USA). HBsAg levels were determined before starting NUC treatment and on-treatment every 12 months.

Results: A total of $95 \mathrm{HBsAg}$-positive, HBeAg-negative patients (M/F: 73/22, median age 50 yrs, $34 \%$ cirrhotic) with stable viral suppression by NUCs, were enrolled. Precisely 56 patients underwent to Tenofovir, 22 Entecavir and 17 Lamivudine. The median treatment duration was 111 months, range $25-183$ months. There was a significant decrease of the HBsAg levels during NUC therapy from $3471 \mathrm{UI} / \mathrm{ml}$ at the baseline to $1758 \mathrm{IU} / \mathrm{ml}$ at the last determination $(\mathrm{p}<0.001)$. The statistically significant HBsAg decrease was also maintained when the patients were clustered according to antiviral therapy, severity of liver disease and previous interferon treatment. HBsAg seroclearance occurred in $18 / 95$ patients $(19 \%)$. The multivariate analysis showed that the only predictive parameter statistically significant of HBsAg seroclearance is the HBsAg level at baseline. Moreover, HBsAg seroconversion to HBsAb occurred in $4 / 18$ patients, in which undetectable $\mathrm{HBsAg}$ value was evidenced at least two years before the seroconversion, and NUC therapy was successfully stopped, without relapse after a mean follow-up period of 24 months.

Conclusion: The results of this study suggest a role of on-treatment $\mathrm{HBsAg}$ quantification in the management of NUC-treated patients. HBsAg measurement would be a useful parameter to optimize antiviral treatment schedule. Disclosure of Interest: All authors have declared no conflicts of interest.

\section{P1348 IMPROVEMENTS IN CHRONIC HEPATITIS B PATIENTS AND THE ALTERATION IN GUT MICROBIOTA AFTER FECAL MICROBIOTA TRANSPLANTATION}

C. Xiao, B. Zhang, J. Ren

Zhongshan Hospital Affiliated To Xiamen University, Department of

Gastroenterology, Xiamen/China

Contact E-mail Address: xiaoxx@xmu.edu.cn

Introduction: Chronic hepatitis B (CHB) is a common liver disease worldwide, and can be progressed to liver cirrhosis and hepatocellular carcinoma. Unfortunately, only a minority of CHB patients could achieve the clearance or seroconversion of hepatitis $\mathrm{B}$ virus e-antigen $(\mathrm{HBeAg})$, the end point of treatment, even after multiple years of antiviral therapy. Therefore, it is urgent to develop new and effective strategy for treatment of $\mathrm{CHB}$ and examine the mechanisms.

Aims \& Methods: In this study, we performed 60 times of fecal microbiota transplantation (FMT) by nasointestinal tube for $20 \mathrm{CHB}$ patients who continued with their previous antiviral treatment, and accordingly measured the $\mathrm{HBeAg}$ level four weeks after each FMT. Fecal samples of CHB patients before (Baseline) and after FMT as well as donors were collected for analyses of gut microbiota by sequencing 16S V3-V4 regions on Illumina MiSeq using PE 250 reagents.

Results: Results showed that HBeAg of 13 patients $(65 \%)$ was cleared or reduced after one to seven times of FMT. Based on OTUs at cutoff of $3 \%$ disimilarity, there were significant (PERMANOVA, $\mathrm{P}=0.001$ ) differences in overall gut bacterial communities among CHB-Baseline, CHB-FMT, and donors, forming three major clusters in PCA ordination. Whereas, no significant differences (ANOVA, $\mathrm{P}>0.05$ ) were detected in $\alpha$-diversity indexes among the three groups, including observed OTU numbers, Shannon index, Simpson index, and Pielou evenness. This implies that it is the taxonomic relative abundance, not taxon number, that contributed to the bacterial community differences. Overall, gut bacteria were mainly composed of Fimicutes (Lachnospiraceae, Ruminococcaceae, Veilloneuaceae), Bacteroidetes (S24-7, bacteroidaceae, Prevotellaceae), and Proteobacteria (Alcaligenaceae, Enterobacteriaceae). More specifically, Actinomyces was significantly higher in $\mathrm{CHB}$ patients (CHB-Baseline) than FMT-treated patients (CHB-FMT) and donors, and was identified as the biomarker of CHB using LEfSe analysis. Conversely, Prevotella and Epulopiscium were significantly decreased in CHB-Baseline after FMT to almost equal to the abundances in donors, and were also identified as biomarkers.

Conclusion: In summary, along with the clearance or remission of $\mathrm{HBeAg}$, the gut bacterial communities of chronic hepatitis B patients were remarkably alerted after fecal microbiota transplantation, with some taxa abundances changed accordingly, which suggested their potential application as targets for clinical diagnosis and treatments in future.

Disclosure of Interest: All authors have declared no conflicts of interest.

\section{P1349 EFFICACY OF PEGYLATED INTERFERON ALFA-2A ADD- ON THERAPY VERSUS NUCLEOSIDE ANALOGUE MONOTHERAPY IN TREATMENT-EXPERIENCED CHRONIC HEPATITIS B PATIENTS: A RANDOMISED, CONTROLLED, OPEN-} LABEL TRIAL

X. Hu${ }^{1}$, J. Ye ${ }^{1}$, W. Yanqin ${ }^{1}$, R. $\mathrm{Li}^{1}$, C. Shao ${ }^{1}$, Z. Bihui

${ }^{1}$ Gastroenterology, the First Affiliated Hospital of Sun Yat-sen University, Guangzhou/China

Contact E-mail Address: 1446989090@qq.com

Introduction: Hepatitis B surface antigen (HBsAg) seroconversion is rarely achieved in chronic hepatitis $\mathrm{B}(\mathrm{CHB})$ patients during nucleoside analogue (NA) monotherapy. The efficacy of pegylated interferon alfa-2a (peg-IFN $\alpha-2 a$ ) add-on therapy in those $\mathrm{CHB}$ patients remains unclear.

Aims \& Methods: We aimed to compare the efficacy of peg-IFN $\alpha$-2a add-on therapy with NA monotherapy in treatment-experienced $\mathrm{CHB}$ patients. We enrolled hepatitis $\mathrm{B}$ e antigen ( $\mathrm{HBeAg}$ )-negative $\mathrm{CHB}$ patients aged older than 16 from the First Affiliated Hospital of Sun Yat-sen University in China. All had received NA monotherapy for 3 years with sustained undetectable plasma HBV DNA. The exclusion criteria included: cirrhosis or other chronic liver diseases, previous immunological therapy, pregnancy and breastfeeding, contraindications for peg-IFN $\alpha-2 \mathrm{a}$, or other serious diseases. The eligible patients were assigned to receive peg-IFN $\alpha$-2a add-on therapy for $48 / 72$ weeks or to continue to receive NA monotherapy for 96 weeks. HBV DNA levels, HBV serologic indicators, liver function, renal function, thyroid function, blood cells count and imaging examination were assessed. The primary end point was HBsAg loss from baseline to week 96.

Results: 71 patients were enrolled ( 22 to peg-IFN $\alpha$-2a add-on therapy group and 49 to NA monotherapy group), of whom 9 in peg-IFN $\alpha$ - 2 a add-on therapy group and 25 in NA monotherapy group completed more than 24 weeks of follow-up, the remaining patients have not yet reached 24 weeks of follow-up. There was no significant difference in age, gender, body mass index (BMI), HBeAg levels, alanine aminotransferase (ALT), or aspartate aminotransferase (AST) between the two groups at baseline. At week 24, HBsAg levels in pegIFN $\alpha$-2a add-on therapy group were significantly lower than the baseline $\left(2.96 \pm 0.14\right.$ vs $\left.2.09 \pm 0.82 \log _{10} \mathrm{IU} / \mathrm{ml}, \mathrm{p}=0.009\right)$, but there was no obvious change in NA monotherapy group $\left(3.43 \pm 0.46\right.$ vs $3.44 \pm 0.44 \log _{10} \mathrm{IU} / \mathrm{ml}$, $\mathrm{p}=0.843$ ). The HBsAg loss in peg-IFN $\alpha$-2a add-on therapy group was significantly higher than in NA monotherapy group at week $24(0.87 \pm 0.76$ vs $\left.-0.01 \pm 0.10 \log _{10} \mathrm{IU} / \mathrm{ml}, \mathrm{p}=0.008\right)$. Among those patients who completed 96 weeks of follow-up, two patients in peg-IFN $\alpha$-2a add-on therapy group $(22.22 \%)$ achieved $\mathrm{HBs} A g$ seroconversion, but none in NA monotherapy group $(0 \%)$. Conclusion: The peg-IFN $\alpha$-2a add-on therapy increased loss of $\mathrm{HBsAg}$ in $\mathrm{HBeAg}$ negative treatment-experienced $\mathrm{CHB}$ patients as compared to NA monotherapy. Disclosure of Interest: All authors have declared no conflicts of interest. 
P1350 HEPATITIS C IN LEBANON: BURDEN OF THE DISEASE AND VALUE OF COMPREHENSIVE SCREENING AND TREATMENT

A. Abou Rached ${ }^{1}$, S. Abi Kheir ${ }^{1}$, J. Saba ${ }^{1}$, S. Assaf ${ }^{2}$, G. Kassis ${ }^{2}$, Y.S. Gonzales ${ }^{3}$, O. Ethgen ${ }^{4}$

${ }^{1}$ Lebanese University, Beirut/Lebanon

${ }^{2}$ Abbvie Biopharmaceuticals GmBH L, Beirut/Lebanon

${ }^{3}$ Abbvie Biopharmaceuticals GmBH L, chicago/United States of America

${ }^{4}$ SERFAN Innovation, Liege/Belgium

Contact E-mail Address: abourachedantoine@gmail.com

Introduction: As few reliable data on the burden of hepatitis $\mathrm{C}$ virus (HCV) are available from the Middle East, we analyzed HCV burden in the Lebanese population and the value of comprehensive screening and treatment at different age groups and fibrosis stages.

Aims \& Methods: A multi-cohort, health-state-transition model was developed to project the number of $\mathrm{HCV}$ patients achieving a sustained virologic response 12 weeks after treatment $\left(\mathrm{SVR}_{12}\right)$ or progressing to compensated cirrhosis (CC), decompensated cirrhosis (DCC), hepatocellular carcinoma (HCC), and liverrelated death (LrD) from 2016 to 2036. Epidemiology and mortality data were extracted from the Ministry of Health bulletin while costs were collected from insurance claims. The proportion of patients screened for HCV was projected to increase to $60 \% / 85 \% / 99 \%$ (low/medium/high screening scenarios) in 2036, with a new cohort of patients being diagnosed each year. $S \mathrm{R}_{12}$ rates were extracted from clinical trials. Separate models were used for 18-39 and 40-80 age groups to account for difference prevalence and screening rates.

Results: Low, medium and high HCV screening scenarios showed that 3838,5665 and 7669 individuals would be diagnosed with HCV infection from 2016 to 2036 , $40 \%$ aged $18-39$ and $60 \%$ aged $40-80$. In the absence of treatment, the projected number of patients reaching CC, DCC, HCC and LrD in 2036 was 899, 147, 131 and 147 respectively for the $18-39$ age groups. In the $40-80$ age groups, these projections were substantially greater: 2828 CC, 736 DCC, 668 HCC and 958 LrD. The overall economic burden of these liver complications would reach 150 million $€$. However, introducing direct-acting antivirals (DAAs) for F0-F4 patients would increase by $43 \%$ and $62 \%$ the proportion of remaining lifeyears (LYs) spent in $\mathrm{SVR}_{12}$ compared to DAAs given to F2-F4 or to F3-F4 only, respectively. Although DAAs for F0-F4 increase the cost of HCV treatment, they also provide the greatest population health benefit and lowest cost per $\mathrm{LY}$ gained in $\mathrm{SVR}_{12}$. Compared to no treatment and screening, adopting the high screening variant and DAAs access to F0-F4 would cost an additional $1,957 €$ for every LY gained in $\mathrm{SVR}_{12}$ for patients aged $18-39$ and $168 €$ for the $40-80$ age group

Conclusion: An enhanced screening policy coupled with broader access to DAAs will diminish the future clinical and economic burden of HCV in the Lebanese population and provide the greatest health benefits per amount invested, among middle-aged and elder adults with a big difference in additional costs between the 2 groups

Disclosure of Interest: All authors have declared no conflicts of interest.

\section{P1351 HEPATITIS C TREATMENT IN HEMODIALYSIS PATIENTS: THE EFFICACY AND SAFETY OF DIRECT-ACTING ANTIVIRALS IN THE REAL LIFE}

L. Carvalho, J. Rodrigues, M.A. Túlio, T. Bana, E Costa, L. Lebre, C. Chagas Gastrenterology, Centro Hospitalar de Lisboa Ocidental, Lisboa/Portugal

Contact E-mail Address: lilipcarvalho@gmail.com

Introduction: Hepatitis C virus (HCV) infection is a prevalent condition in patients with end-stage renal disease (ESRD) and increases the risk of death. Treatment of $\mathrm{HCV}$ in these patients reduces both morbidity and mortality rates and improves the outcomes of one future kidney transplantation. Yet patients with hepatitis $\mathrm{C}$ and chronic kidney disease have few treatment options. Directacting antivirals (DAA) are a well-established, effective and safe treatment regimen of the $\mathrm{HCV}$ infection, but proofs on ESRD patients requiring hemodialysis (HD) are scarce. Additional studies are still necessary in order to evaluate the real impact of these agents in the daily clinical practice.

Aims \& Methods: Our objective was to assess the efficacy and safety of DAA treatment for $\mathrm{HCV}$-infected patients with ESRD under HD, in the daily practice of a tertiary care centre, describing the HCV infection, treatment type, the evolution of virological response and clinical outcomes (anemia and other adverse effects). The HD and HCV-infected patients treated with DAA between April 2015 and February 2017 were analyzed.

Results: Our sample included 16 patients, 13 males (81\%) and 3 females $(19 \%)$ with a mean age of 59 years (45-72 years). Fourteen Caucasians and 2 diabetics patients. Four patients were treatment-experienced $(25 \%)$. The mean viral load was 5.5 $\log 10$ (3.9-7.07 $\log 10)$. Genotype distribution was the following: genotype $1-81 \%(13 / 16)$ and genotype $4-19 \%(3 / 16)$. Distribution according to fibrosis stages was as follows: $\mathrm{F} \leq 2-62 \%(10 / 16), \mathrm{F} 3-19 \%(3 / 16)$ and $\mathrm{F} 4-19 \%(3 / 16)$. All these patients underwent a treatment regimen with ombitasvir/paritaprevir/ ritonavir and dasabuvir (3D). Seven of these patients $(44 \%)$ received concomitant ribavirin at an average dose of $400 \mathrm{mg}$ per week (200-600 $\mathrm{mg}$ per week). The treatment duration was 12 weeks for 13 patients $(81 \%)$ and 24 weeks for others 3 patients $(19 \%)$. Regarding treatment response rates, $81 \%(13 / 16)$ of patients had Rapid Virological Response (RVR) and $100 \%(16 / 16)$ of patients had End of Treatment Response (ETR). The global sustained virologic response (SVR) rate was $100 \%$. Hemoglobin was monitored throughout the treatment, with 2 cases of severe anemia, one led to a $3 \mathrm{D}$ suspension at week 21 and the other one to a ribavirin suspension at week 4 of treatment. There were no other serious adverse effects.

Conclusion: In our sample of ESRD under HD patients, 3D therapy allowed the eradication of $\mathrm{HCV}$ with high efficacy, even in patients who did not meet the expected duration of treatment or dose of ribavirin. The safety of DAA in this group is demonstrated by the non-occurrence of severe adverse effects.

Disclosure of Interest: All authors have declared no conflicts of interest.

\section{Reference}

Carvalho-Filho, R. J., et al. Management of hepatitis C in patients with chronic kidney disease. World Journal of Gastroenterology: WJG, 21(2), 2015, 408-422. Fabrizi, F., et al. Combined antiviral therapy of hepatitis $\mathrm{C}$ virus in dialysis patients: meta-analysis of clinical trials. Journal of viral hepatitis, 2011, 18.7.

\section{P1352 HEPATITIS C TREATMENT IN RENAL} TRANSPLANTATION: THE EFFICACY AND SAFETY OF DIRECTACTING ANTIVIRALS IN THE REAL LIFE

L. Carvalho, J. Rodrigues, M.A. Túlio, T. Bana, E Costa, L. Lebre, C. Chagas Gastrenterology, Centro Hospitalar e Lisboa Ocidental, Lisboa/Portugal

\section{Contact E-mail Address: lilipcarvalho@gmail.com}

Introduction: Hepatitis $\mathrm{C}$ virus (HCV) infection in renal transplant patients predisposes to graft failure and progression of renal disease, increasing mortality. Due to immunosuppression and oscillating glomerular filtration rate (eGFR) in these patients, the treatment of $\mathrm{HCV}$ infection confers a greater challenge. Direct-acting antivirals (DAA) are a well-established, effective and safe treatment regimen of the $\mathrm{HCV}$ infection, however evidences on renal transplantation are scarce. Additional studies are still necessary in order to evaluate the real impact of these agents in the daily clinical practice.

Aims \& Methods: Our objective was to assess the efficacy and safety of DAA treatment for $\mathrm{HCV}$-infected patients with renal transplantation, in the daily practice of a tertiary care centre, describing the HCV infection, treatment type, the evolution of virological response and clinical outcomes (kidney function, anemia and other adverse effects). HCV-infected and renal transplanted patients treated with DAA between April 2015 and February 2017 were analyzed.

Results: Including 19 patients, 10 males $(53 \%)$ and 9 females $(47 \%)$ with a mean age of 57 years (40-70 years). The majority of these patients $(89 \%)$ were treatment-naïve. Genotype distribution was the following: genotype 1-74\% (14/19), genotype 3-16\% (3/19) and genotype 4-10\% (2/19). Distribution according to fibrosis stages was as follows: $\mathrm{F} \leq 2-63 \%(12 / 19), \mathrm{F} 3-21 \%(4 / 19)$ and F4-16\% (3/19). Sixteen patients $(85 \%)$ were treated with sofosbuvir/ledipasvir, $2(10 \%)$ with sofosbuvir/daclatasvir and $1(5 \%)$ with sofosbuvir. Concomitant ribavirin was given in 4 patients. The treatment duration was 12 weeks for 16 patients $(84 \%)$ and 24 weeks for others $2(10 \%)$. One patient realized only 21 weeks of treatment, needing to suspend it due to severe anemia. Regarding treatment response rates, $74 \%(14 / 19)$ of patients had Rapid Virological Response (RVR) and 100\% (19/19) of patients had End of Treatment Response (ETR). The global sustained virologic response (SVR) rate was $100 \%$. Hemoglobin, eGFR and immunosuppression levels were monitored during this period. The eGFR (CKD EPI) did not change significantly, with pre and posttreatment mean values of 66.4 and $65.4 \mathrm{ml} / \mathrm{min} / 1.73 \mathrm{~m} 2$, respectively. There were 2 cases of severe anemia, one that resulted in ADA suspension at week 21 and the other one ribavirin suspension at week 7 of treatment. Immunosuppressive levels remained stable. There were no other serious adverse reactions.

Conclusion: In our sample of HCV-infected with kidney transplant, DAA are effective and well tolerated, even in the more advanced stages of fibrosis, maintaining the integrity and viability of the graft, without interfering with the efficacy of immunosuppressant.

Disclosure of Interest: All authors have declared no conflicts of interest.

\section{References}

Carvalho-Filho RJ, Feldner ACC, Silva AEB, Ferraz MLG. Management of hepatitis $\mathrm{C}$ in patients with chronic kidney disease. World Journal of Gastroenterology: WJG. 2015;21(2):408-422. doi:10.3748/wjg.v21.i2.408.

Rostaing L, Izopet J, Kamar N. Hepatitis C virus infection in nephrology patients. Journal of Nephropathology. 2013;2(4):217-233. doi:10.12860/ JNP.2013.36

\section{P1353 WHAT HAPPENED WITH LIVER STIFFNESS VALUES ASSESSED BY MEANS OF TRANSIENT ELASTOGRAPHY IN PATIENTS WITH HCV LIVER CIRRHOSIS AFTER DAA TREATMENT}

I. Sporea ${ }^{1}$, R. Lupusoru ${ }^{1}$, S.A. Popescu ${ }^{1}$, A. Lazar ${ }^{1}$, R. Mare ${ }^{1}$, L. Gheorghe ${ }^{2}$, S. Iacob ${ }^{2}$, R. Sirli

${ }^{1}$ Department Of Gastroenterology And Hepatology, University of Medicine and Pharmacy Victor Babes Timisoara, Timisoara/Romania

${ }^{2}$ Fundeni Clinical Institute, Bucharest/Romania

Contact E-mail Address: isporea@umft.ro

Introduction: Liver stiffness (LS) measurements by Transient Elastography (TE) has been widely accepted as a tool for fibrosis assessment.

Aims \& Methods: The aim of this study was to assess LS dynamics in a group of patients with HCV liver cirrhosis after DAA treatment. This bicentric clinical 
trial included 276 patients with compensated HCV cirrhosis (all genotype 1b), who received DAA for 12 weeks. All patients were evaluated by means of TE at the beginning and at the end of treatment (EOT), and one subgroup (180 patients) also 12 weeks after EOT, all of them with sustained viral response (SVR 12), and another subgroup (55 patients) also at 24 weeks after EOT (SVR 24). Reliable LS measurements (LSM) were defined as median value of 10 valid $\mathrm{LSM}$, with $\mathrm{IQR}<30 \%$ and $\mathrm{SR} \geq 60 \%$. Both $\mathrm{M}$ and XL probes were used. For diagnosing cirrhosis we used a cut-off value of $12 \mathrm{kPa}$ as proposed by the Tsochatzis meta-analysis. We considered a decrease or increase of more than $10 \%$ in LSM as being significant.

Results: Out of 276 subjects, reliable measurements were obtained in $92.7 \%$, so that the final analysis included 256 patients. The mean LS values decreased significantly after DAA: $25 \pm 11.7 \mathrm{kPa}$ vs. $22.5 \pm 12 . \mathrm{kPa}(\mathrm{p}=0.009)$. Most patients, $(59.7 \%-152 / 256)$ presented more than $10 \%$ decrease in LS values, $23 \%(59 / 256)$ had stable LS values, while in $17.3 \%(45 / 256)$ cases, the LS values increased. In the subgroup of 180 patients where LSM were also performed 12 weeks after EOT (SVR 12), the mean LS values were significantly lower 12 weeks after EOT as compared to baseline: $20.3 \pm 10.8 \mathrm{kPa}$ vs. $25.5 \pm 11.4 \mathrm{kPa}(\mathrm{p}<0.0001)$ and also as compared to EOT: $20.3 \pm 10.8 \mathrm{kPa}$ vs. $22.8 \pm 12.2 \mathrm{kPa},(\mathrm{p}=0.04)$. In the subgroup of 55 patients wher LSM were also performed 24 weeks after EOT (SVR 24), the mean LS values were significantly lower at SVR 12 and SVR 24 as compared to EOT $(18.7 \pm 8.2 \mathrm{kPa}$ vs. $21.6 \pm 7.7 \mathrm{kPa}, \mathrm{p}=0.05$ and $18.3 \pm 6.6 \mathrm{kPa}$ vs. $21.6 \pm 7.7 \mathrm{kPa}, \mathrm{p}=0.01)$

Conclusion: In our group mean liver stiffness values evaluated by TE significantly decreased after antiviral treatment at SVR 12 and SVR 24, as compared to EOT. Overall, in our study almost $60 \%$ of patients had EOT liver stiffness values lower than at baseline, at SVR 12 almost $75 \%$ of patients had liver stiffness values lower than at baseline and at SVR 24 almost $77 \%$ of patients had liver stiffness values lower than at baseline.

Disclosure of Interest: I. Sporea: I hereby confirm that I have received financial support (congress travel grant or speaker fee) from Philips, Siemens, General Electric, Abbvie, Zentiva, Bristol Meyers Squibb

S.A. Popescu: I hereby confirm that I have received financial support (congress travel grants, speaker fee) from: Philips, General Electric, Abbvie, AstraZeneca, Zentiva

R. Sirli: I hereby confirm that I have received financial support (congress travel grant or speaker fee) from Philips, Abbvie, Zentiva

All other authors have declared no conflicts of interest.

\section{References}

1. Messina JP, Humphreys I, Flaxman A et al. Global distribution and prevalence of hepatitis C virus genotypes. Hepatology 2015;61:77-87 doi $10.1002 /$ hep. 27259

2. European Association for the Study of the Liver. EASL Recommendations on Treatment of Hepatitis C 2016. J Hepatol 2017 Jan;66(1):153-194. doi: 10.1016/j.jhep.2016.09.001

3. Tsochatzis EA, Gurusamy KS, Ntaoula S, Cholongitas E, Davidson BR Burroughs AK. Elastography for the diagnosis of severity of fibrosis in chronic liver disease: a meta-analysis of diagnostic accuracy. $J$ Hepatol 2011; 54: 650-659. doi: 10.1016/j.jhep.2010.07.033.

\section{P1354 ACHIEVING SVR AFTER DAA THERAPY FOR HCV DECREASES THE ACCURACY OF BAVENO VI CLASSIFICATION OF HIGH-RISK VARICES IN CIRRHOTIC PATIENTS}

M. I. Rotaru ${ }^{1}$, H. Stefanescu ${ }^{2}$, A. Suciu ${ }^{2}$, R. $\mathrm{Sav}^{3}$, E. Pop ${ }^{3}$, M. Lupsor ${ }^{2}$, B.D. Procopet ${ }^{2}$, A. Seicean ${ }^{4}$, M. Tantau ${ }^{3}$, Z. Sparchez ${ }^{3}$, C. Radu ${ }^{1}$ Hepatology, Regional Institute of Gastroenterology and Hepatology, ClujNapoca, Cluj-Napoca/Romania

${ }^{2}$ Hepatology, Cluj-Napoca University of Medicine and Pharmacy 3rd Medical Clinic, Hepatology and Medical Imaging D, Cluj-Napoca/Romania

${ }^{3}$ Iuliu Hat,ieganu University of Medicine and Pharmacy, Cluj-Napoca/Romania

${ }^{4}$ Regional Institute of Gastroenterology and Hepatology Prof. Dr. Octavian Fodor, Cluj Napoca/Romania

Contact E-mail Address: rotaru.i.magda@gmail.com

Introduction: Baveno VI criteria suggest that cirrhotic patients with platelet count $(\mathrm{PLT})>150,000 / \mu \mathrm{l}$ and liver stiffness measure values $(\mathrm{LSM})<20 \mathrm{kPa}$ bare a low risk of having high-risk esophageal varices (EV) and do not require endoscopic screening. The direct-acting antiviral agents (DAA) determine a very high rate of sustained virologic response (SVR) among patients with HCV induced chronic hepatitis and cirrhosis, and therefore, might decrease the risk of decompensation, including variceal bleeding.

Aims \& Methods: Therefore, the aim of the study was to asses the influence of DAA therapy on the accuracy of the Baveno VI criteria for in cirrhotic HCV patients. All consecutive patients with compensated HCV liver cirrhosis approved for DAA treatment in our center who gave their informed consent were included. All patients were screened for EV before starting the therapy by esogastroduodenoscopy. LSM (FibroScan $\left.{ }^{\circledR}\right)$ and usual biological parameters were evaluated at baseline and when assessing SVR 12

Results: 50 patients were included $(59.5(45-84)$ years, $54 \%$ women) between December 2015 and July 2016. All patients achieved SVR (100\%). At base line, 17/50 patients had EV. After completing DAA therapy, liver enzymes [GOT $(\mathrm{p}<0.0001), \quad$ GPT $(\mathrm{p}<0.0001)], \quad$ GGT $(\mathrm{p}<0.003)$ and LSM $(\mathrm{p}<0.0001)$ significantly decreased, while albumin $(\mathrm{p}=0.014)$ and PLT $(\mathrm{p}=0.39)$ increased. We found a moderate correlation between GOT and LSM both at baseline $(r=0.31 ; p=0.001)$ and at $\operatorname{RVS} 12(r=0.45 ; p=0.004)$, but the amplitude of the decrease appears not to be correlated $[\mathrm{r}(\Delta \mathrm{GOT}$ vs.
$\Delta \mathrm{LSM})=0.149 ; \mathrm{p}=0.3]$. At baseline, according to Baveno VI criteria, 29 patients were classified at low risk of having EV (accuracy $68 \%$, Se $69.7 \%$, Sp $64.7 \%$, PPV $79.31 \%$, NPV 52.38\%). At SVR 12,10 aditional patients entered this class, but the accuracy $(60 \%)$ decreased.

Conclusion: DAA therapy induces a significant decrease in liver inflammation and liver stiffness and also a non-significant increase of platelets count. These changes induce a decrease of the accuracy of classification of patients with low risk of having EV (according to Baveno VI criteria), therefore is not advisable to use Baveno VI criteria as surrogate for endoscopy screening for esophageal varices in patients compensated HCV liver cirrhosis who obtained SVR.

Disclosure of Interest: M.I. Rotaru: This work was partly funded by the Iuliu Hat,ieganu University of Medicine and Pharmacy, Cluj-Napoca under Grant No. 4944/3/08.03.2016 awarded to dr. Corina Radu.

H. Stefanescu: This work was partly funded by the Iuliu Hat,ieganu University of Medicine and Pharmacy, Cluj-Napoca under Grant No. 4944/3/08.03.2016 awarded to dr. Corina Radu.

A. Suciu: This work was partly funded by the Iuliu Hat,ieganu University of Medicine and Pharmacy, Cluj-Napoca under Grant No. 4944/3/08.03.2016 awarded to dr. Corina Radu.

R. Sav: This work was partly funded by the Iuliu Hat,ieganu University of Medicine and Pharmacy, Cluj-Napoca under Grant No. 4944/3/08.03.2016 awarded to dr. Corina Radu.

E. Pop: This work was partly funded by the Iuliu Hatieganu University of Medicine and Pharmacy, Cluj-Napoca under Grant No. 4944/3/08.03.2016 awarded to dr. Corina Radu.

M. Lupsor: This work was partly funded by the Iuliu Hat ieganu University of Medicine and Pharmacy, Cluj-Napoca under Grant No. 4944/3/08.03.2016 awarded to dr. Corina Radu.

B.D. Procopet: This work was partly funded by the Iuliu Hat ieganu University of Medicine and Pharmacy, Cluj-Napoca under Grant No. 4944/3/08.03.2016 awarded to dr. Corina Radu.

A. Seicean: This work was partly funded by the Iuliu Hat,ieganu University of Medicine and Pharmacy, Cluj-Napoca under Grant No. 4944/3/08.03.2016 awarded to dr. Corina Radu.

M. Tantau: This work was partly funded by the Iuliu Hat,ieganu University of Medicine and Pharmacy, Cluj-Napoca under Grant No. 4944/3/08.03.2016 awarded to dr. Corina Radu.

Z. Sparchez: This work was partly funded by the Iuliu Hat,ieganu University of Medicine and Pharmacy, Cluj-Napoca under Grant No. 4944/3/08.03.2016 awarded to dr. Corina Radu.

C. Radu: This work was partly funded by the Iuliu Hat ieganu University of Medicine and Pharmacy, Cluj-Napoca under Grant No. 4944/3/08.03.2016.

\section{P1355 MONOCYTE CHEMOATTRACTANT PROTEIN -1 CONCENTRATIONS IN SERUM DEPEND ON OVERWEIGHT IN CHRONIC HEPATITIS C PATIENTS AND INCREASE AFTER SUSTAINED VIROLOGIC RESPONSE}

I. Valkov ${ }^{1}$, T. Velikova ${ }^{2}$, K. Tumangelova-Yuzeir ${ }^{2}$, D. Kyurkchiev ${ }^{2}$, K. Antonov

${ }^{1}$ Clinic Of Gastroenterology, University Hospital St. Ivan Rilski, Medical University - Sofia, Sofia/Bulgaria

${ }^{2}$ Clinical Immunology, University Hospital St. Ivan Rilski, Medical University Sofia, Sofia/Bulgaria

Contact E-mail Address: ivan.valkov@yahoo.co.uk Introduction: Monocyte chemoattractant protein-1 (MCP-1) is a chemokine mediator in liver inflammation and fibrogenesis. It contributes to the severity of nonalcoholic fatty liver disease and chronic hepatitis $\mathrm{C}(\mathrm{CHC})$ by recruiting proinflammatory activated monocytes and macrophages. Signaling between the mononuclear phagocyte system (MPS) and hepatic stellate cells is key for active fibrogensis. The extent to which metabolic and immune factors are implicated in fatty liver of patients with $\mathrm{CHC}$ is still not clear.

Aims \& Methods: The aim of the study was to explore the associations between serum MCP-1, liver fibrosis, fatty liver and metabolic factors in $\mathrm{CHC}$ patients before, and after antiviral treatment. We included 21 patients in the study (11 men, 10 women, age $42 \pm 9.7$ ) with chronic hepatitis $\mathrm{C}$ virus (HCV) infection-17 with genotype 1 and 4 with genotype 3 . Liver biopsy was done in 19 and histology showed fatty liver in 8 patients. Fatty liver was present in 17 of all patients on ultrasound. Liver fibrosis was scored mild (F1) in 9, significant (F2) in 7 and advanced bridging fibrosis (F3) in 2 cases (METAVIR). Compensated liver cirrhosis (F4) was diagnosed in 3 cases by liver biopsy or endoscopic screening for varices. Weight was normal in 10, overweight in 5 and obese in 6 patients. MCP-1 was quantified by ELISA in serum. Laboratory panel included complete blood count, liver tests, HOMA-IR and serum lipid levels. Seventeen patients received treatment (standard of care peg-interferon alpha, ribavirin and/or direct-acting antivirals). Sustained virologic response (SVR) was attained by 12 and nonresponse/relapse (NVR) by 5 patients. Serum probes were obtained before treatment and after response evaluation. Statistical analysis included Spearman's rho, Mann-Whitney U test, Wilcoxon's test and Student's paired t-test.

Results: MCP-1 in serum correlated with BMI $(r=0.522, p=0.015)$. MCP-1 concentrations in overweight and obese patients were higher than in those with normal weight $(\mathrm{p}=0.020)$. Patients with F3-F4 had higher BMI $(\mathrm{p}=0.010)$ and HOMA-IR $(p=0.042)$. An increase in serum MCP-1 was found in patients after SVR $(\mathrm{p}=0.018)$, while no significant variation from baseline values was found in NVR patients. The result remained significant in subgroup analysis of SVR patients with F1-F2 $(p=0.028)$ and in those with fatty liver $(\mathrm{p}=0.017)$. MCP1 in serum did not show any association with assessment of liver fibrosis, fatty liver, insulin resistance and serum lipid levels. 
Conclusion: MCP-1 concentrations in serum depend on overweight in patients with $\mathrm{CHC}$. Overweight and insulin resistance are associated with progression of CHC. Serum levels of MCP-1 increase after HCV clearance. Fluctuation of the MCP-1 concentration in serum could reflect an antiinflammatory activation of MPS and a gradient dependent dynamic replacement of the profibrotic cell subsets in the liver with proresolution ones after SVR. Fatty liver plays a role for inflammatory responses in CHC patients after SVR.

Disclosure of Interest: All authors have declared no conflicts of interest.

\section{Reference}

Marra F., Tacke F. Gastroenterology. 2014 Sep;147(3):577-594.e1. Roles for chemokines in liver disease.

P1356 HLA -A02, HLA- A03 AND HLA-B15: A NEW RISK FOR
HEPATIC STEATOSIS IN EGYPTIAN CHRONIC HEPATITIS C PATIENTS

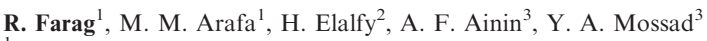

${ }^{1}$ Tropical Medicine, Mansoura University, Mansoura/Egypt

${ }^{2}$ Endemic Medicine And Hepatology, Mansoura University, Mansoura/Egypt

${ }^{3}$ Mansoura Faculty of Medicine, Mansoura/Egypt

Contact E-mail Address: raghda.farag@yahoo.com

Introduction: HCV interferes with the host lipid metabolism leads to insulin resistance and hepatic steatosis. Although it is usually mild in genotype 4, many data suggests that simple steatosis may have the potential to progress to fibrosis, cirrhosis and subsequent hepatocellular carcinoma. Many heritable host factors with observed inter-ethnic variation in the prevalence of steatosis are decumented, and in many cases hepatic steatosis may be detected in absence of all these risk factors; so a role for host genetic factors in development of hepatic steatosis in chronic HCV patients may be suggested.

Aims \& Methods: In this study, we aim to evaluate the association of HLA class $\mathrm{A}-\mathrm{B}$ alleles and presence of steatosis in chronic HCV genotype 4 infected patients. This study included two hundred unrelated non diabetic non obese chronic HCV patients with normal lipid profile, 98 of them had biopsy proven steatosis. Serological testing of HLA class I antigens (HLA-A, and HLA-B alleles) were performed with a standard complement-dependent micro-lymphocytotoxicity assay.

Results: The frequency of A02, A03, B15 and B17 alleles were significantly higher in chronic HCV patients with steatosis $(\mathrm{OR}=1.77,2.64,4.44,5.68)$ and $95 \%$ $\mathrm{CI}=0.96-3.27 ; 1.02-7.04 ; 0.84-31.17 ; 1.12-38.65$ with $\mathrm{P}=0.034,0.022,0.044$ 0.015 respectively. On the other hand, the frequency of A01 and B12 alleles were significantly higher in patients without steatosis $(\mathrm{OR}=0.56,0.41)$ and $95 \%$ CI were $0.30-1.05 ; 0.20-0.83$ and $\mathrm{P}=0.015$ and 0.005 . On logistic regression analysis, patients who carry HLA-A02, A03 and H|LA-B15 alleles may have 2.2, 3.9 and 11.18 fold risk to have hepatic steatosis (B coefficient: $0.79 ; 1.37 ; 2.41$ ) $95 \% \mathrm{CI}=1.09-4.42,1.04-11.05,2.15-58.13 ; \mathrm{P}=0.027,0.009,0.004)$ while carrying HLA-A01 alleles may be protected from having HCV associated hepatic steatosis; $(\mathrm{OR}=0.34,95 \% \mathrm{CI}=0.16-0.72 ; \mathrm{P}=0.005)$ with constant -9.47 and overall accuracy of $69 \%$. In addition, patients who have moderate activity index in liver histopathlogy have 5.9 risk to have hepatic steatosis $(\mathrm{OR}=5.92$, $95 \% \mathrm{CI}=2.92-11.99, \mathrm{P}<0.001)$

Conclusion: In chronic HCV genotype 4 patients, carring HLA-A02, HLA-A03 and HLA-B15 alleles may have a risk for presence of hepatic steatosis while presence of HLA-A01 alleles may have a protective role.

Disclosure of Interest: All authors have declared no conflicts of interest.

\section{Reference}

Khattab MA, Abdel-Fattah ME, Eslam M, Abdelaleem A, Abdelaleem RA, Shatat M, Ali A, Hamdy L, Tawfek H Hepatic Steatosis in Genotype 4 Chronic Hepatitis C Patients: Implication for Therapy. J Clin Gastroenterol. 2010 Feb 25.

\section{P1357 THE VALUE OF 2D-SWE.GE FOR THE EVALUATION OF LIVER FIBROSIS IN PATIENTS WITH HCV COMPENSATED CHRONIC HEPATOPATHIES}

F. Bende, I. Sporea, S.A. Popescu, R. Sirli, M. Danila, R. Mare, A. Stepan Gastroenterology And Hepatology, University of Medicine and Pharmacy "Victor Babes" Timisoara, Timisoara/Romania

Contact E-mail Address: bendefelix@gmail.com

Introduction: Chronic liver diseases are quite frequently encountered in daily practice and are due mainly to chronic viral infections (B or C viruses) and to other conditions such as alcoholic steatohepatitis - (ASH) and to non-alcoholic fatty liver disease (NAFLD). While liver biopsy remains the gold standard method for fibrosis assessment, stage classification and also for necro-inflammatory activity grading, in the last years, non-invasive assessment methods (biological tests and elastographic methods) were developed and they are being used more and more, to the detriment of liver biopsy.

Aims \& Methods: The aim of this study was to evaluate the performance of the 2D-shear wave elastography technique from General Electrics (2D-SWE.GE), for the evaluation of liver fibrosis in patients with HCV chronic hepatopathies, using Transient Elastography (TE) as the reference method. The study included 145 consecutive subjects with HCV compensated chronic hepatopathies, in whom liver stiffness was evaluated in the same session by means of 2 elastographic measurements: TE (FibroScan, EchoSens) and 2D-SWE.GE (LOGIQ E9, GE Healthcare). Reliable LS measurements were defined as follows: for TE-the median value of 10 measurements with a success rate of $\geq 60 \%$ and an interquartile range $<30 \%$ and for 2 D-SWE.GE - the median value of 10 measurements acquired in a homogenous area and an interquartile range (IQR) $<30 \%$. To discriminate between various stages of fibrosis by TE we used the following cut-offs: $F \geq 2: 7.1 \mathrm{kPa}, \mathrm{F} \geq 3: 9.5 \mathrm{kPa}, \mathrm{F}=4: 12.5 \mathrm{kPa}[1]$.

Results: Reliable LS measurements were obtained in 138/145 (95.1\%) subjects by 2D-SWE.GE and in $139 / 145(95.8 \%)$ by TE $(\mathrm{p}=0.9)$. The final analysis was performed on 134 subjects with valid measurements by both methods. We found a good positive correlation between the LS values obtained by the 2 methods: $\mathrm{r}=0.79, \mathrm{p}<0.0001$. Based on TE cut-off values [1] we divided our cohort into 4 groups: F0-F1: 36/134 (26.8\%), F2: 23/134 (17.2\%), F3: 23/134 $(17.2 \%)$ and F4: $52 / 134(38.8 \%)$. The areas under the receiver operating characteristic curve (AUROC) were 0.909 for significant fibrosis $(F \geq 2), 0.954$ for severe fibrosis $(F \geq 3)$ and 0.942 for cirrhosis $(F=4)$. The best cut-off values for $\mathrm{F} \geq 2$ was $7 \mathrm{kPa}$ (Sensitivity 85.7 , Specificity 80.5 ), for $\mathrm{F} \geq 3$ it was $9.2 \mathrm{kPa}$ (Sensitivity 85.3 , Specificity 91.5 ) and $\mathrm{F}=4$ it was $10.7 \mathrm{kPa}$ (Sensitivity 84.6 , Specificity 91.4).

Conclusion: 2D-SWE.GE seems a reliable method for liver fibrosis staging in patients with HCV compensated chronic hepatopathies. The best 2D-SWE.GE cut-off values for $\mathrm{F} \geq 2, \mathrm{~F} \geq 3$ and $\mathrm{F}=4$ in $\mathrm{HCV}$ chronic hepatopathies were 7 , 9.2 and $10.7 \mathrm{kPa}$

Disclosure of Interest: I. Sporea: I hereby confirm that I have received financial support (congress travel grant or speaker fee) from Philips, Siemens, General Electric, Abbvie, Zentiva, Bristol Meyers Squibb

S.A. Popescu: I hereby confirm that I have received financial support (congress travel grants, speaker fee) from: Philips, General Electric, Abbvie, AstraZeneca, Zentiva

R. Sirli: I hereby confirm that I have received financial support (congress travel grant or speaker fee) from Philips, Abbvie, Zentiva

All other authors have declared no conflicts of interest.

\section{Reference}

1. Castera L, Vergniol J, Foucher J, et al. Prospective comparison of transient elastography, FibroTest, APRI, and liver biopsy for the assessment of fibrosis in chronic hepatitis C. Gastroenterology 2005; 128: 343-350

\section{P1358 DE NOVO HEPATOCELLULAR CARCINOMA IN PATIENTS WITH CIRRHOSIS AFTER TREATMENT WITH DIRECT ANTIVIRAL AGENTS}

P. Ruiz ${ }^{1}$, L. Deiss Pascual $^{2}$, L. Buendia ${ }^{1}$, I. Erdozain $^{2}$, I. Esain ${ }^{2}$, S. Blanco ${ }^{1}$, A. Baranda ${ }^{2}$, F. Menendez 2

${ }^{1}$ Hospital Universitario Basurto, Bilbao/Spain

${ }^{2}$ Gastroenterology And Hepatology, Hospital Universitario de Basurto, Bilbaol Spain

Contact E-mail Address: laura.deisspascual@osakidetza.net

Introduction: The risk of developing novo hepatocellular carcinoma (HCC) persists after reaching sustained virological response (SVR) in patients infected with hepatitis virus C. It has been suggested that risk is increased in patients treated with the new direct antiviral agents (DAA). In this prospective study we present our results of incidence and prevalence of novo HCC in cirrhotic patients treated with DAA and SVR, and also, the risk factors involved in its development. Aims \& Methods: We included all cirrhotic patients due to HVC infection without previous HCC who reached SVR after DAA treatment in our hospital from February 2014 until December 2016 ( $n=197$, median of follow-up of 17 months). We evaluated with chi square test the following qualitative variables: age, Child-Pugh stage, alcohol consumption pre-treatment, tobacco consumption pre-treatment, diabetes mellitus (DM) pre-treatment, genotype, radiological and endoscopic portal hypertension features pre-treatment. The quantitative variables were evaluated with student t test: age, no. of platelets pre-treatment, fibroscan value pre-treatment.

Results: During follow-up 11 patients were diagnosed of $\mathrm{HCC}$ (5.6\% prevalence, $3.9 \%$ annual incidence). Among all variables evaluated being in a Child- Pugh B stage vs. an A stage $(\mathrm{p}=0.007)$, pre-treatment $\mathrm{DM}(\mathrm{p}=0.002)$ and presence of radiological portal hypertension $(\mathrm{p}=0.001)$ were associated with developing novo HCC. Among the quantitative variables, we evidenced statistically significant differences in the mean value of platelets $(p=0.015)$

Conclusion: In our group of patients, a worse hepatic function evaluated with the Child-Pugh classification and indirect markers of portal hypertension (platelets and radiological features) and also DM are associated statistically significant with the development of novo HCC. The incidence $(>1.5 \%)$ of novo HCC justifies the screening of $\mathrm{HCC}$.

Disclosure of Interest: All authors have declared no conflicts of interest. 
P1359 IS AN ACUTE LIVER FAILURE REALLY ALWAYS AN ACUTE LIVER FAILURE? PRE-EXISTING LIVER DISEASE IS COMMON IN PATIENTS WITH ALF DIAGNOSIS

A. Gottlieb ${ }^{1}$, J. P. Sowa ${ }^{2}$, M. Kottmann ${ }^{2}$, P. Manka ${ }^{2}$, L. Bechmann ${ }^{1}$, A. Canbay ${ }^{1}$ ${ }^{1}$ Gastroenterologe, Hepatology And Infectious Diseases, University Hospital Magdeburg, Magdeburg/Germany

${ }^{2}$ Gastroenterology And Hepatology, University Hospital Essen, Essen/Germany

Contact E-mail Address: aline.gottlieb@med.ovgu.de

Introduction: An acute insult to the liver in patients without pre-existing chronic liver injury can cause acute liver failure (ALF). ALF is a life-threatening condition and requires specialized intensive care. Acute on chronic liver failure (AOCLF) is a not yet clearly characterized situation, where the acute insult occurs in addition to present chronic liver disease without cirrhosis. Though, various definitions of AOCLF are found in the literature. Discrimination of AOCLF from ALF might be trivial for pre-existing alcoholic or viral hepatitis. However, non-alcoholic steatohepatitis (NASH) in obese or diabetic patients might go unnoticed in the critical situation of an ALF/AOCLF. Thus, patients admitted to liver transplantation centers with the diagnosis of ALF might be suffering from AOCLF instead. It is also unclear if the ALF and AOCLF have different impact on disease course, clinical management and transplant organ allocation.

Aims \& Methods: Aim of this study was to identify possible differences between patients with ALF and AOCLF regarding routine parameters and clinical course. In this retrospective single-center study all patients were recruited, who were admitted to the University Hospital of Essen with the initial diagnosis of ALF between 2008 and 2015. Patients included in this study were fulfilling the criteria of the acute liver failure study group Germany. The diagnosis of AOCLF was established upon retrospective examination of patient records. In total 163 patients were recruited (ALF: 131; AoCLF: 32). Clinical records, in particular demographic data, serum parameters and outcome were analyzed for differences between ALF and AOCLF.

Results: Patients with AOCLF were significantly older $(50.3 \pm 15.1$ vs. $39.8 \pm 16.2, \quad \mathrm{p}=0.0008)$, had a higher BMI $(27.5 \pm 5.1$ vs. $24.5 \pm 6.2$, $\mathrm{p}=0.0014)$ and were more often male $(65 \%$ in AOCLF vs. $34 \%$ in ALF $\mathrm{p}=0.0008)$. In addition, the insults that caused the liver failure in AOCLF were significantly different from those patients with ALF. Significant differences were also found for liver enzymes, which were significantly lower in AOCLF patients (AST $\mathrm{p}=0.01$; ALT $\mathrm{p}=0.001$ ). Cell death markers and the MELD did not differ between ALF and AOCLF. Moreover, the outcome was not different between the two groups neither separated as survived or deceased nor as spontaneous remission or non-spontaneous remission (combined transplantation and deceased). Importantly, MELD and the modified MELD including the cell death marker M65 were similarly effective in predicting outcome for both ALF and AOCLF.

Conclusion: In the present study patients with ALF and AOCLF differed in age and BMI, but did not exhibit differences regarding disease severity (according to MELD) or clinical outcome. While the causes for an acute insult differed between ALF and AOCLF, which might imply a different clinical management, clinical outcome was predictable by common factors for ALF.

Disclosure of Interest: All authors have declared no conflicts of interest.

\section{P1360 EFFECTS OF PREOPERATIVE TRANSARTERIAL CHEMOEMBOLIZATION ON PATIENTS' SHORT TERM PROGNOSIS AFTER LIVER TRANSPLANTATION}

\section{Ma}

Department Of Hepatobiliary Surgery, Ruijin Hospital, Shanghai Jiaotong

University School of Medicine, Shanghai/China

Contact E-mail Address: madi_1978@163.com

Introduction: To explore the influence of preoperative transarterial chemoembolization on short-term prognosis in patients with hepatocellular carcinoma after liver transplantation.

Aims \& Methods: A retrospective study was conducted among patients with hepatocellular carcinoma who received liver transplantation from January 2009 to September 2015 in Ruijin Hospital. A total of 31 patients (Male: 29/Female: 2 ) were included. Among them, 14 patients received preoperative hepatic arterial chemoembolization was incorporated into observation group $(n=14)$, and the other 17 patients were included in the control group $(\mathrm{n}=17)$. The data including TBL/ALT/AST levels in preoperative and 1, 3, 5, 7 postoperative days, operation time, blood loss, postoperative complications happening (incision infection, vascular complications, biliary complications, complications classification), perioperative mortality and hospitalized time was analyzed to compare the differences between the two groups of patients.

Results: No statistical difference was found in the total operation time, blood loss and length of hospital stay between two groups, and the occurrence rate of vascular complications, biliary complications and postoperative infection also showed no difference. Although more patients above Clavien IIIa were in the observation group, there was no statistically significant difference in the perioperative mortality between two groups. Besides, preoperative TACE could effectively reduce complications caused by immune reaction $(P=0.048)$. In terms of postoperative indexs of liver function, TBIL, ALT, AST all had a transient rise during the first 3 days after liver transplantation, but recovered gradually over time. There's no remarkable difference in the liver function recovery level between two groups $(P=0.495 ; P=0.141 ; P=0.101)$. No statistical difference was found in the total operation time, blood loss and length of hospital stay between two groups, and the occurrence rate of vascular complications, biliary complications and postoperative infection also showed no difference. Although more patients above Clavien IIIa were in the observation group, there was no statistically significant difference in the perioperative mortality between two groups. Besides, preoperative TACE could effectively reduce complications caused by immune reaction $(P=0.048)$. In terms of postoperative indexs of liver function, TBIL, ALT, AST all had a transient rise during the first 3 days after liver transplantation, but recovered gradually over time. There's no remarkable difference in the liver function recovery level between two groups $(P=0.495$; $P=0.141 ; P=0.101)$

Conclusion: Preoperative TACE won't affect liver function recovery and perioperative safety after liver transplantation. For some patients, it could also educe complications caused by immune reaction.

Disclosure of Interest: All authors have declared no conflicts of interest.

\section{References}

1. Torre LA, Bray F, Siegel RL, et al. Global cancer statistics, 2012. CA Cancer J Clin. 2015, 65(2):87-108

2. Hasegawa K, Kokudo N, Makuuchi M, et al: Comparison of resection and ablation for hepatocellular carcinoma: a cohort study based on a Japanese nationwide survey. J Hepatol 58: 724-729, 2013.

3. Singal AK, Kamath PS. Live donor liver transplantation for alcoholic hepatitis. HEPATOL INT. 2016.

4. Goel A, Mehta N, Guy J, et al. Hepatic artery and biliary complications in liver transplant recipients undergoing pretransplant transarterial chemoembolization. Liver Transpl. 2014, 20(10):1221-1228.

5. Panaro F, Ramos J, Gallix B, et al. Hepatic artery complications following liver transplantation. Does preoperative chemoembolization impact the postoperative course? CLIN TRANSPLANT. 2014, 28(5):598-605.

6. Choi JW, Kim HC, Lee JH, et al. Transarterial chemoembolization of hepatocellular carcinoma with segmental portal vein tumour thrombus. EUR RADIOL. 2016.

7. Sacco R, Mismas V, Marceglia S, et al. Transarterial radioembolization for hepatocellular carcinoma: An update and perspectives. World $J$ Gastroenterol. 2015,21(21):6518-6525.

\section{P1361 IMPORTANCE OF INTERFERON-GAMMA RELEASE ASSAYS IN EVALUATING CANDIDATES FOR LIVER TRANSPLANTATION IN A COUNTRY ENDEMIC FOR TUBERCULOSIS}

M. C. Lita ${ }^{1}$, C. Ester ${ }^{1}$, S. Iacob ${ }^{2}$, C. Pietrareanu ${ }^{2}$, R. Cerban ${ }^{2}$, C. Gheorghe ${ }^{3}$, L.S. Gheorghe ${ }^{2}$

${ }^{1}$ Gastroenterology And Hepatology, Fundeni Clinical Institute, Bucharest/Romania ${ }^{2}$ Fundeni Clinical Institute, Bucuresti/Romania

${ }^{3} 1$ st Dept. Of Gastroenterology \& Endoscopy, PetCenter of Gastroenterology and Hepatology, Bucharest/Romania

\section{Contact E-mail Address: cerbanrazvan@yahoo.com}

Introduction: Romania has the highest incidence of tuberculosis (TB) in the European Union, representing one quarter of the European TB burden. According to clinical practice guidelines for liver transplantation (LT), the second level of screening for infections consists of screening for Mycobacterium tuberculosis, including history of TB, PPD, Interferon-gamma release assays. Aims \& Methods: The aim was to assess the importance of Quantiferon TB Gold test for evaluating patients included on the wait list for LTin Romanian setting. The study was a single-center retrospective cohort study (the single center for LT)that included 264 patientsadmitted on the wait list for LT from January 1, 2014 to November 18, 2016. All patients underwent mandatory screening for Mycobacterium tuberculosis, either using Quantiferon TB Gold test or skin testing using purified protein derivative (PPD). The variables analyzed using Minitab 17 were: age, gender, etiology of liver disease, biochemical test, MELD score. Results: From a total of 264 patients with liver diseases included on the wait list, $60.6 \%$ were males, the average age at diagnosiswas $47.78 \pm 9.92$ years. The etiology of liver cirrhosis was HCV infection in $31.43 \%$, HBV and HBV-HDV coinfection in $45.06 \%$, and alcoholic liver cirrhosis in $18.93 \% ; 24.62 \%$ of patients have been diagnosed with superimposedhepatocellular carcinoma. Eighty three patients $(31.43 \%)$ had positive results for Quantiferon test, 150 patients $(56.81 \%)$ were negative and 17 patients $(6.43 \%)$ had indeterminate results. Only 14 patients $(5.3 \%)$ were skin tested using PPD, $2(0.75 \%)$ of them with positive results. Comparing the subgroups, positive Quantiferon test was associated with $\mathrm{HCV}$ etiology $(\mathrm{p}$ value $=0.049)$ and lower lymphocyte counts, but did not achieve statistical significance $(\mathrm{p}=0.187)$. Patients with indeterminate Quantiferon associated hyperbilirubinemia $(p=0.044)$, hypoalbuminemia $(p=0.032)$ and had a higher MELD score $(\mathrm{p}=0.019)$. After assessment by a multidisciplinary team that included a pneumologist, $38.63 \%$ of patients were diagnosed with latent TB. Isoniazid chemoprophylaxis along with pyridoxine was postponed after LT, in order to prevent further hepatic dysfunction. $48.5 \%$ of patients underwent LT, followed by TB prophylaxis and no cases of TB reactivation have been reported during a follow-up period of 14 months.

Conclusion: Patients transplanted for HCV-related liver cirrhosis should benefit from a careful follow-up on the WL and prompt TB prophylaxis after LTin order to prevent TB reactivation during early immunosuppression. Delaying TB prophylaxis after LT in order to avoid liver toxicity of anti-bacillary drugs was not associated with $\mathrm{TB}$ reactivation.

Disclosure of Interest: All authors have declared no conflicts of interest. 
P1362 A NATIONAL STUDY OF CANCER DIAGNOSES IN IRISH LIVER TRANSPLANT RECIPIENTS WITH PRIMARY SCLEROSING CHOLANGITIS

S. M. O'Reilly ${ }^{1}$, K. Hartery ${ }^{1}$, M. Walshe ${ }^{1}$, S. Deady ${ }^{2}$, E. O'Leary ${ }^{2}$, G.

A. Doherty ${ }^{1}$, D. Houlihan ${ }^{3}$, G. Cullen ${ }^{1}$, H. Mulcahy ${ }^{1}$, J. Sheridan ${ }^{1}$

${ }^{1}$ Centre For Colorectal Disease, St Vincent's University Hospital, Dublin/Ireland

${ }^{2}$ National Cancer Registry Ireland, Cork/Ireland

${ }^{3}$ National Liver Unit, St Vincent's University Hospital, Dublin/Ireland

Contact E-mail Address: susannemoreilly@gmail.com

Introduction: Primary sclerosing cholangitis (PSC) is associated with an increased risk of cholangiocarcinoma, colorectal cancer (CRC) and gallbladder cancer. Orthotopic liver transplantation (OLT) patients are at increased risk of developing de novo malignancies, however limited and conflicting data exists regarding cancer risk post OLT for PSC.

Aims \& Methods: To examine all recorded malignancies over 2 decades in OLT PSC pts and compare to our non-transplanted PSC cohort. To analyse factors associated with the development of malignancies post OLT. We retrospectively studied PSC patients attending the Irish National Liver Unit (INLU) and the Centre for Colorectal Disease (CCD) at St. Vincent's University Hospital from 1 1/94-30/9/16. We integrated this database with the National Cancer Registry in Ireland. This enabled accurate determination of the no. of malignancies recorded in the PSC cohort. Analyzed data included age of recipient at OLT, gender, primary OLT indication, immunosuppressive regime, de novo malignancy post OLT, time from OLT to diagnosis of malignancy or death. Statistical analysis was primarily descriptive. Cox Proportional Hazard Model was used to analyse factors associated with mortality in the PSC OLT cohort.

Results: 107 of 173 patients had undergone transplant for PSC. $27 / 107$ pts were transplanted for cholangiocarcinoma. 12 post-transplant de novo cancers and 12 $\mathrm{BCC} / \mathrm{SCC}$ carcinomas were found in 107 patients during 737.8 person years of follow-up. Median time to cancer diagnosis post OLT was 5 years (IQR 2.8-5.9). Recurrence of PSC was observed in 21 patients $(19.6 \%)$. Post-transplant lymphoproliferative disease (PTLD) remains a major complication after OLT. Prior studies have reported rates of $1-3 \%$ in adult OLT pts. 5 pts were diagnosed with lymphoma post OLT representing $4.7 \%$ of cohort. Median time to diagnosis was 5.3 yrs [IQR 2.8-10.2]. Regarding CRC, 2 patients developed CRC post OLT. 4 patients developed colonic dysplasia; $3 / 4$ underwent colectomy. All those who developed colonic dysplasia/CRC post OLT had co-existing IBD. All 5 colectomy specimens for dysplasia/CRC showed significant co-existing inflammation. One patient post OLT underwent a completion proctectomy for rectal cancer. As expected, cholangiocarcinoma as indication for OLT $(\mathrm{p}=0.005, \mathrm{RR} 2.573$, 95\% CI 1.3-4.95) and an older age at transplant $(\mathrm{p}=0.05, \mathrm{RR} 1.027,95 \% \mathrm{CI}$ $1-1.054)$ were associated with higher mortality. Table 1

\begin{tabular}{lll}
\hline Cancer & SIR & $95 \%$ CI \\
\hline Any cancer & 3.239 & $1.885-5.186$ \\
Excluding skin cancer & 1.97 & $0.848-3.882$ \\
Lymphoma & 36.574 & $14.65-75.36$ \\
Colorectal cancer & 1.779 & $0.023-9.898$ \\
\hline
\end{tabular}

Conclusion: These findings represent national cancer figures in our PSC OLT cohort. The rate of cancer is more than three times higher in this population than the general population. The rates of PTLD are $>30$ times higher than those in the normal population, and slightly higher than previously reported in unselected liver transplant groups. We could not find any association between the development of PTLD and aggressive immunosuppressive regimes for co-existing IBD post OLT. The study highlights that IBD/PSC patients remain at significant risk of colonic neoplasia after OLT and require intensive surveillance.

Disclosure of Interest: All authors have declared no conflicts of interest.

\section{P1363 OUTCOME OF LIVER TRANSPLANTATION FOR PRIMARY SCLEROSING CHOLANGITIS IN CONTEXT OF HLA-DR MISMATCH: SINGLE CENTRE EXPERIENCE}

P. Drastich ${ }^{1}$, L. Bajer ${ }^{1}$, A. Slavcev ${ }^{2}$, M. Benes ${ }^{1}$, P. Trunecka $^{3}$, J. Spicak $^{1}$ ${ }^{1}$ Department Of Hepatogastroenterology, Institute for Clinical and Experimental Medicine, Prague/Czech Republic

${ }^{2}$ Department Of Immunogenetics, Institute for Clinical and Experimental Medicine, Prague/Czech Republic

${ }^{3}$ Transplantcentre, Institute for Clinical and Experimental Medicine, Prague/Czech Republic

Contact E-mail Address: drastich@hotmail.com

Introduction: Primary sclerosing cholangitis (PSC) is a chronic liver disorder of unknown etiology, characterized by inflammation, fibrosis and stenoses of both extra- and intrahepatic bile ducts. For those who develop end-stage liver disease, orthotopic liver transplantation (OLT) remains the only effective treatment currently available. PSC is accompanied with concomitant ulcerative coilitis (UC) in a significant proportion of patients. Benefits of routine HLA typing in donor and recipient prior to OLT were proved in the past.

Aims \& Methods: The aim of this study was to assess the impact of HLA-DR mismatch on acute cellular rejection (ACR), PSC recurrence (rPSC) and course of UC after OLT. After applying inclusion/exclusion criteria we retrospectively evaluated records of 57 patients transplanted at Institute for Clinical and Experimental Medicine (Prague, Czech Republic) between July 1994 and November 2011. Only patients with proper records \pm 5 years from OLT were included. We evaluated likelihood for each variable (ACR, rPSC, course of UC) in patients with either single, or double mismatch in HLA-DR. Input data were analysed with $\chi 2$ and Fishers exact test using MedCalc statistical software. A p - value $<0.05$ was considered as statistically significant.

Results: Out of 57 patients, $27(47.4 \%)$ had single mismatch ("M1" group) and $30(53.6 \%)$ had double mismatch ("M2" group) in HLA-DR. No patient had full match. 33/57 (57.9\%) patients had ACR: 15/27 (55.6\%) of M1 and 18/30 (60\%) of M2 $(\mathrm{p}=0.94) .4 / 27(14.8 \%)$ of M1 and $2 / 30(6.3 \%)$ of M2 had corticoresistant $\operatorname{ACR}(\mathrm{p}=0.57)$. Multiple-episodes of ACR occurred in $11 / 57(19.3 \%)$ patients: $6 / 27(22.2 \%)$ of $\mathrm{M} 1$ and $5 / 25(20 \%)$ of M2 $(\mathrm{p}=0.74) .12 / 57(21.1 \%)$ had de-novo UC after OLT: $7 / 27(25.9 \%)$ of M1 and $5 / 30(16.7 \%)$ of M2 $(\mathrm{p}=0.60)$. In 37 $(68.5 \%)$ patients, UC was diagnosed prior to OLT. $9 / 16(56.3 \%)$ patients with M1 and $6 / 21(28.6 \%)$ patients with M2 had more severe course of UC as compared to course prior to OLT $(\mathrm{p}=0.17) .38$ patients were evaluated for rPSC, which was diagnosed in $17(44.7 \%)$ individuals. $6 / 19$ patients with $\mathrm{M} 1$ and 11/19 with M2 had rPSC $(\mathrm{p}=0.19)$.

Conclusion: Patients with single mismatch in HLA-DR have slight tendency towards development of rPSC and worsening of UC after OLT as compared to patients with double mismatch. Analysis of combined mismatch in HLADR and HLA-DQ could demonstrate more substantial linkages in respective clinical variables. Therefore, these data have to be considered as preliminary as typing for HLA-DQ from frozen blood samples is currently underway. Supported by Ministry of Health of the Czech Republic, grant nr. 15-28064A. All rights reserved

Disclosure of Interest: All authors have declared no conflicts of interest.

\section{P1364 GRAFT DYSFUNCTION IN POST-LIVER TRANSPLANTATION: UTILITY OF TRANSIENT ELASTOGRAPHY BY FIBROSCAN $\mathbb{R}$}

E. Soares ${ }^{1}$, M. Gravito-Soares ${ }^{1}$, D. Gomes ${ }^{1}$, A. Simão ${ }^{2}$, E. Furtado ${ }^{3}$, L. Tomé ${ }^{1}$ ${ }^{1}$ Gastroenterology, Centro Hospitalar e Universitário Coimbra, Coimbra, Portugal, Coimbra/Portugal

${ }^{2}$ Internal Medicine, Centro Hospitalar e Universitário Coimbra, Coimbra, Portugal, Coimbra/Portugal

${ }^{3}$ Liver Transplantation Unit, Centro Hospitalar e Universitário Coimbra, Coimbra, Portugal, Coimbra/Portugal

Contact E-mail Address: es18497@gmail.com

Introduction: Liver biopsy remains the gold standard in the diagnosis of graft dysfunction in post-liver transplantation (GDPLT). Liver transient elastography is a valid non-invasive method for liver fibrosis evaluation, with a good correlation in chronic liver diseases. The progression of fibrosis represents a major problem in the post-liver transplantation.

Aims \& Methods: We aimed to evaluate the predictive role of liver transient elastography in the evaluation of GDPLT and to determine the predictive factors of liver transplantation fibrosis. This was a retrospective observational cohort study of total of 49 patients with post-liver transplantation status who underwent liver transient elastography by Fibroscan ${ }^{\mathbb{B}}$. Selected patients who underwent percutaneous/transjugular liver biopsy. In case of more than one liver biopsy, it was selected the biopsy closer to Fibroscan ${ }^{\circledR}$. The fibrosis and steatosis evaluated by Fibroscan ${ }^{\circledR}$ were compared with those obtained by liver biopsy. Significant fibrosis was considered if $\geq F 2$. Demographic, analytical and associated liver transplantation variables were evaluated.

Results: A total of 32 patients underwent Fibroscan ${ }^{\circledR}$ and liver biopsy. Mean age of $48.53 \pm 11.20$ years and male gender in $68.8 \%(\mathrm{n}=22)$. The mean time between Fibroscan ${ }^{\circledR}$ and liver biopsy was $29.77 \pm 36.90$ months. The mean elastography score was $13.45 \pm 8.31 \mathrm{KPa}$ with $\mathrm{IQR} /$ med of $17.11 \pm 8.66 \%$. Mean CAP score was $207.12 \pm 57.35 \mathrm{~dB} / \mathrm{m}$. Regarding liver biopsy, $34.4 \%(\mathrm{n}=11)$ had significant fibrosis and $25.0 \%(\mathrm{n}=8)$ presented steatosis. Comparing two methods, there was no concordance for steatosis (kappa $=0.273 ; \mathrm{p}=0.117$ ) or inflammation $($ Kappa $=0.063 ; p=0.710)$. On the contrary, a moderate agreement for significant fibrosis $(\mathrm{kappa}=0.431 ; \mathrm{p}=0.003)$ was verified. The mean elastography score showed an accuracy of $79.7 \%$ in predicting histological fibrosis $(A U R O C=0.797 ; p=0.007)$ to a cut-off value of $11.6 \mathrm{KPa}(\mathrm{S}=81.8 \%$; $\mathrm{Sp}=76.2 \%$ ). In relation to analytical parameters, only serum albumin was predictive of histological fibrosis $(\mathrm{OR}=2.79 ; \mathrm{p}=0.043)$.

Conclusion: Liver transient elastography represents a non-invasive and valid alternative procedure to liver biopsy in the evaluation of post-liver transplantation fibrosis but not steatosis or inflammation. Liver transient elastography scoring $\geq 11.6 \mathrm{KPa}$ and low values of serum albumin are predictors of postliver transplantation fibrosis.

Disclosure of Interest: All authors have declared no conflicts of interest. 
P1365 NEW-ONSET DIABETES AFTER TRANSPLANT (NODAT): INCIDENCE, RISK ANALYSIS AND IMPACT ON SURVIVALNEWONSET DIABETES AFTER TRANSPLANT (NODAT): INCIDENCE, RISK ANALYSIS AND IMPACT ON SURVIVAL

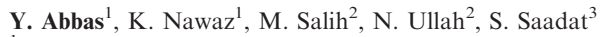

${ }^{1}$ Gastroenterology, Hepatology And Liver Transplant, Shifa International Hospitals, Islamabad/Pakistan

${ }^{2}$ Gastroenterology And Hepatology, Shifa international hospital, islamabad, Islamabad/Pakistan

${ }^{3}$ Nephrology, Shifa International Hospital, Islamabad/Pakistan

\section{Contact E-mail Address: dryasirab@gmail.com}

Introduction: Orthotopic liver transplant has become the standard of care for end-stage liver disease and hepatocellular cancer. Better immunosuppressant paved way for improved survival rates post-transplant. But with this longevity comes a higher prevalence of chronic diseases such as New Onset Diabetes After Transplant (NODAT), Hypertension, metabolic syndrome etc. which have a negative impact on graft function and patient survival.

Aims \& Methods: Primary: To determine the incidence of New Onset Diabetes After Transplant (NODAT), Impaired Fasting Glycaemia (IFG) and post-transplant hyperglycemia in living-donor liver transplant recipients. Secondary: To determine the risk factors associated with NODAT and IFG. To determine impact of NODAT on survival and mortality. It was a retrospective cohort study of 283 living donor liver transplant recipients from 29/4/2011 till 26/4/ 2016. Data was collected from records. Simple means and standard deviation was calculated for continuous variables while frequency statistics were calculated for categorical ones. Risk factors were assessed using binary logistic regression analysis.

Results: A total of 130 post liver transplant patients were analyzed after exclusion. NODAT was present in 41/130 (31.5\%) patients, while 19/130 (14.6\%) patients had impaired fasting glycaemia. Acute cellular rejection, Post-transplant Hyperglycemia and Pre-transplant prediabetes showed increased odds of acquiring NODAT post-transplant. NODAT had significant association with mortality and decreased survival $(\mathrm{p}=0.05)$.

Conclusion: This cohort showed that NODAT is an important post-transplant entity with significant impact on mortality and survival. Early identification of at-risk patients is suggested.

Disclosure of Interest: All authors have declared no conflicts of interest.

\section{References}

Adam R, Hoti E. Liver transplantation: the current situation. Semin Liver Dis 2009; 29 (1): 3-18.

Duffy JP, Kao K, Ko CY et al. Long-term patient outcome and quality of life after liver transplantation: analysis of 20-year survivors. Ann Surg 2010; 252 (4): 652-61.

Simo KA, Sereika S, Bitner N, Newton KN, Gerber DA. Medical epidemiology of patients surviving ten years after liver transplantation. Clin Transplant 2011:25:360-367.

Baid S, Cosimi AB, Farrell ML, et al. Posttransplant diabetes mellitus in liver transplant recipients: risk factors, temporal relationship with hepatitis $\mathrm{C}$ virus allograft hepatitis, and impact on mortality. Transplantation. 2001;72(6):10661072 .

Honda M, Asonuma K, Hayashida S, et al. Incidence and risk factors for newonset diabetes in living-donor liver transplant recipients. Clin Transplant 2013: 27: 426-435

Kuo HT, Sampaio MS, Ye X, Reddy P, Martin P, Bunnapradist S. Risk factors for new-onset diabetes mellitus in adult liver transplant recipients, an analysis of the Organ Procurement and Transplant Network/United Network for Organ Sharing database. Transplantation. 2010;89(9):1134-1140. doi: 10.1097/TP.0b $013 \mathrm{e} 3181 \mathrm{~d} 2 \mathrm{fecl}$

Li DW, Lu TF, Hua XW, et al. Risk factors for new onset diabetes mellitus after liver transplant: a meta-analysis. World J Gastroenterol 2015 May 28;21(20):6329-6340. DOI: 10.3748/wjg.v21.i20.6329

Oufroukhi L, Kamar N, Muscari F, et al Predictive factors for posttransplant diabetes mellitus within one-year of liver transplantation. Transplantation 2008; 85(10): 1436-1442.

American Diabetes Association. Standards of medical care in diabetes-2011. Diabetes Care. 2015;38(suppl 1):S1-S2 | DOI: 10.2337/dc15-S001.

Sharif A, Hecking M, De Vries AP, Porrini E, Hornum M, RasoulRockenschaub S, Berlakovich G, Krebs M, Kautzky-Willer A, Schernthaner G, Marchetti P. Proceedings from an international consensus meeting on posttransplantation diabetes mellitus: recommendations and future directions. American Journal of Transplantation. 2014 Sep 1;14(9):1992-2000.

Kotton $\mathrm{CN}$ et al. Updated International Consensus Guidelines on the Management of Cytomegalovirus in Solid-Organ Transplantation. Transplantation 2013;96: 00-00

\section{P1366 VITAMIN B12 AS A PROGNOSTIC MARKER IN PATIENTS WITH ACUTE ON CHRONIC LIVER FAILURE}

V. L. Anapaz ${ }^{1}$, M. V. Machado ${ }^{2}$, C. Baldaia ${ }^{3}$, A. Valente ${ }^{3}$

${ }^{1}$ Gastroenterology, Hospital Fernando Fonseca, Lisbon/Portugal

${ }^{2}$ Intensive Care Unit Of Gastroenterology And Hepatology, Hospital de Santa

Maria, Lisbon/Portugal

${ }^{3}$ Department Of Gastroenterology And Hepatology, North Lisbon Hospital Center,

University of Lisbon, Portugal, Lisbon/Portugal

Contact E-mail Address: vera_anapaz@hotmail.com
Introduction: Serum vitamin B12 (vB12) levels are increased in myeloproliferative diseases by increased production of the haptocorrin transporter and in liver diseases by release of $\mathrm{vB} 12$ from hepatocytes death.

Aims \& Methods: The aim of this study was to evaluate $\mathrm{vB} 12$ as a prognostic marker in patients with cirrhosis and acute on chronic liver failure (ACLF) induced by infection. Retrospective assessment of 58 patients admitted to an intensive care unit with ACLF in the context of infection (group 1) and 53 patients with compensated hepatic cirrhosis followed as Hepatology outpatients (group 2). Evaluation of $\mathrm{vB12}$ as a predictor of 30 days' mortality.

Results: 111 patients, $68 \%$ male, age $58 \pm 18$ years. Group 1 had more advanced liver disease (CPT $11.9 \pm 0.3$ vs. $6.5 \pm 0.2$ and MELD $27.0 \pm 1.0$ vs. $10 \pm 0.5$ ), higher vB12 $(1413 \pm 149$ vs. $735 \pm 56 \mathrm{pg} / \mathrm{mL})$ and lower survival $(1.6 \pm 0.4$ vs. $6.4 \pm 2.7$ years). VB12 positively correlated with hepatic function scores (CPT: $\mathrm{R}=0.524$; MELD: $\mathrm{R}=0.566$ ). MELD and $\mathrm{vB} 12$ were independent factors of mortality at first month (OR 1.205 and 1.002 for increase of one unit of MELD or vB12). In patients with ACLF, vB12 correlated with liver disease severity (CPT: $R=0.411$; MELD: $R=0.534$ ) and multiorgan failure (number of organ failures: $\mathrm{R}=0.536$, SOFA: $\mathrm{R}=0.553$ ). In group 1 , survival was lower in patients with high vB12 ( $8 \pm 3 v s .37 \pm 11$ months $)$, and 1 month' mortality was associated with vB12, CPT, MELD, number of organ failure, urea, lactates and fibrinogen, in univariate analysis $(\mathrm{p}<0.001)$. In multivariate analysis only fibrinogen maintained statistical significance $(\mathrm{p}<0.001)$.

Conclusion: There was a strong association between high levels of vB12 and clinical decompensation of liver cirrhosis. VB12 correlated with scores of liver function and multiorgan failure, as well as early mortality in patients with ACLD.

Disclosure of Interest: All authors have declared no conflicts of interest.

\section{Reference}

Dou J, Xu W, Ye B, Zhan Y, Mao W; Serum vitamin B12 levels as indicators of disease severity and mortality of patients with acute-on-chronic liver failure; Clin Chim Acta. 2012 Nov 20;413(23-24):1809-12.

\section{P1367 OUTCOMES OF LIVER TRANSPLANTATION IN PATIENTS WITH HEPATOCELLULAR CARCINOMA}

A. Eshraghian ${ }^{1}$, S. Nikeghbalian ${ }^{2}$, S. Ali Malek-Hosseini ${ }^{2}$, B. Geramizadeh ${ }^{2}$ ${ }^{1}$ Gastroenterology And Hepatology, Shiraz University of Medical Sciences, Shiraz/ Iran

${ }^{2}$ Transplant Research Center, Shiraz/Iran

Contact E-mail Address: eshraghiana@yahoo.com

Introduction: Hepatocellular carcinoma (HCC) is the second leading cause of cancer-related mortality worldwide. Patients with viral hepatitis and those with non-alcoholic fatty liver disease (NAFLD) are especially susceptible to HCC. Parallel to an increase in prevalence of NAFLD, the prevalence of HCC is estimated to be increased in next years. Liver transplantation is now considered as a modality of treatment for patients with $\mathrm{HCC}$.

Aims \& Methods: This study aimed to investigate outcomes of liver transplantation in patients with HCC compared to other causes of liver transplantation. In a cross-sectional study patients who had undergone liver transplantation between March 2012 and March 2015 at Shiraz Transplant Center, Shiraz, Iran were included. Patients' characteristics including age, gender, model for end stage liver disease (MELD) score, rejection episodes, laboratory data and information about mortality of patients were recorded. Characteristics of tumor including size, number and vascular invasion were recorded. Based on these findings HCC patients were divided to those within Milan criteria and those beyond Milan criteria. The impact of HCC on post-transplant outcomes was investigated using student t-test and chi-square tests. Multivariate logistic regression was used for analysis of independent risk factors of mortality after liver transplantation. Kaplan-Meier method was used for analysis of survival.

Results: Totally 1014 liver transplant patients were included. 94 patients with HCC underwent liver transplantation. There was no statistically significant difference between those with and without HCC in terms of gender, portal vein thrombosis (PVT), diabetes mellitus (DM), hepatic encephalopathy and hospitalization before liver transplantation $(\mathrm{P}>0.05)$. HCC was significantly more prevalent in cirrhosis due to viral hepatitis $(\mathrm{P}<0.001)$. Acute rejection episodes was not different in patients with and without HCC in early post-transplant period $(\mathrm{OR}=0.563 ; 95 \% \mathrm{CI}: 0.27-1.14, \mathrm{P}=0.108)$. In regression analysis, presence of pre-transplant $\mathrm{DM}(\mathrm{OR}=3.89 ; 95 \% \mathrm{CI}: 1.36-11.11, \mathrm{P}=0.011)$ and acute kidney injury within 30 days after liver transplant $(\mathrm{OR}=4.38$; 95\% CI: $1.44-1327$, $\mathrm{P}=0.009)$ were independent predictors of post-transplant mortality. Mean post-liver transplant survival in HCC patients within Milan criteria was $40.48 \pm 3.69$ months compared to $36.80 \pm 6.28$ months in those beyond Milan criteria $(\mathrm{P}=0.82)$. Mean post-transplant survival in patients with $\mathrm{HCC}+\mathrm{DM}$ was $32.75 \pm 4.7$ months compared to $48.51 \pm 2.60$ months in HCC patients without DM $(\mathrm{P}=0.009)$

Conclusion: Liver transplantation can be used for patients with HCC, however, post-transplant survival seems to be lower. Diabetes mellitus and acute kidney injury were predictors of mortality among our patients with HCC after liver transplantation.

Disclosure of Interest: All authors have declared no conflicts of interest.

\section{References}

1. Menahem B, Lubrano J, Duvoux C, Mulliri A, Alves A, Costentin C, Mallat A, Launoy $G$, Laurent A. Liver transplantation versus liver resection for hepatocellular carcinoma in intention to treat: Attempt to perform an 
ideal meta-analysis. Liver Transpl. 2017 Mar 14. doi: 10.1002/1t.24758. [Epub ahead of print]

2. Zavaglia C, De Carlis L, Alberti AB, Minola E, Belli LS, Slim AO, Airoldi A, Giacomoni A, Rondinara G, Tinelli C, Forti D, Pinzello G. Predictors of long-term survival after liver transplantation for hepatocellular carcinoma. Am J Gastroenterol 2005;100(12):2708-16.

\section{P1368 THE ASSESSMENT OF THE ADC PREDICTIVE VALUE IN SURVIVAL OUTCOMES OF PATIENTS UNDERGOING RADIOFREQUENCY ABLATION FOR METASTATIC COLORECTAL CANCER LIVER TUMORS}

O. Kozak ${ }^{1}$, T. Nowicki ${ }^{1}$, J.M. Pienkowska $^{1}$, E. Izycka-Swieszewska ${ }^{2}$, D. Zadrozny ${ }^{3}$, E. Szurowska ${ }^{1}$

${ }^{1}$ Department Of Radiology, Medical University of Gdansk, Gdansk/Poland ${ }^{2}$ Department Of Pathology And Neuropathology, Medical University of Gdansk, Gdansk/Poland

${ }^{3}$ Clinic Of Oncologic And Liver Surgery, University Warmia and Mazury, Olsztyn/ Poland

\section{Contact E-mail Address: oliwiak@gumed.edu.pl}

Introduction: Liver is one of the most common metastatic sites of colorectal cancer, nearly $50 \%$ patients develop hepatic metastases during the course of their disease. Metastatic spread influences survival rate of those patients. The diffusion weighted imaging (DWI) is MRI sequence designed to detect random movement of water protons in extracellular compartment. Biophysical parameter expressing the flexibility of Brownian motion is the diffusion coefficient (ADC). ADC values for b parameter lower than $300 \mathrm{~s} / \mathrm{mm}^{2}$ are influenced by perfusion whereas ADC values for $\mathrm{b}$ greater than $300 \mathrm{~s} / \mathrm{mm}^{2}$ depend mainly on diffusion. Aggressive malignant process often develops necrotic areas within neoplastic lesion. Necrotic changes are characterized by high ADC values. We suppose that low ADC values correlate with presence of necrosis in highly malignant lesions effecting in lower survival rate.

Aims \& Methods: This is a post hoc analysis of prospective study to assess the predictive value of the ADC in survival outcomes of patients undergoing radiofrequency ablation due to metastatic colorectal cancer lesions in the liver. We analyzed the MRI studies of 52 patients (18 F, 34 M, aged 4383) performed on $1.5 \mathrm{~T}$ scanner one day before the percutaneous RFA treatment. The total number of analyzed lesions was 110 ( 15 per patient), 83 of them were completely ablated, 27 incompletely, what was assessed in follow-up CT studies. The standard protocol of the liver MRI was applied including DWI sequence in $b$ values of 0,15 and $500 \mathrm{~s} / \mathrm{mm}^{2}$. ADC maps were calculated for $\mathrm{b}$ values of 015 and $0500 \mathrm{~s} / \mathrm{mm}^{2}$. The mean ADC value was obtained by threefold marking ROI covering the whole metastatic lesion. In cases of multiple foci only the lesion with the highest ADC value was included into analysis. On basis of ROC analysis the cut-off values of ADC were established: $2.49 \mathrm{~mm}^{2} / \mathrm{s}$ for $\mathrm{b}$ value of $015 \mathrm{~s} / \mathrm{mm}^{2}$ and $1.43 \mathrm{~mm}^{2} / \mathrm{s}$ for $\mathrm{b}$ value of $0500 \mathrm{~s} / \mathrm{mm}^{2}$. The survival outcomes were assessed by mean of Kaplan-Meier estimator. The $\mathrm{p}$ value lower than 0.05 was considered significant.

Results: The statistical analysis included KaplanMeier estimator for 52 patients with 9 censored cases $\left(17.3 \%\right.$ ). In ADC maps for $\mathrm{b}$ value of $0500 \mathrm{~s} / \mathrm{mm}^{2}$, the ADC value $\geq 1.43 \mathrm{~mm}^{2} / \mathrm{s}$ correlated with longer survival time, whereas ADC value $<1.43 \mathrm{~mm}^{2} / \mathrm{s}$ correlated with shorter survival time. Statistically significant differences were identified by $\log$ rank test $=2.6998, \mathrm{p}=0.007$. Such a correlation was not observed for ADC values in ADC maps for $b$ value of $015 \mathrm{~s} / \mathrm{mm}^{2}$ $(\mathrm{p}=0.058)$.

Conclusion: The study showed significant differences in survival rate depending on diffusion influenced ADC values of metastatic lesions.

Disclosure of Interest: All authors have declared no conflicts of interest.

\section{References}

Szurowska E, Nowicki, KT, Izycka-Swieszewska E, Zadrozny D, Markiet K, Studniarek M: Predictive Value of Apparent Diffusion Coefficient of Colorectal Carcinoma Hepatic Metastases' Response to Radiofrequency Ablation. J Magn Reson Imaging,2013 Nov;38(5):1027-32.

Cui Y, Zhang XP, Sun YS, Tang L. Shen: Apparent Diffusion Coefficient: Potential Imaging Biomarker for Prediction and Early Detection of Response to Chemotherapy in Hepatic Metastases. Radiology 2008; 248:894-900.

Koh DM, Scurr E, Collins D et al: Predicting Response of Colorectal Hepatic Metastasis: Value of Pretreatment Apparent Diffusion Coefficients. AJR Am J Roentgenol 2007; 188:1001-1008.

Guiu B, Cercueil JP: Liver diffusion-weighted MR imaging: the tower of Babel? Eur Radio. 12011 Mar;21(3):463-467.

\section{P1369 HOW OFTEN DO WE FIND STEATOSIS AND SEVERE}

\section{FIBROSIS IN TYPE 2 DIABETES MELLITIUS PATIENTS}

I. Sporea ${ }^{1}$, R. Mare ${ }^{1}$, R. Sirli ${ }^{1}$, S.A. Popescu ${ }^{1}$, S. Nistorescu ${ }^{1}$, A. Sima ${ }^{2}$, R. Timar ${ }^{2}$

${ }^{1}$ Department Of Gastroenterology And Hepatology, University of Medicine and

Pharmacy Victor Babes Timisoara, Timisoara/Romania

${ }^{2}$ Diabetes Department, Victor Babes University of Medicine and Pharmacy

Timisoara, Timisoara/Romania

Contact E-mail Address: isporea $@$ umft.ro
Introduction: Type II diabetes and nonalcoholic fatty liver disease (NAFLD) are frequently associated, NAFLD being considered the hepatic expression of the metabolic syndrome.

Aims \& Methods: The aim of the present study was to assess the severity of liver fibrosis and steatosis in a cohort of type 2 diabetic patients, using non-invasive methods:Transient Elastography (TE) and Controlled Attenuation Parameter (CAP). The study included 354 type 2 diabetic patients, who were prospectively randomized (every first 6 patients who were referred to the Metabolic Disease Outpatient Clinic on a consultation day), evaluated in the same session by means of TE and CAP (FibroScan EchoSens) to assess both liver fibrosis and steatosis. Each patient was evaluated for the presence of viral hepatitis $(B, C)$ and an AUDIT-C score was performed to exclude alcohol abuse. Reliableliver stiffness measurements (LSM) were defined as the median value of 10 LSM with an IQR median $<30 \%$. For TE and CAP, M and XL probes were used. A cut-off value of $8.2 \mathrm{kPa}$ [1] was used to define severe fibrosis $(\mathrm{F} \geq 3)$. For differentiation between stages of steatosis we used the following cut-off values [2]: $\mathrm{S} 2$ (moderate) - $255 \mathrm{db} / \mathrm{m}, \mathrm{S} 3$ (severe) - $290 \mathrm{db} / \mathrm{m}$.

Results: Out of 354 diabetic screened we excluded those with associated viral hepatitis, those with an AUDIT-C score $>8$ and those with unreliable LSM. The final analysis included 239 subjects $(59.4 \%$ women, $40.6 \%$ men, mean age $60.4 \pm 9.3 ; \mathrm{BMI}=31.8 \pm 6.1 \mathrm{~kg} / \mathrm{m}^{2}$ ) with reliable LSM. Accordingly to BMI, $10.8 \%$ had normal weight, $26.4 \%$ were overweight and $62.8 \%$ were obese (35.6\% obesity grade I, $17.2 \%$ obesity grade II and $10 \%$ obesity grade III). Moderate and severe steatosis by means of CAP was found in $18.4 \%$ and $69.5 \%$ cases respectively. Severe fibrosis was detected by means of TE $(\mathrm{LSM} \geq 8.2 \mathrm{kPa})$ in $29.3 \%(70 / 239)$ of subjects.

Conclusion: In our group, $87.9 \%$ of diabetic patients had moderate and severe steatosis by CAP and $29.3 \%$ of them had severe fibrosis (TE $\geq 8.2 \mathrm{kPa}$ ), suggesting the need for their systematical assessment.

Disclosure of Interest: I. Sporea: I hereby confirm that I have received financial support (congress travel grant or speaker fee) from Philips, Siemens, General Electric, Abbvie, Zentiva, Bristol Meyers Squibb

R. Mare: I hereby confirm that I have received financial support (congress travel grant) from Philips

R. Sirli: I hereby confirm that I have received financial support (congress travel grant or speaker fee) from Philips, Abbvie, Zentiva,

S.A. Popescu: I hereby confirm that I have received financial support (congress travel grant or speaker fee) from Philips, General Electric, Abbvie, AstraZeneca, Zentiva

All other authors have declared no conflicts of interest.

\section{References}

1. Cassinotto $\mathrm{C}$ et al. Liver stiffness in nonalcoholic fatty liver disease: A comparison of supersonic shear imaging, FibroScan, and ARFI with liver biopsy. Hepatology. 2016;63(6):1817-27.

2. Shi KQ et al. Controlled attenuation parameter for the detection of steatosis severity in chronic liver disease: A meta-analysis of diagnostic accuracy. $J$. GastroenterolHepatol 2014;29(6):1149-58

\section{P1370 LEARNING CURVE EVALUATION USING ELASTPQ}

R. Mare ${ }^{1}$, I. Sporea ${ }^{1}$, S.A. Popescu ${ }^{1}$, R. Sirli ${ }^{1}$, C. Pienar $^{2}$

${ }^{1}$ Gastroenterology And Hepatology, Emergency County Hospital Timisoara.,

Timisoara/Romania

${ }^{2}$ Gastroenterology, Pediatrics, "Victr Babes" University of Medicine, Timisoara Romania

Contact E-mail Address: ruxi2323@yahoo.com

Introduction: Nowadays liver fibrosis can be assessed using non-invasive elastographic techniques. ElastPQ is a quite novel point share wave elastography integrated into an ultrasound system.

Aims \& Methods: The aim of our study was to evaluate the learning curve of obtaining reliable liver stiffness measurements (LSM), using ElastPQ. LSM of a trainee were compared to LSM of an elastography expert (with an experience of more than 500 examinations). Our study group included 50 subjects (mean age- 52.7 years, $66.6 \%$ men, mean $\mathrm{BMI}=25.6 \mathrm{~kg} / \mathrm{m}^{2}$ ). Both the trainee and the expert obtained LSM for each subject, using ElastPQ (EPIQ 7, Philips Healthcare, Bothell, WA, USA). Reliable LSM were defined as the median value of ten measurements acquired in a homogenous area avoiding large vessels and with an IQR/median $<30 \%$. The learning curve was evaluated using the Receiver Operating Curve analysis using the expert's results as reference.

Results: The trainee's performance in obtaining reliable LSM was good (AUC: $0.735,95 \%$ CI $(0.557-0.913), p=0.01)$. The trainee started to have similar results with the elastography expert after the $30^{\text {th }}$ subject. When looking at the IQRs, they became significantly lower after the $30^{\text {th }}$ subject $(2.6 \pm 2.1 \mathrm{kPa}$ vs $6.5 \pm 9.2 \mathrm{kPa}, \mathrm{p}=0.03)$

Conclusion: Obtaining reliable LSM using ElastPQ can be easily achieved after 30 LS examinations.

Disclosure of Interest: R. Mare: I hereby confirm that I have received financial support (congress travel grant) from Philips

I. Sporea: I hereby confirm that I have received financial support (congress travel grant or speaker fee) from Philips, Siemens, General Electric, Abbvie, Zentiva, Bristol Meyers Squibb

S.A. Popescu: I hereby confirm that I have received financial support (congress travel grant or speaker fee) from Philips, General Electric, Abbvie, AstraZeneca, Zentiva 
R. Sirli: I hereby confirm that I have received financial support (congress travel grant or speaker fee) from Philips, Abbvie, Zentiva

All other authors have declared no conflicts of interest.

\section{P1371 ENHANCEMENT PATTERN OF HEPATOCELLULAR} CARCINOMA ON CONTRAST ENHANCED ULTRASOUND EXAMINATION IN PATIENTS WITH CHRONIC LIVER DISEASE

M. Danila ${ }^{1}$, I. Sporea ${ }^{1}$, R. Sirli², A. Popescu ${ }^{2}$, T.V. Moga ${ }^{2}$, A. Stepan ${ }^{2}$ ${ }^{1}$ Department Of Gastroenterology And Hepatology, Victor Babes University of Medicine and Pharmacy Timisoara, Timisoara/Romania

${ }^{2}$ Gastroenterology, University of Medicine and Pharmacy „Victor Babes"

Timisoara, Romania, Timisoara/Romania

Contact E-mail Address: mireladanila@gmail.com

Introduction: In patients with liver cirrhosis, hepatocellular carcinoma (HCC) can be diagnosed by noninvasive imaging methods (contrast-enhanced ultrasound CEUS, contrast CT/MRI)

Aims \& Methods: The aim of this study was to evaluate which is the most common enhancement pattern of HCC on CEUS in all three phases (arterial, portal and late phase). We performed a retrospective study that included patients with a final diagnosis of HCC established by an imaging method (contrast enhanced CT or MRI) or biopsy. A total of 249 patients with HCC were examined (180 men, 69 women, mean age $64 \pm 10$ years); 181 patients had liver cirrhosis and 68 patients chronic hepatopathy with severe fibrosis. All 249 HCCs were evaluated by CEUS using low mechanical index ultrasound, following an intravenous bolus of $2.4 \mathrm{ml}$ SonoVue. CEUS was considered conclusive for HCC if a typical pattern was present following contrast examination (hyperenhancement in the arterial phase accompanied by portal and/or late phases washout). The nodules were classified according to their size in $\leq 3 \mathrm{~cm}$ and $>3 \mathrm{~cm}$. We reevaluated all 249 HCCs CEUS studies using the ACR CEUS LI-RADSv 2016 algorithm.

Results: After CEUS examination a conclusive diagnosis of HCC was obtained in $190 / 249$ cases $(76.3 \%)$. Arterial phase hyperenhancement pattern was present in $227 / 249$ cases $(91.2 \%)$, isoenhancement in the arterial phase was oberved in 17 249 cases $(6.8 \%)$ and hypoenhacement in $5 / 249$ cases $(2 \%)$. In the portal phase washout was observed in $111 / 249$ cases $(44.6 \%)$; in $121 / 249(48.6 \%)$ patients the nodules were isoenhancing and in $17 / 249(6.8 \%)$ the arterial hyperenhancing pattern was maintained. In the late phase washout was observed in 197/249 $(79.1 \%)$ cases. The nodules $\leq 3 \mathrm{~cm}$ were diagnostic conclusive on CEUS in $63.7 \%(72 / 113)$, while nodules $>3 \mathrm{~cm}$ had a conclusive result in $86 \%$ of cases $(117 / 136), \mathrm{p}<.0001$. CEUS examination was conclusive for $\mathrm{HCC}$ in $76.3 \%$ of the cases (190/249), while using the ACR CEUS LI-RADSv 2016 algorithm in $72.2 \%$ of all HCCs $(180 / 249), \mathrm{p}=0.35$

Conclusion: In our study, CEUS arterial hyperenhancement is the most common pattern observed in $\mathrm{HCC}(91.2 \%$ of cases $)$, followed by washout in the late phase ( $79.1 \%$ of cases). The size of the nodule modifies CEUS sensitivity for the diagnosis of $\mathrm{HCC} \mathrm{p}<.0001$

Disclosure of Interest: I. Sporea: I hereby confirm that I have received financial support (congress travel grant or speaker fee) from Philips, Siemens, General Electric, Abbvie, Zentiva, Bristol Meyers Squibb

R. Sirli: I hereby confirm that I have received financial support (congress travel grant or speaker fee) from Philips, Abbvie, Zentiva

A. Popescu: I hereby confirm that I have received financial support (congress travel grants, speaker fee) from: Philips, General Electric, Abbvie, AstraZeneca, Zentiva

All other authors have declared no conflicts of interest.

P1372 DIAGNOSTIC ACCURACY OF CONTRAST-ENHANCED ULTRASOUND ALGORITHM (ACR CEUS LI-RADSV 2016) FOR THE DIAGNOSIS OF HEPATOCELLULAR CARCINOMA IN PATIENTS WITH CHRONIC LIVER DISEASE

A. Stepan ${ }^{1}$, M. Danila ${ }^{1}$, R. Sirli ${ }^{1}$, S.A. Popescu ${ }^{1}$, T.V. Moga ${ }^{1}$, C. S. Ivascu ${ }^{2}$, C. Pienar ${ }^{3}$, I. Sporea $^{1}$

${ }^{1}$ Gastroenterology, University of Medicine and Pharmacy „Victor Babes'

Timisoara, Romania, Timisoara/Romania

${ }^{2}$ Gastroenterology And Hepatology, County Hospital Timisoara, Timisoara Romania

${ }^{3}$ Gastroenterology, Pediatrics, University of Medicine and Pharmacy "Victor

Babes" Timisoara, Timisoara/Romania

Contact E-mail Address: stepan_ana@yahoo.com

Introduction: Contrast-Enhanced Ultrasound (CEUS) is an imaging method that can be used to discriminate between hepatocellular carcinoma (HCC) and other focal liver lesions.

Aims \& Methods: This study aimed to test the latest approved version of a contrast-enhanced ultrasound algorithm (ACR CEUS LI-RADSv 2016) for detecting hepatocellular carcinoma $(\mathrm{HCC})$, in a real-life cohort of high-risk patients. We re-evaluated the CEUS studies of 298 focal liver lesions in patients at high-risk for HCC (liver cirrhosis of any etiology, chronic hepatitis B or C, with severe fibrosis, current or prior HCC) using the ACR CEUS LI-RADSv 2016 algorithm. CEUS LI-RADS categories used for the diagnosis of HCC were: CEUS LR-5 (definitely HCC), CEUS LR-5 V (HCC with macrovascular invasion), CEUS LR-Tr (treated HCC). Contrast-enhanced CT, contrast-enhanced MRI or histology were used as reference methods to evaluate the CEUS LIRADS classification of the 298 lesions.
Results: According to reference method, the 298 lesions were classified as follows: 211 HCCs, 60 non-HCC-non-malignant lesions (fatty infiltration, hemangiomas, simple cysts, regenerative nodules) and 27 non-HCC malignant lesions (liver metastases, cholangiocarcinoma, indeterminate). The diagnostic accuracy of ACR CEUS LI-RADSv 2016 for the diagnosis of hepatocellular carcinoma was $74.4 \%$. Sensitivity, specificity, positive predictive value (PPV) and negative predictive value (NPV) were $65.4 \%, 96.5 \%, 97.8 \%$ and $53.5 \%$, respectively. When we used CEUS alone, a conclusive diagnosis of HCC was obtained in $69.6 \%$ of the cases (147/211), while using the algorithm in $65.4 \%$ of all HCCs $(138 / 211), \mathrm{p}=0.35$

Conclusion: In our study $65.4 \%$ of all HCCs $(138 / 211)$ were correctly diagnosed using ACR CEUS LI-RADSv 2016 algorithm, showing good sensitivity, excellent specificity and PPV for the diagnosis of HCC.

Disclosure of Interest: R. Sirli: I hereby confirm that I have received financial support (congress travel grant or speaker fee) from Philips, Abbvie, Zentiva

S.A. Popescu: I hereby confirm that I have received financial support (congress travel grants, speaker fee) from: Philips, General Electric, Abbvie, AstraZeneca, Zentiva

I. Sporea: I hereby confirm that I have received financial support (congress travel grant or speaker fee) from Philips, Siemens, General Electric, Abbvie, Zentiva, Bristol Meyers Squibb

All other authors have declared no conflicts of interest.

\section{P1373 DICKKOPF-1: AS A SERUM BIOMARKER FOR PREDICTION} OF HEPATOCELLULAR CARCINOMA TREATMENT RESPONSE

\section{A. L. Sharaf ${ }^{1}$, E. G. El-Badrawy ${ }^{1}$, N. A. Khalifa ${ }^{2}$}

${ }^{1}$ Tropical Medicine Department, Zagazig University, Zagazig/Egypt

${ }^{2}$ Clinical Pathology Department, Zagazig University, Zagazig/Egypt

Contact E-mail Address: drahmed_lotfy@hotmail.com

Introduction: Hepatocellular carcinoma ( $\mathrm{HCC}$ ) is the 5th most common cancer worldwide and the 3rd leading cause of cancer-related mortality. In Egypt, HCC is the 2nd most common cancer in men and the 6th most common cancer in women. Egypt has the highest prevalence of $\mathrm{HCV}$ worldwide and has rising rates of HCC. HCC is a disease with fast infiltrating growth and poor prognosis. This bad prognosis is due to the lack of an effective method for early diagnosis. So, it is necessary to find a specific \& sensitive marker for early diagnosis of HCC and for monitoring of treatment response.

Aims \& Methods: The aim of this work is to assess prognostic value of serum DKK1 in predicting treatment response, complication and survival in $\mathrm{HCC}$ patients. This study included 60 Patients divided into two groups. Group A: consisted of 30 patients with liver cirrhosis. Group B: consisted of 30 patients with HCC. Group B patients underwent either radiofrequency ablation or ethanol injection. Clinical assessment, routine laboratory evaluation, CT studies and measurement of serum alpha-fetoprotein (AFP) and DKK1 were performed to all patients and repeated to group $\mathrm{B}$ patients 1 and 3 months after treatment. Results: DKK1 significantly can be used for HCC diagnosis even in $\mathrm{HCC}$ with inconclusive AFP. The optimum cut off value of DKK1 for diagnosis of $\mathrm{HCC}$ was $4.3 \mathrm{ng} / \mathrm{mL}$ (AUC 0.89 , sensitivity $66.7 \%$ and specificity $96.6 \%)(\mathrm{P}<0.001$ ). Serum DKK 1 level significantly decreases after HCC treatment with either radiofrequency ablation or ethanol injection $(\mathrm{P}<0.001)$.

Conclusion: DKK1 has a promising prognostic value and can be used for followup of HCC patients before and after treatment.

Disclosure of Interest: All authors have declared no conflicts of interest.

\section{P1374 EFFECT OF FIBROBLAST GROWTH FACTOR-2 AND ITS RECEPTOR GENE POLYMORPHISMS ON SURVIVAL IN PATIENTS WITH HEPATITIS B VIRUS-ASSOCIATED HEPATOCELLULAR CARCINOMA}

J. Cheong

Gastroenterology, Ajou University Hospital, Suwon/Korea, Republic of

Contact E-mail Address: jaeyoun620@gmail.com

Introduction: Fibroblast growth factor (FGF), vascular endothelial growth factor, and hepatocyte growth factor play a critical role in the pathogenesis of hepatocellular carcinoma (HCC).

Aims \& Methods: We determined the association of single nucleotide polymorphisms (SNPs) in growth factor signaling-related genes with the development and progression of tumors and overall survival in patients with hepatitis B virus (HBV)-associated HCC. We assessed nine SNPs in the FGF1, FGF2, FGF receptor $(F G F R)-2, F l t-1$, and $c$-MET genes in 245 HCC patients and 483 chronic HBV carriers without HCC.

Results: None of the SNPs was associated with the risk of HCC development in chronic HBV carriers. The FGF2 rs308379 A allele was significantly associated with small tumor size, early tumor stage, and less vascular invasion. The Flt-1 rs4771249 C allele was associated with low alpha-fetoprotein levels. KaplanMeier analysis showed that the patients with the FGF2 rs308447 TT genotype had lower survival rates than the patients with the $\mathrm{CC}$ or CT genotype $(P=0.016)$ and that the FGF2 rs308379 A allele carriers had shorter survival rates than those of patients with the TT genotype $(P=0.020)$. The FGFR2 rs1219648 CC genotype was significantly associated with increased survival rates $(P=0.047)$. Multivariate Cox proportional analysis revealed that the FGF2 rs308379 A allele (hazard ratio $=1.663, P=0.004$ ) and advanced tumor stage (hazard ratio $=3.430, P<0.001$ ) were independent prognostic factors for overall survival rates in patients with HCC. 
Conclusion: These observations suggest that the SNPs of the FGF2 and FGFR2 genes can be potential prognostic indicators in patients with $\mathrm{HBV}$-associated HCC.

Disclosure of Interest: All authors have declared no conflicts of interest

\section{References}

1. H.B. El-Serag, Hepatocellular carcinoma, N. Engl. J. Med. 365 (2011) 1118 1127

2. V.T. Nguyen, M.G. Law, G.J. Dore, Hepatitis B-related hepatocellular carcinoma: epidemiological characteristics and disease burden, J. Viral Hepat. 16 (2009) 453-463.

3. D.S. Sandhu, E. Baichoo, L.R. Roberts, Fibroblast growth factor signaling in liver carcinogenesis, Hepatology 59 (2014) 1166-1173.

4. B.R. Zetter, Angiogenesis and tumor metastasis, Annu. Rev. Med. 49 (1998) 407-424.

5. R. Yamaguchi, H. Yano, A. Iemura, et al., Expression of vascular endothelial growth factor in human hepatocellular carcinoma, Hepatology 28 (1998) $68-77$.

\section{P1375 EXTRAHEPATIC HEPATOCELLULAR CARCINOMA METASTASIS: IMPORTANCE OF AN EARLY DIAGNOSIS AND TARGETED THERAPY}

R. Morais ${ }^{1}$, H. Cardoso ${ }^{1}, \mathrm{~S}$. Rodrigues ${ }^{1}$, R. Coelho ${ }^{1}$, R. Liberal ${ }^{1}$, P. Pereira ${ }^{1}$, A. Albuquerque ${ }^{1}$, R. Gonçalves ${ }^{1}, \mathrm{~S}$. Lopes ${ }^{1}$, A.M. Horta, E Vale ${ }^{1}$, G. Macedo ${ }^{2}$ ${ }^{1}$ Gastroenterology, Centro Hospitalar São João, Porto/Portugal

${ }^{2}$ Centro Hospitalar São João, Porto Medical School, Porto/Portugal

Contact E-mail Address: ruimorais20@gmail.com

Introduction: Extrahepatic HCC metastasis are associated with a poor prognosis. Nevertheless there are some effective therapies available.

Aims \& Methods: The aim of this study was to assess the main sites of extrahepatic metastasis in hepatocellular carcinoma (HCC) patients and to evaluate the clinical evolution and treatment. This was a retrospective single-center study in which patients with HCC confirmed extrahepatic metastasis between January 2010 and December 2016 were evaluated.

Results: We evaluated 51 consecutive patients, $80 \%$ male, with a mean age of $64 \pm 11$ years at the time of metastasis. In $41 \%$ of the patients the metastasis were present at the time of HCC diagnosis. In patients with subsequent metastasis, the median time until its development was 9 months (IQR 5-16). The diagnosis of metastasis was incidental in $51 \%$ of the patients. Computed tomography (CT) was the main diagnostic method $(86 \%)$ and in $18 \%$ of the cases histological confirmation was obtained. Nineteen patients underwent thoracic CT and five performed bone scintigraphy prior to metastasis. A total of 70 metastatic sites were identified, the more frequent were lung $(33 \%)$ and bone $(14 \%)$. The MELD score at the time of metastasis was higher than the MELD score at the HCC diagnosis $(\mathrm{p}=0.009)$. Metastasis detection implied changes in $\mathrm{HCC}$ therapy in all patients, $41 \%$ started sorafenib and $55 \%$ were referred for supportive therapy. Seven patients performed metastasis targeted treatment, namely 3 patients underwent radiotherapy. The median overall survival (OS) after metastasis was 4.0 months $(95 \% \mathrm{CI} 2.1-5.8$ months) and the mortality rate was $81 \%$ at 12 months. Patients who underwent metastasis targeted treatment presented a longer OS than those who did not (median 18.5 vs 3.1 months; $p=0.002$ ). In multivariate analysis, MELD score at the time of metastasis $(p=0.004)$ and metastasis treatment $(\mathrm{p}=0.005)$ were independently associated with OS estimation.

Conclusion: A systematic HCC staging, with thoracic CT and bone scintigraphy, may provide an earlier metastasis detection and enable a targeted treatment with a consequent improvement in survival in this difficult-to-treat population. Disclosure of Interest: All authors have declared no conflicts of interest.

\section{Reference}

1. European Association for the Study of the Liver, European Organization for Research and Treatment of Cancer EASL-EORTC Clinical Practice Guidelines: management of hepatocellular carcinoma. $J$ Hepatol. 2012;56(4):908-943.

\section{P1376 MANAGEMENT OF INTERMEDIATE STAGE HEPATOCELLULAR CARCINOMA}

\section{A. Deguchi}

Division Of Gastroenterology, Kagawa Rosai Hospital, Marugame-shi, Kagawa/ Japan

Contact E-mail Address: akihiro41@me.com

Introduction: According to the Barcelona Clinic Liver Cancer (BCLC) staging system, intermediate stage contains very heterogeneous hepatocellular carcinoma (HCC) patients. Recently, subclassification of intermediate stage on the basis of Milano criteria and up to 7 criteria is proposed. In this study, the effectiveness of drug-eluting bead-transarterial chemo-embolization (DEB-TACE) in intermediate stage was investigated.

Aims \& Methods: 120 patients $(\mathrm{M}: \mathrm{F}=90: 30$; median age $=76$; Child A: $\mathrm{B}$ $\mathrm{C}=72: 44: 4$; BCLC stage A: $\mathrm{B}: \mathrm{C}: \mathrm{D}=6: 85: 23: 6)$ with unresectable $\mathrm{HCC}$ who received DEB TACE in our hospital were studied. The objective radiological response was classified according to the modified Response Evaluation Criteria in Solid Tumors (mRECIST) v.1.1 by using dynamic CT at one or two months after therapy. Adverse events were evaluated using NCI CTCAE v. 4.03.
According to Bolondi's subclassification, the patients of BCLC B stage were divided into four groups (B1: 24, B2: 31, B3: 19, B4: 10). The response rate and tumor factor associated response in these patients group were examined. Results: The overall response rate and disease control rate in intermediate stage were $36 \%$ and $89 \%$, respectively. Considering the subclassification, the response rate in B1 group $(61 \%)$ was significantly higher than that of B2 + B3 group $(29 \%)$. Although B2 + B3 group was constituted by the patients who did not satisfy the up to 7 criteria, only in the patients with less than 7 tumors, the response rate $(60 \%)$ was similar to that of $\mathrm{B} 1$ group. Tumor factors associated response and found to be significant on univariate analysis were simple gross classification (simple nodular type) and number of tumor. Tumor diameter was not associated with the response.

Conclusion: For the treatment of intermediate stage of HCC, although DEBTACE is considered to be most effective in $\mathrm{B} 1$ group, it is suggested that DEB-TACE is also effective in the patients with less than 7 tumors in $\mathrm{B} 2+\mathrm{B} 3$ group. In cases with more than 7 tumors, as the response rate is considered to be extremely low, sorafenib and arterial infusion therapy are recommended in $\mathrm{B} 2+\mathrm{B} 3$ group.

Disclosure of Interest: All authors have declared no conflicts of interest.

\section{References}

Bruix, J., et al. (2011). "Management of hepatocellular carcinoma: an update." Hepatology 53(3): 1020-1022.

Bolondi, L., et al. (2012). "Heterogeneity of patients with intermediate (BCLC B) Hepatocellular Carcinoma: proposal for a subclassification to facilitate treatment decisions." Semin Liver Dis 32(4): 348-359.

Kudo, M., et al. (2015). "Subclassification of BCLC B Stage Hepatocellular Carcinoma and Treatment Strategies: Proposal of Modified Bolondi's Subclassification (Kinki Criteria)." Dig Dis 33(6): 751-758.

\section{P1377 HEPATOCELLULAR CARCINOMA RECURRENCE RATE IN HCV INFECTED PATIENTS TREATED WITH DIRECT ANTIVIRAL AGENTS. A SINGLE-CENTER EXPERIENCE}

G.G. Di Costanzo, R. Granata, M. Guarracino, M. Sanduzzi Zamparelli, M. Guarino, G. Cordone, G. D'Adamo, R. Tortora

Hepatology Unit, Cardarelli Hospital, Naples/Italy

Contact E-mail Address: raffaellatortora@live.com

Introduction: In the last few years many $\mathrm{HCV}$ patients with previous diagnosis of hepatocellular carcinoma (HCC) have been treated with direct antiviral agents (DAAs) for HCV infection. However there are conflicting data on HCC recurrence rate after DAAs therapy.

Aims \& Methods: Aim of this study was to prospectively evaluate the rate of HCC recurrence following sustained virological response (SVR) by DAAs. From April 2015 to September 2016 we consecutively enrolled HCV infected patients previously treated for HCC at Liver Unit of Cardarelli Hospital. All patients had a free-disease survival from $\mathrm{HCC}$ of at least 6 months before starting antiviral therapy. The efficacy of HCC therapy was evaluated according to mRecist criteria at CT or MRI. Radiological evaluation was carried out within 30 days from the start of therapy. All patients underwent DAAs therapy, selected on an individual basis according to the recommendation issued by the Italian association of the study of the liver.

Results: A total of 71 patients were enrolled. Among them, 42 patients had available data on SVR status and were considered for the analysis. There were 21 males $(58.3 \%)$ and 15 females. The median age of the patients was 73 years (range:52-85). The median follow-up was 12 months after the beginning of treatment (range: 6-18 months). Genotype distribution was as follows: 36 patients infected with genotype $1(85.7 \%), 5$ with genotype 2 and 1 patients with genotype 3 . SVR was achieved in $38 / 42$ patients $(90.5 \%)$. HCC recurrence was observed in $11 / 38$ patients with SVR $(28.9 \%)$. The median time for recurrence was 9 months from the start of therapy with a range of 1-13 months; with 2 patients who showed recurrence during therapy. Among the patients who did not achieve SVR, $1 / 4$ showed HCC recurrence after 10 months from end of treatment. Conclusion: Treatment with DAAs are highly effective with a SVR of about $90 \%$ even in patients with advanced liver disease. Nonetheless, in patients with previous history of HCC, the eradication of HCV did not reduce the risk of short and medium-term recurrence.

Disclosure of Interest: All authors have declared no conflicts of interest.

\section{P1378 PATTERN OF DISTANT EXTRAHEPATIC METASTASES IN PRIMARY LIVER CANCER: A SEER-BASED STUDY}

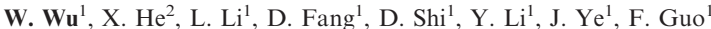

${ }^{1}$ State Key Laboratory for Diagnosis and Treatment of Infectious Diseases, The First Affiliated Hospital, School of Medicine, Zhejiang University, Hangzhou, China, Hangzhou/China

${ }^{2}$ Department of Gastroenterology, Sir Run Run Shaw Hospital, Zhejiang University Medical School, Hangzhou, China, Hangzhou/China

\section{Contact E-mail Address: wwr725@zju.edu.cn}

Introduction: Primary liver cancer is the sixth most common cancer in the world, after cancers of the lung, breast, colorectal, prostate and gastric[1]. However, the extremely poor prognosis for primary liver cancer makes it the second leading cause of cancer-related death globally $(745,000$ deaths, $9.1 \%$ of the total death)[2]. Histologically, the majority of primary liver cancer is either 
hepatocellular carcinoma (HCC) or intrahepatic cholangiocarcinoma (ICC), while combined hepatocellular carcinoma and cholangiocarcinoma is less common[3]. Though these treatments have shown modest improvement in overall survival in early stage disease, the 5-year relative survival for distant metastasis patients is still low $(3.1 \%)$. As we all know, primary liver cancer preferentially metastasize to the portal vein and extrahepatic metastasis include lungs, bones, brain, lymph nodes, and adrenal glands[4-6]. To date, few detailed studies explored extrahepatic metastasis profiles due to rare data of liver cancer metastasis. Patterns of extrahepatic metastasis still need further clarification. Besides, it is unclear whether different metastatic sites would be translated into distinct clinical outcomes.

Aims \& Methods: The objective of this study was to further evaluate extrahepatic metastatic patterns of different histological subtypes and assessed effects of extrahepatic metastasis on survival of advanced disease. Methods: Based on the Surveillance, Epidemiology and End Results (SEER) database, we identified eligible population diagnosed with primary liver cancer. We adopted Chi-squared test to compared metastasis distribution among different histological types. Overall survival (OS) and cancer-specific survival (CSS) were compared between subgroups with different extrahepatic metastasises.

Results: We finally identified 8677 patients who were diagnosed with primary liver cancer from 2010 to 2012 and 1836 patients were in distant metastasis stages. Intrahepatic cholangiocarcinoma was more invasive and had a higher percentage of metastasis compared with hepatocellular carcinoma. Lung was the most common metastatic site and brain was least common site for both hepatocellular carcinoma and intrahepatic cholangiocarcinoma. Extrahepatic metastasis was an independent prognostic factor for liver cancer patients. Patients with brain metastasis had the worst prognosis, compared with other metastasis in OS and CSS analysis.

Conclusion: Different histological subtypes of liver cancer had different metastasis patterns. There were profound differences in risk of mortality among distant extrahepatic metastatic sites. Results from our studies would provide some information for follow-up strategies and future studies.

Disclosure of Interest: All authors have declared no conflicts of interest.

\section{References}

1. Torre LA, Bray F, Siegel RL, Ferlay J, Lortet-Tieulent J and Jemal A Global cancer statistics, 2012. CA Cancer J Clin. 2015; 65(2):87-108.

2. Ferlay J, Soerjomataram I, Dikshit R, Eser S, Mathers C, Rebelo M, Parkin DM, Forman D and Bray F. Cancer incidence and mortality worldwide: sources, methods and major patterns in GLOBOCAN 2012. Int $J$ Cancer. 2015; 136(5):E359-386.

3. Tang D, Nagano H, Nakamura M, Wada H, Marubashi S, Miyamoto A, Takeda Y, Umeshita K, Dono K and Monden M. Clinical and pathological features of Allen's type C classification of resected combined hepatocellular and cholangiocarcinoma: a comparative study with hepatocellular carcinoma and cholangiocellular carcinoma. J Gastrointest Surg. 2006; 10(7):987-998.

4. Olubuyide IO. Pattern of metastasis of primary liver cancer at autopsy: an African series. Trop Gastroenterol. 1991; 12(2):67-72.

5. Lee YT and Geer DA. Primary liver cancer: pattern of metastasis. $J$ Surg Oncol. 1987; 36(1):26-31.

6. Jiang XB, Ke C, Zhang GH, Zhang XH, Sai K, Chen ZP and Mou YG. Brain metastases from hepatocellular carcinoma: clinical features and prognostic factors. BMC Cancer. 2012; 12:49.

\section{P1379 TIME-DEPENDENT EFFECT OF ALFA-FETOPROTEIN AND DISEASE CONTROL ON PATIENTS' SURVIVAL IN BCLC C STAGE HEPATOCELLULAR CARCINOMA}

F.R. Ponziani ${ }^{1}$, I. Spinelli ${ }^{1}$, E. Rinninella ${ }^{1}$, L. Cerrito $^{1}$, A. Saviano ${ }^{1}$,

M. Garcovich ${ }^{1}$, B.E. Annicchiarico ${ }^{1}$, M. Siciliano ${ }^{1}$, L. Riccardi ${ }^{1}$, M.A. Zocco ${ }^{1}$, N. De Matthaeis ${ }^{1}$, M. Biolato ${ }^{1}$, F. Giuliante ${ }^{2}$, R. Iezzi $^{3}$, A.M. De Gaetano ${ }^{3}$, M. Basso ${ }^{4}$, L. Miele ${ }^{1}$, A. Avolio ${ }^{5}$, F. Vecchio ${ }^{6}$, G.L. Rapaccini ${ }^{1}$, A. Grieco ${ }^{1}$, A. Gasbarrini ${ }^{1}$, M. Pompili ${ }^{1}$

${ }^{1}$ Internal Medicine And Gastroenterology, Agostino Gemelli Hospital Dept. of Gastroenterology, Rome/Italy

${ }^{2}$ Hepatobiliary Surgery, Agostino Gemelli Hospital Dept. of Gastroenterology, Rome/Italy

${ }^{3}$ Radiology, Agostino Gemelli Hospital Dept. of Gastroenterology, Rome/Italy

${ }^{4}$ Oncology, Agostino Gemelli Hospital Dept. of Gastroenterology, Rome/Italy

${ }^{5}$ Liver Transplant Surgery, Agostino Gemelli Hospital Dept. of Gastroenterology, Rome/Italy

${ }^{6}$ Histopathology, Agostino Gemelli Hospital Dept. of Gastroenterology, Rome/ Italy

\section{Contact E-mail Address: francesca.ponziani@yahoo.it}

Introduction: BCLC-C stage hepatocellular carcinoma (HCC) includes a wide spectrum of tumor and patients' characteristics. Refining prognosis according to prognostic factors may allow the selection of those subjects who could benefit of curative tratments.

Aims \& Methods: The aim of this study was to investigate the prognostic factors for survival of BCLC-C stage $\mathrm{HCC}$ patients in a real-life setting. Consecutive caucasian cirrhotic patients with BCLC-C stage HCC were included in the analysis. Pre-treatment (Child-Pugh score, performance status (PS), number and maximum size of lesions, vascular invasion, metastases, the combination of vascular invasion and extrahepatic spread, alfafetoprotein (AFP) levels, NIACE score) and post-treatment (the number of treatments after the progression to BCLC-C stage and disease control (DC) considering stable + partial + complete response as the best treatment outcome) variables were considered as prognostic factors. The analysis was adjusted for sex and age.

Results: 116 patients were included in the analysis. After a median follow-up of 22.9 mo (95\% CI 17.3-38.1), the cumulative median survival of the overall population was $13.4 \mathrm{mo}(95 \% \mathrm{CI} 10.6-17)$. At the univariate analysis, tumor size, vascular invasion with or without extrahepatic spread, and AFP $>200$ as pretreatment factors and DC as post-treatment variable were associated with survival. Multivariable cox regression revealed that the only independent predictors of mortality were AFP $>200(\mathrm{HR} 2.2,95 \% \mathrm{CI} 1.27-3.8, \mathrm{p}=0.004)$ and $\mathrm{DC}(\mathrm{HR}$ $0.222,95 \% \mathrm{CI} 0.12-0.4, \mathrm{p}=<0.0001)$. However, the influence of these factors was not homogeneous during time. Indeed, in the early and intermediate period AFP > 200 was an independent predictor of worse outcome (at $6 \mathrm{mo}$ : HR 16.602, $95 \%$ CI $1.97-139.5, \mathrm{p}=0.009$; at 1 yr HR $3.069,95 \%$ CI $1.07-8.76, \mathrm{p}=0.036$ ). As expected, in the multivariable post-treatment prognostic model DC was associated with a better mid- and long-term survival (at $1 \mathrm{yr}$ : HR $0.245,95 \% \mathrm{CI}$ $0.06-0.87, \mathrm{p}=0.030$; at 2 yrs: HR $0.356,95 \% \mathrm{CI} 0.12-1.03 ; \mathrm{p}=0.05$; at 3 yrs: HR $0.094,95 \%$ CI $0.02-0.29, \mathrm{p}=<0.0001)$

Conclusion: In patients with BCLC C HCC, AFP $>200 \mathrm{ng} / \mathrm{mL}$ is a strong prognostic factor in the early and intermediate period, while DC is associated with long-term patients' survival.

Disclosure of Interest: All authors have declared no conflicts of interest.

\section{P1381 INTRAHEPATIC PORTAL HYPERTENSION WITHOUT CIRRHOSIS: EXPERIENCE OF A MORROCAN UNIVERSITY CENTER (33 CASES)}

I. Nakhcha, I. Benelbarhdadi, F.Z. Ajana

Service Medecine C Rabat Maroc, Hopital Ibn Sina Rabat, Rabat/Morocco

Contact E-mail Address: Ibtissamnakhcha2@gmail.com

Introduction: Non-cirrhotic intrahepatic portal hypertension is defined as a portal hypertension (PH) without cirrhosis in liver biopsy and without an obstruction of the portal and the hepatic veins.

Aims \& Methods: This is a retrospective study conducted on the last 19 years in "Medecine C" department at Ibn Sina hospital in Rabat. 33 non-cirrhotic patients with intrahepatic portal hypertension were considered. We analyzed medical data of all patients with hypertension portal without cirrhosis in liver biopsy and without an obstruction of the portal and the hepatic veins.

Results: 33 patients were included in this study, 22 were women. Mean age was 34 years old (18-70). The PH has been revealed by a digestive bleeding in 10 cases $(30 \%)$ and ascitis in 7 cases $(21.2 \%)$. Clinical examination found a splenomegaly in $(75.7 \%)$. Abdominal ultrasonography combined with Doppler showed signs of $\mathrm{PH}$ in all patients and confirmed the absence of obstacle on or above the liver. Upper gastrointestinal endoscopy found esophageal varices in 26 cases $(78.7 \%$ ). Transparietal liver biopsy was performed in 29 cases $(87.9 \%)$.Various etiologies were identified; in $21.2 \%$ ( 7 cases), intrahepatic $\mathrm{PH}$ was due to liver tumors and in $11.7 \%(n=4)$, the etiology was hepatic tuberculosis. Etiological investigations retained: 3 cases $(9 \%)$ of primary biliary cirrhosis in pre cirrhotic stage, 6 cases (18.18) of hepatic portal sclerosis, 4 cases $(12.12 \%)$ of hepatic sarcoidosis, 2 cases $(6 \%)$ with hematological malignancies, 2 cases $(6 \%)$ of polycystic liver, one case $(2.9 \%)$ hepatic angiomatosos liver, a case chronic hepatitis B, a case of Gaucher disease and one case secondary to hamartoma of the liver. Treatment was indicated according the etiology of PH and its severity (beta blockers, ligation). Conclusion: Non-cirrhotic intrahepatic portal hypertension is mostly asymptomatic, related to a heterogeneous group of diseases with a good prognosis excepted solid and hematological malignancies.

Disclosure of Interest: All authors have declared no conflicts of interest.

\section{P1382 CONSERVATIVE MANAGEMENT OF EXTERNAL BILIARY FISTULAS COMPLICATING SURGERY OF HYDATID CYST OF THE LIVER}

O. Baraket, K. Ayed, W. Triki, M. A. Lahmidi, A. Itami, I. Abbassi, S. Bouchoucha

Generla Surgery. Hopital Habibi Bouiguetfa Bizert, Faculty of Medicine of Tunis. University Tunis EIMANAR, Bizerte/Tunisia

Contact E-mail Address: oubaraket@gmail.com

Introduction: Surgical treatment is nowadays the only curative treatment of hydatid cyst of the liver. Surgical approach exposes to many complications especially biliairy fistulas. Many modalities are described for the treatment of these complications.

Aims \& Methods: A retrospective study including 250 patients who underwent a surgical treatment for a hydatid cyst of the liver between 2007 and 2015. The aim of this study is to evaluate the place of conservative management and his benefits in biliairy fistulas.

Results: Conservative surgical treatment oh hydatid cyst was done in 180 cases and radical surgery in 70 cases. A complication occurs in 66 cases. An external biliary fistula occurs in 45 cases $(18 \%)$. Management of all this biliary fistulas was conservative and prolonged drainage of the residual cavity and elevation of the drainage. No major ionic and electrolytic troubles were observed. The fistula healed in 44 of patients with a median time of 4 weeks [range, 1-7]. In one case endoscopic treatment was necessary to heal fistulas. The median hospital stay was 15 days [range 12-60] when biliary fistulas occurred compared to 8 days [6$15]$ when there is no biliary complications. 
Conclusion: External biliary fistulas are a common complication after surgical treatment of hydatid cyst of the liver. Conservative management of this complication is safe and very useful when we respect the indications.

Disclosure of Interest: All authors have declared no conflicts of interest

\section{P1383 THE EVOLUTION OF ESOPHAGEAL VARICES IN NON CIRRHOTIC PORTAL HYPERTENSION CAUSED BY PORTAL VEIN THROMBOSIS}

I. Benelbarhdadi ${ }^{1}$, K. Loubaris ${ }^{2}$, H. Boutallaka ${ }^{3}$, F.Z. Ajana ${ }^{1}$

${ }^{1}$ Medecine C, Ibn Sina University Hospital, Rabat/Morocco

${ }^{2}$ Hepatogastroenterology C (medicine C), Ibn Sina University Hospital, Rabat

Morocco

${ }^{3}$ Gastro-enterology II, Military Hospital Mohamed V, Rabat/Morocco

\section{Contact E-mail Address: ouhabiimane@yahoo.fr}

Introduction: Portal vein thrombosis (PVT) is defined as a partial or complete occlusion of portal vein and/or its tributaries by a thrombus. It exposes to portal hypertension (PHT) by infrahepatic occlusion and consequently to upper digestive hemorrhage, usually due to rupture of gastro esophageal varices.

Aims \& Methods: The aim of this study is to specify the evolution of esophageal varices and thus risk of rebleeding in patients with PHT by PVT unrelated to cirrhosis. It is a retrospective study from January 2010 to February 2017, including 101 patients followed for PHT by PVT without liver disease in the department of hepatogastroenterology (medicine C) at Ibn Sina University hospitaf of Morroco. PVT was diagnosed by abdominal doppler ultrasonography in all patients.

Results: The mean age of patients was $36 \pm 15$ years with extremes ranging from 11 years to 70 years. The sex ratio $\mathrm{M} / \mathrm{F}$ was 0.42 . Five percent of patients had a splenectomy for undocumented reasons before the diagnosis of PHT. Concerning the reasons of hospitalization, $10.9 \%(\mathrm{n}=11)$ were hospitalized for melena, $60.4 \%(n=61)$ for hematemesis and melena and $28.7 \%(n=29)$ for non-specific abdominal pain. Clinical examination was normal in $10.9 \%(n=11)$, showed an ascites in $11.9 \%(\mathrm{n}=12)$, and signs of PHT such as splenomegaly and collateral abdominal venous circulation in $95.1 \%(\mathrm{n}=96)$. Complete blood count showed that $16.8 \%(\mathrm{n}=17)$ had thrombocytopenia, $12.9 \%(\mathrm{n}=13)$ had bicytopenia, and $42.6 \%(n=43)$ had pancytopenia. In all patients, upper GI endoscopy was performed. Hypertensive gastropathy was found in $30.7 \%(\mathrm{n}=31)$, grade I esophageal varices $(\mathrm{OV})$ in $5.9 \%(\mathrm{n}=6)$, grade II in $30.7 \%(\mathrm{n}=31)$, grade III in $48.5 \%$ $(\mathrm{n}=49)$ and gastric varices were noted in $13.9 \%(\mathrm{n}=14)$. These varices were with red spots in $18.8 \%(\mathrm{n}=19)$. All patients had abdominal doppler ultrasonography showing a PVT in $60,3 \%(n=61)$, this was partial in $33.6 \%(n=34)$, complete in $11.9 \%(n=12)$ and extended to the splenic vein in $14.9 \%(n=15)$. Portal cavernoma was found in $39.6 \%(n=40)$. All patients performed an etiologic assessment of thrombosis, myeloproliferative syndrome was found in $8.9 \%(n=9)$, deficiency in inhibitors of coagulation in $31.7 \%(\mathrm{n}=32)$, celiac disease in $4,9 \%$ $(n=5)$, neoplastic leasions in $2.9 \%(n=3)$, no etiology was found in $51.4 \%$ $(\mathrm{n}=52)$. Endoscopic variceal Ligation (EVL) was performed in $70.3 \%$ $(\mathrm{n}=71)$, the mean number of ligation sessions was 3 and eradication of oesophageal varices was noted in $69.3 \%(n=70)$. All patients received anticoagulant therapy except those having portal cavernoma with no obvious cause and $42.6 \%$ of patients $(n=43)$ received beta-blockers for secondary prophylaxis. During follow up, $5.9 \%(n=6)$ of patients have not been seen at consultation and no rebleeding was noted in $89.1 \%(\mathrm{n}=90)$. Concerning portal thrombosis, it dissolved in $49.5 \%(\mathrm{n}=50)$ and stabilized in $10,8 \%(\mathrm{n}=11)$.

Conclusion: The evolution of esophageal varices in non-cirrhotic portal hypertension due to PVT seems to be better than in cirrhotic portal hypertension. Indeed $89.1 \%$ of patients in this study didn't rebleed after eradication of esophageal varices.

Disclosure of Interest: All authors have declared no conflicts of interest.

\section{P1384 EVALUATION OF COMMON BILE DUCT CLEARANCE AFTER ENDOSCOPIC MANAGEMENT OF DIFFICULT BILIARY STONES BY DIRECT PERORAL CHOLANGIOSCOPY: PRELIMINARY RESULTS OF A PILOT STUDY}

A. Anderloni, A. Fugazza, F. Auriemma, R. Maselli, S. Carrara, M. Di Leo, E. Ferrara, P.A. Galtieri, R. Semeraro, G. Lollo, G. Amvrosiadis, A. Repici Digestive Endoscopy Unit, Division Of Gastroenterology, Humanitas Research Hospital, Rozzano, Milan, Italy, Rozzano/Italy

Contact E-mail Address: andrea.anderloni@humanitas.it

Introduction: Incomplete stones clearance after endoscopic management of difficult biliary stones poses the risk of complications such as cholangitis. Confirmation of complete stones clearance is normally confirmed fluoroscopically with obvious risk of false negative/false positive results, leading to possible under/overtreatment. Direct peroral cholangioscopy (POC) refers to the use of nonspecific endoscopes for the direct visualization of the common bile duct (CBD).

Aims \& Methods: We aimed to evaluate the feasibility and safety of POC to confirm CBD complete clearance after endoscopic treatment of difficult biliary stones. From 1st June 2016 to 30 March 2017 all consecutive patients treated with Dilated assisted stone extraction(DASE) for difficult biliary stones at our institution, underwent POC to verify CBD stones complete clearance. Ultraslim (5.9 $\mathrm{mm}$ diameter) or Slim (8.5 mm diameter) endoscopes (FujiFilm EG 530NW or EG 530FP) or standard gastroscope (9.9 mm diameter) (Olympus GIFHQ190), under CO2 insufflation, were used by the peroral route for intubating all accessible bile ducts. Technical success rate, procedural time, outcome and safety of POC were assessed. All adverse events were recorded.

Results: POC was performed in 26 patients $(17 \mathrm{~F} / 9 \mathrm{M}$ mean age 74.6 years \pm 11.9$)$ under propofol sedation (23 patients) or deep sedation ( 3 patients). Mean CBD size was $15 \mathrm{~mm} \pm 3.65$; mean stone diameter $(13.5 \mathrm{~mm} \pm 1.70)$; mean balloon dilation $(13.5 \mathrm{~mm} \pm 2.12)$. Intubation of the papilla and distal biliary duct was successful in $26(100 \%)$ cases (guide-wire assistance in 17 cases, 65.4\%). Hepatic hilum was reached in $13 / 26(50 \%)$ patients with a complete CBD evaluation. Mean investigation time was $6.3 \pm 1.5 \mathrm{~min}$ (range of 5-9 minutes). POC showed persistent large amount of sludge in $2(7.7 \%$ ) cases, completely suctioned through the endoscope, and stones in $4(15.4 \%)$ cases with subsequent endoscopic stones removal. In the remaining patients, POC confirmed complete duct clearance. No adverse events occurred.

Conclusion: CBD complete clearance confirmation using POC is a feasible, quick and safe procedure that can help on clinical decision making (for example obviating the need for possible plastic stent or naso-biliary drainage placement) without substantial increase of time or costs. Our experience, however, is preliminary and was specifically aimed to assess the feasibility of the procedure, which could represent the first step for the development of a possible new indication in the setting of difficult biliary stone management.

Disclosure of Interest: All authors have declared no conflicts of interest.

P1385 LONG-TERM OUTCOMES OF PATIENTS WITH ACUTE CALCULOUS CHOLECYSTITIS AFTER SUCCESSFUL REMOVAL OF GALLBLADDER STONES WITH PERCUTANEOUS TRANSHEPATIC CHOLANGIOSCOPY: A DECADE EXPERIENCE AT A SINGLE TERTIARY CENTER

S. Dong, C.H. Oh, J.R. Moon

Department of Internal Medicine, Kyung Hee University College of Medicine, Seoul/Korea, Republic of

Contact E-mail Address: gidrdong@hanmail.net

Introduction: Percutaneous cholecystostomy (PCT) has been an alternative treatment of acute calculous cholecystitis (ACC) for the patients unsuitable for early cholecystectomy. Lithotomy with percutaneous transhepatic cholangioscopy (PTCS) after PCT track maturation is particularly considered for those patients with gallbladder $(\mathrm{GB})$ stones who are poor surgical candidate. We examined the long-term outcomes of 171 patients with ACC treated by PTCS

Aims \& Methods: This study was a retrospective observational study of 171 consecutive patients who treated with PTCS for ACC in the period from $1 \mathrm{Jan}$ 2005 to 31 Dec 2015. Outcome measures included the success rates, adverse events, recurrence rate and mortality. All data were collected from patients' medical records.

Results: PTCS achieved complete clearance of GB stones in 157 patients $(91.8 \%$ ) The complication rate of of PTCS was $3.5 \%(6 / 171)$. The adverse events included GB perforation $(n=3,1.8 \%)$, hemorrhage $(n=2,1.2 \%)$, disruption of the percutaneous transhepatic biliary drainage fistula $(n=1,0.6 \%)$, and all of which resolved with conservative treatment. The overall recurrence rate of gallstone diseases was $11.5 \%$ during the follow up period. The incidence of recurrent gallstone diseases was significantly higher in those with completely removed GB stones than in those without complete clearance $(10.2 \%, 16 / 157$ vs $21.4 \%$, $3 / 14 ; p<0.05)$. The frequency of recurrence of gallstone disease in patients with contrast passage to the duodenum on cholangiography after PTCS was lower than that in patients without contrast passage.

Conclusion: Gallbladder stone removal with PTCS would be recommanded as an effective and safe treatment modality for the patients with acute cholecystitis who are unsuitable for surgery.

Disclosure of Interest: All authors have declared no conflicts of interest.

\section{P1386 SINGLE DEVICE TECHNIQUE FOR ENDOSCOPIC TREATMENT OF BILE DUCT STONES}

M. Sen-Yo, S. Kaino, S. Amano, S. Shinoda, I. Sakaida Gastroenterology And Hepatology, Yamaguchi University Graduate School of Medicine, Ube/Japan

\section{Contact E-mail Address: mnbs730@gmail.com}

Introduction: Endoscopic retrograde cholangiopancreatography (ERCP) with sphincterotomy is a well-established standard method for treating bile duct stones (BDS). After cannulation of the common bile duct (CBD), a guidewire is placed into the CBD and a sphincterotomy is performed. Subsequently, stone extraction using a balloon or basket catheter is performed. Because of this multistep process, endoscopic treatment for BDS always comes with a risk of losing CBD cannulation. A combination catheter that combines a sphincterotome and a retrieval balloon in a single instrument can eliminate one step in the process of bile duct (BD) clearance and may be able to accomplish BD clearance by using only single device. Additionally, a newly developed 0.025 inch guidewire that is, at once, rigid and flexible enables us to inject contrast agent through the Yconnector smoother. Therefore, the combination catheter with a Y-connector and a 0.025 inch guidewire may become a single device that can facilitate complete BDS clearance. Despite its utility, the success rate of bile duct cannulation and cost benefit requires further analysis.

Aims \& Methods: We aimed to evaluate the therapeutic efficacy of a combined catheter as a single device required for BDS clearance. From January 2016 to July 2016, 11 consecutive patients with BDSs $\leq 10 \mathrm{~mm}$ in size were enrolled in this study (Stonetome group). In all cases, the combined catheter (Stonetome) was 
used for initial bile duct cannulation. Subsequently, sphincterotomy and stone extraction were performed using the combined catheter. The success rate of performing the combined procedure, procedure-related time, adverse events, and the cost of devices were compared with those in 10 patients with BDSs $\leq 10 \mathrm{~mm}$ in size who had undergone endoscopic stone clearance from April 2015 to December 2015 as historical control.

Results: The success rate of selective cannulation and stone clearance did not differ significantly (Stonetome group: $90.9 \%$ and $100 \%$ vs control group: $100 \%$ and $100 \%$, respectively). The median time after bile duct cannulation to complete stone clearance and total procedure time in the Stonetome group were significantly shorter than those in the control group $(401.5 \mathrm{~s}$ versus $982.5 \mathrm{~s}, 645.5 \mathrm{~s}$ versus 1380 s, respectively). In the Stonetome group, delayed bleeding occurred in 1 patient. In the control group, bile duct injury caused by the guidewire occurred in 1 patient. The costs of the used devices did not differ significantly (Stonetome group: \$678 versus control group: \$669).

Conclusion: The combined catheter has the same selective cannulation ability as a conventional catheter and a similar capacity to remove BDSs as common retrieval balloon. Therefore, the combined catheter can reduce the procedure time to remove BDSs.

Disclosure of Interest: All authors have declared no conflicts of interest.

\section{P1387 ACCURACY OF ASGE CRITERIA IN THE IDENTIFICATION OF PATIENTS WITH SUSPECTED CHOLEDOCHOLITHIASIS}

C. Macedo, N. Almeida, D. Gomes, E. Camacho, S. Mendes, L. Tomé, A. M. Ferreira

Gastroenterologia, Centro hospitalar e Universitário de Coimbra, Coimbra/ Portugal

Contact E-mail Address: claudia.macedo.07@hotmail.com

Introduction: The American Society for Gastrointestinal Endoscopy (ASGE) emitted, in 2010, guidelines for the clinical orientation of patients with suspected choledocholithiasis (CL), suggesting the direct refereral to endoscopic retrograde cholangiography (ERC) in certain groups. However, the ERC is an invasive exam and some studies demonstrated that a significant amount of patients classified with very strong risk of CL did not have alterations in ERC.

Aims \& Methods: The aim of this work was to assess the accuracy of the ASGE guidelines in portuguese population. This is a retrospective study that included 212 patients $(52.8 \%$ female sex; $47.2 \%$ male sex; mean age 73.9 ( \pm 14.6 years) admitted to the hospital from 2014 to 2016.

Results: Of the 212 patients, $28(13.2 \%)$ had intermediate risk of CL and 184 $(86.8 \%)$ had high risk, according to the ASGE criteria. These patients were submitted to the following exams/interventions: ERC (154 patients); magnetic resonance cholangiography (50 patients) and endoscopic ultrasound ( 8 patients). In patients initially classified with high risk of CL, this was confirmed in 119 $(64.7 \%)$. The same was seen in $10(35.7 \%)$ of the patients with intermediate risk. The ASGE criteria, when applied to this population, demonstrated an accuracy of $64.3 \%(21.7 \%$ sensitivity; $92.3 \%$ specificity $)$ in the high-risk group, and an accuracy of $35.5 \%$ ( $78.3 \%$ sensitivity; $7.8 \%$ specificity) in the intermediate-risk group. Of the patients with intermediate probability, $12(42.8 \%)$ underwent ERC, and CL was found only in 4 of these patients. The presence of cholangitis, a common bile duct $>6 \mathrm{~mm}$, a common bile duct stone visualized on transabdominal US and a total bilirubin $>4 \mathrm{mg} / \mathrm{dL}$ were strong predictors of CL. The overall ERC complication rate was $13 \%$ (20 patients), of whom 8 had no CL. Conclusion: The ASGE guidelines showed a limited diagnostic accuracy in the identification of patients who actually require ERC, conditioning a significant number of unnecessary procedures with subsequent complications associated with it. It is urgent to rethink the orientation of these patients, with greater use of less invasive diagnostic techniques such as magnetic resonance cholangiography and endoscopic ultrasound

Disclosure of Interest: All authors have declared no conflicts of interest.

\section{Reference}

The role of endoscopy in the evaluation of suspected choledocholithiasis by the American Society for Gastrointestinal Endoscopy, volume 71, No1: 2010 Gastrointestinal Endoscopy

\section{P1388 DOES FIBRIN GLUE APPLIED ON THE CHOLANGIOTOMY IN LAPAROSCOPIC COMMON BILE DUCT EXPLORATION REDUCE THE RISK OF BILE LEAKAGE? A RANDOMIZED STUDY}

B. Darkahi ${ }^{1}$, G. Sandblom ${ }^{2}$, T. Nordén ${ }^{1}$

${ }^{1}$ Surgery Department, Enköping/Sweden

${ }^{2}$ Surgery Department, Uppsala/Sweden

\section{Contact E-mail Address: bahman.darkahi@gmail.com}

Introduction: Laparoscopic choledochotomy as a method of extracting common bile duct stones is a technique with many advantages. One problem, however, is bile leakage along the T-tube. To some extent the leakage may be reduced if the incision is sutured around the T-tube, but this technique has some disadvantages. The aim of this study was to investigate whether application of fibrin glue around the tube results in less leakage than suturing.

Aims \& Methods: Between 2012 and 2016 a total of 1347 cholecystomies were performed in Enköping Hospital. From this group, 42 patients were included in the study and randomized to suturing or fibrin glue for closing the cholangiotomy around the $\mathrm{T}$ tube. Postoperative cholangiography was performed after 7-
10 days after surgery. The amount of flow in the abdominal drain and the level of in the fluid bilirubin was measured daily. In case the flow ceased, the abdominal drain was extracted three days after surgery.

Results: No significant difference between the groups was seen regarding the flow of the abdominal drain or the T-drain for the first three days or operation time Conclusion: Discussion Fibrin glue may be an option to seal cholangiotomy around the T-tube, but studies with greater statistical power are needed to confirm this.

Disclosure of Interest: All authors have declared no conflicts of interest.

\section{P1389 THE IMPACT OF BARIATRIC SURGERY ON ACUTE CHOLANGITIS MORTALITY AND OTHER OUTCOMES: A NATIONWIDE ANALYSIS}

P.T. Kroener ${ }^{1}$, M. S. Abougergi ${ }^{2}$, V. Popov ${ }^{3}$, C. C. Thompson ${ }^{4}$

${ }^{1}$ Mt. Sinai St. Luke's/West, New York/United States of America

${ }^{2}$ Catalyst Medical Consulting, Simpsonville/United States of America/SC

${ }^{3}$ New York University School of Medicine, New York/United States of America

${ }^{4}$ Division Of Gastroenterology, Hepatology, And Endoscopy, Brigham and

Women's Hospital, Boston/United States of America

Contact E-mail Address: thomaskroner@gmail.com

Introduction: Rapid weight loss after bariatric surgery (BS) has been associated with the formation of gallstones, and subsequent acute cholecystitis and cholangitis (AC). However, the complex post-surgical anatomy limits the possibility of performing an ERCP as part of AC treatment. Therefore, the aim of this study was to assess the impact of bariatric surgery on mortality and resource utilization among patients with AC using a national database.

Aims \& Methods: This was a case-control study using the National Inpatient Sample 2013, the largest publically available inpatient database in the United States. All patients with an ICD-9 CM code for a principal diagnosis of AC were included. There were no exclusion criteria. Patients with a past history of BS were identified using the appropriate ICD-9CM codes. The primary outcome was all cause mortality. The secondary outcome was resource utilization: use of ERCP, cholecystectomy, length of hospital stay (LOS), total hospitalization charges and costs. Multivariate regression analyses were used to adjust for the following confounders: Age, sex, race, income in patients' zip code, Charlson Comorbidity Index, hospital region, location, size and teaching status.

Results: A total of 274,775 patients with AC were included in the study, of which $4,240(1.7 \%)$ had undergone BS. The mean patient age was 51 years and $48 \%$ were female. After adjusting for confounders, patients with and without history of bariatric surgery had similar adjusted odds of mortality (adjusted Odds Ratio (aOR): $1.37,95 \% \mathrm{CI}: 0.51-3.65, \mathrm{p}=0.52$ ). As far as resource utilization, patients with bariatric surgery had lower adjusted odds of ERCP (aOR: $0.28,95 \%$ CI: $0.09-0.83, \mathrm{p}=0.02$ ), but higher odds of cholecystectomy (aOR: $3.18,95 \% \mathrm{CI}$ : $1.00-10.05, \mathrm{p}=0.04)$. Both patient groups had similar adjusted length of stay (adjusted mean difference: 1.19 days, 95\% CI: $0.09-0.83, \mathrm{p}=0.16$ ) total hospitalization costs (adjusted mean difference: \$2237, 95\% CI: -\$2308 - \$6782, $\mathrm{p}=0.49$ ), and total hospitalization charges (adjusted mean difference: $\$ 7477$, 95\% CI: $-\$ 9595-\$ 24549, \mathrm{p}=0.39$ )

Conclusion: Bariatric surgery has no impact on inpatient all-cause mortality among patients who develop acute cholangitis, despite its association gallstone acute pancreatitis and limited ERCP performance. In addition, bariatric surgery does not affect resource utilization in this patient population as measured by length of stay and total hospitalization costs and charges.

Disclosure of Interest: All authors have declared no conflicts of interest.

\section{P1391 PATHOLOGICAL, CLINICAL AND RADIOLOGICAL CHARACTERISTICS OF NEOPLASTIC AND NONNEOPLASTIC GALLBLADDER POLYPS}

S. Z. Wennmacker ${ }^{1}$, A. Van Dijk ${ }^{2}$, J. Reassens ${ }^{1}$, N. Hasami ${ }^{1}$, J.P.h. Drenth ${ }^{3}$, C. J.h.m. Van Laarhoven ${ }^{1}$, P. De Reuver ${ }^{1}$, I.D. Nagtegaal ${ }^{4}$

${ }^{1}$ Dept. Of Surgery, Radboud University Medical Centre, Nijmegen/Netherlands

${ }^{2}$ Dept. Of Surgery, Academ. Medisch Centrum Amsterdam, Amsterdam/ Netherlands

${ }^{3}$ Gastroenterology And Hepatology, Radboud University Nijmegen Medical Centre - Gastroenterology and Hepatology, Radboud University Nij, Nijmegen/ Netherlands

${ }^{4}$ Radboud University Nijmegen Medical Center, Radboud University Nijmegen Medical Center Dept. of Pathology, Nijmegen/Netherlands

Contact E-mail Address: sarah.wennmacker@radboudumc.nl Introduction: Prevalence of gallbladder polyps in the Netherlands is 943 per 100,000 cholecystectomies. Histopathologically these gallbladder polyps can be divided in neoplastic polyps (with malignant potential) and nonneoplastic polyps (without malignant potential). Although cholecystectomy is only indicated for neoplastic polyps, $47 \%$ of polyps after cholecystectomy are nonneoplastic.

Further information on the pathological characteristics and subsequent clinical and radiological features could be useful to predict neoplastic or nonneoplastic nature of the gallbladder polyp before surgery.

Aims \& Methods: To assess pathological characteristics of neoplastic and nonneoplastic gallbladder polyps and identify preoperative clinical and radiological predictors for neoplastic and nonneoplastic polyps. Data of the Dutch Pathology Registry was used. In this search 2081 histopathologically proven gallbladder polyps (or (focal) wall thickening $>5 \mathrm{~mm}$ ) were identified in patients of $>18$ years undergoing primary cholecystectomy between 2003 and 2013. Of these 
polyps $56.3 \%$ was neoplastic (adenoma, dysplasia, carcinoma or other malignancies) and $43.7 \%$ nonneoplastic (all other types of polyp). Age and sex of the patient, number of polyps, size of the polyp, coincidence with gallstones and presentation as protruding polyp or wall thickening were extracted from the excerpts. Additional clinical and radiological information was collected from patients' medical records at three hospital in the Netherlands $(n=178)$. The following clinical and radiological predictors were considered: age, gender, ethnicity, BMI, medical history (PSC, Hepatitis, metabolic syndrome, gallbladder disease, Salmonella typhi or Helicobacter pylori infection), family history of gallbladder diseases, presence of abdominal symptoms and radiological features (size, number, shape, surface and echogenicity of the polyp). Associations between possible predictors and gallbladder polyps were assed using univariate and multivariate logistic regression analysis.

Results: Patients with neoplastic polyps were found to be significantly older than patients with nonneoplastic polyps (mean age 65.0 vs 54.2 years, $\mathrm{p}<0.001$ ). Neoplastic polyps were significantly larger (mean size $18.1 \mathrm{~mm}$ (SD 17.9) vs $7.5 \mathrm{~mm}$ (SD 5.9), $\mathrm{p}<0.001$ ), more frequently presented as wall thickening $(29.2 \%$ vs $15.6 \%, \mathrm{p}<0.001)$ or as a single polyp $(88.3 \%$ vs $68.0 \%$, $\mathrm{p}<0.001)$. Gallstones were more frequently found in gallbladders with neoplastic polyps $(50.1 \%$ vs $40.4 \%$, p 0.001$)$. No preoperative clinical features were predictive for neoplastic or nonneoplastic polyps. Presence of a single polyp on ultrasound was a predictor for neoplastic polyps (OR 6.00 (95\% CI 1.32-27.31)). Size and type of polyp were often not mentioned in ultrasound report, or different from histopathological confirmation.

Conclusion: Except for age, no clinical characteristics for neoplastic polyps were identified in this cohort. Although pathological characteristics of neoplastic and nonneoplastic polyps are confirmed, identification of these characteristics on preoperative radiological investigations is poor.

Disclosure of Interest: All authors have declared no conflicts of interest.

\section{P1392 METFORMIN INDUCES APOPTOSIS AND MODULATES PROLIFERATION IN THE BILE DUCT CANCER CELLS}

J. Lee, J.H. Jung, D.H. Koh, S.W. Park, H.J. Jang, M.H. Choi, S. Kae Gastroenterology, Hallym Univerisity Dongtan Sacred Hospital, Gyunggi-Do/ Korea, Republic of

\section{Contact E-mail Address: jinlee@ $@$ hallym.or.kr}

Introduction: Metformin has evidence of antineoplastic activity in some cancer cells.

Aims \& Methods: This study was performed to demonstrate in the bile duct cancer cells whether metformin inhibits the proliferation of cancer cells by inducing apoptosis and affects the expression of gene-related proteins involved in cancer growth, and to identify how metformin affect molecular mechanisms involved in the inhibition of cancer cell growth. Human extrahepatic bile duct cancer cells were cultured. 3-(4,5-Dimethylthiazol-2-yl)-2,5-diphenyltetrazolium bromide (MTT) assays were performed to determine the effect of metformin on cell proliferation. Apoptosis was measured by a cell death detection enzymelinked immunosorbent assay and caspase-3 activity assay. Various protein expressions with or without specific SiRNA transfection were measured by Western blot analysis. The migratory activity of the cancer cells was evaluated by wound healing assay.

Results: 1) Metformin suppressed cell proliferation in bile duct cancer cells by inducing apoptosis. 2) Metformin inhibited mammalian target of rapamycin (mTOR) by activation of pAMPKThr172 - tuberous sclerosis complex 2 (TSC2) pathway, and hyperglycemia impaired metformin-induced AMPKThr172 activation and enhanced phosphorylation of AMPKser485. 3) Metformin blocked inhibitory effect of insulin-like growth factor 1 receptor (IGF-1R), insulin receptor substrate 1 (IRS-1) and AKt pathway on TSC2, and hyperglycemia impaired metformin-induced inhibition of IGF-1R-IRS-1AKt pathway. 4) Metformin modulated invasiveness of bile duct cancer cells, and the effect was impaired by hyperglycemia.

Conclusion: This study shows that metformin has antineoplastic effect in bile duct cancer, and the effect of metformin is attenuated in the hyperglycemic environment. In addition, AMPK and IGF-1R play a key role in the proliferation of bile duct cancer cells, thus they are expected to be important targets for future development of chemotherapy agents.

Disclosure of Interest: All authors have declared no conflicts of interest.

\section{References}

1. Lai SW, Chen PC, Liao KF, Muo CH, Lin CC, Sung FC. Risk of hepatocellular carcinoma in diabetic patients and risk reduction associated with anti-diabetic therapy: a population-based cohort study. Am J Gastroenterol 2012;107:46-52.

2. Chaiteerakij R1, Yang JD, Harmsen WS, Slettedahl SW, Mettler TA, Fredericksen ZS, Kim WR, Gores GJ, Roberts RO, Olson JE, Therneau TM, Roberts LR. Risk factors for intrahepatic cholangiocarcinoma: association between metformin use and reduced cancer risk. HEPATOLOGY 2013;57:648-655

3. Karnevi E, Said K, Andersson R, Rosendahl AH. Metformin-mediated growth inhibition involves suppression of the IGF-I receptor signalling pathway in human pancreatic cancer cells. BMC Cancer 2013;13:235.

\section{P1393 DEREGULATIONS IN BASE EXCISION REPAIR (BER) \\ PATHWAY AND RESULTING OXIDATIVE STRESS AS KEY MODULATOR OF GALL BLADDER ANOMALIES AND PROGRESSION TO CARCINOGENESIS: A NORTHEAST INDIA BASED STUDY}

S. Bose ${ }^{1}$, N. Singh ${ }^{1}$, D. Tiwari ${ }^{1}$, R. Sultana ${ }^{2}$, R. Borkotoky ${ }^{1}$, A. K. Saikia ${ }^{3}$, N. N. Das ${ }^{4}$, S. N. Kazim

${ }^{1}$ Biotechnology, Gauhati University, Guwahati/India

${ }^{2}$ Bioengineering And Technology, Gauhati University, Guwahati/India

${ }^{3}$ Gastroenterology And Medicinal Unit, Central hospital, NF Rly, Guwahati/India

${ }^{4}$ Down Town hospital, Guwahati/India

${ }^{5}$ Jamia Millia Islamia, New Delhi/India

Contact E-mail Address: sujoybose1@gmail.com

Introduction: Molecular pathology of gall bladder anomalies and progression to carcinoma is still obscure and understudied; but is critically relevant to India which harbors the highest number of cases globally, and to ethnically distinct northeast India (NEI) where the cases have been increasing alarmingly.

Aims \& Methods: Evaluate the associative role of genetic alteration(s) and promoter hypermethylation of key BER pathway genes in the predisposition to gall bladder diseases and progression to gall bladder carcinomas (GBC) in the ethnically distinct NEI population. Whole blood and surgically ressected tissue samples were collected from clinically and histopathologically confirmed cases of GBC (adenocarcinomas, $\mathrm{N}=49$ ) along with non-neoplastic control sections, cholelithiasis $(\mathrm{CL}, \mathrm{N}=78$ ) and cholecystitis (CS, N=56); along with blood from voluntary controls $(\mathrm{N}=122)$ with informed consent. The BER pathway genes polymorphisms were screened by PCR-RFLP using DNA samples of the enrolled cases and controls. Differential mRNA expression profile of BER short and long patch pathway genes was studied by RT-PCR. Difference in protein expression was studied by immunofluorescence microscopy. Estimation of oxidative stress in DNA was done using 8-OH-dG EIA kit. The difference in percentage promoter methylation of BER pathway genes was analyzed using RTPCR based HRM method.

Results: The genetic alterations in hOGG1 and XRCC1 gene were highly prevalent in both controls and gall bladder disease cases in NEI population, and was associated with susceptibility and severity of gall bladder anomalies compared to controls; most significantly for XRCC1codon 280 polymorphism in GBC cases compared to controls $[\mathrm{OR}=1.986, \mathrm{p}=0.047]$. Differential mRNA expression profile clearly showed a sharp down-regulation in hOGG1, APE1, pol $\beta$ and PARP1 expression in GBC, CL and CS cases compared to controls; and in GBC compared to $\mathrm{CL}$ and $\mathrm{CS}$ cases. The mRNA expression XRCC1, a component of both short and long patch pathway, was down-regulated in majority of the GBC, CS and CL cases compared to controls; and in GBC cases compared to $\mathrm{CS}$ cases. The protein also showed down-regulation of hOGG1 and XRCC1 in GBC cases compared to controls. The oxidative stress marker 8-oxo-dG estimation showed significantly increased levels in CL, CS and GBC cases DNA compared to controls, the highest being in GBC cases. The higher 8-OH-dG levels in GBC correlated significantly with the variant hOGG1 genotype $(\mathrm{p}<0.001)$. The percent methylation of both XRCC1 and hOGG1 in cases and controls was between $0-30 \%$. Hyper-methylation of XRCC1 and hOGG1 promoter was noticeable in GBC cases compared to controls.

Conclusion: The data indicates an important role of oxidative stress in the pathogenesis of gall bladder diseases and progression to GBC in NEI population; which inturn is due to genetic, expression and epigenetic deregulations in the key genes of the BER short and long patch pathway. The data also suggests the prognostic significance of BER pathway parameters, as well as potential therapeutic targets for the disease, and hence holds clinical relevance.

Disclosure of Interest: S. Bose: No conflict of interest to declare

N. Singh: No conflict of interest to declare

D. Tiwari: No conflict of interest to declare

R. Sultana: No conflict of interest to declare

R. Borkotoky: No conflict of interest to declare

A.K. Saikia: No conflict of interest to declare

N.N. Das: No conflict of interest to declare

S.N. Kazim: No conflict of interest to declare

\section{P1394 THE DEVELOPMENT OF NON-INVASIVE MOLECULAR DIAGNOSIS OF GALLBLADDER CANCER BY BILE JUICE-LIQUID BIOPSY}

H. Kinugasa ${ }^{1}$, K. Nouso ${ }^{2}$, S. Ako ${ }^{2}$, K. Matsumoto ${ }^{2}$, H. Kato $^{2}$, H. Okada ${ }^{2}$ ${ }^{1}$ Internal Medicine, Hiroshima City Hiroshima Citizens Hospital, Hiroshima/ Japan

${ }^{2}$ Dept. Of Gastroenterology, Okayama University, Okayama/Japan

Contact E-mail Address: gyacy14@yahoo.co.jp

Introduction: Gallbladder cancer (GBCa) is often diagnosed at advanced stage resulting poor prognosis, because the patients with early GBCa has not showed any symptoms and the tissue cannot be obtained easily with anatomical reason. To overcome the weakness of the current methods for the diagnosis of GBCa, we focus on the possibility of "liquid biopsy" with bile juice on the concept of noninvasive diagnostic method as circulating tumor DNA in blood. To achieve good treatment effect in future, so called "precision medicine" approach based on the character of the each tumor is mandatory.

Aims \& Methods: Thirty patients with GBCa were enrolled in this study. Bile juice obtained from 24 of 30 patients was analyzed for mutations of 50 oncogenes (Cancer panel; Haloplex, Agilent Technology) by next generation sequencing (NGS; Illumina, San Diego, CA, USA). Tumor tissues from 20 of 30 patients were analyzed as well as bile juice. Each sample was obtained prior to the 
treatment. As negative controls, 19 non-GBCa bile juice and 33 non-GBCa tissue samples were analyzed for mutations of 50 oncogenes in the same way.

Results: The median (range) age was $77(44-90)$ years and the male/female ratio was $0.43(9 / 21)$. Six, six, six, and twelve patients were diagnosed as stage I, II, III, and stage IV, respectively. We set cut-off value at $5 \%$ for rare mutation rate based on the results of healthy samples to avoid false positive. Eleven of $20(55 \%)$ tumor tissue samples were positive for mutation. TP53, MET, SMAD4, CTNNB1 and AR were detected in $7 / 20(35.0 \%), 1 / 20(5.0 \%), 1 / 20(5.0 \%), 1$ $20(5.0 \%)$ and $1 / 20(5.0 \%)$ respectively. In this study, 14 of $30(46.7 \%)$ patients had both tumor tissue samples and bile juice samples. Eight of $14(57.1 \%)$ tumor tissue samples were positive for mutation. In these eight patients, $7(87.5 \%)$ bile juice samples had the same mutation (TP53, ERBB2/3 were detected in $6 / 8$ $(75 \%), 1 / 8(12.5 \%)$, respectively). On the other hand, bile juice samples of other 6 patients without tumor tissue mutation had no mutation. With regard to only bile juice, 14 of $24(58.3 \%)$ bile juice samples with GBCa were positive for mutations. TP53 mutation, ERBB2/3, KRAS were detected in 11/24 (45.8\%), 2 $24(8.3 \%), 1 / 24(4.2 \%)$, respectively. Bile juice analysis for mutations indicated that sensitivity, specificity, positive predictive value (PPV) and negative predict value (NPV) were $58.3 \%, 100 \%, 100 \%$ and $65.5 \%$, respectively. None of negative control samples had any mutations.

Conclusion: Mutations in tumor could be detected in bile juice using NGS. Liquid biopsy with bile juice may help us to diagnose GBCa because of high PPV $(100 \%)$. It may allow us to make new genetic diagnosis of GBCa.

Disclosure of Interest: All authors have declared no conflicts of interest.

\section{P1395 EFFICACY OF INTRADUCTAL RADIOFREQUENCY ABLATION USING A NOVEL ENDOLUMINAL RADIOFREQUENCY ABLATION CATHETER IN A SWINE MODEL}

H. Kim ${ }^{1}$, O. Shin ${ }^{2}$, B. Jeon ${ }^{1}$, H. Choi ${ }^{1}$, S. Kim ${ }^{1}$, S. Kim ${ }^{1}$, H. Chae ${ }^{1}$ Division Of Gastroenterology, Department Of Internal Medicine, The Catholic University of Korea, Uijeongbu St. Mary's Hospital, Uijeongbu/Korea, Republic of ${ }^{2}$ Department Of Hospital Pathology, The Catholic University of Korea, Uijeongbu St. Mary's Hospital, Uijeongbu/Korea, Republic of

Contact E-mail Address: hykkim@catholic.ac.kr

Introduction: Intraductal radiofrequency ablation (RFA) is a new endoscopic ablative technique with direct effect to local tumor have been developed to improve the duration of self-expandable metal stents (SEMS) in the unresectable malignant biliary obstruction. However, there is the concern for the complication such as bile duct perforation or bleeding because the ideal power setting of intraductal RFA has not been clearly revealed still.

Aims \& Methods: Therefore, we aimed to investigate the effects of ablative injury after in vivo intraductal RFA according to the time variation using a novel RFA catheter (ELRA®, STARmed, Goyang, Korea). This novel catheter is a bipolar device and has a temperature sensor within the distal tip, therefore it has a characteristic of target temperature controlled mode. Nine female pigs were divided into three groups according to RFA time variation $(60,90$ and 120 seconds) with the same power setting (10 watts) and RFA target temperature $\left(80^{\circ} \mathrm{C}\right)$. All pigs underwent endoscopic retrograde cholangiography (ERC) and intraductal RFA. Additional cholangiogram was taken immediately after RFA and then a plastic stent was inserted. All the pigs were humanely sacrificed 24 hours after the intraductal RFA. Necropsy was performed and the common bile duct was excised for histologic analysis.

Results: The ERC and application of the intraductal RFA was successful in $100 \%$, and post-RFA cholangiogram did not show the contrast leakage. Median depth of microscopic ablation was significantly different between three groups according to the ablation time ( 60 vs 90 vs 120 seconds $=1.90(1.17-2.23)$ vs. $2.44(2.31-2.60)$ vs. $2.52(2.47-2.64) \mathrm{mm}, \mathrm{p}=0.018)$. There was also a linear relationship between ablation time and microscopic ablation depth $(\mathrm{r} 2=0.552$, $\mathrm{p}=0.002)$. However, there were no statistical differences in macroscopic ablation length and microscopic ablation length. In addition, focal ablation injuries of adjacent liver were found in five out of nine pigs ( 2 of 3 pigs in 60 seconds, 1 of 3 in 90 seconds, 2 of 3 in 120 seconds).

Conclusion: Intraductal RFA using a novel RFA catheter successfully ablated the normal bile duct wall without serious complications in vivo swine model. The optimal time of intraductal RFA was presumed between 90 and 120 seconds at the power setting 10 watts and target temperature of $80^{\circ} \mathrm{C}$. However, focal ablation injury was found in even short ablation time in which microscopic ablation depth was superficial. Further studies are needed to validate the optimal power setting of RFA in a swine model and then human clinical studies are also warranted.

Disclosure of Interest: All authors have declared no conflicts of interest.

\section{References}

1. Zacharoulis D, Lazoura O, Sioka E, et al. Habib EndoHPB: a novel endobiliary radiofrequency ablation device. An experimental study. J Invest Surg 2013;26:6-10.

2. Daglilar ES, Yoon WJ, Mino-Kenudson M, et al. Controlled swine bile duct ablation with a bipolar radiofrequency catheter. Gastrointest Endosc 2013;77:815-819

3. Cho JH, Lee KH, Kim JM, et al. Safety and effectiveness of endobiliary radiofrequency ablation according to the different power and target temperature in a swine model. J Gastroenterol Hepatol 2017;32:521-526.
P1396 PREDICTIVE MODEL FOR THE NEOPLASTIC POTENTIAL OF GALLBLADDER POLYPS

J.K. Lee

Gastroenterology, Samsung Medical Center, Seoul/Korea, Republic of

Contact E-mail Address: jongk.lee@samsung.com

Introduction: While many studies have attempted to define the risk factors for neoplastic potential of gallbladder polyp, precise adaption of the risk factors individually in an real treatment strategy of gallbladder polyp remains elusive. Estimating the probability for neoplastic potential of gallbladder polyp using a combination of several risk factors before surgical resection would be useful in patient consultation.

Aims \& Methods: This study was designed to provide the statistical predictive model for neoplastic potential of gallbladder polyps. We collected data of patients confirmed as GBP through cholecystectomy at Samsung Medical Center between January 1997 and March 2015. Those with a definite evidence for malignancy, such as adjacent organ invasion, metastasis on preoperative imaging studies, polyp larger than $15 \mathrm{~mm}$, and absence of proper preoperative ultrasonography imaging, were excluded. A total of 1976 patients were enrolled. To make and validate the predictive model, we divided the cohort into the modeling group $(n=979)$ and validation group $(n=997)$. Clinical information, ultrasonographic findings, and blood tests were retrospectively analyzed. A prediction model for the probability of neoplasia was fitted from the training set using the logistic regression method equipped with backward elimination with significance level for removal of $P<.05$ for variable selection. The performance of a fitted prediction model was evaluated by the area under the curve (AUC) of a receiver operating characteristic (ROC) curve. A cutoff value of the fitted risk score was chosen by the Youden Index.

Results: Clinical factors of older age, single lesion, sessile shape, and polyp size showed statistical significance of neoplastic potential of GBP in the modeling group. A predictive model* for neoplastic potential of GBP was constructed utilizing the statistical outcome of the modeling group. Statistical validation was performed with the validation group to determine the optimal clinical sensitivity and specificity of the predictive model. Optimal cut-off value for neoplastic probability was $7.4 \%$. *Probability of Neoplastic GB polyp $=\mathrm{e}^{(\mathrm{ps})} / 1+\mathrm{e}^{(\mathrm{ps})}$ where $\mathrm{e}=2.7182$ is the base of the natural logarithm and PS (predictive score) = $-7.3633+0.0374 \times[$ Age $]+0.6667 \times[$ Number $]+1.5784 \times[$ Sessile $]+0.2189 \times$

[Size].

Conclusion: The predictive model for neoplastic potential of GBP may support clinical decision before cholecystectomy.

Disclosure of Interest: All authors have declared no conflicts of interest.

\section{P1397 COMPARISONS OUTCOMES FOR CONTROLLED PHOTODYNAMIC THERAPY IN HIGH GRADE UNRESECTABLE HILAR CHOLANGIOCARCINOMA}

E.T. Park, B.C. Yun, S.U. Lee, B.H. Han

Internal Medicine, Kosin University, Gospel Hospital, Busan/Korea, Republic of

Contact E-mail Address: euntpark@hanmail.net

Introduction: Photodynamic therapy (PDT) provide clinical benefit for patients with unresectable biliary malignancy. In this study, we evaluate the efficacies of controlled PDT for unresectable primary and secondary high-grade hilar cholangiocarcinoma (CC).

Aims \& Methods: In cases of high-grade unresectable hilar CC (Bisthmus type III or IV) and gallbladder (GB) cancer invasion to hilar portion (Group A), we performed controlled PDT. Controlled PDT means malignant stricture dilatation by using balloon catheter up to $10 \mathrm{~mm}$ through percutaneous transhepatic cholangioscopy (PTCS) before PDT. And then applied appropriate laser light source by using straight angled cylindrical diffuser. Finally, gemcitabine-based chemotherapy was done for 6 cycles. We compared to transpaillary approach with non-straight cylindrical diffuser using PDT with gemcitabine-based chemotherapy in similar patients (Group B) in terms of clinical parameters and developing complications.

Results: Between July 2010 and June 2015, 26 cases(31) of high grade unresectable hilar CC (Bisthmus type III or IV), 5 cases of GB cancer with hilar invasion were enrolled for Group A, 23 cases(26) of high-grade unresectable hilar CC (Bisthmus type III or IV), 3 cases of GB cancer with hilar invasion were enrolled for Group B. On three months later, serum bilirubin levels decreased from $7.3 \pm 5.2$ to $2.4 \pm 1.8 \mathrm{mg} / \mathrm{dL}$ in group $\mathrm{A}, 6.9 \pm 5.5$ to $5.2 \pm 3.7 \mathrm{mg} / \mathrm{dL}$ in group $\mathrm{B}(\mathrm{p}<0.05)$. PDT-induced cholangitis is developed in Group A $(3 / 31,9.6 \%)$ and $\mathrm{B}(7 / 26,26.9 \%)$ within seven days $(\mathrm{p}<0.001)$. Progression free survival is superior Group A to Group B that of 14.8 months vs. 8.9 months $(\mathrm{P}<0.05)$.

Conclusion: Controlled PDT with straight applying cylindrical diffuser promise efficacy for clinical parameters and progression free survival compared with transpaillary PDT in unresectable high-grade hilar CC and GB cancer. Additionally, diminished post-PDT cholangitis within 7 days affect improving long term progression free survival.

Disclosure of Interest: All authors have declared no conflicts of interest.

\section{References}

1. Loana Smith, Michel Kahaleh. Biliary tumor ablation with photodynamic therapy and radiofrequency ablation. Gastrointest Endoscopy Clin $\mathrm{N} \mathrm{Am}$. 2015;25:793-80.

2. Jason R., Shiaw H, Nageshwar R., et al. Endoscopic ablation therapy for biliopancreatic malignancies. Clin Endosc. 2015;48:15-19.

3. Tatli S, Tapan U, Morrison PR, Silverman SG. Radiofrequency ablation: technique and clinical applications. Diagn Interv Radiol 2012;18:508-516. 
4. Monga A, Gupta R, Ramchandani M, Rao GV, Santosh D, Reddy DN. Endoscopic radiofrequency ablation of cholangiocarcinoma: new palliative treatment modality (with videos). Gastrointest Endosc 2011;74:935-937.

5. Alis H, Sengoz C, Gonenc M, Kalayci MU, Kocatas A. Endobiliary radiofrequency ablation for malignant biliary obstruction. Hepatobiliary Pancreat Dis Int 2013;12:423-427.

6. Mizandari M, Pai M, Xi F, et al. Percutaneous intraductal radiofrequency ablation is a safe treatment for malignant biliary obstruction: feasibility and early results. Cardiovasc Intervent Radiol 2013;36:814-819.

7. Tal AO, Vermehren J, Friedrich-Rust M, et al. Intraductal endoscopic radiofrequency ablation for the treatment of hilar non-resectable malignant bile duct obstruction. World J Gastrointest Endosc 2014;6:13-19.

\section{P1398 IGG4-ASSOCIATED CHOLANGITIS MIMICKING}

\section{PERIHILAR CHOLANGIOCARCINOMA: A PERSISTENT DILEMMA}

L. M. Hubers ${ }^{1}$, E. Roos ${ }^{2}$, R.J. S. Coelen ${ }^{2}$, J. Verheij ${ }^{3}$, U.H.w. Beuers ${ }^{4}$, T. M. Van Gulik $^{2}$

${ }^{1}$ Gastroenterology \& Hepatology And Tytgat Institute For Liver And Intestinal Research Academic Medical Center, Amsterdam/Netherlands

${ }^{2}$ Surgery, Academic Medical Center, Amsterdam/Netherlands ${ }^{3}$ Pathology, AMC, Amsterdam/Netherlands

${ }^{4}$ Gastroenterology And Hepatology, AMC - Gastroenterology and Hepatology,

AMC; Amsterdam/NL, Amsterdam/Netherlands

Contact E-mail Address: 1.w.hubers@amc.uva.nl

Introduction: IAC is a major manifestation of IgG4-related disease (IgG4-RD), a multi-organ inflammatory disorder of unknown cause. IAC may present with jaundice, abdominal pain and weight loss; immunosuppression is the preferred treatment. Since the disease presentation may closely mimic that of malignancies of the pancreatobiliary tract and accurate diagnostic tests have only recently been developed, misdiagnosis and unnecessary surgery are common as has been consistently reported in literature.

Aims \& Methods: Our aim is to assess the incidence of Immunoglobulin G4 associated cholangitis (IAC) in patients resected for presumed perihilar cholangiocarcinoma (PHC). All patients that underwent resection for presumed $\mathrm{PHC}$ at our institution between 1984 and 2015 were included. Benign histological specimens were re-evaluated by a pathologist and scored according to the international pathological consensus criteria for IgG4-RD. Patients with benign disease who were still alive were re-evaluated to assess IgG4 serum levels and IgG4/IgG RNA ratio to detect activity of IAC.

Results: Between 1984 and 2015, 321 patients underwent liver and bile duct resection for presumed PHC. Of all patients 15\% (47/321) were found to have benign disease on histological examination. $45 \%$ (21/47) of patients with benign disease had evidence of IAC after surgery based on histological criteria $(n=17)$ or laboratory parameters $(n=4)$. The remaining specimens showed unclassified sclerosing inflammation. Out of 12 patients with benign disease that were reevaluated, 9 had ongoing active IgG4-RD requiring immunosuppressive treatment.

Conclusion: Benign biliary disorders mimicking PHC have led to a considerable number of liver and bile duct resections during the last three decades. There was evidence of IAC in $45 \%$ of these patients. When left untreated, IgG4-RD can remain active years after surgery. Novel accurate diagnostic tests for IAC might reduce misdiagnosis and unnecessary surgery.

Disclosure of Interest: All authors have declared no conflicts of interest.

P1399 DIGITAL, SINGLE-OPERATOR CHOLANGIOPANCREATOSCOPY IN THE DIAGNOSIS AND MANAGEMENT OF PANCREATOBILIARY DISORDERS: RESULTS FROM THE MULTICENTER CZECH AND SLOVAK NATIONAL DATABASE

R. Hustak ${ }^{1}$, J. Král ${ }^{2}$, F. Neumann ${ }^{3}$, V. Nosek ${ }^{4}$, E. Skanderova ${ }^{5}$, J. Usak ${ }^{1}$,

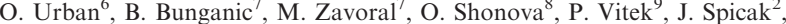
J. Martinek

${ }^{1}$ Internal Clinic, Universitary Hospital Trnava, Trnava/Slovak Republic

${ }^{2}$ Dept. Of Hepatogastroenterology, Institute of clinical and experimental medicine. Prague/Czech Republic

${ }^{3}$ Endoscopy Center, Kolin Hospital, Kolin/Czech Republic

${ }^{4}$ Dept. Of Gastroenterology, Hospital of Jablonec nad Nisou, Lucany nad Nisou/ Czech Republic

${ }^{5}$ Gastroenterology, Hospital Vitkovice, Ostrava/Czech Republic

${ }^{6}$ Vitkovicka Nemocnice Dept. of Gastroenterolgy, Vitkovice/Czech Republic

${ }^{7}$ Department Of Internal Medicine, Military University Hospital, 1st Faculty of

Medicine of Charles University, Prague/Czech Republic

${ }^{8}$ Gastroenterology, Hospital Ceské Budějovice, Ceske Budejovice/Czech Republic

${ }^{9}$ Beskydy Gastrocentre, Internal Department, Faculty Of Medicine, University Of

Ostrava, Hospital Frydek Mistek Internat Dept., Frydek Mistek/Czech Republic

Contact E-mail Address: rhustak@gmail.com

Introduction: Digital cholangioscopy provides higher-resolution imaging of the pancreatobiliary tract compared to the 1st fiberoptic generation. The impact of a new single-operator digital cholangioscope (d-SOC) in diagnosis and treatment of pancreatobiliary diseases has not been intensely assessed.
Aims \& Methods: The aim of this retrospective analysis of prospective case series from 8 centers from the Czech $(n=7)$ and Slovak $(n=1)$ Republics was to assess (1) diagnostic yield of d-SOC visual diagnosis and biopsies in patients with undetermined biliary strictures; (2) the efficacy of d-SOC directed treatment of difficult lithiasis and (3) to analyze procedure related adverse events (AEs). The main outcomes were (1) sensitivity and specificity for visual diagnosis with/without biopsies (2) achievement of a complete duct clearance in patients with difficult lithiasis (3) procedure-related AEs.

Results: A total of 150 patients underwent $166 \mathrm{~d}$-SOC procedures ( 165 cholangioscopies and 1 pancreatoscopy); $81(48.8 \%)$ for diagnostic intents (with biopsy in $66 / 81$ patients $(81.5 \%)$, and $85(51.2 \%)$ for therapeutic intents ( 1 patient had pancreaticolithiasis). The most frequent indication for diagnostic d-SOC was undetermined stenosis $(\mathrm{n}=59)$. Reliable views of a target lesion were obtained in all patients. The sensitivity, specificity and diagnostic accuracy of d-SOC for visual diagnosis of malignant lesion was $88.9 \%(95 \% \mathrm{CI}, 70.8-97.7), 81.2 \%$ (65.6-92.3) and $84.6 \%(73.5-92.4)$. The mean number of biopsies obtained per patient was 4 (range 1-13) and the specimen was adequate for histopathological analysis in $95.5 \%$ of patients $(63 / 66)$. The sensitivity, specificity and diagnostic accuracy of d-SOC guided biopsies for malignancy were $78.3 \%$ (95\% CI, 56.3 $92.5), 96.4 \%(81.6-99.9)$ and $83.3 \%$ (70.7-92.1). The diagnostic accuracy of visual inspection was not statistically influenced by the presence of biliary stent (Yates' p-0.9). Of 63 patients attended for d-SOC-guided stone therapy, a complete intraductal clearance was achieved in one session in 37 and in two sessions in further 10 patients, respectively and the overall success rate was $77 \%$. A total of 11 patients $(11 / 166 ; 6.6 \%)$ experienced an adverse event (cholangitis $n=6$, pancreatitis $n=3$, perforation $n=2$ ), one patient with severe cholangitis died. The incidence of cholangitis was higher among patients who had received prophylactic antibiotics $(n=5)$ compared to those who had not received it $(n=1)$. Conclusion: Our, so far the largest case series analyzing outcomes and safety of a new generation of d-SOC shows that (1) It provides high diagnostic yield in patients with undetermined biliary stenosis; (2) The SOC directed biopsies have a high diagnostic accuracy; (3) d-SOC guided stone lithotripsy is effective in three quarters of patients and (4) Severe adverse events may occur and prophylactic antibiotics may not be effective in preventing post-d-SOC cholangitis.

Disclosure of Interest: All authors have declared no conflicts of interest.

P1400 DISTINCT PROGNOSTIC VALUE AND BIOLOGICAL CHARACTERISTICS OF OSTEOPONTIN IN INTRAHEPATIC CHOLANGIOCARCINOMA

K. Zhou ${ }^{1}$, F. Chen ${ }^{1}$, C. Zhou ${ }^{1}$, L. Cheng ${ }^{2}$, Z. Wang ${ }^{1}$

${ }^{1}$ Liver Cancer Institute, Fudan University, Shanghai/China

${ }^{2}$ Gastroenterology, Zhongshan Hospital Fudan University, Shanghai/China

Contact E-mail Address: 14211210043@fudan.edu.cn

Introduction: Intrahepatic cholangiocarcinoma (ICC) is famous for its poor prognosis. Deepen the knowledge of the mechanism of ICC contributes to improving the treatment. Osteopontin (OPN) is believed to promote tumorigenesis and metastasis in many kinds of cancers, while its role in ICC is controversial.

Aims \& Methods: This study aims to investigate the prognostic value and biological function of OPN in ICC. Human Transcriptome Array 2.0 (HTA 2.0) were used to find out ICC-specific genes. Quantitative PCR was used to investigate the relationship between SPP1 and overall survival (OS) in 85 patients. Immunohistochemistry was used for detecting the prognostic value of OPN, as well as the correlation between OPN and clinicopathological characteristics. Stably transfected ICC cell lines which express either higher or lower level of OPN were conducted by lentivirus. Cell-IQ, the live cell imaging and analysis system, was used for proliferation assay. Wound healing assay and Transwell assay was taken to investigate the ability of migration and invasion among transfected cell lines. PCR array was taken to screen the potential oncogenes regulated by OPN

Results: SPP1 is the most highly expressed genes in ICC with fold change of 43.35 according to HTA 2.0. ICC cell lines express higher level of SPP1 and OPN than normal live cell line $(\mathrm{P}<0.01)$. SPP1 is higher in tumor than adjacent tissues. Patients with higher expression of SPP1/OPN in ICC has better OS than those with low expression $(\mathrm{P}<0.01)$. Both SPP1 and OPN are independent prognostic factor. Higher expression of OPN correlates with better differentiation of ICC and less lymph node metastasis. Compared with negative controlled (NC) cells, ICC cell lines expressing more OPN showed accelerated proliferation rate, stronger ability of migration and invasion. Likewise, ICC cells lines expressing less OPN than NC cells proliferated slower, their capability of migration and invasion was inhibited as well. MMP1, MMP10 and CXCR4 were down regulated in OPN high expression cells and up regulated in OPN low expression cells.

Conclusion: High expression of OPN in ICC tumor tissues indicates less aggressive behavior of ICC and better overall survival for patients after resection. OPN is an advantage prognostic factor in ICC. OPN might function as an inhibitory factor of MMP1, MMP10 and CXCR4 to weaken the capability of migration and invasion in ICC cell lines.

Disclosure of Interest: All authors have declared no conflicts of interest.

\section{References}

1. Ye Q H, Qin L X, Forgues M, et al. Predicting hepatitis B virus-positive metastatic hepatocellular carcinomas using gene expression profiling and supervised machine learning[J]. Nat Med, 2003, 9(4): 416-423. 
2. Terashi T, Aishima S, Taguchi K, et al. Decreased expression of osteopontin is related to tumor aggressiveness and clinical outcome of intrahepatic cholangiocarcinoma[J]. Liver International, 2004, 24(1): 38-45.

3. Sulpice L, Rayar M, Desille M, et al. Molecular Profiling of Stroma Identifies Osteopontin as an Independent Predictor of Poor Prognosis in Intrahepatic Cholangiocarcinoma[J]. Hepatology, 2013, 58(6): 1992-2000.

\section{P1401 PANCREATIC STENT PLACEMENT AFTER ENDOSCOPIC RESECTION OF AMPULLARY TUMORS IS MANDATORY \\ P. Gambitta ${ }^{1}$, F. Iannuzzi ${ }^{1}$, S. Pallotta ${ }^{1}$, A. Ballerini ${ }^{1}$, A. D'Alessandro ${ }^{2}$, E. Bareggi ${ }^{1}$, P. Fontana ${ }^{3}$ \\ ${ }^{1}$ Digestive Endoscopy, ASST Fatebenefratelli-Sacco, Milan/Italy \\ ${ }^{2}$ Clinical Medicine And Surgery, University of Naples Federico II Clinical and \\ Experimental Medicine, Naples/Italy \\ ${ }^{3}$ Endoscopy Unit, Niguarda-Ca' Granda Hospital, Milan/Italy}

Contact E-mail Address: gambitta.pietro@ASST-fatebenefratelli-sacco.it Introduction: Adenoma of the major duodenal papilla is clinically important because this lesion is premalignant and should be resected completely. Endoscopic papillectomy of ampullary adenomas is a promising alternative to surgical resection, however acute and delayed pancratitis represent a major complication of this procedure.

Aims \& Methods: We evaluated the clinical importance of pancreatic duct drainage after endoscopic papillectomy in order to prevent early (acute pancreatitis) and late (pancreatic duct orifice stenosis) complications of this procedure. Our single-centre, retrospective study with a minimal follow-up of 1 year, includes 19 patients who underwent endoscopic ampullectomy between 2012 and 2016. Careful preoperative evaluation was performed by EUS (100\% of patients) and CT/MRI $(94 \%)$. After a collective evaluation between the surgeon and the endoscopist, patients were candidate for endoscopic ampullectomy. Outcome parameters included ampulloma characteristics, bioptical accuracy as well as safety, efficacy, recurrence rate, and survival.

Results: Endoscopic resection was successful in 15 patients $(79 \%)$. Histological review of the resected specimens revealed non specific changes $(10.5 \%)$, low or medium-grade dysplasia $(52.6 \%)$, high-grade dysplasia $(15.8 \%)$ and carcinoma $(21 \%)$. Bioptic accuracy was $68.4 \%$. In 4 cases histologic specimen revealed an invasive carcinoma: 2 patients underwent pancreaticoduodenectomy and two were treated conservatively with placement of biliary and pancreatic stents, due to the high preoperative risk. After complete endoscopic resection (15 patients), pancreatic stents were placed in 10 cases $(66 \%)$. In five cases the positioning of pancreatic stents was not possible due to anatomical difficulties: 2 developed mild pancreatitis after papillectomy; 1 patient developed, as late complication, a stenosis of the pancreatic orifice: the patient died of severe necrotic pancreatitis two years later. Hemorrage was observed in 2 patients, all with pancreatic stents. Recurrence occurred in 2 patients $(10.5 \%)$, both were retreated by snare papillectomy and diathermal ablation (APC).

Conclusion: Pancreatic stent placement after endoscopic ampullectomy is mandatory to prevent acute and delayed pancreatic complications. Preoperative strategy should be accurate by MRI, EUS and ERCP, in order to define the anatomy of the pancreatic duct aiming to improve the success rate of pancreatic stent placement after papillectomy.

Disclosure of Interest: All authors have declared no conflicts of interest.

\section{Reference}

Espinel $\mathbf{J}$ et al. Endoscopic ampullectomy: a technical review. Rev Esp Enferm Dig. 2016 May;108(5):271-8.

\section{P1402 SERUM LEVELS OF HEAVY METALS IN CHOLANGIOCARCINOMA PATIENTS FROM THE NILE DELTA REGION OF EGYPT: A SINGLE-CENTRE STUDY}

M. Abdel-Wahab ${ }^{1}$, M. El-Bendary ${ }^{2}$, M. M. Abdel-Wahab ${ }^{1}$, S. Abed ${ }^{2}$, A. S. Shehatta ${ }^{3}$, M. Salah ${ }^{2}$, A. El-Gilany ${ }^{4}$

${ }^{1}$ Mansoura Surgical Gastroenterology Centre, Mansoura Faculty of Medicine, Mansoura/Egypt

${ }^{2}$ Tropical Medicine And Hepatology, Mansoura Faculty of Medicine- Mansoura University, Mansoura/Egypt

${ }^{3}$ Biochemistry, Mansoura Faculty of Science, Mansoura/Egypt

${ }^{4}$ Public Health, Mansoura Faculty of Medicine, Mansoura/Egypt

Contact E-mail Address: Mahmoud_m_w@hotmail.com

Introduction: Cholangiocarcinoma is a neoplasm arising from the intra- or extrahepatic bile duct linning epithelium. Cholangiocarcinom are presents less than $2 \%$ of human malignancies, however, it is the second common primary hepatic malignancy. Till date, many carcinogens have already been identified and the relevant information with regard to these agents is available. One example is the potentially harmful presence of heavy metals that can cause serious health problems. People may be exposed to heavy metals over the course of their lifetime. The heavy metals in drinking-water pose the greatest threat to public health in this regard. Nile River water is seriously contaminated with heavy metals, pesticides, and hydrocarbons as a result of increasing discharge of untreated industrial wastes and agricultural irrigation wastewater. Several reports on Dakahlia Province show high levels of heavy metal and organocholorine pesticides in the soil and water in this region. High concentrations of heavy metals, including cadmium, are among the pollutants in the water. Plants and fish grown in this water are also contaminated with heavy metals, which can in turn accumulate in humans and animals that feed on these contaminated foods. The serum cadmium levels of residents of Dakahlia Province are almost 10-fold higher than those of residents from cadmium-polluted areas in Cairo and 32 times higher than reference levels for healthy populations in the United States.

Aims \& Methods: We aimed to assess serum levels of Heavy metals namely Zink $(\mathrm{Zn})$, lead $(\mathrm{Pb})$, Cobalt $(\mathrm{Co})$, Cadmium $(\mathrm{Cd})$, Chromium $(\mathrm{Cr})$ and Iron $(\mathrm{Fe})$ as a markers of exposure in cholagiocarcinoma patients and healthy control subjects from the same region in Egypt and its correlation with differentiation of cholangiocarcinoma and tumour marker CA 19-9. This study included 45 patients with cholangiocarcinoma (diagnosed after radiological \&histopathological examination) and 20 healthy control subject attending Mansoura Surgical Gastroenterology centre. All patients and control were permanent residents of North Delta region and the patients were recruited before receiving chemotherapy or radiotherapy. There were no restrictions based on age, sex, or tumor stage. The serum samples were analyzed for concentrations of zinc, lead, cobalt, cadmium, iron and chromium by the acid digestion method followed by using atomic absorption spectrometry. "Buck Scientific Accusys 214 atomic absorption spectrophotometer". The results are reported in $\mathrm{mg} / \mathrm{L}$. in addition to CA 19-9 was assayed by IMMULITE® 2000 XPi immunoassay system supplied by Siemens Healthcare (GmbH Henkestr.Erlangen Germany) using its commercial kits.

Results: The serum levels of $\mathrm{Zn}, \mathrm{Pb}, \mathrm{Co}, \mathrm{Cd}$ and $\mathrm{Fe}$ were significantly higher in patients having cholangiocarcinoma more than control subjects $(\mathrm{P}<0.001)$. Progressive increase in the median values of serum levels of lead $(\mathrm{Pb})$ was found in well differentiated to moderately differentiated to undifferentiated tumours. ( $\mathrm{P}<0.05)$. When correlation was made between the heavy metals and CA-19-9 and the survival of the patients, it was found that Cd only has a positive correlation with CA19-9 and negative correlation with the survival of the patients $(\mathrm{P}<0.5,(\mathrm{P}<0.01)$ respectively.

\begin{tabular}{llll}
\hline & Cholangiocarcinoma & control & Significance test \\
\hline $\begin{array}{l}\text { Sex (N\&\%): } \\
\quad \text { Male Female }\end{array}$ & $22(48.9 \%) 23(51.1 \%)$ & $22(48.9 \%) 23(51.1 \%)$ & $\mathrm{x}^{2}=0.44, \mathrm{P}=0.5$ \\
Age (mean \pm SD & $54.2 \pm 7.9$ & $52.5 \pm 8.99$ & $\mathrm{t}=0.8, \mathrm{P}=0.4$ \\
& Median (Min-Max) & Median (Min-Max) & \\
$\mathrm{Zn}$ & $0.341(0.02-0.92)$ & $0.106(0.002-0.55)$ & $\mathrm{Z}=3.05, \mathrm{P}=0.002$ \\
$\mathrm{~Pb}$ & $1.16(0.067-2.98)$ & $0.353(0.019-0.932)$ & $\mathrm{Z}=4.79, \mathrm{P}<0.001$ \\
$\mathrm{Co}$ & $4.304(0.224-14.1)$ & $0.3255(0.03-2.834)$ & $\mathrm{Z}=5.88, \mathrm{P}<0.001$ \\
$\mathrm{Cd}$ & $0.211(0.0-0.951)$ & $0.0091(0.002-0.11)$ & $\mathrm{Z}=5.53, \mathrm{P}<0.001$ \\
$\mathrm{Fe}$ & $1.991(0.662-5.774)$ & $0.9565(0.0231-2.652)$ & $\mathrm{Z}=3.89, \mathrm{P}<0.001$ \\
$\mathrm{Cr}$ & $0.7063(0.0-2.03)$ & $0.452(0.0663-1.0619)$ & $\mathrm{Z}=1.91, \mathrm{P}=0.057$ \\
\hline
\end{tabular}

Conclusion: The results from this study suggest that cholangiocarcinoma in the Nile Delta region is significantly associated with high serum levels of heavy metals especially Cadmium and lead.

Disclosure of Interest: All authors have declared no conflicts of interest.

\section{References}

AM Kriegel, AS Soliman, Q Zhang, N El-Ghawalby... - Environmental health perspectives, 2006 Dekov VM, Komy Z, Araujo F, Van Put A, Van Grieken R. 1997. Chemical composition of sediments, suspended matter, river water and ground water of the Nile (Aswan-Sohag traverse). Sci Total Environ 201:195-210. Soliman AS, El-Ghawalby N, Ezzat F, Bondy ML, Soultan A, Abdel-Wahab M, et al. 2002. Unusually high rate of youngonset pancreatic cancer in the East Nile Delta region of Egypt. Int J Gastrointes Can 32:143-151.

\section{P1403 SELF-EXPANDLE METAL STENT ARE SUPERIOR TO PLASTIC STENT FOR PREOPERATIVE BILIARY DRAINAGE IN RESECTABLE MALIGNANT DISTAL BILIARY STRICTURE: A SYSTEMATIC REVIEW AND META-ANALYSIS}

A. Tringali ${ }^{1}$, M. Cintolo ${ }^{1}$, L. Cristoferi ${ }^{1}$, E. Forti ${ }^{1}$, F. Pugliese ${ }^{1}$, L. Dioscoridi $^{1}$, R. Manta $^{2}$, M. Mutignani ${ }^{1}$

${ }^{1}$ Endoscopy Unit, Niguarda Hospital, Milano/Italy

${ }^{2}$ Interventional Endoscopy, Ospedale s. Agostino Estense Uo di Endoscopia

Digestiva, Modena/Italy

Contact E-mail Address: albtri10@gmail.com

Introduction: Early surgery is the standard treatment in patients with resectable periampullary or pancreatic head cancer with jaundice. However, early surgery is not always feasible and PBD could be a necesary for patient with jaundice at diagnosis or for those undergoing neoadjuvant treatment. Most studies considered plastic stents for PBD, although SEMS are currently considered superior. A recent RCTS showed that fully covered SEMS are associated with better outcomes compared to plastic stent.

Aims \& Methods: Aim compare the rate of endoscopic reintervention (Stent failure of PBD) before surgery and post operative outcome of metal vs plastic. We conducted a bibliographic search using PUBMED, EMBASE including randomized and non randomized trials. OR using the Manthel-Haenszel method was used for dichotomous variables. Weighted mean differences (WMD) were used as the summary statistic for quantitative analysis of continuous variables, Quantitative synthesis was performed using Review Manager version 5.0. 
Primary outcome was the rate of endoscopic reintervention before surgery. Secondary outcomes were rate of overall complications, 30 days surgical postoperative complications, hospital readmission, overall pancreatic fistula, overall biliary anastomotic leak, overal postoperative mortality. Clinical heterogeneity was assessed by $\mathrm{I}^{2}$ value. where a value exceeding $50 \%$ was indicative of heterogeneity. A random effect model was used in case of heterogeneity.

Results: Three RCTs and five non RCTs were selected including 909 patients. Of these, 300 patients $(33 \%)$ were treated with SEMS and $609(67 \%)$ with plastic stents. The rate of endoscopic reinterventions after PBD was significantly lower in the metal stent $(3.4 \%)$ than in the plastic $(14.8 \%)$ group $(p>0.0001)$. The rate of post operative pancreatic fistula was significantly lower in the metal stent group as well $(5.1 \%$ vs $11.8 \%, \mathrm{p}<0.04)(\mathrm{OR} 0.2395 \% \mathrm{CI} 0.06-0.96)$. The overall pancreatic anastomotic leak was significantly lower in the metal stent group (OR $0.4495 \%$ CI $0.20-0.96)(\mathrm{p}=0.04)$. The rate of postoperative surgical complications, hospital readmission, overall biliary anastomotic fistula and postoperative mortality did not differ between the two groups.

Conclusion: Metal stents are more effective than plastic and should be preferred for PBD in patients with resectable periampullary or pancreatic head tumor when early surgery without PBD is not feasible. However, more RCTS are needed before a firm conclusion could be made.

Disclosure of Interest: All authors have declared no conflicts of interest.

\section{P1404 THE EFFICACY AND SAFETY OF PREOPERATIVE BILIARY DRAINAGE IN PATIENTS WITH OBSTRUCTIVE JAUNDICE: A SYSTEMATIC REVIEW AND META-ANALYSIS}

A. Tringali ${ }^{1}$, M. Cintolo ${ }^{1}$, L. Cristoferi ${ }^{1}$, E. Forti ${ }^{2}$, F. Pugliese ${ }^{1}$, L. Dioscoridi ${ }^{1}$, M. Mutignani ${ }^{1}$

${ }^{1}$ Endoscopy Unit, Niguarda Hospital, Milano/Italy

\section{Contact E-mail Address: albtri10@gmail.com}

Introduction: There is considerable controversy as to whether temporary relief of biliary obstruction prior to major definitive surgery (pre-operative biliary drainage) is of any benefit to the patient. A Cochrane meta-analysis revealed a major morbidity with no difference in mortality in the group subjected to PBD. However, the clinical status of patients was heterogeneous between studies. Aims \& Methods: We aimed to investigate the benefits and harms of pre-operative biliary drainage versus no pre-operative biliary drainage (direct surgery) in patients with obstructive jaundice. A computerized medical literature search was performed by using MEDLINE, EMBASE, Cochrane Library, from 1980 to June 2016 aimed at identifying RCTS comparing PBD versus direct surgery. Data from RCTS related to safety and effectiveness of PBD versus no PBD were extracted by two independent reviewers. Risk ratio or mean differences were calculated with 95 per cent confidence intervals. Clinical heterogeneity was assessed by $\mathrm{I}^{2}$ value. where a value exceeding $50 \%$ was indicative of heterogeneity. A random effect model was used in case of heterogeneity. Outcomes were mortality and morbidity.

Results: Nine trials including 734 patients with malignant or benign obstructive jaundice comparing PBD (375 patients) with no PBD (359) were included in this review. There was no significant difference in mortality (risk ratio $0.89,95 \% \mathrm{CI}$ 067 to $118 ; \mathrm{P}=042$ ) between the two groups. Complications were higher in the PBD group (RR 1.41; 95\% CI 1.09-1.81 $\mathrm{p}=0.008$ ). Overall serious morbidity was higher in the PBD group than in the direct surgery group (RR $1.6695 \% \mathrm{CI}$ $1.28-2.16 \mathrm{p}=0.0002)$. There was no significant difference in length of hospital stay between the two groups: mean difference $4.55(95 \% \mathrm{CI}-1.51$ to 10.61$)$ days $(\mathrm{P}=014)$.

Conclusion: There is currently not sufficient evidence to support or refute the routine pre-operative biliary drainage for patients with obstructive jaundice. $\mathrm{PBD}$ in patients undergoing surgery for obstructive jaundice is associated with similar mortality but increased serious morbidity compared with no PBD. Therefore, PBD should not be used routinely. Further RCTs are needed. Disclosure of Interest: All authors have declared no conflicts of interest.

\section{WEDNESDAY, NOVEMBER 01, 2017}

\section{PANCREAS III - HALL 7}

\section{P1405 INCREASED MORTALITY AMONG PATIENTS WITH ACUTE PANCREATITIS FROM THE MEDITERRANEAN REGION COMPARED WITH THE REST OF SPAIN}

G. García-Rayado ${ }^{1}$, F. Bolado ${ }^{2}$, H.J. Canaval-Zuleta ${ }^{3}$, C. Marra-López ${ }^{4}$, A I. Hernando-Alonso ${ }^{5}$, A. Del-Val-Antonana ${ }^{6}$, H. Sternby $^{7}$, R. Rivera Irigoin ${ }^{8}$, F. J. Grau-Garcia ${ }^{9}$, L. Oms ${ }^{10}$, J. Millastre-Bocos ${ }^{11}$, I. Pascual-Moreno ${ }^{12}$, D. Martínez-Ares ${ }^{13}$, J. A. Rodriguez-Oballe ${ }^{14}$, A. Lopez-Serrano ${ }^{15}$, M. RuizRebollo $^{16}$, A. Viejo-Almanzor ${ }^{17}$, P. Zapater ${ }^{18}$, E. De-Madaria ${ }^{18}$

${ }^{1}$ Gastroenterology Department, University Clinic Hospital, Aragón Health Research Institute (IIS Aragón), Zaragoza/Spain

${ }^{2}$ Gastroenterology, Complejo Universitario de Navarra, Pamplona/Spain ${ }^{3}$ Gastroenterology, Hospital son Llater, Palma de Mallorca/Spain ${ }^{4}$ Gastroenterology, Hospital Universitario Araba, Vitoria-Gasteiz/Spain ${ }^{5}$ Gastroenterology, Hospital Gregorio Maranon, Madrid/Spain

${ }^{6}$ Gastroenterology, Hospital Universitari i Politecnic la Fe, Valencia/Spain ${ }^{7}$ Department Of Surgery, Institution of Clinical Sciences, Malmö/Sweden ${ }^{8}$ Gastroenterology, Hospital Costa del Sol, Marbella/Spain

${ }^{9}$ Gastroenterology, Hospital Arnau de Vilanova, Valencia/Spain

${ }^{10}$ Surgery, Consorci Sanitari de Terrassa, Barcelona/Spain

${ }^{11}$ Hospital Clínico Universitario Lozano Blesa, Zaragoza/Spain

${ }^{12}$ Gastroenterology Department, Hospital Clínico Universitario, Valencia/Spain

${ }^{13}$ Complexo Hospitalario Universitario de Vigo, Vigo/Spain
${ }^{14}$ Gastroenterology, Hospital Universitario Virgen Macarena, Seville/Spain

${ }^{15}$ Hospital Universitario Doctor Peset, Valencia/Spain

${ }^{16}$ Hospital Clinico Universitario, Valladolid/Spain

${ }^{17}$ Gastroenterology, Hospital Puerta del Mar, Cadiz/Spain

${ }^{18}$ Unidad De Gastroenterología, Hospital General Universitario, Alicante/Spain

Contact E-mail Address: guillermogarcia7@hotmail.com

Introduction: Dietary fat consumption affects the human body fat composition. It has been described that unsaturated fatty acids, enriched in human pancreatic necrosis collections, worsen acute pancreatitis (AP) [1]. Preliminary reports analyzing indirect data have also suggested that patients with AP from world regions with a high unsaturated fat intake have a worse outcomes at lower body mass index [2]. We have also described that oleic acid (an unsaturated fat acid) chlorohydrins are associated to worse outcomes [3]. The Mediterranean regions have a higher unsaturated fatty acid intake.

Aims \& Methods: We aimed to compare the incidence of necrotizing AP, persistent organ failure (POF) and mortality among patients with AP from centers located in the Mediterranean region (group M) and patients from centers in other regions (group C). This was a retrospective analysis of a prospective multicenter (23), nation-wide cohort of patients with AP: the ATLANTIS database. Group $\mathrm{M}$ included patients from Spanish autonomous regions in contact with the Mediterranean Sea. Group C included all other autonomous regions. Necrotizing AP and POF were defined according to the revised Atlanta classification. $\mathrm{Chi}^{2}$ was used for univariate analysis. Multivariate analysis was performed by means of binary logistic regression, including in the model: sex, body mass index $>30$, alcoholic etiology and Charlson score (it includes age and comorbidity) $\geq 3$.

Results: We analyzed 1655 patients, $854(52 \%)$ from group M (12 centers) and $801(48 \%)$ from group C (11 centers), $984(60 \%)$ with biliary etiology, $891(54 \%)$ males. The incidence of necrotizing AP was $281(17 \%)$ patients, persistent organ failure: $113(7 \%)$, mortality $70(4 \%)$. The proportion of patients with necrotizing AP was higher in group M: $178(21 \%)$ vs $103(13 \%), p<0.001$. POF was also more frequent in group $\mathrm{M}$ : $74(9 \%)$ vs $39(5 \%), \mathrm{p}=0.002$. Finally, mortality was higher in group M: $49(6 \%)$ vs $21(3 \%), p=0.002$. In multivariate analysis, group $\mathrm{M}$ was independently associated to necrotizing AP [adjusted OR (aOR) 1.7 (1.3$2.3), \mathrm{p}<0.001$ ], to POF [aOR $2(1.3-3.1), \mathrm{p}=0.001$ ] and mortality [aOR 2.6 $(1.5-4.5), \mathrm{p}=0.001]$

Conclusion: Patients from the Mediterranean regions of Spain have a higher incidence of necrotizing AP, POF and mortality. We hypothesize that this disbalance is associated to a higher diet unsaturated fat intake.

Disclosure of Interest: All authors have declared no conflicts of interest.

\section{References}

1. Noel P, Patel K, Durgampudi C, Trivedi RN, de Oliveira C, Crowell MD, et al. Peripancreatic fat necrosis worsens acute pancreatitis independent of pancreatic necrosis via unsaturated fatty acids increased in human pancreatic necrosis collections. Gut. 2014.

2. Patel K, Noel P, Trivedi R, de Oliveira C, Singh VP. 255 High Unsaturated Fat Diets and Unsaturated Visceral Fat Worsen Acute Pancreatitis (AP) Outcomes At Lower Body Mass Index (BMI). Gastroenterology.148(4):S-58.

3. Franco-Pons N, Casas J, Fabrias G, Gea-Sorli S, de-Madaria E, Gelpi E, et al. Fat necrosis generates proinflammatory halogenated lipids during acute pancreatitis. Ann Surg. 2013;257(5):943-51.

\section{P1406 1-DEOXY-SPHINGOLIPIDS, NOVEL BIOMARKERS OF DIABETES, ARE CYTOTOXIC FOR EXOCRINE PANCREATIC CELLS}

R. Chen ${ }^{1}$, T. Hornemann ${ }^{2}$, W. $\mathrm{Yu}^{2}$, S. Camargo ${ }^{3}$, R. Graf ${ }^{1}$, S. Sonda

${ }^{1}$ Visceral \& Transplantation Surgery, University Hospital Zurich, Swiss HPB Centre, Zurich/Switzerland

${ }^{2}$ Institute for clinical chemistry, University Hospital Zurich, Zurich/Switzerland

${ }^{3}$ Institute of Physiology, Universityof Zurich, Zurich/Switzerland

Contact E-mail Address: Rong.Chen@usz.ch

Introduction: Exocrine pancreatic insufficiency and exocrine function alterations are characteristics of pancreatitis. They are frequent in diabetes mellitus (DM) patients with a prevalence up to $50 \%$. Although reduced levels of insulin may explain many of the proposed mechanisms of pancreatic dysfunction following $\mathrm{DM}$, the same phenotype is also detected in insulin-independent DM. This highlights the concept that additional factors are likely to contribute to the pathophysiology of acinar cells. We recently discovered that 1-deoxy-sphingolipids (1deoxySLs), the levels of which increase in DM and metabolic syndrome are cytotoxic for beta cells ${ }^{1}$. Our preliminary results showed that 1-deoxySLs are also cytotoxic for acinar cells in vitro. Furthermore, the high level of 1deoxySLs in diabetic animal model aggravated acinar cell damage whereas lowering levels of 1-deoxySLs improved cell deterioration.

Aims \& Methods: In this research, we investigate molecular and cellular factors that contribute to compromise acinar cell functionality in the context of DM. Based on the endocrine and exocrine pancreas crosstalk, we hypothesize that elevated 1-dSeoxyLs levels affect directly the pancreatic exocrine compartment by compromising pancreatic acinar cells in DM, thus increasing its predisposition to develop exocrine pancreatic diseases. in vivo mouse models with STZ-induced diabetes and cerulein-induced pancreatitis were used in this study. Reduction of 1 -deoxySLs synthesis was achieved by oral L-serine supplementation. Disease severity was assessed with biochemical and immunohistochemical methods. Molecular mechanisms of 1-deoxySL-dependent toxicity were evaluated in vitro on AR42J pancreatic acinar cells and primary acinar cells. 
Results: DM induction resulted in increased 1-deoxySL levels but also atrophy and fibrosis of pancreatic parenchyma. Reduction of 1-deoxySL synthesis by oral L-serine supplementation ameliorated the damage of the exocrine pancreatic tissue, without restoring insulin production in beta cells. This suggests that elevated 1-deoxySLs rather than insulin deficiency contribute to the exocrine damage in DM. In vitro studies showed that treatment with 1-deoxysphinganine, one of the early products of 1-deoxySLs synthesis at low concentration reduced replication and promoted cytotoxicity in pancreatic acinar cells. In addition, this 1-deoxySL-mediated cytotoxicity was associated with mitochondrial dysfunction and endoplasmic reticulum stress.

Conclusion: Our work revealed that 1-deoxySLs are cytotoxic for exocrine pancreatic cells, suggesting a role for these lipids in the exocrine dysfunctions following DM. Oral L-serine supplementation could be a therapeutic treatment for ameliorating exocrine pancreatic diseases in diabetic patients.

Disclosure of Interest: All authors have declared no conflicts of interest.

\section{Reference}

1. Zuellig, R.A., et al., Deoxysphingolipids, novel biomarkers for type 2 diabetes, are cytotoxic for insulin-producing cells. Diabetes, 2014. 63(4): p. $1326-39$.

\section{P1407 ROLE OF THROMBOPHILIA IN SPLANCHNIC VENOUS THROMBOSIS IN ACUTE PANCREATITIS}

S.U. Ahmed ${ }^{1}$, S. S. Rana ${ }^{1}$, J. Ahluwalia ${ }^{2}$, M. Kang ${ }^{3}$, R. Sharma ${ }^{1}$, D. K. Bhasin ${ }^{1}$ ${ }^{1}$ Gastroenterology, Post Graduate Institute of Medical Education and Research, Chandigarh, India, Chandigarh/India

${ }^{2}$ Hematology, Post Graduate Institute of Medical Education and Research, Chandigarh/India

${ }^{3}$ Radiology, Post Graduate Institute of Medical Education and Research, Chandigarh, India, Chandigarh/India

Contact E-mail Address: drsobur1180@gmail.com

Introduction: Splanchnic venous thrombosis (SVT) is a common vascular complication of acute pancreatitis (AP). There is paucity of data on its frequency, risk factors, outcome and natural history. Coagulation abnormality has been implicated but not proven as a cause of SVT in AP.

Aims \& Methods: We aimed to prospectively study the frequency, risk factors and extent of SVT in patients with AP as well as role of trombophilia in causation of SVT. Patients with AP presenting to our centre between January 2015 and June 2016 were prospectively evaluated with contrast enhanced computerized tomography (CECT) abdomen for presence of SVT. These patients were subjected to detail analysis of coagulation parameters. Outcome was assessed in terms of presence or absence of necrosis, severity, organ failure, need for intervention and mortality. None of the patients with SVT were given anticoagulation. Follow-up ultrasound Doppler was performed to look for the status of SVT.

Results: Ninety-six patients with AP (73 males, mean age $31.85 \pm 13.34$ years) were evaluated of which $26(27.1 \%)$ had SVT. Single vessel, two vessels and three vessels were involved in $19(73.1 \%), 3(11.5 \%)$ and $4(15.4 \%)$ patients respectively. Splenic vein, portal vein and superior mesenteric vein involvement were seen in $22(84.6 \%), 11(42.3 \%)$ and $4(15.3 \%)$ patients respectively. Necrotizing pancreatitis, CTSI $>6$ and Modified CTSI $>6$ were found in significantly higher number of patients with SVT than those without SVT $(96.2 \%$ vs $78.6 \%$, $76.9 \%$ vs $47.1 \%$, and $92.3 \%$ vs $67.1 \%$, respectively). Coagulation analysis was performed in 42 patients ( 18 with and 24 without SVT). Protein C, protein S and AT III deficiency were found in $10(23.8 \%), 14(33.3 \%)$ and $13(31.0 \%)$ patients respectively. $\beta_{2}$ GPI and lupus anticoagulant were positive in $2(4.8 \%)$ and $5(11.9 \%)$ patients respectively. Anticardiolipin antibody was negative in all the patients. Factor V Leiden mutation analysis was done in 33 patients (14 with SVT and 19 without SVT) of which $2(6.1 \%)$ were positive. There was no correlation between abnormal coagulation results and outcome of AP. Coagulation abnormality did not differ significantly between the patients with and without SVT. On follow-up, Doppler study done in 7 patients with SVT, spontaneous resolution of SVT occurred in $5(71.4 \%)$ within 1 year. None of the patients had varices on follow-up.

Conclusion: SVT in AP is more common in patient with necrotizing pancreatitis and higher CTSI and MCTSI indices suggesting that local inflammation plays a major role in its causation. Thrombophilia in some form is seen in one third of the patients with AP but does not increase the risk of AP.

Disclosure of Interest: All authors have declared no conflicts of interest.

\section{P1408 EVALUATION OF A PROTOCOL OF AN ENDOSCOPIC ULTRASOUND TRANSMURAL DRAINAGE OF INFECTED PANCREATIC NECROSIS (IPN) WITH LOCAL ANTIBIOTHERAPY THROUGH NASOCYSTIC CATHETER}

J. Lariño Noia ${ }^{1}$, J. López-Díaz ${ }^{2}$, D. De La Iglesia-García ${ }^{2}$, J. Iglesias-Garcia ${ }^{2}$, R. Mejuto-Fernández ${ }^{2}$,F. Macias-Garcia ${ }^{1}$, A. Álvarez-Castro ${ }^{2}$, A. MckenzieBallesteros ${ }^{2}$, S. García-Rodríguez ${ }^{2}$, J. Díaz-Gutiérrez ${ }^{2}$, JE. Dominguez-Muñoz ${ }^{2}$ ${ }^{1}$ Gastroenterology, Health Research Institute of Santiago (IDIS), Santiago de Compostela/Spain

${ }^{2}$ Gastroenterology, University Hospital of Santiago, Santiago de Compostela/Spain

Contact E-mail Address: danieldaigrexa@gmail.com

Introduction: Infected pancreatic necrosis (IPN) is associated to significant morbidity and mortality. Current management of IPN is based on a step-up approach, based on minimally invasive procedures. Our group has recently published a protocol of local infusion of antibiotics for the treatment of IPN (Pancreatology 2016;16:719-25).

Aims \& Methods: We aim at analysing the efficacy of this step-up approach for the treatment of IPN in clinical practice. This was a retrospective single-centre study of patients admitted with acute pancreatitis (AP) between January 2015 and December 2016. The cases with the diagnosis of pancreatic necrosis (NP) and IPN (defined by positive culture of necrosis and clinical, analytical, and/or radiological data of infection) were identified and evaluated. IPN was treated following a step-up approach defined by 1 . intravenous antibiotherapy, 2. Endoscopic ultrasound (EUS) guided transgastric drainage plus local antibiotherapy, 3. endoscopic necrosectomy. Number of patients responding to each therapeutic step was assessed.

Results: 694 cases of AP were included (mean age $79.5 \pm 18.3,355$ male). CT scan was performed only if clinically indicated. 67 patients $(9.6 \%)$ had acute necrotizing pancreatitis (ANP), and 21 of them IPN (31\% of ANP). IPN patients were treated with intravenous antibiotics (imipenem $[\mathrm{n}=15]$ and meropenem $[\mathrm{n}=6]$ ), with good response in 8 ( $38 \%$ of IPN). The remaining 13 cases underwent a EUS guided transmural drainage plus local infusion of antibiotics. Only 3 patients needed endoscopic necrosectomy $(14 \%$ of all IPN). Overall mortality was $1.5 \%$ (11 cases), 6 with edematous pancreatitis $(0.96 \%)$ and 5 with ANP (7.4\% of ANP). Only one patient with IPN died ( $4.8 \%$ of IPN).

Conclusion: By using a step-up approach, nearly two out of three patients with IPN require transgastric drainage. By associating local antibiotherapy through nasocystic catheter to the drainage, only $14 \%$ cases with IPN require endoscopic necrosectomy. With this background, comparative studies are needed to evaluate the impact of local antibiotherapy for IPN.

Disclosure of Interest: All authors have declared no conflicts of interest.

\section{Reference}

González-López J, Macías-García F, Lariño-Noia J, Domínguez-Muñoz JE. Pancreatology. 2016 Sep-Oct;16(5):719-25

\section{P1409 EUS-GUIDED PANCREATIC FLUID COLLECTION DRAINAGE WITH LUMEN-APPOSING METAL STENTS OR PLASTIC DOUBLE PIGTAIL STENTS: A MULTI-FACTORIAL ANALYSIS \\ N. Ge, S. Sun}

Dept. Of Endoscopy, Shengjing Hospital, Shenyang/China

Contact E-mail Address: ge nan2013@163.com

Introduction: We aimed to compare the efficiency of plastic and metal stents for symptomatic pancreatic fluid collection drainage and analyze other main associated factors that affect the outcome of drainage therapy.

Aims \& Methods: Rates of technical and clinical success, procedure-related side effects (hemorrhage, stent migration, and cyst rupture), re-interventions, and duration of hospital stay.

Results: There were 52 patients, 40 who underwent plastic stent placement and 12 who underwent lumen-apposing metal stent placement. The total rate of technical success was $100 \%$. The total rate of clinical success was $100 \%$. The total rate of adverse events was $7.7 \%(4 / 52)$. On multiple logistic regression analysis, the use of plastic stents $(\mathrm{P}<0.05$, Exp $\mathrm{B}=12.168)$ and presence of a large cyst $(\mathrm{P}<0.05$, Exp $\mathrm{B}=1.036)$ were shown to significantly increase the risk of reintervention. On multivariate linear regression analysis, etiology of pseudocyst $(\mathrm{P}<0.05, \mathrm{~B}=-8.427 ;-9.785 ;-5.514)$ was associated with prolonged hospital stent, while stent type was not shown be a factor $(\mathrm{P}>0.05)$.

Conclusion: Both plastic and lumen-apposing metal stents are proven to be highly efficient in pancreatic fluid collection drainage. The lumen-apposing metal stent is superior in preventing complications such as migration and cyst leakage and reducing the rate of re-intervention. Large cyst size is associated with an increased risk of re-intervention and prolonged hospital stay.

Disclosure of Interest: All authors have declared no conflicts of interest.

\section{P1410 EARLY ACHIEVABLE SEVERITY (EASY) INDEX FOR SIMPLE AND ACCURATE EXPEDITE RISK STRATIFICATION IN ACUTE PANCREATITIS}

B. Kui ${ }^{1}$, S. Gódi ${ }^{2}$, J. Bajor ${ }^{3}$, I. Török ${ }^{4}$, M. Macaria ${ }^{4}$, H. Farkas ${ }^{4}$, A. Mickevicius ${ }^{5}$, V. Sallinen ${ }^{6}$, E. R. Maldonado ${ }^{7}$, M. Papp ${ }^{8}$, G. Kovács ${ }^{8}$ P. Sarlós ${ }^{3}$, S. Galeev ${ }^{9}$, B. Nemeth ${ }^{1}$, A. Vincze ${ }^{3}$, P. Pencik ${ }^{10}$, I. Ozola-Zalite ${ }^{11}$, A. Pukitis ${ }^{12}$, A. Litvin ${ }^{13}$, I. Kovalskaya ${ }^{14}$, J. Czimmer ${ }^{3}$, T. Huszár ${ }^{15}$, M. Ihász ${ }^{16}$, G. Pár ${ }^{3}$, I. Hritz ${ }^{17}$, T. Molnár ${ }^{2}$, D. Illés ${ }^{1}$, A. Szentesi ${ }^{2}$, L. Lakatos ${ }^{18}$, G. Capruso ${ }^{19}$, A.V. Patai ${ }^{20}$, E. Stilidi' ${ }^{21}$, G. Peserico ${ }^{22}$, K. Zadorozhna ${ }^{15}$, E. Sonmez ${ }^{23}$, A. Tüzün Ince ${ }^{24}$, S. Vishalkumar ${ }^{25}$, M. Horibe ${ }^{26}$, P. Hegyi ${ }^{2}$ ${ }^{1}$ First Department Of Medicine, University of Szeged, Szeged/Hungary ${ }^{2}$ Institute For Translational Medicine, University of Pécs, Pécs/Hungary ${ }^{3}$ First Department Of Medicine, University of Pécs, Pécs/Hungary

${ }^{4}$ Department Of Gastroenterology, Mures County Emergency Hospital, Targu Mures/Romania

${ }^{5}$ Center Of Hepatology, Gastroenterology And Dietetics, Vilnius University Hospital Santariskiu Klinikos, Vilnius/Lithuania

${ }^{6}$ Department Of Abdominal Surgery, Hospital of Helsinki University Central Hospital, Helsinki/Finland

${ }^{7}$ Department Of General Surgery, Consorci Sanitori del Garraf, Sant Pere de Ribes/Spain 
${ }^{8}$ Department Of Gastroenterology, University of Debrecen, Debrecen/Hungary ${ }^{9}$ Department Of Digestive Surgery, Saint-Luke Clinical Hospital, SaintPetersburg/Russian Federation

${ }^{10}$ Centrum Pece O Zazivaci Trakt, Vitkovicka Nemocnice A. S., Ostrava/Czech Republic

${ }^{11}$ Department Of Gastroenterology, Hepatology And Nutrition, Pauls Stradins Clinical University Hospital, Riga/Latvia

${ }^{12}$ University Hospital, Pauls Stradins Clininic University Hopital Gastroenterology Center, Riga/Latvia

${ }^{13}$ Department Of Surgery, Gomel Regional Clinical Hospital, Gomel/Belarus ${ }^{14}$ General Surgery, Bohomolets National Medical University, Kiev/Ukraine ${ }^{15}$ Department Of Emergency, University of Szeged, Szeged/Hungary

${ }^{16}$ Vas Megyei Markusovszky Egyetemi Oktatókórház, Szombathely/Hungary

${ }^{17}$ I. Department Of Surgery, Semmelweis University, Budapest/Hungary

${ }^{18}$ Department Of Internal Medicine, Csolnoky Ferenc Regional Hospital,

Veszprem/Hungary

${ }^{19}$ Digestive And Liver Disease Unit, Medical School, University La Sapienza, S. Andrea Hospital, Rome/Italy

${ }^{20}$ 2nd Department Of Internal Medicine, Semmelweis University 2nd Dept. of Medicine, Budapest/Hungary

${ }^{21}$ Department Of Therapy And General Practice (family Medicine), Department Of Gastroenterology, Hospital of Medical Academy named after SI Georgievsky, Simferopol/Russian Federation

${ }^{22}$ Department Of Surgery, Oncology And Gastroenterology, University of Padova, Padova/Italy

${ }^{23}$ Department Of Emergency Medicine, Hospital of Bezmialem Vakif, University School of Medicine, Istanbul/Turkey

${ }^{24}$ Department Of Internal Medicine, Gastroenterology Clinic, Hospital of Bezmialem Vakif, University School of Medicine, Istanbul/Turkey

${ }^{25}$ Department Of Surgery, Tan Tock Serg Hospital, Singapore/Singapore

${ }^{26}$ Division Of Gastroenterology And Hepatology Department Of Internal Medicine, Keio University School of Medicine, Tokyo/Japan

Contact E-mail Address: k.kubali@gmail.com

Introduction: Acute pancreatitis (AP) is one of the most common diseases of the gastrointestinal tract associated with significant morbidity and mortality. The assessment of severity is crucial in the management of the disease. The pathomechanism of AP is not well understood, it has no specific therapy. Current methods of risk stratification in AP have a limited value, as they provide little additional information thus may delay appropriate management. Early recognition of severe disease may prevent serious adverse events and improve patient management as well as overall clinical outcome. The EASY trial is an observational, multicenter, prospective cohort study for establishing a simple, easy and accurate clinical scoring system for early prognostication of acute pancreatitis

Aims \& Methods: We aimed to create a new scoring system, which can predict the severity of AP in early phase of disease. Evaluation of simple attainable potential prognostic parameters obtained at admission (or not later than 6-12 hours afterwards) from patients diagnosed with AP will be performed to assess their potential correlation with the disease severity. Approximately $1200(900+300)$ patients from multiple centers will be enrolled into this trial using the Registry. This is an observational prospective cohort study (in which the care or services that patients receive will not be altered); therefore it has a relatively low-risk. The study has an ethical approval by the National Hungarian Ethical Authority (ETT TUKEB). Study management will strictly follow the Ethical Guidelines for Observational Studies.

Results: 600 patients were enrolled in the EASY study from different international centers so far. In early phase of AP vomiting, loss of weight, fever, abdominal tenderness, elevated serum creatinine and lactate dehydrogenase level were more common findings in severe AP, as opposed to the mild and moderate groups.

Conclusion: EASY score may be an easy and accurate system to evaluate the early severity of AP. Although some parameters have shown correlation with the severity of AP in early phase of the disease, we have to include more patients to have reliable results.

Disclosure of Interest: All authors have declared no conflicts of interest.

\section{P1411 EVALUATION OF LOCAL INSTILLATION OF ANTIBIOTICS IN WALLED-OFF PANCREATIC NECROSIS}

M. Werge ${ }^{1}$, S. Roug ${ }^{2}$, E. Feldager ${ }^{1}$, J. D. Knudsen ${ }^{3}$, L.L. Gluud ${ }^{1}$, S. Novovic ${ }^{1}$, P. N. Schmidt ${ }^{1}$

${ }^{1}$ Department Of Gastroenterology And Gastrointestinal Surgery, Hvidovre

Hospital, Copenhagen, Hvidovre/Denmark

${ }^{2}$ Digestive Disease Centre, Bispebjerg Hospital, University of Copenhagen, Copenhagen NV/Denmark

${ }^{3}$ Department Of Clinical Microbiology, Hvidovre Hospital, University of Copenhagen, Hvidovre/Denmark

Contact E-mail Address: hkp835@alumni.ku.dk

Introduction: Infected walled-off pancreatic necrosis (WON) is treated with antibiotics. In order to exert its antibacterial effects in WON, systemically administered antibiotics need to overcome a number of limiting steps. First, antibiotics need to cross the capillary endothelium. Thereafter, antibiotics must diffuse across the interstitial space and finally, the capsule surrounding the WON must be crossed. Therefore, the penetration of the intravenously administered antibiotics into WON can be questioned. Using local antibiotics should theoretically increase the antibiotic concentrations in the necrotic tissue and the efficacy.
Aims \& Methods: The aim was to evaluate the efficacy of local instillation of antibiotics into walled-off pancreatic necrosis. Between 2012 and 2016 we evaluated all patients treated with endoscopic transmural drainage and necrosectomy (EDTN) and concomitant local instillation of antibiotics. We added antibiotics (either gentamicin, vancomycin, or amphotericin B) to the irrigation fluid according to the microbiological findings. The antimicrobial efficacies of local and systemic antibiotics were evaluated using uni- and multivariate logistic regression analyses and Kruskal-Wallis test by stratification of the isolates in sensitive versus not sensitive/antibiotics not given.

Results: Ninety-one patients were included. At the first drainage $81(86 \%)$ patients had infected and 10 sterile WON. A total of 139 isolates were found at the first drainage. Most patients were infected with enterococci $(44 \%)$ or other gram-positive cocci. More than a quarter of the infected patients had fungal species cultured. The infected patients often had polymicrobial infections $(56 \%)$. At the second culture 152 isolated were found. Neither local nor systemic antibiotics were associated with the eradication of microbes between first and second culture. Between second and third culture, the use of local antibiotics was associated with the eradication of microbes $(\mathrm{OR}=2.54, \mathrm{P}=0.01)$, but not systemic antibiotics $(\mathrm{P}=0.33)$ (Table). Between first and second culture 12 patients with fungal infections were treated with local amphotericine B. In all 12 patients the fungus was eradicated. After second culture 20 patients were treated local amphotericine $\mathrm{B}$ and in $17(85 \%)$ patients the fungus was eliminated at the third culture.

\begin{tabular}{|c|c|c|c|c|c|c|}
\hline & \multicolumn{2}{|l|}{ Univariate } & \multicolumn{2}{|l|}{ Multivariate } & \multicolumn{2}{|c|}{ Kruskal-Wallis Test } \\
\hline & OR $(95 \% \mathrm{CI})$ & $\mathrm{P}$-value & OR $(95 \% \mathrm{CI})$ & P-value & $\mathrm{Chi}^{2}$ & P-value \\
\hline \multicolumn{7}{|l|}{ Culture $1->2$} \\
\hline Local Antibiotics & $1.52(0.73-3.17)$ & 0.261 & $1.54(0.74-3.21)$ & 0.249 & 1.26 & 0.261 \\
\hline Systemic Antibiotics & $1.28(0.64-2.59)$ & 0.487 & $1.31(0.64-2.65)$ & 0.458 & 0.48 & 0.488 \\
\hline \multicolumn{7}{|l|}{ Culture $2->3$} \\
\hline Local Antibiotics & $2.54(1.25-5.18)$ & 0.010 & $2.71(1.31-5.59)$ & 0.007 & 6.76 & 0.009 \\
\hline Systemic Antibiotics & $0.72(0.38-1.38)$ & 0.327 & $0.64(0.33-1.25)$ & 0.195 & 0.96 & 0.328 \\
\hline
\end{tabular}

Conclusion: Our data suggest a better efficacy of local antibiotics in the treatment of infected WON compared to systemic antibiotics. The local instillation of antibiotics may be a promising alternative or supplement to systemic administration. Particularly the use of local instillation of amphotericin B appears to be effective in the treatment fungal infections.

Disclosure of Interest: All authors have declared no conflicts of interest.

\section{P1412 CORONARY DISEASE AND CHRONIC OBSTRUCTIVE PULMONARY DISEASE ARE NOT ASSOCIATED WITH WORSE OUTCOME IN ACUTE PANCREATITIS}

J.R. Carvalho, P. Santos, S. Fernandes, M. Moura, T. Antunes, J. Velosa Department Of Gastroenterology And Hepatology, North Lisbon Hospital Center, University of Lisbon, Portugal, Lisbon/Portugal

Contact E-mail Address: joana.rita.carvalho@gmail.com

Introduction: Pancreatitis is a disease of protean manifestations. In its more severe form, involvement of any organ is possible. Cardiovascular and respiratory failure are possible and feared complications.

Aims \& Methods: The aim of this study was to evaluate the effect of chronic ischemic heart disease and chronic obstructive pulmonary disease (COPD) in the outcome of acute pancreatitis (AP), in our population. Retrospective cohort study that included all patients admitted with AP from January 2003 to December 2016, in a tertiary referral centre. Demographic and clinical variables were analyzed by logistic regression (SPSS v23). Clinical outcomes included organ failure $(\mathrm{OF})$, persistent $\mathrm{OF}(>48 \mathrm{~h})$, intensive care unit (ICU) admission and mortality.

Results: A total of 553 patients with AP were included, 58.4\% male, median age $80(18-98)$ years. Most common etiologies included gallstones $(38.9 \%)$ and alcohol $(27.3 \%)$. Twenty-three percent $(n=129)$ developed OF (in $43 \%$ persistent) and $26.8 \%(n=148)$ were admitted in UCI. Mortality rate was $5.6 \%(n=31)$. Fifty-six patients $(10.1 \%)$ had previous history of coronary disease and $5.1 \%$ $(n=28)$ had been diagnosed with COPD. The presence of coronary disease and CODP were not associated with higher Ranson's score $(\geq 3), p=0.076$ and $\mathrm{p}=0.959$, respectively. No association was found between previous history of coronary disease and the development of OF $(\mathrm{p}=0.525)$, persistent $\mathrm{OF}$ $(p=0.287)$, need for ICU admission $(p=0.115)$ and mortality $(p=0.262)$. There was also no association found between previous history of CODP and the development of OF $(p=0.803)$, persistent OF $(p=0.588)$, need for ICU admission $(\mathrm{p}=0.514)$ and mortality $(\mathrm{p}=0.720)$. At multivariate analysis (correcting for age and gender) coronary disease and CODP were not independent predictors of worse outcome in AP

Conclusion: In our population, previous history of coronary disease and CODP were not predictors of worse outcome in AP.

Disclosure of Interest: All authors have declared no conflicts of interest. 


\section{References}

1. Yegneswaran B, Kostis JB, Pitchumoni CS. Cardiovascular manifestations of acute pancreatitis. J Critic Care 2011;225:11-8.

2. Forsmark CE, Vege SS, Wilcox M. Acute pancreatitis. $N$ Engl J Med 2016; 375:1972-1981.

3. Raghu MG, Wig JD, Kochhar R, et al. Lung complications in acute pancreatitis. JOP 2007;8:177-185.

\section{P1413 WORSE OUTCOMES IN ACUTE PANCREATITIS IN PATIENTS WITH TYPE-2 DIABETES MELLITUS}

P. Santos, J.R. Carvalho, S. Fernandes, M. Moura, T. Antunes, J. Velosa Gastrenterologia E Hepatologia, Hospital de Santa Maria - Centro Hospitalar Lisboa Norte, Lisbon/Portugal

\section{Contact E-mail Address: patricia.sants $@$ hotmail.com}

Introduction: Predicting severe pancreatitis is important for early aggressive management of patients with acute pancreatitis (AP). Despite the established role of type-2 diabetes mellitus (DM) in the risk of AP, the impact of DM on the clinical outcome in AP has not been fully elucidated.

Aims \& Methods: Retrospective study including hospital admissions between January 2003 and December 2016 in a single tertiary referral center. Clinical outcomes included organ failure (OF), persistente OF $(>48 \mathrm{~h})$ admission to intensive care unit (ICU) and mortality. Variables were analysed by logistic regression (SPSS v23.0). The objective of this study was to assess the risk of mortality and severity in AP among patients with type-2 DM.

Results: A total of 553 patients $(58.4 \%$ male) with AP were included, median age 80 (18-98) years. Most common etiologies included gallstones (38.9\%) and alcohol $(27.3 \%)$. Twenty three percent developed OF (in $43 \%$ persistent) and $5.6 \%$ $(\mathrm{n}=31)$ were deceased. There were 127 AP patients $(23.0 \%)$ with type-2 DM.Type-2 DM were not associated with higher Ransońs score. There was an association between DM and development of OF (OR 31.7, CI95\% 18.8-53.7, $\mathrm{p}<0.001$ ), persistente OF (OR 45.1, CI95\% 18.7-108.9, p < 0.001), ICU admission (OR 12.3, CI95\% 7.8-19.4, p < 0.001) and mortality (OR 17.1, CI95\% 6.842.8.4, $\mathrm{p}<0.001)$. At multivariate analysis DM was an independent predictor of OF development and ICU admission.

Conclusion: In our population, Type-2 DM was associated with severity and increased mortality in patients with AP. Our findings provide evidence of the potential role of DM in the management of severe AP.

Disclosure of Interest: All authors have declared no conflicts of interest.

\section{References}

1. Working Group IAPAPAAPG. IAP/APA evidence-based guidelines for the management of acute pancreatitis. Pancreatology. 2013;13:e1-15.

2. Wu BU, Johannes RS, Sun X, et al. The early prediction of mortality in acute pancreatitis: a large population-based study. Gut. 2008;57:1698-1703.

3. Girman CJ, Kou TD, Cai B, et al. Patients with type 2 diabetes mellitus have higher risk for acute pancreatitis compared with those without diabetes. Diabetes Obes Metab. 2010;12: 766-771.

4. Lai SW, Muo CH, Liao KF, et al. Risk of acute pancreatitis in type 2 diabetes and risk reduction on anti-diabetic drugs: a population-based cohort study in Taiwan. Am J Gastroenterol. 2011;106:1697-1704.

5. Zhao X, Chang Mei H, Chen L, et al. An increased level of haemoglobin AlC predicts a poorer clinical outcome in patients with acute pancreatitis. Clin Endocrinol (Oxf). 2012;77:241-245.

\section{P1414 ACUTE PANCREATITIS IN LIVER TRANSPLANT} RECIPIENTS: INCIDENCE AND OUTCOME

P. Macinga ${ }^{1}$, K. Poc ${ }^{2}$, P. Trunecka ${ }^{3}$, J. Spicak ${ }^{1}$, T. Hucl ${ }^{1}$

${ }^{1}$ Department Of Gastroenterology And Hepatology, Institute For Clinical And Experimental Medicine, Prague/Czech Republic

${ }^{2}$ First Faculty Of Medicine, Charles University, Prague/Czech Republic

${ }^{3}$ Transplantcentre, Institute for Clinical and Experimental Medicine, Prague/Czech Republic

Contact E-mail Address: peter.macinga@ikem.cz

Introduction: Acute pancreatitis (AP) is an uncommon but potentially devastating condition that may occur in patients with organ transplantation. Reported incidence ranges from 1.5 to $8 \%$ in patients undergoing liver transplantation with significant mortality.

Aims \& Methods: The aim of our study was to assess the incidence, potential risk factors and outcome of AP following liver transplantation in our center. We performed a retrospective analysis of medical records of all adult patients who underwent liver transplantation in our center between September 1996 and November 2014. The diagnosis of AP was defined by combination of clinical manifestation, finding on imaging methods (CT, USG) and elevation of serum amylase and lipase.

Results: Nine hundred and sixty-seven orthotopic liver transplantations were performed in 578 males and 389 females (mean age 51 years, range 18-74). AP occurred in 18 patients $(1.9 \%, 16$ males, 2 females) and resulted in death in 5 patients $(28 \%)$. According to timing of AP we recognized two clinical presentation-early AP ( $<1$ month after liver transplantation) and late ( $>1$ month). Four patients $(22 \%)$ developed early AP, which was severe necrotizing with MODS in all cases and resulted in death in 3 of them $(75 \%)$. Two of them were transplanted for fulminant hepatic failure, one for end-stage liver disease due to chronic hepatitis B infection and one for polycystic liver disease. Two patients were treated by surgical necrectomy and died, the third deceased patient was treated conservatively. In the only surviving patient, a successful EUSguided drainage of walled of pancreatic necrosis and repeated endoscopic necrectomy were performed. We were unable to identify surgical or other factors responsible for development of AP. Late AP occurred in 14 patients $(78 \%)$ with a median delay of 31 months after liver transplant (range 2-176). In 12 patients AP was mild with no mortality and the following etiologies were represented: 4 postERCP, 2 alcoholic, 1 biliary (CBD stones), 1 drug-induced (azathioprine), 1 obstructive (pancreatic cancer); in 3 patients the etiology was unknown. One patient developed a pseudocyst. Two patients with late acute pancreatitis had a severe necrotizing form and both died. One patient with cirrhosis of the liver graft due to chronic rejection had post-ERCP pancreatitis which was complicated by retroperitoneal hemorrhage and graft failure. The other patient with necrotizing pancreatitis of unknown etiology developed MODS and eventually died. Male patients $(\mathrm{p}=0.01)$ and patients transplanted for liver cirrhosis resulting from chronic hepatitis B were at a significantly higher risk of AP development $(\mathrm{p}=0.03)$.

Conclusion: The incidence of AP after liver transplant in our center is low. In it necrotizing form which is more frequent early after liver transplant, it carries a significant risk of mortality exceeding $80 \%$. Male patients and those transplanted for end stage liver disease resulting from chronic hepatitis B are more likely to develop post-liver transplantation pancreatitis.

Disclosure of Interest: All authors have declared no conflicts of interest.

\section{P1415 THE IMPACT OF BILIARY SLUDGE TO DEVELOPMENT OF PAIN AND EFFICACY OF HYMECROMONE IN CHRONIC BILIARY PANCREATITIS}

A. Okhlobystin ${ }^{1}$, N. Ponkratova ${ }^{2}$, O. Okhlobystina ${ }^{2}$, S. Nasonova ${ }^{2}$,

M. Tatarkina ${ }^{2}$, S. A. Budzinsky ${ }^{3}$, V. Ivashkin ${ }^{4}$

${ }^{1}$ Pancreatology And Bowel Diseases, Sechenov University, Moscow/Russian Federation

${ }^{2}$ Vasilenko Clinic Of Internal Diseases Propaedeutic, Gastroenterology And Hepatology, First Moscow State Sechenov Medical University, Moscow/Russian Federation

${ }^{3}$ Department Of Faculty Surgery № ${ }^{2}$ Pirogov Russian National Research Medical University (RNRMU), Moscow/Russian Federation

${ }^{4}$ Internal Diseases Propaedeutic, Hepathology And Gastroenterology Hospital,

First Moscow State Sechenov Medical University, Moscow/Russian Federation

Contact E-mail Address: okhlobystin@mail.ru

Introduction: Biliary sludge (BS) may be one of the factors, related to development of chronic pancreatitis $(\mathrm{CP})$ via sphincter of Oddi dysfunction. As it was demonstrated by Okazaki K et al. in 1988, patients with biliary sludge have higher sphincter of Oddi (SO) pressure and contraction frequency vs. controls. Aims \& Methods: To assess the frequency of CP signs in patients with BS and to investigate the state of major duodenal papilla (MDP) in patients with idiopathic $\mathrm{CP}$ with BS by endoscopic ultrasound (EUS); to evaluate whether antispasmodics can be effective in pain relief at $\mathrm{CP}$, that developed on the background of gallbladder sludge. Protocols of computer tomography, endoscopic and transabdominal ultrasound studies of over 6000 patients of gastroenterological tertiary clinic were examined. Those who had signs of BS were selected to evaluate the presence of at least "mild CP" according to the modified Cambridge classification. Exclusion criteria were: established etiology of CP and signs of pancreatic neoplasm. Patients, who received ursodeoxycholic acid and drugs that affect smooth muscle contractility for less then 3 month prior to the study were excluded. Thirty consecutive patients $(15 \mathrm{~m}, 15 \mathrm{f}$, mean age \pm SD: $52.8 \pm 15.3)$, who had both BS and CP were summoned for physical examination, quality of life assessment, US-cholecystography and endoscopic pancreatobiliary ultrasound with elastography, calculation of MPD surface area $\mathrm{S}_{\mathrm{MDP}}=\left(\mathrm{h}_{\mathrm{MDP}} \times \mathrm{w}_{\mathrm{MDP}}\right) /(2 * 1.27)$ and state of MDP before and after 3 weeks of hymecromone monotherapy $400 \mathrm{mg}$ tid.

Results: Signs of CP were revealed in $6.3 \%$ of BS cases. CP was most common in those who had ointment-like bile $(33.3 \%)$ vs. patients with heterogeneous bile with clots $-7.7 \%$ and hypoechoic particles $-1.7 \%$ (chi-square $38.21, \mathrm{p}<0.00001$ ). Mean $\mathrm{S}_{\mathrm{MDP}}$ was $14.9 \pm 5.2 \mathrm{~mm}^{2}(95 \% \mathrm{CI} 10.9-18.9)$. $\mathrm{S}_{\mathrm{MDP}}$ was below the normal range $\left(20-25 \mathrm{~mm}^{2}\right)$ in $78 \%$ of patents. $\mathrm{S}_{\mathrm{MDP}}$ had positive correlation to the volume of evacuated bile according to US-cholecystography $\left(r_{\mathrm{s}}=0.683\right.$, $\mathrm{p}=0.042)$ and gallbladder contractility coefficient $\left(\mathrm{r}_{\mathrm{s}}=0.817, \mathrm{p}=0.007\right)$. All patients with higher density of MDP at US-elastography had $\mathrm{S}_{\mathrm{MDP}}$ lower than the normal range and were attributed to "fibrosis" group. Only $38 \%$ of patients with CP and BS had normal MDP at EUS. Periampullary diverticula were found in $13 \%$ of the cases, papillary edema - in $38 \%$, fibrosis - in $13 \%$. MDP changes were associated with higher AP level and larger MPD diameter. Hymecromone monotherapy resulted in significant improvement in abdominal pain score $(\mathrm{t}=7.92, \mathrm{p}=0.000)$ and "bodily pain" score of SF-36 questionnaire $(\mathrm{t}=-3.709 \mathrm{p}=0.001)$. Dynamics of "bodily pain" score by SF-36 demonstrated significant negative correlation to the post-treatment level of abdominal pain $\left(r_{s}=-0.395, p=0.037\right)$ Post-treatment pain level had significant negative correlation to the MDP size $\left(r_{S}=-0.867, p=0.002\right)$, though no correlation of pre-treatment pain level to MDP features was found, i.e. patients with less MDP size (most of them had decreased elasticity of papilla of Vater, that was considered as indirect marker of fibrosis) had lower hymecromone efficacy.

Conclusion: BS may cause MDP changes, resulting in development of obstructive $\mathrm{CP}$. Intensity of pain in biliary CP may be related to sphincter of Oddi dysfunction. Efficacy of antispasmodic therapy in these patents could be predicted by the features of MDP at pancreatobiliary EUS. 
Disclosure of Interest: All authors have declared no conflicts of interest.

\section{Reference}

Okazaki K, Yamamoto Y, Nishimori I et al., Motility of the sphincter of Oddi and pancreatic main ductal pressure in patients with alcoholic, gallstone-associated, and idiopathic chronic pancreatitis. Am J Gastroenterol. 1988 Aug;83(8):820-6.

P1416 RETROSPECTIVE ANALYSIS OF EXOCRINE PANCREATIC FUNCTIONALITY IN PATIENTS WITH CHRONIC PANCREATITIS

\section{A. Brandolese ${ }^{1}$, A. Amodio ${ }^{1}$, M.C. Conti Bellocchi ${ }^{2}$, S. Di Stefano ${ }^{1}$,}

P. Campagnola ${ }^{3}$, N. De Pretis ${ }^{1}$, L. Bernardoni ${ }^{2}$, S.F. Crinó ${ }^{1}$, A. Gabbrielli ${ }^{2}$,

L. Frulloni ${ }^{1}$

${ }^{1}$ Medicine, AOUI Verona-Pancreas Center, verona/Italy

${ }^{2}$ Gastroenterology, University of Verona, Verona/Italy

${ }^{3}$ Policlinico G.B. Rossi Dipt. di Gastroenterologia, Verona/Italy

\section{Contact E-mail Address: brandoqui@yahoo.it}

Introduction: Pancreatic exocrine insufficiency is a late complication of chronic pancreatitis; its clinical onset is characterized by steatorrhea and weight loss, borborygmi, flatulence, abdominalpain and malnutrition. Exocrine and endocrine pancreatic function decreases differently in various diseases (autoimmune, paraduodenal, genetic, idiopathic). It has been observed that there has been a recovery of exocrine pancreatic function in autoimmune pancreatitis. In the literature there are no studies analysing the exocrine pancreatic function over time. The fecal elastase test is a good test procedure to evaluate the exocrine pancreatic function

Aims \& Methods: The objective of the retrospective study was to re-evaluate a series of patients with chronic pancreatitis with the aim to evaluate the pancreatic exocrine function over time, in particular, by comparing the exocrine pancreatic function in subgroups of patients with different types chronic pancreatitis Pancreatic exocrine function was estimated through fecal elastase in 143 patients with at least 2 values each (classified into normal, mild and severe exocrine pancreatic insufficiency), the first one taken at the diagnosis of chronic pancreatitis. Patients undergoing surgical pancreatic resection before the second value of fecal elastase were excluded. Etiology was classified in: biliary pancreatitis/sequelae of necrotizing pancreatitis (15), autoimmune (69), paraduodenal (15), genetic (17) and idiopathic (27).

Results: The results show a high frequency of severe exocrine pancreatic insufficiency in the moment of diagnosis of chronic pancreatitis $(38 \%)$ and it appears stable over the following 5 years. Autoimmune and paraduodenal pancreatitis are correlated with severe exocrine pancreatic insufficiency at diagnosis in a high percentage of cases $(51 \%$ and $40 \%)$, biliary/outcomes of necrotizing pancreatitis and idiopathic pancreatitis in an intermediate $(33 \%$ and $26 \%$ ), while genetic in a low percentage $(12 \%)$

Conclusion: The exocrine pancreatic function in patients with autoimmune pancreatitis improved in the first five years of the disease, probably due to the efficacy of steroid/immunosuppressive therapy. Pancreatic endocrine function was less compromised at diagnosis, but showed a progressive deterioration in the first five years. Endocrine and exocrine insufficiency were strictly correlated. Disclosure of Interest: All authors have declared no conflicts of interest.

\section{P1417 "PAINLESS" CHRONIC PANCREATITIS: EPIDEMIOLOGICAL, CLINICAL AND RADIOLOGICAL CHARACTERIZATION}

C. Perini ${ }^{1}$, A. Amodio ${ }^{2}$, L. Bernardoni ${ }^{1}$, A. Gabbrielli ${ }^{1}$, S.F. Crinó ${ }^{1}$, P. Campagnola ${ }^{3}$, D. Costa ${ }^{1}$, F. Capuano ${ }^{3}$, V. Framba ${ }^{1}$, L. Frulloni ${ }^{4}$ ${ }^{1}$ Gastroenterologia Ed Epatologia, Policlinico G.B. Rossi, Verona, Verona/Italy ${ }^{2}$ Gastroenterology, University of Verona, Verona/Italy

${ }^{3}$ Policlinico G.B. Rossi Dipt. di Gastroenterologia, Verona/Italy

${ }^{4}$ Medicine, University of Verona Cattedra di Gastroenterologia Dept. of Medicine Verona/Italy

Contact E-mail Address: claudia.perini@hotmail.it

Introduction: The "painless" chronic pancreatitis $(\mathrm{CP})$ represents a specific subset of CP characterized by the lack of pancreatic pain. So far, scarcity of data has been reported in the literature about this matter and what differentiates this group of patients from those with chronic pancreatitis associated with pancreatic pain.

Aims \& Methods: The aim of the present study is to characterize "painless" CP from the epidemiologic, clinical, radiological, functional, and follow-up standpoint, through a comparison with other forms of chronic pancreatitis presenting with pancreatic pain. The Institutional Database of the Gastroenterology Unit of the Verona University was queried, and all chronic pancreatitis cases were retrieved. Patients were clustered based on the presence of "pancreatic-specific pain" into "painless" and "pain-associated" CP. A retrospective case-control analysis was carried out.

Results: Of 678 patients included from March 2006 to March 2016, 436 were considered eligible for the present study. Of these, $368(84 \%)$ were affected by pain-associated CP, while $68(16 \%)$ had "painless" CP. "Painless" patients were older (median age of $58.5 \pm 10.8 \mathrm{y} / \mathrm{o}$ vs. $42.5 \pm 15.3 \mathrm{y} / \mathrm{o} ; \mathrm{p}<0.001$ ), less frequently presenting with a history of alcohol consumption $(35 \%$ vs. $55 \%$; $\mathrm{p}<0.001)$, more frequently diabetics $(18 \%$ vs. $1 \% ; \mathrm{p}<0.001)$, presenting with steatorrhea $(16 \%$ vs. $2 \% ; \mathrm{p}<0.001)$, and asymptomatic $(63 \%$ vs. $2 \%$; $\mathrm{p}<0.001)$ compared to pain-associated controls. From the radiological standpoint, painless cases were more likely presenting with calcifications at imaging than controls $(90 \%$ vs. $68 \% ; \mathrm{p}<0.001)$. Moreover, in most of painless cases, the $\mathrm{CP}$ cause remained unknown $(56 \%)$. After a median follow-up of $2.6 \pm 2.3$ years, the incidence of diabetes was higher in the painless cases than in controls $(48 \%$ vs. $30 \%$; p < 0.006$)$.

Conclusion: The present study represents the first definition of "painless" CP so far reported in the literature. The "painless" CP is a distinct entity from the epidemiologic, clinical, and radiological standpoint when compared to other forms of CP characterized by the presence of pancreas-specific pain.

Disclosure of Interest: All authors have declared no conflicts of interest.

\section{References}

1. Muniraj T, Aslanian HR, Farrell J, et al. Chronic pancreatitis, a comprehensive review and update. Part I: epidemiology, etiology, risk factors, genetics, pathophysiology, and clinical features. Dis Mon 2014;60:530-50.

2. Whitcomb DC, Frulloni L, Garg P, et al. Chronic pancreatitis: An international draft consensus proposal for a new mechanistic definition. Pancreatology 2016;16:218-24.

3. Conwell DL, Lee LS, Yadav D, et al. American Pancreatic Association Practice Guidelines in Chronic Pancreatitis: evidence-based report on diagnostic guidelines. Pancreas 2014:43:1143-62.

4. Cavallini G, Frulloni L, Pederzoli P, et al. Long-term follow-up of patients with chronic pancreatitis in Italy. Scand J Gastroenterol 1998;33:880-9.

5. Frulloni L, Falconi M, Gabbrielli A, et al. Italian consensus guidelines for chronic pancreatitis. Dig Liver Dis 2010;42 Suppl 6:S381-406.

6. Homma T, Harada H, Koizumi M. Diagnostic criteria for chronic pancreatitis by the Japan Pancreas Society. Pancreas 1997;15:14-5.

7. Mergener K, Baillie J. Chronic pancreatitis. Lancet 1997;350:1379-85.

8. Fasanella KE, Davis B, Lyons J, et al. Pain in chronic pancreatitis and pancreatic cancer. Gastroenterol Clin North Am 2007;36:335-64, ix.

9. Pasricha PJ. Unraveling the mystery of pain in chronic pancreatitis. Nature Reviews Gastroenterology \& Hepatology 2012;9:140-151.

10. Bang UC, Benfield T, Hyldstrup L, et al. Mortality, cancer, and comorbidities associated with chronic pancreatitis: a Danish nationwide matchedcohort study. Gastroenterology 2014;146:989-94.

11. Poulsen JL, Olesen SS, Malver LP, et al. Pain and chronic pancreatitis: a complex interplay of multiple mechanisms. World $J$ Gastroenterol 2013;19:7282-91.

12. Ammann RW, Muellhaupt B. The natural history of pain in alcoholic chronic pancreatitis. Gastroenterology 1999;116:1132-40.

13. Frulloni L, Gabbrielli A, Pezzilli R, et al. Chronic pancreatitis: report from a multicenter Italian survey (PanCroInfAISP) on 893 patients. Dig Liver Dis 2009;41:311-7.

14. Goulston SJ, Gallagher ND. Chronic painless pancreatitis. Gut 1962;3:252-

\section{P1418 LONG-TERM OUTCOMES OF A FULLY COVERED SELF- EXPANDABLE METAL STENT WITH ANTIMIGRATION} PROPERTIES FOR EUS-GUIDED PANCREATIC DUCT DRAINAGE

D. $\mathrm{Oh}^{1}$, D.H. Park ${ }^{2}$, D.H. Cho ${ }^{2}$, T.J. Song ${ }^{2}$, S.S. Lee ${ }^{2}$, D. Seo ${ }^{2}$, S.K. Lee ${ }^{2}$, M. $\mathrm{Kim}^{2}$

${ }^{1}$ Department Of Gastroenterology, Nowon Eulji Medical Center, Eulji University, Seoul/Korea, Republic of

${ }^{2}$ Department Of Gastroenterology, University of Ulsan College of Medicine, Asan Medical Center, Seoul/Korea, Republic of

Contact E-mail Address: dongwook.oh1@gmail.com

Introduction: Recently, EUS-guided pancreatic duct drainage with transmural stenting (EUS-PD) has been used for patients with painful obstructive pancreatitis in whom endoscopic retrograde pancreatography (ERP) has failed. Although the feasibility and safety of EUS-PD with a fully covered self-expandable metal stent (FCSEMS) has been assessed, little is known about the long-term outcomes of EUS-PD with a fully covered self-expandable metal stent (FCSEMS). Removability of an FCSEMS in long-term use and higher cost are the main concerns of EUS-PD with an FCSEMS compared with EUS-PD with a plastic stent.

Aims \& Methods: The aim of this study is to evaluate the procedural and longterm outcomes of EUS-PD with an FCSEMS for patients with painful obstructive pancreatitis who failed ERP. Forty-one consecutive patients with painful obstructive pancreatitis underwent EUS-PD with an FCSEMS after failed ERP. Permanent FCSEMS placement without scheduled stent exchange was considered in malignant MPD strictures or complete MPD obstruction in benign pancreatic stricture. Technical and clinical success, adverse events, and stent patency were assessed. An endoscopic examination and CT scan was performed every 6 months to assess stent patency in benign stricture.

Results: 15 patients had malignant MPD obstruction and 26 patients had benign stricture. EUS-PD was successful in all 41 patients (technical success rate, $100 \%$ ), and symptoms improved in all patients (clinical success rate, $100 \%$ ). EUS-guided pancreaticogastrostomy $(n=39)$ and pancreaticojejunostomy $(n=2)$ were performed. Pain scores improved significantly after FCSEMS placement $(\mathrm{P}=.01)$. Early mild-grade adverse events occurred in 5 patients $(12.2 \%)$ with self-limited abdominal pain, which resolved with conservative treatment. Late adverse events developed in eight patients $(22.2 \%)$, including distal stent fracture $(n=6)$, stent occlusion $(n=2)$. These patients were successfully treated endoscopically. No other adverse events related to FCSEMS, including stent migration, pancreatic sepsis, and stent-induced ductal stricture were observed during follow-up periods. 
Overall mean stent patency duration was 412 days (range 14-1081) during mean follow-up periods (427 days). Median stent patency in malignant stricture was 95 days (range 14-297). Mean stent patency in benign stricture was 525 days (range 121-1081). No patients with malignant strictures required FCSEMS revision or exchange during follow-up periods. FCSEMS removal and exchange was successful in all benign strictures with stent dysfunction. At the end of the follow-up, two patients $(5.6 \%)$ showed resolution of stricture after definite stent removal. Conclusion: EUS-PD with an FCSEMS showed excellent long-term outcomes for patients who failed conventional ERP in both malignant and benign painful obstructive pancreatitis. Endoscopic FCSEMS removal and exchange could be successful in patients with benign strictures until 3-year placement of an FCSEMS. Prospective randomized trial comparing EUS-PD with FCSEMSs and plastic stents may be warranted for painful obstructive pancreatitis after failed ERP.

Disclosure of Interest: All authors have declared no conflicts of interest.

\section{References}

1. Widmer J, Sharaiha RZ, Kahaleh M. Endoscopic ultrasonography-guided drainage of the pancreatic duct. Gastrointest Endosc Clin N Am 2013;23:84761.

2. Giovannini M. EUS-guided pancreatic duct drainage: ready for prime time? Gastrointest Endosc 2013;78:865-7.

3. Park DH, Lee SS, Lee TH, et al. Anchoring flap versus flared end, fully covered self-expandable metal stents to prevent migration in patients with benign biliary strictures: a multicenter, prospective, comparative pilot study (with videos). Gastrointest Endosc 2011;73:64-70.

4. Oh D, Park do H, Cho MK, et al. Feasibility and safety of a fully covered self-expandable metal stent with antimigration properties for EUS-guided pancreatic duct drainage: early and midterm outcomes (with video). Gastrointest Endosc 2016;83:366-73.e2.

5. Dhir V, Isayama H, Itoi T, et al. EUS-guided biliary and pancreatic duct interventions. Dig Endosc 2017.

6. Tyberg A, Sharaiha RZ, Kedia P, et al. EUS-guided pancreatic drainage for pancreatic strictures after failed ERCP: a multicenter international collaborative study. Gastrointest Endosc 2017;85:164-169.

\section{P1419 MONITORING AND OPTIMIZATION OF PANCREATIC ENZYME REPLACEMENT THERAPY IN PATIENTS WITH PANCREATIC EXOCRINE INSUFFICIENCY}

M. Kovacheva-Slavova ${ }^{1}$, S. Siminkovitch ${ }^{1}$, J. Genov $^{1}$, B. Golemanov ${ }^{1}$, R. Mitova ${ }^{1}$, P. Gecov ${ }^{2}$, D. Svinarov ${ }^{3}$, B. Vladimirov ${ }^{1}$

${ }^{1}$ Gastroenterology, University Hospital Tsaritsa Ioanna-ISUL, Sofia/Bulgaria

${ }^{2}$ Medical Imaging, University Hospital Tsaritsa Ioanna-ISUL, Sofia/Bulgaria

${ }^{3}$ Central Laboratory Of Therapeutic Drug Management And Clinical

Pharmacology, Alexandrovska University Hospital, Medical University of Sofia, Sofia, Bulgaria, Sofia/Bulgaria

Contact E-mail Address: kovacheva_mila@abv.bg

Introduction: Fundamental aspects in the treatment of pancreatic exocrine insufficiency (PEI) include pancreatic enzyme replacement therapy (PERT). Monitoring the symptoms of maldigestion as well as the nutritional markers is essential.

Aims \& Methods: To follow-up patients with PEI receiving PERT and to provide normal nutritional status by optimizing the suboptimal PERT if necessary. Study enrolled 142 patients ( 88 males, mean age 52 years): 82 patients had chronic pancreatitis (CP), 30- acute pancreatitis (AP), 30- pancreatic cancer/pancreatic resection. 58 patients were re-monitored 6 months after adjusting suboptimal therapy. Alcohol abuse was most common aetiology. Nutritional status was evaluated by prealbumin and retinol binding protein (RBP) via immunonephelometry, fat-soluble vitamins A, D, E via HPLC and LC-MS/MS; magnesium $(\mathrm{Mg})$ and hemoglobin $(\mathrm{Hb})$. We evaluated in addition albumin; cRP; BMI Imaging morphological data were assessed by Cambridge classification for CT/ MRCP (grade I-IV) and cardiovascular risk (CVR)- by apolipoproteins (apo) AI, B and lipid profile. Statistical analysis was performed via SPSS v22.

Results: Protein malnutrition with prealbumin (mean: $0.208 \pm 0.105 \mathrm{~g} / \mathrm{L}$ ) and RBP (mean: $0.0389 \pm 0.0258 \mathrm{~g} / \mathrm{L}$ ) below reference limits was observed in 67 and 47 of all patients, respectively. We found correlation between prealbumin and $\mathrm{RBP}$ with other nutritional parameters $(\mathrm{Hb}, \mathrm{Mg}$, albumin, $\mathrm{p}<0.01)$, lipid profile and apolipoproteins in patients with $\mathrm{CP}(\mathrm{p}<0.05)$ and negative relationship to CRP $(\mathrm{p}<0.001)$ in AP. Prealbumin and RBP related to age, morphological changes and BMI in CP, p $<0.05$. Mean values of vitamin A, D, E were $527.68 \pm 321.12 \mu \mathrm{g} / \mathrm{L}, 39.74 \pm 24.36 \mathrm{nmol} / \mathrm{L}, 8.07 \pm 6.01 \mu \mathrm{g} / \mathrm{L}$. Vitamin A, D, E insufficiencies were present in $21.95 ; 94.49$ and $40.96 \%$ of all evaluated patients, respectively. Moreover $33.86 \%$ had vitamin D deficiency $(<25 \mathrm{nmol} / \mathrm{L})$. We found lowest vitamin levels in patients with pancreatic cancer/pancreatic resection and correlation with structural changes worsening, $\mathrm{p}<0.05$. Within all patients we observed suboptimal PERT with nutritional deficiencies despite an adequate clinical response (prealbumin, RBP, vitamin A, vitamin D and vitamin $\mathrm{E}$ deficiencies in $44.82,36.73,11.43,92.86$ and $48.57 \%$ of all asymptomatic patients, respectively). During second follow-up (6 months after PERT optimization), we demonstrated clinical improvement with a significant increase in BMI $(\mathrm{p}<0.0001)$ and nutritional markers, $\mathrm{p}<0.01$. CVR was increased in $62 \%$ of patients. Dyslipidemia was more severe in men and in $\mathrm{CP}, \mathrm{p}<0.05$. Optimal non-HDL levels were present in $33.59 \%$ (mean non-HDL levels: $5.23 \pm 2.25 \mathrm{mmol} / \mathrm{L}$ ). Moderate and high heart attack risk according Apo B Apo A-I ratio was observed in $34.35 \%$ and $45.04 \%$ of all patients. In addition, 33 patients suffered from diabetes mellitus.
Conclusion: Proper follow-up and correction of suboptimal PERT as well as CVR screening could reduce the risk of severe malnutrition complications and associated morbidity and mortality by ensuring optimal therapeutic results and better quality of life.

Disclosure of Interest: All authors have declared no conflicts of interest.

\section{P1420 HUR MEDIATED POST-TRANSCRIPTIONAL REGULATION OF HO-1 AND INHIBITORS OF APOPTOSIS PROTEINS IS ASSOCIATED WITH THE POOR CLINICAL OUTCOMES AMONG PATIENTS WITH PANCREATIC CANCER}

Z. Dambrauskas, A. Gulbinas, A. Jakštaite, A. Urboniene

Institute For Digestive Research, Lithuanian University of Health Sciences, Kaunas/Lithuania

Contact E-mail Address: zilvinas.dambrauskas@gmail.com

Introduction: The mRNA binding protein $\mathrm{HuR}$ is involved in the post-transcriptional regulation of cytoprotective molecules, such as COX-2, HO-1 and inhibitors of apoptosis proteins (IAP1, IAP2, XIAP, SURVIVIN), and might be related to poor prognosis in numerous cancer types. However, the association of HuR, COX-2, HO-1 and IAPs family, and their impact on chemoresistance and carcinogenesis in PDAC still remain unclear.

Aims \& Methods: The aim of our study is to assess the relevance and correlation of the IAP regulation by mRNA stabilizing protein $\mathrm{HuR}$ and $\mathrm{HO}-1$ and/or COX2 signaling pathway, and to determine the association with clinicopathological parameters and prognosis of PDAC. Data of 32 patients after pancreatodeduodenectomy for PDAC between 2011-2016 were analyzed. Patient's mRNA expression levels of HuR, COX-2, HO-1, IAP1, IAP2, Survivin and XIAP in PDAC were compared with normal pancreatic tissue obtained from organ donors. Additionally, the correlations among HuR, COX-2, HO-1, IAP1, IAP2, Survivin and XIAP, as well as their respective correlations with clinicopathological parameters were analyzed. The Kaplan-Meier method and log-rank tests were used for univariate analysis. Cox proportional hazard model was applied to indentify prognostic factors that were independently associated with survival.

Results: H0-1, COX-2, HuR, IAP1, IAP2 mRNA expression were accordingly 3fold, 8.8-fold, 1.5-fold, 4.8-fold and 5-fold higher, while XIAP and Survivin mRNA expression were 3.8-fold and 3.4-fold lower when compared to normal pancreatic tissue. Expression of HuR was positively associated with $\mathrm{COX}-2, \mathrm{H} 0$ 1, IAP1, IAP2, XIAP. High expression levels of HuR were significantly correlated with higher $\mathrm{G}$ stage and microvascular invasion, while high levels of XIAP were negatively associated with microvascular and perineural invasion. Univariate analysis revealed that high expression of HuR, lymph-node metastases, tumor differentiation and perineural invasion were significantly associated with overall survival $(0 S)$ of PDAC patients. In multivariate analysis, high levels of HuR, lymph-node metastases, tumor differentiation and perineural invasion were independently correlated with lower OS in patients with PDAC.

Conclusion: Our results suggested that upregulation of HuR in PDAC patients were significantly related with poor outcome. Even though, significant correlation with IAP proteins in PDAC was noticed, more data is needed to analyze the mechanism underlying HuR and IAPs interaction.

Disclosure of Interest: All authors have declared no conflicts of interest.

\section{References}

1. Jakstaite A, Maziukiene A, Silkuniene G, Kmieliute K, Gulbinas A Dambrauskas Z. HuR mediated post-transcriptional regulation as a new potential adjuvant therapeutic target in chemotherapy for pancreatic cancer. World J Gastroenterol. 2015 Dec 14;21(46):13004-19.

2. Berberat PO, Dambrauskas Z, Gulbinas A, Giese T, Giese N, Künzli B, Autschbach F, Meuer S, Büchler MW, Friess H. Inhibition of heme oxygenase-1 increases responsiveness of pancreatic cancer cells to anticancer treatment. Clin Cancer Res. 2005 May 15;11(10):3790-8.

\section{P1421 HYPOXIA INDUCED HIF1A-MEDIATED DIFFERENTIAL O- GALNAC GLYCOSYLATION DIRECTLY REGULATES SIGNALING PATHWAYS IN PANCREATIC CANCER}

B. Mercanoglu, B. T. Hofmann, C. Güngör, J.R. Izbicki, M. Bockhorn, G. Wolters-Eisfeld

Department Of General, Visceral And Thoracic Surgery, University Medical Center Hamburg-Eppendorf, Hamburg/Germany

Contact E-mail Address: ge.wolters@uke.de

Introduction: Hypoxia-induced reprogramming of cell energy metabolism and changes in glycosylation are hallmarks of cancer promoting the induction of an invasive and treatment-resistant phenotype, triggering metastases at an early stage of tumor development. ${ }^{1}$ We examined the impact of hypoxia on $\mathrm{O}$ GalNAc glycosylation in human HEK293, PDAC cell lines and clinical specimens and its link to cancer cell signalling.

Aims \& Methods: We profiled the expression of 88 glycosylation related genes by qPCR in HEK 293 cells subjected to hypoxia either induced by $1 \% \mathrm{O}_{2}$ or $200 \mathrm{mM}$ $\mathrm{CoCl}_{2}$ identifying key O-GalNAc glycosyltransferases downregulated. Functional assays and glycoprotein analysis displayed a pronounced rate of $\mathrm{O}$ GalNAc modified cytosolic proteins derived from hypoxia-treated cells and PDAC specimens. Glycosidase assays could validate specificity of detection method used. Aberrant glycotype could be induced by HIF pathway activator 
ML 228 and inhibited using Echinomycin. PTK and STK analysis of cell lysates displayed correlation between phosphorylation and O-glycosylation in hypoxic samples

Results: Mechanistically we could show, that hypoxia-induced decreased levels of C1GALT1C1 results in reduced T-Synthase activity with subsequent expression of truncated O-glycans (Tn antigen). Differential O-GalNAc glycosylation is inducible using HIF pathway activator ML228 under normoxia and the effect is reversed using $5 \mu \mathrm{M}$ Echinomycin under hypoxia underscoring the role of HIFla regulated transcription. Interestingly, the pattern of Tn antigen modified proteins derived from hypoxic samples differs significantly from engineered COSMC-deficient cells, displaying O-GalNAc moieties in addition to OGlcNAc in cytosolic protein fractions. Further, we could show PI3K/AKT MAPK signalling is depending on the state of cellular O-glycosylation providing a new rationale for the correlation of PDAC Tn antigen glycotype and cancer cell properties. $^{2}$

Conclusion: Our findings point to a novel crosstalk of O-GalNAc and OGlcNAcylation under hypoxia extending the knowledge base of differential OGalNAc glycosylation in pancreatic cancer.

Disclosure of Interest: All authors have declared no conflicts of interest.

\section{References}

1. Erkan, M., Kurtoglu, M. \& Kleeff, J. The role of hypoxia in pancreatic cancer: a potential therapeutic target? Expert Rev Gastroenterol Hepatol 10, 301-316, doi:10.1586/17474124.2016.1117386 (2016).

2. Hofmann, B. T. et al. COSMC knockdown mediated aberrant O-glycosylation promotes oncogenic properties in pancreatic cancer. Mol Cancer 14, 109 doi:10.1186/s12943-015-0386-1 (2015).

\section{P1422 THE EXPRESSION AND FUNCTION OF MIR-195 IN PANCREATIC CANCERTHE EXPRESSION AND FUNCTION OF MIR-195 IN PANCREATIC CANCER}

\section{Y. Zhang, Q. Zhan}

Digestive Department, Wuxi People's Hospital, Wuxi/China

\section{Contact E-mail Address: zhangq33@163.com}

Introduction: Pancreatic cancer is one of the more common malignant tumors in digestive system. The 5-year survival rate is less than about $6 \%$ of pancreatic cancer. Pancreatic cancer with poor prognosis and survival rate is low, due to the early clinical symptom is not easy to find, have a high transfer possible, and the operation difficulty, radiotherapy and chemotherapy is not sensitive. Radical surgical resection is the only opportunity to pancreatic cancer patients get longer survival. Therefore, a deeper understanding of the molecular mechanism of pancreatic cancer occurs, explore new effective treatment is imminent.

Aims \& Methods: Pancreatic cancer is a highly malignant tumor and fourth leading cause of cancer-related death in the world. The median survival after diagnosis is $2-8$ months, and approximately $3-6 \%$ of all patients with pancreatic cancer survive 5 years after diagnosis. This is mostly due to the fact that it is diagnosed at a stage when it is either locally advanced or has already metastasized to the distant organs. Hence, there is a paramount need to understand the molecular mechanisms underlying its initiation, progression and therapy. The recent discovery of microRNAs (miRNAs) has revealed a novel mechanism of gene regulation and provided new ways for cancer research. MicroRNAs are small, non-coding RNA molecules, which regulate the gene expression at posttranscription level. It is widely reported that miRNAs can act as oncogene or tumor suppressor gene. MiR-195 has been recognized as a tumor suppressor gene that is down-regulated in several types of cancer such as HCC, breast cancer, bladder cancer and gastric cancer. In PC, the role of miR-195 remains unclear. The purpose of this experiment was to explore the regulation role of miR-195 in PC development process. We measured miR-195 expression in three pancreatic cancer cells (PANC-1 SW-1990 PANC 03.27) by qRT - PCR, and HPDE cells were used as a control. We performed study of miR-195 by transfecting Panc-1 cells with miR-195 mimic. We used miRNA qRT - PCR to study the transfection efficiency of miR-195 mimic. The biology behaviors of Panc-1 cells transfected with miR-195 and negative control were analysed by CCK-8 proliferation assay, cell cycle, cell migration and invasion assay. We performed Real-Time PCR and western blot to detect the expression of CDK4 Cyclin E1 in Panc-1 cells which were transfected with miR-195 mimic and negative control.

Results: We found that miR-195 was decreased in three pancreatic cancer cells (PANC-1 SW-1990. PANC-03.27).We also found that over-expression of miR195 could suppress the proliferation, migration, invasion and cell cycle of Panc-1 cells. That means the malignancy potential of Panc- 1 cells is inhibited by miR195. Over-expression of miR-195 could down regulate the expression of CDK4 Cyclin E1 in Panc-1 cells.

Conclusion: The expression of miR-195 was down regulated in pancreatic cancer cells. Over expression of miR-195 suppressed cell proliferation, cell cycle, migration and invasion of Panc-1 cells by down regulated the expression of CDK4 and CyclinE1. Moreover, this finding suggests a potential novel strategy for therapeutic interventions of this disease.

Disclosure of Interest: All authors have declared no conflicts of interest.

\section{P1423 TRYPTOPHAN DEGRADATION AS AN ALTERNATIVE} ENERGY SOURCE IN PANCREATIC CANCER

C. Guengoer ${ }^{1}$, C. Kondziella ${ }^{1}$, B. Mercanoglu ${ }^{1}$, G. Wolters-Eisfeld ${ }^{1}$ J.R. Izbicki ${ }^{1}$, M. Bockhorn ${ }^{1}$

${ }^{1}$ Department Of General, Visceral And Thoracic Surgery, University Hospital Hamburg-Eppendorf, Hamburg/Germany

\section{Contact E-mail Address: c.guengoer@uke.de}

Introduction: Pancreatic cancer (PDAC) is one of the most lethal diseases worldwide, still exhibiting almost equal mortality as incidence rates. In spite of strong efforts in clinical and basic research, the molecular mechanisms responsible for this unusual aggressive phenotype still remain not completely understood and insufficient diagnostic and therapeutic tools still substantiate its ranking as the $4^{\text {th }}$-leading cause of cancer-associated deaths in western countries. This devastating prognosis is partially due to frequent occurrence of intrinsic or acquired chemoresistance in PDAC specimens against the nucleoside analogue gemcitabine which is still the standard for chemotherapeutic treatment of locally advanced and metastatic PDAC. In principle, cancer cells are well known for their ability to divide uncontrollably and it was initially thought that high glucose consumption is the main energy-source for proliferating tumor cells, but a large body of evidence suggest that most of the cell mass that makes up new cells resulted from amino-acid metabolism.

Aims \& Methods: The aim of this study was the identification of new biomarkers for chemoresistant pancreatic cancer. A secretome survey of chemoresistant PDAC cells was performed using SILAC-based mass-spectrometric analyses. Relative differences in protein-concentrations among samples were investigated and led to the identification of previously unknown proteins. The impact of RNAi-mediated knockdown of selected genes in proliferating PDAC cells were analyzed using MTT-viability assays. Gene- and protein-expression analyses were performed using Real-Time-PCR and immunohistochemistry using patient-derived PDAC samples.

Results: SILAC-based identification of the Tryptophan degrading enzyme Kynureninase (KYNU) in chemoresistant PDAC cells revealed an overexpressed and secreted form of the KYNU protein, compared to the chemosensitive counterpart. We further identified various stress-related external stimuli (Gemcitabine, IFNg, Hypoxia) as main inductors of KYNU expression/secretion. A RNAi-mediated KYNU knockdown approach was linked to substantially lower proliferation of chemoresistant and aggressive PDAC cells. Global expression analyses using a tissue-microarray of PDAC patient samples $(n=368)$ revealed that high KYNU expression is significantly correlated with a worse outcome in PDAC patients.

Conclusion: The tryptophan degradation pathway member KYNU is overexpressed in a subset of PDAC patients and is linked to substantially increased cancer cell proliferation. Abundant KYNU expression in PDAC patients is linked with a worse clinical outcome. We found that KYNU is a new secreted biomarker of chemoresistant PDAC cells

Disclosure of Interest: All authors have declared no conflicts of interest.

\section{P1424 INTEGRIN A11 IS SPECIFICALLY EXPRESSED IN PANCREATIC TUMOR STROMA AND A KEY TARGET IN REGULATION OF PANCREATIC TUMOR STROMAL} MYOFIBROBLASTS

J. Schnittert ${ }^{1}$, R. Bansal ${ }^{1}$, G. Storm ${ }^{1}$, A. Östman ${ }^{2}$, J. Prakash ${ }^{1}$

${ }^{1}$ Biomaterials Sciene And Technology/Targeted Therapeutics, University of Twente, Enschede/Netherlands

${ }^{2}$ Department Of Oncology-pathology, Karolinska Institute, Stockholm/Sweden

Contact E-mail Address: j.schnittert@utwente.nl

Introduction: The progression of pancreatic ductal adenocarcinoma (PDAC) is promoted by its highly abundant tumor stroma. As one of the main components of the tumor stroma human pancreatic stellate cells (hPSCs), precursor cells of pancreatic tumor stromal myofibroblasts (CAFs), support PDAC progression by enhancing tumor cell growth, invasion and metastasis [1]. The collagen binding transmembrane receptor integrin $\alpha 11$ (ITGA11) is known to be overexpressed by myofibroblasts [2].

Aims \& Methods: The aim of the presented study was to investigate the expression of ITGA11 in human PDAC and to study the role of ITGA11 in CAF regulation. ITGA11 expression was evaluated using immunostaining on human PDAC sections and various other organs. The relation between ITGA11 levels, CAF activation markers and tumor growth was studied in a stroma rich coinjection model in mice. The biological role of ITGA11 in CAF differentiation was studied in hPSCs and hPSCs activated with TGF- $\beta$ or conditioned medium from Panc-1 endothelial tumor cells using qPCR, immunostaining, western blot, wound healing, collagen contraction and cell growth assays.

Results: In this study we have for the first time stained ITGA11 in human PDAC specimens. We found that ITGA11 was highly expressed in stromal myofibroblasts of PDAC patients, as shown by co-localization with the myofibroblasts marker alpha smooth muscle actin ( $\alpha$-SMA). Interestingly, there was no expression in healthy human pancreas and various other tissues from human organs. Furthermore, we induced subcutaneous tumors in mice by injecting Panc-1 or Panc- $1+$ hPSCs and found that ITGA11 was significantly overexpressed in stroma-rich Panc-1 + hPSC tumors. The quantitative gene and protein expression of ITGA11 in subcutaneous tumors, positively correlated with the expression of the CAF markers $\alpha$-SMA, Collal and PDGF $\beta$ R. Activation of hPSCs with TGF- $\beta$ or conditioned medium from Panc-1 resulted in the significant upregulation of ITGA11 and $\alpha$-SMA. Stable ITGA11 knockdown, mediated by shRNA, significantly inhibited hPSC differentiation, migration potential, contractility and cell growth. 
Conclusion: In conclusion, this study introduces ITGA11 to be highly and specifically expressed in the tumor stroma of human PDAC and explores its role in the regulation of the phenotype of pancreatic tumor stromal myofibroblasts. Disclosure of Interest: All authors have declared no conflicts of interest.

\section{References}

1. Apte MV, Wilson JS, Lugea A, Pandol SJ. A starring role for stellate cells in the pancreatic cancer microenvironment. Gastroenterology 144(6), 1210-1219 (2013).

2. Talior-Volodarsky I, Connelly KA, Arora PD, Gullberg D, Mcculloch CA. alpha11 integrin stimulates myofibroblast differentiation in diabetic cardiomyopathy. Cardiovascular research 96(2), 265-275 (2012).

\section{P1425 EFFECT OF ACOUSTIC CAVITATION ON A THREE- DIMENSIONAL CULTURE MODEL OF PANCREATIC ADENOCARCINOMA}

B. Bordacahar ${ }^{1}$, M. Camus ${ }^{1}$, E. Abou Ali ${ }^{2}$, J.L. Mestas ${ }^{3}$, M. Lafond ${ }^{3}$, I. Suarez ${ }^{3}$,

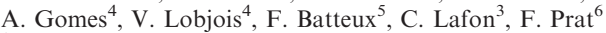

${ }^{1}$ Endoscopy And Digestive Oncology Unit, Hopital Cochin, Paris/France

${ }^{2}$ Department Of Digestive Diseases, APHP Saint Antoine Hospital, Paris/France ${ }^{3}$ Inserm U1032, LabTau, Lyon/France

${ }^{4} I T A V$, Toulouse/France

${ }^{5}$ Equipe Stress Oxydant, INSERM U1016, Paris/France

${ }^{6}$ Hôpital Cochin Dept. of Gastroenterology, Paris/France

Contact E-mail Address: benoit.bordacahar@gmail.com

Introduction: The dismal prognosis of pancreatic ductal adenocarcinoma (PDAC) is mainly due to chemoresistance linked to the tumor microenvironment. Recent developments in therapeutic ultrasound(US), particularly US-induced cavitation, could help overcome chemoresistance by breaking microenvironmental barriers and increase cytotoxic drug availability. Three-dimensional (3D) culture in the form of spheroids is a useful model for reproducing multicellular resistance and analyzing the effects of cavitation.

Aims \& Methods: The objective of this work was to study the effects of acoustic cavitation on a model of PDAC spheroids and to investigate possible potentiation of chemotherapy by US. CAPAN-2 PDAC cell line-derived spheroids were cultured as previously described by Ivascu et al. Four conditions, i.e. control, $400 \mathrm{nM}$-gemcitabine-based chemotherapy (CT) alone, US alone, CT-US combination ( $\mathrm{n}=12$ spheroids per condition), were studied. Experiments were carried out to optimize US settings, in order to observe the occurrence of controlled acoustic cavitation. Comparisons between groups were based on proliferation and growth. Proliferation was evaluated 24 hours after treatment(s) by Uptiblue. Growth was assessed by diameter measurement on light microscopy at day 7 and day 10 .

Results: Compared to the control group, cell proliferation was decreased in spheroids treated with CT $(\mathrm{p}<0.0001)$, but not with US alone. Proliferation was also further impaired in spheroids treated with CT-US combination compared to those treated with CT alone $(\mathrm{p}<0.0001)$, but this synergistic effect of US and CT did not impact growth of spheroid, meaning that spheroid diameter did not decrease after US-CT compared to CT alone.

Conclusion: This study shows the feasibility of applying an ultrasonic treatment (acoustic cavitation) in a three-dimensional culture model of PDAC. The combination of CT and ultrasonic cavitation synergistically reduced cell proliferation. Further analysis of the cytotoxic effects of acoustic cavitation on PDAC spheroids is in progress.

Disclosure of Interest: All authors have declared no conflicts of interest

\section{P1426 PATHOLOGICAL EVALUATION AND REPORTING OF INTRADUCTAL PAPILLARY MUCINOUS NEOPLASMS OF THE PANCREAS: THE CORRELATION AND ANALYSES OF THE HISPATHOTOLOGIC PATTERNS}

C. D. Nava $^{1}$, R. Coudry ${ }^{2}$, M.C. Cesar Machado ${ }^{2}$, L. Meirelles ${ }^{2}$, M.D.C. Gonçalves ${ }^{2}$, G. Ferreira Paduani ${ }^{1}$, J. G. Guerra ${ }^{1}$, J.C. Ardengh ${ }^{1}$ ${ }^{1}$ Gastroenterology And Endoscopy Service, Hospital 9 de julho, São Paulo/Brazil ${ }^{2}$ Pathologic, Hospital Sirio Libanes, Sao Paulo/Brazil

Contact E-mail Address: carolinnava@gmail.com

Introduction: Intraductal papillary-mucinous neoplasm (IPMN) are a heterogeneous group of pancreatic tumors mucin-producing with uncertain biologic behavior. We know, nowadays, that they can be also considered as a precursor of pancreatic carcinoma (PAC), one out of these lesions will develop into a malignancy of different types. There are no established guidelines for pathologic diagnosis/reporting of IPMN.

Aims \& Methods: The aim of this study was to analyze the IPMN subtypes distribution related with clinicopathological, histologic and immunohistochemical and identify which one is more related to development of malignancy. This prospective study reports a 3 -year-follow up, with consecutive patients, where we analyzed the clinical findings, radiological aspects, and morphologic features in patient's suspects of IPMN or PAC undergoing to pancreatic surgery. The lesions were classified based on morphological and immunohistochemically defined by the current WHO criteria.

Results: We analyzed 28 patients (16 women), mean age 66 y-old (range 50-83). $15(53 \%)$ patients were asymptomatic, and $13(47 \%)$ showed abdominal pain (8), chest pain (4), and recurrent acute pancreatitis (1). 10 patients were submitted to subtotal pancreatectomy, 9 to duodenopancreatectomy, 7 to gastroduodenopancreactectomy, 2 subtotal pancreatectomy and splenectomy. The neoplasm was located in head, body and tail, and entire pancreas in 18,9, and 1, respectively. 14 patients $(50 \%)$ had involvement of the main pancreatic + branch ducts (mixed type), $7(25 \%)$ had only the main pancreatic duct involved, $6(21.5 \%)$ had only the branch-duct involved, and $1(3.5 \%)$ had not informed. The mean size of the lesion was $3.3 \mathrm{~cm} \pm 6.1(1-11 \mathrm{~cm})$. Morphologic features showed multi-loculated (23) solid-cystic (4) and solid (1). The immunohistochemically expressed MUC1, MUC5AC, and MUC2 in 18 (64\%), 17 (61\%), and 11 (39\%), respectively. The hispathotologic patterns founded was gastric-type (9), Intestinal-type (3), pancreatobiliary-type (9), mixed-type [6 (Pb-t + I-t (4), and Pb-t + G-t (2)), and oncocytic-type (1) (table 1). The IPMN with a low-grade, and high-grade dysplasia and invasive carcinoma was found in $18(64.2 \%), 6(21.5 \%)$ and $4(14.2 \%)$, respectively. We observed that the all pancreatic intraepithelial neoplasia (PanIN) founded was related to Pb-t $13(46 \%)$. The invasive PAC was presented in 4 patients $(14,2 \%)$ with the follow subtypes (Pb-t (2), and Pb-t $+\mathrm{I}(1))$, and colloid PAC [I-t (1)] (table 2). Was found a synchronic neoplasia in 2 patients (colon adenocarcinoma, and NET).

Conclusion: IPMN of the pancreas is a common cystic lesion located more frequent on both duct (mixed-type). showing more aggressive behaver than others pattern. Pb-t was the most common lesion and were more connecting to invasive PAC and HGD, as well as connected with PanIN. MUC stains are helpful for the diagnosis and papillary histological subtyping. Prospective studies are needed to confirm these findings.

Disclosure of Interest: All authors have declared no conflicts of interest.

\section{Reference}

Correa-Gallego C., Ferrone C. R., Thayer S. P., Wargo J. A., Warshaw A. L., and Fernandez-Del Castillo C 2010. Incidental pancreatic cysts: do we really know what we are watching? Pancreatology 10:144-150. [2004 May;57(5):456-62. Furukawa T, Hatori T, Fujita I, Yamamoto M, Kobayashi M, Ohike N, et al. Prognostic relevance of morphological types of intraductal papillary mucinous neoplasms of the pancreas. Gut. 2011a;60(4):509-16.

P1427 EVALUATION OF LONG TERM SURVIVAL OF CHEMORADIOTHERAPY WITH GEMCITABINE AND S- 1 COMPARED WITH CHEMOTHERAPY ALONE IN THE CASES WITH LOCALLY ADVANCED PANCREATIC CANCER

K. Tomishima ${ }^{1}$, S. Ishii ${ }^{1}$, T. Fujisawa ${ }^{1}$, S. Takahashi ${ }^{1}$, A. Suzuki ${ }^{1}$, R. Kanazawa ${ }^{1}$, H. Saito ${ }^{1}$, H. Isayama ${ }^{1}$, K. Sasai ${ }^{2}$, S. Kawasaki ${ }^{3}$

${ }^{1}$ Departments Of Gastroenterology, Juntendo University, Tokyo/Japan

${ }^{2}$ Department Of Radiology, Juntendo University, tokyo/Japan

${ }^{3}$ Department Of Hepatobiliary-pancreatic Surgery, Juntendo University, Tokyo/ Japan

Contact E-mail Address: tomishim@juntendo.ac.jp

Introduction: Because of the progression of systemic chemotherapies (CT) for locally-advanced pancreas cancer (LA-PC), chemoradiotherapy(CRT) was selected for limited case. However, very long survival cases were reported in CRT and detection of prognostic factors were warranted. In this analysis, we analyzed the LA-PC cases received CRT compared with CT.

Aims \& Methods: Gemcitabine (GEM) and S-1 combination chemoradiotherapy (GS-CRT) was performed according to our previous Phase 1 trial (Journal of Japan Pancreas Society 2010). Till March 2016, 30 LA-PC cases received GSCRT, and the selection criteria were LA-PC with 1) pathological diagnosis, 2) large vessels (CA, SMA, CHA, PV, SMV) invasion, 3) allochronic and concurrency without multiple primary cancer, 4) unexecuted antitumor therapy. The chemotherapy in CRT administration of GEM $\left(200 \mathrm{mg} / \mathrm{m}^{2}\right)$ once a week for 6 weeks, administration of S-1 $\left(80 \mathrm{mg} / \mathrm{m}^{2}\right)$ for 2 weeks a week withdrawal was done twice. Radiation was performed $1.8 \mathrm{~Gy}$ in power of $10 \mathrm{MeV}$ in a week 5-day period for 5.5 weeks, and total dose was $50.4 \mathrm{~Gy}$ (Total 28 times). As after treatment, GEM $1000 \mathrm{mg} / \mathrm{m}^{2}$ was continued until PD. The patients of CT group were also recruited by the same criteria. One of the regimens among GEM alone, S-1 alone and GEM + S-1 was selected for the primary treatment, and total 26 cases were implemented in more than 2 courses.

Results: Baseline characteristics in CRT and CT group were median age (62, 72.5: $\mathrm{p}=0.004)$, male $(20,12: \mathrm{ns})$ and tumor location $\mathrm{Ph} / \mathrm{Pb}(17 / 13,16 / 10 ; \mathrm{ns})$, respectively. Efficacy were disease control rate(DCR) in 3 months after treatment $(90 \%, 57.7 \%: \mathrm{p}=0.01)$, response rate $(\mathrm{RR})(26.7 \%, 0 \%: \mathrm{p}=0.005)$ and conversion surgery $(10 \%, 0 \%: \mathrm{ns})$. There were significant differences in progression free survival (PFS) $(8$ months, 5 months: $\mathrm{p}=0.002)$ and overall survival $(\mathrm{OS})(13 \mathrm{M}$, $9 \mathrm{M}: \mathrm{p}=0.0165)$, respectively. The cases who survived for 18 months and longer were significantly $(\mathrm{p}=0.0495)$ more in CRT $(43.3 \%)$ than CT group $(19.2 \%)$. Grade $3 / 4$ adverse events in CRT group were 13 cases of neutropenia (G4:3 cases) and one case of gastrointestinal symptom, and those in CT group, neutropenia was 11 cases (G4:4 case), interstitial pneumonia (IP) aggravation was one case.

Conclusion: For LA-PC, GS-CRT showed better local tumor control and longer survival, and was considered as good candidate of neo-adjuvant therapy. More long survival cases in CRT than CT was other benefit for LA-PC. We should think about good selection criteria of CRT and improve the survival of LA-PC. Disclosure of Interest: All authors have declared no conflicts of interest. 


\section{Reference}

Chemoradiotherapy in the Management of Locally Advanced Pancreatic Carcinoma: A Qualitative Systematic Review. Florence Huguet, Nicolas Girard, Clotilde Séblain-El Guerche et al.

\section{P1428 HIGH-INTENSITY FOCUSED ULTRASOUND (HIFU) THERAPY FOR UNRESECTABLE PANCREATIC CANCER}

A. Sofuni, Y. Asai, M. Fujita, T. Tsuchiya, S. Tsuji, R. Tanaka, R. Tonozuka, M. Honjo, S. Mukai, K. Yamamoto, Y. Matsunami, K. Kamada, T. Itoi Gastroenterology \& Hepatology, Tokyo Medical University, Tokyo/Japan

\section{Contact E-mail Address: a-sofuni@amy.hi-ho.ne.jp}

Introduction: High-intensity focused ultrasound (HIFU) is expected as new advanced therapy for unresectable pancreatic cancer (PC). HIFU therapy with chemotherapy is being promoted as new method to control local advance by ablation tumor, and mainly achieve relief of pain caused by PC.

Aims \& Methods: We have evaluated the therapeutic effect of HIFU therapy in locally advanced and metastatic PC. We treated PC patients by HIFU as optional local therapy as well as systemic chemo/chemo-radiotherapy, with whom an agreement was obtained in adequate IC, from the end of 2008 in our hospital. This study took approval of member of ethic society of our hospital. HIFU device used is FEP-BY02 (Yuande Bio-Medical Engineering Co.LTD., China). The subjects were 140 PC patients, i.e. 69 cases in stage III, 71 cases in stage IV. Performance status (PS) was PS:0; 79, PS:1; 58, and PS:2; 3 cases. Mean age was $62.3 \pm 10.6$ years, The details of therapy before HIFU treatment (overlap) was chemo-radiotherapy in 34 , chemotherapy in 92, arterial infusion chemotherapy in 5, immunotherapy in 8 , operation in 17, and BSC in 7 cases. Results: All tumors were visualized by HIFU monitor system. Tumor location was head in 38 , uncus in 19 , body in 59 , body $\sim$ tail in 6 , tail in 2 , and others (recurrence) in 16 cases. Treatment data was followed; mean tumor size before and after therapy was $33.5 \pm 10.7$ and $33.7 \pm 11.5 \mathrm{~mm}$, mean treatment sessions: $2.3 \pm 0.7$ times, mean total treatment time: $105 \pm 65.6 \mathrm{~min}$, mean total number of irradiation: $1967.8 \pm 1106$ shots. The effects of HIFU therapy were the following; the rate of complete tumor ablation was $87.9 \%$, the rate of symptom relief effect was $69.4 \%$, the effectiveness of primary lesion was CR:0, PR:21, SD:85, PD:34 cases, primary disease control rate (DCR) more than SD was $75.7 \%$. The therapy after HIFU treatment was operation in 8, chemotherapy in 116, immunotherapies in 4 , and best supportive care (BSC) in 14 cases. MST after diagnosis in HIFU with chemotherapy and chemotherapy alone (38 patients in our hospital) was 1028.3 vs 366.6 days, respectively $(p<0.001)$. MST after HIFU therapy was 606.1 days. Combination therapy of HIFU with chemotherapy was better result than common chemotherapy alone.

Conclusion: This study suggested that HIFU therapy has the potential of new method of combination therapy for PC.

Disclosure of Interest: All authors have declared no conflicts of interest

\section{P1429 EUS AND CT SCAN ACCURACY IN ESTABLISHING THE T STAGE IN PANCREATIC CANCER BASED ON THE UPCOMING TNM $8^{\text {TH }}$ EDITION}

L. Archibugi ${ }^{1}$, M.C. Petrone ${ }^{2}$, S. Crippa ${ }^{3}$, E. Dabizzi ${ }^{2}$, A. Mariani ${ }^{2}$, R. Nicoletti ${ }^{4}$, C. Doglioni ${ }^{5}$, G. Capurso ${ }^{1}$, M. Falconi ${ }^{3}$, P.G. Arcidiacono ${ }^{2}$

${ }^{1}$ Digestive \& Liver Disease Unit, S. Andrea Hospital - University of Rome "La Sapienza", Rome/Italy

${ }^{2}$ Pancreato-biliary Endoscopy And Endosonography Division - Pancreas Center, San Raffaele Scientific Institute Vita Salute San Raffaele University, Milan/Italy ${ }^{3}$ Pancreatic Surgery Department - Pancreas Center, San Raffaele Scientific Institute Vita Salute San Raffaele University, Milano/Italy

${ }^{4}$ Department Of Radiology - Pancreas Center, San Raffaele Scientific Institute, Milan/Italy

${ }^{5}$ Pathology Unit - Pancreas Center, San Raffaele Scientific Institute, Milan/Italy

Contact E-mail Address: livia.archibugi@hotmail.it

Introduction: Pancreatic ductal adenocarcinoma (PDAC) has a dismal prognosis with an overall 5 -year survival rate $<6 \%$. Surgically resected patients, although undergoing a possibly curative treatment, have nevertheless a 5-year survival $<25 \%$. In fact, it has been recently suggested that patients with a tumor of more than $2 \mathrm{~cm}$ might harbor micrometastases at diagnosis. In this view, given the availability of new highly effective chemotherapy regimens that might be employed in the neoadjuvant setting, the correct evaluation of the T stage of pancreatic cancer plays a key role. The new proposed AJCC Staging System for Pancreatic Adenocarcinoma TNM ( $8^{\text {th }}$ edition), in fact, differs from the $7^{\text {th }}$ edition mostly for the evaluation of the $\mathrm{T}$, giving high importance to the diameter of the lesion. This reclassification has already been shown to predict survival differences more efficiently compared to previous editions. In this context, an efficient preoperative evaluation of the $\mathrm{T}$ is of high importance as it might shift the therapeutic decision from upfront surgery to neoadjuvant chemotherapy, that could be preferred in tumors being $>2 \mathrm{~cm}$ (T2). Few studies compared the accuracy of CT scan and EUS in evaluating the dimension of the tumor with heterogeneous results and often dated machines, and no study adopted the new TNM system to evaluate the preoperative staging defined by CT and EUS.

Aims \& Methods: The aim was to evaluate the accuracy of CT scan and EUS, alone and in combination, in establishing the $\mathrm{T}$ stage of surgically resected PDAC as defined by the new upcoming TNM $8^{\text {th }}$ edition and to establish the sensitivity and specificity of the two imaging modalities in discriminating T1 stage from more advanced $\mathrm{T}$ stages. We conducted a retrospective study on a cohort of surgically resected histologically-confirmed PDAC patients at a pancreatic cancer referral center between 2015-2017, who were prospectively included in a dedicated database. Inclusion criteria: a) having both preoperative EUS and CT scan with pancreatic phase evaluation at the centre; b) CT and EUS were performed, at the latest, 30 days apart from each other and from surgical resection; c) no neoadjuvant chemo or radiotherapy was performed. The evaluation of the $\mathrm{T}$ by both imaging modalities was compared to the final pathology $\mathrm{T}$ re-established based on the new TNM $8^{\text {th }}$ edition, in order to calculate specificity and sensitivity. T-test was used for comparison of continuous variables, Fisher's test was used for comparison of categorical variables.

Results: Among the 184 PDAC patients surgically resected between 2015 and 2017 at our center, 30 met inclusion criteria. Of these, $19(63.3 \%)$ were males, with mean age at resection being $67.8 \pm 9.5$ years. The tumor was located in the head in $23 / 30(76.7 \%)$ patients. Mean diameter of the tumor at pathology was $24.9+10.8 \mathrm{~mm}$, mean diameter at EUS was $24.0 \pm 8.6 \mathrm{~mm}(\mathrm{p}=0.74)$, and mean diameter at CT was $25.9 \pm 10.9 \mathrm{~mm}(\mathrm{p}=0.73)$. In $4 / 30(13.3 \%)$ cases CT scan was not able to detect the lesion; mean diameter of undetected lesions was $21.2 \pm 2.9 \mathrm{~mm}$. Accuracy in T assessment was $60 \%(18 / 30)$ for both EUS and CT scan; when considering detection of $\geq \mathrm{T} 2$ lesion in any of the two imaging modalities the accuracy increased to $80 \%(24 / 30)$. The sensitivity and specificity in discriminating $\mathrm{T} 1$ lesions from $\geq \mathrm{T} 2$ lesions was respectively $64.7 \%$ and $76.9 \%$ for EUS and $76.5 \%$ and $72.7 \%$ for CT.

Conclusion: This is the first study evaluating the accuracy of CT and EUS imaging modalities in establishing the T in the setting of the new TNM $8^{\text {th }}$ edition. In our study, CT scan and EUS have both a relatively low accuracy in determining the correct $\mathrm{T}$ stage for pancreatic cancer when used alone, while the accuracy raises significantly when used in combination. CT scan was not able to detect up to $13.3 \%$ of lesions. EUS resulted having a lower sensitivity but higher specificity in discriminating $\mathrm{T} 1$ lesions from $>\mathrm{T} 2$ lesions compared to $\mathrm{CT}$ scan. These preliminary results suggest how EUS, often adopted only as a means to obtain a cytological specimen, has a key role in determining the $\mathrm{T}$ stage, and that the two imaging modalities should be used in combination to better assess the proper therapeutic management as neoadjuvant chemotherapy or upfront surgical resection.

Disclosure of Interest: All authors have declared no conflicts of interest.

\section{P1430 RISK FACTORS AND SURVIVAL IN PANCREATIC}

\section{ADENOCARCINOMA}

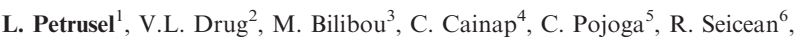
A. Seicean ${ }^{1}$

${ }^{1}$ Dept. Of Internal Medicine, Gastroenterology, Iuliu Hatieganu University of Medicine and Pharmacy, Cluj-Napoca/Romania

${ }^{2}$ Dr., University of Medicine and Pharmacy "Grigore T Popa", Iasi, Romania, Bucharest/Romania

${ }^{3}$ Dept. Of Internal Medicine, Gastroenterology, Regional Institute of Gasroenterology and Hepatology, Iasi/Romania

${ }^{4}$ Oncology, Oncological Institute, Cluj Napoca, Romania, Cluj Napoca/Romania ${ }^{5}$ Dept. Of Internal Medicine, Gastroenterology, Octavian Fodor Regional Institute of Gastroenterology and Hepatology, Cluj-Napoca/Romania

${ }^{6}$ General Surgery, Clinical Emergency County Hospital Cluj-Napoca, Cluj-

Napoca/Romania

Contact E-mail Address: cutas_livia@yahoo.com

Introduction: Pancreatic adenocarcinoma is associated with a $5-6 \%$ survival at 5 years and a poor quality of life. In Romania there are few information about the prognostic influence of known risk factors for pancreatic cancer.

Aims \& Methods: The aim of this study is to evaluate the association between risk factors and the occurrence of pancreatic adenocarcinoma and patients' survival, which may constitute a theoretical basis for screening. We performed a prospective, multicentric study of patients with suspected pancreatic tumors detected in abdominal ultrasound or CT examination, during January 2015-December 2016 , in which were analyzed risk factors and possible association with survival adjusted statistically according to tumor stage (Chi square test, ANOVA, logrank test).

Results: There were 279 patients with pancreatic adenocarcinoma included in the study. Male patients were $58 \%$ from all patients, and the mean age was 63.5 years. Smoking, new-onset diabetes and history of chronic pancreatitis are risk factors for pancreatic adenocarcinoma $(p<0.05)$. At 12 months of follow-up, almost one-third of patients with pancreatic adenocarcinoma died (median survival $=5$ months). It was demonstrated a statistically significant association adjusted for tumor stage between the presence of new-onset diabetes and survival: 5 months vs 3 months with a $\mathrm{HR}=3$. Other risk factors (alcohol, obesity, sex, genetics, coffee intake, some infections and abdominal surgery, history of chronic pancreatitis) had no prognostic role.

Conclusion: In our study, the risk factors for pancreatic cancer were smoking, history of chronic pancreatitis and new-onset diabetes, but the only prognostic factor was diabetes.

Disclosure of Interest: All authors have declared no conflicts of interest.

\section{References}

1. Becker AE, Hernandez YG, Frucht H, Lucas AL. Pancreatic ductal adenocarcinoma: Risk factors, screening, and early detection. World $J$ Gastroenterol 2014 August 28; 20(32): 11182-11198.

2. James TA, Sheldon DG, Rajput A. et al. Risk factors associated with earlier age of onset in familial pancreatic carcinoma. Cancer 2004; 101:2722-2726. 
3. Beg M, Dwivedi AK, Ahmad SA, Ali S, Olowokure O, Impact of diabetes mellitus on the outcome of pancreatic cancer. 2014 May 30;9 (5):e98511

4. Maisonneuve1 P, Lowenfels A. Risk factors for pancreatic cancer: a summary review of meta-analytical studies. International Journal of Epidemiology, 2015, 186-198

5. Lakatos G, Balázs A, s Kui Bet al. Pancreatic Cancer: Multicenter Prospective Data Collection and Analysis by the Hungarian Pancreatic Study Group. J Gastrointestin Liver Dis, June 2016 Vol. 25 No 2: 219-225

6. Li D, Morris JS, Liu J, Hassan MM, Day RS, Bondy ML, Abbruzzese JL. Body mass index and risk, age of onset, and survival in patients with pancreatic cancer. JAMA 2009;301:2553-62.

\section{P1431 PREVALENCE STRATIFICATION OF MALIGNANCY IN RESECTED INTRADUCTAL PAPILLARY MUCINOUS NEOPLASMS INVOLVING MAIN DUCT: IS THE 10 MM WIRSUNG DIAMETER AN ADEQUATE CUTOFF?}

T. Cúrdia Gonçalves ${ }^{1}$, C. Montironi ${ }^{2}$, M. Cuatrecasas ${ }^{2}$, JR. Ayuso ${ }^{3}$, S. Sanchez ${ }^{4}$, H. De Leon ${ }^{5}$, C. Sanchez, G. Fernández-Esparrach ${ }^{6}$, A. Gines ${ }^{6}$, X. Molero ${ }^{7}$, C. Marinho ${ }^{7}$, J. Cotter ${ }^{8}$, E. Vaquero ${ }^{5}$

${ }^{1}$ Gastroenterology Department, Hospital da Senhora da Oliveira, Guimarães/ Portugal

${ }^{2}$ Pathology Department - Centre De Diagnòstic Biomèdic (CDB), Hospital Clínic Barcelona, University of Barcelona, Barcelona/Spain

${ }^{3}$ Department Of Radiology, Hospital Clinic Barcelona, University of Barcelona, Barcelona/Spain

${ }^{4}$ Hepatobiliopancreatic Surgery And Transplant Unit, Department Of Surgery, Hospital Clinic Barcelona, University of Barcelona, Barcelona/Spain

${ }^{5}$ Gastroenterology Department, Hospital Clinic Barcelona, CIBEREHD,

IDIBAPS, University of Barcelona, Barcelona/Spain

${ }^{6}$ Endoscopy Unit, Hospital Clínic, University of Barcelona, Barcelona/Spain

${ }^{7}$ Exocrine Pancreas Research Unit, Hospital Universitari Vall dHebron,

Barcelona/Spain

${ }^{8}$ Pt Government Associate Laboratory, 4 ICVS/3B's, Braga/Guimarães/Portugal

Contact E-mail Address: tiagomcg@ hotmail.com

Introduction: According to the 2012 International guidelines on the management of intraductal papillary mucinous neoplasms (IPMN), main-duct IPMN patients with main pancreatic duct (MPD) diameter of $\geq 10 \mathrm{~mm}$ should have surgical resection, whereas surgery is not always mandatory in those with MDP diameter between 5 and $9 \mathrm{~mm}$.

Aims \& Methods: The aim of the study was to analyze the prevalence of malignancy (high grade dysplasia or invasive carcinoma) in resected IPMN with MPD diameter between 5 and $9 \mathrm{~mm}$ and to identify predictive factors of malignancy. Retrospective analysis of patients with surgically resected IPMN between 2001 and 2016. Demographics, clinical presentation, imaging and histological features were compared between patients with preoperative evidence of MPD diameter between 5-9 mm (Group A) and $\geq 10 \mathrm{~mm}$ (Group B). Malignancy was defined as high-grade dysplasia or invasive carcinoma.

Results: From 122 patients with IPMN submitted to surgery, 66 with MD- or mixed-IPMN entered the final analysis. Mean age was $66 \pm 12$ years and 48 $(72.7 \%)$ patients were men. Group A comprised 47 patients and Group B 19. Abdominal pain was present in $23(34.8 \%)$ patients, jaundice in $19(28.8 \%)$, diabetes in $18(27.3 \%)$, pancreatitis in $15(22.7 \%)$ and weight loss in 12 $(18.2 \%)$, with no statistical differences between study groups. The most common location of the MD-IPMN was the head of pancreas $(60.6 \%)$, and it was multifocal in $34.8 \%$ of the patients. The prevalence of no dysplasia, lowgrade dysplasia, high-grade dysplasia and invasive carcinoma was $10.6 \%, 42.6 \%$, $8.5 \%$ and $38.3 \%$ in group $\mathrm{A}$ and $10.5 \%, 10.5 \%, 21.1 \%$ and $57.9 \%$ in Group $\mathrm{B}$ patients. The overall malignancy rate was $46.8 \%$ in group A and $79 \%$ in group B. Jaundice $(\mathrm{p}=0.017)$, weight loss $(\mathrm{p}=0.035)$, and complete involvement of MPD at pathological analysis $(p=0.018)$ were significantly more common in patients with malignancy.

Conclusion: Almost half of resected IPMN with MPD diameter between 5-9 mm harbor histologically proven malignancy. In these patients, particularly in those with jaundice and weight loss, surgery rather than follow-up should be recommended.

Disclosure of Interest: All authors have declared no conflicts of interest.

\section{P1432 CLINICAL SIGNIFICANCE OF CHEMOTHERAPY FOR ELDERLY UNRESECTABLE PANCREATIC CANCER PATIENTS}

S. Kaino, M. Sen-Yo, S. Shinoda, S. Amano, I. Sakaida

Department Of Gastroenterology \& Hepatology, Yamaguchi University

Gastroenterology \& Hepatology, Ube/Japan

Contact E-mail Address: kaino@yamaguchi-u.ac.jp

Introduction: Pancreatic cancer has poor prognosis despite of improvements in multimodal treatments. As aging of the population advances, it is expected that elderly pancreatic cancer patients increase.

Aims \& Methods: The aim of this study was to investigate the clinical significance of chemotherapy for patients with unresectable pancreatic cancer. At our hospital, 96 patients were diagnosed as having unresectable pancreatic cancer between January 2010 and December 2016. In this study, we defined elderly patients as those older than 75 years. We retrospectively examined the safety and efficacy of chemotherapy in patients with unresectable pancreatic cancer. We analyzed and compared survival periods and adverse events between young and aged patients. Results: Twenty-seven patients were older than 75 years (group A), and 59 were younger than 74 years (group B). We treated $6 / 10 / 0 / 2 / 5 / 4$ patients in group A with GEM/S-1/modified FOLFIRINOX (mFOLFIRINOX)/GEM + nabPTX best supportive care (BSC)/others, respectively. On the other hand, we treated $12 / 14 / 4 / 11 / 5 / 13$ patients in group $B$ with GEM/S-1/mFOLFIRINOX/ GEM + nabPTX/BSC/others, respectively. Severe adverse events (more severe than grade 3 according to CTCAE v4.0) occurred in $18.2 \%$ of the patients in group A and in $33.3 \%$ of the patients in group B. No significant difference was found between the two groups. The median survival time of the patients who were receiving chemotherapies was 197.0 days in group A and 291.0 days in group B. No significant differences were also found between the two groups. The median survival time of the patients who underwent chemotherapy in group A (332.0 days) was significantly longer than that of patients who underwent BSC (71.0 days).

Conclusion: Conclusions: Chemotherapy could be safe and effective for patients older than 75 years who have unresectable pancreatic cancer.

Disclosure of Interest: All authors have declared no conflicts of interest.

\section{P1433 THE USE OF A NEW CORE NEEDLE IN THE ENDOSCOPIC ULTRASOUND ASSISTED TISSUE SAMPLING FOR PANCREATIC SOLID MASSES: A MULTICENTRE PROSPECTIVE STUDY}

S. Carrara ${ }^{1}$, M. Di Leo ${ }^{2}$, L. Bernardoni ${ }^{3}$, D. Rahal ${ }^{2}$, G. Donato ${ }^{4}$, M. Massidda ${ }^{5}$, A. Anderloni ${ }^{2}$, S. Crino ${ }^{3}$, E. Manfrin ${ }^{6}$, M. Ballare ${ }^{4}$, L. Poliani ${ }^{2}$, F. Auriemma ${ }^{2}$, A. Fugazza ${ }^{7}$, P. Occhipinti ${ }^{4}$, A. Gabbrielli ${ }^{3}$, A. Repici ${ }^{2}$

${ }^{1}$ Department Of Gastroenterology, Humanitas Research Hospital, Endoscopic Unit, Rozzano/Italy

${ }^{2}$ Humanitas Research Hospital, Milan, Italy., Rozzano/Italy

${ }^{3}$ Gastroenterology, University of Verona, Verona/Italy

${ }_{5}^{4}$ Azienda ospedaliero-universitaria Maggiore della Carità di Novara, Novara/Italy ${ }^{5}$ Istituto Clinico Humanitas Gavazzeni, Bergamo/Italy

${ }^{6}$ University of Verona, Verona/Italy

${ }^{7}$ Digestive Endoscopy Unit, Division Of Gastroenterology, Humanitas Research

Hospital, Rozzano, Milan, Italy, Rozzano/Italy

Contact E-mail Address: silvia.carrara@hunimed.eu

Introduction: Endoscopic ultrasound-guided biopsy (EUS-biopsy) is considered a reliable, safe, and effective technique for obtaining samples from pancreatic masses with a very high sensitivity and specificity (ranged to $85 \%-92 \%$ and 96-98\%, respectively)[1-3]. A new EUS needle (SharkCore FNB needle, Medtronic, Dublin, Ireland) was introduced in order to improve the tissue acquisition.

Aims \& Methods: The aim of the present study was to evaluate the presence of a histological sample using Shark Core Needles. This study was an observational multicenter prospective non-randomized clinical trial (NCT02946840). All consecutive patients referred for EUS examination and sampling of solid pancreatic masses underwent EUS-guided biopsy with $25 \mathrm{G}$ Shark Core needles. This needle has an innovative tip geometry with a cutting surface designed to acquire cohesive units of tissue and to minimize tissue fracturing. Three passes were performed in every mass. At every pass, a macroscopic on-site quality evaluation (MOSE) was done by endoscopist. If a "worm-like" material was observed at gross visual assessment, it was placed into formalin. If only liquid material was obtained, it was smeared between 2 glass slides, fixed with ethanol, and stained with a Papanicolaou-stain for cytological analysis. Endoscopists recorded macroscopic features of the specimens. Pathologists described macroscopic, microscopic features, immunohistochemical studies. The primary outcome was the procurement rates of histologic core. Pathologists defined core all histological samples with architecturally intact histology, measuring at least $5 \mathrm{~mm}$ in greatest axis. All the other specimens $(<5 \mathrm{~mm})$ were defined as micro-fragments. The final diagnosis was based on surgical resection, and clinical/radiological follow up. The secondary outcomes were diagnostic accuracy and procedure-related adverse events.

Results: Study population included 82 patients, enrolled in three centres, between August 2016 and April 2017, with a mean age of 64.0 (SD 13.7, range 21-84) and $57.3 \%$ female gender. The mean size of the lesions was $27.6 \mathrm{~mm}$ (SD 12.2) and the location was the body and tail in 27 patients $(32.9 \%)$, neck in $11(13.4 \%)$, head and uncinated process in $44(53.7 \%)$. Three needle passages were performed in all but 3 patients who experienced mild bleeding precluding more than one needle passage. At MOSE, endoscopists described presence of "worm-like" material in 192 biopsy samples over $242(79.3 \%)$. In 8 patients only cytological specimens were obtained after 3 needle passes $(9.8 \%)$. Six cases of mild self-limited bleeding were observed $(7.3 \%)$. The pathologists described the presence of a core in 80 samples $(41.7 \%)$, in the other cases, after the specimen preparation, a micro-fragmentation was observed, that didn't affect the histological evaluation. A final histological diagnosis was reached in 73 patients $(90 \%)$ : 50 pancreatic adenocarcinoma, 16 NET, 5 chronic pancreatitis, 2 pancreatic metastasis from other organs.

Conclusion: The new biopsy needle showed a good overall adequacy and a good rate of histological specimens (both core and micro-fragments) during EUSguided tissue acquisition of pancreatic masses, with a minimum number of passes and no major complications. This ability could allow to avoid the use of rapid on-site evaluation and to perform immunohistochemical, molecular and genetic studies on histological samples.

Disclosure of Interest: All authors have declared no conflicts of interest. 


\section{References}

1. Chen J et al., J Cancer Res Clin Oncol 2012;

2. Hewitt MJ et al., GIE 2012;

3. Banafea $\mathrm{O}$ et al., BMC Gastroenterol 2016.

\section{P1434 A 5- YEAR TERTIARY CENTRE EXPERIENCE OF ENDOSCOPIC ULTRASOUND GUIDED FINE NEEDLE ASPIRATION FOR DIAGNOSIS OF SOLID PANCREATIC MASS LESIONS}

T. Skouras ${ }^{1}$, L. Bonnett ${ }^{\circ}$, M. Swaminathan ${ }^{2}$, R. Mcgalliard ${ }^{2}$, A. Metussin ${ }^{2}$, J. Evans ${ }^{3}$, S. Sarkar ${ }^{2}$, T. Giles ${ }^{4}$, A. Cullen ${ }^{4}$, K. Brougham ${ }^{4}$, J. Sheard ${ }^{5}$ ${ }^{1}$ Gastroenterology, Royal Liverpool University Hospital, Liverpool/United Kingdom

${ }^{2}$ Division Of Gastroenterology And Hepatology, Royal Liverpool and Broadgreen University Hospitals NHS Trust, Liverpool/United Kingdom

${ }^{3}$ Interventional Radiology, Royal Liverpool and Broadgreen University Hospitals NHS Trust, Liverpool/United Kingdom

${ }^{4}$ Cytopathology, Royal Liverpool and Broadgreen University Hospitals NHS Trust, Liverpool/United Kingdom

${ }^{5}$ Pathology, Royal Liverpool and Broadgreen University Hospitals NHS Trust, Liverpool/United Kingdom

Contact E-mail Address: Thomas.skouras@nhs.net Introduction: EUS FNA is accepted as the primary modality for tissue diagnosis of solid pancreatic lesions. The presence of on-site cytopathology for immediate evaluation during endoscopic ultrasound-guided fine needle aspiration (EUS FNA) has previously been shown to improve performance characteristics. Due to service pressures EUS FNA is also undertaken in the absence of in-room cytopathology assistance. This is a review of current practice.

Aims \& Methods: We retrospectively assessed 700 consecutive EUS-FNA procedures from January 2011 to January 2016. 459 (65.5\%) solid pancreatic lesions were included in the final analysis after excluding 230 for biliary strictures, hepatic lesions, lymph nodes, gastric, oesophageal lesions, pancreatic cysts and 11 for insufficient information.

Results: In $399(86.9 \%)$ cases on-site cytopathology support was available, while the remaining was unsupported. There were 228 males $(57.1 \%)$ in the supported and $29(48.3 \%)$ in the unsupported group. Mean age was 64.6 (SD: 11.4) and 67.4 (SD: 11.9) respectively. The mean number of passes in the two groups were 2.8 (SD: 1.12$)$ and 1.9 (SD: 1.0$)(\mathrm{P}<0.0001)$. A conclusive diagnosis (malignant, benign, NET, GIST) was made in $84 \%(67 \%, 12 \%, 5 \%, 0 \%)$ of the supported group and in $38 \%(23 \%, 10 \%, 3 \%, 2 \%)$ of the unsupported $(\mathrm{P}<0.0001)$. The mean follow up for the entire cohort was 14.2 months (SD:14.1) and mean survival of in patients diagnosed with malignancy was 10.9 months (SD: 8.7) Overall performance characteristics of EUS FNA were Sensitivity: 90.8\% Specificity: $86.9 \%$ PPV: $91.8 \%$ NPV: $85.4 \%$

Conclusion: This review confirms high performance characteristics of EUS FNA The presence of on-site cytopathologist significantly increases the diagnostic yield.

Disclosure of Interest: All authors have declared no conflicts of interest.

\section{P1435 CLINICAL IMPACT OF GNAS AND KRAS MOLECULAR ALTERATIONS ADDED TO CEA AND CYTOLOGY IN PANCREATIC CYSTIC FLUID OBTAINED BY EUS-FNA}

S. Faias ${ }^{1}$, M. Duarte ${ }^{2}$, C. Albuquerque ${ }^{2}$, R. Roque ${ }^{3}$, J. Pereira Da Silva ${ }^{1}$, R. Fonseca ${ }^{3}$, A. Dias Pereira ${ }^{1}$

${ }^{1}$ Gastroenterology, Instituto Português de Oncologia Francisco Gentil, Lisboa Portugal

${ }^{2}$ CIPM, IPO Lisboa, Lisboa/Portugal

${ }^{3}$ Pathology, IPO Lisboa, Lisboa/Portugal

Contact E-mail Address: sandrarfaias@hotmail.com

Introduction: Pancreatic cystic lesions are a common finding in clinical practice. Classification of cysts as mucinous or non-mucinous cysts, using EUS-FNA with cystic fluid analysis for cytology and CEA became widely used in clinical workup of patients with suspicious pancreatic cysts. Molecular analysis (KRAS and GNAS mutation) is not yet recommended in clinical practice.

Aims \& Methods: We aimed to etermine if mutation in GNAS and KRAS in addition to CEA level and cytology of cystic fluid obtained by EUS-FNA can help in pancreatic cyst classification and decision making. Evaluation of methylation of the GNAS complex locus was performed for cyst classification.

Between 2008-14, 266 EUS were performed for pancreatic cyst evaluation in a single center. We determined the mutational status of GNAS (exons 8 and 9) and KRAS (exons 2 and 3 ) genes by Sanger sequencing in 52 patients, in cystic fluid obtained by EUS-FNA after cytology and CEA analysis. In operated patients, cysts were also analyzed for methylation of the GNAS complex locus.

Results: Mainly female $(67 \%)$, mean age of $59 \pm 15$ years (29-91). Cysts located in head $(42 \%)$, body $(39 \%)$, tail $(17 \%)$ and multiple $(2 \%)$, with a mean size of $3.9 \pm 2.3 \mathrm{~cm}(.8-10)$, and $60 \%>3 \mathrm{~cm}$. Cyst types (as after EUS-FNA + CEA/ cytology): 15 serous cystadenomas (SCAs), 9 pseudocysts, 8 intraductal papillary mucinous neoplasms (IPMNs), 2 mucinous cystic neoplasms (MCNs), 4 adenocarcinomas (ADC), 1 solid pseudopapillary neoplasm (SPN), 1 lymphangioma, 12 non-defined. CEA $>192 \mathrm{ng} / \mathrm{ml}$ in $33 \%$ of patients and cytology with benign cells in $12(23 \%)$, suspicious/malignant in $8(15 \%), 1$ NET $(2 \%)$ and $31(60 \%)$ acellular samples. Surgery in $11(21 \%)$ patients (surgical specimens: 2SCA $3 \mathrm{MCN}, 4 \mathrm{IPMN}, 1$ retention cyst and 1 ADC), chemotherapy/palliation in 6
$(12 \%)$, endoscopic drainage in $1(2 \%)$ and $34(64 \%)$ on f-up. KRAS Mutation in $19 \%$ (9) and in GNAS in 4\%(2) of cyst aspirates. By cyst type, GNAS was mutated in 1 IPMN and 1 ADC. KRAS was mutated in 6 patients on fup (2 IPMNs, 3 ADC, 1 pseudocyst- a unilocular $2 \mathrm{~cm}$ cyst with CEA = 125) and in 3 who had surgery (2 IPMNs and $1 \mathrm{MCN}$ ). Sensitivity and specificity of CEA $>192+$ cytology and KRAS/GNAS mutations for the classification of mucinous and non-mucinous (table 1-1st part) and malignant and non-malignant (table 1-2nd part) are presented in operated cysts.

\begin{tabular}{lll}
\hline Diagnosis of neoplastic mucinous cysts & Sensitivity & Specificity \\
\hline CEA > 192 + citology & $100 \%$ & $67 \%$ \\
KRAS/GNAS mutation & $42 \%$ & $100 \%$ \\
Diagnosis of malignant cysts & & \\
CEA > 192 + citology & $100 \%$ & $33 \%$ \\
KRAS/GNAS mutation & $50 \%$ & $75 \%$ \\
\hline
\end{tabular}

In the surgery group, methylation of the GNAS complex locus was found in $1 / 2$ ADC, $1 / 3$ IPMNs (the malignant IPMN has GNAs mutation and no methylation of the GNAS complex), $1 / 3 \mathrm{MCNs}, 0 / 2 \mathrm{SCA}$ and $0 / 1$ retention cyst.

Conclusion: Sanger sequencing of KRAS and GNAS genes is highly specific for the diagnosis of mucinous neoplastic cysts of the pancreas and may be useful in selected patients. The specificity of Sanger sequencing of KRAS and GNAS genes for the diagnosis of malignant cystic lesions decreases but is higher than CEA/cytology analysis. Methylation in GNAS complex locus was found in malignant cysts but data are preliminary and need further evaluation. More fluid markers are needed to improve pancreatic cyst classification.

All other authors have declared no conflicts of interest.

Disclosure of Interest: S. Faias: This Work was Financed by a Research Grant of Clube Português Do Pâncreas.

\section{P1436 KRAS MUTATION ASSAY ON EUS FNA SPECIMENS FROM} PACIENTS WITH PANCREATIC MASS

M. Laclav ${ }^{1}$, B. Bunganic ${ }^{1}$, T. Halkova ${ }^{2}$, L. Benesova $^{3}$, B. Belsanova ${ }^{3}$, E. Traboulsi ${ }^{4}$, S. Suchanek ${ }^{5}$, M. Minarik ${ }^{3}$, M. Zavoral ${ }^{1}$

${ }^{1}$ Department Of Internal Medicine, Military University Hospital,1st Faculty of Medicine, Charles University, Prague/Czech Republic

${ }^{2}$ Center for Applied Genomics of Solid Tumors (CEGES), Genomac Research Institute, Prague/Czech Republic

${ }^{3}$ Genomac Research Institute Center for Applied Genomics of Solid Tumors,

Prague/Czech Republic

${ }^{4}$ Department Of Pathology, Military University Hospital, Prague/Czech Republic ${ }_{5}^{5}$ Department Of Internal Medicine, 1st Faculty Of Medicine, Charles University, Military University Hospital, Prague/Czech Republic

Contact E-mail Address: martin.laclav@uvn.cz

Introduction: Promising method, which helps to distinguish between chronic pancreatitis and cancer, is point mutations of the proto-oncogene KRAS test. This method is not established in routine clinical practice yet.

Aims \& Methods: Determination of the sensitivity of the KRAS assay using various kinds of samples of patients with pancreatic mass and testing the effect of the presence of KRAS mutations on the prognosis of survival. 147 patients underwent EUS-FNA examination of pancreatic mass, accompanied by blood sampling with subsequent separation of plasma for the detection of circulating tumor DNA. Part of biopsy sample was left native in a stabilizing solution and part as cytological smear. Samples (native aspirates, cytological smears, plasma) were examined for the presence of KRAS mutation by heteroduplex analysis, denaturing capillary electrophoresis.

Results: Among 147 patients with pancreatic masses, $118 \mathrm{x}$ were diagnosed as cancer, 26x chronic pancreatitis, 3x neuroendocrine tumor. In total 147 native aspirates, 118 cytological smears and 94 plasma samples were examined. The highest sensitivity of $K R A S$ mutation was reached in the group of pancreatic cancer patients using cytology, in which $90 \%$ of $K R A S$ mutation was detected $(106 / 118$ of the samples). When using the native cellular aspirates, mutation was detected in $78 \%$ (92/118 samples), and examination of plasma was positive in $27 \%$ (24/90 samples).In four patients with chronic pancreatitis KRAS mutations was detected, although none has been cytologically confirmed as a cancer. Two of these four patients were confirmed in the course of the disease as a cancer, one patient died because of alcoholic delirium and the last one was indicated for surgery recently.

Conclusion: Examination of KRAS mutations can be performed in all patients undergoing EUS-FNA, with the cytology being the most reliable type of sample for genetic tests KRAS examination would be reasonable to introduce into routine clinical practice in a group of patients with unclear differential diagnosis of chronic pancreatitis, especially in those with suspicion of cancer in inflammatory terrain. Financial suport by The Ministry of Defence and Armed Forces of the Czech Republic MO 1012

Disclosure of Interest: All authors have declared no conflicts of interest.

\section{References}

Chari ST, Kelly K, Hollingsworth MA, Thayer SP, Ahlquist DA, Andersen DK, Batra SK, Brentnall TA, Canto M, Cleeter DF, Firpo MA, Gambhir SS, Go VL, Hines OJ, Kenner BJ, Klimstra DS, Lerch MM, Levy MJ, Maitra A, Mulvihill SJ, Petersen GM, Rhim AD, Simeone DM, Srivastava S, Tanaka M, Vinik AI, 
Wong D. Early detection of sporadic pancreatic cancer: summative review. Pancreas. 2015 Jul;44(5):693-712.

Kenner BJ, Chari ST, Cleeter DF, Go VL. Early detection of sporadic pancreatic cancer: strategic map for innovation-a white paper. Pancreas. 2015 Jul;44(5):686-92.

Majumder S, Chari ST, Ahlquist DA. Molecular detection of pancreatic neoplasia: Current status and future promise. World J Gasoenterol. 2015 Oct 28;21(40):11387-95. Review.

Bournet B, Gayral M, Torrisani J, Selves J, Cordelier P, Buscail L. Role of endoscopic ultrasound in the molecular diagnosis of pancreatic cancer. World $J$ Gastroenterol. 2014 Aug 21;20(31):10758-68. Review.

\section{P1437 DO BILIARY STENTS REDUCE THE DIAGNOSTIC PERFORMANCE OF EUS BIOPSY IN PATIENTS WITH A MASS IN THE HEAD OF PANCREAS?}

N. L. Bekkali ${ }^{1}$, M. Nayar ${ }^{1}$, L. Thornton ${ }^{1}$, S. J. Johnson ${ }^{2}$, B. Haugk ${ }^{2}$, A. Darne ${ }^{2}$, J. S. Leeds ${ }^{1}$, R. Charnley ${ }^{3}$, K. Oppong ${ }^{1}$

${ }^{1}$ Hpb-medicine, Freeman Hospital, Newcastle upon Tyne/United Kingdom ${ }^{2}$ Cellular Pathology, Royal Victoria Infirmary, Newcastle upon Tyne/United Kingdom

${ }^{3}$ Hepatobiliary and Pancreatic Surgery, Freeman Hospital, Newcastle Upon Tyne, United Kingdom, newcastle/United Kingdom

Contact E-mail Address: noorbekkali@hotmail.com

Introduction: Self-expanding metal stents (SEMS) are increasingly preferred to plastic stents (PS) for pre-operative drainage and palliation of biliary obstruction secondary to a stricture in the head of pancreas (HOP). Their use has increased over the last 5-6 years. Endoscopic ultrasound (EUS) with fine needle aspiration or biopsy (FNA or FNB) is commonly utilised to make a tissue diagnosis and to aid in staging in those with borderline resectable tumours. Stents may reduce diagnostic performance of FNA/FNB by reducing the visible mass to puncture. There have been 2 studies that assessed the impact of stenting on EUS-FNA performance, one ${ }^{1}$ found no difference in yield and sensitivity among patients with or without stents and between SEMS and plastic. Whilst a more recent study ${ }^{2}$ found accuracy was significantly reduced by the presence of a stent.

Aims \& Methods: The aim was to assess whether stents (SEMS or PS) impair diagnostic performance of EUS tissue acquisition, in a retrospective study of all patients with HOP mass undergoing EUS biopsy between January 2010 and June 2016. Stenting information was obtained from the EUS report and images. Biopsies reported as malignant were considered as such, all other reports were considered benign. A definitive diagnosis of cancer was based on positive pathology or follow up with signs of progression. A benign diagnosis required negative pathology, stable imaging and symptoms for a year or more. Patients with cystic lesions were excluded.

Results: A total of 1861 patients had EUS FNA/FNB of which 731 were for HOP lesions, mean age 65 yrs $(410 \mathrm{~F})$, with tissue sensitivity of $72 \%$ for all types of needles used. Tissue accuracy was significantly different between the 3 groups $(\mathrm{p}=0.0001)$; SEMS $67 \%$, PS $71 \%$ and $83 \%$ in the unstented group. The difference in accuracy was significant between the unstented group versus SEMS $(\mathrm{p}=0.0002)$ and PS $(\mathrm{p}=0.003)$ and not significant between PS and SEMS. Stepwise multi-variable analysis revealed significant difference for accurate tissue diagnosis favouring size needle 25G (OR 1.7 [95\% CI 1.1-2.7] and tumour size (OR 1.04 [1.02-1.07]) and negative affected by presence of stent SEMS (OR $0.3[0.2-0.6]$ ) or PS (OR $0.5[0.3-0.9])$. Other needle sizes (19G or $22 \mathrm{G})$, number of passes or types of needle did not significantly affect tissue accuracy.

Conclusion: Our results show a significant adverse impact of both SEMS and PS on tissue accuracy via EUS FNA/FNB. The effect is greatest with SEMS. These results suggest that where possible EUS and biopsy if required should be performed before stent placement.

Disclosure of Interest: All authors have declared no conflicts of interest.

\section{References}

1. Ranney N, Phadnis M, Trevino J, et al. Impact of biliary stents on EUSguided FNA of pancreatic mass lesions. Gastrointest. Endosc. 2012;76:76-83.

2. Kim JJ, Walia S, Lee SH, et al. Lower Yield of Endoscopic UltrasoundGuided Fine-Needle Aspiration in Patients with Pancreatic Head Mass with a Biliary Stent. Dig. Dis. Sci. 2015;60:543-549.

\section{P1438 SOLID-PSEUDOPAPILLARY NEOPLASM OF THE PANCREAS (SPN) DOES NOT CONTRAINDICATE EUS PONCTION: ABOUT A RETROSPECTIVE SERIES OF FIFTY PATIENTS WITH PREOPERATIVE EUS-FNA (THE SPN G.R.A.P.H.E. SERIES)}

D. Karsenti ${ }^{1}$, S. Koch ${ }^{2}$, L. Poincloux ${ }^{3}$, U. Chaput ${ }^{4}$, G. Vanbiervliet ${ }^{5}$, F. Cholet ${ }^{6}$, M. Wangermez ${ }^{7}$, P. Ah-Soune ${ }^{8}$, P. Bichard ${ }^{9}$, Y. Le Baleur ${ }^{10}$, J. Privat ${ }^{11}$, E. Coron ${ }^{12}$, C. Subtil ${ }^{13}$, M. Palazzo ${ }^{14}$

${ }^{1}$ Digestive Endoscopy Unit, Clinique de Bercy, Charenton-le-Pont/France ${ }^{2}$ Gastroenterology Unit, Chru Minjoz Besancon, Besancon/France

${ }^{3}$ CHU Estaing, service de médecine digestive et hépato-biliaire Clermont Ferrand, Clermont-Ferrand/France

${ }^{4}$ Digestive Endoscopy Unit, CHU St Antoine, Paris/France

${ }^{5}$ Gastroenterology, CHU, Hôpital ĹArchet 2 Gastroentérologie, Nice/France

${ }^{6}$ Gastroenterology, CHU La Cavale Blanche, Brest/France
${ }^{7}$ Dept. De Endoscopie Digestive, CHU Poitiers, Poitiers/France

${ }^{8}$ Digestive Endoscopy Unit, CHU Toulon, Toulon/France

${ }^{9}$ Digestive Endoscopy Unit, CH Genève, Genève/Switzerland

${ }^{10} \mathrm{CHU}$ Henri Mondor, Créteil/France

${ }^{11}$ Gastroenterology, Centre Hospitalier De Vichy, Vichy/France

${ }^{12}$ Digestive Diseases Institute, University of Nantes Institute of Digestive Disease Digestive Diseases Institute, University of $\mathrm{Na}$, Nantes/France

${ }^{13} \mathrm{CHU}$ Bordeaux, Bordeaux/France

${ }^{14}$ Pancreatology, CHU Beaujon, Clichy-la-Garenne/France

Contact E-mail Address: karsenti@club-internet.fr

Introduction: Solid-pseudopapillary neoplasm (SPN) is a rare condition, first described by Frantz in 1959. It occurs mostly in young women, and surgical resection is recommended. Its local recurrence rate is less than $10 \%$ and usually occurs within 4 years after surgery. Before such a surgery, especially in young people, EUS-FNA (Endoscopic ultrasonography with fine needle aspiration) is discussed to confirm diagnosis but rarely performed due to suspected needle tract inoculation by neoplastic cells. The aim of our large multicenter study was to assess the short- and long-term safety of preoperative EUS-FNA in SPN.

Aims \& Methods: This study is a multicenter retrospective register of all SPN diagnosed in the last decade in 14 European expert centers (GRAPHE task force). Inclusion criterion was realization of preoperative EUS-FNA followed by surgical resection. Patient and tumor characteristics were collected, as the EUS-FNA technique (number of passes, needle size, trans-gastric or trans-duodenal access). Immediate or late complications of EUS-FNA and recurrence of SPN were then recorded.

Results: During the period study, 49 patients ( 41 women $/ 8$ men) with preoperative EUS-FNA for SPN were recorded. Mean age of patients was $37 \pm 15$ yo. Tumor location was mostly cephalic $(21 / 36,59 \%)$, but also corporeal in $25 \%$ (9/36), caudal in $25 \%(9 / 36)$, isthmic in $11 \%(4 / 36)$ and in uncus in $5 \%(2 / 36)$. Mean tumor size measured on EUS was $40 \pm 22 \mathrm{~mm}$. Needle used was a $25 \mathrm{G}$ in $3 \%(1 / 37), 22 \mathrm{G}$ in $76 \%(28 / 37), 20 \mathrm{G}$ in $8 \%(3 / 37)$ and $19 \mathrm{G}$ in $13.5 \%(5 / 37)$. Trans-duodenal EUS-FNA was performed in $56 \%(24 / 43)$ and trans-gastric in $44 \%$ (19/43). Mean number of needle passes was $2.2 \pm 0.7$. EUS-FNA allowed certain preoperative diagnosis of SPN in $74 \%$ of cases $(35 / 47)$, probable diagnosis in $6 \%(3 / 47)$, negative in $4 \%(2 / 47)$ and wrong in $6 \%(3 / 47)$. No acute complication of EUS-FNA was reported. With a mean follow-up of $36 \pm 36$ months, only one local recurrence was noted. In this 74 years old man case, a $19 \mathrm{G}$ needle was used ( 2 trans-gastric passes), and recurrence occurred after 84 months.

Conclusion: In this large multicenter retrospective series, a systematic preoperative EUS-FNA did not seem to modify the SPN recurrence rate. Therefore this study allows to validate this attitude as a possible alternative. The data for the series are incomplete at the date of submission of the abstract. The final data will be completed on the day of presentation.

Disclosure of Interest: All authors have declared no conflicts of interest.

\section{P1439 DIAGNOSIS OF PANCREATIC NEUROENDOCRINE TUMOURS USING SUREPATH CYTOLOGY AND IMMUNOHISTOCHEMISTRY WITHOUT NEED FOR EXCISION BIOPSY}

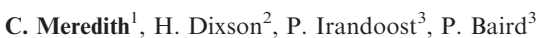

${ }_{1}^{1}$ Gastroenterology And Hepatology, Bankstown-Lidcombe Hospital, Bankstown/ Australia/NSW

${ }^{2}$ Ultrasound And Nuclear Medicine, Bankstown-Lidcombe Hospital, Bankstown Australia/NSW

${ }^{3}$ Cytology, Laverty Pathology, Ryde/Australia/NSW

Contact E-mail Address: drcmeredith@gmail.com Introduction: Pancreatic neuroendocrine tumours (PNETs) are relatively rare, i.e., $\leq 1$ per 100,000 individuals per annum, and account for only $1-2$ percent of all pancreatic tumours. They are separated into 2 major categories: 1) welldifferentiated (WD-NETs) which have round to oval nuclei, coarsely stippled chromatin and finely granular cytoplasm and 2) poorly-differentiated (PDNETs) which have a diffuse architecture with an irregular nucleus and less cytoplasmic granularity. WD-NETs tend to have an indolent course (survival $\sim 67 \%$ at 5 years) but $\sim 50 \%$ have metastasised at the time of diagnosis. PD-NETs are high-grade cancers with an aggressive course resembling NETs arising in lung. WD-NETs contain neurosecretory granules which stain for synaptophysin and or chromogranin. Endoscopic ultrasonography guided fine-needle aspiration biopsy (EUS-FNA) can provide a non-operative cytological diagnosis of PNETs when the pathologist is provided with a good specimen such as the pellet of cells obtained through SurePath (SP).

Aims \& Methods: EUS-FNA samples of pancreatic tumours were collected into a SurePath vial and slides prepared from the cellular pellet. The slides were stained for synaptophysin and Ki67 by immunohistochemistry (IHC) and examined by 2 independent senior cytopathologists.

Results: Sixteen (16) patients with a mean age 65 ( 8 male) were identified by EUS with a suspected PNET. The mean tumour size was $16.2 \mathrm{~mm}+/-4.2 \mathrm{~mm}$. All had the morphology of a PNET and stained positive for synaptophysin.

Conclusion: SP prepared cytology slides from solid pancreatic tumours provides enough diagnostic material for IHC staining for synaptophysin and $\mathrm{Ki} 67$ without the need for a formal excision biopsy. Morphology of SP slides is often diagnostic but a positive stain for synaptophysin makes the diagnosis irrefutable. The 
mitotic index derived from Ki67 staining helps identify WD-NETs which can be monitored and PD-NETs which need aggressive treatment.

Disclosure of Interest: All authors have declared no conflicts of interest.

\section{P1440 EFFECTS OF IGF2BPS ON GROWTH AND PROLIFERATION IN NEUROENDOCRINE PANCREATIC TUMOR CELL LINES}

L. Nietfeld ${ }^{1}$, F. Sperling ${ }^{1}$, K. Theuerkorn ${ }^{1}$, H. Griesmann ${ }^{1}$, S. Krug ${ }^{1}$, S. Hüttelmaier ${ }^{2}$, P. Michl ${ }^{1}$

${ }^{1}$ Internal Medicine I, Martin-Luther-University Halle-Wittenberg, Halle (Saale)/ Germany

${ }^{2}$ Institute Of Molecular Medicine, Martin-Luther-University Halle-Wittenberg, Halle (Saale)/Germany

Contact E-mail Address: lars.nietfeld@uk-halle.de

Introduction: Pancreatic neuroendocrine neoplasms (PNEN) are highly angiogenic tumors which-despite of various targeted options including mTOR and VEGF inhibition-frequently develop secondary drug resistance. IGF2BṔs (IGF2 mRNA-binding proteins) represent a family of canonical RNA-binding proteins (RBP) comprised of three members (IGF2BP1-3) which have been described to promote stem and/or progenitor cell maintenance with reported expression and oncogenic roles in aggressive cancers. IGF2BṔs show a differential expression pattern in various solid tumors including pancreatic neuroendocrine tumors.

Aims \& Methods: We aimed to characterize the role of IGF2BṔs in progression and resistance of pancreatic neuroendocrine neoplasms. We used three different siRNA-pools (IGF2BP1/2/3) to inhibit the different IFG2BP's in pancreatic neuroendocrine BON1 tumor cells. Cellular effects where investigated by Western blot analyses, flow cytometry, clonogenic survival, cell viability and migration assays.

Results: In the pancreatic neuroendocrine tumor cell line BON1, knock-down of IGF2BP1 resulted in a significant reduction of cell viability. Cell cycle analysis by FACS showed a decreased $\mathrm{S}$ phase progression paralleled by a reduction in the proliferation marker PCNA and a markedly reduced MEK/ERK activation. In contrast, Akt signaling was unaffected. Moreover, knock-down of IGF2BP1 significantly reduced clonogenic growth as assessed by colony formation assays and led to decreased cell migration as determined by scratch assays. Interestingly, knock-down of IGF2BP1 was insufficient to induce apoptosis, as assessed by PARP and caspase-3 cleavage as well as annexin-V FACS. Rather, si-IGF2BP1 increased the expression of both the anti-apoptotic and pro-survival factor BCL2 and the cell cycle inhibitor CDKN1B. In contrast to IGF2BP1, knock-down of IGF2BP3 rather induced cell viability, whereas IGF2BP2 modulation had no impact on cell viability and cell cycle progression indicating opposing effects of the three IGF2BṔs on PNEN progression. These in vitro findings were paralleled by distinct expression patterns of IGF2BPs in human and murine PNEN tissues. Elucidation of IGF2BP-modulated RNÁs in PNEN cells is ongoing. Conclusion: In summary, our data suggest that IGF2BP1 promotes tumor progression by enhancing cell cycle progression and clonogenic growth, whereas IGF2BP2 and -3 exert no tumor-promoting role in PNEN.

Disclosure of Interest: All authors have declared no conflicts of interest.

P1441 CONCOMITANT NEUROENDOCRINE TUMOR OF THE PANCREAS AND INTRADUCTAL PAPILLARY MUCINOUS NEOPLASM: IS THERE SOMETHING BEYOND?

L. Venezia ${ }^{1}$, P. Cortegoso Valdivia ${ }^{1}$, C.G. De Angelis ${ }^{1}$

${ }^{1}$ Gastroenterology, A.O.U. Città della Salute e della Scienza, Torino/Italy

Contact E-mail Address: ludovica.venezia@gmail.com

Introduction: Intraductal papillary mucinous neoplasms (IPMNs) and neuroendocrine tumors (NETs) of the pancreas are rare tumors. Up to now 15 patients with association of endocrine and exocrine neoplasms of the pancreas have been reported in the literature, but this association is not expected to be frequent. The aim of this study is to describe a series of nine patients with IPMN and concomitant NET of the pancreas followed in our center and to look for any common findings between them that could help early recognition and further comprehension of this association.

Aims \& Methods: Among all the patients who were followed in our clinic for IPMN or NET, we identified 9 with both diseases, diagnosed on imaging or after biopsies requested for uncertain results of previous investigations. We collected data about the patients (age, sex, symptoms, past and current medical history, blood chemistry, type of imaging) and about the lesions (classification, dimension, localization, imaging features, fine-needle aspiration (FNA) biopsies results and histology).

Results: All patients ( 4 men and 5 women), except one were asymptomatic. Average age at diagnosis was 61 years. All IPMN were branch duct, localized in the head $(n=4)$ and in body-tail $(n=5)$, with a medium diameter of the cysts of $12 \mathrm{~mm}$ (range $8 \mathrm{~mm}-16 \mathrm{~mm}$ ). All NETs underwent endoscopic ultrasound examination with FNA; 8 were non functional G1 with a Ki67 of $1 \%$, positive for Chromogranine A and Neuronospecific Enolase staining. One NET was a symptomatic insulinoma located at the tail (Ki67 of $2 \%, 20 \mathrm{~mm}$ diameter) and underwent distal spleno-pancreasectomy. Among NETs, 6 were in the head and 3 at body-tail, with a medium diameter of $11 \mathrm{~mm}$ (range $6 \mathrm{~m}-15 \mathrm{~mm}$ ). In 4 patients, the first diagnosis was IPMN, followed by identification of NET with second level imaging. In 5 patients, who underwent magnetic resonance imaging for NET, a concomitant IPMN was found

Conclusion: Our study describes 9 cases of endocrine-exocrine pancreatic neoplasms association. In the 15 cases reported by literature one out of the two lesions, had a malignant behavior. In our records, except in one patient, both IPMN and NET in all patients behave in an indolent fashion. All the IPMN were branch type and all the NET, except one, were G1; mainly patients are asymptomatic and a hormone production symptom was observed in only one case. We found neither sex or age predisposition, nor predominant localization or common pathologic background in the patients. No other concomitant neoplastic diseases were found. Although the frequency of association between NET and IPMN is not too large and no diagnostic hallmark were found, it is still to be proven that this association is fortuitous. Further studies in the future are required

Disclosure of Interest: All authors have declared no conflicts of interest.

\section{References}

1. Ishida $\mathrm{M}$, Shiomi $\mathrm{H}$ et al. Concomitant intraductal papillary mucinous neoplasm and neuroendocrine tumor of the pancreas. Oncol Lett 2013, 5(1):6367.

2. Gill KR, Scimeca D et al. Pancreatic neuroendocrine tumors among patients with intraductal papillary mucinous neoplasms: real association or just a coincidence? JOP 2009, 10(5):515-517.

3. Tewari M, Zaitoun AM et al. Three cases of concomitant intraductal papillary mucinous neoplasm and pancreaticneuroendocrine tumour. JOP 2013 Jul 10;14(4):423-7.

\section{P1442 EFFECTS OF LOW-DOSES ASPIRIN ON CLINICAL} OUTCOME AND DISEASE PROGRESSION IN PATIENTS WITH GASTRO-ENTERO-PANCREATIC NEUROENDOCRINE TUMORS: RESULTS OF A MULTICENTRIC RETROSPECTIVE STUDY

S. Massironi ${ }^{1}$, S. Pusceddu ${ }^{2}$, F. Cavalcoli ${ }^{1}$, A. Zilli ${ }^{1}$, G. Tamagno ${ }^{3}$, D. Femia ${ }^{2}$, N. Prinzi ${ }^{2}$, C. Ciafardini ${ }^{1}$, D. Conte ${ }^{1}$

${ }^{1}$ Gastroenterology And Endoscopy Unit, Fondazione IRCCS Ca Granda Ospedale Maggiore Policlinico, Milano/Italy

${ }^{2}$ Medical Oncology, Unit ${ }^{1}$ Enets Center Of Excellence, Fondazione IRCCS Istituto Tumori Milano, Milan/Italy

${ }^{3}$ Department Of Endocrinology/Diabetes, Mater Misericordiae University

Hospital, Dublin/Ireland

Contact E-mail Address: cavalcoli.federica@gmail.com

Introduction: The chemopreventive effect of aspirin (ASA) and other NSAIDs have been observed in the setting of colorectal cancer, showing a reduction in the incidence and mortality. However, the impact of aspirin use on clinical outcome of patients with gastro-entero- pancreatic neoplasms (GEP NEN) has not yet been evaluated.

Aims \& Methods: Aim of the study was to retrospectively evaluate the clinical outcome of GEP NEN patients treated with ASA at three different European referral Centres for NENs. All the GEP NENs patients followed up in three European Centres (Fondazione IRCCS Ca' Granda Ospedale Policlinico Milano, Italy; Fondazione IRCCS Istituto Tumori Milano. Italy; Mater Misericordiae University Hospital, Dublin, Ireland), from January 2005 and September 2016, were retrospectively enrolled. The possible association between ASA and disease grading, staging, primary site, overall OS and PFS were evaluated. At the time of enrolment, clinical data and biochemical parameters were collected for every patient. Chromogranin A $(\mathrm{CgA})$ and specific circulating peptides were evaluated. Morphological and functional imaging (computed tomography, magnetic resonance and Gallium 68PET) were performed to follow up the patients at each Centre.

Results: In the 253 patients included (121 M, median age $64 \mathrm{yrs})$, the primary neuroendocrine tumor was located at the stomach (\#35), pancreas (\#82), small bowel (\#80), appendix (27), colon (\#19) or unknown (\#7). Grading was G1 in 154 patients, G2 in 64, G3 in 5 and not available in 28. TNM staging was I in 99 patients, II in 16, III in 32 and IV in 86 . No clear impact on OS or PFS was observed in patients taking ASA compared to those not taking it. Interestingly, in pNEN an inverse relation was observed between Ki67 and ASA assumption $(\mathrm{r}=-0.35, \mathrm{p}=0.008)$. In small bowel NEN an inverse relation was observed between positive lymphnodes at surgery and ASA assumptions $(r=-0.3$, $\mathrm{p}=0.02$ ). As expected, the intake of ASA was related with the older age of the patients.

Conclusion: According to present data, ASA therapy seems not to have a direct clinical impact on disease progression or survival of NENs, even if it is associated with lower Ki-67 values and less node involvement. Further studies are needed to confirm this observation.

Disclosure of Interest: All authors have declared no conflicts of interest. 


\section{References}

1. Rothwell PM, Fowkes FG, Belch JF, Ogawa H, Warlow CP, Meade TW. Effect of daily aspirin on long-term risk of death due to cancer: analysis of individual patient data from randomised trials. Lancet 2011; 377: 31-41.

2. Rothwell PM, Wilson M, Price JF, Belch JF, Meade TW, Mehta Z. Effect of daily aspirin on risk of cancer metastasis: a study of incident cancers during randomised controlled trials. Lancet 2012; 379: 1591-1601.

3. Spampatti M, Vlotides G, Spöttl G, Maurer J, Göke B, Auernhammer CJ. Aspirin inhibits cell viability and mTOR downstream signaling in gastroenteropancreatic and bronchopulmonary neuroendocrine tumor cells. World $J$ Gastroenterol. 2014; 20: 10038-49

\section{P1443 PANCREATIC LESIONS IN VON HIPPEL-LINDAU SYNDROME: CLINICAL AND EPIDEMIOLOGICAL DATA FROM A SINGLE CENTER}

L. Venezia ${ }^{1}$, P. Cortegoso Valdivia ${ }^{1}$, C.G. De Angelis ${ }^{1}$

${ }^{1}$ Gastroenterology, A.O.U. Città della Salute e della Scienza, Torino/Italy

\section{Contact E-mail Address: cortegosopablo@yahoo.it}

Introduction: Von Hippel-Lindau disease (VHL) is a rare heritable genetic syndrome that may affect different systems and organs: pancreatic manifestations of the disease are frequent during lifetime of the patients. The key feature is the presence of simple cysts, but serous cystadenomas (SCAs) or neuroendocrine tumors (NETs) can be frequently found as well. The aim of this study is to describe pancreatic manifestations in patients with VHL, considering the peculiarity and rarity of this disease.

Aims \& Methods: All patients who referred to the established multi-disciplinary team in our center (Molinette Hospital - Turin) for management and follow-up of VHL disease were included in the study: we then considered the ones with pancreatic involvement (simple cysts, SCAs or pNETs). We collected data about the patients (demographics and medical history), about the lesions (imaging features, hystological and cytological analysis) and about the management.

Results: We enrolled a total of 24 patients, 18 of which $(75 \%)$ had a pancreatic involvement. Multiple simple pancreatic cysts were found in 13 patients, SCAs were found in 2 patients and NETs in 7 patients. The mean age of the patients with pancreatic lesions was $42(\min 25-\max 75), 11$ were males and 7 females (1.6:1 M:F). Simple cysts affected 13 patients, are always multiple (ranging from 12 to $80 \mathrm{~mm}$ ) mostly in the head. 3 patients underwent surgery for symptomatic disease. All pNETs were well differentiated $(\mathrm{G} 1, \mathrm{Ki} 67<2 \%) ; 7$ were located in the head and 2 in the tail ( 2 patients had multiple tumors). 5 out of the $7 \mathrm{pNET}$ patients underwent surgery. The two SCAs were multiple $(\max 65 \mathrm{~mm})$, mostly affecting the head in 1 case and the tail in the other. No surgery was performed. Conclusion: $75 \%$ of our VHL patients showed pancreatic involvement, mostly in males compared to females. $72 \%$ of patients with pancreatic lesions suffered from simple cysts, $39 \%$ from NETs and $11 \%$ from SCAs. To note that all NETs were G1 and behaved in a benign fashion. Surgery was performed only in patients with NETs in the pancreatic head and in patients with symptomatic cystic disease. The mean age of incidence of VHL-related pancreatic lesions was lower than in sporadic cases, thus confirming literature data. Although all lesions in our patients were benign or stable, constant monitoring is needed.

Disclosure of Interest: All authors have declared no conflicts of interest.

\section{Reference}

De Mestier L, Hammel P. Pancreatic neuroendocrine tumors in von HippelLindau disease. Scand J Gastroenterol 2015 Aug;50(8):1054-5

\section{P1444 IMPACT OF TUMOUR SIZE ON THE PROBABILITY OF METASTASIS AND SURVIVAL IN PATIENTS WITH PANCREATIC NEUROENDOCRINE TUMOURS (PNETS): A POPULATION-BASED STUDY}

Y. Liu ${ }^{1}$, B. Ye ${ }^{2}$, X. He ${ }^{3}$, S. Chen ${ }^{3}$, J. Pan ${ }^{4}$, L. Wang ${ }^{5}$, H. Shi ${ }^{6}$

${ }^{1}$ Institute of Gastroenterology, Hangzhou/China

${ }^{2}$ Gastroenterology, Lishui Central Hospital, Zhejiang University Lishui Hospital, Lishui/China

${ }^{3}$ Department Of Gastroenterology, Sir Run Run Shaw Hospital, Zhejiang University, Hangzhou/China

${ }^{4}$ Department Of Endocrinology And Metabolism, Second Affliated Hospital of

Zhejiang University School of Medicine, Hangzhou/China

${ }^{5}$ The second Affiliated Hospital, School of Medicine, Zhejiang University, Hangzhou/China

${ }^{6}$ Lishui Central Hospital, Zhejiang University Lishui Hospital, Lishui/China

\section{Contact E-mail Address: liu_chit@163.com}

Introduction: Neuroendocrine tumours (NET) consist of a diverse group of neoplasms that derive from diffuse neuroendocrine cells throughout the body. Commonly found in gastrointestinal (GI) duct and lung, they also arise in the pancreas. The relationship between tumour size and metastasis rate is poorly recognized in patients with pancreatic neuroendocrine tumours (PNETs). The impact of tumour size on prognosis was controversial in previous investigations. Aims \& Methods: The aim of this study is to evaluate the prognostic impact of tumour size on survival outcomes and its correlation with risk of metastasis in a large PNETs cohort, including all stages. Methods: PNETs cases diagnosed from 1988 to 2013 were retrieved from the Surveillance, Epidemiology, and End
Results (SEER) database. Clinicopathologic features were retrospectively analyzed. Survival was calculated by the Kaplan-Meier method. Multivariable Cox regression models with hazard ratios (HRs) were constructed to analyze survival outcomes and risk factors. Cubic spline analysis was used to assess relationship between tumor size and probability of metastasis.

Results: A total of 5424 patients were identified. There were 1226 patients $(22.6 \%)$ with tumour size of $2 \mathrm{~cm}$ or less. The probability of metastasis increased in a non-linear fashion with increasing tumours size. Univariate analysis showed that tumour size was significantly correlated with survival $(\mathrm{P}<0.001)$, no matter surgery was performed or not. However, subgroup analysis suggested this association to be linear for patients with localized and regional tumours $(P<0.001)$, but stochastic in patients with distant stages $(\mathrm{P}=0.703)$. On multivariate analysis, tumour size was an indicator for metastasis $(\mathrm{HR}=1.010,95 \% \mathrm{CI}$ : 1.008 $1.013, \mathrm{P}<0.001)$ and size $\leq 20 \mathrm{~mm}$ was an independent prognostic factor for good survival $(\mathrm{HR}=1.211,95 \% \mathrm{CI}: 1.048-1.399, \mathrm{P}=0.009$ for size of 21 $40 \mathrm{~mm} ; \mathrm{HR}=1.282,95 \% \mathrm{CI}: 1.116-1.474, \mathrm{P}<0.001$ for size $>40 \mathrm{~mm}$ ). For tumours $\leq 20 \mathrm{~mm}$, surgical treatment was associated with significantly improved survival compared with those patients who did not undergo operation $(\mathrm{P}<0.001)$.

Conclusion: Tumour size affects the probability of metastasis. Its prognostic impact on survival is restricted to patients with localized and regional disease. For patients with tumour size $\leq 20 \mathrm{~mm}$, surgical treatment should be considered preferably.

Disclosure of Interest: All authors have declared no conflicts of interest.

\section{References}

1. McKenna LR, Edil $\mathrm{BH}$, et al. Update on pancreatic neuroendocrine tumours. Gland Surg. 2014, 3 (4): 258-275.

2. Jin K, Luo G, Xu J, Zhang B, Liu C, Ji S, Liu L, Long J, Ni Q, Yu X. Clinical outcomes and prognostic factors of resected pancreatic neuroendocrine neoplasms: A single-center experience in China. Oncology Letters. 2017, 1899.

3. J Franko, W Feng, L Yip, E Genovese, AJ Moser. Non-functional Neuroendocrine Carcinoma of the Pancreas: Incidence, Tumor Biology, and Outcomes in 2,158 Patients. J Gastrointest Surg. 2010, 14: 541-548.

4. National Cancer Institute Surveillance, Epdemiology, and End Results Program. SEER*Stat Database: Incidence-SEER 18 Regs Custom Data (with chemotherapy recode), Nov 2015 Sub (1973-2013 varying). https:// seer.cancer.gov/seerstat/[accessed 25 July 2016]

5. Bettini R, Partelli S, Boninsegna L, et al. Tumor size correlates with malignancy in nonfunctioning pancreatic endocrine tumor. Surgery. 2011, 150: 7582 .

\section{P1445 THE LARGEST FAMILY IN TURKEY WITH MULTIPLE}

\section{ENDOCRINE NEOPLASIA-TYPE 1 AND A NOVEL MUTATION}

\section{A. Cetin ${ }^{1}$, D. G. Duman ${ }^{2}$}

${ }^{1}$ Internal Medicine, Marmara University School of Medicine, Istanbul/Turkey ${ }^{2}$ Gastroenterology, Marmara University Hospital, Istanbul/Turkey

\section{Contact E-mail Address: alicetindr@gmail.com}

Introduction: Multiple Endocrine Neoplasia type-1 (MEN-1) occurs usually sporadically but because it is an autosomal dominant disorder it may affect other family members too. The combination of parathyroid tumors, pancreatic islet cell tumors, and anterior pituitary tumors is characteristic of MEN-1 although it may be accompanied by a substantial number of non-endocrine tumors. The mutations of the MEN-1 result in the alteration of MENIN production which is a protein encoded by this gene and is responsible for tumor-supression under normal circumstances. Herein we present the largest MEN-1 family in Turkey to the best of our knowledge and a newly discovered mutation in MEN-1 that effects this family.

Aims \& Methods: The family inherited the specific MEN-1 mutation is originally S, ebinkarahisar, Giresun and most of the members have moved to Istanbul and Yalova. Consanguineous marriages have been practiced within the family. Family mapping was structured and contained fifty-five members. Among them, eleven patients underwent biochemical, radiologic and if necessary endosonographic evaluation along with the genetic testing. Additionally, we learnt that 2 members of the family had died of pancreatic malignancy. Diagnostic criteria for familial MEN-1 syndrome include; 1- the presence of at least one MEN-1 associated tumor that are from parathyroid, pituitary, or GEP tract origins, 2- at least one first-degree relative with one or more of these endocrine tumors and/or 3-positive genetic testing for abnormal MEN-1 mutation. For our index case, DNA sequencing of the MEN-1 gene performed using Sanger sequencing technique at ABI 3500 sytem. For the other family members only the targeted mutation analysis is performed

Results: Among the family members we had peripheral blood DNA from 9 and we used the tumour tissue DNA from 1 patient. MENIN gene is analyzed with Sanger sequencing. Our index case was ZK who had hypophysis adenoma, parathyroidectomy and pancreatic neuroendocrine tumour and had whipple operation. A three nucleotide deletion p.ser560argfs*3(c.1680 1683 del TGAG) mutation causing a frameshift was detected. Seven out of ten who were analysed tested positive for the mutation. Genetic counseling and information about preimplantation genetic diagnosis (PGD) was given to all patients who are tested positive for the mutation. All 7 patients had p.ser560argfs*3(c.1680_1683 del TGAG) three nucleotide deletion same with that of index case. Out of 15 patients, MEN-1 diagnosis was confirmed in 11. Tumours detected at patients with MEN-1 diagnosis were; nonfunctional pancretic neuroendocrine tumour at three, parathyroid adenoma/hyperplasia at 6 patients and hypophysis adenoma 
at 4 patients. The mean age of our MEN-1 patients were 51 and the age at diagnosis was 41.8 . The mean plasma calcium level was $11.46 \mathrm{mg} / \mathrm{dl}$ and there was no history of renal calculus at any of them.

Conclusion: The family presented here is the one which had the largest number of affected individuals with genetic and clinical properties of MEN-1 at Turkey. Besides the mutation detected here is the novel pathogenic mutation causing loss of function that is described for the first time. Also at MEN-1 families, counseling to prevent the neoplasia development and to prevent the new family member to be effected with PGD has a pivotal importance. We could point out that awareness is the most important caution for prevention.

Disclosure of Interest: All authors have declared no conflicts of interest

\section{P1446 PROGNOSTIC VALUE OF THE DIFFERENT PRE- TREATMENT BIOMARKERS FOR PATIENTS WITH NEUROENDOCRINE TUMORS}

R.R. R. Grigorescu ${ }^{1}$, C. Gheorghe ${ }^{2}$, I. M. Stanel ${ }^{1}$, A. Croitoru ${ }^{3}$, I. Dinu ${ }^{3}$, V. Croitoru ${ }^{4}$

${ }^{1}$ Dept. Of Gastroenterology, Institutul Clinic Fundeni, Bucuresti/Romania

${ }^{2} 1$ st Dept. Of Gastroenterology \& Endoscopy, PetCenter of Gastroenterology and Hepatology, Bucharest/Romania

${ }^{3}$ Dept. Of Oncology, Fundeni Clinical Institute, Bucuresti/Romania

${ }^{4} U M F$ "Carol Davila", Bucharest/Romania

\section{Contact E-mail Address: ralucargrigorescu@yahoo.com}

Introduction: Several inflammatory response materials could be used for prediction of prognosis in cancer patients. The neutrophil lymphocyte ratio (NLR), platelet lymphocyte ratio (PLR), thrombocytosis (the platelets number $>400^{*} 10^{3} / \mathrm{mm}^{3}$ ) have been introduced for prognostic scoring system in various cancers.

Aims \& Methods: The objective of this study was to determine whether the NLR, the PLR or thrombocytosis could predict the clinical outcomes in G1-G2 neuroendocrine tumors. We performed a retrospective review of 31 patients with neuroendocrine tumors with ki 67 below $20 \%$ diagnosed in Fundeni Clinical Institute between 2011-2017. Data about site of the primary tumor, presence of metastasis, NLR, PLR, thrombocytosis (platelet count $>400$ ) and survival were collected and analysed.

Results: The patients characteristics were: primary tumor location was: $61.29 \%$ pancreas, $22.58 \%$ gastrointestinal tract, $16.13 \%$ unknown, $61.29 \%$ had hepatic metastasis, $6.45 \%$ had locally advanced tumor. The primary tumor was resected in $35.48 \%$ patients. The overall 2-year survival rate was $77.42 \%$. The Ki 67 index $(\mathrm{p}<0.04)$, PLR (cut off $>300) \mathrm{p}<0.01$ have statistical significant impact on survival in univariate analysis and on multivariate analysis $(\mathrm{P}<0.05)$. Other factors like ki 67 index, metastatic disease, thrombocytosis and NLR have an impact on survival statistical significant on multivariate analysis.

Conclusion: This study demonstrates the prognostic role of different variables like Ki 67 index, PLR and PLT value, thrombocytosis and metastasis. This factors may be integrated in different scoring systems for prognosis that could guide clinicians for a better management in patients with neuroendocrine tumors.

Disclosure of Interest: All authors have declared no conflicts of interest.

\section{References}

1. in patients with pancreatic cancer. Aliustaoglu M, et Al. Hepato-gastroenterology 2010, 57(99-100):640-645

2. Prognostic Factors in Pancreatic Carcinoma: Serum LDH Levels Predict Survival in Metastatic Disease. Tas, Faruk M.D.; Aykan, Faruk M.D.;et Al. American Journal of Clinical Oncology: December 2001 - Volume 24 Issue 6 - pp 547-550

3. Diagnostic Value Of The Neutrophil To Lymphocyte Ratio And The Platelet To Lymphocyte Ratio In Patients With Gastric Adenocarcinoma Zulfu Bayhan, Sezgin Zeren Fatma Emel Kocak, Cuneyt Kahraman4, Cengiz Kocak5, Sukru Aydin Duzgun Acta Medica Mediterranea, 2016, 32: 111

4. S, ENTURK, Mehmet et al. The role of the mean platelet volume and neutrophil-to-lymphocyte ratio in peritonsillar abscesses,. Braz. j. otorhinolaryngol. [online]. 2016, vol.82, n.6

\section{P1447 FUNCTIONAL RELEVANCE OF THE OVEREXPRESSION OF PLAC8 IN NEUROENDOCRINE PANCREATIC TUMORS}

M. Hutzler ${ }^{1}$, M. Haijat ${ }^{1}$, H. Schmidt ${ }^{1}$, R. Lawlor ${ }^{2}$, A. Scarpa ${ }^{2}$, T.M. Gress ${ }^{1}$, M. Buchholz

${ }_{1}^{1}$ Department Of Gastroenterology, Philipps-University Marburg, Marburg/ Germany

${ }^{2}$ Department Of Pathology And Diagnostics, University and Hospital Trust of Verona, Verona/Italy

\section{Contact E-mail Address: marina.hutzler@staff.uni-marburg.de}

Introduction: Neuroendocrine pancreatic tumors represent the second prevalent entity of malignant tumours of the pancreas and show an overall mortality of about $60 \%$. At the moment surgical resection is the only option of potentially curative therapy, as with the currently available chemo- and radiotherapeutic approaches an inhibition of tumor growth but no regression of the tumor can be achieved. Therefore for about $80 \%$ of pNET patients no curative therapy can be offered. To obtain the identification of novel potential target genes for the development of new therapeutic strategies, primary tissues from pNET patients were analyzed. Amongst others Plac8 (Placenta-specific 8) was identified, which is a small protein of unknown function, showing different forms of cellular localisation depending on the cell type analyzed, indicating at its ability to fulfill a variety of physiological functions.

Aims \& Methods: In the course of this study, the function of Plac8 in neuroendocrine pancreatic tumors is to be unveiled to evaluate its value as a potential target for pNET therapy. Therefore primary tumor tissue of about 100 pNET patients were analyzed for Plac8 expression by quantitative realtime PCR and immunohistochemistry. Furthermore established pNET cell lines from human origin where transfected with siRNAs against Plac8 and there proliferative activity and viability where analyzed by BrdU and MTT assay. Changes in these important characteristics of tumor cells were further examined by westernblot analyzes of key regulators of apoptosis and cell growth.

Results: Plac8 is highly expressed in primary human pNET tissues on RNA- as well as on protein level. Functional in vitro analyses show that the siRNAmediated knockdown of Plac8 not only in human but also in rat cell lines leads to significantly reduced proliferative activity and reduced cell growth. These effects come along with indicative changes in the expression of central regulators of cell cycle while apoptotic pathways seem not to be affected.

Conclusion: Overexpression of Plac8 in neuroendocrine tumors of the pancreas promotes the proliferative phenotype of the tumor cells while the inhibition of Plac8 inhibits cell growth and metabolism. Therefore in the future Plac8 could represent a very interesting target molecule for the treatment of pNETs.

Disclosure of Interest: All authors have declared no conflicts of interest.

WEDNESDAY, NOVEMBER 01, 2017

ENDOSCOPY AND IMAGING III - HALL 7

09:00-14:00

P1448 CLINICAL OUTCOMES OF SUPERFICIAL

LARYNGOPHARYNGEAL CANCER WITH LYMPHO-VASCULAR

INVASION AFTER ENDOSCOPIC LARYNGO-PHARYNGEAL

SURGERY

H. Ishida ${ }^{1}$, R. Nakamura ${ }^{1}$, T. Omori ${ }^{2}$, S. Mayanagi ${ }^{1}$, K. Fukuda ${ }^{1}$, K. Suda ${ }^{1}$,

N. Wada ${ }^{1}$, H. Kawakubo ${ }^{1}$, Y. Kitagawa

${ }^{1}$ Surgery, Keio University School of Medicine, Tokyo/Japan

${ }^{2}$ Departmen Of Surgery, Kawasaki Municipal Kawasaki Hospital, Kawasaki/

Japan

Contact E-mail Address: hishida223@gmail.com

Introduction: Since the majority of laryngopharyngeal carcinomas are detected at an advanced stage, most cases are treated with concurrent chemotherapy and radiation therapy. The key to improving the prognosis and quality of life is early detection of the primary cancer and treatment using minimally invasive surgery. We previously reported the good oncologic outcomes with ELPS (Endoscopic laryngo-pharyngeal surgery) for superficial laryngo-pharyngeal carcinoma. However there is no clinical evidence for an additional treatment nor prognosis about the cases conducted endoscopic resection which were diagnosed to be surficial carcinoma with lympho-vascular invasion histopathologically.

Aims \& Methods: This study aimed to investigate the optimal additional treatment and clinical course for the surficial laryngo-pharyngeal carcinoma with lympho-vascular invasion. We analyzed clinicopathological data in 9 patients showed Lympho-vascular invasion receiving ELPS between 2007 and 2014.

Results: Positive lympho-vascular invasion was found in 9 cases. Detected the tumor depth was SEP in 7 lesions and MP in 2 lesions. Mean alcohol consumption is 9.9 abv units. Average smoking history is 38.9 pack years. 5 cases are lowactivity ALDH2 heterozygotes and have alcohol flushing reaction. Histopathological findings showed 5 cases with lymphatic invasion (ly1, v0), 2 cases with vascular invasion(ly0, v1), and 2cases with lympho-vascular invasion.(ly1, v1). Two patients underwent an additional chemoradiotherapy without recurrence. Four patients had cervical lymph node or local recurrence, two of them were salvage ELPS cases after chemoradiotherapy. One of two salvage cases also had distant metastasis and was given palliative treatment, and finally died. The other one underwent surgical salvage and remained alive. One case with lymphatic and vascular invasion had no adjuvant therapy and remained no recurrence. And the other 2 cases had no recurrence but died of other cause. Conclusion: Lympho-vascular invasion is a risk factor for cervical lymph node metastasis, which has a possibility to a very aggressive disease. In those cases, chemoradiotherapy as an additional treatment is recommended as far as possilble.If patients already had prior radiotherapy, close follow-up is essential to detect recurrence early. In those cases, chemotherapy or additional surgical resection are also considerable if the general conditions are satisfactory.

Disclosure of Interest: All authors have declared no conflicts of interest.

\section{P1449 LONG-TERM OUTCOMES OF EARLY GASTRIC CANCER WITH LATERAL MARGIN POSITIVE AFTER ENDOSCOPIC RESECTION}

H. Kim ${ }^{1}$, D. Yang ${ }^{1}$, J. Park ${ }^{2}$, B.W. Bang 2 , Y.W. Shin ${ }^{2}$

${ }^{1}$ Gastroenterology, Inha University Hospital, Incheon/Korea, Republic of

${ }^{2}$ Division Of Gastroenteroloy, Department Of Internal Medicine, Inha University School of Medicine, Incheon/Korea, Republic of

Contact E-mail Address: kimhg@inha.ac.kr

Introduction: The positive lateral margin after endoscopic resection(ER) of early gastric cancer(EGC), additional surgery or endoscopic submucosal dissection(ESD) are recommended. However, the additional surgery often difficult due to advanced age or patient's comorbid conditions.

Aims \& Methods: The aims of this study is to investigate of appropriate management in patients with positive lateral margin after ER. We analyzed 
retrospectively 103 patients with positive lateral margin after ER from January 2005 to December 2015, in single tertiary center. Clinicopathological data were retrieved to assess the local recurrence rate, survival rate, procedure related adverse events.

Results: Of the 103 patients, 27 patients $(26.4 \%)$ underwent early re-treatment within 3 months after initial ER(17 patients in re-do ESD, 10 patients in additional surgery). And 76 patients $(73.6 \%)$ were observed under close surveillance. Median duration of follow-up period was 45.7(6-132) months. Recurrence rates of early re-treatment group $3.7 \%, \mathrm{n}=1 / 27)$ was lower than surveillance group $(18.4 \%, n=14 / 76 ; p=0.05)$. five-year survival rates not significantly different between the two groups, at $100 \%, 97.4 \%$ respectively. In close surveillance periods, 12 patients were confirmed to local recurrence by follow-up biopsy, then delayed re-treatment was performed (7 patients in re-do ESD, 5 patients in surgery, mean time after initial $E R=27.5$ months). Finally, a total of 24 patients were treated with re-ESD, and 17 patients were treated with additional surgery. Among these two groups, there were no significant difference in recurrence rates $(8.3 \%$ vs. $0 \%)$ and five-year survival rates (both $100 \%)$. However, adverse events that related to treatment was more frequent in additional surgical group ( 2 ileus, 1 umbilical hernia).

Conclusion: The additional treatment should be recommended for patients with positive lateral margin after initial ER for EGCs. In addition, re-ESD which have a similar efficacy and a better quality of life, compared to additional surgery is a favorable option for control of recurrence or residual EGCs.

Disclosure of Interest: All authors have declared no conflicts of interest.

\section{P1450 EFFICACY OF THE FORCED COAGULATION MODE WITH LOW HIGH-FREQUENCY POWER SETTING DURING} ENDOSCOPIC SUBMUCOSAL DISSECTION

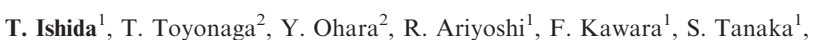
Y. Morita ${ }^{1}$, E. Umegaki ${ }^{1}$, N. Hoshi ${ }^{1}$, T. Azuma ${ }^{1}$

${ }^{1}$ Gastroenterology, Kobe University Graduate School of Medicine, Kobe/Japan

${ }^{2}$ Dept. Of Endoscopy, Kobe University Hospital, Kobe/Japan

Contact E-mail Address: tishida@med.kobe-u.ac.jp

Introduction: Bleeding control is one of the most important factors to success endoscopic submucosal dissection (ESD) in safety. We have reported the endoscopic precoagulation technique using soft coagulation mode (S method) is effective $^{1}$. This method is useful for the prevention of bleeding when relatively large vessels penetrating between muscle layers are dissected. However we have thought that $\mathrm{S}$ method is insufficient especially for large vessels such as more than $2 \mathrm{~mm}$, we have to use hemostatic forceps for preventing hemorrhage despite trying $\mathrm{S}$ method. However we found that forced coagulation mode with low highfrequency power setting (F1-10 method) can exhibit precoagulation function without bursting vessels. It is suggested that F1-10 method is useful for large vessel precoagulation.

Aims \& Methods: We investigated the deference of hemostatic ability between S method and F1-10 method in clinical study and ex vivo study. In clinical study we analyzed retrospectively their hemostatic ability about consecutive six gastric ESD cases in each groups excluded some cases, which have the risk of affecting data for example taking anticoagulation drugs. The diameter of treated vessels, frequency and duration of the compressed vessel, and bleeding frequency after cutting vessels were noted by the recorded videos. In ex vivo study, to investigate the coagulation mechanism of those two different power settings was evaluated the peak voltage, current elapsed time, and electric energy by using the data recording program (VIO DOKU) and the width and depth of coagulation was measured on macro- and microscopic levels using porcine tissues.

Results: In clinical study, the total number of vessels processed by endoknife precoagulation were 49 and 61 vessels in the S and F1-10 methods. The median vessel diameter was $2 \mathrm{~mm}$ in the $\mathrm{S}$ method and $1.5 \mathrm{~mm}$ in the F1-10 method. The median frequency of the compressed vessel was twice in both methods, and the median coagulation time was $9 \mathrm{~s}$ and $10 \mathrm{~s}$ in the S and F1-10 methods, respectively. No significant difference was found between the S and F1-10 methods. The bleeding rate after vessel processing was $18.4 \%(8 / 49)$ and $4.8 \%(3 /$ $62)$ in the $\mathrm{S}$ and F1-10 methods, respectively $(P=0.058$, odds ratio [OR]: 3.84 , $95 \%$ confidence interval $[\mathrm{CI}]: 0.96-15.34)$. No statistically significant differences were found; however, the F1-10 method strongly showed a trend in preventing bleeding after precoagulation compared with the $\mathrm{S}$ method $(P=0.058)$. We further investigated the bleeding rates of the large vessels, defined as $\geq 2 \mathrm{~mm}$ in diameter. There were 26 and 29 large vessels in the S and F1-10 methods, respectively. The bleeding rates of the large vessels were $26.9 \%(7 / 26)$ and $3.4 \%(1 / 29)$ in the S and F1-10 methods, respectively, which were significantly different $(P=0.021$, OR: $10.32,95 \%$ CI: $1.17-90.78)$. In ex vivo study, the median peak voltage was $172 \mathrm{Vp}$ and $560 \mathrm{Vp}$ in the $\mathrm{S}$ and F1-10 methods. The median current elapsed time was $0.22 \mathrm{~s}$ and $1.54 \mathrm{~s}$ in the $\mathrm{S}$ and F1-10 methods. The F1-10 method could keep the electric current longer than the S method. The mean total electrical energy was $6.34 \mathrm{~W}$ and $12.5 \mathrm{~W}$ in the S and F1-10 methods. F1-10 method was able to achieve to give larger electrical energy in the poutine block. And the width and depth of coagulation in F1-10 method spread wider and deeper in macro- and microscopic sections.

Conclusion: F1-10 method is suggested to achieve a stronger hemostatic effect than the $\mathrm{S}$ method in clinical procedures and ex vivo models.

Disclosure of Interest: T. Toyonaga: Dr. Takashi Toyonaga invented the FlushKnife-BT in conjunction with Fujifilm and received royalties from its sale. All other authors have declared no conflicts of interest.

\section{Reference}

1. Tanaka S, Toyonaga T, Morita Y. Endoscopic vessel sealing: a novel endoscopic precoagulation technique for blood vessels during endoscopic submucosal dissection. Dig Endosc. 2013; 25: 341-342.

\section{P1451 EXPOSURE TO ENDOTHERAPY FOR UPPER GASTROINTESTINAL BLEEDING AT THE POINT OF GASTROSCOPY CERTIFICATION-IS IT SUFFICIENT?}

K. Siau, P. Dunckley, J. Anderson, I. Beales, R. Broughton, M. Feeney, A. Haycock, N. Hawkes, R. Valori, C. Wells, S. Thomas-Gibson, B. Mckaig *JAG Working Group For The Quality Assurance Of Training, Joint Advisory Group, London/United Kingdom

\section{Contact E-mail Address: keith@siau.org}

Introduction: Although certification in diagnostic gastroscopy has been established in the UK, there is no formal process for quality assurance (QA) in endotherapy for upper gastrointestinal bleeding (UGIB). Training opportunities are variable, with $11 \%$ of final year UK gastroenterology trainees citing inadequate exposure ${ }^{[1]}$ despite an expectation to independently manage UGIB upon achieving specialist status. Data on endotherapy exposure during endoscopy training is limited. We aimed to assess whether trainees are receiving adequate exposure to endotherapy at the time of gastroscopy certification.

Aims \& Methods: Trainees awarded certification in gastroscopy between September 2009-2016 were identified from the national trainee electronic portfolio (JETS). Trainee inputs and formative assessments (direct observation of procedural skills - DOPS) for UGIB therapy, up to their certification date, were analysed. Only trainees with $>200$ procedures were included, which is the minimum procedural number to allow certification within the UK, thereby excluding those who had submitted baseline information which may have contained therapeutic data. Exposure rates from medical endoscopists (physician and surgical trainees) were compared with non-medical endoscopists (NME). Results: 885 trainee portfolios were analysed (765 medical and 120 NMEs), with a median procedural count of 276 (IQR 124). The median number of therapeutic entries and DOPS were 4 (IQR 11), and 1 (IQR 3) respectively. Overall rates for endotherapy and DOPS were $2.9 \%$ and $0.8 \%$ per procedure. When stratified by therapy, the median exposure to each therapy was either 0 or 1 , with means displayed in Table $1.25 .2 \%$ of trainees had no exposure to any type of endotherapy $(67.5 \%$ of NME and $18.6 \%$ of medical endoscopists, $p<0.0001)$. Of medical endoscopists awarded certification, $37.1 \%$ had not performed band ligation, $50.7 \%$ had not placed a clip, and $54 \%$ had not used heater probe. NME had significantly less exposure to each modality of endotherapy considered (overall odds ratio $0.10, p<0.0001)$.

Table 1: Mean procedural counts at the point of UGI certification.

\begin{tabular}{|c|c|c|c|c|c|c|c|c|}
\hline & Total & $\begin{array}{l}\text { UGIB } \\
\text { Therapy }\end{array}$ & DOPS & Argon & Banding & Clip & $\begin{array}{l}\text { Heater } \\
\text { Probe }\end{array}$ & Injection \\
\hline Medical & 346 & 10.7 & 2.6 & 2.1 & 4.4 & 1.6 & 1.6 & 4.0 \\
\hline Non-medical & 323 & 1.1 & 0.29 & 0.3 & 0.4 & 0.1 & 0.1 & 0.3 \\
\hline $\mathrm{p}$-value & 0.143 & $<0.0001$ & & & & & & \\
\hline
\end{tabular}

Conclusion: Training on endotherapy prior to certification is limited. The current UGI certification process does not ensure competency in endotherapy for UGIB. In response, the JAG QA team have recently released new DOPS forms specific to UGIB, and are consulting on introducing formal certification in endotherapy for UGIB.

Disclosure of Interest: All authors have declared no conflicts of interest.

\section{Reference}

1. GMC National Training Survey Results 2016, Gastroenterology,

\section{P1452 ELECTRONIC CHROMOENDOSCOPY WITH MAGNIFICATION IN THE DETECTION OF DYSPLASIA IN BARRETT'S ESOPHAGUS}

C. Carames ${ }^{1}$, J.C. Carames ${ }^{2}$, M. Xu ${ }^{1}$, M. Carames ${ }^{2}$, M. Gaidhane ${ }^{1}$, M. Kahaleh ${ }^{1}$ ${ }^{1}$ Weill Cornell Medical College, New York/United States of America

${ }^{2}$ Santander Hospital, Reynosa/Mexico

Contact E-mail Address: jccaram@gmail.com

Introduction: Barrett's esophagus (BE) is a known premalignant condition with risk for progression to esophageal adenocarcinoma. Electronic chromoendoscopy with magnification (ECM) is an evolving imaging technology being investigated as a novel imaging tool to identify dysplasia in BE to assist with higher yield BE surveillance and improve the diagnostic yield of esophageal biospies for $\mathrm{BE}$

Aims \& Methods: This is a single-center study to evaluate the efficacy of ECM (iScan by Pentax) in the detection of BE and dysplasic BE in a screening, average risk population with $\mathrm{BE}$ in Northeast Mexico. We conducted a retrospective study of 100 consecutive patients (41 males) with known BE (confirmed by pathology) during surveillance upper endoscopy for dysplasic BE between 
March and September of 2016. All patients underwent EC with iScan. The esophagus was inspected with a ECM capable endoscope (EG- 2990-Zi) and deliberate biopsies were taken from tissue identified by ECM that suggested BE. All biopsies were confirmed by a GI pathologist. Primary endpoint was the correlation between visual inspection diagnosis of dysplasic BE by ECM versus pathologic diagnosis of $\mathrm{BE}$ as the gold standard.

Table 1: Patient characteristics and outcomes

\begin{tabular}{ll}
\hline Characteristics & 100 \\
\hline Age (mean) & 47.7 \\
Male & $41 / 100$ \\
Endoscopic diagnosis: nondysplastic BE & $96 / 100$ \\
Endoscopic diagnosis: BE with LGD & $4 / 100$ \\
Pathologic diagnosis: nondysplastic BE & $94 / 100$ \\
Pathologic diagnosis: BE with LGD & $4 / 100$ \\
Pathologic diagnosis: benign gastric mucosa & $1 / 100$ \\
Pathologic diagnosis: esophageal ulcer & $1 / 100$ \\
Accuracy & $98 \%$ \\
Sensitivity & $100 \%[95 \%$ CI $(96 \%-100 \%)]$ \\
Specificity & $0 \%[95 \%$ CI $(0 \%-84 \%)]$ \\
Positive predictive value & $98 \%[95 \%$ CI $(93 \%-99.7 \%)]$ \\
Negative predictive value & NA
\end{tabular}

Results: In our cohort $41 \%$ were male, with mean age of 47.7 years. Endoscopic diagnoses by ECM were divided into nondysplastic BE (96/100) and suspected dysplastic BE (4/100). On pathology nondysplastic BE was found in 94/100 patients, BE with low-grade dysplasia was found in $4 / 100$ patients. Benign gastric mucosa with no alterations $(1 / 100)$, and ulcerated esophagitis $(1 / 100)$. The overall accuracy of endoscopic diagnoses using ECM against pathology diagnosis was of $98 \%$, with sensitivity of $100 \%$ [ $95 \%$ CI $(96 \%-100 \%)]$, and positive predictive value of $98 \%$ [95\% CI $(93 \%-99.7 \%)]$.

Conclusion: Endoscopic diagnosis of $\mathrm{BE}$ by directed biopsies of esophageal tissue with use of ECM is highly accurate. Future prospective studies are needed to validate our preliminary findings and assess inter-observer variability.

Disclosure of Interest: M. Xu: Grants from BSC, Xlumena, Cook, Olympus, Merit Endotek, Aspire Bariatrics, GI Dynamics, Apollo, Fuji, Pentax, Emcision, Concordia, MI Tech, Maunakea Tech, Ninepoint Medical, W.L. Gore, ASGE.

M. Kahaleh: Grants from BSC, Xlumena, Cook, Olympus, Merit Endotek, Aspire Bariatrics, GI Dynamics, Apollo, Fuji, Pentax, Emcision, Concordia, MI Tech, Maunakea Tech, Ninepoint Medical, W.L. Gore, ASGE.

All other authors have declared no conflicts of interest.

\section{P1453 DOES “INVISIBLE” DYSPLASIA IN BARRETT'S OESOPHAGUS REALLY EXIST?}

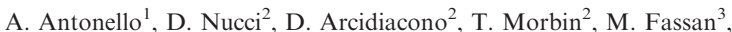

M. Häfner ${ }^{4}$, M. Rugge ${ }^{3}$, S. Realdon ${ }^{2}$

${ }^{1}$ Peterborough City Hospital, Peterborough/United Kingdom

${ }^{2}$ Digestive Endoscopy Unit, Veneto Institute of Oncology, IOV-IRCCS, Padual Italy

${ }^{3}$ Surgical Pathology And Cytopathology Unit, University of Padova, Padua/Italy

${ }^{4}$ Gastroenterology, Physiopathology And Digestive Endoscopy, Hospital of

Bolzano, Bolzano/Italy

Contact E-mail Address: stefano.realdon@iov.veneto.it

Introduction: Barrett's oesophagus (BO) is the main risk factor for oesophageal adenocarcinoma (OAC). International guidelines recommend endoscopic surveillance for early detection of dysplasia (DYS) and/or OAC but current biopsy protocols can miss areas of endoscopically unapparent neoplasia. At present, advanced diagnostic imaging technologies and specific endoscopist and pathologist expertise in detecting the subtle phenotypic changes associated with DYS $\mathrm{OAC}$ are available only in tertiary care referral centres.

Aims \& Methods: The aim of this study is to investigate the diagnosis changes after BO assessment in a tertiary care referral centre. 119 consecutive patients $[$ male $=103$, female $=16$; median age $=58(50 ; 64)]$ referred to the Veneto Institute of Oncology (IOV-IRCCS) BO Unit between $01 / 03 / 2015$ and $01 / 03$ 2017 were considered. Patients were divided in 3 groups according to the admitting diagnosis (AD): BO patients without DYS/OAC or visible lesions (Group 1, $\mathrm{n}=82$ ); BO patients with DYS/OAC but no visible lesions (Group 2, $\mathrm{n}=33$ ); BO patients with DYS/OAC and visible lesions (Group 3, n=4). All patients underwent an endoscopy under deep sedation at IOV with HD magnification endoscopes + NBI + acetic acid chromoendoscopy. Target biopsies and/or EMR were obtained in case of endoscopically visible lesions; 4-quadratic biopsies $/ 2 \mathrm{~cm}$ were obtained otherwise. All the specimens were diagnosed by two expert GI pathologists, who reached a final diagnosis (FD). In Group 2 patients where FD was BO without DYS/OAC, previous histology was reviewed by two expert pathologist.

Results: Results are summarized in Table 1. Group $1(n=82)$ : FD of DYS/OAC with visible lesion was reached in 2 out of 82 patients $(2.4 \%)$, both treated by $\mathrm{EMR}+\mathrm{RFA}$. No one had a FD of DYS/OAC without visible lesions. Group 2 $(n=33)$ : FD of DYS/OAC with visible lesions was revealed in 13 out of 33 patients $(39,4 \%)$ : 11 were treated by EMR + RFA, 1 by surgery and 1 chemio/ radiotherapy. In 20 patients the AD was downgraded to uncomplicated BO: in 19 patients $(57,6 \%)$, a review of previously taken biopsies by expert pathologist excluded neoplasia. In only one patient no lesion was found but the review of original histology slides confirmed a low-grade DYS and the patient was treated by RFA. Group $3(n=4)$ : DYS/OAC was confirmed in all the 4 patients $(2$ treated by EMR + RFA, and 2 by surgery)

Conclusion: When upper endoscopy is performed in a $\mathrm{BO}$ reference center where specific facilities and expertises are available (i.e. deep sedation, HD magnification endoscopes, chromoendoscopy, expert endoscopist and pathologist) "invisible" DYS is a very rare diagnosis.

Disclosure of Interest: All authors have declared no conflicts of interest.

\section{References}

1. Schölvinck DW, van der Meulen K, Bergman JJ, Weusten BL. Detection of lesions in dysplastic Barrett's esophagus by community and expert endoscopists. Endoscopy. 2017; 49:113-120.

2. Shaheen NJ, Falk GW, Iyer PG. et al. ACG Clinical Guideline: Diagnosis and management of Barrett's esophagus. Am J Gastroenterol 2016; 111:30-50

3. Moss A, Bourke MJ, Hourigan LF. et al. Endoscopic resection for Barrett's high-grade dysplasia and early esophageal adenocarcinoma: an essential staging procedure with long-term therapeutic benefit. Am J Gastroenterol 2010; 105:1276-1283

\section{P1454 SAFETY, EFFICACY AND CLOSURE TECHNIQUES OF ENDOSCOPIC FULL THICKNESS RESECTION-INITIAL CLINICAL EXPERIENCE}

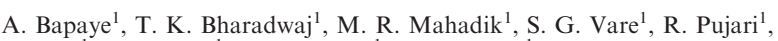
S. Date ${ }^{1}$, N. Dubale ${ }^{1}$, J. A. Bapaye ${ }^{1}$, A. Kulkarni ${ }^{1}$

${ }^{1}$ Shivanand Desai Center For Digestive Disorders, Deenanath Mangeshkar Hospital Digestive Diseases \& Endoscopy, Pune/India

Contact E-mail Address: drtarunbharadwaj@gmail.com

Introduction: Endoscopic full-thickness resection (EFTR) for sub-epithelial lesions (SETs) of GI tract is less frequently described; possibly due to technical challenges involved in dissection and need for resultant defect closure. Current study describes single-center experience of EFTR for treatment of SETs.

Aims \& Methods: Prospective database of patients undergoing EFTR for SETs over 6-years (2011-2017) was abstracted.Patient selection for EFTR-endoscopy, endoscopic ultrasound (EUS) and CECT. Inclusion criteria - encapsulated lesions, predominantly endophytic component and absence of features of invasive malignancy. Exclusion criteria-patients unfit for general anesthesia or major invasive procedure, uncorrectable coagulopathy or high risk features for malignancy. All procedures performed under general anesthesia with endotracheal intubation. High-definition endoscope (GIF-HQ-190 or CIF-HQ-190, Olympus Corp., Japan) with distal transparent hood and carbon dioxide insufflation used in all. After submucosal (SM) elevation by Gelofuscin, mucosal incision and SM dissection performed to expose SET. Encapsulated SET enucleated maintaining intact capsule. Adherent and attached muscularis propria (MP) layer fibers divided. IT or Dual-knife ${ }^{\mathrm{TM}}$ used for dissection and coag-grasper for hemostasis. Resultant MP layer defect closed endoscopically.

Results: Total $\mathrm{N}=18(\mathrm{M} \cdot \mathrm{F}-11: 7)$, mean age-53.6(Range-28-78). Presentation-GI bleed-7(38\%), abdominal pain-4(22\%), non ulcer dyspepsia-1, rectal mass-1 and asymptomatic, incidentally diagnosed-6(33\%). Layer of origin-MP layer in all. Location-stomach-13(72\%), duodenum-2, rectum-2, proximal jejunum1. Mean size of SET $-3.3 \mathrm{~cm}$ (range 1-7). Mean procedure time-182 mins (60$345)$, and mean hospital stay was 4 days. Adverse events-two (11\%)-esophageal laceration during specimen retrieval-1 (closed using endoclips), failure-1 (due to undetected large exophytic component-surgical resection). Histopathology with
Final Diagnosis (FD)

\begin{tabular}{lll}
\hline BO without & $\begin{array}{l}\text { BO with } \\
\text { DYS/OAC but } \\
\text { no visible lesions }\end{array}$ & $\begin{array}{l}\text { BO with } \\
\text { DYS/OAC and } \\
\text { visible lesions }\end{array}$ \\
80 & 0 & 2 \\
19 & 1 & 13 \\
0 & 0 & 4
\end{tabular}


IHC-GIST-9(50\%), neuroendocrine tumor-3(16\%), leiomyoma-1, schwannoma-1, neuroma-1, duplication cyst-1, parietal cell hyperplasia-1. Closure $16 / 18$ ( 2 defects in distal extra peritoneal rectum left open intentionally for healing by secondary intention); techniques-through-the-scope (TTS) clips-7, overthe-scope clip-4, omental patch + clip-2 (TTS-1, OTS- 1), endoscopic suturing1 , endoloop + clip-1. Mortality-nil. Follow up endoscopy at 4 weeks showed healing in all 17 patients. Median follow up -2 months.

Conclusion: EFTR is safe and effective for resection of SETs. Pre EFTR EUS and CECT may be useful to select appropriate candidates. Secure closure of defect is necessary for intraperitoneal full-thickness defects. Further studies comparing EFTR and surgery are recommended

Disclosure of Interest: A. Bapaye: Speaker-Boston scientific corporation, Cook endoscopy, Olympus and Taewoong medical

All other authors have declared no conflicts of interest.

\section{P1455 PROPHYLAXIS OF VARICEAL BLEEDING IN CIRRHOTIC PATIENTS: EFFICACY AND SAFETY OF ENDOSCOPIC VARICEAL LIGATION IN A TERTIARY CENTRE}

\section{E. Soares ${ }^{1}$, M. Gravito-Soares ${ }^{1}$, A. Henriques ${ }^{1}$, N. Almeida ${ }^{1}$, L. Tomé ${ }^{1}$} ${ }^{1}$ Gastroenterology, Centro Hospitalar e Universitário Coimbra, Coimbra, Portugal, Coimbra/Portugal

\section{Contact E-mail Address: es18497@gmail.com}

Introduction: In the natural history of chronic liver disease, variceal bleeding represents a life-threatening complication of portal hypertension, with high risk of mortality and recurrence. Current guidelines define vasoconstrictive therapy or endoscopic variceal ligation (EVL) in primary prophylaxis and the combination of both in secondary prophylaxis.

Aims \& Methods: We aimed to evaluate the efficacy of EVL therapy in both primary and secondary prophylaxis of variceal bleeding in cirrhosis and to establish the patient's clinical outcome. This was a retrospective observational cohort study of a total of 444 EVL procedures performed in 250 cirrhotic patients, who were admitted in a gastroenterology department of a tertiary centre, between 2004-2016, to receive EVL as prophylaxis of variceal bleeding. Sessions of ligation were repeated every two to three weeks in order to reach variceal eradication. The clinical outcome included the recurrence of bleeding (primary endpoint), the eradication success rate of oesophageal varices, EVL-related complications and overall and bleeding-related mortality.

Results: The mean follow-up period for all 250 cirrhotic patients enrolled in the study was $73.2 \pm 41.0$ months, with mean age of $63.9 \pm 10.8$ years and a predominance of male gender $(80.4 \% ; \mathrm{n}=201)$. At initial endoscopy, 237 $(53.4 \%)$ had portal hypertensive gastropathy, being severe in $168(37.8 \% \%)$. EVL was performed as primary prophylaxis in $50.9 \%(\mathrm{n}=226)$ and secondary prophylaxis in $49.1 \%(\mathrm{n}=218)$. Varices were obliterated in $209(83.6 \%)$ patients with mean number of EVL procedures necessary to eradicate varices of $1.8 \pm 0.95$ and a maximum of procedures of 6 . Recurrent bleeding occurred in $11.2 \%(\mathrm{n}=28)$ of cases with a mean time to re-bleeding occurrence of $8.1 \pm 14.2$ months. Major and significant complications were verified in $8.1 \%(\mathrm{n}=36)$ of patients. The main complications were bleeding related to post-banding ulceration $(75.0 \% ; \mathrm{n}=27)$ and infection $(22.2 \% ; \mathrm{n}=8)$, with mean time between EVL and complication occurrence of $11.1 \pm 11.8$ days (minimum:0;maximum:43). Intra-procedure complications occurred in $11(2.5 \%)$ patients with no death, despite of two cases of SengstakenBlakemore balloon necessity. The overall mortality was $5.4 \%(\mathrm{n}=24)$, being $0.4 \%(\mathrm{n}=2)$ related to variceal bleeding.

Conclusion: EVL seems to be an efficient, safe and relatively simple therapeutic procedure in both primary and secondary prophylaxis of variceal bleeding in cirrhotic patients. Since the main complications occurs over 1 week afte EVL procedure, the majority of patients can be safety treated in an ambulatory setting.

Disclosure of Interest: All authors have declared no conflicts of interest.

P1456 THE VALUE OF ENDOSCOPIC FULL-THICKNESS RESECTION FOR GASTRIC AND DUODENAL SUBMUCOSAL TUMORS ORIGINATING FROM THE MUSCULARIS PROPRIA LAYER

M. Xü ${ }^{1}$, C. Zhang ${ }^{1}$, Q. $\mathrm{Li}^{1}$

${ }^{1}$ Endoscopy Center, Zhongshan Hospital, Shanghai/China

Contact E-mail Address: whitelily.chen@aliyun.com

Introduction: Given diminishment of quality of life caused by surgery in the stomach and duodenum, a minimally invasive treatment is desirable for gastric and duodenum submucosal tumors (SMTs).

Aims \& Methods: We aimed to assess the value of endoscopic full-thickness resection (EFTR) technique for gastric and duodenal submucosal tumors (SMTs) originating from the muscular propria (MP) layer. A total of 276 patients with single gastric SMTs originating from the MP layer were performed EFTR between January, 2010 and February, 2014. The tight adhesion of the tumor to gastric or duodenal serosal layer could be seen in every case from endoscopic ultrasound (EUS) before the procedure. The SMTs orientated endoscopically were performed EFTR using a standard ESD technique without laparascopic assistance under direct endoscopic view. The defect of gastric and duodenal wall was closed after resection.

Results: A total of 276 patients included 94 males and 182 females. Their median age was 57.8 years (range, 30-81 years). Among all the 276 SMTs in our study,
165 located in gastric fundus, 96 located in gastric body, 8 located in the antrum, 1 located in the angle of stomach, and 6 located in duodenum. The median lesion size was $1.7 \mathrm{~cm}$ (range $0.7-6.0 \mathrm{~cm})$. The success rate of EFTR was $98.9 \%(273$ 276). EFTR was failed in 3 cases: one case was out of control because of bleeding into enterocoelia, two cases required conversion into laparoscopic surgery because of giant lobulations of the tumor outside the cavity. The median operation time was $65 \mathrm{~min}$ (range, 14-210 min). En bloc resection rate was $98.1 \%$ (268/ $273)$, piecemeal resection rate was $1.9 \%(5 / 273)$. The median length of hospital stays was 4.4 days (range, 1-23 days). Pathological outcomes revealed that the lesions included $137(49.7 \%)$ gastrointestinal stromal tumors (GISTs), 103 $(37.3 \%)$ leiomyomas, $13(4.7 \%)$ schwannomas, $8(2.9 \%)$ calcifying fibrous tumors, $7(2.5 \%)$ glomus tumors, $5(1.8 \%)$ displaced pancreas, and $3(1.1 \%)$ fibroblastomas. The procedure-related complications were as follows. Different degrees of epigastric pain occurred in $168(60.9 \%)$ cases, among which $24(8.7 \%)$ cases required analgesics. Pneumoperitoneum occurred in all the patients and was treated successfully with peritoneocentesis decompression. Seroperitoneum occurred in $15(5.4 \%)$ cases. localized peritonitis occurred in $3(1.1 \%)$ cases, and digestive tract leakage occurred in $1(0.4 \%)$ case. All the cases with above complications recovered spontaneously or after conservative treatments. No massive bleeding or adominal abscess was found after EFTR. None of the 273 cases developed procedure-related death. No tumor residual or recurrence was found during the follow-up period ranging 3-55 months.

Conclusion: EFTR without laparoscopic assistance is minimally invasive, safe, and effective for treating gastric and duodenal SMTs, which originate from the MP layer and adhere tightly to the serosa. High en bloc resection rate could be achieved. However, a larger number of the cases and long-term outcome deserve further research.

Disclosure of Interest: All authors have declared no conflicts of interest.

\section{P1457 BLUE LIGHT IMAGING AND LINKED COLOR IMAGING FOR DETECTION AND CHARACTERISATION OF CHRONIC GASTRITIS}

J. Weigt ${ }^{1}$, A. Link ${ }^{1}$, A. Canbay ${ }^{1}$, P. Malfertheiner ${ }^{1}$

${ }^{1}$ Gastroenterology, Hepatology \& Infectious Diseases, Medizin. Universität

Magdeburg Gastroenterologie und Hepatologie, Magdeburg/Germany

Contact E-mail Address: jochenweigt@gmx.de

Introduction: Current standard in the characterization of gastric mucosal changes is the use of virtual chromoendoscopy with magnification to visualize the pit pattern and vascular changes. The most recent development in light emitting technology is the so called Multi Light Illumination, that composes light out of 4 coloured LED. Blue Light Imaging (BLI) is composed of a continuous spectrum with peaks at 410 and $450 \mathrm{~nm}$ to enhance surface and vascular structures. Linked Color Imaging (LCI) uses BLI light together with post processing that reallocates colour tones resulting in a high contrast of different red tones. Until now only few data exist about the use of BLI and LCI in chronic gastritis (CG).

Aims \& Methods: We aimed to analyse the use of LCI and BLI in detecting and characterization of chronic gastritis and premalignant conditions of the stomach. 24 consecutive patients were investigated. All patients were investigated under conscious sedation. In all cases an endoscope equipped with zoom (Fujifilm EG760Z) was used. Endoscopic classification was based on the following parameters: normal gastric mucosa was defined as mucosa with visible superficial capillary network $(\mathrm{SCN})$ without any focal lesions; atrophy (AG) was defined by whitening of the mucosa with visible deeper vascular architecture in white light, BLI and LCI; intestinal metaplasia (IM) was diagnosed if mucosa had villous or ridged surface with whitening in LCI or white light or light blue crest sign in BLI; CG was diagnosed in case of loss of SCN or focal lesions not matching the definition of other focal lesions or cancer. Biopsies were sampled according to the updated Sidney classification system and in addition of every visible focal lesion. After endoscopy a prediction of histology was made by the endoscopist.

Results: We investigated 24 patients (15 female, 9 male, age 65 yrs (25-87yrs)). H. pylori was detected by histology or urease test in 7 patients. 3 patients showed normal gastric mucosa, 13 patients presented IM or AG either in the antrum or the corpus. According to MAPS criteria 7 patients had extensive disease with premalignant conditions in both, antrum and corpus. The concordance of endoscopic classification and histology was 79.1\% (19/24) in the antrum and corpus each. Despite the inconcordance of histology and endoscopic diagnosis in 5 cases the intervals for surveillance according to MAPS guidelines would have been correctly respected with the use of endoscopic assessment in all cases.

Conclusion: LCI and BLI are accurate in detection and characterization of changes in gastric mucosa with an acceptable concordance to histology. These new imaging modalities are a step towards precise endoscopic diagnosis of gastric mucosal changes and have the potential to reduce the number of unnecessary histologic investigations and offer the possibility for more appropriate endoscopic diagnosis.

Disclosure of Interest: J. Weigt: Research and presenter for Fujifilm.

All other authors have declared no conflicts of interest. 
P1458 LONG-TERM OUTCOMES OF ENDOSCOPIC

SUBMUCOSAL DISSECTION(ESD) FOR RELATIVE INDICATION GROUP OF EARLY ESOPHAGEAL SQUAMOUS CELL CARCINOMA (EESCC) IN AGED PATIENTS

Z. Qi ${ }^{1}$, Y. Zhong ${ }^{1}$, P. Zhou ${ }^{1}$

${ }^{1}$ Endoscpy Center, Zhongshan Hospital Fudan University, Shanghai/China

\section{Contact E-mail Address: poem001@qq.com}

Introduction: According to the Japanese Esophageal Society Guidelines, Early Esophageal Squamous Cell Carcinoma (EESCC) involving the muscularis mucosa or $<200 \mu \mathrm{m}$ invasion of the submucosa, and circumferential extent of $>2 / 3$ were relative indications (RI) for ESD. Additional treatment (AT, including esophagectomy or chemoradiotherapy) may be needed after ESD. But in aged RI patients, most will refuse AT due to higher rates of debilitating symptom in China.

Aims \& Methods: The aim of this study was conducted to evaluate the long-term outcomes of aged RI patients without AT after ESD

Between January 2008 and December 2013, a total of 158 aged EESCC patients were included in the present retrospective study. Prognosis outcomes were analyzed.

Results: 89 patients included in absolute indication (AI) group and 69 in RI group. The baseline characteristics were balanced between two groups. During the follow-up time (median 56 (1-108) months), short-term adverse events $(4.3 \%$ vs $1.1 \%, \mathrm{p}=0.319)$ and postoperative stricture rate $(31.8 \%$ vs $21.3 \%, \mathrm{p}=0.134)$ were higher in RI group than in AI group. 5-year recurrence-free survival rate $(85.8 \%$ vs. $87.2 \%, p=0.561)$, metastasis-free survival rate $(100 \%$ vs. $98.6 \%$, $\mathrm{p}=0.437)$, overall survival $\operatorname{rate}(96.6 \%$ vs $90.0 \%, \mathrm{p}=0.613)$ and cause-specific survival rate( $98.9 \%$ vs $98.5 \%, \mathrm{p}=0.264)$ for $\mathrm{AI}$ group and RI group were comparable.

Conclusion: Aged RI EESCC patients without AT(esophagectomy or chemoradiotherapy) showed comparable prognosis outcomes with AI group after ESD. So follow up may be recommended, substituted for AT in aged RI group.

Disclosure of Interest: All authors have declared no conflicts of interest.

\section{P1459 RETROSPECTIVE ANALYSIS ON SUSPICION OF FOREIGN BODY INGESTION AND FOOD IMPACTION ON GASTROENTEROLOGY EMERGENCIES}

J. T. Correia De Sousa ${ }^{1}$, D. Libanio ${ }^{1}$, P. Lago $^{1}$, R. Marcos-Pinto ${ }^{1}$, I. Pedroto ${ }^{1}$ ${ }^{1}$ Gastroenterology, Centro Hospitalar do Porto, Porto/Portugal

Contact E-mail Address: joao.correia.de.sousa@gmail.com

Introduction: Suspicion of foreign body (FB) and food impaction (FI) are one of the most common motives for endoscopic emergency. This retrospective study reviewed 288 cases of suspicion on FB/FI, by the frequency of endoscopic alterations, predictive factors to presence, types of $\mathrm{FB}$ found, and therapeutic approach.

Aims \& Methods: Unicentric retrospective cohort study of endoscopies preformed during one year of gastroenterology emergency setting.

Results: In 2015, 288 endoscopies were performed on suspicion of FB/FI (22\% of total emergency endoscopies, $\mathrm{n}=1309)$, of them $69.1 \%(\mathrm{~N}=199)$ were performed during the night. Patients' median age was 58 years, and $52.8 \%$ were women. The presence of FB/FI was confirmed in $71.2 \%(\mathrm{n}=205)$; of them $61,5 \%(\mathrm{n}=126)$ were FI. The most frequently found foreign bodies were meat bones $18 \%(\mathrm{n}=37)$ and fish bones $14.6 \%(\mathrm{n}=30)$. Most FB/FI were found on the proximal esophagus $(56.1 \%, \mathrm{n}=115)$. Endoscopic removal was performed on 129 cases $(63.4 \%)$, endoscopic mobilization in $54(26.3 \%)$, and in 22 endoscopic removal wasn't achieved (10, where referred of otolaryngology; 2 for surgery and 10 were deferred to endoscopy with sedation, in operating room). Endoscopy under sedation was performed in 20 cases $(9,7 \%)$. About $1 / 4$ had associated comorbidities, the most common were esophageal ring in 22 $(10.7 \%)$ and benign stenosis in $17(8.3 \%)$ patients. Major complications were rare: 1 perforation $(0.3 \%)$ and 3 deep esophageal lacerations $(1.5 \%)$. Age ( $>55$ years), presence of comorbidities, and previous episodes were associated with presence of FB/FI on Endoscopy (Odds Ratio 2.01, 3.39 and 4.63 respectively).

Conclusion: Endoscopy is frequently preformed for suspicion of $\mathrm{FB} / \mathrm{FI}$ in our emergency setting. Presence is confirmed in the majority of the cases. Predictive factors for presence were identified. Most FB/FI were removed with success with low complication rates. This data favor the endoscopic approach on suspicion of FB/FI.

Disclosure of Interest: All authors have declared no conflicts of interest.

\section{P1460 LEARNING CURVE FOR ENDOSCOPIC SUBMUCOSAL DISSECTION OF GASTRIC NEOPLASMS; LOW-VOLUME SINGLE- CENTER EXPERIENCE}

S.T. Kim, T.H. Kim, S. Na

Internal Medicine, Jeju National University Hospital, Jeju/Korea, Republic of

Contact E-mail Address: panzervor@paran.com

Introduction: Endoscopic submucosal dissection (ESD) has become a standard therapy for early gastric neoplasia. There is no consensus yet about the number of experiences required for performing ESD alone.

Aims \& Methods: We aimed to investigate the learning curve of ESD performed by a single beginner endoscopist focusing on developing the performance of dissection, shortening the procedure time, and preventing complications.
Methods: Records of 120 consecutive ESD procedures performed by a single beginner endoscopist with an ESD knife from March 2012 to Feburary 2016 were collected. For analysis of the learning curve, total procedures were divided into four periods, each comprising 30 sequential ESD. The parameters assessed were the en-bloc resection rate, complete resection rate, procedure time, and related complications.

Results: In the procedure time according to the number of experiences, the procedure time decreased from 30 experience. However, there was no statistical difference from the first $(63.5+54.0)$ to the second quarter $(44.5+31.4$, $\mathrm{p}=0.19)$, to the third quarter $(40.7 \pm 27.8, \mathrm{p}=0.08)$, and to the fourth quarter $(40.8 \pm 23.1, \mathrm{p}=0.09)$. There was no procedure that exceeded 100 minutes from the third quarter. There were a total of seven perforations, four of which were in the first quarter, two in the second, and one in the third. In the procedure time according to the location of the lesions, upper third lesion $(92.0 \pm 43.7)$ showed longer procedure time than middle $(46.6 \pm 40.2, \mathrm{p}<0.01)$ and lower third $(39.5 \pm 27.5, \mathrm{p}<0.01)$ with statistically significant difference. In addition, in the fibrotic lesions, regardless of size and location, all took a very long time, more than 100 minutes.

Conclusion: It needs accumulate experience with the help of a professional expert up to 30 cases, and to the more advanced level, about 90 procedures are needed. And, the location of the lesion is the important factor in determining the difficulty of the procedure. Therefore, it is best to avoid the upper third lesion as far as possible until experience 90 cases or at least 30 procedures.

Disclosure of Interest: All authors have declared no conflicts of interest.

\section{References}

1. Gotoda T, Friedland S, Hamanaka H, et al. A learning curve for advanced endoscopic resection. 2005;62:866-7.

2. Kakushima N, Fujishiro M, Kodashima S, et al. A learning curve for endoscopic submucosal dissection of gastric epithelial neoplasms. 2006;38:991-5.

\section{P1461 COMPARATIVE STUDY OF ESD AND SURGICAI RESECTION FOR GASTRIC SETS ORIGINATED FROM MUSCULARISPROPRIA}

C.B. Ryu ${ }^{1}$, M.S. Lee ${ }^{1}$, J.Y. Bae ${ }^{2}$

${ }^{1}$ Digestive Disease Center And Research Institute, Department Of Internal Medicine, SoonChunHyang University School of Medicine, Bucheon/Korea, Republic of

${ }^{2}$ Department Of Internal Medicine, Seoul Medical Center, Seoul/Korea, Republic of

Contact E-mail Address: ryuchb@gmail.com

Introduction: Endoscopic resection for gastric subepithelialtumors(SETs) originated from the muscularispropria (GSET-PM) has offered less invasive alternatives to surgical resection. The aims of this study were to compare endoscopic subtumoraldissection(ESD) with surgical resection for the removal of GSETPM.

Aims \& Methods: This study involved 17 patients with GSET-PM removed by ESD and 76 patients who underwent curative surgical resection. ESD was attempted in GSET-PM with well marginated tumors which was below $5 \mathrm{~cm}$ and showed an endoluminal growth pattern according to endoscopic ultrasound(EUS) finding.

Results: ESD group were more likely to have upper portion $(10 / 17,58.8 \%)$ and surgery group were more likely to have mid portion $(41 / 76,53.8 \%)(p=0.039)$. ESD group were smaller median tumor size $(25.6 \mathrm{~mm}$ vs $35.9 \mathrm{~mm}, \mathrm{p}=0.037)$ and higher endoluminal ratio $(58.5 \pm 9.1 \%$ vs $45.8 \pm 15.4 \%, \mathrm{p}=0.002)$. ESD group were mostly to have Yamada type III $(10 / 17,58.8 \%)$ and surgery group were mostly Yamada type I $(52 / 76,68.4 \%)(\mathrm{p}<0.001)$. Complete resection by ESD was lower than by surgical resection $(82.4 \%$ vs $100 \%, \mathrm{p}<0.001)$. In ESD group, 3 performed surgical resection after ESD ( 1 incompletely resection and 2 uncontrolled bleeding) and 1 showed perforation was completely resected with endoscopic closure. In surgery group, complications occurred in 6 patients (1 leakage, 1 stricture, 1 hernia and bowel obstruction, 1 wound infection and 2 worsened general condition after surgery). Although surgery group were lower in complication rate than ESD group ( $\mathrm{p}=0.006)$, severity of complications were higher in the surgery group and there were no mortalities in the ESD group compared with 2 in the surgery group. There was no statistical difference of recurrence and the follow-up period between two groups.

Conclusion: Conclusion: ESD can be one of good options for the resection of endoluminal GSET-PM and could be replace treatment by surgical resection in Yamada type III with a high endoluminal ratio.

Disclosure of Interest: All authors have declared no conflicts of interest. 
P1462 CAP ASSISTED UPPER ENDOSCOPY VERSUS SIDE-

VIEWING ENDOSCOPE FOR EXAMINATION OF THE MAJOR DUODENAL PAPILLA: A RANDOMIZED, BLINDED, CONTROLLED, NON-INFERIORITY CROSSOVER STUDY (CAPPAII STUDY)

M. Abdelhafez ${ }^{1}$, V. Phillip ${ }^{1}$, A. Hapfelmeier ${ }^{2}$, M. Elnegouly ${ }^{1}$, M. Dollhopf $^{3}$, A. El Magid Kassem ${ }^{4}$, P. Klare ${ }^{1}$, M. Bajbouj ${ }^{1}$, R.M. Schmid ${ }^{1}$, S. Delius ${ }^{1}$, F. Eckel Florian ${ }^{5}$

${ }^{1}$ Klinik Für Innere Medizin Ii, Technische Universität München, Munich/Germany ${ }^{2}$ Institut Für Medizinische Statistik Und Epidemiologie, Technische Universität München, München/Germany

${ }^{3}$ Gastroenterology And Hepatology, Klinikum Neuperlach, Munich/Germany

${ }^{4}$ Kasr Aliani Hospital, Cairo University, Cairo/Egypt

${ }^{5}$ Inneren Abteilung, RoMed Klinik Bad Aibling, Bad Aibling/Germany

\section{Contact E-mail Address: dr mhafez@hotmail.com}

Introduction: Examination of major duodenal papilla (MDP) by standard forward-viewing is limited and the use of side-viewing endoscope (SVE) is mandatory. Cap assisted esophagogastroduodenoscopy (CA-EGD) utilizes a cap fitted to the tip of the endoscope that can depress the mucosal folds and thus might improve visualization of MDP. The aim of this study was to compare CA-EGD to SVE for complete examination of the MDP.

Aims \& Methods: Prospective, randomized, blinded, controlled, non-inferiority crossover study. Subjects scheduled for elective EGD were randomized to undergo CA-EGD (group A) or SVE (group B) before undergoing a second examination by the alternate method. Images of the MDP were evaluated, after image processing, by three blinded multicenter-experts. Our primary outcome measure was complete examination of the papilla. Secondary outcome measures were image quality of mucosal pattern, ability to obtain an overview of the papilla and overall satisfaction of the evaluators. For secondary outcomes, a score was given from 1 to 101 =poor, $10=$ excellent).

Results: A total of 62 patients were randomized and completed the study. Complete examination of MDP was achieved in 59 patients using CA-EGD compared to 60 patients using SVE (95 vs. $97 \%, p=1.0$ ). CA-EGD had mean scores of $8.7 \pm 1.3,7.1 \pm 0.86$ and $7.9 \pm 1$ regarding mucosal pattern, overview and overall satisfaction, respectively, versus $5.3 \pm 1.6(p<0.001), 8.3 \pm 0.9$ $(p<0.001)$ and $7.6 \pm 0.6$ with $\operatorname{SVE}(p=0.01)$

Conclusion: CA-EGD is non-inferior to SVE for complete examination of MDP. CA-EGD had significantly higher scores than SVE regarding the image quality and overall satisfaction, while SVE had a better overview. CA-EGD is a safe and effective method for examination of MDP and can replace the SVE for diagnostic indications.

Disclosure of Interest: All authors have declared no conflicts of interest.

\section{P1463 (IN)ACCURACY OF CAMBRIDGE PROTOCOL FOR PATIENTS HARBOURING CHD1 MUTATION: A CONSECUTIVE SERIES}

R. Castro, D. Libânio Monteiro, T. Pinto-Pais, M. Dinis-Ribeiro, C. Brandão Department Of Gastroenterology, Instituto Português de Oncologia, Porto, Porto/ Portugal

Contact E-mail Address: rui.arrais.castro@gmail.com

Introduction: Hereditary diffuse gastric cancer (HDGC) accounts for 1 to $3 \%$ of all gastric cancer and it can be caused by a germline mutation of the gene $\mathrm{CDH} 1$. Life time risk for gastric cancer is $80 \%$ with a mean age at diagnosis of 40 years. Affected individuals generally present multiple foci of signet ring cell carcinoma (SRCC) scattered throughout the gastric mucosa, difficulty detected by endoscopy.

Aims \& Methods: The aim of this study was to access the validity of Cambridge protocol with a high-resolution endoscope in patients with proven pathological germline mutation of the gene $\mathrm{CDH} 1$. A prospective cohort study was performed between September 2016 and March 2017 in 11 patients with $\mathrm{CDH} 1$ mutation. They perform a base line high-resolution endoscopy (Olympus-GIF-HQ190) with random biopsies according Cambridge protocol and additional targeted biopsies of any visible lesion. The total number of biopsies and the total number and localization of SRCC foci was registered. For those patients submitted to prophylactic gastrectomy, data was compared with surgical specimen histology. To access the validity of Cambridge protocol with a high resolution endoscope in patients with proven pathological germline mutation of the gene CDH1.

Results: During the 11 endoscopies a total of 353 biopsies (329 random biopsies and 24 targeted biopsies; mean of 32.1 biopsies per patient) were performed. Two patients presented 1 SRCC foci in random biopsies, being that one of them presented positive histology in 2 targeted biopsies. It was necessary to perform 165 random biopsies to identify a single SRCC foci. It was necessary 12 targeted biopsies to identify a SRCC foci associated with a visible lesion. Five patients were submitted to total gastrectomy and every surgical specimens presented SRCC foci.

Conclusion: Despite the use of high-resolution endoscopes and the high number of random biopsies, endoscopic evaluation presents many limitations for the diagnosis of HDGC. According to literature, prophylactic total gastrectomy remains the first option in patients carrying CDH1 mutation.

Disclosure of Interest: All authors have declared no conflicts of interest.

\section{References}

Van der Post, Rachel S et al. "Hereditary Diffuse Gastric Cancer: Updated Clinical Guidelines with an Emphasis on Germline CDH1 Mutation Carriers." Journal of Medical Genetics 52.6 (2015): 361-374. PMC. Web. 27 Apr. 2017.

Brandão, Catarina, and Mário Dinis-Ribeiro. "Early Diagnosis of Hereditary Diffuse Gastric Cancer: (not Only) an Endoscopic Challenge!" Endoscopy International Open 4.12 (2016): E1311-E1312. PMC. Web. 27 Apr. 2017.

\section{P1464 SINGLE-CENTER CLINICAL EXPERIENCE WITH A}

\section{RECENTLY DEVELOPED FULL-THICKNESS ENDOSCOPIC CLIP}

A. Bapaye ${ }^{1}$, S. G. Vare $^{1}$, M. R. Mahadik ${ }^{1}$, T. K. Bharadwaj ${ }^{1}$, R. Pujari ${ }^{1}$, S. Date ${ }^{1}$, N. Dubale ${ }^{1}$, J. A. Bapaye ${ }^{1}$, A. Kulkarni ${ }^{1}$

${ }^{1}$ Shivanand Desai Center For Digestive Disorders, Deenanath Mangeshkar Hospital Digestive Diseases \& Endoscopy, Pune/India

Contact E-mail Address: drtarunbharadwaj@gmail.com

Introduction: Endoscopic clips are used in a variety of clinical situations in GI endoscopy-for arrest of bleeding or for closure of bowel perforations or chronic fistulae. Conventional through-the-scope clips often cannot provide optimum results; and therefore full-thickness (FT) over-the-scope (OTS) clips have been devised.

Aims \& Methods: Current study describes the clinical experience of use of a recently developed FT OTS clip (Padlock ${ }^{\mathrm{TM}}$, Aponos Medical, USA). Data from a prospectively maintained database of all patients undergoing the new FT OTS clip was abstracted. Patient demographics, primary diagnosis, history of previous endotherapy, endoscopic procedure, indications for FT OTS clip usage, technical and clinical success and early and delayed adverse events were recorded. The clip-clip is available in two different sizes for use in upper and lower endoscopy. It is supplied preloaded on a cartridge that fits on the distal end of endoscope. The trip-wire travels alongside the endoscope, enabling additional instruments to be passed through the endoscope channel, and special doublechannel endoscope is not required for its application. Technique of clip application-the clip was loaded on the distal end of the endoscope and endoscope advanced to site of interest. Bowel wall defect or bleeding point was positioned within the clip and strong suction was applied. Clip was fired by closing the handle on the delivery system. Suction was slowly released and site was inspected. Results: Total 21 clips used in 19 patients. M: F-12:7, mean age- 57.9 years (range -24-84 years). Indications for FT OTS clip use-severe GI bleeding-7 (36.8\%) (duodenal ulcer bleed-5, rectal ulcer-1, bleed during ESD for rectal lateral spreading tumor-1); for closure of bowel perforation during endoscopic resections-7 (36.8\%) (gastric-3, duodenum-2, rectum-2); and closure of chronic bowel fistulae-5 $(26.3 \%)$ (esophagus-3, duodenum-1, rectum-1). Previous h/o endotherapy $-3 / 7$ of bleeding patients, primary therapy using OTS clip in remaining 16. Technical success was $100 \%$. Two patients needed two clips each due to large size of defect. Clinical success-bleeding arrested in $7 / 7(100 \%)$, bowel perforation sealed $-7 / 7(100 \%)$; fistula closure successful- $4 / 5(80 \%)$. In one patient of chronic duodenal fistula, fistula reopened 12 weeks after initial sealing of fistula and required surgery. Follow up at 4 weeks revealed no delayed adverse events in any patient.

Conclusion: The new OTS Clip (Padlock ${ }^{\mathrm{TM}}$, Aponos) is safe and effective for treatment of severe bleeding and for closure of post ER full-thickness defects and chronic fistulae. Further studies with larger sample size are recommended. Disclosure of Interest: A. Bapaye: Speaker- Boston scientific corporation, Cook endoscopy, Taewoong medical, Olympus

All other authors have declared no conflicts of interest.

\section{P1465 ENDOSCOPIC AMPULLECTOMY OUTCOMES IN A} TERTIARY ENDOSCOPY DEPARTMENT

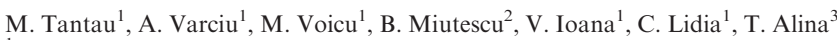
${ }^{1}$ Endoscopy, Regional Institute of Gastroenterology and Hepatology, University of Medicine and Pharmacy Iuliu Hatieganu Cluj-Napoca, Cluj-Napoca/Romania ${ }^{2}$ Department Of Gastroenterology And Hepatology, Victor Babes University of Medicine and Pharmacy Timisoara, Timisoara/Romania

${ }^{3} 4$ th Medical Clinic, University of Medicine and Pharmacy Iuliu Hatieganu ClujNapoca, Cluj-Napoca/Romania

\section{Contact E-mail Address: bmiutescu@yahoo.com}

Introduction: Endoscopic resection of ampullary adenomas has increased in the last decade due to the high morbidity with a high mortality in patients undergoing surgical procedures ${ }^{1}$

Aims \& Methods: This study aims to evaluate the outcome of endoscopic ampullectomy (EA) in a tertiary endoscopy department. We included in the study patients that underwent EA between January 2014 - April 2017 at the Regional Institute of Gastroenterololgy and Hepatology Cluj-Napoca, Romania. All patients had a benign pathological result prior to the EA. Postprocedural complications such as bleeding, perforation, cholangitis, pancreatitis and mortality were analyzed. Data about resection type, post EA histhology and 1 year follow-up was also proccessed. 
Results: 19 patients underwent EA, with a mean age of $63.5 \pm 17.7$ years and a mean size of the tumor of $17.4 \pm 7.8 \mathrm{~mm}$. The male to female ratio is 0.7. "En bloc" resection was done in most cases $15 / 19(78.9 \%)$. Bleeding occured in 6 cases $(31.6 \%)$ and two patients $(10.5 \%)$ developed acute pancreatitis. One patient died due to severe bleeding. The average days of hospitalization after endoscopic ampullectomy were 5.7 with a range from 2 to 25 days. Adenocarcinoma was described in the final histopathological result in $4 / 19$ cases $(21.1 \%)$. One year follow-up noted a reccurence rate of $15.8 \%$ (3/19 cases).

Conclusion: In conclusion, endoscpic ampullectomy is a difficult procedure with an increased risk of complications but performed by experienced endoscopists is safe and surgical interventions can be avoided.

Disclosure of Interest: All authors have declared no conflicts of interest.

\section{Reference}

1. 2015 Sep 26; 5(3): 127-135.

\section{P1466 PER ORAL ENDOSCOPIC MYOTOMY: UPDATED RESULTS FROM A UNITED KINGDOM CASE SERIES}

S. Gulati ${ }^{1}$, A. Emmanuel ${ }^{1}$, H. Inoue ${ }^{2}$, A. Haji ${ }^{1}$, B. Hayee ${ }^{1}$

${ }_{1}^{1}$ Endoscopy, King's Institute of Therapeutic Endoscopy, RS/United Kingdom

${ }^{2}$ Digestive Disease Center, Showa University Koto-Toyosu Hospital, Tokyo/Japan

Contact E-mail Address: shraddha.gulati@nhs.net

Introduction: Per-oral endoscopic myotomy (POEM) has been adopted as a minimally invasive treatment option for achalasia and even spastic oesophageal conditions ${ }^{1}$. The first case of POEM was performed at the King's Institute of Therapeutic Endoscopy (KITE) in 2013. Here we present our initial case series including the first UK case of diffuse oesophageal spasm (DES).

Aims \& Methods: Prospective data was collected for consecutive patients undergoing POEM including demographics, POEM technique, the use of Endoluminal Functional Lumen Imaging Probe (EndoFLIP) and adverse events. Clinical success was defined as a reduction of Eckardt score (ES) to $</=2$ or a reduction in 4 points from baseline. Follow up data at 3 and 12-24 months (m) post-POEM was analysed using the Wilcoxon signed ranks test to compare pre- and post-POEM ES and 4sIRP and pre and post-treatment GORD-HRQoL score. Repeatedmeasures ANOVA was used for multiple time-point comparisons.

Results: POEM was performed in 51 patients $(22 \mathrm{~F}$, age $48.6+/-13.5$ years) Further baseline data is presented in table 1. Median gastric and oesophageal myotomy was $3 \mathrm{~cm}(2-4)$ and $10 \mathrm{~cm} \mathrm{(3-18)} \mathrm{respectively} \mathrm{with} \mathrm{a} \mathrm{selective} \mathrm{circular}$ myotomy in all cases and a posterior approach in 11. POEM was clinically successful in $\mathrm{n}=48 / 51(94 \%)$ eligible for review at $3 \mathrm{~m}$. Reduction in ES at $3 \mathrm{~m} ; 8.5(5-12)$ vs $0(0-6) \mathrm{p}<0.0001$ was sustained in 31 patients with median follow up of $15 \mathrm{~m}(3-36) ; 8.5(5-12)$ vs $2(0-7)$ ANOVA $\mathrm{p}<0.0001$. Reduction in IRP-4 s was observed $24.05+/-10.47 \mathrm{mmHg}$ vs $7.81+/-4.91 \mathrm{mmHg}$ $(\mathrm{p}=0.001)$. Revision of POEM was performed in $\mathrm{n}=3$ at 6,16 and $27 \mathrm{~m}$ with clinical success achieved in all cases at $3 \mathrm{~m}$ review. Pre and post-myotomy EndoFLIP showed an increase in oesophago-gastric junction diameter from $5.87 \mathrm{~mm}$ to $11.27 \mathrm{~mm}(\mathrm{p}<0.001)(\mathrm{n}=11)$ and distensibility improved from $1.14 \mathrm{~mm}^{2} / \mathrm{mmHg}$ to $2.87 \mathrm{~mm}^{2} / \mathrm{mmHg}(\mathrm{p}=0.008)(\mathrm{n}=8)$. Median post-POEM GORD-HRQoL score $(\mathrm{n}=50)$ was $1(0-31) .8$ patients with scores $>12$ improved with medical treatment; $15.5(13-31)$ vs $6.5(1-11, \mathrm{p}=0.0078)$ as did 5 cases of acid reflux identified by $24 \mathrm{hr} \mathrm{pH}$ testing in $\mathrm{n}=19$ at time of analysis. Endoclip displacement was encountered in $\mathrm{n}=2$ and replaced endoscopically including readmission of 1 patient for delayed mucosal incision site healing (Clavien-Dindo Grade IIIb). There were no cases of mortality, perforation, infection/major bleeding.

Table 1: Baseline characteristics

Patient Demographics

Age (mean, SD, range) (years)

$48.6+/-13.5(22-79)$

Male (n) $(\%)$

29 (57)

Female (n) (\%)

22 (43)

Clinical Data

Duration of disease (mean, SD, range) (years)

Eckardt Score (median, range)

$4.4+/-4.2(0.25-25)$

Chicago Subcategorisation

Achalasia Type I (n) (\%)

Achalasia Type II (n) (\%)

Achalasia Type III (n) (\%)

DES

$8(4-12)$

$4(8)$

$42(82)$

2 (4)

$1(2)$

2 (4)

45 (88)

Non-Sigmoid Oesophagus (n) $(\%)$

$6(12)$

Sigmoid Oesophagus (n) $(\%)$

$27(53 \%)$

Prior Achalasia Treatment

$9(18)$

Prior Pneumatic Dilatation; PD (n) (\%) $13(25)$

Prior Heller Myotomy; LHM (n) (\%)
Table 1 Continued

Patient Demographics

Prior POEM (n) $(\%)$

$3(6)$

$>2$ prior treatments (n) $(\%)$

$4(8)$

ASA Physical Status Classification

ASA grade I (n) (\%)

$21(41)$

ASA grade II (n) $(\%)$

$22(43)$

ASA grade III (n) $(\%)$

$8(16)$

Conclusion: This is the largest UK case series of POEM for achalasia including the first successful UK POEM procedure for DES. At our institute, POEM was performed successfully in a potentially more challenging cohort where $52.9 \%$ had prior endoscopic/surgical treatment. Our results are in line with international consortia and ASGE findings that POEM is a safe and efficacious procedure for the treatment of achalasia and oesophageal spastic disorders for both short term and sustained symptomatic benefit.

Disclosure of Interest: All authors have declared no conflicts of interest.

\section{Reference}

1. Stavropoulos S, Desilets D, Fuchs KH et al; Per-oral endoscopic myotomy white paper summary; Gastrointestinal Endoscopy; 2014;1:1-15

\section{P1467 NEW CHALLENGE FOR SAFER ENDOSCOPIC} SUBMUCOSAL DISSECTION USING CO2 LASER

Y. Morita, R. Ariyosh

Gastroenterology, Kobe University, Kobe/Japan

Contact E-mail Address: ymorita@med.kobe-u.ac.jp

Introduction: Endoscopic submucosal dissection (ESD) is increasingly accepted as a minimally invasive treatment for the patients with early gastrointestinal cancers. However, ESD demands high maneuverability technique, and the success of the operation is dependent of each operator's skill. We have developed a novel laser surgery system for ESD to overcome such difficulties of ESD, which is composed of a $\mathrm{CO}_{2}$ laser source and a disposable flexible hollow fiber probe. Compared to conventional ESD (C-ESD) using electric surgical knives, ESD using $\mathrm{CO}_{2}$ laser (L-ESD) method has an advantage of less risk of perforation and massive thermal damage, because the $\mathrm{CO}_{2}$ laser is strongly absorbed by water such as saline or sodium hyaluronate. Farther more, the cutting point can be precisely recognized by another visible guide laser. Due to non-contact laser irradiation and adequate visualization of treatment area, the laser system facilitates more precise and safer treatment and provides high quality and stable dissection. We hypothesized that performing ESD using $\mathrm{CO}_{2}$ laser with a submucosal laser absorber could be a safer and simpler ESD technique.

Aims \& Methods: The aim of this study was to evaluate the feasibility of L-ESD and the quality of the resected specimen obtained by L-ESD in living porcine compared with C-ESD. We performed ESD for a total of 14 hypothetical lesions in three porcine stomachs (L-ESD, 7 lesions; C-ESD 7 lesions) under general anesthesia. En-bloc resection rate, procedure time, adverse events, and the quality of the resected specimen were evaluated. To evaluate the smoothness of the cutting surface in the resected specimens, we compared the length of the resected side of the submucosa (LRS) with the length of the muscularis mucosa (LMM). Results: The en-bloc resection rate was $100 \%$ in both groups. Although the mean L-ESD procedure time was $23.3 \pm 10.8$ minutes, and was significantly longer than in the C-ESD group $(9.4 \pm 6.6$ minutes; $\mathrm{p}<0.05)$, there was no uncontrollable bleeding or perforation in either group. The mean ratio of LRS to LMM was $107 \pm 3.3 \%$ in the L-ESD group, and was significantly lower than that of the CESD group $(138 \pm 28 \%)(\mathrm{P}<0.005)$

Conclusion: ESD using $\mathrm{CO}_{2}$ laser might be a feasible and effective method for the treatment of early gastrointestinal cancers.

Disclosure of Interest: All authors have declared no conflicts of interest.

P1468 LONG-TERM OUTCOME OF ACUTE CORROSIVE INGESTION: A PROSPECTIVE SINGLE-CENTER STUDY

S. Dhar Chowdhury, B. Kumar, R. T. Kurien, S. K. Ghatak, D. Sreekar Gastroenterology, Christian Medical College, Vellore, Vellore/India

Contact E-mail Address: sudipta@cmcvellore.ac.in

Introduction: Acute corrosive ingestion (ACI) is a common and serious medical problem accounting for a number of hospital admissions. ACI causes significant mortality and morbidity. These patients are at risk of developing luminal strictures of the upper gastrointestinal tract in the long term. This is more in patients with high-grade injury.

Aims \& Methods: The present study aimed at assessing the long-term outcomes of high-grade (Zargar's grade $>$ Grade $2 \mathrm{~A}$ ) corrosive-induced injury of upper gastrointestinal tract (1). This was a prospective sudy conducted in the Department of Gastroenterology at Christian Medical College, Vellore. The study period was between January 2008 to December 2014. All patients were managed by a standard protocol which included doing a gastroscopy within 24 hours of ACI. In this study we included patients $\geq 15$ years with high-grade (Zargar's grade 
$\geq$ Grade $2 \mathrm{~A}$ ) corrosive-induced injury of upper gastrointestinal tract. Patients in whom gastroscopy could not be done or who had it done at another hospital were excluded from the study. The study was approved by the Instituitional Ethics committee and was funded by a fluid research grant received from Institutional Review Board at Christian Medical College, Vellore, India. The data was analysed using SPSS version 17. The parametric continuous variables were expressed as, mean $\pm \mathrm{SD}$ and the non parametric continuous variables were expressed as median.Comparison between groups was done using Fisher's exact test.

Results: During the study period a total of 112 patients presented with ACI. In all 82 patients were included in the study. Amongst them, $53 \%$ of the patients were females and the mean age was $36.5 \pm 15.5$ years. The intent of corrosive ingestion was suicidal in $70 \%$ and accidental in $30 \%$. In majority $(50 \%)$ of patients the nature of corrosive was not known. Nasogastric tube placement was done in $50 \%$, nasojejunal tube placement in $32 \%$ and $8 \%$ no tube was placed. Surgery as needed in $19 \%$ (tracheostomy or feeding jejunostomy or a definitive surgery). Amongst the 82 patients who were included in the study, 11 were lost to follow up. Follow up was done in 71 patients in whom the median follow up period was 31 months (range $2 \mathrm{~m}-72 \mathrm{~m}$ ) during which $12(16.9 \%)$ patients expired $(73 \%$ related to ACI). Amongst the 59 patients, that were alive $16(27 \%)$ were symptomatic, $12(20 \%)$ had dysphagia, $5(6 \%)$ had regurgitation, $4(5 \%)$ had chest pain, $6(7 \%)$ had weight loss and $11(18 \%)$ patients required diet modification. In all, $43(73 \%)$ patients underwent barium study during follow up and strictures were noted in $21(36 \%)$. The site of stricture was esophageal in $11(53 \%)$, stomach in $8(38 \%)$ and combined esophageal and stomach in $2(9 \%)$. Esophageal stricture was seen in all patients with Grade III B esophageal injury, $27 \%(6 / 22)$ with Grade III A injury and 19\%(5/27) with Grade II B injury. None of the patients with Grade II A injury developed stricture. Stricture in stomach developed in $25 \%(2 / 8)$ patients with Grade III B injury, 25\% (6/24) with Grade III A injury, $10 \%(1 / 10)$ with II B injury and $20 \%(1 / 5)$ with II A injury.

Conclusion: Acute corrosive ingestion is associated with significant morbidity and mortality.There needs to be stringent control on sale, use and storage of such chemicals.

Disclosure of Interest: All authors have declared no conflicts of interest.

\section{Reference}

1. Zargar SA, Kochhar R, Mehta S, Mehta SK. The role of fiberoptic endoscopy in the management of corrosive ingestion and modified endoscopic classification of burns. Gastrointest Endosc. 1991 Mar-Apr;37(2):165-9.

\section{P1469 PROBE-BASED CONFOCAL LASER ENDOMICROSCOPY (PCLE) FOR IN VIVO DIAGNOSIS OF ESOPHAGEAL AND GASTRIC LESIONS - RESULTS OF A PROSPECTIVE, CONTROLLED, CROSS- OVER STUDY}

M. Kollar ${ }^{1}$, J. Krajciova ${ }^{2}$, J. Maluskova ${ }^{1}$, M. Kment ${ }^{1}$, Z. Vackova ${ }^{2}$, J. Spicak ${ }^{2}$, J. Martinek

${ }^{1}$ Clinical And Transplant Pathology, Institute for Clinical and Experimental Medicine, Prague/Czech Republic

${ }^{2}$ Department Of Hepatogastroenterology, Institute for Clinical and Experimental Medicine, Prague/Czech Republic

\section{Contact E-mail Address: marek.kollar1@seznam.cz}

Introduction: Probe-based confocal laser endomicroscopy (pCLE) provides realtime microscopic visualization with 1000-fold magnification, allowing endoscopic access to the histological evaluation of gastrointestinal lesions. pCLE may thereby be helpful in guidance of endoscopic therapy. However, histopathological assessment still remains a gold standard for histological diagnosis so far, while pCLE-based diagnosis has not been generally accepted yet. Therefore, more studies assessing diagnostic accuracy of pCLE are warranted.

Aims \& Methods: The aim of this study was to compare diagnostic accuracy of pCLE with standard biopsies in the diagnosis of macroscopically visible esophageal and gastric lesions.

A single-center, prospective, controlled and pathologist blinded study. We enrolled all consecutive patients with a visible esophageal or gastric lesion. All patients underwent high-resolution endoscopy and the lesion was examined by pCLE. After pCLE, standard biopsies were taken from the lesion and the specimen was sent for standard histolopathological assessment. Final diagnosis was determined based on assessment of endoscopic or surgical resection specimen (if possible) or standard biopsies. Some of the patients with an obviously malignant lesion underwent endoscopic resection immediately after pCLE without standard biopsies being obtained. Main outcomes of the study were diagnostic accuracy, sensitivity and specificity of pCLE vs. standard biopsies.

Results: We examined 45 lesions (38 esophageal, 7 gastric) in 41 patients.

Definitive diagnoses revealed 28 malignant lesions (21x adenocarcinoma (AC), $7 \mathrm{x}$ squamous carcinoma (SCC)) and 17 benign lesions. In summary, pCLE diagnosis was correct in $76 \%(34 / 45)$ and incorrect in $24 \%(11 / 45)$ while diagnosis based on biopsy was correct in $73 \%(27 / 37)$ and incorrect in $27 \%(10 / 37)$. pCLE confirmed malignant diagnosis in $81 \%(22 / 27)$ of cases and biopsy in $65 \%(10$ 16) (in 6 patients with a definitive diagnosis of $\mathrm{AC}$ and SCC, biopsies showed low- or high-grade dysplasia or necrotic tissue). Benign diagnosis was accurate in $65 \%(11 / 17)$ of lesions based on pCLE vs. $100 \%$ of lesions $(17 / 17)$ based on biopsy. Overall sensitivity, specificity, and diagnostic accuracy of biopsies were 67\% CI 95 (49-88\%), 100\% (54-100\%) and 83\% (68-94); sensitivity, specificity, and accuracy of pCLE for the correct histological diagnosis were $81 \%(62-93 \%)$, $91 \%(59-99 \%)$ and $84 \%(70-93)$, respectively.

Conclusion: pCLE provides satisfactory diagnostic accuracy comparable with standard biopsies in malignant esophageal and gastric lesions in particular.
Supported by a grant from Ministry of Health of the Czech Republic, No. 1627648A. ClinTrial registration: NCT02922049.

Disclosure of Interest: All authors have declared no conflicts of interest.

\section{P1470 COMPARING RISK SCORING SYSTEMS FOR NON- VARICEAL UPPER DIGESTIVE BLEEDING IN THE GASTROENTEROLOGY DEPARTMENT}

D. Lazar $^{1}$, I. Sporea ${ }^{1}$, D. Tornea ${ }^{1}$, L. Girboni ${ }^{1}$, R. Lupusoru ${ }^{1}$, I. Romosan ${ }^{2}$, A. Goldis ${ }^{1}$

${ }^{1}$ Gastroenterology, University of Medicine and Pharmacy, TIMISOARA/Romania

${ }^{2}$ Internal Medicine, University of Medicine and Pharmacy, Timisoara/Romania

Contact E-mail Address: lazar_daniela@yahoo.com

Introduction: Non-variceal upper digestive bleeding (NV-UDB) represents a significant cause of hospital admission. In order to stratify patients according to the risk of the complications (re-bleeding/death), and to predict the need of clinical intervention and patient's outcome, several risk scores have been proposed by international guidelines and implemented in clinical practice.

Aims \& Methods: The aim of the study consisted in the analysis of the accuracy of three risk scoring systems used in non-variceal upper digestive bleeding for assessing patient's prognosis, previously estimated to be predictive for re-bleeding/ death after gastrointestinal bleeding. We assessed prospectively a batch of 1872 patients admitted in the Gastroenterology Department of Emergency County Hospital Timisoara in a 12-year period, in which we calculated 3 risk scoring systems, Rockall, Cedars-Sinai and Baylor, based on clinical and endoscopic data. We compared their accuracy for assessing patient's prognosis, expressed as the need of blood transfusions, number of hospitalization days, re-bleeding, surgery and death. Discriminative ability was assessed using the area under the receiver operating characteristic curve (AUROC).

Results: The batch included $1134(60.6 \%)$ male and $738(39.4 \%)$ female, mean age $62 \pm 7.8$ years. Regarding the need of blood transfusions, the predictive ability of the scores is as follows: Rockall AUROC $0.59 \mathrm{CI}(0.55-0.62)$, sensitivity $(\mathrm{Se})=81.7 \%, \quad$ specificity $(\mathrm{Sp})=35.5 \%, \quad$ positive predictive value $(\mathrm{PPV})=28.4 \%$, negative predictive value $(\mathrm{NPV})=86.1 \% \quad(\mathrm{p}<0.0001)$; Cedars-Sinai AUROC 0.59 CI $(0.55-0.63), \quad \mathrm{Se}=72.4 \%, \quad \mathrm{Sp}=41.3 \%$, $\mathrm{PPV}=28.5 \%, \mathrm{NPV}=82.3 \%(\mathrm{p}<0.001)$; Baylor AUROC $0.56 \mathrm{CI}(0.49-0.63)$, $\mathrm{Se}=41.9 \%, \mathrm{Sp}=75.5 \%, \mathrm{PPV}=40.6 \%, \mathrm{NPV}=76.5 \%$. Number of hospitalization days: Rockall AUROC $0.66 \mathrm{CI}(0.55-0.77), \mathrm{Se}=61.5 \%, \mathrm{Sp}=65.2 \%$, $\mathrm{PPV}=90 \%, \mathrm{NPV}=25 \% \quad(\mathrm{p}=0.003)$; Cedars-Sinai AUROC $0.63 \mathrm{CI}(0.50$ $0.75), \mathrm{Se}=53.1 \%, \mathrm{Sp}=73.9 \%, \mathrm{PPV}=89.5 \%, \mathrm{NPV}=27.4 \%$; Baylor AUROC $0.52 \mathrm{CI}(0.51-0.73), \mathrm{Se}=47.06 \%, \mathrm{Sp}=66.6 \%, \mathrm{PPV}=84.2 \%, \mathrm{NPV}=25 \%$. Rebleeding: Rockall AUROC $0.69 \mathrm{CI}(0.66-0.73), \mathrm{Se}=69.1 \%, \mathrm{Sp}=60.4 \%$, $\mathrm{PPV}=14.2 \%, \quad \mathrm{NPV}=92.8 \% \quad(\mathrm{p}<0.0001) ; \quad$ Cedars-Sinai AUROC 0.73 $\mathrm{CI}(0.69-0.77), \mathrm{Se}=84.4 \%, \mathrm{Sp}=49.02 \%, \mathrm{PPV}=13.7 \%, \mathrm{NPV}=97 \%$; Baylor AUROC $0.54 \mathrm{CI}(0.45-0.63), \quad \mathrm{Se}=35.1 \%, \quad \mathrm{Sp}=81.2 \%, \quad \mathrm{PPV}=16.2 \%$, $\mathrm{NPV}=92.4 \%$. Surgery: Rockall AUROC $0.67 \mathrm{CI}(0.61-0.73), \mathrm{Se}=71.2 \%$, $\mathrm{Sp}=59 \%, \mathrm{PPV}=16 \%, \mathrm{NPV}=98.1 \%$; Cedars-Sinai AUROC $0.72 \mathrm{CI}(0.66$ $0.78), \mathrm{Se}=58 \%, \mathrm{Sp}=77.4 \%, \mathrm{PPV}=9.3 \%, \mathrm{NPV}=97.9 \%$; Baylor AUROC $0.55 \mathrm{CI}(0.41-0.68), \mathrm{Se}=50 \%, \mathrm{Sp}=66.2 \%, \mathrm{PPV}=5.1 \%, \mathrm{NPV}=97.4 \%$. Death: Rockall AUROC $0.85 \mathrm{CI}(0.81-0.89), \mathrm{Se}=84.7 \%, \mathrm{Sp}=76 \%, \mathrm{PPV}=18.2 \%$, $\mathrm{NPV}=99.5 \% \quad(\mathrm{p}<0.0001) ; \quad$ Cedars-Sinai AUROC $0.71 \quad \mathrm{CI}(0.66-0.76)$, $\mathrm{Se}=83.1 \%, \mathrm{Sp}=48.1 \%, \mathrm{PPV}=10.2 \%, \mathrm{NPV}=97.6 \%$; Baylor AUROC 0.75 $\mathrm{CI}(0.67-0.83), \mathrm{Se}=76.09 \%, \mathrm{Sp}=72.3 \%, \mathrm{PPV}=19.2 \%, \mathrm{NPV}=97.2 \%$. There were no statistically significant differences encountered in predicting the need of blood transfusions and surgery between the scores $(\mathrm{p}>0.05)$. Baylor score was superior vs Rockall in estimating the hospitalization period $(\mathrm{p}=0.04)$ and the risk of re-bleeding $(\mathrm{p}=0.0009)$, and Cedars-Sinai proved to be superior to Baylor score in predicting re-bleeding $(\mathrm{p}=0.002)$ and to Rockall score in predicting death $(\mathrm{p}=0.006)$.

Conclusion: On our cohort of patients, Cedars-Sinai score proved to be the best in predicting the re-bleeding and death in patients with NV-UDB in comparison to Rockall and Baylor scores.

Disclosure of Interest: All authors have declared no conflicts of interest.

\section{P1471 PERCUTANEOUS ENDOSCOPIC GASTROSTOMY: A SAFE PROCEDURE EVEN IN CANCER PATIENTS}

J. Pinho' ${ }^{1}$ J. Lage ${ }^{2}$, D. Libanio ${ }^{2}$, S. Ferraz ${ }^{2}$, N. Silva ${ }^{2}$, M. Dinis-Ribeiro ${ }^{2}$, C. Brandão ${ }^{2}$

${ }^{1}$ Gastroenterology, Centro Hospitalar Tondela/Viseu, Viseu/Portugal

${ }^{2}$ Gastroenterology, Instituto Português de Oncologia do Porto, Porto/Portugal

Contact E-mail Address: julianapinho18@gmail.com

Introduction: Dysphagia and malnutrition is a common feature in up to $64 \%$ of patients with head and neck, esophagus and gastro-esophageal cancer, and the need of radiotherapy or chemotherapy often worsens this symptoms. Percutaneous endoscopic gastrostomy (PEG) is the preferred route of feeding and nutritional support in these patients. Although generally considered to be a safe procedure, it has been arguable that PEG tube placement complications in cancer patients may be superior when compared to non-cancer patients.

Aims \& Methods: The aim of this study was to evaluate the complications rate after PEG tube placement in cancer patients. We did a single-centre prospective database including all patients with PEG tube insertion between March 2014 and June 2016, evaluating the complications during 6 months follow-up.

Results: A total of 265 patients $(83 \%$ men, mean age 59 years) underwent PEG tube insertion. 224 patients $(84.5 \%)$ had head and neck cancers and 33 patients 
$(12.5 \%)$ had esophageal cancer; 207 patients $(78.1 \%)$ had stage IV disease. At the time of PEG tube placement, 138 patients $(52 \%)$ had grade 3 dysphagia and the mean body mass index (BMI) was $20.9 \mathrm{Kg} / \mathrm{m} 2$. All the patients underwent antibiotic prophylaxis previous to the procedure. There was an increase on BMI to $23.8 \mathrm{Kg} / \mathrm{m} 2$ at 6 months follow up. Eight patients (3.8\%) had immediate complications after the procedure (bleeding from the PEG tract-6; anesthetic complications - 2). The overall complication rate at the first month of follow up was $14.4 \%$, at the third month $20.5 \%$ and at the sixth month $11.7 \%$. The overall periPEG infection rate was $14 \%$, and was the main complication at the first month of follow up. Development of hyper-granulation tissue was the most frequent complication at the third month of follow-up. Buried bumper syndrome occurred in 10 patients $(3.7 \%)$. None of the patients had tumor seeding at the gastrostomy site. Overall mortality was $26.4 \%$, none of the deaths attributable to PEG tube insertion.

Conclusion: PEG placement is a safe and effective technique in cancer patients. The rate of major complications and tube site infection were similar to the results found in literature for non-cancer patients.

Disclosure of Interest: All authors have declared no conflicts of interest

\section{References}

Rahnemai-Azar AA, Rahnemaiazar AA, Naghshizadian R, Kurtz A, Farkas DT. Percutaneous endoscopic gastrostomy: indications, technique, complications and management. World J Gastroenterol. 2014 Jun 28;20(24):7739-51.

Mansoor H, Masood MA, Yusuf MA. Complications of percutaneous endoscopic gastrostomy tube insertion in cancer patients: a retrospective study. $J$ Gastrointest Cancer. 2014 Dec;45(4):452-9.

\section{P1472 A PROSPECTIVE, SINGLE-CENTER, CROSS-OVER CONTROLLED TRIAL OF CONFOCAL LASER ENDOMICROSCOPY ASSESSMENT OF PERSISTENT OR RECURRENT INTESTINAL METAPLASIA AND RECURRENCE OF NEOPLASIA AFTER ENDOSCOPIC TREATMENT OF BARRETT'S ESOPHAGUS- RELATED NEOPLASIA (BORN)}

J. Krajciova ${ }^{1}$, M. Kollar ${ }^{2}$, J. Maluskova ${ }^{2}$, M. Kment ${ }^{2}$, Z. Vackova ${ }^{1}$, J. Spicak ${ }^{1}$, J. Martinek

${ }^{1}$ Department Of Hepatogastroenterology, Institute for Clinical and Experimental Medicine, Prague/Czech Republic

${ }^{2}$ Clinical And Transplant Pathology, Institute for Clinical and Experimental Medicine, Prague/Czech Republic

\section{Contact E-mail Address: kraj@ikem.cz}

Introduction: Probe-based confocal laser endomicroscopy (pCLE) has been developed to overcome limitations of the current endoscopic sampling techniques. pCLE allows detailed examination of cellular structures and may examine larger areas compared to standard biopsy. Patients after endoscopic treatment of Barrett's esophagus (BE)-related neoplasia (BORN) should undergo endoscopic surveillance with biopsies to detect persistence or recurrence of intestinal metaplasia (IM) or neoplasia (N).

Aims \& Methods: The aim of this prospective study was to evaluate the efficacy of pCLE (vs. standard biopsies) in detection of persistent/recurrent IM/neoplasia in patients after endoscopic treatment of BORN. A single-center, prospective, controlled and pathologist-blinded (still ongoing) study in patients undergoing surveillance endoscopy after endoscopic treatment of BORN. pCLE images were obtained from the neo-Z-line (a few cases including macroscopically visible tongues), the cardia and the esophagus. Thereafter, standard biopsies were taken and sent for histopathological analysis (4 biopsies from macroscopically normal neo-Z-line, 2 biopsies from the cardia and the esophagus and targeted biopsies from visible abnormalities, if present). BE was defined in pCLE as columnarlined epithelium with dark mucin in goblet cells, a villiform pattern, and regularshaped capillaries in the mucosa. The dysplastic BE was characterized by black cells with irregular borders and shapes, high dark contrast to the surrounding tissue, and irregular leaking capillaries in the mucosa.

Results: We examined 29 patients, from these 14 patients $(48 \%)$ had the initial diagnosis of low-grade intraepithelial neoplasia (LGIN), 7 patients $(24 \%)$ had high-grade intraepithelial neoplasia (HGIN) and 8 patients $(28 \%)$ had an early adenocarcinoma (EAC). Persistent/recurrent IM was detected at the level of neo$\mathrm{Z}$-line in 10 patients $(34.5 \%)$ by both standard biopsies and pCLE. pCLE but not biopsies detected persistent/recurrent IM in 2 patients $(6.7 \%)$, another 2 patients had IM present in biopsies but not in pCLE. pCLE diagnosed one patient with recurrent LGIN in a macroscopic visible tongue arising from neo-Z-line, which was not confirmed in biopsies. Sensitivity and specificity of pCLE detection of persistent/recurrent IM was $83.3 \%$ (95\% CI 51.6-97.9) and $89.47 \%(95 \% \mathrm{CI}$ 66.9-98.7), respectively, with positive predictive value $83.3 \%$ (95\% CI 56.1-95.0) and negative predictive value $89.5 \%$ (95\% CI 70.4-96.8). Agreement of pCLE and histopathological findings was $86 \%$.

Conclusion: pCLE seems to be comparable with standard biopsies in detection of persistent/recurrent IM after endoscopic treatment of BORN. Nevertheless, these results need to be confirmed in a larger cohort of patients. Supported by a grant from Ministry of Health of the Czech Republic, No. 16-27648A.

ClinTrial registration: NCT02922049.

Disclosure of Interest: All authors have declared no conflicts of interest
P1473 FLEXIBLE ENDOSCOPIC SEPTUM DIVISION (FESD) OF

ZENKER'S DIVERTICULUM: OUTCOMES FROM A SINGLE TERTIARY CENTER USING A NEW SYMPTOMS SCORE

R. Maselli, G. Lollo, M. Di Leo, R. Nicoletti, E. Finati, P.A. Galtieri, L. Poliani, F. Auriemma, R. Semeraro, A. Fugazza, E.C. Ferrara, A. Anderloni, S. Carrara, A. Repici

Humanitas Researc Hospital, Rozzano/Italy

Contact E-mail Address: roberta.maselli@humanitas.it

Introduction: Symptomatic Zenker's diverticulum (ZD) can be treated by flexible endoscopic septum division (FESD) as a minimally invasive alternative to surgery or to rigid endoscopic procedure. There is still a lack of standardization of the FESD as well as data on late outcome are scarcely reported. Moreover, FESD outcomes are differently reported in available literature and no specific scores have been yet validated.

Aims \& Methods: We aim to report the outcome of all ZD treated by FESD in our institution by using a new symptoms score. We retrospectively reviewed consecutive patients with ZD treated by FESD in a single tertiary-care academic medical center between April 2014 and Feb 2017. All patients were included in a prospectively maintained database. A dedicated new score was created to obtain reliable and objective evaluation of outcome of our patients. This score (MilanoZenker score, MZ-score) is based on the three main ZD symptoms: dysphagia, regurgitation and weight loss. The ZD score (maximum score, 9 ) is the sum of the symptom scores for dysphagia, regurgitation (for both: 0, absent; 1, occasional; 2 , daily; and 3 , each meal), and weight loss (0, no weight loss; $1,<5 \mathrm{~kg} ; 2,5$ $10 \mathrm{~kg}$; and $3,>10 \mathrm{~kg}$ ). The main outcome was clinical success, defined as MZ score $\leq 2$ at the latest follow up. Patient's symptoms were evaluated at 1 month and at every 6 months after the procedure. Adverse events were recorded and classified as intraprocedural, postprocedural ( $<14$ days), and late (15-30 days). Results: 100 patients underwent FESD, with a mean age of $70.3 \pm 11.2$ years (range 39-95) and prevalence of male gender (66\%). Twenty-nine (29\%) of treated ZD were re-intervention: $20(69 \%)$ after previous FESD; $8(27.6 \%)$ after previous surgery; $1(3.4 \%)$ after previous surgery and FESD. Mean initial MZ score was $4.5 \pm 1.8$ (range 1-8). Intraprocedural bleeding was observed in 5 patients $(5 \%)$, with all of successfully treated by clips. No postprocedural/late bleedings were recorded. One perforation was observed and treated conservatively. The mean follow up period was 14 months (range 3-35). Overall clinical success was obtained in $78.5 \%$ of patients. Mean post treatment ZD score was $1.3 \pm 1.5$, significantly lower than initial MZ score $(\mathrm{p}<0.0001)$.

Conclusion: FESD represents a safe and effective treatment for symptomatic patients with Zenker's diverticulum. Although it needs to be validated, proposed $\mathrm{MZ}$ score could represent a useful tool to monitoring post-procedural symptoms and guide subsequent management.

Disclosure of Interest: All authors have declared no conflicts of interest.

\section{P1474 HOW ACCURATE ARE THE SYMPTOMS IN PATIENTS WITH FOOD IMPACTION AND FOREIGN BODIES INGESTION?}

A. Laranjo ${ }^{1}$, I. Mocanu ${ }^{2}$, M. M. Carvalho ${ }^{1}$, S. Pires ${ }^{1}$, N. Veloso ${ }^{1}$, L. Gonçalves $^{1}$, R. Godinho' ${ }^{1}$ I. Medeiros ${ }^{1}$

${ }^{1}$ Gastrenterology Department, Hospital Espirito Santo de Évora, Évora/Portugal ${ }^{2}$ Hospital Espirito Santo, Évora/Portugal

Contact E-mail Address: anamlaranjo@gmail.com

Introduction: Food impaction (FI) and foreign body ingestion (FBI) remain the most frequent indications for emergency esophagogastroduodenoscopy. The symptoms in these patients are often nonspecific and their location is not always related to the endoscopic findings.

Aims \& Methods: The aim of this study was to caracterize the symptomatology of the patients with suspected FI and FBI, to correlate the clinical symptoms with the endoscopic findings and to assess its management and complications. This was a restrospective unicenter cohort study including patients with suspected FI and FBI during three years (2013-2016). Statistic analysis were performed using IBM SPSS Statistics 22 with $\mathrm{p}<0,05$ deemed to be statistically significant.

Results: 198 patients were included ( 90 patients with FI, 70 patients with FBI, 38 patients without endoscopic findings of FI or FBI): $59.1 \%$ men, with a mean age of $60.10 \pm 19$ years. Patients with FI were predominantly men and were significantly older when compared with FBI patients $(p<0.05)$. Dysphagia was the most frequent symptom in FI patients, while foreign body perception and odynophagia were more common in FBI. The symptoms in FI group were localized more frequent in the esophagus $(83.3 \%)$ and FI was identified by esophagogastroduodenoscopy in the distal esophagus in $58.9 \%$ of those patients. In comparison, the symptoms in patients with FBI were predominantly localized in oropharynx $(65.7 \%)$ and FBI was identified in proximal esophagus in $55.7 \%$ of those patients. There was more correlation between the location of the symptoms and the endoscopic findings in patients with FI compared with the patients with FBI (FI $65.6 \%$ VS $47.1 \%$; $<<0.05$ ). In FI patients, the most frequent esophageal abnormalities were peptic stenosis and Schatzki ring. In FBI patients, the most common foreign bodies identified were meat $(47.1 \%)$ and fish bones $(22.9 \%)$. Foreign body forceps was the most used device in FBI patients, while Roth Net was preferred in FI patients. Overall, esophagoduodenoscopy was effective in the resolution of FI in $100 \%$ of the patients and foreign body extraction was not possible in 4 patients $(5.7 \%)$. Major complications occurred in $0.5 \%$ (1 perfuration). 
Conclusion: In FI patients, the location of the symptoms has a better correlation with the endoscopic findings compared with FBI patients. The esophagoduodenoscopy is safe and effective in patients with FI and FBI

Disclosure of Interest: All authors have declared no conflicts of interest.

\section{P1475 GASTROINTESTINAL ENDOSCOPY UNDER SEDATION IS ASSOCIATED WITH PNEUMONIA IN OLDER INPATIENTS- RESULTS OF A RETROSPECTIVE CASE-CONTROL STUDY}

\section{M. Kollmann, W. Schmiegel, T. Brechmann}

Department Of Gastroenterology And Hepatology, Berufsgenossenschaftiches

University Hospital Bergmannsheil Gmbh, Bochum/Germany

Contact E-mail Address: christopher.kollmann@rub.de

Introduction: Apparent aspiration is a notable adverse event during gastrointestinal endoscopy under sedation (GIES) [1,2], but data about the incidence and the role of inapparent aspiration is scarce. Furthermore, patients undergoing endoscopies experience respiratory symptoms such as coughing, shortness of breathing, fever and other respiratory adverse events within 24 hours relatively often in more than 5\% [3]. Since coughing during endoscopy has been attributed to an increased risk of aspiration-related postprocedural infection [4] respiratory infections might be underreported. Additionally, patients in advanced age are not only determined as a high-risk group for GI adverse events following colonoscopies [5], but are also more likely to develop hospital-acquired pneumonia [2] Therefore, the aim of the study was to determine the risk of pneumonia, lower respiratory infection (LRI) and systemic inflammatory activation after GIES. Aims \& Methods: A total of 250 consecutive inpatients who had undergone GIES during a hospital stay of at least three days were included in a retrospective cohort study. Age-, gender- and length of hospital stay-matched controls (ratio 1:1) who had not undergone any invasive procedure or sedation served as controls. Laboratory parameters had to be available before and three and/or seven days after endoscopy. Primary objective was the occurrence of pneumonia in general and older patients ( $\geq 65$ years). Secondary objectives were the development of LRI, elevation of inflammatory markers (CRP and WBC), initiation of antibiotic treatment, pathogen detection and pulmonary infiltration. Statistics included $\chi 2$ test, paired t-test, ANOVA, multiple linear regression analysis. Results: No significant differences for the occurrence of pneumonia (1.6\%, GIES group vs. $0.4 \%$, control group, $\mathrm{p}=.178, \chi 2$ test $)$ and LRI $(4.8 \%$ vs. $2.0 \%$, $\mathrm{p}=.084)$ in general, but in the older age group $(2.6 \%$ vs. $0.0 \%, \mathrm{p}=.041$, and $7.8 \%$ vs. $2.5 \%, \mathrm{p}=.034$, respectively) were detected. Inflammatory parameters were significantly increased after GIES, particularly on day three. GIES patients received antibiotic treatment more frequent while pulmonary infiltration did not differ.

Conclusion: This data confirms a higher risk of pneumonia due to GIES in the advanced aged population. In general, patients are more likely to develop inflammation and to receive antibiotic treatment suggesting an increased risk of radiologically non-visible inflammation due to micro-aspiration.

Disclosure of Interest: All authors have declared no conflicts of interest.

\section{References}

1. Agostoni M, Fanti L, Gemma M, et al. Adverse events during monitored anesthesia care for GI endoscopy: an 8-year experience. Gastrointestinal Endoscopy 2011;74:266-275.

2. Ewan V, Hellyer T, Newton J, Simpson J. New horizons in hospital acquired pneumonia in older people. Age Ageing. 2017 Feb 27:1-7

3. Friedrich K, Scholl SG, Beck S, et al. Respiratory Complications in Outpatient Endoscopy with Endoscopist-Directed Sedation. J Gastrointest Liver Dis 2014;23:255-259.

4. El Chac AH, Eckert G, Rex DK. Prospective description of coughing, hemodynamic changes, and oxygen desaturation during endoscopic sedation. Dig Dis Sci 2012;57:1899-1907.

5. Day LW, Kwon A, Inadomi JM, et al. Adverse events in older patients undergoing colonoscopy: a systematic review and meta-analysis. Gastrointestinal Endoscopy 2011;74:885-896.

\section{P1476 SYMPTOMATIC RESPONSE OF PYLORIC PNEUMATIC DILATATION, BOTOX INJECTION OR COMBINATION THERAPY IN PATIENTS WITH GASTROPARESIS OR DELAYED GASTRIC EMPTYING POST-GASTRIC TRANSPOSITION}

A. Gupta, M. A. Everson, R. Haidry, M. Banks, S. Bown, D. Graham, R. Ganatra, A. Ramasubramoni, L. Lovat, R. Sweis

Department Of Gastroenterology, University College London Hospital, London United Kingdom

Contact E-mail Address: absgupta@aol.com

Introduction: Therapeutic options for gastroparesis or delayed emptying following oesophagectomy with gastric transposition are limited. Although commonly performed, there have been no studies addressing the relative merits of endoscopic dilatation of the pylorus, botulinum toxin (Botox) injection or combination therapy. We assessed the symptomatic response of patients undergoing nonsurgical pyloric intervention at a specialist tertiary centre.
Aims \& Methods: 33 patients (13 male; mean age 45, range 17-80) underwent a total of 51 endoscopic procedures over 2 years. Treatments were either $100 \mathrm{IU}$ units of Botox injected into 4 quadrants of the pylorus or pneumatic dilation (PD) incrementally up to 16-20 mm (Hercules; Cook Medical). Patients with gastric malignancy, previous pyloric surgery or no documented follow-up were excluded. Post-therapeutic responses were assessed at first follow up post-procedure and graded as 'good', 'partial' or 'none/poor'. Patients were grouped according to type of therapy and indication.

Results: There were no immediate or late complications observed. 31 procedures were performed for gastroparesis with a mean post-procedure follow-up of 11 weeks. Overall, a partial or good response was observed in $81 \%(25 / 31)$. Specific treatment with Botox alone (18 procedures) or as part of combination therapy (10 procedures) led to a good or partial response in $86 \%(24 / 28)$ compared to $33 \%(1 / 3)$ who had PD alone $(\mathrm{p}=0.03) .14$ procedures were performed in the post-surgical group with a mean post-procedure follow-up of 10 weeks. Overall, a partial or good response was observed in 93\% (13/14); treatment with Botox alone or as part of combination therapy led to a good or partial response in $100 \%(10 / 10)$ compared to $75 \%(3 / 4)$ who had dilatation alone $(p=0.1) .6$ therapies were performed without a defined indication, 2 patients $(33 \%)$ had a partial or good response, both of which had combination therapy.

Conclusion: Pyloric intervention with Botox, PD or combination therapy are safe and effective treatment options for patients with gastroparesis or delayed gastric emptying following gastric transposition. Subjective treatment without a clear indication shows little improvement.

Disclosure of Interest: All authors have declared no conflicts of interest.

\section{P1477 AN ANALYSIS OF COMPLICATIONS FOLLOWING} ENDOSCOPIC SUBMUCOSAL DISSECTION IN A WESTERN SETTING - MAKING THE CASE FOR A SHORTER LENGTH OF STAY

S. Subramaniam ${ }^{1}$, K. Kandiah ${ }^{1}$, G. Longcroft-Wheaton ${ }^{2}$, P. Bhandari ${ }^{1}$ ${ }^{1}$ Gastroenterology, Queen Alexandra Hospital, Portsmouth/United Kingdom ${ }^{2}$ Gastroenterology, Portsmouth Hospitals NHS trust, Hampshire/United Kingdom

Contact E-mail Address: shar811@googlemail.com

Introduction: Endoscopic submucosal dissection (ESD) is an established technique for the treatment of gastrointestinal (GI) neoplasia in Japan. The high uptake and mastery of the procedure there was in part enabled by the high prevalence of early gastric cancer in Japan. Conventional practice in Japan is to admit patients for 3 to 5 days after the ESD procedure for monitoring in view of the risk of serious complications which is between $1-10 \%$. Cost and resource provision in a publicly-funded Western healthcare setting favours shorter planned stays following ESD

Aims \& Methods: We aimed to identify the type and site of lesions being treated in a Western setting as well as the rate, timing and predictors of complications in order to evaluate current admission practice. An electronic database of all ESD procedures performed in our academic institution from 2012-2017 was analysed. Parameters were the number, type, onset and management of complications following ESD. Significant complications (bleeding and perforation) necessitating hospital admission were categorised as early (within 24 hours) and delayed ( 24 hours to 28 days) post procedure.

Results: A total of 410 ESDs were performed within the time period ( 225 colorectal, 117 oesophageal, 52 gastric and 16 duodenal). There were 21 complications. ESD for gastric and duodenal lesions had a higher complication rate $(11.5 \%$ and $12.5 \%$ respectively). The table below stratifies the complications according to type, location and onset.

\begin{tabular}{llllll}
\hline & & Colorectal & Oesophageal & Gastric & Duodenal \\
\hline Early & Bleeding & $3 / 225$ & $1 / 117$ & $0 / 52$ & $0 / 16$ \\
& Perforation & $2 / 225$ & $0 / 117$ & $2 / 52$ & $1 / 16$ \\
Total (early) & & $5 / 225(2.2 \%)$ & $1 / 117(0.9 \%)$ & $2 / 52(3.9 \%)$ & $1 / 16(6.3 \%)$ \\
Delayed & Bleeding & $5 / 225$ & $1 / 117$ & $4 / 52$ & $1 / 16$ \\
& Perforation & $1 / 225$ & $0 / 117$ & $0 / 52$ & $0 / 16$ \\
Total (delayed) & & $6 / 225(2.7 \%)$ & $1 / 117(0.9 \%)$ & $4 / 52(7.7 \%)$ & $1 / 16(6.3 \%)$ \\
Grand total of & & $11 / 225(4.9 \%)$ & $2 / 117(1.8 \%)$ & $6 / 52(11.5 \%)$ & $2 / 16(12.5 \%)$ \\
$\quad$ all complications & & & & & \\
\hline
\end{tabular}

19 of the 21 total complications $(90.5 \%)$ were successfully managed endoscopically. 1 patient underwent an emergency right hemicolectomy for a delayed colonic perforation and 1 patient had radiological embolisation for a bleed 7 days following a caecal ESD. $72.7 \%(8 / 11)$ of the patients who experienced delayed bleeding presented 7-10 days following ESD. Of this, $50 \%$ occurred following recommencement of anticoagulants/antiplatelets.

Conclusion: ESD in this Western setting was more commonly performed for colonic and oesophageal lesions rather than gastric as seen in Japan. The complication rate is modest and almost all were managed successfully with an endoscopic approach. They occurred more commonly in gastric and duodenal sites. This may be related to the technical difficulties of resection or low volume of procedures performed at these locations. The use of anticoagulants is a risk 
factor for delayed bleeding. Given that the majority of delayed complications occurred more than 5 days post procedure, a standardised 5 day inpatient stay would prove futile in our cohort.

Disclosure of Interest: P. Bhandari: Educational grants from Fujifilm, Olympus and Pentax

All other authors have declared no conflicts of interest.

\section{P1478 PREDICTIVE FACTORS AND MANAGEMENT OF REFRACTORY BENIGN OESOPHAGEAL STRICTURES}

M. Moura, C. Simões, C. Noronha Ferreira, P. Santos, J.P. Freire, J. Lopes, L. Carrilho Ribeiro, J. Velosa

Gastrenterologia E Hepatologia, Hospital de Santa Maria - Centro Hospitalar Lisboa Norte, EPE, Lisboa/Portugal

Contact E-mail Address: cmiguelmour@gmail.com

Introduction: The optimal management and the predictive factors of response to endoscopic dilatation of refractory benign oesophageal strictures remains controversial.

Aims \& Methods: To evaluate the prevalence and factors predicting response to treatment of benign refractory oesophageal strictures with scheduled endoscopic dilatations Retrospective analysis of 75 patients submitted to scheduled endoscopic dilatation of benign oesophageal strictures between October 2010 and November 2016. Strictures were classified as refractory when $\geq 5$ endoscopic dilations were needed with at least one dilation achieving $\geq 15 \mathrm{~mm}$ of diameter during the course of management of the oesophageal strictures.

Results: The study sample included $42(56 \%)$ male patients and the mean age was $57 \pm 18$ years. The dysphagia scale at baseline was: solids (1)-17 $(22.7 \%)$, semisolids (2)-23 (30.7\%), liquids (3)-23 (30.7\%) and complete (4)-12 (16\%). Body mass index (BMI) at baseline was $22 \pm 5 \mathrm{Kg} / \mathrm{m}^{2}$. The aetiology of the benign strictures was: surgical-31 $(41.3 \%)$, peptic-15 $(20 \%)$, caustic-10 $(13.3 \%)$, radiotherapy-10 $(13.3 \%)$ and others $-9(12 \%)$. The location of the oesophageal strictures was as follows: proximal third-34 $(45.3 \%)$, middle third-12 $(16 \%)$, dista third-27 $(36 \%)$ and multiple locations-2 (2.7\%). Stricture type: simple-44 $(58.7 \%)$, complex-31 $(41.3 \%)$. Patients underwent a median of 4 (1-26) endoscopic dilation over a median period of 19 weeks (1-229). Dilations were done with Savary-Gilliard dilators-35 (46.7\%), TTS-balloons-24 (32\%) or both-16 $(21.3 \%)$. The mean diameter of dilation achieved was $15.7 \pm 2.2$ and a dilatation diameter of $>15 \mathrm{~mm}$ was achieved in $56(74.6 \%)$ patients. Local injection of corticosteroid (dexametasone $5 \mathrm{mg}$ ) was performed at least once in $39(52 \%)$ patients and in $\geq 25 \%$ of dilations in $39(52 \%)$ patients. From the study sample, $25(33.3 \%)$ patients fulfilled criteria of refractory strictures. In this subgroup, there was a significant association with post-surgical aetiology $(\mathrm{p}=0.042)$, lower baseline BMI $(\mathrm{p}=0.039)$, higher rate of local injection of corticosteroids $(\mathrm{p}<0.001)$ and higher dilation diameter $(\mathrm{p}<0.001)$. Refractory strictures were significantly associated with the need for local corticoid injection (OR 9.76, $95 \%$ CI $0.035-0.46, p=0.02$ ) by binary logistic regression analysis. However, none of the other factors were found to be independent predictors of response to therapy.

Conclusion: Surgical aetiology was significantly associated with refractory benign oesophageal strictures and these patients were significantly more liked to require local corticosteroid injections during scheduled endoscopic dilatations.

Disclosure of Interest: All authors have declared no conflicts of interest.

P1479 LOCAL CORTICOSTEROIDS IMPROVE EARLY CLINICAL OUTCOMES IN PATIENTS UNDERGOING ENDOSCOPIC DILATION OF BENIGN OESOPHAGEAL STRICTURES

M. Moura, C. Simões, C. Noronha Ferreira, P. Santos, J.P. Freire, J. Lopes, L. Carrilho Ribeiro, J. Velosa

Gastrenterologia E Hepatologia, Hospital de Santa Maria - Centro Hospitalar Lisboa Norte, EPE, Lisboa/Portugal

Contact E-mail Address: cmiguelmour@gmail.com

Introduction: Locally injected corticosteroids have been shown to improve outcomes in patients undergoing endoscopic dilation of peptic strictures.

Aims \& Methods: To evaluate factors predicting early clinical response to endoscopic dilation of benign oesophageal strictures. Retrospective analysis of 75 consecutive patients submitted to scheduled endoscopic dilatations between October 2010 and November 2016. Clinical improvement was defined as dysphagia score $\leq 1$. IBM SPSS $(21$ was used for statistical analysis.

Results: The study sample involved $42(56 \%)$ male patients and the mean age was $57 \pm 18$ years. Dysphagia scale at baseline: solids (1)-17 (22.7\%), semisolids (2) $23(30.7 \%)$, liquids (3) $-23(30.7 \%)$ and complete (4)-12 (16\%). Body mass index at baseline was $22 \pm 5 \mathrm{Kg} / \mathrm{m} 2$. Stricture aetiology: surgical-31 (41.3\%), peptic-15 $(20 \%)$, caustic-10 $(13.3 \%)$, post-radiotherapy-10 $(13.3 \%)$ and others-9 $(12 \%)$. Stricture location: proximal-34 (45.3\%), medium-12 (16\%), distal $27(36 \%)$ and multiple-2 (2.7\%). Stricture type: simple-44 (58.7\%), complex-31 (41.3\%). Patients underwent a median of 4 (1-26) endoscopic dilations over a median period of 19 weeks $(1-229)$. Dilations were performed with Savary-Gilliard dilators-35 $(46.7 \%)$, TTS-balloons-24 $(32 \%)$ or both-16 $(21.3 \%)$. The mean diameter of dilation was $15.7 \pm 2.2$ and dilatation of $\geq 15 \mathrm{~mm}$ was achieved in 56 $(74 \%)$ of patients. Local corticosteroid injection (dexametasone $5 \mathrm{mg}$ ) was performed at least once in $39(52 \%)$ patients and in $\geq 25 \%$ of dilations also in 39 $(52 \%)$ patients. The rate of complications was $13.3 \%$ (perforation $(n=9)$ and bleeding $(=1)$ ). Surgery was performed in 10 patients $(13.3 \%)$ (refractory strictures $(n=7)$ and post dilation perforation $(n=3))$. Improvement of dysphagia symptoms was only associated with the maximum dilation diameter $(\mathrm{p}=0.026)$ and local injection of corticoids $(\mathrm{p}<0.001)$ as was confirmed by binary logistic regression wherein both maximum dilation diameter (OR: 4.92, 95\% IC 1.052.04, $\mathrm{p}=0.027)$ and topical injection of corticosteroids (OR: 7.22, 95\% IC $0.021-0.55, \mathrm{p}=0.007$ ) were strongly associated with improved dysphagia scores. Conclusion: In our study sample with multiple aetiologies of benign oesophageal strictures, only the maximum dilation diameter and local injection of corticosteroids were associated with improved clinical outcomes in patients undergoing endoscopic dilation of benign oesophageal strictures.

Disclosure of Interest: All authors have declared no conflicts of interest.

\section{P1480 TRENDS IN CERTIFICATION FOR GASTROINTESTINAL ENDOSCOPY AND VARIATIONS BETWEEN TRAINEE SPECIALTIES: RESULTS FROM THE UK TRAINEE ENDOSCOPY DATABASE}

K. Siau, R. Broughton, J. Anderson, I. Beales, M. Feeney, A. Haycock, N. Hawkes, G. Johnson, B. Mckaig, R. Pullan, R. Valori, C. Wells, S. ThomasGibson, P. Dunckley

*JAG Working Group For The Quality Assurance Of Training, Joint Advisory Group, London/United Kingdom

Contact E-mail Address: keith@siau.org

Introduction: In the UK, endoscopy certification is overseen by the Joint Advisory Group (JAG). Since 2011, e-certification has been awarded for upper and lower GI endoscopy online via the JAG Electronic Training System (JETS) We aimed to analyse trends in endoscopy e-certification, and assess for differences between trainees in gastroenterology (GI), surgical (GS) and non-medical endoscopists (NME).

Aims \& Methods: We prospectively identified trainees awarded certification for gastroscopy, flexible sigmoidoscopy (FS) and colonoscopy from the JETS database. For each specialty, we collected data on lifetime procedural counts, formative assessments, and key performance indicators (KPIs) at the time of certification. Comparisons between specialties were analysed using a combination of $\mathrm{chi}^{2}$, Mann-Whitney and median tests.

Results: Between June 2011-Dec 2016, 2857 applications were awarded certification. Numbers of gastroscopy and provisional colonoscopy awarded have been in steady state since 2013, whilst numbers for sigmoidoscopy and full colonoscopy continue to increase. Trainees awarded certification comprised mainly of GI $(53.2 \%)$, GS $(28.5 \%)$ and NME $(15.8 \%)$ trainees. With the exception of FS, most certifications were awarded to GI trainees (Figure 2). Median procedural numbers $(\mathrm{p}<0.001)$ and formative DOPS count $(\mathrm{p}<0.001)$ pre-certification varied for each modality in the order of NME $>$ GI $>$ GS. Caecal intubation rates (CIR) at full certification were similar between GI (95.6\%) and GS $(95.6 \%$, $\mathrm{p}=0.81)$, but lower in NME $(93.6 \%, p=0.02$ vs. GS, $p=0.006$ vs. GI), despite no differences at provisional certification (median CIR 95.6\%, $p=0.32$ ). Rates of D2 intubation (median 98.7\%) varied across groups (GS $>$ GI $>$ NME, $\mathrm{p}=0.002)$. Certification awarded at first attempt were similar across specialties (mean $89.4 \%, p=0.19$ ), but varied for gastroscopy (NME $95.5 \%$, GS $90.1 \%$, GI $89.7 \%, p=0.01)$

Conclusion: Despite variations amongst trainee specialties, endoscopy certification is a transparent and robust benchmark for assessing competency, as evidenced by trainee KPIs. Further studies are required to study the impact of recent changes to certification, and if variations in KPIs exist following certification.

Disclosure of Interest: All authors have declared no conflicts of interest.

\section{P1481 EFFICIENCY AND SAFETY OF ENDOSCOPIC PAPILLECTOMY FOR TREATMENT OF DUODENAL PAPILLA TUMORS}

Y. Chu ${ }^{1}$, M. Xu ${ }^{1}$, P. Zhou ${ }^{1}$, C. Zhang ${ }^{1}$

${ }^{1}$ Endoscopy Center And Endoscopy Research Institute, Fudan University Zhongshan Hospital, Shanghai/China

Contact E-mail Address: xu.meidong@zs-hospital.sh.cn

Introduction: A duodenal papilla tumor is an uncommon neoplasm in the upper gastrointestinal tract. In the early stage, patients often have no complaints and the tumors are usually occasional found during gastroduodenoscopy examination. Endoscopic papillectomy can be achieved with curative resection for benign adenoma and some early papillary carcinoma. However, some complications are companied with the procedure, like pancreatitis and bleeding. This retrospective study is to evaluate therapeutic effect and safety of endoscopic papillectomy on duodenal papilla tumors.

Aims \& Methods: From June 2009 to November 2016, the information of patients who received endoscopic papillectomy was recorded, which included basic characteristics and clinical outcomes, such as recurrence rate, bleeding, pancreatitis Results: 37 patients (totally 40 cases) received endoscopic papillectomy. The procedure was completed with gastroscope in 32 cases and duodenoscope in 8 case. Endoscopic mucosal resection (EMR), endoscopic piecemeal mucosal resection (EPMR) and endoscopic submucosal dissection (ESD) was performed in 21, 17 and 2 cases respectively. None of the lesions invaded the submucosal layer. 
Pancreatic stents and biliary stents were inserted in 9 and 12 patients respectively. In general, $5 \%(2 / 40)$ and $12.5 \%(5 / 40)$ cases had intraoperative and postoperative bleeding respectively. $20 \%(8 / 40)$ cases suffered from pancreatitis, of which mild, moderate and severe happened in 3, 4 and 1 cases. Six patients had tumor recurrence. And 3 patients received repeat endoscopic papillectomy, two received pancreatic-coduodenectomy and one received no other treatments with close follow-up. Two patients died from failures of treatments for papillary tumors and one patient died due to other unrelated cause.

Characteristics and adverse events of endoscopic papillary in cases

Sex

Male Female

298

Age (years, mean $\pm \mathrm{SD}$ )

$55.1 \pm 10.0$

Endoscope type

Gastroscope Duodenoscope

328

Resection method

EMR EPMR ESD

Pathological results

LGD, HGD, Tis, Tm, Tsm, Non-tumor

Tumor sizes $(\mathrm{cm})$

Longer diameter, Shorter diameter

Biliary Stent

Yes, No

Pancreatic stent

Yes, No

Hospital stays (days, mean $\pm \mathrm{SD}$ )

Follow-up time (months, mean $\pm \mathrm{SD}$ )

Adverse events

Intraoperative bleeding

Postoperative bleeding

21172

$12,24,0,2,0,2$

$2.02 \pm 0.88,1.50 \pm 0.69$

12,28

9,31

$6.7 \pm 13.4$

$36.6 \pm 28$

$5 \%(2)$

$12.5 \%(5)$

$2.5 \%(1)$

0

Cholangitis

Pancreatitis, Mild, Moderate, Severe

$20 \%(8), 7.5 \%(3), 10 \%(4), 2.5 \%(1)$

$16.2 \%(6)$

$7.9 \%(3)$

$7.9 \%(3)$ $(\mathrm{n}=2296)$ and polypectomy $(\mathrm{n}=370)$ in the 6-months before July 2016 (old DOPS) and after (new DOPS), for trainees at early stages of training (total procedures $<100$ ). To allow analysis, the new DOPS rating scale was aligned to a 4 -point scale, hence a score of 4 on new DOPS $=$ Scores 3 or 4 on old DOPS, and scores on the new and old DOPS compared using the Mann-Whitney U-test. Results: 5117 DOPS $(77.7 \%$ new and $22.3 \%$ old) were included for analysis. Overall, there were variations in distributions of all scores $(p<0.001)$ between forms (Figure 1). Compared to new DOPS, scores of 1 were underutilised on old DOPS $(0.6 \%$ vs $3.0 \%, \mathrm{p}<0.001)$. Frequencies of low scores (pooled scores of $1 \& 2)$ were similar for gastroscopy $(\mathrm{p}=0.53)$ and sigmoidoscopy $(\mathrm{p}=0.34)$, but not for colonoscopy (new $11.9 \%$ vs. old $13.9 \%, \mathrm{p}<0.001$ ) and polypectomy (new $6.8 \%$ vs. $19.9 \%, \mathrm{p}<0.001$ ). Trainees on old DOPS were more likely to be rated as competent (score 3 or 4 ) compared to new DOPS $(86.4 \%$ vs. $55.8 \%, \mathrm{p}<0.001)$. On subgroup analysis, this was evident for gastroscopy $(86.3 \%$ vs. $49.1 \%, \mathrm{p}<0.001)$, colonoscopy $(86.1 \%$ vs. $58.2 \%, \mathrm{p}<0.001)$, sigmoidoscopy $(90.6 \%$ vs. $62.0 \%, \mathrm{p}<0.001)$, but not polypectomy $(80.1 \%$ vs. $67.9 \%, \mathrm{p}=0.12$ ).

Conclusion: Endoscopy assessors are applying a greater range of scores using a new DOPS rating scale based on degree of supervision, in two cohorts of trainees matched for experience. This indicates better construct validity with the new rating scale. Further work is underway to determine the reliability of the new DOPS to inform summative assessment and certification for UK endoscopy trainees.

Disclosure of Interest: All authors have declared no conflicts of interest.

P1484 PROTECTIVE VACUUM SPONGE IMPLANTATION AND CONTINUOUS EVACUATION OF BILE AND PANCREATIC JUICE FOR PREVENTION OF SECONDARY PERFORATION AFTER PRIMARY SUCCESSFUL ENDOSCOPIC RESECTION OF WIDESPREAD D2/D3 DUODENAL AND PAPILLARY ADENOMATA

J. Hochberger ${ }^{1}$, M. Loss ${ }^{2}$, C. Von Heymann ${ }^{3}$, C. Jung ${ }^{4}$, E. Wedi ${ }^{4}$, M. Hofmeyer ${ }^{1}$ ${ }^{1}$ Gastroenterology, Vivantes Klinikum Friedrichshain, Berlin/Germany

${ }^{2}$ Visceral Surgery, Vivantes Klinikum Friedrichshain, Berlin/Germany

${ }^{3}$ Anesthesiology, Vivantes Klinikum Friedrichshain, Berlin/Germany

${ }^{4}$ Service D'endoscopie Digestive, Hôpitaux Universitaires Strassbourg, Strasbourg/ France

Contact E-mail Address: juehochber@mac.com

Introduction: Endoscopic resection of duodenal adenomata carries an increased risk of perforation compared to other locations in the upper or lower GI tract. ${ }^{1,2}$ Additionally in endoscopic resection of widespread adenomata (Spigelman III IV) at the level of D2/D3 there is an increased risk of secondary perforation due to auto-digestion of the denuded duodenal wall by pancreatic enzymes and bile independent of the primary endoscopic resection method. We recently reported of the successful implantation of a mini-vacuum sponge with extended length of the suction tube and reduced in volume compared to a standard esophageal vacuum sponge. ${ }^{3}$

Aims \& Methods: From September $9^{\text {th }}, 2015$ to March $20^{\text {th }}, 2017$ endoscopic resection of wide-spread duodenal or papillary adenomata of $>2 \mathrm{~cm}$ in D2/D3 was performed in five patients. There was a surgical indication for Whipple's resection as primary intervention or in case of failure in all patients. All patients agreed and gave their informed consent to the procedure.

Results: Five patients with widespread duodenal adenomata were included $(2 \mathrm{x}$ papilla, 3x D2/D3 extrapapillary adenomata; 5x tubular; 3x HGIN, 2x LGIN). The macroscopic mean maximum diameter and perpendicular diameter of the lesions were $4 \times 2.8 \mathrm{~cm}$ (largest $7.5 \times 3.7 \mathrm{~cm}$; smallest $2.2 \times 1.8 \mathrm{~cm}$ ). In all cases the implantation of a protective mini-vacuum sponge (EsoVac reduced in volume to $1.2 \times 1.5 \mathrm{~cm}$ (dia, length) with extended suction tube; Braun Corp., Melsungen). Continuous suction was applied over several days $(-125 \mathrm{~mm} \mathrm{Hg}$; ActiVAC, KCI Medical, Wiesbaden) depending on the size of the resection area and healing status $(\mathrm{m}=10$ days, $4-14$ days). An endoscopic/radiologic vacuum sponge exchange was performed every $3-5$ days. In 4 cases additional atraumatic over-the scope-clips (OTSC, Ovesco Tuebingen) were placed during the procedure and in $5 / 5$ cases additional hemoclips were applied to secure the wall and for hemostasis. In $5 / 5$ cases $(100 \%)$ an excellent healing could be observed during follow-up. No patient had to be operated during or following the intervention (FU 2-14 mo.). In all cases the resection was curative with 'en bloc' resection, though in one case the specimen ruptured during retrieval into three parts $(4 \mathrm{x}$ HGIN, 1X LGD; 4x R0, 1x Rx). In one case 10 days after resection an acute bleeding occurred with the need of endoscopic clipping and prophylactic radiologic coiling of the gastroduodenal artery with uneventful course. In a second case a minor bleeding occurred without necessity of transfusion during ablation of an OTSC three mo after the primary intervention. All patients were asymptomatic during follow-up.

Conclusion: The endoscopic resection of large duodenal adenomata in D2/D3 is feasible and was safe in our collective using the application of a duodenal minivacuum sponge for continuous local drainage of bile and pancreatic juice as alterative to Whipple's resection. The results in this first small collective should

Disclosure of Interest: J. Hochberger: Fujifilm Europe: research support, honoraria for lectures Boston Scientific Europe and US: research support, honoraria hence demonstrate validity.

Aims \& Methods: We used the UK trainee endoscopy database (JETS) to collect DOPS scores for gastroscopy $(n=1934)$, sigmoidoscopy $(n=517)$, colonoscopy be reproduced in a prospective multicentric trial. for lectures ERBE Elektromedizin: research support All other authors have declared no conflicts of interest.

Introduction: DOPS are validated tools for assessing competence in endoscopy. Previously, DOPS were scored on a 4-point competence-based scale, with score of 3 and 4 signifying competence. In July 2016, the DOPS rating scale changed to -based scale that has been sh from maximal supervision, up to competent without supervision. We aimed to 


\section{References}

1. Fanning SB, Bourke MJ, Williams SJ et al. Giant laterally spreading tumors of the duodenum: endoscopic resection outcomes, limitations and caveats. Gastrointest Endosc 2012; 75: 805-12

2. Jung JH, Choi KD, Ahn JY et al. Endoscopic submucosal dissection for sessile, nonampullary duodenal adenomas. Endoscopy 2013; 45: 133-5

3. Hochberger J, Wedi E, Tchoumak I, Jung C: Over-the-scope clip placement and endosponge insertion for prevention of pancreatic enzyme- induced duodenal damage after large duodenal endoscopic resection. Endoscopy 2016; 48: E401-E402

\section{P1485 ENDOSCOPIC CLOSURE OF ACUTE PERFORATIONS OF THE GASTROINTESTINAL TRACT IN ANIMAL MODEIS: SYSTEMATIC REVIEW AND META-ANALYSIS}

A. Gabr ${ }^{1}$, N. Ammar ${ }^{2}$, M. El Houssine ${ }^{2}$, M. Rutter ${ }^{3}$

${ }^{1}$ East Kent University Hospitals NHS, Margate/United Kingdom

${ }^{2}$ Public Health Department, Ain Shams University, Cairo/Egypt

${ }^{3}$ Gastroenterology, University Hospital of North Tees, Stockton on Tees/United

Kingdom

Contact E-mail Address: ahmedgabr@hotmail.co.uk

Introduction: Acute perforations are one of the recognized complications of both diagnostic and therapeutic gastrointestinal endoscopy. For decades, surgical treatment has been the standard of care, but endoscopic closure has become a more popular approach, due to feasibility and the reduction of the burden of surgery, combined with the availability of various endoscopic closure devices.

Aims \& Methods: We aimed to assess the technical and clinical success and safety of endoscopic closure, in total, and for each endoscopic device used in closing acute perforations in animal models. Medical literature (Cochrane library, EMBASE, MEDLINE) from 1966 till September 2016 was searched. A systematic review and meta-analysis were performed on studies reporting technical and clinical success of endoscopic closure of acute perforations, according to PRISMA (Preferred Reporting Items for Systematic Reviews and Meta-analyses) guidelines.

Results: 46 studies on animal models were identified. 15 studies, including 4 randomized controlled trials, met our inclusion criteria (acute, less than 24 hours, iatrogenic, no fistulas or leaks, clear documentation of the method of closure, and technical and clinical success and 5 cases or more of endoscopic closure per study), were analysed. A total of 214 endoscopic closures were attempted in these studies. The overall technical success rate was $94.8 \%$ $(\mathrm{n}=201 / 214,95 \%$ CI: 92\%-97.6\%), clinical success was $92.3 \%(\mathrm{n}=189 / 214$ $95 \%$ CI: $88.8 \%-95.8 \%)$, and complication rate was $4.2 \%(n=6 / 214,95 \% \mathrm{CI}$ $1.6 \%-6.8 \%$ ). Technical success for endoclip closure was $84.9 \%$ (95\% CI. $71.4 \%$ $93.6 \%$ ), and clinical success was $83.2 \%$ (95\% CI: $69.5 \%-92.5 \%)$, and complication rate was $2.7 \%(95 \%$ CI: $90.12 \%-99.88 \%)$. For OTSC (Over the scope clip device), technical success was 97\% (95\% CI: 88\%-99.7\%), clinical success was $97 \%$ (95\% CI: $88 \%-99.7 \%$ ), and complication rate was $1.87 \%$ (95\% CI: $91.2 \%$ $98.8 \%$ ). The technical success for endosuturing (endoscopic suturing device) was $92.7 \%(95 \%$ CI: $82 \%$ to $98 \%)$, clinical success was $87 \%(95 \%$ CI: $74.9 \%$ $-94.8 \%)$, complication rate was $1.9 \%(95 \%$ CI: $91.1 \%-98.9 \%)$.

Conclusion: Our study suggests that endoscopic closure is a suitable treatment option for acute iatrogenic gastrointestinal perforations with a reasonable technical and clinical success, and low complication rate. Further confirmation from prospective studies in human is needed.

Disclosure of Interest: All authors have declared no conflicts of interest.

\section{References}

1. Chavez, Y.H.et al (2013). A large international multicentre experience with an over-the-scope clipping device for endoscopic management of Gastrointestinal perforations, fistulae, and leaks in 188 patients. Gastrointestinal Endoscopy, May 2013, vol./is. 77/5 SUPPL. 1(AB148AB149), 0016-5107.

2. Voemarns, R., Moine, O., and Von Rentlen, D. (2012). Efficacy of endoscopic closure of acute Perforations of the Gastrointestinal Tract. Clinical gastroenterology and hepatology 2012; 10:603-608.

3. Jin, Young-Joo. et al (2013).ERCP related duodenal perforation. Endoscopy 2013; 45: 806-812.

4. Fujishiro, M. et al (2006). Nonsurgical management of post-ESD perforation. Endoscopy 2006; 38: 1001 \pm 1006 .

\section{P1486 RISK FACTORS FOR COLORECTAL POLYP IN} ASYMPTOMATIC YOUNG ADULTS UNDER THE AGE OF 50

\section{Y. Myung}

hyundae UVIS hospital, Incheon/Korea, Republic of

Contact E-mail Address: dittomyung@gmail.com

Introduction: Current guidelines recommend that adults age 50 to 75 be screened for colorectal cancer (CRC). However, CRC incidence rates have increased among young adults and have decreased among older adults.

Aims \& Methods: The aim of this study was to investigate the risk factors of colorectal polyps in young adults aged $<50$ years. From January 2016 to December 2016, we compared the risk factors of colorectal polyp group with non-polyp group in patients aged $<50$ years who underwent screening colonoscopy. The risk factors examined included body mass index (BMI), metabolic syndrome, smoking, alcohol consumption, age and gender.

Results: Of the 1862 patients, prevalence of colorectal polyps and adenomatous polyps were $13.1 \%$ and $7.8 \%$, respectively. Multivariate analysis revealed that metabolic syndrome $(\mathrm{OR}, 1.89,95 \% \mathrm{CI}, 1.13-3.17, \mathrm{P}=0.015)$ was independent predictor for colorectal polyps. Age over 40 years old (OR, 2.47, 95\% CI, 1.48 $4.14)$ and metabolic syndrome (OR, $1.59,95 \%$ CI, 0.87-3.30) were independent predictors for adenomatous colorectal polyps.

Conclusion: Metabolic syndrome is risk factor of colorectal polyps in young adults aged $<50$ years. Age over 40 years old is additional risk factor of adenomatous polyps.

Disclosure of Interest: All authors have declared no conflicts of interest.

P1487 COMPARISON BETWEEN AN ASYMMETRIC (SMALL DOSE IN THE MORNING) AND A SYMMETRIC SPLIT-DOSE REGIMEN OF POLYETHYLENE GLYCOL PLUS BISACODYL FOR BOWEI PREPARATION FOR SCREENING COLONOSCOPY: A RANDOMIZED NON-INFERIORITY CLINICAL TRIAL

P. Andreozzi ${ }^{1}$, C. Bezzio $^{2}$, G. De Nucci ${ }^{3}$, S. Saibeni ${ }^{2}$, M. Devani ${ }^{2}$, I. Arena ${ }^{2}$ R. Reati ${ }^{3}$, E. Mandelli ${ }^{3}$, D. Redaelli ${ }^{3}$, D. Morganti ${ }^{3}$, B. Omazzi ${ }^{2}$, G. Manes ${ }^{1}$ Clinical Medicina And Surgery, University Of Naples " Federico Ii", Naples/Italy ${ }^{2}$ ASST Rhodense, Rho/Italy

${ }^{3}$ ASST Rhodense, Garbagnate Milanese/Italy

\section{Contact E-mail Address: paoloandre.85@gmail.com}

Introduction: Bowel cleansing has a critical role to increase the quality and effectiveness of colonoscopy. International guidelines recommend the use of split-dose regimens of PEG solutions. However, the adoption of split dose regimens in clinical practice remains sub-optimal, in particular in early morning.

Aims \& Methods: We aimed to compare the efficacy of bowel preparation using an asymmetric split-dose regimen (approximately $25 \%$ of the dose is given on the day of the procedure and $75 \%$ of the dose is given on the day before) with the standard split-dose regimen in patients undergoing screening colonoscopy. We prospectively enrolled consecutive outpatients undergoing screening colonoscopy. All subjects received a split-dose preparation with a 2L PEG-citratesimethicone plus Bisacodyl. Patients were randomly assigned to: group A, asymmetric split dose regimen (1.5 L of PEG + bisacodyl the day before and $0.5 \mathrm{~L} 3$ hours before colonoscopy); group $\mathrm{B}$, symmetric split dose regimen $(1 \mathrm{~L}$ of PEG + bisacodyl the day before and 1 L 4 hours before colonoscopy). Bowel preparation was evaluated using the Boston Bowel Preparation Scale (BBPS) score. The primary endpoints were the proportion of adequate bowel cleansing (BBPS $\geq 2$ in each segment of the colon). Moreover, all patients filled in a nurseadministered questionnaire assessing compliance, tolerability and safety of bowel preparation. The threshold for statistical significance in this study was $p=0.05$ and a $10 \%$ margin was used to demonstrate non-inferiority of asymmetric vs. symmetric split-dose regimen.

Results: 179 patients were enrolled (mean age $60 \pm 8$ years, males $56 \%$ ), 88 in group A and 91 in group B. Split-dose was taken by $76 / 88$ and by $77 / 91$ patients in group $\mathrm{A}$ and $\mathrm{B}$, respectively $(85.2 \%$ vs $88.5 \%, \mathrm{p}=0.831)$. Failure of cecal intubation occurred in 1 patients for each group. In the ITT analysis, bowel cleansing was considered adequate in $71 / 76(93.4 \%)$ and $70 / 77(90.9 \%)$ patients respectively in group $\mathrm{A}$ and $\mathrm{B}(\mathrm{p}=0.765)$. BBPS total score was similar in group $\mathrm{A}$ and $\mathrm{B}(6.9 \pm 1.2$ vs. $6.9 \pm 1.4 ; \mathrm{p}=0.846)$. No differences were observed regarding adenoma detection rate $[32 / 76(42.1 \%)$ vs $35 / 77(45.4 \%) ; p=0.745)]$ and scores of each colon segment. The full amount of product and adjunctive fluids were taken by $68 / 76(89.4)$ and $71 / 77(92.2 \%)(\mathrm{p}=0.158)$ in group A and $\mathrm{B}$, respectively. Tolerability and occurrence of adverse events were similar in the two groups.

Conclusion: An asymmetric (low morning-dose) split-dose preparation with a low volume formulation with additional Bisacodyl is not inferior to the standard split-dose regimen in achieving an adequate bowel cleansing in patients undergoing screening colonoscopy. A lower amount of preparation in the morning allow to the patients to wake up later; this regimen could be thus preferred by patients undergoing colonoscopy early in the morning. Further study are needed to determine the efficacy and tolerability of the asymmetric preparation for colonoscopy scheduled in early morning.

Disclosure of Interest: All authors have declared no conflicts of interest.

\section{P1488 SINGLE BALLOON OVERTUBE-GUIDED COLORECTAL ENDOSCOPIC SUBMUCOSAL DISSECTION; A NEW APPROACH TO MANAGEMENT OF COLORECTAL ENDOSCOPIC} SUBMUCOSAL DISSECTION

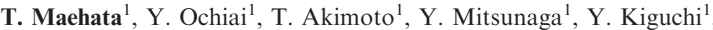
A. Fujimoto ${ }^{1}$, O. Goto $^{1}$, T. Nishizawa ${ }^{1}$, T. Uraoka ${ }^{2}$, N. Yahagi ${ }^{1}$

${ }^{1}$ Cancer Center, Keio University, School of Medicine, Tokyo/Japan

${ }^{2}$ Gastroenterology, National Hospital Organization Tokyo Medical Center, Tokyo/ Japan

Contact E-mail Address: t2maehata@marianna-u.ac.jp

Introduction: Colorectal endoscopic submucosal dissection (ESD) is a technique with remarkably greater difficulty than upper gastrointestinal ESD because of unstable maneuvers and inherited anatomic variability in the colon. Thus, aiming at reducing these restrictions, we have used single balloon (SB) overtube to assist colorectal ESD in cases considered to have difficult operability. In this study, to evaluate the usefulness of a single balloon overtube to assist colorectal ESD. 
Aims \& Methods: The study included 35 patients with 39 colorectal lesions who underwent the SB guided ESD (group SB). The treatment outcome of the group was compared with that of another group that consisted of patients with 39 colorectal lesions who underwent ESD without SB (group NSB). The backgrounds of the patients in group NSB were corrected by using the propensity score matching method. The application of the combined use of SB was determined when the circumferential access to the lesion was difficult, and paradoxical movement was observed.

Results: The characteristics of the lesions in group SB were as follows: proximal, 28 lesions; distal, 11; rectal, 0 ; mean size of tumors, $37.6 \pm 9.8 \mathrm{~mm}$; gross findings of laterally spreading tumor of granular type (LST-G), 11 lesions; non-granular type (NG) tumors, 28; adenomas, 11; mucosal cancers, 22; and submucosal cancers, 6. The mean procedure time was significantly shorter in group SB (SB group vs non-SB group: $67.36 \mathrm{~min}$ vs $106.00 \mathrm{~min}$; $\mathrm{p}<0.05$ ). No significant differences were found in en bloc resection, complete resection, postoperative bleeding, and perforation rates. No accidental symptom associated with balloon endoscopy was observed

Conclusion: Using a single balloon overtube can be expected to improve not only access to the lesion but also facilitate scope manipulation for colorectal ESD. Disclosure of Interest: All authors have declared no conflicts of interest.

\section{P1489 SEDATION IN COLORECTAL ESD: A SYSTEMATIC REVIEW OF WESTERN CLINICAL PRACTICE}

M.E. San Juan Acosta ${ }^{1}$, S. Beg ${ }^{2}$, S. Sansone ${ }^{2}$, K. Ragunath ${ }^{2}$, B. Manorahan ${ }^{2}$

${ }^{1}$ Gastroenterology And Endoscopy Unit, University Hospital Nuestra Señora de Candelaria, Tenerife/Spain

${ }^{2}$ Nottingham Digestive Diseases Biomedical Research Unit, Nottingham University Hospital, Nottingham/United Kingdom

Contact E-mail Address: msanjuanacosta $@$ gmail.com Introduction: Endoscopic Submucosal Dissection (ESD) for colorectal lesions is a highly skilled technique which over the last few years has been adopted by Western countries for the management of colorectal neoplasia. There is no consensus in the literature regarding the technique of anaesthesia/sedation method for ESD. We aim to describe current sedation practices used in ESD in Western Hospitals

Aims \& Methods: A systematic literature search was performed to identify all articles describing colorectal ESD procedure performed in Europe, America and Australia. Electronic databases including PubMed, the Cochrane library and Embase were searched. Original articles or abstracts for congress in English were included. A questionnaire was sent to the authors of the identified articles in order to obtain additional information regarding sedation practice, if this was not explicitly detailed in the original articles. All articles were examined independently for eligibility by two reviewers (S.B. and M.S.). Any Disagreements were resolved by consulting a third reviewer (A.P.)

Results: This review resulted in 18 eligible original articles, of which (5/18) $27.8 \%$ were prospective studies, (7/18) 38.9\% retrospective series and $(6 / 18) 33.3 \%$ were abstracts presented at congresses. The mean number of ESD cases described was 68 (range: 11-302). Most institutions opted to admit patients following the procedure (15) $83.3 \%$, with just one managing patients on an entirely outpatient basis. The mean duration of post procedure observation was 2 days $(0-4)$. The Endoscopy department was the chosen location for colorectal ESD in (12) $66.6 \%$ of institutions and operating room (OR) for (4) $22.2 \%$. In (2) $11.1 \%$ institutions the preference was for OR in selected cases. Deep sedation with propofol or propofol + midazolam $+/$ - fentanyl was used in (8) $44.4 \%$ of the institutions. General anaesthesia was used in (4) $22.2 \%$, while conscious sedation was used in (3) $16.7 \%$. In (3) $16.7 \%$ of institutions deep sedation, conscious sedation or general anaesthesia was used according to the cases and clinician decision. A designated anaesthesiologist was in charge of sedation in (7) $38.9 \%$ of the institutions, with either nurse anaesthesiologist or an anaesthesiologist in (5) $27.8 \%$, a nurse anaesthesiologist alone in (2) $11.1 \%$, the performing endoscopist in (2) $11.1 \%$, endoscopy nurse in (1) $5.5 \%$ and a second physician in (1) $5.5 \%$ of institutions.

Conclusion: The sedation strategy used in colorectal ESD in Western world is heterogeneous. Most institutions opt for deep sedation administered by anaesthesiologists within the endoscopy room. By and large, patients are admitted post procedure for observation. Greater sharing of experience is required to better understand the optimal method of sedation for this technique within the context of western practice.

Disclosure of Interest: All authors have declared no conflicts of interest.

\section{P1490 COMPUTER-AIDED POLYP MEASUREMENT OF 78 POLYPS DURING LIVE COLONOSCOPY (CAPME): A PROSPECTIVE STUDY}

C. Allimant ${ }^{1}$, O. Rouquette ${ }^{1}$, S. Le Roux ${ }^{2}$, B. Pereira ${ }^{3}$, A. Abergel ${ }^{1}$, A. Bartoli ${ }^{4}$ C. Tilmant ${ }^{2}$, L. Poincloux

${ }^{1}$ CHU Estaing, Service de Médecine Digestive et Hépato-biliaire Clermont Ferrand, Clermont-Ferrand/France

${ }^{2}$ Umr 6602 - Cnrs/ubp/sigma, Institut Blaise Pascal, Clermont-Ferrand/France

${ }^{3}$ Centre Hospitalo-universitaire de Clermont-ferrand, Service de Biostatistiques, DRCI, Clermont-Ferrand/France

${ }^{4}$ Umr 6284 Uda-Cnrs, Image Science for Interventional Techniques, ClermontFerrand/France
Contact E-mail Address: callimant@chu-clermontferrand.fr

Introduction: Colonic polyp measurement is an important outcome in current endoscopic practice. Polyp size estimation is mandatory to determine the surveillance interval after polypectomy and useful to anticipate and prevent complications in therapeutic procedures, for it has been proven that polyp size is one of the main risk factor of complications (bleeding, perforation). Nevertheless, a high degree of subjectivity exists (especially overestimation of polyp size) in polyp size estimation because of a lack of validated and reproductible measurement methods. The aim of this study was to compare a computer-aided polyp measurement (CAPME) to an unassisted visual estimation (UVE) and an endoscopic reference measurement (ERM).

Aims \& Methods: This prospective monocentric study was led between November 2015 and July 2016 in the University-affiliated Hospital of Clermont-Ferrand, the Blaise Pascal Institut (BPI) and the Image Science For Interventional Techniques (ISIT) research unit of Clermont-Ferrand (France). Video-endoscopic procedures were recorded and secondly used for the CAPME method. The endoscopic acquisition protocol was standardized with a slow back-andforth movement of the endoscope towards the polyp. A photography of each polyp was taken with an open biopsy forceps placed on the base of the polyp. ERM was then measured thanks to a simple rule of three knowing the fixed size of the open biopsy forceps ( 8 millimetres, mm). UVE was determined on the same photography without any measurement devices by two different endoscopists. All the measurements were realized blinded of the other results. Accuracy of the CAPME and UVE methods was defined by a variation less than $1 \mathrm{~mm}$ with the ERM. We used the Lin concordance correlation coefficient (CCC) to measure the agreement between the variables.

Results: 33 patients and 78 polyps were included in this study. The mean polyp size was $5.3 \mathrm{~mm}$, with $73.1 \%(\mathrm{n}=57)$ of polyp less than $5 \mathrm{~mm}$. The Lin CCC with the ERM was $0.972(95 \% \mathrm{CI}, 0.960-0.983 ; \mathrm{P}<0.001)$ for the CAPME method, and was greater than the UVE Lin CCC $(0.830 ; 95 \%$ CI, $0.797-0.863 ; p<0.001)$. Accurate polyp measurement was obtained for 68 polyps with CAPME method $(87.2 \%)$ versus 56 polyps $(71.8 \%)$ for UVE assessment $(\mathrm{p}<0.01)$. Inaccuracy was increasing proportionally to polyp size $(40.9 \%$ of inaccurate polyp measurement for polyps greater than $10 \mathrm{~mm}$ ) and we observed a systematic overestimation of supracentimetric polyps for UVE method. Unlike UVE, CAPME accuracy was not impacted by polyp size variation in our study $(11.1 \% ; 14.7 \%$ and $10.8 \%$ for polyps of $>10 \mathrm{~mm} ; 5-10 \mathrm{~mm}$ and $<5 \mathrm{~mm}$ respectively).

Conclusion: To our knowledge, this is the first prospective study with significant data analysing a computer-aided polyp measurement in live colonoscopic procedures. In our study, this methods shows more accuracy than the visual estimation, which is the actual gold standard. The main limitation of this study is the endoscopic protocol (back-and-forth movement of the endoscope) that increases the endoscopic procedure. The use of DualFocus might be a solution to overcome this problem.

Disclosure of Interest: All authors have declared no conflicts of interest.

\section{References}

Haute Autorite de Sante - Quand faut-il faire une coloscopie de controle apres une polypectomie?

Lieberman, D. A. et al. Guidelines for colonoscopy surveillance after screening and polypectomy: a consensus update by the US Multi-Society Task Force on Colorectal Cancer. Gastroenterology 143, 844.857 (2012).

Anderson, B. W. et al. Endoscopic overestimation of colorectal polyp size. Gastrointest. Endosc. 83, 201.208 (2016).

Heldwein, W. et al. The Munich Polypectomy Study (MUPS): Prospective Analysis of Complications and Risk Factors in 4000 Colonic Snare Polypectomies. Endoscopy 37, 1116.1122 (2005).

Kim, J. H. et al. Is forceps more useful than visualization for measurement of colon polyp size? World J. Gastroenterol. 22, 3220.3226 (2016).

Mahmud, N., Cohen, J., Tsourides, K. \& Berzin, T. M. Computer vision and augmented reality in gastrointestinal endoscopy. Gastroenterol. Rep. 3, 179.184 (2015).

Chadebecq, F., Tilmant, C. \& Bartoli, A. How big is this neoplasia? Live colonoscopic size measurement using the Infocus-Breakpoint. Med. Image Anal. 19, $58.74(2015)$

\section{P1491 USING THE MULTI-ASSISTANT RATING SCALE (MARS) FOR ENDOSCOPIC NON-TECHNICAL SKILLS PERFORMANCE AUGMENTS USE OF STANDARD KEY PERFORMANCE MEASURES IN INDEPENDENT COLONOSCOPISTS}

N. Hawkes, F. Kokwara, B. Davies, J. Cornish

Gastroenterology, Cwm Taf University Helath Board, $\mathrm{nr}$ Cardiff/United Kingdom

Contact E-mail Address: neilhawkes@aol.com

Introduction: Endoscopic Non-Technical Skills (ENTS) are an essential component of high-quality endoscopy giving information about performance in a team context however these are not routinely assessed as part of the key performance indicators (KPIs) for independently practising colonoscopists. We have developed a validated 360-degree assessment tool based on assistant ratings for ENTS (MARS tool) with acceptable inter-rater agreement providing an assessment in 4 key ENTS domains. We compared measured performance in these areas against existing key performance indicators which measure aspects of insertion and lesion detection skills.

Aims \& Methods: We aimed to add a 360-degree multi-assessor rating scale (MARS) to provide a global assessment of ENTS skills to the existing KPI panel correlating the results against existing caecal intubation rate (CIR) and polyp detection rate (PDR). The validated MARS tool assesses 4 ENTS 
domains-Communication (COMM), Situational Awareness (SITA), Leadership (LEAD) and Judgement \& Decision-making (J\&DM). Each MARS domain was represented by 10 items and is assessed on a 7-point scoring scale - endoscopists should score $>90$ in each domain $(80-90=$ need for improvement, $<80=$ suboptimal performance). CIR and PDR measures are routinely calculated for all colonoscopists using the HICCS Electronic Reporting System with manual validation of these data. Feedback is presented on a quarterly basis to practitionersendoscopists are expected to achieve 90\% CIR and 20\% PDR. Correlation of these factors with practitioners ENTS scores were measured using the Pearson test.

\section{ENTS, CIR and PDR scores by colonoscopist}

\begin{tabular}{lllllll}
\hline Operator & COMM & SITA & LEAD & T\&DM & CIR(\%) & PDR(\%) \\
\hline 1 & 96 & 99 & 97 & 100 & 95.2 & 72.5 \\
2 & 94 & 94 & 87 & 95 & 83.6 & 49 \\
3 & 99 & 100 & 97 & 98 & 90.8 & 51 \\
4 & 100 & 100 & 100 & 99 & 91.5 & 47 \\
5 & 79 & 73 & 73 & 85 & 91.7 & 34.5 \\
6 & 100 & 99 & 99 & 98 & 87.5 & 34.5 \\
7 & 66 & 59 & 62 & 69 & 67.4 & 17 \\
8 & 100 & 100 & 98 & 99 & 83.8 & 20 \\
9 & 74 & 83 & 84 & 86 & 87.5 & 31.5 \\
\hline
\end{tabular}

Results: 9 endoscopists with known variability in standard colonoscopy KPIs consented to an assessment of ENTS using the MARS tool. Their ENTS scores were correlated with existing KPIs for each colonoscopist (Oct 2016Mar 2017). Scatter plots provided an overall positive correlation between ENTS domains and CIR (COMM 0.58; SITA 0.66; LEAD 0.66; J\&DM 0.75) and PDR (COMM 0.49; SITA 0.55; LEAD 0.50; J\&DM 0.60). Three endoscopists were identified as having sub-optimal scores in all of the ENTS domains (operators 5,7,9). Taking into account important KPI thresholds 2 out of 3 $(33 \%)$ of these endoscopists identified were not meeting CIR targets (c.f. $66 \%$ of ENTS competent group) and one (33\%) did not meet PDR targets (c.f. $0 \%$ in ENTS competent group).

Conclusion: The MARS tool is a practical way to measure of ENTS performance designed as a 360 degree feedback and identifies areas for development within independently practitioners that are not currently highlighted by standard colonoscopy KPI measures. There is some correlation with current KPI feedback panels but using a specific validated ENTS assessment tool augments current assessment panels. Both CIR and PDR primarily depend on the colonoscopists' individual skills rather than the team elements required by polypectomy or EMR and these more complex tasks may show stronger correlation with MARS ENTS evaluation.

Disclosure of Interest: All authors have declared no conflicts of interest

P1492 EARLY ECONOMIC MODELLING OF EMI-137 TO IMPROVE

THE DETECTION RATE OF POLYPS IN PATIENTS WITH

INCREASED RISK OF COLORECTAL CANCER

B. Shinkins ${ }^{1}$, A. Smith ${ }^{1}$, N. King ${ }^{1}$, A. Davies ${ }^{2}$, I. Wilson ${ }^{2}$

${ }^{1}$ Test Evaluation Group, Academic Unit Of Health Economics, University of Leeds, Leeds/United Kingdom

${ }^{2}$ Edinburgh Molecular Imaging, Edinburgh/United Kingdom

Contact E-mail Address: b.shinkins@leeds.ac.uk

Introduction: Colorectal Cancer (CRC) is the third most common cancer diagnosed in men and second most common cancer in women, affecting 1,361,000 people worldwide each year. CRC is a slow growing tumour that most commonly develops from polyps which form in the inner lining of the colon or rectum. The identification and removal of polyps whilst still in a precancerous state has been shown to be effective in reducing mortality from CRC. Current clinical practice relies on white light (WL) colonoscopy for detecting colorectal polyps, but this is associated with a detection "miss-rate" of up to $26 \%$ for small $(<10 \mathrm{~mm})$ polyps and $2 \%$ for large polyps (van Rijn et.al., 2006). Missed lesions (false negative results) puts patients at an unnecessary risk of late stage detection, when management has higher costs and worse clinical outcomes. Recent technological developments have resulted in more accurate technologies for polyp detection becoming available. One such technology is the EMI-137 imaging agent (Edinbugh Molecular Imaging (EMI))) which has demonstrated potential to enhance the sensitivity of colonoscopy.

Aims \& Methods: We aimed to evaluate the potential cost-effectiveness of EMI137 compared to standard WL colonoscopy in patients at high risk of colorectal cancer. A de novo economic model for patients participating in the UK national bowel cancer screening programme referred for colonoscopy as a result of a positive faecal occult blood test was developed. The early economic modelling approach consists of 5 stages: 1) literature review, 2) evidence synthesis, 3) care pathway analysis, 4) decision analytic modelling, 5) sensitivity analysis and value of information analysis. The structure of the models has been determined by current recommended practice. The potential benefits of EMI-137 were identified via interviews with endoscopists across Europe and communication with the manufacturer. Early cost-effectiveness and value of information analysis will be conducted to assess the viability of the EMI imaging technology to enhance current NHS patient care, and to inform the necessity and direction of future investments into the research and development of this intervention.

Results: The addition of EMI-137 fluorescent agent to standard WL colonoscopy results in a lifetime per-patient average additional NHS cost of $£ 426$ and an additional 0.04 QALYs, giving an incremental cost-effectiveness ratio (ICER) of $£ 11,245$ per additional QALY. The cost-effectiveness of EMI-137 was sensitive to the time horizon of the model. EMI-137 remained within the NICE recommended cost-effectiveness threshold priced at $\$ 500$ or less. Value of information analyses revealed that uncertainty in the cost-effectiveness of EMI-137 is primarily driven by uncertainty in the assumption that EMI-137 reduces the risk of future polyps after polypectomy. The results of this modelling exercise have since been used to inform the design of Phase II/III clinical studies.

Conclusion: The results of this early economic modelling exercise demonstrated that EMI-137 has the potential to be cost-effective for patients participating in the UK National Bowel Cancer Screen Programme. The value of information analyses have highlighted the key parameters for which further evidence is required, and this will be used to inform the design of future clinical studies.

Disclosure of Interest: A. Davies: I am the CMO at Edinburgh Molecular Imaging

I. Wilson: I am the CEO at Edinburgh Molecular Imaging

All other authors have declared no conflicts of interest.

\section{Reference}

Van Rijn, Jeroen C., et al. "Polyp miss rate determined by tandem colonoscopy: a systematic review." The American journal of gastroenterology 101.2 (2006): 343350 .

\section{P1493 RANDOMIZED CLINICAL TRIAL EVALUATING THE EFFECT OF A VISUAL EDUCATIONAL BOOKLET ON THE PREPARATION OF COLONOSCOPIES IN HOSPITALIZED PATIENTS}

A. Guardiola Arévalo ${ }^{1}$, A. Granja Navacerrada ${ }^{2}$, F.J. Garcia-Alonso ${ }^{3}$, P. Bernal $\mathrm{Checa}^{2}$, M. Hernández Tejero ${ }^{2}$, D. Bonillo ${ }^{1}$, E.M. Andrés Esteban ${ }^{4}$, A. Algaba Garcia $^{1}$, F. Bermejo

${ }^{1}$ Hospital Universitario de Fuenlabrada, Fuenlabrada/Spain

${ }^{2}$ Aparato Digestivo, Hospital Universitario de Fuenlabrada, Fuenlabrada/Spain

${ }^{3}$ Hospital Universitario Río Hortega, Valladolid/Spain

${ }^{4}$ Universidad Autónoma de Madrid, Madrid/Spain

Contact E-mail Address: toniga2011@gmail.com

Introduction: Safety and diagnostic accuracy of colonoscopy depend on the quality of colon cleansing. Several factors have been reported to affect the quality of bowel cleansing, one of them being hospitalized.

Aims \& Methods: We performed a prospective, randomized endoscopist blinded clinical trial between February 2016 and January 2017 included. Our aim was to investigate whether the association of a visual educational booklet improves the level of cleanliness achieved in hospitalized patients who are undergoing a colonoscopy. Hospitalized patients $>18$ years undergoing colonoscopy were included. Exclusion criteria were: previous colonoscopy in the last 3 years, previous colectomy, known inflammatory bowel disease, urgent colonoscopy, dementia or refusal to participate in the study. Both groups received $4 \mathrm{~L}$ polyethyleneglycol solution. The intervention consisted of a visual educational booklet (visual cohort). Demographic data, personal history, reason for admission and for colonoscopy, work shift in which it was performed, and findings at endoscopy were collected. The Boston Bowel Preparation Scale (BBPS) was used to assess the bowel preparation. A BBPS score $<6$ or with at least one segment $<2$, was considered a poor preparation.

Results: One hundred and thirty six patients were included, $51.5 \%$ were male, with a mean age of $64.3 \pm 17.6$ years, and $95.59 \%$ of Spanish nationality. The mean body mass index was $27.3 \pm 5.2 \mathrm{~kg} / \mathrm{m}^{2}$. Educational attainment was below school education in $71.05 \%$. Most patients were submitted from the internal medicine ward $(72.1 \%)$, and the gastroenterology ward $(21.32 \%)$. Anemia $(31.62 \%)$, abdominal radiographic findings $(16.91 \%)$, hematochezia/rectal bleeding $(15.44 \%)$, diarrhea $(11.03 \%)$, and abdominal pain $(8.82 \%)$ were the most frequent indications. Patients characteristics, bowel cleasing and endoscopic findings are shown in table 1 . The educational booklet did not suppose a difference in the bowel cleansing attained. Factors that impacted on the level of well and poorly prepared patients in the bivariable study were: age $(62.1 \pm 18.7$ vs $70.5 \pm 14.8 ; \mathrm{p}=0.0193)$, diabetes mellitus $(22.33 \%$ vs $42.42 \% ; \mathrm{p}=0.024)$, hypertension $(45.63 \%$ vs $69.70 \% ; \mathrm{p}=0.016)$, cardiovascular disease $14.5 \%$ vs $36.3 \%$; $\mathrm{p}=0.006)$, and colorectal cancer on colonoscopy $(99 \%$ vs $81.82 \% ; \mathrm{p}=0.001)$. In the multivariable analysis the only factors associated with a poor bowel cleansing were cardiovascular disease (OR $3.37(1.34-8.46) ; \mathrm{p}=0.010)$, and colorectal cancer on colonoscopy (OR $3.82(1.26-11.61) ; \mathrm{p}=0.018)$

Table 1: Baseline characteristics, bowel cleansing and endoscopic findings.

\begin{tabular}{llll}
\hline & $\begin{array}{l}\text { Standard } \\
\text { management } \\
(\mathrm{n}=70)\end{array}$ & $\begin{array}{l}\text { Educational } \\
\text { booklet } \\
(\mathrm{n}=66)\end{array}$ & $\mathrm{p}$ \\
\hline PATIENTS N=136(n) & $66.2(56.8-78.4)$ & $63.3(53.0-78.3)$ & 0.54 \\
Age(year)* & $51.43 \%$ & $51.52 \%$ & 0.99 \\
Sex(Male) & $26.9(24.4-29.7)$ & $26.9(23.5-29.9)$ & 0.85 \\
BMI $\left(\mathrm{Kg} / \mathrm{m}^{2}\right)^{*}$ & $18(25.71 \%)$ & $19(28.79 \%)$ & 0.69 \\
Diabetes Mellitus (136) & $33(47.14 \%)$ & $33(50 \%)$ & 0.74 \\
Hypercolesterolemia (136) & $19(27.14 \%)$ & $15(22.73)$ & 0.55 \\
Smoking habit (136) & $8(11.59 \%)$ & $4(21.21 \%)$ & 0.13 \\
Alcoholism (135) & $11(15.71 \%)$ & $16(24.24 \%)$ & 0.21 \\
Cardiovascular disease(136) & $5(7.14 \%)$ & $9(13.64 \%)$ & 0.21 \\
Chronic kidney disease(136) & & & \\
\hline
\end{tabular}


Table 1 Continued

\begin{tabular}{llll}
\hline & $\begin{array}{l}\text { Standard } \\
\text { management } \\
(\mathrm{n}=70)\end{array}$ & $\begin{array}{l}\text { Educational } \\
\text { booklet } \\
(\mathrm{n}=66)\end{array}$ & $\mathrm{p}$ \\
PATIENTS N=136 (n) & $6(8.70 \%)$ & $5(7.58 \%)$ & 0.81 \\
\hline Chronic Obstructive Pulmonary Disease $(135)$ & $6(8.57 \%)$ & $3(4.55 \%)$ & 0.34 \\
Obstructive Sleep Apnea Syndrome (136) & $2(2.86 \%)$ & $2(3.03 \%)$ & 0.95 \\
Cirrosis (136) & $8(11.59 \%)$ & $8(12.12 \%)$ & 0.93 \\
Stroke (135) & $3(4.29 \%)$ & $1(1.52 \%)$ & 0.34 \\
Mild dementia (136) & $14(20 \%)$ & $9(13.85 \%)$ & 0.34 \\
Constipation (135) & $13(18.57 \%)$ & $7(10.61 \%)$ & 0.19 \\
Antidepressants (136) & $7(10.45 \%)$ & $8(12.31 \%)$ & 0.74 \\
Family history of CRC (132) & $38(59.37 \%)$ & $32(56.14 \%)$ & 0.81 \\
ASA score $\leq 2$ (121) & $9(13.64 \%)$ & $10(16.13 \%)$ & 0.69 \\
Need for diaper during admission (128) & $18(25.71 \%)$ & $4(6.06 \%)$ & 0.002 \\
Apendicectomy (136) & $3(4.29 \%)$ & $2(3.03 \%)$ & 0.70 \\
Umbilical herniorrhaphy(136) & $8(11.43 \%)$ & $4(6.06 \%)$ & 0.27 \\
Hysterectomy (136) & $1(1.43 \%)$ & $4(6.06 \%)$ & 0.15 \\
Caesarean (136) & $4(5.71 \%)$ & $5(7.58 \%)$ & 0.66 \\
Other gynecological surgery (136) & $21(30 \%)$ & $19(28.79 \%)$ & 0.88 \\
Other surgery (136) & & & \\
COLONOSCOPIES RESULTS: & $58(82.86 \%)$ & $59(89.39 \%)$ & 0.27 \\
Work shift (morning) & $67(95.71 \%)$ & $62(93.94 \%)$ & 0.49 \\
Cecal intubation rates & $7(6-9)$ & $6(5.7-9)$ & 0.17 \\
BBPS* & $2(2-3)$ & $2(2-3)$ & 0.37 \\
BBPS Left Colon* & $2(2-3)$ & $2(2-3)$ & 0.10 \\
BBPS Mild Colon* & $2(2-3)$ & $2(2-3)$ & 0.22 \\
BBPS Right Colon* & $15(45.45 \%)$ & $18(54.55 \%)$ & 0.43 \\
BBPS 55 & $29(41.43 \%)$ & $18(27.27 \%)$ & 0.40 \\
Adenoma detection rate & $9(12.85 \%)$ & $5(7.57 \%)$ & 0.48 \\
Advanced Adenomas & $8(11.43 \%)$ & $8(12.12 \%)$ & 0.90 \\
Colonrectal cancer & & & \\
\hline & & & \\
& & &
\end{tabular}

Conclusion: The use of a visual educational booklet for the preparation of colonoscopies does not provide a significant improvement in hospitalized patients in our health area. Heart disease and/or colon cancer were predictors of poor preparation for colonoscopy. An optimized preparation should be considered for this type of patients.

Disclosure of Interest: All authors have declared no conflicts of interest.

\section{References}

Rosenfeld G1, Krygier D, Enns RA, Singham J, Wiesinger H, Bressler B. The impact of patient education on the quality of inpatient bowel preparation for colonoscopy. Can J Gastroenterol Vol 24 No 9 September 2010.

McNabb-Baltar J, Dorreen A, Al Dhahab H, Fein M, Xiong X, O' Byrne M, et al. Age Is the Only Predictor of Poor Bowel Preparation in the Hospitalized Patient. Can J Gastroenterol Hepatol. 2016;2016:2139264

William F. Ergen, Trisha Pasricha, Francie J. Hubbard, Tina Higginbotham, Tonya Givens, et al. Providing Hospitalized Patients With an Educational Booklet Increases the Quality of colonoscopy Bowel Preparation. Clinical Gastroenterology and Hepatology 2016;14:858-864.

\section{P1494 STUDY OF ULCERATIVE COLITIS COMPLICATED BY PRIMARY SCLEROSING CHOLANGITIS}

S. Murasugi, A. Ito, H. Kambayashi, M. Yonezawa, T. Ohmori, K. Tokushige Institute of Gastroenterology, Tokyo Women's Medical University, Tokyo/Japan

Contact E-mail Address: murashun0305@yahoo.co.jp

Introduction: Primary sclerosing cholangitis (PSC) is often associated with autoimmune diseases, and approximately $70 \%$ of PSC patients in Europe/United States and $32 \%$ in Japan also have ulcerative colitis (UC). While the complication of PSC is confirmed in about $5 \%$ of UC patients, the clinical features of UC associated with PSC differ from those of UC without PSC.

Aims \& Methods: We investigated the clinical and colonoscopic features of colitis associated with PSC. We retrospectively examined the clinical features, including the clinical course and colonoscopic findings, of 25 colitis patients with PSC attending our hospital between 2000 and 2016.

Results: The male-female ratio was 12:13 and the age at diagnosis of PSC was $49 \pm 15$ years. PSC was the initial diagnosis in 12 patients $(48 \%)$, while colitis was the first to be diagnosed in 4 patients $(16 \%)$, and both diseases were found concurrently in 9 patients $(36 \%)$. Among the 21 patients with the diagnosis of PSC first or concurrently, 12 patients (57\%) had no symptoms of PSC and the latter was recognized by screening. There were 12 patients with UC $(52 \%)$ and 11 patients with nonspecific colitis $(48 \%)$. Among the 24 patients in whom the disease extent was assessed, 22 had pancolitis, 1 had left-sided colitis, and 1 had proctitis. Inflammation predominantly affected the right colon in $20 / 22$ patients with pancolitis and also involved the terminal ileum in 9 patients $(48 \%)$. The Mayo score for colonoscopic evaluation of UC was 1 in 16 patients $(64 \%), 2$ in 8 patients $(32 \%)$, and 3 in 1 patient $(4 \%)$. There were no rectal lesions in 10 patients $(40 \%)$. Liver biopsy was performed in 17 patients, and Ludwig's stage was Stage I in 1 patient $(6 \%)$, Stage II in 12 patients $(71 \%)$, Stage III in 3 patients $(18 \%)$, Stage IV in 1 patient $(6 \%)$. Ludwig's stage did not correlate with the Mayo score. All patients with PSC and enterocolitis received oral ursodeoxycholic acid (UDCA), including 13 patients with UDCA only (52\%), 2 patients with combination of salazosulfapyridine (SASP) $(8 \%), 5$ patients in combination with 5-aminosalicylic acid (5-ASA) $(20 \%), 2$ patients in combination with prednisolone (PSL) $(8 \%), 1$ patient with the combination of SASP + PSL $(4 \%)$, and 2 patients with 5 -ASA + PSL $(8 \%)$. The UDCA dose was $300 \mathrm{mg}$ in 2 patients $(8 \%), 600 \mathrm{mg}$ in $15(60 \%)$, and $900 \mathrm{mg}$ in $8(32 \%)$.

Conclusion: In colitis patients with PSC, there was no clear association between colonoscopic disease activity and the severity of PSC. There was no sex difference and the age at diagnosis of PSC showed a bimodal distribution (30 s and $60 \mathrm{~s}$ ). Pancolitis was very frequent and predominantly affected the right colon, but disease activity was low. Rectal lesions were mild or absent. About half of the patients had inflammation of the terminal ileum.

Disclosure of Interest: All authors have declared no conflicts of interest.

\section{P1495 COMPETENCY OF ENDOSCOPIC NON-TECHNICAL} SKILLS (ENTS) DURING ENDOSCOPY TRAINING

K. Siau, P. Dunckley, J. Anderson, I. Beales, R. Broughton, M. Feeney,

N. Hawkes, G. Johnson, B. Mckaig, R. Valori, C. Wells, S. Thomas-Gibson, A. Haycock

${ }^{*} J A G$ Working Group For The Quality Assurance Of Training, Joint Advisory Group, London/United Kingdom

Contact E-mail Address: keith@siau.org

Introduction: Endoscopic non-technical skills (ENTS), comprising of communication and teamwork, situation awareness, leadership and judgement and decision-making, are recognised indicators of quality endoscopy and patient safety. Since July 2016, electronic assessment forms (DOPS) for UK trainee endoscopists have been updated to include ENTS as an assessable domain. We aimed to assess the uptake and distribution of ENTS scoring in DOPS and their correlation with other endoscopic skills, across all assessable endoscopic modalities.

Aims \& Methods: We identified all DOPS submitted between July 2016 and Feb 2017 from the UK endoscopy trainee database (JETS) and acquired data on trainees, procedures and scores. We collated scores for each of the 4 assessable domains (pre-procedural, procedural, post-procedural and ENTs) into overall outcomes of "not competent" (if any domain items required supervision) or "competent", and compared this to the overall competence rating. Statistical analysis was performed using chi2 and regression modelling.

Results: 8601 DOPS were prospectively collected, with ENTS assessed in $99.3 \%$. Competency rates of individual ENTS items are summarised in Table 1. Rates of overall ENTS competency (defined as all items scoring competent) varied across procedures $(\mathrm{p}<0.001)$ : ERCP $39.8 \%$, EUS $44.1 \%$, gastroscopy $59.6 \%$, colonoscopy $62.3 \%$, PEG $71.1 \%$, gastrointestinal bleed $(71.5 \%$ ), sigmoidoscopy $72.4 \%$ and polypectomy $73.2 \%$. Scores by individual ENTS components are displayed in Table 1. Of DOPS awarded overall competency, 5.9\% (240/4077) lacked full competence in ENTS ( $p=0.10$ across modalities). Across trainee specialties and endoscopic modalities, competence was greatest for "communication and teamwork" (77.1\% overall), but least with 'judgement and decision making' $(68.3 \%)$. Competency in ENTS increased with lifetime procedural count (OR 1.008 per increase in procedure, $\mathrm{p}<0.001)$, and correlated strongly with other assessable domains, including overall score $(\mathrm{p}<0.001)$. After adjusting for procedural count, factors predictive of ENTS competence included trainee seniority (OR for ST5 level: 1.96, $\mathrm{p}<0.0001)$, surgical trainees $(\mathrm{OR} 1.21, \mathrm{p}=0.014)$, trainees performing polypectomy (OR 2.02, $\mathrm{p}<0.0001)$, and higher DOPS count (OR 1.03 per increase in DOPS, $\mathrm{p}<0.001)$.

Table 1: Unadjusted ENTS scores by endoscopic modality.

\begin{tabular}{lllll}
\hline & $\begin{array}{l}\text { Communication } \\
\text { and Teamwork }\end{array}$ & $\begin{array}{l}\text { Situational } \\
\text { Awareness }\end{array}$ & Leadership & $\begin{array}{l}\text { Judgement } \\
\text { and Decision } \\
\text { Making }\end{array}$ \\
\hline Colonoscopy & $77 \%$ & $75 \%$ & $70 \%$ & $68 \%$ \\
Dilatation & $78 \%$ & $75 \%$ & $64 \%$ & $64 \%$ \\
Polypectomy & $82 \%$ & $83 \%$ & $81 \%$ & $77 \%$ \\
ERCP & $65 \%$ & $62 \%$ & $54 \%$ & $48 \%$ \\
EUS & $71 \%$ & $71 \%$ & $47 \%$ & $59 \%$ \\
Sigmoidoscopy & $83 \%$ & $81 \%$ & $79 \%$ & $74 \%$ \\
OGD & $73 \%$ & $70 \%$ & $67 \%$ & $64 \%$ \\
PEG & $80 \%$ & $76 \%$ & $78 \%$ & $77 \%$ \\
Gastrointestinal Bleed & $85 \%$ & $83 \%$ & $78 \%$ & $77 \%$ \\
\hline
\end{tabular}

Conclusion: ENTS is an assessable domain within endoscopy training, with scores that correlate with other procedure-related skills, demonstrating construct validity of the ENTS scoring system. Competency of ENTS develop with procedural count, and vary with trainee seniority and specialty. Longer term data are required to assess the impact of ENTS on certification.

Disclosure of Interest: All authors have declared no conflicts of interest. 
P1496 MAKING COLONOSCOPISTS MORE AWARE OF THEIR ENDOSCOPIC NON-TECHNICAL SKILLS-IMPROVING FEEDBACK FORMATS DERIVED FROM THE MULTI-ASSISTANT RATING SCALE (MARS) TOOL

N. Hawkes, F. Kokwara, B. Davies, J. Cornish

Gastroenterology, Cwm Taf University Helath Board, $n r$ Cardiff/United Kingdom

Contact E-mail Address: neilhawkes@aol.com

Introduction: The development of Endoscopic Non-Technical Skills (ENTS) is associated with expert performance and high quality endoscopic outcomes. Whilst ENTS domains have been incorporated into Joint Advisory Group (JAG) Direct Observation of Procedural Skills (DOPS) forms, used as training tools, knowledge of ENTS domains amongst independent practitioners varies. To improve performance in this area of practice requires validated measurement tools and specific feedback against which improvement can be measured. We have previously developed a validated 360-degree multi-assessor rating scale (MARS tool) based on experienced endoscopy assistant ratings for ENTS providing data on 4 main domains of ENTS-each comprising 10 related but independent practice points. Providing an optimised feedback format for this data is likely to maximise the potential benefits of measruing ENTS performance.

Aims \& Methods: We aimed to provide an optimised format for performance enhancing feedback in the ENTS domains and basis for specific auditable outcomes and performance indicators. Local colonoscopists gave consent to application of the ENTS questionnaire. The validated MARS tool assesses 4 ENTS domains-Communication (COMM), Situational Awareness (SITA), Leadership (LEAD) and Judgement \& Decision-making (J\&DM). Each MARS domain in the administered questionnaire was represented by 10 items and is assessed on a 7-point scoring scale. We sought to develop 1) a format to illustrate an individual's overall performance in each of the 4 main ENTS domains in comparison to other operators and 2) a detailed domain breakdown highlighting areas of underperformance 3) Collate feedback on the presentation formats.

Results: 9 endoscopists consented to an assessment of ENTS using the MARS tool. The MARS questionnaires were administered during January 2017-relating to the prior 3 months clinical practice. Acceptable performance thresholds were set as $>90 \%$ good-excellent ratings in each domain. Need for improvement was defined as $80-90 \%$ good-excellent ratings (i.e. 10-20\% average or poor ratings) and sub-optimal performance as $80 \%$ or less good-excellent ratings (i.e. $>20 \%$ average-poor ratings). Good intra- and inter-rater reliability was demonstrated for these cut-off values during validation of the MARS tool. Scatter plots were used to present the overall domain ratings for COMM, SITA, LEAD and J\&DM domains allowing comparison with other endoscopists. To provide more detailed domain-specific feedback to endoscopists an individual report is generated of 4 domain tables summarising the question items and using a 'traffic-light' display to help operators quickly identify those specific skills that require areas for improvement. All endoscopists reported finding the ENTS feedback helpful and indicated that it was 'likely' or 'very likely' to prompt an alteration in practice. A suggestion to add an additional column to the summary table indicated where performance level has changed in subsequent audit rounds is being considered.

Example Feedback table - Situational Awareness Domain for Endoscopist 9

SITA 1 Pre-assessment of potential patient risks GREEN

SITA 2 Recognises problems in a timely fashion

SITA 3 Aware of working environment, minimises disruptions

SITA 4 Effectively troubleshoots technical problems that arise

SITA 5 Recognises and assesses pathological findings effectively

SITA 6 Reacts flexibly and effectively to unexpected or

$$
\text { unforeseen circumstances }
$$

SITA 7 Aware of patient safety parameters during procedure, responds well AMBER

SITA 8 Considers other opinions and guidance when faced with AMBER an untoward event

SITA 9 Predicts well the likely outcome of a situation and plans for this eventuality

SITA 10 Constantly checks patient aiming to minimise procedural discomfort AMBER

Conclusion: The MARS tool is a practical way to measure ENTS performance alongside other key performance measures as part of a robust quality assurance system. The benefits of providing overall comparative data has been established previously in sharing more established key performance indicators. The feedback format for data derived from the MARS tool has additionally provided detailed domain-specific ENTS performance ratings that brings to attention areas for improvement in a clear and understandable way that can be re-tested as part of an integrated audit cycle.

Disclosure of Interest: All authors have declared no conflicts of interest.

\section{P1497 OUTCOMES OF ENDOSCOPIC RESECTION OF COMPLEX COLORECTAL LESIONS REFERRED TO A TERTIARY INSTITUTION AFTER FAILED ATTEMPTS AT RESECTION OR EXTENSIVE MANIPULATION}

A. Emmanuel, S. Gulati, M. Burt, B. Hayee, A. Haji

Endoscopy, King's College Hospital, London/United Kingdom

Contact E-mail Address: aemmanuel@nhs.net
Introduction: Substantial manipulation or sampling of large colorectal lesions prior to endoscopic resection can have significant effects on the feasibility and outcomes of endoscopic treatment. Failed attempts at resection and extensive sampling or tattooing into lesions prior to referral to a specialist centre are common in western practice. However, there are few data defining the scope of the problem and the effects on outcomes following endoscopic resection. We examined the effect of significant prior manipulation on the feasibility and outcomes of endoscopic resection of complex colorectal neoplasms in a UK tertiary referral centre.

Aims \& Methods: Consecutive patients who underwent endoscopic resection of colorectal lesions $\geq 2 \mathrm{~cm}$ were included. All lesions were assessed with magnification chromoendoscopy supplemented by colonoscopic ultrasound in selected cases. A lesion specific approach was used to decide on resection technique. Patients were grouped according to whether they had previous failed attempts at resection, heavy manipulation ( $\geq 6$ biopsies or tattoo into the lesion), or minimal sampling only ( $<6$ biopsies). Outcomes included initial successful endoscopic resection, complications, recurrence and the need for surgery.

Results: 426 of 437 lesions were successfully resected $(97.5 \%)$ using EMR $9 \mathrm{n}=340$ and ESD and Hybrid ESD $(\mathrm{n}=97)$. The mean lesion size was $5.5 \mathrm{~cm}$ $(+/-3.0 \mathrm{~cm}) .225(51 \%)$ lesions had been subjected to failed attempts at resection or heavy manipulation prior to referral. In 97 lesions $(22 \%)$, an average of 1.5 (range 1-5) previous attempts at resection had been made, including 43 attempts at transanal surgical resection in 25 patients. A further 128 lesions $(29 \%)$ had been extensively sampled or tattooed Mean lesion size was $55.6 \mathrm{~mm}+/-30.7 \mathrm{~mm}$. Initial endoscopic resection was deemed successful in $98 \%$ of cases after previous failed attempts, $97 \%$ of cases with prior heavy manipulation and $97 \%$ of other cases $(p=0.86)$. En bloc resection was possible in fewer patients with previous attempts at resection $(14 \%, 31 \%$ and $42 \%$ respectively, $\mathrm{p}<0.001)$. Complication rates were higher for patients with prior heavy manipulation compared to those with failed attempts at resection and minimal sampling (14\% versus $5 \%$ and $3 \%$, $\mathrm{p}<0.001)$. Recurrence rates were $24.1 \%, 14.6 \%$ and $12.2 \%$ respectively $(\mathrm{p}=0.07) .95 \%$ of patients without invasive cancer who had prior failed attempts at resection were free from recurrence and had avoided surgery at last follow up. Conclusion: Failed prior attempts at resection or heavy manipulation of lesions reduces the chance of achieving en bloc resection and increases the risk of complications and recurrence. Nevertheless, specialist management in a dedicated tertiary unit results in safe and successful organ preserving endoscopic treatment of these extremely challenging lesions in over $95 \%$ of cases with few significant complications.

Disclosure of Interest: All authors have declared no conflicts of interest.

P1498 NBI VERSUS BLI! WHICH MODALITY IS BETTER FOR OBSERVATION OF MUCOSAL BLOOD FLOW IN THE SMALL AND LARGE BOWEL USING NBI (NARROW BAND IMAGING) OR BLI (BLUE LASER IMAGING)?

M. Yasuda ${ }^{1}$, Y. Naito ${ }^{2}$, Y. Itoh ${ }^{2}$

${ }^{1}$ Dept. Of Gastroenterology And Hepatology, Uji-Tokushukai Medical Center, Ujicity/Japan

${ }^{2}$ Department Of Gastroenterology And Hepatology, Kyoto Prefectural University of Medicine, Kyoto/Japan

Contact E-mail Address: koutoku7@fa2.so-net.ne.jp

Introduction: In recent years, significant advances and innovations have been made in gastrointestinal endoscopy technology. The special light observation using a narrow band light source different from conventional white light is one such innovation. The special light observation with a magnifying procedure has markedly contributed to improving the diagnosis of lesions in the esophagus, stomach, and large bowel; it is particularly useful for differentiating between benign and malignant lesions and evaluating the depth of invasion. The instruments for narrow band imaging (NBI) were developed by Olympus Co., Ltd., and those for blue laser imaging (BLI) by Fujifilm Co., Ltd. These systems are applied to magnifying endoscopy in clinical practice. Studies have examined the usefulness of magnifying observation with NBI (m-NBI), BLI (m-BLI), and new and brighter BLI (m-BLI bright) for the diagnosis of neoplastic diseases, especially for the early detection of gastrointestinal cancers. However, there are relatively few reports describing the application of these techniques to benign diseases.

Aims \& Methods: This basic study aimed to explore the potential of magnifying endoscopic observation with special light by evaluating the visualization of mucosal blood flow in the small and large bowel. The subjects were selected from among patients who had undergone colonoscopy since April 2016. They were randomized into three groups: patients undergoing examination with ECL600ZP, a high-end instrument manufactured by Fujifilm Co., Ltd. (group F), CF-HQ290ZI, a high-end instrument manufactured by Olympus Co., Ltd. (group O1) or PCF-H290ZI manufactured by Olympus Co., Ltd (group O2). Each group consisted of 25 patients. The visualization of mucosal blood flow in the small and large bowel by magnifying endoscopic observation with special light was evaluated and scored as follows: good visualization 2; partial visualization 1 , and no visualization 0 . The water method and tip attachment were used in all cases.

Results: The respective mean scores for visualization of the small and large bowel were 2 and 2 in group F, 1.32 and 1.24 in group O1, and 1.48 and 1.40 in group $\mathrm{O} 2$. The visualization scores for both the small and the large bowel were significantly higher in group $\mathrm{F}$ than in groups $\mathrm{O} 1$ and $\mathrm{O} 2$. Group $\mathrm{O} 2$ had higher scores than group O1, although the difference was not statistically significant. The endoscope used in group $\mathrm{F}$ has a bright laser light source and maximum optical magnification levels up to 135 times and maximum electronic magnification levels up to 270 times. On the other hand, the endoscopes used in groups O1 
and $\mathrm{O} 2$ have relatively dark xenon light sources and maximum optical magnification levels up to 80 and 110 times, respectively. Therefore, the differences in visualization of mucosal blood flow in the small and large bowel among the groups were considered to be attributable to differences in instrument efficiency. Conclusion: Our results show that magnifying observation with BLI is superior to that with NBI regarding the observation of mucosal blood flow in the small and large bowel. We are planning to conduct a study on the clinical application of magnifying observation with BLI for visualization of mucosal blood flow in the small and large bowel.

Disclosure of Interest: All authors have declared no conflicts of interest.

\section{P1499 MULTIPLE COLORECTAL ADENOMAS WITHOUT APC OR MUTYH GERMLINE MUTATION: A HETEROGENEOUS SUBGROUP OF PATIENTS}

\author{
L. Sanchez Mete, E. Mannisi, A. Martayan, M. Varanese, V. Stigliano \\ Gastroenterology And Digestive Endoscopy, Regina Elena National Cancer \\ Institute, Rome/Italy
}

\section{Contact E-mail Address: lupe.sanchez@ifo.gov.it}

Introduction: Multiple colorectal adenomas (MCRA) can be defined as an endoscopic feature of $\geq 10$ colorectal adenomas in patients (pts) without APC or MUTYH germline mutation. At present its clinical features, management and the presence of extracolonic cancer are not well studied.

Aims \& Methods: The aim of the present study is to better define the clinical characteristes at diagnosis and during follow-up of MCRA affected patients. Forty-eight patients ( 22 Females, 26 Males) with MCRA ( $\geq 10$ colorectal adenomas), without deleterious mutations of APC or MUTYH genes, were recruited for the study. Clinical features at diagnosis and extracolonic manifestations were recorded. Forty patients underwent annual colonoscopy at our division with a mean follow-up of 8.1 years (range 1 to $38 \mathrm{y}$ ). Findings from esophagogastroduodenoscopy were also recorded. Eight patients were lost at follow-up.

Results: The mean age at MCRA diagnosis was $50.1 \pm 14.6$ years (range, 19 to 79 years) and 20 pts $(41.6 \%)$ had at least a first degree relative affected with colorectal neoplasia. Clinical features at diagnosis: the number of polyps ranged between 10 and 20 in $43.7 \%$ of the cases; 21 and 50 in $27.1 \%$; $\geq 50$ in $29.2 \%$; $22.9 \%$ of pts had one or more adenocarcinomas (ADC); $16.6 \%$ had a previously diagnosed extracolonic cancer (breast, endometrial, thyroid, lung, bladder, brain, larynx). Twenty-five pts ( $52 \%)$ needed surgery, ten underwent a subtotal colectomy and fifteen a total colectomy. During follow-up twenty-two (55\%) pts developed recurrent adenomas and two $(5 \%)$ had one or more ADC in the retained colorectum; $12,5 \%$ of pts developed duodenal adenomas, one had a duodenal adenocarcinoma; we recorded one case of abdominal wall desmoid. Conclusion: MCRA patients in the present study had similar clinical characteristics to MUTYH associated Polyposis (MAP) affected patients. They were generally diagnosed at a mean age of $>50$ years, they had more than 20 polyps $(56.3 \%)$ at diagnosis, associated with ADC in $22.9 \%$ of the cases and required surgery in the majority of cases $(52 \%)$. During follow-up, pts also developed recurrent adenomas. Clinical characteristics and family history in these patients support the hypothesis that pathogenic alterations in yet unknown genes may be involved. Next-generation sequencing is a promising technology aimed at this purpose. However, these patients should undergo a closer surveillance than those with sporadic adenomas.

Disclosure of Interest: All authors have declared no conflicts of interest.

\section{P1500 EVALUATION THE RELATIONSHIP BETWEEN NUMBERS OF BIOPSIES PER CASE AND DEGREE OF FIBROSIS IN COLON}

J. Yang ${ }^{1}$, Y. Jung ${ }^{1}$, I. Chung ${ }^{1}$, Y.S. Cho ${ }^{1}$, S.J. Han ${ }^{1}$, T.H. Lee ${ }^{1}$, S. Park ${ }^{1}$, H. Cho ${ }^{2}$, Y. Hwangbo ${ }^{3}$, S. Kim ${ }^{1}$

${ }^{1}$ Division Of Gastroenterology \& Hepatology, Department Of Internal Medicine, Soonchunhyang University College of Medicine, Cheonan Hospital, Cheonan/ Korea, Republic of

${ }^{2}$ Department Of Pathology, Soonchunhyang University College of Medicine, Cheonan/Korea, Republic of

${ }^{3}$ Department Of Preventive Medicine, Soonchunhyang University College of Medicine, Cheonan/Korea, Republic of

Contact E-mail Address: soulfly99@naver.com

Introduction: A degree of fibrosis on targeted lesion is one of the widely known factors which affects the results of Endoscopic mucosal resection (EMR) and Endoscopic submucosal dissection (ESD). Severe fibrosis is associated with complications such as perforation, post-coagulation syndrome and bleeding. Discovering the factors causing fibrosis of tumor could help sorting patients who is suitable for endoscopic removal of colon tumor. Generally a biopsy is performed on a tumor larger than $2 \mathrm{~cm}$ in diameter for a diagnose purpose. Fibrosis can be caused by biopsy performed before resection.

Aims \& Methods: We conducted a study to evaluate the relationship between numbers of biopsies and degree of fibrosis in colon. We retrospectively enrolled 149 lateral spreading tumors (LSTs) from 145 patients who underwent colon ESD or EMR between January 2005 and May 2016. All LSTs were larger than $2 \mathrm{~cm}$ and conducted en-bloc resection. We included elevated or flat type LSTs and excluded lesions of the depressive or ulcerative type. Each data is categorized with prior biopsy status, numbers of biopsies. Degree of fibrosis in resected tumors by EMR or ESD was classified as 0 (no fibrosis), 1 (few), 2 (moderate), 3 (much) by pathologist. Distribution (focal, scattered, and diffuse) and depth (mucosa, submucosa, mucosa and submucosa) of fibrosis were also analyzed.

Results: The mean age of patients was 62.911 .1 years old. Of the total 149 lesions, preprocedural biopsies were performed in 109 lesions $(73.2 \%)$ and biopsy was not performed in 40 lesions $(26.8 \%)$. The size of lesions was $54.4 \%$ in $20-30 \mathrm{~mm}, 28.2 \%$ in $30-40 \mathrm{~mm}$, and $17.4 \%$ in $>40 \mathrm{~mm}$ and the histologic type of lesions was $60.4 \%$ in adenoma, $20.8 \%$ of intramucosal cancer, and $18.8 \%$ of submucosal cancer, respectively. Signs of fibrosis were present on $45.9 \%(50 / 109)$ of patients with prior biopsy, and $40.0 \%(16 / 40)$ of patients without prior biopsy. $(\mathrm{P}=0.326)$ There was no significant difference in degree $(\mathrm{P}=0.791)$, distribution $(\mathrm{P}=0.941)$ and depth $(\mathrm{P}=0.748)$ of fibrosis between groups with or without prior biopsy. In relationship between pathologic results and the presence of fibrosis, the percentage of adenoma, intramucosal cancer, SM-1 $(<1000 \mu \mathrm{m})$ cancer and SM-2 $(>1000 \mu \mathrm{m})$ cancer with fibrosis were $32.31 \%, 66.67 \%, 87.5 \%$ and $90 \%(\mathrm{P}=0.00)$. Additionally, the fibrosis rate for tumors $\leq 3 \mathrm{~cm}$ and $>3 \mathrm{~cm}$ were $44.12 \%$ and $58.97 \%(\mathrm{P}=0.002)$. When biopsies were performed two or more times on a same lesion, it showed significant difference comparing to patients who only underwent biopsy just once. (OR 4.98, 1.20-20.51, 95\% CI, $\mathrm{P}=0.026)$

Conclusion: Degree, distribution and depth of fibrosis were not associated with prior biopsy status. However, the more invasive tumor is, the more presence of fibrosis was found. Moreover, the caution should be needed when performing multiple biopsies because; multiple biopsies are more likely to cause fibrosis. Disclosure of Interest: All authors have declared no conflicts of interest.

\section{References}

1. Lee SP, Kim JH, Sung IK, Lee SY, Park HS, Shim CS, Han HS. Effect of submucosal fibrosis on endoscopic submucosal dissection of colorectal tumors: pathologic review of 173 cases. J Gastroenterol Hepatol. 2015 May;30(5):872-8

2. Jeong JY, Oh YH, Yu YH, Park HS, Lee HL, Eun CS, Han DS. Does submucosal fibrosis affect the results of endoscopic submucosal dissection of early gastric tumors? Gastrointest Endosc. 2012 Jul;76(1):59-66.

3. Makino T, Kanmura S, Sasaki F, Nasu Y, Funakawa K, Tanaka A, Arima S, Nakazawa J, Taguchi H, Hashimoto S, Numata M, Uto H, Tsubouchi H, Ido A. Preoperative classification of submucosal fibrosis in colorectal laterally spreading tumors by endoscopic ultrasonography. Endosc Int Open. 2015 Aug;3(4):E363-7.

4. Matsumoto A, Tanaka S, Oba S, Kanao H, Oka S, Yoshihara M, Chayama $\mathrm{K}$. Outcome of endoscopic submucosal dissection for colorectal tumors accompanied by fibrosis. Scand J Gastroenterol. 2010 Nov;45(11):1329-37.

5. Isomoto H, Nishiyama H, Yamaguchi N, Fukuda E, Ishii H, Ikeda K, Ohnita K, Nakao K, Kohno S, Shikuwa S. Clinicopathological factors associated with clinical outcomes of endoscopic submucosal dissection for colorectal epithelial neoplasms.

2009 Aug;41(8):679-83.

\section{P1501 ADVANCED NEOPLASIA YIELD IN PATIENTS} UNDERGOING COLONOSCOPY AFTER SCREENING FLEXIBLE SIGMOIDOSCOPY: DOES THE DISTAL COLONIC PATHOLOGY PREDICTS THE YIELD IN PROXIMAL COLON?

R. Rameshshanker, F. Purchiaroni, A. Crudeli, A. Rajendran, I. Stasinos, A. Wilson, S. Thomas-Gibson, A. Haycock, A. Humphries, N. Suzuki, B.P. Saunders

Wolfson Endoscopy Unit, St Mark's Hospital, Harrow, London/United Kingdom

Contact E-mail Address: r.rameshshanker@nhs.net

Introduction: Currently patients undergoing a screening flexible sigmoidoscopy (Bowel Scope screening) examination at the age of 55 are referred for colonoscopy if the following polyp criteria are met: polyp $>1 \mathrm{~cm}$, villous histology, high grade dysplasia, 3 or more adenomas or $>20$ hyperplastic polyps (HP).

Aims \& Methods: Objective is to assess the proportion of patients who had an advanced adenoma (size $>1 \mathrm{~cm}$, villous or high grade dysplasia on histology) in the proximal colon when referred for a colonoscopy after a screening flexible sigmoidoscopy. A retrospective cross-sectional study of patients who underwent Bowel Scope screening between July 2013 - July 2016 at St Mark's Bowel Cancer Screening (BCS) Centre was performed. Epidemiology, procedural and polyp data were retrieved from the endoscopy and Bowel Cancer screening database. Results: 9960 patients had a screening flexible sigmoidoscopy in the time period. Descending colon was reached in $82 \%$ of patients. Advanced adenomas were detected in $351(3.2 \%)$ patients. $520(5.2 \%)$ patients had a colonoscopy following screening flexible sigmoidoscopy as per the BCS protocol. Median age was 55 years (male: female ratio 2:1). Caecal intubation was achieved in 98\% (510/520) of cases. At least one adenoma or a sessile serrated adenoma/polyp (SSA/P) was detected, proximal to the extent of flexible sigmoidoscopy examination, in $45 \%$ $(232 / 520)$ of patients (Table 1$)$. Presence of advanced adenoma in the distal colon was an indication for colonoscopy in $351 / 520$ patients $(68 \%)$. Of these, 52 $(14.8 \%)$ had a synchronous proximal colonic advanced adenoma and 20 $(5.7 \%)$ had a synchronous SSA/P. Only $5(1.4 \%)$ patients had an advanced $\mathrm{SSA} / \mathrm{P}$ (polyp $>1 \mathrm{~cm}$ or with dysplasia) in the proximal colon. Presence of distal advanced adenoma was associated with proximal advanced adenoma $(\mathrm{p}=0.0006)$. However, there was no association between presence of distal advanced adenoma and proximal SSA $/ \mathrm{P}(\mathrm{P}=0.47)$ or advanced SSA $/ \mathrm{P}(\mathrm{p}=0.4)$.

Table 1: Proximal colonic pathology during colonoscopy 


\begin{tabular}{|c|c|c|c|c|}
\hline Indication & $\begin{array}{l}\text { Number } \\
\text { of patients }\end{array}$ & $\begin{array}{l}\text { Proximal } \\
\text { Advanced } \\
\text { adenoma }\end{array}$ & $\begin{array}{l}\text { Proximal } \\
\text { SSA/P }\end{array}$ & $\begin{array}{l}\text { Proximal } \\
\text { advanced } \\
\mathrm{SSA} / \mathrm{P}\end{array}$ \\
\hline Indication & $\begin{array}{l}\text { Number } \\
\text { of patients }\end{array}$ & $\begin{array}{l}\text { Proximal } \\
\text { Advanced } \\
\text { adenoma }\end{array}$ & $\begin{array}{l}\text { Proximal } \\
\text { SSA } / \text { P }\end{array}$ & $\begin{array}{l}\text { Proximal } \\
\text { advanced } \\
\text { SSA } / P\end{array}$ \\
\hline Adenoma $>1 \mathrm{~cm}$ & 153 & $14.4 \%$ & $8.5 \%$ & $2.6 \%$ \\
\hline Villous features & 189 & $14.3 \%$ & $3.2 \%$ & $0.5 \%$ \\
\hline High-grade dysplasia & 9 & $36.4 \%$ & $8.3 \%$ & $0 \%$ \\
\hline $\begin{array}{l}\text { Others }(>1 \mathrm{~cm} \text { non adenomatous } \\
\text { polyp, }>20 \text { hyperplastic } \\
\text { polyps, }>3 \text { adenomas) }\end{array}$ & 169 & $5.3 \%$ & $5.8 \%$ & $1.6 \%$ \\
\hline
\end{tabular}

Conclusion: Distal colonic advanced adenomas are a marker of synchronous proximal colonic adenomas and sessile serrated polyps. When colonoscopies were performed for other indications (non-adenomatous polyp $>1 \mathrm{~cm}$, multiple distal HP polyps) the yield in the proximal colon was significantly smaller. These "soft" indications for colonoscopy accounted for a significant additional workload that appears unjustified.

Disclosure of Interest: B.P. Saunders: Advisory board member of Olympus UK All other authors have declared no conflicts of interest.

\section{P1502 LEARNING CURVE FOR OPTICAL DIAGNOSIS OF COLORECTAL POLYPS USING CUMULATIVE SUM ANALYSIS}

R. Rameshshanker, A. Wilson, S. Thomas-Gibson, N. Kamperidis, N. O'Shea, B.P. Saunders

Wolfson Endoscopy Unit, St Mark's Hospital, Harrow, London/United Kingdom

Contact E-mail Address: r.rameshshanker@nhs.net

Introduction: Optical diagnosis for diminutive and small colorectal polyps is an attractive option to reduce costs and streamline patient care. The American Society of Gastrointestinal Endoscopy Preservation and Incorporation of Valuable Endoscopic Innovations (PIVI) established a 90\% diagnostic threshold for real time endoscopic assessment of the histology of diminutive colorectal polyps. For adoption of optical diagnosis in clinical practice, colonoscopists must be trained and show on-going competance. The learning curve for trainees to achieve the competency has not been fully explored.

Aims \& Methods: Aim is to evaluate the minimum number of polyps to achieve and maintain the optical diagnostic thresholds per PIVI standards using an upward CUSUM plot. Four trainees without previous experience in optical diagnosis at our institution participated in this prospective study. Four weeks before the commencement of the study they were given a training module on optical diagnosis (OD). OD was based on NICE and WASP classification. ${ }^{1,2}$ During the study period (January 2016-August 2016), each trainee documented the optical diagnosis of polyps less than $10 \mathrm{~mm}$ in size. Confidence levels of OD were noted at the same time. Patient demographics and polyp details (site, size, Paris classification and histology) were collected prospectively. OD of each polyp was compared against the polyp histology. Polyps without the histological confirmation were excluded from the analysis. Every trainee had on-going feedback on their performance.

Results: A total of 708 polyp observations were performed by trainees during the study period. Total number of adenomas, hyperplastic polyps and sessile serrated adenomas/polyps (SSA/P) were 364,214 and 52 respectively. Trainees OD performance was plotted on a upward CUUM plot.

Table1: Trainees optical diagnostic performance

\begin{tabular}{lllll}
\hline & Trainee & Trainee 2 & Trainee 3 & Trainee 4 \\
\hline Sensitivity & $95 \%$ & $96 \%$ & $94 \%$ & $92 \%$ \\
Specificity & $91 \%$ & $87 \%$ & $83 \%$ & $91 \%$ \\
Positive Predictive Value & $89 \%$ & $94 \%$ & $88 \%$ & $94 \%$ \\
Negative Predictive Value & $92 \%$ & $92 \%$ & $91 \%$ & $93 \%$ \\
\hline
\end{tabular}

All 4 trainees achieved sustained accuracy (90\% threshold) in OD within 12-58 observations. The number of polyps required to reach the plateau varied between 12 to 58 . Every trainee's confidence level improved over time (from $69 \%$ to $89 \%$ ) and the effect was augmented by in-vivo feedback and revision of training module. Table 1 summarises the optical diagnostic performance of all 4 trainees. Negative predictive value for adenomas were above $90 \%$ for all trainees.

Conclusion: The CUSUM scores of all 4 trainees in the study reached the PIVI standards plateau by the 58th polyp observation. In-vivo feedback and continued training appears important to maintain the performance. Our preliminary findings could be used as a guide to plan the certification process for implementation of optical diagnosis.

Disclosure of Interest: B.P. Saunders: Advisory board member - Olympus UK

All other authors have declared no conflicts of interest.
P1503 THE CLINICAL VALUE OF ENDOSCOPIC FULL-

THICKNESS RESECTION FOR THE TREATMENT OF

COLORECTAL SUBMUCOSAL TUMORS ORIGINATING FROM THE

MUSCULARIS PROPRIA: A PROSPECTIVE SINGLE-CENTER

STUDY

M. Xu, C. Zhang, Q. Li

Endoscopy Center, Zhongshan Hospital, Shanghai/China

\section{Contact E-mail Address: xumeidong@aliyun.com}

Introduction: Given diminishment of quality of life caused by colectomy and rectectomy, a minimally invasive treatment is desirable for colorectal submucosal tumors (SMTs).

Aims \& Methods: The aim of the current study was to evaluate the clinical efficacy, safety and feasibility of endoscopic full-thickness resection (EFTR) for colorectal SMTs originating from the MP layer. A prospective study was carried out, including a consecutive cohort of 56 patients who underwent EFTR for colorectal SMTs originating from the MP layer between January 2008 and September 2014 in our center. Among these lesions, 21 located in the colon, 9 located in the intraperitoneal rectum and 26 located in the extraperitoneal rectum. The tight adhesion of the lesion to the serosal layer was identified before EFTR in all cases. EFTR was performed using a standard ESD technique under direct endoscopic view. The defect of colorectal wall was closed after resection in all cases. Complete resection rate, complications and lesion recurrence were evaluated

Results: Successful EFTR was performed in $54(96.4 \%)$ patients. The other 2 patients were transferred to suffer laparoscopic right hemi-colectomy and EFTR combining laparoscopic operation respectively, because the lesions involved the external organs and were too difficult to get en bloc resection endoscopically. The en bloc resection rate and complete resection rate were both $96.4 \%(54 / 56)$. Among 54 cases, 52 of these lesions were performed with EFTR without laparoscopic assistance, while 2 needed laparoscopic assistance to get the defect closed after resection. The median operation time was $45 \mathrm{~min}$ (range, $20-130 \mathrm{~min}$ ). The median maximum diameter of resected tumors was $1.5 \mathrm{~cm}$ (range, $0.5-5.0 \mathrm{~cm}$ ). Accurate histopathologic results were acquired from all the resected lesions, including 18 leiomyomas, 11 gastrointestinal stromal tumors (GISTs), 8 fibrous tumors, 5 schwannomas, 11 granulomas, 2 displaced endometrium, and 1 hamartoma. Three patients had local peritonitis and two patients developed postoperative bleeding. All of them recovered after receiving conservative treatments. No single case developed diffuse peritonitis. No lesion residual or recurrence was found during the follow-up period ranging 2-54 months.

Conclusion: EFTR appears to be a safe, feasible, and effective procedure for providing accurate histopathologic evaluations, as well as a curative treatment for colorectal SMTs originating from the MP layer. However, it should be performed by the very experienced endoscopists.

Disclosure of Interest: All authors have declared no conflicts of interest.

\section{P1504 COLONIC ESD BY UTILIZING SHORT DOUBLE BALLOON ENDOSCOPE HOW TO TREAT DIFFICULT CASES IN COLONIC} ESD

S. Katsuki, T. Fujita, K. Takanashi, E. Waga

Center Of Gastroenterology, Otaru Ekisaikai Hospital Center of Gastroenterology, Otaru/Japan

Contact E-mail Address: sinichi-katuki@otaru-ekisaikai.jp

Introduction: Colonic ESD has been becoming a standard treatment in the world. However, sometimes it is hard to remove the colon tumor during ESD. When we find it difficult to detach the colorectal neoplasm in ESD, we have to consider, the following three points: if we don't have enough experience and skill, we should take the training more. If there are lots of vessels and fibrosis in the submucosal layer, it is necessary to choose adequate tools. And if patients have complicated colon, suitable endoscope need to be selected. In such cases we always use DBE.

Aims \& Methods: We evaluated the outcomes of colonic ESD by using DBE (DBE-ESD). Short DBE we used were EC450BI5, EN530BI and EI580BT (Fujifilm Co., Tokyo, Japan). We've performed DBE-ESD on 211 lesions in 184 patients. We analyzed the lesions located in the proximal colon, and the following items were examined: arrival time, procedure time, rate of negative margin, perforation rate, length of hospital stay and recurrence rate in the 5thyear after the ESD.

Results: There were 159 lesions located in the proximal colons. The median arrival time to the lesion was $7.9 \mathrm{~min}$, operation time $51.1 \mathrm{~min}$, negative rate of horizontal margin $99.4 \%$, vertical margin $99.4 \%$, perforation rate $0 \%$, median length of hospital stay 3.1 days., and recurrence rate in patients with more than 5 year follow-up $0 \%$

Conclusion: Because the balloons and the overtube retained the scope at stable position, we were able to get good working space. Therefore, DBE should be one option for difficult cases in ESD.

Disclosure of Interest: All authors have declared no conflicts of interest. 
P1505 WITHDRAWAL TIME MONITORING AND FULLSPECTRUM ENDOSCOPY IMPROVE ADENOMA DETECTION RATE

G. De Nucci ${ }^{1}$, P. Andreozzi ${ }^{2}$, C. Bezzio ${ }^{3}$, R. Reati ${ }^{1}$, D. Morganti ${ }^{1}$, D. Redaelli ${ }^{1}$, E. Mandelli ${ }^{1}$, B. Omazzi ${ }^{3}$, I. Arena ${ }^{3}$, S. Saibeni ${ }^{3}$, G. Manes ${ }^{1}$

${ }^{1}$ ASST Rhodense, Garbagnate Milanese/Italy

${ }^{2}$ Clinical Medicine And Surgery, University Of Naples " Federico II", Naples/Italy ${ }^{3}$ ASST Rhodense, Rho/Italy

Contact E-mail Address: germanadenucci1@gmail.com

Introduction: Adenoma detection rate (ADR) is a quality indicator of screening colonoscopy. Monitoring withdrawal time (WT) and use of full-spectrum endoscopy (FUSE) have been suggested to increase the ADR since allow an accurate evaluation of the hidden areas of the colon.

Aims \& Methods: We aimed to evaluate whether monitoring of WT alone or in combination with the use of FUSE would be able to increase the ADR. In a prospective non-randomized observational study, consecutive outpatients, aged 18-85 yr, undergoing colonoscopy with different indications were enrolled. In phase 1, endoscopists performed 660 colonoscopies either with standard forwardviewing endoscope $($ SFVE) $(n=330)$ or with FUSE $(n=330)$ without a dedicated WT protocol. In this phase, colonoscopy WTs were measured without the endoscopists' knowledge of being monitored. In phase 2, endoscopists were informed of being monitored and performed further 660 colonoscopies either with SFVE $(\mathrm{n}=330)$ or with FUSE $(\mathrm{n}=330)$.

Results: No differences were observed among the four arms in terms of demographic, clinical features, and indications to colonoscopy. WT was lower in phase 1 arms compared to phase 2 arms (SFVE: $267 \pm 96$ vs. $387 \pm 65, \mathrm{p}=0.001$ FUSE: $293 \pm 112$ vs. $430 \pm 93, \mathrm{p}=0.001)$. When endoscopists were aware of being monitored and used full-spectrum endoscope we observed a higher ADR [phase 1 SFVE $27.3 \%$ (90) phase 1 FUSE $33.0 \%$ (109) phase 2 SFVE $33.6 \%$ (111) phase 2 FUSE $41.8 \%$ (138); $\mathrm{p}=0.001]$ and adenoma per colonoscopy (APC) [phase 1 SFVE $0.43 \pm 0.85$ phase 1 FUSE $0.56 \pm 1.08$ phase 2 SFVE $0.65 \pm 1.24$ phase 2 FUSE $0.71 \pm 1.08 ; \mathrm{p}=0.004]$. The detection rate of adenoma located proximally to the splenic flexure was higher in phase 2 arms (phase 1 SFVE $11.2 \%$ vs. phase SFVE $16.4 \%, p=0.056$; phase 1 FUSE: $12.7 \%$ vs. phase 2 FUSE $18.9 \%, \mathrm{p}=0.033$ ), whereas adenoma located distally to the splenic flexure was higher in the FUSE arms compared to SFVE arms, but these differences were not significant (Phase 1 SVFE $20.0 \%$ vs. Phase 1 FUSE $24.8 \%$, $\mathrm{p}=0.081$; Phase 2 SVFE $21.8 \%$ vs. Phase 2 FUSE $27.0 \%, \mathrm{p}=0.147$ )

Conclusion: Unmonitored endoscopists have a sub-optimal WT, which increases when they are aware to be monitored. Use of full spectrum scopes combined with WT monitoring results in increase of adenoma detection rate. In particular, monitoring WT increases the detection of adenomas in proximal colon, whereas the use of FUSE seems to increase the detection of adenomas in distal colon. Disclosure of Interest: All authors have declared no conflicts of interest.

\section{P1506 HIGH LEVELS OF “PRESUMED POLYP MISS RATE" AT 1 AND 3 YEARS FOLLOWING INDEX SCREENING COLONOSCOPY: NO ROOM FOR COMPLACENCY}

R. Rameshshanker, F. Purchiaroni, A. Wilson, A. Rajendran, S. Thomas-Gibson, A. Humphries, A. Haycock, N. Suzuki, B.P. Saunders

Wolfson Endoscopy Unit, St Mark's Hospital, Harrow, London/United Kingdom

Contact E-mail Address: r.rameshshanker@nhs.net

Introduction: Colonoscopy with polypectomy is considered the optimal method of bowel cancer prevention. Despite improvements in colonoscopy training and technology, it remains as an imperfect tool and the adenoma miss rates vary between $6-27 \%$

Aims \& Methods: Aim is to determine the presumed miss rate for adenomas and sessile serrated adenomas/polyps (SSA/Ps) after a complete screening colonoscopy Methodology A prospective observational study was performed at our bowel cancer screening centre over 12 months from July 2015. Patients who underwent a surveillance colonoscopy following an index colonoscopy were included (one and three-year surveillance). All colonoscopies were performed by 6 experienced, accredited bowel cancer screening colonoscopists. Polyp characteristics and procedural data were prospectively recorded and collected. Polyp histology and epidemiology data were retrieved from our endoscopy database. A polyp was considered as "missed" at the index colonoscopy if at 1 year surveillance it was not adjacent to a scar (a recurrence) or at 3 years if $>5 \mathrm{~mm}$ in size and not adjacent to a scar.

Results: 241 patients underwent a surveillance colonoscopy (male: female 2:1, median age 65 years). 90/241(37.3\%) patients had a one-year surveillance colonoscopy. There was no significant difference in the quality of bowel preparation, caecal intubation rate and total procedure time between index and surveillance procedures. Total number of polyps detected during index and surveillance colonoscopies were 815 and 469 respectively. The presumed miss rate of polyps, adenomas, SSA $/$ Ps and advanced adenomas were $37.8 \%(469 / 1241)$, $22.1 \%(176 / 798), 41.7 \%(20 / 48)$ and $15.2 \%(36 / 236)$ respectively. More adenomas were missed in the proximal colon when compared to distal colon $(26.64 \%$ vs $18.04 \%, p=0.01$ ). Table 1 illustrates the distribution of missed adenomas in each segment of colon. Adenoma miss rates per size as follows: $<5 \mathrm{~mm}, 6-9 \mathrm{~mm}$ and $>10 \mathrm{~mm}$ were 24.27 and $8 \%$ respectively. Higher number of polyps $(>3)$ detected during index colonoscopy independently correlated with high miss rates $(84.3 \%$ vs $72 \%, \mathrm{p}=0.04)$

Table 1: Missed polyps at different colonic segments

\begin{tabular}{lll}
\hline Location & $\begin{array}{l}\text { Adenoma miss } \\
\text { rate (\%) }\end{array}$ & $\begin{array}{l}\text { Sessile serrated } \\
\text { adenoma } \\
\text { miss rate }(\%)\end{array}$ \\
\hline Rectum & 9 & 33 \\
Rectosigmoid junction & 19 & 0 \\
Sigmoid colon & 23 & 50 \\
Descending colon & 10 & 0 \\
Splenic flexure & 26 & 0 \\
Transverse colon & 27 & 30 \\
Hepatic flexure & 30 & 57 \\
Ascending colon & 26 & 50 \\
Caecum & 26 & 0
\end{tabular}

Conclusion: Our study highlights that there is likely to be a significant miss rate for adenomas and SSA/Ps even after careful index colonoscopy. Miss rate was higher when multiple polyps are seen at the index examination. This finding appears to justify the current BSG (British Society of Gastroenterology) guidelines for an early, 1 year colonoscopy when multiple polyps are seen. The presumed polyp miss rate at $1 \& 3$ years may be justified as a new quality metric within screening programmes.

Disclosure of Interest: B.P. Saunders: Advisory board member of Olympus UK All other authors have declared no conflicts of interest.

P1507 IMPACT OF PERIODONTAL DISEASE ON PREVALENCE OF COLORECTAL NEOPLASIA IN PATIENTS UNDERGOING ROUTINE SCREENING COLONOSCOPY

A. Dokladanska ${ }^{1}$, P. Jeschek ${ }^{1}$, E. Waldmann ${ }^{2}$, D. Penz ${ }^{1}$, B. Majcher ${ }^{2}$,

A. Hinterberger ${ }^{2}$ G. Heinze ${ }^{3}, \mathrm{M}$. Ferlitsch ${ }^{4}$

${ }^{1}$ Department Of Internal Medicine III, Division Of Gastroenterology And

Hepatology, Medical University of Vienna, Vienna/Austria

${ }^{2}$ Medical University of Vienna, Vienna/Austria

${ }^{3}$ Section For Clinical Biometrics, Center For Medical Statistics, Informatics And Intelligent Systems, Medical University of Vienna, Vienna/Austria

${ }^{4}$ Quality Assurance Working Group, Austrian Society of Gastroenterology and

Hepatology (OEGGH), Vienna/Austria

Contact E-mail Address: angelika.dokladanska@meduniwien.ac.at

Introduction: Systemic diseases including several types of cancer have been associated with periodontitis, potentially owing to the constant systemic inflammatory state in those patients. Data on a potential association of periodontal disease and colorectal neoplasia is scarce and conflicting.

Aims \& Methods: Data from 25,407 patients undergoing healthy check up assessing periodontal disease according to periodontosis-risk classes (PRC 0 -healthy gingiva, PRC 1 - tatar or plaque, PRC 2 - redness or swelling) and screening colonoscopy between 2009 and 2012 in Austria were included. Colonoscopy outcomes were compared between patients with and without signs of periodontal disease using multivariate models adjusting for age, sex, smoking, alcohol consumption, diabetes and BMI

Results: In multivariate adjusted models, patients with periodontal disease had similar odds for the detection of colorectal polyps as those without signs of periodontal disease [adjOR 1.070; 95\% CI: 0.918; 1.247]. Regarding the prevalence of adenomas, patients with periodontal disease, likewise, had similar odds as those with healthy periodontal tissue [adjOR $1.010 ; 95 \% \mathrm{CI}: 0.840 ; 1.213$ ]. Similarly, those with periodontal disease had comparable odds for colorectal adenomas as those without signs of periodontal disease $[1.055(0.785 ; 1.418)]$. In the table below the adenoma detection rate (ADR) and advanced adenoma detection rate (AADR) divided into the periodontosis-risk classes.

Table 1: ADR (adenoma detection rate) and AADR (advanced adenoma detection rate) according to the periodontosis-risk classes

\begin{tabular}{llll}
\hline & PRC 0 & PRC 1 & PRC 2 \\
\hline Adenoma (ADR) & $19,34 \%$ & $19,56 \%$ & $20,02 \%$ \\
Advanced adenoma (AADR) & $5,42 \%$ & $6,1 \%$ & $6,5 \%$ \\
\hline
\end{tabular}

Conclusion: Conclusion: Periodontal disease has no impact on the adenoma and advanced adenoma detection rates in a large screening colonoscopy cohort. Disclosure of Interest: All authors have declared no conflicts of interest. 


\section{References}

1. Anon. STATISTIK AUSTRIA - Dickdarm, Enddarm. Available at: http:/ www.statistik.at/web_de/statistiken/gesundheit/krebserkrankungen/dickdar$m$ enddarm/[Accessed October 30, 2014].

2. Ferlay J, Shin H-R, Bray F, et al. Estimates of worldwide burden of cancer in 2008: GLOBOCAN 2008. Int. J. Cancer 2010;127:2893-2917.

3. Ferlitsch M. Sex-Specific Prevalence of Adenomas, Advanced Adenomas, and Colorectal Cancer in Individuals Undergoing Screening Colonoscopy. JAMA J. Am. Med. Assoc. 2011;306:1352.

4. Yen AM-F, Lai H, Fann JC-Y, et al. Relationship between Community Periodontal Index and Fecal Hemoglobin Concentration, an Indicator for Colorectal Neoplasm. J. Dent. Res. 2014;93:760-766.

5. Bernstein CN, Blanchard JF, Kliewer E, et al. Cancer risk in patients with inflammatory bowel disease: a population-based study. Cancer 2001;91:854 862

6. Scannapieco FA, Bush RB, Paju S. Associations between periodontal disease and risk for atherosclerosis, cardiovascular disease, and stroke. A systematic review. Ann. Periodontol. Am. Acad. Periodontol. 2003;8:38-53.

\section{P1508 OUTCOMES OF ENDOSCOPIC RESECTIONS OF LARGE NON-POLYPOID LESIONS IN INFLAMMATORY BOWEL DISEASE: A SINGLE UNITED KINGDOM CENTRE EXPERIENCE}

S. Gulati, A. Emmanuel, M. Burt, P. Dubois, B. Hayee, A. Haji

Endoscopy, King's Institute of Therapeutic Endoscopy, RS/United Kingdom

Contact E-mail Address: shraddha.gulati@nhs.net

Introduction: Patients with colitis carry an increased risk for the development of dysplasia compared to those without ${ }^{1}$. The SCENIC consensus statement recommends endoscopic resection of all visible dysplasia ${ }^{2}$. Due to technical challenges and limited experience in the West of large colitis associated non-polypoid endoscopic resections, such patients are often subjected to colectomy.

The King's Institute of Therapeutic Endoscopy (KITE) is a tertiary centre for endoscopic assessment and resection of large/challenging colorectal polyps. Here we present the largest single-centre case series of large non-polypoid resections associated with colitis.

Aims \& Methods: Adults with confirmed colitis (ulcerative colitis extending beyond the rectosigmoid junction and crohn's colitis affecting at least the left colon) with lesions at least $20 \mathrm{~mm}$ in size within the colitis segment were included. Data including demographics, clinical history, lesion characteristics, method of resection and post-resection surveillance were collected prospectively in patients from January 2011 to November 2016. Resection techniques included endoscopic mucosal resection (EMR), endoscopic submucosal dissection (ESD) and hybrid ESD. Surveillance of resection site with magnification chromoendoscopy (mCE) was performed at 3 months with pan colonic $\mathrm{mCE}$ at 1-year post resection and annually thereafter.

Results: Thirteen lesions satisfied the inclusion criteria in 13 patients. Patient demographics and clinical data are presented in table 1 . Mean lesion size was $47.3+/-22.4(20-90) \mathrm{mm}$. All lesions were non polypoid with distinct margins and no ulceration. High-frequency mini-probe ultrasound confirmed intramucosal lesions in 5 cases where pit/vascular pattern was distorted due to inflammation. En bloc resection was achieved in 6 cases. $69 \%$ lesions were deeply scarred of which $66 \%$ had experienced prior instrumentation. Resection of a single lesion was abandoned due to intense fibrosis. Macrosocpic evidence of complete resection was achieved in all remaining cases. Endoscopic diagnosis of pre-cancerous lesions of less than $1000 \mu \mathrm{m}$ submucosal invasion was confirmed histopathologically in $100 \%$ of resected lesions. Complete excision was confirmed in all en bloc resections. A single case of small perforation and another with delayed minor bleeding were both managed endoscopically. Mortality/hospital admission within 30 days post resection was $0 \%$. Median follow up was 28 months (1235 ) with no recurrence. Alternative site dysplasia was detected in 2 patients. All lesions were sub $20 \mathrm{~mm}$ and resected endoscopically. Two patients were referred for colectomy due to a concomitant diagnosis of neuroendocrine tumour and the second with alternate site advanced dysplasia.

Table 1: Baseline characteristics.

Patient Demographics

Age at time of resection (mean, $\mathrm{SD}$, range) (years)

$57.31+/-12.7,30-81$

Male (n) $(\%)$

$10(77)$

Female (n) $(\%)$

$3(23)$

Clinical Data

Duration of disease (mean, SD, range) (years)

Disease extent

Splenic Flexure (n) (\%)

$19.9,14,2,1-50$

Pan-colonic/Extensive (n) (\%)

Primary Sclerosing Cholangitis

$10(77)$

IBD Medication

5-ASA* (n) (\%)

$11(84)$

Azathioprine (n) (\%)

2 (15)

Biologics (n) (\%)

1 (7)
Table 1 Continued

Patient Demographics

ASA grade II (n) (\%)

7 (54)

ASA grade III (n) $(\%)$

$4(31)$

ASA grade IV (n) $(\%)$

2 (15)

Conclusion: This cohort series demonstrates that endoscopic resection of large non-polypoid lesions in association with colitis is feasible using an array of resection methods, safe and has good long term outcomes in a western tertiary endoscopic centre.

Disclosure of Interest: All authors have declared no conflicts of interest.

\section{References}

1. Choi CR, Rutter M, Askari A et al. Forty-year analysis of colonoscopic surveillance program for neoplasia in Ulcerative Colitis: An updated review. Am J Gastroenterol. 2015.110(7):1022-34

2. Laine L, Kaltenbach T, Barkun A, et al. SCENIC international consensus statement on surveillance and management of dysplasia in inflammatory bowel disease. Gastrointest Endosc 2015;81:489-501.

\section{P1509 RESEARCH ON APPLICATION OF TRANSANAL TUBE DECOMPRESSION FOR PREVENTION OF COMPLICATIONS IN COLORECTAL MUCOSAL LESIONS AFTER ESD}

\section{Bing, Z. Qi, Y. Zhong}

Endoscopy Center And Endoscopy Research Institute, Zhongshan Hospital, Fudan University, Shanghai, Shanghai/China

\section{Contact E-mail Address: 13564623481@126.com}

Introduction: Endoscopic submucosal dissection (ESD) has been widely used in the minimally invasive treatment of early colorectal mucosa and submucosal lesions. This technique has made it possible to resect even large mucosal or submucosal lesions en bloc, and the recurrence rate is lower. However, due to the thinner colorectal wall and more abundant blood vessels, postoperative complications after ESD is higher in this site. As a result, how to prevent complications related to ESD for colorectal lesions has raised widespread concern. In recent years, more and more researchers placed transanal tube for patients with colorectal cancer resection or intestinal obstruction to promote the early discharge of the gas and liquid in the intestine. The efficacy of this method to reduce incidence of complications and to promote recovery of intestinal function have been verified by a number of studies. Based on this, we applied transanal tube to some patients with colorectal ESD, hoping to provide new ideas for the prevention and treatment of complications.

Aims \& Methods: We aimed to evaluate transanal tube for prevention of complications in colorectal mucosal lesions after endoscopic submucosal dissection (ESD). Data of 61 patients with colorectal mucosal lesions undergoing ESD from January to December 2016 were reviewed. All patients were followed up and we analyzed the incidence rate of complications after ESD within one month.

Results: The median age of 61 patients was 61(32 83) years. 21 of all lesions were located at right-half colon, 9 at left-half colon and 31 at rectum. The mean diameter of the lesions was $3.26 \pm 2.27(0.8-12.0) \mathrm{cm}$. There were not intraoperative complications including serious bleeding and perforation. Delayed bleeding on the eighth post-ESD day was detected in $1(1.6 \%)$ patient who was cured by transfusion. $3(4.9 \%)$ patients suffered post-ESD electrocoagulation syndrome and perforation did not present in all cases. In this group with transanal tube for decompression, the rates of perforation, delayed bleeding and post-ESD electrocoagulation syndrome were all lower than others which was $1.4 \sim 8.2 \%, 0.5 \% \sim 9.5 \%$ and $12.1 \% \sim 40.2 \%$ respectively in literature reports. Conclusion: The application of transanal tube in colorectal mucosal lesions after ESD could effectively reduce the incidences of complications. However, we should do more research to know whether transanal tube need to be placed routinely after ESD or not.

Disclosure of Interest: All authors have declared no conflicts of interest.

\section{References}

1. Isomoto H, Nishiyama H, Yamaguchi $\mathrm{N}$, et al. Clinicopathological factors associated with clinical outcomes of endoscopic submucosal dissection for colorectal epithelial neoplasms[J]. Endoscopy, 2009,41(8):679-683.

2. Zhao W T, Li N N, He D, et al. Transanal Tube for the Prevention of Anastomotic Leakage After Rectal Cancer Surgery: A Systematic Review and Meta-analysis[J]. World J Surg, 2017,41(1):267-276.

3. Chen T, Yao L Q, Xu M D, et al. Efficacy and Safety of Endoscopic Submucosal Dissection for Colorectal Carcinoids[J]. Clin Gastroenterol Hepatol, 2016,14(4):575-581.

4. Tanaka S, Terasaki M, Kanao H, et al. Current status and future perspectives of endoscopic submucosal dissection for colorectal tumors[J]. Dig Endosc, 2012,24 Suppl 1:73-79.

5. Lee S P, Sung I K, Kim J H, et al. A randomized controlled trial of prophylactic antibiotics in the prevention of electrocoagulation syndrome after colorectal endoscopic submucosal dissection[J]. Gastrointest Endosc, 2016. 
P1510 OUTCOMES FOLLOWING UNDERWATER ENDOSCOPIC

MUCOSAL RESECTION OF > 10MM COLONIC POLYPS: A PROSPECTIVE DUAL-CENTRE STUDY

K. Siau ${ }^{1}$, A. Yusuf ${ }^{2}$, N. Suzuki ${ }^{3}$, S. Ishaq ${ }^{4}$

${ }^{1}$ Royal College Of Physicians, JAG Clinical Fellow, London/United Kingdom ${ }^{2}$ Gastroenterology, East Sussex Healthcare NHS Trust, Eastbourne/United Kingdom

${ }^{3}$ Wolfson Endoscopy Unit, St Mark's Hospital, Harrow, London/United Kingdom ${ }^{4}$ Department Of Gastroenterology, Dudley Group Hospitals NHS Foundation Trust, Dudley/United Kingdom

Contact E-mail Address: keith@siau.org

Introduction: Underwater endoscopic mucosal resection (UEMR) is an alternative to traditional EMR for the resection of colonic polyps. With this technique, water insufflation is used in place of air or $\mathrm{CO}_{2}$, and submucosal lifting is usually not required, as water-immersed submucosa cushions itself from the muscularis propria. ${ }^{[1]}$ Theoretically, this reduces the risk of diathermy-induced injury, ${ }^{[1]}$ and allows for more complete resection margins. ${ }^{[2]}$

Aims \& Methods: In this prospective dual-centre study, we aim to evaluate the safety and efficacy of UEMR for clinically significant $(>10 \mathrm{~mm})$ colonic polyps. Studied outcomes included: 1) completeness of UEMR, 2) intraprocedural and 30-day complication rates, 3) percentage requiring submucosal lift, and 4) rates and predictors of polyp recurrence. Procedures were performed by two screening endoscopists accepting tertiary referrals at St. Mark's Hospital, London, and Russell's Hall Hospital, Dudley, UK. Recurrence was defined as the presence of any polyp tissue at the resection site. Endoscopy records were examined and correlated with histology. Univariate analyses were performed using Pearson's $\mathrm{chi}^{2}$ to identify predictors of measured outcomes.

Results: Between June 2014 and March 2017, and A total of 85 patients (median age 69.5 years, interquartile range [IQR] 11, 50.6\% male) underwent UEMR of 97 colonic polyps (median size $25 \mathrm{~mm}$, IQR $25 \mathrm{~mm}$, range $10-160 \mathrm{~mm}$ ). 13 $(13.4 \%)$ were recurrences following previous conventional EMR. Polyps were predominantly left sided $(66 \%)$ with flat $(63.5 \%)$ or sessile $(35.4 \%)$ morphology. $43.8 \%$ of polyps were removed en bloc, whilst argon plasma coagulation (APC) was used in $13.7 \%$. Histology comprised of: low-grade dysplasia $(80.2 \%)$, highgrade dysplasia $(12.5 \%)$, adenocarcinoma $(3.1 \%)$ and non-dysplastic sessile serrated lesion $(4.2 \%)$. Overall, resection at index UEMR was deemed endoscopically complete in $97.9 \%$. Submucosal lift was required in $27.8 \%$ and positively correlated with polyp size $>30 \mathrm{~mm}$ (OR $3.58,95 \%$ CI $1.37-9.38, \mathrm{p}=0.01$ ), but not morphology (flat vs. sessile, $\mathrm{p}=0.99$ ). The 30 -day complication rate was $4.1 \%(n=4)$, consisting of intraprocedural bleeding $(n=2$, average diameters: $35 \mathrm{~mm})$ and delayed rebleeding $(\mathrm{n}=2$; average diameter: $57.5 \mathrm{~mm})$, with haemostasis achieved for all cases. No cases of perforation or mortality were identified. Of the $60.8 \%(n=59)$ who attended for repeat endoscopy post-UEMR, the rate of recurrent or residual polyp was $14.8 \%(8 / 54)$ at 4 months and $13 / 59(22.0 \%)$ within 1 year. Significant predictors of post-UEMR recurrence included: piecemeal vs. en bloc resection (OR 5.50, 95\% CI 1.10-27.6, $\mathrm{p}=0.03$ ) and recurrent polyp (OR 4.17, 95\% CI 1.02-17.05, $\mathrm{p}=0.04$ ), but not polyp size, site, morphology, dysplasia status, use of submucosal lift, APC, patient age, or study centre. Conclusion: UEMR is a safe alternative to conventional EMR for the management of clinically significant colonic polyps. However, our post-UEMR recurrence rate of $22.0 \%$ appears higher than other studies,${ }^{[2]}$ but may be skewed by the tertiary nature of referrals. Although randomised trials are awaited, our data suggest that those performing UEMR should attempt en bloc resection where possible, and consider wider resection margins for recurrent polyps.

Disclosure of Interest: All authors have declared no conflicts of interest

\section{References}

1. Binmoeller, KF. J Interv Gastroenterol. 2014; 4:4, 113-116.

2. Shenck RJ et al. Surg Endosc. 2017; DOI 10.1007/s00464-017-5474-4

\section{P1511 WATER-AIDED COLONOSCOPY - RESEARCH FOCUS IN THE PAST DECADE AND CURRENT CLINICAL PRACTICE}

\section{F. W. Leung}

Medicine, Sepulveda Ambulatory Care Center, VAGLAHS; David Geffen School of Medicine at UCLA, North Hills/United States of America/CA

\section{Contact E-mail Address: felixleung@socal.rr.com}

Introduction: Water-aided techniques have forged a paradigm shift in endoscopic diagnosis and therapy. The inauguration (10/22/2014) of the International WATERS with memberships worldwide attested to participants' commitment to advance clinical, educational and research missions. To aid planning of future work in each of these areas, a descriptive study of water-aided colonoscopy was performed.

Aims \& Methods: The aims of this study were two-folds. Study 1: To assess the focus of investigations in the past decade. Study 2: To obtain a cross-sectional snapshot of current clinical practice. Study 1: Studies registered at Clinicaltrial.gov were searched for using the search term "water colonoscopy". Study 2: Members of International WATERS voluntarily participated in a survey. After indicating the proportion of patients with different modes of sedation, respondents selected yes (1) or no (0) responses to each of 16 questions related to their practice of water-aided colonoscopy.

Results: Study 1: In the past decade, 48 trials of water-aided colonoscopy were registered at Clinicaltrial.gov. They aimed at evaluation of insertion pain in unsedated, minimally sedated, or on demand sedation patients; assessment of efficacy in difficult colonoscopy; study of the impact on adenoma detection; and underwater mucosal resection or polypectomy. Study 2: Questionnaire responses are summarized in Table 1 . Respondents: $n=23$. Water-aided colonoscopy is used in patients sedated with propofol, minimal sedation or on demand sedation (3-16\%); but more commonly in patients with moderate or no sedation $(30-34 \%)$. During insertion $95.5 \%$ use infusion of water and only $36.4 \%$ leave the air/CO2 pump on. $42.9 \%$ and $33.3 \%$ record volumes infused and suctioned upon arrival to the cecum. $52.4 \%$ remove almost all infused water during insertion. $71.4 \%$ and $59.1 \%$ performed polypectomy $(<20 \mathrm{~mm}$ and $>20 \mathrm{~mm}$, respectively) underwater during withdrawal.

Table 1A: $\%$ of respondent's patients

\begin{tabular}{|c|c|c|c|}
\hline & & Mean & SD \\
\hline \multicolumn{2}{|l|}{ Sedated with propofol } & 16 & 30 \\
\hline \multicolumn{2}{|l|}{ Receive minimal sedation } & 11 & 19 \\
\hline \multicolumn{2}{|l|}{ Are unsedated } & 34 & 35 \\
\hline \multicolumn{2}{|l|}{ Receive on demand sedation } & 3 & 5 \\
\hline \multicolumn{2}{|l|}{ Receive moderate sedation } & 30 & 39 \\
\hline \multicolumn{2}{|l|}{$\begin{array}{l}\text { Table 1B: Proportion of } \\
\text { respondents using the } \\
\text { following approaches }(\%)\end{array}$} & \multicolumn{2}{|l|}{ Mean } \\
\hline \multirow[t]{2}{*}{ Infuse water during } & insertion & \multicolumn{2}{|l|}{95.5} \\
\hline & withdrawal & \multicolumn{2}{|l|}{36.4} \\
\hline \multirow[t]{2}{*}{ Leave air $/ \mathrm{CO}_{2}$ pump on during } & insertion & \multicolumn{2}{|l|}{36.4} \\
\hline & withdrawal & \multicolumn{2}{|l|}{90.0} \\
\hline \multirow[t]{2}{*}{ Keep track of volume of water } & infused at different insertion locations & \multicolumn{2}{|l|}{19.0} \\
\hline & suctioned at different insertion locations & \multicolumn{2}{|l|}{14.3} \\
\hline \multirow[t]{2}{*}{ Keep track of volume of water } & infused upon arrival to the cecum & \multicolumn{2}{|l|}{42.9} \\
\hline & suctioned upon arrival to the cecum & \multicolumn{2}{|l|}{33.3} \\
\hline \multirow[t]{2}{*}{ Keep track of volume of water } & infused when colonoscopy is finished & \multicolumn{2}{|l|}{33.3} \\
\hline & suctioned when colonoscopy is finished & \multicolumn{2}{|l|}{28.6} \\
\hline \multirow{2}{*}{$\begin{array}{l}\text { Perform polypectomy }(<20 \mathrm{~mm}) \\
\text { underwater during }\end{array}$} & insertion & \multicolumn{2}{|l|}{31.8} \\
\hline & withdrawal & \multicolumn{2}{|l|}{71.4} \\
\hline \multirow{2}{*}{$\begin{array}{l}\text { Perform polypectomy }(>20 \mathrm{~mm}) \\
\text { underwater during }\end{array}$} & insertion & \multicolumn{2}{|l|}{13.6} \\
\hline & withdrawal & \multicolumn{2}{|l|}{59.1} \\
\hline \multirow[t]{2}{*}{ Remove almost all infused water during } & insertion & \multicolumn{2}{|l|}{52.4} \\
\hline & withdrawal & \multicolumn{2}{|l|}{57.1} \\
\hline
\end{tabular}

Conclusion: The variable modes of application amongst respondents who profess to use water-aided colonoscopy reflect the versatility and strength of the paradigm-changing approach, which is easily adaptable to meet the diverse needs of individual colonoscopists. Standardization based on results of randomized controlled trials appears to be prudent to permit further assessment of water-aided colonoscopy in clinical, educational and research settings.

Disclosure of Interest: All authors have declared no conflicts of interest.

\section{P1512 ENDOSCOPIC SUBMUCOSAL DISSECTION: RESULTS AND LEARNING CURVE OF A LARGE PROSPECTIVE SERIE OF 183 CASES IN THREE CENTERS}

M. Lopez Gomez ${ }^{1}$, M. Hernández ${ }^{1}$, C.E. González ${ }^{1}$, J. Santiago ${ }^{2}$, C. Bernardo ${ }^{2}$, B. Conde ${ }^{2}$, C. González-Lois ${ }^{2}$, P. Matallanos ${ }^{1}$, J.L. Calleja ${ }^{1}$, L.E. Abreu García ${ }^{2}$, A. Herreros De Tejada ${ }^{3}$

${ }^{1}$ Puerta de Hierro, Madrid/Spain

${ }^{2}$ Hospital Puerta de Hierro, Madrid/Spain

${ }^{3}$ Servicio De Aparato Digestivo, Hospital Puerta de Hierro, Madrid/Spain

Contact E-mail Address: albertoherreros@yahoo.com

Introduction: Endoscopic submucosal dissection (ESD) is a suitable technique used for the endoscopic management of selected early gastrointestinal neoplasms (EGN).

Aims \& Methods: This is a prospective study of patients with EGN eligible for ESD in three tertiary hospitals. The main goal was to evaluate initial therapeutic results and learning curve of ESD. Initial Technical success rates, procedure speed, en-bloc \& R0k resection, R0, speed and complications rates were prospectively evaluated. The results of the learning curve were analyzed by chronological order of blocs of 50 cases. Perforation was established as any disruption of the muscular layer, regardless of size or identification of peritoneal fat. Time of procedure was considered from initial submucosal injection to final detachment of the specimen.

Results: ESD was attempted in 183 lesions from January 2012 to April 2017. Majority of procedures were performed at Puerta de Hierro University Hospital $(160 / 87.4 \%)$. Mean age was 67 (SD 10.6) years, with male proportion $55.2 \%$. Most common location was colorectal $(77.8 \%)$, followed by gastric $(12.8 \%)$ and esophageal $(9.4 \%)$. Success was observed in $96.2 \%$ of patients with en-bloc and R0 resection of $93.9 \%$ and $92.3 \%$ respectively. Mean lesion size was $46.5 \mathrm{~mm}$ (range 8-130) with a mean speed of $9.01 \mathrm{~min} / \mathrm{cm} 2$ (range 1-209). Perforation was 
the main complication (48 $(26.2 \%)$ events), requiring surgery in $5(10.4 \%)$ cases. Perforation was related statistically significant to location $(\mathrm{p}=0.05)$ and LST morphology $(\mathrm{p}=0.05)$. Most frequent location of perforation was transverse colon (OR 88.3; SE 137), followed by descending colon (OR 13.5; SE 19.4) and splenic flexure (OR 6.3, SE 11.8). Perforation was more common in LSTNG lesions vs LST-G (OR 14,1 SE $=19.3$ vs 11.6 SE 15.6). Perforation rates were not statistically associated with the presence of severe submucosal fibrosis compared to absence of fibrosis ( 0.8 SE 0.6 vs 1 SE $1 ; \mathrm{p}=0.9)$. Post-ESD complications were observed in $15(8.2 \%)$ patients (delayed perforation(7), bleeding(4), electrocoagulation syndrome(1), severe esophageal stricture(1), haemoperitoneum(1) and splenic rupture(1)). Six cases $(40 \%)$ were managed with surgery. Results from the learning curve progression according to consecutive chronological blocs of 50 cases (33 last bloc) are summarized in table 1. Initial success increased from $94 \%$ to $100 \%$; speed of ESD decreased after the first 50 cases (15.5 $\mathrm{cm}^{2} / \mathrm{min}$ ), up to 6.7 and $6.5 \mathrm{~cm}^{2} / \mathrm{min}$ in the last 2 blocs. A high perforation rate in the first period $(32 \%)$ was reduced to $18-30.3 \%$ the following periods. Endoscopic treatment was successful in most cases of perforation $(89.6 \%)$. Surgery was required for severe complications, incomplete ESD and/or perforation $(\mathrm{n}, \%)(16$ cases, $8.7 \%)$

Conclusion: On clinical ESD, high rates of success and en-bloc and R0 resection can be achieved along the learning curve. Perforation is the most common complication and is still a challenge for Western countries. However, increasing experience reflects a high success in endoscopic management of perforation. Disclosure of Interest: All authors have declared no conflicts of interest.

\section{References}

Fujiya M et al. (2015) Efficacy and adverse events of EMR and endoscopic submucosal dissection for the treatment of colon neo- plasms: a meta-analysis of studies comparing EMR and endoscopic submucosal dissection. Gastrointest Endosc 583-95

Fu Q et al. (2016) Relevant risk factors of positive lateral margin after en bloc endoscopic submucosal dissection for early gastric adenocarcinoma. J Dig Dis.

Nagata K, Shimizu M (2012) Pathological evaluation of gastrointestinal endoscopic submucosal dissection materials based on Japanese guidelines. World $J$ Gastrointest Endosc 4(11):489-499.

Endoscopic submucosal dissection: European Society of Gastrointestinal Endoscopy (ESGE) Guideline

\section{P1513 ENDOSCOPIC SUBMUCOSAL DISSECTION FOR COLORECTAL NEOPLASIA: THE EXPERIENCE OF A UK TERTIARY REFERRAL CENTRE}

A. Emmanuel, S. Gulati, M. Burt, B. Hayee, A. Haji

Endoscopy, King's College Hospital, London/United Kingdom

\section{Contact E-mail Address: aemmanuel@nhs.net}

Introduction: Despite the advantages of endoscopic submucosal resection (ESD) demonstrated in large series from the far east, the procedure is not commonly practiced in the west and its role in standard practice is still debated. Although limited evidence regarding its efficacy in European practice is emerging, very few centres in the United Kingdom perform ESD regularly, if at all. We report the experience of a UK tertiary referral institution using ESD as part of a lesion specific, pragmatic approach to endoscopic resection in a complex patient cohort. Aims \& Methods: Patients who underwent ESD of colorectal lesions were included. Lesions were assessed with magnification chromoendoscopy supplemented by colonoscopic ultrasound in selected cases. A lesion specific approach was used to decide on resection technique, which included assessment of morphology, pit pattern, risk of submucosal invasion, and presence of submucosal fibrosis or scarring. ESD was used where en bloc resection was deemed essential, or as part of a hybrid procedure to ensure resection of a dominant nodule or suspicious area of a lesion in one piece, or where fibrosis or scarring would make standard EMR impossible. A resection was designated a hybrid procedure if ESD was used to effect submucosal dissection, circumferential incision alone to assist snare resection was not included.

Results: 116 lesions (mean size $58.8 \mathrm{~mm})$ were resected using ESD $(\mathrm{n}=58)$ and hybrid ESD $(\mathrm{n}=58) .82(70.7 \%)$ had been subjected to prior attempts at resection $(n=58)$ or extensive sampling. Only 11 lesions had no prior biopsies performed. En bloc resection was achieved in 93.1\% where ESD was used alone, with a recurrence rate of $4.7 \%$ after a mean follow up of 12.4 months. There were 6 microperforations treated with either endoscopic clips or antibiotics alone with no adverse sequelae, and one clinically significant perforation requiring surgery. However, the resected lesion in this case contained an invasive adenocarcinoma with deep submucosal invasion-there was no residual tumour in the surgical resection specimen. Post- procedure bleeding occurred in 6 patients, none of whom required additional interventional therapy. $71 \%$ of cases were successfully performed as day case procedures. $97 \%$ of patients without invasive cancer were free from recurrence and had avoided surgery at last follow-up.

Conclusion: Colorectal ESD can be used as part of a standard lesion specific approach in a western referral centre to deliver safe and effective organ conserving treatment to patients with large challenging lesions. Knowledge regarding lesion assessment and selection in western practice should be improved to reduce the incidence of prior heavy manipulation and guide appropriate referral.

Disclosure of Interest: All authors have declared no conflicts of interest.

P1514 RISK OF STENOSIS AND OUTCOMES FOLLOWING ENDOSCOPIC RESECTION OF LARGE COLORECTAL LESIONS INVOLVING MORE THAN 75\% OF THE LUMINAL CIRCUMFERENCE

A. Emmanuel, S. Gulati, M. Burt, B. Hayee, A. Haji

Endoscopy, King's College Hospital, London/United Kingdom

Contact E-mail Address: aemmanuel@nhs.net

Introduction: Little is known about the risk of stenosis and outcomes following endoscopic resection of lesions in the colorectum which leave extensive mucosal defects. A limited number of studies suggest significant stenosis rates, although reports on outcomes and suggested management are conflicting. We determined the risk of stenosis and outcomes of endoscopic resection of colorectal lesions leaving mucosal defects $\geq 75 \%$ of the circumference.

Aims \& Methods: Consecutive patients who underwent endoscopic resection of colorectal lesions $\geq 2 \mathrm{~cm}$ were included. Resection technique included EMR, ESD and hybrid techniques involving ESD. Patients were grouped according to circumferential extent of the mucosal defect after resection. Surveillance colonoscopy was performed at 3 and 12 months. Clinicopathological characteristics and outcomes were compared between groups.

Results: 435 colorectal lesions $\geq 2 \mathrm{~cm}$ were resected using EMR $(\mathrm{n}=342)$, ESD $(\mathrm{n}=45)$ or hybrid techniques $(\mathrm{n}=48)$. Circumferential extent of the resulting mucosal defect was $>75 \%$ in 41 patients. 8 lesions were fully circumferential: 1 caecal lesion and the rest in the recto-sigmoid and rectum. 3 of these circumferential lesions contained deeply invasive adenocarcinoma and 1 benign lesion ultimately required surgery. The 41 lesions with a mucosal defect $>75 \%$ of the circumference had a mean size of $100.5 \mathrm{~mm}$ vs $49.0 \mathrm{~mm}$ for other lesions $(\mathrm{p}<0.001)$. These patients had significantly more complications $(16.7 \%$ vs $4.7 \%, \mathrm{p}<0.001)$, including a higher rate of perforation $(8.3 \%$ vs $2.3 \%$, $\mathrm{p}=0.02$ ), although none required surgery, and a significantly higher rate of recurrence $(44.8 \%$ vs $9.2 \%, \mathrm{p}<0.001) .79 \%$ of patients without cancer were free from recurrence and had avoided surgery at last follow-up compared to $97 \%$ of patients with mucosal defects $<75 \%(\mathrm{p}<0.001)$. Stenosis occurred in 7 patients: 4 lesions extensively involving the rectum and recto-sigmoid and 2 lesions involving the sigmoid colon extending to the rectosigmoid. 1 of these involved a mucosal defect of only $50 \%$ of the circumference and 3 were fully circumferential. 1 patient had a symptomatic anorectal stenosis requiring dilatation under anaesthesia, 1 patient was asymptomatic but underwent early dilatation after the first surveillance endoscopy at 3 months. The remaining patients were asymptomatic and managed expectantly. In all these latter cases spontaneous improvement in the stricture was noted at the subsequent surveillance colonoscopy

Conclusion: The majority of patients with these extensive complex lesions can successfully be treated with endoscopic resection and avoid surgery. However, these patients have a significantly greater risk of complications and recurrence and should be managed in a tertiary institution. Although there is a significant risk of stenosis, it appears that most cases are asymptomatic and spontaneously improve with expectant management.

Disclosure of Interest: All authors have declared no conflicts of interest.

P1515 RISK OF HIGH-GRADE DYSPLASIA AND SUBMUCOSAL INVASION IN DIFFERENT MORPHOLOGICAL SUB-TYPES OF LARGE COLORECTAL NEOPLASTIC LESIONS RESECTED AT A UK TERTIARY REFERRAL UNIT

A. Emmanuel ${ }^{1}$, S. Gulati ${ }^{1}$, M. Burt ${ }^{2}$, B. Hayee ${ }^{1}$, A. Haji ${ }^{1}$

${ }^{1}$ Endoscopy, King's College Hospital, London/United Kingdom

${ }^{2}$ Endoscopy, King's Institute of Therapeutic Endoscopy, RS/United Kingdom

Contact E-mail Address: aemmanuel@nhs.net

Abstract: P1512

\begin{tabular}{|c|c|c|c|c|c|}
\hline $\mathrm{N}=183$ & $1-50$ & $51-100$ & $101-150$ & $151-183$ & Total $(\mathrm{n} / \%)$ \\
\hline Colorectal location (n.\%) & $40 / 50(80 \%)$ & $42 / 50(84 \%)$ & $34 / 50(68 \%)$ & $25 / 33(75.7 \%)$ & $141 / 183(77 \%)$ \\
\hline Success(n, \%) & $47 / 50(94 \%)$ & $46 / 50(92 \%)$ & $50 / 50(100 \%)$ & $33 / 33(100 \%)$ & $176 / 183(96.2 \%)$ \\
\hline En bloc(n, \%) & $47 / 50(94 \%)$ & $46 / 50(92 \%)$ & $50 / 50(100 \%)$ & $33 / 33(100 \%)$ & $172 / 183(93.9 \%)$ \\
\hline $\mathrm{R} 0(\mathrm{n}, \%)$ & $45 / 50(90 \%)$ & $45 / 50(90 \%)$ & $48 / 50(96 \%)$ & $31 / 33(94 \%)$ & $169 / 183(92.3 \%)$ \\
\hline Speed $(\mathrm{cm} 2 / \min )$ Mean (SD) & $7.68(5.02)$ & $15.5(37.8)$ & $6.7(5.5)$ & $6.5(5.1)$ & $9.01(19.1)$ \\
\hline Perforation(n, \%) & $16 / 50(32 \%)$ & $13 / 50(26 \%)$ & $9 / 50(18 \%)$ & $10 / 33(30.3 \%)$ & $48 / 183(26.2 \%)$ \\
\hline Surgery due to perforation $(\mathrm{n}, \%)$ & $2 / 16(12.5 \%)$ & $1 / 13(7.7 \%)$ & $0 / 9(0 \%)$ & $2 / 10(20 \%)$ & $5 / 48(10.4 \%)$ \\
\hline
\end{tabular}


Introduction: Although it is well recognised that the risk of invasive carcinoma in apparent benign colorectal neoplastic lesions differs according to morphology, the incidence of invasive cancer varies between studies and there is limited data from large western series to inform practice. The importance of appropriate resection techniques, including the use of ESD, is increasingly recognised in western practice. It is therefore imperative that the risk of submucosal invasion is assessed as accurately as possible to prevent inappropriate attempts at resection. We determined the risk of submucosal invasion and high-grade dysplasia (HGD) in different morphological sub- types of large colorectal lesions subjected to endoscopic resection.

Aims \& Methods: Colorectal lesions $\geq 2 \mathrm{~cm}$ subjected to endoscopic resection were included. Lesions were assessed with magnification chromoendoscopy. Clinicopathological data recorded included morphological type according to Paris classification, sub-types of laterally spreading tumours (LST), degree of dysplasia, presence of submucosal invasion and outcomes following resection.

Results: 435 colorectal lesions $\geq 2 \mathrm{~cm}$ were resected. Mean lesion size was $55.2 \mathrm{~mm}$ (range $20 \mathrm{~mm}-160 \mathrm{~mm}$ ). The frequency of and the incidence of high-grade dysplasia and invasive adenocarcinoma in the different morphological sub-types are shown in Table 1. The incidence of high-grade dysplasia $(8.6 \%)$ and invasive adenocarcinoma $(1.2 \%)$ was very low in LST granular homogenous lesions.

\begin{tabular}{llll}
\hline Morphology & $\begin{array}{l}\text { Mean } \\
\text { size }(\mathrm{mm})\end{array}$ & $\begin{array}{l}\text { High-grade } \\
\text { dysplasia }(\%)\end{array}$ & $\begin{array}{l}\text { Invasive } \\
\text { adenocarcinoma (\%) }\end{array}$ \\
\hline Is & 36.7 & $2(33.3)$ & $1(16.7)$ \\
Isp & 37.8 & $21(27.6)$ & $7(9.2)$ \\
IIa & 27.2 & $5(10)$ & $0(0)$ \\
IIa + IIc & 35.0 & $3(100)$ & $2(66.6)$ \\
LST G H & 54.3 & $15(8.6)$ & $2(1.2)$ \\
LST G MN & 83.3 & $49(43.0)$ & $(12(10.0)$ \\
LST NG & 45.0 & $4(36.4)$ & $2(18.2)$ \\
\hline
\end{tabular}

LST $\mathrm{G}$ H $=$ LST granular homogenous LST G MN = LST granular mixed nodular LST NG $=$ LST non-granular ESD or hybrid ESD was used to resect 97 lesions. $53 \%$ of lesions had been subjected to previous failed attempts at resection, $\geq 6$ biopsies or had tattoo injected at their base prior to referral. Of 29 invasive adenocarcinomas, 9 were deemed to be cured by endoscopic resection (superficial submucosal invasion with no adverse prognostic features). Of the remaining 20, 5 patients refused surgical resection, 5 were unfit for major surgery and 10 had surgery. 2 had residual tumour in the surgical resection specimen, 5 had none and 3 had surgery at their referring institution. Only 3 patients with initially benign lesions developed adenocarcinoma in subsequent lesions-all of these were early cancers without nodal metastases at surgical resection.

Conclusion: In one of the largest European series reporting the incidence of invasive carcinoma in different morphological sub-types of colorectal neoplastic lesions, we confirm that LST granular homogeneous type lesions have a very low incidence of invasive carcinoma and that care should be taken in the choice of resection technique for other sub-types of LSTs which more frequently harbour malignancy. Accurate endoscopic assessment and classification to aid meticulous lesion selection is essential to this process.

Disclosure of Interest: All authors have declared no conflicts of interest.

\section{P1516 POLYP DETECTION INCREASES WITH COLONOSCOPY WITHDRAWAL TIME}

K. Agar, L. Hamzaoui, M. Medhioub, B. Bouchabou, M.M. Azouz Gastro-enterology, Med Taher Maamouri Nabeul, Nabeul/Tunisia

\section{Contact E-mail Address: khaledagar27@gmail.com}

Introduction: Polypectomy of adenomatous polyps during colonoscopy has been shown to decrease the incidence of colorectal cancer (CRC). Withdrawal time during colonoscopy is thought to improve polyp detection. A minimal withdrawal time of six minutes is recommended.

Aims \& Methods: The aim of this study is to evaluate the role of colonoscopy withdrawal time in adenomatous polyp detection. It is a retrospective, descriptive and analytic study collecting all consenting patients who had a colonoscopy in Mohamed Taher Maamouri Hospital of Nabeul, between January 2016 and March 2017. Incomplete colonoscopies or those performed in patients having had segmental colectomy were excluded. We defined two groups of patients: G1: withdrawal time $\geq 7$ minutes - G2: withdrawal time $<7$ minutes Two parameters were evaluated: -Polyp Detection Rate (PDR): the percent of colonoscopies with one or more adenomatous polyps detected -Mean Number of polyps detected per colonoscopy (MNP).

Results: A total of 1059 patients were screened for enrollment; 749 total colonoscopies were studied: 383 men $(51.1 \%)$ and 366 women $(48.9 \%)$. Colic preparation was good (Boston score $>6$ ) in $49.4 \%$ of patients. Colonoscopy was normal in 434 cases $(57.9 \%)$. Colonic polyps were detected in 197 patients $(26.3 \%)$. The mean number of polyps detected per colonoscopy (MNP) was 2.31 (min: 1, max: 25 ). The mean polyp diameter was 6.8 millimeters ( 1 to $50 \mathrm{~mm}$ ). The mean withdrawal time was 8.2 minutes (4 to 20 minutes). Overall PDR was $26 \%$. G1 included 599 colonoscopies and G2 included 150 colonoscopies. PDR in G1 was $28.04 \%$ versus $20 \%$ in G2, the difference between the two groups was not significant (p: 0.7). As for the MNP, it was significantly higher in G1: 2.29 versus 1.86 in $\mathrm{G} 2$ (p: 0.049).
Conclusion: In our study, a withdrawal time exceeding seven minutes was significantly associated with the number of polyps detected in colonoscopy. Further studies may be helpful to confirm these results ideally by comparing these parameters in the same patients.

Disclosure of Interest: All authors have declared no conflicts of interest.

\section{P1517 OUTCOMES OF ENDOSCOPIC RESECTION OF RECURRENT COLORECTAL LESIONS TREATED AT A UK TERTIARY REFERRAL CENTRE}

A. Emmanuel, S. Gulati, M. Burt, B. Hayee, A. Haji

Endoscopy, King's College Hospital, London/United Kingdom

\section{Contact E-mail Address: aemmanuel@nhs.net}

Introduction: Endoscopic resection of large colorectal lesions, especially by piecemeal EMR, carries a significant risk of recurrence. Although several series examine the outcomes and risk of recurrence following endoscopic resection, few focus on the outcomes of patients being treated for recurrence after initial expert resection, and these mostly focus on one technique to deal with recurrence. We evaluated the outcomes after recurrence of colorectal lesions after apparent successful endoscopic resection in a specialised UK tertiary institution employing a range of resection techniques.

Aims \& Methods: Consecutive patients who underwent endoscopic resection of colorectal lesions $\geq 2 \mathrm{~cm}$ were included. All lesions were assessed with magnification chromoendoscopy supplemented by colonoscopic ultrasound in selected cases. A lesion specific approach was used to decide on resection technique. Outcomes were evaluated for patients treated for recurrent lesions.

Results: Of 396 colorectal lesions $\geq 2 \mathrm{~cm}$ initially resected, recurrence occurred in 48 patients. $36 \%$ of these patients had already had a mean of 1.6 previous failed attempts at resection prior to referral to our institution, and $66 \%$ had had either a failed attempt at resection or extensive sampling involving $>6$ biopsies or tattoo placed under the lesion. $69 \%$ of patients were successfully treated with further endoscopic resection and avoided surgery. 27 recurrent lesions larger than $20 \mathrm{~mm}$ were treated with endoscopic resection, with a mean lesion size of $48.3+1-$ $19.1 \mathrm{~mm}$. Techniques used were $\operatorname{EMR}(\mathrm{n}=16), \operatorname{ESD}(\mathrm{n}=2)$, Hybrid ESD and EMR $(\mathrm{n}=9)$. The remaining lesions $<2 \mathrm{~cm}$ were resected using EMR. A mean of $1.4+/-0.75$ procedures were required to achieve successful endoscopic treatment of recurrence. Of 23 patients who were ultimately successfully treated with endoscopic resection, 15 required a single further endoscopic resection after recurrence, 8 patients required 2 or more further resections. 8 patients required surgery, 3 as a result of developing invasive adenocarcinoma with the recurrence. There were no perforations as a result of endoscopic resection of recurrent lesions and only 1 patient was readmitted with post-procedural bleeding which was managed conservatively.

Conclusion: These data demonstrate the challenges of an advanced endoscopic resection service in much of western practice where patients with recurrent lesions represent a particularly complex cohort, most of whom have already had extensive prior manipulation or attempts at resection. Familiarity with a range of resection techniques and appropriate equipment is essential to successfully treat recurrent lesions in this group with endoscopic resection, which can be achieved in the majority of patients without significant complications.

Disclosure of Interest: All authors have declared no conflicts of interest.

\section{P1518 THE EFFECTIVENESS OF NEW TECHNIQUE WITH SELF- EXPANDABLE METALLIC STENT INSERTION IN TREATING RIGHT-SIDED COLONIC OBSTRUCTION}

C.B. Ryu ${ }^{1}$, M.S. Lee ${ }^{1}$, J.Y. Bae ${ }^{2}$

${ }^{1}$ Digestive Disease Center And Research Institute, Department Of Internal Medicine, SoonChunHyang University School of Medicine, Bucheon/Korea, Republic of

${ }^{2}$ Department Of Internal Medicine, Seoul Medical Center, Seoul/Korea, Republic of

Contact E-mail Address: ryuchb@gmail.com

Introduction: Self-expandable metallic stent (SEMS) is widely used to treat malignant colonic obstruction. However, most reports about SEMS insertion have concentrated on the left colon and very tough to insert SEMS on the right colon, especially distal ascending colon.

Aims \& Methods: This study aimed to (1) investigate the effectiveness of new insertion technique with SEMS for right-sided colonic obstruction and (2) compare the safety and technical success of SEMS insertion. The data from ten patients who underwent SEMS with a new technique for malignant obstruction of ascending colon in our hospital were analyzed retrospectively. Initially, we tried to insert with the straight type guiding tube and wire for obstructed area of ascending colon under $\mathrm{CO}_{2}$ insufflation. It defined by difficult cannulation more than 20 minutes manipulation. For difficult cannulation, we change to curved type guiding tube (CleverCut2V/CleverCut3V Double and Triple Lumen sphincterotomes). All cased were difficult cannulation and change to curved type. Cannulation time between straight and curved type guiding tube, technical and clinical success, complications, and technical difficulties were analyzed. We compared the results between SEMS insertion and decompression tube placement in right colons and the outcomes of SEMS insertion between right- and left-sided colonic obstructions.

Results: Cannulation time with a curved type guiding tube decreased of all cases (20 $\mathrm{min}$ vs $8.5 \mathrm{~min}$ ). For ascending colons, the technical and clinical success rate of SEMS insertion with new technique significantly $100 \%(10 / 10)$. There was no 
complitation $(0 / 10)$. Concerning SEMS insertion, the technical difficulty and safety of SEMS insertion were similar between right- and left-sided colonic obstructions.

Conclusion: A new technique of curved type guiding tube with SEMS insertion for right-sided colon, especially distal ascending colon is significantly more effective than straight type guiding tube, and this procedure was safer and less technically challenging than expected. SEMS insertion should be considered for treating right-sided malignant colonic obstruction.

Disclosure of Interest: All authors have declared no conflicts of interest.

\section{P1519 ENDOSCOPIC FULL-THICKNESS RESECTION FOR COLONIC LESIONS. INITIAL EXPERIENCE IN 3 CENTERS OF CATALONIA}

H. Uchima Koecklin ${ }^{1}$, D. Barquero ${ }^{2}$, A. Fernandez-Simon ${ }^{2}$, A. Mata ${ }^{2}$, C. Huertas $\mathrm{Nadal}^{3}$, M. Figa ${ }^{1}, \mathrm{M}$. Hombrados ${ }^{1}$, J. Espinós ${ }^{4}$

${ }^{1}$ Gastroenterology, Endoscopy Unit, Hospital Josep Trueta Girona, Girona/Spain ${ }^{2}$ Gastroenterology And Endoscopy Unit, Hospital Sant Joan Despí Moisès Broggi, BARCELONA/Spain

${ }^{3}$ Hospital Dr. Josep Trueta, Girona/Spain

${ }^{4}$ Gastroenterology, Hospital Mutua Terrassa, Barcelona/Spain

Contact E-mail Address: huchima.girona.ics@gencat.cat

Introduction: Endoscopic full-thickness resection (EFTR) in the colon using the FTRD kit is a recent technique that allows en-bloc resection of colonic lesions that are poor candidates for resection by endoscopic mucosal resection (EMR). It does not require the injection of a solution into the submucosa and allows an enbloc resection of colonic lesions up to approximately $30 \mathrm{~mm}$. We present results of the initial experience in 3 centers of Catalonia.

Aims \& Methods: We retrospectively analyzed the clinical, endoscopic and anatomopathological data of all cases of EFTR performed in 3 centers in Catalonia using the FTRD kit (Ovesco Endoscopy, Tübingen, Germany) during the period between June 2015 and January 2017. All patients underwent the procedure under deep sedation with propofol, all patients were duly informed of the technique and potential complications derived from the procedure, obtaining written informed consent prior to the procedure. All EFTR resections in the colon were performed using the FTRD kit (Ovesco Endoscopy, Tübingen, Germany), grasping and pulling the lesion inside the cap, deploying the OTSC and resecting the tissue over the OTSC with the preloaded snare. Clinical assessment was performed before and after procedures with a complete blood test before procedure. Blood test was not routinely used after procedures. Demographic, clinical, endoscopic and histologic data were collected from each patient, and analysed with STATA software.

Results: 16 endoscopic full-thickness resections of the colon were performed. The mean age of the patients was 69 years (53-79), with men being $88 \%$. The indications were recurrent/residual lesions with non lifting sign $(75 \%)$ and untreated lesions with non lifting sign $(25 \%)$. The locations were juxtavalvular $(n=2)$, hepatic flexure $(n=3)$, transverse colon $(n=4)$, descending colon $(n=3)$, sigma, stump or anastomosis $(\mathrm{n}=2)$. The mean diameter of the resected samples was $20.8 \mathrm{~mm}(13-33 \mathrm{~mm})$. The histology of the lesions was adenoma with low grade dysplasia $(50 \%)$, adenoma with high grade dysplasia $(25 \%)$, sessile serrated polyp without dysplasia $(6 \%)$ and three lesions were adenocarcinoma with deep submucosal invasion $(19 \%)$. There were no clinically relevant complications or emergency surgery. 1 patient had mild self-limited hemorrhage, 6 patients had mild abdominal pain $(38 \%)$ and 3 patients developed postpolypectomy syndrome $(19 \%)$.

Conclusion: Endoscopic full-thickness resection is a safe and feasible technique for selected cases in colon.

Disclosure of Interest: All authors have declared no conflicts of interest.

\section{References}

Schmidt A, Damm M, Caca K. Endoscopic full-thickness resection using a novel over-the-scope device. Gastroenterology 2014; 147: 740-742.e2.

Schmidt A, Bauerfeind P, Gubler C, et al. Endoscopic full-thickness resection in the colorectum with a novel over-the-scope device: first experience. Endoscopy 2015; 47: 719-25.

\section{P1520 A META-ANALYSIS: CHROMOENDOSCOPY OR WHITE LIGHT ENDOSCOPY FOR NEOPLASIA DETECTION IN LYNCH SYNDROME}

O. Har-Noy ${ }^{1}$, D. E. Yung ${ }^{2}$, A. Koulaouzidis ${ }^{2}$, U. Kopylov ${ }^{3}$, L. H. Katz ${ }^{1}$ ${ }^{1}$ Gastroenterology, Department Of Medicine A, Sheba Medical Academic Hospital, Ramat-Gan/Israel

${ }^{2}$ The Royal Infirmary of Edinburgh, Edinburgh/United Kingdom

Contact E-mail Address: liorkatz5346@gmail.com

Introduction: Lynch syndrome (LS) is an autosomal-dominant disease, with increased risk of colorectal cancer (CRC), hence annual surveillance colonoscopy is recommended. do not recommend one over the other.

Aims \& Methods: We aimed to compare the diagnostic yield (DY) of different endoscopic modalities for detection of colorectal neoplasia in patients with LS by performing a meta-analysis of existing literature. We searched Pubmed for prospective studies. For each modality, we performed comparative total per-adenoma analysis and sub-analyses for flat lesions and location (right/left colon).
Meta-analysis was performed using pooled rate ratios (RR) with fixed effects model due to low heterogeneity within the subgroups.

Results: Five studies compared white light endoscopy (WLE) to chromoendoscopy. In four studies compared WLE to chromoendoscopy directly (one study used a "back-to-back" design but lesions were resected only after chromoendoscopy and the other study compared two different sets of patients). Chromoendoscopy $(n=75)$ identified more adenomas in the right colon than WLE ( $\mathrm{n}=73$ patients) RR 3.27 (95\% CI 1.83-5.87). Overall detection of adenomas or flat lesions was not statistically different between the two methods. Conclusion: Chromoendoscopy is superior to WLE for detection of adenomas in Lynch syndrome, especially flat lesions and adenomas in the right colon. Disclosure of Interest: U. Kopylov: research grants- Takeda, Medtronic, Janssen; Consultancy- Jannsen, CTS; Lecture fees- Abbvie, Jannsen, Takeda, CTS All other authors have declared no conflicts of interest.

\section{References}

1. Provenzale, D. et al. Genetic/Familial High-Risk Assessment: Colorectal Version 1.2016, NCCN Clinical Practice Guidelines in Oncology. Journal of the National Comprehensive Cancer Network: JNCCN 14, 1010-1030 (2016)

2. East, J. E. et al. Narrow band imaging for colonoscopic surveillance in hereditary non-polyposis colorectal cancer. Gut 57, 65-70, doi:10.1136/ gut.2007.128926 (2008)

3. Huneburg, R. et al. Chromocolonoscopy detects more adenomas than white light colonoscopy or narrow band imaging colonoscopy in hereditary nonpolyposis colorectal cancer screening. Endoscopy 41, 316-322, doi:10.1055/s0028-1119628 (2009)

4. Hurlstone, D. P. et al. The role of high-magnification-chromoscopic colonoscopy in hereditary nonpolyposis colorectal cancer screening: a prospective "back-to-back" endoscopic study. The American journal of gastroenterology 100, 2167-2173, doi:10.1111/j.1572-0241.2005.41481.x (2005).

5. Lecomte, T. et al. Chromoendoscopic colonoscopy for detecting preneoplastic lesions in hereditary nonpolyposis colorectal cancer syndrome. Clinical gastroenterology and hepatology: the official clinical practice journal of the American Gastroenterological Association 3, 897-902 (2005).

6. Rahmi, G. et al. Impact of chromoscopy on adenoma detection in patients with Lynch syndrome: a prospective, multicenter, blinded, tandem colonoscopy study. The American journal of gastroenterology 110, 288-298, doi:10.1038/ajg.2014.423 (2015).

7. Ramsoekh, D. et al. A back-to-back comparison of white light video endoscopy with autofluorescence endoscopy for adenoma detection in high-risk subjects. Gut 59, 785-793, doi:10.1136/gut.2008.151589 (2010).

8. Stoffel, E. M. et al. Missed adenomas during colonoscopic surveillance in individuals with Lynch Syndrome (hereditary nonpolyposis colorectal cancer). Cancer prevention research (Philadelphia, Pa.) 1, 470-475, doi:10.1158/1940-6207.capr-08-0098 (2008).

9. Bisschops, R., Tejpar, S., Willekens, H., De Hertogh, G. \& Van Cutsem, E. Virtual chromoendoscopy (I-SCAN) detects more polyps in patients with Lynch syndrome: a randomized controlled crossover trial. Endoscopy 49, 342-350, doi:10.1055/s-0042-121005 (2017).

\section{P1521 HOT AVULSION TECHNIQUE - A FIRST-LINE APPROACH FOR TREATMENT OF VISIBLE RESIDUAL NEOPLASIA DURING ENDOSCOPIC MUCOSAL RESECTION OF COLORECTAL POLYPS?}

J. Pinho ${ }^{1}$, R. Castro ${ }^{2}$, D. Libanio ${ }^{2}$, P. Pimentel-Nunes ${ }^{2}$, T. Pinto-Pais ${ }^{2}$, M. DinisRibeiro $^{2}$, R.P. Bastos ${ }^{2}$

Gastroenterology, Centro Hospitalar Tondela/Viseu, Viseu/Portugal

${ }^{2}$ Department Of Gastroenterology, Instituto Português de Oncologia, Porto, Porto/ Portugal

Contact E-mail Address: julianapinho18@gmail.com

Introduction: Endoscopic mucosal resection (EMR) has been shown to be useful in the removal of large colorectal adenomas. These lesions are often resected by using a piecemeal technique, which a risk of recurrence in $10 \%$ to $30 \%$ of cases. Recently, hot avulsion technique (HA) has shown promising results in the resection of residual fragments of large colorectal adenomas, with lower recurrence rate.

Aims \& Methods: The aim of this study was to evaluate the efficacy and safety of $\mathrm{HA}$ at index EMR and at EMR local recurrence. We did a retrospective study based on all the HA performed between June 2015 and February 2017. The endoscopic characteristics, complications and recurrence rate after the initial HA were evaluated.

Results: 33 HA were performed among 29 patients (16 men and 13 women) with an average age of 69 years. The average follow up time was 11 months. HA was used to remove residual adenomatous tissue at 17 index EMR (mean size of the lesion $30 \mathrm{~mm}$ ) and to remove recurrent fibrotic adenomatous tissue at EMR scar in 12 cases (mean size of recurrence tissue $14 \mathrm{~mm}$ ). HA was successful in removing residual/recurrent adenomatous tissue in all patients. There were no immediate or long term adverse events. Comparing the two groups, local recurrence after initial HA occurred in one case at the index EMR group (1/17) and in 2 cases at the local EMR recurrence group $(2 / 12)$. The overall recurrence rate in patients with a minimum 6 months follow up was $15 \%(3 / 20)$

Conclusion: HA is a safe and effective technique to eradicate both residual tissue in large colorectal adenomas and recurrent fibrotic adenomatous tissue at EMR site, with low recurrence rate.

Disclosure of Interest: All authors have declared no conflicts of interest. 


\section{References}

Veerappan SG, Ormonde D, Yusoff IF, Raftopoulos SC. Hot avulsion: a modification of an existing technique for management of nonlifting areas of a polyp (with video). Gastrointest Endosc. 2014 Nov; 80(5):884-8.

Holmes I, Kim HG, Yang D-H, Friedland S. Avulsion is superior to argon plasma coagulation for treatment of visible residual neoplasia during EMR of colorectal polyps (with videos). Gastrointest Endosc. 2016 Nov;84(5):822-9.

P1522 QUALITY IN BOWEL CLEANSING, PERFORMANCE MEASURES AND PATIENTS SATISFACTION USING DIFFERENT PURGATIVES IN SCREENING COLONOSCOPY

E. Waldmann ${ }^{1}$, D. Penz ${ }^{1}$, B. Majcher ${ }^{2}$, A. Dokladanska ${ }^{1}$, A. Hinterberger ${ }^{2}$ H. Sinkovec ${ }^{3}$, G. Heinze ${ }^{3}$, M. Trauner ${ }^{3}$, M. Ferlitsch ${ }^{2}$

${ }^{1}$ Department Of Internal Medicine III, Division Of Gastroenterology \&

Hepatology, Medical University of Vienna, Vienna/Austria

${ }^{2}$ Quality Assurance Working Group, Austrian Society of Gastroenterology and Hepatology (OEGGH), Vienna/Austria

${ }^{3}$ Section For Clinical Biometrics, Center For Medical Statistics, Informatics And Intelligent Systems, Medical University of Vienna, Vienna/Austria

\section{Contact E-mail Address: elisabeth.waldmann@meduniwien.ac.at}

Introduction: Quality of bowel preparation and adenoma detection rate (ADR) are routinely assessed in screening colonoscopy. However, data on patient experience are scarce.

Aims \& Methods: This prospective non-interventional study compared bowel preparation quality according to the Harefield Scale, performance quality measures and patients satisfaction in screening colonoscopies performed within an Austrian quality assurance program.

Results: 5000 screening colonoscopies performed by 20 endoscopists were included in this study. $50.3 \%$ of screened individuals were women. Because of

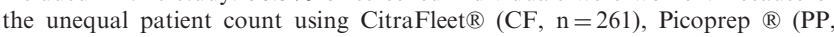
$n=2678)$, Klean-Prep ${ }^{\circledR}(K P, n=804)$ and Moviprep ${ }^{\circledR}(M P, n=1252), P C$ and $\mathrm{CF}$ were grouped as low volume (LV) purgatives. Age and gender adjusted success rates and ADR per purgative were $97.0 \%$ and $23.3 \%$ for $\mathrm{LV}, 97.5 \%$ and $32.5 \%$ for KP and $93.5 \%$ and $26.0 \%$ for MP. Women had higher success rates than men $(\mathrm{p}=0.073)$ and success rate decreased with patients' age $(\mathrm{p}=0.008)$. The compliance regarding consummation of the entire volume was best with LV $(89.2 \%$, KP $87.6 \%$, MP $87.3 \%)$, which had a significant effect on success rate $\mathrm{p}=0.027 .93 .5 \%$ of patients in the $\mathrm{LV}$ group would use the same purgative again compared to $68.4 \%$ in the KP and $73.2 \%$ in the MP group. Conclusion: All investigated purgatives met the required quality standards of $\geq 90 \%$ rate of adequate bowel preparation according to the current ESGE guidelines. Success rates were higher in women and younger patients. Although only $<90 \%$ of patients consumed the whole volume, the majority of patients would use the same purgative again.

Disclosure of Interest: All authors have declared no conflicts of interest.

\section{P1523 DIFFERENCES BETWEEN BOWEL PREPARATION}

\section{QUALITY OF SURVEILLANCE AND SCREENING COLONOSCOPY}

A. K. Hinterberger, D. Penz, E. Waldmann, B. Majcher, A. Dokladanska, A. Szymanska, M. Trauner, M. Ferlitsch

Department Of Internal Medicine III, Division Of Gastroenterology \& Hepatology, Medical University of Vienna, Austria, Vienna/Austria

Contact E-mail Address: anna.hinterberger@meduniwien.ac.at

Introduction: Adequate bowel preparation is necessary for a successful and complete colonoscopy, Minimum of adequate rate (excellent + good + fair) should be $90 \%$ according the actual ESGE guidelines (Kaminski et al, Endoscopy 2017). The aim of study was, to verify if there is a difference at quality of preparation between screening and surveillance colonoscopy. It might be that there is an improvement at surveillance colonoscopy due to better knowledge.

Aims \& Methods: 107,614 examinations from 288 endoscopists were analysed between 2012 and 2017 within the Austrian certificate of screening colonoscopy. For the calculations six different categories were used: excellent, good, fair, poor, unsatisfactory, poor only in the right colon. Data are shown as Mean and SD. Results: 8578 surveillance colonoscopies $(43.88 \%$ female, mean age 66.67$)$ and 99271 screening colonoscopies $(51.04 \%$ female, mean age was 62.31$)$ were included. Within screening colonoscopy $37.59 \%(\mathrm{SD}=31.50)$ were excellent prepared vs. $34.60 \%(\mathrm{SD}=33.98)$ within surveillance colonoscopy, good 47.43 $(\mathrm{SD}=27.28)$ vs. $45.61 \%(\mathrm{SD}=31.28)$, fair $11.39 \%(\mathrm{SD}=11.58)$ vs. $14.39 \%$ $(\mathrm{SD}=21.69)$, poor $2.38 \%(\mathrm{SD}=3.06)$ vs. $3.68 \%(\mathrm{SD}=9.75)$, unsatisfactory $0.77 \%(\mathrm{SD}=1.20)$ vs. $0.89 \%(\mathrm{SD}=3.69)$, poor only in the right colon $0.69 \%$ $(\mathrm{SD}=1.81)$ vs. $0.39 \%(\mathrm{SD}=1.22)$. Calculations revealed no significant differences among screening and surveillance colonoscopies relating the following categories: excellent $(p=0.4357)$, good $(p=0.5911)$, unsatisfactory $(p=0.6282)$ and poor only in the right colon $(\mathrm{p}=0.1457)$. Bowel preparation varies significantly between screening and surveillance in groups of fair $(p=0.0454)$ and poor $(\mathrm{p}=0.0497)$. Bowel preparation influences the adenoma detection rate (ADR). Mean ADR in excellent preparation was $22.16 \%(\mathrm{SD}=16.36 \%)$, in good $25.41 \%(\mathrm{SD}=17.30 \%)$, in fair $24.63 \% \quad(\mathrm{SD}=19.44)$, in poor $19.94 \%$ $(\mathrm{SD}=25.57)$, in unsatisfactory $12.08 \%(\mathrm{SD}=22.77)$ and in poor only in the right colon $24.22 \%(\mathrm{SD}=30.89 \%)$. Adequate rate in screening colonoscopy was $96.22 \% \quad(\mathrm{SD}=3.98 \%) \quad$ vs. $95.07 \% \quad(\mathrm{SD}=10.45 \%) \quad$ in surveillance colonoscopy. Rates correspond to ESGE guidelines and do not differ significantly $(\mathrm{p}=0.1265)$

Conclusion: Data showed distinctions between fair and poor, whereas there were more fair and more poor prepared patients at the surveillance colonoscopy, than at the screening colonoscopy. Age of patients at surveillance colonoscopy was higher, hence patients need improved enlightenment. ADR in excellent, good and fair bowel preparation was adequate. In poor, unsatisfactory was low and ADR at poor only in the right colon is high due to low count $(\mathrm{N}=374)$.

Disclosure of Interest: All authors have declared no conflicts of interest.

\section{P1524 DIFFERENCES IN QUALITY OF BOWEL PREPARATION AT SCREENING COLONOSCOPIES IN PRIVATE PRACTICES AND HOSPITALS}

B. Majcher, D. Penz, E. Waldmann, A. Hinterberger, A. Dokladanska, A. Szymanska, M. Trauner, M. Ferlitsch

Department Of Internal Medicine III, Division Of Gastroenterology \& Hepatology, Medical University of Vienna, Wien/Austria

Contact E-mail Address: barbara.majcher@meduniwien.ac.at

Introduction: Bowel preparation influences the adenoma detection rate and is therefore an important quality parameter in screening colonoscopy. According to actual ESGE guideline "Performance measures for lower gastrointestinal endoscopy: a European Society of Gastrointestinal Endoscopy (ESGE) Quality Improvement Initiative", $90 \%$ of colonoscopies should have adequate bowel preparation. The aim of this study was to investigate whether there is a difference in quality of bowel preparation between private practices and hospitals.

Aims \& Methods: Data from screening colonoscopies performed within quality certificate in Austria (2012 - 2017) provided by 245 endoscopists were evaluated. The recording of the quality of the bowel preparation was described as one of the following categories: excellent, good, fair, poor, poor only in the right colon and unsatisfactory.

Results: From the 125,127 screening colonoscopies included in this study, $72.93 \%$ were performed in private practices, $(50.66 \%$ female, mean age 60.7$)$ and $27.07 \%$ in hospitals $(49.35 \%$ female, mean age 60.35$)$. Significant difference was found between the average values of screening colonoscopies with excellent bowel preparation $(38.00 \%(\mathrm{SD}=30.36)$ in private practices vs. $27.92 \%(\mathrm{SD}=23.11)$ in hospitals, $\mathrm{p}=0.0215)$, fair $(10.68 \% \quad(\mathrm{SD}=10.89) \quad$ vs. $15.18 \% \quad(\mathrm{SD}=9.93)$, $\mathrm{p}=0.0001)$ and unsatisfactory $(0.66 \%(\mathrm{SD}=0.96 \%)$ vs. $1.43 \% \quad(\mathrm{SD}=1.76)$, $\mathrm{p}=0.0003$ ) while there was no statistical significance among the average values from participants with good bowel preparation quality $(47.40 \%(\mathrm{SD}=26.38)$ in private practices vs. $51.78 \%(\mathrm{SD}=19.28)$ in hospitals, $\mathrm{p}=0.6428)$, poor $(2.57 \%$ $(\mathrm{SD}=2.93)$ vs. $2.89(\mathrm{SD}=2.89), \mathrm{p}=0.2987)$ and poor only in the right colon $(0.69(\mathrm{SD}=1.74)$ vs. $0.80 \%(\mathrm{SD}=1.15, \mathrm{p}=0.7721)$. The mean ADR did not show any statistical difference between private practices and hospitals $(23.98 \%$ $(\mathrm{SD}=10.89 \%)$ vs. $25.80 \%(\mathrm{SD}=12.80 \%), \mathrm{p}=0.7652)$

Conclusion: More excellent bowel preparation was found in private practices while the screening colonoscopies in hospitals showed a higher rate of fair and unsatisfactory bowel preparation.

Disclosure of Interest: All authors have declared no conflicts of interest.

\section{P1525 RISK FACTORS FOR RESIDUAL NEOPLASIA AFTER ENDOSCOPIC MUCOSAL RESECTION OF LATERALLY SPREADING TUMORS}

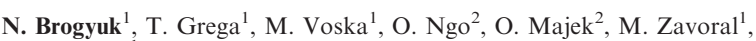
S. Suchanek

${ }^{1}$ Department Of Internal Medicine, 1st Faculty Of Medicine, Charles University, Military University Hospital, Prague/Czech Republic

${ }^{2}$ Institute Of Biostatistics And Analyses, Masaryk University, Faculty of Medicine, Brno/Czech Republic

Contact E-mail Address: Nadiya.Brodyuk@uvn.cz

Introduction: Laterally spreading tumors (LSTs) are important precursors of colorectal carcinoma. They are usually removed by endoscopic mucosal resection (EMR). However, local residual neoplasia (LRN) may occur during follow-up. The aim of the study was to evaluate the occurrence of LRN and the risk factors for its presence.

Aims \& Methods: This retrospective study in a high-volume tertiary-referral center examined patients who had undergone EMR between 2013 and 2015 and who had had at least 1 surveillance colonoscopy after the initial treatment. LRN was defined histologically as the presence of neoplastic tissue in the postEMR site.

Results: 160 laterally spreading tumors were diagnosed in 138 patients $(62 \%$ men, mean age 67 years). Mean follow-up interval for surveillance colonoscopy was 6 months. Residual neoplasia at surveillance endoscopy was present following $21 \%$ of colonic EMRs. Single variate analysis showed evidence of an increased risk of residual neoplasia for $\operatorname{LST} \geq 20 \mathrm{~mm}(\mathrm{p}=0.006)$, villous adenomas $(\mathrm{p}=0.001)$, piecemeal resection $(p=0.011)$ and $G$-type morphology $(p=0.003)$. In multivariate analysis, only size of the lesion $(\mathrm{p}=0.080)$ and villous component $(\mathrm{p}=0.043)$ were found to be a significant risk factor of LRN.

Conclusion: This retrospective study shows that the occurrence of LRN is frequent. Careful colonoscopic surveillance after EMR and the use of new methods to further reduce residual neoplasia are needed.

Disclosure of Interest: All authors have declared no conflicts of interest. 
P1526 ENDOSCOPIC SURVEILLANCE AFTER SURGERY ON

FAMILIAL ADENOMATOUS POLYPOSIS PATIENTS: IT IS EFFECTIVE BUT INTERVALS CAN BE WIDENED

J. Pinho ${ }^{1}$, J. Lage ${ }^{2}$, M. Dinis-Ribeiro ${ }^{2}$, C. Brandão ${ }^{2}$

${ }^{1}$ Gastroenterology, Tondela - Viseu Hospital Center, Viseu/Portugal

${ }^{2}$ Gastroenterology, Instituto Português de Oncologia do Porto, Porto/Portugal

\section{Contact E-mail Address: julianapinho18@gmail.com}

Introduction: Familial adenomatous polyposis (FAP) is an autosomal dominantly inherited syndrome, associated with a lifetime risk for colorectal cancer close to $100 \%$. Thus prophylactic colectomy is recommended for patients with FAP. Colectomy with ileorectal anastomosis (IRA) is the surgical option of choice in the majority of patients, given the complications and morbidity associated with ileal pouch-anal anastomosis. Therefore, annual endoscopic surveillance is recommended after surgery to prevent cancer in the rectal remnant (CRR).

Aims \& Methods: The aim of this study was to determine the impact of endoscopic surveillance on CRR prevention in FAP patients after surgery. We did a retrospective single center study on findings of follow up endoscopies and determination of the cumulative risk of adenomas and prevalence of high risk adenomas (HRA) (villous histology, high-grade dysplasia and $\geq 10 \mathrm{~mm}$ ) and CRR.

Results: 30 patients submitted to IRA were included ( $50 \%$ women), with a mean age of 43 years, 2 patients with attenuated phenotype. Nine patients had adenocarcinoma in the resected colon. Six patients started chemoprophylaxis after surgery (sulindac-4; celecoxib -1). The median time to adenoma appearance was 5 years (95\% CI 3.4-6.6) and to HRA/CRR 12 years (95\% CI 5.2-18.8), with a decreased median time to both adenomas and HRA/CRR in patients under chemoprophylaxis. The cumulative risk of adenomas was $20 \%$ at 1 year after surgery, $34.1 \%$ at 3 years and $57.4 \%$ at 5 years. During the follow up period, HRA/CRR developed in 17 patients $(56.7 \%)$ : HRA-12 patients $(40 \%)$; intramucosal carcinoma-2 patients $(6.7 \%)$; invasive adenocarcinoma-3 patients $(10 \%)$. None of the patients died with CRR. The cumulative risk of HRA/CRR was $21.8 \%$ at 5 years, $46.1 \%$ at 10 years and $66.3 \%$ at 15 years. All the patients with HRA/CRR had rectal involvement prior to surgery $(\mathrm{p}=0.008)$ and a higher number of adenomas resect in the rectal remnant $(\mathrm{p}=0.017)$.

Conclusion: The FAP endoscopic surveillance program allowed detection of HRA/CRR in a high percentage of patients. Based on these results, an intensive surveillance program should be suggested but endoscopic surveillance intervals widen in the first 5 years after surgery.

Disclosure of Interest: All authors have declared no conflicts of interest.

\section{References}

Maehata Y, Esaki M, Nakamura S, Hirahashi M, Ueki T, Iida M, et al. Risk of cancer in the rectal remnant after ileorectal anastomosis in patients with familial adenomatous polyposis: single center experience. Dig Endosc. 2015 May;27(4):471-8.

Syngal S, Brand RE, Church JM, Giardiello FM, Hampel HL, Burt RW. ACG clinical guideline: Genetic testing and management of hereditary gastrointestinal cancer syndromes. Am J Gastroenterol. 2015 Feb;110(2):223-62.

\section{P1527 BOWEL PREPARATION FOR FLEXIBLE SIGMOIDOSCOPY: COMPARISON OF POLYETHYLENE GLYCOL ELECTROLYTE SOLUTION (PEG-ES) AND PHOSPHATE ENEMA IN 4,949 PATIENTS AT TWO UK HOSPITALS}

A. Yusuf, S. Paranahewa, A. Jeevagan, P. Mayhead

Gastroenterology, East Sussex Healthcare NHS Trust, Eastbourne/United Kingdom

\section{Contact E-mail Address: abdyus12@nhs.net}

Introduction: Flexible sigmoidoscopy is increasingly used to examine the left side of the bowel for diagnostic and surveillance purposes, and is now part of the UK bowel cancer screening programme. It is crucial to achieve adequate bowel cleansing in order to optimise the diagnostic yield of the test, and also to minimise the number of repeat procedures. However, the optimum bowel preparation for this procedure has consistently been debated ${ }^{1,2,3}$.

Aims \& Methods: Both phosphate enema and (PEG-ES) are commonly used for bowel preparation in flexible sigmoidoscopy at both hospitals participating in this study. We therefore wanted to compare the outcomes for these two methods. We retrospectively reviewed all the patients who underwent flexible sigmoidoscopy from January 2014 to December 2016 using each hospital's electronic endoscopy reporting system. We analysed their demographics, type of bowel preparation used in each case, and the quality of their individually achieved bowel preparation, subjectively graded as "Excellent, Adequate or Inadequate" by the endoscopist performing the procedure. A chi-squared test was used to calculate p-values.

Results: In total 6196 patients underwent flexible sigmoidoscopy during the study period (males $2885(46.56 \%)$, mean age 62.80 years, range $16-101$ years). 1247 $(20.13 \%)$ patients were excluded from further analysis for the following reasons: No bowel preparation given $(n=451)$, no documentation of the quality of the bowel preparation $(n=657)$, and non-(PEG-ES) oral preparation used $(n=139)$. A total of 4949 patients were included in the study, of whom 2103 had (PEG-ES) $(42.49 \%)$ (males $986(46.89 \%)$, mean age 60.97 years, range $18-95$ years) and
$2846(57.50 \%$ ) (males 1269 (44.59\%), mean age 63.98 years, range $17-101$ years) had phosphate enema. The results are summarised in the table below.

\begin{tabular}{lllll}
\hline $\begin{array}{l}\text { Type of bowel } \\
\text { preparation }\end{array}$ & Excellent & Adequate & $\begin{array}{l}\text { Total Adequate } \\
\text { and Excellent }\end{array}$ & Inadequate \\
\hline $\begin{array}{l}\text { (PEG-ES) }(\mathrm{n}=2103) \\
\begin{array}{l}\text { Phosphate enema } \\
(\mathrm{n}=2846)\end{array}\end{array}$ & $1126(53.54 \%)$ & $770(36.61 \%)$ & $1896(90.16 \%)$ & $207(9.84 \%)$ \\
\begin{tabular}{l} 
Total $(\mathrm{n}=4949)$ \\
\hline
\end{tabular} & & $1297(45.57 \%)$ & $1921(67.50 \%)$ & $925(32.50 \%)$ \\
\hline
\end{tabular}

Conclusion: Our large retrospective study showed that oral preparation with (PEG-ES) gave significantly better results than phosphate enema, which gave acceptable results in only $67.5 \%$ of the patients. As a result of this study, PEG-ES is now the preferred option at our hospitals, if there is no contraindication for this.

Disclosure of Interest: All authors have declared no conflicts of interest.

\section{References}

1. Bini EJ, Unger JS, Rieber JM, et al. Gastrointest Endosc. 2000 Aug;52(2):218-22.

2. Drew PJ1, Hughes M, Hodson R, et al. Eur J Surg Oncol. 1997 Aug;23(4):315-6.

3. Sharma VK, Chockalingham S, Clark V, Kapur A, et al. Am J Gastroenterol. 1997 May;92(5):809-11.

\section{P1528 MANAGEMENT OF RESECTION OF LARGE COLONIC} LESIONS IN A REAL-LIFE SETTING: THE SCALP STUDY

A. Amato ${ }^{1}$, F. Radaelli ${ }^{1}$, V. Cennamo ${ }^{2}$, E. Di Giulio ${ }^{3}$, L. Fuccio ${ }^{4}$, G. Manes ${ }^{5}$ O. Tarantino ${ }^{6}$, G. Fiori ${ }^{7}$, M. De Bellis ${ }^{8}$, A. Buda ${ }^{9}$, F. Pigò ${ }^{10}$, P. Cesaro ${ }^{11}$, A. Anderloni ${ }^{12}$, P. Occhipinti ${ }^{13}$, G. Feliciangeli ${ }^{14}$, P. Dulbecco ${ }^{15}$, A. Musso $^{16}$, G. Gullotti ${ }^{17}$, M. Giardini ${ }^{18}$, B. Mangiavillano ${ }^{19}$, S. Paggi ${ }^{1}$, C. Hassan ${ }^{20}$, A. Repici ${ }^{12}$

${ }_{1}^{1}$ Gastroenterology Division, Valduce Hospital, Como, Italy, Como/Italy

${ }^{2}$ Maggiore Hospital, Bologna/Italy

${ }^{3}$ AO Sant'Andrea, Rome/Italy

${ }^{4}$ Policlinico Sant'Orsola Malpighi, Bologna/Italy

${ }^{5}$ ASST Rhodense, Garbagnate Milanese/Italy

${ }^{6}$ Gastroenterology And Digestive Endoscopy, San Giuseppe Hospital, Empoli/Italy

${ }^{7}$ European Institute of Oncology, Milan/Italy

${ }^{8}$ National Cancer Institute, G. Pascale Foundation, Naples/Italy

${ }^{9}$ Gastroenterology And Digestive Endoscopy Unit, Ospedale di Feltre, Feltre/Italy

${ }^{10}$ Aou Modena, Gastroenterology and Digestive Endoscopy Unit, Modena/Italy

${ }^{11}$ Fondazione Poliambulanza, Brescia/Italy

${ }^{12}$ Humanitas Research Hospital, Milan, Italy., Rozzano/Italy

${ }^{13}$ Gastroenterology Unit, "Maggiore della Caritá" Hospital, Novara/Italy

${ }^{14}$ Macerata Hospital, Macerata/Italy

${ }^{15}$ Genoa University, Genoa/Italy

${ }_{17}^{16}$ Città della Salute e della Scienza, Turin/Italy

${ }^{17}$ AOU Policlinico G. Martino, Messina/Italy

${ }^{18}$ Urbino Hospital, Urbino/Italy

${ }^{19}$ Gastrointestinal Endoscopy Unit, Humanitas - Mater Domini, Castellanza (VA)/ Italy

${ }^{20}$ Gastroenterology, Nuovo Regina Margherita Hospital, italy/Italy

Contact E-mail Address: arnamato@gmail.com

Introduction: Endoscopic resection of large colonic lesions (LCLs, >20 mm) is effective and it is associated with an acceptable incidence of incomplete resection and complications when performed by appropriately trained endoscopists in resourced centers ${ }^{1,2,3}$. Scanty data on the management of these lesions outside referral centers are reported in the literature.

Aims \& Methods: Aim of present study is to evaluate the management of endoscopic resection of LCLs and intra-procedural complications in a real-life setting. In a prospective, multicenter, observational study in 20 centers, data from consecutive endoscopic resections of LCLs performed over a 6-month period were collected by a web-database. All patients undergoing LCLs resection were enrolled at procedure-time and followed-up at 15 days for adverse events and at 6 months for endoscopic/histological recurrence.

Results: 1452 LCLs (mean size $30.6 \mathrm{~mm}$, SD 12.4; $41.4 \%$ lateral spreading tumor, $28.1 \%$ sessile, and $30.5 \%$ pedunculated) removed in 1329 patients $(58 \%$ males, mean age $66 \pm 11.4$ years) were analysed. An endoscopic mucosal resection (EMR) was performed in $57.9 \%$, snare polypectomy in $34.7 \%$, underwater EMR in $1.2 \%$ and endoscopic submucosal dissection in $6.2 \%$ of the lesions. Of patients with LCLs, $19.4 \%$ were on ATT (62.5\% aspirin, $12.2 \%$ thienopyridines, $4.8 \%$ dual antiplatelet, $15.4 \%$ vitamin $\mathrm{K}$ antagonists [VKAs], $5.1 \%$ direct oral anticoagulants [DOACs]). Aspirin and/or thienopyridines were withheld before resection in $53.6 \%$ and $91.7 \%$ of patients, respectively. Overall, intraprocedural bleeding requiring endoscopic therapy occurred in $8.1 \%$ of patients; $28 \%$ of them were on ATT, which had always been withheld, but in $48 \%$ of patients on aspirin. At multivariate analysis, intra-procedural bleeding was correlated with increasing polyp size (Odds Ratio 1.02 95\% Confidence Interval $1.01-1.04)$, and inversely with execution of pre- and post-resection prophylaxis maneuvers (Odds Ratio 0.55 95\% Confidence Interval 0.37-0.82 and Odds Ratio $0.5595 \%$ Confidence Interval $0.37-0.82$ respectively). As concerns 
complications, delayed bleeding occurred in $4.5 \%$ of the subjects, whereas perforation occurred in $1.5 \%(0.9 \%$ early and $0.6 \%$ delayed $)$ of patients, $86.7 \%$ of whom were successfully managed endoscopically. At the moment, 6-months follow-up is available for $35 \%$ of the patients, with a positive endoscopic and/ or histological recurrence documented in $22.8 \%$.

Conclusion: The management of resection of LCLs varies widely. The incidence of intra-procedural bleeding correlates with polyp size and prophylactic maneuvers, and its endoscopic management is successful in most of cases. Overall, complication rate is marginal and efficacy is good, even in a real-life setting. Disclosure of Interest: All authors have declared no conflicts of interest.

\section{Reference}

1. $2014 ; 12: 651-61$

\section{P1529 ENDOSCOPIC REMOVAL OF HIGH-RISK COLORECTAL ADENOMAS: SAFE AND EFFECTIVE?}

M. Rutka ${ }^{1}$, K. Farkas ${ }^{1}$, A. Fabian ${ }^{1}$, R. Bor ${ }^{1}$, A. Balint ${ }^{1}$, Á. Milassin ${ }^{1}$, Z. Szepes ${ }^{1}$, T. Molnar ${ }^{1}$

${ }^{1}$ First Department Of Internal Medicine, University Of Szeged, Szeged/Hungary

Contact E-mail Address: rutka.mariann@gmail.com

Introduction: The incidence and mortality of colorectal cancer (CRC) can be decreased trough the removal of precancerous adenomas. Endoscopic removal of polyps over $2 \mathrm{~cm}$ is considered a high-risk procedure both for complications and malignant transformation.

Aims \& Methods: The aim of this study was to evaluate the outcome and complication rate after endoscopic removal of polyps over $2 \mathrm{~cm}$. In this retrospective study clinical and demographic data of patients undergoing polypectomy due to colorectal adenoma over $2 \mathrm{~cm}$ between 2012 and 2017 were collected. Data on endoscopic procedures, complications of polypectomy and histological assessments of the removed polyp were obtained.

Results: Data of 100 patients (male/female: 58/42) was analyzed in the study. Forty-seven of the 106 removed polyps proved to be pedunculated, 21 were sessile and 34 flat. Six patients had more than one large polyp $(>2 \mathrm{~cm})$. The locations of the removed polyps were rectum in 33 , sigmoid colon in 38 , coecum in 12 and other parts of the colon in 23 patients. In 65 cases, polyps were excised with endoscopic mucosa resection (EMR) or hybrid endoscopic submucosa dissection (ESD), In 41 cases snare was used to remove the polyps in one or more pieces. Based on histological findings $54(50.9 \%)$ polyps were shown to be lowgrade adenomas, $34(32.07 \%)$ high-grade adenomas, $1(0.9 \%)$ polyp was hyperplastic, and $17(16.03 \%)$ proved to be malignant among which complete endoscopic removal was achieved in 9 patients $(52.9 \%)$. Additional smaller polyps were found in 39 patients and a synchronous cancer in 7. During polypectomies 91 hemoclips were deployed to close suspected perforation ( 8 cases) to cease bleeding (19) or for prevention. Postpolypectomy syndrome developed in 8 cases. Second-look colonoscopy was required in 8 cases due to bleeding within a mean of 4 days after the first examination. Hemoclip insertion was needed in 5 cases and epinephrine injection in 1 case. The bleeding stopped spontaneously in 2 cases. Blood transfusion was required in 3 patients. Surgical intervention was not needed in any case.

Conclusion: Malignant transformation was revealed in $16 \%$ of the polyps over the size of $2 \mathrm{~cm}$. Complete endoscopic removal of these polyps was successfully performed in half of the patients. Endoscopic removal of high-risk polyps is safe in experienced hand.

Disclosure of Interest: All authors have declared no conflicts of interest.

\section{P1530 WHAT IMPROVED AND WHAT REMAINS TO BE ACHIEVED IN ORDER TO COMPLY WITH THE NEW RECOMMENDATIONS OF POLYPECTOMY BY THE EUROPEAN SOCIETY OF GASTROINTESTINAL ENDOSCOPY}

J. P. Rodrigues ${ }^{1}$, R. Pinho ${ }^{2}$, L. Alberto ${ }^{2}$, A. Rodrigues ${ }^{2}$, L. Proença ${ }^{2}$, T. Freitas ${ }^{2}$, A. P. Silva ${ }^{2}$, S. Fernandes ${ }^{2}$, S. Leite ${ }^{2}$, J. Silva ${ }^{2}$, A. Ponte ${ }^{2}$, M. Sousa ${ }^{2}$, J. C. Silva ${ }^{2}$, J. Carvalho ${ }^{2}$

${ }^{1}$ Gastroenterology, CHVNG/E, Vila Nova De Gaia/Portugal

${ }^{2}$ Gastroenterology, Centro Hospitalar Vila Nova de Gaia/Espinho, Vila Nova De Gaia/Portugal

\section{Contact E-mail Address: jaimepereirarodrigues@gmail.com}

Introduction: The choice of polypectomy technique differs according to regional preferences and availability. This year, in order to standardize the approach to this techniques, the European Society of Gastrointestinal Endoscopy (ESGE) published recommendations for colorectal polypectomy and endoscopic mucosal resection (EMR). ${ }^{1}$

Aims \& Methods: We aimed to evaluate the recent years evolution of the adherence to the recommendations of colorretal polypectomy and EMR at a tertiary center. We conducted a unicentric analysis of polypectomy and mucosectomy techniques performed consecutively between January and June of 2011 and 2016 at a tertiary center. According to the recommendations, the excision of sessile and flat polyps is considered adequate when performed with cold biopsy forceps or cold snare for polyps $\leq 3 \mathrm{~mm}$, cold snare if $4-9 \mathrm{~mm}$, cold or hot snare if 10 $19 \mathrm{~mm}$ and EMR if $\geq 20 \mathrm{~mm}$. Polypectomy of pedunculated polyps is considered adequate when performed with a diathermic loop in polyps $<20 \mathrm{~mm}$, always in association with any prophylactic therapy when polyp size $\geq 20 \mathrm{~mm}$.
Results: We included 1721 endoscopic procedures of polypectomy and EMR, concerning 696 patients $(64.5 \%$ male; mean age $=64.2 \pm 11.0$ years $) .1381$ $(80.2 \%)$ sessile polyps, $153(8.9 \%)$ flat lesions and $187(20.9 \%)$ pedunculated polyps were identified, with a mean size of $7.9 \pm 7.0 \mathrm{~mm}$. Regarding sessile and flat polyps, one of the recommended excision techniques was performed in: $84.6 \%(\mathrm{n}=270)$ of $\leq 3 \mathrm{~mm}$ polyps $(75.7 \%$ in 2011 vs. $95.8 \%$ in 2016 ; $\mathrm{p}<0.001) ; 22.2 \%(\mathrm{n}=109)$ of $4-5 \mathrm{~mm}$ polyps $(12.5 \%$ vs. $36.5 \% ; \mathrm{p}<0.001)$; $13.4 \%(\mathrm{n}=59)$ of $6-9 \mathrm{~mm}$ polyps $(5.4 \%$ vs. $23.8 \% ; \mathrm{p}<0.001) ; 100 \%(\mathrm{n}=206)$ of $10-19 \mathrm{~mm}$ polyps; and $100 \%(\mathrm{n}=88)$ of $\geq 20 \mathrm{~mm}$ lesions. For pedunculated polyps, the resection technique was adequate in: $99.3 \%(n=134)$ of polyps of size $<20 \mathrm{~mm}(100 \%$ vs. $97.6 \% ; \mathrm{p}>0.05)$ and in $84.6 \%(\mathrm{n}=44)$ of those $\geq 20 \mathrm{~mm}$ $(82.6 \%$ vs. $86.2 \% ; \mathrm{p}>0.05)$. Overall, $52.3 \%(\mathrm{n}=900)$ of endoscopic procedures of polypectomy or EMR were performed as recommended; $42.7 \%(\mathrm{n}=410)$ in 2011 vs. $64.5 \%(\mathrm{n}=490)$ in 2016; $\mathrm{p}<0.001$.

Conclusion: Even before publication of the european recommendations, there has already been an increase in the proportion of polypectomies performed adequately in the different groups of lesions. There is still a need to adjust clinical practice in some subgroups, especially in polyps of size $4-9 \mathrm{~mm}$, in order to strictly comply with the recommendations.

Disclosure of Interest: All authors have declared no conflicts of interest.

\section{Reference}

1. Ferlitsch M, Moss A, Hassan C, et al. Colorectal polypectomy and endoscopic mucosal resection (EMR): European Society of Gastrointestinal Endoscopy (ESGE) Clinical Guideline. Endoscopy. 2017 Mar;49(3):270-297.

\section{P1531 ENDOSCOPIC SUBMUCOSAL DISSECTION (ESD) OF} SUPERFICIAL COLORECTAL NEOPLASMS AT THE ANAL CANAL AND ILEOCECAL VALVE

F. Iacopini ${ }^{1}$, Y. Saito ${ }^{2}$, T. Gotoda ${ }^{3}$, C. Grossi ${ }^{1}$, W. Elisei ${ }^{1}$, F. Montagnese ${ }^{1}$, P. Rigato ${ }^{4}$, A. Cefaro ${ }^{5}$, G. Costamagna ${ }^{6}$

${ }^{1}$ Ospedale S. Giuseppe, Albano L., Rome, Italy, UOC Gastroenterologia ed Endoscopia, Albano Laziale/Italy

${ }^{2}$ Endoscopy Division, National Cancer Center Hospital Endoscopy Division, Tokyo/Japan

${ }^{3}$ Division Of Gastroenterology And Hepatology, Department Of Medicine, Nihon University School of Medicine, Tokyo/Japan

${ }^{4}$ Ospedale S Giuseppe, UOC Pathology and Citology, Marino/Italy

${ }^{5}$ Ospedale $S$ Giuseppe, UOC General Surgery, Albano Laziale/Italy

${ }^{6}$ Digestive Endoscopy Unit, Università Cattolica del Sacro Cuore, Roma/Italy

Contact E-mail Address: federico.iacopini@gmail.com

Introduction: Endoscopic resection of superficial neoplasms at the perineal rectum is difficult due to pain sensibility, narrowness of the anal canal, presence of internal rectal plexus, whereas that of at the ileocecal valve (ICV) due to the variable morphology of the ICV itself and ileal involvement.

Aims \& Methods: Aim was to assess the feasibility and outcomes of ESD in these locations. Prospectively collected database in a single nonacademic center. From 1.2010 to 11.2016 , all consecutive patients scheduled to ESD for a superficial neoplasm in the perineal rectum (distal margin $<30 \mathrm{~mm}$ from the dentate line) and at the ICV were compared to those in the pelvic rectum and in the cecum and ascending colon, respectively. ESD was performed with the standard technique. Follow-up was scheduled at 3 and 6 months within the first year and then yearly. Biopsies were taken from the scar of the resection site if a residual tissue was detected.

Results: A total 16 neoplasms at ICV were compared to 110 neoplasms in the cecum and ascending colon; 30 neoplasms in the perineal rectum were compared to 58 cases in the pelvic rectum (Table). Features of neoplasms in the perineal and pelvic rectum were no different as well as neoplasms at the ICV and cecum and ascending colon. ESD en bloc rates were lower in the perineal rectum and the $\mathrm{ICV}$, but no significant differences were found with the respective control groups $(\mathrm{P}=0.490$ and 0.404 , respectively). ESD R0 rate was significantly lower at the ICV $(\mathrm{P}=0.021)$. Adverse events were not different, although 3 perforations occurred in the cecum and ascending colon. During follow-up (median 36 months; range 24-84): residual tissue was diagnosed at the ICV in $2(13 \%)$ cases, in the cecum and ascending colon in $2(2 \%)$ cases $(P=0.078)$; in the perineal rectum in $4(13 \%)$ cases and pelvic rectum in $2(3 \%)$.

\begin{tabular}{lllll}
\hline & $\begin{array}{l}\text { IC valve } \\
\text { (n.16) }\end{array}$ & $\begin{array}{l}\text { cecum \& } \\
\text { Ascending } \\
\text { (n.110) }\end{array}$ & $\begin{array}{l}\text { perineal } \\
\text { rectum } \\
\text { (n.30) }\end{array}$ & $\begin{array}{l}\text { Pelvic } \\
\text { Rectum } \\
\text { (n.58) }\end{array}$ \\
\hline size, mm median (range & $44(20-85)$ & $35(15-80)$ & $48(23-180)$ & $35(12-70)$ \\
morphology: LST-G/NG & $12 / 4$ & $81 / 22$ & $23 / 3$ & $39 / 7$ \\
scar positive & 0 & 19 & 8 & 10 \\
ESD en bloc & $12(75)$ & $91(83)$ & $22(73)$ & $48(83)$ \\
ESD R0 & 7 & 81 & 15 & 41 \\
ESD R1 (HM/VM) & $5 / 0$ & $10 / 0$ & $6 / 1$ & $4 / 1$ \\
\hline
\end{tabular}

Conclusion: The ESD of neoplasms at the ICV and perineal rectum is feasible and effective. The complete resection rate is low due to the challenging anatomy of 
that precludes conducting a mucosal incision far from tumor margins. A careful endoscopic follow-up is mandatory to detect residual neoplasms.

Disclosure of Interest: All authors have declared no conflicts of interest.

\section{P1532 SELF-EXPANDABLE METALLIC STENT IN THE OCCLUSIVE COLORECTAL CANCER AS PALLIATIVE TREATMENT}

T. Gago, A. Antunes, A.M. Vaz, P. Queirós, J. Roseira, A. C. Cunha, A. Ramos, H. Guerreiro

Serviço De Gastrenterologia De Faro, Centro Hospitalar do Algarve, Faro/ Portugal

Contact E-mail Address: taniagago@gmail.com

Introduction: Colorectal cancer (CRC) is one of the most common malignancies in developed countries, with associated occlusive disease being relatively common. Endoscopic placement of self-expandable metallic stent (SEMS) is the first-line palliative treatment for malignant bowel obstruction.

Aims \& Methods: Evaluate the outcome of endoscopic SEMS placement in CRC obstruction. Retrospective analysis of patients CRC submitted to endoscopic placement of SEMS from 2009 to 2016 in the Gastroenterology Department of Centro Hospitalar do Algarve. Statistical analysis was performed with SPSS version 24.

Results: The study included 23 patients with CRC obstruction, who were submitted to endoscopic SEMS placement, with a mean age of $75.2 \pm 13.47$ years. The stents were placed with a palliative purpose in $69.6 \%$ of cases $(n=16)$ and a transitory procedure before surgery in $30.4 \%$ of cases $(n=7)$. Technical and clinical success was found in $91.3 \%$ of the patients, without any recorded death during the procedure. In patients whose goal was palliative treatment $(75 \%$ men and $25 \%$ women) they had a mean age of $81.6 \pm 9.28$ years. In $43.8 \%$ of the patients the tumor was located in the rectum, $31.6 \%$ in the sigmoid region and $25 \%$ in the recto-sigmoid transition. Being the majority $(75 \%)$ well differentiated. There was a need for dilatation in $31.3 \%$, most of the stents were uncovered $(56.3 \%) .25 \%$ of the patients had complications. After stent placement, about $25 \%$ of the patients did chemotherapy. There was a $75 \%$ mortality rate $(37.5 \%$ died by 6 months and $37.5 \%$ died by 12 months of follow-up). The use of chemotherapy after SEMS placement influenced the complications associated with the procedure $(\mathrm{p}<0.05)$ but none of the other variables had a statistically significant influence on early death (up to 6 months).

Conclusion: SMES is an effective and safe palliative option for unresectable tumors, although the use of chemotherapy after the placement of prostheses may have an influence on the appearance of complications. Malignant colon occlusive of the colon can be treated effectively with the use of endoscopic techniques.

Disclosure of Interest: All authors have declared no conflicts of interest.

\section{References}

1. Van Hooft JE, Van Halsema EE, Vanbiervliet G, Beets-Tan RGH, DeWitt JM, Donnellan F, et al. Self-expandable metal stents for obstructing colonic and extracolonic cancer: European Society of Gastrointestinal Endoscopy (ESGE) Clinical Guideline. Gastrointest Endosc. 2014; 80:747-61.

2. X.-D. Zhao, B.-B. Cai, R.-S. Cao, R.-H. Shi. Palliative treatment for incurable malignant colorectal obstructions: a meta-analysis. World $J$ Gastroenterol, 19 (2013), pp. 5565-5574

3. M. Karoui, A. Charachon, C. Delbaldo, J. Loriau, A. Laurent, I Sobhani. Stents for palliation of obstructive metastatic colon cancer: impact on management and chemotherapy administration. Arch Surg, 142 (2007), pp. 619 623

\section{P1533 RISK FACTORS FOR ADENOMA RECURRENCE AFTER ENDOSCOPIC MUCOSAL RESECTION OF LARGE COLORECTAL POLYPS}

J. Pinho, D. Martins, P. Sousa, R. Araújo, A. Castanheira, E. Cancela, R. Cardoso, P. Ministro, A. Silva

Gastroenterology, Centro Hospitalar Tondela/Viseu, Viseu/Portugal

Contact E-mail Address: julianapinho18@gmail.com

Introduction: Endoscopic mucosal resection (EMR) has been shown to be a safe and effective technique for removal of large colorectal adenomas. However, loca adenoma recurrence remains a significant limitation, with prior published data describing recurrence rates of $10 \%$ to $30 \%$ post EMR.

Aims \& Methods: This study aimed to evaluate the outcomes of EMR for large colorectal adenomas and identify the risk factors for adenoma recurrence. We did a retrospective analysis of the colorectal EMR performed between June 2009 and December 2016. Resected lesions larger than $20 \mathrm{~mm}$ in diameter with at least 3 months follow up were included. Patients referred for surgery were excluded.

Results: During the study period, 201 colorectal lesions $\geq 20 \mathrm{~mm}$ in size were removed in 198 patients (118 men and 80 women, mean age 68 years). Mean lesion size was $35 \mathrm{~mm}$ and $137(68.2 \%)$ were located in the rectum and left colon 66 lesions $(32.8 \%)$ were larger than $40 \mathrm{~mm}$ in diameter. Piecemeal resection was performed in 171 lesions $(85.1 \%$ ). Local adenoma recurrence occurred in 44 cases $(21.9 \%)$ after a mean time of follow up of 7.6 months, and the majority was managed with polypectomy or new EMR. The cumulative risk of adenoma recurrence was $7.5 \%$ at 3 months, $15.5 \%$ at 6 months and $17.1 \%$ at 12 months. In the multivariate analysis, the variables associated with a high risk of recurrence were lesions $\geq 40 \mathrm{~mm}$ in size $(\mathrm{p}=0.0001)$ and intra-procedural bleeding $(p=0.020)$. The recurrence rate was higher in the patients treated with argon plasma coagulation $(\mathrm{p}=0.046)$

Conclusion: After EMR of large colorectal adenomas, local recurrence rate was $21.9 \%$. The risk factors for adenoma recurrence include lesions $>40 \mathrm{~mm}$ and intra-procedural bleeding. Argon plasma coagulation was not associated with lower recurrence rate.

Disclosure of Interest: All authors have declared no conflicts of interest.

\section{References}

Margagnoni G, Angeletti S, D’Ambra G, Pagnini C, Ruggeri M, Corleto VD, et al. Outcome and risk of recurrence for endoscopic resection of colonic superficial neoplastic lesions over $2 \mathrm{~cm}$ in diameter. Dig Liver Dis. 2016 Apr;48(4):399403

Moss A, Williams SJ, Hourigan LF, Brown G, Tam W, Singh R, et al. Longterm adenoma recurrence following wide-field endoscopic mucosal resection (WF-EMR) for advanced colonic mucosal neoplasia is infrequent: results and risk factors in 1000 cases from the Australian Colonic EMR (ACE) study. Gut. 2015 Jan;64(1):57-65.

\section{P1534 THE INFLUENCE OF THE REAL FOLLOW-UP TIMES DURING A COLORECTAL CANCER SCREENING PROGRAM IN} DAILY PRACTICE

E. Perez-Cuadrado-Robles ${ }^{1}$, B. Martínez-Andrés ${ }^{2}$, E.A. Torrella Cortes ${ }^{3}$, E. Pérez-Cuadrado-Martínez ${ }^{2}$,F. Pérez-Riquelme ${ }^{4}$

${ }^{1}$ Gastroenterology Department, Cliniques Universitaires Saint-Luc, Brussels Belgium

${ }^{2}$ Gastroenterology, Morales Meseguer Hospital, Murcia/Spain

${ }^{3}$ Hospital Morales Meseguer, Murcia/Spain

${ }^{4}$ Consejeria de Sanidad, Murcia/Spain

\section{Contact E-mail Address: kikemurcia@gmail.com}

Introduction: European colorectal screening guidelines have modified the followup interval times based on baseline colonoscopy findings in recent years. In addition, the waiting list and individual conditions may modify the real followup times and this could impact in advanced adenoma detection rate in follow-up and patients outcome.

Aims \& Methods: The aim of the present study was to comparatively analyse the risk of advanced lesions (advanced adenoma, invasive cancer) in high-risk patients included in a colorectal cancer screening program with different real follow-up times. One-thousand one-hundred and sixty-six patients (mean age: $60.66 \pm 5.86$ years, $69.1 \%$ men) who underwent a baseline colonoscopy with $>$ 3adenomas and/or $\geq 10 \mathrm{~mm}$ between 2007-2012 were included. A KaplanMeier regression and a comparative subgroup analysis by Long-Rank test were carried out to detect the impact of real times within patients of the same risk. An adjustment for baseline covariates (high-grade dysplasia, villous component) by Cox analysis was also performed.

Results: The real follow-up times in $\geq 3$ adenomas $(n=853,73.16 \%)$ and $\geq 1$ adenoma $\geq 10 \mathrm{~mm}(\mathrm{n}=779,66.81 \%)$ were $38.54 \pm 11.57$ and $38.66 \pm 11.68$ months. The risk of advanced lesions were $0.26 \%, 1.46 \%, 2.83 \%, 9.09 \%$ and $10.38 \%$ $(\mathrm{n}=121$ advanced lesions) in $12,24,36,48$ and $>60$ months respectively. The most important increase was at $3-4$ years $(+0.52 \% /$ month $)$. The proportion of advanced lesions within $1-2$ adenomas and $\geq 3$ adenomas subgroups at 48 months were $5.43 \%$ and $10.43 \%(p<0.001)$, with no differences in small adenomas $<>10 \mathrm{~mm}(p=0.478)$.

Conclusion: The risk of advanced lesions in high-risk patients increased significantly at 36-60 months after baseline colonoscopy, being more important in $>3$ adenomas subgroup. There were no differences for 1-3 years interval.

Disclosure of Interest: All authors have declared no conflicts of interest.

\section{P1535 CLINICAL OUTCOME OF ENDOSCOPIC SUBMUCOSAL DISSECTION OF MALIGNANT NON-PEDUNCULATED COLORECTAL LESIONS}

C. Rönnow ${ }^{1}$, E. Toth ${ }^{2}$, H. Thorlacius ${ }^{3}$

${ }^{1}$ Dept. Og Gastrointestinal Surgery, Lund University Surgery, Malmö/Sweden

${ }^{2}$ Skåne University Hospital, Malmo/Sweden

${ }^{3}$ Dept. Of Gastrointestinal Surgery, Lund University Surgery, Malmö/Sweden

Contact E-mail Address: cf frimand@hotmail.com

Introduction: Conventional endoscopic resection, such as snare polypectomy and endoscopic mucosa resection (EMR) of benign polyps in colon and rectum reduces colorectal cancer $(\mathrm{CRC})$ incidence and mortality but the role of endoscopic resection in the management of patients with early CRC (i.e. submucosal invasion) remain elusive. Large sessile and flat lesions are difficult to remove en bloc with EMR, resulting in a high level of tumor recurrence. Thus, endoscopic resection of large $(>2 \mathrm{~cm})$ sessile and flat malignant lesion with snare or EMR is not recommended. Endoscopic submucosal dissection (ESD) results in high en bloc resection rates of large $(\mathrm{z} 2 \mathrm{~cm})$ benign lesions resulting in low numbers of recurrences. However, there is limited data in the literature on the potential role of ESD in the treatment of patients with early CRC. In the present study, we present our results on performing ESD in patients with large sessile and flat malignant lesion in the colon and rectum in a large European center.

Aims \& Methods: Our aim was to investigate the potential role of ESD in treatment of early colorectal cancer. We retrospectively reviewed medical records of 
254 patients that underwent colorectal ESD at the endoscopy unit at Skane University Hospital in Malmö, Sweden Jan 2014 to Dec 2016. Indications for ESD were flat and sessile lesions larger than $20 \mathrm{~mm}$ in diameter with low or high graded dysplasia (251 cases). Moreover, three patients with known colorectal adenocarcinoma underwent ESD due to significant comorbidity excluding surgery. In total, we identified and included 29 cases of histologically verified submucosal invasive CRC in this study.

Results: This study included 29 patients with median age of 69 years (range 44-89 years). Median tumour size was $40 \mathrm{~mm}$, ranging from $20-70 \mathrm{~mm}$. Tumours were either flat (Paris classification IIa, 6 cases), sessile (Paris classification 1s, 19 cases) or a combination of flat and sessile (4 cases). Half of the lesions were located in the rectum and half in the colon. En bloc resection was achieved in 24 cases $(83 \%)$, piecemeal resection in 4 cases $(14 \%)$ and ESD was incomplete in one case. Median time to complete the procedure was $89 \mathrm{~min}$ (Range 18 to $594 \mathrm{~min})$. Macroscopic complete resection was obtained in 26 cases $(90 \%)$. R0 resection was found in 20 specimen $(69 \%)$, RX was found in 3 cases $(10 \%)$ and $\mathrm{R} 1$ was found in 5 cases $(17 \%)$. Lymphovascular involvement was seen in 6 cases $(21 \%)$. Depth of invasion was as follows; Sm1: 15, Sm2: 7, Sm 3: 7. In total four suspected immediate perforations occurred, three of these were managed conservatively (clips, fasting and antibiotics) and in one case, ESD was aborted and the patient was taken to emergency surgery. Pathological assessment of the resected sigmoid segment revealed T3N0. No acute significant bleeding occurred during the procedures. One patient sought emergency care 12 days after the procedure with rectal bleeding, no colonoscopy was performed to determine the site of bleeding. Five patients underwent additional surgery since the pathological report stated that the resection was $\mathrm{R} 1$. Tumour residue was only found in one of the five resected specimens. 18 patients have undergone endoscopic follow up, to this date without any sign of recurrence. Two patients await surgery and three patients await endoscopic follow up.

Conclusion: We herein present our findings on performing ESD on 29 patients with early CRC. Our results indicate that colorectal ESD is a safe and effective treatment in meticulously chosen patients even with malignant lesions. Further studies with longer follow up is needed.

Disclosure of Interest: All authors have declared no conflicts of interest.

\section{P1536 ENDOSCOPIC SUBMUCOSAL DISSECTION (ESD) VS HYBRID DISSECTION: WHICH TECHNIQUE TO FAVOR IN LARGE COLORECTAL LESIONS?}

M. Guillaumot, S. Leblanc, H. Soliman, B. Brieau, M. Barret, R. Coriat, F. Prat, S. Chaussade

Gastroenterology, Cochin Hospital, Paris/France

Contact E-mail Address: marie-anne.guillaumot@aphp.fr

Introduction: Large colorectal lesions $(>20 \mathrm{~mm}$ ) can be removed endoscopically by endoscopic mucosal resection (EMR), often in a piecemeal fashion resulting in low en bloc and radical (R0)-resection rates. In this indication, submucosal dissection (ESD) allows en bloc resection whatever the size, but still remains technically difficult and time consuming. A hybrid endoscopic technique has been developed, called simplified or hybrid dissection. The aim of our study was to evaluate the results and complications of endoscopic dissection by hybrid technique compared to classical endoscopic submucosal dissection.

Aims \& Methods: Our study was carried out from January 2013 to June 2016 from a prospective database. The 40 lesions removed by hybrid technique were compared to the control group of 109 ESD. The hybrid dissection procedure was performed as follow: submucosal injection around the lesion of macromolecules, circumferential mucosal incision and submucosal dissection using the tip of a single-strand snare by endo-cut Q mode, central submucosal injection of the lesion and final resection with the single-strand snare, if possible in en-bloc. Patient characteristics, tumor location and size, dissection characteristics, "block" resection rate, R0 resection rate (healthy margins), procedure and hospitalization time, and complications were identified and compared with the socalled "classical" ESD technique.

Results: Lesions were more frequently located in the colon (vs rectum) in the hybrid dissection group compared to the ESD group $(72.5 \%$ versus $28.5 \%$, $\mathrm{p}<0.001)$. The lesions were type IIc according to Paris classification in $10 \%$ of the hybrid dissection group and $13.1 \%$ in the ESD group $(\mathrm{p}=0.8)$. The mean size of the lesions was lower in the hybrid dissection group than in the ESD group $(32.4 \mathrm{~mm} \pm 13 \mathrm{~mm}$ compared to $54.4 \mathrm{~mm} \pm 26.7 \mathrm{~mm}, \mathrm{p}<0.001)$. An en bloc resection was performed in $52.5 \%$ and $84.4 \%$ in the hybrid dissection and ESD group, respectively $(\mathrm{p}<0.001)$. The procedure time (including general anesthesia time) was lower in the hybrid dissection group compared to the ESD group ( $103 \mathrm{~min} \pm 62 \mathrm{~min}$ vs $191 \mathrm{~min} \pm 73 \mathrm{~min}, \mathrm{p}<0.001$, respectively). The hospitalization time was lower in the hybrid dissection group than in the ESD group ( 1.1 days \pm 1.13 days vs. 2.8 days \pm 1.8 days, $\mathrm{p}<0.001)$. R0 resection rates were lower in the hybrid dissection group than in the ESD group $(47.5 \%$ and $61 \%$ respectively, $\mathrm{p}<0.001$ ). Hybrid dissection was performed for adenocarcinoma, adenoma with high grade dysplasia and adenoma with low grade dysplasia in $12.5 \%, 42.5 \%$ and $40 \%$, respectively. The rate of adenocarcinoma was lower compared to the ESD group $(12.5 \%$ versus $30.8 \%, p=0.009)$. In the hybrid dissection group, the rate of perforation was lower than the ESD group $(5 \%$ versus $20 \%, \mathrm{p}=0.04)$. There was no significant difference in the rate of bleeding $(1.8 \%$ In the hybrid dissection group and $2.5 \%$ in the ESD group). In case of complication, there was no need of surgical treatment in the hybrid dissection group, but was needed in one patient in the ESD group.
Conclusion: Hybrid dissection is less effective in terms of en bloc resection of large colorectal tumors. Classical endoscopic submucosal resection should be preferred, especially in case of suspected adenocarcinoma despite longer procedure and hospitalisation time.

Disclosure of Interest: All authors have declared no conflicts of interest.

\section{P1537 TRENDS IN STATISTICS REGARDING ELECTIVE ERCP PROCEDURES IN THE VENETO REGION: A RETROSPECTIVE STUDY BASED ON ADMINISTRATIVE DATABASES}

M. Saia ${ }^{1}$, E. Rosa-Rizzotto ${ }^{1}$, E. Guido ${ }^{1}$, D. Caroli $^{1}$, A. Frasson ${ }^{2}$, B. Germaná ${ }^{3}$, P. Pilati ${ }^{2}$, F. De Lazzari ${ }^{1}$

Dipto. Di Gastroenterologia, St. Anthony Hospital Gastroenterology Unit Dept. of Medicine, Padova/Italy

${ }^{2}$ Surgery Unit, Padova/Italy

${ }^{3}$ San Martino Hospital - Ulss1, Gastroenterologic Unit, Belluno/Italy

Contact E-mail Address: d.caroli@libero.it

Introduction: Since its introduction in 1968, Endoscopic retrograde cholangiopancreatography (ERCP) has become a commonly performed endoscopic procedure used to diagnose and to treat conditions associated to the pancreatobiliary system. It is nevertheless associated to the highest risk of complications of all routine endoscopic procedures. It is important to have a thorough understanding of the potential complications and the adverse events that may be associated to ERCP procedures so that these may be managed appropriately should they occur. The aim of this study was to examine the trends in ERCP usage here in the Veneto Region (Northeastern Italian area) and, in particular, the complications and mortality rate associated to it.

Aims \& Methods: Utilizing an anonymous database of hospital discharge records referring to the period between 2007 and 2015, a retrospective study was carried out to examine the complications associated to ERCP. All of the elective hospitalizations for gallstones in the bile duct during which the procedure was carried out within two days of being hospitalized were examined. Hospitalizations for neoplasms were not considered. The study considered the onset of complications or death as outcome indicators as well as the patients' post-procedure status; the threshold value that was utilized was 2 days and the patients who later underwent surgery (e.g. cholecystectomy) were excluded from our analysis; the associations between the type of hospital where the patients were being assisted both with regard to the type of organization (Hub or Spoke model) and the type of management (public/private) were also evaluated

Results: A total of 3,136 admissions out of total of 14,626 hospital days (SD:4.6 \pm 5.8 days) were identified in a total of 40 hospitals, $6(15 \%)$ of which private. The sex ratios were overlapping and the mean age, which was equal to $68.3 \pm 14.2$ (range 6-98 yrs), was higher in the females $(69.1 \pm 14,9$ vs. $67.5 \pm 13,5$ ); significant deviations during the period examined were not noted. A total of 212 complications $(6.8 \%)$ were registered: these included acute pancreatitis $(4.5 \%)$, cholangitis $(1.3 \%)$, sepsis $(0.4 \%)$, acute cholecystitis $(0.3 \%)$, cardiopulmonary complications $(0.2 \%)$, perforations and hemorrhage $(0.2 \%)$. The complications that presented, which were significantly higher in the female $\operatorname{sex}(7.3 \%$ vs. $6.2 \% ; \mathrm{p}<0.05)$, besides a greater average hospital stay in those subjects $(10.9 \pm 13.9$ vs. $4.2 \pm 4.4$ days), the within hospital mortality was $4.1 \%$ (13 cases) and there was a $6.1 \%$ of deaths due to complications. As far as the post-procedure hospital stay was concerned, in $55 \%$ of the cases, the discharge of patients within a two day period was more frequent in those assisted in public hospitals (OR:1.55;IC95\%:1.21-1.89;p < 0.05) in which $90 \%$ of the activities were carried out and no differences linked to their characteristics. The stratification of complications according to the type of hospital (range 2-17\%) did not uncover any significant differences during the period examined.

Conclusion: Study findings uncovered that pancreatitis was the most common post-procedure ERCP complication in the patients studied; the total complication rate was in line with that reported in the literature. That result and the fact that no correlation was found between the the type and percent of complications and the type of hospital can be attributed to the effective regional hospital organization characterized by a capillary network of specialists capable of performing complex endoscopic procedures throughout the region limiting the need to transfer patients from one hospital to another.

Disclosure of Interest: All authors have declared no conflicts of interest.

\section{P1538 EFFECT OF OBESITY, DYSLIPIDEMIA AND DIABETES MELLITUS ON THE RISK OF POST-ERCP PANCREATITIS}

H. Hassine, H. Elloumi, M. Sabbah, N. Bibani, D. Trad, D. Gargouri, A. Ouakaa, J. Kharrat

Gastroenterology, Habib Thameur Hospital, Tunis/Tunisia

Contact E-mail Address: hajer.hassine@gmail.com

Introduction: Risk factors for post-endoscopic retrograde cholangiopancreatography pancreatitis (PEP) have been widely investigated. Nevertheless, studies focusing in metabolic conditions especially obesity, dyslipidemia and diabetes mellitus (DM) are still limited.

Aims \& Methods: The aim of our study was to evaluate the effect of these factors on the frequency of PEP. We retrospectively analyzed all ERCP performed over a 12 months period [January 2015- December 2015] and carried out at the gastroenterology unit of our hospital. All patients were evaluated prospectively for the frequency of PEP based on the consensus criteria.The patients with obesity (Body mass index $\mathrm{BMI}>30 \mathrm{~kg} / \mathrm{m}^{2}$ ), dyslipidemia (triglyceride $>2 \mathrm{~g} / \mathrm{L}$ or LDL-cholesterol $>1.6 \mathrm{~g} / \mathrm{L}$ ) and DM (history of DM or fasting glucose level 
$>1.26 \mathrm{~g} / \mathrm{l})$ were find out before the procedure. The rate of PEP compared separately between patients with and without of these metabolic conditions.

Results: Two hundred twenty three procedures were performed during the study period. The rate of obesity, dyslipidemia and DM was $23.7 \%, 8.1 \%$ and $19.7 \%$ respectively. A PEP occurred in 13 patients with an overall incidence of $5.8 \%$. The rate of PEP was $3.8 \%$ in patients with obesity, $11.1 \%$ in patients with dyslipidemia and $9.1 \%$ in patients with DM. Although PEP was more frequent in dyslipidemia ( $11.1 \%$ vs $5.3 \%)$ and DM (9.1\% vs 5.3\%) groups, results did not rich a statistical significance $(\mathrm{p}=0.30, \mathrm{p}=0.31$ respectively). This could be explained by the small effective of patients. In the other hand, PEP was less frequent in the obesity group $(3.8 \%$ vs $6.9 \%)$ but there was no statistical significance $\mathrm{p}=0.28$.

Conclusion: In our study, metabolic conditions were not associated with an increased risk of PEP. It seems to be wise to evaluate the role of these conditions in larger prospective studies since the expanded prevalence of metabolic syndrome in general population.

Disclosure of Interest: All authors have declared no conflicts of interest.

\section{P1539 PLACE OF ENDOSCOPIC RETROGRADE CHOLANGIOPANCREATOGRAPHY (ERCP) IN THE MANAGEMENT OF HEPATIC HYDATID DISEASE}

H. Hassine, M. Sabbah, N. Bibani, D. Trad, H. Elloumi, D. Gargouri, A. Ouakaa, J. Kharrat

Gastroenterology, Habib Thameur Hospital, Tunis/Tunisia

\section{Contact E-mail Address: hajer.hassine@gmail.com}

Introduction: Hepatic hydatid disease (HHD) is a major endemic health problem in certain areas of the world such as Tunisia. Intrabiliary rupture of a hepatic hydatid cyst is a common complication ranging between 3 and $17 \%$. Furthermore, biliary leakage is the most frequent postoperative complication following surgery for hydatid cysts of liver. Both conditions require per endoscopic biliary drainage.

Aims \& Methods: The aim of this study was to assess the results of ERCP in patients with HHD. We retrospectively analyzed the results and complications of all ERCP performed for HHD whether before or after surgical treatment over a 10 years period [January 2007 - December 2016] and carried out at the gastroenterology unit of our hospital.

Results: Sixty seven procedures were included (mean age 40.4 years [15-82] and sex ratio male/female $31 / 36)$. Of the 67 procedures, $58(86.5 \%$ ) were performed in patients who had undergone previous surgery. The indications of the ERCP were persistent external biliary fistulae in $77.6 \%$, overcome obstruction or cholangitis due to residual materials within bile duct in $20.7 \%$ and secondary biliary strictures in $1.7 \%$. In patients who had not undergone previous surgery $(13.4 \%)$, the indications of the ERCP were cholangitis due to intra-biliary rupture of hydatid cyst in $44.4 \%$ associated with acute pancreatitis in $55.6 \%$. The cannulation of the papilla was impossible in 6 cases $(8.9 \%)$ and the endoscopic sphincterotomy (ES) could not performed. When papilla cannulation was obtained, per endoscopic cholangiographic findings were: dilation of the biliary tract $(21.3 \%)$ with filling defects of varying size and shapes $(52.5 \%)$, leakage of contrast medium into the cyst cavity $(41 \%)$ and distal stenosis $(3.3 \%)$. ES was then performed in all cases with satisfactory results. Thus, hydatid membranes $(36 \%)$ or daughter cysts $(1.6 \%)$ encountered in bile ducts have been emptied out in $93.4 \%$ by biliary occlusion balloon and/or Dormia basket. Nevertheless, two patients required biliary stenting due to stricture of the bile duct and two others required nasobiliary drainage. One patient presented post ERCP pancreatitis $(1.5 \%)$

Conclusion: ERCP is a safe and effective way to manage biliary complications of HHD. In most patients, ES is the most efficient treatment of postoperative external biliary fistulas, jaundice and accompanying cholangitis. In some cases, biliary stenting or nasobiliary drainage may be required.

Disclosure of Interest: All authors have declared no conflicts of interest.

\section{P1540 QUALITY INDICATORS AND POST OPERATIVE OUTCOME OF ERCP PERFORMED IN A LOW RESOURCE SETTING; CAN QUALITY INDICATORS FROM DEVELOPED SETTINGS BE APPLIED?}

I. Kongala Liyanage ${ }^{1}$, E. Thalagala ${ }^{1}$, S. Kulatunge ${ }^{1}$, R. Peiris ${ }^{1}$, N. Nawarathne ${ }^{1}$ Gastroenterology And Hepatology Unit, National Hospital of Sri Lanka, Colombo/Sri Lanka

Contact E-mail Address: Isurujith@gmail.com

Introduction: Endoscopic Regrograde Cholangiopancreatogram (ERCP) is a complex and invasive procedure. Quality control and monitoring for complications is an important part of ensuring patient safety. Gastroenterology and Hepatology unit of the National Hospital of Sri Lanka performs the most number and variety of ERCP procedures in Sri Lanka. Although facilities are limited, detailed patient records and logs were maintained since commencement of ERCP procedures in this unit.

Aims \& Methods: A retrospective analysis was carried out on quality indicators and complications of the ERCP procedures performed in this unit since 2006. Data from written records were entered into an electronic database and analysis was done using STATA version 13. Quality indicators and standards published by the American Society of Gastrointestinal Endoscopy and American College of Gastroenterology in 2015 were used.

Results: A total of 3780 ERCP procedures were performed. Females consisted of $54 \%$ of the patients $(\mathrm{n}=2041)$. Mean age in years was $57.6(\mathrm{SD}=8.4)$. Male patients were older with a mean age of 61.2 years whereas the mean age of females was 54.6 years $(P<0.05)$. Choledocolithiasis was the commonest indication $(85 \%)$ and $68 \%$ out of these patients had active cholangitis at the time of the procedure. Chronic pancreatitis, benign and malignant strictures requiring stenting, postoperative bile duct damage the other common indications. Data were available on 19 out of the 24 quality indicators. A total of 15 quality standards were met ( $7 / 9$ pre procedure, $4 / 5$ intra procedure and $4 / 10$ post procedure). Most prevalent major complications were Post ERCP Pancreatitis (PEP) and post ERCP Cholangitis (PEC) complicating $2.22 \%$ and $4.78 \%$ procedures respectively. Mortality was less than $0.1 \%(\mathrm{n}=3)$. Cardiorespiratory complications and complications of anesthesia were seen in 16 patients $(0.41 \%)$ resulting in one mortality. These were significantly associated with older age and the total number of medical comorbidities the patients had $(\mathrm{p}<0.05)$. Deficiencies practices and information gathering were noted mostly in postoperative care and includes documentation of patient satisfaction rate and long-term complication rates. After the quality review data on all quality indicators are gathered for future quality control purposes.

Conclusion: This supports the fact that centers with minimal resources can monitor quality standards and maintain satisfactory patient safety levels. Low numbers of postoperative complications were noted in this review. Old age and having multiple medical comorbidities were associated with a higher complication risk. Quality checkups help to identify deficiencies in practice as well as documentation and help to adjust future practices, even in low resource settings. Disclosure of Interest: All authors have declared no conflicts of interest.

\section{References}

1. Masci, E., et al., Complications of diagnostic and therapeutic ERCP: a prospective multicenter study. Am J Gastroenterol, 2001. 96(2): p. 417-423.

2. Talukdar, R., Complications of ERCP. Best Practice \& Research Clinical Gastroenterology, 2016. 30(5): p. 793-805.

3. Cotton, P.B., Quality endoscopists and quality endoscopy units. J Interv Gastroenterol, 2011. 1(02): p. 83-87.

4. Cotton, P.B., et al., Excellence in endoscopy: toward practical metrics. Gastrointestinal Endoscopy. 63(2): p. 286-291.

5. Adler, D.G., et al., Quality indicators for ERCP. The American journal of gastroenterology, 2015. 110(1): p. 91.

6. Rizk, M.K., et al., Quality indicators common to all GI endoscopic procedures. The American journal of gastroenterology, 2015. 110(1): p. 48.

7. Early, D.S., et al., Appropriate use of GI endoscopy. Gastrointestinal endoscopy, 2012. 75(6): p. 1127-1131.

8. Kang, S.H. and J.J. Hyun, Preparation and patient evaluation for safe gastrointestinal endoscopy. Clinical endoscopy, 2013. 46(3): p. 212-218.

9. Tang, S.-j. and G. Raju, Endoscopic photography and image documentation. Gastrointestinal Endoscopy. 82(5): p. 925-931.

10. Freeman, M.L., et al., Complications of endoscopic biliary sphincterotomy. New England Journal of Medicine, 1996. 335(13): p. 909-919.

11. Vandervoort, J., et al., Risk factors for complications after performance of ERCP. Gastrointestinal endoscopy, 2002. 56(5): p. 652-656.

12. Tarnasky, P.R., Mechanical prevention of post-ERCP pancreatitis by pancreatic stents: results, techniques, and indications. Jop, 2003. 4(1): p. 58-67.

13. Cheng, C.-L., et al., Risk factors for post-ERCP pancreatitis: a prospective multicenter study. The American journal of gastroenterology, 2006. 101(1): p. 139-147.

14. Chen, Y.K., et al., Endoscopic sphincterotomy-induced pancreatitis: increased risk associated with nondilated bile ducts and sphincter of Oddi dysfunction. American Journal of Gastroenterology, 1994. 89(3).

15. Manolakopoulos, S., et al., Octreotide versus hydrocortisone versus placebo in the prevention of post-ERCP pancreatitis: a multicenter randomized controlled trial. Gastrointestinal endoscopy, 2002. 55(4): p. 470-475.

16. Elmunzer, B.J., et al., A meta-analysis of rectal NSAIDs in the prevention of post-ERCP pancreatitis. Gut, 2008. 57(9): p. 1262-1267.

17. Freeman, M.L., Complications of endoscopic biliary sphincterotomy: a review. Endoscopy, 1997. 29(04): p. 288-297.

18. Baron, T.H., et al., Quality indicators for endoscopic retrograde cholangiopancreatography. The American journal of gastroenterology, 2006. 101(4): p. 892.

\section{P1541 USE OF MICROCATHETERS IN ECHOENDOSCOPY- GUIDED BILIOPANCREATIC RENDEZ-VOUS-INITIAL} EXPERIENCE

J. Pinto, C. Saldaña Dueñas, C. Leitão, I. Fernández-Urién Sainz, J.J. Vila Endoscopy Unit, Biliary And Pancreatic Diseases Unit, Complejo Hospitalario de Navarra, Pamplona/Spain

Contact E-mail Address: jsdiaspinto@gmail.com

Introduction: EUS-guided pancreatic or biliary rendezvous is a technically demanding procedure, and the intraductal manipulation of the guidewire remains the most challenging step. Passing the guidewire through the needle may cause its fragmentation on the sharp metallic bevel. We have described the method of using a microcatheter for EUS-guided rendezvous, which allows easier handling and exchange of the guidewire, while avoiding both the risk of fragmentation and the need of injection of contrast to achieve a complete ductogram.

Aims \& Methods: We aimed to evaluate the early experience of the microcatheter method in EUS-guided rendezvous procedures in the biliary and pancreatic tracts. During EUS-guided biliary or pancreatic rendezvous, initial puncture of the duct of interest was attempted with a 19G needle without stylet and 
previously flushed with contrast. A 0.025 " guidewire was then inserted through the needle into the duct and advanced anterogradely to the papilla. If further manipulation was necessary to entry the duodenum, movements were performed with caution in order to avoid fragmentation of the guidewire. Whenever the passage through the papilla was not achieved or the guidewire movements were hampered by the needle bevel, we performed a microcatheter technique. After removing the needle, leaving the guidewire in situ, a $3 \mathrm{~F}, 150 \mathrm{~cm}$ long microcatheter was inserted over the guidewire into the duct. Then, manipulation of the guidewire, guidewire exchange and contrast injection were performed according to the discretion of the endoscopist. We reviewed the cases of EUS-guided pancreatic or biliary rendezvous performed in our unit using microcatheters from September 2015 to March 2017. Technical success was considered when the rendezvous could be completed.

Results: Nine patients with previous unsuccessful manipulation of the guidewire with the needle during EUS-guided biliary or pancreatic rendezvous underwent a microcatheter-guided attempt on the same procedure. Pancreatic rendezvous was attempted in 5 cases ( 2 chronic pancreatitis, 2 pancreas divisum and 1 pancreatic cancer) and biliary rendezvous in the other 4 (3 biliary stenosis and 1 ampulloma). Technical success was achieved in 7 patients $(78 \%)$ with the microcatheter technique. Technical failure occurred in 1 patient with biliary stenosis in whom a EUS-guided hepaticogastrostomy was performed in the same procedure and in 1 patient with chronic pancreatitis with symptomatic pancreatic duct stenosis There were no adverse events after the procedure, irrespective of technical success.

Conclusion: In our series, using a microcatheter for the indractuctal manipulation of the guidewire increases the EUS-guided rendezvous technical success without increasing the complication rate, irrespective of technical success.

Disclosure of Interest: All authors have declared no conflicts of interest.

\section{Reference}

Vila $\mathbf{J}$ et al. A novel method for endoscopic ultrasound-guided pancreatic rendezvous with a microcatheter. Endoscopy. 2015;47 Suppl 1:E575-6.

\section{P1542 ERCP AND PTCD IN BILIARY TRACT COMPLICATIONS AFTER LIVER TRANSPLANTATION: PREDICTORS OF LONG- TERM OUTCOME}

M. Heinemann ${ }^{1}$, B. Tafrishi ${ }^{1}$, S. Pischke ${ }^{1}$, C. Spehr ${ }^{2}$, A. W. Lohse ${ }^{1}$, L. Fischer ${ }^{3}$, T. Rösch ${ }^{4}$, M. Sterneck ${ }^{1}$, U. Denzer

${ }^{1}$ I Department Of Medicine, University Medical Center Hamburg-Eppendorf,

Hamburg/Germany

${ }^{2}$ University Medical Center Hamburg-Eppendorf, Hamburg/Germany

${ }^{3}$ Department Of Hepatobiliary Surgery And Transplantation, University Medical Center Hamburg-Eppendorf. Hamburg/Germany

${ }^{4}$ Department Of Interdisciplinary Endoscopy, University Medical Center

Hamburg-Eppendorf, Hamburg/Germany

${ }^{5}$ Department Of Endoscopy, University Clinic Marburg, Marburg/Germany

Contact E-mail Address: bita_tafrishi2005@hotmail.de

Introduction: Biliary tract complications (BTC) are the leading problem in patients after orthotope liver transplantation (LT). The present study analysed the results and predictors of treatment outcomes in patients with biliary stenoses undergoing endoscopic retrograde cholangiopancreatography (ERCP) and/or percutaneous transhepatic cholangiodrainage (PTCD) at the University Medical Centre Hamburg-Eppendorf.

Aims \& Methods: All adult patients who received ERCP or PTCD for BTC after LT between 2009 and 2015 were retrospectively analysed. Remission of BTC was defined as no need of intervention for at least 12 months. To identify predictors of endoscopic treatment outcome in patients with biliary stenoses, a multivariate logistic regression analysis was performed after univariate variable selection. Laboratory parameters that were significant in the multivariate analysis, were dichotomised stepwise according to the most informative cut-off predicting outcome. Furthermore, endoscopic techniques were analysed in both the ERCP- and PTCD-subgroup.

Results: Of 144 patients with BTC after LT, 116 were diagnosed with biliary stenoses. Among these, 86 received ERCP, 17 PTCD and 13 both techniques. Long-term remission was achieved in 55 patients $(47 \%$ overall; $53 \%$ in ERCP alone and $30 \%$ when PTCD was applied). Patients with non-anastomotic strictures (NAS) (odds ratio [OR] $0.25,95 \%$ confidence interval [CI] $0.10-0.57$; $\mathrm{p}=0.001)$, requirement of PTCD (OR $0.30,95 \%$ CI $0.10-0.79 ; \mathrm{p}=0.018)$ and higher pre-interventional serum-bilirubin levels (OR $0.88,95 \%$ CI $0.76-0.98$; $\mathrm{p}=0.037$ ) were less likely to achieve remission. The most informative bilirubin cut-off to predict the outcome was $5 \mathrm{mg} / \mathrm{dl}$. This cut-off maintained a significant association with outcome in the multivariate model (OR $0.30,95 \%$ CI $0.08-0.95$; $\mathrm{p}=0.049)$. In the ERCP-subgroup, dilation with a higher maximal balloon-diameter was associated with a favourable outcome $(p=0.043)$.

Conclusion: Both ERCP and PTCD can provide long-term benefit in patients with BTC after LT. However, patients with NAS and requirement of PTCD had less favourable outcomes. The pre-interventional bilirubin level could be a valuable parameter to identify patients at risk of treatment failure. Larger ERCP balloon diameters may benefit remission.

Disclosure of Interest: All authors have declared no conflicts of interest.
P1543 RESULTS OF THE FRENCH NATIONAL OBSERVATIONAL STUDY CONCERNING THE PRACTICE OF PROBE BASED CONFOCAL ENDOMICROSCOPY (CELLVIZIO $®$ )

E. Mahfouz ${ }^{1}$, G. Rahmi ${ }^{2}$, L. Palazzo ${ }^{3}$, R. Gincul ${ }^{4}$, B. Napoléon ${ }^{5}$, M. Giovannini ${ }^{6}$, J. Moreau ${ }^{7}$, M. Levy ${ }^{8}$, G. Perrod ${ }^{2}$, A. Vienne ${ }^{2}$, E. Samaha ${ }^{1}$, J. Canard ${ }^{9}$, F. Caillol ${ }^{10}$, V. Lepilliez ${ }^{11}$, T. Ponchon ${ }^{12}$, E. Coron ${ }^{13}$, C. Cellier ${ }^{2}$ ${ }^{1}$ Paris, HEGP, Paris/France

${ }^{2}$ Gastroenterology And Digestive Endoscopy, Georges-Pompidou European Hospital, Paris/France

${ }^{3}$ Gastro-enterlogie, Cabinet Medical, PARIS/France

${ }^{4}$ Dept. De Endoscopie Digestive, Hopital Edouard Herriot, Lyon/France

${ }^{5} 69$, Hopital Privé Jean Mermoz, Lyon/France

${ }^{6}$ Dept. Of Endoscopy, Paoli-Calmettes, Marseille Cedex/France

Gastroenterology And Nutrition, Centre Hospitalier Universitaire de Toulouse.

Hopital Rangueil, Toulouse/France

${ }^{8}$ Creteil, Hopital Henri Mondor, creteil/France

${ }^{9}$ Trocadero Clinic, Hopital Euopeen Georges Pompidou, Paris/France

${ }^{10}$ Endoscopy, Institut Paoli Calmette, Marseille/France

${ }^{11}$ Hopital Prive Jean Mermoz, Lyon/France

${ }^{12}$ Dept. Of Digestive Diseases, Edouard Herriot University Hospital, Lyon/France

${ }^{13}$ Digestive Diseases Institute, University of Nantes, Nantes/France

Contact E-mail Address: eidfadymahfouz@gmail.com

Introduction: Confocal endomicroscopy is an endoscopic imaging technique permitting the microscopic analysis of the digestive mucosa in real time (esophagus, stomach, duodenum, colon, biliary tract and pancreas) due to injection of fluorescein which is an intravenous contrast. The aim of this national observational study under the guard of SFED is to evaluate the practice of confocal endomicroscopy in France, specifically its indication, histologic correlation, therapeutic benefits depending on the operator and complications.

Aims \& Methods: We executed a multicentric observational prospective study from September 2013 to February 2015. Collection of data was based on a standardized collection sheet. All operators were trained on performing confocal endmicroscopy. The intravenous injection of fluorescein was given either in bolus or in a perfusion method at a dilution of 1 or $10 \%$. Demographic, clinical, endoscopic and endomicroscopic data were collected. For each act the correlation between the confocal endomicroscpy and histology and the outcome of the ECM depending on the operator was reported.

Results: In total 399 procedures of confocal endomicroscopy were done on 399 patients (median age was $59+/-14.5$, males were $52 \%$ ) and these were performed in 12 centers. The main indications were: diagnosis and monitoring of Barrett esophagus 28\% (111/399), surveillance of gastritis 4\% (16/399), characterization of colorectal polyp and searching for dysplasia in IBD patients $17 \%$ (68/399), undetermined biliary stenosis $11 \%$ (42/399), pancreatic cysts $30 \%$ (123/ 399 ) and other rare cases (lymph nodes characterization, pancreatic mass, ampulloma, GIST, celiac disease, control post mucosectomy of gastric and duodenal polyps) $10 \%(39 / 399)$. The quality of imaging was good in $83 \%$ of cases $(331$ $399)$, average in $16 \%(64 / 399)$ and poor in $1 \%(6 / 399)$. The correlation with histology was measured by using Cohen's kappa coefficient. The results were respectively $\mathrm{k}=0.9,0.78,0.82,0.7,0.94,0.93$ for Barrett's esophagus, gastritis, IBD, colorectal polyps, undetermined biliary stenosis and pancreatic cysts. The outcome of the procedure according to the operator was beneficial for three main indications: Barrett's esophagus ( $82 \%$ especially for targeting biopsies), serous pancreatic cysts ( $100 \%$ of cases), and undetermined biliary stenosis $(90 \%$ of cases and especially for real time therapeutic decision). One major side effect was seen during the study, which was an anaphylactic choc after a bolus injection of $2.5 \mathrm{cc}$ of fluorescein $10 \%$ in a $69 \mathrm{y}$ old patient who didn't have any previous history of allergies. This patient was hospitalized for surveillance for three days.

Conclusion: In conclusion, confocal endomicroscopy is an in vivo microscopic imaging technique that is easily performed throughout the digestive tube with a good histologic correlation especially for barrett's esophagus, undetermined biliary stenosis and pancreatic cysts. Its importance in the management of patients remains to be clarified with the advent of new endoscopic magnification techniques.

Disclosure of Interest: All authors have declared no conflicts of interest.

P1544 ENDOSCOPIC RETROGRADE

CHOLANGIOPANCREATOGRAPHY IN PATIENTS WITH SURGICALLY MODIFIED GASTRO-ENTERIC-BILIARY ANATOMY: RESULTS FROM A TERTIARY CENTER

E. Soares, M. Gravito-Soares, N. Almeida, D. Gomes, E. Camacho, S. Mendes, R. Ferreira, L. Tomé

Gastroenterology, Centro Hospitalar e Universitário Coimbra, Coimbra, Portugal, Coimbra/Portugal

Contact E-mail Address: es18497@gmail.com

Introduction: Endoscopic retrograde cholangiopancreatography (ERCP) represents a crucial procedure in the management of biliopancreatic pathology. However, its performance in patients with surgically modified gastro-entericbiliary anatomy (SMGA) is a challenging issue.

Aims \& Methods: We aimed to evaluate the efficacy of this advanced endoscopic technique in patients with SMGA. This was a retrospective observational cohort study of all patients with surgical modification of biliary/pancreatic access undergone ERCP, between 01/2002 and 02/2017. Demographic variables, indications, the breakdown of surgical procedures and technical success rate were evaluated as well as potential predictive factors of therapeutic efficacy rate. Compared patients with successful technique(G1) and therapeutic failure by ERCP 
requiring surgical and/or radiological therapy $(\mathrm{G} 2)$, regarding recurrence of biliopancreatic pathology, major complications and post-procedure 30-days mortality.

Results: A total of 153 ERCP procedures were carried out in 103 patients with SMGA (mean age: $75.4 \pm 11.3$ years, men: $80.6 \%$ ). The breakdown of surgical procedures was: Billroth II-77.7\%(n=80); Billroth I-10.7\% $(\mathrm{n}=11)$; Roux-enY-8.7\%(n $=9)$; post-duodenopancreatectomy-2; gastric sleeve-1. The forwardviewing endoscope was used in $79.8 \%(\mathrm{n}=122)$, duodenoscope in $19.6 \%(\mathrm{n}=30)$ and colonoscope in $0.6 \%(\mathrm{n}=1)$. The success rate was: technical-62.1\%(95/153); therapeutic- $93.7 \%(89 / 95)$. The success rate by type of surgical reconstruction was: gastric sleeve- $100 \%$; Billroth I-94.4\%; Billroth II-60.5\%; post-duodenopancreatectomy- $50 \%$; Roux-en-Y-18.2\%. After multivariate analysis, poor papillary orientation/localization $(\mathrm{OR}=5.618 ; \mathrm{p}=0.006)$ was the only independent predictor of technical failure. On the other hand, high values of total bilirubin $(\mathrm{OR}=1.339 ; \mathrm{p}=0.024)$ and benign etiology of obstructive jaundice $(\mathrm{OR}=1.789 ; \mathrm{p}=0.017)$ were independent predictors of therapeutic success. In 25 patients with no endoscopic technical/therapeutic success, surgical $(n=14)$ or radiological approach $(\mathrm{n}=11)$ was performed. Comparing G1vsG2, there were no differences in the recurrence rate of biliopancreatic disease $(23.1 \% \mathrm{vs} 36.0 \% ; \mathrm{p}=0.205)$ or mortality $(9.0 \% \mathrm{vs} 8.0 \% ; \mathrm{p}=1.000)$. However, G2 group had a high rate of major complications $(12.8 \% \mathrm{vs} 36.0 \% ; \mathrm{p}=0.016)$. Conclusion: The technical and therapeutic success rates of ERCP in patients with SMGA were $62.1 \%$ and $93.7 \%$, respectively (lower in Roux-en-Y). Given the increased number of patients with SMGA and the high rate of complications associated with radiological and surgical therapies, it is essential to optimize ERCP technique rather than conventional endoscopy/duodenoscopy, including the use of balloon-assisted enteroscopy.

Disclosure of Interest: All authors have declared no conflicts of interest.

\section{P1545 NEWLY DEVELOPED BASKET FOR DIGITAL, SINGLE- OPERATOR CHOLANGIOSCOPY FOR MANAGEMENT OF RESIDUAL STONES AFTER LITHOTRIPSY}

J.H. Moon, Y.N. Lee, H.J. Choi, H.W. Lee, T.H. Lee, M.H. Choi, S. Cha, Y.D. Cho, S. Park

Digestive Disease Center And Research Institute, Department Of Internal Medicine, SoonChunHyang University School of Medicine, Bucheon and Seoul Korea, Republic of

Contact E-mail Address: jhmoon@schmc.ac.kr

Introduction: Detection of residual or fragmented stones after lithotripsy for retained common bile duct stones can be improved by digital, single-operator cholangioscopy (SOC) by high resolution imaging quality. However, therapeutic intervention for the removal of residual CBD stones is limited by lack of appropriate accessories.

Aims \& Methods: We evaluated the role of SOC and newly developed dormia basket for the evaluation and removal of residual stones after lithotripsy. From March to October 2016, 34 patients who had undergone lithotripsy for retained CBD stones with no evidence of filling defects in occluded balloon cholangiography were included. After balloon cholangiography, the bile duct was evaluated by SOC for the complete evacuation of stones. Detected residual CBD stones were directly retrieved with newly developed dormia basket inserted into the working channel of SOC. The incidence of residual stones detected by SOC, and the success rate of residual stone retrieval under SOC were investigated. Results: DPOC was successfully performed in all patients. Of these, 11 patients $(32.4 \%)$ had residual CBD stones. The residual stones were successfully removed in 10 patients $(90.9 \%)$ by dormia basket under SOC. $(84.6 \%)$ except residual stones on Lt. hepatic duct. There were no complications associated with SOC or direct stone removal.

Conclusion: Digital SOC combined with newly developed dormia basket is useful for the detection and extraction of residual stones after lithotripsy for retained CBD stones expanding therapeutic intervention of SOC.

Disclosure of Interest: All authors have declared no conflicts of interest.

\section{P1546 ASSOCIATION BETWEEN PREDICTIVE FACTORS AND RADIATION EXPOSURE DURING ENDOSCOPIC RETROGRADE CHOLANGIOPANCREATOGRAPHY}

S. Dong, C.H. Oh, J.R. Moon

Kyung Hee University College of Medicine Dept. of Internal Medicine, Seoul/ Korea, Republic of

\section{Contact E-mail Address: gidrdong@hanmail.net}

Introduction: Endoscopic retrograde cholangiopancreatography (ERCP) relies on the use of ionizing radiation in the form of fluoroscopy. Because use of fluoroscopy has positive relationship with radiation exposure, it makes a risk of the development of cancer and other radiation toxicity. The increasing exposure of patients and endoscopists to radiation is concerning.

Aims \& Methods: The aim of our study was to evaluation of predictive factors of radiation exposure to the patients and endoscopists during endoscopic retrograde cholangiopancreatography. A cross-sectional retrospective analysis of 892 ERCPs performed at tertiary academic hospital over 48-month period by on expert endoscopist was conducted. The primary outcomes of interest were the radiation exposure of patients and endoscopists during procedure as determined by radiation dose in dose area product (DAP), absorbed dose (AD) and fluoroscopy time. And we correlated them with age, sex, body mass index, diagnosis, duration of procedure, procedure name and procedure complexity.
Results: As a result of analysis of the 892 ERCPs performed during 4 years, the mean duration of fluoroscopy time was 5.52 mins (95\% CI, 5.15-5.93). Mean radiation duration were as follows: $\mathrm{CBD}$ stones $(\mathrm{n}=511,5.76 \mathrm{mins})$; malignant stenosis of bile duct $(n=189,5.78$ mins); pancreatic disease $(n=95,5.28$ mins); benign stenosis of bile duct $(\mathrm{n}=51,5.32 \mathrm{mins})$; and periampullary stenosis ( $\mathrm{n}=46,4.89$ mins). Multivariate analysis revealed that prolonged duration of fluoroscopy time was related with specific factors of patient included age, BMI, diagnosis and procedure complexity $($ all $\mathrm{p}<0.05)$. Among the parameters, procedure complexity was the most significant relation with radiation dose. In addition, the following three procedures made prolonged procedure duration: two more procedures performed during ERCP and mechanical lithotripsy (all $\mathrm{p}<0.05)$

Conclusion: ERCPs are associated with significantly higher radiation exposure to patients on the specific procedure. The endoscopists should be aware of the increased dose of radiation required when performing ERCP in patients with increased BMI, old age, and who need two more ERCP procedure.

Disclosure of Interest: All authors have declared no conflicts of interest.

\section{P1547 DEVELOPMENT OF AN ERCP REGISTRY FOR QUALITY CONTROL AND BENCHMARKING}

D. Pécsi ${ }^{1}$, S. Gódi ${ }^{2}$, F. Pakodi ${ }^{2}$, P. Nagy ${ }^{1}$, T. Molnár ${ }^{1}$, P. Hegyi ${ }^{1}$, A. Vincze ${ }^{2}$ ${ }^{1}$ Institute For Translational Medicine, Universitiy of Pécs, Pécs/Hungary ${ }^{2} 1$ st Department Of Medicine, Division Of Gastroenterology, Universitiy of Pécs, Pécs/Hungary

Contact E-mail Address: daniel.pecsi@aok.pte.hu

Introduction: To obtain representative information about invasive endoscopic procedures is a principal goal to monitor efficacy and safety. A web-based, online central registry can serve this aim allowing structured data collection and usage of universally accepted terminology.

Aims \& Methods: We decided to develop a web-based registry which is easy to use and captures relevant procedure related information. The primary aim of the registry is to monitor relevant outcome data of ERCP.

Results: Hungarian experts in ERCP were invited at the initiation of the registry for discussion and consensus. Detailed data collection form was initially developed based on internationally recommended quality parameters. The prospective observational research project was approved by the Scientific and Research Ethics Committee (Budapest, Hungary). A web-based case report form was developed after finalisation and tested from January 2017 at our department. ERCP related data of consecutive patients were collected prospectively after consenting the patients. The data from the first 100 procedures were analysed to demonstrate the usability of the registry. Ninety-two patients (51 females, 41 males) were involved in this preliminary evaluation with an average age of 69.2 years (23-93 years). The number of elective and urgent examinations were almost equal. The indication of ERCP were the following: bile duct disorder based on laboratory or imaging data was the indication in $45 \%$, obstructive jaundice in $31 \%$, cholangitis in $16 \%$, acute biliary pancreatitis in $5 \%$, pancreatic disorder in $3 \%$. The difficulty of procedures was evaluated: grade $1-22 \%$, grade $2-45 \%$, grade $3-30 \%$, grade $4-3 \%$. Fifty-nine procedures were performed in patients with intact papilla of Vater, bile duct access was not successful in one case $(1.7 \%)$. Precut procedure was applied in 14 cases $(23.3 \%)$, all of them resulted successful biliary access at the same procedure. Immediate complications were observed in 8 cases $(8 \%), 6$ of them $(6 \%)$ were bleeding. All were successfully controlled, and none of the patients required blood transfusion or repeated endoscopy. Mild post-ERCP pancreatitis was developed in one patient $(1 \%)$. Cholangitis was observed in 2 cases $(2 \%)$. Follow up was conducted 30 days after the ERCP by a telephone call and/or reviewing health care documentations to observe long term outcome. Four patients $(4 \%)$ died during this period, but only 1 death was related to the procedure, caused by unresolved cholangitis. Conclusion: The ERCP registry is an essential tool for measuring quality indicators. The universal usage will allow benchmarking at individual, institutional and national level and will help in quality improvement. Efficacy, safety and impact on different pancreatobiliary disorders will be also measurable.

Disclosure of Interest: All authors have declared no conflicts of interest.

\section{P1548 DOUBLE-BALLON ENTEROSCOPY FOR ENDOSCOPIC RETROGRADE CHOLANGIOPANCREATOGRAPHY IN PATIENTS WITH SURGICALLY ALTERED UPPER GASTROINTESTINAL ANATOMY}

C. Ghaoui ${ }^{1}$, E. Samaha ${ }^{1}$, G. Rahmi ${ }^{1}$, G. Perrod ${ }^{1}$, H. Benosman ${ }^{1}$, A. Vienne ${ }^{1}$, C. Savale ${ }^{1}$, S. Khater ${ }^{1}$, L. Abbes ${ }^{1}$, J. Danset ${ }^{1}$, G. Malamut ${ }^{1}$, C. Cellier ${ }^{1}$ ${ }^{1}$ Hepato-gastro-enterologie Et Endoscopie Digestive, Hopital Europeen Georges Pompidou, Paris/France

Contact E-mail Address: celineghaoui@hotmail.com

Introduction: Double-balloon enteroscopy-assisted endoscopic retrograde cholangiopancreatography or DBE-ERCP allows access to the biliary ducts of patients with surgically altered Upper Gastrointestinal (GI) anatomy. We studied the feasibility and efficacy of DBE-ERCP at our institution.

Aims \& Methods: This is a retrospective study of all patients with surgically altered GI anatomy who underwent DBE-ERCP at our institution between February 2011 and March 2017. The primary endpoint was the global success rate of DBE-ERCP. The secondary endpoints were (1) the success rate of enteroscopy defined as reaching the desired postsurgical anatomic target, (2) the diagnostic success rate defined as successful cannulation of the native papilla or 
anastomosis, and (3) the therapeutic success rate. We used a $2.2 \mathrm{~m} \mathrm{DBE}$ with a 2.8 or $3.2 \mathrm{~mm}$ of operating channel (EN $450 \mathrm{~T} 5$, or EN580T Fujinon inc Saitama Japan).

Results: A total of 12 patients (sex ratiol/1) with a mean age of 65 [47-82] underwent 14 DBE-ERCP. 7 patients had Roux-en-Y gastro-jejunostomy with a bilio-jejunostomy, 4 patients had Roux-en-Y with a native papilla, and 1 patient had a Billroth II gastric bypass. Enteroscopy success rate was 93\% (13/ 14 procedures). The diagnostic success rate was $85 \%$ (11/13 procedures) with $4 / 5$ of native papillae. Therapeutic interventions including sphincterotomy $(n=4)$, biliary stone extraction $(n=4)$ and biliary dilation $(n=2)$ were needed in $8 / 11$ procedures and their success rate was $100 \%$. The global success rate of DBEERCP was $78 \%$ (11/14 procedures). Our results were comparable to those of the literature (global success rate of $82 \%$ ). The only complication was one case of superficial intestinal lacerations without perforation (complication rate $7 \%$ ). Conclusion: DBE-ERCP in patients with surgically altered upper GI anatomy is a safe and efficient procedure with a global success rate of $78 \%$. Using shorter enteroscopes with wider operating channel in the future might improve the success rate of this technique.

Disclosure of Interest: All authors have declared no conflicts of interest.

\section{Reference}

Skinner M, Popa D, Neumann H, Wilcox CM, Mönkemüller K. ERCP with the overtube-assisted enteroscopy technique: a systematic review. Endoscopy. 2014;46:560-72. doi: 10.1055/s-0034-1365698

\section{P1549 NEEDLE-KNIFE SPHINCTEROTOMY (NKS) VERSUS TRANSPANCREATIC SPHINCTEROTOMY (TPS) FOR DIFFICULT BILIARY CANNULATION: A SYSTEMATIC REVIEW AND META- ANALYSIS}

A. Tringali ${ }^{1}$, M. Cintolo ${ }^{1}$, L. Cristoferi ${ }^{1}$, E. Forti ${ }^{1}$, F. Pugliese $^{1}$, L. Dioscoridi ${ }^{1}$, M. Mutignani ${ }^{1}$

${ }^{1}$ Endoscopy Unit, Niguarda Hospital, Milano/Italy

Contact E-mail Address: albtri10@gmail.com

Introduction: Biliary cannulation may be difficult in $10-15 \%$ of patients (1) and needle-knife sphincterotomy is more often used as a rescue treatment. A more recent technique for difficult cases is transpancreatic sphincterotomy. Both situations are well known as Post-Ercp pancreatitis risk factor (2). To best of our knowledge only few studies compared success rate and adverse events in these techniques (3-.7)

Aims \& Methods: We aimed to compare the efficacy and safety of NKS comparing to TPS in difficult biliary cannulation We conducted a bibliographic search using PUBMED, EMBASE including 2 RCTS and 4 non randomized trials from January 2000 to December 2016. OR using the Manthel-Haenszel method was used for dichotomous variables. Quantitative synthesis was performed using Review Manager version 5.0. Primary outcome was success rate. Secondary outcomes were rate of overall complications, and pancreatitis. Clinical heterogeneity was assessed by $\mathrm{I}^{2}$ value, where a value exceeding $50 \%$ was indicative of heterogeneity. A random effect model was used in case of heterogeneity.

Results: Success rate was higher in NKS group compared to TPS (OR 2.98 $95 \%$ CI $1.01-8.85, \mathrm{p}=0-.05)$. Complications and risk of pancreatitis were similar in both group (OR $0.7495 \% \mathrm{CI} 0.51-1.09 \mathrm{p}=0.13$; OR $1.0995 \% \mathrm{CI} 0.68-1.75$ $\mathrm{p}=0.71$ )

Conclusion: NKS is associated with higher success rate with equal risk of complication and pancreatitis risk compared to TPS. Further and well design RCTs are needed before a firm conclusion could be made

Disclosure of Interest: All authors have declared no conflicts of interest.

\section{References}

1. Tse F, Yuan Y, Moayyedi P et al. Guidewire-assisted cannulation of the ommon bile duct for the prevention of post-endoscopic retrograde cholangiopancreatography (ERCP) pancreatitis. Cochrane Database Syst Rev 2012; 12: CD009662

2. Masci E, Mariani A, Curioni S et al. Risk factors for pancreatitis following endoscopic retrograde cholangiopancreatography: a meta-analysis. Endoscopy 2003; 35: 830-834

3. Catalano MF, Linder JD, Geenen JE. Endoscopic transpancreatic papillary septotomy for inaccessible obstructed bile ducts: Comparison with standard precut papillotomy. Gastrointest Endosc. 2004;60:557-561.

4. Halttunen J, Keränen I, Udd M, Kylänpää L. Pancreatic sphincterotomy versus needle knife precut in difficult biliary cannulation. Surg Endosc. 2009;23:745-749

5. Zang J, Zhang C, Gao J. Guidewire-assisted transpancreatic sphincterotomy for difficult biliary cannulation: a prospective randomized controlled trial. Surg Laparosc Endosc Percutan Tech 2014; 24: 429-433

6. Lee YJ, Park YK, Lee MJ et al. Different strategies for transpancreatic septotomy and needle knife infundibulotomy due to the presence of unintended pancreatic cannulation in difficult biliary cannulation. Gut Liver 2015; 9: 534-539 7. Wang P, Zhang W, Liu F, Li ZS, Ren X, Fan ZN, Zhang X, Lu NH, Sun WS, Shi RH, et al. Success and complication rates of two precut techniques, transpancreatic sphincterotomy and needle-knife sphincterotomy for bile duct cannulation. J Gastrointest Surg. 2010;14:697-704
P1550 TRANSPANCREATIC SPHINCTEROTOMY: A VALUABLE TECHNIQUE FOR GAINING CBD ACCESS

S. Esmaily ${ }^{1}$, M. Elzubier ${ }^{2}$, D. Dwarakanath ${ }^{1}$, V. Mitra ${ }^{3}$, D. Majumdar ${ }^{3}$, B.K. Chaudhury ${ }^{1}$, J. Hancock ${ }^{1}$

${ }^{1}$ Gastroenterology, University Hospital of North Tees, Stockton on Tees/United Kingdom

${ }^{2}$ Gastroenterology, North Tees University Hospital, Stockton on Tees/United

Kingdom

${ }^{3}$ Dept. Of Gastroenterology, James Cook University Hospital, Middlesbrough United Kingdom

Contact E-mail Address: shiranesmaily@doctors.org.uk

Introduction: Transpancreatic Sphincterotomy (TPS) involves wire-guided cannulation of the main pancreatic duct (MPD) followed by standard pull-type sphincterotome cutting towards the main channel. Typically a MPD stent insertion should follow TPS to prevent post-ERCP pancreatitis (PEP). ESGE [1] recommends that in patients with a small papilla that is difficult to cannulate, TPS should be considered if unintentional wire guided MPD cannulation occurs. A previous study had suggested that TPS might be as effective as double guide wire (DGW) technique in achieving biliary cannulation in difficult cases and has a lower pancreatitis rate. [1]

Aims \& Methods: The aim of this study was to review the practice, complications and outcomes of TPS in University Hospital of North Tees (district general hospital in the north-east of UK). All ERCP procedures between January 2014 and October 2016 were reviewed. Endoscopy reports, blood results and discharge letters were used for data collection.

Results: 1365 ERCP procedures were performed in the study period. Overall CBD cannulation rate was $91.3 \%$. 105/1365 (7.7\%) wire guided TPS procedures were carried out. Mean age in the TPS group was 67 years (range: 20-91) - 64/105 $(61 \%)$ male and $41 / 105(39 \%)$ females. 3 senior consultants and a senior endoscopic fellow performed TPS. TPS was used as the initial strategy in the event of MPD cannulation and without using alternative methods such as DGW. Choledocholithiasis was the commonest ERCP indication in TPS group (58 105). Other indications include pancreatic cancer, biliary stricture, cholangiocarcinoma and bile leak post cholecystectomy. CBD cannulation was achieved in 96/ $105(90.5 \%)$ of cases $-81 / 105(77.1 \%)$ during the first ERCP and $15 / 105(14.2 \%)$ at second ERCP [where CBD cannulation was achieved at second attempt, TPS had been done at the first ERCP]. In 9/105 patients CBD cannulation was not achieved. 8/105 $(7.6 \%)$ had a complication. 6/105 (5.7\%) patients had PEP - 5/6 had a prophylactic MPD stent. 1/105 had a post sphincterotomy bleed which was controlled with endoscopic therapy. 1/105 had a perforation and a subsequent long hospital stay. There were no procedure-related deaths.

Conclusion: This study demonstrates that TPS is a safe and effective way of gaining CBD access. Our data suggests that experienced operators in a DGH setting can safely carry out TPS. Our study is the second largest cohort in the literature and the largest cohort in the UK. We would suggest early adoption of TPS if wire access to the pancreatic duct is achieved, this will likely reduce complication rate as a result of less engagement with the papilla and overall reduced time at $\mathrm{CBD}$ cannulation.

Disclosure of Interest: All authors have declared no conflicts of interest.

\section{Reference}

1. Papillary cannulation and sphincterotomy techniques at ERCP: ESGE Clinical Guideline. Endoscopy. 2016 Jul;48(07):657-83.

\section{P1551 ENDOSCOPIC REMOVAL OF BILE DUCT STONES - A RETROSPECTIVE SINGLE-CENTER STUDY}

G. Constantinescu ${ }^{1}$, O.M. Plotogea ${ }^{1}$, M. Ilie $^{1}$, B. Ungureanu ${ }^{2}$,

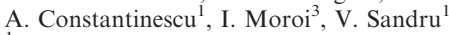

${ }^{1}$ Dept. Of Gastroenterology, SCUB, Bucharest/Romania

${ }^{2}$ Emergency County Hospital, Craiova, Craiova/Romania

${ }^{3}$ Dept. Of Gastroenterology, Clinical Emergency Hospital Bucharest, Bucharest Romania

Contact E-mail Address: gabrielconstantinescu63@gmail.com

Introduction: Choledocholithiasis is a relative frequent condition in patients with gallbladder stones (prevalence of 3-16\%). Endoscopic sphincterotomy and stone extraction is the recommended treatment of bile duct stones. In the case of failed endoscopic therapy, cholecystectomy combined with bile duct exploration or intraoperative endoscopic retrograde cholangiopancreatography should be performed

Aims \& Methods: The main objective of this study was to evaluate the efficacy of endoscopic extraction methods in patients who presented choledocholithiasis. We conducted a retrospective single center study over 7 years from Jan 2009 to Dec 2015. Patients with single or multiple bile duct stones submitted to endoscopic retrograde cholangio-pancreatography (ERCP) were included. We analyzed each technique by considering the following parameters: mean diameter of the stone(s), mean diameter of CBD and success rate defined by number of cases solved endoscopically vs. cases of residual lithiasis/referred to surgery. Every ERCP was performed using Olympus ${ }^{\circledR}$ side-viewing endoscopes. Stone size, number and CBD diameter were documented on the initial diagnostic cholangiogram. Stones were removed using retrieval balloon catheter, Dormia extraction basket, mechanical lithotriptor and $\mathrm{CRE}^{\mathrm{TM}}$ Balloon Dilator (DASE). In case of residual lithiasis, a biliary $10 \mathrm{Fr} / 7 \mathrm{Fr}$ Amsterdam plastic stent was placed and a second ERCP was planned within 2-4 weeks. The efficacy endpoint was the success rate regarding complete clearance of the bile duct. Statistical analysis 
was performed using SPSS version 20.0. A P-value of less than 0.05 was considered statistically significant.

Results: We included 1976 patients who presented choledocholithiasis and underwent different endoscopic extraction techniques. Mean diameter of stone(s) extracted using only the basket (Group A) was $6.8 \mathrm{~mm}$ with a mean CBD diameter of $11.6 \mathrm{~mm}$ for patients with complete clearance of the bile duct compared to $11.8 \mathrm{~mm}$ (mean stone diameter) with $14.3 \mathrm{~mm}$ (mean CBD diameter) for patients solved eventually by surgery $(\mathrm{P}<0.001)$. Only $3.2 \%$ of cases had to be referred to surgery. Stones removed using only a retrieval balloon (Group B) had a mean diameter of $7.6 \mathrm{~mm}$ with a mean CBD of $13 \mathrm{~mm}$ among patients solved endoscopically, significantly lower than those with residual lithiasis: $15.5 \mathrm{~mm}$ (mean stone diameter) with $16 \mathrm{~mm}$ (mean CBD diameter) $(\mathrm{P}<0.001)$. $9 \%$ of cases were referred to surgery. The lithotriptor alone (Group 3) was efficient for a mean stone diameter of $12.3 \mathrm{~mm}$ with a mean CBD diameter of $13.3 \mathrm{~mm}$. By associating the basket with a retrieval balloon (Group 4) we obtained a $100 \%$ success rate of endoscopic removal for a mean stone diameter of $9.6 \mathrm{~mm}$ with a mean CBD diameter of $12.9 \mathrm{~mm}$. We also analyzed the parameters in patients who underwent endoscopic extraction by using a retrieval balloon combined with balloon dilator (Group 5). The success rate in this group was $90 \%$ for a mean stone diameter of $14.8 \mathrm{~mm}$ with a mean CBD diameter of $12.4 \mathrm{~mm}$. In Group 6 we included cases which required combined techniques $(>=3)$. We observed a mean stone diameter of $9.4 \mathrm{~mm}$ with a mean CBD diameter of $13.8 \mathrm{~mm}$ in patients solved endoscopically, compared to those referred to surgery who had a mean stone diameter of $14.2 \mathrm{~mm}$ with a mean CBD diameter of $14.3 \mathrm{~mm}(\mathrm{P}<0.001)$. In this group the success rate was $67.2 \%$. Overall, we had a success rate of $91.3 \%$ for endoscopic removal of choledocholithiasis with a mean stone diameter of $7.1 \mathrm{~mm}$ and a mean CBD diameter of $12.1 \mathrm{~mm}$, compared to $3.8 \%$ of cases referred to surgery with a mean stone diameter of $13.6 \mathrm{~mm}$ and a mean CBD diameter of $14.3 \mathrm{~mm}$ $(\mathrm{P}<0.001)$.

Conclusion: The most successful endoscopic method to remove large stones $\geq 15 \mathrm{~mm}$ was balloon dilator combined with retrieval balloon. Intermmediate stones $7-15 \mathrm{~mm}$ can be successfully removed by using retrieval balloon or lithotriptor or a combination of basket with retrieval balloon \pm balloon dilator. Most CBD stones $<7 \mathrm{~mm}$ were successfully removed by using basket. In conclusion, any diameter $>7 \mathrm{~mm}$ will most probably require more elaborate techniques.

Disclosure of Interest: All authors have declared no conflicts of interest.

\section{Reference}

European Association for the Study of the Liver (EASL). EASL Clinical Practice Guidelines on the prevention, diagnosis and treatment of gallstones. Journal of Hepatology 2016 vol.65;146-181.

\section{P1552 DICLOFENAC AND INDOMETHACIN IN THE PREVENTION OF POST-ERCP PANCREATITIS: A SYSTEMATIC REVIEW AND META-ANALYSIS OF PROSPECTIVE CONTROLLED TRIALS}

A.V. Patai ${ }^{1}$, N. Solymosi ${ }^{2}$, L. Mohácsi ${ }^{3}$, Á. Nagy ${ }^{1}$, A. Patai ${ }^{4}$

${ }^{1}$ 2nd Department Of Internal Medicine, Semmelweis University 2nd Dept. of Medicine, Budapest/Hungary

${ }^{2}$ Bioinformatics Research Group, University of Veterinary Medicine, Budapest/ Hungary

${ }^{3}$ Department Of Computer Science, Corvinus University of Budapest, Budapest/ Hungary

${ }^{4}$ Department Of Medicine And Gastroenterology, Markusovszky Hospital, Szombathely/Hungary

\section{Contact E-mail Address: arpad.patai@gmail.com}

Introduction: Diclofenac and indomethacin are the most studied drugs for preventing post-ERCP pancreatitis (PEP), but their use is controversial.

Aims \& Methods: Our aim was to evaluate of all trials published in full text and studied efficacy of diclofenac or indomethacin prospective controlled with placebo or non-treatment for the prevention of PEP in adult patients undergoing ERCP. Systematic search of databases (PubMed, Scopus, Web of Science, Cochrane) for relevant studies published from inception to 30 June 2016.

Results: Our meta-analysis of 4741 patients from 17 trials showed that diclofenac or indomethacin significantly decreased the risk ratio (RR) of PEP to $0.60(95 \%$ confidence interval $/ \mathrm{CI} / 0.46-0.78, \mathrm{P}=0.0001)$, number needed to treat $(\mathrm{NNT})$ was 20 , and the reduction of RR of moderate to severe PEP was $0.64(95 \% \mathrm{CI}$ $0.43-0.97, \mathrm{P}=0.0339)$. The efficacy of indomethacin compared to diclofenac was similar $(\mathrm{P}=0.98)$. The efficacy of indomethacin or diclofenac did not differ according to timing $(\mathrm{P}=0.99)$ nor between patients with average-risk and high-risk for PEP $(\mathrm{P}=0.6923)$. The effect of non-rectal administration of indomethacin or diclofenac was not significant $(P=0.1507)$, but rectal route was very effective $(\mathrm{P}=0.0005)$ with a NNT of 19 . The administration of indomethacin or diclofenac was avoided in patients with renal failure. Substantial adverse events were not detected.

Conclusion: The use of rectally administered inexpensive and safe diclofenac or indomethacin before or closely after ERCP is recommended in every patient (without renal failure) undergoing ERCP.

Disclosure of Interest: All authors have declared no conflicts of interest.

\section{References}

1. Abu-Safieh Y, et al. Diclofenac vs. placebo in a randomized double blind controlled trial in post ERCP pancreatitis. Am J Clin Med Res 2014
2. Andrade-Dávila VF, et al. Rectal indomethacin vs. placebo to reduce the incidence of pancreatitis after endoscopic retrograde cholangiopancreatography: results of a controlled clinical trial. BMC Gastroenterol 2015

3. Cheon YK, et al. Efficacy of diclofenac in prevention of post-ERCP pancreatitis in predominantly high-risk patients: a randomized double-blind prospective trial. Gastrointest Endosc 2007

4. Döbrönte Z, et al. Effect of rectal indomethacin in the prevention of postERCP acute pancreatitis. Orv Hetil 2012

5. Döbrönte $Z$ et al. Is rectal indomethacin effective in preventing of post-endoscopic retrograde cholangiopancreatography pancreatitis? World J Gastroenterol 2014

6. Elmunzer BJ, et al. A randomized trial of rectal indomethacin to prevent postERCP pancreatitis. N Engl J Med 2012

7. Koshbaten $\mathrm{M}$, et al. Role of diclofenac in reducing post-endoscopic retrograde cholangiopancreatography pancreatitis. J Gastroenterol Hepatol 2008

8. Levenick JM, et al. Rectal indomethacin does not prevent post-ERCP pancreatitis in consecutive patients. Gastroenterology 2016

9. Lua GW, et al. Can rectal diclofenac prevent post endoscopic retrograde cholangiopancreatography pancreatitis? Dig Dis Sci 2015

10. Montaño Loza A, et al. Effect of the administration of rectal indomethacin on amylase serum levels after endoscopic retrograde cholangiopancreatography, and its impact on the development of secondary pancreatitis episodes. Rev Esp Enferm Dig 2007

11. Murray B, et al. Diclofenac may reduce the incidence of acute pancreatitis after endoscopic retrograde cholangiopancreatography. Gastroenterology 2003

12. Otsuka T, et al. Low-dose rectal diclofenac for prevention of post-ERCP pancreatitis: a randomized controlled trial. J Gastroenterol 2012

13. Park SW, et al. Intramuscular diclofenac for the prevention of post-ERCP pancreatitis: a randomized trial. Endoscopy 2015

14. Patai Á, et al. Effect of rectal indomethacin for preventing post-ERCP pancreatitis depends on difficulties of cannulation. Results from a randomized study with sequential biliary intubation. J Clin Gastroenterol 2015

15. Senol A, et al. Efficacy of intramuscular diclofenac and fluid replacement in prevention of post-ERCP pancreatitis. World J Gastroenterol 2009

16. Sotoudehmanesh $\mathrm{R}$, et al. Indomethacin may reduce the incidence and severity of acute pancreatitis after ERCP. Am J Gastroenterol 2007

17. Zhao XW, et al. Effect of diclofenac on the levels of lipoxin A4 and resolvin D1 and E1 in the post-ERCP pancreatitis. Dig Dis Sci 2014

\section{P1553 RECTAL DICLOFENAC AND PANCREATITIS AFTER ENDOSCOPIC RETROGRADE CHOLANGIOPANCREATOGRAPHY \\ L. Del Olmo Martinez ${ }^{1}$, B. Velayos ${ }^{2}$, M. Ruiz-Rebollo ${ }^{2}$ \\ ${ }^{1}$ Gastroenterolgy, Hospital Clinico, Valladolid/Spain \\ ${ }^{2}$ Hospital Clinico Universitario, Valladolid/Spain}

Contact E-mail Address: ldelolmo@yahoo.es

Introduction: Rectal diclofenac or indomethacin reduces the risk of pancreatitis after endoscopic retrograde cholangiopancreatography (ERCP). Most studies of its efficacy included high-risk cohorts and excluded low-risk patients. We investigated the potential of rectal diclofenac to prevent post-ERCP pancreatitis (PEP) in a variety of patients.

Aims \& Methods: A cohort of 1534 ERCPs performed at the Hospital Clínico of Valladolid between 2009 and July 2016 was collected. The median age of the patients was 75 years old (between 12 and 102 years). $54 \%$ were male and $45.9 \%$ female. There were 93 procedures in which cannulation of the desired pathway was not achieved but the papilla had been manipulated so they are patients who have been included in the study. After may 2012, with a few exceptions, patients received diclofenac before their procedure. 730 patients did not receive Diclofenac. PEP was defined by consensus criteria.

Data regarding the factors predisposing to the development of PEP have been collected both in relation to the technique itself and to patient-related factors. A comparative study has been made between both groups using Chi square.

Results: The two groups were similar in age, sex, suspicion of Oddi sphincter dysfunction, recurrent acute pancreatitis, chronic pancreatitis, cannulation time$10 \mathrm{~m}$, use of pre-cut, previous PEP, dilation without sphincterotomy. There were differences in the number of sphincterotomies in which it was greater in the Diclofenac group (p.004). There was also a greater number of Wirsung cannulations in the group treated with Diclofenac (p.004). There were a total of 47 PEP $(3.1 \%)$, being $78.3 \%$ mild acute pancreatitis.

Taken as a whole the patients had no difference in the number of PEP between the two groups, since in those treated with Diclofenac there was $3.4 \%$ and in the non-treated patients $2.8 \%$.

When taking only patients with de novo sphincterotomy, there was no difference between the number of PEP between the two groups being $4.4 \%$ in those treated with Diclofenac versus $4 \%$ in the untreated patients. In those patients who were cannulated Wirsung, an incidence of PEP of $8.2 \%$ was observed in the group without Diclofenac, compared with $6.6 \%$ in the treated group (p.006). There were no differences between those treated with Wirsung's prosthesis and those not treated in both groups. There was no PEP in patients treated with pancreatic prosthesis.

There was a higher incidence of PEP in women in both groups and a trend towards greater number of PEP among those treated with Diclofenac, although without statistical significance. There was also a greater number of PEP in patients under 40 years of age treated with Diclofenac compared to those not treated with $14.3 \%$ versus $7.1 \%$ (p.024).

No differences were found between the groups treated and not treated with Diclofenac when crossing with sphincter dysfunction of Oddi, previous PEP, number and sizes of choledocholithiasis and sizes with the appearance of PEP. 
Conclusion: In this retrospective cohort study of patients undergoing ERCP that included low-risk patients, rectal diclofenac was not associated with a significant decrease in the absolute rate of pancreatitis. In our study, diclofenac decreases the impact of PEP in those patients who are cannulated the pancreas.

Disclosure of Interest: All authors have declared no conflicts of interest.

P1555 A PILOT STUDY OF PROBE-BASED CONFOCAL LASER ENDOMICROSCOPY FOR COMPUTER-AIDED DIAGNOSIS OF BILE DUCT CANCER BY USING THE DEEP LEARNING TECHNOLOGY

K. Furukawa ${ }^{1}$, N. Yokoyama ${ }^{2}$, H. Hashidate ${ }^{3}$

${ }^{1}$ Dept. Of Gastroenterology, Niigata General Hospital Dept. of Gastroenterology, Niigata/Japan

${ }^{2}$ Niigata City General Hospital Dept. of Digestive Surgery, Niigata/Japan

${ }^{3}$ Pathology, Niigata City General Hospital, Niigata/Japan

Contact E-mail Address: furukawa@hosp.niigata.niigata.jp

Introduction: The confocal laser endomicroscope (CLE) is of two types, an endoscope-based CLE (eCLE), which is integrated in the tip of the endoscope, and a probe-based CLE (pCLE), which goes through the accessory channel of the endoscope. The biliary tract, which cannot be reached by using eCLE, is observable with pCLE by using cholangioscopy. pCLE has the advantage of obtaining a magnification image that is like taking a biopsy tissue specimen but noninvasively, without the interference of bleeding and mucus secretion. However, it is sometimes difficult because only few gastroenterologists can achieve the required level of conventional diagnostic accuracy.

Aims \& Methods: We developed a computer-aided diagnosis (CAD) system based on pCLE imaging using deep learning technology. The purpose of this study was to determine the usefulness of this CAD system for the diagnosis of bile duct cancer. We prepared the classifier of the extracted features of the bile duct cancer pCEL images by using the deep learning framework presented by Kyocera communication system Co. Ltd. Japan. The pCLE images by Cellvisio (Mauna Kea Technologies, France) were obtained through the SpyGlass DS (Boston Scientific Corporation, USA). They were compared with the pathological examination results from the surgical specimen and biopsy using SpyBite (Boston Scientific Corporation, USA). Learning sets were constructed by using 49 images of normal area and 23 images of cancer lesion. The test sets of the pCLE images were constructed using 6 images of normal area and 14 images of cancer lesion, separately from the learning set.

Results: The accuracy, sensitivity for cancer diagnosis, specificity, negative-predictive value, positive-predictive value of our CAD system by test set were $69.8 \%, 50 \%, 100 \%, 53.8 \%$, and $100 \%$, respectively. The 7 false-negative diagnoses reduced the sensitivity and negative-predictive value. The images of 6 of the false-negative diagnoses, indicating a 1.0 probability, did not show signs of cancer at all. A constant diagnosis was possible while being extremely small learning sets. The specificity and positive-predictive value were good, and the pCLE image was thought to have a characteristic suitable for CAD by using deep learning technology. However, many false-negative diagnoses with a probability 1.0 may have occurred owing to deflection and the lack of learning sets. To improve precision, a teaching set of adequate quality and quantity is necessary. Conclusion: Automated diagnosis of bile duct cancer can be achieved by using the deep learning technology of pCLE imaging. Our CAD system will be improved with the appropriate learning sets.

Disclosure of Interest: All authors have declared no conflicts of interest.

P1556 COMPARISON OF EUS-GUIDED FINE NEEDLE BIOPSY TECHNIQUES FOR CORE TISSUE ACQUISITION AND DIAGNOSTIC PERFORMANCES IN PANCREATIC SOLID LESIONS

C. Cho ${ }^{1}$, S.J. Yeo ${ }^{1}$, A.N. $\mathrm{Seo}^{2}$, H.I. Bae ${ }^{2}$

${ }^{1}$ Internal Medicine, Kyungpook National University Medical Center, Daegu/ Korea, Republic of

${ }^{2}$ Pathology, Kyungpook National University Medical Center, Daegu/Korea, Republic of

Contact E-mail Address: cmcho@knu.ac.kr

Introduction: The acquisition of core tissue in endoscopic ultrasound-guided tissue sampling (EUS-TS) is necessary for histologic diagnosis and immunohistochemical staining in the diagnosis of some solid mass lesions. Although recent studies revealed the superiority of core biopsy needle in the specimen adequacy, controversy still remains that which EUS-TS techniques would result in better acquisition of core tissue and diagnostic accuracy.

Aims \& Methods: The aim of our study was to evaluate EUS-TS techniques with a ProCore needle using suction and slow pull suction for solid pancreatic lesions without an on-site cytopaphologist.Patients who referred to EUS-TS for pancreatic mass lesion were enrolled. We performed EUS-guided fine needle biopsy (EUS-FNB) using a ProCore needle (Cook Medical, Limerick, Ireland) with two needle passes and applied each pass of different techniques (suction or slow pull suction) which were randomly allocated. EUS-TS specimens were evaluated by one experienced cytopathologist who was blinded to applied techniques. The acquisition of core tissue and diagnostic performances were compared between two techniques.

Results: From Aug. 2014 to Dec. 2016, 94 patients with pancreatic mass were enrolled and 12 patients were excluded due to no final diagnosis $(n=5)$, cystic lesion $(n=5)$ and loss of follow up after EUS-TS $(n=2)$. Finally, 82 patients (48 males; median age, 63 years) with 164 needle passes were included without technical failure and procedure-related adverse events. The median size of the lesion was $26 \mathrm{~mm}$ (range, 11 to $80 \mathrm{~mm}$ ). There were 68 malignant and 14 benign lesions. Overall core tissue acquisition and diagnostic accuracy was $84.8 \%$ (139/ $164)$ and $73.2 \%(120 / 164)$, respectively. There was no significant difference between suction and slow pull suction in the acquisition of core tissue $(85.4 \%$ vs. $84.1 \%, p=1.000)$ and diagnostic accuracy $(72.0 \%$ vs. $74.4 \%, p=0.860)$ Conclusion: Although our study revealed no differences between EUS-TS techniques in the core tissue acquisition and diagnostic accuracy for pancreatic solid lesions, further prospective study including variable lesions and sizes of needle is needed to validate for optimal application and sequences of EUS-FNB techniques.

Disclosure of Interest: All authors have declared no conflicts of interest.

\section{P1560 EUS-GUIDED GALLBLADDER DRAINAGE FOR ACUTECHOLECYSTITIS WITH A SILICONE-COVERED NITINOI SHORT FLARED ENDS STENT: A CASE SERIES}

R. Manta ${ }^{1}$, C. Zulli ${ }^{2}$, F.P. Zito ${ }^{1}$, A. Zullo ${ }^{3}$, E. Forti ${ }^{4}$, A. Tringali,

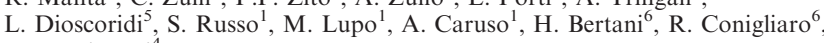
M. Mutignani

Aou Modena, Gastroenterology and Digestive Endoscopy Unit, Modena/Italy

${ }^{2}$ Endoscopy Unit, AOUI San Giovanni di Dio e Ruggi d'Aragona, G. Fucito Center, Mercato San Severino (SA)/Italy

${ }^{3}$ Gastroenterology Unit, Nuovo Regina Margherita' Hospital, Rome/Italy

${ }^{4}$ Diagnostic and Interventional Digestive Endoscopy, Niguarda Ca' Granda Hospital, Milan/Italy

${ }^{5}$ Endoscopy Unit, Niguarda-Ca' Granda Hospital, Milan/Italy

${ }^{6}$ U.O.C. Gastroenterology and Digestive Endoscopy Unit, Nuovo Ospedale Civile Sant'Agostino Estense, Modena/Italy

Contact E-mail Address: zulli.claudio@gmail.com

Introduction: Gallbladder drainage, performed by EUS-guided positioning of specially designed fully covered metal stents, may be consiedered a valid option in patients with cholecystitis unfit for surgery. We describe the first case series of patients with diagnosis of acute cholecistitis treated conservatively using a silicone-covered nitinol stent with bilateral anchor flanges (NAGI-stent).

Aims \& Methods: Our aim was to evaluate the feasibility and clinical impact of EUS-guided drainage with NAGI-stent in patients with acute cholecistitis unfit for surgery. Sixteen consecutive patients ( 9 males; Mean age: 84 years) with diagnosis acute cholecystitis according to Tokyo guidelines criteria, not suitable for surgical approach, were conservative treated and drained with EUS-guided short flared stents positioning. The procedure was performed in 2 tertiary endoscopic centers by 4 experienced endoscopists (>500 EUS/ERCP performed yearly), by using the NAGI-stent. Each attempt to access the gallbladder was firstly performed from the transduodenal position and resulted successful in 13 $(81 \%)$ patients, whilst a transgastric approach was preferred in the remaining 3 patients. Two different approaches were performed for the EUS-guided gallbladder puncture: a) a 0.035 -inch wire was advanced through a $19 \mathrm{G}$-needle into the gallbladder and dilation of the access was achieved with a $10 \mathrm{Fr}$ cystoenterostome; b) a 0.035 -inch wire was advanced through a $10 \mathrm{Fr}$ cystoenterostome directly used in order to achieve gallbladder access. At the end of the procedure, a fully covered metallic stent, $12-16 \mathrm{~mm}$ of diameter and $20-30 \mathrm{~mm}$ of length with bilateral anchor flanges (NAGI-stent) was advanced on the wire by using the fluoroscopy guide. Technical success, clinical success, adverse events, and longterm outcome were assessed.

Results: Technical success was achieved in all cases, clinical success was observed in $15(94 \%)$ patients, whilst in 1 case the procedure failed due to stones impaction into the stent but it resolved with a new stent positioning. Symptoms relief occurred in all patients, 1 day after the procedure in $12(75 \%)$ cases and 2 days later in remaining $4(25 \%)$ patients. A bleeding episode occurred in 2 $(12.5 \%)$ patients, in one case such complication was intra-procedural and it was successfully stopped during the same endoscopic session, in the other case it was a delayed adverse events requiring arterial embolization but the patient died 10 days later. At follow-up, two patients died due to myocardial infarction at 2 and 6 months, one for acute renal failure after 6 months, two for pancreatic cancer after 7 months and one for cholangiocarcinoma after 5 months. In the remaining patients no cholecystitis recurrence or biliary obstruction were observed at median follow-up of 112 days (range 49-180 days).

Conclusion: Our data showed that EUS-guided gallbladder drainage with NAGIstent is feasible and successful in patients with acute cholecystitis unfit for surgery. Since this type of stent is cheaper compared to others, the use of such device may result more attractive as a further endoscopic option for these selected patients.

Disclosure of Interest: All authors have declared no conflicts of interest.

P1561 EFFICIENCY COMPARISON BETWEEN 22 G VERSUS $25 \mathrm{G}$ NEEDLES DURING ENDOSCOPIC ULTRASOUND FINE NEEDLE ASPIRATION FOR SOLID PANCREATIC MASSES ASSESSMENT: A SYSTEMATIC REVIEW AND META-ANALYSIS BASED ON RCTS

H. G. Guedes, R. B. Duarte, D. T.H. De Moura, S. Cheng, M. Coronel, M. E.L. Dos Santos, S.E. Matuguma, D.M. Chaves, W. M. Bernardo, E.G. Hourneaux Moura

Gastrointestinal Endoscopy Unit, Clinical Hospital of São Paulo University Medical School, Sao Paulo/Brazil

Contact E-mail Address: ralphbduarte@hotmail.com 
Introduction: Endoscopic ultrasound guided-fine needle aspiration (EUS-FNA) is considered the gold standard method for assessment solid pancreatic masses. The needles for aspiration currently available are $19 \mathrm{G}, 22 \mathrm{G}$ and $25 \mathrm{G}$ and there is no concrete evidence to prove the benefit of one against another.

Aims \& Methods: We aimed to compare the efficiency in the diagnosis of solid pancreatic lesions through the EUS-FNA with $25 \mathrm{G}$ and $22 \mathrm{G}$ needles. Studies were analyzed from five databases (Medline, Scopus, Cochrane, LILACS and CINAHL), without year or language restriction, using an extensive search strategy. Only randomized trials comparing $22 \mathrm{G}$ and $25 \mathrm{G}$ needles were included. Two independent reviewers went through the literature search and the results were analyzed by fixed and random effects. The diagnostic characteristics were calculated for a $95 \%$ confidence interval.

Results: 504 studies were found in the search, of which 21 were read and then finally 04 randomized studies were selected to be included in the analysis. Thus, a total of 462 patients were evaluated (233: $25 \mathrm{G}$ needle/229: $22 \mathrm{G}$ needle). The sensitivity of the $25 \mathrm{G}$ needle was $93 \%$ (CI, 89-96\%; I2 $0.0 \%$ ), and for the $22 \mathrm{G}$ needle was $91 \%$ (CI, 85-94\%; I2 19.9\%). The specificity of the $25 \mathrm{G}$ needle was $87 \%$ (CI, 73-96\%; I2 41.1\%), and for the $22 \mathrm{G}$ needle was $83 \%$ (CI, 70-93\%; I2 81.1\%). The positive likelihood ratio of the $25 \mathrm{G}$ needle was 4.57 (CI, 2.08-10.03, I2 $0.0 \%$ ), and for the $22 \mathrm{G}$ needle was 4.26 (CI, 0.43-41.88, I $294.7 \%$ ). The post-test probability of the $25 \mathrm{G}$ needle in the study population was $93.85 \%$ and for the $22 \mathrm{G}$ needle was $91.30 \%$. The area under the sROC curve of the $25 \mathrm{G}$ needle was 0.9705 and for the $22 \mathrm{G}$ needle 0.9795 , also showing no statistically significant correlation between them $(p=0.497)$.

Conclusion: Based on randomized studies, this systematic review and meta-analysis did not demonstrate a significant statistical difference between the $22 \mathrm{G}$ and $25 \mathrm{G}$ needles used during EUS-FNA in the diagnosis of solid pancreatic lesions. Disclosure of Interest: All authors have declared no conflicts of interest.

\section{References}

1. Beckingham IJ. ABC of diseases of liver, pancreas, and biliary system. Gallstone disease. BMJ 2001; 322: 91-94

2. Lucas AL, Malvezzi M, Carioli G et al. Global Trends in Pancreatic Cancer Mortality From 1980 Through 2013 and Predictions for 2017. Clin Gastroenterol Hepatol 2016; 14: 1452-1462.e1454

3. de la Santa LG, Retortillo JA, Miguel AC et al. Radiology of pancreatic neoplasms: An update. World J Gastrointest Oncol 2014; 6: 330-343

4. DeWitt J, Devereaux B, Chriswell M et al. Comparison of endoscopic ultrasonography and multidetector computed tomography for detecting and staging pancreatic cancer. Ann Intern Med 2004; 141: 753-763

5. Xu MM, Sethi A. Imaging of the Pancreas. Gastroenterol Clin North Am 2016; 45: 101-116

6. Eloubeidi MA, Decker GA, Chandrasekhara V et al. The role of endoscopy in the evaluation and management of patients with solid pancreatic neoplasia. Gastrointest Endosc 2016; 83: 17-28

7. Eloubeidi MA, Jhala D, Chhieng DC et al. Yield of endoscopic ultrasoundguided fine-needle aspiration biopsy in patients with suspected pancreatic carcinoma. Cancer 2003; 99: 285-292

8. Bang JY, Ramesh J, Trevino J et al. Objective assessment of an algorithmic approach to EUS-guided FNA and interventions. Gastrointest Endosc 2013; 77: 739-744

9. Kamata K, Kitano M, Omoto S et al. New endoscopic ultrasonography techniques for pancreaticobiliary diseases. Ultrasonography 2016; 35: 169 179

10. Madhoun MF, Wani SB, Rastogi A et al. The diagnostic accuracy of 22gauge and 25-gauge needles in endoscopic ultrasound-guided fine needle aspiration of solid pancreatic lesions: a meta-analysis. Endoscopy 2013; 45: 86-92

11. Hewitt MJ, McPhail MJ, Possamai L et al. EUS-guided FNA for diagnosis of solid pancreatic neoplasms: a meta-analysis. Gastrointest Endosc 2012; 75: $319-331$

12. Lee JK, Lee KT, Choi ER et al. A prospective, randomized trial comparing 25-gauge and 22-gauge needles for endoscopic ultrasound-guided fine needle aspiration of pancreatic masses. Scand J Gastroenterol 2013; 48: $752-757$

13. Affolter KE, Schmidt RL, Matynia AP et al. Needle size has only a limited effect on outcomes in EUS-guided fine needle aspiration: a systematic review and meta-analysis. Dig Dis Sci 2013; 58: 1026-1034

\section{P1562 OUTCOMES AND LEARNING CURVES OF EUS-GUIDED GALLBLADDER DRAINAGE WITH LUMEN APPOSING STENTS}

A. Y.B. Teoh ${ }^{1}$, S. M. Chan ${ }^{1}$, H. C. Yip ${ }^{1}$, V. Wong ${ }^{1}$, P.W.Y. Chiu ${ }^{1}$, E. $\mathrm{Ng}^{1}$

${ }^{1}$ Surgery, Chinese University of Hong Kong, Hong Kong/Hong Kong PRC

Contact E-mail Address: anthonyteoh@surgery.cuhk.edu.hk

Introduction: EUS-guided gallbladder drainage (EGBD) is gaining popularity as an alternative method for drainage of the gallbladder in patients suffering from acute cholecystitis that are at high-risk for cholecystectomy. However, the longterm outcomes and the learning curves of the procedure is uncertain.

Aims \& Methods: This was a retrospective review of all patients that received EGBD in the Prince of Wales Hospital between June 2012 to March 2017. Al procedures were performed or supervised by a single operator. EGBD was performed in patients that are at high-risk for cholecystectomy and suffering from acute cholecystitis or on long-term cholecystostomy tube drainage. Outcome parameters included demographics, technical and clinical success, procedural characteristics, adverse events and follow-up duration.
Results: 50 patients were recruited during the study period. The mean (S.D.) age was 82.34 (9.3) years old and 22 patients were male. 42 patients were American Society of Anesthesiologist grading of $\geq 3$ and the mean (S.D.) age-adjusted Charlson comorbidity index was 5.96 (1.82). The mean (S.D.) size of the gallbladder was $9.94(9.83) \mathrm{cm}$. The hospital stay was $6.78(5.36)$ days and follow-up duration was 664.65 (541.58) days. The overall technical success and clinical success rates were $96 \%$ and $86 \%$ respectively. Clinical success was not achieved in 7 patients as they suffered from 30-day mortality. 6 were due to uncontrolled sepsis and multi-organ failure even after adequate gallbladder drainage. 1 patient had aspiration pneumonia resulting in death. 15 patients $(30 \%)$ suffered from 30 day adverse events with 6 of these being attributable to the procedure. Maldeployment of the stent occurred in 6 patients and this was managed by placement of an additional metallic stent to bridge the gallbladder to the gastric or duodenal lumen. Recurrent cholecystitis occurred in 1 patient $(2 \%)$ during follow-up.

When comparing the first 25 procedures to the subsequent 25 procedures, significant differences were observed in the procedural time [29.21 (10.65) vs 15.6 (1.66) minutes, $\mathrm{P}<0.001)$, the need of an additional stent $[24 \%$ vs $0 \%$, $\mathrm{P}=0.022]$ and hospital stay [7.76 (5.13) vs 5.75 (5.50) days, $\mathrm{P}=0.009]$. Whilst no differences were observed in the technical and clinical success rates and adverse events.

Conclusion: EGBD is a safe and effective method for achieving gallbladder drainage in patients that are at high-risk for cholecystectomy. Performance of 25 procedures are required to gain competency in the procedure.

Disclosure of Interest: A.Y.B. Teoh: I am a Consultant for Boston Scientific, Taewoong Medical and Cook medical Corporation

All other authors have declared no conflicts of interest.

\section{P1563 TECHNICAL FEASIBILITY STUDY OF EUS-GUIDED HYDROGEL MICROPARTICLE INJECTION INTO THE PANCREATIC HEAD-DUODENAL WALL INTERFACE IN A CADAVERIC MODEL}

S. Kim ${ }^{1}$, K. Ding ${ }^{2}$, A. Rao ${ }^{2}$, L. Rosati ${ }^{2}$, A. Narang ${ }^{2}$, E.J. Shin ${ }^{1}$

${ }^{1}$ Gastroenterology And Hepatology, Johns Hopkins University School of Medicine, Baltimore/United States of America/MD

${ }^{2}$ Department Of Radiation Oncology And Molecular Radiation Sciences, Johns

Hopkins University School of Medicine, Baltimore/United States of America/MD

Contact E-mail Address: eshin3@jhmi.edu

Introduction: Despite advances in radiotherapy for pancreatic cancer, local gastrointestinal (GI) toxicity still remains one of the major limitations to effective dose delivery and further dose escalation due to the close proximity of the GI wall to the pancreas, particularly in the head region. One potential method to reduce local GI toxicity would be to increase the physical distance between the head of the pancreas and the duodenal wall. A novel, injectable hydrogel, synthesized as iodinated polyethylene glycol microparticles, has been FDA-approved for use as a soft tissue fiducial marker. The hydrogel remains stable for 3 months and is absorbed by 7 months. To date, there has been no reports on the technical feasibility of endoscopic ultrasound (EUS)-guided hydrogel injection into the interface between the head of the pancreas and the duodenal wall to increase the peri-pancreatic space for the course of radiotherapy.

Aims \& Methods: We aimed to evaluate the technical feasibility of EUS-guided hydrogel injection into the interface between the head of the pancreas and the duodenal wall in a cadaveric model. Baseline CT was performed on three unfixed cadaveric specimens. Using a linear EUS scope, the interface between the duodenal wall and the head of the pancreas was identified in a cadaveric model. A 19-gauge FNA needle was used to inject the hydrogel into the peri-pancreatic space with creation of a visible separation between the duodenal wall and the pancreatic parenchyma. The procedure was repeated along the length of the head and uncinate of the pancreas. CT was performed post procedure to confirm location and to measure the distance created between the duodenum and pancreas. Gross dissection of the pancreas and duodenum was performed to evaluate localization of the hydrogel.

Results: All three cadavers underwent successful EUS-guided injection of the hydrogel. Cadaver 1 received a total injection volume of $9.5 \mathrm{cc}$ with creation of peri-pancreatic space along the head of the pancreas measuring $11.77 \mathrm{~mm}$ in maximal diameter. Cadaver 2 received a total injection volume of $27 \mathrm{cc}$ with creation of peri-pancreatic space along the head and uncinate of the pancreas measuring $13.20 \mathrm{~mm}$ in maximal diameter. Cadaver 3 received a total injection volume of $10 \mathrm{cc}$ with creation of peri-pancreatic space along the head of the pancreas measuring $12.89 \mathrm{~mm}$ in maximal diameter. The hydrogel was clearly visualized during EUS with hyperechoic echogenicity and on post-procedure CT images without any artifacts in all cases.

Conclusion: EUS-guided delivery of hydrogel is feasible and results in an increase in the peri-pancreatic space in a cadaveric model. The hydrogel is clearly visualized on EUS and CT without significant artifacts. Further studies are warranted to evaluate feasibility, effectiveness and safety in a clinical model.

Disclosure of Interest: All authors have declared no conflicts of interest. 


\section{P1564 EX-VIVO RADIOFREQUENCY ABLATION OF PORCINE} LIVER: A PRELIMINARY STUDY OF EFFICACY OF A NEW SYSTEM

G. Rossi ${ }^{1}$, M.C. Petrone ${ }^{1}$, E. Dabizzi ${ }^{1}$, A. Mariani ${ }^{1}$, S.G.G. Testoni ${ }^{1}$, M. Traini ${ }^{1}$, L. Archibugi ${ }^{2}$, P. Magnoni ${ }^{1}$, P.G. Arcidiacono

${ }^{1}$ Pancreato-biliary Endoscopy And Endosonography Division, Pancreas

Translational \& Clinical Research Center San Raffaele Scientific Institute IRCCS

Vita Salute San Raffaele University, Milan/Italy

${ }^{2}$ Digestive \& Liver Disease Unit, S. Andrea Hospital - University of Rome "La Sapienza", Rome/Italy

\section{Contact E-mail Address: rossi.gemma@hsr.it}

Introduction: There are few published studies about the use of a novel radiofrequency (RF) system (EUSRA RF needle; VIVA RF generator; STARmed Co, Ltd; Koyang, Korea), with poor standardization of the procedure in terms of ablation powers and ablation times, resulting in great heterogeneity of the results.

Aims \& Methods: To standardize the radiofrequency ablation (RFA) procedure using this new system performing ex-vivo tests on porcine liver in order to find the best ablation power and ablation time to produce the maximum size of coagulative necrosis at histological examination. The system consists in a radiofrequency generator delivering electric energy, a 19 Gauge needle $(150 \mathrm{~cm}$ in length with a $10 \mathrm{~mm}$ monopolar electrode), a peristaltic pump (to perfuse the needle with chilled saline solution, maximizing the ablation volume without tissue charring), an isolating plate and a pedal to deliver RFA. Liver samples were treated at different powers: 10, 20, 30 and 40 Watts (W); each ablation power was applied for a duration of $1,3,5,7$ and 15 minutes, according to Fibonacci escalation dose scheme, used in phase I studies. We registered macroscopically: the size (millimeters) of the global treated area and the size of the central necrotic core. Microscopically an expert pathologist, blinded about ablation powers and times applied, gave the report of the histological examination (millimeters of coagulative necrosis and surrounded zone).

Results: The lower ablation power $(10 \mathrm{~W})$ produced the maximum macroscopic ablated area. RF ablation time at $10 \mathrm{~W}$ showed a good linear correlation with both the sizes of total macroscopic ablated area $(\mathrm{R}=0.92)$ and central macroscopic ablated area $(R=0.89)$. Histologically the pathologist described two different injured zones by thermal effect: a central small and well-demarcated coagulative necrotic area around the needle insertion point (A zone) with a maximum diameter of 4 millimeters and a surrounded larger area of "diaphanization" (B zone), showing mild signs of cellular alterations (cytoplasmic hypochromia) without cellular necrosis. A zone sizes didn't change among different ablation times (mean size: $3.25 \mathrm{~mm}$ ) while B zone diameter increased with the increase RF application at the fixed power of $10 \mathrm{~W}$. At the microscopic analysis the pathologist didn't see any difference in size of coagulative necrosis among the different ablation powers $(\mathrm{R}=0.24)$

Conclusion: RFA with this new system is feasible and effective to produce very small areas of coagulative necrosis (millimeters) well-demarcated in respect to the surrounding parenchyma and could be useful, in the future, to treat, with multiple passes and higher precision, target lesions with a flexible needle. Moreover, the system can produce larger zones of mild cellular alterations at lower ablation powers $(10 \mathrm{~W})$, increasing with the increase of ablation times, but it needs future in-vivo animal studies in order to assess the evolution of these zones (evolving into fibrosis? necrosis? recovering?).

Disclosure of Interest: All authors have declared no conflicts of interest.

\section{References}

1. Wu Y, Tang Z, Fang H, et al. High operative risk of cool-tip radiofrequency ablation for unresectable pancreatic head cancer. J Surg Oncol 2006; 94:3925.

2. Kim HJ, Seo DW, Hassanuddin A, et al. EUS-guided radiofrequency ablation of the porcine pancreas. Gastrointest Endosc 2012; 76:1039-43.

3. Lathikia et al, Ramchandani M, Galasso D, et al EUS-guided radiofrequency ablation for management of pancreatic insulinoma by using a novel needle electrode (with videos). Gastrointest Endosc 2016; 83:234-239.

\section{P1565 THROMBOEMBOLIC DISEASE DIAGNOSED BY ENDOSCOPIC ULTRASOUND IN PANCREATIC CANCER: A CASE SERIES}

C. Atalaia Martins ${ }^{1}$, P. Pinto-Marques ${ }^{1}$, I. Mocanu ${ }^{1}$, J. Cortez-Pinto ${ }^{1}$, D. Serra ${ }^{2}$ ${ }^{1}$ Gastrenterology, Hospital Garcia de Orta, Almada/Portugal

${ }^{2}$ Gastrenterology, Hospital da Luz, Lisboa/Portugal

Contact E-mail Address: catarinatalaiamartins@gmail.com

Introduction: Malignant associated thromboembolic disease (TED) has a complex multifactorial pathogenesis. Tumor cells activate platelets and express procoagulant factores including tissue factor and thrombin; in addition, normal host tissues may express procoagulant activity in response to the tumor. Thrombotic risk varies substantially according to cancer location and pancreatic cancer is one of the leading causes. The clinical spectrum includes migratory superficial thrombophlebitis, arterial thrombosis, deep venous thrombosis, portal vein thrombosis and disseminated intravascular coagulation. We ought to assess the role of endoscopic ultrasound (EUS) diagnosing TED in pancreatic cancer patients.

Aims \& Methods: We perform a retrospective review of all EUS cases for pancreatic cancer in two centers and assess all TED events diagnosed.

Results: In a period of 6 months, a total of 55 EUS for pancreatic neoplasms were performed in two centers. TED was present in 5 patients $(9 \%)$ : 3 were male and the mean age was 70 (range, 46-81). In 1 patient the EUS indication was a large abdominal mass whose origin was not clear, in the remaining 4 the indication was the pancreatic neoplasm. In all of them was performed EUS with fine-needle aspiration. EUS identified 4 arterial pulmonary embolism (PE) and 1 inferior vena caval thrombosis (IVCT) with right atrial extension: $2(3,6 \%)$ had recently been diagnosed by computed tomography (CT) but $3(5,4 \%)$ were not previously known. In all these, CT confirmed diagnosis.

Table 1: Demographic, clinical and ultrasonographic characteristics of the patients.

\begin{tabular}{|c|c|c|c|c|c|c|c|}
\hline & $\begin{array}{l}\text { Gender } \\
(\mathrm{F} / \mathrm{M})\end{array}$ & Age & $\begin{array}{l}\text { Neoplasm } \\
\text { e location }\end{array}$ & $\begin{array}{l}\text { Cytology } \\
\text { results }\end{array}$ & $\begin{array}{l}\text { Thromboembolic } \\
\text { disease }\end{array}$ & $\begin{array}{l}\text { Thromboembolic } \\
\text { disease } \\
\text { previously } \\
\text { known? } \\
\text { (Yes/No) }\end{array}$ & $\begin{array}{l}\text { CT } \\
\text { confirmation? } \\
\text { (Yes/No) }\end{array}$ \\
\hline Case & & 81 & Isthmus & $\begin{array}{l}\text { Leiomyosarcoma } \\
\text { metastasis }\end{array}$ & & Yes & Yes \\
\hline Case & & 70 & Tail & Adenocarcinoma & EP & No & Yes \\
\hline Case & & 81 & Head & Not available yet 1 & EP & No & Yes \\
\hline Case & & 46 & Tale & $\begin{array}{l}\text { Neuroendocrine } \\
\text { tumor }\end{array}$ & IVCT & Yes & Yes \\
\hline Case & & 72 & $\begin{array}{l}\text { Uncinate } \\
\text { process }\end{array}$ & Adenocarcinoma & EP & No & Yes \\
\hline
\end{tabular}

Conclusion: To the best of our knowledge, this is the first case series of EUSbased TED diagnosis in pancreatic cancer patients. This series underlines importance of a systematic, station approach EUS technique, namely in the mediastinum regardless the clinical indication. TED is a common complication of pancreatic cancer and has major therapeutic and prognostic implications. Disclosure of Interest: All authors have declared no conflicts of interest.

\section{References}

1. Robbins D. Pulmonary embolism diagnosed with EUS on a patient with adenocarcinoma of the pancreas. Gastrointest Endosc 2013; 78 (3): 542-43

2. Khorana AA., Fine RL. Pancreatic cancer and thromboembolic disease. Lancet Oncol 2004; 5(11): 655-63

3. Shah MM., Saif MW. Pancreatic cancer and Thrombosis. J Pancreas 2010; 11(4): $331-33$

\section{P1566 THE ROLE OF EARLY ENDOSCOPIC ULTRASONOGRAPHY FOLLOWING TRANSABDOMINAL ULTRASONOGRAPHY IN PATIENTS WITH SUSPECTED BILIARY COLIC}

B. Mangiavillano ${ }^{1}$, L. Frazzoni ${ }^{2}$, R. Grassia ${ }^{3}$, M. Flavia Savarese ${ }^{4}$,

M. Bianchetti ${ }^{1}$, A. Repici ${ }^{5}$, L. Fuccio ${ }^{2}$

${ }^{1}$ Gastrointestinal Endoscopy Unit, Humanitas - Mater Domini, Castellanza (VA)/ Italy

${ }^{2}$ Department Of Medical And Surgical Sciences, S. Orsola-Malpighi Hospital, Bologna/Italy

${ }^{3}$ Gastroenterology, ASST Cremona, Cremona/Italy

${ }^{4}$ Gastrointestinal Endoscopy, Borea Hospital, Sanremo (IM)/Italy

${ }^{5}$ Dept. Of Gastroenterology, Ist. Clinico Humanitas Rozzano Dept. of Gastroenterology, Milano/Italy

Contact E-mail Address: b mangiavillano@hotmail.com

Introduction: Choledocholithiasis is the most common cause of biliary pain, leading to cholangitis and gallstone pancreatitis. Patients affected by cholelithiasis presents an incidence of choledocholithiasis ranging from $8 \%$ to $20 \%$. When the suspicion of choledocholithiasis is confirmed, stones should be removed by ERCP, but this operative measurement is associated with high rates of adverse events as post-ERCP pancreatitis, bleeding or perforation. A correct diagnosis of choledocholithiasis, before ERCP, is mandatory to decrease the operative risk and health care costs. Endoscopic ultrasound (EUS) has a high sensitivity and specificity in the diagnosis of CBD stones and could substitute other imaging modalities as CT-scan or MRCP, when indicated.

Aims \& Methods: The aim of our study was to assess the role of early EUS $(<48$ hours), in patients undergone US in emergency room for suspected biliary colic. We retrospectively evaluated all the patients arrived at first aid for suspected biliary colic (i.e. right upper quadrant pain and/or epigastric region, associated with an elevation in serum ALT, AST, GGT, ALP, or total bilirubin, but in absence of amylase or lipase elevation). All patients, irrespective of the finding at the US, performed an EUS within 48 hours since admission. Data are presented as proportions with $95 \% \mathrm{CI}$ and mean \pm standard deviation (SD). Correlation between categorical variables was evaluated by computing the "phi" coefficient. We computed the number needed to misdiagnose, i.e. the number of patients who need to be tested in order for one to be misdiagnosed by the test, as $1 /$ (1-diagnostic accuracy). All the analyses were run with $\mathrm{R}$ statistical software.

Results: Overall, from January 2016 to December 2016, 88 patients (56\% female; mean age $64 \pm 17$ years) were admitted to our hospital for suspected biliary colic. All of these subjects underwent abdominal ultrasound (US) at admission, which identified gallstones in 47 patients $(71 \%)$; further, US documented common bile duct (CBD) stones in $58(65 \%)$ patients, CBD sludge in $4(5 \%)$ subjects, whereas no choledocholithiasis was found in $26(30 \%)$ patients. At EUS examination CBD stones were found in $70(80 \%)$ patients. Comparing US to EUS, US gave false negative results in $16(18 \%)$ cases and false positive findings (i.e. identifying CBD stones not documented by EUS) in $8(9 \%)$ patients. The two diagnostic procedures showed little correlation $(\mathrm{phi}=0.289)$. The number of 
patients needed to be tested by US in order to provide an incorrect diagnosis was 3.7 (95\% CI: 2.6-5.5).

Conclusion: US performed in the emergency room has a low diagnostic performance compared to EUS, but remains a first-step-approach in patients with right upper quadrant pain and/or in epigastric region, associated with an elevation in liver enzyme. Based on the results of our study, EUS performed within 48 hours from the admission allows an immediate correct endoscopic treatment with significant spare of unnecessary operative procedures, reducing possible related complications and costs.

Disclosure of Interest: All authors have declared no conflicts of interest.

\section{P1567 BILIOPANCREATIC RADIOFREQUENCY ABLATION: COMPARISON OF THE THREE CURRENTLY AVAILABLE DEVICES IN A PIG MODEL}

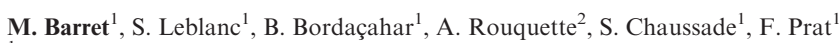
${ }^{1}$ Gastroenterology, Cochin Hospital, Paris/France

${ }^{2}$ Pathology, Cochin Hospital, Paris/France

\section{Contact E-mail Address: maximilien.barret@aphp.fr}

Introduction: Three devices are currently available to perform radiofrequency ablation (RFA) of biliopancreatic lesions. Data from animal models are scarce. Aims \& Methods: Radiofrequency ablation was performed in four live pigs on the common bile duct and the liver parenchyma using an endobiliary probe (endoHBP), on the liver and pancreatic parenchyma using an RFA catheter through an echoendoscope and biopsy needle (EUS RFA) and using a needleshaped RFA catheter (EUSRA) through an echoendoscope. Radiofrequency ablation time and power were allowed to vary. The animals were sacrificed 2 hours after the procedure. Histopathological assessment of the maximal depth of thermal lesions was performed on three representative slides for each RFA impact.

Results: In the common bile duct, the depth of ablation ranged from $215 \pm 47$ $($ Power $=8 \mathrm{~W}$, Time $=90 \mathrm{~s})$ to $330 \pm 43 \mu \mathrm{m}($ Power $=10 \mathrm{~W}$, Time $=30 \mathrm{~s})$, suggesting that power is the most important parameter in this location. Conversely, depth of ablation in the liver parenchyma using the endoHBP probe ranged from $947 \pm 237 \mu \mathrm{m}($ Power $=10 \mathrm{~W}$, Time $=30 \mathrm{~s})$ to $1960 \pm 20 \mu \mathrm{m}($ Power $=10 \mathrm{w}$, Time $=180 \mathrm{~s}$ ), suggesting that time is the most important parameter for RFA in the liver. The EUS RFA probe in the liver parenchyma showed a tissue necrosis increasing with the power setting used, ranging from $1903 \pm 451 \mu \mathrm{m}$ $($ Power $=8 \mathrm{~W}$, Time $=120 \mathrm{~s})$ to $2457 \pm 1047 \mu \mathrm{m}$ (Power $=12 \mathrm{~W}$, Time $=120 \mathrm{~s}$ ). This was not observed in the pancreatic parenchyma, were tissue damage ranged from $3108 \pm 373 \quad$ (Power $=8, \quad$ Time $=120 \mathrm{~s}$ ) to $2305 \pm 78 \mu \mathrm{m}$ (Power $=12$, time $=120 \mathrm{~s}$ ). The EUSRA ablation of the liver parenchyma resulted in tissue damage from $1573 \pm 245 \mu \mathrm{m}($ Power $=30 \mathrm{w}$, Time $=11 \mathrm{~s}$ ) to $1545 \pm 120 \mu \mathrm{m} \quad($ Power $=70 \mathrm{~W}$, Time $=9 \mathrm{~s})$. In the pancreas, ablation depth ranged from $3616 \pm 475 \mu \mathrm{m} \quad($ Power $=30 \mathrm{~s}, \quad$ Time $=15 \mathrm{~s}) \quad$ to $3805 \pm 446 \mu \mathrm{m}$ $($ Power $=70 \mathrm{~W}$, Time $=11 \mathrm{~s})$.

Conclusion: Power and time of ablation have different effects on the extent of tissue necrosis in the common bile duct, the hepatic and pancreatic parenchyma, depending on the type of catheter used to perform RFA. As indications for hepatobiliopancreatic RFA tend to expand, specific ablation protocols should be developed for each tumor location and device.

Disclosure of Interest: All authors have declared no conflicts of interest.

\section{P1568 ENDOSCOPIC ULTRASOUND-GUIDED RENDEZVOUS FACILITATES BILIARY CANNULATION IN CASE OF INACCESSIBLE INTRA-DIVERTICULAR PAPILLA}

D. Kypraios, A. Malachias, L. Theodoropoulos, S.L. Hatzinicolaou, S. Stavrinidis, N. Saribegioglou, G. Sofianidis, K. Tsamakidis,

D. Dimitroulopoulos, D. Xinopoulos

Gastroenterology, Saint Savvas Oncological Hospital, Athens/Greece

\section{Contact E-mail Address: dimkypreos@hotmail.com}

Introduction: Endoscopic ultrasound (EUS)-guided rendezvous techniques facilitate common bile duct (CBD) access during subsequent endoscopic retrograde cholangio-pacreatography (ERCP) in a single session. Cases of initial ERCP failure mainly comprise malignant biliary or ampullary infiltration and altered anatomy of the papilla, the former accounting for the majority of reports in the literature.

Aims \& Methods: We aimed to evaluate the efficacy and safety of EUS-guided rendezvous in a series of distal CBD obstruction with failed initial ERCP, due to inaccessible intra-diverticular papilla. Consecutive patients with distal CBD obstruction, in whom selective biliary cannulation at ERCP was unsuccessful due to large duodenal diverticula, underwent EUS-guided rendezvous. CBD puncture was performed via the transduodenal approach and the guide wire was advanced antegradely across the papilla. The echoendoscope was then exchanged for a duodenoscope and a sphincterotome was inserted through the papilla alongside or over the wire, to allow further manipulations.

Results: In a total of 2480 ERCPs performed over a 4-year period, 18 cases were selected to undergo EUS-guided rendezvous due to the presence of a large ampullary diverticulum. Primary indication for ERCP was CBD stones in 15 patients, pancreatic head cancer in 2 patients and cholangiocarcinoma in 1 patient. Mean age of the patients was 77 years (range 62-91) and mean diameter of the CBD was $16 \mathrm{~mm}$ (range 8-21). Successful CBD puncture with antegrade passing of the wire into the duodenum and subsequent ERCP, in the same session, was achieved in $2 / 3(66.6 \%)$ cases of malignant obstruction and in $13 / 15(86.6 \%)$ cases of lithiasis. Retrograde billiary cannulation during ERCP was performed over the wire in 6 cases and alongside the wire in 12 . The mean procedural time was 80 minutes (range 55-115). A case of inadvertent CBD wall penetration by the sphincterotome, with contrast extravasation, occurred during an over-the-wire cannulation. No major complications, i.e perforation (extra-luminal air or bile leakage), bleeding and pancreatitis occurred. Three cases of amylasemia and transient fever were noted.

Conclusion: EUS-guided rendezvous is an effective salvage technique for failed CBD cannulation via standard ERCP, in cases of inaccessible papilla due to large ampullary diverticula. Along the guide-wire billiary cannulation may prevent inadvertent CBD wall trauma, compared to the over-the-wire approach.

Disclosure of Interest: All authors have declared no conflicts of interest.

\section{References}

1. Tsuchiya T, Itoi T, Sofuni A, Tonozuka R, Mukai S. Endoscopic ultrasonography-guided rendezvous technique. Dig Endosc. 2016 Apr;28 Suppl 1:96101. Review. PubMed PMID: 26786389.

2. Iwashita T, Yasuda I, Mukai T, Iwata K, Ando N, Doi S, Nakashima M, Uemura S, Mabuchi M. Shimizu M. EUS-guided rendezvous for difficult biliary cannulation using a standardized algorithm: a multicenter prospective pilot study (with videos). Gastrointest Endosc. 2016 Feb;83(2):394-400. doi: 10.1016/j.gie.2015.04.043. Epub 2015 Jun 16. PubMed PMID: 26089103.

3. Isayama H, Nakai Y, Kawakubo K, Kawakami H, Itoi T, Yamamoto N, Kogure H, Koike K. The endoscopic ultrasonography-guided rendezvous technique for biliary cannulation: a technical review. $J$ Hepatobiliary Pancreat Sci. 2013 Apr;20(4):413-20. doi: 10.1007/s00534-012-0577-8. Review. PubMed PMID: 23179560

4. Ustundag Y, Alper E, Parlak E. EUS-guided rendezvous instead of precut techniques as a rescue measure when selective biliary cannulation fails: is it replacement or misplacement? Gastrointest Endosc. 2012 Aug;76(2):466-7; author reply 467. doi: 10.1016/j.gie.2012.02.013. PubMed PMID: 22817802.

5. Iwashita T, Lee JG. Endoscopic ultrasonography-guided biliary drainage: rendezvous technique. Gastrointest Endosc Clin N Am. 2012 Apr;22(2):24958, viii-ix. doi: 10.1016/j.giec.2012.04.018. Epub 2012 May 9. Review. PubMed PMID: 22632947

\section{P1569 A RANDOMIZED CONTROL TRIAL ASSESSING THE CONTRAST ENHANCED GUIDED FINE NEEDLE ASPIRATION IN SOLID PANCREATIC NEOPLASMS}

A. Seicean ${ }^{1}$, A. Samarghitan ${ }^{2}$, I. Rusu ${ }^{2}$, M. I. Gheorghiu ${ }^{2}$, C. Pojoga ${ }^{2}$, R. Seicean ${ }^{3}$

${ }^{1}$ University of Medicine and Pharmacy Iuliu Hatieganu, Cluj Napoca/Romania

${ }^{2}$ Regional Institute of Gastroenterology and Hepatology Prof. Dr. Octavian Fodor, Cluj Napoca/Romania

${ }^{3}$ First Surgical Clinic, Iuliu Hatieganu University of Medicine and Pharmacy, ClujNapoca/Romania

Contact E-mail Address: andradaseicean@gmail.com

Introduction: The preoperative differentiation of the solid pancreatic neoplasms by endoscopic ultrasound fine needle aspiration (EUS-FNA) remains around $90 \%$ and different needles or techniques of sampling has been used for improving the results. Data about the progress in diagnosis when the contrast harmonic enhanced EUS- FNA (CH-EUS-FNA) is used are scarced.

Aims \& Methods: We aimed to assess the role of contrast-enhanced EUS-FNA compared to standard EUS-FNA in diagnosing the solid pancreatic neoplasms. Methods: Patients from one tertiary medical center with visible solid pancreatic mass on CT scan were included. EUS-FNA(one pass) and CH-EUS-FNA (one pass) were performed randomly in each patients by using a standard $22 \mathrm{G}$ needles, an Olympus-Aloka equipment and Sonovue as contrast agent. Core histology was assessed separately for each pass by the same pathologist blinded from the randomization process. The final diagnosis was based on the results of EUSFNA and surgery, or the findings after 9 months' follow-up.

Results: The final diagnosis of 40 patients included was adenocarcinoma $(n=36)$, neuroendocrine tumors $(n=2)$, chronic pancreatitis $(n=2)$. The lesions were located in the head of the pancreas $(60 \%)$, body $(32 \%)$ and tail $(8 \%)$. The diagnostic sensitivity and specificity based on core histology was $89 \%$ and $94 \%$ in the CH-EUS-FNA passes and $86 \%$ and $91 \%$ in the EUS-FNA passes and the diagnostic value was significantly better in the CH-EUS-FNA group $(\mathrm{p}=0.0046$, $\mathrm{t}$-test $)$. The visual core size was not significant for the true- positive diagnosis of malignancy.

Conclusion: In a randomized control trial, CH-EUS-FNA improved significantly the diagnosis of solid pancreatic neoplasms over standard EUS-FNA. These techniques are complementary, not competitive, and they can be performed in the same session, resulting the increasing of the diagnostic rate with a minimum of passes.

Disclosure of Interest: All authors have declared no conflicts of interest. 
P1570 EARLY CAPSULE ENDOSCOPY PROVIDES BETTER BLEEDING LOCALIZATION VALUE IN PATIENTS PRESENTING WITH NON-HEMATEMESIS GASTROINTESTINAL BLEEDING WHEN COMPARED TO CLINICAL SYMPTOMS ALONE

S. Jawaid ${ }^{1}$, N. Marya ${ }^{2}$, A. Foley ${ }^{1}$, C. Marshall ${ }^{1}$, K. Bhattacharya ${ }^{1}$, D. Cave ${ }^{1}$ ${ }^{1}$ University of Massachusetts Medical Center, Worcester/United States of America ${ }^{2}$ UCLA, Los Angeles/United States of America

\section{Contact E-mail Address: sajawaid@gmail.com}

Introduction: Traditionally, clinical symptoms such as melena were used as strong predictors for an upper GI bleeding source with primary evaluation with an EGD (esophagogastroduodenoscopy). Little consideration was given to the small bowel. It has been known for decades that melena can originate from the nose to the right colon and hematochezia can originate from the proximal gut to the rectum. Thus, current endoscopic approaches have limited localization value and diagnostic yields. We hypothesize capsule endoscopy (VCE) provides better localization of bleeding when compared to clinical symptoms alone.

Aims \& Methods: In this study, we compare the efficacy of localizing the source of bleed in early VCE versus SOC (standard of care) tests chosen based on clinical symptoms alone. This was a prospective single center randomized trial of 73 consecutive patients presenting to the University of Massachusetts Medical Center with NHGIB (melena, hematochezia/anemia, or guaiac-positive stools anemia). Exclusion criteria included presence of pacemaker, dementia, nonEnglish speaking, hemodynamically significant bleeding. Patients were randomized to SOC arm versus early capsule (EC) deployment. The SC group received a primary diagnostic procedure based on clinical symptoms that was dictated by the gastroenterologist on service, who was at liberty to choose the procedure sequence as they felt appropriate.

Results: Of the 218 patients screened, 73 were included. 2 patients from the initia included group were excluded (one due to technical capsule failure and one was transferred from an outside hospital). Baseline characteristics were similar and depicted in Table 1. The EC group $(n=34)$ had localization of presumed source of bleeding in $67.6 \%(\mathrm{n}=23)$ of patients at the time of the first diagnostic procedure compared to $48.4 \%(\mathrm{n}=16)$ in the SOC group $(\mathrm{p}=0.02)$. Active bleeding or stigmata of recent bleeding at the time of the first procedure was seen in $64.7 \%(\mathrm{n}=22)$ of patients in the EC groups compared to only $30 \%$ $(\mathrm{n}=11)$ in the SOC group $(\mathrm{p}=0.003)$. However, when melena was the presenting symptom in the SOC $(n=26)$ group, EGD was the most commonly chosen primary diagnostic procedure $(n=23)$, but was only diagnostic $52 \%$ of the time. After complete diagnostic evaluation in the SOC group, patients presenting with melena had lesions located in the esophagus $(3.8 \%)$, stomach/duodenum $(46.2 \%)$, small bowel $(11.5 \%)$, colon $(11.5 \%)$, but $27 \%$ had no source identified. EC group had lesions localized to the esophagus $(2.9 \%)$, stomach/duodenum $(35.2 \%)$, small intestine $(8.8 \%)$, colon $(20.5 \%)$, and $32.3 \%$ did not have lesions identified. When hematochezia/anemia were the presenting symptom $(n=7)$, EGD was never diagnostic as a primary procedure, colonoscopy (COLO) had a $50 \%$ diagnostic rate, and VCE was diagnostic $100 \%$ of the time.

Conclusion: VCE used as the first test in patients with NHGIB detects active bleeding significantly more often than the SOC approach, since it examines much more of the GI tract than EGD and COL alone. Detection of the anatomic site of bleeding allows for better therapeutic decisions.

Disclosure of Interest: S. Jawaid: This study was funded by an unrestricted grant from Olympus Tokyo

All other authors have declared no conflicts of interest.

P1571 MUCOSAL HEALING RATES INDUCED BY ADALIMUMAB IN ISOLATED SMALL BOWEL CROHN'S DISEASE: PROSPECTIVE EVALUATION BY CAPSULE ENDOSCOPY

E. Gal ${ }^{1}$, O. Ben-Bassat ${ }^{1}$, Z. Levi ${ }^{1}$, G. M. Fraser ${ }^{1}$, Y. Snir ${ }^{1}$, H. Yanai ${ }^{1}$, I. Dotan ${ }^{2}$, I. Avni-Biron

${ }^{1}$ Gastroenterology, Rabin Medical Center, Petach Tikva/Israel

${ }^{2}$ Division Of Gastroenterology, Rabin Medical Center, Petach Tikva/Israel

Contact E-mail Address: eyal.ga183@gmail.com

Introduction: Data regarding mucosal healing $(\mathrm{MH})$ of isolated small bowe Crohn's Disease (CD) induced by anti-tumor necrosis factor alpha agents are scarce.

Aims \& Methods: 1) To evaluate MH rates by capsule endoscopy (CE) in patients with symptomatic CD and active isolated small bowel (SB) disease treated with adalimumab (ADA). 2) To correlate $\mathrm{MH}$ with clinical and biological indices of remission. This was a prospective observational, single center study. CD patients with isolated (per CE) active (CDAI > 220) SB disease, who were recommended ADA by their treating physician were consecutively recruited; first $\mathrm{CE}$ was performed prior to commencing ADA, and the second -14-week after starting ADA. All enrollees underwent a patency capsule study to confirm patency. Disease severity was assessed by the capsule endoscopy Crohn's Disease activity index (CECDAI) score. $\mathrm{MH}$ was defined as CECDAI score $<3$.

Results: Out of 31 patients screened, 24 were eligible, and 22 completed the study (as two patients developed an allergic reaction to ADA and were withdrawn) Females: $12(54.5 \%)$, median disease duration: 3 years (IQR 1-7), biologic experienced (Infliximab) -3 patients. After 14 weeks of ADA therapy MH was detected in $8 / 22$ patients $(36.4 \%)$, CDAI $<150$ in $11 / 22$ patients $(50 \%)$, normalization of CRP in $7 / 22$ patients $(31.8 \%)$, and normalization of fecal calprotectin in $8 / 22$ patients $(36.4 \%)$. Inflammatory indices significantly decreased within 14 weeks of ADA treatment compared with baseline: median CECDAI 5 (1-16) vs 15 (10-20), $\mathrm{p}=0.001$; median CDAI $150.8(109.8-211.5)$ vs $256(240.5-320.2)$, $\mathrm{p}<0.001$; median CRP $0.14(0.07-0.5)$ vs $1.1(0.2-1.5) \mathrm{mg} / \mathrm{dl}, \mathrm{p}=0.002 ;$ median fecal calprotectin- $83(80-139)$ vs $128(61.5-266.5), \mu \mathrm{g} / \mathrm{gram}, \mathrm{p}=0.014 . \mathrm{MH}$ detected by CE did not correlate with normalization of either CDAI $<150$, CRP or fecal calprotectin levels.

Conclusion: ADA induced $\mathrm{MH}$ in $36 \%$ of $\mathrm{CD}$ patients with isolated active SB disease. MH did not correlate with either clinical or biological remission. Thus, further evaluation should be performed after 52 weeks of maintenance therapy. Disclosure of Interest: All authors have declared no conflicts of interest.

P1572 CORRELATION BETWEEN SMALL BOWEL MRI, FAECAL CALPROTECTIN AND CAPSULE ENDOSCOPY IN THE INVESTIGATION OF INFLAMMATORY BOWEL DISEASE

S. Vijayan ${ }^{1}$, D. E. Yung ${ }^{2}$, H. Suzuki ${ }^{3}$, R. Makins ${ }^{4}$, U. Kopylov ${ }^{5}$, A. Levartovsky ${ }^{5}$, B.J.F. Rosa ${ }^{6}$, J. N. Plevris ${ }^{2}$, A. Koulaouzidis ${ }^{2}$ ${ }^{1}$ Medical School, University of Edinburgh, Edinburgh/United Kingdom

${ }^{2}$ The Royal Infirmary of Edinburgh, Edinburgh/United Kingdom ${ }^{3}$ Southern General Hospital, Glasgow/United Kingdom

${ }^{4}$ Cheltenham General Hospital, Cheltenham/United Kingdom

${ }^{5}$ Gastroenterology, Chaim sheba Medical Center, Ramat Gan/Israel ${ }^{6}$ Gastroenterology, Centro Hospitalar Do Alto Ave, Guimarães/Portugal

Contact E-mail Address: diana.e.yung@gmail.com

Introduction: Capsule endoscopy (CE) is widely used to investigate the small bowel (SB). However, patients with inflammatory bowel disease (IBD) are considered to be at higher risk for capsule retention. The ESGE recommends using dedicated cross-sectional imaging to assess SB patency in patients with known Crohn's Disease (CD) prior to CE. Evidence suggests that a combination of SB magnetic resonance imaging (MRI), faecal calprotectin (FC) and CE together may be more effective in assessing SB inflammation compared to an individual modality alone. We aimed to assess the effectiveness of this approach.

Aims \& Methods: Retrospective, multicentre study; consecutive patients who had undergone both SB MRI and CE within 6 months of each other were included. Data collected: patient demographics, indications for CE and MRI, CE and MRI findings, and FC levels closest to the time of CE. Continuous data is presented as means \pm standard deviation; comparisons were made using $\mathrm{T}$-test $(\mathrm{p}<0.05$ taken to be significant).

Results: 82 patients $(28 \mathrm{M} / 54 \mathrm{~F}$, Mean age $41.4 \mathrm{yrs})$ underwent both $\mathrm{CE}$ and SB MRI at 4 centres in the United Kingdom, Israel and Portugal. Indications included suspected SB inflammation $(n=61)$, IBD reassessment $(n=21)$. Overall, there were 3 incomplete CEs, but no case of SB capsule retention. Of 82 SB MRIs, 4 patients had evidence of SB obstruction, 10 had SB thickening and/or inflammation, 3 had other findings (SB pneumatosis, polyps). 64/82 cases were normal and 1 study had poor contrast, precluding any conclusion. Of the 4 patients with SB MRI evidence of obstruction, only 1 had strictures seen on CE. The other 3 patients had insignificant or normal CE findings. Of the 10 patients with SB thickening and/or inflammation seen on MRI, 6 had corresponding CE findings; in particular, there were 2 cases with strictures on CE and 4 cases with mucosal inflammatory changes. 64 patients had normal SB MRI and $35(54.7 \%)$ had a normal CE. $18 / 64(28.1 \%)$ patients had mucosal inflammatory changes on CE; 2 of them had strictures which were eventually traversed by the capsule. 10 patients had other non-inflammatory findings on CE. Of 18 patients with normal SB MRI but SB inflammation on CE, 9 had a raised FC $(\mathrm{FC}>150 \mu \mathrm{g} / \mathrm{g}), 5$ had borderline FC levels $(50-100 \mu \mathrm{g} / \mathrm{g})$. None of the patients in this group had normal FC levels; the mean FC was $637.5 \pm 844.4 \mu \mathrm{g} / \mathrm{g}$. In the group of patients with both normal SB MRI and CE, 16/35 had raised FC; 7 patients had borderline FC levels. The overall mean FC for these patients was $266.3 \pm 221.9 \mu \mathrm{g} / \mathrm{g}$. The mean FC between patients with SB inflammation seen on CE and patients with normal SB MRI and CE was significantly different $(\mathrm{p}=0.04)$

Conclusion: A significant proportion $(28.1 \%)$ of patients with normal SB MRI to investigate possible SB inflammation had CE findings showing SB inflammation, including 2 patients with strictures. However, no retention occurred in this group. Raised FC was significantly associated with CE findings despite normal SB MRI. Disclosure of Interest: All authors have declared no conflicts of interest.

\section{References}

1. Pennazio M, et al. Small-bowel capsule endoscopy and device-assisted enteroscopy for diagnosis and treatment of small-bowel disorders: European Society of Gastrointestinal Endoscopy (ESGE) Clinical Guideline. Endoscopy. 2015;47:352-86.

2. Kopylov U, et al. Detection of small bowel mucosal healing and deep remission in patients with known small bowel Crohn's disease using biomarkers, capsule endoscopy, and imaging. The American journal of gastroenterology. 2015;110:1316-23.

\section{P1573 HOW MANY CAPSULE ENDOSCOPY STUDIES CAN BE} READ IN A GIVEN SESSION BEFORE ACCURACY IS AFFECTED?

S. Beg ${ }^{1}$, R. Sidhu ${ }^{2}$, T.R. Card ${ }^{1}$, E. Wronska ${ }^{3}$, K. Ragunath ${ }^{1}$

${ }_{1}^{1}$ Gastroenterology, Nottingham Digestive Diseases Centre, UH/United Kingdom ${ }^{2}$ Royal Hallamshire Hospital, Sheffield/United Kingdom

${ }^{3} \mathrm{Dr}$, Gastroenterology, Medical Center for Postgraduate Education and Maria Sklodowska-Curie Memo, Warsaw/Poland

Contact E-mail Address: sabina.beg@nhs.net

Introduction: The interpretation of small bowel capsule endoscopy (SBCE) requires a high level of concentration. An abnormality may be present on just a few of the many thousands of images presented for interpretation. It is 
unknown whether fatigue affects the accuracy of SBCE reporting and how many SBCE studies can be read in one session.

Aims \& Methods: Thirty-two participants (16 experienced SBCE readers and 16 novices) were invited to participate in this study. Each participant was asked to read 6 pre-selected SBCE studies consecutively. These studies were presented in a random order. All readings took place using the single view mode, readers were able to choose the frames per second viewed from a range of speeds. Fatigue was measured subjectively using a Likert scale and objectively using a computerbased psychomotor vigilance test. These measures were performed at prior to commencing the study and after every second capsule read. Accuracy in lesion detection was determined by comparison with a gold standard reading derived from the non-consecutive readings of two expert capsule readers. Accuracy was plotted against the order in which SBCE studies were read. The aim of this study was to determine whether fatigue influences the accuracy of SBCE interpretation and how many cases can be read before accuracy declines.

Results: In keeping with existing literature, high intra-observer variability amongst the participants was observed, with experienced readers reaching kappa values of 0.51 with the gold standard and 0.08 amongst novices. As progressive SBCE studies were read the mean speed increased for both experts and novices, with a mean reduction of 10 minutes between the first and the last study read. This was associated with faster reading speeds selected in progressive studies read. Where accuracy was analyzed with respect to the reading speed chosen, a negative correlation between increasing speed and accuracy was demonstrated, with $31 \%$ of lesions detected when read at 6-10 frames per second, compared to $15 \%$ when using the $22-28$ speed. There was no significant change in accuracy with progressive capsule read when the group was analyzed as a whole. The accuracy of experienced readers declined after just one study read, from $38 \%$ to $27 \%$ and plateaued thereafter. Novice readers demonstrated no significant change across the time points, with a trend towards improvement, perhaps indicating skills acquisition during the study.

Conclusion: The accuracy of SBCE reporting declines after one study reporting in a given period of time by expert SBCE readers. The optimal time interval between study reporting needs to be explored. This does not affect novice readers perhaps demonstrating skill acquisition.

Disclosure of Interest: All authors have declared no conflicts of interest.

\section{P1574 FEASIBILITY OF SAME-DAY COLON CAPSULE ENDOSCOPY (CCE) IN PATIENTS WITH INCOMPLETE COLONOSCOPY}

M. Hussey ${ }^{1}$, G. Holleran ${ }^{2}$, C. Tersaruolo ${ }^{2}$, D. Mcnamara ${ }^{1}$

${ }^{1}$ Gastroenterology, Tallaght Hospital, Dublin/Ireland

${ }^{2}$ Trinity Academic Gastroenterology Group, Trinity College, Dublin/Ireland

\section{Contact E-mail Address: husseyma@tcd.ie}

Introduction: Rates of incomplete colonoscopies (IC) range from $2-19 \%$, requiring repeat procedures or radiological imaging which can often lead to diagnostic delays as well as increased inconvenience for the patient. Same-day CCE may offer a more convenient and cost-effective mode of colonic examination post IC. Aims \& Methods: We aimed to determine the feasibility of same-day CCE post incomplete colonoscopy. A prospective pilot study was performed. Any patient without a contraindication to CCE with an IC for reasons other than poor bowel prep was offered the test following an appropriate recovery time of 1-hour post IC. Informed consent was obtained from all subjects. Upon ingestion of the capsule, where feasible, $10 \mathrm{mg}$ of IV metoclopramide was given to overcome the antimotility effects of fentanyl given during routine colonoscopy. Standard booster protocol for CCE was administered. Patient demographics, procedure indication, sedation levels along with key CCE data including preparation quality, completion, positivity rates and adverse events were all recorded.

Results: To date, 40 same-day CCE have been completed. The mean age was 57 yrs.(22-83 yrs.) and $65 \%$ (26) were female. Indications for OC were; altered bowel habit $33 \%(n=13)$, Iron deficiency anaemia $30 \%(n=12)$, Inflammatory Bowel Disease Assessment 15\% $(\mathrm{n}=6)$, PR bleeding 5\% $(\mathrm{n}=2)$, abdominal pain $5 \%(\mathrm{n}=2)$, polyp surveillance $5 \%(\mathrm{n}=2)$, positive family history of CRC $5 \%$ $(\mathrm{n}=2)$ and abnormal imaging $2 \%(\mathrm{n}=1)$. OC were incomplete due to excessive looping $40 \%(n=16)$, patient intolerance $30 \%(n=12)$ and severe diverticular disease $30 \%(\mathrm{n}=12)$. The mean sedation used during OC was $5 \mathrm{mg}$ midazolam (range $3-10 \mathrm{mg})$ and $75 \mathrm{mcg}$ of fentanyl (range $50-100 \mathrm{mcg})$. In all $84 \%(\mathrm{n}=34)$ of CCE were complete, however full colonic views were obtained in $94 \%(n=37)$. Mean colonic passage time was 222 minutes and overall image quality was deemed to be excellent in $16 \%(n=6)$, good in $31 \%(n=12)$, adequate in $44 \%$ $(\mathrm{n}=18)$ and poor in $9 \%(\mathrm{n}=4)$ of participants. Overall findings were normal $25 \%(n=10)$, polyps $38 \%(n=15)$, inflammation $22 \%(n=9)$, diverticular disease $25 \%(n=10)$, angiodysplasia $3 \%(n=1)$. Amongst the patients who had polyps, 8 required polypectomies and the remaining 7 were put on a surveillance programmes. Based on the CCE findings, 4 of the IBD patients required treatment escalation. In terms of adverse events one patient reported abdominal pain during the procedure and one patient retained the capsule due to an inflammatory stricture.

Conclusion: CCE would appear to be feasible in the majority of patients and significantly detects colonic pathology.

Disclosure of Interest: All authors have declared no conflicts of interest.
P1575 DEDICATED DIFFUSION WEIGHTED MR IMAGING FOR STAGING PERITONEAL METASTASES IN COLORECTAL CANCER; AN ACCURATE PREOPERATIVE SELECTION TOOL FOR CYTOREDUCTION SURGERY (CRS/HIPEC) CANDIDATES

I. Jansen ${ }^{1}$, D.M. Lambregts ${ }^{1}$, S. Chandrasegaram-Shanmuganathan ${ }^{1}$, N. Kok ${ }^{2}$, G.L. Beets ${ }^{2}$, R. G.h. Beets-Tan ${ }^{1}$, A. Aalbers ${ }^{2}$, M. Lahaye ${ }^{1}$

${ }^{1}$ Radiology, The Netherlands Cancer Institute, Amsterdam/Netherlands

${ }^{2}$ Surgery, The Netherlands Cancer Institute, Amsterdam/Netherlands

Contact E-mail Address: M.Lahaye@nki.nl

Introduction: Peritoneal carcinomatosis (PC) is a well-known mechanism of spread of cancer; it is the second-most frequent cause of death in colorectal cancer patients. However, the prognosis of PC patients has dramatically improved in recent years. Nowadays, selected patients benefit from extensive cytoreductive surgery and hyperthermic intraperitoneal chemotherapy (CRSHIPEC). 5-year survival rates of up to $50 \%$ are reported after CRS-HIPEC. Despite this survival gain, CRS-HIPEC has a considerable morbidity rate of $49 \%$ with a severe complication rate of $21 \%^{1}$. Obviously, it is important for patients with a limited life expectancy to avoid pointless and costly aggressive surgical procedures. Hence, recognizing patients with a maximum risk-to-benefit ratio for the procedure is imperative. To select patients who could benefit from CRS-HIPEC the Peritoneal Cancer Index (PCI) is used. The PCI combines the location/size of peritoneal tumours found at surgery at 13 abdominal regions. Each of the 13 regions is scored for implant size on a scale of $0-3(0=$ no visible tumor implants; $1=$ implants $<0.50 \mathrm{~cm} ; 2=0.50-5.0 \mathrm{~cm} ; 3=>5.0 \mathrm{~cm})$. The PCI is the sum of the lesion scores from all 13 areas, and thus can vary between $0-39$. The PCI is widely validated and is a quantitative prognostic indicator for longterm outcome. However, with this surgical staging procedure it is not always feasible to inspect all relevant intra-abdominal regions due to formation of adhesions and/or tumour. So, there is a desperate need for a non-invasive imaging tool that could select those patients who will benefit from CRS-HIPEC. However, currently there is no validated imaging tool that can accurately predict the PCI.

Aims \& Methods: Therefore, the aim of this study was to estimate the PCI preoperatively with diffusion weighted MRI (DW-MRI) and compare this with the PCI found at surgery to assess whether DW-MRI can be used to select CRS HIPEC candidates. In this ongoing study twenty-four consecutive patients (April 2016-April 2017) with histologically proven peritoneal carcinomatosis from colorectal origin were included. Patients were scheduled for exploratory laparoscopy and/or CRS/HIPEC and underwent preoperative dedicated DW-MRI (scantime $=30 \mathrm{~min}$ )

Two independent readers prospectively determined the PCI on DW-MRI. Patients were categorized as low-risk (PCI 0-21) versus high-risk (PCI 22-39); in our center considered operable versus non-operable. Reference standard was PCI found at surgery. Furthermore both readers evaluated whether a R1 resection could be achieved on a 5-point scale combining PCI and other risk factors for an incomplete surgery, like invasion in hepatic hilum, mesenteric vessels, extensive pelvic invasion of liver metastases. Sensitivity and specificity of the test were calculated and receiver-operating characteristic curves (ROC) were constructed. The area-under-the-curve (AUC) was calculated for each reader. Quadratic weighted kappa was used to evaluate the interobserver agreement.

Results: At surgery the mean PCI was 13.8 (range 0-34). For reader 1 the mean PCI was $13.4(0-29)$ and for reader $11.9(0-34)$. Both readers categorized 23 out of 24 patients correctly (accuracy $96 \%$ ) when compared to surgical findings. Both readers understaged the same patient. The sensitivity and specificity for selecting patients with a PCI $<22$ was for both readers $100 \%$ and $75 \%$, respectively. The interobserver agreement was perfect $(\mathrm{k}=1.0)$. The UAC for predicting overall operability was 0.99 for reader 1 and 0.95 for reader 2 .

Conclusion: These data suggest that DW-MRI is a robust and highly accurate selection tool to noninvasively select colorectal cancer patients who could benefit from CRS/HIPEC. Interestingly, no overstaging occurred with DWI-MRI; this means that DWI-MRI did not deprive patients from potential curative surgery. In addition, due to the lack of large studies concerning this subject, our "pilot study' is one the largest DW-MRI study in PC literature.

Disclosure of Interest: All authors have declared no conflicts of interest.

\section{Reference}

1. Chua, T. C., et al (2009) Should the treatment of peritoneal carcinomatosis by cytoreductive surgery and hyperthermic intraperitoneal chemotherapy still be regarded as a highly morbid procedure?: a systematic review of morbidity and mortality, Ann Surg 249, 900-907.

\section{P1576 HISTOLOGICAL PREDICTION OF COLONIC POLYPS BY COMPUTER VISION. PRELIMINARY RESULTS}

C. Sánchez-Montes ${ }^{1}$, J. Sánchez ${ }^{2}$, C. Rodríguez De Miguel ${ }^{1}$, H. Córdova ${ }^{1}$, J. Bernal ${ }^{1}$, M. López-Cerón ${ }^{1}$, J. Llach ${ }^{1}$, G. Fernández-Esparrach ${ }^{1}$

${ }^{1}$ Endoscopy Unit, Hospital Clinic, University of Barcelona, Barcelona/Spain

${ }^{2}$ Center Computer Vision, Universitat Autònoma de Barcelona, Barcelona/Spain

Contact E-mail Address: crsanchez@ clinic.cat

Introduction: During colonoscopy, clinicians perform visual inspection of the polyps to predict histology. Kudo's pit pattern classification is one of the most commonly used for optical diagnosis. These surface patterns present a contrast with respect to their neighboring regions and they can be considered as bright regions in the image that can attract the attention of computational methods.

Aims \& Methods: We aimed to assess the accuracy of a new computational system based on the segmentation and characterization of bright regions as 
cues to obtain an automatic histological classification of colonic polyps. Our automatic histology prediction system is based on the segmentation of textural elements from polyp surface and their correlation with Kudo's pit pattern classification. Textural elements are identified as bright regions in polyp surface and there are characterized according to their shape into tubular and circular: a high presence of tubular patterns is associated to an adenomatous histology whereas the absence of prominent tubular structures is associated to non-adenomatous. Taking this into account, we characterized segmented bright regions using a tubularity metric $(T u b)$ designed to obtain low values for circular shapes and high values for tubular regions of the same area. We tested our method in high definition (HD) white light polyp images which were obtained with a colonoscope Olympus CIF-H190 at Hospital Clinic in Barcelona. Neither conventional nor virtual chromoendoscopy were used. These images were selected to show as much variability in polyp appearance as possible. We used the mean of all Tub values for an image to classify it into two classes: Adenoma and Non-Adenoma. A ROC curve was constructed to select the optimal threshold value of Tub. Then, we compared the histology prediction provided by our system and the actual histology obtained after lesion removal.

Results: 51 polyp images were analyzed: $38(74.5 \%)$ adenoma and $13(25.5 \%)$ non-adenoma. Mean size of polyps was $14 \pm 13$ (range 1-40) and had the following morphology based on Paris classification: 5 (9.8\%) 0-Ip, 27 (52.9\%) 0-Is and $19(37.3 \%)$ 0-IIa. Mean Tub values were different for adenoma compared to Non-adenoma $(19.5 \pm 6.5$ vs $14.1 \pm 6.3 ; \mathrm{p}=0.013)$. An optimal threshold value of $T u b=13.14$ to separate adenoma vs. non-adenoma was selected from the operating point of the ROC curve. With this value, our method was able to provide an accurate histological diagnosis in 44 out of 51 images $(86 \%)$ (table) with a Sensitivity, Specificity, PPV and NPV for the diagnosis of adenoma of $95 \%, 61 \%, 88 \%$ and $80 \%$, respectively. On the other hand, processing of a single HD image took 2.7 seconds making feasible its use in the endoscopy room.

\begin{tabular}{lllll}
\hline & & \multicolumn{2}{l}{ Histological Gold standard } & \\
\cline { 3 - 4 } & & Non-adenoma & Adenoma & total \\
\hline \multirow{2}{*}{ Automatic prediction } & Non-adenoma & 8 & 2 & 10 \\
& Adenoma & 5 & 36 & 41 \\
& total & 13 & 38 & 51 \\
\hline
\end{tabular}

Conclusion: A computer vision system based on bright regions in the image has a high accuracy for on-line prediction of polyp histology during colonoscopy. Though the use of shape characterization is promising, the inclusion of other polyp characteristics (i.e. shape, color, vessels...) as well as enlarging the validation database could improve the robustness of our methodology.

Disclosure of Interest: All authors have declared no conflicts of interest.

\section{P1577 COLORECTAL LATERALLY SPREADING TUMORS DETECTED AT CT COLONOGRAPHY}

M. Iwabuchi, M. Sugimura, S. Toda, K. Ukai

Gastroenterology, Sendai Medical Center, Sendai/Japan

Contact E-mail Address: s7856@snh.go.jp

Introduction: Laterally spreading tumors (LSTs) of the colorectum are classified into the following two subtypes according to their morphology; granular type (LST-G), and non-granular type (LST-NG). Meanwhile, CT colonography (CTC) is a relatively new radiological technique for imaging the entire colorectum and its sensitivity for detecting polyps more than $1 \mathrm{~cm}$ has been reported to be greater than $90 \%$. However, the detectability of LST in CTC has not been fully delineated.

Aims \& Methods: The aim of this study was to determine the detectability in CTC of LSTs focusing on their subtypes. We retrospectively reviewed and matched data on endoscopic and CTC reports in 33 patients ( 35 LSTs) who received a multidetector CT scan using contrast media immediately after total colonoscopy at the Sendai Medical Center, Japan, between May 2012 and December 2016. LSTs were classified into the following two categories: LST-Gs (24 lesions) and LST-NGs (11 lesions).

Results: 35 pathologically proven LSTs were evaluated. Histology included adenomas in 18 , intramucosal cancers in 14 , and submucosal cancer in 3 . The mean diameter of the LSTs was $24.5 \mathrm{~mm}$. Of the 35 LSTs detected on colonoscopy, 30 were observed and matched by CTC $(85.7 \%) .23$ out of $24(95.8 \%)$ LST-Gs were detected, while 7 out of $11(63.6 \%)$ LST-NGs were detected. The detectability of LST-Gs was significantly higher than that of LST-NGs $(\mathrm{p}<0.05)$. The detectability of adenoma $(77.8 \% ; 14 / 18)$ was lower than that of intramucosal $(92.9 \%$; $13 / 14)$ and submucosal $(100 \% ; 3 / 3)$ cancer. Furthermore, the detectability of LSTs tended to increase with increasing tumor size.

Conclusion: CTC can effectively detect LST-Gs, while the detection of LST-NGs is still challenging.

Disclosure of Interest: All authors have declared no conflicts of interest.
P1578 ANALYSIS OF CT COLONOGRAPHY MISS RATE OF LARGE

NEOPLASTIC LESIONS DETERMINED BY COLONOSCOPY ON JAPANESE NATIONAL CT COLONOGRAPHY TRIAL (JANCT)

M. Hirayama ${ }^{1}$, T. Takayama ${ }^{2}$, R. Fujii ${ }^{1}$, T. Minagawa ${ }^{1}$, S. Ohiwa ${ }^{2}$, K. Sakata ${ }^{1}$, M. Yoshida ${ }^{1}$, Y. Okagawa ${ }^{1}$, H. Ihara ${ }^{1}$, T. Sumiyoshi ${ }^{1}$, Y. Tsuji ${ }^{2}$, N. Yoshizaki ${ }^{1}$, H. Kondo ${ }^{1}$, K. Nagata ${ }^{3}$

${ }^{1}$ Gastroenterology, Tonan Hospital, Sapporo/Japan

${ }^{2}$ Oncology, Tonan Hospital, Sapporo/Japan

${ }^{3}$ Center For Public Health Sciences, National Cancer Center, Tokyo/Japan

Contact E-mail Address: otarugi@gmail.com

Introduction: CT colonography (CTC) may be readily used for imaging of the colon in elderly or poor risk patients with colon polyps and cancer because of its non-invasiveness and relatively short inspection time. But, the drawbacks to make a misdiagnosis may be inevitable. Therefore, analysis of misdiagnosis case is crucial on the new modality for the colon cancer screening examination. Aims \& Methods: We evaluated the misdiagnosed case of large neoplastic lesions determined by colonoscopy (CS) from JANCT by CTC with radiologist and gastroenterologist interpretations ${ }^{1)}$. Out of 1257 cases enrolled in JANCT, 1181 cases were actually studied, omitting 76 cases according to the exclusion criteria. More than 16 DAS CT were used for CTC, respectively. Images were retrospectively reconstructed by using a $0.5 \mathrm{~mm}$ section index. The CTC examination was prepared by PEG-C solution before scanning. $\mathrm{CO} 2$ gas as an effervescent agent was then administrated just before scanning. This was used for the contrast medium. CTC images were analyzed by AZE Virtual Place software. The CTC and CS were independently analyzed by endoscopist and radiologist in blind fashion. We investigated misdiagnosed lesions with CTC more than $10 \mathrm{~mm}$ detected by CS. We considered the pseudo-negative lesions misdiagnosed with CTC interpretation (PNL) by radiologist or gastroenterologist and also considered the true pseudo-negative lesions misdiagnosed with CTC interpretation (true PNL) by radiologists and gastroenterologists. Because we conceived true PNL showed the limitation of CTC interpretation instead of PNL involved a human error.

Results: PNL was diagnosed by CS at 0-Ip (8 cases, 8 lesions), 0-Is (10 cases, 12 lesions) and 0-IIa (17cases, 19 lesions), respectively according to the criteria of the Paris classification. True PNL was also diagnosed at 0-Ip (1 case, 1 lesion), 0 Is ( 5 cases, 6 lesions) and 0-IIa ( 11 cases, 13 lesions), respectively. True PNL/PNL ratio was 0 -Ip $12.5 \%, 0$-IIa $50 \%$ and 0 -IIa $68.5 \%$, respectively. There was no PNL at 0 -IIc, Type I, II and III on this study. Most of all true PNL were so called flat lesion not only 0-IIa lesion.

Conclusion: CTC was proven to be a reasonably useful approach to obtain the image of colon diseases without any invasiveness to the patient. On CTC interpretation, lower protruded lesion was considered less detectivity than highly protruded lesion like 0-Ip.

Disclosure of Interest: All authors have declared no conflicts of interest.

\section{Reference}

1. Nagata K, Endo S, Honda T et al.: Accuracy of CT Colonography for Detection of Polypoid and Nonpolypoid Neoplasia by Gastroenterologists and Radiologists: A Nationwide Multicenter Study in Japan. Am J Gastroenterol. 2017 Jan; 112(1):163-171.

\section{P1579 DIFFUSION-WEIGHTED MAGNETIC RESONANCE FOR} ASSESSING FIBROSIS IN CROHN'S DISEASE

A. Caruso ${ }^{1}$, M. Scarpa ${ }^{2}$, G. Schifano ${ }^{3}$, C. Mescoli ${ }^{4}$, M. Rudatis ${ }^{3}$, R. D'Inca ${ }^{5}$, G.C. Sturniolo ${ }^{5}$, C. Lacognata ${ }^{3}$, I. Angriman ${ }^{6}$

${ }^{1}$ Gastroenterology Unit, Ospedale di Gastelfranco Veneto, Castelfranco Veneto $($ TV)/Italy

${ }^{2}$ Esophageal And Digestive Tract Surgical Unit, Regional Centre For Esophageal Disease, Veneto Institute of Oncology (IOV-IRCCS), PadovalItaly

${ }^{3}$ Radiology I, Azienda Ospedaliera di Padova, Padova (PD)/Italy

${ }^{4}$ Department Of Medicine, Dimed, Pathology Unit, University of Padova, Padova $(P D) /$ Italy

${ }^{5}$ Surgery, Oncology And Gastroenterology, Gastroenterology, Padua/Italy

${ }^{6}$ Dept. Of General Surgery, University of Padova, Padova/Italy

Contact E-mail Address: ant.caruso@hotmail.it

Introduction: The formation of fibrotic tissue in intestinal wall of Crohn's Disease (CD) patients is transmural and mucosal biopsies are unrepresentative of real intestinal damage. Magnetic Resonance Enterography (MRE) allows a transmural study of the bowel loops. Recently the percentage of gain of contrast medium has been proved useful to study fibrosis in CD patients. Diffusion Weighted Imaging (DWI) through the restriction of the apparent diffusion coefficient (ADC) allows an accurate evaluation of disease activity in CD patients avoiding contrast agents.

Aims \& Methods: The aim of this study is to investigate if DWI sequence of MRE was able to identify intestinal fibrosis in CD patients candidate to surgery, using the histopathology specimens and percentage of gain as gold-standard. Thirty ileocolonic CD patients candidates to surgery for stricturing disease were consecutively enrolled from October 2010 to November 2015. All patients underwent MRE before the surgery. The histopathological examination was performed using an histological score for inflammation (AIS) and fibrosis in the stenotic segment and in the ileum before the stenosis.

Results: All population had an active disease at MRE. ADC value had a significant correlation with fibrosis score $(r=-0.648 ; p<0.0001)$, AIS $(r=-0.763$; $p<0.0001)$ and percentage of gain $(r=-0.687 ; p<0.0001)$. A strong correlation emerged between wall thickness and fibrosis score $(r=0.671 ; p<0.0001)$. The 
threshold of wall thickness for fibrosis was $>6.3 \mathrm{~mm}$ (specificity $100 \%$ and sensitivity $69.23 \%$ with AUC 0.89 ). The cut-off of ADC value for fibrosis was $<1.1 \times 10^{-3} \mathrm{~mm}^{2} \times \mathrm{s}^{-1}$ with a sensitivity of $71.79 \%$ and specificity of $94.44 \%$ $(\mathrm{AUC}=0.83)$.

Conclusion: The DWI sequence with ADC value can identify fibrosis in intestinal wall of $\mathrm{CD}$ patients avoiding the use of contrast medium.

Disclosure of Interest: All authors have declared no conflicts of interest.

\section{P1580 MOLECULAR IMAGING OF C-MET IN THE CLINICAL MANAGEMENT OF GASTROINTESTINAL CANCERS}

N. Mcdonald, C. Portal, I. Wilson

Edinburgh Molecular Imaging, Edinburgh/United Kingdom

\section{Contact E-mail Address: i.wilson@edinimage.com}

Introduction: The primary indication for c-Met targeted optical imaging agent EMI-137 is enhanced endoscopic detection of lesions during colorectal cancer screening, including flat lesions that are difficult to detect by normal white light endoscopy. We have evaluated the potential benefit of EMI-137 and analogues beyond colorectal cancer screening since c-Met is up-regulated in many other cancers.

Aims \& Methods: We have synthesised analogues of EMI-137 where the fluorescent reporter was replaced by a radionuclide chelating moiety for PET imaging. The use of this agent alongside EMI-137 could enable the PET localization of the lesions prior to surgery guided by fluorescent signals. Through a systematic analysis of scientific literature databases, and the Human Protein Atlas, we have identified more than 13 different types of solid cancer that are amenable to optical imaging for which there is evidence for c-Met as a target. We believe that imaging of c-Met with EMI-137 in these indications has the potential to positively impact critical problems in the existing patient care path and reduce morbidity, mortality, and healthcare costs. We have assessed: 1) the healthcare problem, the impact on patient care path, and the likelihood of adoption by clinicians 2) the hardware landscape; whether imaging hardware required is commercially available or is being developed, and feedback from clinicians in the US and EU 3) our confidence in c-Met as a valid imaging target to address the healthcare problem

Results: We have identified a number of promising applications within Digestive Oncology; gastric cancer, locally-advanced rectal cancer, and bile duct cancer surgery are all life-threatening indications with urgent healthcare problems that could be improved by utilising imaging of c-Met with EMI-137. Compatible imaging systems are commercially available for these indications. There is also strong evidence for c-Met as a biomarker in stratification in Barrett's oesophagus (BO), a potentially precancerous lesion with the risk of progression to oesophageal cancer. Progression rates are low and overall survival rates in BO patients are similar to the general population. However, due to the poor prognosis of oesophageal cancer, patients with $\mathrm{BO}$ lesions are managed by regular endoscopic surveillance and biopsy. This means that there is arguably a disproportionate healthcare burden relative to the level of risk.

Conclusion: Gastric cancer, locally advanced rectal cancer, and bile duct cancer surgery all have strong evidence for c-Met as a valid target, and the healthcare problems are clear and widely recognized, with EMI-137 having the potential to have high impact and improve curative procedures in serious, life-threatening conditions. An imaging agent that enabled more accurate risk stratification of BO patients would lead to a change in patient management, with the potential to remove unnecessary biopsy and to reduce the frequency of surveillance.

Disclosure of Interest: N. McDonald: Employee of Edinburgh Molecular Imaging Ltd.

C. Portal: Employee of Edinburgh Molecular Imaging Ltd

I. Wilson: Employee of Edinburgh Molecular Imaging Ltd

\section{P1581 HOMEMADE FIXATION OF FULLY-COVERED SELF- EXPANDING METAL STENT}

C.M.P.S. Pennacchi, E. H.G. Dominguez, E. Q. Mendonça, J. F.D. Oliveira, B. D.C. Martins, G. De Paulo, F. Kawaguti, C. C. Gusmon, U. Ribeiro Jr., R.

A.A. Sallum, F. Maluf-Filho

Endoscopy, ICESP Sao Paulo Dept. of Endoscopy, Sao Paulo/Brazil

\section{Contact E-mail Address: ktpennacchi@yahoo.com.br}

Introduction: Esophageal self-expandable metal stents are currently used as an alternative for surgical treatment in esophageal neoplasia, benign strictures, fistulas and anastomotic leaks. Migration is a common complication after stent placement and have higher rates when fully covered stents are employed. Covered stents prevent tumor ingrowth and can be removed easely, they can be used in the closure of fistulas and leaks. External fixation of the stent with Shim's technique seems to be efficient in preventing stent migration, but has a high cost and is not always avaliable. Fixation by clipping or sutures has similar limitations. We developed a homemade technique for external fixation of the stent using dental floss to prevent stent migration. We present the results of this technique in a small cohort.

Aims \& Methods: The present study enroled sixteen patients with esophageal malignancies, anastomotic leaks, esophageal fistulas and extrinsecal compression. The stents used in these patients were five partially covered and ten fully covered. We developed a homemade technique using dental floss for external fixation of the stent which prevent stent migration. We pull stripes of dental floss into the stent mesh and using a method similar to exchange of a nasobiliary drainage catheter, the dental floss is drawn out through the nose, tied a knot into it and its loose end is fixed to the patient's earlobe.
Results: Upper gastrointestinal endoscopy was performed after two weeks and the proximal uncovered flange of the stent was evaluetad. If it was embeddeding the esophageal mucosa and did not separate from the esophagus with air insufflation, the external fixation was removed. Otherwise, the fixation was kept for another 2-4 weeks when a new endoscopic evaluation was performed. Patients were evaluated 15-30-60 days after stent placement. In cases of migration of the entire lenghy of the stent into the stomach, the patient received a new stent and the same fixation method was employed. In cases of stents partially migrated through the cardia, the same stent was repositioned and fixed with dental floss stripes as previously described.

Conclusion: According to the results we believe this homemade technique using dental floss for external fixation of stents is a simple and cheap method that can be applied and used to prevent stent migration.

Disclosure of Interest: All authors have declared no conflicts of interest.

\section{P1582 CLINICAL OUTCOME WHEN USING SELF EXPANDING METAL STENT IN OBSTRUCTIVE COLORECTAL CANCER IN 248 PATIENTS; 7 YEARS EXPERIENCE FROM A TERTIARY CENTER}

M. Shafazand, T. Radomski, L. Alkelin, N. Papachrysos

Gastroenterology, Sahlgrenska University Hospital, Gothenburg/Sweden

Contact E-mail Address: morteza.shafazand@vgregion.se

Introduction: The reported incidence of colorectal cancer in Sweden in 2014 was $60-65 / 100,000$ inhabitants and caused $25-30$ deaths $/ 100,000^{1}$. Of all colorectal cancer, approximately $15-20 \%$ debutes with acute obstructive symptoms. Conventional acute surgical procedures in these conditions (open surgery) have been shown to lead to mortality risk up to $20 \%$ and morbidity risk of $45-50 \%$, followed by increased need for intensive care and more infections and stoma complications $^{2}$. Self-expanding Metal Stent (SEMS) for relieving malignant colon obstruction is a treatment option for non-curative cases or for bridging the patient for later surgery. Studies have shown "clinical success" of SEMS at $90 \%$. An article from 2007 concludes that SEMS in acute colon obstruction has better results regarding sickness and side effects compared with acute open surgery ${ }^{3}$

Aims \& Methods: Our compilation covers the years 2010-16, when 248 SEMS interventions $(53 \%$ men, $47 \%$ women, age $28-97)$ were performed at SU/Östra Hospital. In $78 \%$ of cases, the obstruction was located below the left flexure. In $80 \%$, SEMS was made for palliative purposes.

Results: Technically, SEMS succeeded in $98 \%$ of cases and had clinical success in $90 \%$ of cases(absence in need of emergency surgery). Complications (colony perforation) occurred in $6 \%$ of the cases. Mortality within 30 days was $11 \%$ and within 90 days $22 \%$. For all performed interventions, the difference with regard to 90 -day mortality for the indication was palliative vs. bridging, 29 resp. $3 \%$. Based on the clinical outcomes "success" vs "failure", the 90-day mortality rate was 19 resp. $55 \%$

Conclusion: Our interpretation is that SEMS is an effective method of acceptable safety regarding complications in acute malignant colon obstruction. The method is suitable for both intended intestinal relief for palliative purposes, as well as awaiting later curative measure (bridge to surgery)

Disclosure of Interest: All authors have declared no conflicts of interest.

\section{References}

1. http://www.socialstyrelsen.se/Lists/Artikelkatalog/Attachments/20008/201512-26.pdf.

2. Deans GT et al. Malignant obstruction of the left colon. Br j Surg 1994; 81: 1270-6.

3. Watt AM et al. Self-expanding metallic stents for relieving malignant colorectal obstruction: a systematic review. Ann Surg 2007; 246: 24-30.

\section{P1583 ENDOSCOPIC TREATMENT OF ESOPHAGEAL FISTULAS}

D. Bucur ${ }^{1}$, D. Oprisanescu ${ }^{1}$, G. Constantinescu ${ }^{2}$, M. Ilie $^{2}$, V. Sandru ${ }^{2}$, D. Tabacelia ${ }^{2}$

${ }^{1}$ Gastroenterology, Clinical Emergency Hospital Bucharest, Bucharest/Romania ${ }^{2}$ Dept. Of Gastroenterology, Clinical Emergency Hospital Bucharest, Bucharest Romania

\section{Contact E-mail Address: bucur.denisaa@gmail.com}

Introduction: Tracheoesophageal fistulas are severe complications which commonly occur secondary to malignant tumors of the esophagus. Other causes include mediastinal or respiratory tract tumors, surgery, radiotherapy and trauma. Without treatment they often lead to pulmonary and gastroenterological complications, such as pneumonia, acute respiratory distress syndrome and poor nutrition. The purpose of this study was to asses the efficacy of endoscopic treatment performed in our clinic for esophageal fistulas.

Aims \& Methods: We performed a retrospective study on 43 patients admitted in our clinic between January 2015 and April 2017 for esophageal fistulas.

Results: The average age of the patients included in the study was 54 years old. The causes leading to the tracheoesophageal fistulas were the following: esophageal malignancy in 18 cases $(41.8 \%)$, surgery in 8 cases $(18,6 \%)$, respiratory tract malignancy in 6 cases $(14 \%)$, gastric sleeve in 5 cases $(14 \%)$, radiotherapy in 3 cases $(7 \%)$ and trauma in 2 cases $(4.6 \%)$. In every case, the treatment of choice was placement of fully-covered metallic stents. A number of 16 patients needed reintervention, with a mean of 2 reinterventions per patient. Regarding recurrent fistulas, endoclips were used in one case $(2.3 \%)$, additional stenting in 11 cases 
$(25.5 \%)$ and repositioning of the stent in 4 cases $(9.3 \%)$. All cases resulted in fistula closure without additional surgical intervention.

Conclusion: Fully covered metallic stents offered effective treatment for esophageal fistulas, regardless of the cause. Recurrent fistulas were successfully treated by additional stents and in rare cases endoclips.

Disclosure of Interest: All authors have declared no conflicts of interest.

\section{References}

1. Dumonceau JM, Cremer M, Lalmand B, Devière J. Esophageal fistula sealing: choice of stent, practical management, and cost. Gastrointest Endosc. 1999:49:70-78

2. Moyes LH, Mackay CK, Forshaw MJ. The use of self-expanding plastic stents in the management of oesophageal leaks and spontaneous oesophageal perforations. Diagn Ther Endosc. 2011;2011:418103.

3. Qadeer MA, Dumot JA, Vargo JJ, Lopez AR, Rice TW. Endoscopic clips for closing esophageal perforations: case report and pooled analysis. Gastrointest Endosc. 2007;66:605-611.

\section{P1584 LONG-TERM OUTCOME OF ENDOSCOPIC TREATMENT OF SYMPTOMATIC ENTERIC STRICTURES IN CROHN'S DISEASE}

E. Aichinger, M. Koch, K. Rothfuss, C. Schäfer, K. Grün, M. Schneider, J. Peveling-Oberhag, N. Lubomierski, J. Albert

Department Of Gastroenterology, Hepatology And Endocrinology, Robert Bosch Hospital, Stuttgart/Germany

Contact E-mail Address: e.aichinger@alumni.pmu.ac.at

Introduction: Endoscopic treatment of enteric strictures in patients with Crohn's disease (CD) is well established; however, long-term outcome is unknown.

Aims \& Methods: All patients with CD, who had undergone endoscopic therapy of symptomatic strictures at Robert-Bosch-Hospital Stuttgart from 2008-2017, were included in this retrospective cohort study. A follow-up was available for $131 / 135(97 \%)$ patients with a mean of 30.1 months (0 to 103$)$.

Results: A total of 452 endoscopic interventions (mean 3.4 per patient, median 2 per patient, range 1-69 treatments) were performed in 135 patients (female $\mathrm{n}=67 /$ male $\mathrm{n}=68$, mean age 47.5 years, BMI: $22.8 \pm 4.98 \mathrm{~kg} / \mathrm{m}^{2}$, duration of illness: median 25.1 months). In 165 cases, the dominant stricture was located in the ileocecum, in 105 in the colon, esophagus (90), duodenum (54), upper intestine (26), lower intestine (11) or stomach (1). In 166 and 286 cases, there was an anastomotic and non-anastomotic stricture present, respectively. Treatment consisted of hydrostatic balloon dilatation $(n=447)$; bougienage (4), and cSEMS (1). Dilatation was performed to a mean of $14 \mathrm{~mm}$ (SD: 2.4 , range 7 to $24 \mathrm{~mm}$ ). In seven cases complications occurred after endoscopic treatment (bleeding: $\mathrm{n}=5$; infection: $\mathrm{n}=1$; perforation: $\mathrm{n}=1$ ) which resulted in an extension of the hospital stay $(n=5)$, antibiotic therapy (1) and surgery (1). Immediate clinical success was observed in 438 of 452 of cases $(96.9 \%)$. A single intervention was performed in $61 / 135$ patients $(45.2 \%)$, two interventions in $36(26.7 \%)$, and three or more in $38(28.1 \%)$. In 41 of 135 patients $(30.4 \%)$, surgical treatment of the stricture was required in the course of disease.

Conclusion: Endoscopic treatment of symptomatic stricture in CD is safe and effective. Repetitive dilation is feasible with a significant reduction of clinical symptoms, and surgery was required in about $30 \%$ of patients at long-term follow-up.

Disclosure of Interest: All authors have declared no conflicts of interest

\section{References}

1. Morar PS, Faiz O, Warusavitarne J, Brown S, Cohen R, Hind D, Abercrombie J, Ragunath K, Sanders DS, Arnott I, Wilson G, Bloom S, Arebi N; Crohn's Stricture Study (CroSS) Group. Systematic review with meta-analysis: endoscopic balloon dilatation for Crohn's disease strictures. Aliment Pharmacol Ther. 2015 Nov;42(10):1137-48. doi: 10.1111/apt.13388.

2. Sunada K, Shinozaki S, Nagayama M, Yano T, Takezawa T, Ino Y, Sakamoto H, Miura Y, Hayashi Y, Sato H, Lefor AK, Yamamoto H. Long-term Outcomes in Patients with Small Intestinal Strictures Secondary to Crohn's Disease After Double-balloon Endoscopy-assisted Balloon Dilation. Inflamm Bowel Dis. 2016 Feb;22(2):380-6. doi: 10.1097/ MIB.0000000000000627.

\section{P1585 ENDOSCOPIC INTRALESIONAL STEROID INJECTION IS EFFECTIVE IN THE TREATMENT OF BENIGN REFRACTORY OESOPHAGEAL STRICTURE, A META-ANALYSIS}

L. Szapáry ${ }^{1}$, N. Farkas ${ }^{2}$, K. Márta ${ }^{1}$, P. Hegyi ${ }^{1}$, B. Eross ${ }^{1}$

${ }^{1}$ Centre For Translational Medicine, University of Pécs Faculty of Medicine, Pecs/ Hungary

${ }^{2}$ Institute Of Bioanalysis, Universitiy of Pécs, Pécs/Hungary

Contact E-mail Address: lacika.szapary0701@gmail.com

Introduction: Endoscopic dilation is an effective treatment in oesophageal strictures, but recurrences may require frequent and repeated dilations in the long term. Several trials have been conducted to determine the efficacy of intralesional steroid injection in the treatment of benign refactory oesophageal strictures, since the first pediatric case series was published in 1969. However, a meta-analysis has not been carried out yet.
Aims \& Methods: The aim of the analysis is to summarise the results and establish evidence in support or against the complementary treatment.

A meta-analysis was performed using the preferred reporting items for systematic review and meta-analysis protocols (PRISMA-P). Two reviewers conducted a comprehensive search on databases from inception to February 2016, to identify trials, comparing the efficacy of dilatation to dilatation combined with intralesional steroid injection. A meta-analysis was conducted on the data using the random-effects method by DerSimonian and Laird, because of the high level of the heterogeneity.

Results: There were 45 articles found in Embase, 55 in PubMed, and 6 in the Cochrane database. Altogether 11 articles were suitable for analyses, after exclusion of duplicate articles, case reports, results from non-human and pediatric studies. These studies involved 373 patients in total. The periodic dilation index was comparable in 4 studies, where the pooled result showed, that it decreased in the intralesional steroid plus dilation group (standardized mean difference: $-0.717,95 \% \mathrm{CI}:-1.191 ;-0.242$, p-value: 0.003$)$. The total number of repeat dilatations was comparable in 5 studies, where the standardized mean difference was -0.465 as compared to the dilation alone group $(95 \% \mathrm{CI}:-0,777$; $-0,153$, p-value: 0.004$)$. The dysphagia score was comparable in 5 studies, but in this case no significant difference could observed between the two groups (standardized mean difference: $0.274,95 \% \mathrm{CI}:-1.822 ; 1.165$, p-value: 0.510 ).

Conclusion: Our meta-analysis showed a significant improvement in the periodic dilatation index and total number of repeated dilations in the patients treated by intralesional steroid injection as well. We recommend the use of intralesional steroid injection for benign refractory oesophageal strictures.

Disclosure of Interest: All authors have declared no conflicts of interest.

\section{References}

Kochhar R et al Intralesional steroids augment the effects of endoscopic dilation in corrosive esophageal strictures. Gastrointest Endosc 1999; 49: 509-513 Altintas E et al Intralesional steroid injection in benign esophageal strictures resistant to bougie dilation. J Gastroenterol Hepatol 2004; 19: 1388-1391

Rupp $\mathrm{T}$ et al Randomized trial of Savary dilation with/without intralesional steroids for benign gastroesophageal reflux strictures [abstract]. Gastrointest Endosc 1995; 41: 357

Kochhar $\mathrm{R}$ et al Usefulness of intralesional triamcinolone in treatment of benign esophageal strictures. Gastrointest Endosc 2002; 56: 829-834

Camargo MA et al Use of corticosteroids after esophageal dilations on patients with corrosive stenosis:prospective, randomized and double-blind study. Rev Assoc Med Bras 2003;49:286-292.

Nijhawan S et al Aggressive bougie dilatation and intralesional steroids is effective in refractory benign esophageal strictures secondary to corrosive ingestion. Dis Esophagus. 2015 Nov 6.

Kochhar R et al Usefulness of intralesional triamcinolone in treatment of benign esophageal strictures. Gastrointest Endosc 2002;56:829-34.

Orive-Calzada et al (2012) Efficacy of intralesional corticosteroid injection in endoscopic treatment of esophageal strictures. Surg Laparosc Percutan Tech 22:518-522

Pereira-Lima JC et al A prospective randomized trial of intralesional triamcinolone injections after endoscopic dilation for complex esophagogastric anastomotic strictures: steroid injection after endoscopic dilation. Surg Endosc. 2015 May;29(5):1156-60

Ahn Y et al Efficacy of intralesional triamcinolone injections for benign refractory oesophageal strictures at Counties Manukau Health, New Zealand. Dunne D et al Five year follow up of prospective randomized trial of savory dilations with or without intralesional steroids of benign gastrooesophageal reflux strictures. Gastroenterology. 1999

\section{P1586 EFFICACY AND COMPLICATIONS IN PALLITVE OESOPHAGEAL STENTING, EXPERINCES OF A TERTIARY REFERRAL CENTER IN THE UK}

C. Clisby ${ }^{1}$, C. Gordon ${ }^{2}$, B. Eross ${ }^{2}$

${ }^{1}$ Endoscopy, Royal Bournemouth Hospital, Bournemouth/United Kingdom

${ }^{2}$ Gastroenterology, Royal Bournemouth Hospital, Bournemouth/United Kingdom

Contact E-mail Address: c.clisby83@gmail.com

Introduction: Palliative stenting is now established as a major treatment for dysphagia resulting from incurable malignant stricture of the oesophagus. There are multiple possible complications after oesophageal stent insertion; most of these present with increasing dysphagia; however pain, bleeding, and reflux are also common. Although data on rates of various complications are available, there are no agreed standards to audit performance against, nor any requirement to do so. As this is a palliative procedure, success in symptom control is paramount; if this is not being achieved, then the appropriateness of the procedure must be questioned. As with any invasive procedure, complication rates are affected by multiple variables: technique, type of stent, length and site of stricture, operator experience, patient co-morbidities and performance status. As a result of these variables, stated complication rates also vary. Only one study reported a rate of dysphagia (29\%); migration occurred in $5-15 \%$ of cases, tumour ingrowth/overgrowth $5-20 \%$, and food bolus obstruction in 5-15\%. Quoted median survival ranged from $61-104$ days, with a 30-day mortality of $20-28 \%$

Aims \& Methods: Our aim was to scrutinisy the efficacy and the safety of our palliative oesophageal stenting work. A retrospective study of all patients undergoing oesophageal stent insertion at our unit, between 01.01.2012 and 01.04.2016 was undertaken, looking for evidence of complications, repeat interventions, and for survival statistics after stent insertion. Patients who had undergone stent insertion for reasons other than oesophageal malignancy were excluded. 
Results: Between 01.01.2012 and 01.04.2016, 152 stents were inserted in 125 patients for dysphagia in palliative oesophageal cancer; 104 patients had stent inserted once, 16 patients had twice, 4 patients had 3 times and 1 patient had 4 stent insertions. $69.6 \%$ were male and the median age at death was 79 years (SD 10.94). The reported histology for the 125 patients revealed, $85(68 \%)$ adenocarcinoma, $30(24 \%)$ squamous cell carcinoma, $5(4 \%)$ Lung cancer causing external compression of the oesophagus, $2(1.6 \%)$ mesothelioma, $1(0.8 \%)$ externally compressing spindle cell sarcoma, $1(0.8 \%)$ metastatic adenocarcinoma from the colon, $1(0.8 \%)$ externally compressing signet ring adenocarcinoma. Of these stent insertions $52(34.2 \%)$ were documented to have on going or recurrent dysphagia after the procedure, of which the causes were: tumour over- or ingrowth in $9(5.9 \%)$; stent migration in $15(9.9 \%)$; distal obstruction due to gastric folds $2(1.3 \%)$, dysfunction of the anti-reflux valve in $3(2.0 \%)$; and food bolus obstruction in 7 cases $(4.6 \%)$, stent occlusion of unspecified cause $2(1.3 \%)$ and stent disintegration in $1(0.7 \%)$ case. In $13(8.6 \%)$ cases cause for dysphagia was not found or not investigated. $100(65.8 \%)$ stent insertions resulted in complete resolution of the dysphagia. Repeat endoscopy was necessary in $34(27.2 \%)$ patients, who had 98 repeat gastroscopies in total, to deal with minor complications of the stent insertion or to investigate dysphagia. In total there were 13 $(8.7 \%)$ significant complications caused by the stent insertion of which $7(4.6 \%)$ were bleeding, $2(1.3 \%)$ were tracheo-oesophageal fistula formations, $1(0.7 \%)$ was delayed perforation, $1(0.7 \%)$ was a too short stent, $1(0.7 \%)$ was a disintegrating stent and $1(0.7 \%)$ was a compression of the bronchi. Median survival of the 125 patients after stent insertion was 96 days (SD 128) and 30-day mortality was $11.2 \%$ (14 patients). It is important to note that with retrospective data analysis, some data is not available, due to variations in recording at the time and a reliance on the patient to report symptoms to a clinician. Currently 2 patients are still alive.

Conclusion: Palliative stenting at this centre continues to be an effective treatment for patients with dysphagia from oesophageal cancer. On the whole, outcomes from stenting at this unit compare favourably with published data in terms of dysphagia, other complications, and mortality. Steps to improve post-procedure monitoring in the form of a "stent registry" with prospective collection of data by telephone or face-to-face follow-up could be useful in future service development. Disclosure of Interest: All authors have declared no conflicts of interest.

\section{P1587 LONG-TERM RESULTS: WHEN RE-STENOSIS COMES AFTER ESOPHAGEAL STENTING?}

D. Gusev, S. Kashin, N. Akhapkin

Endoscopy, Yaroslavl Regional Cancer Hospital, Yaroslavl/Russian Federation

Contact E-mail Address: denis_gusev83@mail.ru

Introduction: Self-expanding metallic stents (SEMS) is a well-established form of palliative treatment for dysphagia in esophageal cancer. Progression of the tumor after stenting with re-stenosis is a serious problem in patients with better prognosis and longer survival.

Aims \& Methods: The aim of this study was to assess the timing and probability of esophageal re-stenosis after stenting by tumor growth. We performed a retrospective analysis of patients with advanced esophageal cancer who was underwent stenting, as a palliative treatment, for the last 5 years in the Yaroslavl Regional Cancer Hospital. We installed partially covered SEMS of one design from one manufacturer. The length of stents was $80,100,120,140 \mathrm{~mm}$, diameter $20 \mathrm{~mm}$. Patients with follow-up of more than 60 days were included in this study. Results: Of the 165 patients from the database, 97 patients were selected, whose follow-up period was more than 2 months. There was a predominantly of male patients $(78.5 \%)$, mean age of 72 years. To each patient was inserted a same partially covered stent, which is $4 \mathrm{~cm}$ longer than a tumor stenosis. The stent was placed $2 \mathrm{~cm}$ distal and $2 \mathrm{~cm}$ proximal of the stenosis. Of 97 patients, $18(18.5 \%)$ patients had tumor overgrowth with recurrence of dysphagia. Tumor overgrowth was verified as malignancy. Tumor localization before stenting: the upper and middle part of the esophagus - 6 patients, the lower part and the cardia - 12 patients. In 12 cases tumor growth spread out in the proximal direction, in 6 cases-in the distal. Interestingly, the distal tumor overgrowth after stenting was predominant $(66.6 \%)$ in cases of localization in middle and upper part of the esophagus, while the proximal tumor spread $(83.3 \%)$ was noted in cases of localization in the lower part of the esophagus or cardia. Period of re-stenosis was from 2 to 12 months, an average of 5.7 months. The period of re-stenosis in the upper and middle part of the esophagus is 4 months, while in cases of tumor localization in the lower part of the esophagus or cardia is 6.1 months. In almost cases palliation was successfully achieved with re-intervention. Five patients required several sessions of argon-plasma coagulation an average one time in 3-4 weeks.

Conclusion: The use of SEMS in patients with advanced esophageal cancer, with an expected long-term survival, is associated with an increased risk of re-stenosis by tumor overgrowth. Period of re-stenosis was an average of 5.7 months. The direction of spread tumor after stenting and the time of dysphagia recurrence could depend from localization of the tumor. This complication is not fatal and managed by endoscopic methods.

Disclosure of Interest: All authors have declared no conflicts of interest.
P1588 SELF-EXPANDABLE METAL STENTS ARE A VALID OPTION IN PATIENTS WITH LONG-TERM SURVIVAL FROM ADVANCED ESOPHAGEAL CANCER

E. Rodrigues-Pinto ${ }^{1}$, P. Pereira ${ }^{2}$, T.H. Baron ${ }^{3}$, G. Macedo ${ }^{1}$

${ }_{1}^{1}$ Gastroenterology, Centro Hospitalar São João, Porto/Portugal

${ }^{2}$ Gastroenterology, Hospital São João, Oporto/Portugal

${ }^{3}$ Gastroenterology \& Hepatology, University of North Carolina, North Carolina United States of America

Contact E-mail Address: edu.gil.pinto@gmail.com

Introduction: Self-expandable metal stents (SEMS) are often used for palliative treatment of dysphagia in patients with advanced esophageal cancer, with anticipated limited survival. However, due to previous reports of high adverse event (AE) rates when used long-term, concerns have been raised with the use of SEMS in patients with survival longer than 3 months.

Aims \& Methods: We aimed to the role of esophageal SEMS in patients with advanced esophageal cancer and expected survival longer than 6 months. Technical success was defined as successful deployment of the stent in the correct position. Clinical success was defined as relief of dysphagia 1 week after placement.

This was a retrospective study of patients with clinical dysphagia and advanced esophageal cancer who underwent SEMS placement with a stent dwell time of greater than 6 months. In all patients the indication for stent placement was dysphagia due to esophageal malignancy.

Results: Forty-two patients were followed for 298 days (183-861 days). At the end of follow-up the mortality was $93 \%$. The majority of lesions were located at the proximal/middle esophagus $(55 \%)$, and were traversable using an ultrathin gastroscope in $79 \%$ of patients; in no patient could a standard upper endoscope pass. Twenty-nine patients (69\%) underwent further therapy (chemotherapy and or radiotherapy) with SEMS in situ; however, only 14 began therapy before development of an AE (4 patients underwent further therapy after AE). Clinical improvement occurred in all patients, however $59 \%$ of patients $(\mathrm{n}=25)$ experienced an AE (15 migrations, 8 overgrowth/ingrowths and 2 stent-induced fistulae). The median stent patency was 236 days (19-513). Two AEs occurred within 30 days of stenting, 7 occurred between 30-90 days, 7 occurred between 90-180 days, and 9 occurred after 180 days. Endoscopic management was attempted in every SEMS-related AE (20 patients required a second SEMS, 2 had successful SEMS repositioning and 1 was treated with argon plasma; 2 SEMS were removed without the need for further therapy), with a clinical success of $100 \%$, however, in 7 patients the previously treated AE recurred (4 in/overgrowths and 3 migrations). Multivariate analysis showed that strictures traversable with an ultrathin gastroscope were associated with a higher risk of AEs (OR 11.7, 95\% CI [1.2-114.6], $\mathrm{p}=0.035)$.

Conclusion: Long-term esophageal stenting in patients with advanced esophageal cancer is associated with a high prevalence of AEs without an impact on mortality, and most could be managed endoscopically. Strictures traversable only using an ultrathin gastroscope are a risk factor for AEs.

Disclosure of Interest: All authors have declared no conflicts of interest.

\section{References}

McManus K, Khan I, McGuigan J. Self-expanding oesophageal stents: strategies for re-intervention. Endoscopy. 2001;33:601-4

Vakil N, Morris AI, Marcon N, et al. A prospective, randomized, controlled tria of covered expandable metal stents in the palliation of malignant esophageal obstruction at the gastroesophageal junction. Am J Gastroenterol. 2001;96:1791-6

Siersema PD. Treatment options for esophageal strictures. Nat Clin Pract Gastroenterol Hepatol. 2008;5:142-52

Homs MY, Steyerberg EW, Eijkenboom WM, et al. Single-dose brachytherapy versus metal stent placement for the palliation of dysphagia from oesophageal cancer: multicentre randomised trial. Lancet. 2004:364:1497-504.

Schoppmann SF, Langer FB, Prager G, et al. Outcome and complications of long-term self-expanding esophageal stenting. Dis Esophagus. 2013;26:154-8

Spaander MC, Baron TH, Siersema PD, et al. Esophageal stenting for benign and malignant disease: European Society of Gastrointestinal Endoscopy (ESGE) Clinical Guideline. Endoscopy. 2016;48:939-48.

\section{P1589 NOVEL PERCUTENOUS TECHNIQUES IN MANAGEMENT OF POST-CHOLECYSTECTOMY COMPLICATIONS}

M. Ghalab ${ }^{1}$, A. A. Abd El Kerim², S. E. Hegab ${ }^{3}$, O. S. El Aassar², M.H. Emara Elzanan $^{4}$, E. Emara ${ }^{5}$, M. Sharaan ${ }^{6}$

${ }^{1}$ Diagnostic And Intervention Radiology Department, Kafr Elsheikh University, Faculty of Medicine, Kafr Elsheikh/Egypt

${ }^{2}$ Diagnostic And Intervention Radiology Department, Alexandria University, Faculty of Medicine, Alexandria/Egypt

${ }^{3}$ Radiology Department, Alexandria University, Alexandria/Egypt

${ }^{4}$ Tropical Medicine, Zagazig University Dept. of Tropical Medicine Faculty of

Medicine, Zagazig/Egypt

${ }^{5}$ Radiology, Kafr ElSheikh University Hospital, kafr Elsheikh/Egypt

${ }^{6}$ General Surgery, Alexandria University, Faculty of Medicine, Alexandria/Egypt

Contact E-mail Address: drmahmoudghalab@yahoo.com

Introduction: The incidence of iatrogenic bile duct injury (BDI) remains high despite increased awareness of the problem with significant impact on patient's well-being and even survival despite seemingly adequate therapy. Over the past years, endoscopic and percutaneous biliary interventions became readily 
available in many centers and have revolutionized the management of iatrogenic biliary or concomitant vascular injuries.

Aims \& Methods: Evaluate the role of intervention radiology procedures to manage different post-cholecystectomy complications focusing on the novel techniques to improve the final outcome. From June 2014 to June 2016, 30 patients presenting with post cholecystectomy complications were referred to interventional radiology unit in our university hospital. They were 9 males and 21 females (age range: 18-66 years). Patients presented with biliary leaks $(n=12)$, benign biliary strictures with intrahepatic biliary dilatations $(n=21)$, postoperative hernia $(n=2)$, bleeding related to hepatic artery pseudo-aneurysm $(n=1)$. Different types of interventional procedures were performed, including: Percutaneous trans-hepatic drainage $(\mathrm{PTD})(\mathrm{n}=16)$, sequential dilatation of benign stricture with increasing catheter calibers over 6 months followed by manometric studies before catheter withdrawal $(n=6)$, biliary stenting with plastic stent $(n=2)$, Insertion of pigtail catheter $(n=15)$, preoperative progressive pneumo-peritoneum for their adhesolysis effect to manage post-operative huge incisional hernias before their surgical repair $(n=1)$, and selective embolization of bleeding related hepatic artery pseudo-aneurysm $(n=1)$ using tissue adhesive (n-Butyl 2 Cyanoacrylate).

Results: All percutaneous procedures were technically successful. No recorded early or late complications.After manometric studies, all managed cases with benign strictures did not show any clinical evidence of restenosis during 6 months follow-up. Overall, 14 out of 30 patients $(46.7 \%)$ were only managed by different interventional radiology procedures. Second step surgical repair was needed for 13 patients $(43.3 \%)$ and endoscopic managed for 3 patients $(10 \%)$ with biliary leaks.

Conclusion: Minimally invasive interventions were valuable techniques in the management of different post-cholecystectomy complications. In fully equipped centers, expert multidisciplinary teams would achieve high cure rates for iatrogenic biliary injuries.

Disclosure of Interest: All authors have declared no conflicts of interest.

\section{P1590 WHAT IS THE ROLE OF ANGIOGRAPHY IN ACUTE COLONIC AND SMALL BOWEL BLEEDING?}

R. Vale Rodrigues ${ }^{1}$, P. Amaro ${ }^{1}$, M. Ferreira ${ }^{1}$, M. Barros ${ }^{1}$, P. Donato ${ }^{1}$, L. Tomé $^{1}$ ${ }^{1}$ Gastroenterology, Centro Hospitalar e Universitário de Coimbra, Coimbra/ Portugal

Contact E-mail Address: rita.vale.rodrigues@gmail.com

Introduction: Angiography is a diagnostic and therapeutic modality that is widely available for upper gastrointestinal bleeding but is used less frequently when the source of bleeding is placed distally to the Treitz angle.

Aims \& Methods: To assess the usefulness of angiography in the diagnosis of colonic and small bowel bleeding and to determine the efficacy and complications of therapeutic procedures. Retrospective study; we included all patients with colonic and small bowel bleeding that were submitted to arteriography with or without embolization, admitted to the gastroenterology department of a tertiary hospital between February 2006 and November 2016. Statistics: Chi-square/ Fisher exact test, T-student.

Results: Sixty-six patients were evaluated, $63.6 \%$ male, mean age $=75$ years $(29$ 95). Angiography was done for: bleeding recurrence $(36.2 \%)$, hemodynamic instability $(33.3 \%)$, both $(27.3 \%)$ or failure to endoscopic hemostasis $(3 \%)$. The aetiology after angiographic study was: presumed diverticular $(n=28)$, angiectasis $(n=8)$, confirmed diverticular $(n=6)$, tumoral $(n=5)$, post-mucosectomy/polypectomy $(n=4)$, unclarified $(n=12)$, others $(n=3$ : Dieulafoy, ileum ulcers, radiation proctitis). Angiography showed additional clinical information in $25.8 \%$ of patients $(n=17)$. Twenty-three patients $(34.8 \%)$ underwent arterial embolization, all with technical success, with bleeding recurrence in 3 , of which only one was submitted to surgery; there were 2 cases of bowel ischemia. The reasons for deciding not to embolize were: absence of active bleeding $(90.7 \%)$ and end-vessel bleeding $(9.3 \%)$. There were no differences between the groups in demographic data, comorbidities, mortality, source of bleeding, haemoglobin at admission/discharge and creatinine $(\mathrm{p}>0.05)$; Arterial embolization was more frequent if hemodynamic instability $(\mathrm{p}=0.029)$; The average time of hospitalization was lower in the group submitted to embolization ( 8.8 vs 11.5 days, $\mathrm{p}=0.014)$. Overall, 11 patients died, due to: re-bleeding/hypovolemic shock $(n=5)$, exacerbation of comorbidities $(n=3)$, hospital acquired infection $(n=2)$ or post embolization complication $(\mathrm{n}=1)$.

Conclusion: Arteriography was a valid option for the diagnosis of colonic and small bowel bleeding; allowed therapeutic intervention in more than one third of patients, with $87 \%$ of clinical success and reduction of hospitalization time.

Disclosure of Interest: All authors have declared no conflicts of interest.

\section{References}

1. Gralnek IM, Neeman Z, Strate LL. Acute Lower Gastrointestinal Bleeding. $N$ Engl J Med. 2017 Mar 16;376(11):1054-1063.

2. Clerc D, Grass F, Schäfer M, Denys A, Demartines N, Hübner M. Lower gastrointestinal bleeding-Computed Tomographic Angiography, Colonoscopy or both? World J Emerg Surg. 2017 Jan 3;12:1

3. Loffroy R, Favelier S, Pottecher P, Estivalet L, Genson PY, Gehin S, et al. Transcatheter arterial embolization for acute nonvariceal upper gastrointestinal bleeding: Indications, techniques and outcomes. Diagn Interv Imaging. 2015 Jul-Aug;96(7-8):731-44.
WEDNESDAY, NOVEMBER 01, 2017

09:00-14:00 SURGERY III - HALL 7_

\section{P1591 PROGNOSTIC VALUE OF CARDIOPULMONARY EXERCISE TESTING FOR MORBIDITY RISK AND SURVIVAL AFTER OESOPHAGECTOMY FOR CANCER}

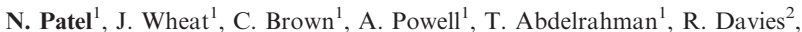
I. Appadurai ${ }^{2}$, W. Lewis

${ }^{1}$ General Surgery, University Hospital Wales, Cardiff/United Kingdom

${ }^{2}$ Anaesthesia, University Hospital Wales, Cardiff/United Kingdom

Contact E-mail Address: neilrama@hotmail.com

Introduction: Surgery for radical treatment of oesophageal cancer carries significant inherent risk. Objectively Identifying patients that are high risk of complications is of importance. The aim of this study was to assess the prognostic value of physical fitness variables determined objectively by cardiopulmonary exercise testing (CPET) in patients undergoing potentially curative surgery for oesophageal cancer (OC) within an enhanced recovery programme.

Aims \& Methods: Consecutive 180 OC patients (106 ACA, 11 SCC, 3HGD) underwent preoperative CPET with prospective recording of morbidity and survival. Non-parametric receiver operating characteristic (ROC) curves and logistic regression were used to assess the relationship between CPET variables and postoperative morbidity severity score (MSS).

Results: Of 180 patients, 120 were included for analysis (median age $65 \mathrm{yr}$., 100 male, 75 neoadjuvant therapy); 60 did not proceed to surgery and were excluded. Postoperative morbidity and mortality occurred in $83(69 \%, \mathrm{CD} \geq 3$ in 27 , $22.5 \%)$ and $4(3.3 \%)$ patients respectively. ROC curve analysis showed oxygen uptake (peak V02) gave an area under the ROC of $0.66(95 \%$ CI 0.55 to 0.77 , $\mathrm{p}=0.009$ ) and optimum cut-off of $17.0 \mathrm{ml} / \mathrm{kg} / \mathrm{min}$ (sensitivity $70 \%$, specificity $53 \%$ ). Anaerobic Threshold (AT) gave an area under the ROC of $0.62(95 \% \mathrm{CI}$ $0.51-0.74, \mathrm{p}=0.048$ ) and optimum cut-off of $10.5 \mathrm{ml} / \mathrm{kg} / \mathrm{min}$ (sensitivity $60 \%$, specificity $44 \%$ ). Multivariable analysis revealed peak V02 to be the only independent factor to predict morbidity severity CD $>3$ (OR $0.85,95 \%$ CI $0.75-0.97$, $\mathrm{p}=0.018)$. Cumulative survival was associated with operative MSS $\left(\mathrm{Chi}^{2} 4.892\right.$, DF $1, \mathrm{p}=0.027$ ) but not with CPET variables.

Conclusion: CPET is a significant predictor of morbidity after oesophagectomy with peak V02 the most important factor.

Disclosure of Interest: All authors have declared no conflicts of interest.

\section{P1592 PROPENSITY SCORE ANALYSIS OF 18-FDG PET/CT ENHANCED STAGING IN PATIENTS UNDERGOING SURGERY FOR OESOPHAGEAL CANCER}

N. Patel ${ }^{1}$, K. Foley ${ }^{2}$, D. Chan ${ }^{2}$, C. Brown ${ }^{1}$, A. Powell ${ }^{1}$, T. Abdelrahman ${ }^{1}$, P. Fielding ${ }^{2}$, A. Roberts ${ }^{2}$, W. Lewis

${ }^{1}$ General Surgery, University Hospital Wales, Cardiff/United Kingdom

${ }^{2}$ Radiology, University Hospital Wales, Cardiff/United Kingdom

\section{Contact E-mail Address: neilrama@hotmail.com}

Introduction: PET/CT has become an integral part of the staging pathway for potentially curable oesophageal cancer (OC), primarily used to identify occult distant metastases unseen by conventional radiological modalities. The aim of this study was to analyze the effect of PET/CT introduction on overall survival and assess patterns of recurrence after oesophagectomy.

Aims \& Methods: Consecutive 496 patients undergoing oesophagectomy for cancer [median age 63 (31-80) yr., 395 male, 425 ACA, 71 SCC, 325 neoadjuvant therapy] were studied. Two hundred and twenty-three patients underwent PET/ CT enhanced staging protocols and the primary outcome measure was overall survival based on intention to treat.

Results: Overall 3-year survival pre-PET/CT was $42.5 \%$ compared with $57.8 \%$ post-PET/CT $\left(\mathrm{Chi}^{2} 6.571\right.$, df $\left.1, \mathrm{p}=0.004\right)$. On multivariable analysis, pT stage (HR 1.486 [95\% CI 1.27-1.74] p < 0.0001), pN stage (HR 1.116 [95\% CI 1.05 1.20] $\mathrm{p}=0.001)$ and PET/CT (HR $0.689[95 \%$ CI $0.53-0.89] \mathrm{p}=0.004)$ were independently associated with duration of overall survival. Recurrent cancer was observed in 125 patients $(51.4 \%)$ pre-PET/CT; compared with 74 patients post- PET/CT $(37.8 \%, \mathrm{p}=0.015)$, and was less likely to be distal in location after PET/CT inception ( 39.5 vs. $27.0 \%, \mathrm{p}=0.006)$.

Conclusion: PET/CT enhanced staging is an important and independent factor associated with improved survival in patients undergoing oesophagectomy for cancer.

Disclosure of Interest: All authors have declared no conflicts of interest.

\section{P1593 PREDICTION OF LYMPH NODE METASTASIS FOR SUPERFICIAL ESOPHAGEAL CANCER WITH USING RANDOM FOREST ANALYSIS}

M. Takeuchi ${ }^{1}$, H. Kawakubo ${ }^{1}$, K. Fukuda ${ }^{1}$, R. Nakamura ${ }^{1}$, K. Suda ${ }^{1}$, N. Wada ${ }^{1}$, Y. Kitagawa

${ }^{1}$ Department Of Surgery, Keio University School of Medicine, Tokyo/Japan

Contact E-mail Address: masaty871222@gmail.com

Introduction: Although surgical techniques and perioperative management for esophageal cancer has been developed, it cannot be still safe to be performed esophagectomy. Therefore, endoscopic submucosal dissection (ESD) for the superficial cancer have been increased. We also need to consider the risk of lymph node metastasis before treatment in each patient and the aim of this study is to predict lymph node metastasis for superficial esophageal cancer. 
Aims \& Methods: Seventy patients who was diagnosed as clinical Tla-MM, T1bSM1 or T1b-SM2 and underwent esophagectomy at the Keio University, Tokyo, Japan between July 2000 and June 2016 were enrolled in this study. Patients who underwent esophagectomy as additional resections after ESD were included. We used random forest analysis to predict lymph node metastasis.

Results: There were 62 men and 8 women in this study. The mean age of all patients was $62.8 \pm 8.2$ years. The main location of the tumor was in the middle thoracic esophagus (Upper: Middle: Lower, 13: 39: 18, respectively). 14 patients had lymph node metastasis in pathological findings; 2 patients $(25 \%)$ were diagnosed as clinical T1a-MM, 2 patients $(6.6 \%)$ were T1b-SM1 and 10 patients $(31.3 \%)$ were T1b-SM2. Random forest technique (2000 trees) resulted in an estimate of error rate of $25.7 \%$. Lymph node metastasis was most associated with the factor of pathological T (relative importance $100 \%$ ) followed by lymphatic invasion $(96.3 \%)$, clinical T $(89.7 \%)$ and multifocal tumor $(72.2 \%)$. Conclusion: Random forest analysis confirmed the predictors for lymph node metastasis such as pathological $\mathrm{T}$ and lymphatic invasion.

Disclosure of Interest: All authors have declared no conflicts of interest.

\section{P1594 PROGNOSTIC SIGNIFICANCE OF CARDIORESPIRATORY FITNESS, BODY COMPOSITION ANALYSIS, AND SYSTEMIC INFLAMMATORY RESPONSE IN UPPER GASTROINTESTINAL CANCER}

N. Patel ${ }^{1}$, P. Blake ${ }^{1}$, J. Wheat ${ }^{1}$, C. Brown ${ }^{1}$, A. Powell ${ }^{1}$, T. Abdelrahman ${ }^{1}$, W. Lewis ${ }^{1}$

${ }^{1}$ General Surgery, University Hospital Wales, Cardiff/United Kingdom

\section{Contact E-mail Address: neilrama $@$ hotmail.com}

Introduction: Malnutrition is associated with higher rates of operative morbidity and therefore represent potentially reversible prognostic risk factors. Bioelectrical Impedance Analysis (BIA) is a non-invasive, easily reproducible and simple means of accurately measuring body composition.

Aims \& Methods: The aim of this study was to assess the prognostic value of body composition variables determined objectively by bioelectrical impedance analysis (BIA) in patients undergoing potentially curative surgery for oesophageal (OC) and gastric cancer (GC) within an enhanced recovery programme (ERP). Consecutive $168 \mathrm{OG}$ patients [median age 65 (24-86) yr., 131 male, 105 OC, 64 GC, 157 ACA, 8 SCC, 3 Neuroendocrine] underwent preoperative measurements of systemic inflammatory response [SIR, including FBC, CRP, Albumin, and modified Glasgow Prognostic Score (mGPS)], Patients underwent multifrequency $(0.5 \mathrm{kHz}, 50 \mathrm{kHz}$ and $100 \mathrm{kHz})$ BIA assessment using a Maltron Bioscan 920 (Maltron International Ltd, Essex, UK), and Cardio Pulmonary Exercise (CPX) assessment was performed selectively (70 OC, 27 GC). Primary outcome measure was Clavien Dindo (CD) morbidity severity score (MSS) of $\geq 3$. Results: Oesophagectomy was performed in 106, gastrectomy in 64, and laparotomy only in 25 patients. Postoperative morbidity and mortality occurred in 75 $(45 \%, \mathrm{CD} \geq 3$ in $35,21 \%)$ and $4(2 \%)$ patients respectively. On univariable analysis, MSS $\geq 3$ was associated with anaerobic threshold $(\mathrm{p}=0.011)$, CRP $(p=0.001)$, mGPS $(p=0.011)$, intra-cellular water $(I C W, p=0.041)$, and extracellular water content $(\mathrm{p}=0.015)$. Multivariable binary logistic regression revealed ICW content [LQ vs. UQ CD $\geq 3,5$ vs. 39\%, OR 1.22 (95\% CI 1.06 1.41) $\mathrm{p}=0.006$ ] and CRP [LQ vs. UQ $\mathrm{CD} \geq 3,7$ vs. $37 \%$, OR $1.03(0.99-1.06)$ $\mathrm{p}=0.076]$ to be independently associated with MSS.

Conclusion: Seven-fold variability in morbidity severity was observed after OG cancer surgery and ICW and SIR were the most important prognostic indicators. Disclosure of Interest: All authors have declared no conflicts of interest.

\section{P1595 COPING AND QUALITY OF LIFE AFTER ESOPHAGECTOMY FOR CANCER}

E. Pinto ${ }^{1}$, F. Cavallin ${ }^{2}$, L.M. Saadeh ${ }^{1}$, R. Alfieri ${ }^{2}$, C. Caberlotto ${ }^{2}$, M C. Bellissimo ${ }^{2}, \mathrm{M} . \mathrm{Cagol}^{2}, \mathrm{C} \cdot \mathrm{Castoro}^{2}, \mathrm{M} . \mathrm{Scarpa}^{2}$

${ }^{1}$ Oncological Surgery, Veneto Institute of Oncology (IOV-IRCCS), Padova/Italy ${ }^{2}$ Esophageal And Digestive Tract Surgical Unit, Regional Centre For Esophageal Disease, Veneto Institute of Oncology (IOV-IRCCS), Padova/Italy

\section{Contact E-mail Address: luca.saadeh@gmail.com}

Introduction: Esophagectomy is one the most challenging and burdening operation for its length and the variety of anatomical districts involved. Nowadays, as consequence of the improved postoperative mortality, effects of esophagetcomy are chronic and quality of life $(\mathrm{QoL})$ has become one of the main outcome measure to evaluate treatment. Coping is the ability of managing stressful situations such as postoperative conditions. At present, there are no studies focusing on coping after esophagectomy for esophageal cancer

Aims \& Methods: The aim of this study is to describe coping styles and QoL after esophageal resection. EORTC QLQ-C30 was used to assess QoL while the COPE-NVI (New Italian Version) was used to measure the coping ability. They are both self-reported questionnaires, validated in Italian language. EORTC C30 functional scales are: PF2 (physical functioning), RF (role functioning), EF (emotional functioning), CF (cognitive functioning) and SF (social functioning). COPE-NVI considers five dimensions: Social Support (SS), Avoidance Strategies (AS), Positive Attitude (PA), Orientation to the Problem (OP), Transcendent Orientation (TO). Non-parametric statistics was used.

Results: Fifty-one EC patients participated in the study and completed the C30 and COPE-NVI questionnaires. Thirty-two patients completed the questionnaires after surgery. Mean COPE-NVI scales were: $\mathrm{SS}=22.3$ (SD 9.5), $\mathrm{AS}=23.2(\mathrm{SD} 4.8), \mathrm{PA}=30.8(\mathrm{SD} 7.9), \mathrm{PO}=22.5(\mathrm{SD} 8.8), \mathrm{TO}=22.1 \quad(\mathrm{SD}$
5.0). Overall, mean C30-QL2 was 72.9 (SD 16.3). Mean C30 functional scales were: $\mathrm{PF} 2=80.8(\mathrm{SD} 16.6), \mathrm{RF}=85.0(\mathrm{SD} 20.7), \mathrm{EF}=81.5(\mathrm{SD} 17.0), \mathrm{CF}=87.4$ $(\mathrm{SD} 14.0)$ and $\mathrm{SF}=90.0$ (SD 14.0). Avoidance Strategies score was negatively correlated with Social Function $(r h o=-0.60, p=0.004)$. The remaining correlations between COPE-NVI scales, C30-QL2 and C30 functional scales were statistically not significant. Nineteen patients completed the questionnaire twice, with 3-6 months between the two fillings. The mean differences of the COPENVI scales were not statistically significant.

Conclusion: In our series, patients used Positive Attitude to manage everyday life changing. Whenever avoidance strategies (i.e. emotional indifference or drugs) were used, social abilities were compromised. Moreover, after surgical intervention coping seemed to be a stable feature. Therefore, in the early follow-up after surgery multidisciplinary team can identify and the coping features of patients in order to improve them to prevent QoL worsening.

Disclosure of Interest: All authors have declared no conflicts of interest.

\section{P1596 A RELIABLE AND ACCURATE ALGORITHM TO QUANTIFY THE TUMOR STROMA QTS AMONG TUMOR ENTITIES: HIGH INFILTRATION OF CD3 ${ }^{+}$AND CD8 ${ }^{+}$LYMPHOCYTES CORRELATES WITH IMPROVED SURVIVAL IN HEPATOCELLULAR CARCINOMA AND PANCREATIC CANCER}

R.C. Miksch ${ }^{1}$, J. Hao ${ }^{1}$, K. Dötzer ${ }^{1}$, F. Schlüter ${ }^{1}$, M. Weniger ${ }^{1}$, S. Yin ${ }^{1}$, S. Ormanns ${ }^{2}$, J.G. D'Haese ${ }^{1}$, M. Guba ${ }^{1}$, J. Werner ${ }^{1}$, B. Mayer ${ }^{1}$, A.V. Bazhin ${ }^{1}$, M.B. Schoenberg ${ }^{1}$

${ }^{1}$ Department Of General, Visceral, And Transplantation Surgery, Ludwig-

Maximilians University of Munich, Munich/Germany

${ }^{2}$ Department Of Pathology, Ludwig-Maximilians University of Munich, Munich/ Germany

Contact E-mail Address: rainer.miksch@med.uni-muenchen.de

Introduction: The tumor micro environment plays a vital role in the growth of malignancies. Through for example tumor-infiltrating lymphocytes (TILs) it influences overall and disease free survival of patients in various cancer entities. There a many studies in the literature that investigate TILs. However, there is great heterogeneity about how to quantify these cells in the tumor tissue. Therefore, we present a novel Quantification of the Tumor Stroma (QTS) Algorithm to reliably and accurately quantify cells of the tumor stroma and to perform a correlation with survival after resection of patients with hepatocellular carcinoma and pancreatic cancer.

Aims \& Methods: Immunohistochemical staining of CD3 and CD8 antigens in frozen sections of metastatic colorectal cancer (mCRC) and ovarian cancer $(\mathrm{OvCa})$ as well as in paraffin sections of hepatocellular carcinoma (HCC) and pancreatic cancer ( $\mathrm{PCa}$ ) was performed. For each entity 10 slides per antigen were examined $(n=80)$. In these different entities reliability and accuracy of computed quantification was tested in order to develop a general algorithm (Figure): First, reliability of identification of hot spots was investigated using two blinded observers. Hot spots were defined as regions with the highest density of TILs. The absolute amounts of cells were compared with the intraclass-correlation coefficient (ICC). Second, accuracy was tested. To examine whether quantification of 1 vs 3 hot spots yields accurate results $\mathrm{CD}^{+} / \mathrm{CD}^{+}$ratio as well as the absolute cell numbers were compared with the ICC respectively. Third, computed counting methods (1) ZEN 2 software counting (ZC), (2) ImageJ software with subjective threshold (ISC) and (3) ImageJ with colour deconvolution (IAC) were compared to a manual counting (gold standard) using a linear regression analysis. Finally, 60 resected tumor tissues of $\mathrm{HCC}$ and 30 of PCa were retrieved. 3 hot spots have been selected for every slide and groups of high/low infiltration of $\mathrm{CD}^{+}$and $\mathrm{CD}^{+}$Lymphocytes have been created according to the median value. Then, statistical correlation with overall survival (OS) and disease free survival (DFS) was performed.

Comparison of manual counting to the computed methods showed mostly excellent accuracy of the obtained results using intraclass correlation with reliability analysis (ICC) and coefficient B with linear regression (B):

$\begin{array}{llll}\mathrm{mCRC} & \mathrm{OvCa} & \mathrm{HCC}\end{array}$

ZC ICC $=0.926, \mathrm{~B}=0.868 \mathrm{ICC}=0.987, \mathrm{~B}=0.968 \mathrm{ICC}=0.869, \mathrm{~B}=0.621 \mathrm{ICC}=0.601, \mathrm{~B}=1.280$ ISC ICC $=0.973, \mathrm{~B}=0.851 \mathrm{ICC}=0.992, \mathrm{~B}=1.030 \mathrm{ICC}=0.990, \mathrm{~B}=1.060 \mathrm{ICC}=0.934, \mathrm{~B}=0.914$ $\mathrm{IACICC}=0.986, \mathrm{~B}=0.945 \mathrm{ICC}=0.990, \mathrm{~B}=1.060 \mathrm{ICC}=0.955, \mathrm{~B}=0.723 \mathrm{ICC}=0.932, \mathrm{~B}=1.327$

Results: Quantification results from 2 blinded observers for reliable detection of hot spots were 0.949 in mCRC, 0.843 in OvCa, 0.805 in HCC and 0.957 in PCa. The ICC for the ratio of CD8/CD3 in 1 hot spot compared to the average from 3 hot spots was consistent in all groups. The absolute cell count in 1 vs 3 hot spots did differ reaching ICC scores reflecting a poor accuracy. ZC in PCa yielded inconsistencies. ISC reached excellent results in all groups. IAC reached excellent reliability in frozen sections and HCC but not in PCa (Table). High infiltration of $\mathrm{CD}^{+}$Lymphocytes correlated significantly with improved DFS in HCC $(\mathrm{p}=0.016)$ and PCa $(\mathrm{p}=0.001)$ as well as improved OS in PCa $(\mathrm{p}=0.046)$. High infiltration of $\mathrm{CD} 8^{+}$Lymphocytes correlated significantly with improved DFS in HCC $(\mathrm{p}=0.028)$ and OS in PCa $(\mathrm{p}=0.006)$

Conclusion: In this we introduce a systematized way to count tumor stroma cells in frozen and paraffin sections. Some rules should be observed: First, subjective selection of hot spots by 1 observer is accurate. Second, if a ratio is to be determined, quantification of 1 hot spot is sufficient. If the absolute cell count is to be determined, quantification of 3 hot spots is recommended. Third, 
computed counting methods achieve mostly excellent accuracy. ISC is accurate for sections with high background staining. IAC can be used in sections with low background staining. With the QTS Algorithm quantification of cells in the tumor stroma is reliable and accurate. Furthermore, clinical correlations after the us of this algorithm show results according to the literature: High infiltration of $\mathrm{CD}^{+}$and $\mathrm{CD}^{+}$Lymphocytes correlates with favorable prognosis in HCC and $\mathrm{PCa}$. The future use of immunoscoring systems may help to predict prognosis after resection.

Disclosure of Interest: All authors have declared no conflicts of interest.

\section{References}

Sun et al., The predictive value of centre tumour CD8(+) T cells in patients with hepatocellular carcinoma: comparison with Immunoscore. Oncotarget, 2015 Karakhanova et al., Prognostic and predictive value of immunological parameters for chemoradioimmunotherapy in patients with pancreatic adenocarcinoma. Br J Cancer, 2015.

\section{P1598 LIVER RESECTION IN OBESE PATIENTS WITH HEPATOCELLULAR CARCINOMA}

H. Iwama, S. Hai, E. Hatano, T. Okada, Y. Asano, N. Uyama, K. Suzumura, M. Tada, J. Fujimoto

Hepato-biliary-pancreatic Surgery, Hyogo College of Medicine, Hyogo/Japan

Contact E-mail Address: iwamax33f167@hotmail.com

Introduction: Lifestyle-related disease has been recognized as a risk factor for hepatocellular carcinoma (HCC). On the other hand, there are few reports concerning liver resection (LR) in obese patients.

Aims \& Methods: We performed curative LR in 471 patients with HCC between 2001 and 2015. In this study, we defined an obesity as no less than 25 of body mass index (BMI). We compared clinicopathological findings, operation details, and surgical outcomes of the obese and non-obese patients. Furthermore, we assessed the safety and the benefit of laparoscopic partial hepatectomy and left lateral segmentectomy in the obese patients.

Results: Among 471 patients, 123 patients $(26.1 \%)$ were defined as obese. Among them, 20 patients $(4.2 \%)$ showed no less than 30 of BMI. Diabetes, hypertension, and hyperlipidemia were significantly more common, and the patients with hepatitis virus positive were less in the obese patients group than in the non-obese patients group $(\mathrm{p}<0.05)$. The two groups showed no differences in the liver function tests except the indocyanine green retention rate at 15 minutes. There were no significant differences between the two patients group in the number of tumors, diameter of tumor, prevalence of cirrhosis, frequency of portal invasion, the operative procedure, operative duration, blood loss, incidence of postoperative complications, postoperative hospital stay, and in-hospital mortality $(3.3 \%$ vs. $1.4 \%$ ). No significant difference was found in relapse-free survival rate, or overall survival rate between the two groups, too. Thirteen patients underwent laparoscopic surgery, and 34 patients had open surgery. The two groups showed no difference in the background, including BMI. However the operation time ( $265 \mathrm{~min}$ vs. $397.5 \mathrm{~min}$ ) and the postoperative hospital stay (14 days vs. 18 days) were sinificantly shorter, and the blood loss $(50 \mathrm{ml}$ vs. $600 \mathrm{ml})$ was less in the laparoscopic surgery group than in the open surgery group $(\mathrm{p}<0.05)$.

Conclusion: Liver resection in the obese patients with $\mathrm{HCC}$ was safe, and laparoscopic liver resection might be more useful for reducing the surgical stress and reducing the hospital stay.

Disclosure of Interest: All authors have declared no conflicts of interest. and amount procedures not completed with minimally invasive technique decreases with time from the last hospitalization to surgery. All p-values $<0.05$. Conclusion: For those patients undergoing elective cholecystectomy after an acute cholecystitis the safety increases if surgery is performed later than 30 days after discharge.

Disclosure of Interest: All authors have declared no conflicts of interest.

P1600 MIXED REALITY SURGERY USING CT-BASED PATIENTSPECIFIC IMMERSIVE 3D HOLOGRAMS ENHANCED SPATIAL AWARENESS IN HEPATO-PANCREATO-BILIARY AND GASTROINTESTINAL SURGERY

\section{Sugimoto}

Graduate School Of Health Welfare Science, International University of Health Welfare, Tokyo/Japan

Contact E-mail Address: sugimotomaki@mac.com

Introduction: We developed a CT-based patient-specific holographic surgical simulation navigation system of immersive mixed reality (MR).

Aims \& Methods: The aim of this study is to identify its benefit of simulating, analyzing and evaluating operative surgical treatment options in gastrointestinal and hepato-pancreato-biliary surgery.

We used our original immersive MR application using HoloLens, that is a pair of MR smartglasses built-in head-mounted display. By reconstructing the patientspecific 3D surface polygons of each organ out of the patient's MDCT images, MR anatomy was displayed on the grasses three-dimensionally during actual surgery. The HoloLens features an inertial measurement unit which includes an accelerometer, gyroscope, and a magnetometer for environment understanding sensors, an energy-efficient depth camera, a 2.4-megapixel photographic video camera, and an ambient light sensor. We performed 10 gastrointestinal and hepato-pancreato-biliary surgeries.

Results: The accurate surgical anatomy of size, position, and depth of the cancers, surrounding organs, and vessels during surgeries could be measured using build-in dual infrared light sensors. The exact location between surgical devices and patient's anatomy could be traced on the pair of MR smartglasses by satellite tracking. The gesture controlled manipulation by surgeons' hands with surgical groves was useful for intraoperative anatomical references of tumors and vascular position under sterilized environment. It allowed the user to manipulate the spatial attributes of the virtual and real anatomies, which can enhance spatial awareness. The use of our systems reduced the length of the operation and discussion time.

MR navigation assistance could support complex procedures with the help of pre- and intra-operative imaging with better visualisation of the surgical anatomy and spatial awareness with visualisation of surgical instruments in relation to anatomical landmarks.

Conclusion: We report illustrative benefits of the immersive MR in surgical planning, simulation, education, and image-guided navigation. These could overcome the limitations of the conventional image-guided surgery.

Disclosure of Interest: All authors have declared no conflicts of interest.

\section{Reference}

1. Maki Sugimoto. Augmented Tangibility Surgical Navigation Using Spatial Interactive 3-D Hologram zSpace with OsiriX and Bio-Texture 3-D Organ Modeling. IEEE Computer Application Technologies (CCATS), 2015 International Conference on. 189-194, 2015. DOI:10.1109/CCATS.2015.53

\section{P1599 TIMING OF ELECTIVE CHOLECYSTECTOMY AFTER ACUTE CHOLECYSTITIS - A POPULATION-BASED STUDY}

\section{A. Popowicz, G. Sandblom}

Department Of Clinical Science, Intervention And Technology, Karolinska Institute, Stockholm/Sweden

\section{Contact E-mail Address: agnieszka.popowicz@ki.se}

Introduction: Acute cholecystectomy as treatment of acute cholecystitis is standard of care. However, many patients are still treated conservatively and undergo elective surgery. Often 6-12 weeks following the primary admittance is postulated as a good timing for an elective surgery but there are no studies on the optimal timing for delayed cholecystectomy.

Aims \& Methods: The aim of our study was to determine when it is most advantageous to perform an elective cholecystectomy after an episode of acute cholecystitis. All patients treated for acute cholecystitis in Sweden during the years of 2006 and 2013 were identified through the Swedish Inpatient Register. This cohort was cross-linked with the Swedish register for gallstone surgery, GallRiks where information on surgical outcome was retrieved. The impact of time from admission to surgery with regards to operative time, percentage of procedures completed with minimally invasive technique, peri and postoperative complications and bile duct injury or bile leakage were analysed with logarithmic transformation of time and multivariate logistic regression adjusting for gender and age.

Results: During the years 2006 to 2013, 31091 patients were treated for acute cholecystitis in Sweden. After exclusion of patients that did not perform surgery, were not registered in GallRiks and patients that were treated with acute cholecystectomy 8532 patients were identified that underwent planned surgery. In multivariate linear regression analysis with adjustment for gender and age the risk for peri- and postoperative complications, bile duct injuries and bile leakage

\section{P1602 EFFICACY OF DOUBLE PIGTAIL STENT FOR POST OESO- GASTRIC SURGICAL LEAKS NON LINKED TO BARIATRIC SURGERY: A PILOT BI-CENTRE RETROSPECTIVE STUDY}

J. Jacques ${ }^{1}$, L. Pagnon ${ }^{1}$, A. Taibi ${ }^{2}$, R. Legros ${ }^{1}$, T. Tabouret ${ }^{1}$, T. Barrioz ${ }^{3}$, M. Wangermez ${ }^{2}$, S. Durand-Fontanier ${ }^{2}$, S. Bouvier ${ }^{2}$, M. Mathonnet ${ }^{2}$, D. Valleix ${ }^{2}$, A. Le -Sidaner ${ }^{2}$, H. Lepetit ${ }^{1}$, V. Loustaud-Ratti ${ }^{2}$, D. Sautereau ${ }^{1}$ ${ }^{1}$ Hepato-gastro-enterology, CHU Limoges - Hepato-Gastro-Enterology, CHU Limoges; Limoges/FR, Limoges/France

${ }^{2}$ Digestive Surgery, $\mathrm{CHU}$ Limoges, Limoges/France

${ }^{3}$ Dept. De Endoscopie Digestive, CHU Poitiers, Poitiers/France

Contact E-mail Address: la.pagnon@gmail.com

Introduction: Post-surgical leaks are the first complication after non-bariatric oeso-gastric surgery in terms of frequency and morbidity. Anastomotic fistula change the prognostic of the operated patient increasing the length of stay, increasing costs, increasing mortality and increasing risks of oncological recurrence. Surgery is still a possibility of treatment in particular in case of severe sepsis but the role of endoscopy has evolved with development of a new armamentarium of endotherapy dedicated to such complications. In cases of post laparoscopic sleeve gastrectomy leaks, a shift is ongoing between closure or diversion methods (Ovesco, clips, covered self expandable metal stents (SEMS)) and internal drainage (double pig tail stents). Two large studies have just reported a high efficiency and a low complication rate with internal drainage with double pig tail stents for post sleeve gastrectomy leaks. No data are available for such an endoscopic internal drainage in fistulas of the upper digestive tract non-linked to bariatric surgery. Here we report the results of a pilot bicentre retrospective study.

Aims \& Methods: A retrospective study of all upper digestive tract leaks non linked to bariatric surgery and treated by double pig-tail stent (DPTS) in two 
tertiary centers has been performed. DPTS are traced by the pharmacies of our two hospitals and all cases of DPTS were analysed thanks to the database of the pharmacies of our two hospitals since May 2014 (first DPTS used for non bariatric upper digestive tract fistula) 7 or 10 french DPTS have been used according to the size of the fistula and the choice of the physician. Technical success was defined as the possibility to place the DPTS within the fistula. Clinical success was a composite endpoint combining clinical amelioration of the patient and healing of the fistula allowing the removal of the DPTS.

Results: 17 patients have been treated by DPTS from May 2014 to March 2017 for an upper digestive tract leaks non linked to bariatric surgery. Fistulas were linked to a lewis-santy surgery in 8 patients $(47 \%)$, total gastrectomy in 4 $(23.5 \%)$, boerhaave syndrome in $2(11.8 \%)$, endoscopic perforation in 2 $(11.8 \%)$ and aortic surgery in 1 (5.9). An infected collection was present in 16 patients $(94 \%)$ and $11(64.7 \%)$ suffer from a clinical sepsis. The mean delay between surgery and the diagnostic of flistula was 10 days and the delay of the diagnosis of fistula and the endoscopic drainage by DPTS was 16,4 days. DPTS were used alone in first intention in 11 cases $(64.7 \%)$, in first intenetion in combination with a SEMS in $11.7 \%$ and in second intention after failure of a SEMS in $3(11.7 \%)$. All patients had a technical success and 15 patients $(88.8 \%)$ had a clinical success. The mean delay for refeeding after DPTS was 17 days. The mean ablation time of the DPTS was 73 days. 3 patients presented a complication (1 stenosis, 1 bleeding and 1 migration).

\begin{tabular}{ll}
\hline & $\mathrm{N}=17$ \\
\hline AGE & $55(25-77)$ \\
ETIOLOGY & \\
$\quad$ Lewis Santy & $8(47 \%)$ \\
total gastrectomy & $4(23,5 \%)$ \\
Boerhaave & $2(11,8 \%)$ \\
Endoscopic perforation & $2(11,8 \%)$ \\
Aortic surgery & $1(5,9 \%)$ \\
ASA score & $2,3(1-4)$ \\
Associated collection & $16(94 \%)$ \\
Clinical sepsis & $11(64,7 \%)$ \\
Biological sepsis & $14(82,3 \%)$ \\
Delay surgery-diagnosis & 10 days \\
Delay Diagnosis-DPTS & 16,4 days \\
Endoscopic procedure & \\
DPTS alone in first intention & $11(64,7 \%)$ \\
DPTS + SEMS in first intention & $2(11,7 \%)$ \\
DPTS in second intention & $4(23,5 \%)$ \\
Technical success & $17(100 \%)$ \\
Clinical success & $15(88,8 \%)$ \\
Delay for refeeding & $17(4-52)$ \\
Delay for ablation of DPTS & $73(36-157)$ \\
Complications & \\
Stenosis & $1(5,9 \%)$ \\
Migration & $1(5,9 \%)$ \\
Bleeding & $1(5,9 \%)$ \\
\hline &
\end{tabular}

Conclusion: Endoscopic internal drainage using DPTS seems to be an interesting therapeutic option for upper digestive tract leaks non-linked to bariatric surgery. DPTS are effective, safe, cheap and could replace SEMS not always well tolerated and with a high rate of migration. Prospective multicenter studies are needed to confirm theses preliminary results.

Disclosure of Interest: All authors have declared no conflicts of interest.

\section{References}

Donatelli G, Dumont J-L, Cereatti F, Ferretti S, Vergeau BM, Tuszynski T, et al. Treatment of Leaks Following Sleeve Gastrectomy by Endoscopic Internal Drainage (EID). OBES SURG. 2015 Jul;25(7):1293-301.

Bouchard S, Eisendrath P, Toussaint E, Le Moine O, Lemmers A, Arvanitakis $\mathrm{M}$, et al. Trans-fistulary endoscopic drainage for post-bariatric abdominal collections communicating with the upper gastrointestinal tract. Endoscopy. 2016 Sep;48(9):809-16.

\section{P1603 ROUTINE USE OF POLYPROPYLENE MESH FOR PROPHYLAXIS OF ABDOMINAL COMPARTMENT SYNDROME FOR PATIENTS AFTER RUPTURED ABDOMINAL AORTIC ANEURYSM OPEN REPAIRING. RESULTS OF THREE YEARS FOLLOW-UP}

O. Popova ${ }^{1}$, O. Sergeev ${ }^{2}$

${ }^{1}$ Department Of Emergency Surgery, Dnipropetrovsk Regional Hospital, Dnipro/ Ukraine

${ }^{2}$ Vascular Surgery, Dnipropetrovsk regional hospital, Dnipro/Ukraine

Contact E-mail Address: oksana.nikolaevna.popova@gmail.com
Introduction: Abdominal compartment syndrome (ACS) is serious complication of big number of surgical interventions. According the data of the World Society of the Abdominal Compartment Syndrome (WSACS), rate of mortality without treatment is more than $90 \%$, after treatment from $25 \%$ to $75 \%$ [1]. Patients with ruptured abdominal aortic aneurysms (rAAA) are the group of high risk regarding this complication. Rate of incidence ACS at these patients is between $8 \%$ to $25 \%$ [2]. According to data of various authors, from one third to one half of them have died [3]. One of the main cause of this is the absence of good monitoring of intraabdominal pressure in this group of patients[4]. Currently, we have one effective way of treatment for this pathology: decompressive laparotomy[5]. But prophylaxis becomes more important point, if we take to attention mortality after start the develop of abdominal compartment.

Aims \& Methods: We aimed to investigate the impact of implantation polypropylene mesh in abdominal wall on rate of development ACS and it severity for patients after open repairing of ruptured abdominal aortic aneurysm. Patients with rAAA (total amount $\mathrm{n}=87$ patients) were operated in standard volume. Before finish of surgery in study group $(n=49,34$ males, 15 females, average age 63 years $+/-14$ years) was performed implantation of polypropylene mesh ("inlay' type) and was sutured skin and subcutaneous tissue only. In control group $(\mathrm{n}=38,26$ males, 12 females, average age 61 years $+/-15$ years $)$ surgery was finished by standard way. In all patients had infrarenal type of rAAA. Middle laparotomy or thoracophrenodiafragmal access was used. After surgery intraabdominal pressure was controlled by intravesical method during first seven days after operation. We fixated rate of complication and mortality in both groups. We followed-up patients after surgery and controlled far outcomes too. First mesh implantation was performed at 24 February 2014, last at 03 March 2017. Results: In study group we fixated 5 cases of ACS $(10.2 \%)$, including 3 cases of light ADS (intraabdominal pressure (IAP) $-12-15 \mathrm{mmHg}$ ), 1 case of modrate ADS (IAP 16-20 mmHg), and 1 case of severe ADS (IAP 21$25 \mathrm{mmHg}$ ).Decompressive laparotomy was performed in one case with satisfactory result. In general, mortality in study group was $18.4 \%$ (9 cases).In control group we fixated 9 cases of ACS $(23,7 \%)$, including 3 cases of light ADS (intraabdominal pressure (IAP) $-12-15 \mathrm{mmHg}$ ), 2 cases of moderate ADS (IAP 16-20 $\mathrm{mmHg}$ ), and 3 cases of severe ADS (IAP 21-25 mmHg) and 1 case with very severe ADS (IAP more than $25 \mathrm{Hgmm}$ ). Decompressive laparotomy was performed in three cases, satisfactory result was ahcieved in 1 case, in two cases patients have died from polyorgan insufficiency. In general, mortality in control group was $28.9 \%$ (11 cases). We did not find any specific complication, related with implantation of polypropylene mesh during all three years of followup.

Conclusion: 1. Impantation of polypropylene mesh is a safe and effective procedure for prophylaxis of ACS for patient with rAAA. 2. Impantation of mesh allows to avoid ACS for patient with rAAA and related with it complication and outcomes. 3. It is possible to implantate polypropylene mesh for other deseases, not only for rAAA, but this point requires further investigations.

Disclosure of Interest: All authors have declared no conflicts of interest.

\section{References}

1. De Waele JJ, Hoste EA. Decompressive laparotomy for abdominal compartment syndrome-a critical analysis. Critical Care 2006;10:R51.

2. Bajardi G1, Pecoraro F. Abdominal compartment syndrome (ACS) after abdominal aortic aneurysm (AAA) open repair. Ann Ital Chir. 2009 SepOct;80(5):369-74.

3. Muresan, M., Muresan. (2017). How much does decompressive laparotomy reduce the mortality rate in primary abdominal compartment syndrome?: A prospective study on 66 patients. Medicine, 96(5), e6006.

4. Choi, J. Y. S., Burton, P., Walker, S. and Ghane-Asle, S. (2008), ABDOMINAL COMPARTMENT SYNDROME AFTER RUPTURED ABDOMINAL AORTIC ANEURYSM. ANZ Journal of Surgery, 78: 648-653.

5. Loftus, I.M. et al.The abdominal compartment syndrome following aortic surgery. European Journal of Vascular and Endovascular Surgery, Volume 25, Issue 2, 97-109

6. Burch, JM, Moore, EE, Moore, FA, Franciose, R. The abdominal compartment syndrome. Surg Clin N Am. 1996;76:833-842.

\section{P1604 ENDOSCOPIC PERORAL DRAINAGE (EPOD) OF PERITONEAL COLLECTIONS AND ABSCESSES SECONDARY TO BARIATRIC SURGERY LEAKS: THE PARADIGM SHIFT OF SEEING PERITONEUM AS AN ORGAN AMENABLE FOR FLEXIBLE ENDOSCOPES INTERVENTIONS}

A.J. Baptista ${ }^{1}$, A. Salinas ${ }^{1}$, M.A. Guzman ${ }^{1}$, A. Gelrud ${ }^{2}$, D. Ramirez ${ }^{1}$, W. García ${ }^{1}$, A. Oropeza ${ }^{1}$, H. Rass ${ }^{1}$, J.F. Piñerúa Gonsálvez ${ }^{1}$ ${ }^{1}$ Hospital de Clínicas Caracas, Caracas/Venezuela

${ }^{2}$ ASGE - American Society for Gastrointestinal Endoscopy, Downers Grove/United States of America

Contact E-mail Address: albertogebaptista@gmail.com

Introduction: Peritoneal collections and abscess after Bariatric Surgery (BS) leaks are dreaded complications. Laparoscopic or open surgery and percutaneous CT drainage are the current indications. Endoscopic management of pancreatic collections is a rationale for Endoscopic Peroral Drainage (EPOD) approach in cases of BS leaks with abdominal collections.

Aims \& Methods: The aim of this study is to evaluate utility and safety of EPOD to treat peritoneal collections and abscesses secondary to BS leaks. Methods: This retrospective study included 65 consecutive patients from 2007 to 2015 at a single center (40 Sleeve gastrectomy, 25 gastric bypass) after 5 to 21 days from 
surgery. Patients presented heart rate over $120 \mathrm{bpm}$. Images from CT showed left sub-phrenic, peri-gastric or free abdominal collections. An Upper GI endoscopy was performed to localize the leak opening and enter to peritoneal cavity. Either 9.8 or $5.8 \mathrm{~mm}$ diameter gastroscope were used. In 10 patients with orifices smaller than $5.8 \mathrm{~mm}$ balloon dilatation of the leak opening allowed peritoneal access. The fluid content was suctioned out (100 to $700 \mathrm{ml})$. Sample was taken for bacterial cultures. The cavity was flushed and suctioned out with sterile saline solution $(200 \mathrm{ml}$ to $1000 \mathrm{ml})$. In cases of inadequate location surgical drains catheters were repositioned or replaced using endoscopic forceps and snares. Fifteen patients had malfunctioning latex drains. Replacement for drainage catheters was performed advancing with endoscopes through the leak all the way down to the skin. Once the tip of the endoscope was outside the peritoneum the latex drains were removed. Catheters were snared or grasped and pulled back into the peritoneum leaving the proximal end close to the fistula opening. In 5 patients without surgical drainage systems one laparoscopic port was localized inside peritoneum and re-opened under endoscopic vision to allow drainage catheters placement. In 8 patients peritoneal adhesions were endoscopically liberation with endoscopic forceps or knifes to facilitate peritoneal navigation.

Results: Heart rate returned to normal within 24 hours and leukocytosis improved after 72 hours. In $50 \%$ of patients heart rate returned to normal immediately. Average time for the whole procedure was 45 minutes. Abdominal catheters were removed between 7 and 18 days once full resolution of the drainage was achieved. Twenty patients were discharged within the first 24 hours. The rest were discharged between 3 and 8 days. Partially covered SEMS were placed for 6 to 8 weeks leading to complete closure of leaks. There were no adverse events related.

Conclusion: EPOD for peritoneal collections and abscesses secondary to BS leaks is feasible, safe and highly effective.

Disclosure of Interest: All authors have declared no conflicts of interest.

\section{P1605 CLINICAL ASSESSMENT OF THE FAILING TO REVERSE A DIVERTING ILEOSTOMY}

A. Sobolewska-Włodarczyk ${ }^{1}$, M. Wlodarczyk ${ }^{1}$, J. Włodarczyk $^{1}$, P. Siwiński ${ }^{1}$, J. Kasprzyk ${ }^{1}$, A. Dziki ${ }^{2}$, t. Dziki ${ }^{2}$

${ }^{1}$ Department Of Biochemistry; Department Of Gastroenterology, Medical University of Lodz, Lodz/Poland

${ }^{2}$ Department Of General And Colorecetal Surgery, Medical University of Lodz, Lodz/Poland

\section{Contact E-mail Address: olasobolewska1@poczta.onet.pl}

Introduction: A diverting loop ileostomy with procedure of intestinal resections and intestinal anastomosis decreases morbidity from anastomotic leaks and may even reduce the risk of anastomotic leak as shown in recent studies. Reversal of a temporary ileostomy is considered a simple surgical procedure presenting with a low morbidity and mortality rates. However, ileostomy reversal may be associated with number of complications requiring reoperation, with anastomotic leak (AL) being most critical often leading to failure in restoration of digestive tract continuity.

Aims \& Methods: The study aimed at evaluating the results of restoration of intestinal continuity in patients primarily operated on for colorectal cancer and inflammatory conditions. We assessed the frequency of failure following the surgical procedure of diverting loop ileostomy closure. The study was conducted at a tertiary referral center. 147 adult patients $(89$ men, mean age $50.5 \mathrm{y} \pm 16.71 \mathrm{y}$ and 58 women, mean age $49.3 \mathrm{y} \pm 16.99 \mathrm{y}$;) were enrolled to this study. All included patients underwent surgical closure of a temporary loop ileostomy at the Department of General and Colorectal Surgery, Lodz, Poland, between 2004 and 2015. The medical data was collected in a retrospective manner basing on hospital records. The analyzed parameters included length of hospitalization, gender, age, BMI, concomitant conditions, American Society of Anesthesiologists classification score (ASA) and the character of postoperative complications. Statistical analysis was used to evaluate the correlation between the variables and postoperative complications.

Results: AL as a postoperative complication with the need to create a new ostomy occurred in 15 patients $(10.2 \%)$. Higher values of BMI and older age have been noted compared to noncomplicated surgeries (27.54 \pm 2.36 vs. $23.02 \pm 3.98$; $\mathrm{p}<0.001 ; 54.53 \pm 12.3$ vs. $50.25 \pm 15.95 ; \mathrm{p}=0.029$ respectively). There was no significant correlation between patients' gender and the incidence of AL $(\mathrm{p}=0.087)$. The number of prior surgical procedures and ASA scale positively correlated with the prevalence of $\mathrm{AL}(\mathrm{p}=0.038 ; \mathrm{p}=0.003$ respectively). There were no significance diference betwenn patient with colorectal cancer and inflammatory conditions $(\mathrm{p}=0.534)$. The average time interval from prior surgery to reversal of the stoma and AL occurrence were not related and did not reveal statistical significance $(8.5 \pm 4.41 \mathrm{vs}$. $7.24 \pm 4.47 ; \mathrm{p}=0.25)$. Univariate logistic regression demonstrated that a BMI levels, patients' age and average time interval from prior surgery to reversal procedure were significantly associated with failure of restoration of intestinal continuity.

Conclusion: AL as a critical postoperative complication is still a major issue related with failure of intestinal continutiy restoration in patients undergoing loop ileostomy clouser. Patients with elevated BMI, older age and shorter average time interval from prior surgery to reversal procedure present higher risk of failure in restoration of digestive tract continuity. The incidence rate of AL was similar in patients operated primarily on for inflammatory conditions and colorectal cancer.

Disclosure of Interest: All authors have declared no conflicts of interest.
P1606 BODY COMPOSITION AS A PREDICTOR OF MORBIMORTALITY FOLLOWING BILIOPANCREATIC CANCER SURGERY

M.P.C. Santos ${ }^{1}$, S. Velho ${ }^{2}$, C. Cunha ${ }^{2}$, F. Costa ${ }^{3}$, L. Agostinho², R. Cruz ${ }^{2}$, R. Maio ${ }^{2}$, M. Cravo ${ }^{1}$

${ }^{1}$ Gastroenterology Service, Hospital Beatriz Ângelo, Loures/Portugal

${ }^{2}$ Hospital Beatriz Ângelo, Loures/Portugal

${ }^{3}$ Hospital da Luz, Lisboa/Portugal

Contact E-mail Address: mariapiacostasantos@gmail.com

Introduction: The impact of body composition on the outcomes following pancreaticoduodenectomy is still unclear.

Aims \& Methods: The aim of this study was to analyze the association between body composition and postoperative complications and 90-day mortality in patients undergoing biliopancreatic cancer surgery. Retrospective study of patients with pancreatic, ampullary or bile duct carcinoma that underwent surgery between March 2012 and October 2016. Body composition (skeletal muscle area, visceral fat area, subcutaneous fat area and muscle radiation attenuation) was assessed in diagnostic or staging computed tomography (CT), in axial images at the level of the $3^{\text {rd }}$ lumbar vertebra. Postoperative complications were recorded according to Clavien-Dindo classification and categorized as minor (grade IIIIa) and major (grade IIIb-V).

Results: Fifty-nine patients were analyzed and 11 were excluded due to unavailable CT scan at our institution. Forty-eight were included, 28 were men, with a mean age of $70.9 \pm 8.5$ years. The incidence of major complications was $25 \%$ and 90-day mortality was $8.3 \%$. On simple logistic regression of factors associated with major complications skeletal muscle area (OR $0.97,95 \%$ CI $0.94-1.00$, $\mathrm{P}=0.09)$ and index (OR 0.91, 95\% CI $0.81-1.00, \mathrm{P}=0.09)$ showed a trend for a protective effect. On multiple logistic regression skeletal muscle index was a protective factor (OR $0.89,95 \%$ CI $0.79-0.99, \mathrm{P}=0.05)$ and longer surgery $(\mathrm{OR}$ $1.01,95 \%$ CI $0.99-1.03, \mathrm{P}=0.07$ ) was associated with higher incidence of major complications. The receiver-operator characteristic (ROC) curve showed an acceptable power of discrimination of major complications using a model with skeletal muscle index and surgery duration as independent variables (area under the curve of 0.736 ). On simple logistic regression surgery duration (OR 1.02, 95\% CI $1.00-1.06, \mathrm{P}=0.05)$, visceral fat area $(\mathrm{OR} 1.02,95 \% \mathrm{CI} 1.00-1.04, \mathrm{P}=0.02)$ and index (OR $1.05,95 \% \mathrm{CI} 1.01-1.12, \mathrm{P}=0.04)$ were associated with higher 90 day mortality whereas muscle radiation attenuation had a protective effect $(\mathrm{OR}$ $0.88,95 \%$ CI $0.76-0.99, \mathrm{P}=0.05$ ).

Conclusion: These results suggest that low values of skeletal muscle and muscle radiation attenuation, as well as high values of visceral fat and longer surgery are associated with worse clinical outcomes following biliopancreatic cancer surgery. Disclosure of Interest: All authors have declared no conflicts of interest.

\section{P1607 GASTROSCHISIS: A 16-YEAR STUDY}

A.M. Bradeanu ${ }^{1}$, L. Balanescu ${ }^{2}$, I. Nenciu ${ }^{3}$

${ }^{1}$ Neonatal Intensive Care Unit, Emergency Children's Clinical Hospital " $G$. Alexandrescu" Bucharest, Bucharest/Romania

${ }^{2}$ Pediatric Surgery, Emergency Children's Clinical Hospital "G. Alexandrescu" Bucharest, Bucharest/Romania

${ }^{3}$ Emergency Care Unit, Emergency Children's Clinical Hospital " $G$. Alexandrescu" Bucharest, Bucharest/Romania

Contact E-mail Address: anamariabrad@yahoo.com

Introduction: Gastroschisis is a ventral body wall defect trough which protrude mainly large and small intestines. The disease's clinical course and prognosis depend on both surgical techniques, severity of the defect, accompanying anomalies and complications.

Aims \& Methods: We performed a retrospective study based on the analysis of patient's records admitted to our hospital Neonatal Intensive Care Unit between January 2000 and December 2016.The aims of this study were to evaluate defect's incidence, management and outcome of patients with gastroschisis in our institution.

Results: During the period 2000-2016 the overall incidence of gastroschisis in our NICU was $5.23 \%$ (140 cases-all transferred from other units). The average birth gestational age was 35.5 weeks and the average birth weight was $2270 \mathrm{~g}$. The abdominal wall defect was identified in antenatal period only in $15.4 \%$ of cases. Also, 23 patients $(16.4 \%)$ presented other associated anomalies: 12 cases with atresia or stenosis of various intestinal segments, 6 cases with renal anomalies and 5 cases with cardiac defects. Surgical techniques performed for abdominal defect closure were: primary closure in 35 cases $(25 \%)$, staged closure in 12 cases $(8.57 \%)$, Gross technique in 44 cases $(31.4 \%)$ and manual reduction and closure (Bianchi procedure) in 49 cases $(35 \%)$. The average period of digestive pause was 12.9 days and the mean length of stay in NICU was 30.4 days. Patients were also mechanical ventilated for an average period of 4.6 days. The most frequent complication was late-onset sepsis $(37.8 \%)$; also 26 neonates $(18.5 \%)$ underwent unplanned reoperation and 7 neonates $(5 \%)$ remained with short bowel. The average mortality in our group was $33 \%$ but it constantly decreased over the years (from $84 \%$ in 2000 to $11 \%$ in 2016). Sepsis, prematurity, low birth weight and associated defects were identified as major risk factors for the unfavorable outcome (death)

Conclusion: The management of neonates with gastroschisis depends on several factors including the status of herniated organs, the size of abdominal cavity, the presence of other associated congenital anomalies and last but not least on the resources and experience of the neonatal interdisciplinary team. Significant changes occurred in the management of gastroschisis in our Unit and as consequence, the outcome of patients with gastroschisis has dramatically improved during the studied period. 
Disclosure of Interest: All authors have declared no conflicts of interest.

\section{References}

1. Miranda da Silva Alves F, Miranda ME, de Aguiar MJ, Bouzada Viana MC Nutritional management and postoperative prognosis of newborns submitted to primary surgical repair of gastroschisis. $J$ Pediatr (Rio J). 2016;92:268-75.

2. Aldrink JH, Caniano DA, Nwomeh BC: Variability in gastroschisis management: a survey of North American pediatric surgery training programs. $J$ Surg Res 176:159, 2012

3. Bradnock TJ et al: Gastroschisis: one year outcomes from national cohort study. BMJ 343:, 2011

WEDNESDAY, NOVEMBER 01, 2017

09:00-14:00

\section{IBD III - HALL 7}

\section{P1608 ASSESSING THE EFFECT OF ETHNICITY ON URINARY}

\section{METABOLIC PROFILES IN INFLAMMATORY BOWEL DISEASE}

L. C. Hicks ${ }^{1}$, S.T.R. Powles ${ }^{2}$, L. W.L. Chong ${ }^{2}$, J. Swann ${ }^{3}$, E. Holmes ${ }^{3}$, H.

R.T. Williams ${ }^{2}$, T. R. Orchard ${ }^{2}$

${ }^{1}$ Gastroenterology, Imperial College Healthcare NHS Trust, London/United

Kingdom

${ }^{2}$ Division Of Digestive Diseases. Department Of Surgery And Cancer, Imperial

College London, London/United Kingdom

${ }^{3}$ Division Of Computational And Systems Medicine. Department Of Surgery And Cancer, Imperial College London, London/United Kingdom

Contact E-mail Address: 1.hicks@imperial.ac.uk

Introduction: Urinary metabolic profiling has been shown to distinguish patients with inflammatory bowel disease (IBD) from healthy controls (HC), and also separate ulcerative colitis (UC) from Crohn's disease (CD) in Caucasian (Cau) cohorts (1). Diet and lifestyle also have an effect on metabolic profiles (2), and these differ in patients from different ethnic backgrounds. Moreover, clinical phenotype varies between Caucasians and South Asians (SA)(3), however discriminatory metabolites have not been studied in different ethnic populations. The aim of this study was to compare the urinary metabolic profiles of IBD patients and healthy controls from Caucasian and South Asian backgrounds.

Aims \& Methods: Samples from 405 IBD patients (283 Caucasian and 122 South Asian) and 137 healthy controls (98 Caucasian and 48 South Asian) were analysed by $\mathrm{H}^{1} \mathrm{NMR}$ spectroscopy. Clinical and dietary data were collected. Orthogonal partial least squares discriminant analysis (OPLSDA) was performed to examine whether there were differences in metabolic data between Cau and SA. $\mathrm{R}^{2}$ (variance), $\mathrm{Q}^{2}$ (quality assessment) and $\mathrm{p}$ values (validity) for each model were described.
Results: The phenotype of South Asian Crohn's disease was not significantly different to Caucasian Crohn's disease in this cohort. In the South Asian UC group there was more pancolitis $(\mathrm{p}=0.051)$ and less proctitis $(\mathrm{p}=0.008)$. There were more vegetarians in the South Asian group. OPLSDA was able to separate patients with IBD from healthy controls, and also UC from Crohn's disease, in the Caucasian cohort, but this separation could not be replicated in South Asians (negative $\mathrm{Q}^{2}$ values).

Conclusion: The separation between Caucasian and South Asian healthy controls may reflect differing lifestyles including diet. Caucasian IBD patients could be separated from healthy controls, and Crohn's disease from UC, replicating previous studies. South Asian IBD patients could not be separated from healthy controls which may be due to lower numbers of South Asian patients in this study, and specifically less Crohn's disease patients where stronger discriminating models have been shown in Crohn's disease in previous studies. In Crohn's disease, Caucasians and South Asians could be separated, but Caucasian and South Asian patients could not be distinguished in the UC cohort, possibly suggesting the metabolic milieu in Crohn's disease is stronger and less influenced by the impact of ethnicity.

Disclosure of Interest: All authors have declared no conflicts of interest.

\section{References}

1. Williams, H.R., et al., Am J Gastroenterol, 2009. 104(6): p.1435-44.

2. Stella, C. et al., J Proteome Res, 2006. 5(10): p.2780-8

3. Walker, D.G., et al., Am J Gastroenterol, 2011. 106(7): p.1281-9.

\section{P1609 EFFECTS OF ACUTE CHANGES IN FERMENTABLE FIBRE} INTAKE ON REGIONAL COLONIC FERMENTATION AND TRANSIT IN PATIENTS WITH QUIESCENT ULCERATIVE COLITIS

C. K. Yao ${ }^{1}$, R. E. Burgell ${ }^{1}$, K. M. Taylor ${ }^{1}$, M. G. $\operatorname{Ward}^{2}$, J. S. Barrett ${ }^{1}$, J G. Muir ${ }^{1}$, P.R. Gibson ${ }^{1}$

${ }^{1}$ Department Of Gastroenterology, Monash University, Melbourne/Australia/VIC ${ }^{2}$ Department Of Gastroenterology, Alfred Hospital, Melbourne/Australia/VIC

Contact E-mail Address: chu.yao@monash.edu

Introduction: Reduced saccharolytic fermentation has been described in patients with quiescent ulcerative colitis (UC). Such defects may differ across colonic regions along with acute variations in dietary fibre intake. These aspects deserve further study.

Aims \& Methods: We aimed to define regional colonic fermentation by direct intestinal $\mathrm{pH}$-transit profiling in patients with quiescent $\mathrm{UC}$ following acute variations in fermentable fiber intake. A randomized, double-blind, crossover trial was performed. Patients with quiescent UC (Partial Mayo Score $\leq 1$; faecal calprotectin $<150 \mu \mathrm{g} / \mathrm{g}$ ) and healthy controls who were not taking any

Abstract: P1608

\begin{tabular}{|c|c|c|c|c|}
\hline & Caucasian ( $\mathrm{n}$ and \%) & South Asian ( $\mathrm{n}$ and \%) & - & - \\
\hline All IBD & 283 & 122 & - & - \\
\hline $\mathrm{CD}$ & $160(57 \%)$ & $42(34 \%)$ & - & - \\
\hline CD Disease location* & L1: 24. L2: 50, L3: 68 & L1: 7, L2: 16, L3: 19 & - & - \\
\hline CD Disease behaviour* & B1: 144, B2: 12, B3: 12 & B1: 26, B2: 7, B3: 4 & - & - \\
\hline $\mathrm{UC}$ & $123(43 \%)$ & $80(66 \%)$ & - & - \\
\hline UC Disease extent* & E1: 29, E2: 32, E3: 45 & E1: 6 , E2: 26, E3: 48 & - & - \\
\hline \multirow[t]{3}{*}{ Controls } & 98 & 39 & - & - \\
\hline & All (Cau and SA) & All (Cau and SA) & All (Cau and SA) & All (Cau and SA) \\
\hline & Separation & P values (100 permutation testing) & $\mathrm{R} 2$ & Q2 \\
\hline Controls vs $\mathrm{IBD}^{+}$ & Yes & $\mathrm{p}=0.001$ & 0.596 & 0.627 \\
\hline Controls vs $\mathrm{CD}^{+}$ & Yes & $\mathrm{p}=0.001$ & 0.659 & 0.623 \\
\hline Controls vs $\mathrm{UC}^{+}$ & Yes & $\mathrm{p}=0.007$ & 0.798 & 0.757 \\
\hline \multirow[t]{3}{*}{$\mathrm{CD}$ vs $\mathrm{UC}^{+}$} & Yes & $\mathrm{p}=0.001$ & 0.880 & 0.282 \\
\hline & Caucasian & Caucasian & Caucasian & Caucasian \\
\hline & Separation & $P$ values (100 permutation testing) & $\mathrm{R} 2$ & Q2 \\
\hline Controls vs $\mathrm{CD}^{+}$ & Yes & $\mathrm{p}=0.003$ & 0.634 & 0.627 \\
\hline Controls vs $\mathrm{UC}^{+}$ & Yes & $\mathrm{p}=0.012$ & 0.815 & 0.769 \\
\hline \multirow[t]{3}{*}{$\mathrm{CD}$ vs $\mathrm{UC}^{+}$} & Yes & $\mathrm{p}=0.008$ & 0.882 & 0.458 \\
\hline & SA & SA & SA & SA \\
\hline & Separation & $P$ values (100 permutation testing) & $\mathrm{R} 2$ & Q2 \\
\hline Controls vs $\mathrm{CD}^{+}$ & $\mathrm{No}^{++}$ & $\mathrm{N} / \mathrm{A}$ & 0.593 & -0.214 \\
\hline Controls vs $\mathrm{UC}^{+}$ & $\mathrm{No}^{++}$ & $\mathrm{N} / \mathrm{A}$ & 0.337 & -0.147 \\
\hline $\mathrm{CD}$ vs $\mathrm{UC}^{+}$ & $\mathrm{No}^{++}$ & $\mathrm{N} / \mathrm{A}$ & 0.217 & -0.071 \\
\hline
\end{tabular}

*Montreal classification

${ }^{+}$OPLSDA model examining the differences in urinary metabolic profiles between these cohorts

${ }^{++} \mathrm{p}$ value cannot be calculated if OPLSDA model has negative $\mathrm{Q}^{2}$ values

OPLSDA models also separated South Asian healthy controls from Caucasians healthy controls, and South Asian Crohn's disease from Caucasian Crohn's disease,

but in UC no robust model could be made. 
antibiotics (including sulfasalazine), probiotics or fiber supplements were recruited. After a 7-day run-in, subjects were fed study meals high $(11 \mathrm{~g}$ oligosaccharides, $2 \mathrm{~g}$ resistant starch) or low $(<1 \mathrm{~g})$ in fermentable fiber over a 12 -h period before ingesting the $\mathrm{pH}$-motility capsule, subjects then crossed over to the other diet 3 days after passage of the capsule. Endpoints were diet-associated differences in overall, caecal and distal colonic $\mathrm{pH}$, and colonic transit time (CTT), taken as time between the ileo-cecal junction and capsule exit. Caecal $\mathrm{pH}$ was defined as the minimum $\mathrm{pH}$ following passage through the ileo-cecal junction whereas maximum $\mathrm{pH}$ was arbitrarily used as distal colonic $\mathrm{pH}$.

Results: 15 UC patients (aged 24-72 y; 9 males) and 9 controls (aged 22-69 years, 4 males) were studied. A decrease in overall and distal colonic $\mathrm{pH}$ was observed in controls with high vs low fiber diet (Table 1). In UC patients, a high fermentable fiber intake reduced caecal $\mathrm{pH}$ but paradoxically tended to increase distal colonic $\mathrm{pH}$. A significant association was observed for UC extent and changes in overall ( $r=0.81 ; p<0.001$, Spearman's correlation) and caecal $\mathrm{pH}(r=0.53$; $p=0.04$ ) after a high fermentable fiber diet (Figure 1). No differences in CTT were observed between diets in either cohorts but subgroup analysis in the UC cohort showed highly heterogeneous responses to a high fermentable fiber diet. $64 \%$ patients had slower CTT whilst $36 \%$ had unchanged or faster CTT. In contrast, majority $(63 \%)$ of controls had no changes in CTT after a high fiber diet.

Table 1: Colonic $\mathrm{pH}$ and transit responses to acute changes in fermentable fiber intake

\begin{tabular}{llllll}
\hline & & $\begin{array}{l}\text { Overall } \\
\text { mean pH } \\
(95 \% \mathrm{CI})\end{array}$ & $\begin{array}{l}\text { Mean } \\
\text { cecal pH } \\
(95 \% \mathrm{CI})\end{array}$ & $\begin{array}{l}\text { Mean distal } \\
\text { colonic pH } \\
(95 \% \mathrm{CI})\end{array}$ & $\begin{array}{l}\text { Median } \\
\text { [IQR] } \\
\text { CTT (h) }\end{array}$ \\
\hline $\mathrm{UC} \mathrm{n}=15$ & Low fiber & $6.4(6.2-6.8)$ & $5.6(5.3-5.7)$ & $7.9(7.6-8.2)$ & $17[9-23]$ \\
& High fiber & $6.3(6.0-6.5)$ & $5.2(5.0-5.4)$ & $8.1(7.6-8.4)$ & $21[16-39]$ \\
& $p$-value & 0.20 & 0.001 & 0.09 & 0.13 \\
Healthy $\mathrm{n}=9$ & Low fiber & $6.9(6.5-7.2)$ & $5.5(5.2-5.8)$ & $8.2(8.0-8.5)$ & $16[15-37]$ \\
& High fiber & $6.3(6.0-6.5)$ & $5.2(4.9-5.5)$ & $7.7(7.4-8.0)$ & $18[15-32]$ \\
& $p$-value $^{1}$ & 0.02 & 0.15 & 0.04 & $0.58^{2}$ \\
\hline
\end{tabular}

${ }^{1}$ paired t-test or ${ }^{2}$ Mann-Whitney test

Conclusion: A high fermentable fiber diet partially increased colonic fermentative activity in patients with quiescent UC compared to controls. Moreover, contrary to controls, UC patients exhibited an increase in distal $\mathrm{pH}$ and heterogeneous colonic transit responses after a high fermentable fiber intake. Our findings suggest that abnormalities in motility and regional defects in the function of the colonic microbiota exist despite quiescent disease.

Disclosure of Interest: C.K. Yao: The Department of Gastroenterology, Monash University benefits financially from the sales of a digital app and booklet on the low FODMAP diet.

R.E. Burgell: Rebecca has received consultancy fees from Allergan. The Department of Gastroenterology, Monash University benefits financially from the sales of a digital app and booklet on the low FODMAP diet.

J.S. Barrett: The Department of Gastroenterology, Monash University benefits financially from the sales of a digital app and booklet on the low FODMAP diet. J.G. Muir: The Department of Gastroenterology, Monash University benefits financially from the sales of a digital app and booklet on the low FODMAP diet. P.R. Gibson: PG has served as consultant or advisory member for AbbVie, Ferring, Janssen, Merck, Allergan, Pfizer, Celgene \& Takeda; research support from AbbVie \& Janssen; speaking honoraria for his institution from AbbVie, Janssen, Ferring, Takeda, Mylan \& Pfizer.

All other authors have declared no conflicts of interest.

\section{P1610 FECAL MICROBIAL DYSBIOSIS IN CHINESE PATIENTS WITH INFLAMMATORY BOWEL DISEASE}

H. Ma, H. Zhang

Department Of Gastroenterology, First Affiliated Hospital of Nanjing Medical University, 300 Guangzhou Rd, Nanjing 210029, China, Jiangsu/China

\section{Contact E-mail Address: mahaiqin2010@163.com}

Introduction: Microbial dysbiosis in the gut has been suggested to play an important role in the pathogenesis of inflammatory bowel disease (IBD).

Aims \& Methods: In this study, we aimed to analyze the fecal microbiota in Chinese patients with IBD. Fecal samples from 15 patients with Crohn's disease (CD), 14 patients with ulcerative colitis (UC) and 13 healthy individuals were subjected to $16 \mathrm{~S}$ rDNA sequencing. The V4 hypervariable regions of 16S rDNA were sequenced by the Illumina MiSeq2500 platform. Quality control and operational taxonomic units (OTUs) were calculated with QIIME software.

Results: Significant differences in community richness and microbial structure were observed both in CD and UC compared with normal controls. At the phylum level, analysis of the microbial compositions revealed that the Proteobacteria percentages, were significant higher in IBD than in controls. While at the genus level, we found that the abundance of 8 genera in CD and 23 genera in UC, particular the Escherichia genus, were significantly different when comparing with controls. We next surveyed the taxonomics composition distribution between different phases of disease, detecting that the abundance of the Bacteroidetes was significant decreased in active CD group compared with inactive CD group. However, the Proteobacteria was only nominally increased in active $C D$ relative to inactive $C D$, which did not hold significance after correction. Furthermore, the relative abundance of the Bacteroidetes, especially the
Bacteroides, was negatively correlated with the calculated Crohn's disease activity index (CDAI) scores of patients.

Conclusion: Our findings showed the specific characteristics and dysbiosis of fecal microbiota within Chinese IBD patients. In addition, the abundance of the Bacteroidetes was significant lower in active $C D$ group than in inactive $C D$ group and it was negatively correlated with CDAI, indicating that the Bacteroidetes could be related with the disease activity.

Disclosure of Interest: All authors have declared no conflicts of interest.

\section{Reference}

1. Knights, D., K.G. Lassen, and R.J. Xavier, Advances in inflammatory bowel disease pathogenesis: linking host genetics and the microbiome. Gut, 2013. 62(10): p. $1505-10$

\section{P1611 HYPOXIA-MEDIATED IRON UPTAKE PREVENTS} INFLAMMATORY GENE EXPRESSION IN THE INTESTINAL EPITHELIUM THROUGH THE REGULATION OF NF-KB BINDING ACTIVITY

S. Simmen, J. Cosin Roger, C. De Valliere, M. Scharl, J. Zeitz, S. Vavricka, G. Rogler, P. A. Ruiz-Castro

Gastroenterology And Hepatology, University Hospital Zurich, University of Zurich, Zurich/Switzerland

Contact E-mail Address: PedroAntonio.Ruiz-Castro@usz.ch

Aims \& Methods: In the present study, we sought to elucidate the effects of iron supplementation on hypoxia-mediated responses in the intestinal epithelium. For this purpose, serum starved Caco-2 monolayers were subjected to normoxia $(21 \% \mathrm{O} 2)$ or hypoxia $(0.2 \% \mathrm{O} 2)$ in the presence and absence of ferric ammonium iron citrate (FAC) and the iron chelator deferoxamine (DFO). Total RNA was isolated and changes in the expression of tumor necrosis factor (TNF), interleukin (IL)-1 $\beta$, DMT-1, FPN and ferritin was assessed by real-time quantitative PCR. Western blot analysis was performed with antibodies against ferritin, pNF- $\kappa$ B, HIF- $1 \alpha$, p-mTOR, p62 and LC3. mRNA synthesis in Caco-2 cells under hypoxia was blocked using actinomycin D. Chromatin immunoprecipitation experiments were carried using antibodies against NF- $\kappa \mathrm{B}$ and primers for promoter binding regions of TNF and IL-1 $\beta$. Healthy volunteers $(n=10)$ were subjected to hypoxic conditions resembling an altitude of $4,000 \mathrm{~m}$ above sea level for $3 \mathrm{~h}$ using a hypobaric chamber. Serum samples were collected the day prior to hypoxia, and one day, one week and one month after hypoxia.

Introduction: Environmental hypoxia has been stablished to influence the development of inflammatory bowel disease (IBD). Adaptive responses to low oxygentension are mediated through hypoxia inducible factor (HIFs), which are tightly regulated by oxygen and iron levels through the action of hydroxylases. Dietary iron is mainly absorbed by duodenal enterocytes through the divalent metal transporter (DMT)-1. Once iron is inside the enterocytes, it is either sequestered into ferritin or transported out of the enterocyte into the circulation by ferroportin (FPN). Regulation of uptake, storage and export of iron is mediated by signals reflecting oxygen and intracellular iron levels in enterocytes, and systemic iron requirements. Central to systemic iron regulation is the liver hormone hepcidin, which regulates and is regulated by systemic iron levels. Hepticidin expression is induced by cytokines and results in anemia of inflammation.

Results: Hypoxia induced the mRNA expression of TNF and IL-1 $\beta$ concomitantly with the iron transporters DMT-1 and FPN in Caco-2 cells under ironstarving conditions, thereby promoting iron uptake. The iron chelator deferoxamine (DFO) induced HIF-1 $\alpha$ stabilization and TNF mRNA expression under both normoxic and hypoxic conditions. Conversely, iron supplementation induced ferritin protein accumulation under normoxic and hypoxic conditions, and reduced TNF and IL-1 $\beta$ mRNA expression. Interestingly, neither iron chelation nor iron supplementation reduced hypoxia-mediated $\mathrm{p}-\mathrm{NF}-\kappa \mathrm{B}$. Iron induced p-mTOR and blocked autophagy. Iron overload enhanced decay of $\mathrm{TNF}$, but not IL-1 $\beta$ mRNA. Iron also prevented binding of NF- $\kappa \mathrm{B}$ to the promoter of TNF and IL-1 $\beta$. Healthy volunteers presented reduced serum levels of iron, as well as transferrin saturation. Ferritin levels were unchanged indicating anemia of inflammation and suggesting enhanced intracellular iron accumulation in enterocytes following hypoxia.

Conclusion: Our results suggest that hypoxia-mediated iron uptake is crucial to counteract hypoxia-induced pro-inflammatory gene expression, and identify iron intracellular uptake and storage as a hypoxia protective mechanism to reduce mucosal inflammation.

Disclosure of Interest: All authors have declared no conflicts of interest.

\section{P1612 OGR1 (GPR68) EXPRESSION IS INCREASED IN INTESTINAL INFLAMMATION AND CORRELATES WITH DISEASE ACTIVITY IN PATIENTS WITH IBD}

J. Cosin Roger ${ }^{1}$, K. Baebler ${ }^{1}$, S. Bengs ${ }^{1}$, S. Lang ${ }^{1}$, M. Turina ${ }^{2}$, A. Rickenbacher ${ }^{2}$, M. Scharl ${ }^{1}$, K. Seuwen ${ }^{3}$, P. A. Ruiz-Castro ${ }^{1}$, B. Misselwitz ${ }^{1}$, G. Rogler ${ }^{1}$, C. De Valliere $^{1}$

${ }^{1}$ Gastroenterology And Hepatology, University of Zurich, University Hospital Zurich, Zurich/Switzerland

${ }^{2}$ University Hospital Zürich, Zürich/Switzerland

${ }^{3}$ Novartis Institutes for Biomedical Research, Basel/Switzerland

Contact E-mail Address: katharina.baebler@usz.ch 
Introduction: In both forms of inflammatory bowel disease (IBD), Crohn's disease (CD) and ulcerative colitis (UC), inflammation of the gut wall is associated with extracellular tissue acidification. Low extracellular $\mathrm{pH}$ stimulates the family of proton-sensing G-protein coupled receptors (GPCRs); ovarian cancer G-protein coupled receptor 1 (OGR1), T-cell death-associated gene 8 (TDAG8 or GPR65) and G-protein coupled receptor 4 (GPR4), which activate second messenger signaling cascades. Recent studies reported a link between IBD and this family of $\mathrm{pH}$-sensing receptors; in genome-wide association studies (GWAS), TDAG8 has been identified as an IBD-risk gene. The mechanism behind the increased risk of developing IBD remains unclear. OGR1 and TDAG8 are alleged to act in opposition by regulation of the inflammatory response; enhancing or inhibiting inflammatory pathways respectively, however the interplay between OGR1 and TDAG8 is unclear.

Aims \& Methods: In this study we aim to investigate the role of OGR1 in IBD patients. Expression of OGR1 in surgical specimens from non-IBD $(n=5), C D$ $(\mathrm{n}=10)$ and UC $(\mathrm{n}=10)$ patients was determined by immunohistochemistry, RT-qPCR and Western blotting. Clinical disease activity was assessed by the Harvey Bradshaw Index (HBI) and the Modified Truelove and Witts activity index (MTWAI) for $\mathrm{CD}$ and UC patients, respectively. Nonparametric Spearman's rank correlation analysis was performed.

Results: OGR1 immunostaining of human surgical samples from non-IBD patients revealed OGR1 expression mainly in lamina propria cells, with weaker staining in epithelial cells. OGR 1 staining in IBD patients was stronger compared to controls; however, in IBD patients OGR1 is highly expressed in both epithelial cells and cells in the lamina propria. Further, paired samples taken at the same time, from non-inflamed and inflamed intestinal tissue from IBD patients showed stronger OGR1 staining in the inflamed mucosa compared to the non-inflamed mucosa. Accordingly, mRNA and protein expression of OGR1 was significantly increased in patients with IBD compared to non-IBD patients. Additionally, a significant positive correlation was observed between OGR1 expression and the clinical score in both the non-inflamed (rs 0.7311, p=0.0069) and the inflamed mucosa (rs $0.7698, \mathrm{p}=0.0034$ )

Conclusion: The expression of OGR1 is significantly increased in patients with IBD. OGR 1 expression correlates with IBD disease activity, suggesting an active role of OGR1 in IBD pathogenesis. OGR1 appears to be a therapeutic target among the $\mathrm{pH}$-sensing receptors involved in IBD.

Disclosure of Interest: All authors have declared no conflicts of interest.

\section{P1613 MICROBIAL PROFILING OF NEWLY DIAGNOSED PATIENTS WITH ULCERATIVE COLITIS DIFFERS WITH ETHNICITY; RESULTS OF AN INCEPTION COHORT TIME SERIES ANALYSIS}

R. Misra ${ }^{1}$, A. Perdones-Montero ${ }^{2}$, N. S. Ding ${ }^{3}$, D. Rees ${ }^{4}$, O. Faiz ${ }^{5}$, J. Marchesi ${ }^{6}$ N. Arebi ${ }^{1}$

${ }^{1}$ Gastroenterology, St. Marks Academic Institute, London/United Kingdom

${ }^{2}$ Centre For Computational Systems Medicine, Imperial College, London/United Kingdom

${ }^{3}$ Gastroenterology, St. Vincents Hospital, Melbourne/Australia

${ }^{4}$ Division Of Women's Health, Kings College, London/United Kingdom

${ }^{5}$ Surgical Epidemiology Trials And Outcomes Unit (setoc), St. Marks Academic Institute, London/United Kingdom

${ }^{6}$ Imperial College London, London/United Kingdom

\section{Contact E-mail Address: rm399@imperial.ac.uk}

Introduction: Ulcerative colitis (UC) phenotype in South Asian (SA) migrants differs to Caucasians with a predominant pan-colonic extent. ${ }^{1}$ A separate study showed different microbial profiles with lower bacterial diversity in the SA group. ${ }^{2}$ The significance of these findings is unclear due to small sample size $(\mathrm{n}=30)$ and single time point analysis.

Aims \& Methods: We aimed to examine microbial profile of SA and Caucasian $\mathrm{UC}$ patients. Faecal samples were collected from $48 \mathrm{UC}$ patients recruited in a prospective inception cohort study at diagnosis (time point 1 ; months $0-3$ ) and 2 further time points over one year (time point 2 : months 4-8, time point 3 : months 9-12). Patients were stratified by ethnic group (SA, Caucasian, Other), treatment (none, 5-ASA, Azathioprine and Steroids) and disease duration. Healthy controls (HC) were recruited locally among the staff at St. Marks Hospital. For 16S pyrosequencing the hypervariable region (V1) of the 16S rRNA gene was amplified by PCR. The amplicons were subjected to sequencing using Illumina MiSeq technology. The sequences were loaded onto the QIIME pipeline. Statistical analysis was performed using STAMP 2.1.3 software with Welch's two-sided ttest for comparing two groups. Microbial richness was calculated based on Chaol index. Weighted Unifrac metrics were applied to construct Principle Coordinate Analysis (PCoA) plots.

Results: Ninety-four faecal samples were collected. Sample collection for all time points included SA $(n=13)$, Caucasian $(n=9)$, healthy SA $(n=11)$ and healthy Caucasian $(\mathrm{n}=12)$. There was no significant difference in relative abundance of bacteria between treatment groups (5-ASA, Azathioprine, Steroids, None) and time points (1 vs 3). Comparing healthy SA and healthy Caucasians, there was only one significant difference in relative abundance of Clostridiales at the family level. (Increased in SA group). The Chaol diversity index showed a trend towards lower diversity in the SA group compared to Caucasians although there was no significant difference. There were significant increases in Bifidobacterium, Rikenellaeceae, Lactobacillus and Streptococcus (Table 1) and significantly decreased relative abundance in Barnessiellacae, Anaerostipes and Ruminococcus.
TABLE 1: Summary of Bacterial Taxonomic Findings in South Asians (SA) and Caucasians with ulcerative colitis. *Increase or decrease in SA relative to Caucasians

\begin{tabular}{|c|c|c|c|c|c|}
\hline Phylum & Change* & Family & Change* & Genus & Change* \\
\hline Actinobacteria & Increased & Bifidobacteriaceae & Increased & Bifidobacterium & $\begin{array}{l}\text { Increased } \\
\text { Increased }\end{array}$ \\
\hline Bacteriodetes & Decreased & Bacteroidales & Decreased & $\begin{array}{l}\text { Barnessiellaceae } \\
\text { Rikenellaceae }\end{array}$ & $\begin{array}{l}\text { Decreased } \\
\text { Increased }\end{array}$ \\
\hline \multirow[t]{3}{*}{ Firmicutes } & & $\begin{array}{l}\text { Lactobacillacae } \\
\text { Streptococcae } \\
\text { Clostridiales } \\
\text { Lachnospiraceae }\end{array}$ & $\begin{array}{l}\text { Increased } \\
\text { Increased } \\
\text { Increased } \\
\text { Decreased }\end{array}$ & $\begin{array}{l}\text { Lactobacillus } \\
\text { Streptococcus }\end{array}$ & $\begin{array}{l}\text { Increased } \\
\text { Increased } \\
\text { Decreased } \\
\text { Decreased }\end{array}$ \\
\hline & & & & Unclassified & Decreased \\
\hline & & $\begin{array}{l}\text { Ruminococcaceae } \\
\text { Veillonellaceae }\end{array}$ & $\begin{array}{l}\text { Decreased } \\
\text { Increased }\end{array}$ & Ruminococcus & Decreased \\
\hline Proteobacteria & & Pasteurellaceae & Decreased & & \\
\hline
\end{tabular}

Conclusion: Increased Bifidobacteria and Lactobacillus in the SA group is consistent with the previous study. ${ }^{2}$ A possible explanation is the consumption of fermented foods in the SA group although there was no difference between healthy SA and Caucasian controls. There is a trend towards lower diversity in the SA group and reduced Bacterioides which are consistent with the UC dysbiosis described in the literature. Functional analysis of this unique microbial profile through metagenomic and metabonomic techniques may explain the different disease behaviour in the SA group.

Disclosure of Interest: All authors have declared no conflicts of interest.

\section{References}

1. Carr I., Mayberry JF. The effects of migration on ulcerative colitis: A threeyear prospective study among Europeans and first- and second-generation South Asians in Leicester (1991-1994). Am $J$ Gastroenterol 1999;94(10):2918-22. Doi: 10.1016/S0002-9270(99)00494-3.

2. Mar JS., LaMere BJ., Lin DL., Levan S., Nazareth M., Mahadevan U., et al. Disease Severity and Immune Activity Relate to Distinct Interkingdom Gut Microbiome States in Ethnically Distinct Ulcerative Colitis Patients. MBio 2016;7(4). Doi: 10.1128/mBio.01072-16

\section{P1614 VITAMIN D SUPPLEMENTATION REDUCES FAECAL CALPROTECTIN AND ALTERS INTESTINAL MICROBIOTA COMPOSITION IN PATIENTS WITH ACTIVE ULCERATIVE COLITIS}

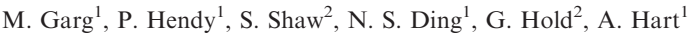

${ }_{1}^{1}$ Ibd Unit, St Mark's Hospital, Harrow/United Kingdom

${ }^{2}$ School Of Medicine, University of Aberdeen, Aberdeen/United Kingdom

Contact E-mail Address: philiphendy14@gmail.com

Introduction: There is evidence for vitamin $\mathrm{D}$ as an immunomodulator in patients with IBD, but results from clinical trials to date are inconclusive. It is uncertain whether vitamin D supplementation may affect the intestinal microbiota.

Aims \& Methods: This study aimed to assess the effect of vitamin D replacement in deficient patients with and without ulcerative colitis (UC) on inflammation and faecal microbiota. Vitamin D was replaced over 8 weeks to patients with active UC, inactive UC, and non-IBD controls with baseline $25(\mathrm{OH})$ vitamin $\mathrm{D}<50 \mathrm{nmol} / \mathrm{L}$, and markers of inflammation and stool collected for microbiota analyses by next generation sequencing.

Results: Eight patients with active UC, 9 with inactive UC and 8 non-IBD controls received 40,000 units of vitamin D weekly over 8 weeks. No demographic differences were noted across the groups. Mean baseline $25(\mathrm{OH})$ vitamin D levels were 34 (range 12-49) nmol/L. Vitamin D supplementation increased mean $25(\mathrm{OH})$ vitamin $\mathrm{D}$ to 111 (range $71-158) \mathrm{nmol} / \mathrm{L}(\mathrm{P}<0.001)$, and reduced parathyroid hormone levels from mean 4.3 to $3.3 \mathrm{pmol} / \mathrm{L}(\mathrm{p}=0.017)$. No change in baseline medications for UC took place in patients with UC, except for one patient with active UC who ceased his 5-aminosalicylate. Faecal calprotectin levels reduced from median 275 to $91 \mu \mathrm{g} / \mathrm{g}(\mathrm{p}=0.023)$ in patients with active colitis, but did not change in patients with inactive colitis or non-IBD controls. Similar improvements in albumin, platelet count and symptomatic disease activity indices were noted. No changes in overall bacterial diversity were noted. There was a trend towards an increase in abundance of Ruminococcus gnavus post vitamin D supplementation in active UC patients, but this did not reach statistical significance.

Conclusion: Vitamin D supplementation was associated with reduced intestinal inflammation in patients with active UC. A randomised controlled trial evaluating vitamin D in IBD is required, along with further investigation of potential mechanisms by which vitamin D may alter specific microbial composition.

Disclosure of Interest: M. Garg: This work was supported by the European Crohn's and Colitis Fellowship awarded to Dr Mayur Garg, and St Mark's Association Grant awarded to Prof Ailsa Hart and Dr Mayur Garg.

All other authors have declared no conflicts of interest. 
P1615 SUPPRESSION OF PHOSPHOLIPASE A AF INTESTINAL MICROBIOTA AMELIORATES MUCOSAL INFLAMMATION IN A GENETIC MOUSE MODEL OF ULCERATIVE COLITIS

W. Stremmel, S. Staffer, N.C. Stuhrmann, A. Gauss, N. Burger, D. Hornuss Abt. Für Innere Medizin Iv, Universitätsklinik Heidelberg, Heidelberg/Germany

Contact E-mail Address: wolfgang_stremmel@med.uni-heidelberg.de Introduction: Attack by commensal microbiota is one component for induction of inflammatory episodes in ulcerative colitis (UC). In UC, the mucus layer is intrinsically devoid of phosphatidylcholine (PC) resulting in low hydrophobicity which facilitates bacterial invasion. Colonic ectophospholipase-carrying bacterial strains are likely candidates to break the PC mucus barrier. Therefore we aimed to evaluate the effect of phospholipase $\mathrm{A}_{2}\left(\mathrm{PLA}_{2}\right)$ inhibitors on inflammation in a genetic UC mouse model.

Aims \& Methods: Attack by commensal microbiota is one component for induction of inflammatory episodes in ulcerative colitis (UC). In UC, the mucus layer is intrinsically devoid of phosphatidylcholine (PC) resulting in low hydrophobicity which facilitates bacterial invasion. Colonic ectophospholipase-carrying bacterial strains are likely candidates to break the PC mucus barrier. Therefore we aimed to evaluate the effect of phospholipase $\mathrm{A}_{2}\left(\mathrm{PLA}_{2}\right)$ inhibitors on inflammation in a genetic UC mouse model.

Results: Luminal UDCA-LPE reduced the PLA 2 activity in stool by $36 \pm 8 \%$. Concomitantly no inflammatory phenotype was observed when compared to kindlin $2^{(-/-)}$mice not treated with UDCA-LPE. The improvement was documented in regard to stool consistency, calprotectin levels in stool, and macroscopic/endoscopic as well as histologic features of the mucosa. The pattern of colonic microbiota distribution obtained in the UC phenotype mice was reversed by UDCA-LPE to the control mice pattern.

Conclusion: The inhibition of the bacterial ectophospholipase $\mathrm{A}_{2}$ activity improves mucosal inflammation in a genetic mouse model of UC. It is assumed that the remaining mucus PC shield is better preserved when luminal PLA 2 is suppressed.

Disclosure of Interest: All authors have declared no conflicts of interest.

\section{P1616 THE IMPACT OF THE RS8005161 POLYMORPHISM ON G PROTEIN-COUPLED RECEPTOR GPR65 (TDAG8) PH-ASSOCIATED SIGNALING IN INTESTINAL INFLAMMATION}

I. Tcymbarevich ${ }^{1}$, K. Baebler ${ }^{1}$, J. Cosin Roger $^{1}$, N. Obialo ${ }^{1}$, M. R. Spalinger ${ }^{1}$, J. J. Eloranta ${ }^{2}$, S. Lang ${ }^{1}$, G. A. Kullak-Ublick ${ }^{2}$, C.A. Wagner ${ }^{3}$, M. Scharl ${ }^{4}$, K. Seuwen ${ }^{1}$, P. A. Ruiz-Castro ${ }^{1}$, G. Rogler ${ }^{1}$, B. Misselwitz ${ }^{1}$, C. De Vallière ${ }^{1}$ ${ }^{1}$ Gastroenterology And Hepatology, University Hospital Zurich, University of Zurich, Zurich/Switzerland

${ }^{2}$ Clinical Pharmacology And Toxicology, University Hospital Zurich, University of Zurich, Zurich/Switzerland

${ }^{3}$ Institute of Physiology, University of Zürich, Zürich/Switzerland

${ }^{4}$ Novartis Institutes for Biomedical Research, Basel/Switzerland

Contact E-mail Address: katharina.baebler@usz.ch

Introduction: Inflammatory bowel diseases (IBDs), Crohn's disease (CD) and ulcerative colitis (UC) are typically associated with a decrease in local $\mathrm{pH}$. Genome-wide association studies (GWAS) identified over 240 non-overlapping single-nucleotide polymorphisms (SNP) associated with IBD. G-protein-coupled receptor 65 (GPR65) or T-cell death associated gene 8 (TDAG8) has recently been reported to be a genetic risk factor for IBD. The T genotype of the lead GPR65 SNP (rs8005161) within the GPR65 gene confers increased IBD risk. In response to extracellular acidification, GPR65 activates second messengers: cAMP, via the Gs signaling pathway, and G12/13/Rho signaling.

Aims \& Methods: In this study, we analyzed the functional relevance of SNP rs8005161 as well as the association of this GRP65 variant with IBD. Discovery: 2064 IBD patients from the Swiss IBD Cohort Study (SIBDCS) were genotyped for IBD risk SNPs by MALDI-TOFF mass spectrometry. Validation: 1138 individuals (591 non-IBD, $203 \mathrm{UC}, 344 \mathrm{CD}$ ) were genotyped for risk SNPs, GPR65 (rs8005161, rs3742704) and GALC (rs1805078), with Taqman SNP Genotyping Assays. 10 IBD patients of each genotype GPR65 rs8005161 TT, CT and wild type/CC (WT/CC) from the SIBDCS and 10 non-IBD controls (CC) were recruited for functional studies. CD14+ cells were isolated from blood samples and subjected to an extracellular acidic $\mathrm{pH}$ shift ( $\mathrm{pH} 6.6 \mathrm{vs} . \mathrm{pH} 7.6$ ) in functional assays and tested for cAMP and RhoA GTPase activation.

Results: Sequenced genotype frequency of rare homozygote rs 8005161 in the SIBDCS was $1.17 \%$, minor allele frequency (MAF) - $10.3 \%$. The variant rs 8005161 was more frequent in UC patients (MAF $14.53 \%$ vs $10.05 \%$ in the non-IBD group), whereas no statistically significant association with IBD, UC or CD was found for the other variants (GPR65 rs3742704, GALC rs1805078) by Taqman genotyping. No significant differences were observed in the cAMP production between IBD (WT/CC, CT, TT) and non-IBD (WT/CC) genotype carriers upon $\mathrm{pH}$ shift from 7.6 to 6.6. A decreased activation of GTPase RhoA was seen in IBD (WT/CC, CT, TT) patients after an acidic $\mathrm{pH}$ shift, but no differences between rs8005161 TT, CT and CC patients was observed.

Conclusion: The minor T allele of the GPR65 associated SNP rs8005161 was significantly enriched in UC patients. RhoA signaling was reduced in IBD patients indicating differential activation of Rho signaling in patients versus controls. However, no differences in cAMP signaling in IBD TT/CT/CC subjects compared to healthy $\mathrm{CC}$ subjects were observed. Our results suggest impaired RhoA signaling in IBD patients upon a $\mathrm{pH}$ shift, indicating a mechanistic explanation for increased IBD risk with GPR65 polymorphisms.

Disclosure of Interest: All authors have declared no conflicts of interest.
P1617 B2-STRICTURING AND B3-PENETRATING PHENOTYPE IN CROHN'S DISEASE: CHARACTERIZATION OF MACROPHAGES POPULATION AND WNT SIGNALING

D. Ortiz Masia ${ }^{1}$, P. Salvador ${ }^{1}$, D. Macias-Ceja ${ }^{2}$, R. Alos $^{3}$, J. Hinojosa Del Val ${ }^{4}$,

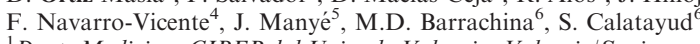

${ }^{1}$ Dept. Medicina, CIBERehd-Univ. de Valencia, Valencia/Spain

${ }^{2}$ Fisabio, Valencia/Spain

${ }^{3}$ Surgery, Hospital de Sagunto, Sagunto, Valencia/Spain

${ }^{4}$ Servicio Gastroenterologia, Hospital de Manises, Valencia/Spain

Ibd Unit, IGTP: Institut d'Investigació en Ciències de la Salut Germans Trias Pujol, Badalona, Barcelona/Spain

${ }^{6}$ Dept. De Farmacologia, CIBERehd-Univ. de Valencia, Valencia/Spain

\section{Contact E-mail Address: m.dolores.ortiz@uv.es}

Introduction: Macrophages contribute to fibrosis through the release of different mediators and the pattern of secretion may vary according to their phenotype. Recent evidences have identified Wnt pathway as an emerging modulator of fibrosis.

Aims \& Methods: The aim of the present study is to analyze the pattern of expression of macrophage markers and Wnt ligands in surgical resections from Crohńs disease (CD) patients with different disease behavior. CD patients were categorized according to Montreal classification (age at diagnosis, location and behavior). mRNA was isolated form resections of patients presenting an stricturing (B2) or a penetrating (B3) behavior or from patients with colorectal cancer (control). The expression of macrophage markers (CD206, CD86, iNOs, Arginase), Wnt ligands (Wnt1, Wnt2, Wnt2b, Wnt3, Wnt3a, Wnt3b, Wnt4, Wnt5a, Wnt5b, Wnt6, Wnt7a, Wnt7b, Wnt8a, Wnt8b, Wnt9a, Wnt9b, Wnt10a, Wnt $10 b$ and Wnt 16 ) and DKK1 (inhibitor of Wnt signaling) was analyzed by RTPCR.

Results: B3-patients seem to present a higher infiltration of macrophages since increased expression of markers classically used to detect pro-inflammatory (CD86) and regulatory/pro-resolving/pro-fibrotic phenotypes (CD206, ARG) was detected in this group. These patients also presented a generalized overexpression of Wnt ligands together with augmented DKK1 mRNA levels. B2patients showed a more complex situation with ligands that present increased (Wnt3), reduced (Wnt2B) or unchanged expression in the absence of significant variations in the levels of macrophage markers (Table). Table. Relative (Gene/bactin) mRNA expression (fold induction vs control group) of genes with detectable levels. Data are expressed as Mean \pm SEM with $n \geq 7$ in all groups and analyzed by ANOVA + Kewman-Keuls test. ( ${ }^{*} \mathrm{p}<0.05$ vs control; \#p $<0.05$ vs B2).

Conclusion: Crohn's disease patients presenting a stricturing (B2) or a penetrating (B3) behavior undergoing surgical resection differ in the pattern of macrophage infiltration and Wnt signaling.

Disclosure of Interest: All authors have declared no conflicts of interest.

P1618 CD16 POSITIVE CELLS EXPRESS TGFB AND MEDIATES MURINE INTESTINAL FIBROSIS

P. Salvador ${ }^{1}$, D. Ortiz-Masia ${ }^{2}$, D. Macias-Ceja ${ }^{3}$, L. Gisbert-Ferrándiz ${ }^{1}$, C. Hernández ${ }^{3}$, J.V. Esplugues ${ }^{3}$, M.D. Barrachina ${ }^{1}$, S. Calatayud ${ }^{1}$

${ }^{1}$ Dept. De Farmacologia, CIBERehd-Univ. de Valencia, Valencia/Spain

${ }^{2}$ Dept. Medicina, CIBERehd-Univ. de Valencia, Valencia/Spain

${ }^{3}$ Fisabio, Valencia/Spain

Contact E-mail Address: pedro.salvador@uv.es

Introduction: M2 macrophages play a key role in injury repair and fibrosis. We have reported that STAT6-dependent macrophages mediate mucosal repair after TNBS-induced acute colitis and that, in a chronic model, STAT6-deficient animals accumulate macrophages expressing the CD16 marker that promote intestinal fibrosis ${ }^{2}$

Aims \& Methods: We aim to analyze whether the expression of the pro-fibrotic mediator TGF $\beta$ is related with this macrophage phenotype and the relevance of these cells in murine intestinal fibrosis. Murine peritoneal macrophages obtained from both WT or STAT6 (-/-) mice were treated with IL-4 $(20 \mathrm{ng} / \mathrm{ml})$, IL-10 $(50 \mathrm{ng} / \mathrm{ml})$ or vehicle and the mRNA expression of CD16 and TGF $\beta$ was analyzed $72 \mathrm{~h}$ later by qPCR. WT or STAT6 (-/-) mice were weekly administered with TNBs $(0.5,0.5,0.75,0.75,1$, and $1 \mathrm{mg}$, intrarectally) or saline and were sacrificed 7 weeks after the first TNBs administration. The mRNA expression of CD16, TGF $\beta$, Vimentin, Colla1, $\alpha$-SMA, MMP2 and TIMP1 was evaluated in murine intestinal mucosa by qPCR. In all cases results were expressed as fold induction $v s$ vehicle and the correlation between different measurements was analyzed using Pearson's correlation coefficient (r).

Results: A positive and significant correlation between CD16 mRNA and TGF $\beta$ mRNA was observed in isolated macrophages from WT mice $(r=0.637$, $\mathrm{P}=0.03)$ and STA6 $(-/-)$ mice $(\mathrm{r}=0.677, \mathrm{P}=0.002)$ receiving different treatments. In the mucosa of WT and STAT6 mice the expression of TGF $\beta$ showed a positive and significant correlation with CD16 mRNA $(r=0.601$, $\mathrm{P}<0.001$ ), and with the expression of several fibrotic markers (vimentin: $\mathrm{r}=0.6537, \quad \mathrm{P}<0.0001 ;$ Colla1: $\mathrm{r}=0.5101, \mathrm{P}=0.0003 ; \quad \alpha$-SMA: $\mathrm{r}=0.4126$, $\mathrm{P}=0.0048$; MMP2: $\mathrm{r}=0.5729, \mathrm{P}<0.0001$; and TIMP1: $\mathrm{r}=0.3958, \mathrm{P}=0.0071$ ). Conclusion: The expression of TGF $\beta$ is associated with CD16 positive cells and is involved in murine intestinal fibrosis.

Disclosure of Interest: All authors have declared no conflicts of interest. 


\section{Reference}

1. Cosín-Roger J, Ortiz-Masiá D, Calatayud S, Hernández C, Esplugues JV, Barrachina MD. Mucosal Immunol. 2016 Jul;9(4):986-98. doi: 10.1038/ mi.2015.123. 2. p087. Increased wnt ligands expression in M2c macrophages is associated with fibrosis in Stat6 knockout mice ECCO 2017 (s120).

\section{P1619 GTS-21, A7 NICOTINIC ACETYLCHOLINE RECEPTOR AGONIST, ATTENUATE DSS-INDUCED COLITIS BY IMPROVING INTESTINAL MUCOSAL BARRIER FUNCTION}

\section{Y. Zhu' ${ }^{1}$, H. Zhang ${ }^{2}$}

${ }^{1}$ Department Of Gastroenterology, First Affiliated Hospital of Nanjing Medical University, NanJing Jiang Su/China

${ }^{2}$ Department Of Gastroenterology, Jiangsu Province Hospital and Nanjing Medical University, Nanjing/China

Contact E-mail Address: zhuyunjuan2008@126.com

Introduction: The intestinal inflammation is reduced by electrical stimulation of the efferent vagus nerve. Cholinergic output may be a target to minimize tissue damage in autoimmune disease. Cholinergic neural output can modulate innate immune responses through stimulation of $\alpha 7$ nicotinic acetylcholine receptors ( $\alpha 7 \mathrm{nAChR}$ ). GTS-21, a selective $\alpha 7 \mathrm{nAChR}$ agonist, has previously demonstrated to inhibit the inflammation associated with rheumatoid arthritis (RA). In this study we investigate whether GTS-21 protects against DSS-induced colitis and its potential mechanism.

Aims \& Methods: Male BABL/c mice $(8-10$ weeks old, $n=32)$ were randomly divided into 4 groups: normal control group, DSS-induced group, GTS-21 treatment control group (DSS-induced mice treated with GTS-21), $\alpha$-BGT group (DSS-induced mice treated with $\alpha$-BGT and GTS-21) $(\mathrm{n}=8$, each group). DSS group was given final concentration of $3.5 \%$ DSS drinking water, the treatment group was treated with GTS-21 (20 mg/kg intraperitoneal injection)per day, $\alpha$ BGT group was pre-treated with a-BGT $(0.1 \mathrm{mg} / \mathrm{kg} / \mathrm{day}$, intraperitoneal injection) for $30 \mathrm{~min}$ prior to GTS-21 injection. and the control group received saline. $\mathrm{Caco} 2$ cells were randomly divided into 4 groups: normal control group, TNF- $\alpha-$ induced group, GTS-21 treatment control group, $\alpha$-BGT group. TNF- $\alpha$ group of Caco 2 cells were exposed by $25 \mathrm{ng} / \mathrm{ml} \mathrm{TNF}-\alpha$, GTS- 21 group were given $100 \mathrm{ng}$ / $\mathrm{ml} \mathrm{GTS}-21$ for $30 \mathrm{~min}$ prior to TNF- $\alpha$; $\alpha$-BGT group pre-treated with a-BGT $(50 \mathrm{ng} / \mathrm{ml})$ for $30 \mathrm{~min}$ prior to GTS-21 injection. BAY 11-2028 (NF- $\kappa$ B inhibitor) group were given $50 \mathrm{ng} / \mathrm{ml}$ BAY-11-2028 for $30 \mathrm{~min}$ prior to TNF- $\alpha$. Disease activity index, macroscopic scores, and colonic damage were determined. The intestinal permeability of mice was measured by fluorescein-isothiocyanate-dextran (FITC-Dextran) method. Western blot was used to detect the tight junction protein and NF- $\kappa \mathrm{B}$ associated protein expression.

Results: Compared with DSS-induce mice, DAI score decreased and colon length improved after administration of GTS-21 $(9.13 \pm 0.74 \mathrm{~cm}$ vs $6.85 \pm 0.53 \mathrm{~cm}$, $\mathrm{P}<0.01)$, HAI score decreased $(1.25 \pm 1.32$ vs $10.5 \pm 2.64, \mathrm{P}<0.05)$. The $\alpha 7 \mathrm{nAChR}$ antagonist $\alpha$-BGT can eliminate those protective effects (Figure 1) 2. The intestinal permeability improved after administration of GTS-21 compared with DSS-induced mice $(49.52 \pm 28.59 \mathrm{ug} / \mathrm{ml}$ vs $157.40 \pm 32.40 \mathrm{ug} / \mathrm{ml}$ $\mathrm{P}<0.05)$; whereas $\alpha$-BGT can block this protection $(115.50 \pm 6.10 \mathrm{ug} / \mathrm{ml}$ vs $49.52 \pm 28.59 \mathrm{ug} / \mathrm{ml} \mathrm{P}<0.05$ ) (Figure 2.3). 3.The expressions and distribution of tight junction protein in DSS-induced mice were enhanced after treatment with GTS-21 $(\mathrm{p}<0.05)$ (Figure 4.5). 4. GTS-21 attenuated the NF- $\kappa$ B activation $(p<0.05)$. a-BGT administration reversed the inhibitory effect of GTS-21 $(\mathrm{p}<0.05)$ (Figure 6). 5 GTS-21 improves the distribution of tight junction proteins in the intestinal epithelial cells induced by TNF- $\alpha$ (Figure 7). 6.GTS-21 reduces nuclear translocation of NF- $\kappa \mathrm{B}$ in $\mathrm{Caco} 2$ cells induced by TNF- $\alpha$ (Figure 8)

Conclusion: The $\alpha 7$ nicotinic acetylcholine receptors agonist GTS-21 can attenuate the intestinal inflammation in DSS-induced mice, which may be due to improving intestinal mucosal barrier function by enhancing the expression of tight junction protein

Disclosure of Interest: All authors have declared no conflicts of interest.

\section{P1620 EXPRESSION OF WNT10B IN M2-MACROPHAGES FROM CROHN'S DISEASE PATIENTS}

D. Ortiz Masia ${ }^{1}$, D. Macias-Ceja ${ }^{2}$, P. Salvador ${ }^{3}$, D. Bernardo ${ }^{4}$, F. NavarroVicente $^{5}$, R. Alos ${ }^{6}$, L. Gisbert-Ferrándiz ${ }^{7}$, C. Hernández ${ }^{2}$, S. Calatayud ${ }^{3}$, M.D. Barrachina ${ }^{3}$

${ }^{1}$ Dept. Medicina, CIBERehd-Univ. de Valencia, Valencia/Spain

${ }^{2}$ Fisabio, Valencia/Spain

${ }^{3}$ Dept. De Farmacologia, CIBERehd-Univ. de Valencia, Valencia/Spain

${ }^{4}$ Gastroenterology, Hospital Universitario La Princesa, Madrid/Spain

${ }_{5}^{5}$ Servicio Gastroenterología, Hospital de Manises, Valencia/Spain

${ }^{6}$ Surgery, Hospital de Sagunto, Sagunto, Valencia/Spain
${ }^{7}$ Dpto. Farmacologia And Ciberehd, Universitat Valencia, Valencia/Spain

Contact E-mail Address: m.dolores.ortiz@uv.es

Introduction: Intestinal fibrosis is a common complication of IBD and macrophages can contribute to fibrosis through the release of different mediators. We have recently reported that Wnt $10 \mathrm{~b}$ increase the deposition of collagen and promotes intestinal fibrosis in TNBs-treated mice (P015 ECCO 2017).

Aims \& Methods: The aim of the present study is to analyze the pattern of expression of macrophages and the expression of Wnt10b in human macrophages from surgical resections from Crohn's disease $(C D)$ patients with a B2-stricturing and B3-penetrating behavior according to Montreal classification (age at diagnosis, location and behavior) as well as from non-inflamed tissue from cancer patients. Resections were immediately processed and lamina propria mononuclear cells characterized by flow cytometry. Macrophages were identified within singlet viable leukocytes as CD $45+$ CD14 + CD 64+. The percentage of CD206, CD16, CD163, CD86 and Wnt10b positive cells was analyzed in macrophages. Data are expressed as Mean \pm SEM $\%$ with $n \geq 5$ in all groups $\left(\mathrm{P}<0.05^{*}\right.$ vs control, Student's t test).

Results: The percentage of macrophages identified as CD $45+$ CD14 + CD64+ constituted the $0.22 \pm 0.03 \%$ of total events in the control mucosa while represented the $1.35 \pm 0.40 \%$ in the mucosa from CD patients $(\mathrm{p}<0.05)$. A high proportion of mucosal macrophages expressed CD206 in both, control $(83.3+4.8 \%)$ and $\mathrm{CD}(89.6+3.9 \%)$. In these CD206+ cells, an increased expression of CD16 in CD mucosa was observed (Control: $34.5 \pm 6.8 \%$; CD: $59.7 \pm 6.4 \% *$ ) while that of CD163 was similar in both groups (control: $72.8 \pm 8.4 \%$; CD: $85.2 \pm 7.3 \%$ ). The analysis of Wnt10b in CD206+ showed a significantly higher expression in $\mathrm{CD}$ patients $(56.6 \pm 4.2 \%)$ than in control samples $(30.1 \pm 10.4 \%)$. Additionally, CD $86+$ macrophages were more abundant in CD mucosa (Control: $8.2 \pm 1.1 \%$; CD: $29.3 \pm 10.2 \% *$ ).

Conclusion: The mucosa of $\mathrm{CD}$ patients accumulate pro-inflammatory macrophages measured as CD86+ cells while those macrophages expressing the M2marker CD206 alter their phenotype increasing the expression of both, CD16 and the profibrotic mediator Wnt10b.

Disclosure of Interest: All authors have declared no conflicts of interest.

\section{Reference}

Ortiz-Masia, D, Salvador P, Macías-Ceja DC, L. Gisbert-Ferrándiz, Hernández $\mathrm{C}$, Esplugues, JV, Calatayud S and Barrachina MD. Wnt10b could mediated fibrosis in IBD patients. P015 ECOO 2017.

\section{P1621 ANAEMIA PREVALENCE AND TREATMENT APPROACH FOR INFLAMMATORY BOWEL DISEASE}

G. Bengi ${ }^{1}$, H. Keyvan ${ }^{2}$, H. Akpinar ${ }^{1}$

${ }^{1}$ Gastroenterology, Dokuz Eylul University Hospital, Izmir/Turkey ${ }^{2}$ Internal Medicine, Dokuz Eylul University Hospital, Izmir/Turkey

\section{Contact E-mail Address: drookselbengi@ hotmail.com}

Introduction: For inflammatory bowel disease (IBD), anaemia is the most frequently observed extra intestinal finding, prevalence of which varies from $6 \%$ to $74 \%$. It's of great importance to determine and treat anaemia as it lowers patients' life quality and leads to labour loss. The main causes of anaemia in IBD are iron deficiency anaemia (IDA) and anaemia of chronic disease (ACD). In this study, we aim to specify the type and prevalence of anaemia along with a treatment approach for inflammatory bowel disease (IBD).

Aims \& Methods: In this study, we aim to specify the type and prevalence of anaemia along with a treatment approach for inflammatory bowel disease (IBD). We conducted a retrospective study on 465 patients, who were diagnosed with IBD and followed up at our hospital from June 2015 to June 2016 (male: 254 female: 211 , average age: $47 \pm 14.4$, Crohn disease (CD): 257 , Ulcerative Colitis (UC): 208). According to WHO criteria, anaemia occurs when haemoglobin value is below $13 \mathrm{~g} / \mathrm{dL}$ in men and $12 \mathrm{~g} / \mathrm{dL}$ in women.

Results: In our study, we determined that $50.3 \%$ of total 465 patients had anaemia, which was more frequent in women then men $(64 \%$ vs. $39 \%, p<0.001)$. Anaemia frequency was higher in $\mathrm{CH}$ cases $(57 \%)$ then in UC cases $(41 \%)$ $(\mathrm{p}=0,001) . \mathrm{CD}$ involvement were as follows: $54.5 \%$ in ileal involvement, $60.4 \%$ in colonic involvement and $58.5 \%$ in ileocolonic involvement. Furthermore, $27.5 \%$ of UC patients had proctitis (E1) involvement while $41 \%$ of them had involvement in left colitis (E2) and $31.5 \%$ had pancolitis involvement. There was no significant relation between anaemia frequency and duration of disease $(\mathrm{p}=0.216)$. We specified the following types of anaemia: IDA only $32.9 \%$ (77), ACD only $5.5 \%$ (13), IDA and ACD combination $6.8 \%$ (16), anaemia stemming from $\mathrm{B}_{12}$ /folic acid deficiency $6.4 \%(15)$, multiple anaemia $17.8 \%$ (41) and anaemia with no etiology $30.7 \%$ (72). $50 \%$ of patients with anaemia received treatment; $23 \%$ of IDA patients had oral iron intake and $41 \%$ of them had parenteral iron treatment while $53 \%$ of patients who were suffering from megaloblastic anaemia got $\mathrm{B}_{12} /$ folic acid treatment.

\begin{tabular}{|c|c|c|c|c|c|c|c|c|c|}
\hline & CD206 & $\mathrm{ARG}$ & CD86 & Wnt2 & Wnt2B & Wnt3 & Wnt9A & Wnt10B & DKK1 \\
\hline CONTROL & $1.9 \pm 04$ & $1.5 \pm 0.3$ & $2.7 \pm 0.6$ & $2.3 \pm 0.6$ & $1.4 \pm 0.2$ & $1.4 \pm 0.2$ & $1.3 \pm 0.2$ & $1.2 \pm 0.3$ & $1.7 \pm 0.2$ \\
\hline B2 & $3.1 \pm 0.8$ & $1.4 \pm 0.4$ & $3.4 \pm 0.7$ & $3.3 \pm 0.7$ & $0.4 \pm 0.1^{*}$ & $2.7 \pm 0.6^{*}$ & $2.7 \pm 0.6$ & $0.6 \pm 0.2$ & $2.1 \pm 0.7$ \\
\hline B3 & $7.7 \pm 1.6^{*} \#$ & $3.2 \pm 0.8^{*} \#$ & $7.0 \pm 1.8^{* \#}$ & $5.1 \pm 1.2$ & $1.7 \pm 0.3 \#$ & $6.0 \pm 1.0 * \#$ & $3.9 \pm 1.7^{*}$ & $2.3 \pm 0.4^{*} \#$ & $6.1 \pm 1.7^{*} \#$ \\
\hline
\end{tabular}


Conclusion: We found out that almost half of all IBD patients $(50.3 \%)$, whom we followed up, had anaemia, the most frequent reason of which was IDA. Almost half of these patients received anaemia treatment. We should increase the treatment rate in our IBD patients that have anaemia.

Disclosure of Interest: All authors have declared no conflicts of interest.

\section{P1622 SUCCINATE RECEPTOR (SUCNR1) MEDIATES} INFLAMMATION IN A MURINE MODEL OF COLITIS

D.C. Macias Ceja ${ }^{1}$, D. Ortiz-Masia ${ }^{2}$, P. Salvador ${ }^{2}$, L. Gisbert-Ferrándiz ${ }^{2}$, S. Coll-Puig ${ }^{2}$, C. Hernández ${ }^{1}$, J.V. Esplugues ${ }^{1}$, S. Calatayud ${ }^{2}$, M.D. Barrachina ${ }^{3}$ ${ }^{1}$ Fisabio, Valencia/Spain

${ }^{2}$ Pharmacology, Universitat Valencia, Valencia/Spain

${ }^{3}$ Dept. De Farmacologia, CIBERehd-Univ. de Valencia, Valencia/Spain

Contact E-mail Address: dulmace@alumni.uv.es

Introduction: IBD is a chronic disorder of the gastrointestinal tract characterized by disruption of epithelial barrier function and gut inflammation. Evidence supports a relevant role of succinate, an intermediate of the tricarboxylic acid cycle ${ }^{1}$, in inflammation and the succinate receptor, SUCNR1, has been recently related with rheumatoid arthritis ${ }^{2}$

Aims \& Methods: To analyze the role of SUCNR1 in a murine model of colitis induced by TNBS. WT or SUCNR $1^{-1-}$ mice received TNBS $(3.5 \mathrm{mg} / 20 \mathrm{~g}$ mice, intrarectally) or vehicle (EtOH $40 \%$ ) and were weighed daily (results are expressed as percentage vs the weight at day 0 ) and mice were sacrificed 2 and 4 days after TNBS administration. Colon length and mucosal histology were evaluated according to Wallace Score (1-10). The mRNA expression levels of $i N O S$, Arginase $I, C O X-2, T N F-\alpha, I L-1 \beta, I L-6$ and $I L-10$ was analysed by qPCR Results: Treatment of mice with TNBS induced a loss of body weight that peaked 2 days after treatment. Subsequently, mice began to recover and, four days after treatment, body weight reached similar values to those of control animals. In TNBS-treated SUCNR1-/- mice compared with TNBS-treated WT mice: a) the loss of body weight was significantly $(\mathrm{P}<0.05)$ attenuated $(96.99 \pm 0.7 \%$ vs $91.78 \pm 1.1 \%)$; b) the reduction in colon length was prevented $(6.6 \pm 0.2$ vs $5.2 \pm 0.2 \mathrm{~cm}) ; \mathrm{c})$ damage score was significantly reduced $(3.5 \pm 0.2$ vs $4.8 \pm 0.5)$ two days after TNBS treatment. d) The increase in the expression of pro-inflammatory molecules was significantly prevented $(\mathrm{P}<0.05)$ (table 1$)$ while no significant differences were detected in the expression of COX-2 (Table 1).

Table1: mRNA expression of different molecules detected in the colon of TNBStreated mice. Results are expressed as fold induction vs the value obtained in WT mice.

\begin{tabular}{llllllll}
\hline & iNOS & ArgI & COX-2 & TNF $\alpha$ & IL-1 $\beta$ & IL-6 & IL-10 \\
\hline WT & $2.6 \pm 0.6$ & $1.6 \pm 0.7$ & $1.1 \pm 0.2$ & $2.6 \pm 0.6$ & $1.7 \pm 0.2$ & $4.2 \pm 1.0$ & $0.8 \pm 0.1$ \\
KO & $1.5 \pm 0.4$ & $2.8 \pm 1.0$ & $1.3 \pm 0.3$ & $1.3 \pm 0.2$ & $1.2 \pm 0.1$ & $1.7 \pm 0.3$ & $1.4 \pm 0.2$
\end{tabular}

Conclusion: Activation of the succinate receptor SUCNR1 mediates murine colitis. These findings highlight the biological significance of SUCNR1 and open the door to novel approaches for IBD treatment.

Disclosure of Interest: All authors have declared no conflicts of interest.

\section{References}

1. Mills, E and O'Neill L.A.J. 2014. Succinate: a metabolic signal in inflammation. Trends Cell Biol. 24:313-320.

2. Littlewood-Evans, A, Sarret, S, Apfel, V, Loesle, P, Dawson, J, Zhang, J, Muller, A, Tigani, B, Kneuer, R, Patel, S, Valeaux, S, Gommermann, N, Rubic-Schneider, T, Junt, T and Carballido, JM. 2016. GPR91 senses extracellular succinate released from inflammatory macrophages and exacerbates rheumatoid arthritis. J. Exp. Med. 213:1655-1662.

\section{P1623 ZIP7 INDUCES DISRUPTION OF THE INTESTINAL BARRIER THROUGH ACTIVATION OF ENDOPLASMIC RETICULUM STRESS IN INFLAMMATORY BOWEL DISEASE}

J. Sun, Q. Zhan, F. An

Wuxi People's Hospital, Nanjing Medical University, wuxi/China

Contact E-mail Address: jingdianjing99@126.com

Introduction: Inflammatory bowel disease (IBD) is a chronic intestinal inflammatory disease with a tendency to recurrence, and intestinal barrier disorders play a key role in its development. The deficiency of zinc is a clinical manifestation and can promote the development of IBD. Zinc Transporter Member 7 (ZIP7) is a gate keeper protein in the intracellular release of zinc in cells. Recent studies revealed that ZIP7 helps to maintain the intestinal mucosal integrity. In addition, ZIP7 can regulate endoplasmic reticulum stress.

Aims \& Methods: Our aim was to investigate the role of ZIP7 in IBD initiation and progression. We investigated the expression of ZIP7 in the intestinal mucosa of IBD patients and in interleukin-10-gene-deficient ( $\mathrm{Il1} 0^{-/-}$) mice, and assessed the relation between ZIP7 and disease activity. ZIP7 upregulated/downregulated lentivirus was used to infect IEC6 and HIEC cells, then we evaluated the expression of inflammatory factors, mucosal tight junction proteins (Occludin and ZO1), and proteins related with endoplasmic reticulum stress (IRE1, XBP1, TRAF2, ASK1 and p-JNK). In addition, we used siRNA to silence IRE1 and SP600125 to inhibit the JNK pathway respectively, then evaluated the effect of endoplasmic reticulum stress on mucosal tight junction proteins.

Results: We found that ZIP7 was downregulated both in the intestinal mucosa of IBD patients and in $1110^{-/-}$mice, which was associated with disease activity. In IEC6 and HIEC cells, the expression of mucosal tight junction proteins was consistent with the level of ZIP7, but the expression of inflammatory factors and endoplasmic reticulum stress associated proteins were on the contrary. After the silence of IRE1 and the inhibition of JNK pathway, the expression of mucosal tight junction proteins was partly resumed in ZIP7 downregulated cells.

Conclusion: ZIP7 induces disruption of the intestinal barrier, which was associated with activation of endoplasmic reticulum stress in IBD. It is expected to provide a novel mechanism of IBD and provide a new target for the treatment of IBD

Disclosure of Interest: All authors have declared no conflicts of interest.

\section{P1624 EFFECT OF T CELL ACTIVATION AND INFLAMMATION ON THE INTERACTION BETWEEN T CELLS AND ENTERIC GLIAL} CELLS

I. Neveu ${ }^{1}$, J. Pabois ${ }^{2}$, T. Durand ${ }^{2}$, J. Gonzales ${ }^{2}$, J. A. Gonzales ${ }^{2}$, M. Neunlist ${ }^{2}$, P. Naveilhan ${ }^{2}$

${ }^{1}$ INSERM U1235, Nantes/France

${ }^{2}$ Inserm 1235 "tens", INSERM, Nantes/France

Contact E-mail Address: julie.pabois@etu.univ-nantes.fr

Introduction: The accumulation of immune cells around the enteric neuronal ganglia of patients affected by Crohn's disease is predictive of disease recurrence after post-operative resection. As cells of the enteric nervous system could be implicated in the formation of these myenteric and submucosal plexitis in chronic inflammatory digestive diseases, inter-relationship between $\mathrm{T}$ cells and enteric glial cells (EGC) was investigated.

Aims \& Methods: To analyse the interactions between immune and enteric neural cells, EGC isolated from the myenteric plexus of the rat digestive tract were cocultured with CFSE-labeled T cells. Impact of T cell activation on neuro-immune interactions was investigated by treating $\mathrm{T}$ cells with anti-CD3/anti-CD28 antibodies. To determine whether inflammatory conditions favored the contacts between glial and immune cells, EGC were treated with LPS or TNF/IL1 prior their exposition to $\mathrm{T}$ cells. After 2 hours, non-adherent cells were removed and the $\mathrm{T}$ cells interacting with $\mathrm{EGC}\left(\mathrm{S}_{100^{+}}\right)$were counted. Immunocytochemistry were also used to characterize the subpopulations of $\mathrm{T}$ cells $\left(\mathrm{CD} 4^{+}, \mathrm{CD} 8^{+}\right)$that contact glial cells.

Results: Analyses reveal that non-activated T lymphocytes are capable of interacting with EGC. They also show that activation of T cells with anti-CD3/antiCD28 antibodies increases the number of T lymphocytes interacting with EGC. Interestingly, an increased number of EGC-T cell interactions was observed after pretreatment of EGC with inflammatory stimuli. This phenomenon was also noted with activated $\mathrm{T}$ cells. Characterization of $\mathrm{T}$ cells show that both CD4 and CD8 cells are capable of contact with EGC.

Conclusion: Our present data reveal that EGC interact with T cells. These contacts are favored by $\mathrm{T}$ cell activation but also by EGC exposure to inflammatory cytokines. Further experimentations are required to characterize these neuroimmune interactions but they suggest that EGC-T cell contact may play a crucial role in case of inflammatory bowel diseases. This work is supported by the Association François Aupetit.

Disclosure of Interest: All authors have declared no conflicts of interest.

\section{P1625 ENTERIC GLIAL CELLS REACTION TO INFLAMMATION IS LOST IN CROHN'S DISEASE}

M. Rolli-Derkinderen ${ }^{1}$, C. Pochard $^{1}$, T. Clairembault ${ }^{1}$, N. Cenac ${ }^{2}$, A. Bourreille ${ }^{1}$ M. Neunlist

${ }^{1}$ U1235 Inserm, TENS, Nantes/France

${ }^{2}$ Digestive Health Research Institute, INSERM UMR-1043 CNRS UMR-5282, Toulouse/France

Contact E-mail Address: malvyne.derkinderen@univ-nantes.fr

Introduction: Enteric glial cells (EGC) are essential to intestinal epithelial barrier (IEB) homeostasis. In healthy intestines, EGC reduce IEB permeability and promote mucosal healing. In inflammatory bowel disease (IBD) such as Crohn's Disease (CD) and Ulcerative Colitis (UC), both EGC phenotype and IEB functions are altered, but putative involvement of EGC in IBD pathogenesis remains unknown. If the astrocyte reactivity is well studied, the reaction of EGC to chronic inflammation is not well documented. We investigated whether EGC impact on IEB permeability was altered in an inflammatory environment and in EGC from IBD patients.

Aims \& Methods: Rat EGC as well as human EGC from control, CD and UC patients were stimulated with the cytomix TI (TNFalpha+IL1beta; 1 to $100 \mathrm{ng}$ $\mathrm{ml}$ ) or LPS for 2 or 4 days. Reactive EGC phenotype where characterized and reactive EGC functional impact on IEB permeability was studied (i) in vitro using human intestinal epithelial cells (IEC) in a non-contact co-culture model, or (ii) in vivo by grafting the treated rat EGC in colon wall of Sprague Dawley rats. Results: Rat and human control EGC induced a significant reduction of IEB paracellular permeability after TI treatment when compared with untreated or LPS treated EGC. LPS or TI treatment had no significant effects on IEC alone. In vivo colon wall grafting with control EGC did not modified the permeability whereas colon wall grafting with EGC preconditioned by TI significantly reduced 
the permeability when compared to control animals. Human EGC from control or UC patients treated with TI induced a decrease in IEB permeability too, but EGC from CD patients did not.

Conclusion: This work is not only the first evidence showing that reactive EGC can have beneficial effects upon IEB permeability, but also shows that EGC from $\mathrm{CD}$ but not UC patients have lost these reactivity. This could define EGC as active players in $\mathrm{CD}$ pathogenesis.

Disclosure of Interest: All authors have declared no conflicts of interest.

\section{P1626 PROSTACYCLIN REVERSES COLITIS THROUGH THE DOWN REGULATION OF INTESTINAL EPITHELIAL PERMEABILITY}

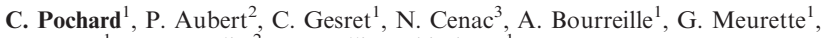
E. Coron ${ }^{1}$, M. Neunlist ${ }^{2}$, M. Rolli-Derkinderen ${ }^{1}$

${ }^{1}$ U1235 Inserm, TENS, Nantes/France

2 Inserm 1235 "tens", INSERM, Nantes/France

${ }^{3}$ Digestive Health Research Institute, INSERM UMR-1043 CNRS UMR-5282, Toulouse/France

Contact E-mail Address: camille.pochard@univ-nantes.fr

Introduction: In inflammatory bowel disease (IBD) both intestinal epithelial barrier (IEB) permeability and PTGIS expression are altered. Nevertheless the role of the lipid mediator PGI2 produced by PTGIS in IEB regulation is unknown. The present study concerns the control of IEB permeability by PGI2 and its involvement in the development of colitis.

Aims \& Methods: PGI2 production from control or IBD biopsies was established using high sensitivity liquid chromatography tandem mass spectrometry. Consequences of flolan PGI2 analogous supplementation were evaluated in a DSS-induced mice model of colitis, measuring disease activity index (DAI), inflammation (pro-inflammatory cytokine mRNA) and IEB permeability (sulfonic acid flux). Molecular mechanisms involved were assessed by quantification of junctional and pro-proliferative $v s$ pro-apoptotic protein expression (western blot and immunostaining). Eventually PGI2 impact on reversing IEB breakdown was assessed ex vivo measuring permeability of mice or human mucosal explants treated with staurosporine apopstosis inducer, or permeability of IBD biopsies both treated or not with flolan.

Results: Biopsies from IBD patients had lower PGI2 production compared to control patients, and addition of flolan reduced their permeability. In vivo PGI2 supplementation significantly reduced DAI, and inflammation (Interferong mRNA) as well as reduced IEB permeability. DSS-induced clivage of Caspase 3 is normalized by flolan. Ex vivo, staurosporine-induced permeability of mice or human mucosal explants is entirely inhibited by flolan.

Conclusion: This study not only presents a role of PGI 2 in controlling IEB permeability through the regulation of apoptosis mechanisms, but also reveals that increased permeability in IBD patients can be fixed by PGI2 supplementation.

Disclosure of Interest: All authors have declared no conflicts of interest.

\section{P1627 7A-HYDROXY-4-CHOLESTEN-3-ONE FOR DIAGNOSIS AND MANAGEMENT OF BILE ACID MALABSORPTION: FIRST YEAR CLINICAL EXPERIENCE}

B. Friedli ${ }^{1}$, F. Brunner ${ }^{1}$, C. Prost $^{2}$, C. Bovet ${ }^{2}$, D. Kröll ${ }^{3}$, A. Macpherson ${ }^{1}$, P. Juillerat ${ }^{1}$

${ }^{1}$ Gastroenterolgy, Inselspital, Bern University Hospital, Bern/Switzerland

${ }^{2}$ University Institut Of Clinical Chemistry, Bern University Hospital, Bern

Switzerland

${ }^{3}$ Chirurgie, University of Bern, Bern/Switzerland

\section{Contact E-mail Address: pascal.juillerat@insel.ch}

Introduction: $7 \alpha$-hydroxy-4-cholesten-3-one (7HCO) is a reliable method to diagnose bile acid malabsorption (BAM). Since 7HCO is an intermediate metabolite in the bile acid synthesis, increased levels reflect bile acid production, which is the case in BAM.

Aims \& Methods: We evaluate retrospectively, prospectively collected clinical data during the first year after implementation of a new test using ultrahigh performance liquid chromatography coupled to mass spectrometry to measure $7 \mathrm{HCO}$ (see reference). In adult patients with clinical suspicion of BAM, unexplained diarrhea and a subgroup with obesity $7 \mathrm{HCO}$ was measured. Levels $<30 \mathrm{ng} / \mathrm{ml}$ are considered as normal values. The decision to treat with cholestyramine was at the discretion of the treating physicians.

Results: We performed $1267 \mathrm{HCO}$ analysis in 112 patients $(62 \%$ female, mean age $51+/-16$ years) with a mean level of $84+/-91 \mathrm{ng} / \mathrm{ml}$. Cholestyramin treatment was more likely initiated in patients with Crohn's disease (RR 1.8; 95\% CI $0.9-3.7$ ) or after ileocecal resection (RR 3.1; 95\% CI 1.7-5.7). Diarrhea improved in $60 \%$ of patients with a $7 \mathrm{HCO}$ level above $40 \mathrm{ng} / \mathrm{ml}$. Thresholds of 60 or $100 \mathrm{ng} / \mathrm{ml}$ do not improve prediction of response to cholestyramin treatment.
7HCO measurement in subgroups

\begin{tabular}{lllll}
\hline Subgroups & Number & Mean $[\mathrm{ng} / \mathrm{ml}]$ & SD & Range\# \\
\hline Diarrhea & 79 & $94^{*}$ & 96 & $<5->300$ \\
No diarrhea & 33 & $59^{*}$ & 71 & $<5->300$ \\
Cholestyramin treated & 27 & $167 \S$ & 105 & $11->300$ \\
Cholestyramin untreated & 85 & $57 \S$ & 67 & $<5->300$ \\
Crohn's disease (CD) & 18 & 182 & 105 & $13->300$ \\
Ileocecal resection (IR) & 26 & 197 & 105 & $28->300$ \\
CD + IR & 13 & 214 & 95 & $41->300$ \\
Obese (mean BMI 39.1 kg/m2) & 21 & 62 & 49 & $6-244$ \\
\hline
\end{tabular}

" $\mathrm{p}<0.05 ; \S \mathrm{p}<0.001 ;$ \#Validation Range 5-300 ng/mL

Conclusion: A 7HCO measurement above $40 \mathrm{ng} / \mathrm{ml}$ seems to be associated with a good response to cholestyramine treatment, which suggests clinical bile acid malabsorption. However, most patients have higher levels, particularly in Crohn's disease after ileocecal resection. These preliminary results warranted confirmation on a larger scale.

Disclosure of Interest: All authors have declared no conflicts of interest.

\section{Reference}

A UHPLC-MS/MS method for the quantification of $7 \alpha$-Hydroxy-4-cholesten-3one to assist in diagnosis of bile acid malabsorption. Jean-Christophe Prost, Felix Brunner, Pascal Juillerat et al. Clinical Mass Spectrometry, Mar 2017. DOI: 10.1016/j.clinms.2017.02.001.

\section{P1628 THE ROLE OF SEVERAL CYTOKINES IN THE PATHOGENESIS OF AUTOIMMUNE INFLAMMATION IN PATIENTS WITH ULCERATIVE COLITIS}

A. Valeeva ${ }^{1}$, R. A. Abdulkhakov ${ }^{2}$, O. V. Skorokhodkina ${ }^{1}$, S.R. Abdulkhakov ${ }^{2}$, S. Khaiboullina ${ }^{2}$, E. Martinova ${ }^{2}$, A. Rizvanov ${ }^{2}$

${ }^{1}$ Clinical Immunology And Allergology, Kazan State Medical University, Kazan/ Russian Federation

${ }^{2}$ Kazan State Medical University, Kazan/Russian Federation

Contact E-mail Address: aliv05@mail.ru

Introduction: Ulcerative colitis (UC) is a clinical type of inflammatory bowel diseases. The pathogenesis of UC remains unclear. Nowadays the role of T-helpers type 17 (Th17) as well as cytokines they release is discussed in pathogenesis of autoimmune inflammation in UC.

Aims \& Methods: The aim of study is to analyze the serum levels of following cytokines: interleukin (IL)-17A and F, 21, 22, 23, 10 in UC patients both in the acute stage of disease and remission.Forty eight UC patients in the acute stage and twenty patients in remission were included into the study. Serum cytokine levels were analyzed using multiplex immunoassay for Th17 cytokines (Bio-Rad, USA). Statistical analysis was performed using STATISTICA 6.0 Software Package. The control group consisted of 11 healthy volunteers.

Results: Statistically significant increase of IL-17A level $(15 \mathrm{pg} / \mathrm{ml}[12.11 ; 23.38])$; $14.68 \mathrm{pg} / \mathrm{ml}[11.29 ; 17.19]$ respectively) was observed in patients with UC both in acute stage and remission compared to controls $(7.36 \mathrm{pg} / \mathrm{ml}[5.18 ; 8.06]$, $\mathrm{p}=0.00007, \mathrm{p}=0.00029$ respectively). The same trend was observed regarding IL-21, which median values were higher both in acute stage $(156.51 \mathrm{pg} / \mathrm{ml}$ [133.44;233.53]) and remission $(144.02 \mathrm{pg} / \mathrm{ml}[133.44 ; 154.43])$ compared to control group $(98.31 \mathrm{pg} / \mathrm{ml}[89.14 ; 124.86])$, and showed statistically significant differences $(p=0.00077, p=0.0054$ respectively). Besides it was revealed that IL$17 \mathrm{~F}$ and IL-22 were also higher in acute stage $(136.5 \mathrm{pg} / \mathrm{ml}[68.25 ; 228.185]$ and $3.76 \mathrm{pg} / \mathrm{ml}[1.5 ; 5.83]$, respectively) compared to controls $(48.7 \mathrm{pg} / \mathrm{ml}[38.7 ; 87.6]$; and $2.6 \mathrm{pg} / \mathrm{ml}[1.7 ; 3.2]$, respectively), however differences were not statistically significant $(p=0.06 ; p=0.172$, respectively). Increase of described cytokines levels could be a sign of Th17 functional overactivity suggesting autoimmune type of inflammation.Statistically significant increase of IL-10 in remission $(27.99 \mathrm{pg} / \mathrm{ml}$ [17.53;33.55]) compared to controls $(4.36 \mathrm{pg} / \mathrm{ml}$ [3.26;15.25], $\mathrm{p}=0.0046$ ) was found as well. IL-10 was also higher in patients with acute stage $(21.93$ [3.61;35.35]) compared to controls, however differences were not statistically significant $(\mathrm{p}=0.065)$. IL-10 as an anti-inflammatory cytokine characterizes the activity of regulatory $\mathrm{T}$-cells which suppress autoimmune inflammation. In addition, it was revealed that the level of IL-23 which stimulates Th17 differentiation was higher both in acute stage $(258.4 \mathrm{pg} / \mathrm{ml}[55 ; 367.49])$ and remission $(244.93 \mathrm{pg} / \mathrm{ml}[200.26 ; 301.93])$ compared to controls $(124.3 \mathrm{pg} / \mathrm{ml}$ [107.9;296.04]), however differences were not statistically significant.

Conclusion: Increase of IL-17A, IL-17F, IL-21, IL-22 levels could be a sign of Th17 functional overactivity suggesting autoimmune type of inflammation. IL17A and IL-21 produced by Th17 cells might be considered as markers of active autoimmune inflammation in UC patients.

Disclosure of Interest: All authors have declared no conflicts of interest. 


\section{P1629 ANP32E IS INVOLVED IN THE STEROID-REFRACTORY}

\section{ULCERATIVE COLITIS}

V. Lorén ${ }^{1}$, A. Garcia-Jaraquemada ${ }^{1}$, J.E. Naves ${ }^{1}$, J. Carmona ${ }^{1}$, E. Cabré ${ }^{1}$, M. Mañosa ${ }^{1}$, J. Manyé ${ }^{1}$, E. Domènech ${ }^{1}$

${ }^{1}$ Gastroenterology, Germans Trias i Pujol Research Institute \& CIBER, Badalona/ Spain

Contact E-mail Address: vloren@igtp.cat

Introduction: The steroid-refractoriness is a common complication of ulcerative colitis (UC), which appears unpredictably. Several mechanism of action (MoA) has been implicated in corticosteroid failure. However, there are no conclusive studies on the molecular functions involved in UC steroid-refractoriness.

Aims \& Methods: Therefore, we decided to know in depth the MoA related to the steroid failure, exploring transcriptomic data from a cohort of patients with UC treated with glucocorticoids. RNA from rectal biopsies was obtained before and on the 3rd day of glucocorticoid treatment. Then, whole-genome expression using microarrays (Illumina, USA) and profiles microRNA by sequencing (Illumina, USA) were analysed. After quality control, omics data were compared between phenotypes. The results of these comparisons were integrated into mathematical models generated by means of Systems Biology.

Results: These models reproduce the updated molecular interactions on glucocorticoids and UC, and integrating our experimental data, we identified a potential MoA that includes 64 key proteins, 18 of them capable of perfectly classifying patients with a good response to glucocorticoids and the non-responders. The biological functions of these proteins have been associated with inflammation (e.g. RelA), glucocorticoid receptor transcription (e.g. NR3C1 and NCOA3) and angiogenesis (e.g. VEGF), mainly. But among these 18 proteins, the ANP32e has never been related to either steroid-refractoriness or ulcerative colitis. ANP32e is a chaperone linked to H2A-H2A.z exchange (H2A.z is part of the nucleosomes flanking DNA regions recognized by the glucocorticoid receptor). Additional WB and immunofluorescence assays confirm differences in the intestinal levels of ANP32e and in the nuclear localization at baseline, between patients with different responses to glucocorticoids.

Conclusion: In conclusion, this study has identified a potential new MoA related with UC steroid-refractoriness involving chromatin remodeling modifications. Disclosure of Interest: All authors have declared no conflicts of interest.

P1630 CO-HOUSING DSS TREATED MICE WITH HEALTHY MICE RESULTS IN FASTER NORMALIZATION OF THE INTESTINAL MICROBIOTA AND PROMOTES RECOVERY

M.R. Spalinger ${ }^{1}$, L. Hering ${ }^{1}$, C. Gottier ${ }^{2}$, S. Lang ${ }^{2}$, G. Rogler ${ }^{2}$, M. Scharl ${ }^{2}$

${ }^{1}$ University Hospital Zürich, Zürich/Switzerland

${ }^{2}$ Gastroenterology And Hepatology, University Hospital Zurich, Zürich/

Switzerland

\section{Contact E-mail Address: marianne.spalinger@usz.ch}

Introduction: The intestine is populated with myriads of bacteria, which form a complex ecosystem and have tremendous impact on our health. In inflammatory bowel disease (IBD), shifts in microbiota composition and a reduction in overall diversity have been described. There are attempts to therapeutically transfer the microbiota from healthy subjects to persons suffering from intestinal disease. While in case of Clostridium difficile infections, this approach proves to be very efficient; the therapeutic value of fecal microbial transfer (FMT) in IBD is still unclear. In mouse models of intestinal inflammation, the effect of FMT has been studied poorly and if so, germ-free or antibiotic-treated animals have been used-models that poorly reflect the situation in human IBD patients. Here, we addressed how transmission of microbiota from healthy to diseased mice affects recovery from acute colitis.

Aims \& Methods: Acute colitis was induced in 12-14 week old C57B6 mice by administration of $2 \%$ DSS in the drinking water for 7 days. Mice with colitis were co-housed with healthy mice after removal of DSS. Due to coprophagy, this results in fast transfer of the microbiota between co-housed mice. To analyse changes in the composition of microbiota over time, stool samples were taken every second day and sequenced for the V4 hyper-variable region in the bacterial 16S DNA.

Results: As expected, DSS treatment resulted in severe weight loss, and even 7 days after withdraw of DSS (day 15), histology confirmed severe colitis. Intestinal inflammation was accompanied by an overall reduction of microbial diversity (decreased Shannon index, $\mathrm{p}<0.01$ ), and a marked shift in the composition of the microbiota (increased abundance of Verrucomicrobia, Cyanobacteria and some families of Firmicutes [mainly Clostridiacea], although overall abundance of Firmicutes was decreased [ $p<0.01$ for all]). However, on day 15 , these changes were less pronounced, indicating a normalization of the microbiota composition upon recovery. DSS-treated mice which were co-housed with healthy littermates after colitis induction, showed faster recovery (earlier weight gain, reduced histological scores, reduced levels of the infiltration marker myeloperoxidase (MPO), less pronounced shortening of the colon, $\mathrm{p}<0.01$ for all) and an earlier normalization of the microbiota composition.

Conclusion: Our results indicate that co-housing of DSS-treated mice with healthy mice results in transfer of healthy microbiota to diseased mice, and promotes recovery from colitis. This indicates that introduction of a "healthy" microbiota might have beneficial effects during intestinal inflammation and opens the possibility to systematically study the effect of genetic alterations in donor and/or recipient on the efficacy of FMT.

Disclosure of Interest: All authors have declared no conflicts of interest.

\section{P1631 ILEAL SULFOMUCINS PREDICT CLINICAL RECURRENCE AFTER ILEO-COLONIC RESECTION FOR CROHN'S DISEASE: A PROSPECTIVE STUDY AT 5 YEARS}

L. Biancone ${ }^{1}$, M. Ascolani ${ }^{2}$, C. Mescoli ${ }^{3}$, G. Sica ${ }^{4}$, G. Palmieri ${ }^{5}$, S. Romeo ${ }^{1}$, L. Albertoni ${ }^{3}$, B. Neri ${ }^{1}$, F. Pallone ${ }^{1}$, M. Rugge

${ }^{1}$ Systems Medicine, Università Tor Vergata Internal Medicine, Roma/Italy ${ }^{2}$ Gi Unit, Department Of Systems Medicine, University "Tor Vergata" of Rome, Italy, Rome/Italy

${ }^{3}$ Department of Medicine, DIMED; Pathology Unit; University of Padova, Padova, Italy, Padua/Italy

${ }^{4}$ Surgical Unit, Department of Surgery, University “Tor Vergata” of Roma, Rome/ Italy

${ }^{5}$ Pathology Unit, Department of Surgery, University "Tor Vergata” of Roma, Rome/Italy

${ }^{6}$ Pathology, Medical School of the Padova University, Padova/Italy

Contact E-mail Address: biancone@med.uniroma2.it

Introduction: In Crohn's Disease (CD), colonic phenotype of the ileum has been reported in severe lesions, but not in $\mathrm{CD}$ ileum with no lesions or with early postoperative recurrence (1)

Aims \& Methods: In a prospective study at 5 years, we aimed to address whether colonic phenotype of the ileum may represent a subclinical marker of clinical postoperative recurrence in $\mathrm{CD}$. At this purpose, clinical recurrence was assessed yearly for 5 years in a cohort of CD patients with colonic phenotype of the ileum already defined at surgery, 6 and at 12 months after ileo-colonic resection (1). A cohort of CD patients with clinical and endoscopic recurrence already assessed and reported at 6 and 12 months after ileo-colonic resection (1), was clinically followed up for additional 4 years. In the 5 years follow up, indication for treatments, haematochemical, radiological and/or endoscopic assessments was given according to current European guidelines (2). Haematochemical assessments included whole blood count, ESR, serum CRP evaluation yearly for 5 years, with possible additional assessments, according to clinical indications. Clinical recurrence $(\mathrm{CDAI}>150)$ was assessed yearly for 5 years. Colonic phenotype of the ileum was already reported at surgery, at 6 and 12 mos, according to the expression of sulfomucins (colon mucin-type) and sialomucins (small intestine mucin-type) (1). In the present study, correlation between the percentage of expression of sulfomucins and clinical recurrence was evaluated yearly for 5 years. Statistical analysis: results expressed as median (range), correlations were assessed by the Spearman correlation test, differences between groups by the unpaired $\mathrm{T}$ test.

Results: After ileo-colonic resection, clinical follow up at 5 years was completed by $17 / 19(89.4 \%)$ CD patients enrolled (12 males, age 41 [17-57]). The percentage of expression of sulfomucins (colon phenotype) as assessed in the ileal surgical specimens was significantly correlated with the CDAI score at 4 years $(r=0.62$; $\mathrm{p}=0.007)$ and at 5 years $(\mathrm{r}=0.6 ; \mathrm{p}=0.010)$. Differently, in the ileal biopsies at 6 months, colonic phenotype of the ileum, evaluated by the expression of sulfomucins was significantly correlated with the CDAI score at 6 monoths $(\mathrm{r}=0.68$; $p=0.003$ ). The percentage of expression of sulfomucins in the ileal biopsies at 12 months was significantly correlated with the CDAI score at 6 months $(r=0.57$; $\mathrm{p}=0.015)$ and at 2 years $(\mathrm{r}=0.53 ; \mathrm{p}=0.02)$. The ileal expression of sulfomucins in the surgical specimens at surgery was higher in patients with vs without clinical postoperative recurrence at 2 years $(40[10-99]$ vs $5[0-50 ; \mathrm{p}=0.04)]$. The expression of colon mucin-type in the ileal biopsies at 12 months was higher in patients with vs without clinical recurrence, both at 12 months $(30$ [1-40] versus $0[0-35]$; $\mathrm{p}=0.02)$ and at 2 years $(30[0-40]$ verus $0[0-35] ; \mathrm{p}=0.029)$. No correlations were observed between the percentage of expression of sulfomucins at surgery, at 6 or at 12 months and the haematochemical parameters considered.

Conclusion: In CD, colonic phenotype of the ileum as assessed by the expression of ileal sulphomucins, may represent a predictive marker of clinical recurrence after ileo-colonic resection for CD

Disclosure of Interest: L. Biancone: The study was not supported by any gran nor funded and any of the below reported disclosures are related to the study. Lecture fees or Advisory Board: Zambon, MS\&D, Takeda, Abbvie, Sofar, Ferring, Wassermann;

F. Pallone: The study was not supported by any grant nor funded and any of the below reported disclosures are related to the study. lecture fees from Zambon, Takeda.

All other authors have declared no conflicts of interest.

\section{References}

1. Ascolani M, Mescoli C, Palmieri G, Sica G, Calabrese E, Petruzzielo C, Onali S, Albertoni L, Lolli E, Condino G, Pallone F. Rugge M, Biancone L. Inflamm Bowel Dis 2014; 20(9):1555-61.

2. Gomollón F, Dignass A, Annese V, et al. J Crohns Colitis 2017; 11(1): 3-25. 
P1632 IMMUNOGENICITY OF A QUADRIVALENT INFLUENZA VACCINE AMONG PATIENTS WITH INFLAMMATORY BOWEL DISEASE

S. Shirai ${ }^{1}$, Y. Sakata ${ }^{1}$, K. Akutagawa ${ }^{2}$, K. Yamanouchi $^{3}$, N. Tsuruoka ${ }^{1}$, H. Endo ${ }^{4}$, R. Shimoda ${ }^{5}$, T. Noda ${ }^{6}$, N. Sugisaki ${ }^{7}$, R. Iwakiri ${ }^{8}$, K. Fujimoto ${ }^{9}$ ${ }^{1}$ Internal Medicine Of Gastroenterology, Saga Medical School, -,Nabeshima, Saga/Japan

${ }^{2}$ Internal Medicine, Taku City Hospital, Taku/Japan

${ }^{3}$ Internal Medicine \& Gastrointestinal Endoscopy, Takagi Hospital, Okawa/Japan ${ }^{4}$ Department Of Internal Medicine, Saiseikai Kratsu Hospital, Karatsu/Japan ${ }^{5}$ Internal Medicine And Endoscopy, Saga Medical School, Saga/Japan

${ }^{6}$ Internal Medicine And Endoscopy, Karatsu Red Cross Hospital, Karatsu/Japan ${ }^{7}$ Graduate School Of Medical Science, Saga University, Saga/Japan

${ }^{8}$ Dept. Of Gastrointestinal Endoscopy, Saga Medical School Dept. of Gastroenterology, Saga/Japan

${ }^{9}$ Dept. Of Internal Medicine, Saga Medical School, Saga/Japan

Contact E-mail Address: totoronin1029@gmail.com

Introduction: Immunization of a quadrivalent influenza vaccine (A/California/7 2009(H1N1) pdm09, A/Switzerland/9715293/2013(NIB-88) (H3N2), B/Phuket $3073 / 2013$, and $\mathrm{B} /$ Texas/2/2013) began in the $2015 / 2016$ season. We assessed the immunogenicity and booster effect of quadrivalent influenza vaccine for adult inflammatory bowel disease patients treated with immunosuppressive drugs and/or anti-TNF- $\alpha$ agents.

Aims \& Methods: Single vaccination group and booster group were randomly assigned to adult patients with crohn's disease or ulcerative colitis, and quadrivalent influenza vaccine was administered subcutaneously. Serum samples were collected at 3 points (before vaccination, 4 weeks after vaccination and after the end of influenza season) in the single group and 4 points in the booster group (before vaccination, 4 weeks after the first vaccination, 4 weeks after the second vaccination and after the end of the influenza season). Antibody titer against each influenza strain was measured by inhibition of hemagglutination.

Results: A total of 132 patients with IBD were randomly assigned to single vaccination and booster groups. Eighteen patients received immunomodulatory monotherapy and 20 received anti-TNF-alpha single agent therapy. Nineteen patients received combination therapy of immunosuppressant and anti-TNF- $\alpha$ agents. No significant difference between the single vaccination group and booster group was observed (geometric mean titers: H1N1: $p=0.81 ; \mathrm{H} 3 \mathrm{~N} 2$ : $\mathrm{p}=0.79$; B/Phuket: $\mathrm{p}=0.82$; $\mathrm{B} /$ Texas: $\mathrm{p}=0.84$ ). In patients treated with infliximab, seroprotection rate $(\mathrm{SP} \%)$ and seroconversion rate $(\mathrm{SC} \%)$ tended to be lower in influenza A strains in patients who maintained blood concentrations $(\mathrm{SP} \%$ H1N1: OR 0.37 (0.11-1.21); H3N2: OR $0.22(0.07-0.68)$, SC\%: H1 N1: 0.23 (0.06-0.91); H3N2: $0.19(0.06-0.56))$

Conclusion: Serological response rate to influenza vaccination was low in IBD patients receiving immunosuppressant therapy, especially infliximab, even with a quadrivalent influenza vaccine.

Disclosure of Interest: All authors have declared no conflicts of interest.

\section{P1633 THE INCIDENCE OF INFLAMMATORY BOWEL DISEASE IN SEVEN ETHNICALLY DIVERSE URBAN POPULATIONS IN ENGLAND; RESULTS FROM THE UK INCEPTION COHORT EPIDEMIOLOGICAL STUDY (UNITE)}

R. Misra ${ }^{1}$, J. Limdi ${ }^{2}$, R. Cooney ${ }^{3}$, M. Brookes ${ }^{4}$, E. Fogden ${ }^{5}$, S. Pattni ${ }^{6}$, N. Sharma ${ }^{7}$, G. Young ${ }^{8}$, J. Burisch ${ }^{9}$, P. Munkholm ${ }^{9}$, N. Arebi

${ }^{1}$ Gastroenterology, St. Marks Academic Institute, London/United Kingdom

${ }^{2}$ Gastroenterology, Pennine NHS Trust, Bury/United Kingdom

${ }^{3}$ Gastroenterology, University Hospitals Birmingham NHS Foundation Trust,

Birmingham/United Kingdom

${ }^{4}$ Department Of Gastroenterology, Royal Wolverhampton Hospitals NHS Trust,

Wolverhampton/United Kingdom

${ }^{5}$ Gastroenterology, Sandwell and Birmingham NHS Trust, Birmingham/United

Kingdom

${ }^{6}$ Gastroenterology, Leicester NHS Trust, Leicester/United Kingdom

${ }^{7}$ Gastroenterology, Heart of England NHS Foundation Trust, Birmingham/United

Kingdom

${ }^{8}$ London North West NHS Trust, London/United Kingdom
${ }^{9}$ Department Of Gastroenterology, North Zealand University Hospital,

Frederikssund/Denmark

Contact E-mail Address: rm399@imperial.ac.uk

Introduction: The global incidence of inflammatory bowel disease (IBD) is increasing. Migration may influence disease epidemiology. The UK demographics are affected by sustained migration from South Asia. The incidence of UC in South Asians (SA) was previously reported as higher than the Caucasian population. ${ }^{1,2}$ These single centre studies were done over 20 years ago. The current epidemiology of IBD in the multiethnic UK is unknown.

Aims \& Methods: We aimed to describe the UK incidence of IBD and distribution within ethnic groups. Census data (2011) was used for background population size, ethnic groups and to identify areas with high SA populations where Indian and Pakistani were the predominant groups. The incidence was calculated by using the number of subjects in each ethnic group in the background population as the denominator. Adult patients ( $>16$ years) with newly diagnosed IBD (fulfilling Copenhagen diagnostic criteria) were prospective recruited over one year in 7 catchment areas with high SA population. Data including demographics ethnic codes and disease phenotype (Montreal classification) was collected onto the Epicom database. Chi-squared test was used to detect differences in IBD incidence between ethnic groups. A p value $<0.05$ was considered significant. Results: Over 1 year IBD was diagnosed in 351 cases; 219 ulcerative colitis (UC), 107 Crohn's disease (CD) and 26 Inflammatory bowel disease unclassified. (IBDU). Collective crude incidence rates were 15.54/100,000 for IBD, 9.69/ 100,000 for UC, $4.80 / 100,000$ for CD and 1.01/100,000 IBDU. (Table 1) Crude IBD incidence rates varied between populations: lowest was $6.81 / 100,000$ in Pennine, North Manchester and highest 26.11/100,000 in Leicester. Overall incidence of UC was higher than CD $(9.69 / 100,000$ vs $4.80 / 100,000)$ and was consistent for all populations except Pennine (3.31/100,000 for CD and 2.76/100,000 UC) Of the total number of IBD cases recruited 298/351 were coded as Caucasian, Indian or Pakistani. IBD, UC and CD incidence was similar between Pakistani and Caucasian groups. UC incidence was significantly higher in the Indian population compared to Caucasians and Pakistanis $(\mathrm{p}<0.001)$. Data for disease phenotype was available for $160 / 219$ patients with UC $(24 \% \mathrm{E} 1,42 \% \mathrm{E} 2$ and $34 \%$ E3). There was no significant difference in disease extent between ethnic groups.

Conclusion: The incidence rates for IBD in seven urban populations in England are similar to recent data from Western Europe (IBD 18.5/100,000, UC 9.8/100/ $000 \mathrm{CD} 6.3 / 100,000$ and IBDU 2.4/100,000). Ethnic-adjusted incidence rates showed that Indians have higher incidence of UC than Caucasians and Pakistanis with highest observed incidence in Northwest London. These findings are consistent with previous data suggesting that Indians have a higher predisposition for UC. Genetic, environmental and dietary factors may be responsible for differences and further analyses are underway. Better understanding of the susceptibility of Indians to UC may lead to the underlying cause of UC.

Disclosure of Interest: All authors have declared no conflicts of interest.

\section{References}

1. Probert CS., Jayanthi V., Pinder D., Wicks AC., Mayberry JF. Epidemiological study of ulcerative proctocolitis in Indian migrants and the indigenous population of Leicestershire. Gut 1992;33(5):687-93.

2. Carr I., Mayberry JF. The effects of migration on ulcerative colitis: A threeyear prospective study among Europeans and first- and second-generation South Asians in Leicester (1991-1994). Am $J$ Gastroenterol 1999;94(10):2918-22.

Abstract: P1633. Table 1: Incidence rates per 100000 for inflammatory bowel disease, Crohn's disease, ulcerative colitis and inflammatory bowel disease unclassified in England for patients aged 16 years or older in 2016/17. CD, Crohn s disease; IBD, inflammatory bowel disease; IBDU, IBD unclassified; UC, ulcerative colitis.

\begin{tabular}{|c|c|c|c|c|c|c|c|c|}
\hline \multirow{2}{*}{ Population } & \multicolumn{3}{|c|}{ Background population } & \multirow[b]{2}{*}{ No. of cases } & \multirow[b]{2}{*}{ IBD crude } & \multirow[b]{2}{*}{ CD crude } & \multirow[b]{2}{*}{ UC crude } & \multirow[b]{2}{*}{ IBDU crude } \\
\hline & Cauc & Ind & Pak & & & & & \\
\hline Birmingham, University Hospital & 520,986 & 52,491 & 92,047 & 31 & 13.03 & 4.46 & 7.48 & 1.09 \\
\hline Birmingham, Heart of England NHS Trust & & & & 70 & & & & \\
\hline Birmingham, Sandwell & & & & 18 & & & & \\
\hline Leicester & 137,910 & 74,098 & 5,584 & 86 & 26.11 & 7.59 & 15.79 & 2.42 \\
\hline London, Brent and Harrow & 170,540 & 103,417 & 16,632 & 78 & 17.74 & 3.64 & 13.42 & 0.64 \\
\hline North Manchester, Pennine NHS Trust & 445,293 & 3,475 & 37,565 & 40 & 6.81 & 3.31 & 2.76 & 0.92 \\
\hline Wolverhampton & 141,044 & 25,370 & 2,719 & 28 & 14.0 & 5.0 & 9.0 & 0.0 \\
\hline All centres & $1,415,773$ & 258,851 & 154,747 & 351 & $15.54(95 \% \mathrm{CI}, \pm 8.42)$ & $4.80(95 \% \mathrm{CI}, \pm 1.90)$ & $9.69(95 \% \mathrm{CI}, \pm 5.55)$ & $1.01(95 \% \mathrm{CI}, \pm 0.89)$ \\
\hline $\begin{array}{l}\text { Incidence by Ethnicity } \\
\text { Caucasian }\end{array}$ & & & & 210 & 14.83 & 5.58 & 8.26 & 0.99 \\
\hline Indian & & & & 62 & 26.22 & 5.07 & 19.45 & 1.69 \\
\hline Pakistani & & & & 26 & 14.67 & 5.64 & 7.33 & 1.69 \\
\hline
\end{tabular}




\section{P1634 IBD-RELATED MALIGNANCIES AND MORTALITIES} OBSERVED BETWEEN 2015-2016-TWO YEARS' RESULTS FROM THE PROSPECTIVE NATIONWIDE HUNGARIAN REGISTRY

T. Molnar ${ }^{1}$, M. Rutka ${ }^{1}$, F. Nagy ${ }^{1}$, P. Fritz ${ }^{2}$, L. Lakatos ${ }^{3}$, P. Miheller ${ }^{4}$, Z. Erdélyi ${ }^{3}$, T. Szamosi ${ }^{5}$, E. Schäfer ${ }^{5}$, A. Vincze ${ }^{6}$, P. Sarlós ${ }^{6}$, J. Banai $^{5}$, Á. Kovács ${ }^{7}$, J. Novák $^{8}$, A. Salamon ${ }^{9}$, A. Szepes ${ }^{10}$, N. Szigeti ${ }^{11}$, M. Juhász ${ }^{12}$, K. Müllner ${ }^{13}$, Z. Barta ${ }^{14}$, O. Kadenczki ${ }^{14}$, A. Gelley ${ }^{15}$, K. Palatka ${ }^{16}$, M. Papp ${ }^{16}$, A. $Z_{\text {Zaránd }}{ }^{17}$, P.L. Lakatos ${ }^{18}$, G.L. Veres ${ }^{19}$, A. Fabian ${ }^{19}$, A. Balint ${ }^{19}$, R. Bor ${ }^{1}$ Z. Szepes ${ }^{1}$, K. Farkas

${ }^{1}$ First Department Of Internal Medicine, University of Szeged, Szeged/Hungary ${ }^{2}$ St. Francis Hospital, Kalocsa/Hungary

${ }^{3}$ Department Of Internal Medicine, Csolnoky Ferenc Regional Hospital, Veszprem/ Hungary

${ }^{4}$ 2nd Dept. Of Medicine, Semmelweis Univerisity 2nd Department of Internal Medicine, Budapest/Hungary

${ }^{5}$ Military Hospital-State Health Centre, Budapest/Hungary

${ }^{6} 1$ st Department Of Medicine, University of Pecs, Pecs/Hungary

${ }^{7}$ Péterfy Sándor Hospital and Trauma Center, Budapest/Hungary

${ }^{8}$ Bekes County Pandy Kalman Hospital, Gyula/Hungary

${ }^{9}$ Department Of Gastroenterology, Tolna County Teaching Hospital, Szekszard/ Hungary

${ }^{10}$ Gastroenterology \& Endoscopy, Bacs-Kiskun Country Hospital Gastroenterology \& Endoscopy, Kecskemét/Hungary

${ }^{11}$ 2nd Department Of Internal Medicine And Nephrology Centr, University of Pécs, Pécs/Hungary

${ }^{12}$ St. Margaret Hospital, Budapest/Hungary

${ }^{13}$ 2nd Dept. Of Medicine, Semmelweis Univerisity, Budapest/Hungary

${ }^{14}$ University of Debrecen, Debrecen/Hungary

${ }^{15}$ Betegápoló Irgalmas Rend Budai Irgalmasrendi Hospital, Budapest/Hungary

${ }^{16}$ Department Of Gastroenterology, University of Debrecen, Debrecen/Hungary

${ }^{17}$ Semmelweis University, Budapest/Hungary

${ }^{18} 1$ st Department Of Medicine, Semmelweis University Faculty of Medicine 1st Dept. of Medicine - 1st Department of Medicine, Semmel, Budapest/Hungary

${ }^{19}$ I. sz. Gyermekklinika, Semmelweis Egyetem, Budapest/Hungary

Contact E-mail Address: molnaribd@hotmail.com

Introduction: Inflammatory bowel diseases (IBD-Crohn's disease (CD); ulcerative colitis (UC)) are lifelong inflammatory conditions of the gastrointestinal tract. IBD-associated colorectal cancer (CRC) accounts for approximately $1-2 \%$ of all cases of CRC. Although data on mortality rates in IBD patients are controversial, CRC has been shown to account for approximately $10-15 \%$ of all deaths among IBD patients. The aim of our nationwide registry was to prospectively collect IBD-related mortalities and all types of malignancies diagnosed in the Hungarian IBD population.

Aims \& Methods: Data on all death and malignancies developed between 2015 and 2016 in IBD patients were recorded. Each members of the Hungarian Society of Gastroenterology were prospectively interviewed 3 monthly by personal emails to report both death and malignancies observed in their patient population. Demographic and clinical data including previous immunosuppressive and biological therapy were also collected.

Results: Fifty-five newly diagnosed malignancies were reported (mean age: 49.3 years old, mean disease duration was 16.9 years; male/female ratio was 34/21): 30 CRC (mean age: 52.5 years, mean disease duration: 19.9 years, male/female ratio was $22 / 8$ ) one of them had 1 pouch cancer previously colectomized because of sigmoid tumor, 4 skin, 3 lung, 2 breast, 2 cervix, 2 pancreas, 2 liver, 1 gallbladder, 1 prostate, 1 esophageal, 1 salivary gland, 1 thyroid, 1 central nervous system, 1 tonsil, laryngeal cancer, 1 B-cell lymphoma and 1 retroperitoneal sarcoma throughout the examined period. Twenty-two of the $30 \mathrm{CRC}$ cases were associated with UC, $60 \%$ with pancolitis. Twenty of the 30 cases were located on the rectosigmoid region, 2 had multiple localization. Nineteen deaths were reported during the examined period. Ten fatal cases were related to IBD (6 males 4 females; $1 \mathrm{CD}$ patient with rectal cancer, 1 patient with pouch cancer, $1 \mathrm{CD}$ patient with pancreas, $1 \mathrm{CD}$ patient with interstitial pneumonia, $1 \mathrm{UC}$ and 2 $\mathrm{CD}$ patients with septic complications, $1 \mathrm{UC}$ patient with meningitis, $1 \mathrm{UC}$ patient with hemorrhagic shock, $1 \mathrm{CD}$ patient with intestinal perforation). Age of death was significantly lower in case of IBD-related mortality compared to the other patients and general population (51.9 vs. 65 vs. 73.4 (data from Central Statistical Office 2013, Hungary) years, $\mathrm{p}<0.05$ ). Immunosuppressive and/or biological therapy was ever given for $4 / 10$ patients with IBD-related mortality. Conclusion: The most frequently observed IBD-related malignancy is CRC, which can be multifocal and mainly involved the distal part of the colorectum typically in UC patients with pancolitis and chronic activity. Malignancy and septic complications were the leader causes of IBD-related mortality characterized by earlier death than in the rest and in the non-IBD population.

Disclosure of Interest: All authors have declared no conflicts of interest.

P1635 ANAL CANAL HUMAN PAPILLOMAVIRUS (HPV) INFECTION IN MEN AND WOMEN UNDERGOING COLONOSCOPY: PREVALENCE AND RISK FACTORS, HIGH BURDEN IN CROHN'S DISEASE PATIENTS

L. Vuitton ${ }^{1}$, E. Jacquin ${ }^{2}$, A. Parmentier ${ }^{3}$, F. Fein ${ }^{4}$, A. Dupont-Gossart ${ }^{4}$,

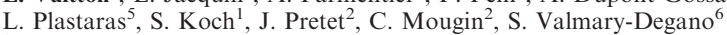
${ }^{1}$ Dept. Of Gastroenterology, Besançon University Hospital Gastroenterology, Besancon/France

${ }^{2}$ Ea 3181, National Reference Center For Hpv, Bourgogne-Franche Comté University, Besancon/France

${ }^{3}$ Dept. Of Methodology, Besançon University Hospital, Besançon/France
${ }^{4}$ Dept. Of Gastroenterology, Besancon University Hospital, Besancon/France

${ }^{5}$ Hepatogastroenterology, Hopital Colmar, Colmar/France

${ }^{6}$ Dept. Of Pathology, Besançon University Hospital, Besancon/France

Contact E-mail Address: lvuitton@chu-besancon.fr

Introduction: The increasing incidence of anal canal (AC) carcinomas in men and women requires better knowledge on Human papillomavirus (HPV) infection at this site and its risk factors. Higher incidence of AC cancers in Crohn's disease (CD) patients is strongly suggested in the literature, without knowledge on HPV involvement. A gastroenterology population offers the opportunity to study a mixed and non-sexually at risk selected population and to study anal HPV infection in CD patients.

Aims \& Methods: The aim of the study was to assess AC HPV infection prevalence and its risk factors in a gastroenterology population. The 'Human PAPILLomavirus ANal infection' - PAPILLAN- study took place in a French university hospital gastroenterology unit. Consecutive patients were prospectively recruited at the occasion of a colonoscopy, whatever the indication. On the colonoscopy day, under general anesthesia, AC smear was sampled with a dedicated brush for molecular analysis. HPV detection and genotyping was performed with the INNO-LiPA assay. Risk factors for any HPV, and high risk (HR) HPV infection were assessed by bivariate and multivariate analysis after logistic regression.

Results: A total of 469 consecutive patients (median age 54 years, $52 \%$ women) had suitable anal samplings for HPV DNA detection. Among them 101 had inflammatory bowel disease (IBD), 70 had CD. 112 patients had at least one immunosuppressive treatment for IBD or another condition. Overall $34 \%$ of the population had a detection of any HPV type in AC smears. HR HPV prevalence was $18 \%$, LR HPV prevalence was $9 \%$ and HPV16 prevalence was $7 \%$. Most prevalent HR HPV types were, by decreasing order: HPV16, HPV51, HPV52 and HPV39. Among all patients with HPV positive or HR HPV positive samples, $65.6 \%$ and $65.9 \%$ were women, respectively $(\mathrm{p}<0.0001 ; \mathrm{p}=0.0035$, compared to men). Regarding medical history, HR HPV and HPV16 prevalence were significantly higher in Crohn's disease patients $(30 \%, \mathrm{p}=0.0051 ; 14 \%, \mathrm{p}=0.0072$, compared to the rest of the study population). Eleven/12 patients $(50 \%)$ with perianal $\mathrm{CD}$ had an AC infection with any HPV. Multivariable analysis associated female gender and history of sexually transmitted disease with the presence of any HPV in AC; and female gender, history of sexually transmitted disease, lifetime and past year number of sexual partners, active smoking and immunosuppressive treatment (OR 5.3) with the presence of HR HPV.

Conclusion: We demonstrated that $\mathrm{CD}$ patients harbor more frequent $\mathrm{AC}$ infection with HR HPVs and that immunosuppressive treatment is an independent factor for HR HPV infection at this site. These findings strongly support prophylaxis with vaccination and adequate screening in our patients.

Disclosure of Interest: L. Vuitton: Speaker for Abbvie, Hospira, MSD, Ferring, Takeda Research grants from MSD, Takeda Consulting fees from Ferring, Abbvie

S. Koch: Speaker for Abbvie, MSD, Norgine, Olympus

All other authors have declared no conflicts of interest.

L. Plastaras: Speaker for Hospira, Abbvie, MSD

\section{P1636 GUT COLONIZATION WITH EXTENDED SPECTRUM BETA- LACTAMASE PRODUCING ENTEROBACTERIA MAY INCREASE DISEASE ACTIVITY IN INFLAMMATORY BOWEL DISEASES OUT- PATIENTS: PRELIMINARY STUDY RESULTS}

V. Skuja ${ }^{1}$, K. Pekarska ${ }^{1}$, H. Dauvarte ${ }^{1}$, E. Vasuka $^{1}$, L. Dobelniece ${ }^{1}$, J. Malina $^{1}$, D. Rudzite ${ }^{2}$, E. Lavrinovica ${ }^{2}$, L. Piekuse ${ }^{1}$, A. Kalcenaua ${ }^{1}$, A. Krumina ${ }^{1}$, A. Lejnieks ${ }^{1}$, A. Derovs ${ }^{3}$

${ }^{1}$ Riga Stradins Univeristy, Riga/Latvia

${ }^{2}$ Riga East Clinical University Hospital, Riga/Latvia

${ }^{3}$ Gastroenterology, Hepatology And Nutrition Clinic, Riga East Clinical University Hospital, Riga/Latvia

Contact E-mail Address: vita@skuja.lv

Introduction: Extended spectrum beta-lactamase producing Enterobacteria (ESBL-E) are the most frequently found multi-drug resistant bacteria colonizing the gut of inflammatory bowel disease (IBD) patients. ${ }^{1}$ Changes in the microbiome may act as a trigger in IBD inflammation process.

Aims \& Methods: The aim of the study was to analyze whether gut colonization with ESBL producing Enterobacteria is associated with clinically relevant disease activity increase in ulcerative colitis (UC) and in Crohn's disease (CD). All consecutive patients with confirmed UC and CD diagnosis, previously hospitalized in two largest tertiary medical care centres in Riga, Latvia during a 7-year period (2010-2016) were included in the study, interviewed, rectal swabs were collected, Enterobacteria were cultured and analyzed for ESBL presence according to EUCAST guidelines. To clinically evaluate disease activity UC patients were evaluated according to Mayo score, Montreal classification, adapted Truelove and Witt's criteria and CD patients according to Crohn's disease activity index (CDAI), suggested by ECCO IBD guidelines (2016).

Results: A total of 101 patients with UC and 47 patients with CD were tested for gut colonization with ESBL-E. We found that $12(11.9 \%)$ of the UC patients and $5(10.6 \%)$ of the CD patients were colonized with ESBL-E. Statistically significant differences were found in all UC clinical disease activity scores between patients with and without gut colonization with ESBL-E and showed tendency towards statistical significance in $\mathrm{CD}$. The mean disease activity according to Mayo score in UC patients without ESBL-E colonization was $3.44(\mathrm{SD}=2.07)$, whereas in patients with ESBL-E colonization it was $5.08(\mathrm{SD}=2.84)(\mathrm{p}=0.015)$. Most of the UC patients without ESBL-E colonization $(n=63 ; 70.8 \%)$ were in clinical remission, whereas half of the patients with ESBL-E colonization $(n=6$; 
$50 \%$ ) had mild to moderate to severe disease activity, according to Montreal classification disease severity section $(\mathrm{p}=0.037)$. Most of the UC patients without ESBL-E colonization $(\mathrm{n}=81 ; 91 \%)$ had mild disease activity, whereas half of the patients with ESBL-E colonization $(n=6 ; 50 \%)$ had moderate disease activity, according to modified Truelove and Witt's criteria $(p<0.001)$. Most of the CD patients without ESBL-E colonization $(n=38 ; 90 \%)$ had moderate disease activity, whereas most of the patients with ESBL-E colonization $(n=3 ; 60 \%)$ had severe disease activity, according to CDAI $(\mathrm{p}=0.05)$.

Conclusion: Gut colonization with ESBL-E might increase disease activity in outpatients with IBD. Such finding could be clinically relevant and help to improve diagnostic and treatment protocols for IBD patients, because eradication of ESBL producing bacteria might reduce IBD disease activity.

Disclosure of Interest: All authors have declared no conflicts of interest.

\section{Reference}

1. Leung, W. et al., 2012. Prevalence and predictors of MRSA, ESBL, and VRE colonization in the ambulatory IBD population. J. Crohns. Colitis 6, 743-9.

\section{P1637 IS SMOKING CESSATION LINKED TO NEW ULCERATIVE COLITIS CASES? A RESTROSPECTIVE COHORT-BASED HYPOTHESIS}

M. Grueber ${ }^{1}$, L. Biedermann ${ }^{2}$, S. Vavricka ${ }^{3}$, A. Schoepfer ${ }^{4}$, A. Macpherson ${ }^{5}$, P. Juillerat ${ }^{6}$, C. Clair Willii ${ }^{4}$, N. Fournier ${ }^{4}$

${ }^{1}$ Gastroenterology, Inselspital, Bern/Switzerland

${ }^{2}$ USZ Zürich, Zürich/Switzerland

${ }^{3}$ Gastroenterology And Hepatology, University Hospital Zurich, University of Zurich, Zurich/Switzerland

${ }^{4}$ CHUV+Universtiy of Lausanne, Lausanne/Switzerland

${ }^{5}$ Uvcm Gastroenterology, University of Bern, Bern/Switzerland

${ }^{6}$ Abt. Gastroenterology, Inselspital Bern University Clinic for Visceral Surgery and Medicin, Bern/Switzerland

Contact E-mail Address: maude.grueber@gmail.com

Introduction: Smoking has a differential effect on inflammatory bowels diseases (IBD); deleterious for Crohn's disease (CD) and protective for ulcerative colitis (UC). Thickness of the mucus layer, immune system (cytokines production), microvasculature and intestinal microbiome are potential mechanistic factors influenced by the nictoine and numerous other substances. It has been hypothestized that smoking cessation is associated with the second peak of diagnosis in UC patients after 50 years old. Our aim was to confirm this hypothesis using data on smoking status at IBD diagnosis.

Aims \& Methods: Adult IBD patients included in the Swiss IBD cohort from November 2006 to November 2015 were asked about their smoking status at diagnosis. We compared the proportion of former smokers in 10-year groups of $\mathrm{UC}$ and CD patients.

Results: 2361 IBD patients $(1366 \mathrm{CD}, 995 \mathrm{UC})$ were included in the analysis. Among them $52 \%$ of $\mathrm{CD}$ ans $24 \%$ of UC patients were smokers at diagnosis (proportion of smokers in Switzerland (2014): 29\%). The higher proportion $(66 \%)$ of former smokers at diagnosis was in the 50 to 60 years old group of UC patients compared to only $26 \%$ in CD patients between 40 to 50 years old $(\mathrm{p}<0.001)$. On a gender basis, the higher proportion of former smokers is particularly significant high among male 50-60 years old with UC $(68 \%)$ and persists among them over 60 years old $(52 \%)$.

Conclusion: The proportion of former smokers at diagnosis increases dramatically and significantly over years in UC patients compared to $\mathrm{CD}$ patients. A peak was reached over 50 years old suggesting an indirect impact of smoking cessation on the second peak of diagnosis in ulcerative colitis.

Disclosure of Interest: All authors have declared no conflicts of interest.

\section{P1638 THE IMPACT OF INFLAMMATORY BOWEL DISEASE ON HARD TEETH TISSUES-PRELIMINARY RESULTS FROM POLIBD STUDY}

D. Piatek ${ }^{1}$, I. Korona-Glowniak ${ }^{2}$, A. Malm² , S. Jarmakiewicz ${ }^{3}$,

S. Pudzianowski ${ }^{4}$, A. Pekala ${ }^{5}$, P. R. Kiela ${ }^{6}$, R. Filip ${ }^{5}$

${ }^{1}$ Conservative Dentistry With Endodontics, Medical University of Lublin, Lublin/

Poland

${ }^{2}$ Pharmaceutical Microbiology, Medical University of Lublin, Lublin/Poland

${ }^{3}$ Medical Faculty, Rzeszów University, Rzeszów/Poland

${ }^{4}$ Dental Clinical Center, Medical University of Lublin, Lublin/Poland

${ }^{5}$ Gastroenterology With The Central Department Of Endoscopy, Clinical Hospital

No.2 Rzeszow, Poland, Rzeszów/Poland

${ }^{6}$ Steele Children's Research Center, Departments Of Pediatrics And

Immunobiology, Arizona Health Sciences Center 6351, University of Arizona,

Tucson/United States of America/AZ

\section{Contact E-mail Address: r.s.filip@wp.pl}

Introduction: In aetiology of inflammatory bowel disease (IBD) role of microorganisms including those from the oral cavity is taken into account. Oral bacteria are known for biofilm formation and enamel demineralisation leading to the formation of caries cavities.

Aims \& Methods: The aim of the study was to determine the state of hard teeth tissues measured by DMFT index in adult patients with ulcerative colitis (UC) and Crohn's disease (CD). The study involved $119 \mathrm{UC}$ and $\mathrm{CD}$ patients aged from 18 to 72 (mean 34.45). Disease phenotype at diagnosis was classified according to the Montreal classification and only the patients who had conducted a complete diagnostic workup of the entire gastrointestinal tract were included. Following, the data on the occurrence of extraintestinal symptoms, the incidence of $I B D$ in the family, type of treatment, including surgery, were also collected. The complete assessment of the hard teeth tissues based on the DMFT index (decayed, missing, filled teeth) was performed.

Results: The study population was characterized by a high DMFT index with mean value 19.35. There was a significantly higher number of filled $(F)$ and lower number of lost teeth (M) among patients who underwent respective surgery. Noticed incidence of IBD in the family was similar in both groups of patients. Type of treatment has no significant impact on DMFT index values. There were no statistically significant differences between UC and CD group in DMFT index, $\mathrm{M}$ and $\mathrm{F}$ values. However, it has been found that these groups differ in $\mathrm{D}$ values which averaged 4,5 and 3,2 for $C D$ and UC groups $(\mathrm{p}<0.05)$. The analysis of both patients' groups with regard to gender showed statistically significant differences in $\mathrm{D}$ and $\mathrm{F}$ values. The lowest mean $\mathrm{D}$ value was noticed in $\mathrm{UC}$ females group $(2,8)$ and the highest in $\mathrm{CD}$ males $(5,2)$; average $\mathrm{F}$ values were opposite-11,4 for UC females and 7,8 for CD males. Statistical analysis of DMFT index among patient from CD and UC groups according to disease phenotype showed no significant differences in DMFT, D and $\mathrm{M}$ in both groups. Average DMFT varied from 18.14 for L1 to 20.50 for L4 patients in CD group and from 17.30 for E1 to 20.55 for E2 in UC group. However, significant difference in $\mathrm{F}$ values was observed in $\mathrm{CD}$ patients: $\mathrm{L} 1$ and $\mathrm{L} 2$ have lower average $F$ than those with L3 and L4 (6.66, 7.46, 10.25 and 14.00 respectively; $p=0.0215)$. $D$ values were significantly higher for patients with none or one extraintestinal symptoms $(\mathrm{p}<0.05)$. Results of $\mathrm{D}$ values obtained in our study were higher than for healthy population aged 35-44 in Poland (mean $\mathrm{D} \sim 2$ ).

Conclusion: The results of preliminary POLIBD study among two groups of IBD patients showed similar values in DMFT index and higher average number of carious teeth in CD patients, especially in men.

Disclosure of Interest: All authors have declared no conflicts of interest.

\section{P1639 RESTING ENERGY EXPENDITURE IN WOMEN WITH CROHN'S DISEASE: A CROSS-SECTIONAL STUDY}

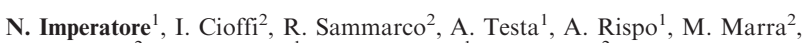
F. Contaldo ${ }^{2}$, N. Caporaso ${ }^{1}$, F. Castiglione ${ }^{1}$, F. Pasanisi ${ }^{2}$

${ }^{1}$ Gastroenterology, Department of Clinical Medicine and Surgery, School of Medicine Federico II of Naples, Naples/Italy

${ }^{2}$ Internal Medicine and Clinical Nutrition Unit, Department of Clinical Medicine and Surgery, Federico II University, Naples, Naples/Italy

Contact E-mail Address: nicola.imperatore@alice.it

Introduction: Crohn's disease (CD) is a chronic inflammatory disease that can affect any section of the gastrointestinal tract. Malnutrition is a common sequel in these patients and many pathogenic mechanisms could be involved such as poor dietary intake, altered energy expenditure, nutrient malabsorption and/or losses.

Aims \& Methods: This cross-sectional study aimed to evaluate the resting energy expenditure (REE) in CD patients, in accordance with clinical status of disease, compared to a control group.

Methods: All consecutive adult CD women were prospectively enrolled, while a group of healthy women, matched for age and weight, served as control group (C). CD women were classified in clinically active disease (CD-A) and clinical remission (CD-R) according to Crohn's Disease Activity Index (CDAI) (>150 and $<150$, respectively). All subjects underwent REE measure by indirect calorimetry with a canopy system, while body composition variables, such as fat-free mass (FFM) and fat mass (FM), were assessed by bio-impedance analysis (BIA). Results: Finally, forty-two women with CD, 23 with clinically active disease (CD$\mathrm{A} ; \mathrm{CDAI}=219 \pm 53)$ and 19 in clinical remission $(\mathrm{CD}-\mathrm{R} ; \mathrm{CDAI}=83 \pm 41)$ were recruited for the study, while 40 matched-healthy women were enrolled as control group (C). We found that body weight, FFM and phase angle (PA) differed among groups; but age, height and FM did not. Post-hoc analysis revealed that body weight was significantly lower for CD-A in comparison with $\mathrm{C}$ (CDA: $55.7 \pm 9.23 \mathrm{~kg}$ vs $\mathrm{C}: 64.5 \pm 6.3 \mathrm{~kg} ; \mathrm{p}=0.02$ ). FFM was reduced in women with CD than C (CD-A: $39.6 \pm 4.32 \mathrm{~kg}$ and CD-R: $39.5 \pm 6.68 \mathrm{~kg}$ vs C: $44.4 \pm 4.68 \mathrm{~kg}$; $\mathrm{p}<0.01)$ while PA was lower for CD-A compared to both CD-R and C (CD-A: $5.5 \pm 0.6$ vs CD-R: $6.0 \pm 0.5$ and $C: 6.1 \pm 0.5 ; p<0.001)$. REE did not differ among groups; nevertheless when it was adjusted for FFM, we observed that $\mathrm{REE} / \mathrm{FFM}$ increased for both CD-A and CD-R groups compared to C (CD-A: $35.9 \pm 4.17 \mathrm{kcal} / \mathrm{kg}, \quad \mathrm{CD}-\mathrm{R}: \quad 35.1 \pm 4.96 \mathrm{kcal} / \mathrm{kg}$ vs $\mathrm{C}: 30.2 \pm 3.38 \mathrm{kcal} / \mathrm{kg}$; $\mathrm{p}<0.01)$.

Conclusion: These preliminary results show that REE, when adjusted for FFM, is increased in women with $\mathrm{CD}$, unrelated to disease activity, compared to healthy subjects and this could negatively affect the energy balance and contribute to weight loss.

Disclosure of Interest: All authors have declared no conflicts of interest. 


\section{P1640 MAGNETIC RESONANCE CHOLANGIOGRAPHY} ABNORMALITIES IN PATIENTS WITH INFLAMMATORY BOWEL DISEASE

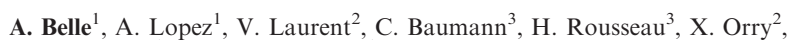
J. Bronowicki ${ }^{1}$, L. Peyrin-Biroulet ${ }^{1}$

${ }^{1}$ Gastroenterology And Hepatology, CHU de Nancy, Vandoeuvre-Les-Nancy/ France

${ }^{2}$ Service De Radiologie, CHRU de Nancy, Vandoeuvre-Les-Nancy/France ${ }^{3}$ Plateforme D'aide à La Recherche Clinique, CHRU de Nancy, Vandoeuvre-LesNancy/France

Contact E-mail Address: arthurbelle@hotmail.fr

Introduction: Primary sclerosing cholangitis (PSC) is a rare and devastating complication of inflammatory bowel disease (IBD). There is no standard for the screening of primary sclerosing cholangitis (PSC) in patients with IBD. Magnetic resonance cholangiography (MRC) may replace liver biopsy in this clinical situation. The main objective of this prospective observational study was to assess the frequency of MRC-detected liver diseases, including PSC, in adult IBD patients with liver function abnormalities and to identify clinical and biological characteristics associated with these findings.

Aims \& Methods: From June 1, 2009 to January 31, 2017, 421 patients were included and screened with MRC: cohort 1 included 206 IBD patients with liver abnormalities; cohort 2 included 28 IBD patients without liver abnormalities; and cohort 3 included 187 non-IBD patients with liver abnormalities. Two senior radiologists independently evaluated MRC findings.

Results: MRC abnormalities were observed in $18 \%$ of patients in the cohort 1 ; $3.6 \%$ in the cohort 2 ; and $31 \%$ in the cohort 3 (Table 1). Based on MRC, we found respectively $11.2 \%, 0 \%$, and $7 \%$ of PSC in cohorts 1,2 , and $3.29 .2 \%$ of IBD patients with liver abnormalities had infra-clinical PSC. A history of intestinal resection $(\mathrm{P}=.0357)$, abnormal gamma-glutamyl transferase values $(\mathrm{P}=.0064)$, and abnormal alkaline phosphatase values $(\mathrm{P}=0.021)$ were significantly associated to suspected PSC.

Table 1: MRC abnormalities in cohorts 1,2 and 3

\begin{tabular}{lllllll}
\hline $\begin{array}{l}\text { Results of } \\
\text { MRC }\end{array}$ & Total & Normal & Ductopenia & Doubt & PSC & Others \\
\hline Cohort 1 & 206 & $150(72.8 \%)$ & $28(13.6 \%)$ & $9(4.4 \%)$ & $23(11.2 \%)$ & 0 \\
Cohort 2 & 28 & $27(96.4 \%)$ & $1(3.6 \%)$ & 0 & 0 & 0 \\
Cohort 3 & 187 & $116(62.0 \%)$ & 0 & 0 & $13(7.0 \%)$ & $58(31.0 \%)$
\end{tabular}

Conclusion: Using MRC in patients with IBD, we found a higher prevalence of PSC than based on clinical symptoms. Systematic screening for PSC using MRC could be recommended in routine practice for IBD patients.

Disclosure of Interest: All authors have declared no conflicts of interest.

\section{P1641 TRUECOLOURS ULCERATIVE COLITIS (TCUC): WILL PATIENTS WITH UC COMPLETE DIGITAL QUESTIONNAIRES IN REAL-TIME?}

A. Walsh ${ }^{1}$, M. Peters ${ }^{2}$, C. Hinds ${ }^{3}$, V. Sexton ${ }^{3}$, A. Kormilitzin ${ }^{4}$, P. Seeva ${ }^{1}$, G. Collins ${ }^{5}$, S. Keshav ${ }^{1}$, O. Brain ${ }^{1}$, H. Uhlig ${ }^{1}$, A. Simmons ${ }^{6}$, J. Geddes ${ }^{3}$, G. Goodwin ${ }^{3}$ S. Travis ${ }^{1}$

${ }^{1}$ Gastroenterology, John Radcliffe Hospital, Oxford, Oxford/United Kingdom

${ }^{2}$ Nuffield Department Of Population Health, University of Oxford, Oxford/United Kingdom

${ }^{3}$ Psychiatry, University of Oxford, Oxford/United Kingdom

${ }^{4}$ Mathematical Institute, University of Oxford, Oxford/United Kingdom

${ }^{5}$ Botnar Research Centre, Centre for Statistics in Medicine, Oxford/United Kingdom

${ }^{6}$ Weatherall Institute of Molecular Medicine, Oxford/United Kingdom

\section{Contact E-mail Address: alissa.walsh@linacre.ox.ac.uk}

Introduction: TCUC is a comprehensive real-time web-based programme for patients with UC. It monitors multiple parameters via electronic questionnaires: symptoms, quality of life (QoL), outcomes (eg emergency department visits) and demographics. Medications are entered and personalised treatment guidance formulated. This information, graphically displayed on a traffic light system, is available to the patient and clinical team via the TCUC website, which is housed on a secure National Health Service server.

Aims \& Methods: A prospective, non-randomised 6-month pilot study recruited patients from the Oxford Inflammatory Bowel Disease service. The aims were to assess feasibility, usability and adherence. Recruitment rate (number recruited/ expression of interest) and retention rates were calculated. At registration, questionnaires were scheduled either daily (simple clinical colitis activity index, SCCAI), fortnightly (QoL, IBD-Control-8, CUCQ-8 and EQ5D-5L), or once only (outcomes,). Patients then received email prompts linked to scheduled ques-

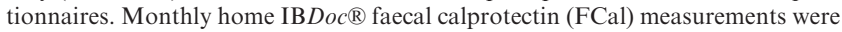
incorporated, monthly blood tests collected and flexible sigmoidoscopy performed at entry and after 6 months. Adherence to all questionnaires and samples was calculated. Usability was assessed via the System Usability Scale (SUS) $(\mathrm{n}=59)$ and qualitative interviews $(\mathrm{n}=28)$. SUS broadly classifies usability of a system from poor $(<70)$ to superior $(>90)$. A deductive approach was used for the qualitative coding and analysis.

Results: Sixty-six patients were recruited (recruitment rate 66/240 invitations delivered, $28 \%)$. Of 66 patients, $29(44 \%)$ were male, median age 40.7 yrs
(IQR 17.0, range 18-65), median duration of disease 5.6 years (IQR 10.7, range $0-30$ ), distribution of disease (E1 18\%, E2 38\%, E3 33\%, unknown $11 \%$ ), activity of disease at entry (remission $38 \%$, mild $35 \%$, moderate $26 \%$, severe $1 \%$ ), tertiary education $58 \%$, biologic use $47 \%$. Retention rate $57 / 66$, $(86 \%)$. Main reason for withdrawal $(5 / 9,55 \%)$ was the need for sigmoidoscopy and monthly blood tests during the trial.

Table 1: Adherence to Questionnaires

\begin{tabular}{llll}
\hline & $\begin{array}{l}\text { Adherence } \\
\text { over } \\
6 \text { months }\end{array}$ & $\begin{array}{l}\text { First } \\
3 \text { months }\end{array}$ & $\begin{array}{l}\text { Last } \\
3 \text { months }\end{array}$ \\
\hline $\begin{array}{l}\text { Questionnaires } \\
\begin{array}{l}\text { Fortnightly QoL questionnaires } \\
\text { (IBD-Control 8, CUCQ8 and EQ-5D) }\end{array}\end{array}$ & $76 \%$ & $81 \%$ & $72 \%$ \\
$\begin{array}{l}\text { Once only demographic and } \\
\text { outcome questionnaires }\end{array}$ & $100 \%$ & $100 \%$ & $100 \%$ \\
\hline
\end{tabular}

Uptake of FCal home testing was 73\% (48/66), median tests 4 (IQR 3, range 16). Median SUS score was 92.5 (IQR 15, range 45-100) which corresponds to a 'superior product'. Qualitative interviews confirmed that TCUC was efficient, effective, easy to learn and well liked. Improvements suggested included optimisation of the graphical display on smartphones and decreasing the number of QoL questionnaires from three to one, with a preference for IBD-Control.

Conclusion: Patients with UC will collect digital data in real-time, with good adherence to symptom, QoL, outcome questionnaires and FCal home testing. Usability was classified as 'superior' but further improvements are possible. Larger studies are required to determine cost effectiveness.

Disclosure of Interest: A. Walsh: An unrestricted educational grant from Abbvie Pharmaceuticals was received for this work. Buhlmann laboratories provided all IBDoc kits for this study.

All other authors have declared no conflicts of interest.

\section{P1642 BODY COMPOSITION AS A PREDICTOR FACTOR OF DISEASE OUTCOME IN INFLAMMATORY BOWEL DISEASE- RESULTS OF 3-YEAR FOLLOW-UP}

A.A. Csontos ${ }^{1}$, A. Molnár ${ }^{2}$, R. Hencz ${ }^{3}$, D. Piri ${ }^{3}$, S. Dakó ${ }^{3}$, T. Ferenci ${ }^{4}$, E. Pálfi ${ }^{5}$, P. Miheller ${ }^{1}$

${ }^{1}$ 2nd Dept. Of Medicine, Semmelweis Univerisity 2nd Department of Internal Medicine, Budapest/Hungary

${ }^{2}$ School Of Phd Studies, Doctoral School Of Pathological Sciences, Health Sciences Research, Semmelweis University, Budapest/Hungary

${ }^{3}$ 2nd Department Of Medicine, Semmelweis University, Budapest/Hungary

${ }^{4}$ John Von Neumann Faculty Of Informatics, Physiological Controls Research

Center, Óbuda University, Budapest/Hungary

${ }^{5}$ Faculty Of Health Sciences, Department Of Dietetics And Nutrition Sciences,

Semmelweis University, Budapest/Hungary

Contact E-mail Address: csontosagnesanna@gmail.com

Introduction: Malnutrition and altered body composition can develop in patients with inflammatory bowel diseases (IBD) for a variety of reasons. Malnutrition and sarcopenia may worsen disease outcome in chronic disorders, raise the risk of infections and hospitalisation.

Aims \& Methods: We followed 198 IBD outpatients (144 CD and 54 UC) for 3 years to indetify potentional risk factors of unfavourable disease outcome. Baseline body composition were measured by bioelectrical impedance analysing (BIA) method to evaluate nutritional status. Penalized logistic regression was used for the multivariate modelling of the outcome, with two sets of - prespecified - predictor variables age, sex, CU/CD, BMI, FFMI.

Results: According to our results $19.2 \%$ of the patients $(n=38)$ were underweight (had BMI $\left.<18.5 \mathrm{~kg} / \mathrm{m}^{2}\right)$ and $29.8 \%(\mathrm{n}=59)$ had alarming low fat-free mass index (FFMI) and were at risk of sarcopenia. Overall $31.5 \%(n=62)$ of the patients needed steroid therapy and $53.5 \%(\mathrm{n}=106)$ was given anti-TNF. Almost third of the participant $(30.8 \%, \mathrm{n}=61)$ were hospitalized due to disease flair or its complication at least once during the follow-up time. The mean period of hospitalization was $19.14 \pm 32.7$ days. $20.2 \%(n=40 \%)$ of all participants have undergone intestinal surgery. Hospitalization was positively associated with sarcopenia risk: alarming low FFMI was associated with an OR of 1.81 (95\% CI: $1.03-3.20, \mathrm{p}=0.04060)$. The risk of operation was higher in patients with lower BMI: $\mathrm{OR}=1.55$ (95\% CI: $1.05-2.29, \mathrm{p}=0.02778)$ for 5 units decrease; no other association was significant in the models.

Conclusion: Our results suggests that low BMI is a risk factor of surgery in inflammatory bowel disease patients. Furthermore alarming low FFMI is a predictor of need of hospitalization and that suggets more serious flares. Identification of malnutrition and altered body composition has notable importance in disease outcome among IBD patients.

Disclosure of Interest: All authors have declared no conflicts of interest. 
P1643 CONTRAST-ENHANCED ULTRASOUND IS HELPFUL IN

THERAPEUTIC DECISION MAKING IN PATIENTS WITH STRICTURING CROHN'S DISEASE

Z. Zelinkova ${ }^{1}$, B. Kadleckova ${ }^{2}$, D. Podmanicky ${ }^{3}$

${ }^{1}$ Department Of Gastroenterology, St Michael's Hospital, Bratislava/Slovak Republic

${ }^{2}$ IBD Center, Bratislava/Slovak Republic

${ }^{3}$ Department Of Surgery, St Michael's Hospital, Bratislava/Slovak Republic

Contact E-mail Address: zuzana.zelinkova@nsmas.sk

Introduction: The majority of Crohn's disease (CD) develop stricturing complications of the disease at some point. The proper selection of patients with potential benefit of therapy escalation is crucial in order to avoid unnecessary bowel damage. Rapid contrast uptake in the affected bowel segment at intravenous contrast-enhanced ultrasound (CEUS) has been shown to correlate with disease activity but there are no data available on the benefit of CEUS for the therapeutic decision making in this clinical setting.

Aims \& Methods: The aim of the study was to evaluate the clinical outcomes of $\mathrm{CD}$ patients with stricturing disease managed based on the CEUS findings. CD patients with stricturing disease were recruited from two IBD centra between June 2015 and February 2017. Patients with penetrating disease complications were excluded. CEUS was performed using $2.4 \mathrm{~mL}$ of intravenous contrast (SonoVue, Bracco Imaging). Patients having rapid uptake (within 20 second after injection) were indicated for therapy escalation, patients without uptake with obstructive symptoms were referred for surgery; patients without uptake and no obstructive symptoms remained at the stable medication. In patients with the minimal follow-up of one year clinical and endoscopic remission was evaluated.

Results: In total, 27 patients were included ( 10 men; median age 37 yrs, range 23 67; 22 pts with ileo-coecal localization, 3 pts with multiple small bowel segments involvement, 2 with colonic disease). Seventeen patients (63\%) had rapid uptake at the CEUS; 13 of these patients had therapy escalation ( 3 pts intensification or switch of another biological; 10 pts had therapy step-up to antiTNF or immunosuppression). Remaining three pts improved subsequently on stable therapy with antiTNF and one patient with longstanding symptomatic colonic stricture was referred for surgery. Ten patients $(37 \%)$ had no rapid uptake at the CEUS seven out of these patients had symptomatic stricturing disease and were referred for surgery. Three patients had no symptoms and no therapeutic changes were made. Twenty-five patients had follow-up longer than 12 months (median 18 months, range 13-23). In the group of patients with rapid uptake, all but two patients achieved sustained remission after therapy escalation. Two patients initially responded to therapy but have lost response and eventually needed surgery. In the group with no rapid uptake, patients without surgery remained in remission without any changes in the medication. Patients who underwent surgery in this group had no changes in therapy after surgery and none of these patients had recurrence at the surveillance colonoscopy at 12 months.

Conclusion: Contrast-enhanced ultrasound might be helpful in guiding the therapeutic decision making between surgery and therapy intensification in patients with stricturing Crohn's disease.

Disclosure of Interest: All authors have declared no conflicts of interest

P1644 FERTILITY AND PREGNANCY IN IBD - OUR EXPERIENCES

E. Schäfer ${ }^{1}$, K. Bursics ${ }^{1}$, T. Szamosi ${ }^{1}$, J. Banai ${ }^{1}$, F. Zsigmond ${ }^{1}$, T. Gyökeres ${ }^{1}$, L. Herszényi ${ }^{2}$

${ }^{1}$ MHEK, Budapest/Hungary

${ }^{2}$ Department Of Gastroenterology, MHEK, Budapest/Hungary

Contact E-mail Address: schafereszter@gmail.com

Introduction: Inflammatory bowel disease (IBD) commonly affects patients during their reproductive years, making the interaction between fertility, pregnancy, and IBD an important issue for both genders. As these are the reproductive years, patients and physicians often have concerns regarding the interaction between IBD, medications and surgery used to treat IBD, and reproduction, pregnancy outcomes, and neonatal outcomes.

Aims \& Methods: We aimed to determine IBD patient's reproductive behaviour and evaluate the factors that affect fear of family planning. Finally we investigated the correlation of disease activity and pregnancy outcome. 207 patients from our IBD population (142 with Crohn's disease, 65 with ulcerative colitis) were reviewed by personal questionnaires.

Results: In all, $53 \%$ of participants were female (the mean age was 31.9 years with mean duration of disease activity of 10.7 years). $47 \%$ of patients were male, (the mean age was 32.5 years with disease duration of 10.2 years). 62 patients (32 female and 30 male) have children (39 and 42) after diagnosing IBD (mean age of childbearing was 30 years. $81 \%$ of reviewed female and $79 \%$ of male patients plan to have more children, although approximately one-third of patients $(33 \%$ of female and $29 \%$ of male patients) reported having fewer children than planned up till now secondary to disease activity, surgical and ongoing medical treatments. The main patient-related reasons for "voluntary infertility" were fear of congenital abnormality secondary to medications of IBD $(68 \%)$ and concern about genetic risk of IBD in child $(56 \%) .50 \%$ of male and $75 \%$ of female patients got adequate information and education from conception do delivery. Pregnancy was planned in $77 \%$ of cases, the childbearing and delivery was without any complication. Prematurity and low birth weight occurred in 6-6 cases $(10 \%-10 \%)$. IBD was in remission in most cases, during pregnancy $25 \%$ of the patients had flare. $40 \%$ of women could breastfeed their baby after 6 months.

Conclusion: The management of IBD in women during their reproductive years should include consideration of their family planning decisions, and education counseling regarding the overall safety of medications and the importance of medication adherence should occur prior to conception. Disease control prior to desired conception and throughout pregnancy is the most important factor to keep in mind when caring for the IBD patients. Educating the patients will help to achieve successful outcomes both for patients and babies.

Disclosure of Interest: All authors have declared no conflicts of interest.

\section{References}

Mountifield R et al: Fear and Fertility in Inflammatory Bowel Disease: A Mismatch of Perception and Reality Affects Family Planning Decisions, Inflamm Bowel Dis 2009;15:720-7

Beaulieu D.B. et al.: Inflammatory bowel disease in pregnancy, World $J$ Gastroenterol 2011 June 14; 17(22): 2696-2701

van der Woude CJ et al.: The Second European Evidenced-Based Consensus on Reproduction and Pregnancy in Inflammatory Bowel Disease, Journal of Crohn's and Colitis, 2015, 1-18

Hosseini-Carroll P. et al.: Pregnancy and inflammatory bowel diseases: Current perspectives, risks and patient management. World J Gastrointest Pharmacol Ther 2015 November 6; 6(4): 156-171

Palomba S. et al.: Inflammatory bowel diseases and human reproduction: A comprehensive evidence-based review. World J Gastroenterol 2014 June 21; 20(23): 7123-7136

Huang VW et al.: From conception to delivery: Managing the pregnant inflammatory bowel disease patient. World J Gastroenterol 2014 April 7; 20(13): 34953506

\section{P1645 DIAGNOSTIC DELAY AND PREDICTIVE FACTORS FOR CROHN'S DISEASE IN AN ALGERIAN POPULATION}

Y. Chelbi, R. Boulares

Constantine, Ben Badis Hospital, Constantine/Algeria

Contact E-mail Address: Yacinechelbi4@yahoo.fr

Introduction: Crohn's disease (CD) is a chronic inflammatory bowel disease whose diagnostic delay (DD) is highly variable. A delay in diagnosis of MC may be detrimental to the patient, delaying appropriate therapeutic management. Factors Influencing SD may be a function of the country's health system, but also linked to the particular clinical and evolutionary profile of the disease. The objective of this study was to measure the $\mathrm{DD}$ of $\mathrm{CD}$, to describe its distribution and evolution over time and to The factors associated with a long DD (>Q3). Aims \& Methods: All patients with certain or probable CD between 2004 and 2016 identified by The department's inflammatory disease hospital registry was included. The socio-demographic characteristics collected included: the patient's residential area at the time of diagnosis in urban, rural or semi-urban, distance from the nearest hospital $(\mathrm{CH})$. Clinical symptoms and phenotype of $\mathrm{CD}$ to diagnosis according to the Montreal classification were collected

Results: Among 247 patients with CD; 90 had a median SD of 03 months. A DD $\geq 7$ months was considered a diagnostic delay observed in most patients is 157 . In univariate and multivariate analysis at diagnosis, the female sex $(54.25 \%)$, young age $(37.24 \%)$, absence of emaciation $(27 \%)$, presence of extra-digestive manifestations $(25.91 \%)$ and Isolated lesions (L1) (34\%) and penetrating phenotype (B3) $(22.67 \%)$ were associated with anopenial lesions $(27.12 \%)$. Diagnostic delay. Socio-demographic characteristics were not associated with delayed diagnosis. Conclusion: This study shows that most of the patients, $63.56 \%$ have a diagnostic delay significantly associated with the female sex, The young age, the absence of weight loss and a localization of the disease limited to the hail the penetrating phenotype of disease. No socioeconomic variables or reflective of access to care were found to influence.

Disclosure of Interest: All authors have declared no conflicts of interest.

\section{Reference}

https://www.ecco-ibd.eu/index.php/publications/ecco-guidelines-science.html http://www.ibdcohort.ch

\section{P1646 TUBERCULOSIS IN INFLAMMATORY BOWEL DISEASE UNDER TUMOUR NECROSIS FACTOR ALPHA ANTAGONIST-THE RISK BEYOND SCREENING}

L. Carvalho, M.A. Túlio, J. Carmo, J. Rodrigues, T. Bana, E Costa, C. Chagas Gastrenterology, Centro Hospitalar Lisboa Ocidental, Lisboa/Portugal

Contact E-mail Address: lilipcarvalho@gmail.com

Introduction: Tumour necrosis factor alpha antagonist (anti-TNF $\alpha$ ) has revolutionized the treatment of the inflammatory bowel disease (IBD). Considering that it plays a central role in immune-mediated modulation, there are some obvious concerns about safety. There is evidence that it increases the risk of opportunistic infections such as tuberculosis, particularly latent infection reactivation. Due to global high incidence of tuberculosis and its commonly severity in immunocompromised patients (extrapulmonary and disseminated pattern), the exclusion of latent tuberculosis (LT) infection is currently part of the screening prior to starting biologic therapy. Despite negative screening, the risk of tuberculosis infection remains active during the immunomodulation therapy. However, only a few cases of life-threatening disseminated tuberculosis have been reported in immunocompromised patients probably related to the increased use of higher accuracy screening tests, such as interferon-gamma release assays (IGRA). Negative screening cases with IGRA are not described. 
Aims \& Methods: We intend to know the incidence of tuberculosis in IBD patients under anti-TNF $\alpha$ therapy in a single tertiary referral centre, analyzing the tuberculosis screening methods and demographic characteristics. IBD patients treated with anti-TNF $\alpha$ therapy between January 2000 and December 2016 were retrospectively analyzed.

Results: During this period 166 patients received anti-TNF $\alpha$ therapy. Before antiTNF $\alpha$ treatment, screening for LT was performed through medical history, chest X-ray, tuberculin skin test (TST) and/or IGRA. Forty-two patients $(25 \%)$ had positive screening and received tuberculosis prophylaxis prior anti-TNF $\alpha$ therapy. Seven patients $(4.2 \%)$ developed tuberculosis while under anti-TNF $\alpha$ treatment (four women, mean age $44+/-7$ years and mean IBD duration $10+/-8$ years). Six of them had a negative LT screening (methods: 4 TST and 2 IGRA) and one patient had positive TST screening, been treated with isoniazid before starting anti-TNF $\alpha$ therapy. During screening three patients were under immunosuppressive and one under corticosteroid therapy. In the IGRA negative screening patients, the diagnosis of tuberculosis occurred within the first 10 weeks after starting anti-TNF $\alpha$. There were five cases of miliary tuberculosis and two of pulmonary disease. Despite difficult diagnosis, all patients were treated successfully, six of whom needed hospitalization.

Conclusion: In our centre the incidence of tuberculosis in IBD patients under anti-TNF $\alpha$ therapy was $4.2 \%$ and most of them presenting with a severe disease pattern. The therapeutic regime of tuberculosis was effective and no mortality was recorded. All this patient had a previously negative screening, two of them with IGRA, been considered a high sensitivity and specificity screening method. Therefore, a surveillance strategy for IBD patients with anti-TNF $\alpha$ therapy is needed.

Disclosure of Interest: All authors have declared no conflicts of interest.

\section{References}

Abreu C, Magro F, Santos-Antunes J, Pilão A, Rodrigues-Pinto E, Bernardes J, et al. Tuberculosis in anti-TNF $\alpha$ treated patients remains a problem in countries with an intermediate incidence: analysis of 25 patients matched with a control population. J Crohns Colitis. 2013;7:e486-92, http://dx.doi.org/10.1016/ j.crohns.2013.03.004

Beglinger C, Dudler J, Mottet C, Nicod L, Seibold F, Villiger PM, et al. Screening for tuberculosis infection before the initiation of an anti-TNF-alpha therapy. Swiss Med Wkly 2007;137:620-2.

Onal IK, Kekilli M, Tanoglu A, Erdal H, Ibis M, Arhan M. Tuberculosis and Crohn's Disease Revisited. J Coll Physicians Surg Pakistan. 2015;25(6):443-448. Van Assche G, Dignass A, Reinisch W, et al. The second European evidencebased Consensus on the diagnosis and management of Crohn's dissesse: Definitions and diagnosis. J Crohn's Colitis. 2010;4(1):7-27.

Targownik LE, Bernstein CN. Infectious and malignant complications of TNF inhibitor therapy in IBD. Am J Gastroenterol. 2013;108(12):1835-1842, quiz 1843 .

\section{P1647 CAN A PATIENT RATE THE ACTIVITY OF THEIR CROHN'S DISEASE THROUGH A MOBILE APP? THE MEDICROHN STUDY}

A. Echarri Piudo ${ }^{1}$, I. Vera Mendoza ${ }^{2}$, J. Guardiola $^{3}$, V. Ollero ${ }^{1}$, B. Castro ${ }^{4}$, F. Gallego ${ }^{5}$, M. Aguas ${ }^{6}$, M. Chaparro ${ }^{7}$, D. Ceballos ${ }^{8}$, C. Arajol ${ }^{3}$, P. Robledo Andrés $^{9}$, S. Riestra ${ }^{10}$, I. Marín-Jiménez ${ }^{11}$, P. Nos $^{12}$, S. García-López ${ }^{13}$

${ }_{1}^{1}$ Gastroenterology, Complejo Hospitalario de Ferrol, Ferrol/Spain

${ }^{2}$ Hospital Puerta de Hierro, Madrid/Spain

${ }^{3}$ Gastroenterology, Hospital Universitari de Bellvitge, Hospitalet de Llobregat/ Spain

${ }^{4}$ Hospital Marqués de Valdecilla, Cantabria/Spain

${ }^{5}$ Hospital de Poniente, Almeria/Spain

${ }^{6}$ Hospital La Fe, Valencia/Spain

${ }^{7}$ Gastroenterology, Hospital Universitario de La Princesa, Madrid/Spain

${ }^{8}$ Departamento De Gastroenterología, Hospital Universitario Dr. Negrin, Las

Palmas/Spain

${ }^{9}$ Hospital San Pedro de Alcantara, Caceres/Spain

${ }^{10}$ Gastroenterology, Hospital Central de Asturias, Oviedo/Spain

${ }^{11}$ Departamento De Gastroenterología, Hospital Gregorio Marañón, Madrid/Spain

${ }^{12}$ Gastroenterology, Hospital Universitario y Politécnico de La Fe and CIBERehd, Valencia/Spain

${ }^{13}$ Department Of Gastroenterology, Hospital Universitario Miguel Servet,

Zaragoza/Spain

Contact E-mail Address: ana.echarri.piudo@sergas.es

Introduction: The MediCrohn study was designed to evaluate the level of agreement between the Harvey Bradshaw Index (HBI) translated into a patient-based questionnaire completed through a mobile app, and the original HBI questionnaire assessed by the clinician (considered as reference)

Aims \& Methods: Patients completed the HBI score through a mobile app designed for both Android and iPhone devices and thereafter $(<48 \mathrm{~h}$ later), the questionnaire was completed onsite by the gastroenterologist who was blinded for the patients' responses. We assessed agreement between HBI scores of the clinician and patient on the total sum score and per item. HBI score $<5$ was considered as inactive disease.

Results: 135 patients participated in the study and completed the HBI trough a mobile app (mean age: $36 \pm 8$ years, $58 \%$ women). The proportion of agreement between clinician and patient assessment, both evaluating $\mathrm{CD}$ as active or in remission was $91.1 \%$. Only in 12 cases $(11 \%)$, the patient classified CD as active, whereas the physician evaluated it as inactive. No active cases remained undetected by the patient evaluation. Sensitivity, specificity, positive and negative predictive values are shown in Table. The highest agreement was seen for the questions: "abdominal mass" and "general well-being" whereas "number of liquid stools per day" was the item with the lowest agreement.

\begin{tabular}{|c|c|c|c|c|}
\hline Patient Rate & $\begin{array}{l}\text { Clinician } \\
\text { Assessment }\end{array}$ & $\begin{array}{l}\text { Clinician } \\
\text { Assessment }\end{array}$ & Total & \\
\hline & Active & Remission & & \\
\hline Active & 26 & 12 & 38 & \\
\hline Remission & 0 & 97 & 97 & \\
\hline Total & 26 & 109 & 135 & \\
\hline Sensitivity ( $\%)$ & Specificity $(\%)$ & $\operatorname{PPV}(\%)$ & NPV (\%) & Agreement \\
\hline 100 & 89 & 68 & 100 & $91.1 \% \mathrm{CI}(95 \%)$ \\
\hline
\end{tabular}

Conclusion: The HBI score self-administered by the patient through a mobile app resulted in a high percentage of agreement with the gastroenterologist evaluation, and high negative predictive value for disease activity. Results of the MediCrohn study encourage the use of this mobile app and gives some hints on its conditions of use as a support for the involvement of patients in the management of their disease. Future studies will help to define its precise role in clinical practice. Disclosure of Interest: All authors have declared no conflicts of interest.

\section{References}

1. Aguas M, Del Hoyo J, Bebia $\mathbf{P}$ et al. Telemedicine in Inflammatory Bowel Disease: Opportunities and aproaches. Inflamm Bowel Dis 2015; 21: 392-399.

2. Marín-Jimenez I, Nos P, Domenech E et al. Diagnostic performance of the Simple Clinical Colitis Activity Index self-administered online at home by patients with ulcerative colitis: CRONICA-UC Study.

3. Jackson, B.D et al. Ehealth technologies in inflammatory bowel disease: a systematic review. J. Crohns Colitis 2016; 10: 1103-1121.

4. Bossuyt P, Pouillon L, Peyrin-Biroulet P. Primetime for e-health in IBD? Nat Rev Gastroenterol Hepatol 2017; 14:133-134.

\section{P1648 HISTOLOGICAL ASSESSMENT OF REMISSION IN ULCERATIVE COLITIS: DISCREPANCIES BETWEEN DAILY PRACTICE AND EXPERT OPINION}

P. Kranenburg ${ }^{1}$, T.E.h. Römkens ${ }^{1}$, A. Van Tilburg ${ }^{2}$, C. Bronkhorst ${ }^{3}$, J.P.h. Drenth ${ }^{1}$, I.D. Nagtegaal ${ }^{2}$, F. Hoentjen ${ }^{1}$

${ }^{1}$ Gastroenterology And Hepatology, RadboudUMC, Nijmegen/Netherlands

${ }^{2}$ Pathology, RadboudUMC, Nijmegen/Netherlands

${ }^{3}$ Pathology, Jeroen Bosch Hospital, 's-Hertogenbosch/Netherlands

Contact E-mail Address: pim.kranenburg@radboudumc.n1

Introduction: Histological remission (HR) has become an important treatment target in ulcerative colitis (UC). However, limited data exist on reliability of histological scoring in daily practice, when it comes to assess minor histological abnormalities. We investigated the reproducibility and reliability of UC histological scores in colonic biopsies assessed as HR by a general pathologist in daily practice. Next, we investigated correlations between the initial histological assessment and the expert review by expert gastrointestinal (GI)-pathologists.

Aims \& Methods: We performed a retrospective single-centre study in a tertiary IBD referral centre. Colonic biopsies of UC patients with mucosal healing $(\mathrm{MH})$ throughout the examined colon were included. All biopsies were re-assessed by three blinded GI-pathologists using three histological scoring indexes (Geboes score (GS), Riley score (RS), Harpaz (Gupta) Index (HGI)) and a global visual scale (GVS). We evaluated inter- and intraobserver variation and correlations between scores and initial histological assessment using Cronbach's alpha and Spearman's rho analysis.

Results: We included 270 biopsies from 39 UC patients. The interobserver concordance for all histological indexes was substantial to almost perfect (GS 0.84; RS 0.91, HGI 0.61GVS 0.74). The correlation between the RS and GS was almost perfect $(\mathrm{R}=0.86)$, but no correlation was found between the primary histological assessment and the GS $(0.00)$, the RS $(-0.01)$, the HGI $(0.03)$ and the GVS $(-0.04)$ as scored by expert GI-pathologists.

Conclusion: Available histological scores for UC are reliable with strong mutual correlations in case of limited histological abnormalities. However, the discrepancy between daily practice histological assessment and dedicated GI-pathologists is significant and this may have important implications for the selection process of a unified histological disease activity score in UC.

Disclosure of Interest: All authors have declared no conflicts of interest. 
P1649 EVALUATION OF MODIFIED MAYO ENDOSCOPIC SCORE AND DUBLIN, NEW ENDOSCOPIC SCORES THAT INCORPORATE ULCERATIVE COLITIS EXTENSION, IN THE PREDICTION OF RELAPSE

J. P. Rodrigues ${ }^{1}$, R. Pinho ${ }^{2}$, S. Fernandes ${ }^{2}$, J. Silva ${ }^{2}$, A. Ponte ${ }^{2}$, M. Sousa ${ }^{2}$, J. C. Silva ${ }^{2}$, S. Leite ${ }^{2}$, T. Freitas ${ }^{2}$, L. Proença ${ }^{2}$, A. P. Silva ${ }^{2}$, L. Alberto ${ }^{2}$,

A. Rodrigues ${ }^{2}$, J. Carvalho ${ }^{2}$

${ }^{1}$ Gastroenterology, CHVNG/E, Vila Nova De Gaia/Portugal

${ }^{2}$ Gastroenterology, Centro Hospitalar Vila Nova de Gaia/Espinho, Vila Nova De Gaia/Portugal

\section{Contact E-mail Address: jaimepereirarodrigues@gmail.com}

Introduction: Current endoscopic activity scores for Ulcerative Colitis (UC) do not take into account the extent of mucosal inflammation. Recently, two endoscopic scores that combine the assessment of severity and disease extension were developed, the Modified Mayo Endoscopic Score (MMES) ${ }^{1}$ and Degree of Ulcerative Colitis Burden of Luminal Inflammation (DUBLIN) ${ }^{2}$.

Aims \& Methods: We aimed to evaluate the relation of the scores with disease activity and as predictive factors of clinical relapse. Patients with UC in clinical remission (partial Mayo score $[\mathrm{pMS}] \leq 1$ ) who underwent colonoscopy between January/2010 and December/2013 were included. MMES and DUBLIN scores were calculated. Analytical and histological activity (defined by Geboes score $\geq 3.1$ and Nancy score $=2-4$ ) as well as predictive factors of relapse and relapse-free time were evaluated. Relapse was defined as $\mathrm{pMS} \geq 2$, therapy to induce remission, hospitalization and/or colectomy.

Results: 82 patients were selected, $51.2 \%(\mathrm{n}=42)$ female, mean age $=49.4 \pm 13.7$ years. MMES ranged between $0-13.8$ and DUBLIN between $0-9$. MMES and DUBLIN scores presented good correlation $(\mathrm{r}=0.945, \mathrm{p}<0.001)$. MMES was higher in patients with histological activity defined by Nancy $(3.7 \pm 4.0$ vs. $0.8 \pm 1.5 ; \mathrm{p}<0.001)$ and Geboes $(4.0 \pm 4.2$ vs. $1.3 \pm 2.4 ; \mathrm{p}=0.005)$. DUBLIN was also higher in patients with histological activity defined by Nancy $(1.9 \pm 2.1$ vs. $0.5 \pm 0.8 ; \mathrm{p}=0.001)$ and Geboes $(2.0 \pm 2.3$ vs. $0.7 \pm 1.2$; $\mathrm{p}=0.008)$. There was no significant correlation between both scores and analytical activity. Relapse occurred in $36.6 \%(\mathrm{n}=30)$ of patients, with a cumulative risk of $9.8,18.4,25.9,31.5$ and $42.0 \%$ at $12,24,36,48$ and 60 months, respectively. Mayo Endoscopic Subscore (MES) $(\mathrm{p}<0.001)$, MMES $(\mathrm{p}<0.001)$, DUBLIN $(p<0.001)$, Geboes $(p=0.03)$ and Nancy scores $(p=0.001)$ presented a significant association with relapse. In multivariate analysis, $\operatorname{MES}(\mathrm{OR}=2.32$; $\mathrm{p}<0.001)$, MMES $(\mathrm{OR}=1.19 ; \mathrm{p}<0.001)$ and DUBLIN $(\mathrm{OR}=1.36 ; \mathrm{p}<0.001)$ were predictive of relapse independently from histology. Areas under the ROC curve were 0.71 (MES, $\mathrm{p}<0.001$ ), 0.75 (MMES, $\mathrm{p}<0.001$ ) and 0.74 (DUBLIN $\mathrm{p}=0.001$ ) for prediction of relapse, with MMES significantly higher than MES by a difference of $0.037(0.002-0.072) ; \mathrm{p}=0.03$.

Conclusion: MMES and DUBLIN scores correlate with each other and with histological activity and are independent predictors of relapse. MMES was superior to MES in the prediction of relapse.

Disclosure of Interest: All authors have declared no conflicts of interest.

\section{References}

1. Lobatón T, Bessissow T, De Hertogh G, et al. The Modified Mayo Endoscopic Score (MMES): A New Index for the Assessment of Extension and Severity of Endoscopic Activity in Ulcerative Colitis Patients. J Crohns Colitis. 2015 Oct;9(10):846-52.

2. Rowan CR, Twomey P, Cullen G, et al. Dublin (Degree of Ulcerative Colitis Burden of Luminal Inflammation) Score, a Simple Method to Quantify Inflammatory Burden in Ulcerative Colitis. Poster presentations: Clinical: Diagnosis and outcome (P250), ECCO 2017.

\section{P1650 USEFULNESS OF MAGNETIC RESONANCE ENTEROGRAPHY ON MEDICAL DECISION-MAKING IN PATIENTS WITH INFLAMMATORY BOWEL DISEASE (IBD) AFTER A 1-YEAR FOLLOW-UP: A MULTICENTER STUDY}

L. Ramos ${ }^{1}$, A. Hernandez Camba ${ }^{2}$, I. Rodríguez - Lago ${ }^{3}$, M. Carrillo Palau ${ }^{1}$, L. Cejas $^{2}$, A. Elorza ${ }^{3}$, I. Alonso Abreu ${ }^{1}$, M. Vela ${ }^{2}$, A. Hidalgo ${ }^{3}$, N. AlvarezBuilla $^{1}$, G.E. Rodriguez ${ }^{2}$, M. Aduna ${ }^{4}$, L. Diaz-Florez ${ }^{5}$, Y. Rodriguez ${ }^{2}$, C. Tardillo ${ }^{2}$, D. Eiroa ${ }^{6}$, J.A. Larena ${ }^{4}$, M.S. Garrido ${ }^{6}$, J.L. Cabriada ${ }^{3}$, E. Quintero Carrion ${ }^{7}$

${ }^{1}$ Gastroenterology, Hospital Universitario de Canarias, San Cristobal de La Laguna/Spain

${ }^{2}$ Gastroenterology, Hospital Universitario Nuestra Señora de Candelaria, Santa Cruz de Tenerife/Spain

${ }^{3}$ Gastroenterology, Hospital de Galdakao, Galdakao/Spain

${ }_{5}^{4}$ Osatek, Hospital de Galdakao, Galdakao/Spain

${ }^{5}$ Radiodiagnostic, Hospital Universitario de Canarias, San Cristobal de La Laguna/Spain

${ }^{6}$ Radiodiagnostic, Hospital Universitario Nuestra Señora de Candelaria, Santa Cruz de Tenerife/Spain

${ }^{7}$ Gastroenterology, Hospital Univ. de Canarias, Santa Cruz de Tenerife/Spain

\section{Contact E-mail Address: Dr.alejandrohc@gmail.com}

Introduction: Magnetic resonance enterography (MRE) is an imaging technique recomended to determinate and confirm the extension and activity of Crohńs disease (CD) in the small bowel and discriminate penetrating disease and complications. MRE diagnosis allows to optimize medical treatment in IBD patients. Aims \& Methods: The aim of this study is to evaluate the impact of MRE on medical decision making in IBD patients and determinate the maintenance of this new treatment along the time. Consecutive MRE studies performed in patients with confirmed or suspected Crohn's disease between January 2011 and August 2014 were included in three tertiary centers. Medical charts were retrospectively reviewed. MRE indication, demographic and IBD data were collected at time of MRE. Three months after MRE, medical decision (conservative approach with maintenance therapy, significant change in medical therapy or surgery) was assessed. After twelve months of follow-up, the treatment decided after MRE was reviewed.

Results: A total of 474 MRE studies were performed and indications for MRE were: assesment of small bowel involvement in $40(8.3 \%)$ patients with indeterminate colitis (IC) and $20(4.2 \%)$ with suspected-IBD patients or evaluation of severity and extension of the disease in $414(87.5 \%) \mathrm{CD}$ patients $(232 \mathrm{~F}$; mean age $37 \pm 13$ years). Only 4 patients with suspected-IBD $(4 / 20 ; 20 \%)$ had involvement of small bowel on MRE confirming the $\mathrm{CD}$ diagnosis. Twenty-one patients with IC $(21 / 40 ; 52.5 \%)$ changed the diagnosis to CD. In 199/474 (40.5\%) MRE determinated a change on medical decision and $140(70.3 \%)$ patients modified maintenance treatment because of MRE findings. Of them, $127(63.8 \%)$ underwent "set-up" treatment by prescribing inmunosupressants (IS) $(\mathrm{n}=45)$, antiTNF agents $(n=22)$, anti-TNF escalation $(n=8)$, adding IS to anti-TNF agents $(n=9)$ and changing anti-TNF agents $(n=5)$. In addition, $13(9.2 \%)$ patients underwent "top-down" therapy due to stop IS $(n=7)$, anti-TNF $(\mathrm{n}=3)$ or anti-TNF de-escalation $(\mathrm{n}=3)$. Surgery was indicated on $62(62 /$ $199 ; 31,1 \%$ ) patients after MRE. After one year of follow-up, the medical decision was maintained on $65.4 \%$ (288/440) of patients.

Conclusion: RE is a very helpful tool for the medical management of $\mathrm{CD}$ patients. MRE provides major information to optimize treatment in the long-term of patients with active $\mathrm{CD}$.

Disclosure of Interest: All authors have declared no conflicts of interest.

\section{P1651 CORRELATION BETWEEN THE LÉMANN INDEX AND THE} INFLAMMATORY BOWEL DISEASE- DISABILITY INDEX IN CROHN'S DISEASE

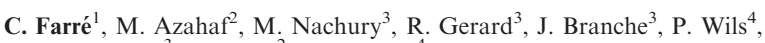
P. Desreumaux ${ }^{3}$, O. Ernst ${ }^{2}$, B. Pariente ${ }^{4}$

${ }^{1}$ Claude Huriez Hospital, Lille/France

${ }^{2}$ Radiology, CHRU Lille, Lille/France

${ }^{3}$ Gastroenterology department, CHRU Lille, Université Lille, Lille/France

${ }^{4}$ Gastroenterology Unit, Hôpital Huriez, Lille University Hospital, Lille/France

Contact E-mail Address: farre.clementine@gmail.com

Introduction: Crohn's disease (CD) is a chronic progressive destructive disease, resulting in cumulative structural bowel damage, which may predict long-term disability. The Lémann Index (LI) has been developed to measure CD-related bowel damage, including bowel surgery, presence of stricturing and penetrating lesions (Pariente and al, Gastroenterology 2015). The first Inflammatory Bowel Disease - Disability Index (IBD-DI) has recently been validated (GowerRousseau, Gut 2015)

Aims \& Methods: The aim of the present study was (1) to identify factors associated with bowel damage and with disability in $\mathrm{CD}$ and (2) to evaluate the correlation between the LI and the IBD-DI. We performed a prospective study in the tertiary referral center of the Claude Huriez Hospital in Lille, from September 2016 to November 2016, including all consecutive CD outpatients. Bowel damage was assessed by the LI calculated according to the published LI protocol. Abdominal and pelvic Magnetic resonance imaging (MRI)s were reviewed and red by the same couple of one gastroenterologist and one radiologist. The IBD-DI was also calculated for all patients. Factors associated with LI and IBD-DI levels were identified by means of bivariate analyses of variance. Results: 130 patients were prospectively and consecutively included. Median age was 34.0 (interquartile range [IQR]: $26.0-46,0$ ) and median disease duration was 10.0 (IQR: 5.0-17.0) years. 65 patients $(50 \%)$ underwent at least one resection surgery. The median LI was 10,8 (IQR: $0.6-17.5)$. Disease duration $(\mathrm{p}<0.0001)$, cumulated anal location $(\mathrm{p}<0,0001)$ and $\mathrm{CD}$ activity $(\mathrm{p}<0.0001)$ were associated with higher LI scores. Median IBD-DI was 25,0 (IQR: 14,7-41,1). Female gender $(p=0,02), C D$ activity $(p<0,0001))$ and current anoperineal lesions assessed by clinical examination and pelvic MRIs $(p=0.001)$ were associated with higher IBD-DI scores. The correlation coefficient between the LI and the IBD-DI was $0,12(-0,05,0,29 ; \mathrm{p}=0,154)$.

Conclusion: In a large cohort of $\mathrm{CD}$ patients from a tertiary referral $\mathrm{CD}$ center, disease duration, anal location and $\mathrm{CD}$ activity are associated with bowel damage assessed by the LI, while female gender, disease activity and current anoperinal lesions are associated with disability assessed by the IBD-DI. Correlation between the LI and the IBD-DI was low.

Disclosure of Interest: All authors have declared no conflicts of interest.

\section{References}

Pariente et al, Gastroenterology 2015

Gower-Rousseau et al, Gut 2015 
P1652 COMPARISON OF CYTOKINES MRNA EXPRESSION IN INFLAMED AND NON-INFLAMED MUCOSA OF PATIENTS WITH INFLAMMATORY BOWEL DISEASE

Z. Lešková ${ }^{1}$, A. Krajcovicova ${ }^{1}$, K. Soltys ${ }^{2}$, S. Stuchlik ${ }^{2}$, I. Sturdik ${ }^{1}$, T. Koller ${ }^{1}$, M. Huorka ${ }^{1}$, T. Hlavaty ${ }^{1}$

${ }^{1} 5$ th Department Of Internal Medicine, Sub Dept. Of Gastroenterology \& Hepatology, Comenius University Faculty of Medicine, University Hospital Bratislava, Bratislava/Slovak Republic

${ }^{2}$ Department Of Molecular Biology, Faculty of Natural Sciences, Comenius University in Bratislava, Bratislava/Slovak Republic

Contact E-mail Address: leskova991@gmail.com

Introduction: The aetiology of Crohn's disease (CD) and ulcerative colitis (UC) is not known. Recent data suggest a different cytokine profile between $C D$ and UC. Aims \& Methods: The aim of this study was to analyse the expression of mRNA of proinflammatory, regulatory anti-inflammatory cytokines, chemokines and their ligands (IL-6,-8,-10,-12, IL-23, TNF $\alpha$, CCR1,- 2-5.-9, IL-10,TLR2,-4,-5, CD 207, -206), and transcription factor FoxP3 in the inflamed and non-inflamed intestinal biopsy samples of mucosa in IBD patients. We performed a crosssectional study. The cohort consisted of 87 consecutive IBD patients (47 CD and 40 UC) who underwent colonoscopy at the IBD centre of University Hospital Bratislava. We took biopsies from non-inflamed and if present also from inflamed mucosa from sigma (CD, UC) and terminal ileum (CD). mRNA was extracted from mucosal biopsy samples, isolated by a RLT buffer and reversely transcribed. We normalized the expression of the target genes to the expression of the house-keeping gene (GAPDH). Finally, we compared the expression of cytokines in inflamed and non-inflamed mucosa separately for CD and UC patients.

Results: In UC patients, we observed higher expression of IL-8 $(\mathrm{p}=0.04)$, IL-23 $(\mathrm{p}=0.019)$ TLR2 $(\mathrm{p}=0.002), \quad$ CCR $1 \quad(\mathrm{p}=0.007)$, CCR2 $(\mathrm{p}=0.037)$, CCR5 $(p \leq 0.001), \operatorname{CD} 206(p=0.011), \operatorname{TNF} \alpha(p=0.002)$ and IL-6 $(p=0.006)$ in the inflamed mucosa from sigma. In CD patients, we observed increased expression of chemokines of CCR1 $(p=0.005)$ and IL- $8(p<0.001)$ in the inflamed mucosa of a terminal ileum and decreased expression of CCL5. Also, in group of patients with $\mathrm{CD}$ we did not observe the difference of the expression of mRNA cytokines between the inflamed and non-inflamed mucosa of sigma.

Conclusion: There was a significant difference in the mRNA cytokine profiles between CD and UC patients.

Disclosure of Interest: All authors have declared no conflicts of interest.

\section{P1653 IMPACT OF THE BALLOON-ASSISTED ENDOSCOPY AND ENDOSCOPIC BALLOON DILATION THERAPY FOR THE SURGICAL RATE OF CROHN'S DISEASE}

T. Nishimoto, K. Mitsui, M. Suzuki, A. Ehara, S. Tanaka, A. Tatsuguchi, S. Fujimori, K. Iwakiri

Dept. Of Gastroenterology, Nippon Medical School, Graduate School of Medicine, Tokyo/Japan

Contact E-mail Address: clubman629@gmail.com

Introduction: The various methods of disease monitoring for Crohn's disease (CD) are available however the quality of those methods differ. High accuracy, low cost, patients-friendly and also physicians-friendly methods are needed. Balloon-assisted enteroscopy (BAE) is labour-intensive method. However, DBE enable a detail inspection and therapeutic capabilities, and could not be substituted for any other modalities. The surgical intervention is one of the poorest outcome in the disease history of CD patients. There is little knowledge whether the monitoring of disease activity and the endoscopic intervention using BAE contribute the preferable outcome of CD.

Aims \& Methods: We conducted a retrospective single-centre cohort study to elucidate whether the on-demand BAE and Endoscopic balloon dilation (EBD) therapy leads to the decreasing of surgery rate for the small bowel and better outcomes for CD patients. Double balloon endoscopy (DBE) examinations were conducted when the attending physicians of each patients were needed. EBD therapy was undergone at the stenosis where the endoscope could not pass and was not contraindication regardless of the presence of symptoms. The 181 examinations of DBE for $55 \mathrm{CD}$ patients since 2004 were enrolled in this study. The primary endpoint is the comparing the differences of surgical rates between using endoscopic balloon dilation therapy and not. The secondary endpoints are cumulative non-surgery rate of CD patients using DBE monitoring and EBD therapy by Kaplan-Meier method.

Results: Subjects were 55 patients, genders were 9 females and 46 males, mean age was 42 years old (SD, 13.0, median 44 range, 22-77). $50(28 \%)$ antegrade, 131 $(72 \%)$ retrograde DBE and $58(32 \%)$ EBD therapy were undergone. EBD was performed in $17(31 \%$, mean 3.4 times per patient) patients. In 17 patients who underwent EBD therapy, only one $(6 \%)$ patient underwent surgery after EBD and the other $16(94 \%)$ of patients did not undergo surgery during follow-up periods (mean 1719 days). In 38 patients who did not undergo EBD therapy, 7 $(18 \%)$ patients underwent surgery during the follow-up periods (mean 1659 days). The surgery rate of EBD group was approximately three times lower than non-EBD group. Cumulative non-surgery rate of $\mathrm{CD}$ patients who were monitored using DBE in on-demand manner were $85 \%$ in 3 years and $82 \%$ in 5years. These results may mean that non-EBD group consist of two major subgroups that one was the patients who had complex strictures that did not meet the indication for EBD and the early surgery was needed, and the other was the patients whose small bowel lesions were mild

Conclusion: In patients who underwent EBD, the necessity for surgery was smaller. Furthermore, in patients who underwent DBE monitoring, the cumulative non-surgery rate was relatively higher than historical control. DBE may show the future necessity for surgery and discriminate the necessity for the strict monitoring from mild or moderate monitoring that leads to better quality of life for CD patients. The scheduled monitoring using enteroscopy would be a further issue to clarify.

Disclosure of Interest: All authors have declared no conflicts of interest.

\section{References}

Hirai F, Beppu T, Takatsu N, Yano Y, Ninomiya K, Ono Y, Hisabe T, Matsui T. Long-term outcome of endoscopic balloon dilation for small bowel strictures in patients with Crohn's disease. Dig Endosc. 2014 Jul;26(4):545-51. PMID: 24528293.

Sunada K, Shinozaki S, Nagayama M, Yano T, Takezawa T, Ino Y, Sakamoto H, Miura Y, Hayashi Y, Sato H, Lefor AK, Yamamoto H. Long-term Outcomes in Patients with Small Intestinal Strictures Secondary to Crohn's Disease After Double-balloon Endoscopy-assisted Balloon Dilation. Inflamm Bowel Dis. 2016 Feb;22(2):380-6. PMID: 26535767.

\section{P1654 THE RELATIONSHIP OF NEUTROPHILIC AND ENDOTHELIAL ACTIVITY MARKERS WITH THE DISEASE ACTIVITY IN INFLAMMATORY BOWEL DISEASE}

S. Boga ${ }^{1}$, A.R. Koksal ${ }^{1}$, H. Alkim ${ }^{1}$, A. A. Ozagari ${ }^{2}$, I. Sen ${ }^{1}$, M. Sahin ${ }^{1}$, C. Alkim

${ }^{1}$ Gastroenterology, Sisli Hamidiye Etfal Education and Research Hospital, Istanbul/Turkey

${ }^{2}$ Pathology, Sisli Hamidive Etfal Education and Research Hospital, Istanbul Turkey

Contact E-mail Address: salihboga@yahoo.com

Introduction: Neutrophil gelatinase-associated lipocalin (NGAL) is a protein that is secreted by neutrophils in increased amounts in inflammatory disorders and its level reflects neutrophilic activity. Endoglin is the receptor and modulator of endothelial TGF- $\beta$ and it is responsible for the endothelial activation secondary to inflammation. Although the increase in endoglin expression with the inflammation in the colonic mucosa of mice was shown before, its relationship with the disease activity in inflammatory bowel disease (IBD) is not searched yet. The relationship of serum NGAL and serum and colonic endoglin levels in ulcerative colitis (UC) and Crohn's disease (CD) patients were investigated in this study. Aims \& Methods: Eighty-six UC, 63 CD, 36 non-IBD (screening, irritable bowel syndrome) and 52 healthy controls who were followed up in Gastroenterology Department of Sisli Hamidiye Etfal Education and Research Hospital between years 2015-2016 were enrolled. Patients were evaluated by endoscopic (Rachmilewitz index for UC, simple endoscopic score for CD (SES-CD) for CD) clinical (clinical colitis activity index (CCAI) for UC and CD activity index (CDAI) for CD), and pathologic activity scores and immuhistochemical staining.

Results: There were no differences between UC and CD patients in terms of serum endoglin and NGAL levels. NGAL and endoglin levels were significantly higher in endoscopically active UC group $(\mathrm{n}=59)(142.8 \pm 68.7 \mathrm{ng} / \mathrm{mL}$ and $546.0 \pm 148.8 \mathrm{pg} / \mathrm{mL})$ compared to inactive UC $(\mathrm{n}=27)(119.7 \pm 26.3 \mathrm{ng} / \mathrm{mL}$ and $476.9 \pm 134.2 \mathrm{pg} / \mathrm{mL})$, to non-IBD $(115.8 \pm 27.2 \mathrm{ng} / \mathrm{mL}$ and $460.6 \pm 103.2 \mathrm{pg} / \mathrm{mL})$ and to controls $(116.7 \pm 31.7 \mathrm{ng} / \mathrm{mL}$ and $457.2 \pm 114.1 \mathrm{pg} /$ $\mathrm{mL})$. Although there were limited number of inactive $C D$ patients $(\mathrm{n}=11)$, serum NGAL and endoglin levels were significantly higher in endoscopically active CD group $(\mathrm{n}=52)(135.0 \pm 28.9 \mathrm{ng} / \mathrm{mL}$ and $555.6 \pm 133.6 \mathrm{pg} / \mathrm{mL})$ compared to inactive CD $(115.2 \pm 35.9 \mathrm{ng} / \mathrm{mL}$ and $458.7 \pm 132.8 \mathrm{pg} / \mathrm{mL})$, to non-IBD and to controls (Figure). Stricturing and fistulizing CD groups had significantly higher endoglin levels compared to inflammatory $C D(p<0.001$ and $p=0.001)$. NGAL levels were significantly increasing with the increasing disease extention in $\mathrm{UC}$ and $\mathrm{CD}(\mathrm{p}=0.012$ and $\mathrm{p}<0.001)$. While the clinical activity subgroups were evaluated, there were significant difference in UC and CD patients in terms of endoglin levels but not NGAL levels (Endoglin: $p=0.002 p=0.574$, NGAL: $\mathrm{p}=0.020, \mathrm{p}=0.171)$. Endoglin levels were more strongly correlated with the pathologic activity scores in both UC and CD groups compared to NGAL levels (Endoglin: $r=0.443, p<0.001 ; r=0.453, p<0.001$ NGAL: $r=0.274$ $\mathrm{p}=0.011 ; \mathrm{r}=0.409, \mathrm{p}=0.001)$ The immunohistochemical staining index of endoglin showed positive correlation with the immunohistochemical staining of vascular endothelial growth factor (VEGF) (UC $\mathrm{r}=0.486, \mathrm{p}<0.001 ; \mathrm{CD} r=0.383$, $\mathrm{p}=0.002$ ). The immunohistochemical staining index of endoglin in the colonic mucosa was correlated with the serum levels of endoglin in both UC and CD patients (UC r $=0.641, \mathrm{p}<0.001$; CD r $=0.437$, $\mathrm{p}<0.001$ )

Conclusion: The present study highlights significant associations between endoglin and NGAL and IBD presence and activity, and demonstrates elevated serum and colonic endoglin levels in patients with active IBD as a novel finding. Disclosure of Interest: All authors have declared no conflicts of interest. 
P1655 MONITORING OF LABORATORY PARAMETERS DURING THIOPURINE MAINTENANCE THERAPY IN PATIENTS WITH INFLAMMATORY BOWEL DISEASE: AN UNNECESSARY BURDEN?

J. E. Kreijne ${ }^{1}$, A. C. De Vries ${ }^{1}$, G. Bouma ${ }^{2}$, G. Dijkstra ${ }^{3}$, R. West ${ }^{4}$, M.J. Pierik ${ }^{5}$, D. J. De Jong ${ }^{6}$, N. K.H. De Boer ${ }^{2}$, CJ. Van Der Woude ${ }^{1}$

${ }^{1}$ Gastroenterology And Hepatology, Erasmus University Medical Center, Rotterdam/Netherlands

${ }^{2}$ Gastroenterology And Hepatology, VU University Medical Center, Amsterdam/ Netherlands

${ }^{3}$ Gastroenterology And Hepatology, University Medical Center Groningen, Groningen/Netherlands

${ }^{4}$ Gastroenterology, Sint Franciscus Gasthuis \& Vlietland, Rotterdam/Netherlands

${ }^{5}$ Gastroenterology And Hepatology, Maastricht University Medical Center, Maastricht/Netherlands

${ }^{6}$ Gastroenterology And Hepatology, Radboud University Medical Center, Nijmegen/Netherlands

\section{Contact E-mail Address: j.kreijne@erasmusmc.nl}

Introduction: Although thiopurine-induced myelotoxicity and hepatotoxicity rarely occur during maintenance thiopurine therapy for inflammatory bowel disease (IBD), current guidelines advise laboratory monitoring every 3 months. This study was performed to assess the current laboratory monitoring regime in thiopurine maintenance therapy with regards to consequences of myelotoxicity and hepatotoxicity.

Aims \& Methods: In this multicenter cohort study, we evaluated adult IBD patients with quiescent disease who were on maintenance thiopurine therapy between 2000-2016. Data collection started after 12 consecutive months of thiopurine treatment. The primary outcome was therapy adjustment, i.e. therapy cessation or dose reduction, due to myelotoxicity (leukocyte count $<4.010 \mathrm{e} 9$ 1, platelet count $<15010 \mathrm{e} 9 / 1$ ) and/or hepatotoxicity (alkaline phosphatase (AP), gamma-glutamyltransferase $(\gamma$-GT), alanine aminotransferase (ALT) and/or aspartate aminotransferase (AST) above the upper limit of normal (ULN)). The secondary outcomes were prevalence of myelotoxicity and hepatotoxicity and additional diagnostic procedures due to this toxicity.

Results: This study included 223 IBD patients $(55 \%$ female, $64 \%$ with Crohn's disease, mean age at diagnosis 27.2 years (SD 11,5)). Median follow-up was 3.2 years (IQR 1.9-4.7). The mean monitoring frequency was 3.3 assessments per treatment year (SD 1.8). Toxicity was observed in 445/2402 laboratory assessments $(18.5 \%)$ in 120 patients. In total, $20(0.8 \%)$ therapy adjustments were performed and 25 laboratory assessments $(1.0 \%)$ led to additional diagnostic procedures. Myelotoxicity, observed in 244 assessments, led to 11 dose reductions and in 3 patients therapy was stopped. For hepatotoxicity, observed in 201 assessments, 2 dose reductions were performed and in 4 patients therapy was stopped. Ninety percent of observed toxicity were mild leukopenia (leukocyte count $3.0-4.0)$ or mild hepatotoxicity $(<2 \mathrm{ULN})$, primarily in the first years of treatment. Dose adjustments were more often associated with moderate leukopenia (leukocyte count $<3.0)$ than with mild leukopenia $(p<0.01)$. In total, 2 complications were recorded, 1 patient was hospitalized because of pancytopenia and received red blood cell transfusion, and 1 patient was treated for a CMV infection. Both patients presented with symptoms in clinic with preceding normal laboratory values. No mortality due to thiopurine-induced toxicity was observed. Conclusion: Although mild toxicity is common during maintenance thiopurine therapy, adjustments based on laboratory assessments are rare. Therefore, a less intensive regime to monitor thiopurine-induced toxicity should be considered.

Disclosure of Interest: N.K.H. de Boer: Nanne de Boer has received a research and travel grant from TAKEDA outside the submitted work and served as principal investigator and consultant for TEVA.

CJ. van der Woude: CJW has served as a speaker and a consultant for Abbot, Abbvie, MSD and as a consultant for Shire and received funding from Janssen Biologics BV.

All other authors have declared no conflicts of interest.
P1656 ULTRASOUND ELASTICITY IMAGING PREDICTS

THERAPEUTIC OUTCOMES IN PATIENTS WITH CROHN'S DISEASE TREATED WITH ANTI-TUMOR NECROSIS FACTOR ANTIBODIES

S. Orlando, M. Fraquelli, M. Coletta, F. Branchi, A. Magarotto, C.B. Conti, S. Mazza, D. Conte, G. Basilisco, F. Caprioli Gastroenterology And Endoscopy Unit, Fondazione IRCCS CaGrande Ospedale Maggiore policlinico di Milano, Milano/Italy

Contact E-mail Address: stefania.orlando86@gmail.com

Introduction: Intestinal fibrosis represents one of the main sources of morbidity for patients with Crohn's disease (CD), as its onset is associated with the development of CD-related complications which increase the likelihood of hospitalization and surgery ${ }^{1}$. Ultrasound elasticity imaging (UEI) is a non-invasive ultrasonographic technique developed to evaluate tissue fibrosis by measuring tissue strain after application of a force ${ }^{2}$. We have recently demonstrated that UEI can reliably detect severe ileal fibrosis in patients with Crohn's disease ${ }^{3}$. We therefore hypothesized that a more severe range of fibrosis might influence the therapeutic response to anti-tumor necrosis factor (TNF) treatment.

Aims \& Methods: The aim of this explorative study was to assess the ability of UEI to predict therapeutic outcome in active CD patients treated with anti-TNF antibodies. 30 patients with ileal or ileocolonic CD ( 20 males, age $38.8 \pm 14.5)$ initiating anti-TNF treatment were enrolled in the study. All patients completed the induction phase and underwent scheduled maintenance therapy with antiTNF for $16.1 \pm 8.5$ months. Patients underwent bowel ultrasound and UEI at baseline and 14 weeks after initiation of anti-TNF. Bowel wall stiffness at UEI was quantified by calculating the strain ratio (SR) between the bowel wall and the surrounding mesenteric tissue. Receiver operating characteristic curve analysis was used to identify the best SR cut off able to predict surgery/bowel obstruction. Transmural healing at 14 weeks was defined as a bowel wall thickness $\leq 3 \mathrm{~mm}$. Frequency of CD-related surgeries or hospitalizations due to bowel obstruction was recorded during the follow-up. The data are given as mean values \pm standard deviation

Results: Five patients $(16.6 \%)$ underwent surgery or hospitalization for bowel obstruction during the follow up. Frequency of CD-related surgeries or hospitalizations was significantly greater in patients with $S R \geq 2$ at baseline than in patients with $\mathrm{SR}<2(\mathrm{p}=0.02)$. A significant reduction in bowel thickness was observed after 14 weeks of anti-TNF treatment (from $5.8 \pm 1.5 \mathrm{~mm}$ to $5.1 \pm 1.7 \mathrm{~mm}, \mathrm{p}<0.005)$, while $\mathrm{SR}$ values remained unaltered (1.5 vs 1.3 , $\mathrm{p}=0.5$ ). A significant inverse correlation was observed between values of strain ratio at baseline and thickness variations following anti-TNF therapy $(\mathrm{p}=0.007)$. Eight out of 30 patients $(27 \%)$ achieved transmural healing at 14 weeks. Baseline SR was significantly lower in patients with transmural healing than in patients not achieving this endpoint $(1.06 \pm 0.16$ vs $1.67 \pm 0.17, p<0.05)$. Conclusion: This explorative study shows that UEI is be able to predict therapeutic outcomes, including CD-related surgeries and transmural healing, in patients with Crohn's disease treated with anti-TNF therapy.

Disclosure of Interest: All authors have declared no conflicts of interest.

\section{References}

1. Jeuring SF, van den Heuvel TR, Liu LY, et al. Improvements in the LongTerm Outcome of Crohn's Disease Over the Past Two Decades and the Relation to Changes in Medical Management: Results from the Population-Based IBDSL Cohort. Am J Gastroenterol 2017;112:325-336.

2. Kim K, Johnson LA, Jia C, et al. Noninvasive ultrasound elasticity imaging (UEI) of Crohn's disease: animal model. Ultrasound Med Biol 2008;34:90212.

3. Fraquelli M, Branchi F, Cribiu FM, et al. The Role of Ultrasound Elasticity Imaging in Predicting Ileal Fibrosis in Crohn's Disease Patients. Inflamm Bowel Dis 2015;21:2605-12. 


\section{P1657 CLINICAL CHARACTERISTICS OF RECTAL-SPARING}

\section{ULCERATIVE COLITIS}

A. Ito, O. Teppei, T. Katutoshi

Dept. Medicine, Inst. Gastroentelorgy, Tokyou Womens Medical University, Tokyo/Japan

Contact E-mail Address: itoayumi@ige.twmu.ac.jp

Introduction: Ulcerative colitis (UC) generally involves the entire large intestine extending from the rectum to the ileocecal junction. However, some patients with moderate or severe UC lack any obvious rectal involvement (known as rectalsparing [RS]-UC).

Aims \& Methods: In this study, we evaluated the differences in the clinical characteristics and disease course between patients with refractory UC with or without RS. Of the 437 inpatients with refractory UC who achieved remission between April 2001 and September 2016 (follow-up period: 915 \pm 53 days, mean \pm SD), 57 patients were classified as RS-UC and 340 patients without RS (standard [S]-UC group). Patients of the two groups were compared for gender, age at onset, site of involvement, disease duration, pretreatment clinical activity index (CAI, Lichtiger score), Hb, C-reactive protein (CRP), total dose of prednisolone (PSL) before the achievement of remission, duration of hospitalization, endoscopic scores (Mayo and UCEIS), and relapse rates (at 100, 300, 500, and 1000 days post-remission). Patients with RS were defined as those without any detectable rectal inflammation despite not receiving local treatment, such as rectal enema/infusion or suppositories. Remission was defined as CAI of $\leq 4$. Relapse was defined as the need to restart remission induction therapy, such as intensive intravenous PSL, initiation of treatment with biological agent, or readministration and dose escalation of tacrolimus to achieve a high blood trough level ( $\geq 10 \mathrm{ng} / \mathrm{dl})$.

Results: RS was observed in $57(10.4 \%)$ patients. There were significant differences in CRP (RS-UC: $5.1 \pm 6.02$, S-UC: $2.3 \pm 3.4 \mathrm{mg} / \mathrm{L}$ ) and pretreatment endoscopic scores (Mayo: RS-UC: $2.7 \pm 0.3$, S-UC: $2.4 \pm 0.5$; UCEIS: RS-UC: $6.1 \pm 1.3$, S-UC: $5.7 \pm 1.4)$ between the two groups $(\mathrm{P}<0.05)$. There was no significant difference in the relapse rate at 100 days after remission (RS-UC: $18 \%$, S-UC: $15 \%$ ). However, the two groups showed significant differences in the relapse rates at 300 days (RS-UC: $38 \%$, S-UC: $17 \%$ ), 500 days (RS-UC: 57\%, S-UC: $48 \%$ ), and 1000 days (RS-UC: $77 \%$, S-UC: $62 \%$ ) after remission. Conclusion: Our results showed that RS is not an uncommon finding among patients with UC. Based on the higher CRP, endoscopic score, and relapse rates in the RS group, than the standard UC group, we recommend aggressive treatment of UC patients with RS.

Disclosure of Interest: All authors have declared no conflicts of interest.

\section{P1658 EVALUATION OF COLONIC MUCOSA WITH FLEXIBLE SPECTRAL IMAGING COLOR ENHANCEMENT (FICE) IN PATIENTS WITH LONG TERM ULCERATIVE COLITIS DURING DYSPLASIA SCREENING}

G. Bengi ${ }^{1}$, S. Kaya Özdinç ${ }^{1}$, H. Akpınar ${ }^{1}$

${ }^{1}$ Gastroenterology, Dokuz Eylul University Hospital, İzmir/Turkey

\section{Contact E-mail Address: sedozdinc@yahoo.com}

Introduction: Ulcerative colitis (UC) associated colorectal cancer risk (CRC) is related to the age of onset and duration and anatomic extent of the disease. The risk of malignancy increases with the duration of the disease (1). Current guidelines recommend beginning the surveillance colonoscopy after eight to ten years of disease; random biopsies should be obtained from 4 quadrants of every $10 \mathrm{~cm}$ of the entire colon. In addition, any suspicious areas should be biopsied (2). Recent endoscopic imaging technologies provide a more detailed visualization of the superficial microstructure of the mucosa and vascularity. Thus it is possible to get targeted biopsies. The purpose of this study is; to evaluate the image patterns of dysplastic changes in ulcerative colitis, and their histopathological correlation, by using a virtual chromoendoscopy technique, FICE and to investigate the effectiveness of this technique.

Aims \& Methods: The purpose of this study is; to evaluate the image patterns of dysplastic changes in ulcerative colitis, and their histopathological correlation, by using a virtual chromoendoscopy technique, FICE and to investigate the effectiveness of this technique. Eighteen follow-up patients of our inflammatory bowel disease polyclinic, with UC at least 8 years of disease history and who are in clinical remission are included to this study. Screening colonoscopy was performed to the patients included in the study. Entire colon and especially dysplasia suspected areas visualised with standart endoscopy first, and then with FICE; random and targeted biopsies were taken. Image patterns were compared with histopathologic diagnosis. Normal, colitis and polyps images acquired by FICE, were evaluated by seven endoscopists and statistical analysis was performed.

Results: In a total of 18 patients, by evaluating 123 colonic segments, 1831 images were taken. 1652 image acquired by FICE, and 179 were white light images. Images taken by FICE separately, from each areas of normal colonic mucosa, polipoid lesions and colitis. A total 584 biopsies were taken, $492(84.2 \%)$ random and $92(15.7 \%)$ targeted biopsies. While there were no dysplasia in random biopsies, low-grade dysplasia was detected in 3 diminutive polyps taken as targeted biopsy. The analysis of the FICE images by voting by seven endoscopists showed that, the best imaging channels are; 2, 6, 9 for normal mucosa; 3, 7, 9 for polyps; and 2, 3, 9 for colitis. When all the images are analyzed, channels 2 and 9, were found to be significantly different from other channels. When the most rated channels, 2 and 9, were compared to the WLE, the FICE image is better for evaluation of the mucosal and vascular structure. However, there were no significant difference between channels 2 and 9.
Conclusion: There were no significant superiority of FICE, in dysplasia screening. Consistent with the literature, this study showed us that FICE is effective in detecting diminutive polyps, and evaluating surface patterns without magnification. In clinical remission in patients with UC, FICE can have a role in the assessment of the severity of inflammation. For this purpose, more clinical trials are needed. Our study is the first study with FICE in UC. Channels 2 and 9 are the best image channels of FICE in UC.

Disclosure of Interest: All authors have declared no conflicts of interest.

\section{References}

Eaden JA, Mayberry JF. Guidelines for screening and surveillance of asymptomatic colorectal cancer in patients with inflammatory bowel disease. Gut 2002;51 Suppl 5:V10-V12.

Eaden JA, Abrams KR, Mayberry JF. The risk of colorectal cancer in ulcerative colitis:a meta-analysis. Gut 48(4), 526-535 (2001).1

\section{P1659 DYSBIOSIS OF THE GUT MICROBIOTA IN RELATION TO DISEASE ACTIVITY IN INFLAMMATORY BOWEL DISEASE}

P. Ricanek ${ }^{1}$, R. Kalla ${ }^{2}$, Y. Ber ${ }^{3}$, S. Vatn ${ }^{1}$, M. K. Karlsson ${ }^{4}$, L. Finnby ${ }^{4}$, D. Bergemalm ${ }^{5}$, A. Carstens ${ }^{5}$, J.D. Söderholm ${ }^{6}$, J. Jahnsen ${ }^{7}$, F. Gomollon ${ }^{3}$, J. Halfvarson ${ }^{5}$, J. Satsangi ${ }^{8}$, C. Casén ${ }^{4}$, M.H. Vatn ${ }^{9}$

${ }_{1}^{1}$ Department Of Gastroenterology, Akershus University Hospital Centre for

Molecular Biology, Neuroscience and Gastroenterology, Oslo/Norway

${ }^{2}$ CGEM, University of Edinburgh, Edinburgh/United Kingdom

${ }^{3}$ Gastroenterology, Hospital Clinico Lozano Blesa, Zaragoza/Spain

${ }^{4}$ Clinical Dept., Genetic Analysis AS, Oslo/Norway

${ }^{5}$ Dept. Of Gastroenterology, Faculty Of Medicine And Health, Örebro University, Örebro/Sweden

${ }^{6}$ University Hospital, Linköping University, Linköping/Sweden

${ }^{7}$ Dept. Of Gastroenterology, Akershus University Hospital, Lorenskog/Norway

${ }^{8}$ University Of Edinburgh, Gastrointestinal Unit, Edinburgh/United Kingdom

${ }^{9}$ Inst. Of Clinical Medicine, University of Oslo, Oslo/Norway

Contact E-mail Address: petr.ricanek@medisin.uio.no

Introduction: The gut microbiome is thought to be relevant to the pathogenesis of inflammatory bowel disease (IBD). We aimed to explore associations between measures of gut microbiota and clinical as well as inflammatory disease activity in an inception cohort of treatment-naïve IBD patients as well as with inflammatory activity in symptomatic non-IBD patients and healthy controls. The term 'dysbiosis' expresses alterations in the gut microbial community.

Aims \& Methods: Patients were diagnosed according to international criteria, including endoscopic and histopathologic assessment. Clinical disease activity in Crohn's Disease (CD) patients was measured by the Harvey-Bradshaw index (HBI), and in ulcerative colitis (UC) patients by the Simple Clinical Colitis Activity Index (SCCAI). Inflammatory activity was assessed by CRP and faecal calprotectin (FCal), (fCAL® ELISA, Bühlmann laboratories AG). Stool samples were collected within 60 days prior to and 14 days after the diagnosis, and stored at $-80^{\circ} \mathrm{C}$. Antibiotic treatment within the last two months was an exclusion criterion. Faecal microbiota profiles were generated by 16S rRNA analyses, using the GA-map ${ }^{\mathrm{TM}}$ Dysbiosis Test. Dysbiosis was defined as non, mild or severe (1). Differences in disease activity between levels of dysbiosis severity were analysed using ANOVA at a significance level of $\mathrm{p}<0.05$, and univariate associations between inflammatory activity and logtransformed microbiota profiles were analysed using ANCOVA. P-values corrected for multiple testing, using Benjamini-Hochberg correction, are presented. Results: Data on dysbiosis, bacteria profiles, and FCal were available in $57 \mathrm{CD}$, 80 UC, 12 IBD-U patients and 100 symptomatic non-IBD patients, and 45 healthy controls. CRP was available for $52 \mathrm{CD}, 74 \mathrm{UC}, 10 \mathrm{IBD}-\mathrm{U}$ patients, and 88 symptomatic non-IBD patients. HBI was available for $50 \mathrm{CD}$ patients, while SCCAI was available for 77 UC patients. Disease activity: No association was found between FCal and dysbiosis in UC patients $(\mathrm{P}=0.08), \mathrm{CD}$ patients $(\mathrm{P}=0.22)$, and healthy controls $(\mathrm{P}=0.57)$. However, an association was found between FCal and dysbiosis in symptomatic non-IBD patients $(\mathrm{P}=0.04)$ and in IBD-U $(P=0.005)$. An association was found between CRP and dysbiosis in CD patients $(\mathrm{P}=0.02)$, while not for $\mathrm{UC}$ and symptomatic non-IBD patients. No association was found between $\mathrm{HBI}$ and dysbiosis in $\mathrm{CD}$ patients $(\mathrm{P}=0.23)$, and between SCCAI and dysbiosis in UC patients $(\mathrm{P}=0.32)$. Microbiota: Increasing dysbiosis severity in UC, CD and non-IBD patients yielded lower abundance of Faecalibacterium prausnitzii, and higher abundance of Proteobacteria, a profile typically observed in gut inflammatory conditions. In addition, the commensal bacteria Bifidobacterium yielded lower abundance with increased dysbiosis severity in UC and non-IBD patients, and in combination with elevated levels of FCal and/or CRP in UC patients. In the healthy controls, increasing dysbiosis severity yielded higher abundance of Proteobacteria.

Conclusion: In conclusion, a relationship between faecal dysbiosis in sub-groups of IBD and non-IBD was found, in CD patients also with CRP. Accordingly, gut bacteria profiles and abundance may potentially be used to differentiate between severity in UC and CD patients, as a non-invasive tool to monitor disease activity in IBD.

Disclosure of Interest: M.K. Karlsson: Employee of Genetic Analysis AS

L. Finnby: Emplyee of Genetic Analysis

C. Casén: An employe of Genetic Analysis AS

All other authors have declared no conflicts of interest. 


\section{Reference}

1. Casén et al. Aliment Pharmacol Ther 2015; 42: 71-83

\section{P1660 SIMPLIFIED MR ENTEROCOLONOGRAPHY CLASSIFICATION BASED ON ENDOSCOPIC FINDINGS FOR ACTIVITY ASSESSMENT OF CROHN'S DISEASE}

T. Fujii ${ }^{1}$, Y. Kitazume ${ }^{2}$, K. Takenaka ${ }^{1}$, M. Kimura ${ }^{1}$, E. Saito ${ }^{1}$, K. Matsuoka ${ }^{1}$, M. Nagahori ${ }^{1}$, K. Ohtsuka ${ }^{1}$, M. Watanabe ${ }^{1}$

${ }^{1}$ Gastroenterology And Hepatology, Tokyo Medical and Dental University, Tokyo/ Japan

${ }^{2}$ Radiology, Tokyo Medical And Dental University, Tokyo/Japan

Contact E-mail Address: tfujii.gast@tmd.ac.jp

Introduction: Crohn's disease (CD) is a lifelong inflammatory bowel disease. Evaluating the extent and severity of the disease is critical to determine appropriate therapeutic strategies in patients with Crohn's disease. MR imaging is one of the most recommended technique for detection of large and small bowel lesions of $\mathrm{CD}$. The aim of this study is to establish clinical utility of the 5point MR enterocolonography (MREC) classification for assessing CD activity based on endoscopic findings.

Aims \& Methods: A total of 120 patients ( 70 for derivation cohort and 50 for validation cohort) with $\mathrm{CD}$ was enrolled and undergone MREC and ileocolonoscopy or balloon-assisted enteroscopy (BAE). MREC was evaluated for each bowel segment; rectum, sigmoid, descending, transverse, ascending colon, terminal, proximal ileum, and jejunum, according to the consensus of two observers in the derivation phase, and independently by three observers in the validation phase, using a 5-point MREC classification based on a lexicon of MR findings. The conventional MR score, or MaRIA, was evaluated simultaneously. Areas under the receiver operating characteristic curves (AUCs) were obtained to assess the accuracy of discriminating deep ulcers. Inter-observer reproducibility was assessed using weighted Kappa coefficients.

Results: BAE was performed in $49(70 \%)$ and $37(74 \%)$ patients in the derivation and validation cohorts. The AUCs of MREC classification were $89.0 \%$ in the derivation phase and $88.5,81.0$, and $77.3 \%$ for three observers in the validation phase. The AUCs of MREC classification were statistically non-inferior to those of MaRIA $(p<0.001)$. The cross-validation accuracy was $81.9 \%$ in the derivation and $81.5 \%$ in the validation phase. The MREC classification showed good Conclusion: For clinical use, radiological reporting systems should be simple and provide appropriate levels of accuracy and reproducibility. The 5-point MREC classification meets these requirements, and is useful for evaluating CD activity in the large to deep small bowel segments.

Disclosure of Interest: All authors have declared no conflicts of interest.

\section{P1661 RISK FACTORS FOR METABOLIC SYNDROME AND ITS COMPONENTS IN INFLAMMATORY BOWEL DISEASE}

K. Efthymakis, M. Serio, M. Fioravante, A. Milano, F. Laterza, A. Bonitatibus, M. Neri

Department Of Medicine And Ageing Sciences And Center For Excellence On Ageing And Translational Medicine (cesi-met), "G. D'Annunzio" University and Foundation, Chieti/Italy

Contact E-mail Address: efkn78@gmail.com

Introduction: Metabolic Syndrome (MetS) is a combination of biochemical and anthropometric disturbances and a recognized risk factor for cardiovascular disease. A higher prevalence of this condition has been previously reported in IBD patients, correlating to age as in the general population.

Aims \& Methods: The aim of this study was to assess the effect of individual disease activity-related putative risk factors for MetS in a group of IBD patients, as well as any protective effects of treatment on MetS or its components. Consecutive IBD patients and age- and sex-matched controls were included during a 1-year period. MetS was diagnosed according to the "harmonized" criteria as the presence of $>3$ criteria among elevated waist circumference, blood pressure, blood glucose, serum tryglicerides, or reduced HDL levels. All subjects underwent colonoscopy; endoscopic disease activity was assessed according to SES-CD and Mayo endoscopic scores. CRP, fecal calprotectin (FC), hemoglobin and ferritin levels were also measured.

Results: We enrolled 145 consecutive IBD patients ( 53 Crohn's disease and 92 ulcerative colitis; $58 \mathrm{M} / 87 \mathrm{~F}$; mean age $51 \pm 18$ ys) and 250 age- and sex-matched controls. Overall MetS prevalence was $37 \%$ in IBD and $21.6 \%$ in controls $(\mathrm{OR}=2.1,95 \% \mathrm{CI}: 1.32-3.39)$. Prevalence according to sex or disease type did not show significant differences. At multivariate analysis, age and BMI $>25$ were associated to an increased probability for a positive MetS status both in IBD (respectively, $\mathrm{OR}=3.41$ and $\mathrm{OR}=6.0$ ) and controls (respectively, $\mathrm{OR}=3.47$ and $\mathrm{OR}=3.74)$. In patients under 50 years, age $(\mathrm{OR}=1.24)$, CRP $(\mathrm{OR}=1.9)$ and $\mathrm{FC}(\mathrm{OR}=1.35)$ positivity were associated to MetS status, while a $\mathrm{BMI}>25$ increased risk at any age $(<50 \mathrm{ys}$ OR $=3.8,>50 \mathrm{ys} \mathrm{OR}=1.56)$. Endoscopic activity did not associate to MetS status at any age. Interestingly, anti-TNF $\alpha$ treatment was protective in both groups, but reached statistical significance only in older subjects $(>50 \mathrm{ys} \mathrm{OR}=0.08)$. Regarding individual MetS components, in the $<50$ ys subgroup, age and CRP positivity associated with an impaired glycemic (respectively, $\mathrm{OR}=1.15$ and $\mathrm{OR}=2.28$ ) and lipidemic status (respectively, $\mathrm{OR}=1.23$ and $\mathrm{OR}=2.3$ ). In older patients, CRP positivity only associated to impaired HDL status $(\mathrm{OR}=5.41)$. Importantly, anti-TNF $\alpha$ treatment favourably associated to HDL status $(\mathrm{OR}=0.2)$.
Conclusion: MetS prevalence is increased in IBD compared to healthy controls at any age. Subjects with increased BMI and/or inflammatory markers are at higher risk for MetS, while anti-TNF $\alpha$ agents appear to be protective. The components associated to MetS are differently distributed according to age, with the inflammatory ones prevailing in subjects $<50$ years and metabolic disturbances in older patients. These results indicate that, while in the short term appropriate treatments may reduce MetS occurrence and associated risks in subjects $>50$ years, in younger patients more effective inflammation control measures may prevent MetS and its related long-term neoplastic and cardiovascular complications. Disclosure of Interest: All authors have declared no conflicts of interest.

\section{P1662 C-REACTIVE PROTEIN/ALBUMIN RATIO IS A GOOD PREDICTOR OF RESPONSE TO INTRAVENOUS CORTICOSTEROIDS IN ACUTE SEVERE ULCERATIVE COLITIS}

S. Monteiro ${ }^{1}$, T. Cúrdia Gonçalves ${ }^{1}$, F. Dias De Castro ${ }^{2}$, S. Leite ${ }^{1}$, M.J. Moreira ${ }^{2}$, J. Cotter

${ }^{1}$ Gastroenterology, Hospital Senhora da Oliveira-Guimarães, Guimarães/Portugal ${ }^{2}$ Icvs/3b's, PT Government Associate Laboratory, Guimarães/Braga/Portugal

\section{Contact E-mail Address: sara.s.o.monteiro $@$ gmail.com}

Introduction: Patients with acute severe ulcerative colitis (ASUC) have a high risk of rescue medical therapy or colectomy. Recently, the C-reactive protein (CRP)/ albumin ratio at the $3 \mathrm{rd}$ day of treatment, with intravenous corticosteroids, has been shown to be a predictor of early colectomy in patients with ASUC.

Aims \& Methods: To evaluate the accuracy of CRP/albumin ratio on admission, to predict response to intravenous corticosteroids in patients with ASUC. Retrospective assessment of systematically hospitalized patients with first episode of ASUC, who required intravenous corticosteroids. Demographic, clinical, laboratory and endoscopic variables were evaluated on admission. The response to intravenous corticosteroids on day 3 was based on Oxford criteria. In unresponsive patients, rescue medical therapy with infliximab or cyclosporine has been instituted. The accuracy of CRP/albumin ratio in predicting non-response to intravenous corticosteroids was assessed by the area under the ROC curve (AUC).

Results: 51 patients were included, $30(58.8 \%)$ of them female, with a mean age $34.3 \pm 14.5$ years. Twelve patients $(23.5 \%)$ required medical rescue therapy. No patient underwent colectomy. The presence of deep ulcers and a shorter evolution of the disease were associated with a lack of response to intravenous corticosteroids, $\mathrm{p}<0.001$ and $\mathrm{p}=0.008$, respectively. Patients with no response to intravenous corticosteroids had higher CRP admission values and lower albumin values, compared to patients with response, 111 vs $67.5(\mathrm{mg} / \mathrm{L}), \mathrm{p}=0.028,2.8$ vs $3.5(\mathrm{~g}$ $\mathrm{dL}), \mathrm{p}=0.005$, respectively. The CRP/albumin ratio was also higher in unresponsive patients 40.06 vs $22.14, p=0.022$, showing a good accuracy for predicting no response to intravenous corticosteroids with an AUC of $0.746, p=0.01$

Conclusion: A high value of CRP/albumin ratio was significantly associated with the absence of response to intravenous corticosteroids, at the 3rd day of treatment. This index may allow a better risk stratification on admission, of patients with acute severe ulcerative colitis.

Disclosure of Interest: All authors have declared no conflicts of interest.

P1663 INSUFFICIENT VARIATION OF MEDIUM CORPUSCULAR VOLUME ( $\triangle$ MCV) IN INFLAMMATORY BOWEL DISEASE UNDER THYOPURINES PREDICTS DIFFICULTY IN ACHIEVING COMBINED DEEP REMISSION OUTCOME EVEN AFTER COMBINATION WITH ANTI-TNF - THE OTHER SIDE OF THE MCV FLOW STUDY

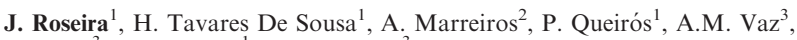
T. Gago $^{3}$, L. Contente ${ }^{1}$, H. Guerreiro

${ }^{1}$ Gastroenterology, Algarve Hospital Center, Portimão/Portugal

${ }^{2}$ Biomedical Science Department, University of the Algarve, Faro/Portugal

${ }^{3}$ Gastroenterology, Centro Hospitalar do Algarve, Faro/Portugal

Contact E-mail Address: jsr_roseira@hotmail.com

Introduction: The MCV flow study confirmed the association $\triangle \mathrm{MCV} \geq 7 \mathrm{fl}$ at week 26-28 of Azathioprine monotherapy (mAza) with favourable outcomes in a Portuguese IBD population.

Aims \& Methods: For this work, our aims were to evaluate the need for step-up therapy in those under mAza with $\triangle \mathrm{MCV}<7$ and to identify predictors of combined deep remission outcomes (DeepRem), at the same timepoint, for the patients who subsequently began combination therapy with Anti-TNF (AzaExperienced + Anti-TNF). Evaluation of patients under mAza with $\triangle \mathrm{VGM}<7$ at key timepoint week 26-28 treatment, included for The $M C V$ flow study. Demographic characterization and severity of pre-treatment disease was evaluated (Montreal classification, previous surgery status, Mayo score and Crohn's disease activity index [CDAI]). $\triangle$ MCV's association with DeepRem [Steroid-free clinical remission $(\mathrm{CDAI}<150$, Mayo $<2)+$ mucosal healing $(\mathrm{MH})+\mathrm{C}$-reactive protein $(\mathrm{CRP})<10]$ and need for biological therapy at the key-timepoint was verified. Evaluation of DeepRem and its independent predictors in patients who subsequently started combination therapy. Statistic: Chisquare test; Binary logistic regression.

Results: A total of 106 IBD patients were evaluated [56.6\% women, mean age $39 \pm 15.2$ years; 58 ad, $14 \%$ operated] at week $26-28$ of mAza. Identified strong association between an average $\Delta \mathrm{VGM}>7(\mathrm{n}=70 ; 66 \%)$ with DeepRem $(\mathrm{p}<0.05)$, while a $\triangle \mathrm{VGM}<7$ was associated with biological therapy need $(\mathrm{p}<0.05) . \quad 45$ patients were later started with Anti-TNF therapy 
(Aza + Infliximab 46.7\%; Aza + Adalimumab 53.3\%) and only 44\% achieved DeepRem at the key-timepoint. A Crohn's A3L2B3 + p phenotype $(p=0.045)$, steroid therapy in the last year $(\mathrm{p}=0.009)$ and $\Delta \mathrm{VGM}<7(\mathrm{p}=0.036)$ were identified as the variables that best explained the difficulty reaching DeepRem. Conclusion: This study confirms the prognostic value of $\triangle \mathrm{VGM}$ in our population. For $\mathrm{mAza}$ patients, $\Delta \mathrm{VGM} \geq 7$ was associated with DeepRem and $\Delta \mathrm{VGM}<7$ was found to be associated with biological therapy need. However, even after starting Anti-TNF, $\triangle \mathrm{VGM}<7$ was identified as a predictor of the difficulty reaching DeepRem.

Disclosure of Interest: All authors have declared no conflicts of interest.

P1664 FECAL CALPROTECTIN PREDICTS SHORT-TERM RELAPSE IN INFLAMMATORY BOWEL DISEASE PATIENTS IN DEEP REMISSION

R. Nakov ${ }^{1}$, V. Nakov ${ }^{1}$, V. Gerova ${ }^{1}$, L. Tankova ${ }^{1}$, T. Kundurzhiev ${ }^{2}$, B. Vladimirov ${ }^{1}$

${ }^{1}$ Gastroenterology, Clinic of Gastroenterology, "Tsaritsa Ioanna - ISUL"

University Hospital, Medical University - Sofia, Sofia/Bulgaria

${ }^{2}$ Department Of Social Medicine And Health Management, Section Of Biostatistic. Medical University, Sofia, Sofia/Bulgaria

Contact E-mail Address: radislav.nakov@gmail.com

Introduction: Most inflammatory bowel disease (IBD) patients with clinically successful treatment seem to have some degree of residual mucosal inflammation. Elevated fecal calprotectin (FC) concentrations can be found despite clinical remission and may indicate relapse risk in asymptomatic IBD.

Aims \& Methods: The aim of this prospective study was to evaluate whether elevated FC values can predict short-term clinical and/or endoscopic relapse. We enrolled 60 IBD patients (30 ulcerative colitis - UC, 30 Crohn's disaese $\mathrm{CD})$ who were in clinical and endoscopic remission. FC was measured using quantitative immunochromatographic point-of-care test (Quantum Blue $\mathbb{R}$ Calprotectin, Bühlmann Laboratories AG, Switzerland). Patients were followed-up by FC examination and clinical activity assessment every second month until relapse or up to 24 months. Ileocolonoscopy was performed at inclusion and at the time of clinical relapse.

Results: During the follow-up $36(60 \%)$ relapsed and $24(40 \%)$ remained in remission. The mean time to relapse in all patients was 13.9 (range 2-20) months. Significant increase in median FC levels was seen 2 months $(\mathrm{p}<0.001)$ before endoscopic relapse. ROC analysis indicated that a cut-off of $>90 \mu \mathrm{g} / \mathrm{g}(\mathrm{OR} 24,95 \% \mathrm{CI}=5-117, \mathrm{p}<0.001)$ in mean $\mathrm{FC}$ values 2 months before relapse could predict relapse in UC patients with $83,3 \%$ sensitivity and $82.9 \%$ specificity. In CD patients a cut-off of $>155 \mu \mathrm{g} / \mathrm{g}(\mathrm{OR} 193,95 \% \mathrm{CI}=22$ $1682, \mathrm{p}<0.001$ ) could predict relapse within two months with $91.7 \%$ sensitivity and $94.6 \%$ specificity. Constantly normal FC values during the follow-up were predictive for deep remission.

Conclusion: It seems that FC elevates two months before clinical and/or endoscopic relapse. FC is a suitable marker for predicting relapse and building a follow-up strategy for IBD patients in clinical practice.

Disclosure of Interest: All authors have declared no conflicts of interest.

P1665 GLYCEMIC CONTROL AND INSULIN RESISTANCE IN PATIENT WITH INFLAMMATORY BOWEL DISEASE PRELIMENARY RESULTS FROM THE POLIBD STUDY

R. Filip ${ }^{1}$, J. Sztembis ${ }^{2}$, P. Kiela ${ }^{3}$, A. Rybak ${ }^{4}$

${ }^{1}$ Gastroenterology With The Central Department Of Endoscopy, Clinical Hospital No.2 Rzeszow, Poland, Rzeszów/Poland

${ }^{2}$ Internal Medicine, Endocrinology And Nephrology, Clinical Hospital No.2

Rzeszow, Poland, Rzeszów/Poland

${ }^{3}$ Arizona Health Sciences Center, University of Arizona Tucson, Tuscon/United States of America

${ }^{4}$ Department Of Laboratory Diagnostics, Clinical Hospital No.2 Rzeszow, Poland, Rzeszów/Poland

Contact E-mail Address: jsztembis@gmail.com

Introduction: Hyperglycemia associated with critical illness - also called stress hyperglycemia or stress diabetes - has been demonstrated with much higher prevalence of severe ill patients. It is connected with many factors, including increased cortizol level, catecholamines uptake, glucagon production, gluconeogenesis, insulin resistance and inflammatory markers.It is not considered as an adaptive responce anymore. Futhermore uncontrolled hyperglycemia is associated with poor outcomes and significantly increases mortality rates. That is why stress hyperglycemia and insulin resistance may be a marker of severe illness. Aims \& Methods: We analysed the data (glycemia, insulin level, HOMA IR level, C-reactive protein level, HbA1C) of 62 patient aged 18 years and older (20 women and 42 men, $32.26+/-13.8$ years of age) with IBD hospitalized in our clinic from 2016 to 2017.16 patient were with Ulcerative Colitis (UC) and 48 patients with Crohn's Disease.

Results: The analysis of patients with Ulcerative Colitis showed that only one of the patients had hyperglycemia within the range of $140 \mathrm{mg}-200 \mathrm{mg}$ (the patient had type $2 \mathrm{DM}$ ) but interestingly $37.5 \%$ of the patients had fasting hyperglycemia over $100 \mathrm{mg}$. Over $86 \%$ of these patient were submitted to our clinic with the exacerbation of the disease and had abnormal level of C-reactive protein, calprotectin $(>1800)$ and fasting insulin level over $10 \mathrm{IU} / \mathrm{ml}$. The analysis of the group of patients with Crohn's Disease showed different results which may be connected with different metabolic profile of these patients. Most of the patients with fasting hyperglycemia (only $12.5 \%$ ) had elevated C-reactive protein level but not fasting insulin level - it was within normal range. The highest level of the fasting insulin (over $10 \mathrm{IU} / \mathrm{ml}$ ) in this group was observed in 3 patients who had to undergo immediate surgical treatment - two of them because of the bowel obstruction and one of them because of the perforation.

Conclusion: In our opinion there is a strong connection beetween fasting glucose, CRP level and exacerbation of the disease in CU patients but not in case of the Crohn's Disease. In this group the elevated fasting insulin may be a marker of severe illness.

Disclosure of Interest: All authors have declared no conflicts of interest.

\section{P1666 THE ROLE OF MR IMAGING IN ASSESSMENT OF LEMANN} INDEX IN THE COURSE OF CROHN'S DISEASE

J.M. Pienkowska, O. Kozak, T. Nowicki, E. Szurowska

Radiology, Medical University of Gdansk, Gdansk/Poland

Contact E-mail Address: oliwiak@gumed.edu.p

Introduction: Crohn's disease (CD) is a progressive, chronic and destructive inflammatory bowel process which, during its course, can lead to complications such as strictures and penetrating lesions (fistulas and abscesses), which may consequently require operative treatment. In some patients, bowel damage is present at the moment of diagnosis. The aim of the study is to assess the initial Lemann Index (LI), which comprehensively evaluates the entire gastrointestinal tract damage in patients with newly diagnosed Crohn's disease.

Aims \& Methods: In 209 patients with clinical suspicions of Crohn's disease MR imaging, endoscopic examination and histopathology were performed. In 151 patients with confirmed active/chronic CD the Lemann Index has been calculated on the basis of radiological and clinical information for initial assessment of cumulative digestive tissue damage. To create the Lemann Index the gastrointestinal tract was divided into 4 organs: upper digestive tract, small bowel, colon rectum and anus. Each organ was divided into segments ( 3 for the upper digestive tract, 6 for the colon/rectum and 1 for anus). Strictures and penetrating lesions were assessed at each segment on 4-degree scale (0-3) according to the severity of lesions.

Results: Based on the findings of the initial radiological examination, active inflammation process was found in 76 patients and chronic process in 75 patients. The baseline study demonstrated such complications as strictures in 14 patients, fistulas in 15 and abscesses in 4 patients. For all patients the LI was calculated. The obtained values were within the range from 0 to 22 .

Conclusion: Over the years, the progression of Crohn's disease leads to an increase in the value of Lemann Index, therefore, it seems that the evaluation of this repetitive parameter already in the first, baseline study and in all following control MR examinations will allow for a more complete assessment of patients in terms of progressive bowel damage and modification of the therapeutic process.

Disclosure of Interest: All authors have declared no conflicts of interest.

\section{References}

1. Pariente B, Mary J-Y, Danese S, et al. Development of the Lemann Index to Assess Digestive Tract Damage in Patients With Crohn's Disease. Gastroenterology 2015; 148:52-63

2. Gilletta C, Lewin M, Bourrier A, et al. Changes in the Lemann Index Values During the First Years of Crohn's Disease. Clin Gastroenterol Hepatol 2015 13(9): 1633-40

3. Pariente B, Cosnes J, Danese S, et al. Development of the Crohn's Disease Digestive Damage Score, the Lemann Score. Inflamm Bowel Dis 2011; 17(6):1415-22

4. Fiorino G, Bonifacio C, Peyrin-Biroulet L, et al. Preventing Collateral Damage in Crohn's Disease: The Lemann Index. J Crohns Colitis 2016; 10(4):495-500

\section{P1667 OPTICAL CHARACTERIZATION OF LESIONS IN IBD} COLITIS: A SYSTEMATIC REVIEW AND META-ANALYSIS

R. Lord ${ }^{1}$, N. Burr ${ }^{2}$, N. Mohammed ${ }^{2}$, V. Subramanian ${ }^{3}$

${ }^{1}$ Gastroenterology, Endoscopic research fellow/Gastroenterology registrar research, Leeds/United Kingdom

${ }^{2}$ Gastroenterology, Leeds Institute for Biomedical and Clinical Sciences, Leeds/ United Kingdom

${ }^{3}$ Gastroenterology, Leeds Teaching Hospitals NHS Trust, Leeds/United Kingdom

\section{Contact E-mail Address: richard.lord2@nhs.net}

Introduction: Optical imaging is being increasingly advocated for characterization of polyps during colonoscopy. The accuracy of these techniques during surveillance colonoscopy in colonic inflammatory bowel disease (IBD-C) is unclear and with variable results reported. We aimed to perform a systematic review and meta-analysis of the diagnostic accuracy of optical imaging techniques including dye based and virtual chromoendoscopy, magnification endoscopy and confocal laser endomicrosocpy.

Aims \& Methods: We searched Medline and Embase for relevant papers. Full articles or abstracts were eligible when characterization performance of dyebased chromoendoscopy (DCE), virtual chromoendoscopy (VCE) (narrowband imaging [NBI], i-scan, Fujinon intelligent chromoendoscopy [FICE]), magnification endoscopy and confocal laser endomicroscopy (CLE) had been compared with histopathology, as the reference standard. Enough information had to 
Pooled Results

\begin{tabular}{|c|c|c|c|c|c|c|c|c|c|}
\hline & \multirow[b]{2}{*}{$\begin{array}{l}\text { Number of } \\
\text { studies }\end{array}$} & \multirow[b]{2}{*}{$\begin{array}{l}\text { Number of } \\
\text { lesions }\end{array}$} & \multirow[b]{2}{*}{$\begin{array}{l}\text { Number of } \\
\text { dysplastic } \\
\text { lesions }(\%)\end{array}$} & \multicolumn{6}{|l|}{ Pooled Results } \\
\hline & & & & $\begin{array}{l}\text { Sensitivity } \\
(95 \% \mathrm{CI})+\mathrm{I}^{2}\end{array}$ & $\begin{array}{l}\text { Specificity } \\
(95 \% \text { CI })+I^{2}\end{array}$ & $\begin{array}{l}\text { +ve Likelihood } \\
\text { Ratio } \\
(95 \% \mathrm{CI})+\mathrm{I}^{2}\end{array}$ & $\begin{array}{l}\text {-ve Likelihood } \\
\text { Ratio } \\
(95 \% \text { CI })+I^{2}\end{array}$ & $\begin{array}{l}\text { Diagnostic } \\
\text { Odds Ratio } \\
(95 \% \mathrm{CI})+\mathrm{I}^{2}\end{array}$ & $\begin{array}{l}\text { AUROC } \\
\text { (SE AUC) Q }\end{array}$ \\
\hline $\begin{array}{l}\text { Virtual } \\
\text { chromoendoscopy }\end{array}$ & 5 & 987 & $\begin{array}{l}121 \\
(12.3 \%)\end{array}$ & $\begin{array}{l}0.803 \\
(0.72-0.87) \\
\mathrm{I}^{2}=78 \%\end{array}$ & $\begin{array}{l}0.833 \\
(0.80-0.86) \\
\mathrm{I}^{2}=95.4 \%\end{array}$ & $\begin{array}{l}5.331 \\
(2.78-10.2) \\
\mathrm{I}^{2}=92.9\end{array}$ & $\begin{array}{l}0.201 \\
(0.06-0.59) \\
\mathrm{I}^{2}=87.2 \%\end{array}$ & $\begin{array}{l}28.21 \\
(6.08-130) \\
\mathrm{I}^{2}=86.4 \%\end{array}$ & $\begin{array}{l}0.9137 \\
(0.056) 0.84\end{array}$ \\
\hline $\begin{array}{l}\text { Dye based } \\
\text { chromoendoscopy }\end{array}$ & 6 & 1767 & $\begin{array}{l}249 \\
(14 \%)\end{array}$ & $\begin{array}{l}0.558 \\
(0.49-0.62) \\
\mathrm{I}^{2}=85.6 \%\end{array}$ & $\begin{array}{l}0.89 \\
(0.87-0.91) \\
\mathrm{I}^{2}=84.4 \%\end{array}$ & $\begin{array}{l}4.21 \\
(2.27-7.8) \\
\mathrm{I}^{2}=86.7 \%\end{array}$ & $\begin{array}{l}0.48 \\
(0.29-0.80) \\
\mathrm{I}^{2}=81.8 \%\end{array}$ & $\begin{array}{l}9.92 \\
(3.71-26.5) \\
\mathrm{I}^{2}=80 \%\end{array}$ & $\begin{array}{l}0.838 \\
(0.071) 0.76\end{array}$ \\
\hline $\begin{array}{r}\text { Magnification } \\
\text { endoscopy }\end{array}$ & 5 & 226 & $\begin{array}{l}79 \\
(13.3 \%)\end{array}$ & $\begin{array}{l}0.899 \\
(0.81-0.96) \\
\mathrm{I}^{2}=37.1 \%\end{array}$ & $\begin{array}{l}0.856 \\
(0.82-0.89) \\
\mathrm{I}^{2}=44.9 \%\end{array}$ & $\begin{array}{l}6.55 \\
(3.86-11.1) \\
I^{2}=62.0 \%\end{array}$ & $\begin{array}{l}0.143 \\
(0.06-0.33) \\
\mathrm{I}^{2}=35.6 \%\end{array}$ & $\begin{array}{l}53.01 \\
(14.1-199) \\
\mathrm{I}^{2}=54.7 \%\end{array}$ & $\begin{array}{l}0.9304 \\
(0.025) 0.865\end{array}$ \\
\hline $\begin{array}{l}\text { Confocal laser endomi- } \\
\text { croscopy } \\
\text { (CLE) }\end{array}$ & 8 & 1179 & $\begin{array}{l}86 \\
(7 \%)\end{array}$ & $\begin{array}{l}0.826 \\
(0.72-0.89) \\
\left.\mathrm{I}^{2}=60.6 \%\right)\end{array}$ & $\begin{array}{l}0.947 \\
(0.93-0.95) \\
\mathrm{I}^{2}=83.4 \%\end{array}$ & $\begin{array}{l}13.11 \\
(5.3-31.2) \\
\mathrm{I}^{2}=85.7\end{array}$ & $\begin{array}{l}0.20 \\
(0.08-0.46) \\
\mathrm{I}^{2}=66.7 \%\end{array}$ & $\begin{array}{l}78.1 \\
(17.8-341) \\
\mathrm{I}^{2}=74.2 \%\end{array}$ & $\begin{array}{l}0.959 \\
(0.031) 0.903\end{array}$ \\
\hline $\begin{array}{l}\text { Real-time Kudo pit } \\
\text { pattern }\end{array}$ & 10 & 2724 & $\begin{array}{l}357 \\
(13 \%)\end{array}$ & $\begin{array}{l}0.624 \\
(0.48-0.76) \\
\mathrm{I}^{2}=88.2 \%\end{array}$ & $\begin{array}{l}0.88 \\
(0.83-0.93) \\
\mathrm{I}^{2}=92.8 \%\end{array}$ & $\begin{array}{l}5.64 \\
(3.52-9.05) \\
I^{2}=90.1 \%\end{array}$ & $\begin{array}{l}0.34 \\
(0.20-0.57) \\
\mathrm{I}^{2}=86.8 \%\end{array}$ & $\begin{array}{l}20.0 \\
(7.9-50.6) \\
\mathrm{I}^{2}=85.4 \%\end{array}$ & $\begin{array}{l}0.917 \\
(0.039) 0.85\end{array}$ \\
\hline Real-time CLE & 6 & 402 & $\begin{array}{l}47 \\
(11.7 \%)\end{array}$ & $\begin{array}{l}0.872 \\
(0.70-1.0) \\
\mathrm{I}^{2}=60.8 \%\end{array}$ & $\begin{array}{l}0.96 \\
(0.93-0.99) \\
\mathrm{I}^{2}=41.3 \%\end{array}$ & $\begin{array}{l}15.4 \\
(6.29-37.8) \\
I^{2}=60.5 \%\end{array}$ & $\begin{array}{l}0.154 \\
(0.03-0.62) \\
\mathrm{I}^{2}=71.1 \%\end{array}$ & $\begin{array}{l}108.74 \\
(21.3-552) \\
\mathrm{I}^{2}=54.2 \%\end{array}$ & $\begin{array}{l}0.97 \\
(0.024) 0.92\end{array}$ \\
\hline
\end{tabular}

be required to obtain a $2 \times 2$ contingency table. Pooled analysis was done using a random-effects model. Sub-group analysis was performed at real-time Kudo pit pattern based and real-time CLE for characterization of visible lesions. Heterogeneity was assessed using Chi squared and $\mathrm{I}^{2}$ statistics.

Results: Our search strategy identified 172 studies of which only 20 met the inclusion criteria. The pooled results are outlined in the table.

Conclusion: Real-time CLE and magnification endoscopy had the best performance characteristics. However there was a lot of heterogeneity in the results. Most CLE and magnification studies were single centre, single expert user which could explain these results. CLE studies were also affected by attrition bias with some studies reporting non-interpretable mages in a significant proportion. Disclosure of Interest: All authors have declared no conflicts of interest.

P1668 EVALUATION OF SEVERITY SCORE IN CROHN'S DISEASE

C. Gouveia ${ }^{1}$, M. P. Santos ${ }^{1}$, C. Palmela ${ }^{1}$, C. Gomes ${ }^{2}$, J. Torres ${ }^{1}$, C. Fidalgo ${ }^{1}$, L. Glória ${ }^{1}$, M. Cravo ${ }^{1}$

${ }^{1}$ Gastrenterologia, Hospital Beatriz Ângelo, Lisboa/Portugal

${ }^{2}$ Hospital Beatriz Ângelo, Lisboa/Portugal

Contact E-mail Address: catarinagouveia228@gmail.com

Introduction: The need to stratify patients with Crohn's disease (CD) according to the risk of developing complications is essential to delineate therapeutic approach. A score that evaluates the severity of DC (Siegel et al, Gut 2016) has recently been developed and published, taking into account intestinal lesions, disease activity and complications during the course of the disease, ranging from 0 to 100 .

Aims \& Methods: We aimed to evaluate the performance of the aforementioned score at the time of diagnosis and its relation to the need for surgery and hospitalizations in patients with $\mathrm{CD}$. We performed a retrospective study that included patients diagnosed with DC in our hospital between 01/2012-12/2015. The score was calculated at the time of diagnosis and information on the course of the disease was collected. Statistical analysis was performed in SPSS (v23).

Results: A total of 57 patients $(52.6 \%$ women) with a mean age of $33.74 \pm 15.8$ years were included. The median severity score at diagnosis was $16 \pm 16.5$ ranging from 4 to 50 . Twenty-four patients $(40.7 \%)$ required surgery and $29(49.2 \%)$ were hospitalized for reasons associated with the disease. At diagnosis, the score was higher in $\mathrm{A} 1$ patients ( 26 vs 19.2 and $13.7, \mathrm{p}=0.05$ ), with a penetrating phenotype ( 25.5 vs. 19 and $12, p=0.02)$, and in patients requiring surgery during the course of their disease ( 21 vs $14, \mathrm{p}=0.1$ ). There was a positive correlation between the score at diagnosis and the number of surgeries during follow-up $(r=0.43 ; p=0.05)$. Survival analysis showed a trend towards shorter time for surgery or hospitalization in patients with a score above the median at diagnosis (p logrank $=0.09$ ).

Conclusion: The new severity score seems to be a promising tool for stratification and prognosis of patients with $\mathrm{CD}$ at diagnosis, and its utility should be validated in prospective studies.

Disclosure of Interest: All authors have declared no conflicts of interest.

\section{P1669 ENDOSCOPIC FINDINGS AND COLONOSCOPIC PERFORATION IN MICROSCOPIC COLITIS; A SYSTEMATIC REVIEW OF THE LITERATURE}

W.M. Marlicz ${ }^{1}$, I. Łoniewski ${ }^{2}$, K. Skoniecznał̇decka ${ }^{2}$, D. E. Yung ${ }^{3}$, A. Koulaouzidis ${ }^{3}$

${ }^{1}$ Department Of Gastroenterology, Pomeranian Medical University, Szczecin/ Poland

${ }^{2}$ Department Of Biochemistry And Human Nutrition, Pomeranian Medical University, Szczecin/Poland

${ }^{3}$ The Royal Infirmary of Edinburgh, Edinburgh/United Kingdom

Contact E-mail Address: diana.e.yung@gmail.com

Introduction: Microscopic colitis (MC) is a clinical syndrome of severe watery diarrhoea with few or no endoscopic abnormalities. The incidence of MC is reportedly similar to that of other inflammatory bowel diseases. The need for histological confirmation of MC frequently guides reimbursement health policies. With the advent of high-definition (HD) colonoscopes, the incidence of distinct endoscopic findings reported in MC has risen. This has the potential to improve diagnosis times, increase cost-effectiveness of MC management and diminish the workload and costs of busy modern endoscopy units.

Aims \& Methods: Publications on distinct endoscopic findings in MC available until $31^{\text {st }}$ March 2017 were searched systematically (electronic and manual) in PubMed. The following search terms/descriptors were used: collagenous colitis(CC) OR lymphocytic colitis(LC) AND endoscopy, colonoscopy, findings, macroscopic, erythema, mucosa, vasculature, scars, lacerations, fractures. An additional search for MC AND perforation was made.

Results: Eighty $(n=80)$ articles, predominantly single case reports $(n=45)$, were retrieved. Overall, 1,582 (1,159 female; $61.6 \pm 14.1$ years) patients(pts) with MC and endoscopic findings were reported. The majority of articles $(n=62)$ were on CC (756 pts; $77.5 \%$ female). We identified 16 papers comprising 779 pts $(68.9 \%$ female) with LC and 7 articles describing 47 pts ( $72.3 \%$ female) confirmed to have MC. The youngest patient was 10 and the oldest 97 years old. Aside from diarrhoea, symptoms included abdominal pain, weight loss, bloating, flatulence and oedema. In the study group we found $616(38.9 \%)$ pts with macroscopic findings. The most common colonoscopic findings were non-ulcerous lesions i.e. pseudomembranes, a variable degree of vasculature pruning $\&$ dwindling, mucosal lacerations \& abnormalities such as erythema/edema/nodularity, or surface textural alteration $(\mathrm{n}=537 ; 87.2 \% \mathrm{pts})$. Isolated linear ulcerations were identified in 5 pts $(0.8 \%)$ and in conjunction with non-ulcer lesions in 74 pts $(12.0 \%)$. The location of endoscopic findings was not reported in 26 articles. Distinct endoscopic findings were described in the left (descending, sigmoid, rectum $-23 / 20 / 10$ studies), right (cecum, ascending-7/21 studies) \& transverse colon (14), as well as duodenum (4), and terminal ileum (2). Colonic perforation was reported in 9 patients $(0.57 \%)$.

Conclusion: Endoscopic findings are recognized with increased frequency in pts with MC. This could improve MC diagnosis by prompting a more extensive biopsy protocol in such cases and an earlier initiation of treatment. Procedurerelated perforation has been reported in this group; therefore, cautious air insufflation is advisable when endoscopic findings are recognised.

Disclosure of Interest: All authors have declared no conflicts of interest.

\section{References}

1. Yung DE, Koulaouzidis A, Fineron P, Plevris JN. Microscopic colitis: a misnomer for a clearly defined entity? Endoscopy. 2015;47(8):754-7

2. Koulaouzidis A, Saeed AA. Distinct colonoscopy findings of microscopic colitis: not so microscopic after all? World $J$ Gastroenterol. 2011;17(37):4157-65 


\section{P1670 PREVALENCE AND QUANTITATIVE ASSESSMENT OF} LIVER STEATOSIS IN INFLAMMATORY BOWEL DISEASE PATIENTS

D.V. Balaban ${ }^{1}$, I. Enache ${ }^{2}$, B. Macadon 2 , M. Patrasescu' 2 , G.C. Robu ${ }^{2}$, A. Zoican ${ }^{2}$, S. Bucurica ${ }^{3}$, R.S. Costache ${ }^{3}$, P. Nuta ${ }^{2}$, F. Ionita Radu' ${ }^{2}$, M. Jinga ${ }^{3}$ 1"Carol Davila" University of Medicine and Pharmacy, Bucharest/Romania ${ }^{2 "}$ Dr. Carol Davila" Central Military Emergency University Hospital, Bucharest/ Romania

${ }^{3}$ University of Medicine and Pharmacy Carol Davila, Bucharest/Romania

Contact E-mail Address: iuliaenache.89@gmail.com

Introduction: It is well recognized that patients with inflammatory bowel disease (IBD) are at risk for nonalcoholic fatty liver disease (NAFLD). Our aim was to evaluate the prevalence and to quantify hepatic steatosis in IBD patients using the controlled attenuation parameter (CAP).

Aims \& Methods: We prospectively recruited all IBD patients presenting for a disease flare or follow-up visit in our clinic, during a 18 month period. Patients who reported chronic alcohol intake more than $20 \mathrm{~g} /$ day and those with coexisting viral hepatitis were excluded from analysis. Clinical characteristics and laboratory data were recorded. Hepatic steatosis was evaluated by conventional ultrasound, hepatic steatosis index (HSI) and transient elastography with CAP (Fibroscan, Echosens, Paris). Significant steatosis $(S \geq 1)$ was defined for a CAP value over 236 [1], and the cut-off of HSI for detecting NAFLD was set at $\geq 36[2]$.

Results: Altogether 62 IBD patients (35 ulcerative colitis, UC and 27 Crohn's disease, $C D$ ), mean age $45 \pm 15$ years, $50 \%$ female, were included in the analysis. The two groups (UC, CD) were similar regarding disease activity (remission/ flare $-48.5 / 51.4 \%$ in the UC group, $55.6 / 44.4 \%$ in the CD group), BMI (24.1 and 24.4 respectively) or inflammatory markers (ESR 21 and $21.1 \mathrm{~mm} / \mathrm{h}$, fibrinogen 498 and $513 \mathrm{mg} / \mathrm{dl}$ ). UC patients had higher mean cholesterol values (205.9 vs. $176.4 \mathrm{mg} / \mathrm{dl}$ ) and $11 \%$ of them were diabetic (compared to none in the CD group). Mean CAP was higher in CD compared to UC-246 vs. $225 \mathrm{~dB} / \mathrm{m}$, while HSI was similar among the two groups $34.9 \pm 4.9$ and $34.6 \pm 5.4$, respectively. US identified 18/62 (29\%) patients with fatty liver, HSI detected 3 more patients (21/ $62,33.9 \%)$ and CAP even 2 more $(23 / 62,37.1 \%)$, yielding an extra $8 \%$ detection rate. NAFLD-IBD patients were more likely to have CD phenotype, history of resection, steroid use and longer disease duration.

Conclusion: In our cohort, about one in three IBD patients had fatty liver disease, as quantified by CAP. Diagnostic performance of CAP was better than conventional ultrasound and HSI in detecting fatty liver in IBD patients.

Disclosure of Interest: All authors have declared no conflicts of interest.

\section{References}

1. Imajo K, Kessoku T, Honda Y, et al. Magnetic Resonance Imaging More Accurately Classifies Steatosis and Fibrosis in Patients With Nonalcoholic Fatty Liver Disease Than Transient Elastography. Gastroenterology 2016; 150:626-637.e7

2. Bessissow T, Le NH, Rollet K, et al. Incidence and predictors of nonalcoholic fatty liver disease by serum biomarkers in patients with inflammatory bowel disease. Inflamm Bowel Dis. 2016; 22(8):1937-44.

\section{P1671 INTESTINAL MICROBIOTA BIOMARKERS AS A NEW TOOL TO SUPPORT IRRITABLE BOWEL SYNDROME DIAGNOSTICS}

J. Amoedo ${ }^{1}$, L. Torrealba ${ }^{2}$, S. Ramió-Pujol ${ }^{1}$, L. Oliver ${ }^{1}$, A. Bahí ${ }^{3}$, M. Serrano ${ }^{1}$, M. Malagón ${ }^{3}$, D. Busquets ${ }^{2}$, P. Gilabert ${ }^{4}$, J. Guardiola ${ }^{4}$, M. Serra-Pagès ${ }^{1}$, J. Garcia-Gil ${ }^{1}$, X. Aldeguer ${ }^{1}$

${ }^{1}$ GoodGut SL, Girona/Spain

${ }^{2}$ Hospital Universitari Dr. Josep Trueta, Girona/Spain

${ }^{3}$ Institut d'Investigació Biomédica de Girona Dr. Josep Trueta, Salt/Spain

${ }^{4}$ Hospital Universitari de Bellvitge, Hospitalet del Llobregat/Spain

Contact E-mail Address: joan.amoedo@goodgut.eu

Introduction: Irritable Bowel Syndrome (IBS) is a common gastrointestinal disorder that affects around $11 \%$ of global population. Despite the high prevalence of IBS, the cause of this disorder remains unknown and the criteria used to diagnose IBS are still unclear. In recent years, disturbances in the intestinal microbiota have been associated to the pathophysiology of IBS. Recently, two accurate biomarkers: Faecalibacterium prausnitzii (Fpra) and Escherichia coli (Eco) have been shown to discriminate between Inflammatory Bowel disease (IBD) and Healthy subjects (H). Therefore, the purpose of this study was to verify the capability of Fpra and Eco abundances to distinguish among healthy subjects, IBS, and IBD patients, in order to create a non-invasive system of diagnostic support for IBS patients.

Aims \& Methods: A cohort consisting of $33 \mathrm{H}$ and 14 IBS was enrolled. IBS patients were separated by subtypes: IBS with constipation (C-IBS), IBS with diarrhea (D-IBS), and alternate IBS (A-IBS). Rome IV criteria were used to diagnose IBS patients. Moreover, 29 ulcerative colitis (UC) and 15 Crohn's disease (CD) patients were also included. All subjects were recruited by the Gastroenterology Services of the Hospital Universitari Dr. Josep Trueta (Girona, Spain) and Hospital Universitari de Bellvitge (Hospitalet del Llobregat, Spain). Fpra total and Eco abundances were quantified by qPCR.

Results: We found lower abundance values of Fpra in IBS patients when compared with $\mathrm{H}(\mathrm{P}=0.005)$. In contrast, Eco abundance was higher in IBS patients, although the differences observed were not significant $(\mathrm{P}=0.221)$. When comparing among subtypes of IBS (C-IBS, D-IBS, and A-IBS) no significant differences were observed, although Fpra abundance was lower in C-IBS. We also used Fpra in combination with Eco as a complementary indicator of dysbiosis (Ratio
Fpra/Eco). This ratio allows a good discrimination between $\mathrm{H}$ and IBS $(\mathrm{P}=0.041)$. When it comes to discrimination between IBS and IBD patients, significant differences were observed in Fpra/Eco ratio between UC and IBS patients $(P=0.008)$, but not between IBS and $C D$ patients $(P=0.775)$. Concerning disorders different to IBS, significant differences were also observed between $\mathrm{H}$ and $\mathrm{CD}(\mathrm{P}>0.001)$, between $\mathrm{H}$ and $\mathrm{UC}(\mathrm{P}=0.037)$, and between $\mathrm{CD}$ and $\mathrm{UC}(\mathrm{P}=0.027)$.

Conclusion: Fpra abundance is a good biomarker to discriminate between healthy subjects and IBS patients. The use of Fpra/Eco ratio allows to distinguish IBS from $\mathrm{H}$ and $\mathrm{UC}$ patients. In contrast, none of the used biomarkers was able to differentiate IBS and CD patients. These results show that IBS and CD patients share similar dysbiosis parameters opening the need of further study to stablished any eventual pathogenic link.

Disclosure of Interest: All authors have declared no conflicts of interest.

\section{P1672 DISTINGUISH BETWEEN ULCERATIVE COLITIS AND CROHN DISEASE USING AN ELECTRONIC NOSE AND DATA MINING: PRELIMINARY STUDY}

J. Pelegri-Sebastia ${ }^{1}$, E. Climent ${ }^{2}$, J.B. Talens ${ }^{1}$, L. Torse ${ }^{3}$, A.B. Beltran Niclos ${ }^{3}$, T. Sogorb ${ }^{1}, \mathrm{P}$. Nos ${ }^{4}$

${ }^{1}$ Igic, Campus De Gandia, Universitat Politecnica de Valencia, Grao de Gandia/ Spain

${ }^{2}$ Electronic Engineering, Universitat Politecnica de Valencia, Grao de Gandia/

Spain

${ }^{3}$ Iis $\mathrm{La} \mathrm{Fe}$, Hospital Universitari La Fe, Valencia/Spain

${ }^{4}$ Gastroenterology, Hospital Universitario y Politécnico de La Fe and CIBERehd, Valencia/Spain

Contact E-mail Address: Belenbeltranniclos@gmail.com

Introduction: Inflammatory bowel disease (IBD) and irritable bowel syndrome (IBS) may present in a similar manner [1] [2]. Measuring faecal calprotectin concentration is often recommended to rule out inflammatory bowel disease, however, there are no tests to positively diagnose irritable bowel syndrome and invasive tests are still used to rule out other pathologies [3,4]. There is a chance; therefore, for novel, non-invasive diseasespecific biomarkers. Volatile organic compounds (VOCs), originating from physiological metabolic processes in the human body, are excreted as waste products through stool samples. For this reason, several biological, non-invasive, simple and low-cost biological markers of inflammation that are useful in clinical practice for both diagnostic screening and therapeutic or course response monitoring are being evaluated in recent years Evolution of the disease. In this sense, stool markers, and especially calprotectin, have become of great importance in recent years as screening to select patients requiring more diagnostic studies and as a marker of activity for therapeutic follow-up [5]. Can the VOCs from stool samples show differences between ulcerative colitis and Crohn's disease?

Aims \& Methods: Five healthy individuals (control group- $\mathrm{CON}$ ) and nineteen patients diagnosed with IBD were selected for the analysis of their stool VOCs. Healthy participants Healthy control samples (Control) $(n=5)$ were collected from healthy voluntaries workers in the Digestive Diseases Area and they reported no illnesses related to the gastrointestinal tract and had not undergone antibiotic treatment in the 3 months before sampling. Active patients were defined as a Mayo Clinical score of 3 or more for ulcerative colitis (UC) and a Harvey Bradshaw clinical index of 4 or more for Crohn's disease (CD). In both cases with a calprotectin $>300 \mathrm{micrg} / \mathrm{gr}$ or relevant endoscopic lesions. Patients were classified according Montreal and ECCO criteriai. This preliminary study is based in a group of CD-UC-CON where was analyzed $10 \mathrm{CD}$ patients, $9 \mathrm{UC}$ patients and 5 controls with 455 samples. Data from stool samples was obtained using eNose MOOSY32 [6].

Results: Figure 1 shows the scatter 3D plot with three voltage parameters for the group CD-UC-CON. These parameters mean the voltage from the eNose's signals on saturation slope $(\mathrm{vB})$ and late saturation $(\mathrm{cV})$ and the number of sensor from the MOOSY32. Table 1 shows the comparative between relative error absolute and classification.

Table 1: Different algorithms test for matrix classification by WEKA software. Classification Relative absolute error

\begin{tabular}{|c|c|c|c|}
\hline MLP Cross & 10 & $92.0 \%$ & $16.5 \%$ \\
\hline MLP & $30 \%$ Train $+70 \%$ Test & $94.1 \%$ & $12.2 \%$ \\
\hline BayesNet Cross & 10 & $89.8 \%$ & $5.8 \%$ \\
\hline BayesNet & $30 \%$ Train $+70 \%$ Test & $86.0 \%$ & $22.1 \%$ \\
\hline J48 Cross & 10 & $89.6 \%$ & $16.7 \%$ \\
\hline $\mathrm{J} 48$ & $30 \%$ Train $+70 \%$ Test & $89.7 \%$ & $17.1 \%$ \\
\hline
\end{tabular}

Conclusion: In this preliminary research to distinguish between Ulcerative Colitis and Crohn's Disease, the best algorithm for patient's classification was the MLP with $30 \%$ to train and $70 \%$ to test. Although the high classifications result it is hopeful is necessary continue working to understanding how the eNose's signals affect to the relative absolute error and improving the algorithms to decrease the error.

Disclosure of Interest: All authors have declared no conflicts of interest. 


\section{References}

1. Van Assche G, Dignass A, Panés J, et al. The second European evidence-based consensus on the diagnosis and management of Crohńs disease: definitions and diagnosis. J Crohns Colitis 2010; 4(1): 7-27.

2. Stange E, Travis S, Vermeire S, et al. European evidence-based consensus on the diagnosis and management of ulcerative colitis: definitions and diagnosis. $J$ Crohns Colitis 2008; 2(1): 1-23.

3. Mowat C, Cole A, Windsor A, et al. Guidelines for the management of inflammatory bowel disease in adults. Gut 2011; 60(5): 571-607.

4. Konikoff MR, Denson LA. Role of fecal calprotectin as a biomarker of intestinal inflammation in inflammatory bowel disease. Inflamm Bowel Dis. 2006; 12: 524-34

5. D'Haens G, Ferrante M, Vermeire S, et al. Fecal calprotectin is a surrogate marker for endoscopic lesions in inflammatory bowel disease. Inflamm Bowel Dis. 2012; 18:, Sept. 2011

6. M. Cupane, 'Food Industry Production', IEEE Instrum. Meas. Mag., pp. 2733

\section{P1673 CZECH REGISTRY OF INFLAMMATORY BOWEL DISEASE PATIENTS ON BIOLOGICAL THERAPY: RESULTS FROM THE FIRST YEAR}

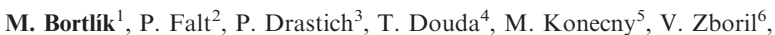
M. Lukas

${ }^{1}$ IBD Clinical And Research Center, ISCARE I.V.F. a.s., Prague/Czech Republic ${ }^{2}$ Digestive Diseases Center, Vitkovice Hospital, Ostrava/Czech Republic

${ }^{3}$ Department Of Hepatogastroenterology, Institute for Clinical and Experimental Medicine, Prague/Czech Republic

${ }^{4}$ 2nd Department Of Internal Medicine - Gastroenterology, Faculty of Medicine and University Hospital Hradec Kralove, Charles University, Hradec Kralovel Czech Republic

${ }^{5}$ 2nd Department Of Internal Medicine, University Hospital Olomouc, Olomouc/ Czech Republic

${ }^{6}$ University Hospital Brno, Department of Internal Medicine -

Hepatogastroenterology, Brno/Czech Republic

${ }^{7}$ Institute Of Medical Biochemistry And Laboratory Diagnostics, 1st Faculty of

Medicne and General University Hospital, Charles University, Prague/Czech

Republic

Contact E-mail Address: MBortlik@seznam.cz

Introduction: Czech Registry of Inflammatory Bowel Disease (IBD) Patients on Biological Therapy (CREdIT) was launched in March, 2016 in order to collect and analyze the data from Czech centers appointed to provide biological therapy to IBD patients.

Aims \& Methods: The objective is to provide information on the CREdIT registry after its first year of operation. Basic demographic data (sex, age, diagnosis, Montreal classification or disease extent) were collected together with information on disease activity, therapeutic regimen, concomitant medication and adverse events. Patients who begun biologic therapy prior to enrolment into the registry as well as those who start their treatment are included, and labeled as "retrospective" and "prospective" cohort, respectively.

Results: Among 30 centers providing biological therapy to patients with Crohńs disease (CD) and ulcerative colitis (UC) in the Czech Republic, 24 participated as of the end of March 2017. The centres enrolled 2129 IBD patients (CD, 1595, $74.9 \%$; UC, 505, 23.7\%; IBD-U, 29, 1.4\%), with median of 63 (range 4-528) patients per center. Majority belong to the retrospective cohort $(65 \%)$, the remaining $35 \%$ represent the prospective cohort. According to the latest data check, patients are treated with infliximab $(1273,59.8 \%)$, adalimumab (739, $34.7 \%$ ), vedolizumab $(73,3.4 \%)$, and golimumab $(42,2 \%)$. Treatment intensification (at any time of therapy) was used in 348 patients $(19.1 \%)$. Infliximab was the most frequently used drug in the first line of biological treatment of both diseases, while adalimumab and vedolizumab were the most used drugs in the second and third line, respectively. When started with biological therapy, majority of CD patients was on concomitant azathioprine $(55.4 \%)$, while UC patients used most frequently mesalazine $(72.7 \%)$. Among $\mathrm{CD}$ and $\mathrm{UC}$ patients, the proportion of patients on concomitant corticosteroids decreased from $42.7 \%$ and $67.9 \%$ to $10.2 \%$ and $24.5 \%$, respectively. $76(3.5 \%)$ patients experienced at least one adverse event while on biologic treatment.

Conclusion: The new Czech national registry collects and provides data on IBD patients treated with biological therapy. Majority of patients enrolled have Crohńs disease, and the mostly used drug is infliximab. Therapy is effective with low frequency of adverse events.

Disclosure of Interest: All authors have declared no conflicts of interest.

P1674 THE DIAGNOSTIC VALUE OF MIRNA-320A AS A BIOMARKER IN PATIENTS WITH INFLAMMATORY BOWEL DISEASE

F. Cordes ${ }^{1}$, C. Ruhs ${ }^{1}$, M. Brückner ${ }^{1}$, T.M. Nowacki ${ }^{1}$, F. Lenze ${ }^{1}$, M.A. Schmidt ${ }^{2}$, C. Cichon ${ }^{2}$, D. Bettenworth

${ }^{1}$ Department Of Medicine B, University Hospital Münster, Münster/Germany

${ }^{2}$ Institute Of Infectiology, Centre For Molecular Biology Of Inflammation (zmbe), University of Münster, Münster/Germany

Contact E-mail Address: annafriederike.cordes@ukmuenster.de
Introduction: In patients with inflammatory bowel diseases (IBDs), comprising Crohn's disease (CD) and Ulcerative colitis (UC), a patient-tailored therapy is an unmet need that requires accurate monitoring of the intestinal disease activity. We demonstrated recently, that the expression of microRNA (miR)-320a follows the disease activity in murine colitis models ${ }^{1}$. In this prospective study we evaluated the potential of miR-320a as a biomarker to monitor the disease activity in IBD patients as well as its potential to distinguish UC/CD from infectious colitis. Aims \& Methods: The miR-320a was measured by qRT-PCR analysis in peripheral blood samples from $36 \mathrm{CD}$ und $34 \mathrm{UC}$ patients with acute flare of disease $(n=51)$ and in remission $(n=37)$ as well as in healthy control patients $(n=20)$ and in patients with infectious colitis $(n=9)$. Disease activity was assessed clinically applying the Crohn's disease activity index (CDAI) and the partial Mayo score (pMayo) for UC patients as well as the simple endoscopic score Crohn's disease (SES-CD) and the endoscopic Mayo score (eMayo) to score endoscopic disease activity.

Results: Both in CD and in UC patients, miR-320a expression in remission was significantly increased as compared to healthy controls $(49 \pm 8 / 87 \pm 17$ vs. $17 \pm 3$; both $\mathrm{p}<0.001)$ but distinctly lower as in $\mathrm{CD} / \mathrm{UC}$ patients with acute flare $(1718 \pm 488 ; p=0.006 ; 531 \pm 107, p=0.001)$. In CD patients with clinical acute flare (CDAI > 220), miR-320a expression level were significantly increased as compared to $\mathrm{CD}$ patients in clinical remission (CDAI $<150 ; 2677 \pm 637$ vs. $57 \pm 9 ; \mathrm{p}<0.001)$ and showed a strong correlation with endoscopic disease activity $\left(\mathrm{r}^{2}=0.70\right)$. Similarly, in UC patients, miR-320a also revealed a significant increase in patients with low (pMayo 3-4), moderate (pMayo 5-6) and severe clinical disease activity (pMayo $>6$ ) as compared to UC patients in remission $(259 \pm 47 ; 281 \pm 26 ; 1090 \pm 204$ vs. $76 \pm 13$, all $\mathrm{p}<0.001)$. Furthermore, we detected a significantly enhanced miR-320a expression with increasing endoscopic disease activity (eMayo 1: $89 \pm 14$ vs. eMayo 2: $301 \pm 50, \mathrm{p}=0.006$; vs. eMayo 3: $775 \pm 245 ; \mathrm{p}=0.02$ ). Most importantly, miR-320a expression in CD and UC patients with acute flare of disease was significantly increased as compared to patients with infectious colitis $(53 \pm 12, \mathrm{p}<0.001)$.

Conclusion: The miR-320a expression in peripheral blood from IBD patients follows the clinical and endoscopic disease activity and may distinguish between IBD and infectious colitis. Therefore, miR-320a might serve as biomarker to non-invasively assess the disease activity in IBD patients.

Disclosure of Interest: All authors have declared no conflicts of interest.

\section{Reference}

1. Cordes F, Bruckner M, Lenz P, et al. MicroRNA-320a strengthens intestinal barrier function and follows the course of experimental colitis. Inflamm Bowel Dis 2016;22:2341-55.

\section{P1675 COST EFFECTIVENESS OF IBDOC FAECAL} CALPROTECTIN AS A SURROGATE MARKER OF MUCOSAL HEALING POST INDUCTION OF BIOLOGICAL AGENTS IN INFLAMMATORY BOWEL DISEASE

G. El-Safi, K. Sugrue, D. Fitzgerald, C. Byron, L. Barry, M. Farman, A. Alhanaee, M. Buckley, J. Mccarthy

Dept. Of Gastroenterology, Mercy University Hospital, Cork/Ireland

Contact E-mail Address: jafaralsafi@hotmail.com

Introduction: Traditionally in our unit all IBD patients started on anti-TNF therapy are followed up at 3 months in the clinic and we aim to do a colonoscopy at 6 months to assess for mucosal healing. Recently we have started using a relatively new technology, called IBDoc, which allows testing the faecal calprotectin at home using a smart phone application and the results are automatically updated in our database.

Aims \& Methods: We aimed to evaluate the cost effectiveness of using IBDoc faecal calprotectin post induction of biological agents. The data was collected retrospectively from ourIBDoc data base. All patients that were commenced on anti-TNF therapy for IBD and trained in using IBDoc at home were included. IBDoc faecal calprotectin was tested at 3 and 6 month post induction of biological agents.

Results: Total number included in the study was 131 patients. $40 \%$ had normal calprotectin at 3 month saving 53 follow up clinic visits (cost of clinical visit 128 euro), and $75 \%$ had a normal faecal calprotectin at 6 months saving 40 routine colonoscopy (cost of colonoscopy 337 euro). 78 patients had a raised faecal calprotectin at 3 month, of which $28 \%$ had a normal faecal calprotectin at 6 month saving 22 follow-up colonoscopy. In total 53 clinical visits and 62 colonoscopies were avoided.

Conclusion: This study demonstrate a significant cost effectiveness of using IBDoc faecal calprotectin post induction of anti-TNF therapy, as well as reducing the waiting time for both clinic visits and colonoscopies.

Disclosure of Interest: G. El-Safi: Abbvie

All other authors have declared no conflicts of interest.

P1676 DOSE OPTIMISATION OF INFLIXIMAB: COMPARATIVE STUDY OF A RAPID TEST AND A ESTABLISHED ELISA METHOD

A. Echarri Piudo ${ }^{1}$, G. Molina ${ }^{1}$, A. Lopez Insua ${ }^{1}$, H. Ferreiro-Mazón ${ }^{2}$, B. Fernandez Saya ${ }^{2}$, J. Cid Fernandez ${ }^{3}$, V. Ollero ${ }^{4}$

${ }_{1}^{1}$ Gastroenterology, H. arquitecto marcide, Ferrol/Spain

${ }^{2}$ Inmunology, $H$. arquitecto marcide, Ferrol/Spain

${ }^{3}$ Inmunology, Hospital Teresa Herrera, Coruña/Spain

${ }^{4}$ Gastroenterology, Complejo Hospitalario de Ferrol, Ferrol/Spain 
Contact E-mail Address: ana.echarri.piudo@sergas.es

Introduction: Infliximab (IFX) levels have been associated with clinical and endoscopic outcomes in patients with inflammatory bowel disesase (IBD). Therapeutic drug monitoring (TDM) and a treat-to-target therapeutic approach may arise as a clinical strategy to optimise infliximab efficacy, safety and cost. The current IFX assay test and the cut-off levels used in practice, are based on a high time consuming ELISA procedure, processed in a batch-like method (tecnical delay) and requiring high expertise. An easy to use, rapid point-of-care test would dramatically reduce the turnaround time for results, allowing a faster decision. The aim of this work is to compare a newly developed point-of-care diagnostic test (Quantum Blue ${ }^{\circledR}$ assay) with the standard ELISA method (Promonitor ${ }^{\circledR}$ assay) determining a correlation factor that permits therapeutic management in a similar way.

Aims \& Methods: A total of 135 serum sample from patients with CD and UC. IFX concentration in serum samples were determined using a well established IFX-especific ELISA assay (Promonitor ${ }^{\circledR}$ ) and the tested assay Quantum Blue ${ }^{\circledR}$. According to the manufacturer, the lower and upper limits of quantification are: In the Quantum Blue assay $0.4 \mu \mathrm{g} / \mathrm{ml}$ and $20 \mu \mathrm{g} / \mathrm{ml}$, respectively In the Promonitor assay $0.035 \mu \mathrm{g} / \mathrm{ml}$ and $14.4 \mu \mathrm{g} / \mathrm{ml}$, respectively. All statistics were carried out using the statistical programs IBM SPSS statistics 21 and Epidat version 4.2 .

Results: The IFX levels measured by the point-of-care method were higher than those measured by established ELISA (Promonitor level: mean 4.67, median 3.2 s.d. 4.39 (0.035-14.4)); Quantum Blue level mean 6.31; median 3.7 s.d. 6.27 $(0.4-20)$. The intraclass correlation coefficients were $0.81(\mathrm{p}<0.001)$. The standard ELISA had a high $(\mathrm{r}=0.89)$ and significant $(\mathrm{p}<0.001)$ correlation with the Quantum Blue $\mathbb{R}$ assay. A Bland-Altman analysis showed a bias of $1.88 \%$ confirming the overall excellent correlation of the two methods. The results for each method were stratified according to a commonly accepted therapeutic range (3$7 \mathrm{ug} / \mathrm{ml}$; lower than 3 and higher than 7) with a high agreement. We estimated a simplified score to convert the "point-of-care" level into "Promonitor" level and facilitate dose management: "Nivel de Promonitor $=0,793+0,615 *$ Nivel QB". Conclusion: Quantum Blue $($ assay is a really useful technique enabling the fast and friendly quantitative determination of IFX levels to ensure correct dosing in IBD.

Disclosure of Interest: All authors have declared no conflicts of interest.

\section{P1677 RISK FACTORS ASSOCIATED WITH A HIGH RISK OF COLORECTAL CANCER IN PATIENTS WITH ULCERATIVE COLITIS. PRELIMINARY RESULTS FROM THE IBSEN COHORT}

P. Klepp ${ }^{1}$, M. C. Smaastuen ${ }^{2}$, M. Hoivik ${ }^{1}$, B. Mom ${ }^{1}$, I. Study Group ${ }^{1}$

${ }^{1}$ Clinical Medicine, University of Oslo, Oslo/Norway

${ }^{2}$ University of Oslo, Oslo/Norway

Contact E-mail Address: pasklepp@gmail.com

Introduction: The significance of established risk factors for colorectal cancer in inflammatory bowel disease remains debated.

Aims \& Methods: Our aim was to evaluate how individual disease variables contribute to the development of colorectal cancer. A population-based inception cohort of patients with ulcerative colitis patients diagnosed between 1990-1994 have been prospectively followed at one, five, 10 and 20 years after inclusion. In total we identified 517 patients, $266(51.5 \%)$ were males, aged median 37.4 (min 4 , max 88) years at inclusion. The following variables were analyzed: age at ulcerative colitis diagnosis, gender, presence of low or high grade dysplasia, use of azathioprine and 5-amino salicylic acid, extent of mucosal inflammation, first-grade relative with colorectal cancer.

Results: We identified 10 patients diagnosed with colorectal cancer after being diagnosed with ulcerative colitis. Age at ulcerative colitis diagnosis and low grade dysplasia were significantly associated with higher risk of CRC (HR $=1.06$, $95 \%$ CI [1.02-1.11], $\mathrm{p}=0.003$ and $\mathrm{HR}=86.495 \% \mathrm{CI}[10.1-740.3], \mathrm{p}<0.001$ for age and low grade dysplasia respectively). Patients who were older than 40 years at diagnosis had 4 times higher risk of developing colorectal cancer compared to patients diagnosed with ulcerative colitis at a younger age. $\mathrm{HR}=4.08$, $95 \%$ CI $[1.02,16.39], \mathrm{p}=0.047$. Male gender $(\mathrm{HR}=1.949$, use of azathioprine $(\mathrm{HR}=2.50)$, use of 5 -amino salicylic acid $(\mathrm{HR}=2.92)$ and extent $(\mathrm{HR}=1.78)$ were also associated with higher risk of CRC, however the estimates did not reach the level of statistical significance. Males diagnosed with UC when older than 40 years and with extent of inflammation had $8.4 \%$ probability of developing CRC within 20 years after their diagnosis

Conclusion: Our results show that the presence of low grade dysplasia significantly increases the risk of colorectal cancer. The study was limited by the sample size.

Disclosure of Interest: All authors have declared no conflicts of interest.

\section{P1678 MANAGEMENT OF COMPLEX PERIANAL FISTULAE: WHEN TO SUSPECT INFLAMMATORY BOWEL DISEASE?}

M. F. Cardoso ${ }^{1}$, J. C. Branco ${ }^{1}$, A. M. Oliveira ${ }^{1}$, C. G. Rodrigues ${ }^{1}$, L. Santos ${ }^{1}$, C. Carneiro ${ }^{2}$, J. Reis ${ }^{1}$

${ }^{1}$ Gastroenterology, Hospital Professor Doutor Fernando Fonseca, Amadora/

Portugal

${ }^{2}$ Surgery, Hospital Professor Doutor Fernando Fonseca, Amadora/Portugal

Contact E-mail Address: marianafcardoso@gmail.com

Introduction: Perianal fistulae are a frequent manifestation of inflammatory

bowel disease (IBD) often associated with significant morbidity, which may precede or appear simultaneously with intestinal disease. ${ }^{1}$ The approach to patients with perianal fistulae and no intestinal symptoms is poorly defined, and it remains to elucidate if there is a subgroup of patients in whom a more extensive search for IBD is warranted.

Aims \& Methods: Unicentric retrospective cohort study including patients evaluated between 2012 and 2016 for complex perianal fistulae, defined according to the American Society of Colon and Rectal Surgeons ${ }^{2}$. Patients with known IBD, malignancy or history of pelvic radiation where excluded. Patients in whom the diagnosis of IBD was established were compared to the others.

Results: 124 patients were included (mean age $48.2 \pm 17.8$ years; $71.0 \%$ men), with a mean follow-up of 26 months. 18 patients $(14.5 \%)$ were diagnosed with IBD, namely Crohn's disease. The classification of the fistulous tracts was as follows: intersphicteric $(71.8 \%)$, transphincteric $(38.7 \%)$, extrasphincteric $(7.3 \%)$, suprasphincteric $(5.6 \%)$, horseshoe $(24.2 \%)$ and with an abscess $(44.4 \%)$. The majority of patients with IBD presented intestinal symptoms (15/ 18 ), in 8 cases preceding the perianal disease but not motivating investigation in the past. In $3 / 18(16.7 \%)$ patients diagnosed with IBD, perianal fistula was the sole clinical manifestation of the disease. Patients with IBD were younger (30.1 vs 51.4 years, $\mathrm{p}<0.001)$, had a higher incidence of perianal abscesses $(88.9 \%$ vs $36.8 \%, \mathrm{p}<0.001)$, more persistent $/$ recurrent disease $(88.9 \%$ vs $50.6 \%, \mathrm{p}=0.003)$ and a tendency to a higher number of fistulous tracts $(38.9 \%$ had $\geq 5$ tracts vs $17.9 \%$ in the group without IBD; $\mathrm{p}=0.059)$.

Conclusion: In this cohort of patients with complex perianal fistulae, although in the majority of cases the diagnosis of IBD was suggested by the presence of intestinal symptoms, these were absent in $16.7 \%$ patients. There should be a higher suspicion of IBD in young patients and in those who have perianal disease of greater complexity.

Disclosure of Interest: All authors have declared no conflicts of interest.

\section{References}

1. Gionchetti P, Dignass A, Danese S, et al. 3rd EUROPEAN Evidence-based consensus on the diagnosis and management of Crohn's disease 2016: Part 2: Surgical management and special situations. $J$ Crohns Colitis. 2017;11(2):135-149.

2. Vogel JD, Johnson EK, Morris AM, Paquette IM, Saclarides TJ, Feingold DL, Steele SR. Clinical Practice Guideline for the Management of Anorectal Abscess, Fistula-in-Ano, and Rectovaginal Fistula. Dis Colon Rectum. 2016 Dec;59(12):1117-1133.

\section{P1679 THE ADDITION OF AN IMMUNOSUPPRESSANT IS AN EFFECTIVE OPTIMIZATION STRATEGY AFTER LOSS OF RESPONSE TO ANTI-TNF ALPHA MONOTHERAPY IN PATIENTS WITH INFLAMMATORY BOWEL DISEASE: A TWO-YEAR STUDY}

F.S. Macaluso ${ }^{1}$, C. Sapienza ${ }^{1}$, M. Ventimiglia ${ }^{1}$, S. Renna ${ }^{1}$, G. Rizzuto ${ }^{1}$, R. Orlando ${ }^{1}$, M. Di Pisa ${ }^{2}$, M. Affronti ${ }^{1}$, E. Orlando ${ }^{1}$, M. Cottone ${ }^{1}$, A. Orlando ${ }^{1}$ ${ }^{1}$ Division of Internal Medicine, "Villa Sofia-Cervello" Hospital, Palermo/Italy ${ }^{2}$ Gastroenterology and Endoscopy Unit, "Villa Sofia-Cervello" Hospital, Italy, Palermo/Italy

\section{Contact E-mail Address: fsmacaluso@gmail.com}

Introduction: In patients with inflammatory bowel disease (IBD) the addition of an immunosuppressant (IM) after loss of response to anti-TNF alpha monotherapy is regarded as an emerging strategy of therapeutic optimization. However, few clinical data have been reported to date.

Aims \& Methods: The aim of this study was to evaluate efficacy and tolerability of this "selective" combination therapy in patients with IBD. All consecutive patients with loss of response to anti-TNF alpha monotherapy despite an intensive dose optimization who added an IM from October 2014 to October 2016 were entered in a prospectively maintained database. The steroid-free remission and the clinical response, this latter defined as a clinical improvement (reduction of Harvey-Bradshaw Index $\geq 3$ for Crohn's disease and of Mayo Partial Score $\geq 2$ for ulcerative colitis compared with baseline) with a concomitant reduction of steroid dosage compared with baseline and discontinuation within twelve weeks, were set as clinical end points.

Results: Among 630 patients treated with biologics during the study period, 46 (33 Crohn's disease and 13 ulcerative colitis) were included (table 1). A total of 31 patients $(67.4 \%)$ were treated with an intravenous anti-TNF $\alpha$ (infliximab, as originator product or biosimilar), while $15(32.6 \%)$ with a subcutaneous anti$\mathrm{TNF} \alpha$ agent (10 adalimumab and 5 golimumab). The mean doses of thiopurines used in combination therapy were below those regarded as therapeutic in IBD, methotrexate was mostly employed at a dose of $15 \mathrm{mg} /$ week, and all patients treated with mycophenolate mofetil were able to tolerate the target dose of $1500 \mathrm{mg} /$ day. The mean duration of follow-up was $12.8 \pm 7.3$ months. Twentyone patients $(45.7 \%)$ remained on combination therapy at the end of follow-up: $15(32.6 \%)$ maintained a steroid-free remission, and 6 patients $(13.0 \%)$ achieved a clinical response. In patients who experienced a treatment success, median value of C-reactive protein decreased from the baseline to the end of follow-up $(13.2$ vs. $3.0, p=0.01$; normal values $<5 \mathrm{mg} / \mathrm{L})$. Adverse events leading to treatment discontinuation were reported in 8 out of 46 patients $(17.4 \%)$. 
Table 1

Variable
Age (years), mean \pm S.D.
Male gender, n (\%)
Smokers, n (\%) Never Current Ex
Type of Disease, n (\%) Crohn's Disease Ulcerative Coliti
Duration of disease (years), mean \pm S.D.
Localization of the disease, n (\%) Crohn's Disease Ileal
Ileocolic Colic Upper gastrointestinal tract ${ }^{1}$ Perianal
Disease involvement Ulcerative Colitis Proctitis Left-
sided Extensive

Behavior (Crohn's Disease), n (\%) Inflammatory Stricturing Fistulizing

Previous resections (Crohn's Disease), n (\%)

Disease Activity Crohn's Disease Harvey-Bradshaw Index $(\mathrm{HBI})$, mean \pm S.D. Ulcerative Colitis Mayo Partial score, mean \pm S.D. C-Reactive Protein, mean \pm S.D. (n.v. $<5 \mathrm{mg} / \mathrm{L}$ )

Endoscopy within six months of combination therapy initiation $(n=26)$ Crohn's Disease SES-CD, mean \pm S.D. Rutgeerts score, $0 / 1 / 2 / 3 / 4$ Ulcerative Colitis Mayo Endoscopic score, $0 / 1 / 2 / 3$

Extraintestinal manifestations, $\mathrm{n}(\%)$

Concurrent prednisone at baseline, n (\%)

Line of anti-TNF $\alpha$ therapy, n (\%) First Second Third

Experienced to the IM used in combination therapy, n (\%)

Time between start of anti-TNF $\alpha$ therapy and addition of IM (months), median (I.Q.I.)

Combination therapy, $\mathrm{n}(\%) \mathrm{IFX}+\mathrm{AZA}$ IFX + 6-MP IFX + MTX IFX + MMF TOTAL IV ADA + AZA ADA + MTX GOL + AZA GOL + MMF TOTAL SC

\section{$\mathrm{N}=46$}

$45.6 \pm 13.0$

$22(47.8 \%)$

$40(87.0 \%)$

$4(8.7 \%)$

$2(4.3 \%)$

$33(71.7 \%)$

$13(28.3 \%)$

$12.4 \pm 8.4$

$10(30.4 \%)$

$18(54.5 \%)$

$3(9.1 \%)$

$2(6.0 \%)$

$6(18.2 \%)$

$0(0.0 \%)$

$6(46.2 \%)$

$7(53.8 \%)$

$15(45.5 \%)$

$14(42.4 \%)$

$4(12.1 \%)$

$17(53.1 \%)$

$7.7 \pm 3.6$

$4.1 \pm 1.6$

$16.6 \pm 25.6$

$9.9 \pm 5.8$

$0 / 1 / 2 / 0 / 5$

$0 / 0 / 4 / 6$

$21(45.7 \%)$

$20(43.5 \%)$

$10(21.7 \%)$

$34(73.9 \%)$

$2(4.4 \%)$

$13(28.3 \%)$

7 (13.5)

$11 / 46(23.9 \%)$ $5 / 46(10.9 \%)$

$12 / 46(26.1 \%)$

$3 / 46(6.5 \%)$

$31 / 46(67.4 \%)$

$2 / 46(4.3 \%)$

$8 / 46(17.4 \%)$

$2 / 46(4.3 \%)$

$3 / 46(6.5 \%)$

$15 / 46(32.6 \%)$

Conclusion: In patients with IBD the addition of an immunosuppressant is an effective and safe optimization strategy after loss of response to anti-TNF alpha monotherapy. Low doses of IM are sufficient to achieve a clinical response in this setting.

Disclosure of Interest: F.S. Macaluso: Lecture grants from MSD and Takeda Pharmaceuticals

S. Renna: advisory board member for AbbVie and MSD; lecture grants from AbbVie, MSD, Takeda Pharmaceuticals, Zambon.

M. Cottone: Received financial support for the organization of a second level Master degree in inflammatory bowel disease from AbbVie, MSD, Takeda Pharmaceuticals and Sofar

A. Orlando: Advisory board member for AbbVie, MSD, Takeda Pharmaceuticals; lecture grants from AbbVie, MSD, Takeda Pharmaceuticals, Sofar, Chiesi.

All other authors have declared no conflicts of interest.

P1680 CORRELATION BETWEEN EXTRA-INTESTINAL

MANIFESTATIONS AND ANTI-DRUG ANTIBODIES

DEVELOPMENT IN CROHN'S DISEASE PATIENTS

M. Crespi ${ }^{1}$, G. Bodini ${ }^{1}$, E.G. Giannini ${ }^{1}$, V. Savarino ${ }^{1}$, A. Jain ${ }^{2}$, E. Savarino ${ }^{3}$ ${ }^{1}$ Department Of Internal Medicine, IRCCS San Martino DIMI, Genova (GE) Italy

${ }^{2}$ Prometheus Laboratories Inc., San Diego/United States of America

${ }^{3}$ Uo Gastroenterology Department Of Surgical, Oncological And

Gastroenterological Sciences, University Of Padova, Padova Italy, University of

Padova, Padova/Italy

Contact E-mail Address: mattiacrespi4@gmail.com

Introduction: Extra-Intestinal Manifestations (EIM) are frequently (up to 40\%) encountered in patients with Crohn's Disease (CD). Commonly, their presence is associated to a more severe degree of luminal disease and lower response to conventional therapy (i.e. immunosuppressants). Drug trough levels are associated with biological drug response, while the role of Anti-drug Antibodies (AAA) is still debated. Moreover, the predicting factors associated with AAA development have not been thoroughly studied yet. To the best of our knowledge, there are no studies correlating the presence of EIM and AAA development.

Aims \& Methods: The aim of our prospective study was to identify an association between the presence of EIM and the development of AAA in CD patients treated with biological therapy. We prospectively enrolled $60 \mathrm{CD}$ patients $(32$ males, median age $=46 y$, range $=21-72$ ) treated either with adalimumab (ADA n $39,65 \%$; IFX n $21,35 \%$ ) with a median follow-up of 80 (range 14-206) weeks. Blood samples were drawn at standardized time pointsassessed using an homogenous mobility shift assay (HMSA; Prometheus Lab, San Diego, United States). Results: AAA were detected in $27(45 \%)$ patients and their development has proved to be more frequent in subjects treated with IFX rather than those in therapy with ADA (n 14, 66.6\% vs $13,33.3 \%, P=0.017$ ). EIM were observed in $26(43.3 \%)$ patients, without any significant difference between ADA and IFX patients (n 17, 51.5\% vs $\mathrm{n}=9,42.9 \%, P=1$ ). We found that ADA treated patients with EIM were more likely to develop AAA $(\mathrm{n}=9,52.9 \%$ versus $\mathrm{n}=4,18.2 \%, P=0.039$ ) while no statistically significant association between EIM and AAA development was observed in IFX treated patients $(n=5$, $55.5 \%$ versus $\mathrm{n}=9,75 \%, P=0.4$ ).

Conclusion: We found that ADA-treated patients with EIM tend to develop more frequently AAA. Assuming that the presence of AAA reduces the effectiveness of biological therapy, the presence of EIM may be considered a predictive factor for loss of response to biological therapy with anti-tumor necrosis factor alpha drugs.

Disclosure of Interest: All authors have declared no conflicts of interest.

P1681 THE PRESENCE OF IRRITABLE BOWEL SYNDROME-TYPE SYMPTOMS IN MICROSCOPIC COLITIS IS ASSOCIATED WITH INCREASED PSYCHOLOGICAL COMORBIDITY AND IMPAIRED QUALITY OF LIFE

J. S. Kane ${ }^{1}$, A. J. Irvine ${ }^{1}$, Y. Derwa ${ }^{1}$, O. Rotimi ${ }^{2}$, A.C. Ford ${ }^{1}$

${ }^{1}$ Leeds Gastroenterology Insitute, St James's University Hospital, Leeds/United Kingdom

${ }^{2}$ Department Of Histopathology, St James's University Hospital, Leeds/United Kingdom

Contact E-mail Address: jackjohnkane@googlemail.com

Introduction: Patients with microscopic colitis (MC) often present with abdominal pain and diarrhoea ${ }^{1}$, and small cross-sectional surveys suggest that up to one-third may meet diagnostic criteria for irritable bowel syndrome (IBS) ${ }^{2}$. However, the impact of IBS-type symptoms in patients with MC, in terms of their effect on psychological health and quality of life has not been assessed.

Aims \& Methods: We conducted a cross-sectional survey of individuals with MC. We analysed demographic data, symptoms that met the Rome III criteria for IBS, mood (via hospital anxiety and depression scale (HADS)) and somatoformtype behaviour (via patient health questionnaire-15 (PHQ-15)), and quality of life (QOL) (via SF-36) in order to examine risk factors for, and impact of, IBS-type symptoms in patients with MC.

Results: In total, 157 individuals with $\mathrm{MC}$ returned completed questionnaires, 53 $(36.6 \%)$ of whom met the Rome III diagnostic criteria for IBS. The commonest histological subtype of MC was collagenous colitis $(52.9 \%, \mathrm{n}=83)$, followed by lymphocytic colitis $(38.2 \%, \mathrm{n}=60)$, and MC-not otherwise specified $(8.9 \%$, $\mathrm{n}=14$ ). Individuals meeting the Rome III criteria for IBS had significantly higher levels of anxiety (HADS-anxiety score 8.6 vs. 5.0, $\mathrm{P}<0.001$ ), depression (HADS-depression score 6.1 vs. $3.5, \mathrm{P}=0.001$ ), and somatoform-type behaviour (PHQ-15 score 12.5 vs. $7.9, \mathrm{P}<0.001$ ) compared with individuals who did not. Individuals meeting the Rome III criteria scored significantly worse on all domains of the SF-36, except for physical functioning. There were also trends towards these individuals being younger ( 65 years vs. 69.2 years, $\mathrm{P}=0.011$ ) or taking proton-pump inhibitors $(58.5 \%, \mathrm{n}=31$ vs. $42.4 \%, \mathrm{n}=39, \mathrm{P}=0.062)$.

Conclusion: More than one-third of individuals with MC met diagnostic criteria for IBS and these individuals reported higher levels of anxiety, depression, and somatisation plus impaired quality of life. Management strategies for these symptoms are required.

Disclosure of Interest: J.S. Kane: This work was supported by an investigatorinitiated grant from Dr. Falk Pharma UK Ltd.

A.C. Ford: This work was supported by an investigator-initiated grant from Dr. Falk Pharma UK Ltd.

All other authors have declared no conflicts of interest.

\section{References}

1. Pardi DS, Kelly CP. Microscopic Colitis. Gastroenterology 2011;140(4):1155-1165.

2. Kamp EJ, Kane JS, Ford AC. Irritable Bowel Syndrome and Microscopic Colitis: A Systematic Review and Meta-analysis. Clin Gastroenterol Hepatol 2016;14(5):659-68. 
P1682 ANEMIA IN INFLAMMATORY BOWEL DISEASES

S. Bernardo ${ }^{1}$, J.R. Carvalho ${ }^{1}$, S. Fernandes ${ }^{1}$, A. Costa ${ }^{1}$, L. Branco ${ }^{1}$, A.R. Gonçalves ${ }^{1}$, C. Baldaia ${ }^{1}$, A. Valente ${ }^{1}$, P. M. Santos ${ }^{1}$, A. Oliveira ${ }^{2}$, L. Correia ${ }^{1}$, J. Velosa ${ }^{1}$

${ }^{1}$ Gastroenterology, Hospital Santa Maria, Centro Hospitalar Lisboa Norte, Lisbon/Portugal

${ }^{2}$ Hematology, Hospital Santa Maria, Centro Hospitalar Lisboa Norte, Lisbon/ Portugal

Contact E-mail Address: soniamcb@hotmail.com

Introduction: Anaemia is one of the most common extraintestinal manifestations in inflammatory bowel disease (IBD), with iron deficiency being the most frequent cause.

Aims \& Methods: To characterize anemia in a population of patients with IBD and to evaluate the efficacy of anti-TNF and carrier therapy. We retrospectively analyzed 169/1166 patients with IBD followed in a tertiary center between 2010 2016. All the patients received replacement therapy with ferric carboxymaltose, ferric saccharum or transfusional support. We analyzed the effect of immunosuppressive therapy with and without anti-TNF: group 1 and 2 respectively. Active disease was defined as C-reactive protein (CRP) $\geq 0.5 \mathrm{mg} / \mathrm{dl}$, faecal calprotectin $(\mathrm{FC}) \geq 50 \mathrm{ug} / \mathrm{ml}$ or presence of ulcers in colonoscopy. Anaemia was defined acording to ECCO criteria (iron deficiency anaemia (IDA), anaemia of chronic disease (ACD) and mixed anaemia (MA). Multifactorial anaemia (MFA) was diagnosed when there was also vitamin B12 or folate deficiency.

Results: 169 patients were included: 111 with Crohn's Disease (CD), 54 with Ulcerative Colitis (UC) and 4 with unclassified IBD. 44.4\% were male and the mean age was 32.2 years (range $7-82$ ). $98.2 \%$ had anemia and $1.8 \%$ had only iron deficiency; $3.5 \%$ and $18.3 \%$ had a deficit of vitamin $\mathrm{B} 12$ and folate. The most common etiologies of anemia found in CD and UC were IDA $(54.1 \%$ and $46.3 \%)$, followed by MFA (19.8\% and $24.1 \%)$, ACD $(15.3 \%$ and $24.1 \%)$ and MA $(8.1 \%$ and 5.6\%). Female gender (OR 3.743 95\% CI 1.554-9.018, $\mathrm{p}=0.003)$, previous surgery (OR $2.84595 \%$ CI $1.111-7.284, \mathrm{p}=0.02)$ and penetrating pattern in $\mathrm{CD}(\mathrm{OR} 8.25295 \% \mathrm{CI} 1.289-52.919, \mathrm{P}=0,026)$ were predictors of normal hemoglobin ( $\mathrm{Hb}$ ). CRP was associated with highest ferritin values $(294.85 \pm 302.00$ vs $102.10 \pm 127.75, \mathrm{p}<0.001)$. Hb and CRP had a negative correlation (p.c-0.176, p =0.022). In UC, CRP and FC normalization was associated with higher $\mathrm{Hb}$ and transferrin saturation $(13.63 \pm 1.66$ vs $12.47 \pm 1.38$, $\mathrm{p}=0.005$ and $51.33 \pm 26.50$ vs $27.63 \pm 9.96, \mathrm{p}=0.048$ ). There was no difference in the parameters evaluated comparing groups 1 and 2 . There was also no significant difference in the variation of $\mathrm{Hb}$ and iron values after treatment with ferric saccharum or ferric carboxymaltose.

Conclusion: In a population of patients with IBD, iron deficiency was the main cause of anaemia and the two forms of intravenous iron replacement were equally effective. In the group of patients with UC, elevated CRP was associated with anaemia.

Disclosure of Interest: All authors have declared no conflicts of interest.

\section{References}

1. Axl U Dignass et al.European Consensus on the Diagnosis and Management of Iron Deficiency and Anaemia in Inflammatory Bowel Diseases, ECCO guideline. Journal of Crohn's and Colitis, 2015, 211-222.

2. Thomas W. Lee et al. Iron replacement therapy in inflammatory bowel disease patients with iron deficiency anemia: A systematic review and meta-analysis. Journal of Crohn's and Colitis, (2012) 6, 267-275.

3. Anna Testa et al. The burden of anaemia in patients with inflammatory bowel diseases. Digestive and Liver Disease 48 (2016) 267-270.

\section{P1683 TROUGH LEVELS AND ANTIBODIES TO USTEKINUMAB ARE NOT CORRELATED TO RESPONSE TO USTEKINUMAB TREATMENT IN CROHN'S DISEASE PATIENTS}

C. Painchart ${ }^{1}$, S. Brabant ${ }^{2}$, N. Duveau ${ }^{3}$, P. Wils ${ }^{1}$, M. Boualit ${ }^{1}$, M. Nachury ${ }^{1}$, J. Branche ${ }^{1}$, R. Gerard ${ }^{1}$, P. Desreumaux ${ }^{1}$, M. Labalette ${ }^{2}$, B. Pariente ${ }^{1}$ ${ }^{1}$ Gastroenterology department, CHRU Lille, Université Lille ${ }^{2}$ Lille/France ${ }^{2}$ Immunology laboratory, CHRU Lille, Université Lille ${ }^{2}$ Lille/France ${ }^{3}$ Gastroenterology department, CHR Roubaix, Roubaix/France

\section{Contact E-mail Address: claire.painchart@laposte.net}

Introduction: Ustekinumab (UST) has been shown to be effective in refractory Crohn's disease (CD) in phase III trials. The aim of the present study was to prospectively evaluate the association between UST trough levels and anti-ustekinumab antibodies, with the response and the remission to induction and maintenance UST treatment in CD patients.

Aims \& Methods: We performed a prospective study including all CD patients refractory to anti-TNF who received subcutaneous UST from September 2015 to November 2016 in the tertiary French referral center of Gastroenterology in Claude Huriez hospital in Lille. During induction patients received $90 \mathrm{mg}$ of SC UST at week 0, 4 and 12. During the maintenance phase patients received $90 \mathrm{mg}$ of SC UST every 8 weeks that could be optimized by shortening injection interval to every 4 weeks in case of loss of response. Clinical response was defined by a decreased Harvey Bradshaw Index (HBI) by 3 points, clinical remission by HBI $<5$, loss of response by new increase of HBI. UST trough levels and antibodies were dosed at 12 weeks, and at a single time-point for patients who had received more than 3 months of UST. The results of dosage were obtained by enzyme-Linked ImmunoSorbent Assay technique. We evaluated the correlation between clinical and biological response and remission to UST, and UST through levels and antibodies concentrations. Differences between independent groups were traced with the use of the Mann-Whitney exact test.
Results: Forty-eight patients with active disease received at least three UST injections and were prospectively included. At time of ustekinumab introduction $77 \%$ of patients received concomitant immunosuppressant and $42 \%$ received corticosteroids. At the end of the induction phase (week 12) clinical response was observed in $57 \%$ patients. There was no significant difference in mean UST trough levels in patients who responded to UST induction (median $1160 \mathrm{ng} / \mathrm{ml}$; IQR: 603-1644) as compared to patients who did not respond (median $1556 \mathrm{ng} /$ ml; IQR: $494-2758, p=0.24)$. Forty-three $(90 \%)$ patients received at least 4 injections of UST, with 12 patients who were optimized at the time of dosages. Clinical response was observed in 30/43 $(70 \%)$ patients. Median UST concentration in clinical responder was $1359 \mathrm{ng} / \mathrm{ml}$ (IQR: 554-2068) and $2392 \mathrm{ng} / \mathrm{ml}$ in non-responder (IQR: 1496-3494), with no significant difference between the two groups of patients $(\mathrm{p}=0.20)$. UST antibodies were undetectable for the 48 patients.

Conclusion: We confirmed that UST treatment is effective in the majority of CD patients refractory to anti-TNF agents. Median trough levels to UST are not correlated to response and remission to UST induction and maintenance treatment, with no antibodies developed against UST.

Disclosure of Interest: All authors have declared no conflicts of interest.

\section{P1684 ECONOMIC IMPLICATIONS IN INFLAMMATORY BOWEL DISEASES: RESULTS FROM A RETROSPECTIVE ANALISYS IN AN ITALIAN CENTRE}

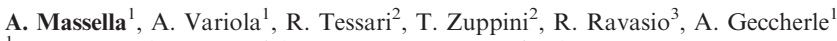
${ }_{1}^{1}$ Gastroenterology, Ospedale Sacro Cuore, Negrar/Italy

${ }^{2}$ Pharmacy, Ospedale Sacro Cuore, Negrar/Italy

${ }^{3}$ Health Publishing \& Services, Milano/Italy

Contact E-mail Address: arianna.massella@gmail.com

Introduction: Inflammatory bowel diseases (IBDs) are chronic conditions characterized by elevated costs (both direct and indirect). Over the last years also a significative healthcare burden associated with IBD has emerged, due to an increasing use of biological therapies and hospitalization costs. Despite the creation of local or regional databases in Italy data regarding healthcare expenditure are lacking.

Aims \& Methods: The aim of this study was to evaluate costs comprehensive of biological treatments and hospitalizations in a series of patients with ulcerative colitis (UC) and Crohn's disease (CD) and their correlation with demographic and clinical variables. Disease severity was evaluated by clinical scores (partial Mayo score for UC, Harvey Bradshaw Index for CD). We analyzed retrospectively patients treated by biologics referred to our IBD Unit between May 2015 and April 2016 who underwent at least six months follow-up (last visit October 2016). As regards biological therapies costs burdened by our Centre pharmacy for each drug (Infliximab, Adalimumab, Golimumab, Vedolizumab) and for single patient were evaluated. About hospitalizations the average costs of hospital care specific for a department through fares for homogeneous groups of patients i.e. Diagnosis-Related Group (DRG) were collected. The mean overall monthly expenditure for each case was then evaluated.

Results: We collected clinical-economical data of 142 patients in biological treatment in the selected period ( $52 \mathrm{UC}$, mean age 44.3 years and male $40.4 \%$; $90 \mathrm{CD}$, mean age 38.8 years and male $56.7 \%)$. About half of $C D$ patients $(48.9 \%)$ underwent previous intestinal surgery. The disease severity was higher in UC group vs $\mathrm{CD}$ one. In UC group Infliximab was the most prescribed biologic $(51.9 \%)$, followed by Golimumab (29.9\%) and Adalimumab (21.2\%). While CD patients were treated with Adalimumab in $54.4 \%$ and Infliximab in $45.6 \%$. The average cost for month of treatment was $1235.41 \pm 358.38 €$ for UC and $1148.92 \pm 337.36$ $€$ for CD (no statistical differences between the groups). In both groups expenditure due to biologics amounts for more than $80 \%$. We analyzed costs in groups different for sex, age and disease activity (only the last one was associated with increased costs with $\mathrm{R}^{2}=0.84$ for $\mathrm{UC}$ and 0.95 for $\mathrm{CD}$ ). The cost increases in patients with more lines of therapy in UC (not in $\mathrm{CD}$ ) but the difference wasn't significative.

Conclusion: In our study the main cost is due to biological therapy but the subjects enrolled were the most severe in comparison to the whole IBD population under conventional therapy. No differences were found between the type of biologic administered and the way of administration (intravenous or subcutaneous) so the therapeutical choice could be driven by clinical reasons and not only economic ones.

Disclosure of Interest: All authors have declared no conflicts of interest. 
P1685 THE SICILIAN NETWORK FOR INFLAMMATORY BOWEL DISEASE (SN-IBD): PRELIMINARY DATA ON EFFICACY OF BIOLOGICAL THERAPY

A. Orlando ${ }^{1}$, F.S. Macaluso ${ }^{1}$, W. Fries ${ }^{2}$, A.C. Privitera ${ }^{3}$, M. Cappello ${ }^{4}$, S. Siringo $0^{5}$, G. Inserra ${ }^{6}$, A. Magnano ${ }^{6}$, R. Di Mitri ${ }^{7}$, N. Belluardo ${ }^{8}$, G. Scarpulla ${ }^{9}$, G. Magri ${ }^{10}$, A. Trovatello ${ }^{11}$, A. Carroccio ${ }^{12}$, S. Genova $^{13}$, C. Bertolami ${ }^{14}$, R. Vassallo ${ }^{15}$, C. Romano ${ }^{16}$, S. Pellegrino ${ }^{17}$, M. Citrano ${ }^{18}$, S. Accomando ${ }^{19}$, M. Ventimiglia ${ }^{1}$, S. Renna ${ }^{1}$, R. Orlando ${ }^{1}$, G. Rizzuto $^{1}$, E. Vinci ${ }^{20}$, M. Cottone ${ }^{1}$

${ }^{1}$ Division of Internal Medicine, "Villa Sofia-Cervello" Hospital, Palermo/Italy ${ }^{2}$ Inflammatory Bowel Disease Unit, A.O.U. Policlinico "G. Martino", Messina, Italy., Messina/Italy

${ }^{3}$ IBD Unit, A.O. "Cannizzaro", Catania, Italy, Catania/Italy

${ }^{4}$ Gastroenterology And Hepatology Unit, A.O.U. Policlinico "G. Giaccone",

Palermo University, Palermo/Italy

${ }^{5}$ Gastroenterology Unit, ARNAS "Garibaldi", Catania/Italy

"Internal Medicine Unit, A.O.U. Policlinico "Vittorio Emanuele", Catania, Italy., Catania/Italy

${ }^{7}$ Gastroenterology And Endoscopy Unit, ARNAS Civico-Di Cristina-Benfratelli Hospital, Palermo/Italy

${ }^{8}$ Gastroenterology Unit, A.O. "Guzzardi", Vittoria, Italy., Vittoria/Italy

${ }^{9}$ A.O. S.Elia di Caltanissetta, P.O. Raimondi U.O.C. di Gastroenterologia,

Caltanissetta/Italy

${ }^{10}$ Gastroenterology Unit, A.O. "Santa Marta e S. Venera", Acireale, Italy.,

Acireale/Italy

${ }^{11}$ Surgery Unit, A.O. "Umberto I", Siracusa, Italy, Siracusa/Italy

${ }^{12}$ Internal Medicine, Giovanni Paolo II Hospital, Agrigento/Italy

${ }^{13}$ Gastroenterology and Endoscopy Unit, A.O. "S. Antonio Abate", Trapani, Italy,

Trapani/Italy

${ }^{14}$ Gastroenterology Unit, A.O.O.R. "Papardo Piemonte", Messina, Italy, Messina/ Italy

${ }^{15}$ Gastroenterology, Ospedale "Bucchieri la Ferla" Fatebenefratelli, Palermo/Italy ${ }^{16}$ Pediatric Gastroenterology And Endoscopy, Pediatric Department, Pediatric Department, Messina/Italy

${ }^{17}$ Pediatric Department, Gastroenterology Unit, University of Messina, Messina/ Italy

${ }^{18}$ Pediatric Unit, A.O.O.R. "Villa Sofia-Cervello", Palermo, Italy, Palermo/Italy ${ }^{19}$ Pediatric Unit, A.O.U. Policlinico "G. Giaccone", Palermo, Italy, Palermo/Italy ${ }^{20}$ IBD Unit, Azienda Ospedaliera per l'Emergenza Cannizzaro, Catania/Italy

Contact E-mail Address: ambrogiorlando@gmail.com

Introduction: The monitoring of appropriateness, costs, and clinical outcomes of biological therapy in inflammatory bowel disease (IBD) is a relevant need.

Aims \& Methods: We aimed to evaluate all these issues in Sicily through a webbased network of all prescribing centers. The Sicilian Network for Inflammatory Bowel Disease (SN-IBD) is composed by a super Hub coordinator centre and five Hub plus ten Spoke centres. From January 2013, all IBD patients starting a biological agent (incident cases) or already on treatment (prevalent cases) were entered in a web based software. Herein we report data of incident cases about the efficacy of biological therapy after twelve weeks and one year of treatment. As clinical end-point, we set remission (corresponding to a Mayo Partial Score $<2$ for UC, and to a Harvey-Bradshaw Index $<5$ for $\mathrm{CD}$ ), and response (reduction of Harvey-Bradshaw Index $\geq 3$ for $C D$ and Mayo Partial Score $\geq 2$ for UC compared with baseline).

Results: From January 2013 to January 2017, 1578 patients were included. Incident cases were 1151 (808 Crohn's disease [CD], 335 ulcerative colitis [UC], 8 unclassified colitis). Considering that $22.2 \%$ of patients experienced more than one line of therapy, a total of 1407 treatments were reported. CD: there was a higher proportion of patients naïve to biologics among those on adalimumab (ADA) compared with infliximab (IFX) and vedolizumab (VDZ) $(89.3 \%$ vs. $53.3 \%$ vs. $30.6 \%, p<0.001)$. At week 12 , there was a higher rate of response remission for adalimumab (ADA) compared with infliximab (IFX) and vedolizumab (VDZ) $(79.8 \%$ vs. $71.8 \%, \mathrm{p}=0.005$, and $79.8 \%$ vs. $47.2 \%, \mathrm{p}<0.001$, respectively), and a higher efficacy of IFX compared with VDZ $(71.8 \%$ vs. $47.2 \%, p=0.004)$. The superiority of ADA over IFX remained significant in naïve patients $(81.5 \%$ vs. $73.7 \%$, OR $1.581, \mathrm{p}=0.026)$, but not in non-naïve. At week 52, ADA had a higher rate of response compared to IFX $(65.4 \%$ vs. $56.0 \%, p=0.018)$. However, there was no statistical difference between the two drugs when patients were stratified in naïve and non-naïve, while ADA was superior over IFX in patients with ileo-colic disease $(68.8 \%$ vs. $48.4 \%$, OR $2.282, p=0.001)$. UC: there was a higher proportion of patients naive to biologics among those on IFX compared with GOL and ADA $(91.6 \%$ vs. $44.1 \%$ vs. $55.4 \%, \mathrm{p}<0.001)$. At week 12, there was a higher rate of response/remission for IFX compared with golimumab (GOL) $(71.8 \%$ vs. $56.6 \%, p=0.034)$, but this difference was lost when patients were stratified in naïve and non-naïve. At week 52 , IFX had a higher rate of response/remission compared to GOL $(58.2 \%$ vs. $38.2 \%, \mathrm{p}=0.039)$ and ADA $(58.2 \%$ vs. $33.9 \%, \mathrm{p}=0.002)$. However, IFX was superior to GOL in naive patients only $(60.2 \%$ vs. $26.7 \%$, OR $4.165, p=0.018$; interaction test: $p=0.02$ ), but non in non-naïve, and IFX was superior to ADA in naive patients only $(60.2 \%$ vs. $37.8 \%$, OR $2.650, \mathrm{p}=0.029)$. For both $\mathrm{CD}$ and UC, no significant difference in efficacy was observed between IFX originator and biosimilars. Several factors were identified as predictor of response - independently of the drug employed - at multivariable logistic regression analysis (table 1).

Table 1

\begin{tabular}{llll}
\hline DISEASE-TIME POINT & VARIABLE & OR & $\mathrm{p}$ \\
\hline CD-12 WEEKS & Naive to biologics & 1.989 & $<0.001$ \\
& Age (continuous variable) & 0.982 & $<0.001$ \\
& Upper GI tract involvement & 0.388 & 0.002 \\
& Perianal disease & 0.650 & 0.029 \\
CD-52 WEEKS & Naive to biologics & 2.262 & $<0.001$ \\
& Upper GI tract involvement & 0.394 & 0.005 \\
UC-12 WEEKS & Naive to biologics & 2.188 & $<0.001$ \\
UC-52 WEEKS & Naive to biologics & 2.338 & $<0.001$ \\
& Current smoking & 1.562 & 0.007 \\
\hline
\end{tabular}

Conclusion: In one of the largest "real-life" series of IBD patients on biological therapy reported to date, ADA in CD had a higher success compared to IFX at both 12 and 52 weeks; however, this results could be influenced by the preference of ADA as first-line anti-TNF drug in CD. IFX in UC was superior to GOL and ADA at 52 weeks; once again, this result could be influenced by the preference of IFX as first-line anti-TNF agent in UC; no difference was found between GOL and ADA in UC. Being naïve to biologics is a relevant predictor of response in both $\mathrm{CD}$ and $\mathrm{UC}$ at any time point. No significant difference in efficacy was observed between IFX originator and biosimilars.

Disclosure of Interest: A. Orlando: Advisory board member for AbbVie, MSD, Takeda Pharmaceuticals; lecture grants from AbbVie, MSD, Takeda Pharmaceuticals, Sofar, Chiesi.

F.S. Macaluso: Lecture grants from MSD and Takeda Pharmaceuticals All other authors have declared no conflicts of interest.

\section{P1686 SINGLE-CENTRE EXPERIENCE WITH BIOLOGICAL TREATMENT IN BUDESONIDE-REFRACTORY MICROSCOPIC COLITIS PATIENTS}

N. Daferera ${ }^{1}$, S. Ignatova ${ }^{2}$, A. Münch ${ }^{2}$

${ }^{1}$ Gastroenterology, University Hospital of Linkoping, Linköping/Sweden

${ }^{2}$ Gastroenterology, Dept. of Gastroenterology, Linköping/Sweden

Contact E-mail Address: andreas.munch@regionostergotland.se

Introduction: Microscopic colitis (MC) is a common cause of chronic watery diarrhoea which is effectively treated with budesonide. However, in rare cases patients are refractory to budesonide leading to a severely deteriorated quality of life. Data on treatment options for budesonide refractory MC patients are sparse. We retrospectively evaluated the outcome of MC patients who have received biological therapy at our centre

Aims \& Methods: All patients with MC treated with biological therapy at the Department of Gastroenterology, University hospital Linkoping, Sweden were selected and the outcome recorded. Patients were investigated according to sex, age, disease subtype, clinical remission (defined as $<$ mean 3 and no watery stools/day/week), clinical response (defined as $50 \%$ reduction of mean stool frequency/day/week), side effects and long-term outcome.

Results: 14 patients (10 women) with mean age at diagnosis of 49 years (range 19$76)$, thereof 12 with collagenous colitis and 2 with lymphocytic colitis were investigated. All 14 patients had received anti-TNF agents (11 adalimumab (ADA), 3 infliximab (IFX). ADA was given as induction treatment (160-80-40 mg) and IFX $5 \mathrm{mg} / \mathrm{kg}$ at week 0,2 and 6 . Six patients achieved clinical remission. Of these, 2 patients maintained clinical remission for several years after induction treatment, 2 patients are on maintenance treatment with $40 \mathrm{mg} \mathrm{ADA} / \mathrm{eow}$, one patient on $400 \mathrm{mg}$ INF every 8 weeks and one patient had loss of response and did not gain clinical remission despite the use of a third anti-TNF agent (certolizumab). Four patients had a clinical response but stopped treatment due to side effects (vomiting, anxiety, fatigue and eczema-all reversible). Four patients had no effect on anti-TNF despite the fact that 2 patient had even tested both antiTNF agents. Of these 4 patients who failed anti-TNF, 3 patients received treatment with vedolizumab (300 mg week 0,2 and 6$)$ without effect. One patient had further treatment with rituximab $(1000 \mathrm{mg}$ twice) and another patient tested ustekinumab (390 mg once); both did not achieve any clinical improvement.

Conclusion: Budesonide refractory MC patients can achieve clinical remission or response on anti-TNF agents. In the cases that failed anti-TNF, further treatment with vedolizumab $(n=3)$, rituximab $(n=1)$ and ustekinumab $(n=1)$ did not improve the clinical condition. Prospective studies with long duration are necessary to evaluate the efficacy of various biological treatments in budesoniderefractory MC.

Disclosure of Interest: All authors have declared no conflicts of interest. 
P1687 BENEFICIAL EFFECT OF A LOW FODMAPS DIET IN DIFFERENT GASTROINTESTINAL DISORDERS

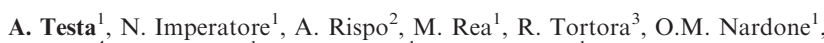
L. Lucci $^{4}$, G. Accarino ${ }^{1}$, N. Caporaso ${ }^{1}$, F. Castiglione ${ }^{1}$

${ }^{1}$ Gastroenterology, Department of Clinical Medicine and Surgery, School of Medicine Federico II of Naples, Naples/Italy

${ }^{2}$ Hepatology Unit, Cardarelli Hospital, Naples/Italy

${ }^{3}$ Nutrition, Department of Clinical Medicine and Surgery, School of Medicine Federico II of Naples, Naples/Italy

Contact E-mail Address: annatesta82@virgilio.it

Introduction: Recent studies have shown that FODMAPs (Fermentable Oligosaccharides, Disaccharides, Monosaccharides, and Polyols)-free diet is effective in subjects with Irritable Bowel Syndrome (IBS). Patients with Inflammatory Bowel Diseases (IBD), and celiac disease (CD) can experience functional gastrointestinal symptoms unrelated to inflammation, but data about the use of low FODMAPs diet in these settings is still scarce.

Aims \& Methods: To evaluate the usefulness of a low FODMAPs diet on patients with IBS, non-active IBD, and CD on strict gluten-free diet (GFD), we performed a dietetic interventional prospective study evaluating the effect of a low FODMAPs diet on subjects affected by IBS, CD following at least a 1-year-GFD and IBD who had been experiencing abdominal symptoms without signs of active inflammation. Each subject was put on a low FODMAPs diet after being evaluated by filling out questionnaires concerning on quality of life and symptoms experienced (IBS-SSS and SF-36), and was re-evaluated twice, first after 1 month and second after 3 months.

Results: 127 subjects were enrolled: 56 with IBS, 30 with IBD and 41 with CD. The analysis of the IBS-SSS survey showed that abdominal symptoms improved after 1 month and 3 months of low FODMAPs diet in all subjects, with statistically significant difference within each group at T0 (average score in IBS: $293 \pm 137 \mathrm{SD}$, average score in IBD: $206 \pm 86 \mathrm{SD}$, average score in CD: $222 \pm 65 \mathrm{SD}, \mathrm{p}<0.001)$. Furthermore, by analysing the SF-36 questionnaire, while we did not observe any significant difference between the three groups, in terms of response to diet $(\mathrm{p}=\mathrm{NS})$, we observed a clinical improvement from T0 to T3, after the start of the diet, for most of the questionnaire's domains. Conclusion: A low FODMAPs diet could be a valid option to counter abdominal symptoms in patients with IBS, non-active IBD or CD on GFD, and, thus improve their quality of life and social relations.

Disclosure of Interest: All authors have declared no conflicts of interest.

P1688 EFFICACY OF VEDOLIZUMAB ON INTESTINAL AND ARTICULAR SYMPTOMS: REAL-LIFE DATA FROM THE SICILIAN NETWORK FOR INFLAMMATORY BOWEL DISEASE (SN-IBD)

F.S. Macaluso ${ }^{1}$, R. Orlando ${ }^{1}$, W. Fries ${ }^{2}$, M. Scolaro ${ }^{2}$, A. Magnano ${ }^{3}$, D. Pluchino ${ }^{3}$, M. Cappello ${ }^{4}$, G. C. Morreale, S. Siringo ${ }^{5}$, A.C. Privitera ${ }^{6}$, C. Ferracane ${ }^{6}$, N. Belluardo ${ }^{7}$, N. Alberghina ${ }^{7}$, M. Ventimiglia ${ }^{1}$, S. Renna ${ }^{1}$, M. Di Pisa $^{8}$, M. Cottone ${ }^{1}$, A. Orlando ${ }^{1}$

${ }^{1}$ Division of Internal Medicine, "Villa Sofia-Cervello" Hospital, Palermo/Italy "Inflammatory bowel disease Unit, A.O.U. Policlinico "G. Martino", Messina, Italy, Messina/Italy

${ }^{3}$ Gastroenterology Unit, A.O.U. Policlinico "Vittorio Emanuele", Catania, Italy, Catania/Italy

${ }^{4}$ Di.bi.mis., Gastroenterology and Hepatology Unit, Palermo/Italy

${ }^{5}$ Gastroenterology Unit, ARNAS "Garibaldi", Catania/Italy

"IBD Unit, A.O. "Cannizzaro", Catania, Italy, Catania/Italy

${ }^{7}$ Gastroenterology Unit, A.O. "Guzzardi”, Vittoria, Italy, Vittoria/Italy

${ }^{8}$ Gastroenterology and Endoscopy Unit, "Villa Sofia-Cervello" Hospital, Italy,

Palermo/Italy

Contact E-mail Address: fsmacaluso@gmail.com

Introduction: Vedolizumab (VDZ) is a new biologic agent approved for the treatment of adult patients with moderately to severely active ulcerative colitis (UC) and Crohn's disease (CD)

Aims \& Methods: The Sicilian Network for Inflammatory Bowel Disease (SNIBD) is a group composed by all Sicilian centres prescribing biologics. These centres continuously enter in a web based software all real-life data about prescriptions ond outcomes of biological therapy in patients with inflammatory bowel disease (IBD). Herein we report data on efficacy of VDZ on intestinal and articular symptoms after 10 and 24 weeks of treatment. As clinical end-point, we set steroid-free remission (corresponding to a Mayo Partial Score $<2$ for UC, and to a Harvey-Bradshaw Index $<5$ for $\mathrm{CD}$ ), and clinical response (reduction of Harvey-Bradshaw Index $\geq 3$ for $C D$ and Mayo Partial Score $\geq 2$ for UC compared with baseline with a concomitant reduction of steroids dosage at week 10, and complete discontinuation at week 24).

Results: From July 2016 to April 2017, 163 patients ( 84 with CD and 79 with UC) were included (table 1). At week 10, a steroid-free remission was obtained in 71 patients $(43.6 \%)$, while a clinical response in $37(22.7 \%)$. Out of 71 patients reaching 24 weeks of follow-up, $29(40.8 \%)$ were in steroid-free remission, and $10(14.1 \%)$ had a clinical response. No significant difference in terms of clinical benefit (rate of remission plus clinical response) among patients with $\mathrm{UC}$ and $\mathrm{CD}$ was reported at week $10(68.4 \%$ vs. $64.3 \%$, respectively; $\mathrm{p}=0.58)$ and at week 24 $(54.3 \%$ vs. $55.6 \%$, respectively; $\mathrm{p}=0.91)$, and no difference was observed comparing naïve and non naïve patients, neither at week $10(61.5 \%$ vs. $67.7 \%$, respectively; $\mathrm{p}=0.48)$ nor at week $24(30.0 \%$ vs. $59.0 \%$, respectively; $\mathrm{p}=0.11)$. At multiple logistic regression analysis, a longer duration of disease (OR 0.961 , $\mathrm{p}=0.047)$ and presence of steroid-dependence (OR $0.189, \mathrm{p}=0.033$ ) were predictors of reduced rates of clinical benefit at week 10, while a low serum level of C-reactive protein at baseline $(\mathrm{OR} 0.950, \mathrm{p}=0.031)$ was predictor of clinical benefit at week 24. An improvement of articular symptoms was reported in $39.5 \%$ of patients with active spondyloarthritis at baseline at week 10 , and in $45.4 \%$ of patients at week 24 . The only factor associated with articular response was the coexistence of clinical benefit on intestinal symptoms, both at week 10 $(\mathrm{OR} 8.471, \mathrm{p}=0.05)$ and week $24(\mathrm{OR} 5.600, \mathrm{p}=0.08)$. Three inductions or flares of spondyloarthritis during treatment with VDZ were reported. Table 1

\begin{tabular}{ll}
\hline Variable & $\mathrm{N}=163$ \\
\hline
\end{tabular}

Age (years), mean \pm S.D.

$50.6 \pm 16.0$

Male gender, n (\%)

$94(57.7 \%)$

$134(82.2 \%)$

$17(10.4 \%)$

$12(7.4 \%)$

Type of Disease, n (\%) Crohn's Disease Ulcerative $84(51.5 \%)$ $79(48.5 \%)$ Colitis

Duration of disease (years), mean \pm S.D.

Localization of the disease, n (\%) Crohn's Disease Ileal Ileocolic Colic Upper gastrointestinal tract ${ }^{1}$ Perianal Disease Ulcerative Colitis Proctitis Leftsided Extensive

$11.7 \pm 8.5$

$22(26.2 \%)$

$50(59.5 \%)$

$8(9.5 \%)$

$4(4.8 \%)$

$12(14.3 \%)$

$1(1.3 \%)$

$25(31.6 \%)$

$53(67.1 \%)$

Behavior (Crohn's Disease), n (\%) Inflammatory Stricturing Fistulizing

Previous resections (Crohn's Disease), n (\%)

Disease Activity Harvey-Bradshaw Index, Crohn's Disease - mean \pm S.D. Mayo Partial score, Ulcerative Colitis - mean \pm S.D. C-Reactive

Protein, mean \pm S.D. (n.v. $<5 \mathrm{mg} / \mathrm{L}$ )

Endoscopy within three months of initiation of VDZ $(\mathrm{n}=)$ Crohn's Disease SES-CD, mean \pm S.D. Rutgeerts score, $0 / 1 / 2 / 3 / 4$ Ulcerative Colitis Mayo Endoscopic score, $0 / 1 / 2 / 3$

Extraintestinal manifestations IBD-associated SpA Past history (inactive at initiation of VDZ) Peripheral arthropathy Axial arthropathy Active at initiation of VDZ Peripheral arthropathy Axial arthropathy Cutaneous Ocular $36(42.9 \%)$ $42(50.0 \%)$ $6(7.1 \%)$

$51(60.7 \%)$

$8.3 \pm 4.6$

$3.9 \pm 2.4$

$9.8 \pm 15.7$

$7.6 \pm 7.5$

$1 / 3 / 4 / 5 / 13$

$2 / 4 / 40 / 24$

$7(4.3 \%)$

$0(0 \%)$

$40(24.5 \%)$

$15(9.2 \%)$

$4(2.5 \%)$

$1(0.6 \%)$

$124(76.1 \%)$

Previous biological treatments Yes No (naïve to biologics)

Indication for treatment with VDZ Failure of anti-

$\mathrm{TNF} \alpha$ therapy Contraindications to anti-TNF $\alpha$ therapy

Steroid-dependent, n (\%)

$39(23.9 \%)$

$109(66.9 \%)$

$54(33.1 \%)$

$144(88.3 \%)$

$103(63.2 \%)$

$13(8.0 \%)$

Concurrent therapy with immunosuppressant, $\mathrm{n}$ $(\%)$

Conclusion: In this large cohort of Sicilian IBD patients, VDZ showed good efficacy after 10 and 24 weeks of treatment, particularly in those with a shorter duration of disease and a limited inflammatory burden. A subset of patients reported improvement also on articular symptoms, probably as a consequence of the concomitant control of gut inflammation.

Disclosure of Interest: F.S. Macaluso: Lecture grants from MSD and Takeda Pharmaceuticals

S. Renna: advisory board member for AbbVie and MSD; lecture grants from AbbVie, MSD, Takeda Pharmaceuticals, Zambon.

M. Cottone: financial support for the organization of a second level Master degree in inflammatory bowel disease from AbbVie, MSD, Takeda Pharmaceuticals and Sofar

A. Orlando: Advisory board member for AbbVie, MSD, Takeda Pharmaceuticals; lecture grants from AbbVie, MSD, Takeda Pharmaceuticals, Sofar, Chiesi.

All other authors have declared no conflicts of interest. 
P1689 POSITIVE PHARMACOKINETIC EFFECT OF AZATHIOPRINE CO-MEDICATION ON INFLIXIMAB TROUGH LEVELS IS DOSE-DEPENDENT

B. Kadleckova ${ }^{1}$, K. Otottova ${ }^{1}$, J. Lucenicova $^{2}$, P. Bozek ${ }^{2}$, P. Mikus ${ }^{3}$,

Z. Zelinkova ${ }^{4}$

${ }^{1}$ IBD Center, Bratislava/Slovak Republic

${ }^{2}$ Department Of Biochemistry \& Hematology, St Michael's Hospital, Bratislaval Slovak Republic

${ }^{3}$ Nuclear Pharmacy, Pharmaceutical Faculty of Comenius University, Bratislava/ Slovak Republic

${ }^{4}$ Department Of Gastroenterology, St Michael's Hospital, Bratislava/Slovak Republic

Contact E-mail Address: zuzana.zelinkova@nsmas.sk

Introduction: Combined immune suppression of anti-tumour necrosis factor (antiTNF) biologicals and thiopurines is superior to respective monotherapies in remission induction and maintenance of response in inflammatory bowel disease (IBD). One of the putative mechanism of this clinical benefit is mutually positive pharmacokinetic effect of thiopurines on antiTNF levels and vice versa. It has been suggested that for this synergistic effect, reduced dose of thiopurines might be sufficient but the data supporting this hypothesis are still limited. Aims \& Methods: The aim of the study was to assess the differences of infliximab trough levels according to the dose of concomitantly used thiopurines. All IBD patients treated with infliximab (Remicade $\AA$ ) in two IBD centra between November 2015 and April 2017 were eligible. Infliximab trough levels were routinely measured in all patients with maintenance infliximab therapy using com-

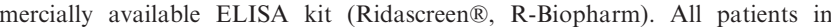
remission with stable dose regimen of $5 \mathrm{mg} / \mathrm{kg}$ every 8 weeks at the time of the first assessment of infliximab trough levels were identified retrospectively from the medical records. The differences in the proportion of patients with adequate trough levels $(3-12 \mu \mathrm{g} / \mathrm{mL})$ between patients on infliximab monotherapy, reduced (below $2 \mathrm{mg} / \mathrm{kg}$ ) azathioprine (AZA) dose vs. full ( 2 to $2.5 \mathrm{mg} / \mathrm{kg}$ ) AZA dose were analyzed statistically.

Results: Out of a total of 214 IBD patients treated with infliximab, there were 154 in remission at the time of the first assessment of infliximab trough levels. After excluding patients with previously intensified dose regimen, 125 patients were further analyzed. Among these $125 \mathrm{pts}, 41 \mathrm{pts}(33 \%)$ were on infliximab monotherapy, 58 pts $(46 \%)$ were using combined immune suppression with a reduced dose of AZA and $26(21 \%)$ were using the full AZA dose concomitantly with infliximab. Both groups, patients with infliximab monotherapy as well as patients using a reduced AZA dose had significantly lower percentage of patients with therapeutic levels of infliximab compared with the group using the full dose AZA co-medication $(41 \%$ vs. $64 \%$ vs. $81 \%$; infliximab monotherapy, reduced AZA dose and full AZA dose, respectively; $\mathrm{p}<0.001$ for both comparisons, infliximab monotherapy vs. AZA full dose and reduced AZA dose vs. full AZA dose)

Conclusion: The proportion of patients with adequate infliximab trough levels is significantly higher in patients with full dose of concomitant azathioprine compared with the patients using a reduced dose of azathioprine. Thus, in order to maximize the chances for sustained benefit from infliximab maintenance treatment, the combined immune suppression should comprise full dose of azathioprine.

Disclosure of Interest: Z. Zelinkova: Speaker's fee from Abbvie, MSD, Takeda, Janssen

All other authors have declared no conflicts of interest.

P1690 C $C_{60}$ FULLERENES ATTENUATE THE INTENSITY OF COLON DAMAGE AND EXTRAINTESTINAL MANIFESTATIONS ON RAT ACUTE ULCERATIVE COLITIS MODEL

H. Kuznietsova ${ }^{1}$, I. Byelinska ${ }^{2}$, N. Dziubenko ${ }^{1}$, O. Gurniak ${ }^{1}$, O. Lynchak ${ }^{1}$, Y. Prylutskyy ${ }^{1}$, V. Rybalchenko

${ }^{1}$ Educational Scientific Center "Institute Of Biology And Medicine", Taras Shevchenko National University of Kyiv, Kyiv/Ukraine

${ }^{2}$ Department Of Membranology And Cytology, Taras Shevchenko National University of Kyiv, Kyiv/Ukraine

Contact E-mail Address: biophyz@gmail.com

Introduction: The primary drugs used for inflammatory bowel disease (IBD) treatment have some adverse effects and often are ineffective, so the need fo more potent and more reliable medications is clear. A significant role at all the stages of the inflammatory process is attributed to reactive oxygen species, therefore the use of antioxidants is a promising direction of the IBD therapy.

Aims \& Methods: $\mathrm{C}_{60}$ fullerenes are the effective free radicals scavengers [1] therefore the evaluation of possible protective properties of water-soluble pristine $\mathrm{C}_{60}$ fullerenes using the simulation of acute ulcerative colitis (UC) in rats was aimed to be discovered. The pristine $\mathrm{C}_{60}$ fullerene aqueous colloid solution $\left(\mathrm{C}_{60} \mathrm{FAS}\right.$; initial concentration $\left.0.15 \mathrm{mg} / \mathrm{ml}\right)$ was prepared according to the protocols developed before [2]. UC was simulated by acetic acid intracolonic instillation. $\mathrm{C}_{60}$ FAS was intraperitoneally or intrarectally applied at dose of $0.5 \mathrm{mg} / \mathrm{kg}$ $\mathrm{C}_{60}$ daily for 2 times after UC induction. The colon injury was estimated semiquantitatively on macro- and light microscopy levels using 10- and 14- grade scales respectively, and the grade of total injury (GTI) was calculated, permeability of colon epithelium was estimated by phenol red dye daily excretion. The states of the colon, liver, pancreas and spleen were assessed by histological (hematoxylin-eosin staining) and biochemical (plasma blood liver enzymes activity) methods, quantitative blood indices were calculated and leucograms were analyzed.

Results: Colon wall edema, hyperemia and hemorrhage on bowel internal surface, ulcers of different size, epithelium desquamation and inflammatory features manifested by submucosa lymphoid and histiocytic infiltration and extravasation appeared under UC condition. GTI was equal to $12\left(\mathrm{GTI}_{\mathrm{contol}}=0\right)$. Rat diurnal phenol red dye excretion exceeded the control one 6.6 times suggesting the damage of colon epithelial barrier. Degenerative features of exocrine pancreas and liver injury were also observed in UC rats. Anemia features manifested by hemoglobin concentration and red blood cell count decrease, reticulocytes volume, hematocrit and the platelet count increase could be caused by intestinal hemorrhages and subsequent erythro- and megakariocytopoiesis following active release of "young" erythrocytes (reticulocytes) and platelets into the blood. The last could be confirmed with histological assay (rise of the erythroblastic islets and megakaryocytes numbers in the spleen). Increase the number of neutrophils and spleen lymphatic follicles suggested the inflammatory process in the organism. The application of $\mathrm{C}_{60} \mathrm{FAS}$ by both ways caused GTI drop down to 6 points, restored the mucosa epithelial barrier integrity, attenuated the signs of anemia and normalized the platelet and neutrophils counts, AST and the state of spleen. In addition, $\mathrm{C}_{60} \mathrm{FAS}$ intraperitoneally restored the state of the pancreas but increased liver functional upload (as evidenced by elevated ALT activity), whereas its rectal application normalized both ALT and AST activities but didn't affect the pancreas. Moreover, $\mathrm{C}_{60}$ FAS rectally increased monocytes number, which could be explained by involving the latest in mucosa healing process.

Conclusion: $\mathrm{C}_{60} \mathrm{FAS}$ corrects local and systemic morphofunctional changes, conditioned by the induction of acute UC. The protective properties of $\mathrm{C}_{60}$ fullerenes against bowel, hematopoietic system and liver due to its local application are more expressed compared to their systemic one, but their impact on pancreas is controversial. Thus, water-soluble pristine $\mathrm{C}_{60}$ fullerenes could be used as efficient therapeutic agents at ulcerative colitis.

Disclosure of Interest: All authors have declared no conflicts of interest.

\section{References}

1. Prylutska S, et. al. Fullerenes, Nanotubes, Carbon Nanostruct, 2008; 16(5-6): 698-705.

2. Ritter U, et. al. Fullerenes, Nanotubes, Carbon Nanostruct, 2015; 23(6): 530-4.

\section{P1691 EARLY CLINICAL REMISSION BY WEEK 2 PREDICTS GOOD SHORT- AND LONG-TERM EFFICACY OF TACROLIMUS THERAPY IN PATIENTS WITH MODERATE TO SEVERE STEROID- REFRACTORY ULCERATIVE COLITIS}

N. Oshima, S. Ishihara, K. Kawashima, H. Sonoyama, N. Yamashita,

N. Fukuba, Y. Mishima, I. Moriyama, T. Yuki, Y. Kinoshita

Gastroenterology And Hepatology, Shimane University School of Medicine,

Izumo/Japan

Contact E-mail Address: si360405@med.shimane-u.ac.jp

Introduction: Tacrolimus, a calcineurin inhibitor, has been shown to be safe and effective when used as salvage therapy for steroid-refractory ulcerative colitis (UC). Its pharmacological effect has been reported to be dependent on trough level in blood, though little is known regarding predictive factors in relation to the clinical efficacy of tacrolimus in UC patients.

Aims \& Methods: The aim of this study was to identify factors related to prediction of short- and long-time efficacy of tacrolimus for UC. We retrospectively reviewed the medical records of patients with moderate to severe steroid-refractory UC who were treated with tacrolimus as induction therapy at Shimane University Hospital between January 2010 and March 2016. Oral tacrolimus was administered at a whole-blood trough level of $10-15 \mathrm{ng} / \mathrm{mL}$ to induce remission and then $5-10 \mathrm{ng} / \mathrm{mL}$ to maintain remission. Following tacrolimus therapy for 3 months, patients in clinical remission were given azathioprine for maintenance at an appropriate dosage. Using the Rachmilewitz clinical activity index (CAI), clinical remission was defined as a score of $\leq 4$. Predictive factors associated with short- and long-term tacrolimus efficacy were analyzed by evaluating various clinical parameters.

Results: Thirty-six patients received oral tacrolimus for induction, of whom 22 $(61.1 \%)$ and $27(75 \%)$ experienced clinical remission at 2 and 12 weeks, respectively, after starting therapy. In patients showing short-term efficacy, the clinical remission rate at 2 weeks was significantly associated with CAI at 12 weeks. Interestingly, of the 22 patients in clinical remission within 2 weeks, 21 $(95.5 \%)$ remained in remission at 12 weeks. In contrast, only 6 of $14(42.9 \%)$ who did not achieve clinical remission at 2 weeks were not in clinical remission at 12 weeks. For evaluating the long-term efficacy of tacrolimus induction therapy, relapse-free periods were assessed using the Kaplan-Meier method. The relapsefree rate at 48 weeks was higher in patients who achieved clinical remission within 2 weeks as compared to those without remission at that time point $(79.0 \%$ vs. $60.0 \%$ ).

Conclusion: Tacrolimus induction therapy was effective for patients with moderate to severe steroid-refractory UC Our results clearly indicate that clinical remission achievement within 2 weeks is useful for predicting both short- and long-term outcome in UC patients treated with that therapy.

Disclosure of Interest: All authors have declared no conflicts of interest. 


\section{P1692 INFLIXIMAB (IFX) IN MODERATE TO SEVERE}

ULCERATIVE COLITIS (UC): COMPARISON BETWEEN

SCHEDULED TREATMENT STRATEGY AND BRIDGE STRATEGY

A.M. Sambuelli ${ }^{1}$, A. H. Gil ${ }^{1}$, S. M. Negreira ${ }^{1}$, M. I. Bellicoso ${ }^{1}$, S. Goncalves ${ }^{1}$, S.P. Huernos ${ }^{1}$, P.R. Tirado ${ }^{1}$, P. Chavero ${ }^{1}$, A. Cabanne ${ }^{2}$

${ }^{1}$ IBD Section - Medicine, Bonorino Udaondo Hospital, Buenos Aires/Argentina

${ }^{2}$ Pathology, Bonorino Udaondo Hospital, Buenos Aires/Argentina

Contact E-mail Address: alicia.sambuelli@gmail.com

Introduction: UC is a potentially severe disease that carries an increased risk of complications and colectomy. Immunosuppressant and biological therapies are relevant tools for complex patients. The ACCENT study showed that in Crohn's disease (CD), scheduled IFX infusions vs. episodic are associated with greater efficacy. In UC, historical difficulties of economic access had conditioned to our IBD center, to use IFX in moderate to severe UC as a bridge to thiopurines in pts $6 \mathrm{mp} /$ aza naïve. In UC, the mentioned strategy was insufficiently compared with a regimen of scheduled IFX treatment, that currently we use.

Aims \& Methods: Our aim was to compare (retrospectively) in moderate to severe UC the results of induction with IFX (in thiopurine naïve pts.) continuing with $6 \mathrm{mp} /$ aza maintenance vs. similar induction followed by scheduled IFX maintenance strategy.

We included a cohort of moderate to severe UC treated with IFX in an IBD center (2006 to 2015) comparing results between IFX bridge followed by thiopurines (re-induction when available for moderate to severe relapse) vs. scheduled IFX (induction $0,2,6 \mathrm{wks}$ and $8 \mathrm{wks}$ ' interval infusions maintenance) Optimization (by frequency of intervals) was allowed in both modalities. Comparisons: Kaplan Meier/Log rank test: a) Cumulative incidence of colectomy, b) survival times relapse free, c) survival times corticosteroid free. Results: We identified 135 UC patients receiving IFX for moderate to severe UC (M 60, F 75, Age (mean \pm SD) $35.9 \pm 13.2$, UC duration $5.8 \pm 5.9$ yrs, Extent: extensive $58.5 \%$, Left-sided $41.5 \%$, Activity: severe $78.5 \%$, moderate $21.5 \%$, mean Mayo score: $10.1 \pm 1.8$. Primary no responders at week 12 (n 25: 18 $5 \%$ ) were not considered in maintenance comparisons, which were performed in 110 out of the 135 pts. (Follow-up $37.5 \pm 24.0$ months) Groups: IFX bridge $(\mathrm{n}=51)$ and scheduled IFX ( $\mathrm{n} 59)$, which were not different in extent, and mean age, UC duration, Mayo score. Cumulative incidence of colectomy was significantly lower in the scheduled strategy (HR: 6.8805, 95\% CI 1.7207 to 27.5133 , $\mathrm{p}=0.0349$ ), survival times free of relapse (HR 3.1026, 95\% CI 1.8368 to 5.2405 , $\mathrm{p}<0.0001$ ) and free of corticosteroids (HR 2.6057, 95\% CI 1.5516 to 4.3757 , $\mathrm{p}=0.0003$ ). A proportion of UC significantly higher within the re-induced pts (IFX bridge) required a shift of biological drug compared with the scheduled strategy $(\mathrm{p}=0.016$, Fisher), despite of similar rates of optimization. Infusion reactions needing definitive IFX suspension were more frequent as a trend $(\mathrm{p}<0.06)$ in re-induced $\mathrm{pts}$

Conclusion: Similarly to was described for CD pts, in the CU, the scheduled IFX treatment strategy regimen, after a moderate-severe outbreak, seem to be associated with better long-term outcome regarding colectomy requirement, relapses, and need for corticosteroids, compared with a bridge IFX strategy followed by thiopurines

Disclosure of Interest: All authors have declared no conflicts of interest.

\section{P1693 ILLNESS PERCEPTIONS, COPING STRATEGIES, OUTCOMES AND THEIR CHANGES OVER TIME IN IBD PATIENTS WITH ARTHROPATHIES: A 12-MONTH FOLLOW-UP STUDY}

S. J.h. Van Erp ${ }^{1}$, M. Van Der $\mathrm{Have}^{2}$, H.H. Fidder ${ }^{2}$, D. Van Der Heijde ${ }^{3}$, R. Wolterbeek ${ }^{4}$, D. W. Hommes ${ }^{5}$, A. A. Kaptein 6 , A. E. Van Der Meulen - De Jong ${ }^{1}$

${ }^{1}$ Gastroenterology And Hepatology, Leiden University Medical Center, Leiden/ Netherlands

${ }^{2}$ Gastroenterology And Hepatology, University Medical Center Utrecht, Utrecht/ Netherlands

${ }^{3}$ Rheumatology, Leiden University Medical Center, Leiden/Netherlands ${ }^{4}$ Medical Statistics, Leiden University Medical Center, Leiden/Netherlands ${ }^{5}$ Center For Inflammatory Bowel Diseases, University of California Los Angeles, Los Angeles/United States of America

${ }^{6}$ Medical Psychology, Leiden University Medical Center, Leiden/Netherlands

Contact E-mail Address: s.j.h.van_erp@lumc.nl

Introduction: Arthropathies are the most common extra-intestinal manifestation (EIM) in patients with inflammatory bowel disease (IBD). Psychological and behavioural factors, including illness perceptions and coping strategies, perceived by IBD patients with arthropathies may differ from patients without arthropathies and may change during follow-up. Understanding these differences and changes over time, creates knowledge for health care professionals about interventions that may be effective in IBD patients with arthropathies.

Aims \& Methods: This longitudinal follow-up study evaluates the differences in illness perceptions (individual cognitions and emotions about a disease), coping strategies (behavioral efforts and strategies to deal with a chronic disease) and illness outcomes between IBD patients with and without arthropathies, and examines the changes of these variables in IBD patients with arthropathies after 12 months.

In total, 204 IBD patients with $(n=123)$ and without $(n=81)$ arthropathies completed questionnaires at baseline and after 1 year follow-up to assess illness perceptions (Revised Illness Perception Questionnaire), coping strategies (Coping with Rheumatic Stressors Questionnaire) and illness outcomes including quality of life (Short Form-36), and activity and work impairment (Work Productivity Activity Index). Linear regression analyses were used to assess the impact of arthropathies on illness perceptions, coping and illness outcomes compared with IBD patients without arthropathies. Mixed model analyses were used to evaluate the change of these factors in IBD patients with arthropathies over time. Results: Linear regression models demonstrated that arthropathies in IBD were associated with the illness perceptions strong 'illness identity' ( $B 1.15(95 \% \mathrm{CI}$ $0.31-1.98), p=0.007)$, strong 'cyclical timeline' (B 1.33 (95\% CI $0.33-2.34)$, $\mathrm{p}=0.010)$, more 'consequences' $(B 2.00(95 \% \mathrm{CI} 0.60-3.42), \mathrm{p}=0.006)$, stronger 'emotional representations' (B $1.58(95 \%$ CI $0.08-3.08), \mathrm{p}=0.039)$ and a low 'coherence' (B-1.29 $(95 \% \mathrm{CI}-2.45-(-0.14)), \mathrm{p}=0.029)$. In addition, the coping strategies increased 'diverting attention' ( $B \quad 1.34$ (95\% CI $0.02-2.66)$, $\mathrm{p}=0.047)$ and increased 'consideration' (B $1.18(95 \% \mathrm{CI} 0.10-2.27), \mathrm{p}=0.033)$, as well as the illness outcomes low physical (B -7.22 (95\% CI $-9.68-(-4.77))$, $\mathrm{p}<0.001)$ and mental $(B-3.10(95 \% \mathrm{CI}-5.99-(-0.23)), \mathrm{p}=0.035)$ health and increased activity impairment $(B 0.15(95 \%$ CI $0.07-0.23), \mathrm{p}<0.001)$ were related with arthropathies in IBD. After 12 months follow-up, lower scores on the illness perception dimension 'treatment control' $(p=0.001)$ and increased scores on the coping strategy 'pacing' $(\mathrm{p}=0.03)$ were found in IBD patients with arthropathies compared with baseline scores.

Conclusion: Suffering from arthropathies in IBD is strongly associated with different illness perceptions, coping strategies and illness outcomes and changes of these factors over time. As a gastroenterologist, addressing the maladaptive illness perceptions, coping strategies and related poor illness outcomes in these patients, may provide an important target for behavioural and physical interventions including cognitive behavioural therapy (CBT) or physical exercise. Disclosure of Interest: All authors have declared no conflicts of interest.

\section{P1694 INFLIXIMAB BIOSIMILAR CT-P13 THERAPY IS EFFECTIVE IN MAINTAINING ENDOSCOPIC REMISSION IN ULCERATIVE COLITIS-RESULTS FROM MULTICENTRE OBSERVATIONAL COHORT}

K. Farkas ${ }^{1}$, A. Balint ${ }^{1}$, M. Rutka ${ }^{1}$, M. Kolar ${ }^{2}$, M. Bortlik ${ }^{3}$, D. Duricova ${ }^{2}$, V. $\operatorname{Hruba}^{2}$, M. Lukas ${ }^{2}$, K. Mitrova ${ }^{2}$, K. Malickova ${ }^{2}$, M. Lukas ${ }^{2}$, Z. Szepes ${ }^{1}$

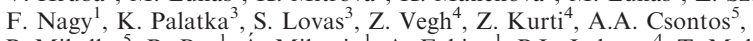
P. Miheller ${ }^{5}$, R. Bor ${ }^{1}$, Á. Milassin ${ }^{1}$, A. Fabian ${ }^{1}$, P.L. Lakatos ${ }^{4}$, T. Molnar ${ }^{1}$ ${ }^{1} 1$ st Department Of Medicine, University of Szeged, Szeged/Hungary

${ }^{2}$ IBD Clinical And Research Center, ISCARE I.V.F. a.s., Prague/Czech Republic ${ }^{3}$ Department Of Gastroenterology, University of Debrecen, Debrecen/Hungary ${ }^{4} 1$ st Department Of Medicine. Semmelweis University Faculty of Medicine $1 \mathrm{st}$ Dept. of Medicine - 1st Department of Medicine, Semmel, Budapest/Hungary ${ }^{5}$ 2nd Dept. Of Medicine, Semmelweis Univerisity 2nd Department of Internal Medicine, Budapest/Hungary

Contact E-mail Address: farkas.klaudia@gmail.com

Introduction: CT-P13, the first biosimilar monoclonal antibody to infliximab (IFX) has previously been confirmed to be efficacious in inducing mucosal healing in ulcerative colitis (UC) patients.

Aims \& Methods: The aim of this study was to evaluate the efficacy of CT-P13 therapy in maintaining mucosal healing in UC. Patients diagnosed with UC, who were administered CT-P13 from June 2014 at 4 Hungarian and one Czech IBD Centre were prospectively enrolled. Sigmoideoscopy was performed at week 14 and week 54 to assess mucosal healing. Mucosal healing was defined as Mayo endoscopic subscore of 0 or 1 . Complete mucosal healing was defined as Mayo endoscopic subscore of 0 . CT-P13 trough levels, antibody positivity, serum inflammatory markers as CRP level, fecal calprotectin at weeks 14 and 54, concomitant steroid and azathioprine therapy at the time of induction therapy and at weeks 14 and 54, previous use of anti TNF drug and the need of dose intensification as possible predictive factors for mucosal healing at week 54 were evaluated. Results: Seventy-five UC patients were included in the study of which 74 patients completed the induction therapy and 54 patients had already completed the 54week treatment period. Mucosal healing was shown in $55.4 \%$ of the patients at week 14 and in $61.7 \%$ at week $54(\mathrm{p}=0.33)$. Complete mucosal healing was present in $24.3 \%$ at week 14 , but in $36.2 \%$ at week 54 . The median values of $\operatorname{CRP}(p=0.017)$, leukocytes $(p=<0.001)$, thrombocytes $(p=<0.001)$, and albu$\min (\mathrm{p}=0.002)$ showed significant difference at baseline and week 54 . Mean trough level of CT-P13 was $5.02 \mu \mathrm{g} / \mathrm{ml}$ and $4.4 \mu \mathrm{g} / \mathrm{ml}$ at week 14 and 54 . Serum antibody positivity was proved in $7.7 \%$ and $26.2 \%$ of cases at week 14 and 54 , respectively. Dose escalation was necessary in one third of patients. None of the patients need surgery who completed week 54 , however 4 subjects who stopped CT-P13 therapy after induction regimen required colectomy.

Conclusion: Mucosal healing was revealed in two-third of the patients during CTP13 maintenance therapy. Our study confirmed the long-term efficacy of CT-P13 therapy on mucosal healing in UC.

Disclosure of Interest: All authors have declared no conflicts of interest.

P1695 THE USE OF ANTI-TNFS IN INDUCING CLINICAL RESPONSE AND REMISSION IN ULCERATIVE COLITIS: A COMPARATIVE ANALYSIS IN THE REAL-LIFE EXPERIENCE OF A SINGLE REFERRAL CENTER

B. Scrivo, M.G. Cilluffo, G. C. Morreale, V. Calvaruso, M. Mendolaro, A. Vitello, S. Peralta, M. Cappello

Gastroenterology And Hepatology, Policlinico Paolo Giaccone, Palermo/Italy

Contact E-mail Address: barbara.scrivo@gmail.com

Introduction: Anti-tumor necrosis factor (anti-TNF) agents, infliximab (IFX) and more recently adalimumab (ADA) and golimumab (GOL), have been shown effective and safe in the treatment of moderate-to-severe ulcerative colitis 
(UC). Lack of head-to-head RCTs makes the choice among the three anti-TNFs difficult and indirect comparisons by network metanalyses lead to discrepant results.

Aims \& Methods: Our aim was to compare efficacy of IFX, ADA and GOL in inducing clinical response and remission in a prospective cohort of patients with moderate to severe UC. From June 2015 to October 2016, 61 consecutive UC patients were treated with anti-TNFs: 19 with IFX, 25 with ADA and 17 with GOL. Disease activity was assessed by Mayo Score. Clinical response and/or remission were evaluated at week 8 and at week 16 . We also recorded: indications to biologic therapy, previous immunosuppressive or anti-TNF therapy and rate of anti-TNF discontinuation.

Results: Among the 61 patients, 36 were males; mean age was $43,6 \pm 15$, no significant difference was present in baseline characteristics (extent and disease activity); 39 patients were thiopurine failure; 38 were navve to anti-TNFs, most were treated with IFX $(p=0.001)$. ADA and GOL were more often used as a second-line or third-line. The principal indication for steroid resistance patients was IFX. No significant difference was observed between IFX and ADA both at week 8 (response $\mathrm{p}=0.28$; remission $\mathrm{p}=0.68$ ) and at week 16 (response $\mathrm{p}=0.5$; remission $\mathrm{p}=0.97$ ), though there was a trend towards a higher rate of response at week 8 with IFX ( $79 \%$ vs $64 \%$ ). IFX and ADA were more effective than GOL at week 8 (response: IFX vs GOL $p=0.020$; remission: ADA vs GOL $p=0.027$ ). At week 16 only IFX seems to be more effective than GOL in inducing clinical response $(p=0.048)$ but not remission. No significant difference among the three drugs was observed in patients naïve to anti-TNFs. Treatment was discontinued in 2 patient in IFX group and in 6 patients in GOL group and in 6 patients with ADA because of persistent disease activity.

Conclusion: This single-center study shows that IFX is more effective than GOL both in the induction ( 8 weeks) and in the maintenance of response (16 weeks). ADA is more effective than GOL in inducing remission at 8 weeks but no significant difference is observed in the medium-term. However, GOL was used mainly as a second or third-line. In naïve patients, efficacy among anti-TNFs is comparable. Our results may help clinicians in the choice of an anti-TNF in UC: IFX should be preferred in steroid-resistant patients to get a faster response, ADA and GOL should be the first option in steroid-dependant patients naïve to anti-TNFs.

Disclosure of Interest: All authors have declared no conflicts of interest.

\section{Reference}

1. 2014 Apr;39(7):660-71. doi: 10.1111/apt.12644. Epub 2014 Feb 9.

\section{P1696 REAL WORLD SAFETY OF VEDOLIZUMAB IN}

\section{INFLAMMATORY BOWEL DISEASE: A META-ANALYSIS}

S. Schreiber ${ }^{1}$, A. Dignass ${ }^{2}$, L. Peyrin-Biroulet ${ }^{3}$, M. Mosli $^{4}$, G. Hather ${ }^{5}$,

D. Demuth ${ }^{6}$, A. Blake ${ }^{6}$, R. Curtis ${ }^{6}$, J. M. Khalid ${ }^{6}$, E. V. Loftus Jr ${ }^{7}$

${ }^{1}$ Christian-Albrechts-University Kiel, Kiel/Germany

${ }^{2}$ Agaplesion Markus Hospital, Frankfurt/Germany

${ }^{3}$ Nancy University Hospital, Les Nancy/France

${ }^{4}$ King Abdulaziz University, Jeddah/Saudi Arabia

${ }^{5}$ Takeda Oncology, Takeda Global Research and Development, Boston/United

States of America

${ }^{6}$ Takeda Development Centre Europe, London/United Kingdom

${ }^{7}$ Mayo Clinic College of Medicine, Rochester/United States of America/MN

Contact E-mail Address: s.schreiber@mucosa.de

Introduction: Vedolizumab (VDZ), a gut-selective monoclonal anti- $\alpha_{4} \beta_{7}$-integrin antibody, is used for treatment of Crohn's disease (CD) and ulcerative colitis (UC). Data from large real-world cohorts can further characterise safety events not fully elucidated in a clinical trial setting, such as the risk of serious infections, as identified with anti-tumour necrosis factor-alpha (TNF $\alpha$ ) therapy in the TREAT $^{1}$, GISEA $^{2}$ and ENCORE ${ }^{3}$ registries.

Aims \& Methods: We conducted a systematic review and meta-analysis of realworld safety outcomes reported for VDZ in UC and CD. MEDLINE-, Cochrane-, and EMBASE-indexed publications and conference abstracts $(\mathrm{n} \geq 10)$ from May 1, 2014-January 10, 2017 were searched for studies reporting real-world VDZ safety outcomes. Reports for patients $<18$ years of age or for off-label VDZ use were excluded. A meta-analysis was conducted using the DerSimonian-Laird random effects method to obtain a weighted mean of adverse event (AE) rates.

Results: Two hundred and eighteen published studies were identified, with 33 reporting safety rates on 2857 VDZ-treated patients (CD: 1532; UC: 829; unspecified/other: 36 ; three studies $[\mathrm{n}=460]$ did not report individual UC/CD data) over a VDZ exposure/follow-up period ranging 0.5-18 months (20 studies). Among included studies, the mean age of patients ranged from 21 to 67 years, with mean disease duration ranging from 7 to 16 years. Most VDZ-treated patients $(62-100 \%)$ had prior exposure to $>1$ anti-TNF $\alpha$ therapy and $6-64 \%$ of VDZ-treated patients were receiving concomitant corticosteroids and immunomodulators. The most common non-infectious AEs were acne or acne-like lesions $(7 \% ; 95 \%$ confidence interval $[\mathrm{CI}] 5-11 \%)$, fatigue $(6 \% ; 95 \%$ CI $3-$ $15 \%$ ) and arthralgia $(5 \% ; 95 \%$ CI $3-10 \%$ ) (Table 1$)$. The most common infectious AEs were upper respiratory tract infections $(6 \% ; 95 \% \mathrm{CI}, 3-11 \%)$ and sinusitis $(4 \% ; 95 \%$ CI $<1-19 \%)$ (Table 1$)$. Infusion-related reactions occurred in $2 \%(95 \% \mathrm{CI}<1-4 \%)$ of patients $(\mathrm{n}=811)$, and malignancies were reported in $<1 \%$ of patients $(<1-4 \% ; 2$ studies). Overall, the pooled AE rate reported in VDZ-treated patients was $21 \%(95 \%$ CI $14-32 \%) ; 10 \%(95 \%$ CI $6-16 \%)$ for infections, $8 \%(95 \%$ CI 6-10\%) for serious AEs and 7\% (95\% CI 3-13\%) for serious infections (Table 1).
Table 1: Pooled real-world adverse event rates of vedolizumab in inflammatory bowel disease

Non-infectious adverse events ( $\geq 2 \%$ of patients)

\begin{tabular}{|c|c|c|c|}
\hline Event & $\mathrm{n}$ & Rate, \% & $\begin{array}{l}95 \% \text { Confidence } \\
\text { Interval }(\mathrm{CI})\end{array}$ \\
\hline Acne or acne-like lesions & 290 & 7.2 & $4.8-10.9$ \\
\hline Fatigue & 569 & 6.3 & $2.6-14.6$ \\
\hline Arthralgia & 1356 & 5.2 & $2.7-9.9$ \\
\hline $\begin{array}{l}\text { Exacerbation of IBD } \\
\text { symptoms }\end{array}$ & 674 & 4.9 & $2.1-11.1$ \\
\hline Muscle pains & 147 & 4.8 & $2.3-9.7$ \\
\hline Headache & 937 & 4.7 & $3.0-7.2$ \\
\hline Dizziness & 222 & 4.5 & $1.4-13.6$ \\
\hline Cough & 185 & 4.0 & $0.3-39.7$ \\
\hline $\begin{array}{l}\text { Other skin and subcuta- } \\
\text { neous-related }^{\mathrm{a}}\end{array}$ & 900 & 3.7 & $1.6-8.0$ \\
\hline Nausea & 623 & 3.2 & $1.2-8.5$ \\
\hline Paraesthesia & 526 & 2.9 & $1.0-7.9$ \\
\hline $\begin{array}{l}\text { Respiratory, thoracic and } \\
\text { mediastinal-related }^{\mathrm{b}}\end{array}$ & 70 & 2.9 & $0.7-10.7$ \\
\hline Hives & 146 & 2.1 & $0.7-6.3$ \\
\hline Liver-related $^{c}$ & 468 & 2.0 & $0.1-23.9$ \\
\hline Memory impairment & 136 & 2.0 & $0.4-10.2$ \\
\hline Other musculoskeletal and & 498 & 2.0 & $0.3-12.5$ \\
\hline
\end{tabular}

connective tissue-

related $^{\mathrm{d}}$

Infectious adverse events ( $\geq 2 \%$ of patients)

Upper respiratory tract $\quad 960$ infection

Sinusitis

Other respiratory infections ${ }^{\mathrm{e}}$

Genitourinary tract infections

Flu or flu-like infection

Perianal abscess

Clostridium difficile

Folliculitis

960

Any adverse events

Event

Adverse events

328

Rate, \%

$95 \% \mathrm{CI}$

6.0

$3.2-10.8$

Serious adverse events

Infections

409

4.2

$0.8-19.1$

Serious infections

450

284

1101

146

3.0

$1.7-5.7$

1.1-8.0

2.8

2.5

2.1

$0.8-8.8$

$1.1-5.6$

$1.2-3.6$

$0.7-6.3$

${ }^{a}$ Includes paradoxical skin manifestations, acute generalised exanthematous pustulosis, dry skin, erythema nodosum, palmar erythema ${ }^{\mathrm{b}}$ Includes spontaneous nose bleed 'Includes liver test abnormalities (transient transaminitis), druginduced liver injury (not specified) ${ }^{\mathrm{d}}$ Includes severe musculoskeletal syndrome, exacerbation of pre-existing enteropathic arthritis ${ }^{\mathrm{e}}$ Includes pneumonia, lower respiratory tract infections, respiratory tract infection (not specified)

Conclusion: Pooled analysis of AE rates across multiple studies support the favourable, long-term benefit-risk profile of VDZ in real-world clinical practice, with low rates of infusion-related reactions, serious infections and malignancies reported, and no identification of new safety signals. These results are consistent with integrated safety data reported for VDZ in six clinical trials ( $>4000$ patientyears) ${ }^{4}$ despite the selection of complex patients failing previous immunosuppressive or biologic therapies. Limitations of incidental reporting in real-world studies include potential underestimates of $\mathrm{AE}$ rates and the reporting of AEs not regularly observed in clinical trials, ${ }^{3}$ for example, due to the variability in comedication use and sub-optimal screening of prior infections.

Disclosure of Interest:

Dr Edward Loftus has received financial support for research from AbbVie, Janssen, UCB, Takeda, Pfizer, GlaxoSmithKline, Amgen, Bristol-Myers Squibb, Genentech, Robarts Clinical Trials, Gilead, Receptos; and has served as a consultant for AbbVie, Janssen, UCB, Takeda, Immune Pharmaceuticals, Celgene, MedImmune, Theradiag, Genentech, Seres Health, Sun Pharmaceuticals, Bristol-Myers Squibb

S. Schreiber: Has received on-spot consultancy fees from AbbVie, MSD, and Takeda/Millennium for participation in expert advisory activities.

A. Dignass: Has served as a speaker and consultant for Ferring, Falk Pharma, Mundipharma, Hospira, Sandoz, Otsuka, Abbvie, Janssen, Takeda, MSD, Vifor, and Pharmacosmos.

L. Peyrin-Biroulet: Received consulting fees from Merck, Abbvie, Janssen, Genentech, Mitsubishi, Ferring, Norgine, Tillots, Vifor, Therakos, Pharmacosmos, Pilège, BMS, UCB-pharma, Hospira, Celltrion, Takeda, Biogaran, Boerhinger Ingelheim, Lilly, Pfizer, HAC-Pharma, Index 
M. Mosli: I have received consultation and speaker fees from Takeda, Abbvie and Janssen

G. Hather: Employee of Takeda Development Center

D. Demuth: Employee of Takeda Development Center Europe Ltd

A. Blake: Employee of Takeda Development Center Europe Ltd

R. Curtis: Employee of Takeda Development Center Europe Ltd

J.M. Khalid: Employee of Takeda Development Center Europe Ltd

E.V. Loftus Jr: Disclosure has been added to references section of abstract

\section{References}

1. Lichtenstein GR et al. Am J Gastroenterol 2012;107:1409

2. Atzeni F et al. Autoimmun Rev 2012;12:225

3. D'Haens $\mathrm{G}$ et al. J Crohns Colitis 2016: Epub ahead of print

4. Colombel JF et al. Gut 2017; 66:839

\section{P1697 COST-UTILITY ANALYSES OF BIOLOGICS FOR REFRACTORY ULCERATIVE COLITIS}

S. Campbell Davies ${ }^{1}$, C. Inserra $^{1}$, R. Salerno ${ }^{2}$, A. Cassinotti ${ }^{2}$, G. Muserra ${ }^{1}$, M. Piacenza ${ }^{1}$, S. Ardizzone ${ }^{2}$

${ }^{1}$ Pharmacy, ASST Fatebenefratelli Sacco, Milan/Italy

${ }^{2}$ Gastroenterology, ASST Fatebenefratelli Sacco, Milan/Italy

Contact E-mail Address: secampbelldavies@gmail.com

Introduction: Although many biologics (Bs) have been approved for the treatment of moderate-to-severe Ulcerative Colitis (UC) in patients who have responded inadequately to conventional therapy, the selection of Bs is controversial due to the lack of head-to-head trials. Indirect economic comparisons of these costly drugs are available from National Healthcare perspectives that are not the Italian ones.

Aims \& Methods: The objective is to evaluate cost-utility of Bs for the treatment of refractory moderate-to-severe UC both in Italy and in the Lombardy Region. A Markov model (considering 3 transition states: remission, clinical response, relapse) was constructed using the software R 3.3.1 markovchain-package to evaluate incremental cost-utility ratios (ICUR) of adalimumab (ADA), infliximab (IFX), infliximab biosimilar (IFX-B), golimumab (GOL) and vedolizumab (VED) treatments of patients over a 10-year time horizon from the perspective of the Italian $(\mathrm{N})$ and Lombardy Region $(\mathrm{R})$ healthcare system. Clinical parameters were derived from clinical trials. Costs (actualised by-1,5\%) were obtained from the National database and Regional public tender. Utility was expressed as QALY (Quality Adjusted Life Years)

Results: Costs per treatment were different from a $\mathrm{N}$ and $\mathrm{R}$ perspective (ADA $-55 \%$; IFX - $16.7 \%$; IFX-B - 29.6\%; GOL - 9.6\%; VED - 10\%). Direct healthcare costs (treatment cost, visits, lab tests, hospital admissions) were calculated over 10 years of treatment per patient: ADA (N: $€ 114,227, \mathrm{R}: € 68,314$ $-40.2 \%)$, IFX (N: $€ 130,595, \mathrm{R}: € 103,081,-21 \%)$, IFX-B (N: $€ 110,438, \mathrm{R}$ : $€ 78,852,-28.6 \%)$, GOL (N: $€ 118,602, \mathrm{R}: € 96,922,-18.3 \%)$, VED (N: $€ 113,852, \mathrm{R}: € 102,932,-9.6 \%$ ) with associated QALY respectively of 6.68 $6.66,6.66,6.70,7.02$. From a $\mathrm{N}$ perspective, IFX-B was dominating compared to all other treatments. The ICUR of VED/IFX-B was $€ 9,483$ for 10 years (willingness to pay $€ 948 / \mathrm{QALY}$ ). From a R perspective, ADA was dominating compared to all other treatments. The ICUR of VED/ADA was $€ 101,818$ for 10 years (WTP $€ 10,182 /$ QALY).

Conclusion: National and Regional cost-utility analyses produced different results. As Regional price discounts can occur, local analysis is needed to estimate the economic impact of therapies to ensure optimal choice.

Disclosure of Interest: All authors have declared no conflicts of interest.

\section{P1698 ENDOSCOPIC SUBMUCOSAL DISSECTION OF ULCERATIVE COLITIS-ASSOCIATED DYSPLASIA: A SINGLE CENTER-BASED EXPERIENCE}

D. Yang, S. Lee, K. Chang, E.M. Song, S.W. Hwang, S.H. Park, B.D. Ye, J. Byeon, S. Myung, S. Yang

Gastroenterology, Asan Medical Center, University of Ulsan College of Medicine, Seoul/Korea, Republic of

Contact E-mail Address: gi.dr.yang@gmail.com

Introduction: Dysplasia is considered as the precursor of colitis-associated cancer in the long standing ulcerative colitis (UC). Although endoscopic submucosal dissection (ESD) has been suggested as an endoscopic resection technique for non-polypoid dysplasia, only a few studies investigated the feasibility of ESD as a treatment option of non-polypoid dysplasia.

Aims \& Methods: We aimed to investigate the feasibility of ESD for the resection of visible, non-polypoid dysplasia in UC. From August 2009 to January 2017, 19 UC patients with low grade dysplasia (LGD), high grade dysplasia (HGD) or early colon cancer were admitted for ESD and their medical records were retrospectively reviewed.

Results: Mean age of the 19 patients was $55.5 \pm 15.4$ years and mean duration between UC diagnosis and dysplasia detection was $13.7 \pm 6.5$ years. Nine were male. Five of 19 patients directly underwent colectomy without ESD trial due to non-lifting sign $(n=4)$ or surface ulceration $(n=1)$. Of these, preoperative single HGD $(n=3)$ or ECC $(n=1)$ patients showing non-lifting sign were diagnosed as invasive cancer and a single preoperative LGD case with non-lifting sign had multifocal LGDs in the colectomy specimen. As a result, ESD was performed for 14 of 19 patients. Major and minor axes of the lesion was $23.4 \pm 9.0 \mathrm{~mm}$ and
$18.1 \pm 9.1 \mathrm{~mm}$, respectively. The lesions were located at the rectum $(\mathrm{n}=9)$, sigmoid colon $(n=2)$, descending colon $(n=1)$, and transverse colon $(n=1)$. The gross morphology showed Paris IIa $(n=7)$, IIb $(n=4)$, Is $(n=1)$, and IIb + Is $(n=2)$. No lesions contained ulcerations. The borders were distinct in 6 and vague but endoscopically assumable in 8 cases. Mean UC endoscopic index of severity scores of the surrounding mucosa was $0.3 \pm 0.5$. Mean resection time was $54.8 \pm 25.7$ minutes. En bloc resection and R0 resection rates was $92.9 \%$ and $71.4 \%$, respectively. There was no perforation or clinically significant bleeding. Mean hospitalization period after ESD was $1.14+0.36$ days. Final histology of ESD specimens revealed 1 indefinite for dysplasia (IND), 1 sessile serrated adenoma/polyp, 7 LGDs, 3 HGDs, and 1 intramucosal adenocarcinoma. Synchronous lesions were present in 5 patients. Synchronous dysplasia in 4 patients were removed endoscopically. However, colectomy was done in one patient having synchronous adenocarcinoma and endoscopically unresectable dysplasia diagnosed by the surveillance biopsy specimens taken during ESD. Metachronous or recurrent dysplasia was identified in 2 of 9 patients who underwent follow-up colonoscopy after ESD (median follow-up period 12.3 months, range 6.3-38.2 months). One developed metachronous LGD at 18 months after ESD and the other developed both metachronous and recurrent LGDs at 8 months after ESD.

Conclusion: According to our ESD series for dysplasia, ESD seems to be feasible for the endoscopic resection of UC-associated dysplasia. However, meticulous surveillance colonoscopy is mandatory to monitor local recurrence and metachronous dysplasia. Non-lifting sign and surface ulceration are highly suggestive of invasive colitic cancer.

Disclosure of Interest: All authors have declared no conflicts of interest.

\section{P1699 EVALUATION OF ADHERENCE TO INFLIXIMAB THERAPY IN IBD PATIENTS}

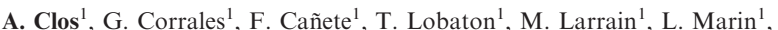
M. Mañosa ${ }^{1}$, E. Cabré ${ }^{1}$, E. Domènech

${ }^{1}$ Gastroenterology, Hospital Germans Trias I Pujol, Badalona/Spain

\section{Contact E-mail Address: ariadna.closparals@gmail.com}

Introduction: Biological therapies are effective treatments for inflammatory bowel disease (IBD) but represent an important economic burden to the healthcare system. Adherence surveillance is necessary to optimize the efficacy of treatment and its costs. This issue has been evaluated just in a fewer studies.

Aims \& Methods: We aim to describe the adherence to infliximab in patients with IBD and identify causes and factors associated with poor adherence We identified all IBD patients treated with infliximab in a single center since 2009. Fulfillment of the prescribed schedule was assessed for every single infliximab infusion. For every patient, we grouped infusions in "courses of treatment" defined as the administration of infliximab at the same dose and schedule for a minimum of six months. Therefore, restarting the treatment after a holiday of more than 4 months, or changing the interval of doses were considered as a new course of treatment. We defined "infusion well administrated" when it was done within seven days before or after the date prescribed.

Results: We included a total of 147 courses of treatment, administered to 100 patients. Seventy-four per cent of courses were Crohn disease patients, and $25 \%$ in ulcerative colitis patients. In $89 \%$ of courses combo therapy with immunosuppressants was used. The prescribed regimes were: every 8 weeks $(76.2 \%)$, every 10 weeks $(7.5 \%)$, every 6 weeks $(8.8 \%)$, every 12 weeks $(5.4 \%)$ or every 4 weeks $(2 \%)$. The mean duration per course was 23 months (range: $6-103)$. Only 69 out of 1714 infusions ( $4 \%$ ), were not properly administered. The reported causes for that included: 36 "unknown" $(52 \%), 18$ "change requested by patient" $(26 \%), 15$ "due to logistic reasons" $(15 \%)$ and 4 "other" $(5.7 \%)$. In 107 courses $(73 \%)$ all the infusions were well administered; in $143(97 \%)>80 \%$ of infusions were well administered and only 4 courses $(2.7 \%)$ had less than $<80 \%$ of adherence. In more than an half of the infusions the cause could not been identified, for that reason analysis of predictive factors could not be performed.

Conclusion: The adherence to the scheduled infliximab regime was very high and it would contribute to maintain the drug efficacy. The reasons for changing the date of administration should be indicated in the clinical history to identify associated factors and minimize the lack of adherence.

Disclosure of Interest: All authors have declared no conflicts of interest.

\section{P1700 TNF-EXPRESSION OF MONOCYTES IS A PREDICTIVE MARKER FOR RESPONSE TO INFLIXIMAB TREATMENT}

D. Lissner, B. Jessen, E. Sonnenberg, F. Schmidt, A. Kuehl, B. Siegmund Department For Gastroenterology, Infectious Diseases And Rheumatology, Charité-Universitätsmedizin Berlin, Campus Benjamin Franklin, Berlin/Germany

Contact E-mail Address: donata.lissner@ charite.de

Introduction: One-third of all patients with inflammatory bowel diseases (IBD) do not respond to initial treatment with the anti-TNF-antibody Infliximab. Thus, predictive markers for response to anti-TNF-treatment are required.

Aims \& Methods: The study's aim was to investigate whether levels of TNF produced by peripheral blood mononuclear cells (PBMCs) can predict response to anti-TNF-treatment. Fourteen patients with proven Crohn's disease (CD) or ulcerative colitis (UC) without treatment with biologicals in the past six months were included prior to first Infliximab infusion. Disease activity was measured by the use of Harvey-Bradshaw-Index (HBI) or partial Mayo Score, C-reactive protein (CRP) and ultrasound (Limberg Score). TNF-expression of LPS-stimulated PBMCs was measured by ELISA before treatment. Additionally PBMCs' 
intracellular TNF-expression was analysed by flow cytometry. According to a cut-off of $300 \mathrm{pg} / \mathrm{ml}$, patients were divided into low- and high-TNF-producers. Primary endpoint was clinical response, secondary endpoints were decrease in CRP and Limberg Score. Clinical response was defined as a decline in Score of $\geq 2$ (HBI) or $\geq 3$ (partial Mayo Score). A HBI $<5$ or a partial Mayo-Score $<2$ was defined as remission. Results were analysed using the Fisher's exact test.

Results: Nine patients reached the endpoint at week 6 and were available for further analysis (5 patients with $\mathrm{CD}, 4$ patients with UC). The median TNFexpression was $653.84 \mathrm{pg} / \mathrm{ml}(49.53-1154.78 \mathrm{pg} / \mathrm{ml})$. TNF was mainly produced by $\mathrm{CD} 14^{+}$monocytes. Four patients were identified as low-producers and five as high-producers. All high-producers responded well to the treatment regarding clinical scoring as compared to only half of the low-producers, but statistical significance was not reached due to the small number of patients (high: $100 \%$ vs. low: $50 \%$ clinical response, $\mathrm{p}=0.167$ ). However, remission rates after 6 weeks were significantly higher in high-producers compared to low-producers (high: $80 \%$ vs. low: $0 \%$ remission; $\mathrm{p}=0.048$ ). Secondary endpoints showed no significant difference in the two groups.

Conclusion: Quantification of TNF-expression in PBMCs and the resulting classification in low- and high-producers could be a potential predictive marker for response to anti-TNF-treatment in IBD patients.

Disclosure of Interest: D. Lissner: Donata Lissner received a research grant from Pfizer and lecture fees from Falk and Abbvie

B. Siegmund: Britta Siegmund received a research grant from Pfizer, served as consultant for Janssen, MSD, Abbvie, Takeda, Hospira and received lecture fees from Abbvie, Falk, Ferring, MSD, Merck, Takeda; all money went to the institution

All other authors have declared no conflicts of interest.

\section{P1701 EVALUATION OF CONCOMITANT CORTICOSTEROID AND VEDOLIZUMAB USE IN PATIENTS WITH INFLAMMATORY BOWEL DISEASE (IBD) IN REAL-LIFE CLINICAL PRACTICE}

T. Ylisaukko-Oja ${ }^{1}$, J. Aaltonen ${ }^{1}$, S. Torvinen ${ }^{1}$, A. Eberl $^{2}$, H. Nuutinen ${ }^{3}$,

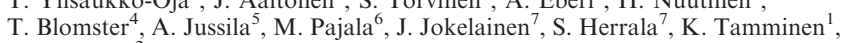
T. Sipponen ${ }^{2}$

${ }^{1}$ Takeda Oy, Helsinki/Finland

${ }^{2}$ Department Of Gastroenterology, Helsinki University Hospital and University of Helsinki, Helsinki/Finland

${ }^{3}$ Department Of Medicine, Turku University Hospital, University of Turku, Turku/ Finland

${ }^{4}$ Department Of Medicine, Division Of Gastroenterology, Oulu University

Hospital, Oulu/Finland

${ }^{5}$ Department Of Gastroenterology And Alimentary Tract Surgery, Tampere University Hospital, Tampere/Finland

${ }^{6}$ Department Of Internal Medicine, Kuopio University Hospital, Kuopio/Finland ${ }^{7}$ MedEngine Oy, Helsinki/Finland

\section{Contact E-mail Address: taina.sipponen@hus.fi}

Introduction: Corticosteroids (CS) are often used concominantly with biologics in treatment of inflammatory bowel disease (IBD). However, their side-effect profile causes significant clinical and economic burden in long-term treatment. In this study, we investigated the impact of concomitant CS use on vedolizumab treatment persistence in patients with Crohn's disease (CD) and ulcerative colitis (UC).

Aims \& Methods: This was a nationwide (Finland), retrospective, non-interventional, multi-center chart review. From 27 centers, we included adult $(\geq 18$ years of age) IBD patients who received at least one vedolizumab infusion since 2014 . Data were collected from medical charts in a standardized case report form. The key data collection points were at baseline, week 14 and month 6 of vedolizumab treatment. The main aim of the study was to analyze vedolizumab treatment persistence among IBD patients using CS in real-world clinical setting.

Results: 247 patients (CD 108, UC 139) were included. At baseline, 47 (43.5\%) $\mathrm{CD}$ and $84(60.4 \%)$ UC patients were using CS. Higher percentage of patients using CS at baseline discontinued vedolizumab during the 6-month follow-up compared to CS non-users (CD, 14/47 (29.8\%) vs. 13/61 (21.3\%); UC, 31/84 $(36.9 \%)$ vs. $16 / 55(29.1 \%))$. CS users had less vedolizumab discontinuations due to primary inefficacy $(\mathrm{p}=0.04)$ and more discontinuations due to adverse events $(\mathrm{p}=0.04)$, than CS non-users. Over half of the patients on CS at baseline and who persisted on vedolizumab were able to discontinue CS before 6 months timepoint (CD, 18/33 (54.5\%); UC, 37/53 (69.8\%)). Among CD patients, CS users had higher baseline disease activity than non-users. Such difference was not observed in UC. CS users had shorter disease duration in both CD and UC. There was no difference in the number of prior TNF-alpha inhibitors between CS users and non-users.

Conclusion: Use of CS at the time of initiating vedolizumab treatment was more common in UC than in CD. Vedolizumab treatment persistence was lower in CS users than in non-users in both CD and UC. The data suggests that CS users have less vedolizumab discontinuations due to primary inefficacy and more discontinuations due to adverse events, than CS non-users. The majority of patients on CS at baseline who persisted on vedolizumab were steroid-free by 6 months, potentially relieving the burden of CS-induced side-effects for both patients and society.
Disclosure of Interest: T. Ylisaukko-oja: TY is owner of MedEngine Oy and consultant for Takeda Oy.

J. Aaltonen: JA is employee of Takeda Oy.

S. Torvinen: ST is employee of Takeda Oy.

J. Jokelainen: JJ is employee of MedEngine Oy.

S. Herrala: $\mathrm{SH}$ is employee of MedEngine Oy.

$\mathrm{K}$. Tamminen: KT is employee of Takeda Oy.

All other authors have declared no conflicts of interest.

\section{P1702 VEDOLIZUMAB TROUGH LEVELS PREDICT CLINICAL}

\section{OUTCOMES IN INFLAMMATORY BOWEL DISEASE}

L. Guidi ${ }^{1}$, D. Pugliese ${ }^{1}$, T. Panici Tonucci ${ }^{1}$, B. Tolusso ${ }^{2}$, C. Felice ${ }^{1}$, A. Papa ${ }^{1}$, I. De Vitis ${ }^{1}$, E. Gremese ${ }^{2}$, A. Gasbarrini ${ }^{3}$, G.L. Rapaccini ${ }^{1}$, A. Armuzzi ${ }^{1}$ ${ }^{1}$ Internal Medicine And Gastroenterology IBD Unit Presidio Columbus, Fondazione Policlinico Universitario A.Gemelli Università Cattolica del Sacro Cuore, Rome/Italy

${ }^{2}$ Institute of Rheumatology, Fondazione Policlinico Universitario A. Gemelli Catholic University of the Sacred Heart, Rome, Italy, Rome/Italy

${ }^{3}$ Internal Medicine, Gastroenterology And Liver Diseases, Gemelli Hospital Dept. of Internal Medicine Dept. of Gastroenterology, Rome/Italy

Contact E-mail Address: danipug@libero.it

Introduction: Vedolizumab is an alpha4beta7 integrin antagonist for the treatment of inflammatory bowel disease (IBD). The role of Vedolizumab drug monitoring, based on the assessment of Vedolizumab trough levels (VTL) and antiVedolizumab antibodies (AVA) has not been clarified yet. In this study we investigated the correlation between VTL and AVA and clinical response.

Aims \& Methods: Consecutive IBD patients who started therapy with Vedolizumab at our centre were prospectively enrolled. Each patient underwent $300 \mathrm{mg}$ infusion at weeks $0,2,6$ and 14; additional doses at week 10 and then every 4 weeks were given to no-responders at week 6 . Clinical activity was evaluated at baseline and week 6, 14 and 22 by Harvey Bradshaw Index (HBI) and partial Mayo score (pMayo). C-reactive protein (CRP) was measured at weeks 6, 14, 22. VTL and AVA were assayed by ELISA (Theradiag, Marne La Vallee, France) at weeks 6, 10 and 14. Limits of detection for VTL and AVA were $2 \mathrm{ug} /$ $\mathrm{ml}$ and $35 \mathrm{ng} / \mathrm{ml}$, respectively. Clinical response was defined as at least $30 \%$ reduction of activity scores from baseline and remission was defined as $\mathrm{HBI}<5$ or pMayo $<2$. Statistics was performed by Mann Whitney test, Spearman's rho, receiver operating characteristic (ROC) curve analysis.

Results: We included 50 patients (mean age 45.5 y; male $56 \%$ ) with Crohn's disease $(\mathrm{CD}, \mathrm{n}=28)$ and Ulcerative colitis $(\mathrm{UC}, \mathrm{n}=22), 44(88 \%)$ IBD patients had previous anti-TNF $\alpha$ therapy. Baseline median HBI was 8 (5-16) and median pMayo was 6 (5-7). Median VTL (Interquartile range, IQR) at weeks 6, 10 and 14 were $38.6(20.3-53.5), 25.4(13.4-45.6)$ and $17.7(10.1-33.7) \mathrm{ug} / \mathrm{ml}$, respectively. VTL measured at week 6 were significantly higher in clinical responders as compared to non-responders: median (IQR) 50.7 (31.8-56.8) vs $33.1(19.4-42)$ $\mathrm{ug} / \mathrm{ml}, \mathrm{p}=0.04$. Week 6 VTL were also higher in CRP responders $(<5 \mathrm{mg} / \mathrm{l})$ : median (IQR) $48.1(32.5-55.8)$ vs $32.8(19.7-44.3) \mathrm{ug} / \mathrm{ml}, \mathrm{p}=0.04$. Week 6 VTL were inversely correlated with CRP (rho $-0.39, \mathrm{p}=0.006$ ). By ROC curve analysis we identified a cut off value for VTL of $44.3 \mathrm{ug} / \mathrm{ml}$ for clinical response at week 6 (AUC 0.677 , sensitivity $61.9 \%$, specificity $79.3 \%, p=0.02$ ). Week 6 VTL were significantly higher in patients in clinical remission at their last follow-up (mean 20 weeks) compared to non-remitters: median (IQR) 55.8 (46-64.1) vs 33.3 $(25.9-40.4) \mathrm{ug} / \mathrm{ml}, \mathrm{p}=0.0035$. The ROC curve analysis identified a cut off of 44.3 $\mathrm{ug} / \mathrm{ml}$ (AUC 0.813 sensitivity $88.9 \%$, specificity $73.2 \%, \mathrm{p}=0.0006$ ). Week 14 VTL were also significantly higher in patients in clinical remission at 22 weeks compared to non-remitters: median (IQR) 38.3 (20.5-49.8) vs 13.4 (8.4-20.6) ug/ $\mathrm{ml}, \mathrm{p}=0.0035$. The cut off identified by ROC curve analysis for this outcome was $16.4 \mathrm{ug} / \mathrm{ml}$ (AUC 0.820 sensitivity $100 \%$ specificity $63 \%, \mathrm{p}=0.0019$ ). AVA were detected in $2 \%$ of patients at week 6 , in $5.9 \%$ at week 10 and in $4.5 \%$ at week 14 and were not correlated with clinical response.

Conclusion: These preliminary data suggest that obtaining a VTL of $44.3 \mathrm{ug} / \mathrm{ml}$ after the first 2 Vedolizumab infusions is correlated with early clinical and biological (CRP) response and with clinical remission at a mean follow-up of 20 weeks. Week 14 VTL are correlated with clinical remission at week 22 and the identified cut off is $16.4 \mathrm{ug} / \mathrm{ml}$. Immunogenicity of Vedolizumab is low in these patients.

Disclosure of Interest: L. Guidi: Lecture fees by AbbVie, Merck, Takeda, Mundipharma, Zambon

D. Pugliese: Lecture fees by Takeda, AbbVie

A. Armuzzi: Lecture fee and consulting fee: AbbVie, Chiesi, Ferring, Hospira, Janssen, Lilly, Merck, Mundipharma, Nikkiso, Pfizer, Samsung, Takeda, Zambon

All other authors have declared no conflicts of interest. 
P1703 EFFICACY, SAFETY AND LONG-TERM OUTCOME OF ENDOSCOPIC DILATION THERAPY FOR PRIMARY CROHN'S DISEASE STRICTURES OF THE UPPER GASTROINTESTINAL TRACT-AN INTERNATIONAL MULTICENTER COMBINED ANALYSIS

D. Bettenworth ${ }^{1}$, M. M. Mücke ${ }^{2}$, A. Singh ${ }^{3}$, R. Lopez ${ }^{4}$, M. Goetz ${ }^{5}$, T. Matsui ${ }^{6}$, J.G. Karstensen ${ }^{7}$, N. S. Ding ${ }^{8}$, T. B. Qui ${ }^{8}$, J. Hampe ${ }^{9}$, K. Matthes ${ }^{9}$, P.V. Valli ${ }^{10}$, G. Rogler ${ }^{11}$, F. Rieder ${ }^{12}$

${ }^{1}$ Department Of Medicine B, University Hospital Münster, Münster/Germany ${ }^{2}$ Department Of Internal Medicine ${ }^{1}$ University Hospital Frankfurt, Frankfurt a.M./Germany

${ }^{3}$ Department Of Hospital Medicine, Cleveland Clinic Foundation, Cleveland/ United States of America

${ }^{4}$ Department Of Quantitative Health Sciences, Cleveland Clinic Foundation, Cleveland/United States of America

${ }_{5}^{5}$ Interdisziplinäre Endoskopie, Universitätsklinikum Tübingen, Tübingen/Germany ${ }^{6}$ Gastroenterology, Fukuoka University Chikushi Hospital, chikushino/Japan ${ }^{7}$ Dept. Of Gastroenterology, Copenhagen University Hospital Herlev, Herlev/ Denmark

${ }^{8}$ Gastroenterology, St. Vincents Hospital, Melbourne/Australia

${ }^{9}$ Gastroenterologie Und Hepatologie, University Hospital Dresden, Dresden

Germany

${ }^{10}$ University Hospital Zurich, Zürich/Switzerland

${ }^{11}$ Gastroenterology And Hepatology, University of Zurich, University Hospital

Zurich, Zurich/Switzerland

${ }^{12}$ Department Of Gastroenterology, Hepatology \& Nutrition, Digestive Diseases And Surgery Institute, Cleveland Clinic Foundation, Cleveland/United States of America

Contact E-mail Address: dominik.bettenworth@ukmuenster.de

Introduction: Strictures in Crohn's disease (CD) may occur in different segments along the gastrointestinal (GI) tract. In contrast to ileocecal strictures, endoscopic balloon dilation (EBD) for CD-associated strictures of the upper gastrointestinal (UGI) tract is rarely reported. We therefore performed a combined efficacy and safety analysis of EBD for UGI CD-associated strictures in this multi-centre cohort study.

Aims \& Methods: Individual patients characteristics were retrieved from electronic case report forms. Upper GI tract was defined as esophagus, stomach and duodenum up to the ligament of Treitz. Time-to-event analysis was performed to assess symptom recurrence, re-dilation or surgery. Kaplan-Meier estimates were used to calculate event rates. For the multivariable Cox regression models, all variables that were available for at least $85 \%$ of subjects were considered for inclusion and the score method was used to choose the best model with two factors for each outcome.

Results: A total of $73 \mathrm{CD}$ patients and 127 performed dilation procedures were included. Stricture locations were: duodenum $n=46$, stomach $n=14$; esophagus $\mathrm{n}=9$; stomach and duodenum $\mathrm{n}=4$. Technical success rate was $94.1 \%$ resulting in clinical efficacy in $88.9 \%$ of patients. Major complications, defined as perforation, bleeding or dilation-related surgery, occurred in $2.9 \%$ of all procedures. During a median follow up period of 36 months, $89.4 \%$ of patients underwent redilation and $29.8 \%$ required surgical intervention. The multivariable Cox regression analyses indicated that a younger age at diagnosis was associated with symptom recurrence (HR $0.82(0.71,0.95), \mathrm{p}=0.008)$, mucosal inflammation with need for re-dilation (HR $2.7(1.4,5.2), \mathrm{p}=0.003)$ and use of anti-TNF with need for surgery (HR $4.8(1.8,12.4), \mathrm{p}=0.001)$.

Conclusion: EBD for CD-associated strictures of the upper gastrointestinal tract has a high rate of short-term technical and clinical success with moderate longterm efficacy and acceptable complication rates.

Disclosure of Interest: All authors have declared no conflicts of interest

\section{P1704 POST-MARKETING SAFETY EXPERIENCE OF VEDOLIZUMAB IN PATIENTS WITH PRE-EXISTING VIRAL \\ HEPATITIS}

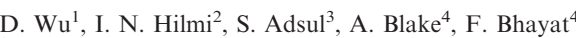

${ }^{1}$ Chung-ho Memorial Hospital, Kaohsiung Medical University, Kaohsiung/Taiwan

${ }^{2}$ University Malaya Medical Centre, Kuala Lumpur/Malaysia

${ }^{3}$ Takeda Pharmaceuticals (Asia Pacific), Singapore/Singapore

${ }^{4}$ Takeda Development Centre Europe, London/United Kingdom

Contact E-mail Address: Fatima.Bhayat@takeda.com

Introduction: Systemic immunosuppressive treatment, such as anti-tumour necrosis factor-alpha $(\mathrm{TNF} \alpha)$ therapy, can reactivate latent hepatitis virus. Vedolizumab (VDZ), a humanised monoclonal antibody, blocks gut-specific lymphocyte trafficking by binding to $\alpha_{4} \beta_{7}$ integrin and is approved in moderately to severely active ulcerative colitis or Crohn's disease. The gut selectivity of VDZ may be associated with a lower risk of virus reactivation than anti-TNF $\alpha$ agents. The VDZ European Product Label advises caution regarding VDZ use in patients with controlled chronic severe infection or a history of recurring severe infection. ${ }^{2}$ As VDZ clinical trials excluded patients with chronic viral hepatitis $\mathrm{B}$ or hepatitis $\mathrm{C}$ infection, there are no clinical trial data available in this population.

Aims \& Methods: Here, we describe the safety experience of VDZ use in patients with pre-existing hepatitis $\mathrm{B}$ or hepatitis $\mathrm{C}$ in the post-marketing setting. Adverse events (AEs) were identified from the VDZ Global Safety database (data-cut May 20, 2014 to Nov 19,2016) and included if patients' medical history or concurrent conditions included a Medical Dictionary for Regulatory Activities (MedDRA) preferred term suggestive of hepatitis B or hepatitis $\mathrm{C}$ infection.
Results: In the context of $\sim 77.382$ patient-years of VDZ exposure and a total of 30.470 AEs in the VDZ database, 51 events $(n=13$ serious [non-fatal]; $n=38$ non-serious) occurred in 15 patients with pre-existing viral hepatitis $B(n=5$, including 2 chronic cases) or hepatitis $C(n=10)$. Of the 15 patients, six had ulcerative colitis, seven had Crohn's disease, and in two the indication was not reported (NR). Eight patients received prior/concomitant anti-TNF $\alpha$ therapy; $N R n=2$. Events reported were reflective of the general VDZ safety profile in patients without viral hepatitis. Liver-related events were reported in two patients with hepatitis $\mathrm{C}-$ one patient who was a smoker reported hepatic neoplasm; the other reported hepatic mass and had a history of skin cancer, cholecystectomy, bladder tumour removal and right radical orchiectomy. Both events resulted in VDZ discontinuation. Of events with a reported outcome, $22 / 26(84.6 \%)$ were resolved or resolving at the time of reporting and $4 / 26(15.4 \%)$ were unresolved $\mathrm{NR} n=25$. VDZ treatment was continued in $10 / 14(71.4 \%)$ patients and discontinued in $4 / 14(28.6 \%)$; NR $n=1$.

Conclusion: In the post-marketing setting, there was no evidence of increased risk of viral reactivations in patients with hepatitis $\mathrm{B}$ or hepatitis $\mathrm{C}$ receiving VDZ. Limitations associated with post-marketing safety reporting (e.g. voluntary nature of reporting and incomplete patient medical history) and currently limited availability of VDZ in regions with endemic hepatitis B and hepatitis $\mathrm{C}$ infection should be considered when interpreting these results.

Disclosure of Interest: I.N. Hilmi: No conflict of interest

S. Adsul: Employee of Takeda Pharmaceuticals (Asia Pacific)

A. Blake: Employee of Takeda Development Center Europe Ltd

F. Bhayat: Employee of Takeda Development Center Europe Ltd

All other authors have declared no conflicts of interest.

\section{References}

1. Sansone S et al. World J Gastroenterol 2014;20:3516-3524

2. Takeda Pharma A/S. ENTYVIO ${ }^{\circledR}$ (vedolizumab) summary of product characteristics. Accessed March 2017

\section{P1705 AMINOSALICYLATES FOR MAINTENANCE THERAPY IN ULCERATIVE COLITIS: IS THE ADHERENCE REALLY IMPORTANT?}

D. Martí, M.P. Ballester, M. Fullana, P. Navarro, M.M. Boscá, J. Tosca, F. Mora, M. Minguez

Digestive Disease, University Clinic Hospital of Valencia, Valencia/Spain

\section{Contact E-mail Address: mapibafe@gmail.com}

Introduction: The goal of maintenance therapy in Ulcerative Colitis (UC) is to keep clinical and endoscopic steroid-free remission. 5-aminosalicylate (5-ASA) represents the first line maintenance therapy ${ }^{1}$. Non-adherence to 5-ASA is associated with an increased risk of disease relapse, colorectal cancer and worsening of quality of life $\mathrm{e}^{2-3}$. Adherence rate has been analysed in several studies with controversial results $4-5$

Aims \& Methods: The aim of this study is to quantify the prevalence of adherence to 5-ASA, to identify risk factors to non-adherence and its correlation with the course of the disease. Observational, analytical, retrospective, single tertiary centre, cohort study of all UC patients followed-up in our IBD unit, until January 2016, with 5-ASA maintenance treatment prescribed by an electronic management program. Adherence was considered when $80 \%$ of the prescribed 5ASA was dispense at pharmacy, during one year (2016), assuming that dispensed 5-ASA is equivalent to medication intake. The study analysed, according to the existence and degree of 5-ASA adherence, UC phenotypic expression (age, sex, smoking habit, Montreal classification, extraintestinal manifestations, complications), disease course (moderate-severe relapse rate that require corticosteroid therapy), 5-ASA properties (dose, administration regime, formulation), consumption of other UC and non-UC chronic drugs. A multivariable logistic regression was applied to discriminate adherent subjects including the risk factors of the univariable analysis. A Spearman's rank coefficient analysis was performed to correlate percentage of adherence with relapse rate.

Results: The study cohort included 433 patients, $55 \%$ males with a median age at the first 5-ASA prescription analysed of 49 years (IQR 39-61). 17\% had a proctitis, $31 \%$ a left-side colitis and $52 \%$ an extended disease. $30 \%$ of patients suffered from extraintestinal manifestations and 8 from a complication. The mean dose of 5-ASA taken was $2.6 \mathrm{~g}$ /day (range $0.7-4.8$ ) distributed in a daily dose in $61 \%$ of the patients. Adherence prevalence to 5-ASA was $63 \%$. Adherent group had a higher median age $(52( \pm 11)$ vs $43( \pm 13), \mathrm{p}=0.001)$ and received more non-UC chronic drugs $(\mathrm{OR}=2.3,95 \mathrm{CI}=1.5-3.4, \mathrm{p}=0.001)$. The independent variables, age and intake of other non-UC drugs, included in the multivariate analysis reached a predictive capacity of $65 \%$ outcomes of adherence. There was no significant reduction in the risk of moderate-severe flares that required corticosteroid therapy when comparing both adherent and non-adherent groups and rank correlation showed no relationship between adherence to 5ASA and relapse rate $(r=0.06 ; p=0.2)$. Patients treated with adjuvant maintenance therapy (bitherapy with thiopurines or antiTNF or triple therapy with both) presented a significant reduction of flares $(\mathrm{OR}=0.6,95 \mathrm{CI}=0.7-0.9$, $\mathrm{p}=0.019)$.

Conclusion: Adherence to 5-ASA in our population is low. Older patients that take other non-UC chronic treatments show higher adherence. With a one year prescribed and dispensed analysis, non-adherence to 5-ASA is not related with a higher risk of flares that require corticosteroid therapy. Patients treated with adjuvant maintenance therapy, show better results throughout the course of the disease.

Disclosure of Interest: All authors have declared no conflicts of interest. 


\section{References}

Dignass, A., et al. Second European evidence-based consensus on the diagnosis and management of ulcerative colitis Part 2: Current management. Journal of Crohn's and Colitis, 2012, 6(10), 991-1030.

Kawakami, A., et al. Relationship between non-adherence to aminosalicylate medication and the risk of clinical relapse among Japanese patients with ulcerative colitis in clinical remission: A prospective cohort study. Journal of Gastroenterology, 2012. 48(9), 1006-1015.

Kane, S., et al. Medication nonadherence and the outcomes of patients with quiescent ulcerative colitis. The American Journal of Medicine, 2003. 114(1), 39-43.

Kane, S. V., et al. Prevalence of nonadherence with maintenance mesalamine in quiescent ulcerative colitis. The American Journal of Gastroenterology, 2001 96(10), 2929-2933.

Bermejo, F., et al. Factors that modify therapy adherence in patients with inflammatory bowel disease. Journal of Crohn's and Colitis, 2010. 4(4), 422-426.

P1706 DEVELOPMENT AND FEASIBILITY OF A WEB-BASED REGISTRY FOR MULTICENTRE SURVEILLANCE OF EFFECTIVENESS AND SAFETY OF NOVEL IBD-DRUGS IN THE NETHERLANDS

V. B.c. Biemans ${ }^{1}$, CJ. Van Der Woude ${ }^{2}$, G. Dijkstra ${ }^{3}$, A. E. Van Der Meulen - De Jong ${ }^{4}$, K.h.n (. De Boer ${ }^{5}$, C.Y. Ponsioen ${ }^{6}$, B. Oldenburg ${ }^{7}$, F. Hoentjen ${ }^{8}$, M.J. Pierik ${ }^{9}$

${ }^{1}$ Gastroenterology And Hepatology, Radboudumc, Nijmegen/Netherlands

${ }^{2}$ Dept. Of Gastroenterology, Erasmus Medisch Centrum Dept. of

Gastroenterology, Rotterdam/Netherlands

${ }^{3}$ UMC Groningen Gastroenterology and Hepatology, Groningen/Netherlands

${ }^{4}$ Leiden University Medical Center Dept. of Gastroenterology, Leiden/Netherlands

${ }^{5}$ Gastroenterology And Hepatology, VU University Medical Center, Amsterdam/ Netherlands

${ }^{6}$ Gastroenterology \& Hepatology, AMC Amsterdam Gastroenterology and

Hepatology - Gastroenterology \& Hepatology, AMC Amsterdam Gastroe,

Amsterdam/Netherlands

${ }^{7}$ University Medical Center Utrecht, Utrecht/Netherlands

${ }^{8}$ Dept Of Gastroenterology And Hepatology, Universitair Medisch Centrum St.

Radboud, Nijmegen/Netherlands

${ }^{9}$ Dept Of Gastroenterology And Hepatology, Maastrich University Medical Center Dept. of Gastroenterology, Maastricht/Netherlands

Contact E-mail Address: vince.biemans@radboudumc.nl

Introduction: Randomized controlled trials provide efficacy data of novel IBD drugs. The majority of patients included in these trials however, especially for novel biologics, are highly selected patients from referral centres and are included in a variety of countries with very different health care systems. Inclusion criteria and follow-up protocols are strict and do not reflect routine care. Long-term country specific effectiveness and safety data for novel drugs are therefore warranted. Development and implementation of a novel protocol and electronic case reporting registry for every new compound is however time consuming and expensive. Therefore, the Initiative on Crohn and Colitis (ICC) aimed to develop a web-based registry suitable for capturing, managing, and reporting data for all drugs and all IBD phenotypes in everyday practice in all centres.

Aims \& Methods: Here, we aim to test the feasibility of the web-based registry in patients starting vedolizumab. With a structured iterative process with IBD-specialist from the ICC, case report forms and lab-evaluation forms were developed to assess key elements of disease activity, safety and a PROM. Furthermore the ICC decided on a uniform follow-up protocol reflecting everyday practise. A web-based registry for capturing, managing and reporting follow-up data of IBD patients starting a new drug was developed (ICC-case series). The registry automatically reminds the treating physician or nurse prior to novel follow-up visits. Feasibility of the ICC-case series was assessed in 6 centres in the Netherlands in patients who started vedolizumab. To test data extraction and reporting, the baseline characteristics of the first cases were assessed. The characteristics were compared to baseline characteristics of subjects in the vedolizumab registration studies.

Results: A total of 230 IBD (4 IBD-U) patients starting vedolizumab were included. All users found the ICC-case series easy to use and received the reminder mails for follow-up visits. Baseline characteristics were successfully extracted and are reported in table 1.

Table 1: Baseline characteristics of ICC cohort and GEMINI trials

\begin{tabular}{lllll}
\hline & $\begin{array}{l}\text { ICC } \\
\text { cohort CD } \\
(\mathrm{N}=146)\end{array}$ & $\begin{array}{l}\text { GEMINI } \\
2(1) \\
(N=1115)\end{array}$ & $\begin{array}{l}\text { ICC } \\
\text { cohort UC } \\
(\mathrm{N}=80)\end{array}$ & $\begin{array}{l}\text { GEMINI } \\
1(2) \\
(N=895)\end{array}$ \\
\hline Age - yr & $39 \pm 13.7$ & $36.1 \pm 12.1$ & $43.7 \pm 16.5$ & $40.3 \pm 13.1$ \\
Male - no. (\%) & $50(34.2)$ & $520(46.6)$ & $50(62.5)$ & $525(58.7)$ \\
Current smoker - no. (\%) & $38(25.9)$ & $298(26.7)$ & $1(1.3)$ & $55(6.1)$ \\
Disease duration-yr & $13.6 \pm 12.5$ & $9.0 \pm 7.8$ & $8.6 \pm 7.8$ & $6.9 \pm 6.4$ \\
Median CRP - mg/L (IQR) & $7(4-20)$ & 11.5 & $6(2-15)$ & - \\
Median fecal calprotectine - & $881(287-1800)$ & 686.0 & $1551(441-2519)$ & $899(341-2127)$
\end{tabular}

(287-1800) 686.0 $\mathrm{ug} / \mathrm{g}(\mathrm{IQR})$

Disease location $\mathrm{CD}, \mathrm{n}(\%)$

Ileum only

$31(21.2) \quad 181(16.2)$

(continued)
Table 1 Continued

\begin{tabular}{|c|c|c|c|c|}
\hline Characteristic & $\begin{array}{l}\text { ICC } \\
\text { cohort CD } \\
(\mathrm{N}=146)\end{array}$ & $\begin{array}{l}\text { GEMINI } \\
2(1) \\
(N=1115)\end{array}$ & $\begin{array}{l}\text { ICC } \\
\text { cohort UC } \\
(\mathrm{N}=80)\end{array}$ & $\begin{array}{l}\text { GEMINI } \\
1(2) \\
(N=895)\end{array}$ \\
\hline Colon only & $36(24.7)$ & $316(28.3)$ & - & - \\
\hline Ileum and colon & $56(38.4)$ & $618(55.4)$ & - & - \\
\hline Ileum and upper GI & $11(7.5)$ & - & - & - \\
\hline $\begin{array}{l}\text { Ileum and colon and upper } \\
\text { GI }\end{array}$ & $4(2.7)$ & - & - & - \\
\hline \multicolumn{5}{|l|}{ Disease behavior } \\
\hline Inflammatory & $89(61)$ & - & - & - \\
\hline Stricturing & $35(24)$ & - & - & - \\
\hline Penetrating & $12(8.2)$ & - & - & - \\
\hline Perianal fistula & $13(8.9)$ & - & - & - \\
\hline \multicolumn{5}{|l|}{ Disease location UC, n (\%) } \\
\hline Proctitis & - & - & $5(6.3)$ & $116(13.0)$ \\
\hline Left-sided & - & - & $29(36.3)$ & $448(50.1)$ \\
\hline Pancolitis & - & - & $37(46.3)$ & $331(37.0)$ \\
\hline \multicolumn{5}{|l|}{$\begin{array}{l}\text { Concomittant medications - } \\
\text { no. }(\%)\end{array}$} \\
\hline Glucocorticosteroids only & $59(40.4)$ & $381(34.2)$ & $37(46.3)$ & $332(37.1)$ \\
\hline $\begin{array}{l}\text { Immunosuppressive agents } \\
\text { only }\end{array}$ & $28(19.2)$ & $181(16.2)$ & $12(15)$ & $159(17.8)$ \\
\hline $\begin{array}{l}\text { Both immunosuppressive } \\
\text { and }\end{array}$ & $16(11)$ & - & $14(17.5)$ & $149(16.6)$ \\
\hline \multicolumn{5}{|l|}{ Corticosteroid } \\
\hline \multicolumn{5}{|l|}{$\begin{array}{l}\text { immusuppressive agents } \\
\text { Prior TNF antagonist ther- } \\
\text { apy }(\%)\end{array}$} \\
\hline$\geq 1$ & $145(99.3)$ & $645(57.8)$ & $69(86.3)$ & $367(41.0)$ \\
\hline$\geq 2$ & $114(78.1)$ & $398(35.7)$ & $37(46.3)$ & - \\
\hline Prior IBD surgery $(\%)$ & $72(49.3)$ & $466(41.8)$ & - & - \\
\hline Perianal surgery $(\%)$ & $27(18.5)$ & - & - & - \\
\hline IPAA (\%) & - & - & $3(3.8)$ & - \\
\hline
\end{tabular}

Conclusion: The ICC developed a uniform web-based registry to study postmarketing safety and effectiveness of novel IBD-drugs. A feasibility study with 230 patients starting vedolizumab showed successful data-capture, managing, and reporting with the ICC-case series in 6 centres. Table 1 shows clear differences between baseline characteristics of real-life Dutch patients and patients in the GEMINI studies underlining the importance of country specific post-marketing data.

Disclosure of Interest: All authors have declared no conflicts of interest

\section{References}

1. Sandborn WJ, Feagan BG, Rutgeerts P, Hanauer S, Colombel JF, Sands BE, et al. Vedolizumab as induction and maintenance therapy for Crohn's disease. $N$ Engl J Med. 2013;369(8):711-21.

2. Feagan BG, Rutgeerts P, Sands BE, Hanauer S, Colombel JF, Sandborn WJ, et al. Vedolizumab as induction and maintenance therapy for ulcerative colitis. N Engl J Med. 2013;369(8):699-710.

\section{P1707 SIX-YEAR EFFICACY AND SAFETY OF AZATHIOPRINE TREATMENT IN THE MAINTAINANCE OF STEROID-FREE REMISSION IN INFLAMMATORY BOWEL DISEASE PATIENTS}

C. Cassieri ${ }^{1}$, R. Pica ${ }^{1}$, E. V. Avallone ${ }^{1}$, G. Brandimarte ${ }^{2}$, M. Zippi ${ }^{1}$, P. Crispino ${ }^{1}$, D. De Nitto ${ }^{1}$, G. P. Lecca ${ }^{2}$, P. Vernia ${ }^{1}$, P. Paoluzi ${ }^{1}$, E. S. Corazziari ${ }^{1}$ ${ }^{1}$ Department Of Internal Medicine And Medical Specialties, "gastrointestinal Unit", Sapienza University of Rome Dep. of Gastroenterology, Roma/Italy ${ }^{2}$ Division of Internal Medicine and Gastroenterology, "Cristo Re" Hospital, Rome, Italy, Roma/Italy

Contact E-mail Address: claudio.cassieri@libero.it

Introduction: Azathioprine (AZA) and thiopurine are widely used for induction and maintenance of remission in patients steroid-resistant or dependent with inflammatory bowel disease (IBD). The treatment must be withdrawn in 5 $30 \%$ of patients due to the occurrence of adverse events.

Aims \& Methods: Aim of this study has been to investigate its efficacy and safety in maintaining steroid-free remission in steroid dependent IBD patients six year after the institution of treatment. Data from consecutive IBD outpatients referred in our Institution, between 1985-2015, were reviewed and all patients treated with AZA were included in this retrospective study. AZA was administered at the recommended dose of $2-2.5 \mathrm{mg} / \mathrm{kg}$. Blood chemistry was analysed before administration of the drug, every 10-15 days for the first 3 months and then every 1-2 months following the institution of treatment.

Results: Out of 2722 consecutive IBD outpatients visited in the index period, AZA was prescribed to 415 patients, $227(54.7 \%)$ were affected by Crohn's disease (CD) and $188(45.3 \%)$ by ulcerative colitis (UC). One hundred and fifty-eight patients with a follow-up $<72$ months were excluded from the study. Two hundred and fifty-seven patients were evaluated, $143(55.6 \%)$ with $\mathrm{CD}$ and $114(44.4 \%)$ with UC. One hundred and forty-two $(55.2 \%)$ were male 
and $115(44.8 \%)$ female (average age of $35.68 \pm 14.22$ SD years, range $14-74$ y.). Six year after the institution of treatment, $130(50.6 \%)$ patients still were in steroid-free remission ( $85 \mathrm{CD}$ vs $45 \mathrm{UC}, 59.5 \%$ and $39.5 \%$, respectively, $\mathrm{p}=0.0017), 71(27.6 \%)$ had a relapse requiring retreatment with steroids $(29$ $\mathrm{CD}$ vs $42 \mathrm{UC}, 20.3 \%$ and $36.8 \%$, respectively, $\mathrm{p}=0.0048), 56(21.8 \%)$ discontinued the treatment due to side effects ( $29 \mathrm{CD}$ vs $27 \mathrm{UC}, 20.2 \%$ and $23.7 \%$, respectively). Loss of response from $1^{\text {st }}$ to $6^{\text {rd }}$ year of follow-up was low, about $20 \%$.

Conclusion: Six years after the onset of treatment $56 \%$ of patients did not require further steroid courses. After the first year loss of response was low in five subsequent years. In the present series the maintenance of steroid-free remission was significantly higher in CD than in UC patients. The occurrence of side effects leading to the withdrawal of AZA treatment has been low.

Disclosure of Interest: All authors have declared no conflicts of interest.

\section{P1708 CLINICAL EFFICACY AND SAFETY OF ANTI-TNF THERAPY IN INFLAMMATORY BOWEL DISEASE IN THE ELDERLY: A UK TERTIARY REFERRAL CENTRE EXPERIENCE}

\section{J. Digby-Bell}

Dept. Of Gastroenterology, St. Thomas' Hospital, London/United Kingdom

Contact E-mail Address: jdigbybell@doctors.org.uk

Introduction: Many patients, especially the elderly or those with comorbidities, are excluded from clinical drug trials and little real-life data exists on the safety and efficacy of anti-TNF.

Aims \& Methods: We aimed to compare the clinical efficacy and safety of antiTNF therapy in patients over 60 years in a tertiary IBD centre in London, UK. We interrogated our IBD biologics database from January 2009 to November 2015 and performed retrospective data analysis until end of follow up in April 2017. Data was collected on demographics, endoscopy, calprotectin, CRP, clinical scores, serious infections, malignancy, drug levels and anti-drug antibodies. Patients with an age of ' $\geq 60$ ' when starting anti-TNF therapy were identified and ' $<60$ ' comparators were selected at random in a 2:1 ratio. Primary endpoints: week 14 and week 54 steroid free clinical remission (Harvey Bradshaw Index $<5$ or Simple Colitis Activity index $<3$ ) Secondary endpoint: proportion of patients remaining on anti-TNF at the end of follow up

Results: See table.

Conclusion: Only a small number of ' $\geq 60$ ' patients started anti-TNF ( 29 out of greater than 650). This may reflect our local population or that clinicians favour non anti-TNF therapies in this older group. Overall there was similar clinica efficacy at weeks 14 and 54 of anti-TNF therapy between the 'young' and 'old' groups. There was a higher discontinuation rate after 1 year of therapy in the older group $(p=0.043)$. There were more adverse events in the older group $(7 / 29)$ including 3 new cancer diagnoses compared with the younger group $(3 / 58)$. 4 patients had detectable anti-drug antibodies in the older group despite 2 of them having therapeutic thiopurine suggesting that the elderly may have more immunogenicity than the young. Further studies with more patients across multiple sites are required to clarify safety and efficacy in the elderly.

Disclosure of Interest: All authors have declared no conflicts of interest.

\section{P1709 TACROLIMUS IN REFRACTORY ULCERATIVE COLITIS-12 MONTH OUTCOME IN A SINGLE-CENTRE UK DISTRICT} HOSPITAL

N. Jayasooriya, L. Everson, S. Mann

Gastroenterology, Barnet and Chase Farm Hospital, Royal Free Trust NHS, Barnet/United Kingdom

Contact E-mail Address: nishani.jayasooriya@nhs.net

Introduction: Rescue therapy is required for patients with moderate - severe ulcerative colitis (UC) who have failed to respond to steroids and thiopurines. Anti-Tumour Necrosis Factor agents (Anti-TNFs) are widely used before considering a colectomy. Calcineurin inhibitors such as ciclosporin and Tacrolimus may be considered as alternatives to biologics. There have been some case series in assessing the use of Tacrolimus in such patients although the United Kingdom experience is limited. $(1,2)$

Aims \& Methods: We aimed to review the outcome of patients who received Tacrolimus as rescue and subsequent maintenance therapy for refractory symptoms of UC. This was a retrospective single-centre case review series. All patients who were refractory to standard medical therapies and being considered for a colectomy were reviewed by a Gastroenterologist with an interest in Inflammatory Bowel Disease. Demographic data, indications for treatment, clinical course and outcomes were reviewed from Electronic Patient Records (EPR). Results: Fourteen patients $(\mathrm{F}=6$; mean age of 54 years) received Tacrolimus. 8 patients $(57 \%)$ had evidence of pancolitis and six patients $(43 \%)$ had distal colitis. All patients had previously received thiopurines and 11 patients $(78.6 \%)$ had also received anti-TNFs. Three patients declined Anti-TNF treatment. All patients were steroid-dependent prior to commencing Tacrolimus. One patient received ciclosporin before the switch. The remaining 13 patients were initiated on Tacrolimus in the out-patient setting at a starting dose of $0.1 \mathrm{mg} / \mathrm{kg}$ day in 2 divided doses. Patients took Tacrolimus for a mean period of 18.8 months (range: 2 months to 49 months). Eight patients $(57 \%)$ achieved a steroid-free remission within 6 months. An additional 3 patients $(23 \%)$ had a clinical response within 6 months, but required one course of steroids during this time period. Three patients $(23 \%)$ failed to respond to Tacrolimus; 1 patient remains steroid-dependent and does not wish to proceed to surgery, 1 patient was switched to infliximab and 1 patient proceeded at 10 months to have an elective sub-total colectomy. Tacrolimus was withdrawn in all 3 non-responders. Of the $11(78.6 \%)$ initial responders, 12-month outcome included withdrawal of Tacrolimus in 7 patients $(63.6 \%)$. Reasons for withdrawal included: $n=1$ renal impairment; $\mathrm{n}=1$ started on infliximab; $\mathrm{n}=3$ referred for leucapharesis; $\mathrm{n}=1$ restarted on Azathioprine and $n=1$ referred for proctocolectomy. Three patients $(21.4 \%)$ remain in steroid-free clinical remission with a good quality of life and

\begin{tabular}{|c|c|c|}
\hline & $<60$ years & $\geq 60$ years \\
\hline Total & $\mathrm{n}=58$ & $\mathrm{n}=29$ \\
\hline $\begin{array}{l}\text { Week } 14 \text { steroid free remis- } \\
\text { sion }(\mathrm{HBI}<5 \\
\text { SCCAI }<3)\end{array}$ & $28 / 41(68.3 \%)$ & $8 / 16(50 \%)$ \\
\hline $\begin{array}{l}\text { Week } 54 \text { steroid free remis- } \\
\text { sion }(\mathrm{HBI}<5 \\
\text { SCCAI }<3)\end{array}$ & $24 / 40(60 \%)$ & $8 / 15(53.3 \%)$ \\
\hline $\begin{array}{l}\text { Remain on anti-TNF at } \\
\text { week } 54\end{array}$ & $46 / 58(79.3 \%)$ & $23 / 28(82.1 \%)$ \\
\hline $\begin{array}{l}\text { Reasons for stopping anti- } \\
\text { TNF before week } 54\end{array}$ & $\begin{array}{l}7 \text { primary non-response } 2 \text { secondary loss of response with } \\
\text { detectable anti-drug antibodies } 1 \text { infusion reaction } 1 \\
\text { clinical \& endoscopic remission }\end{array}$ & $\begin{array}{l}2 \text { primary non-response } 2 \text { secondary loss of response with } \\
\text { detectable anti-drug antibodies } 1 \text { infusion reaction }\end{array}$ \\
\hline $\begin{array}{r}\text { Remain on anti-TNF at end } \\
\text { of follow up (April 2017) }\end{array}$ & $38 / 58(65.5 \%)$ & $12 / 29(41.4 \%) \mathbf{p}<\mathbf{0 . 0 5}$ \\
\hline $\begin{array}{l}\text { Reasons for stopping bio- } \\
\text { logic during study } \\
\text { period }\end{array}$ & $\begin{array}{l}8 \text { primary non-response } 4 \text { secondary loss of response } 3 \text { sec- } \\
\text { ondary loss of response with detectable anti-drug anti- } \\
\text { bodies } 1 \text { infusion reaction } 1 \text { clinical and endoscopic } \\
\text { remission } 2 \text { infections (skin and respiratory) } 1 \text { stopped } \\
\text { attending }\end{array}$ & $\begin{array}{l}4 \text { primary non-response } 2 \text { secondary loss of response } 3 \text { sec- } \\
\text { ondary loss of response with detectable anti-drug anti- } \\
\text { bodies } 1 \text { infusion reaction } 1 \text { clinical and endoscopic } \\
\text { remission } 1 \text { infection (ophthalmic) } 1 \text { new diagnosis } \\
\text { cancer (colorectal) } 1 \text { severe fatigue } 1 \text { peripheral neuro- } \\
\text { pathy } 1 \text { moved away } 1 \text { stopped attending }\end{array}$ \\
\hline $\begin{array}{l}\text { Length of time on anti-TNF } \\
\text { if stopped (months) }\end{array}$ & Range: 3-73 Median: 12 & Range: 3-63 Median: 18 \\
\hline $\begin{array}{l}\text { Anti-drug antibodies } \\
\text { detectable during follow } \\
\text { up }\end{array}$ & $\begin{array}{l}3 / 58(5.2 \%)-3 \text { infliximab weeks } 14,34 \text { and } 762 \text { no conco- } \\
\text { mitant } 1 \text { subtherapeutic TGNs } 1 \text { prior exposure to } \\
\text { infliximab }\end{array}$ & $\begin{array}{l}4 / 29(13.8 \%)-3 \text { infliximab, } 1 \text { adalimumab weeks } 14,48,52 \\
\text { and } 542 \text { concomitant with therapeutic TGNs } 2 \text { no con- } \\
\text { comitant } 0 \text { prior exposure to anti-TNF }\end{array}$ \\
\hline $\begin{array}{l}\text { Adverse events throughout } \\
\text { follow up }\end{array}$ & $\begin{array}{l}1 \text { new diagnosis cancer (testicular) } 1 \text { infusion reaction } 1 \\
\text { infection (dental abscess) }\end{array}$ & $\begin{array}{l}3 \text { new diagnosis cancer (prostate, colorectal \& thyroid) } 1 \\
\text { spontaneous ileal perforation requiring emergency sur- } \\
\text { gery } 1 \text { infusion reaction } 2 \text { infections (chest infection and } \\
\text { shingles) }\end{array}$ \\
\hline
\end{tabular}


no adverse effects on maintenance treatment with Tacrolimus. 11 patients $(78.6 \%)$ have avoided a colectomy during the first 12-months of follow-up. Conclusion: Tacrolimus should be considered as an alternative treatment for patients with refractory UC in the out-patient setting. This is particularly useful if the patient is unwilling to cosider a colectomy. With close monitoring and adherence to protocols, it is safe and effective allowing patients an alternative immunosuppresant which may either avoid the need for a colectomy or, give some time to adjust to its implications.

Disclosure of Interest: All authors have declared no conflicts of interest.

\section{References}

1. Yamamoto $\mathrm{S}$ et al. Long term effect of tacrolimus therapy in patients with refractory ulcerative colitis. Aliment Pharmacol Therap 2008;28:589-597

2. Ogata $\mathrm{H}$ et al. Double-blind, placebo-controlled trial of oral tacrolimus (FK506)in management of hospitalized patients with steroid-refractory ulcerative colitis. Inflamm Bowel Dis 2012.18:803-8

\section{P1710 A REAL LIFE COMPARISON OF THE EFFICACY OF ADALIMUMAB VERSUS GOLIMUMAB IN MODERATE-TO-SEVERE ULCERATIVE COLITIS. A MULTICENTER EXPERIENCE FROM THE SICILIAN NETWORK FOR INFLAMMATORY BOWEL DISEASE (SN-IBD)}

S. Renna ${ }^{1}$, F. Mocciaro $^{2}$, M. Ventimiglia ${ }^{1}$, F.S. Macaluso ${ }^{1}$, R. Orlando ${ }^{1}$, M. Billeci ${ }^{1}$, M. Cappello ${ }^{4}$, M. Mendolaro ${ }^{5}$, A.C. Privitera ${ }^{6}$, C. Ferracane $^{6}$, W. Fries ${ }^{7}$, V. Pisana ${ }^{7}$, A. Magnano ${ }^{8}$, D. Pluchino ${ }^{8}$, G. Inserra ${ }^{9}$, G. Scarpulla ${ }^{10}$ S. Garufi ${ }^{10}$, A. Carroccio ${ }^{11}$, S. Siringo ${ }^{12}$, R. Dimitri ${ }^{2}$, M. Cottone ${ }^{1}$, A. Orlando ${ }^{1}$ Division Of Internal Medicine, "Villa Sofia-Cervello"' Hospital, Palermo University, Palermo/Italy

${ }^{2}$ Gastroenterology And Endoscopy Unit, ARNAS Civico-Di Cristina-Benfratelli

Hospital, Palermo/Italy

${ }^{4}$ Di.bi.mis - University Of Palermo - Italy, UOC Gastroenterologia ed Epatologia Di.Bi.Mis - University of Palermo, Palermo/Italy

${ }^{5}$ Gastroenterology And Hepatology Unit, A.O.U. Policlinico "G. Giaccone", Palermo University, Palermo/Italy

"IBD Unit, A.O. "Cannizzaro", Catania, Italy, Catania/Italy

${ }^{7}$ Inflammatory bowel disease Unit, A.O.U. Policlinico "G. Martino", Messina,

Italy, Messina/Italy

${ }^{8}$ Gastroenterology Unit, A.O.U. Policlinico "Vittorio Emanuele", Catania, Italy, Catania/Italy

${ }^{9}$ Policlinico, Catania, Catania/Italy

${ }^{10}$ A.O. S.Elia di Caltanissetta, P.O. Raimondi U.O.C. di Gastroenterologia, Caltanissetta/Italy

${ }^{11}$ Division of Internal Medicine, A.O. "G. Paolo II", Sciacca/Italy

${ }^{12}$ Gastroenterology Unit, ARNAS "Garibaldi”, Catania/Italy

Contact E-mail Address: sararenna.md@gmail.com

Introduction: Adalimumab (ADA) and golimumab (GOL) are effective in the induction and maintenance treatment of moderate-to-severe ulcerative colitis (UC). No comparable data between the 2 drugs are available up to now.

Aims \& Methods: We reported the Sicilian Network experience on the comparative efficacy of ADA and GOL in patients (pts) with moderate-to-severe UC From June 2015 until April 2017, 197 consecutive pts with moderate to severe UC were treated with ADA or GOL. The efficacy was evaluated at 8 week and at the end of the follow up considering "clinical response" (reduction of at least 2 points of Partial Mayo Score with concomitant steroid reduction or discontinuation) and "clinical remission" (Partial Mayo Score $<2$ without steroids). The presence of clinical response or clinical remission was defined as "clinical benefit". Endoscopic Mayo Score was evaluated at the end of the follow up in pts who underwent colonoscopy.

Results: 118 pts were treated with ADA and 79 with GOL for a median follow up of 40.21 [20.32, 69.14] weeks for ADA and 34.00 [17.43, 54.79] weeks for GOL $(\mathrm{p}=0.08)$. Eighty-eight $\mathrm{pts}$ were naïve to anti-TNF $\alpha$ (59 ADA, 29 GOL, $\mathrm{p}=0.09$ ). No difference in Mayo Score value was observed between the 2 groups at the time of first drug injection $(p=0.92)$. After 8 weeks clinical benefit was achieved in 93/118 (78.8\%) pts treated with ADA and $50 / 79(63.3 \%)$ pts treated with GOL $(\mathrm{p}=0.026)$. Clinical remission was achieved in $48 / 118(40.7 \%)$ pts treated with ADA and $20 / 79(25.3 \%)$ pts treated with GOL $(p=0.038)$. At the end of the follow up clinical benefit was achieved in $79 / 118(66.9 \%)$ pts treated with ADA and $37 / 79(46.8 \%)$ pts treated with GOL $(\mathrm{p}=0.008)$. Clinical remission was achieved in 50/118 $(42.4 \%)$ pts treated with ADA and $23 / 79(29.1 \%)$ pts treated with GOL $(\mathrm{p}=0.082)$. No difference was observed in clinical outcomes at 8 weeks and at the end of the follow up between naive and non naive pts $(p=0.187)$. At the end of the follow up the median Endoscopic Mayo Score was $3.00[0.00,5.00]$ in pts treated with ADA and 4.00 [1.00, 7.00] in pts treated with GOL $(\mathrm{p}=0.025)$. Univariable analysis revealed that age $>40$ years at the time of first drug injection and age $<40$ years at the diagnosis were associated with higher remission rate in pts treated with ADA respect to pts treated with GOL at 8 weeks and at the end of the follow up $(\mathrm{p}=0.034$ and $\mathrm{p}=0.016$ respectively). Disease duration $>5$ years was associated with a higher remission rate in pts treated with ADA respect to pts treated with GOL at 8 weeks and at the end of the follow up $(\mathrm{p}=0.017)$.

Conclusion: This is the first study where the comparable efficacy of ADA and GOL was evaluated. These real life data confirmed the efficacy of subcutaneous anti-TNF $\alpha$ in the treatment of moderate to severe UC. ADA resulted to be more effective than GOL in inducing and maintaining clinical benefit.
Larger prospective studies with longer follow up are warranted to confirm this data.

Disclosure of Interest: S. Renna: Abbvie, MSD, Takeda.

F. Mocciaro: Abbvie, MSD

F.S. Macaluso: MSD, Abbvie, Takeda

A. Orlando: Abbvie, MSD, Takeda

All other authors have declared no conflicts of interest.

\section{P1711 REAL-LIFE STUDY (GORE-UC) EVALUATING THE} EFFECTIVENESS OF GOLIMUMAB FOR THE TREATMENT OF ULCERATIVE COLITIS: AN INTERIM ANALYSIS FROM ITALIAN GROUP FOR THE STUDY OF INFLAMMATORY BOWEL DISEASE (IG-IBD)

D. Pugliese ${ }^{1}$, A. Variola ${ }^{2}$, A.C. Privitera ${ }^{3}$, M. Allocca ${ }^{4}$, F. Bossa ${ }^{5}$, M. Cappello ${ }^{6}$, G. Lorenzon ${ }^{7}$, S. Mazzuoli ${ }^{8}$, M. Principi ${ }^{9}$, R. Sablich ${ }^{10}$, A. Ferronato ${ }^{11}$, S. Festa ${ }^{12}$, L. Moser ${ }^{13}$, G. Tapete ${ }^{14}$, G. Bodini ${ }^{15}$, M. Di Girolamo ${ }^{16}$, L. Grossi $^{17}$ F. Mocciaro ${ }^{18}$, C. Ricci $^{19}$, S. Saibeni ${ }^{20}$, R. Spagnuolo ${ }^{21}$, E. Capoferro ${ }^{2}$, A. Armuzzi ${ }^{1}$

${ }^{1}$ IBD Unit, Fondazione Policlinico Universitario A. Gemelli - Catholic University of the Sacred Heart, Rome, Italy, Rome/Italy

${ }^{2}$ Gastroenterologia, Ospedale Sacro cuore DOn Calabria, Negrar (VR)/Italy

IBD Unit, A.O. "Cannizzaro", Catania, Italy, Catania/Italy

${ }^{4}$ Ibd Center, IRCCS Humanitas, Rozzano/Italy

${ }^{5}$ Gastroenterology And Digestive Endoscopy, Casa Sollievo della Sofferenza Hospital, San Giovanni Rotondo/Italy

${ }^{6}$ Gastroenterology Section, DiBiMis, University of Palermo, Palermo/Italy

${ }^{7}$ Surgery, Oncology And Gastroenterology, Gastroenterology, Padua/Italy ${ }^{8}$ Gastroenterology \& Artificial Nutrition, San Nicola Pellegrino" Hospital, Trani/ Italy

${ }^{9}$ Emergency And Organ Transplantation, University of Bari, Bari/Italy

${ }^{10}$ SC di Gastroenterologia AAS5 - Ospedale S.Maria degli Angeli, Pordenone/Italy ${ }^{11}$ Digestive Endoscopy Unit, ULSS4 Alto Vicentino, Santorso/Italy

${ }^{12}$ GI Unit, AO S.Filippo Neri, Rome, Rome/Italy

${ }^{13}$ Gastroenterologia B, Azienda Ospedaliera Universitaria Integrata di Verona, Verona/Italy

${ }^{14}$ UO Gastroenterologia Universitaria- Azienda Ospedaliera Universitaria pisana, Pisa/Italy

${ }^{15}$ Department Of Internal Medicine, IRCCS San Martino DIMI, Genova/Italy ${ }^{16}$ AOU Policlinico di Modena, UOC Gastroenterologia, Modena/Italy

${ }^{17} G$ d́Annunzio University of Chieti-Pescara, c/o Fisiopatologia Digestiva Ospedale Spirito Santo, Pescara/Italy

${ }^{18}$ Gastroenterology And Endoscopy Unit, Gastroenterology And Endoscopy Unit, Arnas Civico-Di Cristina-Benfratelli Hospital, Palermo, Italy., Palermo/Italy

${ }^{19}$ Dept. Of Experimental And, University of Brescia, Brescia/Italy

${ }^{20}$ Gastroenterology Unit, Rho Hospital, ASST Rhodense, Rho/Italy

${ }^{21}$ Department of Medical And Surgery Sciences, IBD Unit, "Magna Grecia",

University, Catanzaro, Catanzaro/Italy

Contact E-mail Address: danipug@libero.it

Introduction: The efficacy of golimumab for the induction and maintenance of clinical remission in adult subjects moderately to severely active Ulcerative Colitis (UC) has been studied in two completed clinical trials. ${ }^{1-2}$ However, patients enrolled in clinical trials are not entirely representative of those encountered in the clinical practice setting. In Italy, golimumab is on the market for treating UC from March 2015. The aim of our study is to evaluate the durability and safety of golimumab in the context of real-life clinical practice.

Aims \& Methods: An observational, multicenter, retrospective-prospective, phase IV study, enrolling all patients starting golimumab from March to December 2015, from 21 IG-IBG centers. This study consists of two different parts: 1) retrospective, regarding data until December 2016 and 2) a prospective one, still ongoing, that will be concluded at the end of 2017 . The co-primary outcomes were the overall durability of treatment with golimumab, defined as persistence on golimumab therapy because of sustained clinical benefit, and safety. Results for the first 54-weeks period are reported.

Results: 121 patients ( $47 \%$ female), with mean age of 45.7 years $( \pm$ SD 14.3$)$ and a median duration of disease of 8 years, (range 0-28) were included. Sixty-seven patients $(55.4 \%)$ had severe endoscopic activity (Mayo 3). Clinical activity was defined as moderate (Partial Mayo Score (PMS) $5-6)$ in 55 patients $(45.5 \%)$ and severe (PMS 7-9) in 66 patients $(54.5 \%)$ Previous exposure to anti TNF- $\alpha$ was reported in $52 \%$ of patients ( 38 Infiximab, 4 Adalimumab, 21 both). Steroiddependence and refractoriness were reported in $78.5 \%$ and $16.5 \%$ of patients, respectively. After 54 weeks, the cumulative persistence on golimumab therapy was $31 \%$. Seventy-seven patients withdrew from treatment, without significant difference among anti TNF- $\alpha$ naïve vs exposed patients $(55.2 \%$ vs $71.4 \%$, $\mathrm{p}=0.11$ Chi-Square test). Among $90 \%$ of patients who completed week 8 , $48 \%$ of patients were still on golimumab therapy at week 54 . Thirty patients $(39 \%)$ withdrew within the first 14 weeks. Among the remaining patients, at week 54 the persistence on golimumab therapy was $57.1 \%$. Ten patients reported an adverse event, but only 6 of them withdrew from treatment. Four patients reported paradoxical skin lesions, unresponsive to topical therapies. Fifteen patients (12.4) underwent surgery within the first 54 weeks, with a greater percentage among anti TNF- $\alpha$ exposed $(20.6 \%$ vs $3.4 \%, \mathrm{p}=0.02$ Chi-Square test).

Conclusion: This preliminary real-life data study endorses golimumab's promising results, showing $57.1 \%$ of durability treatment at week 54 in those patients 
who completed first 14 weeks of treatment and confirming it as a safe drug. Anti TNF- $\alpha$ naïve patients were more likely to avoid colectomy.

Disclosure of Interest: D. Pugliese: Lecture fees from AbbVie and Takeda.

M. Allocca: Speaker's fees: Janssen, Pfizer Consultant's fee: Nikkiso Europe

M. Di Girolamo: Speaker for AbbVie and Takeda

All other authors have declared no conflicts of interest.

\section{References}

1 Sandborn WJ et al, Gastroenterology. 2014;146(1):85-95.

2 Sandborn WJ et al, Gastroenterology. 2014;146(1):96-109.

\section{P1712 PREVALENCE OF CIPROFLOXACIN RESISTANCE IN INFLAMMATORY BOWEL DISEASE PATIENTS WITH GUT COLONIZATION WITH EXTENDED SPECTRUM BETA- LACTAMASE PRODUCING ENTEROBACTERIA ACCORDING TO BACTERIAL PLASMID GENES}

V. Skuja ${ }^{1}$, K. Pekarska ${ }^{1}$, H. Dauvarte ${ }^{1}$, E. Vasuka ${ }^{1}$, L. Dobelniece ${ }^{1}$, J. Malina $^{1}$, D. Rudzite ${ }^{2}$, E. Lavrinovica ${ }^{2}$, L. Piekuse ${ }^{1}$, A. Kalcenaua ${ }^{1}$, A. Krumina ${ }^{1}$, A. Lejnieks ${ }^{1}$, A. Derovs ${ }^{3}$

${ }^{1}$ Riga Stradins Univeristy, Riga/Latvia

${ }^{2}$ Riga East Clinical University Hospital, Riga/Latvia

${ }^{3}$ Gastroenterology, Hepatology And Nutrition Clinic, Riga East Clinical University Hospital, Riga/Latvia

\section{Contact E-mail Address: vita@skuja.lv}

Introduction: Ciprofloxacin is one of the most frequently used antibiotics in hospitalized inflammatory bowel disease (IBD) patients. Also discrepancies between clinical guidelines and real clinical situations are observed in terms of antibiotics use in patients with IBD. In the last few years an emerging resistance to ciprofloxacin, ranging from $43 \%$ to $82 \%$, has been described in extended spectrum beta-lactamase producing bacteria (ESBL-E) colonizing the gut. ${ }^{1,2}$ Aims \& Methods: The objective of this study was to evaluate the gut colonization with ESBL-E in IBD patients, determine the resistance to ciprofloxacin and bacterial plasmid genes associated with that. Rectal swabs were collected from all consecutive patients with confirmed ulcerative colitis (UC) and Crohn's disease (CD) hospitalized in two largest tertiary medical care centres in Riga, Latvia during a 7-year period (2010-2016). Enterobacteria were cultured and analyzed for ESBL presence according to EUCAST guidelines, resistance to ciprofloxacin and bacterial plasmid genes CTX-M, TEM and SHV were detected.

Results: A total of 148 patients with confirmed IBD diagnosis were included in the study-101 (68\%) with UC, $47(32 \%)$ with CD. We found that $12(12 \%)$ of the UC patients and $5(11 \%)$ of the CD patients were colonized with ESBL-E. The isolated ESBL producing strains from UC patients included Escherichia coli $(\mathrm{n}=10)$, Klebsiella oxytoca $(\mathrm{n}=1)$ and Escherichia hermanii $(\mathrm{n}=1)$. The isolated ESBL producing strains from CD patients included only Escherichia coli $(\mathrm{n}=5)$. The isolated bacterial plasmid genes associated with ESBL production in UC included CTX-M $(\mathrm{n}=11 ; 92 \%)$, TEM $(\mathrm{n}=4 ; 33 \%)$, SHV $(\mathrm{n}=1 ; 8 \%)$, in CD CTX-M $(n=4 ; 80 \%)$ and TEM $(n=3 ; 60 \%)$. In UC $6(50 \%)$ and in CD $1(20 \%)$ of the isolated ESBL-E were resistant to ciprofloxacin. In 1 case of the ciprofloxacin resistance CTX-M, TEM and SHV gene combination was observed, in 1 case CTX-M and TEM gene combination was observed, in 4 cases only CTX-M gene was present and in 1 case only TEM gene was present.

Conclusion: 1. Higher gut colonization rate with ESBL-E in IBD patients, mostly with E. coli, expressing CTX-M gene was found comparing with the literature. 2. Higher resistance to ciprofloxacin was found in ESBL-E isolated from UC patients, comparing to UC patients. 3. CTX-M and TEM genes are associated with resistance to ciprofloxacin.

Disclosure of Interest: All authors have declared no conflicts of interest.

\section{References}

1. Lübbert, Christoph et al. 2015. "Colonization with Extended-Spectrum Beta-Lactamase-Producing and Carbapenemase-Producing Enterobacteriaceae in International Travelers Returning to Germany.' International Journal of Medical Microbiology 305(1):148-56.

2. Vervoort, J. et al. 2014. "High Rates of Intestinal Colonisation with Fluoroquinolone-Resistant ESBL-Harbouring Enterobacteriaceae in Hospitalised Patients with Antibiotic-Associated Diarrhoea." European journal of clinical microbiology \& infectious diseases: official publication of the European Society of Clinical Microbiology 33(12):2215-21.

\section{P1713 PATTERNS OF ANTI-TNF USE IN CROHN'S DISEASE PATIENTS IN FRANCE: RESULTS FROM AN ANALYSIS OF A FRENCH ADMINISTRATIVE DATABASE (GENERAL SAMPLE OF HEALTH INSURANCE BENEFICIARIES) AND HOSPITAL DISCHARGE DATA}

R. Toueg ${ }^{1}$, I. Bureau ${ }^{2}$, C. Laurendeau ${ }^{2}$, L. Lamarsalle ${ }^{3}$, M. Allez ${ }^{4}$

${ }^{1}$ Janssen Cilag, Issy-Les-Moulineaux/France

${ }^{2}$ Cemka, Bourg-la-reine/France

${ }^{3}$ Heva Heor, Lyon/France

${ }^{4}$ Gastroenterology, Hopital Saint-Louis APHP, Université Denis Diderot Paris

${ }^{7}$ Paris/France

Contact E-mail Address: rtoueg@its.jnj.com
Introduction: Anti-TNFs are well-established in therapeutic management of Crohn's disease (CD). Real-life data on their pattern of use in a French clinical setting are, however, limited to this day.

Aims \& Methods: The objective of this study was to examine for characteristics of CD patients and anti-TNF use in a real-life setting in France through the general sample of health insurance beneficiaries (EGB database) which includes reimbursement data from a sampled $1 / 97^{\text {th }}$ of the French population. A cohort of 1280 patients with CD in the EGB database between $01 / 01 / 2010$ and $31 / 02 / 2014$ was retrospectively constituted, of which $189(14.8 \%)$ initiated an anti-TNF treatment (infliximab, adalimumab) during that period and were studied for the analysis. An additional analysis was performed based on French hospital discharge data (medical information systems program [PMSI]) from $8142 \mathrm{CD}$ patients to compare results from the EGB database but only support infliximab use due to its exclusive hospital availability in France (adalimumab can be prescribed in both hospital and retail markets)

Results: $48.7 \%$ of anti-TNF treated patients were male and the mean age at the initiation of an anti-TNF treatment was 38 . The mean duration between diagnosis (measured through the assignment to an ALD [Long Term Illness]) and start of anti-TNF treatment was 6 years. Concomitant treatments such as corticosteroids and immunosuppressants were prescribed at least once in $63 \%$ and $47 \%$ of patients respectively. Around $57 \%$ of patients initiated a treatment with infliximab and $43 \%$ with adalimumab. Results at 12 months after anti-TNF initiation are presented in the table below:

Table 1: Anti-TNF use in patients initiating an anti-TNF treatment with at least 12 months of follow-up At 12 months, $13.6 \%$ of patients underwent surgery. Results from the hospital discharge database confirmed some of our observations. Optimization rate for infliximab 12 months after initiation was similar $(33.1 \%$ at 12 months for infliximab). Treatment discontinuation rates were also within the same range observed and stable over time, with $10 \%$ of patients discontinuing infliximab treatment each year and a discontinuation rate after 12 months of treatment of $27.2 \%$.

\begin{tabular}{lll}
\hline Anti-TNF use at 12 months after initiation & N & Results \\
\hline Drug survival rate for the first line anti-TNF & 108 & $69.8 \%[62.3-76.1]$ \\
Survival rate for first line adalimumab & 47 & $71.7 \%[59.9-80.6]$ \\
Survival rate for first line infliximab & 61 & $66.3 \%[55.9-74.8]$ \\
Anti-TNF dose optimization rate & 85 & $41.5 \%[34.5-49.3]$ \\
Dose optimization rate for adalimumab & 53 & $33.6 \%[25.2-43.8]$ \\
Dose optimization rate for infliximab & 63 & $38.2 \%[30.1-47.8]$ \\
Switch rate to another anti-TNF & 126 & $17.5 \%[12.6-24.1]$ \\
Switch rate from adalimumab to infliximab & 70 & $13.6 \%[8.1-22.4]$ \\
Switch rate from infliximab to adalimumab & 88 & $14.9 \%[9.6-23.0]$ \\
Anti-TNF treatment discontinuation rate & 108 & $31.4 \%[25.0-38.9]$ \\
Discontinuation rate for adalimumab & 47 & $28.2 \%[19.4-40.0]$ \\
Discontinuation rate for infliximab & 61 & $33.7 \%[25.2-44.0]$ \\
\hline
\end{tabular}

Conclusion: The general sample of health insurance beneficiaries' database provides a unique representative sample to analyze and describe real-life usage of anti-TNF in Crohn's disease patients in France.

Disclosure of Interest: R. Toueg: Raphael Toueg is an employee of Janssen I. Bureau: Isabelle BUREAU is an employee of CEMKA, which has received honoraria from Janssen

C. LAURENDEAU: Caroline LAURENDEAU is an employee of CEMKA, which has received honoraria from Janssen

L. Lamarsalle: Ludovic LAMARSALLE is an employee of HEVA HEOR, which has received honoraria from Janssen

M. Allez: Matthieu Allez has received honoraria from Novo Nordisk, MSD, Abbvie, Ferring, Genentech, TxCell, Janssen, Pfizer, GSK, Hospira and UCB

\section{P1714 PREDICTIVE FACTORS OF RESPONSE TO ANTI-TNF A TREATMENT OF COMPLEX ANO-PERINEAL FISTULAS IN CROHN'S DISEASE}

M. Hafi ${ }^{1}$, M. Fekih ${ }^{1}$, A. Laabidi ${ }^{1}$, N. Ben Mustapha ${ }^{1}$, J. Boubaker ${ }^{1}$, A. Filali ${ }^{1}$ ${ }^{1}$ Gastroenterology, la rabta hospital, tunis/Tunisia

Contact E-mail Address: maroua.hafi@gmail.com

Introduction: Ano-perineal fistulas (APF) are a common location of Crohn's disease $(C D)$. Their treatment is still disappointing. Identifying the predictive factors of response could guide the practitioner to adapt the anti-TNF $\alpha$ treatment of each patient.

Aims \& Methods: We performed a descriptive, longitudinal and retrospective study over a period of 14 years. We included all patients with a definite diagnosis of complex APF of CD treated with anti-TNF $\alpha$ with a minimum follow-up of one year. Patients less than 16 years of age or over 70 years were excluded and non-observing patients were also excluded. A univariate and multivariate statistical analysis was then carried out using the SPSS software to identify the predictive factors of response to the treatment.

Results: A total of 49 patients had complex APF treated with anti-TNF $\alpha .10 \%$ of the patients had also recto-vaginal fistulas. The mean age was 31 years. The sex ratio women/men was 1.35 . All of the patients had an MRI at diagnosis. Patients had concomitant antibiotics and seton drainage in all cases. $76 \%$ of the patients recieved azathioprin. After the induction phase, $53 \%$ of the patients 
achieved clinical remission, $31 \%$ a partial clinical response and $12 \%$ a primary failure. $53 \%$ of the patients maintaned a clinical remission after a year of maintenance therapy. After a mean time of 13 months, $42 \%$ of the patients had a loss of response. The analytical study found that the absence of recto-colic involvement, CRP negativity and normalization of platelet count under treatment and achievement of clinical remission after the induction phase were predictive factors of long term good response to anti-TNF $\alpha$ treatment. Clinical remission after the induction phase was the only independent predictive factor of long-term remission under maintenance treatment after multivariate analysis. However, partial clinical response was predictive of loss of response as well as the presence of a recto-vaginal fistula and young age at diagnosis.

Conclusion: According to our results, the type of response obtained after the induction phase seems to be closely related to the subsequent development of our patients. Prospective studies assessing early therapeutic adaptations could better evaluate this perspective in the event of a partial clinical response. In addition, rectal involvement and recto-vaginal fistulas are factors of poor response for which aggressive and specific treatment is essential.

Disclosure of Interest: All authors have declared no conflicts of interest

\section{P1715 COMPARISON OF ORIGINAL AND BIOSIMILAR INFLIXIMAB IN PATIENTS WITH INFLAMMATORY BOWEL DISEASE: A RETROSPECTIVE AND MULTICENTRIC STUDY IN SPAIN}

H. Martínez Lozano ${ }^{1}$, J. Miranda Bautista ${ }^{1}$, K. Villa ${ }^{2}$, Y. González-Lama ${ }^{2}$, P. López Serrano ${ }^{3}$, J. L. Pérez Calle ${ }^{3}$, P. Pérez Galindo ${ }^{4}$, D. Carpio López V. Matallana ${ }^{2}$, M. Calvo ${ }^{2}$, M.I. Vera ${ }^{2}$, I. Marín-Jiménez ${ }^{1}$, L. Menchén Viso ${ }^{1}$

${ }^{1}$ Gastroenterology, University General Hospital Gregorio Marañón, Madrid/Spain ${ }^{2}$ Gastroenterology, University Hospital Puerta de Hierro Majadahonda, Madrid/ Spain

${ }^{3}$ Gastroenterology, Universitary Hospital Fundación Alcorcón, Madrid/Spain ${ }^{4}$ Gastroenterology, University Hospital Complex of Pontevedra, Pontevedra/Spain

Contact E-mail Address: helena18_3@hotmail.com

Introduction: The management of chronic inflammatory bowel disease (IBD) has experienced significant advance with the development of biologic therapy. Infliximab (IFX) was the first monoclonal antibody approved for IBD. The patent expiry of biologics and their relatively high costs that result in a significant economic burden on the healthcare system, had led to the development of biosimilar agents. The biosimilar IFX has been authorised for use in all the indications as the reference IFX. The demonstration of biosimilar IFX efficacy and safety equivalence was based on two pivotal clinical trials in rheumatic diseases. As a result of the extrapolation to IBD, there is growing controversy regarding the appropriate use of biosimilar IFX. The efficacy and safety of infliximab reference in inducing and maintaining remission in IBD has been extensively proven in clinical trials. However, the role of biosimilar IFX, has not been systematically investigated in clinical practice.

Aims \& Methods: We aimed to compare the safety and efficacy in inducing and maintaining remission in IBD, between the reference IFX group and biosimilar IFX group. This retrospective, multicenter study was carried out at 4 tertiary hospitals, from January 2013 to December 2016. The analysis included 2 cohorts of consecutive IBD patients. One cohort composed of patients who were started original IFX since 2013. The second cohort included patients who were treated from the introduction of biosimilar IFX. Adverse events (AEs), demographic, clinical, endoscopic and laboratory data were collected on all patients. Efficacy was assessed according to response and remission at 14th, 54th week. For CU, response was defined as a decrease in partial Mayo score of 2 or more from baseline and a partial Mayo score of 1 or less was used to remission. For CD, response was defined as a decrease in Harvey-Bradshaw score of 3 or more from baseline, and a Harvey-Bradshaw score of 4 or less was used to remission. We used Student's t test for independent samples and Chi-square test. Time to withdrawal due to adverse effects was estimated using Kaplan-Meier survival analysis, and the log rank test was used to test for treatment group differences.

Results: The analysis included 346 consecutive IBD patients, 104 treated with original IFX and 242 with biosimilar IFX. 103 patients were diagnosed with CU, 238 with $\mathrm{CD}$ and 5 with indeterminate colitis. Overall median follow-up was 21 months. Baseline clinical activity scores were not significantly different among the 2 groups. Frequency of concomitant azathioprine and systemic steroids were not different among both groups. Patients in biosimilar infliximab group were more likely to experience previous biologic treatment failure $(29.2 \%$ versus $20.2 \%$ in the original IFX, $\mathrm{p}=0.0163$ ). There were no significant differences in patients achieving response and remission at weeks 14 and 54 . There were no significant differences in rate of withdrawals among the 2 groups $(37.1 \%$ versus $38.8 \%$ for biosimilar IFX, $\mathrm{p}=0.811$ ). There were no significant differences in cumulative discontinuation rate due to AEs in original IFX and biosimilar IFX (42.42, (95\% CI 39.49-45.34) months versus 44.61 (95\% CI 42.66-46.56) months, log-rank test $\mathrm{p}=0.292$ ).

Conclusion: Our clinical experience showed similar efficacy and safety profile of biosimilar IFX compared to original IFX.

Disclosure of Interest: All authors have declared no conflicts of interest.
P1716 DOES SEVERE ENDOSCOPIC COLITIS PREDICT STEROID REFRACTORY DISEASE IN ACUTE SEVERE COLITIS?

F. Er-Rabie, S. Elmahjoubi, E.M. Amine, W. Khannoussi, Z. Ismaili, G. Kharrasse

Gastroenterology, Oujda University Hospital, Oujda/Morocco

Contact E-mail Address: fadoua.errabie@gmail.com

Introduction: Acute severe Colitis (ASC) is a severe complication of inflammatory bowel disease (IBD), for which there is no consensus definition. Its diagnosis is based on clinico-biological and/or endoscopic criteria. Low endoscopy is essential for the positive diagnosis of ASC as well as for the diagnosis of IBD

Aims \& Methods: The objective of this study is to describe the endoscopic aspect of ASC and its interest in therapeutic management in our series of 48 cases it is a prospective descriptive and analytical study of a series of 48 cases of acute severe colitis (ASC) collected during a period of 3 years (2014-2016) in the gastroenterology department.

Results: The average age of our patients is 39.8 years with extremes ranging from 14 to 65 years, a female predominance was found with a sex ratio, M/F of 0.77 . The ASC was inaugural in $20(41.66 \%)$, while 28 cases $(58.33 \%)$ are known to have IBD, with 24 cases of UC $(85.7 \%)$. Initial endoscopy was performed in all patients. The average time to perform endoscopy (from the onset of symptoms) was 37 days ( 2 to 75 days). Severe endoscopic aspects were present in 30 patients: deep ulcer (29 cases), spontaneous bleeding (4 cases), friability (4 cases). Other endoscopic lesions found were erythema (12 cases), erosions (5 cases), superficial ulcer ( 25 cases), pseudo polyps (14 cases), contact bleeding (27 cases). Biopsy was performed in all patients, histology was in favor of UC in $64.58 \%$ cases. CMV viral inclusions were found in $2.08 \%$ of cases. First-line medical treatment is based mainly on parenteral corticosteroid therapy, has been established in all patients. A second-line treatment with anti-TNF was introduced in 5 cases $(10.41 \%)$ while surgical treatment was indicated in 15 cases $(31.25 \%)$ of which 12 cases had severe endoscopic colitis. steroid refractory disease was associated with endoscopic severe colitis $(\mathrm{p}=0.04)$. In mono-varied analysis, endoscopic severe colitis was found in males more than females (53.6 vs 43.3) With a statistically significant difference $\mathrm{p}=0.020$

Conclusion: Endoscopy in ASC occupies an important place to specify the morphological severity and thus make the positive diagnosis, the severe endoscopic colitis constitutes one of the predictive elements of steroid refractory disease requiring the use of a second therapeutic pallium.

Disclosure of Interest: All authors have declared no conflicts of interest.

\section{P1717 BASELINE CALPROTECTIN DOES NOT PREDICT}

\section{RESPONSE TO BIOLOGICAL THERAPY IN ULCERATIVE COLITIS}

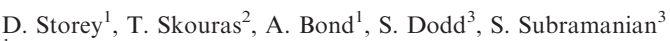

${ }^{1}$ Division Of Gastroenterology And Hepatology, Royal Liverpool and Broadgreen University Hospitals NHS Trust, Liverpool/United Kingdom

${ }^{2}$ Gastroenterology, Royal Liverpool University Hospital, Liverpool/United

Kingdom

${ }^{3}$ Royal Liverpool University Hospital, Liverpool/United Kingdom

Contact E-mail Address: Thomas.skouras@nhs.net

Introduction: Response to biological drugs in ulcerative colitis (UC) is variable with induction response rates of $64.5 \%$ (vs $29.3 \%$ for placebo), $50.4 \%$ (vs $34.6 \%$ for placebo), $51.0 \%$ (vs $30.3 \%$ for placebo), $47.1 \%$ (vs $25.5 \%$ for placebo) for infliximab, adalimumab, golimumab and vedolizumab, respectively. Apart from prior exposure to anti-tumour necrosis factor (anti-TNF) agents and concurrent immunomodulatory therapy, predictors of clinical response and remission to biological drugs have not been identified. We sought to investigate the utility of baseline faecal calprotectin (FC) and early change in FC in predicting clinical response and remission to biological therapy in UC.

Aims \& Methods: Patients who were commenced on any biological therapy for $\mathrm{UC}$ and had a baseline FC at the time of commencement were included in this retrospective study. Disease activity was monitored serially by calculation of Simple Clinical Colitis Activity Index (SCCAI) or by Physician global assessment (PGA) or by treatment persistence. Clinical response was defined as decrease in $\mathrm{SCCAI} \geq 3$ or a decrease in PGA score from baseline to follow up. The ability of $\mathrm{FC}$ and fold change in FC to predict response and remission at 6 months was estimated using Mann-Whitney test.

Results: A total of 94 patients were commenced on biological therapy of who 70 commenced anti-TNF therapy and 24 commenced vedolizumab with a mean age of $41.8(\mathrm{SD}:+18.2)$. Fifty-one $(72 \%)$ and $39(55 \%)$ patients commencing antiTNF therapy were on concurrent immunomodulators (IM) and steroids respectively compared to $9(38 \%)$ and $16(67 \%)$ patients respectively for vedolizumab. Sixteen $(67 \%)$ patients treated with vedolizumab had prior exposure to anti-TNF agents compared to $4(6 \%)$ in the antiTNF treated group. The 6-month response among patients treated with vedolizumab and anti-TNF agents was $77.2 \%$ and $73.5 \%$ respectively. The calprotectin values were similar for responders $(428.5$ $(72,2100)[n=16])$ and non-responders $(600(107,2100)[n=5])$ to vedolizumab $(\mathrm{P}=0.56)$. Similarly, responders $(909(13,2100)[\mathrm{n}=28])$ and non-responders $(850(240,2100)[\mathrm{n}=13])$ to antiTNF agents had comparable calprotectin values at baseline, $\mathrm{P}=0.93$

Conclusion: In a single-centre series of biologic treated UC patients, baseline FC did not predict clinical response at 6 months.

Disclosure of Interest: S. Subramanian: Advice report member for Abbvie, Janssen and Behringer-ingelheim On speaker bureau for Dr Falk, Abbvie and MSD.

All other authors have declared no conflicts of interest. 


\section{P1718 EIGHT YEARS EXPERIENCE OF DRUG EFFICACY IN CROHN'S DISEASE PATIENTS: A PROSPECTIVE MULTICENTER REAL-LIFE STUDY}

M. Lodyga ${ }^{1}$, P. Eder ${ }^{2}$, M. Gawron-Kiszka ${ }^{3}$, M. Hartleb ${ }^{3}$, J. Kierkus $^{4}$, M. Klopocka ${ }^{5}$, M. Kukulska ${ }^{6}$, M. Grzymislawski ${ }^{2}$, E. Malecka-Panas ${ }^{7}$, E. Poniewierka ${ }^{6}$, I. Smola ${ }^{6}$, T. Rawa $^{8}$, J. Regula ${ }^{9}$, G. Rydzewska ${ }^{10}$

${ }^{1}$ Department Of Internal Medicine And Gastroenterology With IBD Subdivision, CSK MSWiA, Warsaw/Poland

${ }^{2}$ Department Of Gastroenterology, Human Nutrition And Internal Diseases,

Poznan University of Medical Sciences, Poznan/Poland

${ }^{3}$ Department Of Gastroenterology And Hepatology, Medical University of Silesia, Katowice/Poland

${ }^{4}$ Department Of Gastroenterology, Hepatology, And Feeding Disorders, Children's Memorial Health Institute, Warsaw/Poland

${ }^{5}$ Gastroenterology Nursing Unit, Centre For Therapeutic Endoscopy, University Hospital No2 Collegium Medicum in Bydgoszcz, Nicolaus Copernicus University, Torun/Poland

${ }^{6}$ Department Of Gastroenterology And Hepatology, Medical University, Wroclaw/ Poland

${ }^{7}$ Department Of Digestive Tract Diseases, Medical University of Lodz, Lodz/

Poland

${ }^{8}$ Department Of Gastroenterology And Hepatology, Medical Centre for Postgraduate Education, Warsaw/Poland

${ }^{9}$ Department Of Gastroenterology, Hepatology And Oncology, Medical Center for Postgraduate Education, Warsaw, Poland, Warsaw/Poland

${ }^{10}$ Faculty Of Medicine And Health Science, Uniwersytet Jana Kochanowskiego, Kielce/Poland

Contact E-mail Address: mlodyga@op.pl

Introduction: The prevalence of Crohn's disease is important for planning of health care and allocation of clinical resources. In 2005, a National Patient's Registry in Poland was established to collect demographic and clinical data. To quantify efficacy of medications in real-life treatment during the study period, data regarding medical treatment were collected from Registry.

Aims \& Methods: The aim of the study was assess the efficacy and tolerance of different medications in reference to demographic data and disease location and behaviour. 6030 of patients have been enrolled to the Polish National CD Patient's Registry, conducted in 95 gastroenterology centers in Poland. Patient's phenotype according to: Montreal classification, demographics, smoking, alcohol consumption, extraintestinal manifestation and medical treatment have been evaluated. The impact of demographic factors on the use of drugs from different groups (mesalamine, prednisone, azathioprine, methotrexate, antiTNF), and medications efficacy and tolerance was assessed. The efficacy assessment was evaluated according to subjective 4-step scale. Similarly treatment tolerance was assessed according to 2-step scale.

Results: No gender effects were observed on the use or efficacy of individual drug classes, although greater tolerability of prednisone and azathioprine was observed in men (respectively 95.56 vs 93.82 and 93.94 vs. 91.65, both $\mathrm{p}<0.05$ ). Smoking did not affect the effectiveness and tolerability of the used medications. However surprisingly fewer smokers were treated with azathioprine, methotrexate, and anti-TNF in comparison to non-smokers ( 38 vs $45 \%, 0.5$ vs $1.55 \%, 6.5$ vs $11 \%$, all $\mathrm{p}<0.05$ ) In patient's declaring casual alcohol use, the efficacy and tolerability of prednisone was significantly better than in patient's declaring abstinence ( 89 vs 84 and 96 vs $93 \%$; p < $<.05$ ). Referring to the Montreal classification, efficacy of mesalamine, prednisone and azathioprine was significantly higher in A1 group with the lowest in A2 patients (A1: 90, A2:83, A3: 86 for prednisone, $\mathrm{p}<0.05$ ) Regarding relation to localization of the disease, the efficacy of treatment with immunosuppressive agents (azathioprine and methotrexate) in L3 was significantly lower compared with L1 and L2, despite of increased use of immunosuppressive drugs in L1 (76 vs $82 \%, \mathrm{p}<0.05)$. As predicted, the use of immunosuppressive and anti-TNF drugs was higher in complicated disease behavior (B2 and B3) than in B1, however efficacy of infliximab was similar in B1 in comparison to others (B3: $15.5 \%$ B1: $7 \%$ of infliximab use).

Conclusion: This is the first study comparing efficacy and tolerability of treatment methods used in "real-life" practice in Poland during last 8 years. Most observations are in compliance with data from clinical trials. Positive effect of casual alcohol consumption on efficacy of medications requires further observation. Interestingly some unexpected relationships, concerning similar efficacy of infliximab in different disease behavior was found. This effect requires also further observations in regards to more frequent use of anti-TNF drugs in last years.

Disclosure of Interest: All authors have declared no conflicts of interest.
P1719 EFFICACY OF VEDOLIZUMAB INDUCTION THERAPY IN PATIENTS WITH SEVERE, THERAPEUTIC RESISTANT INFLAMMATORY BOWEL DISEASE

R. Bor ${ }^{1}$, K. Farkas ${ }^{1}$, P. Miheller ${ }^{2}$, K. Palatka ${ }^{3}$, T. Szamosi ${ }^{4}$, P.L. Lakatos ${ }^{5}$ K.B. Gecse ${ }^{6}$, P.A. Golovics ${ }^{5}$, A. Vincze ${ }^{7}$, L. Lakner ${ }^{8}$, M. Rutka ${ }^{1}$, A. Balint ${ }^{1}$, A. Fabian ${ }^{1}$, A. Milassin ${ }^{1}$, Z. Szepes ${ }^{1}$, T. Molnar ${ }^{1}$

${ }^{1}$ First Department Of Medicine, University of Szeged First Department of Internal Medicine First Department of Internal M, Szeged/Hungary

${ }^{2}$ Second Department Of Internal Medicine, Semmelweis University, Budapest/ Hungary

${ }^{3}$ Department Of Gastroenterology, University of Debrecen, Debrecen/Hungary ${ }_{5}^{4}$ Military Hospital-State Health Centre, Budapest/Hungary

${ }^{5} 1$ st Department Of Medicine, Semmelweis University Faculty of Medicine 1st Dept. of Medicine - 1st Department of Medicine, Semmel, Budapest/Hungary

${ }^{6} 1$ st Department Of Medicine, Semmelweis University, Budapest/Hungary

${ }^{7} 1$ st Department Of Medicine, University of Pecs, Pecs/Hungary

${ }^{8}$ Csolnoky Ferenc Veszprém County Hospital, Veszprém/Hungary

Contact E-mail Address: bor.reni86@gmail.com

Introduction: Vedolizumab (VDZ) is the first gut-specific monoclonal antibody alternative to anti-tumor necrosis factor alpha therapy in patients with moderateto-severe inflammatory bowel disease (IBD). It has been registered since 2016 in Hungary, but currently the high treatment costs are considerably limiting the availability of VDZ. All newly initiated VDZ therapy is individualized, it should be approved by the steering committee of five Hungarian IBD-specialists. This results in that VDZ therapy is available exclusively for patients in whom conventional treatment was ineffective or contraindicated.

Aims \& Methods: The aim of our non-interventional retrospective study was to assess the efficacy of induction VDZ therapy. 41 patients (16 with Crohn's disease (CD) and 25 with ulcerative colitis (UC) received VDZ induction therapy between September 2016 and April 2017 in Hungary. Efficacy of induction therapy was assessed based on the changes of activity indices on week 14 .

Results: 40 of 41 enrolled IBD patients were therapeutic failure or intolerant for infliximab and/or adalimumab therapy. The mean age was 38.6 years (range 18 67; median 40) and the average disease duration was 11.7 years (range 1-36; median 10). In 16 cases moderate and in 25 cases severe disease activity was observed. Extra intestinal manifestations occurred in 6 patients, and in 6 cases the IBD was associated with primary sclerosing cholangitis (PSC). Rate of the therapeutic responders for VDZ induction therapy was $80.49 \% \quad(\mathrm{~N}=33)$. Complete clinical remission was observed in 19 cases $(46.34 \%) 8$ cases $(19.51 \%)$ of which were steroid-free remission. In one case VDZ therapy had to be interrupted due to development of IBD associated colorectal cancer and in one case due to MCV infection.

Conclusion: Our results suggest that induction VDZ therapy is effective and it is a safe therapeutic option in anti-tumor necrosis factor alpha failure or intolerant IBD patients with moderate or severe disease activity.

Disclosure of Interest: All authors have declared no conflicts of interest.

\section{P1720 OUTCOMES OF TREATMENT FOR LATENT} TUBERCULOSIS INFECTION IN PATIENTS WITH INFLAMMATORY BOWEL DISEASE RECEIVING BIOLOGIC THERAPY

G. Piovezani Ramos ${ }^{1}$, G. Stroh ${ }^{1}$, B. Al Bawardy ${ }^{2}$, W. A. Faubion², K. A. Papadakis ${ }^{2}$, P. Escalante

${ }^{1}$ Internal Medicine, Mayo Clinic, Rochester/United States of America/MN ${ }^{2}$ Gastroenterology And Hepatology, Mayo Clinic, Rochester/United States of America/MN

${ }^{3}$ Pulmonary And Critical Care Medicine, Mayo Clinic, Rochester/United States of America/MN

Contact E-mail Address: ramos.guilherme@mayo.edu

Introduction: Tuberculosis (TB) reactivation is of particular concern in patients with inflammatory bowel disease (IBD) treated with biologic therapies. Screening for latent tuberculosis infection (LTBI) is indicated prior to initiating treatment. Despite the reduction of TB reactivation following treatment for LTBI the risk of reactivation still exists. The efficacy of LTBI treatment in IBD patients receiving biologic therapy and the timing of biologic therapy initiation has not been extensively studied.

Aims \& Methods: In order to evaluate the effectiveness of LTBI treatment in IBD patients receiving biologic, we conducted a retrospective review of all IBD patients diagnosed with LTBI following a tuberculin skin test (PPD) or interferon gamma release assay (IGRA) and who received biologic therapy between January 1996 and August 2016. Data extracted included patient demographics, $\mathrm{TB}$ risk factors, chest $\mathrm{x}$-ray findings, biologic agent used, prior and concomitant therapies, and LTBI treatment regimen. TB reactivation after completion of LTBI treatment was the primary outcome of the study. Risk of TB reactivation was calculated using McGill University's "The Online TST/IGRA Interpreter." Results: A total of 35 IBD patients (27 Crohn's; 8 ulcerative colitis) were included in the study. Their mean age was $38.3 \pm-14.4$ years and $68.6 \%$ were male (Table $1)$. The median time from diagnosis of IBD to LTBI was 9 years $(0-48$ years). Prior IBD therapies included corticosteroids $(86 \%)$, aminosalicylates $(83 \%)$, other immunosuppressants $(69 \%)$. At least $43 \%$ of patients have been previously exposed to at least 1 biologic agent. The most common LTBI treatment regimen was isoniazid (INH) for 9 months $(n=26,74 \%)$. Biologic therapy used were infliximab $(\mathrm{n}=14,40 \%)$, adalimumab $(\mathrm{n}=10,29 \%)$, vedolizumab $(\mathrm{n}=7$, $20 \%)$, and certolizumab pegol $(n=4,11 \%)$. Combination therapy with an immunomodulator and a biologic agent was administered in $57 \%$ of cases $(n=20)$. All patients were treated for LTBI and the majority $(83 \%)$ was treated prior to 
starting biologic therapy. The median time from initiation of LTBI treatment to biologics was 43 days (4-3653). The mean duration of follow-up was $2.9 \pm 3.3$ years. The median calculated annual risk of developing active TB without treatment was $0.52 \%(0.08 \%-1.3 \%)$. Of the cohort studied, only one patient taking adalimumab monotherapy after completing 6 months of INH therapy developed reactivation of $\mathrm{TB}$. The estimated $\mathrm{TB}$ reactivation rate in our cohort was 0.98 cases per 100 patient-years of follow up.

Table 1: Cohort Characteristics and Estimated Post-treatment Tuberculosis Reactivation Rate

\begin{tabular}{lc}
\hline Mean Age & $38.3( \pm 14.4)$ years \\
Male Sex & $24 / 35$ patients \\
Type of Inflammatory & Ulcerative Colitis $(23 \%)$ \\
Bowel Disease (IBD) & Crohn's Disease $(77 \%)$ \\
Mean Time since IBD & 9 years (range: $0-48)$ \\
Diagnosis & \\
Type of Biologic Therapy & Infliximab $(40 \%)$ Adalimumab \\
& $(29 \%)$ Vedikuzumab $(20 \%)$ \\
Type Latent Tuberculosis & Certolizumab $(11 \%)$ \\
Therapy & Isoniazid (INH) for $9-$ months \\
& $(74 \%)$ INH for $6-$ months \\
& $(11 \%)$ Rifampin $4-$ months \\
& $(9 \%)$ INH + Rifampin for 3- \\
median time to initiate & months $(3 \%)$ Others $(3 \%)$ \\
biologic therapy & 43 days (range: $4-3653)$ \\
Mean duration of follow-up & \\
Mean Pre-treatment Risk of & $2.9 \pm 3.3$ years \\
Development of Active & $0.52 \% /$ year $\quad$ (range: $0.08 \%$ - \\
Tuberculosis & $1.3 \% /$ year) \\
Estimated Post-treatment & \\
Tuberculosis & 0.98 cases per 100 patient-years \\
Reactivation Rate & \\
\hline
\end{tabular}

Conclusion: Treatment for LTBI in patients with IBD treated with biologics is effective, but does not eliminate the risk of reactivation, which occurred at a rate of 0.98 cases per 100 patient-years in our cohort. Additional studies with extended follow-up are warranted to further characterize the efficacy of LTBI treatment in these patients.

Disclosure of Interest: All authors have declared no conflicts of interest.

\section{P1721 CLINICAL CHARACTERISTICS AND MANAGEMENT OF CROHN'S DISEASE IN PATIENTS WITH RESIDUAL DISEASE AFTER SURGERY COMPARED WITH CURATIVE SURGERY. RESULTS FROM PRACTICROHN STUDY}

E. Domènech ${ }^{1}$, M.D. Martín Arranz ${ }^{2}$, A. Gutiérrez ${ }^{3}$, M. Iborra ${ }^{4}$, V. GarcíaSánchez ${ }^{5}$, M. Barreiro-De Acosta ${ }^{6}$, L. Cea-Calvo ${ }^{7}$, C. Romero ${ }^{7}$, B. Juliá De Páramo

${ }^{1}$ Gastroenterology Unit, Hospital Germans Trias i Pujol, Barcelona/Spain ${ }^{2}$ Gastroenterology Unit, Hospital Universitario La Paz, Madrid/Spain ${ }^{3}$ Gastroenterology, Hospital General Universitario de Alicante, Alicante/Spain ${ }^{4}$ Gastroenterology Unit, Hospital Politécnico La Fe and Ciber-EHD, Valencia/ Spain

${ }^{5}$ Gastroenterology, Hospital Universitario Reina Sofía, Córdoba/Spain ${ }^{6}$ Gastroenterology Unit, Complejo Hospitalario Universitario de Santiago, Santiago de compostela/Spain

${ }^{7}$ Medical Department, MSD, Madrid/Spain

Contact E-mail Address: eugenidomenech@gmail.com

Introduction: Resection in Crohn's disease (CD) intends to be a curative surgery, but sometimes it is not possible to remove completely the lesions of the damaged gut. The aim of our study was to describe the characteristics and management of patients with residual disease after surgery (RD) and to compare these with patients with curative surgery (CS) in post operative $C D$ patients.

Aims \& Methods: PRACTICROHN was a retrospective study that included adult patients from 26 Spanish hospitals who underwent CD-related ileocolonic resection with ileocolonic or ileorectal anastomosis between January 2007 and December 2010. Clinical data was retrospectively collected from clinical charts. RD was defined when lesions were still present after surgical resection. Postoperative recurrence (POR) was defined by clinical symptoms (diarrhea, abdominal pain) and endoscopic Rutgeerts score $\geq i 2$, and/or CT or MRI confirmation of disease activity. Categorical variables were compared with the $\chi^{2}$ test or Fisher's exact test Kaplan-Meier method was used to assess time to clinical recurrence and a log-rank test to obtain statistical significance.

Results: Three hundred and sixty-four patients were analyzed (mean age 40 years [SD 13], 50\% men). Of these, $27(7.5 \%)$ had RD after surgery. Median age at diagnosis was shorter in patients with RD than CS: 23 (IQR 19-34) years vs 29 (IQR 23-40), $\mathrm{p}=0.02$. At the time of resection $\mathrm{B} 1(+\mathrm{p})$ behavior was more frequent in RD than in CS: $6(22 \%)$ vs $26(8 \%), p=0.05$; and location was mainly $\mathrm{L} 1( \pm \mathrm{L} 4)$ in CS $(190,57 \%)$ and $\mathrm{L} 3( \pm \mathrm{L} 4)$ in $\mathrm{RD}(19.70 \%), \mathrm{p}=0.02$. Four $(16 \%)$ patients in RD were receiving immunomodulators at the time of surgery vs 132 $(41 \%)$ of CS, $p=0.02$. More patients in RD vs CS presented postoperative complications $(12(44 \%)$ vs $87(26 \%), p=0.06)$ as well as hospitalizations the first year after surgery $(10(37 \%)$ vs $42(12 \%) \mathrm{p}=0.001)$. No differences in smoking habit, perianal disease or length of resection were found between the two groups. More patients were performed an endoscopy within the first year after surgery in the RD vs CS: $16(59 \%)$ vs $122(36 \%), \mathrm{p}=0.03$ but no difference in prophylactic treatment were found in RD vs CS groups. POR was more frequent among patients with RD $(69 \%$ vs. $29 \%, \mathrm{p}=0.001)$. Median time to POR was longer in patients who received prophylaxis vs those who didn't received it $(698$ vs 392 days; $\mathrm{p}=0.4) .81 / 275(29 \%)$ in the CS presented POR with median time to POR being longer in patients who received prophylaxis (no median found vs 1529 days) $\mathrm{p}=0.04$. Table 1 .

Table 1

\begin{tabular}{|c|c|c|c|c|c|c|c|}
\hline \multirow{2}{*}{\multicolumn{2}{|c|}{ Residual disease patients }} & \multirow[b]{2}{*}{$\mathrm{N}$} & \multirow[b]{2}{*}{ Events } & \multirow{2}{*}{$\begin{array}{l}\text { Median } \\
\text { time to POR }\end{array}$} & \multicolumn{2}{|c|}{ CI $95 \%$} & \multirow[b]{2}{*}{ p-value } \\
\hline & & & & & LL & UL & \\
\hline \multirow[t]{2}{*}{ Prophylaxis } & Yes & 15 & 8 & 698 & 217 & - & \multirow[t]{2}{*}{0.4} \\
\hline & No & 8 & 8 & 392 & 287 & - & \\
\hline \multirow{2}{*}{\multicolumn{2}{|c|}{ Curative surgery patients }} & \multirow[t]{2}{*}{$\mathrm{N}$} & \multirow[t]{2}{*}{ Events } & \multirow{2}{*}{$\begin{array}{l}\text { Median time } \\
\text { to POR }\end{array}$} & \multicolumn{2}{|c|}{ CI $95 \%$} & p-value \\
\hline & & & & & LL & UL & \\
\hline \multirow[t]{2}{*}{ Prophylaxis } & Yes & 187 & 48 & - & - & - & \multirow[t]{2}{*}{0.04} \\
\hline & No & 88 & 33 & 1529 & 839 & - & \\
\hline
\end{tabular}

Conclusion: Residual disease is a rare situation after intestinal resection in CD. Patients with residual disease after surgery, are more likely followed-up endoscopically within the first year. Conversely, similar proactive recurrence prevention was observed compared to curative surgery. In the case of residual disease although prophylactic treatment is useful, most of the patients will present POR.RD is a factor of poor prognosis in post-operative CD patients.

Disclosure of Interest: L. Cea-Calvo: MSD employee

C. Romero: msd employee

B. Juliá De Páramo: MSD employee

All other authors have declared no conflicts of interest.

\section{P1722 SEVERITY OF BILE ACID MALABSORPTION CORRELATES WITH LENGTH OF ILEAL RESECTION IN CROHN'S DISEASE}

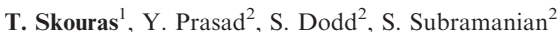

${ }^{1}$ Gastroenterology, Royal Liverpool University Hospital, Liverpool/United Kingdom

${ }^{2}$ Royal Liverpool University Hospital, Liverpool/United Kingdom

Contact E-mail Address: Thomas.skouras@nhs.net

Introduction: Bile acid malabsorption (BAM) is a common cause of diarrhoea in Crohn's disease (CD) patients with ileal resection and can lead to complications such as renal and biliary stone disease. BAM is usually diagnosed by selenium labelled homotaurocholic acid test $\left({ }^{75} \mathrm{SeHCAT}\right)$ but its availability is limited. Thus, a large proportion of resected $C D$ patients either remain underdiagnosed or subject to empirical therapy. There is a paucity of studies examining the correlation between length of ileal resection and severity of BAM which will be of particular use to clinicians with no recourse to diagnostic testing for BAM.

Aims \& Methods: We identified all CD patients with a prior surgical resection who underwent ${ }^{75} \mathrm{SeHCAT}$ testing at our institute. Testing was based on the treating clinician's discretion. The length of resected ileum was recorded from histopathology report. We conducted a Spearman's correlation test to check for correlation between length of resected ileum and percentage retention on ${ }^{75} \mathrm{SeHCAT}$. Response to treatment with bile salt sequestrant and ${ }^{75} \mathrm{SeHCAT}$ retention values was tested using Mann-Whitney test.

Results: A total of 97 patients were identified with a mean age of 46.4 (SD 14.5). The median length of resected ileum was $22.5 \mathrm{cms}$ (range $1.5-95 \mathrm{cms}$ ) with a median of 1 resection (range $1-4)$. Overall, 90 patients $\left(92.8 \%\right.$ ) had ${ }^{75}$ SeHCAT retention values of $<5 \%, 5(5.2 \%)$ patients between $5-10 \%$ and only 2 patients had values of $>15 \%$. There was moderate correlation between ${ }^{75} \mathrm{SeHCAT}$ retention and length of ileal resection (Spearman's rho: 0.4041, P $<0.001$ ). Data on response to treatment was available for 60 patients, of who $41(42 \%)$ responded and $19(19 \%)$ failed to respond to bile salt sequestrants. The ${ }^{75} \mathrm{SeHCAT}$ retention values was comparable among responders (median $0.02 \%$, range $0.1-6.6$ ) and non-responders (median $0.02 \%$, range $0.1-6.6$, Mann-Whitney test, $\mathrm{P}=0.72$ ).

Conclusion: There was moderate correlation between length of ileal resection and severity of BAM as defined by ${ }^{75} \mathrm{SeHCAT}$ retention values. Response to bile salt sequestrant therapy was not dependent on ${ }^{75} \mathrm{SeHCAT}$ retention values.

Disclosure of Interest: S. Subramanian: Advice report member for Abbvie, Janssen and Behringer-ingelheim On speaker bureau for Dr Falk, Abbvie and MSD

All other authors have declared no conflicts of interest. 


\section{WEDNESDAY, NOVEMBER 01, 201709:00-14:00}

\section{OTHER LOWER GI DISORDERS III - HALL 7}

\section{P1723 A MICROBIAL SIGNATURE OF PSYCHOLOGICAL DISTRESS IN IRRITABLE BOWEL SYNDROME}

J. Peter, C. Fournier, L. Knoblich, B. Keip, G. Moser

Internal Medicine III Dept Gastroenterology And Hepaatology, Medical

University of Vienna, Vienna/Austria

Contact E-mail Address: gabriele.moser@meduniwien.ac.at

Introduction: Irritable Bowel Syndrome (IBS) is associated with alterations along the brain-gut-microbiota axis. Previous studies have suggested a parallel segregation of microbial features with psychological burden in IBS $(1,2,3)$.

Aims \& Methods: The study aimed at examining microbial correlates of psychological distress, anxiety, depression and stress perception. 16s rRNA faecal microbial analyses (Illumina MiSeq, V1-2 amplified from total DNA) in 48 IBS patients (Rome-III criteria, mean age 42 years, 35 female subjects, 25 diarrhoea-dominant, 5 constipation-dominant and 18 alternating-type IBS). Assessment of psychological and clinical variables with validated questionnaires, microbial analysis via QIIME. Machine learning to predict psychological distress through a composite model of bacterial features. Correlational analysis and comparisons in bacterial abundance between subgroups defined by thresholds in psychological variables.

Results: Thirty-one patients (65\%) showed psychological distress, $22(31 \%)$ anxiety, and 10 depression $(21 \%)$. Psychological distress was uncorrelated with IBS severity (Spearman's $\rho=0.05, \mathrm{p}=0.736$ ). Microbial beta diversity was significantly associated with distress and depression $\mathrm{q}=0.044$ each). A random forest model using 148 microbial estimators was able to correctly classify patients regarding presence of psychological distress $($ AUROC $=0.98)$. Patients exceeding thresholds of distress, anxiety, depression and stress perception showed significantly higher abundances of Proteobacteria $(\mathrm{LDA}=2.5)$. Patients with anxiety were characterized by higher abundances of Bacteroidaceae (LDA =3.0). Depression correlated inversely with Lachnospiraceae $(\rho=-0.58, \mathrm{q}=0.018)$, anxiety positively with Anaerotruncus $(\rho=0.65, \mathrm{q}=0.001)$.

Conclusion: A microbial signature accurately predicted the presence of psychological distress. Psychological variables significantly segregated gut microbial features, underscoring the role of brain-gut-microbiota interaction in IBS Supported by Austrian Society of Gastroenterology and Hepatology (ÖGGH) and funds of the Oesterreichische Nationalbank, Fund project number: 16506 Disclosure of Interest: All authors have declared no conflicts of interest.

\section{References}

1. Moloney RD, Johnson AC, O'Mahony SM, et al. Stress and the Microbiota-Gut-Brain Axis in Visceral Pain: Relevance to Irritable Bowel Syndrome. CNS Neurosci Ther 2016;22(2):102-17.

2. Tap J, Derrien $\mathbf{M}$, Törnblom $\mathbf{H}$, et al. Identification of an Intestinal Microbiota Signature Associated With Severity of Irritable Bowel Syndrome. Gastroenterology 2016;152(1):111-23.

3. Liu Y, Zhang L, Wang X, et al. Similar fecal microbiota signatures in patients with diarrhea-predominant irritable bowel syndrome and patients with depression. Clin Gastroenterol Hepatol 2016;14(11):1602-11.

\section{P1724 SACCHAROMYCES BOULARDII CNCM I-745 LOWERS FECAL CHOLIC ACID CONCENTRATIONS DURING ANTIBIOTHERAPY IN HEALTHY VOLUNTEERS: A NEW POTENTIAL MECHANISM IN THE PROTECTION AGAINST CLOSTRIDIUM DIFFICILE INFECTION}

H. Duboc ${ }^{1}$, C. Chong Nguyen ${ }^{1}$, T. Kabbani ${ }^{2}$, D. Rainteau ${ }^{1}$, L. Humbert ${ }^{1}$, S. Dowd ${ }^{3}$, L. Palmieri ${ }^{1}$, B. Coffin ${ }^{1}$, K. Pallav ${ }^{4}$, A. Bado ${ }^{1}$, C. Kelly ${ }^{1}$ Department of Hepato Gastro Enterology and University Paris Diderot, Sorbionne Paris Cité, INSERM UMR 1149, Louis Mourier Hospital, APHP, Colombes, France, Colombes/France

${ }^{2}$ BIDMC \& Harvard Medical School, Boston/United States of America/MA ${ }^{3} 3$ Molecular Research, Shallowater, TX, USA, Shallowater/United States of America

${ }^{4}$ Harvard School of Public Health, Boston, MA, USA, Boston/United States of America

${ }^{5}$ Division of Gastroenterology, Department of Medicine, Beth Israel Deaconess Medical Center, Boston, MA, USA, Boston/United States of America

Contact E-mail Address: henri.duboc@gmail.com Introduction: Saccharomyces boulardii (SB) CNCM I-745 demonstrated clinical efficacy in the secondary prevention of post-antibiotic Clostridium difficile infection (CDI), but the mechanism remains unclear. Cholic acid (CA) is a primary bile acid (BA), synthesized by the liver which triggers the germination of $\mathrm{C}$. difficile spores in the intestine. Physiologically, the gut microbiota transforms primary BAs (cholic acid and chenodeoxycholic) into secondary (deoxycholic and lithocholic). CA loses its germinating properties after transformation and appears to become protective against CDI. The goals of this work in healthy volunteers (HV) were to: A) Describe the effect of antibiotics on the quantity of 'pro-germination' primary BA including CA in human stools B) Describe whether SB enhances transformation to 'protective' secondary BA in human.

Aims \& Methods: This work was an ancillary of a previous study conducted in 4 groups of $\mathrm{HV}$ at Beth Israel Deaconess Medical Center and Harvard Medical School, Boston, Massachusetts. The previous results of this work showed that SB CNCM I-745 can modulate shifts in the microbiota and reduces diarrhea during an antibiotic therapy. Group $1(\mathrm{n}=12)$ received SB CNCM I-745 $500 \mathrm{mg}$ twice daily for 14 days. Group $2(n=12)$ received Amoxicillin-Clavulanate (AC) $(875$ $125 \mathrm{mg}$, twice daily) for 7 days. Group $3(\mathrm{n}=12)$ received $\mathrm{AC}$ for 7 days and SB for 14 days. Group $4(n=12)$ did not receive any treatment. Groups $1,2,3$ had successive stool samples at D-28, 0, 3, 7, 10, 13,21. Group 4 had stool samples at D0, 7 and 21. The fecal concentrations of 28 BAs were measured by HPLC/MS, and expressed as \% of total BA concentration.

Results: AC alone (group 2) significantly reduced the rate of fecal secondary BA at day 7 compared to control (group 4) $(54.8 \pm 10.1$ vs $83.1 \pm 7.4 \%, p=0.017)$. In group 3 (AC plus $\mathrm{SB}$ ), the decrease in secondary BA rate was significantly less than for $\mathrm{AC}(71.23 \pm 7.4$ versus $54.20 \pm 9 \%, \mathrm{p}=0.04)$, and this difference was prolonged over time. Similarly, the AC $+\mathrm{SB}$ group showed a significantly lower (and sustained) increase in $\mathrm{CA}$ than in the $\mathrm{AC}$ alone group.

Conclusion: Antibiotics alter the transformation of BA by microbial enzymes into the intestine. This increases primary BA while reducing secondary BA in feces. Cholic Acid rates, a primary BA which facilitates $C$. difficile spore germination in vitro, increases in stool during antibiotic therapy. The concomitant administration of SB during AC treatment significantly reduces this CA peak. These results highlight new human data on a potential mechanism for post-antibiotic CDI: alteration of the microbiota can encourage germination of $C$. difficile spores via increased CA concentrations and reduced concentrations of secondary BAs. The effectiveness of SB in preventing recurrent CDI may be explained, in part, through modulation of microbiota changes that influence the balance of pro- and anti-germination BA concentrations

Disclosure of Interest: H. Duboc: I worked with Biocodex as an advisor for the developement of a free smartphone App for patients suffering of constipation. C. Kelly: Scientific advisor and consultant to: Merck, Seres Therapeutics and Summit

All other authors have declared no conflicts of interest.

\section{P1725 ASSOCIATION OF FUSOBACTERIUM NUCLEATUM IN} ORAL CAVITY AND COLORECTAL CARCINOMAS

Y. Komiya ${ }^{1}$, T. Higurashi ${ }^{2}$, A. Nakajima ${ }^{2}$

${ }^{1}$ Gastrointestinal Medicine, Yokohama City University, Yokohama/Japan

${ }^{2}$ Department Of Gastroenterology And Hepatology, Yokohama City University Yokohama/Japan

Contact E-mail Address: komiyacoco@yahoo.co.jp

Introduction: While particular imbalances in the gut microbiota have been linked to colorectal adenomas and cancers, some specific bacterium have been identified as a related factor. Recent studies have reported a high abundance of Fusobacterium nucleatum ( $F$. nucleatum) in colorectal cancer (CRC) subjects compared to normal subjects ${ }^{1,2}$. F. nucleatum is also known as a pathogenic species of oral microbiota, but it is not known if $F$. nucleatum plays a role in other part of the digestive tract. $F$. nucleatum may affect metabolic pathways for the carcinogenesis $^{3}$. We examined whether there is relationship between $F$. nucleatumin oral cavity and CRC

Aims \& Methods: We assessed the abundance of Fusobacterium in CRC, colorectal mucosa and saliva. We extracted DNA from mucosal biopsies and measured bacterial levels by quantitative PCR of the 16S ribosomal RNA gene. We also investigated the homology of $F$. nucleatum in oral cavity and CRC.

Results: In 51 CRC cases, Fusobacterium positivity was significantly higher in CRC compared to controls $(\mathrm{p}<0.05)$. Fusobacterium was more detected in CRC $(12.9 \%)$ than in normal tissues $(3.9 \%)$ respectively. The detection rate of F. nucleatum was $96 \%$ in saliva and $93 \%$ in CRC by next-generation sequencer. A total of 15 patients with $\mathrm{CRC}$ were included to check the homology of $\mathrm{F}$. nucleatum in saliva and CRC. From the 15 patients, 9 were F.nucleatum-positive in CRC. 8 of 9 from these patients were F.nucleatum-positive in saliva. From these patients who were F.nucleatum-positive in saliva and CRC, we next looked for the results of AP-PCR and 6 patients have shown common band patterns. Conclusion: The results support a link between the abundance of $F$. nucleatum in oral cavity and CRC. Our data also indicate that there may be a route from the oral cavity to the CRC in $F$. nucleatum positive cases. We are now identifying DNA sequences, specific for the objective strains.

Disclosure of Interest: All authors have declared no conflicts of interest.

\section{References}

1. Castellarin, M., Warren, R. L., Freeman, J. D., Dreolini, L., Krzywinski, M., Strauss, J., Barnes, R., Watson, P., Allen-vercoe, E., Moore, R. A. \& Holt, R. A. 2012. Fusobacterium nucleatum infection is prevalent in human colorectal carcinoma. Genome Res, 22, 299-306.

2. Kostic, A. D., Chun, E., Robertson, L., Glickman, J. N., Gallini, C. A., Michaud, M., Clancy, T. E., Chung, D. C., Lochhead, P., Hold, G. L., Elomar, E. M., Brenner, D., Fuchs, C. S., Meyerson, M. \& Garrett, W. S. 2013. Fusobacterium nucleatum potentiates intestinal tumorigenesis and modulates the tumor-immune microenvironment. Cell Host Microbe 14, 207-15.

3. Ito, M., Kanno, S., Nosho, K., Sukawa, Y., Mitsuhashi, K., Kurihara, H., Igarashi, H., Takahashi, T., Tachibana, M., Takahashi, H., Yoshii, S., Takenouchi, T., Hasegawa, T., Okita, K., Hirata, K., Maruyama, R., Suzuki, H., Imai, K., Yamamoto, H. \& Shinomura, Y. 2015. Association of Fusobacterium nucleatum with clinical and molecular features in colorectal serrated pathway. Int J Cancer, 137, 1258-68. 
P1727 HUMAN MILK OLIGOSACCHARIDES; A NEW STRATEGY AGAINST POST-ANTIBIOTIC CLOSTRIDIUM DIFFICILE

\section{INFECTION?}

\section{K. Vigsnaes}

Business Development, Glycom A/S, Horsholm/Denmark

Contact E-mail Address: louise.vigsnaes@glycom.com

Introduction: Human Milk Oligosaccharides (HMOs) are a family of complex carbohydrates found in high concentrations in human milk and which are now becoming commercially available. In clinical studies, in both infants and adults, HMOs powerfully and specifically modulate the gut microbiota by increasing bifidobacteria and reducing certain pathogenic bacteria $(1,2)$. Also, HMO bacterial consumption results in the production of beneficial metabolites such as short chain fatty acids and the lowering of $\mathrm{pH}$. Hence, the selective growth of bifidobacteria on HMOs can create an ecological niche that is more colonization resistant against pathogens. Bifidobacteria may also have a direct impact on microbial toxins by reducing their level and cytotoxic effect (3). Antibiotics, especially broad spectrum antibiotics, dramatically impact the microbiota and its balance, and have been implicated in the pathogenesis of many health conditions including gastrointestinal symptoms such as diarrhoea (4). The most commonly cited mechanism for antibiotic-associated diarrhoea is intestinal overgrowth of the pathogenic bacterium, Clostridium difficile.

Aims \& Methods: The aim of this study is to investigate, in in vitro models of $C$ difficile infection, (i) the impact of HMOs on the microbial community and activity (e.g. bacterial metabolites and $\mathrm{pH}$ ), and (ii) the anti-pathogenic activity of HMOs against $C$. difficile, with a focus on preventing recurrence of the infection. Two in vitro models, each using human faecal microbiota infected with $C$. difficile, were used to examine the impact of HMOs on bacterial metabolite production and $C$. difficile infection. One model is a 48 hour batch fermentation while the other is a simulated gut model, run for 3 weeks post infection, which simulates the infection cycle of $C$. difficile after antibiotic treatment.

Results: The study revealed that the HMOs increase the level of bifidobacteria, increase the concentration of beneficial metabolites such as short chain fatty acids and decrease $\mathrm{pH}$ compared to a control with no added HMOs. Additionally, HMOs reduced the level of $C$. difficile; in some cases completely eradiating $C$. difficile below detection limits. This antimicrobial effect of HMOs on $C$. difficile was $\mathrm{pH}$-independent, hence another mechanism is causing the antipathogenic activity of HMOs.

Conclusion: Conclusively, the results show that HMOs can impact $C$. difficile infection in an in vitro system, which suggests HMOs as a potential approach to reduce risk of antibiotic associated diarrhoea and post-antibiotic $C$. difficile infection.

Disclosure of Interest: L.K. Vigsnaes: I am a Glycom A/S employee working as Preclinical Development Manager in the Business Development department.

\section{References}

1. Puccio et al., 2017, J. Ped Gastro Nutr. 64:624-631

2. Elison et al., 2016, Br J Nutr. 116: 1356-1368

3. Valdés-Varela, L., et al., Frontiers in microbiology, 2016, 7

4. Langdon et al., 2016, Genome Medicine. 8:39

\section{P1728 CHANGES IN GUT MICROBIATA ASSOCIATED WITH AGING IN OBESE INDIVIDUALS}

R. Tanaka ${ }^{1}$, M. Matsuzaka ${ }^{1}$, S. Nakaji ${ }^{2}$, Y. Sasaki

${ }^{1}$ Medical Informatics, Hirosaki University, Hirosaki/Japan

${ }^{2}$ Hirosaki University, Hirosaki/Japan

Contact E-mail Address: r-tana@hirosaki-u.ac.jp

Introduction: It has been reported that the composition of human gut microbiota changes with aging, body mass index (BMI), diet and other environmental factors. In particular, the relationship between gut microbiota and obesity has been underlined frequently because intervention in the microbiota may reduce body fat. In this study, we investigated the relationship between obesity and composition of gut microbiota in healthy Japanese population.

Aims \& Methods: Participants were 1.082 healthy Japanese adults (410 males, 672 females) who participated in the Iwaki Health Promotion Project in 2014. Faecal samples were analysed by $16 \mathrm{~S}$ rRNA gene-targeted sequencing to determine family composition of gut microbiota. They were classified into obese group $(B M I \geq 25)$ and normal weight group $(B M I<25)$ according to Japanese standard and were stratified into 7 age groups, 19-29, 30-39, 40-49, 50-59, 60-69, 70-79 and 80-90. The family composition of gut microbiota in each age group was compared between obese and normal group.

Results: There were 235 obese participants, and 847 normal ones. The proportion of Bacteroidaceae decreased substantially, and Ruminococcaceae increasing slightly with aging in obese group. The proportion of Bifidobacteriaceae, Lachnospiraceae and Porphyromonadaceae decreased gradually with aging in both groups

Conclusion: Changes in composition of gut microbiota with aging were different between obese and normal group. Some previous researches observed differences of composition of gut microbiota between them, but many of the researches did not take aging into consideration. Our study indicated that different intervention stratified with age could be needed.

Disclosure of Interest: All authors have declared no conflicts of interest.
P1729 BACTERIOCIN PRODUCTION BY MUCOSAL BACTERIA IN COLORECTAL NEOPLASIA

D. Kohoutova ${ }^{1}$, M. Forstlova ${ }^{2}$, P. Moravkova ${ }^{1}$, S. Rejchrt ${ }^{1}$, D. Smajs ${ }^{3}$, J. Bures ${ }^{1}$ ${ }^{1}$ 2nd Department Of Internal Medicine - Gastroenterology, Charles University, Faculty of Medicine and University Hospital in Hradec Kralove, Hradec Kralove/ Czech Republic

${ }^{2}$ Department $O$ f Clinical Microbiology, Charles University, Faculty of Medicine and University Hospital in Hradec Kralove, Hradec Kralove/Czech Republic

${ }^{3}$ Department Of Biology, Masaryk University, Faculty of Medicine, Brno/Czech Republic

Contact E-mail Address: darina.kohoutova $@$ seznam.cz

Introduction: Due to its high incidence, sporadic colorectal cancer (CRC) remains a huge medical and socioeconomical burden in many countries worldwide including the Czech Republic. The exact contribution of large intestinal bacteria to the pathogenesis of CRC has not been elucidated yet, still the mucosal, not the luminal, microbiota seem to play the crucial role. Bacteriocins are small proteins, which are produced by the Enterobacteriaceae bacteria, especially by Escherichia coli. Bacteriocins are divided into more groups, colicins and microcins are the most important ones. Bacteriocins possess antibacterial, antineoplastic, proapoptotic and probiotic effect.

Aims \& Methods: The aim of this prospective study was to evaluate bacteriocin production by mucosal large intestinal bacteria in colorectal neoplasia. We used an original methodology reported by our group (1). Mucosal biopsies were taken in the caecum, transverse colon and rectum within the colonoscopy in patients with non-advanced colorectal adenoma, non-a-A (11 men, 10 women, mean age $63 \pm 10$ ), advanced colorectal adenoma, a-A (which was defined as neoplasia larger than $10 \mathrm{~mm}$ and/or containing villous component and/or containing high grade dysplasia; 13 men, 7 women, mean age $65 \pm 9$ ), sporadic colorectal carcinoma, CRC (12 men, 10 women, mean age $70 \pm 10)$ and in the controls (average risk population with normal findings on colonoscopy and with negative history of colorectal neoplasia and/or inflammatory bowel disease; 7 men, 13 women, mean age $57 \pm 14$ ). After appropriate microbiological culture bacteriocin production by each strain was investigated by PCR methods.

Results: A total of 249 mucosal biopsies were taken (60 controls, 63 non-a-A, 60 a-A, 66 CRC, ) and samples were further investigated. Colicin producing strains were isolated in 22\% (13/60) controls, 59\% (37/63) non-a-A, 55\% (33/60) a-A and in $76 \%(50 / 66)$ CRC. Significantly higher production of colicins was observed in non-a-A, a-A and CRC group when compared to controls, $p<0.001$. Significantly higher production of colicins was confirmed in patients with CRC compared to patients with $a-A, p=0.016$. Microcin producing strains were isolated in $23 \%(14 / 60)$ controls, $56 \%(35 / 63)$ non-a-A, $78 \%(47 / 60)$ a-A and in $62 \%(41 / 66)$ CRC. Significantly higher production of microcins was observed in non-a-A compared to controls, $\mathrm{p}=0.002$, in a-A and CRC group when compared to controls, $\mathrm{p}<0.001$. Microcins were produced more frequently in patients with a-A compared to those with non-a-A, $\mathrm{p}=0.008$.

Conclusion: Strains isolated from large bowel mucosa in patients with colorectal neoplasia produce bacteriocins more frequently compared to those with normal findings on colonoscopy. We presume, that mucosal large intestinal microbiota with their products including bacteriocins play an important role during the development of colorectal neoplasia.

Disclosure of Interest: All authors have declared no conflicts of interest.

\section{Reference}

BMC Infect Dis 2014; 14: 733.

P1730 CARBOXYLIC AND AMINO ACIDS MIXTURE IDENTICAL TO THE METABOLITES OF THE PROBIOTIC ESCHERICHIA COLI M17 INDUCES BACTERIOCIN SYNTHESIS IN PROBIOTIC LACTOBACILLUS HELVETICUS D75 AND D76 STRAINS AND ENHANCES THEIR ANTIMICROBIAL ACTIVITY AGAINST TEST PATHOGENS

T. Vakhitov ${ }^{1}$, V. Toropov ${ }^{1}$, O. Shalaeva ${ }^{1}$, S. Sitkin ${ }^{2}$, E. Tkachenko ${ }^{3}$ ${ }^{1}$ Dept. Of Microbiology, State Research Institute of Highly Pure Biopreparations, St. Petersburg/Russian Federation

${ }^{2}$ Dept. Of Internal Diseases, Gastroenterology \& Dietetics, North-Western State Medical University named after I.I. Mechnikov, St. Petersburg/Russian Federation ${ }^{3}$ Kirov Military Medical Academy, St. Petersburg/Russian Federation

Contact E-mail Address: tkachenkoe@mail.ru

Introduction: The production of bacteriocins is considered as the key metabolic function of gut microbiota and as the inherent property of probiotic strains. Bacteriocins and metabolites of probiotic microorganisms (metabiotics) can optimize host-specific physiological functions related to human health.

Aims \& Methods: This study aims to: (a) detect the bacteriocin genes of probiotic strains Lactobacillus helveticus D75 (NCBI Reference Sequence NZ_CP020029.1) and Lactobacillus helveticus D76 (NCBI Reference Sequence NZ_CP016827.1) and (b) evaluate in vitro effects of the carboxylic and amino acids mixture identical to the metabolites of the probiotic Escherichia coli strain M17 (components of Actoflor ${ }^{\circledR}-\mathrm{S}$ dietary supplement). The antagonistic activity of Lactobacillus helveticus D75 and Lactobacillus helveticus D76 strains was estimated by the deferred antagonism method. The identification of bacteriocin genes was performed by PCR using helveticin $\mathrm{J}$ gene primers. Amplified fragments were sequenced using ABI PRISM ${ }^{\circledR} 310$ Genetic Analyzer and were analyzed using NCBI/BLASTX

Results: The identical sequences of $537 \mathrm{bp}$ homologous to gene fragment of helveticin of Lactobacillus helveticus DPC 4571 (lhv_1632 gene) were detected 
in DNA of both probiotic strains. Sequencing of these fragments showed difference in three nucleotides compared to the reference DNA of DPC 4571 strain (A instead of $\mathrm{G}$ at position 46, C instead of $\mathrm{T}$ at position 249 and $\mathrm{A}$ instead of $\mathrm{T}$ at position 537), but all these replacements do not lead to changes in the amino acid sequence of a bacteriocin. For Lactobacillus acidophilus D76 another bacteriocin gene fragment of $283 \mathrm{bp}$ was identified (in addition to $537 \mathrm{bp}$ fragment). The latter had $95 \%$ homology with the helveticin $\mathbf{J}$ gene of Lactobacillus helveticus R0052 (R0052 09025 gene). In NCBI/BLASTX database the sequences homologous to the helveticin gene of Lactobacillus helveticus DPC 4571 were found in 11 microorganisms related to Lactobacillus acidophilus, Lactobacillus amylovorus, Lactobacillus crispatus, Lactobacillus gallinarum, Lactobacillus helveticus and Lactobacillus kitasatonis. The addition of the carboxylic and amino acids mixture (Actoflor ${ }^{\circledR}$-S) results in 2-2.5-fold enhanced antimicrobial activity of both tested probiotic strains against test pathogens Escherichia coli O75 and Salmonella Enteritidis 209, most likely due to an increase in bacteriocin gene expression.

Conclusion: Study shows that there are at least two bacteriocins in Lactobacillus helveticus D76 and one bacteriocin in Lactobacillus helveticus D75. Carboxylic and amino acids mixture identical to the metabolites of the probiotic Escherichia coli strain M17 probably induces bacteriocin synthesis in probiotic strains Lactobacillus helveticus D75 and Lactobacillus helveticus D76 and enhances their antimicrobial activity against test pathogens Escherichia coli O75 and Salmonella enteritidis 209. However, the true potential of gut microbial metabolites mixture and identified probiotic bacteriocins for human health has yet to be realized

Disclosure of Interest: All authors have declared no conflicts of interest.

\section{References}

1. Pranckutė R, Kaunietis A, Kuisienè N, Čitavičius DJ. Combining prebiotics with probiotic bacteria can enhance bacterial growth and secretion of bacteriocins. Int $J$ Biol Macromol. 2016 Aug;89:669-76. doi: 10.1016/ j.ijbiomac.2016.05.041

2. Dobson A, Cotter PD, Ross RP, Hill C. Bacteriocin production: a probiotic trait? Appl Environ Microbiol. 2012 Jan;78(1):1-6. doi: 10.1128/AEM.0557611.

3. Fernandez B, Le Lay C, Jean J, Fliss I. Growth, acid production and bacteriocin production by probiotic candidates under simulated colonic conditions. J Appl Microbiol. 2013 Mar;114(3):877-85. doi: 10.1111/jam.12081.

4. Foster LM, Tompkins TA, Dahl WJ. A comprehensive post-market review of studies on a probiotic product containing Lactobacillus helveticus R0052 and Lactobacillus rhamnosus R0011. Benef Microbes. 2011 Dec 1;2(4):31934. doi: 10.3920/BM2011.0032.

5. Shenderov BA. Metabiotics: novel idea or natural development of probiotic conception. Microb Ecol Health Dis. 2013 Apr 12;24. doi: 10.3402/ mehd.v24i0.20399. eCollection 2013.

\section{P1731 LONG-TERM SAFETY AND EFFECT ON GASTROINTESTINAL SYMPTOMS OF FECAL MICROBIOTA TRANSPLANTATION}

P. Arkkila ${ }^{1}$, A. Hillamaa ${ }^{2}$, J. Jalanka ${ }^{3}$, E. Mattila ${ }^{4}$, V. Anttila ${ }^{4}$, R. Satokari ${ }^{3}$ ${ }^{1}$ Gastaroenterology, Helsinki University Hospital, Helsinki/Finland

${ }^{2}$ Univeristy of Tarto, Tarto/Estonia

${ }^{3}$ Helsinki Univeristy, Helsinki/Finland

${ }^{4}$ Infectious Diseases, Helsinki University Hospital, Helsinki/Finland

Contact E-mail Address: perttu.arkkila@hus.fi

Introduction: Fecal microbiota transplantation (FMT) ihas been shown to be effective treatemtn for recurrent Clostridium difficile infection. Concern has been raised about the long-term safety of FMT.

Aims \& Methods: The aim of this study was to determinate the long-term safety of fecal microbiota transplantation (FMT), and its effect on gastrointestina symptoms (GI) in Clostridium difficile (CDI) patients. We studied 84 patients of which 45 received an FMT treatment via colonoscopy and 39 served as controls receiving antibiotic treatment $(\mathrm{AB})$ for the recurrent $\mathrm{CDI}$ and followed their recovery on average of 3.8 yrs. All together 130 patients ( 55 patients in the FMT group and 75 patients in the $\mathrm{AB}$ group were sent a 45 -item questionnaire collecting information about the patient demographics, their physical and mental health, including allergies, infections, gastroenterological conditions such as IBD and IBS, diabetes, autoimmune diseases, neurological disorders, mental wellbeing and malignancies. Response rate for the questionnaire was $64.6 \%$.

Results: There were no differences in the incidence of severe diseases between the groups including the incidence of IBD, diabetes, diseases of the nervous system, autoimmune disease, incidence of colon polyps and cancer. Change of weight was neither different between groups (kg;SD); FMT + 2.5 (5.6) and $\mathrm{AB}+1.3$ (5.6), $\mathrm{p}=0.51$. The $\mathrm{AB}$ treated subjects recorded more frequently that their bowel function had become worse and more irregular after the treatment $(\mathrm{AB}=35.9 \%, \mathrm{FMT}=11.1 \%, \mathrm{P}=0.001)$ compared to $\mathrm{FMT}$ group. $77.8 \%$ of the patients treated with FMT experienced GI symptoms related to IBS whereas $92.3 \%$ of antibiotic-treated patients recorded these symptoms $(P=0.06)$. AB patients experienced more symptoms of the upper intestinal tract than the FMT-treated patients $(\mathrm{AB}=51.3 \%, \mathrm{FMT}=31.1, \mathrm{p}=0.045$. In this cohort $97.6 \%$ of the FMT-treated patients and $60 \%$ of $\mathrm{AB}$ treated patients would prefer in the future that their initial treatment to be FMT instead of antibiotics. Conclusion: FMT is a rational, durable, safe, and acceptable treatment option for patients with recurrent CDI. No severe diseases appeared after FMT and FMT seem to relieve GI symptoms better than antibiotic treatment. FMT and AB treated patients would prefer in the future that their initial treatment for recurrent CDI to be FMT instead of antibiotics.
Disclosure of Interest: All authors have declared no conflicts of interest.

\section{References}

1. Mattila E, Uusitalo-Seppälä R, Wuorela M, Lehtola L, Nurmi H, Ristikankare M, Moilanen V, Salminen K, Seppälä M, Mattila PS, Anttila VJ, Arkkila P. Fecal transplantation, through colonoscopy, is effective therapy for recurrent Clostridium difficile infection. Gastroenterology. 2012 Mar;142(3):490-6.

2. Cammarota G, Ianiro G, Tilg H, Rajilić-Stojanović M, Kump P, Satokari R, Sokol H, Arkkila P, Pintus C, Hart A, Segal J, Aloi M, Masucci L, Molinaro A, Scaldaferri F, Gasbarrini G, Lopez-Sanroman A, Link A, de Groot P, de Vos WM, Högenauer C, Malfertheiner P, Mattila E, Milosavljević T, Nieuwdorp M, Sanguinetti M, Simren M, Gasbarrini A; European FMT Working Group European consensus conference on faecal microbiota transplantation in clinical practice. Gut. 2017 Apr;66(4):569-580.

\section{P1732 CLOSTRIDIUM DIFFICILE-ASSOCIATED DISEASE IN A PORTUGUESE HOSPITAL CENTER}

T. Gago, A. Antunes, A.M. Vaz, P. Queirós, J. Roseira, A. C. Cunha, A. Ramos, H. Guerreiro

Serviço De Gastrenterologia De Faro, Centro Hospitalar do Algarve, Faro/

Portugal

Contact E-mail Address: taniagago@gmail.com

Introduction: Clostridium difficile-associated disease (CDAD) is an infection caused by Clostridium difficile, gram-positive, anaerobic, spore-forming and toxin-producing bacteria. Infection is recognized as the leading cause of diarrhea associated with health care services in the developed countries. In Portugal epidemiological data are limited.

Aims \& Methods: Characterize Clostridium difficile-associated disease episodes in a Portuguese Hospital Center. Retrospective analysis of 250 hospitalized patients with CDAD, in Centro Hospitalar do Algarve, between 2011 and 2015. The data was obtained from clinical processes and statistical analysis was performed with SPSS version 23 .

Results: The patients were mostly women $(52 \%)$. The mean incidence of CDAD was $0.21 \%$ and the patients had an associated mortality of $28 \%$. The year with the highest incidence was $2015(0.53 \%)$ but with a lower associated mortality rate. CDAD was mostly acquired at the hospital level $(75.6 \%)$ and the mean length of hospital stay was 33 days. About $82.4 \%$ of the cases were first occurrences and the remaining $(18.6 \%)$ were recurrences of CDAD. The majority of the population under study performed Proton Pump Inhibitors-IBP $(52.8 \%)$ and antibiotic therapy $(74.4 \%)(26.8 \%$ made a single antibiotic, and $23.6 \% 2$ or more distinct antibiotics). Penicillin antibiotic class was the most used, followed by Cephalosporins $(21.5 \%)$, Fluoroquinolones $(11.4 \%)$ and Macrolides $(10.1 \%)$. Conclusion: A significant increase in the incidence of CDAD was observed in this study. This increase may be related to several factors, such as the improvement of laboratory diagnostic methods, increased antibiotic prescription, hospital contamination with Clostridium difficile spores or with the appearance of new and more virulent Rybotypes.

Disclosure of Interest: All authors have declared no conflicts of interest.

\section{References}

Shin JH, High KP, Warren CA. Older Is Not Wiser, Immunologically Speaking: Effect of Aging on Host Response to Clostridium difficile Infections. J Gerontol A Biol Sci Med Sci 2016

Surawicz CM, Brandt LJ, Binion DG, et al. Guidelines for diagnosis, treatment, and prevention of Clostridium difficile infections. Am J Gastroenterol 2013;108(4):478-98; quiz 99.

Carmo J, Marques S, Chapim I, et al. Leaping Forward in the Treatment of Clostridium Difficile Infection: Update in 2015. GE Portuguese Journal of Gastroenterology 2015;22(6):259-67.

Davies KA, Longshaw CM, Davis GL, et al. Underdiagnosis of Clostridium difficile across Europe: the European, multicentre, prospective, biannual, point-prevalence study of Clostridium difficile infection in hospitalised patients with diarrhoea (EUCLID). Lancet Infect Dis 2014;14(12):1208-19.

\section{P1733 THE EFFICACY OF SELECTIVE ARTERIAL EMBOLIZATION IN THE MANAGEMENT OF DIVERTICULAR BLEEDING}

Y. Fukami ${ }^{1}$, S. Sakita ${ }^{2}$, T. Mochida ${ }^{2}$, C. Kamitsuma ${ }^{2}$, A. Igarashi ${ }^{2}$, J. Tsuchiya ${ }^{2}$, K. Yasuda ${ }^{2}$, M. Ike ${ }^{2}$, N. Kozawa ${ }^{2}$, M. Kaneshiro ${ }^{2}$, H. Ikemiyagi $^{2}$, K. Yoshino ${ }^{2}$, M. Watanabe

${ }^{1}$ Digestive Disease Center, Yokohama city Minato Red Cross Hospital, Yokohama/Japan

${ }^{2}$ Gastroenterology, Yokohama-city Red Cross Hostpital, Yokohama/Japan ${ }^{3}$ Gastroenterology, Tokyo Medical and Dental University, Graduate School of Medical Science, Tokyo/Japan

Contact E-mail Address: yfukami.gast@gmail.com

Introduction: Colonic diverticular bleeding is the most common cause of lower gastrointestinal bleeding. Persistent bleeding or acute massive bleeding of presenting with hemodynamic disorders requires an interventional treatment. The question of what is the best treatment for acute diverticular bleeding remains 
unanswered. In our institution, we gastroenterologists perform interventional radiology for. Retrospective study of transarterial embolization (TAE) for colonic diverticular bleeding was performed.

Aims \& Methods: The aim of this study is to clarify the efficacy of TAE for colonic diverticular bleeding. 229 patients were diagnosed as diverticular bleeding from Jan 2010 to Dec 2016 in our institution. Bleeding stopped spontaneously in 126 patients. 103 patients were performed colonoscopy. Overt bleeding occurred in 8 patients after colonoscopy, and those were eligible for this study who underwent TAE. Conservative management or endoscopic procedure were not successful in all the patients. 7 patients were male and 1 was female with a median age of 62.6 years (range 39-85 years). The average opportunity for enhanced CT was 2.1, 7 patients were in shock, and all of 8 the patients were treated with blood transfusion. Those who were extravasation-positive in enhanced CT underwent angiogram from vasa recta near the extravasation at least 3 times. In case radiopaque clips were placed at the bleeding site via colonoscopy to mark embolization site, regardless of whether or not active extravasation was identified on angiogram, coil embolization was performed using 0.010 inch coils in vasa recta. Technical success rate and complications were evaluated. Technical success was defined as immediate complete cessation of bleeding confirmed by digital subtraction angiography showing no further contrast extravasation at the end of each TAE. Clinical success was defined as no recurrent bleeding in observation period.

Results: Technical success rate was $88 \%(7 / 8)$, and clinical success was also $88 \%(7 / 8) .6$ patients were extravasation-positive in enhanced CT, and 5 patients were extravasation-positive in angiogram. Although 3 patients were extravasation-negative in angiogram, 2 patients underwent TAE. After TAE, no recurrence of bleeding was observed. Severe adverse events such as bowel infraction did not occur in all cases.

Conclusion: As the microcatheter technique has recently improved further, adverse events are rare. Therefore superselective coil embolization could be first choice for diverticular bleeding with extravasation-positive in enhanced CT. Disclosure of Interest: All authors have declared no conflicts of interest.

\section{P1734 ACCURACY OF THE NASOGASTRIC TUBE AND THE BUN/ CREATININE RATIO FOR DISTINGUISHING BETWEEN UPPER AND LOWER SOURCES OF GASTROINTESTINAL BLEEDING. A SYSTEMATIC REVIEW}

S. Machlab, E. Martinez-Bauer, P. Garcia-Iglesias, A. Lira, C. Marmol, G. Llibre, J.P. Da Costa, M. Gallach, L. Melcarne, V. Puig-Divi, F. Junquera, R. Campo, E. Brullet, X. Calvet

Gastroenterology, Hospital Universitari Parc Tauli, Sabadell/Spain

Contact E-mail Address: stmachlab@tauli.cat

Introduction: The insertion of a nasogastric tube (NGT) and assessment of the BUN/creatinine ratio were recommended as initial measures to distinguish between upper and lower gastrointestinal bleeding (American College of Gastroenterology 2016). As the nasogastric tube is one of the most bothersome interventions for the patient, we evaluated the evidence supporting these recommendations.

Aims \& Methods: The aim of the study was to identify the diagnostic accuracy (sensitivity, specificity, positive predictive value, negative predictive value and likelihood ratios) of the NGT and the BUN/creatinine ratio for distinguishing between upper and lower sources of gastrointestinal (GI) bleeding. We conducted a systematic review of the literature in order to identify studies assessing the diagnostic accuracy of the NGT or BUN/creatinine in patients with melena, hematochezia or rectorrhagia without hematemesis. The search was performed in November 2016 in five data bases (Pubmed, Scopus, Web of Science, Cochrane Plus Library and OpenGrey).

Results: Four studies met the selection criteria (two evaluating the NGT, one BUN/creat and one both). The two methods had a low sensitivity for detecting upper GI bleeding source. Both a positive NGT aspiration and BUN/creatinine ratio above 30 markedly increased the probability of an upper GI source with a positive likelihood ratio ranging from 2 to 11 . Unfortunately, the sensitivity of both tests for upper GI bleeding was very low (negative likelihood ratios around 0.6).Characteristics and results of the studies selected are shown in table 1 .

Conclusion: For patients with gastrointestinal bleeding without hematemesis, BUN/Creat $\geq 30$ indicates a high probability of an upper GI source. Nasogastric tube aspiration provides little additional information and so is not indicated. Neither test reliably rules out an upper GI source of bleeding.
Therefore, in cases of uncertainty, an upper GI endoscopy will be necessary to reliably rule out an upper GI source.

Disclosure of Interest: All authors have declared no conflicts of interest.

\section{P1735 GLASGOW-BLACHFORD SCORE ACCURATELY PREDICTS THE NEED OF TRANSFUSION IN ACUTE LOWER GASTROINTESTINAL BLEEDING. A DIAGNOSTIC ACCURACY EVALUATION STUDY}

S. Machlab ${ }^{1}$, E. Martinez-Bauer ${ }^{2}$, P. Garcia-Iglesias ${ }^{1}$, A. Lira ${ }^{1}$, C. Marmol ${ }^{1}$, G. Llibre ${ }^{1}$, J.P. Da Costa ${ }^{1}$, M. Gallach ${ }^{1}$, L. Melcarne ${ }^{1}$, V. Puig-Divi ${ }^{1}$ F. Junquera ${ }^{2}$, R. Campo ${ }^{1}$, X. Calvet ${ }^{1}$, E. Brullet ${ }^{1}$

${ }^{1}$ Gastroenterology, Hospital Universitari Parc Tauli, Sabadell/Spain

${ }^{2}$ Corporación Parc Tauli, Sabadell/Spain

Contact E-mail Address: stmachlab@tauli.cat

Introduction: The incidence of acute lower gastrointestinal bleeding (LGB) is increasing in Western countries, but the predictors of its outcome are not well studied and defined.

Aims \& Methods: The aim of this study was to compare the accuracy of GlasgowBlachford score (GBS) with three available risk scores (State, Velayos and Newman) for predicting the need of any clinical intervention (endoscopic therapy, vascular embolization, surgery and need of transfusion) in patients admitted for acute LGB. Retrospective study from January 2013 to December 2015 in a university tertiary care hospital. Patients with acute LGB were identified using the International Classification of Diseases (9th Revision) and Clinical Modification codes for admission diagnosis. Scores were retrospectively calculated according to clinical reports data. Area under the receiver operating characteristic curve (AUROC), sensitivity, specificity, positive and negative predictive values were calculated for four scores. Also the best cut-off of each score was chosen from using the AUROC curve values.

Results: A total of $298(51 \%$ men) consecutive patients with acute LGB were identified. Median age was 76.1 years (range 25.4-96.5), $201(67.4 \%)$ of patients were older than 70 years. Five patients $(1.7 \%)$ died, $18(6 \%)$ developed recurrent bleeding, $89(29.9 \%)$ needed transfusion, $30(12.1 \%)$ received endoscopic therapy, and $3(1 \%)$ underwent transcatheter arterial embolization. No patient required any surgical intervention. AUROC of GBS score was 0.87 (95\% CI:0.82-0.91) for the need of transfusion, and 0.82 (95\% CI:0.76-0.87) for the need of any clinical intervention. AUROC for the need of transfusion and clinical intervention were 0.68 (95\% CI:0.61-0.74) and 0.67 (95\% CI:0.60-0.73) for the Strate score, 0.77 (95\% CI:0.71-0.83) and 0.74 (95\% CI:0.68-0.80) for the Velayos score and 0.78 (95\% CI:0.72-0.85) and 0.74 (95\% CI:0.68-0.81) for the Newman score, respectively. GBS was significantly more accurate than LGB risk scores for predicting the need of transfusion. Although AUROC of GBS was also numerically better for predicting the need of any clinical intervention, the difference was only significant when comparing with the Strate score. All the risk scores were more accurate for determining the need for transfusion than for the need of clinical intervention. Sensitivity, specificity and negative and positive predictive values for each score are shown in table 1.

Table 1: Sensitivity (S), specificity (Sp), Positive (PPV) and negative (NPV) predictive values of the different socres for detecting the need of trasnsfusion (TRF) or clinical intervention (CI). *Best cut-off scores for Blachford secore were 6 for transfusion and 4 for clinical intervention. Values are expresed as $\%$

\begin{tabular}{lcccccccc}
\hline Score & \multicolumn{7}{c}{ TRF S TRF Sp TRF PPV TRF NPV CI S CI Sp CI PPV CI NPV } \\
\hline Blachford* $^{*}$ & 80 & 78 & 61 & 90 & 89 & 59 & 50 & 91 \\
Strate $>1$ & 69 & 58 & 41 & 81 & 66 & 58 & 86 & 78 \\
Velayos $>0$ & 95 & 46 & 41 & 96 & 90 & 46 & 44 & 90 \\
Newman $>192$ & 40 & 39 & 93 & 89 & 40 & 32 & 88 \\
\hline
\end{tabular}

Conclusion: The GBS was superior to the 3 LGB risk scores for predicting the need for transfusion and clinical intervention. The GBS may be an useful tool for risk stratification in acute LGB.

Disclosure of Interest: All authors have declared no conflicts of interest.

Abstract No: P1734

Table 1: Characteristics and results of the studies

\begin{tabular}{|c|c|c|c|c|c|c|c|c|}
\hline Estudy (year) & Design/Period & Sample size/Testee & $\begin{array}{l}\text { Sensitivity } \\
(\%)\end{array}$ & $\begin{array}{l}\text { Specificity } \\
(\%)\end{array}$ & $\begin{array}{l}\text { Predictive } \\
\text { positive value } \\
(\%)\end{array}$ & $\begin{array}{l}\text { Predictive } \\
\text { negative } \\
\text { value }(\%)\end{array}$ & $\begin{array}{l}\text { Negative } \\
\text { Likelihood ratio }\end{array}$ & $\begin{array}{l}\text { Positive } \\
\text { Likelihood ratio }\end{array}$ \\
\hline Richards 1990 & $\begin{array}{l}\text { Retrospective 1981- } \\
\quad 1990\end{array}$ & $\begin{array}{l}126 \quad \text { BUN/creat } \\
(\text { Cut off } \geq 36)\end{array}$ & 37 & 100 & 100 & 53 & 0.63 & +++++ \\
\hline Aljebreen 2004 & $\begin{array}{l}\text { Retrospective 1999- } \\
\quad 2001\end{array}$ & $520 \mathrm{NGT}$ & 68 & 54 & 41 & 78 & 0.61 & 1.44 \\
\hline Witting 2006 & $\begin{array}{l}\text { Retrospective 1997- } \\
\quad 2002\end{array}$ & $\begin{array}{l}325 \quad \text { BUN/creat } \\
(\text { Cut off } \geq 30) \text { NGT }\end{array}$ & 3942 & 9491 & 8181 & 7061 & 0.650 .56 & 6.411 .1 \\
\hline Kessel 2016 & $\begin{array}{l}\text { Retrospective 2011- } \\
\quad 2014\end{array}$ & 386 NGT & 28 & 86 & 99 & 2 & 0.84 & 2 \\
\hline
\end{tabular}




\section{References}

Kwak MS, Cha JM, Han YJ, et al. The Clinical Outcomes of Lower Gastrointestinal Bleeding Are Not Better than Those of Upper Gastrointestinal Bleeding.@BULLET J Korean Med Sci 2016; 31: 1611-1616.

Strate LL, Gralnek IM. ACG Clinical Guideline: Management of Patients With Acute Lower Gastrointestinal Bleeding. Am J Gastroenterol. 2016; May;111(5):755.

Zakko L, Laurse SB, Stanley A, et al. Upper gastrointestinal bleeding scores predict outcomes for patients presenting with hemodinamically significant hematochezia?. Gastroenterology. 2016; 150 Suppl 1: Sa1738.

\section{P1736 ACUTE LOWER GASTROINTESTINAL BLEEDING IN PATIENTS TREATED WITH NON-VITAMIN K ANTAGONIST ORAL ANTICOAGULANTS COMPARED WITH WARFARIN IN CLINICAL PRACTICE: CHARACTERISTICS AND CLINICAL OUTCOME}

G. Diamantopoulou ${ }^{1}$, C. Konstantakis ${ }^{1}$, G. Theocharis ${ }^{1}$, V. Theopistos ${ }^{1}$, G. Skroubis ${ }^{2}$, C. Triantos ${ }^{1}$, V. Nikolopoulou ${ }^{1}$, K. Thomopoulos

${ }_{1}^{1}$ Gastroenterology, University Hospital of Patras, Patras/Greece

${ }^{2}$ Surgery, University Hospital of Patras, Patras/Greece

\section{Contact E-mail Address: geodiamant@hotmail.com}

Introduction: Acute lower gastrointestinal bleeding (ALGIB) occurs in patients taking anticoagulants either warfarin or non Vitamin $\mathrm{K}$ oral anticoagulants (NOACs). The use of NOACs has been increasing compared with warfarin in recent years. We investigated patients with ALGIB on anticoagulation therapy and we analyzed characteristics, management and clinical outcome in patients treated with NOACs versus warfarin.

Aims \& Methods: All patients with ALGIB on anticoagulation therapy treated in our hospital during a seven year period were evaluated. Characteristics and clinical outcome were compared between patients on warfarin and patients on NOACs.

Results: Out of 587 patients with ALGIB, 43 (7.3\%) were on NOACs and 68 $(11.6 \%)$ on warfarin with an age $75.9 \pm 9.5$ vs $77.1 \pm 7.9$. The bleeding site was in the small bowel in $2 / 43$ and $6 / 68$ respectively. Causes of bleeding were not different between two groups except for polyps/neoplasia $(8 / 43$ vs $6 / 68, p=0.003)$. Endoscopic hemostasis was more commonly needed in patients on NOACs $17 / 43$ vs $14 / 68(\mathrm{p}=0.049)$, while they required less hospitalisation days $(6.1 \pm 4.2 \mathrm{vs}$ $4.5 \pm 3.6, \mathrm{p}=0.04)$. Blood transfusions and need for other interventions (embolization and/or surgery) were not different. Also recurrence of bleeding (4/43 vs $11 / 68)$ and mortality (3/43 vs $0 / 68)$ were low and not statistically different between the two groups.

Conclusion: ALGIB in patients on NOACs although presents some differences it has a similar clinical outcome to patients with ALGIB on warfarin.

Disclosure of Interest: All authors have declared no conflicts of interest.

\section{References}

Flack KF, Desai $\mathbf{J}$ et al.Major Gastrointestinal Bleeding Often Is Caused by Occult Malignancy in Patients Receiving Warfarin or Dabigatran to Prevent Stroke and Systemic Embolism From Atrial Fibrillation. Int $J$ Colorectal Dis. 2016 Feb; 31(2):175-88.

Nagata N, Niikura R, Sakurai T, Shimbo T, Aoki T, Moriyasu S, Sekine K, Okubo H, Imbe K, Watanabe K, Yokoi C, Yanase M, Akiyama J, Uemura N. Safety and Effectiveness of Early Colonoscopy in Management of Acute Lower Gastrointestinal Bleeding on the Basis of Propensity Score Matching Analysis. Clin Gastroenterol Hepatol. 2016 Apr; 14(4):558-64

Farrell JJ, Friedman LS. Review article: the management of lower gastrointestinal bleeding. Aliment Pharmacol Ther. 2015; 21:1281-1298.

Chong V, Hill AG, MacCormick AD. Accurate triage of lower gastrointestinal bleed (LGIB) - A cohort study. Int J Surg. 2016 Jan; 25:19-23.

Bresci G, Parisi G, Bertoni M, Tumino E, Capria A. The role of video capsule endoscopy for evaluating abscure gastrointestinal bleeding: usefulness of early use. J Gastroenterol. 2005; 40:256-259.

Limsrivilai J, Srisajjakul S, Pongprasobchai S, Leelakusolvong S, Tanwandee T.A Prospective Blinded Comparison of Video Capsule Endoscopy Versus Computed Tomography Enterography in Potential Small Bowel Bleeding: Clinical Utility of Computed Tomography Enterography. J Clin Gastroenterol. 2016

Moss AJ, Tuffaha H, Malik A. Lower GI bleeding: a review of current management, controversies and advances. Int J Colorectal Dis. 2016 Feb; 31(2):175-88

P1737 NOBLADS - THE NEW RISK SCORE TO PREDICT THE SEVERITY OF ACUTE LOWER GASTROINTESTINAL BLEEDING

M. Patita, G. Nunes, R. Barosa, L. Ramos, C. Fonseca

Gstrenterology, Hospital Garcia de Orta, Almada/Portugal

Contact E-mail Address: martapatita21@gmail.com

Aims \& Methods: We aimed to evaluate the accuracy of the NOBLADS score to predict severe LGIB and the outcome of patients admitted by LGIB. We performed a retrospective, observational and unicentric study. Including patients admitted for acute LGIB and submitted to endoscopic evaluation between January/2015 and March/2016. LGIB was classified as severe if $\geq 2$ units of erythrocyte concentrate (UCE) were required and/or if hematocrit drop
$>20 \%$. Total score ranges from $0-8$; when total score is $\geq 2$, it is considered high risk for severe LGIB.

Introduction: A new risk score for acute lower gastrointestinal bleeding (LGIB) has recently been validated, based on 8 admission criteria-nonsteroidal antiinflammatory drugs use, absence of diarrhea, absence of abdominal tenderness, systolic blood pressure $\leq 100 \mathrm{mmHg}$, antiplatelet drugs use, albumin level $<3 \mathrm{~g} /$ $\mathrm{dL}$, disease score $\geq 2$ (Charlston comorbidity index) and syncope (NOBLADS). Results: 173 patients were included (male: $50.3 \%$, mean age: $69 \pm 17$ years), with LGIB manifested by hematochezia $(91.9 \%)$ or melena. Endoscopic evaluation was performed $1.7 \pm 2$ days after admission, with the most frequent findings being diverticular hemorrhage $(n=53)$ and ischemic colitis $(n=29)$; no lesions were found in $8.3 \%$ of cases. Thirty-three patients required intervention (endoscopic $n=27$, radiological $n=2$, surgical $n=4)$ and $36(20.8 \%)$ repeated endoscopic evaluation. $28.9 \%$ of patients presented severe LGIB and the NOBLADS score determined the severity of LGIB with an area under the curve value of $0.92 \pm 0.018$. Overall, higher score values were associated with a requirement for transfusion support, intervention and longer hospitalization $(\mathrm{p}<0.001$ for trend test). Patients at high risk for severe LGIB (score $\geq 2, \mathrm{n}=39$ ) presented a significantly higher number of transfused UCEs (3.6 vs $0.08, p<0.001)$, intervention $(38 \%$ vs $13 \%, \mathrm{p}<0.001)$ and days of hospitalization $(12.8$ vs 3 days, $\mathrm{p}<0.001)$

Conclusion: The NOBLADS score is simple and quick to apply. It predicts with high accuracy the risk of severe LGIB and allows to identify patients that are more likely to require transfusional support, intervention and prolonged hospitalization. In clinical practice, NOBLADS score may be useful to select on admission patients who will benefit from hospitalization or from earlier intervention.

Disclosure of Interest: All authors have declared no conflicts of interest.

\section{Reference}

Aoki T, Nagata N, et al. Development and Validation of a Risk Scoring System for Severe Acute Lower Gastrointestinal Bleeding. Clin Gastrenterol Hepatol. 2016 Nov; 14(11):1562-1570.e2.

\section{P1739 ENDOSCOPIC MUCOSAL RESECTION OF COLORECTAL POLYP IN ANTICOAGULATED PATIENTS}

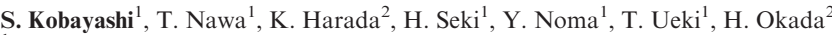
${ }^{1}$ Fukuyama City Hospital, Fukuyama/Japan

${ }^{2}$ Okayama University Hospital, Okayama/Japan

Contact E-mail Address: sayo44@hotmail.co.jp

Introduction: The management of antithrombotic agents during the peri-endoscopic period concerns the risks of bleeding and thromboembolism. The Japan Gastrointestinal Endoscopy Socaiety (JGES) guidelines revised in 2012 emphasizes the risk of thromboembolism rather than bleeding. So heparin bridge of anticoagulants is recommended at high-bleeding-risk procedure such as endoscopic mucosal resection (EMR). However heparin bridge in colorectal EMR raises the bleeding rate to approximately $20 \%$, that is very high-rate incident compared with the bleeding rate of $0.3-6.1 \%$ generally. It is doubtful whether heparin bridge is appropriate.

Aims \& Methods: The aim of this study to clarify the safeness of colorectal EMR under taking anticoagulants without a heparin bridge in anticoagulated patients. This is a prospective intervention study in our hospital and other nine multicenters (UMIN000021416). The subjects are colorectal polyps resectable by EMR. Inclusion criteria of patient is good performance status (ECOG) $0-1$, without sever organ failure. Warfarin is continued as usual if PT-INR is less than 2.6, and direct oral anticoagulants (DOAC) is continued until an evening of the day before and is discontinued only on the day. Primary endopoint is postEMR bleeding required any interventions (major bleeding). Major bleeding is defined as active bleeding or adherent clot on the resection site by emergent colonoscopy, or conservative treatment required blood transfusion. Conservative bleeding with no intervension (minor bleeding), thromboembolism, and comparison between warfarin and DOAC are clarified as secondary endopoint. The procedure-related period is defined as up to 30 days after EMR or is finished for two weeks after hemostasis.

Results: 41 patients (154 lesions) were perfomed EMR from February 2015 to March 2017. Patients' characteristics were as follow, the median age was 74 years (range 61-88) and the average of polyp size was 11.6 (range 5-22) $\mathrm{mm}$ in diameter. Anticoagulants were devided into 19 cases of warfarin and 22 cases of DOAC. The incidence of major bleeding was none in all cases. On the other hand, minor bleeding which did not require any intervention was recognized at $14.6 \%(6 / 41)$, but there was no case to interrupt anticoagulants. The rate of minor bleeding is no difference between warfarin and DOAC. There was no correlation between minor bleeding and PT-INR value in three cases of warfarin. All three cases of DOAC were medicine once a day. Thromboembolism was not observed.

Conclusion: In colorectal EMR, we clarify the safeness with continuation of warfarin and short discontinuation of DOAC on that day, compared with heparin bridge. We expect to lead to reconsideration of current guideline for safer treatment.

Disclosure of Interest: All authors have declared no conflicts of interest.

\section{References}

Akira H. Removal of small colorectal polyps in anticoagulateds patients;a prospective randomized comparison of cold snare and conventional polypectomy; Gastroenterological Endoscopy Vol79 No.3 2014 
Takuya Inoue, Clinical features of post-polypectomy bleeding associated with heparin bridge therapy; Digestive Endoscopy 2014;26:243-249

James D. Douketis, M.D., Perioperative Bridging Anticoagulation in Patients with Atrial Fibrillation; New England Journal of medicine. 2015;373:823-33

Multicenter study on hemorrhagic risk of heparin bridging therapy for periendoscopic thromboprophylaxis ; Matsumoto et al. BMC Gastroenterology (2015) 15:89

\section{P1740 RISK OF COLORECTAL CANCER IN ASYMPTOMATIC INDIVIDUALS WHOSE FIRST DEGREE RELATIVES WERE AFFECTED BY CRC AT DIFFERENT AGES OF ONSET: A SYSTEMATIC REVIEW AND META-ANALYSIS OF 9.28 MILLION SUBJECTS}

J.L. L. Huang, M. C. Wong, C. Chan, J. Lin, W. W. Cheung, M. Liang, Y. Fang, C. Yu, D. Fung

Chinese University of Hong Kong, Hong Kong/Hong Kong Prc

Contact E-mail Address: martin_wong@cuhk.edu.hk

Introduction: The current literature is mixed regarding whether first-degree relatives (FDRs) of patients who suffered from colorectal cancer (CRC) at much earlier age are at substantially increased risk of CRC.

Aims \& Methods: The present systematic review and meta-analysis examined the $\mathrm{CRC}$ risk conferred by family history of CRC in FDRs according to their age of onset. We searched Ovid Medline, EMBASE and grey literature from their inception to December 2016, and included all screening studies that investigated the relationship between family history of CRC in FDR and incidence/prevalence of CRC. Two reviewers independently worked on selection, assessment and data extraction of eligible articles. A random effects meta-analysis was employed to pool relative risks (RR) and odds ratios. Subgroup analyses were performed according to the age of onset of CRC in FDRs of asymptomatic subjects ( $<40$ vs. $\geq 40 ;<50$ vs. $\geq 50 ;<60$ vs. $\geq 60$ years). Statistical heterogeneity was assessed by the $\mathrm{I}^{2}$ statistics. Publication bias was evaluated by an inverted funnel plot analysis with Begg's regression model.

Results: Fifty-six case-control and seven cohort studies involving 9.28 million subjects were included in the analysis. A family history of CRC in FDRs of asymptomatic subjects conferred a significantly higher risk of CRC $\left(\mathrm{RR}=1.76,95 \% \mathrm{CI}=1.57-1.97 ; \mathrm{p}<.001, \mathrm{I}^{2}=95.7 \%\right)$. Earlier age of onset of CRC in FDRs was associated with significantly higher risk of CRC in index subjects $(\mathrm{RR}=3.29,95 \%$ C.I. $1.67-6.49$ for $<40$ years vs. $\mathrm{RR}=1.42,95 \%$ C.I. $1.24-1.62$ for $\geq 40$ years, $\mathrm{p}=0.017$; $\mathrm{RR}=2.81,95 \%$ C.I. $1.94-4.07$ for $<50$ years vs. $\mathrm{RR}=1.47,95 \%$ C.I. $1.28-1.69$ for $>50$ years, $\mathrm{p}=0.001)$. The Begg's regression test did not identify any publication bias (Kendall's tau $=0.122, \mathrm{p}=0.159)$

Conclusion: A family history of CRC in FDRs whose age of onset is earlier than 40 or 50 years conferred a significantly higher risk of CRC to asymptomatic individuals, implying that age of onset could potentially enhance the discriminatory capability of $\mathrm{CRC}$ prediction scores.

Disclosure of Interest: All authors have declared no conflicts of interest.

\section{P1741 IS THERE ANY DIFFERENCE IN RISK OF COLORECTAL CANCER AMONG ASYMPTOMATIC SUBJECTS WHOSE SIBLINGS VS. PARENTS WERE AFFECTED? A SYSTEMATIC REVIEW AND META-ANALYSIS}

J.L. L. Huang, M. C. Wong, C. Chan, J. Lin, W. W. Cheung, M. Liang, Y. Fang, D. Fung, C. Yu

Chinese University of Hong Kong, Hong Kong/Hong Kong Prc

Contact E-mail Address: martin_wong@cuhk.edu.hk

Introduction: Few studies compared the risk of colorectal cancer (CRC) among individuals with probands who were parents, siblings, and those with two or more probands.

Aims \& Methods: This systematic review and meta-analysis tested the hypothesis that the risk of CRC conferred by family history of CRC in parents vs. siblings vs. $\geq 2$ first-degree relatives (FDRs) was similar. The Ovid Medline, EMBASE and grey literature were searched from their inception to December 2016, and all screening studies that examined the association between detection of CRC and family history of CRC in FDR were included. Two reviewers independently searched, assessed and extracted data from eligible studies. The relative risks (RR) and odds ratios were pooled based on a random effects meta-analysis We conducted subgroup analyses according to the identity of FDRs affected (parents vs. siblings vs. $\geq 2$ FDRs), and examined statistical heterogeneity by the $I^{2}$ statistics. Potential publication bias was explored by funnel plot analysis with Begg's regression test.

Results: We identified 56 case-control and 7 cohort studies, consisting of 9.28 million subjects who were finally included in the meta-analysis. Asymptomatic individuals with siblings affected $(\mathrm{RR}=2.44,95 \% \mathrm{CI}=1.90-3.13)$; parents affected $(\mathrm{RR}=2.18,95 \% \mathrm{CI}=1.95-2.45)$ and $\geq 2$ FDRs affected $(\mathrm{RR}=2.68$, $95 \% \mathrm{CI}=1.92-3.74$ ) had statistically similar risk of CRC. We did not identify any publication bias based on the Begg's regression test $(\mathrm{p}=0.159)$.

Conclusion: The risk of CRC was similar among subjects whose siblings; parents or $\geq 2$ FDRs were affected by CRC. Information on the identity of the FDRs affected does not seem to be necessary when the risk of CRC in asymptomatic individuals is predicted.

Disclosure of Interest: All authors have declared no conflicts of interest.

P1742 GILBERT SYNDROME IS NOT THAT INNOCENT!

N. Arber ${ }^{1}$, M. Menachem ${ }^{1}$, L. Ari ${ }^{2}$, G. Lior ${ }^{1}$, B. Ezra ${ }^{1}$, H. Gil ${ }^{1}$, H.G. Amira ${ }^{1}$ J. Maayan ${ }^{1}$, S. Shapira ${ }^{1}$

${ }^{1}$ Health Promotion Center And Integrated Cancer Prevention Center, Tel Aviv

Medical Center, Tel Aviv/Israel

${ }^{2}$ Tel-Aviv Sourasky Medical Center, Tel Aviv/Israel

Contact E-mail Address: nadira $@$ tlvmc.gov.il

Introduction: Gilbert's syndrome is considered to be entirely benign. Some studies have shown a reduced risk for cardiovascular disease (CVD). There is conflicting data regarding cancer risk among these patients

Aims \& Methods: We aimed to evaluate the association of Gilbert syndrome with CVD and cancer. Clinical and epidemiological data was obtained from consecutive healthy subjects undergoing annual screening at the Integrated Cancer Prevention Center in Tel Aviv. The annual check-up includes: thorough examination by specialists in internal medicine, surgery, dermatology/plastic surgery, OBGYN, urology, oncology, oral surgery, gastroenterology. Blood work (smac 24 , blood count, TSH, CRP, PSA), vaginal US, PSA and mammography ( $>40 \mathrm{yrs}$ ), LDCT in heavy smokers and all needed imaging when clinically indicated. Peripheral blood DNA was extracted from all subjects. Gilbert syndrome was determined by clinical criteria (normal liver function tests but to mild elevation in unconjugated bilirubin $<3 \mathrm{mg} / \mathrm{dl}$ without any hemolysis. In the majority of the cases the diagnosis was confirmed genetically by the homozygous mutation (TA)7TAA in the promoter region of UGT1A1 enzyme. Prevalence of CVD and cancer were compared between subjects with/without Gilbert syndrome. Mortality data was obtained from the Israeli ministry of health and cancer incidence from the Israeli registry.

Results: A total of $6258(49 \%)$ men and 6461 (51\%) women, mean age $47.0 \pm 11.5$ years, were included of which 1.019 had clinical gilbert. Gilbert was significantly more common among men $(11.5 \%$ versus $4.6 \% \mathrm{P}<0.001)$. The rate of gilbert disease was equal in Sephardic and Ashkenazi Jews. Malignancy and CVD were diagnosed in $678(5.3 \%)$ and $1.837(14.4 \%)$ subjects respectively. The prevalence of any CVD was significantly higher in the Gilbert group (OR 1.23 $95 \% \mathrm{CI} 1.04-1.46 \mathrm{p}=0.017$ ), as well as hypertension (OR $1.3795 \%$ CI $1.12-1.68$ $\mathrm{p}=0.003)$ and CVA $(1.1 \%$ versus $0.6 \% \mathrm{p}=0.06)$. Higher rate of kidney and bladder cancers (OR 2.64, 1.22-5.70, $\mathrm{p}=0.019)$ was also observed in the gilbert group. In contrast, the prevalence of breast cancer was much lower among patients with Gilberts (OR 0.36, 0.13-0.97, P=0.034)

Conclusion: In Israel Gilbert syndrome is not that innocent. In a large cohort it seems to be associated with increased risk of

hypertension, CVD and CVA. Bladder cancer is higher but females are protected from breast cancer. Further studies

are mandated in order to better understand these findings and determine proper screening and surveillance practices

in Gilbert disease.

Disclosure of Interest: N. Arber: Bayer Bio-view Gi-View Micro-medic Checkcap

All other authors have declared no conflicts of interest.

\section{P1743 CHARACTERISTICS AND PREDICTORS OF INTERVAL}

\section{CANCER: A CASE-CONTROL STUDY}

I. Laish ${ }^{1}$, J. Mizrahi ${ }^{2}$, F. Konikoff ${ }^{1}$

${ }^{1}$ Meir Medical Center, Kfar Saba/Israel

${ }^{2}$ Stony Brook University Hospital, New York/United States of America

Contact E-mail Address: ido.laish@gmail.com

Introduction: Interval colorectal cancer is largely related to a poor endoscopic performance (missed lesions, incomplete polyp resection) or different biology in the development of the polyp (accelerated growth). Thus, quality endoscopic measures and Lynch syndrome were highly investigated for their association with interval cancer. However, most reports came from the Western world and not the Middle East, and differences in ethnicity or environmental factors might potentially have impact on the biology of tumor progression. In addition, patient-related factors were less investigated for their association with interval cancer. The aim of this study was thus to assess tumor and patient characteristics and predictors of interval cancer in a population from Israel

Aims \& Methods: This retrospective cohort study included all patients that were diagnosed with colon cancer in our institution between 2005-2014. Cases included patients with a previous colonoscopy within 1-10 years before the diagnosis of cancer, with either negative findings or benign polyps. Only full colonoscopies with at fair or good preparation were included. Interval cancer was defined on an individual basis, when cancer occurred within the recommended surveillance interval according to accepted guidelines. Cases were further stratified according to time since index colonoscopy ( $<3$ years, 3-10 years). "Positive" controls were cancer patients without previous colonoscopy, and "negative" controls were sex- and age-matched patients with two negative colonoscopies within the study period who were randomly selected on a 5:1 ratio. Tumor characteristics (location, staging) and patient-related features (age, gender, positive family history of colon cancer, aspirin use, diabetes, diverticulosis) were compared between cases and control groups. 
Results: 845 patients were diagnosed with a colon cancer within the study period, and 83 cases $(9.8 \%)$ were found to have interval cancer. Among them, 51 patients $(61.5 \%)$ had negative findings at index colonoscopy, while $22(38.5 \%)$ had either non-advanced adenomas $(12 \%)$ or advanced adenoma $(26.5 \%)$. Compared to "positive" controls with primary cancer (575 patients with full data), patients with interval cancer were older $(84 \%$ above $60 y$ vs. $65 \%$, p- 0.03$)$ and had proximal (cecal to splenic flexure) tumor location ( $57 \%$ vs. $34 \%$, p- 0.0001 ), but gender ( $47 \%$ vs. $53 \%$ males) and tumor staging $(78 \%$ vs. $70 \%$ in stage 0 2, p- 0.12) were not different. Compared to "negative" healthy controls (255 patients), cases with interval cancer and negative findings at index colonoscopy (51 patients) had higher prevalence of diabetes $(33 \%$ vs. $15 \%, \mathrm{p}-0.002)$ but the same rate of family history, aspirin use and diverticulosis. There were no significant differences in all these characteristics between patients with interval cancer $<3$ years and 3-10 years.

Conclusion: Patients with interval cancer tend to be older and have proximal tumor location than patients with primary colon cancer, and have higher prevalence of diabetes. A close surveillance or the use of better endoscopic techniques (e.g. use of NBI, longer retrieval time) should be considered for patients with these characteristics.

Disclosure of Interest: All authors have declared no conflicts of interest.

\section{P1744 THE RELATIONSHIP BETWEEN QUANTITATIVE FIT RESULTS AND NEOPLASTIC FINDINGS}

J.S. Mcgrath, M. Borgaonkar, D. Pace, S. Antle, S. Stone, E. Randell, J. Quinlan Dept. Of Gastroenterology, Memorial University, St. Johńs/Canada

Contact E-mail Address: jmcgrath@mun.ca

Introduction: Fecal Immunochemical Testing (FIT) is currently used in most Canadian provinces to screen for colorectal cancer. Newfoundland and Labrador has implemented a provincial population based colon cancer screening program over the past five years. Newfoundland and Labrador selects patients for colonoscopy if one of two FIT values $\geq 100 \mathrm{ng} / \mathrm{mL}$.

Aims \& Methods: The goal of this study is to assess the effectiveness of different FIT cut-offs and number of FIT tests for detecting adenomas and colorectal cancer.

Results: Data for this study were obtained in a prospective fashion using the Newfoundland and Labrador Colon Cancer Screening Program. 21,371 patients enrolled in the study were between the ages of 50-74 and at average risk for colon cancer between July 1, 2012 and June 30, 2016. 16,152 participants returned their FIT tests. 1831 were positive on at least one FIT kit and underwent colonoscopy. The positive FIT values ranged from 100.0 to 54,017 . The mean FIT was 942.3 (25th percentile: 145 , 50th percentile: 260,75 th percentile: 576 ). Of the 1831 participants who had a colonoscopy $73(4.0 \%)$ were found to have colorectal cancer and $1092(59.6 \%)$ were found to have an adenoma. By using only one FIT test at a cut off of 100 , our program would have missed $8.2 \%$ of cancers. An additional 541 colonoscopies were required to detect these cancers. If we stratified patients according to number of FIT tests we found that $83.5 \%$ of colon cancers detected were positive on both FIT kits at a quantitiative cut off of 100 . If a FIT cut off of 200 was used and applied to our patients our program would have missed $9.6 \%$ of cancers based on one of two tests being positive, $24.7 \%$ of cancers is only one FIT kit were supplied to patients and $38.4 \%$ of cancers based on both FIT kits being positive. An additional 1133 colonoscopies were required to detect the additional cancers by using two FIT kits as opposed to one. There was a substantial decline in detection of cancer, advanced adenoma and simple adenoma as FIT cut off was increased and with a reduction in FIT kits complted from two to one. The positive predictive value for cancer increased as the quantitative FIT cutoff was increased but this came at the expense of case detected. Conclusion: Two FIT tests are more effective than one at screening. Patients with two FIT positive results are more likely to have: colon cancer, an advanced adenoma and a simple adenoma. Further triaging of colonoscopy wait lists could be considered based on quantitative FIT values and number of positive tests. Provinces and health authorities need to be cautious when determining the number of FIT kits a participant should complete as well as setting the quantitative cut off. Increasing the FIT cut-off results in a higher probability of colon cancer or adenoma but there are overall less cancers detected.

Disclosure of Interest: All authors have declared no conflicts of interest.

\section{P1745 METABOLIC RISK FACTORS AND THEIR IMPACT IN COLORECTAL CANCER SCREENING: MULTICENTER PROSPECTIVE STUDY}

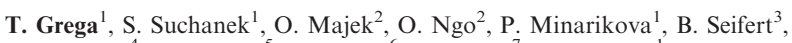
O. Shonova ${ }^{4}$, K. Balihar ${ }^{5}$, J. Cyrany ${ }^{6}$, M. Benes ${ }^{7}$, M. Zavoral ${ }^{1}$

${ }^{1}$ Department Of Internal Medicine, 1st Faculty Of Medicine, Charles University, Military University Hospital, Prague/Czech Republic

${ }^{2}$ Institute Of Biostatistics And Analyses, Masaryk University, Faculty of Medicine, Brno/Czech Republic

${ }^{3}$ Department Of General Practice, Department of GP First Faculty of Medicine Charles University, Prague/Czech Republic

${ }^{4}$ Gastroenterology, Hospital České Budějovice, Ceske Budejovice/Czech Republic ${ }^{5}$ Gastro, Fakultni nemocnice Plzen, Plzen/Czech Republic

${ }^{6}$ 2nd Dept. Of Internal Medicine, University Hospital Hradec Kralove, Hradec Kralove/Czech Republic

${ }^{7}$ Department Of Hepatogastroenterology, Institute for Clinical and Experimental Medicine, Prague/Czech Republic

\section{Contact E-mail Address: togrega@gmail.com}

Introduction: Screening of colorectal cancer is focused on the population with average risk of this disease. Individuals with metabolic syndrome represent a population with a higher risk of developing colorectal cancer, but they are not included in colorectal cancer screening standards.

Aims \& Methods: To identify the metabolic risk factors associated with colorectal neoplasia (advanced adenomas and cancers) and evaluated their impact in colorectal cancer screening. Prospective, multicenter study was performed from January 2013 to December 2015 at eight high-quality colonoscopy centers. Demographic characteristics, anthropometric measurements, metabolic risk factors, and colonoscopic pathologic findings were assessed in patients with metabolic syndrome (target group) who underwent preventive colonoscopy (FIT positive or screening colonoscopy). This data was compared with consecutive patients without metabolic syndrome (control group). All patients meet the screening population criteria (asymptomatic, without family or personal history of colorectal neoplasia).

Results: 1500 individuals were enrolled; 726 persons (494 men, 68\%) in the target group (metabolic syndrome) and 774 persons $(353 \mathrm{men}, 46 \%)$ in the control group (without metabolic syndrome). The significantly higher prevalence of advanced adenomas was observed in the target group (18\%; $95 \%$ CI $15-21 \%$ ) comparing to the control group $(9 \% ; 95 \%$ CI $7-11 \%)$; OR $1.8 ; \mathrm{p}=0.002$. Similarly, the prevalence of all adenomas was higher in the target group $(48 \%$; $95 \%$ CI $44-51 \%)$ than in the control group $(35 \%, 95 \%$ CI $32-38 \%)$; however, the difference was not statistically significant $(\mathrm{p}=0.179)$. Individuals with isolated high cardiovascular risk (SCORE $\geq 10 \%$ ) had higher prevalence of both, non-advanced adenomas $(51 \%, 95 \%$ CI $46-56 \% ; \mathrm{p}=0.327)$ and advanced adenomas $(22 \%, 95 \%$ CI $18-26 \% ; \mathrm{p}=0.049)$ comparing to the individuals with isolated DM2. Advanced adenomas were more likely in patients aged 65-75 years.

Conclusion: Colorectal neoplasia is positively associated with metabolic syndrome. Cardiovascular risk factors (SCORE $\geq 10 \%$ ) is a stronger risk factor than the presence of diabetes mellitus type 2 . Individuals with SCORE $\geq 10 \%$ should be considered as a risk group of colorectal carcinomas, and therefore colonoscopy as the primary screening method is appropriate. This project has been supported by the Czech Ministry of Health grant NT 13673 and MO 1012. Disclosure of Interest: All authors have declared no conflicts of interest.

\section{P1746 PREVALENCE OF SERRATED POLYPOSIS SYNDROME IN AVERAGE-RISK SCREENING COLONOSCOPIES IN GERMANY}

C. Schramm ${ }^{1}$, K. S. Janhsen ${ }^{2}$, J. Dinter ${ }^{1}$, F. Kuetting ${ }^{1}$, T. Goeser ${ }^{1}$, H. Steffen ${ }^{1}$ ${ }^{1}$ University Hospital Cologne, Cologne/Germany

${ }^{2}$ St.-Katharinen-Hospital, Frechen/Germany

\section{Contact E-mail Address: christoph.schramm@uk-koeln.de}

Introduction: Serrated polyps (SPs) have been recognized as precursors of colorectal cancer (CRC), accounting for up to $30 \%$ of CRCs via the serrated neoplasia pathway. SPs are classified into hyperplastic polyps (HPs), sessile serrated polyps (SSPs) with or without dysplasia and traditional serrated adenomas (TSAs). ${ }^{1}$ The serrated polyposis syndrome (SPS) is characterised by multiple SPs throughout the colon. ${ }^{1}$ It is associated with an increased risk of developing CRC. ${ }^{2}$ Published data on prevalence of SPS ranged from 0.03 in the UK to 0.8 in Spain. ${ }^{3}$

Aims \& Methods: The aim of the study was to determine the prevalence of SPS in average-risk individuals participating in the German CRC screening programme. We retrospectively analyzed screening colonoscopies performed by 11 gastroenterologists in 4 medical practices and 1 tertiary academic hospital between $01 / 01 /$ 2011 and 14/12/2016. Individuals < 50 years, with an increased risk for CRC (i.e. inflammatory bowel disease, history of CRC, cancer syndromes), and with previous or incomplete colonoscopy (i.e. procedures not reaching the cecum) were excluded. SPS diagnostic criteria according to the WHO definition from 2010 were: I) $\geq 5$ SPs proximal to the sigmoid colon, of which two are $\geq 10 \mathrm{~mm}$, II) any number of SPs proximal to the sigmoid colon in an individual who has a firstdegree relative with SPS, or III) $\geq 20$ SPs of any size distributed through-out the colon. ${ }^{1}$

Results: A total of 3089 individuals were analyzed. $47.5 \%$ were male, median age was 62 years (interquartile range 56, 70). Overall adenoma detection rate was $33.9 \%$. Detection rates for SPs, HPs, SSAs and TSAs were $21 \%, 18 \%, 4 \%$, and $0.2 \%$. Of all individuals with detected SPs none fullfilled the diagnostic criteria for SPS. Hence, we determined a prevalence of SPS of $0 \%$ in our cohort.

Conclusion: In our study, the prevalence of SPS in average-risk individuals undergoing screening colonoscopy was $0 \%$. Because overall ADR was above the recommended values for screening colonoscopies, we conclude that this low prevalence might be attributed to a lack of awareness for SPS rather than to low-quality procedures. However, we were not able to obtain information about first-degree relatives with SPS from medical records of individuals with SPs to check the second diagnostic criterium for SPS. We also included only one colonoscopy per individual. It has been shown that prevalence increases when followup colonoscopies are included as well. ${ }^{3}$ Both facts might have caused an underestimation of the true prevalence of SPS in our cohort.

Disclosure of Interest: All authors have declared no conflicts of interest.

\section{References}

1. Snover DC, Ahnen DJ, Burt RW, et al. Serrated polyps of the colon and rectum and serrated polyposis. In: Bosman T, Carneiro F, Hruban R, et al. WHO classification of tumours of the digestive system. Lyon, 2010:160-5.

2. Boparai KS, Mathus-Vliegen EMH, Koornstra JJ, et al. Increased colorectal cancer risk during follow-up in patients with hyperplastic polyposis syndrome: a multicentre cohort study. Gut 2010;59:1094-100. 
3. Ijspeert JEG, Bevan R, Senore C, et al. Detection rate of serrated polyps and serrated polyposis syndrome in colorectal cancer screening cohorts: a European overview. Gut 2016;0:1-8. doi:10.1136

\section{P1747 CONTRIBUTION OF GERMLINE MUTATIONS TO NON} FAMILIAL EARLY ONSET CANCERS

A. Mannucci ${ }^{1}$, F. Calabrese ${ }^{1}$, R. A. Zuppardo ${ }^{1}$, U. Elmore $^{2}$, F. Aleotti ${ }^{2}$, M. Lemma ${ }^{2}$, A. Tamburini ${ }^{2}$, M. Reni ${ }^{3}$, M. Ronzoni ${ }^{3}$, V. Burgio ${ }^{3}$, E. Mazza ${ }^{3}$, M.G. Patricelli ${ }^{4}$, A. Russo Raucci ${ }^{4}$, R. Rosati ${ }^{2}$, P.A. Testoni ${ }^{1}$, G.M. Cavestro ${ }^{1}$ ${ }^{1}$ Gastroenterology And Gastrointestinal Endoscopy Unit, Università Vita-Salute San Raffaele, Milano/Italy

${ }^{2}$ Gastroenterological Surgery Unit, San Raffaele Hospital, Vita-Salute University, Milano/Italy

${ }^{3}$ IRCCS Ospedale San Raffaele, Milano/Italy

${ }^{4}$ Division Of Genetics And Cell Biology, IRCCS San Raffaele, Milano/Italy

Contact E-mail Address: alessandromannucci11@gmail.com

Introduction: Early onset gastroenterological cancers lacking a positive family history are an increasingly worrisome entity. On one hand, early onset is the cornerstone of genetically determined oncological problems, but on the other negative family history does not support the suspect of familial syndromes.

Aims \& Methods: We addressed the contribution of germline mutations to non familial early onset cancers. Patients with pancreatic, gastric, esophageal, duodenal and colorectal cancers were enrolled from 2015 to 2017 at the Gastrointestinal Personalized Medicine unit. Eligibility criteria were the juvenile onset and the negativity for clinical criteria of hereditary cancer syndromes. Early onset colorectal cancer was defined as $<45$ yrs. For the other cancers, the threshold was defined at 50 . Eligible patients provided informed consent. Genes were sequenced by means of a validated Next Generation Sequencing panel of oncological susceptibility genes and confirmed by means of Sanger sequencing.

Results: Among 12 colorectal cancer patients $(7 \mathrm{~F}, 5 \mathrm{M})$, NGS analysis showed: 2 mutations of MSH2 and MSH6 occurring de novo, given the absence of family history; 3 variants of unknown significance (VUS) (2 MSH2 and 1 MLH1); and 7 were negative. Age-stratification revealed that, among those $\leq 35$ years $(n=4), 1$ had MSH2 gene mutation and 3 were negative. In the $36-40$ age group $(n=3), 1$ had a VUS in MLH1 and 2 were negative. In the age group 41-45 $(n=5), 1$ MSH6 mutation and 2 VUS were found, alongside 2 negative results.

Among the colorectal cancers, $17 \%$ of patients had a de novo mutation of Lynch Syndrome, $25 \%$ had a VUS, and $58 \%$ were negative.

2 gastric diffuse cancer underwent NGS analysis (40 and $45 \mathrm{yrs}, 1 \mathrm{M}$ and $1 \mathrm{~F}$ ), both negative.

Among 2 pancreatic cancer patients $(<50 \mathrm{yrs}, 2 \mathrm{~F})$, one tested negative and the other had a VUS on PMS2.

NGS analysis performed on 1 esophageal cancer (46 yrs, M) was negative.

1 duodenal cancer (46 yrs, F) has a MSH2 mutation and 2 VUS (MSH6 and PMS2).

Conclusion: A significant percentage $(17 \%)$ of early onset colorectal cancers resulted in Lynch Syndrome even when family history is not suggestive of hereditary cancer. We reliably infer the determinant role of genetics, even when the family history does not support the hypothesis. Elsewhere, our results suggest that the already known susceptibility genes seldom contribute to sporadic early onset cancers. Other genes and mechanisms may explain the early onset phenotype. Our data show that NGS is often non conclusive in early onset GI cancers, and further development is needed to better classify VUS $(25 \%)$

Disclosure of Interest: All authors have declared no conflicts of interest.

\section{P1748 IMPACT OF COLIBACTIN-PRODUCING ESCHERICHIA COLI ON IMMUNE MICROENVIRONMENT IN PRECLINICAL COLORECTAL CANCER MODELS}

A. Lopes ${ }^{1}$, A.H. Casse ${ }^{2}$, J. Veziant ${ }^{3}$, E. Cardamone ${ }^{1}$, D. Pezet $^{3}$, N. Barnich ${ }^{1}$, E. Miot-Noirault ${ }^{4}$, S. Naimi ${ }^{2}$, E. Billard ${ }^{1}$, B. Dumas ${ }^{5}$, M. Bonnet $^{1}$

${ }^{1}$ M2isH, Inserm UMR 1071/Université d'Auvergne/INRA USC 2018, ClermontFerrand/France

${ }^{2}$ Histopathology and Bio-Imaging Group, Translational Sciences, Sanofi R\&D,

Vitry sur seine/France

${ }^{3}$ Chirurgie digestive, Centre Hospitalier Universitaire, Clermont-ferrand/France

${ }^{4}$ INSERM UMR 1240/Université d'Auvergne, Clermont Ferrand/France

${ }^{5}$ Research Biologics, Sanofi R\&D, Vitry-sur-seine/France

Contact E-mail Address: amelie.lopes0703@gmail.com

Introduction: Increasing evidence links the immune microenvironment, microbiota and colorectal cancer (CRC). Colibactin-producing-E.coli are more frequently detected on mucosa CRC patients and exhibit procarcinogenic properties on CRC murine models. Aim of this work was to evaluate the impact of chronical infection by colibactin-producing $E$. coli on immune cells in $\mathrm{APC}^{\mathrm{min} /+}$ mice models.

Aims \& Methods: Min mice were per os inoculated with a CRC-colibactin-producing E. coli strain (11G5), non pathogenic E. coli (K-12 MG1655) or PBS. Using optical in vivo imaging (IVIS spectrum), we evaluated oxidative stress induction with a bioluminescent inflammation probe in Min mice chronically infected. After 7 weeks, number and volume of polyps were evaluated and colonic samples were histogically analysed. Detection of immune cells was quantified by immunofluorescent labelling using a specific and innovative algorithm created with Tissue Studio software. Then, the density and localization of immune cells were performed in the three colon regions of interest: lymphoid follicle, mucosa and tumor.
Results: Using optical imaging, we detected a significant increase of luminescent signal in the gut of the infected group suggesting an increase of oxidative stress and inflammation. Histological analyses showed no difference about intratumoral immune infiltrate density on $11 \mathrm{G} 5$ and K12-infected mice. However, using our specific algorithm, we observed a significant increase of lymphoid follicle size in the gut of mice infected with the 11G5 strain compared to mice feeding with non-pathogenic K12 strain. Interestingly, follicle size was positively correlated with tumor volume, on the $11 \mathrm{G} 5$ infected group suggesting an association between pro-carcinogenic proprieties of this strain and gut immune response. In addition, we observed an increase of neutrophils ( $\mathrm{Ly} 6 \mathrm{G}^{+}$cells) on mucosa and lymphoid follicle of mice infected with 11G5 compared to K12 and non-infected mice. These results can be linked with our in vivo optical imaging observations and our results about the increase of neutrophils chemo-attractants CXCL1 and CCL20 measured by qRT-PCR after infection. Analyses of T cells, macrophages, B cells and myeloid suppressive cells are in progress.

Conclusion: Here we can observe an increase of lymphoid follicle associated with tumor volume after colibactin-producing $E$. coli infection. Our first results suggest that neutrophils can be one of the immune cells implicated in this process. This work shows a link between immune microenvironment, pathogenic E. coli and tumor development.

Disclosure of Interest: All authors have declared no conflicts of interest.

\section{P1749 INVESTIGATING THE DIRECT INTERACTION BETWEEN} CD24 AND B-CATENIN IN INTESTINAL TUMORIGENESIS

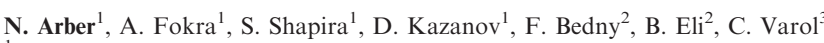
${ }^{1}$ Health Promotion Center And Integrated Cancer Prevention Center, Tel Aviv

Medical Center, Tel Aviv/Israel

${ }^{2}$ Tel Aviv Medical Center, Tel Aviv/Israel

${ }^{3}$ Gastroenterology, Tel-Aviv Sourasky Medical Center, Tel-Aviv/Israel

Contact E-mail Address: nadira $@$ tlvmc.gov.il

Introduction: CD24 is a glycosylphosphatidylinositol-linked protein that functions as an adhesion molecule and is overexpressed at an early stage of CRC (Sagiv et al., 2006). The Wnt/b-catenin signaling pathway plays an important role in the CRC carcinogenesis process. C57BL6/J mice carrying the ApcMin mutation develop $\sim 24.3 \pm 3.7$ adenomas and several carcinomas in the small intestine by the age of 16 weeks compared to the $\sim 7 \pm 1.7$ polyps that ApcMin/CD24 -/ ("double KO") mice developed. Mice colonoscopy showed a significant reduction in the number and size of polyps upon depletion of CD24 alleles. The ApcMin mice displayed severe splenomegaly $(355 \pm 68 \mathrm{mg})$ compared $(141 \pm 49 \mathrm{mg})$ in double KO mice similar to WT mice. Hb level in the ApcMin was $3.8 \pm 2.5$, significantly lower than in the double KO mice $(8.2 \pm 0.9)$ and their WT littermate.

Aims \& Methods: We aimed to study the cellular interactions between CD24 and $\beta$-catenin, and effects of their interaction on intestinal tumorigenesis. CD24inducible 293T-Rex cells previously developed in our lab (Shapira et al., 2011) and SW480 CRC cells stably transfected with CD24 (Naumov et al., 2014) were used to study this interaction in vitro. Coimmunoprecipitation and immunofluorescent staining were used to investigate the interaction between the two

proteins. Far western blotting (WB) analysis was used to confirm this direct interaction by probing the standard WB membrane with the purified CD24 protein.

Results: In vitro: Western blotting analyses showed that expression of CD24 in 293T-Rex cells induced the activation of $\beta$-catenin, while down-regulation of CD24 in SW480 cells caused a decrease in the level of active $\beta$-catenin. Cytoplasmic/nuclear fractionation showed that more active $\beta$-catenin entered the nucleus in cells that expressed CD24 (clone1) as compared to control cells (clone 4). In addition, in both cell lines, TOP/FOP luciferase reporter assay showed a significant increase in Luciferase activity upon CD24 expression induction. Co-immunoprecipitation studies of CD24 and $\beta$-catenin indicated that these two proteins might be interacting. In addition, In HEK-293T cells and SW480 cells, immunofluorescent staining of CD24 and $\beta$-catenin showed that these two proteins co-localize on the cellular membrane. Furthermore, far western blotting analysis suggests that a direct interaction between the proteins exist.

Conclusion: 1. CD24 plays a major role in intestinal tumorigenesis. 2. CD24 interacts with the Wnt pathway by

activating $\beta$-catenin. 3. CD24 interacts directly with $\beta$-catenin 4. Down-regulation of CD24 may be an important aim in

the therapy of CRC.

Disclosure of Interest: N. Arber: Bio-view Micro-Medic Check-cap Gi-View Bayer

All other authors have declared no conflicts of interest.

\section{Reference}

Sagiv, E., Memeo, L., Karin, A., Kazanov, D., Jacob-Hirsch, J., Mansukhani, M., Rechavi, G., Hibshoosh, H. \& Arber, N. 2006. CD24 is a new oncogene, early at the multistep process of colorectal cancer carcinogenesis. Gastroenterology, 131, 630-9 
P1750 YM155 AS AN INHIBITOR OF CANCER STEMNESS

SIMULTANEOUSLY INHIBITS AUTOPHOSPHORYLATION OF

EGFR AND G9A-MEDIATED STEMNESS IN EGFR-POSITIVE

CANCER CELLS

C. Cheng ${ }^{1}$, A. $\mathrm{Ho}^{2}$

${ }^{1}$ Hematology And Oncology, Mackay Memorial Hospital, Taipei/Taiwan

${ }^{2}$ Division Of Gastroenterology, Cheng Hsin General Hospital, Taipei/Taiwan

Contact E-mail Address: aisheng49@gmail.com

Introduction: Cancer stem cells survive as the leading reason to tumor recurrence after tumor repressive treatments. Therefore, it is worth discovering specific and efficient inhibitors against cancer stemness for applications in reducing tumor recurrence. Previously, literature has indicated that YM155 can significantly reduce the stemness-derived tumorsphere formation of gastric cancer and suppress EGFR activity. However, the pharmaceutical mechanism of YM155 is not completely clear.

Aims \& Methods: The aim of this study attempted to investigate the potential mechanism of YM155 against cancer stemness in EGFR-positive cancers. The tumorspheres derived from EGFR-mutant HCC827 and EGFR-wild-type HCT116 and A549 cells expressing higher cancer stemness markers, CD133, were used as cancer stemness models.

Results: We found that higher EGFR autophosphorylation (Y 1068) in HCC827A549-, and HCC116- derived tumorspheres compared to the parental cells, which induced tumorsphere formation through activating G9a-mediated stemness property. YM155 was demonstrated to inhibit the tumorsphere formation by unexpectedly blocking the autophosphorylation of EGFR and EGFR/G9amediated stemness pathway. The chemical and genetic inhibitions of EGFR and G9a revealed the significant role of EGFR-G9a pathway in maintaining the cancer stemness property.

Conclusion: In conclusion, this study not only revealed that EGFR triggered the formation of tumorspheres through elevating the G9a-mediated stemness, but also demonstrated that YM155 inhibited the formation of tumorspheres by simultaneously blocking autophosphorylation of EGFR and activity of G9a as a potent anti-stemness agent against EGFR-positive cancers.

Disclosure of Interest: All authors have declared no conflicts of interest.

\section{References}

1. Na YS, Yang SJ, Kim SM, Jung KA, Moon JH, et al. (2012) YM155 induces EGFR suppression in pancreatic cancer cells. PLoS One 7: e38625.

2. Cheng XJ, Lin JC, Ding YF, Zhu L, Ye J, et al. (2016) Survivin inhibitor YM155 suppresses gastric cancer xenograft growth in mice without affecting normal tissues. Oncotarget 7: 7096-7109.

P1753 COLORECTAL CANCER CELLS INDUCE NEUROGENESIS IN THE ENTERIC NERVOUS SYSTEM OF THE TUMOR MICROENVIRONMENT VIA A NGF-DEPENDENT PATHWAY

F. Drissi, A. Bessard, M. Touvron, H. Boudin, F. Cossais, L. Van Landeghem, M. Neunlist, E. Duchalais

Inserm U1235, University of Nantes, Nantes/France

Contact E-mail Address: farouk.drissi@gmail.com

Introduction: Several studies have demonstrated that the colorectal cancer (CRC) microenvironment harbors an increased neurite density as compared to healthy areas. Moreover recent findings indicate that specific stimuli activate neurogenesis in the enteric nervous system (ENS), in adult mice. However whether tumor epithelial cells (TEC) induce neuritogenesis and/or neurogenesis in the ENS during CRC remains unknown.

Aims \& Methods: Human colon specimens from patients with CRC and from $\mathrm{AOM} / \mathrm{DSS}$ treated mice were whole-mount dissected in order to isolate the myenteric and submucosal plexus from healthy area and tumor margin. The number of enteric neurons per ganglia was compared between healthy area and tumor margin after anti-Hu immunostaining in both plexus. Human CRC supernatants, TEC (Caco-2 cells)-conditioned medium or NGF were added to primary culture of rat enteric nervous system and primary culture of rat enteric neurons. In fixed primary cultures, the number of enteric neurons per ganglia and the length of neurites were calculated after anti-Hu and anti-tubulin-III immunostaining respectively. In order to determine the role of NGF in CRC supernatants and TEC-conditioned medium effects, anti-NGF blocking antibodies were added to primary cultures.

Results: Immunofluorescence studies on whole-mount dissected ENS plexus demonstrate that the densities of neuronal bodies were significantly increased in the margins of both human CRC and murine AOM/DSS-induced dysplasia compared to healthy areas $(n=7$ and $n=5$ respectively; $p<0.05)$. The increase in neuronal density was similar in low-grade and high-grade dysplasia as well as carcinoma $(\mathrm{n}=5)$. In vitro human CRC supernatants and TEC-conditioned medium enhanced the densities of neurites and neuronal cell bodies in primary cultures of ENS (pcENS) as compared to healthy mucosa supernatants and control medium, respectively $(n=6 ; p<0.05)$. Finally, nerve growth factor (NGF) induced an increase in the density of neuronal bodies in pcENS $(n=4$ $\mathrm{p}<0.05$ ), and our preliminary data show that the addition of an anti-NGF blocking antibody to human CRC supernatant and TEC-conditioned medium inhibits their impact on neurogenesis and neuritogenesis $(n=6 ; p<0.05)$.
Conclusion: Altogether these results indicate that the increased neuronal density in CRC margin may result from the activation of neurogenesis in the ENS induced by TEC via a NGF-dependent pathway.

Disclosure of Interest: All authors have declared no conflicts of interest.

\section{P1754 FGF14 IS A FUNCTIONAL TUMOR SUPPRESSOR THROUGH INHIBTING AMPK/MTOR PATHWAY IN COLORECTAL CANCER}

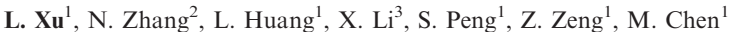

${ }^{1}$ Department Of Gastroenterology, The First Affiliated Hospital of Sun Yat-sen University, Guangzhou/China

${ }^{2}$ Sun Yat-sen University, Guangzhou/China

${ }^{3}$ State Key Laboratory Of Oncology In South China, Sun Yat-sen University

Cancer Center, Guangzhou/China

Contact E-mail Address: lixiaxu320@163.com

Introduction: Promoter hypermethylation-induced epigenetic silencing of tumor related genes played a key role in the initiation and development of colorectal cancer (CRC). Using Methylated DNA Immunoprecipitation (MeDIP), we identified that Fibroblast Growth Factor 14 (FGF14) was preferentially methylated in CRC.

Aims \& Methods: We aimed to investigate the epigenetic regulation and biological function of FGF14 in CRC.The expression of FGF14 in 10 CRC cell lines and 24 pairs of $C R C$ tissues and paired adjacent normal tissues by real-time PCR. CRC cells were treated with DNA demethylating agent 5-aza-2'-deoxycytidine (5-Aza). The methylation status of FGF14 in CRC cell lines and CRC were determined by real-time MSP. The biological function of FGF14 in CRC was interrogated by cell viability assay, colony formation, immunofluorescence and flow cytometry, as well as in vivo study.

Results: FGF14 was downregulated or silence in all (10/10) CRC cell lines, while it was readily expressed in normal colonic tissues. The expression of FGF14 was significantly lower in primary CRCs as compared to their adjacent normal tissues $(P<0.01)$. The loss of FGF14 gene expression was restored by treatment with DNA demethylating agent 5-Aza. Re-expression of FGF14 in CRC cell lines inhibited colony formation, suppressed cell viability, and induced cell apoptosis via AMPK/mTOR pathway, accompanied with enhanced protein expression of cleaved caspase-3, cleaved caspase-7, cleaved caspase-9 and PARP. In xenograft mouse model, overexpression of FGF14 significantly reduced tumor growth $(P<0.001)$

Conclusion: FGF14, which induces cell apoptosis via AMPK/mTOR pathway, is a novel tumor suppressor down-regulated by epigenetic inactivation.

Disclosure of Interest: All authors have declared no conflicts of interest.

\section{References}

Xicola RM, Llor X. DNA methylation defects in sporadic and hereditary colorectal cancer. Gastroenterol Hepatol 2012,35(7):480-7.

Taby R. Issa JP. Cancer epigenetics. CA Cancer J Clin 2010, 60(6): 376-92.

Raju K, David W. Comprehensive Molecular Characterization of Human Colon and Rectal Cancer. Nature 2012,487(7407): 330-7

Obata T, Toyota M, Satoh A, Sasaki Y, Ogi K, Akino K, Suzuki H, Murai M, Kikuchi T, Mita H, Itoh F, Issa JP, Tokino T, Imai K. Identification of HRK as a target of epigenetic inactivation in colorectal and gastric cancer. Clin Cancer Res 2003,9(17):6410-8

Wang Z, Yuan X, Jiao N, Zhu H, Zhang Y, Tong J. CDH13 and FLBN3 gene methylation are associated with poor prognosis in colorectal cancer. Pathol Oncol Res 2012,18(2):263-70.

Akai J, Storey K. Brain or brawn: how FGF signaling gives us both. Cell 2003 , 115(5): 510-2.

Goldfarb M. Fibroblast growth factor homologous factors: evolution, structure, and function. Cytokine Growth Factor Rev 2005,16(2):215-20.

Lin X, Buff EM, Perrimon N, Michelson AM. Heparan sulfate proteoglycans are essential for FGF receptor signaling during Drosophila embryonic development. Development 1999,126(17):3715-23.

Smallwood PM, Munoz-Sanjuan I, Tong P, Macke JP, Hendry SH, Gilbert DJ, Copeland NG, Jenkins NA, Nathans J. Fibroblast growth factor (FGF) homologous factors: new members of the FGF family implicated in nervous system development. Proc Natl Acad Sci USA 1996,93(18):9850-7.

Olsen SK, Garbi M, Zampieri N, Eliseenkova AV, Ornitz DM, Goldfarb M, Mohammadi M. Fibroblast growth factor (FGF) homologous factors share structural but not functional homology with FGFs. J Biol Chem 2003, 278(36):34226-36.

Goldfarb M, Schoorlemmer J, Williams A, Diwakar S, Wang Q, Huang X, Giza J, Tchetchik D, Kelley K, Vega A, Matthews G, Rossi P, Ornitz DM, D'Angelo E. Fibroblast growth factor homologous factors control neuronal excitability through modulation of voltage-gated sodium channels. Neuron 2007,55(3):449 63. 


\section{P1755 CHARACTERISTICS OF HYPERMUTATOR IN DIGESTIVE}

\section{SYSTEM CANCERS}

H. Matsubayashi ${ }^{1}$, H. Ishiwatari ${ }^{2}$, N. Kawata ${ }^{3}$, K. Takizawa ${ }^{3}$, N. Kakushima ${ }^{3}$

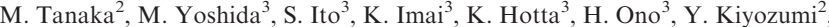
Y. Horiuchi ${ }^{2}$, K. Urakami ${ }^{4}$, M. Kusuhara ${ }^{4}$

${ }^{1}$ Endoscopy, Shizuoka Cancer Center, Suntogun/Japan

${ }^{2}$ Shizuoka Cancer Center, Suntogun/Japan

${ }^{3}$ Division Of Endoscopy, Shizuoka Cancer Center, Nagaizumi/Japan

${ }^{4}$ Research Institute, Shizuoka Cancer Center, Suntogun/Japan

Contact E-mail Address: h.matsubayashi@scchr.jp

Introduction: A cancer with a number of somatic mutations is defined as "hypermutator", and shows therapeutic features, such as high sensitivity to immune checkpoint inhibitor. However, to date, analyses of hypermutator have not been done with a large number of cases.

Aims \& Methods: The aim of this study is to analyze the incidences and characteristics of hypermutator in digestive system cancers. We analyzed somatic mutation of digestive system cancers in 1145 cases (age: 67.4 \pm 11.3 yrs., $\mathrm{M}: \mathrm{F}=755: 390$ ), those underwent surgery after full informed consent during 2014 to 2015. Genomewide sequencing was performed on 47 inherited cancerassociated genes and 411 cancer-associated genes using next generation sequencer (Ion Torrent Proton sequencer and Ion AmpliSeq Exome RDY kit, Thermo Fisher Scientific). Hypermutator was defined when a tumor having $>500$ mutations in the somatic DNA.

Results: The 1145 subjects included 583 colorectal cancers (CRC), 229 gastric cancers (GC), 103 metastatic liver tumors, 100 hepatocellular carcinomas (HCC), 45 pancreatic cancer, 23 GISTs, 15 esophageal cancers and 14 neuroendocrine tumors, etc. Hypermutator was recognized in 66 cases $(5.8 \%)$. Age and gender were not different by the hypermutation. Hypermutator was recognized in $6.2 \%$ (36 cases) of CRCs, $11.8 \%$ (27 cases) of gastric cancers, $2 \%$ ( 2 cases) of HCCs, and one case of small intestinal cancer. Within the hypermutator group, multiple cancers developed in $13.9 \%$ of CRC patients and $25.9 \%$ of GC patients. Mutation of $B R A F$ was detected in $33.3 \%$ (22 cases), that of mismatch repair genes (either of $M L H 1, M S H 2, M S H 6, P M S 2$ ) in $13.6 \%, P O L E$ in $9.1 \%$, and $P O L D 1$ in $4.5 \%$, respectively, in the hypermutator group.

Conclusion: Hypermutator was recognized in 5-10\% of digestive system cancers, predominantly seen in CRCs and GCs. Cases of hypermutator sometimes develops multiple cancers, associated with a somatic mutation of mismatch repair genes. Further research must be needed to clarify the characteristic of hypermutator of the digestive organs in the therapeutic aspects.

Disclosure of Interest: All authors have declared no conflicts of interest.

P1756 UNBIASED ANALYSIS OF REGULATION OF TRANSCRIPTION FACTORS UPON ER STRESS IN THE LS174T COLORECTAL CANCER CELL LINE EXPOSES CTBP2 AS A POTENTIAL REGULATOR OF STEMNESS

B. J. Meijer ${ }^{1}$, B. F. Westendorp ${ }^{1}$, J. H.m. Van Der Meer ${ }^{1}$, W. L. Smit ${ }^{1}$, J. Heijmans ${ }^{1}$, V. Muncan ${ }^{1}$, G.R. Van Den Brink

${ }^{1}$ Tytgat Institute For Liver And Intestinal Research, Academic Medical Center Amsterdam/Netherlands

Contact E-mail Address: b.j.meijer@amc.nl

Introduction: In the intestinal epithelium, stem cells are located at the bottom of the crypt and their maintenance and differentiation is essential for sufficient organ function. Accumulation of mis- or unfolded proteins in the endoplasmic reticulum, so-called ER-stress, leads to an unfolded protein response (UPR) which can force an intestinal epithelial stem cell (iESC) into differentiation. However, the molecular mechanism of this differentiation process upon ER stress is largely unknown. A prominent feature of the UPR is translation inhibition via PERK-eIF2a phosphorylation and we have previously shown that the differentiation upon ER-stress is dependent on PERK-eIF2a phosphorylation ${ }^{1}$ Therefore we hypothesize that ER stress influences stem cell differentiation on a translational level by downregulating key transcription factors (TFs) that serve an important role in maintaining stem cell fate.

Aims \& Methods: Our aim is to identify transcription factors important for stem cell maintenance using a transcriptomics approach. On thapsigargin treated LS174T-cells, a cell line with a transcriptional profile resembling intestinal stem cells, we performed a TF DNA-binding assay (catTFRE) in which TFs present in cellular lysate are bound to plasmid DNA, co-extracted and quantified using mass-spectrometry ${ }^{2}$. We confirmed downregulation using immunoblots in colorectal cancer cell lines. Additionally, we examined CtBP2 expression in mouse intestine using in situ hybridization, epithelial FACS sorting and immunohistochemistry. To study CtBP2 function, we transfected a LS174T cell line with shRNA CtBP2 and analyzed stemcell and differentiation markers.

Results: By using the CatTFRE assay we quantified the binding activity for $>1000$ transcription factors. Forty-three TFs showed significant downregulated binding to template DNA upon thapsigargin treatment compared to control. We confirmed downregulation of eight of these TFs on protein level, suggesting a loss of binding due to translation inhibition upon ER stress. We identified transcriptional corepressor $\mathrm{CtBP} 2$ as the most significantly downregulated protein. Interestingly, in mouse small intestine, CtBP2 is expressed in the proliferative compartment where stem cells reside. Additionally, mRNA expression analysis showed abundance of $\mathrm{CtBP} 2$ in iESCs. We next investigated the effect of $\mathrm{CtBP} 2$ knockdown on stemness in the LS174T cell line. Three days upon shCtBP2 transfection we showed a significant decrease in $m R N A$ levels of stem cell markers $\operatorname{Lgr} 5$ and Olfm 4 compared to control and an increase in differentiation markers Villin and P21, suggesting induced iESC differentiation.
Conclusion: Using an unbiased transcriptomics approach we identified transcription factors that are lost on protein level upon ER stress. Furthermore, our data suggests that the significant loss of the transcriptional regulator CtBP2 contributes to intestinal epithelial stem cell differentiation.

Disclosure of Interest: All authors have declared no conflicts of interest.

\section{References}

1. Heijmans J, et al. ER stress causes rapid loss of intestinal epithelial stemness through activation of the unfolded protein response. Cell reports. 2013;3(4):1128-39.

2. Ding C, et al. Proteome-wide profiling of activated transcription factors with a concatenated tandem array of transcription factor response elements. Proceedings of the National Academy of Sciences of the United States of America. 2013;110(17):6771-6.

P1757 MICROBIOTA A NEW INDICATOR OF COLORECTAL CANCER (CRC) HETEROGENEITY

I. Sobhani ${ }^{1}$, E. Bergstein ${ }^{1}$, A. Ghozlane ${ }^{2}$, S. Kennedy

${ }_{1}^{1}$ Dept. De Hepato-gastroenterologie, Universite Paris 12 Hopital H. Mondor Dept. of Hepato-Gastroenterology, Créteil/France

Statistics, C3b, Insitut Pasteur, Paris/France

Contact E-mail Address: iradj.sobhani $@$ aphp.fr

Introduction: Location and somatic gene signature of CRCs may impact prognosis and therapy response. A relative specific CRC-related dysbiosis has been characterized.

Aims \& Methods: The aim was to characterize colon microbiota in CRC patients regarding location, gene markers and outcome. Patients $(N=173)$ signed consent for whole metagenome (shot gun sequencing on Illumina HiSeq2500) analysis of stool DNA: 72 CRC (53 sporadic-S, 19 Lynch-L), 87 asymptomatic subjects (normal colonoscopy), 14 first degree healthy relatives from Lynch families. "MOCAT" pipeline was used, library sorted (Phred quality score 20 Alientrimmer v0.4.0) after exclusion of $<35 \mathrm{nt}$, human genes or phage sequences. Quality sequences were aligned (REFMG.V13) and most abundant genes constructed (MBMA program v0.1). The Shaman program (shaman.c3bi.pasteur.fr) was used. The number of bacteria was estimated (REFMG program). The linear model (GLM) was implemented in the DESeq2 $\mathrm{R}$ kit. Differences between Control $(\mathrm{N}=87)$ and CRCs $(\mathrm{N}=69)$, between $\mathrm{L}(\mathrm{N}=19)$ and $\mathrm{S}$ CRCs $(\mathrm{N}=50)$, and between LCRC $(\mathrm{N}=19)$ and Healthy Lynch relatives were obtained after interaction of age, BMI and gender was considered (GLM model). The $\mathrm{P}<0.1$ value was retained after correction (Benjamini and Hochberg). The specific taxonomic composition of the control and CRC groups was subjected to random analysis (Caret's $\mathrm{R}$ package) with two optimization parameters (precision and kappa) in the model.

Results: There was no difference for gender, age $(p=0.08)$ and BMI $(p=0.187)$ in the L and S CRCs. Significant differences were observed between Normal and CRCs, C-CRC and L-CRC, L-CRC and first degree relatives based on the common component (similarity of sequences): 13 species differentiated Normal and CCRs, two were more prevalent in L-CRCs. The panels of bacteria linked with location, MSI, Ras mutations, methylation phenotypes and survival were identified. No significant link was observed with TNM Staging: I ( N =17, 2L and $15 \mathrm{~S})$, II $(\mathrm{N}=12,5 \mathrm{~L}$ and $7 \mathrm{~S})$, III $(\mathrm{N}=20,10 \mathrm{~L}, 10 \mathrm{~S})$, IV $(\mathrm{N}=22.1 \mathrm{~L}, 21 \mathrm{~S})$. DFS might be dysbiosis dependent.

Conclusion: CRC dysbiosis is location-dependent Several bacteria are associated with Ras mutation, MSI, and methylation status. They may directly or through therapies impact the prognosis. Microbiota signature should be taken in consideration in trials.

Disclosure of Interest: All authors have declared no conflicts of interest.

\section{Reference}

Zeller et al; Potential of fecal microbiota for early-stage detection of colorectal cancer. 2014 Nov; 10(11): 766

\section{P1758 EPIGENETIC SILENCING OF SMOC1 IS ASSOCIATED WITH DEVELOPMENT OF COLORECTAL TRADITIONAL SERRATED ADENOMAS}

H. Aoki ${ }^{1}$, E. Yamamoto ${ }^{2}$, A. Takasawa ${ }^{3}$, T. Niinuma $^{1}$, H. Yamano ${ }^{2}$, H. Matsushita ${ }^{4}$, T. Harada ${ }^{1}$, T. Sugai ${ }^{5}$, H. Suzuki ${ }^{1}$

${ }_{1}^{1}$ Dept. Of Molecular Biology, Sapporo Medical University, Sapporo/Japan ${ }^{2}$ Department Of Gastroenterology And Hepatology, Sapporo Medical University, Sapporo/Japan

${ }^{3}$ Department of Molecular and Cellular Pathology, Sapporo Medical University Sapporo/Japan

${ }^{4}$ Department of Gastroenterology, Akita Red Cross Hospital, Akita/Japan ${ }^{5}$ Department of Molecular Diagnostic Pathology, Iwate Medical University, Morioka/Japan

Contact E-mail Address: hironori_a1123@yahoo.co.jp

Introduction: Colorectal serrated lesions (SLs) include hyperplastic polyp (HP), traditional serrated adenoma (TSA) and sessile serrated adenoma/polyp (SSA/P). SSA/Ps are well-known precursors of colorectal cancer (CRC) characterized by BRAF mutation and microsatellite instability (MSI), whereas the molecular characteristics of TSAs are not fully understood. 
Aims \& Methods: We aimed to identify epigenetic alterations associated with the development of TSAs and to clarify the associations between clinical, pathological and molecular characteristics in colorectal lesions. The genome-wide DNA methylation status in TSAs consisting of protruding and flat components was analyzed using an Infinium HumanMethylation450 BeadChip, and changes in DNA methylation during the development of TSAs were identified. Methylation of identified genes and CIMP markers (MINT1, -2, -12, -31, p16 and MLH1) and $\mathrm{BRAF} / \mathrm{KRAS}$ mutations were analyzed in 847 colorectal lesions and 61 samples of normal colonic tissue. Effects of ectopic expression on CRC cell growth in a prevalently methylated gene in TSAs were assessed in vitro and in vivo.

Results: BeadChip analysis revealed 11 genes in which methylation levels were progressively increased during development of TSAs. Among them, SMOC1 was prevalently methylated in TSAs, but was rarely methylated in SSA/Ps $(\mathrm{p}<0.001)$. RT-PCR revealed that SMOC1 is abundantly expressed in normal colon and SSA/Ps, but is significantly downregulated in TSAs. Immunohistochemical analysis showed that SMOC1 was expressed in the epithelium of normal colonic tissues and SSA/Ps, but that expression is significantly reduced in TSAs. Ectopic expression of SMOC1 suppressed proliferation, colony formation and in vivo tumor formation by CRC cells. Analysis of colorectal lesions revealed that SMOC1 is frequently methylated in TSAs and CRCs, and that SMOC1 methylation is strongly associated with KRAS mutation and CIMP-low.

Conclusion: Methylation of SMOC1 is associated with TSA development but is rarely observed in SSA/Ps. Immunohistochemical analysis of SMOC1 may be a useful marker to discriminate between SSA/Ps and TSAs. Our data suggests SMOC1 methylation may play a role in the neoplastic pathways arising in TSAs. Disclosure of Interest: All authors have declared no conflicts of interest.

\section{P1759 DEVELOPMENT AND VALIDATION OF PREDICTIVE MODEL FOR PARTICIPATION IN COLORECTAL CANCER SCREENING IN KOREA}

\section{J. Chung ${ }^{1}$, J.J. Park ${ }^{2}$, J.Y. Yoon ${ }^{3}$}

${ }^{1}$ National Medical Center Division of Gastroenterology, Seoul/Korea, Republic of ${ }^{2}$ Department Of Internal Medicine, Gangnam Severance hospital, Seoul/Korea, Republic of

${ }^{3}$ Gastroneterology, Kyung Hee University Hospital at Gang Dong, Seoul/Korea, Republic of

\section{Contact E-mail Address: drbeatrix@hanmail.net}

Introduction: The number of individuals partaking in colorectal cancer (CRC) screening still remains to be low even after the implementation of the Korean Government's National Cancer Screening Program for CRC. The aim of this study is to identify factors associated with partaking in CRC

Aims \& Methods: The Korean National Health and Nutrition Examination Survey (KNHANES) $2007 \sim 2010$ datasets were used to develop a CRC screening participation screening score. 10,527 individuals aged $\geq 50$ who completed the survey and not previously diagnosed with CRC were selected. Both logistic regression (LR) analysis and artificial neural network (ANN) were used to develop predictive models. Multilayer perception ANN was constructed based on 16 clinical variables. We then validated the models using the KNHANES 2011 and $2012(n=5986)$ datasets and compared them with each other.

Results: Out of 10,527 individuals selected, $57.0 \% \quad(n=6005)$ responded unscreened for CRC. Among various demographic and socioeconomic factors, following 8 parameters including, age, household income, marital status, education level, private health insurance, self-reported depression, self-reported health status, and residence were found to be independently associated with CRC screening participation. LR analysis produced screening score (range 0-10.3), and a cutoff point of $\geq 5.5$ defined $49 \%$ as unscreened for CRC and yielded area under the curve (AUC) of 0.626. When validated with KNHANES 2011 and 2012 datasets, the AUC of the defined LR model was 0.663 , meanwhile the AUC of ANN based predictive model was 0.743 .

Conclusion: The ANN produced better performing model than LR analysis based model in identifying population with low CRC screening participation. Sensible approaches should be implemented to encourage partaking in CRC screening in the identified individuals.

Disclosure of Interest: All authors have declared no conflicts of interest.

\section{P1760 CD24 PREDICTIVE LEVELS- A SIMPLE NOVEL BLOOD}

\section{TEST FOR EARLY DETECTION OF VARIOUS MALIGNANCIES}

N. Arber ${ }^{1}$, S. Shapira ${ }^{1}$, D. Kazanov ${ }^{1}$, A. Fokra ${ }^{1}$, Z. Sally ${ }^{2}$, S. Zigdon ${ }^{2}$, J. Maayan ${ }^{1}$, L. Ari ${ }^{2}$, K. Roni ${ }^{2}$, L. Alissa ${ }^{2}$, I. Ofer ${ }^{2}$, E. Liberman

${ }^{1}$ Health Promotion Center And Integrated Cancer Prevention Center, Tel Aviv

Medical Center, Tel Aviv/Israel

${ }^{2}$ Tel-Aviv Sourasky Medical Center, Tel Aviv/Israel

Contact E-mail Address: nadira@tlvmc.gov.il

Introduction: Background: CD24, a mucin-like cell surface molecule, highly expressed in large variety of solid tumors and hematological malignancies (HM) (Gastro 2006, Clin Can Res 2007, Can Res 2008). mAb to CD24 were found to inhibit the growth CD24 cancer cells (Gastro 2009). We have shown that a simple non-invasive blood test evaluating CD24 levels on PBL had good sensitivity and specificity for detecting colorectal neoplasia in subjects undergoing screening colonoscopy (Kraus et al., 2009).
Aims \& Methods: We aimed to improve a simple, noninvasive blood test that could reliably identify individuals with different types of cancer.

Blood was taken from patients with various malignancies (CRC, Pancreatic Cancer (PC), gastric cancer (GC), sarcoma and HM), that was confirmed by histology. Age, gender and ethnic matched healthy individuals served as controls. Healthy subjects were indeed very healthy. They underwent a thorough and extensive workout at the Integrated cancer prevention center at Tel Aviv Medical Center (Eur J Intern Med. 2013) All samples were collected and processed identically. For each sample, 20,000 leukocytes were analyzed by flow cytometry for the expression of CD24. An initial template file has been generated using gates within the software to create a hierarchical population tree at the beginning of the screen. All additional analyses were accomplished after data acquisition have been completed. The template file include compensation adjustment, which is uniformly applied to all the data collected in order to minimize fluorescence overlap between detection channels

Results: The novel assay was improved significantly. distinguished healthy from CRC (Fig.1a) ( $\mathrm{P}<0.013)$, PC (Fig.1b) $(\mathrm{P}<0.018)$, biliary tract $(\mathrm{P}<6.45 \mathrm{E}-12)$, sarcoma $(\mathrm{P}<0.036)$, multiple myeloma (Fig $2 \mathrm{a})(\mathrm{P}<0.4 \mathrm{E}-08)$, $\operatorname{MDS}(\mathrm{P}<0.1)$ and Lymphoma $(\mathrm{P}<2.1 \mathrm{E}-07)$ patients. $\mathrm{CD} 24$ expression levels were higher by up to $25 \%$ in cancer cases as compared to normal subjects. The sensitivity and specificity for CRC were $79.2 \%$ and $74.7 \%$, and for PC $70.0 \%$ and $75.9 \%$, respectively.

The positive (PPV) and negative predictive (NPV) values of CD24 for the detection of CRC was $38 \%$ and $94.8 \%$, and for PC $17.1 \%$ and $97.3 \%$, respectively. Specificity and sensitivity for HM were also statistically significant (data not shown). The CD24 test could not discriminate between patients with cervical, stomach and lung cancers and healthy subjects.

Conclusion: Conclusions: CD24 expression in PBLs is a promising blood test for the early detection of CRC, PC and HM.

Disclosure of Interest: N. Arber: Bayer Bio-view Gi-View Micro-medic Checkcap

All other authors have declared no conflicts of interest.

\section{P1761 FACTORS ASSOCIATED WITH OPTIMIZING} PREPARATION FOR COLONOSCUPOY USING SPLIT DOSE

\section{PICOLAX}

E. Half ${ }^{1}$, R. Leiba ${ }^{2}$, T. Hanania ${ }^{3}$, I. Chermesh ${ }^{3}$, E. Hag ${ }^{1}$

${ }_{1}^{1}$ Rambam Health Care Campus Dept. of Gastroenterology, Haifa/Israel

${ }^{2}$ Epidemiology, Rambam Health Care Campus, Haifa/Israel

${ }^{3}$ Gatroenterology, Rambam Health Care Campus Dept. of Gastroenterology,

Haifa/Israel

\section{Contact E-mail Address: eisahag@yahoo.com}

Introduction: Colonoscopy is considered the gold standard for prevention and early detection of colorectal cancer (CRC), however its effectiveness is directly related to quality of bowel preparation. Two of the quality measures of colonoscopy, cecal intubation rate and adenoma detection rate, are both associated with adequate bowel prepation. Data on factors associated with quality of preparation using Picolax ${ }^{\circledR}$ are limited.

Aims \& Methods: We aimed to evaluate factors associated with a good bowel preparation using Picolax ${ }^{\circledR}$ (Sodium picosulfate magnesium citrate) in the Israeli heterogeneous population. Consecutive outpatients referred for colonoscopy were prospectively assessed by a nurse practitioner filling out a questionair. Bowel preparation consisted of Bisacodyl $10 \mathrm{mg} / \mathrm{d} 3$ days before colonoscopy and split dose picolax. Demographics, medical history, Bisacodyl and water consumption, time of total preparation and time between end of prep to colonoscopy were evaluated. Quality was assessed using the Boston Bowel Preparation Score (BBPS). Bowel prep was considered "good" if BBPS was $\geq 6$ and $\geq 2$ in each 1 segment or "low" if BBPS was $<6$ or $<2$ in any colonic segment.

Results: A total of 452 patients were included in the study $(\mathrm{M}=54 \%$, mean age $56.5 \pm 16.3 \mathrm{yrs}), 366452(81 \%)$ achieved a "good" bowel preparation, and 86 $(19 \%)$ were classified as "not good." No significant difference was observed between ethnicities and gender in terms of achieving a good bowel preparation $(\mathrm{p}=0.77, \mathrm{p}=0.054)$. There was a significant difference among diabetics $(\mathrm{n}=93$, $20.6 \%)$ and non-diabetics $(n=359,79.4 \%)$ in quality of the bowel prep. While $69.9 \%$ of diabetics achieved a good bowel preparation only $83.8 \%$ of non diabetics achieved a good bowel preparation $(\mathrm{p}=0.004)$. In the univariant analysis, Bisacodyl had no effect on bowel preparation $(\mathrm{p}=0.83)$ except in the diabetics, where those who took an average of $5.3+1.9$ (median =6) vs. $4.31+1.5$ (median $=4)$ tablets fared better $(\mathrm{p}=0.018)$. Other chronic diseases had no effect on bowel preparation. Drinking 3-7 vs 8-15 glasses of water achieved good preparation in $72.7 \%$ vs $83.8 \%$ of cases, respectively $(\mathrm{p}=0.01)$. Time from end of preparation to colonoscopy $<8 \mathrm{hrs}$ achieved significantly better prep vs $>8 \mathrm{hrs}$ $(\mathrm{p}=0.002)$. In the multivariant model for prediction of quality of bowel preparartion that included the time between sachets of picolax, number of water cups consumed, diabetes, gender, and age we found that all, excluding age, were good predictors of bowel preparation. Women had a better chance of achieving an adequate bowel preparation $(\mathrm{OR} 1.68, \mathrm{p}=0.045,95 \% \mathrm{CI}=1.01-2.79)$. Patients without diabetes had a better chance of achieving an adequate bowel preparation $(\mathrm{OR}=2.05, \mathrm{p}=0.014,95 \% \mathrm{CI}=1.16-3.63)$. Patients who had 5-9 hours between the 2 sachets of picolax had a lower chance of achieving an adequate bowel preparation as compared to those who had 9 to 24 hours between the 2 sachets $(\mathrm{OR}=0.375, \mathrm{p}=0.009,95 \% \mathrm{CI}=0.180-0.785)$. Lastly, drinking fewer than 8 cups lowers the chance of achieving an adequate bowel preparation $(\mathrm{OR}=0.461, \mathrm{p}=0.003,95 \% \mathrm{CI}=0.275-0.775)$. 
Conclusion: Diabetics require a more intense bowel preparation aided by Bisacodyl, which does not help others. Time between both doses should exceed 8 hours and preparation should end no later than 8 hours prior to colonoscopy. Patients should be instructed to drink a minimum of 8 glasses of water with each dose of picolax.

Disclosure of Interest: All authors have declared no conflicts of interest.

\section{P1762 IMPROVED ADENOMA DETECTION WITH ELUXEO}

LINKED COLOR IMAGING (LCI) AS COMPARED TO CONVENTIONAL WHITE-LIGHT HIGH-DEFINITION COLONOSCOPY-A RANDOMIZED CONTROLLED TRIAL

\section{Szalai ${ }^{1}$, L. Oczella ${ }^{1}$, Z. Dubravcsik ${ }^{2}$, A. Szepes ${ }^{2}$, L. Madacsy ${ }^{3}$}

${ }^{1}$ Endo-Kapszula Private Endoscopy Unit, Székesfehérvár/Hungary

${ }^{2}$ Gastroenterology \& Endoscopy, Bacs-Kiskun Country Hospital Gastroenterology \& Endoscopy, Kecskemét/Hungary

${ }^{3}$ Department Of Gastroenterology, Bacs-kiskun County Hospital, Hungary, Endocam LTD, Szekesfehervar/Hungary

\section{Contact E-mail Address: dr.szalai.milan@gmail.com}

Introduction: Colonoscopy is the gold standard method of colorectal cancer and polyp screening, but polyps are missed during a colonoscopic examination at a rate that varies from $6 \%$ to $27 \%$. Improved adenoma detection rates can be achieved with optimized endoscopic visualization methods. A recently developed new Fujinon endoscope system, Eluxeo carries a new function of electronic chromoendoscopy, Linked Color Imaging (LCI), that enhances the coloring and contrast of mucous membranes and blood vessels which are difficult to see with the conventional endoscopes. In our prospective randomized study, we evaluated the effectiveness of LCI, a new endoscopic visualization technique that may enhance image quality to improve colonic adenoma detection.

Aims \& Methods: Up till now 247 eligible patients, elder than 45 years, admitted for screening outpatient colonoscopy were randomly enrolled to undergo highdefinition white-light colonoscopy (WLC) or LCI colonoscopy during instrument withdrawal. The colonoscopic procedures were performed by three experienced endoscopists with Fujinon 7000 processor and with either the conventional highdefinition Fujinon EC 590Z or a new EC 760Z VS Eluxeo colonoscope. All of the colonoscopic procedures were made under Propofol deep sedation guided by an anesthesiologist team. The minimum withdrawal time was defined as more than 6 minutes. All colonoscopies were routinely assisted with pure $\mathrm{CO} 2$ insufflation. The primary outcome parameter of our study was to assess and compare the polyp and adenoma detection rate with the two endoscopic technology.

Results: A total of 247 patients were randomized (mean age 58.7 years), 101 patients enrolled in the WLC group and 146 patients in the LCI group. No significant differences have been observed in the patient demographics and colonoscope withdrawal time between the two groups. Patients having both colorectal polyps and adenomas were detected more frequently in the LCI group than in the control group: $60.9 \%$ and $43.8 \%$ versus $55.4 \%$ and $33.6 \%$ respectively, however, this was not statistically significant $(\mathrm{p}=0.32$ and 0.16$)$. In contrast, the total number of adenomas relative to the total number of polyps detected with LCI withdrawal were significantly higher than with conventional WLC: 105 vs. 159 and 55 vs. 102, respectively $(\mathrm{p}<0.0498)$

Conclusion: The LCI enhancement of the Fujinon Eluxeo colonoscopy system was superior to the conventional HD-WLC in detecting patients with colorectal adenomas, which was mainly due to the ability of the more sensitive detection of minute (less than $5 \mathrm{~mm}$ ) adenomas.(Study was supported by ECT grant GINOP 2.1.1.-15- 2015-00128.

Disclosure of Interest: All authors have declared no conflicts of interest.

P1763 COMPREHENSIVE ANALYSIS OF LONG NON-CODING RNAS WITH CHARACTERISTIC EXPRESSION LEVEL ALTERATION IN COLORECTAL ADENOMAS AND CANCERS

A. Kalmár ${ }^{1}$, Z.B. $\mathrm{Nagy}^{1}$, O. Galamb ${ }^{2}$, B. Wichmann ${ }^{1}$, B.K. Bartak ${ }^{1}$, G. Valcz ${ }^{2}$, K. Szigeti ${ }^{1}$, Z. Tulassay ${ }^{1}$, P. Igaz $^{1}$, B. Molnar ${ }^{1}$

${ }^{1}$ 2nd Dept. Of Internal Medicine, Semmelweis University, Budapest/Hungary ${ }^{2}$ Semmelweis University, 2nd Departemnt of Internal Medicine, Cell Analysis Laboratory, Budapest/Hungary

\section{Contact E-mail Address: alexandra.kalmar@gmail.com}

Introduction: Long non-coding RNAs (lncRNAs) play role in colorectal cancer (CRC) development, however, lncRNA expression profile in CRC and its relation to the epigenetic regulatory system still remain incomplete.

Aims \& Methods: We aimed the perform whole genomic lncRNA expression profiling and the analysis of underlying functional interactions of aberrantly expressed lncRNAs. IncRNA expression levels were analyzed on 60 colonic biopsy samples (20 CRCs, 20 adenomas, 20 normals $)$ by Human Transcriptome Array (HTA) 2.0. Expression alteration of certain candidates was verified by qPCR. Furthermore, in silico validation was performed on HGU133 Plus 2.0 array data and also on TCGA COAD dataset. miRNA targets of lncRNAs were predicted with the miRCODE algorithm and miRNA expression was analyzed using miRNA 3.0 Array. Comprehensive IncRNA-mRNA coexpression pattern analysis was also performed.

Results: According to HTA results in adenomas 12 lncRNAs (e.g. LINC00278) were upregulated and 6 lncRNAs (e.g. RP11.747D18.1) were downregulated compared to normals, while in CRCs 1 lncRNA (UCA1) was overexpressed and 8 lncRNAs (e.g. LINC00350) were underexpressed compared to adenomas $(\mathrm{p}<0.05 ;-2 \geq \mathrm{Fc} \geq 2)$. In CRC samples 8 lncRNAs (e.g. AC123023.1) were overexpressed and 9 IncRNAs (e.g. RP13-497K6.1) were downregulated compared to normals. $42 \%$ of lncRNAs upregulated in CRC samples showed elevated expression $(\mathrm{p}<0.05)$ already in adenomas (e.g. overexpressed CCAT1, downregulated LINC01133). In line with aberrant expression of certain lncRNAs in tumors, miRNA and mRNA targets' expression showed systematic alterations, e.g. UCA1 upregulation in CRC samples in parallel with miR-1 downregulation accompanied by CMET target mRNA overexpression $(\mathrm{p}<0.05)$. Conclusion: The defined lncRNA sets (e.g. CCAT1, UCA1) may have a regulatory role in adenoma and CRC development and in tumor cell growth pathways. A subset of CRC-associated lncRNAs showed significant differential expression in precancerous samples, that raise the possibility to develop potential adenomaspecific markers and achieve early detection of colon lesions.

Disclosure of Interest: All authors have declared no conflicts of interest.

\section{P1764 A1-ANTITRYPSIN (SERPIN-A1) AS A PUTATIVE} BIOMARKER FOR COLORECTAL CANCER

D. Kypraios ${ }^{1}$, S.L. Hatzinicolaou ${ }^{1}$, A. Malachias ${ }^{1}$, L. Theodoropoulos ${ }^{1}$, A. Vakrakou ${ }^{2}$, G. Papachristopoulou ${ }^{2}$, D. Dimitroulopoulos ${ }^{1}$, M. Talieri ${ }^{2}$, D. Xinopoulos 1

${ }^{1}$ Department Of Gastroenterology, St. Savvas Athens Cancer Hospital, Athens/ Greece

${ }^{2}$ G. Papanicolaou Research Center of Oncology, Saint Savvas Cancer Hospital, Athens/Greece

Contact E-mail Address: matinahatzinicolaou@gmail.com

Introduction: Serine protease inhibitors (Serpins) play an important role in the regulation of enzymes involved in proteolytic cascades. Members of the family are: alphal-antitrypsin, alphal-antichymotrypsin, $\mathrm{Cl}$ inhibitor, antithrombin and neuroserpin. Kallikrein-related peptidases (KLKs) are involved in proteolytic cascades of different tissues. KLK14, acting via PAR-2, represents an autocrine/paracrine regulator of colon tumorigenesis and alphal-antitrypsin is a natural inhibitor of KLK14. Therefore its role in regulating the proteolytic cascade in colorectal tumorigenesis is of great importance.

Aims \& Methods: The aim of this study was to analyze A1-antitrypsin (AAT) expression in tissue samples at different stages in the process of colon cancer development. We examined a total of 245 colon samples. Of those, there were 101 colorectal carcinoma tissues, for 70 of which paired normal mucosa was also examined. A total of 74 colorectal adenomas were examined. Quantitative real time PCR was used to measure AAT expression. Clinical evaluation of AAT levels was demonstrated in terms of disease-free survival (DFS) and overall survival (OS).

Results: Alpha1-antitrypsin expression was found to be significantly associated with TNM stage $(\mathrm{p}=0.028)$. Cox proportional hazard regression model using univariate analysis revealed that high status alphal-antitrypsin expression is a significant factor for disease-free survival (DFS) $(\mathrm{p}=0.002)$ and overall survival (OS) $(\mathrm{p}=0.026)$ in patients with colorectal cancer. Kaplan-Meier survival curves demonstrated that low alphal-antitrypsin expression is significantly associated with longer DFS $(\mathrm{p}=0.001)$ as well as OS $(\mathrm{p}=0.021)$.

Conclusion: Our data suggests that alphal-antitrypsin expression could be considered as a potential biomarker of unfavorable prognosis for colorectal cancer. Disclosure of Interest: All authors have declared no conflicts of interest.

\section{References}

1. Pérez-Holanda et al. Serum concentration of alpha-1 antitrypsin is significantly higher in colorectal cancer patients than in healthy controls, $B M C$ Cancer 2014, 14:355

2. Luis Bujanda et al. Evaluation of Alpha 1-Antitrypsin and the Levels of mRNA Expression of Matrix Metalloproteinase 7, Urokinase Type Plasminogen Activator Receptor and COX-2 for the Diagnosis of Colorectal Cancer, PLOS ONE 2013

3. Xie LQ, Zhao Ch, Cai SJ, et al. Novel proteomic strategy reveal combined al antitrypsin and cathepsin $\mathrm{O}$ as biomarkers for colorectal cancer early screening, Journal of Proteome Research 2010

4. Talieri M, Papadopoulou S, Xynopoulos D, et al. Cathepsin B and Cathepsin D expression i $\eta$ the progression of colorectal adenoma to carcinoma. Cancer letters 2004; 205: 97- I 06

5. Herszényi L, Farinati F, Cardin R, István G, Molnár LD, Hritz I, De Paoli M, Plebani M, Tulassay Z. BMC Cancer. 2008 Jul 10;8:194

P1765 DIFFERENTIATION BETWEEN NEOPLASTIC AND NONNEOPLASTIC DIMINUTIVE COLORECTAL POLYPS WITH FUJINON ELUXEO-BLI VERSUS FICE ELECTRONIC CHROMOENDOSCOPY WITH AND WITHOUT OPTICAL MAGNIFICATION-A RANDOMIZED PROSPECTIVE STUDY

L. Madacsy ${ }^{1}$, M. Szalai ${ }^{2}$, L. Oczella ${ }^{2}$, Z. Dubravcsik ${ }^{3}$, P. Novak ${ }^{3}$, K. Zsobrak ${ }^{2}$, A. Szepes ${ }^{3}$

${ }^{1}$ Department Of Gastroenterology, Bacs-kiskun County Hospital, Hungary,

Endocam LTD, Szekesfehervar/Hungary

${ }^{2}$ Endo-Kapszula Private Endoscopy Unit, Székesfehérvár/Hungary

${ }^{3}$ Gastroenterology \& Endoscopy, Bacs-Kiskun Country Hospital Gastroenterology \& Endoscopy, Kecskemét/Hungary

Contact E-mail Address: lmadacsy@mail.fmkorhaz.hu

Introduction: Real-time differentiation between neoplastic and non-neoplastic colorectal lesions may be crucial during colonoscopy. While adenomas are 
neoplastic, and therefore should be resected, hyperplastic polyps never turn malignant and do not require specific endoscopic therapy. The aim of our prospective, randomized study was to distinguish subcentimetric hyperplastic and adenomatous polyps based on Fujinon FICE versus Eluxeo BLI electronic chromoendoscopic technology with high-definition colonoscopy with and without optical magnification.

Aims \& Methods: In order to create a video and digital picture library of polyps, patients undergoing screening or diagnostic colonoscopy were considered for inclusion. Patients with at least one histologically verified $<10 \mathrm{~mm}$ polyp were included. A short $(20 \mathrm{sec})$ video-clip and at least one still picture of each polyp without and with $50 \mathrm{x}$ optical zoom at standard white-light (WLI), and with FICE-light or BLI-light were recorded with Fujinon EC 590Z and EC760Z endoscopes and stored in an anonymized database. Once the video-library was completed, each of our 5 colonoscopic experts (ML, SZM, OL, DZS, and SZA) independently and randomly reviewed all of the cases with a standardized electronic questionnaire. In each cases, all of the observers had to assess the color, the vascularization and the surface of the polyps, and the pit pattern was also analyzed according to the Kudo classification. Finally, with the degree of confidence (low/medium/high on VAS), the histological prediction and the final decision has been clarified on each lesion as neoplastic or non-neoplastic (hyperplastic).

Results: Up till now 115 polyps were enrolled and recorded into our digital webbased library, 59 were assigned into the FICE and 56 into the BLI group. All of the detected 115 polyps were endoscopically removed and histologically analyzed and this was regarded as gold standard. The overall accuracy with WLI versus FICE versus BLI technology of the 5 experts without zoom and with 50x times magnification to differentiate between hyperplastic and adenomatous lesions were $77.62 \%$ and $84.51 \%$, vs. $74.58 \%$ and $83.90 \%$ vs. $83.93 \%$, and $88.84 \%$, respectively. There was an excellent correlation between the histopathological results and our KUDO classification with both FICE and BLI technology. Both 50x times optical zoom and BLI technology were independently and significantly improved our confidence rate that was associated with a more precise histological prediction as compared to non-zoom, WLI or FICE endoscopic polyp assessment.

Conclusion: The new electronic chromoendoscopic technology with Eluxeo BLI significantly improved the reliability of the histology prediction as compared to FICE technology of Fujinon. High-confidence predictions for the differentiation of neoplastic and non-neopastic polyps with Eluxeo BLI electronic chromoendoscopy provide a potential for real-time endoscopic diagnosis of hyperplastic polyps to support resect and discharge strategy. (Study was supported by ECT grant GINOP 2.1.1.-15- 2015-00128)

Disclosure of Interest: All authors have declared no conflicts of interest.

\section{P1766 HOW FREQUENTLY DO SECONDARY CARE CLINICIANS MISS COLORECTAL CANCER?}

V. Theis ${ }^{1}$, S. Priestley ${ }^{2}$, A. Bassi ${ }^{2}$, E. Berryman ${ }^{2}$, I. Vasiliadou ${ }^{1}$, B. Richards ${ }^{1}$, H. Parry ${ }^{1}$

${ }^{1}$ St Helens and Knowsley NHS Trust, DR/United Kingdom

${ }^{2}$ Gastroenterology, St Helens \& Knowsley Teaching Hospitals NHS Trust, DR/ United Kingdom

Contact E-mail Address: beth.richards@doctors.org.uk

Introduction: Colorectal cancer (CRC) is the 4th commonest cancer in the UK but has an excellent prognosis upon early diagnosis accounting for the development of bowel cancer screening. However not all patients are eligible for this. Furthermore, colorectal investigations have the potential to miss the diagnosis and lack of appropriate investigation of alarm symptoms may also delayed diagnosis.

Aims \& Methods: We aimed to determine the miss rate of previous investigations and reviews for colorectal symptoms and iron deficiency anaemia (IDA) over the preceding 3 years prior to CRC diagnosis at our institution. 215 patients were identified from the local CRC registry between $1 / 10 / 15-30 / 9 / 16$ and electronic records reviewed to determine if any review for lower GI symptoms or IDA had occurred in the preceding 3 years. Missed diagnoses were defined as patients having prior endoscopic or cross sectional imaging viewing the affected area or having prior clinic/inpatient review failing to adequately investigate colorectal alarm symptoms.

Results: Of the 215 patients $37(17 \%)$ were deemed to have undergone possible delay in their diagnosis causing an average 9.6 and median 5 months delay (1.533 months). Cancers were right sided in 18 patients (49\%), Dukes C or above in $76 \%$, with $57 \%$ only suitable for palliative therapy. Of the 37 patients with a delayed diagnosis $22(59 \%)$ were deemed possible missed diagnoses. The remaining 15 delays $(41 \%)$ were due to patient choice $(11 \%)$ or delayed clinic review investigation $(30 \%)$; in this latter group the delay was a mean 3.9 months (1.5-7 months). Missed diagnosis occurred at: 1) Endoscopy in $10(48 \%) ; 8$ colonoscopies (median 18 (4.5-27) months earlier), 2 sigmoidoscopies (1 and 3 months earlier), 2) Radiology: CTvC missed 2 CRC (15 and 2 months earlier but laparoscopy for a presumed benign lesion was planned for the latter); CTAP/CTKUB missed 8 cases (median 5(2-26) months previously), 3) Clinical assessment: missed appropriate inpatient/outpatient investigation based on presenting with colorectal alarm symptoms or IDA occurred in 7 cases (median 3(2-16) months delay). 2 patients were a miss on both $\mathrm{CT}$ and endoscopy and 3 on $\mathrm{CT}$ and clinic review. Delayed follow-up upon re-referral compounded delayed diagnosis in 11 $(50 \%)$ of the 22 missed cases.

Conclusion: Our data suggest a delay in CRC diagnosis occurred in almost a fifth of cancer diagnoses. Delayed cancer diagnosis tended to occur in right-sided lesions and resulted in a median 5-month delay with lesions generally advanced at diagnosis. In $59 \%$ the diagnosis could have been expedited by earlier follow-up and/or investigation. While miss rates for endoscopic and imaging modalities were low, $19 \%$ of missed cancer diagnosis were due to lack of appropriate investigations following outpatient or inpatient review for colorectal alarm symptoms or IDA; this might be improved by increasing specialist inpatient in-reach services and senior review of outpatient cases.

Disclosure of Interest: All authors have declared no conflicts of interest.

\section{References}

1. Cancer Research UK

2. Frenette CT, Strum WB. Relative rates of missed diagnosis for colonoscopy, barium enema, and flexible sigmoidoscopy in 379 patients with colorectal cancer. J Gastrointest Cancer. 2007;38:148-153.

\section{P1767 ENDOCYTOSCOPIC VASCULAR PATTERN FOR COLORECTAL LESION IS HELPFUL IN PREDICTING PATHOLOGICAL DIAGNOSIS}

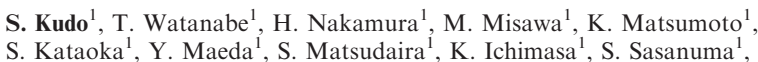
N. Toyoshima ${ }^{1}$, Y. Mori ${ }^{1}$, T. Kudo ${ }^{1}$, T. Hisayuki ${ }^{1}$, K. Wakamura $^{1}$, T. Hayashi ${ }^{1}$, T. Baba ${ }^{1}$, H. Miyachi ${ }^{1}$, F. Ishida ${ }^{1}$, H. Inoue ${ }^{2}$

${ }^{1}$ Digestive Disease Center, Showa University Northern Yokohama Hospital,

Yokohama/Japan

${ }^{2}$ Digestive Disease Center, Showa University Koto-Toyosu Hospital, Tokyo/Japan

Contact E-mail Address: kudos@med.showa-u.ac.jp

Introduction: Till now, narrow-band imaging (NBI) could make it possible to analyze the surface microvessels of colorectal lesions for differentiating neoplasms from non-neoplasms and for predicting the histropathological diagnosis. Endocytoscopy (EC) is the next generation of ultramagnification endoscopy that allow visualization of the glandular structure and cellular atypia. EC has visualized living tumor cells in vivo and obtained an ultra-magnification pathological image simply by applying the scope to the target mucosa during an endoscopic examination. However, in order to visualize cell nuclei, dye staining (i.e. methyleneblue) is always required. Since dye staining complicates the procedure, new observation method without use of dye has been strongly desired. On the other hand, EC with NBI (EC-NBI) allows ultra-magnified microvessel observation without using any dye solution.

Aims \& Methods: The aim of this study was to validate the evidence whether the observation of surface microvessels using EC-NBI was useful in predicting the histopathology of colorectal lesions. The study included 438 patients who underwent complete colonoscopy and endoscopic or surgical treatment between April 2006 and June 2015. A total of 576 lesions (45 Non-neoplastic polyps, 304 adenomas, 71 intramucosal cancer, 21 slightly invasive submucosal cancer (SMs) and 135 massively invasive submucosal cancer) were retrospectively evaluated. We used the Kudo classification for the degree of submucosal invasion and classified cancers accordingly. SMs cancer without vessel permeation does not metastasize. In contrast, SMm lesions show a substantial proportion $(\sim 10 \%)$ of lymph node metastasis. We named the ultra-magnified microvessel findings as endocytoscopic vascular (ECV) pattern and classified into the following 3 groups: EC-V1, the surface microvessels were very fine obscure; EC-V2, the surface microvessels were more clearly seen and showed a regular vessel network, and their caliber and arrangement were uniform; and EC-V3, the surface microvessels were thick, and their caliber and arrangement were non-homogeneous.

Results: The sensitivity, specificity and accuracy of EC-V1 for diagnosis of hyperplastic polyp were $88.9 \%, 98.5 \%$ and $97.7 \%$, respectively. As regards the sensitivity, specificity and accuracy of EC-V3 for diagnosis of SMm were $82.2 \%$, $98.0 \%$ and $94.3 \%$, respectively.

Conclusion: Endocytoscopic vascular pattern was helpful in predicting the histopathology of colorectal lesions.

Disclosure of Interest: All authors have declared no conflicts of interest.

\section{Reference}

1. Kudo S. Endoscopic mucosal resection of flat and depressed types of early colorectal cancer. Endoscopy. 1993;25(7):455-61. Epub 1993/09/01.

\section{P1768 CONSIDERATION OF RECTAL NEUROENDOCRINE TUMOR IN OUR HOSPITAL}

M. Akita

Internal Medicine, Mitoyo General Hospital, Kanonji City/Japan

Contact E-mail Address: m_akita1976@yahoo.co.jp

Introduction: Although patients with endoscopic treatment indications for rectal neuroendocrine tumours (NETs) are recommended to undergo endoscopic therapy, those with a tumour diameter of $\leq 10 \mathrm{~mm}$ and up to the deep submucosal layer. However, no clear guideline has been established on the radical criteria and additional resection. Prognosis of the disease is also unclear. The validity of the diagnosis and treatment recommended in the guideline for rectal NETs will be examined in the cases treated at our hospital.

Aims \& Methods: We examined the macroscopic features, pathological features, treatment methods, and prognosis of 22 patients diagnosed as having a rectal NET and treated at our hospital between 2007 and May 2016.

Results: The mean age of the patients was 65.2 years (range, 49-88 years); maleto-female ratio, 15:7; diagnosis opportunity, 21 asymptomatic cases and 1 
symptomatic condition (lumbago); lesion site (Rs/Ra/Rb), 1/2/19 cases; mean tumour diameter, $8.2 \mathrm{~mm}$; presence of biopsy, $14 / 8$ cases; biopsy diagnosis rate, $11 / 14(78.5 \%)$; presence/absence of endoscopic ultrasonography, 11/11 cases; and $\mathrm{M} / \mathrm{SM} / \mathrm{MP}, 1 / 19 / 1$ cases. The TNM classification of the cases was as follows: T1a, 16 cases; T1b, 5 cases; T2, 1 case; N1, 1 case; and M1, 1 case. The treatment was endoscopic mucosal resection/endoscopic mucosal resection with ligation/ endoscopic mucosal resection/endoscopic submucosal dissection/surgery/drug therapy in $5 / 5 / 7 / 2 / 1 / 1$ case. Of 19 endoscopic treatment cases, 15 corresponded to a tumor diameter of $\leq 10 \mathrm{~mm}$, with negative resection margin and vascular invasion as criteria for curative resection, and 3 cases of unknown stump were recognised. In the EMRL group, all cases were negative. In all the cases except the case of other-disease death, it elapsed without recurrence. Both surgical cases showed a positive vascular invasion, and one case was a confirmed N1, but neither of the patients survived without a relapse. In the case with hepatic and bone metastases, medication was administered, and the effect was temporarily effective, but the patient died a year and a half later.

Conclusion: Endoscopic treatments are considered appropriate for rectal NETs with no evidence of ulcer or depression, no apparent metastasis, and not $>10 \mathrm{~mm}$ in diameter. Among the treatment options, EMRL is considered useful and well tolerated. However, follow-up observation and case accumulation seem necessary to determine the long-term prognosis in NET, which may recur after years of surgery.

Disclosure of Interest: All authors have declared no conflicts of interest.

\section{P1769 SELECTIVE ERADICATION OF K-RAS MUTATED CANCER CELLS BY DELIVERY OF BACTERIAL TOXINS}

N. Arber ${ }^{1}$, B. Ilana ${ }^{2}$, D. Kazanov ${ }^{1}$, S. Shapira ${ }^{1}$

${ }^{1}$ Health Promotion Center And Integrated Cancer Prevention Center, Tel Aviv

Medical Center, Tel Aviv/Israel

${ }^{2}$ Tel-Aviv Sourasky Medical Center, Tel Aviv/Israel

Contact E-mail Address: nadira@tlvmc.gov.il

Introduction: Inactivation of TP53 is the most frequent genetic damage in human cancer. In addition, hyperactivation of the RAS pathway is common in many human malignancies (Lung (LC) 40\%, pancreatic (PC) $>95 \%$ and colorectal cancer (CRC) 50\%). Despite multiple attempts, targeting these pathways for the treatment of cancer, for example through the development of RAS pathway inhibitors has not proven to be effective thus far. Herein, we propose to exploits the hyperactive RAS pathway and TP53 mutation status of human cancer to deliver targeted antitumor herapy. We had previously reported that a recombinant adenovirus, carrying a pro-apoptotic gene (PUMA) under the regulation of RAS-responsive elements (PY4) effectively suppressed the growth of human cancer cells harboring hyperactive RAS (Giladi et al, 2007). Furthermore, we had shown, both in vitro and in vivo, that replacing the pro-apoptotic gene with a bacterial toxin can improve the efficacy of this system (Shapira et al, 2015).

Aims \& Methods: We aimed to establish a tight regulated dual system by expressing a toxin under PY4 elements in cancer cells, while sparing normal cells by expressing the anti-toxin under p53 responsive elements (RGC) specifically in non-malignant cells. Adenoviral vectors carrying the toxin (PY4-MazF-mcherry) and the antitoxin (RGC-MazE-GFP) were designed under the regulation of RAS and 553 responsive elements, respectively. A virus carrying the toxin but lacking the PY4 serves as a control virus. Those three constructs were cloned into a "first generation" $\Delta \mathrm{E} 1 / \Delta \mathrm{E} 3$ human type 5 adenoviral vector. Virus particles were produced, their titer was calculated by the End-Point Dilution Assay and their potency was tested in vitro. Cell death was measured qualitatively by using the fluorescent microscopy and was quantified by the enzymatic MTT assay. HCT116+/+;Rasmut/p53wt and HCT116-/-;Rasmut/p53 mut CRC cell lines, as well as, A549;Rasmut/p53wt and H2030 Rasmut/p53wt LC cells were used for testing the potency and safety of the system

Results: Massive cell death was induced in a dose-dependent manner $(\sim 60 \% ; 7.5$ MOI) in CRC cells harboring mutated Ras after co-infection with the toxin and the antitoxin viruses. The antidote was able to protect $\sim 70 \%$ of the cells that were affected by background low level expression of the toxin construct, due to promoter leakage [Fig1]. Similar results were obtained in a colony formation assay and FACS analysis of co-infected LC cells. A549 harboring mutated Ras showed greater sensitivity ( $\sim 82 \%$ early apoptotic cells) compared to H1650 cells harboring WT Ras ( $\sim 27 \%$ early apoptotic cells) [Fig2]. These results indicate that the antitoxin indeed actively protects cells with WT p53.

Conclusion: Exploiting the activated RAS pathway and mutation in p53 holds promise for effective and safe therapy that could specifically target tumor cells while sparing normal tissues.

Disclosure of Interest: N. Arber: Bayer Bio-view Gi-View Micro-medic Checkcap

All other authors have declared no conflicts of interest.

\section{P1770 USE OF A COMBINATION OF LENTIVIRUS PARTICLES AND A SPECIFIC PEPTIDE FOR ERADICATION OF CD24- EXPRESSING}

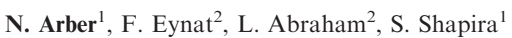

${ }^{1}$ Health Promotion Center And Integrated Cancer Prevention Center, Tel Aviv

Medical Center, Tel Aviv/Israel

${ }^{2}$ KAplan Medical Center, Hadassah-Hebrew University, Rehovot/Israel

Contact E-mail Address: nadira@tlvmc.gov.il
Introduction: Lentiviral replication is driven by a molecular motor consisting of the three viral enzymes: the reverse transcriptase, protease and integrase (IN). The genomic RNA of the virus is used to produce a copy of viral DNA by reverse transcription, and the integrase catalyses the covalent insertion of this DNA into the chromosomes of the infected cells. Integration of the viral DNA -which is flanked by LTR sequences- proceeds through a combination of cleavage and ligation resulting in the appearance of double-stranded breaks in the host genome that eventually leads to apoptosis. CD24 is a heavily glycosylated cellsurface GPI-anchored protein. We have previously shown that CD24 is an important player in the multistep process of GI carcinogenesis (Gastro 2006, Clin Can Res 2007, Can Res 2008) as well as in many other human malignancies (Cervical, Bladder, Esophageal squamous cell carcinoma, Glioma, Breast etc.). Aims \& Methods: We aimed to develop and specifically direct lentiviruses towards target cells for effective and selective eradication by stimulation of the viral integrase. Integrase derived peptides (designated as INS) were synthesized as well as control peptide. Humanized Anti-CD24 antibody fragment ( $\mathrm{scFv}$ ) was engineered and fused to the lentivirus envelope. Cell death was measured qualitatively by using fluorescent microscopy and was quantified by the enzymatic MTT assay. Human colorectal, pancreatic, lung and triple negative breast cancer cells were used for testing the potency of the lentiviral-based system.

Results: INS was able to stimulate the viral Integrase enzyme in test tubes and in viral infected cells. The anti-CD24 antibody fragment has a high affinity and specificity toward CD24, allowing targeted precision of viral transduction (Figure 1). These Lentivirus particles contain DNA molecules with flanked LTRs allowing their integration into the CD24-expressing target cells DNA and formation of double-strand breaks due to the action of the particles integrase whose activity was stimulated by the IN derived peptides. Massive cell death was induced upon exposure of the infected cells to the INS peptide compared to the control peptide.

Conclusion: The use of IN derived peptides together with the CD24-targeted lentitherapy approach suggest a novel strategy to specifically promote death of CD24-expressing cancer cells

Disclosure of Interest: N. Arber: Bayer Bio-view Gi-View Micro-medic Checkcap

All other authors have declared no conflicts of interest.

\section{P1771 ILF3 STABILIZES AND ACTIVATES EGFR-MEDIATED G9A PATHWAY FOR MAINTAINING CANCER STEMNESS PROPERTY IN EGFR-POSITIVE CANCERS}

\section{Cheng ${ }^{1}$, A. $\mathrm{Ho}^{2}$}

${ }^{1}$ Hematology And Oncology, Mackay Memorial Hospital, Taipei/Taiwan

${ }^{2}$ Division Of Gastroenterology, Cheng Hsin General Hospital, Taipei/Taiwan

Contact E-mail Address: aisheng49@gmail.com

Introduction: A specific inhibitor of interleukin enhancer binding factor 3 (ILF3), YM155, suppresses EGFR phosphorylation and significantly inhibits the formation of cancer stemness tumorspheres in vitro, suggesting that ILF3 as an oncogene participates in the maintaining of cancer stem cell property through stabilizes EGFR-mediated stemness pathway. Since cancer stemness cell is the leading reason for tumor recurrence in the tumor repressive treatments, and EGFR enhances the formation of cancer stemness, it is worthy of investigating the function of ILF3 for maintaining the cancer stemness property in the EGFRpositive cancers.

Aims \& Methods: The tumorspheres derived from EGFR-wild-type and KRASmutant colorectal HCT116 and lung A549 cells expressing higher cancer stemness markers, CD133, were used as cancer stemness models in this study. RNAseq was utilized to search for the putative genes involving in the formation of tumorspheres as the cancer stemness markers. Meanwhile, the differentiating stemness markers were also compared between ILF3-knockdowned and the control shLuc cells. Then, the protein level and phosphorylation of EGFR were investigated in the YM155-treated and ILF3-knockdowned cells for validating that EGFR was regulated and stabilized by ILF3. The ILF3-knockdowned cells were also transplanted into SCID mice for evaluating the function of ILF3 in vivo.

Results: We found that higher EGFR autophosphorylation (Y1068) in HCC116and A549-derived tumorspheres compared to the parental cells. The results of RNAseq evaluated that CD133 was a positive stemness marker, whereas MARCH4 as a negative marker. Knockdown of ILF3 reduced the cell proliferation in A549 cells in vitro and in vivo, demonstrating that ILF3 was an oncogene involving in cancer cell survival. Moreover, inhibition of ILF3 by YM155 blocked the autophosphorylation of EGFR and inhibited the EGFR-downstream G9a activation, leading to a reduction of stemness property. Moreover, Knockdown of G9a reduced the CD133 expression and increased MARCH4 expression, revealing that G9a was essential for maintaining for cancer stemness property in the EGFR-positive cancers.

Conclusion: In conclusion, this study demonstrated that ILF3 played an important role maintaining the EGFR-mediated cancer stemness property in HCC116 and A549 EGFR-positive cancer cells. We demonstrated that ILF3 stabilized and phosphorylated EGFR to enhance the activation of G9a, leading to increasing CD133 and decrease MARCH4 expressions. Therefore, we suggested that the ILF3 inhibitor, YM155, was potential for utilization in cancer therapy against the EGFR-positive cancers.

Disclosure of Interest: All authors have declared no conflicts of interest.

\section{References}

1. Na YS, Yang SJ, Kim SM, Jung KA, Moon JH, et al. (2012) YM155 induces EGFR suppression in pancreatic cancer cells. PLoS One 7: e38625. 
2. Cheng XJ, Lin JC, Ding YF, Zhu L, Ye J, et al. (2016) Survivin inhibitor YM155 suppresses gastric cancer xenograft growth in mice without affecting normal tissues. Oncotarget 7: 7096-7109.

3. Nakamura N, Yamauchi T, Hiramoto M, Yuri M, Naito M, et al. (2012) Interleukin enhancer-binding factor $3 / \mathrm{NF} 110$ is a target of YM155, a suppressant of survivin. Mol Cell Proteomics 11: M111 013243

4. Wang XK, He JH, Xu JH, Ye S, Wang F, et al. (2014) Afatinib enhances the efficacy of conventional chemotherapeutic agents by eradicating cancer stemlike cells. Cancer Res 74: 4431-4445.

5. Liu S, Ye D, Guo W, Yu W, He Y, et al. (2015) G9a is essential for EMTmediated metastasis and maintenance of cancer stem cell-like characters in head and neck squamous cell carcinoma. Oncotarget 6: 6887-6901.

\section{P1772 EFFICACY AND SAFETY OF TWELVE CHEMOPREVENTIVE REGIMENS FOR THE RECURRENCE OF COLORECTAL ADENOMAS: A NETWORK META-ANALYSIS \\ H. Chen \\ Jiangsu Procince Hospital, Nanjing/China}

Contact E-mail Address: chenhan068@hotmail.com

Introduction: Although various pharmacological agents have been trialed for recurrent colorectal adenomas, their comparative effectiveness remains unknown. We conducted both direct and indirect comparisons of twelve chemopreventive agents for recurrent colorectal adenomas.

Aims \& Methods: MEDLINE, EMBASE, Scopus, Web of Science, and Cochrane Central Register of Controlled Trials, and ClinicalTrials. gov were searched up to May 1, 2016. RCTs were assessed by a random-effects model within a Bayesian framework. Agents for each outcomes were ranked by surface under the cumulative ranking area (SUCRA). This study is registered with PROSPERO, number CRD42016041923.

Results: 33 RCTs were eligible, enrolling 44,647 participants treated by twelve regimens: 9 aspirin and other NSAIDs, 11 antioxidants, 4 dietary supplements, 3 clacium, 4 folic acid, 2 calcium plus antioxidants, 2 aspirin plus folic acid and 1 each metformin, ursodeoxycholic acid (UDCA), aspirin plus calcium with vita$\min \mathrm{D}$, and difluoromethylornithine (DMFO) plus sulindac. For recurrent colorectal adenomas, findings were significantly better for sulindac plus difluoromethylornithine than other tested agents. Aspirin/NSAIDs was more effective than placebo in both pairwise (OR, 0.73 [95\%CI, 0.59 to 0.90$]$ ) and NMA (OR, 0.75 [95\% CrI, 0.57 to 0.98$]$ ). Subgroup analysis showed the highest probability of aspirin $(<160 \mathrm{mg} / \mathrm{L})$ to be the most efficacious agent among al NSAIDs (SUCRA $=71.7 \%$ ). For safety profiles, the top three ranked agents were metformin $(86.7 \%)$, antioxidants $(82.0 \%)$ and dietary supplements $(65.9 \%)$, but none reached statistically significance when compared with placebo. Aspirin/NSAIDs performed the worst $(16.4 \%)$ with significantly more serious adverse events than placebo (pairwise: OR, 1.25 [95\%CI 1.14 to 1.38$]$ ); NMA (OR, $1.26[95 \%$ CrI 1.12 to 1.44$])$. Other regimens were not significantly different to each other in both pairwise and network comparisons, these agents include antioxidants, dietary supplements, calcium as well as folic acid.

Conclusion: For individuals with presumed increased risk of CRC, moderate-tolow dose aspirin and sulindac-associated combination therapy are both effective for recurrent colorectal adenomas. Future studies are required to provide more precise estimates of the optimal NSAIDs with an effective dose and low adverse events.We also suggest the further evaluation of NSAIDs-associated combination regimens and other novel agents (e.g.metformin)in the chemoprevention of $\mathrm{CRC}$

Disclosure of Interest: All authors have declared no conflicts of interest.

\section{References}

1. Torre LA, Bray F, Siegel RL, et al. Global Cancer Statistics, 2012. Ca Cancer J Clin 2015;65:87-108

2. Fearon ER. Molecular genetics of colorectal cancer. Annu Rev Pathol 2011;6:479-507.

3. Arber N, Levin B. Chemoprevention of colorectal cancer: ready for routine use? Recent Results. Cancer Res 2005;166:213-30.

4. Atkin WS, Saunders BP, et al. Surveillance guidelines after removal of colorectal adenomatous polyps. Gut. 2002;51 Suppl 5:V6-9.

5. Brand L, Munding J, Pox CP, et al. b-catenin, Cox-2 and p53 immunostaining in colorectal adenomas to predict recurrence after endoscopic polypectomy. Int J Colorectal Dis 2013;28:1091-1098.

\section{P1773 COX-2 MEDIATES RESISTANCE TO EICOSAPENTAENOIC ACID IN COLORECTAL CANCER CELLS}

M. Volpato, L. Coletta, M.A. Hull

Leeds Institute Of Biomedical And Clinical Sciences, University of Leeds, Leeds/ United Kingdom

Contact E-mail Address: m.volpato@leeds.ac.uk

Introduction: The omega-3 polyunsaturated fatty acid eicosapentaenoic acid (EPA) has anti-colorectal cancer (CRC) activity. Several mechanisms of action have been described, including lipid mediators metabolism and surface receptor signalling. Aspirin is a known cyclo-oxygenase (COX) inhibitor with cancer preventive properties.

Aims \& Methods: The aim of this study was to identify possible mediators of resistance to EPA in CRC cells. Human and mouse CRC cell lines were screened for their sensitivity to EPA by MTT assay in vitro $(\mathrm{n}=3)$. The MC38/MC38r and CT26 wt/COX2 CRISPR/Cas9 knocked out (COX2ko, gifted by Dr Zelenay, Manchester, UK) isogenic mouse CRC line pairs were used to further investigate the molecular basis of resistance to EPA. COX-1/2 protein expression was measured by western blot in vitro and by immunohistochemistry in vivo. Aspirin was used at a non-cytotoxic dose of $500 \mu \mathrm{M}$ to provide COX inhibition in combination studies $(\mathrm{n}=3)$. In vivo studies were performed using female CD1-nude mice, fed diets supplemented with 7\%(v/w) control corn oil, 6.1\%(v/w) EPA-triglyceride oil $+0.9 \%(\mathrm{v} / \mathrm{w})$ control oil, $600 \mathrm{ppm}$ aspirin or a combination of both EPA and aspirin $(\mathrm{n}=14)$ for 4 weeks. After the first 2 weeks, $5 \times 10^{6}$ MC38 and MC38r cells were injected as subcutaneous tumours on the right flank, before sacrifice 2 weeks later. Tumour volumes were measured by calipers.

Results: The human and mouse CRC cell lines tested displayed varying levels of sensitivity to EPA ( IC $_{50}$ range: $36 \pm 1 \mu \mathrm{M}$ to $242 \pm 2 \mu \mathrm{M}$ ). HT29 cells were sensitive human CRC cells $\left(\mathrm{IC}_{50}=89 \pm 1 \mu \mathrm{M}\right)$ and $\mathrm{CaCo} 2$ cells were the least sensitive $\left(\mathrm{IC}_{50}=228+1 \mu \mathrm{M}\right)$. In particular, MC38 mouse CRC cells were the most sensitive to EPA $\left(\mathrm{IC}_{50}=36 \pm 1 \mu \mathrm{M}\right)$ of all cell lines tested, whilst isogenic MC38r cells were 3.9-fold more resistant $\left(\mathrm{IC}_{50}=163 \pm 1 \mu \mathrm{M}\right)$. We found a strong increase in COX1 and COX2 protein expression in EPA-resistant MC38r compared to MC38 cells. Genetically modified CT26 COX2ko cells were used to assess whether COX2 specifically played a role in EPA resistance. Indeed, CT26 COX2ko cells were 3 times more sensitive to EPA $\left(\mathrm{IC}_{50}=78 \pm 1 \mu \mathrm{M}\right)$ than parental CT26 cells ( $\mathrm{IC}_{50}=242 \pm 2 \mu \mathrm{M}$, high $\mathrm{COX} 2$ expression). Aspirin was then evaluated as a potential combination treatment with EPA. A decrease in $\mathrm{IC}_{50}$ values for EPA was observed in 8 out 10 CRC cell lines tested (1.1-fold to 4 -fold increase in sensitivity to EPA). Whilst the response of MC38 cells to EPA was increased 2-fold $\left[\mathrm{IC}_{50}(\mathrm{EPA}+\right.$ aspirin $\left.)=19 \pm 1 \mu \mathrm{M}\right], \mathrm{MC} 38 \mathrm{r}$ cell response was increased 3.8-fold $\left[\mathrm{IC}_{50}(\mathrm{EPA}+\right.$ aspirin $\left.)=42 \pm 1 \mu \mathrm{M}\right]$. Aspirin increased CT26 wt response to EPA $\left[\mathrm{IC}_{50} \quad(\mathrm{EPA})=242 \pm 1 \mu \mathrm{M}\right.$ compared with $\mathrm{IC}_{50}$ $(\mathrm{EPA}+$ aspirin $)=99 \pm 1 \mu \mathrm{M}]$ but did not affect the EPA response of CT26 COX2ko cells $\left[\mathrm{IC}_{50}(\mathrm{EPA})=78 \pm 1 \mu \mathrm{M}\right.$ and $\mathrm{IC}_{50}(\mathrm{EPA}+$ aspirin $\left.)=84 \pm 1 \mu \mathrm{M}\right]$. We measured EPA incorporation in these cells and observed an increased accumulation of EPA in CT26wt cells treated with both EPA and aspirin compared to EPA alone. A similar pattern of response was measured in vivo. EPA administration exerted anti-tumour activity in MC38 subcutaneous tumour-bearing CD1-nude mice $(n=14,37 \%$ decrease in mean tumour volumes, $\mathrm{p}=0.02)$, which was not augmented by combination with aspirin. However, EPA alone did not significantly impact on tumour volumes in EPA-resistant MC38r tumour bearing mice whilst the EPA-aspirin combination was associated with a $42 \%$ reduction in tumour volume compared with control $(\mathrm{n}=14, \mathrm{p}=0.003)$. Administration of aspirin, alone or in combination with EPA, did not alter COX $1 / 2$ protein expression in tumour tissues.

Conclusion: Human and mouse CRC cells display differential sensitivity to EPA in vitro. Our data indicate that $\mathrm{COX} 2$ mediates resistance to EPA in CRC cells by a mechanism that likely involves EPA substrate utilisation, thereby reducing EPA levels. Aspirin combination sensitises CRC cells to EPA in vitro and in vivo and may be a clinically relevant candidate for prevention and treatment of CRC in combination therapy.

Disclosure of Interest: All authors have declared no conflicts of interest.

\section{P1774 PROGNOSTIC ROLE OF GLASGOW PROGNOSTIC SCORE IN PATIENTS WITH COLORECTAL CANCER: EVIDENCE FROM POPULATION STUDIES}

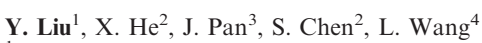

${ }_{1}^{1}$ Institute of Gastroenterology, Hangzhou/China

${ }^{2}$ Department of Gastroenterology, Sir Run Run Shaw Hospital, Zhejiang University Medical School, Hangzhou, China, Hangzhou/China

${ }^{3}$ Department Of Endocrinology And Metabolism, Second Affliated Hospital of

Zhejiang University School of Medicine, Hangzhou/China

${ }^{4}$ The Second Affiliated Hospital, School of Medicine, Zhejiang University,

Hangzhou/China

Contact E-mail Address: liu_chit@163.com

Introduction: Colorectal cancer (CRC) is the third most common cancer worldwide and accounts for $10 \%$ of all newly diagnosed cancers 1 . Although the therapies have dramatically developed, the long-term survival rate of patients with CRC remains low. In recent years, great efforts have been made to identify inflammation-related factors for precise prediction of disease prognosis. Glasgow Prognostic Score (GPS) is such an inflammation-based factor, defined by the combination of the level of serum C-reactive protein (CRP) and albumin, which are indicators of systematic inflammatory response and nutritional status respectively. Growing evidence suggested that GPS was served as an independent prognostic index in a variety of malignant cancers. For patients with CRC, the GPS system was also widely studied, but the results were controversial.

Aims \& Methods: To investigate the correlation between GPS and prognosis of patients with $\mathrm{CRC}$ to further clarify its clinical significance. A comprehensive search of Pubmed, Embase, Cochrane library, Web of Science, ChinaInfo and Chinese National Knowledge Infrastructure was performed to identify eligible studies, from which the risk of overall survival (OS) and cancer-specific survival (CSS) were extracted. A random-effect model was adopted to combine hazard ratio (HR) and $95 \%$ confidence interval (CI). Heterogeneity and publication bias among studies were assessed.

Results: 25 articles with a total of 5660 participants were included. The pooled results indicated that elevated GPS was associated with poor OS $(\mathrm{HR}=2.83$, $95 \% \mathrm{CI}: \quad 2.00-4.00, \quad \mathrm{P}<0.01)$ and $\mathrm{CSS}(\mathrm{HR}=1.94,95 \% \mathrm{CI}: \quad 1.51-2.49$, $\mathrm{P}<0.01)$. This correlation was confirmed both in primary operable and advanced inoperable patients. Increased GPS was also closely related to advanced tumour-node-metastasis (TNM) stage (odds ratio $[\mathrm{OR}]=1.44,95 \%$ 
CI: $1.010-2.065, \mathrm{P}<0.05)$ and elevated level of serum carcinoembryonic antigen $(\mathrm{OR}=2.252,95 \% \mathrm{CI}: 1.508-3.362, \mathrm{P}<0.01)$. Subgroup analysis revealed a significant association between high GPS and poor survival outcome according to the factors of sample size, study of region and cut-off value of GPS level.

Conclusion: GPS could serve as a reliable predictive index for patients with CRC. Disclosure of Interest: All authors have declared no conflicts of interest.

\section{References}

1. Global Burden of Disease Cancer Collaboration, et al. The global burden of cancer 2013. JAMA Oncol 1, 505-27 (2013).

2. Grivennikov, S.I., Greten, F.R. \& Karin, M. Immunity, inflammation, and cancer. Cell 140, 883-899 (2010).

3. McMillan, D.C. The systemic inflammation-based Glasgow Prognostic Score: a decade of experience in patients with cancer. Cancer Treat Rev 39, 534-540 (2013).

4. Roxburgh, C.S., McMillan, D.C. Cancer and systemic inflammation: treat the tumour and treat the host. Br J Cancer 110,1409-1412 (2014)

5. Ishizuka, M. et al. Impact of an inflammation-based prognostic system on patients undergoing surgery for hepatocellular carcinoma: a retrospective study of 398 Japanese patients. Am J Surg 203, 101-106 (2012).

6. Vashist, Y.K. et al. Glasgow Prognostic Score is a predictor of perioperative and long-term outcome in patients with only surgically treated esophageal cancer. Ann Surg Oncol 18, 1130-1138 (2011).

7. Lamb, G.W., Aitchison, M., Ramsey,.S, Housley, S.L. \& McMillan, D.C. Clinical utility of the Glasgow Prognostic Score inpatients undergoing curative nephrectomy for renal clear cell cancer: basis of new prognostic scoring systems. Br J Cancer 106, 279-83 (2012).

8. Torre, M.L. et al. The Glasgow Prognostic Score as a predictor of survival in patients with potentially resectable pancreatic adenocarcinoma. Ann Surg Oncol 19, 2917-2923 (2012).

9. Son, H.J., et al. Preoperative plasma hyperfibrinogenemia is predictive of patients with nonmetastatic colon cancer. Annals of surgical oncology 20, 2908-2913 (2013)

\section{P1775 COST EFFECTIVENESS OF THE FIRST SURVEILLANCE COLONOSCOPY IN POPULATION WITH ADVANCED COLORECTAL POLYPS OR MULTIPLE POLYPS FROM COLORECTAL CANCER SCREENING PROGRAM}

L. Carot $^{1}$, M. González ${ }^{1}$, M. Batlle ${ }^{1}$, A. Buron ${ }^{2}$, X. Bessa Casserras ${ }^{2}$, M. Andreu Garcia $^{2}$, A. Álvarez-Urturi ${ }^{1}$

${ }^{1}$ Gastroenterology, Hospital del Mar, Barcelona/Spain

${ }^{2}$ Hospital del Mar, Barcelona/Spain

Contact E-mail Address: 1.carot.lc@parcdesalut.mar

Introduction: The implementation of the CRC screening program has generated an increase in surveillance colonoscopies. However, the intermediate-high risk group that included advanced lesions (size $\geq 10 \mathrm{~mm}$, villous component or high grade dysplasia) or the presence of 3 or more polyps, has a low incidence of metachronous risk lesions when performing colonoscopy at 3 years according to the current recommendations. Identifying predictors of metachronous lesions would provide a better risk stratification and improve the efficiency of surveillance programs.

Aims \& Methods: We aimed to identify the cost effectiveness of the first surveillance colonoscopy and the predictive factors of metachronous lesions at 3 years in individuals with advanced lesions or $\geq 3$ polyps detected at baseline screening colonoscopy. This was an analysis of all cases with advanced polyps and/or multiplicity from CRC screening program population of Barcelona detected at baseline colonoscopy during the years 2010-2011 and with a performed colonoscopy after 3 years. Epidemiological and clinical data of all individuals were collected as well as the morphological data of all polyps. For the statistical study, a bivariate analysis and logistic regression were performed.

Results: 638 cases were identified, with mean age of 64 years. 342 were men $(66.2 \%) .23 .8 \%$ required more than one colonoscopy for the complete removal all the polyps. A complete surveillance colonoscopy at 3 years was performed in 518 cases $(82 \%)$ with an average surveillance time of 38 months [15-75]. Mean fecal hemoglobin was $440 \mathrm{ng} / \mathrm{ml}$. $51.8 \%$ suffered from hypertension, $15 \%$ from diabetes mellitus, $46.5 \%$ from dyslipidemia and $12.3 \%$ from chronic obstructive pulmonary disease. $45.8 \%$ of individuals were overweight (BMI $\geq 25)$ and $34.7 \%$ were obese $(\mathrm{BMI} \geq 30)$. Surveillance colonoscopy was normal or with low-risk polyps in 420 cases $(80.1 \%)$; and advanced polyps or multiplicity were identified in 98 cases $(18.9 \%) 73$ advanced adenoma in 59 cases $(11.4 \%), \geq 3$ adenomas in 62 cases $(11.9 \%), \geq 3$ adenomas and/or serrated in 71 cases $(13.7 \%)$. The presence of $\geq 3$ adenomas and/or serrated polyps was the only variable that was associated with increased risk of the diagnosis of advanced adenomatous or serrated lesions in surveillance colonoscopy $(p<0.001)$

Conclusion: In individuals with advanced polyps and/or multiplicity the incidence of metachronous risk lesions at 3 years is low. Assessment a quality baseline colonoscopy with complete removal of all the polyps could allow to increase the interval of surveillance, maintaining and ensuring the compliance of the surveillance at 3 years in the cases with multiplicity in the basal colonoscopy Disclosure of Interest: All authors have declared no conflicts of interest.
P1776 OUTCOMES OF ENDOSCOPIC SUBMUCOSAL

DISSECTION FOR COLORECTAL LESIONS: A SINGLE EUROPEN CENTER EXPERIENCE

R. Maselli ${ }^{1}$, P.A. Galtieri ${ }^{1}$, L. Lamonaca ${ }^{1}$, M. Di Leo ${ }^{1}$, R. Semeraro ${ }^{2}$, L. Poliani ${ }^{1}$, F. Auriemma ${ }^{1}$, A. Fugazza ${ }^{2}$, G. Lollo ${ }^{2}$, G. Amvrosiadis ${ }^{1}$, E.C. Ferrara ${ }^{2}$, A. Anderloni ${ }^{2}$, S. Carrara ${ }^{1}$, A. Repici ${ }^{1}$

${ }^{1}$ Humanitas Research Hospital, Milan, Italy., Rozzano/Italy

${ }^{2}$ Humanitas Researc Hospital, Rozzano/Italy

Contact E-mail Address: roberta.maselli@humanitas.it

Introduction: Colorectal Endoscopic Submucosal Dissection (ESD) is recommended for superficial neoplastic lesions at high risk of submucosal invasion. ESD allows an en-bloc resection but ESD experience is still limited in Western countries.

Aims \& Methods: The aim of this retrospective study was to evaluate technical and clinical outcomes of colorectal ESD, in a single tertiary European center. We retrospectively analyzed all consecutive patients treated by ESD for colorectal lesion at Humanitas Research Hospital (Milan, Italy) from January 2011 to September 2016. The primary outcomes were technical success, defined as enbloc resection and clinical success, defined as curative resection (R0) without need for surgery. Secondary study outcomes were complication rate and adenoma/carcinoma recurrence. Complications were divided in early ( $\leq 24$ hours) and delayed ( $>24$ hours) and included bleeding and perforation. Recurrences were identified as the presence of adenoma or carcinoma at the endoscopic follow-up performed at 6 months, 1,3 and 5 years. Data were analyzed by STATA 14 statistical software.

Results: A total of 185 lesions in 185 patients (M/F 97/59, mean age $67.6 \pm 11.5$ ). Lesions were located in the rectum $(64.3 \%)$, left colon $(9.7 \%)$, transverse $(11.9 \%)$ and right colon $(14 \%)$. Mean lesion size was $39.3 \pm 16.7 \mathrm{~mm}$. Histology revealed low-grade dysplasia (LGD) in $64(34.6 \%)$, high-grade dysplasia (HGD) in 78 $(42.2 \%)$ and adenocarcinoma in $42(22.7 \%)$ cases. Only 1 NET $(0.5 \%)$ was found. En bloc resection was achieved in $88 \%$ of lesions. Clinical success (R0) was obtained in $84.8 \%$ of patients. The remaining 28 patients $(15.2 \%)$ needed surgery for unfavorable histology. A total of 12 complications were reported: 10 early complications ( 8 perforations and 2 bleeding) and 2 delayed complications ( 1 bleeding and 1 perforation). All but one adverse events were managed endoscopically. Surgery was required in the only case of delayed perforation. All patients untreated with surgery $(\mathrm{n}=156)$ underwent follow-up evaluation. In particular, all patients had 6 months follow up, 97 patients had 1 y follow-up, 26 patients had 3 y follow-up, 9 patients had 5 y follow-up. Adenoma recurrences were found in $3.8 \%$ at 6 months and $2 \%$ at 1 year. We did not find any adenoma recurrence at 3 and 5 years. No cancer recurrence occurred in the follow-up period.

Conclusion: ESD is a feasible strategy to manage superficial colorectal tumors. This study demonstrates favorable technical and short-term clinical outcome of colorectal ESD, but further studies are needed to confirm the long-term efficacy. Disclosure of Interest: All authors have declared no conflicts of interest.

\section{P1777 POPULATION-BASED COLORECTAL CANCER SCREENING IN THE CZECH REPUBLIC-FIRST RESULTS OF THE NEW PROGRAM SETTINGS}

S. Suchanek ${ }^{1}$, T. Grega ${ }^{1}$, O. Majek ${ }^{2}$, O. $\mathrm{Ngo}^{2}$, B. Seifert ${ }^{3}$, L. Dusek ${ }^{2}$, M. Zavoral

${ }^{1}$ Department Of Gastroenterology, Military University Hospital, Prague/Czech Republic

${ }^{2}$ Institute Of Biostatistics And Analyses, Masaryk University, Faculty of Medicine, Brno/Czech Republic

${ }^{3}$ Department Of General Practice, Department of GP First Faculty of Medicine Charles University, Prague/Czech Republic

Contact E-mail Address: stepan.suchanek@uvn.cz

Introduction: The organized non-population based National Colorectal Cancer (CRC) Screening Program in the Czech Republic has been running since year 2000. In January 2014, the transition to population based setting has been implemented. Currently, the annual immunochemical FOBT (FIT) is offered at the age $50-54$, followed by FIT + colonoscopy, if positive. In age of 55, there is a choice of either FIT biannually or screening colonoscopy in 10 years' interval.

Aims \& Methods: Main aim was to assess the impact of the first 30 months of the population-based CRC screening program on the target population participation and colorectal neoplasia detection. The data from National Reference Center (health insurance companies database) and Preventive Colonoscopies Registry in years 2013 (non-population based settings) and 2014-2015 (population-based settings) were evaluated and compared.

Results: In year 2014, there were 1,500,897 individuals (53\% of all CRC screening target population) invited to FIT or colonoscopy, with $17.3 \%$ response. It resulted in the coverage increase by 4.3 percent points in comparison with year 2013 (31.5\% in year 2014). This trend continued in year 2015 with the target population participation at $33.1 \%$. This has influenced the increase of number of detected adenomas (by $42 \%$ ) and cancers (by $20 \%$ ) detection in year 2014. In year 2015 , all the results remained stable (detailed results in the table). 


\begin{tabular}{llllll}
\hline Year & $\begin{array}{l}\text { Colonoscopy } \\
\text { (numbers) }\end{array}$ & $\begin{array}{l}\text { Adenomas } \\
\text { (numbers) }\end{array}$ & Ratio & $\begin{array}{l}\text { Cancers } \\
\text { (numbers) }\end{array}$ & Ratio \\
\hline 2013 & 26940 & 10126 & $37.6 \%$ & 867 & $3.2 \%$ \\
2014 & 38128 & 14416 & $37.8 \%$ & 1042 & $2.7 \%$ \\
2015 & 37330 & 14081 & $37.7 \%$ & 969 & $2.6 \%$ \\
\hline
\end{tabular}

Conclusion: The transition to population-based program resulted in the improvement of target population participation followed by increase in colorectal neoplasia detection.

Disclosure of Interest: All authors have declared no conflicts of interest.

Supported by the projects MO1012 and PRVOUK-P27/LF1/1

\section{P1778 LONG-TERM OUTCOMES OF ENDOSCOPIC \\ SUBMUCOSAL DISSECTION FOR EARLY CANCER AND HIGH GRADE DYSPLASIA IN COLORECTUM \\ P. Zhou ${ }^{1}$, T. Chen ${ }^{1}$ \\ ${ }^{1}$ Endoscopy Center, Zhongshan Hospital, Fudan University, Shanghai/China \\ Contact E-mail Address: chentao66@yahoo.com}

Introduction: Although endoscopic submucosal dissection (ESD) is a widely accepted treatment for colorectal neoplasia, little is known about large consecutive studies evaluating long-term outcomes of early cancer and high grade dysplasia. We investigated the efficacy and safety of ESD for early cancer and high grade dysplasia in colorectum and evaluated the long-term outcomes, including local recurrence and metastasis.

Aims \& Methods: We performed a retrospective analysis of data collected from 514 consecutive patients with 520 colorectal early cancer and high-grade dysplasia treated with ESD between January 2007 and December 2013. Histology and patient data were collected during an average follow-up time of more than 5 years to determine tumor stage and type, resection status, complications, tumor recurrence, and distant metastasis.

Results: The overall rates of en bloc resection, complete resection, R0 resection, major complications were $94.4 \%, 91.3 \%, 89.2 \%$ and $2.1 \%$, respectively. Large tumors and snare-assisted ESD were independent factors of piecemeal resection. ESD of colon tumors increased the risk for complications. During the follow-up period, all patients remained free from metastasis. However, local recurrence occurred in 4 patients $(0.8 \%)$; large tumors and piecemeal resection were risk factors.

Conclusion: ESD is effective and safe for resection of early cancer and high grade dysplasia in colorectum and long-term outcomes are favorable. ESD is indicated for the treatment of colorectal early cancer and high grade dysplasia to obtain curative resection and prevent the local recurrence.

Disclosure of Interest: All authors have declared no conflicts of interest.

\section{P1779 LOW UPTAKE OF PSYCHOLOGICAL THERAPIES AMONG} PATIENTS WITH IRRITABLE BOWEL SYNDROME IN SECONDARY CARE

O. Craig ${ }^{1}$, C. Black ${ }^{1}$, L. Houghton ${ }^{2}$, A.C. Ford ${ }^{1}$

${ }^{1}$ Leeds Gastroenterology Institute, St. James's University Hospital, Leeds/United Kingdom

${ }^{2}$ Leeds Institute Of Biomedical \& Clinical Sciences, St.James's University

Hospital, Leeds/United Kingdom

Contact E-mail Address: ofcraig@gmail.com

Introduction: Patients with irritable bowel syndrome (IBS) often have co-existent mood disorder and psychological illness. Meta-analyses of randomised controlled trials consistently demonstrate that psychological therapies, such as cognitive behavioural therapy (CBT) and hypnotherapy, are effective treatments for IBS. In the UK the National Institute for Health and Care Excellence (NICE) recommends considering the use of these in patients with no response to pharmacological therapies, and for refractory symptoms.

Aims \& Methods: We performed a cross-sectional survey to examine willingness of patients with IBS to engage with psychological therapies. We collected complete symptom data from consecutive, unselected referrals to secondary care seen in a specialist IBS clinic. All participants completed the validated Rome IV questionnaire for IBS, the IBS severity scoring system (IBS-SSS), the hospital anxiety and depression scale (HADS) to assess mood, and the patient health questionnaire-12 (PHQ-12) to examine somatoform-type behaviour. They also provided their opinion on possible treatment options, and were asked to rank medical therapies, dietitian input, psychological therapies, including CBT and hypnotherapy, and explanation of the condition and/or reassurance in order of preference.

Results: Among 93 adults with confirmed IBS (74 (79.6\%) female, mean age 36.0 years (range 16 to 77 years)), $35(37.6 \%)$ had high levels of anxiety, $22(23.7 \%)$ had high levels of depression, and $23(24.7 \%)$ had severe levels of somatoformtype behaviour. Despite this, only $10(10.8 \%)$ of 93 patients ranked psychological therapies as their first-choice treatment option. In total, $8.8 \%$ of patients with high levels of anxiety ranked psychological therapies as their first-choice treatment option, versus $13.2 \%$ without $(\mathrm{P}=0.64), 9.5 \%$ of those with high levels of depression, versus $12.1 \%$ of those without $(\mathrm{P}=0.91)$, and $17.4 \%$ of patients with severe somatoform behaviour, versus $9.2 \%$ of those without $(P=0.26)$. Those with severe symptoms according to the IBS-SSS were no more likely to select psychological therapies as their first-choice treatment option than those with mild or moderate symptoms $(7.7 \%$ versus $21.7 \%, \mathrm{P}=0.10)$

Conclusion: Despite high levels of psychological comorbidity and NICE recommendations, patients with IBS in a specialist clinic were generally reluctant to consider psychological therapies such as CBT or hypnotherapy. Those with anxiety, depression, somatoform-type behaviour, or severe symptoms were no more willing to consider these therapies than those without.

Disclosure of Interest: All authors have declared no conflicts of interest.

\section{P1780 GUT SYMPTOMS AND TRANSIT DISTURBANCE IN PARKINSON'S DISEASE ARE PAN-ENTERIC BUT NOT UBIQUITOUS: A WIRELESS MOTILITY CAPSULE STUDY}

V. Passananti, N. Zarate Lopez, R. Sweis, A. V. Emmanuel

GI Physiology Unit, University College London Hospital, London/United Kingdom

Contact E-mail Address: valentinapassananti@gmail.com

Introduction: Symptoms of gastrointestinal dysfunction are among the most common non-motor complaints in Parkinson's patients. These may involve muscles from the oropharynx to the anorectum, and the autonomic and enteric nervous system are often involved, resulting in secondary bowel dysmotility.

Aims \& Methods: The objectives of this study were to evaluate a technology measuring the spectrum of gut dysfunction, the Wireless Motility Capsule (WMC), in Parkinson's disease. We also wanted to correlate transit measures with gastrointestinal symptoms. Fifteen PD patients and 7 controls (table1) were included. PD severity were scored with the modified Hoehn and Yahr (H\&Y) staging scale. GI symptom burden was identified by Wexner constipation score and Gastroparesis Cardinal Symptom Index (GCSI). Acidity, motility and transit data were obtained, as standard, by WMC. All medications affecting $\mathrm{pH}$ and motility, including L-dopa, were discontinued for 5 days before and for the duration of the WMC study. Were analyzed data about gastric emptying time (GET), small bowel transit time (SBTT), colonic transit time (CTT) and whole gut transit time(WGTT).

Results: One patient could not swallow the capsule, and of the 14 patients completing the study, 8 reported GI symptoms. Compared to non-symptomatic patients, those with GI symptoms showed significant delayed transit in the stomach, colon and whole gut (table 1). However, small bowel transit did not significantly differ. GI dysfunction was not correlated with H\&Y score in this small study. GCSI and Wexner constipation scores were correlated, suggesting a pan-enteric problem in symptomatic individuals. There was a significant correlation between the Wexner constipation score and CTT in all patients $(\mathrm{p}<0.01)$ but not GCSI and GET $(\mathrm{p}>0.10)$. The results of Wireless Motility Capsule did not differ between non-symptomatic PD and controls.

\begin{tabular}{llll}
\hline & $\begin{array}{l}\text { Non } \\
\text { symptomatic } \\
\text { PD }(\mathrm{n}=6)\end{array}$ & $\begin{array}{l}\text { Symptomatic } \\
\text { PD }(\mathrm{n}=8) \text { [p value vs } \\
\text { Non symptomatic PD] }\end{array}$ & $\begin{array}{l}\text { Controls }(\mathrm{n}=7) \\
\text { [p value vs Non } \\
\text { symptomatic PD] }\end{array}$ \\
\hline Sex (M:F) & $5: 1$ & $6: 2$ & $5: 2$ \\
Age & $61.2 \pm 10.9$ & $69.6 \pm 13.4[0.2744]$ & $56.8 \pm 13.1[0.0924]$ \\
GCSI & $3.3 \pm 1.5$ & $22.6 \pm 9.6[0.0017]$ & $2.4 \pm 1.4[0.5704]$ \\
Wexner & $2.2 \pm 2.0$ & $14.3 \pm 3.2[0.0003]$ & $2.1 \pm 3.0[0.6428]$ \\
GET & $2.74 \pm 1.05$ & $5.18 \pm 0.58[0.0007]$ & $2.58 \pm 1.14[0.4713]$ \\
SBTT & $4.08 \pm 0.71$ & $4.17 \pm 0.34[0.7193]$ & $4.10 \pm 0.40[0.7294]$ \\
CTT & $27.44 \pm 7.89$ & $57.82 \pm 30.61[0.0426]$ & $28.3 \pm 6.90[0.5156]$ \\
\hline
\end{tabular}

Conclusion: We have shown that Parkinson's patients with gut symptoms have both upper and lower complaints. Symptomatic PD patients also have markedly delayed transit times throughout the whole gut compared to asymptomatic PD patients and controls. Whilst severity of constipation is related to delayed colonic transit no such relationship is present between gastroparesis symptoms and gastric emptying. The implication is that treating symptomatic Parkinson's patients should address the whole gut, whether with prokinetics or dual therapy. Disclosure of Interest: All authors have declared no conflicts of interest.

\section{P1781 OUTLET DYSFUNCTION IS PREVALENT IN SEVERE FUNCTIONAL BLOATING: PRELIMINARY REPORT FROM A MULTICENTER ITALIAN STUDY}

P. Iovino ${ }^{1}$, M.C. Neri ${ }^{2}$, L. D'Alba ${ }^{3}$, S. Gallotta ${ }^{1}$, G. Chiarioni ${ }^{4}$

${ }^{1}$ Medicine And Surgery, University of Salerno, Baronissi, SA/Italy

${ }^{2}$ Istituto Pio Albergo Trivulzio, Milan/Italy

${ }^{3}$ Gastroenterology And Digestive Endoscopy, Azienda Ospedaliera San Giovanni Addolorata, Rome/Italy

${ }^{4}$ Gastroenterology, AOUI Verona - Gastroenterology, AOUI Verona; Verona/IT, Verona/Italy

Contact E-mail Address: piovino@unisa.it

Introduction: Bloating and abdominal distension are common and bothersome symptoms and a frequent complaint of patients affected by functional gastrointestinal disorders (FGID). Recent studies demonstrated that an impairment in 
the handling of gas is a relevant underlining mechanism in FGID patients with bloating. However, solid data on defecation pattern in these patients are lacking. Aims \& Methods: Our aim is to study the relationship between the defecation pattern, the severity of bloating and the abdominal girth measurements in FGID patients consulting for bloating as primary complain with/without visible abdominal distension unresponsive to diet advise. We performed a prospective, multi-center study of patients with severe abdominal bloating (VAS score $\geq 24$ on a $100-\mathrm{mm}$ scale) as primary complain with/without visible abdominal distension. Patients were recruited at 4 gastroenterology outpatient clinics in Italy. Comorbid FGID were grouped according to Rome III criteria. All patients were prescribed a lactose-free diet supplemented by dietary advice according to the NICE guidelines for two weeks. A belt around the abdomen at standardized sites provided assessment of abdominal girth measurements. During the 2-week run-in period patients completed a daily diary log including abdominal bloating and pain/discomfort scores (100-mm VAS), Bristol Stool Form and stool frequency. At randomization visit, all patients filled in a questionnaire on adequate relief of bloating on a Likert scale and a further abdominal bloating 100-mm VAS. A belt around the abdomen at standardized sites provided assessment of abdominal girth two hours after a meal. All patients reporting insufficient adequate relief of abdominal bloating at the end of the run-in period underwent a standardized balloon expulsion test (BET) scored as either successful or failed. A straining questionnaire was also administered.

Results: 76 patients ( 66 female, $39.8 \pm 12.2$ mean age, 6 IBS-D, 6 IBS-M, 30 IBSC, 9 IBS-U, 6 FC, 16 FB, 3 FD) completed the 2-week run-in period. A significant negative correlation was found between adequate relief and both bloating and abdominal girth changes $(r=-.53$ and $-.52, \mathrm{p}<0.001$, respectively). 53/76 $(70 \%)$ patients reported inadequate relief (worse or no improvement). Among the non-responders the vast majority $(68 \%)$ failed the BET. Multiple regression analysis showed that BET (successful or failed), as dependent variable, was significantly related to bloating severity. No relationship was demonstrated for abdominal girth changes, FGID diagnosis and straining questionnaire.

Conclusion: In this prospective, multicenter trial simple diet advise was of benefit in approximately $30 \%$ of FGID patients consulting for severe bloating. In the non-responders outlet dysfunction was prevalent and correlated with subjective bloating perception. The study is ongoing, but our data may support bowel retraining as potential treatment option for functional bloating.

Disclosure of Interest: S. Gallotta: Serena Gallotta received research funding from Fondazione Torsoli Premio Irene Habib premio.habib@fondazionetorsoli.it

All other authors have declared no conflicts of interest.

\section{References}

Azpiroz F, Malagelada JR. Abdominal bloating. Gastroenterology. 2005 Sep;129(3):1060-78

Chiarioni G, Kim SM, Vantini I, Whitehead WE. Validation of the balloon evacuation test: reproducibility and agreement with findings from anorectal manometry and electromyography. Clin Gastroenterol Hepatol. 2014;12:2049-54

P1782 PATHOPHYSIOLOGY ASSESSMENT OF FECAL INCONTINENCE AND RISK FACTORS ASSOCIATED. RESULTS OF A TEN YEARS RETROSPECTIVE STUDY

L. D’Alba ${ }^{1}$, E. Ribichini ${ }^{2}$, R. Urgesi ${ }^{1}$, M. A. Vitale ${ }^{1}$, L. Pallotta ${ }^{1}$, M. C. Di Paolo $^{1}$, G. Villotti ${ }^{1}$, A. De Cesare ${ }^{1}$, S. Cocca ${ }^{3}$, P. Zaccari ${ }^{4}$, M. G. Graziani ${ }^{1}$ Gastroenterology And Digestive Endoscopy, Azienda Ospedaliera San Giovann Addolorata, Rome/Italy

${ }^{2}$ Gastroenterology, Department Of Internal Medicine And Medical Specialties, Sapienza University Of Rome, Policlinico Umberto I, Rome, Italy, Rome/Italy ${ }^{3}$ Campus Bio Medico University, Roma/Italy

${ }^{4}$ Gastroenterology, Policlinico Umberto I, Rome, Italy, Rome/Italy

\section{Contact E-mail Address: emanuela.ribichini@gmail.com}

Introduction: Faecal Incontinence (FI) is a common and socially disabling condition, more prevalent among females over 50 years old. Detailed anatomical and physiological assessment of each patient is important to determine the correct cause of FI and selection the most appropriate therapy. Conventional and High Resolution (HR) Anorectal Manometry (ARM) is a useful tool to categorize anal and/or rectal dysfunction in addition to provide physiological assessment of both anal sphincters and rectum.

Aims \& Methods: To evaluate symptoms and anorectal function of patients affected by FI, we included 358 patients with FI (77\% female (F) and $23 \%$ men (M), mean age 63 (range 22-92 year) referring to the outpatient unit of Digestive Pathophysiology of S. Giovanni-Addolorata Hospital, Rome from January 2006 to Dicember 2016. Clinical presentation (history, symptom profile and severity) and anorectal physiological evaluation (digital examination, manometry, rectal sensory testing, balloon evacuation test) were analyzed. The manometric parameters obtained with conventional and HR-ARM were: resting pressure, squeeze pressure, rectal compliance, rectal sensibility and the anorectal pattern during the defecatory maneuvers.

Results: 114 out of 358 patients (32\%) reported both FI and difficulty evacuating stools. 168 with FI had also urinary incontinence UI $(47 \%)$. Proctological surgery (n $122,34 \%$ ), pelvic surgery (n $77,21 \%$ ) and traumatic anal or vaginal history (n 144, 40\%) were statistically associated with FI $(p \leq 0.05)$. Normal manometric parameters were found in 16 patients $(4 \%)$. Manometric alterations observed were: internal anal sphincter (IAS) dysfunction: $228(64 \%)$; isolated external anal sphincter (EAS) dysfunction: $274(76 \%)$; combined sphincter dysfunction IAS and EAS: $198(55 \%)$; isolated dyssynergic defecation: $100(28 \%)$; rectal hypersensitivity: $130(36 \%)$.
Conclusion: In our study, in accordance with the literature, we observed a female prevalence in FI. FI is significantly associated with UI. This may be expired by risk factors in common with these clinical conditions. In effect FI is significantly associated with previous proctological/pelvic surgery and traumatic anal/vaginal delivery. Furthermore, patients with FI referred difficulty evacuating stools, too. In fact in patients with dyssynergic-type constipation, the FI may be confused with an encopresis. Finally we observed these prevalent manometric alterations: combined dysfunction IAS and EAS, and rectal hypersensitivity. Manometric findings could help physicians to identify appropriated patients for a biofeedback therapy.

Disclosure of Interest: All authors have declared no conflicts of interest.

\section{References}

1. Management of fecal incontinence. Current Treatment Approaches and Future Perspectives. Springer. 2016.

2. Townsend DC, Carrington EV, Grossi U, Burgell RE, Wong JY, Knowles CH, Scott SM Neurogastroenterol Motil. 2016 Oct;28(10):1580-8. doi: 10.1111/nmo.12858.

\section{P1783 RETROSPECTIVE STUDY: ROLE OF SEHCAT TEST IN THE DIAGNOSIS OF BILE ACID MALABSORPTION AS A CAUSE OF CHRONIC DIARRHOEA AND POTENTIAL RISK FACTORS ASSOCIATED}

C. Barber Caselles ${ }^{1}$, B. Lobo Alvarez ${ }^{2}$, J.L. Mosquera ${ }^{2}$, S. Aguade-Bruix ${ }^{3}$, F. Azpiroz ${ }^{2}$ J. Santos ${ }^{2}$

${ }^{1}$ Research Unit Gastroenterology Department., Hospital General Vall d́Hebron, Vall d Hebron Research Institute, Barcelona/Spain

${ }^{2}$ Neuro-immuno-gastroenterology. Research Unit Gastroenterology Department., Hospital General Vall dHebron, Vall d Hebron Research Institute, CIBERehd, Barcelona/Spain

${ }^{3}$ Nuclear Medicine Department, Hospital General Vall d Hebron, Barcelona/Spain

\section{Contact E-mail Address: claudiabarbercaselles@gmail.com}

Introduction: Bile acid malabsorption (MAB) is a common and frequently underinvestigated cause of chronic diarrhoea. Most of the cases of chronic diarrhoea after excluding organic disorders are labelled as functional diarrhoea or irritable bowel syndrome (IBS). The most commonly used diagnostic test is Selenium homocholic acid taurine (SeHCAT) scan due to its sensitivity, specificity, safety and low cost. However this test is not frequently used in the algorithm for the diagnosis of chronic diarrhoea.

Aims \& Methods: We aimed to evaluate the usefulness of SeHCAT scan in evaluating patients with chronic diarrhoea and identify potential risk factors associated to MAB. We retrospectively reviewed all patients who had SeHCAT scan between june 2014 and Octuber 2016 in a University Hospital. BAM was defined as SeHCAT retraction of less than $15 \%$. We collected the following variables: demographic characteristics, IBS-D Rome III criteria, duration of diarrhoea (months), stool culture, parasitic investigation of stool specimens, background of comorbid gastrointestinal and other comorbid conditions, positive HLA-DQ2 and DQ8 haplotype.

Results: 137 patients referred to clinic for chronic diarrhoea underwent SeHCAT testing over the reviewed period. $42 \mathrm{M} ; 95 \mathrm{~F}$, median age 46 y (95\% C.I $44.0-50.1)$, median BMI $25.34 \mathrm{~kg} / \mathrm{m} 2$ (95\% C.I $24.88-27.00), 70.4 \%$ of patients met IBS-D Rome III criteria, median duration of diarrhoea 48 months (95\% C.I 43.10 59.24). Background of co-morbid gastrointestinal conditions $45.3 \%(62 / 136)$, other co morbid conditions $55.3 \%$ (73/136). History of previous positive stool culture $1.5 \%$ and parasitic investigation of stool specimens $13 \%(17 / 131)$. Percentage of positive HLA-DQ2 and DQ8 haplotypes were $27.8 \%(35 / 126)$ and $10.2 \%(13 / 127)$, respectively. SeHCAT test was positive for BMA in $48.9 \%$ (67/137): $25.4 \%$ (mild $10-15 \%$ ); $31.3 \%$ (moderate $5-10 \%$ ), and $43.3 \%$ $($ severe $<5 \%)$. Patient characteristics between positive and negative SeHCAT test were similar (Table 1). Interestingly, patients with SeHCAT test exhibited longer periods of diarrhoea.

Table 1

\begin{tabular}{lll}
\hline & $\begin{array}{l}\text { Positive } \\
\text { SeHCAT test }\end{array}$ & $\begin{array}{l}\text { Negative } \\
\text { SeHCAT test }\end{array}$ \\
\hline Sex $(\mathrm{M} ; \mathrm{F})$ & $30 ; 37$ & $12 ; 58$ \\
Age y (median; 95\% C.I $)$ & $48.00(44.52-53.4)$ & $40.50(40.69-49.4)$ \\
BMI (median; 95\% C.I) & $26.84(25.56-28.78)$ & $23.64(23.31-25.98)$ \\
Duration of diarrhoea & $60.00(43.51-66.95)$ & $24.00(35.53-58.34)$
\end{tabular}

(months; median; 95\% C.I)

IBS-D Rome III criteria $(\%)$

Abdominal surgery (\%)

Cholecystectomy (\%)

Gastrointestinal conditions $(\%)$

Other co morbid conditions $(\%)$

$67.2 \%(45 / 67)$

$71.4 \%(50 / 70)$

$34.3 \%(23 / 67)$

$14.3 \%(10 / 70)$

$14.9 \%(10 / 67)$

0

$41.8 \%(28 / 67)$

$48.6 \%(34 / 70)$

$57.1 \%(36 / 63)$

$53.6 \%(37 / 69)$ 
Table 1 Continued

\begin{tabular}{lll}
\hline & $\begin{array}{l}\text { Positive } \\
\text { SeHCAT test }\end{array}$ & $\begin{array}{l}\text { Negative } \\
\text { SeHCAT test }\end{array}$ \\
\hline HLA-DQ2 Haplotype $(\%)$ & $23.8 \%(15 / 63)$ & $31.7 \%(20 / 63)$, \\
HLA-DQ8 Haplotype $(\%)$ & $14.1 \%(9 / 64)$ & $6.3 \%(4 / 63)$ \\
\hline
\end{tabular}

Patients who exhibited MAB (confirmed by SeHCAT test) were treated with colestyramine, $37.1 \%(23 / 67)$ exhibited partial response, $33.9 \%(21 / 67)$ exhibited total response, $3.2 \%(2 / 67)$ exhibited no good tolerance, we had no information in $21 \%(13 / 67)$.

Conclusion: SeHCAT scanning must be considered as a diagnostic tool for the for the diagnosis of chronic diarrhoea, specially in those patients with long-standing diarrhoea.

Disclosure of Interest: All authors have declared no conflicts of interest.

\section{P1784 INTAKE OF FERMENTABLE OLIGO-, DI- AND MONO- SACCHARIDES AND POLYOLS (FODMAPS) INCREASES THE RISK OF IRRITABLE BOWEL SYNDROME (IBS) IN INDIVIDUALS EXPOSED TO PSYCHOSOCIAL STRESS IN THE COMMUNITY: RESULTS OF A LARGE, PROSPECTIVE, POPULATION BASED STUDY}

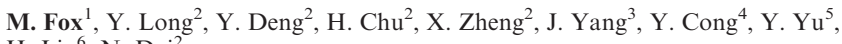
H. Liu ${ }^{6}$, N. Dai ${ }^{2}$

${ }^{1}$ Abdominal Center: Gastroenterology, St. Claraspital, Basel/Switzerland

${ }^{2}$ Gastroenterology, Department of Gastroenterology, Sir Run Run Shaw Hospital, School of Medicine, Zhejiang University, Hangzhou, Zhejiang Province, China, Hangzhou/China

${ }^{3}$ Department of Gastroenterology, The First People's Hospital of Hangzhou, Hangzhou, Zhejiang Province, China, Hangzhou, China, Hangzhou/China ${ }^{4}$ Gastroenterology, Department of Gastroenterology, Zhejiang Hospital, Hangzhou, Zhejiang Province, China, Hangzhou/China

${ }^{5}$ Epidemiology \& Statistics, Department of Epidemiology \& Statistics, School of Medicine, Zhejiang University, Hangzhou, Zhejiang Province, China, Hangzhou/ China

${ }^{6}$ Zhejiang Provincial Key Laboratory of Laparoscopic Technology, Sir Run Run Shaw Hospital, School of Medicine, Zhejiang University, Hangzhou, Zhejiang Province, China, Hangzhou/China

Contact E-mail Address: dr.mark.fox@gmail.com

Introduction: The cause of IBS is uncertain; however, food intolerance shares many features with this condition. Consumption of FODMAPs has been shown to induce IBS-type symptoms (Shephard 2008) and clinical trials have shown that a low FODMAP diet can improve symptoms in this patient group (Halmos 2014). However, FODMAP intake is not higher in IBS than in health (Bohn 2013) and it is not proven that the outcome of low FODMAP diet is better than standard dietary advice in this condition (Bohn 2015). Recent, experimental research has shown that psychological factors are associated with increased postprandial symptoms in IBS patients (Zhu 2013, Van Oudenhove 2016). This study was designed to assess the relative importance of, and interaction between, psychiatric disease, social stress and diet in the aetiology of IBS in the general community.

Aims \& Methods: This population based study tested the hypothesis that high FODMAP intake increases the risk of IBS more in individuals with psychiatric disease and/or life event stress than other members of the community.

Subjects aged 16-74 were randomly selected from five South-Chinese communities. All subjects completed questionnaires by face-to-face inquiry with investigators including demographic information, gastrointestinal symptoms (Rome III), dietary intake (food frequency chart validated in Chinese community), psychiatric disease (HADS), life event stress (LES) and quality of life (SF-8).

Results: From 1999/2115 (94.7\%) members of the community that completed study questionnaires, $117(5.9 \%)$ had IBS by Rome III criteria. The IBS group ingested less lactose than the "No-IBS" group $(\mathrm{P}=0.024)$. Intake of other FODMAPs was similar in both groups $(\mathrm{P}=0.346)$. Compared to the "NoIBS" group, individuals with IBS had a greater likelihood of depression (OR $1.5(0.97-2.32) ; \mathrm{p}=.05)$, anxiety $(2.84(1.84-4.39), \mathrm{p}<0.001)$, recent life event stress $(1.5(1.03-2.20) ; \mathrm{P}=0.03)$ or medical and/or surgical co-morbidity (OR $2.90(1.30-5.45), \quad \mathrm{P}<0.001)$. The IBS group also had lower quality of life $(\mathrm{P}<0.001)$. Joint risk analysis identified high intake of total FODMAP intake as a risk factors for IBS only in subjects with psychiatric disease and/or life event stress (table). Similar effects were seen for individual symptoms, in particular bloating (OR 2.4(1.25-4.60), $\mathrm{p}<0.008$ ). Increased risk of IBS was identified with ingestion of high intake of individual FODMAPs (e.g. fructose, fructans, lactose) in combination with psychosocial factors, but not with sucrose (control) in any group.
Table: Joint effects of psychiatric disease, life stress \& total FODMAP intake on relative risk of IBS in community

\begin{tabular}{lllllll}
\hline \multicolumn{2}{l}{ Psych Disease } & FODMAP Life Stress IBS & No IBS & \multicolumn{2}{l}{ Adjusted* OR p-value } \\
\hline No & Low & Low & $19(5.1)$ & $356(94.9)$ & 1.0 & - \\
$"$ & " & High & $23(6.8)$ & $315(93.2)$ & $1.2(0.6-2.4)$ & 0.530 \\
$"$ & High & Low & $14(3.5)$ & $383(96.5)$ & $0.6(0.3-1.3)$ & 0.213 \\
$"$ & " & High & $16(4.5)$ & $342(95.5)$ & $0.9(0.5-1.9)$ & 0.886 \\
Yes & Low & Low & $9(7.6)$ & $109(92.4)$ & $1.6(0.7-3.8)$ & 0.274 \\
$"$ & " & High & $16(9.5)$ & $152(90.5)$ & $1.9(0.9-3.9)$ & 0.094 \\
$"$ & High & Low & $5(4.9)$ & $97(95.1)$ & $1.0(0.4-2.9)$ & 0.932 \\
$"$ & " & High & $15(10.5)$ & $128(89.5)$ & $2.3(1.1-4.8)$ & 0.029 \\
\hline
\end{tabular}

*Adjusted variables: age, sex, marital status, education, job, income, smoking, drinking, and medical history.

Conclusion: FODMAP intake was similar in IBS and No-IBS groups in the community (lactose intake was lower in IBS subjects, likely due to avoidance of dairy products (Long 2017)). However, as expected, IBS patients in the community had a greater likelihood of psychiatric disease, life event stress and clinical co-morbidity. Joint effects analysis demonstrated that high FODMAP intake alone was not associated with abdominal symptoms; however, IBS was more common in those with a high FODMAP intake and concomitant psychosocial factors known to increase visceral sensitivity to digestive dysfunction (Zhu 2013).(ClinicalTrials: NCT01286597)

Disclosure of Interest: All authors have declared no conflicts of interest.

References

Shepherd, S. "Dietary triggers of abdominal symptoms in IBS patients: randomized placebo-controlled evidence." Clin Gastroenterol Hepatol 2008.

Halmos, E. "A diet low in FODMAPs reduces symptoms of IBS." Gastroenterology 2014

Bohn, L. "Nutrient intake in patients with IBS compared with the general population." Neurogastroenterol Motil 2013.

Bohn, L. "Diet low in FODMAPs reduces symptoms of IBS as well as traditional dietary advice: a randomized controlled trial." Gastroenterology 2015.

Zhu, Y. "Bloating and distention in IBS: the role of gas production and visceral sensation after lactose ingestion in a population with lactase deficiency." Am J Gastroenterol 2013.

Van Oudenhove, L. "Depression and Somatization Are Associated With Increased Postprandial Symptoms in IBS Patients." Gastroenterology 2016.

Long, Y. "Prevalence and risk factors for functional bowel disorders in South China." Neurogastroenterol Motil 2017.

\section{P1785 CHARACTERIZING IBS PATIENTS WITH ANXIETY OR DEPRESSION}

I. Pontén ${ }^{1}$, M. Simrén ${ }^{1}$, H. Törnblom ${ }^{1}$

${ }^{1}$ Department Of Internal Medicine And Clinical Nutrition, Sahlgrenska Academy, University of Gothenburg, Gothenburg/Sweden

Contact E-mail Address: irina.ponten@gu.se

Introduction: A large proportion of patients with irritable bowel syndrome (IBS) suffer from anxiety or depression, but the associations with pathophysiological findings and overall symptom reporting are not clear.

Aims \& Methods: We included 772 patients with IBS (Rome III criteria) who attended a university hospital-based outpatient clinic specialized in functional GI disorders between 2005 and 2015. The patients underwent examinations to investigate oro-anal transit time (OATT) and visceral sensitivity (rectal balloon distension and a lactulose challenge test), and they also completed questionnaires to assess anxiety and depression (HAD), overall IBS symptoms (IBS-SSS), bowel habits (BSF), quality of life (IBSQOL), extraintestinal somatic symptoms (PHQ12), sense of coherence (SOC), fatigue (MFI), GI-specific anxiety (VSI) and physical and sexual abuse.

Results: Based on validated HAD cut-off levels $(\geq 8)$, anxiety and depression were present in $55 \%$ and $26 \%$ of the IBS patients, respectively. More women were anxious $(\mathrm{p}<0.001)$, but for depression no gender differences were detected $(p=0.76)$. IBS patients with anxiety or depression were younger $(p<0.001$, $p=0.01$ ), and more commonly reported sexual and/or physical abuse $(\mathrm{p}<0.001)$ than IBS patients without anxiety or depression. The presence of anxiety or depression did not differ between IBS subgroups based on the predominant bowel habit $(p=0.41, p=0.18)$. For an overview of comparisons of data from questionnaires and pathophysiological examinations, see table 1. Both the presence of anxiety and of depression were associated with reports of more severe GI and extraintestinal symptoms, GI-specific anxiety, fatigue, and lower sense of coherence. Regarding pathophysiological examinations, the findings were more inconsistent. OATT was similar between groups, as was stool form and frequency. Visceral sensitivity tended to be higher in patients with anxiety, and depressed patients reported more severe pain during the lactulose challenge 
test. Quality of life (IBSQOL) was reduced for all domains in patients with anxiety and depression ( $\mathrm{p}<0.001$ for all comparisons).

Table 1: Characterization of IBS patients with anxiety or depression

\begin{tabular}{lllllll}
\hline Variable & Anxiety & Median & P-value & Depression & Median & P-value \\
\hline IBS-SSS & No Yes & 283331 & $<0.001$ & No Yes & 298330 & $<0.001$ \\
VSI & No Yes & 3352 & $<0.001$ & No Yes & 3956 & $<0.001$ \\
SOC & No Yes & 153124 & $<0.001$ & No Yes & 146111 & $<0.001$ \\
PHQ-12 & No Yes & 69 & $<0.001$ & No Yes & 710 & $<0.001$ \\
MFI & No Yes & 1414 & $<0.001$ & No Yes & 1519 & $<0.001$ \\
OATT (days) & No Yes & 1.31 .4 & 0.67 & No Yes & 1.31 .4 & 0.52 \\
Stool form (BSF) & No Yes & 44 & 0.93 & No Yes & 4.04 .0 & 0.49 \\
$\begin{array}{l}\text { Stool frequency (stools/day) } \\
\text { Lactulose challenge test, } \\
\quad \text { perceived pain (AUC) }\end{array}$ & No Yes & 1.71 .7 & 0.53 & No Yes & 1.71 .8 & 0.36 \\
$\begin{array}{c}\text { Balloon distension, pain } \\
\quad \text { threshold (mmHg) }\end{array}$ & No Yes & 2824 & 0.06 & No Yes & 7051710 & 0.01 \\
\hline
\end{tabular}

Conclusion: The presence of anxiety and depression seems to clearly potentiate the already substantial disease burden in IBS patients. However, the association with other pathophysiological findings is less distinct. This group of patients with complex and severe symptoms will benefit from a holistic management approach. Disclosure of Interest: M. Simrén: Magnus Simrén has received unrestricted research grants from Danone and Ferring Pharmaceuticals, and served as a Consultant/Advisory Board member for AstraZeneca, Danone, Nestlé, Menarini, Almirall, Allergan, Albireo, Glycom and Shire, and as. All other authors have declared no conflicts of interest.

H. Törnblom: Hans Törnblom has served as Consultant/Advisory Board member for Almirall and Allergan as a speaker for Tillotts, Takeda, Shire and Almirall.

P1786 THE ASSOCIATION BETWEEN IRRITABLE BOWEL SYNDROME AND LACTOSE INTOLERANCE

R. Dabak ${ }^{1}$, T. Arslan Kucuk ${ }^{1}$, S. Tuzun ${ }^{1}$, H. Cetin ${ }^{1}$, E. Ahıshalı C. Dolapcioglu ${ }^{2}$

${ }^{1}$ Family Medicine, Dr. Lutfi Kırdar Kartal Research And Training Hospital, Istanbul/Turkey

${ }^{2}$ Gastroenterology, Dr.Lutfi Kırdar Kartal Training and Research Hospital,

Istanbul/Turkey

Contact E-mail Address: resat_dabak@hotmail.com

Introduction: Irritable bowel syndrome (IBS) and lactose intolerance may co-exist and readily cause diagnostic confusion due to similar symptomatology $(1,2)$.

Aims \& Methods: This study aim to examine the incidence of lactose intolerance in healthy controls and in subjects diagnosed with IBS based on Roma III criteria, as an effort to investigate the association between IBS and lactose intolerance. The patient population consisted of individuals between 18 and 80 years of age who attended between June-December 2013. Patients diagnosed with IBS based on Roma III criteria comprised the IBS group, and subtypes of IBS. Control subjects were healthy volunteers over 18 years of age with no IBS-like symptoms. All participants ingested $25 \mathrm{~g}$ of lactose dissolved in $250 \mathrm{ml}$ of water within 5 minutes after 8 hours of fasting, in order to evaluate the lactose intolerance via hydrogen breath test $(0,15,30,60,90$, and 120 minutes). Additionally, symptoms arising during the test were assessed.

Results: Of the total 200 participants, $100(50 \%)$ were in IBS and $100(50.0 \%)$ were in control group. There were 133 females $(66.5 \%)$, and the mean age was $40.5 \pm 12.3$ years. Of the total 70 patients $(35.0 \%)$ with lactose intolerance, 47 $(47.0 \%)$ were in IBS and $23(23.0 \%)$ were in control groups $(p=0.001)$ Symptoms related to IBS were more common in participants with lactose intolerance in both groups $(\mathrm{p}=0.001, \mathrm{p}=0.001$ respectively). A comparison of the two groups with regard to symptomatology after the test showed the presence of complaints in $35(35.0 \%)$ patients in IBS group as compared to $24(24.0 \%)$ subjects among controls $(\mathrm{p}=0.092)$. The incidence of lactose intolerance in patients with IBS subtypes of diarrhea-predominant IBS, constipation-predominant IBS, mixed IBS, and unspecified IBS were $27(57.4 \%), 7(4.9 \%), 10(21.3 \%)$, and $3(6.4 \%)$, respectively, with no significant differences $(\mathrm{p}=0.161, \mathrm{p}=0.124$, $\mathrm{p}=1.000$, and $\mathrm{p}=0.661$ respectively).

Conclusion: A significantly increased frequency of lactose intolerance was found among IBS patients than in controls. In additional, symptoms associated with lactose intake occurred at a higher frequency in IBS patient, although the difference was insignificant.

Disclosure of Interest: All authors have declared no conflicts of interest.

\section{References}

1. Gupta D, Ghoshal UC, Misra A, Misra A, Choudhuri G, Singh K. Lactose intolerance in patients with irritable bowel syndrome from northern India: A case-control study. Journal of Gastroenterology and Hepatology 2007;22:22612265 .
2. Farup PG, Monsbakken KW, Vandvik PO. Lactose Malabsorption in a Population with Irritable Bowel Syndrome: Prevalence and Symptoms. A Case-Control Study. Scand J Gastroenterol 2004;39:645-649.

P1788 THE PREVALENCE AND IMPACT OF OVERLAPPING ROME IV FUNCTIONAL GASTROINTESTINAL DISORDERS ON SOMATISATION, QUALITY OF LIFE, AND HEALTHCARE UTILISATION: RESULTS FROM A THREE-COUNTRY GENERAL POPULATION STUDY

I. Aziz ${ }^{1}$, O.S. Palsson ${ }^{2}$, H. Törnblom ${ }^{1}$, A. Sperber ${ }^{3}$, W.E. Whitehead ${ }^{4}$, M. Simrén ${ }^{1}$

${ }^{1}$ Department Of Internal Medicine And Clinical Nutrition, Sahlgrenska Academy, University of Gothenburg, Gothenburg/Sweden

${ }^{2}$ Dept. Of Medicine, University of North Carolina, Chapel Hill, NC/United States of America

${ }^{3}$ Ben-gurion University Of The Negev, Faculty of Health Sciences, Beer-Sheva/ Israel

${ }^{4}$ Dept. Of Medicine, University of North Carolina at Chapel Hill, Chapel Hill, NC/ United States of America

Contact E-mail Address: imy_aziz9@yahoo.co.uk

Introduction: The population prevalence of Rome IV functional gastrointestinal disorders (FGIDs) and their cumulative effect on health impairment is unknown. We sought to address this issue.

Aims \& Methods: An Internet-based health survey was completed by 5931 of 6300 general population adults from three English-speaking countries (2100 each from US, Canada, and UK). The survey included questions on demographics, medication, surgical history, somatisation, quality of life, doctor-diagnosed organic GI disease, and criteria for the Rome IV FGIDs. Comparisons were made between those with Rome IV FGIDs against non-GI and organic GI disease controls.

Results: The number of subjects having symptoms compatible with a FGID was $2083(35 \%)$ compared to $3421(57.7 \%)$ non-GI and $427(7.2 \%)$ organic GI disease controls. The most frequently met diagnostic criteria for FGIDs was bowel disorders $(n=1665,28.1 \%)$, followed by gastroduodenal $(n=627,10.6 \%)$, anorectal $(n=440,7.4 \%)$, oesophageal $(n=414,7 \%)$, and gallbladder disorders $(\mathrm{n}=10,0.2 \%)$. On average, the 2083 individuals who met FGID criteria qualified for 1.5 FGID diagnoses, and 742 of them $(36 \%)$ qualified for FGID diagnoses in more than one anatomic region. The presence of FGIDs in multiple regions was associated with increasing somatisation, worse mental and physical quality of life, greater use of medical therapies, and a higher prevalence of abdominal surgeries; all $\mathrm{p}<0.001$, see table. Notably, individuals with FGIDs in multiple regions had worse somatisation and quality of life scores than organic GI disease controls.

\begin{tabular}{|c|c|c|c|c|c|c|}
\hline & $\begin{array}{l}\text { No } \\
\text { FGID } \\
\text { (healthy) }\end{array}$ & $\begin{array}{l}\text { One } \\
\text { FGID } \\
\text { region }\end{array}$ & $\begin{array}{l}\text { Two } \\
\text { FGID } \\
\text { regions }\end{array}$ & $\begin{array}{l}\text { Three } \\
\text { FGID } \\
\text { regions }\end{array}$ & $\begin{array}{l}\text { Four } \\
\text { FGID } \\
\text { regions }\end{array}$ & $\begin{array}{l}\text { Organic } \\
\text { GI-disease }\end{array}$ \\
\hline Number of subjects & 3421 & 1341 & 493 & 166 & 83 & 427 \\
\hline $\begin{array}{l}\text { Mean number of somatic } \\
\text { symptoms }\end{array}$ & 2.8 & 4.6 & 5.6 & 6.3 & 7.3 & 4.7 \\
\hline Mean PHQ-12 score & 3.3 & 6 & 7.5 & 9.1 & 10.9 & 6.3 \\
\hline Mean SF8-MCS QOL & 52.1 & 45.7 & 42.8 & 38.6 & 37.2 & 47.9 \\
\hline Mean SF8 PCS-QOL & 51.9 & 47.4 & 44.1 & 40.2 & 38.6 & 43.8 \\
\hline GI-related medication $(\%)$ & $35 \%$ & $59 \%$ & $74 \%$ & $84 \%$ & $93 \%$ & $71 \%$ \\
\hline Abdominal surgery $(\%)$ & $19 \%$ & $26 \%$ & $31 \%$ & $37 \%$ & $54 \%$ & $53 \%$ \\
\hline
\end{tabular}

Conclusion: Roughly a third of the general adult population fulfils diagnostic criteria for a Rome IV FGID. In a third of this subset multiple GI regions are involved and this overlap is associated with increased health impairment. Study Support: The Rome Foundation

Disclosure of Interest: All authors have declared no conflicts of interest.

P1789 WITHIN-PERSON CORRELATIONS BETWEEN GASTROINTESTINAL AND PSYCHOLOGICAL FEATURES OF THE IRRITABLE BOWEL SYNDROME

E. Clevers ${ }^{1}$, J. Tack ${ }^{2}$, H. Törnblom ${ }^{3}$, G. Ringström ${ }^{4}$, L. Van Oudenhove ${ }^{5}$, M. Simrén 6

${ }^{1}$ KU Leuven, Leuven/Belgium

${ }^{2}$ Gastroenterology, University of Leuven, University Hospital Gasthuisherg, Leuven/Belgium

${ }^{3}$ Dept Of Gastroenterology And Hepatology, Sahlgrenska Academy Faculty of Medicine, Gothenburg/Sweden 
${ }^{4}$ Institute Of Medicine, Sahlgrenska Academy, University of Gothenburg, Gothenburg/Sweden

${ }^{5}$ Translational Research Center For Gastrointestinal Disorders (targid), Katholieke Universiteit Leuven, Leuven/Belgium

${ }^{6}$ Dept Of Internal Medicine, Sahlgrenska University Hospital - Dept of Internal Medicine, Sahlgrenska University Hospital; Gothe, Gothenburg/Sweden

Contact E-mail Address: egbert.clevers@kuleuven.be

Introduction: Although correlations between features of irritable bowel syndrome (IBS) have been reported, these were based on between-person rather than within-person variation. We investigated the longitudinal within-person correlations between features of IBS.

Aims \& Methods: We used a longitudinal cohort of 276 IBS patients, who filled out questionnaires once annually over five years on the following features: gastrointestinal (GI) symptom severity (GSRS), quality of life (QOL), GI specific anxiety (VSI), general anxiety and depression (HADS), coping resources (CRI), and sense of coherence (KASAM). For each participant, scores were centered on their own mean, and within-person correlations were computed for all pairs of features.

Results: Aggregate within-person correlations are shown in figure 1. Withinperson correlations were strong for the triad GI symptom severity, GI specific anxiety, and QOL (|r|: 0.47 to 0.64). Another set of features was comprised of general anxiety, depression, coping resources, and sense of coherence (|r|: 0.39 to $0.57)$. Within-person correlations between the two sets were weak $(|\mathrm{r}|: 0.00$ to $0.37)$. However, within-person correlations tended towards bimodal distributions across the population, especially for GI symptom severity and depression $(\mathrm{r} \sim 0.6$ for half of participants, and $\mathrm{r} \sim-0.4$ for the other half)

Conclusion: Here we show that, within individual IBS patients, GI symptom severity is strongly correlated with GI specific anxiety and QOL, but not with four other psychological features. The presence of negative within-person correlations in some individuals may imply a lack of relation, but could also signal long-term causative processes.

Disclosure of Interest: J. Tack: Jan Tack has given Scientific advice to Abide Therapeutics, AlfaWassermann, Allergan, Christian Hansen, Danone, Genfit, Ironwood, Janssen, Kiowa Kirin, Menarini, Mylan, Novartis, Nutricia, Ono Pharma, Rhythm, Shionogi, Shire, SK Life Sciences, Takeda.

H. Törnblom: Hans Törnblom has served as Consultant/Advisory Board member for Almirall and Allergan as a speaker for Tillotts, Takeda, Shire and Almirall.

L. Van Oudenhove: Lukas Van Oudenhove has received grant support from Abide Therapeutics and Nestlé and has given scientific advice to Grünenthal.

M. Simrén: Magnus Simrén has received unrestricted research grants from Danone and Ferring Pharmaceuticals, and served as a Consultant/Advisory Board member for AstraZeneca, Danone, Nestlé, Menarini, Almirall, Allergan, Albireo, Glycom and Shire, and as a.

All other authors have declared no conflicts of interest.

\section{P1790 IRRITABLE BOWEL SYNDROME WITH CONSTIPATION: IMPACT OF SYMPTOM SEVERITY ON HEALTH-RELATED QUALITY OF LIFE: A POST HOC ANALYSIS OF DATA FROM TWO PHASE 3 TRIALS OF LINACLOTIDE}

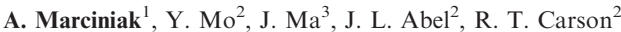

${ }^{1}$ Allergan plc, Marlow/United Kingdom

${ }^{2}$ Allergan plc, Jersey City/United States of America/NJ

${ }^{3}$ Allergan plc, Bridgewater/United States of America/NJ

Contact E-mail Address: Anne.Marciniak@Allergan.com

Introduction: Irritable bowel syndrome with constipation (IBS-C) is a chronic gastrointestinal disorder associated with significant impairment in health-related quality of life (HRQoL). However, information on the impact of IBS-C symptom severity on patients' HRQoL is lacking.

Aims \& Methods: This post hoc analysis assessed the burden of IBS-C on HRQoL according to symptom severity among adult patients meeting the modified Rome II criteria for IBS based on baseline data from two Phase 3 clinical trials of linaclotide. HRQoL was evaluated based on the disease-specific Irritable Bowel Syndrome Quality of Life questionnaire (IBS-QOL) and the EuroQol-5Dimension (EQ-5D). The IBS-QOL includes eight subscales (dysphoria, activity interference, body image, health worry, food avoidance, social reaction, sexual, relationships), with total and subscale scores calculated on a $0-100$ scale and higher scores indicating better IBS-specific HRQoL. A within-person change of $\geq 10$ points is considered clinically meaningful. The EQ-5D assesses five dimensions of general health (mobility, self-care, usual activities, pain/discomfort, anxiety/depression), generating an overall index score of between 0 (worst possible health state) and 1 (best possible health state); a within-person change of 0.05 is considered meaningful. Symptom severity was assessed during the pre-treatment period based on the global symptom score (GSS) on a scale of $0-4$, where $0=$ no symptoms and $4=$ very severe symptoms. Data from both trials were pooled and scores for both instruments were assessed for all patients based on GSS $(<3$ or $\geq 3$ ), as well as age ( $<65$ or $\geq 65$ years) and sex.

Results: A total of 1602 IBS-C patients were included for analysis. Compared to patients with GSS $<3$, those with GSS $\geq 3$ had lower HRQoL as indicated by lower mean IBS-QOL overall and subscale scores, with the greatest differences seen for body image (15-point difference), dysphoria and health worry (both 13- point difference) subscales (Table). Patients with GSS $\geq 3$ also had a lower EQ$5 \mathrm{D}$ index score compared to those with GSS $<3$ ( 0.67 vs 0.72 ) (Table). Women reported a slightly lower mean total IBS-QOL score compared to men, but had a notably lower score (14-point difference) on the body image subscale (Table). No difference in mean EQ-5D score was observed between sexes. IBS-QOL total and EQ-5D index scores were similar between patients aged $<65$ and $\geq 65$ years, though the younger subgroup generally had lower scores on the IBS-QOL, including on the food avoidance and sexual subscales (Table).

Table

\begin{tabular}{|c|c|c|c|c|c|c|c|}
\hline \multirow{3}{*}{$\begin{array}{l}\text { Instrument } \\
\text { IBS-QOL }\end{array}$} & \multicolumn{7}{|c|}{ Pooled Phase 3 trials baseline data } \\
\hline & \multirow[b]{2}{*}{ Total } & \multicolumn{2}{|l|}{ Sex } & \multicolumn{2}{|l|}{ Age } & \multicolumn{2}{|l|}{ GSS } \\
\hline & & $\mathrm{F}$ & M & $<65$ & $\geq 65$ & $<3$ & $\geq 3$ \\
\hline $\mathrm{n}$ & 1595 & 1437 & 158 & 1511 & 84 & 92 & 1503 \\
\hline Total score, mean & 61.1 & 60.8 & 63.8 & 60.9 & 65.0 & 71.5 & 60.4 \\
\hline Dysphoria & 62.3 & 62.2 & 62.8 & 62.2 & 64.2 & 74.6 & 61.5 \\
\hline Activity interference & 68.0 & 68.2 & 66.4 & 68.1 & 67.1 & 76.4 & 67.5 \\
\hline Body image & 48.2 & 46.8 & 61.0 & 47.8 & 55.5 & 62.0 & 47.4 \\
\hline Health worry & 44.9 & 44.4 & 49.3 & 44.7 & 49.5 & 57.3 & 44.2 \\
\hline Food avoidance & 50.1 & 49.7 & 54.1 & 49.6 & 59.5 & 59.0 & 49.6 \\
\hline Social reaction & 67.1 & 66.7 & 70.0 & 66.8 & 71.5 & 75.5 & 66.5 \\
\hline Sexual & 67.9 & 67.0 & 75.5 & 67.2 & 80.4 & 77.8 & 67.2 \\
\hline $\begin{array}{l}\text { Relationships } \\
\text { EQ-5D }\end{array}$ & 73.4 & 73.6 & 71.6 & 73.3 & 75.3 & 80.6 & 72.9 \\
\hline $\mathrm{n}$ & 1598 & 1440 & 158 & 1513 & 85 & 93 & 1505 \\
\hline Index score, mean & 0.68 & 0.68 & 0.67 & 0.68 & 0.70 & 0.72 & 0.67 \\
\hline
\end{tabular}

Conclusion: Among this patient population, IBS-C patients with higher symptom severity reported greater impairments in HRQoL. These results indicate that symptom severity may be an important consideration for disease management and emphasise the need for IBS-C treatments that improve both symptom burden and HRQoL.

Disclosure of Interest: A. Marciniak: Anne Marciniak is an employee of Allergan plc and shareholder in Pfizer, Amgen, and Allergan plc.

Y. Mo: Yifan Mo is an employee of Allergan plc.

J. Ma: Julia Ma is an employee of Allergan plc.

J.L. Abel: Jessica L. Abel is an employee of Allergan plc and shareholder in Allergan plc.

R.T. Carson: Robyn T. Carson is an employee of Allergan plc and shareholder in Allergan plc.

\section{P1791 HEALTH CARE UTILIZATION FOR ROME IV IRRITABLE BOWEL SYNDROME; A THREE-COUNTRY SURVEY IN THE GENERAL POPULATION}

N. Jossan ${ }^{1}$, H. Törnblom ${ }^{1}$, M. Simrén ${ }^{1}$, I. Aziz ${ }^{1}$, O.S. Palsson ${ }^{2}$,

W.E. Whitehead ${ }^{2}$, A. Sperber ${ }^{3}$

${ }^{1}$ Department Of Internal Medicine And Clinical Nutrition, Institute of Medicine, Sahlgrenska University hospital, Gothenburg/Sweden

${ }^{2}$ Dept. Of Medicine, University of North Carolina, Chapel Hill, Chapel Hill, NC United States of America/NC

${ }^{3}$ Ben-gurion University Of The Negev, Faculty of Health Sciences, Beer-Sheva/ Israel

Contact E-mail Address: navkiran.jossan@gu.se

Introduction: No population-based studies have investigated the symptom burden and healthcare utilization associated with Rome IV defined irritable bowel syndrome (IBS)

Aims \& Methods: The aim of the current study was to assess these variables in IBS subjects in the general population. An Internet survey was completed by 6300 individuals distributed equally between United States, United Kingdom and Canada. Equal sex, age and education distribution across the countries was ensured by use of quota-based sampling. The survey included questions on demographics, the Rome IV diagnostic questionnaire, the patient health questionnaire (PHQ-12), the 8-item Short Form (SF-8) quality of life (QOL) questionnaire, health care utilization and past gastrointestinal (GI) disease diagnoses by doctors. Respondents with an organic GI disease were excluded from the IBS population.

Results: From the 6300 individuals who completed the survey, 369 were excluded due to inconsistent responses, leaving 5931 (49.2\% female; mean age $47.4 \pm 17.1$ years) to be included for analysis (1949 US, 1994 UK, 1,988 Canada). $305(5.1 \%)$ of these fulfilled Rome IV diagnostic criteria for IBS, after 36 individuals were excluded due to organic disease. Compared to the non-IBS population, the IBS population was younger (mean-age $44.7 \pm 14.5$ years; $p=0.004$ ) and predominantly female $(66 \% ; \mathrm{p}<0.001)$. Apart from reporting more frequent GI 
symptoms, IBS subjects also reported more somatic symptoms; $103(34 \%)$ had abdominal pain at least 3 times/week, $232(76 \%)$ subjects reported sensation of bloating at least 3 times/month, and $232(76 \%)$ scored $\geq 7$ on PHQ-12, indicating high somatic symptom burden ( $\mathrm{p}<0.001$ for all). They also had poorer self-rated overall health, more general body pain, and more health-related impairment of social activities $(\mathrm{p}<0.001$ for all). See table 1 for details. IBS subjects also reported more frequent visits to the doctor compared to the non-IBS population, both for non-GI and GI related problems; 241 (79\%) vs. $3133(56 \%)$ reported visiting a doctor at least once/year for any health issue and $195(63 \%)$ vs. 1144 $(20.5 \%)$ had seen a doctor for GI problems ( $\mathrm{p}<0.001$ for all). IBS subjects also reported higher frequency of visits to gastroenterologists, gynaecologists and surgeons in secondary care; $\mathrm{p}<0.001$ for all. The use of medication for pain (both subscribed and over the counter), GI related symptoms, depression and anxiety was increased amongst subjects with IBS, as was the rate of abdominal surgery $(\mathrm{p}<0.001$ for all), appendectomy excluded. See table 1 for details.

\begin{tabular}{|c|c|c|c|}
\hline & $\begin{array}{l}\text { IBS } \\
\mathrm{n}=305 \\
\mathrm{n}(\%)\end{array}$ & $\begin{array}{l}\text { Not IBS } \\
\mathrm{n}=5590 \\
\mathrm{n}(\%)\end{array}$ & p-value \\
\hline \multicolumn{4}{|l|}{ GI symptoms } \\
\hline $\begin{array}{l}\text { Abdominal pain }>3 \text { times/week } \\
\text { Bloating }>3 \text { times a month }\end{array}$ & $\begin{array}{l}103(33.8) \\
232(76.1)\end{array}$ & $\begin{array}{l}88(1.6) \\
849(15.2)\end{array}$ & $\begin{array}{l}<0.001 \\
<0.001\end{array}$ \\
\hline $\begin{array}{l}\text { Somatization PHQ-score } 7 \text { or } \\
\text { above }\end{array}$ & $323(76.1)$ & $1381(24.7)$ & $<0.001$ \\
\hline \multicolumn{4}{|l|}{ Quality Of Life } \\
\hline $\begin{array}{l}\text { Overall estimation of health past } \\
4 \text { weeks (SF-8). Very poor/poor } \\
\text { Fair/good Very good/excellent }\end{array}$ & $\begin{array}{l}90(29.5) \\
182(59.7) \\
33(10.8)\end{array}$ & $\begin{array}{l}273(4.9) \\
3026(54.1) \\
2291(41.0)\end{array}$ & $<0.001$ \\
\hline $\begin{array}{l}\text { Bodily pain past } 4 \text { weeks (SF- } \\
8 \text { ) None/very mild Mild/moder- } \\
\text { ate Severe/very severe }\end{array}$ & $\begin{array}{l}41(13.4) \\
168(55.1) \\
96(31.5)\end{array}$ & $\begin{array}{l}3567(63.8) \\
1707(30.5) \\
316(5.6)\end{array}$ & $<0.001$ \\
\hline $\begin{array}{l}\text { Limitation in social activities due } \\
\text { to physical health or emotional } \\
\text { problems past } 4 \text { weeks ( } S F-8) \\
\text { Not at all Very little/somewhat } \\
\text { Quite a lot/could do no social } \\
\text { acticity }\end{array}$ & $\begin{array}{l}53(17.4) \\
144(47.2) \\
108(35.4)\end{array}$ & $\begin{array}{l}3084(55.2) \\
2021(36.2) \\
485(8.7)\end{array}$ & $<0.001$ \\
\hline $\begin{array}{l}\text { Frequency of doctor visits At } \\
\text { least once/year }\end{array}$ & $241(79)$ & $3133(56)$ & $<0.001$ \\
\hline \multicolumn{4}{|l|}{ Medication use } \\
\hline $\begin{array}{l}\text { For constipation For diarrhea } \\
\text { Pain, prescribed Pain, over } \\
\text { the counter Gas/bloating } \\
\text { Anti-depressive Anti-anxiety }\end{array}$ & $\begin{array}{l}76(24.9) \\
62(20.3) \\
122(40) \\
114(37.4) \\
67(22.0) \\
107(35.1) \\
92(30.2)\end{array}$ & $\begin{array}{l}274(4.9) \\
163(2.9) \\
775(13.9) \\
972(17.4) \\
249(4.5) \\
669(12.0) \\
557(10.0)\end{array}$ & $\begin{array}{l}<0.001 \\
<0.001 \\
<0.001 \\
<0.001 \\
<0.001 \\
<0.001 \\
<0.001\end{array}$ \\
\hline \multicolumn{4}{|l|}{ Surgery } \\
\hline $\begin{array}{l}\text { Cholecystectomy } \\
\text { Appendectomy } \\
\text { Hysterectomy Other } \\
\text { abdominal surgery }\end{array}$ & $\begin{array}{l}52(17.9) \\
38(12.5) \\
31(10.2) \\
53(17.4)\end{array}$ & $\begin{array}{l}412(7.4) \\
553(9.9) \\
334(6.0) \\
399(7.1)\end{array}$ & $\begin{array}{l}<0.001 \\
0.18 \\
0.005 \\
<0.001\end{array}$ \\
\hline
\end{tabular}

Conclusion: Individuals fulfilling the Rome IV criteria for IBS in the general population have increased GI and non-GI healthcare utilization in primary and secondary care, excess non-GI symptom burden and impaired QOL. [Support: Rome Foundation]

Disclosure of Interest: All authors have declared no conflicts of interest.

\section{P1792 ISCHAEMIC COLITIS AND VITAMIN K DEFICIENCY: A HYPOTHESIS}

A. G. Tsimperidis, I. A. Linardou, A. N. Kapsoritakis, A. K. Psychos, S. P. Potamianos

Department Of Gastroenterology, Faculty of Medicine, School of Health Sciences, University of Thessaly, Larissa/Greece

\section{Contact E-mail Address: atsimperidis@yahoo.com}

Introduction: Ischaemic colitis (IC) is the most common vascular disease of the colon, although its exact pathophysiological mechanisms remain unclarified. ${ }^{1}$

Aims \& Methods: The aim of this study was to evaluate routine coagulation parameters associated with vitamin $\mathrm{K}$ in patients with IC and to compare them with a group of controls (individuals with known predisposing factors for IC but without evidence of the disease). This study was carried out in the context of a study evaluating thrombophilia among IC patients. The present study used the same study groups as the latter. ${ }^{2}$ History of malignancy, systemic inflammation, liver disease-cirrhosis or use of anticoagulants were exclusion criteria for both patients and controls. The control group was using more antihypertensive drugs compared to the patients' group, while no difference was observed in any other drug category. Fifty-six cases of IC (admitted from September 2007 until June 2011 at the tertiary Department of Gastroenterology in central Greece) and 44 controls (subjects admitted for upper gastrointestinal bleeding, choledocholithiasis, and colon polyps) with known predisposing factors but no evidence of IC were evaluated. Coagulation indices measured were: prothrombin time (PT), activated partial thromboplastin time (aPTT) and lupus anticoagulant (LA). Venous blood collected from all IC patients and controls at the second or third day of hospitalization (1-3 days from the onset of symptoms).

Table 1: Coagulation tests in IC and control groups.

\begin{tabular}{llllll}
\hline Parameter & IC group & Controls & Normal range & Units & p value \\
\hline PT & $14.5[13.7-15.7]$ & $13.1[12.3-13.9]$ & $9.0-14.0$ & sec & .0005 \\
INR & $1.15[1.09-1.22]$ & $1.02[0.96-1.07]$ & $0.85-1.15$ & & .0005 \\
aPTT & $28.7[26.7-31.7]$ & $26.4[24.9-28.6]$ & $25.0-35.0$ & sec & .0005 \\
LA & $1.05[0.97-1.13]$ & $1.04[1.03-1.10]$ & $0.8-1.22$ & & .948 \\
\hline
\end{tabular}

Results: Significant differences were recorded in the levels of PT (median: 14.5 vs $13.1 \mathrm{sec}, \mathrm{p}=.0005$ ) and aPTT (median: 28.7 vs $26.4 \mathrm{sec}, \mathrm{p}=.0005$ ) between patients and controls, respectively. Prolongation over the upper limit of normal of PT $(>14 \mathrm{sec})$ was most common in IC patients, $67.9 \%$ vs $18.2 \%$ $(\mathrm{p}=.0005)$ as well as the prolongation of aPTT $(>35 \mathrm{sec}), 12.2 \%$ vs $6.2 \%$ $(\mathrm{p}=.469)$. The presence of LA was characterized as weakly present in 5 of 6 patients with the aPTT prolongation $(9.6 \%$, normalized LAC ratio: $1.2-1.5)$ and moderately present in 1 patient $(1.9 \%$, normalized LAC ratio: $1.5-2.0)$.

Conclusion: Prolongation of PT and aPTT, possibly indicating a chronic Vitamin $\mathrm{K}$ deficiency state, may be implicated in the pathophysiological mechanisms of IC.

Disclosure of Interest: All authors have declared no conflicts of interest.

\section{References}

1. Feuerstadt P, Brandt LJ. Update on Colon Ischemia: Recent Insights and Advances. Curr Gastroenterol Rep 2015; 17: 45.

2. Tsimperidis AG, Kapsoritakis AN, Linardou IA et al. The role of hypercoagulability in ischemic colitis. Scand J Gastroenterol 2015; 50: 848-855.

\section{P1794 ARTERIAL PH AND LACTATE HAVE SIGNIFICANT IMPACT ON THE OUTCOMES OF PATIENTS WITH ISCHAEMIC COLITIS}

C. Simões ${ }^{1}$, V. Magno Pereira ${ }^{2}$, M. Silva ${ }^{3}$, J. Carvão ${ }^{2}$, A. Oliveira ${ }^{2}$, C. Baldaia ${ }^{2}$, G. Serrão ${ }^{2}$, L. Jasmins ${ }^{2}$, J. Velosa ${ }^{1}$

${ }^{1}$ Department Of Gastroenterology And Hepatology, North Lisbon Hospital Center, University of Lisbon, Lisbon/Portugal

${ }^{2}$ Gastrenterology, Hospital Central do Funchal, Funchal/Portugal

${ }^{3}$ Gastroenterology, Centro Hospitalar São João, Porto Medical School, Porto/

Portugal

Contact E-mail Address: magnovitorp@gmail.com

Introduction: Ischaemic colitis (IC) encompasses a number of clinical entities resulting in insufficient blood supply to the colon. The incidence of adverse outcome in patients with IC remains high.

Aims \& Methods: We conducted a multicenter retrospective cohort study including consecutive patients with IC diagnosed between January 2013 and December 2016, according to the Modified Brandt and Boley criteria (clinical, colonoscopy, pathology consistent with IC and negative culture). The following data were collected: age, sex, clinical symptoms, comorbidities, organ failure, laboratory (hemoglobin, platelet number, leucocytes, C-reactive protein, creatinine, sodium, potassium, arterial $\mathrm{pH}$ and arterial lactate and $\mathrm{LDH}$ ), endoscopic and $\mathrm{CT}$ findings, surgery and death. Logistic regression was used to study association with poor outcomes - surgery and overall mortality.

Results: In this study, 349 patients were registered and 193 fulfilled the predetermined criteria: $121(62.7 \%)$ were females and mean age was 72-years-old (IQR: 23-94). At admission a total of $165(85.5 \%)$ patients presented with hematochezia, $112(58 \%)$ with abdominal pain, $82(42.5 \%)$ with diarrhea, $27(11.9 \%)$ with shock and $7(3.6 \%)$ with occlusion. Twenty three $(11 \%)$ required surgery. In-hospital, 30-day and overall follow-upmortality rates (up to 205 weeks) were $7.3 \%, 6.7 \%$ and $14 \%$, respectively. On multivariate analysis, surgery was independently associated with shock (OR: 4.8; $\mathrm{p}=0.011)$ intestinal occlusion(OR: $3.5 ; \mathrm{p}=0.046)$, and arterial lactate (OR: $1.3 ; \mathrm{p}=0.012)$. On univariate analysis, hemoglobin, hematocrit, C-reactive protein, $\mathrm{LDH}$, the number of risk factors, arterial $\mathrm{pH}$, and the need of red blood support IQ were significantly $(\mathrm{p}<0.05)$ associated with in-hospital and 30-day mortality. Creatinine was also associated with intra-hospital mortality. The variables that correlated independently in the multivariate analysis with in-hospital mortality were male gender (OR:4.5; $\mathrm{p}=0.03)$, and arterial $\mathrm{pH}(\mathrm{OR}: 0.001 ; \mathrm{p}=0.036)$ and with 30 -day mortality was the need of red blood cells support (OR: 2.1; $\mathrm{p}=0.0086$ ).

Conclusion: In our cohort of predominant elderly patients, arterial $\mathrm{pH}$, shock and lactate were significantly associated with need of surgery. Arterial pH on admission was also independently associated with in-hospital mortality. Early identification of these factors can anticipate decision management in this context and select the best care level for each patient.

Disclosure of Interest: All authors have declared no conflicts of interest. 
P1795 THE RISK PREDICTIVE VALUES OF ACG CLASSIFICATION IN A COHORT OF ISCHEMIC COLITIS-REFINING THE DEFINITION OF MILD DISEASE

V. Magno Pereira ${ }^{1}$, C. Simões ${ }^{2}$, M. Silva ${ }^{3}$, J. Carvão $^{1}$, A. Oliveira ${ }^{1}$, C. Baldaia ${ }^{2}$, G. Serrão ${ }^{1}$, L. Jasmins ${ }^{1}$, J. Velosa ${ }^{5}$

${ }^{1}$ Gastrenterology, Hospital Central do Funchal, Funchal/Portugal

${ }^{2}$ Department Of Gastroenterology And Hepatology, North Lisbon Hospital Center, University of Lisbon, Lisbon/Portugal

${ }^{3}$ Gastroenterology, Hospital São João, Oporto/Portugal

Contact E-mail Address: carolinabaptistasimoes@gmail.com

Introduction: Although most cases of colon ischemia (IC) are mild and self-limiting, when severe it implies high mortality rates. We aimed to evaluate the risk predictive value of the classification of disease severity proposed by American College of Gastroenterology (ACG) guidelines (2015), created to provide a management algorithm for these patients and select the level of care.

Aims \& Methods: A retrospective multicenter study was conducted on adult patients with definite CI (clinical, colonoscopic, pathologic and culture criteria), between 2013 and 2016. Data was collected on clinical presentation, comorbidities, organ failure, management and outcome. Each case was classified according to ACG guidelines after assessment of the number of risk factors (gender, systolic blood pressure $<90 \mathrm{~mm} \mathrm{Hg}$, heart rate $>100$ beats per min, abdominal pain without rectal bleeding, $\mathrm{BUN}>20 \mathrm{mg} / \mathrm{dl}, \mathrm{Hgb}<12 \mathrm{~g} / \mathrm{dl}, \mathrm{LDH}>350 \mathrm{U} / 1$, serum sodium $<136 \mathrm{mEq} / 1(\mathrm{mmol} / \mathrm{l}), \mathrm{WBC}>15 \times 109 / \mathrm{cmm})$. Patients were then classified as mild ( 0 risk factors (RF)), moderate ( $1-3$ risk factors), and severe (more than 3 risk factors or any of the following: peritoneal signs, pneumatosis or portal venous gas, gangrene on colonoscopic examination and pan-colonic or isolated right-colon ischemia involvement on imaging by colonoscopy or computed tomography)

Results: 349 cases with the clinical diagnosis of IC were analyzed.193 patients met the inclusion criteria of definitive diagnosis of CI (62.7\% females; mean age 72 years $( \pm 13)$. ACG classification of mild, moderate and severe disease was attributed respectively to $21 \%$ of patients ( 0 intra-hospital deaths), $45 \%$ ( 2 deaths) and $34 \%$ (12 deaths). The number of ACG RF was: $40 \%$ with 0 RF, $8 \%$ with $1,9 \%$ with $2,15 \%$ with $3,16 \%$ with $4,8 \%$ with $15 \%, 4 \%$ with 6 and $1 \%$ with 7 . No patient with 0 or $1 \mathrm{RF}$ died. Only 1 patient with $2 \mathrm{RF}$ died. The remaining 13 deaths were verified with at least $3 \mathrm{RF}$. The univariate analysis revealed a statistical correlation between RF and intra-hospital or 30-day mortality as well as the need for surgery $($ mean $=4.06, \mathrm{sd}=1.85)$. ACG classification presented high predictive accuracy for in-hospital and 1-month mortality with an AUROC of 0.78 and $0.79(\mathrm{p}<0.001)$, respectively. For a cutoff of 2 ACG RF, the sensibility (SE) for death was $100 \%$, specificity (SP) $52 \%$, with a positive predictive value (PPV) of $14 \%$ and negative predictive value (NPV) of $100 \%$. For 3 ACG RF the results were: SE $93 \%$, SP $61 \%$, PPV $16 \%$ and NPV $99 \% .3$ or more risk factor had an odds ratio of 20.2 (confidence interval (CI) 2.59-158) for intra-hospital mortality and 18.42 for 1-month mortality (CI 2.34-144).

Conclusion: No patient in this cohort with less than 2 ACG RF died, suggesting that the ACG classification as mild disease may include 0 and 1 risk factor without changing the prognosis. Short-term mortality risk increases significantly in patients with at least $3 \mathrm{ACG}$ RF.

Disclosure of Interest: All authors have declared no conflicts of interest.

\section{Reference}

Brandt LJ, Feuerstadt P, Longstreth GF, et al. ACG clinical guideline: Epidemiology, risk factors, patterns of presentation, diagnosis, and management of colon ischemia (CI). Am J Gastroenterol 2015; 110: 18-44.

P1796 HOW COST AFFECTS THE TREATMENT CHOICE FOR IRRITABLE BOWEL SYNDROME WITH DIARRHEA PATIENTS: A COST-EFFECTIVENESS ANALYSIS OF TRICYCLIC AGENTS AND RIFAXIMIN

E. D. Shah, E. Andraska, J. Li, S. Zhang, W.D. Chey

Division Of Gastroenterology, University of Michigan, Ann Arbor/United States of America

Contact E-mail Address: ershah@med.umich.edu

Introduction: Drug pricing and third party payer coverage exert a profound effect on access to prescription therapies in patients with irritable bowel syndrome with diarrhea (IBS-D). We performed a cost-effectiveness analysis to assess the tradeoffs associated with treating IBS-D patients with a tricyclic agent (TCA) or rifaximin.

Aims \& Methods: We constructed a decision analytic model evaluating three treatment strategies for IBS-D in the United States healthcare system: first-line therapy with TCA-only, first-line rifaximin followed by second-line TCA for nonresponders, and first-line TCA followed by second-line rifaximin for nonresponders. This model accounted for direct and indirect costs of therapy (Medicaid NADAC database and Healthcare Blue Book) and work-productivity loss (published literature and US Bureau of Labor) with a 3\% per annum discount rate. Rifaximin was administered in 4-month treatment cycles based on published clinical experience. Responder and discontinuation rates were derived from clinical trial data, and validated health utility values were assigned to terminal health states. Base-case analysis was performed to determine incremental cost-effectiveness ratios (ICER) for both rifaximin strategies. Threshold analysis assessed rifaximin pricing at contemporary willingness-to-pay (WTP) levels per quality adjusted life year (QALY). Appropriate sensitivity analyses were conducted. Analysis was performed with a 1-year time horizon from societal and payer perspectives.

Results: Based on the average acquisition cost of rifaximin (USD \$29.78/pill), second-line rifaximin could be cost-effective from a societal perspective (Table 1). However, at contemporary WTP thresholds neither rifaximin strategy was costeffective from a payer perspective despite greater effectiveness than TCA alone. Dependent on WTP, a $12-62 \%$ price reduction (USD $\$ 18.46-\$ 26.34 /$ pill) would enable the first-line TCA followed by second-line rifaximin to be more costeffective than a TCA-only strategy (Table 1 ). An $84-88 \%$ price reduction (USD \$3.53-\$4.71/pill) would enable first-line rifaximin followed by secondline TCA to be more cost-effective than TCA-only, though first-line TCA followed by second-line rifaximin would remain the more cost-effective strategy. Our model was robust in tornado analysis and most influenced by rifaximin treatment interval. Sensitivity analysis on rifaximin retreatment interval suggests that current pricing may be based on longer retreatment intervals than those found in clinical literature (Fig 1a). Sensitivity analysis with a lower TCA responder rate could enable first-line rifaximin to be the preferred strategy, albeit at a reduced price (Fig 1b).

Conclusion: Rifaximin is an effective therapy for IBS but is less cost-effective than TCA as currently priced. We propose an evidence-based pricing strategy which would maximize the cost-effectiveness of rifaximin in IBS-D patients.

Disclosure of Interest: W.D. Chey: Dr. Chey is a consultant for Ironwood Pharmaceuticals and Allergan.

All other authors have declared no conflicts of interest.

\section{P1797 PREDICTIVE FACTORS FOR BETTER OUTCOMES IN} COLONOSCOPY-ASSOCIATED PERFORATION

B. Kim, S. Park, M.A. Yang, S.H. Yun, Y. Lee, J.W. Kim, J. Cho Gastroenterology Division, Internal Medicine Department, Presbyterian Medical Center, Jeonju-si/Korea, Republic of

Contact E-mail Address: cccagape@hanmail.net

Introduction: Colonoscopy has been widely used for diagnostic and therapeutic purposes. Although the incidence is very low, perforation is one of the most serious complications. It is important to decide whether to try endoscopic clipping or to perform prompt surgical management.

Aims \& Methods: We retrospectively reviewed charts of all patients who experienced colonoscopy-associated perforation in a single center between May 2009 and July 2015 , and totally 45 patients were enrolled.

Table: The risk factors surgical treatment in colonoscopy-associated perforation

\begin{tabular}{llll}
\hline variable & $\begin{array}{l}\text { Surgery group } \\
(\mathrm{N}=18)\end{array}$ & $\begin{array}{l}\text { Conservative group } \\
(\mathrm{N}=27)\end{array}$ & P value \\
\hline Age(year) & 66 & 67 & .688 \\
Sex(M/F) & $10 / 8$ & $14 / 13$ & .807 \\
Purpose of colonoscopy & & & .000
\end{tabular}

Therapeutic

Diagnostic

9

Location of perforation

Rectum

Sigmoid

Descending

Transverse

Ascending

Endoscopic clipping

Yes

No

2

.002

Results: Diagnostic cases in purpose, sigmoid colon in location and non-clipping status were significantly more common in surgery group than conservative group (Table). Endoscopic clipping was performed in 31 cases (immediate; 23, delayed; 8 ), and immediate clipping group had significantly lower rate of operation $(p=0.013)$ and better clinical outcome (duration of antibiotics: $p=0.006$, hospital stay: $\mathrm{p}=0.001$ ). Among 18 surgical cases, 13 patients had primary closure and 5 patients had complex surgery ( 2 ; segmental resection, 3; Hartmann's procedure). The early $(<24 \mathrm{hr})$ surgical management significantly decreased the possibility of complex surgery $(\mathrm{p}=0.002)$, as well as had better clinical outcomes such as duration of antibiotic use, fasting time and length of hospital stay $(\mathrm{p}=0.003, \mathrm{p}=0.001, \mathrm{p}=0.005$, respectively). In therapeutic cases, all five perforated patients who had surgery within 1 day could be managed by simple primary closure, but all four patients who had surgery after 1 day required complex surgery. 
Abstract No: P1796

Table 1: Comparative cost-effectiveness of treatment approaches with and without rifaximin in irritable bowel syndrome with diarrhea (IBS-D)

\begin{tabular}{|c|c|c|c|c|c|}
\hline Strategy & $\begin{array}{l}\text { Total cost } \\
\text { (USD/yr) }\end{array}$ & $\begin{array}{l}\text { Total } \\
\text { effectiveness } \\
\text { (QALY gained) }\end{array}$ & $\begin{array}{l}\text { Incremental } \\
\text { cost (USD) }\end{array}$ & $\begin{array}{l}\text { Incremental } \\
\text { effectiveness } \\
\text { (QALY) }\end{array}$ & $\begin{array}{l}\text { ICER } \\
\text { (USD/QALY gain) }\end{array}$ \\
\hline $\begin{array}{l}\text { Societal perspective } \\
\text { TCA only }\end{array}$ & $\$ 4,355$ & 0.017 & & & \\
\hline Rifaximin as first-line for IBS-D & $\$ 7,608$ & 0.020 & $\$ 428$ & +0.0029 & $\$ 1,138,254$ (dominated $^{*}$ ) \\
\hline Rifaximin as second-line for IBS-D & $\$ 4,783$ & 0.022 & $\$ 3,252$ & +0.0052 & $\$ 82,375$ \\
\hline $\begin{array}{l}\text { Payer perspective } \\
\text { TCA only }\end{array}$ & $\$ 728$ & 0.017 & & & \\
\hline Rifaximin as first-line for IBS-D & $\$ 4,177$ & 0.020 & $\$ 894$ & +0.0029 & $\$ 1,207,136$ (dominated $*$ ) \\
\hline Rifaximin as second-line for IBS-D & $\$ 1,622$ & 0.022 & $\$ 3,449$ & +0.0052 & $\$ 171,850$ \\
\hline
\end{tabular}

*first-line rifaximin strategy was dominated (less effective and more expensive) than a second-line rifaximin strategy at all price points. ICER =incremental cost effectiveness ratio; QALY = quality adjusted life year; TCA = tricyclic antidepressant; IBS-D = irritable bowel syndrome with diarrhea; USD = US dollar

Conclusion: In colonoscopy-associated perforation, immediate endoscopic clipping decreases the possibility of operation and shows better clinical outcomes, and early surgical approach decreases complex operation rate. Especially, early operation need be considered in diagnostic perforation regardless of endoscopic clipping.

Disclosure of Interest: All authors have declared no conflicts of interest.

P1798 PATIENTS' AND CLINICIANS' VIEWS OF AND EXPERIENCE WITH A NOVEL CLINICAL PATHWAY FOR FUNCTIONAL GASTROINTESTINAL DISORDERS

E. C. Linedale $^{1}$, A. Mikocka-Walus ${ }^{1}$, P.R. Gibson ${ }^{2}$, J. M. Andrews ${ }^{1}$

${ }^{1}$ Department Of Medicine, The University of Adelaide, Adelaide/Australia/SA ${ }^{2}$ Gastroenterology, Monash University, Melbourne/Australia/VIC

Contact E-mail Address: ecushla.linedale@adelaide.edu.au

Introduction: Despite diagnostic criteria and effective management options for functional gastrointestinal disorders (FGID), confidence in managing these disorders in primary care is low, and long waiting lists for specialist care are common. New models which efficiently transfer specialist-held expertise to primary care practitioners is needed.

Aims \& Methods: We aimed to explore and describe the patient and primary healthcare provider (PHCP) experience of a novel non-specialist-dependent algorithm-based approach to the diagnosis and management of FGID (ADAMFGID). Consecutive patients triaged to the 'routine waitlist' of an Australian public hospital Gastroenterology Department over 2 years, with non-specific gastrointestinal symptoms (no alarms) were randomised to waitlist control or the algorithm (2:1). Algorithm patients were screened for organic disease with an alarms-based questionnaire and panel of routine blood/stool tests. When patients had clinical alarms or abnormal tests, data were reviewed by a gastroenterologist and, if appropriate, prompt gastroenterologist appointment offered. All others were classified using Rome III criteria. and received a letter explaining their FGID diagnosis and dietary/psychological management options. Waitlist control patients were not screened. All participants completed follow-up surveys at 6,26 and 52 weeks. Referring doctors of the algorithm group were sent a feedback survey at study completion.

Results: 89 participants were screened (42 years [SD 14], 62\% female). 35 had clinical alarms warranting gastroenterologist review and 45 were diagnosed with FGID (9 excluded). At 6 week follow up: 35/36 FGID respondents had read the diagnostic/management letter, and most $(n=25)$ found it useful (17 useful, 8 partially useful). Few discussed it with their PHCP $(n=13)$, yet most $(n=28)$ commenced management ( $\mathrm{n}=33$ by 12 months). Dietary management options were preferred over psychological therapies $(\mathrm{n}=30 \mathrm{vs} \mathrm{n}=16)(\mathrm{p}=.001)$, as were do-it-yourself options above therapist consultation. $61 \%(n=22)$ of patients saw symptom improvement at 6 weeks, (18/21 at 12 months). 2 did not accept the diagnosis, and 3 consulted a specialist privately. 19/36 participants had no concerns at 6-week follow-up (19/21 at 12 months). Common concerns included symptom persistence $(6-\mathrm{wk} \mathrm{n}=10,12$-mth $\mathrm{n}=10)$ and fear of missed pathology $(6$-wk $n=4,12$-mth $n=4)$. Most respondents $(74 \%, 46 / 62)$ found the approach acceptable (FGID group: acceptable 12/36, moderately acceptable 10/36; Screen Fail Group: acceptable 20/26, moderately acceptable 4/26). Others expressed dissatisfaction with the healthcare system $(n=4)$, diagnosis $(n=3)$, screening questionnaire $(\mathrm{n}=1)$ and lack of improvement in symptoms $(\mathrm{n}=1)$. All responding PHCPs (23/80; 14 FGID group, 9 screen-fail group) found the approach acceptable (13 acceptable, 12 moderately acceptable).

Conclusion: An algorithm-based approach to the diagnosis and management of FGIDS, which does not rely on specialist input is feasible, acceptable and effective. A larger scale randomised controlled trial of this new clinical pathway for the diagnosis and management of FGIDs, in primary care is warranted.

Disclosure of Interest: P.R. Gibson: AbbVie, Ferring, Janssen, Ferring, Fresenius Kabi, Mylan Merck, Nestle Health Science, Danone, Allergan, Pfizer and
Takeda. Research grants from AbbVie, Janssen, Falk Pharma, Danone and A2 Milk Company.

J.M. Andrews: JMA has served as a speaker, a consultant and/or an advisory board member for Abbott, Abbvie, Allergan, Celgene, Ferring, Takeda, MSD, Shire, Janssen, Hospira and Pfizer, and has received research funding from Abbott, Abbvie, Ferring, MSD, Shire, Janssen.

All other authors have declared no conflicts of interest.

\section{P1799 ANNUAL FECAL IMMUNOLOGICAL TESTING IS LESS COSTLY THAN COLONOSCOPY EVERY 5 YEARS AND REDUCES MORTALITY IN FAMILIAL COLORECTAL CANCER SCREENING}

D. Nicolás Pérez ${ }^{1}$, I. Castilla-Rodríguez ${ }^{2}$, M. Salgado-Fernández ${ }^{3}$, M. Ponce ${ }^{3}$, J.M. Herrero-Rivas ${ }^{5}$, T. Ocaña ${ }^{6}$, M.P. Roncales ${ }^{7}$, F. Sopeña-Biarge ${ }^{7}$, J. Santiago ${ }^{8}$, C. Alvarez Urturi ${ }^{9}$, E. Andreu-García ${ }^{10}$, O. Castaño-Fernández ${ }^{11}$, P. Muñoz-Garrido ${ }^{12}$, M. Rodríguez-Soler ${ }^{10}$, M. Carrillo Palau ${ }^{1}$, M. LlanosMuñoz ${ }^{14}$, N. González-López ${ }^{1}$, J. Cubiella $^{3}$, F. Balaguer ${ }^{16}$, L. Bujanda Fernández De Piérola ${ }^{17}$, A. Lanas ${ }^{18}$, A. Suárez González ${ }^{11}$, A. Herreros De Tejada $^{19}$, R. Jover ${ }^{10}$, X. Bessa Casserras ${ }^{20}$, A. Gimeno Garcia ${ }^{1}$, E. Quintero Carrion ${ }^{1}$

${ }^{1}$ Servicio De Aparato Digestivo, Hospital Universitario de Canarias, Santa Cruz de Tenerife/Spain

${ }^{2}$ Departamento de Ingeniería Informática y de Sistemas. Universidad de La Laguna. Health Services Research on Chronic Patients Network (REDISSEC). Centre for Biomedical Research of the Canary Islands (CIBICAN), Santa Cruz de Tenerife, Spain., Santa Cruz de Tenerife/Spain

${ }^{3}$ Complexo Hospitalario Universitario de Ourense, Ourense/Spain

${ }^{5}$ Complexo Hospitalario Universitario de Ourense, Orense/Spain

${ }^{6}$ Hospital Clinic Barcelona, Barcelona/Spain

${ }^{7}$ Servicio De Aparato Digestivo, Hospital Clínico Universitario Lozano Blesa, Zaragoza/Spain

${ }^{8}$ Hospital Puerta de Hierro, Madrid/Spain

${ }^{9}$ Hospital del Mar - Parc de Salut Mar, Barcelona/Spain

${ }^{10}$ Servicio De Aparato Digestivo, Hospital General Universitario de Alicante, Alicante/Spain

${ }^{11}$ Servicio De Aparato Digestivo, Hospital Universitario Central de Asturias, Oviedo/Spain

${ }^{12}$ Hospital Universitario Donostia, Donostia/Spain

${ }^{14}$ Servicio De Oncología Médica, Hospital Universitario de Canarias, Santa Cruz de Tenerife/Spain

${ }^{16}$ Gastroenterology, Hospital Clinic Barcelona, Barcelona/Spain

${ }^{17}$ Servicio De Aparato Digestivo, Hospital de Donostia, Donostia/Spain

${ }^{18}$ Dept. Medicine \& Gastroenterology, University of Zaragoza University Hospital Dept. of Medicine-Gastroenterology, Zaragoza/Spain

${ }^{19}$ Servicio De Aparato Digestivo, Hospital Puerta de Hierro, Madrid/Spain

${ }^{20}$ Servicio De Aparato Digestivo, Hospital del Mar - Parc de Salut Mar.

Barcelona/Spain

Contact E-mail Address: dnicolasp@telefonica.net

Introduction: Colonoscopy every 5 years, starting at the age of 40 years, is considered the first-choice screening strategy in first degree relatives (FDR) of patients with colorectal cancer CRC, as these individuals are considered at higher risk of developing CRC than average-risk individuals. However, this practice has a low adherence and remains opportunistic. Recently, it has been suggested that annual fecal immunochemical testing (FIT) might be a valid alternative to colonoscopy in this setting. However, there are scarce data regarding cost-effectiveness of these strategies from the perspective of healthcare services. Aims \& Methods: This study was aimed to compare the cost-effectiveness of annual FIT and colonoscopy every 5 years, to reduce CRC mortality, in FDR of patients with CRC. A Markov model was constructed to simulate the efficacy and cost of annual FIT (cut-off $10 \mu \mathrm{g} \mathrm{Hb} / \mathrm{g}$ feces) or colonoscopy every 5 years of 
previously unscreened FDR, starting at age 40 years and ending at age 75. A $50 \%$ adherence for each strategy was assumed. The model was adjusted to the incidence of CRC in Spain and real prevalence of advanced adenoma and CRC in the familial-risk population (http://dx.doi.org/10.1371/journal.pmed.1002008.g001). The main outcomes were quality-life-year (QALY) gained compared with no screening, lifetime burden of colonoscopy, lifetime number of colonoscopy complications, and the incremental cost-effectiveness ratio (ICER). We applied a willingness-to-pay threshold of $€ 25,000$ per QALY gained. Data from a prospective EuroQoL survey carried out on 920 Spanish patients at different disease stages were used for QALY measurement. Sensitivity analysis was performed to evaluate the robustness of the model.

Results: In a hypothetical cohort of 10,000 asymptomatic FDR, annual FIT and colonoscopy every 5 years were cost-effective over no screening. Taking no screening as comparator, ICER for annual FIT and colonoscopy every 5 years was 1989 and 4472 euros/QALY, respectively. Compared to no screening, annual FIT and colonoscopy every 5 years reduced CRC mortality by $59 \%$ and $81 \%$, respectively. The annual FIT strategy saved $33 \%$ of colonoscopies and was associated with a lower number of complications compared to colonoscopy every 5 years. The results were robust in sensitivity analyses.

Conclusion: Assuming a $50 \%$ adherence, annual FIT is less costly than colonoscopy every 5 years for CRC screening and reduces mortality in the familial-risk population. These data suggest that FDR of patients with CRC could be included in organized nationwide FIT-based screening programs.

Disclosure of Interest: All authors have declared no conflicts of interest.

\section{References}

1. Quintero E, Carrillo M, Leoz ML, Cubiella J, Gargallo C, Lanas A, Bujanda L, Gimeno-García AZ, Hernández-Guerra M, Nicolás-Pérez D, AlonsoAbreu I, Morillas JD, Balaguer F, Muriel A; Oncology Group of the Asociación Española de Gastroenterología (AEG) Risk of Advanced Neoplasia in First-Degree Relatives with Colorectal Cancer: A Large Multicenter Cross-Sectional Study. PLoS Med. 2016 May 3;13(5):e1002008. doi: 10.1371/journal.pmed.1002008. eCollection 2016 May. PubMed PMID: 27138769; PubMed Central PMCID: PMC4854417.

2. Schoen RE, Razzak A, Yu KJ, Berndt SI, Firl K, Riley TL, Pinsky PF. Incidence and mortality of colorectal cancer in individuals with a family history of colorectal cancer. Gastroenterology. 2015 Nov;149(6):1438-1445.e1. doi: 10.1053/j.gastro.2015.07.055. Epub 2015 Aug 5. PubMed PMID: 26255045; PubMed Central PMCID: PMC4628587.

3. Quintero E, Carrillo M, Gimeno-García AZ, Hernández-Guerra M, NicolásPérez D, Alonso-Abreu I, Díez-Fuentes ML, Abraira V. Equivalency of fecal immunochemical tests and colonoscopy in familial colorectal cancer screening. Gastroenterology. 2014 Nov;147(5):1021-30.e1; quiz e16-7. doi: 10.1053 j.gastro.2014.08.004. Epub 2014 Aug 13. PubMed PMID: 25127679.

4. Castells A, Castellví-Bel S, Balaguer F. Concepts in familial colorectal cancer: where do we stand and what is the future? Gastroenterology. 2009 Aug;137(2):404-9. doi: 10.1053/j.gastro.2009.06.015. Epub 2009 Jun 21. Review. PubMed PMID: 19540838.

\section{P1800 COMBINATION OF FOBT AND FECAL CALPROTECTIN MAY BE USEFUL FOR REDUCING UNNECESSARY COLONOSCOPIES IN SYMPTOMATIC PATIENTS}

C. Sostres ${ }^{1}$, A. Lué ${ }^{1}$, M.V. Barra Pardos ${ }^{2}$, G. Hijos ${ }^{2}$, A. Perales ${ }^{3}$, J.J. Puente ${ }^{2}$ A. Lanas ${ }^{1}$, F. Gomollon ${ }^{4}$

${ }^{1}$ Biosanitary Research Institute Aragón (IIS Aragón), Zaragoza/Spain

${ }^{2}$ Hospital Clinico Universitario Lozano Blesa, Zaragoza/Spain

${ }^{3}$ Medicine, Zaragoza University, Zaragoza/Spain

${ }^{4}$ Gastroenterology, Hospital Clínico Universitario Lozano Blesa, IIS Aragón and CIBERehd, Zaragoza/Spain

Contact E-mail Address: carlossostres@gmail.com

Introduction: Faecal occult blood test (FOBT) is a non-invasive and easily performed test which has demonstrated to reduce CRC incidence and mortality in the populations. Faecal calprotectin (FCP) has good evidence for detecting inflammatory bowel disease but its value in CRC and adenoma detection in the symptomatic population is less well studied. Most patients with symptoms have benign pathology but in an effort to detect most cancer or pre-cancerous lesions, almost all patients undergo colonoscopy.

Aims \& Methods: To evaluate and compare the diagnostic accuracy of the combination of FOBT plus FCP versus each test alone in symptomatic patients referred for diagnostic colonoscopy. A total of 171 patients who completed colonic investigations and returned stool samples, were prospectively recruited and included in the final analysis. FOBT was performed by SENTi FIT 270 test (Sentinel Diagnostics, Milan, Italy) and FCP by the EliA Calprotectin immunoassay (Thermo Fisher Scientific, Waltham, United States). Reference cut-off levels for abnornal test were $117 \mathrm{ng} / \mathrm{ml}$ for FOBT and $50 \mu \mathrm{g} / \mathrm{g}$ for FCP respectively. The diagnostic accuracy of FOBT and FCPwere evaluated by a logistic regresion model. CRC, advanced adenoma, IBD and angiodysplasia were considered as relevant pathology. Positive and negative predictive values, sensitivity and specificity were calculated. MedCalc was used for the ROC curve analysis. Results: 171 patients $(42.7 \%$ female; median age 62 years, IQR: $51-68)$ were included. $37(21.6 \%)$ had relevant colonic pathology. The most frequent indications for colonoscopy were previous episode of rectal bleeding in $71(42 \%)$ patients, change of bowel habits in $28(16 \%)$ and anaemia in $22(13 \%)$ Diagnostic accuracy of FOBT, FCP and combination of both are summarized in table 1.
PPV: predictive positive value; NPV: predictive negative value

\begin{tabular}{lllllll}
\hline Test & Normal test & Abnormal test & PPV & NPV & Sensitivity & Specificity \\
\hline FOBT & 131 & 40 & $55 \%$ & $89 \%$ & $59 \%$ & $89 \%$ \\
FCP & 81 & 90 & $30 \%$ & $88 \%$ & $73 \%$ & $53 \%$ \\
Combination & 114 & 57 & $56 \%$ & $96 \%$ & $86 \%$ & $81 \%$ \\
\hline
\end{tabular}

AuROC curves for relevant colonic pathology were 0.777 (95\% CI: $0.708-0.837$ $\mathrm{P}<0.0001)$ for FOBT, $0.692(95 \% \mathrm{CI}: 0.617-0.760 ; \mathrm{P}=0.0005)$ for $\mathrm{FCP}$ and 0.848 (95\% CI: $0.785-0.898 ; \mathrm{P}<0.0001)$ for combination of both tests respectively. Combination of both test have a significant better diagnostic accuracy compared to either test alone ( $\mathrm{P}=0.043$ vs FOBT and $\mathrm{P}=0.002$ vs $\mathrm{FCP}$ ) with a higher NPV. No significant difference was observed between FOBT and FCP $(\mathrm{P}=0.221)$.

Conclusion: Our model based on combination of FOBT and FCP has a better diagnostic accuracy performance compared to each test alone for the detection of relevant colonic pathology. Because of high NPV, performing FOBT and FCP in symptomatic patients before colonoscopy could be a feasible strategy in order to avoid unnecessary procedures

Disclosure of Interest: A. Lanas: Angel Lanas is advisor to Sysmex Spain All other authors have declared no conflicts of interest.

\section{P1801 CLINICAL PRACTICE, MONITORING, AND PATIENT} SAFETY DURING PROCEDURAL SEDATION IN FIVE COUNTRIES

R. Saunders ${ }^{1}$, J. Davis ${ }^{1}$, R. Weissbrod ${ }^{2}$, P. Kranke ${ }^{3}$, J. R. Lightdale ${ }^{4}$, D. Whitaker ${ }^{5}$

${ }^{1}$ Health Economics, Coreva Scientific, Freiburg/Germany

${ }^{2}$ Medtronic, Jerusalem/Israel

${ }^{3}$ University of Würzburg, Würzburg/Germany

${ }^{4}$ University of Massachusetts, Worcester/United States of America

${ }^{5}$ Manchester Royal Infirmary, Manchester/United Kingdom

Contact E-mail Address: jason@coreva-scientific.com

Introduction: Procedural sedation is considered an integral part of gastrointestinal endoscopy in many countries. The use of sedation does, however, vary internationally and can present patient safety concerns. It has been questioned whether trials assessing patient safety in endoscopy can be generalized worldwide given variations in sedation practice and adverse events rates.

Aims \& Methods: We conducted an international survey to examine procedural sedation practices and the incidence of adverse events (AEs) during procedural sedation in France, Germany, Italy, UK, and USA. Data collection from providers (nurses, physicians, and anaesthesiologists) was via online surveys. Respondents were screened to assure that they had the expertise and experience to complete the survey. The survey covered topics such as guidelines, sedation agents, monitoring, patient throughput, and $\mathrm{AE}$ incidence, treatment, and outcomes as defined by World SIVA ${ }^{1}$. Data analysis took a global approach with subgroup analysis by location.

Results: 101 providers completed the survey, with 20 responses per country, excepting the USA with 21 . More than $62 \%$ of respondents were gastroenterologists and anaesthesiology nurses. The main sedation agents reported employed were midazolam (93 respondents), propofol (90), fentanyl (75), ketamine (57), and meperidine (15). Respondents from France reported higher ketamine and lower fentanyl use than other countries $(\mathrm{p}=0.03)$. Standard of care monitoring was generally reported to be comprised of pulse oximetry plus blood pressure and/or heart rate. Capnography use varied by country, and was standard of care for $46 \%$ of respondents (ranging from $15 \%$ in Italy to $67 \%$ in the US). The most desired property of a monitoring modality across all countries was one that "provides an early warning of patient compromise". "Data displays and alarms relat[ing] to clinical events" was ranked second globally and in all countries apart from Italy. All respondents reported experience with patient safety issues in the last year, with hypotension the most common (67), followed by mild desaturation (63), bradycardia (63) and prolonged desaturation (52). AE incidence was influenced by monitoring modalities used. Among German respondents, for example, 7 of 11 who did not use capnography as standard of care reported severe oxygen desaturation events, compared with versus 0 of 9 who routinely use it $(\mathrm{p}=0.005)$. Providers also differed in their reported adverse event incidence. Gastroenterologists most commonly reported mild oxygen desaturation to occur, while anaesthesiologists and nurses reported hypotension to be the most common AE experienced during procedural sedation (Table).

Conclusion: Clinical sedation practices are relatively consistent across countries, as are the occurrence of adverse events. Pulse oximetry monitoring is almost universally used during sedation, while capnography use is more variable. Sedation monitoring that provides an actionable early warning of patient compromise remains a priority.

Disclosure of Interest: R. Saunders: Rhodri Saunders is the owner of Coreva Scientific $\mathrm{GmbH} \& \mathrm{Co} \mathrm{KG}$, which has received consultancy fees from Medtronic Inc and Covidien AG

J. Davis: Jason Davis is an employee of Coreva Scientific GmbH \& Co KG, which received consultancy fees for designing and undertaking this research

R. Weissbrod: Rachel Weissbrod is an employee of Medtronic

P. Kranke: Peter Kranke has advised for multiple pharmaceutical and medical device companies. He did not receive any remuneration for work performed on this research project. 
Abstract No: P1801

Table: Most commonly reported adverse events by provider

\begin{tabular}{|c|c|c|c|}
\hline Rank & Anaesthesiologist & Sedation Nurse & Gastroenterologist \\
\hline 1 (Most frequent) & Hypotension & Hypotension & Mild desaturation (short) \\
\hline 2 & Bradycardia & Bradycardia & Bradycardia \\
\hline 3 & Tachycardia & Tachycardia & Tachycardia \\
\hline 4 & Mild desaturation (short) & Mild desaturation (short) & Hypotension \\
\hline 5 & Airway obstruction & Apnoea (short) & Mild desaturation (prolonged) \\
\hline 6 & Mild desaturation (prolonged) & Mild desaturation (prolonged) & Hypertension \\
\hline 7 & Apnoea (short) & Hypertension & Severe desaturation \\
\hline 8 & Hypertension & Failed sedation & Airway obstruction \\
\hline 9 & Prolonged apnoea & Allergy & Allergy \\
\hline 10 & Failed sedation & Airway obstruction & Apnoea (short) \\
\hline 11 & Allergy & Prolonged apnoea & Failed sedation \\
\hline 12 & Severe desaturation & Severe desaturation & Prolonged apnoea \\
\hline 13 & Cardiac arrest & Cardiovascular collapse & Cardiovascular collapse \\
\hline 14 & Cardiovascular collapse & Cardiac arrest & Seizure \\
\hline 15 (Least Frequent) & Seizure & Seizure & Cardiac arrest \\
\hline
\end{tabular}

J.R. Lightdale: Jenifer Lightdale did not receive any remuneration for participation in this research project. She has previously consulted for Medtronic and other medical device companies.

D. Whitaker: David Whitaker has consulted for Medtronic and Covidien. He did not receive any remuneration for participation in this research project.

\section{Reference}

1. Mason et al. BJA. 2012;108(1):13-20

P1802 PERFORMANCE OF THE MOTUS PURE-VU SYSTEM - A NOVEL DEVICE FOR ACHIEVING ADEQUATE BOWEL PREP IN POORLY PREPPED PATIENTS

P.D. Siersema ${ }^{1}$, K. Van Keulen ${ }^{1}$, H. Neumann ${ }^{2}$, M.C.w. Spaander ${ }^{3}$ ${ }^{1}$ Gastroenterology \& Hepatology, University Merdical Center Gastroenterology \& Hepatology - Gastroenterology \& Hepatology, University, Nijmegen/Netherlands ${ }^{2}$ Interventional Endoscopy Center, I. Medizinische Klinik Und Poliklinik, Universität Erlangen-Nürnberg, Mainz/Germany

${ }^{3}$ Gastroenterology \& Hepatology, Erasmus Medical Center Rotterdam, Rotterdam Netherlands

Contact E-mail Address: kelly.vankeulen@radboudumc.n

Introduction: The success of colonoscopy depends on the quality of the bowel preparation, which is estimated to occur in as many as $25 \%$ of colonoscopy procedures. The MOTUS GI Pure-Vu ${ }^{\mathrm{TM}}$ System (Tirat Carmel, Israel) is an FDA cleared device designed to improve visualization in an inadequately prepared colon by facilitating intra-procedural cleaning.

Aims \& Methods: This study aims to evaluate the performance of the Pure- $\mathrm{Vu}$ System in cleansing a poorly prepared colon, assess the system's usability, patient satisfaction and safety. Forty-seven cases were planned to be enrolled at three clinical sites, of which 32 had completed the study so far. Pure-Vu was used in subjects with a partially prepped colon after $2 \times 10 \mathrm{mg}$ Bisacodyl, diet restrictions (no dried fruit, seeds or nuts) starting 2 days before the procedure and a 24-hour clear liquid diet prior to the colonoscopy. Study endpoints were: (1) Safety, 2) Improvement of colon cleansing level as per the Boston Bowel Preparation Scoring (BBPS) when comparing before and after Pure-Vu use, (3) Pure-Vu usability via questionnaire and (4) Patients' satisfaction via questionnaire.

Results: Thirty-two subjects (41\% males), aged $31-74$ years and with a mean BMI of $26 \pm 3.9$. were included in the analysis. Indications for colonoscopy included family history of CRC (56\%) and polyp surveillance $(44 \%)$. No serious adverse events were reported. The Pure-Vu significantly increased the number of subjects with an adequate cleansing level (BBPS $>=2$ for all 3 colon segments) from $25 \%$; CI $95 \%[11 \%, 43 \%]$ at baseline to $100 \%$; CI $95 \%[89 \%, 100 \%]$ afte Pure-Vu and the cecum was reached and visualized in all study cases (i.e., $100 \%$; CI $95 \%[89 \%, 100 \%])$. Mean post-treatment BBPS score was $8.5 \pm 0.8$ vs. $3.38 \pm 2.3$ prior to Pure-Vu use. Physicians were satisfied with the device's general use of ease and found it in most cases acceptable to good or excellent to insert and to angulate the colonoscope. No major difficulties were experienced when performing polypectomy. Thirty of $32(94 \%)$ patients reported that they would recommend Pure-Vu to theirs friends and family members who need a colonoscopy. Thirty $(94 \%)$ of the patients found the overall bowel preparation as very tolerable $(55 \%)$ or acceptable $(39 \%)$. Seventy-nine percent of patients who had a previous colonoscopy procedure reported that the Pure-Vu bowel preparation was more tolerable as compared to their previous colonoscopy preparation and $14 \%$ of the patients reported it to be about the same.

Conclusion: The Pure-Vu System was found to be safe and effective in cleaning inadequately prepared colons to an adequate level for a thorough exam. Based upon these early results it is expected that the device may play a role in patients with an inadequately prepared colon which may help to improve the overall quality of colonoscopy.

Disclosure of Interest: All authors have declared no conflicts of interest.

\section{P1803 THE EUROPEAN SOCIETY OF GASTROINTESTINAL ENDOSCOPY KEY PERFORMANCE MEASURES FOR COLONOSCOPY IN THE POLISH COLORECTAL CANCER SCREENING PROGRAM}

M. Bugajski ${ }^{1}$, P. Wieszczy ${ }^{2}$, M. Rupinski ${ }^{1}$, J. Regula ${ }^{2}$, M.F. Kaminski ${ }^{1}$

${ }^{1}$ Department Of Gastroenterological Oncology, The Maria Sklodowska-Curie Memorial Cancer Centre and Institute of Oncology, Warsaw, Poland, Warsaw Poland

${ }^{2}$ Department Of Gastroenterology, Hepatology And Oncology, Medical Center for Postgraduate Education, Warsaw, Poland, Warsaw/Poland

Contact E-mail Address: marek.bugajski@gmail.com

Introduction: Recently, the European Society of Gastrointestinal Endoscopy (ESGE) published guidelines on key performance measures for colonoscopy (1). We analyzed feasibility of monitoring these measures and whether the proposed standards were met in the Polish Colonoscopy Screening Program (PCSP). Aims \& Methods: We analyzed database records for 40,644 participants aged 55 to 64 years, who between 2014 and 2015, underwent screening colonoscopy in 24 centers of population-based PCSP. We used the ESGE guideline definitions to calculate values of all seven key performance measures. We compared key performance measures within the PCSP against proposed standards on the program and center level. Data on adequacy of bowel preparation was routinely assessed with the Boston Bowel Preparation Scale, whereas data on patient experience with the validated Gastronet questionnaire (2). Data on complication rates were collected from the National Health Fund database and Personal Identification Number Registry.

Results: Overall, on the program level, all minimum standards for colonoscopy key performance measures were met. Rate of adequate bowel preparation was $92.1 \%$ for the whole program, ranging $80.9-99.2 \%$ per individual center, with 7 centers $(29.2 \%)$ not reaching minimum standard of $90 \%$ and 9 centers $(37.5 \%)$ reaching the target standard of $95 \%$. Cecal intubation rate was $97.4 \%$ (range 93.4-99.4\%), with all centers reaching minimum standard of $90 \%$ and only one center not reaching target standard of $95 \%$. Adenoma detection rate was $29.9 \%$ (range $19.1-39.1 \%)$, with 7 centers $(29.2 \%)$ not reaching minimum standard of $25 \%$. Appropriate polypectomy technique was applied in case of $90.9 \% 6$ to $9 \mathrm{~mm}$ polyps (range $64.3-100 \%$ ) with only 2 centers not reaching minimum standard of $80 \%$ and $48.2 \%$ of 4 to $5 \mathrm{~mm}$ polyps (range $0-100 \%$ ) with only 6 centers reaching minimum standard of $80 \%$. Target standard of $90 \%$ was reached in 15 centers for polyps 6 to $9 \mathrm{~mm}$ in diameter and only 2 centers for polyps 4 to $5 \mathrm{~mm}$ in diameter. For the whole program, 7-day hospitalization rate after screening colonoscopy was $0.3 \%$ (122 cases) and 30-day all-cause mortality was $0.02 \%$ ( 9 cases). Gastronet questionnaire coverage is assumed to be $100 \%$, however the response rate was $65.3 \%$ (range $7.6 \%-81.8 \%$ ), with painful colonoscopy rate of $19.2 \%$. No minimum standard is set, however target standard of $90 \%$ of procedures with measured patient's experience was not met. Appropriate post-polypectomy surveillance, based on the European guidelines, was proposed in $95.4 \%$ of cases (range $84.9-99.7 \%$ ). Target standard of $95 \%$ was met in 15 centers, the minimum standard is not set.

Conclusion: Monitoring ESGE performance measures for colonoscopy is feasible in colonoscopy programmatic screening setting 6 of 7 performance measures were easy to monitor with PCSP database, however monitoring complications needs further development to avoid extracting data from external registries. PCSP meets proposed minimum standards on program level, however some centers need additional interventions to meet the quality standards. Applying appropriate polypectomy technique for polyps ranging 4 to $5 \mathrm{~mm}$ in diameter 
is currently the biggest issue in PCSP and further training is needed to reach minimum standard for this performance measure.

Disclosure of Interest: All authors have declared no conflicts of interest.

\section{References}

1. Kaminski MF, Thomas-Gibson S, Bugajski M et al. Performance measures for lower gastrointestinal endoscopy: a European Society of Gastrointestinal Endoscopy (ESGE) Quality Improvement Initiative. Endoscopy. 2017 Apr;49(4):378-397.

2. Hoff G1, Bretthauer M, Huppertz-Hauss G et al. The Norwegian Gastronet project: Continuous quality improvement of colonoscopy in 14 Norwegian centres. Scand J Gastroenterol. 2006 Apr;41(4):481-7.

\section{P1805 VALIDATION OF THE "FAILURE TO PROVIDE ADEQUATE RELIEF" (F-PAR) SCALE IN A SPECIALIST CLINIC SETTING}

V. Passananti, N. Zarate Lopez, A. V. Emmanuel

GI Physiology Unit, University College London Hospital, London/United Kingdom

\section{Contact E-mail Address: valentinapassananti@gmail.com}

Introduction: Treatment of chronic idiopathic constipation is somewhat empiric, but based on step-wise approach[1]. If first-line conservative treatment (lifestyle advice and laxatives) do not relieve symptoms sufficiently, secondary approaches with prokinetic or secretagogue drugs are used before considering hospital-based care (biofeedback, psychosocial support, transanal irrigation (TAI), surgery). Nevertheless, patients are often dissatisfied with care[2] and fail to progress to adequate levels of therapy. The 5-point Failure to Provide Adequate Relief (FPAR) scale[3] was developed to facilitate the recognition of when to move from one step to the next.

Aims \& Methods: The aim of this study was to validate F-PAR in a tertiary clinic setting. We studied 403 consecutive consultations of 331 patients (262 women, mean age 41) in our specialist clinic. All fulfilled Rome III/IV diagnostic criteria for chronic constipation. Immediately prior to each face-to-face clinical assessment by one of 2 experienced physicians, participants completed the F-PAR scale; patients were seen blind to the F-PAR result. Standard clinic assessment was undertaken to identify efficacy of the current management as the gold standard.

Results: Of the 403 consultations, clinical assessment identified inadequate relief with current therapy was identified in 200 . Neither duration nor type of treatment were correlated with relief. The table stratifies, by clinical gold standard, each item of the F-PAR and in the lower panel the total number of F-PAR items replied to positively.

Table: Positive F-PAR items correlated to clinical assessment of relief

\begin{tabular}{lll}
\hline & $\begin{array}{l}\text { Adequate relief } \\
\text { (Clinical) } \\
\mathrm{n}=203\end{array}$ & $\begin{array}{l}\text { Inadequate } \\
\text { relief (Clinical) } \\
\mathrm{n}=200\end{array}$ \\
\hline Bowel frequency inadequate & 5 & 71 \\
Strain most occasions & 6 & 89 \\
Stool hardness & 3 & 21 \\
Onset other symptom & 2 & 57 \\
Current therapy poor tolerable & 8 & 80 \\
0 FPAR replies & 187 & 1 \\
1 FPAR replies & 10 & 41 \\
2 FPAR replies & 4 & 67 \\
3 FPAR replies & 2 & 22 \\
4 FPAR replies & 0 & 8 \\
5 FPAR replies & 0 & 9 \\
\hline
\end{tabular}

Conclusion: Our findings showed that the F-PAR with only five questions can be considered sufficient to provide clinical evidence of treatment failure. The use of standardized process to investigate the efficacy of treatment may reduce the time and improve the quality of managing for the chronic constipation patients.

Disclosure of Interest: All authors have declared no conflicts of interest.

\section{References}

1. Tack et al Neurogastroenterol Motil. 2011 Aug;23(8):697-710

2. Emmanuel et al United European Gastroenterol J. 2013 Oct;1(5):375-84

3. Tack et al Aliment Pharmacol Ther. 2017 Feb;45(3):434-442
P1806 ADHERENCE WITH TRANSANAL IRRIGATION USING THE NAVINA ${ }^{\text {TM }}$ SYSTEM IS ASSOCIATED WITH PERSONALITY TRAITS EVEN WHEN THERE IS IMPAIRED HAND FUNCTION

V. Passananti, J. Storrie, N. Zarate Lopez, A. V. Emmanuel

GI Physiology Unit, University College London Hospital, London/United Kingdom

Contact E-mail Address: valentinapassananti@gmail.com

Introduction: Transanal irrigation has become a key therapeutic modality in managing patients with neurological diseases who experience constipation and or faecal incontinence. Such neurogenic bowel dysfunction (NBD) complicates over three quarters of patients with spinal cord injury (SCI) and multiple sclerosis (MS). Approximately $60 \%$ of patients who start TAI continue with long-term treatment. A common cause of treatment cessation is impaired hand ${ }^{(1)}$. Training of the patient is a key aspect of TAI therapy and requires patients to be willing to manage their health themselves: self-efficacy.

Aims \& Methods: We wished to study whether use of a novel TAI system, Navina $^{\mathrm{TM}}$ Smart, which has an electronic pump component allows patients with impaired levels of hand function to adhere to TAI therapy. We also wished to identify if there were physiological or psychological correlates of adherence. Twenty-eight consecutive patients (19 SCI and 9 MS; 17 male, mean age 42) were studied. All patients scored greater than 18 on the Cochin Hand Function questionnaires were completed to assess anxiety/depression and locus of control respectively. Anorectal physiology (manometry, sensation and rectal compliance) was undertaken at baseline. Training in TAI was undertaken by the same experienced nurse, with weekly follow up until a stable regime was established. Adherence with therapy at 12 weeks was identified.

Results: At 12 weeks, 16/28 (57\%) of patients were still using Navina TAI, similar proportions with SCI (11/19) and MS (5/9). There was no difference in baseline scores for HAD-anxiety $(6.4 \pm 2.3$ vs $5.9 \pm 2.9 ; \mathrm{p}=0.37)$ and HAD-depression $(8.6 \pm 3.9$ vs $8.8 \pm 4.2 ; p=0.46)$ and were similar in both those who were and were not still using TAI (mean $\pm \mathrm{SD}$ respectively). The Rotter score for nonadherers was significantly greater than adherers (14.2 \pm 6.7 vs $10.6 \pm 5.9$ respectively; $p=0.008$ ). There was no difference in any of the anorecyal parameters between those who did or did not adhere with TAI.

Conclusion: Navina Smart TAI is an effective therapy in $57 \%$ of NBD patients with significant hand dysfunction. Anorectal physiology, anxiety and depression did not predict likelihood of treatment adherence. An external locus of control, reflecting a belief that health events occur because of outside forces (such as fate, chance, or powerful others), is associated with reduced treatment success. The results suggest that future studies of TAI should consider locus of control as an important potential predictor of outcome

Disclosure of Interest: All authors have declared no conflicts of interest.

\section{References}

1. Emmanuel et al PLoS One. 2016 Aug 24;11(8):e0159394

2. Duruöz, et al $J$ Rheumatol. 1996;23:1167-72

3. Zigmond, AS; Snaith, RP Acta Psychiatrica Scandinavica. 1983. 67: 361-370

4. Rotter, Julian B. 1966. Psychological Monographs 80 (1): 1-28

\section{WEDNESDAY, NOVEMBER 01, 201709:00-14:00} OESOPHAGEAL, GASTRIC AND DUODENAL DISORDERS III - HALL 7.

\section{P1807 THE DUODENAL MUCOSA RETAINS A DIVERSE} MICROBIOTA FOLLOWING BOWEL PREPARATION

E. Shanahan ${ }^{1}$, A. Shah ${ }^{1}$, A. Do ${ }^{2}$, P. Ghasemi ${ }^{1}$, T. Hansen ${ }^{1}$, N. Koloski ${ }^{1}$, M. Morrison ${ }^{3}$, G. Holtmann ${ }^{1}$

${ }^{1}$ Gastroenterology And Hepatology, Princess Alexandra Hospital, Brisbane/ Australia

${ }^{2}$ Faculty Of Medicine, University of Queensland Translational Research Institute, Brisbane/Australia/QLD

${ }^{3}$ University of Queensland, Diamantina Institute, Microbial Biology and Metagenomics, Brisbane/Australia

Contact E-mail Address: e.shanahan@uq.edu.au

Introduction: The microbiota inhabiting the gastrointestinal (GI) tract plays an essential role in gut health. Although mucosal biopsies are increasingly used for microbiota studies, these are subject to variations introduced through sampling technique and patient preparation. The impact of bowel preparation on the mucosa-associated microbiota (MAM) is of particular interest given it results in complete emptying of bowel contents via laxative ingestion. Although bowel preparation does not appear to induce long term changes to stool microbiota [1], it can induce short-term changes to the colonic MAM [2]. While improvements in clearance of the small intestine after bowel preparation have been reported [3], the impact on the upper GI microbiota is currently unknown. Given patients may undergo both upper GI endoscopy and colonoscopy consecutively, a subset of endoscopy patients will have consumed bowel preparation prior to their procedure, representing a potential bias in MAM analyses. Therefore, this study aimed to assess the impact of bowel preparation on the duodenal MAM.

Aims \& Methods: Individuals undergoing upper GI endoscopy, with or without concurrent colonoscopy, were recruited consecutively with ethical approval. Individuals underwent upper GI endoscopy following overnight fast $(\mathrm{n}=58)$, or both upper endoscopy and colonoscopy following polyethylene glycol bowel preparation $(\mathrm{n}=48)$. Participants were undergoing screening for iron deficiency anaemia or GI symptoms with no evidence of mucosal disease/inflammation $(\mathrm{n}=88)$, or with diagnosed Crohn's disease $(\mathrm{n}=18)$. Duodenal biopsies were obtained and gDNA extracted. Amplicon libraries of the 16S rRNA gene were sequenced (Illumina MiSeq). Sequencing of reagent controls enabled exclusion of 
non-duodenal sequences. Bioinformatics and statistics were performed in QIIME and Calypso.

Results: A diverse microbiota was observed in duodenal mucosal samples from all subjects, following overnight fasting or bowel preparation. Overall the duodenal microbiota was dominated by the genus Streptococcus, followed by Prevotella, Veillonella and Neisseria. Microbial diversity within samples was not significantly different with and without bowel preparation (Chaol metric). Principal coordinates analysis (weighted UniFrac) revealed substantial overlap between the two groups, and no significant clustering was observed (ADONIS) based on whether patients had undergone overnight fasting or bowel preparation. Similar findings were obtained when these analyses were repeated with exclusion of the Crohn's disease population.

Conclusion: This study reveals a diverse duodenal MAM is retained following bowel preparation. The comparison of overnight fasting and bowel preparation indicates these differences in patient preparation do not substantially alter the duodenal MAM. Thus patients undergoing concurrent upper GI endoscopy and colonoscopy can be included in study cohorts investigating the upper GI MAM without risk of a substantial confounding effect.

Disclosure of Interest: All authors have declared no conflicts of interest.

\section{References}

1. O'Brien, CL et al. PLoS One 2013. 8(5) e62815

2. Shobar, RM et al. Clin Transl Gastroenterol 2016. 7, e143

3. Rokkas, T et al. Am J Gastroenterol 2009. 104:219-27

\section{P1808 PERFORMANCE OF GLASGOW-BLACTHFORD, ROCKALL AND AIMS65 SCORES TO PREDICT OUTCOMES AND TO IDENTIFY THE LOW-RISK GROUP AFTER UPPER GI BLEEDING IN PATIENTS WITH CANCER}

M. C. Franco ${ }^{1}$, S. Jang 2 , B. D.C. Martins ${ }^{1}$, T. Stevens ${ }^{2}$, V. Jairath ${ }^{3}$, R. Lopez ${ }^{2}$, J. J. Vargo ${ }^{2}$, A. Barkun, F. Maluf-Filho

${ }^{1}$ Gastrointestinal Endoscopy Unity, Cancer Institute of University of São Paulo, São Paulo/Brazil

${ }^{2}$ Department Of Gastroenterology And Hepatology, Cleveland Clinic, Cleveland/ United States of America

${ }^{3}$ Western University, London/Canada

${ }^{4}$ Division Of Gastroenterology, McGill University and the McGill University

Health Centre, Montreal/Canada

Contact E-mail Address: mcavalcantefranco@gmail.com

Introduction: Upper gastrointestinal bleeding (UGIB) in patients with cancer presents a unique and difficult challenge as these patients are at higher risk for rebleeding and mortality. ${ }^{1}$ Currently available prognostic scoring systems for UGIB for the general population have produced variable accuracy in their validation studies. ${ }^{2}$ An effective method of stratification for cancer patients to identify the high-risk group for early hospital-based intervention and death could enhance the outcomes of this specific population.

Aims \& Methods: The primary aim of this study was to compare the GlasgowBlatchford score (GBS), Rockall score (RS) and AIMS65 score for predicting ICU admission, blood transfusion, hemostatic therapy, rebleeding, and in-hospital mortality in cancer patients with UGIB. The secondary aim was to assess the above cited scores in correctly identifying low-risk patients that can be effectively managed as an outpatient. An IRB-approved, prospective study was conducted at the Cancer Institute of Sao Paulo, Brazil. Consecutive patients with known cancer admitted with UGIB were enrolled. Pre-endoscopic clinical parameters, endoscopic findings pertinent to the scoring systems, hemostasis techniques, and outcomes were collected into a prospective registry. Patients were followed for at least 30 days or until the day of discharge, whichever was longer. The low-risk group was defined as those without blood transfusion, hemostatic therapy (by endoscopy, radiotherapy, angiographic or surgical intervention), rebleeding or mortality in 30 days. Multiple logistic regression with receiver operating characteristics analysis was done to assess the predictive ability of each scoring system for the above outcomes.

Results: From April 2015 to May 2016, 394 consecutive patients were screened, while 259 patients met the inclusion criteria. A total of 243 patients were considered for the final analysis, after excluding 16 patients due to missing data or lost to follow up (Table 1). Predicting outcomes: The AIMS65 score (area under curve [AUC] 0.85) was significantly better for predicting ICU admission than GBS (AUC 0.79; $\mathrm{p}=0.04$ ), both the total and clinical RS (AUC 0.71 and 0.66; $\mathrm{p}<0.001$ for both). The GBS best predicted the need for blood transfusion (AUC 0.82 , sensitivity $71 \%$ and specificity $80 \%$ for GBS $\geq 12$ ) compared with the other prognostic scores. All scores performed poorly in predicting the need for hemostatic therapy and risk of rebleeding. The AIMS65 score best predicted in-hospital mortality (AUC 0.84) compared to the GBS (AUC 0.75; p =0.004), both the total and clinical RS (AUC 0.70 and $0.69 ; \mathrm{p}<0.001$ for both). Among patients with confirmed bleeding at EGD, there was no difference in 30-day mortality if the etiology of bleeding was tumoral or non-tumoral disease $(38.1 \%$ vs $31.9 \% ; p=0.46)$. Identifing low-risk group: With GBS score of 0 as the cut-off value, its specificity was $100 \%$ with sensitivity of $5.8 \%$. When GBS $\leq 2$ was used as cut-off, its specificity was maintained at $100 \%$, while sensitivity was increased to $23.5 \%$. This change increased the proportion of the patients from $1 \%$ to $5 \%$ without erroneously discharging high-risk patients. In comparison, when an AIMS65 value of 0 was chosen as definition for low-risk, this tool misclassified 20 patients who needed hospital interventions (specificity of $53 \%$ and sensitivity of $89.5 \%$ ). Finally, head-to-head comparison between GBS vs. RS, and GBS vs. AIMS65 scoring system revealed GBS to be superior to both the clinical RS $(\mathrm{p}<0.001)$ and AIMS65 $(\mathrm{p}=0.001)$ in correctly identifying low-risk patients.

Table 1: Demographic and Clinical Characteristics

\begin{tabular}{ll}
\hline Factor & Total $(\mathrm{n}=243)$ \\
\hline Age (years) & $60.6 \pm 13.6$ \\
Female/Male & $71(29.2 \%) / 172(70.8 \%)$ \\
Outpatient/Inpatient & $178(73.3 \%) / 65(26.7 \%)$ \\
Cancer in the Upper GI Tract & $74(30.5 \%)$ \\
Cancer Stage: & \\
I or II & $17(7.0 \%)$ \\
III & $48(19.8 \%)$ \\
IV & $177(73.1 \%)$ \\
Hemoblobin & $8.1 \pm 2.9$ \\
Albumin & $2.8 \pm 0.75$ \\
Rebleeding & $24(9.9 \%)$ \\
RBC Transfusion & $147(60.5 \%)$ \\
ICU & $107(44.0 \%)$ \\
Hemostatic Therapy & $104(42.8 \%)$ \\
30-day Mortality & $66(27.2 \%)$ \\
Follow-up time (days) & $30.0[22.0,30.0]$ \\
Clinical Rockall & $4.6 \pm 1.2$ \\
Total Rockall & $7.0 \pm 2.0$ \\
AIMS65 & $1.7 \pm 1.2$ \\
Glasgow-Blatchford & $10.8 \pm 4.2$ \\
&
\end{tabular}

Conclusion: The AIMS65 score was superior to other scoring systems in predicting in-hospital mortality and ICU admission in patients with cancer and UGIB, whereas the GBS was superior for predicting the need for blood transfusion. All scores performed poorly in prediction of hemostatic therapy and rebleeding. The GBS was superior in accurately identifying low-risk patients. Furthermore, the cut-off $\leq 2$ in GBS score displays increased sensitivity without compromising specificity, effectively increasing the number of patients who can be safely managed as an outpatient.

Disclosure of Interest: All authors have declared no conflicts of interest.

\section{References}

1. Maluf-Filho F et al. United Eur Gastroenterol J 2013.

2. Stanley AJ et al. BMJ 2017.

\section{P1809 THE EFFECTS OF ANTICOAGULANTS ON THE CLINICAL} OUTCOME OF ENDOSCOPIC TREATMENT

K. Namikawa ${ }^{1}$, T. Yoshio ${ }^{1}$, H. Tomita ${ }^{2}$, T. Yamada ${ }^{3}$, J. Fujisaki ${ }^{4}$ ${ }_{1}^{1}$ Gastroenterology, Cancer Institute Hospital Ariake, Tokyo/Japan

${ }^{2}$ Gastroenterology, Ehime Medical Center Hospital, Matsuyama/Japan

${ }^{3}$ Gastroenterology \& Hepatology, Osaka National Hospital, Osaka/Japan

${ }^{4}$ Gastroenterology, Cancer Institute Hospital JFCR, Tokyo/Japan

Contact E-mail Address: ken.namikawa@jfcr.or.jp

Introduction: Endoscopists are more frequently performing endoscopic resection (ER) in patients on antiplatelet or anticoagulant therapy and nowdays patients have increasingly started taking direct oral anticoagulant (DOAC) therapies, including direct anti-Xa and thrombin inhibitors. Major guidelines recommend the cessation of anticoagulants before ER and heparin bridging therapy (HBT) for high thrombotic risk cases, although these are still controversial. A recent study has suggested that HBT may be associated with a higher post-endoscopic resection bleeding (PEB) rate in patients on anticoagulants.

Aims \& Methods: This study aimed to evaluate the effect of anticoagulants on PEB rate. This was a retrospective study based on medical records from three centers. PEB was defined as bleeding that occurred $6 \mathrm{~h}$ to 10 days after ER, which required endoscopic hemostasis. We reviewed 108 gastric tumors including adenoma and early cancer in 97 patients on anticoagulant therapy who underwent endoscopic submucosal dissection (ESD) in our hospitals between June 2008 and February 2016. Further, we reviewed 69 colorectal polyps including adenoma and early cancer in 69 patients on anticoagulant therapy who underwent ER in our hospitals between October 2013 and September 2016. ER included polypectomy, endoscopic mucosal resection (EMR), and ESD. Patients were divided into two groups: those prescribed warfarin and patients prescribed DOAC. The management of antithrombotics was based on the Japanese Gastroenterological Endoscopy Society guidelines published in 2005 and 2012. The anticoagulants used during the study period were warfarin, dabigatran, rivaroxaban, apixaban, and edoxaban. Warfarin was discontinued 4-5 days before ER, whereas the others were stopped $24-48 \mathrm{~h}$ prior to the procedure. For patients at a high thrombotic risk, intravenous unfractionated heparin was administered after ceasing anticoagulants.

Results: Warfarin and DOAC were prescribed to $73(75 \%)$ and $24(25 \%)$ patients, respectively. Apixaban was administered to 1 (1\%), dabigatran to 12 $(12 \%)$, rivaroxaban to $11(11 \%)$ patients. There were no significant differences between the DOAC and warfarin groups in terms of clinical characteristics or 
PEB rate. Overall, 57 patients $(78 \%)$ in the warfarin group underwent HBT. and were significantly associated with $\mathrm{PEB}$ rate compared to the patients who did not undergo HBT in the warfarin group $(36 \%$ vs. $0 \%, \mathrm{p}<0.05)$. Although there was no significant difference in the PEB rate between the group that did and did not undergo HBT in the DOAC group, the PEB rate was higher among rivaroxaban recipients than dabigatran recipients $(45 \%$ vs. $0 \%$; p < 0.05$)$. Multivariate analysis revealed that HBT, rivaroxaban, and anticoagulants plus antiplatelet therapy were associated with PEB $(\mathrm{P}<0.05)$. Warfarin and DOAC were prescribed to $72(57 \%)$ and $54(43 \%)$ patients, respectively. Dabigatran was administered to $16(13 \%)$, rivaroxaban to $18(14 \%)$, apixaban to $18(14 \%)$, edoxaban to $2(1.6 \%)$ patients. Patients underwent polypectomy $(n=50)$, EMR $(n=62)$, or ESD $(\mathrm{n}=10)$. PEB occurred in $5(4.0 \%)$ patients (one polypectomy and four EMRs. Among them, warfarin was prescribed to one patient $(1.4 \%)$ who also underwent HBT. Rivaroxaban and apixaban were prescribed to two patients $(11 \%)$ each. PEB rate was higher in the DOAC group than in the warfarin group $(7.4 \%$ vs. $1.4 \%)$

Conclusion: Patients on HBT, rivaroxaban, and anticoagulants plus antiplatelet therapy are at an increased PEB risk after ESD for gastric tumors. In patients who underwent ER for colorectal polyps, PEB was more common in DOAC than that in warfarin recipients. That was the difference between ER for colorectal polyps and ESD for gastric tumors. We suggested that the PEB rate differs among DOACs because each drug has its own blood concentration and metabolization. Most clinicians have a limited knowledge on PEB during DOAC therapy because these are relatively new drugs, and because PEB has a low prevalence. Therefore, comparative data on larger patient series are needed to address this issue.

Disclosure of Interest: All authors have declared no conflicts of interest.

P1810 ENDOSCOPIC ALLPICATION OF MUCOADHESIVE POWDER (NEXPOWDER $\left.{ }^{\circledR}\right)$ FOR HEMOSTASIS IN PATIENTS WITH GASTROINTESTINAL BLEEDING

B.W. Bang ${ }^{1}$, K.S. Kwon ${ }^{1}$, H.K. Kim ${ }^{1}$, Y.W. Shin ${ }^{1}$, S.J. Hong ${ }^{2}$, J. Park ${ }^{1}$, J.H. Moon ${ }^{3}$, J.J. Hwang ${ }^{2}$

${ }^{1}$ Division Of Gastroenteroloy, Department Of Internal Medicine, Inha University School of Medicine, Incheon/Korea, Republic of

${ }^{2}$ Digestive Disease And Research Institute, SoonChung Hyang University School of Medicine, Bucheon/Korea, Republic of

${ }^{3}$ Gastroenterology, Digestive Disease Center, SoonChunHyang University School of Medicine, Bucheon/Korea, Republic of

Contact E-mail Address: bangbyoung@naver.com

Introduction: Although endoscopic hemostasis is usually effective in controlling gastrointestinal (GI) hemorrhage, some have difficulty in achieving successful hemostasis depending on the location and severity of hemorrhage. NEXPOWDER ${ }^{\circledR}$ (Next Biomedical, Incheon, South Korea) is a biocompatible and biodegradable powder and the hemostatic effects are accomplished by physical barrier when this powder immediately forms mucoadhesive hydrogel after contacting blood or water. It shows high adhesiveness and persistency of gel on ulcer base. In addition, new powder delivering device was developed to reduce catheter clogging during endoscopic application.

Aims \& Methods: The aims of this study were to confirm 1) success rate of

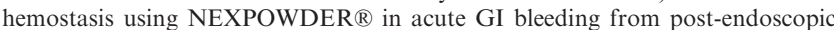
procedure or various causes, 2) re-bleeding rate on second-look endoscopy at 1 or 3 days after the procedure, 3) persistent rate of hydrogel on ulcer base at followup endoscopy, and 4) clogging rate of catheter during spraying powder. The NEXPOWDER $₫$ was delivered by newly developed spraying device through an 8 Fr catheter. We defined the initial hemostatic success as when the bleeding disappeared within 10 minutes. A second-look endoscopy was performed in one and three days after the procedure.

Results: A total of 57 patients were enrolled. The bleeding developed in 46 patients with post-endoscopic resection ulcers (41 ESD induced ulcers and 5 EMR induced ulcers), 8 patients with peptic ulcers and 3 patients with other causes. 1) Success rates of hemostasis in acute bleeding were $96.5 \%(55 / 57)$ of

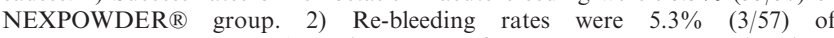
NEXPOWDER $®$ group. 3) Persistent rate of NEXPOWDER $®$ on ulcer base was $70.3 \%(26 / 37) 1$ day after the procedure, and $38.5 \%(15 / 38) 3$ days after the procedure. 4) Clogging rate of spraying catheter was $3.5 \%(2 / 57)$.

Conclusion: The endoscopic application of NEXPOWDER $®$ is effective for the several types of acute GI bleeding. This effective hemostatic action of NEXPOWDER ${ }^{\circledR}$ might be achieved by physical barrier of mucoadhesive and persistent hydrogel on ulcer base. And new powder delivering device shows low rate of catheter clogging. In addition, a newly developed powder delivering device shows low scattering and targeted spraying properties onto bleeding site. Disclosure of Interest: All authors have declared no conflicts of interest.

\section{References}

1. Bustamante-Balén M, Plumé G. Role of hemostatic powders in the endoscopic management of gastrointestinal bleeding. World $J$ Gastrointestinal Pathophysiol 2014:5:284-292

2. Chen YI, Barkun AN. Hemostatic powders in gastrointestinal bleeding: A systematic review. Gastrointest Endosc Clin A Am 2015;25:535-552
P1811 CLINICAL FEATURES OF DELAYED BLEEDING AFTER ENDOSCOPIC SUBMUCOSAL DISSECTION FOR GASTRIC NEOPLASMS IN HIGH-RISK AND LOW-RISK PATIENTS

T. Inoue, Y. Maegawa, S. Ito, T. Shimizu, Y. Yokoyama, S. Tawara, S. Ishii, Y. Haruna, T. Yakushijin, A. Inoue

Gastroenterology, Osaka General Medical Center, Osaka/Japan

Contact E-mail Address: taktaktakkun@gmail.com

Introduction: Antithrombotic drugs are administered to patients undergoing endoscopic treatment at high risk for thromboembolism. However, antithrombotic drugs have been also known as a cause of delayed bleeding associated with endoscopic treatment, including endoscopic submucosal dissection (ESD). We previously reported the clinical features of postpolypectomy bleeding associated with heparin bridge therapy (1), and then various risk factors of delayed bleeding after endoscopic treatment have been reported.

Aims \& Methods: The aims of the present study are to investigate the risk factors of delayed bleeding after gastric ESD and to clarify the clinical features of delayed bleeding in high-risk and low-risk patients. Data of consecutive inpatients who underwent ESD for gastric neoplasms in Osaka General Medical Center between January 2009 and December 2016 were retrospectively investigated. Independent risk factors of delayed bleeding were analyzed by using a multivariate analysis by logistic regression model, and three predictors of delayed bleeding were selected. Patients were categorized into a high-risk group or lowrisk group for bleeding, and the clinical features of post-procedural bleeding in each group were investigated.

Results: A total of 717 patients with 781 gastric neoplasms were identified. Mean age was 74.6 , and $71.6 \%$ was male. With regard to comorbidity, the proportion of hypertension, diabetes, chronic liver disease, and hemodialysis was $50.2 \%$, $19.2 \%, 2.7 \%$, and $6.1 \%$, respectively. Total 188 patients have taken oral antithrombotic drugs, and of them, 50 patients treated by gastric ESD under heparin bridge therapy. Two-thirds lesions were located in gastric body and median tumor size (range) was $15(3-80) \mathrm{mm}$. En-bloc resection was achieved in 751 lesions $(96.2 \%)$, and no uncontrollable bleeding occurred. Forty-nine patients $(6.8 \%)$ experienced delayed bleeding after gastric ESD. Hospital stay was significantly longer in bleeding cases than in non-bleeding cases [median hospital stay (range) $11(3-20)$ vs. $9(9-25), p=0.007]$. A univariate and multivariate analysis showed heparin bridge therapy, antiplatelet therapy, and hemodialysis, and age $(75<)$ were the independent risk factors for delayed bleeding. We defined patients with heparin bridge therapy, antiplatelet therapy, and hemodialysis as a high-risk group for bleeding, and the remainder as a low-risk group. The incidence of delayed bleeding was significantly higher in the high-risk group than in the low-risk group $(14.3 \%$ vs. $3.6 \%, p<0.001)$. Severe bleeding requiring transfusion and recurrent bleeding were more often in the high-risk group than in the low-risk group. Median onset (range) of delayed bleeding was POD1 (0-16) in low-risk group and POD6 (0-15) in high-risk group. No thromboembolism occurred in each group.

Conclusion: Bleeding high-risk patients with heparin bridge therapy, antiplatelet therapy, and hemodialysis should be carefully observed after gastric ESD while early hospital discharge is acceptable for bleeding low-risk patients.

Disclosure of Interest: All authors have declared no conflicts of interest.

\section{Reference}

1. Inoue T, Nishida T, Maekawa A, et al. Clinical features of post-polypectomy bleeding associated with heparin bridge therapy. Digestive Endosc 2014;26:243-249

\section{P1812 EFFICACY AND SAFETY OF FERRIC CARBOXYMALTOSE TREATMENT IN PATIENTS HOSPITALIZED FOR ACUTE GASTROINTESTINAL BLEEDING NOT ASSOCIATED WITH PORTAL HYPERTENSION}

G. Torres-Vicente ${ }^{1}$, M. Planella-De Rubinat ${ }^{2}$, R. Ballester-Clau ${ }^{2}$, L. López-

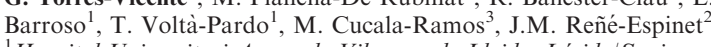
${ }^{1}$ Hospital Universitari Arnau de Vilanova de Lleida, Lérida/Spain

${ }^{2}$ Hospital Universitari Arnau de Vilanova de Lleida. Institut de Recerca Biomèdica de Lleida, Lérida/Spain

${ }^{3}$ Vifor Pharma España, Barcelona/Spain

Contact E-mail Address: gisela.tv@hotmail.com

Introduction: There are few studies of the efficacy of parenteral ferric carboxymaltose (FCM) treatment in acute gastrointestinal bleeding (GIB) of different origins. Few data are available on its use to treat anaemia post-acute haemorrhage.

Aims \& Methods: To determine the efficacy and safety of FCM treatment in patients with acute GIB not associated with portal hypertension. A retrospective descriptive 3-year study of patients with acute GIB (anemia with evident bleeding and/or hemodynamic instability) treated with FCM as part of our hospital's habitual clinical practice.

Results: Analysis of 84 patients admitted with acute GIB $(69.0 \%$ male, mean age 68.0 years [SD 6.9]), with a Charlson index $>3$ in $67.1 \%$ of cases $(>5$ in $31.6 \%)$ $15.5 \%$ had previously suffered acute GIB due to peptic ulcer. There were 86 hospital admissions for acute GIB; $93.8 \%$ were upper GIB (above the angle of Treitz). The most frequent clinical presentation was melena, in $76.7 \%$ of cases. $25.6 \%$ presented hemodynamic instability at admission. The mean GlasgowBlatchford index score was 10.1 (SD 2.7) and the mean Rockall score postendoscopy was 4.2 (SD 1.7). The most common causes of bleeding were: $36.0 \%$ duodenal ulcus, $29 \%$ gastric ulcus, $9.3 \%$ gastritis/erosions, and $7.3 \%$ angiodysplasia of the colon. The mean $\mathrm{Hb}$ at admission was $9.0 \mathrm{~g} / \mathrm{dL}$ (SD 2.2) 
and the mean of the lowest $\mathrm{Hb}$ during admission was $7.6 \mathrm{~g} / \mathrm{dL}$ (SD 1.3). The most common total dose of FCM administered was $1000 \mathrm{mg}$. During admission, a mean $\mathrm{Hb}$ increase of $0.8 \mathrm{~g} / \mathrm{dL}$ (SD 2.3) was observed in a mean period of 5.7 days (median: 4.0) after treatment with FCM, with an increase of $4.2 \mathrm{~g} / \mathrm{dL}$ (SD 2.6) 30 days after acute GIB. After FCM administration, the mean $\mathrm{Hb}$ increased significantly $(\mathrm{p}<0.0001)$ in patients $\geq 75$ years $(2.1 \mathrm{~g} / \mathrm{dL}$ [SD 1.7]), in patients with Charlson index $\geq 3(1.9 \mathrm{~g} / \mathrm{dl}$ [SD 1.6]), and when $\mathrm{Hb}$ level during admission was $<10 \mathrm{~g} / \mathrm{dL}(2.0 \mathrm{~g} / \mathrm{dL}$ [SD 1.7]). No adverse reactions were observed.

Conclusion: In patients with acute GI bleeding the administration of ferric carboxymaltose improves $\mathrm{Hb}$ levels promptly and safely, especially in patients of advanced age and with associated comorbidities.

Disclosure of Interest: M. Cucala-Ramos: Mercedes Cucala is employee of Vifor Pharma.

J.M. Reñé-Espinet: Reñé-Espinet, Josep Maria received research grant from Vifor Pharma.

All other authors have declared no conflicts of interest.

\section{P1813 NOVEL EUS-GUIDED TREATMENT OF GASTRIC VARICES WITH A LIQUID NON-ADHESIVE NEUROVASCULAR EMBOLIZATION AGENT}

A.J. Baptista, M.A. Guzman, H. Rass, J.F. Piñerúa Gonsálvez, E. Richards Hospital de Clínicas Caracas, Caracas/Venezuela

\section{Contact E-mail Address: albertogebaptista@gmail.com}

Introduction: Endoscopic Injection of adhesive agents such as N-Butyl-2Cyanoacrylate $(\mathrm{NBC})$ is an accepted option for the management of gastric varices. Recently the combination of NBC and coils has been used with endoscopic ultrasound (EUS) assistance. Nevertheless adhesive properties of the polymer can cause blockage of instrumentation material and damage to endoscopes. Some adverse events include ulcers, vascular wall necrosis, rebleeding and distal embolism. Ethylene-vinyl alcohol (EVOH) has been extensively used in interventional radiology to treat cerebral arteriovenous malformations and has the advantage of being radiopaque.

Aims \& Methods: We aimed to demonstrate a novel gastric varices embolization therapy with EUS-guided injection of a composite non-adhesive endovascular liquid agent EVOH combined with Dimethylsulfoxide (DMSO) as a primer. Five cases of EUS-guided Injection under fluoroscopic vision with EVOH is described in 3 men and 2 women, aged 50-65 years with gastric fundus varices, portal hypertension, Child-B hepatic cirrhosis and previous episodes of bleeding. The ecoendoscope was advanced to the gastroesophageal junction. The selected gastric varix was punctured using a 22 Gauge needle. EVOH injected volume ranged between 1.5 and $3 \mathrm{cc}$. Vascular flow progressive obliteration was real time monitored by EUS. Ceftriaxone was intravenously administered during the procedure. Patients were discharged on the same day. The mean follow-up was 6 months. Endoscopic and radiological control was performed at 1 and 3 months. The average procedure time was $20 \mathrm{~min}$.

Results: All patients presented mild epigastric pain during the first 12 hours effectively managed with oral analgesics The obliteration of variceal flow was achieved in all patients in a single session. There were no new episodes of bleeding or complications related to the technique.

Conclusion: EUS-guided embolization of gastric varices with EVOH can be considered as an efficient alternative. The procedure promises effective advantages in terms of number of sessions required, local or systemic adverse events and endoscopes damage. Prospective multicenter study with greater number of cases and cost evaluation against coils alone or combined with NBC are required. Disclosure of Interest: All authors have declared no conflicts of interest.

\section{P1814 PEPTIC ULCER BLEEDING IN THE ELDERLY: CLINICAL OUTCOMES AND IN-HOSPITAL MORTALITY}

D. Matei ${ }^{1}$, I. Groza ${ }^{2}$, S. Pasca ${ }^{2}$, D. Negrutiu ${ }^{1}$, B. Furnea ${ }^{1}$, C. Bocsan ${ }^{2}$, C. Cruciat ${ }^{1}$, A. Seicean ${ }^{2}$, M. Tantau ${ }^{2}$

${ }^{1}$ Regional Institute of Gastroenterology and Hepatology Prof. Dr. Octavian Fodor, Cluj Napoca/Romania

${ }^{2}$ University of Medicine and Pharmacy Iuliu Hatieganu, Cluj Napoca/Romania

\section{Contact E-mail Address: dmatei68@gmail.com}

Introduction: The increase in life expectancy and the increased use of antiplatelets, oral anticoagulants and nonsteroidal anti-inflammatory drugs (NSAIDs) may account for the increasing frequency of older patients with upper gastrointestinal bleeding.

Aims \& Methods: The aim of this study was to assess the clinical and endoscopic aspects, hospital course and mortality rate in the elderly population with peptic ulcer bleeding. In this prospective study we enrolled all patients diagnosed with peptic ulcer bleeding, who were hospitalized in a tertiary medical center over a period of 24 months (January 2015- December 2016). Patients were divided into two groups, according to their age: patients with age below and over 75 years old. The following characteristics were analyzed in both groups: antiplatelets, oral anticoagulants and NSAID's uses, smoking, alcohol consumption, presence of comorbidities, (quantified by Charlson score), endoscopic aspect of ulcer (Forrest IA, IB, IIA, IIB were quantified as high risk endoscopic stigmata, while Forrest IIC and III were considered as low risk endoscopic stigmata) and the number of ulcers. We also analyzed comparatively the need of transfusion, hospitalization duration, rebleeding, need for surgery and in-hospital mortality.
Results: 431 patients have been included in this study. $24.8 \%$ of patients were over 75 years old. The following differences have been observed by comparing the two groups of patients (over and below 75 years old): female $47.7 \%$ vs $25.3 \%$ $(\mathrm{p}<0.001)$; antiplatelets use $40.2 \%$ vs $20.1 \%(\mathrm{p}<0.001)$; oral anticoagulants use $20.6 \%$ vs $7.7 \%(\mathrm{p}<0.001)$; NSAIDs use $25.2 \%$ vs $33.6 \%(\mathrm{p}=0.133)$; smoking $5.6 \%$ vs $35.2 \% \quad(p<0.001)$; alcohol consumption $17.8 \%$ vs $45.4 \%$ $(\mathrm{p}<0.001)$; one or more comorbidities $77.6 \%$ vs $62 \%(\mathrm{p}=0.005)$; high risk endoscopic stigmata $61.7 \%$ vs $64.4 \%(\mathrm{p}=0.724)$; multiple ulcers $41.1 \%$ vs $31.8 \%(\mathrm{p}=0.099)$; need for blood transfused $59.8 \%$ vs $58 \%(\mathrm{p}=0.832)$; hospitalization duration $8.4 \pm 6.1$ vs $7.8 \pm 6.0$ days $(\mathrm{p}=0.382)$; rebleeding $10.3 \%$ vs $11.4 \%(\mathrm{p}=0.745)$; need for surgery $2.8 \%$ vs $4.6 \%(\mathrm{p}=0.589)$ and in-hospital mortality $13.1 \%$ vs $6.2 \%(\mathrm{p}=0.021)$. In most of the cases $(88.2 \%)$, the cause of death was other than hemorrhagic shock $(92.9 \%$ vs $85.0 \%, \mathrm{p}=0.484)$. Using multivariate analysis, three out of these factors were identified as representing independent factors significantly associated with the age over 75 years old: oral anticoagulants use $(\mathrm{OR}=2.40,95 \% \mathrm{CI}: 1.24-4.62, \mathrm{p}=0.009)$, antiplatelet use $(\mathrm{OR}=2.33,95 \% \mathrm{CI}: 1.43-3.81, \mathrm{p}=0.001)$ and in-hospital mortality $(\mathrm{OR}=2.09$, $95 \% \mathrm{CI}: 1.27-4.47, \mathrm{p}=0.048)$.

Conclusion: The use of oral anticoagulants and antiplatelet was significantly higher in older patients, compared to the younger group. Elderly patients with peptic ulcer bleeding do not have a different rebleeding, need for surgery, need of transfusion or hospitalization duration than younger patients. In-hospital mortality was higher in elderly patients due to more frequent association of comorbidities.

Disclosure of Interest: All authors have declared no conflicts of interest.

\section{References}

González-González JA, Monreal-Robles R, García-Compean D, Paz-Delgadillo J, Wah-Suárez M, Maldonado-Garza HJ. Nonvariceal upper gastrointestinal bleeding in the elderly: clinical outcomes and prognostic factors. J Dig Dis. 2017 Feb 16

Zullo A, Hassan C, Campo SM, Morini S. Bleeding peptic ulcer in the elderly: risk factors and prevention strategies. Drugs Aging. 2007;24(10):815-28. Review

\section{P1815 PREDICTIVE FACTORS FOR IN-HOSPITAL MORTALITY IN PATIENTS WITH PEPTIC ULCER BLEEDING}

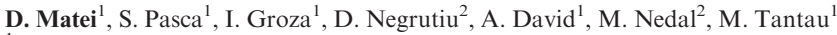
${ }^{1}$ University of Medicine and Pharmacy Iuliu Hatieganu, Cluj Napoca/Romania ${ }^{2}$ Regional Institute of Gastroenterology and Hepatology Prof. Dr. Octavian Fodor. Cluj Napoca/Romania

\section{Contact E-mail Address: dmatei68@gmail.com}

Introduction: Peptic ulcers are the most frequent cause of upper gastrointestinal bleeding. In different population based surveys regarding all-cause UGIB, mortality ranges between $3 \%$ and $14 \%$

Aims \& Methods: The aim of this study was to assess in-hospital mortality in patients with peptic ulcer bleeding and to evaluate the risk factors associated with mortality. In this prospective study we enrolled all patients diagnosed with peptic ulcer bleeding, who were hospitalized in a tertiary medical center over a period of 24 months (January 2015- December 2016). Patients were divided into two groups - those who died and those who survived - and the following parameters were compared: age, signs of hemodynamic instability (hypotension, tachycardia), presence of comorbidities (quantified by Charlson score), endoscopic aspect of ulcer (Forrest IA, IB, IIA, IIB were quantified as high risk endoscopic stigmata, while Forrest IIC and III were considered as low risk endoscopic stigmata), number of ulcers, Rockall score, Blatchford score, degree of anemia, coagulation disorders, renal function. We also analyzed comparatively rebleeding, the need of transfusion and need for surgery.

Results: The study included 431 patients. In-hospital mortality rate was $7.9 \%$. The following differences have been observed by comparing patients who died and those who survived: age $>75$ years $41.2 \%$ vs $23.4 \%(\mathrm{p}=0.036)$; hypotension $17.6 \%$ vs $2.3 \%(\mathrm{p}<0.001)$; tachycardia $47.1 \%$ vs $21.4 \%(\mathrm{p}=0.001)$; one or more comorbidities $94.1 \%$ vs $63.5 \%(\mathrm{p}=0.001)$; high risk endoscopic stigmata $79.4 \%$ vs $62.2 \%(\mathrm{p}=0.070)$; multiple ulcers $41.2 \%$ vs $33.5 \%(\mathrm{p}=0.473)$; Rockall score $\geq 5$ points $94.1 \%$ vs $46.9 \%(\mathrm{p}<0.001)$; Blatchford score $>10$ points $91.2 \%$ vs $66.2 \%(\mathrm{p}=0.005)$; hemoglobin $<9.5 \mathrm{~g} / \mathrm{dl} 70.6 \%$ vs $41.1 \%(\mathrm{p}=0.002) ;$ INR $\geq 2.5 \quad 17.6 \%$ vs $5.8 \% \quad(\mathrm{p}=0.022) ;$ creatinine $\geq 1.5 \mathrm{mg} / \mathrm{dl} \quad 38.2 \%$ vs $10.8 \%$ $(\mathrm{p}<0.001)$; rebleeding $20.6 \%$ vs $10.3 \%(\mathrm{p}=0.123)$; need for blood transfusion $82.4 \%$ vs $56.4 \%(\mathrm{p}=0.006)$; need for surgery $11.8 \%$ vs $3.5 \%(\mathrm{p}=0.063)$. In most cases $(88.2 \%)$, the cause of death was other than hemorrhagic shock. Using multivariate analysis, three out of these factors were identified as representing independent factors significantly associated with in-hospital mortality: tachycardia $(\mathrm{OR}=2.83,95 \% \mathrm{CI}: 1.21-6.58, \mathrm{p}=0.016)$, Rockall score $\geq 5 \quad(\mathrm{OR}=6.65$, $95 \% \mathrm{CI}: 1.46-30.16, \quad \mathrm{p}=0.014)$ and creatinine $\geq 1.5 \mathrm{mg} / \mathrm{dl} \quad(\mathrm{OR}=1.25$, $95 \% \mathrm{CI}: 1.25-7.73, \mathrm{p}=0.014)$.

Conclusion: In-hospital mortality rates in patients with peptic ulcer bleeding was $7.9 \%$. Tachycardia, Rockall score $\geq 5$ and creatinine $\geq 1.5 \mathrm{mg} / \mathrm{dl}$ were the independent risk factors significantly associated with in-hospital mortality. Disclosure of Interest: All authors have declared no conflicts of interest.

\section{References}

Sostres C, Lanas A. Epidemiology and demographics of upper gastrointestinal bleeding: prevalence, incidence, and mortality. Gastrointest Endosc Clin N Am. 2011 Oct;21(4):567-81.

Rahman SI, Saeian K. Nonvariceal Upper Gastrointestinal Bleeding. Crit Care Clin. 2016 Apr;32(2):223-39. 
Nahon S, Pariente A; group of investigators of the ANGH (Association Nationale des Gastroentérologues des Hôpitaux Généraux) Impact of comorbidity on mortality in patients with peptic ulcer bleeding: confirmative data of a French prospective study. Am J Gastroenterol. 2013 Sep;108(9):1532-3

\section{P1816 ANAEMIA AND UPPER GI BLEEDING: A LOCAL EXPERIENCE}

M. Ding, R. Prawiradiradja, Z. Arastu, H. Sabri, M. Smith Gastroenterology, Royal shrewsbury hospital, Shrewsbury/United Kingdom

Contact E-mail Address: Michaeldjw@gmail.com

Introduction: There has been significant research recently on the use of blood transfusions in upper GI bleeding (UGIB) [1] with recent evidence advocating a restrictive approach to blood transfusions as well as the use of iron therapy[2] for anaemia post UGIB. Our team conducted a local retrospective analysis on patients admitted with UGIB over a six month period and analysed the use of blood transfusions at our trust which consists of two District General Hospitals. Patient data over a period of up to 12 months post discharge was collected to monitor their anaemia.

Aims \& Methods: Our aim was to monitor the appropriateness of transfusions in Upper GI Bleeding as well as monitoring the response to iron therapy following discharge. All inpatients that had an Upper GI endoscopy for UGIB were analysed. Electronic patient records were obtained from our endoscopy software and hospital database. Patients were selected over a time period of six months from 1 / $6 / 2015$ to $31 / 12 / 2015$. A Student's T-Test was used to compare the average increase in haemoglobin $(\mathrm{Hb})$ for patients discharged with iron therapy against those who were not.

Results: There were 148 patients, 81 male and 67 female. The mean age was 69.3 , minimum 20 and maximum of 98 . The average $\mathrm{Hb}$ on admission was $103 \mathrm{~g} / \mathrm{L}$ $(\min =32 \mathrm{~g} / \mathrm{L}, \max =178 \mathrm{~g} / \mathrm{L}) .78$ out of $148(52.7 \%)$ patients presenting with UGIB received a blood transfusion. The mean amount of blood received for those transfused was 3.7 units. 48 out of $78(61.5 \%)$ of blood transfusions were given when $\mathrm{Hb}$ was below $70 \mathrm{~g} / \mathrm{L}$. 30 of $78(38.5 \%)$ were transfused above a $\mathrm{Hb}$ of $70 \mathrm{~g} / \mathrm{L} .(36.7 \%, \mathrm{n}=11)$ of those who were transfused with $\mathrm{Hb}$ above 70 had cardiac risk factors. The mortality rate in those transfused above $\mathrm{Hb}$ of 70 was $13.3 \%(\mathrm{n}=4)$ vs $10.4 \%(\mathrm{n}=5) 41.5 \%(\mathrm{n}=44)$ patients who were anaemic post-UGIB were discharged with iron therapy. The average rise in $\mathrm{Hb}$ was $26.5 \%$ for those discharged on iron vs $12.1 \%$ for those who did not. There was a statistically significant rise in $\mathrm{Hb}$ for those discharged with iron therapy $(\mathrm{p}<0.005)$ on follow-up versus those who did not receive it $(n=62)$. The anaemia related readmission rates were similar for patients discharged on iron or not $(9.1 \% \mathrm{n}=4$ vs $9.7 \% \mathrm{n}=6)$.

Conclusion: The data obtained supports a restrictive transfusion policy (mortality rate of $13.3 \%$ vs $10.4 \%$ ). $58.5 \%$ of patients who were anaemic on discharge did not receive any iron therapy. On follow up, there was a statistically significant rise in $\mathrm{Hb}$ level in the group discharged on iron. Our data affirms recent evidence favouring iron therapy post UGIB. Further education is needed to improve outcome in patients presented with GI bleed.

Disclosure of Interest: All authors have declared no conflicts of interest.

\section{References}

1. Villanueva $\mathrm{C}$, et al. Transfusion strategies for acute upper gastrointestinal bleeding. $N$ Engl J Med, 368 (2013), pp. 11-21

2. Bager P and Dahlerup JF. Randomised clinical trial: Oral vs. intravenous iron after upper gastrointestinal haemorrhage - a placebo-controlled study. Aliment Pharmacol Ther 2014 Jan; 39:176.

\section{P1817 THE RELATIONS AMONG SERUM GHRELIN, MOTILIN, CIRCULATING ANTIMYENTERIC ANTIBODIES AND GASTRIC EMPTYING AND AUTONOMIC NERVOUS SYSTEM FUNCTION IN PATIENTS WITH AUTOIMMUNE GASTRITIS}

Ç. Kalkan, F. Karakaya, I. Soykan

Gastroenterology, Ankara University, Ankara/Turkey

\section{Contact E-mail Address: mfkarakaya@yahoo.com}

Introduction: Autoimmune gastritis (AIG) is an organ-specific autoimmune disease of the stomach marked by autoantibodies directed to hydrogen/potassiumATPase and intrinsic factor. Gastric emptying of solids is delayed and autonomic nerve dysfunction is detected in patiens with autoimmune gastritis. This may be a cause of symptoms and also there is a close relation between autonomic nerve dysfunction and delayed gastric emptying. As ghrelin and motilin are putative regulators of gastric emptying and, some autoimmune gastritis patients may have delayed gastric emptying leading upper gastrointestinal symptoms.

Aims \& Methods: The aims of this study were to: (i) compare serum levels of ghrelin and motilin in patients with delayed to normal gastric emptying and (ii) investigate whether circulating antimyenteric antibodies, serum levels of ghrelin and motilin have any effect on autonomic nerve function and gastric emptying. Forty-one patients with AIG were included into this study. Autoimmune gastritis was diagnosed depending upon histopathological findings in gastric biopsy specimens. Non-invasive cardiovascular reflex tests were used in order to evaluate autonomic nervous system function. Sympathetic nerve function was evaluated with two tests: blood pressure response to standing and handgrip test. Parasympathetic nerve function was evaluated with three tests: valsalva manoeuvre, heart rate response to deep breathing (E/I ratio) and orthostatic blood pressure testing. Dysfunction was considered to exist if at least two tests were positive. Gastric emptying time was evaluated by a standard 2-hour scintigraphic test using a solid meal. A half-time of longer than 110 minutes was chosen in the present study to further investigate patients with delayed gastric emptying. Serum ghrelin and motilin levels were tested by ELISA and circulating antimyenteric antibodies were tested by IFA.

Results: Forty-one patients ( 27 women), mean age $56.61 \pm 11.79$ years with AIG were included into the study. Overall, $22(53.6 \%)$ patients showed delayed GE and 19 patients showed normal GE (GET 1/2: 241.19 \pm 199 vs $90 \pm 19$ mins, $\mathrm{p}<0.001)$. Serum ghrelin and motilin levels of patients with delayed GE were significantly decreased compared to patients with normal GE, respectively $(67.55 \pm 8.81$ vs $126.79 \pm 25.81 \mathrm{pg} / \mathrm{mL}, \quad \mathrm{p}<0.001$ and $279.59 \pm 111.12$ vs $500.42 \pm 155.95 \mathrm{pg} / \mathrm{mL}, \mathrm{p}<0.001)$. In all, $26(63.4 \%)$ patients showed autonomic nervous system dysfunction and, $15(36.5 \%)$ patients had normal autonomic nervous system test findings (total autonomic test score: $0.8 \pm 0.25$ vs $5.65 \pm 1.74, p<0.001)$. Serum ghrelin and motilin levels of patients with deranged autonomic nervous system function were significantly decreased compared to patients with normal autonomic nervous system function, respectively $(80.73 \pm 28.46$ vs $127.79 \pm 28.06 \mathrm{pg} / \mathrm{mL}, \quad \mathrm{p}<0.001$ and $316.92 \pm 160.47$ vs $490.20 \pm 141.02 \mathrm{pg} / \mathrm{mL}, \mathrm{p}<0.001)$. In multivariate analysis, plasma motilin level was found as an independent factor that affected serum ghrelin level $(\mathrm{r}=0.623, \mathrm{p}=0.019)$. However, serum ghrelin $(\mathrm{r}=0.623, \mathrm{p}=0.019)$ and gastrin $(\mathrm{r}=-0.70, \mathrm{p}<0.001)$ levels were found as independent factors that affected plasma motilin level. We also investigated the presence of antimyenteric antibodies, however all the patients were negative by means of antimyenteric antibodies therefore, no further relationship was sought.

Conclusion: Mean fasting serum ghrelin and plasma motilin levels in autoimmune gastritis patients with delayed GE and deranged autonomic nerve function were significantly decreased. None of the patients in our study were positive for circulating antimyenteric antibodies. These decreased serum ghrelin and plasma motilin levels in patients with autoimmune gastritis suggest a potential role for ghrelin and motilin in explaining the finding of the delayed gastric emptying observed in an important part ofautoimmune gastritis patients. We believe that these new observations supply more insight into the pathophysiology of autoimmune gastritis.

Disclosure of Interest: All authors have declared no conflicts of interest.

\section{Reference}

Kalkan $\mathrm{C}$ et al. Relationships between autonomic nerve function and gastric emptying in patients with autoimmune gastritis. Clin Auton Res 2016:189-96.

\section{P1818 EFFECTS OF FAECAL MICROBIOTA TRANSPLANTATION ON NEUROGENIN 3, MUSAHSI 1 AND ENTEROENDOCRINE CELLS IN THE DUODENUM OF PATIENTS WITH IRRITABLE BOWEL} SYNDROME

\section{T. Mazzawi ${ }^{1}$, M. El-Salhy ${ }^{2}$, G.A. Lied ${ }^{3}$, T. Hausken ${ }^{4}$}

${ }_{1}^{1}$ Gastroenterology-medicine, Haukeland University Hospital, Bergen/Norway ${ }^{2}$ Gastroenterology-medicine, Stord Hospital Helse-Fonna, Stord/Norway ${ }^{3}$ Center For Nutrition, Clinical Medicine, University of Bergen, Bergen/Norway ${ }^{4}$ Haukeland University Hospital, National Centre for Functional Gastrointestinal Disorders, Bergen/Norway

\section{Contact E-mail Address: tarek.mazzawi@med.uib.no}

Introduction: The interaction between gut microbiota and enteroendocrine cells alterations is believed to play an important role in the pathophysiology of irritable bowel syndrome (IBS). The densities of the duodenal enteroendocrine cells are abnormal in IBS patients, which appears to be caused by a reduced stem cells density and their differentiation into endocrine cells (1).

Aims \& Methods: The aim is to investigate the effects of faecal microbiota transplantation (FMT) on the differentiation of the stem cells into enteroendocrine cells as detected by neurogenin 3, the stem cells as detected by Musashi 1 and the enteroendocrine cells in the duodenum of patients with IBS. The study included 16 IBS patients according to Rome III criteria and four patients were excluded. The remaining patients $(n=12,4$ females and 8 males, age range $20-44$ years) were divided according to the cause of IBS into PI-IBS patients $(n=6)$ and idiopathic IBS $(n=6)$ and received FMT donated from their relatives. The patients completed the IBS-symptom severity scoring system (IBS-SSS) before and 3 weeks after FMT. The patients underwent gastroscopies with biopsies taken from the descending part of the duodenum at baseline and 3 weeks after FMT. The biopsies were immunostained for neurogenin 3, Musashi 1 and all types of duodenal enteroendocrine cells, and quantified by computerized image analysis.

Results: The score of IBS symptoms as assessed by IBS-SSS was significantly reduced 3 weeks after $(240.2 \pm 33.6)$ compared to before $(326.6 \pm 22.3)$ receiving FMT, $P=0.0009$. The scores of IBS-SSS before and 3 weeks after FMT for PIIBS are $300.8 \pm 27.8$ and $210 \pm 41$, respectively $(\mathrm{P}=0.025)$, and for idiopathic IBS are $352 \pm 34$ and $270.3 \pm 54$, respectively, $(P=0.034)$. The densities of neurogenin 3, Musashi 1 and enteroendocrine cells in the duodenum of IBS patients before and 3 weeks after receiving FMT are presented in Table 1 .

Conclusion: Faecal microbiota transplantation improved the symptoms in IBS patients, both PI and idiopathic. This improvement was associated with a change in the enteroendocrine cell density. The changes in the enteroendocrine cell density does not appear to be caused by changes in the stem cells or their early progenitors, but rather by changes in the differentiation progeny as detected by changes in neurogenin 3 .

Disclosure of Interest: All authors have declared no conflicts of interest. 


\section{Reference}

1. El-Salhy M, Hatlebakk JG and Hausken T: Reduction in duodenal endocrine cells in irritable bowel syndrome is associated with stem cell abnormalities. World journal of gastroenterology: WJG 21: 9577-9587, 2015.

\section{P1819 THE EFFECT OF ESOPHAGEAL ACID EXPOSURE ON NMDA RECEPTOR SUBUNITS EXPRESSION AND D-SERINE IN PREFRONTAL CORTEX AND HIPPOCAMPUS}

W. Zhang ${ }^{1}$, L. Duan ${ }^{2}$, K. Wang ${ }^{2}$, X. Wang ${ }^{3}$

${ }^{1}$ Gastroenterology, Peking University Third Hospital, Beijing/China

${ }^{2}$ Department Of Gastroenterology, Peking University Third Hospital, Beijing

China

${ }^{3}$ Pharmacology, Peking University Health Science Center, Beijing/China

Contact E-mail Address: weifang@bjmu.edu.cn

Introduction: Neuronal plasticity has been reported to develop following nociceptive emotional experience in prefrontal cortex (PFC) and hippocampus. The $\mathrm{N}$-methyl-D-aspartate receptor (NMDAR) and D-serine, the endogenous coagonist of NMDAR1, may mediate the neural plasticity. However, whether the neural plasticity participates in the mechanism of esophageal visceral hypersensitivity is little known.

Aims \& Methods: This study aims to investigate the expression of NMDAR and the alteration of D-serine after neonatal and adult esophageal acid exposure. All rats were exposed to esophageal acid or saline at postnatal days 7-15(P7-P15), and most rats underwent acute acid or saline exposure again at adult time (P60).All rats were randomly distributed to 5 groups, including $\mathrm{P} 7 \mathrm{~S}, \mathrm{P} 7 \mathrm{H}$, $\mathrm{P} 7 \mathrm{H}+\mathrm{P} 60 \mathrm{H}, \mathrm{P} 7 \mathrm{H}+\mathrm{P} 60 \mathrm{~S}, \mathrm{P} 7 \mathrm{~S}+\mathrm{P} 60 \mathrm{H}(\mathrm{P} 7$ : postnatal 7-15 days; P60: adult at postnatal 60 days; H: $0.1 \mathrm{~N} \mathrm{HCL}$ infusion; S: saline control). The tissue harvest was conducted at P60. We examined the expression of subunits of NMDAR (including NR1, NR2A, and NR2B), c-fos, and serine racemase in PFC, dorsal hippocampus $(\mathrm{DH})$ and ventral hippocampus $(\mathrm{VH})$. We also determinated the Dserine and L-serine in PFC and hippocampus by LC-MS analysis. Statistic comparisons were performed by General Linear Model and one way ANOVA in SPSS.

Results: In PFC, compared with adult saline treatment (AS, including $\mathrm{P} 7 \mathrm{H}+\mathrm{P} 60 \mathrm{H}$ and $\mathrm{P} 7 \mathrm{~S}+\mathrm{P} 60 \mathrm{H}$ group) and without adult treatment (A-, including P7S and $\mathrm{P} 7 \mathrm{H}$ group), adult acid exposure $(\mathrm{AH})$ increased the expression of $\mathrm{NR} 1$ $(\mathrm{P}=0.052, \quad \mathrm{P}=0.298), \quad \mathrm{NR} 2 \mathrm{~B} \quad(\mathrm{P}=0.035, \quad \mathrm{P}=0.045)$, and serine racemase $(\mathrm{P}=0.022, \mathrm{P}=0.017)$ significantly. In ventral hippocampus, compared with adult treatment absence, adult acid exposure caused increasing expression of NR2B $(P=0.012)$ and NR1 $(P=0.024)$ significantly. In PFC, the expression of serine racemase in the $\mathrm{P} 7 \mathrm{~S}+\mathrm{P} 60 \mathrm{H}$ group was obviously higher than that of other groups $(P=0.008)$. See Table1. In dorsal hippocampus, there was statistical significance on the level of $\mathrm{c}-\mathrm{fos}$ between the $\mathrm{P} 7 \mathrm{~S}+\mathrm{P} 60 \mathrm{H}$ group and other groups $(\mathrm{P}=0.008)$. Table1. In PFC, the LC-MS analysis results that $\mathrm{D}$-serine (AH vs A$: \mathrm{P}=0.000$, AS vs $\mathrm{A}-: \mathrm{P}=0.042$, AH vs $\mathrm{AS}: \mathrm{P}=0.081)$ and $\mathrm{L}$-serine $(\mathrm{AH}$ vs $\mathrm{A}$ $: \mathrm{P}=0.000$, AS vs $\mathrm{A}-\mathrm{P}=0.015$, $\mathrm{AH}$ vs $\mathrm{AS}: \mathrm{P}=0.082$ ) decreased in the $\mathrm{AH}$ and AS group, comparing with A- group.

The expression of serine racemase in PFC and c-fos in $\mathrm{VH}$

\begin{tabular}{|c|c|c|c|c|}
\hline \multirow{2}{*}{$\begin{array}{l}\text { Group } \\
(\mathrm{n}=8 / \text { group })\end{array}$} & \multicolumn{2}{|c|}{ PFC Serine Racemase } & \multicolumn{2}{|l|}{ VH c-fos } \\
\hline & $($ mean $\pm \mathrm{SD})$ & $\begin{array}{l}\mathrm{P} \text { value } \\
(\text { vs } \mathrm{P} 7 \mathrm{~S}+\mathrm{P} 60 \mathrm{H})\end{array}$ & $($ mean $\pm \mathrm{SD})$ & $\begin{array}{l}\mathrm{P} \text { value } \\
(\text { vs } \mathrm{P} 7 \mathrm{~S}+\mathrm{P} 60 \mathrm{H})\end{array}$ \\
\hline $\mathrm{P} 7 \mathrm{~S}$ & $0.139 \pm 0.131$ & 0.03 & $0.035 \pm 0.008$ & 0.021 \\
\hline
\end{tabular}

(continued)
Continued

The expression of serine racemase in PFC and c-fos in $\mathrm{VH}$

\begin{tabular}{|c|c|c|c|c|}
\hline \multirow{2}{*}{$\begin{array}{l}\text { Group } \\
(\mathrm{n}=8 / \text { group })\end{array}$} & \multicolumn{2}{|c|}{ PFC Serine Racemase } & \multicolumn{2}{|l|}{ VH c-fos } \\
\hline & $($ mean $\pm \mathrm{SD})$ & $\begin{array}{l}\mathrm{P} \text { value } \\
(\text { vs } \mathrm{P} 7 \mathrm{~S}+\mathrm{P} 60 \mathrm{H})\end{array}$ & $($ mean $\pm \mathrm{SD})$ & $\begin{array}{l}\mathrm{P} \text { value } \\
(\text { vs } \mathrm{P} 7 \mathrm{~S}+\mathrm{P} 60 \mathrm{H})\end{array}$ \\
\hline P7H & $0.141 \pm 0.083$ & 0.034 & $0.036 \pm 0.015$ & 0.026 \\
\hline $\mathrm{P} 7 \mathrm{H}+\mathrm{P} 60 \mathrm{H}$ & $0.166 \pm 0.066$ & 0.119 & $0.035 \pm 0.011$ & 0.023 \\
\hline $\mathrm{P} 7 \mathrm{H}+\mathrm{P} 60 \mathrm{~S}$ & $0.124 \pm 0.031$ & 0.014 & $0.040 \pm 0.015$ & 0.057 \\
\hline $\mathrm{P} 7 \mathrm{~S}+\mathrm{P} 60 \mathrm{H}$ & $0.300 \pm 0.149$ & - & $0.083 \pm 0.060$ & - \\
\hline
\end{tabular}

Conclusion: Acute esophageal acid exposure may increase the expression of NMDAR in PFC and ventral hippocampus. We also found the first acid exposure at adult stage may enhance the expression of serine racemase in PFC and cfos in ventral hippocampus, but this phenomenon may be absent in those rats having the experience of acid exposure in early life. Those long-term and transitional molecular alterations may mediate the development of acid exposure related esophageal visceral hypersensitivity.

Disclosure of Interest: All authors have declared no conflicts of interest.

\section{P1820 1.8. UPPER GI NERVE- GUT AND MOTILITY: TRANSMITTERS/SIGNALS/RECEPTORS/ENTERIC NERVOUS} SYSTEM

L. Jia ${ }^{1}$, L. Yao ${ }^{1}$, C. Dong-Yun ${ }^{1}$, L. Wei-Dong ${ }^{1}$, X. Jian ${ }^{2}$

${ }^{1}$ Department Of Gastroenterology, Guangzhou First People's Hospital, Guangzhou Medical University, Guangzhou/China

${ }^{2}$ Department Of Psychology, Guangzhou Nansha Central Hospital, Guangzhou/ China

Contact E-mail Address: 13925012853@139.com

Introduction: Globus pharyngeus, a sensation of a lump or tightness in the throat, is a well- defined clinical symptom that is usually long-lasting, difficult to treat, and has a tendency to recur. More than half of globus patients suffered from probable psychological disorders, such as anxiety and depression 1 Antidepressants are used in the treatment of functional gastrointestinal disorders (FGIDs) and showed a promising efficacy. Our study manifested that low-dose amitriptyline is well tolerated and effective for general globus pharyngeus patients ${ }^{2}$. Our anterior study had ever speculated that AMT could modify brain-gut axis function, up-regulating brain-gut peptides, reducing the visceral sensitivity and regulating the secretory and motor functions of the gastrointestinal tract ${ }^{3}$, so that gastrointestinal symptoms as well as emotional well-being could be significantly improved. As we known, serotonin (5-hydroxtryptamine, 5 -HT) is an important factor in gut function, playing key role in intestinal peristalsis, secretion, and sensory signaling in the brain-gut axis ${ }^{4-5}$. Several studies have investigated the association between SLC6A4 and functional gastrointestinal disorders, including IBS and FD. Besides, the association between various complex behavioral traits and disorders were also studied, including anxiety, major depression, suicide, smoking behavior, alcohol dependence. A single gene (SLC6A4), located on thehuman chromosome 17q11.2. 17q12, is coded by serotonin transporter (5-HTT). The polymorphism of this gene is characterized by the insertion or deletion of the 44- $b p$ sequence and this is related to the different transcriptional activity of the gene. Allele with $44-b p$ deletion (short allele) is characterizedby a three times lower transcriptional activity than allele with 44- $b p$ insertion (long allele). Compared to other FIGDs, the researches about globus are rare. The pathogenesis of globus pharyngeus is still unknown.

Abstract No: P1818

Table 1: Densities of stem cells and enteroendocrine cells in the duodenum of total IBS group, PI-IBS and idiopathic IBS patients before and after receiving FMT

\begin{tabular}{|c|c|c|c|c|c|c|c|c|c|}
\hline \multirow[b]{2}{*}{ Markers/Hormones } & \multicolumn{6}{|c|}{ Immunoreactive cells densities } & \multirow[b]{2}{*}{$* P$-value } & \multirow[b]{2}{*}{$* * P$-value } & \multirow[b]{2}{*}{$* * * P$-value } \\
\hline & $\begin{array}{l}\text { Total IBS, } \\
\text { before }\end{array}$ & $\begin{array}{l}\text { Total IBS, } \\
\text { after }\end{array}$ & $\begin{array}{l}\text { PI-IBS, } \\
\text { before }\end{array}$ & $\begin{array}{l}\text { PI-IBS, } \\
\text { after }\end{array}$ & $\begin{array}{l}\text { Idiopathic IBS, } \\
\text { before }\end{array}$ & $\begin{array}{l}\text { Idiopathic } \\
\text { IBS, after }\end{array}$ & & & \\
\hline Neurogenin 3 & $222.3 \pm 13.8$ & $394.3 \pm 30.7$ & $214.2 \pm 18.5$ & $430.5 \pm 28.9$ & $230.5 \pm 21.5$ & $358.2 \pm 52.9$ & 0.0006 & 0.0007 & 0.1 \\
\hline Musashi 1 & $5.7 \pm 0.4$ & $5 \pm 0.5$ & $5.3 \pm 0.7$ & $5.2 \pm 0.8$ & $6 \pm 0.4$ & $4.8 \pm 0.7$ & 0.42 & 0.9 & 0.2 \\
\hline Chromogranin A & $370.3 \pm 21$ & $269.8 \pm 22$ & $340.8 \pm 34$ & $422.7 \pm 31$ & $399.8 \pm 20.9$ & $316.8 \pm 10.2$ & 0.98 & 0.0006 & 0.0065 \\
\hline Serotonin & $135.1 \pm 14.7$ & $142 \pm 12.8$ & $100.5 \pm 7.1$ & $160.7 \pm 16.6$ & $169.7 \pm 20.6$ & $123.3 \pm 17.3$ & 0.7 & 0.012 & 0.034 \\
\hline Somatostatin & $58.6 \pm 4$ & $66.2 \pm 6.3$ & $52.3 \pm 2.7$ & $78.5 \pm 8.1$ & $64.8 \pm 7$ & $53.8 \pm 6.8$ & 0.3 & 0.011 & 0.017 \\
\hline Cholecystokinin & $122.8 \pm 6.7$ & $110.7 \pm 8.1$ & $113 \pm 10.4$ & $126.5 \pm 0.5$ & $132.5 \pm 7.2$ & $94.8 \pm 9$ & 0.2 & 0.052 & 0.0006 \\
\hline Secretin & $83.8 \pm 4.9$ & $86.7 \pm 5.9$ & $80.5 \pm 8.8$ & $89.7 \pm 10.7$ & $87.2 \pm 4.8$ & $83.7 \pm 5.9$ & 0.5 & 0.099 & 0.6 \\
\hline Gastric inhibitory peptide & $65.1 \pm 3.8$ & $70.3 \pm 6.2$ & $60 \pm 3 \pm 3.7$ & $84 \pm 7.1$ & $69.8 \pm 6.3$ & $57.2 \pm 7$ & 0.5 & 0.014 & 0.2 \\
\hline
\end{tabular}


To the best of our knowledge, our findings are the first to establish an association between SLC6A4 gene polymorphism and globus.

Aims \& Methods: 84 patients diagnosed with globus according to Rome III and 160 healthy controls were genotyped for 5-HTTLPR polymorphism by PCR amplification and agarose gel electrophoresis. All globus patients were studied with high-resolution manometry pre-therapy. Globus patients were randomized into paroxetine group; amitriptyline group for 6-week treatment, and were asked to complete the following questionnaires pre- and post-therapy: Glasgow Edinburgh Throat Scale (GETS), Pittsburgh Sleep Quality Index, Hamilton Rating Scale Anxiety/Depression. Treatment response was defined as a $>50 \%$ reduction in GETS scores.

Results: The significant difference was shown in globus performed $\mathrm{S} / \mathrm{S}$ genotype with anxiety when compared to without $\left(\mathrm{X}^{2}=14.579, P=0.006\right)$. The $\mathrm{L} / \mathrm{S}$ genotype was significant difference between high upper esophageal sphincter pressure $(>104 \mathrm{mmHg})$ and non-high upper esophageal sphincter pressure patients $\left(\mathrm{X}^{2}=14.433, P=0.006\right)$. There was significant association between the $\mathrm{S} / \mathrm{S}$ genotype and the response to antidepressants treatment, while patients with sleep disorders or depression not.

Conclusion: A significant association was observed between $\mathrm{S} / \mathrm{S}$ genotype of SLC6A4 polymorphism and globus pharyngeus, suggesting that SLC6A4 is a potential candidate gene involved in the pathogenesis of globus pharyngeus. Disclosure of Interest: All authors have declared no conflicts of interest.

\section{References}

1. Tang B, Jia L, Liu J, et al. Clinical-psychological characteristics of refractory globus patients in China. Dig Liver Dis. 2016; 48(4):381-4.

2. You LQ, Liu J, Jia L, et al. Effect of low-dose amitriptyline on Globus Pharyngeus and its side effects. World J Gastroenterol. 2013; 19(42):7455-60.

3. $2013 ; 19(26): 4214-20$.

4. Khan WI, Ghia JE. Gut hormones: emerging role in immune activation and inflammation. Clin Exp Immunol. 2010;161(1):19-27.

5. Kim DY, Camilleri M. Serotonin: a mediator of the brain-gut connection. Am J Gastroenterol. 2000; 95(10):2698-709.

\section{P1821 DIAGNOSTIC YIELD OF PROVOCATIVE TESTS ON ESOPHAGEAL HIGH RESOLUTION MANOMETRY (HRM) \\ S. Hasak ${ }^{1}$, S.A. Basit ${ }^{2}$, V. Naravadi ${ }^{3}$, A. Lazarescu ${ }^{4}$, C.P. Gyawali ${ }^{1}$ ${ }_{1}^{1}$ GI, Washington University in St. Louis, St. Louis/United States of America ${ }^{2}$ University of Nevada, Reno/United States of America/NV \\ ${ }^{3}$ Loyola University Chicago, Maywood/United States of America/IL \\ ${ }^{4}$ University of Alberta, Edmonton/Canada}

Contact E-mail Address: hasak.s@wustl.edu

Introduction: Multiple rapid swallows (MRS) and rapid drink challenge (RDC) are provocative tests that can enhance the diagnostic value of esophageal HRM. The discriminatory characteristics of these provocative tests were evaluated in symptomatic patients referred for esophageal HRM.

Aims \& Methods: Consecutive patients presenting for esophageal HRM over a 2month period underwent provocative testing with MRS and RDC in addition to the standard manometric protocol. Integrated relaxation pressure $>15 \mathrm{mmHg}$ identified outflow obstruction; those without outflow obstruction were further analyzed using software tools (IRP, distal contractile integral, DCI; intrabolus pressure, IBP) for peristaltic reserve (MRS or RDC DCI > mean DCI from wet swallows), and obstruction (IBP $>30 \mathrm{mmHg}$ during MRS or RDC). All patients completed symptom questionnaires addressing reflux symptoms (GERD-Q), dysphagia (Mayo Dysphagia Questionnaire, MDQ) and global symptom severity (GSS) on a $100 \mathrm{~mm}$ visual analog scale (VAS). Univariate and multivariate analyses were performed to evaluate overall yield (proportions with MRS/RDC findings not seen with the standard HRM protocol) and to assess association of presenting symptoms with results of MRS and RDC in patients without outflow obstruction.

Results: 149 patients $(55.4 \pm 1.2 \mathrm{yr}, 68 \% \mathrm{~F})$ fulfilled inclusion criteria and had no outflow obstruction on HRM. Of these, $91(61 \%)$ had peristaltic reserve on MRS, $19(12.6 \%)$ on RDC ( $<<0.001)$, only 10 were concordant. Obstruction was noted in $16(9.8 \%)$ on MRS, $8(4.9 \%)$ on RDC $(p=0.09)$, and 5 were concordant; of these, only 2 patients had panesophageal compartmentalization of pressure, and only 1 had elevated IRP during provocative measures. Within ineffective esophageal motility, peristaltic reserve was noted in $59.3 \%$. Within dysphagia presentations, obstruction was noted in $7.7 \%$, and absent peristaltic reserve in $50 \%$. Thus, the overall yield of MRS was $80.5 \%$, and RDC $19.8 \%$ $(\mathrm{p}<0.001) .131$ patients had adequate questionnaire data. Findings on provocative tests did not predict presenting symptoms (GERD-Q or MDQ). Obstruction on RDC predicted higher GSS (odds ratio 5.56, 95\% CI 1.04-29.72). Conclusion: MRS identifies peristaltic reserve better than RDC and has higher overall clinical value. While both MRS and RDC identify outflow obstruction, only RDC obstruction predicts higher symptom burden. Neither MRS nor RDC findings correlate with presenting symptoms.

Disclosure of Interest: All authors have declared no conflicts of interest.
P1822 THE INCIDENCE AND PREVALENCE OF ACHALASIA IN ENGLAND IN TWO NATIONAL DATABASES

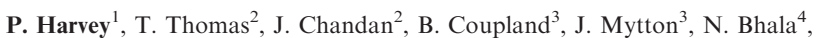
P. Patel ${ }^{3}$, K. Nirantharakumar ${ }^{2}$, N. Trudgill ${ }^{1}$

${ }^{1}$ Gastroenterology, Sandwell and West Birmingham NHS Trust, Birmingham/ United Kingdom

${ }^{2}$ Institute Of Applied Health Research, University of Birmingham, Birmingham/ United Kingdom

${ }^{3}$ Health Informatics, University Hospital Birmingham, Birmingham/United Kingdom

${ }^{4}$ Gastroenterology, University Hospitals Birmingham NHS Foundation Trust, Birmingham/United Kingdom

Contact E-mail Address: Philipharvey@nhs.net

Introduction: Achalasia is an uncommon condition of failed lower oesophageal sphincter relaxation. Data regarding the incidence and prevalence are limited. The aim of this study was to provide accurate, contempory epidemiological data utilising two national databases.

Aims \& Methods: Hospital Episode Statistics (HES) includes demographic and diagnostic data for all English hospital admissions. The Health Improvement Network (THIN) database includes primary care records of $7 \%$ of the UK population, representative of national demographics. Both were searched for incident cases and THIN for prevalent cases of achalasia.

Results: There were 10,509 and 711 new achalasia subjects in HES and THIN respectively. The incidence per 100,000 population in HES was $1.99(95 \%$ CI $1.87-2.11)$ and $1.53(1.42-1.64)$ per 100,000 person years in THIN. The prevalence measured in THIN was 27.1 (25.4-28.9) per 100,000 population.

Table 1: Annual incidence and prevalence of achalasia

\begin{tabular}{llllll}
\hline & $\begin{array}{l}\text { Incidence } \\
\text { rate HES } \\
\text { (per 100,000 } \\
\text { population) }\end{array}$ & 95\% CI & $\begin{array}{l}\text { Incidence rate } \\
\text { THIN } \\
\text { (per 100,000 } \\
\text { person years) }\end{array}$ & 95\% CI & $\begin{array}{l}\text { Prevalence } \\
\text { THIN } \\
\text { (per 100,000 } \\
\text { population) }\end{array}$ \\
\hline 2006 & 1.733 & $1.62-1.85$ & 1.409 & $1.08-1.81$ & 21.14 \\
2007 & 1.798 & $1.69-1.92$ & 1.656 & $1.39-2.08$ & 21.72 \\
2008 & 1.789 & $1.68-1.91$ & 1.550 & $1.21-1.96$ & 22.49 \\
2009 & 1.853 & $1.74-1.97$ & 1.696 & $1.34-2.12$ & 23.16 \\
2010 & 2.015 & $1.90-2.14$ & 1.663 & $1.31-2.09$ & 23.66 \\
2011 & 1.781 & $1.67-1.90$ & 1.424 & $1.09-1.82$ & 24.62 \\
2012 & 2.032 & $1.91-2.16$ & 1.549 & $1.20-1.96$ & 25.23 \\
2013 & 2.179 & $2.06-2.31$ & 1.618 & $1.26-2.05$ & 26.06 \\
2014 & 2.421 & $2.29-2.56$ & 1.476 & $1.12-1.91$ & 26.34 \\
2015 & 2.236 & $2.11-2.36$ & 1.342 & $0.96-1.80$ & 27.10
\end{tabular}

Conclusion: The incidence of oesophageal achalasia was approximately 15 to 20 per 1 million population. There were approximately 17,500 patients with achalasia in UK. In 2015 the above data represents the largest published epidemiological investigation of achalasia. The variation of findings between the databases likely results from differences in coding practice and marginally different population structures.

Disclosure of Interest: All authors have declared no conflicts of interest.

\section{P1823 ACHALASIA DESPITE NORMAL INTEGRATED} RELAXATION PRESSURE WITH 5ML WATER SWALLOWS

S. Sanagapalli ${ }^{1}$, M. Duffy ${ }^{1}$, A. V. Emmanuel ${ }^{2}$, A. Raeburn ${ }^{1}$, M. Banks ${ }^{3}$, R. Haidry ${ }^{3}$, L. Lovat ${ }^{3}$, R. Sweis ${ }^{1}$

${ }^{1}$ GI Physiology, University College London Hospital, London/United Kingdom ${ }^{2}$ University College London, London/United Kingdom

${ }^{3}$ Department Of Gastroenterology, University College London Hospital, London/ United Kingdom

Contact E-mail Address: rami.sweis@nhs.net

Introduction: Resistance to bolus flow across the lower esophageal sphincter (LES) is a hallmark of achalasia. Presently the gold standard of diagnosis is by high-resolution manometry (HRM) demonstration of raised integrated relaxation pressure (IRP) following ten $5 \mathrm{~mL}$ water swallows; however, this does not replicate normal swallowing behavior. It has been demonstrated that the addition of adjunctive tests improves sensitivity of identifying relevant dysmotility. Such tests include multiple water swallows (MWS; $200 \mathrm{~mL}$ water drunk freely) and solid swallows. In addition, the timed barium esophagram (TBE) measures esophageal emptying. This study describes a cohort of patients who have been treated as having achalasia based on resistance to flow not exhibited with single water swallows.

Aims \& Methods: Inclusion criteria were all patients between October 2014-2016 with normal mean and median IRP with $5 \mathrm{~mL}$ water swallows but considered to have achalasia due to resistance to flow demonstrated by pan-esophageal pressurization (PEP) during MWS or solid swallows and/or a persistent column at 5 minutes during TBE. Outcome following treatment was based on the Eckardt symptom score (ES). 
Results: 14 patients ( 9 male) fulfilled inclusion criteria. 7 were treatment-naïve and 7 treatment-experienced (3 myotomy, 4 dilatation). Mean resting LES pressure was $14.6 \pm 7.4 \mathrm{mmHg}$. In all patients, mean and median IRP values for ten $5 \mathrm{~mL}$ water swallows were non-raised (mean $9.1 \pm 4.3$ and $8.7 \pm 4.5 \mathrm{mmHg}$ respectively). Of the 7 treatment-naïve patients, 5 demonstrated PEP on MWS, 3 on solid swallows and 6 had a positive TBE at 5 minutes. In treatment-experienced patients, 5 had PEP on MWS, 1 on solid swallows and all had a positive TBE. Of the 13 who had resistance to flow on TBE, $10(77 \%)$ also had resistance demonstrated during MWS and/or solid swallows. Mean height of the 5-minute column of barium at baseline was $16.5 \pm 8.9 \mathrm{~cm}$. 8 patients have (so far) undergone therapy based on these findings; one per-oral endoscopic myotomy and 7 pneumatic dilatations. The median baseline ES was 7.5 (IQR 5-8). The median ES at minimum 3 months (range 3-15 months) following treatment was 1 (IQR $0-2.5 ; P<0.01$ cf. baseline). Similarly, there was significant improvement in TBE findings post-therapy (mean 5-minute column height $3.5 \pm 4.1 \mathrm{~cm}$; $P=0.04 \mathrm{cf}$. baseline).

Conclusion: A normal IRP for water swallows does not preclude a diagnosis of achalasia. The addition of free drinking/solids during HRM or the TBE can identify pathology that might have been missed with standard $5 \mathrm{~mL}$ water swallows alone as normal, clinically relevant swallowing behavior is reproduced. Patients treated based on this algorithm exhibit excellent treatment outcomes, validating this approach. Further, the close correlation of HRM adjunctive testing with TBE supports its routine inclusion in clinical practice.

Disclosure of Interest: All authors have declared no conflicts of interest.

\section{P1824 200ML RAPID DRINK CHALLENGE DURING HIGH RESOLUTION MANOMETRY PREDICTS OESOPHAGOGASTRIC JUNCTION OBSTRUCTION IN DYSPHAGIA, AND IS CORRELATED WITH SYMPTOM SEVERITY IN ACHALASIA}

S. Gabieta-Sonmez ${ }^{1}$, P. Woodland ${ }^{2}$, J. Arguero ${ }^{1}$, J.L.S. Ooi ${ }^{1}$, K. Nakagawa ${ }^{1}$, E. Glasinovic ${ }^{1}$, E. Yazaki ${ }^{1}$, D. Sifrim ${ }^{1}$

${ }^{1}$ Wingate Institute For Neurogastroenterology, Barts and the London School of Medicine, Queen Mary University, London/United Kingdom

${ }^{2}$ School Of Medicine, Queen Mary University Barts \& the London Dept. of Medicine and Dentis., London/United Kingdom

Contact E-mail Address: Shirley.Sonmez@bartshealth.nhs.uk

Introduction: In patients with dysphagia, the identification of obstruction at the OGJ level determines the need for further endoscopic or surgical therapy. Timed barium oesophagogram (TBO) is regularly used to detect impaired oesophageal emptying in due to OGJ obstruction in patients with dysphagia and achalasia. "Volume loading" the oesophagus with a $200 \mathrm{ml}$ rapid drink challenge (RDC) has recently been shown to help with the diagnosis of oesophageal motility disorders. We predict that this test can evaluate OGJ emptying at HRM without need for further TBO study. As such we hypothesised that RDC can predict pathological barium column at TBO, and associates with symptom severity in achalasia.

Aims \& Methods: 39 patients with untreated dysphagia (mean age 55yr) were evaluated. All patients underwent HRM with standard protocol plus RDC, followed by TBO. In patients with achalasia, symptoms were recorded by Eckhard score. Analysis of HRM followed the Chicago Classification v3 algorithm. During TBO, oesophageal emptying was assessed at min 1, 3 and 5. OGJ obstruction was diagnosed by persistent barium column at 5 minutes. During RDC, the mean IRP was measured during the rapid drinking period.

Results: HRM diagnosis was normal or minor peristaltic abnormality $(n=5)$, achalasia type I $(n=3)$, achalasia type II $(n=22)$, achalasia type III $(n=2)$, oesophagogastric junction outflow obstruction $(\mathrm{n}=3)$, absent contractility $(\mathrm{n}=2)$ and Jackhammer $(\mathrm{n}=2)$. ROC curve analysis showed that a mean IRP during RDC $>17 \mathrm{mmHg}$ had a sensitivity of $100 \%$ and specificity of $87 \%$ for an abnormal TBO. In patients with achalasia, only mean IRP during RDC correlated with Eckhardt score $(r=0.42, p=0.03)$. No standard HRM parameters or TBO findings correlated with Eckhard score.

Conclusion: $200 \mathrm{ml}$ rapid drink challenge during HRM is a useful adjuvant in assessment of oesophageal motility disorders. It can predict an abnormal TBO with excellent sensitivity and specificity in patients with dysphagia. In patients with achalasia, a higher mean IRP during $200 \mathrm{ml}$ rapid drink challenge is the only HRM metric that correlates with worse symptom burden. These findings suggest that that the rapid drink challenge is a clinically relevant parameter.

Disclosure of Interest: D. Sifrim: research grant from Sandhill Scientific (Denver, USA) research grant from Reckkit Benkiser (Hull, UK)

All other authors have declared no conflicts of interest.

\section{P1825 THE TIMED BARIUM ESOPHAGRAM SURFACE AREA CORRELATES WITH SYMPTOM IMPROVEMENT BETTER THAN COLUMN HEIGHT FOLLOWING TREATMENT IN ACHALASIA}

S. Sanagapalli ${ }^{1}$, J. Maynard $^{1}$, A. Plumb ${ }^{2}$, A. V. Emmanuel ${ }^{3}$, A. Raeburn ${ }^{1}$, M. Banks ${ }^{4}$, R. Haidry ${ }^{4}$, L. Lovat ${ }^{4}$, R. Sweis ${ }^{1}$

${ }_{1}^{1}$ GI Physiology, University College London Hospital, London/United Kingdom ${ }^{2}$ Radiology And Imaging, University College London Hospital, London/United Kingdom

${ }^{3}$ University College London, London/United Kingdom

${ }^{4}$ Department Of Gastroenterology, University College London Hospital, London/ United Kingdom
Introduction: The timed barium esophagram (TBE) is an objective measurement of esophageal emptying used in the assessment of achalasia. Post-therapy reduction of the maximum height of the residual barium column has been found to correlate imperfectly with short-term symptomatic outcomes, but carries longterm prognostic implications. We hypothesize that the surface area (SA) of the barium column may be more accurate than height; firstly, by incorporating improvement in esophageal width that often occurs post-therapy, but also by correcting for artificially higher height values due to esophageal (longitudinal) contraction occurring during a single image. We aimed to compare the correlation of TBE outcome measures of height and SA with symptom improvement post-therapy.

Aims \& Methods: Inclusion criteria were achalasia patients who underwent therapy between August 2015-6 and had TBE and Eckardt score (ES) performed at baseline as well as within 6 months post-therapy. With TBE upright single images were acquired at 1,2 and 5 minutes following ingestion of $100-200 \mathrm{~mL}$ of lowdensity barium sulfate. Barium height was measured between the gastro-esophageal junction and the superior extent of any residual barium column. After manually defining the column boundaries, software was used to calculate SA (AGFA IMPAX surface area tool (Figure)). Adequate symptom relief was defined as reduction in ES to $\leq 3$. On TBE, metrics of adequate emptying evaluated were i) post-therapy column height $<5 \mathrm{~cm}$, ii) $>50 \%$ reduction in column height from pre to post-therapy and iii) $>50 \%$ reduction in column SA from pre to post-therapy. Associations between symptom improvement and TBE measures of emptying were assessed using Pearson's correlation (R). Paired $t$-tests compared TBE measures before and after therapy.

\begin{tabular}{llll}
\hline & $\begin{array}{l}\text { Cut-off value } \\
\text { for adequate } \\
\text { emptying }\end{array}$ & $\begin{array}{l}\text { Concordance } \\
\text { between adequate TBE \& } \\
\text { symptom relief (based } \\
\text { on ES) n/total (\%) }\end{array}$ & $\begin{array}{l}\text { Pearson's } \\
\text { correlation } \\
\text { between TBE \& } \\
\text { post-therapy ES R }\end{array}$ \\
TBE parameter & $<5 \mathrm{~cm}$ & $7 / 18(39 \%)$ & -0.05 \\
\hline $\begin{array}{l}\text { Post-therapy 5-min barium } \\
\text { column }\end{array}$ & $8 / 18(44 \%)$ & 0.20 \\
$\begin{array}{l}\% \text { Reduction in 5-minute } \\
\text { barium height post-therapy } \\
\text { from baseline }\end{array}$ & $>50 \%$ & $12 / 18(67 \%)$ & 0.36 \\
$\begin{array}{l}\% \text { Reduction in SA of 5- } \\
\text { min barium post-therapy } \\
\text { from baseline }\end{array}$ & & & \\
\hline
\end{tabular}

Results: 18 patients (9 male; 6 Type I, 11 Type II, 1 Type III) were included. 11 had dilatation and 7 endoscopic myotomy. Reductions with therapy of both mean 5-minute barium column height $(14.7 \pm 8.7$ to $7.9 \pm 6.0 \mathrm{~cm} ; P=0.01)$ and SA $\left(52.7 \pm 43.5\right.$ to $\left.24.5 \pm 26.0 \mathrm{~cm}^{2} ; \quad P=0.02\right)$ were noted. Symptoms also improved with treatment; median baseline ES of 7 (IQR 5.25-8) improved to 0 (IQR $0-1$ ) post-therapy. Only 2 patients had inadequate symptom relief and are awaiting further treatment. However there was poor concordance between posttherapy barium column height and symptomatic relief (i.e. post-therapy column $>5 \mathrm{~cm}$ despite $\mathrm{ES} \leq 3$ or vice versa), and the correlation (R) between these two variables was poor (Table). Similar poor concordance was seen when adequate emptying was defined by $>50 \%$ reduction in column height, but $>50 \%$ reduction in SA paralleled symptom relief most closely

Conclusion: In TBE performed on achalasia patients post-therapy, reduction in SA of the residual barium column compared with baseline values parallels symptomatic relief more closely than reduction of column height.

Disclosure of Interest: All authors have declared no conflicts of interest.

\section{P1826 NUTRIENTS ENHANCE BELCHING AND REDUCE INTESTINAL PROPULSION OF GASTRIC GAS IN HEALTHY HUMANS}

E.N. Caballero De Garcia, B. Benslaiman Seyid-Jebari, J. Serra Pueyo Gut Motility Unit, Hospital Unversitary Germans Trias i Pujol, Badalona/Spain

Contact E-mail Address: noecaballerosuarez@gmail.com

Introduction: During meal intake, large volumes of gas can be ingested together with nutrients, and patients with gas-related abdominal symptoms often refer symptom exacerbation by meals. Previous studies have shown that under fasting conditions gastric gas is rapidly eliminated from the stomach, either via gas emptying to the small bowel and colon, or via belching. Nutrients have several effects on gastric and intestinal motor function that could modulate gas transport and symptoms.

Aims \& Methods: We aimed to determine the effect of gastric nutrients on transport of gastric gas, and its relationship with abdominal symptoms. In 7 healthy volunteers without gastrointestinal symptoms (4 women and 3 men, age-range 21-25 yrs), a mixture of non-absorbable gases was infused into the stomach, $5-\mathrm{cm}$ caudal to the lower margin of the LES, at $25 \mathrm{~mL} / \mathrm{min}$ during $60 \mathrm{~min}$ (Total gas infused: $1500 \mathrm{ml}$ ). In each subject two gas infusion tests were performed on separate days, with simultaneous infusion of nutrients (Nutridrink $1.5 \mathrm{Kcal} / \mathrm{ml}$, total $315 \mathrm{Kcal}$ ) or saline. Belching, by an esophageal multilumen impedance manometry catheter, rectal gas evacuation, via a rectal tube connected to a barostat, and epigastric and abdominal symptoms, by specific questionnaires (from 0-6), were continuously recorded from the start of gas infusion until $30 \mathrm{~min}$ after gas infusion stopped. (Total recording time: $90 \mathrm{~min}$ ).

Results: During saline infusion, participants evacuated via the rectum virtually all the infused gas $(1613 \pm 87 \mathrm{ml})$, with exceptional belching $(1.1 \pm 0.8$ belches $)$ and mild epigastric perception (score $1.7 \pm 0.6$ at the end of infusion), that decreased during the $30 \mathrm{~min}$ following infusion stop (score $1.1 \pm 0.4 ; \mathrm{p}=0.051$ vs infusion 
stop). Gastric nutrients reduced significantly rectal gas evacuation $(1220 \pm 187 \mathrm{ml} ; \mathrm{p}=0.016 \mathrm{vs}$ saline $)$, increased the number of recorded belches $(3.3 \pm 1.3$ belches; $\mathrm{p}=0.018$ vs saline), and produced greater epigastric perception (score $2.7 \pm 0.6 ; \mathrm{p}=0.030$ ) that decreased during the $30 \mathrm{~min}$ following infusion stop (score $1.6 \pm 0.7 ; \mathrm{p}=0.042$ vs infusion stop). By contrast to epigastric perception, abdominal perception was only somewhat higher during gas infusion with nutrients (score 2.6 \pm 0.5 ) than during saline (score $2.1 \pm 0.5 ; \mathrm{p}=0.100$ vs nutrients).

Conclusion: Gastric nutrients modulate transit of gastric gas, by reducing gas propulsion to the distal gut, and enhancing retrograde gas evacuation via belching.

Disclosure of Interest: All authors have declared no conflicts of interest.

\section{P1827 MODIFICATIONS OF THE ECKARDT SCORE PARAMETERS AFTER PERORAL ENDOSCOPIC MYOTOMY}

M. Barret ${ }^{1}$, S. Leblanc ${ }^{1}$, M. Gaudric ${ }^{1}$, M. Guillaumot ${ }^{1}$, A. Oudjit ${ }^{2}$, M. Dhooge ${ }^{1}$,

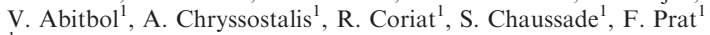

${ }^{1}$ Gastroenterology, Cochin Hospital, Paris/France

${ }^{2}$ Radiology, Cochin Hospital, Paris/France

\section{Contact E-mail Address: maximilien.barret@aphp.fr}

Introduction: Peroral endoscopic myotomy (POEM) is a recently developed technique or the treatment of lower esophageal sphincter achalasia. POEM could be as efficient as surgical Heller myotomy, while associated with lower morbidity. Currently, the Eckardt score is the clinical score that is the most widely used to assess the outcomes after the treatment of achalasia, clinical success being defined by a score below 4 . However, POEM might not equally improve all four parameters of the Eckardt score.

Aims \& Methods: All consecutive patients undergoing POEM for achalasia at our institution performed by 3 operators with at least 6 months follow-up were prospectively included. Demographic, clinical, procedural, manometric and radiological data were collected.

Results: Between March 2013 and July 2016, 62 POEM procedures were performed in 59 patients (Male/female 33/26; Median age $\pm \mathrm{SD}=47 \pm 17$, range (15-77)). Median (IQR) follow-up time was 8 (3-13) months. Achalasia was diagnosed for a median (IQR) of 24 (13-62) months, and $42 \%$ of the patients had received a previous treatment, by botulinum toxin injection $(8 \%)$, pneumodilation $(32 \%)$, or Heller myotomy $(7 \%)$. Achalasia subtypes were type I/II/III in $37 \% / 47 \% / 15 \%$ of cases. Median Eckardt score and integrated resting pressure were 7 (6-8) and 24 (19-33) $\mathrm{mmHg}$, respectively. An anterior myotomy was done in $60 \%$ of cases, and a posterior myotomy in $40 \%$ of cases. Median myotomy length was $14(12-16) \mathrm{cm}$, and hospital stay was $3(2-4)$ days. Severe complications occurred in $5 \%$ of cases ( 1 pleural effusion requiring drainage, 2 pneumonias with more than 10 days of hospital stay). Success rates were similar between patients treated by anterior or posterior myotomy $(92 \%$ vs $92 \%, \mathrm{p}=1)$, or between treatment naïve or pretreated patients $(88 \%$ vs $96 \%, \mathrm{p}=0.32)$. Six treatment failures were treated by redo POEM in 3 cases, pneumodilation in 2 cases, and esophagectomy in one case. Median Eckardt score varied from 7 (6-8) to $1(0-2)$ at 3 months and $2(0-3)$ at 12 months $(\mathrm{p}<0.0001)$. Dysphagia score varied from $3(3-3)$ to $0(0-1)$ and $1(0-3)(\mathrm{p}<0.0001)$, while regurgitations varied from $2(1-3)$ to $0(0-1)$ and $1(0-1)(\mathrm{p}<0.0001)$, chest pain varied from $1(0-2)$ to $0(0-1)$ and $0(0-1)(\mathrm{p}=0.006)$, and weight loss from $2(1-3)$ to $0(0-0)$ and $0(0-0)(\mathrm{p}<0.0001)$. At three months, median (range) drop of the integrated resting pressure was $16(12 ; 22) \mathrm{mmHg}$. At last follow-up visit, $86 \%$ of patients still had clinical success, and $31 \%$ reported gastro-esophageal reflux symptoms. Conclusion: Our results confirm the efficacy of the POEM as a first line or rescue treatment for achalasia, with a low complication rate. POEM is most effective on dysphagia and weight loss, while chest pain and regurgitations tend to persist after treatment.

Disclosure of Interest: All authors have declared no conflicts of interest.

\section{P1828 SUBGROUP ANALYSES OF CLINICAL TRIALS ON A} HERBAL MEDICINE IN FD, STW 5

J. Müller ${ }^{1}$, O. Kelber ${ }^{1}$, B. Vinson ${ }^{1}$, C. Fink ${ }^{1}$, S. Rabini ${ }^{1}$, K. Kraft ${ }^{2}$, M.A. Storr ${ }^{3}$ ${ }^{1}$ Innovation \& Development, Phytomedicines Supply And Development Center, Bayer Consumer Health, Steigerwald Arzneimittelwerk GmbH, Darmstadt/ Germany

${ }^{2}$ Chair For Naturopathy, Center for Internal Medicine, Rostock/Germany

${ }^{3}$ Center of Endoscopy, Starnberg/Germany

Contact E-mail Address: olaf@kelber.org

Introduction: Well-proven therapeutic options for the therapy of functional gastrointestinal diseases are rare and therefore gain high attention. One of these is STW 5 (Iberogast), for which more than five decades of therapeutic experience in more than 73 Mio patients are available. For determining whether its efficacy in functional dyspepsia proven by meta-analyses is comparable in male and female as well as patients of different age groups, sub-group analyses were conducted. Aims \& Methods: After meta-analyses of randomized placebo-controlled double blind trials with STW 5 have proven compliance to modern standards for a proof of efficacy, now sub-group analyses were conducted. The analyses (ANCOVA) were based on the original single patient data from the trials, including demografic data and primary endpoints.

Results: As the primary outcome variable, the validated gastrointestinal symptom score (GIS) [1], as well as the therapeutic dose $(3 \times 20$ drops/day) were identical in all trials, a uniform evaluation was possible. The full analysis set (FAS) included 557 patients ( 272 resp. 285 for placebo resp. verum). The mean age (48 resp. 49 years), the mean body size (in both groups $168.7 \mathrm{~cm}$ ), the mean body weight (72.0 resp. $72.2 \mathrm{~kg}$ ), the BMI (25.35 resp. 25.54), the gender distribution $(67.3$ resp. $69.5 \%$ females $)$, the duration of the disease at the time of inclusion and the baseline of the GIS (11.6 resp. 11.5 points) were very well comparable between both groups. For the primary variable GIS the difference between placebo and verum after 28 days of treatment showed a highly significant $(\mathrm{p}<0.0001)$ difference between placebo and verum $(6.7$ resp. 4.7 points) The analyses in different age groups (up to $30,30-40,40-50,50-60$, above 60) and in male and female patients did show a comparable efficacy in all these groups.

Conclusion: These meta-analyses therefore clearly show the efficacy of STW 5 (Iberogast) and its therapeutic usefulness irrespective of age and gender. Given also the very good tolerability shown by the study data and the pharmacovigilance data, STW 5 is well suitable also in self-medication. Additional insights can be expected from additional sub group analyses, as e.g. the evaluation of subgroups with specific predominant symptoms.

Disclosure of Interest: J. Müller: Employee, Steigerwald Arzneimittelwerk GmbH, Darmstadt, Germany

O. Kelber: Employee, Steigerwald Arzneimittelwerk GmbH, Darmstadt, Germany

B. Vinson: Employee, Steigerwald Arzneimittelwerk GmbH, Darmstadt, Germany

C. Fink: Employee, Steigerwald Arzneimittelwerk GmbH, Darmstadt, Germany S. Rabini: Employee, Steigerwald Arzneimittelwerk GmbH, Darmstadt, Germany

K. Kraft: Travel grants and honorary from Steigerwald Arzneimittelwerk GmbH, Darmstadt, Germany

M.A. Storr: Travel grants and honorary from Steigerwald Arzneimittelwerk GmbH, Darmstadt, Germany

\section{Reference}

1. Adam et al. Aliment Pharmacol Ther 2005; 22: 357-363

\section{P1829 THE TREATMENT OF ACHALASIA IN PATIENTS WITH OESOPHAGEAL VARICES: AN INTERNATIONAL CASE SERIES}

C. Magee $^{1}$, R. Holloway ${ }^{2}$, C.P. Gyawali ${ }^{3}$, S. Roman ${ }^{4}$, M. Pioche ${ }^{5}$, E. Savarino ${ }^{6}$, F. Quader ${ }^{3}$, N. Zarate Lopez ${ }^{7}$, A.J. Bredenoord ${ }^{8}$, R. Sweis ${ }^{7}$

${ }^{1}$ University College London Hospitals, London/United Kingdom

${ }^{2}$ Royal Adelaide Hospital, Adelaide/Australia

${ }^{3}$ Washington University in St. Louis, St Louis/United States of America/MO

${ }^{4}$ Digestive Physiology, Hôpital Edouard Herriot, Lyon/France

${ }^{5}$ Hepatogastroenterology, Hopital Edouard Herriot, LYON/France

${ }^{6}$ Uo Gastroenterology Department Of Surgical, Oncological And

Gastroenterological Sciences, University Of Padova, Padova Italy, University of

Padova, Padova/Italy

${ }^{7}$ Gi Physiology Unit, University College London Hospital, London/United

Kingdom

${ }^{8}$ Dept. Of Gastroenterology, Academisch Med. Centrum Amsterdam, Amsterdam Netherlands

Contact E-mail Address: cormac.magee@ nhs.net

Introduction: Achalasia is a chronic condition presenting with dysphagia, regurgitation, chest pain and/or weight loss. Management options include Heller's myotomy, Botox, pneumatic dilatation and Per-Oral Endoscopic Myotomy (POEM). Treatments carry risks of bleeding and perforation. Conomitant portal hypertension with varices is very rare and achalasia treatmentin this context has only been described in single case reports.

Aims \& Methods: Experience from physicians/surgeons treating these disorders was sought through the International Manometry Working Group.

Results: 13 patients with portal hypertension from 6 international centres have been collected; mean age $61 \pm 9$ years. The median pre- therapy Eckardt score was 7 (IQR 6-9). 9/13 (69\%) patients had a barium swallow and 12/13 (92\%) had oesophageal physiology studies performed. There were 3 Type I, 6 Type II, 2 Type III achalasia and 2 with oesophago-gastric outflow obstruction. Varices were identified endoscopically in 7 patients, radiologically in 5 and in 1 patient varices were first noted during surgical myotomy. 2 patients had grade 3 varices, 3 had grade 2 and 3 had grade 1 varices (grading not provided for the rest). Cirrhosis was due to alcohol in 7 patients, non-alcoholic steatohepatitis in 3, cryptogenic in 2 and 1 had hepatitis C cirrhosis. $75 \%$ were Child-Pugh A and $25 \%$ were Child-Pugh B. Patients had diverse treatments for their achalasia. 4 were treated with Botox injections ( 1 with EUS), 4 had dilation alone, 3 received a POEM, another had POEM then dilation and 1 patient had Botox followed by Heller's myotomy. 3 patients underwent variceal eradication in advance; all had banding first but in 2 patients superficial eradication was followed by a transjugular intrahepatic portosystemic shunt (TIPSS) before endoscopic dilation. All patients had symptomatic improvement with median Eckardt score post intervention $=1($ IQR $0-2) \mathrm{p}<0.0001$ compared to baseline. A matched group of 20 patients who underwent treatment for achalasia (all subtypes) but without varices had similar outcomes $(\mathrm{p}=\mathrm{NS})$. No patients had recorded complications of bleeding or perforation; however both patients who had TIPSS had temporary hepatic decompensation.

Conclusion: This reports 13 patients from international centres who have had interventions for achalasia on the background of oesophageal varices. None had bleeding complications despite only 3 having had variceal eradication. Symptom response mirrored those who undergo standard achalasia therapy, 
regardless of the severity of varices, treatment modality provided or achalasia subtype.

Disclosure of Interest: All authors have declared no conflicts of interest.

\section{P1830 THE NATURAL HISTORY OF ACHALASIA: EVIDENCE OF A CONTINUUM-THE PATTERN-EVOLUTIVE STAGING THEORY}

R. Salvador, G. Voltarel, E. Pesenti, E. Savarino, G. Capovilla, A. Perazzolo, L. Nicoletti, S. Merigliano, M. Costantini

Dept. Of Surgical, Oncological And Gastroenterological Sciences, University Hospital of Padova, Padova/Italy

Contact E-mail Address: m.costantini@unipd.it

Introduction: Esophageal achalasia is classified into three clinically relevant patterns at High Resolution Manometry (HRM) and according to Chicago Classification. Currently, it is unclear whether they represent distinct entities or are part of a disease continuum.

Aims \& Methods: The aims of this study were: a) to test the hypothesis that the three manometric patterns represent different stages in the evolution of esophageal achalasia and b) to investigate whether manometric patterns change after Laparoscopic Heller-Dor (LHD). We evaluated the patients who had a diagnosis of achalasia and underwent LHD as first treatment from 1992 to May 2016. Symptoms were scored using a detailed questionnaire for dysphagia, food-regurgitation, and chest pain; barium swallow, endoscopy, and esophageal manometry (conventional or High Resolution technique) were performed, before and 6 months after surgical treatment. All conventional manometric tracings, before 2010 , were reviewed and re-classified according to the manometric-pattern classification, whereas after 2010 the HRM data were prospectively collected.

Results: Five-hundred and eleven consecutive achalasia patients ( $\mathrm{M}: \mathrm{F}=283: 228)$ represented the study population. Based on their manometric findings, 231 patients $(45.2 \%)$ were classified as having pattern I, $241(47.2 \%)$ had pattern II, and $39(7.6 \%)$ had pattern III. Demographic and clinical data showed that pattern III cases had a shorter duration of symptoms, a more incidence of chest pain, and a less dilated gullet $(\mathrm{p}<0.001)$. Further, all patients with a sigmoidshaped mega-esophagus (radiological grade IV) had pattern I achalasia. One patient with diagnosis of pattern III achalasia, who refused any treatment evolved to pattern II at a follow-up manometry performed for a progressive worsening dysphagia after 36 months. At a median follow-up of 30 months (IQR 12-56), the outcome of surgery was positive in 479 patients $(91.7 \%)$. The only predictor of final outcome was the preoperative manometric pattern $(\mathrm{p}<0.001)$. All patients $(42)$ whose surgical treatment failed underwent one or more endoscopic pneumatic dilations using Rigiflex balloons ( 30,35 or $40 \mathrm{~mm}$ ) The overall success rate of the combined treatment (LHD plus endoscopic dilations where necessary) was $98 \%$. All patients with pattern I preoperatively had the same pattern after LHD, whereas more than $50 \%$ of patients with pre-treatment pattern III had patterns I or II after surgery. There were no cases showing the opposite trend (Table 1). Five patients showed signs of a partial recovery of peristalsis (all patients had a pattern II before LHD).

Table 1: Changing manometric patterns after LHD. $* 5$ patients had a recovery of peristalsis

\begin{tabular}{llll}
\hline & Pattern 1 post & Pattern 2 post & Pattern 3 post \\
\hline Pattern 1 pre & $159(100 \%)$ & 0 & 0 \\
Pattern 2 pre* & $65(29.5 \%)$ & $149(67.7 \%)$ & 0 \\
Pattern 3 pre & $7(24.1 \%)$ & $8(27.6 \%)$ & $8(48.3 \%)$ \\
\hline
\end{tabular}

Conclusion: The data of this study strongly support the hypothesis/theory that the different manometric patterns of achalasia could represent different evolutive stages of the disease - where pattern III is the earlier stage, pattern II an intermediate stage, and pattern I the end stage.

Disclosure of Interest: All authors have declared no conflicts of interest.
P1831 ROLE OF A SERUM BIOMARKERS PANEL

(GASTROPANELR) IN NON-INVASIVE DIAGNOSIS OF UPPER GI DISEASE: DATA BY A PRIMARY CARE POPULATION OF NORTHEAST ITALY

G. Baldassarre ${ }^{1}$, M. Franceschi ${ }^{1}$, M.P. Panozzo ${ }^{2}$, F. Tomba ${ }^{1}$, A. Ferronato ${ }^{1}$, S. Grillo ${ }^{3}$, D. Sella ${ }^{1}$, M. Santacatterina ${ }^{2}$, A. Antico ${ }^{2}$, F. Di Mario ${ }^{4}$

${ }^{1}$ Endoscopic Unit - Department Of Surgery, Ulss 7 Pedemontana, Santorso/Italy

${ }^{2}$ Department Of Clinical Pathology, Ulss 7 Pedemontana, Santorso/Italy

${ }^{3}$ Gastroenterology, University of Parma, Italy, PARMA/Italy

${ }^{4}$ University Of Parma, Department of Clinical and Experimental Medicine, section of Gastroenterology, Parma/Italy

Contact E-mail Address: francesco.tomba@aulss7.veneto.it

Introduction: The development of non-invasive methods to detect the presence of H. pylori, and to estimate the extent and severity of gastritis, have reduced the need for diagnostic endoscopy in asymptomatic individuals. However, it is not known whether the use of non-invasive diagnostic methods is effective in dyspeptic patients.

Aims \& Methods: To use a non-invasive blood test with four stomach-specific biomarkers to assess the prevalence of different stomach conditions: gastroesophageal reflux disease (GERD), H. pylori (HP) infection, chronic atrophic gastritis (CAG), and the efficacy of proton pump inhibitor (PPI) therapy in a primary care population. A cohort of 2583 dyspeptic patients (male $36 \%$, mean age 44.0 yrs, range 6-95) was selected in a primary care population and examined with a panel of biomarkers [Pepsinogen-I (PG-I) and -II (PG-II), amidated gastrin-17 (G-17), and HP IgG (Biohit, Finland)]. A standard questionnaire, including upper gastrointestinal symptoms and PPI use, was administered. Exclusion criteria were dysphagia, anemia, weight loss and vomiting. CAG patients underwent to endoscopy and histological examination.

Results: Healthy stomach was found in $21.2 \%$ patients. Prevalence of CAG increased with patient's age in both sexes as well as the use of PPIs $(p=0.0001)$. Table 1 shows the serum biomarkers values divided according to five categories: healthy stomach $(\mathrm{H}), \mathrm{GER}, \mathrm{HP}, \mathrm{CAG}$, and PPIs therapy. Conclusion: The combination of data on the levels of PG-I, PG-II, G-17 and HP IgG allow to diagnose different pathological conditions such as HP- and non HPrelated gastritis, the appropriateness of PPI administration, GERD and CAG, a precancerous condition.

Disclosure of Interest: All authors have declared no conflicts of interest.

\section{P1832 SUSTAINED TREATMENT EFFECTS OF MENTHACARIN ON SYMPTOMS AND QUALITY OF LIFE IN PATIENTS WITH FUNCTIONAL DYSPEPSIA 8 WEEKS AFTER THE END OF A 4-WEEK PLACEBO-CONTROLLED TRIAL}

G.J. Holtmann ${ }^{1}$, B. Stracke ${ }^{2}$

${ }^{1}$ Gastroenterology, Royal Adelaide Hospital, Adelaide/Australia

${ }^{2}$ Clinical Research, Dr. Willmar Schwabe GmbH \& Co. KG, Karlsruhe/Germany

Contact E-mail Address: g.holtmann@uq.edu.au

Introduction: Functional dyspepsia (FD) is one of the most common functional gastrointestinal disorders characterised by chronic or relapsing symptoms without structural or biochemical abnormalities that can be identified in the routine clinical setting. Thus, treatment targets symptoms. Very little is known about prolonged treatment for more than 4 weeks.

Aims \& Methods: The aim of the additional data analysis of a previous randomized placebo-controlled trial was to explore post-treatment effects that occurred after continuation of therapy after a 4-week randomized placebo controlled treatment with Menthacarin ${ }^{1}$ with regard to disease-specific symptoms and QoL in FD patients. After the 4-week randomised placebo-controlled treatment period, patients were allowed to continue the treatment. The treatment was given in a double-blind fashion and allocation of treatment followed the initial randomisation. 114 adult FD outpatients were initially treated and received twice a day one enteric-coated Menthacarin capsule or a matched placebo capsule for 4 weeks. Fifty-four of them participated in the optional follow-up phase and received Menthacarin (34) or placebo (20) for further 8 weeks according to their original randomization. The results of these 54 patients are reported here. Outcomes were assessed utilising the validated self-rating Nepean Dyspepsia Index (NDI). Intra-individual differences between baseline and week 4/week 12 for NDI sub-scores for pain (sum of the NDI items "pain or ache in upper

Abstract No: P1831

\begin{tabular}{|c|c|c|c|c|c|}
\hline & $\mathrm{H}$ & GER & $\mathrm{HP}$ & $\mathrm{CAG}$ & PPI \\
\hline Patients & $548(21.2 \%)$ & $784(30.4 \%)$ & $353(13.7 \%)$ & $138(5.3 \%)$ & $760(29.4 \%)$ \\
\hline $\operatorname{Sex}(M / F)$ & $162 / 386$ & $311 / 473$ & $111 / 242$ & $43 / 95$ & $294 / 466$ \\
\hline Age (yrs) & $39.6 \pm 14.0$ & $40.3 \pm 13.0$ & $45.0 \pm 13.6$ & $59.1 \pm 16.8^{\mathrm{c}}$ & $47.8 \pm 15.6^{\mathrm{d}}$ \\
\hline PG1 (ug/L) & $79.1 \pm 28.5$ & $72.3 \pm 23.9^{\mathrm{a}}$ & $92.2 \pm 40.8^{\mathrm{b}}$ & $12.3 \pm 9.7^{\mathrm{c}}$ & $167.4 \pm 107.7^{\mathrm{d}}$ \\
\hline PG2 (ug/L) & $6.5 \pm 3.1$ & $6.3 \pm 2.5^{\mathrm{a}}$ & $11.8 \pm 9.0^{\mathrm{b}}$ & $9.6 \pm 10.2^{\mathrm{c}}$ & $15.1 \pm 11.9^{\mathrm{d}}$ \\
\hline PG1/PG2 & $13.1 \pm 4.2$ & $12.3 \pm 3.9$ & $9.3 \pm 3.7^{\mathrm{b}}$ & $1.7 \pm 1.9^{\mathrm{c}}$ & $12.9 \pm 5.1$ \\
\hline $\mathrm{G} 17(\mathrm{pmol} / \mathrm{L})$ & $2.8 \pm 2.4$ & $0.4 \pm 0.3^{\mathrm{a}}$ & $4.8 \pm 8.4^{\mathrm{b}}$ & $86.6 \pm 99.7^{\mathrm{c}}$ & $12.2 \pm 16.0^{\mathrm{d}}$ \\
\hline HP IgG (EIU) & $8.9 \pm 6.5$ & $\begin{array}{l}9.8 \pm 6.8 \\
H \text { vs } G E R{ }^{a} p=0.0001\end{array}$ & $\begin{array}{l}85.2 \pm 34.7^{\mathrm{b}} \\
\mathrm{H} \text { vs } \mathrm{P}^{\mathrm{b}} \mathrm{p}=0.0001\end{array}$ & $\begin{array}{l}22.5 \pm 23.7^{\mathrm{c}} \\
\mathrm{H} \text { vs } \mathrm{CAG}^{\mathrm{c}} \mathrm{p}=0.0001\end{array}$ & $\begin{array}{l}24.5 \pm 34.3^{\mathrm{d}} \\
\mathrm{H} \text { vs PPI }{ }^{\mathrm{d}} \mathrm{p}=0.0001\end{array}$ \\
\hline
\end{tabular}


abdomen', 'discomfort in upper abdomen', 'cramps in upper abdomen' and 'bloating in upper abdomen') and discomfort (sum of the NDI items 'pressure in upper abdomen' and 'fullness after eating or slow digestion') and for QoL by NDI total score were compared and descriptively tested by means of WilcoxonMann-Whitney U-tests.

Results: After the initial 4 weeks, 54/114 patients opted for an extension of therapy. Interestingly, 34 out of 52 patients had been on active therapy while only 20 had received placebo. Until week 4, the NDI sub-score for pain had decreased by $7.5 \pm 3.9$ points during Menthacarin treatment as compared to $5.0 \pm 4.1$ points during placebo treatment $(\mathrm{p}=0.0371)$. After the follow-up, overall reduction for Menthacarin ( $8.7 \pm 4.9$ points) was also significantly better as compared to placebo $(5.1 \pm 4.9$ points, $\mathrm{p}=0.005)$. The NDI sub-score for discomfort had decreased until week 4 by $3.5 \pm 2.1$ points during active therapy as compared to $1.2 \pm 2.1$ points during placebo treatment $(\mathrm{p}=0.0003)$. For the 12 week therapy, the score had declined by $3.7 \pm 2.5$ points and $1.3 \pm 2.6$ for Menthacarin and placebo, respectively $(\mathrm{p}=0.0014)$. Overall QoL improvement was better for active medication for 4 and 12 weeks as compared to placebo. Conclusion: After 4 weeks of randomised double-blind, placebo-controlled treatment with either Menthacarin or placebo, patients who received active medication are more likely to opt for a continuation of therapy as compared to patients on placebo. The gain over placebo remained significant even after 12 weeks of treatment. ${ }^{1}$ Menthacarin ${ }^{\circledR}$ is a proprietary combination of essential oils of a specified quality from Mentha $\times$ piperita L. ( $90 \mathrm{mg}$ Peppermint oil WS ${ }^{\circ} 1340$ ) and Carum carvi (50 mg Caraway oil WS $₫ 1520$ )

Disclosure of Interest: G.J. Holtmann: Financial support for research and lecture fees from Dr. Willmar Schwabe GmbH \& Co. KG

B. Stracke: Employee of Dr. Willmar Schwabe GmbH \& Co. KG

\section{P1833 IMPROVEMENT OF APPROPRIATENESS OF PROTON PUMP INHIBITOR (PPI)-THERAPY PRESCRIPTION WITH USE OF SEROLOGICAL MARKERS (GASTROPANEL) IN A PRIMARY CARE POPULATION}

M. Franceschi ${ }^{1}$, M.P. Panozzo ${ }^{2}$, A. Ferronato ${ }^{1}$, F. Tomba ${ }^{1}$, D. Sella ${ }^{1}$, S. Landi ${ }^{3}$, A. Antico ${ }^{2}$, F. Di Mario ${ }^{4}$, G. Baldassarre

${ }^{1}$ Endoscopic Unit - Department Of Surgery, Ulss 7 Pedemontana, Santorso/Italy ${ }^{2}$ Department Of Clinical Pathology, Ulss 4 Alto Vicentino, Santorso, Italy, Hospital ULSS4 Alto Vicentino, Santorso/Italy

${ }^{3}$ Department Of Medicine And Surgery, University of Parma, Parma/Italy

${ }^{4}$ University Of Parma, Department of Clinical and Experimental Medicine, section of Gastroenterology, Parma/Italy

Contact E-mail Address: francesco.tomba@aulss7.veneto.it

Introduction: The introduction of proton pump inhibitors (PPIs) into clinical practice has revolutionized the management of acid-related diseases. Studies in primary care and emergency settings suggest that PPIs are frequently inappropriately prescribed or used in clinical conditions with little benefit.

Aims \& Methods: To evaluate the role of Gastropanel in relation to the appropriateness of PPI-therapy prescription. 2583 dyspeptic patients (male $36 \%$, mean age of 44.0 yrs, range 6-95) with no alarm symptom (i.e., dysphagia, anemia, weight loss and vomiting) from a primary care population were included in the study. For each patient a blood sample was collected for serum Pepsinogen I (PG-I) and II (PG-II), Gastrin 17 (G-17) and IgG HP (Biohit, Oyj, Finland); moreover, a clinical questionnaire searching for symptoms and presence of HP, and PPI therapy was filled out. We have evaluated the following serological profiles: HP infection, chronic atrophic gastritis (CAG), response to PPI therapy. The results obtained were used to evaluate the appropriateness of PPI-therapy prescription

Results: $1015 / 2583(39.3 \%)$ received PPI therapy up to three months before serum sampling and were included in the study. Among 1015 patients under PPI therapy, $294(29.0 \%)$ received half-dose PPIs, 709 (69.8\%) full-dose PPIs and $12(1.2 \%)$ an exceeding-dose PPIs. 38 patients under PPIs therapy $(3.7 \%)$ showed a serological status compatible with body CAG (the definitive diagnosis was confirmed histologically). 68 (6.7\%) presented HP infection. Table 1 shows the values of PG-I and G-17 values according to the response to PPI therapy. Conclusion: An appropriate prescription of PPI should be preceded by the assessment of gastric functional status. In particular, patients with HP infection should be eradicated before PPI therapy, while CAG patients should not receive PPI therapy because they are no responder. Patients who do not respond to PPI therapy should be further investigated (compliance, diagnosis).

Disclosure of Interest: All authors have declared no conflicts of interest.
P1834 DUODENAL ACID PERFUSION INCREASES DUODENAL PERMEABILITY AND ACTIVATES THE DUODENOGASTRIC REFLEX, INDEPENDENTLY FROM MAST CELL ACTIVATION

H. Vanheel ${ }^{1}$, R. Farré ${ }^{1}$, D. Beeckmans ${ }^{1}$, M. Vicario ${ }^{2}$, J. Tack ${ }^{1}$, T. Vanuytsel ${ }^{1}$ ${ }^{1}$ Targid, KU Leuven, Leuven/Belgium

${ }^{2}$ Vall d'Hebron Institut Recerca Gastroenterology, Barcelona/Spain

Contact E-mail Address: hanne.vanheel@med.kuleuven.be

Introduction: We recently reported that functional dyspepsia patients show impaired duodenal integrity, associated with low-grade inflammation (Vanheel, Gut 2014). A potential cause underlying this phenomenon may be the increased duodenal acid exposure that has been demonstrated in some of these patients. Aims \& Methods: Our aim was to evaluate the effect of duodenal acid perfusion on duodenal permeability in healthy volunteers and to investigate whether mast cell activation is required for acid-induced impairment of mucosal integrity. As it has already been shown that duodenal acid activates duodenogastric reflex pathways, we also assessed intragastric pressure (IGP). This study consisted of 2 parts, each including 10 healthy volunteers. 1) An infusion tube was positioned in the second part of the duodenum and a high resolution manometry probe was positioned in the stomach to measure IGP. $\mathrm{HCl} 0.1 \mathrm{~N}$ or saline was infused in the duodenum during $30 \mathrm{~min}(5 \mathrm{~mL} / \mathrm{min})$ in a randomized, double-blind manner. Duodenal biopsy specimens were obtained after infusion to measure transepithelial electrical resistance (TEER) and paracellular passage (fluorescein-labeled dextran, $4 \mathrm{kDa}$ ) in Ussing chambers. Expression of cell-to-cell adhesion proteins (claudin 1-4, occludin, zonula occludens $1-3, \beta$-catenin, E-cadherin, desmocollin2, desmoglein-2) in biopsies was evaluated by PCR, western blot and/or immunofluorescence. The number of mast cells and eosinophils was counted using immunohistochemistry for tryptase and eosinophilic major basic protein respectively, and by evaluating the expression of these proteins using PCR and western blot. 2) The participants were orally treated with placebo or with the mast cell stabilizer disodiumcromoglycate (DSCG) $200 \mathrm{mg}$ qid for 2 weeks. After treatment, the study design was as described above (although only acid perfusion was performed).

Results: IGP was significantly lower during acid perfusion compared with saline perfusion $(p=0.003)$. Acidification also resulted in decreased TEER $(p=0.005)$, increased passage $(\mathrm{p}=0.007)$ and lower protein expression of claudin 3 $(p=0.0006)$. No difference in mast cell $(p=0.34)$ and eosinophil $(p=0.34)$ counts were detected, but an increased protein expression of tryptase $(\mathrm{p}=0.0008)$ was found after acid perfusion. In the placebo and the DSCG group, acidification induced a similar drop in IGP $(p=0.68)$. There was also no difference in TEER $(\mathrm{p}=0.70)$ and passage $(\mathrm{p}=0.21)$ after acid perfusion between both pretreatments.

Conclusion: Duodenal acid perfusion in healthy volunteers disrupts epithelial integrity and activates an inhibitory duodenogastric reflex. Although this effect seems to be independent from mast cell activation, increased duodenal acid exposure in functional dyspepsia is a potential pathophysiological mechanism contributing to abnormalities in duodenal and gastric structure and function observed in patients with functional dyspepsia.

Disclosure of Interest: All authors have declared no conflicts of interest.

P1835 APPROPRIATE USE OF PPI IN THE ELDERLY: EVALUATION OF ACID SECRETION AND ATROPHIC GASTRITIS BY MEANS OF A NON-INVASIVE TEST

M. Franceschi ${ }^{1}$, S. Speroni ${ }^{2}$, R. De Bastiani ${ }^{3}$, A. Antico ${ }^{4}$, M.P. Panozzo ${ }^{4}$, G. Baldassarre ${ }^{1}$, S. Scida ${ }^{5}$, C. Miraglia ${ }^{5}$, L. Franzoni $^{5}$, V. Corrente ${ }^{5}$, M. Rugge ${ }^{6}$, F. Di Mario ${ }^{2}$, C. Scarpignato ${ }^{2}$, A. Pilotto

${ }^{1}$ Endoscopic Unit - Department Of Surgery, Ulss 7 Pedemontana, Santorso/Italy

${ }^{2}$ Department Of Clinical \& Experimental Medicine, Clinical Pharmacology \&

Digestive Pathophysiology Unit, University of Parma, Parma/Italy

${ }^{3}$ Italian Association for Gastroenterology in Primary Care (GICA-CP), Feltre/ Italy

${ }^{4}$ Department Of Clinical Pathology, Ulss 4 Alto Vicentino, Santorso, Italy,

Hospital ULSS4 Alto Vicentino, Santorso/Italy

${ }^{5}$ Department Of Medicine And Surgery, University Of Parma, Italy, University of Parma, Parma/Italy

${ }^{6}$ Pathology, Medical School of the Padova University, Padova/Italy

${ }^{7}$ Department Of Geriatric Care, Orthogeriatrics And Rehabilitation, Frailty Area, E.O. Galliera Hospital, National Relevance \& High Specialization Hospital, Genova/Italy

Contact E-mail Address: silvia.speroni@studenti.unipr.it

Introduction: Gastric acid secretion is believed to decrease in the aging stomach, but the number of elderly patients on proton pump inhibitor (PPI) therapy is

\begin{tabular}{|c|c|c|c|c|c|c|}
\hline & $\mathrm{N}$ & $\begin{array}{l}\text { Therapy } \\
\text { Half PPI n.,\% }\end{array}$ & Full PPI n.,\% & Excess PPI n.,\% & $\begin{array}{l}\text { Gastric Functionl status } \\
\text { PG-I (ug/L) means+/-DS }\end{array}$ & $\begin{array}{l}\text { Gastric Functionl status } \\
\text { G-17 (pmol/L)means }+/-D S\end{array}$ \\
\hline All & 1015 & 294 & 709 & 12 & $137.0+/-84.7$ & $11.7+/-21.1$ \\
\hline Good response G-17 > 7 & 351 & $83(23.6)$ & $259(73.8)$ & $9(2.6)$ & $194.5+/-121.1$ & $22.1+/-17.9$ \\
\hline Low response G-17 1-7 & 421 & $141(33.5)$ & $279(66.3)$ & $1(0.2)$ & $127.1+/-8.3$ & $3.1+/-1.76$ \\
\hline No response $\mathrm{G} 17<1$ & 205 & $64(31.2)$ & $140(68.3)$ & $1(0.5)$ & $91.7+/-40.9$ & $0.38+/-0.28$ \\
\hline CAG & 38 & $6(15.8)$ & $31(68.3)$ & $1(2.6)$ & $16.6+/-14.8$ & $70.3+/-55.2$ \\
\hline
\end{tabular}


increasing. Pepsinogen I (PGI) $<30 \mu \mathrm{g} / \mathrm{L}$, PGI/PGII $<3$ and gastrin-17 (G17) $>10 \mathrm{pmol} / \mathrm{L}$ are non-invasive serological markers surrogate to explore gastric function, with a negative predictive value for chronic atrophic gastritis (CAG) of $96 \%$.

Aims \& Methods: Aim of the study was to evaluate gastric function by means of serology (PGI, PGII, G-17 and IgG-antibodies against Helicobacter pylori) in very elderly patients, including centenarians. A total of 379 patients were prospectively enrolled $(\mathrm{M}=126, \mathrm{~F}=253$, mean age $=83.6 \pm 8.7$, range $70-106)$. They were divided in four groups: 132 subjects with an age between 70 and 79 years old (first group), 146 subjects between 80 and 89 (second group), 76 subjects between 90 and 99 (third group) and 25 subjects between 100 and 106 (fourth group). Demographics and drug intake, particularly the PPI intake, were collected. For all patients, serological markers were determined in fasting blood by using Gastropanel® (Biohit Oyj, Finland; normal values: PGI: 30 $120 \mu \mathrm{g} / \mathrm{L}$; PGII: 2-15 $\mu \mathrm{g} / \mathrm{L}$; PGI/PGII ratio: > 3; G17: 1-9 pmol/L; H.p.-IgG: $<30$ EIU).

Results: In the first group (age 70-79), 18.2\% of the subjects showed H. pylori infection (PGII $>10 \mu \mathrm{g} / \mathrm{L}$, IgG against H.p. $>30$ EIU), $22.7 \%$ had CAG (PGI $<30 \mu \mathrm{g} / \mathrm{L}$ and PGI/PGII $<3$ ) and $53.8 \%$ were under PPI therapy. $16.9 \%$ of the patients on PPI therapy had CAG. In the second group (age $80-89$ ), 32.9\% of the subjects showed $H$. pylori infection, $8.9 \%$ had CAG and $48.6 \%$ were under PPI therapy. $8.5 \%$ of the patients on PPI therapy had CAG. In the third group (age $90-99$ ), $22.4 \%$ of the subjects showed $H$. pylori infection, $10.5 \%$ had CAG and $48.7 \%$ were under PPI therapy. $8.1 \%$ of the patients on PPI therapy had CAG. In the fourth group (age 100-106), 44.0\% of the subjects showed $H$. pylori infection, $16.0 \%$ had CAG and $72.0 \%$ were under PPI therapy. $16.7 \%$ of the patients on PPI therapy had CAG

Conclusion: Acid secretion is preserved in most of the elderly and very elderly subjects, even in centenarians. Serological markers may be helpful to identify patients affected by CAG in which the administration of PPI is inappropriate, especially in the elderly.

Disclosure of Interest: All authors have declared no conflicts of interest

\section{P1836 THE PSYCHOLOGICAL CHARACTERISTICS OF REFLUX HYPERSENSITIVITY-A PILOT STUDY BASED ON SCL-90 QUESTIONNAIRE AND 24 HOUR PH-IMPEDANCE MONITORING}

K. Wang, Z. Xu, Z. Xia, Y. Ge, L. Duan

Department Of Gastroenterology, Peking University Third Hospital, Beijing/China

\section{Contact E-mail Address: duanlp@bjmu.edu.cn}

Introduction: Reflux hypersensitivity (RHV) was lately defined as a functional esophageal disorder by Rome IV workshop. The clinical and psychological characteristics are still unknown.

Aims \& Methods: The aim of this study was to assess the reflux and psychological characteristics of RHV. Patients who underwent $24 \mathrm{~h} \mathrm{pH}$-impedance monitoring were screened from Jan $1^{\text {st }} 2011$ to Nov $31^{\text {st }} 2015$. The patients with heartburn or chest pain $\geq 2$ days/week for more than 6 months were enrolled. Healthy volunteers (HV) were enrolled too. All subjects fulfilled the SCL-90 questionnaire, underwent gastroscopy to exclude upper gut organic diseases and underwent HRM test to exclude main motility disorders. The patients with normal esophageal mucosal but overload acid, weakly acid or non-acid reflux were diagnosed as non-erosive reflux disease (NERD). The patients with normal esophageal mucosal and normal reflux but positive symptom index (SI) or symptom association possibility (SAP) were diagnosed as RHV. And the patients with normal mucosal, normal reflux, normal SI or SAP and negative PPI test results were enrolled in functional heartburn (FH) group. The reflux and psychological characteristics were compared among NERD, RHV and FH.

Results: Total 231 patients were enrolled. 107 were NERD $(48.25 \pm 1.18$ yrs, $\mathrm{M}: \mathrm{F}=55: 52), 92$ were $\mathrm{FH}(48.30 \pm 1.27 \mathrm{yrs}, \mathrm{M}: \mathrm{F}=9: 83), 32$ were RHV $(48.41 \pm 2.36 \mathrm{yrs}, \mathrm{M}: \mathrm{F}=4: 28) .28 \mathrm{HVs}(47.21 \pm 2.27, \mathrm{M}: \mathrm{F}=8: 20)$ were enrolled as controls. NERD presented significantly higher acid exposure time $(\mathrm{pH}>4.2)$ than those of FH, RHV and HV $(6.55 \pm 0.71,1.25 \pm 0.12,1.90 \pm 0.33,2.95 \pm 0.53$, respectively, $\mathrm{p}<0.005)$. The acid reflux and weakly acid reflux were both higher in NERD than that in FH, RHV and HV $(p<0.001)$. The total scores of SCL-90 of each group of patients were significantly higher than HV (NERD:HV, $133.15 \pm 3.68$ vs. $108.61 \pm 4.51, \mathrm{p}=0.004 ; \quad \mathrm{FH}: \mathrm{HV}, \quad 133.15 \pm 3.68$ vs.
$108.61 \pm 4.51, \mathrm{p}=0.003 ; \quad$ RHV:HV, $142.67 \pm 8.91 \mathrm{vs} . \quad 108.61 \pm 4.51, \mathrm{p}=0.002)$. RHV patients presented higher compulsive factor score than FH $(1.85 \pm 0.16$ vs. $1.58 \pm 0.06, \mathrm{p}=0.047)$ and $\mathrm{HV}(1.85 \pm 0.16$ vs. $1.37 \pm 0.09, \mathrm{p}=0.004)$. The patients of NERD, FH and RHV presented significantly higher depression, anxiety, hostile factor scores than those of HV $(\mathrm{p}<0.05)$.

Conclusion: NERD, RHV and FH patients showed higher scores than HVs in total and most factors of SCL-90. Furtherly, RHV patients presented higher compulsive score than FH, NERD and HVs. The role of senseless thought, impulse, and act of compulsive behavior in the pathogenesis of RHV needs to be studied furtherly.

Disclosure of Interest: All authors have declared no conflicts of interest.

\section{P1837 HIGH-RESOLUTION ESOPHAGEAL MANOMETRY:} EVALUATION OF NEW SYSTEMS FOR THE ACQUISITION AND ANALYSIS

G. Capovilla ${ }^{1}$, A. Costantini ${ }^{2}$, G. Voltarel $^{1}$, E. Pesenti ${ }^{1}$, A. Perazzolo ${ }^{1}$, L. Nicoletti ${ }^{1}$, R. Salvador ${ }^{1}$, M. Costantini ${ }^{1}$

${ }^{1}$ Of Surgical, Oncological And Gastroenterological Sciences, University Hospital of Padova, Padova/Italy

${ }^{2}$ Università Cattolica del Sacro Cuore, Rome/Italy

\section{Contact E-mail Address: m.costantini@unipd.it}

Introduction: High-Resolution Manometry (HRM) has recently became the gold standard for the evaluation of esophageal motility. A new classification of esophageal motility disorders (Chicago Classification, v. 3.0) has been developed, based on the findings from a given hardware and software. Different systems for HRM and new features of the existing ones have recently been developed.

Aims \& Methods: In this study we aimed to evaluate a new solid-state HRM system and a new 3-D catheter and system for the study of lower esophageal sphincter (LES). Fifteen healthy volunteers $(7 \mathrm{~m}, 8 \mathrm{f}$, median age 27$)$ underwent two consecutive Esophageal HRM studies by using two different solid state systems (ManoScan, Medtronic, Minneapolis, USA and Medica SpA, Italy with Unisensor AG, Attikon, Switzerland catheter). The studies were performed in a random order using the standard protocol. Furthermore, a new 3-D catheter for the study of sphincters was evaluated in $12 / 15$ volunteers.

Results: Table 1 reports the findings obtained with the Medica system compared to the consolidated Medtronics system. The data of the 3-D evaluation are also reported. The data are expressed as medians (and $5^{\text {th }}-95^{\text {th }}$ percentiles).

Conclusion: Significant differences were recorded in most of the considered parameters obtained by the two HRM systems. This is particularly relevant in the evaluation of the LES relaxation, the cardinal point in the hierarchical approach of the Chicago Classification, probably due to different analysis used by the two systems (the lowest residual pressure vs IRP). Furthermore, the differences found in the measurement of the LES resting pressure and abdominal length may rely either on the different technology (3-D vs linear transducers) or on the different algorithms and thresholds used. The latter may probably also apply to the differences found with the two systems in the duration of the esophageal contractions and in the new contractile parameters (i.e. DCI and DL) introduced by the Chicago Classification. This study emphasizes the need for a careful validation of any new system for esophageal manometry. The acquisition of new sets of normal values, to be used to compare the data measured in patients, is therefore mandatory. The results of our study may represent the reference normal values for other esophageal laboratories that are using the HRM systems and devices we tested here.

Disclosure of Interest: All authors have declared no conflicts of interest.

\begin{tabular}{|c|c|c|c|c|c|c|c|}
\hline & $\begin{array}{l}\text { LES Resting } \\
\text { pressure } \\
(\mathrm{mmHg})\end{array}$ & $\begin{array}{l}\text { LES Overall } \\
\text { length }(\mathrm{cm})\end{array}$ & $\begin{array}{l}\text { LES Abdominal } \\
\text { Length }(\mathrm{cm})\end{array}$ & $\begin{array}{l}\text { LES Residual } \\
\text { pressure/IRP } \\
(\mathrm{mmHg})\end{array}$ & $\begin{array}{l}\text { Amplitude } \\
3-5 \mathrm{~cm} \\
(\mathrm{mmHg})\end{array}$ & $\begin{array}{l}\text { Amplitude } \\
7-9 \mathrm{~cm} \\
(\mathrm{mmHg})\end{array}$ & $\begin{array}{l}\text { Amplitude } \\
11-13 \mathrm{~cm} \\
(\mathrm{mmHg})\end{array}$ \\
\hline A. Medica/Unisensor & $17.5(6.5-31.5)$ & $3.7(2.8-5.3)$ & $1.0(0-2.3)$ & $0(-0.7-4.5)$ & $111(56-224)$ & $74(37-134)$ & $75(33-112)$ \\
\hline B. Medtronics & $33.1(16-56)$ & $4.1(2.0-5.0)$ & $1.9(0.1-2.8)$ & $14.1(3.8-15.9)$ & $94(61-155)$ & $80.8(50-154)$ & $67(40-114)$ \\
\hline C. 3-D Medtronics & $39.3(26-60)$ & $4.0(2.9-5.6)$ & $2.35(0.6-3.8)$ & $14.2(1.4-25)$ & - & - & - \\
\hline$p A$ vs $B=p B$ vs $C=$ & $<0.01<0.01$ & 0.940 .48 & 0.010 .06 & $<0.0010 .46$ & 0.9 & 0.15 & 0.17 \\
\hline & $\begin{array}{l}\text { Duration } 3-5 \mathrm{~cm} \\
\quad(\mathrm{sec})\end{array}$ & $\begin{array}{l}\text { Duration } 7-9 \mathrm{~cm} \\
\quad(\mathrm{sec})\end{array}$ & $\begin{array}{l}\text { Duration } 11-13 \mathrm{~cm} \\
\quad(\mathrm{sec})\end{array}$ & $\begin{array}{l}\text { Distal Contractile } \\
\text { Index (DCI) }\left(\mathrm{mmHg}^{*} \mathrm{~s} * \mathrm{~cm}\right)\end{array}$ & $\begin{array}{l}\text { Distal Latency } \\
\quad(\mathrm{DL})(\mathrm{sec})\end{array}$ & \multicolumn{2}{|c|}{$\begin{array}{l}\text { IntraBolus } \\
\text { Pressure (IBP) (mmHg) }\end{array}$} \\
\hline A. Medica/Unisensor & $3.1(1.7-4.6)$ & $1.7(1.0-3.1)$ & $2.4(1.4-3.3)$ & $3000(1167-6200)$ & $8.8(7.45-12.5)$ & \multicolumn{2}{|l|}{$7.1(0.2-19)$} \\
\hline B. Medtronics & $3.7(2.8-5.1)$ & $3.2(2.6-4.3)$ & $3.0(2.4-4.2)$ & $1583(650-2760)$ & $6.4(4.7-8.9)$ & \multicolumn{2}{|l|}{$10.4(8.7-14.95)$} \\
\hline$p A$ vs $B=$ & 0.01 & 0.002 & 0.004 & $<0.001$ & $<0.001$ & \multicolumn{2}{|l|}{0.13} \\
\hline
\end{tabular}


P1838 PROTON PUMP INHIBITOR THERAPY IMPROVES

ESOPHAGEAL SYMPTOMS BY RESTORING A NORMAL ESOPHAGEAL PERISTALSIS IN PATIENTS WITH PROTON PUMP INHIBITOR-RESPONSE ESOPHAGEAL EOSINOPHILIA

M. Della Coletta ${ }^{1}$, N. De Bortoli ${ }^{2}$, O. Bartolo ${ }^{3}, \mathrm{~S}_{\text {. Tolone }}^{4}$, G. Bodini ${ }^{5}$, E. Marabotto ${ }^{6}$, P. Zentilin ${ }^{7}$, A. Mauro ${ }^{8}$, R. Penagini ${ }^{9}$, V. Savarino ${ }^{10}$, E. Savarino ${ }^{11}$

${ }^{1}$ Division Of Gastroenterology, Department Of Surgery, Oncology And Gastroenterology, University of Padua, Padua/Italy

${ }^{2}$ Division Of Gastroenterology, Department Of Internal Medicine, University of Pisa, Pisa/Italy

${ }^{3}$ Dept. Of Gastroenterology, University of Padova, Padova/Italy

${ }^{4}$ Surgery, Second University of Naples, Naples/Italy

${ }^{5}$ Department Of Internal Medicine, IRCCS San Martino DIMI, Genova/Italy

${ }^{6}$ University of Genoa, Genoa, Italy, Genoa/Italy

${ }^{7}$ Dept. Of Internal Medicine, University of Genoa, Genova/Italy

${ }^{8}$ Gastroenterology And Endoscopy Unit, University of Milan, Milano/Italy

${ }^{9}$ Dipartimento Di Scienze Mediche, Università degli Studi di Milano Dipto. di Gastroenterologia, Milan/Italy

${ }^{10}$ Dept Internal Medecine, Universita di Genova, Genova/Italy

${ }^{11}$ Department Of Surgery, Oncology And Gastroenterology, University of Padua, Padua/Italy

Contact E-mail Address: edoardo.savarino@gmail.com

Introduction: Proton Pump Inhibitor-response esophageal eosinophilia (PPI-

REE) is a condition characterised by symptoms of esophageal dysfunction in the setting of eosinophilic inflammation on esophageal biopsies responding to a course of 8 weeks of PPI therapy. Recent data collected by using esophageal high resolution manometry (HRM) documented that patients with PPI-REE present frequently motility abnormalities, mostly weak peristalsis and hypotensive esopahgogastric junction (EGJ). Data on the effect of PPIs in improving these motor abnormalities are lacking.

Aims \& Methods: We aimed to prospectively compare HRM features of patients with PPI-REE before and after a course of PPI therapy. Consecutive patients with symptoms suggestive of EoE underwent upper endoscopy to assess the presence of at least $15 \mathrm{eos} / \mathrm{hpf}$ on oesophageal biopsies at mid/proximal esophagus and, then, were treated with twice-daily PPIs for at least 8 weeks. Thereafter, patients repeated upper endoscopy and PPI-REE was identified in case of less than $15 \mathrm{eos} / \mathrm{hpf}$ and a $50 \%$ decrease from baseline. Patients with PPI-REE underwent HRM at the time of the diagnosis (off-PPI) and after the course of PPIs (on-PPI). Patients with achalasia and absent peristalsis were excluded (Chicago Classification v.3)

Results: Twenty-eight patients [23M/5F; mean age 35] reporting dysphagia $(93 \%)$, bolus impaction $(68 \%)$ and chest pain $(25 \%)$ were diagnosed with PPIREE. After anti-secretory therapy, most of the patients reported complete resolution of esophageal symptoms directly linked to esophageal infiltration $(p<0.001)$, namely dysphagia, bolus impaction and chest pain. Compared to HRM features at baseline, HRM after PPI therapy showed that patients with PPI-REE had higher median EGJ resting pressure [baseline 11 (1-34) vs. postPPI 17 (1-34); $\mathrm{p}<0.05$ ], greater mean distal contraction integral [1094 (483$5281)$ vs. 2634 (495-6450); $\mathrm{p}<0.01$ ], and less frequent panesophageal pressurization [6 $(21 \%)$ vs. $0(0 \%) ; \mathrm{p}=0.02)]$. No differences were observed in terms of distal latency and rate of different EGJ subtypes $(\mathrm{p}=\mathrm{ns})$. As to the manometric diagnoses, after PPI therapy patients with PPI-REE showed a reduced rate of ineffective motility or fragmented peristalsis $[16(57 \%)$ vs. $7(25 \%), \mathrm{p}=0.02]$ and increased frequency of normal peristalsis [ $9(32 \%)$ vs. $18(64 \%), p=0.03$ ] No differences were observed in terms of frequency of distal esophageal spasm and outflow obstruction diagnoses $(\mathrm{p}=\mathrm{ns})$

Conclusion: In most PPI-REE patients, PPI therapy restores the impairment of esophageal motility as expressed by ineffective or fragmented peristalsis, thus favouring the return to a normal motility pattern. This finding, paralleled with symptoms improvement in the same subjects, seems to emphasize the important role of inflammation linked to the eosinophilic infiltration of the esophageal wall in inducing motor dysfunction and related symptoms.

Disclosure of Interest: V. Savarino: Consulting fee from Malesci, Reckitt, AlfaWasserman, Abbvie

E. Savarino: Consulting fee from Medtronic, Sofar, Takeda, Abbvie, MSD

All other authors have declared no conflicts of interest.

\section{P1839 EOSINOPHILIC ESOPHAGITIS: MANAGEMENT IN THE DAILY CLINICAL DUTCH PRACTICE}

B. Vermeulen ${ }^{1}$, A. Bogte ${ }^{2}$, P.D. Siersema ${ }^{1}$

${ }^{1}$ Gastroenterology And Hepatology, Radboudumct, Nijmegen/Netherlands

${ }^{2}$ University Medical Centre Utrecht, Utrecht/Netherlands

Contact E-mail Address: bram.vermeulen@radboudumc.nl

Introduction: In recent years, new guidelines and recommendations have been published regarding the diagnostic criteria and therapeutic management of eosinophilic esophagitis (EoE).

Aims \& Methods: The aim of this study was to assess the diagnostic and therapeutic management of patients diagnosed with EoE in daily clinical Dutch practice and whether this was according to guidelines and recommendations.

A population-based, retrospective cohort study was conducted using data from the Dutch national pathology registry (PALGA), medical records, and telephone interviews of patients diagnosed with EoE in two academic and two non-academic hospitals in the period 2004-2014. Data regarding demographics, clinical manifestations, endoscopic results, histologic samples and therapeutic strategies were collected. Standard statistical analyses were performed to summarize the patient characteristics.

\begin{tabular}{llllll}
\multicolumn{5}{l}{ Initial treatment after diagnosis EoE } \\
$\begin{array}{lllll}\text { Treatment, } \\
\mathrm{n}(\%)\end{array}$ & $\begin{array}{l}\text { Total, }(\%) \\
(\mathrm{n}=119)\end{array}$ & $\begin{array}{l}\text { AC 1, } \\
\mathrm{n}(\%) \\
(\mathrm{n}=49)\end{array}$ & $\begin{array}{l}\text { AC 2, } \\
\mathrm{n}(\%) \\
(\mathrm{n}=27)\end{array}$ & $\begin{array}{l}\text { Non AC 1, } \\
\mathrm{n}(\%) \\
(\mathrm{n}=25)\end{array}$ & $\begin{array}{l}\text { Non AC 2, } \\
\mathrm{n}(\%) \\
(\mathrm{n}=18)\end{array}$ \\
\hline TCS & $36(30.3)$ & $17(34.7)$ & $5(18.5)$ & $11(44.0)$ & $3(16.7)$ \\
PPI & $35(29.4)$ & $16(32.7)$ & $8(29.6)$ & $4(16.0)$ & $7(38.9)$ \\
PPI + TCS & $12(10.1)$ & $5(10.2)$ & $1(3.7)$ & $4(16.0)$ & $2(11.1)$ \\
Dilation & $3(2.5)$ & 0 & $2(7.4)$ & 0 & $1(5.6)$ \\
Prednisone & $2(1.7)$ & 0 & $1(3.7)$ & $1(4.0)$ & 0 \\
TCS + dilation & $1(0.8)$ & $1(2.0)$ & 0 & 0 & 0 \\
Diet & 0 & 0 & 0 & 0 & 0 \\
None & $24(20.2)$ & $9(18.4)$ & $8(29.6)$ & $4(16.0)$ & $3(16.7)$ \\
Missing & $6(5.0)$ & $1(2.0)$ & $2(7.4)$ & $1(4.0)$ & $2(11.1)$ \\
\hline
\end{tabular}

Results: In total, 119 patients were diagnosed with EoE and included in this study. The median age at onset of symptoms was 29 years (IQR, 15-42) and the median age at diagnosis was 38 years (IQR, 23-51 years), leading to a median diagnostic delay of 6.5 years (IQR, 2-14 years). The median delay in diagnosis between first contact in the hospital and diagnosis was 1.0 year (IQR, 2-14 years). The incidence of patients newly diagnosed with EoE increased steadily over a period of 11 years. Criteria for the microscopic diagnosis of EoE varied between pathologists in each hospital. Initial treatment included topical corticosteroids $(30.3 \%)$, proton pump inhibitors (PPIs) $(29.4 \%)$ or a combination of both $(10.1 \%)$. A follow-up endoscopy was performed in $40.3 \%$ of patients. During follow-up, treatment included PPIs (76.0\%), topical corticosteroids $(59.6 \%)$ or a combination of both $(45.4 \%)$

Conclusion: Diagnostic and therapeutic discrepancies between daily clinical practice and recommendations from current and past guidelines were observed. Remarkably, the diagnostic entity PPI-responsive EoE was only used in one center, follow-up endoscopy was performed in less than half of patients and all pathologists used different criteria for the microscopic diagnosis of EoE. Moreover, varying therapeutic strategies were utilized in the participating centers. Our results show that apart from developing guidelines, efforts should be undertaken to implement them in daily clinical practice.

Disclosure of Interest: All authors have declared no conflicts of interest.

\section{References}

Furuta GT, Liacouras CA, Collins MH, Gupta SK, Justinich C, Putnam PE, et al. Eosinophilic esophagitis in children and adults: a systematic review and consensus recommendations for diagnosis and treatment. Gastroenterology 2007;133: 1342-63.

Liacouras CA, Furuta GT, Hirano I et al. Eosinophilic esophagitis: updated consensus recommendations for children and adults. J Allergy Clin Immunol $2011 ; 128:$ 3-20.e6

Dellon ES, Gonsalves N, Hirano I, Furuta GT, Liacouras CA, Katzka DA. ACG clinical guideline: evidenced based approach to the diagnosis and management of esophageal eosinophilia and eosinophilic esophagitis (EoE). Am J Gastroenterol 2013; 108: 679-92

Schoepfer AM, Safroneeva E, Bussmann C, Kuchen T, Portmann S, Simon HU, et al. Delay in diagnosis of eosinophilic esophagitis increases risk for stricture formation, in a time-dependent manner. Gastroenterology 2013; 145:1230-6, e1-2.

\section{P1840 IGG4 EXPRESSION IS ELEVATED IN PATIENTS WITH EOSINOPHILIC ESOPHAGITIS COMPARED TO PATIENTS WITH GASTROESOPHAGEAL REFLUX DISEASE}

S. Weidlich ${ }^{1}$, S. Nennstiel ${ }^{1}$, K. Brockow ${ }^{2}$, M. Jesinghaus ${ }^{3}$, J. Slotta-Huspenina ${ }^{4}$, M. Bajbouj ${ }^{5}$, R.M. Schmid ${ }^{5}$, C. Schlag

${ }^{1}$ Gastroenterology Department, Klinikum Rechts der Isar TU München, München/ Germany

${ }^{2}$ Dermatology Department Biederstein, Technical University of Munich, München Germany

${ }^{3}$ Institute for Pathology, Technical University of Munich, München/Germany ${ }^{4}$ Institute For General Pathology And Pathological Anatomy, Technical University Munich, München/Germany

${ }^{5}$ Klinik Für Innere Medizin II, Technische Universität München, München/ Germany

Contact E-mail Address: simon.weidlich@mri.tum.de

Introduction: Eosinophilic Esophagitis (EoE) is a chronic immune disease of the esophagus, which is presented by esophageal dysfunction und histologically characterized by a predominant eosinophilic inflammation. EoE is mainly found in patients with atopic conditionsHowever, recently an association with IgG4 but not with IgE has been reported. Gastroesophageal reflux disease (GERD) is the most important differential diagnosis of EoE. In this study we measured systemic serum IgG4 and IgE levels of EoE patients before and a after a topic steroid therapy, correlated them to esophageal IgG4-positive plasma cells and compared them to GERD patients. 
Aims \& Methods: Serum levels of IgG4 and IgE of 19 EoE patients were measured before and after eight weeks of therapy with budesonide (1 mg twice a day). Biopsies were taken from the esophagus before and after therapy for histological and immunhistochemical evaluation. 14 patients with GERD without histological proof of eosinophilic granulocyte infiltration were taken a as control group. Serum levels of IgG4 and IgE of 19 EoE patients were measured before and after eight weeks of therapy with budesonide ( $1 \mathrm{mg}$ twice a day). Biopsies were taken from the esophagus before and after therapy for histological and immunhistochemical evaluation. 14 patients with GERD without histological proof of eosinophilic granulocyte infiltration were taken a as control group.

Results: Serum IgG4 levels of EoE patients were significantly higher than in GERD patients (mean: $121.0 \mathrm{mg} / \mathrm{dL}$ vs. $71.2 \mathrm{mg} / \mathrm{dL}, \mathrm{p}=0.034$ ). In contrast, no significant difference of $\mathrm{IgE}$ levels in EoE and GERD patients was observed. In EoE patients, the number of eosinophilic granulocytes in histology was decreased at a significant level after topic steroid therapy (mean: 51.9 eosinophiles/high power field (hpf) vs. 6.4 eosinophiles $/ \mathrm{hpf} \mathrm{p}<0.001)$. After therapy lower levels of IgG4-serum-levels could be measured (mean: $121.0 \mathrm{mg} / \mathrm{dL}$ vs. $104.2 \mathrm{mg} / \mathrm{dL}$ $\mathrm{p}=0.019$ ), whereas IgE levels did not show a significant difference.The esophageal biopsies of EoE patients showed a high number of IgG4-positive plasma cells (mean expression of $27.4 \mathrm{IgG} 4$-positive plasma cells of 46.4 stromal plasma cells/hpf).

Conclusion: EoE patients show higher systemic IgG4- but not IgE-serum levels compared to GERD-patients. These elevated levels normalize under effective topic steroid therapy. Additionally high local expression of IgG4- positive plasma cells can be seen in EoE patients. These findings might be further evidence for a possible IgG4-association of EoE.

Disclosure of Interest: All authors have declared no conflicts of interest.

\section{P1841 SYSTEMATIC REVIEW: HEALTH-RELATED QUALITY OF LIFE IN CHILDREN AND ADULTS WITH EOSINOPHILIC ESOPHAGITIS: MEASURE INSTRUMENTS AND DETERMINANT FACTORS}

A. J. Lucendo Villarin, L. Arias-González, J. Molina-Infante, Á. Arias Dept. De Gastroenterologia, Hospital General de Tomelloso Dept. of Digestive Health Dept. of Gastroenterology, Tomelloso/Spain

\section{Contact E-mail Address: alucendo $@$ vodafone.es}

Introduction: Measurement of Health-related quality of life (HRQoL) with generic or specific intruments has been increasingly used in patients suffering from EoE to support both research and clinical care. Generic instruments aim at measuring the overall HRQoL of patients across several conditions, being useful to compare HRQoL across different disease states and for evaluating health economics outcomes. Disease-specific instruments assess domains specific to a given disease and are considered more sensitive to changes in the patient's health state. An up-to-date systematic review will provide a useful resource for researchers and EoE specialists to ensure they can select an appropriate HRQOL measure for patients in their practice in order to identify correctable factors determining an impaired perception and to improve treatment outcomes.

Aims \& Methods: We aim to systematically review the current HRQoL measures for patients with EoE and to appraise their measurement properties using a robust evaluation methodology checklist. We also sought to identify diseasespecific determinant factors for HRQoL in children and adults with EoE, and the effect of investigations and interventions for EoE on HRQoL. A search strategy was designed to identify and retrieve all documents dealing with the relationship between HRQoL and EoE in children and adults. This systematic literature search was performed in 5 major databases (PubMed, EMBASE, Scopus, PsycInfo and Web of Science) for the period up to March 2017. The measure properties of each specific EoE instrument identified and their performance properties were assessed using the quality properties checklist proposed by Terwee et al. Levels of the HRQoL measure establishment or use in literature: we used Cohen's criteria. Cohort studies, case series and case reports were evaluated for the risk of bias with the aid of the Joanna Briggs Institute critical appraisa checklist. A descriptive summary with data tables was produced to summarize the literature. Quantitative pooling of data was not meaningful so a narrative synthesis of the data was undertaken.

Results: Of the 596 references identified, data was collected from 34 studies including a total of 1,842 individual patients. Three disease-specific HRQoL measures in EoE covering different aspects of patients' lives and developed in English were scored positive regarding measurement properties. Respectively, the PedsQL inventory (including parent and child report forms) and the Peds-QoLä EoE module were the generic and specific instruments respectively used in children, while the SF-36 and EoE-QoL-A were the most used questionnaires in adults. EoE significantly impairs HRQoL of affected children and adolescents, which manifests in the normal development of their daily activities, their physical health and their mental status, with parents generally underestimating the impact of the disease regarding children declared HRQoL. Regarding determinant factors, age was not associated with HRQoL. Number and severity of symptoms negatively correlated with child-reported and Parent proxy-reported PedsQL score and family impact score. Disease duration was identified as a risk factor for a low SF-36 score. EoE impacts on a number of domains including frustration, embarrassment or fear about the disease outcomes. No significant differences in the overall QoL score were found in adult patients managed with dietary or pharmacological therapy, with specific treatment modalities having a negligible influence on overall EoE-specific QoL. Symptoms scores determined exerted an increased impact on swallowing anxiety and emotional impact subscales. Female gender negatively influenced QoL scores. Disease activity, as expressed by endoscopic findings, were also significant determinants of QoL. A higher educational level was also found to be a strong determinant for a worse QoL.
Emotional impact was the only dimension with a significantly worse score in patients under dietary restrictions.

Conclusion: HRQoL is a relevant outcome that should be considered in clinical practice and research of EoE. Further validation studies in several languages and populations are required to support the use of disease-specific HRQoL measures. Disclosure of Interest: All authors have declared no conflicts of interest.

\section{P1842 TREATMENT SATISFACTION OF ADULT EOSINOPHILIC ESOPHAGITIS PATIENTS}

E. Safroneeva ${ }^{1}$, D. Hafner ${ }^{1}$, C. Kuehni ${ }^{1}$, M. Zwahlen ${ }^{1}$, S. Trelle ${ }^{1}$, A. Straumann ${ }^{2}$, A. Schoepfer ${ }^{3}$

${ }^{1}$ University of Bern, Bern/Switzerland

${ }^{2}$ Gastroenterology Fmh, Swiss EoE Research Network, Olten/Switzerland

${ }^{3}$ CHUV + Universtiy of Lausanne, Lausanne/Switzerland

Contact E-mail Address: ekaterina.safroneeva@ispm.unibe.ch

Introduction: Available treatment options for adult EoE patients include drugs (proton-pump inhibitors [PPI], swallowed topical corticosteroids [STC]), food elimination diets, and esophageal dilation. Knowledge about patients' view regarding the different therapeutic options is very limited.

Aims \& Methods: We aimed to systematically assess adult EoE patients' satisfaction with different EoE-specific treatment modalities. We first created a questionnaire that included items that queried general demographic characteristics $(7$ items), EoE-specific patient history and presence of atopic disease ( 8 items), past and present EoE-specific therapy ( 9 items), concomitant medication use ( 7 items), important considerations for choice of therapy (2 items), as well as treatment satisfaction with various therapies recalled over a period of 12 months (assessed using the validated "Treatment Satisfaction Questionnaire for Medication" [TSQM], 52 items). The TSQM consists of 14 items falling into 4 scales: effectiveness ( 3 items), side effects ( 5 items), convenience ( 3 items), and overall satisfaction ( 3 items). The score for each TSQM scale ranges from 0 (dissatisfied) to 100 (satisfied). In analogy with other conditions, a score above 66.6 and 83.3 identifies patients that are 'satisfied' and 'very satisfied' with therapy, respectively. The psychologist-guided focus groups with adult EoE patients were conducted to inform the content and the structure of the questionnaire and ensure that patient understand the items, instructions, and response options. The questionnaire was sent to 148 patients in Switzerland. Data were double-entered by two researchers into an EpiData database (version 3.1, the EpiData Association, Odense, Denmark). Dataset was then imported into Stata (version 13, College Station, Texas, USA) for analysis.

Results: Patient response rate was $73.5 \%(108 / 147)$. Mean patient age at inclusion was $46.3 \pm 15.9$ years, $85 / 108(78.8 \%)$ of patients were male, and mean disease duration (from the time of diagnosis to the time of enrollment) was $6.8 \pm 5.1$ years. In the last 12 months, $11.1 \%, 48.1 \%, 10.2 \%$, and $28.7 \%$ of patients reported to have suffered from symptoms of asthma, rhinoconjunctivitis, eczema, and food allergy, respectively. In the last 12 months, $25.0 \%, 3.7 \%$, $77.8 \%, 1.9 \%, 19.4 \%$, and $13.0 \%$ were treated with PPI, STC in the form of a syrup, STC in the form of a powder, STC in a form of a spray, diet, and esophageal dilation, respectively $(37.0 \%$ patients received more than one treatment; $7.4 \%$ of patients did not receive any treatment). Patients identified the following considerations as important for the choice of therapy: the effect of the treatment on the symptoms $(88.9 \%)$, the effect of treatment on esophageal inflammation $(75.9 \%)$, possible side effects $(69.4 \%)$, ease of therapy use $(58.3 \%)$, recommendation of the physician $(50.0 \%)$, and compatibility of therapy with lifestyle $(46.3 \%)$. When asked about single most important criterion for the choice of therapy, $48.5 \%, 33.7 \%, 11.9 \%, 3.0 \%$ and $2.0 \%$ of patients chose the effect of treatment on symptoms AND esophageal inflammation, the effect of the treatment on the symptoms, the effect of treatment on esophageal inflammation, compatibility of therapy with lifestyle, possible side effect, and recommendation of the physician, respectively, as deciding factor. The TSQM scales scores as well as average TSQM values for patients on PPI, STC, and diet are shown in Table 1. Conclusion: Adult EoE patients consider both effect of medication on symptoms as well as inflammation as most important criteria, when choosing EoE therapy. EoE patients appear to be 'satisfied' with PPI, STC, and dietary therapy and 'very satisfied' with STC, if the therapy is taken twice daily.

Disclosure of Interest: All authors have declared no conflicts of interest.

P1843 SYSTEMATIC ANALYSIS OF SUBEPITHELIAL EOSINOPHIL COUNTS INCREASES DIAGNOSTIC YIELD IN ADULTS WITH EOSINOPHILIC ESOPHAGITIS

A. Schoepfer ${ }^{1}$, A. Simko $^{2}$, C. Bussmann ${ }^{3}$, E. Safroneeva ${ }^{4}$, M. Zwahlen ${ }^{4}$, T. Greuter ${ }^{5}$, L. Biedermann ${ }^{5}$, S. Vavricka ${ }^{5}$, S. Godat ${ }^{6}$, A. Reinhard ${ }^{2}$, C. Saner ${ }^{2}$, H. Maye $^{2}$, C. Sempoux ${ }^{2}$, C. Brunel ${ }^{2}$, C. Blanchard ${ }^{7}$, D. Simon ${ }^{4}$, H. Simon ${ }^{4}$

A. Straumann

${ }^{1}$ CHUV+Universtiy of Lausanne, Lausanne/Switzerland

${ }^{2}$ University of Lausanne, Lausanne/Switzerland

${ }^{3}$ Viollier Pathology Basel, Basel/Switzerland

${ }^{4}$ University of Bern, Bern/Switzerland

${ }^{5}$ Gastroenterologie Und Hepatologie, University Hospital Zurich, Zürich Switzerland

${ }^{6}$ Endoscopy, CHU Lausanne, Lausanne/Switzerland

${ }^{7}$ Nestlé Research Center, Lausanne/Switzerland

${ }^{8}$ Gastroenterology Fmh, Swiss EoE Research Network, Olten/Switzerland

Contact E-mail Address: alain.schoepfer@ chuv.ch 
Introduction: For technical reasons, the histologic characterization of eosinophilic esophagitis (EoE)-specific alterations is almost exclusively based on those found in the esophageal epithelium, whereas little is known about subepithelial abnormalities.

Aims \& Methods: In this study, we aimed to systematically assess the nature of subepithelial histologic alterations, analyze their relationship with epithelial histologic findings, endoscopic features, and symptoms, and evaluate the diagnostic impact of subepithelial eosinophil counts in patients with an epithelial peak eosinophil count of $<15 / \mathrm{hpf}$. We prospectively included in this cohort study adult EoE patients who underwent assessment of clinical, endoscopic, and histologic disease activity using scores.

Results: We included 200 EoE patients (mean age $43.5 \pm 15.7$ years, $74 \%$ males) with a median peak count of 36 intraepithelial eosinophils/hpf [IQR 14-84]. The following histologic features were identified in the subepithelial layer: eosinophilic infiltration (median peak count of 20 eosinophils/hpf [IQR 10-51]), eosinophil degranulation $(43 \%)$, fibrosis $(82 \%)$, and lymphoid follicles $(56 \%)$. Peak intraepithelial eosinophil counts were higher, identical, and lower when compared to the subepithelial layer in $62.5 \%, 7 \%$, and $30.5 \%$ of patients, respectively. Subepithelial histologic activity correlated with epithelial histologic activity (rho $0.331, \mathrm{p}<0.001$ ), endoscopic severity (rho 0.208, $\mathrm{p}=0.003$ ), and symptom severity (rho $0.179, \mathrm{p}=0.011)$. Forty percent $(21 / 52)$ of patients with $<15$ intraepithelial eosinophils/hpf had subepithelial peak counts of $\geq 15 / \mathrm{hpf}$.

Conclusion: In one third of patients subepithlial peak eosinophilc counts are higher than epithelial eosinophil counts. Systematic assessment of subepithelial eosinophil counts can aid in diagnosing EoE in additional $40 \%$ of all patients with epithelial eosinophils $<15 / \mathrm{hpf}$.

Disclosure of Interest: All authors have declared no conflicts of interest.

\section{P1844 GASTROESOPHAGEAL REFLUX DISEASE PATIENTS REFLUXATE TYPE INFLUENCE ON MACROPHAGE PHENOTYPE}

S. Lyamina ${ }^{1}$, S. Kalish ${ }^{1}$, A. Paraskevova ${ }^{2}$, O. Storonova ${ }^{2}$, S. Pirogov ${ }^{3}$, A. Ponomarev ${ }^{4}$, D. Rumyantseva ${ }^{2}$, A.S. Troukhmanov ${ }^{2}$, V. T. Ivashkin ${ }^{5}$, I. Maev ${ }^{6}$, I. Malyshev ${ }^{1}$

${ }^{1}$ Pathophysiology, Moscow State University of Medicine and Dentistry, Moscow/ Russian Federation

${ }^{2}$ Vasilenko Clinic Of Internal Diseases Propaedeutic, Gastroenterology And Hepatology, First Moscow State Sechenov Medical University (Sechenov

University), Moscow/Russian Federation

${ }^{3}$ Endoscopy, P.A. Herzen Moscow Cancer Research Institute, Moscow/Russian Federation

${ }^{4}$ Pathological Anatomy Department, First Moscow State Sechenov Medical University, Moscow/Russian Federation

${ }_{5}^{5}$ Dept. Of Gastroenterology, director Of The Clinic, Sechenov University, Moscow Russian Federation

${ }^{6}$ Department Of Internal Diseases Propaedeutic And Gastroenterology, Moscow State University of medicine and dentistry, Moscow/Russian Federation

\section{Contact E-mail Address: svlvs@mail.ru}

Introduction: The modern understanding of gastroesophageal reflux disease (GERD) pathogenesis includes cell and molecular mechanisms of associated esophagus mucosal inflammation and Th1/Th2 immune response imbalance due to the recurrent exposure to acidic and nonacidic refluxate of gastric contents. Th1 or Th2 immune response is mainly predetermined by innate and adaptive immune response cells - macrophages. Considering the concept of M1/M2 macrophage phenotype programming and paradigm of $\mathrm{Th} 1 / \mathrm{Th} 2$ immune response M1/M2 macrophage phenotype imbalance is one of the main cell components of GERD mucosal inflammation.

Aims \& Methods: We aimed to assess the influence of refluxate type on macrophage phenotype and compare the data with predominated macrophage phenotype in systemic circulation in GERD patients. 68 patients with different forms of GERD and Barrett's esophagus were included in the study. Macrophage phenotype was assessed in vitro by adding acidic and nonacidic refluxates of patients $(\mathrm{n}=68)$ with different $\mathrm{pH}(4.6-6.6, \mathrm{n}=31$ (group I); 6.7-7.2, $\mathrm{n}=28$ (group II); $7.3-8.1, n=6$ (group III)) to peritoneal macrophages of C57/BL6 mice $(n=68)$ with the following macrophage culturing in standard conditions $(10 \%$ FBS, RPMI1640) for 36 hours. Monocytes were isolated from the patients' blood samples and cultured to macrophages (monocyte derived macrophages, MDM) in standard conditions -RPMI 1640 medium, $10 \% \mathrm{FBS}, 37^{\circ} \mathrm{C}, 5 \% \mathrm{CO}_{2}$. Pooled analysis of macrophage and MDM phenotype included assessment of secretory activity (Th1/Th2 cytokine production in culture medium, Antigenix, USA), typical M1/M2 surface macrophage CD markers (CD25, CD80 and CD163, CD206, respectively) performed by flow cytometry (Beckman Coulter FC500). Results: Analysis of cytokine macrophage production revealed the prevalence of Th1 and Th1/Th2 bivalent (IL-2, IL-6) cytokines as compared to Th2. The most significant changes due to the influence of refluxate $\mathrm{pH}$ were observed in Th1 cytokine IL-8, TNF $\alpha$ and TNF $\beta$. Increasing of $\mathrm{pH}$ resulted in 2 times-fold, 1.8 times-fold and 3.5 times-fold fall of these cytokines production respectively $(\mathrm{p}<0.05)$. Th2 cytokine macrophage production increased with increasing $\mathrm{pH}$, and most significantly changed in IL10: $8.43 \pm 3.13 \mathrm{pg} / \mathrm{ml}$ in group I vs. $27.7 \pm 8.65 \mathrm{pg} / \mathrm{ml}$ in group III $(\mathrm{p}<0.05)$. The expression of surface M1/M2 macrophage $\mathrm{CD}$ markers significantly varied depending on the acidity of refluxate. Mild-alkaline $\mathrm{pH}$ (III group) resulted in increasing expression of $\mathrm{M} 2$ markers CD163/CD206 as compared to M1-CD80/CD25, but changing M1/M2 index of $\mathrm{CD}$ expression still showed the prevalence of $\mathrm{M} 1$ phenotype in groups: $2.49 \pm 0.15$ (II) vs $1.29 \pm 0.12$ (III) $(\mathrm{p}<0.05$ ). Analysis of MDM phenotype showed the prevalence of M1 phenotype in all groups.

Conclusion: Pooled analysis of GERD patients refluxate type influence on macrophage phenotype showed the prevalence of M1 proinflammatory activated macrophages (Th1) with increased expression of M1 surface markers and predominantly increased production of Th1 cytokines (IL-8, TNF $\alpha$, TNF $\beta$ ). The changes were more expressed in macrophages exposed to acid refluxate. The effect of mild-alkalotic refluxate resulted in definite trend of increasing expression of CD M2 markers and increased production of Th2 cytokines as compared to the other refluxate types. Analysis of MDM phenotype showed the prevalence of $\mathrm{M} 1$ phenotype regardless of refluxate $\mathrm{pH}$.

Disclosure of Interest: All authors have declared no conflicts of interest.

\section{P1845 THE LOCATION OF OESOPHAGEAL MUCOSAL AFFERENT NERVES ARE MORE SUPERFICIAL IN PATIENTS WITH NERD THAN IN HEALTHY VOLUNTEERS AND PATIENTS WITH BARRETT'S OESOPHAGUS}

P. Woodland ${ }^{1}$, J.L.S. Ooi ${ }^{1}$, F. Grassi ${ }^{1}$, C. Lee ${ }^{1}$, J. Evans ${ }^{2}$, N. Koukias ${ }^{3}$, C. Triantos ${ }^{4}$, S. A.C. Mcdonald ${ }^{2}$, M. Peiris ${ }^{1}$, R. Aktar ${ }^{1}$, A. Blackshaw ${ }^{1}$ D. Sifrim ${ }^{1}$

${ }^{1}$ Wingate Institute For Neurogastroenterology, Barts and the London School of Medicine, Queen Mary University, London/United Kingdom

${ }^{2}$ Centre For Tumour Biology, Barts Cancer Institute, Barts and the London School of Medicine, Queen Mary University, London/United Kingdom

${ }^{3}$ Gastroenterology, University Hospital of Patras, Patras/Greece

${ }^{4}$ School Of Medicine, University of Patras, Patras/Greece

Contact E-mail Address: p.woodland@qmul.ac.uk

Introduction: The pathophysiology of heartburn perception in gastro-esophageal reflux disease (GERD) remains unclear. The degree of reflux-induced epithelial change seldom predicts symptom severity, as evidenced by the greater symptom burden seen in non-erosive reflux disease (NERD) compared to patients with Barrett's esophagus (BE). Existing models of acid hypersensitivity are inadequate to explain this discordance.

Aims \& Methods: To test the hypothesis that differences in peripheral esophageal mucosal innervation may be relevant, we studied the distribution of mucosal nerve fibers in patients with NERD and BE and compared the results with that of healthy subjects. 13 patients with NERD undergoing reflux testing and 16 patients with $\mathrm{BE}$ undergoing endoscopic surveillance were prospectively recruited. Biopsies were obtained from the proximal and distal esophageal mucosa in NERD patients and the distalmost squamous epithelium in BE patients, then examined immunohistochemically for presence and location for calcitonin gene-related peptide (CGRP)-immunoreactive nerve fiber. The results were compared with those from 10 healthy volunteers (HV) previously studied by our group.

Results: The distribution of mucosal CGRP-immunoreactive nerves is equidistant from the distal esophageal lumen in HV and BE (median 25.5 cell layers to surface [IQR 21.4-28.8] vs $21.5[16.1-27.5]$ respectively, $\mathrm{p}=0.155)$. Mucosal innervation is significantly more superficial in NERD both distally $(9.5$ cell layers [1.5-13.3], $\mathrm{p}<0.0001$ vs both $\mathrm{BE}$ and $\mathrm{HV})$ and proximally (5.0 [2.5-9.3], $\mathrm{p}=0.0098$ vs HV).

Conclusion: The acid hypersensitivity seen in NERD may be partially explained by the increased proximity of mucosal afferents to the esophageal lumen, and therefore greater exposure to noxious substances in refluxate. Conversely, the relative acid hyposensitivity in $\mathrm{BE}$ may be attributed to the deeper location of mucosal nerves.

Abstract No: P1842

Table 1: TSQM scales scores for PPI, STC (in powder form), and different diets in adult EoE patients.

\begin{tabular}{|c|c|c|c|c|c|}
\hline TSQM scales & $\begin{array}{l}\text { PPI } \quad(n=27 ; \text { median } \\
\text { treatment duration } 6 \\
\text { years [3-9] }\end{array}$ & $\begin{array}{l}\text { STC }(n=84 ; \text { median } \\
\text { treatment duration } 5 \\
\text { years }[2-6]\end{array}$ & $\begin{array}{l}\text { STC only }(n=17 \text {; once } \\
\text { daily; median treatment } \\
\text { duration } 5 \text { years [3-6] }\end{array}$ & $\begin{array}{l}\text { STC only }(\mathrm{n}=22 \text {; twice } \\
\text { daily; median treatment } \\
\text { duration } 3.5 \text { years }[1-6]\end{array}$ & $\begin{array}{l}\text { Diet }(\mathrm{n}=21 \text {; median } \\
\text { treatment duration } 2 \\
\text { years [1-4.5] }\end{array}$ \\
\hline Effectiveness & $66.7[38.9-77.8] 100$ & $83.3[66.7-94.4] \quad 100$ & $\begin{array}{lll}77.8 & {[61.1-94.4]} & 100\end{array}$ & {$[72.2-100]$} & $77.8[50-88.9] 100[100$ \\
\hline Side-effects & {$[100-100] \quad 88.9 \quad[77.8-$} & {$[100-100] \quad 83.3 \quad[66.7-$} & {$\left[\begin{array}{lll}{[100-100]} & 72.2 & {[61.1-}\end{array}\right.$} & {$[100-100] 75$ [61.1-100] } & $100] 50[33.3-66.7] 78.6$ \\
\hline Convenience & $100] 71.4[50-85.7] 79.8$ & $100] \quad 78.6 \quad[64.3-92.9]$ & $100] \quad 78.6 \quad[57.1-92.9]$ & $85.7 \quad[64.3-92.9] \quad 83.8$ & {$[57.1-92.9] \quad 76.6 \quad[59.8$} \\
\hline Overall satisfaction & {$[69.4-85.5]$} & $84.4[72.8-92.3]$ & $80.1[71.3-89.4]$ & [69.9-94.4] & 81.9] \\
\hline
\end{tabular}


Disclosure of Interest: P. Woodland: Research grant Reckkit Benckiser (Hull UK

D. Sifrim: research grant Sandhill Scientific (Denver USA) research grant Reckkit Benckiser (Hull UK)

All other authors have declared no conflicts of interest.

\section{P1846 BELCHING PATTERNS IN PATIENTS WITH ISOLATED PATHOLOGICAL UPRIGHT REFLUX AND PATHOLOGICAL BIPOSITIONAL REFLUX}

J.M. Conchillo, J. T.w. Snijkers, J. Santos Fernandez, A. A.m. Masclee Gastroenterology, Maastricht UMC, Maastricht/Netherlands

Contact E-mail Address: j.conchillo@mumc.nl

Introduction: Belching is a commonly occurring symptom in patients with gastroesophageal reflux disease (GERD). Belching may elicit reflux. It is unknown whether GERD patients with isolated pathological upright reflux (UP) have belching patterns that are different from GERD patients with pathological bipositional reflux (BIP)

Aims \& Methods: Aim of this study was therefore to examine the belching patterns of UP reflux patients as compared with BIP reflux patients. We included 50 consecutive patients with pathological reflux and typical symptoms who underwent 24-h pH-impedance monitoring at the Maastricht University Medica Centre from 2015 to 2017. Patients referred for excessive belching were excluded. A group of 25 UP reflux patients (10 male, mean age 52.9 years (range 22-77)) and 25 BIP reflux patients (11 male, mean age 47.9 years (range 18-77)) were enrolled. All 24-h pH-impedance tracings were analysed manually. We classified belches according to: a) physiological mechanism: supragastric vs. gastric; and b) their temporal relationship with a liquid reflux episode: isolated belch, preceding or during a liquid reflux episode. Symptom-association analysis was performed to assess a relationship between reported symptoms and reflux episodes.

Results: BIP patients showed higher acid reflux time $(17.8 \pm 2.4 \%$ vs. $7.3 \pm 0.6 \%$, $\mathrm{p}<0.001)$ and higher number of total reflux episodes $(121 \pm 9$ vs. $97 \pm 8$, $\mathrm{p}=0.05)$ than UP patients. Notably, both the proportion of reflux episodes with belches of any type and the proportion of belches preceding liquid reflux were higher in UP patients than in BIP patients $(51.7 \pm 3.6 \%$ vs. $32.1 \pm 3.7 \%$, $\mathrm{p}<0.001$ and $27.3 \pm 3.1 \%$ vs. $17.8 \pm 2.9 \%, \mathrm{p}=0.03$, respectively). No difference was found in the proportion of both supragastric and gastric belches between groups. During 24-h $\mathrm{pH}$-impedance monitoring UP patients reported more symptoms $(21 \pm 6$ vs. $12 \pm 3, \mathrm{p}=0.16)$ and had more positive symptoms with belches $(60.2 \pm 7.1 \%$ vs. $39.0 \pm 6.6 \%, \mathrm{p}=0.03)$ than BIP patients. Of the total number of belches that were detected using 24-h $\mathrm{pH}$-impedance, more belches were symptomatic in UP patients than in BIP patients $(24.4 \pm 6.4 \%$ vs. $11.1 \pm 2.5 \%, \mathrm{p}=0.06)$.

Conclusion: In our study, GERD patients with isolated pathological upright reflux had more often (symptomatic) belches than GERD patients with pathological bipositional reflux. Therefore, examination of belching patterns can assist diagnostic and therapeutic strategic planning in GERD patients who are refractory to medical therapy.

Disclosure of Interest: All authors have declared no conflicts of interest.

\section{P1847 IS REFLUX DURING NAPS WORSE THAN DURING NIGHT- TIME SLEEP?}

C. Teruel Sánchez-Vegazo, M. J. De Higes Ruiz, A. Albillos Gastroenterology, Hospital Ramón y Cajal, Madrid, Madrid/Spain

Contact E-mail Address: cteruelvegazo@yahoo.es

Introduction: Gastroesophageal reflux in the recumbent period is related to a higher risk of developing oesophageal lesions (severe esophagitis or Barrett oesophagus). The only study to date that analysed reflux during daytime naps suggests that it is worse than that which occurs during the night-time sleep. Our objective was to determine if reflux during naps is more severe than during nighttime sleep.

Aims \& Methods: Between February 2015 and November 2016 patients that underwent ambulatory 24-hour oesophageal $\mathrm{pH}$ monitoring at our motility unit were screened. Those who slept an afternoon nap in the recumbent position in addition to the regular night-time sleep, and had a pathological acid exposure (deMeester score $>14.72$ ) were included. We excluded those patients with previous foregut surgery or sleep apnea, those taking sleeping medication and those who were in recumbent position more than twice during the study. All studies were done off-therapy. Number of refluxes, number of refluxes per hour, reflux duration and oesophageal acid exposure (AET) time were compared between the two recumbent periods. Correlation between AET and meal-to-bed time was analysed, both for naps and night-time sleep.

Results: A total of 32 patients were selected $(59.4 \%$ women, mean age $51.31 \pm 14.59$, median BMI 26.48 (range 21.63-38.71)). Indication was typica GERD symptoms in $68.8 \%$ and atypical symptoms in $32.3 \%$. Oesophageal manometry revealed normal oesophageal body motility in $65.6 \%$, inefficient peristalsis in $28.1 \%$, and a median lower oesophageal sphincter pressure of $8 \mathrm{mmHg}$ (range 1-24). Median nap duration was shorter than that of the night-time sleep: 108 mins (range 30-375) vs 454 mins (range 240-593) $(\mathrm{p}=0.00)$. Median meal-to-bed time was also shorter for naps: 30.5 mins (range 4-185) vs 110.5 mins (range 9-247) $(\mathrm{p}=0.00)$. Median number of refluxes per hour and median recumbent AET were similar in both periods: 1.71 (range 0 26.5 ) vs 1.85 (range $0-12.8)(\mathrm{p}=0.45$ ) and 1.55 (range $0-61$ ) vs 5.9 (range $0-36.1$ ) $(\mathrm{p}=0.57)$, respectively. $87 \%$ of total recumbent reflux took place during the night. Correlation between AET and meal-to-bed time trended to be significant for naps (Spearman rho $=0.33, \mathrm{p}=0.065$ ), and was not significant for night-time sleep (Spearman rho $=0.02, \mathrm{p}=0.94$ ). All 5 patients who waited more than an hour after lunch to lay down had an AET during the nap of less than $1 \%$.

Conclusion: Naps are not associated with a more severe acid reflux than nighttime sleep, what does not support a specific recommendation against taking daytime naps for GERD patients. Avoiding short meal-to-bed time specifically for naps could be advisable.

Disclosure of Interest: All authors have declared no conflicts of interest.

\section{References}

Hila A, Castell DO. Nighttime reflux is primarily an early event. $J$ Clin Gastroenterol 2005;39:579-583.

Allen L, Poh CH, Gasiorowska A et al. Increased oesophageal acid exposure at the beginning of the recumbent period is primarily a recumbent-awake phenomenon. Aliment Pharmacol Ther 2010;32:787-794.

Dean BB, Aguilar D, Johnson LF et al. The relationship between the prevalence of nighttime gastroesophageal reflux disease and disease severity. Dig Dis Sci 2010;55:952-959.

Fujiwara Y, Arakawa T, Fass R. Gastroesophageal reflux disease and Sleep. Gastroenterol Clin N Am 2013;42:57-70.

Nasrollah L, Maraday-Romero C, Jha LK et al. Naps are associatedmore commonly with gastroesophageal reflux, compared with nocturnal sleep. Clin Gastroenterol Hepatol 2015;13:94-99.

\section{P1848 TREATMENT WITH PROTON PUMP INHIBITORS (PPI) DOES NOT REDUCE ACIDIC LARYNGOPHARYNGEAL REFLUX (LPR) DESPITE REDUCING DISTAL ACIDIC GASTROESOPHAGEAL REFLUX AND IMPROVING SYMPTOMS IN PATIENTS WITH LPR}

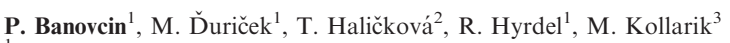

${ }^{1}$ Internal Gastroenterological Clinic, Jessenius Faculty of Medicine, Martin/Slovak Republic

${ }^{2}$ Clinic Of ENT And Head And Neck Surgery, Central Military Hospital, Ružomberok/Slovak Republic

${ }^{3}$ Asthma And Allergy Center. The Johns Hopkins University School of Medicine, Baltimore/United States of America/MD

Contact E-mail Address: pbanovcin@gmail.com

Introduction: PPI improve LPR symptoms in many patients. It is implicitly assumed that this effect is due to reduction of acidic LPR by PPI. Here we tested this assumption. We evaluated LPR and distal gastroesophageal reflux by using simultaneous $\mathrm{pH} /$ impedance monitoring in laryngopharyngeal segment and in distal esophagus before and after PPI treatment.

Aims \& Methods: Patients referred for suspected LPR were screened and those with positive reflux finding score (RFS $>7$, determined by ENT physician) and or positive reflux symptom index (RSI $>13$ ) and at least one acidic LPR episode during $24 \mathrm{~h} \mathrm{pH}$-impedance study were enrolled. The RSI, RFS and dual $\mathrm{pH} /$ impedance study of LPR and distal reflux were completed before and after 3 months therapy with PPI twice a day. Appropriate distance between $\mathrm{pH}$ sensors was chosen based on manometrically determined LES and UES so that the proximal $\mathrm{pH}$ sensor was positioned $1 \mathrm{~cm}$ above UES and distal sensor was positioned $4-6 \mathrm{~cm}$ above LES. By definition each LPR event was preceded by reflux detection in the distal esophagus.

Results: 18 patients $(11 \mathrm{M} / 7 \mathrm{~F}, 51 \pm 10 \mathrm{yrs})$ completed the study. In this group the PPI treatment substantially improved the symptoms of LPR. Reflux finding score (RFS) was decreased by $40 \%$ from $9 \pm 1$ to $5 \pm 1$ ( $\mathrm{P}<0.01$ ). Reflux symptom index (RSI) was decreased by $40 \%$ from $23 \pm 3$ to $14 \pm 3(\mathrm{P}<0.01)$. As expected distal acidic reflux was dramatically (by $75 \%$ ) decreased by PPI treatment $(\mathrm{P}<0.01)$. The number of distal acidic reflux episodes was reduced from $17 \pm 2$ to $3 \pm 1(P<0.01)$. Surprisingly, acidic LPR was not decreased by PPI treatment. The number of $\mathrm{LPR}$ with $\mathrm{pH}<5.0$ was $2 \pm 0.5$ vs. $3 \pm 1, \mathrm{P}=\mathrm{NS}$, and the number of LPR episodes with $\mathrm{pH}<4.0$ was $0.7 \pm 0.1$ vs. $0.6 \pm 0.2, \mathrm{P}=\mathrm{NS}$ ). Surprisingly, the number of acidic LPR episodes with $\mathrm{pH}=5.0-6.0$ was even increased $(14 \pm 2$ vs. $21 \pm 3, \mathrm{P}<0.05)$ leading to an increase in the overall number of LPR episodes by approximately $50 \%$ from $16 \pm 2$ to $24 \pm 3$ $(\mathrm{p}<0.05)$. PPI treatment did not decrease laryngopharyngeal time the $\mathrm{pH}$ was $<6.0(258 \pm 48 \mathrm{~s}$ vs. $301 \pm 64 \mathrm{~s}, \mathrm{P}=\mathrm{NS})$ and did not alter the composition of laryngopharyngeal reflux ( $\mathrm{P}=\mathrm{NS})$.

Conclusion: Proton pump inhibitor treatment does not reduce acidic laryngopharyngeal reflux despite substantially improving symptoms in patients with objectively established LPR. This suggests that PPI treatment influences some aspects of pathogenesis of LPR symptoms that are not readily detected by laryngopharyngeal $\mathrm{pH} /$ impedance monitoring.

Disclosure of Interest: All authors have declared no conflicts of interest. 


\section{P1849 EFFECT OF L-MENTHOL ON ESOPHAGEAL PERISTALTIS} AND LOWER ESOPHAGEAL SPHINCTER IN HEALTHY

\section{VOLUNTEERS}

P. Banovcin ${ }^{1}$, M. Duriček ${ }^{1}$, P. Lipták ${ }^{2}$, R. Hyrdel $^{1}$, M. Kollarik ${ }^{3}$

${ }_{1}^{1}$ Internal Gastroenterological Clinic, Jessenius Faculty of Medicine, Martin/Slovak Republic

${ }^{2}$ Clinic Of Gastroenterological Internal Medicine, University Hospital in Martin, Martin/Slovak Republic

${ }^{3}$ Asthma And Allergy Center, The Johns Hopkins University School of Medicine, Baltimore/United States of America/MD

Contact E-mail Address: pbanovcin@gmail.com

Introduction: Menthol is widely used as a food flavourant. In general, it is considered a trigger of gastroesophageal reflux symptoms. It has been assumed that menthol reduces the tone of the lower esophageal sphincter (LES) as a consequence of direct effect on the smooth muscle of the LES. Evidence has been provided that menthol sprayed on the gastric mucosa significantly suppresses gastric peristalsis (1). However, there is no clear evidence of the impact of menthol on the esophageal peristalsis and LES tone.

Aims \& Methods: The aim of the study was to evaluate the effect of the menthol infusion into the esophagus on the esophageal peristalsis and lower esophageal sphincter pressure in healthy volunteers. High-resolution manometry and the parameters of esophageal pressure topography were used to quantitatively evaluate the certain components of esophageal motility. 13 healthy volunteers without esophageal symptoms were enrolled. High-resolution manometry catheter with a thin silicon tube attached were placed transnasally so that the distal end of the tube was $5 \mathrm{~cm}$ above the LES. After a $5 \mathrm{~min}$. adaptation period the measurement was performed in the supine position according to the protocol as follows: after the baseline recording 10 water swallows of $5 \mathrm{ml}$ and $3+3$ water swallows of 10 and $15 \mathrm{ml} 15 \mathrm{ml}$. After that a $20 \mathrm{~min}$. infusion challenge with $3 \mathrm{mM}$ menthol $8 \mathrm{ml} / \mathrm{min}$. was carried out and subsequently the water swallows in order described above were repeated. HRM tracings were manually analyzed using ManoScan software and parameters used in the Chicago classification (v3.0) were evaluated. Integrated relaxation pressure (IRP), nadir LES pressure and distal contractile integral (DCI) values from $5 \mathrm{ml}, 10 \mathrm{ml}$ and $15 \mathrm{ml}$ swallows were obtained. These were compared before and after the menthol infusion. Paired parametric tests were used for statistical analysis.

Results: None of the subjects had any motility disorder defined by the Chicago Classification v3.0. Few volunteers reported only mild cold sensation during infusion presumed to be of the esophageal origin. The nadir LES pressure before and after menthol infusion was $7.5 \pm 0.8 \mathrm{mmHg}$ vs. $7.3 \pm 0.7 \mathrm{mmHg}$, respectively $(\mathrm{p}>0.7)$. IRP of $5 \mathrm{ml}$ swallows was $2.8 \pm 0.6 \mathrm{mmHg}$ vs. $2.1 \pm 0.5 \mathrm{mmHg}$ showed significance $(\mathrm{p}=0.01)$. However, difference of IRP of $10 \mathrm{ml}$ and $15 \mathrm{ml}$ swallows was not significant ( $\mathrm{p}>0.1, \mathrm{p}>0.5$, respectively). Average DCI pressure (5 ml swallows) was $737.8 \pm 126.2 \mathrm{mmHg}$ and $814.2 \pm 116.2 \mathrm{mmHg}$ before and after menthol infusion, respectively $(\mathrm{p}>0.5)$. We found no difference in DCI in $10 \mathrm{ml}$ and $15 \mathrm{ml}$ swallows before and after menthol infusion. Menthol seemed to have had only a marginal insignificant effect on IRP and DCI in rapid swallow test

Conclusion: We quantified the effect of menthol on the esophageal function and LES pressure in healthy volunteers using high resolution manometry. The analysis of HRM tracings revealed that menthol has no effect on particular parameters of the LES pressure and esophageal peristalsis.

Disclosure of Interest: All authors have declared no conflicts of interest.

\section{Reference}

1. Hiki N, Kaminishi M, Yasuda K, Uedo N, Honjo H, Matsuhashi N, Hiratsuka T, Sekine C, Nomura S, Yahagi N, Tajiri H, Suzuki H. Antiperistaltic effect and safety of L-menthol sprayed on the gastric mucosa for upper GI endoscopy: a phase III, multicenter, randomized, double-blind, placebo-controlled study. Gastrointest Endosc. 2011 May;73(5):932-41. doi: 10.1016/j.gie.2010.12.013

P1850 ANALYSIS OF THE RELATIONSHIP BETWEEN GLOBUS PERCEPTION AND ACIDIC LARYNGOPHARYNGEAL REFLUX BY DUAL PHARYNGEAL AND ESOPHAGEAL 24-HOUR PH/ IMPEDANCE MONITORING

L. Bubernáková, M. Ďuriček, P. Banovcin, R. Hyrdel

Internal Gastroenterological Clinic, Jessenius Faculty of Medicine, Martin/Slovak Republic

Contact E-mail Address: lenka.bubernakova@gmail.com

Introduction: Globus is considered to be related to the gastroesophageal reflux disease/laryngopharyngeal reflux (LPR). However, a substantial part of subjectiveness and self-perception of globic sensation which is impossible to measure objectively makes this symptom difficult to study. Visceral hypersensitivity and/ or altered functional state of the afferent nerve endings in the hypopharynx resulting from reflux have been suggested responsible for the development of globus. We hypothesized differences in the reflux burden and esophageal/pharyngeal acid exposure between the patients with LPR symptoms with globus compared to the patients with LPR symptoms without globus.

Aims \& Methods: Patients referred for suspected LPR were screened and those with positive reflux symptom index $($ RSI $>13)$ and at least one acidic LPR episode during $24 \mathrm{~h} \mathrm{pH} /$ impedance study were enrolled. We recruited patients that were at least 30 days without PPI treatment. Appropriate distance between $\mathrm{pH}$ sensors was chosen based on manometrically determined LES and UES so that the proximal $\mathrm{pH}$ sensor was positioned $1 \mathrm{~cm}$ above UES and distal sensor was positioned 4-6 $\mathrm{cm}$ above LES. For each LPR event we determined the maximum drop of $\mathrm{pH}$ on the $\mathrm{pH}$ levels $<5.5$ and $<5.0$. For these $\mathrm{pH}$ levels we determined pharyngeal acid exposure time calculated as cumulative time of pharyngeal reflux below that $\mathrm{pH}$ level during $24 \mathrm{~h}$. According to the question 8 (sensations of sticking/lump in the throat) of self-evaluated RSI questionnaire the patients were divided into globus positive group (tick 4-5 in the RSI) or globus negative (tick $0-1$ in the RSI).

Results: $19(13 \mathrm{M} / 6 \mathrm{~F})$ completed the study. The number of globus positive and negative patients was 11 and 8 , respectively. There were no major differences between groups. Disregarding the question about globus in the RSI, there was no significant difference of the RSI between the globus positive and negative group $(25 \pm 2$ vs. $21 \pm 2$, respectively, $\mathrm{p}=\mathrm{NS})$. As for the reflux in distal esophagus, we observed no difference in the acid exposure time between the globus positive and negative patients $(2.2 \pm 0.5 \%$ vs. $3.9 \pm 1.1 \%$, respectively, $\mathrm{p}=\mathrm{NS})$. We therefore assumed differences in the results from the hypopharyngeal $\mathrm{pH}$ sensor. However, the number of LPR events with $\mathrm{pH}$ drop to $<5.5$ showed no significant difference between the globus positive and negative patients, either for the number of LPR events $(5[2.5-6.5]$ vs. $3.5[1.8-6.3]$, respectively, $p=N S)$, or for the pharyngeal acid exposure time $(45 \pm 14 \mathrm{~s}$ vs. $88 \pm 42 \mathrm{~s}$, respectively, $\mathrm{p}=\mathrm{NS})$. We speculated that more acidic LPR events $(\mathrm{pH}$ drop to $<5)$ might be of greater relevance. However, no significant difference was found between the globus positive and negative patients either in terms of the number of these LPR events $(1[0-2]$ vs. $1[1-1]$, respectively, $\mathrm{p}=\mathrm{NS})$, or the pharyngeal acid exposure time $(16 \pm 6 \mathrm{~s}$ vs. $42 \pm 36 \mathrm{~s}$, respectively, $\mathrm{p}=\mathrm{NS})$.

Conclusion: We found no significant difference in the reflux burden in the distal esophagus/hypopharynx between the groups of symptomatic LPR patients with and without globus using $24 \mathrm{~h}$ dual channel $\mathrm{pH} /$ impedance. Other factors, e.g. visceral hypersensitivity might play a role in the development of globus symptoms, even in patients with objectively established LPR.

Disclosure of Interest: All authors have declared no conflicts of interest.

\section{P1851 GASTRIN-17 AS A NON-INVASIVE MARKER FOR GERD: A PROSPECTIVE STUDY ON SAMPLE OF 777 CONSECUTIVE PATIENTS}

G. Masciangelo ${ }^{1}$, F. Di Mario ${ }^{1}$, L. Franzoni ${ }^{1}$, M. Franceschi ${ }^{3}$, S. Landi ${ }^{1}$, T.B. Tene Fokam ${ }^{1}$, S. Grillo ${ }^{1}$, S. Scida ${ }^{1}$, E. Savarino ${ }^{4}$, N. De Bortoli ${ }^{5}$, C. Miraglia ${ }^{2}$, A. Bertelè ${ }^{6}$, V. Corrente ${ }^{7}$, C. Scarpignato ${ }^{2}$

${ }^{1}$ Department Of Medicine And Surgery, University of Parma, Parma/Italy ${ }^{3}$ Endoscopic Unit, Department Of Surgery, Ulss4, Hospital ULSS4 Alto Vicentino, Santorso/Italy

${ }^{4}$ Department Of Surgery, Oncology And Gastroenterology, University of Padua, Padua/Italy

${ }^{5}$ Division Of Gastroenterology, Department Of Internal Medicine, University of Pisa, Pisa/Italy

${ }^{6}$ Department Of Clinical \& Experimental Medicine, Clinical Pharmacology \& Digestive Pathophysiology Unit, University of Parma, Parma/Italy

University Hospital, Parma (Italy), Parma/Italy

Contact E-mail Address: francesco.dimario@unipr.it

Introduction: Due to a negative feed-back mechanism, gastrin-17 (G17) can be considered a mirror of acid secretion: the lower G17 values, the higher gastric acid secretion. Some studies suggest serum G17 low levels as a marker of acidrelated conditions, like Gastroesophageal Reflux Disease (GERD). Aim of the study was to evaluate the property of low levels of G17 in GERD diagnosis, in comparison with clinical and instrumental GERD gold-standards (typical symptoms, esophagitis at endoscopy, a positive Demeester score at $\mathrm{pH}$-metry).

Aims \& Methods: We enrolled 777 consecutive patients $(M=369$; mean age $=55.3$ ys; range $=33-76$ y y) affected by upper gastrointestinal (GI) troubles, showing low levels of serum G17 (BioHit Oyj, Finland; mean values: 1-9 pmol/1). Exclusion criteria included: current Proton Pump Inhibitors, previous history of surgery, neoplasms, current Helicobacter Pylori infection. Both typical, atypical and extra esophageal GERD symptoms, according with Montreal Classification were collected. All patients underwent upper GI endoscopy and esophagitis was classified according with Los Angeles criteria. In a subgroup of 221 patients $(\mathrm{M}=103$; mean age $=52.5$ ys; range $=35-69$ ys $)$ with Non Erosive Reflux Disease (NERD) a 24 hours pH-metry was performed using the Demeester score of 14 to establish a condition of acid reflux.

Results: Typical symptoms (heartburn and/or regurgitation) have been detected in 547 patients, being in 479 ones heartburn the prevalent symptom and in 68 ones regurgitation. Extra-esophageal symptoms (mainly chronic cough) were present in 303 patients. At the endoscopical evaluation, 349 subjects showed a picture of esophagitis with different degrees of severity of Los Angeles score. One hundred and seventy out 221 patients which underwent $24 \mathrm{~h} \mathrm{pH}$-metry showed a positive Demeester score for acid gastroesophageal reflux. Summarizing, 700 out of the 777 subjects included in the study showed at least one of the criteria (clinical, endoscopical or functional) accepted to support the diagnosis of GERD.

Conclusion: By using low levels of G17 as a non-invasive marker of GERD, in more than $90 \%$ (700 out of $777 \mathrm{pts}$ ) the diagnosis of reflux disease was confirmed, according with the current clinical or instrumental gold standard criteria, suggesting the use of this simple method to identify subjects with suspected GERD almost when typical symptoms are lacking or a NERD picture is find. Disclosure of Interest: All authors have declared no conflicts of interest. 
P1852 HIGH RESOLUTION MANOMETRY SHOULD BE CONSIDERED THE BEST TEST TO DIAGNOSE SLIDING HIATAL HERNIA

I. Martinucci ${ }^{1}$, N. De Bortoli ${ }^{1}$, E. Savarino ${ }^{2}$, S. Tolone ${ }^{3}$, E. Marabotto ${ }^{4}$,

M. Frazzoni ${ }^{5}$, L. Frazzoni ${ }^{6}$, M. Bellini ${ }^{1}$, V. Savarino ${ }^{7}$, S. Marchi ${ }^{1}$

${ }^{1}$ Gastroenterology Unit, University of Pisa, Pisa/Italy

${ }^{2}$ Department Of Surgery, Oncology And Gastroenterology, University of Padua,

Padua/Italy

${ }^{3}$ Surgery, Second University of Naples, Naples/Italy

${ }^{4}$ University of Genoa, Genoa, Italy, Genoa/Italy

${ }^{5}$ Digestive Pathophysiology Unit, Baggiovara Hospital, Modena/Italy

${ }^{6}$ Department Of Medical And Surgical Sciences, S. Orsola-Malpighi Hospital, Bologna/Italy

${ }^{7}$ Dept Internal Medecine, Universita di Genova, Genova/Italy

Contact E-mail Address: martinucci.irene@gmail.com

Introduction: Sliding hiatal hernia $(\mathrm{HH})$ is a frequent diagnosis during upper endoscopy (UE) in patients with GERD-related symptoms. Recently, high resolution manometry (HRM) allowed an accurate evaluation of the esophago-gastric junction (EGJ) and its sub-types (Chicago Classification V3.0; CCv3). Few data are available comparing the diagnostic accuracy of HRM and UE to detect $\mathrm{HH}$.

Aims \& Methods: The aim of this study was to compare the prevalence of $\mathrm{HH}$ obtained with UE and HRM and to determine the role of this finding by diagnosing gastroesophageal reflux disease (GERD) on the basis of impedance and $\mathrm{pH}(\mathrm{MII}-\mathrm{pH})$ monitoring. We enrolled consecutive patients with heartburn and $\mathrm{HH}$ diagnosed with UE. After UE, all patients underwent HRM and MII-pH to investigate GERD. All tests were performed previous a 20-day wash-out from proton pump inhibitors. Erosive esophagitis (ERD) was diagnosed according to the Los Angeles Classification, and $\mathrm{HH}$ was diagnosed when the separation between the squamo-columnar junction and the diaphragmatic impression was greater than $2 \mathrm{~cm}$. Patients with achalasia or major disorders of peristalsis or previos surgery were excluded. MII-pH monitoring allowed to sub-classify patients with non-erosive GERD in: NERD (abnormal AET), hypersensitive esophagus (normal AET, reflux events but positive symptom-reflux association, HE) and functional heartburn (normal AET, reflux events and negative symptom-reflux association, $\mathrm{FH}$ ). All patients underwent barium X-ray to measure $\mathrm{HH}$. Recognition of two rings larger than $2 \mathrm{~cm}$ (recumbent position) between EGJ and diaphragmatic hiatus was necessary to detect $\mathrm{HH}$

Results: We evaluated 151 patients (94 females) with mean age of $56.2 \pm 15.4$ yrs. ERD was diagnosed in 34 patients $(22.5 \%)$. MII-pH allowed to subgroup patients in: $48(31.8 \%)$ NERD, $43(28.5 \%) \mathrm{HE}$, and $26(17.2 \%) \mathrm{FH}$. As expected, MII-pH showed a higher AET in ERD and NERD group $(\mathrm{p}<0.001)$. HRM was normal in $131 / 151(86.8 \%)$ and $20 / 151(13.2 \%)$ had ineffective motility. HH was confirmed in $83 / 151$ patients (HRM $55 \%$ vs UE $100 \%$; p < 0.05 ) with HRM and in $88 / 151$ patients (barium $58.3 \%$ vs UE $100 \%$; $<<0.05$ ) with barium X-ray. The mean length of $\mathrm{HH}$ during endoscopy $(5.4 \pm 2.6)$ was reported greater than that during HRM $(3.9 \pm 2.2)$ and Barium X-ray $(4 \pm 2.5)(\mathrm{p}<0.05)$. All details are reported in Table I.

Table I: Characteristic of the enrolled population stratified for GERD diagnosis

\begin{tabular}{llllll}
\hline & ERD (34) & NERD (48) & HE (43) & FH (26) & $p$ \\
\hline EGJ pressure (mean) & $25.86 \pm 11.8$ & $30.3 \pm 13.2$ & $31.4 \pm 12.6$ & $33.1 \pm 13.7$ & 0.635 \\
DCI & $1001 \pm 683$ & $1431 \pm 1632$ & $1577 \pm 1365$ & $1799 \pm 1453$ & 0.042 \\
IRP & $11.8 \pm 5.1$ & $12.6 \pm 7.2$ & $13.1 \pm 7.8$ & $11.4 \pm 7.1$ & 0.57 \\
DL & $6.8 \pm 1.6$ & $6.8 \pm 1.1$ & $6.6 \pm 1.4$ & $6.9 \pm 1.5$ & 0.836 \\
AET (\%) & $7.7 \pm 2.9$ & $5.1 \pm 2.3$ & $2.7 \pm 1.4$ & $1.1 \pm 0.9$ & $<0.001$ \\
Reflux number & $77.8 \pm 23.7$ & $66.9 \pm 31.4$ & $24.6 \pm 9.1$ & $17.9 \pm 7.5$ & $<0.001$ \\
Hiatal hernia (HRM) & $33(97.1 \%)$ & $34(70.8 \%)$ & $16(37.2 \%)$ & 0 & $<0.001$ \\
Hiatal hernia (X-ray) & $34(100 \%)$ & $36(75 \%)$ & $17(39.5 \%)$ & $1(3.8 \%)$ & $<0.001$ \\
\hline
\end{tabular}

Conclusion: HRM and barium X-ray showed similar diagnostic accuracy to detect HH. Thus, HRM might be considered the test of chioce during pre-surgical evaluation for laparoscopic antireflux surgery.

Disclosure of Interest: All authors have declared no conflicts of interest.

\section{P1853 HIGH RESOLUTION MANOMETRY CAN BE PREDICTIVE OF GERD AS CONFIRMED BY IMPEDANCE-PH MONITORING: DEVELOPMENT AND INTERNAL VALIDATION OF A PREDICTIVE MODEL}

S. Tolone ${ }^{1}$, L. Frazzoni ${ }^{2}$, E. Savarino ${ }^{3}$, M. Frazzoni ${ }^{4}$, N. De Bortoli ${ }^{5}$, I. Martinucci ${ }^{5}$, G. Bodini ${ }^{6}$, M. Furnari ${ }^{7}$, R. Penagini ${ }^{8}$, A. Mauro ${ }^{9}$, V. Savarino ${ }^{10}$ L. Docimo

${ }^{1}$ Surgery, University of Campania "Luigi Vanvitelli",Naples, Naples/Italy

${ }^{2}$ Department Of Medical And Surgical Sciences, S. Orsola-Malpighi Hospital,

Bologna/Italy

${ }^{3}$ Department Of Surgery, Oncology And Gastroenterology, University of Padua, Padua/Italy

${ }^{4}$ Digestive Pathophysiology Unit, Baggiovara Hospital, Modena/Italy

${ }^{5}$ Division Of Gastroenterology, Department Of Internal Medicine, University of Pisa, Pisa/Italy

${ }^{6}$ Department Of Internal Medicine, IRCCS San Martino DIMI, Genova/Italy
${ }^{7}$ Di.m.i., Gastroenterology Unit, DiMI, Gastroenterology Unit, University of Genoa., Genoa/Italy

${ }^{8}$ Dipartimento Di Scienze Mediche, Università degli Studi di Milano Dipto. di Gastroenterologia, Milan/Italy

${ }^{9}$ Gastroenterology And Endoscopy Unit, University of Milan, Milano/Italy

${ }^{10}$ Dept Internal Medecine, Universita di Genova, Genova/Italy

Contact E-mail Address: salvatore.tolone@unicampania.it

Introduction: In patients with symptoms suggestive of gastroesophageal reflux disease (GERD) candidate to anti-reflux surgery or in those refractory to antacid therapy, it is recommended to perform function tests in order to objectively diagnose GERD. Esophageal manometry is currently considered the gold standard method to assess esophageal motility. However, it has been shown limited capability to diagnose GERD. With the advent of high-resolution manometry (HRM), new metrics have been developed to investigate esophageal motor function, esophagogastric junction (EGJ) morphology and theirfunction. In particular, the antireflux barrier function of EGJ can now be assessed evaluating the contraction integral of the EGJ.

Aims \& Methods: Our aim was to assess the differences in terms of HRM characteristics between patients with typical reflux symptoms with GERD and those with functional heartburn (FH), classified by means of upper GI endoscopy and impedance-pH (MII-pH) monitoring off-medication. We also aimed to develop a predictive model for distinguishing $\mathrm{FH}$ from GERD by using the prevalence of these HRM features. Consecutive patients with heartburn and/or regurgitation and a recent endoscopic assessment were enrolled. All patients underwent HRM to assess the EGJ and 10 single water swallows to evaluate esophageal peristalsis and EGJ function and one multirapid swallow (MRS). The tracings were analyzed according to the Chicago Classification 3.0. EGJ pressure, EGJ contractile integral (EGJ-CI), EGJ morphology, mean and peak distal contractile integral (DCI), MRS DCI/mean DCI ratio and motility pattern were measured. Moreover, all patients underwent MII-pH off-therapy. We measured the esophageal acid exposure time (AET), number of total impedance reflux episodes and symptom association analysis using both symptom association probability (SAP+ if $>95 \%$ ) and symptom index (SI+ if $>50 \%$ ). GERD was diagnosed in case of one or more abnormal parameters at MII-pH. To analyze the differences between patients with MII-pH positive and negative for GERD, MannWhitney's $U$ test for continuous variables was used and significance was set at $\mathrm{P}<0.05$. A multivariate logistic regression analysis was then carried out to identify factors independently associated with MII-pH positivity for GERD, significance settled at $\mathrm{P}<0.05$. According to Hosmer's purposeful selection of covariates, a predictive model based on HRM variables was built by logistic regression analysis. The model was tested for discriminative performance by computing the area under ROC curve (AUROC), and calibration was assessed by Hosmer-Lemeshow goodness-of-fit test.The bootstrap re-sampling method was used to evaluate the internal validity of the model and to correct for overfitting.

Results: Overall, 68 patients ( $39 \mathrm{~F} / 29 \mathrm{M}$, median age 42.3) underwent HRM and MII-pH.HRM findings stratified according to MII-pH positivity for GERD are provided in Table 1. At univariate analysis, statistically significant differences were found in EGJ pressure, EGJ-CI, mean DCI and MRS DCI/mean DCI ratio between GERD and FH. Based on logistic regression analysis and according to Hosmer's purposeful selection of covariates, we developed a predictive model which ultimately encompassed two HRM variables, i.e. DCI MRS/mean DCI ratio (OR 1.614, 95\% CI 0.993-2.895) and EGJ-CI (OR 0.952, 95\% CI $0.913-$ 0.985). The model had a fair discriminative performance (AUROC: 0.798, 95\% CI: $0.650-0.894$ ) between MII-pH positivity and negativity for GERD and was well calibrated according to Hosmer-Lemeshow goodness-of-fit test $(p=0.769)$. The internal validation of the model by bootstrap method showed AUROC $=0.779$. Considering as cut-off the predicted probability of MII-pH for GERD which maximized sensitivity and specificity (i.e. $67.4 \%$ ), sensitivity and specificity of the predictive model in predicting GERD against $\mathrm{FH}$ were $80.8 \%$ and $76.2 \%$, respectively. Table 1

\begin{tabular}{|c|c|c|c|}
\hline HRM variable & $\begin{array}{l}\text { GERD group } \\
(\mathrm{n}=47)\end{array}$ & $\begin{array}{l}\text { No GERD group } \\
(\mathrm{n}=21)\end{array}$ & $P$ value \\
\hline $\begin{array}{l}\text { EGJ resting pressure, } \\
\text { mmHg (median, IQR) }\end{array}$ & $17.6(16.9)$ & $22.7(19.1)$ & $0.028^{\circ}$ \\
\hline $\begin{array}{l}\text { Peak DCI, } \mathrm{mmHg} * \mathrm{~cm} * \\
\text { sec (median, IQR) }\end{array}$ & 991 (1887) & $1632(2160)$ & $0.018^{\circ}$ \\
\hline $\begin{array}{l}\text { Mean DCI, } \mathrm{mmHg} * \mathrm{~cm} * \\
\quad \text { sec (median, IQR) }\end{array}$ & $590(1299.5)$ & $1191(1166)$ & $0.004^{\circ}$ \\
\hline $\begin{array}{l}\text { DCI MRS, } \mathrm{mmHg} * \mathrm{~cm} * \\
\quad \text { sec (median, IQR) }\end{array}$ & 1039 (1983) & 842 (1764) & $0.465^{\circ}$ \\
\hline $\begin{array}{l}\text { DCI MRS/mean DCI, } \\
\mathrm{mmHg} * \mathrm{~cm} * \mathrm{sec} \\
\text { (median, IQR) }\end{array}$ & $1.723(2.299)$ & $1.113(1.212)$ & $0.012^{\circ}$ \\
\hline$\underset{(\text { median, IQR) }}{\text { EGJ CI, } \mathrm{mmHg} * \mathrm{~cm} * \mathrm{sec}}$ & $7(18)$ & $22(17.6)$ & $0.004^{\circ}$ \\
\hline $\begin{array}{l}\text { EGJ morphology (type II } \\
\text { and III vs. type I), n (\%) }\end{array}$ & $14(29.8)$ & $3(14.3)$ & $0.232^{\wedge}$ \\
\hline
\end{tabular}

Conclusion: Our data indicate that HRM can be useful in detecting GERD, with our predictive model allowing a high level of suspicion for reflux disease. In particular the role of the EGJ-CI in GERD pathophysiology has been 
emphasized highlighted. Further larger studies considering different GERD phenotypes are needed.

Disclosure of Interest: All authors have declared no conflicts of interest.

P1854 GORD PATIENTS ARE FREQUENTLY DISSATISFIED ON LONG-TERM PPI: EXPLORING THE REASON(S) AND MANAGEMENT IN ROUTINE CLINICAL CARE (LOPA II STUDY)

J. Labenz ${ }^{1}$, G. Labenz ${ }^{2}$, M. Müller ${ }^{3}$, D. Stephan ${ }^{4}$, F. Willeke ${ }^{4}$

${ }^{1}$ Abt. Für Innere Medizin, Diakonie Klinikum Abt. für Innere Medizin - Abt. für

innere Medizin, Diakonie Klinikum Abt. für Inne, Siegen/Germany

${ }^{2}$ Reflux Center Siegerland, Burbach/Germany

${ }^{3}$ Diakonie Klinikum Jung-Stilling-Krankenhaus, Siegen/Germany

${ }^{4}$ St. Marienkrankenhaus Siegen, Siegen/Germany

\section{Contact E-mail Address: j.labenz@t-online.de}

Introduction: Randomized controlled trials report about $30 \%$ of GORD patients complain of bothersome remaining symptoms (heartburn, regurgitation) despite PPI. The LOPA (Lost Patients) I Study of 333 GORD patients seen in general practice revealed $46 \%$ of patients experienced heartburn or regurgitation symptoms at least twice per week despite PPI. A total of $20 \%$ were dissatisfied with their treatment. Few patients had received specific GORD diagnostics or recommended other options $(<10 \%)$.

Aims \& Methods: The LOPA II study is a prospective, multicenter, observationa study conducted in 17 general practice clinics. Patients with chronic GORD, taking PPI therapy for at least 1 year, and not satisfied with their treatment were asked to complete a questionnaire. Patients were asked the duration of their PPI therapy, satisfaction with their current condition, frequency of symptoms in the last week, whether they had previously received diagnostic evaluation or surgical consult related to GORD, whether they plan to consult a reflux specialist for further diagnostics, and reasons for dissatisfaction with their current medication treatment. "Lost Patients" were defined as those with a satisfaction score of 1 or 2 on a 5-point Likert scale (1: very dissatisfied; 2: dissatisfied), GerdQ score at least 8, and have not previously received specialized GORD diagnostics.

Results: 510 consecutive patient responses were collected within one year Patients suffered from GORD an average of 9.6 years and prescribed PPI therapy for an average duration of 7.9 years. $70 \%$ were dissatisfied or very dissatisfied on their current PPI therapy (score of 1 or 2 ). $83 \%$ reported heartburn or regurgitation at least 2 days in the prior week ( $53 \%$ 4-7 days). $49 \%$ reported using additional medication other than their prescribed PPI at least 2 days per week (34\% 4-7 days). In patients dissatisfied on PPI, most cited insufficient symptom control $(87 \%)$ as a reason for dissatisfaction. In addition, $31 \%$ cited concern with long-term use of drugs and $27 \%$ the need for daily medication. $92 \%$ of patients had received an upper endoscopy, $12 \%$ had a prior $\mathrm{pH}$-metry, $7 \%$ manometry, and $9 \%$ received prior surgical consult for GORD. Of patients who never received surgical consult, $48 \%$ were not aware of any surgical anti-reflux methods, $25 \%$ were concerned about possible complications, $18 \%$ felt their condition is not serious enough, $6 \%$ were recommended against anti-reflux procedure by their doctor.

Conclusion: Chronic GORD patients who are dissatisfied with their PPI therapy are rarely offered specialized GORD diagnostic procedures or treatment alternatives. Half of the patients took medication in addition to PPI to control their reflux. In addition to persistent symptoms, concerns of long-term PPI use and burden of daily medication play a role in patient dissatisfaction with PPI therapy. Disclosure of Interest: All authors have declared no conflicts of interest.

\section{P1855 PREVALENCE AND PATHOPHYSIOLOGY OF GASTRO- ESOPHAGEAL REFLUX DISEASE IN PATIENTS WITH AUTOIMMUNE GASTRITIS}

G. Maddalo $^{1}$, V. Pilotto ${ }^{1}$, E. Savarino ${ }^{1}$, C. Orlando ${ }^{1}$, M. Fassan ${ }^{2}$, D. Basso ${ }^{2}$, M. Rugge ${ }^{2}$, F. Farinati ${ }^{1}$

${ }^{1}$ Surgery, Oncology And Gastroenterology, Gastroenterology Unit, University Of Padua, Padua/Italy

${ }^{2}$ Medicine, University of Padua, Padua/Italy

Contact E-mail Address: gemma.maddalo@gmail.com

Introduction: Autoimmune gastritis (AIG) is characterized by corpus-predominant atrophy with consequent hypo-achlorhydria. In AIG patients dyspepsia is frequent but acid reflux symptoms not uncommon, with few data available regarding gastroesophageal reflux disease (GERD) in AIG.

Aims \& Methods: Our study was aimed to define the prevalence of reflux symptoms in AIG patients, to evaluate the serological, histological and clinical differences in AIG patients with or without reflux symptoms and to investigate the physiopathology behind these symptoms. One hundred and fifty AIG outpatients were evaluated and 87 were included in the study: 29 AIG patients with reflux symptoms (AIG-R) and 58 without (controls), selected with similar age and gender distribution. AIG-R underwent a $\mathrm{pH}$-impedance $(\mathrm{pH} / \mathrm{I})$ and high resolution manometry (HRM). Serum biomarkers, EGDS, histology and anamnestic data were evaluated in both groups. Statistics was performed as indicated.

Results: AIG-R were $19 \%$ overall and $28 \%$ of them showed endoscopic esophageal lesions, with more frequent hiatal hernia than in controls $(\mathrm{p}<0.02) . \mathrm{pH} / \mathrm{I}$ diagnosed acid reflux, esophageal hypersensitivity and a normal pattern in $7 \%$, $28 \%$ and $66 \%$ patients, respectively. The number of non-acid reflux (NAR) was higher when compared with acid ones $(\mathrm{p}<0.0001)$, moreover NAR and NAR proximal extension were associated with endoscopic lesions $(\mathrm{p}<0.03$ and $\mathrm{p}<0.05$, respectively). HRM revealed normal pattern in $62 \%$ of patients, minor peristalsis disorders in $24 \%$, and outflow obstruction in $14 \%$. According to the new Rome IV criteria, $55 \%$ of patients presented "functional esophageal disorders" (Rome IV-IN). No differences were detected in serological marker and clinical presentation. AIG-R presented lower antrum gastritis $(p<0.006)$ and a trend toward lower atrophy stage $(p=0.07)$ when compared with controls. The two patient with acid GERD were an OLGA 0 with mild gastrin increase and an OLGA I with short segment Barrett's esophagus. Lower OLGA stages, lower corpus atrophy $(\mathrm{p}<0.02)$ and more frequent response to PPI $(p<0.05)$ were associated with Roma IV-OUT status.

Conclusion: AIG-R patients are not uncommon despite the hypo-achlorhydria. Acid reflux is rare in AIG, while motility and "functional" disorders are frequent. Lower corpus atrophy and OLGA stage in Rome IV-OUT patients, with an at least partly preserved secretion, is likely relevant in the pathogenesis of symptoms. Treatment should consider use of proton pump inhibitor drugs only in specific patients.

Disclosure of Interest: All authors have declared no conflicts of interest.

\section{P1856 DIET IS MORE EFFECTIVE THAN ANTACIDS IN RELIEVING REFLUX SYMPTOMS IN MILD GERD}

M. Furnari ${ }^{1}$, S. Cento ${ }^{2}$, S. Tolone ${ }^{3}$, M. Frazzoni ${ }^{4}$, E. Savarino ${ }^{5}$, L. Frazzoni ${ }^{6}$, E. Marabotto ${ }^{1}$, G. Bodini ${ }^{1}$, V. Savarino ${ }^{1}$, N. De Bortoli ${ }^{7}$, S. Marchi ${ }^{7}$, P. Minale $^{2}$ ${ }^{1}$ Department Of Internal Medicine, Gastroenterolgoy Unit, University of Genoa, Genoa/Italy

${ }^{2}$ Division Of Allergy, University of Genoa, Genoa/Italy

${ }^{3}$ Division of General and Bariatric Surgery, Department of Surgery, Second University of Naples, Naples-Italy, Naples/Italy

${ }^{4}$ Digestive Pathophysiology Unit, Baggiovara Hospital, Modena/Italy

${ }^{5}$ Department Of Surgery, Oncology And Gastroenterology, University of Padua,

Padua/Italy

${ }^{6}$ Department Of Medical And Surgical Sciences, S. Orsola-Malpighi Hospital, Bologna/Italy

Dipartimento Di Medicina Interna - Università Degli Studi Di Pisa, Clinica Medica I/II Complesso S. Chiara, Pisa/Italy

\section{Contact E-mail Address: manuelefurnari@gmail.com}

Introduction: Gastroesophagel reflux disease (GERD) is a common disorder commonly overlapping with several functional or allergic/immunological disorders, whose symptoms are related to food intake. Awareness of health care costs and potential side effects of long-term acid suppression has increased the attention in non-pharmacologic treatment for alleviating reflux symptoms.

Aims \& Methods: The aim of our prospective study was to evaluate the noninferiority of a controlled diet compared to antacid compounds in relieving reflux symptoms in patients suffering from mild GERD. We considered 500 consecutive patients referred to the Division of Allergy and Clinical Immunology of AziendaIST IRCCS San Martino of Genova for gastrointestinal symptoms associated to food intake. Patients with a clinical and instrumental diagnosis of food allergy, food intolerance, Irritable Bowel Syndrome (IBS) and Small intestinal Bacterial Overgrowth (SIBO) were excluded. Patients with a diagnosis of GERD based on clinical history represented our study population. Basal metabolic rate and calories need of patients was assessed by means of Harry-Benedict equation corrected for their physical activity. Patients were asked to take antacids for one month. Subsequently patients discontinued medication consumption and followed an elimination diet of food commonly associated to eliciting reflux symptoms, based on their calories need. Efficacy of the two treatments was evaluated by means of a validated symptomatic questionnaire (RDQ administered at baseline, after antacids course (1 month) and after diet (1 month). A treatment was considered effective if a $50 \%$ improvement in the symptomatic score was recorded.

Results: After investigations 261 patients out of $500(52.2 \%)$ were excluded because of IBS (140), celiac disease (6), nickel allergy (25), lactose intolerance (60), SIBO (10), and allergy to other foods (20). The remaining 239 patients were diagnosed as affected by mild GERD (median age 47; BMI 24; $132 \mathrm{~F} / 107 \mathrm{M}$; no erosive reflux disease) and were included in our interventional prospective study. Among them, antacids treatment was effective in 180 cases $(75.3 \%)$. Symptoms relief was reported by 220 patients $(92.1 \%)$ after diet based on calories need. No differences were noted between responders and not responders in terms of BMI, age and gender. Diet was more effective than antacids treatment in relieving reflux symptoms in mild GERD $(\mathrm{p}<0.01)$

Conclusion: A structured diet regimen, tailored on the metabolic need of the patients, apperas more effective than antacids alone in relieving reflux symptoms in patients with mild GERD. Further controlled studies are mandatory to confirm these preliminary data.

Disclosure of Interest: All authors have declared no conflicts of interest.

\section{P1857 INTERIM RESULTS OF A PROSPECTIVE MULTICENTER REGISTRY OF LOWER OESOPHAGEAL SPHINCTER STIMULATION FOR GORD: THE LESS-GORD REGISTRY}

J. Labenz ${ }^{1}$, A. Leodolter ${ }^{2}$, J. Pedersen ${ }^{3}$, A. Nieponice ${ }^{4}$, R. Weise ${ }^{5}$, N. Bouvy ${ }^{6}$, S. Schoppmann ${ }^{7}$, C. Gutt ${ }^{8}$, A. Madisch ${ }^{9}$

${ }^{1}$ Abt. Für Innere Medizin, Diakonie Klinikum Abt. für Innere Medizin - Abt. für innere Medizin, Diakonie Klinikum Abt. für Inne, Siegen/Germany

${ }^{2}$ Evangelisches Krankenhaus Herne, Herne/Germany

${ }^{3}$ Aarhus University Hospital, Aarhus/Denmark 
${ }^{4}$ Hospital Universitario, Fundacion Favaloro, Buenos Aires/Argentina ${ }^{5}$ St.-Marien-Hospital, Friesoythe/Germany

${ }^{6}$ Clinical Research, Maastricht University Medical Center, St. Louis/Netherlands ${ }^{7}$ Allgemeines Krankenhaus der Stadt Wien, Wien/Austria

${ }^{8}$ Klinikum Memmingen, Memmingen/Germany

${ }^{9}$ Medical Department I, Klinikum Siloah, Hannover/Germany

Contact E-mail Address: j.labenz@t-online.de

Introduction: Safety and efficacy of electrical stimulation of the lower oesophageal sphincter (ES-LOS) using the EndoStim ${ }^{\circledR}$ LOS Stimulation System (Nijmegen, The Netherlands) has been demonstrated in clinical trials up to $>5$ years. Data on outcomes in routine clinical practice is growing.

Aims \& Methods: An ongoing, prospective international multicenter web-based registry is collecting data in patients with disruptive GORD symptoms, treated with ES-LOS in clinical practice. Data is collected at baseline and at routine follow-ups for 5 years. Demographics, adverse events, GORD symptoms, GORD health related quality of life (GORD-HRQL) scores, use of proton pump inhibitors (PPIs) and physiological data (oesophageal $\mathrm{pH} / \mathrm{manometry}$ ) are collected when available.

Results: 180 patients at 13 sites in Europe and Latin America have been enrolled. Follow-up data up to 2 years is available. Median (IQR) age at the time of implant was $51(41-60), 57 \%$ were male. All patients were taking prescription PPI at baseline. At their last follow-up between 6 and 24 months post op, $70 \%$ (84/121) were completely off PPI ( $\mathrm{p}<0.001)$. Median (IQR) composite GORDHRQL score improved from $23(17-29)$ preoperatively to $8(4-15)$ at 6 months, 7 $(2-12)$ at 12 months, and $8(4-15)$ at 24 months $(\mathrm{p}<0.001$ at all time points, $\mathrm{n}=154,121,66,33$ at baseline, M6, M12, M24, respectively). Oesophageal pH testing post-op was performed by a few sites either as standard of care or in patients with residual symptoms. Median (IQR)\% 24-hour oesophageal acid exposure improved from $8.2 \%(4.6-18.4)$ at baseline to $4.7 \%(1.4-14.5)$ at 6 months $(\mathrm{p}=0.26)$ and $3.6 \%(1.0-5.8)$ at 12 months $(\mathrm{p}=0.04)(\mathrm{n}=120,39,10$ at baseline, M6, M12, respectively). The proportion of patients with moderate to severe regurgitation decreased from $64 \%$ at baseline to $22.5 \%$ after 6 and $13.4 \%$ after 12 months. Extra-oesophageal symptoms (recurrent cough, pneumonitis, shortness of breath) and sleep disturbances also decreased substantially. Overall, dysphagia and gas were less common at 12 months than preoperatively. Four serious adverse events were reported in 3 patients. One myocardial infarction related sudden death at 11-month post-op, not related to the device or procedure; 1 event of asymptomatic electrode erosion into the oesophagus detected during routine endoscopy and the device safely removed during laparoscopic fundoplication; and 2 events of gastroparesis in 1 patient requiring hospitalization, possibly related to the device, were reported.

Conclusion: ES-LOS is safe and effective in treating patients with disruptive GORD symptoms despite PPI in routine clinical practice. ES-LOS should be considered a viable treatment option for treating these patients.

Disclosure of Interest: J. Labenz: Consulting fees - EndoStim BV

All other authors have declared no conflicts of interest.

P1858 ANTI-REFLUX MUCOSECTOMY (ARMS) FOR REFRACTORY GERD-INITIAL CLINICAL EXPERIENCE

A. Bapaye ${ }^{1}$, M. R. Mahadik ${ }^{1}$, R. Pujari ${ }^{1}$, T. K. Bharadwaj ${ }^{1}$, S. G. Vare ${ }^{1}$, N. Dubale ${ }^{1}$, S. Date ${ }^{1}$, J. A. Bapaye

${ }^{1}$ Shivanand Desai Center For Digestive Disorders, Deenanath Mangeshkar Hospital Digestive Diseases \& Endoscopy, Pune/India

Contact E-mail Address: amolbapaye@gmail.com

Introduction: PPI therapy remains the mainstay for treatment of gastroesophageal reflux disease (GERD), however laparoscopic fundoplication is recommended in refractory patients. Various endoscopic methods have been attempted with variable success. Anti-reflux mucosectomy (ARMS) is a recently introduced endoscopic therapy for refractory GERD

Aims \& Methods: The current study describes initial clinical experience of ARMS.Data from a prospectively maintained database of consecutive patients undergoing ARMS for refractory GERD was abstracted. Inclusion criteriaGERD symptoms $>=1$ year, daily PPI usage $>=6$ months; absence of hiatus hernia $>3 \mathrm{~cm}$ on EGD and normal esophageal body motility on highresolution manometry (HRM). Exclusions-hiatus hernia $>3 \mathrm{~cm}$, poor or absent esophageal body motility, poor-risk candidates for anesthesia or invasive procedure. Pre-ARMS evaluation-EGD to assess Hill's grade of flap valve, esophageal manometry, 24-hour ambulatory esophageal $\mathrm{pH}$ studies, PPI requirement and GERD-HRQL questionnaire. ARMS performed using cap EMR technique. Follow up protocol-EGD at 2-4 weeks, GERD-HRQL questionnaire, pH studies and PPI requirement at 4-6 weeks. Parameters for data analysis - pre-and post-ARMS GERD-HRQL questionnaire and Deemester scores, Hill's grading of gastroesophageal valve on EGD, PPI requirement and procedure-related adverse events (AE)

Results: $\mathrm{N}=15$, duration-12-months. Mean age- 40.8 years (Range 22-69); M: F-11: 4. HRM-normal esophageal body motility in all. Mean GERD-HRQL score improved significantly from pre-ARMS-40.4 to post-ARMS-7.6 $(\mathrm{p}<0.05)$. Mean Deemester score decreased from 85.8 pre-ARMS to 5.9 postARMS $(\mathrm{p}<0.05)$. Mean Hill's valve grade decreased pre-ARMS $=2.8$; postARMS $=1.6(\mathrm{p}<0.05)$. Three AE's-muscle injury-2 (treated by endoclips), grade I dysphagia-1. At 4 weeks follow up, $11 / 15$ patients $(73.3 \%)$ had discontinued PPI, 4/11 (36.3\%) had $>50 \%$ reduction in PPI dosage.

Conclusion: Current study shows impressive short-term results for ARMS Significant symptom resolution and acid exposure reduction occurred in all patients. $100 \%$ patients could discontinue or reduce PPI usage. AE's were minor. Larger randomized studies with longer term follow up are recommended.
Disclosure of Interest: A. Bapaye: Speaker- Boston scientific corporation, Cook medical, Taewoong medical, Olympus

All other authors have declared no conflicts of interest.

\section{P1859 EFFECTIVENESS OF SELECTIVE SEROTONIN REUPTAKE INHIBITORS IN PATIENTS WITH NON-EROSIVE REFLUX} DISEASE: A RANDOMIZED TRIAI

\author{
I. Biriuchenko, N. Bondarenko, A. Svintsitskyy
}

National Medical University, Kyiv/Ukraine

Contact E-mail Address: doctor.gastro@yahoo.com

Introduction: To date a large quantity of $5-\mathrm{HT}_{4}$ receptors were found in esophageal mucosa that indicates on an important role of this neuromediator in pathogenesis of non-erosive reflux disease (NERD) and gastroesophageal reflux disease (GERD). Antidepressants seems to be effective, as they have central analgesic effect, reduce chemical and mechanical sensitivity and have local effect on gastrointestinal tract.

Aims \& Methods: Our aim was to assess the superiority of combined treatment using proton pump inhibitor (PPI) plus antidepressant by comparison with PPI for patients with NERD. Methods: In this randomized superiority study adult patients (18-65 years) with confirmed diagnosis of NERD were eligible to participate. Exclusion criteria: the presence of "red flag" signs and other comorbidities that could explain the symptoms. Patients were assessed in clinically and psychologically. Psychological testing was done using validated short-form version of the depression anxiety stress scales (DASS-21) and Toronto alexithymia scale (TAS). All the patients were randomly divided into two arms: patients of the first arm received PPI (pantoprasole) $40 \mathrm{mg}$ once a day plus escitalopram as follows: initial dose $-5 \mathrm{mg}$ /day for the first two weeks of treatment; depending on individual response the dose was increased to $10 \mathrm{mg} / \mathrm{day}$. The second arm received only PPI $40 \mathrm{mg}$ once a day. Patients were assessed on the $4^{\text {th }}$ and $8^{\text {th }}$ week of treatment. The superiority was shown if there was more lasting decrease in heartburn severity, reduced anxiety (A), depression (D) and alexithymia levels. Results: Of 75 randomized patients 39 were allocated to the first arm and 36 to the second one. The groups were statistically comparable in age and sex. Treatment results showed reduction of heartburn severity in both groups on the $8^{\text {th }}$ week, however more significant in the first group (1group-89.7\%, 2 group-61.1\%, $\mathrm{P}<0.001)$. Also both $\mathrm{A}$ and $\mathrm{D}$ levels were much lower in the first group compared to the second one $(\mathrm{P}<0.001)$. Compering the levels alexithymia between two arms on the $8^{\text {th }}$ week after the initiation of treatment we found that alexithymic type of personality prevailed in patients that received PPI only $(\mathrm{P}<0.001)$

Conclusion: The combination of PPI plus antidepressant demonstrates superiority to PPI therapy along, showing more lasting symptoms regression and improved psychological and emotional condition of patients.

Disclosure of Interest: All authors have declared no conflicts of interest.

\section{P1860 QUALITY OF ESOPHAGEAL MUCOSAL HEALING IN EROSIVE REFLUX DISEASE: A RANDOMIZED COMPARATIVE TRIAL WITH LANSOPRAZOLE ALONE OR COMBINED WITH REBAMIPIDE}

K. Song ${ }^{1}$, Y.C. Lee ${ }^{2}$, S.J. Hong ${ }^{3}$, S.W. Jeon ${ }^{4}$

${ }^{1}$ Gastroenterology \& Hepatology, Konyang University Hospital, Deajon/Korea, Republic of

${ }^{2}$ Gastroenterology, Severance Hospital, Yonsei University College of Medicine,

Seoul/Korea, Republic of

${ }^{3}$ Soonchunhyang University Hospital, Bucheon/Korea, Republic of

${ }^{4}$ Kyungpook National University Hospital, Daegu/Korea, Republic of

Contact E-mail Address: postit2@daum.net

Introduction: The quality of histologic healing or inflammatory cytokine-related change in erosive reflux disease (ERD) had been verified in few studies. Rebamipide is an anti-inflammatory drug promoting gut mucosal healing.

Aims \& Methods: We conducted double-blinded comparative study to assess endoscopic, histologic quality of mucosal healing in ERD, following 4-week medical treatment with lansoprazole combined with rebamipide or not. The patients with ERD in modified LA classification grade A-D were enrolled via four referral institutes, regardless of GERD related symptoms. The enrolled subjects were randomly allocated to ingest $30 \mathrm{mg}$ lansoprazole alone or ingest lansoprazole $30 \mathrm{mg}$ with rebamipide $100 \mathrm{mg}$ three times in a day for 4 weeks. Baseline and follow up endoscopies were performed to assess endoscopic healing (regressed into minimal change or normal), and to obtain esophageal biopsy specimens (at 3 o'clock direction, $3 \mathrm{~cm}$ proximal area from squamocolumnar junction). Additional tissue samples were obtained to measure tissue inflammatory cytokines (IL-8, PAF, at 9 o'clock direction, $3 \mathrm{~cm}$ proximal area from squamocolumnar junction).

Results: Overall 109 patients were enrolled and randomly allocated to lansoprazole group $(\mathrm{N}=54)$ or combination group $(\mathrm{N}=55)$. Demographic data, smoking or drinking habits were not significantly different between both groups. The endoscopic healing rate at 4 -week was not different significantly $(75.0 \%$ vs $78.9 \% ; P=0.686$, Difference $3.95 \%, 95 \%$ CI $[-15.24,23.13])$. The histologic changes of basal layer thickness, intraepithelial infiltration of inflammatory cells (eosinophils, neutrophils) were normalized in subsets of patients regardless of the fate of endoscopic healing, and were not different in both groups. In contrast, papillary length, intraepithelial infiltration of $\mathrm{T}$ lymphocyte and the dilated intercellular space (DIS) was normalized significantly in endoscopically 
healed combination group. When both group were pooled in a group (proton pump inhibitor administration), the papillary length and DIS was significantly improved in endoscopically healed patients. Tissue level of IL-8, but not LysoPAF, was significantly decreased in lansoprazole alone group.

Conclusion: Adjuvant therapy of rebamipide to lansoprazole failed to reveal additional esophageal healing effect by endoscopic or histologic evaluation. Papillary length and DIS was more evident parameter of quality of mucosal healing in patient of erosive reflux disease treated with proton pump inhibitor. Long term follow-up data are needed on whether these histologic parameters of mucosal healing can help predict the prognosis of gastroesophageal reflux disease.

Disclosure of Interest: All authors have declared no conflicts of interest.

\section{References}

Gastroesophageal Reflux Might Cause Esophagitis Through a CytokineMediated Mechanism Rather Than Caustic Acid Injury, Souza RF et al. GASTROENTEROLOG Y 2009;137:1776-1784

The Benefits of Combination Therapy with Esomeprazole and Rebamipide in Symptom Improvement in Reflux Esophagitis: An International Multicenter Study. Hong SJ et al. Gut and Liver, Vol. 10, No. 6, November 2016, pp. 910-916

\section{P1861 LONG-TERM RESULTS OF RADIOFREQUENCY ABLATION (RFA) IN PATIENTS WITH BARRETT'S ESOPHAGUS RELATED NEOPLASIA}

J. Krajciova ${ }^{1}$, J. Maluskova ${ }^{2}$, M. Kollar ${ }^{2}$, Z. Vackova $^{1}$, J. Spicak ${ }^{1}$, J. Martinek ${ }^{1}$ Department Of Hepatogastroenterology, Institute for Clinical and Experimental Medicine, Prague/Czech Republic

${ }^{2}$ Clinical And Transplant Pathology, Institute for Clinical and Experimental

Medicine, Prague/Czech Republic

\section{Contact E-mail Address: kraj@ikem.cz}

Introduction: Radiofrequency ablation (RFA) with or without endoscopic resection (ER) is an established endoscopic treatment of early Barrett's esophagus (BE) related neoplasia (BORN). After successful treatment, follow-up is still required as recurrences may occur. The aim of this prospective single-center case series was to assess the long-term efficacy of endoscopic treatment (RFA with or without ER) for BORN. Main outcomes were complete remission of neoplasia $(\mathrm{CR}-\mathrm{N})$ and intestinal metaplasia (CR-IM) and recurrence of IM (RIM) and neoplasia (R-N).

Aims \& Methods: A total of 99 consecutive patients with BORN have been treated since 2009 . Of those, 87 patients ( 75 men, mean age 64 , range $22-91$ ) completed the treatment and were included into this analysis. The patients had been followed up during 296 patient-years (mean 3.4 years, range 0.5-6). Thirtythree patients were diagnosed with adenocarcinoma (38\%), 24 patients with highgrade dysplasia $(28 \%)$ and 30 patients with low-grade dysplasia $(34 \%)$. Prior to RFA, ER for visible lesions was performed in 57 patients $(66 \%)$. Mean length of the Barrett's esophagus (BE) was $4.6 \mathrm{~cm}$ (range $1-13 \mathrm{~cm}$ ). After treatment, the patients have undergone regular endoscopic surveillance with multiple biopsies. Results: Complete remission of IM (CR-IM) and complete remission of neoplasia (CR-N) were achieved in 54 patients (54/83 pts, 65.1\%; 95\% CI 54.3-74.5) and 82 patients $(98.8 \% ; 95 \%$ CI 92.8-99.9), respectively. All patients who did not achieve CR-IM had macroscopically normal neo-Z-line without visible abnormalities except one patient where macroscopic eradication of BE was not achieved due to giant hiatal hernia and who was referred for anti-reflux surgery. During the follow-up, 18 patients $(33.3 \%, 18 / 54$ pts) experienced a recurrence of IM and 3 patients $(3.7 \%, 3 / 82 \mathrm{pts})$ had a recurrence of neoplasia (LGD 2x, HGD 1x). We did not encounter any patient with a subsquamous neoplasia. All recurrences occurred at the level of neo-Z-line and 6 patients with recurrent IM had also macroscopic recurrence of $\mathrm{BE}$. A total of 13 patients underwent endoscopic retreatment: $7 \mathrm{x}$ probe-based re-RFA, 5x escape argon plasma coagulation (APC) and $1 x$ ER. After retreatment, we achieved 100\% CR-N and 54\% (7/13 pts) CR-IM. Treatment-related adverse events occurred in 22 patients $(25 \%)$ : 12x chest pain and 10x stricture. Two patients with a stricture had to undergo surgical resection - first patient due to perforation during balloon dilatation of a postRFA stricture, the second because of refractory post-RFA stricture after 20 sessions of dilatation.

Conclusion: RFA combined with ER for patients with BORN achieves a high success rate of CR-N with durable results. Recurrence of IM occurs in approximately one-third of patients and supports continuous endoscopic surveillance even after complete eradication. Nonetheless, the majority of recurrent IM occurs within a normally appearing neo-Z-line with questionable clinical relevance.

Disclosure of Interest: All authors have declared no conflicts of interest.
P1862 METHODS OF MEASURING BARRETT'S MUCOSAL THICKNESS WITH VOLUMETRIC LASER ENDOMICROSCOPY (VLE), AS A BIOMARKER TO GUIDE TO TREATMENT CHOICE

I. Levink ${ }^{1}$, G. Tearney ${ }^{2}$, H. C. Wolfsen ${ }^{1}$, S. Schlachter ${ }^{3}$, P.D. Siersema ${ }^{4}$, M. B. Wallace ${ }^{1}$

${ }_{1}^{1}$ Gastroeneterology, Mayo Clinic, Jacksonville/United States of America/FL

${ }^{2}$ Wellman Center Of Photomedicine, Massachussets general hospital, Boston/ United States of America/MA

${ }^{3}$ Ninepoint Medical, Boston/United States of America/MA

${ }^{4}$ Gastroenterology \& Hepatology, University Merdical Center Gastroenterology \&

Hepatology - Gastroenterology \& Hepatology, University, Nijmegen/Netherlands

Contact E-mail Address: irislevink@hotmail.com

Introduction: Barrett's Esophagus (BE) is a premalignant condition, in which prolonged gastroesophageal reflux results in intestinal metaplasia, leading to an annual risk of adenocarcinoma $0.12 \%$ year $^{1}$. The current standard of treatment is Endoscopic Resection (ER) of visible nodular lesions and Radiofrequency Ablation (RFA) of flat Barrett's, which has a success rate of $92 \%{ }^{2}$. For refractory cases, wide field ER, cryotherapy and other methods are used, but may have higher adverse events. We hypothesize that thicker Barrett's tissue is less likely to respond to RFA and have developed methods to precisely measure tissue thickness with Volumetric Laser Endomicroscopy (VLE). These methods may facilitate future studies, correlating tissue thickness with response to therapy and prediction of optimal treatment.

Aims \& Methods: We performed a nested cohort study from the U.S. VLE Registry (which comprises 1000 patients) of patients with BE, who had a baseline VLE scan, followed by RFA ablation and had at least one follow up exam. We excluded patients who had any ablative therapy prior to baseline VLE. The primary outcome was the percentage reduction of Prague length after the first treatment. Secondary outcomes were: 1 . complete remission of intestinal metaplasia (CRIM) during 12 months after baseline procedure, 2. complete remission of dysplasia (CRD) during 12 months after baseline procedure, and 3. number of RFA treatments necessary for complete response of intestinal metaplasia. We estimated the thickness of BE mucosal layers, by measuring the distance between the esophageal surface to the deepest edge of the lamina propria. In order to do so, we developed an algorithm (ImageJ software; imagej.nih.gov/ij/) that automatically adjusts every clockwise image into a high-resolution vertical scan with enhanced contrast. We used two measurement protocols: subjective, by drawing a line from the surface to the edge of the lamina propria (LP) and by plotting a grayscale density plot at the same location, using the sharp drop off in density to indicate the lamina propria.

Results: We included 92 patients with BE (at least C0M1), who had a baseline VLE scan, followed by RFA, and had no prior ablative therapy. These patients were divided in three patient groups: without prior EMR and treated with only RFA $(\mathrm{n}=70$, mean Prague length $(\mathrm{M})=5.399, \mathrm{SEM}=0.520)$, without prior EMR treated with combined EMR and RFA at baseline $(\mathrm{n}=3$, mean Prague length $(M)=6.67, \mathrm{SEM}=1.67)$, with prior EMR and RFA at baseline $(n=19$, mean Prague length $(M)=3.816, \mathrm{SEM}=0.616$ ). After determination of gastroesophageal junction (GEJ) location on VLE, we measured mucosal thickness in 8 segments at $0.5 \mathrm{~cm}$ intervals from GEJ to the top of the Barrett's tissue. The mean amount of selected cross sections per scan in the first 40 patients was 6.33 $(\mathrm{SEM}=0.50 ; \mathrm{n}=40)$. The measurements of thickness, using the subjective and objective protocol in one patient is shown in Table 1 .

Conclusion: We developed an algorithm, that automatically adjusts raw VLE images in order to recognize superficial layers of the esophageal wall and measure the thickness of these different layers in Barrett's tissue. We performed both subjective and objective methods for thickness calculation. This is the first step to the production of a biomarker for the prediction of treatment response in patients with Barrett's Esophagus. Further research is needed to demonstrate which of these two measurement protocols can predict the response to RFA and ER.

Disclosure of Interest: G. Tearney: Tearney G is the co-inventor of the VLE scan; he contributed VLE expertise to be able to do the measurements. The measurements itself were done by Iris Levink

S. Schlachter: Schlachter, $\mathrm{S}$ is an employee at Ninepoint Medical and manages the data of the Ninepoint registry. Schlachter, S had no influence on the outcomes of the measurements. The measurements were done by Levink, I.

All other authors have declared no conflicts of interest.

\section{References}

1. Hvid-Jensen F, Pedersen L, Drewes AM, et al. Incidence of adenocarcinoma among patients with Barrett's esophagus. N Engl J Med 2011;365:1375-83.

2. Fleischer DE, Overholt BF, Sharma VK, et al. Endoscopic radiofrequency ablation for Barrett's esophagus: 5-year outcomes from a prospective multicenter trial. Endoscopy 2010;42:781-9.

Abstract No: P1862

Table 1: The measurements of Barrett's thickness in one patient, using the two different measurement protocols.

\begin{tabular}{|c|c|c|c|c|c|c|c|c|}
\hline Pt. & $\begin{array}{l}\text { Age } \\
\text { (years) }\end{array}$ & SEX & BMI & $\begin{array}{l}\text { Highest grade } \\
\text { prior biopsy }\end{array}$ & $\begin{array}{l}\text { Prior } \\
\text { Treatment }\end{array}$ & $\begin{array}{l}\text { Prague Length, } \\
\text { circumferential and } \\
\text { maximum extend } \\
\text { in } \mathrm{cm}\end{array}$ & $\begin{array}{l}\text { Thickness } \\
\text { subjective measured, } \\
\text { pixels [SEM, number } \\
\text { of measurements] }\end{array}$ & $\begin{array}{l}\text { Thickness } \\
\text { objective measured, } \\
\text { pixels [SEM, number } \\
\text { of measurements] }\end{array}$ \\
\hline 1 & 85 & Male & 25.8 & High grade dysplasia & None & C16M16 & $317.38[9.96,65]$ & $286.85[8.96,65]$ \\
\hline
\end{tabular}


P1863 SEVEN-YEAR PROSPECTIVE FOLLOW-UP RESULTS OF RADIOFREQUENCY ABLATION FOR BARRETT'S ESOPHAGUS WITH HIGH-GRADE DYSPLASIA AND EARLY CANCER

K. Belghazi ${ }^{1}$, B.L.a.m. Weusten ${ }^{1}$, S. L. Meijer ${ }^{2}$, J.J. G.h.m. Bergman ${ }^{1}$, R. E. Pouw ${ }^{1}$

${ }^{1}$ Gastroenterology And Hepatology, Academic Medical Center, Amsterdam/ Netherlands

${ }^{2}$ Pathology, Academic Medical Center, Amsterdam/Netherlands

Contact E-mail Address: k.belghazi@amc.uva.nl

Introduction: Radiofrequency ablation (RFA) of Barrett's esophagus (BE), with or without prior endoscopic resection (ER) of focal lesions, results in complete eradication of intestinal metaplasia (CE-IM) and complete eradication of neoplasia (CE-neo) in $93-100 \%$ and $96-100 \%$, respectively.

Aims \& Methods: The aim of this study was to assess if the excellent results after successful RFA for BE with high-grade dysplasia (HGD) or early cancer (EC) are sustained on the long term. We screened all patients treated with RFA, and ER in case of visible lesions, for BE with histologically proven HGD/EC, who were previously enrolled in 5 consecutive cohort studies in a tertiary referral center in the Netherlands. All patients who had reached endoscopic and histologically confirmed CE-neo and CE-IM after RFA were included for evaluation of long-term follow-up (FU). Primary outcome: recurrence of $\mathrm{HGD} / \mathrm{EC}$; recurrence of endoscopically visible Barrett's mucosa. Secondary outcomes: Buried Barrett's glands; IM in biopsies obtained distal to a normal appearing neo squamocolumnar junction (neo-SCJ); need for retreatment; sustained CE-IM and CE-neo at last FU.

Results: 68 patients were included (55 men, median 64 yrs, median BE C5M6). In 53/68 patients ER was performed (worst pathology: low-grade dysplasia (LGD) $(\mathrm{n}=3)$, HGD $(\mathrm{n}=23)$, EC $(\mathrm{n}=27))$. Worst pathology pre-RFA (after any ER) was: non-dysplastic IM $(n=9)$, LGD $(n=27)$, HGD $(n=32)$. Median FU was 85 months (IQR 58-96) with a median of 7 FU endoscopies per patient. Recurrence of $\mathrm{HGD} / \mathrm{EC}$ was found in 2 patients $(3 \%)$ : one patient with a T1m2 EAC $3 \mathrm{~cm}$ above the neo-SCJ after 44 months and one patient had a visible lesion at the neo-SCJ with HGD after 22 months, both were treated successfully with ER. Recurrence of endoscopically visible Barrett's mucosa was seen in 22 patients $(32 \%)$ after a median of 20 months: small Barrett island $(n=10)$, BE tongue $(<1 \mathrm{~cm} \mathrm{n}=9, \leq 2 \mathrm{~cm} \mathrm{n}=1)$, circumferential $\mathrm{BE}<2 \mathrm{~cm}(\mathrm{n}=2)$. In 3 patients Buried Barrett's glands were detected (overall 3/448 FU endoscopies, 0.7\%). IM in a normal appearing neo-SCJ was found in 19 patients $(28 \%)$, and this was not reproduced in $84 \%$. In 2 patients LGD without IM was found in the neoSCJ. Eleven patients required retreatment: APC for small areas of visible Barrett's mucosa $(n=5)$, six patients had additional ER $(1 \mathrm{x}$ T1m2, 1x HGD, $2 \mathrm{x}$ LGD, $2 \mathrm{x}$ visible Barrett's islands), RFA for LGD without IM in the neo-SCJ $(\mathrm{n}=1)$. CE-neo and CE-IM (excluding IM in the neo-z-line) at the last FU endoscopy was seen in $100 \%$ and $96 \%$ respectively.

Conclusion: With 7-years of follow-up, this study presents the longest published follow-up data on RFA for BE with $\mathrm{HGD} / \mathrm{EC}$ to date. Our long-term outcomes show that after successful RFA recurrence of $\mathrm{HGD} / \mathrm{EC}$ is rare $(3 \%)$. Recurrence of endoscopically visible BE was found in $32 \%$ of patients, however it was confined to small islands or tongues $\leq 1 \mathrm{~cm}$ in the vast majority of patients.

Disclosure of Interest: B.L.A.M. Weusten: Financial support for research: Covidien/Medtronic, C2Therapeutic.

J.J.G.H.M. Bergman: Research support: Olympus, Fujifilm, Erbe, Ninepoint Medical ,C2 therapeutics, Cernostics, Interpace, Lumen-R, Medtronic, Boston Scientific. Speakers fee: Olympus, Fujifilm, WATTS-3D, Medtronic, Boston Scientific.

All other authors have declared no conflicts of interest.

\section{P1864 ACTION IS REQUIRED TO IMPROVE UNDERSTANDING AND REDUCE ANXIETY LEVELS AMONGST PATIENTS IN THE BARRETT'S SURVEILLANCE PROGRAMME}

V. Sehgal, A. Kumar, D. S. Kalsi, P. Jegatheesvaran, J. Walton, J. Sadeghian, S. Murray

Gastroenterology, Homerton University Hospital NHS Foundation Trust, London/ United Kingdom

\section{Contact E-mail Address: vsehgal@doctors.org.uk}

Introduction: Barrett's oesophagus (BE) is the only identifiable pre-cursor condition for oesophageal adenocarcinoma. Endoscopic surveillance is performed in $\mathrm{BE}$ to detect dysplasia as it likely to be amenable to curative therapy. There is data to suggest that a diagnosis of BE has a negative impact on the quality of life of patients. To our knowledge, no guidance exists on the counselling of patients entered into endoscopic surveillance for BE. The aim of this study was to check patient understanding of their diagnosis of BE and associated anxiety levels at a district general hospital.

Aims \& Methods: An in-house database was used to identify patients with BE over a 10-year period (2006-17). A simple, 14-point based questionnaire was devised and answers obtained via a telephone consultation (Table 1).

Results: 163 patients with BE were identified. In total, 104 patients ( 70 male, 34 female) were recruited ( 13 deceased, 38 did not answer, 8 declined). 2 patients had previously undergone therapy for BE (1 RFA, 1 fundoplication). Results are displayed in Table 1. Less than a third of patients remembered meeting a clinician to discuss their diagnosis and the rationale for follow-up. Consequently, only $41 \%$ of patients understood their diagnosis and $44 \%$ the rationale for surveillance. Although almost all patients $(92 \%)$ were on a regular proton pump inhibitor, less than half $(48 \%)$ understood why. Only $11 \%$ of patients were aware of the overall cancer progression risk and even fewer $(7 \%)$ of the treatment options that are currently available. Interestingly, half of all patients admitted feeling anxious about their diagnosis with the majority (82\%) admitting that further counselling would benefit in this regard.

Table 1: A 14-point based questionnaire which was used to check the understanding amongst patients with Barrett's Oesophagus (BE-Barrett's Oesophagus, OAC-Oesophageal adenocarcinoma PPI-Proton pump inhibitor).

\begin{tabular}{lcc}
\hline & Yes $(\%)$ & No $(\%)$ \\
\hline 1. Did you receive a letter or see someone in & $32(31)$ & $72(69)$
\end{tabular}

clinic to discuss your diagnosis and plans for $72(69)$ future follow-up?

2. If yes, did you understand this?

3. Broadly speaking, do you understand what

$\mathrm{BE}$ is?

4. Do you understand that chronic acid reflux into the lower oesophagus is the most likely cause of BE?

5. Are you on a regular PPI?

6. Do you know what the overall risk of progression to cancer is?

7. Are you aware of the term 'dysplasia' and how this helps to risk stratify your condition and interval length for surveillance endoscopy? 8. Do you understand what the rationale for endoscopic surveillance in BE is?

$96(92)$

9. Have you ever been told if you have a short or long segment of $\mathrm{BE}$ and the importance of this? 10. Are you aware of any treatment options for $\mathrm{BE}$ ?

11. If yes, do you know when this indicated?

12. Does or has anyone in your family suffered with $\mathrm{BE}$ or OAC?

13. Do you feel or have you ever felt anxious about your diagnosis of BE?

14. Do you think it would be useful for your understanding or reduce your anxiety if you either sat down with someone in clinic or spoke to someone over the phone regarding your BE?

\section{$6(6)$}

Conclusion: We have demonstrated that patients with BE have a relatively poor understanding of their diagnosis and the treatment options that are available to them. Further efforts need to be made to address this and help empower a group of patients who are understandably anxious about their diagnosis.

Disclosure of Interest: All authors have declared no conflicts of interest.

\section{P1865 BARRETT'S ESOPHAGUS IS ASSOCIATED WITH TOTAL SERUM ADIPONECTIN IN WOMEN, BUT NOT WITH OTHER INFLAMMATORY OR METABOLIC BIOMARKERS}

M. Jovani ${ }^{1}$, Y. Cao ${ }^{1}$, D. Feskanich ${ }^{2}$, B. C. Jacobson ${ }^{3}$, A. T. Chan ${ }^{1}$

${ }^{1}$ Clinical And Translational Epidemiology Unit, Massachusetts General Hospital, Boston/United States of America/MA

${ }^{2}$ Channing Division Of Network Medicine, Department Of Medicine, Brigham and Women's Hospital and Harvard Medical School, Boston/United States of America/ $M A$

${ }^{3}$ Boston University Medical Center and Boston University School of Medicine, Boston/United States of America/MA

Contact E-mail Address: manol.jovani@mail.harvard.edu

Introduction: Data on the association between inflammatory and metabolic biomarkers and Barrett's esophagus (BE) are scant and conflicting.

Aims \& Methods: We aimed to study the association between circulating inflammatory biomarkers (interleukin-6 [IL-6], high-resolution C-reactive protein [hrCRP], intra-cellular adhesion molecule [ICAM], tumor necrosis factor receptor-2 [TNF-R2]) and metabolic biomarkers (leptin, adiponectin, C-peptide, insulin-like growth factor 1 [IGF-1], and insulin-like grow factor binding proteins -1, -2 and -3 [IGFBP-1, -2 and -3$]$ ) with BE. This was a case-control study, nested within two female-only prospective cohort studies (Nurses' Health Study 1 and 2) and one male-only prospective cohort (Health Professional Follow-up Study). Participants of provided biennial detailed information on demographic, lifestyle, dietary and medical factors, including endoscopy use. Overall, 80,437 participants enrolled in these cohorts provided a prediagnostic blood specimen between 1989 and 1995. Among these participants, through 2012, we identified 283 cases of BE (163 females and 120 males). Two study physicians, blinded to biomarkers results, reviewed the medical records of patients reporting $\mathrm{BE}$. We matched $\mathrm{BE}$ participants with 626 controls who underwent upper endoscopy during the same time period and did not have BE (361 females and 265 males) on year of birth, year of blood collection, month of blood collection, fasting status and "am or pm" blood draw. We used multivariable conditional logistic regression models, adjusting for known and putative risk factors for BE, to assess the association between each biomarker and the risk of BE. We used the lowest quintile as reference, and assessed linear trend across exposure categories using the median of each quintile as a continuous variable.

Results: In women, plasma adiponectin was significantly associated with BE $\left(\mathrm{p}_{\text {-trend }}=0.01\right)$. When compared to the lowest quintile $\left(\mathrm{Q}_{1}\right)$, the the multivariate odds ratio $(\mathrm{OR})$ for the highest quintile $\left(\mathrm{Q}_{5}\right)$ of adiponectin was $0.39(95 \% \mathrm{CI}$ $0.17,0.88)$. This association was not materially altered after further adjustment 
for leptin. The association did not differ according to subgroups defined by BMI $\left(\mathrm{p}_{\text {-interaction }}=0.33\right)$ or regular aspirin use $(\mathrm{p}$ - interaction $=0.82)$. Among men, we observed that adiponectin was not associated with BE. However, we observed an OR of $0.41 ; 95 \%$ CI $0.17,0.99$ comparing extreme quintiles of IGFBP-3 $(\mathrm{p}$-trend $=0.11)$. Among both women and men, we did not observe any other significant associations between inflammatory or metabolic biomarkers and BE. Conclusion: There was little evidence that pre-diagnostic inflammatory or metabolic biomarkers are associated with risk of BE. Only adiponectin was associated with a decreased risk of $\mathrm{BE}$ in women but not in men. These findings may serve to provide input for clinical risk assessment for $\mathrm{BE}$ and shed light on potential mechanisms of its pathogenesis.

Disclosure of Interest: All authors have declared no conflicts of interest.

\section{P1866 OUTCOMES OF TREATMENT OF PATIENTS WITH EARLY- STAGE ADENOCARCINOMA OF THE ESOPHAGUS WITH INCIPIENT SUBMUCOSAL INVASION, RETROSPECTIVE ANALYSIS OF 19 CASES FROM A TERTIARY REFERRAL CENTER IN THE UK}

\section{B. Eross ${ }^{1}$, C. Clisby $^{2}$, C. Gordon ${ }^{1}$}

${ }^{1}$ Gastroenterology, Royal Bournemouth Hospital, Bournemouth/United Kingdom

${ }^{2}$ Endoscopy, Royal Bournemouth Hospital, Bournemouth/United Kingdom

\section{Contact E-mail Address: xerobal@yahoo.it}

Introduction: Endoscopic mucosal resection (EMR) is an established diagnostic and treatment tool in the management of Barrett's oesophagus (BO) with early neoplasia. However there is not enough clinical evidence on the management of the patients, in whom the EMR's histologic assessment identifies early-stage adenocarcinoma of the oesophagus with incipient submucosal invasion (pT1b $\mathrm{sm} 1)$.

Aims \& Methods: We have conducted a retrospective analysis using our electronic database for endoscopic procedures for patients with BO, who underwent EMR from October 2010 to December 2016. We investigated the size of the EMRs, the complication rates of the EMRs, the histological features and the resection margins of the EMR specimens and also the outcomes with the mortality.

Results: A total of 99 patients underwent 134 EMR procedures, and the histology identified early adenocarcinoma with incipient invasion of the submucosa in 25 patients. $23(92 \%)$ were male, the mean age at the EMR was 71 years (SD: 8.1). In all 25 EMRs $7(28 \%)$ patients had a single piece, $7(28 \%)$ patients a 2 piece, 7 $(28 \%)$ patients 3 piece and $4(16 \%)$ patients 4 piece EMR. The median length of the circumferential and maximum extent of the BO segments were 2 and $5 \mathrm{~cm}$ respectively (interquartile range (IQR) $2-4)$. We observed $6(24 \%)$ intra-procedural bleedings and $2(8 \%)$ patient needed admissions with post procedural bleeding and $1(4 \%)$ of them required transfusion. Stricture was endoscopically detectable but not causing any symptoms in $1(4 \%)$ patient and another patient (4\%) had slight dysphagia post EMR, but did not need dilation. Histology showed lymphovascular invasion in $6(24 \%)$ patients and vascular invasion in $1(4 \%)$ patient. Of all 25 early adenocarcinomas $7(28 \%)$ were reported as poorly differentiated, $11(44 \%)$ as moderately differentiated and $3(12 \%)$ as well differentiated. In $4(16 \%)$ cases differentiation was not reported. All resection margins were reported as being clear from dysplasia or cancer in $7(28 \%)$ cases, radial resection margins were reported with dysplasia in $3(12 \%)$ cases and with cancer in $15(60 \%)$ cases, but this included the multiple piece EMRs. The deep margin was reported as being clear in $18(72 \%)$ cases, with dysplasia in $1(4 \%)$ case and with cancer in $6(25 \%)$ cases. There were $15(60 \%)$ patients with cancer invasion on the radial and/or deep margin of the EMR specimen, of these patients $9(60 \%)$ had oesophagectomy and in the histologic assessment of these specimens, lymph node involvement was observed in 2 cases $(22.2 \%$ of all oesophagectomies and $9.5 \%$ of all surviving and currently cancer-free patients). There was no residual cancer in $3(33.3 \%)$ of the surgical specimens. Radical radio-chemotherapy was given in $1(6.7 \%)$ patient and $5(33.3 \%)$ patients did not have radical treatment for clinical reasons. There were $10(40 \%)$ patients without cancer invasion of the EMR resection margins, of these $4(40 \%)$ had oesophagectomy and $1(10 \%)$ radical radio-chemotherapy. The histologic assessment of these surgical specimens showed residual cancer in $3(30 \%)$ cases and high-grade dysplasia in 1 $(10 \%)$ case. Of the 25 patients $5(20 \%)$ met the criteria and had radio frequency ablation of the residual Barrett's oesophagus. Of the $13(52 \%)$ patients who have had oesophagectomy $1(7.7 \%)$ patient died of the deterioration precipitated by the oesophagectomy, and sadly in this case the oesophagectomy specimen did not show any residual cancer. Of the $12(48 \%)$ patients who have not had oesophagectomy $3(25 \%)$ died since their EMR, $1(8.3 \%)$ of cardiac arrest, $1(8.3 \%)$ of chronic obstructive pulmonary disease and $1(8.3 \%)$ of advanced oesophageal cancer, 18 months after the EMR, and the $9(75 \%)$ surviving patient are all cancer free on follow up investigations, one after radical chemo/radiotherapy. The median survival of all $21(84 \%)$ patients currently alive is 25 months (range: 2-68 months; SD: 22.2).

Conclusion: In this retrospective analysis we have found that the clinical outcomes are very difficult to predict for patients with early adenocarcinoma and incipient invasion of the submucosa. Clinical decision making remains very challenging and has to be individualised for all patient, until further in depth studies gives us more useful prognostic factors.

Disclosure of Interest: All authors have declared no conflicts of interest.
P1867 THE USE OF ENDOCYSTOSCOPY FOR THE EARLY DETECTION OF ESOPHAGEAL NEOPLASM

S. M. Chan ${ }^{1}$, P.W.Y. Chiu ${ }^{2}$, A. Y.B. Teoh ${ }^{1}$, H.C. Yip ${ }^{1}$, V. Wong ${ }^{1}$, E. Ng${ }^{1}$, J.Y. Lau $^{2}$

${ }^{1}$ Surgery, The Chinese University of Hong Kong, HK/Hong Kong PRC

${ }^{2}$ Institute of Digestive Disease Room $940207 / F$, Lui Che Woo Clinical Sciences Building, Prince of Wales Hospital, Shatin, N.T., Hong Kong, Hong Kong/Hong Kong PRC

Contact E-mail Address: shannonchan@surgery.cuhk.edu.hk

Results: From July 2015 to March 2017, forty-four patients were included in the study. Seventeen of the forty-four $(38.6 \%)$ patients had histological confirmed cancer of the esophagus. There were sixteen patients who had normal finding and nine patients with esophagitis. The positive predictive value for malignancy (ECA 4 and 5) was $89.5 \%$; the negative predictive value was $100 \%$. Sensitivity was $100 \%$ and specificity was $92.6 \%$. Similar findings were noted with IPCL on magnifying NBI. The positive predictive value for malignancy (IPCL 4 and 5) was $89.5 \%$; negative predictive value was $100 \%$. Sensitivity and specificity were also similar at $100 \%$ and specificity $92.6 \%$ respectively. To compare the diagnostic accurary of endocystoscopy and magnifying NBI, the McNemar test was performed. The McNemar chi-squared statistic is NaN, and the McNemar chisquared statistics with Yates correction of 0.5 is infinity, meaning that the two tests have the same diagnostic accurary.

Conclusion: Endocystoscopy had a high positive predictive value and sensitivity for esophageal malignancy. Its diagnostic accuracy was comparable to magnifying NBI. It may be helpful as an adjunct for better characterization of esophageal lesions. However, further studies on interobserver variability is required.

Introduction: Early detection of esophageal cancers can significantly reduce the morbidity and improve the prognosis of these patients. A new prototype scope incorporating the endocystoscopy function into a magnifying endoscope has been designed. Previously, Inoue et al have published a pilot trial on evaluating the use of this endocystoscope in various types of benign and malignant pathology in the esophagus. An endocytoscopic atypia (ECA) classification was proposed. The sensitivity and specificity of this classification system was evaluated. Aims \& Methods: All consecutive patients who had esophagogastroduodenoscopy (EGD) arranged for screening of the esophagus during the period July 2015 to March 2017 were recruited into the study. EGD with narrow band imaging and endoscocystoscopy were performed in these patients. During the procedure, the esophageal mucosa was stained with $0.5 \%$ methylene blue and then with crystal violet. The endocystoscopic findings were graded from 1 to 5 according the Inoue et al's ECA classification. The esophageal mucosa was also evaluated with narrow band imaging (NBI) and the findings were classified according to the Intrapapillary capillary loop (IPCL) pattern classification. These findings were compared against the gold standard of histopathological diagnosis which was based on the Vienna classification.

Disclosure of Interest: All authors have declared no conflicts of interest.

\section{Reference}

Inoue H, Sasajima K, Kaga M et al. Endoscopic in vivo evaluation of tissue atypia in the esophagus using a newly designed integrated endocystoscope: a pilot study. Endoscopy 2006; 38(9):891-895.

\section{P1868 CLINICAL OUTCOMES OF ENDOSCOPIC RESECTION FOR ACHALASIA-ASSOCIATED SUPERFICIAL ESOPHAGEAL CANCER}

Y. Nishikawa, K. Goda, S. Kono, K. Sumi, M. Ominami, Y. Ikebuchi, I. Haruo, M. Onimaru, H. Ito, H. Inoue

Gastroenterology, Showa University Koto-Toyosu Hospital, Tokyo/Japan

Contact E-mail Address: nishikawa6@med.showa-u.ac.jp

Introduction: Esophageal achalasia is considered to be a high-risk factor for superficial esophageal cancer. But there are few reports of endoscopic resection for this cancer, and the outcome is unclear. In our hospital, we have performed over 1300 Per-Oral Endoscopic Myotomy procedures for esophageal achalasia and related diseases. In this process, we diagnosed 10 superficial esophageal cancers in patients with achalasia. We performed endoscopic resection for all cases and report relatively long-term outcome.

Aims \& Methods: We aimed to evaluate clinicopathological findings and outcomes of endoscopic resection for 10 achalasia-associated superficial esophageal cancer. This is a case series study at our hospital. Between August 2010 and February 2017, 10 achalasia patients with superficial esophageal cancer underwent endoscopic resection. We performed in all cases upper gastrointestinal endoscopy using lugol's solution and narrow band imaging, and we included all patients that had early cancers that were eligible for endoscopic resection in this series. At 2 and 12 months after treatment, we performed follow-up endoscopy in all cases. After this, we performed long-term endoscopic follow-up every year. In the case that the tumor invasion depth is to the muscularis mucosa(MM) on histopathology, we performed endoscopic follow-up every 6 months and CT scan.

Results: There were 6 men and 4 women and their average age was 61.7 years. 8 patients were diagnosed with lesions before POEM. We performed endoscopic resection $(\mathrm{ESD} / \mathrm{EMR} ; 9 / 1)$ in all cases after POEM. None of the patients had a severe adverse event. The mean tumor diameter was $30 \mathrm{~mm}$ (range: $5-80 \mathrm{~mm}$ ). The pathological diagnosis was 8 SCC, 2 high grade intraepithelial neoplasia. Out of the SCC cases, 7 were found with superficial lesion with depth of Tis-EP to T1a-LPM, and 1 with depth of T1a-MM, without lymphatic invasion(ly0) or venous invasion(v0). Follow-up surveillance mean term was 32 months (range: 1- 
67 months). Ectopic lesions were detected in 2 cases, and these patients underwent endoscopic resection. All patients survived in this term.

Conclusion: Endoscopic resection for achalasia-associated superficial esophageal cancer invasive to the MM on histopathology is safe and effective, and relatively long term outcome is good.

Disclosure of Interest: All authors have declared no conflicts of interest.

\section{P1869 USEFULNESS OF TRIAMCINOLONE INJECTION TO PREVENT STRICTURE AFTER CIRCUMFERENTIAL ESOPHAGEAL ESD FOR SCC AND EAC}

T. Oyama ${ }^{1}$, A. Takahashi ${ }^{1}$

${ }^{1}$ Endoscopy, Saku Central Hospital Advansed Care Center, Nagano/Japan

Contact E-mail Address: oyama@coral.ocn.ne.jp

Introduction: ESD is a standard treatment for superficial esophageal cancer in Japan. Stricture is one of important complication of esophageal ESD, and it makes quality of life of the patient worse. Usefulness of Triamcinolone (TA) injection to prevent stricture after semi-circumferential ESD has been reported. However, usefulness of TA injection after circumferential esophageal ESD is still unclear.

Aims \& Methods: The aim of this study is to clarify the usefulness of triamcinolone injection to prevent stricture after circumferential ESD. A total of forty-four patients treated by circumferential esophageal ESD from 2004 to 2016 in Saku Central Hospital Advanced Care Center were enrolled to this retrospective study. The patients treated from 2004 to 2009 were followed up without TA injection (Non-TA group). And, local injection of TA was performed for the patients after 2009 (TA group). The number of patient in Non-TA and TA group was 16 and 28 , respectively. Age of both groups was $65(30-83)$ and 61 (42-82) years old. The length of circumferential resection was $75(50-10)$ and $76(55-111) \mathrm{mm}$, respectively. There was no significant difference in the background of both groups. Fifty mg TA was injected into submucosal layer just after ESD, and TA injection was repeated in two-weeks interval by the ESD ulcer healed. When the length of circumferential ESD was $50 \mathrm{~mm}$ or longer, $100 \mathrm{mg}$ Triamcinolone was injected just after ESD, and $50 \mathrm{mg}$ TA injection was repeated in two-weeks interval. Fifteen $\mathrm{mm}$ endoscopic balloon dilatation (EBD) was performed when the scope couldn't pass the ESD ulcer. The primary endpoint was the number of balloon dilatation. The secondary endpoints were duration from ESD to ulcer healing, complications and the difference between Barrett's esophageal adenocarcinoma (EAC) and squamous cell carcinoma (SCC).

Results: 1. Number of EBD in Non-TA and TA group were 20 (13-33) and 5.1 $(0-23)$, respectively $(\mathrm{p}<0.01) 2$. Duration from ESD to ulcer healing were 10 (3$23)$ and $6.9(1-24)$ months. respectively. $(\mathrm{p}=0.47) 3$. Complications: Perforation rate due to EBD was $6.3 \%(1 / 16)$ and $3.6 \%(1 / 28)$. Both patients were treated by conservative therapy. 4. Difference between EAC and SCC: There were four EAC and 24 SCC patients in TA group. The number of EBD were $5.3(5-11)$ and $5.4(3-11)$, respectively. Duration of ulcer healing were 10 (4-14) and $6.5(1-$ $24)$, respectively. There was no significant difference between two groups. Conclusion: Triamcinolone injection is safe and effective treatment to prevent stricture after circumferential ESD, not only for SCC but also for EAC. Disclosure of Interest: All authors have declared no conflicts of interest.

\section{P1870 OUTCOME OF ENDOSCOPIC SUBMUCOSAL DISSECTION FOR SUPERFICIAL PHARYNGEAL SQUAMOUS CELL CARCINOMA}

T. Oyama ${ }^{1}$, A. Takahashi ${ }^{1}$

${ }^{1}$ Endoscopy, Saku Central Hospital Advansed Care Center, Nagano/Japan

\section{Contact E-mail Address: aurevoireurope $@$ yahoo.co.jp}

Introduction: Superficial pharyngeal squamous cell carcinoma (SCC) has been increasing in Japan. And, such SCC could be treated by endoscopic submucosal dissection (ESD). However, the outcome of pharyngeal ESD is unknown.

Aims \& Methods: The aim of this study is to clarify the outcome and prognosis of pharyngeal SCC treated by ESD. 89 pharyngeal SCC in 68 patients treated by ESD from Jan. 2006 to Jan. 2017 in Saku Central Hospital Advanced Care Center were enrolled to this retrospective study. CT and neck US were performed for the preoperative staging. All ESD were performed using a Hook knife under general anesthesia with tracheal intubation. Clip with line or forceps was used to make traction during ESD. Annual endoscopy, CT scan and neck US were performed as surveillance after ESD, and these examinations were recommended twice a year for the patients who had subepithelial (SEP) SCC. Male/Female was $67 / 1$. Mean age was $67.1(40-92)$. Mean size of tumor and specimen were 12.9 (1$50)$ and $26.8(5-65) \mathrm{mm}$, respectively.

Results: 1. En bloc resection rate was $100 \%$. 2. Complication: Delayed bleeding rate was $1.5 \%(1 / 68)$. Re-intubation was needed for the hemostasis. Dysphasia was shown in $4.4 \%(3 / 68)$. Two patients had mild dysphagia and improved in two weeks. The remaining one patient had severe dysphagia. The patient had a big SCC, $50 \mathrm{~mm}$ in size, and the SCC extend from light piriform sinus to aryepiglotic fold. The patient could keep vocal function. However, eating was impossible for mis-swallowing. 3. Invasion depth: Epithelial (EP) and subepithelial (SEP) SCC were 60\% (53/89) and 39\% (35/89), respectively. One case was inflammation. 4. The rate of lymph-duct involvement of EP and SEP were $0 \%$ and $5.7 \%(2 / 35)$, respectively. LNM was found in one of two patients who had lymph duct involvement, and treated by lymph node dissection. The other patient was followed up without additional therapy, and free from LNM for one year. 5 . Venous involvement of EP and SEP were $0 \%$ and $2.9 \%(1 / 35)$, respectively. The only patient who had venous involvement was 92 years old, and followed up without an additional therapy. 6. Local recurrent rate was $0 \%, 7$. LNM rate of EP and SEP was $0 \%$ and $5.7 \%(2 / 35)$, respectively. One of two patients who had LNM was a 76-year-old male. He had SEP SCC without lymph duct involvement. A cervical LNM was diagnosed 6 months after ESD. Lymph node dissection and chemo-radio therapy (CRT) was performed for the patient. The patient died of other disease without recurrence of pharyngeal SCC. The other patient also had a cervical LNM and treated by lymph node dissection + CRT. The patient is alive without recurrence for 10 years after ESD. 8. Prognosis No patient died of pharyngeal SCC after ESD.

Conclusion: ESD is a safe and useful treatment for superficial pharyngeal SCC. However, surveillance of LNM is important for the patients who had SEP SCC. Disclosure of Interest: All authors have declared no conflicts of interest.

\section{P1871 SHOULD ANTITHROMBOTIC AGENTS BE DISCONTINUED PRIOR TO ESOPHAGEAL ENDOSCOPIC SUBMUCOSAL DISSECTION?}

N. Kawata, M. Tanaka, K. Takizawa, N. Kakushima, M. Yoshida, S. Ito, K. Imai, K. Hotta, H. Ishiwatari, H. Matsubayashi, H. Ono Division Of Endoscopy, Shizuoka Cancer Center, Shizuoka/Japan

Contact E-mail Address: n.kawata@scchr.jp

Introduction: Endoscopic submucosal dissection (ESD) has been widely performed as a minimally invasive treatment for superficial esophageal squamous cell neoplasms (ESCNs) in Japan and Asian countries. According to the current guidelines[1], ESD is classified as a high bleeding risk procedure. However, these guidelines have not been fully validated.

Aims \& Methods: The aim of this study was to identify the risk factors of bleeding associated with esophageal ESD, and to clarify whether antithrombotic agents increase the risk of bleeding for esophageal ESD. A total of 458 ESCNs in 411 patients who underwent ESD at our hospital from January 2013 to October 2016 were analyzed in this retrospective study. ESD was performed using an IT knife nano (Olympus, Tokyo, Japan). Hemostatic forceps (Coagrasper; Olympus, Tokyo, Japan) were used when hemostasis during ESD proved difficult with the IT knife nano. Longer hemostatic time during ESD was defined as more than 120 secs required for hemostasis with hemostatic forceps. We analyzed the relationship between risk factors for longer hemostatic time during ESD and the following factors using univariate and multivariate analyses: age $(<75$ or $\geq 75$ years), sex, body mass index $(<25$ or $\geq 25)$, treatment for synchronous multiple ESCNs, previous radiation therapy, antithrombotic agents, lesion location (upper or middle or lower), lesion size $(<2 \mathrm{~cm}$ or $\geq 2 \mathrm{~cm})$, lesion circumference $(<3 / 4$ or $\geq 3 / 4)$, and the endoscopist's experience of esophageal ESD $(<40$ or $\geq 40$ procedures).

Results: Twenty-nine ESCNs $(6 \%)$ were treated by ESD without discontinuation of antithrombotic agents. Hemostatic forceps were used for 116 lesions (25\%), median forceps use time was 73 secs (range: $8-1200$ secs), and the median number of forceps application during procedures was 2 (range: 1-9 times). Of these, 41 lesions $(9 \%)$ met our definition for longer hemostatic time. Univariate analysis revealed that lesion size $(\geq 2 \mathrm{~cm})$, lesion circumference $(\geq 3 / 4)$, and the endoscopist's experience $(\geq 40)$ were significantly associated with longer hemostatic time. Multivariate logistic regression analysis revealed that a lesion size $\geq 2 \mathrm{~cm}$ (OR 2.4 [95\% CI 1.1-5.1], $P=0.02$ ) was an independent risk factor for longer hemostatic time. Postoperative bleeding occurred 20 days after ESD in one patient $(0.2 \%)$ receiving the continuous administration of warfarin and aspirin.

Conclusion: Our results suggest that continuous use of antithrombotic agents does not increase the risk of bleeding during esophageal ESD, and that postoperative bleeding was a rare occurrence. Discontinuation of antithrombotic agents may therefore not be necessary prior to esophageal ESD

Disclosure of Interest: All authors have declared no conflicts of interest.

\section{Reference}

1. Veitch AM, Vanbiervliet G, Gershlick AH et al. Endoscopy in patients on antiplatelet or anticoagulant therapy, including direct oral anticoagulants: British Society of Gastroenterology (BSG) and European Society of Gastrointestinal Endoscopy (ESGE) guidelines. Endoscopy 2016; 48: 385-402

\section{P1872 EXPLORATORY STUDY OF PREDICTIVE BIOMARKER FOR DEFINITIVE CHEMORADIOTHERAPY USING BIOPSY SPECIMENS OF PATIENTS WITH ESOPHAGEAL SQUAMOUS CELL} CARCINOMA

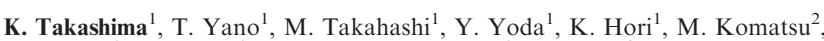

T. Kojima ${ }^{3}$, H. Daiko ${ }^{4}$, K. Minashi ${ }^{5}$, M. Muto ${ }^{6}$, H. Sasaki $^{2}$, S. Fujii

${ }^{1}$ Gastroenterology And Endoscopy, National Cancer Center Hospital East, Kashiwa/Japan

${ }^{2}$ Translational Oncology, National Cancer Center Research, Chuo-ku/Japan ${ }^{3}$ Gastroenterology And Oncology, National Cancer Center Hospital East, kashiwa/ Japan

${ }^{4}$ Esophageal Surgery, National Cancer Center Hospital East, Kashiwa/Japan

${ }^{5}$ Clinical Trial Promotion, Chiba Cancer Center, Chiba/Japan

${ }^{6}$ Therapeutic Oncology, Kyoto University, Kyoto/Japan

${ }^{7}$ Pathology, Exploratory Oncology Research And Clinical Trial Center, National

Cancer Center Hospital East, Kashiwa/Japan

Contact E-mail Address: ktakashi@east.ncc.go.jp 
Introduction: While definitive chemoradiotherapy (CRT) showed high efficacy for esophageal squamous cell carcinoma (ESCC), approximately $40 \%$ of patients develop local failure, resulting in poor long-term survival ${ }^{1}$. However, there is no definitive biomarker which is useful to predict survival outcome after CRT for ESCC. Several studies have investigated the correlation of expression of CD24, cytokeratin 4 (CK4), or podoplanin (PDPN) with prognosis of patients with various malignant tumors who underwent surgical resection ${ }^{2,3}$. However, it remains unclear whether the expression of these proteins can predict the outcome of CRT for patients with ESCC.

Aims \& Methods: The aim of this study was to clarify the predictive values of expression of CD24, CK4, and PDPN for ESCC patients who received CRT. Among patients with ESCC who received CRT or curative esophagectomy with extended lymph node dissection (OPE) as an initial treatment between 2005 and 2009 at our institution, cohorts were selected based on the following criteria: clinical stage II, III (UICC-TNM classification 6th editon), age of 75 years old or younger, ECOG Performance Status $0-1$, and no prior or concurrent other cancers. The method of immunohistochemistry (IHC) was utilized to examine the protein expression of CD24, CK4, and PDPN in pretreatment biopsy specimens of ESCC. The cut-off values for CD24, CK4, and PDPN expression were used hazard ratio for overall survival (OS). The prognostic factor of CD24, CK4, and PDPN expression were statistically analyzed. OS was calculated from the date of CRT or OPE to the date of death or last follow-up, using the Kaplan-Meier method. The survival predictors identified by univariate analysis was assessed by multivariate analysis using a Cox's proportional hazards model.

Results: 148 ESCC patients (CRT group, $n=83$; OPE group, $n=65$ ) were analyzed. In the CRT group, 40 patients had stage II and 43 patients had stage III, and the 5-year OS was 52\%. In the OPE group, 32 patients had stage II and 33 patients had stage III, and the 5-year OS was $66 \%$. By univariate analysis, there were no significant clinical variables for OS in differences between the CRT and OPE group. The cut off value for CD24, CK4, and PDPN expression were $20 \%$, $10 \%$, and $20 \%$, respectively. While the expression equal to the cut off value or more was defined as strong, the expression less than the cut off value was defined as weak. The frequency of strong protein expression was $50 \%$ for CD24, $12 \%$ for CK4, 65\% for PDPN, respectively. In the CRT group, the OS of patients with strong CD24 expression was significantly better than that of patients with weak CD24 expression $(P=0.015$; strong/weak 5 -year: OS $65 \% / 43 \%)$. On the other hand, patients with strong CD 24 expression was poorer OS comparing with patients with weak expression in the OPE group, however there was no significant difference $(\mathrm{P}=0.286$; strong/weak 5 -year OS: $57 \% / 74 \%)$. As for patients with strong CD24 expression, there was no significant difference between CRT group and OPE group $(\mathrm{p}=0.446)$, however there was significant difference between CRT and OPE group in patients with weak CD 24 expression $(p=0.009)$. There were also no significant differences of the OS based on expression of CK4 or PDPN between the CRT and OPE group, respectively. Multivariate analyses revealed the strong CD24 expression in the CRT group $(\mathrm{P}=0.012 ; \mathrm{HR}=2.787 ; 95 \% \mathrm{CI}: 1.253-6.200)$ as an independent variable for favorable outcome.

Conclusion: CD24 expression was significantly associated with the survival outcome of patients with ESCC when they were treated with CRT. Furthermore, weak CD24 expression might be a useful predictive biomarker of poor outcome for CRT in ESCC patients.

Disclosure of Interest: All authors have declared no conflicts of interest.

\section{References}

1. Kato, K, et al. Int J radial Oncol Biol Phys, 1:81(3):684-90, 2011

2. Sano A, et al. Ann Surg Oncol. 16:506-514, 2009

3. Tanaka M, et al. Molecular Medicine reports 12: 4029-4036, 2015

\section{P1873 ENDOSCOPIC TREATMENT OF PATIENTS WITH HIGH- RISK EARLY ESOPHAGEAL CANCER}

M. Kollar ${ }^{1}$, J. Krajciova ${ }^{2}$, J. Maluskova ${ }^{1}$, E. Honsova ${ }^{3}$, A. Pazdro ${ }^{4}$, T. Harustiak ${ }^{4}$, D. Kodetova ${ }^{5}$, Z. Vackova ${ }^{2}$, J. Spicak ${ }^{2}$, J. Martinek ${ }^{2}$ ${ }^{1}$ Clinical And Transplant Pathology, Institute for Clinical and Experimental Medicine, Prague/Czech Republic

${ }^{2}$ Department Of Hepatogastroenterology, Institute for Clinical and Experimental Medicine, Prague/Czech Republic

${ }^{3}$ Institute for Clinical and Experimental Medicine, Prague/Czech Republic

${ }^{4}$ Third Department Of Surgery, 1st Faculty of Medicine, Charles University in Prague and Motol, Praha/Czech Republic

${ }^{5}$ Department Of Pathology And Molecular Medicine, University Motol Hospital, Prague, Praha/Czech Republic

Contact E-mail Address: marek.kollar1@seznam.cz

Introduction: Endoscopic treatment is a standard therapeutic approach for patients with Tla early esophageal cancer (EEC). In patients with 'high-risk' Tla cancer (e.g. advanced grading or invasion to blood/lymphatic vessels) and in patients with any submucosal (sm) invasion (T1b), surgery is recommended as a standard of care. However, recent data suggest, that endoscopic treatment might be curative in selected patients with 'high-risk' EEC

Aims \& Methods: The aim of this study was to assess outcomes of endoscopic treatment in patients with 'high-risk' EEC. 'High-risk' cancer was defined as any cancer with sm invasion or mucosal cancer with at least one of the following: poor differentiation $(\mathrm{G} 3 / \mathrm{G} 4)$, invasion to blood $(\mathrm{A}+)$ or lymphatic vessels $(\mathrm{L}+)$ and high tumor cell dissociation (TCD3). The main outcome measurement was tumor-free survival.

A single-center, retrospective analysis of prospectively collected data. Patients with EEC underwent endoscopic resection (ER) or endoscopic submucosal dissection (ESD). Based on histopathological staging, patients with 'high-risk' EEC without contraindications were referred for surgery. Remaining patients continued in endoscopic treatment consisting of further sessions of ER and/or radiofrequency ablation if necessary. The patients have been followed up for a median of 39 months (range 2-156).

Results: A total of 56 patients with 'high-risk' EEC underwent endoscopic treatment: 21 patients $(41 \%)$ had T1a cancer with 'high-risk' features and 35 patients $(59 \%)$ had T1b cancer with sm invasion (sm1: 15, sm2: 9, sm3: 11); 45 patients had adenocarcinoma (EAC), 11 patients had squamous carcinoma (SCC); 19 patients were referred for surgery. A total of 37 patients $(66 \%)$ continued in endoscopic treatment. Complete local remission (CLR) of neoplasia was achieved in $35 / 37$ patients $(95 \%)$ ). Two patients without CLR continued endoscopic therapy with palliative intent. Tumor generalization occurred in 2 patients (one of them achieved CLR) 24 months after endoscopic treatment (both patients had sm 3 invasion, $\mathrm{A}+, \mathrm{L}+$ ) and these patients are undergoing oncological treatment. All remaining patients with CLR $(n=33)$ have experienced neither local relapse nor generalization. One patient had to undergo surgery due to endoscopy related perforation. Tumor-free survival was $89 \%$ (CI 79-99\%) in patients treated endoscopically and endoscopy related mortality was $0 \%(0 / 37)$. Among 19 patients who were referred for esophagectomy, one patient presented with tumor generalization revealed during the operation. The remaining 18 patients underwent esophagectomy; local residua of malignancy were present in $5 / 18$ patients $(28 \%$,$) . Lymph node (LN) metastases have not been detected in any patient$ among the 337 examined LNs. Surgery related mortality was $6 \%(1 / 18)$.

Conclusion: Endoscopic treatment provides long-term remission or cure in a considerable number of patients with 'high-risk' EEC and it may thus represent a valid alternative to surgery. Broadening of indications for radical endoscopic treatment of early EEC should be reconsidered.

Disclosure of Interest: All authors have declared no conflicts of interest.

\section{P1874 DIAGNOSTIC ACCURACY OF ENDOSCOPIC} ULTRASONOGRAPHY FOR ESOPHAGEAL SUBMUCOSAL GLAND DUCT INVOLVEMENT ACCOMPANIED BY EARLY ESOPHAGEAL CANCER

Y. Jie, L. Ying

Digestive Department, TheAffiliated Drum Tower Hospital, Nanjing University

Medical School, Nanjing, Jiangsu Province, Ch, Nanjing/China

Contact E-mail Address: 13770755008@126.com

Introduction: Normally, resided within the submucosal layer of esophagus, each esophageal submucosal gland will culminate in a single duct. The esophageal submucosal gland ducts (ESMGDs) can traverse the subepithelial connective tissue and muscularis mucosa, and deliver the acinar serections to the esophageal lumen. However, the clinicopathological features of the esophageal submucosal gland duct involvement (ESMGDI) and its precursor lesion have not been comprehensively evaluated so far, and the series study focusing on endoscopic features of this lesion has not been reported widely. While since the 1990s, the esophageal lesions presumed to originate from ESMGDs had been described constantly in various case reports. Currently, in addition to the gold standard of histopathology, almost no more useful modality could be applied to this lesion. In our study, we considered that the ESMGDI had a correlation with early esophageal cancer, and we noted that the ESMGDI had special features under the endoscopic ultrasonography (EUS). The typical ultrasonic images of EUS could help diagnose ESMGDI.

Aims \& Methods: In order to investigate the clinical value of EUS for diagnosing ESMGDI accompanied by early esophageal cancer, which were suggested by conventional endoscopy or biopsy, this study retrospectively analyzed the consecutive patients with early esophageal cancer diagnosed in the Endoscopy Center at the Affiliated Drum Tower Hospital, Nanjing, China from September 2009 to November 2016. The clinical data of 519 patients were included in this study, and all of them had already underwent EUS combined with Endoscopic Submucosal Dissection(ESD). The EUS preoperative diagnosis were compared with the results of postoperative pathology from ESD.

Results: According to the pathological results, all patients (371 males and 148 females, with a mean age of $67.5 \pm 4$.5years) had been diagnosed with early esophageal cancer with different invasive depth. Out of 519 patients, about 478 patients were not found ESMGDI by both examinations. Besides, postoperative pathology confirmed that 40 patients were identified with ESMGDI, 34 patients of which were completely consistent with the preoperative diagnosis of EUS Approximately $98.7 \%(512 / 519)$ of ESMGDI were diagnosed exactly by EUS. Another six cases were estimated as flase negative inaccurately, including two squamous cell carcinoma and four high-grade intraepithelial neoplasia. One case was regarded as ESMGDI by EUS while confirmed not by pathology. Therefore, the EUS values for sensitivity and specificity for the diagnosis of ESMGDI were $85.0 \%(34 / 40)$ and $99.8 \%(478 / 479)$ respectively. Furthermore, the positive predictive value was $97.1 \%(34 / 35)$, and the negative predictive value was $98.8 \%$ (478/484).

Conclusion: The esophageal submucosal gland duct involvement is a kind of lesion performed as a hypoechoic sonographic pattern located in the thickened mucosa. EUS has a satisfactory diagnostic accuracy for ESMGDI as well as good sensitivity and specificity.

Disclosure of Interest: All authors have declared no conflicts of interest. 
P1875 PRETREATMENT NEUTROPHIL TO LYMPHOCYTE RATIO IS NOT A PREDICTOR OF RESPONSE TO NEOADJUVANT THERAPY IN ESOPHAGEAL CANCER

G. Pirozzolo ${ }^{1}$, S.S. Gisbertz ${ }^{2}$, O. De Simoni ${ }^{1}$, A. Slaman $^{2}$, C. Castoro ${ }^{3}$,

M. Scarpa ${ }^{1}$, M.I. Van Berge Henegouwen ${ }^{2}$

${ }^{1}$ Esophageal And Digestive Tract Surgical Unit, Regional Centre For Esophageal

Disease, Veneto Institute of Oncology (IOV-IRCCS), Padova/Italy

${ }^{2}$ Surgical Department, Academic Medical Centre, Amsterdam/Netherlands

${ }^{3}$ Venesto Institute of Oncology, Padova/Italy

\section{Contact E-mail Address: giovanni.pirozzolo@gmail.com}

Introduction: Preoperative Neutrophil to Lymphocyte Ratio (NLR) has been proposed as a prognostic marker in several solid tumors (Templeton 2014). A recent retrospective study of 60 patients showed the prognostic relevance of NLR as a predictor of response in esophageal cancer patients treated with chemoradiotherapy. The aim of this study is to assess the NLR prognostic strength in a retrospective series of two high-volume centers.

Aims \& Methods: A retrospective review of two prospective esophageal cancer database was conducted. Neutrophil to lymphocyte ratio was defined as the prechemoradiotherapy serum neutrophil count divided by lymphocyte count. We dichotomized the NLR data using as cut-off values 2,5 and 3 respectively. Univariable logistic regressions were performed to determine the effect of NLR on response after neoadjuvant treatment. Survival curves were constructed with Kaplan Meier method and compared with the long rank test.

Results: We included 280 patients. The analysis of NLR as predictor of pathologic complete response (pCR) showed a OR of $0.963(95 \%$ CI $0.531-1.746$, $\mathrm{p}=901)$ and $1.161(95 \%$ CI $0.647-2.081, \mathrm{p}=0.617)$ considering as cut-off values 2.5 and 3 respectively. In our large series, NLR did not result as a predictive marker neither in terms of Overall Survival nor in terms of Disease Free Survival ( $\mathrm{p}=0.997$ and $\mathrm{p}=0.672$ respectively).

Conclusion: Our results did not confirm NLR as a significant marker of $\mathrm{pCR}$. Moreover, the survival analysis did not reveal significant differences using NLR as pre-treatment prognostic marker. The heterogeneity of treatments, the complexity of the disease, the absence of a validated and pre-defined NLR cut-off value in the available literature are the main limits to our analysis. Further studies are needed to assess the clinical relevance of NLR as a predictive marker of response to neoadjuvant treatment.

Disclosure of Interest: All authors have declared no conflicts of interest.

\section{References}

Templeton et al (2014) J Natl Cancer Inst

McLaren et al (2017) J Gastrointes Surg

Sharaiha RZ et al (2011) Ann Surg Oncol

Moyes LH, et al (2009) Br J Cancer

\section{P1876 CAN THE USE OF A COMPUTER DECISION SUPPORT SYSTEM PREVENT COMPLICATED ULCER AMONG PATIENTS TREATED WITH NSAID OR ASPIRIN? A RANDOMISED} CONTROLLED CLUSTER TRIAL IN GENERAL PRACTICE

J.M. Petersen ${ }^{1}$, D. E. Jarbøl ${ }^{2}$, J. Hallas ${ }^{3}$, M. R. Munch ${ }^{2}$, O.B. Schaffalitzky De Muckadell $^{4}$, J.M. Hansen ${ }^{4}$

${ }^{1}$ Dept. Of Medical Gastroenterology, University of Southern Denmark, Odense Denmark

${ }^{2}$ Research Unit Of General Practice, University of Southern Denmark, Odense C/ Denmark

${ }^{3}$ Institute Of Clinical Pharmacology, University of Southern Denmark, Odense M/ Denmark

${ }^{4}$ Dept. Of Gastroenterology, Odense University Hospital, Odense/Denmark

Contact E-mail Address: johanna.martina.petersen@rsyd.dk

Introduction: Background consumption of non-steroidal-anti-inflammatorydrugs (NSAID) and Aspirin is high in Denmark and the majority of these drugs are prescribed in General Practice. Risk factors for complicated ulcer are well established and include NSAID, Aspirin, age, prior ulcer, Helicobacter pylori, anticoagulants, selective-serotonin-reuptake-inhibitors (SSRIs), Adenosine-Di-Phosphate (ADP)-inhibitors and glucocorticoids. Proton Pump Inhibitors (PPIs) reduce the risk of complication but preventive treatment with PPI is only given to a third of the people at risk. The mortality from an ulcer complication is $10 \%$ and above.

Aims \& Methods: The aim was first in a randomised cluster design to test if a Computer Decision Support System (CDSS) - based on a risk profile for the individual patient - reduced the frequency of ulcer complications. Second to investigate if use of the CDSS changed the prescription pattern in general practice with regard to prescriptions of NSAID, Aspirin and PPI.

Results: Ninety-six GPs responsible for the treatment of 52,649 patients were randomised to the CDSS-group and 90 GPs responsible for the treatment of 43,861 patients to the control group. No significant differences were found between the two patients groups regarding peptic ulcer complications, uncomplicated ulcer, gastro-oesophageal reflux or usage of upper gastrointestinal(GI)endoscopies. The patients' risk profile was identical in the two groups. During the intervention period lasting from April 2013 to September 2014 a significantly higher prescription rate of NSAID and PPI was found in the CDSS group. In addition a significantly higher co-prescription of NSAID and PPI was found in the CDSS group-particularly in high-risk patients. No significant difference was found in co-prescription of Aspirin and PPI when comparing CDSS-group to the control-group.

Conclusion: Conclusion A CDSS based on a risk profile for the individual patient had no impact on the main outcomes ulcer complications, uncomplicated ulcer, reflux or endoscopies. Usage of CDSS increased the amount of co-prescription of PPI and NSAID in medium and high-risk groups. A similar pattern was not found for Aspirin. Triggering and the timing of the CDSS and its implementation in the Electronical Health Record should be optimized.

Disclosure of Interest: All authors have declared no conflicts of interest.

\section{References}

1. Hansen JM, Pedersen O, Bytzer P, Scaffalitzky de Muckadell O,. Changing characteristics of patients with bleeding peptic ulcer. Gastroenterology. 2002;121:A-477.

2. Christensen S, Riis A, Norgaard M, Sorensen HT, Thomsen RW. Short-term mortality after perforated or bleeding peptic ulcer among elderly patients: a population-based cohort study. BMC Geriatr. 2007;7:8.

3. Zimmerman J, Siguencia J, Tsvang E, Beeri R, Arnon R. Predictors of mortality in patients admitted to hospital for acute upper gastrointestinal hemorrhage. Scandinavian journal of gastroenterology. 1995;30(4):327-31.

4. Hansen JM, Hallas J, Lauritsen JM, Bytzer P. Non-steroidal anti-inflammatory drugs and ulcer complications: a risk factor analysis for clinical decisionmaking. Scandinavian journal of gastroenterology. 1996;31(2):126-30.

5. Hallas JL, J. Dalgard Villadsen, H. Nonsteroidal Anti-inflammatory Drugs and Upper Gastrointestinal Bleeding, Identifying High-Risk Groups by Excess Risk Estimates. Scandinavian journal of gastroenterology. 1995;30:438-44.

6. Laine L, Bombardier C, Hawkey CJ, Davis B, Shapiro D, Brett C, et al. Stratifying the risk of NSAID-related upper gastrointestinal clinical events: results of a double-blind outcomes study in patients with rheumatoid arthritis. Gastroenterology. 2002;123(4):1006-12.

7. Pilotto A, Franceschi M, Maggi S, Addante F, Sancarlo D. Optimal management of peptic ulcer disease in the elderly. Drugs \& aging. 2010;27(7):545-58.

8. Sturkenboom MC, Burke TA, Dieleman JP, Tangelder MJ, Lee F, Goldstein JL. Underutilization of preventive strategies in patients receiving NSAIDs. Rheumatology. 2003:42 Suppl 3:iii23-31.

9. JM Petersen JH, OB Scaffalitzky De Muckadell, M Dall, J Hallas. A model to estimate the risk of NSAID/Aspirin-related upper gastrointestinalbleeding for the individual patient based on risk factors.

\section{P1877 ACCELERATION OF HEALING OF PREEXISTING GASTRIC ULCERS BY CARBON MONOXIDE RELEASING MOLECULE -2 (CORM-2). INVOLVEMENT OF HEME OXYGENASE, OXIDATIVE STRESS AND PROINFLAMMATORY MARKERS}

T. Brzozowski, K. Magierowska, S. Kwiecien, M. Magierowski, M. HubalewskaMazgaj, D. Wojcik, R. Pajdo, A. Chmura

Physiology, Jagiellonian University Medical College, Cracow/Poland

Contact E-mail Address: katarzyna.magierowska@uj.edu.pl

Introduction: Carbon monoxide $(\mathrm{CO})$ is produced endogenously in the body as a by-product of heme degradation via activity of the enzyme heme oxygenase (HO)-1. This gaseous mediator with multidirectional biological activity exerts antiproliferative, anti-inflammatory and immunomodulatory properties. A newly discovered class of compounds, named CO-releasing molecules (CORMs), is capable of liberating CO gaseous molecule that can be useful as pharmacological tool to assess the physiological role of $\mathrm{CO}$ under experimental conditions. CORM-2 was implicated in gastroprotection against formation of acute gastric lesions but the contribution of $\mathrm{CO}$ to the mechanism of gastric ulcer healing has not been fully elucidated.

Aims \& Methods: We determined the effect of daily treatment with vehicle or CORM-2, on healing of preexisting gastric ulcers induced by serosal application of acetic acid (ulcer area $=28 \mathrm{~mm}^{2}$ ) in rats. Our second goal was to examine the mechanism of CO released from its donor by the determination of the CORM-2induced alterations in gastric blood flow $(\mathrm{GBF})$ at ulcer margin, the parameters of oxidative stress and the gastric mucosal expression of pro-inflammatory and anti-inflammatory factors. Groups of seventy rats with gastric ulcers (A-D) received daily treatment with $\mathrm{A}$ ) vehicle (saline), B) CORM-2 in doses from 1 up to $10 \mathrm{mg} / \mathrm{kg}$ i.g., C) the HO-1 inductor, hemin $(5 \mathrm{mg} / \mathrm{kg}$ i.p.), D) the HO-1 activity inhibitor, zinc protoporphirin IX (ZnPP IX) $(5 \mathrm{mg} / \mathrm{kg}$ i.p.). After 9 days of treatment, the ulcer area was measured by planimetry, the gastric blood flow (GBF) at ulcer margin was determined by Laser Doppler technique, plasma TNF- $\alpha$ and IL- $1 \beta$ levels were monitored by ELISA and IL-1 $\beta-$ TNF- $\alpha$, HO- 1 , HO-2, COX-1, COX-2, iNOS, cNOS mRNAs were analyzed by RT-PCR and Western blot. Gastric mucosal samples were collected for the assessment of MPO activity, level of reduced glutathione (GSH) and lipid peroxidation products (MDA + 4HNE) by spectrophotometry.

Results: Treatment with CORM-2 significantly reduced the area of gastric ulcers and significantly raised GBF at ulcer margin. The dose accelerating ulcer healing by $50 \%$ (ID50) and significantly raising GBF was $10 \mathrm{mg} / \mathrm{kg}$ as compared with vehicle-control animals. The administration of hemin significantly reduced the area of gastric ulcers and raised GBF at ulcer margin but the treatment with ZnPP IX significantly increased the area of gastric ulcers and significantly decreased the GBF at ulcer margin. The decrease in gastric ulcer healing by 
CORM-2 was accompanied by a significant decrease in plasma levels of IL-1B and TNF-alpha comparing to vehicle-control group. The expression of IL-1B, TNF-alpha, COX-2 and iNOS mRNA was strongly upregulated in vehicle-treated gastric mucosa but expression of these factors was significantly attenuated in CORM-treated animals. The increased mucosal expression of mRNA for HO-1 but not HO-2 was detected in vehicle control group and these effects were ameliorated by treatment with CORM-2. The gastric mucosal MPO activity and themucosal content of MDA $+4 \mathrm{HNE}$ in gastric mucosa were elevated in vehicle-control group and these effects were significantly inhibited by CORM-2.

Conclusion: $\mathrm{CO}$ released from its donor CORM-2 accelerates the healing of preexisting gastric ulcerations by the mechanism involving an increase in gastric microcirculation at ulcer margin, the inhibition of inflammatory response as manifested by the attenuation of enhancing synthesis of the proinflammatory markers IL-1B, TNF-alpha, COX-2 and iNOS as well as by antioxidative properties of CORM-2 releasing CO limiting lipid peroxidation.

Disclosure of Interest: All authors have declared no conflicts of interest.

\section{P1878 PROTON PUMP INHIBITORS INAPPROPRIATE USE IN PATIENTS ADMITTED IN A TERTIARY GREEK HOSPITAL CREATES SIGNIFICANT DIRECT COSTS BURDEN AND EXPOSURE OF PATIENTS TO THE RISK OF UPPER GASTROINTESTINAL \\ COMPLICATIONS}

G. Vlachonicolou, L. Bounou, A. Theodorou-Kanakari, S. Kalousios, M. Roma, E.A. Mavroeidi, G. D. Dimitriadis, K. Triantafyllou

2nd Dept Of Internal Medicine And Research Institute, National and Kapodistrian University of Athens, Medical School, Athens/Greece

Contact E-mail Address: ktriant@med.uoa.gr

Introduction: There is evidence of proton pump inhibitors (PPIs) misuse in the community and in the hospitals causing significant direct and indirect costs burden for the health care system.

Aims \& Methods: We aimed to evaluate the frequency of inappropriate PPIs administration in hospitalized patients, to measure the direct in-hospital costs of PPIs overuse and to calculate the number of patients exposed to the risk of upper gastrointestinal (UGI) complications due to medication underuse. This was a prospective, cross-sectional, prescription-indication drug-utilization, chart-review study in hospitalized patients with follow-up until discharge, in a tertiary 500 beds hospital in Athens, Greece. We recorded data of all patients admitted (intensive care, psychiatric, pediatric, obstetrics and day clinic admission were excluded) during three consecutive on-call days of the hospital in March 2017 regarding PPIs utilization before admission, during hospitalization and at discharge. We thereafter calculated the direct hospital costs of PPIs overuse and the number of patients at risk of UGI complications due to PPIs underuse for 1 year period, using a simulation model.

Results: We included data from 470 patients aged $67 \pm 19$ yrs; $32.5 \%$ were prescribed a PPI before admission, $65.9 \%$ during hospitalization and $32.8 \%$ at discharge. PPIs overutilization was detected in $15.7 \%, 41.3 \%$ and $12.6 \%$ of the patients before, during and after the admission, while medications underutilization was detected in $10.2 \%, 8.1 \%$ and $9.5 \%$ of them, respectively. Admission at internal medicine and orthopedics clinics was associated with the highest unadjusted ORs (1.68 [95\%CI 1.63-1.72] and 1.68 [1.59-1.78]) for PPIs misuse. $80 \%$ of the 193 over treated patients received PPIs iv $(80 \%$ of them od, $20 \%$ bid) while the rest were treated with PPIs per os $(90 \%$ of them od, $10 \%$ bid) during their 8 days of mean hospitalization length. This accounts for 1480 PPI iv and 344 PPI per os doses inappropriately given during the observation period. Taking into account in our simulation model that there are 90 on-call days of our hospital annually, the cost of each PPI dose is 3.435 and 0.235 euros for iv and per os preparations and assuming a similar to that of our sample case distribution for the next 12 months, we calculated the direct hospital costs burden of inappropriate PPIs use at 154940 euros per year. Using the same model, 1200 patients would be at risk of UGI complications annually, due to under prescription of PPIs at discharge.

Conclusion: Hospitalization does not represent an opportunity for optimization of PPIs utilization. On the contrary, the frequency of PPIs inappropriate use during hospitalization is higher than that before admission, causing significant direct costs for the hospital and exposing patients to the risks of UGI complications.

Disclosure of Interest: All authors have declared no conflicts of interest.

\section{P1879 CONTINUOUS INCREASE IN PREVALENCE OF FUNDIC GLAND POLYPS WITH THE LENGTH OF PROTON PUMP INHIBITORS USE. IS THERE ANY CLINICAL CONSEQUENCE?}

R. Kroupa ${ }^{1}$, M. Dastych ${ }^{1}$, S. Konecny ${ }^{1}$, J. Dolina ${ }^{1}$

${ }^{1}$ Department Of Internal Medicine and Gastroenterology, University Hospital and Faculty of Medicine Masaryk's University, Brno/Czech Republic

Contact E-mail Address: kroupa.radek@fnbrno.cz

Introduction: Proton pump inhibitor (PPI) usage is associated with an increased risk of development of fundic gland polyps (FGP). The trend of change in the prevalence with increasing length of PPI exposure over 5 years is not known. Theoretical risk of FGP seems to be very low for clinical consequence in management.

Aims \& Methods: To evaluate the relationship between the length of PPI use and risk of fundic gland polyps. Prospective cohort study in patients referred for upper gastrointestinal endoscopy between years 2015-2016 was performed. The length of PPI use was ascertained from direct patient interview and confirmed with medical records. The use of PPI in history not continuing at the time of endoscopy was also inquired. FGP were determinated both endoscopically and histologically. Odds ratios for subsequent intervals of length of PPI use were calculated. Clinically relevant complications-dysplasia and bleeding from large polyps were recorded.

Results: During study period 1525 patients (mean age 59.2 years, $53 \%$ male) were included. Only $612(40 \%)$ of patients had no history of any PPI use. Fundic gland polyps were identified in 170 patients $(11 \%)$ and only 13 of them $(7.6 \%)$ were without PPI use. The prevalence of FGP in subgroups of patients according to the length of PPI use was: $10.6 \%$ of 161 patients using PPI less than 1 year, $9.4 \%$ of 405 (1-4 years of PPI), $25.2 \%$ of 230 (5-9 years of PPI), $35.1 \%$ of $94(10-15$ years of PPI) and $47.8 \%$ of 23 patients using PPI more than 15 years. The appropriate odds ratios were 5.4 (CI 2.6-11.4), 4.8 (CI 2.5-9.1), 15.5 (CI 8.329.0 ), 24.9 (CI 12.4-49.9) and 42.2(CI 15.7-113.1) respectively, all with statistical significance $(\mathrm{p}<0.0001)$. Only 1 case of low grade dysplasia within FGP was observed in the patient with familiar adenomatous polyposis. Polyps larger than $15 \mathrm{~mm}$ with signs of bleeding determinated as a cause of sideropenic anemia were diagnosed in 6 patients.

Conclusion: The prevalence of FGP is permanently growing during prolonged use of PPI. Complex source of informations regarding history of PPI use identified quite low proportion of patient with FGP and without prior PPI use. Rather rare complications like bleeding from FGP might be clinically significant in increasing number of long term PPI users. The requirement of repetitive endoscopies and therapeutic interventions may burden gastroenterologists and sources.

Disclosure of Interest: All authors have declared no conflicts of interest.

\section{Reference}

Martin F, Chenevix-Trench G, Yeomans N. Systematic review with meta-analysis: fundic gland polyps and proton pump inhibitors. Alimentary Pharmacology \& Therapeutics [serial online]. November 2016;44(9):915-925.

\section{P1880 ENDOSCOPIC RESECTION OF ADVANCED AMPULLARY ADENOMAS: A SINGLE-CENTER 14-YEAR RETROSPECTIVE COHORT STUDY}

S. E. Van Der Wiel, J.W. Poley, M.J. Bruno, A.D. Koch Gastroenterology And Hepatology, Erasmus University Medical Center, Rotterdam/Netherlands

Contact E-mail Address: s.e.vanderwiel@erasmusmc.nl

Introduction: Adenomas of the ampulla of Vater are rare. Endoscopic ampullectomy has been recognized as a safe and reliable treatment of selective tumors of the ampulla of Vater and is associated with lower morbidity and mortality rates than surgical resection. However, the success rates for endoscopic ampullectomy range from 61 to 92 percent, with recurrence described in up to 33 percent of patients. Despite the increasing number of studies concerning endoscopic resection of ampullary tumors, data evaluating endoscopic resection of the more advanced ampullary adenomas are limited.

Aims \& Methods: The aim of our study was to evaluate the technical success, complications and recurrence of endoscopic resection for treating patients with ampullary adenomas with intraductal extension (AIE), and patients with lateral spreading adenomas (LSA). Between January 2002 and November 2016, all patients referred to the Erasmus Medical Center, Rotterdam, for endoscopic resection of an ampullary lesion were retrospectively identified. Cases were selected by using ENDOBASE and we provided a search in the our local PALGA database. We included patients with a histological diagnosis of adenoma. Endoscopic resection was performed by 5 experienced endoscopists. Endoscopic success was defined as complete excision of the adenoma, irrespective of the number of attempts, and in the absence of recurrence. All patients underwent endoscopic follow-up. Early and late complications were registered.

Results: We included 84 patients, 56 patients $(67 \%)$ had an adenoma confined to the ampulla (ACA), 17 patients $(20 \%)$ had a LSA and 11 patients $(13 \%)$ were treated for adenomas that demonstrated growth pattern with intraductal extension. Fifty-five percent of the patients were men and the median age was 65.4 years (range 32-89). The median lesion size was $24.6 \mathrm{~mm}$ (range 5-80) for patients with ACA, $34.8 \mathrm{~mm}$ (range 23-50) for LSA and $16.3 \mathrm{~mm}$ (range 10 $20)$ for patients with an AIE $(\mathrm{P}=0.039)$. Complications occurred in 26 patients $(30.9 \%)$, of which hemorrhage was most seen in $17.9 \%$, followed by perforation in $5.9 \%$ of the patients. Complications were equally divided over these three groups $(\mathrm{P}=0.775)$. The mean follow-up duration was 31.1 months (range 0 129) for ACA, 23.1 months (range $0-127$ ) for LSA and 11.9 months (range 037) for IEA $(P=0.136)$. Endoscopic resection was curative in $87.5 \%$ of patients with a localized adenoma, $82.3 \%$ in patients with a lateral spreading adenoma and in only $9.1 \%$ of patients with an intraductal extended tumor $(\mathrm{P}<0.000)$. Recurrence occurred in 9 patients $(10.7 \%), 5$ of them had a localized adenoma, 3 patients with a lateral spread adenoma and 1 patient with an intraductal extended adenoma $(\mathrm{P}=0.875)$.

Conclusion: Endoscopic ampullectomy is a safe and successful treatment in patients with an adenoma with or without a lateral spreading growth pattern. In case of an intraductal extended adenoma endoscopic success rates are significantly lower.

Disclosure of Interest: All authors have declared no conflicts of interest. 
P1881 FACTORS ASSOCIATED WITH TECHNICAL DIFFICULTY

OF ENDOSCOPIC SUBMUCOSAL DISSECTION FOR EARLY GASTRIC CANCER WHICH MET EXPANDED INDICATION CRITERIA; POST HOC ANALYSIS USING DATA OF MULTIINSTITUTIONAL PROSPECTIVE CONFIRMATORY TRIAL

\section{(JCOG0607)}

T. Yano ${ }^{1}$, N. Hasuike ${ }^{2}$, H. Ono ${ }^{3}$, N. Boku ${ }^{4}$, G. Ogawa ${ }^{5}$, J. Mizusawa ${ }^{5}$, I. Oda ${ }^{6}$, H. Doyama ${ }^{7}$, S. Hori ${ }^{8}$, H. Iishi', K. Takizawa ${ }^{3}$, H. Fukuda ${ }^{5}$, M. Muto ${ }^{10}$ ${ }_{1}^{1}$ Gastroenterology And Endoscopy, National Cancer Center Hospital East, Kashiwa/Japan

${ }^{2}$ Sano Hospital, Kobe/Japan

${ }^{3}$ Division Of Endoscopy, Shizuoka Cancer Center, Shizuoka/Japan

${ }^{4}$ National Cancer Center Hospital, Tokyo/Japan

${ }^{5}$ JCOG Data Center, Tokyo/Japan

${ }^{6}$ Endoscopy Division, National Cancer Center Hospital, Tokyo/Japan

${ }^{7}$ Dept. Of Gastroenterology, Ishikawa Prefectural Central Hosp., Kanazawa/Japan ${ }^{8}$ Shikoku Cancer Center, Matsuyama/Japan

${ }^{9}$ Department Of Gastrointestinal Oncology, Osaka International Cancer Institute, Osaka/Japan

${ }^{10}$ Therapeutic Oncology, Kyoto University, Kyoto/Japan

\section{Contact E-mail Address: toyano@east.ncc.go.jp}

Introduction: There are few reports about the technical difficulty of gastric endoscopic submucosal dissection (ESD) which were investigated through the prospective trial.

Aims \& Methods: The aim of this study was to evaluate the factors associated with the technical difficulty of ESD for early gastric cancer (GC) which met expanded indication criteria using data from JCOG0607. The major inclusion criteria of JCOG 0607 was as follows: 1) histologically proven intestinal-type adenocarcinoma; 2) cT1aN0M0; 3) lesion without finding of ulcer (UL negative) and $>2 \mathrm{~cm}$ in size, or UL positive and $\leq 3 \mathrm{~cm}$ in size; 4) age 20-75. ESD were performed by certified endoscopists or under the supervision of certified endoscopists who had experienced 100 cases or more. The difficult case was defined that ESD took 120 minutes ( $\mathrm{min}$ ) or longer, and/or developed perforation during procedure. Multivariate analysis was performed to investigate the clinical factors associated with technical difficulty of ESD.

Results: Between June 2007 and Oct. 2010, a total of 470 patients (pts) (male female: $385 / 85$, median age $65 y-0$ (range: $40-75)$ ) were enrolled from 29 institutions. Tumor location was upper (U) of stomach in 71 , middle in 255 , and lower in 144 pts, respectively. The median tumor size was $2.5 \mathrm{~cm}$ (range: $0.5-13$ ), and 207 lesions were judged as UL positive whereas the other 263 were assessed as UL negative. And, 152 of 263 UL negative lesions were $3 \mathrm{~cm}$ or less in size, and the other 111 of them were larger than $3 \mathrm{~cm}$. Median procedure time was $79 \mathrm{~min}$ (range: 14-462), and it took $120 \mathrm{~min}$ or longer in 127 pts. 12 pts developed perforation during ESD, and the procedure time took $120 \mathrm{~min}$ or longer in 9 of them. Therefore, 130 pts $(27.7 \%)$ were classified in difficult case group. The proportion of difficult case was $23.2 \%(48 / 207)$ in UL positive and $\leq 3 \mathrm{~cm}$ cases, $18.4 \%(28 / 152)$ in UL negative and $\leq 3 \mathrm{~cm}$, and $48.6 \%(54 / 111)$ in UL negative and $>3 \mathrm{~cm}$, respectively. Multivariable showed UL negative and $>3 \mathrm{~cm}$ (vs. UL negative and $\leq 3 \mathrm{~cm}$, odds ratio, $4.66 ; 95 \%$ confidence interval, 2.59-8.37, $\mathrm{p}<0.0001)$ was the most significant factor associated with technical difficulty, and $U$ of stomach, and age $\leq 60$ were also associated with difficulty.

Conclusion: Lesion larger than $3 \mathrm{~cm}$ without finding of ulcer, $U$ of stomach, age $\leq 60$ were independent factors associated with technical difficulty of ESD for GC which met expanded indication criteria.

Disclosure of Interest: All authors have declared no conflicts of interest.

\section{Reference}

Hasuike N, et al. A non-randomized confirmatory trial of an expanded indication for endoscopic submucosal dissection for intestinal-type gastric cancer (cT1a): the Japan Clinical Oncology Group study (JCOG0607). Gastric Cancer. 2017 Feb 21.

\section{P1882 COMPARATIVE EFFECTIVENESS OF NOVEL FINE- NEEDLE BIOPSY DEVICE VS CONVENTIONAL FINE-NEEDLE ASPIRATION FOR ENDOSCOPIC ULTRASOUND DIAGNOSIS OF GASTRIC SUBMUCOSAL LESIONS}

M. Mizrahi ${ }^{1}$, E. U. Yee ${ }^{2}$, I. Nasser ${ }^{1}$, J. Cohen ${ }^{1}$, J. Sheridan ${ }^{1}$, R. Chuttani ${ }^{1}$, T. Berzin ${ }^{1}$, M. Sawhney ${ }^{1}$, D. Pleskow ${ }^{1}$

${ }^{1}$ Beth Israel Deaconess Medical Center, Harvard Medical School, Boston/United States of America

${ }^{2}$ Department of Pathology. Beth Israel Deaconess Medical Center, Boston/United States of America/MA

Contact E-mail Address: mmizrahi@ bidmc.harvard.edu

Introduction: Endoscopic ultrasound with fine-needle aspiration (EUS-FNA) is commonly used for the diagnosis of various gastrointestinal lesions. Recently, a novel fine-needle biopsy (FNB) system (SharkCore, Medtronic) was developed to acquire cohesive units of tissue to increase the diagnostic yield of EUS.

Aims \& Methods: Our study objective was to compare the diagnostic yield of EUS-FNA using a conventional needle vs. EUS-FNB using the novel needle for gastric submucosal lesions. We conducted a prospective analysis of patients undergoing diagnostic EUS from November 2014 to October 2015. Each patient underwent 3 FNA passes followed by 3 FNB passes, without onsite cytologic evaluation. Data gathered included demographics, size and location of the lesion, needle size, and complications. Pathology and cytology were reviewed separately by two blinded, expert gastrointestinal pathologists. Diagnostic yield was defined as the ratio between the number of significant findings detected by EUS tissue acquisition and the total number of EUS examinations performed for a given indication.

Results: During the study period 1487 EUS procedures were performed in our clinic. Thirty patients with gastric submucosal lesions were enrolled to participate in the study, $66 \%$ were female, mean age was 62 years (range $31-88$ ). Mean lesion size was $27 \mathrm{~mm}$ (range $12-60 \mathrm{~mm}$ ). Twenty-two-gauge needles were used in 14 cases $(55 \%)$ and twenty-five-gauge needles were used in 12 cases $(45 \%)$. Overall diagnostic yield for 3 needle passes was higher for FNB vs. FNA for gastric submucosal lesions ( $78 \%[\mathrm{~N}=20]$ vs. $44 \%[\mathrm{~N}=12], \mathrm{P}=0.04)$. Analyzing each of the different passes, the diagnostic yield was: $61 \%, 67 \%$ and $78 \%$ with the first, second, and third FNB pass, respectively. In evaluation of tissue quantity and quality of FNB specimens, the mean tissue length was $7.3 \pm 5.2 \mathrm{~mm}$, with a mean lesional tissue proportion between 51 to $75 \%$ of the total tissue procured (determined as the percent of the surface are occupied by lesion over the surface area of the entire tissue on one slide). No complications occurred during the study period.

Conclusion: FNB using a novel core needle system is safe and effective for diagnosis of gastric submucosal lesions. When performed without on-site cytologic evaluation, EUS-FNB has a higher diagnostic yield than FNA and may represent an advance for endoscopic ultrasound guided gastrointestinal biopsies. Disclosure of Interest: T. Berzin: Consultant for Medtronic

D. Pleskow: Consultant for Medtronic

All other authors have declared no conflicts of interest.

\section{P1883 A COMPARISON OF SUBMUCOSAL TUNNELING ENDOSCOPIC RESECTION AND ENDOSCOPIC FULL-THICKNESS RESECTION FOR GASTRIC FUNDUS SUBMUCOSAL TUMORS}

T. Duan, Y. Tan, X. Wang, L. Liang, D. Liu

Department Of Gastroenterology, The second Xiangya Hospital of Central South University, Changsha/China

\section{Contact E-mail Address: tianyingduan@163.com}

Introduction: Submucosal tumors (SMT) refer to protuberant lesion covered with intact mucosa, and most of SMT found in stomach are small and asymptomatic. While a half of these gastric SMTs are considered to be gastrointestinal stromal tumors (GIST) with malignant potential, especially for those with large diameters $^{[1]}$. One of the strategies for SMTs $<2 \mathrm{~cm}$ is periodical surveillance by esophagogastroduodenoscopy (EGD) and endoscopic ultrasonography (EUS), but it involves issues related to the patient's compliance and stress, cost-effectiveness, and the risk associated with repeated endoscopic procedures and delayed diagnosis of malignancy ${ }^{[1]}$. Therefore, it is necessary to remove these SMTs and endoscopic resection could be considered as an alternative method. Endoscopic full-thickness resection (EFTR) is a safe and effective endoscopic method for gastric fundus SMT originating from the muscularis propria (MP) layer with a high rate of complete resection ${ }^{[2]}$. Submucosal tunneling endoscopic resection (STER) is a novel endoscopic technique and has demonstrated to be safe and effective for the treatment of gastric SMTs ${ }^{[3]}$. However, little is known about the comparison between STER and EFTR for treating SMTs in gastric fundus. In this retrospective study, we aimed to compare both endoscopic techniques for treating gastric fundus SMTs.

Aims \& Methods: Both submucosal tunneling endoscopic resection (STER) and endoscopic full-thickness resection (EFTR) are effective methods for gastric fundus submucosal tumors (SMTs), however, little is known about the comparison between these two resections. The aim of this study was to compare the safety and efficacy of STER and EFTR for treating SMTs in gastric fundus. We retrospectively collected the clinical data about patients with gastric fundus submucosal tumors who received submucosal tunneling endoscopic resection or endoscopic full-thickness resection at our hospital from April 2011 to May 2016. Epidemiological data (gender, age), tumor size, procedure-related parameters, complications, length of stay, cost and follow-up data were compared.

Results: A total of 43 patients were enrolled, and 15 of them received submucosal tunneling endoscopic resection, while the other 28 cases received endoscopic fullthickness resection. There was no significant difference between the two groups in terms of gender, age, tumor size, en bloc resection rate, operation time, pathohistological results, hospital stay and cost $(p>0.05)$. However, patients who received endoscopic full-thickness resection had a longer suture time and needed more clips to close the gastric wall defect $(p<0.05)$. No recurrence was noted in the submucosal tunneling endoscopic resection and endoscopic full-thickness resection groups during a mean follow-up of 12.1 and 22.8 months, respectively.

Comparison of clinical characteristics and therapeutic outcomes between STER and EFTR

\begin{tabular}{llll}
\hline & STER $(\mathrm{n}=15)$ & EFTR $(\mathrm{n}=28)$ & $\mathrm{P}$ \\
\hline Sex, M/F & $5 / 10$ & $13 / 15$ & 0.407 \\
Age, year & $48.4 \pm 11.2$ & $53.4 \pm 9.7$ & 0.136 \\
Concomitant disease, \% & $20 \%(3 / 15)$ & $21.4 \%(6 / 28)$ & 1.000 \\
Tumor size, mm & $19.0 \pm 8.3$ & $15.3 \pm 7.0$ & 0.126 \\
Operation time, min & $76.7 \pm 38.0$ & $63.3 \pm 24.4$ & 0.200 \\
Suture time, sec & $296.7 \pm 97.0$ & $383.4 \pm 104.0$ & 0.011 \\
\hline
\end{tabular}


Continued

Comparison of clinical characteristics and therapeutic outcomes between STER and EFTR

\begin{tabular}{llll}
\hline & STER $(\mathrm{n}=15)$ & EFTR $(\mathrm{n}=28)$ & $\mathrm{P}$ \\
\hline No. of clips for suture & $5.8 \pm 1.4$ & $7.6 \pm 1.6$ & 0.001 \\
Complications, \% & $6.7 \%(1 / 15)$ & $14.3 \%(4 / 28)$ & 0.643 \\
En bloc resection, \% & $6.7 \%(1 / 15)$ & $3.6 \%(1 / 28)$ & 1.000 \\
GIST/Leiomyoma/Schwannoma & $11 / 4 / 0$ & $25 / 2 / 1$ & 0.173 \\
Length of stay, d & $6.1 \pm 1.5$ & $6.2 \pm 2.0$ & 0.856 \\
Cost, USD & $3260.9 \pm 618.3$ & $3237.5 \pm 615.8$ & 0.906 \\
Follow-up time, mon & $12.1 \pm 12.2$ & $22.8 \pm 18.4$ & 0.052 \\
\hline
\end{tabular}

Conclusion: The treatment efficacy between submucosal tunneling endoscopic resection and endoscopic full-thickness resection for treating gastric fundus submucosal tumors was comparable, but submucosal tunneling endoscopic resection offers advantages over endoscopic full-thickness resection in terms of shorter suture time and smaller number of clips needed to close the gastric wall defect. Disclosure of Interest: All authors have declared no conflicts of interest.

\section{References}

1. Nishida T, Kawai N, Yamaguchi S, et al. Submucosal tumors: comprehensive guide for the diagnosis and therapy of gastrointestinal submucosal tumors. Dig Endosc. 2013;25:479-489.

2. Schlag C, Wilhelm D, von Delius S, et al. EndoResect study: endoscopic fullthickness resection of gastric subepithelial tumors. Endoscopy. 2013; 45(1): 4 11.

3. Xu MD, Cai MY, Zhou PH, et al. Submucosal tunneling endoscopic resection: a new technique for treating upper GI submucosal tumors originating from the muscularis propria layer (with videos). Gastrointest Endosc. 2012;75:195-199.

\section{P1884 COMPARISON OF THE DIAGNOSTIC YIELDS OF ENDOSCOPIC ULTRASOUND-GUIDED FINE NEEDLE ASPIRATION FOR DUODENAL AND GASTRIC SUBEPITHELIAL LESIONS}

\section{K. Nakamura}

Gastroenterology, St.Luke's International Hospital, Chuo-ku/Japan

Contact E-mail Address: nakaken@luke.ac.jp

Introduction: The progress of diagnosis and treatment for gastrointestinal subepithelial lesions (SELs) have been remarkable by the wide use of Endoscopic Ultrasound-Guided Fine Needle Aspiration (EUS-FNA) and the development of Laparoscopy and Endoscopy Cooperative Surgery. EUS-FNA is used widely for SELs that occur in the digestive tract. There are many reports on gastric SELs, but there are no studies on a large group of patients focusing on duodenal SELs.

Aims \& Methods: The aim of this study was to investigate the usefulness and safety of EUS-FNA for duodenal SELs, comparing them with gastric SELs. Cross-sectional study was conducted using 41 patients who underwent EUSFNA at teritary medical center in Tokyo between April, 2012 and February, 2017. We divited into 2 groups: 6 patients with duodenal SELs (group D) and 35 patients with gastric SELs (group G), who were diagnosed as SELs located on the 4th layer by pre-operative EUS and were performed EUS-FNA consecutively. We retrospectively evaluated the patient characteristics and outcome of the technique on the subjects.

Results: Age (median, [range]); D: 61, [42-63], G: 60, [32-85]; male/female ratio: D, 1/5, G, 18/17; tumor size (median, [range])(mm): D, 16, [14-45], G, 24, [1367]; type of needle (22-gauge/19-gauge): D, 5/1, G, 30/6 (One case was used two type of needles.); number of needle passes: D, 5, [4-5], G, 4, [3-7]; procedure time (median, [range])(min): D, 34, [22-52], G, 42, [20-67]; diagnostic yield: D, 100\%, G, $91.4 \%$; complication: D, $0 \%, G, 2.9 \%$. There were no significant differences into 2 groups, but the size of duodenal SELs tended to be smaller than gastric SELs. Histological diagnosis of EUS-FNA using immunohistochemical analysis showed duodenal SELs were 5 GIST, 1 neurinoma, and gastric SELs were 25 GIST, 4 ectopic pancreas, 3 neurinoma, 3 indeterminate.Two of the indeterminates were followed up by CT, one was SEL like cancer diagnosed by conventional biopsy.

Conclusion: Although five of the six cases of duodenal SELs were small lesions of less than $20 \mathrm{~mm}$, immunohistochemical staining was performed for all the cases, and diagnosis was made. EUS-FNA for duodenal SELs was as useful and safe as gastric SELs.

Disclosure of Interest: All authors have declared no conflicts of interest.

\section{References}

1. Wiersema MJ, Vilmann P, Giovannini M, Chang KJ, Wiersema LM Endosonography-guided fine-needle aspiration biopsy: diagnostic accuracy and complication assessment. Gastroenterology. 1997 Apr;112(4):1087-95.

2. Larghi A, Fuccio L, Chiarello G, Attili F, Vanella G, Paliani GB, Napoleone M, Rindi G, Larocca LM, Costamagna G, Ricci R. Fine-needle tissue acquisition from subepithelial lesions using a forward-viewing linear echoendoscope. Endoscopy. 2014 Jan;46(1):39-45
P1885 SHORT-TERM OUTCOME OF ENDOSCOPIC SUBMUCOSAL DISSECTION FOR EARLY GASTRIC CANCER IN ELDERLY PATIENTS AND LONG-TERM OUTCOME AFTER NONCURATIVE RESECTION

H. Sakaguchi ${ }^{1}$, Y. Ohara ${ }^{2}$, T. Toyonaga ${ }^{2}$, H. Kaku ${ }^{1}$, K. Matsuoka ${ }^{1}$, T. Sako ${ }^{1}$,

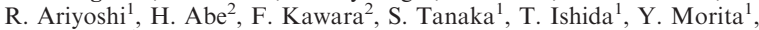
E. Umegaki ${ }^{1}$

${ }^{1}$ Division Of Gastroenterology, Department Of Internal Medicine, Graduate School of Medicine, Kobe University, Kobe/Japan

${ }^{2}$ Endoscopy, Kobe University Hospital, Kobe/Japan

Contact E-mail Address: valencia.0601@gmail.com

Introduction: Endoscopic submucosal dissection(ESD) is widely used as a standard treatment for superficial tumors in the GI tract and its safety has been established. Opportunities for elderly patients to undergo ESD for early gastric cancer is increasing due to the continued improvement in life expectancy. However, short-term and long-term outcome of ESD for elderly patients is still unclear because of the high frequency of comorbidities and possible increased risk of complications related to ESD in this population.

Aims \& Methods: Therefore, we investigated the safety, efficacy and short-term outcome of gastric ESD for patients over 80 years old. Additionally, we evaluated the long-term outcome of non-curative resections according to both age groups 1056 lesions in 886 patients treated with ESD between January 2011 and December 2015 in our hospital were retrospectively reviewed. They were classified into two groups; elderly group - older than 80 years old (246 lesions in 201 patients) and non-elderly group - 79 years old and younger (810 lesions in 685 patients). The patient demographics, lesion characteristics, short-term ESD outcome, complications (perforation, postoperative bleeding, postoperative delirium, and pneumonia), were examined and compared between the two groups. Concerning the long-term outcome of non-curative ESD, cases performed between 2011 and 2013 were assessed.

Results: The median age was 83 years old (range: $80-92$ ) in the elderly group and 70 years old (36-78) in the non-elderly group. The ratio of female patients was significantly higher in the elderly group $(30.9 \%$ vs. $17.0 \%$ : $p<0.0001)$ Comorbidities were significantly higher in the elderly group including heart disease $(24.8 \%$ vs. $10.5 \%$ : $p<0.0001)$, lung disease $(13.8 \%$ vs. $7.4 \%: \mathrm{p}=0.002)$ and dementia $(3.3 \%$ vs. $1.0 \%: p=0.01)$. Tumor location was not significantly different between the two groups. Median specimen and tumor size were the same in both groups with no significant difference: $43 \mathrm{~mm}$ and $15 \mathrm{~mm}$, respectively. The en bloc resection rates $(96.3 \%$ and $97.8 \%$ ) and the curative resection rates $(82.5 \%$ and $84.6 \%)$ were not significantly different. The perforation rates were not statistically different $(2.44 \%$ and $3.21 \%)$. However, the postoperative bleeding rate $(5.28 \%$ vs. $2.72 \%: \mathrm{p}=0.05)$, postoperative delirium $(2.0 \%$ vs. $0.25 \%$ : $\mathrm{p}=0.009)$ and pneumonia $(2.0 \%$ vs. $0.25 \%: \mathrm{p}=0.009)$ were significantly higher in the elderly group. In multivariate analysis, age over 80 was not an independent risk factor for postoperative bleeding, however it was the independent risk factor for postoperative delirium and postoperative pneumonia. Nineteen elderly patients and 54 non-elderly patients with non-curative resections were followed-up for 3 years after ESD for the long-term outcome analysis. The percentage of patients who underwent additional surgery after ESD was $26.3 \%(5 / 19)$ and $51.85 \%(28 / 54)$ respectively $(\mathrm{p}=0.05)$. Neither disease specific death nor progression to advanced gastric cancer was found in each age group. Overall survival rate 3 years after ESD was $68.4 \%$ and $85.2 \%$ respectively $(\mathrm{p}=0.17)$.

Conclusion: ESD is a safe and effective treatment for early gastric neoplasia even in patients over 80 years old. However, because postoperative delirium and postoperative pneumonia were observed more often in the elderly patients, more careful attention to these conditions during perioperative care may be necessary. Elderly patients over 80 years, with non-curative resections, underwent less frequent additional surgery without any impact on the disease specific death and global mortality.

Disclosure of Interest: All authors have declared no conflicts of interest.

\section{References}

Akasaka, T., Nishida, T., Tsutsui, S., Michida, T., Yamada, T., Ogiyama, H., . . Hayashi, N. (2013). Short-term outcomes of endoscopic submucosal dissection (esd) for early gastric neoplasm: Multicenter survey by osaka university esd study group. Gastroenterological Endoscopy, 55(1), 77-83.

Toyokawa, T., Fujita, I., Morikawa, T., Okamoto, A., Miyasaka, R., Watanabe, K., ... Tomoda, J. (2011). Clinical outcomes of ESD for early gastric neoplasms in elderly patients. European Journal of Clinical Investigation, 41(5), 474-478.

\section{P1886 GASTRIC INTESTINAL METAPLASIA OUTCOMES: RESULTS FROM A 17 YEAR TERTIARY CENTRE UPPER GI SURVEILLANCE PROGRAMME IN IRELAND}

M. Mc Nally ${ }^{1}$, I. Cretu ${ }^{1}$, A. O'Connor

${ }^{1}$ Trinity Academic Gastroenterology Group, Naas General Hospital, Kildare/ Ireland

Contact E-mail Address: maireadnf@gmail.com

Introduction: Adenocarcinoma of the stomach is the second leading cause of cancer related death in the world. Gastric intestinal metaplasia (GIM) is an important intermediate stage in the gastric cancer cascade through a series of well-defined precursor lesions including nonatrophic gastritis, multifocal atrophic gastritis, intestinal metaplasia, and dysplasia. The prevalence of GIM is unclear in many parts of the world and few studies have evaluated the rate of progression to gastric cancer in patients with GIM. There is a lack of clarity in 
published guidelines regarding appropriate surveillance of patients with GIM and there is wide disparity in the management of this premalignant condition. Aims \& Methods: This study aimed to analyze surveillance practice and characterize the natural history of this premalignant condition by identifying all patients with GIM on an upper GI surveillance programme and reviewing follow up data. This is a retrospective study of patients with GIM who are currently enrolled in an upper GI surveillance programme. Patients with a history of GIM identified at any time during an 18 year surveillance period (from 1998 to 2016) were included in the study. Patient characteristics, endoscopy data including histology, rates of Helicobacter pylori infection, Barrett's oesophagus association and outcomes were reviewed.

Results: 160 patients (including those with Barrett's oesophagus, GIM and family history of gastric cancer) were enrolled on the surveillance programme. 42 patients with GIM were identified-20 females $(47.6 \%)$ and 22 males $(52.3 \%)$. The mean age at which GIM was first diagnosed was 60.6 years (range from 17.9 to 71.5 years). $15 / 42$ patients $(35.7 \%$ ) had co-existent Barrett's oesophagus and Helicobacter pylori was identified in $6 / 42(14.3 \%)$. The follow-up period ranged from 0.5 to 17.3 years. 27 patients had repeat gastroscopies following initial diagnosis. 15 patients are still awaiting a repeat gastroscopy. A large degree of variability in the number and frequency of follow-up gastroscopies was observed. The average interval of follow-up gastroscopies was 3.3 years per person. 14/27 patients $(51.8 \%)$ had no evidence of GIM on most recent gastroscopy, $7 / 27$ patients $(26 \%)$ had repeat findings of persistent focal GIM, 5/27 patients $(18.5 \%)$ progressed to extensive GIM. No cases of dysplasia were recorded but 1 patient $(3.7 \%)$ developed gastric cancer.

Conclusion: This study suggests a low apparent risk of progression of gastric intestinal metaplasia in a small western cohort. Further studies may be necessary to address if the applicability of published surveillance guidelines can be generalised to regions with low gastric cancer prevalence.

Disclosure of Interest: All authors have declared no conflicts of interest.

\section{References}

Correa, $\mathrm{P}$ et al. (1990). Gastric precancerous process in a high risk population: cohort follow-up. Cancer Res, 50(15), 4737-40.

denHoed, $\mathrm{C}$ et al. (2011). The prevalence of premalignant gastric lesions in asymptomatic patients: predicting the future incidence of gastric cancer. Eur $J$ Cancer, 47(8), 1211-8.

deVries, A et al. (2008). Gastric cancer risk in patients with premalignant gastric lesions: a nationwide cohort study in the Netherlands. Gastroenterology, 134(4), 945-52.

Dinis-Ribeiro, M et al. (2012). Management of precancerous conditions and lesions in the stomach (MAPS): guideline from the European Society of Gastrointestinal Endoscopy (ESGE), European Helicobacter Study Group (EHSG), European Society of Pathology (ESP), and the Sociedade Portuguesa de Endoscopia Digestiva (SPED). Endoscopy, 44(1), 74-94.

Fennerty, M et al. (1992). Gastric intestinal metaplasia in ethnic groups in the southwestern United States. Cancer Epidemiol, Biomarkers Prev, 1(4), 293-296. Gonzales, C et al. (2010). Gastric cancer occurrence in preneoplastic lesions: a long-term follow-up in a high-risk area in Spain. Int J Cancer, 127(11), 2654-60. O'Connor, A., McNamara, D., \& O’Morain, C. (2013). Surveillance of gastric intestinal metaplasia for the prevention of gastric cancer. Cochrane Database Syst Rev, 9.

Sipponen, P., \& Kimura, K. (1994). Intestinal metaplasia, atrophic gastritis and stomach cancer: trends over time. Eur J Gastroenterol Hepatol, 6, s79-83.

\section{P1887 DIFFERENT RELATIONSHIP BETWEEN STAGE OF GASTRIC CANCER AND GENOTYPE OF TGFB1 BASED ON FIRST- DEGREE RELATIVE OF GASTRIC CANCER}

M. Kwon ${ }^{1}$, N. Kim ${ }^{2}$, C. Yun ${ }^{1}$, Y.J. Hwang ${ }^{1}$, Y.J. Choi ${ }^{2}$, H. Yoon ${ }^{1}$, C.M. Shin ${ }^{2}$, Y.S. Park ${ }^{1}$, D.H. Lee

${ }^{1}$ Gastroenterology, Seoul National University Bundang Hospital, Seoul/Korea, Republic of

${ }^{2}$ Department Of Internal Medicine, Seoul National University Bundang Hospital, Seongnam/Korea, Republic of

\section{Contact E-mail Address: tsm3wa@naver.com}

Introduction: Previously we reported that direct family history of gastric cancer (GC) as a risk factor of GC and genetic polymorphisms of TGFB1 (transforming growth factor- $\beta 1$ ) was associated with the development of GC in the first-degree relative of GC. The aim of this study is to investigate relationship between stage of gastric cancer and genetic polymorphism of TGFB1 regarding first-degree relative of $\mathrm{GC}$

Aims \& Methods: From January 2006 to March 2017, 1090 gastric cancer patients were enrolled at Seoul National University Bundang Hospital in whom stage of GC was obtained from surgery, endoscopic submucosal dissection (ESD), endoscopic mucosal resection (EMR), and computer tomography (CT) and positron emission tomography (PET)-CT images. 203 patients $(18.6 \%)$ had direct family history GC and $887(81.4 \%)$ did not have. Genotype of TGFB1-509 was measured by the polymerase chain reaction-restriction fragment length polymorphism. Relationship between $T G F B$ polymorphism and stage or GC tissue type was analyzed.

Results: Proportion of stage $1 \& 2$ was statistically higher in the group with direct family history GC $(170,83.7 \%)$ than without direct family history $(660,74.4 \%)$. $(\mathrm{P}=0.005)$. When $\mathrm{GC}$ stage was analyzed regarding direct family history and $T G F B 1$ genetic polymorphism the ratio of gastric cancer stage I to TGFB1509 T carrier was significantly higher than that of stage II or higher $(P=0.008)$, only in male. However, this difference was not found in female. In addition no significant difference was found in GC patients without direct family history. Lauren classification and $T G F B 1$ genotype did not show any statistically significant results even in the group with direct family history.

Conclusion: Family history of GC affects the stage of GC and the genotype of TGFB1-509 could be underlying mechanism in case of male. Survival analysis is undergoing.

Table: Differences of gastric cancer stage according to TGFB1-509 polymorphism and family history $(\mathrm{Hx})$ of gastric cancer

\begin{tabular}{lllllll}
\hline & & & Stage 1 & Stage $2 \leq$ & & \\
& & TGFB1-509 & N $(\%)$ & N $(\%)$ & Total & P-value \\
\hline Family Hx. (+) & Female & C/C & $18(78.3)$ & $5(21.7)$ & 23 & 0.243 \\
& & T carrier & $27(64.3)$ & $15(35.7)$ & 42 & \\
& Male & C/C & $20(52.6)$ & $18(47.4)$ & 38 & $\mathbf{0 . 0 0 8}$ \\
& & T carrier & $76(76.0)$ & $24(24.0)$ & 100 & \\
& Total & C/C & $38(62.3)$ & $23(37.7)$ & 61 & 0.146 \\
& & T carrier & $103(72.5)$ & $39(27.5)$ & 142 & \\
Family Hx. (-) & Female & C/C & $46(61.3)$ & $29(38.7)$ & 75 & 0.781 \\
& & T carrier & $137(63.1)$ & $80(36.9)$ & 217 & \\
& \multirow{2}{*}{ Male } & C/C & $91(61.9)$ & $56(38.1)$ & 147 & 0.568 \\
& & T carrier & $289(64.5)$ & $159(35.5)$ & 448 & \\
& Total & C/C & $137(61.7)$ & $85(38.3)$ & 222 & 0.529 \\
& & T carrier & $426(64.1)$ & $239(35.9)$ & 665 & \\
\hline
\end{tabular}

Disclosure of Interest: All authors have declared no conflicts of interest.

\section{P1888 GASTRIC ADENOCARCINOMA AND PROXIMAL POLYPOSIS OF THE STOMACH. A GENETIC STUDY OF A NEWLY DIAGNOSED FAMILY}

I. Tachecí ${ }^{1}$, M. Podhola ${ }^{2}$, M. Leško ${ }^{3}$, M. Minarik ${ }^{4}$, L. Benesova ${ }^{5}$

M. Kopáčová ${ }^{2}$, S. Rejchrt ${ }^{2}$, J. Bures ${ }^{2}$

${ }^{1}$ 2nd Dpt Of Internal Medicine, Charles University Faculty of Medicine 2nd Dpt of Internal Medicine, Hradec Kralove/Czech Republic

${ }^{2}$ The Fingerland Department Of Pathology, Charles University in Praha, Faculty of Medicine at Hradec Kralove, University Teaching Hospital, Hradec Kralove/ Czech Republic

${ }^{3}$ Charles University in Praha, Faculty of Medicine at Hradec Kralove, University Teaching Hospital, Hradec Kralove/Czech Republic

${ }^{4}$ Center For Applied Genomics Of Solid Tumors (ceges), Genomac Research Institute, Praha/Czech Republic

${ }^{5}$ Genomac Research Institute Center for Applied Genomics of Solid Tumors, Praha/ Czech Republic

Contact E-mail Address: tacheci $@$ gmail.com

Introduction: Gastric adenocarcinoma and proximal polyposis of the stomach (GAPPS) has to been described recently only in a few families worldwide (only one in Europe so far). Three different point mutations in promoter $1 \mathrm{~B}$ of the APC gene were identified as causal (c.-191T $>$ C, c.-192A $>$ G, and c.-195A $>$ C). We diagnosed GAPPS in the second Czech white family (not related to that one published previously-ref. 1).

Aims \& Methods: We diagnosed GAPPS across 3 generations in a new Czech white family. A genetic analysis of the family was performed.

Results: The Proband (a 43-year-old male) was endoscopically regularly surveyed from his 34 years of age because of fundic-gland polyposis with predominant involvement of the gastric fundus and body (with relative sparing of the lesser curve) and microcytic anaemia. Polyposis slowly progressed with the intestinal differentiated low-grade dysplasia in polypectomy specimens 10 years after the diagnosis. As the GAPPS criteria were fulfilled (ref. 2), he and his family underwent genetic testing and bi-directional Sanger sequencing of promoter 1B revealed a point mutation (c.-191 T $>$ C). The same type of mutation was described in his father (63 years old), sister (41 years old), nephew (son of his sister, 6 years old), uncle (father's brother, 51 years old) and 2 cousins (uncle's daughters, 23 and 27 years old), all have been asymptomatic. No gastric cancer in the family history was mentioned. The Proband underwent preventive total gastrectomy, histology of the surgical specimen confirmed severe involvement of gastric body with fundic gland polyps, low-grade and focal high-grade dysplasia. The microcytic anaemia improved rapidly after surgery. The rest of family is scheduled for gastroscopy. The fundic-gland polyposis of similar distribution (with significantly lower number of polyps, without any dysplastic changes) was recently diagnosed in the 23-year-old cousin.

Conclusion: The second European family with GAPPS is presented. The recently described mutations in promoter $1 \mathrm{~B}$ of the APC gene does not automatically mean a faster progression of the disease as suggested earlier. GAPPS can be presented with various phenotypes with a different course of disease, the prevalence can be higher than previously reported. Acknowledgement: The study was supported by the Research Project PROGRES Q40-15.

Disclosure of Interest: All authors have declared no conflicts of interest. 


\section{References}

1. Repak R, Kohoutova D, Podhola M, Rejchrt S, Minarik M, Benesova L, Lesko M, Bures J. The first European family with gastric adenocarcinoma and proximal polyposis of the stomach: case report and review of the literature. Gastrointest Endosc. 2016 Oct;84(4):718-25.

2. Worthley DL, Phillips KD, Wayte N, Schrader KA, Healey S, Kaurah P, Shulkes A, Grimpen F, Clouston A, Moore D, Cullen D, Ormonde D, Mounkley D, Wen X, Lindor N, Carneiro F, Huntsman DG, ChenevixTrench G, Suthers GK. Gastric adenocarcinoma and proximal polyposis of the stomach (GAPPS): a new autosomal dominant syndrome. Gut. 2012 May;61(5):774-9

\section{P1889 ENDOSCOPIC TREATMENT FOR LATERAL SPREADING SUPERFICIAL ESOPHAGEAL SQUAMOUS CELL CARCINOMA}

H. Kawakubo ${ }^{1}$, T. Omori ${ }^{2}$, K. Suda ${ }^{3}$, R. Nakamura ${ }^{4}$, N. Wada ${ }^{1}$, N. Yahagi ${ }^{5}$, Y. Kitagawa

${ }^{1}$ Department Of Surgery, Keio University School OF Medicine, Tokyo/Japan ${ }^{2}$ Kawasaki Municipal Kawasaki Hospital, Kawasaki/Japan

${ }^{3}$ Department Of Surgery, Keio University School of Medicine, Tokyo/Japan

${ }^{4}$ Keio University School of Medicine, Tokyo/Japan

${ }^{5}$ Cancer Center, Keio University, School of Medicine, Tokyo/Japan

Contact E-mail Address: hkawakubo@z3.keio.jp

Introduction: ESD is the one of the options of treatment even for lateral spreading (size is over $5 \mathrm{~cm}$ ) esophageal squamous cell carcinoma (ESCC). Endoscopic diagnosis is developed by magnified endoscopy, however accuracy of diagnosis for lateral spreading ESCC is not high. Some patients have to undergo additional treatment because tumor is invaded to submucosal layer or lymphvascular invasion. On the other hand, wide resection by ESD could cause the delay of additional treatment because of the treatment for esophageal stricture after ESD. Thus, treatment strategy for lateral spreading ESCC has to include additional treatment after ESD. Aim of this study is to evaluate our treatment strategy for lateral spreading superficial ESCC.

Aims \& Methods: From January 2010 to December 2014, 49 cases of lateral spreading superficial ESCC were resected by surgery or ESD. Diagnosis, treatment methods and outcomes are evaluated. Our indications for additional treatment after ESD are the cases of over pT1b (SM2) or lymphvascular invasion. Results: In 49 cases of lateral spreading superficial ESCC, 32 cases were treated by ESD and 17 case were treated by surgery. All lesions of 32 cases are completely resected by ESD. Average size of tumor treated by ESD is $59.4 \mathrm{~mm}(50$ $85 \mathrm{~mm}$ ). Accuracy of estimated depth of invasion by endoscopy for ESD cases is $65.7 \%$. Four of 32 cases of ESD underwent additional therapy ( 3 for surgery and 1 for CRT) because of pT1b (SM2) or lymphvascular invasion, and one case has lymph-node metastasis. Rate of stricture after ESD is $20.0 \%$ for sub-circumference ESD and $77.8 \%$ for circumference ESD instead of steroid injection. Average time and duration for control of esophageal stricture by Baloon Bougie is 13.5 times and 18 weeks. In 17 surgical cases, all cases are treated by thoracoscopic esophagectomy. Average size of tumor treated by surgery is $76.5 \mathrm{~mm}(50-130 \mathrm{~mm})$. Accuracy of estimated depth of invasion by endoscopy for surgical cases is $47.1 \%$. Seven cases in $17(41.2 \%)$ have lymph node metastasis. Rate of lymph node metastasis is $42.9 \%$ for pT1a-MM, $100 \%$ for pT1bSM1 and $42.9 \%$ for pT1b-SM2. One case died by recurrence after surgery and 48 cases were survived without any recurrences.

Conclusion: Accuracy of estimated depth of invasion by endoscopy for lateral spreading superficial ESCC is cute low compared to normal superficial ESCC. Most of strictures after sub-circumference ESD could be prevented by steroid injection. However control of strictures after circumference ESD is difficult. Thus, diagnostic ESD should not be performed for circumferencial lesions of lateral spreading superficial ESCC for the patients who will select CRT for additional treatment, and CRT should be selected for first treatment of these cases. Long survival could be obtained by ESD or surgery for the patients of lateral spreading ESCC by our treatment strategy.

Disclosure of Interest: All authors have declared no conflicts of interest.

\section{P1890 DIAGNOSTIC ACCURACY OF ENDOSCOPIC ULTRASONOGRAPHY FOR ESOPHAGEAL SUBMUCOSAL GLAND DUCT INVOLVEMENT ACCOMPANIED BY EARLY ESOPHAGEAL CANCER}

Y. Jie, L. Ying

Digestive Department, The Affiliated Drum Tower Hospital, Nanjing University

Medical School, Nanjing, Jiangsu Province, China, Nanjing/China

Contact E-mail Address: 13770755008@126.com

Introduction: Normally, resided within the submucosal layer of esophagus, each esophageal submucosal gland will culminate in a single duct. The esophageal submucosal gland ducts (ESMGDs) can traverse the subepithelial connective tissue and muscularis mucosa, and deliver the acinar serections to the esophageal lumen. However, the clinicopathological features of the esophageal submucosal gland duct involvement (ESMGDI) and its precursor lesion have not been comprehensively evaluated so far, and the series study focusing on endoscopic features of this lesion has not been reported widely. While since the 1990s, the esophageal lesions presumed to originate from ESMGDs had been described constantly in various case reports. Currently, in addition to the gold standard of histopathology, almost no more useful modality could be applied to this lesion. In our study, we considered that the ESMGDI had a correlation with early esophageal cancer, and we noted that the ESMGDI had special features under the endoscopic ultrasonography (EUS). The typical ultrasonic images of EUS could help diagnose ESMGDI.

Aims \& Methods: In order to investigate the clinical value of EUS for diagnosing ESMGDI accompanied by early esophageal cancer, which were suggested by conventional endoscopy or biopsy, this study retrospectively analyzed the consecutive patients with early esophageal cancer diagnosed in the Endoscopy Center at the Affiliated Drum Tower Hospital, Nanjing, China from September 2009 to November 2016. The clinical data of 519 patients were included in this study, and all of them had already underwent EUS combined with Endoscopic Submucosal Dissection (ESD). The EUS preoperative diagnosis were compared with the results of postoperative pathology from ESD.

Results: According to the pathological results, all patients (371 males and 148 females, with a mean age of $67.5 \pm 4.5$ years) had been diagnosed with early esophageal cancer with different invasive depth. Out of 519 patients, about 478 patients were not found ESMGDI by both examinations. Besides, postoperative pathology confirmed that 40 patients were identified with ESMGDI, 34 patients of which were completely consistent with the preoperative diagnosis of EUS. Approximately $98.7 \%(512 / 519)$ of ESMGDI were diagnosed exactly by EUS. Another six cases were estimated as flase negative inaccurately, including two squamous cell carcinoma and four high-grade intraepithelial neoplasia. One case was regarded as ESMGDI by EUS while confirmed not by pathology. Therefore, the EUS values for sensitivity and specificity for the diagnosis of ESMGDI were $85.0 \%(34 / 40)$ and $99.8 \%(478 / 479)$ respectively. Furthermore, the positive predictive value was $97.1 \%(34 / 35)$, and the negative predictive value was $98.8 \%$ (478/484).

Conclusion: The esophageal submucosal gland duct involvement is a kind of lesion performed as a hypoechoic sonographic pattern located in the thickened mucosa. EUS has a satisfactory diagnostic accuracy for ESMGDI as well as good sensitivity and specificity.

Disclosure of Interest: All authors have declared no conflicts of interest.

\section{P1891 PEPSINOGENS AND GASTRIN-17 FOR IDENTIFICATION OF GASTRIC CANCER PRECURSOR LESIONS: THE RESULTS FROM THE GISTAR PILOT STUDY}

C. Robles ${ }^{1}$, D. Rudzite ${ }^{2}$, I. Polaka ${ }^{3}$, L. Tzivian $^{3}$, I. Kikuste $^{3}$, A. Vanags ${ }^{4}$ S. Isajevs ${ }^{5}$, I. Liepniece-Karele ${ }^{5}$, S. Parshutin ${ }^{3}$, J. Young Park ${ }^{6}$, R. Murillo ${ }^{6}$ R. Herrero ${ }^{6}$, M. Leja ${ }^{7}$

${ }^{1}$ Prevention And Implementation Group, International Agency for Research on Cancer, Lyon/France

${ }^{2}$ Riga East Clinical University Hospital, Riga/Latvia

${ }^{3}$ Institute Of Clinical And Preventive Medicine \& Faculty Of Medicine, University Of Latvia, Riga/Latvia

${ }^{4}$ Digestive Diseases Centre GASTRO, LV/Latvia

${ }^{5}$ Academic Histology laboratory, Riga/Latvia

${ }^{6}$ International Agency for Research on Cancer, Lyon/France

${ }^{7}$ Department Of Research, Riga East University Hospital, Riga/Latvia

\section{Contact E-mail Address: ikikuste@gmail.com}

Introduction: Few major international guidelines consider pepsinogen tests as the best available non-invasive tests to detect precancerous lesions (in particular, corpus atrophy) in the stomach mucosa. Gastrin-17 (G-17) has been suggested as indirect marker for antral atrophy. Different tests are used in Europe and Asia. Recommendations from the European Commission expert group consider the need for additional studies before any screening with biomarkers can be recommended for implementation.

Aims \& Methods: Generally healthy 40-65 years aged participants of the GISTAR pilot study referred for upper endoscopy according to the pilot study protocol were enrolled. Pepsinogen (Pg) I and II were assessed from plasma samples by two methods-ELISA (Biohit Plc.) and latex-agglutination (Eiken Chemical Co.) test systems. G-17 and IgG group antibodies to H.pylori infection were assessed by Biohit Plc. ELISA test systems. The following cutoff values were considered characteristic for atrophy: Pg I/II $<3$ for ELISA, Pg I/II $<3$ and PgI $<70 \mathrm{ng} / \mathrm{ml}$ for latex-agglutination, and $\mathrm{G}-17<1 \mathrm{pmol} / 1$ (in a plasma sample obtained in fasting condition). Biopsies were sampled and read by two independent pathologists according to the updated Sydney system. OLGA and OLGIM scoring systems were also applied. Cancer, dysplasia, OLGA/OLGIM III-IV taken together were considered high-risk lesions.

Results: Altogether 1044 subjects $(55 \%$ females, mean age $=52,67.7 \%$ with positive $H$. pylori antibodies) were included to the study. The sensitivity and specificity for detecting moderate to severe atrophy in the corpus for Biohit ELISA test was $47.2 \%$ and $92.3 \%$, but for Eiken latex-agglutination test$81.1 \%$ and $62.1 \%$, respectively. The corresponding values for G-17 to detect atrophy in the antrum were $29.2 \%$ and $58.9 \%$. High-risk lesions were detected by Biohit ELISA pepsinogen testsystem with $22.8 \%$ sensitivity and $91.6 \%$ specificity, but with Eiken latex-agglutination test-with $67.4 \%$ and $62.5 \%$ sensitivity and specificity.

Conclusion: Due to the high specificity, pepsinogens (e.g. ELISA) could be potentially used for screening of precancerous lesions if relatively low sensitivity could be accepted. G-17 does not seem to provide any additional benefit. Disclosure of Interest: All authors have declared no conflicts of interest. 
P1892 MIR-211 INHIBITS PROLIFERATION AND MIGRATION OF GASTRIC CANCER CELLS BY TARGETING FOXC1

X. He ${ }^{1}$, Y. Ding ${ }^{1}$, Z. Jin ${ }^{1}$, L. Sun ${ }^{1}$, J. Si ${ }^{1}$

${ }^{1}$ Department Of Gastroenterology, Sir Run Run Shaw Hospital, hangzhou/China

Contact E-mail Address: hexingkang@zju.edu.cn

Introduction: miRNAs are a group of small non-coding RNAs that dysregulate in various types of cancers [1-3]. They play a pivotal role in several pathological processes. Growing evidence show that miRNAs can act as either tumour oncogenes or suppressors in different types of cancers. Previous studies revealed that miR-211-5p could suppress melanoma proliferation, migration, and invasion [4] However, the contrasting result suggested that miR-211-5p was a potential oncogene in non-small cell lung cancer by targeting SRCIN [5]. Therefore, the functional role of miR-211-5p is still unclear in the pathogenesis of gastric cancer. Aims \& Methods: We aimed to investigate and characterize the biological roles of miR-211 in the development of gastric cancer. The expression level of miR-211$5 \mathrm{p}$ was measured in paired primary gastric cancer with corresponding adjacent gastric mucosa by RT-PCR. Furthermore, we investigate the biological role of miR-211-5p in proliferation, apoptosis, migration of gastric cancer cell lines in vitro.

Results: The expression levels of miR-211-5p were significantly decreased in gastric cancer and low expression of miR-211-5p correlates with poor prognosis in gastric cancer patients. Ectopic expression of miR-211-5p suppressed proliferation, migration and induced apoptosis in gastric cancer cells in vitro. Bioinformatics and quantitative analysis revealed that FoxC1 might be a target of miR-211-5p. Downregulation of $\mathrm{FoxCl}$ inhibited proliferation and migration of gastric cancer cells and Overexpression of FoxCl partly abrogated the inhibitory effects of miR-211-5p on gastric cancer cell proliferation and motility. Conclusion: We demonstrate that miR-211-5p acted as a tumour suppressor by targeting $\mathrm{FoxC} 1$ in gastric cancer and miR-211-5p might be a potential target for the treatment of gastric cancer.

Disclosure of Interest: All authors have declared no conflicts of interest.

\section{References}

1. Jiang G, Cui Y, Yu X, Wu Z, Ding G and Cao L. miR-211 suppresses hepatocellular carcinoma by downregulating SATB2. Oncotarget. 2015; 6(11):9457-9466.

2. Xu D, Liu S, Zhang L and Song L. MiR-211 inhibits invasion and epithelialto-mesenchymal transition (EMT) of cervical cancer cells via targeting MUC4. Biochem Biophys Res Commun. 2017; 485(2):556-562.

3. Zhu J, Wang S, Chen Y, Li X, Jiang Y, Yang X, Li Y, Wang X, Meng Y, Zhu M, Ma X, Huang C, Wu R, Xie C, Geng S, Wu J, et al. miR-19 targeting of GSK3beta mediates sulforaphane suppression of lung cancer stem cells. J Nutr Biochem. 2017; 44:80-91

4. Bell RE, Khaled M, Netanely D, Schubert S, Golan T, Buxbaum A, Janas MM, Postolsky B, Goldberg MS, Shamir R and Levy C. Transcription factor/microRNA axis blocks melanoma invasion program by miR-211 targeting NUAK1. J Invest Dermatol. 2014; 134(2):441-451.

5. Ye L, Wang $\mathrm{H}$ and Liu B. miR-211 promotes non-small cell lung cancer proliferation by targeting SRCIN1. Tumour Biol. 2016; 37(1):1151-1157.

\section{P1893 CONGENITAL OR METAPLASTIC: EVALUATION OF GASTROESOPHAGEAL NEO-JUNCTIONS TO ASSESS CARDIAC TYPE EPITHELIUM ORIGIN}

J. Castela, D. Vinha Pereira, S. Mão De Ferro, R. Casaca, R. Fonseca, P. Chaves, A. Dias Pereira

Núcleo Regional do Sul da Liga Portuguesa Contra o Cancro, Lisboa/Portugal

Contact E-mail Address: joanarocastela@gmail.com

Introduction: The dramatic rise in the incidence of gastroesophageal junction (GEJ) adenocarcinoma in the Western countries, has promoted an incresead interest about the etiopathogenesis and natural history of GEJ. The cardiac epithelium (CE), which integrates the morphological spectrum of Barrett's esophagus, is frequently present in endoscopically normal GEJ in children and adults. Its congenital versus metaplastic origin still needs to be clarified. The gastroesophageal neo-junction after esophagectomy seems the ideal model to study the development of GEJ epithelia, reproducing "in-vivo" its natural history.

Aims \& Methods: The aim of this study was to evaluate the prevalence of metaplastic CE in the gastroesophageal neo-junction. Prospective study of patients undergoing esophagectomy due to esophageal/GEJ neoplasia between November/2015 and November/2016. Upper gastrointestinal endoscopy (UGIE) (Olympus, GIF-HQ 190) was performed 3 months after surgery; the neo-junction was evaluated with white light and Narrow band imaging (NBI); protocolated biopsies were made (suspected areas of $\mathrm{CE}$, randomized in the anastomosis if endoscopic absence of CE, $2 \mathrm{~cm}$ above and below the anastomosis). Endoscopic CE defined by the presence of circular pattern with NBI in the anastomosis. Histological evaluation performed by 3 pathologists with gastrointestinal expertise. A questionnaire for gastroesophageal reflux (GER) symptoms evaluation was applied.

Results: 20 patients were included (9 adenocarcinomas), 19 men, mean age $60 \pm 11$ years, 9 under proton-pump inhibitor and 10 with RGE symptoms. UGIE: unable to pass a standard scope in anastomosis due to stenosis: $3 / 20$; esophagitis: 5/20; columnar epithelium of the esophagus: $1 / 20$. Epithelium suggestive of CE: present in 17/20, suspect in $2 / 20$ and absent in $1 / 20$. Histologically: CE confirmed in $18 / 20$ patients; additionally identified oxyntocardiac epithelium in $12 / 20$ and intestinal metaplasia in $2 / 20$. The endoscopic diagnosis of $\mathrm{CE}$ revealed a sensitivity, specificity, positive and negative predictive value of $94.4 \%, 100 \%, 100 \%$ and $66.7 \%$, respectively.

Conclusion: The identification of metaplastic CE in neo-junctions is a very frequent and early event and its presence corroborates the hypothesis of metaplasia, to the detriment of a congenital origin. The good endoscopic and histological correlation observed for $\mathrm{CE}$ will allow the definition of endoscopic patterns, essential for its recognition.

Disclosure of Interest: All authors have declared no conflicts of interest.

\section{References}

1. Odze RD. Unraveling the mystery of the gastroesophageal junction: a pathologist's perspective. Am J Gastroenterol. 2005 Aug;100(8):1853-67.

2. Chandrasoma PT, Der R, Ma Y, et al. Histology of the gastroesophageal junction: an autopsy study. Am J Surg Pathol. 2000 Mar; 24(3):402-9.

3. Langner C, Schneider NI, Plieschnegger W, et al. Cardiac mucosa at the gastro-oesophageal junction: indicator of gastro-oesophageal reflux disease? Data from a prospective central European multicentre study on histological and endoscopic diagnosis of oesophagitis (histoGERD trial). Histopathology. 2014 Jul; 65(1):81-9.

4. Lord RV, Wickramasinghe K, Johansson JJ, et al. Cardiac mucosa in the remnant esophagus after esophagectomy is an acquired epithelium with Barrett's-like features. Surgery. 2004 Sep; 136(3):633-40.

5. Peitz U, Vieth M, Pross M, et al. Cardia-type metaplasia arising in the remnant esophagus after cardia resection. Gastrointest Endosc. 2004 Jun; 59(7):810-7.

6. Boeriu A, Dobru D, Pascarenco O, et al. Magnifying Endoscopy and Chromoendoscopy in Upper Gastrointestinal tract: clinical applications, New Techniques in Gastrointestinal Endoscopy, Prof. Oliviu Pascu. Intechopen. 2011.

\section{P1894 ENDOSCOPIC MUCOSAL RESECTION OF NON- AMPULLARY SPORADIC DUODENAL ADENOMAS: LONG TERM RESULTS}

G. Valerii ${ }^{1}$, A. Tringali ${ }^{1}$, I. Boskoski ${ }^{1}$, P. Familiari ${ }^{1}$, V. Bove ${ }^{1}$, R. Landi $^{2}$, V. Perri ${ }^{1}$, G. Costamagna ${ }^{1}$

${ }^{1}$ Digestive Endoscopy Unit, Catholic University of Rome-Fondazione Policlinico A. Gemelli, Rome, Italy, Roma/Italy

${ }^{2}$ Dipartimento Uoc Endoscopia Digestiva Chirurgica, Policlinico A. Gemelli, Rome, Italy, Roma/Italy

Contact E-mail Address: vinbove@alice.it

Introduction: Duodenal adenomas are rare epithelial tumors and represent $25 \%$ of the benign lesions diagnosed in the small bowel. Non-ampullary sporadic duodenal adenomas (NASDA) are usually asymptomatic and their diagnosis is mostly incidental. NASDA are benign epithelial tumors with a potential for malignant transformation via the adenoma-carcinoma sequence; nevertheless this risk is lower compared to ampullary or duodenal adenomas in the context of genetic syndromes. Results of Endoscopic Mucosal Resection (EMR) of NASDA are assessed in a large series after long-term follow-up.

Aims \& Methods: Consecutive patients undergoing EMR of NASDA between May 2002 and December 2016 were identified from an electronic database. Patients with a genetic polyposis syndrome (FAP or Peutz-Jeghers) and/or adenoma of the major or minor duodenal papilla were excluded. Preoperative biopsy were performed at operator discretion, considering that the majority of the patients were referred from other centers. In case of doubt for a possible involvement of the ampulla of Vater, duodenoscopy with a side-viewing endoscope was also performed. EUS was not systematically done before duodenal EMR. Size, site of the lesion, pre- and post-EMR histology, adverse events, local recurrence and survival rates were retrospectively analysed. En-bloc resection was preferred, when possible, for lesions $<20 \mathrm{~mm}$; bigger lesions were removed piecemeal. Blended "endocut" current was used in all the cases. All resected specimens were retrieved for histological examination. $\mathrm{CO}_{2}$ insufflation was routinely used during duodenal EMR after 2013. Argon plasma coagulation was used to eradicate residual adenomatous tissue at discretion of the operator. Endoscopic follow-up was scheduled after 3,6 and 12 months for the first year, and then yearly for up to 5 years.

Results: EMR of 75 NASDA was performed in 68 patients $(56 \%$ en-bloc resection, $44 \%$ piecemeal). The mean size was $14.4 \mathrm{~mm}$ and $29.9 \mathrm{~mm}$ for lesions resected en-bloc and piecemeal, respectively. Histopatological findings were: low-grade dysplasia $(n=27,36 \%)$, high-grade dysplasia $(n=34,45.4 \%)$, highgrade dysplasia with focal adenocarcinoma $(\mathrm{n}=12,16 \%)$, intramucosal adenocarcinoma $(\mathrm{n}=2,2.6 \%)$. Pre-EMR biopsy tended to downgrade the lesion in $44.4 \%(16 / 36)$. Retroperitoneal perforations occurred in $3 / 75(4.0 \%)$ procedures and were treated by surgical $(n=2)$ or percutaneous $(n=1)$ drainage; delayed bleeding was reported in $13 / 75(17.3 \%)$ resections and was successfully managed by endoscopy $(n=12)$ or radiologic embolization $(n=1)$. There was no procedure-related mortality. Follow-up was available in $61 / 68$ patients $(89.7 \%)$ after a median time of 59 months (range 3-147) from resection. Residual and recurrent adenoma were diagnosed in 9 and 6 cases, respectively; all but one were successfully retreated endoscopically.

Conclusion: The present series reports the results of duodenal EMR for NASDA after more than 4-year median follow-up. When biopsies had been performed before duodenal EMR for NASDA, downstaging of the lesion was recorded more than $40 \%$ cases in our series, suggesting that biopsies are not routinely necessary before EMR. EMR for NASDA is effective with favorable long-term outcomes. The main limitation of duodenal EMR is the high incidence of residual/recurrent adenoma which was $27.3 \%$ in our series. Piecemeal EMR was 
associated, in our series, with a higher incidence of residual/recurrent adenoma, when compared to en-bloc resection. These results are similar to those reported in the literature. Residual and recurrent duodenal adenomas were successfully retreated by EMR in all of them but one. Mortality related to NASDA was absent in our series after a median follow-up of 59 months (range 1-147). Management of adverse events after EMR for NASDA requires the availability of interventional radiologists and surgeons with experience in retroperitoneal surgery. In our experience colorectal adenomas was correlated to NASDA $(33.3 \%)$, colonoscopy is considered part of the pre-EMR assessment when NASDA is diagnosed. A recall system and patient's compliance to endoscopic follow-up are mandatory to detect recurrences and their prompt treatment.

Disclosure of Interest: G. Costamagna: Grant/research support from Olympus Japan Member of advisory committees or review panels for Cook, Inc., Boston Scientific Corp., and Taewoong Medical, Inc., Speaker and teacher for Boston Scientific, Corp. and Given Imaging.

All other authors have declared no conflicts of interest.

\section{P1895 CROSSTALK BETWEEN THE G PROTEIN-COUPLED RECEPTOR 39 AND RECEPTOR TYROSINE KINASES IN HUMAN GASTRIC CANCER}

S. Leal-López ${ }^{1}$, B. Otero-Alen ${ }^{1}$, C. S. Seonae-Mosteiro ${ }^{2}$, L. S. Estevez-Perez ${ }^{1}$, T. Garcia-Caballero ${ }^{3}$, R. Gallego-Gomez ${ }^{3}$, JE. Dominguez-Muñoz ${ }^{4}$, J. P. PerezCamiña $^{1}$, Y. Pazos-Randulfe ${ }^{1}$

${ }^{1}$ Gastroenterology, Health Research Institute of Santiago (IDIS), Santiago de Compostela/Spain

${ }^{2}$ Endocrinology, Health Research Institute of Santiago (IDIS), Santiago de Compostela/Spain

${ }^{3}$ Morphological Sciences, University Hospital of Santiago, Santiago de

Compostela/Spain

${ }^{4}$ Gastroenterology, University Hospital of Santiago, Santiago de Compostela/Spain

Contact E-mail Address: info@fienad.org

Introduction: Obestatin is a bioactive peptide with a well-defined function on cell proliferation mediated by the GPR39 receptor. Our data involve this system in several processes located in the stomach: from pepsinogen secretion in healthy stomach to malignant proliferation in gastric adenocarcinomas (1). A key step in obestatin signaling is the transactivation process GPR39-RTK through MMPs activation. This step could imply a key mechanism by which MMPs regulate these diseases. Numerous studies have shown that alterations in the expression and/or mutations in members of the family of receptors EGFR/ErbB are present in tumours, and these changes may contribute to the progression of cancer. In this sense, obestatin is added to the group of MMPs regulator factors, which have been implicated in diverse human diseases, such as inflammatory diseases and cancer $(2,3)$.

Aims \& Methods: The aim of the present study is to elucidate the detailed activation/regulation mechanism of GPR39-RTK cross-talk triggered by obestatin. Results: Obestatin triggers phosphorylation of RTKs that belong mainly to the family of EGFR (EGFR, 126.7 $\pm 1.1 \%$ ), insulin (InsulinR, 128.6 $\pm 1.3 \%$ ), Trk $($ TrkC, $170.9 \pm 4.6 \%)$, VEGF (VEGFR3, $114.9 \pm 3.1 \%$ ) and the ephrin receptors (EphA1, $135.8 \pm 5.2 \%$ ), both in AGS and KATO-III cell lines. Furthermore, we observed an augment in the expression of proteases in lysates and secretomes after obestatin treatment both in AGS and KATO-III cell lines. The most outstanding increments were located in the families of: ADAMs (ADAM9, $133.9 \pm 0.1 \%$; ADAM8, 406.9 $\pm 19.6 \%$ ), CTSs (CTSB, $193.4 \pm 3.9 \%$; CTSV $189.2 \pm 4.9 \%$ ), KLKs (KLK3, 358.4 $\pm 12.6 \%$; KLK13, 365.1 $\pm 7.1 \%$ ) and MMPs (MMP1, 690.6 $\pm 10.0 \%$; MMP13, $273.1 \pm 0.2 \%$ ). In human tissues, the obtained data were heterogeneous. However, it was observed an overexpression for EphA1 and InsulinR in adenocarcinomas when compared to healthy stomach, while it was observed a decrease for Axl. Regarding the proteases, ADAMTS13 y PSEN1 were found overexpressed in healthy tissue compared to gastric adenocarcinomas. Besides, CTSX $/ \mathrm{Z} / \mathrm{P}$ expression augmented with the differentiation of the tumour.

Conclusion: These data corroborated obestatin involvement in proliferation, migration and apoptosis and in diverse oncogenic process. Besides, the GPR39-RTK crosstalk is not only limited to the EGFR, but instead depends on the existing RTKs present in the tissues, and regulated the expression of proteases involved in processes and pathways already described for obestatin. Disclosure of Interest: All authors have declared no conflicts of interest.

\section{References}

1. Alen BO, et al. Oncotarget. 2016;7:5957-71.

2. Pazos Y, et al. Growth Factors 2007;25:373-81.

3. Alvarez CJP, et al. Endocr-Relat Cancer. 2009;16:599-611.

\section{P1896 INDOMETHACIN INDUCES LYSOSOMAL DYSFUNCTION IN GASTRIC CANCER CELLS AND INCREASES THEIR SENSITIVITY TO CYTOTOXIC DRUGS}

J. Vallecillo-Hernández ${ }^{1}$, L. Gisbert-Ferrándiz ${ }^{1}$, P. Salvador ${ }^{1}$, D. Ortiz-Masia ${ }^{2}$, D.C. Macias-Ceja ${ }^{3}$, M.D. Barrachina ${ }^{1}$, S. Calatayud ${ }^{1}$, C. Hernández ${ }^{3}$

${ }^{1}$ Dept. Farmacologia, CIBERehd-Univ. de Valencia, Valencia/Spain

${ }^{2}$ Dept. Medicina, CIBERehd-Univ. de Valencia, Valencia/Spain

${ }^{3}$ FISABIO, Valencia/Spain
Introduction: NSAIDs have demonstrated chemopreventive activity against gastrointestinal cancers but their toxicity limits their use as prophylactics. They have now been postulated as possible adjuvants to surgery or chemotherapy in order to prevent the formation of new lesions and reduce disease progression. Cancer cells enhance their resistance to the cytotoxic action of antineoplastic drugs by activating autophagy, which is a catabolic process that degrades superfluous and damaged organelles in the lysosome. We previously showed that indomethacin inhibits autophagy in gastric cancer cells by acting at a late stage in this catabolic process.

Aims \& Methods: The aim of this study is to analyze whether indomethacin modulates lysosomal function and oxaliplatin-induced cell death in these cells. Gastric AGS cells were treated with increasing concentrations of indomethacin for 2, 6 and 20 hours. Lysosomal $\mathrm{pH}$ was assessed by using Lysotracker Red and acridine orange fluorescent probes (static cytometry). Cathepsin activity was determined by using Omnicathepsin fluorescence substrate. In cells treated with indomethacin for 2 hours LAMP2 immunostaining was also carried out. In another set of experiments AGS cells were treated with increasing doses of the antineoplastic drug oxaliplatin and indomethacin. Cell viability was measured by an MTT assay, the rate of apoptosis/necrosis was analyzed by means of the Apoptosis Detection Kit, and autophagy by p62 immunoblotting.

Results: Treatment of AGS cells with indomethacin decreased Lysotracker fluorescence in AGS cells after 2, 6 and 20 hours. Indomethacin also produces an acute reduction in lysosome-derived fluorescence of acridine orange after 2 and 6 hours of treatment. In addition, we observed a significant reduction of cathepsin enzymatic activity in cells treated with indomethacin for 6 or 20 hours. Furthermore, there was an altered distribution of LAMP2-positive dots from the perinuclear position observed in control cells to a peripheral position in cells treated with indomethacin. Taken together, these data suggest that indomethacin inhibits the activity of lysosomal acidic enzymes by increasing lysosomal $\mathrm{pH}$. On the other hand, oxaliplatin decreased cell viability in a dosedependent manner after 48 hours of treatment, and treatment of cells with indomethacin during the last 24 hours further decreased cell viability. In addition, indomethacin also increased the rate of apoptosis and necrosis in AGS cells treated with oxaliplatin. Finally, indomethacin blocked the autophagic degradation of p62 protein induced by oxaliplatin.

Conclusion: Indomethacin inhibits lysosomal function in gastric cancer cells. This could explain the inhibitory action on autophagy and the resultant increase in susceptibility to cytotoxic drugs.

Disclosure of Interest: All authors have declared no conflicts of interest.

\section{P1897 HOXD10 METHYLATION IN PLASMA WAS POTENTIALLY SERVED AS BIOMARKER OF GASTRIC CANCER AND PRECANCEROUS LESIONS}

L. Wang, L. Wang, M. Luo

The Second Affiliated Hospital, School of Medicine, Zhejiang University, Hangzhou/China

Contact E-mail Address: wanglj76@hotmail.com

Introduction: Gastric cancer (GC) is the fifth most common cancer and the third leading cause of cancer death worldwide. Our previous studies have demonstrated that HoxD10 functioned as a candidate tumor suppressor in GC, which is silenced through promoter hypermethylation. The diagnostic value of HoxD10 methylation in the plasma of GC patients was not determined.

Aims \& Methods: We aim to evaluate whether HoxD10 promoter methylation in plasma could serve as noninvasive biomarker of GC. Methylated specific PCR (MSP) assays were used to measure HoxD10 promoter methylation in plasma of 38 healthy volunteers, 55 patients with gastric intraepithelial neoplasia (GIN), $123 \mathrm{GC}$ patients and $17 \mathrm{GC}$ after surgery patients.

Results: We demonstrated that the rate of HoxD10 methylation was $0 \%$ in healthy controls, $34.55 \%$ in GIN, $46.34 \%$ in GC and $17.65 \%$ in GC after surgery group, respectively. The methylation rate was significantly higher in GIN or GC than in the normal control group $(\mathrm{p}<0.01)$. The methylation rate of the posttreatment group was significantly lower than that in the GC group $(\mathrm{p}<0.05)$. In GC patients, HoxD10 promoter methylation was correlated to tumor invasion depth $(\mathrm{p}<0.05)$, while no association was found in sex, age, tumor size, TNM stage, CEA level and $H$. pylori infection $(\mathrm{p}>0.05)$.

Conclusion: It demonstrated that the HoxD10 promoter methylation rate in plasma dissociated DNA was increased with the progression from precancerous lesions to GC. Combined testing of HoxD10 methylation and CEA may be a great significant method for the early diagnosis of GC in high-risk populations. Disclosure of Interest: All authors have declared no conflicts of interest.

\section{References}

1. Fock K M. Review article: the epidemiology and prevention of gastric cancer. Alimentary Pharmacology \& Therapeutics. 2014; 40(3): 250-260.

2. Soetikno R, Kaltenbach T, Yeh R, et al. Endoscopic mucosal resection for early cancers of the upper gastrointestinal tract. Journal of Clinical Oncology Official Journal of the American Society of Clinical Oncology. 2005; 23(20): 4490-4498.

3. Hartgrink H H, Jansen E P M, Grieken N C T V, et al. Gastric cancer. Lancet. 2009; 374(9688): 477-490.

4. Liangjing W, Shujie C, Meng X, et al. Homeobox D10 Gene, a Candidate Tumor Suppressor, Is Downregulated through Promoter Hypermethylation and Associated with Gastric Carcinogenesis. Molecular Medicine. 2012; 18(1): 389 . 


\section{P1898 ACETYLCHOLINE INDUCES CANCER STEM CELL- LIKE}

\section{PHENOTYPE IN GASTRIC CANCER CELLS OF DIFFUSE TYPE}

P.H. Nguyen ${ }^{1}$, Y. Touchefeu ${ }^{2}$, E. Duchalais ${ }^{1}$, T. Durand ${ }^{3}$, P. Aubert ${ }^{1}$, P. Girot ${ }^{2}$, M. Peron ${ }^{2}$, S. Bruley Des Varannes ${ }^{2}$, C. Varon ${ }^{3}$, M. Neunlist ${ }^{1}$, T. Matysiak Budnik $^{2}$

${ }^{1}$ University Of Nantes, INSERM U1235, Nantes/France

${ }^{2}$ Hepato-gastroenterology, IMAD, CHU de Nantes, Nantes/France

${ }^{3}$ Université De Bordeaux, INSERM U1053, Bordeaux/France

Contact E-mail Address: tamara.matysiakbudnik@chu-nantes.fr

Introduction: The mechanisms of gastric carcinogenesis, especially of diffuse type of gastric cancer (GC) are poorly understood. The cancer stem cells (CSC) represent a particular fraction of cancer cells, considered at the origin of cancer and responsible for tumor development. The enteric nervous system (ENS) is a newly recognized component of tumor microenvironment and its role in carcinogenesis has been recently suggested. In particular, the role of acetylcholine (ACh), a major neuromediator released by enteric nerves, has been suggested in gastric carcinogenesis but its effect on gastric CSC remains to be established.

Aims \& Methods: Our aim was to study the effect of ACh on gastric cancer cells, and in particular its capacity to induce the stem cell phenotype, and to study the mechanisms involved. Adenocarcinoma gastric epithelial cells MKN-45 were first cultured in adherent conditions in the presence of ACh $(0.1-1-10 \mu \mathrm{M})$, before being cultured in non- adherent condition in order to favour expansion of CSC and formation of tumorspheres (T). The effect of ACh on $\mathrm{T}$ formation was evaluated under microscope by quantifying the number and size of $\mathrm{T}$ using the System snapshot file in INCell analyzer 2200/6000. The involvement of different cholinergic (muscarinic and nicotinic) receptors in ACh- induced responses was studied by pharmacological approach using selective agonists and antagonists. The role of nitregic pathway was studied using SNP (NO donor) and L-Name (nitric oxide synthesis inhibitor). Finally, the effect of $\mathrm{ACh}$ on the expression of CSC and epithelial-mesenchymal transition (EMT) markers was studied by immunofluorescence, RT-qPCR and flow cytometry. Statistical analysis was performed by Man-Whitney test, Kruskal-Wallis test, or two-way non-parametric ANOVA test using SPSS16.0 F software

Results: ACh at concentrations of 0.1 and $1 \mu \mathrm{M}$ significantly increased the number and size of $\mathrm{T}$ as compared to control conditions $(\mathrm{p}<0.001)$ Bethanecol, a selective muscarinic receptor agonist, increased the number and size of T, while the stimulatory effect of ACh on T was significantly reduced by selective muscarinic receptor antagonist, atropine. Similarly, DMPP, a selective nicotinic receptor agonist, increased the number and size of T, while hexamethonium, a nicotinic receptor antagonist, significantly inhibited stimulatory effect of $\mathrm{ACh}$ on $\mathrm{T}$ ( $\mathrm{p}<0.001$ as compared to ACh-stimulated cells). Interestingly, LName, significantly inhibited the effects of ACh on the number of T. Conversely, SNP, at 0.1 and $1 \mu \mathrm{M}$, increased the number and size of T. Finally, ACh induced the expression of CSC markers (CD44, ALDH) and EMT markers (Snail, Zebl and Vimentin) on gastric cancer cells.

Conclusion: This study shows that $\mathrm{ACh}$ induces CSC properties of gastric cancer cells of diffuse type via activation of muscarinic and nicotinic pathways. It also shows that Ach effects are, at least in part, mediated by nitregic pathway. These results suggest that ENS may be a new actor in gastric carcinogenesis.

Disclosure of Interest: All authors have declared no conflicts of interest.

P1899 SYSTEMATIC REVIEW AND META-ANALYSIS OF THE PROGNOSTIC SIGNIFICANCE OF CPG ISLAND METHYLATOR PHENOTYPE IN GASTRIC CANCER

A. Powell ${ }^{1}$, S. Soul ${ }^{2}$, N. Patel ${ }^{3}$, C. Brown ${ }^{3}$, W. Lewis ${ }^{3}$

${ }^{1}$ Division Of Cancer And Genetics, Cardiff University, Cardiff/United Kingdom

${ }^{2}$ Abertawe Bro Morgannwg University Health Board. Swansea/United Kingdom ${ }^{3}$ General Surgery, University Hospital of Wales, Cardiff/United Kingdom

Contact E-mail Address: powella16@cardiff.ac.uk

Introduction: $\mathrm{CpG}$ Island Methylator Phenotype (CIMP) has been identified as a distinct molecular subtype of gastric cancer, yet associations with survival are conflicting. A meta-analysis was performed to estimate its potential prognostic significance.

Aims \& Methods: A systematic review of Embase, Medline, PubMed, PubMed Central and Cochrane databases related to CIMP in gastric was performed and the primary outcome measure was cumulative 5 -year survival.

Results: A total of 967 patients from 10 studies were included, and the median rate of tumour CIMP-H was $40.9 \%$ (range $5.3-62.7 \%$ ). Pooled analysis suggested that specimens exhibiting CIMP-H were associated with poorer 5-year survival (OR 1.49, 95\% CI 1.11-2.01, p < 0.05). Significant heterogeneity was observed between studies $\left(\mathrm{I}^{2}=88 \%, \mathrm{p}<0.001\right)$. Sub-analysis related to poor $(n=5)$ or improved outcomes $(n=5)$, revealed that CIMP was associated with both poor (OR $8.15,95 \%$ CI $4.65-14.28, \mathrm{p}<0.05$, study heterogeneity $\mathrm{I}^{2}=52 \%$, $\mathrm{p}=0.08)$ and improved survival (OR $0.42,95 \%$ CI $0.27-0.65, \mathrm{p}<0.05$, study heterogeneity $\left.\mathrm{I}^{2}=0 \%, \mathrm{p}=0.960\right)$.

Conclusion: There was significant heterogeneity in the gene panels used to identify CIMP which may explain the survival differences. Consensus regarding CIMP methodology would be welcome to better facilitate the assessment of its prognostic value.

Disclosure of Interest: All authors have declared no conflicts of interest.
WEDNESDAY, NOVEMBER 01, 201709:00-14:00

H. PYLORI III - HALL 7

\section{P1900 THE VALIDITY OF BREATH COLLECTION BAGS USING THE NOVEL BREATHID ${ }^{\circledR} H P$ LAB SYSTEM: A MULTICENTER CLINICAL STUDY IN 257 SUBJECTS}

H. Shirin ${ }^{1}$, J. Gonzalez ${ }^{2}$, S. Hazan ${ }^{3}$, G. Gottlieb ${ }^{4}$, K. Friedenberg ${ }^{5}$, D. Gatof ${ }^{6}$, R. Ganeshappa ${ }^{7}$, S. Delgado ${ }^{8}$, D. Abramowitz ${ }^{1}$, R. Hardi ${ }^{9}$, A. Coates ${ }^{10}$, M. Haq ${ }^{11}$, N. Mehta ${ }^{12}$, B. Jones ${ }^{13}$, S. Moss ${ }^{14}$

${ }^{1}$ Assaf Harofeh Medical Center, Tel-Aviv University, Zerifin/Israel

${ }^{2}$ Palmetto Research, LLC, Hialeah/United States of America

${ }^{3}$ Ventura Clinical Trials, Ventura/United States of America/CA

${ }^{4}$ Del Sol Research Management, Tuscon/United States of America/AZ

${ }^{5}$ Great Lakes Gastroenterology, Mentor/United States of America/OH

${ }^{6}$ Innovative Clinical Research, Lafayette/United States of America/CO

${ }^{7}$ Digestive Disease Center of South Texas, P.L.L.C, San Antonio/United States of America/TX

${ }^{8}$ Barzilai Medical Center, Ben Gurion University of the Negev, Ashkelon/Israel ${ }^{9}$ Chevy Chase Clinical Research, Chevy Chase/United States of America/MD ${ }^{10}$ West Michigan Clinical Research Center, Wyoming/United States of America $M I$

${ }^{11}$ Hope Clinical Research, Kissimmee/United States of AmericalFL

${ }^{12}$ Digestive Disease Care PC, New Hyde Park, Hyde Park/United States of America $/ N Y$

${ }^{13}$ Innovative Clinical Research, Rapid City/United States of America/SD

${ }^{14}$ Rhode Island Hospital, Brown University, Providence/United States of America/ $R I$

Contact E-mail Address: haimsh@asaf.health.gov.il

Introduction: The BreathID $\mathbb{R} H p$ urea breath test (UBT) system provides several advantages over other ${ }^{13} \mathrm{C}$ breath analyzers for the detection of Helicobacter pylori (H. pylori), including: higher accuracy, operator independence and immediacy of results. However, there are occasions when mailing or transporting saved breath samples may be preferable to real time analysis, especially in centers requiring a large quantity of automated analyses where continuous sampling from a single patient can provide a bottle neck preventing multiple other users from accessing the device.

Aims \& Methods: To evaluate the sensitivity and specificity of a new BreathID ${ }^{\circ} \mathrm{Hp}$ Lab System (Exalenz Bioscience Ltd, Modiin, Israel), a ${ }^{13} \mathrm{C}-$ UBT system using breath sampling bags, for the diagnosis of $H$. pylori in a multi-center international clinical study. A total of 257 subjects with evaluable results for UBT and biopsy [rapid urease testing (RUT) and histology] were enrolled into two study groups: 189 naïve subjects with unknown $H$. pylori status were included in the pre-therapy group, and 68 subjects who had completed eradication therapy comprised the post-eradication therapy group. To assess the stability of the breath samples in the breath sample bags, each pair of breath sample bags was analyzed at a different time point up to 14 days after collection. Analytical studies were conducted to evaluate the reproducibility and repeatability of the ${ }^{13} \mathrm{C}-\mathrm{UBT}$ results using a cut-off of 5 Delta Over Baseline (DOB).

Results: Among the pre-therapy subjects evaluated with the composite results from the two biopsy based methods (RUT and histology/immunohistochemistry), 176 results ( 37 positive, 139 negative) matched those of the UBT resulting in an overall agreement of $98.3 \%(95 \%$ CI: $95.2 \% ; 99.7 \%)$ with a sensitivity of $100 \%(95 \%$ CI: $90.6 \% ; 100.00 \%)$ and specificity of $97.9 \%(95 \%$ CI: $94.0 \%$; $99.3 \%$ ). The overall agreement between the UBT and the biopsy diagnosis in the post-eradication therapy cohort (55 negative, 13 positive) was $98.5 \%(95 \%$ CI: $92.1 \% ; 100.0 \%)$. The sensitivity of the UBT in this cohort was $92.3 \%(95 \%$ CI: $66.7 \% ; 98.6 \%)$ and its specificity was $100.0 \%(95 \%$ CI: $93.5 \% ; 100.0 \%)$. The overall stability evaluated in samples of 191 (45 positive, 146 negative) subjects from the pre-therapy cohort was excellent, with positive agreement in $97.8 \%$ $[95 \%$ CI $(88.43,99.61)])$ and negative agreement in $100.0 \%$ [95\% CI $(97.44$, $100.0)$ ]. Similarly there was uniformly high overall reproducibility of the test results over different batches, when analyzed on different days and under different storage conditions.

Conclusion: The validation studies of the BreathID $\mathbb{B} H p$ Lab System described above show it is a highly accurate and dependable method for the diagnosis of $H$. pylori infection.Based on the current study, BreathID $\mathbb{R}$ Hp Lab System received on November 2016 U.S. Food and Drug Administration (FDA) marketing clearance for $H$. pylori bacterium detection.

Disclosure of Interest: H. SHIRIN: Haim Shirin received grants and stock options from Exalenz

All other authors have declared no conflicts of interest.

\section{References}

1. Chey WD, Wong BC, Practice Parameters Committee of the American College of Gastroenterology. American College of Gastroenterology guideline on the management of Helicobacter pylori infection. Am J Gastroenterol. 2007;102:1808-1825.

2. Management of Helicobacter pylori infection - the Maastricht V/Florence Consensus Report. P Malfertheiner, F Megraud, C A O'Morain, J P Gisbert, E J Kuipers, A T Axon, F Bazzoli, A Gasbarrini, J Atherton, D Y Graham, R Hunt, P Moayyedi, T Rokkas, M Rugge, M Selgrad, S Suerbaum, K Sugano, E M El-Omar, on behalf of the European Helicobacter and Microbiota Study Group and Consensus panel. Gut. 2016;0:1-25. 
3. Schmilovitz-Weiss H, Sehayek-Shabat V, Eliakim R, Skapa E, Avni Y, Shirin H. Applicability of a short/rapid 13C-urea breath test for Helicobacter pylori: retrospective multicenter chart review study. $B M C$ Gastroenterology. 2012;12:8

4. Broide E, Shirin H. Evaluation of Exalenz Bioscience's BreathID for Helicobacter pylori detection. Expert Rev Mol Diagn. 2015;15(3):299-312.

\section{P1901 MACHINE-LEARNING-BASED AUTOMATIC DIAGNOSIS SYSTEM FOR HELICOBACTER PYLORI INFECTION USING LINKED COLOR IMAGING}

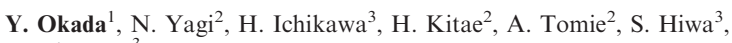
T. Hiroyasu ${ }^{3}$

${ }^{1}$ Life And Medical Science, Graduate School of Doshisha University, Kyotanabe/ Japan

${ }^{2}$ Gastroenterology, Murakami Memorial Hospital, Asahi University, Gifu/Japan

${ }^{3}$ Faculty Of Life And Medical Science, Doshisha University, Kyotanabe/Japan

Contact E-mail Address: yokada@mis.doshisha.ac.jp

Introduction: Linked color imaging (LCI), a recently developed endoscopic technique, emphasizes diffuse redness, which is a characteristic of Helicobacter pylori $(\mathrm{Hp})$ infection. However, the diagnosis of $\mathrm{Hp}$ infection does not have objective indicators; it depends on medical doctors' experience. Therefore, it is necessary to construct objective indicators to diagnose $\mathrm{Hp}$ infection.

Aims \& Methods: The aims of this study are to determine objective indicators for the presence or absence of Hp infection to support medical doctors' diagnoses by constructing an automatic diagnostic system. In the proposed system, first, a region with a high hue in LCI images is defined as a region of interest (ROI). Images with a wide ROI and images with a narrow ROI are classified as high and low hue images, respectively. As a result, LCI images are classified into two types in which inflammation due to $\mathrm{Hp}$ infection presents as red and purple. Then, the presence or absence of $\mathrm{Hp}$ infection is learned by machine learning for each type of LCI image. The feature values used in the learning process are the ratio of the ROI, the average and median hue values in high hue images, and the mode saturation value and the median and variance of the hue in low hue images. Then, the trained classifiers diagnose the presence or absence of $\mathrm{Hp}$ infection automatically. In this paper, the constructed system was evaluated using 128 images (32 patients) in which endoscopic examination (LCI observation) and $\mathrm{Hp}$ infection diagnosis were performed at Murakami Memorial Hospital of Asahi University. Furthermore, support vector machines were used as classify learners for diagnosis

Results: In the previous system [1], 29 out of 32 cases were automatically diagnosed correctly. In contrast, all cases were automatically diagnosed correctly with the proposed system. This result demonstrates that classifying LCI images into two types based on color improves the accuracy of this system.

Conclusion: The proposed system can automatically diagnose the presence or absence of $\mathrm{Hp}$ infection with the same precision as medical doctors with sufficient experience. Therefore, the proposed system can support the diagnosis of medical doctors with less experience.

Disclosure of Interest: All authors have declared no conflicts of interest.

\section{Reference}

1. Hiroyasu T, Okada Y, et al. Helicobacter pylori Infection Identification from Gastroscopy Images: Reliability and Validity of Endoscopy Linked Color Imaging. Proceedings of The Twenty-Second International Symposium on Artificial Life and Robotics 2017. 2017; 595-598

\section{P1902 HELICOBACTER PYLORI DETECTION BY $\Gamma$ - GLUTAMYLTRANSPEPTIDASE-ACTIVATED FLUORESCENT PROBE}

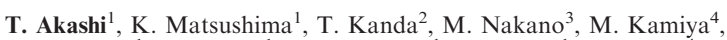

Y. Akazawa ${ }^{1}, \mathrm{~K}$. Ohnita ${ }^{1}, \mathrm{~F}$. Takeshima ${ }^{1}, \mathrm{~K}$. Nakao ${ }^{1}$, Y. Urano ${ }^{4}, \mathrm{H}$. Isomoto ${ }^{4}$ ${ }^{1}$ Department Of Gastroenterology And Hepatology, Nagasaki university hospital, Nagasaki/Japan

${ }^{2}$ Division Of Medicine And Clinical Science, Tottori University Hospital, Yonago/ Japan

${ }^{3}$ International Health, Institute Of Tropical Medicine, Nagasaki University, Nagasaki/Japan

${ }^{4}$ Laboratory Of Chemical Biology And Molecular Imaging, Graduate School Of Medicine, The University of Tokyo, Tokyo/Japan

Contact E-mail Address: fuk_naga_ta23@yahoo.co.jp

Introduction: $\gamma$-glutamyltranspeptidase (GGT) is a cell surface-associated enzyme that is not highly expressed in normal cell. However GGT is overexpressed in various type of human cancers. It is known that Helicobacter pylori $(H$. pylori) also produce GGT. Urano et al have developed an enzymatically activatable fluorescent probe, $\gamma$-glutamyl hydroxymethyl rthodamine green (gGluHMRG), which is nonfluorescent under a neutral $\mathrm{pH}$ and normal cellular environment, but turns to be highly fluorescent upon reaction with $\mathrm{GGT}^{1)}$. Aim of this study is to consider if gGlu-HMRG can be useful for diagnosing infection $H$. pylori.
Aims \& Methods: In this study, we investigated whether activation of gGluHMRG fluorescence detects a wild-type of $H$. pylori (WT) and a ggt gene-disrupted mutant of $H$. pylori (ggt mutant). In addition, we investigated whether activation of gGlu-HMRG fluorescence was suppressed in $H$. pylori culture solution which was co-incubated with an inhibitor of GGT (GGsTop). Furthermore, we applied gGlu-HMRG to biopsy specimens which were taken from antrum and corpus of stomach in $H$. pylori positive patients $(\mathrm{n}=13)$ and $H$. pylori negative patients $(\mathrm{n}=14)$. We then observed the increase of fluorescence intensity over time $(1 \mathrm{~min}, 5 \mathrm{~min}, 10 \mathrm{~min}, 15 \mathrm{~min})$. Fluorescence intensity was quantified by Image J2 software (National Institutes of Health, Rockville, Maryland $)^{2}$.

Results: Activation of gGlu-HMRG fluorescence was detected in WT strain, but was not in ggt mutant strain. Activation of gGlu-HMRG fluorescence was inhibited by GGsTop. There was significant difference of the increase of fluorescence intensity between $H$. pylori positive and negative both in antrum corpus of stomach (antrum $\mathrm{p}=0.0008$, corpus $\mathrm{p}=0.047$ ).

Conclusion: GGT-activated fluorescent probe can be useful for $H$. pylori infection diagnosis.

Disclosure of Interest: All authors have declared no conflicts of interest.

\section{References}

1. Urano Y, Sakabe M, Kosaka N, et al. Rapid cancer detection by topically spraying a $\gamma$-glutamyltranspeptidase-activated fluorescent probe. Sci Transl Med 2011;3:110ra119.

2. Mizushima T, Ohnishi S, Shimizu Y, et al. Fluorescent imaging of superficial head and neck squamous cell carcinoma using a $\gamma$-glutamyltranspeptidaseactivated targeting agent: a pilot study. BMC Cancer 2016;16:411.

\section{P1903 SEROLOGICAL CHANGES AFTER EQUIVOCAL HELICOBACTER PYLORI-SEROLOGY TEST FINDINGS DEPEND ON THE GASTRIC SECRETING ABILITY}

J.H. Kim, S. Lee, S.P. Lee, J.H. Kim, I. Sung, H.S. Park, C.S. Shim Internal Medicine, Gastroenterology, Konkuk University Hospital, Seoul/Korea Republic of

Contact E-mail Address: kjhcess@gmail.com

Introduction: The serum anti-Helicobacter pylori (H. pylori) IgG and serum pepsinogen (PG) assays are widely used for gastric cancer screening. An equivocal serology test finding indicates $\mathrm{IgG}$ titer between the positive and negative test findings.

Aims \& Methods: The study aim was to evaluate the long-term, follow-up result after an equivocal test finding on the serum anti-H. pylori $\operatorname{IgG}$ assay. Koreans above 18 years-old with an equivocal serum anti- $H$. pylori $\mathrm{IgG}$ assay finding were included. Subjects were excluded if they did not undergo $H$. pylori serology test, serum PG assay, and upper gastrointestinal (UGI) endoscopy on the same day at our center. Annual test findings were followed up using the same methods.

Results: Of the 7,178 subjects who underwent the serum assays and UGI endoscopy on the same day, $274(3.8 \%)$ subjects showed an equivocal H. pylori serology test finding. Of the 98 followed-up subjects, $58(59.2 \%)$ showed seropositive finding at the mean follow-up period of $30.6 \pm 12.4$ months. Subjects with seroconversion showed a higher initial serum PG I $(\mathrm{p}=0.023)$ and PG II $(\mathrm{p}=0.036)$ levels than the subjects without seroconversion.

Conclusion: An equivocal $H$. pylori serology test finding is not rare $(3.8 \%)$ in Korean adults, and $60 \%$ of the equivocal subjects show seroconversion within 3 years. Higher seroconversion rates in the subjects with high PG I and PG II levels suggest that intact gastric secreting ability play a role for the survival of $H$. pylori. Therefore, equivocal subjects with increased serum PG levels should be considered as a potential seropositive subjects.

Disclosure of Interest: All authors have declared no conflicts of interest.

\section{References}

1. Sung JK. Diagnosis and treatment of Helicobacter pylori infection. Korean $J$ Med 2015;89:149-156.

2. Mizuno S, Miki I, Ishida T, et al. Prescreening of a high-risk group for gastric cancer by serologically determined Helicobacter pylori infection and atrophic gastritis. Dig Dis Sci 2010;55:3132-3137.

3. Choi HS, Lee SY, Kim JH, Sung IK, Park HS, Shim CS. Combining the serum pepsinogen levels and Helicobacter pylori antibody test is not useful for detecting neoplasms with advanced histology. J Dig Dis 2014;15:293-298.

4. Samloff IM, Varis K, Ihamaki T, et al. Relationships among serum pepsinogen I, serum pepsinogen II, and gastric mucosal histology. A study in relatives of patients with pernicious anemia. Gastroenterology 1982;83:204-209.

5. Salmonff IM. Cellular localization of group I pepsinogen in human gastric mucosa by immunofluorescence. Gastroenterology 1971:61:185-188.

6. Samloff IM, Liebman WM. Cellular localization of the group II pepsinogens in human stomach and duodenum by immunofluorescence. Gastroenterology 1973;65:36-42

7. Lee SY. Endoscopic gastritis, serum pepsinogen assay, and Helicobacter pylori infection. Korean J Intern Med 2016;31:835-844.

8. McColl KE. Clinical practice. Helicobacter pylori infection. $N$ Engl J Med 2010;362:1597-1604. 
9. Ekstrom AM, Held M, Hansson LE, Engstrand L, Nyrén O. Helicobacter pylori in gastric cancer established by CagA immunoblot as a marker of past infection. Gastroenterology 2001;121:784-791.

10. Graham DY, Nurgalieva ZZ, El-Zimaity HM, et al. Noninvasive versus histologic detection of gastric atrophy in a Hispanic population in North America. Clin Gastroenterol Hepatol 2006;4:306-314.

11. Kokkola A, Rautelin H, Puolakkainen P, et al. Diagnosis of Helicobacter pylori infection in patients with atrophic gastritis: comparison of histology, 13C-urea breath test, and serology. Scand J Gastroenterol 2000;35:138-141.

\section{P1904 WE CAN JUDGE THE EXISTENCE OF PRESENT OR PAST H. PYLORI INFECTION WITH ONLY ONE ENDOSCOPIC CARDIAC IMAGES (WHALE SHARK SIGN: WSS)}

T. Yamasaki ${ }^{1}$, T. Sakurai ${ }^{1}$, J. Mitobe ${ }^{1}$, M. Mitsunaga ${ }^{1}$, Y. Amano ${ }^{2}$, Y. Tokai ${ }^{3}$, M. Saruta ${ }^{1}$

${ }^{1}$ Department Of Gastroenterology And Hepatology, The Jikei University, Tokyo/ Japan

${ }^{2}$ Department Pf Gastroenterology, Saitama Kyodo Hospital, Kawaguchi/Japan

${ }^{3}$ Department Pf Gastroenterology, Cancer Institute Hospital, Tokyo/Japan

Contact E-mail Address: takusan.yamasan@gmail.com

Introduction: Several $H$. pylori (HP)infection related gastric findings (mucosa atrophy, metaplastic change, diffuse redness, spotty redness and nodular change of the antrum etc.) are so important sign of HP infection on endoscopic examination. On the other hand, we have confused with various newly endoscopic findings (patchy redness and map-like redness etc.) were seen on the posteradicated stomach. On this time, we have found out a new other ultimate useful finding showing HP infection related gastritis at gastric cardia (EG junction) including present and post HP infection. The endoscopic image of gastric cardia is the first gastric view through the esophagus on each endoscopic examination.

Aims \& Methods: Our aim of this study is to elucidate possibility of judgement with only this cardiac endoscopic view about presence or absence with HP infection. We have found out so useful and specific cardiac image (We call Whale Shark Sign: WSS) closely related to HP infection. We have examined the presence of WSS on 4, 268 cases that have been able to overviewed on their endoscopic profiles. We have judged the presence of HP infection with serum HP antibody titers on each case. The 3,233 cases their serum HP antibody titers were measured from Jan. 2012 to Oct. 2016. A total of 2,810 patients (HP positive) were enrolled. Fisher's exact test was used in all statistical analyses. The judgement of HP present or past infection was done more than serum HP antibody $3 \mathrm{U} / \mathrm{ml}$ to avoid false negative results.

Results: Mean age of patients was 52.4 years old. In case of WSS positive, al their serum HP antibody titers showed more than cut-off level $(3 \mathrm{U} / \mathrm{ml})$. This means that the presence of WSS closely related to HP related gastritis. The positive predictive value (PPV) of WSS was surprisingly high (98\%). According to this high PPV, we can think WSS positive cases are high risk of gastric carcinoma. This WSS mean that the presence of irregular gastric mucosa surface pattern and the presence of lymphoid hyperplasia, that showing HP infectious stomach. Especially presence of lymphoid hyperplasia at gastric cardia is most important specific sign of HP related gastritis. This lymphoid hyperplasia at gastric cardia were recognized small round whitish nodules on white light endoscopy. And this was more emphasized with image-enhanced endoscopy (Narrow Band Imaging: NBI), it looks like Whale Shark. This WSS sign is very simple and easy for every gastroenterologist. It is so useful to know gastric cancer risk at gastric entrance (cardia) with the presence of very easy simple sign.

Conclusion: We have been able to judge the presence of HP infection with only cardiac endoscopic images (WSS), we should take care of seeing the presence of
WSS sign. Since this sign is very easy and simple, everyone will be able to judge the presence of HP infection and gastric cancer risk.

Disclosure of Interest: All authors have declared no conflicts of interest.

\section{P1905 SERUM PEPSINOGEN II AS A NON-INVASIVE MARKER FOR DIAGNOSIS OF HELICOBACTER PYLORI INFECTION: A} PROSPECTIVE STUDY IN A COHORT OF DYSPEPTIC PATIENTS

F. Di Mario ${ }^{1}$, C. Miraglia ${ }^{1}$, S. Scida ${ }^{1}$, A. Violi ${ }^{1}$, M. Franceschi ${ }^{2}$, G. Baldassarre ${ }^{3}$,

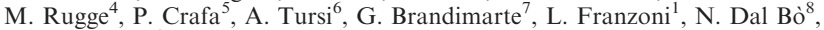
R. Cannizzaro ${ }^{9}$, C. Scarpignato

${ }^{1}$ Department Of Medicine And Surgery, University of Parma, Italy, University of Parma, Parma/Italy

${ }^{2}$ Endoscopic Unit, Department Of Surgery, Ulss4, Hospital ULSS4 Alto Vicentino, Santorso/Italy

${ }^{3}$ Digestive Endoscopy Unit, ULSS4 Alto Vicentino, Santorso/Italy

${ }^{4}$ Pathology, Medical School of the Padova University, Padova/Italy

${ }^{5}$ Department Of Medicine And Surgery, University of Parma, Parma/Italy

${ }^{6}$ Gastroenterology Service, ASL BAT Gastroenterology Service, Andria/Italy

"Division of Internal Medicine and Gastroenterology, "Cristo Re" Hospital, Rome. Italy, Roma/Italy

${ }^{8}$ Gastroenterological Unit, Treviso Hospita, Treviso/Italy

${ }^{9}$ Oncological Gastroenterology, Centro di Riferimento Oncologico di Aviano

S.O.C. di Gastroenterologia, Aviano/Italy

Contact E-mail Address: francesco.dimario@unipr.it

Introduction: The diagnosis of Helicobacter pylori (H.p.) infection is currently made by means of non-invasive (Urea Breath Test or HpSA) or invasive (gastric biopsy or culture) methods. Serological assessment of H.p. infection by using levels of $\mathrm{IgG}$ is not recommended. Pepsinogen II (PGII) is validated in the literature as a non-invasive marker of gastric inflammation. Aim of the study was to assess in a population of dyspeptic patients the clinical availability of PGII determination in singling out subjects infected by H.p. in comparison with noninfected ones.

Aims \& Methods: A cohort of 880 consecutive dyspeptic patients (F 439; mean age 55.5 ys; range: 29.83 ys) were enrolled in the study. Exclusion criteria: previous surgery, previous H.p. eradication therapy, concomitant PPI. In all patients the diagnosis of H.p. infection was made by means of upper GI endoscopy and at least one of these two methods: UBT, HpSA. All patients underwent blood sample for determination of serum levels of PGII (Biohit Oyj; Finland; normal values: $2-15 \mu \mathrm{g} / 1$ ). In a group of $670 \mathrm{pts}$ a course of antibiotics (triple, concomitant or sequential therapy) to cure H.p. infection was performed and PGII levels were assessed at baseline (T0) and after two months (T1) from the end of the antibiotic therapy. The search for the most appropriate cut-off of PGII in diagnosis of H.p. infection was assessed by using the ROC curve method.

Results: 430 out of 860 dyspeptic patients (F 245;mean age 52.3 ys; range $32-69$ ys) showed an H.p-related gastritis (group1) in comparison with 430 (F 261; mean age 57.3 ys; range 38-74 ys) resulted negative for H.p infection (group 2). The mean value of PGII in group 1 was $20.9 \pm 6.1$ opposite to $7.2 \pm 1.7$ in group $2 ; \mathrm{p}<0.0001$. 415 out of 670 patients treated with antibiotics schedules were cured from H.p infection, opposite with 255 non-eradicated ones. In the group of cured patients, the PGII values at T0 were 16.5. in comparison with 8.6 at T1; $p<0.001$. The cut-off for PGII in the diagnosis of H.p infection, by comparing the $430 \mathrm{H} . \mathrm{p}$ positive pts and the 430 negative ones with method of ROC curve identified the mean value of $10.6 \mu \mathrm{g} / 1$, youden index $\mathrm{J}=0.997$.

Conclusion: serum PGII levels seem able to perform diagnosis of H.p infection in dyspeptic patients, as well as the efficacy of antibiotics treatment for H.p eradication, being $10.6 \mu \mathrm{g} / 1$ the best cut-off in singling out infected from non-infected subjects.

Disclosure of Interest: All authors have declared no conflicts of interest.

Abstract No: P1903. Table: Different characteristics of the subjects with an equivocal $H$. pylori test finding according to the repeated $H$. pylori serology test findings

\begin{tabular}{|c|c|c|c|}
\hline Variables & $\begin{array}{l}\text { Seropisitive finding on the } \\
\text { follow-up test }(\mathrm{n}=58)\end{array}$ & $\begin{array}{l}\text { Seronegative or equivocal } \\
\text { finding on the follow-up test }(n=40)\end{array}$ & p-value \\
\hline Age (years-old) & $52.1 \pm 9.8$ & $54.0 \pm 11.2$ & 0.386 \\
\hline Gender (male) & $43(74.1 \%)$ & $23(57.5 \%)$ & 0.084 \\
\hline Follow-up preriod (months) & $32.1 \pm 13.0$ & $28.6 \pm 11.3$ & 0.165 \\
\hline Past H. pylori eradication & $6(10.3 \%)$ & $10(25.0 \%)$ & 0.054 \\
\hline Initial serum pepsinogen I level $(\mathrm{ng} / \mathrm{ml})$ & $61.2 \pm 32.0$ & $48.5 \pm 15.6$ & 0.023 \\
\hline Initial serum pepsinogen II level $(\mathrm{ng} / \mathrm{ml})$ & $12.3 \pm 8.6$ & $9.3 \pm 3.5$ & 0.036 \\
\hline Initial serum pepsinogen $\mathrm{I} / \mathrm{II}$ ratio & $5.5 \pm 1.5$ & $5.5 \pm 1.4$ & 0.985 \\
\hline Body mass index $\left(\mathrm{kg} / \mathrm{m}^{2}\right)$ & $24.6 \pm 3.4$ & $23.8 \pm 2.6$ & 0.231 \\
\hline Cigarette smoking Current smoker Past smoker Non-smoker & $18(31.0 \%) 16(27.6 \%) 24(41.4 \%)$ & $5(12.5 \%) 13(32.5 \%) 22(55.0 \%)$ & 0.101 \\
\hline Alcohol drinking Heavy drinker* Social drinker Non-drinker & $5(8.6 \%) 40(69.0 \%) 13(22.4 \%)$ & $6(14.3 \%) 23(59.5 \%) 11(26.2 \%)$ & 0.451 \\
\hline Upper gastrointestinal symptom & $18(31.0 \%)$ & $10(25.0 \%)$ & 0.516 \\
\hline Recent intake of drug(s) & $14(24.1 \%)$ & $8(20.0 \%)$ & 0.629 \\
\hline Comorbidity Hypertension Diabetes mellitus Others & $17(29.3 \%) 5(8.6 \%) 19(32.8 \%)$ & $13(32.5 \%) 4(10.0 \%) 13(32.5 \%)$ & 0.7360 .8160 .979 \\
\hline
\end{tabular}

Statistically significant values are highlighted in bold. Continuous variables are shown as mean value \pm standard deviation using the Student's $t$-test. Categorical variables are shown in frequency (\%) using the Chi-square test or Fisher's exact test. *Criteria for heavy drinking was $\geq 15$ drinks/week for men and $\geq 8$ drinks/week for women. Social drinker was defined as those who drink alcohol, but less than heavy drinkers. 


\section{P1906 CAN THE UREA BREATH TEST PREDICT HELICOBACTER}

\section{PYLORI ERADICATION?}

D. Brennan ${ }^{1}$, C. Dalton ${ }^{1}$, P. Murray ${ }^{1}$, J. O’Toole ${ }^{1}$, H. Temperley ${ }^{1}$, C. O’Morain ${ }^{2}$, S. Smith ${ }^{1}$, D. Mcnamara ${ }^{1}$

${ }^{1}$ Trinity Academic Gastroenterology Group, Trinity College Dublin, Dublin/Ireland ${ }^{2}$ Faculty Of Health Sciences, Trinity College Dublin - Faculty of Health Sciences, Trinity College Dublin; Dublin/IE, Dublin/Ireland

Contact E-mail Address: dbrenna9@tcd.ie

Introduction: The Urea Breath Test (UBT) is considered the gold standard noninvasive test for detection of Helicobacter pylori infection in Ireland. In Ireland, eradication rates for standard clarithromycin-based triple therapy have fallen below the $80 \%$ deemed acceptable for a given treatment. With this in mind, it is important to optimise management of $H$. pylori infection. It has been suggested that the DOB value is reflective of the amount of bacteria present in the stomach and could predict whether the infection is eradicated.

Aims \& Methods: The aim of this study was to determine whether there is an association between DOB and eradication of $H$. pylori infection in an Irish cohort. Treatment naïve adult patients undergoing UBT were included. Patients were deemed to be $H$. pylori positive if a Delta Over Baseline (DOB) value of $>4.0 \%$ was obtained. Positive patients were categorised into low $(<16$ $\%$ ), intermediate $(16-35 \%$ ), and high ( $>35 \%$ ) DOB groups. A random subset of positive patients was given clarithromycin-based triple therapy for 7 days. A follow-up breath test was performed at least 8 weeks post-treatment to confirm eradication of $H$. pylori in all patients. The three DOB groups were compared with respect to age, gender and eradication rates.

Results: Out of 860 of UBTs assessed (mean age $43.5 \pm 15.2$ years, $39 \%$ male), $289(33.6 \%$ ) were $H$. pylori positive (mean age $43.1 \pm 14.9$ years, $41.9 \%$ male). Of the total positive patients, $91(31.5 \%)$ returned for a follow-up UBT to confirm eradication of $H$. pylori. When patients were categorised into low, intermediate and high UBT groups, there was no significant difference in age and gender between the three groups $(\mathrm{p}=0.06$ for age, $\mathrm{p}=0.3$ for gender). Eradication rates in the low, intermediate and high UBT groups were $70.5 \%, 63.0 \%$ and $50.0 \%$ respectively $(\mathrm{p}=0.3)$. Patients were then categorised according to eradication status. When eradication was successful, the average DOB value was significantly lower, at $20.6 \%$ compared to $29.8 \%$ when eradication was unsuccessful ( $\mathrm{p}=0.03,95 \%$ CI 0.69 to 17.5$) .46(50.5 \%)$ patients were given clarithromycin-based triple therapy for 7 days. When this subset of patients was categorised into low, intermediate and high UBT groups, eradication rates were $76.2 \%, 75 \%$ and $70 \%$ respectively. When these rates were compared to respective rates in those whose treatment was not known, no difference was observed. The subset was also categorised according to eradication status. When eradication was successful, the average DOB value was lower, at $22.0 \%$ compared to $25.8 \%$ when eradication was unsuccessful $(\mathrm{p}=0.6)$. Similarly, when these DOB values were compared to respective values in those whose treatment was not known, no difference was observed.

Conclusion: As the DOB value increases in the UBT, the eradication rate of $H$ pylori decreases, regardless of treatment regimen. When categorised according to eradication status, the DOB value was significantly lower when eradication was successful (20.6\% vs $29.8 \%$, $\mathrm{p}=0.03)$. The $\mathrm{DOB}$ value could be a useful value in stratifying patients with $H$. pylori infection; especially as histology and antimicrobial resistance information is unavailable in patients undergoing non-invasive testing for $H$. pylori infection.

Disclosure of Interest: All authors have declared no conflicts of interest.

P1907 HIGH-DOSE CAM WITH VONOPRAZAN (P-CAB) PLUS AMX TRIPLE THERAPY REGIMEN IS THE STRONGEST H. PYLORI ERADICATION THERAPY EVEN IF CAM RESISTANCE

T. Yamasaki ${ }^{1}$, T. Sakurai ${ }^{1}$, J. Mitobe ${ }^{1}$, M. Mitsunaga ${ }^{1}$, Y. Amano ${ }^{2}$, Y. Tokai ${ }^{3}$, M. Chibai ${ }^{4}$, M. Saruta ${ }^{1}$

${ }^{1}$ Department Of Gastroenterology And Hepatology, The Jikei University, Tokyo/ Japan

${ }^{2}$ Department Pf Gastroenterology, Saitama Kyodo Hospital, Kawaguchi/Japan

${ }^{3}$ Department Pf Gastroenterology, Cancer Institute Hospital, Tokyo/Japan

${ }^{4}$ Surgery, Heiwadai Clinic, Tokyo/Japan

Contact E-mail Address: takusan.yamasan@gmail.com

Introduction: Success or failure of Amoxicillin (AMX) and Clarithromycin (CAM)-based $H$. pylori (HP) eradication therapy mainly depends on sensitivity of CAM for HP. In Japan, we are permitted to prescribe AMX and CAM as antibiotics of $1^{\text {st }}$ eradication. So far now, several proton pomp inhibitors (PPIs) have been used in HP eradication therapy, most of reports said that the success rate of eradication approximately from 70 to $80 \%$ on AMX and CAM-based triple therapy regardless of CAM dose $(400$ or $800 \mathrm{mg} /$ day $)$ in Japan. Recently the ratio of CAM resistance in Japan comes up to over $30 \%$, we have to overcome CAM resistance. On the other hand, recently it is considered that the stability of continuous gastric acid suppression is one of most important factors. At the point of gastric acid suppression, we expect stronger acid suppressive drug rather than PPIs. Since Feb. 2015 we have used P-CAB (Potassium-Competitive Acid Blocker) instead of PPIs in AMX and CAM-based HP eradication therapy to improve success rate of eradication.

Aims \& Methods: The aim of this study is to elucidate effects of CAM dose on success rate of P-CAB Vonoprazan (VPZ) and one of PPI Rabeprazole (RPZ) based triple therapy regimens (VAC, RAC).

This study is a multicenter center ( 7 hospitals), prospective case study from Jan. 2012 to Oct. 2016. A total of 1,310 patients (HP positive) were enrolled. Mean age of patients was 52.4 years old. Fisher's exact test was used in all statistical analyses. Regimen of VAC (400 or 800) was VPZ $(40 \mathrm{mg} /$ day $)$ b.i.d., AMX (1.500 mg/day) b.i.d. plus CAM $(400$ or $800 \mathrm{mg}$ day) b.i.d. for 7 days. Regimen of RAC (400 or 800) was RPZ (20 mg/day) b.i.d., AMX (1.500 mg/day) b.i.d. plus CAM (400 or $800 \mathrm{mg} /$ day) b.i.d. for 7 days. The judgement of success or failure on eradication was done with urea breath test on 3 months later after eradication therapy to avoid false negative results.

Results: Success rate of VAC 800 showed significantly high $(416 / 428=97.2 \%$, PPS) rather than VAC $400(373 / 423=88.2 \%$, PPS $)(\mathrm{p}<0.001)$. The average success rate of VAC regimens was $92.7 \%(789 / 851$, PPS). On the other hand, success rate of RAC 800 showed high $(190 / 245=77.6 \%$, PPS ) rather than RAC $400(140 / 198=70.7 \%$, PPS $)(p=0.125)$. The average success rate of RAC regimens was $74.5 \%(330 / 443$, PPS). The average success rate of VAC regimens $(789 / 851=92.7 \%)$ were significantly high rather than the average success rate of RAC regimens $(330 / 443=74.5 \%)(p<0.001)$. These results suggest that using high dose CAM with VPZ (P-CAB) plus AMX regimen might be the strongest $H$. pylori eradication triple therapy regimen to overcome CAM resistance.

Conclusion: High-dose CAM with Vonoprazan (P-CAB) plus AMX triple therapy regimen is the strongest $H$. pylori eradication therapy even if CAM resistance. We will be able to expect significantly high success rate (more than $97 \%$ ) as HP $1^{\text {st }}$ eradication therapy.

Disclosure of Interest: All authors have declared no conflicts of interest.

\section{P1908 COMPARISON OF THE EFFICACY BETWEEN BISMUTH AND ALTERNATING RIFAXIMIN ON SECOND-LINE QUADRUPLE REGIMEN OF HELICOBACTER PYLORI ERADICATION}

J. Park, J.S. Lee, J. Kim, S.J. Hong, H.G. Kim, T.H. Lee, S.R. Jeon, J. Cho Digestive Disease Center, Institute For Digestive Research, Soonchunhyang University College of Medicine, Seoul/Korea, Republic of

Contact E-mail Address: junspark@schmc.ac.kr

Introduction: Bismuth is a heavy metal which has antimicrobial activity through regulating iron uptake profile of bacteria. Helicobacter pylori (H. pylori) is also highly susceptible to bismuth. So the Korean guideline preferably recommends the regimen contains bismuth for the patients who failed on $H$. pylori eradication with the primary regimen consists of proton pump inhibitors, amoxicillin, and clarithromycin. Rifaximin is one of derivatives of rifamycin with antimicrobial activity against $H$. pylori. It can achieve high concentrations within the gastrointestinal tract and remains active in acidic environment. So rifaximin has been studied as a treatment for persistent $H$. pylori infection. Rifaximin has been prescribed for replacing the bismuth of the regimen concurrently uses PPI, metronidazole, and tetracycline in Soonchunhyang University Hospital, Seoul for a while. So we reviewed the clinical outcomes of the 2 different regimens.

Aims \& Methods: From May $1^{\text {st }} 2003$ to October $31^{\text {st }} 2015$, six thousand and five hundred ninety-five patients were treated their $H$. pylori infection in Soonchunhyang University Hospital, Seoul. And their prescriptions and result of eradication were retrospectively reviewed on the medical records. The patients had clarified pre-and post-eradication result, which can be assured by the rapid urease test or the urea breath test performed within 100 days of eradication were enrolled. And statistical analyses were performed to the patients who had secondline treatment to compare the efficacy of the bismuth containing regimen and the rifaximin containing one.

Results: During the periods over 12 years, two thousand and seven hundred patients were prescribed the standard triple eradication regimen and 2,109 $(78.11 \%)$ patients showed the successful treatment result. One hundred twentysix of treatment failure group were consecutively treated with second-line regimens. Thirty-five patients were prescribed the bismuth-containing regimen and $34(97.14 \%)$ of them showed successful eradication result. Other 91 patients were treated with the rifaximin-containing regimen and showed $92.31 \%$ of eradication rates. These treatment success rates are not different significantly in statistics. (Fishers' exact test, p-value $=0.442$ )

Conclusion: Alternative rifaximin containing regimen of second-line $H$. pylori treatment didn't showed inferiority on standard bismuth-containing one. This suggest that the use of rifaximin is possible in the patients who are not suitable to bismuth use and failed with primary eradication.

Disclosure of Interest: All authors have declared no conflicts of interest.

\section{P1909 COMPARISON OF 10-DAY STANDARD TRIPLE THERAPY AND LEVOFLOXACIN BASED THERAPY FOR HELICOBACTER PYLORI ERADICATION: RANDOMIZED CONTROLLED TRIAL}

M.K. Kang, S. Lee, M.C. Kim, K.H. Kim, K.O. Kim, B.I. Jang, T. Kim Internal Medicine, Yeungnam University College of Medicine, Daegu/Korea, Republic of

Contact E-mail Address: kmggood111@naver.com

Introduction: Standard triple therapy (STT) has been widely used in Helicobacter pylori infection, but eradication rate is decreasing because of clarithromycin resistance. Recently, Levofloxacin-based therapy (LBT) has been evaluated to overcome the low eradication rate of standard triple therapy and reported eradication rated over $80 \%$.

Aims \& Methods: We compared the efficacy and safety of STT group and LBT group for Koreans. Between April 2014 and April 2016, 49 patents in the STT group (amoxicillin $1 \mathrm{~g}$ bid, clarithromycin $500 \mathrm{mg}$ bid and esomeprazole $20 \mathrm{mg}$ bid for 10 days) and 48 in the LBT group(levofloxacin $500 \mathrm{mg}$ bid, amoxicillin $1 \mathrm{~g}$ 
bid and esomeprazole $20 \mathrm{mg}$ bid for 10 days) were enrolled, prospectively. $H$. pylori eradication rate as the primary endpoint and serious adverse effects as the secondary endpoint were defined.

Results: $H$. pylori eradication rate as the primary endpoint was higher in the LBT group than in the STT group, but there was no statisfically significant difference between the two groups $(82.0 \%$ vs. $84.8 \%, \mathrm{P}=0.927)$. Serious adverse effects as the secondary endpoint tended to be more frequent in the LBT group, but there was no significant difference $(6.1 \%$ vs. $15.2 \%, \mathrm{P}=0.267)$. The overall rates of adverse effects were not different between two groups.

Conclusion: In comparison of $H$. pylori eradication rate of our study, LBT was not significantly higher than STT, but it may be an alternative treatment if STT eradication rate is lowered in Korea.

Disclosure of Interest: All authors have declared no conflicts of interest.

\section{P1910 TREATMENT OF HELICOBACTER PYLORI INFECTION: WILL TAILORING THERAPY FIRST TIME OVERCOME INCREASING FAILURE OF STANDARD TRIPLE THERAPY?}

D. Brennan, M. Hussey, D. Tighe, C. O'Morain, S. Smith, D. Mcnamara Trinity Academic Gastroenterology Group, Trinity College Dublin, Dublin/Ireland

Contact E-mail Address: dbrenna9@tcd.ie

Introduction: In Ireland, Helicobacter pylori infection has become increasingly resistant to commonly used antibiotics, such as clarithromycin. Concurrently, eradication rates for standard clarithromycin-based triple therapy have fallen below the $80 \%$ deemed acceptable for a given treatment.

Aims \& Methods: The aim of this study was to compare eradication rates of standard clarithromycin-based triple therapy with those of tailored therapy based on antimicrobial susceptibility as a first-line treatment for $H$. pylori infection. Treatment-naïve adult patients undergoing endoscopy were prospectively recruited. Biopsies from $H$. pylori-positive patients (assessed by CLO test) were processed for sensitivity testing by E-testing and genotyping by the GenoType HelicoDR assay (Hain). Patients randomly received either clarithromycin-based standard triple therapy or tailored treatment based on antibiotic sensitivities, for $7 / 14$ days. A follow-up breath test was performed at least 8 weeks post-treatment. Results: To date 889 patients have undergone endoscopy and $186(21 \%)$ were $H$. pylori positive. Infected patients were significantly younger (mean age 53 vs 49 years, $\mathrm{p}=0.002)$ and tended to be male $(43 \%$ vs $53 \%, \mathrm{p}=0.02)$. Of $186 \mathrm{H}$. pyloripositive patients, $112(60 \%)$ were treatment naïve. Culture of $H$. pylori was successful in $57 \%(64 / 112)$ of samples and primary clarithromycin resistance was $47 \%(30 / 64)$ by E-test. Genotypic resistance data was available for $93 \%$ (104/112) patients and 55\% (61/104) strains were clarithromycin resistant. Thus far, $99(88 \%)$ treatment naïve patients have been enrolled in the study, $92(93 \%)$ have completed the study. Of these, $45(46 \%)$ have received standard triple therapy and $54(54 \%)$ have received tailored therapy. In the tailored arm, $25(46 \%)$ patients received standard triple therapy, $14(26 \%)$ received levofloxacin-based triple therapy, $12(22 \%)$ received a PPI and combination of antibiotics based on their sensitivities (e.g. levofloxacin, clarithromycin, rifampicin, tetracycline or metronidazole), and $3(6 \%)$ bismuth quadruple therapy. The eradication efficacy of tailored therapy by intention-to-treat analysis was higher at $74 \%$ (40/ 54) compared to $67 \%(\mathrm{n}=30 / 45)$ for standard therapy $(\mathrm{p}=0.5)$. The eradication efficacy by per-protocol analysis was also higher, at $82 \%(40 / 49)$ for tailored versus $70 \%(30 / 43)$ for standard therapy $(p=0.2)$. Patients in each arm were further categorised by clarithromycin resistance status, phenotypically by Etest or genotypically by GenoType HelicoDR when culture was unsuccessful Of note, in clarithromycin resistant patients, tailored therapy achieved a better eradication rate per protocol analysis than standard triple therapy ( $83 \%$ vs $57 \%$ per protocol, $\mathrm{p}=0.09$ ).

The eradication rates of standard triple therapy and tailored therapy according to clarithromycin resistance status

\begin{tabular}{ccllll}
\hline & & \multicolumn{2}{l}{ Standard Arm $(\mathrm{n}=45)$} & \multicolumn{2}{l}{ Tailored Arm $(\mathrm{n}=54)$} \\
\hline & & $\begin{array}{l}\text { Resistant } \\
\text { strains } \\
(\mathrm{n}=22)\end{array}$ & $\begin{array}{l}\text { Sensitive } \\
\text { Strains } \\
(\mathrm{n}=23)\end{array}$ & $\begin{array}{l}\text { Resistant } \\
\text { strains } \\
(\mathrm{n}=26)\end{array}$ & $\begin{array}{l}\text { Sensitive } \\
\text { Strains } \\
(\mathrm{n}=28)\end{array}$ \\
\hline $\begin{array}{c}\text { Eradication } \\
\text { Rate }\end{array}$ & ITT analysis & $12 / 22=55 \%$ & $18 / 23=78 \%$ & $19 / 26=73 \%$ & $21 / 28=75 \%$ \\
& PP analysis & $12 / 21=57 \%$ & $18 / 22=82 \%$ & $19 / 23=83 \%$ & $21 / 26=81 \%$ \\
\hline
\end{tabular}

Conclusion: In those who are sensitive to clarithromycin, standard clarithromycin-based triple therapy achieves an acceptable eradication rate of approximately $81 \%$. However, a high primary clarithromycin resistance rate was observed in this study $(47 \%)$. In those who are resistant to clarithromycin, prescribing a regimen based antibiotic susceptibilities increases eradication rates to $83 \%$, compared to those treated with standard triple therapy $(57 \%, \mathrm{p}=0.09)$ Disclosure of Interest: All authors have declared no conflicts of interest.
P1911 TREATING HELICOBACTER PYLORI INFECTION SECOND

TIME ROUND: IS TAILORING THERAPY BASED ON ANTIMICROBIAL SUSCEPTIBILITY THE BEST OPTION?

D. Brennan, M. Hussey, D. Tighe, C. O'Morain, S. Smith, D. Mcnamara

Trinity Academic Gastroenterology Group, Trinity College Dublin, Dublin/Ireland

Contact E-mail Address: dbrenna9@tcd.ie

Introduction: Due to increasing prevalence of antibiotic-resistant Helicobacter pylori, the number of patients who require rescue treatment (after 1 or more failed eradication attempts) is increasing. The Irish Helicobacter pylori Working Group consensus for the diagnosis and treatment of H. pylori infection in adult patients in Ireland recommendeds that second-line and subsequent treatments depend on the first-line treatment and should not be the same treatment. First-line treatment for H. pylori is not standardised in Ireland, therefore it's difficult to recommend a specific rescue treatment. Prescribing a tailored regimen based on antibiotic sensitivities upon first eradication failure may be most effective.

Aims \& Methods: The aim of this study was to examine the efficacy of a tailored regimen based on antimicrobial susceptibility as a rescue treatment for $H$. pylori infection. Patients previously treated for $H$. pylori and undergoing endoscopy were prospectively recruited. Biopsies from $H$. pylori-positive patients (CLO test) were processed for sensitivity testing by E-testing and genotyping by the GenoType HelicoDR assay (Hain). Patients received treatment based on antibiotic sensitivities, for $7 / 14$ days. A follow-up breath test was performed 8 weeks post-treatment

Results: Of 889 gastroscopies done between April 2013- February 2017, 186 $(20.9 \%)$ were $H$. pylori positive. Of these, $74(39.8 \%)$ were previously treated: $39(52.7 \%)$ received one prior treatment and $35(47.3 \%)$ received $>1$. Thus far, 55 patients have agreed to participate and been eligible for the study; $44(80 \%)$ patients have completed the study. $27(49 \%)$ patients have received levofloxacin triple therapy; $14(26 \%)$ a PPI and 2 antibiotics based on their sensitivities; 10 $(18 \%)$ bismuth quadruple and $4(7 \%)$ clarithromycin triple therapy. The efficacy of tailored treatment by intention-to-treat and per protocol analysis was poor, at $47.3 \%(26 / 55)$ and $59.1 \%(26 / 44)$ respectively. Patients who received one previous treatment were significantly more likely to achieve eradication than those who received $>1$ previous treatment $(76.2 \%$ vs $43.5 \%, p=0.04)$.

Conclusion: Rescue eradication rates are disappointing. Eradication rates in patients who received more than one previous treatment were significantly lower than those who had just one previous treatment $(43.5 \%$ vs $76.2 \%$, $\mathrm{p}=0.04)$. This emphasizes the importance of eradicating $H$. pylori infection the first time round, before more virulent or antimicrobial resistant strains are selected for.

Disclosure of Interest: All authors have declared no conflicts of interest.

\section{Reference}

Smith S,. et al. "The Irish Helicobacter pylori Working Group consensus for the diagnosis and treatment of $H$. pylori infection in adult patients in Ireland." European Journal of Gastroenterology and Hepatology 29(5): 552-559 2017

\section{P1912 ARE PROBIOTICS USEFUL AS ADJUVANTS IN ERADICATION THERAPY OF HELICOBACTER PYLORI INFECTION?}

M. Gravito-Soares, E. Gravito-Soares, D. Gomes, N. Almeida, L. Tomé Gastroenterology, Centro Hospitalar e Universitário Coimbra, Coimbra, Portugal, Coimbra/Portugal

Contact E-mail Address: ms18498@gmail.com

Introduction: Helicobacter pylori $(\mathrm{Hp})$ successful eradication has been considered since it contributes to several gastrointestinal disorders. Sequential therapy has been used widely as the first approach in $\mathrm{Hp}$ eradication therapy (HpET). However, its fails in $10-45 \%$. The addition of probiotics has been considered because of potential benefit in the improvement of efficacy and reduction of side effects during HpET.

Aims \& Methods: We aimed to evaluate the effect of probiotics, as adjuvant to sequential HpET on treatment efficacy, side effects and patient compliance. This was a prospective study of total of 1159 patients followed in a gastroenterology outpatient clinic. Selected patients undergone Hp screening for unexplained gastrointestinal symptoms or disorders with $\mathrm{HpET}$ indication. Compared patients undergone sequential therapy (10-day treatment of 5 days of pantoprazole + amoxicilin followed by further 5 days of pantoprazole + clarithromycin + metronidazole-G1:n $=85$ ) and patients with additional supplement of Lactobacillus reuteriprotectis therapy 2tablets/day in previous two weeks and during treatment $(\mathrm{G} 2 ; \mathrm{n}=77)$, since it was approved for this indication. Screening $\mathrm{Hp}$ test and indication, eradication rate, auto-reported side effects and patient compliance were evaluated.

Results: Of 265(22.9\%) patients underwent screening Hp test, $147(55.5 \% ; 147 /$ $265)$ were positive, being the majority obtained by gastric biopsy $(86.0 \% ; \mathrm{n}=228)$. The mean age was $58.6 \pm 15.8$ years with women predominance $(60.8 \% ; \mathrm{n}=161)$. The main indications for Hp screening were dyspepsia $(27.9 \%)$, epigastric pain $(24.2 \%)$, gastroduodenal peptic ulcerous disease $(19.2 \%)$ and gastroesophageal reflux disease $(15.8 \%)$. At gastric biopsies, chronic gastritis was present in $61.5 \%(\mathrm{n}=163)$, gastric atrophy in $17.0 \%(\mathrm{n}=45)$ and intestinal metaplasia in $7.9 \% \quad(\mathrm{n}=21)$, with $\mathrm{Hp}$ mild colonization in most cases $(58.5 \% ; 76 / 130)$. Eradication rate was significantly higher in patients who had probiotic supplement $(\mathrm{G} 1-74.1 \% \mathrm{vsG} 2-92.2 \% ; \mathrm{p}=0.002 ; \mathrm{OR}=4.132)$. No significant difference was verified between two groups in relation to side effects (G1$15.3 \% \mathrm{vsG} 2-9.1 \% ; \mathrm{p}=0.094)$ or patient non-compliance (G1-2.4\%vsG2- 
$0.0 \% ; \mathrm{p}=0.106$ ). However, significantly higher rates of diarrhea were noted in treated group without probiotics $(\mathrm{G} 1-5.9 \% \mathrm{vs} 0.0 \% ; \mathrm{p}=0.031 ; \mathrm{OR}=1.063)$. Of G2 patients, $19(24.5 \%)$ performed sequential therapy first, with eradication rate improvement of $78.9 \%(15 / 19)$. The remaining cases that didn't respond were submitted to 14-day of quadruple therapy with PPI + amoxicilin + metronidazole + levofloxacin with success.

Conclusion: Co-adjuvant sequential therapy with probiotic Lactobacillus reuteri protectis, within previous two weeks to final of treatment, is associated with higher HpET rate, initially or after sequential therapy failure, and lower rate of diarrhea-associated treatment.

Disclosure of Interest: All authors have declared no conflicts of interest

\section{P1913 THE IMPACT OF CLOSTRIDIUM BUTYRICUM MIYAIRI-588 ON HELICOBACTER PYLORI ERADICATION THERAPY}

S. Takayama, O. Handa, R. Mukai, Y. Suyama, A. Majima, Y. Onozawa, O. Dohi, T. Okayama, N. Yoshida, K. Kamada, K. Katada, K. Uchiyama, T. Ishikawa, T. Takagi, H. Konishi, Y. Naito, Y. Itoh

Department Of Gastroenterology And Hepatology, Kyoto Prefectural University of Medicine, Kyoto/Japan

Contact E-mail Address: shunt02@koto.kpu-m.ac.jp

Introduction: As a country with high incidence of gastric cancer, the elimination of Helicobacter pylori (HP) is useful strategy for the prevention of gastric cancer in Japan. And the eradication therapy for HP -infected gastritis was approved as an insurance indication since 2013, and virtually all HP -infected patients were subject to eradication. In Japan, triple therapy using proton pump inhibitor (PPI)/amoxicillin (AMPC)/clarithromycin (CAM) has been used as a regimen for the primary eradication therapy. Since HP has rapidly acquired the resistant character against CAM, the eradication rate has gradually been decreasing. Recently, vonoprazan (VPZ), a novel potassium competitive acid blocker, has been approved for HP eradication therapy. Recently, higher HP-eradication ratio by VPZ + AMPC + CAM than PPI based triple therapy has been reported. However, there might be some concern for the use of VPZ; higher serum gastrin, decrease in the diversity of intestinal microbiota and increase in colitis. Therefore, PPI-based triple therapy is still used, and additive effect of probiotics has been reported in these therapy.

Aims \& Methods: The aim of this study is to investigate the effect of probiotics, Clostridium butyricum Miyairi-588 (Miya-BM®:MBM) on PPI-based triple therapy comparing the eradication rate and side effect with VPZ-based triple therapy. From January 2015 to December 2016, patients who received HP primary eradication therapy in our hospital were retrospectively evaluated. They were divided into three groups; 1) patients who received PPI + AMPC + CAM therapy (PPI group), and 2) patients who received VPZ + AMPC + CAM therapy (VPZ group), 3) patients who received $\mathrm{PPI}+\mathrm{AMPC}+\mathrm{CAM}+\mathrm{MBM}$ therapy (PPI + MBM group), and the eradication rate and side effects were evaluated. Results: The number of patients enrolled in this study were $468 ; 150$ cases in the PPI group, 271 cases in the VPZ group and 47 cases in the PPI + MBM group. Successful rate of HP eradication was $81.7 \%$ in the PPI group, $89.8 \%$ in the VPZ group and $89.1 \%$ in the PPI + MBM group. The eradication rate in VPZ group was significantly higher than PPI group. However, the eradication rate of VPZ group was not significantly higher than that of PPI + MBM group. The rate of side effect was $14.7 \%$ in the PPI group, $10.0 \%$ in the VPZ group and $19.1 \%$ in the PPI + MBM group, and there was no significant difference between 3 groups. Conclusion: The study shows that Clostridium butyricum Miyairi-588 can have additive effects in PPI-based triple therapy for HP.

Disclosure of Interest: O. Handa: Lecture fee from AstraZeneca K.K. and DAIICHI SANKYO COMPANY

Y. Naito: Lecture fee and scholarship donations from EA Pharma Co. Lecture fee from Takeda Pharmaceutical Company

All other authors have declared no conflicts of interest.

\section{P1914 THE EFFECTS OF SACCHAROMYCES BOULARDII SUPPLEMENTATION ON HELICOBACTER PYLORI ERADICATION RATE AND SIDE EFFECTS DURING SEQUENTIAL THERAPY: A PROSPECTIVE RANDOMISED CONTROLLED STUDY}

H. Boutallaka ${ }^{1}$, H. Seddik ${ }^{1}$, K. Loubaris ${ }^{1}$, F. Nejjari ${ }^{2}$, S. Berrag ${ }^{1}$, I. Elkoti ${ }^{1}$, A. Benkirane

${ }^{1}$ Gastro-enterology II, Military Hospital Mohamed V, Rabat/Morocco

${ }^{2}$ Gastroenterology, HMIMV RABAT, Rabat/Morocco

\section{Contact E-mail Address: hanaeboutallaka@gmail.com}

Introduction: The eradication of Helicobacter Pylori remains crucial because of constantly evolving data. The recent recommendations of Mastricht V stipulate that the concomitant quadritherapy and the bismuth quadruple therapy are more efficient than the sequential therapy because of a higher rate of eradication $(90 \%$ vs $82 \%$ ), but with more important side effects. The aim of our study is to investigate the effects of the Saccharomyces boulardii supplementation to the sequential therapy on Helicobacter pylori eradication rate and associated therapy side effects.

Aims \& Methods: One hundred ninety nine patients with Helicobacter pylori infection documented on a histological study of gastric biopsies were enrolled from May 2013 to May 2016, on a single center, prospective, controlled and randomized study, performed in the Gastro Enterology II department on the military hospital of Rabat. Using a permuted block randomization, our patients were assigned to one of the following groups: a control group receiving the standard sequential therapy, and an experimental group receiving in addition

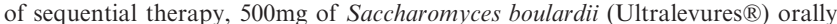
and daily during the ten days of regimen. All the patients were reviewed in the end of the therapy to evaluate the adherence to treatment and the incidence and severity of side effects. The eradication of Helicobacter pylori was evaluated by 13C-urea breath 4 to 6 weeks after the end of the protocol. Statistical analysis was performed by the software SPSS 20.0. A model of logistic regression was performed to analyse the effects of Saccharomyces boulardii supplementation on eradication rate and side effects.

Results: There was no significant difference between the two groups on age (middle age $=44.3+/-13.8$ years vs $43+/-13.2$ years), gender (Sex ratio M/ $\mathrm{F}=1.15$ vs 1.05 ), medical antecedents, smoking, endoscopic and histological datas. In Intention To Treat ITT and Per Protocol PP analysis, the eradication rate was significantly higher in the experimental group ( $86.6 \%$ ITT, $87.5 \% \mathrm{PP}$ ), comparing to the control group $(78.2 \%$ ITT $74.7 \%$ PP), $\mathrm{p}=0.02$. Moreover, the Saccharomyces boulardii supplementation allowed a significant reduction of the incidence of overall side effects ( $\mathrm{RR}=0.26$, IC 95\% [0.14-0.47], $\mathrm{p}<0.001)$, and the incidence of antibiotic-associated diarrhea $(R R=0.07, \operatorname{IC} 95 \%[0.028-0.20]$, $\mathrm{p}<0.001$ ). The incidence of nausea and vomiting, dizziness, asthenia and metallic taste was also lower in the experimental group, although the differences were not statiscally significant. In the multivariate analysis, the Saccharomyces boulardii supplementation is associated with an optimization of the eradication rate $(\mathrm{RR}=2.4$, IC95\% $=[0.19-1.09], \mathrm{p}=0.02)$, and with a reduction of the antibioticassociated diarrhea AAD (RR $=0.07, \mathrm{IC} 95 \%=[0.02-0.26], \mathrm{p}<0.001)$.

Conclusion: Our study shows that the Saccharomyces boulardii treatment during sequential therapy in Helicobacter pylori regimen is associated with a significant reduction of side effects and particularly the antibiotic-associated diarrhea, and allows an optimization of the eradication rate of Helicobacter pylori. Disclosure of Interest: All authors have declared no conflicts of interest.

\section{P1915 “CONCOMITANT" OR "SEQUENTIAL" THERAPY FOR THE ERADICATION OF HELICOBACTER PYLORI: WHICH REGIMEN COMES FIRST IN MOROCCO?}

A. Ait Errami, Z. Samlani, S. Oubaha, K. Krati

Hepatogastroenterology, CHU Mohammed VI, Marrakech/Morocco

Contact E-mail Address: adil.ae $@$ hotmail.fr

Introduction: Helicobacter pylori (H. pylori) infection, the major cause of peptic ulcer disease, is a bacterial infection that can lead to inflammation and ulcers in the lining o the stomach and the upper part of the small intestine. It has been proved that curative treatment of $H$. pylor infection markedly reduces relapse of peptic ulcers, bleeding, and gastric cancer. The decline of Helicobacter pylori $(H$. pylori) eradication rates with standard triple therapy resulted in a search for novel therapies for first-line therapy of H.pylori infection

Aims \& Methods: The objective of this study is to compare, in Morocco, an African country, the efficacy rates of the concomitant versus the sequential $H$. pylori eradication therapy. Our prospective randomized study included 164 patients with newly diagnosed $H$. pylori infection, randomized to receive a 14 day concomitant or 10-day sequential therapy. Treatment outcome was assessed by C13-urea breath test at least 4 weeks after therapy. Intention to treat (ITT) and per protocol (PP) analysis of the eradication rates were performed. Secondary end points included patient compliance and safety.

Results: The concomitant therapy group achieved statistically significant higher eradication rates when compared with the sequential treatment group, both in the ITT and in the PP analysis $\left(86.6 \%\right.$ versus $79.9 \%, \mathrm{p}^{1 / 40} 0.002$, and $90.6 \%$ versus $72.1 \%, \mathrm{p}^{1 / 4} 0.001$, respectively), after adjusting for age, gender, smoking status, and the presence or not of ulcer and/or non-ulcer dyspepsia. Both groups displayed excellent compliance rates $(99.5 \%$ for the concomitant therapy group and

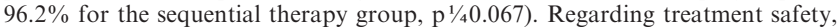
major adverse events that led to the discontinuation of both regimens were few, with no statistical difference between the two groups $(6.2 \%$ for the concomitant therapy group and $3.1 \%$ for the sequential therapy group).

Conclusion: Concomitant therapy led to statistically significant higher eradication rates over sequential therapy. Both therapies showed excellent compliance and an acceptable safety profile. The 14-day quadruple concomitant scheme should be the adopted for first-line $H$. pylori eradication in Morocoo.

Disclosure of Interest: All authors have declared no conflicts of interest.

P1916 PROSPECTIVE COMPARATIVE STUDY OF TWO FIRSTLINE REGIMENS FOR HELICOBACTER PYLORI ERADICATION: 14DAYS NON-BISMUTH QUADRUPLE OPTIMIZED CONCOMITANT THERAPY VERSUS 10-DAYS BISMUTH-CONTAINING QUADRUPLE THERAPY USING A THREE-IN-ONE CAPSULE

J. Alcedo, M. Gracia, P. García-Cámara, C. Palacín, S. Frago, I. Aured, L. Luzón, S. Gallego, C. Jimeno-Ayllón, D. Pérez-Milián, D. Villafranca Department Of Digestive Diseases, Miguel Servet University Hospital, Saragossa/ Spain

Contact E-mail Address: jalcedo@telefonica.net

Introduction: The Maastricht V/Florence Consensus Report recommends bismuth quadruple or non-bismuth quadruple concomitant therapies as first-line treatments for $H$ pylori infection, in areas where clarithromycin resistance is high $(>15 \%)$. Head-to-head studies between both therapies are needed.

Aims \& Methods: We aimed to compare compliance, efficacy and adverse effects of two first-line $H$ pylori eradication therapies in a high clarithromycin resistance 
area, and in clinical practice. A prospective study was performed in a Spanish center recruiting consecutive naïve adult patients, candidates to $H$ pylori eradication. Omeprazole $40 \mathrm{mg}$, Clarithromycin $500 \mathrm{mg}$, Amoxicillin $1 \mathrm{~g}$ and Metronidazole $500 \mathrm{mg}$, all drugs b.i.d, for 14 days (OCAM); or Omeprazole $20 \mathrm{mg}$ b.i.d and 3-in-1 capsule with Bismuth $140 \mathrm{mg}+$ Tetracycline $125 \mathrm{mg}+$ Metronidazole $125 \mathrm{mg}$, 3 capsules q.i.d, for 10 days (3-1-OBMT) were prescribed according to physician criteria. Compliance was assessed by striking the consumed doses in a patient filled template, and adverse effects using a specific questionnaire with a 1-3 intensity scale. Efficacy was determined by $13 \mathrm{C}$-urea breath test. A descriptive study and analysis of efficacy by intention to treat (ITT) were performed. Cases with poor therapeutic compliance $(<80 \%)$ or no available data were excluded in per-protocol (PP) analysis. Chi2, Student's $\mathrm{t}$, and Mann-Whitney $\mathrm{U}$ tests with significance level $\mathrm{p}<0.05$ were applied. The protocol was approved by the Ethics Committee.

Results: 216 patients (63.43\% women; mean age 51.53 -range $19-84$ years-) were included. OCAM were prescribed in 103 and 3-1-OBMT in 113. No differences in age, sex and functional dyspepsia as indication to eradicate were observed between groups. Main indications for treatment were functional dyspepsia $(39.35 \%)$, gastroduodenal ulcer $(19.44 \%)$ and non-investigated dyspepsia $(13.89 \%)$. Compliance was $<80 \%$ in 11 patients and unknown in 7 . The efficacy outcome was unavailable in 9 subjects. Compliance $\geq 80 \%$ was obtained in $96 \%$ with OCAM and in $93.53 \%$ with $3-1$-OBMT $(\mathrm{p}=0.64)$. The ITT rates were $82.52 \%$ vs $85.84 \% \quad(\mathrm{p}=0.63)$, and PP $89.47 \%$ vs $96.04 \% \quad(\mathrm{p}=0.13)$, for OCAM and 3-1-OBMT respectively. The outcomes of adverse effects (frequency, number, duration and intensity) are shown in the Table.

\begin{tabular}{|c|c|c|c|c|c|c|c|}
\hline & \multirow{2}{*}{$\begin{array}{l}\text { Frequency } \\
{[\%]}\end{array}$} & \multirow{2}{*}{$\begin{array}{l}\text { Number } \\
\text { [Mean (SD)] }\end{array}$} & \multirow{2}{*}{$\begin{array}{l}\text { Days } \\
\text { [Mean (SD)] }\end{array}$} & \multicolumn{4}{|c|}{$\begin{array}{l}\text { Maximum } \\
\text { Intensity [\%] }\end{array}$} \\
\hline & & & & None & Mild & Moderate & Intense \\
\hline OCAM & 96.97 & $4.08(2.49)$ & 10.07 (4.6) & 3.41 & 20.45 & 22.73 & 53.41 \\
\hline OBMT & 91.51 & $3.92(2.6)$ & $6.02(3.43)$ & 7.14 & 19.39 & 40.82 & 32.65 \\
\hline p-value & 0.17 & 0.70 & $<0.0001$ & 0.0149 & & & \\
\hline
\end{tabular}

Conclusion: In a high clarithromycin resistance area, 14-days OCAM and 10-days 3-1-OBMT regimens achieve high and similar compliance and efficacy rates, but 3-1-OBMT provides a superior safety profile.

Disclosure of Interest: All authors have declared no conflicts of interest.

\section{Reference}

Malfertheiner P, Megraud F, O'Morain AC et al.Management of Helicobacter pylori infection - the Maastricht V/Florence Consensus Report. Gut 2017; 66:630 .

\section{P1917 MANAGEMENT OF HELICOBACTER PYLORI INFECTION AT PRIMARY CARE LEVEL. THE IMPLEMENTATION OF SPECIFIC COUNSELLING IMPROVES ERADICATION RATES}

V. Laredo ${ }^{1}$, T. Arroyo Villarino ${ }^{2}$, E. Alfaro ${ }^{1}$, C. Sostres ${ }^{3}$, A. Lanas ${ }^{3}$

${ }^{1}$ University Hospital Lozano Blesa, Zaragoza/Spain

${ }^{2}$ University of Zaragoza School of Medicine, Zaragoza/Spain

${ }^{3}$ Biosanitary Research Institute Aragón (IIS Aragón), Zaragoza/Spain

\section{Contact E-mail Address: vlaredodelatorre@gmail.com}

Introduction: We have detected a large increase of urea breath test (UBT) requests for Helicobacter pylori $(\mathrm{Hp})$ diagnosis by primary care physicians (PCP). In this way, most $\mathrm{Hp}$-infected patients are now being managed at primary care level. However, little is known about outcomes of $\mathrm{Hp}$ infection by PCP. Aims \& Methods: 1 . To evaluate and compare the eligibility of UBT indications, treatment regimens and eradication rates between PCP and gastroenterology specialist (GS). 2. To evaluate the effect of introduction of specific counselling to PCP in the management profile of $\mathrm{Hp}$ infection. First, we prospectively included 500 consecutive UBT indicated by PCP (250) and GS. Appropriate UBT indications were considered those included in the $3^{\text {rd }}$ Spanish Consensus Conference on Helicobacter pylori infection (1). Hp treatment prescribed and eradication rates were collected retrospectively. Finally, we analyzed another consecutive 240 UBT and treatment outcomes after the introduction of specific counselling to PCP (a personal letter with the accepted indications for UBT and treatment issued by their referent GS). Statistical analysis was performed using SPSS (version 22.0).

Results: We have analyzed 740 UBT (500 pre-intervention and 240 post-intervention). $66.89 \%$ women, mean age of $48.94 \pm 16.6$ years. Most $(68.4 \%)$ of preintervention UBT were performed for the initial diagnosis of $\mathrm{Hp}$ infection, and the rest were indicated after previous eradication treatment. Inappropriate indication of UBT in the pre-intervention cohort was significantly higher in those test requested by PCP compared to GS $(36.4 \%$ vs $7.2 \%$; p < 0.001$)$. Also, inappropriate treatment regimens were significantly higher in the PCP group $(65 \%$ vs $26.4 \% ; \mathrm{p}<0.0001)$. Consequently, eradication rates were significantly lower in PCP compared to GS group $(57.1 \%$ vs $81.1 \%$; p < 0.001$)$. A significant increase in the adherence to appropriate treatment regimens (71\% vs $35 \%$; $\mathrm{p}<0.0001)$ and eradication rates $(78 \%$ vs $57.1 \% ; \mathrm{p}<0.0001)$ were observed in the PCP group after the implementation of specific counselling based on national guidelines.
Conclusion: Hp infection management at primary care level is inappropriate with very low $\mathrm{Hp}$ infection eradication rates. The introduction of a specific counselling to PCP has significantly improved these outcomes. These data should encourage the implementation of interventional strategies in order to reduce the actual increase in antibiotic resistance.

Disclosure of Interest: All authors have declared no conflicts of interest.

\section{Reference}

1. Gisbert JP, Calvet X, Bermejo F, Boixeda D, Bory F, Bujanda L, et al. III Spanish Consensus Conference on Helicobacter pylori infection. Gastroenterol Hepatol. 2013;36:340-374.

\section{WEDNESDAY, NOVEMBER 01, 201709:00-14:00 SMALL INTESTINAL III - HALL 7}

\section{P1918 VALPROATE AND CHIR 99201 AMELIORATES RADIATION-} INDUCED INTESTINAL EPITHELIAL INJURY IN MOUSE MODEL

Y. Kim, J.S. Shin

Internal Medicine, Division Of Gastroenterology, Korea Cancer Center Hospital, Seoul/Korea, Republic of

Contact E-mail Address: younjoo282@gmail.com

Introduction: Radiation-induced gastrointestinal syndrome (RIGS) stems from the clonogenic loss of crypt cells and villi depopulation and results in mucosal barrier disruption, bacterial invasion, inflammation and sepsis. Valproate (VPA) is the one of the popular anti-convulsants, recently its Notch signal modulatory effect has been reported. Notch signal pathway is the essential to maintain intestinal stem cells and to differentiate to secretory cells such as goblet cells. Moreover, It has been reported that combination of VPA and CHIR 99021 (GSK3B inhibitor) has powerful proliferatory effect for instestinal stem cells, such as Lgr 5+ cells. One of the major difficulties for RIGS studies is the fact that crypts are not easily accessed and cultured with traditional means. Ex-vivo culture techniques for single crypt or a stem cell derived enteroid, with essential features of the in vivo tissue architecture, have been recently developed. Thus, we have adopted the 3D-cultured enteroids for RIGS ex-vivo model, and proved the effect of VPA and SCFAs for RIGS.

Aims \& Methods: We have adopted the 3D-cultured enteroids for RIGS ex-vivo model, and proved the effect of VPA and SCFAs for RIGS. To culture enteroid, ten centimeters segments of jejunum were procured from 9-13 week-old C57BL6 mice. Crypts were isolated by EDTA chelation, suspended in Matrigel and grown in culture media containing epidermal growth factor, noggin, R-spondin 1. After 1 day in culture, the enteroids were treated (or not) 3 mM CHIR 99021 (GSK3B inhibitor) and $1 \mathrm{mM}$ VPA. On day 3 , the enteroids were irradiated as a dose dependent manner. The evaluation of irradiated enteroids was performed by measuring MTT assay, budding efficiency of enteroid, and EdU staining. On post-irradiation Day 2 and Day 7, RT-PCR was performed.

Results: Enteroid from mouse had multiple crypts ('budding') with well-differentiated goblet, Paneth cells, +4 stem cells (quiescence stem cells, BMI1 is expressed), Lgr5+ stem cells. In the response of radiation, irradiated enteroid decreased proliferation rate in a dose dependent manner, as measured by MTT assay and budding efficiency of enteroids. Irradiated enteroids with VPA +CHIR 99021 could maintain their +4 stem cells even in $10 \mathrm{~Gy}$ of irradiation, lethal dose of mouse intestinal epithelium., and they were able to proliferation. Combination of VPA + CHIR 99021 did not have an effect on paneth cells, enterochromaffin cells and goblet cells.

Conclusion: VPA and CHIR 99021 may ameliorate RIGS in ex-vivo mouse enteroid, through +4 reservoir stem cell preservation and stimulatory action for epithelial proliferation. Considering their clinical application such as safety, they could be possible strategy for prevention and treatment of RIGS.

Disclosure of Interest: All authors have declared no conflicts of interest.

\section{References}

1. i, 2009. 459(7244): p. 262-5. Single Lgr5 stem cells build crypt-villus structures in vitro without a mesenchymal niche.

2. Gut, 2014. 63(8): p. 1345-54. Ex vivo culture of the intestinal epithelium: strategies and applications.

3. Science, 2013. 340(6137): p. 1190-4.Growing self-organizing mini-guts from a single intestinal stem cell: mechanism and applications.

4. Nat Methods. 2014 Jan;11(1):106-12. Niche-independent high-purity cultures of Lgr5+ intestinal stem cells and their progeny.

5. World J Stem Cells. 2013 Oct 26;5(4):106-11. New insights for pelvic radiation disease treatment: Multipotent stromal cell is a promise mainstay treatment for the restoration of abdominopelvic severe chronic damages induced by radiotherapy.

6. Cancer Biother Radiopharm. 2009 Dec;24(6):689-99. HDAC inhibitor, valproic acid, induces p53-dependent radiosensitization of colon cancer cells

7. Gastroenterology 2012;143:1266-.1276 Crypt Base Columnar Stem Cells in Small Intestines of Mice Are Radioresistant 


\section{P1919 PREVALENCE OF CELIAC DISEASE AMONG RELATIVES}

\section{IN ALGERIA}

F. Lounes ${ }^{1}$, N. Aliarous ${ }^{1}$, S. Salah ${ }^{2}$, K. Belanteur ${ }^{2}$, M.G. Mokhtech ${ }^{3}$, Z.C. Amir ${ }^{3}$, D. Hannoun ${ }^{4}$, R. Ouldgougam ${ }^{2}$, Y. Chikhi ${ }^{1}$, S. Cheraitia ${ }^{1}$, F. Asselah ${ }^{3}$,

H. Asselah ${ }^{1}$, M. Lahcene

${ }^{1}$ Gastroenterology, Bologhine Hospital, Algiers/Algeria

${ }^{2}$ Immunology, Pasteur Institute, Algiers/Algeria

${ }^{3}$ Pathology, Mustapha hospital, Algiers/Algeria

${ }^{4}$ Epidemilogy, National Institute of Health, Algiers/Algeria

${ }^{5}$ Internal Medecine, Bologhine hospital, Algiers/Algeria

Contact E-mail Address: lounesfadila@yahoo.fr

Introduction: Celiac disease (CD) is an important health problem worldwide. It is characterized by a high prevalence $(1 \%)$, non specific morbidity, long-term complications and epidemiological progression. However, mass screening is not recommended. Currently, the strategy of 'Targeted screening,' defined as screening of high-risk groups, is widely practiced and recommended by learned Societies, in particular, in relatives of CD patients. In the world, targeted screening studies related to CD showed prevalence ranging from 4.2 to $13 \%$.

Aims \& Methods: The main aim of our study is to determine the prevalence of $\mathrm{CD}$ in the first degree relatives in Algerian population. This was a descriptivetransversal study with prospective recruiting. This is a screening of first-degree relatives of patients known and treated for $\mathrm{CD}$. Relatives are screened by using antitransglutaminase (TTG) antibodies in the serum. Upper digestive endoscopy and duodenal biopsy are performed in all sero-positive relatives and graded as per Marsh modified by Oberhuber classification to confirm the diagnosis. 546 first degree relatives of 107 families are included in our study.

Results: Among the 546 first-degree relatives, we have $18.5 \%$ of parents, $57.5 \%$ of siblings and $24 \%$ of children; 44 cases have positive anti TTG antibodies. The prevalence of $\mathrm{CD}$ among first-degree relatives is $8.1 \%$ with Confident Interval (CI) at $95 \%$ [5.8-10.4]. The prevalence of CD in first-degree relatives with positive serology and positive duodenal biopsy is $7.3 \%$ with CI at $95 \%$ [5.2-9.4] Histo-pathological study of duodenal biopsies of new cases, detected by targeted screening, shows 5 cases of Marsh I, 5 cases Marsh IIIa, 27 cases Marsh IIIb and 3 cases Marsh IIIc. 44 patients are screened with CD; they are divided as follows: $9 \%$ Parents (4 mothers), $11 \%$ of children (son 3 and 5 girls) and $72 \%$ of siblings (brothers and 23 sisters 9 ). The average age of screening cases is 31.8 years with CI at $95 \%$ [ 27.2 to 36.4$]$ and extremes of [3-71] years. The mean duration of symptoms before diagnosis is 3.4 years, with CI at $95 \%$ [2.8 to 4.0 ] years. Among the 44 new cases detected by targeted screening, whereas $11.4 \%$ are asymptomatic, $88.6 \%$ are symptomatic. This is a classical form in one case and atypical form in $86.4 \%$ of cases.

Conclusion: The present work entitled "Prevalence of celiac disease in relatives" is the first screening study of CD in the first-degree relatives carried out in Algeria. Among the 546 first-degree relatives, it is showed that 44 patients present positive serology which corresponds to the prevalence of $\mathrm{CD}$ in first degree relatives of $8.1 \%$ with CI at $95 \%$ [5.8-10.4]. Our investigation supports the idea that the development of an extensive screening approach is needed to promote early diagnosis and to prevent complications of $\mathrm{CD}$ in first-degree relatives.

Disclosure of Interest: All authors have declared no conflicts of interest.

P1920 LONG-TERM HEALTH AND LIFESTYLE OUTCOMES IN ADULT CELIAC DISEASE PATIENTS DIAGNOSED IN CHILDHOOD BECAUSE OF CLINICAL SUSPICION OR BY SCREENING

L. Kivelä ${ }^{1}$, S. Alin ${ }^{2}$, S. Kröger ${ }^{2}$, K. Kaukinen ${ }^{2}$, K. Kurppa

${ }^{1}$ Tampere Center For Child Health Research, University of Tampere and Tampere University Hospital, University of Tampere/Finland

${ }^{2}$ School Of Medicine, University of Tampere, Tampere/Finland

Contact E-mail Address: laura.kivela@fimnet.fi

Introduction: Celiac disease affects $1-2 \%$ of population, but due to diverse presentation most patients remain unrecognized. Diagnostic efficiency could be improved by screening of at-risk groups, but long-term benefits of this approach are unclear. To shed light to this issue, we compared a variety of celiac diseaserelated and other parameters in large cohorts of adult patients diagnosed in childhood either because of clinical suspicion or by screening.

Aims \& Methods: A questionnaire about current health and lifestyle, adherence to gluten-free diet (GFD) and follow-up of celiac disease was sent to 564 adults with a childhood diagnosis. Further, the participants fulfilled validated Gastrointestinal Symptom Rating Scale (GSRS) and Psychological General Well-Being (PGWB) surveys for symptoms and quality of life. Clinical and histological presentation at diagnosis and other relevant medical data were confirmed from patient records. All variables were compared between screendetected and clinically detected patients.

Results: Altogether 235 (42\%) adults completed the questionnaires. At diagnosis, screen-detected patients $(\mathrm{n}=49)$ were older $(11.3 \mathrm{vs} 8.8 \mathrm{yr}, \mathrm{p}=0.016)$ and had less symptoms $(44 \%$ vs $85 \%, \mathrm{p}<0.001)$ and poor growth $(17 \%$ vs $51 \%$, $\mathrm{p}<0.001)$ than clinically detected patients $(\mathrm{n}=186)$. They also had a trend to have less often total villous atrophy $(18 \%$ vs $32 \%, \mathrm{p}=0.075)$ and anemia $(18 \%$ vs $32 \%, \mathrm{p}=0.072$ ). The groups did not differ in gender, current age (median 26.5 vs $27.1 \mathrm{yr}, \mathrm{p}=0.245)$, time from the diagnosis, self-experienced health or concern about health, clinical symptoms, strict GFD $(74 \%$ vs $80 \%, p=0.161)$, lifestyle restrictions caused by GFD, presence of celiac disease-related complications, physical activity, fertility or GSRS and PGWB scores. However, screen-detected patients smoked less $(4 \%$ vs $15 \%, p=0.037)$ and had more often celiac disease in relatives $(78 \%$ vs $58 \%, \mathrm{p}=0.011)$

Conclusion: Diagnostic approach and presentation of celiac disease in childhood do not seem to affect the long-term health outcomes or attitude towards the disease in adulthood. Lack of difference in the dietary adherence and lifestyle restrictions gives further support for active screening and early diagnosis of celiac disease.

Disclosure of Interest: All authors have declared no conflicts of interest.

\section{P1921 REVIEW OF SERVICE PROVISION OF NATIONAL INSTITUTE FOR HEALTH AND CARE EXCELLENCE (NICE) RECOMMENDED QUALITY STANDARDS FOR COELIAC DISEASE AT A BIG DISTRICT GENERAL HOSPITAL}

F.K. Shaikh, D. Vani, M. Sprakes, F. Greenhalgh

Gastroenterology, Mid Yokshire Hospitals NHS Trust, Wakefield/United

Kingdom

Contact E-mail Address: sheikh177@yahoo.com

Introduction: In the UK, 1 in 100 people are affected with coeliac disease. NICE published quality standards (QS134) for coeliac disease in October 2016 based on NICE guidelines NG20 (September 2015). This quality standard covers the recognition, assessment and management of coeliac disease in children, young people and adults. The quality standard is expected to contribute to improvements in the diagnosis of coeliac disease, growth in children and young people, health-related quality of life, incidence of osteoporosis, intestinal lymphoma, vitamin D deficiency, and iron deficiency

Aims \& Methods: We audited our departmental practices against NICE quality standards for coeliac disease. It is a retrospective data analysis of patients, 16 years and above, with positive coeliac serology from April 2016 to September 2016. The main parameters examined were source of referral, value of tissue transglutaminase (tTG) antibodies, type of referral (new or follow up), timing of OGD (less than or more than 6 weeks), whether or not seen by dietician, offer of annual review, and DEXA scan.

Results: Total of 95 cases were examined. The General Practitioner was the main source of referral for tTG antibody $(76 \%)$ test followed by gastroenterology outpatients (OP) (13\%), 2\% each was shared by surgical OP and inpatient gastroenterology services. Other referrers contributed $3 \%$ each. $88 \%$ (84 out of 95 ) positive tTG results was more than $20 \mathrm{U} / \mathrm{ml} .53$ out of $95(56 \%)$ were new referrals, $30(31 \%)$ were follow-up and $12(13 \%)$ referrals had no further record on system. 11 of $53(21 \%)$ new patients had OGD in less than 6 weeks; 30 of $53(56 \%)$ had OGD in more than 6 weeks, 22 of $95(23 \%)$ had no record of OGD on system. 56 of $95(59 \%)$ patients had dietician review. 26 of $95(27 \%)$ were offered or had annual review. 42 of $95(44 \%)$ had DEXA scan to assess bone density.

Conclusion: The incidence and prevalence of coeliac disease in our study are 30.28 per 100,000 persons-years and 8 per 100,000 population respectively. The data demonstrates that Mid Yorkshire NHS Trust is not providing required service standards for optimum care of coeliac patients in the Mid Yorkshire region. We need to keep in mind that some gastroscopies would have been performed in the primary care sector, the records of which were not available on our system. This factor could have potentially affected our results. Due to several constraints in our service provision, time frames stipulated in the NICE guidance are challenging as evidenced by the audit results. Inspite of our unique local access to endoscopy units in primary care, we are still not able to deliver the required standards of care. Given the potential disease sequelae of coeliac disease, such as osteoporosis and lymphoma, it is important that these issues are addressed by the development of local referral and management pathways to ensure that all coeliacs are captured, investigated, and followed up appropriately.

Disclosure of Interest: All authors have declared no conflicts of interest.

\section{Reference}

NICE Quality Standards (QS134) for Coeliac Disease (October 2016) NICE Guidelines NG20 (September 2015)

\section{P1922 SERUM MICROBIAL MARKERS IN NONRESPONSIVE} CELIAC DISEASE

L. M. Viitasalo ${ }^{1}$, K. Kurppa ${ }^{1}$, H. Huhtala ${ }^{2}$, M. Mäki ${ }^{1}$, K. Kaukinen ${ }^{3}$, S. Iltanen ${ }^{4}$ ${ }^{1}$ Center For Child Health Research, University of Tampere and Tampere University Hospital, Tampere/Finland

${ }^{2}$ Faculty Of Social Sciences, University of Tampere, Tampere/Finland ${ }^{3}$ Department Of Internal Medicine, Tampere University Hospital, Faculty of

Medicine and Life Sciences, University of Tampere, Tampere/Finland

${ }^{4}$ Kanta-Häme Central Hospital, Hämeenlinna/Finland

Contact E-mail Address: liisa.viitasalo@uta.fi

Introduction: In nonresponsive celiac disease (NRCD) the symptoms and duodenal damage persists despite a gluten-free diet (GFD). We have previously shown an association between dysbiotic microbiota and persistent symptoms in treated CD (1). Furthermore, serum microbial antibodies to Saccharomyces cerevisiae (ASCA), Pseudomonas fluorescens-associated sequence (I2) and Bacteroides caccae TonB-linked outer membrane protein $(\mathrm{OmpW})$ were found to be gluten-sensitive (2) and present already at early stages of CD development (3). We hypothesized that increased seroreactivity to microbial antibodies is associated also with NRCD

Aims \& Methods: Serum ASCA, I2 and OmpW were measured in $20 \mathrm{CD}$ patients with persistent villous atrophy and mucosal inflammation despite strict GFD and negative celiac serology (NRCD group). Corresponding GFD responsive patients served as CD controls (58 samples at diagnosis and 55 on GFD) and 
80 healthy blood donors as non-CD controls. Kruskal-Wallis test was used to compare antibody titers and Dunn-Bonferroni for post hoc pairwise comparisons.

Results: At least one serum microbial marker was positive in $80 \%$ of NRCD patients, in $97 \%$ of untreated and $87 \%$ of treated CD patients and in $44 \%$ of non-CD controls. NRCD patients had the highest frequency of ASCA positivity $(64 \%$ vs $52 \%, 20 \%$ and $0 \%$, respectively) and also significantly higher ASCA $\mathrm{IgA}$ (median $14.5 \mathrm{U} / \mathrm{ml})$ and $\mathrm{IgG}(32.5 \mathrm{U} / \mathrm{ml})$ titers than treated $\mathrm{CD}$ patients $(7.0$ $\mathrm{U} / \mathrm{ml}, 13.0 \mathrm{U} / \mathrm{ml})$ and non-CD controls $(4.5 \mathrm{U} / \mathrm{ml}, 5.8 \mathrm{U} / \mathrm{ml})$. There was no difference in ASCA between NRCD and untreated CD. The frequencies of I2 $(65 \%)$ and OmpW $(45 \%)$ were lower in NRCD than in untreated CD $(86 \%$, $59 \%$, respectively), while I2 titers were higher in NRCD (median absorbance $0.76)$ and untreated (1.0) and treated (0.83) CD than non-CD controls $(0.32)$ OmpW was elevated in untreated (1.1) and treated (0.94) CD patients compared with non-CD controls $(0.79)$

Conclusion: Seropositivity and high titers of ASCA were associated with NRCD and might thus serve as additional follow-up tool for histological recovery in CD. Disclosure of Interest: All authors have declared no conflicts of interest

\section{References}

1. Wacklin P, Laurikka P, Lindfors K, Collin P, Salmi T, Lahdeaho ML, et al. Altered duodenal microbiota composition in celiac disease patients suffering from persistent symptoms on a long-term gluten-free diet. $A m \quad J$ Gastroenterol 2014 Dec;109(12):1933-1941.

2. Ashorn S, Valineva T, Kaukinen K, Ashorn M, Braun J, Raukola H, et al. Serological Responses to Microbial Antigens in Celiac Disease Patients During a Gluten-Free Diet. J Clin Immunol 2009 March;29(2):190-195.

3. Viitasalo L, Niemi L, Ashorn M, Ashorn S, Braun J, Huhtala H, et al. Early microbial markers of celiac disease. J Clin Gastroenterol 2014 Aug;48(7):620 624.

\section{P1923 CORRELATION BETWEEN OXIDATIVE STRESS AND DUODENAL ATROPHY IN CELIAC DISEASE}

F. Ferretti ${ }^{1}$, F. Branchi ${ }^{2}$, L. Roncoroni ${ }^{2}$, S. Moretti ${ }^{3}$, A. Vezzoli ${ }^{3}$, S. MrakicSposta $^{3}$, V. Lombardo ${ }^{1}$, D. Conte ${ }^{4}$, L. Elli ${ }^{2}$

${ }^{1}$ Center For The Prevention And Diagnosis Of Celiac Disease, Gastroenterology And Endoscopy Unit, Fondazione IRCCS Ca' Granda Ospedale Maggiore Policlinico, Milan/Italy

${ }^{2}$ Gastroenterology And Endoscopy Unit, Fondazione Irccs Cà Granda Ospedale Maggiore Policlinico, Center for the Diagnosis and Prevention of Celiac Disease, Milan/Italy

${ }^{3}$ Institute of Bioimaging and Molecular Physiology, National Council of Research $(C N R)$, Segrate $(M I) /$ Italy

${ }^{4}$ Department Of Pathophysiology And Transplantation, Università Degli Studi Di Milano, Milan- Italy, Gastroenterology and Endoscopy Unit, Fondazione IRCCS Ca' Granda Ospedale Maggiore Policlinico, Milan/Italy

Contact E-mail Address: francesca.ferretti01@gmail.com

Introduction: High levels of reactive oxygen species (ROS) and impaired antioxidant defense systems lead to oxidative stress $(\mathrm{OxS})$ and tissue injury in different intestinal and extraintestinal conditions, including celiac disease (CD). A possible effect of gluten ingestion on intracellular oxidative imbalance has been suggested. Aims \& Methods: The first aim of the study was to investigate the effects of OxS in CD, evaluating the levels of ROS and oxidative damage biomarkers in sera of naïve patients (N-CD), coeliac patients on a gluten-free diet (GFD) including responders (CD-GFD) or non-responders (NRCD) to dietary treatment. The second aim was to look for new serological biomarkers corresponding to morphological/functional alterations detected in biopsies according to MarshOberhuber classification. Finally, a possible correlation between ROS production and/or biomarkers of $\mathrm{OxS}$ and/or hematological data was investigated. Analysis were conducted on small intestinal biopsy specimens and peripheral blood samples of celiac patients (N-CD, CD-GFD and NRCD). The methods included Electron Paramagnetic Resonance (EPR) technique for ROS detection, High Performance Liquid Chromatography (HPLC) analysis of erythrocytes glutathione (GSH), enzymatic assays for oxidative damage biomarkers (lipid peroxidation measured by thiobarbituric acid-reactive substances (TBARS) method; protein oxidation, measured by a protein carbonyl (PC) assay kit; total antioxidant capacity (TAC), measured by an enzymatic kit; nitric oxides metabolites in sera, assessed by the Griess method).

Results: Overall, blood samples and biopsies from 54 patients affected by CD were collected (44 F; median age $43.98 \pm 13.44$ years; range $19-80$ years; $17 \mathrm{~N}$ $\mathrm{CD}, 18 \mathrm{CD}-\mathrm{GFD}$ and $19 \mathrm{NRCD}$ ). Hemoglobin and haematocrit levels were significantly lower in NRCD and N-CD than in CD-GFD group $(\mathrm{p}<0.05)$. In our study, a significantly increased production of ROS, lipid peroxidation and oxidized protein levels, plasma nitrate concentrations were reported in NRCD and N-CD compared to CD-GFD. On the contrary, the TAC and GSH levels were significantly decreased in N-CD and NRCD groups compared to CD-GFD. Data are summarized in Table 1. A significant direct relationship between Marsh subtypes and ROS production rate $\mathrm{R}^{2}=0.19 ; \mathrm{p}<0.001$ ), TBARS $\left(\mathrm{R}^{2}=0.20 ; \mathrm{p}<0.001\right)$ and $\mathrm{PC}\left(\mathrm{R}^{2}=0.17 ; \mathrm{p}<0.001\right)$ was found by Pearson's product-moment correlation while an inverse correlation between Marsh subtypes and TAC $\left(\mathrm{R}^{2}=0.23 ; \mathrm{p}<0.001\right)$ and $\mathrm{GSH}\left(\mathrm{R}^{2}=0.34\right.$; $\mathrm{p}<0.0001)$ was identified. In all groups of patients, at higher ROS production rate levels corresponded to greater plasma TBARS concentrations and lower erythrocytic GSH levels.

Conclusion: Several defense mechanisms are implied in maintaining the cell integrity and tissue homeostasis. According to our results, the presence of higher levels of ROS, oxidative damage biomarkers and nitric oxides metabolites in naïve and non-responders patients suggest an implication of $\mathrm{OxS}$ in the functional and histological damage of $\mathrm{CD}$. In parallel, the antioxidant capacity of celiac patients with the active form of the disease is significantly reduced. On the contrary, in patients responding to GFD, the pro-oxidant/antioxidant balance seems to be greatly recovered.

Disclosure of Interest: All authors have declared no conflicts of interest.

P1924 CIRCULATING EXTRACELLULAR VESICLES, A NOVEL MECHANISM OF ENDOCRINE CELLULAR CROSS-TALK, ARE INCREASED IN NEWLY DIAGNOSED CELIAC DISEASE PATIENTS

K. Efthymakis, G. Bologna, P. Lanuti, C. Pipino, A. Milano, F. Laterza, P. Simeone, A. Bonitatibus, A. Pandolfi, M. Marchisio, S. Miscia, M. Neri Department Of Medicine And Ageing Sciences And Center For Excellence On Ageing And Translational Medicine (cesi-met), "G. D'Annunzio" University and Foundation, Chieti/Italy

Contact E-mail Address: efkn78@gmail.com

Introduction: Extracellular vesicles (EVs) have been recently hypothesized to represent a major peripheral mechanism of cellular cross-talk. EVs carry surface receptors and proteins characteristic of their cells of origin and shuttle molecules (proteins, RNAs, microRNAs) potentially controlling physiological and pathological systemic processes. Recent studies have demonstrated an increased number of circulating EVs in a variety of conditions characterized by multiorgan impairment and/or damage such as insulin-resistance, atherosclerosis and obesity. Celiac disease (CD) is an immune-mediated inflammatory enteropathy, elicited by gluten ingestion in genetically susceptible individuals. It is frequently associated with a variety of systemic conditions both autoimmune and potentially immune-mediated in nature.

Aims \& Methods: The aim of this study was to assess and characterize patterns of circulating EVs in newly diagnosed CD patients. We enrolled consecutive adult anti-tTG positive, biopsy proven CD patients. Circulating EVs were identified untouched on whole blood samples by a no-lyse/no-wash method, combined with EVs volumetric count (FACSVerse, BD), based on a novel six-colour flow cytometry panel, in order to identify and enumerate both the whole EV compartment and different EVs subpopulations. Data are expressed as mean $\pm \mathrm{SD}$ and statistical differences were evaluated by means of T-test.

Results: We evaluated 12 celiac adults (mean age $=42.9 \pm 19.1$ vs. $40.8 \pm 15.9$ years, $\mathrm{F} / \mathrm{M}=4: 1$ ) at diagnosis and 12 age- and sex-matched healthy controls. Histology was considered positive for lesions of grade $>\mathrm{B} 1$ according to the Corazza-Villanacci classification. Mean anti-tTG levels at diagnosis were $6.9 \pm 3.5$ times ULN. Mean number of total circulating EVs was significantly higher in CD than in controls $(59895 \pm 72482$ vs $14383 \pm 10018 \mathrm{EV} / \mathrm{microL}$, $p=0.035$ ). Subgroup analysis showed that EpCAM + EVs, of epithelial origin, and CD $41+$ platelet-derived EVs were not significantly different between CD and controls ( $894 \pm 1004$ vs. $548 \pm 1237$ and $3052 \pm 1563$ vs. $1734 \pm 1810$ respectively, $p=\mathrm{ns}$ ). On the contrary, CD $45+\mathrm{EVs}$, of leucocyte origin, showed a significantly higher number in CD patients vs. controls $(460 \pm 492$ vs. $119 \pm 150$ $p=0.026$ ).

\section{Abstract No: P1923}

Data on levels of ROS and oxidative damage biomarkers in sera of naïve patients (N-CD), coeliac patients on a gluten-free diet (GFD) including responders (CDGFD) or non-responders (NRCD) to treatment.

\begin{tabular}{|c|c|c|c|c|}
\hline & $\mathrm{N}-\mathrm{CD}$ & NRCD & CD-GFD & $P$ value \\
\hline ROS production $\left(\mu \mathrm{mol} \cdot \mathrm{min}^{-1}\right)$ & $0.21 \pm 0.03^{*}$ & $0.22 \pm 0.04^{\wedge}$ & $0.17 \pm 0.03^{* \wedge}$ & $*^{* \wedge}<0.05$ \\
\hline TAC levels (mM) & $1.07 \pm 0.30^{*}$ & $1.16 \pm 0.47^{\wedge}$ & $1.68 \pm 0.54^{* \wedge}$ & $*<0.01,{ }^{\wedge}<0.05$ \\
\hline GSH levels $\left(\mu \mathrm{mol} . \mathrm{L}^{-1}\right)$ & $534.40 \pm 37.46^{*}$ & $507.80 \pm 81.73^{\wedge}$ & $634.00 \pm 187.80^{* \wedge}$ & $*<0.001, \wedge<0.0001$ \\
\hline Peroxidized lipid levels $(\mu \mathrm{M})$ & $3.59 \pm 0.67^{*}$ & $3.46 \pm 0.87^{\wedge}$ & $2.82 \pm 0.47^{* \wedge}$ & $*<0.01, \wedge<0.05$ \\
\hline Oxidized proteins levels (nmol. $\mathrm{mg}^{-1}$ protein) & $1.42 \pm 0.43^{*}$ & $1.23 \pm 0.53^{\wedge}$ & $0.91 \pm 0.20^{* \wedge}$ & $*<0.001, \wedge<0.05$ \\
\hline Plasma nitrates concentrations ( $\mu$ mol.L-1) & $99.74 \pm 30.76^{*}$ & $54.61 \pm 14.57^{\wedge}$ & $22.21 \pm 6.92^{* \wedge}$ & $*<0.001, \wedge<0.01$ \\
\hline
\end{tabular}


Conclusion: Celiac disease patients at diagnosis show higher numbers of circulating EVs than age- and sex-matched controls. Phenotypical assessment suggests that this increase is not primarily driven by epithelial or endothelial damage. On the contrary, the increased numbers of leucocyte-derived EVs, suggest their potential implication in systemic signaling.

Disclosure of Interest: All authors have declared no conflicts of interest.

\section{P1925 COELIAC DISEASE AND REPRODUCTIVE DISORDERS: IS THERE ANY CORRELATION}

H. Boutallaka, I. Benelbarhdadi, F.Z. Ajana

Medecine C, Ibn Sina University Hospital, Rabat/Morocco

\section{Contact E-mail Address: hanaeboutallaka@gmail.com}

Introduction: The coeliac disease is an autoimmune enteropathy induced by the ingested gluten proteins (Corn, barley, rye), that occurs in people who are genetically predisposed primarily affecting the small intestine inducing atrophic lesions, which are regressive with a gluten-free diet. The classic form is actually a minority of patients. The extradigestive forms are currently the most found, with varieted manifestations including reproductive disorders. The aim of our study is to evaluate the frequency of these disorders in the coeliac disease and their evolution under gluten-free diet.

Aims \& Methods: It's a single-center, retrospective and descriptive study including 241 patients with coeliac disease enrolled within period of 17 years from 1995 to 2016 in the department of Gastroenterology « Medecine C » in Ibn Sina University Hospital.

Results: About 241 patients suffering from coeliac disease, 58 patients presented reproductive disorders, either $28.9 \%$. Recruting 53 women and 5 men, with a sex ratio $\mathrm{M} / \mathrm{F}$ of $10 / 6$. The mean age was 32.25 years ranging from 13 to 59 years old. The diagnosis of coeliac disease was based on: Histology (severe or partial Villous atrophy with intraepithelial lymphocytosis exceeding $30 \%$ ), the antiendomysial antibodies and/or antitransglutaminase antibodies positive. The reproductive disorders were never isolated but always associated with digestive or extradigestive signs at the time of the diagnosis of coeliac disease. These disorders were manifested by: retarding puberty in 11 cases $(19 \%)$, secondary amenorrhea in 13 cases $(22.4 \%)$, Metrorrahgia in 12 cases $(20.6 \%)$, absence of developpement of secondary sexual caracters in 8 cases $(12.5 \%)$, spontaneous abortion in 7 cases $(10.9 \%)$, menometrorrahgia in 4 cases $(13.8 \%)$, primary sterility in 5 cases $(8.6 \%)$, precocious menopause in 6 cases $(10.3 \%)$, premature labour and/or IUGR in 3 cases $(5 \%)$, primary amenorrhea in 2 cases $(3.4 \%)$, and intrauterine Fœtal death IUFD in one case $(1.7 \%)$. All our patients benefited from a glutenfree diet. 15 patients were excluded from the study, 2 patients died, and 12 patients are still under follow-up. Of the 29 patients stayed, the evolution of the reproductive disorders under gluten-free diet was good in 26 cases $(90 \%)$, with normalization of the cycles in 15 cases, The cycle was returned in 6 cases, development of secondary sexual characters in 2 cases, fertility was returned in one case, one case developed her cycle after primary amenorrhea, and one case was delivered a baby in term after a repeated premature deliveries. The evolution was good in 3 cases as regard missed abortion four years after the gluten-free diet in 1 patient, and amenorrhea continued in 2 cases.

Conclusion: Reproductive disorders related to the coeliac disease were frequent and variable. In our study, these disorders well responded to the gluten-free diet in $90 \%$ of cases, and these disorders were reversible under gluten-free diet. Disclosure of Interest: All authors have declared no conflicts of interest.

\section{P1926 SEVERITY OF MUCOSAL DAMAGE AND TISSUE TRANSGLUTAMINASE ANTIBODY LEVELS CORRELATE WELL IN ADULT CELIAC DISEASE IRRESPECTIVE OF CLINICAL FEATURES}

R. Maxim ${ }^{1}$, A. Trifan ${ }^{2}$, A. Plesa ${ }^{2}$, S. C. Oana Cristina ${ }^{1}$, I. Ciortescu ${ }^{2}$,

I. Girleanu ${ }^{2}$, C. Stanciu ${ }^{3}$

${ }^{1}$ University of Medicine and Pharmacy "Grigore T. Popa", Faculty of Medicine, IASI/Romania

${ }^{2}$ Institute Of Gastroenterology And Hepatology, "Grigore T. Popa" University of Medicine and Pharmacy, Iasi/Romania

${ }^{3}$ Institute of Gastroenterology and Hepatology, Iasi/Romania

Contact E-mail Address: ancatrifan@yahoo.com

Introduction: Celiac disease (CD) is a chronic imune-mediated enteropathy that occurs in genetically predisposed individuals. The clinical phenotypes ranges from classical gastrointestinal manifestations to only atypical signs, thus making the clinical diagnosis a challenge. The aim of the study was to investigate the relationship between duodenal histology, specific antibody levels and clinical presentation in adult CD Romanian patients.

Aims \& Methods: Study design: retrospective retrieval of information prospectively entered into a structured database including 81 adult patients diagnosed with CD hospitalized at the Institute of Gastroenterology and Hepatology, "St. Spiridon" Hospital, Iasi between January, 2012- December, 2016 admitted with symptoms of abdominal disturbances (diarrhoea, heartburn, nausea, vomiting, regurgitation, abdominal pain). Demographic, clinical, serological, and histological characteristics of individuals with $\mathrm{CD}$ were reviewed.

Results: The study group included 81 adult patients with a female: male ratio of $3: 1,60(71.1 \%)$ female patients, mean age $40.02 \pm 12.14$ years. A total of $48.1 \%$ patients presented with gastrointestinal (GI) complaints and $51.9 \%$ of patients presented mostly with non-GI manifestations, and advanced age of symptom onset in the latter category (38yrs vs 47yrs). Marh-Oberhuber classification was used to assess mucosal injury and Marsh 3c lesions were found in $25(30.9 \%)$ cases. When assessing the serological parameters, IgA anti-tissue transglutaminase (IgA-tTG) antibody $(61.45 \pm 76.458 \mathrm{u} / \mathrm{mL}$ vs $162.02 \pm 106.179 \mathrm{u} / \mathrm{mL}$, $P=0.001)$ and IgA anti-gliadine antibodies (IgA-AGA) levels $(61.83 \pm 69.41 \mathrm{u} /$ $\mathrm{mL}$ vs $77.15 \pm 71.02 \mathrm{u} / \mathrm{mL}, P=0.001$ ) correlated with intestinal villous atrophy (Marsh 3a and 3c) in CD patients by Spearman rank correlation. Among symptoms, abdominal distention and diarrhea were associated with abnormal histology. Hemoglobin levels were evaluated and anemia was diagnosed in $61.7 \%$ patients among patients with elevated IgA-tTG levels $(\mathrm{r}=-0.316 ; P=0.004)$, IgA-AGA $(\mathrm{r}=-0.301 ; P=0.006)$ and Marsh $3 \mathrm{~b}-3 \mathrm{c}$ lesions $(P=0.0048)$. Among biological markers included in the statistical analysis, low iron levels (cut off $30 \mathrm{mg} / \mathrm{dl}$ ), hypocholesterolemia and low protein levels were associated with Marsh 3 b lesions $(P=0.006)$ and elevated tTG-IgA titers $(\mathrm{r}=-0.384 ; P=0.001)$. Conclusion: IgA-tTG and AGA levels correlate with duodenal villous atrophy in adult CD patients. An IgA-tTG titer $>160$ was nearly always associated with severe CD histopathology. GI and non-GI symptoms are not reliable predictors of $\mathrm{CD}$.

Disclosure of Interest: All authors have declared no conflicts of interest.

\section{P1927 ASSOCIATION OF CELIAC DISEASE AND PATENT FORAMEN OVALE}

M. Sezikli ${ }^{1}$, G. Dindar ${ }^{1}$, D. Karacimen ${ }^{2}$, S.B. Acikgoz ${ }^{3}$

${ }^{1}$ Department Of Gastroenterology, Health Sciences University Kocaeli Derince Education and Research Hospital, Kocaeli/Turkey

${ }^{2}$ Department Of Cardiology, İzmit Seka State Hospital, Kocaeli/Turkey

${ }^{3}$ Internal Medicine, Health Sciences University Kocaeli Derince Education and Research Hospital, Kocaeli/Turkey

Contact E-mail Address: drsezikli@hotmail.com

Introduction: Celiac disease is an immunologically-mediated enteropathy that triggered by the intake of gluten-containing foods in genetically predisposed individuals. It causes intestinal and extraintestinal problems. Extraintestinal findings are observed in many systems. The prevalence of extraintestinal findings in the cardiovasculer system of celiac disease is not clearly known and the correlation between them has not been yet obviously explained. There is also no data in the literature regarding the association of patent foramen ovale (PFO) and celiac disease, which is $10-25 \%$ common in the general population. We performed an echocardiography study to determine the frequency of accompanying cardiac findings in our patients with celiac disease. In this article, we aimed to share the frequency of the PFO detected in celiac patients with high results.

Aims \& Methods: Between May-June 2015, 65 patients who applied to the gastroenterology clinic of Derince Education and Research Hospital and followed up with celiac diagnosis were identified. The sociodemographic characteristics, celiac disease diagnosis duration, symptoms and complaints, accompanying diseases, drug use histories, hemogram and biochemical parameters of these patients were recorded. The patients underwent saline contrast transthoracic echocardiography in the cardiology clinic. Patients' data were recorded. The obtained data were evaluated with appropriate statistical methods.

Results: Sixty-five celiac patients were included in the study, $21(32.3 \%)$ male and $44(67.7 \%)$ female. The mean age of the patients was $41.5 \pm 14.1$ years. PFO was detected in $39(60 \%)$ of the patients. There was no difference in the incidence of PFO in between male and female patients. $(61.9 \%$ and $59.1 \%$ respectively, p:0.829). Compared with the frequency of PFO in the general population, the incidence of PFO in celiac patients was higher. (25\% and $60 \%$ respectively)

Conclusion: As a result, the incidence of $\mathrm{PFO}$ is more prevalent in celiac patients than in the general population. For this reason, the evaluation and treatment of the PFO, which may be the cause of cerebrovascular disease in clinical follow-up of patients, are required. In addition, the high incidence of PFO in celiac patients suggests that celiac disease is a factor affecting the development of patients from intrauterine period. Because our patients did not undergo transesophageal echocardiography, this rate may be less than real exist. For this reason, further evaluation is required with a wider patient group and by transesophageal echocardiography.

Disclosure of Interest: All authors have declared no conflicts of interest.

\section{P1928 USEFULNESS OF BULB BIOPSY SAMPLES IN CELIAC DISEASE DIAGNOSIS IN ADULTS}

D.V. Balaban ${ }^{1}$, A. Popp ${ }^{2}$, J. Taavela ${ }^{2}$, K. Laurila ${ }^{2}$, M. Mäki², M. Jinga "Carol Davila" University of Medicine and Pharmacy, Bucharest/Romania ${ }^{2}$ Center For Child Health Research, University of Tampere and Tampere University Hospital, Tampere/Finland

Contact E-mail Address: vbalaban@yahoo.com

Introduction: Currently available celiac disease (CD) guidelines recommend sampling of both the bulb and distal duodenum for diagnostics. This has been reinforced by the recent data on ultra-short CD [1]. However, it has been previously shown in pediatric $\mathrm{CD}$ that bulb specimens are frequently of poor quality and that morphological injury is common in the duodenal bulb in non-celiac patients also, and it can lead to false-positive diagnoses [2]. Our aim was to address the same issue in adult $\mathrm{CD}$, using the same validated morphometric methods [3].

Aims \& Methods: We prospectively recruited cases of clinically recommended upper GI endoscopy; all patients also had signs and symptoms of CD and were checked for CD serology (serum tissue transglutaminase 2 antibodies and endomysial antibodies) and biopsy sampled according with current 
recommendations. Paraffin embedded biopsy samples were assessed for villous height $(\mathrm{VH})$, crypt depth $(\mathrm{CrD})$ and $\mathrm{VH}$ to $\mathrm{CrD}$ ratio $(\mathrm{VH}: \mathrm{CrD})$. The corresponding frozen duodenal samples were assesed for duodenal IgA deposits targeting transglutaminase 2 (TG2-IgA), density of CD3 (cut-off 37 cells $/ \mathrm{mm}$ epithelium) and $\gamma \delta \mathrm{T}$ cell receptor bearing intraepithelial lymphocytes/IELs (cut-off 4.3 cells $/ \mathrm{mm}$ epithelium). The study was approved by the Local Ethical Committee.

Results: Altogether 41 patients, mean age $45 \pm 14$ years, $61 \%$ female, were recruited. Among these, 21 were finally diagnosed as adult CD (mean tTG 156 U/1, median EMA 1:500, and crypt hyperplastic mucosal lesion in distal duodenum) and the rest 20 were non-CD controls (serum negative and normal on distal duodenal biopsy). All patients were on a gluten-containing diet. Quality of bulb biopsy samples was unsatisfactory and unreadable in $67 \%$ of $\mathrm{CD}$ cases and $50 \%$ of controls, even after reorientations and recuttings. All CD patients had, when measurable, $\mathrm{VH}: \mathrm{CrD}<2$ in the anatomical bulb (average 0.31 , range $0.02-0.61$ ). On the other hand also non-CD controls had a crypt hyperplastic diseased bulb mucosa in $80 \%$ of patients (average $\mathrm{VH}$ :CrD 1.65, range $0.7-4.1$ ), but the injury was not that severe as in $C D(p=0.0006)$. Inflammation was significantly higher in CD compared to controls (CD3 81.87 vs. 34.05, $\mathrm{p}<0.01 ; \gamma \delta$ IELSs 29.12 vs. $6.44, \mathrm{p}<0.01)$. Bulb IgA deposits were positive in all $\mathrm{CD}$ patients and was able to discriminate $\mathrm{CD}$ cases from disease controls.

Conclusion: As reported in children, bulb biopsy samples in adults are frequently of poor quality and not reliable for accurate histological measurements. Also, interpretation of results from bulb samples should be done with caution, as non$\mathrm{CD}$ patients may have mild injury in the bulb lining and could be misinterpreted as CD. Assessment of bulb TG2-IgA subepithelial deposits is a powerful tool to confirm CD

Disclosure of Interest: All authors have declared no conflicts of interest.

\section{References}

1. Mooney PD, Kurien M, Evans KE, et al. Clinical and immunologic features of ultra-short celiac disease. Gastroenterology. 2016; 150: 1125-1134

2. Taavela J, Popp A, Korponay-Szabo IR, et al. Prospective study on the usefulness of duodenal bulb biopsies in celiac disease diagnosis in children: urging caution. Am J Gastroenterol. 2016;111:124-133.

3. Taavela J, Koskinen O, Huhtala $\mathrm{H}$, et al. Validation of morphometric analyses of small-intestinal biopsy readouts in celiac disease. PLoS One 2013;8(10):e76163.

\section{P1929 PROSPECTIVE MULTI-CENTER-STUDY TO EVALUATE A FINGER PRICK-BASED POINT-OF-CARE-TEST (POCT) FOR DIAGNOSIS OF COELIAC DISEASE}

P. H. Tangermann ${ }^{1}$, F. Branchi ${ }^{1}$, W. Spitz ${ }^{2}$, U. Möhler ${ }^{2}$, J. Aschenbeck ${ }^{3}$,

S. Schubert ${ }^{4}$, A. Schroeder ${ }^{5}$, F. Heller ${ }^{3}$, S. Trenkel ${ }^{6}$, M. Schmitt ${ }^{7}$, B. Siegmund ${ }^{1}$, C. Bojarski ${ }^{1}$, M. Schumann ${ }^{1}$

${ }^{1}$ Gastroenterology, Charité Berlin, Berlin/Germany

${ }^{2}$ Praxis am Mexicoplatz, Berlin/Germany

${ }^{3}$ Gastroenterologische Praxis, Berlin/Germany

${ }^{4}$ Praxis am Bayrischen Platz, Berlin/Germany

${ }^{5}$ Praxis am Hohenzollerndamm, Berlin/Germany

${ }^{6}$ Pädiatrie, Klinikum Westbrandenburg, Potsdam/Germany

${ }^{7}$ Pädiatrie Ludwigfelde, Ludwigfelde/Germany

\section{Contact E-mail Address: michael.schumann@ charite.de}

Introduction: Celiac disease (CD) is a chronic inflammatory disorder triggered by the ingestion of gluten in genetically susceptible people. The prevalence varies in between European countries and averages at $1 \%$ within whole Europe. It is anticipated that the number of undetected and therefore untreated cases is high. Since untreated CD is associated with a high morbidity, further diagnostic modalities to uncover undiagnosed coeliacs are currently explored. In this setting, we evaluate Simtomax ${ }^{\mathrm{TM}}$, a POCT detecting deamidated gliadin peptide antibodies, with the goal to identify patients at need for duodenal biopsies prior to gastroscopy. Thus, the goal was to establish a test allowing to guide the endoscopist in his decision to collect duodenal biopsies.

Aims \& Methods: Prospective investigator-initiated multi-center study in six adult gastroscopy and two pediatric gastroscopy centers in Berlin and Brandenburg, Germany, approved by the local ethical committees. Finger prick blood of patients registered for gastroscopy and eligible for the study (exclusion: defective coagulation, established celiac disease or on gluten-free diet) was analyzed by the POCT (IgA and IgG for deamidated gliadin peptides; Simtomax ${ }^{\text {TM }}$ test, Tillots, Switzerland). Test results were compared with duodenal histology (Marsh classification). In POCT-positive individuals transglutaminase-IgA serology was performed.

Results: Analysis was performed at $\mathrm{n}=721$ adult patients (average age: $48 \mathrm{yrs}$ ) and $\mathrm{n}=108$ pediatric patients (average age: $11 \mathrm{yrs}$ ). In the adult cohort 45 POCtests were judged as "positive". Within the POCT positives, 6 Marsh III case were detected. None of the 676 POCT-negative individuals revealed CD. Therefore, the prevalence of CD in this population was $0.8 \%$. The POCT-specificity in this group was $94 \%(95 \%$-CI $92-96 \%)$, the sensitivity was $100 \%(95 \%$ CI $51-100 \%$ ). In the pediatric cases 21 POCTs were judged as "positive" with 13 being true-positive. Of the 87 negative POCTs, 68 were true-negative, but 4 were false-negatives. Thus, the prevalence of $\mathrm{CD}$ in the pediatric gastroscopy was $16 \%$. However, sensitivity in this group was only $76 \%$ (CI $50-93 \%$ ) and specificity $91 \%$ (CI $83-96 \%$ ). Examiners at various centers suggested, that "faint" bands in the POCT analysis might contribute to interpretation failures.

Conclusion: A screening test-like a POCT in CD-needs to perform optimally especially in sensitivity. In the adult population all CDs were detected by the
POCT. However, the CD prevalence in this group was low. In the pediatric group the test revealed a critically lower sensitivity, either due to a sensitivity problem with this serology in the pediatric population or secondary to a suboptimal $\operatorname{IgA}$ band expression of the POCT.

Disclosure of Interest: All authors have declared no conflicts of interest.

\section{P1930 QUALITY STANDARDS IN COELIAC DISEASE: A} RETROSPECTIVE EVALUATION IN A SINGLE SPECIALIST CLINIC

\section{Fitzpatrick ${ }^{1}$, S. Nichols ${ }^{2}$, E. Soilleux ${ }^{2}$, S. Travis ${ }^{3}$}

${ }^{1}$ Translational Gastroenterology Unit, Nuffield Department of Medicine, University of Oxford, Oxford/United Kingdom

${ }^{2}$ Department Of Nutrition And Dietetics, John Radcliffe Hospital, Oxford/United Kingdom

${ }^{3}$ Gastroenterology Department, John Radcliffe Hospital, Oxford/United Kingdom

Contact E-mail Address: mebfitz@gmail.com

Introduction: Quality standards in coeliac disease management were recently published by the National Institute for Health and Care Excellence ${ }^{1}$. These specify a new 6-week target for the time from referral to endoscopy, which was previously covered by the 18 -week referral to treatment (RTT) pathway ${ }^{2}$. They also state that all newly-diagnosed patients should discuss a gluten-free diet with a specialist dietitian. We retrospectively evaluated practice in the Oxford University Hospitals NHS Foundation Trust coeliac clinic against these criteria, and against national guidelines (duodenal bulb sampling at endoscopy and screening for nutritional deficiency) $)^{3}$.

Aims \& Methods: The medical records of 110 patients referred to our clinic between September 2015 and September 2016 were examined. The date of referral and endoscopy were recorded, along with relevant demographic, clinical and laboratory information. Data were collected and analysed in Microsoft Excel. Results: Eighty-five patients $(68 \%$ female, median age 34$)$ were seen with suspected or newly-diagnosed coeliac disease, of whom $76(89 \%)$ were referred with positive coeliac serology and would be subject to the 6-week target. Six patients declined or delayed endoscopy, and endoscopy or referral information were not available for 4 patients. For the remaining 66 patients, median time from referral to endoscopy was 12 weeks 2 days (SD 37 days), with 59 patients $(89 \%)$ within 18 weeks, but only 11 patients $(17 \%)$ within 6 weeks (Figure 1$)$. Duodenal bulb biopsies were taken at endoscopy in 31 patients $(44 \%)$. A diagnosis of coeliac disease was made in $74(87 \%)$ of all patients referred, of whom $67(90 \%)$ were referred to a specialist dietitian. Haematinics (iron studies, vitamin B12 and folate) were measured in 67 patients $(90 \%)$, bone densiometry was measured in 51 patients $(69 \%)$ and all patients were offered a follow-up appointment in the coeliac clinic. Iron deficiency was found in 31 patients $(45 \%)$ of patients tested, folate deficiency in 12 patients $(18 \%)$, vitamin $\mathrm{B} 12$ deficiency in 5 patients $(7 \%)$ and vitamin D deficiency in 23 patients $(38 \%)$ ). Osteoporosis was diagnosed in 5 patients $(10 \%)$ and osteopenia in 10 patients $(20 \%)$.

Conclusion: Appropriate dietitian referral, specialist follow-up and screening for nutritional deficiency and bone disease occur within the Oxford coeliac disease service. Compliance with recommended biopsy protocols was only $44 \%$. Whilst most referrals met the previous 18-week RTT pathway, few would have met the new quality standards.

Disclosure of Interest: M. FitzPatrick: Michael FitzPatrick is supported by an Oxford-Celgene Research Fellowship funded by Celgene Corporation

All other authors have declared no conflicts of interest.

\section{References}

1. National Institute for Health and Care Excellence, 2016. Coeliac Disease: NICE quality Standard QS134. London: NICE.

2. Department of Health, 2015. The NHS constitution for England. London: Department of Health. Available at: < http://www.gov.uk/government/publications/the-nhs-constitution-for-england $>$ [accessed 14 February 2017].

3. Ludvigsson JF, Bai JC, Biagi F, et al. 2002. Diagnosis and management of adult coeliac disease: guidelines from the British Society of Gastroenterology. Gut. 63:1210-28.

\section{P1931 MANAGEMENT OF OCCULT OBSCURE GASTROINTESTINAL BLEEDING PATIENTS BASED ON LONG- TERM OUTCOMES}

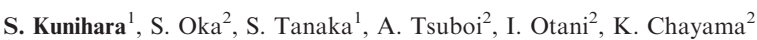
${ }^{1}$ Department Of Endoscopy, Hiroshima University Hospital, Hiroshima/Japan ${ }^{2}$ Department Of Gastroenterology And Metabolism, Hiroshima University Hospital, Hiroshima/Japan

Contact E-mail Address: sayokok@hiroshima-u.ac.jp

Introduction: We previously reported that small-bowel capsule endoscopy (CE) is effective in diagnosing small-bowel lesions with occult obscure gastrointestinal bleeding (OGIB) (Gastroenterol Res Pract. 2013). However, there is no consensus regarding the management of occult OGIB patients without bleeding source lesions.

Aims \& Methods: We aimed to consider management of occult OGIB patients based on the long-term outcomes. A total of 357 consecutive occult OGIB patients (203 men; mean age: 59.7 years) who underwent CE at Hiroshima University Hospital before March 2016 and whose entire small-bowel could be observed and followed-up by $\mathrm{CE}$ for at least 12 months, were enrolled. We examined each patient to confirm the positive CE findings rate, the detection rate of bleeding source lesions, the details of bleeding source lesions, the overt 
bleeding rate with or without treatment, the rate of anaemia exacerbation (hemoglobin levels decreased $>2.0 \mathrm{~g} / \mathrm{dL}), 5$ year overall survival rate (OS), and 5 year disease specific survival rate (DSS). Occult OGIB was defined as recurrent or persistent iron deficiency anaemia with or without a positive faecal occult blood test and no bleeding findings by esophagogastroduodenoscopy and colonoscopy. Results: Results: The positive CE findings rate was 44\% (157/357) and the detection rate of bleeding source lesions was $27 \%(98 / 357)$. All of the treated bleeding source lesions (Group A) were as follows: angioectasia 61 patients (YanoYamamoto classification Type 1a 37 patients, Type 1b 24 patients), non-specific ulcer 10 patients, nonsteroidal anti-inflammatory drugs-indeeded ulcer 8 patients, hemangioma 5 patients, Crohn's disease 3 patients, primary cancer 2 patients, metastatic cancer 2 patients, gastrointestinal stromal tumour 2 patients, malignant lymphoma 2 patients, others 3 patients. Lesions that were not regarded as bleeding source and were followed-up without treatment (Group B) were as follows: angioectasia 25 patients (Type la without oozing 25 patients), erythema 31 patients, others 3 patients. There were no patients with overt bleeding in Group B. Although 6 patients $(10 \%)$ had anaemia exacerbation in Group $\mathrm{B}$, they had angioectasia (Type 1a) that was not a bleeding source lesion. OS in both Group A and Group B was $90 \%$. DSS in Group A was $99 \%$ and in Group B was $100 \%$. One patient in Group A died of a primary small-bowel cancer. Conclusion: Conclusion: Long-term outcomes with occult OGIB patients were good except malignant tumor, because overt bleeding and/or anaemia exacerbation did not occur within the follow-up period. Thus, occult OGIB patients without bleeding source lesions, including Type la angioectasia without oozing, and erythema, are unnecessary to follow-up with CE in occult OGIB patients.

Disclosure of Interest: All authors have declared no conflicts of interest.

\section{P1932 A PILOT STUDY EXPLORING THE VALUE OF FAECAL IMMUNOCHEMICAL TEST (FIT) WHEN INVESTIGATING ANAEMIA OR OCCULT GASTROINTESTINAL BLEEDING WITH SMALL BOWEL CAPSULE ENDOSCOPY}

D. Tighe ${ }^{1}$, C. Judge ${ }^{1}$, P. Walsh ${ }^{2}$, M. Kelly ${ }^{2}$, A. O'Connor ${ }^{1}$, N. Breslin ${ }^{1}$, B. Ryan ${ }^{1}$, G. Boran ${ }^{2}$, D. Mcnamara

${ }^{1}$ Department Of Gastroenterology Trinity Academic Gastroenterology Group

(TAGG), AMNCH Tallaght, Dublin/Ireland

${ }^{2}$ Department Of Clinical Chemistry, AMNCH Tallaght, Dublin/Ireland

Contact E-mail Address: donaltighe83@gmail.com

Introduction: Small bowel capsule endoscopy (SBCE) is a very useful method of investigating iron-deficient anaemia, or occult gastrointestinal (GI) bleeding. It can help diagnose sources of anaemia or bleeding, such as angiodysplasia, small bowel Crohn's disease, polyps, lymphoma, and malignant lesions. There is however a need to improve the diagnostic yield, particularly where resources and access to capsule endoscopy are restricted. Faecal immunochemical test (FIT) has an established role, in investigating large bowel bleeding, and is incorporated into a number of bowel cancer screening programmes.

Aims \& Methods: The aim of our study was to investigate whether FIT could help predict likelihood of small bowel bleeding or other significant pathology at time of small bowel capsule endoscopy. This was a prospective pilot study, performed at our centre from September 2016-April 2017. Indications for enrolment were patients referred for SBCE with the indication of anaemia or occult GI bleeding. Baseline patient characteristics were obtained including age, gender, history of renal disease, transfusion requirements and use of antiplatelet/anti-coagulants. Patient haemoglobin $(\mathrm{Hb})$ level was checked on the day of SBCE where possible. Patients were asked to return one completed FIT for further analysis. A cut of $50 \mathrm{ng} / \mathrm{ml}$ was chosen, as this is the standard cut-off used, in the Irish National Bowel Cancer Screening programme.

Results: A total of 40 patients were enrolled, mean age 55.4 years (range 18-77), $64 \%$ were female. A total of $27.6 \%$ of patients were on anti-platelet agents or anti-coagulants. $34 \%$ of patients had a blood transfusion within the last year. Mean $\mathrm{Hb}$ for the cohort was $12.8 \mathrm{~g} / \mathrm{dL}$ (range $7.8-15.9 \mathrm{~g} / \mathrm{dL}$ ). The average FIT reading was $459 \mathrm{ng} / \mathrm{ml}$ (range $0-4426 \mathrm{ng} / \mathrm{ml}$ ). $30 \%$ of patients had a FIT level $>50 \mathrm{ng} / \mathrm{ml} .46 \%$ of patients, had positive findings at SBCE. $9 / 12(75 \%)$ of patients with a FIT level $>50 \mathrm{ng} / \mathrm{ml}$ had positive findings at capsule endoscopy compared to $5 / 28(17.8 \%)$ for FIT $<50 \mathrm{ng} / \mathrm{ml}$, p value $=0.002,95 \%$ C.I. 0.29 0.86 O.R. 0.16. These included $4 / 12(33 \%)$ new cases of Crohns, $3 / 12(25 \%)$ angiodysplasia, $3 / 12(33 \%)$ non-IBD enteritis, $1 / 12(16.7 \%)$ small bowel tumour, and $1 / 12(16.7 \%)$ melaena, with no clear source. In addition there was a good correlation between FIT and Haemoglobin levels. $60 \%$ of patients with FIT $>50 \mathrm{ng} / \mathrm{ml}$ were anaemic $(\mathrm{Hb}<11.5 \mathrm{~g} / \mathrm{dL})$, compared to $17 \%$ with FIT $<50 \mathrm{ng} / \mathrm{ml}$, p value $=0.0295 \%$ C.I $0.09-0.76$ O.R 0.14. Combining $\mathrm{Hb}$ and FIT levels, was also informative and predictive of small bowel pathology. $83 \%$ of patients, who were anaemic and had a FIT $>50 \mathrm{ng} / \mathrm{ml}$ had clinically significant findings at SBCE compared to $21 \%$ pick up rate in patients with normal $\mathrm{Hb}$ and FIT levels, $\mathrm{p}$ value $=0.0595 \%$ C.I $0.22-1.03$ O.R. 0.05 . Overall the sensitivity for a FIT $>50 \mathrm{ng} / \mathrm{ml}$ for detecting small bowel pathology was $83 \%$ with a specificity of $92 \%$, giving a positive predictive value of $83.3 \%$ ( $95 \%$ C.I. $56 \%-95 \%)$. Antiplatelet usage was not predictive of a positive FIT, as $16.7 \%$ of patients with a FIT $>50 \mathrm{ng} / \mathrm{ml}$ were on anti-platelet agents, compared to $83.3 \%$ who weren't.

Conclusion: FIT is useful at predicting clinically significant small bowel pathology at the time of capsule endoscopy. It may help better identify and prioritise patients who would best benefit from referral.

Disclosure of Interest: All authors have declared no conflicts of interest.

\section{P1933 VIDEO CAPSULE ENDOSCOPY IN THE ASSESSMENT OF} PORTAL HYPERTENSIVE ENTEROPATHY

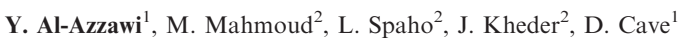
${ }^{1}$ Gastroenterology, University of Massachusetts Medical School, Worcester/United States of America/MA

${ }^{2}$ Gastroenterology, UMass Medical Center, Worcester/United States of America

\section{Contact E-mail Address: yasir.alazzawi@umassmemorial.org}

Introduction: The features of the portal hypertension enteropathy (PHE) vary from mild mucosal changes to varices with or without bleeding. The prevalence and the correlation of factors that predict the development of PHE are not fully understood.

Aims \& Methods: Our aim in this study is to examine the prevalence of the different manifestations portal hypertensive enteropathy and it's correlation with the Child-Pugh score (CTP) in cirrhosis using video capsule endoscopy (VCE). At a single center, we performed a retrospective chart review study of patients between the age of 18 and 80 with cirrhosis, who had VCE study between January 2010 and January 2016. Based on the published literature, we divided the portal hypertensive enteropathy lesions in our study into vascular lesions (arteriovenous malformation (AVM), red spots, bleeding or varices) and mucosal lesions (mild inflammatory changes or severe inflammatory changes which are a mosaic, congested and friable mucosa).

Results: 100 patients with cirrhosis had a VCE study. The mean age was 62.82 years. Male gender was predominant with a $64 \%$, while the Caucasians represented $82 \%$ of the cohort. The most common etiologies of the cirrhosis were chronic alcohol abuse followed by chronic hepatitis $\mathrm{C}$ virus (HCV) and nonalcoholic steatohepatitis (NASH)table 1A. The average MELD score was $13.86( \pm 0.66)$. VCE detected small bowel lesions in $71 \%$ of the patients while the features of portal hypertensive enteropathy were found in $65 \%$ from the total cohort. AVMs and Inflammatory changes were the most common findings, followed by bleeding. 13 patients found to have two or more lesions by VCE. More than $50 \%$ of the lesions were vascular in nature (table1A). The percentage of the CTP A, CTP B and CTP C was $46 \%, 42 \%$ and $12 \%$ respectively. The odds of finding portal hypertensive enteropathy in decompensated cirrhosis is twice that in compensated cirrhosis (odd ratio of 2.0) table 1B. 45 patients had negative EGD exam for any active bleeding, esophageal varices (EV), portal hypertensive gastropathy(PHG) or gastric varices(GV). 31 of them $(69 \%)$ had features of portal hypertension enteropathy in their VCE. table 1B. Table $1 \mathrm{~A}$

\begin{tabular}{|c|c|}
\hline & Number \& $(\%)$ \\
\hline Total number of patients & 100 \\
\hline \multicolumn{2}{|l|}{ Causes of cirrhosis: } \\
\hline Congestive Hepatopathy & $4(4 \%)$ \\
\hline Cryptogenic & $8(8 \%)$ \\
\hline $\mathrm{HCV}$ & $22(22 \%)$ \\
\hline Alcohol & $37(37 \%)$ \\
\hline Hemochromatosis & $2(2 \%)$ \\
\hline NASH & $18(18 \%)$ \\
\hline PSC & $2(2 \%)$ \\
\hline PBC & $6(6 \%)$ \\
\hline $\mathrm{AIH}$ & $1(1 \%)$ \\
\hline Age & $62.82 \pm 2$ \\
\hline MELD score & $13.86( \pm 0.66)$ \\
\hline Demographic: Male & $64(64 \%)$ \\
\hline Female & $36(36 \%)$ \\
\hline Whites & $82(82 \%)$ \\
\hline Asian & $2(2 \%)$ \\
\hline African-American & $1(1 \%)$ \\
\hline Others & $15(15 \%)$ \\
\hline Small intestine lesions & $71(71 \%)$ \\
\hline Portal hypertensive enteropathy (PHE) & $65(65 \%)$ \\
\hline Vascular lesions: AVMs & $18(18 \%)$ \\
\hline Varices & $1(1 \%)$ \\
\hline Bleeding & $15(15 \%)$ \\
\hline Red spot & $9(9 \%)$ \\
\hline Inflammatory changes: Mild & $18(18 \%)$ \\
\hline Severe & $15(15 \%)$ \\
\hline No Portal hypertension enteropathy & $35(35 \%)$ \\
\hline
\end{tabular}

Table 1B

\begin{tabular}{llllll}
\hline & Number & PHE & No PHE & O.R & P-value \\
\hline CTP-A & 46 & 26 & 20 & 0.5 & 0.01 \\
CTP-B +C & 54 & 39 & 15 & 2.0 & 0.01 \\
\hline
\end{tabular}


Continued

\begin{tabular}{llllll}
\hline & Number & PHE & No PHE & O.R & P-value \\
\hline Alcohol & 37 & 20 & 17 & 0.4 & 0.04 \\
HCV & 22 & 13 & 9 & 0.4 & 0.02 \\
NASH & 18 & 14 & 4 & 2.1 & 0.1 \\
Cholestatic liver disease(PSC + PBC) & 8 & 6 & 2 & 1.6 & 0.4 \\
Thrombocytopenia & 69 & 48 & 21 & 1.8 & 0.09 \\
Male & 64 & 42 & 22 & 1.1 & 0.7 \\
Female & 36 & 23 & 13 & 0.9 & 0.7 \\
EGD + (EV, PHG or GV) & 55 & 34 & 21 & 0.7 & 0.5 \\
EGD & 45 & 31 & 14 & 1.3 & 0.5 \\
\hline
\end{tabular}

Conclusion: VCE detected small bowel lesions in $71 \%$ in our cohort. There is a high prevalence of PHE in patients with decompensated cirrhosis. Vascular lesions are the most common finding in the small bowel of this population. Disclosure of Interest: All authors have declared no conflicts of interest.

\section{References}

Mekaroonkamol P, Cohen R, Chawla S. Portal hypertensive enteropathy. World Journal of Hepatology. 2015;7(2):127-138. doi:10.4254/wih.v7.i2.127.

Abdelaal UM, Morita E, Nouda S, Kuramoto T, Miyaji K, Fukui H, Tsuda Y, Fukuda A, Murano M, Tokioka S, et al. Evaluation of portal hypertensive enteropathy by scoring with capsule endoscopy: is transient elastography of clinical impact? J Clin Biochem Nutr. 2010;47:37-44.

De Palma GD, Rega M, Masone S, Persico F, Siciliano S, Patrone F, Matantuono L, Persico G. Mucosal abnormalities of the small bowel in patients with cirrhosis and portal hypertension: a capsule endoscopy study. Gastrointest Endosc. 2005;62:529-534

P1934 MULTICENTER PROSPECTIVE CASE-CROSSOVER STUDY ON THE ASSOCIATION BETWEEN OVERT SMALL-BOWEL BLEEDING AND DRUGS USING CAPSULE ENDOSCOPY DATABASE IN JAPAN

N. Ohmiva ${ }^{1}$, S. Fujimori ${ }^{2}$, M. Nakamura ${ }^{3}$, A. Yamada ${ }^{4}$, K. Maki ${ }^{2}$, S. Oka ${ }^{5}$,

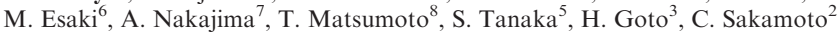
${ }^{1}$ Department Of Gastroenterology, Fujita Health University School of Medicine, Toyoake/Japan

${ }^{2}$ Department Of Gastroenterology, Nippon Medical School, Graduate School of Medicine, Tokyo/Japan

${ }^{3}$ Department Of Gastroenterology And Hepatology, Nagoya University Graduate School of Medicine, NagoyalJapan

${ }^{4}$ Department Of Gastroenterology, The University of Tokyo, Tokyo/Japan

${ }^{5}$ Endoscopy, Hiroshima University Hospital, Hiroshima/Japan

${ }^{6}$ Department Of Medicine And Clinical Science, Graduate School of Medical

Sciences Kyushu University, Fukuoka/Japan

${ }^{7}$ Division Of Gastroenterology And Hepatology, Yokohama City University School of Medicine, Yokohama/Japan

${ }^{8}$ Division Of Gastroenterology, Department Of Internal Medicine, Iwate Medical University, Morioka/Japan

Contact E-mail Address: nohmiya@med.nagoya-u.ac.jp

Introduction: Small-bowel capsule endoscopy (SBCE) have been useful in managing obscure gastrointestinal bleeding. We previously reported that the use of oxicams and diclofenac was associated with an increased risk of nonsteroidal anti-inflammatory drug (NSAID)-induced small-bowel injury (Aliment Pharmacol Ther 2014). However, the etiology and temporal development of drug-associated small-bowel bleeding (SBB) has not been well characterized.

Aims \& Methods: The aim of this study is to determine the risk of drugs associated with overt SBB using a case-crossover design. The Japanese Association for Capsule Endoscopy developed a prospectively recorded database of outpatients and inpatients who underwent SBCE at 18 medical centers in Japan, and data were collected from 1052 patients with obscure gastrointestinal bleeding (OGIB) between December 2010 and June 2016. This database includes patient characteristics, drugs used, SBCE findings, and final diagnosis. Drugs used were identified during a "case period" 4 weeks before the overt SBB, and a "control period" 24-5 weeks before the overt SBB. Drug adherence was classified into 4 groups: 1) $100 \%$, 2) $50 \%$ or higher, 3) lower than $50 \%$, and 4) $0 \%$. Using conditional logistic regression, odds ratios (ORs) and $95 \%$ confidential intervals (CIs) were estimated using Mantel-Haenszel estimator in discordant cases.

Results: Of 1052 patients with OGIB (male/female: 678/374, age of onset: $68 \pm 87$ ), 455 with SBB (male/female: 291/164, age of onset: $70 \pm 91$ ) were enrolled. Final diagnoses were vascular lesions $(n=169,37 \%)$, followed by inflammatory lesions $(n=148,33 \%)$, drug injuries $(n=64,14 \%$ : NSAID 47 , aspirin 15 , both 1 , anticancer drug 1$)$, tumors $(n=48,11 \%$ : malignant 32 , benign 16), diverticula $(n=13,3 \%)$, etc. Data from 133 patients on 346 drugs which could be identified 24 weeks before overt SBB were analyzed. ORs $(95 \%$ Cis $)$ of enteric-coated aspirin $(n=51)$, warfarin $(n=27)$, clopidogrel $(\mathrm{n}=24)$, and loxoprofen (a propionic acid derivative, $\mathrm{n}=21)$ were $5.7(1.3$ $24.3), 3.7(0.8-16.6), 313406(2.3 \mathrm{E}-145-4.3 \mathrm{E}+155)$, and 18.0(2.3-139.6). Aspirinassociated SBB was caused by aspirin-induced injuries, angiodysplasias, Meckel's diverticula, and polyps. Loxoprofen-associated SBB was caused by mostly loxoprofen-induced injuries.
Conclusion: Enteric-coated aspirin, clopidogrel, and loxoprofen were identified as agents causing overt SBB during a relatively short period after administration. Disclosure of Interest: All authors have declared no conflicts of interest.

\section{Reference}

Risk factors of symptomatic NSAID-induced small intestinal injury and diaphragm disease. Ishihara $M$, Ohmiya $N$, Nakamura $M$, Funasaka $K$, Miyahara R, Ohno E, Kawashima H, Itoh A, Hirooka Y, Watanabe O, Ando T, Goto H. Aliment Pharmacol Ther. 2014 Sep;40(5):538-47.

\section{P1935 META-ANALYSIS REVEALS SIMILAR REBLEEDING RATES AMONG EASTERN AND WESTERN POPULATIONS UP TO FIVE YEARS AFTER INDEX VIDEO CAPSULE ENDOSCOPY} EXAMINATION FOR OBSCURE GASTROINTESTINAL BLEEDING

G. Tziatzios, P. Gkolfakis, G. D. Dimitriadis, K. Triantafyllou

2nd Dept Of Internal Medicine And Research Institute, National and Kapodistrian University of Athens, Medical School, Athens/Greece

\section{Contact E-mail Address: ktriant@med.uoa.gr}

Introduction: Video capsule endoscopy (VCE) is the first-line diagnostic modality for obscure gastrointestinal bleeding (OGIB) investigation and different re-bleeding rates have been published among Western and Eastern studies.

Aims \& Methods: Aim of this meta-analysis was to examine the differences in rebleeding rates in patients with OGIB after index VCE, as measured in Western and Eastern studies. A comprehensive literature search in MEDLINE was conducted to identify all studies examining re-bleeding rate after VCE for OGIB. Meta-analysis assessed the pooled proportion of re-bleeding events after VCE for OGIB according to study's origin (Western vs. Eastern) as the primary end point. Pooled odds ratios for re-bleeding after positive vs. negative index VCE and after long ( $>24$ months) vs. short ( $<24$ months) follow-up in the two studies origins, comprised the secondary endpoints. Study outcomes effect sizes were calculated using RevMan 5.3 software random effect model and they are presented as rate $[95 \% \mathrm{CI}]$ or $\mathrm{OR}[95 \% \mathrm{CI}]$. Heterogeneity was measured using the $\mathrm{I}^{2}$ statistics and publication bias risk was examined with Funnel plots inspection.

Results: Thirty-eight (14 Eastern and 24 Western) studies were included in the analysis with 5197 patients followed from 6 to 52 months. We detected significant heterogeneity with no evidence for publication bias in the meta-analyzed studies. While the overall, pooled rate of re-bleeding after VCE was $25(21-29) \%$, $\mathrm{I}^{2}=93 \%$, similar re-bleeding rates were detected among Eastern and Western populations $\left[22(16-28) \%, \mathrm{I}^{2}=93 \%\right.$ vs. $\left.28(22-35) \%, \mathrm{I}^{2}=95 \%\right]$. The re-bleeding risk after positive compared to negative VCE index examination was higher $\left[1.89(1.10-3.24), \mathrm{I}^{2}=72 \%\right]$ in Eastern population studies, while a similar difference was not detected in the Western studies [1.46(0.72-2.94), $\left.\mathrm{I}^{2}=88 \%\right]$. When only studies with short-term follow-up were analyzed, the OR of re-bleeding after positive vs. negative VCE was $1.23[0.58-2.61], \mathrm{I}^{2}=77 \%$ and $1.93[0.90-4.13]$, $\mathrm{I}^{2}=71 \%$ in Western and Eastern studies, respectively. For studies with longterm follow-up, no significant difference in the OR of re-bleeding after positive vs. negative index VCEs was detected either in the East [2.03(0.96-4.29), $\left.\mathrm{I}^{2}=71 \%\right]$ or in the West $\left[2.29(0.46-11.37), \mathrm{I}^{2}=96 \%\right]$.

Conclusion: Our analysis shows that patients undergoing VCE for OGIB have similar re-bleeding rates in the East and in the West, regardless of the length of the follow-up. An increased re-bleeding risk after positive vs. negative index VCE was noted only in studies originating from the East.

Disclosure of Interest: All authors have declared no conflicts of interest.

\section{P1936 LONG-TERM OUTCOMES AFTER NEGATIVE DOUBLE- BALLOON ENTEROSCOPY FOR SUSPECTED OVERT SMALL BOWEL BLEEDING (OBSCURE-OVERT GASTROINTESTINAL BLEEDING)}

R. Hashimoto ${ }^{1}$, T. Matsuda ${ }^{1}$, M. Nakahori ${ }^{1}$

${ }^{1}$ Dept. Of Gastroenterology, Sendai Kosei Hospital, Sendai/Japan

Contact E-mail Address: rinhashimoto@gmail.com

Introduction: There are very few reports about long-term outcomes in patients with negative balloon assisted enteroscopy for suspected overt small bowel bleeding (obscure-overt gastrointestinal bleeding).

Aims \& Methods: The aim of this study is to evaluate long-term outcomes and risk factors of re-bleeding after negative double balloon enteroscopy (DBE) for suspected overt small bowel bleeding. We investigated 297 patients undergoing DBE for suspected overt small bowel bleeding between December 2004 and April 2016 at Sendai Kousei Hospital. Prospectively collected data were reviewed, and 83 patients $(27.9 \%)$ showed negative results in the first antegrade and/or retrograde DBE. For these patients, letter and telephone interviews were conducted in April 2017. As a result, a cohort of 64 patients could be followed. The primary outcome measurement is overt rebleeding and necessity for clinical assessment after negative DBE.

Results: Rebleeding was observed in 19 of 64 patients $(29.7 \%)$ with 76 months follow-up period. The mean period during the first DBE and the first rebleeding episode were 11.6 months(2day-48months). Three patients showed rebleeding after more than three years of the first DBE. At the time of rebleeding, emergent endoscopy including DBE (within less than 48 hours) and/or contrast-enhanced computed tomography (CECT) was performed in all patients. The bleeding source was identified in 17 of 19 patients $(89.4 \%)$. The bleeding source were duodenum $(n=8)$, jejunoileum $(n=7)$ and colon $(n=2)$, respectively. One 
patient died due to uncontrollable duodenal bleeding. Blood transfusion before the first DBE was associated with rebleeding (odds ratio 22.5, $\mathrm{p}=0.0005$ ), but total enteroscopy rate, use of capsule endoscopy, use of anti-thrombotic agents and use of NSAIDs were not associated with rebleeding.

Conclusion: Rebleeding after negative DBE for suspected overt small bowel bleeding is not rare. Blood transfusion before the first DBE may predict rebleeding. Emergent endoscopy including DBE and concomitant CECT use could help the diagnosis on re-bleeding.

Disclosure of Interest: All authors have declared no conflicts of interest.

\section{P1937 DOES DISCONTINUATION OF ANTITHROMBOTIC AGENTS AFFECT DIAGNOSTIC YIELD OF SMALL BOWEL CAPSULE ENDOSCOPY IN PATIENTS WITH OBSCURE GASTROINTESTINAL BLEEDING?}

T. Watanabe ${ }^{1}$, S. Shimada ${ }^{1}$, Y. Nadatani ${ }^{1}$, K. Otani ${ }^{1}$, F. Tanaka ${ }^{1}$, Y. Nagami ${ }^{1}$, N. Kamata ${ }^{1}$, H. Yamagami ${ }^{1}$, T. Tanigawa ${ }^{1}$, M. Shibatoge ${ }^{1}$, T. Miyazaki ${ }^{2}$,

S. Nakamura ${ }^{2}$, Y. Fujiwara ${ }^{1}$

${ }^{1}$ Dept. Of Gastroenterology, Osaka City University Graduate School of Medicine Dept. of Gastroenterology, Osaka/Japan

${ }^{2}$ Department Of Inflammatory Bowel Disease, Division Of Internal Medicine,

Hyogo College of Medicine, Nishinomiya/Japan

Contact E-mail Address: watanabet@med.osaka-cu.ac.jp

Introduction: Capsule endoscopy (CE) is a useful and noninvasive modality for investigation of the small intestine, and currently, it has become the first-line modality for the diagnosis of obscure gastrointestinal bleeding (OGIB). Use of antithrombotic agents including antiplatelets and anticoagulants is associated with gastrointestinal bleeding. Antithrombotic users account for a large portion of patients with OGIB, and those with OGIB often undergo CE. It should be noted that some patient with over OGIB discontinue antithrombotic agents at the time of CE, which may affect endoscopic findings.

Aims \& Methods: To examine the effect of discontinuation of antithrombotic agents on the diagnostic yield of $\mathrm{CE}$, and to assess the predictive factors associated with positive $\mathrm{CE}$ findings in patients using antithrombotics who develop overt OGIB. Between March 2004 and December 2015, 130 consecutive patients (75 male; mean age, 71.9 years) taking antithrombotics who underwent CE for overt OGIB were enrolled, whereas patients who underwent double-balloon endoscopy prior to $\mathrm{CE}$ were excluded. Findings were considered positive if the observed lesions could explain the bleeding, while findings including isolated red spots and a single small polyp were considered negative. The primary endpoint was the difference in the rate of positive CE findings between patients who continued and those who discontinued antithrombotic agents. Furthermore, a propensity score analysis was performed to reduce the effects of selection bias and potential confounding factors. The secondary endpoint was to assess the predictive factors for the positive $\mathrm{CE}$ findings by using multiple logistic regression.

Results: Of the 73 patients who continued antithrombotic agents, $36(49.3 \%)$ patients had positive findings in the small intestine (ulcer/erosion $[n=24]$, angioectasia $[n=7]$, tumor $[n=4]$, and blood pooling $[n=1])$, while of the 57 patients who discontinued these agents, $35(61.4 \%)$ patients had positive findings (ulcer/erosion $[\mathrm{n}=17]$, angioectasia $[\mathrm{n}=11]$, tumor $[\mathrm{n}=3]$, and blood pooling $[\mathrm{n}=4]$ ). The rates of positive CE finding did not differ between the two groups. Even after propensity score matching, discontinuation of antithrombotic agents did not affect the rate of positive CE finding. In multivariate analysis, the lowest hemoglobin level before CE examination was an independent predictive factor associated with positive CE findings. The odds ratio per $1 \mathrm{~g} / \mathrm{dL}$ increase in the lowest hemoglobin level was 0.84 (95\% confidence interval, $0.71-0.98)$. However, other factors, including sex, age, and discontinuation of antithrombotic agents, were not associated with positive $\mathrm{CE}$ findings.

Conclusion: Discontinuation of antithrombotic agents did not affect the diagnostic yield of CE in patients with overt OGIB, and lowest hemoglobin level was associated with positive $\mathrm{CE}$ findings

Disclosure of Interest: All authors have declared no conflicts of interest.

\section{P1938 EFFICACY OF REBAMIPIDE TO PREVENT LOW-DOSE ASPIRIN-INDUCED SMALL INTESTINAL INJURY}

\section{S. S. Vyalor}

Gastroenterology, Peoples Friendship University of Russia, Moscow/Russian

Federation

Contact E-mail Address: svialov@mail.ru

Introduction: Long-term use of low-dose aspirine (LDA) is associated with development of peptic ulcers, gastrointestinal bleeding, enteropathy. For prevention of LDA-induced gastroduodenal mucosal injury, proton pomp inhibtor (PPI) are the first-line therapy according to several guidelines. However, gastric acid suppresants, like PPI, do not prevent small intestinal mucosal injury. The latest clinical trials sjowed that rebamipide stimulates the production of prostaglandines, thereby preventing mucosal injury. This could improve mucosal breaks in small intestine in patients recieve LDA.

Aims \& Methods: We aimed to investigate the protective role and efficacy of rebamipide for prevention low-dose aspirin-induced small intestinal mucosal injury and enteropathy. Subjects comprised patients undergoing longlife lowdose aspirin therapy prescribed by cardiologist. Patients with a high-risk of gastrointestinal bleeding were excluded. This trial was performed as a randomised open-labelled clinical study with the permission of an institutional review board. The trial was included 100 patients (50 cases in each group) received enteric-coated low-dose aspirin $100 \mathrm{mg}$. The Group PPI received LDA plus pantoprazole $40 \mathrm{mg}$, the Group RBD received plus rebamipide $300 \mathrm{mg}$. Before starting therapy, we checked the background characteristics of each patient (H.pylori, use of LDA, NSAID, bismuth, PPI; and endoscopic findings). Gastroduodenoscopy and capsule endoscopy were performed, and the fecal occult blood reaction and fecal calprotectin levels were measured before, two and four weeks after drug administration. After the therapy, we has asked physicians and patients about medication compliance and side effects. Capsule endoscopy was then repeated. The primary endpoint was the change in the number of mucosal breaks from baseline to 4 weeks. The secondary endpoints were the rates of side effects.

Results: The fecal calprotectin levels increased significantly in Group PPI, they did not increase in Group RBD. The mean number of small intestinal mucosal injuries by capsule endoscopy in Group PPI increased significantly up to 3,9 after 4 weeks of LDA treatment. There was not detected new small intestinal injuries in Group RBD. Stomach ulcer, bleeding or stenosis were not found in any subject. There were no significant differences in the presence of fecal occult blood in both groups. There were no significant side effects in Group RBD. Conclusion: In conclusion, rebamipide is effective and sufficient for preventing mucosal injury of the small intestine induced by low-dose aspirin. These results show the gastroprotective and enteroprotective effects of rebamipide, suggesting that it may be a good choice in low-dose aspirin users with gastrointestinal toxicity that is not suppressed by acid suppressants alone.

Disclosure of Interest: All authors have declared no conflicts of interest.

\section{References}

1. Watanabe T, Takeuchi T, Handa O, et al. A Multicenter, Randomized, Double-Blind, Placebo-Controlled Trial of High-Dose Rebamipide Treatment for Low-Dose Aspirin-Induced Moderate-to-Severe Small Intestinal Damage. Green J, ed. PLOS ONE. 2015;10(4):e0122330. doi:10.1371/journal.pone.0122330.

2. Ota K, Takeuchi T, Nouda S, et al. Determination of the adequate dosage of rebamipide, a gastric mucoprotective drug, to prevent low-dose aspirininduced gastrointestinal mucosal injury. Journal of Clinical Biochemistry and Nutrition. 2016;59(3):231-237. doi:10.3164/jcbn.16-49.

3. Yamamoto T, Isono A, Mishina Y, et al. Gastroduodenal Mucosal Injury in Patients Taking Low-Dose Aspirin and the Role of Gastric Mucoprotective Drugs: Possible Effect of Rebamipide. Journal of Clinical Biochemistry and Nutrition. 2010;47(1):27-31. doi:10.3164/jcbn.09-103.

4. Iwamoto J, Mizokami Y, Saito Y, et al. Small-bowel mucosal injuries in lowdose aspirin users with obscure gastrointestinal bleeding. World Journal of Gastroenterology: WJG. 2014;20(36):13133-13138. doi:10.3748/ wjg.v20.i36.13133.

5. Mo C, Sun G, Wang Y-Z, Lu M-L, Yang Y-S. PPI versus Histamine H2 Receptor Antagonists for Prevention of Upper Gastrointestinal Injury Associated with Low-Dose Aspirin: Systematic Review and Meta-analysis. Green J, ed. PLoS ONE. 2015;10(7):e0131558. doi:10.1371/ journal.pone.0131558.

\section{P1939 CAPSOCAM SV-1 VERSUS PILLCAM SB 3 IN THE DETECTION OF OBSCURE GASTROINTESTINAL BLEEDING: RESULTS OF A PROSPECTIVE RANDOMIZED COMPARATIVE MULTI-CENTER STUDY}

L. L. Zwinger ${ }^{1}$, B. Siegmund ${ }^{1}$, A. Stroux ${ }^{3}$, A. Adler ${ }^{4}$, W. Veltzke-Schlieker ${ }^{5}$ R. Wentrup ${ }^{1}$, C. Jürgensen ${ }^{4}$, B. Wiedenmann ${ }^{4}$, F. Wiedbrauck ${ }^{7}$, S. Hollerbach ${ }^{8}$, T. Liceni ${ }^{9}$, C. Bojarski

${ }^{1}$ Medizinische Klinik Mit Schwerpunkt Gastroenterologie, Infektiologie, Rheumatologie, Charité, Berlin/Germany

${ }^{3}$ Institut Für Epidemiologie Und Statistik, Charité Universitätsmedizin Berlin, Berlin/Germany

${ }^{4}$ Medizinische Klinik Mit Schwerpunkt Gastroenterologie Und Hepatologie, Charité Universitätsmedizin Berline, Berlin/Germany

${ }^{5}$ University Hospital, Charite Berlin, Berlin/Germany

${ }^{7}$ Klinik Für Gastroenterologie, Allgemeines Krankenhaus Celle, Celle/Germany

${ }^{8}$ Gastroenterology, AKH Celle, Celle/Germany

${ }^{9} \mathrm{MVZ}$ für Gastroenterologie, Berlin/Germany

Contact E-mail Address: lilli.zwinger@gmx.de

Introduction: Capsule endoscopy allows high-quality imaging of the small bowel. Newer capsule with a panoramic viewing mode is available and might increase the detection rate of bleeding lesions in patients with obscure gastrointestinal bleeding. Furthermore, an improved patient acceptance rate is expected.

Aims \& Methods: In a randomized prospective comparative multi-center study, patients with obscure gastrointestinal bleeding were included and examined either with CapsoCam SV-1 or with PillCam SB 3. Detection of bleeding lesions, transit and evaluation time and adverse events were evaluated. Physicians were interviewed about their experience with both capsules and the evaluation software. A detailed subject questionnaire analyzed acceptance of each capsule system. Three months after initial capsule endoscopy follow-up procedures were documented.

Results: One hundred eighty-one patients with obscure gastrointestinal bleeding were recruited into the study. After exclusion of 28 patients 153 patients were randomized and CapsoCam SV-1 $(n=78)$ or PillCam SB $3(n=75)$ was administered. CapsoCam SV-1 detected more cases of bleeding (31/79, diagnostic yield $39.7 \%$ ) compared to PillCam SB $3(26 / 75$, diagnostic yield $34.6 \%$, n.s.). Transit time of both capsules was not different. Evaluation time with PillCam SB 
3 was superior to CapsoCam SV-1 (27 min. vs. $40 \mathrm{~min}, \mathrm{p}=0.01) .95 \%$ of the physicians were satisfied with each capsule system and evaluation software. The acceptance rate of the patients to retrieve the CapsoCam SV-1 was high. Adverse events/SAEs were $17.9 \% / 1.3 \%$ with CapsoCam SV-1 and $16 \% / 0 \%$ with PillCam SB 3. Re-bleeding rate was $28.75 \%$ within 3 months.

Conclusion: Both capsules allow high-quality imaging of the small bowel CapsoCam SV-1 detected more lesions, however, relevant bleeding sources were visualized by both capsules. Physician's satisfaction was high with both capsule systems and evaluation software. Patient's acceptance with CapsoCam SV-1 was unexpectedly high. SAEs were $0 \%$ with PillCam SB 3 and $1.3 \%$ with CapsoCam SV-1.

Disclosure of Interest: All authors have declared no conflicts of interest.

\section{P1940 VALIDATION OF A SCORE CHART TO PREDICT THE RISK OF CHRONIC MESENTERIC ISCHEMIA: A DISCRIMINATIVE AND USEFUL TOOL IN CLINICAL DECISION-MAKING}

L. J.d. Van Dijk ${ }^{1}$, D. Van Noord ${ }^{2}$, R. H. Geelkerken ${ }^{3}$, S. A. Berendsen ${ }^{1}$, A C. De Vries ${ }^{1}$, A. Moelker ${ }^{4}$, H. J.m. Verhagen ${ }^{5}$, J.J. Kolkman ${ }^{6}$, M.J. Bruno', On Behalf Of The Dutch Mesenteric Ischemia Study Group (Dmis) ${ }^{7}$

${ }^{1}$ Gastroenterology And Hepatology, Erasmus University Medical Center, Rotterdam/Netherlands

${ }^{2}$ Department Of Gastroenterology And Hepatology, Franciscus Gasthuis \& Vlietland, Rotterdam/Netherlands

${ }^{3}$ Vascular Surgery, Medical Spectrum Twente and Experimental Center of Technical Medicine, Faculty Science and Technology, University Twente, Enschede/Netherlands

${ }^{4}$ Radiology, Erasmus University Medical Center, Rotterdam/Netherlands

${ }^{5}$ Vascular Surgery, Erasmus University Medical Center, Rotterdam/Netherlands ${ }^{6}$ Gastroenterology And Hepatology, Medical Spectrum Twente, Enschede/ Netherlands

${ }^{7}$ multidisciplinary study group, studygroup/Netherlands

Contact E-mail Address: 1.vandijk@erasmusmc.nl

Introduction: Chronic mesenteric ischemia (CMI) is the result of insufficient mucosal perfusion of the gastrointestinal tract, mostly caused by atherosclerotic stenosis of the mesenteric arteries. Other causes of CMI are vasculitis, median arcuate ligament syndrome or non-occlusive ischemia (NOMI) due to decreased cardiac output or hypo-oxygenation. The diagnosis of CMI remains challenging as chronic abdominal pain is common and mesenteric artery stenoses are frequently observed in the general population but not necessarily related. Harki et al.(1) designed a score chart to predict the risk of CMI based on a cohort of CMI suspected patients. This score chart consists of patient characteristics (female $1 \mathrm{pt}$, weight loss $1 \mathrm{pt}$, cardio-vascular disease $1 \mathrm{pt}$ ) and radiologic evaluation $(50-70 \%$ celiac artery (CA) stenosis $1 \mathrm{pt},>70 \%$ CA stenosis $4 \mathrm{pts}, 50-70 \%$ superior mesenteric artery (SMA) stenosis $1 \mathrm{pt}$ and $>70 \%$ SMA stenosis $3 \mathrm{pts}$ ). A total score of $0-2$ pts predicts an absolute risk of CMI of $0-21 \%, 3-6$ pts a $22-$ $46 \%$ risk and $\geq 7$ pts a risk of $>79 \%$. We aimed to validate this prediction model in a prospective large multicenter patient cohort.

Aims \& Methods: Patients suspected of CMI referred to two Dutch specialized CMI referral centers were included consecutively from January 2014 to August 2016. After diagnostic work-up of medical history taking, mesenteric CT-angiography or MR-angiography, and a mucosal ischemia test (visible light spectroscopy or tonometry), all patients were discussed in a specialized CMI multidisciplinary meeting resulting in an expert based consensus diagnosis. All patients with a CMI consensus diagnosis were planned for treatment (revascularization for occlusive disease and medication for NOMI). A definitive diagnosis of CMI was made if successful treatment resulted in durable symptom relief. The score chart to predict the risk of CMI was computed for each patient.

Results: A total of 246 patients were included and consensus diagnosis of CMI was made in $108(44 \%)$ patients, which resulted in $96(39 \%)$ patients with a definitive diagnosis of CMI after a positive response therapy. A definitive diagnosis of CMI was made in $9 \%$ of the patients with low risk, in $40 \%$ of the patients with intermediate risk and in $94 \%$ of the patients with high risk of CMI according to the score chart, respectively. Etiology and vascular lesions are specified for each risk group in the table. The discriminative ability of the score chart was strong (C-Statistic 0.87).

\begin{tabular}{llll}
\hline & $\begin{array}{l}\text { Low risk } \\
(0-2 \mathrm{pts}) n=91\end{array}$ & $\begin{array}{l}\text { Intermediate } \\
\text { risk }(3-6 \mathrm{pts}) \\
n=107\end{array}$ & $\begin{array}{l}\text { High risk } \\
(\geq 7 \mathrm{pts}) \\
n=48\end{array}$ \\
\hline $\begin{array}{l}\text { Diagnosis } \\
\text { Consensus diagnosis CMI }\end{array}$ & $9(9.9 \%)$ & $53(49.5 \%)$ & $46(95.8 \%)$ \\
Definitive diagnosis CMI & $8(8.8 \%)$ & $43(40.2 \%)$ & $45(93.8 \%)$ \\
Vascular lesion & & & \\
No significant vascular lesion ${ }^{\#}$ & $73(80.2 \%)$ & $20(18.7 \%)$ & $0(0.0 \%)$ \\
Single vessel & $17(18.7 \%)$ & $69(64.5 \%)$ & $5(10.4 \%)$ \\
CA stenosis & $11(64.7 \%)$ & $50(72.5 \%)$ & $5(100.0 \%)$ \\
SMA stenosis & $0(0.0 \%)$ & $18(26.1 \%)$ & $0(0.0 \%)$ \\
IMA stenosis & $6(35.3 \%)$ & $1(1.4 \%)$ & $0(0.0 \%)$ \\
Multi vessel & $1(1.1 \%)$ & $18(16.8 \%)$ & $43(89.6 \%)$ \\
CA and SMA stenosis & $0(0.0 \%)$ & $7(38.9 \%)$ & $19(44.2 \%)$ \\
\hline
\end{tabular}

Continued

\begin{tabular}{llll}
\hline & $\begin{array}{l}\text { Low risk } \\
(0-2 \text { pts }) n=91\end{array}$ & $\begin{array}{l}\text { Intermediate } \\
\text { risk }(3-6 \text { pts }) \\
n=107\end{array}$ & $\begin{array}{l}\text { High risk } \\
(\geq 7 \mathrm{pts}) \\
n=48\end{array}$ \\
\hline CA and IMA stenosis & $0(0.0 \%)$ & $3(16.7 \%)$ & $\begin{array}{l}1(2.3 \%) \\
1(2.3 \%) \\
\text { SMA and IMA stenosis }\end{array}$ \\
$\begin{array}{ll}\text { CA, SMA and IMA stenosis } \\
\text { Etiology }\end{array}$ & $1(1.1 \%)$ & $1(5.5 \%)$ & $22(51.2 \%)$ \\
No sign. vascular lesion ${ }^{\#}$, & $68(74.7 \%)$ & $19(17.8 \%)$ & $0(0.0 \%)$ \\
$\quad$ no ischemia & $7(7.7 \%)$ & $53(49.5 \%)$ & $42(87.5 \%)$ \\
Atherosclerosis & $8(8.8 \%)$ & $32(29.9 \%)$ & $2(4.2 \%)$ \\
MALS & $0(0.0 \%)$ & $0(0.0 \%)$ & $3(6.3 \%)$ \\
MALS and atherosclerosis & $0(0.0 \%)$ & $1(0.9 \%)$ & $0(0.0 \%)$ \\
Vasculitis & $5(5.5 \%)$ & $1(0.9 \%)$ & $0(0.0 \%)$ \\
NOMI & $3(3.3 \%)$ & $0(0.0 \%)$ & $1(2.1 \%)$ \\
NOMI and atherosclerosis & $0(0.0 \%)$ & $1(0.9 \%)$ & $0(0.0 \%)$ \\
NOMI and MALS & &
\end{tabular}

Conclusion: The score chart for CMI based on patient characteristics and anatomy is a reliable tool to discriminate the risk of CMI and useful for clinical decision-making, for example to adopt a wait-and-see policy in patients with a low risk and immediate vascular intervention in patients with high risk of CMI. Disclosure of Interest: All authors have declared no conflicts of interest.

\section{Reference}

1. Harki J, Vergouwe Y, Spoor JA, Mensink PB, Bruno MJ, van Noord D, Kuipers EJ, Tjwa ET. Diagnostic Accuracy of the Combination of Clinical Symptoms and CT or MR Angiography in Patients With Chronic Gastrointestinal Ischemia. J Clin Gastroenterol. 2016.

\section{P1941 LONG-TERM SYMPTOM RELIEF AFTER REVASCULARIZATION IN PATIENTS WITH SINGLE ARTERY CHRONIC MESENTERIC ISCHEMIA}

L. J.d. Van Dijk ${ }^{1}$, L.M.g. Moons ${ }^{2}$, D. Van Noord ${ }^{3}$, A. Moelker ${ }^{4}$, H. J.m. Verhagen ${ }^{5}$, M.J. Bruno ${ }^{1}$, E. V. Rouwet ${ }^{5}$

${ }^{1}$ Gastroenterology And Hepatology, Erasmus University Medical Center, Rotterdam/Netherlands

${ }^{2}$ Gastroenterology And Hepatology, University Medical Center Utrecht, Utrecht/ Netherlands

${ }^{3}$ Department Of Gastroenterology And Hepatology, Franciscus Gasthuis \& Vlietland, Rotterdam/Netherlands

${ }^{4}$ Radiology, Erasmus University Medical Center, Rotterdam/Netherlands ${ }^{5}$ Vascular Surgery, Erasmus University Medical Center, Rotterdam/Netherlands

Contact E-mail Address: 1.vandijk@erasmusmc.nl

Introduction: Isolated stenosis of the celiac artery (CA) or the superior mesenteric artery (SMA) is frequently detected in patients with abdominal complaints. These patients may suffer from chronic mesenteric ischemia (CMI) causing nonspecific abdominal complaints as postprandial pain, nausea or diarrhea. However, the existence of single artery mesenteric ischemia is a topic of continuous clinical debate and reports on the effectiveness of single mesenteric artery revascularization are scarce. We evaluated the long-term clinical success rates for single CA or SMA revascularization in patients with gastrointestinal symptoms and confirmed mucosal ischemia.

Aims \& Methods: Data were collected from all 97 consecutive patients with gastrointestinal symptoms and a single mesenteric artery stenosis referred to the outpatient clinic of our tertiary care institution for analysis of CMI between January 2006 and October 2010. All patients underwent a standardized diagnostic work-up for CMI at baseline consisting of medical history taking and physical examination, imaging of the gastrointestinal arteries with either CT- or MRangiography and/or conventional catheter angiography, and a functional test for detecting mucosal ischemia using either tonometry or visible light spectroscopy. All cases were discussed in a multidisciplinary meeting attended by a vascular surgeon, interventional radiologist and gastroenterologist, all specialized in CMI, leading to an expert based consensus diagnosis. Patients with consensus diagnosis of CMI underwent surgical or endovascular revascularization. The primary outcome was clinical response to revascularization, defined as relief of presenting symptoms as experienced by the patient.

Results: Consensus diagnosis of CMI was obtained in 62/97 patients and all consensus patients were revascularized. Isolated CA stenosis was present in 55/ 62 patients $(89 \%$ ) (31 vascular disease; 24 median arcuate ligament syndrome, MALS) and isolated atherosclerotic SMA stenosis in 7 patients. After a mean follow-up of $5.5 \pm 3.0$ years, $42 / 62$ patients $(68 \%)$ experienced sustained symptom relief. Responders to revascularization had a BMI increase during follow-up in contrast to the non-responders $\left(+0.43 \pm 2.5\right.$ versus $-1.06 \pm 2.4 \mathrm{~kg} / \mathrm{m}^{2}$, $\mathrm{p}=0.033$ ). Response to revascularization was not related to lesion localization (CA $67 \%$ versus SMA $71 \%, p=0.825$ ) or lesion etiology (MALS $63 \%$ versus vascular disease $71 \%, \mathrm{p}=0.483$ ). See table. 
$\mathrm{CA}=$ celiac artery; $\mathrm{SMA}=$ superior mesenteric artery; $\mathrm{MALS}=$ median arcuate ligament syndrome

\begin{tabular}{lll}
\hline & Symptom relief & No symptom relief \\
\hline $\begin{array}{l}\text { All patients } \\
\text { Vascular lesion }\end{array}$ & $42 / 62(68 \%)$ & $20 / 62(32 \%)$ \\
CA stenosis & $37 / 55(67 \%)$ & $18 / 55(33 \%)$ \\
SMA stenosis & $5 / 7(71 \%)$ & $2 / 7(29 \%)$ \\
Etiology & & \\
MALS & $15 / 24(63 \%)$ & $9 / 24(38 \%)$ \\
Vascular disease & $27 / 38(71 \%)$ & $11 / 38(29 \%)$ \\
\hline
\end{tabular}

Conclusion: Revascularization of the CA or SMA provides long-term symptom relief in $68 \%$ of patients with chronic gastrointestinal symptoms and confirmed mucosal ischemia due to single mesenteric artery stenosis. This provides the opportunity to help patients with otherwise unexplained, refractory abdominal complaints.

Disclosure of Interest: All authors have declared no conflicts of interest.

\section{P1942 UNDERUTILIZATION OF ENDOSCOPIC ARGON PLASMA COAGULATION FOR TREATMENT OF BLEEDING GASTROINTESTINAL ANGIODYSPLASIAS: AN INTERNATIONAL MULTICENTRE COHORT STUDY}

K. Grooteman ${ }^{1}$, M. Matheeuwsen ${ }^{1}$, G. Holleran ${ }^{2}$, E. Van Geenen ${ }^{1}$,

D. Mcnamara ${ }^{2}$, J.P.h. Drenth

${ }^{1}$ Gastroenterology And Hepatology, Radboudumc, Netherlands, Nijmegen/

Netherlands

${ }^{2}$ Department of Clinical Medicine, Trinity College, Dublin/Ireland

Contact E-mail Address: karinagrooteman@gmail.com

Introduction: Endoscopic argon plasma coagulation (APC) is the first-line treatment in patients with iron deficiency anaemia or overt bleeding resulting from gastrointestinal angiodysplasias. In the minority of patients active bleeding angiodysplasias are seen during endoscopy, but in contrast non-bleeding angiodysplasias can be an incidental finding. This can make the decision whether to treat endoscopic detected angiodysplasias with APC difficult.

Aims \& Methods: The aim of this study is to investigate the need for repeat endoscopies with APC in patients with angiodysplasias who were left untreated during the index endoscopy performed for iron deficiency anaemia or overt bleeding. We initiated an international, multicentre cohort study to collect clinical, laboratory and endoscopic data from angiodysplasia patients. Cases were identified through a systematic search in endoscopy reports from 2010-2015 with follow-up until July 2016. Inclusion criteria was endoscopic detection of angiodysplasia in the context of overt bleeding or iron deficiency anaemia. Exclusion criteria were other vascular anomalies and angiodysplasias as incidental finding. The primary outcome was repeat endoscopy with APC.

Results: A total of 197 patients with proven angiodysplasia as cause for anaemia or bleeding were included (mean age $=68$ years; $58 \%$ male). Median follow-up was 37 months (range $18-57)$. In $52 \%$ of the cases $(n=103)$ APC treatment for bleeding angiodysplasia(s) was performed at the index endoscopy. Repeat endoscopy with APC was necessary in 17 patients $(18 \%)$ in whom angiodysplasias were detected but left untreated during the index endoscopy. Median time between index and repeat endoscopy was 21 weeks. A total of 48 patients $(51 \%)$ who received a purely diagnostic index endoscopy were in need of other treatment modalities (e.g. iron supplementation, blood transfusion, stop anticoagulants). Anaemia and/or overt bleeding resolved spontaneously in 24 patients $(26 \%)$.

Conclusion: A substantial proportion of patients with clinical symptomatic angiodysplasia bleeding do not receive APC at the index endoscopy and continue to be dependent on iron supplementation, blood transfusion or undergo repeat endoscopy with APC.

Disclosure of Interest: All authors have declared no conflicts of interest.

P1943 DIGESTIVE INVOLVEMENT IN SYSTEMIC DISEASES: A

\section{UNIVERSITY HOSPITAL EXPERIENCE}

W. Khannoussi ${ }^{1}$, K. Chhita ${ }^{1}$, H. Bachir ${ }^{2}$, G. Kharrasse $^{1}$, A. El Mekkaoui ${ }^{1}$, S. Hamaz ${ }^{2}$, H. B. Alaoui ${ }^{2}$, K. Serraj ${ }^{2}$, Z. Ismaili ${ }^{3}$

${ }^{1}$ Hepato Gastroenterology, University Hospital Mohammed VI, Oujda/Morocco

${ }^{2}$ Internal Medecine, Mohammed VI University Hospital, Oujda/Morocco

${ }^{3}$ Hepatogastroenterology, CHU Med VI Oujda, Oujda/Morocco

Contact E-mail Address: wkhannoussi@yahoo.com

Introduction: Digestive manifestations in systemic diseases including vasculitis and granulomatosis is broad and can affect any segment of the digestive tract and related organs. The clinical symptoms are not specific and it can be challenging for diagnosis. The other difficulty remains the interference of digestive side effects of medication used.

Aims \& Methods: We aimed to review various digestive manifestations of systemic diseases. This was a retrospective study from Feb 2009 to Sep 2016 in internal medicine and gastroenterology departments. The exclusion criteria was incomplete data considering the diagnosis of the systemic disease.

Results: patients were included, sex ratio $0.38(101 \mathrm{~F} / 39 \mathrm{M})$, mean age at inclusion was 40 years old $[13,79]$. The following chart summarize the $\%$ of digestive manifestations by disease:

\begin{tabular}{lll}
\hline Systemic disease & $\begin{array}{l}\text { Number of } \\
\text { cases }\end{array}$ & $\begin{array}{l}\text { Digestive } \\
\text { manifestations (\%) }\end{array}$ \\
\hline Systemic Lupus erythematosus & 49 & $10.36 \%$ \\
Systemic sclerosis & 27 & $8.33 \%$ \\
Behcet's disease & 27 & $4.39 \%$ \\
Sjogren syndrome & 14 & $7.37 \%$ \\
Wegener's granulomatosis & 2 & $21.60 \%$ \\
Antiphospholipid Anti body Syndrome & 3 & $22.58 \%$ \\
Amyloidosis & 1 & $45.16 \%$ \\
Churg-Strauss syndrome & 2 & $20.96 \%$ \\
Dermatomyositis & 2 & $25.80 \%$ \\
Microscopic Polyangiitis & 1 & $0 \%$ \\
Horton's disease & 2 & $8.06 \%$ \\
Takayasu arteritis & 3 & $3.22 \%$ \\
Cryoglobulinaemic vasculitis & 2 & $0 \%$ \\
Henoch-Schönlein purpura & 3 & $30.10 \%$ \\
Leucocytoclasic vasculatis & 2 & $12.9 \%$ \\
\hline
\end{tabular}

Patients symptoms and clinical findings were: Abdominal pain in 32 cases $(22.9 \%)$, Nausea and vomiting in 7 cases $(5 \%)$, stool modification 15 cases $(10.7 \%)$, jaundice in 4 cases $(2.9 \%)$,dysphagia in cases $(7.1 \%)$, hepatomegaly in 4 cases $(2.9 \%)$, splenomegaly in 8 cases $(5.7 \%)$, ascitis in 13 cases $(9.3 \%)$, digestive bleeding 17 cases $(12.1 \%)$. Laboratory findings: elevated liver enzymes 18 cases $(15.9 \%)$, alkaline phosphatase elevation 15 cases $(19.2 \%)$. Radiological findings: 17 ascitis $(14.4 \%), 12$ digestive thickening $(10.16 \%), 6$ hepatomegaly $(5.08 \%), 14$ splenomegaly $(11.86 \%), 2$ portal cavernoma $(1.6 \%), 3$ portal thrombosis $(2.5 \%), 4$ esophageal distension $(3.4 \%)$, acalculous cholecystitis in 2 cases $(1.6 \%), 2$ portal hypertension $(1.6 \%)$, steatosis in 3 cases $(2.5 \%), 3$ hepatic angioma $(2.5 \%)$, acute pancreatitis in 2 cases $(1.6 \%), 2$ mesenteric ischemia $(1.6 \%), 1$ budd chiari $(0.8 \%), 1$ hepatic carcinoma $(1.8 \%)$. Upper gastrointestinal Endoscopy findings: hiatus hernia in 3 cases $(8.6 \%)$, esophagitis in 11 cases $(31.4 \%)$, esophageal varices in 2 cases $(5.7 \%)$, Gastritis in 37 cases, duodenitis in cases 16 cases, ulcers in 3 cases $(8.6 \%), 4$ celiac disease $(11.42 \%)$. Lower GI endoscopy: Crohn's desease in 1 case $(8.3 \%)$, ulcerative colitis in 1case $(8.3 \%)$, 1 hyperplastic polyps $(8.3 \%), 2$ colitis $(16.6 \%)$. Eosophagal manometry: motility disorder in 4 cases. The most used drugs were immunosuppresive drugs, steroids and hydrochloroquine, all causing digestive side effects mainly abdominal pain. Conclusion: The digestive involvement is around $10 \%$ of cases in the most represented systemic diseases, the main symptom is abdominal pain probably related to medication, endoscopic modifications are mainly non specific but we found some association with celiac disease and IBD. Liver involvement was noticed in $15 \%$ of cases.

Disclosure of Interest: All authors have declared no conflicts of interest.

\section{WEDNESDAY, NOVEMBER 01, 201709:00-14:00}

\section{NUTRITION III - HALL 7}

\section{P1944 CHANGES IN LEVELS OF VITAMIN D IN OBESE PATIENTS} SUBMITTED TO BARIATRIC SURGERY

A. Vaz ${ }^{1}$, R. Guerreiro ${ }^{2}$, D. Sousa ${ }^{1}$, P. Queirós ${ }^{3}$, T. Gago $^{1}$, J. Roseira $^{3}$,

H. Guerreiro ${ }^{1}$

${ }^{1}$ Gastroenterology, Centro Hospitalar do Algarve, Faro/Portugal

${ }^{2}$ Clinical Pathology, Centro Hospitalar do Algarve, Faro/Portugal

${ }^{3}$ Gastroenterology, Centro Hospitalar do Algarve, Portimão/Portugal

Contact E-mail Address: anam_vaz@hotmail.com

Introduction: An association between obesity and vitamin D deficiency has been reported in several studies. This may be explained, among other things by the sequestration of the fat-soluble vitamin D in the adipose tissue. Bariatric surgery, including Roux-en-Y gastric bypass (RYGB) is an effective treatment for more extreme cases of obesity, promoting significant weight loss and consequently reduction in some obesity-related health problems. However, the problem of vitamin D deficiency doesn't seem to be solved after RYGB and can even be exacerbated by the changes in digestion and absorption of this nutrient after the surgery.

Aims \& Methods: The aim of this study was to analyze the prevalence of vitamin $\mathrm{D}$ deficiency (VDD) and vitamin D insufficiency (VDI) in a population of obese patients, before and after being submitted to RYGB. We included patients patients selected to undergo RYGB for obesity. We measured anthropometric values and the levels of 25-hydroxy-vitamin $\mathrm{D}(25[\mathrm{OH}] \mathrm{D})$ before and 1 year after the procedure. VDD was defined as serum $25(\mathrm{OH}) \mathrm{D}<20 \mathrm{ng} / \mathrm{mL}$ and VDI as serum $25(\mathrm{OH}) \mathrm{D}$ concentrations between $20-30 \mathrm{ng} / \mathrm{mL}$. Levels of $25(\mathrm{OH}) \mathrm{D}$ $>30 \mathrm{ng} / \mathrm{mL}$ were considered normal.

Results: We included 39 patients. Thirty-two were females $(82.1 \%)$ and the mean age was $43.6 \pm 11.3$ years. The mean BMI before surgery was $41.6 \mathrm{~kg} / \mathrm{m}^{2}$. There was a significant decrease in BMI after surgery and, on average, the mean total body weight loss was $34 \%$ and the mean excess BMI loss was $90 \%$. Before 
bariatric surgery, $52.3 \%$ of patients had VDD and $36.8 \%$ had VDI. After surgery, the number of VDD increased to $71.1 \%(\mathrm{p}=0.079)$. The mean levels of $25(\mathrm{OH}) \mathrm{D}$ decreased significantly from $19.8 \mathrm{ng} / \mathrm{mL}$ before surgery to $16.6 \mathrm{ng} / \mathrm{mL}$ after surgery $(\mathrm{p}<0.05)$. There was no correlation between the amount of weight loss and the changes in the levels of $25(\mathrm{OH}) \mathrm{D}$ in our study.

Conclusion: There is a high prevalence of vitamin D deficiency in obese patients eligible for bariatric surgery. The level of deficiency tends to increase after RYGB. This population of patients should, therefore, be offered an adequate level of vitamin D supplementation, especially after the procedure.

Disclosure of Interest: All authors have declared no conflicts of interest.

\section{P1946 INTRAGASTRIC BALLOON: A CRITICAL VIEW IN NON ELECTIVE BARIATRIC SURGERY PATIENTS}

R.J.F. Fernandez ${ }^{1}$, E. Usuy ${ }^{2}$, C.F. Diestel ${ }^{1}$, S. Barrichello ${ }^{1}$, A. F. Teixeira ${ }^{1}$, M. Galvao Neto ${ }^{3}$

${ }^{1}$ Dept. Of Bariatric Endoscopy, Endogastro Rio, Rio de Janeiro/Brazil

${ }^{2}$ Dept. Of Gastroenterology, Usuy Clinic, Florianopolis/Brazil

${ }^{3}$ Bariatric Endoscopy, Gastro Obeso Center, São Paulo/Brazil

Contact E-mail Address: ricfittipaldi@hotmail.com

Introduction: Bariatric surgery is established as an excellent therapy for obesity. However, lower degrees of overweight without surgical indication also impact on patients' health and quality of life, and the intragastric balloon(IGB) may be a treatment option.

Aims \& Methods: We aimed to assess the efficacy of excess weight treatment with an IGB in patients with overweight and grade I obesity at EndogastroRio Clinic. A total of 717 patients were analyzed. A liquid filled IGB was used. The patients had initial body mass index (BMI) between 27 and $34.9 \mathrm{~kg} / \mathrm{m}^{2}$. The level of significance was set at $\mathrm{p}<0.05$.

Results: Results 615 patients were women. 131 patients had overweight and 586 had grade I obesity. Mean age was 37.97 years (17-75). Weight loss results and treatment success rates are shown on table 1 . Percent excess weight loss (\%EWL) was higher in overweight group $(\mathrm{p}<0.0001)$ and percent total body weight loss $(\% \mathrm{TBWL})$ was higher in the grade I obesity group $(\mathrm{p}=0.0009) .96(73.28 \%)$ overweight patients and $132(22.52 \%)$ grade I obesity patients reached a normal $\operatorname{BMI}(<25 \mathrm{~kg} / \mathrm{m} 2)$.

\begin{tabular}{|c|c|c|c|}
\hline & $\begin{array}{l}\text { Total group } \\
(\mathrm{n}=717)\end{array}$ & $\begin{array}{l}\text { Overweight } \\
(\mathrm{n}=131)\end{array}$ & $\begin{array}{l}\text { Grade I Obesity } \\
(\mathrm{n}=586)\end{array}$ \\
\hline \multicolumn{4}{|c|}{ Body weight(kg) } \\
\hline Baseline & $88.55 \pm 10.14$ & $78.90 \pm 6.56$ & $90.67 \pm 9.56$ \\
\hline Final & $73.20 \pm 10.78$ & $66.73 \pm 8.13$ & $74.62 \pm 10.73$ \\
\hline Reduction & $15.35 \pm 6.49$ & $12.16 \pm 4.76$ & $16.05 \pm 6.53$ \\
\hline$\%$ TBWL & $17.36 \pm 7.08$ & $15.51 \pm 6.11$ & $17.76 \pm 7.11$ \\
\hline \multicolumn{4}{|l|}{$\mathrm{BMI}(\mathrm{kg} / \mathrm{m} 2)$} \\
\hline Baseline & $32.05 \pm 2.04$ & $28.73 \pm 0.94$ & $32.78 \pm 1.38$ \\
\hline Final & $26.46 \pm 2.43$ & $24.26 \pm 1.85$ & $26.95 \pm 2.51$ \\
\hline Reduction & $5.59 \pm 2.36$ & $4.46 \pm 1.86$ & $5.83 \pm 2.37$ \\
\hline \multicolumn{4}{|c|}{ Excess weight (kg) } \\
\hline Baseline & $19.77 \pm 6.04$ & $10.52 \pm 2.7$ & $21.81 \pm 4.45$ \\
\hline Final & $4.42 \pm 7.44$ & $-1.65 \pm 5.09$ & $5.77 \pm 2.37$ \\
\hline$\%$ EWL & $83.97 \pm 41.89$ & $122.77 \pm 57.89$ & $75.36 \pm 31.33$ \\
\hline \multicolumn{4}{|l|}{$\%$ TBWL } \\
\hline$<10 \%$ & $106(14.78 \%)$ & $22(15.27 \%)$ & $83(14.16 \%)$ \\
\hline$\geq 10 \%$ & $611(85.22 \%)$ & $109(83.21 \%)$ & $503(85.84 \%)$ \\
\hline \multicolumn{4}{|c|}{$\%$ EWL(n;\%) } \\
\hline$<25 \%$ & $32(4.46 \%)$ & $2(1.52 \%)$ & $30(5.12 \%)$ \\
\hline$\geq 25 \%$ & $685(95.54 \%)$ & $129(98.48 \%)$ & $556(94.88 \%)$ \\
\hline \multicolumn{4}{|l|}{ BMI(n;\%) } \\
\hline$<25 \mathrm{~kg} / \mathrm{m}^{2}$ & $213(29.71 \%)$ & $96(73.28 \%)$ & $132(22.52 \%)$ \\
\hline
\end{tabular}

${ }^{*} \mathrm{p}<0.0001$ for all comparisons between values at baseline and at the end of the study. IGB = intragastric balloon; $\mathrm{BMI}=$ body mass index; TBWL $=$ total body weight loss; $\mathrm{EWL}=$ excess weight loss. Success rates (criteria: $\geq 10 \% \mathrm{TBWL}$ or $\geq 25 \%$ EWL)

Conclusion: Endoscopic treatment of obesity with an IGB shows to be an excellent therapeutic option to non elective patients for bariatric surgery according to BMI criterion

Disclosure of Interest: M. Galvao Neto: I declare that I have received personal fees from FRACTYL LABS, GI WINDOWS, APOLLO ENDO SURGERY, GI DYNAMICS, ETHICON ENDO SURGERY, not related to the present study.

All other authors have declared no conflicts of interest.
P1947 SPATZ3® ADJUSTABLE INTRAGASTRIC BALLOON

TREATMENT: A BRAZILIAN MULTICENTRIC EXPERIENCE

R.J.F. Fernandez ${ }^{1}$, C.F. Diestel ${ }^{1}$, M. Galvao Neto $^{2}$, A. F. Teixeira ${ }^{1}$, E. Usuy ${ }^{3}$, S. Barrichello

${ }^{1}$ Dept. Of Bariatric Endoscopy, Endogastro Rio, Rio de Janeiro/Brazil

${ }^{2}$ Bariatric Endoscopy, Gastro Obeso Center, São Paulo/Brazil

${ }^{3}$ Dept. Of Gastroenterology, Usuy Clinic, Florianopolis/Brazil

Contact E-mail Address: ricfittipaldi@hotmail.com

Introduction: Intragastric balloons (IGB) are already used worldwide in the treatment of overweight and obesity, with established success. The Spatz3 ${ }^{\circledR}$ adjustable balloon brings the possibility of balloon volume control during all the treatment, possibly reducing the risk of early removals due to intolerance and greater weight loss when compared to traditional IGBs.

Aims \& Methods: We aimed to analyze the initial 25 months results regarding weight loss and complications with Spatz $3 \AA$ adjustable intragastric balloon in Brazil. In this retrospective longitudinal study were included patients submitted to Spatz3 ${ }^{\circledR}$ adjustable IGB treatment between October 2014 to April 2017 in four private clinics in Brazil. The IGB Spatz3 ${ }^{\circledR}$ was filled with a standard volume of $600 \mathrm{ml}$ that was downward or upward adjusted when necessary. The patients presented a minimum body mass index (BMI) of $27 \mathrm{~kg} / \mathrm{m}^{2}$. Were analyzed the complications of Spatz3 ${ }^{\circledR}$ treatment and BMI reduction, percent total body weight loss (\% TBWL) and percent excess weight loss ( $\%$ EWL). Data were analyzed using descriptive statistic and the Student $t$ test. The level of significance was set at $\mathrm{p}<0.05$.

Results: 422 patients underwent implant Spatz $3 \circledR$ balloon in the period. The complications $(14.28 \%)$ at the present study were: early balloon removal $(6.89 \%)$, gastric ulcer $(3.94 \%)$, spontaneous deflation $(1.48 \%)$, gas production inside the balloon $(0.98 \%)$, gastric perforation $(0.23 \%)$ and Malory Weiss Syndrome $(0.23 \%)$. There was no death at the present study. Twenty-eight patients underwent downward adjustment due to intolerance (mean volume reduction: $162.86 \mathrm{~mL}$ ) and all of then kept in the treatment (no early removals). 180 patients have completed the treatment (minimum 9 months of gastric balloon stay). The BMI decreased from 37.69 to $31.51 \mathrm{~kg} / \mathrm{m}^{2}$ ( $\left.\mathrm{p}<0.0001\right)$, body weight decreased from 107.67 to $90.16 \mathrm{~kg}(\mathrm{p}<0.0001)$ and excess weight dimished from 36.79 to $19.27 \mathrm{~kg}(\mathrm{p}<0.0001)$. Eighty-six patients underwent upward adjustment. The adjustment resulted in a further mean weight loss of $4.2 \mathrm{~kg}$ ( -9 to $20 \mathrm{~kg})$, the range of upward volume was $281.73 \pm 66.58 \mathrm{ml}(100-420 \mathrm{ml})$ and the moment of the procedure was $7.06 \pm 1.64$ months. The group of patients that did the upward adjustment dont have a higher \% TBWL, \%EWL or a a higher BMI reduction when compared to the group that did not $(p=0.4413, p=0.9245$, $\mathrm{p}=0.2729$, respectively).

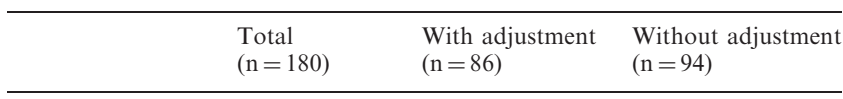

Body weight $(\mathrm{kg})$

Fination

Final

Reduction

$\%$ TBWL

BMI $\left(\mathrm{kg} / \mathrm{m}^{2}\right)$

Baseline

Final

Reduction

$107.67 \pm 23.65$

$108.66 \pm 25.12$

$106.76 \pm 22.3$

$90.16 \pm 22.53 \quad 90.41 \pm 23.39$

$89.94 \pm 21.83$

$17.51 \pm 11.67 \quad 18.26 \pm 10.99$

$16.83 \pm 12.28$

$16.22 \pm 9.74 \quad 16.81 \pm 9.09$

$15.68 \pm 10.33$

Excess weight $(\mathrm{kg})$

Baseline

Final

$\%$ EWL

$\%$ TBWL

$<10 \%$

$\geq 10 \%$

$37.69 \pm 6.16$

$38.26 \pm 6.56$

$37.16 \pm 5.77$

$31.51 \pm 6.11$

$31.55 \pm 6.11$

$31.29 \pm 6.14$

$6.18 \pm 4.07$

$6.51 \pm 3.91$

$5.87 \pm 4.21$

$\% \operatorname{EWL}(\mathrm{n} ; \%)$

$<25 \%$

$\geq 25 \%$

$36.79 \pm 19.07 \quad 38.15 \pm 20.15$

$35.58 \pm 18.04$

$21.79 \pm 19.33 \quad 19.89 \pm 18.45$

$23.25 \pm 19.39$

$56.68 \pm 40.12 \quad 56.98 \pm 34.18$

$56.41 \pm 42.86$

$52(28.89 \%)$

$19(22.09 \%)$

$33(35.11 \%)$

$128(71.11 \%) \quad 67(77.91 \%)$

$61(64.89 \%)$

$40(22.22 \%)$

$16(18.6 \%)$

$24(25.53 \%)$

BMI (n;\%)

$23-28 \mathrm{~kg} / \mathrm{m}^{2}$

$140(77.78 \%) \quad 7081.4 \%)$

$70(74.47 \%)$

$21(11.67 \%) \quad 10(11.63 \%) \quad 11(11.7 \%)$

Conclusion: This study shows that Spatz $3 \circledR$ IGB treatment is an effective procedure for weight reduction, without mortality but with higher morbidity rates when compared to tradition IGBs. Even more, the downward adjustment shows to be effective in preventing the early balloon removal, although the upward adjustment does not show to be able in providing a greater weight loss. Disclosure of Interest: M. Galvao Neto: I declare that I had received personal fees from FRACTYL LABS, GI WINDOWS, APOLLO ENDO SURGERY, GI DYNAMICS, ETHICON ENDO SURGERY, not related to the present study. All other authors have declared no conflicts of interest. 
P1948 VOMITING FREQUENCY AFTER INTRAGASTRIC BALLOON PLACEMENT AND INTRAVENOUS HYDRATION REQUIREMENT

S. Barrichello ${ }^{1}$, R.J.F. Fernandez ${ }^{2}$, E. Usuy ${ }^{3}$, A. F. Teixeira ${ }^{1}$, M. Galvao Neto $^{4}$, T. F. Souza ${ }^{5}$, E. Grecco 5

${ }^{1}$ Endoscopy, FMABC, São paulo/Brazil

${ }^{2}$ Dept. Of Digestive Endoscopy, Endogastro Med Service, Rio de Janeiro/Brazil

${ }^{3}$ Dept. Of Gastroenterology, Usuy Clinic, Florianopolis/Brazil

${ }^{4}$ Bariatric Endoscopy, Gastro Obeso Center, São Paulo/Brazil

${ }^{5}$ Bariatric Endoscopy, Faculdade de Medicina do ABC - Hospital Mário Covas, São Paulo/Brazil

\section{Contact E-mail Address: sergio.barrichello@healthme.com.br}

Introduction: The endoscopic treatment of obesity and over weight with intragastric balloon (IGB), is a safe and effective option. Nausea and Vomiting are the most commons side effects after IGB implant.

According to A. Scudero Sanches, $71.1 \%$ of patients experience nause and $57.9 \%$ had vomiting. Iñaki Imaz et al, in a systematic review with more than 3000 patients showed that nausea and vomiting are the fifth leading cause of early acessory explantation. The reported symptoms are more common in first 3 days post implant.

Regarded as one of the major causes of gastric balloon early removal, is important to evaluate in daily practice the frequency of patients requiring intravenous hydration to quell nausea and vomiting during the first day os the accessory use. Aims \& Methods: Evaluation of Frequency of vomiting and number of patients requiring intravenous hydration (HEV) after gastric balloon placement. This was a retrospective analysis of medical records of 340 obese and over weight patients treated with intragastric balloon, between November 2015 to December 2016 in the bariatric endoscopy division of the Mario Covas Hospital in São Paulo. The implant was performed by 3 endoscopists with experience in bariatric endoscopy, all procedure were performed under moderate sedation and anesthetist's care. The patients used omeprazole $40 \mathrm{mg}$ once a day, and antiemetic drugs (dimenhydrinate) $8 / 8$ hours the first 5 days as well. The data recorded were about the numbers and frequency of vomiting and if there was need for intravenous hydration during the first three days after intragastric balloon implant

Results: There were 340 patients treated with intragastric balloon betwen 2015 and 2016.

The present study showed $74.41 \%$ vomiting until the third day after placement of the BIG, and it is in accordance with literature. Those 252 patients who had vomiting, $67.58 \%$ presents frequence of 0 to 5 times per day and $32.42 \%$ of the group 5 to 10 times per day. Approximately $9.48 \%$ of the patients required intravenous hydration and there were no early explant.

Conclusion: Gastric balloon causes vomiting in the early days post implant in $74.41 \%$ of patients and cause dehydration requiring Intravenous fluid therapy in $7.06 \%$ of all patients who underwent implantation of gastric balloon (IGB)

Disclosure of Interest: M. Galvao Neto: Apollo endosurgery consultant

Safety and effectiveness of the intragastric balloon for obesity. A meta-analysis. Imaz I, Martínez-Cervell C, García-Alvarez EE, Sendra-Gutiérrez JM, González-Enríquez J. Obes Surg. 2008 Jul;18(7):841-6. doi: 10.1007/s11695007-9331-8. Epub 2008 May 6. Review. PMID:18459025

All other authors have declared no conflicts of interest.

P1949 BODY MASS IMPROVEMENT AND METABOLIC CHANGE RATE AFTER INTRAGASTRIC BALLOON TREATMENT

S. Barrichello ${ }^{1}$, R.J.F. Fernandez ${ }^{2}$, E. Usuy ${ }^{3}$, T. F. Souza ${ }^{4}$, M. Galvao Neto ${ }^{5}$, A. F. Teixeira ${ }^{1}$, E. Grecco ${ }^{4}$

${ }^{1}$ Endoscopy, FMABC, São paulo/Brazil

${ }^{2}$ Dept. Of Digestive Endoscopy, Endogastro Med Service, Rio de Janeiro/Brazil ${ }^{3}$ Dept. Of Gastroenterology, Usuy Clinic, Florianopolis/Brazil

${ }^{4}$ Bariatric Endoscopy, Faculdade de Medicina do ABC - Hospital Mário Covas, São Paulo/Brazil

${ }^{5}$ Bariatric Endoscopy, Gastro Obeso Center, São Paulo/Brazil

Contact E-mail Address: sergio.barrichello@healthme.com.br

Introduction: While monitoring weight loss in patients undergoing very low calorie diets (VLCD), regardless of which method used, mostly patients experience significant weight reduction, but also a significant fat-free mass (FFM) loss. The fat-free mass loss is associated a decrease in basal metabolic rates, a fact confirmed in patients undergoing treatment with intragastric balloon (IGB).

Aims \& Methods: Retrospective study was conducted with analysis of medical records of obese patients treated with intragastric balloon betwem 2013 to 2015 in a supplementary health service. Patients were evaluated at three different moments: before intragastric balloon placement, 45 days post implant and after BIG removal (30 days).

Results: We evaluated 29 patients, mean age of 40.07 years $( \pm 10.35$ years), pretreatment weight $97.44 \mathrm{~kg}( \pm 16.70 \mathrm{~kg})$, pre BMI $35.51 \mathrm{~kg} / \mathrm{m} 2(4.3 \mathrm{~kg} / \mathrm{m} 2)$ and a bioimpedance showing a $\%$ of fat-free mass (FFM) of 63.15 and $36.84 \%$ fat mass (FM) with a metabolic pretreatment rate of $1893.24( \pm 337.21)$. Forty five days after gastric balloon placement, bioimpedance avaluation showed $64.9 \%$ of FatFree Mass, $35.1 \%$ of Fat Mass and metabolic rate in 1731, associated with a weight loss of $9.46 \mathrm{~kg}$ or $27.37 \%$ overweight In the third assessment, there were a significant improvement in BMI, weight and metabolic rate with $\mathrm{p}<0.05$. The final metabolic rate was 1694.67 , FFM was $69.7 \%$ with $30.3 \%$ of FM, a reduction about $17 \%$ of the initial weight with the decrease of $44.29 \%$ overweight. Conclusion: Intragastric balloon was effective as minimally invasive treatment of obesity and over weight.
Patients' body mass distribution had a clear improvement, with a significant increase in the percentage of Fat-Free Mass. A significant reduction of the basal metabolic rate of 1893.24 to 1694.67 was noted. Endoscopic approach with gastric balloon provides a significant weight loss and helps patients acquiring healthy habits

Disclosure of Interest: M. Galvao Neto: Apollo endosurgery consultant

All other authors have declared no conflicts of interest.

\section{P1950 A LARGE BRAZILIAN EXPERIENCE WITH THE INTRAGASTRIC BALLOON TREATMENT IN HIGH VOLUME OF PATIENTS CENTERS}

R.J.F. Fernandez ${ }^{1}$, C.F. Diestel ${ }^{1}$, M. Galvao Neto ${ }^{2}$

${ }^{1}$ Dept. Of Bariatric Endoscopy, Endogastro Rio, Rio de Janeiro/Brazil

${ }^{2}$ Florida International University, Miami/United States of America/FL

Contact E-mail Address: ricfittipaldi@hotmail.com

Introduction: Endoscopic methods, especially the intragastric balloon (IGB), have been shown to be effective for the treatment of excess weight.

Aims \& Methods: We aimed to assess the efficacy and complications of excess weight treatment with a non adjustable IGB.

A liquid-filled IGB with a volume of 600 to $700 \mathrm{ml}$ was used. The patients had a minimum initial body mass index (BMI) of $27 \mathrm{~kg} / \mathrm{m} 2$ and were followed up by a multidisciplinary team consisting of a nutritionist, a doctor and a psychologist. For statistical analysis, the patients were divided into groups according to sex and degree of excess weight (overweight and grade I, II and III obesity). Data were analyzed using descriptive statistical methods, the Student t-test, and analysis of variance followed by the Tukey post-test. The level of significance was set at $\mathrm{p}<0.05$.

Results: A total of 5874 patients were analyzed. The incidence of complications was $7.32 \%(n=430)$, as listed below: $299(5.09 \%)$ early IGB removal, $58(0.98 \%)$ absence of weight loss or weight gain. The incidence of gas production inside the balloon was $0.20 \%(n=12)$ and the incidence of leakage was $0.54 \%(n=32)$; pregnancy was $0.32 \%(n=19)$; gastric perforation was $0.06 \%(n=4)$; upper digestive bleeding was $0.05 \%(n=3)$; Wernick Korsakoff syndrome due to excessive vomiting was $0.01 \%(\mathrm{n}=1)$, pancreatitis and esophagus perforation was $0.01 \%$ each $(\mathrm{n}=1)$. Of the 5444 remaining patients, $4081(74.9 \%)$ were women and $1363(25.1 \%)$ were men. Mean age was 38.38 years. The patients showed a significant weight loss, with a significantly lower final BMI (mean: $30.08 \pm 5.06 \mathrm{~kg} / \mathrm{m} 2$ ) than the initial BMI (mean: $36.94 \pm 5.67 \mathrm{~kg} / \mathrm{m} 2$ ) $(\mathrm{p}<0.0001)$. Mean BMI reduction was $6.85 \pm 3.06 \mathrm{~kg} / \mathrm{m} 2$ (range: $0.25-29.79)$. Mean percent total body weight loss (TBWL) was $18.42 \pm 7.25 \%$ and mean percent excess weight loss (EWL) was $65.66 \pm 36.24 \%$ (range $3.99-336.14$ ). The weight loss in kilograms was $19.13 \pm 8.86$. The treatment success rate (\%EWL > 25) was $93.0 \%$, as follow: overweight was $99.0 \%$, grade I obesity was $95.83 \%$, grade II obesity $93.65 \%$ and grade III obesity was $86.09 \%$. Percent EWL was higher in the overweight group (OVW) $(131.54 \%$ EWL), followed by grade I obesity $(\mathrm{G} 1 \mathrm{O})(76.67 \%)$, grade II obesity $(\mathrm{G} 2 \mathrm{O})(56.01 \%)$ and grade III obesity $(\mathrm{G} 3 \mathrm{O})(45.45 \%)$ sequentially $(\mathrm{p}<0.0001)$. Percent EWL was also higher in women $(69.71 \%$ EWL) than in men $(53.39 \% \mathrm{EWL})(\mathrm{p}<0.0001)$. Results are better shown in table 01 .

\begin{tabular}{|c|c|}
\hline & $\mathrm{n}=5444$ \\
\hline \multicolumn{2}{|l|}{$\mathrm{BMI}(\mathrm{Kg} / \mathrm{m} 2)$} \\
\hline initial & $36.94 \pm 5.67$ \\
\hline final* & $30.08 \pm 5.06$ \\
\hline reduction & $6.85 \pm 3.06$ \\
\hline \multicolumn{2}{|l|}{ Body weight } \\
\hline reduction $(\mathrm{Kg})$ & $19.13 \pm 8.86$ \\
\hline$\% \mathrm{TBWL}$ & $18.42 \pm 7.25$ \\
\hline \multicolumn{2}{|l|}{ Excess weight } \\
\hline$\%$ EWL (total group) & $65.66 \pm 36.24$ \\
\hline \%EWL (OVW) & $131.54 *$ \\
\hline$\%$ EWL (G1O) & $76.67 *$ \\
\hline$\%$ EWL (G2O) & $56.01 *$ \\
\hline$\%$ EWL (G3O) & $45.45^{*}$ \\
\hline \multicolumn{2}{|l|}{ Treatment Success Rate $(\% \mathrm{EWL}>25)$} \\
\hline total group & $93.0 \%$ \\
\hline OVW & $99.0 \%$ \\
\hline G1O & $95.83 \%$ \\
\hline $\mathrm{G} 2 \mathrm{O}$ & $93.65 \%$ \\
\hline $\mathrm{G} 3 \mathrm{O}$ & $86.09 \%$ \\
\hline
\end{tabular}

${ }^{*} \mathrm{p}<0.0001$ for all comparisons between values at baseline and at the end of the study. body mass index(BMI); total body weight loss(TBWL); excess weight loss(EWL); overweight (OVW); grade I obesity (G1O); grade II obesity (G2O); grade III obesity $(\mathrm{G} 3 \mathrm{O})$

Conclusion: Endoscopic treatment of excess weight with an IGB has been established as an excellent therapeutic option. 
Disclosure of Interest: M. Galvao Neto: I received personal fees from FRACTYL LABS, personal fees from GI WINDOWS, personal fees from APOLLO ENDO SURGERY, personal fees from GI DYNAMICS, personal fees from ETHICON ENDO SURGERY, outside the submitted work.

All other authors have declared no conflicts of interest.

\section{P1951 FRUCTO-OLIGOSACCHARIDE EXACERBATES STRESS- INDUCED VISCERAL HYPERALGESIA AND GUT INFLAMMATION IN A MURINE MODEL}

B. Chen ${ }^{1}$, L. Du ${ }^{1}$, H. He ${ }^{1}$, J.J. Kim ${ }^{1}$, N. Dai

${ }_{1}^{1}$ Department Of Gastroenterology, Sir Run Run Shaw Hospital, School of

Medicine, Zhejiang University, Hangzhou/China

Contact E-mail Address: binrui@zju.edu.cn

Introduction: Many factors contribute to the development of irritable bowe syndrome (IBS) including the altered visceral perception, intestinal low-grade inflammation and psychosocial factors. Recent research has revealed a relationship between intake of FODMAPs (Fermentable Oligosaccharides, Disaccharides, Monosaccharides, and Polyols) and abdominal complaints. A diet low in FODMAPs can reduce symptoms in patients with IBS but mechanisms were poorly understood.

Aims \& Methods: We aim to explore the role of FODMAPs in triggering IBS symptoms by investigating visceral sensitivity, intestinal inflammation and short chain fatty acid (SCFA) in stress induced IBS mice model. Fructo-oligosaccharide (FOS) as one of the most frequently exposed FODMAPs in daily life was used in this study. Mice were subjected to water avoidance stress (WAS condition; $1 \mathrm{~h} /$ day for 10 days) or sham stress (basal condition; $1 \mathrm{~h} /$ day for 10 days) with an oral gavage of saline or saline solution containing FOS $(8 \mathrm{~g} / \mathrm{kg})$ for 2 weeks. Then visceral sensitivity was measured by abdominal withdrawal reflex (AWR) in response to colorectal distension (CRD) and histologic analyses were used to evaluate mucosal inflammation. Immunohistochemistry, reverse transcription, and gas chromatography were used to estimate mucosal mast cell, levels of cytokines (IL-6, IL-23, TNF- $\alpha$, IL-10, IL-1 $\beta$ ) and SCFA, respectively.

Results: Stress induced visceral hyperalgesia and low-grade inflammation in WAS mice as a model of IBS. In WAS condition, increased visceral sensitivity and mucosal mast cell $(12.3 \pm 2.61$ vs. $8.33 \pm 3.55, P<0.01)$ were observed in FOS-administered mice compared with saline-administered mice. In WAS condition, cytokine expression were mediated by FOS with increased IL-23 $(3.17 \pm 2.11$-fold, $P<0.05)$ in ileum and IL-1 $\beta(2.45 \pm 1.55$-fold, $P<0.05)$ in colon compared with saline. In addition, the average concentrations of acetic $(2.48 \pm 0.62$ vs. $1.04 \pm 0.10, P<0.01)$, propionic $(0.48 \pm 0.09$ vs. $0.33 \pm 0.0$, $P<0.01)$, butyric $(0.27 \pm 0.09$ vs. $0.19 \pm 0.003, P<0.05)$ and total SCFA $(3.61 \pm 0.89$ vs. $1.79 \pm 0.17, P<0.01)$ significantly increased in FOS-administered mice compared with saline-administered mice in WAS condition. In basal condition, no difference of visceral sensitivity, intestinal inflammation and SCFA were observed between mice treated with FOS or saline.

Conclusion: Oral gavage of FOS leads to both an increase in visceral sensitivity and gut inflammation in stress induced IBS mice. These effects are link with the production of SCFA in the gut which involved in the regulation of sensitivity and intestinal immune activation. These findings support the hypothesis that visceral hypersensitivity and intestinal inflammation aggravated by certain FODMAPs may be responsible for IBS symptom generation, and indicate an alternative mechanism of the efficacy of the low-FODMAP diet for IBS patients.

Disclosure of Interest: All authors have declared no conflicts of interest.

\section{References}

1. Takahashi T, Nakade Y, Fukuda H, et al. Daily intake of high dietary fiber slows accelerated colonic transit induced by restrain stress in rats Digestive diseases and sciences 2008: 53; 1271-1277

2. Halmos EP, Power VA, Shepherd SJ, et al. A diet low in FODMAPs reduces symptoms of irritable bowel syndrome Gastroenterology 2014: 146; 67-75.e65

\section{P1952 GENOMIC ANALYSIS OF THE MULTISPECIES PROBIOTIC PRODUCT VSL\#3}

D. Mora ${ }^{1}$, W. M. De Vos ${ }^{2}$, F. P. Douillard ${ }^{2}$

${ }^{1}$ Department Of Food Environmental And Nutritional Sciences, University of Milan, Milan/Italy

${ }^{2}$ Rpu Immunobiology And 3 Dept Of Food Hygiene, University of Helsinki, Helsinky/Finland

Contact E-mail Address: diego.mora@unimi.it

Introduction: Several formulations consisting of live lactic acid bacteria, including bifidobacteria, are marketed as probiotic products. However, these products are often poorly defined and insight into their mode of action is lacking. This hampers further application in treating diseases, limits comparative studies, and prevents predicting their efficacy. We have previously addressed this by providing genomic and functional characterization of single commercial strains (Kainkainen et al 2009, Douillard et al 2013, Tytgat et al 2016). The multispecies product VSL\#3 is marketed globally for treating colitis ulcerosa, pouchitis, and irritable bowel syndrome. To provide a rational basis for understanding the function of VSL\#3 and generate a baseline for future studies, the genomes of all 8 strains that make up this multispecies product were determined and used to predict their function. The strains were provided by the facility which is currently producing both the single strains and the blend mix (CSL-SACCO System, Zelo Buon Persico - Lodi-ITALY)
Aims \& Methods: The individual strains of multispecies product VSL\#3 were cultured on MRS-based media and DNA was extracted using established methods. The DNA was subject to paired-end Illumina sequencing using a HiSeq2000 platform, assembled and annotated as previously described (Douillard et al 2013).

Results: The next generation sequencing provided high quality genomes of all 8 strains that are components of the multispecies product VSL\#3. Detailed phylogenetic and genomic analysis confirmed the species composition to be as indicated in the VSL\#3 product specification and showed the 8 strains of this multispecies product to belong the species Streptococcus thermophilus, Lactobacillus acidophilus, Lactobacillus (para)casei, Lactobacillus plantarum, Lactobacillus helveticus, Bifidobacterium breve and $B$. animalis subsp. lactis (this species included two strains). The species $L$. paracasei and $L$. casei are highly related and in need for further official taxonomic resolution. The annotated genes of the assembled genomes were used to identify genes involved in potential probiotic functions. Full sets of genes for the production of tight adherence pili were observed in the Bifidobacterium spp. and are known to produce pseudopilins that we recently identified to mediate microbe-host crosstalk, promote intestinal integrity, and influence host cell development (O'Connell Motherway et al 2011). Moreover, a series of signaling proteins were identified in the genomes of the Lactobacillus spp., including surface layer proteins and sortase-dependent pili proteins that we showed to interact with the mucosal surfaces and dendritic cells (Konstantinov et al 2008; Kankainen et al 2008; Tygat et al 2016).

Conclusion: The genomic analysis of the VSL\#3 strains confirmed the product specifications, defined the baseline genetic coding capacity, and predicted a number of probiotic mechanisms that could explain the efficacy of this multispecies product.

Disclosure of Interest: All authors have declared no conflicts of interest.

\section{References}

Douillard FP et al (2013) Microbial Biotechnol 6:576-87.

Kankainen M et al (2009) Proc Natl Acad Sci USA 106:17193-8.

Konstantinov SR et al (2008). Proc Natl Acad Sci USA 105: 19474-478.

Tytgat HL et al (2016) Appl Environ Microbiol 82:5756-62.

O'Connell Motherway M et al (2011) Proc Natl Acad Sci USA 108:11217-22.

\section{P1953 RAISING PUBLIC AWARENESS OF GASTROINTESTINAL DISEASES: AN INNOVATIVE STRATEGY FOR A NATIONAL CAMPAIGN}

L. Franzoni ${ }^{1}$, P. $\mathrm{Crafa}^{2}$, A. Bertelè ${ }^{3}$, V. Corrente ${ }^{4}$, S. Grillo ${ }^{5}$, S. Landi ${ }^{6}$, C. Miraglia ${ }^{1}$, S. Scida ${ }^{1}$, F. Di Mario

${ }^{1}$ Department Of Medicine And Surgery, University Of Parma, Italy, University of Parma, Parma/Italy

${ }^{2}$ Department Of Pathology, University Hospital, Parma, Italy; University

Hospital, Parma, Italy, ParmalItaly

${ }^{3}$ Department Of Clinical \& Experimental Medicine, Clinical Pharmacology \&

Digestive Pathophysiology Unit, University of Parma, Parma/Italy

${ }^{4}$ University Hospital, Parma (Italy), Parma/Italy

${ }^{5}$ Gastroenterology, University of Parma, Italy, PARMA/Italy

${ }^{6}$ University of Parma, Parma/Italy

Contact E-mail Address: francesco.dimario@unipr.i

Introduction: Disease prevention and high public awareness are fundamental to reduce morbidity, mortality and health-related costs. Extensive research led to identification of causes of gastrointestinal (GI) diseases, nevertheless it is still difficult to get population involved. The goals of our pilot campaign are: (a) to raise awareness about GI diseases, risk factors, signs, symptoms, in order to convince people to change behavior and to prompt them with concerns to visit doctors as early as possible; (b) to facilitate communication between healthcare providers and population; (c) to determine the knowledge of health personnel about the appropriate diagnostic investigations. Any information we can share may also benefit patients and their families, in recognition of the many people who suffer with the pain and discomfort caused by GI disorders.

Aims \& Methods: We organized population-based events, out of health facilities, during which: (a) giant inflatable anatomical models of GI organs that can be visited inside, were installed; (b) educational panels and brochures were set and explained both inside and outside the models; (c) videos illustrating endoscopic exams and histopathology examinations were projected and discussed; (d) clinical cases, also mimicking patient encounter, were simulated.

Results: We started an innovative strategy focused on the keywords: multidisciplinary team, scientific rigor but simple words, people attraction, curiosity inducing communication, in Parma and neighbouring Cities. Specialists in Gastroenterology, Anatomic Pathology, Radiology, Surgery, Biochemistry, Nutrition, together with pre- and post-graduate Students, discussed various aspects of gastrointestinal diseases. Selected people were examined by ultrasonography. Municipality and civil society were also involved to ensure organizational efficiency. The most discussed topics regarded dyspepsia, gastritis, helicobacter pylori infection, gastroesophageal reflux disease, alcohol abuse, cirrhosis, hepatitis, irritable bowel syndrome, intestinal polyp and polyposis, GI cancer, food allergy and intolerance, optimal nutrition in health and disease. The event performed in the main square of Parma lasted two full consecutive days and was attended by about 3,000 people, most of which also filled a questionnaire. A total of 120 ultrasound examinations were performed. In neighbouring Cities the events were organized for one day; as a consequence, the number of participants was lower in proportion, but very satisfactory. 
Conclusion: The events were educational and enjoyable for all age groups. The interactive approach and the environment out of health-care centres facilitated population to feel comfortable and eager to learn, as well as clinical cases simulation provided a valuable entertaining experience. This strategy of raising public awareness of GI diseases seems promising, we are refining the model for a national campaign.

Disclosure of Interest: All authors have declared no conflicts of interest.

\section{P1954 ALTERED PLASMA PROFILE AND URINE EXRECTION OF AMINO ACIDS IN COELIAC DISEASE CHILDREN AFTER ADMINISTRATION OF OLIGOFRUCTOSE-ENRICHED INULIN INTO GLUTEN-FREE DIET-RESULTS OF RANDOMIZED, PLACEBO-CONTROLLED PILOT TRIAL}

N. Drabińska ${ }^{1}$, E. Jarocka-Cyrta ${ }^{2}$, U. Krupa-Kozak ${ }^{3}$

${ }^{1}$ Department Of Chemistry And Biodynamics Of Food, Institute of Animal

Reproduction and Food Research of Polish Academy of Sciences, Olsztyn/Poland ${ }^{2}$ Faculty Of Medical Science, Department Of Clinical Pediatrics, University of Warmia \& Mazury, Olsztyn/Poland

${ }^{3}$ Institute of Animal Reproduction and Food Research Polish Academy of Sciences, Olsztyn/Poland

\section{Contact E-mail Address: n.drabinska@pan.olsztyn.pl}

Introduction: Amino acids are essential metabolites which play a role as protein constituents. Moreover, amino acids and their metabolites feature in regulation of anabolic and catabolic metabolism, detoxification processes, and act as neurotransmitters. Concentration of circulating amino acids reflects about dietary protein intake and can be an indicative of malnutrition. Changes in the amino acid homeostasis are observed in coeliac disease (CD) [1] due to malabsorption caused by enteropathy but also because of the treatment with gluten-free diet (GFD) shown to be low in the important nutrients.

Aims \& Methods: A randomized, placebo-controlled interventional trial was designed to assess the influence of an oligofructose-enriched inulin (OEI) as a supplement of GFD on plasma profile and urine excretion of amino acids in CD children strictly following GFD at least 1 year. We randomized 34 children diagnosed with $\mathrm{CD}$ into a group receiving $10 \mathrm{~g}$ of OEI daily and a placebo (maltodextrin) group during a 12-week nutritional intervention. Amino acid profiles in urine and plasma was analysed via EZ:Faast ${ }^{\mathrm{TM}}$ derivatisation method followed by gas chromatography/mass spectrometry detection.

Results: At the baseline and after supplementation, 22 and 27 amino acids were identified in plasma and urine, respectively in both groups of children. Significantly higher levels $(\mathrm{p}<0.05)$ of alanine, serine, asparagine, glutamine, threonine, phenylalanine, lysine, histidine, $\alpha$-aminoadipic acid, $\alpha$-aminopimelic acid, hydroxylysine and cystine were determined in urine of OEI group after intervention as compared to the baseline and placebo group. The analysis of amino acid profiles in plasma samples showed a significant increase $(p<0.05)$ of majority of amino acids in both OEI and placebo groups after intervention. However, the concentrations of glutamine and glutamic acid were significantly higher $(\mathrm{p}<0.05)$ in the OEI supplemented group.

Conclusion: Our study showed that supplementation of GFD with OEI impacts the amino acid homeostasis in CD children. OEI in GFD increased a total concentration of amino acids in both urine and plasma. Higher excretion of amino acids in urine accompanied with increased amino acid content in plasma may indicate the improved absorption or/and stimulated de novo synthesis of proteins. Increase in the concentration of glutamine can stimulate recovery of the intestinal mucosa by itself or by the involvement in the synthesis of citrulline known as negatively correlated with intestinal mucosal damage [1], therefore we can suspect that OEI added to GFD can help in restoring of the intestinal barrier integrity.

Disclosure of Interest: All authors have declared no conflicts of interest. The research was supported by statutory funds of the Department of Chemistry and Biodynamics of Food in the IAR\&FR PAS and by the National Science Centre, Poland (project number: 2016/21/N/NZ9/01510).

\section{Reference}

1. Senvic et al. Nurt. Hosp. 2015; 32(1): 139-143.

\section{P1955 LONG-TERM TRENDS IN HEMATOLOGICAL AND NUTRITIONAL STATUS AFTER GASTRECTOMY FOR GASTRIC CANCER \\ J. Kim \\ General Surgery, The Catholic University of Korea, Seoul/Korea, Republic of}

\section{Contact E-mail Address: doctor2003@catholic.ack kr}

Introduction: The quality of life and nutritional management after gastrectomy are major issues for gastric cancer patients, as is oncological surveillance. Weight loss usually follows gastric resection, with reported losses ranging from $10 \%$ to $30 \%$ of the preoperative weight. This loss has been attributed to inadequate oral intake, malabsorption, rapid intestinal transit time, and bacterial overgrowth. Timely, appropriate nutritional intervention can minimize diet intolerance, weight loss, and micronutrient deficiencies that often follow surgery.

Aims \& Methods: This study investigated long-term trends in hematological and nutritional parameters after gastrectomy for gastric cancer and evaluated the influence of the reconstruction type on these trends. The medical records of 558 patients who underwent curative gastrectomy with standard lymph node dissection for stage I gastric cancer between January 2006 and December 2013 were reviewed. The hematological and nutritional parameters evaluated included hemoglobin, ferritin, vitamin $\mathrm{B}_{12}$, total protein, albumin, total cholesterol, triglyceride, and calcium. The patients were followed up for 6 months postoperatively and then annually until death, cancer recurrence, or follow-up loss.

Results: In the long term, ferritin and triglyceride gradually decreased after gastrectomy, while the other parameters decreased slightly or were stable. In the comparisons according to reconstruction type, the Roux-en-Y group had the lowest levels of hemoglobin, ferritin, vitamin $\mathrm{B}_{12}$, total protein, albumin, and total cholesterol beginning 6 months postoperatively compared with the Billroth I and II groups. However, only ferritin and vitamin $\mathrm{B}_{12}$ had significant differences in the 5-year cumulative incidences of deficiency according to the reconstruction type, whereas albumin, triglyceride, total cholesterol and calcium did not.

Conclusion: Although malabsorption and malnutrition are common in patients after a gastrectomy, most nutritional parameters were stable or decreased slightly in the long-term and were not markedly influenced by the reconstruction type or extent of gastrectomy. Therefore, for more accurate nutritional assessment after gastrectomy, multidirectional monitoring should be considered rather than simply measuring biochemical parameters.

Disclosure of Interest: All authors have declared no conflicts of interest.

\section{References}

H.J. Lee, J.M. Chung, E.H. Seo, et al. Clinicopathologic characteristics of gastric cancer diagnosed at health screening. Korean J Med 2008; 75(6): 665-72.

Jun JH, Yoo JE, Lee JA, et al. Anemia after gastrectomy in long-term survivors of gastric cancer: A retrospective cohort study. Int J Surg 2016; 28: 162-8.

Kim AR, Cho J, Hsu YJ, et al. Changes of quality of life in gastric cancer patients after curative resection: a longitudinal cohort study in Korea. Ann Surg 2012; 256(6): 1008-13.

Bozzetti F, Ravera E, Cozzaglio L, et al. Comparison of nutritional status after total or subtotal gastrectomy. Nutrition 1990; 6(5): 371-5.

Bae JM, Park JW, Yang HK, et al. Nutritional status of gastric cancer patients after total gastrectomy. World J Surg 1998; 22(3): 254-60; discussion 60-1.

\section{P1956 EVOLUTION OF REPRODUCTIVE DISORDERS RELATED TO CELIAC DISEASE UNDER GLUTEN -FREE DIET}

A. Aomari $^{1}$, M. Firwana ${ }^{1}$, A. Amjahdi ${ }^{1}$, I. Benelbarhdadi ${ }^{2}$

${ }^{1}$ CHU Rabat, Rabat/Morocco

${ }^{2}$ Rabat, Ibn Sina Hospital, Rabat/Morocco

Contact E-mail Address: ayoub.medinterne@gmail.com

Introduction: Celiac disease is an autoimune enteropathy inducted by the ingestion of gluten. the classical form has become a minority. Currently, the most frequent forms of presentation are extradigestive with various manifestations, among others, reproductive disorders. The aim of our study is to assess the frequency of these disorders in celiac disease and their evolution under glutenfree diet.

Aims \& Methods: Descriptive retrospective study of 173 patients with celiac disease followed in the departement of diseases of the digestive tract medecine c of the Ibn Sina Hospital in Rabat, over a period of 18 years.

Results: In 173 patient with celiac disease, 58 patients had reproductive disorders. there are 53 women and 5 men. The average age was 32.25 years; the diagnosis of celiac disease is based on histology and serology. The reproductive problems were never isolated but always associated with other digestive signs at the time of diagnosis of celaic disease. These disorders are represented by: delayed puberty in 11 cases, secondary amenorrhea in 13 cases, irregular menstrual in 12 cases, absence of development of secondary sex characteristics in 8 cases, spontaneous abortions in 7 cases, menometrorrhagia in 4 cases, primary sterility in 5 cases early menopause in 6 cases, premature delivery in 3 cases primary amenorrhea in 2 cases and intrauterine fetal death in 1 case. All our patients have had a glutenfree diet. 15 Patients lost to follow-up, 2 patients died, and 12 patients undergowing follow-up. The remaining 29 patients, the evolution of reproductive disorders under gluten-free diet was favorable in 26 cases $(90 \%)$, with the normalization of cycles in 15 cases, resumption of cycles in 6 cases development of secondary sex characteristics in 2 cases, fertility resumption in one case, initiation of cycles after primary amenorrhea in one case and delivery of a newborn at term after premature deliveries in one case. The evolution was unfavorable in 3 cases with the notion of miscarriage 4 years after the start of the gluten-free diet in one patient and the absence of cycle resumption in 2 cases.

Conclusion: The reproductive disorders associated with celiac disease are frequent and varied. In our study, these disorders responded very well under a gluten-free diet conducted in $90 \%$ of the case. these disorders are thus reversible under this diet.

Disclosure of Interest: All authors have declared no conflicts of interest.

\section{P1957 ROLE OF VAGAL AFFERENTS ON HIGH FAT DIET} INDUCED ALTERATIONS IN RAT BEHAVIOUR AND GUT MOTILITY

Y. Öztürk ${ }^{1}$, B. Akgün ${ }^{1}$, O. Çetin ${ }^{1}$, H. Ö. Karatas, ${ }^{1}$, B. Güney ${ }^{1}$, Z. N. Özdemir $\mathrm{Kumral}^{2}$, D. Özbeyli ${ }^{2}$, S. Arabacı Çetin ${ }^{2}$, H. Zortul ${ }^{3}$, I. Peker ${ }^{4}$, F. Arıcıoğlu ${ }^{3}$, C. Erzik ${ }^{4}$, B. Çağlayan Yeğen ${ }^{2}$, N. Imeryüz ${ }^{5}$

${ }^{1}$ Third Grade Student, Marmara University, School of Medicine, İstanbul/Turkey ${ }^{2}$ Physiology, Marmara University, School of Medicine, İstanbul/Turkey 
${ }^{3}$ Pharmacology, Marmara University Faculty of Pharmacy, İstanbul/Turkey ${ }^{4}$ Medical Biology, Marmara University, School of Medicine, İstanbul/Turkey ${ }_{5}^{5}$ Gastroenterology And Physiology, Marmara University, School of Medicine, İstanbul/Turkey

Contact E-mail Address: yonca-ozturk@hotmail.com

Introduction: High -fat diet induces depression and anxiety, alters gastrointestinal motility in rats but operative mechanisms are not clear. Vagal nerve conducts physiological information about luminal content to the higher brain centres.

Aims \& Methods: Is there any role of vagal afferent nerves on high fat diet (HFD)-induced alterations in cognitive functions and gut motility? Ten-week old male Sprague-Dawley rats $(\mathrm{n}=38)$ were treated with either perivagal $1 \%$ capsaicin $(n=19)$ or vehicle $(10 \%$ Tween 80 in oil $)(n=19)$. After 3 weeks of recovery, rats were pair-fed with chow (fat $2-7 \%)(n=18)$ or HFD $(\% 45$ fat) $(\mathrm{n}=20)$ for 5 weeks until decapitation. In between 4 th and 5 th weeks of HFD rats were subjected to open field tests (anxiety and depression-like behaviour); novel object recognition, passive avoidance tests (memory). Food and water intake were measured after $16 \mathrm{hrs}$. of food and $24 \mathrm{hrs}$. of water deprivation. Weight of faeces for $16 \mathrm{hrs}$. and transit time with charcoal were measured. Data was expressed as mean standard deviation, comparison between groups were done with two- way ANOVA.

Results: VAD increased body weight significantly $(\mathrm{p}<0.05)$ during feeding period irrespective of fat content of the diet. Fat content and VAD had no effect on $1 \mathrm{hr}$ food intake after food deprivation. HFD decreased water intake $(\mathrm{p}<0.0075)$, VAD blunted this effect $(\mathrm{p}<0.05)$. Both HFD and VAD decreased faeces weight significantly ( $p<0.0001$ and $p<0.05$ respectively) but there was not any change in intestinal transit. HFD impaired short -term memory $(\mathrm{p}<0.02)$, whereas VAD compromised spatial learning $(\mathrm{p}<0.04)$. HFD rats were more anxious in OFT $(\mathrm{p}<0.01)$.

Conclusion: HFD-induced alterations in memory and anxiety were not affected by VAD but VAD blunted effect of HFD on water intake and faeces weight, suggesting that their operating mechanisms are different. VAD by itself impaired spatial memory that requires further investigation.

Disclosure of Interest: All authors have declared no conflicts of interest.

P1958 OPTIMAL NUTRITIONAL ROUTE FOLLOWING TOTAL GASTRECTOMY: A SYSTEMATIC REVIEW AND META-ANALYSIS OF RANDOMIZED CONTROLLED TRIALS

S. Govil ${ }^{1}$, D. Chan ${ }^{2}$, T. Abdelrahman ${ }^{2}$, A. Foliaki ${ }^{2}$, W. Lewis ${ }^{2}$, G. Clark ${ }^{2}$, G. Blackshaw ${ }^{2}$

${ }^{1}$ Cardiff University, Cardiff/United Kingdom

${ }^{2}$ General Surgery, University Hospital of Wales, Cardiff/United Kingdom

Contact E-mail Address: govils@cardiff.ac.uk

Introduction: Total gastrectomy can profoundly influence patients' nutritional status and controversy surrounds the various nutritional options available postoperatively, namely total parenteral nutrition (TPN), or enteral nutrition (EN) either via the nasojejunal (NJ) or feeding jejunostomy (JEJ) route. The aim of this review was to determine the optimal nutritional route after total gastrectomy for cancer.

Aims \& Methods: PubMed, MEDLINE and the Cochrane Library (January 1985 to January 2017) were systematically searched for randomized controlled trials comparing various nutritional routes after total gastrectomy. The primary outcome measure was overall morbidity.

Results: Six studies involving 353 patients (median age 62 years, 217 males, 170 TPN, 96 NJ, 87 JEJ) who underwent total gastrectomy were analysed. Overall morbidity was significantly greater in patients after TPN compared with EN [odds ratio (OR) 1.95, 95\% Confidence Interval (CI) 1.16-3.28, $\mathrm{p}=0.01$ ]. There was a trend towards greater mortality in patients who received TPN compared with EN (OR 1.90, 95\% CI $0.64-5.70, \mathrm{p}=0.25)$. When EN was sub analyzed according to NJ or JEJ, morbidity was significantly greater in patients who received TPN compared with JEJ (OR 4.59, 95\% CI 1.74-12.10, $\mathrm{p}=0.002$ ) but not in patients who received NJ feeding (OR $1.23,95 \%$ CI $0.64-2.36, \mathrm{p}=0.53$ ). Conclusion: Enteral nutrition in the form of feeding jejunostomy was associated with lower morbidity compared with TPN following total gastrectomy for cancer.

Disclosure of Interest: All authors have declared no conflicts of interest

\section{References}

1. Parkin DM, Bray F, Ferlay J, Pisani P. Global cancer statistics, 2002. CA Cancer J Clin. 2005 Mar-Apr;55(2):74-108.

2. Mazaki T, Ebisawa K. Enteral versus parenteral nutrition after gastrointestinal surgery: a systematic review and meta-analysis of randomized controlled trials in the English literature. J Gastrointest Surg. 2008 Apr;12(4):739-55.

3. Liu X, Wang D, Zheng L, Mou T, Liu H, Li G. Is early oral feeding after gastric cancer surgery feasible? A systematic review and meta-analysis of randomized controlled trials. PLoS One. 2014;9(11):e112062.

4. Moher D, Liberati A, Tetzlaff J, Altman DG. Preferred reporting items for systematic reviews and meta-analyses: the PRISMA statement. Bmj. $2009 \mathrm{Ju}$ 21;339:b2535.

5. Jadad AR, Moore RA, Carroll D, Jenkinson C, Reynolds DJ, Gavaghan DJ, et al. Assessing the quality of reports of randomized clinical trials: is blinding necessary? Control Clin Trials. 1996 Feb;17(1):1-12.

6. Cochrane Handbook for Systematic Reviews of Interventions version 5.1.0. In: Higgins J, Green S, editors: The Cochrane Collaboration; 2011.

7. Baigrie RJ, Devitt PG, Watkin DS. Enteral versus parenteral nutrition after oesophagogastric surgery: a prospective randomized comparison. Aust $N Z J$ Surg. 1996 Oct;66(10):668-70.
8. Heylen AM, Lybeer MB, Penninckx FM, Kerremans RP, Frost PG. Parenteral versus needle jejunostomy nutrition after total gastrectomy. Clinical nutrition. 1987;6(3):131-6.

9. Kamei H, Hachisuka T, Nakao M, Takagi K. Quick recovery of serum diamine oxidase activity in patients undergoing total gastrectomy by oral enteral nutrition. American Journal of Surgery. 2005;189(1):38-43.

10. Li J, Ji Z, Yuan C, Zhang Y, Chen W, Ju X, et al. Limited efficacy of early enteral nutrition in patients after total gastrectomy. Journal of Investigative Surgery. 2011;24(3):103-8.

11. Nomura E, Lee SW, Kawai M, Hara H, Nabeshima K, Nakamura K, et al. Comparison between early enteral feeding with a transnasal tube and parenteral nutrition after total gastrectomy for gastric cancer. HepatoGastroenterology. 2015;62(138):536-9.

12. Sand J, Luostarinen M, Matikainen M. Enteral or parenteral feeding after total gastrectomy: prospective randomised pilot study. Eur J Surg. 1997 Oct;163(10):761-6.

\section{P1959 TAUROLIDINE PREVENTS CATHETER-RELATED BLOODSTREAM INFECTIONS IN PATIENTS ON HOME PARENTERAL NUTRITION-A RANDOMIZED CONTROLLED TRIAL}

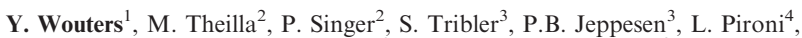
L. Vinter-Jensen ${ }^{5}$, H.H. Rasmussen ${ }^{6}$, F. Rahman ${ }^{7}$, G. Wanten ${ }^{8}$

${ }^{1}$ Radboudumc, Nijmegen/Netherlands

${ }^{2}$ Rabin Medical Center Beilinson ICU and Nutrition Institute, Petah Tikva/Israel ${ }^{3}$ Department Of Medical Gastroenterology, University Hospital of Copenhagen,

Rigshospitalet, Copenhagen/Denmark

${ }^{4}$ Medical And Surgical Science, University of Bologna, Bologna/Italy

${ }^{5}$ Dept. Of Medical Gastroenterology, Aalborg University Hospital Dept. of

Medical Gastroenterology, Aalborg/Denmark

${ }^{6}$ Aalborg Hospital, Aalborg/Denmark

${ }^{7}$ GI Medicine, University College London Hospitals, London/United Kingdom

${ }^{8}$ Department Of Gastroenterology And Hepatology, Radboud University Nijmegen, Nijmegen/Netherlands

Contact E-mail Address: Yannick.Wouters@radboudumc.nl

Introduction: Patients on home parenteral nutrition (HPN) are exposed to a lifelong risk of catheter-related bloodstream infections (CRBSI), which threaten catheter and patient survival. Both taurolidine $2 \%$ and saline $0.9 \%$ solution are used as catheter lock solutions (CLS) to prevent CRBSI. The optimal agent however, remains unclear. We hypothesized that taurolidine as CLS is superior to saline in preventing CRBSI in HPN patients.

Aims \& Methods: We hypothesized that taurolidine $2 \%$ as CLS is superior to saline $0.9 \%$ in preventing CRBSI in HPN patients. This multicenter double blinded trial randomly assigned HPN patients to use either the CLS taurolidine $2 \%$ or saline $0.9 \%$ for one year. Primary outcome was the number of CRBSI/ 1000 catheter days. Secondary outcomes included time to CRBSI or catheter removal due to CRBSI, number of catheter removals due to CRBSI, exit-site infections, catheter occlusions, and (serious) adverse events.

Results: Of 105 randomized patients, 102 were enrolled as modified intention-totreat population. With taurolidine, 5 CRBSI occurred during 15318 catheter days. In the saline arm 18 CRBSI occurred over 12493 catheter days. CRBSI 1000 catheter days were 0.33 and 1.44 in the taurolidine and saline groups, respectively (relative risk, $0.23 ; 95 \% \mathrm{CI}, 0.07$ to $0.63 ; \mathrm{P}=0.002$ ). The cumulative proportion of CRBSI-free patients after one year was $88 \%$ in the taurolidine group and $49 \%$ in the saline group $(\mathrm{P}=0.002)$. The number of catheter removals due to CRBSI was two $(4 \%)$ in the taurolidine group and eight $(16 \%)$ in the saline arm $(\mathrm{P}=0.049)$. The cumulative proportion of patients without a catheter removal due to CRBSI was higher in the taurolidine group $(\mathrm{P}=0.025)$. Exit-site infection and catheter occlusion rates were similar in both groups. Except for occurrence of CRBSI $(\mathrm{P}=0.002)$, there was no difference in (serious) adverse events between groups. Drug-related adverse events were rare and generally mild to moderate.

Conclusion: Taurolidine $2 \%$ decreased the risk for CRBSI by more than four times in HPN patients compared to saline $0.9 \%$. Given its favorable safety profile and lack of evidence for altering microbial susceptibility, taurolidine locking therefore seems a key strategy to prevent CRBSI

Disclosure of Interest: Y. Wouters: Financial support for study from Geistlich Pharma AG.

M. Theilla: Financial support for study from Geistlich Pharma AG.

P. Singer: Financial support for study from Geistlich Pharma AG

S. Tribler: Financial support for study from Geistlich Pharma AG.

P.B. Jeppesen: Financial support for study from Geistlich Pharma AG.

L. Pironi: Research Support from: Baxter, B. Braun, and NPS/Nycomed and Shire. Financial support for study from Geistlich Pharma AG. Consultant for: Baxter, B. Braun, and NPS/Nycomed and Shire.

L. Vinter-Jensen: Financial support for study from Geistlich Pharma AG. H.H. Rasmussen: Financial support for study from Geistlich Pharma AG. F. Rahman: Financial support for study from Geistlich Pharma AG. G. Wanten: Research Support from: Baxter, B. Braun, and Fresenius. Financial support for study from Geistlich Pharma AG. Consultant for: Baxter, B. Braun, and Fresenius. 
P1960 REPAIR OF DAMAGED CENTRAL VENOUS CATHETERS SUBSTANTIALLY PROLONGS DEVICE SURVIVAL IN PATIENTS ON HOME PARENTERAL NUTRITION

Y. Wouters ${ }^{1}$, R. Vissers ${ }^{1}$, W. Kievit ${ }^{2}$, G. Wanten

${ }^{1}$ Radboudumc, Nijmegen/Netherlands

${ }^{2}$ Radboud University Medical Center, Nijmegen/Netherlands

Contact E-mail Address: Yannick.Wouters@radboudumc.n

Introduction: Patients with severe intestinal failure depend on life-long home parenteral nutrition (HPN) support. Repeated central venous catheter (CVC) loss due to complications, including mechanical damage, compromises vascular access. It remains unclear whether repair of damaged CVCs is an effective strategy to extend catheter life, avoid surgical replacement and maintain venous access.

Aims \& Methods: The objective of this study was to characterize patients who underwent catheter repair and to evaluate effects on catheter survival and describe complications. This study concerns a retrospective analysis of all catheter repairs that were performed in HPN patients in the Radboud University Medical Center between January 2000 and May 2017. Primary endpoint was the difference in catheter survival in the presence or absence of catheter repair. To this end, a non-parametric survival analysis was performed. Secondary outcomes included localization of catheter damage and frequency of repair-related complications within 1 month after catheter repair.

Results: A total of 50 repairs in $38 \mathrm{CVCs}$ of $32 \mathrm{HPN}$ patients were included in the analysis. 16 CVCs (32\%) were damaged at the distal end, near the screw thread of the catheter, $25 \mathrm{CVCs}(50 \%)$ at the junction between the rigid and flexible part of the catheter, and $9 \mathrm{CVCs}(18 \%)$ at the flexible part of the catheter. The mean time to catheter repair after placement was 2.2 years $(95 \% \mathrm{CI}=1.55-2.89)$. The mean catheter survival after repair was extended by 1.4 years to 3.6 years $(95 \%$ $\mathrm{CI}=2.69-4.46 ; \mathrm{p}=0.01)$. No repair-related complications occurred within 1 month after catheter repair.

Conclusion: Repair of damaged CVCs significantly extends catheter life in HPN patients and maintains venous access. Catheter repair is a safe procedure.

Disclosure of Interest: Y. Wouters: Previous financial support for prospective study from Geistlich Pharma AG.

G. Wanten: Research Support from: Baxter, B. Braun, and Fresenius. Financial support for study from Geistlich Pharma AG. Consultant for: Baxter, B. Braun, and Fresenius.

All other authors have declared no conflicts of interest.

\section{P1961 LONG-TERM CLINICAL OUTCOMES OF PATIENTS ON HOME PARENTERAL NUTRITION USING TAUROLIDINE CATHETER LOCKS}

Y. Wouters ${ }^{1}$, B. Roosenboom ${ }^{2}$, W. Kievit ${ }^{1}$, G. Wanten ${ }^{2}$

${ }^{1}$ Radboudumc, Nijmegen/Netherlands

${ }^{2}$ Department Of Gastroenterology And Hepatology, Radboud University Nijmegen, Nijmegen/Netherlands

Contact E-mail Address: Yannick.Wouters@radboudumc.nl

Introduction: Catheter-related complications (CRCs) in home parenteral nutrition (HPN) patients are a threat to both catheter and patient survival. Taurolidine $2 \%$, an antimicrobial catheter lock solution (CLS), is an effective agent for the prevention of catheter-related bloodstream infections (CRBSI). Aims \& Methods: The aim of this retrospective study was to evaluate long-term clinical outcomes of our HPN patient cohort that uses the CLS taurolidine. Between 2008 and 2016, all adult HPN patients requiring a central venous catheter (CVC) or port-a-cath (PAC) who used taurolidine as CLS were included. CRC incidence rates/1000 catheter days were described. Kaplan-Meier analysis was used to determine the time until a first CRC. Cox proportional hazard analysis was performed to identify risk factors for a first CRC.

Results: In 221 HPN patients, 658 CVCs (418 Hickmans, 172 PACs, and 28 nontunneled CVCs) were inserted, comprising 261252 catheter days. Median survival for Hickmans, PACs and non-tunneled catheters was 175 (43-544), 310 (61-827) and 14 (7-19) days, respectively. During eight years of follow-up, 176 CRBSI occurred and 80 catheter-related occlusions (CRO). CRBSI and CRO rates/1000 catheter days were 0.74 and 0.34 , respectively. In $47 \%$ and $32 \%$ of patients, at least one CRBSI and CRO occurred, respectively. Median time to a first CRBSI or CRO was 246 (54-817) and 215 catheter days (5-2070). Numerically, but not significantly, CRBSI and CRO rates decreased over time. The sole use of intravenous fluids was associated with a significantly lower risk for CRBSI (RR 0.32). Twenty patients reported adverse events (5 grade 1,13 grade 2 and 2 grade 3 ) which were possibly related to the use of taurolidine.

Conclusion: This study describes the largest cohort of HPN patients to date on long-term taurolidine $2 \%$ as CLS. Overall, CRC incidence rates were low when compared with the literature. We found no evidence for a decreased effect of taurolidine over time.

Disclosure of Interest: Y. Wouters: Financial support for previous study from Geistlich Pharma AG.

G. Wanten: Research Support from: Baxter, B. Braun, and Fresenius. Financial support for study from Geistlich Pharma AG. Consultant for: Baxter, B. Braun, and Fresenius.

All other authors have declared no conflicts of interest.
P1962 INTESTINAL DYSBIOSIS IN PATIENTS WITH SHORT BOWEL SYNDROME DEPENDENT ON TOTAL PARENTERAL NUTRITION IS REFLECTED BY ALTERED METABOLOME IN FAECES

L. Bajer ${ }^{1}$, M. Kostovcik ${ }^{2}$, J. Hradecky ${ }^{3}$, T. David ${ }^{4}$, P. Wohl ${ }^{1}$, J. Spicak ${ }^{1}$, P. Drastich ${ }^{1}$, M. Cahova ${ }^{1}$

${ }^{1}$ Institute for Clinical and Experimental Medicine, Prague/Czech Republic

${ }^{2}$ Institute of Microbiology of the ASCR, Prague/Czech Republic

${ }^{3}$ University of Chemistry and Technology, Prague/Czech Republic

${ }^{4}$ Faculty of Science, Charles University, Prague/Czech Republic

Contact E-mail Address: lukasbajer1@gmail.com

Introduction: Patients with short bowel syndrome (SBS) exhibit substantial disturbances in gut microbiota composition, which implicates significant alterations of intestinal metabolome.

Aims \& Methods: The aim of this study was to perform genetic and metabolomic analysis of stool samples collected from SBS patients totally dependent on parenteral nutrition (TPN). We analysed the stool samples from 8 healthy individuals and 8 patients with SBS dependent on TPN. Faecal microbiota composition was assessed by sequencing of variable V3 and V4 regions of $16 \mathrm{~S}$ rRNA gene using Illumina MiSeq TM platform. Library preparation, template preparation and template sequencing was performed according to manufacturer's protocols. Obtained data were filtered by quality and length and processed for alpha and beta diversity analyses using QIIME software package. SCFA profile was measured using solid phase microextraction (SPME) coupled to gas chromatography and high resolution mass spectrometry employing time of flight mass analyser (Pegasus GC-HRT; LECO, USA). D-lactate content was determined using Megazyme kit.

Results: Weighted Unifrac analysis revealed significant differences between control and TPN subjects. In healthy controls, most abundant phylum was Firmicutes $(64.2 \pm 7.5 \%)$, followed by Bacteroidetes $(22.5 \pm 9.1 \%)$ and Actinobacteria $(8.9 \pm 4.5 \%)$. Proteobacteria were found only in one control sample. In TPN group, Firmicutes represented $66.4 \pm 29 \%$ of microbiota but Bacteroidetes were absent and Actinobacteria significantly reduced $(1.6 \pm \%)$. Proteobacteria were found in all samples $(23.6 \pm 15 \%)$. The most abundant metabolites in control stool samples were short - chain fatty acids (SCFA): acetate, propionate and butyrate. In the TPN group, lactate predominated significantly, while SCFA were absent in the intestinal content of these patients.

Conclusion: Long-term dependence on total parenteral nutrition results in dysbiosis of the intestine residuum characterized by extinction of Bacteroidetes and appearance of Proteobacteria. This shift was reflected by the changes in the composition of prevailing metabolites in stool.

Disclosure of Interest: All authors have declared no conflicts of interest.

Supported by Ministry of Health of the Czech Republic, grant nr. 15-28745A. All rights reserved.

\section{P1963 COMPARING RISKS OF ADVERSE EVENTS ASSOCIATED WITH PERCUTANEOUS ENDOSCOPIC GASTROSTOMY (PEG) PLACEMENT BETWEEN THE MODIFIED INTRODUCER TECHNIQUE AND THE OVERTUBE ASSISTED PULL TECHNIQUE}

H. Matsui ${ }^{1}$, H. Inomata ${ }^{1}$, H. Horiuchi ${ }^{1}$, N. Tamai ${ }^{1}$, K. Sumiyama ${ }^{1}$

${ }^{1}$ Department Of Endoscopy, Jikei University School of Medicine, Tokyo/Japan

\section{Contact E-mail Address: hiro24tht80@yahoo.co.jp}

Introduction: Techniques of percutaneous endoscopic gastrostomy (PEG) placement can be divided into two techniques, the pull technique or the introducer technique. Although the development of the pull technique greatly improved procedural safety for the gastrostomy placement, the pull technique, in which the PEG catheter is delivered through the oral cavity and the hypopharynx, inevitably contaminates the peristomal site. A series of studies directly comparing the newly developed modified introducer technique using a blunt cannula compared with the conventional pull technique demonstrated that the modified introducer technique was more preferable regarding procedure related adverse events. ${ }^{1.2)}$ Meanwhile, use of an overtube while guiding the catheter into the stomach in pull technique succeeded the reduction of the bacterial implantation. It is still unclear if the modified introducer technique or the overtube assisted pull technique would reduce risks of adverse events.

Aims \& Methods: In this study, we retrospectively investigated risks of adverse events associated with the modified introducer technique and the overtube assisted pull method.Outcomes of patients who underwent the PEG placement from Jan 2013 to Oct 2016 at Jikei University Hospital were analyzed. The following data were collected from clinical records: age, gender, technique of PEG, reasons for PEG, lab tests and prognostic nutritional index (PNI).

Results: During the study period, 236 PEG placements were done in 234 patients. The average age was $69.3 \pm 12.5$. The modified introducer technique was applied in 167 procedures $(70.8 \%)$ and the overtube assisted technique was applied in 69 procedures $(29.2 \%)$. The PEG was placed aiming for nutrition supports for cancer patients in 132 procedures, cerebrovascular accident in 51 procedures, aspiration pneumonia in 32 procedures, and others such as infection and disuse atrophy in 21 procedures. Age (the overtube assisted pull technique $>$ the modified introducer group), Gender and preoperative serum C-reactive protein level were significantly different between the two groups $(\mathrm{p}<0.05)$. Overall, adverse events were observed in $19(8.1 \%)$ procedures, although there was no procedure related mortality in the both groups. The risks of clinically significant adverse events were not different between the two groups. There was no significant difference in the types and the rate of adverse events between the two groups. However, severe peristomal bleedings were observed only in the modified introducer technique group. Four patients required suture placements and 3 
patients required blood transfusion for the peristomal bleeding. In a univariate analysis, age, the rate of aspiration pneumonia as the reason for the PEG placement were higher in patients encountering adverse events $(\mathrm{p}<0.05)($ table 1$)$. Also, serum platelet level, serum albumin and the rate of nutrition supports for cancer as the reason of the PEG placement was lower in patients encountering adverse events $(\mathrm{p}<0.05)$. In a multivariate analysis, lower serum platelet level was solely recognized as a relevant predictive factor for adverse events $(p<0.05)$.

The types of the technique used were not relevant to risks of adverse events.

Clinical backgrounds of patients with and without adverse events.

\begin{tabular}{llll}
\hline & $\begin{array}{l}\text { with adverse } \\
\text { events }(\mathrm{n}=19)\end{array}$ & $\begin{array}{l}\text { without adverse } \\
\text { events }(\mathrm{n}=217)\end{array}$ & $\mathrm{p}$ \\
\hline $\begin{array}{l}\text { Age (mean } \pm \text { SD) } \\
\text { Sex (male/female) }\end{array}$ & $77.4 \pm 7.9$ & $68.6 \pm 12.6$ & $\mathrm{p}<0.05$ \\
$\begin{array}{l}\text { Technique for PEG (intro- } \\
\text { ducer/pull) }\end{array}$ & $13 / 6$ & $162 / 55$ & n.s. \\
$\begin{array}{l}\text { Reasons for PEG (cancer/ } \\
\quad \text { cerebrovascular acci- } \\
\text { dent/aspiration pneu- }\end{array}$ & $5 / 5 / 6 / 3$ & $154 / 63$ & n.s. \\
$\quad$ monia/others) & & $127 / 46 / 26 / 18$ & $\mathrm{p}<0.05$ \\
$\begin{array}{l}\text { Lab tests (mean } \pm \text { SD) } \\
\text { ChE (U/L) }\end{array}$ & $184.9 \pm 60.8$ & $214.9 \pm 78.9$ & n.s. \\
\hline
\end{tabular}

(continued)
Continued

Clinical backgrounds of patients with and without adverse events.

\begin{tabular}{llll}
\hline & $\begin{array}{l}\text { with adverse } \\
\text { events }(\mathrm{n}=19)\end{array}$ & $\begin{array}{l}\text { without adverse } \\
\text { events }(\mathrm{n}=217)\end{array}$ & $\mathrm{p}$ \\
\hline $\mathrm{TP}(\mathrm{g} / \mathrm{dL})$ & $6.1 \pm 0.9$ & $6.4 \pm 0.8$ & n.s. \\
$\mathrm{Alb}(\mathrm{g} / \mathrm{dL})$ & $2.9 \pm 0.6$ & $3.1 \pm 0.6$ & $\mathrm{p}<0.05$ \\
$\mathrm{CRP}(\mathrm{mg} / \mathrm{dL})$ & $1.5 \pm 2.0$ & $2.0 \pm 3.3$ & n.s. \\
$\mathrm{WBC}(/ \mu \mathrm{L})$ & $7336.8 \pm 3162.1$ & $6696.8 \pm 3086.6$ & n.s. \\
$\mathrm{Hb}(\mathrm{g} / \mathrm{dL})$ & $10.4 \pm 2.2$ & $11.3 \pm 1.9$ & n.s. \\
$\mathrm{Plt}(/ \mu \mathrm{L})$ & $20.7 \pm 8.4$ & $25.6 \pm 8.8$ & $\mathrm{p}<0.05$ \\
$\mathrm{TLC}(/ \mu \mathrm{L})$ & $1400 \pm 797.9$ & $1387.6 \pm 967.4$ & n.s. \\
$\mathrm{PNI}$ & $35.7 \pm 8.5$ & $38.4 \pm 8.5$ & n.s. \\
\hline
\end{tabular}

Conclusion: There was no significant difference in overall risks of adverse events between the modified introducer technique group and the overtube assisted pull technique group. However, the modified introducer technique may be associated with higher risks of severer adverse events. Especially, special care should be taken in patients with lower serum platelet level.

Disclosure of Interest: All authors have declared no conflicts of interest.

References

1. Gastrointest. Endosc. 2003; 57: 837-41.

2. Home Health Care Endosc. Ther. Qual. Life 2005; 9: 79-83. 